


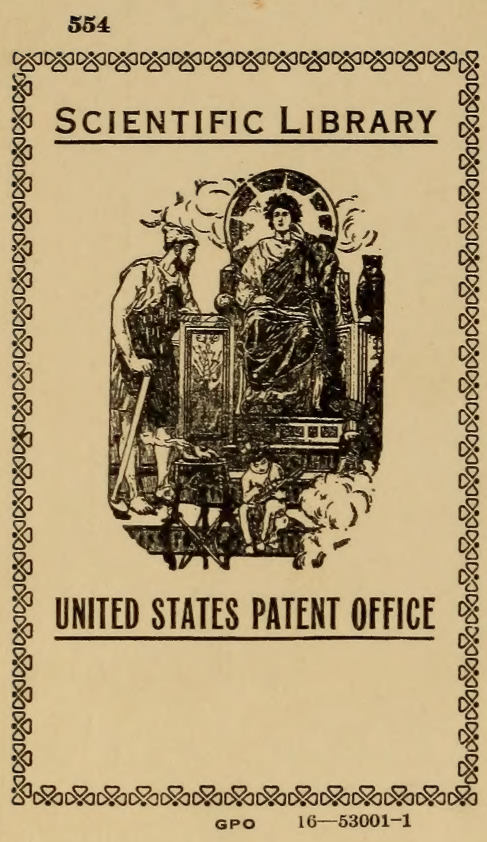



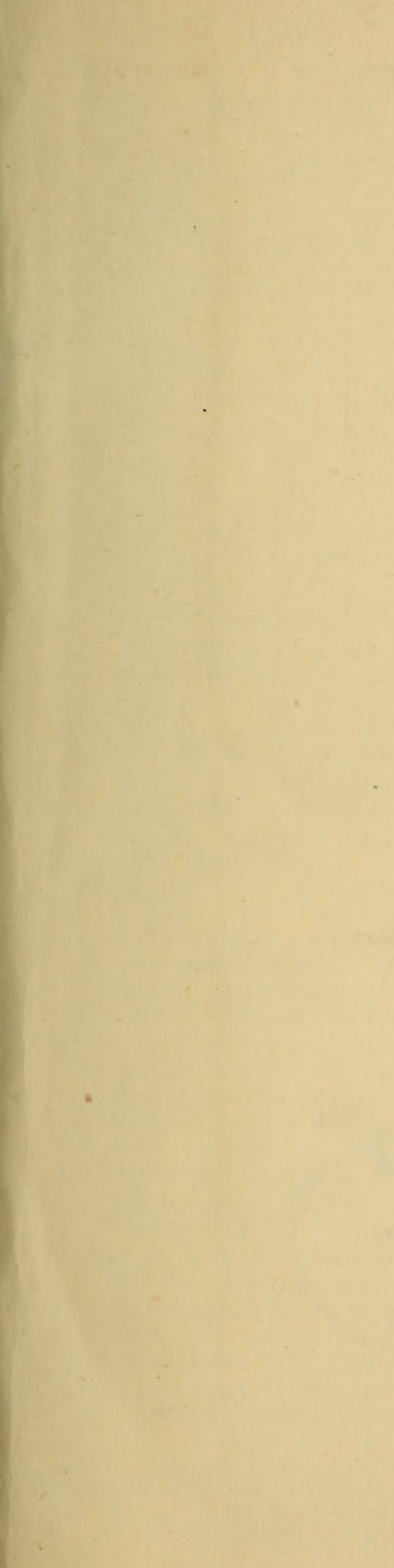


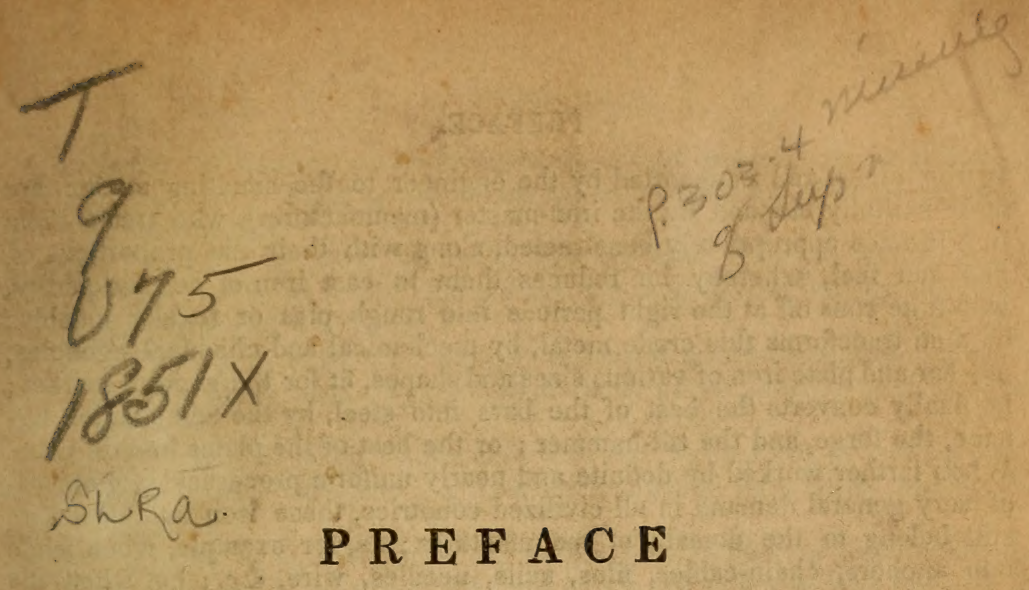

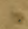

IT is the business of operative industry to produce, transform, and dis. sribute all such material objects as are suited to satisfy the wants of mankind. The primary production of these objects is assigned to the husbandman, the fisherman, and the miner; their transformation to the manufacturer and artisan; and their distribution to the engineer, shipwright, and sailor.* The unworked or raw materials are derived, - 1 . from the organic processes of vegetables and animals, conducted either without or with the fostering care of man; 2 . from the boundless stores of mineral and metallic wealth arranged upon or within the surface of the earth by the benignant Par of our being, in the fittest condition to exercise our physical and intellectu. powers in turning them to the uses of life.

The task which I have undertaken in the present work, is to describe and explain the transformations of these primary materials, by mechanical and chemical agencies, into general objects of exchangeable value; leaving, on the one hand, to the mechanical engineer, that of investigating the motive powers of transformation and transport; and, on the other hand, to the handicraftsman, that of tracing their modifications into objects of special or local demand. Contemplated in this view, an art or manufacture may be defined to be that species of industry which effects a certain change in a substance, to suit it for the general market, by combining its parts in a new order and form, through mechanical or chemical means. Iron will serve thie purpose of illustrating the nature of the distinctions here laid down, between mechanical engineering ; arts and manufactures ; and handicraft trades. The engineer perforates, the ground with a shaft, or a drift, to the level of the ore, erects the pumps for drainage, the ventilating, and hoisting apparatus, along with the requisite steam or water power; he constructs the roads, the bridges, oanals, railways, harbors, docks, cranes, \&c., subservient to the transport of the ore and metal; he mounts the steam or water-power, and bellows for working the blast-furnaces, the forges, and the cupolas; his principal end and aim on all occasions being to overcome he forces of inertia, gravity, and cohesion. The ores extracted and sorted

* For correct and copious information upon agricultural production, I have great pleasure in referring my readers to Mr. Loudon's elaborate Encyclopedias of Agricullure, Gardening, and Plants; and for mercantile production and distribution, to $\mathrm{Mr}$. $\mathrm{M}^{5} \mathrm{Cul}$. loch's excellent Dictionary of Commerce and Commercial Navigation. 
by the mirier, and transported by the engineer to the smelting station, are there skilfully blended by the iron-master (manufacturer), who treats them in a furnace appropriately constructed, along with their due proportions of flux and fuel, whereby he reduces them to cast iron of certain quality, which he runs off at the right periods into rough pigs or regular moulds; he then transforms this crude metal, by mechanical and chemical agencies, into bar and plate irrn of various sizes and shapes, fit for the general market; he finally converts the best of the bars into steel, by the cementation furnace, the forge, and the tilt-hammer; or the best of the plates into tin-plate. When farther worked by definite and nearly uniform processes into objects of very general demand in all civilized countries, these iron and steel bars still belong to the domain of manufactures; as, for example, when made into anchors, chain-cables, files, nails, needles, wire, \&c.; but when the iron is fashioned, into ever varying and capricious forms, they belong either to the general business of the founder and cutler, or to the particular calling of some handicraft, as the locksmith, gratesmith, coachsmith, gunsmitn, tinman, \&c.

Such are the principles which have served to guide me in selecting art1cles for the present volume. By them, as a clew, I have endeavored to hold a steady course through the vast and otherwise perplexing labyrinth of arts, manufactures, and mines; avoiding alike engineering and mechanical arts, which cause no change in the texture or constitution of matter, and handicraft operations, which are multiform, capricious, and hardly susceptible of scientific investigation. In fact, had such topics been introduced into the volume, it would have presented a miscellaneous farrago of incongruous articles, too numerous to allow of their being expounded in a manner either interesting or instructive to the manufacturer and the metallurgist. I readily acknowledge, however, that $I$ have not been able to adhere always so rigorously as I could have wished to the above rule of selection; having been constrained by intelligent and influential friends to introduce a few articles which I would gladly have left to the mechanical engineer. Of these Printing is one, which, having had no provision made for it in my original plan, was too hastily compiled to admit of my describing, with suitable figures, the flat-printing automatic machine of Mr. Spottiswoode, wherewith the pages of this volume were worked off; a mechanism which I regard as the most elegant, precise, and productive, hitherto employed to execute the best style of letter press.

I have imbodied in this work the results of my long experience as a Professor of Practical Science. Since the year 1805, when I entered at an early age upon the arduous task of conducting the schools of chemistry and manufactures in the Andersonian Institution, up to the present day, I have been assiduously engaged in the study and improvement of most of the chemical and many of the mechanical arts. Consulted professionally by proprietors of factories, workshops, and mines of various descriptions, both in this country and abroad, concerning derangements in their operations, or defects in their products, I have enjoyed peculiar opportunities of becoming familiar with their minutest details; arid have frequently had the good fortune to rectify what was amiss, or to supply what was wanting. Of the stores of information thus acquired, I have availed myself on the prosent occasion; careful, meanwhile, to neglect no means of knowledge which my extensive intercourse with foreign nations affords.

I therefore humbly hope that this work will prove a valuable contribution to the literature of science, serving -

In the first place, to instruct the Manufacturer, Metallurgist, and Tradesman, in the princ iples of their respective processes, so as to render them in 
reality the masters of their business, and to emancipate them from a state of bondage to such as are too commonly the slaves of blind prejudice and vicious routine.

Secondly, to afford to Merchants, Brokers, Drysalters, Druggists, and Officers of the Revenue, characteristic descriptions of the commodities which pass through their hands.

Thirdly, by exhibiting some of the finest developments of chemistry and physics, to lay open an excellent practical school to students of these kindred sciences.

Fourthly, to teach Capitalists, who may be desirous of placing their funds in some productive bank of industry, to select judiciously among plausible claimants.

Fifthly, to enable Gentlemen of the Law to become well acquainted with the nature of those patent schemes which are so apt to give rise to litigation.

Sixthly, to present to our Legislators such a clear exposition of our staple manufactures, as may dissuade them from enacting laws which obstruct industry, or cherish one branch of it to the injury of many others : and,

Lastly, to give the General Reader, intent chiefly on intellectual cultivation, a view of many of the noblest achievements of science, in effecting those grand transformations of matter to which Great Britain owes her paramount wealth, rank, and power among the kingdoms.

The latest statistics of every important object of manufacture is given from the best, and, usually, from official authority, at the end of each article.*

The following summary of our manufactures is extracted from $\mathrm{Mr}$. Macqueen's General Statistics of the British Empire, published in 1836. It shows the amount of capital embarked in the various departments of manufacturing industry, and of the returns of that capital :-

\begin{tabular}{|c|c|c|c|c|c|c|c|}
\hline \multirow[b]{2}{*}{ Cotton manufactures } & \multirow[b]{2}{*}{-} & \multirow[b]{2}{*}{ - } & \multirow[b]{2}{*}{ - } & \multirow[b]{2}{*}{-} & & Capital. & Produce. \\
\hline & & & & & - & $\stackrel{£}{\stackrel{E}{40,973,872}}$ & $\stackrel{f}{52,513,586}$ \\
\hline Woollen ditto & - & - & - & . & - & $36,000,000$ & $44,250,000$ \\
\hline Silk & - & - & - & . & - & $8,000,000$ & $10,000,000$ \\
\hline Linen & - & - & - & . & - & $12,000,000$ & $15,421,186$ \\
\hline Leather & - & - & - & - & - & $13,000,000$ & $16,000,000$ \\
\hline ditto, to maki & ing pig & iron & - & - & - & $10,000,000$ & $7,098,000$ \\
\hline Iron, hardware, cutlery, 8 & $\& c$. & - & - & . & - & $25,000,000$ & $31,072,600$ \\
\hline Copper and brass ditto & - & - & . & - & - & $3,600,000$ & $4,673,186$ \\
\hline China, glass, \&c. - & & - & - & - & - & $8,600,000$ & $10,892,794$ \\
\hline Paper, furniture, books, 8 & $\& c$. & - & - & & & $10,000,000$ & $14,000,000$ \\
\hline Spirits (British), ales, soa & $a p, \& c$ & & . & . & & $37,600,000$ & $47,163,847$ \\
\hline Sundries additional - & & - & . & . & - & & $9,000,000$ \\
\hline Totals & - & - & 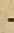 & . & - & $201,773,872$ & $262,085,199$ \\
\hline
\end{tabular}

In consequence of an arrangement with $\mathrm{Mr}$. William Newton, patent agent, and proprietor of the London Journal of Arts, Sciences, and Manufactures, I have been permitted to enrich this Dictionary with many interesting descriptions and illustrative figures of modern patent inventions and improvements, which I could not otherwise have presented to my readers. Mr. Newton has lately enhanced the value of his Journal by annexing to it a catalogue raisonnée, entitled "An Analytical Index to the Subjects contained in the 23 Volumes," which constitute the first and second series. The

- The statistics of agriculture, trade, and manufactures is ably and fully discussed in Mr. M'Culloch's Dictionary already referred to. 
subsequent 22 volumes, of his Conjoined Series, are of still superior interest ; and the whole form a vast storehouse of Mechanical and Chemical Invention.

Although I am conscious of having used much diligence for many years in collecting information for this work, from every quarter within my reach, the utmost pains in preparing it for publication, and incessant vigilance during its passage through the press, yet I am fully aware that it must contain several errors and defects. These I have studied to rectify, in the text of this edition, and more completely in a compendious Supplement.

Since this book is not a Methodical Treatise, but a Dictionary, one extensive subject may be necessarily dispersed through many articles. Thus, for example, information upon the manufacture of Colors will be found under azure; black pigment ; bone-black ; bronze ; brown dye ; calico-printing ; carmine ; carthamus ; chromium ; cochineal ; crayons ; dyeing ; enamels ; gold ; gilding ; gamboge ; gray dye ; green dye ; green paints ; indigo ; kermes; lac dye; lakes; madder; massicot; mercury; periodide of; $\mathrm{Na}$ ples yellow; orange dye ; orpiment; paints ; grinding of ; ochres ; paperhangings; pastes; pearl white; Persian berries; pottery pigments ; Prus. sian blue; purple of Cassius ; red lead ; rouge; Scheele's green; Schweinfurth green; stained glass ; terra di Sienna ; ultramarine ; umber ; verditer ; vermilion; vitrifiable colors, weld, white lead; woad; yellow, king's.

A casual consulter of the Dictionary, who did not advert to this distribution, might surmise it to be most deficient, where it is in reality most copious

The elaborate and costly Encyclopedias, and Dictionaries of Arts, which have appeared from time to time in this country, and abroad, have, for the most part, treated of the mechanical manufactures more fully and correctly than of the chemical. The operations of the former are, in fact, tolerably obvious and accessible to the inspection of the curious; nor are they diffcult to transfer into a book, with the aid of a draughtsman, even by a person but moderately versed in their principles. But those of the latter are not unfrequently involved in complicated manipulations, and depend, for their success, upon a delicate play of affinities, not to be understood without an operative familiarity with the processes themselves. Having enjoyed the best opportunities of studying the chemical arts upon the greatest scale in this kingdom and on the Continent, I may venture, without the imputation of arrogance, to claim for my work, in this respect, more precision and copiousness than its predecessors possess. I have gone as far in describing several curious processes, hitherto veiled in mystery, as I felt warranted, without breach of confidence, to go ; regarding it as a sacred duty never to publish any secret whatever, without the consent of its proprietor. During my numerous tours through the factory districts of Great Britain, France, \&c., many suggestions, however, have been presented to my mind, which I am quite at liberty to communicate in private, or carry into execution, in other districts too remote to excite injurious competition against the original inventors. I am also possessed of many plans of constructing manufactories, of which the limits of this volume did not permit me to avail myself, but which I am ready to furnish, upon moderate terms, to proper applicants. I conclude by pointing attention to the very insecure tenure by which patents for chemical or chemico-mechanical inventions are held; of which there is hardly one on record which may not be readily evaded by a person skilled in the resources of practical chemistry, or which could stand the ordeal of a court of law, directed by an experienced chemist. The specifications of such patents stand in need of a thorough reform; being for the most part not only discreditable and delusive to the patentees, but calculated co involve them in one of the greatest of evils - a chancery suit.

LoNDon: 13 Charlotte Street, Bedford Square, August, 1843 . 


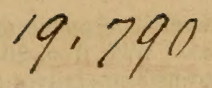

A

DI C TIONAR Y

OF

\section{ARTS, MANUFACTURES, AND MINES}

ABB-WOOL. Among clothiers, this term signifies the woof or weft.

ACETATE. (Acétate, Fr.; Essigsaure, Germ.) Any saline compound of which the acetic is the acid constituent; as acetate of soda, of iron, of copper, \&c.

ACETATE OF ALUMINA, see Red Liquor and Mordant; of Cofier, see Copper ; of Iron, see Iron; of Lead, see Lead ; of Lime, see Pyroligneous Acid.

ACETIC ACID (Acide Acétique, Fr.; Essigsaure, Germ.) is the name of the sour principle which exists in vinegar. It occurs, ready formed, in several products of the vegetable kingdom, and is generated during the spontaneous fermentation of many vegetable and animal juices. The sambucus nigra, or black elder, the phonix dactilifera, and the rhus typhinus are plants which afford a notable quantity of vinegar. It is found, likewise, in the sweat, urine, milk, and stomach of animals. All infusions of animal or vegetable matters in water, when exposed for some time to the air, at a moderate temperature, ferment into vinegar; and most vegetables, when subjected to decomposition by fire, give off condensable vapors of acetic acid. All liquids containing alcohol are susceptible of passing into the state of vinegar; but the pre-existence of alcohol is not necessary to this change, as we learn from the acetification of vegetable soups, infusion of cabbage, starch - paste, \&c.

Vinegar may be distinguished into four varieties, according to the mode of its production, though all of them are capable of being converted, by chemical means, into one identical acetic acid. 1. Wine vinegar. 2. Malt vinegar. 3 Sugar vinegar. 4. Wood vinegar, or pyroligneous acid. Fermentation is the source of the acid in the first three varieties. Here alcohol is first generated, and is next converted into vinegar by the influence of the air at a genial temperature; a change which will be investigated under Fermentation. But the conversion of spirit of wine into acetic acid may be demonstrated by direct experiment. When the vapor of alcohol is brought into contact in the atmosphere with the black powder obtained by mixing muriate of platina, potash, and alcohol, vinegar is rapidly formed at the expense of the alcohol. In Germany, where crude alcohol bears a low price, the manufacture of vinegar has been arranged upon that principle, which, as throwing some light on the process of acetification, I shall briefly describe. See Platinum for the mode of preparing the above powder.

Under a large case, which for experimental purposes may be made of glass, several saucer-shaped dishes of pottery or wood are to be placed in rows, upon shelves over each other, a few inches apart. A portion of the black platina powder moistened being suspended over each dish, let as much vinous spirits be put into them as the oxygen of the included air shall be adequate to acidify. This quantity may be inferred from the fact, that 1000 cubic inches of air can oxygenate 110 grains of absolute alcohol, converting them into 122 grains of absolute acetic acid, and $64 \frac{1}{2}$ grains of water.

The above simple apparatus is to be set in a light place (in sunshine, if convenient), at a temperature of from $68^{\circ}$ to $86^{\circ} \mathrm{Fahr}$., and the evaporation of the alcohol is to be promoted by hanging several leaves of porous paper in the case, with their bottom edges dipped in the sprrit. In the course of a few minutes, a most interesting phenomenon will be perceived. The mutual action of the platina and the alcohol will be displayed by an increase of temperature, and a generation of acid vapors, which, condensing on the sides of the glass-case, trickle in streams to the bottom. This striking transformation continues till all the oxygen of the air be consumed. If we wish, then, to renew the process, we must open the case for a little, and replenish it with air. With a boz of 12 cubic feet in capacity, and with a provisicn of 7 or 8 ounces of the platina powder, 
we can, in the course of a day, convert one pound of alcohol into pure acetic art, fit tor every purpose, culinary or chemical. With from 20 to 30 pounds of the platina powder (which does not waste), we may transform, daily, nearly 300 pounds of bad spirits intc the finest vinegar. Though our revenue laws preclude the adoption of this elegant process upon the manufacturing scale in this country, it may be regarded as one of the greatest triumphs of chemistry, where art has rivalled nature in one of her most mysterious operations.

To readers acquainted with chemical symbols, the following numerical representation of the conversion of alcohol into acetic acid may be accepiable :-

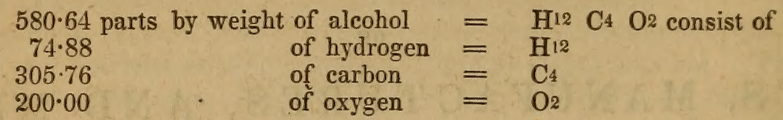

If we combine with this mixture, 400 parts of oxygen $=\mathrm{O}_{4}$, we have,-

$$
\begin{array}{lll}
\text { of water } & =337 \cdot 44=\mathrm{H}_{6} \mathrm{O}_{3} \\
\text { acetic acid } & =643 \cdot 20=\mathrm{H}_{6} \mathrm{C}_{4} \mathrm{O}_{3}
\end{array}
$$

Hence, in this formation of vinegar, 100 parts by weight of alcohol take 68.89 parts of oxygen; and there are produced 58.11 parts of water, and 110.78 of acetic acid.

These beautiful experiments prove, that when in a mere mixture of alcohol and water, under the influence of the atmospheric air and heat, some vinegar comes to be formed after a considerable time, the same formation of vinegar takes place in a similar, but more effective, manner, when a ferment is present, which acts here in a somewhat analogous way to the platina powder in the preceding case. Several azotized substances serve as re-agents towards the acetous fermentation,-such as vinegar readymade, vinegar-yeast, or lees, barley bread, leaven, beer barm, and similar vegetable matters, which contain gluten. The best and purest ferment is, however, vinegar itself. With this ferment we must conjoin, as an essential condition of acetification, the íree access of atmospheric air.

It is a well-known fact, that spirituous liquors, as weak brandy, wine, and beer, \&c., may be preserved for years in close vessels, without undergoing the acetous fermentation, even when they repose upon a layer of lees. It is equally well known, that these very liquors, if they stand for some time in open vessels, become readily sour, especially if exposed, also, to a somewhat high temperature. If we fill a flask with common brandy, and subject it, without a stopper, to the influence of air and warmth, the contained liquor may, at the end of many weeks, discover no sensible acidity; if we add to the same brandy a ferment, and stop the flask air-tight, everything will still remain unchanged; but if we leave a portion of air in the flask, or leave it uncorked, vinegar will soon make its appearance in the brandy.

If we investigate the nature of the air which remains over brandy in the act of acetification, we shall find that it consists entirely of carbonic acid and azote, the oxygen being absorbed and combined in the acetic acid and water formed.

Since this absorption of oxygen from the air can take place only at the surface of the fermenting liquors, we thus see the necessity and the practical importance of amplifying that surface, in order to accelerate and complete the acetification by multiplying the points of contact between the alcohol and the oxygen. The essence of the new German method of rapid acetification depends upon this principle.

Temperature has also a remarkable influence on the formation of vinegar. The acid fermentation proceeds very feebly in the cold, but takes an accelerated pace as the heat is raised. It would even appear that spirituous vapors brought by themselves in contact with atmospheric air, without the aid of any ferment, are capable of being converted into acetic acid, since it has happened in the rectification of brandy, in a still furnished with a large capital and adopter pipe into which air was allowed to enter, that vinegar made its appearance. Hence, warmth does not seem to act as a promoter of the combination of alcohol with oxygen in a merely chemical point of view, but it acts, so to speak, physically. Over the warm liquor a stratum of spirit vapor appears to float, which, coming there into conflict with the atmospherical oxygen, probably causes the generation of some acetic acid, and thus accelerates the operation, much more than by the mere contact of the oxygen with the liquid surface.

When we expose any spirituous liquors, as wine, beer, \&c., with the requisite ferment, to the external air, at a temperature of from $64^{\circ}$ to $68^{\circ} \mathrm{Fahr}$., the fluid, however clear before, becomes soon turbid; filamentous slimy particles begin to appear moving in the middle and on the sides of the vessel, and then form a scum on the top of the liquor. When this scum has acquired a certain thickness and consistence, it falls in a sediment to the bottom. The G'ermans call it the vinegar mother, as it serves to excite acetification in fresh liquors. Meanwhile, the liquor has become warmer than the sur 


\section{ACETIC ACID.}

rounding air, and the vinegar process betrays itself by diffusing a peculiar aroma in the apartment. Whenever all the alcohol present has been ccnverted into acetic acid, the liquor comes into a state of repose; its temperature sinks to the pitch of the atmosphere; it becomes bright, and is the article well known by its taste and smell under the name of vinegar.

Genuine wine or raisin vinegar differs from .tat formed either from apples, or sugar, haer, \&c., in containing wine-stone or tartar; by which peculiarity it may be distinguished, except in those cases where crude tartar has been artificially added to the other vinegars, as a disguise. Barley-malt vinegar contains some phosphoric acid, in the state of phosphate of lime or magnesia, derived from the grain.

After these general observations upon acetification, we shall now proceed to describe the process for manufacturing vinegar on the commercial scale.

1. Wine vinegar.-The first consideration with a vinegar-maker is a good fermenting room, in which the wines may be exposed to a steady temperature, with an adequate cupply of atmospherical air. As this air is soon deprived of its oxygenous constituent, facilities ought to be provided for a renewal of it by moderate ventilation. The airholes for this purpose ought to be so contrived that they may be shut up when the temperature begins to fall too low, or in windy weather. The best mode of communicating the proper warmth to a chamber of this kind is by means of fire-flues or hot water pipes, running along its floor at the sides and ends, as in a hot-house; the fireplace being on the outside, so that no dust may be created by it within. The flue is best made of bricks, and may have a cross section of 10 or 12 inches by 15 deep. The soot deposited, even when coals are burned, will find ample space in the bottom of the flue, without interfering essentially with the draught, for a very long period, if it be made of the above dimensions. Low-roofed apartments are preferable to high ones; and those built with thick walls, of imperfectly conducting materials, such as bricks, lined with lath and plaster work. Should the chamber, however, have a high ceiling, the fermenting tuns must be raised to a suitable height on scaffolding, so as to benefit by the warmest air. Sometimes the vinegar vessels are placed at different levels; in which case the upper ones acetify their contents much sooner than the under, unless they are emptied and filled alternately, which is a good plan.

Orleans is the place most famous for vinegars. The building there destined to their manufacture is called a vinaigrerie, and is placed, indifferently, either on the ground floor or the floor above it; but it has always a southern exposure, to receive the influence of the sunbeams. The vessels employed for carrying on the fermentation are casks, called molhers. Formerly they were of a large capacity, containing about 460 litres (150 gallons, Eng.); but at the present day they are barrels of half that capacity, or somewhat less than an old English hogshead. It is now known that the wine passes sooner into vinegar the smaller the mass operated upon, the more extensive its contact with the air, and the more genial its warmth. These casks were formerly arranged in three ranks by means of massive scaffolding; they are now set in four ranks, but they rest on much smaller rafters, sustained by uprights, and ean be packed closer together. The casks, which are laid horizontally, are pierced at the upper surface of their front end with two holes: one, to which the name of eye is given, is two inches in diameter; it serves for putting in the charge, and drawing off the vinegar when it is made; the other hole is much smaller, and is placed immediately alongside; it is merely an air-hole, and is necessary to allow the air to escape, because the funnel completely fills the other hole in the act of filling the cask.

When new vessels are mounted in a vinegar work, they must be one third filled with the best vinegar that can be procured, which becomes the true mother of the vinegar to be made; because it is upon this portion that the wine to be acidified is successively added. At the ordinary rate of work, they put at first upon the mother, which occupies one third of the vessel, a broc of ten litres of red or white wine; eight days afterwards they add a second broc; then a third, and a fourth, always observing the same interval of time, 8 days. After this last charge, they draw off about forty litres of vinegar, and then recommence the successive additions.

It is necessary that the vessel be always one third empty if we wish the acetification to go on steadily; but as a portion of the tartar and the lees forms and accumulates in the lower part of the cask, so as eventually to counteract the fermentation, the time arrives when it is requisite to interrupt it, in order to remove this residuum, by clearing ou: all the contents. The whole materials must be renovated every 10 years; but the casks, if well made and repaired, will serve for 25 years.

We have mentioned a definite period at which the vinegar may be dratwn off; but that was on the supposition that the process had all the success we could wish; there are circumstances, tifficult to appreciate, which modify its progress, as w'e shall presently show. We ought, therefore, before discharging the vinegar, to test and see if the fer 
mentation has been somplete. We proceed as follows: we plunge into the 1quor white stick or rod, bent at one end, and then draw it out in a horizontal direction: if it be covered with a white thick froth, to which is given the name of work (travail), we judge that the operation is terminated; but if the work, instead of being white and pearly, be red, the manufacturers regard the fermentation to be unfinished, and they endeavor to make it advance, by adding fresh wine, or by increasing the heat of the apartment.

It is not always easy to explain why the fermentation does not go on as rapidly in one case as in another. There are even certain things which seem at present to be entirely inexplicable. It happens sometimes, for example, that although all the vessels have been equally charged, and with the same wine, yet the fermentation does not form in the same manner in the whole; it will move rapidly in some, be languid, or altogether inert, in others. This is a very puzzling anomaly; which has been ascribed to electrical and other obscure causes, because it is not owing to want of heat, the casks in the warmest positions buing frequently in fault; nor to the timber of the cask. It, lowerer, paralyzes the process so completely that the most expert vinegar makers have nothing else for it, when this accident happens, than to empty entirely what they call the lazy cask, and to fill it with their best vinegar. The fermentation now begins, and proceeds as well in it as in the others. See Fermentation.

We must here make an important remark, relatively to the temperature which should prevail in the fermentation room. In many chemical works we find it stated, that the heat should not exceed $18^{\circ} \mathrm{R}$., or $65^{\circ} \mathrm{Fahr}$, for fear of obtaining bad products. But the vinegar makers constantly keep up the heat at from $24^{\circ}$ to $25^{\circ} \mathrm{R}$., $75^{\circ}$ to $77^{\circ} \mathrm{F}$.; when the acetification advances much more rapidly, and the vinegar is equally strong. The best proof of this heat not being too high is, that under it, the vessels in the upper part of the room, work best and quickest. In Orleans, cast-iron stoves and wood fuel are used for communicating the requisite warmth.

Before pouring the wine into the mothers, it is clarified in the following manner. There are tuns which can contain from 12 to 15 pieces of wine. Their upper end has at its centre an opening of four or five inches diameter, which may be closed afterwards with a vooden cover; this opening is for the purpose of receiving a large funnel. The inside of the tun is filled with chips of beechwood, well pressed down. The wine is poured upon these chips, allowed to remain for some time, and then gently drawn off by a pipe in the lower part of the vessel. The lees are deposited upon the chips, and the wine runs off quite clear. However, it happens sometimes, notwithstanding this precaution, that the vinegar, after it is made, requires to be clarified, more particularly if the wine employed had been weak. The vinegar must be filtered in the same way; and it derives an advantage from it, as the products of different casks get thereby mixed and made uniform.

By this Orleans method several weeks elapse before the acetification is finished; but a plan has been lately devised in Germany to quicken greatly the acid fermentation by pe. culiar constructions. This system is called, the quick vinegar work, because it will com plete the process in the course of 2 or 3 days, or even in a shorter time. It depends, chiefly, upon the peculiar construction of the fermenting vessels, whereby the vinous liquor is exposed on a vastly expanded surface to the action of the atmospheric air.

An oaken tub, somewhat narrower at the bottom than the top, from 6 to 7 feet high and 3 feet in diameter, is furnished with a well-fitted grooved, but loose, cover. About half a foot from its mouth, the tub has a strong oak or beech hoop fitted to its inside surface, sufficiently firm to support a second cover, also well fitted, but moveable. The space under this second cover is destined to contain the vinous liquor, and in order to bring it very amply into contact with the atmosphere, the following contrivances have been resorted to: This cover is perforated, like a sieve, with small holes of from 1 to 2 lines in diameter, and about $1 \frac{1}{2}$ inch apart. Through each of these holes a wick of packthread or cotton is drawn, about 6 inches long, which is prevented from falling through by a linot on its upper end, while its under part hangs free in the lower space. The wiclis must be just so thick as to allow of the liquor poured above the corer passing chrough the holes in drops. The edges of the lid must be packed with tow or hemp to prevent the liquor running down through the interval.

The whole lower compartment is now to be filled with chips of beechwood up to nearly the periorated cover. The liquor, as it trickles through the holes, diffuses itself over the chips, and, sinking slowly, collects at the bottom of the tub. The cnips should be prepared for this purpose by being repeatedly scalded in boiling water, then dried, and imbued with hot vinegar. The same measures may also be adopted for the tub. To protide for the renewal of the air, the tub is perforated at about a foot from its bottom with eight holes, set equally apart round the circumference, two thirds of an inch wide, and sloping down, through which the air may enter into this lower compartment, without the trickling liquor being allowed to flow out. In order that the foul air which has 
become useless may escape, four large holes are pierced in the sieve cover, at equal distan. ecs asunder and from the centre, whose united areas are rather smaller than the total areas of the holes in the side of the tub. Into these four holes open glass tubes must be inseried, so as to stand some inches above the cover, and to prevent any of the liquor from running through them. The proper circulation of the air takes place through these. draught holes. This air may afterwards pass off through a hole of $2 \frac{1}{2}$ inches dianuter in the uppermost cover, in which a funnel is placed for the supply of liquor as it is wanted to keep up the percolation.

The temperature of the fermenting compartment is ascertained by means of a thermometer, whose bulb is inserted in a hole through its side, and fastened by a perforated cork. The liquor collected in the under vessel runs off by a syphon inserted near its bottom, the leg of which turns up to nearly the level of the ventilating air pipes before it is bent outwards and downwards. Thus the liquor will begin to flow out of the under compartment only when it stands in it a little below the sieve cover, and then it will run slowly off at the inclined mouth of the syphon, at a level of about 3 inches below the lower end of the glass tubes. There is a vessel placed below, upon the ground, to receive it. The tub itself is supported upon a wooden frame, or a pier of brickwork, a foot or 18 inches high.

A tub constructed like the above is called a GRADUATION vESSEL, which see. It $\$$ worked in the following way:-The vinegar room must be, in the first place, heated to froin $100^{\circ}$ to $110^{\circ} \mathrm{F}$., or till the thermometer in the graduation vessel indicates at least $77^{\circ}$. The heat may then be modified. We now pour through the uppermost cover of the tub a mixture, warmed to $144^{\circ} \mathrm{F}$., of 8 parts proof spirits, 25 parts soft water, 15 parts of good vinegar, and as much clear wine or beer. The water should be first heated, and then the vinegar, spirits, and wine may be added to it. Of this mixture, so much should be poured in as is necessary to cover over the second lid, 2 or 3 inches deep, with the liquor; after which, the rest may be poured slowly in, as it is wanted.

When the liquor has run for the first time through the graduation vessel, it is not yet sufficiently acidified; but the weak vinegar collected in the exterior receiving cistern must be a second time, and, if need be, a thir' time, passed through the graduation tub, in order to convert all the alcohol into acetic acid. In general, we may remark, that the stronger the vinous liquor the more difficult and tedious is its conversion into vinegar, but it is so much the stronger. To lessen this difficulty somewhat, it would be well not to put all the spirits at first into the wash, or mixed liquors, but to add a little more of it at the second and the third running, especially when we desire to have very strong vinegar.

After the graduation vessel has been some days at work, it is no longer necessary to add vinegar to the mixture of spirits and water, since the sides of the graduation tub, the beech chips, and the packthreads, are all impregnated with the ferment, and supply its place. The mixture must, however, be always maintained at the temperature of $100^{\circ}$.

Instead of the above mixture of brandy, water, and wine, we may employ, according to Dingier, a clear fermented wort of malt, mixed with a little spirits. The perfect vinegar, which collects in the receiving cistern, may be immediately racked off into the store casks for sale.

It has been objected to this process, that, in consequence of the mixture of saccharine and glutinous materials, which are contained in beer or worts, along with the acetous fermentation, there is also, partially, a vinous fermentation, and much carbonic acid, thereby disengaged, so as to obstruct the acetification. This obstruction may be remedied by a freer circulation of air, or by the exposure of quicklime in the chamber. It is a more substantial objection, that, from the addition of beer, \&c., more Iees, or dregs, are deposited in the graduation tub, whereby a more frequent cleansing of it, and of the beech chips, with a loss of time and vinegar, becomes necessary. The only mode of obviating this difficulty is, to take well-clarified fermented wash.

Another evil attendant on the quick process is, the evaporation of the spirituous liquors. Since, in the graduation tub, there is a temperature of $110^{\circ}$, it is impossible to avoid a loss of spirit from the circulation and efflux of the air. The air, indeed, that issues from the top hole in the uppermost cover, might be conducted over an extensive surface of fresh water, where its spirit would be condensed in a great measure. But, after all, this fear of great loss is, I believe, groundless; because the spirit is rapidly acidified by the oxygen of the air, and thereby rapidly loses its volatility.

The supply of the warm wash should be drawn from a cistern placed near the ceiling, where the temperature of the apartment is hottest; and it may be replenished from the partly acetified liquor in the cistern on the floor. With this view, two cisterns should be placed above, so that one of them may always contain liquor sufficiently hot, and thus the process will suffer no interruption.

When malt wash is used for this quick process, the resulting vinegar must be clarified 
iri a tun with beech chips, as above described. In two or three days the inpurities will be deposited, and the fine vinegar may be racked off.

The folluwing prescription, for preparing what he calls malt wine, is given by Dr. Kastner. Eighty pounds of pale barley malt, and 40 pounds of pale wheat malt, are to be crushed together. These 120 pounds are to be infused with 150 quarts of water, at the temperature of $122^{\circ} \mathrm{Fahr}$, afterwards with 300 quarts of boiling water, and the whole body is to be mashed thoroughly, till all the lumps disappear. It is then to be .eft at rest in a large covered tub, for two or three hours, to allow the grains to settle down, from which the wort is to be drawn off. When it has fallen to the temperature of $64^{\circ}$ Fahr., 15 pounds of good yeast are to be stirred in, and it must now be left for two or three days to ferment, in a loosely covered tun. When the vinous fermentation has taken place, the clear liquor must be drawn off by a tap hole, a little above the bottom, so as to leave the lees and scum in the tun. This malt wine, he adds, may be kept for a long time in close vessels, and is always ready for making quick vinegar.

2. Mall Vinegar. - The greater part of British vinegar is made from malt, oy the following process :-1 boll of good barley malt, properly crushed, is to be mashed with water at $160^{\circ} \mathrm{Fahr}$. The first water should have that temperature; the second must be hotter than $160^{\circ}$, and the third water, for the extraction of all the soluble matter, may be boiling hot. Upon the whole, not more than 100 gallons of wort should be extracted. After the liquor has cooled to $75^{\circ}$ Fahr., 3 or 4 gallons of beer yeast are poured in, and well mixed with a proper stirrer. In 36 or 40 hours, according to the temperature of the air, and the fermenting quality of the wash, it is racked of into caslis, which are laid upon their sides in the fermenting apartment of the vinegar work, which should be kept at a temperature of $70^{\circ}$ at least; in summer partly by the heat of the sun, but in general by the agency of proper stoves, as above described. The bung-hole should be left open, and the casks should not be full, in order that the air may acl over an extensive surface of the liquor. It would be proper to secure a freer circulation to the air, by boring a hole in each end of the cask, near its upper edge. As the liquor, by evaporation, would be generally a few degrees colder than the air of the apartment, a circulation of air would be established in at the bung-hole, and out by the end holes. By the ordinary methods, three months are required to make this vinegar marketable, or fit for the manufacture of sugar of lead.

In making vinegar for domestic purposes, the casks are usually set on their ends; and they have, sometimes, a false bottom, pierced with holes, placed about a foot above the true one. On this bottom, a quantity of rape, or the refuse raisins, \&c. from the making of British wines, is laid. The malt liquor has a proper quantity of yeast added to it. In about 24 hours it becomes warm, and is then racked off into another similar cask. Aftes some time, this racking process is discontinued, and the vinegar is allowed to complete its fermentation quietly. The proper temperature must always be kept up, by placing the cask in a warm situation. A little wine-stone (argal) added to the malt wash, would make the vinegar liker that made from wine. Sometimes a little isinglass is employed to clarify vinegar. A portion of sulphuric acid is often added to it.

3. Sugar Vinegar.-By pursuing the following plan, an excellent sugar vinegar may bo made. In 158 quarts of boiling water dissolve 10 pounds of sugar, and 6 pounds of wine-stone; put the solution into a fermenting cask, and when it is cooled to the temperature of from $75^{\circ}$ to $80^{\circ}$, add 4 quarts of beer yeast to it. Stir the mixture well, then cover the vessel loosely, and expose it for 6 or 8 days to the vinous fermentation, at a temperature of from $70^{\circ}$ to $75^{\circ} \mathrm{Fahr}$. When it has become clear, draw off the vinous liquor, and either acetify it in the graduation tub above described, or by the common vinegar process. Before it is finished, we should add to it 12 quarts of strong spirits (brandy), and 15 quarts of good vinegar, to complete the acetous fermentation. With a graduation tub which has been used, this addition of vinegar is unnecessary.

The following simpler prescription for making sugar vinegar deserves attention. For every gallon of hot water take 18 ounces of sugar; and when the sirup has cooled to $\mathbf{7 5}$, add 4 per cent., by measure, of yeast. When the vinous fermentation is pretty well advanced, in the course of 2 or 3 days, rack off the clear wash from the lees into a proper cask, and add 1 ounce of wine-stone, and 1 of crushed raisins, for every gallon of water. Fxpose it in a proper manner, and for a proper time, to the acetifying process; and then rack off the vinegar, and fine it upon beech chips. It should be afterwards put into bottles, which are to be well corked.

Vinegar obtained by the preceding methods has always a yellowish or brownish color. It may be rendered colorless by distillation. For nicer chemical purposes, this is done in a glass retort; bat on a large scale, it is usually performed in a clean copfer still, furnished with a capital and worm-refrigeratory, either of silver or block tin. It is volatile at the boiling temperature of water; and if the process be carried on briskly, it will not sensibly corrode the copper. But we can never obtain', in this way, a strong article; for, as soon as the vinegar gets concentrated to a certain degree, we 
cannot force off the remainder by heat, for fear of giving it an empyreumatic odor; be. cause the sluten, coloring matter, \&c. begin to adhere to the bottom of the still. We are, therefore, obliged to suspend the operation at the very time when the acid is acquiring strength. It has been also proposed to concentrate vinegar by the process of con gelation; but much of it remains entangled among the frozen water; and common distilled vinegar is so weak, that it congeals it one mass.

Before the process for pyroligneous acid, or wood vinegar, was known, there was only one method of obtaining strong vinegar practised by chemists; and it is still followed by some operators, to prepare what is called radical or aromatic vinegar. This consists in decomposing, by heat alone, the crystallized binacetate of copper, commonly, but improperly, called distilled verdigris. With this view, we take a stoneware retort ( fig. 1 ),

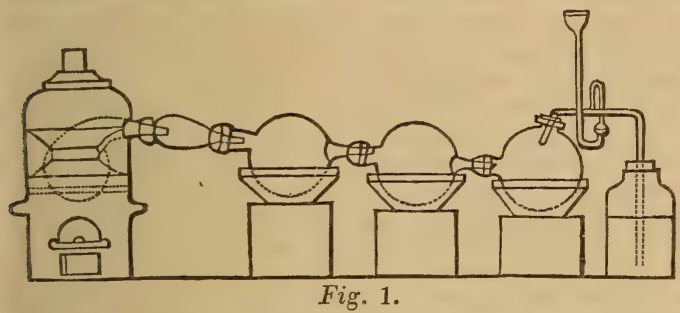
of $a$ size suited to the quantity we wish to operate upon; and coat it with a mixture of fire clay and horsedung, to make it stand the heat better. When this coating is dry, we introduce into the retort the crystallized acetate slightly bruised, but very dry; we fill it as far as it will hold without spilling when the beak is considerably inclined. We then set it in a proper furnace. We attach to its neck an adopter pipe, and two or three globes with opposite tubulures, and a last globe with a vertical tubulure. The apparatus is terminated by a Welter's tube, with a double branch; the shorter issues from the last globe, and the other dips into a flask filled with distilled vinegar. Everything being thus arranged, we lute the joinings with a putty made of pipeclay and linseed oil, and cover them with glue paper. Each globe is placed in a separate basin of cold water, or the whole may be put into an oblong trough, through which a constant stream of cold water is made to flow. The tubes must be allowed a day to dry. Next day we proceed to the distillation, tempering the heat very nicely at the beginning, and increasing it by very slow degrees till we see the drops follow each other pretty rapidly from the neck of the retort, or the end of the adopter tube. The vapors which pass over are very hot, whence a series of globes are necessary to condense them. We should renew, from time to time, the water of the basins, and keep moist pieces of cloth upon the globes; but this demands great eare, especially if the fire be a little too brisk, for the vessels become, in that case, so hot, that they would infallibly be broken, if touched suddenly with cold water. It is always easy for us to regulate this operation, according to the emission of gas from the extremity of the apparatus. When the air bubbles succeed each other with great rapidity, we must damp the fire.

The liquor which passes in the first half hour is weakest; it proceeds, in some measure, from a little water sometimes left in the crystals, which when well made, however, ought to be anhydrous. A period arrives towards the middle of the process when we see the extremity of the beak of the retort, and of the adopter, covered with crystals of a lamellar or needle shape, and of a pale green tint. By degrees these crystals are carried into the condensed liquid by the acid vapors, and give a color to the product. These crystals are merely some of the cupreous salt forced over by the heat. As the process approaches its conclusion, we find more difficulty in raising the vapors; and we must then augment the intensity of the heat, in order to continue their disengagement. Finally, we judge that the process is altogether finished, when the globes become cold, notwithstanding the furnace is at the hottest, and when no more vapors are evolved. The fire may then be allowed to go out, and the retort to cool.

As the acid thus obtained is slightly tinged with copper, it must be rectified before bringing it into the market. For this purpose we may make use of the same apparatus, only substituting for the stoneware retort a glass one, placed in a sand bath. All the globes ought to be perfectly clean and dry. The distillation is to be conducted in the usual way. If we divide the product into thirds, the first yields the feeblest acid, and the third the strongest. We should not push the process quite to diryness, because there remain in the last portions certain inpurities, which would injure the flavor of the acid.

The tctal acid thus obtained forms nearly one half of the weight of the acetate employed, and the residuum forms three tenths; so that about two tenths of the acid have been decomposed by the heat, and are lost. As the oxyde of copper is readily reduced to the metallic state, its oxygen goes to the elements of one part of the acid, anc forms water, which mingles with the products of carbonic acid, carbureted hydrogen, and 
carbonic oxyde gases which are disengaged : and there remains in the retort some char coal mixed with metallic copper. These two combustibles are in such a state of division, that the residuum is pyrophoric. Hence it often takes fire the moment of its being removed from the cold retort. The very considerable loss experienced in this operation has induced chemists to try different methods to obtain all the acid contained in the ace. tate. Thus, for example, a certain addition of sulphuric acid has been prescribed; but, besides that the radical vinegar obtained in this way always contains sulphurous acid, from which it is difficult to free it, it is thereby deprived of that spirit called the pyroacetic, which tempers the sharpness of its smell, and gives an agreeable aroma. It is to be presumed, therefore, that the preceding process will continue to be preferred for making aromatic vinegar. Its odor is often further modified by essential oils, such as those of rosemary, lavender, \&c.

4. Pyroligneous Acid, or Wood Vinegar.-The process for making this acid is founded upon the general property of heat, to separate the elements of vegetable substances, and to unite them anew in another order, with the production of compounds which did not exist in the bodies subjected to its action. The respective proportion of these products varies, not only in the different substances, but also in the same substance, according as the degree of heat has been greater or less, or conducted with more or less skill. When we distil a vegetable body in a close vessel, we obtain at first the included water, or that of vegetation; there is next formed another portion of water, at the expense of the oxygen and hydrogen of the body; a proportional quantity of charcoal is set free, and, with the successive increase of the heat, a small portion of charcoal combines with the oxygen and hydrogen to form acetic acid. This was considered, for some time, as a peculiar acid, and was accordingly called pyroligneous acid. As the proportion of carbon becomes preponderant, it combines with the other principles, and then some empyreumatic oil is volatilized, of little color, but which becomes thicker, and of a darker tint, always getting more loaded with carbon.

Several elastic fluids accompany these different products. Carbonic acid comes over, but in small quantity, much carburreted hydrogen, and, towards the end, a considerable proportion of carbonic oxyde. The remainder of the charcoal, which could not be carried off in these several combinations, is found in the retort, and preserves, usually, the form of the vegetable body which furnished it. Since mankind have begun to reason on the different operations of the arts, and to raise them to a level with scientific researches, they have introduced into several branches of manufacture a multitude of improvements, of which, formerly, they would harlly have deemed them susceptible. Thus, in particular, the process for carbonizing wood has been singularly meliorated, and in reference to the preceding observations, advantage has been derived from several products that formerly were not even collected.

The apparatus employed for obtaining crude vinegar from wood, by the agency of heat, Fig. 2. are large iron cylinders. In this country they are made of cast iron, and are laid horizontally in the furnace; in France, they are made of sheet iron riveted together, and they are set upright in the fire. Fig. 2 will give an accurate idea of the British plan, which is much the same as that adopted for decomposing pit coal in gas works, only that the cylinders for the pyroligneous acid manufacture are generally larger, being frequently 4 feet in diameter, and 6 or 8 feet long, and built horizontally in brickwork, sc that the flame of one furnace may play around two of them. It would probably answer better, if their size were brought nearer the dimensions of the gas-light retorts, and if the whole system of working them were assimilated to that of coal gas.

The foliowing arrangement is adopted in an excellent estabiishment in Glasgow, where the above large cylinders are 6 feet long, and both ends of them project a very little beyond the brickwork. One end has a disc or round plate of cast iron, well fitted, and firmly bolted to it, from the centre of which disc an iron tube, about 6 inches diameter, proceeds and enters, at a right angle, the main tube of refrigeration. The diameter of this tube may be from 9 to 14 inches, according to the number of cylinders. The other end of the cylinder is called the mouth of the retort ; this is closed by a disc of iron, smeared round its edge by clay lute, and secured in its place by fir wedges. The charge of wood for such a cylinder is about $8 \mathrm{cwt}$. The hard woods-oak, ash, birch, and beech-are alone used; fir does not answer. The heat is kept up during the day-time, and the furnace is allowed to cool during the night. Next morning the door is opened, the charcoal removed, and a new charge of wood is introduced. The average product of crude vinegar called pyroligncous acid, is 35 gallons. It is much contaminated with tar, is of a deep brown color, 
and has a sp. or. of 1.025 . Its total weight is therefore about 300 lbs., but the residuary charcoal is found to weigh no more than one fifth of the wood employed; hence nearly one half of the ponderable matter of the wood is dissipated in incondensable gases. Count Rumford states, that the charcoal is equal in weight to more than four tenths of the wood from which it is made. The count's error seems to have arisen from the slight heat of an oven to which his wood was exposed in a glass cylinder. The result now given, is the experience of an eminent manufacturing chemist.

The crude pyroligneous acid is rectified by a second distillation in a copper still, in the body of which about twenty gallons of viscid tarry matter are left from every 100. It has now become a transparent brown vinegar, having a considerably empyreumatic smell, and a sp. gr. of 1.013 . Its acid powers are superior to those of the best household vinegar, in the proportion of three to two. By redistillation, saturation with quicklime, evaporation of the liquid acetate to dryness, and conversion into acetate of soda by suiphate of suda, the empyreumatic matter is so completely dissipated, that on decomposing the pure acetate of soda by sulphuric acid, a perfectly colorless and grateful vinezar rises in distillation. Its strength will be proportionable to the concentration of the decomposing acid.

The acetic acid of the chemist may be prepared also in the fllowing modes :-1. Two parts of fused acetate of potash, with one of the strongest oil of vitriol, yield, by slow distillation from a glass retort into a refrigerated receiver, concentrated acetic acid. A small portion of sulphurous acid, which contaminates it, may be removed by redistillation from a little acetate of lead. 2. Or four parts of good sugar of lead, with one part of sulphuric acid, treated in the same way, afford a slightly weaker acetic acid. 3. Gently calcined sulphate of iron, or green vitriol, mixed with sugar of lead, in the pre rortion of 1 of the former to $2 \frac{1}{2}$ of the latter, or with acetate of copper, and carefully distilled from a porcelain retort into a ccol receiver, may be aiso considered an economical process. But that with binacetate of copper above described, is preferable to any of these.

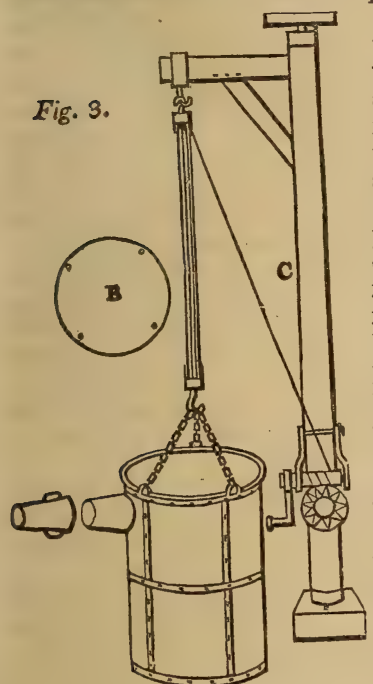

The manufacture of pyroligneous acid is conducted in the following way in France. Into large cylindrical vessels ( $f$ g. 3 ) made of riveted sheet iron, and having at their top and side a small sheet iron cylinder, the wood intended for making charcoal is introduced. To the upper part of this vessel a cover of sheet iron, B, is adapted, which is fixed with bolts. This vessel, thus closed, represents, as we see, a vast retort. When it is prepared, as we have said, it is lifted by means of a swing crane, c, and placed in a furnace, D ( fig. 4), of a form relative to that of the vessel, and the openirg of the furnace is covered with a dome, $\mathrm{E}$, made of masonry or brickwork. The whole being thus arranged, heat is applied in the furnace at the bottom. The moisture of the wood is first dissipated, but by degrees the liquor ceases to be transparent, and becomes sooty. An adopter tube, A, is then fitted to the lateral cylinder. This adopter enters into another tube at the same degree of inclination which commences the condensing apparatus. The means of condensation vary according to the localities. In certain works they cool by means of air, by making the vapor pass through a long series of cylinders, or sometimes, even, through a series of casks connected together; but most usually water is used for condensing, when it can be easily procured in abundance. The most simple

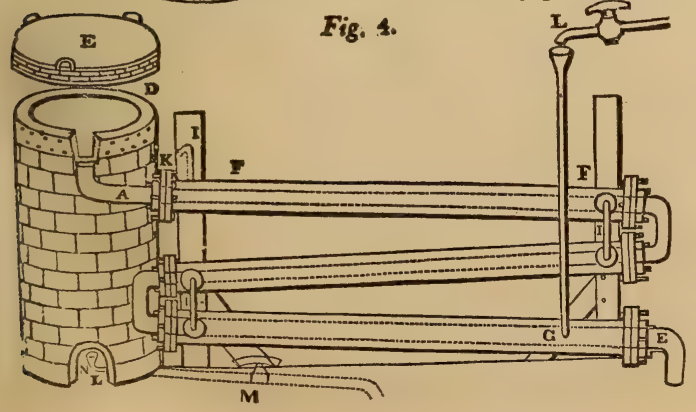
apparatus employed for this purpose consists of two cy linders, F, F (fig. 4), the one within the other, and which leave between them a sufficient space to allow a considerable body of water to circulate along and cool the vapors. This double cylinder is adapted to the distilling vessel, and placed at a certain inclina. tion. To the first double tube, F, F, a second, and. 
sometimes a third, entirely similar, are connected, which, to save space, return upon them. selves in a zigzag fashion. The water is set in circulation by an ingenious means now adopted in many different manufactories. From the lower extremity, G, of the system of condensers, a perpendicular tube rises, whose length should be a littie more than the most elevated point of the system. The water, furnished by a reservoir, L, enters by means of the perpendicular tube through the lower part of the system, and fills the whole space between the double cylinders. When the apparatus is in action, the vapors, as they condense, raise the temperature of the water, which, by the column in $\mathrm{L}, \mathrm{G}$, is pressed to the upper part of the cylinders, and runs over by the spout $\mathrm{K}$. To this point a very short tube is attached, which is bent towards the ground, and serves as an overflow.

The condensing apparatus is terminated by a conduit in bricks covered and sunk in the ground. At the extremity of this species of gutter is a bent tube, E, which discharges the liquid product into the first cistern. When it is full, it empties itself, by means of an orrerflow pipe, into a great reservoir : the tube which terminates the gutter plunges into the liquid, and thus intercepts communication with the inside of the apparatus. The aisengaged gas is brought back by means of pipes $M$ L, from one of the sides of the conduit to the under part of the ash pit of the furnace. These pipes are furnished with stopcocks $\mathrm{M}$, at some distance in front of the furnace, for the purpose of regulating the jet of the gas, and interrupting, at pleasure, communication with the inside of the apparatus. The part of the pipes which terminates in the furnace rises perpendicularly several inches above the ground, and is expanded like the rose of a watering can, $\mathrm{N}$. The gas, by means of this disposition, can distribute itself uniformly under the vessel, without suffering the pipe which conducts it to be obstructed by the fuel or the ashes.

The temperature necessary to effect the carbonization is not considerable: however, at the last it is raised so high as to make the vessels red hot; and the duration of the process is necessarily proportional to the quantity of wood carbonized. For a vessel which shall contain about 5 meters cube (nearly 6 cubic yds.), 8 hours of fire is sufficient. It is known that the carbonization is complete by the color of the flame of the gas: it is inrst of a vellowish red; it becomes afterwards blue, when more carbonic oxyde than carbonic hydrogen is evolved; and towards the end it becomes entirely white, - a circumstance owing, probably, to the furnace being more heated at this period, and the combustion being more complete. There is still another means of knowing the state of the process, to which recourse is more frequently had; that is the cooling of the first tubes, which are not surrounded with water: a few drops of this fluid are thrown upon their surface, and if they evaporate quietly, it is judged that the calcination is sufficient. The adopter tube is then unluted, and is slid into its junction pipe; the orifices are immediately stopped with plates of iron and plaster loam. The brick cover, $\mathrm{E}$, of the furnace is first removed by means of the swing crane, then the cylinder itself is lifted out and replaced immediately by another one previously charged. When the eylinder which has been taken out of the furnace is entirely cooled, its cover is removed, and the charcoal is emptied. Five cubic meters of wood furnish about 7 chaldrons (voies) and a half of charcoal. (For modifications of the wood-vinegar apparatus, see CHArCoAL and Pyroligneous ACID.)

The different qualities of wood employed in this operation give nearly similar products in reference to the acid; but this is not the case with the charcoal, for it is better the harder the wood; and it has been remarked that wood long exposed to the air furnishes a charcoal of a worse quality than wood carbonized soon after it is cut.

Having described the kind of apparatus employed to obtain pyroligneous acid, I shall now detail the best mode of purifying it. This acid has a reddish brown color; it holds in solution a portion of empyreumatic oil and of the tar which were formed at the same lime, another portion of these products is in the state of a simple mixture : the latter may be separated by repose alone. It is stated above, that the distilling apparatus terminates in a subterranean reservoir, where the products of all the vessels are mixed. A common pump communicates with the reservcir, and sinks to its very bottom, in order that it may draw off only the stratum of tar, which, according to its greater density, occupies the lower part. From time to time the pump is worked to remove the tar as it is deposited. The reservoir has at its top an overflow pipe, which discharges the clearest acid into a cistern, from which it is taken by means of a second pump.

The pyroligneous acid thus separated from the undissolved tar is transferred from this cistern into large sheet iron boilers, where its saturation is effected either by quicklime or loy chalk, the latter of which is preferable, as the lime is apt to take some of the iar into combination. The acid parts by saturation with a new portion of the tar, which is removed by skimmers. 'The neutral solution is then allow ed to rest for a sufficient time to let its clear parts be drawn off by decantation.

The acetate of lime thus obtained indicat's by the hydrometer, before being mixed witt. the waters of edulcoration, a degree corresposding to the acidimetric degree of the acid 
employed. This solution must be evaporated till it reaches a specific gravity of $1 \cdot 114$ ( $15^{\circ}$ Baumé), after which there is added to it a saturated solntion of sulphate of soda. The acids exchange bases; sulphate of lime precipitates, and acetate of soda remains in solution. In sume manufactures, instead of pursuing the above plan, the sulphate of soda is dissolred in the hot pyroligneous acid, which is afterwards saturated with chalk or lime. By this means no water need be employed to dissolve the sulphate, and acordingly the liquor is obtained in a concentrated form without evaporation. In both modes the sulphate of lime is allowed to settle, and the solution of acetate of soda is decanted. The residuum is set aside to be edulcorated, and the last waters are employed for washing fresh portions.

The acetate of soda which results from this double decomposition is afterwards evaporated till it attains to the density of $1 \cdot 225$ or $1 \cdot 23$, according to the season. This solution is poured into large crystallizing vessels, from which, at the end of 3 or 4 days, according to their capacity, the mother waters are decanted, and a first crystallizaticin is obtained of rhomboidal prisms, which are highly colored and very bulky. Their facettes are finely polished, and their edges very sharp. The mother waters tre submitted to successive evaporations and crystallizations till they refuse to crystallize, and they are then burnt to convert them into carbonate of soda.

To avoid guesswork proportions, which are always injurious, by the loss of time which they occasion, and by the bad results to which they often lead, we should determine experimentally, beforehand, the quantities absolutely necessary for the reciprocal decomposition, especially when we change the acid or the sulphate. But it may be remarked that, notwithstanding all the precautions we can take, there is always a notable quantity of sulphate of soda and acetic acid, which disappear totally in this decomposition. This arises from the circumstance that sulphate of soda and acetate of lime do not completely decompose each other, as I have ascertained by experiments on a very considerable scale; and thus a portion of each of them is always lost with the mother waters. It might be supposed that by calcining the acetate of lime we could completely destroy its empyreumatic oil; but, though I have made many experiments with this view, I never could abtain an acetate capable of affording a tolerable acid. Some manufacturers prefer to make the acetate of soda by direct saturation of the acid with the alkali, and think that the higher price of this substance is compensated by the economy of time and fuel which it produces.

'The acetate of soda is easily purified by crystallizations and torrefaction; the latter process, when well conducted, freeing it completely from every particie of tar. This torrefaction, to which the name of fusion may be given, requires great care and dexterity. It is usually done in shallow cast iron boilers of a hemispherical shape. During all the time that the heat of about $500^{\circ} \mathrm{Fahr}$. is applied, the fused mass must be diligently worked with rakes; an operation which continues about 24 hours for half a ton of materials We must carefully avoid raising the temperature so high as to decompose the acetate and be sure that the heat is equally distributed; for if any point of the mass enters inte decomposition, it is propagated with such rapidity, as to be excessively difficult to stop its progress in destroying the whole. The heat should never be so great as to disengage any smoke, even when the whole acetate is liquefied. When there is no more frothing up, and the mass flows like oil, the operation is finished. It is now allowed to cool in a body, or it may be ladled out into moulds, which is preferable.

When the acetate is dissolved in water, the charcoaly matter proceeding from the decomposition of the tar must be separated by filtration, or by boiling up the liquor to the specific gravity $1 \cdot 114$, when the carbonaceous matter falls to the bottom. On evaporating the clear liquor, we obtain an acetate perfectly fine, which yields beautiful crystals on cooling. In this state of purity it is decomposed by sulphuric acid, in order to separate its acetic acid.

This last operation, however simple it appears, requires no little care and skill. The acetate of soda crystallized and ground is put into a copper, and the necessary quantity of sulphuric acid of 1.842 (about 35 per cent. of the salt) to decompose almost, but not all, the acetate, is poured on. The materials are left to act on each other; by degrees the acetic acid quits its combination, and swims upon the surface; the greater part of the resulting sulphate of soda falls in a pulverulent form, or in small granular crystals, to the bottom. Another portion remains dissolved in the liquid, which has a specific gravity of 1.08. By distillation we separate this remainder of the sulphate, and finally obtain acetic acid, having a specific gravity of 1.05 , an agreeable taste and smell, though towards the end it becomes a little empyreumatic, and colored; for which reaso! , the last portions must be kept apart. The acid destined for table use ought to be ditilled in an alembic whose capital and condensing worm are of silver; and to make it very fine, it may be afterwards infused over a little washed bone-black. It is usually obtained in a pretty concentrated state; but when we wish to give it the highest degree of concen. tration, we mix with it a quantity of dry muriate of lime, and distil anew. This acid may 
be afterwards exposed to congelation, when the strongest will crystallize. It is decanted, and the crystals are melted by exposing them to a temperature of from $60^{\circ}$ to $70^{\circ} \mathrm{Fahr}$.; this process is repeated till the acid congeals without remainder, at the temperature of $55^{\circ}$ Fahr. It has then attained its maximum strength, and has a specific gravity of 1.063 .

We shall add an observation on the above mode of decomposing the acetate of soda by sulphuric acid. Many difficulties are experienced in this process, if the sulphuric acid be poured on in small quantities at a time; for then such acrid fumes of acetic acid are disengaged, that the workmen are obliged to retire. This inconvenience may be saved by adding all the sulphuric acid at once; it occupies the lower part of the vessel, and decomposes only the portion of the acetate in contact with it; the heat evolved in consequence of this reaction is diffused through a great mass, and produces no sensib?e effect. When the sulphuric acid forms an opening, or a species of little crater, the workinan, by means of a rake, depresses the acetate into it by degrees, and then the decomposition proceeds as slowly as he desires.

The acetic acid, like the nitric, chloric, and some others, has not hitherto been obtained free from water, and the greatest degree of concentration which we have been able to give it is that in which it contains only the quantity of water equivalent to the atomic weight of another oxydized body; a quantity which amounts to 14.89 per cent. The processes prescribed for preparing concentrated acetic acid sometimes tend to deprive it of that water without which it could not exist : hence, in all such cases, there is a part of the acid itself decomposed to furnish the water necessary to the constitution of the remainder. The constituent principles of the decomposed portion then form a peculiar, intoxicating, highly inflammatory liquid, called the PYRO-ACETIC SPIRIT.

The most highly concentrated acid of 1.063 becomes denser by the addition of a certain quantity of water up to a certain point. According to Berzelius, the prime equivalent of this acid is $643 \cdot 189$, oxygen being reckoned 100 . Now, the above strongest acid consists of one prime of acid, and one of water $=1124.79$. When it contains three atoms of water, that is, $337 \cdot 437$ parts to $643 \cdot 189$, or $34 \cdot 41$ to 65.59 in 100 , it then has taken its maximum density of 1.075 ; after which the further addition of water diminishes its specific gravity, as the following table of Mollerat shows. His supposed anhye drous or dry acid contains, at $1 \cdot 0630,0 \cdot 114$ parts of water.

Table of Acetic Acid.

\begin{tabular}{r|c|c|c|}
$\begin{array}{r}\text { Water in } \\
100 \text { parts. }\end{array}$ & $\begin{array}{c}\text { Specific } \\
\text { gravity. }\end{array}$ & $\begin{array}{c}\text { Water in } \\
100 \text { parts. }\end{array}$ & $\begin{array}{c}\text { Specific } \\
\text { gravity. }\end{array}$ \\
\cline { 2 - 4 } 0.00 & 1.0630 & $33 \cdot 83$ & $1 \cdot 0742$ \\
$8 \cdot 37$ & 1.0742 & 37.60 & 1.0728 \\
17.00 & 1.0770 & 47.00 & 1.0658 \\
23.00 & 1.0791 & 50.00 & 1.0637 \\
28.10 & 1.0763 & 51.80 & 1.063 \\
\hline
\end{tabular}

Acetic acid readily takes fire when it is heated in open vessels to the boiling point, and it burns with a blue flaine, nearly like alcohol. It must be kept in close vessels, otherwise it loses its strength, by attracting humidity from the air. When concentrated, it is used only as a scent, or pungent exciter of the olfactory organs, in sickness and fainting fits. Its anti-epidemic qualities are apocryphal. What is met with in the shops under the name of salts of vinegar is nothing but sulphate of potash, put up in small vials, and impregnated with acetic acid, sometimes rendered aromatic with oil of rosemary or lavender. of

Acetic acid, in its dry state, as it exists in fused acetate of potash or soda, is composed

$$
\begin{aligned}
& 47 \cdot 536 \text { carbon } \\
& 5 \cdot 822 \text { hydrogen } \\
& 46 \cdot 642 \text { oxygen }
\end{aligned}
$$

$100 \cdot 000$

And its symbol by Berzelius is $\mathrm{H}_{6} \mathrm{C}_{4} \mathrm{O}_{3}=\mathrm{A}$. We must bear in mind that his atomic weight for hydrogen is only one half of the number usually assigned to it by British chemists, in consequence of his making water a compound of two atoms of hydrogen and one of oxygen.

When the vapor of acetic acid is made to traverse a red-hot tube of iron, it is con. verted into water, carbonic acid, carbureted hydrogen, but chiefly pyro-acetic spirit. 
Acetic acid is a solvent of several organic products; such as camphor, gluten, gum-re. sins, resins, the fibrine of blood, the white of egg, \&c.

It is an important problem to ascertain the purity and strength of vinegar. Spurious acidity is too often given to it by cheaper acids, such as the sulphuric and the nitric. The former may most surely be detected by the nitrate of baryta, or even by acetate of lead, which occasion a white precipitate in such adulterated vinegar. For the case of nitric, which is more insidious, the proper test is, a bit of gold leaf, wetted with a few drops of muriatic acid. If the leaf dissolves, on heating the mixture in a watch glass, we may be sure that nitric acid is present.

Specific gravity, if determined by a sensible hydrometer, is a good test of the strength of the genuine vinegar; and the following table of Messrs. Taylor is nearly correct, or sufficiently so for commercial transactions.

Revenue proof vinegar, called by the English manufacturer No. 24, has a specific gravity of

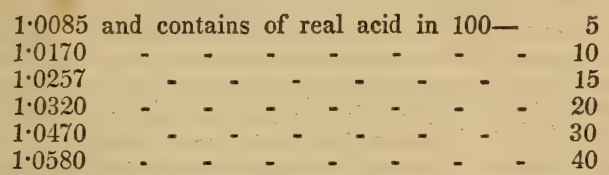

An excise duty of $2 d$. is levied on every gallon of the above proof vinegar. Its strength is not, however, estimated directly by its specific gravity, but by the specific gravity which it assumes when saturated with quicklime. The decimal fraction of the specific gravity of the calcareous acetate is very nearly the double of that of the pure vinegar; or, 1.009 in vinegar becomes 1.018 in acetate of lime. The vinegar of malt contains so much mucilage or gluten, that when it has only the same acid strength as the above, it has a density of 1.0014 , but it becomes only 1.023 when converted into acetate of lime: indeed, 0.005 of its density is due to mucilaginous matter. This fact shows the fallacy of trusting to the kydrometer for determining the strength of vinegars, which may be more or less loaded with vegetable gluten. The proper test of this, as of all other acids, is, the quantity of alkaline matter which a given weight or measure of it will saturate. For this purpose the bicarbonate of potash, commonly called, in the London shops, carbonate, may be employed very conveniently. As it is a very uniform substance, and its atomic weight, by the hydrogen radix, is 100.584 , while the atomic weight of acetic acid, by the same radix, is $51 \cdot 563$, if we estimate 2 grains of the bicarbonate as equivalent to 1 of the real acid, we shall commit no appreciable error. Hence, a solution of the carbonate containing 200 grains in 100 measures, will form an acetimeter of the most perfect and convenient kind ; for the measures of test liquid expended in saturating any measure,-for instance, an ounce or 1000 grains of acid,-will indicate the number of grains of real acetic acid in that quantity. Thus, 1000 grains of the above proof, would require 50 measures of the acetimetrical alkaline solution, showing that it contains 50 grains of real acetic acid in 1000, or 5 per cent.

It is common to add to purified wood vinegar, a little acetic ether, or caramelized (burnt) sugar to color it, also, in France, even wine, to flavor it. Its blanching effect upon red cabbage, which it has been employed to pickle, is owing to a little sulphurous acid. This may be removed by redistillation with peroxyde of manganese. Indeed, Stoltze professes to purify the pyroligneous acid solely by distilling it with peroxyde of znanganese, and then digesting it with bruised wood charcoal ; or by distilling it with a nixture of sulphuric acid and manganese. But much acid is lost in this case by the formation of acetate of that metal.

Birch and beech afford most pyroligneous acid, and pine the least. It is exclusively employed in the arts, for most purposes of which it need not be very highly purified. It is mich used in calico printing, for preparing acetate of iron called IRON LrQUoR, and acelate of alumina, called RED LIQUOR; which see. It serves also to make sugar of lead; yet when it contains its usual quantity, after rectification of tarry matter, the acetate of lead will hardly crystallize, but forms cauliflower concretions. This evil may be remedied, I believe, by boiling the saline solution with a very little nitric acid, which causes the precipitation of a brown granular substance, and gives the liquor a reddish tinge. The solution being afterwards treated with bruised charcoal, becomes colorless, and furnishes regular crystals of acetate or sugar of lead.

Pyroligneous acid possesses, in a very eminent degree, anti-putrescent properties. Flesh steeped in it for a few hours may be afterwards dried in the air without corrupting; but it becomes hard, and somewhat leather-like : so that this mode of preservation does not answer well for butcher's meat. Fish are sometimes cured with it. See Pyro-AcETTc Spirit; Pyroxilic Ether; Pyroxolic Spirit; Pyroligneous Acid and Vinegar. 
ACETIMETER. An apparatus for determining the strength of vinegar. See the conclusion of the preceding article for a description of m.y simple method of acetimetry. ACETONE. The new chemical name of pyro-acetic spirit.

ACID OF ARSENIC. (Acide Arsenique, Fr.; Arseniksaure, Germ.)

ACIDS. A class of chemical substances characterized by the property of combining with and neutralizing the alkaline and other bases, and of thereby forming a peculiar class of bodies called salts. The acids which constitute objects of special manufacture for commercial purposes are the following:-acetic, arsenious, carbonic, chromic, citric, malic, muriatic, nitric, oxalic, phosphoric, sulphuric, tartaric, which see.

ACROSPIRE. (Plumule, Fr.; Blattkeim, Germ.) That part of a germinating seed which botanists call the plumula, or plumes. See BEER and MatT.

ADDITIONS. Such articles as are added to the fermenting wash of the distiller are distinguished by this trivial name.

ADIPOCIRE. Fr. (Fettuachs, Germ.) The fatty matter generated in dead bodies buried under peculiar circumstances. In 1786 and 1787, when the churchyard of the Innocents, at Paris, was cleaned out, and the bones transported to the catacombs, it was discovered that not a few of the cadavres were converted into a saponaceous white substance, more especially many of those which had been interred for fifteen years in one pit, to the amount of 1500 , in coffins closely packed together. These bodies were flattened, in consequence of their mutual pressure; and, though they generally retained their shape, there was deposited round the bones of several a grayish white, somewhat soft, flexible substance. Fourcroy presented to the Academy of Sciences, in 1789, a comprehensive memoir upon this phenomenon, which appeared to prove that the fatty body was an ammoniacal soap, containing phosphate of lime; that the fat was similar to sper. maceti, as it assumed on slow cooling a foliated crystalline structure; as also to wax, as, when rapidly cooled, it became granular: hence he called it Adipocire. Its melting point was $52.5^{\circ} \mathrm{C}$. $\left(126.5^{\circ} \mathrm{Fahr}\right.$.). He likewise compared this soap to the fat of gall-stones, and supposed it to be a natural product of the slow decomposition of all animal matter, except bones, nails, and hairs.

This substance was again examined by Chevreul in 1812, and was found by him to contain margaric acid, oleic acid, combined with a yellow coloring, odorous matter, besides ammonia, a little lime, notash, oxyde of iron, salts of lactic acid, an azotized substance; and was therefore considered as a combination of margaric and oleic acids, in variable proportions (whence arose its variable fusibility), but that it was not analogous with either spermaceti or cholesterine (gallstones). These fat acids are obviously generated by the reaction of the ammonia upon the margarine and oleine, though they eventually lose the greater part of that volatile alkali.

According to the views of both Gay Lussac and Cherreul, this adipocire proceeds solely from the pre-existing fat of the dead body, and not from the flesh, tendons, or carsilages, as had been previously imagined; which had led to some expensive and abortive attempts, upon the great scale of manufacture, to conrert the dead bodies of cattle into adipocire, for the purposes of the candle-maker or soap-boiler, by exposing them for some time to the action of moisture.

Von Hartkol made experiments during 25 years upon this subject, from which he inferred, that there is no formation of adipocire in bodies buried in dry ground; that in moist earth the fat of the dead body does not increase, but changes into a fetid saponaceous substance, incapable of being worked into either soap or candles; that the dead bodies of mammalia immersed in running water, leave behind after 3 years a pure fat, which is more abundant from young than from old anmials; that the intestines afford more fat than the muscles; that from this fat, without any purification, candles may be made, as void of smell, as hard, and as white, as from bleached wax; that from cadavers immersed for 3 years in stagnant water, more fat is procured than from those in running water, but that it needs to be purified beiore it can be made into soap or candles.

The cause of the difference between Hartkol's and Chevreul's results cannot be assigned, as the latter has not published his promised remarks upon the subject. At any rate, dead animal matter can be worked up more profitably than in making artificial adipocire.

ADIT. The horizontal entrance of a mine. It is sometimes called the drift. See Mining and Metallurgy.

ADULTERATION. The debasing any product of manufacture, especially chemical, by the introduction of cheap materials. The art of ascertaining the genuineness of the several products will be taught under the specific objects of manufacture.

列THER. See ETHER.

AFFINITY. The chemical term denoting the peculiar attractive force which produces the combination of dissimilar substances; such as of an alkali with an acid, or of sulphur with a metai 
AGARIC. A species of boletus or fungus, which grows in dunghills; with the salts of iron it affords a black dye. It is said to be convertible into a kind of china ink. AGATE. A silicious mineral which is cut into seals and other forms for the coarser kinds of jewellery. See GEM.

AIR. See VENTILATION.

ALABASTER, is a stone usually white, and soft enough to be scratched by iron. There are two kinds of it : the gypseous, which is merely a natural semi-crystalline sulphate of lime; and the calcarcous alabaster, which is a carbonate of lime. The oriental alabaster is always of the latter kind, and is most esteemed, because it is agreeably variegated with lively colors, and especially with zones of honey-yellow, yellow-brown, red, \&c. ; it is, moreover, susceptible of taking a marble polish.

The fireness of the grain of alabaster, "the uniformity of its texture, the beauty of its polished surface, and its semi-transparency, are the qualities which render it valuable to the sculntor and to the manufacturer of ornamental toys.

The limestone alabaster is frequently found as a yellowish-white deposite in certain fountains. The most celebrated spring of this kind is that of the baths of San Filippo, in Tuscany. The water, almost boiling hot, runs over an enormous mass of stalactites, which it has formed, and holds the carbonate of lime in solution by means of sulphureted hydrogen (according to M. Alexandre Brongniard), which escapes by contact of the atmosphere. Advantage has been taken of this property to make basso relievos of considerable hardness, by placing moulds of sulphur very obliquely, or almost upright, in wooden tubs open at the bottom. These tubs are surmounted at the top with a large woolen cross. The water of the spring, after having deposited in an external conduit or cistern the coarser sediment, is made to flow upon this wooden cross, where it is scattered into little streamlets, and thence lets fall, upon the sulphur casts, a precipitate so much the finer the more nearly vertical the mould. From one to four months are required for this operation, according to the thickness of the deposited crust. By analogous processes, the artists have succeeded in moulding vases, figures of animals, and other objects, in relief, of every different form, which require only to be trimmed a little, and afterwards polished.

The cummon alabaster is composed of sulphuric acid and lime, though some kinds of it effervesce with acids, and therefore contain some carbonate of lime. This alabaster occurs in many different colors, and of very different degrees of hardness, but it is always softer than marble. It forms, usually, the lowest beds of the gypsum quarries. The sculptors prefer the hardest, the whitest, and those of a granular texture, like Carrara marble, and so like that they can only be distinguished by the hardness.

The alabaster is worked with the same tools as marble; and as it is many degrees softer, it is so much the more easily cut; but it is more difficult to polish, from its little solidity. After it has been fashioned into the desired form, and smoothed down with pumice stone, it is polished with a pap-like mixture of chalk, soap, and milk; and, last of all, finished by friction with flannel. It is apt to acquire a yellowish tinge.

Besides the harder kinds, employed for the sculpture of large figures, there is a softer alabaster, pure white and semi-transparent, from which sinall ornamental objects are made, such as boxes, vases, lamps, stands of time-pieces, \&c. This branch of business is mueh prosecuted in Florence, Leghorn, Milan, \&c., and employs a great many turning lathes. Of all the alabasters, the Florentine merits the preference, on account of its beauty and uniformity, so that it may be fashioned into figures of considerable size : for which purpose there are large work-shops where it is cut with steel saws into blocks and masses of various shapes. Other sorts of gypsum, such as that of Salzburg and Austria, contain sand veins, and hard nodules, and require to be quarried by clearing and blasting operations, which are apt to crack it, and unfit it for all delicate objects of sculpture. It is, besides, of a gray shade, and often stained with darker colors.

The alabaster best adapted for the fine arts is pretty white when nevly broken, and becomes whiter on the surface by drying. It may be easily cut with the knife or chisel, and formed into many pleasing shapes by suitable steel tools. It is worked either by the hand alone, or with the aid of a turning lathe. The turning tools should not be too thin or sharp-edged; but such as are employed for ivory and brass are most suitable for alabaster, and are chiefly used to shave and to scratch the surface. The objects which cannot be turned may be fashioned by the rasping tools, or with minute files, such as variegated foliage. Fine chisels and graving tools are also used for thelpiter pieces of statuary.

For polishing such works, a peculiar process is required: pumice stone, in fine powder, serves to snooth down the surfaces very well, but it soils the whiteness of the alabaster. To take away the unevennesses and roughnesses dried shave-grass (equisetum) anstrers best. Frictions with this plant and water polish down the asperities left by the chisel : the fine streaks left by the grass may be removed by rubbing the pieces with slalied lime, finely pulverized and sifted, made into a paste, or putty, with water. The 
polish and satin-lustre of the surface are communicated by friction, first with soap-wate and lime, and finally with powdered and elutriated tale or French chalk.

Such articles as consist of several pieces are joined by a cement composed of quicklime and white of egg, or of well-calcined and well-sifted Paris plaster, mixed with the least possible quantity of water.

Alabaster objects are liable to become yellow by keeping, and are especially injured by smoke, dust, \&c. They may be in some measure restored by washing with soap and water, then with clear water, and again polished with shave-grass. Grease spots may be removed either by rubbing with talc powder, or with oil of turpentine.

The surface of alabaster may be etched by covering over the parts that are not to be touched with a solution of wax in oil of turpentine, thickened with white lead, and immersing the articles in pure water after the varnish has set. The action of the water is continued from 20 to 50 hours, more or less, according to the depth to which the etching is to be cut. After removing the varnish with oil of turpentine, the etched places, which are necessarily deprived of their polish, should be rubbed with a brush dipped in finely-powdered gypsum, which gives a kind of opacity, contrasting well with the rest of the surface.

Alabaster may be stained either with metallic solutions, with spirituous tinctures of dyeing plants, or with colored oils, in the same way as marbles.

This substance has been hardened, it is said, by exposing it to the heat of a baker's oven for 10 or 20 hours, after taking it out of the quarry, and giving it the figure, roughly, which it is intended to have. After this exposure, it must be dipped for two minutes in running water; when it is cold, it must be dipped a second time for the same period. On being exposed to the air for a few days, alabaster so treated acquires a marble-like hardness. I doubt the truth of this statement.

ALBUM GRÆCUM. The white dung of dogs, sometimes used to soften leather in the process of dressing it after the depilatory action of lime.

ALCARAZZAS. A species of porous earthenware, made in Spain, for cooling liquors. See Pottery.

ALCOHOL. The well-known intoxicating liquor procured by distillation from various vegetable juices, and infusions of a saccharine nature, which have undergone the vinous fermentation. Common alcohol, or proof spirit, as it is called, contains about one half its weight of water. It may be concentrated till its specific gravity becomes so low as 0.825 , by simple redistillation at a steam or water-vath heat; but to make it stronger, we must mix with it, in the still or retort, dry carbonate of potash, muriate of lime, or some other substances strongly attractive of water, and then it may be obtaines of a specific gravity so low as 0.791 at $16^{\circ}$ Reaumur $\left(68^{\circ}\right.$ Fahr.), water being 1.000 . At 0.825 , it contains, still, 11 per cent. of water; and in this state it is as volatile as absolute alcohol, on account of the inferior density of the aqueous vapor, compared to the alcoholic. Indeed, according to Yelin and Fuchs, the boiling point of anhydrous alcohol is higher than of that which contains 2 or 3 per cent. of water; henee, in the distillation of alcohol of 94 per cent., the first portions that come over are more aqueous than the following. Absolute alcohol has its boiling point at $168 \frac{1}{2}^{\circ} \mathrm{Fahr}$; but when it holds more than 6 per cent. of water, the first portions that come over are richest in alcohol, and the temperature of the boiling point, or of the spirituous vapor, is always higher the longer the distillation continues. According to Gröning's researches, the following temperatures of the alcoholic vapors correspond to the accompanying contents of alcohol in per centage of volume, which are disengaged in the boiling of the spirituous liquid.

\begin{tabular}{|c|c|c|c|c|c|}
\hline Temperature. & $\begin{array}{l}\text { Alcoholic con- } \\
\text { tent of the } \\
\text { vapor. }\end{array}$ & $\begin{array}{l}\text { Alcoholic con- } \\
\text { tent of the boil- } \\
\text { ing liquid. }\end{array}$ & Temperature. & $\begin{array}{l}\text { Alcoholic con- } \\
\text { tent of the } \\
\text { vapor. }\end{array}$ & $\begin{array}{l}\text { Alcoholic con- } \\
\text { tent of the lioil } \\
\text { ing liquid. }\end{array}$ \\
\hline Fahr. $\begin{aligned} 170 \cdot 0 \\
171 \cdot 8 \\
172 \\
172 \cdot 8 \\
174 \\
174 \cdot 6 \\
176 \\
178 \cdot 3 \\
180 \cdot 8 \\
183 \\
185 \\
187 \cdot 4\end{aligned}$ & $\begin{array}{l}93 \\
92 \\
91 \\
90 \frac{1}{2} \\
90 \\
89 \\
87 \\
85 \\
82 \\
80 \\
78 \\
76\end{array}$ & $\begin{array}{l}92 \\
90 \\
85 \\
80 \\
70 \\
70 \\
65 \\
50 \\
40 \\
35 \\
30 \\
25\end{array}$ & $\begin{aligned} & \text { Fahr. } 189 \cdot 8 \\
& 192 \cdot 0 \\
& 164 \\
& 196 \cdot 4 \\
& 198 \cdot 6 \\
& 201 \\
& 203 \\
& 205 \cdot 4 \\
& 207 \cdot 7 \\
& 210 \\
& 212\end{aligned}$ & $\begin{array}{r}71 \\
68 \\
66 \\
61 \\
55 \\
50 \\
42 \\
36 \\
28 \\
13 \\
0\end{array}$ & $\begin{array}{r}20 \\
18 \\
15 \\
12 \\
10 \\
7 \\
5 \\
3 \\
2 \\
1 \\
0\end{array}$ \\
\hline
\end{tabular}


Gröning undertook this investigation in order to employ the thermometer as an alcoholmetci in the distillation of spirits; for which purpose he thrust the bulb of the thermorweter through a cork, inserted into a tube fixed in the capital of the still. The state of the barometer ought also to be considered in making comparative experiments of this kind. Since, by this method, the alcoholic content may be compared with the temperature of the vapor that passes over at any time, so, also, the contents of the whole distillation inay be found approximately; and the method serves as a convenient means of making continual observations on the progress of the distillation.

The temperature, corresponding to a certain per centage of alcohol in vapor, suggests the employment of a convenierit method for obtaining, at one process, a spirit as free from water as it can be made by mere distillation. We place over the top of the capital a water-bath, and lead up through it a spiral pipe from the still, which there passes obliquely downwards, and proceeds to the refrigeratory. If this bath be maintained, by a constant influx of cold water, at a certain temperature, only the alcoholic vapor corresponding to that temperature will pass over, and the rest will be recondensed and returned into the still. If we keep the temperature of the water at $174^{\circ}$, for example, the spirituous vapor which passes over will contain 90 per cent. of absolute alcohol, according to the preceding table. The skilful use of this principle constitutes the main improvement in modern distilleries. See Distillation and Still.

Another method for concentrating alcohol is that discovered by Sömmering, founded upon the property of ox bladders to allow water to pass through and evaporate out of them, but not to permit alcohol to transpire, or only in a slight degree. Hence, if an ox's bladder is filled with spirit of wine, well tied at the mouth, and suspended in a warm nlace, the water will continually exhale, and the alcohol will become nearly anhydrous; for in this way alcohol of 97 or 98 per cent. may be obtained.

According to Sömmering, we should take for this purpose the bladder of an ox or a calf, soak it for some time in water, then inflate it and free it from the fat and the attached vessels; which is to be also done to the other surface, by turning it inside out. After it is again inflated and dried, we must smear over the outer side twice, and the inner side four times, with a solution of isinglass, by which its texture is made closer, and the concentration of the alcohol goes on better. A bladder so prepared may serve more than a hundrea times. It must be charged with the spirits to be concentrated, leaving a small space vacant; it is then to be tightly bound at the mouth, and suspended in a warm situation, at a temperature of $122^{\circ} \mathrm{Fahr}$, over a sand-bath, or in the neighborhood of an oven. The surface of the bladder remains moist with the water, as long as the sp. gr. of the contained spirit is greater than 0.952 . Weak spirit loses its water quicker than strong; but in from 6 to 12 hours the alcohol may be concentrated, when a sutitable heat is employed. This economical method is particularly applicable in obtaining alcohol for the preparation of varnishes. When the alcohol is to serve for other purposes, it must be freed, by distillation, from certain matters dissolved out of the bladder. Alcohol may likewise be strengthened, as Sömmering has ascertained, when the vessel that contains the spirit is bound over with a bladder which does not come into contact with the liquid. Thus, too, all other liquors containing alcohol and water, as wine, cider, \&c., may be made more spirituous.

To procure absolute alcohol, we must take chloride of calcium recently fused, reduce it to coarse powder, and $\mathrm{mix}$ it with its own weight of spirit of wine, of sp. gr. 0.833 , in a bottle, which is to be well stoppered, and to be agitated till the salt is dissolved. The clear solution is to be poured into a retort, and half of the volume of the alcohol employed, or so much as has the sp. gr. 0.791 at $68^{\circ} \mathrm{Fahr}$., is to be distilled off at a gentle heat. Quicklime has also been employed for the same purpose, but it is less powerful and convenient. Alcohol, nearly free from water, may be obtained without distillation, by adding dry carbonate of potash to a spirit of wine, of sp. gr. $0 \cdot 825$. The water combines with the potash, and falls to the bottom in a dense liquid, while the pure spirit floats on the surface. This contains, however, a little alkali, which can only be separated by distillation.

Antiddrous alcohol is composed by weight of 52.66 carbon, 12.90 hydrogen, and 34.44 of oxygen. It has a very powerful attraction for water, and absorbs it from the atmosphere; therefore it must be kept in well-closed vessels. It also robs vegetable and animal bodies of their moisture; and hence common alcohol is employed for preserving anatomical preparations. Alcohol is a solvent for many substances: resins, essential oils, camphor, are abundantly dissolved by it, forming varnishes, perfumed spirits, \&c. The solution of a resin or essential oil in alcohol becomes milky on the addition of water, which, by its attraction for alcohol, separates these substances. Several salts, especially the deliquescent, are dissolved by it, and some of them give a color to its flame; thas, the solutions of the salts of strontia in alcohol burn with a crimson flame, those of copper and borax green, lime reddish, and baryta yellow.

When water is mixed with alcohol, heat and a condensation of volume are the result; 
these effects being greatest with 54 per cent. of alcohol and 46 of water, and thence de creasing with a greater proportion of water. For alcohol which contains 90 per cent. of wate; , this condensation amounts to 1.94 per cent. of the volume : for 80 per cent., 2.87 ; for 70 per cent., 3.44 ; for 60 per cent., 3.73 ; for 40 per cent., 3.44 ; for 30 per cent., 2.72 ; for 20 per cent., 1.72 ; for 10 per cent., 0.72 . Hence, to estimate the quantity of alcohol in any spirit it is necessary that the specific gravity be ascertained for each determinate proportion of alcohol and water that are mixed together. When this is done, we may, by means of an areometer constructed for liquids lighter than water, determine the strength of the spirit, either by a scale of specific gravities or by an arbitrary graduation corresponding to certain commercial objects, and thus we may determine the per centage of alcohol in whiskey or brandy of any strength or purity. An areometer intended for this use has been called an alcoholmeter, in particular when the scale of it is so graduated that, instead of the specific gravity, it indicates immediately the per centage of anhydrous alcohol in a given weight or volume of the liquid. The scale graduated according to the per centage of pure alcohol by weight, constitutes the alcoholmeter of Richter; and that by the per centage in volume, the alcoholmeter of Tralles and Gay Lussac.

As liquors are sold in general by the measure, not by the weight, it is convenient, therefore, to know the alcoholic content of the mixtures in the per centage by volume. Tralles has constructed new tables upon the principles of those of Gilpin, in which the proportion is given by volume, and anhydrous alcohol is assumed for the basis; which, at $60^{\circ}$ Fahr., has a specific gravity of 0.7939 compared with water at its maximum density, or a specific gravity of 0.7946 compared with water of the temperature of $60^{\circ}$ Fahr. Gilpin's aleohol of 0.825 contains 92.6 per cent. by volume of anhydrous alcohol.

The following table exhibits the per centage of anhydrous alcohol by volume, at a temperature of $60^{\circ} \mathrm{Fahr}$, in correspondence with the specific gravities of the spirits, water being considered at $60^{\circ} \mathrm{Fahr}$. to have a specific gravity of 0.9991 .

Alcoholmetrical Table of Tralles.

\begin{tabular}{|c|c|c|c|c|c|}
\hline $\begin{array}{l}\text { Alcohol in } 100 \\
\text { measures of } \\
\text { spirit. }\end{array}$ & $\begin{array}{l}\text { Specific gravity } \\
\text { at } 60^{\circ} \text { Fahr. }\end{array}$ & $\begin{array}{l}\text { Difference of } \\
\text { the sp. gr. }\end{array}$ & $\begin{array}{l}\text { Alcohol in } 100 \\
\text { measures of } \\
\text { spirit. }\end{array}$ & $\begin{array}{l}\text { Specific gravity } \\
\text { at } 60^{\circ} \text { Fahr. }\end{array}$ & $\begin{array}{l}\text { Difference of } \\
\text { the sp. gr. }\end{array}$ \\
\hline 0 & 9991 & & 51 & 9315 & 20 \\
\hline 1 & 9976 & 15 & 52 & 9295 & 20 \\
\hline 2 & 9961 & 15 & 53 & 9275 & 20 \\
\hline 3 & 9947 & 14 & 54 & 9254 & 21 \\
\hline 4 & 9933 & 14 & 55 & 9234 & 20 \\
\hline 5 & 9919 & 14 & 56 & 9213 & 21 \\
\hline 6 & 9906 & 13 & 57 & 9192 & 21 \\
\hline 7 & 9893 & 13 & 58 & 9170 & 22 \\
\hline 8 & 9881 & 12 & 59 & 9149 & 22 \\
\hline 9 & 9869 & 12 & 60 & 9126 & 22 \\
\hline 10 & 9857 & 12 & 61 & 9104 & 28 \\
\hline 11 & 9845 & 12 & 62 & 9082 & 22 \\
\hline 12 & 9834 & 11 & 63 & 9059 & 23 \\
\hline 13 & 9823 & 11 & 64 & 9036 & 23 \\
\hline 14 & 9812 & 11 & 65 & 9013 & 23 \\
\hline 15 & 9802 & 10 & 66 & 8989 & 24 \\
\hline 16 & 9791 & 11 & 67 & 8965 & 24 \\
\hline 17 & 9781 & 10 & 68 & 8941 & 24 \\
\hline 18 & 9771 & 10 & 69 & 8917 & 24 \\
\hline 19 & 9761 & 10 & 70 & 8892 & 25 \\
\hline 20 & 9751 & 10 & 71 & 8867 & 25 \\
\hline 21 & 9741 & 10 & 72 & 8842 & 25 \\
\hline 22 & 9731 & 10 & 73 & 8817 & 25 \\
\hline 23 & 9720 & 11 & 74 & 8791 & 26 \\
\hline 24 & 9710 & 10 & 75 & 8765 & 26 \\
\hline 25 & 9700 & 10 & 76 & 8739 & 26 \\
\hline 26 & 9689 & 11 & 77 & 8712 & 27 \\
\hline 27 & 9679 & 10 & 78 & 8685 & 27 \\
\hline 28 & 9668 & 11 & 79 & 8658 & 27 \\
\hline 29 & 9657 & 11 & 80 & 8631 & 27 \\
\hline 30 & 9646 & 11 & 81 & 8602 & 28 \\
\hline 31 & 9634 & 12 & 82 & 8575 & 28 \\
\hline 32 & 9622 & 12 & 83 & 8547 & 28 \\
\hline 33 & 9609 & 13 & 84 & 8518 & 29 \\
\hline
\end{tabular}


Alcoholmetrical Table of Tralles (continued).

\begin{tabular}{|c|c|c|c|c|c|}
\hline $\begin{array}{l}\text { Aleohol in } 100 \\
\text { measures of } \\
\text { spirit. }\end{array}$ & $\begin{array}{l}\text { Specific gravity } \\
\text { at } 60^{\circ} \mathrm{Fahr} \text {. }\end{array}$ & $\begin{array}{l}\text { Difference of } \\
\text { the sp. gr. }\end{array}$ & $\begin{array}{c}\text { Alcuhol in } 100 \\
\text { measures of } \\
\text { spirit. }\end{array}$ & $\begin{array}{l}\text { Specific gravity } \\
\text { at } 60^{\circ} \text { Fahr. }\end{array}$ & $\begin{array}{l}\text { Difference of } \\
\text { the sp gr. }\end{array}$ \\
\hline 34 & 9596 & 13 & - 85 & 8488 & 30 \\
\hline 35 & 9583 & 13 & 86 & 8458 & 30 \\
\hline 36 & 9570 & 13 & 87 & 8428 & 30 \\
\hline 37 & 9556 & 14 & 88 & 8397 & 31 \\
\hline 38 & 9541 & 15 & 89 & 8365 & 32 \\
\hline 39 & 9526 & 15 & 90 & 8332 & 33 \\
\hline 40 & 9510 & 16 & 91 & 8299 & 33 \\
\hline 41 & 9494 & 16 & 92 & 8265 & 34 \\
\hline 42 & 9478 & 16 & 93 & 8230 & 35 \\
\hline 43 & 9461 & 17 & 94 & 8194 & 36 \\
\hline 44 & 9444 & 17 & 95 & 8157 & 37 \\
\hline 45 & 9427 & 17 & 96 & 8118 & 39 \\
\hline 46 & 9409 & 18 & 97 & 8077 & 41 \\
\hline 47 & 9391 & 18 & 98 & 8034 & 43 \\
\hline 48 & 9373 & 18 & 99 & 7988 & 46 \\
\hline 49 & 9354 & 19 & 100 & 7939 & 49 \\
\hline 50 & 9335 & 19 & & & \\
\hline
\end{tabular}

Remarks on the preceding Table of Alcohol.

The third column of this table exhibits the differences of the specific gravities, which give the denominator of the fraction for such densities as are not found sufficiently near in the table; and the difference of their numerators is the next greatest to the density found in the table. For example: if the specific gravity of the liquor found for $60^{\circ}$ Fahr. $=9605$ (the per centage will be between 33 and 34 , the difference from 9609 (which is the next greatest number in the table) $=4$, and the fraction is $\frac{4}{13} \cdot$ therefore the true per centage is $33 \frac{4}{13}$. From the construction of this table the per centage of alcohol by weight may also be found. For instance : we multiply the number representing the volumes of alcohol (given in the table for any determinate specifie gravity of the mixture) by the specific gravity of the pure alcohol, that is, by 7939 , and the product is the number of pounds of alcohol in so many pounds as the specific gravity multiplied by 100 gives. Thus, in the mixture of 9510 specific gravity, there are 40 measures of alcohol; hence there are also in 95,100 pounds of this spirit $7939+40=31.756$ pounds of alcohol; and in 100 pounds of the spirits of 0.9510 specific gravity, $33 \cdot 39$ pounds of alcohol are contained.

As the preceding table gives the true alcoholic content when the portion of spirit under trial has the normal temperature of $60^{\circ} \mathrm{Fahr}$., the following table gives the per centage of alcohol for the specific gravities corresponding to the accompanying temperatures.

For example : if we have a spirituous liquor at $80^{\circ} \mathrm{Fahr}$., whose specifie gravity is 0.9342 , the alcohol present is 45 per cent. of the volume, or that specific grarity at that temperature is equal to the specific gravity 0.9427 at the normal temperature of $60^{\circ}$ Fahr. This table may also be employed for every degree of the thermometer and

\begin{tabular}{|c|c|c|c|c|c|c|c|c|c|c|c|c|c|}
\hline \multirow{2}{*}{$\begin{array}{c}\text { Alcohol } \\
\text { per cent. }\end{array}$} & \multicolumn{6}{|c|}{ Temperature. } & \multirow{2}{*}{$\begin{array}{c}\text { Alcohol } \\
\text { per cent. }\end{array}$} & \multicolumn{6}{|c|}{ Temperature. } \\
\hline & $30^{\circ} \mathrm{F}$. & $35^{\circ} \mathrm{F}$. & $40^{\circ} \mathrm{F}$ & $45^{\circ} \mathrm{F}$. & $50^{\circ} \mathrm{F}$. & $55^{\circ} \mathrm{F}$. & & $60^{\circ} \mathrm{F}$. & $65^{\circ} \mathrm{F}$. & $70^{\circ} \mathrm{F}$. & $\approx 5^{\circ} \mathrm{F}$. & $80^{\circ} \mathrm{F}$. & $85^{\circ} \mathrm{F}$. \\
\hline 0 & 9994 & $999 \tau$ & 9997 & 9998 & 9997 & 9994 & 0 & 9991 & 9987 & 9991 & $99 \approx 6$ & 9970 & 9.962 \\
\hline 5 & 9924 & 9926 & 9926 & 9926 & 9925 & 9922 & 5 & 9919 & 9915 & 9909 & 9903 & 9897 & 9559 \\
\hline 10 & 9568 & 9369 & 93003 & 9367 & $9 \$ 65$ & 9861 & 10 & 9357 & 9352 & 9345 & $9 \times 39$ & 9831 & 9523 \\
\hline 15 & $9 \times 23$ & $9 \$ 22$ & 9520 & 9817 & 9813 & $9 s 0 \tau$ & 15 & 9802 & 9796 & 9788 & $97 \% 9$ & $9 \div 71$ & 9761 \\
\hline 20 & 9786 & 9782 & $9 \approx \%$ & $9 \div \div 2$ & $9 \div 66$ & $9 \div 59$ & 20 & $9 \% 51$ & 9743 & 9733 & 9722 & $9 \div 11$ & $9 \approx 00$ \\
\hline 25 & 9753 & 9746 & 9738 & $9 \div 29$ & 9720 & 9709 & 25 & 9700 & 9690 & 9678 & 9665 & 9652 & 9633 \\
\hline 30 & $9 \tau 1 \tau$ & 9707 & 9695 & 9684 & 9672 & 9639 & 30 & 9646 & 9632 & 9618 & 9603 & 9588 & $95 \% 2$ \\
\hline 35 & $96 \% 1$ & 9658 & 9641 & 9629 & 9614 & 9599 & 35 & $95 \$ 3$ & 9566 & 9549 & 9532 & 9511 & 9495 \\
\hline 40 & 9615 & 9593 & 9581 & 9563 & 9546 & 4528 & 40 & 9510 & 9191 & 9472 & 9452 & 9433 & 9412 \\
\hline 45 & 9544 & 9525 & 9506 & 9486 & 9467 & 9447 & 45 & 9427 & 9406 & 9385 & 9364 & $93 \div 2$ & 9320 \\
\hline 50 & 9460 & 9440 & 9420 & 9399 & 9378 & 9356 & 50 & 9335 & 9313 & 9290 & 9267 & 9241 & 9221 \\
\hline 55 & 9369 & 9347 & 9325 & 9302 & 9279 & 9256 & 55 & 9234 & 9211 & 9187 & 9163 & 9139 & 9114 \\
\hline 60 & $926 \pi$ & 9245 & 9222 & 9199 & 9174 & 9150 & 60 & 9126 & 9102 & $90 \div 6$ & 9051 & 9026 & 9000 \\
\hline 65 & $916 ?$ & 9133 & 9113 & 9085 & 9063 & 9038 & 65 & 9013 & 8958 & 8962 & 8936 & 8909 & 8852 \\
\hline 70 & 9016 & 9021 & 8996 & 8970 & 8944 & 8917 & 70 & 8892 & 8866 & 8839 & 8312 & 8754 & 8756 \\
\hline 75 & 8925 & 8599 & $88 \div 3$ & 8847 & 8820 & 8792 & 75 & 8765 & 8738 & $8 \% 10$ & 8651 & 8652 & 8622 \\
\hline 80 & 8795 & 8771 & 8744 & 5716 & 8689 & 8659 & 80 & 8631 & 8602 & 8573 & 8514 & 8514 & 8493 \\
\hline 85 & 8663 & S635 & 8606 & $857 \%$ & 8547 & 8517 & 85 & 8458 & 8458 & 8427 & 8396 & 8365 & 8333 \\
\hline 90 & 8517 & 8486 & 8455 & 8425 & 8395 & 8363 & 90 & 8332 & 8300 & $826 \mathrm{~S}$ & 8236 & 8201 & 8171 \\
\hline
\end{tabular}


every per centage, so as to save computation for the intervals. It is evident from in spection that a difference of $5^{\circ} \mathrm{Fahr}$. in the temperature changes the specific gravity of the liquor by a difference nearly equal to 1 volume per cent. of alcohol; thus at 35 and $85^{\circ}$ Fahr. the very same specific gravity of the liquor shows nearly 10 volumes per cent. of alcohol more or less; the same, for example, at 60 and 40 per cent.

The importance of extreme accuracy in determining the density of alcoholic mixtures in the United Kingdom, on account of the great revenue derived from them to the State, and their consequent high price in commerce, induced the Lords of the Treasury a few years ago to request the Royal Society to examine the construction and mode of applying the instrument now in use for ascertaining and charging the duty on spirits. This instrument, which is known and described in the law as Sikes's hydrometer, possesses, in many respects, decided advantages over those formerly in use. The committee of the Royal Society state, that a definite mixture of alcohol and water is as invariable in its value as absolute alcohol can be; and can be more readily, and with equal accuracy, identified by that only quality or condition to which recourse can be had in practice, namely, specific gravity. The committee further proposed, that the standard spirit be that which, consisting of alcohol and water alone, shall have a specific gravity of 0.92 at the temperature of $62^{\circ}$ Fahr., water being unity at the same temperature; or, in other words, that it shall at $62^{\circ}$ weigh $\frac{92}{100}$ or $\frac{23}{2} \frac{3}{5}$ of an equal bulk of water at the same temperature.

This standard is rather weaker than the old proof, which was $\frac{12}{13}$, or 0.923 ; or in the proportion of nearly $1 \cdot 1$ gallon of the present proof spirit per cent. The proposed standard will contain nearly one half by weight of absolute alcohol. The hydrometer ought to be so graduated as to give the indication of strength; not upon an arbitrary scale, but in terms of specific gravity at the temperature of $62^{\circ}$.

The committee recommend the construction of an equation table, which shall indicate the same strength of spirit at every temperature. Thus in standard spirit at $62^{\circ}$ the hydrometer would indicate 920 , which in this table would give proof spirit. If that same spirit were cooled to $40^{\circ}$, the hydrometer would indicate some higher number; but which, being combined in the table with the temperature as indicated by the thermometer, should still give proof or standard spirit as the result.

It is considered advisable, in this and the other tables, not to express the quality of the spirit by any number over or under proof, but to indicate at once the number of gallons of standard spirit contained in, or equivalent to, 100 gallons of the spirit under examination. Thus, instead of saying 23 over proof, it is proposed to insert 123 ; and in place of 35.4 under proof, to insert its difference to 100 , or $64 \cdot 6$.

It has been considered expedient to recommend a second table to be constructed, so as to show the bulk of spirit of any strength at any temperature, relative to a standard bulk of 100 gallons at $62^{\circ}$. In this table a spirit which had diminished in volume, at any given temperature, 0.7 per cent., for example, would be expressed by 99.3 ; and a spirit which had increased at any given temperature 0.7 per cent., by 100.7 .

When a sample of spirit, therefore, has been examined by the hydrometer and thermometer, these tables will give first the proportion of standard spirit at the observed temperature, and next the change of bulk of such spirit from what it would be at the standard temperature. Thus, at the temperature of $51^{\circ}$, and with an indication (sp. gr.) of 8240,100 gallons of the spirit under examination would be shown by the first table to be equal to $164 \cdot 8$ gallons of standard spirit of that temperature; and by the second table it would appear that 99.3 gallons of the same spirit wauld become 100 at $62^{\circ}$, or in reality contain the 164.8 gallons of spirit in that state only in which it is to be taxed.

But as it is considered that neither of these tables can alone be used for charging the duty (for neither can express the actual quantity of spirit of a specific gravity of 0.92 at $62^{\circ}$ in 100 gallons of stronger or weaker spirit at temperatures above or below $62^{\circ}$ ), it is considered essential to have a third table, combining the two former, and expressing this relation directly, so that upon mere inspection it shall indicate the proportion of standard spirit in 100 gallons of that under examination in its then present state. In this table the quantities should be set down in the actual number of gallons of standard spirit at $62^{\circ}$, equivalent to 100 of the spirit under examination; and the column of quantities may be expressed by the term value, as it in reality expresses the proportion of the only valuable substance present. As this will be the only table absolutely necessary to be used with the instrument for the purposes of the excise, it may, perhaps, be thought unnecessary to print the former two. 
The following specimen table has been given by the committee :-

\begin{tabular}{|c|c|c|c|c|c|}
\hline \multicolumn{3}{|c|}{ Temperature $45^{\circ}$. } & \multicolumn{3}{|c|}{ Temperature $75^{\circ}$. } \\
\hline Indication.* & Strength. & Value. & Indication. & Strength. & Value. \\
\hline 9074 & $114 \cdot 5$ & & 8941 & $114 \cdot 5$ & \\
\hline 7 & $114 \cdot 3$ & & 4 & $114 \cdot 3$ & \\
\hline 9 & $114 \cdot 2$ & & 5 & $114 \cdot 2$ & \\
\hline 81 & $114 \cdot 0$ & & 8 & $114 \cdot 0$ & \\
\hline 3 & $113 \cdot 9$ & & 9 & $113 \cdot 9$ & \\
\hline 5 & $113 \cdot 7$ & & 52 & $113 \cdot 7$ & \\
\hline 6 & $113 \cdot 6$ & & 3 & $113 \cdot 6$ & \\
\hline 9 & $113 \cdot 4$ & & 6 & $113 \cdot 4$ & \\
\hline 90 & $113 \cdot 3$ & & 7 & $113 \cdot 3$ & \\
\hline 3 & $113 \cdot 1$ & & 9 & $113 \cdot 1$ & \\
\hline
\end{tabular}

The mixture of alcohol and water, taken as spirit in Mr. Gilpin's tables, is that of which the specific gravity is 0.825 at $60^{\circ} \mathrm{Fahr}$, water being unity at the same temperature. The specific gravity of water at $60^{\circ}$ being 1000 , at $62^{\circ}$ it is 99,981 . Hence, in order to compare the specific gravities given by Mr. Gilpin with those which would result when the specific gravity of water at $62^{\circ}$ is taken at unity, all the former numbers must be divided by 99,981 .

Table of the Specific Gravities of different Mixtures, by Weight, of Alcohol and Water, at different Temperatures; constructed by Mr. Gilpin, for the use of the British Revenue on Spirits.

\begin{tabular}{|c|c|c|c|c|c|c|c|c|c|c|c|}
\hline 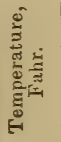 & $\begin{array}{c}\text { Pure } \\
\text { Alcohol }\end{array}$ & $\begin{array}{c}100 \\
\text { Alcohol } \\
5 \\
\text { Water. }\end{array}$ & $\begin{array}{c}100 \\
\text { Alcohol } \\
10 \\
\text { Water. }\end{array}$ & $\begin{array}{c}100 \\
\text { Alcohol } \\
15 \\
\text { Water. }\end{array}$ & $\begin{array}{c}100 \\
\text { Alcohol } \\
20 \\
\text { Water. }\end{array}$ & $\begin{array}{c}100 \\
\text { Alcohol } \\
25 \\
\text { Water. }\end{array}$ & $\begin{array}{c}100 \\
\text { Alcohol } \\
30 \\
\text { Water. }\end{array}$ & $\begin{array}{c}100 \\
\text { Alcohol } \\
35 \\
\text { Water. }\end{array}$ & $\begin{array}{c}100 \\
\text { Alcohol } \\
40 \\
\text { Water. }\end{array}$ & $\begin{array}{c}100 \\
\text { Alcohol } \\
45 \\
\text { Water. }\end{array}$ & $\begin{array}{c}100 \\
\text { Alcohol } \\
50 \\
\text { Water. }\end{array}$ \\
\hline $\begin{array}{c}\text { Deg. } \\
30 \\
35 \\
40 \\
45 \\
50 \\
55 \\
60 \\
65 \\
70 \\
75 \\
80 \\
85 \\
90 \\
95 \\
100 \\
\end{array}$ & $\begin{array}{r}0.83896 \\
.836 \% 2 \\
.83145 \\
.83214 \\
.829 \% 7 \\
.82736 \\
.82500 \\
.82262 \\
.82023 \\
.81780 \\
.81530 \\
.81291 \\
.81044 \\
.80 \tau 94 \\
.80548 \\
\end{array}$ & $\begin{array}{r}0.84995 \\
.84769 \\
.84539 \\
.84310 \\
.84076 \\
.83834 \\
.83599 \\
.83362 \\
.83124 \\
.82578 \\
.82631 \\
.82396 \\
.82150 \\
.81900 \\
.81657 \\
\end{array}$ & \begin{tabular}{|r|}
0.85957 \\
.85729 \\
.85507 \\
.85277 \\
.85042 \\
.84802 \\
.84568 \\
.84334 \\
.84092 \\
.83851 \\
.83603 \\
.83371 \\
.83126 \\
.82877 \\
.82639 \\
\end{tabular} & $\begin{array}{r}0.86825 \\
.86507 \\
.86361 \\
.86131 \\
.85902 \\
.85664 \\
.85430 \\
.85193 \\
.84951 \\
.84710 \\
.84467 \\
.84243 \\
.84001 \\
.83753 \\
.83513 \\
\end{array}$ & $\begin{array}{r}0.87585 \\
.87357 \\
.87184 \\
.86905 \\
.86676 \\
.86441 \\
.86208 \\
.85976 \\
.85736 \\
.85496 \\
.85248 \\
.85036 \\
.84797 \\
.84550 \\
.84038 \\
\end{array}$ & $\begin{array}{r}0.88282 \\
.88059 \\
.87838 \\
.87613 \\
.87384 \\
.87150 \\
.86918 \\
.86680 \\
.86451 \\
.86212 \\
.85966 \\
.85757 \\
.85518 \\
.85272 \\
.85031 \\
\end{array}$ & $\begin{array}{r}0.88921 \\
.88701 \\
.85481 \\
.88255 \\
.86030 \\
.87796 \\
.87569 \\
.87337 \\
.87105 \\
.86864 \\
.86622 \\
.86411 \\
.86172 \\
.85928 \\
.85688 \\
\end{array}$ & $\begin{array}{r}0.89511 \\
.89294 \\
.890 \% 3 \\
.88819 \\
.88626 \\
.88393 \\
.88169 \\
.87938 \\
.87705 \\
.87466 \\
.87228 \\
.87021 \\
.86797 \\
.86542 \\
.86302 \\
\end{array}$ & $\begin{array}{r}0.90054 \\
.89339 \\
.89617 \\
.89396 \\
.89174 \\
.88945 \\
.88 \% 20 \\
.88490 \\
.83254 \\
.88018 \\
.87776 \\
.87590 \\
87360 \\
.87114 \\
.86879 \\
\end{array}$ & $\begin{array}{r}0.90558 \\
.90345 \\
.90127 \\
.89909 \\
.89681 \\
.89458 \\
.89232 \\
.89006 \\
.88773 \\
.88538 \\
.88301 \\
.88120 \\
87889 \\
.87654 \\
.87421 \\
\end{array}$ & $\begin{array}{r}0.91023 \\
.90811 \\
.90596 \\
.90380 \\
.901+j 0 \\
.89933 \\
.89707 \\
.89479 \\
.89252 \\
.89018 \\
.88781 \\
.58609 \\
.88376 \\
.88146 \\
.87915 \\
\end{array}$ \\
\hline Ten & $\begin{array}{l}\text { rature, } \\
\text { ir. }\end{array}$ & $\begin{array}{c}100 \\
\text { Alcohol } \\
55 \\
\text { Water. }\end{array}$ & $\begin{array}{c}100 \\
\text { Alcoho? } \\
60 \\
\text { Water. }\end{array}$ & $\begin{array}{c}100 \\
\text { Alcohol } \\
65 \\
\text { Water. }\end{array}$ & $\mid \begin{array}{c}100 \\
\text { Alcohol } \\
70 \\
\text { Water. }\end{array}$ & $\mid \begin{array}{c}100 \\
\text { Alcohol } \\
\approx 5 \\
\text { Water. }\end{array}$ & $\begin{array}{c}100 \\
\text { Alcohol } \\
80 \\
\text { Water. }\end{array}$ & $\begin{array}{c}100 \\
\text { Alcohol } \\
85 \\
\text { Water. }\end{array}$ & $\begin{array}{c}100 \\
\text { Alcohol } \\
90 \\
\text { Water. }\end{array}$ & $\begin{array}{c}100 \\
\text { Aleohol } \\
95 \\
\text { Water. }\end{array}$ & $\mid \begin{array}{c}100 \\
\text { Alcohol } \\
100 \\
\text { Water }\end{array}$ \\
\hline & $\begin{array}{l}\text { Deg. } \\
30 \\
35 \\
40 \\
45 \\
50 \\
55 \\
60 \\
65 \\
70 \\
75 \\
80 \\
85 \\
90 \\
95 \\
100\end{array}$ & $\begin{array}{r}0.91449 \\
.91241 \\
.91026 \\
.90812 \\
.90596 \\
.90367 \\
.90144 \\
.89920 \\
.89695 \\
.89164 \\
.89225 \\
.89043 \\
.88817 \\
.88588 \\
.83571\end{array}$ & \begin{tabular}{|}
0.91847 \\
.91640 \\
.91428 \\
91211 \\
.90997 \\
.90768 \\
.90549 \\
.90328 \\
.90104 \\
$.898 \% 2$ \\
.89639 \\
.89460 \\
.89230 \\
.89003 \\
$88 \% 69$
\end{tabular} & $\begin{array}{r}0.92217 \\
\cdot 92009 \\
\cdot 91799 \\
\cdot 91584 \\
.91370 \\
\cdot 91144 \\
90927 \\
.90707 \\
.90481 \\
.90252 \\
\cdot 90021 \\
.89843 \\
.89617 \\
.89390 \\
.89158\end{array}$ & $\begin{array}{r}0.92563 \\
.92355 \\
.92151 \\
.91937 \\
.91723 \\
.91502 \\
.91287 \\
.91066 \\
.90847 \\
.90617 \\
.90385 \\
.90209 \\
.89988 \\
.89 \% 63 \\
.89536\end{array}$ & $\begin{array}{r}0.92889 \\
.92680 \\
.92476 \\
.92264 \\
.92051 \\
.91837 \\
.91622 \\
.91400 \\
.91181 \\
.90952 \\
.90723 \\
.90558 \\
.90342 \\
.90119 \\
.89889\end{array}$ & $\begin{array}{r}0.93191 \\
.92986 \\
.92783 \\
.92570 \\
.92358 \\
.92115 \\
.91933 \\
.91715 \\
.91493 \\
.91270 \\
.91046 \\
.90882 \\
.90688 \\
.90443 \\
.90215\end{array}$ & $\begin{array}{r}0.93474 \\
.93274 \\
.93072 \\
.92859 \\
.926+7 \\
.92436 \\
.92225 \\
.92010 \\
.91793 \\
.91569 \\
.91340 \\
.91186 \\
.90967 \\
.90747 \\
.90522\end{array}$ & $\begin{array}{r}0.93741 \\
.93541 \\
.95341 \\
.93131 \\
.92919 \\
.92707 \\
.92499 \\
.92283 \\
.92069 \\
.91849 \\
.91622 \\
.91465 \\
.91248 \\
.91029 \\
.90805\end{array}$ & $\begin{array}{r}0.93991 \\
.93790 \\
.93592 \\
.93382 \\
.93177 \\
.92963 \\
.92758 \\
.92546 \\
.92333 \\
.92111 \\
.91891 \\
.91729 \\
.91511 \\
.91290 \\
.91066\end{array}$ & $\begin{array}{c}0.94222 \\
.94025 \\
.93827 \\
.93621 \\
.93419 \\
.93208 \\
.93002 \\
.92794 \\
.92580 \\
.92364 \\
.92142 \\
.91969 \\
.91751 \\
.91531 \\
.91310\end{array}$ \\
\hline
\end{tabular}

* By specific gravity. 
Table of the Specific Gravities of different Mixtures, \&c. (continued).

\begin{tabular}{|c|c|c|c|c|c|c|c|c|c|c|}
\hline 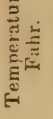 & $\begin{array}{c}95 \\
\text { Alcohol } \\
100 \\
\text { Water. }\end{array}$ & $\begin{array}{c}90 \\
\text { Alcohol } \\
100 \\
\text { Water. }\end{array}$ & $\begin{array}{c}85 \\
\text { Alcohol } \\
100 \\
\text { Water. }\end{array}$ & $\begin{array}{c}80 \\
\text { Atcohol } \\
100 \\
\text { Water. }\end{array}$ & $\begin{array}{c}75 \\
\text { Alcohol } \\
100 \\
\text { Water. }\end{array}$ & $\begin{array}{c}\tau 0 \\
\text { Alcohol } \\
100 \\
\text { Water. }\end{array}$ & $\begin{array}{c}65 \\
\text { Alscohol } \\
100 \\
\text { Water. }\end{array}$ & $\begin{array}{c}60 \\
\text { Alcohol } \\
100 \\
\text { Water. }\end{array}$ & $\begin{array}{c}55 \\
\text { Alcohol } \\
100 \\
\text { Water. }\end{array}$ & $\begin{array}{c}50 \\
\text { Alcohod } \\
100 \\
\text { Water. }\end{array}$ \\
\hline $\begin{array}{l}\text { Deg. } \\
30 \\
35 \\
40 \\
45 \\
50 \\
55 \\
60 \\
65 \\
70 \\
75 \\
80 \\
\end{array}$ & \begin{tabular}{|}
0.94447 \\
.91249 \\
.94055 \\
.93860 \\
.93658 \\
.93452 \\
.93247 \\
.93040 \\
.92528 \\
.92613 \\
.92393 \\
\end{tabular} & $\begin{array}{r}0.94675 \\
.94484 \\
.94295 \\
.94096 \\
.93597 \\
.93696 \\
.93493 \\
.93285 \\
.93076 \\
.92 \lessdot 65 \\
.92646 \\
\end{array}$ & $\begin{array}{r}0.94920 \\
.94734 \\
.94547 \\
.9434 \mathrm{~s} \\
.94149 \\
.93948 \\
.93749 \\
.93546 \\
.93337 \\
.93132 \\
.92917 \\
\end{array}$ & $\begin{array}{r}0.95173 \\
.949 ธ 8 \\
.94802 \\
.94605 \\
.94414 \\
.94213 \\
.94018 \\
.93522 \\
.93616 \\
.93413 \\
.93201 \\
\end{array}$ & $\begin{array}{r}0.95429 \\
.95246 \\
.95060 \\
.94871 \\
.61683 \\
. .4486 \\
.44296 \\
.94099 \\
.93898 \\
.93695 \\
.93488 \\
\end{array}$ & $\begin{array}{r}0.95681 \\
.95502 \\
.95328 \\
.95143 \\
.94958 \\
.94767 \\
.94579 \\
.94388 \\
.94193 \\
.93989 \\
.93 \approx 85 \\
\end{array}$ & $\begin{array}{r}0.95944 \\
.957 \% 2 \\
.95602 \\
.95123 \\
95213 \\
.9505 \pi \\
.948 \% 6 \\
.94609 \\
.94500 \\
.94301 \\
.94102 \\
\end{array}$ & $\begin{array}{r}0.96209 \\
.96048 \\
95879 \\
.95703 \\
95534 \\
.95357 \\
.95181 \\
.95000 \\
.94513 \\
.94623 \\
.94431 \\
\end{array}$ & $\begin{array}{r}0.96170 \\
.96315 \\
.96159 \\
.95993 \\
.95531 \\
.95562 \\
95493 \\
.95318 \\
.95139 \\
.94957 \\
.94768 \\
\end{array}$ & $\begin{array}{l}0.96719 \\
.96579 \\
96434 \\
96280 \\
.9 t 126 \\
.95966 \\
.95804 \\
95635 \\
.95169 \\
.95292 \\
.95111 \\
\end{array}$ \\
\hline \multicolumn{2}{|c|}{$\begin{array}{c}\text { Temperature, } \\
\text { Falır. }\end{array}$} & $\begin{array}{c}45 \\
\text { Alcohol } \\
100 \\
\text { Water. }\end{array}$ & $\begin{array}{c}40 \\
\text { Alcohol } \\
100 \\
\text { Water. }\end{array}$ & $\begin{array}{c}35 \\
\text { Alcuhol } \\
100 \\
\text { Water. }\end{array}$ & $\begin{array}{c}30 \\
\text { Alcohol } \\
100 \\
\text { Water. }\end{array}$ & $\begin{array}{c}25 \\
\text { Alcohol } \\
100 \\
\text { Water. }\end{array}$ & $\begin{array}{c}20 \\
\text { Alcohol } \\
100 \\
\text { Water. }\end{array}$ & $\begin{array}{c}15 \\
\text { Alcohol } \\
100 \\
\text { Water. }\end{array}$ & $\begin{array}{c}10 \\
\text { Alcoho] } \\
100 \\
\text { Water. }\end{array}$ & $\begin{array}{c}5 \\
\text { Alenhol } \\
100 \\
\text { Watcr. }\end{array}$ \\
\hline \multicolumn{2}{|c|}{$\begin{array}{c}\text { Degrees. } \\
30 \\
35 \\
40 \\
45 \\
50 \\
55 \\
60 \\
65 \\
70 \\
75 \\
80 \\
\end{array}$} & $\begin{array}{r}0.96967 \\
.96840 \\
.96706 \\
.96563 \\
.96420 \\
.96272 \\
.96122 \\
.95962 \\
.95802 \\
.95638 \\
.95467 \\
\end{array}$ & $\begin{array}{r}0.97200 \\
.97086 \\
.96967 \\
.96840 \\
.96708 \\
.96575 \\
.9643 \tau \\
.96288 \\
.96143 \\
.95987 \\
.95826 \\
\end{array}$ & $\begin{array}{r}0.97418 \\
.97319 \\
.97220 \\
.97110 \\
.96995 \\
.96877 \\
.96752 \\
.96620 \\
.96484 \\
.96344 \\
.96192 \\
\end{array}$ & $\begin{array}{r}0.97635 \\
.97556 \\
.97472 \\
.97384 \\
.97284 \\
.97181 \\
.97074 \\
.96959 \\
.96836 \\
.96708 \\
.96568 \\
\end{array}$ & $\begin{array}{r}0.97860 \\
.97801 \\
.9773 z \\
.97666 \\
.97589 \\
.97500 \\
.97410 \\
.97309 \\
.97203 \\
.97086 \\
.96963 \\
\end{array}$ & $\begin{array}{r}0.98108 \\
.98076 \\
.98033 \\
.97980 \\
.97920 \\
.97847 \\
.97771 \\
.97688 \\
.97596 \\
.97495 \\
.97385 \\
\end{array}$ & $\begin{array}{r}0.98412 \\
.98397 \\
.98373 \\
.98335 \\
.95293 \\
.98239 \\
.98176 \\
.98106 \\
.98028 \\
.97943 \\
.97845 \\
\end{array}$ & $\begin{array}{r}0.98804 \\
.98804 \\
.98795 \\
.98774 \\
.98745 \\
.98702 \\
.98654 \\
.98594 \\
.98527 \\
.98454 \\
.98367 \\
\end{array}$ & $\begin{array}{l}0.99334 \\
.99344 \\
.99345 \\
.99338 \\
.99316 \\
.99284 \\
.99244 \\
.99194 \\
.99134 \\
.99066 \\
.98991 \\
\end{array}$ \\
\hline
\end{tabular}

Experiments were made, by direction of the committee, to verify Gilpin's tables, which showed that the error introduced in ascertaining the strength of spirits by tables founded on Gilpin's numbers must be qnite insensible in the practice of the revenue. The diserepancies thus detected, on a mixture of a given strength, did not amount in any one instance to unity in the fourth place of decimals. From a careful inspection of such documents the committee are of opinion, that Gilpin's tables possess a degree of accuracy far surpassing what could be expected, and sufficiently perfect for all practical or scientific purposes.

The following table is given by Mr. Lubbock, for converting the apparent specific gravity, or indication, into true specific gravity.

\begin{tabular}{|c|c|c|c|c|c|c|c|c|c|c|c|c|c|}
\hline \multirow{2}{*}{ 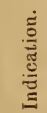 } & \multicolumn{4}{|c|}{ 一 } & \multicolumn{3}{|c|}{ Temperature. } & \multicolumn{4}{|c|}{+} & & \multirow{2}{*}{ 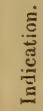 } \\
\hline & $30^{\circ}$ & $32^{\circ}$ & $37^{\circ}$ & $42^{\circ}$ & $47^{\circ}$ & $52^{\circ}$ & $57^{\circ}$ & $62^{\circ}$ & $67^{\circ}$ & $\tau 2^{\circ}$ & $77^{\circ}$ & $80^{\circ}$ & \\
\hline .82 & .00083 & .00078 & .00065 & .00052 & .00039 & .00025 & .00012 & & .00011 & .00024 & 00035 & .00012 & .82 \\
\hline .83 & $\cdot 00084$ & .00079 & .00066 & 00052 & .00039 & .00026 & .00012 & & .00012 & .00024 & 00036 & .00042 & .83 \\
\hline .84 & . 00055 & .00080 & $\cdot 00066$ & 00053 & .00039 & .00026 & 00013 & & .00012 & .00024 & .00036 & .00043 & .84 \\
\hline .85 & $.000 \leq 6$ & $.000 \leq 1$ & .00067 & .00054 & $.000 \pm 0$ & $.0002 \hat{~}$ & .00013 & & .00012 & .00025 & 1.00037 & $.000+3$ & 85 \\
\hline 86 & $.0008 i$ & 1.00032 & 100065 & .00054 & 00040 & $.0002 \%$ & .00013 & & .00012 & .00025 & .00037 & .00044 & .86 \\
\hline .87 & $.000 \leq 8$ & .00083 & 00069 & .00055 & .00041 & .00027 & .00013 & & .00012 & .00025 & 100037 & 00044 & 87 \\
\hline .86 & $\cdot .000 \leqslant 9$ & .00084 & .00070 & .00055 & .00041 & .00027 & .00013 & & .00012 & .00026 & 00038 & 00045 & 88 \\
\hline .59 & .00090 & - cous5 & .00070 & .00055 & .00042 & .00028 & 00013 & & .00012 & .00026 & 00038 & .00045 & 89 \\
\hline .90 & .00091 & .00055 & .00071 & .00056 & .00042 & .00028 & $\cdot 00014$ & & .00013 & 00026 & $\cdot 00039$ & 00046 & 90 \\
\hline .91 & .00092 & .00086 & .00072 & .00057 & .00043 & .00028 & .00014 & & .00013 & .00026 & .00039 & .00046 & .91 \\
\hline .92 & - 00093 & .00087 & .00073 & .00058 & .00043 & -00029 & 00014 & & .00013 & .00027 & .00040 & 00047 & .92 \\
\hline .93 & .00094 & - oudses & .00073 & .00059 & .00044 & .00029 & .00014 & & .00013 & 00027 & 00040 & .00047 & .93 \\
\hline .94 & .00095 & - 00059 & $\cdot 00074$ & .00059 & .00044 & .00029 & .00014 & & .00013 & .00027 & .00040 & .00048 & .94 \\
\hline .95 & .00096 & .00090 & .00075 & .00060 & .00045 & .00029 & $\cdot .00014$ & & .00013 & .00028 & .00041 & 00048 & .95 \\
\hline .96 & .00097 & .00091 & .00076 & .00060 & .00045 & - 0 mo30 & .00014 & & .00013 & .00028 & .00011 & $000+9$ & .96 \\
\hline .97 & - 011098 & - 0 (1) 192 & .00077 & .00061 & .00046 & $.00 \$ 30$ & .00015 & & .00014 & 00028 & .00042 & .00049 & .97 \\
\hline .98 & $\cdot 00099$ & - onogis & $.000 \pi \%$ & .00062 & $\cdot 00040$ & .00030 & .00015 & & .00014 & 00028 & .00042 & 00050 & .98 \\
\hline .99 & .00100 & .00094 & .00078 & .00062 & .00047 & .00031 & .00015 & & .00014 & .00029 & .00043 & .00050 & .99 \\
\hline$\cdot 100$ & 1.00101 & .00095 & .00079 & .00063 & .00047 & .00031 & .00015 & & & & & & 100 \\
\hline
\end{tabular}


The hydrometer constructed, under the directions of the Commissioners of Excise, by Mr. Bate, has a scale of 4 inches in length divided into 100 parts, and 9 weights. It has thus a range of 900 divisions, and expresses specific gravities at the temperature of $62^{\circ} \mathrm{Fahr}$. In order to render this instrument so accurate a measurer of the specific gravity, at the standard temperature, as to involve no error of an appreciable amount, Mr. Bate has constructed the weights (which in this instrument are immersed in the fluid of different specific gravities) so that each successive weight should have an increase of bulk over the preceding weight equal to that part of the stem occupied by the scale, and an increase of weight sufficient to take the whole of the scale, and no more, down to the liquid. This arrangement requires great accuracy of workmanship, and enhances the price of the instrument. But it allows of increased strength in the ball, where it is very much required, and it gives, upon inspection only, the indication (apparent specific gravity) by which the general table is to be examined and the result ascertained. Fig. 5 represents this instrument and two of its nine ballast weights. It comprehends all specific gravities between 820 and 1000. It indicates true specific gravity with almost perfect accuracy at the temperature of $62^{\circ} \mathrm{Fahr}$.; but it does not exclude other instruments from being used in conjunction with tables. The latter are, in fact, independent of the instrument, and may be used with gravimeters, or any instrument affording indications by specific gravity at a given temperature.

The cominercial value of spirituous liquors being much lower in France than in England, a less sensible instrument becomes sufficient for the wants of that country. Baumé's and Cartier's hydrometers,

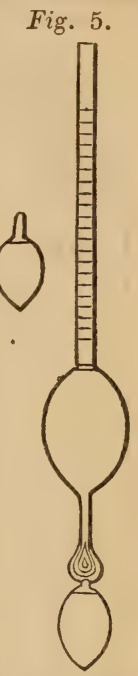
with short arbitrary scales, are very much employed, but they have been lately super seded by an ingenious and ready instrument contrived by M. Gay Lussac, and called by him an alcoometre. He takes for the term of comparison pure alcohol by volume, at the temperature of $15^{\circ}$ Cent., and represents the strength of it by 100 centimes, or by unity. Consequently, the strength of a spirituous liquid is the number of centimes in volume of pure alcohol which that liquid contains at the temperature of $15^{\circ}$ Cent. The instrument is formed like a common hydrometer, and is graduated for the temperature of $15^{\circ}$ Cent. Its scale is divided into 100 parts or degrees, each of which denotes a centime of alcohol; the division 0 at the bottom of the stem corresponds to pure water, and the division 100 at its top, to pure alcohol. When immersed in a spirituous liquor at $15^{\circ}$ Cent. $\left(59^{\circ} \mathrm{Fahr}\right.$.) it announces its strength directly. For example : if in spirits supposed at the temperature of $15^{\circ}$ Cent. it sinks to the division 50 , it indicates that the strength of this liquor is 50 per cent., or that it contains 50 centimes of pure alcohol. In our new British proof spirit, it would sink to nearly 57, indicating 57 by volume of pure alcohol, allowing for condensation, or 50 by weight. A table of correction is given for temperature, which he calls "Table of real strength of spirituous liquors." The first vertical column of this table contains the temperatures, from $0^{\circ}$ to $30^{\circ}$ Cent., and the first horizontal line the indications of the alcoomètre. In the same table we have most ingeniously inserted a correction for the volume of the spirits when the temperature differs from $15^{\circ}$ Cent. If we take 1000 litres or gallons, measured at the temperature of $2^{\circ}$, of a spirituous liquor whose apparent strength is $44^{\mathrm{c}}$; its real strength at $15^{\circ}$ will from the preceding mode of correction be 49c. On heating this liquid to $15^{\circ}$, in order to find its real specific gravity or strength, its bulk will become greater; and, instead of 1000 litres or gallons, which it measured at $2^{\circ}$, we shall have 1009 at $15^{\circ} \mathrm{C}$. This number is inscribed in smaller characters in the same square cell with the real force, precisely under $49 \mathrm{c}$. All the numbers in small characters, printed under each real strength, indicate the volume which 1000 litres of a spirituous liquor would have, when measured at the temperature at which its apparent strength is taken. In the above example, the quantity in litres or gallons of pure alcohol contained in 1000 litres or gallons of the spirits, measured at the temperature of $2^{\circ}$, will be, therefore, 1009 lit. $\times 0.49=494$ lit. 41 .

This quantity of pure alcohol, thus estimated, is called richness of spirit in alcohol, or simply richness.

Let us take an example similar to the preceding, but at a higher temperature than $15^{\circ}$ Cent. Suppose we have 1000 litres measured, at the temperature of $25^{\circ}$, of spirits whose apparent strength is $53 \mathrm{c}$, what is the real quantity of pure alcohol which this spirit contains at the temperature of $15^{\circ}$ ? We shall find in the table, first of all, that the real strength of the spirits is $49 \mathrm{c} \cdot 3$. As to its bulk or volume, it is very clear that the 1000 litres in cooling from $25^{\circ}$ to $15^{\circ}$, will occupy a smaller space. This volume will be 993 litres; it is inscribed directly below $49 c \cdot 3$, the real strength. We 
shall therefore have of purc alcohol, contained in the 1000 litres of spirits measured at the temperature of $25^{\circ}$, or their richness, 993 lit. $\times 0.493=489$ lit. 55 .

Alcometrical Table of real Strength, by M. Gay Lussac.

\begin{tabular}{|c|c|c|c|c|c|c|c|c|c|c|}
\hline $\begin{array}{c}\text { Temperature. } \\
\text { C. }\end{array}$ & 3lc & $32 c$ & $33 c$ & $34 \mathrm{c}$ & $35 \mathrm{c}$ & $36 \mathrm{c}$ & $37 \mathrm{c}$ & $38 \mathrm{c}$ & $39 \mathrm{c}$ & $40 c$ \\
\hline $\begin{array}{l}\text { Deg. } \\
10\end{array}$ & $\begin{array}{l}33 \cdot 0 \\
1002\end{array}$ & $\begin{array}{c}34 \\
1002\end{array}$ & $\begin{array}{c}35 \\
1003\end{array}$ & $\begin{array}{c}36 \\
1003\end{array}$ & $\begin{array}{c}37 \\
1003\end{array}$ & $\begin{array}{c}38 \\
1003\end{array}$ & $\begin{array}{c}39 \\
1003\end{array}$ & $\begin{array}{c}40 \\
1003\end{array}$ & $\begin{array}{c}41 \\
1003\end{array}$ & $\begin{array}{c}42 \\
1003\end{array}$ \\
\hline 11 & $\begin{array}{l}32 \cdot 6 \\
1002\end{array}$ & $\begin{array}{l}33 \cdot 6 \\
1002\end{array}$ & $\begin{array}{l}34 \cdot 6 \\
1002\end{array}$ & $\begin{array}{l}35 \cdot 6 \\
1002\end{array}$ & $\begin{array}{l}36 \cdot 6 \\
1002\end{array}$ & $\begin{array}{l}37 \cdot 6 \\
1002\end{array}$ & $\begin{array}{l}3 S \cdot 6 \\
1002\end{array}$ & $\begin{array}{l}39 \cdot 6 \\
1002\end{array}$ & $\begin{array}{l}40 \cdot 6 \\
1003\end{array}$ & $\begin{array}{l}41 \cdot 6 \\
1003\end{array}$ \\
\hline 12 & $\begin{array}{l}32 \cdot 2 \\
1001\end{array}$ & $\begin{array}{l}33 \cdot 2 \\
1001\end{array}$ & $\begin{array}{l}34 \cdot 2 \\
1002\end{array}$ & $\begin{array}{l}35 \cdot 2 \\
1002\end{array}$ & $\begin{array}{l}36 \cdot 2 \\
1002\end{array}$ & $\begin{array}{l}37 \cdot 2 \\
1002\end{array}$ & $\begin{array}{l}38 \cdot 2 \\
1002\end{array}$ & $\begin{array}{l}39 \cdot 2 \\
1002\end{array}$ & $\begin{array}{l}40 \cdot 2 \\
1002\end{array}$ & $\begin{array}{l}41 \cdot 2 \\
1002\end{array}$ \\
\hline 13 & $\begin{array}{l}31 \cdot 8 \\
1001\end{array}$ & $\begin{array}{l}32 \cdot 8 \\
1001\end{array}$ & $\begin{array}{l}33 \cdot 8 \\
1001\end{array}$ & $\begin{array}{l}34 \cdot 8 \\
1001\end{array}$ & $\begin{array}{l}35 \cdot 8 \\
1001\end{array}$ & $\begin{array}{c}36 \cdot S \\
1001\end{array}$ & $\begin{array}{l}37 \cdot 8 \\
1001\end{array}$ & $\begin{array}{c}38 \cdot 8 \\
1001\end{array}$ & $\begin{array}{l}39 \cdot 8 \\
1001\end{array}$ & $\begin{array}{l}40 \cdot 8 \\
1001\end{array}$ \\
\hline 14 & $\begin{array}{l}31 \cdot 4 \\
1001\end{array}$ & $\begin{array}{l}32 \cdot 4 \\
1001 \\
\end{array}$ & $\begin{array}{l}33 \cdot 4 \\
1001\end{array}$ & $\begin{array}{l}34 \cdot 4 \\
1001\end{array}$ & $\begin{array}{l}35 \cdot 4 \\
1001\end{array}$ & $\begin{array}{c}36 \cdot 4 \\
1001\end{array}$ & $\begin{array}{l}37 \cdot 4 \\
1001\end{array}$ & $\begin{array}{l}3 S \cdot 4 \\
1001\end{array}$ & $\begin{array}{l}39 \cdot 4 \\
1001\end{array}$ & $\begin{array}{l}40 \cdot 4 \\
1001\end{array}$ \\
\hline 15 & $\begin{array}{c}31 \\
1000\end{array}$ & $\begin{array}{c}32 \\
1000\end{array}$ & $\begin{array}{c}33 \\
1000\end{array}$ & $\begin{array}{c}34 \\
1000\end{array}$ & $\begin{array}{c}35 \\
1000\end{array}$ & $\begin{array}{c}36 \\
1000\end{array}$ & $\begin{array}{c}37 \\
1000\end{array}$ & $\begin{array}{c}38 \\
1000\end{array}$ & $\begin{array}{c}39 \\
1000\end{array}$ & $\begin{array}{c}40 \\
1000\end{array}$ \\
\hline 16 & $\begin{array}{l}30 \cdot 6 \\
1000\end{array}$ & $\begin{array}{l}31 \cdot 6 \\
1000\end{array}$ & $\begin{array}{c}32 \cdot 5 \\
999\end{array}$ & $\begin{array}{c}33 \cdot 5 \\
999\end{array}$ & $\begin{array}{c}34 \cdot 5 \\
999\end{array}$ & $\begin{array}{c}35 \cdot 5 \\
999\end{array}$ & $\begin{array}{c}36 \cdot 5 \\
999\end{array}$ & $\begin{array}{c}37 \cdot 5 \\
999\end{array}$ & $\begin{array}{c}38 \cdot 5 \\
999\end{array}$ & $\begin{array}{c}39 \cdot 5 \\
999\end{array}$ \\
\hline 17 & $\begin{array}{c}30 \cdot 2 \\
999\end{array}$ & $\begin{array}{c}31 \cdot 2 \\
999\end{array}$ & $\begin{array}{c}32 \cdot 1 \\
999\end{array}$ & $\begin{array}{c}33 \cdot 1 \\
999\end{array}$ & $\begin{array}{c}34 \cdot 1 \\
999\end{array}$ & $\begin{array}{c}35 \cdot 1 \\
999\end{array}$ & $\begin{array}{c}36 \cdot 1 \\
999\end{array}$ & $\begin{array}{c}37 \cdot 1 \\
999\end{array}$ & $\begin{array}{c}38 \cdot 1 \\
999\end{array}$ & $\begin{array}{c}39 \cdot 1 \\
999\end{array}$ \\
\hline 18 & $\begin{array}{c}29 \cdot 8 \\
999\end{array}$ & $\begin{array}{c}30 \cdot 8 \\
999\end{array}$ & $\begin{array}{c}31 \cdot 7 \\
998\end{array}$ & $\begin{array}{c}32 \cdot 7 \\
998\end{array}$ & $\begin{array}{c}33 \cdot 7 \\
998\end{array}$ & $\begin{array}{c}34 \cdot 7 \\
998\end{array}$ & $\begin{array}{c}35 \cdot 7 \\
998\end{array}$ & $\begin{array}{c}36 \cdot 7 \\
998\end{array}$ & $\begin{array}{l}37 \cdot 7 \\
998\end{array}$ & $\begin{array}{c}38 \cdot 7 \\
998\end{array}$ \\
\hline 19 & $\begin{array}{c}29 \cdot 4 \\
998\end{array}$ & $\begin{array}{c}30 \cdot 4 \\
998\end{array}$ & $\begin{array}{c}31 \cdot 3 \\
998\end{array}$ & $\begin{array}{c}32 \cdot 3 \\
993\end{array}$ & $\begin{array}{c}33 \cdot 3 \\
998\end{array}$ & $\begin{array}{c}34 \cdot 3 \\
998\end{array}$ & $\begin{array}{c}35 \cdot 3 \\
998\end{array}$ & $\begin{array}{c}36 \cdot 3 \\
998\end{array}$ & $\begin{array}{c}37 \cdot 3 \\
997\end{array}$ & $\begin{array}{c}38 \cdot 3 \\
997\end{array}$ \\
\hline 20 & $\begin{array}{l}29 \\
998\end{array}$ & $\begin{array}{c}30 \\
998\end{array}$ & $\begin{array}{l}30 \cdot 9 \\
997\end{array}$ & $\begin{array}{c}31.9 \\
997\end{array}$ & $\begin{array}{c}32 \cdot 9 \\
997\end{array}$ & $\begin{array}{c}33 \cdot 9 \\
997\end{array}$ & $\begin{array}{l}34 \cdot 9 \\
997\end{array}$ & $\begin{array}{c}35 \cdot 9 \\
997\end{array}$ & $\begin{array}{l}36 \cdot 9 \\
997\end{array}$ & $\begin{array}{c}37 \cdot 9 \\
997\end{array}$ \\
\hline 21 & $\begin{array}{c}28 \cdot 6 \\
997\end{array}$ & $\begin{array}{c}29 \cdot 6 \\
997\end{array}$ & $\begin{array}{l}30 \cdot 5 \\
997\end{array}$ & $\begin{array}{c}31 \cdot 5 \\
997\end{array}$ & $\begin{array}{l}32 \cdot 5 \\
997\end{array}$ & $\begin{array}{c}33 \cdot 5 \\
997\end{array}$ & $\begin{array}{c}34 \cdot 5 \\
997\end{array}$ & $\begin{array}{c}35 \cdot 5 \\
996\end{array}$ & $\begin{array}{c}36 \cdot 5 \\
996\end{array}$ & $\begin{array}{c}37 \cdot 5 \\
996\end{array}$ \\
\hline 22 & $\begin{array}{c}28 \cdot 2 \\
997\end{array}$ & $\begin{array}{c}29 \cdot 2 \\
997\end{array}$ & $\begin{array}{c}30 \cdot 1 \\
996\end{array}$ & $\begin{array}{c}31 \cdot 1 \\
996\end{array}$ & $\begin{array}{c}32 \cdot 1 \\
996\end{array}$ & $\begin{array}{c}33 \cdot 1 \\
996\end{array}$ & $\begin{array}{c}34 \cdot 1 \\
996\end{array}$ & $\begin{array}{c}35 \cdot 1 \\
996\end{array}$ & $\begin{array}{c}36 \cdot 1 \\
996\end{array}$ & $\begin{array}{c}37 \cdot 1 \\
996\end{array}$ \\
\hline 23 & $\begin{array}{c}27 \cdot 8 \\
996\end{array}$ & $\begin{array}{c}28 \cdot 8 \\
\text { y96 }\end{array}$ & $\begin{array}{c}29 \cdot 7 \\
996\end{array}$ & $\begin{array}{c}30 \cdot 7 \\
996\end{array}$ & $\begin{array}{c}31 \cdot 7 \\
996\end{array}$ & $\begin{array}{c}32 \cdot 7 \\
996\end{array}$ & $\begin{array}{c}33 \cdot 7 \\
996\end{array}$ & $\begin{array}{c}34 \cdot 7 \\
995\end{array}$ & $\begin{array}{l}35 \cdot 7 \\
995\end{array}$ & $\begin{array}{l}36 \cdot 7 \\
995\end{array}$ \\
\hline 24 & $\begin{array}{c}27 \cdot 4 \\
996\end{array}$ & $\begin{array}{c}28 \cdot 4 \\
996\end{array}$ & $\begin{array}{c}29 \cdot 3 \\
995\end{array}$ & $\begin{array}{c}30 \cdot 3 \\
995\end{array}$ & $\begin{array}{c}31 \cdot 3 \\
995\end{array}$ & $\begin{array}{c}32 \cdot 3 \\
995\end{array}$ & $\begin{array}{c}33 \cdot 3 \\
995\end{array}$ & $\begin{array}{c}34 \cdot 3 \\
995\end{array}$ & $\begin{array}{l}35 \cdot 3 \\
995\end{array}$ & $\begin{array}{c}36 \cdot 3 \\
994\end{array}$ \\
\hline 25 & $\begin{array}{l}27 \\
995\end{array}$ & $\begin{array}{l}28 \\
995\end{array}$ & $\begin{array}{c}28 \cdot 9 \\
995 \\
\end{array}$ & $\begin{array}{c}29 \cdot 9 \\
995 \\
\end{array}$ & $\begin{array}{c}30 \cdot 9 \\
995 \\
\end{array}$ & $\begin{array}{c}31 \cdot 9 \\
994\end{array}$ & $\begin{array}{l}32 \cdot 9 \\
994\end{array}$ & $\begin{array}{c}33 \cdot 9 \\
994 \\
\end{array}$ & $\begin{array}{c}34 \cdot 9 \\
994 \\
\end{array}$ & $\begin{array}{c}35 \cdot 9 \\
994 \\
\end{array}$ \\
\hline $\begin{array}{c}\text { Temperature. } \\
\text { C. }\end{array}$ & $41 \mathrm{c}$ & $42 c$ & $43 c$ & $44 \mathrm{c}$ & $45 c$ & $46 c$ & $47 \mathrm{c}$ & $48 c$ & $49 c$ & $50 \mathrm{c}$ \\
\hline $\begin{array}{l}\text { Deg. } \\
10\end{array}$ & $\begin{array}{c}43 \\
1003\end{array}$ & $\begin{array}{c}44 \\
1004\end{array}$ & $\begin{array}{c}45 \\
1004\end{array}$ & $\begin{array}{c}46 \\
1001\end{array}$ & $\begin{array}{l}46 \cdot 9 \\
1004\end{array}$ & $\begin{array}{l}47 \cdot 9 \\
1004\end{array}$ & $\begin{array}{l}48 \cdot 9 \\
1004\end{array}$ & $\begin{array}{l}49 \cdot 9 \\
1004\end{array}$ & $\begin{array}{l}50 \cdot 9 \\
1004\end{array}$ & $\begin{array}{l}51 \cdot 8 \\
1004\end{array}$ \\
\hline 11 & $\begin{array}{l}42 \cdot 6 \\
1003 \\
\end{array}$ & $\begin{array}{l}43 \cdot 6 \\
1003\end{array}$ & $\begin{array}{l}44 \cdot 6 \\
1003\end{array}$ & $\begin{array}{l}45 \cdot 6 \\
1003\end{array}$ & $\begin{array}{l}46 \cdot 6 \\
1003\end{array}$ & $\begin{array}{l}47 \cdot 6 \\
1003\end{array}$ & $\begin{array}{l}48 \cdot 6 \\
1003\end{array}$ & $\begin{array}{l}49 \cdot 5 \\
1003\end{array}$ & $\begin{array}{l}50 \cdot 5 \\
1003\end{array}$ & $\begin{array}{l}51 \cdot 5 \\
1003\end{array}$ \\
\hline 12 & $\begin{array}{l}42 \cdot 2 \\
1002\end{array}$ & $\begin{array}{l}43 \cdot 2 \\
1002\end{array}$ & $\begin{array}{l}44 \cdot 2 \\
1002\end{array}$ & $\begin{array}{l}45 \cdot 2 \\
1002\end{array}$ & $\begin{array}{l}46 \cdot 2 \\
1002\end{array}$ & $\begin{array}{l}47 \cdot 2 \\
1002\end{array}$ & $\begin{array}{l}48 \cdot 2 \\
1002\end{array}$ & $\begin{array}{l}49 \cdot 2 \\
1002\end{array}$ & $\begin{array}{l}50 \cdot 2 \\
1002\end{array}$ & $\begin{array}{l}51 \cdot 1 \\
1002\end{array}$ \\
\hline 13 & $\begin{array}{l}41 \cdot 8 \\
1001\end{array}$ & $\begin{array}{l}42 \cdot 8 \\
1001\end{array}$ & $\begin{array}{l}43 \cdot 8 \\
1001\end{array}$ & $\begin{array}{l}44 \cdot 8 \\
1002\end{array}$ & $\begin{array}{l}45 \cdot 8 \\
1002\end{array}$ & $\begin{array}{l}46 \cdot 8 \\
1002\end{array}$ & $\begin{array}{l}47 \cdot 8 \\
1002\end{array}$ & $\begin{array}{l}4 S \cdot 8 \\
1002\end{array}$ & $\begin{array}{l}49 \cdot S \\
1002\end{array}$ & $\begin{array}{l}50 \cdot 8 \\
1002\end{array}$ \\
\hline 14 & $\begin{array}{l}41 \cdot 4 \\
1001\end{array}$ & $\begin{array}{l}42 \cdot 4 \\
1001\end{array}$ & $\begin{array}{l}43 \cdot 4 \\
1001\end{array}$ & $\begin{array}{l}44 \cdot 4 \\
1001\end{array}$ & $\begin{array}{l}45 \cdot 4 \\
1001\end{array}$ & $\begin{array}{l}46 \cdot 4 \\
1000\end{array}$ & $\begin{array}{l}47 \cdot 4 \\
1001\end{array}$ & $\begin{array}{l}48 \cdot 4 \\
1001 .\end{array}$ & $\begin{array}{l}49 \cdot 4 \\
1001\end{array}$ & $\begin{array}{l}50 \cdot 4 \\
1000\end{array}$ \\
\hline 15 & $\begin{array}{c}41 \\
1000\end{array}$ & $\begin{array}{c}42 \\
1000\end{array}$ & $\begin{array}{c}43 \\
1000\end{array}$ & $\begin{array}{c}44 \\
1000\end{array}$ & $\begin{array}{c}45 \\
1000\end{array}$ & $\begin{array}{c}46 \\
1000\end{array}$ & $\begin{array}{c}47 \\
1000\end{array}$ & $\begin{array}{c}4 S \\
1000\end{array}$ & $\begin{array}{c}49 \\
1000\end{array}$ & $\begin{array}{c}50 \\
1000\end{array}$ \\
\hline 16 & $\begin{array}{c}40 \cdot 6 \\
999\end{array}$ & $\begin{array}{c}41 \cdot 6 \\
999\end{array}$ & $\begin{array}{c}42 \cdot 6 \\
999\end{array}$ & $\begin{array}{c}43 \cdot 6 \\
999\end{array}$ & $\begin{array}{c}44 \cdot 6 \\
999\end{array}$ & $\begin{array}{l}45 \cdot 6 \\
999\end{array}$ & $\begin{array}{c}46 \cdot 6 \\
999\end{array}$ & $\begin{array}{c}47 \cdot 6 \\
999\end{array}$ & $\begin{array}{c}48 \cdot 6 \\
999\end{array}$ & $\begin{array}{c}49 \cdot 6 \\
999\end{array}$ \\
\hline
\end{tabular}


ALCOHOL.

Aleometrical Table of real Strength, by M. Gay Lussac (continued).

\begin{tabular}{|c|c|c|c|c|c|c|c|c|c|c|}
\hline $\begin{array}{c}\text { Temperature. } \\
\text { C. } \\
\end{array}$ & $41 \mathrm{c}$ & $42 \mathrm{c}$ & $43 \mathrm{c}$ & $44 \mathrm{c}$ & $45 \mathrm{c}$ & $46 c$ & $47 \mathrm{c}$ & $48 c$ & $49 c$ & $50 \mathrm{c}$ \\
\hline $\begin{array}{c}\text { Deg. } \\
17\end{array}$ & $\begin{array}{l}40 \cdot 2 \\
999\end{array}$ & $\begin{array}{c}41 \cdot 2 \\
999\end{array}$ & $\begin{array}{l}42 \cdot 2 \\
999\end{array}$ & $\begin{array}{c}43 \cdot 2 \\
998\end{array}$ & $\begin{array}{c}44 \cdot 9 \\
998\end{array}$ & $\begin{array}{c}45 \cdot 2 \\
998\end{array}$ & $\begin{array}{c}46 \cdot 2 \\
998\end{array}$ & $\begin{array}{c}47 \cdot 2 \\
998\end{array}$ & $\begin{array}{c}48 \cdot 2 \\
998\end{array}$ & $\begin{array}{c}49 \cdot 2 \\
993\end{array}$ \\
\hline 18 & $\begin{array}{c}39 \cdot 8 \\
998\end{array}$ & $\begin{array}{c}40 \cdot 8 \\
998\end{array}$ & $\begin{array}{c}41 \cdot 8 \\
998\end{array}$ & $\begin{array}{c}42 \cdot S \\
998\end{array}$ & $\begin{array}{c}43 \cdot 8 \\
998\end{array}$ & $\begin{array}{c}44 \cdot 9 \\
998\end{array}$ & $\begin{array}{c}45 \cdot 9 \\
998\end{array}$ & $\begin{array}{c}46 \cdot 9 \\
998\end{array}$ & $\begin{array}{c}47 \cdot 9 \\
998\end{array}$ & $\begin{array}{c}48 \cdot 9 \\
998\end{array}$ \\
\hline 19 & $\begin{array}{c}39 \cdot 4 \\
99 \tau\end{array}$ & $\begin{array}{c}40.4 \\
997\end{array}$ & $\begin{array}{c}41 \cdot 4 \\
997\end{array}$ & $\begin{array}{c}42 \cdot 5 \\
997\end{array}$ & $\begin{array}{c}43 \cdot 5 \\
997\end{array}$ & $\begin{array}{c}44 \cdot 5 \\
997\end{array}$ & $\begin{array}{c}45 \cdot 5 \\
997\end{array}$ & $\begin{array}{c}46 \cdot 5 \\
997\end{array}$ & $\begin{array}{c}47 \cdot 5 \\
997\end{array}$ & $\begin{array}{c}48 \cdot 5 \\
997\end{array}$ \\
\hline 20 & $\begin{array}{c}39 \\
997\end{array}$ & $\begin{array}{l}40 \\
997\end{array}$ & $\begin{array}{c}41 \\
997\end{array}$ & $\begin{array}{l}42 \cdot 1 \\
997\end{array}$ & $\begin{array}{c}43 \cdot 1 \\
996\end{array}$ & $\begin{array}{c}44 \cdot 1 \\
996\end{array}$ & $\begin{array}{l}45 \cdot 1 \\
996\end{array}$ & $\begin{array}{c}46 \cdot 1 \\
996\end{array}$ & $\begin{array}{c}47 \cdot 2 \\
996\end{array}$ & $\begin{array}{c}48 \cdot 2 \\
996\end{array}$ \\
\hline 21 & $\begin{array}{c}38 \cdot 6 \\
996\end{array}$ & $\begin{array}{c}39 \cdot 6 \\
996\end{array}$ & $\begin{array}{c}40 \cdot 6 \\
996\end{array}$ & $\begin{array}{c}41 \cdot 7 \\
996\end{array}$ & $\begin{array}{c}42 \cdot 7 \\
996\end{array}$ & $\begin{array}{c}43 \cdot 7 \\
996\end{array}$ & $\begin{array}{c}44 \cdot 8 \\
996\end{array}$ & $\begin{array}{l}45 \cdot 8 \\
996\end{array}$ & $\begin{array}{l}46 \cdot 8 \\
995\end{array}$ & $\begin{array}{l}47 \cdot 8 \\
995\end{array}$ \\
\hline 22 & $\begin{array}{c}38 \cdot 2 \\
996\end{array}$ & $\begin{array}{c}39 \cdot 2 \\
995\end{array}$ & $\begin{array}{c}40 \cdot 2 \\
995\end{array}$ & $\begin{array}{c}41 \cdot 3 \\
995\end{array}$ & $\begin{array}{c}42 \cdot 3 \\
995\end{array}$ & $\begin{array}{c}43 \cdot 3 \\
995\end{array}$ & $\begin{array}{c}44 \cdot 3 \\
995\end{array}$ & $\begin{array}{c}45 \cdot 3 \\
995\end{array}$ & $\begin{array}{c}46 \cdot 4 \\
995\end{array}$ & $\begin{array}{c}47 \cdot 4 \\
995\end{array}$ \\
\hline 23 & $\begin{array}{c}37 \cdot 8 \\
995\end{array}$ & $\begin{array}{c}38 \cdot 8 \\
995\end{array}$ & $\begin{array}{c}39 \cdot 8 \\
995\end{array}$ & $\begin{array}{c}40 \cdot 9 \\
994\end{array}$ & $\begin{array}{c}41 \cdot 9 \\
994\end{array}$ & $\begin{array}{c}42 \cdot 9 \\
994\end{array}$ & \begin{tabular}{|c|}
$43 \cdot 9$ \\
994
\end{tabular} & $\begin{array}{c}44 \cdot 9 \\
994\end{array}$ & $\begin{array}{c}46 \\
994\end{array}$ & $\begin{array}{c}47 \\
994\end{array}$ \\
\hline 24 & $\begin{array}{c}37 \cdot 4 \\
994\end{array}$ & $\begin{array}{c}38 \cdot 4 \\
994\end{array}$ & $\begin{array}{c}39 \cdot 4 \\
994\end{array}$ & $\begin{array}{c}40 \cdot 5 \\
994\end{array}$ & $\begin{array}{c}41 \cdot 5 \\
994\end{array}$ & $\begin{array}{c}42 \cdot 5 \\
994\end{array}$ & $\begin{array}{c}43 \cdot 6 \\
994\end{array}$ & $\begin{array}{c}44 \cdot 6 \\
994\end{array}$ & $\begin{array}{c}45 \cdot 6 \\
993\end{array}$ & $\begin{array}{c}46 \cdot 6 \\
993\end{array}$ \\
\hline 25 & $\begin{array}{c}37 \\
994 \\
\end{array}$ & $\begin{array}{l}38 \\
994 \\
\end{array}$ & $\begin{array}{c}39 \\
993 \\
\end{array}$ & $\begin{array}{c}40 \cdot 1 \\
993 \\
\end{array}$ & $\begin{array}{c}42 \cdot 1 \\
993 \\
\end{array}$ & $\begin{array}{c}42 \cdot 2 \\
993\end{array}$ & $\begin{array}{c}43 \cdot 2 \\
993 \\
\end{array}$ & $\begin{array}{c}44 \cdot 2 \\
593\end{array}$ & $\begin{array}{c}45 \cdot 2 \\
993\end{array}$ & $\begin{array}{c}46 \cdot 3 \\
993\end{array}$ \\
\hline $\begin{array}{c}\text { Temperature. } \\
\text { C. }\end{array}$ & $51 \mathrm{c}$ & $52 \mathrm{c}$ & $53 c$ & $54 \mathrm{c}$ & $55 \mathrm{c}$ & $56 \mathrm{c}$ & $5 \pi \mathrm{c}$ & $58 \mathrm{c}$ & $59 \mathrm{c}$ & $60 \mathrm{c}$ \\
\hline $\begin{array}{l}\text { Deg } \\
10\end{array}$ & $\begin{array}{l}52 \cdot 8 \\
1004\end{array}$ & $\begin{array}{c}53 \cdot 8 \\
1004 \\
\end{array}$ & $\begin{array}{c}54 \cdot 8 \\
1004\end{array}$ & $\begin{array}{c}55 \cdot 8 \\
1004\end{array}$ & $\begin{array}{c}56 \cdot 8 \\
1004\end{array}$ & $\begin{array}{l}57 \cdot 8 \\
1004\end{array}$ & $\begin{array}{l}58 \cdot 8 \\
1004\end{array}$ & $\begin{array}{c}59 \cdot 7 \\
1004\end{array}$ & $\begin{array}{l}60 \cdot 7 \\
1004\end{array}$ & $\begin{array}{l}61 \cdot 7 \\
1004\end{array}$ \\
\hline 11 & $\begin{array}{l}52 \cdot 5 \\
1003\end{array}$ & $\begin{array}{r}53 \cdot 5 \\
1003 \\
\end{array}$ & $\begin{array}{c}54 \cdot 4 \\
1003\end{array}$ & $\begin{array}{c}55 \cdot 4 \\
1003\end{array}$ & $\begin{array}{c}56 \cdot 4 \\
1003\end{array}$ & $\begin{array}{l}57 \cdot 4 \\
1003\end{array}$ & $\begin{array}{l}58 \cdot 4 \\
1003 \\
\end{array}$ & $\begin{array}{l}59 \cdot 4 \\
1003 \\
\end{array}$ & $\begin{array}{l}60 \cdot 4 \\
1003\end{array}$ & $\begin{array}{c}61 \cdot 4 \\
1003 \\
\end{array}$ \\
\hline 12 & $\begin{array}{l}52 \cdot 1 \\
1002\end{array}$ & $\begin{array}{l}53 \cdot 1 \\
1002\end{array}$ & $\begin{array}{l}54 \cdot 1 \\
1002\end{array}$ & $\begin{array}{c}55 \\
1002\end{array}$ & $\begin{array}{c}56 \\
1002\end{array}$ & $\begin{array}{c}57 \\
1002\end{array}$ & $\begin{array}{c}58 \\
1002\end{array}$ & $\begin{array}{c}59 \\
1002\end{array}$ & $\begin{array}{c}60 \\
1002\end{array}$ & $\begin{array}{c}61 \\
1002\end{array}$ \\
\hline 13 & $\begin{array}{c}51.8 \\
1002\end{array}$ & $\begin{array}{l}52 \cdot 7 \\
1002\end{array}$ & $\begin{array}{l}53 \cdot 7 \\
1002\end{array}$ & $\begin{array}{c}54 \cdot 7 \\
1002\end{array}$ & $\begin{array}{l}55.7 \\
1002\end{array}$ & $\begin{array}{l}56 \cdot 7 \\
1002\end{array}$ & $\begin{array}{l}57 \cdot 7 \\
1002\end{array}$ & $\begin{array}{c}5 S \cdot 7 \\
1002\end{array}$ & $\begin{array}{l}59 \cdot 7 \\
1002\end{array}$ & $\begin{array}{l}60 \cdot 7 \\
1002\end{array}$ \\
\hline 14 & $\begin{array}{l}51 \cdot 4 \\
1001\end{array}$ & $\begin{array}{c}52 \cdot 3 \\
1001\end{array}$ & $\begin{array}{c}53 \cdot 3 \\
1001\end{array}$ & $\begin{array}{c}54 \cdot 3 \\
1001\end{array}$ & $\begin{array}{c}55 \cdot 3 \\
1001\end{array}$ & $\begin{array}{l}56 \cdot 3 \\
1001\end{array}$ & $\begin{array}{c}57 \cdot 3 \\
1001\end{array}$ & $\begin{array}{l}58 \cdot 3 \\
1001\end{array}$ & $\begin{array}{l}59 \cdot 3 \\
1001\end{array}$ & $\begin{array}{l}60 \cdot 3 \\
1001\end{array}$ \\
\hline 15 & $\begin{array}{c}51 \\
1000\end{array}$ & $\begin{array}{c}52 \\
1000\end{array}$ & $\begin{array}{c}53 \\
1000\end{array}$ & $\begin{array}{c}54 \\
1000\end{array}$ & $\begin{array}{c}55 \\
1000\end{array}$ & $\begin{array}{c}56 \\
1000\end{array}$ & $\begin{array}{c}57 \\
1000\end{array}$ & $\begin{array}{c}58 \\
1000\end{array}$ & $\begin{array}{c}59 \\
1000\end{array}$ & $\begin{array}{c}60 \\
1000\end{array}$ \\
\hline 16 & $\begin{array}{c}50 \cdot 6 \\
999\end{array}$ & $\begin{array}{c}51 \cdot 6 \\
999\end{array}$ & $\begin{array}{c}52 \cdot 6 \\
999\end{array}$ & $\begin{array}{c}53 \cdot 6 \\
999\end{array}$ & $\begin{array}{c}54 \cdot 6 \\
999\end{array}$ & $\begin{array}{c}55 \cdot 6 \\
999\end{array}$ & $\begin{array}{c}56 \cdot 6 \\
999\end{array}$ & $\begin{array}{c}57 \cdot 6 \\
999\end{array}$ & $\begin{array}{c}58 \cdot 6 \\
999\end{array}$ & $\begin{array}{c}59 \cdot 6 \\
999\end{array}$ \\
\hline 17 & $\begin{array}{c}50 \cdot 3 \\
998\end{array}$ & $\begin{array}{c}51 \cdot 3 \\
998\end{array}$ & $\begin{array}{c}52 \cdot 3 \\
995\end{array}$ & $\begin{array}{c}53 \cdot 3 \\
998\end{array}$ & $\begin{array}{c}54 \cdot 3 \\
998\end{array}$ & $\begin{array}{c}55 \cdot 3 \\
998\end{array}$ & $\begin{array}{c}56 \cdot 3 \\
998\end{array}$ & $\begin{array}{c}57 \cdot 3 \\
998\end{array}$ & $\begin{array}{c}58 \cdot 3 \\
998\end{array}$ & $\begin{array}{c}59 \cdot 3 \\
998\end{array}$ \\
\hline 18 & $\begin{array}{c}49 \cdot 9 \\
998\end{array}$ & $\begin{array}{c}50 \cdot 9 \\
998\end{array}$ & $\begin{array}{c}51 \cdot 9 \\
998\end{array}$ & $\begin{array}{c}52 \cdot 9 \\
998\end{array}$ & $\begin{array}{c}53 \cdot 9 \\
998\end{array}$ & $\begin{array}{c}54 \cdot 9 \\
998\end{array}$ & $\begin{array}{c}55 \cdot 9 \\
998\end{array}$ & $\begin{array}{c}56 \cdot 9 \\
997\end{array}$ & $\begin{array}{c}57 \cdot 9 \\
997\end{array}$ & $\begin{array}{c}58 \cdot 9 \\
997\end{array}$ \\
\hline 19 & $\begin{array}{c}49 \cdot 5 \\
997\end{array}$ & $\begin{array}{l}50 \cdot 6 \\
997\end{array}$ & $\begin{array}{l}51 \cdot 6 \\
997\end{array}$ & $\begin{array}{c}52 \cdot 6 \\
997\end{array}$ & $\begin{array}{c}53 \cdot 6 \\
997\end{array}$ & $\begin{array}{c}54 \cdot 6 \\
997\end{array}$ & $\begin{array}{c}55 \cdot 6 \\
997\end{array}$ & $\begin{array}{c}56 \cdot 6 \\
997\end{array}$ & $\begin{array}{c}57 \cdot 6 \\
997\end{array}$ & $\begin{array}{l}58 \cdot 6 \\
997\end{array}$ \\
\hline 20 & $\begin{array}{c}49 \cdot 2 \\
996\end{array}$ & $\begin{array}{l}50 \cdot 2 \\
996\end{array}$ & $\begin{array}{l}51 \cdot 2 \\
996\end{array}$ & $\begin{array}{c}52 \cdot 2 \\
996\end{array}$ & $\begin{array}{c}53 \cdot 2 \\
996\end{array}$ & $\begin{array}{c}54 \cdot 2 \\
996\end{array}$ & $\begin{array}{c}55 \cdot 2 \\
996\end{array}$ & $\begin{array}{c}56 \cdot 2 \\
996\end{array}$ & $\begin{array}{c}57 \cdot 2 \\
996\end{array}$ & $\begin{array}{l}58 \cdot 2 \\
996\end{array}$ \\
\hline 21 & $\begin{array}{c}48 \cdot 8 \\
995\end{array}$ & $\begin{array}{c}49 \cdot 8 \\
995\end{array}$ & $\begin{array}{c}50 \cdot 8 \\
995\end{array}$ & $\begin{array}{c}51 \cdot 8 \\
995\end{array}$ & $\begin{array}{c}52 \cdot 9 \\
995\end{array}$ & $\begin{array}{c}53 \cdot 9 \\
995\end{array}$ & $\begin{array}{c}54 \cdot 9 \\
995\end{array}$ & $\begin{array}{c}55 \cdot 9 \\
995\end{array}$ & $\begin{array}{c}56 \cdot 9 \\
995\end{array}$ & $\begin{array}{c}57 \cdot 9 \\
995\end{array}$ \\
\hline 22 & $\begin{array}{c}48 \cdot 4 \\
995\end{array}$ & $\begin{array}{c}49 \cdot 4 \\
995\end{array}$ & $\begin{array}{c}50 \cdot 4 \\
995\end{array}$ & $\begin{array}{c}51 \cdot 4 \\
994\end{array}$ & $\begin{array}{c}52 \cdot 5 \\
994\end{array}$ & $\begin{array}{c}53 \cdot 5 \\
994\end{array}$ & $\begin{array}{c}54 \cdot 5 \\
994\end{array}$ & $\begin{array}{c}55 \cdot 5 \\
994\end{array}$ & $\begin{array}{c}56 \cdot 5 \\
994\end{array}$ & $\begin{array}{c}57 \cdot 5 \\
994\end{array}$ \\
\hline 23 & $\begin{array}{l}48 \\
994\end{array}$ & $\begin{array}{c}49 \cdot 1 \\
994\end{array}$ & $\begin{array}{c}50 \cdot 1 \\
994\end{array}$ & $\begin{array}{c}51 \cdot 1 \\
994\end{array}$ & $\begin{array}{c}52 \cdot 1 \\
994\end{array}$ & $\begin{array}{c}53 \cdot 1 \\
994\end{array}$ & $\begin{array}{c}54 \cdot 1 \\
994\end{array}$ & $\begin{array}{c}55 \cdot 1 \\
993\end{array}$ & $\begin{array}{c}56 \cdot 1 \\
993\end{array}$ & $\begin{array}{c}57 \cdot 1 \\
993\end{array}$ \\
\hline 24 & $\begin{array}{c}47 \cdot 6 \\
993\end{array}$ & $\begin{array}{c}48 \cdot 7 \\
993\end{array}$ & $\begin{array}{c}49 \cdot 7 \\
993\end{array}$ & $\begin{array}{c}50 \cdot 7 \\
993\end{array}$ & $\begin{array}{c}51 \cdot 8 \\
993\end{array}$ & $\begin{array}{c}52 \cdot 8 \\
993\end{array}$ & $\begin{array}{c}53 \cdot 8 \\
993\end{array}$ & $\begin{array}{c}54 \cdot 8 \\
993\end{array}$ & $\begin{array}{c}55 \cdot 8 \\
993\end{array}$ & $\begin{array}{c}56 \cdot 8 \\
992\end{array}$ \\
\hline 25 & $\begin{array}{c}47 \cdot 3 \\
099 \\
\end{array}$ & $\begin{array}{c}48 \cdot 3 \\
392 \\
\end{array}$ & $\begin{array}{c}49 \cdot 3 \\
902 \\
\end{array}$ & $\begin{array}{c}50 \cdot 3 \\
992 \\
\end{array}$ & $\begin{array}{c}51 \cdot 4 \\
992 \\
\end{array}$ & $\begin{array}{c}52 \cdot 4 \\
992 \\
\end{array}$ & $\begin{array}{c}53 \cdot 4 \\
992 \\
\end{array}$ & $\begin{array}{c}54 \cdot 4 \\
992 \\
\end{array}$ & $\begin{array}{c}55 \cdot 5 \\
992 \\
\end{array}$ & $\begin{array}{c}56 \cdot 5 \\
992 \\
\end{array}$ \\
\hline
\end{tabular}


ALCOHOL.

Alcometrical Table of real Strength, by M. Gay Lussac (continued).

\begin{tabular}{|c|c|c|c|c|c|c|c|c|c|c|}
\hline $\begin{array}{c}\text { Temperature. } \\
\text { C. }\end{array}$ & $6 \mathrm{lc}$ & $62 \mathrm{c}$ & $63 c$ & $64 \mathrm{c}$ & $65 c$ & $66 c$ & $6 \pi \mathrm{c}$ & $6 * x$ & $69 \mathrm{c}$ & $\tau 0 c$ \\
\hline $\begin{array}{l}\text { Deg. } \\
10\end{array}$ & $\begin{array}{l}62 \cdot 7 \\
1004\end{array}$ & $\begin{array}{r}63 \cdot 7 \\
1004\end{array}$ & $\begin{array}{l}64 \cdot 7 \\
1004\end{array}$ & $\begin{array}{l}65 \cdot 7 \\
1004\end{array}$ & $\begin{array}{l}66 \cdot 7 \\
1004\end{array}$ & $\begin{array}{l}67 \cdot 6 \\
1004\end{array}$ & $\begin{array}{l}68 \cdot 6 \\
1001\end{array}$ & $\begin{array}{l}69 \cdot 6 \\
1004\end{array}$ & $\begin{array}{l}70 \cdot 6 \\
1004\end{array}$ & $\begin{array}{l}71 \cdot 6 \\
1004\end{array}$ \\
\hline 11 & $\begin{array}{c}62 \cdot 4 \\
1003\end{array}$ & $\begin{array}{l}63 \cdot 4 \\
1003\end{array}$ & $\begin{array}{c}64 \cdot 4 \\
1003\end{array}$ & $\begin{array}{c}65 \cdot 4 \\
1003\end{array}$ & $\begin{array}{c}66 \cdot 4 \\
1003\end{array}$ & $\begin{array}{l}67 \cdot 3 \\
1003\end{array}$ & $\begin{array}{l}68 \cdot 3 \\
1003\end{array}$ & $\begin{array}{l}69 \cdot 3 \\
1004\end{array}$ & $\begin{array}{l}70 \cdot 3 \\
1004\end{array}$ & $\begin{array}{l}71 \cdot 3 \\
1004\end{array}$ \\
\hline 12 & $\begin{array}{c}62 \\
1002\end{array}$ & $\begin{array}{c}63 \\
1002\end{array}$ & $\begin{array}{c}64 \\
1002\end{array}$ & $\begin{array}{c}65 \\
1002\end{array}$ & $\begin{array}{c}66 \\
1002\end{array}$ & $\begin{array}{c}67 \\
1002\end{array}$ & $\begin{array}{c}68 \\
1003\end{array}$ & $\begin{array}{c}69 \\
1003\end{array}$ & $\begin{array}{c}70 \\
1003\end{array}$ & $\begin{array}{c}71 \\
1003\end{array}$ \\
\hline 13 & $\begin{array}{l}61.7 \\
1002\end{array}$ & $\begin{array}{l}62 \cdot 7 \\
1002\end{array}$ & $\begin{array}{c}63 \cdot 7 \\
1002\end{array}$ & $\begin{array}{l}64 \cdot 7 \\
1002\end{array}$ & $\begin{array}{l}65 \cdot 7 \\
1002\end{array}$ & $\begin{array}{l}66 \cdot 7 \\
1002\end{array}$ & $\begin{array}{l}67 \cdot 7 \\
1002\end{array}$ & $\begin{array}{c}68 \cdot 7 \\
1002\end{array}$ & $\begin{array}{c}69 \cdot 6 \\
1002\end{array}$ & $\begin{array}{l}70 \cdot 6 \\
1002\end{array}$ \\
\hline 14 & $\begin{array}{c}61 \cdot 3 \\
1001\end{array}$ & $\begin{array}{c}62 \cdot 3 \\
1001\end{array}$ & $\begin{array}{c}63 \cdot 3 \\
1001\end{array}$ & $\begin{array}{l}64 \cdot 3 \\
1001\end{array}$ & $\begin{array}{c}65 \cdot 3 \\
1001\end{array}$ & $\begin{array}{l}66 \cdot 3 \\
1001\end{array}$ & $\begin{array}{l}67 \cdot 3 \\
1001\end{array}$ & $\begin{array}{l}68 \cdot 3 \\
1001\end{array}$ & $\begin{array}{c}69 \cdot 3 \\
1001\end{array}$ & $\begin{array}{l}70 \cdot 3 \\
1001\end{array}$ \\
\hline 15 & $\begin{array}{c}61 \\
1000\end{array}$ & $\begin{array}{c}62 \\
1000\end{array}$ & $\begin{array}{c}63 \\
1000\end{array}$ & $\begin{array}{c}64 \\
1000\end{array}$ & $\begin{array}{c}65 \\
1000\end{array}$ & $\begin{array}{c}66 \\
1000\end{array}$ & $\begin{array}{c}67 \\
1000\end{array}$ & $\begin{array}{c}68 \\
1000\end{array}$ & $\begin{array}{c}69 \\
1000\end{array}$ & $\begin{array}{c}70 \\
1000\end{array}$ \\
\hline 16 & $\begin{array}{c}60 \cdot 6 \\
999\end{array}$ & $\begin{array}{c}61 \cdot 7 \\
999\end{array}$ & $\begin{array}{c}62 \cdot 7 \\
999\end{array}$ & $\begin{array}{c}63 \cdot 7 \\
999\end{array}$ & $\begin{array}{c}64 \cdot 7 \\
999\end{array}$ & $\begin{array}{c}65 \cdot 7 \\
999\end{array}$ & $\begin{array}{c}66 \cdot 7 \\
999\end{array}$ & $\begin{array}{c}67 \cdot 7 \\
999\end{array}$ & $\begin{array}{c}68 \cdot 7 \\
999\end{array}$ & $\begin{array}{c}69 \cdot 7 \\
999\end{array}$ \\
\hline 17 & $\begin{array}{c}60 \cdot 3 \\
998\end{array}$ & $\begin{array}{c}61 \cdot 3 \\
998\end{array}$ & $\begin{array}{c}62 \cdot 3 \\
995\end{array}$ & $\begin{array}{c}63 \cdot 3 \\
998\end{array}$ & $\begin{array}{c}64 \cdot 3 \\
998\end{array}$ & $\begin{array}{c}65 \cdot 3 \\
998\end{array}$ & $\begin{array}{c}66 \cdot 3 \\
998\end{array}$ & $\begin{array}{c}67 \cdot 3 \\
998\end{array}$ & $\begin{array}{c}68 \cdot 3 \\
998\end{array}$ & $\begin{array}{c}69 \cdot 3 \\
998\end{array}$ \\
\hline 18 & $\begin{array}{c}59 \cdot 9 \\
997\end{array}$ & $\begin{array}{c}61 \\
997\end{array}$ & $\begin{array}{c}62 \\
997\end{array}$ & $\begin{array}{c}63 \\
997\end{array}$ & $\begin{array}{c}64 \\
997\end{array}$ & $\begin{array}{c}65 \\
997\end{array}$ & $\begin{array}{l}66 \\
997\end{array}$ & $\begin{array}{c}67 \\
997\end{array}$ & $\begin{array}{c}68 \\
997\end{array}$ & $\begin{array}{c}69 \\
997\end{array}$ \\
\hline 19 & $\begin{array}{c}59 \cdot 6 \\
997\end{array}$ & $\begin{array}{c}60 \cdot 6 \\
997\end{array}$ & $\begin{array}{c}61 \cdot 6 \\
997\end{array}$ & $\begin{array}{c}62 \cdot 7 \\
997\end{array}$ & $\begin{array}{c}63 \cdot 7 \\
997\end{array}$ & $\begin{array}{c}64 \cdot 7 \\
997\end{array}$ & $\begin{array}{c}65 \cdot 7 \\
997\end{array}$ & $\begin{array}{c}66 \cdot 7 \\
997\end{array}$ & $\begin{array}{c}67 \cdot 7 \\
996\end{array}$ & $\begin{array}{c}68 \cdot 7 \\
\therefore 96\end{array}$ \\
\hline 20 & $\begin{array}{c}59 \cdot 2 \\
996\end{array}$ & $\begin{array}{c}60 \cdot 3 \\
996\end{array}$ & $\begin{array}{c}61 \cdot 3 \\
996\end{array}$ & $\begin{array}{c}62 \cdot 3 \\
996\end{array}$ & $\begin{array}{c}63 \cdot 3 \\
996\end{array}$ & $\begin{array}{c}64 \cdot 3 \\
996\end{array}$ & $\begin{array}{c}65 \cdot 4 \\
996\end{array}$ & $\begin{array}{c}66 \cdot 4 \\
996\end{array}$ & $\begin{array}{c}67 \cdot 4 \\
996\end{array}$ & $\begin{array}{c}68 \cdot 4 \\
996\end{array}$ \\
\hline 21 & $\begin{array}{c}58 \cdot 9 \\
995\end{array}$ & $\begin{array}{c}59 \cdot 9 \\
995\end{array}$ & $\begin{array}{c}61 \\
995\end{array}$ & $\begin{array}{c}62 \\
995\end{array}$ & $\begin{array}{c}63 \\
995\end{array}$ & $\begin{array}{c}64 \\
995\end{array}$ & $\begin{array}{c}65 \\
995\end{array}$ & $\begin{array}{c}66 \\
995\end{array}$ & $\begin{array}{c}67 \\
995\end{array}$ & $\begin{array}{c}68 \cdot 1 \\
995\end{array}$ \\
\hline 22 & $\begin{array}{c}58 \cdot 5 \\
994\end{array}$ & $\begin{array}{c}59 \cdot 5 \\
994\end{array}$ & $\begin{array}{c}60 \cdot 6 \\
994\end{array}$ & $\begin{array}{c}61 \cdot 6 \\
994\end{array}$ & $\begin{array}{c}62 \cdot 7 \\
994\end{array}$ & $\begin{array}{c}63 \cdot 7 \\
994\end{array}$ & $\begin{array}{c}64 \cdot 7 \\
994\end{array}$ & $\begin{array}{c}65 \cdot 7 \\
994\end{array}$ & $\begin{array}{c}66 \cdot 7 \\
994\end{array}$ & $\begin{array}{c}67 \cdot 8 \\
994\end{array}$ \\
\hline 23 & $\begin{array}{c}58 \cdot 1 \\
993\end{array}$ & $\begin{array}{c}59 \cdot 2 \\
993\end{array}$ & $\begin{array}{c}60 \cdot 2 \\
993\end{array}$ & $\begin{array}{c}61 \cdot 3 \\
993\end{array}$ & $\begin{array}{c}62 \cdot 3 \\
993\end{array}$ & $\begin{array}{c}63 \cdot 3 \\
993\end{array}$ & $\begin{array}{c}64 \cdot 3 \\
993\end{array}$ & $\begin{array}{c}65 \cdot 4 \\
993\end{array}$ & $\begin{array}{c}66 \cdot 4 \\
993\end{array}$ & $\begin{array}{c}67 \cdot 4 \\
993\end{array}$ \\
\hline 24 & $\begin{array}{c}57 \cdot 8 \\
992\end{array}$ & $\begin{array}{c}58 \cdot 9 \\
992\end{array}$ & $\begin{array}{c}59 \cdot 9 \\
992\end{array}$ & $\begin{array}{c}61 \\
992\end{array}$ & $\begin{array}{c}62 \\
992\end{array}$ & $\begin{array}{c}63 \\
992\end{array}$ & $\begin{array}{c}64 \\
992\end{array}$ & $\begin{array}{c}65 \\
992\end{array}$ & $\begin{array}{c}66 \\
992\end{array}$ & $\begin{array}{c}67 \cdot 1 \\
992\end{array}$ \\
\hline 25 & $\begin{array}{c}57 \cdot 5 \\
992 \\
\end{array}$ & $\begin{array}{c}58 \cdot 5 \\
992\end{array}$ & $\begin{array}{c}59 \cdot 5 \\
992\end{array}$ & $\begin{array}{c}60 \cdot 6 \\
991 \\
\end{array}$ & $\begin{array}{c}61 \cdot 6 \\
991\end{array}$ & $\begin{array}{c}62 \cdot 6 \\
991\end{array}$ & $\begin{array}{c}63 \cdot 7 \\
991\end{array}$ & $\begin{array}{c}64 \cdot 7 \\
991\end{array}$ & $\begin{array}{c}65 \cdot 7 \\
991 \\
\end{array}$ & $\begin{array}{c}66 \cdot 7 \\
991 \\
\end{array}$ \\
\hline $\begin{array}{c}\text { Temperature. } \\
\text { C. }\end{array}$ & 7lc & $72 \mathrm{c}$ & $73 \mathrm{c}$ & $74 \mathrm{c}$ & $75 \mathrm{c}$ & $76 \mathrm{c}$ & $\pi \tau \mathrm{c}$ & $78 c$ & $79 s$ & $80 \mathrm{c}$ \\
\hline $\begin{array}{l}\text { Deg. } \\
10\end{array}$ & $\begin{array}{l}72 \cdot 6 \\
10 \times 4\end{array}$ & $\begin{array}{l}73 \cdot 5 \\
1004\end{array}$ & $\begin{array}{l}74 \cdot 5 \\
1005\end{array}$ & $\begin{array}{c}75 \cdot 5 \\
1005\end{array}$ & $\begin{array}{l}76 \cdot 5 \\
1005\end{array}$ & $\begin{array}{l}77 \cdot 5 \\
1005\end{array}$ & $\begin{array}{l}78 \cdot 5 \\
1005\end{array}$ & $\begin{array}{l}79 \cdot 5 \\
1005\end{array}$ & $\begin{array}{l}80 \cdot 5 \\
1005\end{array}$ & $\begin{array}{l}81 \cdot 5 \\
1005\end{array}$ \\
\hline 11 & $\begin{array}{l}72 \cdot 3 \\
1004\end{array}$ & $\begin{array}{l}73 \cdot 2 \\
1004\end{array}$ & $\begin{array}{l}74 \cdot 2 \\
1004\end{array}$ & $\begin{array}{l}75 \cdot 2 \\
1004\end{array}$ & $\begin{array}{l}76 \cdot 2 \\
1004\end{array}$ & $\begin{array}{l}77 \cdot 2 \\
1004\end{array}$ & $\begin{array}{l}78 \cdot 2 \\
1004\end{array}$ & $\begin{array}{l}79 \cdot 2 \\
1004\end{array}$ & $\begin{array}{l}50 \cdot 2 \\
1004\end{array}$ & $\begin{array}{l}81 \cdot 2 \\
1004\end{array}$ \\
\hline 12 & $\begin{array}{c}72 \\
1003\end{array}$ & $\begin{array}{l}72 \cdot 9 \\
1003\end{array}$ & $\begin{array}{c}73 \cdot 9 \\
1003\end{array}$ & $\begin{array}{c}74 \cdot 9 \\
1003\end{array}$ & $\begin{array}{l}75 \cdot 9 \\
1003\end{array}$ & $\begin{array}{l}76 \cdot 9 \\
1003\end{array}$ & $\begin{array}{l}77 \cdot 9 \\
1003\end{array}$ & $\begin{array}{l}78 \cdot 9 \\
1003\end{array}$ & $\begin{array}{l}79 \cdot 9 \\
1003\end{array}$ & $\begin{array}{l}80 \cdot 9 \\
1003\end{array}$ \\
\hline 13 & $\begin{array}{l}71 \cdot 6 \\
1002\end{array}$ & $\begin{array}{l}72 \cdot 6 \\
1002\end{array}$ & $\begin{array}{l}73 \cdot 6 \\
1002\end{array}$ & $\begin{array}{l}74 \cdot 6 \\
1002\end{array}$ & $\begin{array}{c}75 \cdot 6 \\
1002\end{array}$ & $\begin{array}{l}76 \cdot 6 \\
1002\end{array}$ & $\begin{array}{l}77 \cdot 6 \\
1002\end{array}$ & $\begin{array}{l}78 \cdot 6 \\
1002\end{array}$ & $\begin{array}{l}79 \cdot 6 \\
1002\end{array}$ & $\begin{array}{l}80 \cdot 6 \\
1002\end{array}$ \\
\hline 14 & $\begin{array}{l}71 \cdot 3 \\
1001\end{array}$ & $\begin{array}{c}72 \cdot 3 \\
1001\end{array}$ & $\begin{array}{l}73 \cdot 3 \\
1001\end{array}$ & $\begin{array}{l}74 \cdot 3 \\
1001\end{array}$ & $\begin{array}{l}75 \cdot 3 \\
1001\end{array}$ & $\begin{array}{l}76 \cdot 3 \\
1001\end{array}$ & $\begin{array}{l}77 \cdot 3 \\
1001\end{array}$ & $\begin{array}{l}78 \cdot 3 \\
1001\end{array}$ & $\begin{array}{l}79 \cdot 3 \\
1001\end{array}$ & $\begin{array}{l}80 \cdot 3 \\
1001\end{array}$ \\
\hline 15 & $\begin{array}{c}71 \\
1000\end{array}$ & $\begin{array}{c}72 \\
1000\end{array}$ & $\begin{array}{c}73 \\
1000\end{array}$ & $\begin{array}{c}74 \\
1000\end{array}$ & $\begin{array}{c}75 \\
1000\end{array}$ & $\begin{array}{c}76 \\
1000\end{array}$ & $\begin{array}{c}77 \\
1000\end{array}$ & $\begin{array}{c}78 \\
1000\end{array}$ & $\begin{array}{c}79 \\
1000\end{array}$ & $\begin{array}{c}80 \\
1000\end{array}$ \\
\hline 16 & $\begin{array}{c}70 \cdot 7 \\
999\end{array}$ & $\begin{array}{c}71 \cdot 7 \\
999\end{array}$ & $\begin{array}{c}72 \cdot 7 \\
999\end{array}$ & $\begin{array}{c}73 \cdot 7 \\
999\end{array}$ & $\begin{array}{c}74 \cdot 7 \\
999\end{array}$ & $\begin{array}{c}75 \cdot 7 \\
999\end{array}$ & $\begin{array}{c}76 \cdot 7 \\
999\end{array}$ & $\begin{array}{c}77 \cdot 7 \\
999\end{array}$ & $\begin{array}{c}78 \cdot 7 \\
999\end{array}$ & $\begin{array}{c}79 \cdot 7 \\
999\end{array}$ \\
\hline 17 & $\begin{array}{c}70 \cdot 3 \\
998\end{array}$ & $\begin{array}{c}71 \cdot 3 \\
998\end{array}$ & $\begin{array}{c}72 \cdot 3 \\
998\end{array}$ & $\begin{array}{c}73 \cdot 3 \\
998 \\
\end{array}$ & $\begin{array}{c}74 \cdot 3 \\
998\end{array}$ & $\begin{array}{c}75 \cdot 4 \\
998\end{array}$ & $\begin{array}{c}76 \cdot 4 \\
998\end{array}$ & $\begin{array}{c}77 \cdot 4 \\
998\end{array}$ & $\begin{array}{c}78 \cdot 4 \\
998\end{array}$ & $\begin{array}{c}79.4 \\
998\end{array}$ \\
\hline
\end{tabular}


ALCOHOL.

Alcometrical Table of real Strength, by M. Gay Lussac (continued).

\begin{tabular}{|c|c|c|c|c|c|c|c|c|c|c|}
\hline $\begin{array}{c}\text { Temperature. } \\
\text { C. }\end{array}$ & $\pi 1 \mathrm{c}$ & $\pi 2 \mathrm{c}$ & $73 \mathrm{c}$ & $74 \mathrm{c}$ & $75 c$ & $76 \mathrm{c}$ & $7 \pi \mathrm{c}$ & $78 \mathrm{c}$ & $79 r$ & $80 \mathrm{c}$ \\
\hline $\begin{array}{l}\text { Deg. } \\
18\end{array}$ & $\begin{array}{l}70 \\
99 \pi\end{array}$ & $\begin{array}{l}71 \\
997\end{array}$ & $\begin{array}{l}72 \\
997 \\
\end{array}$ & $\begin{array}{l}73 \\
99 i \\
\end{array}$ & $\begin{array}{l}74 \\
99 \tau\end{array}$ & $\begin{array}{l}75 \cdot 1 \\
997\end{array}$ & $\begin{array}{c}76 \cdot 1 \\
997\end{array}$ & $\begin{array}{l}77 \cdot 1 \\
997\end{array}$ & $\begin{array}{c}78 \cdot 1 \\
997\end{array}$ & $\underset{997}{79 \cdot 1}$ \\
\hline 19 & $\begin{array}{c}69 \cdot 7 \\
996\end{array}$ & $\begin{array}{c}70 \cdot 7 \\
996\end{array}$ & $\begin{array}{c}71 \cdot 7 \\
996\end{array}$ & $\begin{array}{c}72 \cdot 7 \\
996\end{array}$ & $\begin{array}{c}73 \cdot 7 \\
996\end{array}$ & $\begin{array}{c}74 \cdot 7 \\
996\end{array}$ & $\begin{array}{c}75 \cdot 8 \\
996\end{array}$ & $\begin{array}{c}76 \cdot 8 \\
996\end{array}$ & $\begin{array}{c}77 \cdot 8 \\
996\end{array}$ & $\begin{array}{c}78 \cdot 8 \\
996\end{array}$ \\
\hline 20 & $\begin{array}{c}69 \cdot 4 \\
996\end{array}$ & $\begin{array}{c}70 \cdot 4 \\
996\end{array}$ & $\begin{array}{c}71 \cdot 4 \\
995\end{array}$ & $\begin{array}{c}72 \cdot 4 \\
995\end{array}$ & $\begin{array}{c}73 \cdot 4 \\
995\end{array}$ & $\begin{array}{c}74 \cdot 4 \\
995\end{array}$ & $\begin{array}{c}75 \cdot 5 \\
995\end{array}$ & $\begin{array}{c}76 \cdot 5 \\
995\end{array}$ & $\begin{array}{c}77 \cdot 5 \\
995\end{array}$ & $\begin{array}{c}78 \cdot 5 \\
995\end{array}$ \\
\hline 21 & $\begin{array}{c}69 \cdot 1 \\
995\end{array}$ & $\begin{array}{l}70 \cdot 1 \\
995\end{array}$ & $\begin{array}{c}71 \cdot 1 \\
995\end{array}$ & $\begin{array}{c}72 \cdot 1 \\
994\end{array}$ & $\begin{array}{c}73 \cdot 1 \\
994\end{array}$ & $\begin{array}{c}74 \cdot 1 \\
994\end{array}$ & $\begin{array}{c}75 \cdot 2 \\
994\end{array}$ & $\begin{array}{c}76 \cdot 2 \\
994\end{array}$ & $\begin{array}{c}77 \cdot 2 \\
994\end{array}$ & $\begin{array}{c}78 \cdot 2 \\
994\end{array}$ \\
\hline 22 & $\begin{array}{c}68 \cdot S \\
994 \\
\end{array}$ & $\begin{array}{c}69 \cdot 8 \\
994\end{array}$ & $\begin{array}{l}70 \cdot 8 \\
994\end{array}$ & $\begin{array}{c}71 \cdot 8 \\
994\end{array}$ & $\begin{array}{c}72 \cdot 8 \\
993 \\
\end{array}$ & $\begin{array}{c}73 \cdot 8 \\
993 \\
\end{array}$ & $\begin{array}{c}74 \cdot 8 \\
993\end{array}$ & $\begin{array}{c}75 \cdot 9 \\
993\end{array}$ & $\begin{array}{l}76 \cdot 9 \\
993\end{array}$ & $\begin{array}{c}77 \cdot 9 \\
993\end{array}$ \\
\hline 23 & $\begin{array}{c}68 \cdot 4 \\
993 \\
\end{array}$ & $\begin{array}{c}69 \cdot 4 \\
993\end{array}$ & $\begin{array}{c}70 \cdot 5 \\
993 \\
\end{array}$ & $\begin{array}{c}71 \cdot 5 \\
993 \\
\end{array}$ & $\begin{array}{c}72 \cdot 5 \\
992 \\
\end{array}$ & $\begin{array}{c}73 \cdot 5 \\
992 \\
\end{array}$ & $\begin{array}{c}74 \cdot 5 \\
992 \\
\end{array}$ & $\begin{array}{c}75 \cdot 5 \\
992\end{array}$ & $\begin{array}{c}76 \cdot 6 \\
992\end{array}$ & $\begin{array}{c}77 \cdot 6 \\
992\end{array}$ \\
\hline 24 & $\begin{array}{c}68 \cdot 1 \\
992\end{array}$ & $\begin{array}{c}69 \cdot 1 \\
992\end{array}$ & $\begin{array}{c}70 \cdot 1 \\
992\end{array}$ & $\begin{array}{c}71 \cdot 2 \\
992 \\
\end{array}$ & $\begin{array}{c}72 \cdot 2 \\
992 \\
\end{array}$ & $\begin{array}{c}73 \cdot 2 \\
992 \\
\end{array}$ & $\begin{array}{c}74 \cdot 2 \\
992 \\
\end{array}$ & $\begin{array}{c}75 \cdot 2 \\
991\end{array}$ & $\begin{array}{c}76 \cdot 3 \\
991\end{array}$ & $\begin{array}{c}77 \cdot 3 \\
991\end{array}$ \\
\hline 25 & $\begin{array}{c}67 \cdot 8 \\
991 \\
\end{array}$ & $\begin{array}{c}68 \cdot 8 \\
991\end{array}$ & $\begin{array}{c}69 \cdot \mathrm{S} \\
991 \\
\end{array}$ & $\begin{array}{c}70 \cdot 8 \\
991 \\
\end{array}$ & $\begin{array}{c}71 \cdot 8 \\
991 \\
\end{array}$ & $\begin{array}{c}72 \cdot 8 \\
991 \\
\end{array}$ & $\begin{array}{c}73 \cdot 9 \\
991 \\
\end{array}$ & $\begin{array}{c}74 \cdot 9 \\
991 \\
\end{array}$ & $\begin{array}{r}76 \\
991 \\
\end{array}$ & $\begin{array}{r}77 \\
991 \\
\end{array}$ \\
\hline $\begin{array}{c}\text { Temperature. } \\
\text { C. }\end{array}$ & $81 c$ & $82 \mathrm{c}$ & $83 c$ & $84 c$ & $85 \mathrm{c}$ & $86 c$ & $87 \mathrm{c}$ & $88 c$ & $89 c$ & $90 c$ \\
\hline $\begin{array}{l}\text { Deg. } \\
10\end{array}$ & $\begin{array}{c}82 \cdot 4 \\
1005\end{array}$ & $\begin{array}{c}83 \cdot 4 \\
1005\end{array}$ & $\begin{array}{l}84 \cdot 4 \\
1005 \\
\end{array}$ & $\begin{array}{c}85 \cdot 4 \\
1005 \\
\end{array}$ & $\begin{array}{c}86 \cdot 4 \\
1005\end{array}$ & $\begin{array}{l}87 \cdot 4 \\
1005 \\
\end{array}$ & $\begin{array}{l}88 \cdot 3 \\
1005 \\
\end{array}$ & $\begin{array}{l}89 \cdot 3 \\
1005 \\
\end{array}$ & $\begin{array}{l}90 \cdot 2 \\
1005 \\
\end{array}$ & $\begin{array}{l}91 \cdot 2 \\
1005 \\
\end{array}$ \\
\hline 11 & $\begin{array}{l}82 \cdot 2 \\
1004\end{array}$ & $\begin{array}{r}83 \cdot 1 \\
1004 \\
\end{array}$ & $\begin{array}{l}84 \cdot 1 \\
1004 \\
\end{array}$ & $\begin{array}{c}85 \cdot 1 \\
1004 \\
\end{array}$ & $\begin{array}{l}86 \cdot 1 \\
1004\end{array}$ & $\begin{array}{l}87 \cdot 1 \\
1004\end{array}$ & $\begin{array}{c}88 \\
1004\end{array}$ & $\begin{array}{c}89 \\
1004\end{array}$ & $\begin{array}{c}90 \\
1004\end{array}$ & $\begin{array}{c}91 \\
1004\end{array}$ \\
\hline 12 & $\begin{array}{l}81 \cdot 9 \\
1003 \\
\end{array}$ & $\begin{array}{l}82 \cdot 9 \\
1003 \\
\end{array}$ & $\begin{array}{l}83 \cdot 9 \\
1003 \\
\end{array}$ & $\begin{array}{c}84 \cdot 8 \\
1003 \\
\end{array}$ & $\begin{array}{l}85 \cdot 8 \\
1003 \\
\end{array}$ & $\begin{array}{l}86 \cdot 8 \\
1003 \\
\end{array}$ & $\begin{array}{l}87 \cdot 8 \\
1003 \\
\end{array}$ & $\begin{array}{l}88 \cdot 7 \\
1003\end{array}$ & $\begin{array}{l}89 \cdot 7 \\
1003\end{array}$ & $\begin{array}{l}90 \cdot 7 \\
1003\end{array}$ \\
\hline 13 & $\begin{array}{r}81.6 \\
1002 \\
\end{array}$ & $\begin{array}{l}82 \cdot 6 \\
1 \cap 02 \\
\end{array}$ & $\begin{array}{c}83 \cdot 6 \\
1002 \\
\end{array}$ & $\begin{array}{c}84 \cdot 6 \\
1002 \\
\end{array}$ & $\begin{array}{l}8.5 \cdot 5 \\
1002 \\
\end{array}$ & $\begin{array}{l}86 \cdot 5 \\
1002\end{array}$ & $\begin{array}{l}87 \cdot 5 \\
1002 \\
\end{array}$ & $\begin{array}{r}88 \cdot 5 \\
1002 \\
\end{array}$ & $\begin{array}{l}89 \cdot 5 \\
1002 \\
\end{array}$ & $\begin{array}{l}90 \cdot 5 \\
1002\end{array}$ \\
\hline 14 & $\begin{array}{l}81 \cdot 3 \\
1001 \\
\end{array}$ & $\begin{array}{l}82 \cdot 3 \\
1001 \\
\end{array}$ & $\begin{array}{r}83 \cdot 3 \\
1001 \\
\end{array}$ & $\begin{array}{l}84 \cdot 3 \\
1001 \\
\end{array}$ & $\begin{array}{l}85 \cdot 3 \\
1001 \\
\end{array}$ & $\begin{array}{l}86 \cdot 3 \\
1001 \\
\end{array}$ & $\begin{array}{c}87 \cdot 3 \\
1001 \\
\end{array}$ & $\begin{array}{l}88 \cdot 2 \\
1001\end{array}$ & $\begin{array}{l}89 \cdot 2 \\
1001\end{array}$ & $\begin{array}{c}90 \cdot 2 \\
1001\end{array}$ \\
\hline 15 & $\begin{array}{c}81 \\
1000 \\
\end{array}$ & $\begin{array}{c}82 \\
1000\end{array}$ & $\begin{array}{c}83 \\
1000 \\
\end{array}$ & $\begin{array}{c}84 \\
1000 \\
\end{array}$ & $\begin{array}{c}85 \\
1000\end{array}$ & $\begin{array}{c}86 \\
1000\end{array}$ & $\begin{array}{c}87 \\
1000\end{array}$ & $\begin{array}{c}88 \\
1000\end{array}$ & $\begin{array}{c}89 \\
1000\end{array}$ & $\begin{array}{c}90 \\
1000\end{array}$ \\
\hline 16 & $\begin{array}{c}80 \cdot 7 \\
499\end{array}$ & $\begin{array}{c}81 \cdot 7 \\
999\end{array}$ & $\begin{array}{c}82 \cdot 7 \\
999 \\
\end{array}$ & $\begin{array}{c}83 \cdot 7 \\
999 \\
\end{array}$ & $\begin{array}{c}84 \cdot 7 \\
999\end{array}$ & $\begin{array}{c}85 \cdot 7 \\
999\end{array}$ & $\begin{array}{c}86 \cdot 7 \\
999\end{array}$ & $\begin{array}{c}87 \cdot 7 \\
999\end{array}$ & $\begin{array}{c}88 \cdot 7 \\
999\end{array}$ & $\begin{array}{c}89 \cdot 7 \\
999\end{array}$ \\
\hline 17 & $\begin{array}{c}80 \cdot 4 \\
998\end{array}$ & $\begin{array}{c}81 \cdot 4 \\
998\end{array}$ & $\begin{array}{c}82 \cdot 4 \\
998 \\
\end{array}$ & $\begin{array}{c}83 \cdot 4 \\
998 \\
\end{array}$ & $\begin{array}{c}84 \cdot 4 \\
998\end{array}$ & $\begin{array}{c}85 \cdot 4 \\
998\end{array}$ & $\begin{array}{c}86 \cdot 4 \\
998\end{array}$ & $\begin{array}{c}87 \cdot 4 \\
998\end{array}$ & $\begin{array}{c}88 \cdot 4 \\
998\end{array}$ & $\begin{array}{c}89 \cdot 5 \\
998\end{array}$ \\
\hline 18 & $\begin{array}{c}80 \cdot 1 \\
997 \\
\end{array}$ & $\begin{array}{c}81 \cdot 1 \\
997\end{array}$ & $\begin{array}{c}82 \cdot 1 \\
997 \\
\end{array}$ & $\begin{array}{l}83 \cdot 1 \\
997\end{array}$ & $\begin{array}{c}84 \cdot 1 \\
997 \\
\end{array}$ & $\begin{array}{c}85 \cdot 2 \\
997 \\
\end{array}$ & $\begin{array}{c}86 \cdot 2 \\
997 \\
\end{array}$ & $\begin{array}{c}87 \cdot 2 \\
997\end{array}$ & $\begin{array}{c}88 \cdot 2 \\
997\end{array}$ & $\begin{array}{c}89 \cdot 2 \\
997\end{array}$ \\
\hline 19 & $\begin{array}{c}79 \cdot 8 \\
996 \\
\end{array}$ & $\begin{array}{c}80 \cdot 8 \\
996 \\
\end{array}$ & $\begin{array}{c}81 \cdot 9 \\
996 \\
\end{array}$ & $\begin{array}{c}82 \cdot 9 \\
996 \\
\end{array}$ & $\begin{array}{c}83 \cdot 9 \\
996 \\
\end{array}$ & $\begin{array}{c}84 \cdot 9 \\
996 \\
\end{array}$ & $\begin{array}{c}85 \cdot 9 \\
996 \\
\end{array}$ & $\begin{array}{c}86 \cdot 9 \\
996 \\
\end{array}$ & $\begin{array}{c}87 \cdot 9 \\
996 \\
\end{array}$ & $\begin{array}{c}88 \cdot 9 \\
996 \\
\end{array}$ \\
\hline 20 & $\begin{array}{c}79 \cdot 5 \\
995 \\
\end{array}$ & $\begin{array}{c}80 \cdot 5 \\
995 \\
\end{array}$ & $\begin{array}{c}81 \cdot 6 \\
995 \\
\end{array}$ & $\begin{array}{c}82 \cdot 6 \\
995 \\
\end{array}$ & $\begin{array}{c}83 \cdot 6 \\
995 \\
\end{array}$ & $\begin{array}{c}84 \cdot 6 \\
995 \\
\end{array}$ & $\begin{array}{c}85 \cdot 6 \\
995 \\
\end{array}$ & $\begin{array}{c}86 \cdot 6 \\
995 \\
\end{array}$ & $\begin{array}{c}87 \cdot 7 \\
995 \\
\end{array}$ & $\begin{array}{c}88 \cdot 7 \\
995 \\
\end{array}$ \\
\hline 21 & $\begin{array}{c}79 \cdot 2 \\
994 \\
\end{array}$ & $\begin{array}{c}80 \cdot 2 \\
994 \\
\end{array}$ & $\begin{array}{c}81 \cdot 3 \\
994 \\
\end{array}$ & $\begin{array}{c}82 \cdot 3 \\
994 \\
\end{array}$ & $\begin{array}{c}83 \cdot 3 \\
994 \\
\end{array}$ & $\begin{array}{c}84 \cdot 3 \\
994 \\
\end{array}$ & $\begin{array}{c}85 \cdot 3 \\
994 \\
\end{array}$ & $\begin{array}{c}86 \cdot 4 \\
994 \\
\end{array}$ & $\begin{array}{c}87 \cdot 4 \\
994 \\
\end{array}$ & $\begin{array}{c}88 \cdot 4 \\
994 \\
\end{array}$ \\
\hline 22 & $\begin{array}{c}78 \cdot 9 \\
993 \\
\end{array}$ & $\begin{array}{c}79 \cdot 9 \\
993 \\
\end{array}$ & $\begin{array}{c}81 \\
993 \\
\end{array}$ & $\begin{array}{c}82 \\
993 \\
\end{array}$ & $\begin{array}{c}\delta 3 \\
993 \\
\end{array}$ & $\begin{array}{c}84 \\
993 \\
\end{array}$ & $\begin{array}{c}85 \\
993 \\
\end{array}$ & $\begin{array}{c}86 \cdot 1 \\
993\end{array}$ & $\begin{array}{c}87 \cdot 1 \\
993 \\
\end{array}$ & $\begin{array}{c}88 \cdot 2 \\
993 \\
\end{array}$ \\
\hline 23 & $\begin{array}{c}78 \cdot 6 \\
992 \\
\end{array}$ & $\begin{array}{c}79 \cdot 6 \\
992 \\
\end{array}$ & $\begin{array}{c}80 \cdot 7 \\
992 \\
\end{array}$ & $\begin{array}{c}81 \cdot 7 \\
992 \\
\end{array}$ & $\begin{array}{c}82 \cdot 7 \\
992 \\
\end{array}$ & $\begin{array}{c}83 \cdot 8 \\
992 \\
\end{array}$ & $\begin{array}{c}84 \cdot 8 \\
992 \\
\end{array}$ & $\begin{array}{c}85 \cdot 8 \\
992 \\
\end{array}$ & $\begin{array}{c}86 \cdot 8 \\
992 \\
\end{array}$ & $\begin{array}{l}87 \cdot 9 \\
992\end{array}$ \\
\hline 24 & $\begin{array}{c}78 \cdot 3 \\
991 \\
\end{array}$ & $\begin{array}{c}79 \cdot 3 \\
991 \\
\end{array}$ & $\begin{array}{c}80 \cdot 4 \\
991 \\
\end{array}$ & $\begin{array}{c}81 \cdot 4 \\
991 \\
\end{array}$ & $\begin{array}{c}82 \cdot 4 \\
991 \\
\end{array}$ & $\begin{array}{c}83 \cdot 5 \\
991 \\
\end{array}$ & $\begin{array}{c}84 \cdot 5 \\
991 \\
\end{array}$ & $\begin{array}{c}85 \cdot 5 \\
991 \\
\end{array}$ & $\begin{array}{c}86 \cdot 5 \\
991 \\
\end{array}$ & $\begin{array}{c}87 \cdot 6 \\
991\end{array}$ \\
\hline 25 & $\begin{array}{r}78 \\
991 \\
\end{array}$ & $\begin{array}{r}79 \\
991 \\
\end{array}$ & $\begin{array}{c}80 \cdot 1 \\
990 \\
\end{array}$ & $\begin{array}{c}81 \cdot 1 \\
990 \\
\end{array}$ & $\begin{array}{c}8 \% \cdot 1 \\
990\end{array}$ & $\begin{array}{c}83 \cdot 2 \\
990\end{array}$ & $\begin{array}{c}84 \cdot 2 \\
990 \\
\end{array}$ & $\begin{array}{c}85 \cdot 2 \\
990 \\
\end{array}$ & $\begin{array}{c}86 \cdot 3 \\
990 \\
\end{array}$ & $\begin{array}{c}87 \cdot 4 \\
990 \\
\end{array}$ \\
\hline
\end{tabular}


I consicier the preceding table, which I have extracted from the longer tables of $\mathbf{M}$. Jay Lussac, as an important addition to the resources of British dealers and manufac. turing chemists. With the aid of his little instrument, which may be got for a trifte from its ingenious malier, M. Collardeau, Rue Faubourg St. Martin, at Paris, or constructed by one of the London hydrometer artists, the per centage of real alcohol, and the real value of any spirituous liquor, may be determined to sufficient nicety for mo*: purposes, in a far easier manner than by any instruments now used in this country. It has been alonted by the Swedish government, with M. Gay Lussac's tables.

M. Gay Lussac's table gives, by inspection, the true bulk of the spirits as corrected for temperature; that is, their volume, if of the normal temperature of $15^{\circ} \mathrm{Cent}$ ( $59^{\circ}$ Fahr.). Now this is important information; for, if a person buys 1000 gallons of spirits in hot weather, and pays for them exactly according to their strength corrected for temperature, he will not have 1000 gallons when the weather is in its mean state. He may lose, in this way, several gallons without heing aware of it from his hydrometer.

Sometimes, after moist autumns, when damaged grain abounds, the alcohol distilled from its fermented wash contams a peculiar rolatile body. When we apply nur nose to thir species of spirits in its hot state, the volatile substance dissolved in it irritates the eves and nostrils: it has very nearly the same smell as an alcoholic solution of cyanogen, as any chemist may discover by standing near the discharge pipe of the refrigeratory worm of a raw-grain whisliey still. Such spirits intoxicate more strongly than pure spirits of the same strength, and excite, in many persons, even temporary phrensy. It is a volatile fatty matter, of a very fetid odor, when obtained by itself, as I have pro. cured it in cold weather at some of the great distilleries in Scotland. It does not combine with bases. At the end of a few months, it spontaneously decomposes in the spirits, and leaves them in a less nauseous and noxious state. By largely diluting the spirits with water, and distilling at a moderate temperature, the greater part of this oil may be separated. Part of it comes over with the strongest alcohol, and part with the latter runnings, which are called by the distillers strong and weak feints. The intermediate portion is purer spirit. The feints are always more or less opalescent, or become so on dilution with water, and then throw up an oily pellicle upon their surface. The charcoals of light wood, such as pine or willow, well calcined, and infused in sufficient quantity with the spirits prior to rectification, will deprive them of the greater part of that oily contamination. Animal charcoal, well calcined, has also been found useful; but it must be macerated for some time with the empyreumatic spirits, before distillation. Another mode of separating that offensive oil is, to agitate the impure spirits with a quantity of a fat oil, such as olive oil, or oil of almonds, to decant off the oil, ard re-distil the spirits with a little water.

Some foreign chemists direct empyreumatic or rank spirits to be rectified with the addition of chloride of lime. I have tried this method in every way, and on a considerable scale, but never found the spirits to be improved by it. Ther were rather deteriorated. See Brandy, Distillation, Fermentation, Gin, Rum, Whiskey.

Anhydrous or absolute alcohol, when swallowed, acts as a mortal poison, not only by its peculiar stimulus on the nervous system, but by its abstracting the aqueous particles from the soft tissue of the stomach, with which it comes in contact, so as to destroy its organization. Alcohol of 0.812 consists, by experiments, of 3 atoms of carbon, 6 of hydrogen, and 2 of oxygen; absolute alcohol consists, probably, of 2 of carbon, 3 of hydro gen, and 1 of oxygen.

ALE. The fermented infusion of pale malted barley, usually combined with infusion of hops. See BEER.

ALEMBIC, a STrLt; which see.

ALEMBROTH, salt of. The salt of wisdom, of the alchemists; a compound of bichloride of mercury and sal ammoniac, from which the old white precipitate of mercury is made.

ALGARO'TH, powder of. A compound of oxyde and chloride of antimony, being a precipitate obtained by pouring water into the acidulous chloride of that metal.

ALIZARINE. See MADDER.

ALKALI. A class of chemical bodies, ảistinguished chiefly by their solubility in water, and their powe: of neutralizing acids, so as to form saline compounds. The allialis of manufacturing importance are, ammonia, potash, soda, and quinia. These alkilis change the purple color of red cabbage and radishes to a green, the reddened tincture of litmus to a purple, and the color of turmeric and many other yellow dyes to a brown. Even when combined with carbonic acid, the first three alkalis exercise this diculoring power, which the allialine earths, lime, and barytes, do not. The same three allialis have an acrid, and somewhat urinous taste; the first two are energetic solvents of animal matter; and the three combine with oils, so as to form soaps. They unite with water in every proportion, and also with alcohol; and the first three combine with water after being carbonated. 
ALISALIMETER. An instrument for measuring the alkaline force or purity of any of the alkalis of commerce. It is founded on the principle, that the quantity of real alkali present in any sample, is proportional to the quantity of acid which a given weight of it can neutralize. See the individual alkalis, PотAsH and SodA.

ALKANA, is the natne of the root and leaves of Lausania inermis, which have been long employed in the East, to dye the nails, teeth, hair, garments, \&cc. The leaves, ground and mixed with a little limewater, serve for dyeing the tails of horses in Persia and Turkey.

ALKANET, the root of. (Anchusa tinetoria.) A species of bugloss, cultivated chiefly in the neighborhood of Montpellier. It affords a fine red color to alcohol and oils; but a dirty red to water. Its principal use is for coloring ointments, cheeses, and pommades. The spirituous tincture gives to white marble a beautiful deep stain.

ALLIGATION. An arithmetical formula, useful, on many occasions, for ascertaining the proportion of constituents in a mixture, when they have undergone no change of volume by chemical action. When alcoholic liquors are mixed with water, there is a condensation of bulk, which renders that arithmetical rule inapplicable. The same thing holds, in some measure, in the union of metals by fusion. See Alloy.

ALLOY. (Alliage, Fr.; Legirung, Germ.) This term formerly signified a crmpound of gold and silver, with some metal of inferior value, but it now means any compound of any two or more metals whatever. Thus, bronze is an alloy of copper and tin; brass, in alloy of copper and zine; and type metal, an alloy of lead and antimony. All the alloys possess metallic lustre, even when cut or broken to pieces; they are opaque; are excellent conductors of heat and electricity ; are frequently susceptible of crystallizing; are more or less ductile, malleable, elastic, and sonorous. An alloy which consists of metals differently fusible is usually malleable in the cold, and brittle when hot, as is exemplified with brass and gong metal.

Many alloys consist of definite or equivalent proportions of the simple component metals, though some alloys seem to form in any proportion, like combinations of salt or sugar with water. It is probable that peculiar properties belong to the equivalent or atomic ratio, as is exemplified in the superior quality of brass made in that proportion.

One metal does not alloy indifferently with every other metal, but it is governed in this respect by peculiar affinities; thus, silver will hardly unite with iron, but it combines readily with gold, copper, and lead. In comparing the alloys with their constituent metals, the following differences may be noted; in general, the ductility of the alloy is less than that of the separate metals, and sometimes in a very remarkable degree; on the contrary, the alloy is usually harder than the mean hardness of its constitnents. The mercurial alloys or amalgams are, perhaps, exceptions to this rule.

The specific grarity is rarely the mean between that of each of its constituents, but is sometimes greater and sometimes lese, indicating, in the former case, an approximation, and in the latter, a recedure, of the particles from each other in the act of their union. The following tables of binary alloys exhibit this circumstance in experimental detail :-

Alloys having a density greater than the mean of their constituents.

Gold and zinc

Gold and tin

Gold and bismuth

Gold and antimony

Gold and cobalt

Silver and zinc

Silver and lead

Silver and tin

Silver and bismuth

Silver and antimony

Copper and zinc

Copper and tin

Copper and palladium

Copper and bismuth

Lead and antimony

Platinum and molyblinum

Palladium and bismuth.
Alloys having a density less than the mean of their constituents.

Gold and silver

Gold and iron

Gold and lead

Gold and copper

Gold and iridium

Gold and nickel

Silver and copper

Silver and lead

Iron and bismuth

Iron and antimony

Iron and lead

Tin and lead

Tin and palladium

Tin and antimony

Nickel and arsenic

Zine and antimony.

It would he hardly possible to infer the melting point of an alloy from that of each of Its constituent metals; but, in general, the fusibility is increased by mutual affisity in their state of combination. Of this, a remarkable instance is afforded in the fusible metal consisting of 8 parts of bismuth, 5 of lead, and 3 of tin, which melts at the 
heat of boiling water, or $212^{\circ} \mathrm{Fahr}$, though the melting point deduced from the mean of its components should be $514^{\circ}$. This alloy may be rendered still more fusible hy adding a very little mercury to it, when it forms an excellent material for certain anatomical injections, and for filling the hollows of carious teeth. Nor do the colors of alloys depend, in any considerable degree, upon those of the separate metals; thus, the color of copper, instead of being rendered paler by a large addition of zinc, is thereby converted into the rich-looking pinchbeck metal.

By means of alloys, we multiply, as it were, the numbers of useful metals, and sometimes give usefulness to such as are separately of little value. Since these compounds can be formed only by fusion, and since many metals are apt to oxydize readily at their melting temperature, proper precautions must be taken in making alloy's to prevent this occurrence, which is incompatible with their: formation. Thus, in combining tin and lead, rosin or grease is usually put on the surface of the melting metals, the carbon produced by the decomposition of which protects them, in most cases, sufficiently from oxydizement. When we wish to combine tin with iron, as in the tinning of cast-iron tea kettles, we rub sal ammoniac upon the surfaces of the hot metals in contact with each other, and thus exclude the atmospheric oxygen by means of its fumes. When there is a notable difference in the specific gravities of the metals which we wish to combine, we often find great difficulties in obtaining homogeneous alloys; for each metal may tend to assume the level due to its density, as is remarkably exemplified in alloys of gold and silver made without adequate stirring of the melting metals. If the mass be large, and slow of cooling, after it is cast in an upright cylindrical form, the metals sometimes separate, to a certain degree, in the order of their densities. Thus, in casting large bells and cannons with copper alloys, the bottom of the casting is apt to contain too much copper and the top too much tin, unless very dexterous manipulation in mixing the fused materials have been employed immediately before the instant of pouring out the melted mass. When such inequalities are observed, the objects are broken and re-melted, after which they form a much more homogeneous alloy. This artifice of a double melting is often had recourse to, and especially in casting the alloys for the specula of telescopes.

When we wish to alloy three or more metals, we often experience difficulties, either because one of the metals is more oxydable, or denser, or more fusible, than the others, or because there is no direct affinity between two of the metals. In the latter predicament, we shall succeed better by combining the three metals, first in pairs, for example, and then melting the two pairs together. Thus, it is difficult to unite iron with bronze directly; but if, instead of iron, we use tin plate, we shall immediately succeed, and the bronze, in this manner, acquires valuable qualities from the iron. Thus, also, to render brass better adapted for certain purposes, a small quantity of lead ought to be added to it, but this cannot be done directly with advantage : it is better to melt the lead first along with the zinc, and then to add this alloy to the melting copper, or the copper to that alloy, and fuse them together.

We have said that the difference of fusibility was often an obstacle to metallic combination; but this circumstance may also be turned to advantage in decomposing certain alloys by the process called eliquation. By this means silver may be separated from copper, if a considerable quantity of lead be first alloyed with the said copper; this alloy is next exposed to a heat just sufficient to melt the lead, which then sweats out, so to speak, from the pores of the copper, and carries along with it the greater part of the silver, for which it has a strong affinity. The lead and the silver are afterwards separated from each other, in virtue of their very different oxydability, by the action of heat and air.

One of the alloys most useful to the arts is brass; it is more ductile and less easily oxydized than even its copper constituent, notwithstanding the opposite nature of the zinc. This alloy may exist in many different proportions, under which it has different names, as tombac, similor, pinchbeck, \&c. Copper and tin form, also, a compound of remarkable utility, known under the names of hard brass, for the bushes, steps, and bearings of the axles, arbors, and spindles in machinery; and of bronze, bell-metal, \&c. Gold and silver, in their pure state, are too soft and flexible to form either vessels or coins of sufficient strength and durability; but when alloyed with a little copper, they acquire the requisite hardness and stiffness for these and other purposes.

When we have occasion to unite several pieces of the same or of different metals, we employ the process called soldering, which consists in fixing together the surfaces by means of an interposed alloy, which must be necessarily more fusible than the metal or metals to be joined. That alloy must also consist of metals which possess a strong affinity for the substances to be soldered together. Hence each metal would seem to require a particular kind of solder, which is, to a certain extent, true. Thus, the solder for gold trinkets and plate is an alloy of gold and silver, or gold and copper; that of silver trinkets, is an alloy of silver and copper; that of copper is either fine tin, for pieces that must not be exposed to the fire, or a brassy alloy called hard solder, of which 
the zine forms a considerable proportion. The solder of lead and tinplate is an ailoy of lead and tin, and that of tin is the same alloy with a little bismuth. Tinnung, gilding, and silvering may also be reckoned a species of alloys, since the tin, gold, and silver are superficially united in these cases to other metals.

Metallic alloys possess usually more tenacity than could be inferred from their constituents; thus, an alloy of twelve parts of lead with one of zinc has a tenacity double that of zinc. Metallic alloys are much more easily oxydized than the separate metals, a phenomenon which may be ascribed to the increase of affinity for oxygen which results from the tendency of the one of the oxydes to combine with the other. An alloy of tin and lead heated to redness takes fire, and continues to burn for some time like a piece of bad turf.

Every alloy is, in reference to the arts and manufactures, a new metal, on account of its chemical and physical properties. A vast field here remains to be explored. Not above sixty alloys have been studied by the chemists out of many hundred which may be made; and of these very few have yet been practically employed. Very stight modifications often constitute very valuable improvements upon metallic bodies. Thus, the brass most esteemed by turners at the lathe contains from two to three per cent. of lead; but such brass does not work well under the hammer; and, reciprocally, the brass which is best under the hammer is too tough for turning.

That metallic alloys tend to be formed in definite proportions of their constituents is clear from the circumstance that the native gold of the auriferous sands is an alioy with silver, in the ratios of 1 atom of silver united to $4,5,6,12$ atoms of gold, but never with a fractional part of an atom. Also, in making an amalgam of 1 part of silver with 12 or 15 of mercury, and afterwards squeezing the mixture through chamois leather, the amalgam separates into 2 parts : one, containing a small proportion of silver and much mercury, passes through the skin; and the other, formed of 1 of silver and 8 of mercury, is a compound in definile proportions, which crystallizes readily, and remains in the knot of the bag. An analogous separation takes place in the tinning of mirrors ; for on loading them with the weights, a liquid amalgam of tin is squeezed out, while another amalgam remains in a solid form compesed of tin and mercury in uniform atomic proportions. But, as alloys are generally soluble, so to speak, in tach other, this definiteness of combination is masked and disappears in most cases.

M. Chaudet has made some experiments on the means of detecting the metals of alloys by the cupelling furnace, and they promise useful applications. The testing depends upon the appearances exhibited by the metals and their alloys when heated on a cupel. Pure tin, when heated this way, fuses, becomes of a grayish black color, fumes a little, exnibits incandescent points on its surface, and leaves an oxyde, which, when withdrawn from the fire, is at first lemon-yellow, but when cold, white. Antimony meilts, preserves its brilliancy, fumes, and leaves the vessel colored lemon-yellow when hot, but colorless when coid, except a few spots of a rose tint. Zinc burns brilliantly, forming a cone of oxyde; and the oxyde, much increased in volume, is, when hot, greenish, but when cold, perfectly white. Bismuth fumes, becomes covered with a coat of melted oxyde, part of which sublimes, and the rest enters the pores of the cupel; when cold, the cupel is of a fine yellow color, with spots of a greenish hue. Lead resembles bismuth very much; the cold cupel is of a lemon-yellow color. Copper melts, and becomes eovered with a coat of black oxyde; sometimes spots of a rose tint remain on the cupel.

Alloys.-Tin 75, antimony 25, melt, become covered with a coat of black oxyde, have very few incandescent points; when cold, the oxyde is nearly black, in consequence of the action of the antimony : a $\frac{1}{400}$ part of antimony may be ascertained in this way in the alloy. An alloy of antimony, containing tin, leaves oxyde of tin in the cupel : a $\frac{1}{100}$ part of tin may be detected in this way. An alloy of tin and zinc gives an oxyde which, while hot, is of a green tint, and resembles philosophic wool in appearance. An alloy containing 99 tin, 1 zine, did not present the incandescent points of pure tin, and gave an oxyde of greenish tint when cold. Tin 95, bismuth 5 parts, gave an oxyde of a gray color. Tin and lead give an oxyde of a rusty brown color. An alloy of lead and tin, containing only 1 per cent. of the latter metal, when heated, does not expose a clean surface, like lead, but is covered at times with oxyde of tin. Tin 75, and copper 25 , did not melt, gave a black oxyde : if the heat be much elevated, the under part of the oxyde is white, and is oxyde of tin; the upper is black, and comes from the copper. The cupel becomes of a rose color. If the tin be impure from iron, the oxyde produced by it is marked with spots of a rust color.

The degree of affinity between metals may be in some measure estimated by the greater or less facility with which, when of different degrees of fusibility or volatility, they unite, or with which they can, after union, be separated by heat. The greater or less tendency to separate into differently proportioned alloys, by long-continued fusion, may also give some information upon the subject. Mr. Hatchett remarked, in his 
elaborute researches on metallic alloys, that gold made standard with the usual precau tions, by silver, copper, lead, antimony, \&c., and then cast, after long fusion, intu vertical bars, was by no means a uniform compound; but that the top of the bar, corresponding to the metal at the bottom of the crucible, contained the larger proportion of gold. Hence, for a more thorough combination, two red-hot crucibles should be employed, and the liquefied metals should be alternately poured from the one into the other. To prevent unnecessary oxydizement from the air, the crucibles should contain, besides the metal, a mixture of common salt and pounded charcoal. The metallic alloy should also be occasionally stirred up with a rod of pottery ware.

The most direct evidence of a chemical change having been effected in alloys is, when the compound melts at a lower temperature than the mean of its ingredients. Iron, which is nearly infusible, acquires almost the fusibility of gold when alloved with this precious metal. The analogy is here strong with the increase of solubility which salts acquire by mixture, as is exemplified in the difficulty of crystallizing residuums of saline solutions, or mother waters, as they are called.

In common cases the specific gravity affords a good criterion whereby to judge of the proportion of two metals in an alloy. But a very fallacious rule has been given in some respectable works for computing the specific gravity that should result from the alloying of given quantities of two metals of known densities, supposing no chemical condensation or expansion of volume to take place. Thus, it has been taught, that if gold and copper be united in equal weights, the computed specific gravity is merely the arithmetical mean between the numbers denoting the two specific gravities. Whereas the specific gravity of any alloy must be computed by dividing the sum of the two weights by the sum of the two volumes, compared, for convenience sake, to water reckoned unity. Or, in another form, the rule may be stated thus:-Multiply the sum of the weights into the products of the two specific-gravity numbers for a numerator; and multiply each specific gravity-number into the weight of the other body, and add the two products together for a denominator. The quotient obtained by dividing the said numerator by the denominator, is the truly computed mean specific gravity of the alloy. On comparing with that density, the density found by experiment, we shall see whether expansion or condensation of volume has attended the metallic combination. Gold having a specific gravity of $19 \cdot 36$, and copper of $8 \cdot 87$, when they are alloyed in equal weights, give, by the fallacious rule of the arithmetical mean of the densities $\frac{19 \cdot 36+8 \cdot 87}{2}=14 \cdot 11$;

whereas the rightly computed mean density is only $12 \cdot 16$. It is evident that, on comparing the first result with experiment, we should be led to infer that there had been a prodigious condensation of volume, though expansion has actuaily taken place. Let W, w be the two weights; $P$, p the two specific gravities, then $\mathbf{M}$, the mean specific gravity, is given by the formula-

$$
\mathrm{M}=\frac{(\mathrm{W}+\mathrm{w}) \mathrm{Pp}}{\mathrm{Pw}+\mathrm{pW}}:: 2 \triangle=-\frac{(\mathrm{P}-\mathrm{p})^{2}}{\mathrm{P}+\mathrm{p}}=\text { twice }
$$

the error of the arithmetical mean; which is therefore always in excess.

ALMOND. (Amande, Fr.; Mundel, Germ.) There are two kinds of almond which do not differ in chemical composition, only that the bitter, by some mysterious re-action of its constituents, generates in the act of distillation a quantity of a volatile oil, which contains hydrocyanic acid. Vogel obtained from bitter almonds 8.5 per cent. of husks. After pounding the kernels, and heating them to coagulate the albumen, he procured, by expression, 28 parts of an unctuous oil, which did not contain the smallest particle of hydrocyanic acid. The whole of the oil could not be extracted in this way. The expressed mass, treated with boiling water, afforded sugar and gum, and, in consequence of the heat, some of that acid. The sugar constitutes 6.5 per cent. and the gum 3 . The vegetable albumen extracted, by means of caustic potash, amountea to 30 parts: the vegetable fibre to only 5. The poisonous aromatic oil, according to Robiquet and Boutrin-Charlard, does not exist reaciy-formed in the bitter almond, but seems to be produced under the influence of ebullition with water. These chemists have shu wn that bitter almonds deprived of their unctuous oil by the press, when treated first by alcohol, and then by water, aftord to neither of these liquids any volatile oil. But alcohol dissolves out a peculiar white crystalline body, without smell, of a sweetish taste at first, and afterwards bitter, to which they gave the name of amygdaline. This substance does not seem convertible into volatile oil.

Sweet almonds, by the analysis of Boullay, consist of 54 parts of the bland almond oil, 6 of uncrysiallizable sugar, 3 of gum, 24 of vegetable albumen, 24 of woody fibre, 5 of huskis, 3.5 of water, 0.5 of acetic acid, including loss. We thus see that sweet almonds contain nearly twice as much oil as bitter almonds do.

AT.MOND OIL. A bland fixed oil, obtained usually from bitter almonds by the ac tion of a hydraulic press, either in the cold, or aided by hot iron plates. See Orr. 
ALOE. A series of trials has been made within a few years at Paris to ascertain the comparative strength of cables made of hemp and of the aloe from Algiers; and they are said to have all turned to the advantage of the aloe. Of cables of equal size, that made of aloe raised a weight of 2,000 kilogrammes ( 2 tons nearly); that made of heinp, a weisht of only 400 kilogrammes. At the exposition of ohjects of national industry, two years ago, in Brussels, I saw aloe cordage placarded, as being far preferable to hempen. See Rork.

ALUDEL. A pear-shaped vessel open at either end, of which a series are joined for distilling mercury in Spain. See MEkcory.

ALUM. (Alun, Fr.; Alaum, Germ.) A saline body, consisting of the earth of clay, called alumina by the chemists, combined with sulphuric acid and potash, or sulphuric acid and ammonia, into a triple compound. It occurs in the crystallized form of octahedrons, has an acerb subacid taste, and reddens the blue color of litmus or red cabbage.

Alum worlis existed many centuries ago at Roccha, formerly called Edessa, in Syria, whence the ancient name of Roch alum given to this salt. It was afterwards made at Foya Nova, near Smyrna, and in the neighborhood of Constantinople. The Genoese, and other trading people of Italy, imported alum from these places into western Europe, for the use of the dyers of red cloth. About the middle of the fifteenth century, alum began to be manufactured at La Tolfa, Viterbo, and Volaterra, in Italy; after which time the importation of oriental alun was prohibited by the pope, as detrimental to the interests of his dominions. The manufacture of this salt was extended to Germany at the beginning of the sixteenth century, and to England at a somewhat later period, by Sir Thomas Chaloner, in the reign of Elizabeth. In its pure state, it does not seem to have been known to the ancients; for Pliny, in speaking of something like plumose alum, says, that it struck a black color with pomegranate juice, which shows that the green vitriol was not separated from it. The stypteria of Dioscorides, and the alumen of Pliny, comprehended, apparently, a variety of saline substances, of which sulphate of Iron, as well as alumina, was probably a constituent part. Pliny, indeed, sars, that a substance called in Greek ' $Y_{\gamma} \sigma a$, or watery, probably from its very soluble nature, which was milk-white, was used for dyeing wool of bright colors. This may have been the mountain butter of the German mineralogists, which is a native sulphate of alumina, of a soft texture, waxy lustre, and unctuous to the touch.

The only alum manufactories now worked in Great Britain, are those of Whitby, in England, and of Hurlett and Campsie, near Glasgow, in Scotland; ana these derive the acid and earthy constituents of the salt from a mineral called alum slate. This mineral has a blueish or greenish-black color, emits sulphurous fumes when heated, and acquires thereby an aluminous taste. The alum manufactured in Great Britain contains potash as its allialine constituent; that made in France contains, commonly, ammonia, either alone, or with variable quantities of potash. Alum may in general be examined by water of ammonia, which separates from its watery solutions its earthy basis, in the form of a light flocculent precipitate. If the solution be dilute, this precipitate will float long as an opalescent cloud.

If we dissolve alum in 20 parts of water, and drop this solution slowly into water or caustic ammonia till this be nearly, but not entirely, saturated, a bulky white precipitate will fall down, which, when properly washed with water, is pure aluminous earth or clay, and dried forms 10.82 per cent. of the weight of the alum. If this earth, while still moist, be dissolved in dilute sulphuric acid, it will constitute, when as neutral as possible, the sulphate of alumina, which requires only two parts of cold water for its solution. If we now decompose this solution, by pouring, into it water of ammonia, there appears an insoluble white powder, which is subsulphate of alumina, or basic alum; and contains three times as much earth as exists in the neutral sulphate. If, however, we pour into the solution of the neutral sulphate of alumina a solution of sulphate of potash, a white powder will fall if the solutions be concentrated, which is true alum ; but if the solutions be dilute, by evaporating their mixture, and cooling it, crystals of alum will be obtained.

When newly precipitated alumina is boiled in a solution of alum, a portion of the edrth enters into combination with the salt, constituting an insoluble compound, which falls in the form of a white powder. The same combination takes place. if we decompose a boiling hot solution of alum with a solution of potash, till the mixture appears nearly neutral by litmus paper. This insoluble or basic alum exists native in the alum-stone of Tolía. near Civita Vecchia, and it consists in 100 parts of $19 \cdot 72$ parts of sulphate of potash, 61.99 basic sulphate of alumina, and 18.29 water. When this mineral is treated with a due quantity of sulphuric acid, it dissolves, and is converted into the crystallizable alum of commerce.

These experimental facts develop the principles of the manufacture of alum, which is prosecuted under various modifications, for its important uses in the arts. Alum seldom occurs ready-formed in nature; occasionaliy, as an efflorescence on stones, and in 
certain mineral waters in the East Indies. The alum of European commerce is fabricated artificially, either from the alum schists or stones, or from clay. The mode of manufacture differs according to the nature of these earthy compounds. Some of them, such as the alum-stone, contain all the elements of the salt, but mixed with other matters from which it must be freed. The schists contain only the elements of two of the constituents, namely, clay and sulphur, which are convertible into sulphate of alumina, and this may be then made into alum by adding the alkaline ingredient. To this class belong the alum s.ates, and other analogous schists, containing brown coal.

1. Manufacture of Alum from the Alum Stone.-The alum-stone is a rare mineral, being found in moderate quantity at Tolfa, and in larger in Hungary, at Bereghszasz, and Muszag, where it forms entire beds in a hard substance, partly characterized by numerous cavities, containing drusy crystallizations of alum-stone or basic alum. The larger lumps contain more or fewer flints disseminated through them, and are, according to their quality, either picked out to make alum, or are thrown away. The sorted pieces are roasted or calcined, by which operation apparently the hydrate of alumina, associated with the sulphate of alumina, loses its water, and, as burnt clay, loses its affinity for alum. It becomes, therefore, free; and during the subsequent exposure to the weather the stone gets disintegrated, and the alum becomes soluble in water.

The calcination is performed in common lime-kilns in the ordinary way. In the regulation of the fire it is requisite, here, as with gypsum, to prevent any fusion or running together of the stones, or even any disengagement of sulphuric or sulphurous acids, which would cause a corresponding defalcation in the product of alum. For this reason the contact of the ignited stones with carbonaceous matter ought to be avoided.

The calcined alum-stones, piled in heaps from 2 to 3 feet high, are to be exposed to the weather, and meanwhile they must be continually kept moist by sprinkling them with water. As the water combines with the alum the stones crumble down, and fall, eventually, into a pasty mass, which must be lixiviated with warm water, and allowed to settle in a large cistern. The clear supernatant liquor, being drawn off, must be evaporated, and then crystallized. A second crystallization finishes the process, and furnishes a marketable alum. Thus the Roman alum is made, which is covered with a fine red film of peroxyde of iron.

2. Alum Manufaclure from Alum Schist.-The greater portion of the alum found in British commerce is made from alum-slate and analogous minerals. This slate contains more or less iron pyrites, mixed with coaly or bituminous matter, which is occasionally so abundant as to render them somewhat combustible. In the strata of brown coal and bituminous wood, where the upper layers lie immediately under clay beds, they consist of the coaly substance rendered impure with clay and pyrites. This triple mixture constitutes the essence of all good alum schists, and it operates spontaneously towards the production of sulphate of alumina. The coal serves to make the texture open, and to allow the air and moisture to penetrate freely, and to change the sulphur and iron present into acid and oxyde. When these schists are exposed to a high temperature in contact with air, the pyrites loses one half of its sulphur, in the form of sublimed sulphur or sulphurous acid, and becomes a black sulphuret of iron, which speedily attracts oxygen, and changes to sulphate of iron, or green vitriol. The brown coal schists contain, commonly, some green vitriol crystals, spontaneously formed in them. The sulphate of iron transfers its acid to the clay, progressively, as the iron, by the action of the air with a little elevation of temperature, becomes peroxydized; whereby sulphate of alumina is produced. A portion of the green vitriol remains, however, undecomposed, and so much the more as there may happen to be less of other salifiable bases present in the clay slate. Should a little magnesia or lime be present, the vitriol gets more completely decomposed, and a portion of Epsom salt and gypsum is produced.

The manufacture of alum from alum schists may be distributed under the six following heads :-1. The preparation of the alum slate. 2. The lixiviation of the slate. 3 . The evaporation of the lixivium. 4. The addition of the saline ingredients, or the precipitation of the alum. 5. The washing of the aluminous salts; and, 6. The crys tallization.

1. Preparation of the Alum Slate.-Some alum slates are of such a nature that, being piled in heaps in the open air, and moistened from time to time, they get spontaneously hot, and by degrees fall into a pulverulent mass, ready to be lixiviated. The greater part, however, require the process of ustulation, from which they derive many advantages. The cohesion of the dense slates is thereby so much impaired that their decomposition becomes more rapid; the decomposition of the pyrites is quickened by the expulsion of a portion of the sulphur; and the ready-formed green vitriol is partly decomposed by the heat, with a transference of its sulphuric acid to the clay, and the production of sulphate of alumina.

Such alrum-slates as contain too little bitumen or coal for the roasting process must be mterstratified with layers of small coal or brushwood over an extensive surface. At 
Whitby the alum rock, broken into small pieces, is laid upon a horizontal bed of fuel. composed of brushwood; but at Hurlett small coal is chiefly used for the lover bed. When about four feet of the rock is piled on, fire is set to the bottom in various parts; and whenever the mass is fairly kindled, more rock is placed over the top. At Whitby this pling process is continued till the calcining heap is raised to the height of 90 or 100 feet. The horizontal area is also augmented at the same time till it forms a great bed nearly 200 feet square, having therefore aboat 100,000 yards of solid measurement. The rapidity of the combustion is tempered by plastering up the crevices with small schist moistened. When such an immense mass is inflamed, the heat is sure to rise too high, and an immense waste of sulphur and sulphuric acid must ensue. This evil has been noticed at the Whitby works. At Hurlett the height to which the heap is piled is only a few feet, while the horizontal area is expanded; which is a much more judicious arrangement. At Whitby 130 tons of calcined schist produce on an average 1 ton of alum. In this humid climate it would be advisable to pile up on the top of the horizontal strata of brushwood or coal, and schist, a pyramidal mass of schist, which having its surface plastered smooth, with only a few air-holes, will protect the mass from the rains, and at the same time prevent the combustion from becoming too vehement. Should heavy rains supervene, a gutter must be scooped out round the pile for receiving the aluminous lixi= vium, and conducting it into the reservoir.

It may be observed, that certain alum schists contain abundance of combustible matter, to keep up a suitable calcining heat after the fire is once kindled; and therefore nothing is needed but the first layer of brushwood, which, in this case, may be laid over the first bed of the bituminous schist.

A continual, but very slow heat, with a smothered fire, is most beneficial for the ustulation of alum slate. When the fire is too brisk, the sulphuret of iron may run with the earthy matters into a species of slag, or the sulphur will be dissipated in vapor, by both of which accidents the product of alum will be impaired. Those bituminous alum schists which have been used as fuel under steam boilers have suffered such a violent combustion that their ashes yield almost no alum. Even the best regulated calcining piles are apt to burn too briskly in high winds, and should have their draughtholes carefully stopped under such circumstances. It may be laid down as a general rule, that the slower the combustion the richer the roasted ore will be in sulphate of alumina. When the calcination is complete, the heap diminishes to one half its original bulk; it is covered with a light reddish ash, and is open and porous in the interior, so that the air can circulate freely throughout the mass. To favor this access of air, the masses should not be too lofty; and in dry weather a little water should be occasionally sprinkled on them, which, by dissolving away some of the saline matter, will make the interior more open to the atmosphere.

When the calcined mineral becomes thoroughly cold, we may proceed to the lixiviation. But as, from the first construction of the piles or beds till their complete calcination, many weeks, or even months, may elapse, care ought to be taken to provide a suffieient number or extent of them, so as to have an adequate supply of material for carrying on the lixiviating and crystallizing processes during the course of the year, or at least during the severity of the winter season, when the calcination may be suspended, and the lixiviation becomes unsatisfactory. The beds are known to be sufficiently decomposed by the efflorescence of the salt which appears upon the stones, from the strong aluminous taste of the ashes, and from the appropriate chemical test of lixiviating an aliquot average portion of the mass, and seeing how much alum it will yield to solution of muriate or suiphate of potash.

2. The Lixiviation.-The lixiviation is best performed in stone-built cisterns; those of wood, however strong at first, are soon decomposed, and need repairs. They onght to be erected in the neighborhood of the calcining heaps, to save the labor of transport, and so ar. ranged that the solutions from the higher cisterns may spontaneously flow into the lower In this point of view, a sloping terrace is the best situation for an alum work. In the lowest part of this terrace, and in the neighborhood of the boiling-house, there ought to be two or more large deep tanks, for holding the crude lixivium, and they should be protected from the rain by a proper shed. Upon a somewhat higher level the cisterns of the clear lixivium may be placed. Into the highest range of cisterns the calcined mineral is to be put, taking care to lay the largest lumps at the bottom, and to cover them with lighter ashes. A sufficient quantity of water is now to be run over it, and allowed to rest for some time. The lixivium may then be drawn off, by a stopcock connected with a pipe at the bottom of the cistern, and run into another cistern at a somewhat lower level. Fresh water must now be poured on the partly exhausted schist, and allowed to remain for a sufficient times This lixivium, being weak, should be run off into a separate tank. In some cases a third addition of fresh water may be requisite, and the weak lixivium which is drawn off may be reserved for a fiesh pottion of calcined mineral. In order to save evaporation, it is always requisite to strengthen weals 
leys by employing them instead of water for fresh portions of ealcined schist. Upon the ingenious disposition and form of these lixiviating cisterns much of the economy and suc. cess of an alum work depend. The hydrometer should be always used to determine the degree of concentration which the solutions acquire.

The lixiviated stone, being thus exhausted of its soluble ingredients, is to be removed from the cisterns, and piled up in a heap in any convenient place, where it may be left either spontaneously to decompose, or, after drying, may be subjected to another calcination.

The density of the solution may be brought, upon an average, up to the sp. gr. of from 1.09 to $1 \cdot 15$. The latter density may always be obtained by pumping up the weaker solutions upon fresh calcined mine. This strong liquor is then drawn off, when the sulphate of lime, the oxyde of iron, and the earths are deposited. It is of advantage to leave the liquor exposed for some time, whereby the green vitriol may pass into a persulphate of iron with the deposition of some oxyde, while the liberated acid may combine with some of the clay present, so as to increase the quantity of sulphate of alumina. The manufacture of alum is the more imperfect, as the quantity of sulphate of iron left undecomposed is greater, and therefore every expedient ought to be tried to convert the sulphate of iron into sulphate of alumina.

3. The evaporation of the Schist Lixivium.-As the aluminous liquors, however well settled at first, are apt, on the great scale, to deposite earthy matters in the course of their concentration by heat, they are best evaporated by a surface fire, such as that employed at Hurlett and Campsie. A water-tight stone cistern must be built, having a layer of well rammed clay behind the flags or tiles which line its bottom and sides. This cistern may be 4 - or 6 feet wide, 2 or 3 feet deep, and 30 or 40 feet long, and it is covered in by an arch of stone or brickwork. At one extremity of this tunnel, or covered canal, a fire-grate is set, and at the other a lofty chimney is erected. The cistern being filled to the brim with the alum ley, a strong fire is kindied in the reverberatory grate, and the flame and hot air are forced to sweep along the surface of the liquor, so as to keep it in constant ebullition, and to carry off the aqueous parts in rapor. The soot which is condensed in the process falls to the bottum, and leaves the body of the liquor clear. As the concentration goes on, more of the rough lixivium is run in from the settling cistern, placed on a somewhat higher level, till the whole gets charged with a clear liquor of a specific gravity sufficiently high for transferring into the proper lead boilers.

At Whitb;, the lead pans are 10 feet long, 4 feet 9 inches wide, 2 feet 2 inches deep at the one end, and 2 feet 8 inches deep at the other. This increase of depth and corresponding slope facilitates the decantation of the concentrated lixivium by means of a syphon, applied at the lower end. The bottom of the pan is supported by a series of parallel iron bars, placed very near each other. In these lead pans the liquor is concentrated, at a brisk boiling heat, by means of the flame of a flue beneath them. Every morning the pans are emptied into a settling cistern of stone or lead. The specific gravity of the liquor should be about 1.4 or 1.5 , being a saturated solution of the saline matters present. The proper degree of density must vary, however, with different linds of lixivia, and according to the different views of the manufacturer. For a liquor which consists of two parts of sulphate of alumina, and one part of sulphate of iron, a specifie gravity of 1.25 may be sufficient; but for a solution which contains two parts of sulphate of iron to one of sulphate of alumina, so that the green vitriol must be withdrawn first of all by erystallization, a specific yravity of 1.4 may be requisite.

The construction of an evaporating furnace well adapted to the concentration of aluminous and other crude lixivia, is described under SoDA. The liquor basin may be made of tiles or flags puddled in clay, and secured at the seams with a good hydraulic cement. A mortar made of quicklime mixed with the exhausted schist in powder, and iron turnings, is said to answer well for this purpose. Sometimes over the reverberatory furnace a flat, pan is laid, instead of the arched top, into which the crude liquor is put for neutralization and partial concentration. In Germany, such a pan is made of copper, because iron would waste too fast, and lead would be apt to melt. From this preparation basin the under evaporating trough is gradually supplied with hot liquor. At one side of this lower trough there is sometimes a door, through which the sediment may be ralied out as it accumulates upon the bottom. Such a contrivance is convenient for this mode of evaporation, and it permits, also, any repairs to be readily made; but, indeed, an apparatus of this kind, well mounted at first, will serve for many years.

In the course of the final concentration of the liquors, it is customary to add some of the mother waters of a former process, the quantity of which must be regulated by a proper analysis and knowledge of their contents. If these mother waters contain much free sulphuric acid, from the peroxydation of their sulphate of iron, they may prove useful in dissolving a portion of the alumina of the sediment which is always present in greated or less quantity. 
4. The precipitation of the Alum by adding Alkaline Salts.-As a general rule, it is most advantageous to separate, first of all, from the concentrated clear liquors, the alum in the state of powder or small crystals, by addition of the proper alkaline matter, and to leave the mingled foreign salts, such as the sulphate of iron or magnesia, in solution, instead of trving to abstract these salts by a previous crystallization. In this way we not only simplify and accelerate the manufacture of alum, and leave the mother waters to be worked up at any convenient season, but we also avoid the risk of withdrawing any of the sulphate of alumina with the sulphate of iron or magnesia. On this account, the concentration of the liquor ought not to be pushed so far as that, when it gets cold, it shonld throw out crystals, but merely to the verge of this point. This density may be determined by suitable experiments.

The clear liquor should now be run off into the precipitation cistern, and have the proper quantity of sulphate or muriate of potash, or impure sulphate or carbonate of ammonia added to it. The sulphate of potash, which is the best precipitant, forms $18 \cdot 34$ parts out of 100 of crystallized alum; and therefore that quantity of it, or its equivalent in muriate of potash, or other potash or ammoniacal salts, must be introduced into the aluminous liquor. Since sulphate of potash takes 10 parts of cold water to dissolve it, but is much more soluble in boiling water, and since the precipitation of alum is more abundant the more concentrated the mingled solutions are, it would be prudent to add the sulphate solution as hot as may be convenient; but, as muriate of potash is fully three times more soluble in cold water, it is to be preferred as a precipitant, when it can be procured at a cheap rate. It has, also, the advantage of decomposing the sulphate of iron present into a muriate, a salt very difficult of erystallization, and, therefore, less apt to contaminate the crystals of alum. The quantity of alkaline salts requisite to precipitate the alum, in a granular powder, from the lixivium, depends on their richness in potash or ammonia, on the one hand, and on the richness of the liquors in sulphate of alumina on the other; and it must be ascertained, for each large quantity of product, by a preliminary experiment in a precipitation glass. Here, an aliquot neasure of the aluminous liquor being taken, the liquid precipitant must be added in successive portions, as long as it causes any cloud, when the quantity added will be indicated by the graduation of the vessel. A very exact approximation is not practicable upon the great scale; but, as the mother waters are afterwards mixed together in one cistern, any excess of the precipitant, at one time, is corrected by excess of aluminous sulphate at another, and the resulting alum meal is collected at the bottom. When the precipitated saline powder is thoroughly settled and cooled, the supernatant mother water must be drawn off by a pump, or rather a syphon or stopcock, into a lower cistern. The more completely this drainage is effected, the more easily and completely will the alum be purified.

This mother liquor has, generally, a specific gravity of 1.4 at a medium temperature of the atmosphere, and consists of a saturated solution of sulphate or muriate of black and red oxyde of iron, with sulphate of magnesia, in certain localities, and muriate of soda, when the soaper's salt has been used as a precinitant, as also a saturated solution of sulphate of alumina. By adding some of it, from time to time, to the fresh lixivia, a portion of that sulphate is converted into alum; but, eventually, the mother water must be evaporated, so as to obtain from it a crop of ferruginous crrstals; after which it becomes capable, once more, of giving up its alum to the alkaline precipitants.

When the aluminous lixivia contain a great deal of sulphate of iron, it may be good policy to withdraw a portion of it by crystallization before precipitating the alum. With this view, the liquors must be evaporated to the density of $1 \cdot 4$, and then run off into crystallizing stone cisterns. After the green vitriol has concreted, the liquor should be pumped back into the evaporating pan, and again brought to the density of $1: 4$. On adding to it, now, the alkaline precipitants, the alum will fall down from this concentrated solution, in a very minute crystalline powder, very easy to wash and purify. But this method requires more vessels and manipulation than the preceding, and should only be had recourse to from necessity; since it compels us to carry on the manufacture of both the raluable alum and the lower priced salts at the same time; moreover, the ennperas extracted at first from the schist liquors carries with it, as we have said, a portior of the sulphate of alumina, and acquires thereby a dull aspect; whereas the copperas obtained after the separation of the alum is of a brilliant appearance.

5. The washing, or edulcoration, of the Alum Powder.-This crystalline pulverulent matter has a brownish color, from the admixture of the ferruginous liquors; but it may be freed from it by washing with very cold water, which dissolves not more than one sixteenth of its weight of alum. After stirring the powder and the water well together, the former must be allowea to settle, and then the washing must be drawn uff. A second washing will render the alum nearly pure. The less water is employed, and the more effectually it is drained off, the more complete is the process. The second water may be used in the first washing of another portion of 
alum powder, in the place of pure water. These washings may be added to the schist lixivia.

6. The crystallization.-The washed alum is put into a lead pan, with just enough water to dissolve it at a boiling heat; fire is applied, and the solution is promoled by stirring. Whenever it is dissolved in a saturated state, it is run off into the crystallizing vessels, which are called roching casks. These casks are about five feet high, three feet wide in the middle, somewhat narrower at the ends; they are made of very strong staves, nicely fitted to each other, and held together by strong iron hoops, which are driven on pro lempare, so that they may be easily knocked off again, in order to take the stares asunder. The concentrated solution, during its slow cooling in these close vessels, forms large regular crystals, which hang down from the top, and project from the sides, while a thick layer or cake lines the whole interior of the cask. At the end of eight or ten days more or less, according to the weather, the hoops and staves are removed, when a cask' of apparently solid alum is disclosed to view. The workman now pierces this mass with a pickaxe at the side near the bottom, and allows the mother water of the interior to run off on the sloping stone floor into a proper cistern, whence it is taken and added to another quantity of washed powder to be crystallized with it. The alum is next broken into lumps, exposed in a proper place to dry, and is then put into the finished bing for the market. There is sometimes a little insoluble basic alum (subsulphate) left at the bottom of the cask. This being mixed with the former mother liquors, gets sulphuric acid from them; or, being mixed with a little sulphuric acid, it is equally converted into alum.

When, instead of potash or its salts, the ammoniacal salts are used, or putrid urine, with the aluminous lixivia, ammoniacal alum is produced, which is perfectly similar to the potash alum in its appearance and properties. At a gentle heat both lose their water of erystallization, amounting to $45 \frac{1}{2}$ per cent. for the potash alum, and 48 for the ammoniacal. The quantity of acid is the same in both, as, also, very nearly the quantity of alumina, as the following analyses will show :-

Potash alum.
Sulphate of potash -
Sulphate of alumina
Water - $-18 \cdot 34$

Or otherwise, Potash alum. 1 atom sulphate of potash $-1089 \cdot 07$ 1 24 water $\begin{array}{r}\text { alumina } \\ -\quad-2149 \cdot 80 \\ \hline 5938 \cdot 39 \\ \hline\end{array}$

\begin{tabular}{|c|c|c|c|}
\hline \multicolumn{4}{|c|}{ Ammonia alum. } \\
\hline Sulphate & of ammonia & & - 12.88 \\
\hline Sulphate & of alumina & - & $38 \cdot 64$ \\
\hline Water & - & - & $-48 \cdot 48$ \\
\hline & & & $100 \cdot 00$ \\
\hline
\end{tabular}

Ammonia alum. 1 atom sulphate of ammonia $\quad-716 \cdot 7$ 1 alumina $-2149 \cdot 8$ 24 water - - $\quad-\quad-2699 \cdot 5$ $5566 \cdot 0$

\begin{tabular}{|c|c|c|c|c|c|}
\hline & $\mathrm{r}, \mathrm{I}$ & tas & & & \\
\hline Alumina & - & - & - & & $10 \cdot 82$ \\
\hline Potash & - & - & - & - & $9 \cdot 94$ \\
\hline Sulphuric aci & & - & - & 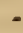 & $33 \cdot 77$ \\
\hline Water - & - & - & - & - & $45 \cdot 47$ \\
\hline
\end{tabular}

When heated pretty strongly, the ammoniacal alum loses its sulphuric acid and ammonia, and only the earth remains. This is a very convenient process for orocuring pure alumina. Ammoniacal alum is easily distinguished from the other by the smell of ammonia which it exhales when triturated with quicklime. The Roman alum, made from alum-stone, possesses most of the properties of the schist-made alums, but it has a few peculiar characters : it crystallizes always in opaque cubes, whereas the common alum erystallizes in transparent octahedrons. It is probable that Roman alum is a sulphate of alumina and potash, with a slight excess of the earthly ingredient. It is permanent when dissolved in cold water; for after a slow evaporation it is recovered in 2 cubical form. But when it is dissolved in water heated to $110^{\circ}$ Fahr. and upwards, of when its solution is heated above this pitch, subsulphate of alumina falls, and on evaporation octahedral crystals of common alum are obtained. The exact composinon of the Roman alum has not been determined, as far as I know. It probably differs from the other also in its water of crystallization. The Roman alum contains, according to MM. Thenard and Roard, only $\frac{1}{220}$ of sulphate of iron, while the common commercial 
alums contain $\frac{1}{1000}$. It may be easily purified by solution, granulation, crystallization, and washing, as has been aiready explained.

Alum is made extensively in France from an artificial sulphate of alumina. For this purpose clays are chosen as free as possible from carbonate of lime and oxyde of iron. They are calcined in a reverberatory furnace, in order to expel the water, to peroxydize the iron, and to render the alumina more easily acted on by the acid. The expulsion of the water renders the clay porous and capable of absorbing the sulphuric acid by capillary attraction. The peroxydation of the iron renders it less soluble in the sulphuric acid; and the silica of the clay, by reacting on the alumina, impairs its aggregation, and makes it more readily attracted by the acid. The clay shonld, therefore, be moderately calcined; but not so as to indurate it like pottery ware, for it would then suffer a species of silicious combination which would make it resist the action of acids. The clay is usually calcined in a reverberatory furnace, the flame of which serves thereafter to heat two evaporating pans and a basin for containing a mixture of the calcined clay and sulphuric acid. As soon as the clay has become friable in the furnace it is taken out, reduced to powder, and passed through a fine sieve. With 100 parts of the pulverized clay, 45 parts of sulphuric acid, of sp. gr. 1.45 , are well mixed, in a stone basin, arched over with brickwork. The flame and hot air of a reverberatory furnace are made to play along the mixture, in the same way as described for evaporating the schist liquors. See Soda. The mixture, being stirred from time to time, is, at the end of a few days, to be raked out, and to be set aside in a warm place, for the acid to work on the clay, during six or eight weeks. At the end of this time it must be washed, to extract the sulphate of alumina. With this view, it may be treated like the roasted alum ores above described. If potash alum is to be formed, this sulphate of alumina is evaporated to the specific gravity of $1 \cdot 38$; but if ammonia alum, to the specific gravity of only $1 \cdot 24$; because the sulphate of ammonia, being soluble in twice its weight of water, will cause a precipitation of pulverulent alum from a weaker solution of sulphate of alumina than the less soluble sulphate of potash could do.

The alum stone, from which the Roman alum is made, contains potash. The following analysis of alunite, by M. Cordier, places this fact in a clear light :-

$\begin{array}{llllll}\text { Sulphate of potash } & - & - & - & - & 18.53 \\ \text { Sulphate of alumina } & - & - & - & - & 38.50 \\ \text { Hydrate of alumina } & - & - & - & - & 42.97 \\ & & & & & 100 \cdot 00\end{array}$

To transform this compound into alum, it is merely necessary to abstract the hydrate of alumina. The ordinary alum stone, however, is rarely so pure as the above analysis would seem to show; for it contains a mixture of other substances; and the above are in different proportions.

Alum is very extensively employed in the arts, most particularly in dyeing, lake making, dressing sheep-skins, pasting paper, in clarifying liquors, \&c. Its purity for the dyer may be tested by prussiate of potash, which will give solution of alum a blue tint in a few minutes if it contain even a very minute portion of iron. A bit of nut-gall is also a good test of iron.

AMADOU. The French name of the spongy combustible substance, called in German zundersehwamm, prepared from a species of agaric, the boletus igniarius, a kind of mushroom, which grows on the trunks of old oaks, ashes, beeches, \&c. It must be plucked in the months of August and September. It is prepared by removing the outer bark with a knife, and separating carefully the spongy substance of a yellow brown color, which lies within it, from the ligneous matter below. This substance is cut into thin slices, and beat with a mallet to soften it, till it can be easily pulled asunder between the fingers. In this state the boletus is a valuable substance for stopping oozing hemorrhages, and some other surgical purposes. To convert it into tinder it must receive a finishing preparation, which consists in boiling it in a strong solution of nitre; drying it, beating it anew, and putting it a second time into the solution. Sometimes, indeed, to render it very inflammable, it is imbued with gunpowder, whence the distinction of black and brown amadou.

AL the puff balls of the lycopodium genus of plants, which have a fleshy or filamentous structure, yield a tinder quite ready for soaking in gunpowder water. The Hindoos employ a leguminous plant, which they call solu, for the same purpose. Its thick spongy stem, being reduced to charcoal, takes fire like amadou.

AMALGAM. When mercury is alloyed with any metal, the compound is called an amalgam of that metal; as, for example, an amalgam of tin, bismuth, \&c.

AMALGAMATION. This is a process used extensively in extracting silver and gold from certain of their ores, founded on the property which mercury has to dissolve these 
netals as disseminated a the minerals, and thus to separate them from the earthy natters. See MIERctry, Metalltrgi, and Silver.

AMBER. (Succin, Fr.; Bernstein. Germ.) A mineral solid, of a tellow color, If rarious shades, which burns quite awar with flame, and consists of carbon, hỵdrogen, tnd oxygen, in nearly the same proportions, and the same state of combination, as regetable resin. Its specific grarity raries, br my trials, from 1.080 to 1.085 . It becomes negatively and powerfully electrical by friction. When applied to a lighted candle it takes fire, swells considerably, and exhales a white smoke of a pungent odor: but dres not run into drops. Copal, which resembles it in several respects, differs in being softer, and in melting into drops at the flame; and mellite, or honer-stone, which is a mineral of a similar color, becomes white when laid on a red-hot coal.

The texture of amber is resino-ritreous, its fracture conchoidal, and lustre glassy, It is perfectly homogeneous; sufficiently hard to scratch grpsum, and to take a fine polish. It is, howerer, scratched by calcareous spar. When amber is distilled in a retort, crrstalline needles of succinic acid sublime into the dome, and oil of amber drops from the bea $i$ into the receirer. Fossil resins, such as that of Highgate, found in the London clay formation, do not afford succinic acid br heat: nor does copal. Al ber is occasionally found of a whitish and brownish color.

The most interesting fact relatire to this regeto-mineral is its geological position, which is rery characteristic and well determined. It is found almost uniformly in separate nodules, disseminated in the sand, clar, or fragments of lignite of the plastic clar, and lignite formation, situated between the calcuire grossier (crag limestone) of the tertiart strata above, and the white chalk below. The size of these nodules raries from a nut to a man's head; but this magnitude is rert rare in true amber. It does not occur either in continuous beds, like the chalk flints, nor in reins; but it lies at one time in the earthr or friable strata, which accompanr or include the lignites; at another, entangled in the lignites themselres; and is associated with the minerals which constitute this formation, principally the pyrites, the most abundant of all. The pieces of amber found in the sands, and other formations evidently allurial, those met with on the seacoasts of certain countries, and especially Pomerania, come undonbtedly from the above geological formation; for the organic matters found still adhering to the amber leare no doubt as to its primitire place. Amber does not, therefore, belong to ant postdiluvian or modern soil, since its natire bed is corered br three or four series of strata, often of considerable thickness, and well characterized; proceeding upwards from the plastic clay which includes the amber: these are, the crag limestone, the bone grpsum, with its marls, the marly limestone, the upper marl sandstone, which covers it, and, lastly, the fresh water or lacustrine formation, often so thick, and composed of calcareous and silicious rocks.

The amber bed is not, however, alwars corered with all these strata; and it is eren rare to see a great mass of one of them abore the ground which contains it; because, were it buried under such strata, it would be difficult to meet with such circumstances as would lay it spontaneously open to the day. But by comparing observations made in different places, relatively to the patches of these formations, which corer the amber deposites, we find that no other mineral formations have been ever seen among them except those abore detailed, and thus learn that its geological locality is completely determined.

The proper yellow amber therefore, or the Borussic, from the country where it has been most abundantly found, belongs to the plastic clay formation, intermediate, in England, between the chalk and the London clar. It is sometimes interposed in thin plates between the layers of the lignites, but more towards the bark of the fibrous lignites, which retain the form of the wood, than towards the middle of the trurk of the tree; a position analogous to that of the resinous matters in our existing ligneous regetables. The fibrous lignites which thus contain amber belong to the dicotrledinous woods. Hence that substance seems to have been formed during the life of the regetable upon which it is now incrusted. It must be remembered that the grounds containing the amber are often replete with the sulphates of iron, alumina, and lime, or at least with the prritore elements of these salts. Some specimens of amber hare a surface figured with irregular meshes, indicating a sort of shrinkage from consolidation, and consequently a matter that was at one time fluid, risuid, or merely soft. From optical examination, Dr. Brewster has concluded amber to be of vegetable origin.

The different bodies included in the amber, distinguishable from its transparence, demonstrate, indeed, in the most conrincing manner, its primitire state of liquidity or softness. These bodies hare long exercised the skill of naturalists. They are generally insects, or remnins of insects, and sometimes leares, stalks, or other portions of regetables. Certain families of insects occur more abundantly than others. Thus the hymenopiera, or insects with four naked mernbranaceons wings, as the bee and wasp, and the diplera, or insects with two wings, as gnats, flies, gadflies, \&cc.; then come the spider tribe; 
some coleoptera (insects with crustaceous shells or elytra, which shut together, and form a longitudinal suture down the back), or beetles, principally those which live on trees; such as the eluterides, or leapers, and the chrysomelida. The lepidoptera, or insects with four membranaceous wings, and pterigostea covered with mail-like scales, are very rare in amber. We perceive from this enumeration, which results from the labors of Germar, Schweiger, \&c., that the insects enveloped in this resinous matter are in general such as sit on the trunks of trees, or live in the fissures of their bark. Hitherto, it has not been found possible to refer them to any living species; but it has been observed in general that they resemble more the insects of hot climates than those of the temperate zones.

The districts where amber occurs in a condition fit for mining operations are not nuınerous; but those in which it is met with in small scattered bits are very abundant. Its principal exploitation is in Eastern Prussia, on the coasts of the Baltic Sea, from Meinel to Dantzick, particularly in the neighborhood of Konigsberg, along the shore which runs north and south from Grossdirschheim to Pillau, and in several other places near Dantzick.

It is collected upon this coast in several ways; 1 . In the beds of small strearns which run near the viliages, and in rounded fragments without bark, or in the sand-banks of rivers, in pieces thrown back by the sea, and rounded by the waves. 2 . If the pieces thrown up by the waters are not numerous, the fishers, clothed in a leather dress, wade into the sea up to the neck, seek to discover the amber by looking along its surface, and seize it with bag nets, hung at the end of very long poles. They conclude that a great deal of amber has been detached from the cliffs by the sea, when many pieces of lignite (wood coal) are seen afloat. This mode of collecting amber is not free from danger, and the fishers, therefore, advance in troops, to lend each other aid in case of accident; but their success, even thus, is most precarious. 3. The third method of searching for amber is a real mining operation : it consists in digging pits upon the borders of the sandy downs, sometimes to a depth of more than 130 feet. 4. The last mode is by exploring the precipitous sea cliffs in boats, and detaching masses of loose soil from them with long poles terminating in iron hooks; a very hazardous employment. They search the cliffs with great care at the level, where the amber nodules commonly lie, and loosen the seams with their hoolis; in which business the boats are sometimes broken against the precipices, or sunk by an avalanche of rubbish.

Amber occurs in Sicily, disseminated in beds of clay and marl, which lie below the crag limestone. It is accompanied with bitumen; and, though a scanty deposite, it is mined for sale. The pieces are coated with a kind of whitish bark, present a variety of colors, and include many insects. Amber is found in a great many places in the sandy districts of Poland, at a very great distance from the sea, where it is mixed with cones of the pine. In Saxony it is met with in the neighborhood of Pretsch and Wittemberg, in a bituminous clay mingled with lignite. At the embouchure of the Jenissey, in Siberia, it occurs likewise along with lignite; as also in Greenland.

Fine amber is considerably valued for making ornamental objects, and the coarser kinds for certain uses in chemistry, medicine, and the arts. The oriental nations prize more highly than the people of Europe trinkets made of amber; and hence the chief commerce of the Pomeranian article is with Turkey. The Prussian government is said to draw an annual revenue of 17,000 dollars from amber. A good piece of a pound weight fetches 50 dollars. A mass weighing 13 pounds was picked up not long since in Prussia, for which 5000 dollars were offered, ana which would bring, in the opinion of the Armenian merchants, from 30,000 to 40,000 dollars at Constantinople. At one time it was customary to bake the opaque pieces of amber in sand, at a gentle heat, for several hours, in order to make it transparent, or to digest it in hot rapeseed oil, with the same view; but how far these processes were advantageous does not appear.

When amber is to be worked into trinkets, it is first split on a leaden plate at a lathe (see Gerrs, Cutting of), and then smoothed into shape on a Swedish whetstone. It is polished on the lathe with chalk and water, or vegetable oil, and finished by friction with flannel. In these processes the amber is apt to become highly electrical, very hot, and even to fly into fragments. Hence, the artists work the pieces time about, so as to keep each of them crol, and feebly excited. The men are often seized with nervous tremors in their wrisis and arms from the electricity. Pieces of amber may be neatly joined by smearing their edges with linseed oil, and pressing them strongly together, while they are held over a charcoal fire. Solid specimens of amber, reported to have been altogether fused by a particular application of heat, are now shown in the roya] cabinet of Dresden.

A strong and durable varnish is made by dissolving amber in drying linseed oil. For this purpose, however, the amber must be previously heated in an iron pot, over a clear red fire, till it soften and be semi-liquefied. The oil, previously heated, is to be now noured in, with much stirring, in the proportion of 10 aunces to the pound of amber; 
and after the incorporation is complete, and the liquid somewhat cooled, a pound of oil of turpentine must be added. Some persons prescribe 2 ounces of melted shellac, though by this means they are apt to deepen the color, already rendered too dark by the roasting.

The fine black varnish of the coachmakers is said to be prepared by melting 16 ounces of amber in an iron pot, adding to it half a pint of drying linseed oil, boiling hot, of powdered resin and asphaltum 3 ounces each: when the materials are well united, by stirring over the fire, they are to be removed, and, after cooling for some time. a pint of warm oil of turpentine is to be introduced.

The oil of amber enters into the composition of the old perfume called eau de luce; and is convertible, by the action of a small quantity of strong nitric acid, into a riscid mass like shoemakers' rosin, which has a strong odor of musk, and, under the name of artificial musk, has been prescribed, in acoholic solution, as a remedy against hooping cough, and other spasmodic diseases.

Acid of amber (succinic acid) is a delicate reagent, in chemistry, for separating red oxyde of iron from compound metallic solutions.

AMBERGRIS. (Ambregric, Fr.; Ambra, Germ.)-A morbid secretion of the liver of the spermaceti whale (physeter macrocephalus), found usually swimming upon the sea. It occurs upon the coasts of Coromandel, Japan, the Moluccas, and Madagascar, and has sometimes been extracted from the rectum of whales in the South sea fishery. It has a gray-white color, often with a black streak, or is marbled, yellow and black; has a strong but rather agreeable smell, a fatty taste, is lighter than water, melts at $50^{\circ}$ C. $\left(140^{\circ} \mathrm{F}\right.$. $)$, dissolves readily in absolute alcohol, in ether, and in both fat and rolatile oils. It contains 85 of the fragrant substance called ambreine. This is extracted from ambergris by digestion with alcohol of $0 \cdot 827$, filtering the solution, and leaving it to spontaneous evaporation. It is thus obtained in the form of delicate white tufts: which are convertible into ambreic acid by the action of nitric acid. Ambergris is used in perfumery.

AMIANTHUS. A mineral in silky filaments, called also AsBestus.

AMMONIA. A chemical compound, called also rolatile alkali. This substance, in its purest state, is a highly pungent gas, possessed of all the mechanical properties of the air, but very condensable with water. It consists of 3 volumes of hydrogen and 1 of azote condensed into two volumes; and hence its density is 0.591 , atmospheric air being 1.000 . By strong compression and refrigeration it may be liquefied into a fluid, whose specific gravity is 0.76 compared to water 1.000 .

Ammonia gas is composed by weight of 82.53 azote and 17.47 hydrogen in 100 parts. It is obtained by mixing muriate of ammonia, commonly called sal ammoniac, with quicklime, in a retort or still, applying a moderate heat, and receiving the gas either over-mercury for chemical experiments, or in water to make liquid ammonia for the purposes of medicine and the arts. Woulfe's apparatus is commonly employed for this condensation.

Ammonia is generated in a great many operations, and especially in the decomposition of many organic substances, by fire or fermentation. Urine left to itself for a few days is found to contain much carbonate of ammonia, and hence this substance was at one time collected in great quantities for the manufacture of certain salts of ammonia, and is still used for its alkaline properties in making alum, scouring wool, \&c. When woollen rags, horns, bones, and other animal substances are decomposed in close ressels by fire, they evolve a large quantity of ammonia, which distils over in the form of a carbonate. The main source of ammonia now in this country, for commercial purposes, is the coal gas works. A large quantity of watery fluid is condensed in their tar pits, which contains, chiefly, ammonia combined with sulphureted hydrogen and carbonic acid. When this water is saturated with muriatic acid and evaporated it yields muriate of ammonia, or sal ainmoniac, somewhat impure, which is afterwards purified by sublimation. See Carbonate of Ammonia and Sal Ammoniac.

The soot of chimneys where coal is burned contains both sulphate and carbonate of ammonia, and was extensively employed, at one time, to manufacture these saits.

In making water of ammonia on the great scale, a cast iron still should be preferred, and equal weights of quicklime and sal ammoniac should be brought to the consistence of a pap, with water, before the heat is applied. In this case a refrigeratory worm or globe should be interposed between the adopter tube of the capital of the still and the bottles of Woulfe's apparatus. The muriate of lime, or chloride of calcium, which is left in the still when the whole ammonia is expelled, is of no value. Water is capable of condensing easily about one third of its weight of ammonia gas, or 460 times its bulk. The following table of the quantity of ammonia in 100 parts by weight of its aqueous combinations, at successive densities, is the result of very careful experiments made by me, and recorded in the Philosophical Magazine for March, 1821. 
ANCHOR.

Table of Water of Ammonia or Volatile Alkali, by Dr. Ure.

\begin{tabular}{|c|c|c|c|c|c|c|}
\hline $\begin{array}{c}\text { Water } \\
\text { of } \\
0.900\end{array}$ & $\begin{array}{c}\text { Ammonia } \\
\text { in } \\
100\end{array}$ & $\begin{array}{l}\text { Water } \\
\text { in } \\
100\end{array}$ & $\begin{array}{c}\text { Specific } \\
\text { gravity by } \\
\text { experiment. }\end{array}$ & $\begin{array}{l}\text { Mean } \\
\text { specific } \\
\text { gravity. }\end{array}$ & \multicolumn{2}{|c|}{ Equivalent primes. } \\
\hline 100 & $26 \cdot 500$ & $73 \cdot 500$ & 0.9000 & & & \\
\hline 95 & $25 \cdot 175$ & $74 \cdot 825$ & 0.9045 & $0 \cdot 90452$ & & Wat. $A m$. \\
\hline 90 & $23 \cdot 850$ & $76 \cdot 150$ & 0.9090 & 0.90909 & $24+76$ & 6 to 1 \\
\hline 85 & $22 \cdot 525$ & $77 \cdot 475$ & $0 \cdot 9133$ & 0.91370 & & \\
\hline 80 & $21 \cdot 200$ & $\tau 8.800$ & 0.9177 & 0.91838 & $21 \cdot 25+78 \cdot 75$ & 7 to 1 \\
\hline 75 & $19 \cdot 875$ & $80 \cdot 125$ & 0.9227 & 0.92308 & & \\
\hline 70 & $18 \cdot 550$ & $81 \cdot 450$ & 0.9275 & 0.92780 & $19 \cdot 1+80 \cdot 9$ & 8 to 1 \\
\hline 35 & $17 \cdot 225$ & $82 \cdot 775$ & 0.9320 & $0 \cdot 93264$ & $17 \cdot 35+82 \cdot 65$ & 9 to 1 \\
\hline 60 & $15 \cdot 900$ & $84 \cdot 100$ & 0.9363 & 0.93750 & $15 \cdot 9+84 \cdot 1$ & 10 to 1 \\
\hline 55 & $14 \cdot 575$ & $85 \cdot 425$ & 0.9410 & $0 \cdot 94241$ & $14 \cdot 66+85 \cdot 34$ & 11 to 1 \\
\hline 50 & $13 \cdot 250$ & $86 \cdot 750$ & 0.9455 & $0 \cdot 94737$ & $13 \cdot 60+86 \cdot 40$ & 12 to 1 \\
\hline 45 & $11 \cdot 925$ & 88.075 & 0.9510 & 0.95238 & $11 \cdot 9+88.1$ & 14 to 1 \\
\hline 40 & $10 \cdot 600$ & $89 \cdot 400$ & 0.9564 & 0.95744 & $11 \cdot 2+88.8$ & 15 to 1 \\
\hline 35 & $9 \cdot 275$ & $90 \cdot 725$ & 0.9614 & 0.96256 & & \\
\hline 30 & $7 \cdot 950$ & $92 \cdot 050$ & 0.9662 & 0.96774 & $8 \cdot 63+91 \cdot 37$ & 20 to 1 \\
\hline 25 & $6 \cdot 625$ & $93 \cdot 375$ & 0.9716 & 0.97297 & $7+93$ & 25 to 1 \\
\hline 20 & $5 \cdot 300$ & $94 \cdot 700$ & 0.9768 & 0.97826 & $6+94$ & 30 to 1 \\
\hline 15 & $3 \cdot 975$ & $96 \cdot 025$ & 0.9828 & $0 \cdot 98360$ & $4 \cdot 5+95 \cdot 5$ & 40 to 1 \\
\hline 10 & $2 \cdot 650$ & $97 \cdot 350$ & 0.9887 & 0.98900 & $3+97$ & 60 to 1 \\
\hline 5 & $1 \cdot 325$ & $98 \cdot 675$ & 0.9945 & 0.99447 & & \\
\hline
\end{tabular}

AMMONIAC, gum-resin. This is the inspissated juice of an umbelliferous plant (the dorema armeniacum) which grows in Persia. It comes to us either in small white tears clustered together, or in brownish lumps, containing many impurities. It possesses a peculiar smell, somewhat like that of asafœtida, and a bitterish taste. It is employed in medicine. Its only use in the arts is for forming a cement to join broken pieces of china and glass, which may be prepared as follows: Take isinglass 1 ounce, distilled water 6 ounces, boil together down to 3 ounces, and add $1 \frac{1}{2}$ ounce of strong spirit of wine; boil this mixture for a minute or two; strain it; add, while hot, first, half an ounce of a milky emulsion of gum ammoniac, and then five drams of an alcoholic solution of resin mastic. This resembles a substance sold in the London shops, under the name of diamond cement. The recipe was given me by a respectable dispensing chemist.

AMORPHOUS. Without shape. Said of mineral and other substances which occur in forms not easy to be defined.

ANALYSIS. The art of resolving a compound substance or machine into its constituent parts. Every manufacturer should so study this art, in the proper treatises, and schools of Chemistry or Mechanies, as to enable him properly to understand and regulate his business.

ANCHOR. (Ancre, Fr.; Anker, Germ.) An iron hook of considerable weight and strength, for enabling a ship to lay hold of the ground, and fix itself in a certain situation by means of a rope called the cable. It is an instrument of the greatest importance to the navigator, since upon its taking and keeping hold depends his safety upon

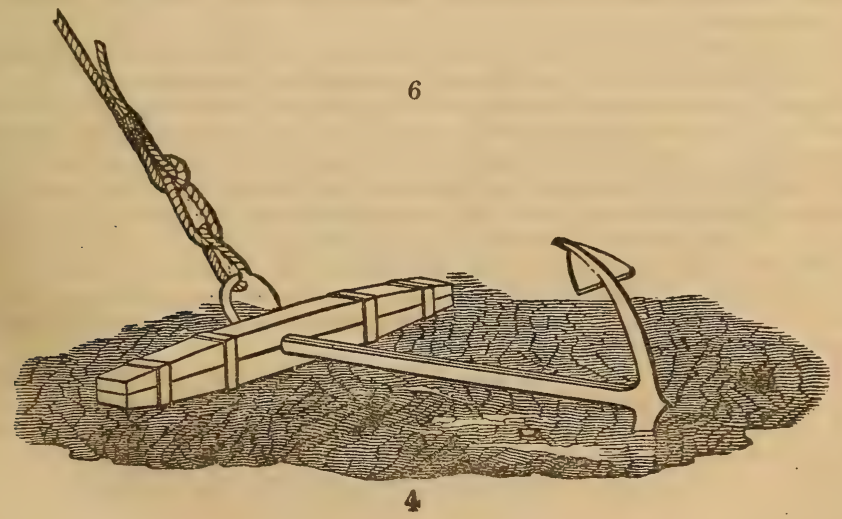


many occasions, especially near a lee shore, where he might be otherwise stranded or shipwrecked. Anchors are generally made of wrought iron, except among nations who cannot work this metal well, and who therefore use copper. The mode in which an anchor operates will be understood from inspection of fig. 6 , where, from the direction of the strain, it is obvious that the anchor cannot move without ploughing up the ground in which its hook or fluke is sunk. When this, however, unluckily takes place, from the nature of the ground, from the mode of insertion of the anchor, or from the violence of the winds or currents, it is called dragging the anchor. When the hold is good, the cable or the buried arm will sooner break than the ship will drive. Anchors are of different sizes, and have different names, according to the purposes they serve; thus there are, sheet, best bower, small bower, spare, streum, and kedge anchors. Ships of the first class have seven anchors, and smaller vessels, such as brigs and schooners, three.

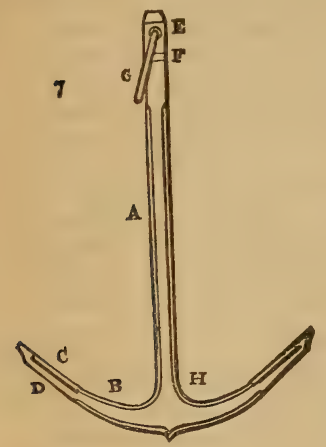

The manufacture of anchors requires great knowledge of the structure of iron, and skill in the art of working it. I shall give, here, a brief notice of the improved system introduced by Mr. Perring, clerk of the cheque at Plymouth, in which the proportions of the parts are admirably adapted to the strains they are likely to suffer. In fig. 7, A is the shank; B, the arm or fluke; C, the palm; D, the blade; $\mathrm{E}$, the square; $\mathrm{F}$, the nut ; $\mathrm{G}$, the ring; $\mathrm{H}$, the crown.

Formerly the shank was made of a number of square iron rods, laid parallel together in a cylindrical form, and bound by iron hoops. When they were welded into one bar, the exterior rods could not fail to be partially burned and wasted by the strong heat. Mr. Perrin abated this evil by using bars of the whole breadth of the shank, and placing them right over each other, hooping them and welding them together at two heats into one solid mass. To any one who has seen the working of puddled iron, with a heavy mill hammer, this operation will not appear difficult.

He formed the crown with bars similarly distributed with those of the shank. His mode of uniting the flukes to the crown is probably the most valuable part of his invertion. The bars and half the breadth of the anchor are first welded separately, and then placed side by side, where the upper half is worked into one mass, while the lower part is left disunited, but has carrier iron bars, or porters, as these prolongation rods are commonly called, welded to the extremity of each portion. The lower part is now heated and placed in the clamping machine, which is merely an iron plate firmly bolted to a mass of timber, and bearing upon its surface four iron pins. One end of the crown is placed between the first of these pins, and passed under an iron strap; the other end is brought between the other pins, and is bent by the leverage power of the elongated rods or porters.

Thus a part of the arm being formed out of the crown gives much greater security that a true union of fibres is effected, than when the junction was made merely by a short scarf.

The angular opening upon the side opposite B H, fig. 7, is filled with the chock, formed of short iron bars placed upright. When this has been firmly welded, the trusspiece is brought over it. This piece is made of plates similar to the above, except that their edges are here horizontal. The truss-piece is half the breadth of the arm; so that, when united to the crown, it constitutes, with the other parts, the total breadth of the arms at those places.

The shank is now shut upon the crown; the square is formed, and the nuts welded to it ; the hole is punched out for the ring, and the shank is then fashioned.

The blade is made much in the way above described. In making the palm, an iron rod is first bent into the approximate form, notching it so that it may more readily take the desired shape. To one end a porter rod is fastened, by which the palm is carried and turned round in the fire during the progress of the fabrication. Iron plates are next laid side by side upon the rod, and the joint at the middle is broken by another plate laid over it. When the mass is worked, its under side is filled up by similar plates, and the whole is completely welded; pieces being added to the sides, if necessary, to form the angles of the palm. The blade is then shut on to the palm, after which the part of the arm attached to the blade is united to that which constitutes the crown. The smith-work of the anchor is now finished.

The junction, or shutting on, as the workmen call it, of the several members of an anchor, is effected by an instrument called a monkey, which is merely a mass of iron raised to a certain height, between parallel uprights, as in the pile engine or vertical ram, and let fall upon the metal previously brought to a welding heat. 
The monkey and the hercules, both silly, trivial names, are similar instruments, and are usually worked, like a portable pile engine, by the hands of several laborers, pulling separate ropes. Many oither modes of manufacturing anchors have been devised, in which mechanical power is more extensively resorted to.

The upper end of the shank $\mathrm{F}$ ( $f \mathrm{~g} .7$ ) is squared to receive and hold the stock steadily, and keep it from turning. To prevent it shifting along, there are two knobs or tenon-like projections. The point of the angle $\mathrm{H}$, between the arms and the shank, is sometimes called the throat. 'The arm B c generally makes an angle of $56^{\circ}$ with the shank A; it is either round or polygonal, and about half the length of the shank.

The stock of the anchor ( $f \mathrm{~g} .6$ ) is made of oak. It consists of two beams which em. brace the square, and are firmly united by iron bolts and hoops, as shown in the figure. The stock is usually somewhat longer than the shank, has in the middle a thickness about one twelfth of its length, but tapers at its under side to nearly one half this thickness at the extremities. In small anchors the stock is frequently made of iron; but in this case it does not embrace the anchor, but goes through a hole made in the square, which is swelled out on purpose.

The weight of anchors for different ressels is proportioned to the tonnage; a good rule being to make the anchor in hundred weights one twentieth of the number of tons of the burden. Thus a ship of 1000 tons would require a sheet anchor of $50 \mathrm{cwts}$. Ships of war are provided with somewhat heavier anchors.

Several new forms and constructions of anchors were proposed under Mr. Piper's patent of November, 1822, by the adoption of which great advantages as to strengihi were anticipated over every other form or construction previously made.

The particular object was to preserve such a disposition of the fibres of the metal as should afford the greatest possible strength; in doing which the crossing or bending of the fibres at the junctions of the shank, flukes, and crown, where great strength is required, has been avoided as much as possible, so that the fibres are not disturbed or injured.

In this respect most anchors are defective ; for in connecting the shanks to the crownpieces, the grain of the metal is either crossed, or so much curved, as to strain the fibre, and consequently induce a wealiness where the greatest strength is required. And, further, the rery considerable thicknesses of metal which are to be brought into inmediate contact by means of the hammer in forging anchors upon the old construction, render it highly prohable that faulty places may be left within the mass, though they be externally imperceptible. Mr. Piper's leading principle was, that the fibre of the metal should run nearly straight in all the parts where strength is particularly required.

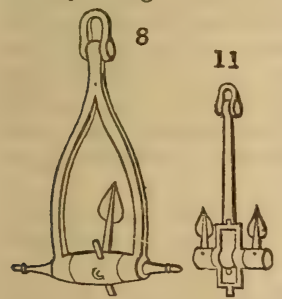

Fig. 8 shows an anchor with one tumbling fluke, which passes through the forked or branched part of the shank. The lower part of this anchor, answering to the crown, has a spindle through it, upon which the fluke turns, and a pin is there introduced for the purpose of confining the fluke when in a holding position. This shank is formed of a solid piece of wrought iron, the fibres of which run straight, and at the crown holes are pierced, which merely bulge the metal without bending the fibres round so as to strain them. The arm and fluke, also, are formed of one piece punched through without curling or crossing the fibre, and the spindle which holds the arm to the crown is likewise straight. This spindle extends some distance on each side of the anchor, and is intended to answer the purpose of a stock; for when either of the ends of the spindle comes in contact with the ground, the anchor will be thrown over into a holding position; or an iron stock may be introduced near the shackle, instead of these projecting ends. In the descent of the anchor, the fluke will fall over towards that side which is nearest the ground, and will there be ready to take hold when the anchor is drawn forward.

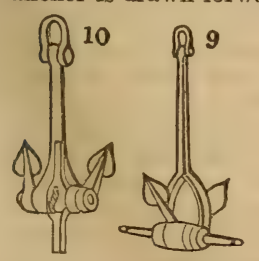

Fig. 9 is another anchor upon the same principle, but slightly varied in form from the last. In this the forked part of the shank is closer than in the former, and there are two arms or flukes connected to the crown-pieces, one of which falls into its holding position as the anchor comes to the ground, and is held at its proper angle by the other fluke stopping against the shank.

Fig. 10 represents another variation in the form of these improved anchors, having two tumbling flukes, which are both intended to take hold of the ground at the same time. The shank is here, as before, made without crossing the grain of the iron, and the eyes for admitting the bolt at the crown and at the shackle are punched out of the solid, not formed by welding or turning the iron round. In this form a guard is introduced at 
the crown, to answer the purpose of a stock, by turning the flukes over into a holding position. The arms and flulies are made, as before described, of the straight fibre of the iron punched through, and the flukes are fixed to the spindle, which passes through the crown-piece.

Fig. 11 has a shank without any fork, but formed straight throughout; the guard here is an elongated frame of iron, for the same purpose as a stock, and is, with the tumbling flukes, fastened to the spindle, which passes through the crown of the anchor, and causes the flukes to fall into their holding position.

The principles of these new anchors are considered to consist in shanks which are made of straight lengths of metal, and finished so that the fibres of the iron shall not be injured by cross-shuts or uncertain welding; also each arm and palm is made in one solid piece, and finished in straight lines, so that the fibres will not be altered, and the shaft-pin or spindle will also be in one straight line; and this is the improvement claimed. These anchors, being made in separate pieces, gire a great adrantage to the morliman to execute each part perfectly; for he will not have such heary weights to lift when hot, which will render these anchors much stronger, with less weight; and if any accident should happen to them, any part may be takien separate from the others to be repaired, and sereral of those parts of the anchor which may be likely to break may be carried on board, in case of accident. This anchor is so contrived that one of thirty hundred weight mas be taken to pieces and put together again, by one man, in twenty minutes; it may also be dismounted, and stowed in any part of the ship, in as little room as straight bars of iron, and speedily put together again.

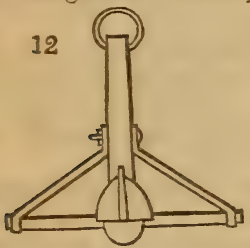

The anchor ( $f$ ig. 12 ) patented by Mr. Brunton, in February, 1822 , has its stock introduced at the crown part, for the purpose of turning it over into a holding position. The shank is perforated through the solid, in two places, with elliptical apertures, for the purpose of giving it a greater stability, and more effectually resisting the strain to which the anchor may be subjected. The stock is a cylindrical iron rod, held at its extremities by lateral braces, which are bolted to the shank.

Fig. 12 shows the form of the anchor. The shank is seen upright, with one of the flukes projecting in its front; the horizontal iron stock is at bottom; and the oblique braces are bolted to both shank and stock. The ends of the stock, from the shoulder, are formed dore-tailed, and oral in the rertical direction, and

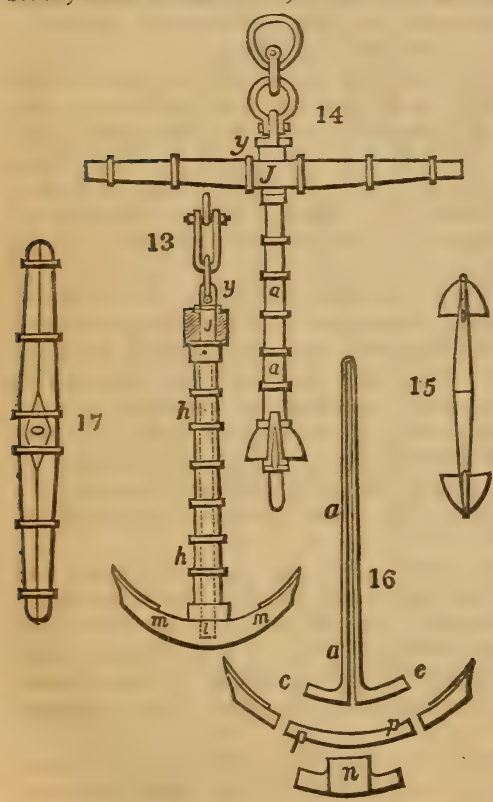
are protruded through apertures in the braces, also oval, but in the horizontal direction, and counter sunk. When the ends of the stock have been thus introduced through the holes, the braces are securely bolted to the shank, the ends of the stock are then spread, br hammering into the counter-sunk holes of the braces, and by that means they ar made firm.

An anchor of this description is consider ed by the patentee to possess considerable advantage, particularly in point of stability over the ordinary construction of anchors and is economical, inasmuch as a less weigh of metal will give, upon this plan, an eque degree of strength.

An ingenious form of anchor was mad. the subject of a patent, by Lientenant Rodg ers, of the Royal Navy, in 1828, and ws afterwards modified by him in a second pa tent, obtained in August, 1829. The who't of the parts of the anchor are to be bound together by means of iron bands or hoops, in place of bolts or pins.

Fig. 13 is a side view of a complete anchor, formed upon his last improved construction, and fig. 14, a plan of the same; fig. 15, an end view of the crown and flukes, or arms; fig. 16 represents the two principal iron plates, $a$, $a$, of which the shank is constructea, but so as to form parts of the stump arms to which the Rukes are to be connected.

The crown piece is to be welded to the stump piece, $c, c$, fig. 16 , as well as to the 
. centre piece $h h$, and the scarfs $m m$ are to be cut to receive the arms or end 3. "Previously, however, to uniting the arms or flukes with the stump arms, the crown and throat of the anchor are to be strengthened, by the application of the crown slabs $n u$, fig. 16, which are to be welded upon each side of the crown, overlapping the end of the pillar $h$, and the throat or linees of the stump arms and the crown piece. The stump arms are then to be strengthened in a similar manner, by the thin flat pieces $p p$, which are to be welded upon each sile. The palms are united to the flukes in the ustual way, and the flukes are also united to the stump arms by means of the long scarfs $m m$. When the shank of the anchor has been thus formed, and united with the flukes, the anchor smith's work may be said to be complete.

Another of the improvements in the construction of anchors, claimed under this patent, consists in a new method of affixing the stock upon the shank of the anchor, which is effected in the following manner: in fig. 14, the stock is shown affixed to the anchor; in fig. 17, it is shown detached. It may be made either of one or two pieces of timber, as may be found most convenient. It is, however, to be observed that the stock is to be completed before fitting on to the shank. After the stock is shaped, a hole is to be made through the middle of it, to fit that part of the shank to which it is to be affixed. Two stock plates are then to be let in, one on each side of the stock, and made fast by counter sunk nails and straps, or hoops; other straps or hoops of iron are also to be placed round the stork, as usual.

In place of nuts, formed upon the shank of the anchor, it is proposed to secure the stock by means of a hoop and a key, shown above and below J, in fig. 14 . By this contrivance, the stock is prevented from going nearer to the crown of the anchor than it ought to do, and the key prevents it from sliding towards the shackle.

Since fitting the stock to the shank of an anchor, by this method, prevents the use of a ring, as in the ordinary manner, the patentee says that he in all cases substitutes a shackle for the ring, and which is all that is required for a chain cable; but, when a heinpen cable is to be used, he connects a ring to the usual shackle, by means of a joining shackle, as in figs. 13 and 14.

MIr. Rodgers proposes, under another patent, dated July, 1833, to alter the size and form of the palms; having found from experience that ancinors with small palms will not only hold better than with large ones, but that the arms 3 in the anchor, even without any palms, have been found to take more secure hold of the ground than anchors of the old construction, of similar weight and length. He has, accordingly, fixed upon onefifth of the length of the arm; as a suitable proportion for the length or depth of the palm. He makes the palms, also, broader than they are long or deep.

ANIME'. A resin of a pale brown yellow color, transparent and brittle. It exudes from the courbaril of Cayenne, a tree which grows also in various parts of South America. It oeeurs in pieces of various sizes, and it often contains so many insects belonging to living species, as to have merited its name, as being animated. It contains about a fifth of one per cent. of a volatile oil, which gives it an agreeable odor. Alcohol does not dissolve the genuine animé, as I have ascertained by careful experiments; nor does caoutchoucine; but a mixture of the two, in equal parts, softens it into a tremulous jelly, though it will not produce a liquid solution. When reduced to this state, the insects can be easily picked out, without injury to their mosc delicate parts.

The specifie gravity of the different specimens of animé which I tried, varied from 1.054 to 1.057 . When exposed to heat, in a glass retort over a spirit flame, it softens, and, by careful management, it may be brought into liquid fusion, without discoloration. It then exhales a few white vapors, of an' ambrosiacal odor, which being condensed in water, and the liquid being tested, is found to be succinic acid. Author.

It is extensively used by the varnish makers, who fuse it at a pretty high heat, and in this state combine it with their oils, or other varnishes.

ANKER. A liquid measure of Amsterdam, which contains 32 gallons English.

ANNEALING or NEALING. (Le recuit, Fr.; das anlassen, Germ.) A process by which glass is rendered less frangible; and metals, which have become brittle, either in consequence of fusion, or long-continued hammering, are again rendered malleable. When a glass ressel is allowed to cool immediately after being made, it will often sustain the shock of a pistol-bullet, or any other blunt body falling into it from a considerable height; while a small splinter of flint, or an angular fragment of quartz, dropped gently into it, makes it sometimes immediately, sometimes after a few minutes, fly to pieces with great violence. This extreme fragility is prevented by annealing, or placing the vessels in an oven, where they take several hours or even some days to cool. Similar phenomena are exhibited in a higher degree by glass-tears, or Prince Rupert's drops. They are procured by letting drops of melted glass fall into cold water. Their form resembles that of a pear, rounded at one extremity, and tapering to a very slender tail at the other. If a part of the tail be broken off, the whole drop flies to pieces with a loud explosion; and yet the tail of a drop may be cut away by a glass-cutter's wheel, or the thick end 
may be struck smartly with a hammer, without the fear of sustaining any injury.

heated to redness, and permitted to cool gradually in the open air, they lose these peculiarities, and do not differ sensibly from common glass.

The properties of unannealed glass depend on a peculiar structure, extending uniformly through its whole substance; -and the bursting of a glass drop by breaking off the tail, or of an unannealed glass vessel, by dropping a piece of flint into it, arises from a crack being thus begun, which afterwards extends its ramifications in different directions throughout the glass.

When metals have been extended to a certain degree under the hammer, they become brittle, and incapable of being further extended without cracking. In this case the workman restores their malleability by annealing, or heating them red-hor. The rationale of this process seems to be, that the hammering and extension of the metal destroy the kind of arrangement which the particles of the metal had previous to the hammering; and that the annealing, by softening the metal, enables it to recover its original structure.

Of late years a mode has been discovered of rendering cast iron malleable, without subjecting it to the action of puddling. The process is somewhat similar to that employed in annealing glass. The metal is liept for several hours at a temperature a little below its fusing point, and then allowed to cool slowly. In this manner vessels are made of cast iron which can sustain considerable violence, without being broken. See STEEL, softening of.

ANNOTTO. (Rocou, or roucou, Fr. ; orleans, Germ.) A somewhat dry and hard paste, brown without, and red within. It is usually imported in cakes of two or three pounds weight, wrapped up in leaves of large reeds, packed in casks, from America, where it is prepared from the seeds of a certain tree, the bixa orellana, of Linnæus.

The pods of the tree being gathered, their seeds are taken out and bruised; they are then transferred to a vat, which is called the steeper, where they are mixed with as much water as covers them. Here the substance is left for several weeks, or even months; it is now squeezed through sieves placed above the steeper, that the water containing the coloring matter in suspension may return into the vat. The residuum is preserved under the leaves of the anana (pine-apple) tree, till it becomes hot by fermentation. It is again subjected to the same operation, and this treatment is continued tiil no more color remains.

The substance thus extracted is passed through sieves, in erder to separate the remainder of the seeds, and the color is allowed to subside. The precipitate is boiled in coppers till it be reduced to a consistent paste; it is then suffered to cool, and dried in the shade.

Instead of this long and painful labor, which occasions diseases by the putrefaction in. duced, and which affords a spoiled product, Leblond proposes simply to wash the seeds of annotto till they be entirely deprived of their color, which lies wholly on their surface; to precipitate the color by means of vinegar or lemon juice, and to boil it ap in the ordinary manner, or to drain it in bags, as is practised with indigo.

The experiments which Vauquelin made on the seeds of annotto imported by Leblond, confirmed the efficacy of the process which he proposed; and the dyers ascertained that the annotto obtained in this manner was worth at least four times more than that of commerce; that, moreover, it was more easily employed; that it required less solvent; that it gave less trouble in the copper, and furnished a purer color.

Annotto dissolves better and more readily in alcohol than in water, when it is introduced into the yellow varnishes for communicating an orange tint.

The decoction of annotto in water has a strong peculiar odor, and a disagreeable taste. Its color is yellowish-red, and it remains a little turbid. An alkaline solution renders its orange-yellow clearer and more agreeable, while a small quantity of a whitish substance is separated from it, which remains suspended in the liquid. If annotto be boiled in water along with an alkali, it dissolves much better than when alone, and the liquid has an orange hue.

The acids form with this liquor an orange-colored precipitate, soluble in alkalies, which communicate to it a deep orange color. The supernatant liquor retains only a pale yellow hue.

When annotto is used as a dye, it is always mixed with alkali, which facilitates its solution, and gives it a color inclining less to red. The annotto is eut in pieces, and boiled for some instants in a copper with its own weight of crude pearl ashes, prorided the shade wanted do not require less alkali. The cloths may be thereafter dyed in this bath, either by these ingredients alone, or by adding others to modify the color; but anuotto is seldom used for woollen, because the colors which it gives are too fugitive, and may be obtained by more permanent dyes. Hellot employed it to dye a stuff, prepared with alum and tartar; but the color acquired had little permanence. It is almost solely used for silks. 
For silks intended to become aurora and orange, it is sufficient to scour them at the rate of 20 per cent. of soap. When they have been well cleansed, they are immersed In a bath prepared with water, to which is added a quantity of alkaline solution of annotto, more or less considerable according to the shade that may be wanted. This bath should have a mean temperature, between that of tepid and boiling water.

When the silk has become uniform, one of the hanks is taken out, washed, and wrung, to see if the color be sufficiently full; if it be not so, more solution of annotto is added, and the silk is turned again round the sticks: the solution keeps without alteration.

When the desired shade is obtained, nothing remains but to wash the silk, and give it two beetlings at the river, in order to free it from the redundant annotto, which would injure the lustre of the color.

When raw silks are to be dyed, those naturally white are chosen, and dyed in the annotto bath, which should not be more than tepid, or even cold, in order that the alkali may not attack the gum of the silk, and deprive it of the elasticity which it is desirable for it to preserve.

What has been now said regards the sillss to which the aurora shades are to to be given; but to make an orange hue, which contains more red than the aurora, it is requisite, after dyeing with annotto, to redden the silks with vinegar, alum, or lemon juice. The acid, by saturating the allali employed for dissolving the annotto, destroys the shade of yellow that the alkali had siven, and restores it to its natural color, which inclines a good deal to red.

For the deep shades, the practice at Paris, as Macquer informs us, is to pass the silks through alum; and if the color be not red enough, they are passed through a faint bath of brazil wood. At Lyons, the dyers who use carthamus, sometimes employ old baths of this ingredient for dipping the deep oranges.

When the orange hues have been reddened by alum, they must be washed at the river; but it is not necessary to beetle them, unless the color turns out too red.

Shades may be obtained also by a single operation, which retain a reddish tint, employing for the annotto bath a less proportion of alkali than has been pointed out.

Guhliche recommends to avoid heat in the preparation of annotto. He directs it to be placed in a glass vessel, or in a glazed earthen one; to cover it with a solution of pure alkali; to leave the mixture at rest for 24 hours; to decant the liquor, filter it, and add water repeatedly to the residuum, leaving the mixture ezch time at rest for two or three days, till the water is no longer colored; to mix all these liquors, and preserve the whole for use in a well-stopped vessel.

He macerates the silk for 12 hours in a solution of alum, at the rate of an eighth of this salt for one part of silk, or in a water rendered acidulous by the aceto-citric acid above described; and he wrings it well on its coming out of this bath

Silk thus prepared is put into the annotto bath quite cold. It is kept in agitation there till it has taken the shade sought for; or the liquor may be maintained at a heat far below ebullition. On being taken out of the bath, the silk is to be washed and dried in the shade.

For lighter hues, a liquor less charged with color is taken; and a little of the acid liquid which has served for the mordant may be added, or the dyed silk may be passed through the acidulous water.

We have seen the following preparation employed for cotton velvet:-one part of quicklime, one of potash, two of soda.

Of these a ley is formed, in which one part of annotto is dissolved; and the mixture is boiled for an hour and a half. This bath affords the liveliest and most brilliant auroras. The buff (chamois) fugitive dye is also obtained with this solution. For this purpose only a little is wanted; but we must never forget, that the colors arising from annotto are all fugitive.

Dr. Joln found in the pulp surrounding the unfermented fresh seeds, which are about the size of little peas, 28 parts of coloring resinous matter, 26.5 of vegetable gluten, 20 of ligneous fibre, 20 of coloring extractive matter, 4 formed of matters analogous to vege. table gluten and extractive, and a trace of spicy and acid matters.

The Cloucestershire cheese is colored with annotto, in the proportion of one cwt. to an ounce of the dye.

When used in calico-printing, it is usually mixed with potash or ammonia and starch.

It is an appropriate substance for tinging varnishes, oils, spirits, \&c.

The import duty upon annotto is $1 s$. per cwt. for flag, and $4 s$. for other sorts. In 1834, $252,981 \mathrm{lbs}$. were imported; and in 1835, 163,421 lbs. The revenue from this drug in these two years, was $180 l$. and $98 l$. respectively.

ANTHRACITE, from $\alpha_{i} \theta \omega a \xi$, coal, is a species of coal found in the transition rock formation, and is often called stone coal. It has a grayish black, or iron bluck color; an imperfectly metallic lustre, conchoidal fracture, and a specific gravity of from 1.4 to $1 \cdot 6$, being, therefore, much denser than the coal of the proper coal measures. It consists 
wholly of carbon, with a small and variable proportion of iron, silica, and alumina. It is difficult to kindle in separate masses, and burns when in heaps or grates without smell or smolie, leaving sometimes an earthy residuum. It has been little explored or worked in the old world; but is extensively used in the United States of America, and has become of late years a most valuable mineral to that country, where it is burned in peculiar grates, adapted to its difficult combustion. In Pennsylvania the anthracite coa] formation has been traced through a tract many miles in width, and extending acros the two entire counties of Luzerne and Schuyllill. At Maunch Chunk, upon the Le high, 800 men were employed, so far back as 1825 , in digging this coal. In that yea. 750,000 bushels were despatched for Philadelphia. It is worked there with little cost or labor, being situated on hills from 300 to 600 feet above the level of the neighboring rivers and canals, and existing in nearly horizontal beds, of from 15 to 40 feet in thickness, corered by only a few feet of gravelly loam. At Portsmouth, in Rhode Island, an extensive stratum of this coal has been worked, with some interruptions, for 20 years; and more recently a mine of anthracite has been opened at Worcester, in . Massachusetts, at the head of the Blackstone canal. It has been of late employed in South Wales, for smelting iron, and in a cupola blast furnace, by Mr. Crane.

ANTIGUGGLER. A small syphon of metal, which is inserted into the mouths of casks, or large bottles, called carboys, to admit air over the liquor contained in them, and thus to facilitate their being emptied without agitation or a guggling noise.

ANTIMONY. (Antimoine, Fr.; Spiessglanz, or Spiessglass, Ger.) The only ore of this metal found in sufficient abundance to be smelted, is the sulphuret, formerly called crude antimony. It occurs generally in masses, consisting of needles closely aggregated, of a metallic lustre, a lead-gray color, inclining to steel-gray, which is unchanged in the streak. The needles are extremely brittle, and melt eren in the flame of a candle, with the exhalation of a sulphureous smell. The powder of this sulphuret is very black, and was employed by women in ancient times to stain their eyebrow's and eyelids. This ore consists in 100 parts of 72.86 metal, and $27 \cdot 14$ sulphur. Specific gravity from $4 \cdot 13$ to $4 \cdot 6$.

The veins of sulphuret of antimony occur associated with gangues of quartz, sulphate of barytes, and carbonate of lime; those of Allemont occur in the numerous fissures of a mica schist, evidently primitive.

In treating the ore to obtain the metal, the first object is to separate the gangue, which was formerly done by filling crucibles with the mixed materials, placing them on the hearth of an oven, and exposing them to a moderate heat. As the sulphuret easily melts, it ran out through a hole in the bottom of the crucible into a po: placed beneath, and out of the reach of the fire. But the great loss from breakage of the crucibles, has caused another method to be adopted. In this the broken ore, being sorted, is laid on the bottom of a concave reverberatory hearth, where it is reduced.

Figs. 18, 19, represent a wind or flame furnace, for the reduction of antimony. The

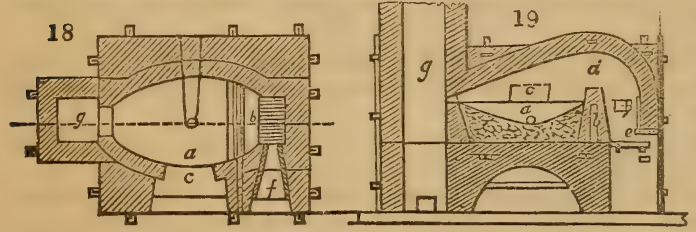
hearth is formed of sand and clay solidly beat together, and slopes from all sides towards the middle, where it is connected with the orifice $a$, which is closed with dense coal-ashes; $b$ is the air channel up through the bridge; $c$, the door for introducing the prepared ore, and running off the slags; $d$, the bridge; $e$, the grate; $f$, the fire or fuel door; $g$, the chimney. With 2 or 3 cwt. of ore, the smelting process is completed in from 8 to 10 hours. The metal thus obtained is not pure enough, but must be fused under coal dust, in portions of 20 or 30 pounds, in erucibles, placed upon a reverberatory hearth.

To obtain antimony free from iron, it should be fused with some antimonic oxyde in a crucible, whereby the iron is oxydized and separated. The presence of arsenic in antimony is detected by the garlic smell, emitted by such an alloy when heated at the blow-pipe; or, better, by igniting it with nitre in a crucible; in which case, iisoluble antimonite and antimoniate of potash will be formed along with soluble arseniate. Water digested upon the mixture, filtered, and then tested with nitrate of silver, will afford the brown-red precipitate characteristic of arsenic acid.

According to Berthier, the following materials afford, in smelting, an exccllent product of antimony : 100 parts of sulphuret; 60 of hammerschlag (protoxyde of iron from the shingling or rolling mills); 45 to 50 of carbonate of soda; and 10 of charcoal powder. From 65 to 70 parts of metallic antimony or regulus should be obtained. Glauber salts may be used instead of soda. For another mode of smeiting antimony, at Malbosc, in the department of Ardèche, in France, see LIQUATIon.

In the works where antimonial ores are smelted, by means of tartar (argol), the alka- 
ine scoriæ, which cover the metallic ingots, are not rejected as useless, for thry hold a certain quantity of antimonial oxyde in combination; a property of the potash flux, which is propitious to the purity of the metal. These scoriæ, consisting of sulphuret of potassium and antimonite of potash, being treated with water, undergo a reciprocal decomposition; the elements of the water act on those of the sulphuret, and the resulting alkaline hydro-sulphuret reacts on the antimonial solution, so as to form a species of kermes mineral, which precipitates. This is dried, and sold at a low price as a veterinary medicine, under the name of kermes, by the dry way.

Metallic antimony, as obtained by the preceding process, is the antimony of commerce, but is not absolutely pure; containing frequently minute portions of iron, lead, and even arsenic; the detection and separation of which belong to the sciences of chemistry and pharmacy. Antimony is a brittle metal, of a silvery white color, with a tinge of blue, a lamellar texture, and crystalline fracture. When heated at the blowpipe, it melts with great readiness, and diffuses white vapors, possessing somewhat of a garlic smell. If thrown in this melted state $\mathrm{n}$ a sheet of flat paper, the globule sparkles, and bursts into a multitude of small spheroids, which retain their incandescence for a long time, and run about on the paper, leaving traces of the white oxyde produced during the combustion. When this oxvde is fused with borax, or other vitrifying matter, it imparts a yellow color to it. Metallic antimony, treated with hot nitric acid and in a concentrated state, is converted into a powder, called antimonions acid, which is altogether insoluble in the ordinary acid menstrua; a property by which the chemist can separate that metal from lead, iron, copper, bismuth, and silver. According to Bergman, the specific gravity of antimony is 6.86 ; but Haidinger makes the $s$ wedish native metal only $6 \cdot 646$. The alchemists had conceived the most brilliant hopes of this metal. The facility with which it is alloyed with gold, sinee its fumes alone render this most ductile metal immediately brittle, led them to assign to it a royal lineage, and to distinguish it by the title of regulus, or the little king.

Its chief employment now is in medicine, and in making the alloys called type metal, stereotype metal, music plaies, and Britannia metal; the first consisting of 6 of lead and 2 of antimony; the second of 6 of lead and 1 of antimony; the third of lead, tin, and antimony; and the fourth also of lead, tin, and antimony, with occasionally a little copper and bismuth.-For Glass of antimony, see PAstes.

ANTISEPTICS. Substances which counteract the spontaneous decomposition of animal and vegetable substances. These are chiefly culinary salt, nitre, spices, and sugar, which operate partly by inducing a change in the animal or vegetable fibres, and partly by rendering the aqueous constituent unsusceptible of decomposition. See Provisions, CURING OF.

ANVIL. A mass of iron, having a smooth and nearly flat top surface of stcel; upon which blacksmiths, and various other artificers, forge metals with the hammer. The common anvil is usually made of seven pieces : 1 , the core, or body ; $2,3,4,5$, the four corner pieces which serve to enlarge its base; 6 , the projecting end, which has a square hole for the reception of the tail or shank of a chisel on which iron bars may be cut through; and 7 , the beak, or horizontal cone round which rods or slips of metal may be turned into a circular form, as in making rings. These 6 pieces are welded separately to the first, or core, and then hammered into a uniform body. In manufacturing large anvils two hearths are needed, in order to bring each of the two pieces to be welded, to a proper heat by itself; and several men are employed in working them together briskly in the welding state, by heavy swing hammers. The steel facing is applied by welding in the same manner. The anvil is then hardened by heating it to a cherry red, and plunging it into cold water; a running stream being preferable to a pool or cistern. The facing should not be too thick a plate, for, when such, it is apt to crack in the hardening. The face of the anvil is now smoothed upon a grindstone, and finally polished with emery and crocus, for all delicate purposes of art.

The blacksmith, in general, sets his anvil loosely upon a wooden block, and in preference on the root of an oak. But the cutlers and file-makers fasten their anvils to a large block of stone, which is an advantage, for the more firmly and solidly this tnol is connected to the earth, the more efficacious will be the blows with the hammer on any object placed upon it.

AQUAFORTIS. Nitric acid, somewhat dilute, was so named by the alchemists on account of its strong solvent and corrosive operation upon many mineral, vegetable, and animal substances. See Nitric Acrd.

AQUA REGIA. The name given by the alchemists to that mixture of nitric and muriatic acids which was best fitted to dissolve gold, styled by them the king of the metals. It is now called nitro-muriatic acid.

AQUA VIT E. The name very absurdly given to alcohol, when used as an intexicating beverage. It has been the aqua mortis to myriads of the human racc; and will, probably, ere long destroy all the native tribes of North America and Australia. 
ARCHIL. A violet red paste used in dyeing, of which the substance called cudbear in Scotland (from Cuthbert, its first preparer in that form), is a modification. Two kinds of archil are distinguished in commerce, the archil plant of the Canaries, and that of Auvergne. The first is most esteemed: it is prepared from the lichen rocellus, which grows on rocks adjoining the sea in the Canary and Cape de Verd Islands, in Sardinia, Minorca, \&c., as well as on the rocks of Sweden. The second species is prepared from the lichen parellus, which grows on the basaltic rocks of Auvergne.

There are several other species of lichen which might be employed in producing an analogous dye, were they prepared, like the preceding, into the substance called archil. Hellot gives the following method for discovering if they possess this property. A little of the plant is to be put into a glass vessel; it is to be moistened with ammonia and lime-water in equal parts; a little muriate of ammonia (sal ammoniac) is added; and the small vessel is corked. If the plant be of a nature to afford a red dye, after three or four days, the small portion of liquid, which will run off on inclining the vessel, now opened, will be tinged of a crimson red, and the plant itself will have assumed this color. If the liquor or the plant does not take this color, nothing need be hoped for; and it is useless to attempt its preparation on the great scale. Lewis says, however, that he has tested in this way a great many mosses, and that most of them afforded him a yellow or reddish-brown color; but that he obtained from only a small number a liquor of a deep reả, which communicated to cloth merely a yellowish-red color.

Prepared archil gives out its color very readily to water, ammonia, and alcohol. Its solution in alcohol is used for filling spirit-of-wine thermometers; and when these thermometers are well freed from air, the liquor loses its color in some years, as Abbé Nollet observed. The contact of air restores the color, which is destroyed anew, in vacuo, in process of time. The watery infusion loses its color, by the privation of air, in a few days; a singular phenomenon, which merits new researches.

The infusion of archil is of a crimson bordering on violet. As it contains ammonia, which has already modified its natural color, the fixed alkalies can produce little change on it, only deepening the color a little, and making it more violet. Alum forms in it a precipitate of a brown red; and the supernatant liquid retains a yellowish-red color. The solution of tin affords a reddish precipitate, which falls down slowly; the supernatant liquid retains a feeble red color. The other metallic salts produce precipitates which offer nothing remarkable.

The watery solution of archil, applied to cold marble, penetrates it, communicating a beautiful violet color, or a blue bordering on purple, which resists the air much longer than the archil colors applied to other substances. Dufay says, that he has seen marble tinged with this color preserve it without alteration at the end of two years.

To dye with archil, the quantity of this substance deemed necessary, according to the quantity of wool or stuff to be dyed, and according to the shade to which they are to be brought, is to be diffused in a bath of water as soon as it begins to grow warm. The bath is then heated till it be ready to boil, and the wool or. stuff is passed through it without any other preparation, except keeping that longest in, which is to have the deepest shade. A fine gridelin, bordering upon violet, is thereby obtained; but this color has no permanence. Hence archil is rarely employed with any other view than to modify, heighten, and give lustre to the other colors. Hellot says, that having employed archil on wool boiled with tartar and alum, the color resisted the air no more than what had received no preparation. But he obtained from herb archil (l'orseille d'herbe) a much more durable color, by putting in the bath some solution of tin. The archil thereby loses its natural color, and assumes one approaching more or less to scarlet, according to the quantity of solution of tin emplnyed. This process must be executed in nearly the same manner as that of scarlet, except that the dyeing may be performed in a single bath.

Archil is frequently had recourse to for varying the different shades and giving them lustre; hence it is used for violets, lilachs, mallows, and rosemary flowers. To obtain a deeper tone, as for the deep soupes au vin, sometimes a little alkali or milk of lime is mixed with it. The suites of this browning may also afford agates, rosemary flowers, and other delicate colors, which cannot be obtained so beautiful by other processes. Alum cannot be substituted for this purpose; it not only does not give this lustre, but it degrades the deep colors.

The herb-archil is preferable to the archil of Auvergne, from the greater bloom which it communicates to the colors, and from the larger quantity of coloring matter. It has, besides, the advantage of bearing ebullition. The latter, moreover, does not answer with alum, which destroys the color; but the herb archil has the inconvenience of dyeing in an irregular manner, unless attention be given to pass the cloth through hot water as soon as it comes out of the dye.

Archil alone is not used for dyeing silk, unless for lilachs; but silk is frequently passed tırough a bath of archil, either before dyeing it in otier baths or after it has been dyed, in order to modifv different colors, or to give them lustre. Examples of this 
will be given in treating of the compound colors. It is sufficient here to point out now white silks are passed through the archil bath. The same process is performed with a bath more or less charged with this color, for silks already dyed.

Archil, in a quantity proportioned to the color desired, is to be boiled in a copper. The barr liquid is to be run off quite hot from the archil bath, leaving the sediment at the turned rouna tub of proper size, in which the silks, newly scoured with soap, are to be After this iein-sticks with much exactness, till they have attained the wishedArchil is in general a very ust receive one betling at the river.

communica es an alluring bloom, noredient in dyeing; but as it is rich in color, and ers $u_{1}$ iften tempted to abuse it, and to exceed the proportions that can add to the beauty without at wo same time injuring in a dangerous manner the permanence of the colors. Nevertheless, tho color obtained when solution of tin is employed, is less fugitive than without this addition : it is red, approaching to scarlet. Tin appears to be the only ingredient which can increase its durability. The solution of tin may be employed, not only in the dyeing bath, but for the preparation of the silk. In this case, by mixing the archil with other coloring substances, dyes may be obtained which have lustre with sufficient durability.

We have spoken of the color of the archil as if it were natural to it ; but it is, really, due to an alkaline combination. The acids make it pass to red, either by saturating the alkali, or by substituting themselves for the alkali.

The lichen which produces archil is subjected to another preparation, to make turnsole (litmus). This article is made in Holland. The lichen comes from the Canary Islands, and also from Sweden. It is reduced to a fine powder by means of a mill, and a certain proportion of potash is mixed with it. The mixture is watered with urine, and allowed to suffer a species of fermentation. When this has arrived at a certain degree, carbonate of lime in powder is added, to give consistence and weight to the paste, which is afterwards reduced into small parallelopipeds that are carefully dried.

The latest researches on the lichens, as objects of manufacture, are those of Westring of Stockholm. He examined 150 species, among which he found several which might be rendered useful. He recommends that the coloring matter should be extracted in the places where they grow, which would save a vast expense in curing, package, carriage, and waste. He styles the coloring substance itself cutbear, persio, or turnsole; and distributes the lichens as follows:-1st. Those which, left to themselves, exposed to moderate heat and moisture, may be fixed without a mordant upon wool or silk; such are the L. cinereus, amatonta, ventosus, corallinus, westringii, saxatilis, conspassus, barbatus, plicatus, vulpinus, \&c.

2. Those which develop a coloring matter, fixable likewise without mordant, but which require boiling and a complicated preparation; such are the lichens subcarneus, dillenii, farinaceus, jubatus, furfuraceus, pulmonareus, cornigalus, cocciferus, digilalus, ancialis, aduncus, \&c. Saltpetre or sea-salt is requisite to improve the lustre and fastness of the dye given by this group to silk.

3. Those which require a peculiar process to develop their color; such as those which become purple throngh the agency of stale urine or ammonia. Westring employed the following mode of testing:- He put three or four drachms of the dried and powdered lichen into a flask; moistened it with three or four measures of cold spring water; put the stuff to be dyed into the mixture, and left the flask in a cool place. Sometimes he added a little salt, saltpetre, quicklime, or sulphate of copper. If no color appeared, he then moistened the lichen with water containing one twentieth of sal ammoniac, and one tenth of quicklime, and set the mixture aside in a cool place from eight to fourteen days. There appeared in most cases a reddish or violet colored tint. Thus the lichen cinereus dyed silk a deep carmelite, and wool a light carmelite the $l$. physodes gave a yellowish-gray; the pustulatus, a rose red; sanguinarius, gray; tartareus, found on the rocks of Norway, Scotland, and England, dyes a crimson-red. In Jutland, cutbear is made from it, by grinding the dry lichen, sifting it, then setting it to ferment in a close vessel with ammonia. The lichen must be of the third year's growth to yield an abundant dye; and that which grows near the sea is the best. It loses half its weight by drying. A single person may gather from twenty to thirty pounds a day in situations where it abounds. No less than 2,239,685 pounds were manufactured at Christiansand, Flekkefiort, and Fakrsund, in Norway, in the course of the six years prior to 1812. Since more solid dyes of the same shade have been invented, the archil has gone much into disuse. Federigo, of Florence, who revived its use at the beginning of the fourteenth century, male such an immense fortune by its preparation, that his family became one of the grandees of that city, under the name of Oricellarii, or Rucellarii. For more than a century Italy possessed the exclusive art of making archil, obtaining the lichens from the islands of the Mediterranean. According to an official report of 1831 , Teneriffe furnished annually 500 quintals (cwts.) of lichen; 
the Canary Isles, 400; Fuerta Santura, 300 ; Lancerot, 300 ; Gomera, 300 ; isle of Fe: ro, 800. This business belonged to the crown, and brought it a revenue of 1500 piastres. The faimers paid from 15 to 20 reals for the right to gather each quintal. At that time the quintal fetched in the London market $4 l$. sterling.

Archil is perhaps too much used in some cloth factories of F.ngland, to the discredit of our dyes. It is said, that by its aid one third of the indigo may be saved in the hiveh of the black cloth by means of archil is also deceptive fine soft tint indiff of cutbrar will dye one pound of woollen cloth. A crimson red : vraned by adding to the decoctior: of archil a little salt of tin (muriate suru passing the cloth throush the bath, after it has been prepared by a mordap or tin and tartar. It must be aftet wards passed through hot water.

ARDENT SPIRIT. A Koonol of moderate strength.

AREOMETER OF BAUME'. This scale is much used by the French authors.

Specific Gravity Numbers corresponding with Baumé's Areometric Degrees.

\begin{tabular}{|c|c|c|c|c|c|c|c|c|c|}
\hline \multicolumn{6}{|c|}{ Liquids denser than Water. } & \multicolumn{4}{|c|}{ Less dense than Winter. } \\
\hline $\begin{array}{c}\text { De- } \\
\text { grees. }\end{array}$ & $\begin{array}{l}\text { Specific } \\
\text { gravity. }\end{array}$ & $\begin{array}{c}\text { De- } \\
\text { grees. }\end{array}$ & $\begin{array}{l}\text { Specific } \\
\text { gravity. }\end{array}$ & $\begin{array}{c}\text { De- } \\
\text { grees. }\end{array}$ & $\begin{array}{l}\text { Specific } \\
\text { gravity. }\end{array}$ & $\begin{array}{c}\text { De- } \\
\text { grees. }\end{array}$ & $\begin{array}{l}\text { Specifie } \\
\text { gravisy. }\end{array}$ & $\begin{array}{c}\text { De- } \\
\text { grees. }\end{array}$ & $\begin{array}{l}\text { Speificic } \\
\text { gravity. }\end{array}$ \\
\hline 0 & $1 \cdot 0000$ & 26 & $1 \cdot 2063$ & 52 & $1 \cdot 5200$ & 10 & $1 \cdot 0000$ & 36 & 0.5468 \\
\hline I & $1 \cdot 0066$ & 27 & $1 \cdot 2160$ & 53 & $1 \cdot 5353$ & 11 & 0.9932 & $3 \pi$ & $0 \cdot-39$ \\
\hline 2 & J.0133 & 28 & $1 \cdot 2258$ & 54 & 1.5510 & 12 & 0.9865 & 38 & 0.8391 \\
\hline 3 & 1.0201 & 29 & $1 \cdot 2358$ & 55 & $1.56 \tau 1$ & 13 & 0.9799 & 39 & $0 .-313$ \\
\hline 4 & 1.0270 & 30 & $1 \cdot 2459$ & 56 & 1.5833 & 14 & 0.9733 & 40 & 0.8295 \\
\hline 5 & $1 \cdot 0310$ & 31 & $1 \cdot 2562$ & 57 & $1 \cdot 6000$ & 15 & 0.9669 & 41 & 08219 \\
\hline 6 & $1 \cdot 0411$ & 32 & $1 \cdot 2667$ & 58 & $1 \cdot 61 \% 0$ & 16 & 0.9605 & 42 & $0 \cdot 5202$ \\
\hline 7 & $1 \cdot 0153$ & 33 & $1 \cdot 2 \pi 73$ & 59 & $1 \cdot 6344$ & $1 \%$ & 0.9542 & 43 & 0.5156 \\
\hline 8 & $1 \cdot 0556$ & 34 & 1.2881 & 60 & 1.6522 & 18 & 0.9180 & 44 & $(1-3111$ \\
\hline 9 & 1.0630 & 35 & $1 \cdot 2992$ & 61 & $1 \cdot 6 \% 05$ & 19 & 0.9120 & 45 & $0.5 n 66$ \\
\hline 10 & $1 \cdot 0 \% 04$ & 36 & $1 \cdot 3103$ & 62 & $1.68=9$ & 2) & 0.9359 & 46 & 0.8022 \\
\hline 11 & $1 \cdot 0 \div 50$ & 37 & $1 \cdot 3217$ & 63 & $1 . \% 0 \% 9$ & 21 & 0.9300 & $4 \pi$ & 0.7978 \\
\hline 12 & $1 \cdot 0857$ & 38 & $1 \cdot 3333$ & 64 & $1 \cdot \div 2 \div 3$ & 22 & 0.9241 & 48 & 0.7935 \\
\hline 13 & $1 \cdot 0935$ & 39 & $1 \cdot 3151$ & 65 & 1. & 23 & 0.9153 & 49 & $0 \cdot \div-92$ \\
\hline 14 & 11014 & 40 & $1 \cdot 3571$ & 66 & $1 . \div 6 \% 4$ & 24 & 0.9125 & 50 & $0.784 ?$ \\
\hline 15 & $1 \cdot 1095$ & 41 & $1 \cdot 3694$ & $6 \tau$ & $1 \cdot 7882$ & 25 & $0 \cdot 9068$ & 51 & $0 \% \div 80 \%$ \\
\hline 16 & $1 \cdot 1176$ & 42 & $1 \cdot 3818$ & 68 & $1 \cdot 8095$ & 26 & 09012 & 52 & $0 \cdot \% 66$ \\
\hline 17 & 1.1259 & 43 & $1 \cdot 3945$ & 69 & $1 \cdot 8313$ & 27 & 0.8957 & 53 & $0 \div \div-75$ \\
\hline 18 & $1 \cdot 1343$ & 44 & $1 \cdot 40 \div 4$ & 70 & $1 \cdot 853 \pi$ & 28 & 0.5902 & 54 & $0 \div 681$ \\
\hline 19 & $1 \cdot 1428$ & 45 & $1 \cdot 4206$ & $\pi 1$ & $1 \cdot 8 \approx 65$ & 29 & $0.68+8$ & 55 & $0 \cdot \div 643$ \\
\hline 20 & $1 \cdot 1515$ & 46 & $1 \cdot 4339$ & 72 & $1 \cdot 9000$ & 30 & $0.8 \% 95$ & 56 & 0.7604 \\
\hline 21 & $1 \cdot 1603$ & 47 & $1 \cdot 44 \div 6$ & 73 & 1.9241 & 31 & 0.8742 & 57 & 0.7656 \\
\hline 22 & $1 \cdot 1692$ & 45 & $1 \cdot 4615$ & 74 & 1.9457 & 32 & 0.8690 & $5 S$ & 0.7526 \\
\hline 23 & $1 \cdot 1 / 83$ & 49 & 1.4758 & 75 & $1 \cdot 9 ; 10$ & 33 & 0.8639 & 59 & $0.745 \%$ \\
\hline 24 & $1 \cdot 18 \div 5$ & 50 & 1.4902 & 76 & 2.0000 & 34 & 0.8558 & 60 & 0.7149 \\
\hline 25 & 11908 & 51 & 1.4951 & & & 35 & 0.8538 & 61 & $0-7411$ \\
\hline
\end{tabular}

ARGILLACEOUS EARTH. The earth of clay, called in chemistry aluriina, because it is obtained in greatest purity from alum.

ARGOL. Crude tartar; which see.

ARMS. Weapons of war. See Frre-ARMs for an account of this manufacture.

ARRACK. A kind of intoxicating beverage made in India, by distilling the fermented juice of the cocna-nut, the palmyra tree, and rice in the husk.

ARROW ROOT. The root of the maranta arundinacea, a plant which grows in the West Indies, furnishes, by pounding in mortars and elutriation through sieres, a peculiar species of starch, commonly, but improperly called arrow root. It is reckined fnore nourishing than the starch of wheat or potatnes, and is generally also freer from peculiar taste or flaror. The fresh root consists, according to Benzon, of 0.07 of rolatile oil; 26 of starch (23 of which are obtained in the form of powder, while the other 3 must be extra ted from the parenchyma in a paste by boiling water); 1.58 of regetahle albumen; 0.6 of a gummy extract; 0.25 of chloride of calcium; 6 of insoluble fibrine; and $65 \cdot 6$ of water.

The import duty upon arrow root from our own colonies is 1s. per ewt.; from foreign parts $2 \%$. per lib. In $1835,987,966$ lbs. were imported, of which only 6267 were exported; leaving 895,406 for home consumption. The total revenue derived that year from arrow root, was $518 l$. See Starch.

AR:ENIC. This metal occurs native in the state of oxyde, and also combined with salphur under the improper name of yellow and red arsenic, or orpiment and realgar - Arsenic is associated with a great many metallic ores; but it is chiefly extracted from those of cobalt, by roasting, in which case the white oxyde of arsenic, or, more correctly, the arsenions acid is obtained. This acid is introduced occasionally in small auantitios 
Into the materials of flint glass, either before their fusion, or in the melting pot. It serves to peruxydize the iron oxyde in the sand, and thereby to purify the budy of the glass: but an excess of it makes the glass milky.

Schec'e's green is a combination of this arsenious acid with oxyde of copper, or an arsenite of copper, and is described under this metal.

Ar:eniate of potash is prepared, in the small way, by exposing to a moderate heat in a crucible, a mixture of equal parts of white arsenic and nitre in powder. After fusion, the crucible is to be cooled; the contents being dissolved in hot water, and the solution filtered, will afford regular crystals on cooling. According to M. Berzelius, they are composed of arsenic acid, 63.87 ; potash, 26.16 ; and water, 9.97 . It is an acidulous salt, and is hence usually called the binarseniate, to denote that its compostion is 2 atoms of arsenic acid, and 1 of potash. This article is prepared upon the great scale, in Saxony, by melting nitre and arsenious acid together in a cylinder of cast-ron. A neutral arseniate also is readily formed, by saturating the excess of acid in the abore salt with potash; it does not crystallize. The acid arseniate is occasionally used in calico printing, for preventing certain points of the cotton cloth from taking on the mordant; with which view it is mixed up with gum water and pipe-clay into a paste, which is applied to such places with a block.

The extraction of arsenic from the cobalt ores, is performed at Altenberg and Reichenstein, in Silesia, with an apparatus, excellently contrived to protect the health of the smelters from the vapors of this most noxious metallic sublimate.

Figs. 20 to 23 represent the arsenical furnaces at Altenberg. Fig. 20 is a vertical section of the poison tower; fig. 21, a longitudinal section of the subliming furnace $A_{2}$ with the adjoining vault $\mathbf{B}$, and the poisun tower in part at $n$; fig. 22, the transverse section of the furnace A, of fig. 21 ; fig. 23, ground plan of the furnace A, wher the left half shows the part above, and the right the part below the muffle or oblong retort; $\mathbf{B}^{\prime}$ is the upper view, $\mathbf{B}^{\prime \prime}$ the ground plan of the vault $\mathbf{B}$, of fig. $21 ; m, n$, the base of the poison tower. In the several figures the same letters denote the same objects;
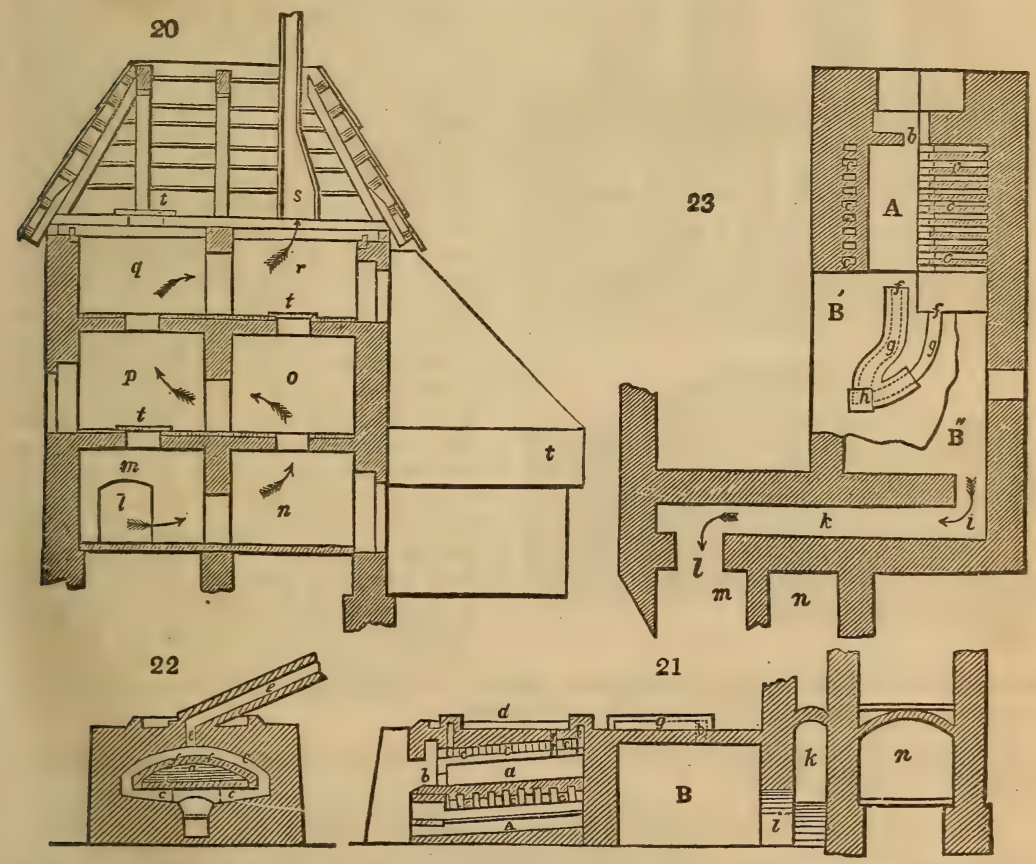

$a$ is the muffle; $b$ is its mouth for turning over the arsenical schlich, or ground ore c $c$, fire draughts or flues; $d$, an aperture for charging the muffle with fresh schlich; $c$, the smoke chimney; $f$, two channels or flues for the ascent of the arsenious fumes, which proceed to other two flues $g$, and then terminate both in $h$, which conducts the fumes into the vault в. They issue by the door $i$, into the conduit $k$, thence by $l$ into the spaces $m, n, 0, p, q, r$, of the tower. The incondensable gases escape by the chimney, $s$. 
The cover $t$, is removed after completion of the process, in order to push down the precipitate into the lower compartments.

The arsenious schlichs, to the amount of 9 or $10 \mathrm{cwt}$. for one operation (1 roast-post, or roasting round), are spread 2 or 3 inches thick upon the bottom of the muffle, heated with a brisk fire to redness, then with a gentler heat, in order to oxydize completely, before subliming, the arsenical ore. With this view the air must have free entrance, and the front aperture of the muffe must be left quite open. After 11 or 12 hours, the calcined materials are raked out by the mouth of the mufle, and fresh ones are introduced by the openings indicated above, which are closed during the sublimation.

The arsenious acid found in these passages is not marketable till it be re-sublimed in large iron pots, surmounted with a series of sheet iron drums or cast-iron cylit. Jers, upon the sides of which the arsenic is condensed in its compact glassy form. The top cylinder as furnished with a pipe, which terminates in a condensing chamber.

Figs. 24, 25, represent the arsenic refining furnaces at Reichenstein. Fig. 24 shows

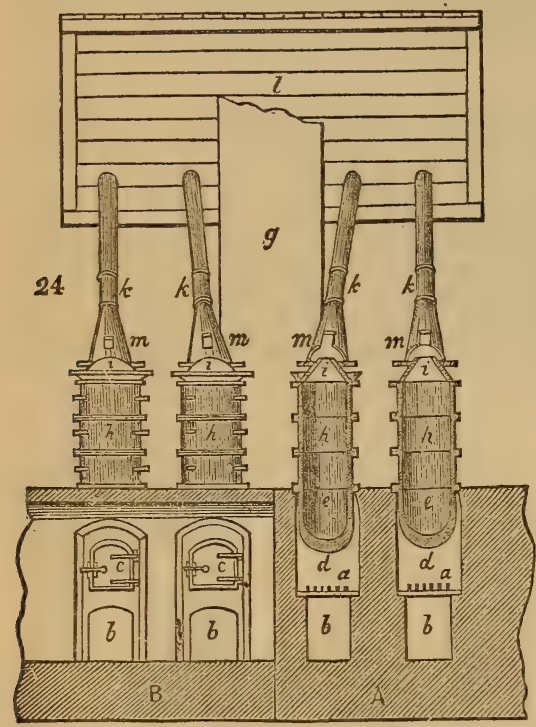

25

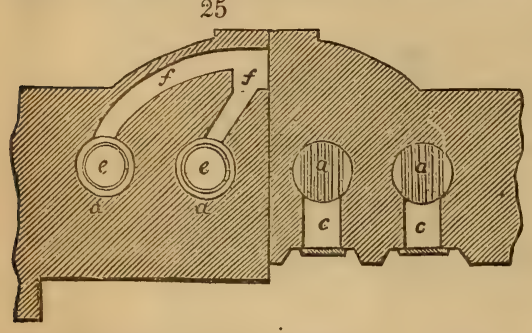
at $A$, a vertical section of the furnace, the kettle, and the surmounting drums or cylinders; over $\mathbf{B}$ it is seen in elevation; fig. 25 is a ground plan of the four fireplaces. $a$ is the grate; $b$, the ash-pit; $c$, the openings for firing; $d$, the fire-place; $e$, iron pots or liettles which are charged with the arsenious powder; $f$, the fire-flues proceeding to the common chimney $g ; h$, iron cylinders; $i$, caps; $k$, pipes leading to the poison vent $l ; m$, openings in the pipes for introducing the probing wires.

The conduct of the process is as follows:-The pot is filled nearly to its brim with $3 \frac{1}{2} \mathrm{cwt}$. of the arsenic meal, the cylinders are fitted on by means of their handles, and luted together with a mixture of loam, blood, and hair ; then is applied first a gentle, and after half an hour, a strong fire, whereby the arsenic is raised partly in the form of a white dust, and partly in crystals ; which, by the continuance of the heat, fuse together into a homogeneous mass. If the fire be too feeble, only a sublimate is obtained; but, if too violent, much of the arsenic is volatilized into the pipes. The workmen judge by the heat of the cylinders whether the operation be going on well or not. After 12 hours the furnace is allowed to cool, provided the probe wires show that the sublimation is over. The cylinders are then lifted off, and the arsenious glass is detached from their inner surface. According to the quality of the poison flour, it yields from $\frac{3}{4}$ to $\frac{7}{8}$ of its weight of the glass or enamel. Should any dark particles of metallic arsenic be intermixed with the glass, a fresh sublimation must be had recourse to.

The following is the product in cwts. of arsenious acid, at Altenberg and Reichenstein, in Silesia, in the years

\begin{tabular}{|l|r|r|r|r|r|r|r|r}
\hline & 1825. & 1826. & 1827 & 1828 & 1829. & 1830. & 1831. & 1832. \\
\cline { 2 - 6 } & & & & & & & & \\
White arsenic in a glassy & 2632 & 1703 & 2686 & 1900 & 2070 & 2961 & 3337 & 2730 \\
Sublimed arsenic in pow- & & & & & & \\
der - - - - & - & 27 & 33 & 31 & 30 & 44 & 69 & 38 \\
Yellow arsenical glass - & 112 & 11 & 56 & - & 86 & 313 & 60 & 219 \\
Red arsenical glass - & 3 & - & - & - & 28 & & & \\
\hline
\end{tabular}


ARTESIAN WELLS. Under this name is designated a cylindrical perforation, bored vertically down through one or more of the geological strata of the earth, till it passes into a porous gravel bed containing water, placed under such incumbent pressure as to make it mount up through the perforation, either to the surface or to a height con venient for the operation of a pump. In the first case, these wells are called spouting or overflowing. This property is not directly proportional to the depth, as might at first sight be supposed, but to the subjacent pressure upon the water. We do not know exactly the period at which the borer or sound was applied to the investigation of subterranean fountains, but we believe the first overflowing wells were made in the ancient French province of Artois, whence the name of Artesian. These wells, of such importance to arriculture and manufactures, and which cost nothing to keep them in condition, have been in use, undoubtedly, for several certuries in the northern departments of France, and the north of Italy; but it is not more than 50 or 60 years since they became known in England and Germany. There are now a great many such wells in London and its neighborhood, perforated through the immensely thick bed of the London clay, and even through some portions of the subjacent chalk. The boring of such wells has given much insight into the geological structure of many districts.

The formation of Artesian wells depends on two things, essentially distinct from each other: 1. On an acquaintance with the physical constitution, or nature, of the mineral structure of each particular country; and, 2. On the skilful direction of the processes by which we can reach the water level, and of those by which we can promote its ascent in the rube. We shall first treat of the best method of making the well, and then offer some general remarks on the other subjects.

The operations employed for penetrating the soil are entirely similar to those daily practised by the miner, in boring to find metallic veins ; but the well excavator must resort to peculiar expedients to prevent the purer water, which comes from deep strata, mingling with the cruder waters of the alluvial beds near the surface of the ground, as also to prevent the small perforation getting eventually filled with rubbish.

The cause of overflowing wells has been ascribed to a variety of circumstances. But, as it is now generally admitted that the numerous springs which issue from the ground proceed fiom the infiltration of the waters progressively condensed in rain, dew, snow, \&c. upon the surface of our globe, the theory of these interior streamlets becomes by no means intricate; being analogous to that of syphons and water jets, as expounded in the treatises on physics. The waters are diffused, after condensation, upon the surface of the soil, and percolate downwards, through the various pores and fissures of the geological strata, to be again united subterraneously in veins, rills, streamlets, or expanded films, of greater or less magnitude, or regularity. The beds traversed by numerous disjunctions will give occasion to numerous interior currents in all directions, which cannot be recovered, and brought to the day ; but when the ground is composed of strata of sand, or gravei very permeable to water, separated by other strata nearly impervious to it, reservoirs are formed to our hand, from which an abundant supply of water may be spontaneously raised. In this case, as soon as the upper stratum is perforated, the waters may rise, in consequence of the hydrostatic pressure upon the lower strata, and even overflow the surface in a constant stream, provided the level from which they proceed be proportionally higher.

The sheets of water occur principally at the separation of two contiguous formations; and, if the succession of the geological strata be considered, this distribution of the water will be seen to be its necessary consequence. In fact, the lower beds are frequently composed of compact sandstone or limestone, and the upper beds of clay. In level countries, the formations being almost always in horizontal beds, the waters which feed the Artesian wells must come from districts somerwat remote, where the strata are more elevated, as towards the secondary and transition rocks. The copious streams condensed upon the sides of these colder lands may be therefore regarded as the proper reservoirs of our wells.

Fig. 26 represents the manner in which the condensed water of the heavens dis-

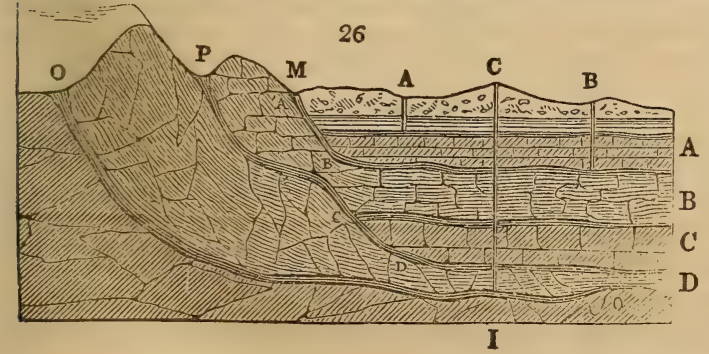
tributes itself under the surface of our globe. Here we have a geological section, showing the succession of the several formations, and the sheets or laminæe of water that exist at their boundaries, as well as in their sandy beds. The figure shows also very plainly that the height 
to which the water reascends in the bore of a well depends upon the height of the re servoir which supplies the sheet of water to which the well is perforated. Thus the well $A$, having gone down to the aqueous expanse $a a$, whose waters of supply are derived from the percolation $\mathrm{M}$, will afford rising waters, which will come to the surface; while in the well $\mathrm{B}$, supplied by the sheet $\mathrm{P}$, the waters will spout above the surface, and in the well $\mathrm{c}$ they will remain short of it. The same figure shows that these wells often traverse sheets of water, which rise to different heights. Thus, in the well c there are five columns of ascending waters, which rise to heights proportional to the points whence they take their origin. Several of these will be spouting or overflowing, but some will remain beneath the surface.

The situation of the intended well being determined upon, a circular hole is generally dug in the ground, about 6 or 8 feet deep, and 5 or 6 feet wide. In the centre of this hole the boring is carried on by two workmen below, assisted by a laborer above, as shown in fig. 27.

The handle ( $f g .28)$ having a female screw in the bottom of its iron shank, with a wooden bar or rail passing through the socket of the shank, and a ring at top, is the general agent to which all the boring implements are to be attached. A chisel

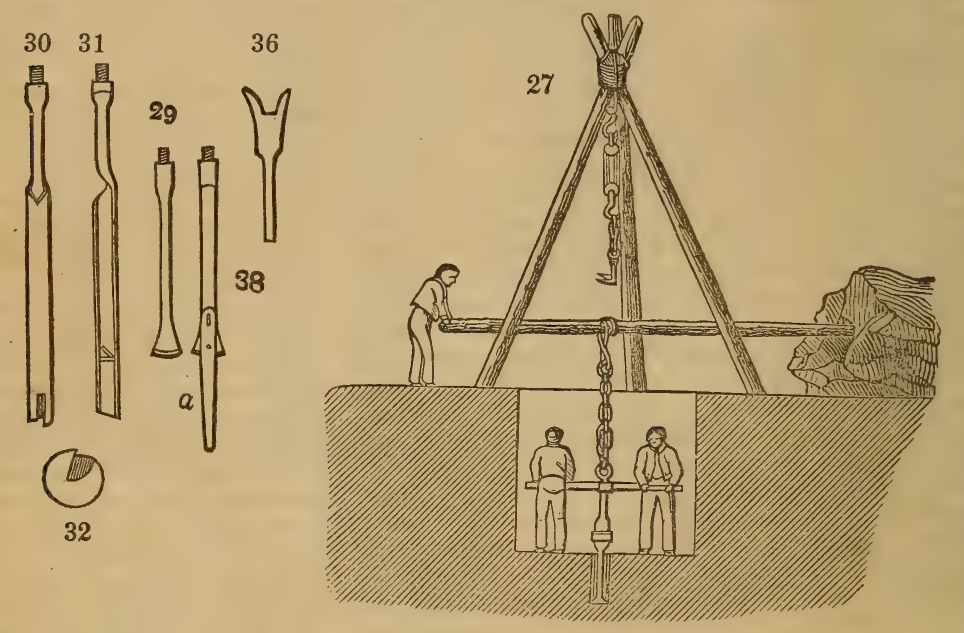

(fig. 29) is first employed, and connected
to this handle by its screw at top. If the
ground is tolerably soft, the weight of the
two workmen bearing upon the cross bar, and
occasionally forcing it round, will soon cause
the chisel to penetrate; but if the ground is
hard or strong, the workmen strike the chisel
down with repeated blows, so as to peck their
way, often changing their situation by walk-
ing round, which breaks the stones, or other
hard substances, that may happen to obstruet
its progress.
The labor is very considerably reduced by means of an elastic wooden pole, placed horizontally over the well, from which a chain is brought down, and attached to the ring of the handle. This pole is usually made fast at one end, as a fulcrum, by being set into a heap of heavy loose stones; at the other end the laborer above gives it a slight up and down vibrating motion, corresponding to the beating motion of the workmen below, by which means the elasticity of the pole in rising lifts the handle and pecker, and thereby very considerably diminishes the labor of the workmen. See fig. 27 .

When the hole has been thus opened by a chisel, as far as its strength would permit, the chisel is withdrawn, and a sort of cylindrical auger $(f i z .30)$ attached to the handle ( $f$ g. 28), for the purpose of drawing up the dirt or broken stones which have been disturbed by the chisel. A section of this auger is shown in fig. 31, by which the internal valve will be seen. The auger being introduced into the hole, and turned round by the workman, the dirt or broken stones will pass through the aperture at bottom (shown at 
fig. 32), and fill the cylinder, which is then drawn up, and discharged at the top of the auger, the valve preventing its escape at bottom.

In order to penetrate deeper into the ground, an iron rod, as $a$, fig. 33 , is now to be attached to the chisel, fig. 29, by screwing on to its upper end, and the rod is also fastened to the handle, fig. 28, by screwing into its socket. The chisel having thus become lengthened by the addition of the rod, it is again introduced into the hole; and the operation of pecking or forcing it down, is carried on by the workmen as before. When the ground has been thus perforated, as far as the chisel and its rod will reach; they must be withdrawn, in order again to introduce the auger, fig. 30, to collect and bring up the rubbish; which is done by attaching it to the iron rod, in place of the chisel. Thus, as the hole becomes deepened, other lengths of iron rods are added, by connecting them together, as $a b$ are in fig. 34. The necessity of frequently withdrawing the rods from the holes, in order to collect the mud, stones, or rubbish, and the great friction produced by the rubbing of the tools against its sides, as well as the lengths of rods augmenting in the progress of the operation, sometimes to the extent of several hundred feet, render it extremely inconvenient, if not impossible, to raise them by hand. A tripedal standard is therefore generally constructed by three scaffolding poles tied together, over the hole, as shown fig. 27, from the centre of which a wheel and axle, or a pair of pully blocks is suspended, for the purpose of hauling up the rods, and from which hangs the fork, fig. 35. This fork is to be brought down under the shoulder, near the top of each rod, and made fast to it by passing a pin through two little holes in the claws. The rods are thus drawn up, about seven feet at a time, which is the usual distance between each joint, and at every haul a fork, fig. 36, is laid hori. zontally over the hole, with the shoulders of the lower rod resting between its claws, by which means the rods are prevented from sinking down into the hole again, while the upper length is unscrewed and removed. In attaching and detaching these lengths of rod, a wrench, fig. 37, is employed, by which they are turned round, and the screws forced up to their firm bearing.

The boring is sometimes performed for the first sixty or a hundred feet, by a chisel of $2 \frac{1}{2}$ inches wide, and cleared out by a gouge of $2 \frac{1}{4}$ diameter, and then the hole is widened by a tool, such as is shown at fig. 38. This is merely a chisel, as fig. 29, four inches wide, but with a guide, $a$, put on at its lower part, for the purpose of keeping it in a perpendicular direction; the lower part is not intended to peck, put to pass down the hole previously made, while the sides of the chisel operate in enlarging the hole to four inches. The process, however, is generally performed at one operation, by a chisel of four inches wide, as fig. 29, and a gouge of three inches and three quarters, as $f i g .30$.

It is obvious that placing and displacing the lengths of rod, which is done every time that the auger is required to be introduced or withdrawn, must, of itself, be extremely troublesome, independent of the labor of boring, but yet the operation proceeds, when no unpropitious circumstances attend it, with a facility almost incredible. Sometimes, however, rocks intercept the way, which require great labor to penetrate; but this is always effected by pecking, which slowly pulverizes the stone. The most unpleasant circumstance attendant upon this business is the occasional breaking of a rod into the hole, which sometimes creates a delay of many days, and an incalculable labor in drawing up the lower portion.

When the water is obtained in such quantities and of such quality as may be required, the hole is dressed or finished by passing down it a diamond chisel, funnel mouthed, with a triangular bit in its centre; this makes the sides smooth previous to putting in the pipe. This chisel is attached to rods, and to the handle, as before described; and, in its descent, the workmen continually walk round, by which the hole is made smooth and cylindrical. In the progress of the boring, frequent veins of water are passed through; but, as these are small streams, and perhaps impregnated with mineral substances, the operation is carried on until an aperture is made into a main spring, which will flow up to the surface of the earth. This must, of course, depend upon the lerel of its source, which, if in a neighboring hill, will frequently cause the water to rise up, and produce a continued fountain. But if the altitude of the distant spring happens to be below the level of the surface of the ground where the boring is effected, it sometimes happens that a well of considerable capacity is obliged to be dug down to that level, in order to form a reservoir, into which the water may flow, and whence it must be raised by a pump; while, in the former instance, a perpetual fountain may be obtained. Hence, it will always be a matter of doubt, in level countries, whether water can be procured which would flow near to or over the surface; if this cannot be effected, the process of boring will be of little or no advantage, except as an experiment to ascertain the fact.

In order to keep the strata pure and uncontaminated with mineral springs, the hole is cased, for a considerable depth, with a metallic pipe, about a quarter of an inch 
smaller than the bore. This is generally made of tin (though sometimes of copper or lead) in convenient lengths; and, as each length is let down, it is held by a shoulder resting in a fork, while another length is soldered to it; by which means a continuous pipe is carried through the bore, as far as may be found necessary, to exclude land springs, and to prevent loose earth or sand from falling in, and choking the aperture.

Mr. John Good, of Tottenham, who had been extensively employed in boring the earth for water, obtained a patent, in Aug. 1823, for certain improved implements contrived by him to facilitate his useful labors; a description of which cannot fail to be interesting.

The figures annexed exhibit these ingenious tools ; fig. 39 is an auger, to be connected

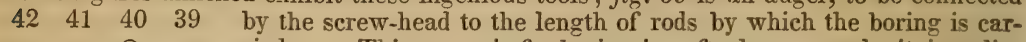

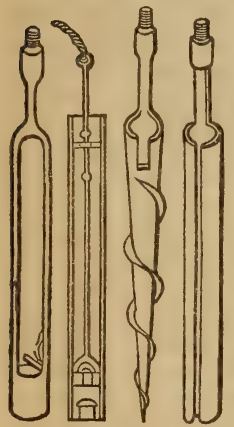
ried on. This auger is for boring in soft clay or sand; it is cylindrical, and has a slit or opening from end to end, and a bit, or cutting-piece at bottom. When the earth is loose or wet, an auger of the same form is to be employed, but the slit or opening reduced in width, or even without a slit or opening. A similar auger is used for cutting through chalk; but the point or bit at bottom should then project lower, and, for that purpose, some of these cylindrical augers are made with moveable bits, to be attached by serews, which is extremely desirable in grinding them to cutting edges. Fig. 40 is a hollow conical auger, for boring loose sandy soils; it has a spiral cutting edge coiled round it, which, as it turns, causes the loose soil to ascend up the inclined plane, and deposite itself in the hollow within. Fig. 41 is a hollow cylinder or tube, shown in section, with a foot-valve, and a bucket to be raised by a rod and cord attached at the top; this is a pumping tool, for the purpose of getting up water and sand that would not rise by the auger. When this cylinder is lowered to the bottom of the bore, the bucket is lifted up by the rod and cord, and descends again by its own gravity, having a valve in the bucket, opening upwards, like other lift pumps; which, at every stroke, raises a quantity of water and sand in the cylinder equal to the stroke; the ascent and descent of the bucket being limited by a guide-piece at the top of the cylinder, and two small knobs upon the rod which stop against the cross-guide. Fig. 42 is a tool for getting up broken rods. It consists of a small cylindrical piece at bottom, which the broken rod slips through when it is lowered, and a small catch with a knife-edge, acted upon by a back-spring. In rising, the tool takes hold of the broken rod, and thereby nables the workman at top to draw it up. Another tool for the same purpose, is shown at fig. 43, which is like a pair of tongs ; it is intended to be slidden down the bore, and for the broken rod to pass between the two catches, which, pressed by back-springs, will, when drawn up, take fast hold of the broken rod.

Fig. 44 is a tool for widening the hole, to be connected, like ail the others, to the end

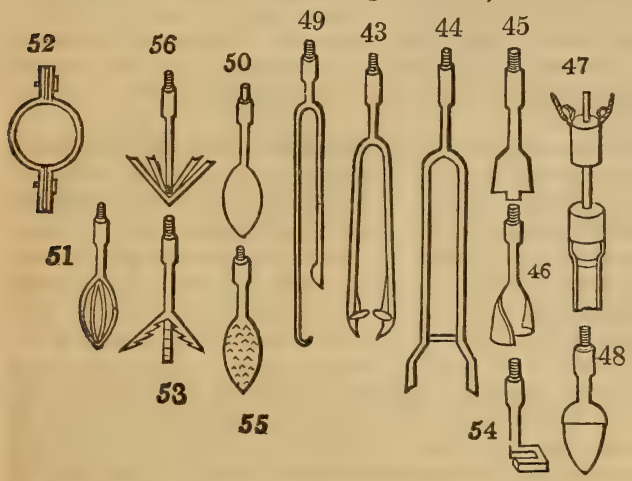
of the length of rods passed down the bore; this tool has two cutting-pieces extending on the sides at bottom, by which, as the tool is turned round in the bore, the earth is peeled away. Fig. 45 is a chisel, or punch, with a projecting piece to be used for penetrating through stone; this chisel is, by rising and falling, made to peck the stone, and pulverize it ; the small middle part breaking it away first, and afterwards the broad part coming into action. Fig. 46 is another chisel, or punching tool, twisted on its cutting edge, which breaks away

a greater portion of the stone as it beats against it.

The manner of forcing down lengths of cast-iron pipe, after the bore is formed, is shown at fig. 47; the pipe is seen below in the socket, at the end of which a block is inserted; and from this hlock a rod extends upwards, upon which a weight at top slides. To this weight cords are shown to be attached, reaching to the top of the bore; where the workmen alternately raise the weight and let it fall, which, by striking upon the block in its middle, beats down the pipe by a succession of strokes; and when one length of pipe has, by these means, been forced down, another length is introduced nto 
the socket of the former. Another tool for the same purpose is shown at fig. 48 , which is formed like an acorn; the raised part of the acorn strikes against the edge of the pire, and by that means, it is forced down the bore. When it happens that an auger breaks in the hole, a tool similar to that shown at fig. 49 is introduced; on one side of this tool a curred piece is attached, for the purpose of a guide, to conduct it past the cylindrical auger; and at the end of the other side is a hook, which, taking hold of the bottom edge of the auger, enables it to be drawn up.

Wrought iron, copper, tin, and lead pipes, are occasionally used for lining the bore; and as these are subject to bends and bruises, it is necessary to introduce tools for the purpose of straightening their sides. One of these tools is shown at fig. 50, which is a bow, and is to be passed down the inside of the pipe, in order to press out any dents. Another tool, for the same purpose, is shown at fig. 51, which is a double bow, and may be turned round in the pipe for the purpose of straightening it all the way down; at fig. 52, is a pair of clams, for turning the pipe round in the hole while driving.

When loose stones lie at the bottom of the hole, which are too large to be brought up by the cylindrical auger, and cannot be conveniently broken, then it is proposed to introduce a triangular claw, as fig. 53, the internal notches of which take hold of the stone, and as the tool rises, bring it up. For raising broken rods, a tool lilie fig. 54 is sometimes employed, which has an angular claw that slips under the shoulder of the rod, and holds it fast while drawing up.

In raising pipes it is necessary to introduce a tool into the inside of the pipe, by which it will be held fast. Fig. 55 is a pine-apple-tool for this purpose; its surface is cut like a rasp, which passes easily down into the pipe, but catches as it is drawn up; and by that means brings the pipe with it. Fig. 56 is a spear for the same purpose, which easily enters the pipe by springing; at the ends of its prongs there are forks which stick into the metal as it is drawn up, and thereby raise it.

These are the new implements, for which the patent was granted. In the process of boring, there does not appear to be anything new proposed; but that these several tools are to be employed for boring, packing, and otherwise penetrating, raising the earth, and extracting broken or injured tools. There are also suggestions for employing long buckets, with valves opening upward in their bottoms, for the purpose of drawing water from these wells when the water will not flow over the surface; also lift pumps, with a succession of buckets for the same purpose. But as these suggestions possess little if any novelty, it cannot be intended to claim them as parts of the patent.

ASPHALTUM. Native bitumen, so called from the lake Asphaltites.

ASSAY and ASSAYING. (Coupellation, Fr.; Abtreiben auf der capelle, Germ.) This is the process by which the quality of gold and silver bullion, coin, plate, or trinkets is ascertained with precision, or by which the quantity of either or both these precious metals is determined in any given alloy. It is, therefore, a case of chemical analysis, in which peculiar methods are employed to attain the object in view with accuracy and despatch. Assaying has been also extended, of late years, to determine the quantity of palladium and platina in certain bullion and gold dust brought from Brazil.

The art of assaying gold and silver by the cupel, is founded upon the feeble affinity which these metals have for oxygen, in comparison with copper, tin, and the other cheaper metals; and on the tendency which the latter metals have to oxydize rapidly in contact with lead at a high temperature, and sink with it into any porous earthy ressel in a thin glassy or vitriform state. The porous vessel may be made either of woodashes, freed from their soluble matter by washing with water; or, preferably, of burned bones reduced to a fine powder.

The lead added to the silver or gold to be assayed, serves chiefly to dissolve the oxydized copper, whence it appears that the quantity of lead requisite for silver assays, ought to be directly proportional to the quantity which the silver and copper would separately require. It has been found by experiment, that 16 parts of lead are quite sufficient to pass 1 of copper through the cupel; and that $\frac{3}{10}$ of lead presents the most suitable proportion for passing one of silver. From these principles, however, if we should always regard the dose of lead to be employed for any alloy as being equal to $16 \times \mathrm{C}+\frac{3}{30} \times \mathrm{S}$ we should certainly commit an error. The phenomena of cupellation is of a more complex nature. Long practice and delicate trials alone can guide to the proper quantity of lead to be employed for every various state of the alloy. The following Table contains the results of M. D'Arcet's elaborate experiments upon this subject :- 


\begin{tabular}{|c|c|c|c|}
\hline \multicolumn{2}{|c|}{ Alloy. } & \multirow{2}{*}{ Lead for 1 of Alloy. } & \multirow{2}{*}{$\begin{array}{c}\text { Ratio of the Copper to } \\
\text { the Lead. }\end{array}$} \\
\hline Silver. & Copper. & & \\
\hline 1000 & 0 & $\frac{3}{10}$ & 0 \\
\hline 950 & 50 & 3 & $1: 60$ \\
\hline 900 & 100 & 7 & $1: 70$ \\
\hline 800 & 200 & 10 & $1: 50$ \\
\hline 700 & 300 & 12 & $1: 40$ \\
\hline 600 & 400 & 14 & $1: 35$ \\
\hline 500 & 500 & 16 or 17 & $1: 32$ \\
\hline 400 & 600 & $16-17$ & $1: 26 \cdot 7$ \\
\hline 300 & 700 & $16-17$ & $1: 22.9$ \\
\hline 200 & 800 & $16-17$ & $1: 20$ \\
\hline 100 & 900 & $16-17$ & $1: 1 \% \cdot 8$ \\
\hline 0 & 1000 & $16-17$ & $1: 16$ \\
\hline
\end{tabular}

Bismuth may be used as a substitute for lead in cupellation; two parts of it being nearly equivalent to three $\cdot$ of lead. But its higher prices will prevent its general introduction among assay masters.

We begin this assay process by weighing, in a delicate balance, a certain weight of the metallic alloy; a gramme (=15.444 gr.) is usually taken in France, and 12 grains in this country. This weight is wrapped up in a slip of lead foil or paper, should it consist of several fragments. This small parcel, thus enveloped, is then laid in a watch glass or a capsule of copper, and there is added to it the proportion of lead suited to the quality of alloy to be assayed; there being less lead, the finer the silver is presumed to be. Those who are much in the habit of cupellation can make good guesses in this way; though it is still guess work, and often leads to considerable error, for if too much lead be used for the proportion of baser metal present, a portion of the silver is wasted; but if too little, then the whole of the copper, \&c. is not carried off, and the button of fine silver remains more or less impure. The most expert and experienced assayer by the cupel, produces merely a series of approximate conjectural results which fall short of chemical demonstration and certainty in every instance. The lead must be, in all cases, entirely free from silver, being such as has been revived from pure litharge; otherwise errors of the most serious kind would be occasioned in the assays.

The best cupels weigh $12 \frac{1}{2}$ grammes, or 193 grains. The cupels allow the fused oxydes to flow through them as through a fine sieve, but are impermeable to the particles of metals; and thus the former pass readily down into their substance while the latter remain upon their surface; a phenomenon owing to the circumstance of the glassy oxydes moistening, as it were, the bone-ash powder, whereas the metals can contract no adherence with it. Hence also the liquid metals preserve a hemispherical shape in the cupels, as quicksilver does in a cup of glass, while the fused oxyde spreads over, and penetrates their substance like water. A cupel may be regarded, in some measure, as a filter permeable only to certain liquids.

If we put into a cupel, therefore, two metals, of which the one is unalterable in the air, the other susceptible of oxydizement, and of producing a very fusible oxyde, it is obvious that, by exposing both to a proper degree of heat, we shall succeed in separating them. We should also succeed, though the oxyde were infusible, by placing it in contact with another one, which may render it fusible. In both cases, however, the metal from

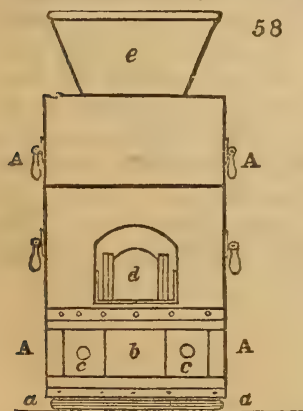
which we wish to part the oxydes must not be volatile; it should also melt, and form a button at the heat of cupellation; for otherwise it would continue disseminated, attached to the portion of oxyde spread over the cupel, and incapable of being collected.

The furnace and implements used for assaying in the Royal Mint and the Goldsmiths' Hall, in the eity of Lon don, are the following:-

A A A A, fig. 58 , is a front elevation of an assay furnace; a $a$, a view of one of the two iron rollers on which the furnace rests, and by means of which it is moved forward or backward; $b$, the ash-pit; $c c$ are the ash-pit dampers, which are moved in a horizontal direction towards each other for regulating the draught of the furnace; $d$, the door, or opening, by which the cupels and assays are introduced into the muffle; $e$, a moveable funnel or chimney by which the draught of the furnace is increased. 
В в в В, fig. 59 , is a perpendicular section of fig. $58 ; a$, end vice of the rollers;

59

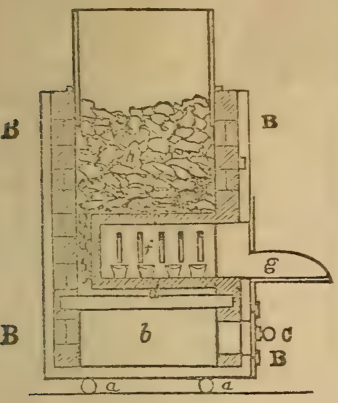

$b$ the ash-pit; $c$ one of the ash-pit dampers; $d$ the grate, over which is the plate upon which the muffle rests, and which is covered with loam nearly one inch thick; $f$ the muffle in section representing the situation of the cupels; $g$ the mouth-plate, and upon it are laid pieces of charcoal, which during the process are ignited, and heat the air that is allowed to pass over the cupels, as will be more fully explained in the sequel; $h$ the interior of the furnace, exhibiting the fuel.

The total height of the furnace is 2 feet $6 \frac{1}{2}$ inches; from the bottom to the grate, 6 inches; the grate, muffle, plate, and bed of loam, with which it is covered, 3 inches; from the upper surface of the grate to the commencement of the funnel e, fig. 58, $21 \frac{1}{2}$ inches; the funnel $e, 6$ inches. The square of the furnace which receives the muffle and fuel is $11 \frac{3}{4}$ inches by 15 inches. The external sides of the furnace are made of plates of wrought iron, and are lined with a 2-inch fire-brick.

c c c c, fig. 60 , is a horizontal section of the furnace over the grate, showing the width
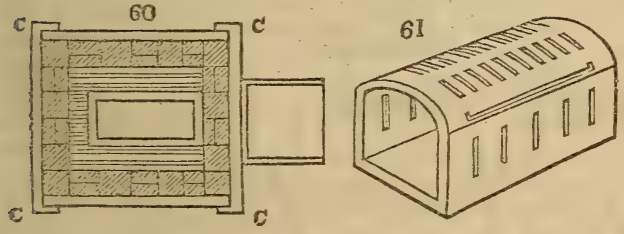
of the mouth-piece, or plate of wrought iron, which is 6 inches, and the opening which receives the mufle-plate.

Fig. 61, represents the muffle or pot, which is 12 inches long, 6 inches broad inside; in the clear $6 \frac{3}{4}:$ in height $4 \frac{3}{2}$ inside measure, and nearly $5 \frac{1}{2}$ in the clear.

Fig. 62, the muffle-plate, which is of the same size as the bottom of the muffle.

Fig. 63, is a representation of the sliding-door of the mouth-plate, as shown at $d$, in jig. 58.

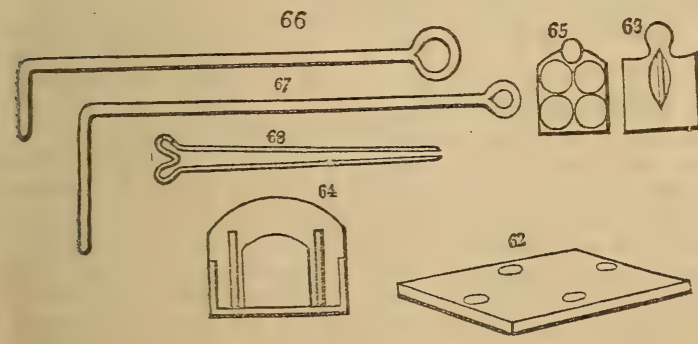

69

\begin{tabular}{|l|l|l|l|l|}
\hline 41 & 42 & 43 & 44 & 45 \\
\hline 36 & 37 & 58 & 33 & 40 \\
\hline 31 & 32 & 33 & 34 & 35 \\
\hline 26 & 27 & 28 & 25 & 30 \\
\hline 21 & 22 & 23 & 24 & 25 \\
\hline 16 & 17 & 15 & 19 & 20 \\
\hline 11 & 12 & 13 & 14 & 15 \\
\hline 6 & 7 & 8 & 9 & 10 \\
\hline 1 & 2 & 3 & 4 & 5 \\
\hline
\end{tabular}

Fig. 64 , a front view of the mouth-plate or piece, $d$, fig. 58 .

Fig. 65, a representation of the mode of making, or shutting up with pieces of char. coal, the mouth of the furnace.

Fig. 66, the teaser for cleaning the grate.

Fig. 67, a larger teaser, which is introduced at the top of the furnace, for keeping a complete supply of charcoal around the muffle.

Fig. 68 , the tongs used for charging the assays into the cups.

Fig. 69, represents a board of wood used as a register, and is divided into 45 equal compartments, upon which the assays are placed previously to their being introduced into the furnace. When the operation is performed, the cupels are placed in the furnace in situations corresponding to these assays on the board. By these means all confusion is aroided, and without this regularity it would be impossible to preserve the accuracy which the delicate operations of the assayer require.

I shall now proceed to a description of a small assay furnace, invented by Messrs. Anfrye and d'Arcet, of Paris. They term it, Le Petit Fourneau à Coupelle. Fig. 70 represents this furnace, and it is composed of a chimney or pipe of wrought iron $a$, and of the furnace B. It is $17 \frac{1}{2}$ inches high, and $7 \frac{1}{4}$ inches wide. The furnace is formed of three pieces; of a dome $\mathrm{A}$; the hody of the furnace $\mathrm{B}$; and the ash-pit $\mathrm{c}$, which is 
used as the base of the furnace, figs. 70 and 71. The principal piece, or body of the furnace, $B$, has the form of a hollow tower, or of a hollow cylinder, flattened equally at the two opposite sides parallel to the axis, in such a manner that the horizontal section is elliptical. The foot which supports it is a hollow truncated cone flattened in like manner upon the two opposite sides, and having consequently for its basis two ellipses

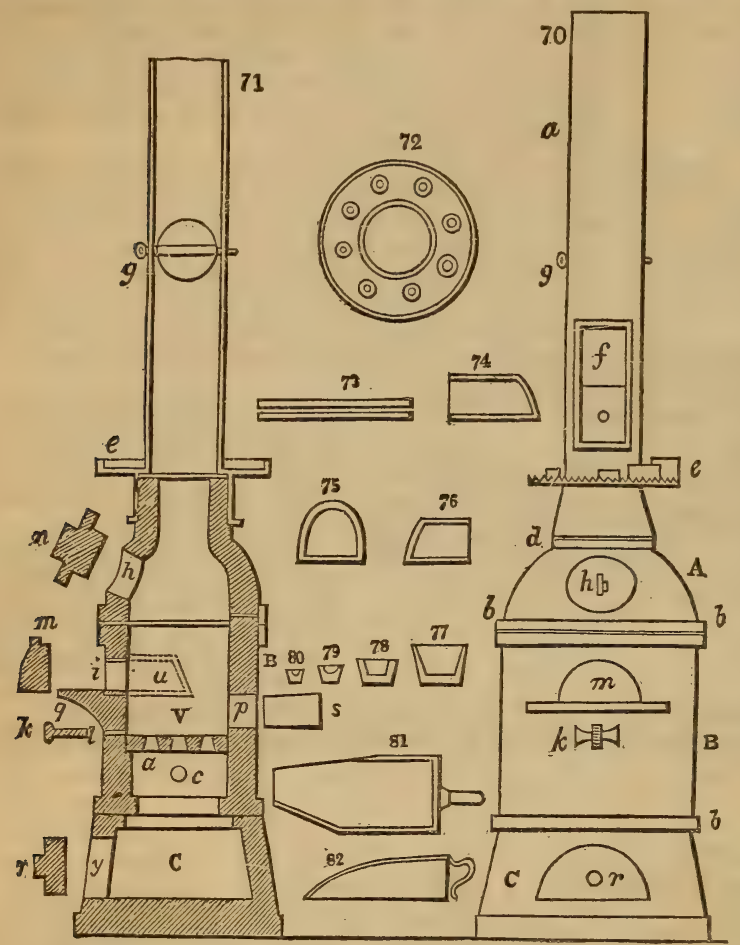
of different diameters; the smallest ought to be equal to that of the furnace, so that the bottom of the latter may exactly fit it. The dome, which forms an arch above the furnace, has also its base elliptical, while that of the superior orifice by which the smoke goes out preserves the cylindrical form. The tube of wrought iron is 18 inches long and $2 \frac{1}{2}$ inches diameter, having one of its ends a little enlarged, and slightly conical, that it may be exactly fitted or jointed upon the upper part of the furnace dome $d$, fig. 70. At the union of the conical and cylindrical parts of the tube, there is placed a small gallery of iron, e, fig. 70, 71. See also a plan of it, fig. 72. This gallery is both ingenious and useful.

Upon it are placed the cupels, which are thus annealed during the ordinary work of the furnace, that they may be introduced into the muffe, when it is brought into its proper degree of heat. A little above this gallery is a door $f$, by which, if thought proper, the charcoal could be introduced into the furnace; abore that there is placed at $\mathrm{g}$ a throttle valve, which is used for regulating the draught of the furnace at pleasure. Messrs. Anfrye and d'Arcet say, that, to give the furnace the necessary degree of heat so as to work the assays of gold, the tube must be about 18 inches abore the gallery, for annealing or heating the cupels. The circular opening $h$, in the dome, fig. 70 , and as seen in the section, fig. 71, is used to introduce the charcoal into the furnace: it is also used to inspect the interior of the furnace, and to arrange the charcoal round the muffle. This opening is kept shut during the working of the furnace, with the mouthpiece, of which the face is seen at $n, f i g .71$.

The section of the furnace, fig. 71 , presents sereral openings, the principal of which is that of the muffle; it is placed at $i$; it is shut with the semicircular door $m$, fig. 70 , and seen in the section $m$, fig. 71. In front of this opening, is the table or shelf, upon which the door of the muffle is made to advance or recede; the letter $q$, fig. 71 , shows the face, side, and cross section of the shelf, which makes part of the furnace. Immediately under the shelf, is a horizontal slit, $l$, which is pierced at the level of the upper part of the grate, and used for the introduction of a slender rod of iron, that the grate may be easily kept clean. This opening is shut at pleasure, by the wedge represented at $k$, figs. 70 , and 71 .

Upon the back of the furnace is a horizontal slit $p$, fig. 71, w:ich supports the firebrick, $s$, and upon which the end of the muffle, if necessary, may rest ; $u, f i g .71$, is the opening in the furnace where the muffle is placed.

The plan of the grate of the furnace is an ellipse: fig. 73 , is a horizontal riew of it. The dimensions of that ellipsis determine the general form of the furnace, and thicliness of the grate. To give strength and solidity to the grate, it is encircled by a bar or honp of 
iron. There is a groovc in which the hoop of iron is fixed. The holes of the grate are truncated cones, haring the greater base below, that ti.e ashes may more easily fall into the ash-pit. The letter $v$, fig. 71 is ows the form of these holes. The grate is support. ed by a small bank or sheif, making part of the furnace, as seen at $a$, fig. 71 .

The ash-pit, $\mathrm{c}$, has an opening $y$ in front, $f i g .71$; and is shut when necessary by the mouth-piece $r$, figs. 70 and 71 .

To give strength and solidity to the furnace, it is bound with hoops of iron, at $b, b, b, b$, fiร. 70 .

Figs. 74, 75, 76, are views of the muffle.

Fig. 77 is a view of a crucible for annealing gold.

Figs. 78, 79, 80, are cupels of various sizes, to be used in the furnace. They are the same as those used by assayers in their ordinary furnaces.

Figs. 81 and 82 are views of the hand-shovels, used for filling the furnace with charcoal; they should be made of such size and form as to fit the opening $h$, in figs. 70 and 71.

The smaller pincers or tongs, by which the assays are charged into the cupels, and by which the latter are withdrawn from the furnace, as well as the teaser for cleaning the grate of the furnace, are similar to those used in the British Mint.

In the furnace of the Mint above described, the number of assays that can be made at one time is 45. The same number of cupels are put into the muffle. The furnace is then filled with charcoal to the top, and upon this are laid a few pieces already ignited. In the course of three hours, a little more or less, according to circumstances, the whole is ignited; during which period, the muffle, which is made of fire-clay, is gradually beated to redness, and is prevented from cracking; which a less regular or more sudden uncrease of temperature would not fail to do : the cupels, also, become properly annealed. All moisture being dispelled, they are in a fit state to receive the piece of silver or gold to be assayed.

The greater care that is exercised in this operation, the less liable is the assayer to accidents from the breaking of the muffle; which is both expensive and troublesome to fit properly into the furnace.

The cupels used in the assay process, are made of the ashes of burnt bones (phosphate of lime). In the Royal Mint, the cores of ox-horn are selected for this purpose; and the aashes produced are about four times the expense of the bone-ash, used in the process of cupellation upon a large scale. So much depends upon the accuracy of an assay of gold or silver, where a mass of $15 \mathrm{lbs}$. troy in the first, and 60lbs troy in the second instance is determined by the analysis of a portion not exceeding 20 troy grains, that every precaution which the longest experience has suggested, is used to obtain an accurate result. Hence the attention paid to the selection of the most proper materials for making the cupels.

The cupels are formed in a circular mould made of cast steel, very nicely turned, by which means they are easily freed from the mould when struck. The bone-ash is used moistened with a quantity of water, sufficient to make the particles adhere firmly together. The circular mould is filled, and pressed level with its surface; after which, a pestle or rammer, having its end nicely turned, of a globular or convex shape, and of a size equal to the degree of concavity wished to be made in the cupel for the reception of the assay, is placed upon the ashes in the mould, and struck with a hammer until the cupel is properly formed. These cupels are allowed to dry in the air for some time before they are used. If the weather is fine, a fortnight will be sufficient.

An assay may prove defective for several reasons. Sometimes the button or bead sends forth crystalline vegetations on its surface with such force, as to make one suppose a portion of the silver may be thrown out of the cupel. When the surface of the bead is dull and flat, the assay is considered to have been too hot, and it indicates a loss of silver in fumes. When the tint of the bead is not uniform, when its inferior surface is bubbly, when yellow scales of oxyde of lead remain on the bottom of the cupel, and the bead adheres strongly to it, by these signs it is judged that the assay has been too cold, and that the silver retains some lead.

Lastly, the assay is thought to be good if the bead is of a round form, if its upper surface is brilliant, if its lower surface is granular and of a dead white, and if it separates readily from the cupel.

After the lead is put into the cupel, it gets immediately covered with a coat of oxyde, which resists the admission of the silver to be assayed into the melted metal; so that the alloy cannot form. When a bit of silver is laid on a lead bath in this predicament, we see it swim about for a long time without dissolving. In order to avoid this result, the silver is wrapped up in a bit of paper; and the carbureted hydrogen generated by its combustion reduces the film of the lead oxyde, gives the bath immediately a bright metallic lustre, and enables the two metals readily to combine.

As the heat rises, the oxyde of lead flows round about over the surface, till it is ab- 
sorbed by the cupel. When the lead is wasted to a certain degree, a very thin film ot if only remains on the silver, which causes the iridiscent appearance, like the colors of soap-bubbles; a phenomenon, called by the old chemists, fulguration.

When the cupel cools in the progress of the assay, the oxygenation of the lead ceases; and, instead of a very liquid vitreous oxyde, an imperfectly melted oxyde is formed, which the cupel cannot absorb. To correct a cold assay, the temperature of the furnace ourht to be raised, and pieces of paper ought to be put into the cupel, till the oxyde of lead which adheres to it be reduced. On keeping up the heat, the assay will resume its ordinary train.

Pure silver almost always vegetates. Some traces of copper destroy this property, which is obviously due to the oxygen which the silver can absorb while it is in fusion, and which is disengaged the moment it solidifies. An excess of lead, by removing all the copper at an early stage, tends to cause the vegetation.

The brightening is caused by the heat evolved, when the button passes from the liquid to the solid state. Many other substances present the same phenornenon.

In the above operation it is necessary to employ lead which is very pure, or at least free from silver. That kind is called poor lead.

It has been observed at all times, that the oxyde of lead carries off with it, into the cupel, a little silver in the state of an oxyde. This effect becomes less, or even disappears, when there is some copper remaining; and the more copper, the less chance there is of any silver being lost. The loss of silver increases, on the other hand, with the dose of lead. Hence the reason why it is so important to proportion the lead with a precision which, at first sight, would appear to be superfluous. Hence, also, the reason of the attempts which have, of late years, been made to change the whole system of silver assays, and to have recourse to a method exempt from the above causes of error.

M. d'Arcet, charged by the Commission of the Mint in Paris, to examine into the justice of the reclamations made by the French silversmiths against the public assays, ascertained that they were well founded; and that the results of cupellation gave for the alloys betwen 897 and 903 thousandths (the limits of their standard coin) an inferior standard, by from 4 to 5 thousandth parts, from the standard or title which should result from the absolute or actual alloy.

The mode of assay shows, in fact, that an ingot, experimentally composed of 900 thousandths of fine silver, and 100 thousandths of copper, appears, by cupellation, to be only, at the utmost, 896 or 897 thousandths; whereas fine silver, of 1000 thousandths, comes out nearly of its real standard. Consequentiy a director of the Mint, who should compound his alloy with fine silver, would be obliged to employ 903 or 904 thousandths, in order that, by the assay in the laboratory of the Mint, it should appear to have the standard of 900 thousandths. These 3 or 4 thousandths would be lost to him, since they would be disguised by the mode of assay, the definitive criterion of the quantity of silver, of which the government keeps count from the coiner of the money.

From the experiments subsequently made by M. d'Arcet, it appears that silver assays always suffer a loss of the precious metal, which varies, however, with the standard of the alloy. It is 1 thousandth for fine silver,

$4 \cdot 3$ thousandths for silver of 900 thousandths,

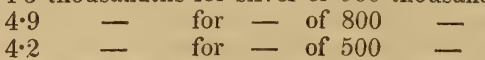

and diminishes thereafter, progressively, till the alloy contains only 100 thousandths of silver, at which point the loss is only $0 \cdot 4$.

Assays requested by the Commission of the Paris Mint, from the assayers of the principal Royal Mints in Europe, to which the same alloys, synthetically compounded, were sent, afforded the results inscribed in the following table.

\begin{tabular}{|c|c|c|c|c|c|c|}
\hline \multirow{2}{*}{\multicolumn{3}{|c|}{ Names of the Assayers. }} & \multirow{2}{*}{$\begin{array}{l}\text { Cities where they } \\
\text { reside. }\end{array}$} & \multicolumn{3}{|c|}{ Standards found for the Mathematical Alloys } \\
\hline & & & & 950 mill. & 900 mill. & 800 mill. \\
\hline \multicolumn{3}{|c|}{ F. de Castenhole, Mint Assayer } & Vienna & $946 \cdot 20$ & $898 \cdot 40$ & $795: 10$ \\
\hline \multicolumn{3}{|c|}{$\begin{array}{l}\text { A. R. Vervaëz, ditto - } \\
\text { D. M. Cabrera, Assayer in }\end{array}$} & Madrid & $944 \cdot 40$ & $893 \cdot 70$ & $789 \cdot 20$ \\
\hline \multicolumn{3}{|c|}{ Spain - - - . - - } & Ditto & $944 \cdot 40$ & $893 \cdot 70$ & $788 \cdot 60$ \\
\hline \multirow{2}{*}{\multicolumn{3}{|c|}{$\begin{array}{l}\text { Assayer - - - - - } \\
\text { Mr. Bingley, Assay Master - }\end{array}$}} & Amsterdam & $947 \cdot 00$ & $895 \cdot 00$ & $795 \cdot 00$ \\
\hline & & & London & $946 \cdot 25$ & $896 \cdot 25$ & $794 \cdot 25$ \\
\hline \multicolumn{3}{|c|}{ Mr. Johnson, Assiyer - - } & Ditto & $933 \cdot 33$ & $883 \cdot 50$ & $783 \cdot 33$ \\
\hline \multicolumn{3}{|c|}{ Inspector of the Mint - - } & Utrecht & $945 \cdot 00$ & $896 \cdot 50$ & $799 \cdot 00$ \\
\hline Assayer of the Mint & - & - & Naples & $945 \cdot 00$ & $891 \cdot 00$ & $787 \cdot 00$ \\
\hline \multirow{2}{*}{$\begin{array}{l}\text { Assayer of Trade - } \\
\text { Assayer of the Mint }\end{array}$} & - & - & Ditto & $945 \cdot 00$ & $891 \cdot 00$ & $787 \cdot 00$ \\
\hline & - & - & Hamburgh & $946 \cdot \frac{13}{72}$ & $897 \cdot \frac{4}{7} \frac{1}{2}$ & $798 \cdot \frac{4}{7} \frac{4}{2}$ \\
\hline Ditto - - - & - & - & Altona & $942 \cdot \frac{1^{2}}{4}$ & $894 \cdot 00$ & 790 \\
\hline
\end{tabular}


These results, as well as those in still greater numbers, obtained from the ablest Parisian assayers, upon identical alloys of silver and copper, prove that ine mocle of assay apjuied to them brings out the standard too low; and further, that the quantity of silver masked or disguised, is not uniform for these different eminent assay masters. An alloy, for example, at the standard of 900 thousandths is judged at

the Mint of Paris to have a standard of $895^{\circ} 6$

At that of Vienna _ _ $\quad 898 \cdot 4$

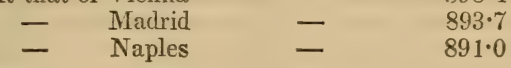

The fact thus so clearly made out of a loss in the standard of silver bullion and coin, meriv the most serious attention; and it will appear astonishing, perhaps, that a thing recurring every day, should have' remained for so long a time in the dark. In reality, however, the fact is not new; as the very numerous and well-made experiments of Tillet, from 1760 to 1763 , which are related in the memoirs of the Academy of Sciences, show, in the silver assays, a loss still greater than that which was experienced lately in the laboratory of the Commission of the French Mint. But he thonght that, as the error was common to the nations in general, it was not worth while or prudent to introduce any innovation.

A mode of assaying, to give, with certainty, the standard of silver bullion, should be entirely. independent of the variable circumstances of temperature, and the unknown proportions of copper, so difficult to regulate by the mere judgment of the senses. The process by the humid way, recommended by me to the Royal Mint in 1829, and exhibited as to its principles before the Right Honorable John Herries, then Master, in 1830 , has all the precision and certainty we could wish. It is founded on the well-known property which silver has, when dissolved in nitric acid, to be precipitated in a chloride of silver quite insoluble, by a solution of sea salt, or by muriatic acid; but, instead of determining the weight of the chloride of silver, which would be somewhat uncertain and rather tedious, on account of the difficulty of drying it, we take the quantity of the solution of sea salt which has been necessary for the precipitation of the silver. To put the process in execution, a liquor is prepared, composed of water and sea salt in such proportions that 1000 measures of this liquor may precipitate, completely, 12 grains of silver, perfectly pure, or of the standard 1000, previously dissolred in nitric acid. The liquor thus prepared, gives, immediately, the true standard of any alloy whatever, of silver and copper, by the weight of it which may be necessary to precipitate 12 grains of this alloy. If, for example, 905 measures have been required to precipitate the 12 grains of alloy, its standard would be 905 thousandths.

The process by the humid way is, so to speak, independent of the operator. The manipulations are so easy; and the term of the operation is rery distinctly announced by the absence of any sensible nebulosities on the affusion of sea salt into the silver solution, while there remains in it $\frac{1}{2}$ thousandth of metal. The process is not tedious, and in experienced hands it may rival the cupel in rapidity; it has the advantage over the cupel of being more within the reach of ordinary operators, and of not requiring a Iong apprenticeship. It is particularly useful to such assayers as have only a few assays to make daily, as it will cost them very little time and expense.

By agitating briskly during two minutes, or thereby, the liquid rendered milky by the precipitation of the chloride of silver, it may be sufficiently clarified to enable us to appreciate, after a few moments of repose, the disturbance that can be produced in it by the addition of 1000 of a grain of silver. Filtration is more efficacious than agitation, especially when it is employed afterwards; it may be sometimes used; but agitation, which is much more prompt, is generally sufficient. The presence of lead and copper, or any other metal, except mercury, has $\mathbf{n}$ perceptible intluence on the quantity of sea salt necessary to precipitate the silver; that is to say, the same quaritity of silver, pure or alloyed, requires for its precipitation a constant quantity of the solution of sea salt.

Supposing that we operate upon a gramme of pure silver, the solution of sea salt ought to be such that 100 centimetres cube may precipitate exactly the whole silver. The standard of an alloy is given by the number of thousandths of solution of sea salt necessary to precipitate the silver contained in a gramme of the alloy.

When any mercury is accidentally present, which is, however, a rare occurrence, it is made obrious by the precipitated chloride remaining white when exposed to daylight, whereas when there is no mercury present, it becomes speedily first gray and then purple. Silver so contaminated must be strongly ignited in fusion before being assayed, and its loss of weight noted. In this case, a cupel assay must be had recourse to.

Preparation of the Normal Solution of Sea Salt, when it is measured by Weight.-Supposing the sea salt pure as well as the water, we have only to take these two bodies in the proportion of $0.5427 \mathrm{k}$. of salt to $99.4573 \mathrm{k}$. of water, to have $100 \mathrm{k}$. of solution, 
of which 100 grammes will precipitate exactly one gramme of silver. But instead of pure salt, wh ch is to be procured with difficulty, and which besides may be altered readily by absorbing the humidity of the air, a concentrated solution of the sea salt of commerce is to be preferred, of which a large quantity may be prepared at a time, to be kept in reserve for use, as it is wanted. Instruction de Gay Lussac.

Prepuration of the Normal Solution of Sea Salt, when measured by Volume.-The measure by weight has the advantage of being independent of tencerature, of having the same degree of precision as the balance, and of standing in nced of no correction. The measure by volume has not all these advantages; but, by giving it sufficient precision, it is more rapid, and is quite sufficient for the numerous daily assays of the mint. This normal solution is so made, that a volume equal to that of 100 gra.nmes of water, or 100 centimetres cube, at a determinate temperature, may precipitate exactly one gramme of silver. The solution may be kept at a constant temperature, and in this case the assay stands in want of no correction; or if its temperature be variable, the assay must be corrected according to its influence. These two circumstances make no change in the principle of the process, but they are sufficiently important to occasion some modifications in the apparatus. Experience has decided the preference in favor of applying a correction to a variable temperature.

We readily obtain a volume of 100 cubic centimetres by means of a pipette, fig. 83 ,

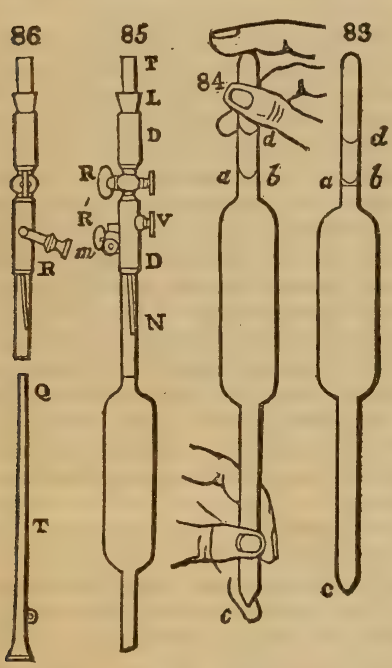
so gauged that when filled with water up to the mark $a, b$, and well dried at its point, it will rur out, at a continuous efflux, 100 grammes of watel at the temperature of $15 \mathrm{C}$. (59 Fah.). We say purposely at one efflux, because after the cessation of the jet, the pipette may still furnish two or three drops of liquid, which must not be counted or reckoned upon. The weight of the volume of the normal solution, taken in this manner with suitable precautions, will be uniform from one extreme to another, upon two centimetres and a half, at most, or to a quarter of a thousandth, and the difference from the mean will be obviously twice less, or one half. Let us indicate the most simple manner of taking a measure of the normal solution of sea salt.

After having immersed the beak $c$ of the pipette in the solution, we apply suction by the mouth, to the upper orifice, and thereby raise the liquid to $d$, above the circular line $a b$. We next apply neatly the forefinger of one hand to this orifice, remove the pipette from the liquid, and seize it as represented in fig. 84. The mark $a b$ being placed at the level of the eye, we make the surface of the solution become exactly a tangent to the plane $a b$. At the instant it becomes a tangent, we leave the beak $c$ of the pipette open, by taking away the finger that had been applied to it, and without changing anything else in the position of the hands, we empty it into the bottle which should receive the solution, taking care to remove it whenever the efflux has run out.

If, after filling the pipette by suction, any one should find a difficulty in applying the forefinger fast enough to the upper orifice, without letting the liquid run down below the mark $a, b$, he should remove the pipette from the solution with its top still closed with his tongue, then apply the middle finger of one of his hands to the lower orifice; after which he may withdraw his tongue, and apply the forefinger of the other hand to the orifice previously wiped. This mode of obtaining a measure of normal solution of sea salt is very sirple, and requires no complex apparatus : but we shall indicate another manipulation still easier, and also more exact.

In this new process the pipette is filled from the top like a bottle, instead of being filled by suction, and it is moreover fixed. Fig. 85 represents the apparatus. D and $\mathbf{D}^{\prime}$ are two sockets separated by a stop-cock $\mathbf{R}$. The upper one, tapped interiorly, receives, by means of a cork stopper $L$, the tube $T$, which admits the solution of sea salt. The lower socket is cemented on to the pipette; it bears a smal air-cock $\mathrm{R}^{\prime}$, and a screw plug $\mathrm{v}$, which regulates a minute opening intended to let the air enter very slowly into the pipette. Below the stop-cock $\mathrm{R}^{\prime}$, a silver tube $\mathrm{N}$, of narrow diameter, soldered to the socket, leads the solution into the pipette, by allowing the air, which it displaces, to escape by the stop-cock $\mathrm{R}^{\prime}$. The screw plug; with the milled head $\mathrm{v}^{\prime}$, replaces the ordinary serer $b_{y}$ which the key of the stop-cock may be made to press vith more or less force, upon its conical seat. 
Fig. 86 represents, in a side view, the apparatus just described. We here remark an air-cock $\mathrm{R}$, and an opening $m$. At the extremity $Q$ of the same figure, the conical pipe $T$ enters, with friction. It is by this pipe that the air is sucked into the pipette when it is to be filled from its beak.

The pipette is supported by two horizontal arms $\mathrm{H} \mathrm{K}$ ( $f$ ig. 87 ) moveable about a

89

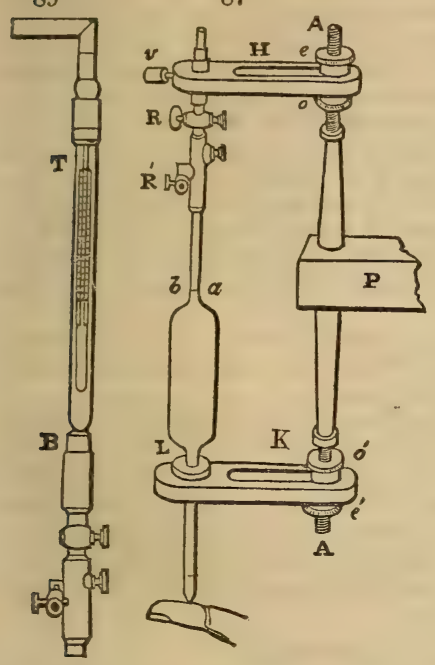
common axis A A, and capable of being drawn out or shortened by the aid of two longitudinal slits. They are fixed steadily by two screw nuts $e$ é, and their distance may be varied by means of round bits of wood or cork interposed, or even by opposite serew nuts o ó. The upper $\operatorname{arm} \mathbf{H}$ is pierced with a hole, in which is fixed, by the pressure of a wooden serew $v$, the socket of the pipette. The corresponding hole of the lower arm is larger; and the beak of the pipette is supported in it by a cork stopper L. The apparatus is fixed by its tail-piece $P$, by means of a screw, to the corner of a wall, or any other prop.

The manner of filling the pipette is very simple. We begin by applying the fore-finger of the left hand to the lower aperture $c$; we then open the two stop-cocks $R$ and $R^{\prime}$. Whenever the liquor approaches the neck of the pipette, we must temper its influx, and when it has arrived at some millimetres above the mark $a b$, we close the two stop-cocks, and remove our fore-finger. We have now nothing more to do than to regulate the pipette; for which purpose the liquid must touch the line $a b$, and must simply adhere externally to the beak of the pipette.

This last circumstance is easily adjusted. After taking away the finger which closed the aperture $c$ of the pipette, we apply to this orifice a moist sponge $m$, fig. 88 , wrapped up in a linen rag, to absorb the superfluous

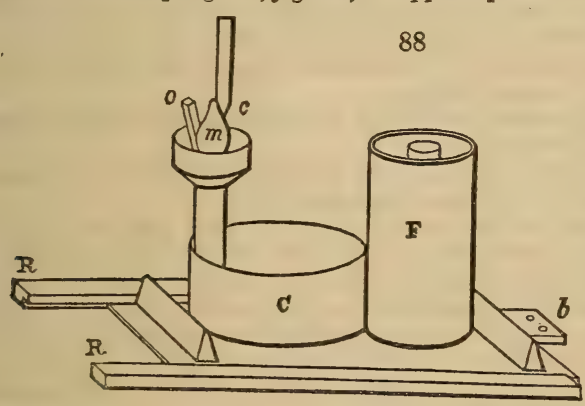
liquor as it drops out. This sponge is called the handkerchief (mouchoir), by M. Gay Lussac. The pipette is said to be wiped when there is no liquor adhering to its point exteriorly.

For the convenience of operating, the handkerchief is fixed by friction in a tube of tin plate, terminated by a cup, open at bottom to let the droppings flow off into the cistern $c$, to which the tube is soldered. It may be easily removed for the purpose of washing it; and, if necessary, a little wedge

of wood, $o$, can raise it toward the pipette.

To complete the adjustment of the pipette, the liquid must be made merely to descend to the mark $a, b$. With this view, and while the handkerchief is applied to the beak 0 the pipette, the air must be allowed to enter very slowly by unscrewing the plug $\mathrm{v}$, fig. 85 ; and at the moment of the contact the handkerchief must be removed, and the bottle $F$, destined to receive the solution, must be placed below the orifice of the pipette, fig. 88. As the motion must be made rapidly, and without hesitation, the bottle is placed $n$ a cylinder of tin-plate, of a diameter somewhat greater, and forming one body with the cistern and the handkerchief. The whole of this apparatus has for a basis a plate of tinned iron, moveable between two wooden rulers $\mathrm{R} R$, one of which bears a groove, under which the edge of the plate slips. Its traverses are fixed by two abutments $b b$, placed so that when it is stopped by one of them, the beak of the pipette corresponds to the centre of the neck of the bottle, or is a tangent to the handkerchief. This arrangement, very convenient for wiping the pipette and emptying it, gives the apparatus sufficient solidity, and allows of its being taken away and replaced without deranging anything. It is obvious that it is of advantage, when once the entry of the air into the pipette has been regulated by the screw $\mathrm{v}$, to leave it : nstantly open, because the 
motion from the handkerchief to the bottle is performed with sufficient rapidity to prevent a drop of the solution from collecting and falling down.

Temperature of the Solution.-After haring described the manner of measuring by volume the normal solution of the sea salt, we shall indicate the most convenient means of taking the temperature. The thermometer is placed in a tube of glass T, fig. 89, which the solution traverses to arrive at the pipette. It is suspended in it by a piece of cork, grooved on the four sides to afford passage to the liquid. The scale is engraved upon the tube itself, and is repeated at the opposite side, to fix the eye by the coincidence of this double division at the level of the thermometric column. The tube is joined below to another narrower one, through which it is attached by means of a cork stopper $B$, in the socket of the stop-cock of the pipette. At its upper part it is cemented into a brass socket, screw-tapped in the inside, which is connected in its turn by a cock, with the extremity, also tapped, of the tube above $T$, belonging to the reservoir of the normal solution. The corks employed here as connecting links between the parts of the apparatus, give them a certain flexibility, and allow of their being dismounted and remounted in a very short time; but it is indispensable to make them be traversed by a hollow tube of glass or metal, which will hinder them from being crushed by the pressure they are exposed to. If the precaution be taken to grease them with a little suet, and to fill their pores, they will suffer no leakage.

Preservation of the Normal Solution of Sea Salt in metullic Vessels.-M. Gar Lussac uses for this purpose a cylindrical vessel or drum of copper, of a capacity of about 110 litres, having its inside covered with a rosin and wax cement.

Preparation of the Normal Solution of Sea Salt, measuring it by Volume.-If the drum contains 110 litres, we should put only 105 into it, in order that sufficient space may be left for agitating the liquor without throwing it out. According to the principle that 100 centimetres cube, or $\frac{1}{10}$ of a litre of the solution should contain enough of sea salt to precipitate a gramme of pure silver ; and, admitting, moreover, 13.516 for the prime equivalent of silver, and $7 \cdot 335$ for that of sea salt, we shall find the quantity of pure salt that should be dissolved in the 105 litres of water, and which corresponds to $105 \times 10=1050$ grammes of silver, to be by the following proportion:-

$$
13 \cdot 516: 7 \cdot 335:: 1050 \text { gramm. : } x=569 \cdot 83 \mathrm{gr} \text {. }
$$

And as the solution of the sea salt of commerce, formerly mentioned, contains approximately 250 grammes per hilogramme, we must take $2279 \cdot 3$ grammes of this solution to have 569.83 gram. of salt. The mixture being perfectly made, the tubes and the pipetle must be several times washed by running the solution through them, and putting it into the drum. The standard of the solution must be determined after it has been well agitated, supposing the temperature to remain uniform.

To arrive more conveniently at this result, we begin by preparing two decimes solutions; one of silver, and another of sea salt.

The decime solution of silver is obtained by dissolving 1 gramme of silver in nitric acid, and diluting the solution with water till its volume becomes a litre.

The decime solution of sea salt may be obtained by dissolving 0.543 grammes of pure sea salt in water, so that the solution shall occupy a litre; but we shall prepare it even with the normal solution which we wish to test, by mixing a measure of it with 9 measures of water; it being understood that this solution is not rigorously equiralent to that of silver, and that it will become so, only when the normal solution employed for its preparation shall be finally of the true standard. Lastly, we prepare beforehand several stoppered rials, in each of which we dissolve 1 gramme of silver in 8 or 10 grammes of nitric acid. For brevity's sake we shall call these tests.

Now to inrestigate the standard of the normal solution, we must transfer a pipcte of it into one of these test vials; and we must agitate the liquors brishly to clarify them. After some instants of repose, we must pour in 2 thousandths of the decime solution of sea salt, which, we suppose, will produce a precipitate. The normal liquor is consequently too feeble; and we should expect this, since the sea sait emplored was not perfectly pure. We agitate and add 2 fresh thousandths, which will also produce a precipitate. We continue thus by successive additions of 2 thousandths, till the last produces no precipitation. Suppose that we have added 16 thousandths : the last two should not be reckoned, as they produced no precinitate; the preceding two were necessary, but only in part; that is to say, the useful thousandths added are above 12 and below 14, or otherwise they are on an average equal to 13.

Thus, in the condition of the normal solution, we require 1013 parts of it to precipitate one gramme of silver, while we should require only 1000 . We shall find the quantity of concentrated solution of sea salt that we should add, by noting that the quantity of solution of sea salt, at first employed, viz. 2279.3 grammes, produced a standard of only 987 thousandths $=1000-13$; and by using the following proportion . $987: 2279 \cdot 3:: 13: x=30 \cdot 02$ grammes. 
This quantity of the strong solution of salt, mixed with the normal solution in the drum, will correct its standard, and we shall now see by how much.

After having" washed the tubes and the pipette with the new solution, we must repeat the experiment upon a fresh gramme of silver. We shall find, for example, in proceeding only by a thousandth at a time, that the first causes a precipitate, but not the second. The standard of the solution is still too weak, and is comprised between 1000 and 1001 ; that is to say, it may be equal to $1000 \frac{1}{2}$, but we must make a closer approximation.

We pour into the test bottle 2 thousandths of the decime solution of silver, which will destroy, perceptibly, two thousandths of sea salt, and the operation will have retrograded by two thousandths; that is to say, it will be brought back to the point at which it was first of all. If, after having cleared up the liquor, we add half a thousandth of the decime solution, there will necessarily be a precipitate, as we knew beforehand, but a second will cause no turbidity. The standard of the normal liquor will be consequently comprehended between 1000 and $1000 \frac{1}{2}$, or equal to $1000 \frac{1}{4}$.

We should rest content with this standard, but if we wish to correct it, we may remark that the two quantities of solution of salt added, viz. 2279.3 gr. +30.02 gr. $=$ 2309.32 gr. have produced only $999 \cdot 75$ thousandths, and that we must add a new quantity of it corresponding to $\frac{1}{4}$ of a thousandth. We make, therefore, the proportion

$$
999 \cdot 75: 2309 \cdot 32:: 0 \cdot 25: x \text {. }
$$

But since the first term differs very little from 1000 , we may content ourselves to have $\mathrm{x}$ by taking the $\frac{0 \cdot 25}{1000}$ of $2309 \cdot 32$, and we shall find $0.577 \mathrm{gr}$. for the quantity of solution of sea salt to be added to the normal solution.

It is not convenient to take exactly so small a quantity of solution of sea salt by the balance, but we shall succeed easily by the following process. We weigh 50 grammes of this solution, and we dilute it with water; so that it occupies exactly half a litre, or 500 centimetres cube. A pipette of this solution, one centimetre cube in volume, will give a decigramme of the primitive solution, and as such a small pipette is divided into twenty drops, each drop, for example, will represent 5 milligrammes of the solution. We should arrive at quantities smaller still by diluting the solution with a proper quantity of water; but greater precision would be entirely needless.

The testing of the normal liquor just described, is, in reality, less tedious than might be supposed. It deserves also to be remarked, that liquor has been prepared for more than 1000 assays; and that, in preparing a fresh quantity, we shall obtain directly its true standard, or nearly so, if we bear in mind the quantities of water and solution of salt which had been employed.

Correction of the Standard of the Normal Solution of Sea Salt, when the Temperature changes.-We have supposed, in determining the standard of the normal solution of sea salt, that the temperature remained uniform. The assays made in such circumstances, have no need of correction; but if the temperature should change, the same measure of the solution will not contain the same quantity of sea salt. Supposing that we have tested the solution of the salt at the temperature of $15^{\circ} \mathrm{C}$.; if, at the time of making the experiment, the temperature is $18^{\circ} \mathrm{C}$., for example, the solution will be too weak on account of its expansion, and the pipette will contain less of it by weight; if, on the contrary, the temperature has fallen to $12^{\circ}$, the solution will be thereby concentrated and will prove too strong. It is therefore proper to determine the correction necessary to be made, for any variation of temperature.

To ascertain this point, the temperature of the solution of sea salt was made successively to be $0^{\circ}, 5^{\circ}, 10^{\circ}, 15^{\circ}, 20^{\circ}, 25^{\circ}$, and $30^{\circ} \mathrm{C}$; ; and three pipettes of the solution were weighed exactly at each of these temperatures. The third of these weighings gave the mean weight of a pipette. The corresponding weights of a pipette of the solution, were afterwards graphically interpolated from degree to degree. These weights form the second column of the following table, entitled, Table of Correction for the Varialions in the Temperature of the Normal Solution of the Sea Salt. They enable us to correct any temperature between 0 and 30 degrees centigrade $\left(32^{\circ}\right.$ and $86^{\circ} \mathrm{Fahr}$.) when the solution of sea salt has been prepared in the same limits.

Lct us suppose, for example, that the solution has been made standard at $15^{\circ}$, and that, at the time of using it, the temperature has become $18^{\circ}$. We see by the second column of the table, that the weight of a measure of the solution is 100.099 gr. at $15^{\circ}$, and 100.065 at $18^{\circ}$; the difference $0.034 \mathrm{gr}$., is the quantity of solution less which has been really taken; and of course we must add it to the normal measure, in order to make it equal to one thousand milliemes. If the temperature of the solution had fallen to 10 degrees, the difference of the weight of a measure from 10 to 15 degrees would be 0.019 gr., which we must on the contrary deduct from the measure, since it had been taken tou large. These differences of weight of a measure of solution at $15^{\circ}$, from that of a 
measure at any other temperature, form the column $15^{\circ}$ of the table, where they are expressed in thousandths; they are inscribed on the same horizontal lines as the temperatures to which each of them relates, with the sign + plus, when they must be added, and with the sign - minus, when they must be subtracted. The columns $5^{\circ}, 10^{\circ}, 20^{\circ}$, $25^{\circ}, 35^{\circ}$, have been calculated in the same manner for the cases in which the normal solution may have been graduated to each of these temperatures. Thus, to calculate the column 10 , the number $100 \cdot 118$ has been taken of the column of weights for a term of departure, and its difference from all the numbers of the same column has been sought.

Table of Correction for the Variations in the Temperature of the Normal Solution of the Sea Salt.

\begin{tabular}{|c|c|c|c|c|c|c|c|}
\hline Temperature. & Weight. & $5^{\circ}$ & $10^{\circ}$ & $15^{\circ}$ & $20^{\circ}$ & $25^{\circ}$ & $30^{\circ}$ \\
\hline 4 & $\begin{array}{c}\text { gram. } \\
100,109\end{array}$ & $\begin{array}{l}\text { mill. } \\
0.0\end{array}$ & $\stackrel{\text { mill. }}{-0.1}$ & $\begin{array}{l}\text { mill. } \\
+0.1\end{array}$ & $\begin{array}{l}\text { mill. } \\
+0.7\end{array}$ & $\begin{array}{l}\text { mill. } \\
+1.7\end{array}$ & $\begin{array}{l}\text { mill. } \\
+2.7\end{array}$ \\
\hline 5 & 100,113 & 0.0 & -0.1 & +0.1 & +0.7 & -1.7 & $\begin{array}{r}2.8 \\
+2.8\end{array}$ \\
\hline 6 & 100,115 & $0 \cdot 0$ & 0.0 & +0.2 & +0.8 & $+1 \cdot 7$ & +2.8 \\
\hline 7 & 110,118 & +0.1 & $0 \cdot 0$ & +0.2 & +0.8 & +1.7 & +2.8 \\
\hline 8 & 100,120 & +0.1 & $0 \cdot 0$ & +0.2 & -0.8 & $\begin{array}{r}1.8 \\
+1\end{array}$ & +2.8 \\
\hline 9 & 100,120 & +0.1 & $0 \cdot 0$ & +0.2 & +0.8 & $-1 \cdot 8$ & +2.8 \\
\hline 10 & 100,118 & +0.1 & $0 \cdot 0$ & +0.2 & +0.8 & $+1 \cdot 7$ & +2.8 \\
\hline 11 & 100,116 & $0 \cdot 0$ & $0 \cdot 0$ & -0.2 & +0.8 & +1.7 & +2.8 \\
\hline 12 & 100,114 & 0.0 & $0 \cdot 0$ & +0.2 & +0.8 & +1.7 & $-2 \cdot 8$ \\
\hline 13 & 100,110 & $0 \cdot 0$ & -0.1 & +0.1 & +0.7 & $+1 \cdot 7$ & -2.7 \\
\hline 14 & 100,106 & -0.1 & -0.1 & +0.1 & +0.7 & +1.6 & $+2 \cdot 7$ \\
\hline 15 & 100,099 & -0.1 & -0.2 & -0.0 & +0.6 & $-1 \cdot 6$ & $+2 \cdot 6$ \\
\hline 16 & 100,090 & -0.2 & -0.3 & -0.1 & +0.5 & -1.5 & +2.5 \\
\hline 17 & 100,078 & -0.4 & -0.4 & -0.2 & +0.4 & $+1 \cdot 3$ & $+2 \cdot 4$ \\
\hline 18 & 100,065 & -0.5 & -0.5 & -0.3 & +0.3 & +1.2 & $+2 \cdot 3$ \\
\hline 19 & 100,053 & -0.6 & -0.7 & -0.5 & +0.1 & $+1 \cdot 1$ & $+2 \cdot 2$ \\
\hline 20 & 100,039 & -0.7 & -0.8 & $-0 \cdot 6$ & 0.0 & -1.0 & $+2 \cdot 0$ \\
\hline 21 & 100,021 & -0.9 & $-1 \cdot 0$ & -0.8 & -0.2 & +0.8 & +1.9 \\
\hline 22 & 100,001 & $-1 \cdot 1$ & $-1 \cdot 2$ & -1.0 & -0.4 & +0.6 & $+1 \cdot 7$ \\
\hline 23 & 99,983 & $-1 \cdot 3$ & $-1 \cdot 4$ & $-1 \cdot 2$ & -0.6 & -0.4 & +1.5 \\
\hline 24 & 99,964 & -1.5 & $-1 \cdot 5$ & $-1 \cdot 4$ & -0.8 & +0.2 & $+1 \cdot 3$ \\
\hline 25 & 99,944 & $-1 \cdot 7$ & $-1 \cdot 7$ & $-1 \cdot 6$ & $-1 \cdot 0$ & $0 \cdot 0$ & $-1 \cdot 1$ \\
\hline 26 & 99,924 & $-1 \cdot 9$ & -1.9 & $-1 \cdot 8$ & $-1 \cdot 2$ & -0.2 & +0.9 \\
\hline 27 & 99,902 & $-2 \cdot 1$ & -2.2 & $-2 \cdot 0$ & $-1 \cdot 4$ & -0.4 & +0.7 \\
\hline 28 & 99,879 & $-2 \cdot 3$ & $-2 \cdot 4$ & $-2 \cdot 2$ & $-1 \cdot 6$ & -0.7 & +0.4 \\
\hline 29 & 99,858 & $-2 \cdot 6$ & $-2 \cdot 6$ & $-2 \cdot 4$ & $-1 \cdot 8$ & -0.9 & $+0 \cdot 2$ \\
\hline 30 & 99,836 & $-2 \cdot 8$ & -2.8 & $-2 \cdot 6$ & -2.0 & $-1 \cdot 1$ & 0.0 \\
\hline
\end{tabular}

Several expedients have been employed to facilitate and abridge the manipulations. In the first place, the vials for testing or assaying the specimens of silver should all be of the same height and of the same diameter. They should be numbered at their top, as well as on their stoppers, in the order 1,2,3, \&c. They may be ranged successively in tens ; the stoppers of the same series being placed on a support in their proper order. Each two vials should, in their turn, be placed in a japanned tin case ( $f i g .90)$, with ten compartments duly numbered. These compartments are cut out anteriorly to about half their height, to allow the bottoms of the bottles to be seen. When each vial has received its portion of alloy, through a wide-beaked funnel, there must be poured into it about 10 grammes of nitric acid, of specific gravity $1 \cdot 28$, with a pipette, containing that quantity; it is then exposed to the heat of a water bath, in order to facilitate the solution of the alloy. The water bath is an oblong vessel made of tin plate, intended to receive the vials. It has a moveable double bottom, pierced with small holes, for the purpose of preventing the vials being broken, as it insulates them from the bottom to which the heat is applied. The solution is rapid; and, since it emits nitrous vapors in abundance, it ought to be carried on under a chimney.

The agitator.-Fig. 91 gives a sufficiently exact idea of it, and may dispense with a lengthened description. It has ten cylindrical compartments, numbered fiom 1 to 10. The vials, after the solution of the alloy, are arranged in it in the order of their num. bers. The agitator is then placed within reach of the pipette, intended to measure out the normal solution of sea salt, and a pipette full of this solution is put in each vial. Each is then closed with its glass stopper, previously dipped in pure water. They are fixed in the cells of the agitator by wooden wedges. The agitator is then suspended 
to a spring $\mathrm{R}$, and, seizing it with the two hands, the operator gives an alternating rapir movement, which agitates the solution, and makes it, in less than a minute, as

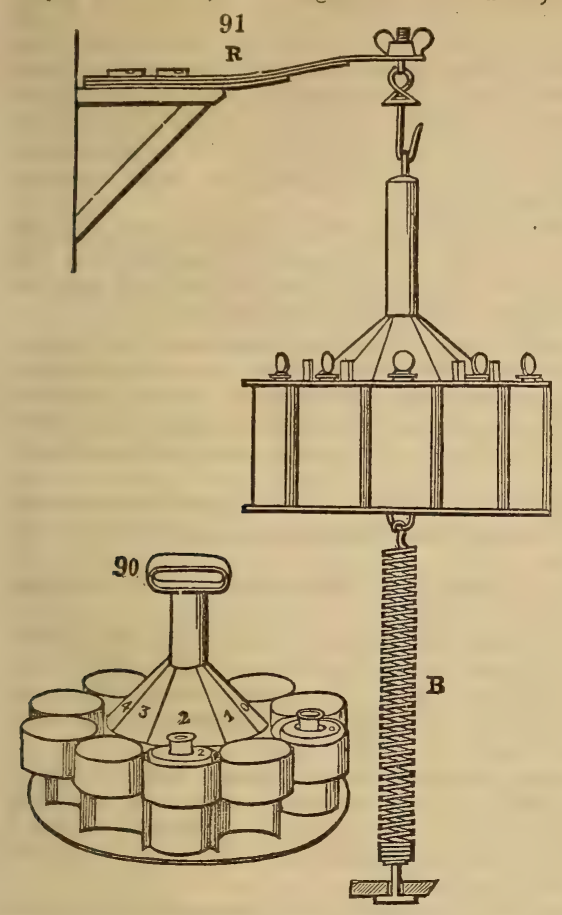

limpid as water. This movement is promoted by a spiral spring, $B$, fixed to the agitator and the ground, but this is seldom made use of, because it is convenient to be able to transport, the agitator from one place to another. When the agitation is finished, the wedges are to be taken out, and the vials are placed in order upon a table furnished with round cells destined to receive them, and to screen them from too free a light.

When we place the vials upon this table, we must give them a brisk circular motion, to collect the chloride of silver scattered round their sides; we must lift out their stoppers, and suspend them in wire rings, or pincers. We next pour a thousandth of the decime solution into each vial; and before this operation is terminated, there is formed in the first vials, when there should be a precipitate, a nebulous stratum, very well marked, of about a centimetre in thickness.

At the back of the table there is a black board divided into compartments numbered from 1 to 10 , upon each of which we mark, with chalk, the thousandths of the decime liquor put into the correspondent vial. The thousandths of sea salt, which indicate an augmentation of standard, are preceded by the sign + , and the thou-

sandths of nitrate of silver by the sign -.

When the assays are finished, the liquor of each vial is to be poured into a large vessel, in which a slight excess of sea salt is kept; and when it is full, the supernatant clear liquid must be run off with a syphon.

The chloride of silver may be reduced without any perceptible loss. After having washed it well, we immerse pieces of iron or zinc into it, and add sulphuric acid in sufficient quantity to keep up a feeble disengagement of hydrogen gas. The mass must not be touched. In a few days the silver is completely reduced. This is easily recognised by the color and nature of the product; or by treating a small quantity of it with water of ammonia, we shall see whether there be any chloride unreduced; for it will be dissolved by the ammonia, and will afterwards appear upon saturating the ammonia with an acid. The chlorine remains associated with the iron or the zinc in a state of solution. The first washings of the reduced silver must be made with an acidulous water, to dissolve the oxyde of iron which may have been formed, and the other washings with common water. After decanting the water of the last washing, we dry the mass, and add a little powdered borax to it. It must be now fused. The silver being in a bulky powder, is to be put in successive portions into a crucible as it sinks down. The heat should be at first moderate; but towards the end of the operation it must be pretty strong to bring into complete fusion the silver and the scoriæ, and to effect their complete separation. In case it should be supposed that the whole of the silver had not been reduced by the iron or zinc, a little carbonate of potash should be added to the borax. The silver may also be reduced by exposing the chloride to a strong heat, in contact with chalk and charcoal.

The following remarks by M. Gay Lussac, the author of the above method, upon the effect of a little mercury in the humid assay, are important :-

It is well known that chloride of silver blackens the more readily as it is exposed to an intense light, and that even in the diffused light of a room, it becomes soon sensibly colored. If it contains four to five thousandths of mercury, it does not blacken; It remains of a dead white: with three thousandths of mercury, there is no marked discoloring in diffused light; with two thousandths it is slight; with one it is much more marked, but still it is much less intense than with pure chloride. With lislf a 
thousandth of mercury the difference of color is not remarkable, and is perceived only in a very moderate light.

But when the quantity of mercury is so small that it cannot be detected hy the difference of color in the chloride of silver, it may be rendered quite evident by a very simple process of concentration. Dissolve one gramme of the silver supposed to contain $\frac{1}{4}$ of a thousandth of mercury, and let only $\frac{1}{4}$ of it be precipitated, by alding only $\frac{2}{4}$ of the common salt necessary to precipitate it entirely. In thus operating, the $\frac{1}{4}$ thousandth of mercury is concentrated in a quantity of chloride of silver four times smaller: it is as if the silver having been entirely precipitated, four times as much mercury, equal to two thousandths, had been precipitated with it.

In taking two grammes of silver, and precipitating only $\frac{1}{4}$ by common salt, the precipitate would be, with respect to the chloride of silver, as if it amounted to four thousandths. By this process, which occupies only five minutes, because exact weighing is not necessary, $\frac{1}{10}$ of a thousandth of mercury may be detected in silver.

It is not useless to observe, that in making those experiments the most exact manner of introducing small quantities of mercury into a solution of silver, is to weigh a minute globule of mercury, and to dissolve it in nitric acid, diluting the solution so th:it it may contain as many cubic centimetres as the globule weighs of centigrammes. Each cubic centimetre, taken by means of a pipette, will contain one milligramme of mercury.

If the ingot of silver to be assayed is found to contain a greater quantity of mercury, one thousandth for example, the hnmid process ought either to be given up in th s case, or to be compared with cupellation.

When the silver contains mercury, the solution from which the mixed chlorides are precipitated does not readily become clear.

Silver containing mercury, put into a small crucible and mixed with lamp-black, to prevent the volatilization of the silver, was heated for three quarters of an hour in a muffle, but the silver increased sensibly in weight. This process for separating the mercury, therefore, failed. It is to be observed, that mercury is the only metal which has thus the power of disturbing the analysis by the humid way.

Assaying of GoLD.-In estimating or expressing the fineness of gold, the whole mass spoken of is supposed to weigh 24 carats of 12 grains each, either real, or merely proportional, like the assayer's weights; and the pure gold is called fine. Thus, if gold be said to be 23 carats fine, it is to be understood, that in a mass, weighing 24 carats, the quantity of pure gold amounts to 23 carats.

In such small work as cannot be assayed by scraping off a part and cupelling it, the assayers endeavor to ascertain its fineness or quality by the touch. "This is a method of comparing the color and other properties, of a minute portion of the metal, with those of small bars, the composition of which is known. These bars are called touch needles, and they are rubbed upon a smooth piece of black basaltes or pottery, which, for this reason, is called the touchstone. Black flint slate will serve the same purpose. Sets of gold needles may consist of pure gold; of pure gold, 23즐 carats with $\frac{1}{2}$ carat of silver; 23 carats of gold with one carat of silver; $22 \frac{1}{2}$ carats of gold with $1 \frac{1}{2}$ carat of silver; and so on, till the silver amounts to four carats; after which the additions may proceed by whole carats. Other needles may be made in the same manner, with copper instead of silver; and other sets may have the addition, consisting either of equal parts of silver and copper, or of such proportions as the occasions of business require. The examination by the touch may be advantageously employed previous to quartation, to indicate the quantity of silver necessary to be added.

In foreign countries, where trinkets and small work are required to be submitted to the assay of the touch, a variety of needles is neccssary; but they are not much used in England. They afford, however, a degree of information which is more considerable than might at first be expected. The attentive assayer compares not only the color of the stroke made upon the touchstone by the metal under examination, with that produced by his needle, but will likewise attend to the sensation of roughness, dryness, smoothness, or greasiness, which the texture of the rubbed metal excites, when abraded by the stone. When two strokes perfectiy alike in color are made upon the stone, he may then wet them with aquafortis, which will affect them very differently, if they be not similar compositions; or the stone itself may be made red-hot by the fire, or by the blowpipe, if thin black pottery be used; in which case the phenomena of oxydation will differ according to the nature and quantity of the alloy. Six principal circumstances appear to affect the operation of parting; namely, the quantity of acid used in parting, or in the first boiling; the concentration of this acid; the time employed in its application: the quantity of acid made use of in the reprise, or second operation; its concentration; and the time during which it is applied. From experiment it has been shown, that each of these unfavorable circumstances might easily occasion a loss of from the half of 
a thirty-second part of a carat, to two thirty-second parts. The assayers explain their technical langnage by observing, that in the whole mass consisting of twenty-four carats, this thirty-second part denotes 1-768th part of the mass. It may easily be conceived, therefore, that if the whole six circumstances were to exist, and be productive of errors, falling the same way, the loss would be very considerable.

It is therefore indispensably necessary, that one uniform process should be followed in the assays of gold; and it is a matter of astonishment, that such an accurate process should not have been preseribed by government for assayers, in an operation of such great commercial importance, instead of every one being left to follow his own judgment. The process recommended in the old French official report is as follows :twelve grains of the gold intended to be assayed must be mixed with thirty grains of fine silver, and cupelled with 108 grains of lead. The cupellation must be carefully attended to, and all the imperfect buttons rejected. When the cupellation is ended, the button must be reduced, by lamination, into a plate of $1 \frac{1}{2}$ inches, or rather more, in length, and four or five lines in breadth. This must be rolled up upon a quill, and placed in a matrass capable of holding about three ounces of liquid, when filled up to its narrow part. Two ounces and a half of very pure aquafortis, of the strength of 20 degrees of Baumé's areometer, must then be poured upon it; and the matrass being placed upon hot ashes, or sand, the acid must be kept gently boiling for a quarter of an hour: the acid must then be cautiously decanted, and an additional quantity of $1 \frac{1}{2}$ ounces must be poured upon the metal, and slightly boiled for twelve minutes. This being likewise carefully decanted, the small spiral piece of metal must be washed with filtered river water, or distilled water, by filling the matrass with this fluid. The vessel is then to be reversed, by applying the extremity of its neck against the bottom of a crucible of fine earth, the internal surface of which is very smooth. The annealing must now be made, after having separated the portion of water which had fallen into the crucible; and, lastly, the annealed gold must be weighed. For the certainty of this operation, two assars must be made in the same manner, together with a third assay upon gold of twenty-four carats, or upon gold the fineness of which is perfectly and generally known.

No conclusion must be drawn from this assay, unless the latter gola shouid prove to be of the fineness of twenty-four carats exactly, or of its known degree of fineness; for: if there be either loss or surplus, it may be inferred that the other two assays, having undergone the same operation, must be subject to the same error. The operation being made according to this process by several assayers, in circumstances of importance, such as those which relate to large fabrications, the fineness of the gold must not be depended upon, nor considered as accurately lnown, unless all the assayers have obtained a uniform result, without communication with each other. This identity must be considered as referring to the accuracy of half the thirty-second part of a carat. For, notwithstanding every possible precaution or uniformity, it very seldom happens that an absolute agreement is obtained between the different assays of one and the same ingot recause the ingot itself may differ in its fineness in different parts of its mass.

The phenomena of the cupellation of gold are the same as of silver, onig the operation is less delicate, for no gold is lost by evaporation or penetration into the boneash, and therefore it bears safely the highest heat of the assay furnace. The button of gold never regetates, and need not therefore be drawn out to the front of the muffle, but may be left at the further end till the assay is complete. Copper is retained more strongly by gold than it is by silver; so that with it 16 parts of lead are requisite to sweat out 1 of copper; or, in general, twice as much lead must be taken for the copper alloys of gold, as for those of silver. When the copper is alloyed with very small quantities of gold, cupellation would afford very uncertain results; we must then have recourse to liquid analysis.

M. Vauquelin recommends to boil 60 parts of nitric acid at $22^{\circ}$ Baumé, on the spiral slip or cornet of gold and silver alloy, for twenty-five minutes, and replace the liquid afterwards by acid of $32^{\circ}$, which must be boiled on it for eight minutes. This process is free from uncertainty when the assay is performed upon an alloy containing a considerable quantity of copper. But this is not the case in assaying finer gold; for then a little silver always remains in the gold. The surcharge which occurs here is 2 or 3 thousandths; this is too much, and it is an intolerable error when it becomes greater, which often happens. This evil may be completely avoided by employing the following process of M. Chaudet. He takes 0.500 of the fine gold to be assayed; cupels it with 1.500 of silver, and 1.000 of lead; forms, with the button from the cupel, a riband or strip three inches long, which he rolls into a cornet. He puts this into a matrass with acid at $22^{\circ} \mathrm{B}$., which he boils for 3 or 4 minutes. He replaces this by acid of $32^{\circ} \mathrm{B}$., and boils for ten minutes; then decants off, and boils again with acid of $32^{\circ}$, which must be finally boiled for 8 or 10 minutes.

Gold thus treated is very pure. He washes the cornet, and puts it entire into a small 
crucible permeable to water; heats the crucible to dull redness under the muffle, when the coll assumes the metallic lustre, and the cornet becomes solid. It is now taken out of the crucible and weighed.

When the alloy contains platinum, the assay presents greater difficulties. In general, to separate the platinum from the gold with accuracy, we must avail ourselves of a pecurliar property of platinum; when alloyed with silver, it becomes soluble in nitric acid. Therefore, by a proper quartation of the alloy by cupellation, and boiling the button with nitric acid, we may get a residuum of pure gold. If we were to treat the button with sulphuric acid, however, we should dissolve nothing but the silver. The copper is easily removed by cupellation. Hence, supposing that we have a quaternary compound of copper, silver, platinum, and gold, we first cupel it, and weigh the button obtained; the loss denotes the copper. This button, treated by sulphuric acid, will suffer a loss of weight equal to the amount of silver present. The residuum, by quartation with silver and boiling with nitric acid, will part with its platinum, and the gold will remain pure. For more detailed explanations, see Platinum.

ATOMIC WEIGHTS on ATOMS, are the primal quantities in which the different objects of chemistry, simple or compound, combine with each other, referred to a common body, taken as unity. Oxygen is assumed by some philosophers, and hydrogen by others, as the standard of comparison. Every chemical manufacturer should be thoroughly acquainted with the combining ratios, which are, for the same two substances, not only definite, but multiple; two great truths, upon which are founded not merely the rationale of his operations, but also the means of modifying them to useful purposes. The discussion of the doctrine of atomic weights, or prime equivalents, belongs to pure chemistry; but several of its happiest applications are to be found in the processes of art, as pursued upon the greatest scale. For many instructive examples of this proposition, the various chemical manufactures may be consulted in this Dictionary.

ATtar OF Roses. See Oils, Volatile, and Perfumery.

AURUM MUSIVUM. Mosaic gold, a preparation of tin; which see.

AUTOMATIC, a term which I have employed to designate such economic arts as are carried on by self-acting machinery. The word "manufacture," in its etymological sense, means any system, or objects of industry, executed by the hands; but in the vicissitude of language, it has now come to signify every extensive product of art which is made by machinery, with little or no aid of the human hand, so that the most perfect manufacture is that which dispenses entirely with manual labor. ${ }^{*}$ It is in our modern cotton and flax mills that automatic operations are displayed to most advantage; for there the elemental powers have been made to animate millions of complex organs, infusing into forms of wood, iron, and brass, an intelligent ageney. And as the philosophy of the fine arts, poetry, painting, and music, may be best studied in their individual master-pieces, so may the philosophy of manufactures in these its noblest creations. $\dagger$

The constant aim and effect of these automatic improvements in the arts are philanthropic, as they tend to relieve the workmen either from niceties of adjustment, which exhaust his mind and fatigue his eyes, or from painful repetition of effort, which distort and wear out his frame. A well-arranged power-mill combines the operation of many work-people, adult and young, in tending with assiduous skill a system of productive machines continuously impelled by a central force. How vastly conducive to the commercial greatness of a nation, and the comforts of mankind, human industry can become, when no longer proportioned in its results to muscular effort, which is by its nature fitful and capricious, but when made to consist in the task of guiding the work of mechanical fingers and arms regularly impelled, with equal precision and velocity, by some indefatigable physical agent, is apparent to every visiter of our cotton, flax, silk, wool, and machine factories. This great era in the useful arts is mainly due to the genius of Arkwright. Prior to the introduction of his system, manufactures were everywhere feeble and fluctuating in their development; shooting forth luxuriantly for a season, and again withering almost to the roots like annual plants. Their perennial growth then began, and attracted capital, in copious streams, to irrigate the rich domains of industry. When this new career commenced, about the year 1770, the annual consumption of cotton in British manufactures was under four millions of pounds weight, and that of the whole of Christendom was probably not more than ten millions. Last year the consumption in Great Britain and Ireland was about two hundred and seventy millions of pounds, and that of Europe and the United States together, four hundred and eighty millions. In our spacious factory apartments the benignant power of steam summons around him his myriads of willing menials, and assigns to each the regulated task, substituting, for painful muscular effort upon their part, the energies of his own gigantic arm, and demanding, in return, only attention and dexterity to correct such little aberrations as casually occur in his workmanship. Under his auspices, 
and in obedience to Arkwright's polity, magnificent edifices, surpassing far in number, ralue, usefulness, and ingenuity of construction, the boasted monuments of Asiatic, Egyptian, and Roman despotism, have, within the short period of fifty years, risen up in this kingdom, to show to what extent capital, industry, and science, may augment the resources of a state, while they meliorate the condition of its citizens. Such is the automatic system, replete with prodigies in mechanics and political economy, which promises, in its future growth, to become the great minister of civilization to the terraqueous globe, enabling this country, as its heart, to diffuse, along with its commerce, the life-blood of linowledge and religion to myriads of people still lying "in the region and shadow of death." ${ }^{* *}$ Of these truths, the present work affords decisive evidence in almost every page.

AUTOMATON. In the etymological sense, this word (self-working) signifies every mechanical construction which, by virtue of a latent intrinsic force, not obvious to common eyes, can carry on, for some time, certain movements more or less resembling the results of animal exertion, without the aid of external impulse. In this respect, all kinds of clocks and watches, planetariums, common and smoke jacks, with a vast number of the machines now employed in our cotton, silk, flax, and wool factories, as well as in our dyeing and calico printing works, may be denominatea automatic. But the term, automaton, is, in common language, appropriated to that class of mechanical artifices in which the purposely concealed power is made to imitate the arbitrary or voluntary motions of living beings. Human figures, of this kind, are sometimes styled Androides, from the Greek term, like a man.

Although, from what we have said, clock-work is not properly placed under the head automaton, it cannot be doubted that the art of making clocks, in its progressive improvement and extension, has given rise to the production of automata. The most of these, in their interior strueture, as well as in the mode of applying the moving power, have a distinct analogy with clocks; and these automata are frequently mounted in connexion with watch work. Towards the end of the 13 th century, several tower clocks, such as those at Strasburg, Lubec, Prague, Olmutz, had curious mechanisms attached to them. The most careful historical inquiry proves that automata, properly speaking, are certainly not older than wheel-clocks; and that the more perfect structures of this kind are subsequent to the general introduction of spring-clocks. Many accounts of ancient automata, such as the flying doves of Archytas of Tarentum, Regiomontanus's iron flies, the eagle which flew towards the emperor Maximilian, in Nuremburg, in the year 1470 , were deceptions, or exaggerated statements; for, three such masterpieces of art would form now, with every aid of our improved mechanisms, the most difficult of problems. The imitation of flying creatures is extremely difficult, for several reasons. There is very little space for the moving power, and the only material possessed of requisite strength being metal, must have considerable weight. Two automata, of the celebrated French mechanician, Vaucauson, first exhibited in the year 1738, have been greatly admired; namely, a flute-player, five and a half feet high, with its cubical pedestal, which played several airs upon the German flute; and that, not by any interior tube-work, but through the actual blowing of air into the flute, the motion of the tongue, and the skilful stopping of the holes with the fingers; as also a duck, which imitated many motions of a natural kind in the most extraordinary manner. This artist has had many imitators, of whom the brothers Droz of Chaux de Fonds were the most distinguished. Several very beautiful clock mechanisms of theirs are known. One of them with a figure which draws; another playing on the piano; a third which writes, besides numerous other combined automata. Frederick Von Knauss completed a writing machine at Vienna, in the year 1760 . It is now in the model cabinet of the Polytechnic Institute, and consists of a globe 2 feet in diameter, containing the mechanism, upon which a figure 7 inches high sits, and writes upon a sheet of paper fixed to a frame, whatever has been placed beforehand upon a regulating cylinder. At the end of every line, it rises and moves its hand sideways, in order to begin a new line.

Very complete automata have not been made of late years, because they are very expensive; and by soon satisfying curiosity, they cease to interest. Ingenious mechanicians find themselves better rewarded by directing their talents to the self-acting machirery of modern manufactures. We may notice here, however, the mechanical trumpeter of Mälzl, at Vienna, and a similar work of Kauffmann, at Dresden. In French Switzerland som artists continue to make minute automata which excite no little wonder; such as singing canary birds, with various movements of a natural kind; also little birds, sometimes hardly three quarters of an inch long, in snuff-boxes and watches of enamelled gold. Certain artificial figures which have been denominated automata, hardly deserve the name; since trick and ounfederacy are more or less con- 
cerned in their operation. To this head belong a number of figures apparently spealsing by mechanism; a clock which begins to strike, or to play, when a person malies a sign of holding up his finger; this effect being probably produced by a concealed green-finch, or other little bird, instructed to set off the détenle of the wheel-worli at a signal. It is likely, also, that the chess player of Von Kempelen, which excited so much wonder in the last century, had a concealed confederate. Likewise, the very ingenious little figures of Tendler, father and son, which imitated English horsemen and rope-dlancers, constructed at Eisenerz, in Styria, are probably no more true automata than the fantoccini, or figures of puppets which are exhibited in great perfection in many towns of Italy, especially at Rome.

The moving power of almost all automata is a wound-up steel spring ; because in comparison with other means of giving motion, it takes up the smallest room, is easiest concealed, and set a-going. Weights are seldom employed, and only in a partial way. The employment of other moving powers is more limited; sometimes fine sand is made to fall on the circumference of a wheel, by which the rest of the mechanism is moved. For the same purpose water has been employed; and, when it is made to fall into an air-chamber, it causes sufficient wind to excite musical sounds in pipes. In particular cases quicksilver has been used, as, for example, in the Chinese tumblers, which is only a phjsical ap paratus to illustrate the doctrine of the centre of gravity.

Figures are frequently constructed for playthings which move by wheels hardly visible An example of this simplest kind of automaton which may be introduced here, as illustrating the self-acting principles of manufactures, is shown in the figure.

Fig. 92 exhibits the outlines of an automaton, representing a swan, with suitably combined movements. The mechanism may be described, for the sake of clearness

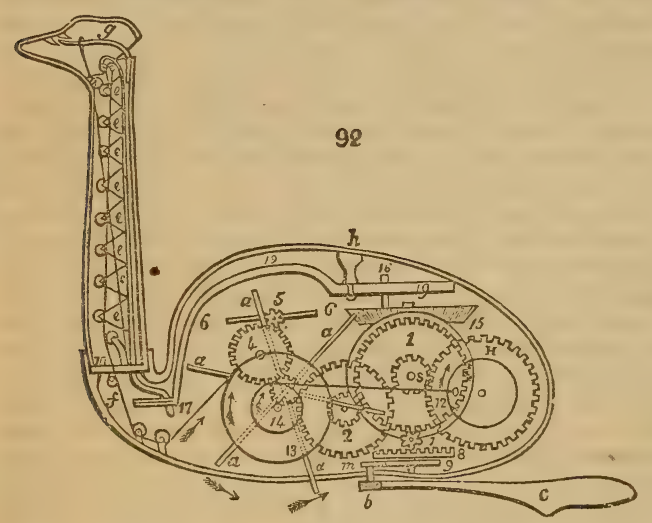
of explanation, under distinct heads. The first relates to the motion of the whole figure. By means of this part it swims upon the water, in directions changed from time to time without exterior agency. Another construction gives to the figure the faculty of bending its neck on several occasions, and to such an extent that it can plunge the bill and a portion of the head under water. Lastly, it is made to move its head and neck slowly from side to side.

On the barrel of the spring, exterior to the usual ratchet wheel, there is a main-wheel, marked 1, which works into the pinion of the wheel 2. The wheel 2 moves a smaller one, shown merely in dotted lines, and on the long axis of the latter, at either end there is a rudder, or water-wheel, the paddles of which are denoted by the letter $a$. Both of these rudder-wheels extend through an oblong opening in the bottom of the figure down into the water. They turn in the direction of the arrow, and impart a straight-forward movement to the swan. The chamber, in which these wheels revolve, is made water tight, to prevent moisture being thrown upon the rest of the machinery. By the wheel 4, motion is conveyed to the fly-pinion 5 ; the fly itself 6 , serves to regulate the working of the whole apparatus, and it is provided with a stop bar not shown in the engraving, to bring it to rest, or set it a-going at pleasure. Here, as we may imagine, the path pursued is rectilinear, when the rudderwheels are made to work in a square direction. An oblique bar, seen only in section at $b$, moveable about its middle point, carries at each end a web foot $c$, so that the direction of the bar $b$, and of both feet towards the rudder wheels, determines the form of the path which the figure will describe. The change of direction of that oblique bar is effected without other agency. For this purpose the wheel 1 takes into the pinion 7, and this carries round the crown-wheel 8 , which is fixed, with an eccentric dise 9, upon a common axis. While the crown-wheel moves in the direction of the arrow, it urns the smaller eccentric portion of the elliptic disc towards the lever $m$, which, pressed upon incessantly by its spring, assumes, by degrees, the position corresponding with the middle line of the figure, and afterwards an oblique position; then it goes back again, and reaches its first situation; consequently, through the reciprocal turning of the bar $h$, and the swim-foot, is determined and varied the path which the swan must pursue. This construction is available with all automata, which work by wheels; and it is ob- 
vious, that we may, by different forms of the disc 9, modify, at pleasure, the direction and the relocity of the turnings. If the disc is a circle, for instance, then the changes will take place less suldenly; if the disc has an outward and inward currature, upon whose edge the end of the lever presses with a roller, the morement will take place in a serpentine line.

The neck is the part which requires the most carefnl workmanship. Its outward case must be flexible, and the neck itself should therefore be made of a tube of spiral wire, corered with leather, or with a feathered bird-slin. The double line in the interior, where wre see the triangles $e, e, e$, denotes a steel spring made fast to the plate 10 , which forms the bottom of the neck; it stands loose, and needs to be merely so strong as to keep the neck straight, or to bend it a little backwards. It should not be equally thick in all points, but it should be weaker where the first graceful bend is to be made; and, in general, its stiffness ought to correspond to the curvature of the neck of this bird. The triangles $e$ are made fast at their base to the front surface of the spring; in the points of each there is a slit, in the midule of which a moreable roller is set, formed of a smoothly turned steel rod. A thin catgut string $f$, runs from the upper end of the spring, where it is fixed over all these rollers, and passes through an aperture pierced in the middle of 10 , into the inside of the rump. If the catgut be drawn straight back towards $f$, the spring, and consequently the neck, must obviously be bent, and so much the more, the more tightly $f$ is pulled, and is shortened in the hollow of the neck. How this is accomplished by the wheel-work will presently be shown. The wheel 11 receives its motion from the pinion $s$, connected with the main-wheel 1 . Upon 11 there is, moreover, the disc 12, to whose circumference a slender chain is fastened. When the wheel 11 turns in the direction of the arrow, the chain will be so much pulled onwards through the corresponding advance at the point at 12 , till this point has come to the place opposite to its present situation, and, consequently, 11 must have performed half a revolution. The other end of the chain is hung in the groove of a very moveable roller 14 ; and this will be turned immediately by the unwinding of the chain upon its axis. There turns, in connexion with it, however, the large roller 13, to which the catgut $f$ is fastened; and as this is pulled in the direction of the arrow, the neck will be bent until the wheel 11 has made a half revolution. Then the drag ceases again to act upon the chain and the catgut; the spring in the neck comes into play: it becomes straight, erects the neck of the animal, and turns the rollers 13 and 14, back into their first position.

The roller 13 is of considerable size, in order that through the slight motion of the roller 14 , a sufficient length of the catgut may be wound off, and the requisite shortening of the neck may be effected; which results from the proportion of the diameters of the rollers 11,13 , and 14 . This part of the mechanism is attached as near to the side of the hollow body as possible, to make room for the interior parts, but particularly for the paddle-wheels. Since the catgut, $f$, must pass downwards on the middle from 10 , it is necessary to incline it sideways and outwards towards 13 , by means of some small rollers.

The head, constituting one piece with the neck, will be depressed by the complete flexure of this; and the bill, being turned downtwards in front of the breast, will tonch the surface of the water. The head will not be motionless; but it is joined on both sides by a very moveable hinge, with the light ring, which forms the upper part of the clothing of the neck. A weak spring, g, also fastened to the end of the neck, tends to turn the head backwards; but in the present position it cannot do so, because a chain at $g$, whose other end is attached to the plate 10 , keeps it on the stretch. On the bending of the neck, this chain becomes slack; the spring g comes into operation, and throws the head so far back, that, in its natural position, it will reach the water.

Finally, to render the turning of the head and the neck practicable, the latter is not closely connected with the rump, while the plate 10 can turn in a cylindrical manner upon its axis, but cannot become loose outwardly. Moreover, there is upon the axis of the wheel 1, and behind it (shown merely as a circle in the engraving) a betel wheel, which works into a second similar wheel, 15 , so as to turn it in a horizontal direction. The pin 16, of the last wheel, works upon a two-armed lever 19, moveable round the point $h$, and this lever moves the neck by means of the pin 17. The shorter arm of the lever 19 has an oval aperture in which the pin 16 stands. As soon as this, in consequence of the morement of the berel wheel 15 , comes into the dotted position, it pushes the oval ring outwards on its smaller diameter, and thereby turns the iever upon the point $h$, into the oblique direction shown by the dotied lines. The pin 16 , haring come on its way right opposite to its present position, sets the lever again straight. Then the lerer, by the further progress of the pin in its circular path, is directed outwards to the opposite side; and, at last, when 15 has made an entire revolution, it is quite straight. The longer arm of the lever follows, of course, these alternating morements, so that it turns the neck upon its plate 10, by means of the pin 17; and, as 18 denotes the bill, 
this enmes into the dotted position. It may be remarked in conclusion, that the drawing of fig. 9: represents about balf the size of which the automaton may be con structed, and that the body may be formed of thin sheet-copper or brass.

Figs. 93 94 95, show the plan of a third autonaton. A horse which mores its fost
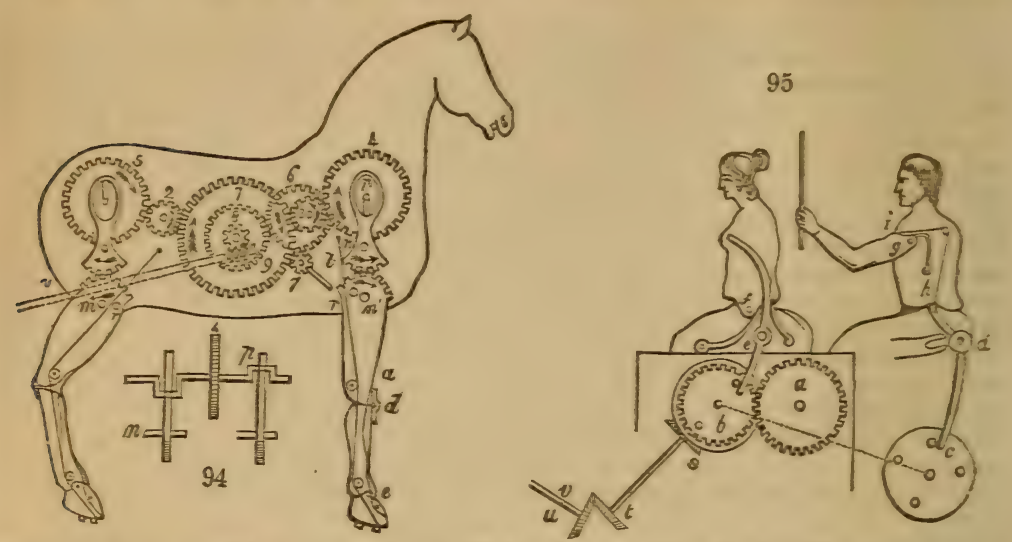

in a natural war, and draws a carriage with two figures sitting in it. The man aprears to drive the horse with a whip; the woman bends formard from him in front. The four wheels of the carriage hare no connexion with the moring mechanism. In fig. 05 , some parts are represented upon a larger scale. The wheel 1 , in fig. 93, operates through the two carrier wheels upon the wheels marked 4 and 5 . BT means of the axis of these two wheels, the feet are set in moticn. The left fore-foot, $a$, then the right hinder foot, more themselres backwards, and take hold of the ground with small tacks in their hoofs, while the two other legs are bent and raised, but no motion of the body takes place. The carriage, howerer, with which the horse is connected, advances npor its whecls. B: studying the mechanism of the foot, $a$, and the parts connected with it, we can readily understand the principles of the morement. The axis of wheel $t$ is crank-shaped on both sides, where it has to operate directlr on the fore feet: but for each foot, it is bent in an opposite direction, as is obrions in the froat riew fig. 94. This crank, or properly its part furthest from the axis, sertes instead of the pin if, in the swan, and mores like it in an oral spot, p, fig. 93, a two-armed lerer, which gives motion through tooth-work, but not as in the swan, by means of a second pin. This wheelwork renders the motion smoother. The above lever has its fulcrum at $n$, fig. 93 , about which it turns alternatelr, to the one and the other side, by virtue of the rotation of the wheel 4. The toothed arch, or the half-wheel on the under side, lars hold of a shorter lever, in a similar arch, upon the upper joint of the foot, which is mored formard and backward upon the pirot $m$. In tirtue of the motions in the direction of the arrow, the foot $a$ will move itself first obliquely bachwards, without bending, and the body will therebr bend itself forward. When the right hand foot makes the same motion. both the other feet are raised and bent. The joints of the foot at $d$ and $\epsilon$ are formed of hinges, which are so constructed that ther can rield no farther than is necessary at evert oblique position of the foot. With the continued rotation of the wheel th the lever turns itself about $n$, in an inverted direction in wards, and impels the uppermest foot-joint forward, so that it forms an acute angle with the bodr in front. The frot is now twice bent upon its joints. This takes place by the traction of the chain $\%$, with is led orer rollers (as the draming shows) to the foot, and is there fastened. As its upper end has its fixed point in the interior of the bodt, it is therefore drawn b: the eccentric pin $r$ standing in the ricinity of $m$, and thus bends the foot at the hinges. If there was space for it, a roller would answer better than a rin. By the recedure of the uppermost joint into the first position, the tension of the chain $t$ ceases again of itself, while the pin $\tau$ remores from it, and the foot is again extended in a straight line by the small springs operating upon its two under parts, which were previously bent stiffy by the cbain. By the aid of the figures, with this exrlanation, it will be apparent that all the fore feet have a similar construction, that the proper succession of motions wili be effected through the toothed arcs, and the position of the cranks on the aris of the wheels 4 and 5 , and hence the adrance of the figure must follow. The wheel 6 puts the fly $\tau$ in motion, by means of the small wheel marked 1 ; in the fixed points of the 4 chains, by means of a ratchet-wheel and a catch, the 
necessary tension will again be produced when the chains have been drawn out a little. There is sufficient room for a mechanism which could give motion to the head and cars, were it thought necessary.

The proper cause of the motions.may now be explained. In fig. 95, $a$ is a wheel connected with the wound-up spring, by which the motion of the two human figures, and also, if desired, that of the horse may be effected. The axis of the wheel $b$ carries a disc with pins, which operate upon the two-armed lever with its fulcrume, and thus cause the bending of the upper part of one of the figures, which has a hinge at $f$. On the axis of that wheel there is a second disc $c$, for giving motion to the other figure; which, for the salie of clearness, is shown separate, although it should sit alongside of its fellow. On the upper end of the double-armed lever $d$, there is a cord whose other end is connected with the moving arm, in the situation $i$, and raises it whenever a pin in the disc presses the under part of the lever. A spring $h$ brings the arm back into the original position, when a pin has passed from the lever, and has left it behind. The pins at $c$ and $d$ may be set at different distances from the middle of the disc, whereby the motions of the figures by every contact of another pin are varied, and are therefore not so uniform, and consequently more natural.

For the connexion of both mechanisms, namely, the carriage with the horse, various arrangements may be adopted. Two separate traction springs should be employed; one at $a$, fig. 95 , in the coach-seat; the other in the body of the horse. In the coachseat at $b$, the fly with its pinion, as well as a ratchet-wheel, is necessary. By means of the shaft, the horse is placed in connexion with the wagon. It may, however, receive its motion from the spring in the carriage, in which case one spring will be sufficient. Upon the latter plan the following construction may be adopted:-To the axis of $b$, fig. 95, a bevel wheel is to be attached, and from this the motion is to be transmitted to the bottom of the carriage with the help of a second bevel wheel $s$, connected with a third bevel wheel $t$. This again turns the wheel $u$, whose long axis $v$ goes to the middle of the horse's body, in an oblique direction, through the hollow shaft. This axis carries an endless screw 9, fig. 93, with very oblique threads, which works into the little wheel 8 , corresponding to the wheel 1 , through an opening in the side of the horse, and in this way sets the mechanism of the horse a-going. With this construction of $f \mathrm{~g}$. 95, a spring of considerable strength is necessary, or if the height of the carriage-seat does not afford sufficient room, its breadth will answer for placing two weaker springs along-side of each other upon a common barrel.

AXE. A tool much used by carpenters for cleaving, and roughly fashioning, blocks of wood. It is a flat iron wedge, with an oblong steel edge, parallel to which, in the short base, is a hole for receiving and holding fast the end of a strong wooden handle. In the cooper's adze, the oblong edge is at right angles to the handle, and is slightly curved up, or inflected towards it.

AXLES, of carriages; for their latest improvements, see WheEl CARriages.

AXUNGE. Hog's lard; see FAT and OrLs.

AZOTIZED, said of certain vegetable substances, which, as containing azote, were supposed at one time to partake, in some measure, of the animal nature; most animal bodies being characterized by the presence of much azote in their composition. The vegetable products, indigo, cafeinc, gluten, and many others, contain abundance of azote.

AZURE, the fine blue pigment, commonly called smalt, is a glass colored with oxyde of cobalt, and ground to an impalpable powder.

The manufacture of azure, or smalt, has been lately improved in Sweden, by the adoption of the following process :-

The cobalt ore is first roasted till the greater part of the arsenic is driven off. The residuary impure black oxyde is mixed with as much sulphuric acid (concentrated) as will make it into a pasto. which is exposed at first to a moderate heat, then to a cherryred ignition for an hour. The sulphate thus obtained is reduced to powder, and dis solved in water. To the solution, carbonate of potash is gradually added, in order to separate the remaining portion of oxyde of iron; the quantity of which depends upon the previous degree of calcination. If it be not enough oxydized, the iron is difficult to be got rid of.

When, from the color of the precipitate, we find that the potash separates merely carbonate of cobalt, it is allowed to settle, the supernatant liquor is decanted, and precipitated, by means of a solution of silicate of potash, prepared as follows :-

Ten parts of potash are carefully mixed with fifteen parts of finely ground flints or sand, and one part of pounded charcoal. This mixture is melted in a crucible of brick clay, an operation which requires steady ignition during 5 or 6 hours. The mass, when melted and pulverized, may be easily dissolved in boiling water, adding to it, by little at a time, the glass previously ground. The filtered solution is colorless, and keeps well in the air, if it contains one part of glass for 5 or 6 of water. The silicate 
of cobalt, which precipitates upon mixing the two solutions, is the preparation of cobalt most suitable for painting upon porcelain, and for the manufacture of blue glass. S'ee Cobalt.

B.

BABLAH. The rind or shell which surrounds the fruit of the mimosa cineraria ; it comes from the East Indies, as also from Senegal, under the name of Neb-neb. It contains gallic acid, tannin, a red coloring matter, and an azotized substance; but the proportion of tanuin is smaller than in sumach, galls, and knoppern (gall-nuts of the common oak) in reference to that of gallic acid, which is considerable in the bablah. It has been used, in dyeing cotton, for producing various shades of drab; as a substitute for the more expensive astringent dye-stuffs.

BAGASSE. The sugar-cane, in its dry, crushed state, as delivered from the sugarmill. It is much employed for fuel in the colonial sugar-houses.

BAKING. (Cuire, Fr. Backen, Germ.) The exposure of any body to such a heat as will dry and consolidate its parts without wasting them. Thus, wood, pottery, and porcelain, are baked, as well as bread.

BALANCE. To conduct arts, manufactures, and mines, with judgment and success, recourse must be had, at almost every step, to a balance. Experience proves that all material bodies, existing upon the surface of the earth, are constantly solicited by a force which tends to bring them to its centre, and that ther actually fall towards it when they are free to move. This force is called gravity. Though the bodies be not free, the effort of gravity is still sensible, and the resultant of all the actions which it exercises upon their material points, constitutes what is popularly called their ueight. These weights are, therefore, forces which may be compared together, and by means of machines may be inade to correspond or be counterpoised.

To discover whether two weights be equal, we must oppose them to each other in a machine where they act in a similar manner, and then see if they maintain an equilibrium; for example, we fulfil this condition if we suspend them at the two extremities of a lever, supported at its centre, and whose arms are equal. Such is the general idea of a balance. The beam of a good balance ought to be a bar of well-tempered steel, of such form as to secure perfect inflexibility under any load which may be fitly applied to its extremities. Its arms should be quite equal in weight and lenoth upon each side of its point of suspension; and this point should be placed in a vertical line over the centre of gravity; and the less distant it is from it, the more delicate will be the balance. Were it placed exactly in that centre, the beam would not spontaneously recover the horizontal position when it was once removed from it. To render its indications more readily commensurable, a slender rod or needle is fixed to it, at right angles, in the line passing through its centres of gravity and suspension. The point, or rather edge of suspension, is made of perfectly hard steel, and turns upon a bed of the same. For common uses the arms of a balance can be made sufficiently equal to gire satisfactory results; but, for the more refined purposes of science, that equality should never be presumed nor trusted to ; and, fortunately, exact weighing is quite independent of that equality. To weigh a body is to determine how many times the weight of that body contains another species of known weight, as of grains or pounds, for example. In order to find it out, let us place the substance, suppose a piece of gold, in the left hand scale of the balance; counterpoise it with sand or shot in the other, till the index needle be truly vertical, or stand in the middle of the scale, proving the beam to be horizontal. Now remove gently the piece of gold, and substitute in its place standard multiple weights of any graduation, English or French, till the needle again resumes the vertical pesition, or till is oscillations upon either side of the zero point are equal. These weights will represent precisely the weight of the gold, since they are placed in the same circumstances precisely with it, and make the same equilibrium with the weight laid in the other scale.

This method of veighing is obviously independent of the unequal length as well as the unequal weight of the arms of the beam. For its perfection two requisites only are indispensable. The first is that the points of suspension should be rigorously the same In the two operations; for the power of a given weight to turn the beam being uncqual, according as we place it at different distances from the centre of suspension, did that point vary in the two consecutive weighings, we would require to employ, in the second, a different weight from that of the piece of gold, in order to form an equilib: um with the sand or shot originally put in the opposite scale; and as there is nothing to indicate such inequality in the states of the beam, great errors would result from it. The best mode of securing against such inequality is to suspend the cords of the scales from sharp-edged rings, upon knife edges, at the ends of the beam, both made of steel so 
hard tempered as to be incapable of indentation. The second condition is, that the balance should be very sensible, that is, when in equilibrium and loaded, it may be disturbed, and its needle may oscillate, by the smallest weight put into cither of the seales. This sensibility depends solely upon the centre or nail of suspension; and it will be the more perfect the less friction there is between that knife-edge surface and the plane which supports it. Both should therefore be as hard and highly polished as possible; and should not be suffered to press against each other, except at the time of weighing. Every delicate balance of moderate size, moreover, should be suspended within a glass case, to protect it from the agitations of the air, and the corruding influence of the weather. In some balances a ball is placed upon the index or needle (whether that index stand above or below the beam), which may be made to approach or recede from the beam by a fine-threaded screw, with the effect of varying the centre of gravity relatively to the point of suspension, and thereby increasing, at will, either the sensibility, or the stability of the balance. The greater the length of the arms, the less distant the centre of gravity is beneath the centre of suspension, the better polished its central knife-edge of $30^{\circ}$, the lighter the whole balance, and the less it is loaded; the greater will be its sensibility. In all cases the arms must be quite inflexible. A balance made by Ramsden for the Royal Society, is capable of weighing ten pounds, and turns with one hundredth of a grain, which is the sevenmillionth part of the weight. In pointing out this balance to me one evening, Dr. Wollaston told me it was so delicate, that Mr. Pond, then astronomer royal, when making some observations with it, found its indications affected by his relative position before it, although it was enclosed in a glass case. When he stood opposite the right arm, that end of the beam preponderated, in consequence of its becoming expanded by the radiation of heat from his body; and when he stood opposite the left arm, he made this preponderate in its turn. It is probable that Mr. Pond had previously adjusted the centres of gravity and suspension so near to each other as to give the balance its maximum sensibility, consistent with stability. Were these centres made to coincide, the beam, when the weights are equal, would rest in any position, and the addition of the smallest weight would overset the balance, and place the beam in a vertical position, from which it would have no tendency to return. The sensibility in this case would be the greatest possible; but the other two requisites of level and stability would be entirely lost. The case would be even worse if the centre of gravity were higher than the centre of suspension, as the balance when deranged, if free, would make a revolution of no less than a semi-circle. A balance may be made by a fraudulent dealer to weigh falsely, though its arms be equal, proviued the suspension be as low as the centre of gravity, for he has only to toss his tea, for instance, forcibly into one scale to cause 15 ounces of it, or thereby, to counterpoise a pound weight in the other. Inspectors of weights, \&c. are not au fait to this fruitful source of fraud among hucksters.

BALSAMS (Baumes, Fr. Balsame, Germ.) are native compounds of ethereal or essential oils, with resin, and frequently benzoic acid. Most of them have the consistence of honey; but a few are solid, or become so by keeping. They flow either spontaneously, or by incisions made from trees and shrubs in tropical climates. They possess peculiar powerful smells, aromatic hot tastes, but lose their odoriferous properties by long exposure to the air. They are insoluble in water; soluble, to a considerable degree, in ether; and completely in alcohol. When distilled with water, ethereal oil comes over, and resin remains in the retort.

1. Balsams With Benzurc ACID :-

Balsam of Peru is extracted from the myroxylon peruiferum, a tree which grows in Peru, Mexico, \&c.; sometimes by incision, and sometimes by evaporating the decoction of the bark and branches of the tree. The former kind is very rare, and is imported in the husk of the cocoa-nut, whence it is called balsam en coque. It is brown, transparent only in thin layers, of the consistence of thick turpentine; an agreeable smell, an acrid and bitter taste; formed of two matters, the one liquid, the other granular, and somewhat crystalline. In 100 parts, it contains 12 of benzoic acid, 88 of resin, with traces of a volatile oil.

The second sort, the black balsam of Peru, is much more common than the preeeding, translucent, of the consistence of well-boiled sirup, very deep red-brown color, an almost intolerably acrid and bitter taste, and a stronger smell than the other balsam. Stoltze regards it as formed of 69 parts of a pecuilar oil, 20.7 of a resin, little soluble in alcohol, of 6.4 of benzoic acid, of 0.6 of extractive matter, and 0.9 of water.

From its high price, balsam of Peru is often adulterated with copaiba, oil of turpentine, and olive oil. One thousand parts of good balsam should, by its benzoic acid, saturate 75 parts of crystallized carbonate of soda. It is employed as a perfume for pomatums, tinctures, lozenges, sealing-wax, and for chocolate and liqueurs, instead of vanilla, when this happens to be very dear 
Liquid amber, Storax or Styrax, flows from the leaves and trunk of the liquid amber styruciflua, a tree which grows in Virginia, Louisiana, and Mexico. It is brownish ash-gray, of the consistence of turpentine, dries up readily, smells agreeably, like benzoin, has a bitterish, sharp, burning taste; is soluble in 4 parts of alcohol, and contains only 1.4 per cent. of benzoic acid.

Balsam of Tolu flows from the trunk of the myroxylon toluiferum, a tree which grows in South America; it is, when fresh, of the consistence of turpentine, is brownish-red, dries into a yellowish or reddish brittle resinous mass, of a smell like benzoin; is soluble in alcohol and ether; affords, with water, benzoic acid.

Chinese varnish flows from the bark of the Augia sinensis; it is a greenish yellow turpentine-like substance, smells aromatic, tastes strong and rather astringent, in thin layers dries soon into a smooth shining lac, and consists of resin, ethereous oil, and benzoic acid. It is soluble in alcohol and ether; and has been employed, immemorially, in China, for lackering and varnishing surfaces, either alone or colored.

BALSAMS WITHOUT BENZOIC ACID :-

Copaiva balsam, balsam of copahu or capivi, is obtained from incisions made in the trunk of the Copaifera officinalis, a tree which grows in Brazil and Cayenne. It is pale yellow, middling liquid, clear transparent, has a bitter, sharp, hot taste; a penetrating disagreeable smell; a specific gravity of from 0.950 to 0.996 . It dissolves in absolute alcohol, partially in spirit of wine, forms with alkalis, crystalline compounds. It consists of 45.59 ethereous oil, $52 \cdot 75$ of a yellow brittle resin, and 1.66 of a brown viscid resin. The oil contains no oxygen, has a composition like oil of turpentine, dissolves caoutchouc (according to Durand), but becomes oxydized in the air, into a peculiar species of resin. This balsam is used for making paper transparent, for certain lackers, and in medicine.

Mecca balsam, or opobalsam, is obtained both by incisions of, and by boiling, the branches and leaves of the Bulsamodendron Gileadense, a shrub which grows in Arabia Felix, Lesser Asia, and Egypt. When fresh it is turbid, whitish, becomes, by degrees, transparent; yellow, thickish, and eventually solid. It smells peculiar, but agreeable; tastes bitter and spicy; does not dissolve completely in hot spirit of wine, and contains 10 per cent. of ethereous oil, of the specific gravity 0.876 .

Japan lac varnish flows from incisions in the trunk of the Rhus Vernix (Melanorrhea usitata) which is cultivated in Japan, and grows wild in North America. The juice becomes black in the air; when purified, dissolves in very little oil; and, mixed with coloring matter, it constitutes the celebrated varnish of the Japanese.

For Benzoin and Turpentine. see these articles in their alphabetical places.

BANDANNA. A style of calico printing, in which white or brightly colored spots are produced upon a red or dark ground. It seems to have been practised from time immemorial in India, by binding up firmly with thread, those points of the cloth which were to remain white or yellow, while the rest of the surface was freely subjected to the dyeing operations.

The European imitations have now far surpassed, in the beauty and precision of the design, the oriental patterns; having called into action the refined resources of mechanical and chemical science. The general principles of producing bright figures upon dark grounds, are explained in the article CALICO-PRINTING; but the peculiarities of the Bandanna printing may be conveniently introduced here. In Brande's Journal for July, 1823, I described the Bandanna gallery of Messrs. Monteith at Glasgow, which, when in full action some years ago, might be reckoned the most magnificent and profitable printing apartment in the world. The white spots were produced by a solution of chlorine, made to percolate down through the Turkey red cotton cloth, in certain points defined and circumscribed by the pressure of hollow lead types in plates, in a hydraulic press. Fig. 96 is an elevation of one press; A, the top or entablature; $\mathrm{B} \mathrm{B}$, the cheeks or pillars; C, the upper block for fastening the upper lead perforated pattern to; $D$, the lower block to which the fellow pattern is affixed, and which moves up and down with the piston of the press; $\mathrm{E}$, the piston or ram; $\mathrm{F}$, the sole or base; G, the water-trough, for the discharged or spotted calico to fall into; $H$, the small cistern, for the aqueous chlorine or liquor-meter, with glass tubes for indicating the height of liquor inside of the cistern; $e e$, glass stopcocks, for admitting the liquor into that cistern from the general reservoir; $f f$, stopcocks for admitting water to wash out the chlorine; $g$; , the pattern lead-plates, with screws for setting the patterns parallel to each other; $m \mathrm{~m}$, projecting angular pieces at each corner, perforated with a half-inch hole to receive the four guide-pins rising from the lower plate, which serve to secure accuracy of adjustment between the two faces of the lead pattern plates; $h h$, two rollers which seize and pull through the discharged pieces, and deliver them into the watertrough. To the left of $\mathrm{D}$ there is a stopcock for filling the trough with water; $l$, is the waste tube for chlorine l:quor and water of washing. The contrivance for blowing a stream of air across the cloth, through the pattern tubes, is not represented in the figure. 
Sixteen engines similar to the above, each possessing the power of pressing wrth several hundred tons, are arranged in one line, in subdivisions of four; the spaces

96

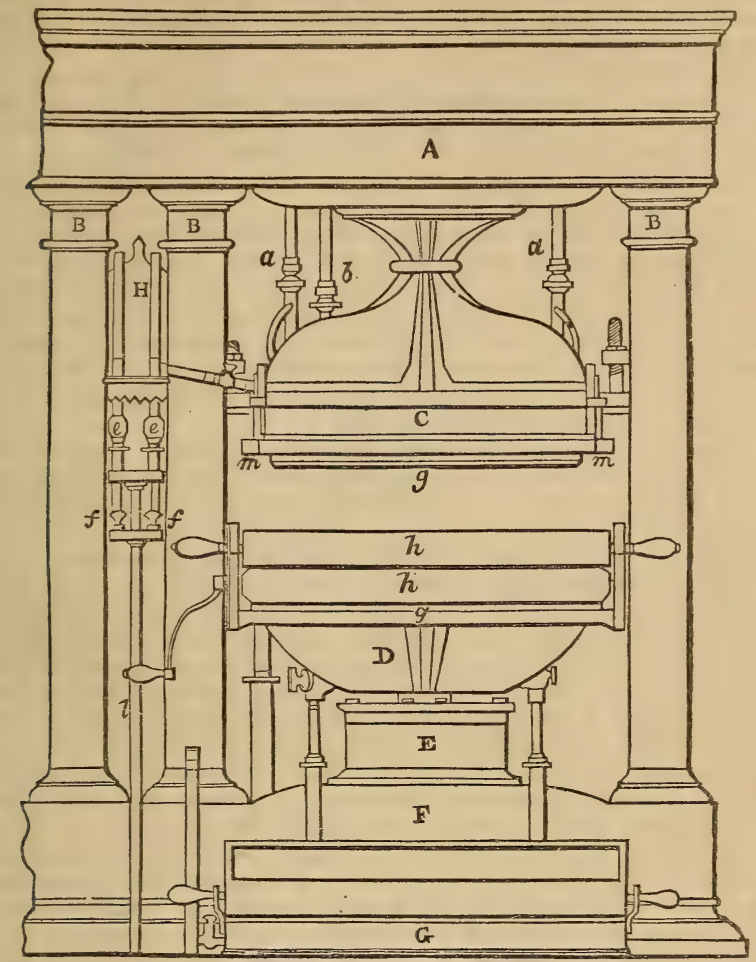

between each subdivision serving as passages to allow the workmen to go readily irom the front to the back of the presses. Each occupies twenty-five feet, so that the total length of the apartment is 100 feet.

To each press is attached a pair of patterns in lead, (or plates, as they are called,) the manner of forming which will be described in the sequel. One of these plates is fixed to the upper block of the press. This block is so contrived, that it rests upon a kind of universal joint; which enables this plate to apply more exactly to the under fellowplate. The latter sits on the moveable part of the press, commonly called the sill. When this is forced up, the two patterns close on each other very nicely, by means of the guide-pins at the corners, which are fitted with the utmost care.

The power which impels this great hydrostatic range is placed in a separate apartment, called the machinery room. This machinery consists of two press cylinders of a peculiar construction, having solid rams accurately fitted to them. To each of these cylinders, three little force-pumps, worked by a steam-engine, are connected.

The piston of the large cylinder is eight inches in diameter, and is loaded with a top-weight of five tons. This piston can be made to rise about two feet through a leather stuffing or collar. The other cylinder has a piston of only one inch in diameter, which is also loaded with a top-weight of five tons. It is capable, like the other, of being raised two feet through its collar.

Supposing the pistons to be $a^{r}$ cheir lowest point, four of the six small force-pumps are put in action by the steam-engine, two of them to raise the large piston, and two the little one. In a short time, so much water is injected into the cylinders, that the loaded pistons have arrived at their highest points. They are now ready for working the hydrostatic discharge-presses, the water pressure being conveyed from the one apartment to the other, under ground, through strong copper tubes, of small calibre.

Two valves are attached to each press, one opening a communication between the large 
driving-evlinder and the cylinder of the press, the other between the small driving-cylinder and the press. The function of the first is simply to lift the under-block of the press into contact with the upper-block; that of the second, is to give the requisite compression to the cloth. A third valve is attached to the press, for the purpose of discharging the water firm its cylinder, when the press is to be relaxed, in order to remove or draw through the cloth.

From twelve to fourteen pieces of cloth, previously dyed Turkey-red, are stretched over each other, as parallel as possible, by a particular machine. These parallel layers are then rolled round a wooden cylinder, called by the workmen, a drum. This cylinder is no:v placed in its proper situation at the back of the press. A portion of the fourteen layers of cloth, equal to the area of the plates, is next drawn through between them, by hooks attached to the two corners of the webs. On opening the valve connected with the eightinch driving-cylinder, the water enters the cylinder of the press, and instantly lifts its lower block, so as to apply the i!nder plate with its cloth, close to the upper one. This valve is then shixt, and the other is opened. The pressure of five tons in the one inch prime-cylinder, is now brought to bear on the piston of the press, which is eight inches in diameter. The aflective force here will, therefore, be 5 tons $\times 82=320$ tons; the areas of cylinders being to each other, as the squares of their respective diameters. The cloth is thus conden.ed between the leaden pattern-plates with a pressure of 320 tons, in a couple of seconds; - a splendid example of automatic art.

The next step, is to admit the blanching or discharging liquor (aqueous chlorine, obtained by adding sulphuric acid to solution of chloride of lime) to the cloth. This liquor is contained in a large cistern, in an adjoining house, from which it is run at pleasure into small lead cisterns $\mathrm{H}$ attached to the presses; which cisterns hare graduated index tubes, for regulating the quantity of liquor according to the pattern of discharge. The stopcocks on the pipes and cisterns containing this liquor, are all made of glass.

From the measure-cistern $\mathrm{H}$, the liquor is allowed to flow into the hollows in the upper lead-plate, whence it descends on the cloth, and percolates through it, extracting in its passage the Turliey-red dye. The liquor is finally conveyed into the waste pipe, from a groove in the under block. As soon as the chlorine liquor has passed through, water is atmitted in a similar manner, to wash away the chlorine; otherwise, upon relaxing the pressure, the outline of the figure discharged would become ragged. The passage of the discharge liquor, as well as of the water through the cloth, is occasionally aided by a pneumatic appáratus, or blowing machine; consisting of a large gasometer, from which air subjected to a moderate pressure may be allowed to issue, and act in the direction of the liquid upon the folds of the cloth. By an occasional twist of the air stopcock, the morkman also can ensure the equal distribution of the discharging liquor, over the whole excavations in the upper plate. When the demand for goods is very brisk, the air apparatus is much employed, as it enables the workman to double his product.

The time requisite for completing the discharging process in the first press is sufficient to enable the other three workmen to put the remaining fifteen presses in play. The discharger proceeds now from press to press, admits the liquor, the air, and the water; and is followed at a proper interval by the assistants, who relax the press, more forwards another square of the cloth, and then restore the pressure. Whenerer the sixteenth press has been liquored, \&c., it is time to open the first press. In this routine, about ten minutes are employed; that is, 224 handkerchiefs $(16+14)$ are discharged every ten minutes. The whole cloth is drawn successively forward, to be successively treated in the above method.

When the cloth escapes from the press, it is passed between the two rollers in fiont ; from which it ' 'ls into a trough of water placed below. It is finally carried off to the washing and bleaching department, where the lustre of both the white and the red is considerably brightened.

By the above arrangement of presses, 1600 pieces, consisting of 12 yards each $=19,200$ yards, are converted into Bandannas in the space of ten hours, by the lal.ar of fcar workmen.

The patterns, or plates, which are put into the presses to determine the white figures on the cloth, are made of lead in the following way. A trellis frame of cast-iron, one inch thick, with turned-up edges, forming a trough rather larger than the intendeu lead pattern, is used as the solid ground-work. Into this trough, a lead plate about one half inch thick, is firmly fixed by screw nails passing up from below. To the elges of this lead plate, the borders of the piece of sheet-lead are soldered, which covers the whole outer surface of the iron frame. Thus a strong trough is formed, one inch deep. The upright border gives at once great strength to the plate, and serves to confine the liquor. A thin sheet of lead is now laid on the thick lead-plate, in the manner of a veneer on toilettables, and is soldered to it round the edges. Both sheets must be made rery smooth beforehand, by hammering them on a smooth stone table, and then finishing with a plane: the surface of the thin sheet (now attached) is to be covered with drawing paper, pasted 
on, and upon this the pattern is drawn. It is now ready for the cutter. The first thing which he does, is to fix down with brass pins all the parts of the pattern which are tc be lef solid. He now proceeds with the little tools generally used by block-cutters which are fitted to the different curvatures of the pattern, and he cuts perpendicularly quite through the thin sheet. The pieces thus detached are easily lifted out; and thus the channels are formed which design the white figures on the red cloth. At the bottom of the channels, a sufficient number of small perforations are made through tne thicher sheet of lead, so that the discharging liquor may have free ingress and egress. Thus one plate is finished ; from which, an impression is to be taken by means of printers' ink, on the paper pasted upon another plate. The impression is taken in the hydrostatic press. Each pair of plates constitutes a set, which may be put into the presses, and removed at pleasure.

BARBERRY. The root of this plant contains a yellow coloring matter, which is soluble in water and alcohol, and is rendered brown by alkalis. The solution is employed in the manufacture of Morocco leather.

B.IRILLA. A crude soda, procured by the incineration of the salsola soda, a plant cultivated for this purpose in Spain, Sicily, Sardinia, \&c. Good barilla usually contains, according to my analysis, 20 per cent. of real alkali, associated with muriates and sulphates, chiefly of soda, some lime, and alumina, with very little sulphur. Caustic leys male from it, are used in the finishing process of the hard soap manufacture. 125,068 cwts. were imported in 1835 , of which only 5.807 were exported. The duty is $2 \mathrm{~s}$. per cwt. Of the above quantity, 64,174 came from Spain and the Balearic islands, 39,943 from the Canaries, and 20,432 from Italy and the Italian islands.

BARIUM, the metallic basis of Baryta.

BARK OF OAK, for tanning. Unfortunately, the Tables of Revenue published by the Board of Trade, mix up this bark and the dyeing barks together, and give the sum of the whole for 1835 , at 826,566 cwts., of which only 2,264 were re-exported. The duty is 1d. per cwt. from British possessions, and $8 d$. from other parts.

BARLEY (Orge, Fr.; Gerste, Germ.) English barley is that with two-rowed ears, or the hordeum vulgare distichon of the botanists; the Scotch beer or bigg, is the hordeum vulgare hexastichon. The latter has two rows of ears, but 3 corns come from the same point, so that it seems to be six-eared. The grains of bigg are smaller than those of barley, and the husks thinner. The specific gravity of English barley varies from 1.25 to 1.33 ; of bigg from 1.227 to 1.265 ; the weight of the husk of barley is $\frac{1}{6}$. that of bigg $\frac{2}{9}$. 1000 parts of barley flour contain, according to Einhof, 720 of starch, 56 sugar, 50 mucilage, 36.6 gluten, 12.3 vegetable albumen, 100 water, 2.5 phosphate of lime, 68 fibrous or ligneous matter. Sp. gravity of barley is 1.235 by my trials.

BAR.M. The yeasty top of fermenting beer. See Beer, Distillatron, FermenTATION.

BARYTA or BARYTES, one of the simple earths. It may be obtained most easily by dissolving the native carbonate of barytes (Witherite) in nitric acid, evaporating the neutral nitrate till crystals be formed, draining and then calcining these in a covered platina crucible, at a bright red heat. A less pure baryta may be obtained bv igniting strongly a mixture of the carbonate and charcoal, both in fine powder and moistened. It is a grayish white earthy looking substance, fusible only at the jet of the oxy-hydrogen blowpipe, has a sharp caustic taste, corrodes the tongue and all animal matter, is poisonous even in small quantities, has a very powerful alkaline reaction; a specific gravity of $4 \cdot 0$; becomes hot, and slakes violently when sprinkled with water, falling into a fine white powder, called the hydrate of baryta, which contains $10 \frac{1}{2}$ per cent. of water, and dissolves in 10 parts of boiling water. This solution lets fall abu $u_{i-}$ dant columnar crystals of hydrate of baryta as it cools; but it still retains one twentieth its weight of baryta, and is called baryta water. The above crystals contain 61 per cent. of water, of which, by drying, they lose 50 parts. This hydrate may be fused at a red heat without losing any more water. Of all the bases, baryta has the strongest affinity for sulphuric acid, and is hence employed either in the state of the above water, or in that of one of its neutral salts, as the nitrate or muriate, to detect the presence, and determine the quantity of that acid present in any soluble compound. Its prime equivalent, according to Berzelius, is 956,880 , oxygen being 100 ; or 76,676 , hydrogen being 1,000 . Native sulphate of baryta, or heavy spar, is fraudulently used to adulterate white lead by the English dealers to a shameful extent.

BASSORINE. A constituent part of a species of gum which comes from Bassora, as also of gum tragacanth, and of some gum resins. It is semi-transparent, difficult to pulverize, swells considerably in cold or boiling water, and forms a thick mucilage without dissolving. Treated with ten times its weight of nitric acid, it affords nearly 23 per cent. of its weight of mucic acid, being much more than is obtainable from gum arabic or cherry-tree gum. Bassorine is very soluble in water slightly acidulated with. 
nitric or muriatic acid. This principle is procured by soaking gum Bassora in a great quantity of cold water, and in removing, by a filter, all the soluble parts.

BATHS. (Bains, Fr. Baden, Germ.) Warm baths have lately come into very general use, and they are justly considered as indispensably necessary in all modern houses of any magnitude, as also in club-houses, hotels, and hospitals. But the mode of constructing these baths, and of obtaining the necessary supplies of hot and cold water, does not appear to have undergone an improrement equal to the extension of their employment.

The several points in regard to warm baths, are,

1. The materials of which they are constructed.

2. Their situation.

3 . The supply of cold water.

4. The supply of hot water.

5. Minor comforts and conveniences.

1. As to the materials of which they are constructed. Of these the best are slabs of polished marble, properly bedded with good water-tight cement, in a seasoned wooden case, and neatly and carefully united at their respectire edges. These, when vriginally well constructed, form a durable, pleasant, and agreeable-looking bath; but the expense is often objectionable, and, in upper chambers, the weight may prore inconvenient. If of white or reined marble, they are also apt to get jellow or discolored by frequent use, and cannot easily be cleansed; so that large Dutch tiles, as they are called, or square pieces of white earthenware, are sometimes substituted; which, however, are difficultly kept water-tight; so that, upon the whole, marble is preferable.

Where there are reasons for excluding marble, copper or tinned iron plate is the usual material resorted to. The former is most expensive in the outfit, but far more durable than the latter, which is, moreover, liable to leakage at the joints, unless most carefully made. Either the one or the other should be well corered, outside and inside, with several coats of paint, which may then be marbled or otherwise ornamented.

Wooden tubs, square or oblong, and oval, are sometimes used for warm baths; and are cheap and convenient, but neither elegant nor cleanly. The wood always contracts a mouldy smell; and the difficulty and nuisance of keeping them water-tight, and preventing shrinkage, are such as to exclude them from all except extemporaneous application.

2. As to the situation of the bath, or the part of the house in which it is to be placed.-In hotels, and club-houses, this is a question easily determined: several baths are usually here required, and each should have annexed to it a properly warmed dressing-room. Whether they are up stairs or down stairs, is a question of convenience, but the basement story, in which they are sometimes placed, should alwars be avoided; there is a coldness and dampness belonging to it, in almost all weathers, which is neither agreeable nor salubrious.

In hospitals, there should be at least two or three baths on each side of the house (the men's and women's), and the supply of hot water should be ready at a moment's notice. The rooms in which the baths are placed should be light, and comparatively large and airy; and such conveniences for getting into and out of the bath should be adopted, as the sick are well known to require. The dimensions of these baths should also be larger than usual.

In private houses, the fittest places for warm baths are dressing-rooms annexed to the principal bed-rooms; or, where such convenience cannot be obtained, a separate bath-room, connected with the dressing-room, and always upon the bed-room floor. All newly-built houses should be properly arranged for this purpose, and due attention should be paid to the warming of the bath-room, which ought also to be properly rentilated. A temperature of $70^{\circ}$ may easily be kept up in it, and sufficient rentilation is absolutely requisite, to prevent the deposition of moisture upon the walls and furniture.

The objection which formerly pevailed, in respect to the difficulty of obtaining adequate supplies of water, in the upper rooms, has been entirely obviated, by having cisterns at or near the top of the house; and we would just hint that these should be so contrived, as to be placed out of the reach of frost; a provision of the utmost importance in every point of view, and very easily effected in a newly-built house, though it unfortunately happens, that architects usually regard these matters as trifles, and treat them with neglect, as indeed they do the warming and ventilation of buildings generally.

3. The supply of water of proper quality and quantity, is a very important point, as connected with the present subject. The water should be soft, clean, and pure; and as free as possible from all substances mechanically suspended in it. In many cases, it answers to dig a well for the exclusive supply of a large house with water. In most 
parts of London this may effectually be accomplished, at a comparatively moderale expense; and, if the well be deep enough, the water will be abundant, soft, and pellucid. The labor of forcing it by a pump to the top of the house, is the only drawback: this, however, is very easily done by a horse-engine, or there are people enough about town, glad to undertake it at a shilling a day. I am led to these remarks by observing the filthy state of the water usually supplied, at very extravagant rates, by the water companies. It often partakes more of the appearance of pea-soup than of the pure element; fills our cisterns and pipes with mud and dirt, and, even when cleared by subsidence, is extremely unpalatable. It deposites its nastiness in the pipes connected with warm baths, and throws down a slippery deposite upon the bottom of the vessel itself to such an extent, as often to preclude its being used, at least as a luxury, which a clear and clean bath really is. This inconvenience may, in some measure, be avoided, by suffering the water to throw down its extraneous matters upon the bottom of the cistern, and drawing our supplies from pipes a little above it ; there will, however, always be more or less deposite in the pipes themselves; and every time the water runs into the cistern, the grouts are stirred up, and diffused through its mass : this, from some cause or other, has lately become an intolerable nuisance; and he who reflects upon the miscellaneous contents of Thames water, will not have his appetite sharpened by a draught of the Grand Junction beverage, nor feel reanimated and refreshed by bathing in a compound so heterogeneous and unsavory.

4 and 5. In public bathing establishments, where numerous and constant baths are re rired, the simplest and most effective means of obtaining hot water for their supply consists in drawing it directly into the baths from a large boiler, placed somewhere above their level. This boiler should be supplied with proper feeding-pipes and gauges; and, above all things, its dimensions should be ample; it should be of wrought iron or copper, except where sea water is used, in which case the latter metal is sometimes objectionable. The hot water should enter the bath by a pipe at least an inch and a half in diameter; and the cold water by one of the same dimension, or somewhat larger, so that the bath may not be long in filling. The relative proportions of the hot and cold water are, of course, to be adjusted by a thermometer, and every bath should have a two inch waste-pipe, opening about two inches from the top of the bath, and suffering the excess of water freely to run off; so that when a person is immersed in the bath, or when the supplies of water are accidentally left open, there may be no danger of an overflow.

Where there is a laundry in the upper story of the house, or other convenient place for erecting a copper and its appurtenances, a plan similar to the above may often be conveniently adopted in private houses, for the supply of a bath upon the principal bedroom floor. An attempt is sometimes made to place boilers behind the fires of dressing-rooms, or otherwise to erect them in the room itself, for the purpose of supplying warm water; but this plan is always objectionable, from the complexity of the means by which the supply of water is furnished to the boiler, and often dangerous from the flues becoming choaked with soot, and taking fire. Steam is also apt, in such cases, to escape in quantities into the room; so that it becomes necessary to search for other methods of heating the bath; one or two of the least objectionable of which I shall describe.

1. A contrivance of some ingenuity consists in suffering the water for the supply of the bath to flow from a cistern above it, through a leaden pipe of about one inch diameter, which is conducted into the kitchen or other convenient place, where a large boiler for the supply of hot water is required. The bath-pipe is immersed in this boiler, in which it makes many convolutions, and, again emerging, ascends to the bath. The operation is $\operatorname{simp}^{7}$ this :- the cold water passing through the convolutions of that part of the pipe whicn is immersed in the boiling water, receives there sufficient heat for the purpose required, and is delivered in that state by the ascending pipe into the bath, which is also supplied with cold water and waste-pipes as usual. The pipe may be of lead, as far as the descending and ascending parts are concerned, but the portion forming the worm, or convolutions immersed in the boiler, should be copper, in order that the water within it may receive heat without impediment.

This plan is economical only where a large boiler is constantly kept at work in the lower part of the house; otherwise, the trouble and expense of heating such a boiler, for the mere purpose of the bath, render it unavailable. The worm-pipe is also apt to become furred, upon the outside, by the deposition of the earthy impurities of the water in which it is immersed; it then becomes a bad conductor of heat, is cleansed with difficulty, and the plan is rendered ineffective. This system, however, has been adopted, in some particular cases, with satisfaction.

2. A much more simple, economical, and independent mode of heating a warm bath, by a fire placed at a distance from it, is the following, which is found to answer perfectly in private houses, as well as upon a more extended scale in large establish- 
ments. It is certainly open to some objections, but these are overbalanced by its advantages. A wagon-shaped boiler, holding about six gallons of water, is properly placed over a small furnace, in any convenient and safe part of the house, as the kitchen, scullery, servants' hall, or wash-house. The bath itself, of the usual dimensions and construction, is placed where it is wanted, with a due supply of cold water from above. Two pipes issue from within an inch of the bottom of the bath at its opposite extremities; one at the head of the bath, about one inch, and the other at the foot, an inch and cule eighth in diameter. These tubes descend to the boiler, the smaller one entering it at the bottom, and the larger one issuing from its top.

Under these circumstances, supposing the pipes and boiler everywhere perfectly tight, when the bath is filled, the water will descend into and expel the air from the builer, and completely fill it. Now, upon making a gentle fire under the boiler, an ascending current of warm water will necessarily pass upwards through the larger pipe which issues from its top, and cold water will descend by the pipe which enters at the bottom; and thus, by the establishment of currents, the whole mass of water in the bath will become heated to the desired point; or, if above it, the temperature may easily be low ered by the admixture of cold water.

The advantages of this form of bath are numerous. The shorter the pipes of communication the better, but they may extend forty or fifty feet without any inconvenience beyond that of expense; so that there is no obstacle to the bath being near the bed-room while the boiler is on the basement story. There is but little time required for heating the bath; the water in which may, if requisite, be raised to about $100^{\circ}$ in about half an hour from the time of lighting the fire. The consumption of fuel is also trifling.

The following are the chief disadvantages attendant upon this plan, and the means of obviating them :-

It is necessary, when the water has acquired its proper temperature, to withdraw the fire from the boiler, or not to use the bath immediately, as it may go on acquiring some heat from the boiler, so that we may become inconveniently hot in the bath. When, therefore, this bath is used, we may proceed as follows:- heat the water in it an hour before it is wanted, to about $100^{\circ}$, and then extinguish the fire. The water will retain its temperature, or nearly so, for three or four hours, especially if the bath be shut up with a cover; so that when about to use it, cold water may be admitted till the temperature is lowered to the required point, and thus all the above inconveniences are avoided.

Another disadvantage of this bath arises from too fierce a fire being made under the boiler, so as to occasion the water to boil within it, a circumstance which ought always to be carefully avoided. In that case, the steam rising in the upper part of the boiler, and into the top pipe, condenses there, and occasions violent concussions, the noise of which often alarms the whole house, and leads to apprehensions of explosion, which, however, is very unlikely to occur; but the concussions thus produced injure the pipes, and may render them leaky; so that in regard to these, and all other baths, \&.e., we may remark, that the pipes should pass up and down in such parts of the house as will not be injured if some leakage takes place; and under the bath itself should be a sufficiently large leaden tray with a waste-pipe, to receive and carry off any accidental drippings, which might injure the ceilings of the rooms below. In all newly-built houses, two or three flues should be left in proper places for the passage of ascending and descending water-pipes; and these flues should in some way receive at their lower part a little warm air in winter, to prevent the pipes freezing; the same attention should also be paid to the situation of the cisterns of water in houses, which should be kept within the house, and always supplied with a very ample waste-pipe, to prevent the danger of overflow. Cisterns thus properly placed, and carefully constructed, should be supplied from the water-mains by pipes kept under ground, till they enter the house, and not carried across the area, or immediately under the pavement, where they are liable to freeze.

3. Baths are sometimes heated by steam, which has several advantages : it may either be condensed directly into the water of the bath, or, if the bath be of copper or tinned iron, it may be conducted into a casing upon its outside, usually called a jacket; in the latter case there must be a proper vent for the condensed water, and for the escape of air and waste steam. Steam is also sometimes passed through a serpentine pipe, placed at the bottom of the bath. But none of these methods are to be recommended for adoption in private houses, and are only advisable in hospitals, or establishments where steam boilers are worked for other purposes than the mere heating of baths.

Many copper and tin baths have been lately constructed in London, with a little furnace attached tc one end, and surrounded with a case or jacket, into which the water flows and circulates backwards and forwards till the whole mass in the bath gets heated to the due degree. One of the best of these is that constructed by Mr. Benham, 
of Wigmore street. The bath must be placed near the fire-grate, and the smoke-pipe of the attached furnace be conducted up the chimney a certain way to secure a sufficient draught to maintain combustion. The above bath, well managed, heats the water from $50^{\circ}$ to $98^{\circ}$ in about 20 or 25 minutes, as $I$ have experimentally proved. When the proper temperature is attained, the fire must of course be extinguished.

BDELLIUM. A gum resin, produced by an unknown plant which grows in Persia and Arabia. It comes to us in yellowish or reddish pieces, smells faintly, luke myrrh, and consists of 59 resin, $9 \cdot 2$ gum, $30 \cdot 6$ bassorine, and $1 \cdot 2$ ethereous oil.

BEER. (Bière, Fr.; Bier, Germ.) The fermented infusion of malted barley, flavored with hops, constitutes the best species of beer; but there are many beverages of inferior quality to which this name is given, such as spruce beer, ginger beer, molasses beer, \&c. all of which consist of a saccharine liquor, partially advanced into the vinous fermentation, and flavored with peculiar substances.

The ancients were acquainted with beer, and the Romans gave it the appropriate name of Cerevisia (quasi Ceresia), as being the product of corn, the gift of Ceres. The most celebrated liquor of this kind in the old time, was the Pelusian potation, so called from the town where it was prepared at the mouth of the Nile. Aristotle speaks of the intoxication caused by beer; and Theophrastus very justly denominated it the wine of barley. We may, indeed, infer from the notices found in historians, that drinks analogous to our beer were in use among the ancient Gauls, Germans, and in fact almost every people of our temperate zone; and they are still the universal beverage in every land where the vine is not an object of rustic husbandry.

The manufacture of beer, or the art of brewing, may be conveniently considered under five heads :-

1. An examination of the natural productions which enter into its composition ; or of barley and hops.

2. The changes which barley must undergo to fit it for making beer ; or the processes of malting and mashing.

3. The formation of a proper wort from the mashed malt and hops.

4. The fermentation of that wort; and,

5. The fining, ripening, and preservation of the beer.

I. Of the materials.

1. Barley, wheat, maize, and several other linds of corn are capable of undergoing those fermentative changes, by which beer may be made; but the first substance is by far the fittest. There are two species of barley, the hordeum vulgare or common barley, having two seeds arranged in a row on its spikes; and the hordeum hexastichon, in which three seeds spring from one point, so that its double row has apparentiy six seeds. The former is the proper barley, and is much the larger sized grain; the latter is little known in England, but is much cultivated in Scotland under the name of bear or bigg; being a hardy plant adapted to a colder country. The finer the climate in which barley grows, the denser and larger its seed, and the thinner its husk; thus the Norfolk and Suffolk barley is distinguished in these respects from that of Aberdeenshire. Bigg is a less compact grain than barley; the weight of a Winchester bushel ( 2150.42 cubic inches) of the former is only about $47 \mathrm{lbs}$., while that of a bushel of the latter is nearly $51 \mathrm{lbs}$. Their constiluents, however, bear much the same proportion to each other.

The quality of barley is proved not only by its density when dry, but by the increase of volume which it acquires when steeped in water. Thus,

100 measures of average English barley thereby swell into 124.

100 - of - Scotch ditto, - - - 121.

100 - of - - bigg or bear, - 118.

Nay, 100 of very fine Suffolk barley have swollen into $\quad-\quad 183$.

While 100 of an inferior Scotch bigg became no more than - 109.

This circumstance indicates so nearly the probable yield of malt, that it is carefully attended to by the officers of excise, who gauge the steep cistern, and levy their duty in conformity with the largest volume. 100 pounds of good barley become almost one half heavier by the absorption of moisture; and weigh upon an average 147 pounds; the best of course taking up most water.

By chemical analysis barley flour seems to consist of $6 \% \cdot 18$ parts of hordeine, or starch and gluten intimately combined, $7 \cdot 29$ of vegetable fibre, $1 \cdot 15$ of coagulated alburaen, 3.52 parts of gluten, 5.21 of sugar, 4.62 of gum, 0.24 of phosphate of lime, and 9.37 of water. The loss amounted to $1 \cdot 42$. To these principles should be added a peculiar volatile oil of a concrete nature, which is obtained during the process of distilling fe: * mented malt wash. (See WHIskey.) It may also be extracted from barley flour, by the solvent action of alcohol; and never amounts to more than a few parts in the thousand. The husk also contains some of that fetid oil. Proust thought that he had discovered in barley a peculiar principle, to which he gave the name of hordeine; and which he separated from starch by the action of both cold and boiling water. He found that by treat- 
ing barley meal successively with water, he obtained from 89 to 90 parts of a farinaceous substance, composed of from 32 to 33 of starch, and from 57 to 58 of hordeine. Einhof oblained from barley seeds, 70.05 of flour, 18.75 of husks or bran, and $11 \cdot 20$ of water.

According to Proust, hordeine is a yellowish powder, not unlike fine saw-dust. It contains no azote, for it affords no ammonia by distillation, and is therefore very dissimilar to gluten. In the germination of barley, which constitutes the process of malting, the proportion of hordeine is greatly diminished by its conversion into sugar and starch. Other chemists suppose that the hordeine of Proust is merely a mixture of the bran of the barley with starch and gluten. It is obvious that the subject stands in need of new chemical researches. In barley the husk constitutes from one fourth to one fifth of the whole weight; in oats it constitutes one third; and in wheat one tenth. From the analysis of barley flour recently made, it appears to consist in 1000 parts : of water, 100 ; albumen, 22.3 ; sugar, 56 ; gum or mucilage, 50 ; gluten, $37 \cdot 6$; starch, 720 ; phosphate of lime, 2.5 .

2. The hop, humulus lupulus, the female flowers of the plant. Ives first directed attention to a yellow pulverulent substance which invests the scales of the catkins, amounting to about one eighth of their weight ; and referred to it the valuable properties which hops impart to beer. We may obtain this substance by drying the hops at a temperature of $86^{\circ}$ F., introduring them into a coarse canvass bag, and shaking it so that the yellow powder shall pass through the pores of the canvass. This powder bears some resemblance to lycopodium. Of the 13 parts in 100 of this powder, 4 parts are foreign matters, derived from the scales of the cones; leaving 9 parts of a peculiar granular substance. When distilled with water, this substance affords two per cent. of its weight $\left(\frac{2}{10}\right.$ for 100 times the weight of hops) of a volatile colorless oil, to which the plant owes its peculiar aroma. This oil dissolves in water in considerable quantity. It appears to contain sulphur (for it blackens solutions of silver), and also acetate of ammonia. No less than 65 per cent. of the yellow dust is soluble in alcohol. This solution, treated with water and distilled, leaves a resin, which amounts to 52.5 per cent. It has no bitter taste, and is soluble in alcohol and ether. The watery solution from which the resin was separated contains the bitter substance which has been called lupuline by Payen and Chevallier, mixed with a little tannin and malic acid. To obtain this in a state of purity, the free acid must be saturated with lime, the solution evaporated to dryness, and the residuum must be treated with ether, which removes a little resin; after which the lupuline is dissolved out by alcohol, which leaves the malate of lime. On evaporating away the alcohol, the lupuline remains, weighing from $8 \cdot 3$ to 12.5 per cent. It is sometimes white, or slightly yellowish, and opaque, sometimes orange yellow and transparent. At ordinary temperatures it is inodorous, but when heated strongly it emits the smell of hops. It possesses the characteristic taste and bitterness of the hop. Water dissolves it only in the proportion of 5 per cent., but it thereby acquires a pale yellow color. Lupuline is neither acid nor alkaline; it is acted upon neither by the dilute acids nor alkalis, nor by the solutions of the metallic salts; it is quite soluble in alcohol, but hardiy in ether. It contains apparently no azote, for it affords no ammonia by destructive distillation; but only an empyreumatic oil.

The yellow dust of hops contains, moreover, traces of a fatty matter, gum, a small quantity of an azotized substance, and several saline combinations in minute quantity. Boiling water dissolves from 19 to 31 per cent. of the contents of the dust, of which a large proportion is resin. Ives thought that the scales of the catkins of hops, when freed from the yellow powder, contained no principles analogous to it; but Payen and Chevallier have proved the contrary. The cones of hop give up to boiling alcohol 36 per cent. of soluble matter; while the same cones, stripped of their yellow powder, yield only 26 per cent.; and further, these chemists found the same principles in the different parts of the hop, but in different proportions.

The packing of the hop catkins or cones is one of the most important operations towards the preservation of this plant; and is probably the cause of the enormous difference in value between the English and French hops after a few years keeping. The former, at the end of six years, possess still great value, and may be sold as an aricle only two or three years old; while the latter have lost the greater part of their value in three years, and are no more saleable at the end of four. In France, it is packed merely by tramping it with the feet in sacks. Under this slight pressure, large interstitial spaces are left amid the mass of the hops, through which the air freely circulates, carrying off the essential oil, and oxygenating some of the other proximate principles, so as to render them inert. By the English method, on the contrary, the hops, after being well rammed into strong sacks hung in frames, are next subjected to the action of a hydraulic press. The valuable yellow powder thus enclosed on every side by innumerable compact scales, is completely screened from the contact of the atmosphere, and from all its vicissitudes of humidity. Its essential oil, in particular, the basis of its flavor is preserved without decay. 
According to the experiments of Chevallier and Payen upon the hops of England, Flanders, the Netherlands, and the department of the Vosges, those of the county of Kent aftorded the largest cones, and were most productive in useful secreted and soluble matters. Next to them were the hops of Alost.

The best hops have a golden yellow color, large cones, an agreeable aroma : when rubbed between the hands, they leave yellow traces, powerfully odoriferous, without any broken portions of the plant, such as leaves, stems, and scaly fragments. When alcohol is digested on good hops, from 9 to 12 per cent. of soluble yellow matter may be obtained by evaporating it to dryness. This is a good test of their quality.

The best-flarored and palest hops are packed in sacks of fine canvass, which are called pockets, and weigh about $1 \frac{1}{2} \mathrm{cwt}$. each. These are bought by the ale brewer. The stronger-flavored and darker-colored hops are packed in bags of a very coarse texture like door-mats, called hop bags : these contain generally about 3 cwt., and are sold to the porter and beer brewers. After the end of a year or two, hops are reckoned to have lost much of their marketable value, and are then sold to the second-rate porter brewers, under the name of old hops. The finest hops are grown in the neighborhood of Canterbury; but those of Worcester have an agreeable mildness of flavor, greatly admired by many ale drinkers. When the bitter and aromatic principles disappear, the hops are no better than so much chaff; therefore, an accurate chemical criterion of their principles would be a great benefit to the brewer.

II. Malting.-This process consists of three successive operations; the steeprag; the couching, sweating, and flooring; and the kiln-drying.

The steeping is performed in large cisterns made of wood or stone, which being filled with clear water up to a certain height, a quantity of barley is shot into them, and well stirred about with rakes. The good grain is heavy, and subsides; the lighter grains, which float on the surface, are damaged, and should be skimmed off; for they would injure the quality of the malt, and the flavor of the beer made with it. They seldom amount to more than two per cent. More barley is successively emptied into the steep cistern, till the water stands only a few inches, about five, above its surface; when this is levelled very carefully, and every light seed is removed. The steep lasts from forty to sixty hours, according to circumstances; new barley requiring a longer period than old, and bigg requiring much less time than barley.

During this steep, some carbonic acid is evolved from the grains, and combines with the water, which, at the same time, acquires a yellowish tinge, and a strawy smell, from dissolving some of the extractive matter of the barley husks. The grain imbibes about one half its weight of water, and increases in size by ahout one fifth. By losing this extract, the husk becomes about one seventieth lighter in weight, and paler in color.

The duration of the steep depends, in some measure, upon the temperature of the air, and is shorter in summer than in winter. In general from 40 to 48 hours will be found sufficient for sound dry grain. Steeping has for its object to expand the farina of the barley with humidity, and thus prepare the seed for germination, in the same way as the moisture of the earth prepares for the growth of the radicle and plumula in seed sown in it. Too long continuance in the steep is injurious; because it prevents the germination at the proper time, and thereby exhausts a portion of the vegetative power: it causes also an abstraction of saccharine matter by the water. The maceration is known to be complete when the grain may be easily transfixed with a needle, and is swollen to its full size. The following is reckoned a good test :-If a barley-corn, when pressed between the thumb and fingers, continues entire in its husk, it is not sufficiently steeped; but if it sheds its flour upon the fingers, it is ready. When the substance exudes in the form of a milky juice, the steep has been too long continued, and the barley is spoiled for germination.

In warm weather it sometimes happens that the water becomes acescent before the grain is thoroughly swelled. This accident, which is manifest to the taste and smell, must be immediately obviated by drawing off the foul water through the tap at the bottom of the cistern, and replacing it with fresh cold water. It does no harm to renew it two or three times at one steep.

The couch.-The water being drawn off, and occasionally a fresh quantity passed through, to wash away any slimy matter which may have been generated in warm weather, the barley is now laid upon the couch floor of stone flags, in square heaps from 12 to 16 inches high, and left in that position for 24 hours. At this period, the bulk of the grain being the greatest, it may be gauged by the revenue officers if they think fit. The moisture now leaves the surface of the barley so completely, that it imparts no dampness to the hand. By degrees, however, it becomes warm; the temperature rising $10^{\circ}$ above the atmosphere, while an agreeable fruity smell is evolved. At this time, if the hand be thrust into the heap, it not only feels warm, but it gets bedewed with moisture. At this sweating stage, the germination begins; the fibrils of the radicle furst sprout forth from the tip of every grain, and a white elevation appears, that soon 
separates into three or more racisles, which grow rapidly larger. About a day after this appearance, the plumula peeps forth at the same point, proceeding thence beneath the husk to the other end of the seed, in the form of a green leaflet.

The greatest heat of the couch is usually about $90^{\circ}$ hours after the barley has been talien out of the steep. In consequence, the radicles tend to increase in length with very great rapidity, and must be checked by artificial means, which constitute the chief art of the maltster. He now begins to spread the barley thinner on the floor, and turns it over several times in the course of a day, bringing the portions of the interior into the exterior surface. The depth, which was originally 15 or 16 inches, is lowered a little at every turning over, till it be brought eventually down to three or four inches. Two turnings a day are generally required. At this period of spreading or flooring, the temperature in England is about $62^{\circ}$, an: in Scotland 5 or 6 degrces lower.

About a day after the radicles appear, the rudiments of the stem, or of the plumula, sprout forth, called by the English maltsters the acrospire. It issues from the same end of the seed as the radicle, but turns round, and proceeds within the husk towards the other end, and would there come forth as a green leaf, were its progress not arrested. The malting, however, is complete before the acrospire becomes a leaf.

The barley couch absorbs oxygen and emits carbonic acid, just as animals do in breathing, but to a very limited extent; for the grain loses only three per cent. of its weight upon the malt floor, and a part of this loss is due to waste particles. As the acrospire creeps along the surface of the seed, the farina within undergoes a remarkable alteration. The gluten and mucilage disappear, in a great measure, the color becomes whiter, and the substanee becomes so friable that it crumbles into meal between the firgers. This is the great purpose of malting, and it is known to be accomplished when the plumula or acrospire has approached the end of the seed. Now the further growth mus! be completely stopped. Fourteen days may be reckoned the usual duration of the germinating stage of the malting operations in England; but in Scotland, where the temperature of the couch is lower, eighteen days, or even twenty-one, are sometimes required. The shorter the period within the above limits, the more advantageous is the process to the maltster, as he can turn over his capital the sooner, and his malt is also somewhat the better. Bigg is more rapid in its germination than barley, and requires to be still more carefully watched. In dry weather $\mathrm{j}_{\mathrm{t}}$ is sometimes necessary to water the barley upon the couch.

Occasionally the odor disengaged from the couch is offensive, resembling that of rotten apples. This is a bad prognostic, indicating either that the barley was of bad quality, or that the workmen, through careless shovelling, have crushed a number of the grains in turning them over. Hence when the weather causes too quick germination, it is better to check it by spreading the heap out thinner than by turning it too frequently over. On comparing different samples of barley, we shail find that the best develop the germ or acrospire quicker than the radicles, and thus occasion a greater production of the saccharine principle; this conversion advances along with the acrospire, and keep: pace with it, so that the portion of the seed to which it has not reached is still in its un altered starchy state. It is never complete for any single barleycorn till the acrospire has come to the end opposite to that from which it sprung; hence one part of the corn may be sugary, while the other is still insipid. If the grain were allowed to regetate beyond this term, the radicles being fully one third of an inch long, the future stem would become visibly green in the exterior; it would shoot forth rapidly, the interior of the grain would become milky, with a complete exhaustion of all its useful constituents, and nothing but the husk would remain.

In France, the brewers, who generally malt their barley themselves, seldom leave it on the couch more than 8 or 10 days, which, even taking into account the warmer climate of their country, is certainly too short a period, and hence they make inferior wort to the English brewer, from the same quantity of malt.

At the end of the germination, the radicles have become $1 \frac{1}{2}$ longer than the barley, and are contorted so that the corns hook into one another, but the acrospire is just beginning to push through. A moderate temperature of the air is best adapted to malting; therefore it cannot be carried on well during the heat of summer or the colds of winter. Malt-floors should be placed in substantial thick-walled buildings, without access of the sun, so that a uniform temperature of $59^{\circ}$ or $60^{\circ}$ may prevail inside. Some recommend them to be sunk a little under the surface of the ground, if the situation be dry.

During germination a remarkable change has taken place in the substance of the grain. The glutinous constituent has almost entirely disappeared, and is supposed to have passed into the matter of the radicles, while a portion of the starch is converted into sugar and mucilage. The change is similar to what starch undergoes when dissolved in water, and digested in a heat of about $160^{\circ} \mathrm{F}$. along with a little gluten. 
The thick paste becomes gradually liquid, transparent, and sweet tasted, and the solution contains now, sugar and gum, mixed with some unaltered starch. The gluten suficrs a change at the same time, and becomes acescent, so that only a certain quantity of starch can be thus converted by a quantity of gluten. By the artificial growth upon the maltfloor, all the gluten and albumen present in barley is not decomposed, and only about one half of the starch is converted into sugar; the other half, by a continuance of the germination, would only go to the growth of the roots and stems of the plant; but it receires its nearly complete conversion into sugar without any notable waste of substance in the brewer's operation of mashing.

The kiln-drying.-When the malt has become perceptibly dry to the hand upon the floor, it is taken to the kiln, and dried hard with artificial heat, to stop all further growth, and enable it to be kept, without change, for future use, at any time. The malt-kiln, which is particularly described in the next page, is a round or a square chamber, covered with perforated plates of cast iron, whose area is heated by a stove or furnace, so that not merely the plates on which the malt is laid are warmed, but the air which passes up through the stratum of malt itself, with the effect of carrying off very rapidly the moisture from the grains. The layer of malt should be about 3 or 4 inches thick, and eveniy spread, and its heat should be steadily kept at from the 90 th to the 100th degree of Fahrenheit's scale, till the moisture be mostly exhaled from it. During this time the malt must be turned over at first frequently, and latterly every three or four hours. When it is nearly dry, its temperature should be raised to from $145^{\circ}$ to $165^{\circ} \mathrm{F}$., and it must be kept at this heat till it has assumed the desired shade of color, which is commonly a brownish-yellow or a yellowish-brown. The fire is now allowed to die ont, and the malt is left on the plates till it has become completely cool; a result promoted by the stream of cool air, which now rises up through the bars of the grate; or the thoroughly dry browned malt may, by damping the fire, be taken hot from the plates, and cooled upon the floor of an adjoining apartment. The prepared malt must be kept in a dry loft, where it can be occasionally turned over till it is used. The period of liln-drying should not be hurried. Many persons employ two dars in this operation.

According to the color and the degree of drying, malt is distributed into three sorts , pale, yellow, and brown. The first is produced when the highest heat to which it has been subjected is from $90^{\circ}$ to $100^{\circ} \mathrm{F}$.; the amber yellow, when it has suffered a heat of $122^{\circ}$; and the brown when it has been treated as above described. The black malt used by the porter brewer to color his beer, has suffered a much higher heat, and is partially charred. The temperature of the kiln should, in all cases, be most gradually raised, and most equably maintained. If the heat be too great at the beginning, the busk gets hard dried, and hinders the evaporation of the water from the interior sub. stance; and should the interior be dried by a stronger heat, the husk will probably split, and the farina become of a horny texture, very refractory in the mash-tun. In general, it is preferable to brown malt, rather by a long-continued moderate heat, than by a more violent heat of shorter duration, which is apt to carbonize a portion of the mucilaginous sugar, and to damage the article. In this way, the sweet is sometimes converted into a bitter principle.

During the kiln-drying, the roots and acrospire of the barley become brittle, and fall off; and are separated by a wire sieve whose meshes are too small to allow the malt itself to pass through.

A quantity of good barley, which weighs 100 pounds, being jur ciously malted, will weigh, after drying and sifting, 80 pounds. Since the raw grain, dried by itself at the same temperature as the malt, would lose 12 per cent. of its weight in water, the malt process dissipates out of these remaining 88 pounds, only 8 pounds, or 8 per cent. of the raw barley. This loss consists of-

$$
\begin{aligned}
& 1 \frac{1}{2} \text { per cent. dissolved out in the steep water, } \\
& 3 \text { - dissipated in the kiln, } \\
& \frac{2}{2}-\text { by the falling of the fibrils, } \\
& \text { of waste. }
\end{aligned}
$$

The bulk of good malt exceeds that of the barley from which it was made, by about 8 or 9 per cent.

The operation of kiln-drying is not confined to the mere expulsion of the moisture from the germinated seeds; but it serves to convert into sugar a portion of the starch. which renuained unchanged, and that in a twofold way; first, by the action of the gluten upon the fecula at an elevated temperature, as also by the species of roasting which the starch undergoes, and which renders it of a gummy nature. (See STARCH.) We shall have a proof of this explanation, if we dry one portion of the malt in a raturally dry atmosphere, and another in a moderately warm kiln; the former will yield less saccharine extract than the latter. Moreover, the kiln-dried malt has a peculiar, agreeable, and faintly burned taste, probably from a small portion of empy. 
reumatic oil formed in the husk, and which not only imparts its flavor to the heer, but also contributes to its preservation. It is therefore obvious, that the skilful preparation of the malt must have the greatest influence both on the quantity and quality of the worts to be made from it. If the germination be pushed too far, a part of the extractible matter is wasted; if it has not advanced far enough, the malt will be too raw, and too mucb of its substanee will remain as an insoluble starch; if it is too highly kiln-dried, a portion of its sugar will be caramelized, and become bitter; and if the sweating was imperfect or irregular, much of the barley may be rendered lumpy and useless. Good malt is distinguishable by the following characters:-

The grain is round and full, breaks freely between the teeth, and has a sweetish taste, an agreeable smell, and is full of a soft flour from end to end. It affords no unpleasant flavor on being chewed; it is not hard, so that when drawn along an.oaken table across the fibres, it leaves a white streak, like chalk. It swims upon water, while unmalted barley sinks in it. Since the quality of the malt depends much on that of the barley, the same sort only should be used for one malting. New barley germinates quicker than old, which is more dried up; a couch of a mixture of the two would be irregular, and difficult to regulate.

Description of the malt-kiln.-Figs. 97, 98, 99, 100, exhibit the construction of a wellcontrived malt-kiln. Fig. 97, is the ground plan : fig. 98 is the vertical section; and figs. 99 and 100, a horizontal and vertical section in the line of the malt-plates. The same letters denote the same parts in each of the figures. A cast-iron cupola-shaped oven is 98
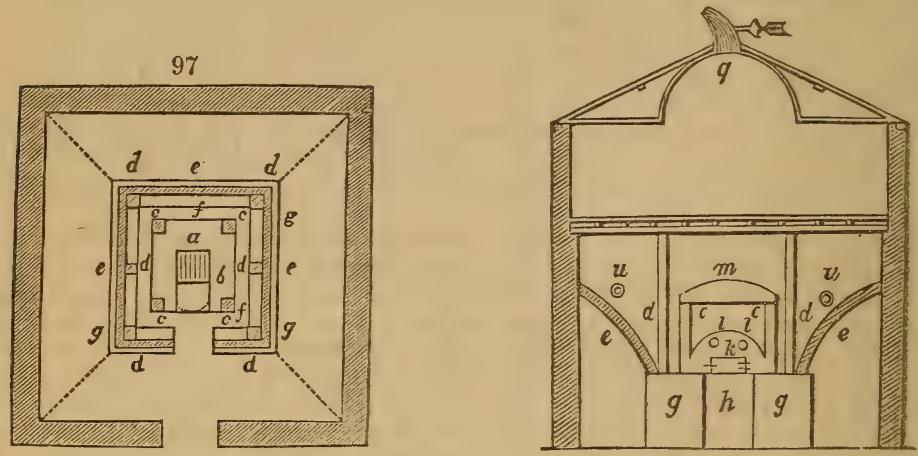

99
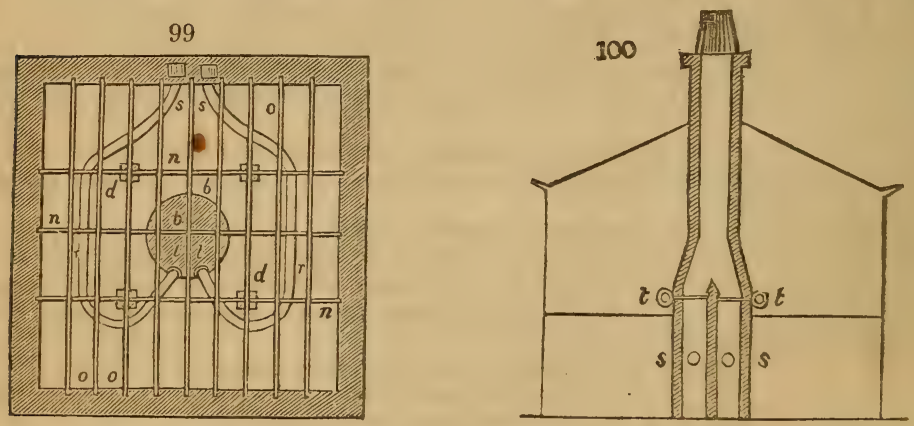

supported in the middle, upon a wall of brickwork four feet high; and beneath it, are the grate and its ash-pit. The smoke passes off through two equi-distant pipes into the chimney. The oven is surrounded with four pillars, on whose top a stone lintel is laid: $a$ is the grate, 9 inches below the sole of the oven $b ; c c c c$ are the four nine-inch strong pillars of brickwork which bear the lintel $m ; d d d d d d$ are strong nine-inch pillars, which support the girder and joists upon which perforated plates repose; $e$ denoles a vaulted arch on each of the four sides of the oven; $f$ is the space between the kiln and the side arch, into which a workman may enter, to inspect and clean the kiln; $g g$, the walls on either side of the kiln, upon which the arches rest; $h$, the space for the ashes to fall ; $k$, the fire-door of the kiln; $l l$, junction-pieces to connect the pipes $r r$ with the kiln; the mode of attaching them is shown in fig. 99. These smolie-pipes lie about three feet under the iron plates, and at the same distance from the side walls; they are supported upon iron props, which are made fast to the arches. In $f i g .98, u$ 
shows their section; at $s s$, fig. 99 , they enter the chimney, which is provided with two register or damper plates, to regulate the draught through the pipes. These registers are represented by $t$, fig. 100, which shows a perpendicular section of the chimney. $m$, fig. YS, is the lintel which causes the heated air to spread laterally instead of ascending in one mass in the middle, and prevents any combustible particles from falling upon the iron cupola. $n n$ are the main girders of iron for the iron beams 00 , upon which the perforated plates $p$ lie; $q, f i g .98$, is the vapor pipe in the middle of the roof, which allows the steam of the drying malt to escape. The kiln may be heated either with coal or wood.

The size of this kiln is about 20 feet square; but it may be made proportionally either smaller or greater. The perforated floor should be large enough to receive the contents of one steep or couch.

The perforated plate might be conveniently heated by steam pipes, laid zig-zag, or in parallel liwes under it; or a wire-gauze web might be stretched upon such pipes. The wooden joists of a common floor would answer perfectly to support this steam-range, and the heat of the pipes would cause an abundant circulation of air. For drying the pale malt of the ale brewer, this plan is particularly well adapted.

The kiln-dried malt is scmetimes ground between stones in a common corn mill, like oatmeal; but it is more generaliy crushed between iron rollers, at least for the purposes of the London brewers.

The crushing mill.-The cylinder malt-mill is constructed as shown in figs. 101, 102. I is the sloping-trough, by which the malt is let down from its bin or floor to the

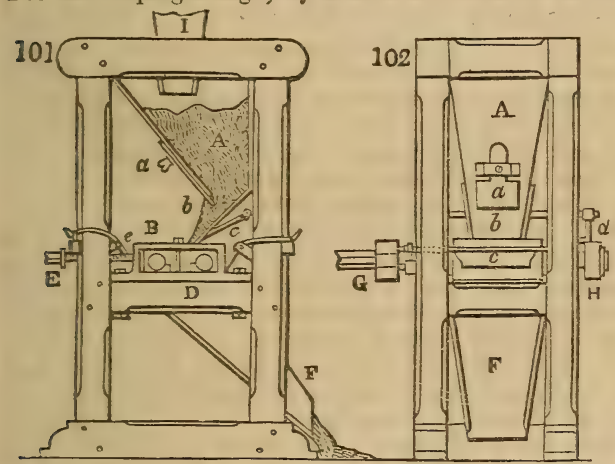
hopper A of the mill, whence it is progressively shaken in between the rollers B D. The rollers are of iron, truly cylindrical, and their ends rest in bearers of hard brass, fitted into the side frames of iron. A screw E goes through the upright, and serves to force the bearer of the one roller towards that of the other, so as to bring them closer together when the crushing effect is to be increased. G is the square end of the axis, by which one of the rollers may be turned either by the hand or by power; the other derives its rotatory motion from a pair of equal-toothed wheels $H$, which are fitted to the other end of the axes of the rollers. $d$ is a catch which works into the teeth of a ratchet-wheel on the end of one of the rollers (not shown in this riew.) The lever $c$ strilies the trough $b$ at the bottom of the hopper, and gives it the shaking motion for discharging the malt between the rollers, from the slide sluice $a$. e e, fig. 101, are scraper-plates of sheet iron, the edges of which press by a weight against the surfaces of the rollers, and keep them clean.

Instead of the cylinders, some employ a crushing mill of a conical-grooved form like a coffee-mill, upon a large scale. (See the general plan, infrà.)

The mashing and boiling.-Mashing is the operation by which the wort is extracted, or eliminated from the malt, and whereby a saccharo-mucilaginous extract is made from it. The malt should not in general be ground into a fine meal, for in that case it would be apt to form a cohesive paste with hot water, or to set, as it is called, and to be difficult to drain. In crushed malt, the husk remains nearly entire, and thus helps to keep the farinaceous particles open and porous to the action of the water. The bulk of the crushed malt is about one fifth greater than that of the whole, or one bushel of malt gives a bushel and a quarter of crushed malt. This is frequently allowed to lie a few days in $c$. cool place, in order that it may attract moisture from the air, which it does very readily by its hygrometric power. Thus, the farinaceous substance which had been induated in the kiln, becomes soft, spongy, and fit for the ensuing process of watery extraction.

Mashing has not for its object merely to dissolve the sugar and gum already present in the malt, but also to convert into a sweet mucilage the starch which had remained unchanged during the germination. We have already stated that starch, mixed with gluten, and digested for some time with hot water, becomes a species of sugar. This conversion takes place in the mash-tun. The malted barley contains not only a portion of gluten, but diastase more than sufficient to convert the starch contained in it, by this means, into sugar. 
The researches of Payen and Persoz show, that the mucilage formed by the reaction of malt upon starch, may either be converted into sugar, or be made into permanent gum, according to the temperature of the water in which the materials are digested. We take of pale barley malt, ground fine, from 6 to 10 parts, and 100 parts of starch; we heat, by means of a water-bath, 400 parts of water in a copper, to about $80^{\circ} \mathrm{F}$.; we then stir in the malt, and increase the heat to $140^{\circ} \mathrm{F}$., when we add the starch, and stir well together. We next raise the temperature to $158^{\circ}$, and endeavor to maintain it constantly at that point, or at least to keep it within the limits of $167^{\circ}$ on the one side, and $158^{\circ}$ on the other. At the end of 20 or 30 minutes, the original milky and pasty solution becomes thinner, and soon after as fluid nearly as water. This is the moment in which the starch is converted into gum, or into that substance which the French chemists call dextrine, from its power of polarizing light to the right hand, whereas common gum does it to the left. If this merely mucilaginous solution, which seems to be a mixture of gum with a little liquid starch and sugar, be suitably evaporated, it may serve for various purposes in the arts to which gum is applied, but with this view, it must be quickly raised to the boiling point, to prevent the farther operation of the malt upon it. If we wish, on the contrary, however, to promote the saccharine fermentation, for the formation of beer, we must maintain the temperature at between $158^{\circ}$ and $167^{\circ}$ for three or four hours, when the greatest part of the gum will have passed into sugar, and by evaporation of the liquid at the same temperature, a starch sirup may be obtained like that procured by the action of sulphuric acid upon starch. The substance, which operates in the formation of sugar, or is the peculiar ferment of the sugar fermentation, may be considered as a residuum of the gluten or vegetable albumen in the germinating grain : it is reckoned by Payen and Persoz, a new proximate principle called diastase, which is formed during malting, in the grains of barley, oats, and wheat, and may be separated in a pure state, if we moisten the malt flour for a few minutes in cold water, press it out strongly, filter the solution, and heat the clear liquid in a water bath, to the temperature of $158^{\circ}$. The greater part of that albuminous azotized substance is thus coagulated, and is to be separated by a fresh filtration; after which, the clear liquid is to be treated with alcohol, when a flocky precipitate appears, which is diastase. To purify it still further, especially from the azotized matter, we should dissolve it in water, and precipitate again with alcohol. When dried at a low temperature, it appears as a solid white substance, which contains no azote; is insoluble in alcohol, but dissolves in water and proof spirit. Its solution is neutral and tasteless; when left to itself, it changes with greater or less rapidity according to the temperature, and becomes sour at a temperature of from $149^{\circ}$ to $167^{\circ}$. It has the property of converting starch into gum (dextrine) and sugar, and indeed, when sufficiently pure, with such energy that one part of it disposes 2000 parts of dry starch to that change, but it operates the quicker the greater its quantity. Whenever the solution of diastase with starch or with dextrine is heated to the boiling point, it loses the sugar-fermenting property. One hundred parts of well-malted starch appear to contain about one part of this substance.

We can now understand the theory of malting, and the limits between which the temperature of the liquor ought to be maintained in this operation; namely, the range between $157^{\circ}$ and $160^{\circ} \mathrm{F}$. It has been ascertained as a principle in mashing, that the best and soundest extract of the malt is to be obtained, first of all, by beginning to work with water at the lowest of these heats, and to conclude the mash with water at the highest. Secondly, not to operate the extraction at once with the whole of the water that is to be employed; but with separate portions and by degrees. The first portion is added with the view of penetrating equally the crushed malt, an l of extracting the already formed sugar; the next for effecting the sugar fermentation by the action of the diastase. By this means, also, the starch is not allowed to run into a cohesive paste, and the extract is more easily drained from the poorer mass, and comes off in the form of a nearly limpid wort. The thicker, moreover, or the less diluted the mash is, so much the easier is the wort fined in the boiler or copper by the coagulation of the albuminous matter: these principles illustrate, in every condition, the true mode of conaucting the mashing process ; but different kinds of malt require a different treatment. Pale and slightly kilned malt requires a somewhat lower heat than malt highly kilned, because the former has more undecomposed starch, and is more ready to become pasty. The former also, for the same reason, needs a more leisurely infusion than the latter, for its conversion into mucilaginous sugar. The more sugar the malt contains, the more is its saccharine fermentation accelerated by the action of the diastase. What has been here said of pale malt, is still more applicable to the case of a mixture of raw grain with malt, for it requires still gentler heats, and more cautious treatment.

III. The mash-tun is a large circular tub with a double bottom; the uppermost of which is called a false bottom, and is pierced with many holes. There is a space of about 2 or 3 inches between the two, into which the stopcocks enter, for letting in the water and drawing off the wort. The holes of the false bottom should be burned, and not bored, 
to prevent the chance of their filling up by the swelling of the wood, which would obstruct the drainage: the holes should be conical, and largest below, being about $\frac{3}{8}$ of an inch there, and $\frac{1}{8}$ at the upper surface. The perforated bottom must be fitted truly at the sides of the mash-tun, so that no grains may pass through. The mashed liquor is let off into a large back, from which it is pumped into the wort coppers. The mash-tun is provided with a peculiar rotatory apparatus for agitating the crushed grains and water together, which we shall presently describe. The size of the wort copner is proportional to the amount of the brewing, and it must, in general, be at least so large as to operate upon the whole quantity of wort made from one mashing; that is, for every quarter of malt mashed, the copper should contain 140 gallons. The mash-tun ought to be at least a third larger, and of a conical form, somewhat wider below than above. The quantity of water to be employed for mashing, or the extraction of the wort, depends upon the greater or less strength to be given to the beer. The seeds of the crushed mait, after the wort is drawn off, retain still about 32 gallons of water for every quarter of malt. In the boiling, and evaporation from the coolers, 40 gallons of water are dissipated from one quarter of malt; constituting 72 gallons in all. If 13 quarters of barley be taken to make 1500 gallons of beer, 2400 gallons of water must therefore be required for the mashing. This example will give an idea of the proportions for an ordinary quality of beer.

When the mash is to begin, the copper must be filled with water, and heated. As sonn as the water has attained the heat of $145^{\circ} \mathrm{m}$ summer, or $167^{\circ}$ in winter, 600 gallons of it are to be run off into the mash-tun, and the 13 quarters of crushed malt are to be gradually thrown in and well intermixed by proper agitation, so that it may be uniformly moistened, and no lumps may remain. After continuing the agitation in this way for one half or three quarters of an hour, the water in the copper will have approached to its boiling point, when 450 gallons at the temperature of about $200^{\circ}$ are to be run into the mash-tun, and the agitation is to be renewed till the whole assumes an equally fluid state: the tun is now to be well covered for the preservation of its heat, and to be allowed to remain at rest for an hour, or an hour and a half. The mean temperature of this mash may be reckoned at about $145^{\circ}$. The time which is necessary for the transmuting heat of the remaining starch into sugar depends on the quality of the malt. Brown malt requires less time than pale malt, and still less than a mixture with raw grain, as already explained. After the mash has rested the proper time, the tap of the tun is opened, and the clear wort is to be drawn out into the under back. If the wort that first flows is turbid, it must be returned into the tun, till it runs clear. The amount of this first wort may be about 675 gallons. Seven hundred and fifty gallons of water, at the temperature of $200^{\circ}$, are now to be introduced up through the drained malt, into the tun, and the mixture is to be agitated till it becomes uniform, as before. The mashtun is then to be covered, and allowed to remain at rest for an hour. The temperature of this mash is from $167^{\circ}$ to $174^{\circ}$. While the second mash is making, the worts of the first are to be pumped into the wort copper, and set a-boiling as speedily as possible. The wort of the second mash is to be drawn off at the proper time, and added to the copper as fast as it will receive it, without causing the ebullition to stop.

A third quantity of water amounting to 600 gallons, at $200^{\circ}$, is to be introduced into the mash-tun, and after half an hour is to be drawn off, and either pumped into the wort copper, or reserved for mashing fresh malt, as the brewer may think fit.

The quantity of extract, per barrel weight, which a quarter of malt yields to wort, amounts to about $84 \mathrm{lbs}$. The wort of the first extract is the strongest; the second contains, commonly, one half the extract of the first; and the third, one half of the second; according to circumstances.

To measure the degrees of concentration of the worts drawn off from the tun, a particular form of hydrometer, called a saccharometer, is employed, which indicates the number of pounds weight of liquid contained in a barrel of 36 gallons imperial measure. Now, as the barrel of water weighs $360 \mathrm{lbs}$., the indication of the instrument, when placed in any wort, shows by how many pounds a barrel of that wort is heavier than a barrel of water; thus, if the instrument sinks with its poise till the mark 10 is upon a line with the surface of the liquid, it indicates that a barrel of that wort weighs ten pounds more than a barrel of water. See SAccharometer.

Or, supposing the barrel of wort weighs $396 \mathrm{lbs}$., to convert that number into specific gravity, we have the following simple rule :-

$$
360: 396:: 100: 1 \cdot 100
$$

at which density, by my experiments, the wort contains 25 per cent. of solid extract.

Having been employed to make experiments on the density of worts, and the ferment ative changes which they undergo, for the information of a committee of the House of Commons, which sat in July and Angust, 1830, I shall here introduce a short abstract of that part of my evidence which bears upon the present subject.

My first object was to clear up the difficulties which, to common apprehension, hung 
over the matter, from the difference in the scales of the saccharometers in use among the brewers and distillers of England and Scotland. I found that one quarter of good malt would yield to the porter-brewer a barrel Imperial measure of wort, at the concentrated specific gravity of $1 \cdot 234$. Now, if the decimal part of this number be multiplied by 360 , being the number of pounds weight of water in the barrel, the product will denote the excess in pounds, of the weight of a barrel of such concentrated wort, over that of a barrel of water; and that product is, in the present case, 84.24 pounds.

Mr. Martineau, jun., of the house of Messrs. Whitbread and Company, and a gentleman connected with another great London brewery, had the kindness to inform me that their average product from a quarter of malt was a barrel of $84 \mathrm{lbs}$. gravity. It is obvious, therefore, that by taking the mean operation of two such great establishments, I must have arrived very nearly at the truth.

It ought to be remarked that such a high density of wort as 1.234 is not the result of any direct experiment in the brewery, for infusion of malt is never drawn off so strong; that density is deduced by computation from the quantity and quality of several successive infusions ; thus, supposing a first infusion of the quarter of malt to yield a barrel of specific gravity $1 \cdot 112$, a second to yield a barrel at $1 \cdot 091$, and a third a barrel at $1 \cdot 031$, we shall have three barrels at the mean of these three numbers, or one barrel at their sum, equal to $1 \cdot 234$.

I may here observe that the arithmetical mean or sum is not the true mean or sum of the two specific gravities; but this difference is either not known or disregarded by the brewers. At low densities this difference is inconsiderable, but at high densities it would lead to serious errors. At specific gravity $1 \cdot 231$, wort or sirup contains one half of its weight of solid pure saccharum, and at $1 \cdot 1045$ it contains one fourth of its weight; but the brewer's rule, when here applied, gives for the mean specific gravity $1 \cdot 1155=$ $\frac{1 \cdot 231+1 \cdot 000}{}$. The contents in solid saccharine matter at that density are however $27 \frac{\pi}{4}$ per cent., showing the rule to be $2 \frac{1}{4}$ lbs. wrong in excess on 100 lbs., or 9 lbs. per barrel.

The specific gravity of the solid dry extract of malt wort is 1.264 ; it was taken in oil of turpentine, and the result reduced to distilled water as unity. Its specific volume is 0.7911 , that is, $10 \mathrm{lbs}$. of it will occupy the volume of $7.911 \mathrm{lbs}$. of water. The mean specific gravity, by computation of a solution of that extract in its own weight of water, is 1.1166 ; but by experiment, the specific gravity of that solution is $1 \cdot 216$, showing considerable condensation of volume in the act of combination with water.

The following Table shows the relation between the specific gravities of solutions of malt extract, and the per-centage of solid extract they contain :

\begin{tabular}{|cc|c|c|c|c|}
\hline \multicolumn{2}{|c|}{ Extr Malt. } & Water. & Malt Extract in 100. & Sugar in 100. & Specific gravity. \\
\hline 600 & + & 600 & $50 \cdot 00$ & $47 \cdot 00$ & $1 \cdot 2160$ \\
600 & + & 900 & $33 \cdot 3$ & $37 \cdot 00$ & $1 \cdot 16 \% 0$ \\
600 & - & 1200 & $28 \cdot 57$ & 31.50 & $1 \cdot 1350$ \\
600 & + & 1500 & $25 \cdot 00$ & $26 \cdot 75$ & $1 \cdot 1130$ \\
600 & + & 1800 & $24 \cdot 00$ & $1 \cdot 1000$ \\
\hline
\end{tabular}

The extract of malt was evaporated to dryness, at a temperature of about $250^{\circ} \mathrm{F}$., without the slightest injury to its quality, or any empyreumatic smell. Bate's tables have been constructed on solutions of sugar, and not with solutions of extract of malt, or they agree sufficiently well with the former, but differ materially from the latter. Allan's tables give the amount of a certain form of solid saccharine matter extracted from malt, and dried at $175^{\circ} \mathrm{F}$., in correspondence to the specific gravity of the solution; but I have found it impossible to make a solid extract from infusions of malt, except at much higher temperatures than $175^{\circ} \mathrm{F}$. Indeed, the numbers on Allan's saccharometer scale clearly show that his extract was by no means dry: thus, at $1 \cdot 100$ of gravity he assigns 29.669 per cent. of solid saccharine matter; whereas there is at that density of solid extract only 25 per cent. Again, at $1 \cdot 135$, Allan gives 40 parts per cent. of solid extract, whereas there are only $33 \frac{1}{3}$ present.

By the triple mashing operations above described, the malt is so much exhausted that it can yield no further extract useful for strong beer or porter. A weaker wort might no doubt still be drawn off for small beer, or for contributing a little to the strength of the next mashing of fresh malt. But this I believe is seldom practised by respectable brewers, as it impoverishes the grains which they dispose of for feeding cattle.

The wort should be transferred into the copper, and made to boil as soon as possible, for if it remains long in the under-back it is apt to become acescent. 'The steam moreover raised from it in the act of boiling serves to screen it from the oxygenating or acidifying influence of the atmosphere. Until it begins to boil, the air should be excluded by some kind of a cover, 
Sometimes the first wort is brewed by itself into strong ale, the second by itself into an intermediate quality; and the third into small beer; hut this practice is not much followed in this country.

We shall now treat of the boiling in of the hops. The wort drawn from the mashtun, whenever it is pumped into the copper, must receive its allowance of hops. Besides evaporating off a portion of the water, and thereby concentrating the wort, boiling has a twofold object. In the first place, it coagulates the albuminous matter, partly by the heat, and partly by the principles in the hops, and thereby causes a general clarification of the whole mass, with the effect of separating the muddy matters in a flocculent form. Secondly, during the ebullition, the residuary starch and hordeine of the malt are converted into a limpid sweetish mucilage, the dextrine above described; while some of the glutinous stringy matter is rendered insoluble by the tannin principle of the hops, which favors still further the clearing of the wort. By both operations the keeping quality of the beer is improved. This boil must be continued during several hours; a longer time for the stronger, and a shorter for the weaker beers. There is usually one seventh or one sixth part of the water dissipated in the boiling copper. This process is known to have continued a sufficient time, if the separation of the albuminous flocks is distinet, and if these are found, by means of a proof gauge suddenly dipped to the bottom, to be collected there, while the supernatant liquor has become limpid. Two or three hours' boil is deemed long enough in many well-conducted breweries; but in some of those in Belgium, the boiling is continued from 10 to 15 hours, a period certainly detrimental to the aroma derived from the hop.

Many prefer adding the hops when the wort has just come to the boiling point. Their effect is to repress the further progress of fermentation, and especially the passage into the acetous stage, which would otherwise inevitably ensue in a few days. In this respect, no other vegetable production hitherto discovered can be a substitute for the hop. The odorant principle is not so readily volatilized as would at first be imagined; for when hop is mixed with strong beer wort and boiled for many hours, it ean still impart a very considerable degree of its flavor to weaker beer. By mere infusion in hot beer or water, without boiling, the hop loses very little of its soluble principles. The tannin of the hop combines, as we have said, with the vegetable albumen of the barley, and helps to clarify the liquor. Should there be a deficiency of albumen and gluten, in consequence of the mashing having been done at such a heat as to have coagulated them beforehand, the defect may be remedied by the addition of a little gelatine to the wort copper, either in the form of calf's foot, or of a little isinglass. If the hops be boiled in the wort for a longer period than 5 or 6 hours, they lose a portion of their fine flavor; but if their natural flavor be rank, a little extra boiling improves it. Many brewers throw the hops in upon the surface of the boiling wort, and allow them to swim there for some time, that the steam may penetrate them, and open their pores for a complete solution of their principles when they are pushed down into the liquor. It is proper to add the hops in considerable masses, because, in tearing them asunder, some of the lupuline powder is apt to be lost.

The quantity of hop to be added to the wort varies according to the strength of the beer, the length of time it is to be kept, or the heat of the climate where it is intended to be sent. For strong beer, $4 \frac{1}{2} \mathrm{lbs}$. of hops are required to a quarter of malt, when it is to be highly aromatic and remarkably clear. For the stronger kinds of ale and porter, the rule, in England, is to take a pound of hops for every bushel of malt, or 8 lbs. .o a quarter. Common beer has seldom more than a quarter of a pound of hops to the bushel of malt.

It has been attempted to form an extract of hops by boiling in covered vessels, so as not to lose the oil, and to add this instead of the hop itself to the beer. On the great scale this method has no practical advantage, because the extraction of the hop is perfectly accomplished during the necessary boiling of the wort, and because the hop operates very beneficially, as we have explained, in clarifying the beer. Such an extract, moreover, could be easily adulterated.

Of the Coolers. - The contents of the copper are run into what is called the hopback, on the upper part of which is fixed a drainer, to keep back the hops. The pump is placed in the hop-back, for the purpose of raising the wort to the coolers, usually placed in an airy situation upon the top of the brewery. Two coolers are indispensable when we make two kinds of beer from the same brewing, and even in single brewings, ealled gyles, if small beer is to be made. One of these coolers ought to be placed above the level of the other. As it is of great consequence to cool the worts down to the fermenting pitch as fast as possible, various contrivances have been made for effecting this purpose. The common cooler is a square wooden cistern, about 6 inches deep, and of such an extent of surface that the whole of one boil may only xcupy 2 inches, or thereabouts, of depth in it. For a quantity of wort equal to about 1500 gallons its area should be at least 54 feet long and 20 feet wide. The seams of 
the cooler must be made perfectly water-tight and smooth, so th at no liquor may lodge in them when they are emptied. The utmost cleanliness is required, and an occasional sweetening with lime-water.

The hot wort reaches the cooler at a temperature of from $200^{\circ}$ to $208^{\circ}$, according to the power of the pump. Here it should be cooled to the proper temperature for the fermenting tun, which may vary from $54^{\circ}$ to $64^{\circ}$, according to circumstances. The ref igeration is accomplished by the evaporation of a porion of the liquor: it is more rapud in proportion to the extent of the surface, to the low tcmperature, and the dryness of the atmosphere surrounding the cooler. The renewal of a body of cool dry air by the agency of a fan, may be employed with great adrantage. The cooler itself must be so placed that its surface shall be freely exposed to the prevailing wind of the district, and be as free as possible from the eddy of surrounding buildings. It is thought by many that the agitation of the wort during its cooling is hurtful. Were the roof made moreable, so that the wort could be readily exposed, in a clear night, to the aspect of the sky, it would cool rapidly by evaporation, on the principles explained by Dr. Wells, in his "Essay on Dew."

When the cooling is effected by eraporation alone, the temperature falls very slowly, even in cold air, if it be loaded with moisture. But when the air is dry, the evaporation is rigorous, and the moisture exhaled does not remain incumbent on the liquor, as in damp weather, but is diffused widely in space. Hence we can understand how wort cools so rapidly in the spring and autumn, when the air is generally dry, and even more quickly than in winter, when the air is cooler, but loaded with moisture. In fact, the cooling process goes on better when the atmosphere is from $50^{\circ}$ to $55^{\circ}$, than when it falls to the freezing point, because in this case, if the air be still, the vapors generated remain on the surface of the liquor, and prevent further evaporation. In summer the cooling can take place only during the night.

In consequence of the eraporation during this cooling process, the bulk of the worts is considerably reduced; thus, if the temperature at the beginning was $208^{\circ}$, and if it be at the end $64^{\circ}$, the quantity of water necessary to be evaporated to produce this refrigeration would be nearly $\frac{2}{5}$ of the whole, putting radiation and conduction of heat out of the question. The effect of this will be a proportional concentration of the beer.

The period of refrigeration in a well-constructed cooler, amounts to 6 or 7 hours in favorable weather, but to 12 or 15 in other circumstances. The quality of the beer is much improved by shortening this period; because, in consequence of the great surface which the wort exposes to the air, it readily absorbs oxgyen, and passes into the acetous fermentation with the production of various mouldy spots; an eril to which ill-hopped beer is particularly liable. Various schemes have been contrived to cool wort, by transmitting it through the convolutions of a pipe immersed in cold water. The best plan is to expose the hot wort for some hours freely to the atmosphere and the cooler, when the loss of heat is most rapid by evaporation and other means, and when the temperature falls to $100^{\circ}$, or thereby, to transmit the liquor through a zig-zag pipe, laid almost horizontally in a trough of cold water. The various methods described under Refrigerator are more complex, but they may be practised in many situations with considerable advantage.

Whilst the wort reposes in the cooler, it lets fall a slight sediment, which consists partly of fine flocks of coagulated albumen combined with tannin, and partly of starch, which had been dissolved at the high temperature, and separates at the lower. The wort should be perfectly limpid, for a muddy liquor never produces transparent beer. Such beer contains, besides mucilaginous sugar and gum, usually some starch, which eren remains after the fermentation, and hinders its clarifying, and gives it a tendency to sour. The wort contains more starch the hotter it has been mashed, the less hops have been added, and the shorter time it has been boiled. The presence of starch in the wort may be made manifest by adding a little solution of iodine in alcohol to it, when it will become immediately blue. We thus see that the tranquil cooling of wort in a proper ves. sel has an adrantage over cooling it rapidly by a refrigeratory apparatus. When the wort is sufficiently cool, it is let down into the fermenting tun. In this transfer the cooling might be earried several degrees hwer, were the wort made to pass down throngh a tube enclosed in another tube, along which a stream of cold water is flowing in the opposite direction, as we have described in the sequel of AcETic AcrD. These fermenting tuns are commonly called gyle-tuns, or working tuns, and are either square or circular, the latter being preferable on many accounts.

IT. Of the Fermentation. - In the great London breweries, the size of these fermenting tuns is such that they contain from 1200 to 1500 barrels. The quantity of wort introduced at a time must, however, be considerably less than the capacity of the ressel, to allow room for the head of yeast which rises during the process; if the vessel be eylindrical, this head is proportional to the depth of the worts. In certain kinds of 
fermentation, it may rise to a third of that depth. In general, the fermentation proceecs more uniformly and constantly in large masses, because they are little influenced br ri cissitudes of temperature; smaller vessels, on the other hand, are more easily handled. The general view of fermentation will be found under that title; I shall here make a few remarks on what is peculiar 10 beer. During the fermentation of wort, a portion of its saccharine matter is converted into alcohol, and wort thus changed is beer. It is necessary that this conversion of the sugar be only partial, for beer which contains no undecomposed sugar would soon turn somr, and even in the caslis its alcohol undergoes a slow fermentation into vinegar. The amount of this excess of sugar is greater in proportion to the strength of the wort, since a certain quantity of alcohol, already formed, prevents the operation of the ferment on the remaining wort. Temperature has the greatest influence upon the fermentation of wort. A temperature of from $55^{\circ}$ to $60^{\circ}$ of the liquor, when that of the atmosphere is $55^{\circ}$, is most advantageous for the commencement. The warmth of the wort as it comes into the gyle-tun mast be modified by that of the air in the apartment. In winter, when this apartment is cold, the wort should not be cooled under $64^{\circ}$ or $60^{\circ}$, as in that case the fermentation would be tedious or interrupted, and the wort liable to spoil or become sour. In summer, when the temperature of the place rises to above $75^{\circ}$, the wort should be cooled, if possible, down to $55^{\circ}$, for which purpose it should be let in by the system of double pipes, above mentioned. The higher the temperature of the wort, the sooner will the fermentation begin and end, and the less is it in our power to regulate its progress. The expert brewer must steer a middle course between these two extremes, which threaten to destroy his labors. In some breweries a convoluted pipe is made to traverse or go round the sides of the gyle-tun, through which warm water is allowed to flow in winter, and cold in summer, so as to modify the temperature of the mass to the proper fermenting pitch. If there be no contrivance of this kind, the apartment may be cooled in summer, by suspending wet canvass opposite the windows in warm weather, and kindling a small stove within it in cold.

When the wort is discharged into the gyle-tun, it must receive its dose of yeast, which has been previously mixed with a quantity of the wort, and left in a warm place till it has begun to ferment. This mixture, called $l o b b$, is then to be put into the tun, and stir red well through the mass. The yeast should be taken from similar beer. Its quantity must depend upon the temperature, strength, and quantity of the wort. In general, one gallon of yeast is sufficient to set 100 gallons of wort in complete fermentation. An excess of yeast is to be avoided, lest the fermentation should be too violent, and be finished in less than the proper period of 6 or 8 days. More yeast is required in winter than in summer; for, at a temperature of $50^{\circ}$, a double quantity may be used to that at $68^{\circ}$.

Six or eight hours after adding the yeast, the tun being meanwhile covered, the fermentation becomes active : a white milky-looking froth appears, first on the middle, and spreads gradually over the whole surface; but continues highest in the middle, forming a frothy elevation, the height of which increases with the progress of the fermentation, and whose color gradually changes to a bright brown, the result, apparently, of the oxydation of the extractive contained in this yeasty top. This covering screens the wort from the contact of the atmospherical air. During this time, there is a perpetual disengagement of carbonic acid gas, which is proportional to the quantity of sugar converted into alcohol. The warmth of the fermenting liquid increases at the same time, and is at a maximum when the fermentation has come to its highest point. This increase of temperature amounts to from $9^{\circ}$ to $14^{\circ}$ or upwards, and is the greater the more rapid the fermentation. But in general, the fermentation is not allowed to proceed so far in the gyle-tun, for after it is advanced a little way, the beer is cleansed, that is, drawn off into other vessels, which are large barrels set on end, with large open:ngs in their top, furnished with a sloping tray for discharging an excess of yeast into the wooden trough, in which the stillions stand. These stillions are placed in communication with a store-tub, which keeps them always full, by hydrostatic pressure, so that the head of yeast may spontaneously flow over, and keep the body of liquor in the cask clean. This apparatus will be explained in describing the brewery plant. See the figures, infrà.

It must be observed, that the quantity of yeast, and the heat of fermentation, differ for every different quality of beer. For mild ale, when the fermentation has reached $75^{\circ}$ its first flavor begins; at $80^{\circ}$ the flavor increases; at $85^{\circ}$ it approaches the high flavor; at $90^{\circ}$ it is high ; but it may be carried to $100^{\circ}$ and upwards, for particular purposes. A wort of 30lbs. per barrel (sp. gr. 1.088), ought to increase about $15^{\circ}$, so that in order to arrive at $80^{\circ}$, it should be set at $65^{\circ}$. The quantity of yeast for such

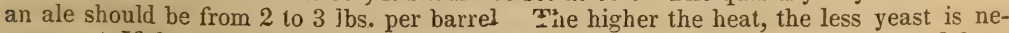
cessary. If the heat of the fermentation should at any time fall, it must be raised by a supply of fresh yeast, well itirred in; but this practice is not advisable in general, because rousing the worts in the gyle-tun is apt to communicate a rank flavor of yeast to the ale. It is the practice of many experienced brewers to look every 2 hours into the 
gyle-tun, chiefly with the view of observing the progress of the heat, which is low at first, but afterwards often increases half a degree per hour, and subsequently declines, as the fermentation approaches its conclusion, till at length the heat becomes uniform, or sometimes decreases, before the fermentation is finished, especially where the quanitity operated upon is small.

Some brewers recommend, when the fermentation is carried to its utmost period, to add about 7 lbs. of wheat or bean flour to a gyle-tun of 25 or 30 barrels, at the time of cleansing, so as to quicken the discharge of the yeast, by disengagement of more carbonic acid. The flour should be whisked up in a pail, with some of the beer, till the lumps are broken, and then poured in. By early cleansing, the yeast is preserved longer in a state proper for a perfect fermentation than by a contrary practice.

For old ale, which is to be long kept, the heat of the fermentation should not exceed $75^{\circ}$, but a longer time is required to complete the fermentation and ensure the future good flavor of the ale.

For porter, the general practice is, to use from 4 to $4 \frac{1}{2} \mathrm{lbs}$. of hops per barrel for keeping; though what is termed mild or mixing porter, has not more than 3 or $3 \frac{1}{2}$ lbs. The heat of fermentation must not exceed $70^{\circ}$, and begin about $60^{\circ}$. If the heat tend to increase much above that pitch in the gyle-tun, the porter should be cleansed, by means of the stillions. At this period of the fermentation, care should be taken that the sweetness of the malt be removed, for which purpose more yeast may be used than with any other beer of the same strength. The quantity is from 3 to $4 \mathrm{lbs}$. per barrel, rousing the wort in the gyle-tun every 2 hours in the day-time.

When the plan of cleansing casks is not employed, the yeast is removed from the surface of the fermenting tun by a skimmer, and the clear beer beneath is then drawn off into the ripening tuns, called store-vats, in which it is mixed up with different brewings, to suit the taste of the customers. This transfer must take place whenever the extrication of carbonic acid has nearly ceased; lest the alcohol formed should dissolve some of the floating yeast, acquire thereby a disagreeable taste, and pass partially into the acetous state.

In this process, during the formation of vinous spirit at the expense of the sugar, the albumen and gluten diffused through the beer, being acted upon by the alcohol, become insoluble; one portion of them is buoyed to the top with the carbonic acid gas, to form the frothy yeast; and another portion falls to form the bottom barm. The former consists of the same materials as the wort, with a large proportion of gluten, which forms its active constituent; the latter is a peculiar deposite, consisting of the same gluten mixed with the various dense impurities of the wort, and nlay be also used as a ferment, but is cruder than the floating yeast. The amount of yeast is proportional to the activity of the fermentation, or extrication of earbonic acid gas, as also to the heat of the mashing process, and the quantity of starch or flour unaltered by germination. Pale malt affords, usually, more yeast than malt highly kilned. When the yeast becomes excessive, from too violent fermentation, it should be skimmed off from time to time, which wili tend to cool the liquor and moderate the intestine changes.

After the beer is let down into the close store-tuns in the cellar, an obscure fermentation goes on, for a considerable period, in its body, which increases its spurtutuous strength, and keeps up in it a constant impregnation of carbonic acid gas, so as to render it lively and agreeable to the taste, when it is casked off for sale. It would appear that beer is never stationary in quality, while it is contained in the tuns; for the moment when it ceases to improve by the decornposition of its residuary sugar, it begins to degenerate into vinegar. This result may be produced either by the exhaustion of the saccharine, or by the fermentative matter. The store cellar should therefore be under ground, free from alternations of temperature, vibrations of carriages, and as cool as possible. In the great London breweries the fermentation is rendered very complete in the cleansing butts; so that a slow and steady ripening is ensured in the great store-tuns. The gyletuns are too capacious to permit the fermentation to be finished, with either safety or sufficient despatch in them.

V. OF RIFENING DIFFERENT KINDS OF BEER.-The varieties of beer depend either upon the difference of their materials, or from a different management of the brewing processes.

With regard to the materials, beers differ in the proportion of their malt, hops, and water; and in the different kinds of malt or other grain. To the class of table or small beers, all those sorts may be referred whose specific gravity does not exceed 1.025 , which contain about 5 per cent. of malt extract, or nearly 18 pounds per barrel. Beers of middling strength may be reckoned those between the density of 1.025 and 1.040 ; which contain at the average 7 per cent. or 25 pounds per barrel. The latter may be made with 400 quarters of malt to 1500 barrels of beer. Stronger beers have a specific gravity of from 1.050 to $1 \cdot 080$, and take from 45 to 75 quarters of malt to the same quantity of beer. The strongest beer found in the market is some of the English and Scotch ales, for which from 18 to 27 quarters of malt are taken for 1500 
gallons of beer. Good porter requires from 16 to 18 quarters for that quantity. Bee:s are sometimes made with the addition of other farinaceous matter to the malt; but when the latter constitutes the main portion of the grain, the malting of the other kinils of corn becomes unnecessary, for the diastase of the barley-malt changes the starch into sugar during the mashing operation. Even with entirely raw grain, beer is made in some parts of the Continent, the brewers trusting the conversion of the starch into sugar to the action of the gluten alone, at a low mashing temperature, on the principle of Saussure's and Kirchoff's researches.

The color of the beer depends upon the color of the malt, and the duration of the boil in the copper. The pale ale is made, as we have stated, from steam or sun-dried malt, and the young shoots of the hop; the deep yellow ale from a mixture of pale yellow and brown malt; and the dark brown beer from well-kilned and partly carbonized malt, mixed with a good deal of the pale, to give body. The longer and more strongly heated the malt has been in the kiln, the less weight of extract, cateris paribus, does it afford. In making the fine mild ales, high temperatures ought to be avoided, and the yeast ought to be skimmed off, or allowed to flow very readily from its top, by means of the cleansing butt system, so that little ferment being left in it to decompose the rest of the sugar, the sweetness may remain unimpaired. With regard to porter, in certain breweries, each of the three kinds of malt employed for it is separately mashed, after which the first and the half of the second wort is boiled along with the whole of the hops, and thence cooled and set to ferment in the gyle-tun. The third drawn wort, with the remaining half of the second, is then boiled with the same hops, saved by the drainer, and, after cooling, added to the former in the gyle-tun, when the two must be well roused together.

It is obvious, from the preceding development of principles, that all amylaceous and saccharine materials, such as potatoes, beans, turnips, as well as cane and starch sirup, molasses, \&c., may be used in brewing beer. When, however, a superior quality of brown beer is desired, malted barley is indispensable, and even with these substitutes a mixture of it is most advantageous. The washed roots of the common carrot, of the red and yellow beet, or of the potato, must be first boiled in water, and then mashed into a pulp. This pulp must be mixed with water in the copper, along with wheaten or oat meal, and the proper quantity of hops, then boiled during 8 or 9 hours. This wort is to be cooled in the usual way, and fermented, with the addition of yeast. A much better process is that now practised, on a considerable scale, at Strasbourg, in making the ale, for which that city is celebrated. The mashed potatoes are mixed with from a twentieth to a tenth of their weight of finely ground barley malt, and some water. The mixture is exposed, in a water-bath, to a heat of $160^{\circ} \mathrm{F}$. for four hours, whereby it passes into a saccharine state, and may then be boiled with hops, cooled, and properly fermented into good beer.

Maize, or Indian corn, has also been employed to make beer; but its malting is somewhat difficult on account of the rapidity and vigor with which its radicals and plumula sprout forth. The proper mode of causing it to germinate is to sover it, $\mathrm{n}$ few inches deep, with common soil, in a garden or field, and to leave it there till the bed is covered with green shoots of the plant. The corn must be then lifted, washed, and exposed to the kiln.

The Difference of the Fermentation.-The greater or less rapidity with which the worts are made to ferment has a remarkable influence upon the quality of the beer, especially in reference to its fitness for keeping. The wort is a mucilaginous solution in which the yeasty principles, eliminated by the fermentation, will, if favored by regular and slow intestine movements, completely rise to the surface, or sink to the bottom, so as to leave the body fine. But, when the action is too violent, these barmy glutinous matters get comminuted and dispersed through the liquor, and can never afterwards be thoroughly separated. A portion of the same feculent matter becomes, moreover, permanently dissolved, during this furious commotion, by the alcohol that is generated. Thus the beer loses not merely its agreeable flavor and limpidity, but is apt to spoil from the slightest causes. The slower, more regularly progressive, and less interrupted, therefore, the fermentation is, so much better will the product be.

Becr, in its perfect condition, is an excellent and healthful beverage, combining, in some measure, the virtues of water, of wine, and of food, as it quenches thirst, stimulates, cheers, and strengthens. The vinous portion of it is the alcohol, proceeding from the fermentation of the malt sugar. Its amount, in common strong ale or beer, is about 4 per cent., or four measures of spirits, specific gravity 0.825 in 100 measures of the liquor. The best brown stout porter contains 6 per cent., the strongest ale even 8 per cent.; but common beer only one. The nutritive part of the beer is the undecomposed gum-sugar, and the starch-gum, not changed into sugar. Its quantity is very variable, according to the original starch of the wort, the length of the fermentation. and the age of the beer. 
The main feature of good beer is fine color and transparency; the productio of which is an object of great interest to the brewer. Attempts to clarify it in thr cask seldorm fail to do it harm. The only thing that can be used with advantage for fining foul or muddy beer, is isinglass. For porter, as commonly brewed, it is frequently had recourse to. A pound of good isinglass will make about 12 gallons of fining.s. It is cut into slender shreds, and put into a tub with as much vinegar or hard beer as will cover it, in order that it may swell and dissolve. In proportion as the solution proceeds, more beer must be poured upon it, but it need not be so acidulous as the first, hecause, when once well softened by the vinegar, it readily dissolves. The mixture should be frequently agitated with a bundle of rods, till it acquires the uniform consistence of thin treacle, when it must be equalized still more by passing through a tammy cloth, or a sieve. It may now be made up with beer to the proper measure of dilution. The quantity generally used is from a pint to a quart per barrel, more or less, according to the foulness of the beer. But before putting it into the butt, it should be diffused through a considerable volume of the beer with a whisk, till a frothy head be raised upon it. It is in this state to be poured into the cask, briskly stirred abont; after which the cask must be bunged down for at least 24 hours, when the liquor should be impid. Sometimes the beer will not be improved by this treatment; but this should be ascertained beforehand, by drawing off some of the beer into a cylindric jar or vial, and adding to it a little of the finings. After shaking and setting down the glass, we shall observe whether the feculencies begin to collect in flocky parcels, which slowly subside; or whether the isinglass falls to the bottom without making any impression upon the beer. This is always the case when the fermentation is incomplete, or a secondary decomposition has begun. Mr. Jackson has accounted for this clarifying effect of isinglass in the following way.

The isinglass, he thinks, is first of all rather diffused mechanically, than chemically dissolved, in the sour beer or vinegar, so that when the finings are put into the foul beer, the gelatinous fibres, being set free in the liquor, attract and unite with the floating feculencies, which before this union were of the same specific grarity with the beer, and therefore could not subside alone; but having now acquired additional weight by the coating of fish glue, precipitate as a flocculent magma. This is Mr. Jackson's explanation; to which I would add, that if there be the slightest disengagement of carbonic acid gas, it will keep up an obscure locomotion in the particles, which will prevent the said light impurities, either alone or when coated with isinglass, from subsiding. The becr is then properly enough called stubborn by the coopers. But the true theory of the action of isinglass is, that the tannin of the hops combines with the fluid gelatine, and forms a flocculent mass, which envelopes the muddy particles of the beer, and carries them to the bottom as it falls, and forms a sediment. When, after the finings are poured in, no proper precipitate ensues, it may be made to appear by the addition of a little decoction of hop.

Mr. Richardson, the author of the well-known brewer's saccharometer, gives the following as the densities of different kinds of beer :-

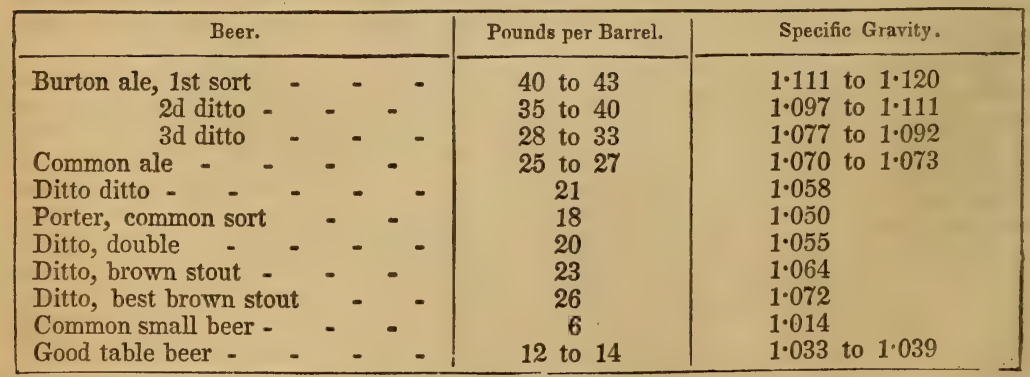

Of Returns or Malt Residuums. - When small beer is brewed after ale or porter, only one mash is to be made; but where this is not done, there may be two mashes, in order to economize malt to the utmost. We may let on the water at $160^{\circ}$ or $165^{\circ}$, in any convenient quantity, infuse for an hour or thereby, then run it off, and pump into the copper, putting some hops into it, and causing it to boil for an instant; when it mar be transferred to the cooler. A second mash or return may be made in the same manner. but at a heat $5^{\circ}$ lower; and then disposed of in the boiler with some hops, which mar remain in the copper during the night at a scalding heat, and may be discharged into the conler in the morning. These two returns are to be let down into the underback immediately before the next brewing, and thence heated in the copper for the next 
mashing of fresh malt, instead of hot water, commonly called liquor, in the breweries. But allowance must be made, in the calculation of the worts, for the quantity of fermentable matter in these two returns. The nett aggregate saving is estimated from the gravity of the return taken when cold in the cooler. A slight economy is also made in the extra boiling of the used hops. The lapse of a day or two between the consecutive brewinss is no objection to the method of returns, because they are too weak in saccharine matter to run any risk of fermentation.

In conclusion, it may be remarked that Mr. Richardson somewhat underrates the grarity of porter, which is now seldom under 20lbs. per barrel. The criterion for transfesring from the gyle-tun to the cleansing butts is the attenuation caused by the production of alcohol in the beer : when that has fallen to 10lbs. or 11lbs., which it usually does in 48 hour's, the cleansing process is commenced. The heat is at this time generally $75^{\circ}$, if it was pitched at $65^{\circ}$; for the heat and the attenuation go hand in hand.

About thirty years ago, it was customary for the London brewers of porter to keep immense stocks of it for eighteen months or two years, with the view of improving its quality. The beer was pumped from the cleansing butts into store-vats, holding from twenty to twenty-five gyles or brewings of several hundred barrels each. The store-vats had commonly a capacity of 5000 or 6000 barrels; and a few were double, and one was treble, this size. The porter, during its long repose in these vats, became fine, and by obscure fermentation its saccharine mucilage was nearly all converted into vinous liquor, and dissipated in carbonic acid. Its hop-bitter was also in a great degree decomposed. Good hard beer was the boast of the day. This was sometimes softened by the publican, by the addition of some mild new-brewed beer. Of late years, the taste of the metropolis has undergone such a complete revolution in this respect, that nothing but the mildest porter will now go down. Hence, six weeks is a long period for beer to be kept in London; and much of it is drunk when only a fortnight old. Ale is for the same reason ' me greatly into vogue; and the two greatest porter houses, Messrs. Barclay, Perkins, \& Co., and Truman, Hanbury, \& Co., have become extensive and successful brewers of mild ale, to please the changed palate of their customers.

We shall add a few observations upon the brewing of Scotch ale. This beverage is characterized by its pale amber color, and its mild balsamic flavor. The bitterness of the hop is so mellowed with the malt as not to predominate. The ale of Preston Pans is, in fact, the best substitute for wine which barley has hitherto produced. The low temperature at which the Scotch brewer pitches his fermenting tun restricts his labors to the colder months of the year. He does nothing during four of the summer months. He is extremely nice in selecting his malt and hops; the former being made from the best English barley, and the latter being the growth of Farnham or East Kent. The yeast is carefully looked after, and measured into the fermenting tun in the proportion of one gallon to 240 gallons of wort.

Only one mash is made by the Scotch ale brewer, and that pretty strong; but the malt is exhausted by eight or ten successive sprinklings of liquor (hot water) over the goods (malt), which are termed in the vernacular tongue, sparges. These waterings percolate through the malt on the mash-tun bottom, and extract as much of the saccharine matter as may be sufficient for the brewing. By this simple method much higher specific gravities may be obtained than would be practicable by a second mash. With malt, the infusion or saccharine fermentation of the diastase is finished with the first mash; and nothing remains but to wash away from the goods the matter which that process has rendered soluble. It will be found on trial that 20 barrels of wort drawn from a certain quantity of malt, by two successive mashings, will not be so rich in fermentable matter as 20 barrels extracted by ten successive sparges of two barrels each. The grains always remain soaked with wort like that just drawn off, and the total residual quantity is three fourths of a barrel for every quarter of malt. The gravity of this residual wort will on the first plan be equal to that of the second mash; but on the second plan, it will be equal only to that of the tenth sparge, and will be more attenuated in a very high geometrical ratio. The only serious objection to the sparging systern is the loss of time by the successive drainages. A mash-tun with a steam jacket promises to suit the sparging system well; as it would keep up a uniform temperature in the goods, without requiring them to be sparged with very hot liquor.

The first part of the Scotch process seems of doubtful economy; for the mash liquor is heated so high as $180^{\circ}$. After mashing for about half an hour, or till every particle of the malt is thoroughly drenched, the tun is covered, and the mixture left to infuse about three hours; it is then drained off into the under-back, or preferably into the wort copper.

After this wort is run off, a quantity of liquor (water), at $180^{\circ}$ of heat, is sprinkled uniformaly over the surface of the malt; being first dashed on a perforated circular board, suspended horizontally over the mash-tun, wherefrom it descends like a shower 
upon the whole of the goods. The percolating wort is allowed to flow off, by three or more small stopcocks round the circumference of the mash-tun, to ensure the equal dif fusion of the liquor.

The first sparge being run off in the course of twenty minutes, another simslar one is affused; and thus in succession till the whole of the drainage, when mixed with the first mash-wort, constitutes the density adapted to the quality of the ale. Thus, the strong worts are prepared, and the malt is exhausted either for table beer, or for a return, as pointed out above. The last sparges are made $5^{\circ}$ or $6^{\circ}$ cooler than the first.

The quantity of hops seldom exceeds four pounds to the quarter of malt. The manner of boiling the worts is the same as that above described; but the conduct of the fermentation is peculiar. The heat is pitched at $50^{\circ}$, and the fermentation continues from a fortnight to three weeks. Were three brewings made in the week, seven or eight working tuns would thus be in constant action; and, as they are usually in one room, and some of them at an elevation of temperature of $15^{\circ}$, the apartment must be pro. pitious to fermentation, however low its heat may be at the commencement. No mors yeast is used than is indispensable; if a little more be needed, it is made effective by rousing up the tuns twice a day from the bottom.

When the progress of the attenuation becomes so slack as not to exceed half a pound in the day, it is prudent to cleanse, otherwise the top-barm might re-enter the body of the beer, and it would become yeast-bitten. When the ale is cleansed, the head, which has not been disturbed for some days, is allowed to float on the surface till the whole of the then pure ale is drawn off into the casks. This top is regarded as a sufficient preservative against the contact of the atmosphere. The Scotch do not skim their tuns, as the London ale brewers commonly do. The Scotch ale, when so cleansed, does not require to be set upon close stillions. It throws off little or no yeast, because the fermentation was nearly finished in the tun. The strength of the best Scotch ale ranges between 32 and 44 pounds to the barrel; or it has a specific gravity of from 1.088 to $1 \cdot 122$, according to the price at which it is sold. In a good fermentation, seldom more than a fourth of the original gravity of the wort remains at the period of the cleansing. Between one third and one fourth is the usual degree of attenuation. Scotch ale soon becomes fine, and is seldom racked for the home market. The following table will show the progress of fermentation in a brewing of good Scotch ale:

20 barrels of mash-worts of $42 \frac{1}{2}$ pounds gravity $=860 \cdot 6$

$20-\quad$ returns $6 \frac{1}{10} \quad \begin{aligned} 10 \\ \end{aligned}$

$12) \overline{982 \cdot 6}$

Founds weight of extract per quarter of malt $=\overline{81}$
March 24. pitched the tun at $51^{\circ}$ : yeast 4 gallons.

March 24. pitched the tun at $51^{\circ}$ : yeast 4 gallons.

$\begin{array}{rrr} & \text { Temp. } & \text { Gravity. } \\ 25 . & 52^{\circ} & 41 \text { pounds. } \\ 28 . & 56^{\circ} & 39 \\ 30 . & 60^{\circ} & 34 \\ \text { April } & 62^{\circ} & 32 \\ 4 . & 65^{\circ} & 29 \text { added } 1 \text { lb. of yeast. } \\ 5 . & 66^{\circ} & 25 \\ 6 . & 67^{\circ} & 23 \\ 7 . & 67^{\circ} & 20 \\ 8 . & 66^{\circ} & 18 \\ 9 . & 66^{\circ} & 15 \\ 10 . & 64^{\circ} & 14.5 \text { cleansed.* }\end{array}$

The following table shows the origin and the result of fermentation, in a number of practícal experiments :-

\begin{tabular}{|c|c|c|c|c|}
\hline $\begin{array}{l}\text { Original Gravity of } \\
\text { the Worts. }\end{array}$ & $\begin{array}{l}\text { Lbs. per Barrel of } \\
\text { Saccharine Matter. }\end{array}$ & $\begin{array}{l}\text { Specific Gravity of } \\
\text { the Ale. }\end{array}$ & $\begin{array}{l}\text { Lbs. per Barrel of } \\
\text { Saccharine Matter. }\end{array}$ & $\begin{array}{l}\text { Attenuation, or Sac- } \\
\text { charum decomposed. }\end{array}$ \\
\hline $1 \cdot 0950$ & $88 \cdot 75$ & 1.0500 & $40 \cdot 25$ & 0.478 \\
\hline $1 \cdot 0918$ & $85 \cdot 62$ & $1 \cdot 0420$ & $38 \cdot 42$ & 0.552 \\
\hline $1 \cdot 0829$ & $78 \cdot 125$ & $1 \cdot 0205$ & $16 \cdot 87$ & 0.787 \\
\hline $1 \cdot 0862$ & $80 \cdot 625$ & $1 \cdot 0236$ & $20 \cdot 00$ & 0.757 \\
\hline $1 \cdot 0780$ & $73 \cdot 75$ & $1 \cdot 0280$ & $24 \cdot 25$ & 0.698 \\
\hline 1.0700 & 65.00 & $1 \cdot 0285$ & $25 \cdot 00$ & 0.615 \\
\hline $1 \cdot 1002$ & $93 \cdot 75$ & $1 \cdot 0400$ & $36 \cdot 25$ & $0 \cdot 613$ \\
\hline
\end{tabular}

* Brewina (Society for diffusing Useful Knowledge), p. 156. 
Fermentation Table - continued.

\begin{tabular}{|c|c|c|c|c|}
\hline $\begin{array}{c}\text { Original Gravity of } \\
\text { the Worts. }\end{array}$ & $\begin{array}{l}\text { I bs. per Barrel of } \\
\text { Saccharine Matter. }\end{array}$ & $\begin{array}{l}\text { Specific Gravity of } \\
\text { the Ale. }\end{array}$ & $\begin{array}{l}\text { Lus. per Barrel of } \\
\text { Saccharine Matter. }\end{array}$ & $\begin{array}{l}\text { Altenuation, or Sar:- } \\
\text { rharum decomposed. }\end{array}$ \\
\hline $1 \cdot 1025$ & $95 \cdot 93$ & 1.0420 & $38 \cdot 42$ & 0.600 \\
\hline 1.0978 & $91 \cdot 56$ & $1 \cdot 0307$ & $2 \tau \cdot 00$ & 0.705 \\
\hline $1 \cdot 0956$ & $89 \cdot 37$ & $1 \cdot 0358$ & $32 \cdot 19$ & 0.640 \\
\hline $1 \cdot 1130$ & $105 \cdot 82$ & $1 \cdot 0352$ & 31.87 & 0.661 \\
\hline $1 \cdot 1092$ & $102 \cdot 187$ & $1 \cdot 0302$ & $26 \cdot 75$ & 0.605 \\
\hline $1 \cdot 1171$ & $110 \cdot 00$ & $1 \cdot 0400$ & $36 \cdot 25$ & 0.669 \\
\hline $1 \cdot 1030$ & $96 \cdot 40$ & $1 \cdot 0271$ & $23 \cdot 42$ & 0.757 \\
\hline $1 \cdot 0660$ & $61 \cdot 25$ & $1 \cdot 0214$ & $17 \cdot 80$ & 0.709 \\
\hline
\end{tabular}

The second column here does not represent, I believe, the solid extract, but the pasty extract obtained as the basis of Mr. Allen's saccharometer, and therefore each of its numbers is somewhat ton high. The last column, also, must be in some measure erroneous, on account of the quantity of acohol dissipated during the process of fermentation. It must be likewise incorrect, because the density due to the saccharine matter will be partly counteracted, by the effect of the alcohol present in the fermented liquor. In fact, the attenuation does not correspond to the strength of the wort; being greatest in the third brewing, and smallest in the first. The quantity of yeast for the abore ale brewings in the table was, upon an average, one gallon for 108 gallons; but it varied with its quality, and with the state of the weather, which, when warm, permits much less to be used with propriety.

The good quality of the malt, and the right management of the mashing, may be tested by the quantity of saccharine matter contained in the successively drawn worts. With this riew, an aliquot portion of each of them should be eraporated by a safety-bath heat to a nearly concrete consistence, and then mixed with twice its rolume of strong spirit of wine. The truly saccharine substance will be dissolved, while the starch and other matters will be separated; after which the proportions of each may be determined by filtration and evaporation. Or an equally correct, and much more expeditious method of arricing at the same result would be, after agitating the viscid extract with the alcohol in a tall glass cylinder, to allow the insoluble fecula to subside, and then to determine the specific gravity of the supernatant liquid by a hydrometer. The additional density which the alcohol has acquired will iridicate the quantity of malt sugar which it has receired. The following table, constructed by me, at the request of Henry Warburton, Esq., M. P., chairman of the Molasses Committee of the House of Commons in 18.30, will show the brewer the principle of this important inquiry. It exhibits the quantity in grains weight of sugar requisite to raise the specific gravity of a gallon of spirit of different densities to the gravity of water $=1 \cdot 000$ :

\begin{tabular}{cc}
$\begin{array}{c}\text { Specific Gravity of } \\
\text { Spirit. }\end{array}$ & Grains ; Weight of Sugar in the \\
0.995 & Gallon Imperial. \\
0.990 & 980 \\
0.985 & $1 \cdot 890$ \\
0.980 & $2 \cdot 800$ \\
0.975 & 3.710 \\
0.970 & 4.690 \\
0.965 & $5 \cdot 600$ \\
0.960 & 6.650 \\
0.955 & $7 \cdot 070$ \\
0.950 & $8 \cdot 400$ \\
\hline
\end{tabular}

The immediate purpose of this table was to show the effect of saccharine matter in disguising the presence or amount of alcohol in the weak feints of the distiller. But a similar table might easily be constructed, in which, taking a uniform quantity of alcohol of 0.825 , for example, the quantity of sugar in any wort-extract would be shown by the increase of specific gravity which the alcohol received from agitation with a ceriain weight of the wort, inspissated to a nearly solid consistence by a safety-pan, made on the principle of my patent sugar-pan. (See Stgar.) Thus, the normal quantities being 1000 grain measures of alcohol, and 100 grains by weight of inspissated mashextract, the hydrometer would at once indicate, by help of the table, first, the quantity per cent. of truly saccharine matter, and next, by subtraction, that of farinaceous matter present in it.

Plan, Machinery, and Utensils of a great Brewery.-Figs. 103 and 104 represent the arrangement of the utensils and machinery in a porter brewery on the largest scale; in which, howerer, it must be observed that the elevation fig. 103 is in a great degree imsginary as to the plane upon which it is taken; but the different vessels are arranged so as 
- to explain their uses most readily, and at the same time to preserve, as neariy as possib]e the relative positions which are usually assigned to each in works of this nature.

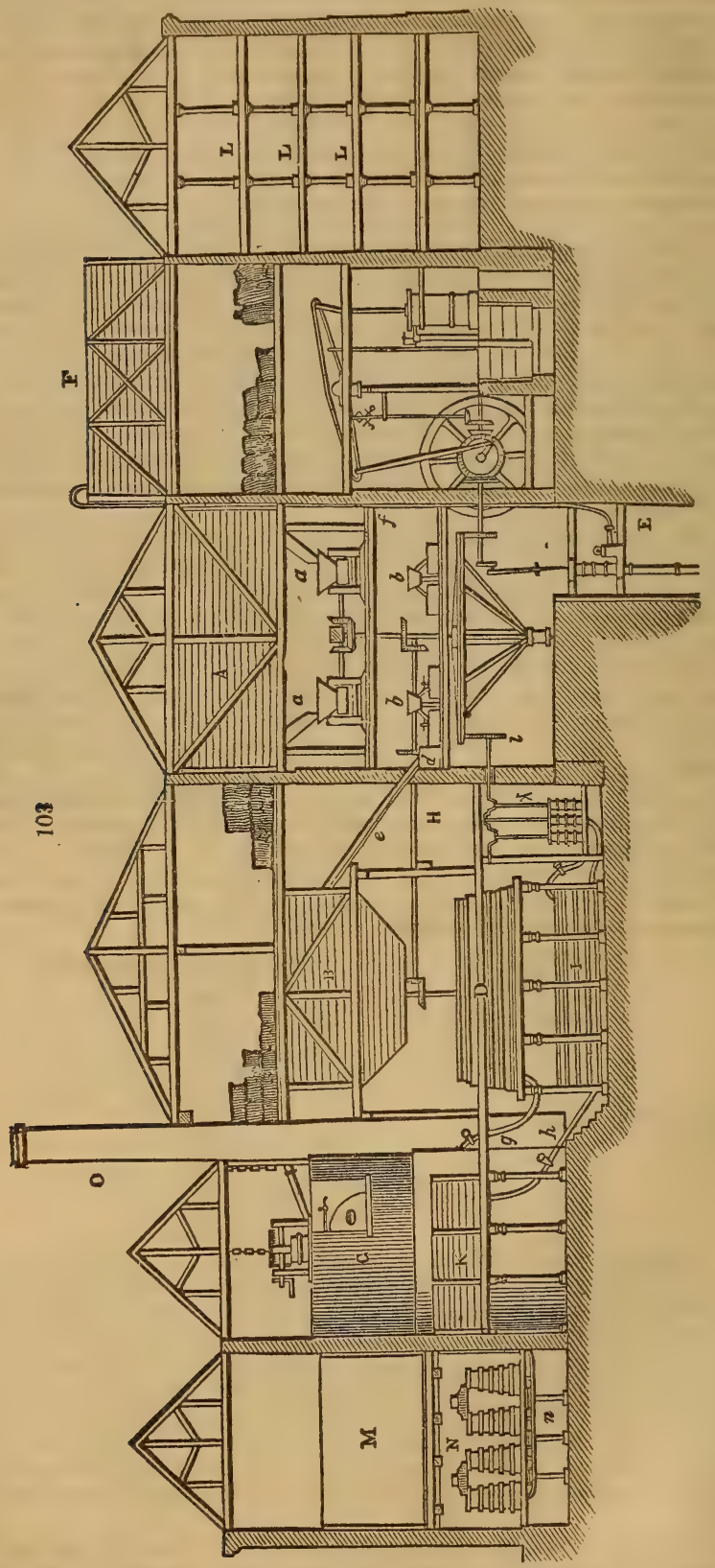

The malt for the zapply of the brewery is stored in vast granaries or malt-lofts, usually situated in the uppe: part of the buildings. Of these, I have been able to represent only one, at A, fig. i0s: the others, which are supposed to be on each side of it, cannot 
be seen in this view. Immediately beneath the granary $A$, on the ground floor, is the mill; in the upper story above it, are two pairs of rollers, figs. 101, 102, and 103, under $a, a$, for bruising or crushing the grains of the malt. In the floor beneath the rollers are the mill-stones $b, b$, where the malt is sometimes ground, instead of being merely bruised by passing between the rollers, under $a, a$.

The malt, when prepared, is conveyed by a trough into a chest $d$, to the right of $b$, from which it can be elevated by the action of a spiral screw, fig. 105, enclosed in the sioping tube $e$, into the large chest or bin $\mathrm{B}$, for holding ground malt, situated immediately over the mash-tun $\mathrm{D}$. The malt is reserved in this bin till wanted, and it is then let down into the mashing-tun, where the extract is obtained by hot water supplied from the copper $\mathrm{G}$, seen to the right of $\mathrm{B}$.

The water for the service of the brewery is obtained from the well $\mathrm{E}$, seen beneath the mill to the left, by a lifting pump worked by the steam engine; and the forcing-pipe $f$ of this pump conveys the water up to the large reservoir or water-back $F$, placed at the top of the engine-house. From this cistern, iron pipes are laid to the copper G (on the right-hand side of the figure), as also to every part of the establishment where cold water can be wanted for cleaning and washing the vessels. The copper $\mathrm{G}$ can be filled with cold water by merely turning a cock; and the water, when boiled therein, is conveyed by the pipe $g$ into the bottom of the mash-tun $D$. It is introduced beneath a false bottom, upon which the malt lies, and, rising up through the holes in the false bottom, it extracts the saccharine matter from the malt; a greater or less time being allowed for the infusion, according to circumstances. The instant the water is drawn off from the copper, fresh water must be let into it, in order to be ready for boiling the second mashing; because the copper must not be left empty for a moment, otherwise the intense heat of the fire would destroy its bottom. For the convenience of thus letting down at once as much liquor as will fill the lower part of the copper, a pan or second boiler is placed over the top of the copper, as seen in fig. 103 ; and the steam rising from the copper communicates a considerable degree of heat to the contents of the pan, without any expensc of fuel. This will be more minutely explained hereafter. (See fig. 107.)

During the process of mashing, the malt is agitated in the mash-tun so as to expose every part to the action of the water. This is done by a 'mechanism contained within the mash-tun, which is put in motion by a horizontal shaft above it, $\mathrm{H}$, leading from the mill. The mash machine is shown separately in fig. 106. When the operation of mash$i_{\mathrm{i} g}$ is finished, the wort or extract is drained down from the malt into the vessel $\mathrm{I}$, called the under-back, immediately below the mash-tun, of like dimensions, and situated always on a lower level, for which reason it has received this name. Here the wort does not remain longer than is necessary to drain off the whole of it from the tun above. It is then pumped up by the three-barrelled pump $k$, into the pan upon the top of the copper, by a pipe which cannot be seen in this section. The wort remains in the pan until the water for the succeeding mashes is discharged from the copper. But this delay is no loss of time, because the heat of the copper, and the steam arising from it, prepare the wort, which had become cooler, for boiling. The instant the copper is emptied, the first wort is let down from the pan into the copper, and the second wort is pumped up from the under-back into the upper pan. The proper proportion of hops is thrown into the copper through the near hole, and then the door is shut down, and screwed fast, to keep in the steam, and cause it to rise up through pipes into the pan. It is thus forced to blow up through the wort in the pan, and communicates so much heat to it, or water, called $l i$ qeior bझ the brewers, that either is brought near to the boiling point. The different worts succeed each other through all the different vessels with the greatest regularity, so that there is no loss of time, but every part of the apparatus is constantly employed. When the ebullition has continued a sufficient period to coagulate the grosser part of the extract, and to evaporate part of the water, the contents of the copper are run off through a large cock into the jack-back $\mathrm{K}$, below $\mathrm{G}$, which is a vessel of sufficient dimensions to contain it, and provided with a bottom of cast-iron plates, perforated with small holes, through which the wort drains and leaves the hops. The hot wort is drawn off from the jackback through the pipe $h$ by the three-barrelled pump, which throws it up to the coolers $\tau,:, x$; this pump being made with different pipes and cocks of communication, to serve ali the purposes of the brewery except that of raising the cold water from the well. The coolers $\mathrm{L}, \mathrm{L}, \mathrm{L}$, are very shallow vessels, built over one another in several stages : and that part of the building in which they are contained is built with lattice-work or shutter flaps, on all sides, to admit free currents of air. When the wort is sufficiently cooled to be put to the first fermentation, it is conducted in pipes from all the different coolers to the iarge fermenting ressel or gyle-tun $M$, which, with another similar vessel behind it, is of sufficient capacity to contain all the beer of one day's brewings.

Whenever the first fermentation is concluded, the beer is drawn off from the great fermenting vessel $M$, into the small fermenting casks or cleansing vessels $N$, of which there are a great number in the brewery. They are placed four together, and to each four a com- 
BEER.

mon spout is provided to carry off the yeast, and conduct it into the troughs $u$, placed beneath. In these cleansing vessels the beer remains till the fermentation is completed; and it is then put into the store-vats, which are cashs or tuns of an immense size, where

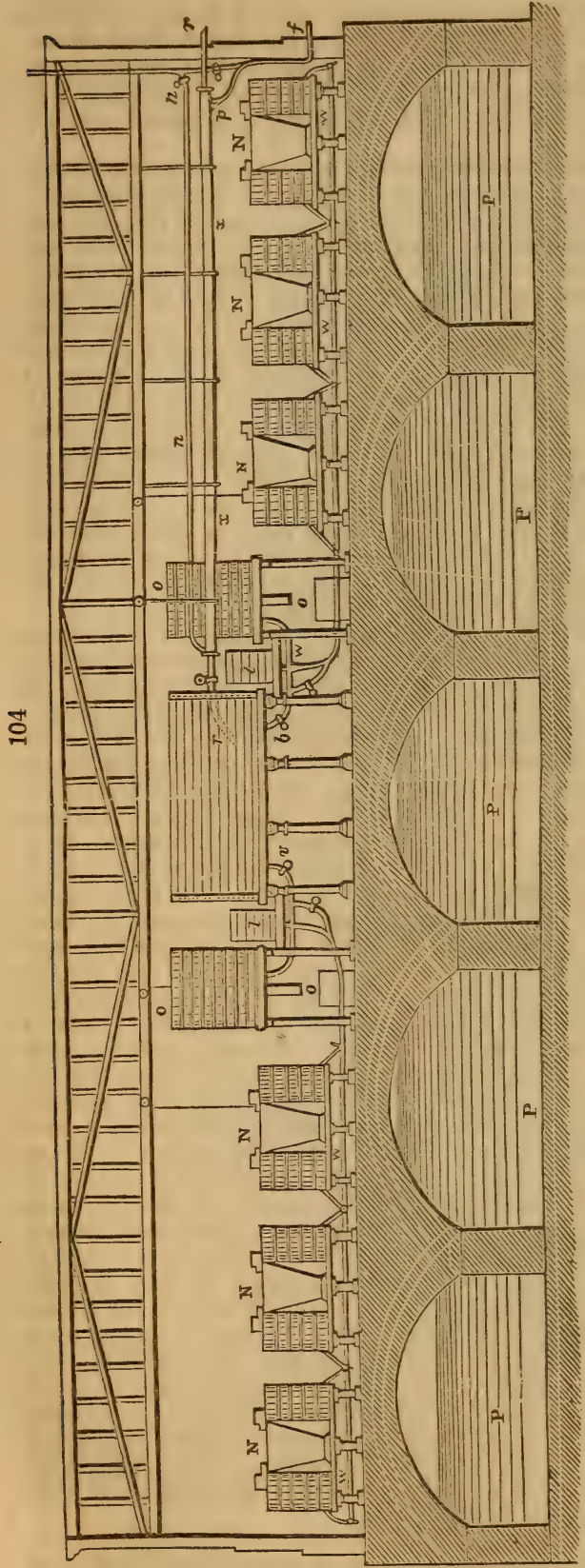
it is kept till wanted, and is finally drawn off into barrels, and sent away from the brewery. The store-vats are not represented in the figure: they are of a conical shape, and of different dimensions, from fifteen to twenty feet diameter, and usually from fifteen to twenty feet in depth. The steam-engine which puts all the machine in motion is exhibited in its place, on the left side of the figure. On the axis of the large fly-wheel is a bevelled spurwheel, which turns another similar wheel upon the end of a horizontal shaft, which extends from the engine-house to the great horse-wheel, set in motion by means of a spur-wheel. The horse-wheel drives all the pinions for the mill-stones $b \quad b$, and also the horizontal axis which works the three-barrelled pump $k$. The rollers $a, a$, are turned by a bevel wheel upon the upper end of the axis of the horse-wheel, which is prolonged for that purpose; and the horizontal shaft $H$, for the mashing engine, is driven by a pair of bevel whecls. There is likewise a sack-tackle, which is not represented. It is a machine for drawing up the sacks of malt from the court-yard to the highest part of the building, whence the sacks are wheeled on it truek to the malt-loft $A$, and the contents of the sacks are discharged.

The horse-wheel is intended to be driven by horses occasionally, if the steam-engine should fail ; but these engines are now brought to such perfection that it is vers seldom any recourse of this kind is needed.

Fig. 104 is a representation of the fermening house at the brewery of Messrs. Whitbread and Company, Chiswell Street, London, which is one of the most complete in its arrangement in the world : it was erected after the plan of Mr. Richardson, who conducts the brewing at those works. The whole of fig. 104 is to be considered as devoted to the same object as the large vessel $M$ and the casks N, fig. 103. In fig. 104 $r r$ is the pipe which leads from the different coolers to convey the wort to the great fermenting ressels or squares $\mathrm{M}$, of which there are two, one behind the other; $f f$ represents a part of the great pipe which conveys all the water from the well E, jig. 103, up to the water cistern 
r. This pipe is conducted purposely up the wall of the fermenting-house, fig. 104, and has a cock in it, near $r$, to stop the passage. Just beneath this passage a branch-pipe $p$ proceeds, and enters a large pipe $x \quad x$, which has the former pipe $r$ withinside of it. From the end of the pipe $x$, nearest to the squares $\mathrm{m}$, another branch $n n$ proceeds, and returns to the original pipe $f$, with a cock to regulate it. The object of this arrangement is to make all, or any part, of the cold water flow through the pipe $x x$, which snrrounds the pipe $r$, furmed only of thin copper, and thus cool the wort passing through the pips. $r$, until it is found by the thermometer to have the exact temperature which is desirab!s: before it is put to ferment in the great square $M$. By means of the cocks at $n$ and $p$, thie quantity of cold water passing over the surface of the pipe $r$ can be regulated at pleasure, whereby the heat of the wort, when it enters into the square, may be adjusted within half a degree.

When the first fermentation in the squares $\mathbf{M} \mathbf{M}$ is finished, the beer is drawn off from them by pipes marked $v$, and conducted by its branches $\mathrm{w} \mathrm{w} \mathrm{w}$, to the different rows of fermenting-tuns, marked $\mathrm{N} \mathrm{N}$, which occupy the greater part of the building. In the hollow between every two rows are placed large troughs, to contain the yeast which they throw off. The figure shows that the small tuns are all placed on a lower level than the bottom of the great vessels $\mathrm{M}$, so that the beer will flow into them, and, by hydrostatic equilibrium, will fill them to the same level. When they are filled, the communication-cock is shut; but, as the working off the yeast diminishes the quantity of beer in each vessel, it is necessary to replenish them from time to time. For this

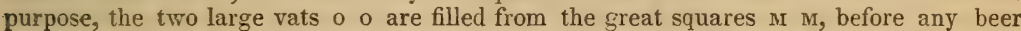
is drawn off into the small casks $\mathrm{N}$, and this quantity of beer is reserved at the higher level for filling up. The two vessels o o are, in reality, situated between the two squares M M; but I have been obliged to place them thus in the section, in order that they may be seen. Near each filling-up tun o is a small cistern $t$ communicating with the tun o by a pipe, which is closed by a float-valve. The small cisterns $t$ are always in communication with the pipes which lead to the small fermenting vessels $\mathrm{N}$; and therefore the surface of the beer in all the tuns, and in the cisterns, will always be at the same level; and as this level subsides by the working off of the yeast from the tuns, the float sinks and opens the valve, so as to admit a sufficiency of beer from the filling-up tuns 0 , to restore the surfaces of the beer in all the tuns, and also in the cistern $t$, to the original level. In order to carry off the yeast which is produced by the fermentation of the beer in the tuns o 0, a conical iron dish or funnel is made to float upon the surface of the beer which they contain; and from the centre of this funnel a pipe, $o$, descends, and passes through the bottom of the tun, being packed with a collar of leather, so as to be water-tight; at the same time that it is at liberty to slide down, as the surface of the beer descends in the tun. The yeast flows over the edge of this funnel-shaped dish, and is conveyed down the pipe to a trough beneath.

Beneath the fermenting-house are large arched vaults, $\mathbf{r}$, built with stone, and lined with stucco. Into these the beer is let down in casks when sufficiently fermented, and is kept in store till wanted. These vaults are used at Mr. Whitbread's brewery, instead of the great store-vats of which we have before spoken, and are in some respects preferable, because they preserve a great equality of temperature, being beneath the surface of the earth.

The mait-rollers, or rnachines for bruising the grains of the malt, figs. 101, 102, have been already described. The malt is shot down from A, fig. 103, the malt-loft, into the hopper; and from this it is let out gradually through a sluice or sliding shuttle, $a$, fig. 103, and falls between the rollers.

Fig. 105, is the screw by which the ground or bruised malt is raised up, or conveyed from one part of the brewery to another. $\mathrm{k}$ is an inclined box or trough, in the centre of which the axis of the screw $\mathrm{H}$ is placed; the spiral iron plate or worm, which is fixed projecting from the axis, ard which forms. the screw, is made very nearly to fill the inside of the box. By this means, when the screw is turned round by the wheels $\mathrm{E} F$, or by any other means, it raises up the malt from the box $d$, and delivers it at the spout $\mathrm{G}$.

This serew is equally applicable for conveying the malt horizontally in the trough $\mathbf{K}$, as slantingly; and similar machines are employed in various parts of breweries for conveying the malt wherever the situation of the works requires.

Fig. 106, is the mashing-machine. $a$ a is the tun, made of wood staves, hooped together. In the centre of it rises a perpendicular shaft, $b$, which is turned slowly round by means of the bevelled wheels $t u$ at the top. $c c$ are two arms, projecting from that axis, and supporting the short vertical axis $d$ of the spur-wheel $x$, which is turned by the spur-wheel $w$; so that, when the central axis $b$ is made to revolve, it will carry the thick short axle $d$ round the tun in a circle. That axle $d$ is furnished with a number of arms, $e e$, which have blades placed obliquely to the plane of their 
motion. When the axis is turned round, these arms agitate the malt in the tun, and give it a constant tendency to rise upward from the bottom.

The motion of the axle $d$ is produced by a wheel, $x$, on the upper end of it, which is

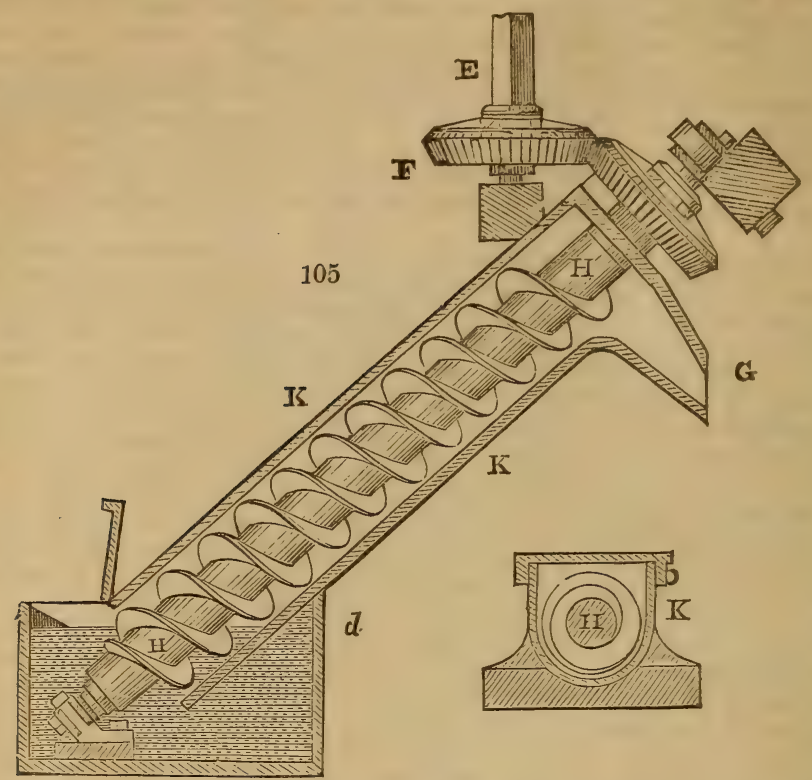

turned by a wheel, $w$, fastened on the middle of the tube $b$, which turns freely round upon its central axis. Upon a higher point of the same tube $b$ is a berel wheel, $o$, receiving motion from a bevel wheel, $q$, fixed upon the end of the horizontal axis $n n_{3}$

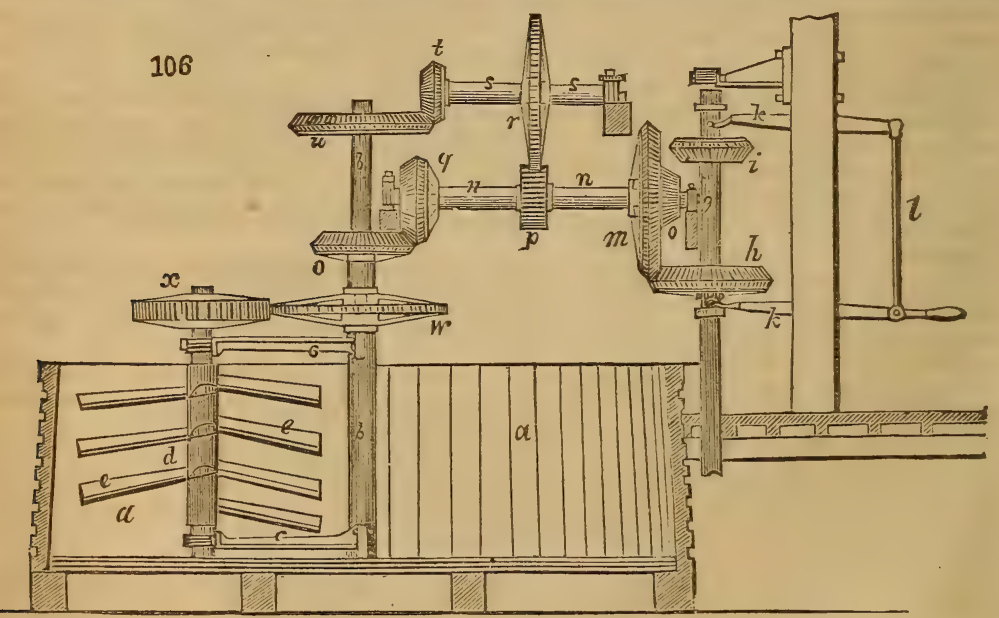

which gives motion to the whole machine. This same axis has a pinion, $p$, upon it, which gires motion to the wheel $r$, fixed near the middle of a horizontal axle, which, at its left hand end, has a bevel pinion, $t$, working the wheel $u$, before mentioned. By these means, the rotation of the central axis $b$ will be very slow compared with the motion of the axle $d$; for the latter will make seventeen or eighteen revolutions on its own axis in the same space of time that it will be carried once round the tun by the motion of the shaft $b$. At the beginning of the operation of mashing, the machine is made to turn with a slow motion; but, after having wetted all the malt by one revo. lution, it is driven quicker. For this purpose, the ascending shaft $f g$, which gives 
motion to the machine, has two level wheels, $h i$, fixed upon a tube, $f g$, which is, fitted upon a central shaft. These wheels actuate the wheels $m$ and $o$, upon the e'nd of the horizontal shaft $n n$; but the distance between the two wheels $h$ and $i$ is such. that they cannot be engaged both at once with the wheeis $m$ and $o$; but the tube $f g$, to which they are fixed, is capable of sliding up and down on its central axis sufficiently to bring enther wheel $h$ or $i$ into gear with its corresponding wheel o or $m$, upon the horizontal shaft; and as the diameters of $n o$, and $i m$, are of very different proportions, the velocity of tice motion of the machine can be varied at pleasure, by using one or other. $k$ and $k$ are $\imath$ wo levers, which are forked at their extremities, and embrace collars at the ends of the tube $f g$. These levers being united by a rod, $l$, the handle $k$ gives the means of moving the tube $f g$, and its wheels $h i$, up or down, to throw either the one or the other wheel into gear.

The object of boiling the wort is not merely evaporation and concentration, but extraction, coagulation, and, finally, combination with the hops; purposes which are better accomplished in a deep confined copper, by a moderate heat, than in an open shallow pan with a quick fire. The copper, being incased above in brickwork, retains its digesting temperature much longer than the pan could do. The waste steam of the close kettle, moreover, can be economically employed in communicating heat to water or weak worts; whereas the exhalations from an open pan would prove a nuisance, and would need to be carried off by a hood. The boiling has a four-fold effect: 1 . it concentrates the wort; 2. during the earlier stages of heating, it converts the starch into sugar, dextrine, and gum, by means of the diastase; 3 . it extracts the substance of the hops diffused through the wort; 4 . it coagulates the albuminous matter present in the grain, or precipitates it by means of the tannin of the hops.

The degree of evaporation is regulated by the nature of the wort, and the quality of the beer. Strong ale and stout for keeping, require more boiling than ordinary porter or table-beer brewed for immediate use. The proportion of the water carried off by eraporation is usually from a seventh to a sixth of the volume. The hops are introduced during the progress of the ebullition. They serve to give the beer not only a bitter aromatic taste, but also a keeping quality, or they counteract its natural tendency to become sour; an effect partly due to the precipitation of the albumen and starch, by their resinous and tanning constituents, and partly to the antifermentable properties of their lupuline, bitter principle, ethereous oil, and resin. In these respects, there is none of the bitter plants which can be substituted for hops with advantage. For strong beer, powerful fresh hops should be selected; for weaker beer, an older and weaker article will suffice.

The hops are either boiled with the whole body of the wort, or extracted with a portion of it; and this concentrated extract added to the rest. The stronger the hops ase, the longer time they require for extraction of their virtues; for strong hops, an hour and a half or two hours boiling may be proper; for a weaker sort, half an hour or an hour may be sufficient; but it is never advisable to push this process too far, lest a disagreeable bitterness, without aroma, be imparted to the beer. In our breweries, it is the practice to boil the hops with a part of thie wort, and to filter the decoction through a drainer, called the jack hop-back. The proportion of hops to malt is very various; but, in general, from a pound and a quarter to a pound and a half of the former are taken for $100 \mathrm{lbs}$. of the latter in making good table-beer. For porter and strong ale, 2 pounds of hops are used, or even more; for instance, one pound of hops to a bushel of malt, if the beer be destined for the consumption of India.

During the boiling of the two ingredients, much coagulated albuminous matter, in various states of combination, makes its appearance in the liquid, constituting what is called the breaking or curdling of the wort, when numerous minute flocks are seen floating in it. The resinous, bitter, and oily-ethereous principles of the hops combine with the sugar and gum, or dextrine of the wort; but for this effect they require time and heat; showing that the boil is not a process of mere evaporation, but one of chemical reartion. A yellowish-green pellicle of hop-oil and resin appears upon the surface of the boiling wort, in a somewhat frothy form: when this disappears, the boiling is presumed to be completed, and the beer is strained off into the cooler. The residuary hops may be pressed and used for an inferior quality of been; or they may be boiled with fresh wost, and be added to the next brewing charge.

Figs. 107,108, represent the copper of a London brewery. Fig. 107 is a vertical section; fig. 108, a ground-plan of the fire-grate and flue, upon a smaller scale : $a$ is the close copper kettle, having its bottom convex within; $b$ is the open pan placed upon its top. From the upper part of the copper, a wide tube, $c$, ascends, to carry off the stean generated during the ebullition of the wort, which is conducted through four downwards-slanting tubes, $d d$ (two only are visible in this section), into the liquor of the pan $b$, in order to warm its contents. A vertical iron shaft or spindle, $e$, passes down through the tube $c$, nearly to the bottom of the copper, and is there mounted with an iron arm, called as 
rouser, which carries round a chain hung in loops, to prevent the hops from adhering to the bottom of the boiler. Three bent stays, $f$, are stretched across the interior, to support the shaft by a collet at their middle junction. The shaft carries at its upper end a bevel

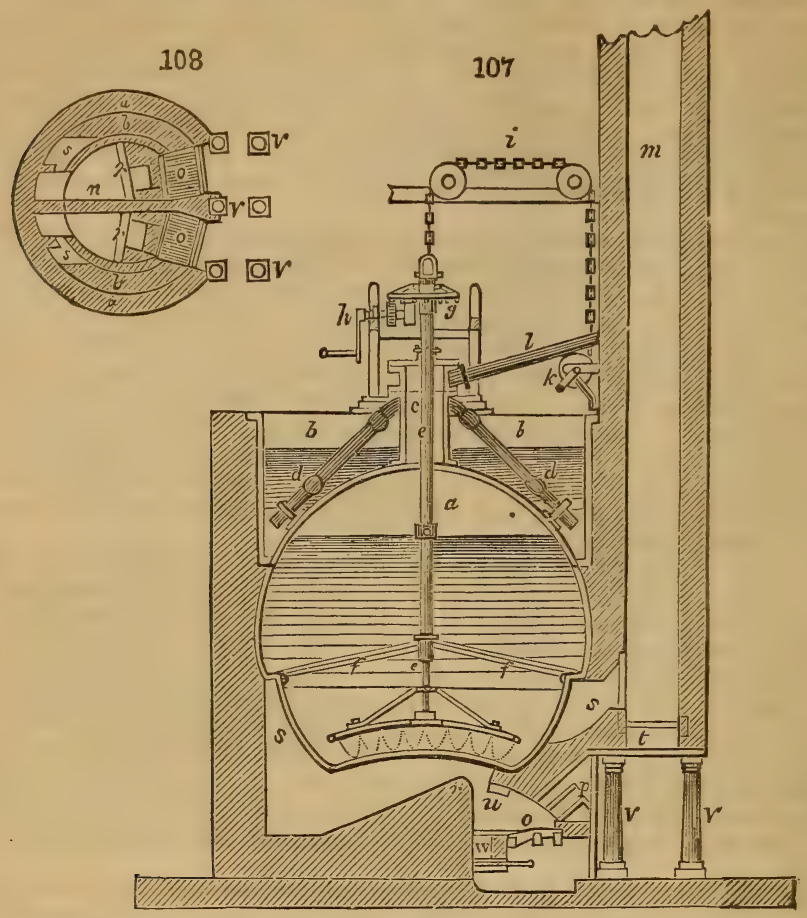

wheel, $g$, working into a bevel pinion upon the axis $h$, which may be turned either by power or by hand. The rouser shaft may be lifted by means of the chain $i$, which, going over two pulleys, has its end passed round the wheel and axle $k$, and is turned by a winch : $l$ is a tube for conveying the waste steam into the chimney $m$.

The heat is applied as follows:-For heating the colossal coppers of the London breweries, two separate fires are required, which are separated by a narrow wall of brickwork, $n$, figs. 107,108 . The dotted circle $\alpha^{\prime} a^{\prime}$ indicates the largest circumference of the copper, and $b^{\prime} b^{\prime}$ its bottom; 00 are the grates upon which the coals are thrown, not throngh folding doors (as of old), but through a short slanting iron hopper, shown at p, fig. 107, built in the wall, and kept constantly filled with the fuel, in order to exclude the air. Thus the lower stratum of coals gets ignited before it reaches the grate. Above the hopper $p$, a narrow channel is provided for the admission of atmospherical air, in such quantity merely as may be requisite to complete the combustion of the smoke of the coals. Behind each grate there is a fire-bridge, $r$, which reflects the flame upwards, and causes it to play upon the bottom of the copper. The burnt air then passes round the copper in a semicircular flue, $s s$, from which it flows off into the chimney $m$, on whose under end a sliding damper-plate, $t$, is placed for tempering the draught When cold air is admitted at this orifice, the combustion of the fuel is immediately checked. There is, besides, another slide-plate at the entrance of the slanting flue into the vertical chimney, for regulating the play of the flame under and around the copper. If the plate $t$ be opened, and the other plate shut, the power of the fire is suspended, as it ought to be, at the time of emptying the copper. Immediately over the grate is a brick arch, $u$, to protect the front edge of the copper from the first impulsion of the flame. The chimney is supported upon iron pillars, $v, v$; $w$ is a cavity closed with a slide-plate, through which the ashes may be taken out from behind, by means of a long iron hook.

Fig. 109 represents one of the sluice-cocks, which are used to make the communications of the pipes with the pumps, or other parts of the brewery. B B represents the pipe in which the cock is placed. The two parts of this pipe are screwed to the side of a box, c c, in which a slider, A, rises and falls, and intercepts, at pleasure, the passage of the pipe. The slider is moved by the rod $a$. This passes through a stuffing- 
box, in the top of the box which contains the slider, and has the rack $b$ fastened to it. The rack is moved by a pinion fixed upon the axis of a handle $e$, and the rack and pinion are contained in a frame $d$, which is supported by two pillars. The frame contains a small roller behind the rack, which bears it up towards the pinion, and keeps its teeth up to the teeth of the pinion. The slider $\mathrm{A}$ is made to fit accurately against the internal surface of the box c, and to bear against this surface by the pressure of a spring, so as to make a perfectly close fitting.

Fig, 110 is a small cock to be placed in the side of the great store vats, for the purpose of drawing off a small quantity of beer, to taste and try its quality. A is a
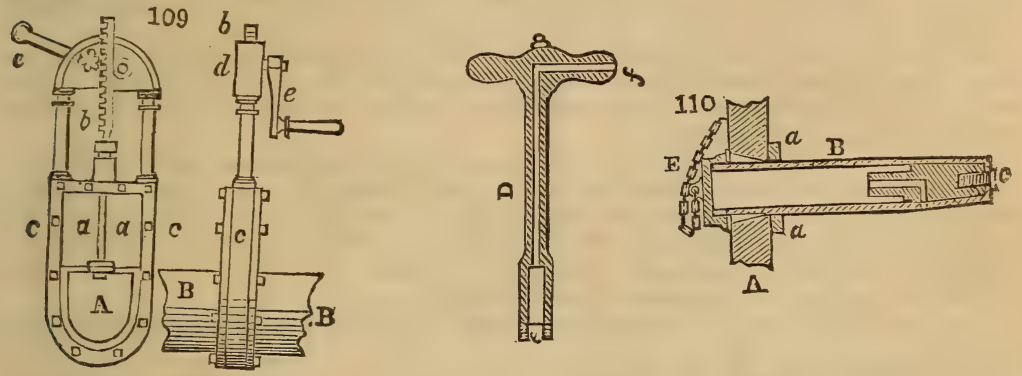

part of the stave or thickness of the great store vat; into this the tube $\mathrm{B}$ of the cock is fitted, and is held tight in its place by a nut, $a, a$, screwed on withinside. At the other end of the tube B, a plug, $\mathrm{c}$, is fitted, by grinding it into a cone, and it is kept in by a screw. This plug has a hole up the centre of it, and from this a hole proceeds sidewise, and corresponds with a hole made through the side of the tube when the cock is open; but when the plug $c$ is turned round, the hole will not coincide, and then the cock will be shut. D is the handle or key of the cock, by which its plug is turned to open or shut it: this handle is put up the bore of the tube (the cover $\mathrm{E}$ being first unscrewed and removed), and the end of it is adapted to fit the end of the plug of the cock. The handle has a tube or passage bored up it, to convey the beer away from the cock when it is opened, and from this the passage $f$, through the handle, leads, to draw the beer into a glass or tumbler. The hole in the side of the plug is so arranged, that, when the handle is turned into a perpendicular direction, with the passage $f$ downwards, the cock will be open. The intention of this contrivanee is, that there shall be no considerable projection beyond the surface of the tun; because it sometimes happens that a great hoop of the tun breaks, and, falling down, its great weight would strike out any cock which had a projection; and, if this happened in the night, much beer might be lost before it was discovered. The cock above described, being almost wholly withinside, and having scarcely any projection beyond the outside surface of the tun, is secure from this accident.

Fig. 111 is a small contrivance of a vent peg, to be screwed into the head of a common cask when the beer is to be drawn off from it, and it is necessary to admit some air to

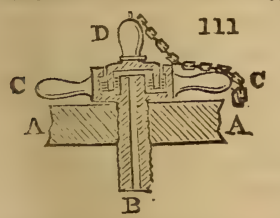
allow the beer to flow. A A represents a portion of the head of the cask into which the tube в is screwed. The top of this tube is surrounded by a small cup, from which project the two small handles $\mathrm{c}$, by which the peg is turned round to screw it into the cask. The cup round the other part of the tube is filled with water; into this a small cup, $\mathrm{D}$, is inverted; in consequence, the air can gain admission into the cask when the pressure within is so far diminished, that the air will bubble up through the water, and enter beneath the small cup $D$.

The most efficient substance for fining beer hitherto discovered is isinglass, which is prepared by solution in vinegar or old stale beer, and this solution is afterwards reduced with thin mild beer generally brewed for the purpose, in all large establishments, from a raw or return wort. It must next be passed through a fine hair sieve, by means of rubbing it down with a hard hair-brush, and brought to the proper consistency by thin mild beer. If properly made, it will be clear, transparent, and free from feculencies. Finings serve excellently to remove any extraneous matter that may be found floating in the beer, and thus changes it from bright to brilliant. The common quantity used is from a pint to a quart per barrel, according to the nature of the beer.

To ascertain whether the beer is in a fit state for fining, put it into a long giass cylindric vessel, and add to it a teaspoonful, or thereby, of the fining; then give the mixture a good shake, by turning the vessel up and down, after closing its mouth with 
the palm of the hand. If the beer has been well brewed, its aptitude to become bright will be soon shown by the mixture getting thick and curdy; a bright portion will generally show itself at the bottom or middle; after which the finings will gradually mount to the top, taking up all the impurities along with them, till the whole becomes brilliant. Some have said that the finings should carry the impurities down to the bottom; but this, according to Mr. Black, ${ }^{*}$ takes place only with stubborn beer, which would not become thoroughly bright with any quantity of finings which could be introduced. Finings have usually a specific gravity of from 1.010 to $1 \cdot 016$, and, when added to beer in a fit condition for fining, invariably go to the top, and not to the bottom. In fining beer in a barrel laid on its side, if the finings do not make their appearance at the bung-hole, the beer will not become bright. The isinglass must not be dissolved with heat, nor in hot water.

Beer brewed from imperfectly malted grain, or from a mixture of malt and raw corn, gives a fermentation quite different in flavor from that of beer from sound malt. The nose is, in fact, the best guide to the experienced brewer for ascertaining whether his process is going on well or ill.

Ropiness is a morbid state of beer, which is best remedied, according to Mr. Black, by putting the beer into a vat with a false bottom, and adding, per barrel, 4 or 5 pounds of hops, taken gradually away after the first boilings of the worts; and to them may be added about half a pound per barrel of mustard-seed. Rouse the beer as the hops are gradually introduced, and, in some months, the ropiness will be perfectly cured. The beer should be drawn off from below the false bottom.

For theoretical views, see FERMENTATION; and for wort-cooling apparatus, see REFRIGERATOR.

BEET-ROO'T SUGAR. See Sugar.

BELL-METAL, an alloy of copper and tin. See Copper.

BELloWS. See Metallurgy.

BEN OIL. See OIL oF BEN.

BENGAL STRIPES. Ginghams; a kind of cotton cloth woven with colored stripes.

BENJAMIN or BENZOIN. (Benjoin, Fr.; Benzöe, Germ.) A species of resin used chiefly in perfumery. It is extracted by incision from the trunk and branches of the styrax benzoin, which grows in Java, Sumatra, Santa Fé, and in the kingdom of Siam. The plant belongs to the decandria monogynia of Linnæus, and the natural family of the ebenaceæ. It hardens readily in the air, and comes to us in brittie masses, whose fracture presents a mixture of red, brown, and white grains of various sizes, which, when white, and of a certain shape, have been called amygdaloid, from their resemblance to almonds The sorted benzoin is, on the other hand, very impure.

The fracture of benzoin is conchoidal, and its lustre greasy : its specific gravity varies from 1.063 to 1.092 . It has an agreeable smell, somewhat like vanilla, which is most manifest when it is ground. It enters into fusion at a gentle heat, and then exhales a white smoke, which may be condensed into the acicular crystals of benzoic acid, of which it contains 18 parts in the hundred. Stoltze recommends the following process for cxtracting the acid. The resin is to be dissolved in 3 parts of alcohol, the solution is to be introduced into a retort, and a solution of carbonate of soda dissolved in dilute alcohol is to be gradually added to it, till the fiee acid be neutralized; and then a bulk of water equal to double the weight of the benzoin is to be poured in. The alcohol being drawn off by distillation, the remaining liquor contains the acid, and the resin floating upon it may be skimmed off and washed, when its weight will be found to amount to about 80 per cent. of the raw material. The benzoin contains traces of a volatile oil, and a substance soluble in water, at least through the agency of carbonate of potash. Ether does not dissolve benzoin completely. The fat and volatile oils dissolve very little of it.

Unverdorben has found in benzoin, besides benzoic acid, and a little volatile oil, no less than three different kinds of resin, none of which has, however, been turned as yet to any use in the arts.

Benzoin is of great use in perfumery, as it enters into a number of preparations ; among which may be mentioned fumigating pastilles, fumigating cloves (called also nails), poudre à la maréchale, \&c. The alcoholic tincture, mixed with water, forms virginal milk. Benzoin enters also into the composition of certain varnishes employed for snuff-boxes and walking-sticlis, in order to give these objects an agreeable smeli when they become heated in the hand. It is likewise added to the spirituous solution of isinglass with which the best court plaster is made.

BERLIN BLUE. Prussian blue. See Brue.

BERRIES OF AVIGNON, und Persian Berries. (Graines d'Avignon, Fr.; Gelbbeeren, Germ.) A yellowish dye-drug, the fruit of the rhamnus infectorius, a plant 
cultivated in Proverce, Languedoc, and Dauphiné, for the sake of its berries, which are plucked before they are ripe, while they have a greenish hue. Another variety comes from Persia, whence its trivial name : it is larger than the French kind, and has : 11prror properties. The principal substances contained in these berries are, 1. A coloring matter, which is united with a matter insoluble in ether, little soluble in concentrated aleohol, and very soluble in water: it appears to be volatile. 2. A matter remarkable for its iitterness, which is soluble in water and alcohol. 3. A third principle, in small quantity. A decoetion of one part of the Avignon or Persian berry in ten of water affords a brownyellow liquor bordering upon green, having the smell of a vegetable extract, and a slightly bitter taste.

With gelatine that decoction gives, after some time, a slight precipitate, -

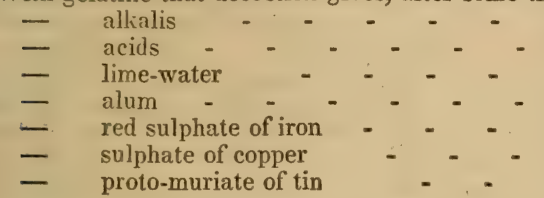

a yellow hue,

a slight muddiness,

a greenish-yellow tint,

- a yellow color, an olive-green color, an olive color, proto-muriate of tin a greenish yellow with a slight precipitate. (See CALICo Printing.)

BERYL. A beautiful mineral i $r$ gem, of moderate price, usually of a green color of various shades, passing into honey-yellow and sky blue.

BEZOAR. The name of certain concretions found in the stornachs of animals, to which many fanciful virtues were formerly ascribed. They are interesting only to the chemical pathologist.

BILE. (Bile, Fr.; galle, Germ.) The secreted liquor of the liver in animals. For an account of the uses of animal bile in the arts, see GALL.

BIRDLIME. (Glu, Fr.; Vogelleim, Germ.) The best birdlime may be made from the middle bark of the holly, boiled seven or eight hours in water, till it is soft and tender, then laid by heaps in pits under ground, covered with stones after the water is drained from it. There it must be left during two or three weeks, to ferment in the summer season, and watered if necessary, till it passes into a mucilaginous state. It is then to be pounded in a mortar to a paste, washed in running water, and kneaded till it be free from extraneous matters. It is next left for four or five days in earthen vessels to ferment and purify itself, when it is fit for use. Birdlime may be made by the same process from the mistletoe (viburnum lantana), young shoots of elder, and the barks of other vegetables. as well as from most parasite plants.

Good birdlime is of a greenish color, and sour flavor, somewhat resembling that of linseed oil; gluey, stringy, and tenacious. By drying in the air it becomes brittle, and may be powdered; but its viscosity may be restored by moistening it. It has an acid reaction with litmus paper. It contains resin, mucilage, a little free acid, coloring and extractive matter. The resin has been called. VIScINE.

BISMUTH. (Bismuth, Fr.; Wismuth, Germ.) Called also marcasite and tin-glass. It was shown to be a metal somewhat different from lead, by G. Agricola, in 1546; Stahl and Dufay proved its peculiarity; but it was more minutely distinguished by l.ott and Geoffroy, about the middle of the last century. It is a rare substance, occurring native, as an oxyde, under the name of bismuth ochre; as a sulphuret, called bismuth glance; as a sulphuret with copper, called copper bismuth ore; as also with copper and lead, callea needle ore. It is found associated likewise with selenium and tellurium. The native metal occurs in various forms and colors, as white, reddish, and variegated; in primitive and floetz formations, along with the ores of cobalt, nickel, copper, silver, and bismuth ochre; at the Saxon Erzegebirge, near Shneeberg, and Joh. Georgenstadt; also in Bohemia, Baden, Wurtemberg, Hessia, Sweden, Norway, England, and France.

The production of this metal is but a limited object of the smelting-works of the Saxon Erzegebirge at Schnceberg. It there occurs, mixed with cobalt speiss, in the proportion of about 7 per cent. upon the average, and is procured by means of a peculiar furnace of lirguation, which is the most economical method, both as to saving fuel, and oxydizement of the bismuth.

The bismuth eliquation furnace at Schneeberg is rêpresented in figs. 112, 113, and 114, of which the first is a view from above, the second a view in front, and the third a transverse section in the dotted line A B of fig. 112. $a$ is the ash-pit; $b$, the findor; quation pupes; $d$, the grate of masonry or brickwork, upon which the fublfintswint throngh the fire-door $e e$. The anterior deeper lying orifice of the eliquation pipes is closed with the clay-plate $f$; which has beneath a small circular groove, through which the liquefici metal flows off. $g$ is a wall extending from the hearth-sole mearly to the anterior orifice of the eliquation pipes, in which wall there are as many fuckoles, $h$, as there are pipes in the furnace: $i$ are iron pans, which receive the fluid metal; $h$, a wooden water- 
trough, in which the bismuth is granulated and cooled; $l$, the posterior and higher lying apertures of the eliquation pipes, shut merely with a sheet-iron cover. The granulations of bismuth drained from the posterior openings fall upon the flat surfaces $m$, and then
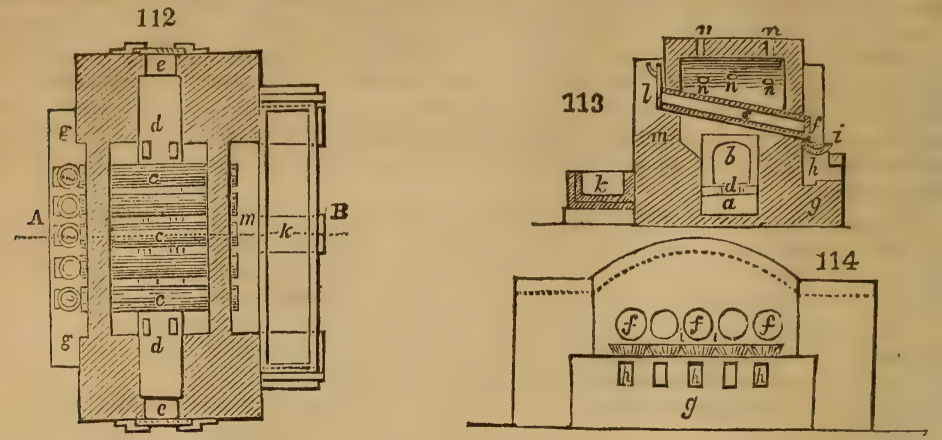

into the water-trough. $n n$ are draught-holes in the vault between the two pipes, which serve for increasing or diminishing the heat at pleasure.

The ores to be eliquated (sweated) are sorted by hand from the gangue, broken into pieces about the size of a hazel nut, and introduced into the ignited pipes; one charge consisting of about $\frac{1}{2} \mathrm{cwt}$. ; so that the pipes are filled to half their diameter, and three fourths of their length. The sheet-iron door is shut, and the fire strongly urged, whereby the bismuth begins to flow in ten minutes, and falls through the holes in the clay-plates into hot pans containing some coal-dust. Whenever it runs slowly, the ore is stirred round in the pipes, at intervals during half an hour, in which time the liquation is usually finished. The residuum, called bismuth barley (graupen), is scooped out with iron rakes into a water trough; the pipes are charged afresh ; the pans, when full, have their contents cast into moulds, forming bars of from 25 to 50 pounds weight. Abont 20 cwt. of ore are smelted in 8 hours, with a consumption of 63 Leipzic cubic feet of wood. The total production of Shneeberg, in 1830, was $9800 \mathrm{lbs}$. The bismuth thus procured by liquation upon the great scale, contains no small admixture of arsenic, iron, and some other metals, from which it may be freed by solution in nitric acid, precipitation by water, and reduction of the subnitrated oxyde by black flux. By exposing the crude bismuth for some time to a dall red heat, under charcoal, arsenic is expelled.

Bismuth is white, and resembles antimony, but has a reddish tint; whereas the latter metal has a blueish cast. It is brilliant, crystallizes readily in small cubical facets, is very brittle, and may be easily reduced to powder. Its specific gravity is 9.83 ; and by hammering it with care, the density may be increased to 9.8827 . It melts at $480^{\circ} \mathrm{Fahr}$., and may be cooled 6 or 7 degrees below this point without fixing; but the moment it begins to solidify, the temperature rises to $480^{\circ}$, and continues stationary till the whole mass is congealed. When heated from $32^{\circ}$ to $212^{\circ}$, it expands $\frac{1}{71} 0$ in length. When pure it affords a very valuable means of adjusting the scale of high-ranged thermometers. At strong heats bismuth volatilizes, may be distilled in close vessels, and is thus obtained in crystalline laminæ.

The alloy of bismuth and lead in equal parts has a density of $10 \cdot 709$, being greater than the mean of the constituents; it has a foliated texture, is brittle, and of the same color as bismuth. Bismuth, with tin, forms a compound more elastic and sonorous than the tin itself, and is therefore frequently added to it by the pewterers. With 1 of bismuth and 24 of tin, the alloy is somewhat malleable; with more bismuth, it is brittle. When much bismuth is present, it may be easily parted by strong muriatic acid, which dissolves the tin, and leaves the bismuth in a black powder. It has been said, that an alloy of tin, bismuth, nickel, and silver hinders iron from rusting. (Erdmann's Journal.) The alloy of bismuth with tin and lead was first examined by Sir I. Newton, and has been called ever since fusible metal. Eight parts of bismuth, 5 of lead, and 3 of tin, melt at the moderate temperature of $202^{\circ} \mathrm{F}$.; but 2 of bismuth, 1 of lead, and 1 of tin, melt at $200 \cdot 75^{\circ} \mathrm{F}$. according to Rose. A small addition of mercury of course aids the fusibility. Such alloys serve to take casts of anatomical preparations. An alloy of 1 bismuth, 2 tin, and 1 lead, is employed as a soft solder by the pewterers; and the same has been proposed as a bath for tempering steel instruments. Cake-moulds, for the manufacturers of toilet soaps, are made of the same metal; as also excellent clichés for stereotype, of 3 lead, 2 tin, and 5 bismuth; an alloy which melts at $199^{\circ} \mathrm{F}$. This compound should be allowed to cool upon a piece of pasteboard, till it becomes of a doughy consistence, before it is applied to the mould, to receive the impress of the stamp. 
The employment of plates of fusible metal as safety rondelles, to apertures in the tons of steam boilers, has been proposed in France, because they would inelt and give way at elevations of temperature under those which would endanger the bursting of the vessel; the fusibility of the alloy being proportioned to the quality of steam required for the engine. It has been found, however, that boilers, apparently secured in this way, burst, while the safety dises remained entire; the expansive force of the steam causing explosion so suddenly, that the fusible alloy had not time to melt or give way.

There are two, perhaps three, oxydes of bismuth; the first and the third, or the suboxyde and super-oxyde, are merely objects of chemical curiosity. The oxyde proper occurs native, and may be readily formed by exposing the metal to a red-white heat in a muffle, when it takes fire, burns with a faint blue flame, and sends off fumes which condense into a yellow pulverulent oxyde. But an easier process than that now mentioned is to dissolve the bismuth in nitric acid, precipitate with water, and expose the precipitate to a red heat. The oxyde thus obtained has a straw yellow color, and fuses at a high heat into an opaque glass of a dark-brown or black color; but which becomes less opaque and yellow after it has cooled. Its specific gravity is so high as $8 \cdot 211$. It consists of 89.87 of metal and 10.13 oxygen in 100 parts. The above precipitate, which is a sub-nitrate of bismuth, is called pearl-white, and is employed as a flux for certain enamels; as it augments their fusibility without imparting any color to them. Hence, it is used sometimes as a vehicle of the colors of other metallic oxydes. When well washed, it is employed in gilding porcelain; being added in the proportion of one fifteenth to the gold. But pearl-white is most used by ladies as a cosmetic for giving a brilliant tint to a faded complexion. It is called blanc de fard, by the French. If it contains, as bismuth often does, a little silver, it becomes gray or dingy colored on exposure to light. When the oxyde is prepared, by dropping the nitric solution into an alkaline ley in excess, if this precipitate is well washed and dried, it forms an excellent medicine; and is given, mixed with gum tragacanth, for the relief of cardialgia, or burning and spasmodic pains of the stomach.

Another sort of pearl-powder is prepared by adding a very dilute solution of common salt to the above nitric solution of bismuth, whereby a pulverulent sub-chloride of the metal is obtained in a light flocculent form. A similar powder of a mother-of-pearl aspect may be formed by dropping dilute muriatic acid into the solution of nitrate of bismuth. The arsenic always present in the bismuth of commerce is converted by nitric acid into arsenic acid, which, forming an insoluble arseniate of bismuth, separates from the solution, unless there be such an excess of nitric acid as to re-dissolve it. Hence the medicinal oxyde, prepared from a rightly-made nitrate, can contain no arsenic. If we write with a pen dipped in that solution, the dry invisible traces will become legible on plunging the paper in water.

It has been proposed to substitute bismuth for lead in assaying silver, as a smaller quantity of it answers the purpose, and, as its oxyde is more fluent, can therefore penetrate the cupel more readily, and give a more rapid result. But, independently of the objection from its high price, bismuth has the disadvantage of boiling up, as well as of rocking or vegetating, with the silver, when the cupellation requires a high heat. In extracting the silver from the galena found in the copper-mine of Yahlun, it has happened sometimes that the silver concreted towards the end of the operation, and produced a cauliflower excrescence, which had to be cupelled again with a fresh dose of lead. It was observed that, in this case, a portion of the silver had passed into the cupel. Berzelius detected in a sample of silver thus concreted the presence of bismuth.

The nitrate of bismuth, mixed with solution of tin and tartar, has been employed as a mordant for dyeing lilach and violet in calico printing.

BISTRE. (Bistre, Fr.; bister, Germ.) A brown color which is used in water colors, in the same way as China ink. It is prepared from wood-soot, that of beech being preferred. The most compact and best burned parcels of soot are collected from the chimney, pulverized, and passed through a silk sieve. This powder is infused in pure water, and stirred frequently with a glass ruler, then allowed to settle, when the water is decanted. If the salts are not all washed away, the process may be repeated with warm water. The paste is now to be poured into a long narrow vessel filled with water, stirred well, and left to settle for a few minutes, in order to let the grosser parts subside. The supernatant part is then to be poured off into a similar vessel. This process may be repeated twice or thrice, to obtain a very good bistre. At last the settled deposite is sufficiently fine, and when freed from its supernatant water, it is mixed with gum-water, moulded into proper cakes, and dried. It is not used in oil painting, but has the same effect in water-colors as brown pink has in oil.

BITUMEN, or ASPHALTUM. (Bitume, Fr.; Erdpech, Germ.) A black substance found in the earth, externally not dissimilar to pit-coal. It is composed of carbon, hydrogen, and oxygen, like organic bodies; but its origin is unknown. It 
has not been observed among the primitive or older strata, but only in the secondary and alluvial furmations. It constitutes sometimes considerable beds, as in the isle of Trinidad, where it occurs over an extensive district, in scattered masses. The greater part of the asphaltum to be met with in commerce comes from the Dead Sea, on whose shores it is cast up and gathered; whence it has got the name of Jewish bitumen. In its black color and fracture it resembles ordinary pitch. By friction it affords ncgative electricity. Its average density is $1 \cdot 16$. It melts at the temperature of boiling water, kindles very readily at the flame, burns brightly with a thick smoke, and leaves little ashes. Distilled by itself, it yıelds a peculiar bituminous oil, very little water, some combustible gases, and traces of ammonja. It leaves about one third of its weight of charcoal after combustion, and ashes, containing silica, alumina, oxyde of iron, sometimes a little lime, and oxyde of manganese. According to John, asphaltum may be decomposed, by different solvents, into three distinct substances. Water dissolres nothing; alcohol (anhydrous) dissolves out a yellow resin equal to 5 per cent. of the meight of the asphaltum; that resin is soluble in dilute alcohol and in ether. The portion not soluble in the alcohol gires up a brown resin to ether, amounting to 70 per cent. of the weight of the asphaltum. On evaporating off the ether, the resin remains of a brownish-black color, which dissolves readily in the rolatile oils, and in the oil of petroleum. The portion of asphaltum which does not dissolve in ether is very soluble in oil of turpentine, and in oil of petroleum; but less so in oil of lavender. These three resinous principles dissolve all together by digestion in the oils of anise, rosemary, turpentine, olire, hemp-seed, nut, and linseed. Caustic potash dissolves a notable quantity of asphaltum; but carbonate of potash has no effect upon it.

Asphaltum enters into the composition of hydraulic cements, and into that of black varnishes, called japans, for coating iron trays, \&c. A similar varnish may be prepared by dissolving 12 parts of fused amber, 2 parts of rosin, and 2 parts of asphaltum, in 6 parts of linseed oil varnish, to which 12 parts of oil of turpentine have been added.

There is a kind of bitumen found at Aniches, in France, in the department of the north, which is black, very fusible, and soft. It burns with flame. Alcohol, ether, and oil of turpentine extract from it a fatty substance, which may be saponified with allikalis.

The bitumen of Murindò, near Choco, in Columbia, is of a brownish-blaci color, soft, and has an earthy fracture. It has an acrid taste, burns with a smell of vanilla, and is said to contain a large quantity of benzoic acid. It appears to be the result of the decomposition of trees containing benzoin.

Asphaltum occurs abundantly at the surface of the salt lake Asphaltites, in Judea, produced from springs in the neighborhood; it is floated down, gathers consistence and accumulates upon the surface of the lake; the winds drive it on the shores, and the inhabitants collect it for sale. Its inspissation diffuses a disagreeable smell in the air of that region, which is supposed by the natives to be powerful enough to kill birds when they attempt to fly across the lake.

But probably the most remarkable locality of asphaltum in the world is the entire basin, or rather plain of it, in the island of Trinidad, called the Tar Lake. It lies on the highest land in the island, and emits a strong smell, sensible at ten miles' distance. Its first appearance is that of a lake of water, but when viewed more nearly, it seems to be a surface of glass. In hot weather its surface liquefies to the depth of an inch, and it cannot then be walked upon. It is of a circular form, about three miles in circumference, and of a depth not ascertained. Large fissures frequently open and close up in it, whence the pitch has been supposed to float upon a body of water. The soil, for a considerable distance round it, consists of cinders and burnt earth, and presents in many points indications of contulsions by subterranean fire. In several parts of the neighboring woods, there are round holes and fissures in the ground, containing liquid bitumen to the depth of two inches.

Mr. Hatchett examined some specimens from Trinidad, and concluded that what had been heretofore supposed to be a pure mineral pitch was in reality only a porous stone of the argillaceous kind, much impregnated with bitumen.

These rarious bitumens belong exclusively to the secondary and tertiary geological formations, and are not found among primitive rocks, except very rarely in veins. They occur most generally in calcareous, argillaceous, and sandy strata, and also in volcanic districts. Petroleum frequently floats on the waters which issue from the volcanic mountains, or which lie at their base; even the sea is at times covered with it near the rolcanic islands of Cape Verd. Mr. Breislack observed a petroleum spring rising from the bottom of the sea near the southern base of Vesuvius.

The substance with which bitumen seems to have the most constant and most remarkable relations, is sea-salt; so that almost all the countries most abundant in petroleum, as Italy - Transylvania, Persia, the envirnns of Babylon, the region of the Dead Sea, \&c., contain salt mines, or lakes, or exhibit saline efflorescences. Iron pyrites is often impregnated with petroleum, or contains a bituminous nucleus. 
The origin of bitumen is as little known as that of most of the productions of nature. Some regard it as an empyreumatic oil, a matter analogous to liquid resin os essential oil, resulting from the destruction of that astonishing multitude of animals and regetables buried in the earth, whose solid remains are daily brought to view in mineral researches. It has been also supposed that naphtha and petroleum are the product of coals decomposed either by the fire of volcanoes, by the subterranean combustion of coal itself, or by the decomposition of pyrites. The latter opinion is not supported by any direct evidence, but the two former are sufficiently probable.

Elastic Bitumen is a rare substance, found hitherto only near Castleton, in Derbyehire, in fissures of slaty clay.

Bituminous mastic, or cement, has been of late extensively employed in France for sot ering roofs and terraces, and lining water cisterns. The mineral bitumen used for the composition of this mastic is procured chiefly from the Obsanr (Bas-Rhin), from the Parc (department de l'Ain), and from the Puy-de-la-Poix (department of Puy-deDome.) But boiled coal-tar answers equally well. In the neighborbood of these localities, there is a limestone impregnated with bitumen, which suits for giving consistence to the cement. This is well dricd, ground to powder, sifted, and stirred while hot, in about one fifth its weight of melted asphaltum, contained in a cast-iron boiler. Dry chalk or briclis, ground and sifted, will suit equally well. As soon as this paste is made quite homogeneous, it is lifted out with an iron shovel or spoon, and spread in rectangular moulds, secured with pegs at the joints, fastened to a kind of platform of smoothed planks, covered with strong sheet-iron. The sides of these moulds should be previously smeared over with a thin coat of loam-paste, to prevent their adhesion to the mastic. Whenever the cake is cold, the frame is taken asunder, and it is removed from the iron plate by an oblong shovel, or strong spatula of iron. These cakes or bricks are usually 18 inches long, 12 broad, and 4 thick, and weigh about $70 \mathrm{lbs}$.

BI ¿TER PRINCIPLE. (Amère, Fr.; Bitterstoff, Germ.) This principle has not been insulated hitherto by the chemist from the other proximate principles of plants, but its existence is sufficiently reconised by the taste. The following list contains the principal bitter substances, many of which have been used in the arts and in medicine.

\begin{tabular}{|c|c|c|c|}
\hline Name. & Part employed. & Country. & Observations. \\
\hline $\begin{array}{l}\text { Quassia } \\
\text { Wormwood } \\
\text { Aloe } \\
\text { Angustura } \\
\text { Orange } \\
\text { Ditto } \\
\text { Acorus } \\
\text { Carduus Benedictus } \\
\text { Cascarilla } \\
\text { Centaury } \\
\text { Chamomile } \\
\text { Colocynth } \\
\text { Colombo } \\
\text { Fumitory } \\
\text { Gentiana lutea } \\
\text { Ground Ivy } \\
\text { Walnut } \\
\text { Island moss }\end{array}$ & $\begin{array}{l}\text { Wood } \\
\text { Herb } \\
\text { Inspissated juice } \\
\text { Bark } \\
\text { Unripe fruit } \\
\text { Peel } \\
\text { Root } \\
\text { Herb } \\
\text { Bark } \\
\text { Herb } \\
\text { Flowers } \\
\text { Fruit } \\
\text { Root } \\
\text { Herb } \\
\text { Root } \\
\text { Herb } \\
\text { Peels }\end{array}$ & $\begin{array}{l}\text { Surinam, East Indies } \\
\text { Great Britain } \\
\text { South Africa } \\
\text { South America } \\
\text { South of Europe } \\
\text { Ditto } \\
\text { Ditto } \\
\text { Greek Archipelago } \\
\text { Jamaica } \\
\text { Great Britain } \\
\\
\text { Levant } \\
\text { East Africa } \\
\text { Great Britain } \\
\text { Switzerland } \\
\text { Great Britain }\end{array}$ & $\begin{array}{l}\text { Powerfully bitter } \\
\text { Ditto } \\
\text { Ditto } \\
\text { Ditto } \\
\text { Aromatic bitter } \\
\text { Ditto } \\
\text { Ditto } \\
\text { Intolerably bitter } \\
\text { Very bitter } \\
\text { Very bitter } \\
\text { With tannin } \\
\text { With starch }\end{array}$ \\
\hline Hops & $\left.\begin{array}{c}\text { Scales of the fe- } \\
\text { male flowers }\end{array}\right\}$ & Great Britain & Aromatic bitters \\
\hline $\begin{array}{l}\text { Milfoil } \\
\text { Large-leaved Satyrion } \\
\text { Rhubarb } \\
\text { Rue } \\
\text { Tansy } \\
\text { Bitter trefoil } \\
\text { Simarouba }\end{array}$ & $\begin{array}{l}\text { Herbs flowers } \\
\text { Herb } \\
\text { Root } \\
\text { Herb } \\
\text { Herb flowers } \\
\text { Herb } \\
\text { Bark }\end{array}$ & $\begin{array}{l}\text { Great Britain } \\
\text { Great Britain } \\
\text { China } \\
\text { Great Britain } \\
\text { Ditto } \\
\text { Ditto } \\
\text { Guyans }\end{array}$ & $\begin{array}{l}\text { Disagreeable odor } \\
\text { Bitter and sharp } \\
\text { Bitter and offensive }\end{array}$ \\
\hline Bryony & Root & Great Britain & $\left\{\begin{array}{r}\text { Sharp, bitter, } \\
\text { nauseous }\end{array}\right.$ \\
\hline Coffee & Seeds & Arabia & \\
\hline
\end{tabular}

BLACK DYE. (Teinte noire, Fr.; Schwartze farbe, Germ.) For 1 cwt. of cloth, there gre pul into a boiler of middle size $18 \mathrm{lbs}$. of logwood, with as much Aleppo galls in 
powder, and the whole, being enclosed in a bag, is boiled in a sufficient quantity of water for 12 hours. One third of this bath is translerred into another boiler with two pounds of verdigris; and the stuff is passed through this solution, st rring it continually during two hours, taking care to keep the bath very hot without boiling. The stuff is then lifted out, another third of the bath is added to the boiler, along with eight pounds of sulphate of iron or green vitriol. The fire is to be lowered while the sulphate dis. solves, and the bath is allowed to cool for half an hour, after which the stufl is intruduced, and well moved about for an hour, after which it is taken out to air. Lastly, the remaining third of the bath is added to the other two, taking care to squeeze the bag well. 18 or $22 \mathrm{lbs}$. of sumach are thrown in; the whole is just brought to a boil, and then refreshed with a little cold water; two pounds more of sulphate of iron are added, after which the stuff is turned through for an hour. It is thereafter washed, aired, and put again into the bath, stirring it continually for an hour. After this, it is carried to the river, washed well, and then filled. Whenever the water runs off clear, a bath is prepared with weld, which is made to boil for an instant; and after refieshing the bath the stuff is turned in to soften, and to render the black more fast. In this manner, a very beautiful black is obtained, without rendering the eloth too harsh.

Commonly more simple processes are employed. Thus the blue cloth is simply turned through a bath of gall-nuts, where it is boiled for two hours. It is next passed through a bath of logwood and sulphate of iron for two hours, without boiling, after which it is washed and fulled.

Hellot has found that the dyeing might be performed in the following manner :-For 20 yards of dark blue cloth, a bath is made of two pounds of fustic (morus tinctoria), $4 \frac{1}{4} \mathrm{lbs}$. of logrood, and $11 \mathrm{lbs}$. sumach. After boiling the cloth in it for three hours it is lifted out, $11 \mathrm{lbs}$. of sulphate of iron are thrown into the boiler, and the cloth is then passed through it during two hours. It is now aired, and put again in the bath ior an hour. It is, lastly, washed and scoured. The black is less relvety than that of the preceding process. Experience convinced him that the maddering prescribed in the ancient regulations only gives a reddish cast to the black, which is obtained finer and more velvety without madder.

A black may be dyed likewise without having given a blue ground. This method is employed for cloths of little value. In this case they are rooted; that is to say, they receive a dun ground with walnut husks, or the root of the walnut-tree, and are afterwards made black in the manner above described, or in some other way; for it is obvious that a black may be obtained by several processes.

According to Lewis, the proportions which the English dyers most generally adopt are, for one hundred and twelve pounds of woollen cloth previously dyed of a dark blue, about five poinds of sulphate of iron, as much gall-nuts, and thiriy pounds of logwood. They begin by zalling the cloth, they then pass it through the decoction of lngwood, to which the sulphate of iron has been added.

When the cloth is completely dyed, it is washed in the rirer, and passed through the fulling-mill till the water runs off clear and colorless. Some persons recommend, for fine cloths, to full them with soap water. This operation requires an expert workman, who can free the cloth thoroughly from the soap. Several recommend at its coming from the fulling to pass the cloth through a bath of weld, with the view of giving soft. ness and solidity to the black. Lewis says, that passing the cloth through weld, after it has been treated with soap, is absolutely useless, although it may be beneficial when this operation has been neglected.

Different operations may be distinguished in dyeing silk black; the boiling of the silk, its galling, the preparation of the bath, the operation of dyeing, the softening of the black.

Silk naturally contains a substance called gum, which gives it the stiffness and elasticity peculiar to it in its native state; but this adds nothing to the strength of the silk, which is then styled raw; it rather renders it, indeed, more apt to wear out by the stiffness which it communicates; and although raw silk more readily takes a black color, yet the black is not so perfect in intensity, nor does it so well resist the re-agents capable of dissolving the coloring particles, as silk which is scoured or deprived of its gum.

To cleanse silk intended for black, it is usually boiled four or five hours with one fifth of its weight of white soap, after which it is carefully beetled and washed.

For the galling, nut-galls equal nearly to three fourths of the weight of the silk are boiled during three or four hours; but on account of the price of Aleppo galls, more or less of the white gall-nuts, or of even an inferior kind called galon, berry or apple galls, are used. The proportion commonly employed at Paris is two parts of Aleppo galls to from eight to ten parts of galon. After the boiling, the galls are allowed to settle foi about two hours. The silk is then plunged into the bath, and left in it from twelve to thirty-six hours, after which it is taken out and washed in the river.

Silk is capable of combining with quantities, more or less considerable, of the astrin- 
gent principle; whence results a considerable increase of weight, not only from the weight of the astringent principle, but also from that of the coloring particles, which subsequently fix themselves in proportion to the quantity of the astringent principle which had entered into combination. Consequently the processes are varied according to the degrec of weight which it is wished to communicate to the silk; a circumstance requiring some illustration.

The commerce of silk goods is carried on in two ways; they are sold either by the weight, or by the surface, that is, by measure. Thus the trade of Tours was formerly distinguished from that of Lyons; the sills of the former being sold by weight, those of the latter, by measure. It was therefore their interest to surcharge the weight at Tours, and, on the contrary, to be sparing of the dyeing ingredients at Lyons; whence came the distinction of light black and heavy black. At present, both methods of dyeing are practised at Lyons, the two modes of sale having been adopted there.

Silk loses nearly a fourth of its weight by a thorough boiling, and it resumes, in the light black dye, one half of this loss; but in the heavy black dye, it talies sometimes upwards of a fifth more than its primitive weight; a surcharge injurious to the beauty of the black, and the durability of the stuff. The surcharged kind is denominated English black, because it is pretended that it was first practised in England. Since silk dyed with a great surcharge has not a beautiful black, it is usually destined for weft, and is blended with a warp dyed of a fine black.

The peculiarity of the process for obtaining the heavy black consists in leaving the silk longer in the gall liquor, in repeating the galling, in passing the sillk a greater number of times through the dye, and even letting it lie in it for some time. The first galling is usually made with galls which have served for a preceding operation, and fresh gall-nuts are employed for the second. But these methods would not be sufficient for giving a great surcharge, such as is found in what is called the English black. To give it this weight, the silk is galled without being ungummed; and, on coming out of the galls, it is rendered supple by being worked on the jack and pin.

The silk-dyers keep a black vat, and its very complex composition varies in different dye-houses. These vats are commonly established for many years : and when their black dye is exhausted it is renovated by what is called in France a brevet. When the deposite which has accumulated in it is too great, it is taken out, so that at the end of a certain time nothing remains of the several ingredients which composed the primitive bath, but which are not employed in the brevet.

For the dyeing of raw silk black, it is galled in the cold, with the bath of galls which has already served for the black of boiled silk. For this purpose, silk, in its native yellow color, is made choice of. It shonld be remarked, that when it is desired to preserve a portion of the gum of the silk, which is afterwards made flexible, the galling is given with the hot bath of gall nuts in the ordinary manner. But here, where the whole gum of the silk, and its concomitant elasticity, are to be preserved, the galling is made in the cold. If the infusion of galls be weak, the silk is left in it for several days.

Silk thus prepared and washed takes very easily the black dye, and the rinsing in a little water, to which sulphate of iron may be added, is sufficient to give it. The dye is made in the cold; but, according to the greater or less strength of the rinsings, it requires more or less time. Occasionally three or four days are necessary; after which it is washed, it is beetled once or twice, and it is then dried without wringing, to avoid softening it.

Raw silk may be more quickly dyed, by shaking it round the rods in the cold bath after the gaising, airing it, and repeating these manipulations several times, after which it is washed and dried as above.

Macquer describes a more simple process for the black by which velvet is dyed at Genoa; and he says that this process, rendered still simpler, has had complete success at Tours. The following is his description.

For $\hat{i}$ cwt. (50 kilogrammes) silk, 22 lbs. (11 kilogrammes) of Aleppo galls, in powder, are bolled for an hour in a sufficient quantity of water. The bath is allowed to settle till the galls have fallen to the bottom of the boiler, from which they are withdrawn; after which $32 \mathrm{lbs}$. of English vitriol (or copperas) are introduced, with $13 \mathrm{lbs}$. of iron filings, and $22 \mathrm{lbs}$. of country gum, put into a kind of two-handled cullender, pierced every where with holes. This kettle is suspended by two rods in the boiler, so as not to reach the bottom. The gum is left to dissolve for about an hour, stirring it from time to time. If, after this time, some gum remains in the kettle, it is a proof that the bath, which contains two hogsheads, has taken as much of it as is necessary. If, on the contrary, the whole gum is dissolved, from one to $4 \mathrm{lbs}$. more may be added. This cullender is left constantly suspended in the boiler, from which it is removed only when the dyeing is going on; and thereafter it is replaced. During all these operations the boiler must be kept hot, but without boiling. The galling of the silk is performed with one 
third of Aleppo galls. The silk is left in it for six hours the first tıme, then for twelve hours. The rest, secundum artem.

Lewis states that he has repeated this process in the small way; and that by adding sulphate of iron progressively, and repeating the immersions of the silk a great number of times, he eventually obtained a fine black.

Astringents differ from nne another as to the quantity of the principle which enters into combination with the oxyde of iron. Hence, the proportion of the sulphate, or of any other salt of iron, and that of the astringents, should vary according to the astringents made use of, and according to their respectire quantities. Gall-nut is the sulstance which contains most astringent; sumach, which seems second to it in this respect, throws down (decomposes), however, only half as much sulphate of iron.

The most suitable proportion of sulphate of iron appears to be that which corresponds to the quantity of the astringent matter, so that the whole iron nrecipitable by the astringent may be thrown down, and the whole astringent may be taken up in combination with the iron. As it is not possible, however, to arrive at such precision, it is better that the sulphate of iron should predominate, because the astringent, when in excess, counteracts the precipitation of the black coloring particles, and has the property of even dissolving them.

This action of the astringent is such that, if a pattern of black eloth be boiled with gallnuts, it is reducible to gray. An observation of Lewis may thence be explained. If cloth be turned several times through the coloring bath, after it has taken a good black color, instead of acquiring more body, it is weakened, and becomes brownish. Too consilerable a quantity of the ingredients produces the same effect; to which the sulphuric acid, set at liberty by the precipitation of the oxyde of iron, contributes.

It is merely the highly oxydized sulphate which is decomposed by the astringent; whence it appears, that the sulphate will produce a different effect according to its state of oxydizement, and call for other proportions. Some advise, therefore, to follow the method of Proust, employing it in the oxydized state ; but in this case it is only partially decomposed, and another part is brought, by the action of the astringent, into the lower degree of oxydizement.

The particles precipitated by the mixture of an astringent and sulphate of iron have not at first a deep color; but they pass to a black by contact of air while they are moist.

Under dyeing I shall show that the black dye is only a very condensed color, and that it assumes more intensity from the mixture of different colors likewise deep. It is for this reason advantageous to unite several astringents, each combination of which produces a different shade. But blue appears the color most conducive to this effect, and it corrects the tendency to dun, which is remarked in the black produced on stuffs by the other astringents.

On this property is founded the practice of giving a blue ground to black eloths, which acquire more beauty and solidity the deeper the blue. Another advantage of this practice is to diminish the quantity of sulphuric acid which is necessarily disengaged by the precipitation of the black particles, and which would not only counteract their fixation, but would further weaken the stuff, and give it harshness.

For common stuffs, a portion of the effect of the blue ground is produced by the rooting.

The mixture of logrvood with astringents contributes to the beauty of the black in a twofold way. It produces molecules of a hue different from what the astringents do, and particularly blue molecules, with the oxyde of copper, commonly employed in the black dyes; which appears to be more useful the more acetate the verdigris made use of contains.

The boil of weld, by which the dye of black cloth is frequently finished, may also contribute to its beauty, by the shade peculiar to its combination. It has, moreover, the advantage of giving softness to the stufis.

The processes that are employed for wool, yield, according to the observation of Levis, only a rusty black to silk; and cotton is hardly dyed by the processes proper for wool and silk. Let us endeavor to ascertain the conditions which these three varieties of dyeing demand.

Wool has a great tendency to combine with coloring substances; but its physical nature requires its combinations to be made in general at a high temperature. The combination of the black molecules may therefore be directly effected in a bath, in proportion as they form; and if the operation be prolonged by subdividing it, it is only with the view of changing the necessary oxydizement of the sulphate, and augmenting that of the coloring particles themselves.

Silk has little disposition to unite with the black particles. It seems to be merely by the ageney of the tannin, 'vith which it is previously impregnated, that these particles can fix themselves on $i^{*}$, especially after it has been scoured. For this reason, silk baths 
should be old, and have the coloring particles accumulated in them, but so feebly suspended as to yield to a weak affinity. Their precipitation is counteracted by the addition of gum, or other mucilaginous substances. The obstacle which might arise from the sulphuric acid set at liberty is destroyed by iron filings, or other basis. Thus, baths of a very different composition, but with the essential condition of age, may be proper for this dye. For cotton black dye, see Calico Printing.

BLACK PIGMENT. The finest light black is prepared principaïy for the manufacturing of printers' ink. In Messrs. Martin and Grafton's patent process, the black is obtained by burning common coal-tar, which should, however, be previously divested, as much as possible, of the ammoniacal liquor and acid mixed with it in the tank.

For this purpose, it is proposed that forr casks should be employed, each capable of holding 130 gallons, and into every one of them are to be put about 60 gallons of the rough impure tar, to which an equal quantity of lime-water is to be added, and then agitated by machinery or manual labor until the lime-water is completely mixed with the tar. The vessels should next be suffered to rest for about six hours, by which time the tar will settle at the bottom of the casks, and the water may be drawn off. The casks containing the tar should now be filled with hot water, which may be supplied from the boiler of a steam engine, and the whole again agitated as before. This process may be repeated three times, suffering the tar to subside between each; and twelve hours should be allowed for settling from the last water, so that the whole of the tar and water may become separated, the water rising to the top of the cask, and the tar being left at the bottom in a pure state.

But, as some of the water will yet remain mechanically combined with the tar, it is proposed that the tar should be subjected to the process of distillation. For this purpose, a still, capable of holding 120 gallons, may be employed, in which about 50 gallons at one time may be operated upon; when, by a gentle heat, the water, and other impurities which the tar may have retained, will be driven off. As soon as the water appears to have evaporated, and the spirit runs fine and clear, the process of distillation skould be stopped; and, when cold, the pure tar may be drawn off, and set apart for the purpose of being employed as contemplated in the patent.

The tar thus purified may be now converted into black, or it may be subjected to further rectification to divest it of the mineral pitch, or asphaltum, which is combined with the oil and spirit: the latter is to be preferred, because the mineral pitch, or asphaltum, is only inflammable at a high temperature, which renders it more troublesome to use in the process here contemplated, and also would cause the apparatus to require frequent cleaning from the carbonized pitch deposited. In order, therefore, to get rid of the mineral pitch, or asphaltum, forty gallons of the tar are to be introduced into a still, as before; and, instead of stopping the operation, as soon as the spirit begins to come over, the distillation is continued with a strong heat, so as to force over the whole of the oil and spirit, leaving the' residuum of asphaltum in the still; this process, however, is known to every chemist, and need not be further explained.

In fig. 115 , is exhibited a rude representation of the apparatus employed in preparing and collecting the fine light spirit black, produced by the combustion of the oil and

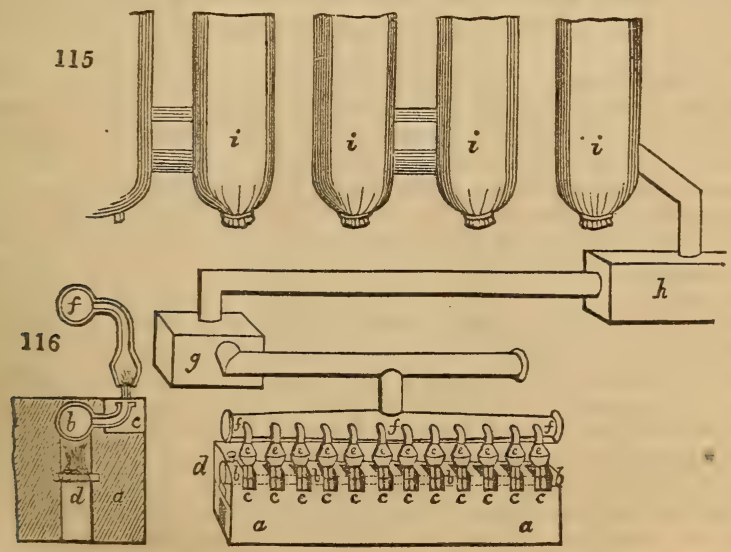

mptrit of coal-tar, after it has been purified as above described. a is the briclimurk which supports a number of burners issuing from a tube, $b$, within, and here shown bj 
dots, as passing along its whole length. Frg. 116 is a section of the brickwork, with the tube, burner, and receiver, as will be described hereafter. The tube may be called the tar main, as it is intended to be filled with tar: it is constructed of cast iron, and from it issue several (in this figure twenty-four) :ets or burners, $c, c, c$; any other numbur may be employed. $d$ is a furnace under the tar main, the flue of which extends along, for the purpose of heating the tar to the boiling point, in order to facilitate the piocess. From the main, $b$, the tar flows into the jets $c$; wicks are introduced into the jets, and, when set fire to, by a red-hot stick, will burn and emit a very considerable quantity of smoke; which it is the object of this apparatus to conduct through many passages, for the purpose of collecting its sooty particles.

There are a number of hoods, $e, e, e$, or bonnets, as they are termed, all of which, through their pipes, have communication with, or lead into a main chimney, $f, f$. Into these hoods or bonnets the smoke of the burners ascends, and from thence passes into the main chimney $f$, and thence through the smoke tubes into the box $g$ : here the heaviest particles of the black deposite themselves; but, as the smoke passes on through the farther pipes, a deposite of the second, or finer, particles of black takes place in the box $h$. From hence the smoke proceeds through other pipes into a series of canvass bags, $i, i, i$, which are proposed to be about eighteen feet long, and three in diameter. These bags are connected together at top and bottom alternately, and through the whole series the smoke passes up one bag and down the next, depositing fine black, called spirit black, upon the sides of the canvass. After the jets have continued burning for several days, the bags are to be beaten with a stick, so that the black may fall to the bottom; and, when a sufficient quantity has accumulated, the bags may be ernptied and swept out. Thus seventy or eighty bags may be employed; so that the smoke should pass through a length of about 400 yards, the farthest of which will be found to contain the finest black. The last bag should be left open, in order to allow the vapor to escape into the open air.

The main tar tube will require to be emptied every four or five days, in order to clear it from the pitchy matter that may have subsided from the burners, and they also will require to be frequently poked with a wire, to clear off the black which forms upon the edges, and to drive down the carbonized tar which attaches itself to the upper part of the jets.

BLEACHING (Blanchiment, Fr.; Bleichen, Germ.) is the process by which the textiie filaments, cotton, flax, hemp, wool, silk, and the cloths made of them, as well as various vegetable and animal suinstances, are deprived of their natural color, and renilered nearly or altogether white. The term bleaching comes from the French verb blanchir, to whiten. The word blanch, which has the same origin, is applied to the whitening of living plants by making them grow in the dark, as when the stems of celery are covered over with mould.

The operations which the bleacher has recourse to differ according to the nature of the bleaching means, the property of - the stuff to be bleached, and local customs or circumstances; and the result is also obtained with more or less rapidity, certainty, economy, and perfection. The destruction of the coloring matters attached to the bodies to be bleached is effected either by the action of the air and light, of chlorine, or sulphurous acid; which may be considered the three bleaching powers employed for manufacturing purposes.

Bleaching by the influence of air and sunshine is the most ancient, and stin the most common, method in several civilized countries; it is also supposed by many to be the leas' injurious to the texture of yarn and cloth. The operations it involves are rery simple, consisting in the exposure of the goods upon a grass-plat to the sky, with their occasional aspersion with moisture if necessary, in addition to the rain and dew. The atmospheric air effects the bleaching by means of its oxygenous constituent, which sombines with the coloring matter, or its elements carbon and hydrogen, and either makes it nearly white, or converts it into a substance easily soluble in water and alkaline solutions. This natural process is too slow to suit the modern demands of the cotton and Jinen manufac urers. Fortunately for them, a new bleaching agent, unknown to our forefathers, has been discovered in chlorine, formerly called oxymuriatic acid, an agent modified by chemistry so as to give an astonishing degree of rapidity, economy, and perfection, to this important art. It is, however, not a little surprising, that the science which has so greatly advanced its practical part should have left its theory far from complete, and should affura no satisfactory answers to the two foliowing questions.-What is the action of the solar rays upon the coloring matter? How do air and chlorine operate upon this principle? Some suppose that light predisposes the coloring matter to combine with oxygen; others fancy that it acts merely in the manner of a high temperature, so as to determine a reaction between the elements of that substance, and to cause a new combination possessed of peculiar properties. It is generally admitted at the present day, that a portion of the oxygen of the air passes into the coloring matter, and changes its con. 
stitution. This is, however, probably not the part which oxygen plays, nor is it the only principle in ltie atmosphere which exercises a bleaching influence. Neither is the action of chlorine such as has been commonly represented in our chemical systems.

But if authors offer us only vague hypotheses concerning the three principal agents, light, oxygen, chlorine, they afford no information whatever concerning the phenomena due to greasy spots so frequently found upon cotton cloth, and so very troublesome to the bleacher. It has indeed been sometimes said in bleach-works, that fatty substances are no longer soluble in alkalis, when they are combined with oxygen. The very reverse of this statement is probably nearer the truth.

The object of bleaching is to separate from the textile fibre, by suitable operations, all the substances which mask its intrinsic whiteness : or which, in the course of ulterior dyeing operations, may produce injurious effects. In this latter respect, cotton deserves especial consideration. This substance is covered with a resinous matter, which obstructs its absorption of moisture, and with a yellow coloring matter in very small quantity, often so inconsiderable in some cottons, that it would be unnecessary to bleach them, before submitting them to the dyer, were it not that the manipulations which they undergo introduce certain impurities which are more or less injurious, and must be removed. It is in fact a circumstance well known in the factories, that unbleached cottons may be dyed any dark color, provided they are deprived of that matter which makes them difficult to moisten. The substances present in cotton goods are the following :-

1. The resinous matter natural to the cotton filaments.

2. The proper coloring matter of this vegetable.

3. The paste of the weaver.

4. A fat matter.

כ. A cupreous soap.

6. A calcareous soap.

7. The filth of the hands.

8. Iron, and some earthy substances.

1. The matter which prevents the moistening of cotton wool may be separated by means of alcohol, which, when evaporated, leaves thin yellowish scales, soluble in alkalis, in acids, and even in a large quantity of boiling water. For a long time the bleaching process commenced with the removal of this resinous stuff, by passing the cloth or the yarn through an alkaline ley. This was called scouring; it is now nearly laid aside.

2. The coloring matter of cotton seems to be superficial, and to have no influence on the strength of the fibres; for the yarn is found to be as strong after it has been stripped by caustic soda of its resinous and coloring matters, as it was before. The coloring matter is slightly soluble in water, and perfectly in alkaline leys. When gray calico is boiled in lime-water, it comes out with a tint darker than it had before ; whence it might be supposed that the coloring matter was not dissolved out, even in part. This, however, is not the case; for if we filter the liquor, and neutralize it with an acid, we shall perceive light flocks, formed of the resinous substance, united with the coloring matter. The dark color of the cloth is to be ascribed solely to the property which lime possesses of browning certain vegetable colors. This action is here exercised upon the remaining color of the cloth.

It may be laid down as a principle, that the coloring matter is not directly soluble by the alkalis; but that it becomes so only after having been for some time exposed to the joint action of air and light, or after having been in contact with chlorine. What change does it thereby experience, which gives it this solubility? Experiments made upon pieces of cloth placed in humid oxygen, in dry oxygen, in moist chlorine, and in dry chlorine, tend to show that hydrogen is abstracted by the atmosphere; for in these experiments proofs of dis-hydrogenation appeared, and of the production of carbonic acid. In all cases of bleaching by chlorine, this principle combines immediately with the hydrogen of the coloring matter, and forms muriatic acid, while the carbon is eliminated. Undoubtedly water has an influence upon this phenomenon, since the bleaching process is quicker with the humid chlorine than with the dry; but this liquid seems to act here only mechanically, in condensing the particles of the gas into a solution. We should also take into account the great affinity of muriatic acid for water.

3. The weaver's dressing is composed of farinaceous matters, which are usually allowed to sour before they are employed. It may contain glue, starch, gluten; which last is very soluble in lime-water.

4. When the dressing gets dry, the hand-weaver occasionally renders his warp-threads more pliant by rubbing some cheap kind of grease upon them. Hence it happens, that the cloth which has not been completely freed from this fatty matter will not readily imbibe water in the different bleaching operations; and hence, in the subsequent dyeing or dunging, these greasy spots, under peculiar circumstances, somewhat like lithosraphic stones, strongly attract the aluminous and iron mordants, as well as the dye-stuffo, and 
occasion stains which it is almost impossible to discharge. The acids act differently upon the fatty matters, and thence remarkable anomalies in bleaching take place. Whep oil is treated with the acetic or muriatic acid, or with aqueous chlorine, it evolves n. gas, as it does with the sulphuric and nitric acids, but it combines with these substances 80 as to form a compound which cannot be dissolved by a strong boiling ley of raustic soda. Carbonic acid acts in the same way with oil. On the other hand, when the oils and fats are sufficiently exposed to the air, they seize a portion of its oxygen, and become thereby capable of saponfication, that is, very soluble in the alkalis.

5. When the hand-weaver's grease continues in contact for a night with the copper dents of his reed, a kind of cupreous soap is formed, which is sometimes very difficult to remove from the web. Lime-water does not dissolve it; but dilute sulphuric acid carries off the metallic oxyde, and liberates the margaric acid, in a state ready to be acted on by alkalis.

6. When cloth is boiled with milk of lime, the grease which is uncombined unites with that alkaline earth; and forms a calcareous soap, pretty soluble in a great excess of lime-water, and still more so in caustic soda. But all fats and oils, as well as the soaps of copper and lime, cease to be suluble in alkaline leys, when they have remained a considerable time upon the goods, and have been in contact with acetic, carbonic, muriatic acids, or chlorine. These results have been verified by experiment.

7. Cotton goods are sometimes much soiled, from being sewed or tamboured with dirty hands; but they may be easily cleansed from this filth by hot water.

8. Any ferruginous or earthy matters which get attached to the goods in the course of bleaching, are readily remováble.

We are now prepared to understand the true principles of bleaching cotton goods, for the most delicate operations of the calico printer.

1. The first process is steeping, or rather boiling, the goods in water, in order to remove all the substances soluble in that liquid.

2. The next step is to wash or scour the goods by the dash-wheel or the stocks. This is of great importance in the course of bleaching, and must be repeated several times; so much so, that in winter, when the water of the dash-wheel is cold, the bleaching is more tedious and difficult. Yarn and very open fabrics do not much need the dashwheel.

By these first two operations, the woven goods lose about sixteen per cent. of their weight, while they lose only two parts out of five hundred in all the rest of the bleaching.

3. In the third place the calicoes are boiled with milk of lime, whereby they are stripped of their gluten, and acquire a portion of calcareous soap. Formerly, and still in many bleach-works, the gluten was got rid of by a species of fermentation of the farinaceous dressing; but this method is liable to several objections in reference to the calico printer. 1. The fermentative action extends sometimes to the goods and weakens their texture, especially when they are piled up in a great heap without being previously washed. 2. The spots of grease, or of the insoluble soaps, become thereby capable of resisting the caustic alkalis, and are rendered in some measure indelible; an effect due to the acetic and carbonic acids generated during fermentation, and which will be easily understood from what has been said concerning the action of acids on fatty substances. It is not, therefore, without good reason that many practical men throw some spent leys into the fermenting vats, to neutralize the acids which are formed. Were it not for the presence of fat, fermentation, skilfully conducted, would be an excellent means of carrying off the gluten; and the steep is therefore applicable to power-loom goods, which are not polluted with grease.

4. The goods are now subjected to a caustic soda ley, which dissolves out the soaps of lime and copper, as well as that portion of the coloring matter which is sufficiently dis-hydrogenated to be capable of combining with it. This bucking with ley, which is repeated several times upon the goods, in order to purge them completely from the fatty matter present in the hand-loom webs, and also partially introduced in the spinning, is almost the only operation to which yarns for Turkey red are subjected. After being boiled in a caustic soda ley, they are passed through solutions of chloride of hime, and afterwards through the acid steep.

5 . When the goods are sufficiently bucked in the leys, they are either exposed to chlorine, or laid out on the grass; sometimes both are had recourse to for delicate work. These different modes of action have the same influence on the coloring matter, but they give rise to different effects in reference to sreasy stains.

The goods are dipped in a solution of chloride of lime, which should be kept tepid by means of steam. Alongside of the chlorine eistern, there is another filled with dilute suiphuric or muriatic acid. When the goods are taken out of the chlorine, they are Jrained on the tep of its cistern till no more liquid runs off them, and they are then plunged into the sour. The action of the acid in the present case may be easily ex. 
plained. In proportion as a salt of lime is formed, this base quits the chlorine, and allows it to act fieely upon the coloring matter. Thus we prevent the development of two great a quantity of chlorine at once, which would be apt to injure the fibres; and we pursue both a prudent and economical plan. Only so much chlorine as is strictly necessary is called forth, and hence it excites no smell in the apartment.

The chlorine serves to acidify the coloring matter, by abstracting a portion of its hydrogen; but we must take the greatest care that there is no grease upon the goods before immersion in it, for the consequence would be, as above shown, very troublesome spots. When the cluth is laid out upon the grass, it is the oxygen of the air which acidifies the coloring matter; for which reason, the dew, which contains much air rich in oxygen, singularly accelerates the bleaching process. It is likewise, by absorbing oxrgen fiom the atmosphere, that fats or oils pass to the state of margaric and oleic acids, and become most easily saponified. Should the goods, however, be left too long on the grass, the fats absorb carbonic acid, and become insoluble in leys.

6. The goods must now receive a new soda ley, to dissolve out that portion of the coloring matter which has been dis-hydrogenated in the chlorine of the air, as well as the grease, if any perchance remained in the soluble state. These last two operations are to be several times repeated, because the coloring matter should be removed only by degrees, for fear of injuring the texture of the goods, by subjecting them to too much chlorine at a time.

7. We finish with the dilute sulphuric acid, which should be very weak and tepid. It dissolves out the iron, and some earthy matters occasionally found upon cotton. The goods must be most carefully washed at the dash-wheel, or in a stream of water on quitting the sour ba $\mathrm{h}$, for if the acid were allowed to dry in them, it would infallibly injure their texture by its concentration. In winter, if the goods are allowed to get frozen with the acid upon them, they may likewise be damaged.

We may here observe, that when the goods are not to remain white, their bleaching may be completed with a ley; for though it leaves a faint yellow tint, this is no inconvenience to the dyer. But when they are to be finished with a starching after the last ley, they must have another dip of the chlorine to render the white more perfect. An immersion in the dilute acid has nearly the same effect.

The principles expounded above lead to this important consequence, that when we wish to bleach goods that are free from greasy stains, as is the case generally with the better kinds of muslins, or when we wish to bleach even greasy goods for the starch finish, we may content ourselves with the following operations:-

1. Boiling in water.

2. Scouring by the stocks or the dash-wheel.

3. Bucking with milk of lime.

4. Passing through chlorine, or exposure on the grass.

5. Bucking, or bouking with milk of lime. These two latter operations require to be alternated several times, till the whole of the coloring matter be removed.

6. Souring.

The bleaching of goods, which are never laid down on the green, and which are not dried between two operations, may be completed in a couple of days. They answer as well for the printer as the others, and they are as white. Cotton fibres or yarns suffer no diminution of iliei: sirength, when the cloth has been properly treated in the above described processes.

Accurate experiments har demonstrated that their strength is not impaired by being boiled in milk of line for two hours at the ordinary pressure, provided thev se ennstantly kept covered with liquid during the whole eouiiiiion, and that they be well washed im. mediately afterwards ; cr, by being boiled in pure water under the rressure of ten atmospheres of stearn; or by being boiled under the same pressure in a caustic soda ley, marking $3^{\circ}$ of Tweedale, or specific gravity $1 \cdot 015$, though it has increased to double the density in the course $\approx f$ the bcil, by the escape of the steam; or by being boiled under the atmospheric pressure at $14^{\circ}$ of Tweedale, or specific gravity of 1.070 ; or by being immersed for eight hours in chloride of lime, capable of decoloring three times its bulk, of test solution of indigo (See CHLORINE); and by being afterwards dipped in sulphuric acid of specific gravity 1.067 , Tweedale $14^{\circ}$; or by being steeped for eighteen hours in sulphuric or muriatic acid of specific gravity $1.035,7^{\circ}$ Tweedale.

In other well-conducted bleach-works the following is the train of operations:1. Cleansing out the weaver's dressing by steeping the cloth for twelve hours in cold water, and then washing it at the stocks or the dash-wheel. 2. Boiling in milk of line, of a strength suited to the quality of the goods, but for a shorter time than with the soda ley; two short operations with the lime, with intermediate washing, bcing preferable to one of greater duration. 3 and 4. Two consecutive leys of ten or twelve hours' boiling, with about two pounds of soda chrystals for $1 \mathrm{cwt}$. of cloth. 5. Exposure to the air for six or eight days, or the application of the chloride of lime and the sulphurie 
acid. 6. A ley of caustic soda, like the former, sometimes with less alkali. 7. Exrosure to the air for six or eight days, or chlorine and the sour, as above. 8. Caustic sodla ley, as before. 9. Chlorine and the sour. 10. Rinsing in hot water, or scouring at the dash-wheel.

If the numoer of vessels to be heated exceeds four or fire, there is an economy in using steam as the medium of heat; but under this number there is an adrantage in the dilect application of fire to a boiling or bucking apparatus; since when only two vessels are in activity, there is a waste of fuel by the extra steam power. It deserres to be remarked, aiso, that the increase of the bulk of the liquid by the condensation of the steam, does not permit the spent white ley to be turned to use for the green goods, on account of its excessire dilution. With the milk of lime boil, however, this dilution wouli be rather an adrantage.

It has been found that the introduction of bran into the fermenting steep (when this is used) endangers the texture of the goods, by causing a putrefactive fermentation in some places.

When in the milk of lime boil there is too much of this caustic earth, or when it is purred in on the top of the goods, they are apt to suffer damage. The milli of lime should be introduced from beneath into the under compartment of the bucking apparatus. For the same reason, after the caustic soda ley, the ressel should be filled up with water, if the goods be not immediately transferred to the dash-wheel. When they are allowed to become partially dry on the top, they are easily injured. The copper of the buching apparatus ought to be of a size proportioned to that of the surmounting crib or vat; for when it is too small, the liquid is too long of being brought into proper circulation, and the goods may be meanwhile injured. In a bucking apparatus, which requires fire or six hours to be brought into full plar, those goods are very apt to be injured, which lie immediately under the overflow pipe.

When the chloride of lime steep is too strong, sometimes small round holes are made in the calico, just as if they had been cut out by a punch, especially in the borders or thicker parts of the goods. This accident is owing to the presence of bubbles of chlorine. From the saturated state of the liquid, they remain gaseous a sufficient lenath of time for corroding the parts of the eloth with which they are in contact. These will be obriously the denser parts, for they confine the gas most completelr, or prerent its diffusion through the mass. This evil is prevented by diluting the chloride steep to the proper degree, and moving the goods through it.

The greasy spots, described abore, show themselves in the maddering by attracting the dye-stuff more copiously than the pure parts of the cloth, so as to mottle it; ther are also recognised in the white goods by being somewhat repulsive of moisture. When the combination of fatty matters with chlorine takes place at the surface of cotton goods, it is of a nature to resist the action of alkalis. It is the stearine, or the principle of suet, particularls, which, br this means, acquires such a strong affinity for cottons ; the elaine, or the principle of oils, has no such remarkable affinity. Lime, in some circumstances, seems to act as a mordant to greasy matters, and to fix them fast. Hence the weaver should be prohibited, in all cases, from allowing candle-grease to touch his web. Goods soiled with it should never be allowed to lie by in the ware-house, but be immediately cleansed before the air has fixed the stearine br converting it into margaric acid. Lime should, in these cases, be prudently employed; chlorine should never be used till the greasy stains are thoroughly removed; and the bleacher shonld never warrant his pieces for the printer till he has verified some of them by the water test.

I shall conclude this general analysis of the principles of bleaching by a few precepts. Avoid lime, at the first ley, for goods which contain greasy spots ; but use it freely after one or two soda leys, and apply two soda leys after it. Do not apply chlorine between these leys, but reserve it for the final operation. Br this plan the goods will be well bleached and very little worn. Use the souring steeps freely, giring them after each ley, whether of lime or soda, since the calcareous base, with which the greasy spots get charged merely from hard water, is an obstacle to the further action of the leys.

I shall now give some-ractical instructions concerning the several steps of the tleaching process, as applied to cotton, linen, silk, and wool.

The first thing which the cotton bleacher does, is to mark the pieces with i.ie initials of the owner, by means of a stamp imbued with coal tar. The linen bleacher marks with nitrate of silver, a far more expensire substance, but one which resists better the severer treatment which his goods are destined to undergo.

The cotton goods are generally singed before they are sent to the bleacher, and this is done either by passing them rapidly over a red-hot semi-cylinder of iron, or orer a row of gas flames, by Mr. Hall's ingenious contrivance. (See Sirgervg.) Each piece is next creased together lengthwise like a rope, folded into a bundle. and fixed br a noose at the end. In this open state it is easily penetrated by the water of the soaling cistern into which it is thrown. It is then scoured by the dash or wash-wheel. It is now ready for 
the bucking or steaming apparatus, where it is treated with milk of lime. The steam chamber resembles the bucking ressel, without its buttom copper; that is to say, a few inches below the grated bottom of the bucking tub, there is a close iron sole, through the centre of which the steam is admitted by several small apertures, for the purpose of diffusing it throughout the goods, and cansing a liquid circulation by its pressure, as the steam does in the proper bucking boiler. One pound of lime previously made into a cream consistenced mixture, and passed through a siere, is used for evely thirty or forty - pounds of cloth, according to its color and texture; and this cream mixed with more water is interstratified with the pieces, as they are laid regularly in the ressel. Whenerer this is stocked with goods, all their interstices are filled up with water. After the lime Jucking, the cloth is transferred to the dash-wheel.

A pound of cloth requires for its whitening about half a pound of good average chloride of lime or bleaching powder, as it is commonly called, and this ought to be dissolved in about three gallons of water. Mr. Crum of Thorniebank, near Glasgow, an extensive and excellent bleacher, has so modified Dr. Dalton's ingenious plan of testing the power of bleaching liquors by green sulphate of iron, as to give it much greater precision for the bleacher's use, than the discoloration of indigo originally proposed by Berthollet. Mr. Crum dissolves four ounces of fresh green ritriol in hot water, and then adds the solution of bleaching powder by small quantities at a time, till the iron becomes wholly peroxydized, when the smell of chlorine will become perceptible. When the bleacher has once found by trial the proper blanching power which his chlorine steep ought to have, he can rerify is standard, by seeing how much of it must be added to an ounce, or any given weight of fresh copperas, dissolved in hot water, to cause the peroxydizement and the exhalation of the peculiar odor. M. Gar Lussac's new method by arsenious acid will be described under chlorine. From the experiments which 1 made some years ago,* upon indigo, it will be seen that this dye stuff is so variable in its quantity of coloring matter, that no two chemists operating with it independently, as a test for chloride of lime, could arrive at the same result. They musi provide themselves with absolute indigo, by an expensive and troublesome process, not suited to the busy bleacher. The vitriolage, as the French term it, or the souring of the English bleacher, consists in immersing the goods for four hours in dilute sulphuric acid, containing one gallon of oil of vitriol to from 25 to 30 of water, thoroughly intermixed by stirring; for the density of the acid is an obstacle to its equal distribution through the woter. This dilute acid will have a density of from 1.047 to $1 \cdot 040$, and will contain from 7 to $6 \frac{1}{2}$ per cent. by weight of the oil of vitriol.

The goods are now washed, and then boiled for eight or nine hours in an alkaline ley, containing about two pounds of crystals of soda, or their equivalent in soda ash or pearl-ash, for every 100 lbs. of cloth. The ley must be made previously caustic by quick lime. A washing in the wheel follows this boil; and then a chlorine steep for five hours in a liquor two thirds of the strength of the former. It is next soured in the dilute sulphuric acid, for two, three, or four hours, according to the color and quality of the cotton, and then thoroughly washed.

The cioth is now bleached white, but cannot be presented in the market till it undergoes certain finishing processes. The piece is elongated from the folds which it contracts during the rotation of the dash-wheel by being thrown into a stream of water in a cistern, terminated by the squeezing r.llers, which take in the end of the piece, and run it through between them, with the effect of making it nearly dry. Two pieces of cloth pass simultaneously through the rollers, and are disentangled spontaneously, so to speak, without the help of hands.

The sciueezing rollers or squeezers, for discharging the greater part of the water from the yarns and goods in the process of bleaching, are represented in figs. 117, 118, the

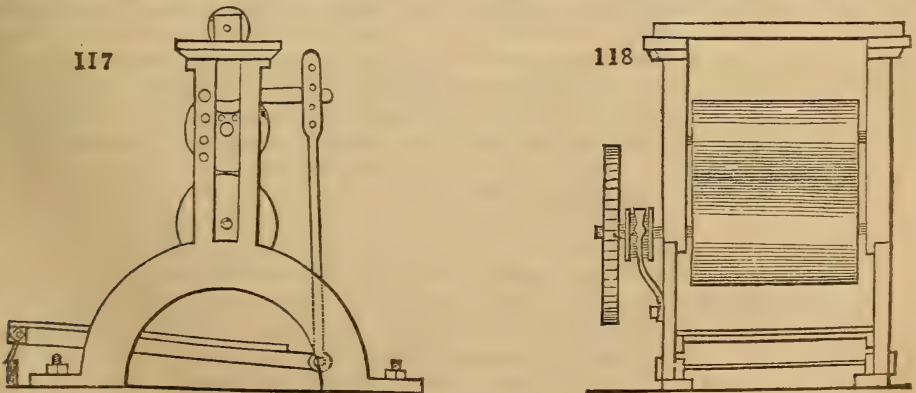

* Quarterly Juurnal of Science, Literature, and the Arts, vol. vil. p. 160. 
former being a cire.riew, to show how the roller gudgeons lie in the slots of the frame, and how the shaft of the upper roller is pressed downward by a weighted lever, through a vertical junction rol, juinted at the bottom to a nearly horizontal bar, on whose end the proper weight is hung. In fig. 118, these rollers of birch-wood are shown in race; the under one receiving motion through the loothed wheel on its shaft, from any suitable power of water or steam. Upon the shaft of the latter, between the toothed wheel and the rolier, the lever and pul!ey for putting the machine into and out of gear are visible. The under roller makes about 25 revolutions in the minute, in which time three pieces of croods, stitched tndwise, measuring 28 yards each, may be run through the machine, from a water trough on one side, to a wooden grating upon the other.

When the goods are run through, they are carried off upon a grated wheelbarrow, in a nearly diy state, and transferred to the spreading machine, called at Manchester a camiroy. In many bleach-works, however, the creased pieces are pulled straisht by the hands of women, and are then strongly beat against a wooden stock to smooth out the edges. This being done, a number of pieces are stitched endwise together, preparatory to being mangled.

Culender.-Fig. 120 is a cross section of this machine, and figs. 119, 121, are front views broken off. The goods are first rolled upon the wooden cylinder $u$, near the

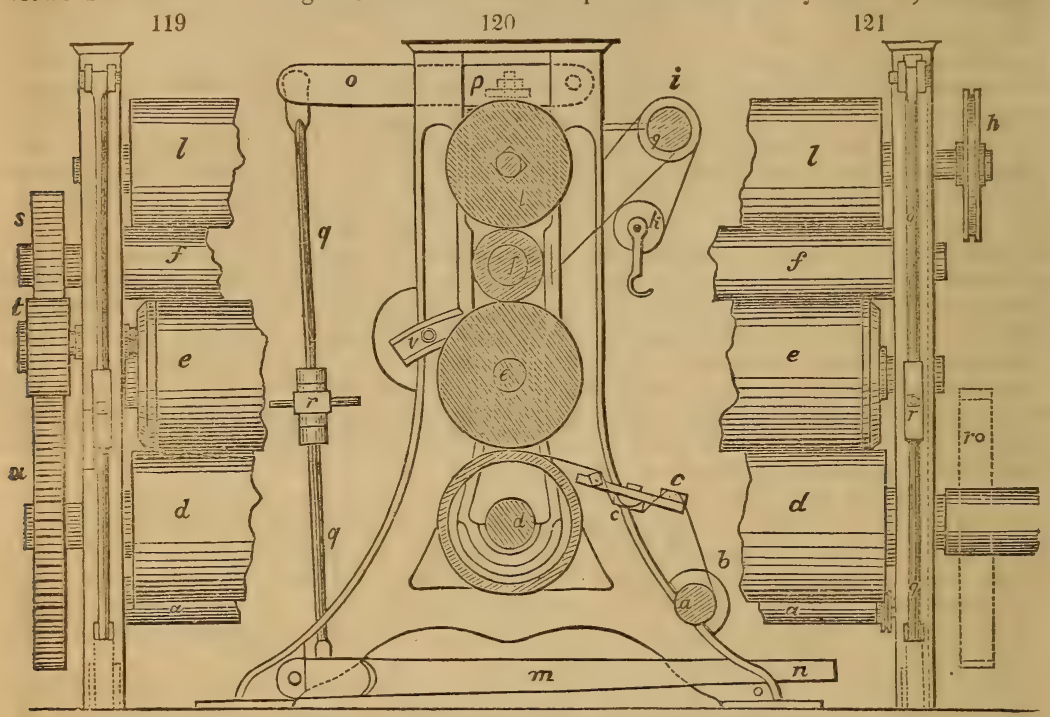

ground; by the tension roller $b$, upon the same cylinder, the goods receive a proper degree of stretching in the winding off. They then pass over the spreading bars $c c c$, by which they are still more distended; next round the hollow iron cylinder $d, 16$ inches diameter, and the paper cylinder $e$, of like dimensions; thence they proceed under the second massive iron cylinder $f$, of 8 inches diameter, to be finally wound about the projecting wooden roller $g$. This is set in motion by the pulleys $h$, fig. 121, and $i, f i g .120$, and receives its proper tension from the hanging roller $k ; l$ is a press cylinder, of 14 inches diameter, made of plane-tree wood. By its means we can at all times secure an equal degree of pressure, which would be hardly possible did the weighted lever press immediately upon two points of the calender rollers. The compression exercised by the crlinders may be "ncreased at pleasure by the bent lever $m$, weights being applied to it at $n$. The upper branch of the lever 0 is made fast by screws and bolts at $p$, to the upper press-cylinder. The junction leg $q$ is attached to the intermediate piece $r$, by left and right-handed screws, so that accorling as that piece is turned round to the right or the left, the pressure of the weighted roller will be either increased or diminished. By turning it still more, the piece will get detached, the whole pressure will be removed, and the press-roller may be taken off; which is a main object of this mechanism.

The unequable movwnent of the cylinders is produced by the wheels $s t u$, of which ine undermost has 69 , the uppermost has 20 , and the carrier-wheel $t$, either 33,32 , or 20 tecth, according to the difference of speed required. The carrier-wheel is bolted on at $\boldsymbol{v}$, and adjusted in its proper place by means of a slot. To the undermost iron cylinder, the first molion is communicated by any power, for which purpose either a rigger (driving valley) is applied to its shaft at $u$, or a crank motion. If it be decired to 
onerate with a heated calender, the undermost hollow cylinder may be filled with hot steam, admitted through a stuffing-box at one end, and discharged through a stuning-box at the other, or by a red-hot iron roller.

Pure starch would be too expensive a dressing for common calico shirtings, and therefore an extemporaneous starch is made by mixing one pound of flour with one gallon of water, and allowing the mixture to ferment in a warm place for twenty-four hours. In this way, a portion of lactic acid is formed, which dissolves the gluten, or separates it from the starch; so that when the whole is thrown upon a sieve, a liquid paste passes through, which, being boiled, answers well for stiffening the goods, without giving them a gray tinge. The paste is thinned with water to the desired degree, and faintly tinged with solution of indigo. The starch, which is sometimes thickened with porcelain clay, Paris plaster, or Spanish white, is put into a trough, and is evenly imparted to the cloth as this is drawn down through it, by the traction of rollers. There is a roller near the bottom of the trough, round which the cloth is made to run, to secure its full impregnation; while the upper rollers serve to expel its excess of the starch, and throw it back into the cistern. See Starching Apparatus.

The goods are next dried in an apartment heated by two, three, or more flues, running along the floor, and covered usually with fire-tiles. At first the heat is moderate, but it is gradually raised to upwards of $110^{\circ} \mathrm{F}$.

The goods must now be passed again through the calender, in order to receive their final smoothness and lustre. They are, in the first place, damped with a peculiar machine, furnished with a circular brush, whose points revolve in contact with water in a trough placed beneath them, and sprinkle drops of water upon the goods as they are drawn forward by a pair of cylinders. They are then subjected to the powerful pressure of the calender rollers.

The calendered pieces are neatly folded into compact parcels, and stamped with the marks of each particular manufacturer, or various devices to suit the markets for which they are designed. They are finally piled on the sole of an hydravlic press, with a sheet of pasteboard between each piece; but with occasional plates of iron to secure uniformity of pressure throughout. When sufficiently condensed by the press, they are taken out, and despatched to their respective manufacturers in a state ready for sale.

There are no less than 25 steps in the bleaching of calicoes, many of them effected with expensive machinery; yet the whole do not produce to the bleacher more than 10 pence per piece of 24 yards.

The following system was pursued, a few years back, by a skilful bleacher of muslins near Glasgow :-

"In fermenting muslin goods, we surround them with our spent leys, from the temperature of $100^{\circ}$ to $150^{\circ} \mathrm{F}$, according to the weather, and allow them to ferment for 36 hours. In boiling $112 \mathrm{lbs}$. $=112$ pieces of yard-wide muslin, we use 6 or 7 lbs. of pearl-ashes, and 2 lbs. of soft soap, with 360 gallons of water, and allow them to boil for 6 hours; then wash them, and boil them again with $5 \mathrm{lbs}$. of pearl-ashes and $2 \mathrm{lbs}$. of soft soap, and allow them to boil 3 hours; then wash them with water, and immerse them into the solution of oxymuriate of lime, at 5 on the test-tube, and allow th.em to remain from 6 to 12 hours; next wash them, and immerse them into dilute sulphuic acid at the specific gravity of $3 \frac{1}{2}$ on Tweedale's hydrometer $=1 \cdot 0175$, and allow them to remain an hour. They are now well washed, and boiled with $2 \frac{1}{2}$ lbs. of pearl-askes, and 2 lbs. of soft soap for half an hour; afterwards washed and immersed into the oxymuriate of lime as before, at the strength of 3 on the test-tube, which is stronger than the former, and allowed to remain for 6 hours. They are again washed, and jinmersed in diluted sulphuric acid at the specific gravity of 3 on Tweedale's hydrometer $=1 \cdot 015$. If the goods be strong, they will require another boil, steep, and sour. At any rate, the sulphuric acid must be well washed out before they receive the finishing operation with starch.

"With regard to the lime, which some use instead of alkali immediately after ferment. ing, the same weight of it is employed as of pearl-ashes. The goods are allowed to boil in it for 15 minutes, but no longer, otherwise the lime will injure the fabric."

More recently the plan adopted is as follows; by which the purest whites are produced for the London market.

" Lime is seldom used for our finer muslin goods, as it is found to injure their fabric, and the colors do not keep for any length of time.

"An alkaline ley is made by boiling equal weights of lime and soda together for an hour: this alkali is used for boiling goods the same as potash, but without soap.

"In finishing jaconets or muslins, after washing them from the sour, they are run tnrough spring-water containing a little fine smalts, which give them a clear shade; if of a coarse fabric, a little well-boiled starch is added to the water. From this they are wrung or pressed, and taken up by the selvage for the breadthing frame, and are run off it upon a tin cylinder heated by steam, by which the piece is completely dried 
in 15 minutes: it is then stripped from the cylinder, neatly folded and pressed, which finishes the piece for the market. From $6 \dot{u}$. to $9 d$. per piece of 12 yards is obtained for the bleaching and finishing of those goods.

c: Buok muslins, after being washed from the sour, are wrung or pressed; then they are hung up to dry in a heated stove, previous to being put into starch, prepared by boiling 3 lbs. of it to every 5 gallons of water, with 20 ounces of smalts : they are wrung out of this starch, and taken to a room heated to $110^{\circ} \mathrm{F}$; the starch is wrought into the piece till clear, then taken into a cold room, and the selvages dressed or set, before being put on the breadthing frame in the heated stove, where the piece is stretched to its length, while three or four persons at each selvage keep the piece to its breadth. If a stiff finisb is wanted, they lieep exactly opposite each other; but in breadthing the piece of elastic, they cross the piece in breadthing, which gives it a springy elastic finish. From $9 d$. to $15 \dot{i}$. per piece of 12 yards is obtained for the bleaching and finishing of these goods.

"Seweit trimmings, flounces, and dresses are run through spring water containing fine smalts with a little well-boiled starch. They are then taken to the drying-store, where they are stented till dry, wlich finishes the piece for the market. From $6 d$. to $8 d$. per piece is obtained for trimmings and flounces, and from $9 d$. to $1 \mathrm{~s}$. for dresses, bleaching and finishing."

In the bleaching of cotton cloth, where fixed colors are previously dyed in the yarn before it is woven into cloth, such as the Turkey or Adrianople red, and its compounds of lilach or purple, by the addition of iron bases, various shades of blue fiom indigo, together with buff and gold color, tinged with the oxydes of iron, great care is necessary.

The common process of bleaching pulicates, into which permanent colors are woven, is, to wash the dressing or starch well out in cold water; to boil them gently in soap, and, after again washing, to immerse them in a moderately strong solution of the oxymuriate of potash; and this process is followed until the white is good: they are then soured in dilute sulphuric acid. If the goods are attended to in a proper manner, the colors, in place of being impaired, will be found greatly improved, and to have acquired a delicacy of tint which no other process can impart to them.

Pulicates, or ginghams, which have been woven along with yarn which has been previously bleached, are first freed by washing from the starch or dressing: they are then washed, or slightly boiled with soap. After which, they are completely rinsed in pure spring water, and then soured.

Besides iliese cuiiumion processes for bleaching, another was some time ago introduced, which consisted in immersing the cotton or linen goods in pretty strong solution of caustic alkali, and afterwards exposing them to the action of steam in a close vessel. It is now generally abandoned.

The cotton or linen goods, having been previously cleaned by steeping and washing, were, after being well drained, steeped in a solution of caustic alkali of the specific grarity of 1020 . After the superfluous alkaline ley had been drained from them, they were arranged on a grating in a receiver. The cover was then placed on the vessel, and firmly screwed down; and the steam was admitted by turning the stopcock of the pipe which communicated with a steam boiler of the common construction.

The stains which come out upon maddered goods, in consequence of defective bleaching, are called in this country spangs. Their origin is such as I have described above, as the following statement of facts will show. The weaver of calicoes receives frequentiy a fine warp so tender from bad spinning or bad staple in the cotton, that it will not bear the ordinary strain of the heddles, or friction of the shuttle and reed, and he is obliged to throw in as much weft as will compensate for the weakness or thinness of the warp, and make a good marketable cloth. He of course tries to gain his end at the least expense of time and labor. Hence, when his paste dressing becomes dry and stiff, he has recourse to such greasy lubricants as he can most cheaply procure; which are commonly either tallow or butter in a rancid state, but the former, being the lowest priced, is preferred. Accordingly, the weaver, having heated a lump of iron, applies it to a piece of tallow held over the warp in the loom, and causes the melted fat to drop in patches upon the yarns, which he afterwards spreads more evenly by his brush. It is obvious, however, that the grease must be very irregularly applied in this way, and be particularly thick on certain spots. This irregularity seldom faic to appear when the goods are bleached or dyed by the common routine of work. Printed calicoes examined by a sliilful eye will be often seen to be stained with large blotches evidently occasioned by this vile practice of the weaver. The ordinary workmen call these copper stains, believing them to be communicated in the dyeing copper. Such stains on the cloth are extremely injurious in dyeing with the indigo vat. The following plan is adopted by some Scotch bleachers, with the effect, it is said, of effectually counteracting spangs from grease.

The goous having been singed and steeped in pure water, as is customary in common bleaching, they are passed through a pair of rollers to press out the impurities which have been loosened by the steeping. It must here, however, be observed, that where the 
expense of one extra drying can be afforded, the process might be very much improved by steeping the brown calicoes for thirty or forty hours before singeing, because this would separate much of that impurity which usually becomes fixed in the stuff on its being pas:ed orer the hot cylinders. When the pieces have been thus singed, steeped, and pressed, they are boiled four times, ten or twelve hours at each time, in a solution of caustic potash, of the specitic gravity of from 1.0127 to 1.0156 , washing them carefully and thoroughly in pure water between each of these boilings. They are then immersed in a solution of the chloride of potash, originally of the strength of 1.0625 , and afierwards reduced with twenty-four times its measure with water.

When the preparation is good, these proportions will whiten cotton goods completely in eight hours. In this steep they are, however, generally suffered to remain twelve hours. It has been supposed that the common bleaching liquor (chloride of lime) cannot, without injury, be substituted for chloride of potash, but I believe this to be a mistake.

Some printers take the pieces from this solution, and, while wet, lay thein upon the grass, and there expose them to the sun and weather for two or three days. They are thence remored to the sours, made of the specific gravity of about 1.0254 at the temperature of $110^{\circ}$ of Fahrenheit. In bleaching common goods, and such as are not designed for the best printing, the specific gravity of the sours is varied from that of 1.0146 to that of 1.0238 , if weighed when they become of the temperature of the atmosphere. In these they are suffered to lie for five or six hours, after which they are taken to the dash-wheei and washed thoroughly. When this operation is finished, they are submitted to four more boilings as before, with a solution of caustic potash; taking care to wash well between each of these boilings. Sometimes pearl-ash, made caustic, is used for the last of these boilings. lest the sulphur, which always exists in the porashes of commerce, should impair the whites. They are next immersed in the diluted chloride of potash, of the strength before mentioned; after which they are well washed in pure water, and then winched for half an hour in common sours. The last process is that of careful washing in plenty of clean water, after which they are not put into the stove, but are immediately hung up in the airing sheds to dry gradually. The water must be good, and abundant.

The number of operations, as here described, is great; but I know of no other mode of procedure by which perfect bleaching is so likely to be effected at all times and in all seasons, without disappointment. It must here be remarked, that, for the best purposes of printing, it would not be sufficient to take goods which have been bleached in the common way and finish these by the better process; because the sulphate of lime deposited in the cloth by that operation will be apt to spoil them for maduer colors; at least, a printer who is curious in his business would hesitate to work up such cloth.

Bucking or Bowking.-This is one of the most important operations in the bleaching of both cotton and linen goods. There are several methods whereby this process is carried on; but of these we shall select only two, distinguishing them as the old and new method of bucking. In the former way, the cloths have been steeped in the alkaline ley, as before described, and afterwards well washed, are regularly arranged in a large wooden vat, or kieve; a boiler of sufficient capacity is then filled with caustic alkaline ley, which is heated to the temperature of blood. The boiler is then emptied by a stop-cock upon the linens in the kieve, until they are covered with the liquor. After having remained on the cloth for some time, it is run off by a stop-cock, at the bottorn of the kieve, into an iron boiler sunk in the ground, from whence it is raised into the boiler by a pump. The heat is now elevated to a higher temperature, and the ley again run upon the goods in the kieve; from whence it is returned into the boiler, as before described: and these operations are continued, always increasing the heat, until the alkaline ley is completely saturated with the coloring matter taken fiom the cloth, which is known by its having acquired a completely offensive smell, and losing its causticity.

When we consider the effect which heated liquids have upon colored vegetable matter, we shall see the propriety of the temperature of the alkaline ley being gradually increased. Thus, when vegetable substances are hastily plunged into boiling liquids, the coloring matter, in place of being extracted, is, by this higher temperature, fixed into them. It is on this principle that a cook acts in the culinary art, when the green color of vegetables is intended to be preserved: in place of putting them into water when cold, they are kept back until the water is boiling; because it is well known that, in the former case, the green color would be entirely extracted, whereas, when the regetables are not infused until the water is boiling, the color is completely preserved or fixed. On the same principle, when the temperature of the alkaline ley is gradually raised, the extractive and coloring matter is more effectually taken from the cloth; and the case is reversed when the ley is applied at the boiling temperature: so much so, that linen which has been so unfortunate as to meet with this treatment, can never be brought to a good white.

When the alkaline ley is saturated with coloring matter, it is run off as unfit for 
further use in this operation; but, were the goods to be instantly tazen out of the kieve, and carried to be washed in the dash-wheel while hot, a certain portion of the coloring matter would be again fixed into them, which is extremely difficult to eradicate. In order to prevent this, the most approved bleachers run warm water upon the cloth as soon as the impure ley is run off : this combines with and carries off part of the remaining impurities. A stream of water is then allowed to run upon the cloth in the kieve, until it comes off almost transparent. The goods are now to be taken to the wash stocks, or to the dash-wheel, to be further cleaned, with the greatest efficacy.

The improved mode of bowking was the invention of Mr. John Laurie, a native of Glasgow. It is now practised by many bleachers in Lancashire, some on more perfect plans than others; but we shall give the description of the kind of apparatus approved of by those whose experience and skill have rendered them the most competent judges.

In fig. 122, A B C D is the wooden kieve; or kier, containing the cloth; C E F D

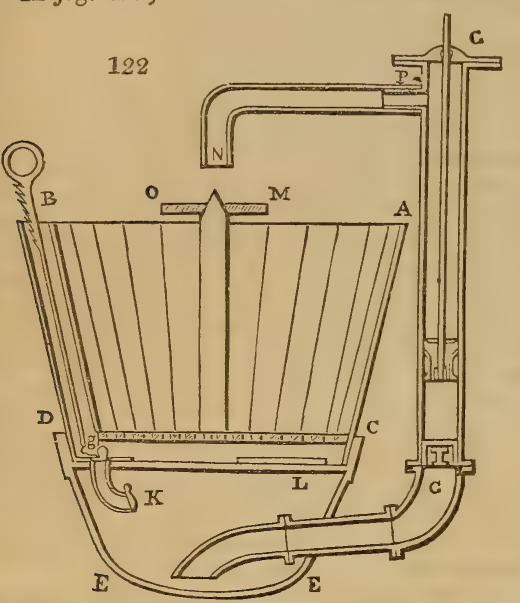
represents the cast-iron-boiler; $G \mathrm{G}$, the pump; $g, \mathbf{K}$, the pipe of communication between the kieve and the boiler. This pipe has a valve on each of its extremities; that on the upper extremity, when shut, prevents the ley from running into the boiler, and is regulated by the attendant by means of the rod and handle $g$ B. The valye at $\mathrm{K}$ admits the ley; but, opening inwards, it prevents the steam from escaping through the pipe $g$ к. The boiler has a steam-tight iron cover, $g \mathbf{L}$; and at $\mathrm{C} \mathrm{D}$, in the kieve, is a wooden grating, a small distance above the cover of the boiler.

At $\mathrm{MO}$ is a broad plate of metal, in order to spread the ley over the cloth. It is hardly necessary to say that the boiler has a furnate, as usual, for similar purposes.

While the ley is at a low temperature, the pump is worked by the mill or steamengine. When it is sufficiently heated, the elasticity of the steam forces it up through the valves of the pump, in which case it is disjoined from the moving power.

N P is a copper spout, which is removed at the time of taking the cloth out of the kieve.

The boilers A, fig. 123, used in bleaching, are of the common form, having a stop-

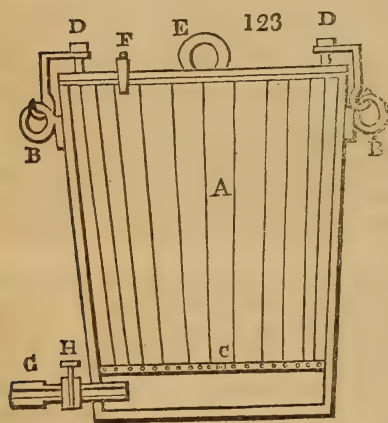
cock, $H_{\mathrm{G}}$, at bottom, for running off the waste ley. They are commonly made of cast-iron, and are capable of containing from 300 to 600 gallons of water, according to the extent of the business done. In order that the capacity of the boilers may be enlarged, they are formed so as to admit of a crib of wood, strongly hooped, or, what is preferable, of cast-iron, to be fixed to the upper rim or edge of it. To keep the goods from the bottom, where the heat acts most forcibly, a strong iron ring, covered with netting made of stout rope, $\mathrm{c}$, is allowed to rest six or eight inches above the bottom of the boiler. Four double ropes are attached to the ring $\mathrm{E}$, for withdrawing the goods when sufficiently boiled, which have each an eye for admitting hooks from the running tackle of a crane. Where more boilers than one are employed, the crane is so placed, that, in the range of its sweep, it may withdraw the goods from any of them. For this purpose, the crane tarns on pivots at top and bottom; and the goods are raised or lowered at pleasure, with double pulleys and sheaves, by means of a cylinder noved by castiron wheels. The lid is secured by the screw bolts $\mathrm{D} \mathrm{D}$, and rings в B. F is a safety valve.

The efficacy of Laurie's bowking apparatus is remarkable. While the heat is gradually rising, a current of fresh ley is constantly presented to the difierent surfaces for saturating the goods, so as to increase its detersive powers. Besides, the manner in 
which the apparatus is worked, first by the water-wheel or steam-engine, and then by its intrinsic operation, puts it completely out of the power of servants to slight the work; not to speak of the great saving of alkali, which, in many cases, has been found to amount to 25 per cent.

A simple modification of the bowkng apparatus is shown in figs. $124,125,126$; the

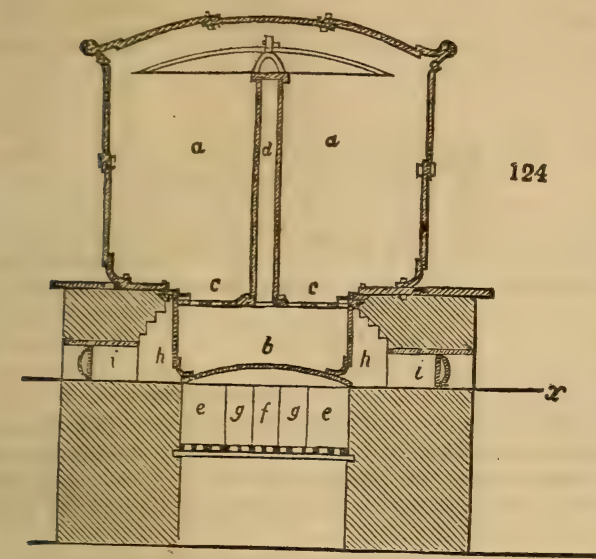
first being a vertical section, the second, a horizontal section in the line $x$ of the first. It consists of two parts: the upper wide part, $a$, serves for the reception of the goods, and the lower or pot, $b$, for holding the ley; $c$ c is an irop grating, shown apart in fig. 126. The grating has numerous square apertures in the middle of the disc, to which the rising pipe $d$ is screwed fast. The upper cylinder is formed of cast iron, or of sheet iron well riveted at the edges; or sometimes of wood, this being secured at its under edge into a groove in the top edge of the leypot. The mouth of the cylinder is constructed usually of sheet iron. $e e$ is the fire-grate, whose upper surface is shown in fig. 125 ; it is made of cast iron, in three

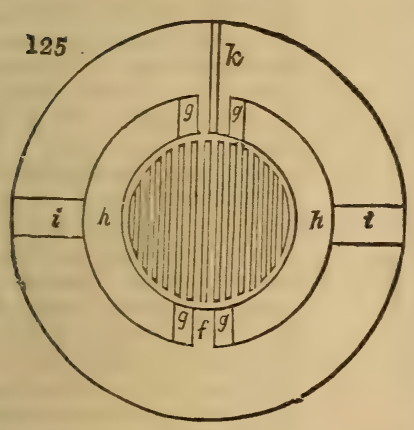
pieces. The flame is parted at $f$, and passes through the two apertures $g g$, into the flues $h h$, so as to play round the pot, as is visible in fig. 125; and escapes by two outlets into the chimney. The apertures $i i$ serve for occasionally sleaning out the flues $h h$, and are, at other times, shut with an iron plate. In the partition $f$, which separates the two openings $g g$, and the flues $h h$, running round the pot, there is a circular space at the point marked with $k$, fig. 125, in which the large pipe for discharging the waste ley is lodged. The upper large cylinder should be incased in wood, with an intermediate space filled with sawdust, to confine the heat. The action of this apparatus is exactly the same as of that already explained.

Besides the boiling, bucking, and other apparatus above described, the machinery and utensils used in bleaching are various, according to the business done by the bleacher. When linen or

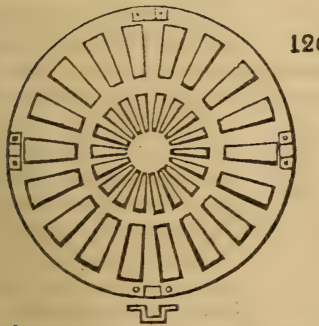

126 heavy cotton cloths are whitened, and the business is carried on to a considerable extent, the machines are both complicated and expensive. They consist chiefly of a water-wheel, sufficiently powerful for giving motion to the wash-stocks, dash-wheels, squeezers, \&c., with any other operations where power is required.

Figs. 127,128 , represent a pair of wash-stocks. A A are called the stocks, or feet. They are suspended on iron pivots at $B$, and receive their motion from wipers on the revolving shaft c. The cloth is laid in at $D$, and by the alternate strokes of the feet, and the curved form of the turnhead 5 , the cloth is washed and gradually turned. At the same time, an abundant stream of water rushes on the cloth throughout holes in the upper part of the turnhead. Wash-stocks are much used in Scotland and in Ireland. In the latter country they are often made with double feet, suspended above and below two turnheads, and wrought with cranks instead of wipers. Wash-stocks, properly constructed, make from 24 to 30 strokes per minute.

This mode of washing is now entirely givén up in Lancashire, where a preference is given to what are called dash-wheels and squeezers. The dash are small water-wheels, the inside of which is divided into four compartmunts, and closed ap, leaving cnly a hole in each compartment for putting in the cloth. 
There are, besides, smaller openings for the free admission and egress of the water em. ployed in cleansing. The cloth, by the motion of the wheel, is raised up in one part of
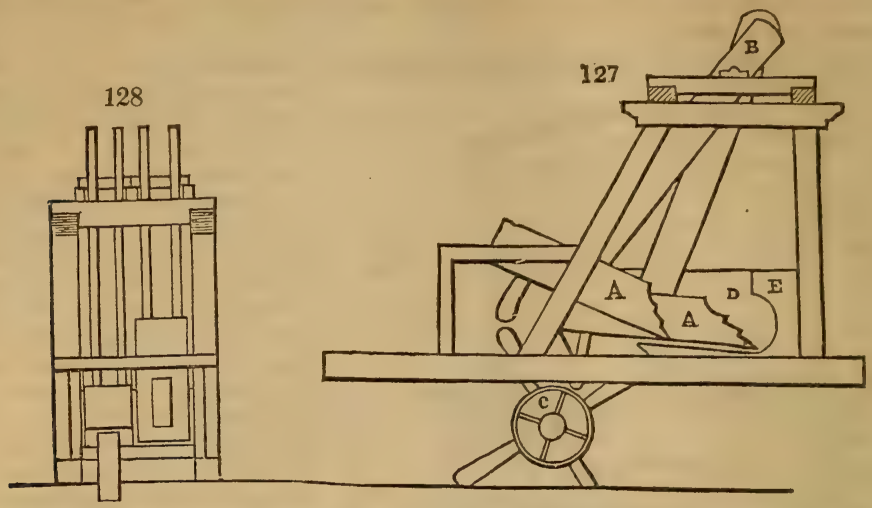

the revolution of the wheel; while, by its own weight, it falls in another. This kind of motion is very effectual in washing the cloth, while, at the same time, it does not injure its strength. The plan, however, where economy of water is of any importance, is very objectionable; because the wheel must move at by far too great a velocity to act to advantage as a water-wheel.

The wash or dash-wheel, now driven by power in all good bleach and print-

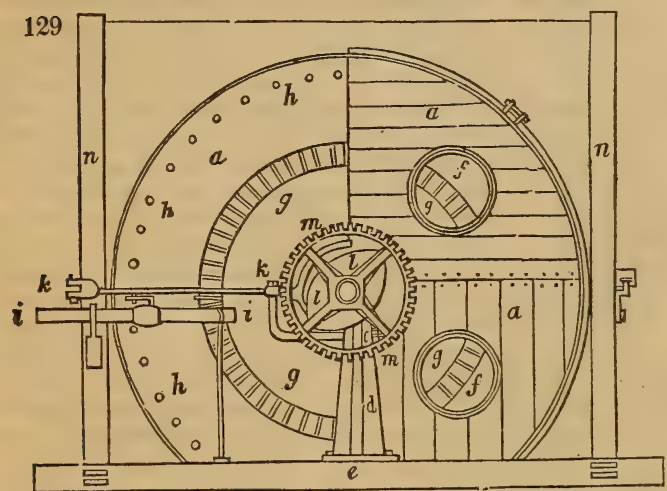
works, is represented in fig. 129, upon the left side in a back view, and upon the right side in a front view (the sketch being halved). Fig. 130 is a ground plan.

$a a$ is the washing-wheel; $b b$ its shaft-ends ; $c c$ their brass bearings or plummerblocks, supported upon the iron pillars $d d$. The frame is made of strong beams of wood, $e e$, bound together by cross bars with mortises. $f f$, two of the circular apertures, each leading to a quadrantal compartment within the dash-wheel. In

the back view (the left-hand half of the figure) the brass grating $g \mathrm{~g}$, of a curvilinear form, is seen, through which the jets of water are admitted into the cavity of the

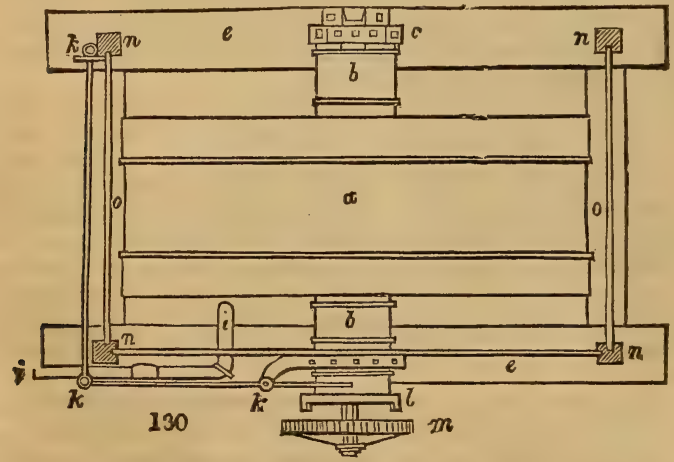
wheel; $h h$, are the round orifices, through which the foul water runs off, as each quadrant passes the lower part of its revolution; $i$, a water-pipe, with a stop-cock for regulating the washingjets; $k k$, the lever for throwing the driving-crab $l$, or coupling-box, into or out of gear with the shaft of the wheel. This machine is so constructed, that the watercock is opened or shut by the same leverage which throws the wheel into or out of gear. $m$, a wheel, fixed upon the round extremity of the shaft of the dash-wheel, which works into the toothed pinion connected 
with the prime mover. When the end of the lever $k$, whose fork embraces the couplingbox upon the square part of the shaft, is pushed forwards or backwards, it shifts the clutch into or out of gear with the toothed wheel $m$. In the latter case, this wheel turns with its pinion without affecting the dash-wheel. $n n$, holdfasts fixed upon the wooden frame, to which the boards $o 0$ are attached, for preventing the water from being thrown about by the centrifugal force.

The dash-wheel is generally from 6 to 7 feet in diameter, about 30 inches wide, and re quires the power of about two horses to drive it.

From one to two pieces of calico may be done at once in each quadrantal compartment, in the course of 8 or 10 minutes; hence, in a day of 13 hours, with two such wheels 1200 pieces of yard-wide goods may be washed.

After the process of washing by the dash-wheel, the water is expressed from the cloth by means of the squeezers already described.

Bleaching of Linen.-Linen contains much more coloring matter than cotton. The former loses nearly a third of its weight, while the latter loses not more than a twentieth. The fibres of flax possess, in the natural condition, a light gray, yellow, or blond color. By the operation of rotting, or, as it is commonly called, water-retting, which is employed to enable the textile filaments to be separated from the boon, or woody matter, the color becomes darker, and, in consequence probably of the putrefaction of the green matter of the bark, the coloring substance appears. Hence, flax prepared without rotting is much paler, and its coloring matter may be in a great measure removed by washing with soap, leaving the filaments nearly white. Mr. James Lee obtained a patent in 1812, as having discovered that the process of steeping and dew-retting is unnecessary, and that flax and hemp will not only dress, but will produce an equal if not greater quantity of more durable fibre, when cleaned in the dry way. Mr. Lee stated that, when hemp or flax plants are ripe, the farmer has nothing more to do than to pull, spread, and dry them in the sun, and then to break them by proper machinery. This promising improvement has apparently come to naught, having been many years abandoned by the patentee himself, though he was favored with a special act of parliament, which permitted the specification of his patent to remain sealed up for seven years, contrary to the general practice in such cases.

The substance which gives steeped flax its pecnliar tint is insoluble in boiling water, in acids, and in alkalis ; but it possesses the property of dissolving in caustic or carbonated alkaline leys, when it has possessed the means of dehydrogenation by previous exposure to oxygen. Hemp is, in this respect, analogous to flax. The bleaching of both depends upon this action of oxygen, and upon the removal of the acidified dye, by means of an alkali. This process is effected generally by the influence of air in combination with light and moisture acting on the linen cloth laid upon the grass : but chlorine will effect the same object more expeditiously. In no case, however, is it possible to acidify the color completely at once, but there must be many alternate exposures to oxygen or chlorine, and alkali, before the flax becomes white. It is this circumstance alone which renders the bleaching of linen an apparently complicated business.

Having made these preliminary observations with regard to the method of applying the alkaline leys used in bleaching linen cloth, I shall now bring the whole into one point of view, by detailing the connexion of these processes, as carried on at a bleach-field, which has uniformly been successful in returning the cloth of a good white, and has otherwise given satisfaction to its employers; and I shall only remark, that I by no means hold it up as the best process which may be employed, as every experienced bleacher knows that processes must be varied, not only according to existing circumstances, but also according to the nature of the linens operated upon.

In order to avoid repetition, where washing is mentioned, it must always be understood that the linen is taken to the wash-stocks or dash-wheel, and washed well in them for some hours. This part of the work can never be overdone; and on its being properly executed between every part of the bucking, boiling, steeping in the chloride of lime solution, and souring, not a little of the success of bleaching depends. By exposure is meant, that the linen cloth is taken and spread upon the bleach-green for four, six, or eight days, according as the routine of business calls for the return of the cloth, in order to undergo further operations.

A parcel of goods consists of 360 pieces of those linens which are called Britannias. Each piece is 35 yards long ; and they weigh, on an average, $10 \mathrm{lbs}$. each : the weight of the parcel is, in consequence, about $3600 \mathrm{lbs}$. avoirdupois weight. The linens are first washed, and then steeped in waste alkaline ley, as formerly described under these pro. cesses; they then undergo the following operations:-

$\begin{array}{llllll}\text { 1st, } & \text { Bucked with } 60 \text { lbs. pearl-ashes, washed, exposed on the field. } \\ 2 \mathrm{~d} \text {, } & \text { Ditto } & 80 & \text { ditto } & \text { ditto ditto } & \text { ditto. } \\ \text { 3d, Ditto } & 90 & \text { potashes } & \text { ditto ditto } & \text { ditto. } \\ \text { 4th, Ditto } & 80 & \text { ditto } & \text { ditto ditto } & \text { ditto. }\end{array}$


5th, Bucked with 80 lbs. pearl-ashes, washed, exposed on the field. 6 th, Ditto 50 ditto ditto ditto ditto. 7th, Ditto 70 ditto ditto ditto ditto. 8th, Ditto 70 ditto ditto ditto ditto. 9th, Soured one night in dilute sulphuric acid, washed.

$10 \mathrm{th}$, Bucked with $50 \mathrm{lbs}$. pearl-ashes, washed, exposed on the field. 11 th, Immersed in the chloride of potash or lime 12 hours.

12 th, Boiled with 30 lbs. pearl-ashes, washed, exposed on the field. 13th, Ditto 30 ditto ditto ditto ditto.

14 th, Soured, washed.

The linens are then taken to the rubbing-board, and well rubbed with a strong lather of black soap, after which they are well washed in pure spring water. At this period they are carefully examined, and those which are fully bleached are laid aside to be blued, and made up for the market; while those which are not fully white are returned to be boiled, and steeped in the chloride of lime or potash; then soured, until they are fully white.

By the above process, 690 lbs. weight of alkali is taken to bleach 360 pieces of linen, each piece consisting of 35 yards in length; so that the expenditure of alkali would be somewhat less than 2 lbs. for each piece, were it not that some parts of the linens are not fully whitened, as above noted. Two pounds of alkali may therefore be stated as the average quantity employed for bleaching each niece of goods.

The method of bleaching linens in Ireland is similar to the foregoing; any alteration in the process depending upon the judgment of the bleacher in increasing or diminishing the quantity of alkali used. But it is common, at most bleach-fields, to steep the linens in the chloride of lime or potash at an early stage of the process, or after the goods have undergone the fifth or sixth operation of bucking. By this means those parts of the flax which are most difficult to bleach are more easily acted upon by the alkali; and, as before noticed, souring early in very dilute sulphuric acid, assists greatly in forwarding the whitening of the linens. Mr. Grimshaw, calico-printer, near Belfast, was the first who recommended early souring, which has since been very generally adopted.

The bleaching of Silk.-Silk in its raw state, as spun by the worm, is either white or yellow of various shades, and is covered with a varnish, which gives it stiffness and a degree of elasticity. For the greater number of purposes to which silk is applied, it must be deprived of this native corering, which was long considered to be a sort of gum. The operation by which this coloring matter is removed is called scouring, cleansing, or boiling. A great many different processes have been proposed for freeing the silk fibres from all foreign impurities, and for giving it the utmost whiteness, lustre, and pliancy; but none of the new plans has superseded, with any advantage, the one practised of old, which consists essentially in steeping the silk in a warm solution of soap; a circumstance placed beyond all doubt by the interesting experiments of $M$. Roard. The alkalis, or alkaline salts, act in a marked manner upon the varnish of silk, and effect its complete solution; the prolonged agency of boiling water, alone answers the same purpose; but nothing agrees so well with the nature of silk, and preserves its brilliancy and suppleness so perfectly, as a rapid boil with soap-water. It would appear, howerer, that the Chinese do not employ this method, but something that is preferable. Probably the superior beauty of their white silk may be owing to the superiority of the raw material.

The most ancient method of scouring silk consists of three operations. For the first, or the ungumming, thirty per cent. of soap is first of all dissolred in clean river water by a boiling heat; then the temperature is lowered by the addition of a little cold water, by withdrawing the fire, or at least by damping it. The hanks of silk, suspended upon horizontal poles over the boiler, are now plunged into the soapy solution, kept at a heat somewhat under ebullition, which is an essential point; for if hotter, the soap would attack the substance of the silk, and not only dissolve a portion of it, but deprive the whole of its lustre. The portions of the hanks plunged in the bath get scoured by degrees; the varnish and the coloring matter come away, and the silk assumes its proper whiteness and pliancy. Whenerer this point is attained, the hanks are turned round upon the poles, so that the portion formerly in the air may be also subjected to the bath. As soon as the whole is completely ungummed, they are taken out, wrung by the peg, and shaken out; after which, the next step, called the boil, is commenced. Into bags of coarse canvass, called pockets, about $25 \mathrm{lbs}$. or $35 \mathrm{lbs}$. of ungummed silk are enclosed, and put into a similar bath with the preceding, but with a smaller proportion of soap, which may therefore be raised to the boiling point without any danger of destroying the silk. The ebullition is to be kept up for an hour and a half, during which time the bags must be frequently stirred, lest those near the bottom should suffer an undue degree of heat. The silk experiences in these two operations a loss of about 25 per cent. of its weight.

The third and last scouring operation is intended to give the silk a slight tinge, which 
renders the white more agreeable, and better adapted to its various uses in trade. In this way we distinguish the China white, which has a faint cast of red, the silver white, the azure white, and the thread white. To produce these different shades, we hegin by preparing a soap-water so strong as to lather by agitation; we then add to it, for the China white, a little annotto, mixing it carefully in; and then passing the silk properly through it, till it has acquired the wished for tint. As to the other shades, we need only azure them more or less with a fine indigo, which has been previously washed severa! times in hot water, and reduced to powder in a mortar. It is then diffused through boiling water, allowed to settle for a few minutes, and the supernatant liquid, which contains only the finer particles, is added to the soap bath in such proportion as may be requisite. The silk, on being taken out of this bath, must be wrung well, and stretched upon perches to dry; after which it is introduced into the sulphuring chamber, if it is to be made use of in the white state. At Lyons, however, no soap is employed at the third operation: after the boil, the silk is washed, sulphured, and azured, by passing through very clear river water properly blued.

The silks intended for the manufacture of blonds and gauzes are not subjected to the ordinary scouring process, because it is essential, in these cases, for them to preserve their natural stiffness. We must therefore select the raw silk of China, or the whitest raw silks of other countries; steep them, rinse them in a bath of pure water, or in one containing a little soap; wring them, expose them to the vapor of sulphur, and then pass them through the azure water. Sometimes this process is repeated.

Before the memoir of M. Roard appeared, extremely vague ideas were entertained about the composition of the native varnish of silk. He has shown that this substance, so far from being of a gummy nature, as had been believed, may be rather compared to bees' wax, with a species of oil, and a coloring matter, which exists only in raw silks. It is contained in them to the amount of from 23 to 24 per cent., and forms the portion of weight which is lost in the ungumming. It possesses, however, some of the properties of vegetable gums, though it differs essentially as to others. In a dry mass, it. is firiable and has a vitreous fracture; it is soluble in water, and affords a solution which lathers like soap; but when thrown upon burning coals, it does not soften like gum, but burns with the exhalation of a fetid odor. Its solution, when left exposed to the open air, at first of a golden yellow, 'jecomes soon greenish, and ere long putrefies, as a solution of animal matter would do in similar circumstances. M. Roard assures us that the city of Lyons alone could furnish several thousand quintals of this substance per annum, were it applicable to any useful purpose.

The yellow varnish is of a resinous nature, altogether insoluble in water, very soluble in aleohol, and contains a little volatile oil, which gives it a rank smell. The color of this resin is easily dissipated, either by exposure to the sun or by the action of chlorine : it forms about one fifty-fifth of its weight.

Bees' wax exists also in all the sorts of silk, even in that of China; but the whiter the filaments, the less wax do they contain.

M. Roard has observed that, if the silk be exposed to the soap baths for some time after it has been stripped of its foreign matters, it begins to lose body, and has its valuable qualities impaired. It becomes dull, stiff, and colored in consequence of the solution more or less considerable of its substance; a solution which takes place in all liquids, and even in boiling water. It is for this reason that silks cannot be alumed with heat; and that they lose some of their lustre in being dyed brown, a color which requires a boiling hot bath. The best mode, therefore, of avoiding these inconveniences, is to boil the silks in the soap-bath no longer than is absolutely necessary for the scouring process, and to expose them in the various dyeing operations to as moderate temperature as may be requisite to communicate the color. When silks are to be dyed, much less soap should be used in the cleansing, and very little for the dark colors. According to M. Roard, raw silks, white or yellow, may be completely scoured in one hour, with 15 lbs. of water for one of silk, and a suitable proportion of soap. The soap and the silk should be put into the bath half an hour before its ebullition, and the latter should be turned about frequently. The dull silks, in which the varnish has already undergone some alteration, never acquire a fine white until they are exposed to sulphureous acid gas. Fxposure to light has also a very good effect in whitening silks, and is had recourse to, it is said, with advantage by the Chinese.

Carbonate of soda has been proposed to be used instead of soap in scouring silk, but it has never come into use. The Abbé Collomb, in 1785, scoured silk by eight hours' boiling in simple water, and he found the silks bleached in this way to be stronger than by soap, but they are not nearly so white. A patent has been taken out in England for bleaching them by steam, of which an account will be found under the article SrLK.

It appears that the Chinese do not use soap in producing those fine white silks which are imported into Europe. Michel de Grubbens, who resided long at Canton, saw and 
practiset himself the operation there, which he published in the Memours of the Academy of Stockholm in 1803. It consists in preparing the silk with a species of white beans, smaller than the Turkey beans, with some wheat flour, common salt, and water. The proportions are 5 parts of beans, 5 of salt, 6 of flour, and 25 of water, to form this vegetable bath. The beans must be previously washed. It is difficult to discover what chemical action can occur between that decoction and the varnish of raw silk; possibly some acid may be developed, which may soften the gummy matter, and facilitate its separation.

Baumé contrived a process which does not appear to have received the sanction of experience, but which may put us in the right way. He macerates the yellow raw silk in a mixture of alcohol at $36^{\circ}$ (sp. gr. 0837) and one thirty-second part of pure muriatic acid. At the end of forty-eight hours, it is as white as possible, and the more so, the better the quality of the silk. The loss which it suffers in this menstruum is only one fortieth; showing that nothing but the coloring matter is abstracted. The expense of this menstruum is the great obstacle to Baumé's process. The alcohol, however, might be in a very great measure recovered, by saturating the acid with chalk, and redistillation.

Bleaching of Wool.-Wool, like the preceding fibrous matter, is covered with a peculiar varnish, which impairs its qualities, and prevents it from being employed in the raw state for the purposes to which it is well adapted when it is scoured. The English give the name yolk, and the French suint, to that native coat: it is a fatty unctuous matter, of a strong smell, which apparently has its chief origin in the cutaneous perspiration of the sheep; but which, by the agency of external bodies, may have undergone some changes which modify its constitution. It results from the experiments of $M$. Vauquelin, that the yolk is composed of several substances; namely, 1 , a soap with basis of potash, which constitutes the greater part of it; 2, of a notable quantity of acetate of potash; 3 , of a small quantity of carbonate, and a trace of muriate, of potash; 4 , of a little lime in an unknown state of combination; 5, of a species of sebaceous matter, and an animal substance to which the odor is due. There are several other accidental matters present on sheeps' wool.

The proportion of yolk is variable in different kinds of wool, but in general it is nore abundant the finer the staple; the loss by scouring being 45 per cent. for the finest wools, and 35 per cent. for the coarse.

The yolk, on account of its soapy nature, dissolves readily in water, with the exception of a little free fatty matter, which easily separates from the filaments, and remains floating in the liquor. It would thence appear sufficient to expose the wools to simple washing in a stream of water; yet experience shows that this method never answers so well as that usually adopted, which consists in steeping the wool for some time in simple warm water, or in warm water mixed with a fourth of stale urine. From 15 to 20 minutes of contact are sufficient in this case, if we heat the bath as warm as the hand can bear it, and stir it well with a rod. At the end of this time the wool may be taken out, set to drain, then placed in large baskets, in order to be completely rinsed in a stream of water.

It is generally supposed that putrid urine acts on the wool by the ammonia which it contains, and that this serves to saponify the remainder of the fatty matter not combined with the potash. M. Vauquelin is not of this opinion, because he found that wool steeped in water, with sal ammoniac and quick lime, is not better scoured than an equal quantity of wool treated with mere water. He was hence led to conclade that the good effects of putrefied urine might be ascribed to anything else besides the ammonia, and probably to the urea. Fresh urine contains a free acid, which, by decomposing the potash soap of the yolk, counteracts the scouring operation.

If wools are better scoured in a small quantity of water than in a great stream, we can conceive that this circumstance must depend upon the nature of the yolk, which, in a concentrated solution, acts like a saponaceous compound, and thus contributes to remove the free fatty particles which adhere to the filaments. It should also be observed that too long a continuance of the wool in the yolk water, hurts its quality very much, by weakening its cohesion, causing the filaments to swell, and even to split. It is said then to have lost its nerve. Another circumstance in the scouring of wool, that shonld always be attended to, is never to work the filaments together to such a degree as to occasion their felting; but in agitating we must merely push them slowly round in the vessel, or press them gently under the feet. Were it at all felted, it would neither card nor spin well

As the heat of boiling water is apt to decompose woollen fibres, we should be careful never to raise the temperature of the scouring bath to near this point, nor, in fact, to exceed $140^{\circ} \mathrm{F}$. Some authors recommend the use of alkaline or soapy baths for scouring wool, but practical people do not deviate from the method above described.

When the washing is completed, all the wool which is to be sent white into the mar- 
ket, must be exposed to the action of sulphurous acid, either in a liquid or a gaseous state. In the latter case, sulphur is burned in a close chamber, in which the wools are hung up or spread out; in the former, the wools are plunged into water, moderately impregnated with the acid. (See Surphuring.) Exposure on the grass may also contribute to the bleaching of wool. Some fraudulent dealers are accused of dipping wools in butter-milk, or chalk and water, in order to whiten them and increase their weight.

Wool is sometimes whitened in the feece, and sometimes in the state of yarn; the latter affording the best means of operating. It has been observed that the wool cut from certain parts of the shecp, especially from the groins, never bleaches well.

After sulphuring, the wool has a harsh crispy feel, which may be removed by a weak soap bath. To this also the wool comber has recourse when he wishes to cleanse and whiten his wools to the utmost. He generally uses a soft or potash soap, and after the wool is well soaked in the warm soap bath, with gentle pressure he wrings it well with the help of a hook, fixed at the end of his washing tub, and hangs it up to dry.

Bleaching of rags, and paste for paper making. - After the rags are reduced to what is called half stuff, they should have the greater part of the floating water run off, leaving just enongh to form a stir-about mass. Into this a clear solution of chloride of lime should be poured, of such a strength as is suited to the color of the rags, which should have been previously sortel; and the engine is kept going so as to churn the rags with the bleaching agent. After an hour, the water may be returned upon the engine, and the washing of the paper resumed. From two to four pounds of good chloride if lime are reckoned sufficient to bleach one hundred weight of rags.

When the rags consist of dyed or printed cottons, after being well washed and reduced to half stuff, they should be put into a large cask or butt, supported horizontally by iron axles upon cradle bearings, so that it may be made to revolve like a barrelchurn. For each hundred weight of the colored rags, take a solution containing from four to eight pounds of chloride of lime; add it to the liquid mixture in the butt along with half a pound of sulphuric acid for every pound of the chloride; and after inserting the bung, or rather the square valve, set the vessel in siow revolution backwards and forwards. In a short time the rags will be colorless. The rags and paper paste ought to be very well washed, to expel all the chlorine, and perhaps a little muriatic acid might be used with advantage to dissolve out all the calcareous matter, a portion of which is apt to remain in the paper, and to operate injuriously upon both the pens and the ink. Some of the French paper manufacturers bleach the paste with chlorine gas. Paper prepared from such paste, well washed, is not apt to give a brown tint to maps, as that carelessiy bleached with chloride of lime is known to do.

BLENDE. ( Fr. and Germ.) Sulphuret of zinc, so named from the German blenden, to dazzle, on account of its glistening aspect. It is called black jack from its usual color. Its lustre is pearly adamantine. Spec. gravity from $3 \cdot 7$ to $4 \cdot 2$. It contains frequently iron, copper, arsenic, cadmium, and silver, all associated with sulphur. It is worked up partly into metallic zinc, and partly into the sulphate of zinc, or white vitriol. It consists of 66.72 zine, and 33.28 sulphur; being nearly by weight as two to one. See Zinc.

BLOCK MANUFACTURE. Though the making of ships' blocks belongs rather to a dictionary of engineering than of manufactures, it may be expected that I should give some account of the automatic machinery for making blocks so admirably devised and mounted by M. I. Brunel, Esq. for the British navy, in the dock-yard of Portsmouth.

The series of machines and operations are as follows:-

1. The straight cross-cutting saw.-The log is placed horizontally on a very low bench, which is continued through the window of the mill into the yard. The saw is exactly over the place where the $\log$ is to be divided. It is let down, and suffered to rest with its teeth upon the log, the back still being in the cleft of the guide. The crank being set in motion, the saw reciprocates backwards and forwards with exactly the same motion as if worked by a carpenter, and quickly cuts through the tree. When it first begins to cut, its back is in the cleft in the guide, and this causes it to move in a straight line; but before it gets out of the guide, it is so deep in the wood as to guide itself: for in cutting across the grain of the wood, it has no tendency to be diverted from its true line by the irregular grain. When the saw has descended through the tree, its handle is eaught in a fired stop, to prevent its cutting the bench. The machine is thrown out of gear, the attendant lifts up the saw by a rope, removes the block cut off, and advances the tree to receive a fresh cut.

2. The circular cross-cutting saw.-This saw possesses universal motion; but the axis is always parallel to itself, and the saw in the same plane. It can be readily raised or lowered, by inclining the upper frame on its axis; and to move it sidewise, the saw frame must swing sidewise on its joints, which connect it with the upper frame. These movements are effected by two winches, each furnished with a pair of equal pinions, working a pair of racks fixed upon two long poles. The spindles of these winches are fixed in two 
vertical posts, which support the axis of the upper frame. One of these pairs of poles is jointed to the extreme end of the upper frame; therefore by turning the handle belonging to them, the frame and saw is elevated or depressed; in like manner, the other pair is attached to the lower part of the saw frame, so that the saw can be moved sidewise by means of their handles, which then swing the saw from its vertical position.

These two handles give the attendant a complete command of the saw, which we suppose to be in rapid motion, the tree being brought forward and properly fixed. By one handle, he draws the saw against one side of the tree, which is thus cut into (perhaps half through); now, by the other handle, he raises the saw up, and hy the first-mentioned handle he draws it across the top of the tree, and cuts it half through from the upper side; he then depresses the saw and cuts half through from the next side; and lastly a trifling cut of the saw, at the lower side, completely divides the tree, which is then advanced to take another cut.

The great reciprocaling saw is on the same principle as the saw-mill in common use in America.

3. The circular ripping saw is a thin circular plate of steel, with teeth similar to those of a pit saw, formed in its periphery. It is fixed to a spindle placed horizontally, at a small distance beneath the surface of a bench or table, so that the saw projects through a crevice a few inches above the bench. The spindle, being supported in proper collars, has a rapid rotatory motion communicated to it by a pulley on the opposite end, round which an endless strap is passed from a drum placed overhead in the mill. The block cut by the preceding machine, from the end of the tree, is placed with one of the sides flat upon the bench, and thus slides forward against the revolving saw, which cuts the wood with a rapidity incredible to any one who has not seen these or similar machines.

4. Boring machine.-The blocks, prepared by the foregoing saws, are placed in the machine represented in fig. 131. This machine has an iron frame, $\mathrm{A} \mathrm{A}$, with three legs, 131

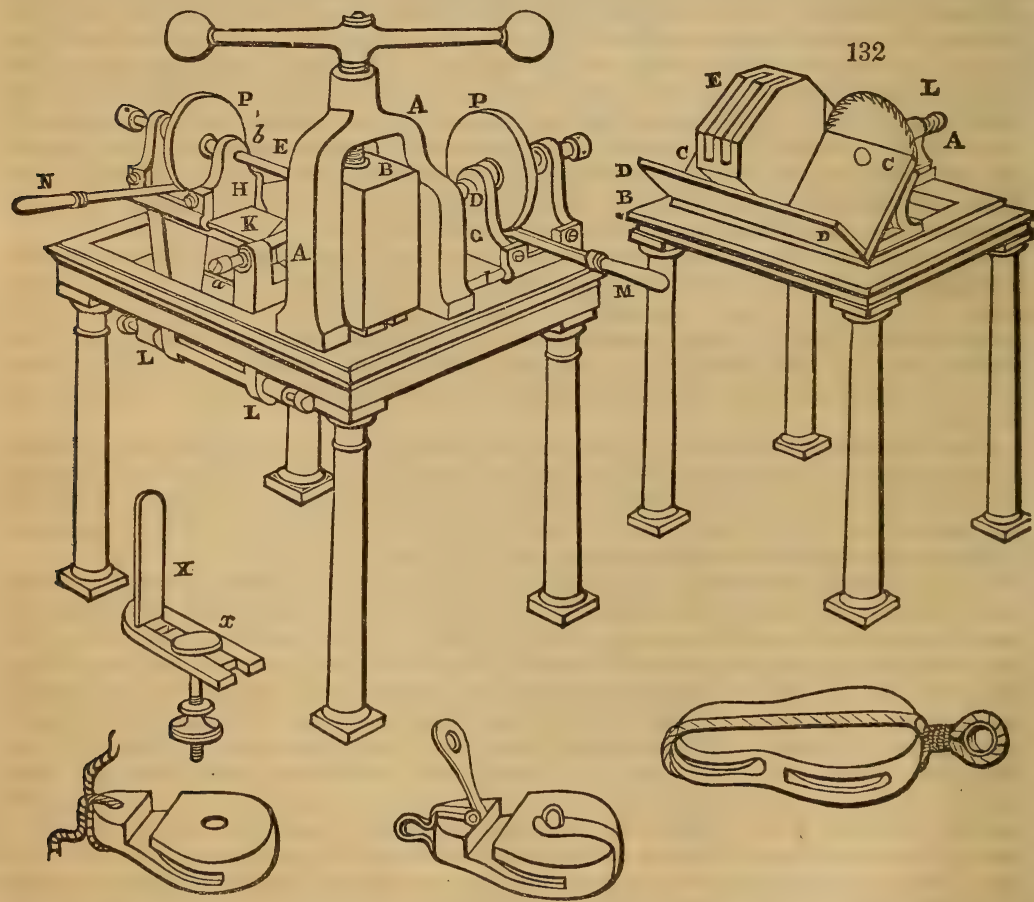

beneath which the block is introduced, and the screw near в being forced down upon it, confines it precisely in the proper spot to receive the borers $D$ and $E$. This spot is determined by a piece of metal fixed perpendicularly just beneath the point of the borer $\mathbf{E}$, shown separately on the ground at $\mathbf{x}$; this pjece of metal adjusts the position for the borer $\mathrm{L}$, and its height is regulated by resting on the head of the screw $x$, which fastens the piece $\mathrm{x}$ down to the frame. The sides of the block are kept in a parallel position, by being applied against the heads of three screws tapped into the double leg of the frame 
4. The borer $\mathrm{D}$ is adapted to bore the hole for the centre pin in a direction exactly perpendicular to the surface resting against the three screws; the other, at $\mathrm{F}$, perforales the holes for the commencement of the sheave holes. Both borers are constructed in nearly the same manner; they are screwed upon the ends of small mandrels, mounted in frames similar to a lathe. These frames, $\mathrm{G}$ and $\mathrm{H}$, are fitted with sliders upon the angular edges of the flat broad bars, $I$ and $K$. The former of these is screwed fast to the frame; the latter is fixed upon a frame of its own, moving on the centre screws, at $\mathrm{L} L$, beneath the principal frame of the machine. By this means the borer $\mathrm{E}$ can be mored within certain limits, so as to bore holes in different positions. These limits are determined by two screws, one of which is seen at $a$; the other, being on the opposite side, is invisible. They are tapped through fixed pieces projecting up from the frame. A projecting piece of metal, from the under side of the slider $\mathrm{K}$ of the borer $\mathrm{E}$, stops against the ends of these screws, to limit the excursion of the borer. The frames for both borers are brought up towards the block by means of levers $\mathrm{M}$ and $\mathrm{N}$. These are centred on a pin, at the opposite sides of the frame of the machine, and have oblong grooves through them, which receive screw pins, fixed into the frames $\mathrm{G}$ and $\mathrm{H}$, beneath the pulleys $\mathrm{P} P$, which give motion to the spindles.

5. The mortising machine is a beautiful piece of mechanism, but too complicated for description within the limits prescribed to this article.

6. The corner saw, fig. 132, consists of a mandrel, mounted in a frame A, and carrying a circular saw $L$ upon the extreme end of it. This mandrel and its frame being exactly similar to those at $\mathrm{G}$ and $\mathrm{H}, \mathrm{fig}$. 131, does not require a separate view, although it is hid behind the saw, except the end of the screw, marked A. This frame is screwed down upon the frame $\mathrm{B}$ B of the machine, which is supported upon four columns. c c, D D, is an inclined bench, or a kind of trough, in which a block is laid, as at E, being supported on its edge by the plane $\mathrm{c}$ c of this bench, and its end kept up to its position by the other part of the bench $\mathrm{D} D$.

By sliding the block along this bench, it is applied to the saw, which cuts off its angles, as is evident from the figure, and prepares it for the shaping engine. All the four angles are cut off in succession, by applying its different sides to the trough, or bench. In the figure, two of them are drawn as being cut, and the third is just marked by the saw. This machine is readily adapted to different sizes of blocks, by the simple expedient of laying pieces of wood of different thickness against the plane D D, so as to fill it up, and keep the block nearer to or farther from the saw ; for all the blocks are required to be cut at the same angle, though, of course, a larger piece is to be cut

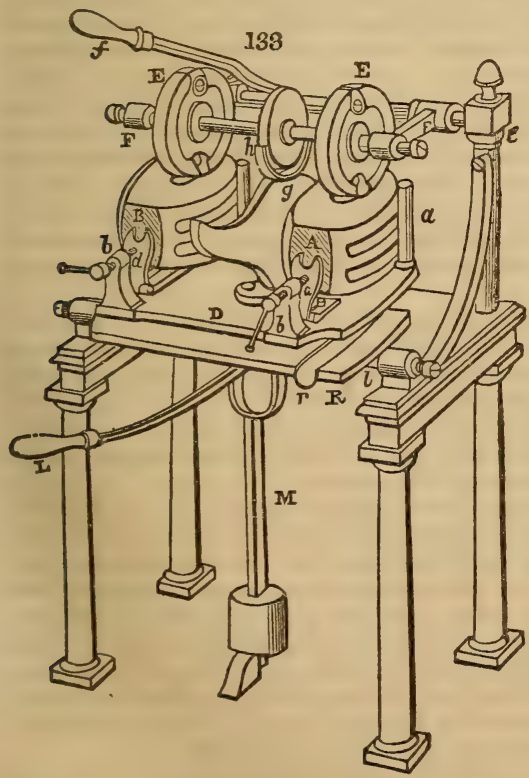
from large than from small blocks. The block reduced to the state of $\mathbf{E}$ is now taken to

7. The shaping machine.-A great deal of the apparent complication of this figure arises from the iron cage, which is provided to defend the workmen, lest the blocks, which are revolving in the circles, or chuck, with an immense velocity, should be loosened by the action of the tool, and fiy out by their centrifugal force. Without this provision, the consequences of such an accident would be dreadful, as the blocks would be projected in all directions, with an inconceivable force.

8. The scoring engine receives two blocks, as they come from the shaping engine, and forms the groove round their longest diameters for the reception of their ropes or straps, as represented in the two snatch blocks and double block, under figs. 131, 132.

A, B, fig. 133, represent the above two blocks, each held between two small pillars $a$ (the other pillar is hid behind the block), fixed in a strong plate $D$, and pressed against the pillars by a screw $b$, which acts on a clamp $d$. Over the blocks a pair of circular planes or cutters, E E, are situated, both being fixed on the same spindle, which is turned by a pulley in the middle of it. The spindle is fitted in a frame $F F$, moving in centres at $e e_{\text {, so }}$ 
as to rise and fall when moved by a handle $f$. This brings the cutters down upon the jlocks; and the depth to which they can cut is regulated by a curved shape $g$, fixed by screws upon the plate $\mathrm{D}$, between the blocks. Upon this rests a curved piece of metal $h$, fixed to the frame $F$, and enclosing, but not touching, the pulley. To admit the cutiers to traverse the whole length of the blocks, the plate D (or rather a frame beneath it) is sustained between the points of two centres. Screws are seen at $l$, on these centres. The frame inclines when the handle $L$ is depressed. At $\mathrm{m}$ is a lever, with a weight at the end of it, counterbalancing the weight of the blocks, and plate $\mathrm{D}$, all which are above the centre on which they move. The frame $\mathrm{F}$ is also provided with a counterpoise to balance the cutters, \&c. The cutters $\mathbf{E} \mathbf{E}$ are circular wheels of brass, with round edges. Each has two notches in its circumference, at opposite sides; and in these notches chisels are fixed by screws, to project beyond the rim of the wheel, in the manner of a plane iron before its face.

This machine is used as follows:- In order to fix the block, it is pressed between the two pins (only one of which at $a$, can be seen in this view), and the clamp $d$, screwed up against it, so as just to hold the block, but no more. The clamp has two claws, as is seen in the figure, each furnished with a ring entering the double prints previously made, in the end of the block. These rings are partly cut away, leaving only such a segment of each as will just retain the block, and the metal between them is taken out to admit the cutter to operate between them, or nearly so. In putting the blocks into this machine, the workman applies the double prints to the ends of the claws of the clamps, but takes care that the blocks are higher between the pins $a$ than they should be: he then takes the handle $f$, and by it presses the cutters $\mathrm{E} E$ (which we suppose are standing still) down upon the blocks, depressing them between their pins at the same time, till the descent of the cutters is stopped by the piece $h$ resting on the shape $g$. He now turns the screws $b b$, to fix the blocks tight. The cutters being put in motion cut the scores, which will be plainly seen by the mode of adjustment just described, to be of no depth at the pin-hole; but by depressing the handle $\mathrm{L}_{\text {, }}$ so as to incline the blocks, and keeping the cutters down upon their shape $g$, by the handle $f$, they will cut any depth towards the ends of the blocks, which the shape $g$ admits.

By this means one quarter of the score is formed; the other is done by turning both blocks together half round in this manner. The centres $l$ are not fitted into the plate $\mathbf{D}$ itself, but into a frame seen at $\mathrm{R}$ beneath the plate, which is connected with it by a centre pin, exactly midway between the two blocks $\mathrm{A}$ в. A spring catch, the end of which is seen at $r$, confines them together; when this catch is pressed back, the plate $D$ can be tumed about upon its centre pin, so as to change the blocks, end for end, and bring the unscored quarters ( $i$. $e$. over the clamps) beneath the cutters; the workman taking the handles $f$ and $L$, one in each hand, and pressing them down, cuts out the second quarter. This might have been effected by simply lifting up the handle $L$; but in that case the cutter would have struck against the grain of the wood, so as to cut rather roughly; but by this ingenious device of reversing the blocks, it always cuts clean and smooth, in the direction of the grain. The third and fourth quarters of the score are cut by turning the other sides of the blocks upwards, and repeating the above operation. The shape $g$ can be removed, and another put in its place, for different sizes and curves of block; but the same pins $a$, and holding clamps $d$, will suit many different sizes.

By these machines the shells of the blocks are completely formed, and they are next polished and finished by hand labor; but as this is perfurmed by tools and methods which are well known. it is needless to enter into any explanation: the finishing required being only a smoothing of the surfaces. The machines cut so perfectly true as to require no wood to be removed in the finishing; but as they cut withont regard to the irregularity of the grain, knots, \&c., it happens that many parts are not so smooth as might be wished, and for this purpose manual labor alone can be employed.

The lignum vitæ for the sheaves of the blocks, is cut across the grain of the wooc by two crnss-cutting saws, a circular and straight saw, as before mentioned. These machines do not essentially differ in their principle from the great cross-cutting saws we have described, except that the wood revolves while it is cutting, so that a small saw will reach the centre of a large tree, and at the same time cut it truly flat. The limits prescribed for our plates will not admit of giving drawings of these machines, and the idea which could be derived from a verbal description would not be materially different from the cross-cutting saws before mentioned. These machines cut of their plates for the end of the tree, which are exactly the thickness for the intended sheave. These pieces are of an irregular figure, and must be rounded and centred in the crown saw.

9. The crown saw is represented in fig. 134, where $\mathrm{A}$ is a pulley revolving by means of an endless strap. It has the crown or trepan saw $a$ fixed to it, by a screw cut within the piece, upon which the saw is fixed, and which gives the ring or hoop of the 
Eaw sufficient stability to perform its office. Both the pulleys and saw revolve together upon a truly cylindrical tube $b$, which is stationary, being attached by a flatunch $c$ to a

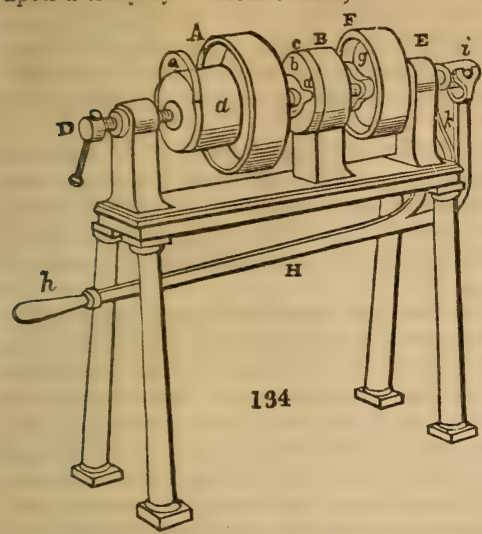
fixed puppet $B$, and on this tube as an axis the saw and pulley turn, and may be slid endwise by a collar fitted round the centrepiece of the pulley, and having two iron rods (only one of which can be seen at $d$ in the figure), passing through holes made through the flaunch and puppet $\mathrm{B}$. When the saw is drawn back upon its central tube, the end of the latter projects beyond the teeth of the saw. It is by means of this fixed ring or tube within the saw, that the piece of wood $e$ is supported during the operation of sawing, being pressed forcibly against it by a screw $\mathrm{D}$, acting through a puppet fixed to the frame of the machine. At the end of this screw is a cup or basin which applies itself to the piece of wood, so as to form a kind of vice, one side being the end of the fixed tube, the other the cup at the end of the screw $\mathrm{D}$. Within the tube $b$ is a collar for supporting a central axis, which is perfectly cylindrical. The other end of this axis, (seen at $f$,) turns in a collar of the fixed puppet $\mathbf{E}$. The central axis has a pulley $\mathbf{F}$, fixed on it, and giving it motion by a strap similar to the other. Close to the latter pulley a collar $g$ is fitted on the centre piece of the pulley, so as to slip round freely, but at the same time confined to move endways with the pulley and its collar. This collar receives the ends of the two iron rods $d$. The opposite ends of these rods are, as above mentioned, connected by a similar collar, with the pulley $\mathrm{A}$ of the saw $a$. By this conncxion, both the centre bit, which is screwed into the end of the central axis $f$, and the saw sliding upon the fixed tube $b$, are brought forward to the wood at the same time, both being in rapid motion by their respective pulleys.

10. The Coaking Engine.-This ingenious piece of machinery is used to cut the three semicircular holes which surround the hole hored by the crown saw, so as to produce $\mathbf{a}$ cavity in the centre of the disc.

11. Face-turining Lathe-The sheave is fixed against a flat chuck at the end of a mandrel, by a universal chuck, similar to that in the coaking engine, except that the centre pin, instead of having a nut, is tapped into the flat chuck, and turned by a screwdriver.

BLOOD. (Sang, Fr.; Blut, Germ.) The liquid which circulates in the arteries and veins of animals; bright red in the former and purple in the latter, among all the tribes whose temperature is considerably higher than that of the atmosphere. It consists, 1. of a colorless transparent solution of several substances in water; and, 2 . of red, undissolved particles diffused through that solntion. Its specific gravity varies with the nature and health of the animal ; being from 1.0527 to 1.0570 at $60^{\circ} \mathrm{F}$. It has a saline sub-nauseous taste, and a smell peculiar to each animal. When fresh drawn from the vessels, it rapidly coagulates into a gelatinous mass, called the clot, cruor, or crassamentum, from which, after some time, a pale yellow fluid, passing into yellowish green, oozes forth, called the serum. If the warm blood be stirred with a bundle of twigs, as it flows from the veins, the fibrine concretes, and forms long fibres and knots, while it retains its usual appearance in other rispects. The clot contains fibrine and coloring matter in various proportions. Berzelius found in 100 parts of the dried clot of blood, 35 parts of fibrine; 58 of coloring matter; 1.3 of carbonate of soda ; 4 of an animal matter soluble in water, along with some salts and fat. The specific gravity of the serum varies from 1.027 to 1.029. It forms about three fourths of the weight of the blood, has an alkaline reaction, coagulates at $167^{\circ} \mathrm{F}$. into a gelatinous mass, and has for its leading constituent albumen to the amount of 8 per cent, besides fat, potash, soda, and salts of these bases. Blood does not seem to contain any gelatine.

The red coloring matter called hematine, may be obtained from the cruor by washing with cold water and filtering.

Blood was at one time largely employed for clarifying sirup, but it is very sparingly used by the sugar refiners in Great Britain of the present day. It may be dried by evaporation at a heat of $130^{\circ}$ or $140^{\circ}$, and in this state has been transported to the colonies for purifying cane juice. It is an ingredient in certain adhesive cements, coarse pigments for protecting walls from the weather, for making animal charcoal in the Prussian blue works, and by an after process, a decoloring carbon. It is used in some Turkey red dye-works. Blood is a powerful manure. 
BLowing Machine. See Iron, Metallurgy, Ventilation.

BLOWPIPE. (Chalumeau, Fr.; Lothröhre, Germ.) Jewellers, mineralogists, chem. ists, enamellers, \&c., make frequent use of a tube, usually bent near the end, terminated with a finely pointed nozzle, for blowing through the flame of a lamp, candle, or gas-jet, and producing thereby a small conical flame possessing a very intense heat. Modifications of blow pipes are made with jets of hydrogen, oxygen, or the two gases mixed in due proportions.

BLUE DYES. (Teint, Germ. See Enamex.) The materials employed for this purpose are indigo, Prussian blue, logwood, bilberry, (vaccinium myrtillus,) elder berries, (sambucus nigra, ) mulberries, privet berries, (ligustrum vulgare, ) and some other berries whose juice becomes blue by the addition of a small portion of alkali, or of the salts of copper. For dyeing with the first three articles, see them in their alphabetical places. I shall here describe the other or minor blue dyes.

To dye blue with such berries as the above, we boil one pound of them in water, adding one ounce of alum, of copperas, and of blue vitriol, to the decoction, or in their stead equal parts of verdigris and tartar, and pass the stuffs a sufficient time through the liquor. When an iron mordant alone is employed, a steel blue tint is obtained; and when a tin one, a blue with a violet cast. The privet berries which have been employed as sap colors by the card painters, may be extensively used in the dyeing of silk. The berries of th $\mathrm{r}$ African night-shade (solanum guineense) have been of late years considerably applied to silk on the continent in producing various shades of blue, violet, red, brown, \&c. but particularly violet. With alkalis and acids these berries have the same habitudes as bilberries; the former turning them green, the latter red. They usually come from Italy compressed in a dry cake, and are infused in hot water. The infusion is merely filtered, and then employed without any mordant, for dyeing silk, being kept at a warm temperature by surrounding the bath vessel with hot water. The goods must be winced for six hours through it in order to be saturated with color; then they are to be rinsed in running water and dried. One pound of silk requires a pound and a half of the berry, cake. In the residuary bath, other tints of blue may be given. Sometimes the dyed silk is finished by running it through a weak alum water. A color approaching to indigo in permanence, but which differs from it in being soluble in alkalis, though incapable of similar disoxydizement, is the gardenia genipa and aculeata of South America, whose colorless juice becomes dark blue with contact of air; and dyes stuffs, the skin, and nails, of an unchangeable deep blue color, but the juice must be applied in the colorless state.

BLUE PIGMENTS. Several metallic compounds possess a blue color; especially those of iron, cobalt, and molydenum. The metallic pigments, little if at all employed, but which may be found useful in particular cases, are the molybdate of mercury, the hydro-sulphuret of tungsten, the prussiate of tungsten, the molybdate of tin, the oxyde of copper darkened with ammonia, the silicate of copper, and a fine violet color formed from manganese and molybdenum. The blues of vegetable origin, in common use, are indigo, litmus, and blue cakes. The blue pigments of a metallic nature found in commerce are the following: Prussian blue; mountain blue, a carbonate of copper mixed with more or less earthy matter; Bremen blue or verdiler, a greenish blue color obtained from copper mixed with chalk or lime; iron blue, phosphate of iron, little employed; cobalt blue, a color obtained by calcining a salt of cobalt with alumina or oxyde of tin; smalt, a glass colored with cobalt and ground to a fine powder; charcoal blue, a deep shade obtained by triturating carbonized vine stalks with an equal weight of potash in a crucible till the mixture ceases to swell, then pouring it upon a slab, putting it into water, and saturating the alkali with sulphuric acid. The liquor becomes blue, and lets fall a dark blue precipitate, which becomes of a brilliant blue color when heated.

Molybdenum blue is a combination of this metal, and oxyde of tin or phosphate of lime. It is employed both as a paint, and an enamel color. A blue may also be obtained by putting into molybdic acid, (made by digesting sulphuret of molybdenum with nitric acid,) some filings of tin, and a little muriatic acid. The tin deoxydizes the molybdic acid to a certain degree, and converts it into the molybdous, which, when eraporated and heated with alumina recently precipitated, forms this blue pigment. Ultramarine is a beautiful blue pigment, which see.

BLUE VITRIOL; sulphate of copper.

BOMBAZINE. A worsted stuff, sometimes mixed with silk.

BONES. (Os, Fr.; Knochen, Germ.) They form the frame work of animal bodies, commonly called the skeleton; upon which the soft parts are suspended, or in which they are enclosed. Bones are invested with a membrane styled the periosteum, which is composed of a dense tissue affording glue; whence it is convertible into jelly, by ebullition with water. Bones are not equally compact throughout their whole substance; the long ones have tubes in their centres lined with a kind of periosteum, of more importance to the life of the bones than even their external coat. The flat, as 
well as the short and thick bones, exhibit upon their surface an osseous mass of a dense nature, while their interior presents a cavity divided into small cellules by their bony partitions.

In reference to the composition of bones, we have to consider two principal constituents; the living portion or the osseous cartilage, and the inorganic or the earthy salts of the bones.

The osseous cartilage is obtained by suspending bones in a large vessel full of dilute muriatic acid, and leaving it in a cool place at about $50^{\circ} \mathrm{Fahr}$. for example. The acid dissolves the earthy salts of the bones without perceptibly attacking the cartilage, which, at the end of a short time, becomes soft and translucid, retaining the shape of the bones; whenever the acid is saturated, before it has dissolved all the earthy salts it should be renewed. The cartilage is to be next suspended in cold water, which is to be frequently changed till it has removed all the acidity. By drying, the cartilage shrinks a little, and assumes a darker hue, but without losing its translucency. It becomes, at the same time, hard and susceptible of breaking when bent, but it possesses great strength.

This cartilage is composed entirely of a tissue passing into gelatine. By boiling with water, it is very readily convertible into a glue, which passes clear and colorless through the filter, leaving only a small portion of fibrous matter insoluble by further boiling. This matter is produced by the vessels which penetrate the cartilage, and carry nourishment to the bone. We may observe all these phenomena in a very instructive manner, by macerating a bone in dilute muriatic acid, till it has lost about the half of its salts; then washing it with cold water, next pouring boiling water upon it, leaving the whole in repose for 24 hours, at a temperature a few degrees below $212^{\circ} \mathrm{Fahr}$.

The eartilage, which has been stripped of its earthy salts, dissolves, but the small vessels which issue from the undecomposed portion of the bone remain under the form of white plumes, if the water has received no movement capable of crushing or breaking them. We may then easily recognise them with a lens, but the slightest touch tears them, and makes them fall to the bottom of the vessel in the form of a precipitate; if we digest bones with strong hot muriatic acid so as to accelerate their decomposition, a portion of the cartilage dissolves in the acid with a manifest disengagement of carbonic acid gas, which breaks the interior mass, and causes the half-softened bone to begin to split into fibrous plates, separable in the direction of their length. According to Marx, these plates, when sufficiently thin, possess, like scales of mica, the property of polarizing light, a phenomenon which becomes more beautiful still when we soak them with the essential oil of the bark of the Laurus Cassia. The osseous cartilage is formed before the earthy part. The long bones are then solid, and they become hollow only in proportion as the earthy salts appear. In the new-born infant, a large portion of the bones is but partially filled with these salts, their deposition in cartilage takes place under certain invariable points of ossification, and begins at a certain period after conception, so that we may calculate the age of the fœtus according to the progress which ossification has made.

The earthy parts of bones are composed principally of the phosphate and carbonate of lime in various proportions, variable in different animals, and mixed with small quantities, equally variable, of phosphate of magnesia and fluate of lime. The easiest means of procuring the earthy salts of bones consists in burning them to whiteness, but the earthy residuum procured in this manner, contains substances which did not exist beforehand in the bones, and which did not form a part of their earthy salts; as, for example, sulphate of soda, produced at the expense of the sulphur of the bones and the alkaline carbonate, proceeding from the cartilage with which it was combined. On the other hand, the greater part of the lime has lost its carbonic acid. As the sulphuric acid is the product of combustion, it is obvious that an acidulous solution of a fresh bone can afford no precipitate with muriate of barytes. The phosphate of lime contained in the bone-salts is a subphosphate, consisting, according to Berzelius, of three prime equiva. nts of the acid, and eight of the base; or of 2,677 parts of the former, and 2,818 of the latter. It is always obtained when we precipitate the phosphate of lime by an excess of ammonia. When calcined bones are distilled in a retort with their own weight of sulphuric acid, a little fluoric acid is disengaged, and it acts on the surface of the glass. The following analyses of the bones of men and horned cattle, are given by Berzelius. They were dried after being stripped of their fat and periosteum till they lost no more weight. 


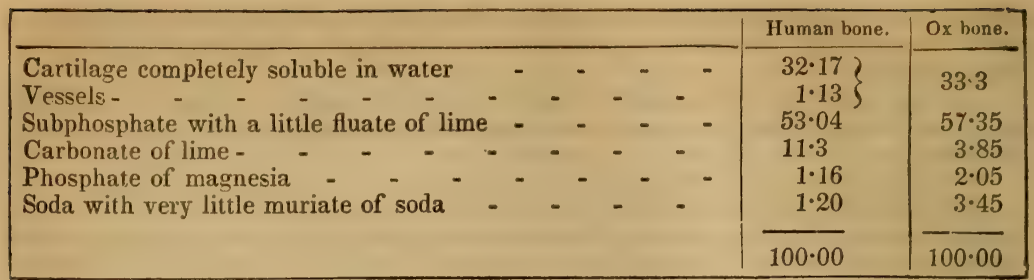

The most essential difference in the composition of these bones is that those of man contain three times as much carbonate of lime as those of the ox; and that the latter are richer in phosphate of lime and magnesia in the same proportion. Fernandez de Barros has established a comparison between the phosphate and carbonate of lime in the bones of different animals. He found in 100 parts of earthy salt of the bones of the following animals :-

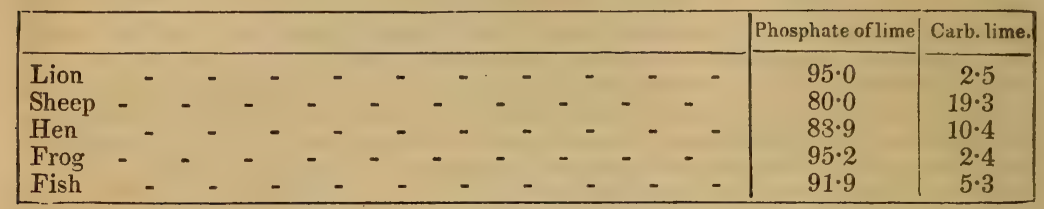

The bones of fish are divided into those which contain earthy salts and those which have none, called cartilaginous fishes. The enamel of the teeth is composed as follows :-

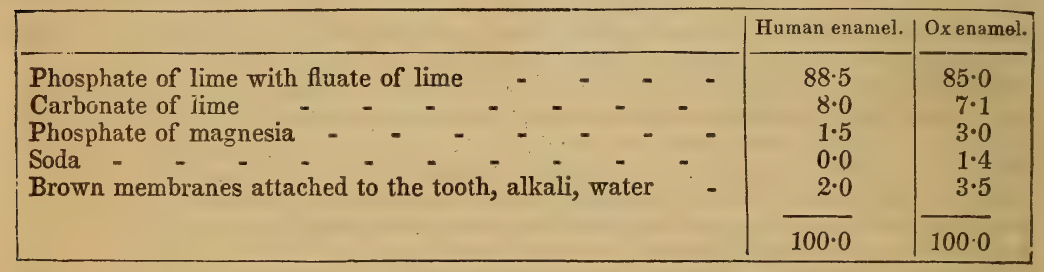

In the arts, the bones are employed by turners, cutlers, manufacturers of animal charcoal, and, when calcined, by assayers for making cupels. In agriculture, they are employed as a manure, for which purpose they should be ground in a mill, and the powder sowed along with the seeds in a drill. It is supposed, in many cases, to increase the crop in weight of grain and straw together, by from 40 to 50 per cent. In France, soup is extensively made by dissolving bones in a steam-heat of two or three days' continuance. The shavings of hartshorn, which is a species of bone, afford an elegant jelly: the shavings of calves' bones may be used in their stead.

Living bones acquire a red tinge when the animals receive madder with their food; but they lose it when the madder is discontinued for some time.

BONE BLACK (Noir d'os, Fr.; Knochenschwartz, Germ.), or Animal charcoal, as it is less correctly called, is the black carbonaceous substance into which bones are converted by calcination in close vessels. This kind of charcaol has two principal applications : to deprive various solutions, particularly sirups, of their coloring matters, and to furnish a black pigment. The latter subject will be treated of under Ivorx BLACK.

The discovery of the antiputrescent and decoloring properties of charcoal in general, is due to Lowitz, of Petersburg; but their modifications have occupied the attention of many chemists since his time. Kels published, in 1798, some essays on the discoloring of indigo, saffron, madder, sirup, \&c. by means of charcoal, but he committed a mistake in supposing bone black to have less power than the charcoal of wood. The first useful application of charcoal to the purification of raw colonial sugar was made by M. Guillon, who brought into the French markets considerable quantities of fine sirups, which he discolored by ground wood charcoal, and sold them to great advantage, as much superior to the cassonades of that time. In 1811, M. Figuier, an apothecary at Montpelijer, published a note about animal charcoal, showing that it blanched vinegars and wines with much more energy than vegetable charcoal; and, lastly, in 1812, 
M. Derosnes proposed to employ animal charcoal in the purification of sirups and sugar refining. The quantities of bone black left in the retorts employed by MM. Payen, for producing crude carbonate of ammonia, furnished abundant materials for making the most satisfactory experiments, and enabled these gentlemen soon to obtain ten per cent. more of refined sugar from the raw article than had been formerly extracted, and to improve, at the same time, the characters of the lumps, bastards, treacle, \&c.

The calcination of bones is effected by two different systems of apparatus; by heating them in a retort similar to that in which coal is decomposed in the gas works, or in small pots piled up in a kiln. For the description of the former, see Gas-Light. On the second plan, the bones, broken into pieces, are put into small cast-iron pots of the form shown in fig. 135, about three eighths of an inch thick, two of which are dexterously placed with their mouths in contact, and then luted together with loam. The lip of the upper pot is made to slip inside of the under one. These double vessels, containing together about fifty pounds of bones, are arranged alongside, and over each other, in an oven, like a potter's kiln, till it be filled. The oven or kiln may be either oblong or upright. The latter is represented in figs. 136, 137, 138. A is the fireplace or grate for the fuel; c c are the openings in the dome of the furnace through which the flame flows; the divisions of these orifices are shown in fig. 138 . B is the wall of brick-work. D the space in which the pots are distributed. $\mathbf{E}$ is the door by which the workman carries in the pots, which is afterwards built up with fire-bricks, and plastered over with loam. This door is seen in fig. 136. F F are the lateral flues for conveying the disengaged gases into the air.

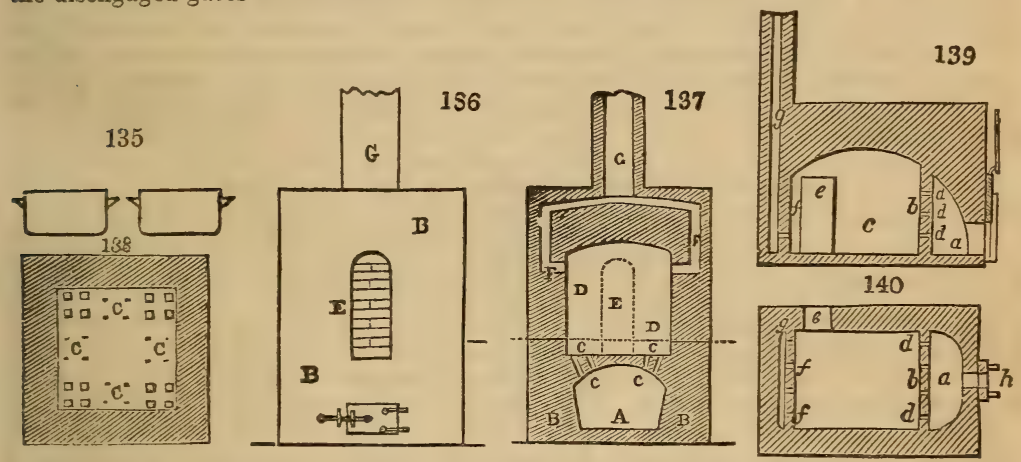

Fig. 139 is a longitudinal section, and fig. 140, a ground plan of a horizontal kiln for calcining bones. $a$ is the fire-chamber, lying upon a level with the sole of the kiln; it is separated by a pillar $b$, from the calcining liearth $c$. In the pillar or wall, several rows of holes $d$, are left at different heights; $e$ is the entrance door; $f$, the outlet vents for the gases, vapors, and smoke, into the chimney $g$; $h$, a sliding damper-plate for regulating the admission of the air into the fire in the space $a$.

By this arrangement the offensive emanations are partly consumed, and partly carried off with the smoke. To destroy the smell completely, the smoke should be made to pass through a second small furnace.

The number of pots that may be put into a kiln of this kind depends, of course, upon its dimensions; but, in general, from 100 to 150 are piled up over each other, in columns, at once; the greatest heat being nearest the roof of the kiln; which resembles, in many respects, that used for baking pottery ware.

In both kilns the interior walls are built of fire-bricks. In the oblong one, the fiercest heat is near the vaulted roof; in the upright one, near the sole; and the pots, containing the larger lumps of bones, should be placed accordingly near the top of the former, and the bottom of the latter. Such a kiln may receive about seventy double pots, containing in the whole thirty-five cwt. of bones.

After the earth is filled with the pots, and the entrance door is shut, the fire is applied at first moderately, but afterwards it must be raised and maintained, at a brisk heat, for eight or ten hours. The door of the ash-pit and the damper may now be nearly closed, to moderate the draught, and to keep up a steady ignition for six or eight hours longer, without additional firing; after which the doors must be all opened to cool the furnace. When this is done, the brick-work of the entrance door musi be taken down, the kiln must be emptied, and immediately filled again with a set of pots previously filled with bones, and luted together; the pots which have been ignited may, in the course of a short time, be opened, and the contents put into the magazine. But 
in operating with the large decomposing cylinder retort, the bones being rakel out hot, must be instantly tossed into a receiver, which can be covered in air-tight till they are cool.

The bones lose upon the average about one half of their weight in the calcination. In reference to the quality of the black, experience has shown that it is so much more powerful as a discoloring agent, as the bones from which it was made have been freer from adhering fatty, fleshy, and tendinous matters.

The charcoal is ground in a mill, either to a fine powder and sifted, or into a coarse granular state, like gunpowder, for the preparation of which two sieves are required, one with moderately fine meshes, to allow the small dust to pass through, and one with large meshes, to separate the proper-sized grains from the coarser lumps. Either a corn-mill, an edgestone mill, or a steel cylinder mill, may be employed for grinding bone-black, and it is generally damped in the operation to keep down the fine dust.

Bone-black, as found in commerce, is very variable in its discoloring power, which arises from its having been exposed either to too great a heat which has glazed its carbon, or too low a heat which has left its albumen imperfectly decomposed. A steady ignition of due continuance is the proper decomposing temperature. Its composition is generally as follows :-

Phosphate of lime, with carbonate of lime; and a little sulphuret of iron, or oxyde of iron, 88 parts; iron in the state of a silicated carburet, 2 parts; charcoal containing about one fifteenth of azote, 10 parts. None of the substances present, except the charcoal, possesses separately any discoloring power.

The quality may be tested by a solution of brown sugar, or molasses, or of indigo in sulphuric acid. The last is generally preferred by the French chemists, who have occupied themselves most with this subject, and it contains usually one thousandth part of its weight of this dye-drug of the best quality. Other animal substances yield a charcoal, possessed of very considerable discoloring properties. The following table by M. Bussy exhibits an interesting comparison of almost every kind of charcoal in this point of view.

Table of the discoloring powers of different charcoals.

\begin{tabular}{|c|c|c|c|c|c|}
\hline Species of Charcoal. & Weight. & $\begin{array}{l}\text { Indigo test } \\
\text { consumed. }\end{array}$ & $\begin{array}{c}\text { Molasses iest } \\
\text { consumed. }\end{array}$ & $\begin{array}{l}\text { Blanehing by } \\
\text { indigo. }\end{array}$ & $\begin{array}{l}\text { Power by } \\
\text { molasses. }\end{array}$ \\
\hline Blood calcined with potash & $\underset{1}{\text { Gramme. }}$ & $\begin{array}{l}\text { Litres. } \\
1 \cdot 60\end{array}$ & $0 \cdot 18$ & 50 & 20 \\
\hline Ditto with chalk - - - & 1 & 0.57 & $0 \cdot 10$ & 18 & 11 \\
\hline Ditto with phosphate lime & 1 & 0.38 & 0.09 & 12 & 10 \\
\hline Gelatine ditto with potash & 1 & $1 \cdot 15$ & $0 \cdot 14$ & 36 & $15 \cdot 5$ \\
\hline Albumen ditto ditto - - & 1 & 1.08 & $0 \cdot 14$ & 34 & $15 \cdot 5$ \\
\hline Starch ditto ditto - - & 1 & 0.34 & 0.08 & $10 \cdot 6$ & $8 \cdot 8$ \\
\hline Charcoal from acet. potash & 1 & $0 \cdot 18$ & $0 \cdot 04$ & $5 \cdot 6$ & $4 \cdot 4$ \\
\hline Ditto from carb. soda by & & & & & \\
\hline phosphorus - - - & 1 & $0 \cdot 38$ & 0.08 & 12 & 8.8 \\
\hline Calcined lamp black - - & 1 & $0 \cdot 128$ & 0.03 & 4 & $3 \cdot 3$ \\
\hline Ditto ditto potash - - - & 1 & 0.55 & 0.09 & $15 \cdot 2$ & $10 \cdot 6$ \\
\hline Bone black treated with & & & & & \\
\hline $\begin{array}{l}\text { mur. acid and potash - } \\
\text { Bone black ditto }\end{array}$ & 1 & $1 \cdot 45$ & $0 \cdot 18$ & 45 & 20 \\
\hline $\begin{array}{l}\text { Bone black ditto with mur. } \\
\text { acid }\end{array}$ & 1 & $0 \cdot 06$ & 0.015 & $1 \cdot 87$ & $1 \cdot 6$ \\
\hline Oil calcined with phosph. & & & & & \\
\hline of lime --- & 1 & $0 \cdot 064$ & $0 \cdot 017$ & 2 & $1 \cdot 9$ \\
\hline Crude bone black - - - & 1 & $0 \cdot 032$ & 0.009 & 1 & 1 \\
\hline
\end{tabular}

With regard to the mode of operation of bone black on colored liquids, M. Payen showed in his prize essay, 1. That the decoloring power of charcoal depends in general upon its state of division; 2. That in the various charcoals, the crubonaceous matter acts only upon the coloring matters, combining with and precipitating them; 3 . That in the application of charcoal to the refining of sugar, it acts also upon the gluten, for it singularly promotes crystallization; 4. That according to the above principles, the decoloring action of charcoals may. be so modified, as to make the most inert become the most active; 5 . That the distinction between animal and vegetable charcoals is improper, and that we may substitute for it that of dull and brilliant charcoals; 6 . That of the substances present in charcoal besides carbon, and particularly animal charcoal, those which favor the decoloring action, have an influence relative only to the carbon; they serve as auxiliaries to it, by insulating its particles, and presenting them more freely to the action of the coloring matter; 7. That animal charcoal, besites its decoloring power, has the valuable property of taking lime in solution from water and 
sirup; 8. That neither vegetable, nor other charcoals, besides the animal, have this power of abstracting lime; 9. That by the aid of the decolorimeter, or graduated tube charged with test solution of indigo or molasses, it is easy to appreciate exactly the de coloring properties of all kinds of charcoal.

Different rarieties of lignite (fossilized wood) or even pit coal, when well carbonized in close ressels, afiord a decoloring charcoal of considerable value. Br reducing 100 parts of clay into a thin paste with water, kneading into it 20 parts of tar, and 500 of finely-ground pit coal, drying the mixed mass, and calcining it out of contact of air, a charcoally matter may be obtained not much inferior to bone-black in whitening sirups.

The restoration of animal charcoal from burnt bones, for the purpose of sugar refining, has been long practised in France. Mr. W. Parker has lately made the following process the subject of a patent. The charcoal, when taken from the vessel in which it has been employed for the purposes of clarifying the sugar, is to be thoroughly washed with the purest water that can be obtained, in order to remove all the saccharine matter adhering to it. When the washing process has been completed, the charcoal is laid out to dry, either in the open air or in a suitable stove, and when perfectly free from moisture, it is to be separated into small pieces and sifted through a sieve, the wires or meshes of which are placed at distances of about two and a half in every inch. This sifting will not only divide the charcoal into small pieces, but will cause any bits of wood or other improper matters to be separated from it.

The charcoal, thus prepared, is then to be packed lightly in cylindrical vessels called crucibles, with some small quantity of bones, oil, or other animal matter mixed with it. The crucibles are then to be closed by covers, and luted at the joints, leaving no other opening but one small hole in the centre of the corer, through which any gas, generated within the vessel when placed in the oven or furnace, may be allowed to escape.

The crucibles are now to be ranged round the oven, and placed, one upon another, in vertical positions; and when the oren is properly heated, gas will be generated within each crucible, and issue out from the central hole. The gas thus emitted, being of an inflammable quality, will take fire, and assist in heating the crucibles; and the operation being carried on until the crucibles become of a red heat, the oven is then to be closed. and allowed to cool; after which the crucibles are to be removed, when the charcoal will be fuund to have become perfectly renovated, and fit for use as before.

BORAX. A native saline compound of boracic acid and soda, found abundantly in Thibet and in South America. The crude product from the former locality was impoited into Europe under the name of tincal, and was purified from some adhering fatty matter by a process kent a long time secret by the Venetians and the Dutch, and which consisted chiefly in boiling the substance in water with a little quicklime.

Gmelin found borax, in prismatic crystals, to contain 46.6 per cent. of water; and Arvredson, in the calcined state, to consist of 68.9 of acid and 31.1 soda, in 100 parts. M. Payen describes an octahedral borax, which contains only 30.64 per cent. of water, and is therefore preferred by the hrasiers in their soldering processes.

Borax has a sweetish, somewhat lixivial taste, and affects vegetable colors like an alkali; it is soluble in 12 parts of cold and 2 of boiling water. It effloresces and becomes opaque in a dry atmosphere, and appears luminous, by friction, in the dark. It melts at a heat a little abote that of boiling water, and gires out its water of crystallization, after which it forms a spongy mass, called calcined borax. The octahedral borax, which is prepared by crystallization, in a solution of $1.256 \mathrm{sp}$. gr., hept up at $145^{\circ} \mathrm{F}$., is not efflorescent. Then borax is ignited, it fuses into a glassy-looking substance.

The following is the improved mode of purifying borax. The crude crystals are to be broken into small lumps, and spread upon a filter lined with a lead grating, under which a piece of cloth is stretched upon a wooden frame. The lumps are piled up to the height of 12 inches, and washed with small quantities of a caustic soda ley of $5^{\circ} \mathbf{B}$. (sp. gr. 1.033) until the liquor comes off nearly colorless; they are then drained, and put into a large copper of boiling water, in such quantities that the resulting solution standa $20^{\circ}$ B. (sp. gr. 1.160.) Carbonate of soda, equivalent to 12 per cent. of the borax, must now be added; the mixed solution is allowed to settle, and the clear liquid syphoned off into crystallizing ressels. Whenever the mother waters get foul, they must be evaporated to dryness in cast-iron pots, and roasted, to burn away the viscid coloring matter.

Borax is sometimes adulterated with alum and common salt; the former addition may be readily detected by a few drops of water of ammonia, which will throw down its alumina; and the latter by nitrate of silver, which will give with it a precipitate insoluble in nitric acid.

The native boracic acid obtained from the lakes of Tuscany, which has been manufactured in France into borax, has greatly lowered the price of this article of commerce. When MM. Payen and Cartier first began the business, they sold the crystals at the same price as the Dutch, viz., 7 francs the kilogramme ( $2 \frac{1}{5} \mathrm{lbs}$, avoird.); but, in a fev 
years, they could obtain only 2 francs and 60 centimes, in consequence of the market getting overstocked. The annual consumption of France in 1823 was 25,000 kilos., and the quantity produced in M. Payen's works was 50,000. The mode of making borax from the acid is as follows:-The lake water is evaporated in graduation houses, and then concentrated in boilers till it crystallizes. In that state it is carried to Marseilles. About 500 kilogrammes of water are made to boil in a copper, and 600 kilo. grammes of crystallized carbonate of soda are dissolved in it by successive additions of 20 kilogrammes. The solution being maintained at nearly the boiling point, 500 kilogrammes of the crystallized boracic acid of Tuscany are introduced, in successive portions. At each addition of about 10 kilogrammes, a lively effervescence ensues, on which account the copper should be of much greater capacity than is sufficient to contain the liquors. When the whole acid has been added, the fire must be damped by being covered up with moist ashes, and the copper must be covered with a tight lid and blankets, to preserve the temperature uniform. The whole is left in this state during 30 hours; the clear liquor is then drawn off into shallow crystallizing vessels of lead, in which it should stand no higher than 10 or 12 inches, to favor its rapid cooling. At the end of three days in winter, and four in summer, the crystallization is usually finished. The mother water is drawn off, and employed, instead of simple water, for the purpose of dissolving fresh crystals of soda. The above crystals are carefully detached with chisels, redissolved in boiling water, adding for each 100 kilos., 10 kilos. of carbonate of soda. This solution marks $20^{\circ} \mathrm{B}$. (sp. gr. $1 \cdot 160$ ); and, at least, one ton (1000 kilos.) of borax should be dissolved at once, in order to obtain crystals of a marketable size. Whenever this solution has become boiling hot, it must be rur. off into large crystallizing lead chests of the form of inverted truncated pyramids, furnished with lids, enclosed in wooden frames, and surrounded with mats to confine the heat. For a continuous business, there should be at least 18 vessels of this kind; as the solution takes a long time to complete its crystallization, by cooling to $30^{\circ} \mathrm{C}$. $\left(86^{\circ} \mathrm{F}\right.$.) The borax crystals are taken out with chisels, after the liquor has been drawn off, and the whole has become cold.

One hundred parts of the purest acid, usually extracted from the lakes of Tuscany, contain only fifty parts of the real boracic acid, and yield no more, at the utmost, than 140 or 150 of good borax.

Dry borax acts on the metallic oxydes at a high temperature, in a very remarkable manner, melting and vitrifying them into beautiful colored glasses. On this account, it is a most useful reagent for the blowpipe. Oxyde of chrome tinges it of an emerald green; oxyde of cobalt, an intense blue; oxyde of copper, a pale green ; oxyde of tin, opal ; oxyde of iron, bottle-green and yellow ; oxyde of manganese, violet; oxyde of nickel, pale emerald green. The white oxydes impart no color to it by themselves. In the fusion of metals, borax protects their surface from oxydizement, and even dissolves away any oxydes formed upon them; by which twofold agency it becomes an excellent flux, invaluable to the goldsmith in soldering the precious metals, and to the brasier in soldering copper and iron.

Borax absorbs muriatic and sulphurous acid gases, but no others, whereby it becomes, in this respect, a useful means of analysis.

The strength or purity of borax may be tested by the quantity of sulphuric acid requisite to neutralize a given weight of it, as indicated by tincture of litmus.

When mixed with shell-lac in the proportion of one part to five, borax renders that resinous body soluble in water, and forms with it a species of varnish.

Boracic acid is a compound of 31.19 of boron, and 68.81 oxygen, in 100 parts. Its prime equivalent referred to oxygen 100 , is 871.96 .

The following process for refining the native Indian borax or tincal, has been published by MM. Robiquet and Marchand:-

It is put into large tubs, covered with water for 3 or 4 inches above its surface, and stirred through it several times during six hours. For 400 pounds of the tincal, there must now be added one pound of quicklime diffused through two quarts of water. Next day the whole is thrown upon a sieve, to drain off the water with the impurities, consisting, in some measure, of the fatty matter combined with the lime, as an insoluble soap. The borax, so far purified, is to be dissolved in $2 \frac{1}{2}$ times its weight of boiling water, and eight pounds of muriate of lime are to be added for the above quantity of borax. The liquor is now filtered, evaporated to the density of $18^{\circ}$ or $20^{\circ} \mathrm{B}$. $(1 \cdot 14$ to $1 \cdot 16$ sp. grav.), and set to crystallize in vessels shaped like inverted pyramids, and lined with lead. At the end of a few days, the crystallization being completed, the mother waters are drawn off, the crystals are detached and dried. The loss of weight in this operation is about 20 per cent.

The quantity of borax imported into the United Kingdom in 1835 was 335,224 pounds; whereof 122,022 pounds were exported. The duty is $10 \mathrm{~s}$. upon the refined, and $4 \mathrm{~s}$ unrefined. 
BOOKBINDING is the art of sewing together the sheets of a book, and securms hem with a back and side boards. Binding is distinguished from stitching, which is merely sewing the leaves without bands or backs; and from half-binding, which con. rists in securing the back only with leather, the pasteboard sides being coverel with blue or marble paper; whereas in binding, both the back and sides are covered with .eather.

Boolibinding, according to the present mode, is performed in the following manner: - The sheets are first folded into a certain number of leaves, according to the form in which the book is to appear; viz., two leaves for folios, four for quartos, eight for octavos, twelve for duodecimos, \&c. This is done with a slip of ivory or boxwood, called a folding-stick; and in the arrangement of the sheets the workmen are directer by the catch-words and signatures at the bottom of the pages. When the leaves are thus folded and arranged in proper order, they are usually beaten upon a stone with a heavy hammer, to make them solid and smooth, and are then condensed in a press. After this preparation they are sewed in a sewing press, upon cords or packthreads called bands, which are kept at a proper distance from each other, by drawing a thread through the iniddle of each sheet, and turning it round each band, beginning with the first and proceeding to the last. The number of bands is generally six for folios, and five for quartos, or any smaller size. The backs are now glued, and the ends of the bands are opened, and scraped with a knife, that they may be more conveniently fixed to the pasteboard sides; after which the back is turned with a hammer, the book being fixed in a press between boards, called backing boards, in order to make a groove for admitting the pasteboard sides. When these sides are applied, holes are made in them for drawing the bands through, the superfluous ends are cut off, and the parts are hammered smooth. The book is next pressed for cutting; which is done by a particular machine called the plough, to which is attached a knife. See the figures and descriptions infra. It is then put into a press called the cutting press, betwixt two boards, one of which lies even with the press, for the knife to run upon; and the other above for the knife to cut against. After this the pasteboards are cut square with a pair of iron shears; and last of all, the colors are sprinkled on the edges of the leaves, with a brush made of hog's bristles; the brush being held in the one hand, and the hair moved with the other.

Diflerent kinds of binding are distinguished by different names, such as law binding, marble binding, French binding, Dutch binding, \&c. In Dutch binding, the backs are vellum. In French binding a slip of parchment is applied over the back between each band, and the ends are pasted upon the inside of each pasteboard. This indorsing, as it is called, is peculiar to the French binders; who are enjoined, by special ordonnunce, to back their books with parchment. The parchment is applied in the press, after the back has been grated to make the paste take hold. The Italians still bind in a coarse thick paper, and this they call binding alla rustica. It is extremely inconvenient, as it is liable to wear without particular care.

A patent was obtained in 1799 by Messrs. John and Joseph Williams, stationers in London, for an improved method of binding books of every description. The improvement consists of a back, in any curved form, turned a little at the edges, and made of iron, steel, copper, brass, tin, or of ivory, bone, wood, vellum, or, in short, any material of sufficient firmness. This back is put on the book before it is bound, so as just to cover without pressing the edges; and the advantage of it is that it prevents the book, when opened, from spreading on either side, and causes it to rise in any part to nearly a level surface. In this method of binding the sheets are prepared in the usual manner, then sewed on vellum slips, glued, cut, clothed, and boarded, or half boarded; the firm back is then fastened to the sides by vellum drawn through holes, or secured by enclosing it in vellum or ferret wrappers, or other materials pasted down upon the boards, or drawn through them.

A patent was likewise obtained in 1800 by Mr. Ebenezer Palmer, a London stationer, for an improved way of binding books, particularly merchants' account-books. This improvement has been described as follows:-let several small bars of metal be provided, about the thickness of a shilling or more, according to the size and thickness of the book; the length of each bar being from half an inch to several inches, in proportion to the strength required in the back of the book. At each end of every bar let a pivot be. made of different lengths, to correspond to the thickness of two links which they are to receive. Each link must be made in an oval form, and contain two holes proportioned to the size of the pirots, these links to be the same metal as the hinge, and each of thern nearly equal in length to the width of two bars. The links are then to be riveted on the pirots, each pivot receiving two of them, and thus holding the hinge together, on the principle of a link-chain or hinge. There must be two holes or more of different sizes, as may be required, on each bar of the hinge or chain; by means of these holes eacl section of the book is strongly fastened to the hinge which operates with the back 
of the book. when bound, in such a manner as to make the different sections parallel with each other, and thus admit writing without inconvenience on the ruled lines, close to the back.

The leather used in covering books is prepared and applied as follows: being first moistened in water, it is cut to the size of the book, and the thichness of the eilge is paired oft on a marble stone. It is next smeared over with paste made of what llour, streiched over the pasteboard on the outside, and doubled over the eilges within. The book is then corded, that is, bound firmiy betwixt two boards, to make the cover stick strongly to the pasteboard and the back; on the exact performance of which the neatness of the book in a great measure depends. The back is then warmed at the fire to soften the glue, and the leather is rubbed down with a bodkin or folding stich, to set and fix it close to the back of the book. It is now set to dry, and when dry, the buards are removed; the book is then washed or sprinkled over with a little paste and water, the edges and squares blacked with ink, and then sprinkled fine with a brush, by striking it against the hand or a stick: or with large spots, by being mixed with solution of green vitriol, which is called marbling. Two blank leaves are then pasted down to the cover, and the leaves, when dry, are burnished in the press, and the cover rolled on the edges. The cover is now glazed twice with the white of an egg, filleted, and, last of all, polished, by passing a hot iron over the glazed color.

The employment in bookbinding of a rolling press for smoothing and condensing the leaves, instead of the hammering which books have usualiy reeeired, is an improvement introduced several years ago into the trade by Mr. W. Burn. His press consists of two iron cylinders about a foot in diameter, adjustable in the usual way, by means of a screw, and put in motion by the power of one man or of two, if need be, applied to one or two winch-liandles. In front of the press sits a boy who gathers the sheets into pacliets, by placing two, three, or four, upon a piece of tin plate of the same size, and corering them with another piece of tin plate, and thus proceeding by alternating tin plates and bundles of sheets till a sufficient quantity hare been put together, which will depend on the stiffness and thickness of the paper. The packet is then passed between the rollers and receired by the man who turns the winch, and who has time to lar the sheets on one side, and to hand orer the tin plates by the time that the boy bas prepared a second packet. A minion Bible may be passel through the press in one minute, whereas the time necessary to beat it would be twenty minutes. It is not, howerer, merely a saring of time that is gainei br the use of the rolling-press; the paper is made smoother than it

1.11

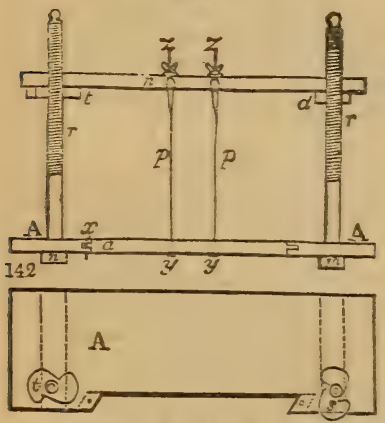
would have been by beating, and the compression is so much greater, that a rolled book will be reduced to about five sixths of the thickness of the same book if beaten. A shelf, therefore, that will hold fifty books bound in the usual way would hold nearly sixty of those bound in this manner, a circumstance of no small importance, when it is considered how large a space even a moderate library occupies, and that book-cases are an expensive article of furniture. The rolling-press is now substituted for the hammer by several considerable bookbinders.

Fig. 141 represents the sewing-press, as it stands upon the table, before which the bookbinder sits. Fig. 142 is a ground plan, without the parts $a$ and $n$ in the former figure. $A$ is the base-board, supported upon the cross bars $m n$, marked with dotted lines in fig. 14\%. Upon the screw rods $r r$, fig. 111, the nuts $t d$ serve to fix the flat upper bar $n$, at any desired distance from the base. That bar has a slit along its middle, through which the hooks below $z=$ nass down for receiring the ends of the sewing cords $p p$,

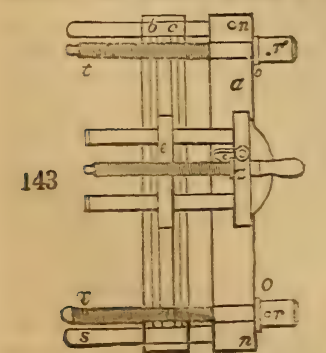
fixed at $y y$, and stretched by the thumb-screws $z z$. The bar $y$ y is let into an oblong space cut out of the front edge of the base-board, and fixed there by a moveable pin $a$, and a fixed pin at its other end, round which it turns.

Fig. 143 is the bookbinder's cutting-press, which is set upright upon a sort of chest for the reception of the paper parings; and consists of three sides, being open above and to the left hand of the workman. The pressbar, or beam $a$, has two holes $n \boldsymbol{n}$ upon its under surface, for securing it to two pegs standing on the top of the chest. The screw rods $t t$ pass through two tapped holes in the bar, marked with $b c$ at its apper and; their heads $r r$ being held by the shoulders 00 . The heads are pierced with 
noles into which lever pins are thrust for screwing the rods hard up. The heavy beam a remains immoveable, while the parallel bar with the book is brought home towards it by the two screws. The two rulers $s s$ serve as guides to preserve the motions truly parallel; and the two parallel lath bars $b c$ guide between them the end bar $\varepsilon$, of the plough, whose knife is shown at $i$, with its clamping screw $z$.

Mr. Ollham, printing engineer of the Bank of England, distinguished for mechanical ingenuity, has contrived a convenient machine for cutting the edges of books, banknotes, \&c., either truly square or polygonal, with mathematical precision. Fig. 144, represents an end elevation of the machine. Fig. 145, a side view of the same, the letters of reference indicating the same parts of the machine in each of the figures.

$a$, is the top cross bar with rectangular grooves $b b ; c c$, are side posts ; $d d$, cross feet to the same, with strengthenin $r$ brackets; $e e$, a square box, in which the press stands, for holding wasle cuttings. Fig. 146, is a cross section of the upright posts, $c c$, taken horizontally. There are rectangular grooves in the upright posts, for the projecting ends of the cast iron cross bracket $f$, to slide up and down in. In the middle of the under-side of this piece $f$, there is a boss, within which is a round recess, to receive the top of the screw $g$, which works in the cast iron cross piece $h$, similarly made with the former, but bolted firmly to the posts $c c$. Upon the screw $g$, there is a circular handle or ring $i$, for

144

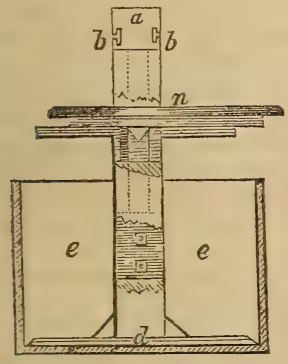

145

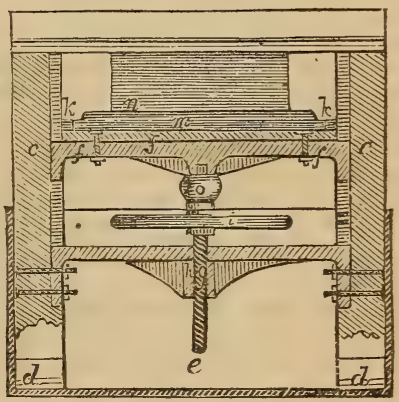

gertially turning the screw, and immediately over it cross holes for tightening the press by means of a lever bar. Upon the cross piece $f$, is bolted the board $j$, and upon each end of this board is made fast the rabbeted pieces $k k$, for another buard $l$, to slide in.

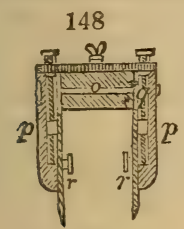

149
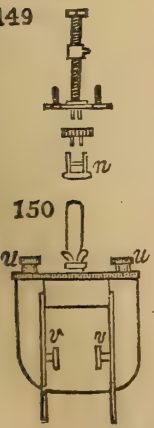

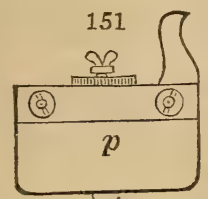

152
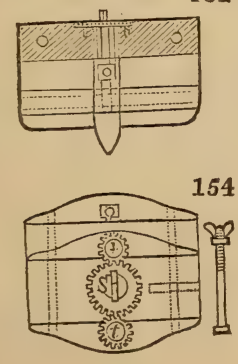

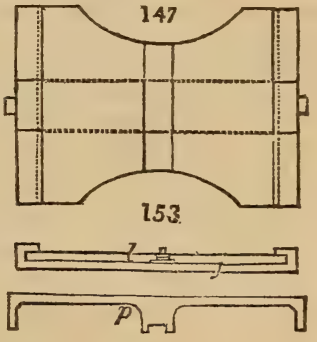

116

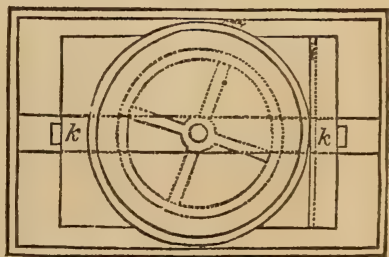

Across the middle of this board, and parallel to the pieces $k k$, the tongue piece $m$ is made fast, which fits into a groove in the bottom of board $l$. A horizontal representation of this is seen at fig. 147, and immediately under this view is also seen an end view of $l$ and $f$, connected together, and a side view of $f$ by itself. In the middle of the board $h$ 
is a pin for a circular board $n$, to turn upon, and upon this letter board is placed the " material to be cut," with a saving piece between it, and the circular piece which is to be divided upon its edge into any number of parts required, with a stationary index on the board $l$, to point to each.

It will now be understood that the "material to be cut," may be turned round upon the centre pin of the board $n$, and also that both it and the board can be shifted backward and forward under the top cross piece $a$, and between the side slide slips $k l k$, the surfaces of which should also be divided into inches and tenths.

The plough, fig. 148, shown in several positions, is made to receive two knives or cutters as the "material to be cut" may require, and which are situated in the plough as I now describe. The plough is composed of three principal parts, namely, the top, and its two sides. The top 0 , is made the breadth of the cross piece $a$, and with a handle made fast thereon. The sides $p p$, are bolted thereto, with bolts and nuts through corresponding holes in the top and sides. The figures below give inside views, and cross sections of the details of the manner in which the cutters and adjustments are mounted. A groove is cut down each cheek or side, in which are placed serews that are held at top and bottom from moving up and down, but by turning they cause the nuts upon them to do so; they are shown at $q q$. These nuts have each a pin projecting inwards, that go into plain holes made in the top ends of cutters $r$. The 148th and follewing figs. are $\frac{1}{4}$ in scale.

The cutters, and the work for causing them to go up and down, are sunk into the cheeks, so as to be quite level with their inner surfaces. Fig. 149 shows one of those screws apart, how fixed, and with moveable nut and projecting pin. The top of each screw terminates with a round split down, and above it a pinion wheel and boss thereon, also similarly split. This pinion fits upon the split pin. Above, there is a cross section of a hollow coupling cap with steel tongue across, that fits into both the cuts of the screw pin and pinion boss, so that when lowered upon each other, they must all turn together. In the middle and on the top of the upper piece 0 , the larger wheel s, runs loose upon its centre, and works into the two pinion-wheels $t t$. The wheel $s$ has a fly-nut with wings mounted upon it.

It will now be seen, when the plough is in its place as at fig. 150, that if it be pushed to and fro by the right hand, and the nut occasionally turned by the left, the knives or cutters will be protruded downwards at the same time, and these either will or will not advance as the coupling caps $u u$ are on or off. The ribs $v v$, run in the grooves $b b$, fig. 144, and keep the cutters to their duty, working steadily. The top cross bar $a$, is the exact breadth of a bank-note, by which means both knives are made to cut at the same time. The paper is cut uniformly to one length, and accurately square.

By the use of this machine, the air-pump paper-wetting apparatus, and appendant press, the paper of 45,000 notes is fully prepared in one hour and a half by one person, and may then be printed. It is not so much injured by this process as by the ordinary method of clipping by hand, soaking it, \&c., which more or less opens and weakens the fabric, especially of bank-note paper.

One of the greatest improvements ever made in the art of bookbinding is, apparently, that for which $\mathrm{Mr}$. William Hancock has very recently obtained a patent. After folding the sheets in double leaves, he places them vertically, with the edges forming the back of the book downwards in a concave mould, of such rounded or semi-cylindrical shape as the back of the book is intended to have. The mould for this purpose consists of two parallel upright boards, set apart upon a cradle frame, each having a portion or portions cut out vertically, somewhat deeper than the breadth of the book, but of a width nearly equal to its thickness before it is pressed. One of these upright boards may be slidden nearer to or farther from its fellow, by means of a guide bar, attached to the sole of the cradle. Thus the distance between the concave bed of the two vertical slots in which the book rests, may be varied according to the length of the leaves. In all cases about one fourth of the length of the book at each end projects beyond the board, so that one half rests between the two boards. Two or three packthreads are now bound round the leaves thus arranged, from top to bottom of the page in different lines, in order to preserve the form given to the back of the mould in which it lay. The book is next subjecte to the action of the press. The back, which is left projecting very slightly in front, is tisn smeared carefully by the fingers with a solution of caoutchouc, whereby each paper-edge receives a small portion of the cement. In a few hours it is sufficiently dry to take another coat of a somewhat stronger caoutchouc solution. In 48 hours, 4 applications of the caoutchouc may be made and dried. The back and the adjoining part of the sides are next covered with the usual band or fillet of cloth, glued on with caoutchouc; after which the book is ready to have the boards attached, and to be covered with ieather or parchment as may be desired.

We thus see that Mr. Hancock dispenses entirely with the operations of stitching, sewing, sawing-in, hammering the back, or the use of paste and glue. Instead of leaves 
attached by thread stitches at 2 or 3 points, we have them agglutinated securely along their whole length. Buolis bound in this way open so perfectly flat upon a table without strait or resilience, that they are equally comfortable to the student, the musician, and the merchant. The caoutchouc eement moreover, being repulsive to insects, and not affected by humidity, gives this mode of binding a great superiority over the old method with paste or glue, which attracted the ravages of the moth, and in damp situations allowed the book to fall to rieces. For engravings, atlases, and legers, this binding is admirably adapted, because it allows the pages to be displayed most freely, without the risk of dislocating the volume; but for security, 3 or 4 stitches should be made. The leaves of music-books bound with caoutchouc, when turned over, lie flat at their whole extent, as if in loose sheets, and do not torment the musician like the leaves of the ordinary books, which are so ready to spring back again. Manuscripts and collections of letters which happen to have little or no margin left at the back for stitching them by, ma! be bound by Mr. Hancock's plan without the least encroachment upon the writing. The thicliest legers, thus bound, open as easily as paper in quire, and may be written on up to the innermost margin of the book without the least inconvenience.

Haring inspected various specimens of Mr. Hancock's workmanship, I willingly bear testimony to the truth of the preceding statement. See Cloth Binning.

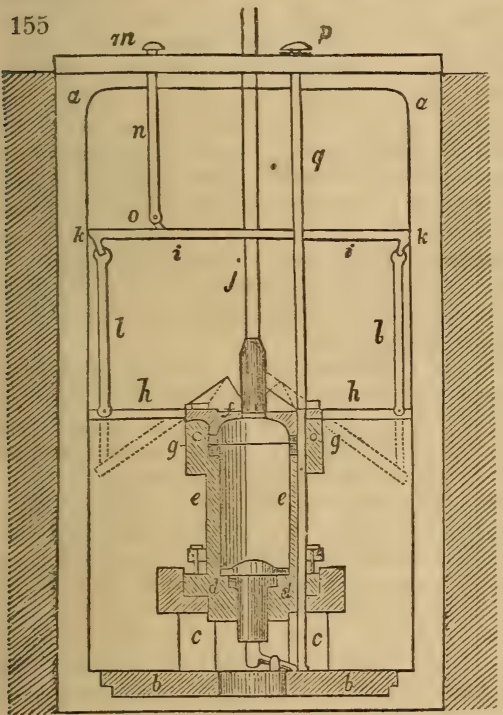

BOTTLE MANUFACTURE. The following mechanism for moulding bottles forms the subject of a patent obtained by Henry Rickets of Bristol, in 1822. Fig. 15E is a section of the apparatus, consisting of a square frame, $u a$, of iron or wood; this is fixed in a pit formed in the floor; $b b$ is the base of the frame, with an aperture for knocking up the bottom of the bottle; $c c$ are four legs secured to the frame-floor $b$, upon which the mould is supported. The platform or stand of the mould $d d$ has an opening in its centre for the introduction of the bottom of the mould, which is raised against the bottom of the bottle by the knocker-up; $e e$ are the sides of the mould; and $f f$ is the top of the mould in two pieces, turning over upon the joints at $g \mathrm{~g}$, so as to form the neck of the bottle; $h h$ are levers or arms for raising and depressing the top pieces; $i i$ is a horizontal shaft or axle, turning in bearings at each end, from which shaft two levers, $k k$, extend; these levers are connected by upright rods, $l l$, to the levers or arms, $h h$, of the top pieces $f f$.

The wcight of the arms $h h$, and rods $l l$, will, by their gravity, cause the top pieces to open, as shown by the dotted lines; in this situation of the mould, the melted glass is to be introduced by a tube as usual. The workman then steps with one foot upon the knob $m$, which forces down the rod $n$, and by means of a short lever 0 , extending from the shaft $i$, forces down the top pieces $f$, and closes the mould, as seen in the figure; the glass is then made to extend itself to the shape of the mould, by blowing as usual, so as to form the bottle, and the workman at this time putting his other foot upon the knob $p$, depresses the rod $q$, and hence raises the bottom of the mould by means of the knockernp, $r$, so as to form the bottom of the bottle.

At the bottom of the mould a ring is introduced of any required thickness, for the purpose of regulating the capacity of the bottle; upon which ring it is proposed to raise letters and figures, as a mould to imprint the maker's name and the size of the bottle. These moulds can be removed and changed at pleasure. Under the knob $p$, a collar or washer is to be introduced, of any required thickness, to regulate the knocling up of the bottom, by which a perfect symmetry of form is presented. In order to make bottles of different sizes or forms, the mould is intended to be removed, and its place supplied by another mould of different dimensions and figure; the lower parts of all the moulds being made to fit the same frame. Such a mould ought to be prescribed by legislative enactment, with an excise stamp to define the capacity of every bottle, and thereby put an end to the interminable frauds committed in the measure of wine and all other liquors sold by the bottle.

BOUGIF. A smooth, flexible, elastic, slender cylinder, introduced into the uretnra, rectum, or æsophagus, for opening or dilating it, in cases of stricture and other diseases. 
The invention of this instrument is clained by Aldereto, a Portuguese physician, but its form and uses were first described by his pupil Amatus, in the year 1554. Some are solid, and some hollow; some corrosive, and some mollifying. They generally owe their elasticity to linseed oil, inspissated by long boiling, and rendered drying by litharge. This viscid matter is spread upon a very fine cord or tubular web of cotton, flax, or silk, which is rolled upon a slab when it becomes nearly solid by drying, and is nnally polished in the same way.

Pickel, a French professor of medicine, published the following recipe for the com position of bougies. Take 3 parts of boiled linseed oil, one part of amber, and one of oil of turpentine; melt and mix these ingredients well together, and spread the compound at three successive intervals upon a silk cord or web. Place the pieces so coated in a stove heated to $150^{\circ} \mathrm{F}$; ; leave them in it for 12 hours, adding 15 or 16 fresh layers in succession, till the instruments have acquired the froper size. Polish them first with pumice-stone, and finally smooth with tripoli and oil. This process is the one still employed in Paris, with some slight modifications; the chief of which is dissolving in the oil one twentieth of its weight of caoutchouc to render the substance more solid. For this purpose the caoutchouc must be cut into slender shreds, and added gradually to the hot oil. The silk tissue must be fine and open, to admit of the composition entering freely among its filaments. Each successive layer ought to be dried first in a stove, and then in the open air, before another is applied. This process takes two months for its completion, in forming the best bougies called elastic; which ought to bear twisting round the finger without cracking or scaling, and extension without giving way, but retracting when let go. When the bougies are to be hollow, a mandril of iron wire, properly bent with a ring at one end, is introduced into the axis of the silk tissue. Some bougies are made with a hollow axis of tin foil rolled into a slender tube. Bougies are also made entirely of caoutchouc, by the intervention of a solution of this substance in sulphuric ether, a menstruum sufficiently cheap in France, on account of the low duty upon alcohol. There are medicated bougies, the composition of which belongs to surgical pharmacy. The manufacture of these instruments of various kinds forms a separate and no inconsiderable branch of industry at Paris. MM. Feburger and Lamotte are eminent in this line.

BRACES. (Bretelles, Fr.; Hosenträger, Germ.) Narrow fillets or bands of leather or textile fabric, which pass over the shoulders, and are attached behind and before to the waistbands of pantaloons and trousers, in the act of wearing them, for supporting their weight, and bracing them up to the body. It is a useful modern invention, superseding the necessity of girding the belly with a tight girdle, as in former times.

BRAIDING MACHINE. (Machine à lacets, Fr.; Bortenwerkerstuhl, Germ.) This being employed not only to manufacture stay-laces, braid, and upholsterers' cord, but to cover the threads of caoutchouc for weaving brace-bands, deserves a description in this work. Three threads at least are required to make such a knitted lace, but 11, 13, or 17, and even 29 threads are often employed, the first three numbers being preferred. They are made by means of a frame of a very ingenious construction, which moves by a continuous rotation. We shall describe a frame with 13 threads, from which the structure of the others may be readily conceived. The basis of the machine consists of
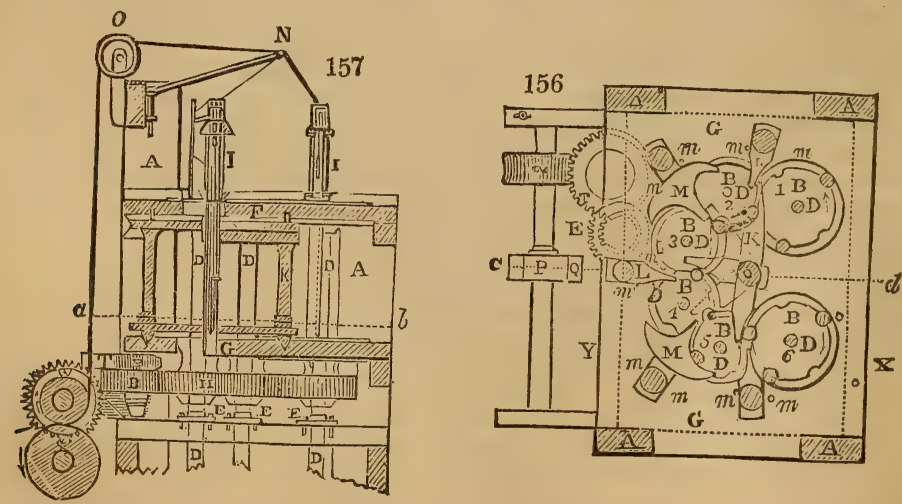

four strong wooden uprights, A, figs. 156, 15\%, 158, occupying the four angles of a rectangle, of which one side is 14 inches long, the other 18 inches, and the height of the rectangle about 40 inches. Fig. 156 is a section in a horizontal plane, passing through the line $a b$ of $f i g .157$, which is a vertical section in a plane passing through the centre of 
the machine $\mathrm{c}$, according to the line $c d$, fig. 156. The side $\mathrm{x}$ is supposed to be the front of the frame; and the opposite side, $\mathbf{Y}$, the back. B, six spindles or skewers, numbered, from 1 to 6 , placed in a vertical position upon the circumference of a circle, whose centre coincides with that of the machine at the point c. These six spindles are composed, 1. Of so many iron shafts or axes $\mathrm{D}$, supported in brass collets $\mathbf{E}$ ( $f \mathrm{~g}$. 157 ), and extended downwards within six inches of the ground, where they rest in brass steps fixed upon a horizontal beam. 2. Wonden heads, made of horn-beam or nut-tree, placed, the first upon the upper end of each spindle, opposite the cut-out beam $F$, and the second opposite the second beam G. 3. Wooden-toothed wheels, H, reciprocally working together, placed between the beam G and the collet-beam $\mathbf{E}$. The toothed wheels and the lower heads for each spindle are in one piece.

The heads and shafts of the spindles No. 1 and 6 , are one fifth stronger than those of the other spindles; their heads have five semicircular grooves, and wheels of 60 teeth, while the heads of the others have only four grooves, and wheels of 48 teeth; so that the number of the grooves in the six spindles is 26 , one half of which is occupied.with the stems of the puppets $I$, which carry the 13 threads from No. 1 to 13 . The toothed wheels, which give all the spindles a simultaneous movement, but in different directions, are so disposed as to bring their grooves opposite to each other in the course of rotation.

$\mathbf{x}$, the middle winglet, triple at bottom and quintuple at top, which serves to guide the puppets in the direction they ought to pursue.

$\mathrm{L}$, three winglets, single at top and bottom, placed exteriorly, which serve a like purpose.

M, two winglets, triple at bottom and single at top, placed likewise exteriorly, and which serve the same purposes as the preceding; $m$ are iron pins inserted in the cut-out beam $\mathrm{G}$, which serve as stops or limits to the oscillations of the exterior winglets.

Now, if by any moving power (a man can drive a pair) rotation be impressed upon the large spindle No. 1, in the direction of the arrow, all the other spindles will necessarily pursue the rotatory movement indicated by the respective arrows. In this case, the 13 puppets worling in the grooves of the heads of the spindles will be carried round simultaneously, and will proceed each in its turn, from one extremity of the machine to the opposite point, crossing those which have a retrograde movement. The 13 threads united at the point $\mathrm{N}$, situated above the centre of the machine, will form at that point ${ }^{d}$ the braid, which, after having passed over the pulley 0 , comes between the two rollers $P$, and is squeezed together, as in a flatting-mill, where the braid is calendered at the same time that it is delivered. It is obvious that the roller $\mathrm{P}$ receives its motion from the toothed wheel of the spindle No. 3, and from the intermediate wheels $\mathrm{R}, \mathrm{s}, \mathrm{T}$, as well as from the endless screw $z$, which drives at proper speed the wheel w, fixed upon the shaft of the roller $\mathrm{P}$.

The braid is denser in proportion as the point $\mathrm{N}$ is less elevated above $g$ the tops of the puppets; but in this case, the eccentric motion of these puppets is much more sensible in reference to that point towards which $b$ all the threads converge than when it is elevated. The threads, which múst be always kept equally stretched by means of a weight, as we shall presently see, are considerably strained by the traction, occasioned by the constantly eccentric movement of the puppets. From this cause, braiding machines must be worked at a moderate velocity. In general, for fine work, 30 turns of the large spindle per minute are the utmost that can safely be made.

The puppet or spindle of this machine, being the most important piece, I have represented it in section, upon a scale one fourth of its actual size, fig. 158. It is formed of a tube, $a$, of strong sheet iron well brazed; $b$ is a disc, likewise of sheet iron, from which a narrow fillet, $c$, rises vertically as high as the tube, where both are pierced with holes, $d e$, through which the thread $f$ is passed, as it comes from the bobbin, $g$, which turns freely upon the tube $a$. The top of this bobbin is conical and toothed. A small catch or detent, $h$, moveable in a vertical direction round $i$, falls by its own weight into the teeth of the crown of the bobbin, in which case this cannot revolve; but when the detent is raised so far as to disengage the teeth, and at the same time to pull the thread, the bobbin turns, and lets out thread till the detent falls back into these same teeth.

A skewer of iron wire, $k$, is loaded with a small weight, $l$, melted upon it. The top of this skewer has an eye in it, and the bottom is recurvel as is shown in fig. 158, so that supposing the thread comes to break, this skewer falls into the actual 
position in the ligure, where we see its 'ower end extend ag beyond the tube $a$, by about $\frac{1}{4}$ of an inch; but as long as the thread is unbroken, the skewer $k$, which serves to keep it always tense, during the eccentric movement of the puppet, does not pass out below the tube.

This disposition has naturally furnished the means of causing the machine to stop, whenever one of the threals brealis. This inferior protrusion of the skewer pushes in its progress a detent, which instantly causes the band to slide from the driving pulley to the loose pulley. Thus the machine carnot operate unless all the threads be entire. It is the business of the operative, who has 3 or 4 under her charge, to mend the threads as they break, and to substitute full bobbins for empty ones, whenever the machine is stopned.

The braiding frame, though it does not move quickly, makes a great deal of noise, and would make still more, were the toothed wheels made of metal instead of wood. For them to act well, they should be inade with the greatest precision, by means of appropriate toobls for forming the teeth of the wheels, and the other peculiar parts.

BRAN. (Son, Fr.; Kleie, Germ.) The husky portion of ground wheat, separated by the bolter from the flour. It is advantageously emplosed by the calico printers, in the clearing process, in which, by boiling in bran-water, the coloring matters adhering to the non-mordanted parts of maddered goods, as well as the dun matters which cloud the mordanted portions, are removed. A valuable series of researches concerning the operation of bran in such cases was made a few years ago by that distinguished chemist and calico printer, M. Daniel Kœchlin Schouch, and published in the ninth number of the Bulletin de la Societé Industrielle de Mulhausen. Nine sets of experiments are recorded, which justified the following conclusions.

1. The dose of two bushels of bran for 10 pieces of calico is the best, the ebullition being kept up for an hour. A boil for the same time in pure water had no effect in clearing either the grounds or the figures.

2. Fifteen minutes boiling are sufficient when the principal object is to clear white grounds, but in certain cases thirty minutes are requisite to brighten the dyed parts. If, by increasing the charge of bran, the time of the ebullition could be shortened, it would be in some places, as Alsace, an economy; because for the passage of ten pieces through a copper or vat heated with steam, $1 \mathrm{cwt}$. of coal is consumed in fuel which costs from $2 \frac{1}{2}$ to 3 francs, while two bushels of bran are to be bought for one franc.

3. By increasing the quantity of water from 12 to 24 hectolitres with two bushels of bran, the clearing effect upon the ten pieces was impaired. It is therefore advantageous not to use too much water.

4. Many experiments concur to prove that flour is altogether useless for the clearing boil, and that finer bran is inferior for this purpose to the cuarser.

5. The white ground of the calicoes boiled with wheat bran, are distinguishable by their superior brightness from that of those boiled with rye bran, and especially with barley bran; the latter having hardly any effect.

6. There is no advantage in adding soap to the bran boil; though a little potash or soda may be properly introduced when the water is calcareous.

7. The pellicle of the bran is the most powerful part, the flour and the starch are of no use in clearing goods, but the mucilage which forms one third of the weight of the bran has considerable efficacy, and seems to act in the following way. In proportion as the mucilaginous substance dissolves the coloring and tawny matters upon the cloth, the husky surface attracts and fixes upon itself the greater part of them. Accordingly, when used bran is digested in a weak alkaline bath, it gives up the color which it had absorbed from the cloth.

The following chemical examination of bran is interesting. A pound of it was "niled at successive times with water; the decoctions, being filtered, let fall in cooling a grayish deposite, which was separated by decantation. The clear liquor afiorded by evaporation to dryness four ounces of a brownish, brittle matter, composed chiefly of mucilage, a little gluten, and starch. The gray deposite of the above filtered liquor amounted to half an ounce. Nine ounces of the cortical portion of the bran were obtained. The loss amounted to $2 \frac{1}{2}$ ounces, being in some measure the hygrometric water of the bran itself:

When boiled with distilled water, goods are cleared pretty well without bran. Cer* tain delicate dyes must be boiled only a few minutes in a strong decoction of bran previously made.

BRANDY. The name given in this country to ardent spirits distilled from wine and possessed of a peculiar taste and flavor, due to a minute portion of a peculias volatile oil. Each variety of alc shol has an aroma characteristic of the fermented subance from which it is procurel; whether it be the grape, cherries, sugar-cane, rice, corn, or potatoes; and it may be distinguished even as procured from different growths of the vine. The brandies of Languedoc, Bordeaux, Armagnac, Cognac, Aunis, 
Saintmee, Rcchelle, Orleans, Barcelona, Naples, \&c being each readily recognisable by

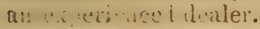

Autugier shwwed, by experiments, that the disagreeable taste of the spirits distilled frum the marc of the grape is owing to an essential oil, contained in the skin of the giape; and foun:l that the oil, when insulated, is so energetic that a few drops are suff. cient to taint a pipe of 600 litres of fine flavored spirit.

The most celebrated of the French brandies, those of Cognac and Armagnac, are slightly rectified to only from 0.935 to 0.922 ; they contain more than half their weight of water, and come over therefore highly charged with the fragrant essential oil of the husk of the grape. When, to save expense of carriage, the spirit is rectified to a much higher degree, the dealer, on receiving it at Paris, reduces it to the market proof by the addition of a little highly-flavored weak brandy and water; but he cannot in this way produce so finely-flavored a spirit, as the weaker product of distillation of the Cognac wine. If the best Cognac brandy be carefully distilled.at a low heat, and the strong spirit be diluted with water, it will be found to have suffered much in its flavor.

Genuine French brandy evinces an acid reaction with litmus paper, owing to a minute portion of vinegar; it contains, besides, some acetic ether, and, when long kept in oak casks, a lic.se astringent matter. The following formula may be proposed for converting a silent or flavorless corn spirit, into a factitious brandy. Dilute the pure alcohol to the proof pitch, add to every hundred pounds weight of it from half a pound to a pound of argol (crude winestone) dissolved in water, a little acetic ether, and French wine-vinegar, some bruised French plums, and flavor-stuff from Cognac; then distil the mixture with a gentle fire, in an alembic furnished with an agitator.

The spirit which comes over may be colored with nicely burned sugar (caramel) to the desired tint, and roughened in taste with a few drops of tincture of catechu or oakbark.

The above recipe will afford a spirit free from the deleterious drugs too often used to disguise and increase the intoxicating power of British brandies; one: which may be reckoned as wholesome as alcohol, in any shape, can ever be.

BRASS. (Laiton, cuivre jaune, Fr.; Messing, Germ.) An alloy of copper and zinc. It was formerly manufactured by cementing granulated copper, called bean-shot, or copper clippings, with caicined calamine (native carbonate of zinc) and charcoal, in a crucible, and exposing them to bright ignition. Three parts of copper were used for three of calamine and two of charcoal. The zinc reduced to the metallic state by the agency of the charcoal, combined with the copper, into an alloy which formed, on cooling, a lump at the bottom of the crucible. Several of these, being remelted and cast into moulds, constituted ingots of brass for the market. James Emerson obtained a patent, in 1781, for making brass by the direct fusion of its two metallic elements, and it is now usually manufactured in this way.

It appears that the best proportion of the constituents to form fine brass is one prime equivalent of copper $=63 \frac{1}{2}+$ one of zinc $=32 \cdot 3$; or very nearly 2 parts of copper to 1 of zinc. The bright gold colored alloy, called Prince's, or Prince Rupert's metal, in this country, consists apparently of two primes of zinc to one of copper, or of nearly equal parts of each. Brass, or hard solder, consists of two parts of brass and one of zine melted together, to which a little tin is occasionally added; but when the solder must be very strong, as for brass tubes that are to undergo drawing, two thirds of a part of zine are used for two parts of brass. Mosaic gold, according to the specification of Parker and Hamilton's patent, consists of 100 parts of copper, and from 52 to 55 of zine; which is no atomic proportion. Bath metal is said to consist of 32 parts of brass and 9 parts of zinc.

The button manufacturers of Birmingham make their platin with 8 parts of brass and 5 of zinc; but their cheap buttons with an alloy of copper, tin, zinc, and lead.

Red brass, the Tombak of some, (not of the Chinese, for this is white copper, consists of more copper and less zinc than go to the composition of brass; being from $2 \frac{1}{2}$ to 8 or 10 of the former to 1 of the latter. At the famous brass works of Hegermühl, to be presently described, 11 parts of copper are alloyed with 2 of zinc into a red brass, from which plates are made that are afterwards rolled into sheets. From such an alloy the Dutch foil, as it is called, is manufactured at Nürnberg; Pinchbeck, Similor, Mannheim gold, are merely different names of alloy similar to Prince's metal. The last consists of 3 of copper and 1 of zinc, separately melted, and suddenly incorporated by stirring.-Wiegleb.

In the process of alloying two metals of such different fusibilities as copper and zinc, a considerable waste of the latter metal by the combustion, to which it is so prone, might be expected; but, in reality, their mutual affinities seem to prevent the loss, in a great measure, by the speedy absorption of the zinc into the substance of the copper. Indeed, copper plates and rods are often brassed externally by exposure, at a high temperature, to the fumes of zinc, and aftewards laminated or drawn. The spurious 
gold wire of Lyons is made from such ; ods. Copper vessels may be superficially conver.ed into brass by boiling them in dilute muriatic acid containing some wine-stone and zine amalgum.

The first step in making brass is to plunge slips of copper into melted zinc till an alloy of somewhat diffic alt fusion be formed, to raise the heat, and add the remaining pronortion of the copper.

The brass of the first fusion is broken to pieces, and melted with a fresh quantity of zine, to obtain the finished brass. Each melting takes about 8 or 9 hours. The metal is now cast into plates, about 40 inches long by 26 inches broad, and from one third to one half inch thick. The moulds are, in this case also, slabs of granite mounted in an iron frame. Granite appears to be preferred to every thing else as a mould, because it preserves the heat long, and by the asperities of its surface, it keeps hold of the clay lute applied to secure the joinings.

The cast plates are most usually rolled into sheets. For this purpose they are cut into ribands of various breadths, commonly about $6 \frac{1}{2}$ inches. The cylinders of the brass rolling-press are generally 46 inches long, and 18 inches in diameter. The ribands are first of all passed cold through the cylinders; but the brass soon becomes too hard to laminate. It is then annealed in a furnace, and, after cooling, is passed afresh through a rolling press. After paring off the chipped edges, the sheets are laminated two at a time: and if they are to be made very thin, even eight plates are passed through together. The brass in these operations must be annealed 7 or 8 times before the sheet arrives at the required thinness. These successive heatings are very expensive; and hence they have led the manufacturers to try various plans of economy. The annealing furnaces are of two forms, according to the size of the sheets of brass. The smailer are about 12 feet long, with a fire-place at each end, and about 13 inches wide. The arch of the furnace has a cylindrical shape, whose axis is parallel to its small side. The hearth is horizontal, and is made of bricks set on edoe. In the front of the furnace there is a large door, which is raised by a lever, or chain, and counterweight, and slides in a frame between two cheeks of cast iron. This furnace has, in general, no chimney, except a vent'slightly raised above the door, to prevent the workmen being incommoded by the smoke. Sometimes the arch is perforated with a number of holes. The sheets of brass are placed above each other, but separated by parings, to allow the hot air to circulate among them, the lowest sheet resting upon two bars of cast iron placed lengthwise.

The large furnaces are usually 32 feet long, by $6 \frac{1}{2}$ feet wide, in the body, and 3 feet at the hearth. A grate, 13 inches broad, extends along each side of the hearth, through its whole length, and is divided from it by a small wall, 2 or 3 inches high. The vault of the furnace has a small curvature, and is pierced with 6 or 8 openings, which allow the smoke to pass off into a low bell-chimney above. At each end of the furnace there is a cast-iron door, which slides up and down in an iron frame, and is poised by a counterweight. On the hearth there is a kind of railway, composed of two iron bars, on the grooves of which the carriage moves with its loads of sheets of brass.

These sheets, being often 24 feet long, could not be easily moved in and out of the furnace; but as brass laminates well in the cold state, they are all introduced and moved out together. With this view, an iron carriage is framed with four bars, which rest on four wheels. Upon this carriage, of a length nearly equal to that of the furnace, the sheets are laid, with brass parings between them. The carriage is then raised by a crane to a level with the furnace, and entered upon the grooved bars which lie upon the hearth. That no heat may be lost, two carriages are provided, the one being ready to put in as the other is taken out; the furnace is meanwhile uniformly kept hot. This method, however convenient for moving the sheets in and out, wastes a good deal of fuel in heating the iron carriage.

The principal places in which brass is manufactured on the great scale in England, are Bristol, Birmingham, and Holywell, in North Wales.

The French writers affirm, that a brass, containing 2 per cent. of lead, works more freely in the turning lathe, but does not hammer so well as a mere alloy of copper and zinc.

At the brass manufactory of Hegermühl, upon the Finon canal near Potsdam, the following are the materials of one charge; 41 pounds of old brass, 55 pounds refined copper (gahrkupfer) granulated; and 24 pounds of zinc. This mixture, weighing 120 pounds, is distributed into four crucibles, and fused in a wind furnace with pitcoal fuel. The waste varies from $2 \frac{1}{2}$ to 4 pounds upon the whole.

Fig. 159 represents the furnace as it was formerly worked there with chareoal; $a$, the laboratory in which the crucibles were placed. It was walled with fire-bricks. The foundations and the filling-in walls were formed of stone rubbish, as being bad conductors or heat; sand and ashes may be also used; $b$, cast-iron circular grating plates pierced with 
12 holes (see fig. 160), over them a sole of loam, $c$, is beat down, and perforated with holes corresponding to those in the iron dises; $d$, the ash-pit; $e$, the bock, a draught flue which

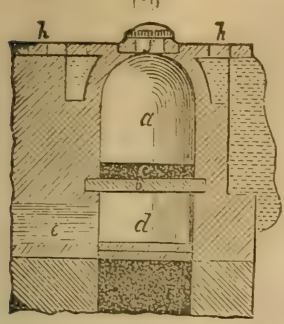

160
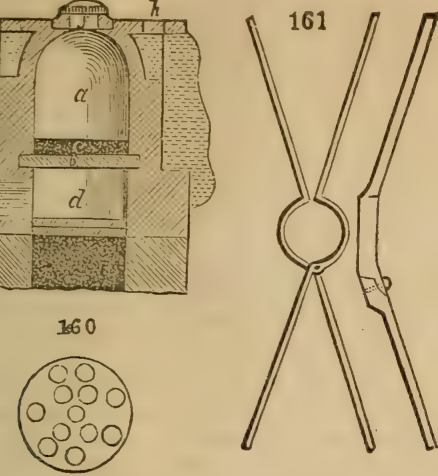
conducts the air requisite to the combustion, from a sunk tunnel, in communica. tion with several melting furnaces. The terrace or crown of the furnace, $f$, lies on a level with the foundry floor, $h, h$, and is shut with a tile of fire-clay, g, which may be moved in any direction by means of hooks and eyes in its binding iron ring. Fig. 161, the tongs for putting in and taking out the charges, as viewed from above and from the side.

Figs. 162, 163, represent the furnaces constructed more recently for the use of pitcoal fuel; fig. 162, being an upright section, and fig. 163, the ground plan. In this furnace the crucibles are not surrounded with the fuel, but they receive the requisite melting heat from the flame proceeding from the grate upon which it is burned. The crucibles stand upon 7 binding arches, $a$, which unite in the middle at the liel-stone $b$, fig. 16.5 ; between the arches are spaces through which the flame rises from

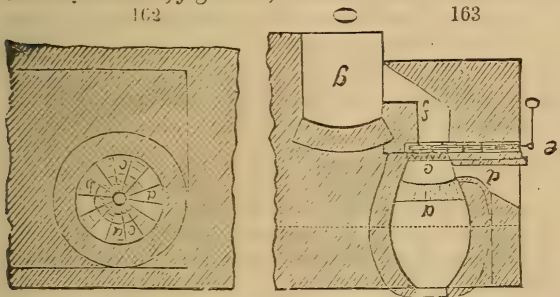

the grate $c ; d$ is the fire-door; $e$ a sliding tile or damper for regulating or shutting off the air-draught; $f$ an inclined plane, for carrying off the cinders that fall through the grate, along the draught tunnel g, so that the air in entering below may not be heated by them.

The crucibles are 16 inches deep, $9 \frac{1}{2}$ wide at the mouth, $6 \frac{1}{2}$ at the bottom; with a thickness in the sides of 1 inch, and $1 \frac{1}{2}$ below; they stand from 40 to 50 meltings. The old brass, which fills their whole capacity, is first put in and melted down; the crucibles are now taken out, and are charged with the half of the zinc in pieces of from 1 to 3 cubic inches in size, covered over with coal ashes; then one half of the copper charge is introduced, again coal-dust; and thus the layers of zinc and copper are distributed alternately with coal-ashes betwixt them, till the whole charge gets finally fused. Over all, a thicker layer of carbonaceous matter is laid, to prevent oxdizement of $t$ he brass. Eight crucibles filled in this way are put into the furnace between the 11 holes of the grate-shelf; and over them two empty crucibles are laid to be heated for the casting operation. In from $3 \frac{1}{2}$ to 4 hours the brass is ready to be poured out. Fifteen English bushels of coals are consumed in one operation; of which six are used at the introduction of the crucibles, and four gradually afterwards.

When sheet brass is to be made the following process is pursued :-

An empty crucible, called a caster (giesser), is taken out of the furnace through the crown with a pair of tongs, and is kept red hot by placing it in a hollow hearth (mundal), surrounded with burning coals; into this crucible the contents of four of the melting pots are poured; the dross being raked out with an iron scraper. As soon as the melting pot is emptied, it is immediately re-charged in the manner above described, and replaced in the furnace. The surface of the melted brass in the caster is swept with the stump of a broom, then stirred about with the iron rake, to bring up any light foreign matter to the surface, which is then skimmed with a little scraper; the crucible is now seized with the casting tongs, and emptied in the following way :-

The mould or form for casting sheet brass consists of two slabs of granite, $a$ a, figs 164, 165. They are $5 \frac{1}{2}$ feet long, 3 feet broad, 1 foot thick, and, for greater security, girt with iron bands, $b$ b, 2 inches broad, $1 \frac{1}{2}$ thick, and joined at the four corners with bolts and nuts. The mould rests upon an oaken block, $c, 3 \frac{1}{2}$ feet lon, $2 \frac{1}{6}$ broad, and $1 \frac{1}{4}$ thick, which is suspended at each end upon gudgeons, in bearing blocks, placed under the foundry floor, $d d$, in the casting pit, $e e$. This is lined with bricks; and is $6 \frac{3}{4}$ feet long, $5 \frac{1}{2}$ broad, and 2 deep; upon the two long side walls of the pit, the bearing blocks are laid, which support the gudgeons. The swing-blocks are 10 inches long, 18 inches broad, 15 inches thick, and are somewhat rounded upon their back edge, so that the casting frame may slope a little to the horizon. To these blocks two cross wooden arms, $f f$, are mortised, upon which the under slab rests freely, but so as to project about 5 inches 
backwards over the block, to secure an equipoise in the act of casting. $g$ g are bara placed at both of the long sides, and one of the ends, between the slabs, to determine the
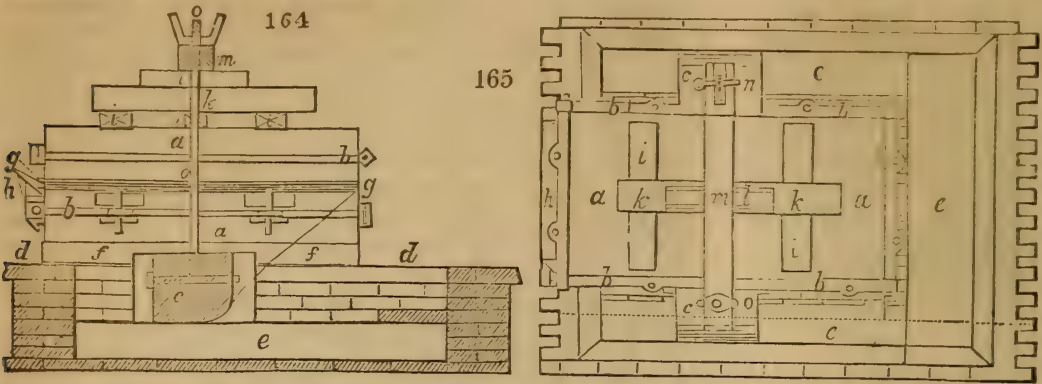

thickness of the brass-plate. Upon the other slab the gate $h$ is fastened, a sheet of iron 6 inches broad, which has nearly the shape of a parallel trapezium (lozenge), and slopes a little towards the horizon. It serves for setting the casting pot upon in the act of pouring out, and renders its emptying more convenient. That gate (steinmaul) is coated with a mixture of loam and hair. The upper slab is securea to the under one in its slanting position by an armor or binding. This consists of the tension bars of wood, ik $l \mathrm{~m}$, of the iron bars $n$, ( 3 to $3 \frac{1}{2}$ inches broad, $1 \frac{1}{2}$ inch thick, see the top view, fig. 165, ) of a rod with holes and pins at its upper end, and of the iron screw spindie 0 . The mode in which these parts act may be understood from inspection of the figure. In order to lift the upper slab from the under one, which is effected by turning it round its edge, a chain is employed, suspending two others, connected with the slab. The former passes orer a puliey, and may be pulled up and down by means of a wheel and axle, or with the aid of a counterweight. Upon each of the two long sides of the slab there are two iron rings, to which the ends of the chains may be hooked. The casting faces of the slab must be coated with a layer of finely ground loam; the thinner the better.

When calamine is employed, $\frac{1}{2} \mathrm{cwt}$. of copper, $\frac{3}{4} \mathrm{cwt}$. of calamine, and $\frac{1}{2}$ the volume of both of charcoal mixed, are put into 7 crucibles, and exposed to heat during 11 or 12 hours; the product being from 70 to $72 \mathrm{lbs}$. of brass.

Brass-plate rolling. At Hegermühl there are two re-heating or annealing furnaces, one larger, 18 feet long, and another smaller, $8 \frac{1}{2}$; the hot chamber is separated from the fire place by iron beams, in such a way that the brass castings are played upon by the fames on both their sides. After each passage through the laminating press (rolls) they are heated anew, then cooled and laminated afresh, till they have reached the proper length. The plates are besmeared with grease before rolling.
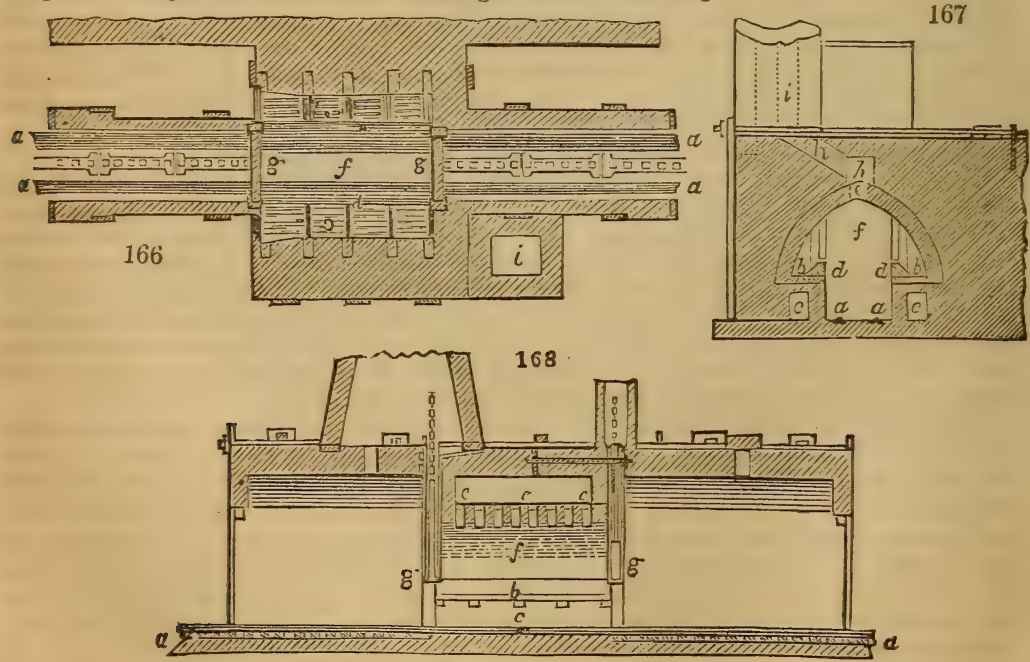

Fig. 166 shows the ground plan of the furnace and its railway; fig. 167, the cross section; and $f \mathrm{~g} .168$, the section lengthwise; $a a$, the iron way bars or rails upon the floor of the 
foundry, for enabling the wheels of the wagon-frame to move readily backward and forwart; $b b$, the two grates; $c c$, the ash pits; $d d$, the fire beams; $e$ e e, vents in the roof of the hot chamber $f ; g g$, two plates for shutting the hot chamber; $h$, the flue; $\imath$, the chimney. After the rolling, the sheets, covered with a blacli oxyde of copper, are plunged into a mother water of the alum works for a few minutes, then washed in clean water, and, lastly, smeared with oil, and scraped with a blunt linife.

In rough brass and brass wares, no less than $16,210 \mathrm{cwts}$. were manufactured in the Prussian States in the year 1832.

For musical purposes, the brass wire made in Ber in has acquired great and merited celebrity ; but that of Birmingham is now preferred, even by foreigners.

BRAsS CoLor, for staining glass, is prepared by exposing for several days thin plates of brass upon tiles in the leer or annealing arch of the glass-house, till it be oxydized into a black powder, aggregated in lumps. This being pulverized and sifted, is to be again well calcined for several days more, till no particles remain in the metallic state; when it will form a fine powder of a russet brown color. A third calcination must now be given, with a carefully regulated heat; its quality being tested from time to time by fusion with some glass. If it makes the glass swell, and intumesce, it is properly prepared; if not, it must be still farther calcined. Such a powder communicates to glass, greens of various tints, passing into turquoise.

When thin narrow strips of brass are stratified with sulphur in a crucible, and calcined at a red heat, they become friable, and may be reduced to powder. This being sifted and exposer upon tiles in a reverberatory furnace for ten or twelve days, becomes fit for use, and is capable of imparting a calcedony, red, or yellow tinge to glass by fusion, according to the mode and proportion of using it.

The glass-makers' red color may be prepared by exposing small plates of brass to a moderate heat in a reverberatory furnace, till they are thoroughly calcined, when the substance becomes pulverulent, and assumes a red color. It is then ready for immediate use.

Brass Color, as employed by the colormen to imitate brass, is of two tints, the red or bronze, and the yellow like gilt brass. Copper filings, mixed with red ochre or bole, constitute the former; a powdered brass imported from Germany, is used for the latter. Both must be worked up with varnish after being dried with heat, and then spread with a flat camel-hair brush evenly upon the surface of the object. The best varnish is composed of 20 ounces of spirits of wine, 2 ounces of shellac, and 2 ounces of sandarach, properly dissolved. See VARNish. Only so much of the brass powder and varnish should be mixed at a time as is wanted for immediate use.

Brass FoIL. Dutch leaf, called Knitter or Rauschgold in Germany, is made from a very thin sheet brass, beat out under a hammer worked by water power, which gives 300 or 400 strokes per minute : from 40 to 80 leaves being laid over each other. By this treatment, it acquires its characteristic solidity and lustre. See above, the process for converting the copper superficially into brass by the fumes of zinc.

BRAZING. (Braser, Fr.; Messing-lothung, Germ.) The soldering together of edges of iron, copper, brass, \&c., with an alloy consisting of brass and zinc, sometimes with a little tin or silver. The surfaces to be thus united must be filed perfectly bright, and not be soiled with the fingers or in any other way. The granular or nearly pulverulent alloy is usually wetted with a paste of ground borax and water, applied in this state, dried, and then exposed carefully to bright ignition at a clear forge fire. Some workmen enclose the part to be soldered in a clay lute, but others prefer leaving it uncovered, that they may see when the solder has flowed freely, and entered into all the seams.

BRAZIL-WOOD. (Bois de Fernambouc, Fr.; Brasilienholz, Germ.) This dye-wood derives its name from the part of America whence it was first imported. It has also the names Fernambuca, wood of Saint Martha, and of Sapan, according to the places which produce it. Linnæus distinguishes the tree which furnishes the Brazil-wood by the name of Casaipinia crista. It commonly grows in dry places among rocks. Its trunk is very large, crooked, and full of knots. It is very hard, susceptible of a fine polish, and sinks in water. It is pale when newly cleft, but becomes red on exposure to the air.

It has different shades of red and orange. Its goodness is determined particularly by its density. When chewed, a saccharine taste is perceived. It may be distinguished from red saunders wood, as the latter does not yield its color to water.

Boiling water extracts the whole coloring matter of Brazil-wood. If the ebullition be long enough continued, it assumes a fine red color. The residuum appears black. In this case, an alkali may still extract much coloring matter. The solution in alcohol or ammonia is still deeper than the preceding.

The decoction of Brazil-wood, called juice of Brazil, is observed to be less fit for dyeing when recent, than when old or even fermented. By age, it takes a yellowish- 
red color. For making this decoction, Hellot recommends to use the hardest water; but it should be remarked, that this water deepens the color in proportion to the earthy salts which it contains. After boiling this wood reduced to chips, or, what is preferabie, to powder, for three hours, this first decoction is poured into a cask. Fresh water is poured on the wood, which is then made to boil for three hours, and mixed with the former. When Brazil-wood is employed in a dyeing bath, it is proper to enclose it in a thin linen hag, as well as all the dye-woods in general.

Wool immersed in the juice of Brazil takes but a feeble tint, which is speedily destroyed. It must receive some preparations.

The wool is to be boiled in a solution of alum, to which a fourth or even less of tartar is added, for a larger proportion of tartar would make the color yellowish. The wool is kept impregnated with it for at least eight days, in a cool place. After this, it is dyed in the Brazil juice with a slight boiling. But the first coloring particles that are deposited, afford a less beautiful color; hence it is proper to pass a coarser stuff previously through the bath. In this manner a lively red is procured, which resists pretty well the action of the air.

Brazil-wood is made use of for dyeing silk what is called false crimson, to distinguish it from the crimson made by means of cochineal, which is much more permanent.

The silk should be boiled at the rate of 20 parts of soap per cent., and then alumed. The aluming need not be so strong as for the fine crimson. The silk is refreshed at the river, and passed through a bath more or less charged with Brazil juice, according to the shade to be given. When water free from earthy salts is employed, the color is too red to imitate crimson; this quality is given it by passing the silk through a slight alkaline solution, or by adding a little alkali to the bath. It might, indeed, be washed in a hard water till it had taken the desired shade.

To make deeper false crimsons of a dark red, juice of logwood is put into the Brazil bath after the silk has been impregnated with it. A little alkali may be added, according to the shade that is wanted.

To imitate poppy or flame color, an annotto ground is given to the silk, deeper even than when it is dyed with carthamus. It is washed, alumed, and dyed with juice of Brazil, to which a little soap water is usually added.

The coloring particles of Brazil-wood are easily affected, and made yellow by the action of acids.

They thus become permanent colors. But what distinguishes them from madder and izermes, and approximates them to cochineal, is their reappearing in their natural color, when they are thrown down in a state of combination with alumina, or with oxyde of tin. These two combinations seem to be the fittest for rendering them durable. It is rcquisite, therefore, to inquire what circumstances are best calcriated to promote the formation of these combinations, according to the nature of t?ie stuff.

The astringent principle, likewise, seems to contribute to the permanence of the coloring matter of Brazil-wood; but it deepens its hue, and can only be employed for light shades.

The coloring particles of Brazil-wood are very sensible to the action of alkalis which give them a purple hue; and there are several processes in which the alkalis, either fixed or volatile, are used for forming violets and purples. But the colors obtained by these methods, which may be easily varied according to the purpose, are perishable, and possess but a transient bloom. The alkalis appear not to injure the colors derived from madder, but they accelerate the destruction of most other colors.

In England and Holland the dye-woods are reduced to powder by means of mills erected for the purpose.

The bright fugitive red, called fancy red, is given to cotton by Nicaragua, or peachwood, a cheap kind of Brazil-wood.

The cotton being scoured and bleached, is boiled with sumach. It is then impregnated with a solution of tin (at $5^{\circ}$ Baumé, according to Vitalis). It should now be washed slightly in a weak bath of the dyeing wood, and, lastly, worked in a somewhat stale infusion of the peach or Brazil wood. When the temperature of this is lukewarm, the dye is said to take better. Sometimes two successive immersions in the bath are given. It is now wrung out, aired, washed in water, and dried.

M. Vitalis says, that his solution of tin is prepared with two ounces of tin and a pound of aqua regia made with two parts of nitric acid at $24^{\circ}$ Baumé, and three parts of muriatic acid at $22^{\circ}$.

For a rose color, the cotton is alumed as usual, and washed from the alum. It then gets the tin mordant, and is again washed. It is now turned through the dye-bath, an operation which is repeated if necessary.

For purple, a little alum is added to the Brazil bath.

1. For amaranth, the cotton is strongly galled, dried, and washed. 
2. It is passed through the black cask (tonne au noir,) see Brack Dye, till it has take a strong gray shade.

3. It receives a bath of lime-water.

4. Mordant of tin.

5. Dreing in the Brazil-wood kath.

6. The two last operations are repeated.

Dingler has endeavored to separate the coloring matter of the different sorts of Brazil. wood, so as to obtain the same tint from the coarser as from the best Pernambuco. His process consists in treating the wood with hot water or steam, in concentrating the decostion so as to obtain 14 or 15 pounds of it from 4 pounds of wood, allowing it to cool, and pouring into it two pounds of skim milk; agitating, then boiling for a few minutes, and filtering. The dun coloring matters are precipitated by the coagulation of the caseous substance. For dyeing, the decoctions must be diluted with water ; for printing they must be concentrated, so that 4 pounds of wood shall furnish only 5 or 6 pounds of decoction, and the liquor may be thickened in the ordinary way. These decoctions may be employed immediately, as by this treatment they have acquired the same property as they otherwise could get only by being long kept. A slight fermentation is said to improve the color of these decoctions; some ground wood is put into the decoction to favor this process.

As gelatine prodnces no precipitate with these decoctions, they consequently contain no tannin. Gall-nuts, however, sumach, the bark of birch or alder, render the color of Brazil-wood more durable, upon alumed linen and cotton goods, but the shade is a little darker.

In dyeing wool with Pernambuco, the temperature of the bath should never be above $150^{\circ}$ Fahr., since higher heats impair the color.

According to Dingler and Kurrer, bright and fast scarlet reds may be obtained upon wool, by preparing a decoction of 50 pounds of Brazil-wood in three successive boils, and setting the decoction aside for 3 or 4 weeks in a cool place ; 100 pounds of the wool are then alumed in a bath of 22 pounds of alum and 11 pounds of tartar, and afterwards rinsed in cold water. Meanwhile we fill two thirds with water, a copper containing 30 pails, and heated to the temperature of $150^{\circ}$ or $160^{\circ} \mathrm{F}$. We pour in 3 pailfuls of the.decoction, heat to the same point again, and introduce 30 pounds of wool, which does not take a scarlet, but rather a crimson tint. This being removed, 2 pails of decoction are put in, and 30 pounds of wool, which becomes scarlet, but not so fine as at the third dip. If the dyer strenothens the color a little at the first dip, a little more at the second, and adds at the third and fourth the quantity of decoction merely necessary, he will obtain a uniform scarlet tint. With 50 pounds of Pernambuco 1000 pounds of wool may be dyed scarlet in this war, and with the deposites another 100 may be dyed of a tile color. An addition of weld renders the color faster but less brilliant.

Karkutsch says the dye may be improved by adding some ox-gall to the bath.

In dyeing cotton the tannin and gallic acid are two necessary mordants, and the color is particularly bright and durable, when the cloth has been prepared with the oily process of Turkey red.

It is said that stale urine heightens the color of the Brazil dye when the ground wood is moistened with it.

The quantity of Brazil or Nicaragua wood imported into the United Kingdom in 1835, was 6,242 tons, whereof 1,811 were exported; of Brazilietto 230 tons. The duty upon the first article is $5 s$. per ton.

BREAD (Pain, Fr.; Brod, Germ.) is the spongy mass produced by baking the leavened or fermented dough of wheat or rye flour, at a proper heat. It is the principal food of highly civilized nations. The skilful preparation of this indispensable article constitutes the art of the Baker. Dough baked without being fermented constitutes cakes or biscuits; but not bread strictly speaking.

Pliny informs us, that barley was the only species of corn at first used for food; and even after the method of reducing it to flour had been discovered, it was long before mankind learned the art of converting it into cakes.

Ovens were first invented in the East. Their construction was understood by the Jews, the Greeks, and the Asiatics, among whom baking was practised as a distinct profession. In this art, the Cappadovians, Lydians, and Phœnicians, are said to have particularly excelled. It was not till about 580 years after the foundation of Rome, that these artisans passed into Europe. The Roman armies, on their return from Macedonia, brought Grecian bakers with them into Italy. As these bakers had handmills beside their ovens, they still continued to be called pistores, from the ancient practice of bruising the corn in a mortar; and their bakehouses were denominated pistoria. In the time of Augustus there were no fewer than 329 public bakehouses in Rome; almost the whole of which were in the hands of Greeks, who long continued the only persons in that city acquainted with the art of baking good bread. 
In nothing, serhaps, is the wise and cautious policy of the Roman government more remarkably displayed, than in the regulations which it imposed on the bakers within the city. To the foreign bakers who came to Rome with the army from Macedonia, a number of freedmen were associated, forming together an incorporation from which neither they nor their children could separate, and of which even those who married the daughters of bakers were obliged to become members. To this incorporation were in trusted all the mills, utensils, slaves, animals, every thing, in short, which belonged to the former bakehouses. In addition to these, they received considerable portions of land; and nothing was withheld, which could assist them in pursuing, to the best advantage, their highly prized labors and trade. The practice of condemning criminals and slaves, for petty offences, to work in the bakehouse, was still continued; and even the judges of Africa were bound to send thither, every five years, such persons as had incurred that kind of chastisement. The bakehouses were distributed throughout the fourteen divisions of the city, and no baker could pass from one into another without special permission. The public granaries were committed to their care; they paid nothing for the corn employed in baking bread that was to be given in largess to the citizens; and the price of the rest was regulated by the magistrates. No corn was given out of these granaries except for the bakehouses, and for the private use of the prince. The bakers had besides private granaries, in which they deposited the grain, which they had taken from the public granaries for immediate use ; and if any of them happened to be convicted of having diverted any portion of the grain to another purpose, he was condemned to a ruinous fine of five hundred pounds weight of gold.

Most of these regulations were soon introduced among the Gauls; but it was long before they found their way into the more northern countries of Europe. Borrichius informs us that in Sweden and Norway, the only bread known, so late as the middle of the 16th century, was unleavened cakes kneaded by the women. At what period in our own history the art of baking became a separate profession, we have not been able to ascertain; but this profession is now common to all the countries in Europe, and the process of baking is also nearly the same.

The French, who particularly excel in the art of baking, have a great many different kinds of bread. Their pain bis, or brown bread, is the coarsest kind of all, and is made of coarse groats mixed with a portion of white flour. The pain bis blanc, is a kind of bread between white and brown, made of white flour and fine groats. The pain blanc, or white bread, is made of white flour, shaken through a sieve after the finest flour has been separated. The pain mollet, or soft bread, is made of the purest flour without any admixture. The pain chaland, or customers' bread, is a very white kind of bread, made of pounded paste. Pain chapele, is a small kind of bread, with a well-beaten and very light paste, seasoned with butter or milk. This name is also given to a small bread, from which the thickest crust has been removed by a knife. Pain cornu, is a name given by the French bakers to a kind of bread made with four corners, and sometimes more. of all the kinds of small bread, this has the strongest and firmest paste. Pain à la reine, queen's bread, pain à la Sigovie, pain chapelé, and pain cornu, are all small kinds of bread, differing only in the lightness or thickness of the paste. Pain gruau is a small very white bread made now in Paris, from the flour separated after a slight grinding from the best wheat. Such flour is in hard granular particles.

In this country we have fewer varieties of bread, and these differ chiefly in their degrees of purity. Our white or fine bread is made of the purest flour; our wheaten bread, of flour with a mixture of the finest bran; and our household bread, of the whole substance of the grain without the separation either of the fine flour or coarse bran. We have also symnel bread, manchet or roll bread, and French bread, which are all made of the purest flour from the finest wheat; the roll bread being improred by the addition of milk, and the French bread by the addition of eggs and butter. To these may be added gingerbread, a cake made of flour, with almonds, liquorice, aniseed, rose-water, and sugar or treacle; and mastlin bread, made of wheat and rye, or sometimes of wheat and barley. We have various kinds of small bread, having various names, according to their various forms. They are, in general, extremely light, and are sweetened with sugar, currants, and other palatable ingredients. In Scotland there is a cake called short bread, made from a pretty thick dough, enriched with butter, sweetened with sugar, and seasoned with orange peel, or other kinds of spices.

The process of making bread is nearly the same in all the countries of modern Europe; though the materials of which it is composed vary with the farinaceous productions of different climates and soils. The flour of wheat is most generally employed for this pur. pose, wherever that vegetable can be reared. This flour is composed of a small portion of mucilaginous saccharine matter, soluble in cold water, from which it may be separated by evaporation; of a great quantity of starch, which is scarcely soluble in cold water, but capable of combining with that fluid by means of heat; and an adhesive gray substance called gluten, insoluble in water, ardent spirit, oil, or ether, and resembling an animal 
substance in many of its properties. Flour kneaded with water, forms a tongh and rather indigestible paste containing all the constituent parts which we have tnumerated. Heat produces a considerable change on the glutinous part of this compound, and renders it more easy of mastication and digestion. Still, however, it continues heavy and tough, compared with bread which is raised by leaven or yeast. Leaven is nothing more than a piece of dough, kept in a warm place till it undergoes a process of fermentation; swelling, becoming spongy, or full of air bubbles, at length disengaging an acidulo-spirituous vapor, and contracting a sour taste. When this leaven is mingled in proper proportions with fresh-made dough, it makes it rise more readily and effectually than it would do alone, and gives it at the same time a greater degree of firmness. Upon the quality of the leaven employed, the quality of the bread materially depends.

The principal improvement which has been made on bread in müern :imes, is the substitution of yeast or barm in place of common leaven. This yeast is the viscid froth that rises to the surface of beer, in the first stage of its fermentation. When mixed with the dough, it makes it rise much more speedily and effectually than ordinary leaven, and the bread is of course much lighter, and freer from that sour and disagreeable taste which may often be perceived in bread raised with leaven, either because too much is mingled with the paste, or because it has been allowed to advance too far in the process of fermentation.

Bread properly raised and baked differs materially from unleavened cakes, not only in being less compact and heavy, and more agreeable to the taste, but in losing its tenacious and glutinous qualities, and thus becoming more salutary and digestible.

We possess several analyses of wheat flour. Ordinary wheat (triticum hybernum mixed with triticum turgidum) contains, according to the analyses made by Vauquelin of several species of wheat flour, the following substances:-

\begin{tabular}{|c|c|c|c|c|c|c|c|c|}
\hline Species of Wheat. & Water. & Gluten. & Starch. & Sugar. & Gum. & Bran. & Total. & $\begin{array}{c}\text { Water } \\
\text { of dough }\end{array}$ \\
\hline $\begin{array}{l}\text { French wheat flour - - } \\
\text { Hard wheat of Odessa }\end{array}$ & $10 \cdot 0$ & $10 \cdot 96$ & $71 \cdot 49$ & $4 \cdot 72$ & $3 \cdot 32$ & - & $100 \cdot 49$ & $50 \cdot 3$ \\
\hline $\begin{array}{l}\text { flour- }-\overline{-}- \\
\text { Soft wheat of Odessa }\end{array}$ & $12 \cdot 0$ & $14 \cdot 55$ & 56.50 & $8 \cdot 48$ & $4 \cdot 90$ & $2 \cdot 3$ & $98 \cdot 73$ & $51 \cdot 2$ \\
\hline flour - - - - - & $10 \cdot 0$ & $12 \cdot 00$ & $62 \cdot 00$ & $7 \cdot 56$ & $5 \cdot 80$ & $1 \cdot 2$ & $98 \cdot 42$ & $54 \cdot 8$ \\
\hline Same sort of flour - - & $8 \cdot 0$ & $12 \cdot 10$ & $70 \cdot 84$ & $4 \cdot 90$ & $4 \cdot 60$ & - & $100 \cdot 41$ & $37 \cdot 4$ \\
\hline Same sort of flour - - & $12 \cdot 0$ & $7 \cdot 30$ & $72 \cdot 00$ & $5 \cdot 42$ & $3 \cdot 30$ & - & $100 \cdot 02$ & $37 \cdot 2$ \\
\hline $\begin{array}{l}\text { Wheat of the French } \\
\text { bakers - - } \\
\text { Flour of the Paris hos- }\end{array}$ & $10 \cdot 0$ & $10 \cdot 20$ & $72 \cdot 80$ & $4 \cdot 20$ & $2 \cdot 80$ & - & $100 \cdot 00$ & $40 \cdot 6$ \\
\hline pitals (2d quality) - & $8 \cdot 0$ & $10 \cdot 30$ & $71 \cdot 20$ & $4 \cdot 80$ & $3 \cdot 60$ & - & $97 \cdot 90$ & $37 \cdot 8$ \\
\hline Ditto (3d quality) - - & $12 \cdot 0$ & $9 \cdot 02$ & $67 \cdot 78$ & $4 \cdot 80$ & $4 \cdot 60$ & $2 \cdot 0$ & $100 \cdot 21$ & $37 \cdot 8$ \\
\hline
\end{tabular}

The following table of analyses merits also a place here.

\begin{tabular}{|c|c|c|c|c|c|c|c|}
\hline Species of Flour. & Water. & Gluten. & Starch. & Sugar. & Gummigluten. & Albumen. & Bran. \\
\hline $\begin{array}{l}\text { Flour of the triticum spelta } \\
\text { Ditto triticum hybernum } \\
\text { Ditto common wheat - } \\
\text { Ditto wheat and rye } \\
\text { mixed (mastlin) - }\end{array}$ & $\begin{array}{l}1 \\
1 \\
-\end{array}$ & $\begin{array}{l}22 \cdot \\
24 \cdot \\
12 \cdot 5 \\
9 \cdot 80\end{array}$ & $\begin{array}{l}74 \cdot \\
68 \cdot \\
74 \cdot 5 \\
75 \cdot 50\end{array}$ & $\begin{array}{l}5 \cdot 50 \\
5 \cdot 0 \\
12 \cdot \\
4 \cdot 22\end{array}$ & $\begin{array}{l}1 \cdot \\
1 \bullet \\
2 \cdot \\
3 \cdot 28\end{array}$ & $\begin{array}{l}1 \cdot 50 \\
1 \cdot 50\end{array}$ & $1 \cdot 2$ \\
\hline
\end{tabular}

The first two of the above analyses were made by Vogel, the third by Proust, and the fourth by Vauquelin.

Analyses of the flour of some other corns.

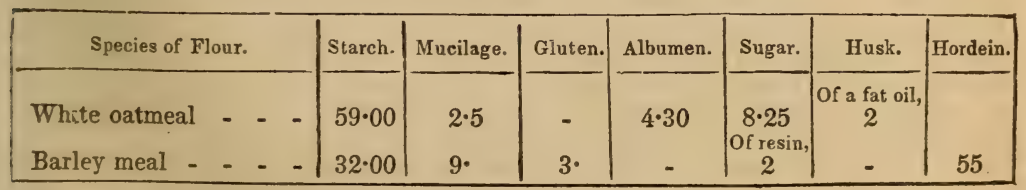

The first analysis is by Vogel, the second by Proust.

It deserves to be remarked, that the flour of Odessa contains a much greater quantity of sugar than the French flour. The substance indicated in the preceding table by the name of gluten, is the gluten of Beccaria; that is to say, a mixture of gluten and vegetable 
albumen. The gum of wheat is not quite identical with ordinary gum. It is a brown azotized substance, which, when treaied by nitric acid, affords no mucic acid, but oxalio acid and the bitter pıinciple of Welter. It contains besides superphosphate of lime.

The last column of the first table exhibits the quantity of water necessary to convert the flour into dough of the ordinary consistence, and it is usually proportional to the quantity of gluten. The hard wheat of Odessa forms an exception in this respect; the reason of the difference being that the starch contained in this flour is not as in.ordinary flour in a fine powder, but in small transparent grains, which resemble pounded gum, and absorb less water than pulverulent starch.

The triticum monococcon, according to Zenneck, contains in its unsifted flour, 16.334 of gluten and vegetable albumen; 64.838 of starch; 11.347 of gum, sugar, and extractive; 7.481 of husks. The sifted flour affords 15.536 of gluten and vegetable albumen; $\mathbf{7 6 . 4 5 9}$ of starch; $7 \cdot 198$ of sugar, gum, and extractive; 0.807 of husky matter. It is difficult to conceive how such great quantities of gluten, albumen, and extractive matter could disappear in the sifting. The triticurn spelta contains in 100 parts of the finest flour, 22.5 of a soft and humid gluten, mixed with vegetable albumen; 74 of starch, and 5.5 of sugar. Here we have an excess of 2 parts in the 100 .

Wheat furnishes very little ashes by incineration, not more than 0.15 per cent. of the weight; containing superphosphates of soda, lime, and magnesia.

The object of baking is to combine the gluten and starch of the flour into a homogeneous substance, and to excite such a vinous fermentative action, by means of its saccharine matter, as shall disengage abundance of carbonic acid gas in it for making an agreeable, soft, succulent, spongy, and easily digestible bread The two evils to be avoided in baking are hardness on the one hand, and pastiness on the other. Well-made bread is a chemical compound, in which the gluten and starch cannot be recognised or separated, as before, by a stream of water. When flour is lineaded into a dough, and spread into a cake, this cake, when baked, will be horny if it be thin, or if thick, will be tough and clammy; whence we see the value of that fermentalive process, which generates thousands of little cells in the mass or crumb, each of them dry, yet tender and succulent, through the intimate combination of the moisture. By this constitution it becomes easily soluble in the juices of the stomach, or, in other words, light of digestion. It is moreover much less liable to turn sour than cakes made from unfermented dough.

Rye, which also forms a true spongy bread, though inferior to that of wheat, consists of similar ingredients ; namely, 61.07 of starch; 9.48 of gluten; 3.28 of vegetable albumen; 3.28 of uncrystallizable sugar; 11.09 of gum; 6.38 of vegetable fibre; the loss upon the 100 parts amounted to $5 \cdot 62$, including an acid whose natare the analyst, M. Einhof, did not determine. Rye flour contains also several salts, principally the phosphates of lime and magnesia. This kind of grain forms a dark-colored bread reckoned very wholesome; comparatively little used in this country, but very much in France, Germany, and Belgium.

Dough fermented with the aid either of leaven or yeast, contains little or none of the saccharine matter of the flour, but in its stead a certain portion, nearly half its weight, of spirit, which imparts to it a vinous smell, and is rolatilized in the oven; whence it might be condensed into a crude weak alcohol, on the plan of $\mathrm{Mr}$. Hick's patent, were it worth while. But the increased complexity of the baking apparatus will probably prove an effectual obstacle to the commercial success of this project, upon which already upwards of $£ 20,000$ sterling have been squandered.

That the sugar of the flour is the true element of the fermentation preposterously called panary, which dough undergoes, and that the starch and gluten have nothing to do with it, may be proved by decisive experiments. The vinous fermentation continues till the whole sugar is decomposed, and no longer; when, if the process be not checked by the heat of baking, the acetous fermentation will supervene. Therefore, if a little sugar be added to a flour which contains little or none, its dough will become susceptible of fermenting, with extrication of gas, so as to make spongy succulent bread. But since this sponginess is produced solely by the extrication of gas, and its expansion in the heat of the oven, any substance capable of emitting gas, or of being converted into it under these circumstances, will answer the same purpose. Were a solution of bicarbonate of ammonia obtained by exposing the common sesqui-carbonate in powder for a day to the air, incorporated with the dough, in the subsequent firing it will be converted into vapor, and in its extrication render the bread very porous. Nay, if water highly impregnated with carbonic acid gas be used for kneading the dough, the resulting bread will be somewhat spongy. Could a light article of food be prepared in this way, then as the sugar would remain undecomposed, the bread would be so much the sweeter, and the more nourishing. How far a change propitious to digestion takes place in the constitution of the starch and gluten, during the fermentative action of the dough, has ot been hitherto ascertained by precise experiments. Medical practitioners, who 
derive an enormous revenue from dys epsia, should taike some pains to investigate this subject.

Dr. Colquhoun, in his able essay upon the art of maling bread, has shown that its textme, when prepared by a sudden formation and disengagement of elastic fluid generated within the oven, differs remarkably from that of a loaf which has been made after the preparatory fermentation with yeast. Bread which has been raised with the common carbonate of ammonia, as used by the pastry-cooks, is porous no doubt, but not spongy with vesicu. lar spaces, like that made in the ordinary way. The former kind of bread never presents that air-cell stratification which is the boast of the Parisian baker, but which is alinost unknown in London. I have found it, moreover, very difficult to expel by the oren the last portion of the ammonia, which gives both a tinge and a taste to the bread. I believe, however, that the bicarbonate would be nearly free from this objection, which operates so much against the sesqui-carbonate of the shops.

In opposition to Mr. Edlin's account of the excellent quality of bread made by im. pregnating dough with carbonic acid gas, ${ }^{*}$ Dr. Colquhoun adduces Vogel's experiments, which show that such dough, when baked, after having been kept in a warm sitruation during the usual time, afforded nothing better than a hard cake, which had no resemblance to common bread. Vogel further states, as illustrative of the general necessity of providing a sufficient supply of disengaged elastic fluid within the dough, before baking it at all, that when he made various attempts to form a well-raised vesicular loaf, within the oven, by mixing flour with carbonate of magnesia, or with zinc filings, and then kneading it into a paste by means of water, acidulated with sulphuric acid, he always met with complete failure and disappointment. Dr. Colquhoun performed a series of well-devised experiments on this subject, which fully confirmed Vogel's results, and prove that a proper spongy bread cannot be made by the agency of either carbonic acid water, or of mixtures of sesqui-carbonate of soda, and tartaric acid. The bread proved doughy and dense in every case, though less so with the latter mixture than the former. No loaf bread can, indeed, be well made by any of these two extempozaneous systems, because they are inconsistent with the thorough kneading of the dough. It is this process which renders dough at once elastic enough to expand when carbonic acid gas is generated within it, and cohesive enough to confine the gas when it is generated. The whole gas of the loaf is disengaged in its interior by a continuous fermentation, after all the processes of kneading have been finished; for the loaf, after being kneaded, weighed out, and shaped, is set aside till it expands gradually to double its bulk, before it is put into the oren. But when a dough containing sesqui-carbonate of soda is mixed with one containing muriatic acid, in due proportions to form the just dose of culinary salt, the yas escapes during the necessary incorporation of the two, and the bread formed from it is dense and hard. Dr. Whiting has, however, made this old chemical process the subject of a new patent for baking bread.

When the baker prepares his dough, he takes a portion of the water needed for the batch, having raised its temperature to from $70^{\circ}$ to $100^{\circ} \mathrm{F}$., dissolves a certain proportion of his salt in it, then adds the yeast, and a certain quantity of his flour. This mixture, called the sponge, is next covered up in the small kneading-trough, alongside of the large one, and let alone for setting in a warm situation. In about an hour, signs of vinous fermentation appear, by the swelling and heaving up of the sponge, in consequence of the generation of carbonic acid; and if it be of a semi-liquid consistence, large air bubbles will force their way to the surface, break, and disappear in rapid suecession. But when the sponge has the consistence of thin dough, it confines the gas, becomes thereby equably and progressively inflated to double its original volume; when no longer capable of containing the pent-up air, it bursts and subsides. This process of rising and falling alternately might be carried on during twenty-four hours, but the baker has learned by experience to guard against allowing full scope to the fermentative principle. He generally interferes after the first, or at furthest after the second or third dropping of the sponge; for were he not to do so, the bread formed with such dough would be invariably found sour to the taste and the smell. Therefore he adds at this stage to the sponge the reserved proportions of flour, salt, and water, which are requisite to make the dough of the desired consistence and size; and next incorporates the whole together by a long and laborious course of kneading. When this operation has been continued till the fermenting and the fresh dough have been intimately blended, and till the glutinous matter of both is worked into such union and consistence that the mass becomes so tough and elastic as to receive the smart pressure of the nand without adhering to it, the kneading is suspended for some time. The dough is now abandoned to itself for a few hours, during which it continues in a state of active fermentation throughout its entire mass. Then it is subjected to a second but much less laborious kneading, in order to distribute the generated gas as evenly as possible 
among its parts, so that they may all partake equally of the vesicular structure. Afte? this second kneading, the dough is weighed out into the portions suitable to the size of bread desired; which are of course shaped into the proper forms, and once more set aside in a warm situation. The continuance of the fermentation soon disengages a fresh quantity of carbonic acid gas, and expands the lumps to about double their pristine volume. These are now ready for the oven, and when they finally quit it in the baked state, are about twice the size they were when they went in. The generation of the due quantity of gas should be complete before the lumps are transferred to the oven; because whenever they encounter its heat, the process of fermentation is arrested; for it is only the previously existing air which gets expanded throughout every part of the loaf, swells out its volume, and gives it the piled and vesicular texture. Thus the well-baked loaf is composed of an infinite number of cellules filled with carbonic acid gas, and apparently lined with a glutinous membrane of a silky softness. It is this which gives the light, elastic, porous constitution to bread.

After suffering the fermentative process to exhaust itself in a mass of dough, and the dough to be brought into that state in which the addition of neither yeast, nor starch, nor gluten will produce any effect in restoring that action, if we mix in 4 per cent. of saccharine matter, of any kind, with a little yeast, the process of fermentation will immediately re-commence, and pursue a course as active and lengthened as at first, and cease alout the same period.*

This experiment, taken in connexion with the facts formerly stated, proves that what was called panary fermentation, is nothing but the ancient and well-known process of the vinous fermentation of sugar, which generates alcohol. There seems to be but one objection to the adoption of this theory. After the loaf is baked, there is found in its composition nearly as much saccharine matter as existed in the flour before fermentation. M. Vogel states that in the baked bread there remains 3.6 parts of sugar, out of the 5 parts which it originally contained. Thus, in 100 parts of loaf bread prepared with wheaten flour, distilled water, and yeast without the admixture of any common salt, he found the following ingredients :-

$\begin{array}{lr}\text { Sugar } & \\ \text { Torrefied or gummy starch } & 3.6 \\ \text { Starch } & 18.0 \\ \text { Gluten, combined with a little starch, } & 53.5 \\ \text { 20.75 }\end{array}$

Exclusive of carbonic acid, muriate of lime, phosphate of lime, \&c.

It must be borne in mind that in every loaf the process of fermentation has been prematurely checked by the baker's oven, and therefore the saccharine constituent can never be wholly decomposed. It seems certain, also, that by the action of gluten upon the starch in the early stage of the firing, a quantity of sugar will be formed by the saccharine fermentation; which we have explained in treating of BEER.

Several masses of dough were prepared by Dr. Colquhoun in which pure wheat starch was mixed with common flour, in various proportions. In some of the lumps this starch had been gelatinized, with the minimum of hot water, hefore it was added to the flour. After introducing the usual dose of salt, the dough was thoroughly kneaded, set apart for the proper period, allowed to ferment in the accustomed way, and then baked in the oren. In outward appearance, increase of bulk, and vesicular texture, none of them differed materially from a common loaf, baked along with them for the sake of comparison; except that when the starch considerably exceeded the proportion of flour in the lump, the loaf, though whiter, had not risen so well, being somewhat less vesicular. But, on tasting the bread of each loaf, those which contained most gelatinized starch were unexpectedly found to be the sweetest. The other loaves, inio which smaller quantities of the gelatinized starch had been introduced, or only some dry starch, had no sweetish taste whatever to distinguish them from ordinary bread. These facts seem to establish the conclusion, that the presence of gelatinous starch in bread put into the oven, is a means of forming a certain portion of saccharine matter within the loaf, during the baking process. Now it is more than probable that gelatinized starch does exist, more or less, in all loaves which have been fermented by our usual methods, and hence a certain quantity of sugar will necessarily be generated at its expense, by the action of heat. Thus the difficulty started by $M$. Vogel is sufficiently solved; and there remains no doubt that, in the saccharine principle of flour, the fermentation has its origin and end, while dough is under fermentation.

The source of the sourness which supervenes in bread, under careless or unskilful hands, had been formerly ascribed to each of all the constituents of flour; to its gluten, its starch, and its sugar; but erroneously, as we now see : for it is merely the result of the second fermentation which always succeeds the vinous, when pushed improperly too far. It has been universally taken for granted by authors, that the acid thus generated 
in dough is the acetic. But there appear good grounds to believe that it is frequently a less rolatiliz acil, probably the lactic, particularly when the process has been tardy, from the im: fection of the yeast or the bad quality of the flour. The experiments of Vogel. Braconnot, and others, prove that the latter acid is generated very readily, and in considerable quantity during the spontaneous decomposition of a great many regetable substances, when in a state of humility. The presence of lactic acid would account for the curi $1 \mathrm{~s}$ fact, that the acidity of unbaked dough is much more perceptible to the taste than to the smell: while the sourness of the same piece of bread, after coming out of the oren. is, on the contrary, much more obvious to the olfactory organs than to the palate. But this is exactly what ought to happen, if the lactic acid coutributes, in conjunction with the acetic, to produce the acescence of the dough. At the ordinary temperature of a bakehouse, the former acid, though rery perceptible in the mouth, is not distinguishable br the nostrils; but as it is easily decomposed br heat, no sooner is it exposed in the high temperature of the oren, than it is resolred, in a great measure, into acetic acid. ${ }^{*}$ and thus becomes more manifest to the sense of smell, and less to that of taste. This theory seems to explain satisfactorily all the phenomena accompanying the prozress of fermentation in baker's dough, and also some of its results in the process of baking which do not easily admit of any other solution.

There are extremely simple and effectual methods for enabiing the baker to adopt measures either to prerent or correct the evil of acescence, and these are to neutralize the ac d by the due exhibition of an alkali, such as soda; or an alkaline earth, such as magnesia or cialk. And it affords a striking proof of how much the artisan has been accustomed to plod, uninquiring and uninformed, orer the same ground, that a remedy so safe and so economical, should remain at this day unthought of and unemploted by most of the manufacturers of bread in the Lnited Kingdom. The introduction of a small portion of carbonate of soda will rectify anr occasional error in the result of the so called panary fermentation, and will, in fact, restore the dough to its pristine sweetness. The quantity of acetate of soda, which will be thus present in the bread, will be altogether inconsiderable: and as it has no disagreeable taste, and is merely aperient to the bowels in a rert mild degree, it can form no objection in the ere of the public police. The restoration of dough thus tainted with acid, and its conversion into pleasant and wholesome bread, has been sufficiently rerified br experiment. But, accordinz to Mr. Elmund DarT, carbonate of magnesia mas be used with still greater adrantage, as during the slow action of the acid upon it, the carbonic acid erolved serves to open up and lighten bread which would otherwise be dense and doughr from the indifferent quality of the flour. Here, howerer, the dangerous temptation lies with a sor ili baker to use cleap or damaged flour, and to rectify the bread made of it br enemical agents, innocent in ihemselves, but injurious as masks of a bad raw material. When sour reast must be used, as sometimes happens with the country bakers, or in private houses at a distance from beer breweries, there can be no harm. but, on the contrars, much propriets, in correcting its acidity, by the addition of as much carbonate of soda to it as will effect its neutralization, but nothing more. When sour yeast has been thus corrected, it has been found, in practice, to possess its fermentative power unimpaire1, and to be equally efficacious with fresh formed reast, in making good palatable loares.

We hare seen that, in baking, about one fourth of the starch is conrerted into a matter possessing the properties of British gum (see STARCH), and also that the gluten, though not decomposed, has its particles disunited, and is not so tough and adhesire as it is in the flour. This principle is also, as we hare said, useful in cementing all the particles of the dough into a tenacious mass, capable of confining the elastic fluid generated by the rinous fermentation of the sugar. Starch is the main constituent, the basis of nourishment in bread, as well as in all farinaceous articles of food. The albumen al. so of the wheat, baing coagulatel by the heat of the oren, contributes to the setting of the bread into a consistent elastic body.

In the mills in the neighborhood of London, no less than seren distinct sorts of floue are ground out of one quantity of wheat. These are for one quarter-

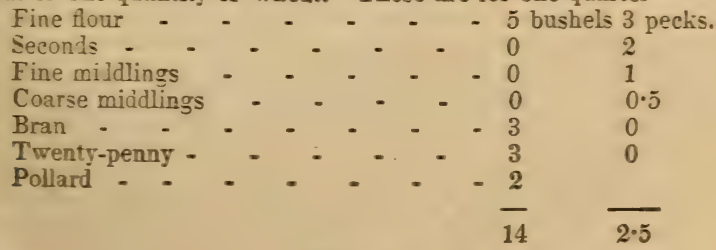

- Berzelius 
So that we have nearly a double bulk of flour, or 14 bushels and $2 \frac{1}{2}$ pecks from 8 bushels of wheat. In the sifting of the flour through the bolter, there is a fine white angular meas obtained called sharps, which forms the central part of the grain. It is consumed partly by the fine biscuit bakers. The bakers of this country were formerly bound by law to bake three kinds of bread, the wheaten, standard wheaten, and the household; marked respectively with a W, S W, and $\mathrm{H}$, and if they omitted to make these marks on their bread they were liable to a penalty. The size of the loaves were usually peck, half-peck, quartern, and half-quartern; the weights of which, within 48 hours of their being baked, should have been respectively 17 lbs. 6 oz.; 8 Jbs. 11 oz.; 4 lbs. 5 oz. 8 dr.; and 4 Jbs. $2 \mathrm{oz} .14 \mathrm{dr}$. In general they weigh about one seventh more before they enter the oven, or they lose one seventh of their weight in baking. The French bread loses fully one sixth in the oven, owing chiefly to its more oblong thin shape, as compared to the cubical shape of the English bread. But this loss of weight is very variable, being dependant upon the quality of the wheaten flour, and the circumstances of baking. The present law in England defines the quartern loaf at 4 lbs., and subjects the baker to a penalty if the bread be one ounce lighter than the standard. Hence it leaves the baker, in self-defence, to leave it in rather a damp and doughy state. But there is much light bread sold in London. I have met with quartern loaves of $3 \mathrm{lbs} .10 \mathrm{oz}$. A sack of flour weighing $280 \mathrm{lbs}$. was presumed by the framers of our former parliamentary acts, for the assize of bread, to be capable of being baked into 80 loaves. If this proportion had been correct, one fifth part of our quartern loaf must consist of water and salt, and four lifths of flour. But in general, of good wheaten flour, three parts will take up one part of water; so that the sack of flour should have turned out, and actually did turn out, more than 80 loaves. At present with $4 \mathrm{lb}$. bread it may well yield 92 loaves.

The following statement of the system of baking at Paris, I received in 1835 from a very competent judge of the business.

1,000 kilogrammes of wheat $=5$ quarters English, cost $200 \mathrm{fr}$., and yield 800 kilos. of flour of the best white quality, equivalent to $5 \frac{1}{10}$ sacks French. Hence the sack of flour costs 40 francs at the mill, and including the carriage to Paris, it costs 45 or 46 franes.

The profit of the flour dealer is about $3 \frac{1}{2}$ franes, and the sale price becomes from 43 to 50 francs.

\section{Bread manufactured from the above.}

One day's work of an ordinary baker, who makes four batches in a day, consists of 3 sacks at 50 francs, or $2 l$. sterling each

Salt $2 \frac{3}{4}$ lbs. at $2 d$. per lb.

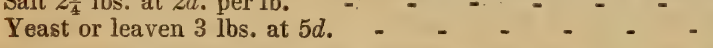

Total cost of materials - - Expenses of Baking. - $£$ s. $d . \quad £$ s. $d$.

Three workmen at different rates of wages, 15 francs - - 012

Fire-wood 0, as the charcoal produced pays for it - _ -

General expenses, such as rent, taxes, interest of capital, \&c.

For this sum 315 loaves are made, being 105 for every sack $\begin{array}{lll}0 & 12 & 0\end{array}$ of flour weighing 156.66 kilos or $344 \frac{2}{2}$ lbs. avoird. One loaf contains therefore $3 \frac{44 \frac{6}{10}}{10}=3.282 \mathrm{lbs}$., and as $100 \mathrm{lbs}$. of flour in Parisian baking are reckoned to produce 127 lbs. of bread, each loaf will weigh $4 \cdot 168$ lbs., avoird., and will cost $7 l$. 5 s. $8 \frac{1}{2} d$. divided by $315=5 \frac{1}{2} d$. very nearly. The value of 315 loaves at the sale price of $6 \dot{d}$. will be

Upon this day's work the clear profit is therefore $\quad \begin{array}{lll}0 & 11 & 6 \frac{1}{3}\end{array}$

$140=\frac{140}{7581}$

6000

$\begin{array}{lll}0 & 0 & 5 \frac{1}{2}\end{array}$

$\begin{array}{lll}0 & 1 & 3\end{array}$

$6 \quad 1 \quad 8 \frac{1}{2}$ A new baking establishment has been recently formed at the Royal Clarence Victualling Establishment at Weevil, near Portsmouth, upon a scale of magnitude nearly sufficient to supply the whole royal navy with biscuits, and that of a very superior description. The following account of it is taken from the United Service Journal. "It having been discovered that the flour supplied to government by contract, had in many instances been most shamefully adulterated, the corn is ground at mills comprised within the establishment, by which means the introduction of improper ingredients is prevented, and precisely the proportion of bran which is requisite in the composition of good sea-biscuit is retained, and no more. The flour-mill is furnished with ten pairs of 
stones, by which 40 bushels of flour may be ground and dressed ready for baking, in an hour. The baking establishment consists of 9 ovens, each 13 feet long by 11 feet wite, and $17 \frac{1}{2}$ inches in height. These are each heated by separate furnaces, so constructed that a blast of hot air and fire sweeps through them, and gives to the interior the requisite dose of heat in an incredible short space of time. The first operation in maling the biscuits consists in mixing the flour, or rather meal and water; 13 gallons of water are first introduced into a trough, and then a sack of the meal, weighing 280 lbs. When the whole has been poured in by a channel communicating with an upper room, a bell rings, and the trough is closed. An apparatus consisting of two sets of what are called linives, each set ten in number, are then made to revolve amongst the flour and water by means of machinery. This mixing operation lasts one minute and a half, during which time the double set of knives or stirrers makes twenty-six revolutions. The next p'ccess is to cast the lumps of dough under what are called the breaking-rollers,huge crlinders of iron, weighing $14 \mathrm{cwt}$. each, and moved horizontally by the machinery along stout tables. The dough is thus formed into large rude masses 6 feet long by 3 feet broad, and several inches thick. At this stage of the business, the kneading is still very imperfect, and traces of dry flour may still be detected. These great masses of dough are now drawn out, and cut into a number of smaller masses about a for $t$ and a half long by a foot wide, and again thrust under the rollers, which is repeated until the mixture is so complete that not the slightest trace of any inequality is discoverable in any part of the mass. It should have been stated that two workmen stand one at each side of the rollers, and as the dough is flattened out they fold it up, or double one part upon another, so that the roller at its next passage squeezes these parts together, and forces them to mix. The dough is next cut into small portions, and being placed upon large flat boards, is, by the agency of machinery, conveyed from the centre to the extremity of the baking-room. Here it is received by a workman, who places it under what is called the sheet roller, but which, for size, color, and thickness, more nearly resembles a blanket. The kneading is thus complete, and the dough only requires to be cut into biscuits before it is committed to the oven. The cutting is effected by what is called the cutting-plate, consisting of a net-work of 52 sharp-edged hexagonal frames, each as large as a biscuit. This frame is moved slowly up and down by machinery, and the workman, watching his opportunity, slides under it the above-described blanket of dough, which is about the size of a leaf of a dining-table; and the cutting-frame in its descent indents the sheet, but does not actually cut it through, but leaves sufficient substance to enable the workman at the mouth of the oven to jerk the whole mass of biscuits nnbroken into it. The dough is prevented sticking to the cutting-frame by the following ingenious device : between each of the cutter-frames is a small flat open frame, moveable up and down, and loaded with an iron ball, weighing several ounces. When the great frame comes down upon the dough, and cuts out 52 biscuits, each of these minor frames yields to the pressure, and is raised up; but as soon as the great frame rises, the wejght of the balls, acting upon the little frames, thrusts the whole blanket off, and allows the workmen to pull it out. One quarter of an hour is sufficient $\checkmark$ bake the biscuit, which is afterwards placed for three days in a drying room, heated io $85^{\circ}$ or $90^{\circ}$, which completes the process." The following statement of the performance of the machinery is taken from actual experiment; in 116 days, during 68 of which the work was continued for only $7 \frac{1}{2}$ hours; and during 48 , for only $5 \frac{3}{4}$ hours each day, in all 769 working hours, equal to 77 days of 10 hours each; the following quantity of biscuit was baked in the 9 ovens, viz. : $12,307 \mathrm{cwt} .=1,378,400 \mathrm{lbs}$. The wages of the men employed in baking this quantity amounted to $273 \mathrm{l} .10 \mathrm{~s} .9 \frac{1}{2} d$. ; if it had been made by hand, the wages would have been $933 l .9 s .10 d$. ; sasing in the wages cf labor, 659l. 7 s. $0 \frac{1}{2} d$. In this, is not included any part of the interest of the sum laid out upon the machine, or expended in keeping it in order. But in a very few years, at such an immense rate of saving, the cost of the engine and other machinery will be repaid. This admirable apparatus is the invention of T. T. Grant, Esq., storekeeper of the Royal Clarence Victualling Establishment, who, we believe, has been properly rewarded, by a grant of 2,000l. from government.

The labor of incorporating the ingredients of bread, viz., flour, water, and salt, or kneading dough, is so great as to have led to the contrivance of various mechanical modes of producing the same effect. Ore of the most ingenious is that for which a patent was obtained in Angust, 1830 , by Mr. Edwin Clayton. It consists of a rotatory kneading trough, or rather barrel, mounted in bearings with a hollow axle, and of an interior frame of cast iron made to revolve by a solid axle which passes through the hollow one; in the frame there are cutters diagonally placed for kneading the dough. The revolving frame and its barrel are made to turn in contrary directions, so as greatly to save time and equalise the operation. This double action represents kneading by the two hands, in which the dough is inverted from time to time, torn asunder, and reunited in every different form. The mechanism will be readily understood from the following description. 
Fig. 169 exhibits a front elevation of a rotatory kneading trough, constructed according to improvements specified by the patentee, the barrel being shown in section; $a$ is the

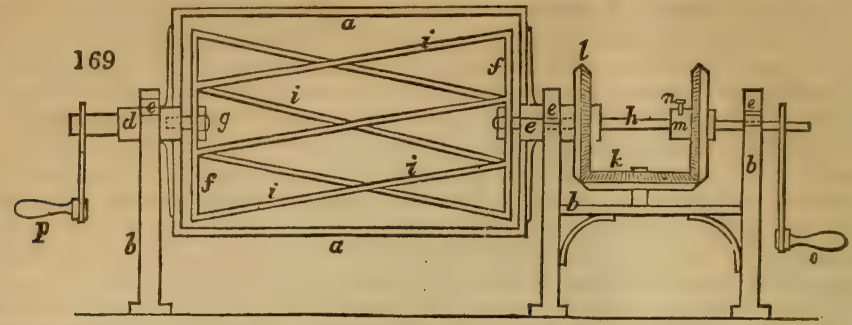

barrel, into which the several ingredients, consisting of flour, water, and yeast, are put, which barrel is mounted in the frame-work $b$, with hollow axles $c$ and $d$, which hollow axles turn in suitable bearings at $e ; f$ is the revolving frame which is mounted in the interior of the barrel $a$, by axles $g$ and $h$. The ends of this revolving frame are fastened, or braced together by means of the oblique cutters or braces $i$, which act upon the dough when the machine is put in motion, and thus cause the operation of kneading.

Either the barrel may be made to revolve without the rotatory frame, or the rotatory frame without the barrel, or both may be made to revolve together, but in opposite ways. These several motions may be obtained by means of the gear-work, shown at $k, l$, and $m$, as will be presently described.

If it be desired to have the revolving motion of the barrel and rotatory frame together, but in contrary directions, that motion may be obtained by fastening the hollow axle of the wheel $m$, by means of a screw $n$, to the axle $h$, of the rotatory frame $f$, tight, so as they will revolve together, the other wheels $k$ and $l$ being used for the purpose of reversing the motion of the barrel. It will then be found that by turning the handle 0 , the two motions will be obtained.

If it be desired to put the rotatory frame $f$, only, into motion, that action will be obtained by loosening the screw $n$, upon the axle of the wheel $m$, when it will be found that the axle $h$ will be made to revolve freely by means of the winch $o$, without giving motion to the wheels $k, l$, and $m$, and thus the barrel will remain stationary. If the rotatory action of the barrel be wanted, it will be obtained by turning the handle $p$, at the reverse end of the machine, which, although it puts the gear at the opposite end of the barrel into motion, yet as the hollow axle of the wheel $m$ is not fastened to the axle $h$, by the screw $n$, these wheels will revolve without carrying round the frame $f$.

M. Kuhlmann, Professor of Chemistry at Lille, having heen called upon several times by the courts of justice to examine by chemical processes bread suspected of containing substances injurious to health, collected some interesting facts upon the subject, which were published under the direction of the central council of salubrity of the department $d u$ Nord.

For some time public attention had been drawn to an odious fraud committed by a great many bakers in the north of France and in Belgium-the introduction of a certain quantity of sulphate of copper into their bread. When the flour was made from bad grain this adulteration was very generaliy practised, as was proved by many convictions and confessions of the guilty persons. When the dough does not rise well in the fermentation (le pain pousse plat), this inconvenience was found to be obviated by the addition of blue vitriol, which was supposed also to cause the flour to retain more water. The quantity of blue water added is extremely small, and it is never done in presence of strangers, because it is reckoned a valuable secret. It occasions no economy of yeast, but rather the reverse. In a litre (about a quart) of water, an ounce of sulphate of copper is dissolved; and of this solution a wine-glass full is mixed with the water necessary for 50 quartern or 4 pound loaves.

M. Kuhlmann justly observes, that there can be no sofety whatever to the public when such a practice is permitted, because ignorance and avarice are always apt to increase the quantity of the poisonous water. In analyses made by him and his colleagues, portions of bread were several times found so impregnared with the above salt that they had acquired a blue color, and presented occasionally even small crystals of the sulphate. By acting on the poisoned bread with distilled water, and testing the water with ferro-cyanate (prussiate) of potash. the reddish brown precipitate or tint characteristic of copper will appear even with small quantities. Should the noxiois impregnation be still more mint te, the bread should be treated with a very dilute nitric acid, either directly or after incineration in a platinum capsule, and the solution, when concentrated by evaporation, should be tested by the ferro-cyanate of potash. In this way, a one seventy thousandth part of sulphate of copper may be detected. 
M. Kuhlmann deduces, from a series of experiments on baking with various small quantities of sulphate of copper, that this salt exercises an extremely energetic action upon the fermentation and rising of the dough, even when not above one seventy thousandth part of the weight of the bread is employed; or one grain of sulphate for ten pounds of bread. The proportion of the salt which makes the bread rise best is one twenty thuusandth, or one grain in three pounds of bread. If much more of the sulphate be added, the bread becomes moist, less white, and acquires a peculiar disagreeable smell like that of leaver. The increase of weight by increased moisture may amount to one sixteenth without the bread appearing softer, in consequence of the solidifying quality of the copper; for the acid does not seem to have any influence; as neither sulphate of soda, sulphate of iron, nor sulphuric acid have any analogous power. Alum operates like blue vitriol on bread, but larger quantities of it are required. It keeps water, and raises well to use the bakers' terms.

When alum is present in bread it may be detected by treating the bread with distilled water, filtering the water first through calico, and next through filtering paper, till it becomes clear; then diriding it into two portions, and into the one pouring a few drops of nitrate or muriate of barytes, and into the other a few drops of water of ammonia. In the former a heary white precipitate indicating sulphuric acid will appear, and in the latter a light precipitate of alumina, redissoluble by a few drops of solution of caustic potash.

When chalk or Paris plaster is used to sophisticate flour, they may be best detected by incinerating the bread made of it, and examining the ashes with nitric acid, which will dissolve the chalk with effervescence, and the Paris plaster without. In both cases the calcareous matter may be demonstrated in the solution, by oxalic acid, or better by oxalate of ammonia.

In baking puff-paste the dough is first kneaded along with a certain quantity of butter, then rolled ont into a thin layer, which is coated over with butter, and folded face-wise many times together, the upper and under surfaces being made to correspond. This stratified mass is again rolled out into a thin layer, its surface is besmeared with butter, and then it is folded face-wise as before. When this process is repeated ten or a dozen times, the dough will consist of many hundred parallel laminæ, with butter interposed between each pair of plates. When a moderately thick mass of this is put into the oven, the elastic vapor disengaged from the water and the butter, diffuses itself between each of the thin laminæ. and causes them to swell into what is properly called puff-paste, being an assemblage of thin membranes, each dense in itself, but more or less distinct from the other, and therefore forming apparently, but not really, light bread.

One of the most curious branches of the baker's craft is the manufacture of gingerbread, which contains such a proportion of molasses, that it cannot be fermented by means of yeast. Its ingredients are flour, molasses or treacle, butter, common potashes, and alum. After the butter is melted, and the potashes and alum are dissolved in a little hot water, these three ingredients, along with the treacle, are poured among the flour, which is to form the body of the bread. The whole is then incorporated by mixture and kneading into a stiff dough. Of these five constituents the alum is thought to be the least essential, although it makes the bread lighter and crisper, and renders the process more rapid; for gingerbread dough requires to stand orer sereral dars, sometimes 8 or 10 , before it acquires that state of porosity which qualifies it for the oren. The action of the treacle and alum on the potashes in erolring carbonic acid, seems to be the gasefying principle of gingerbread; for if the carbonate of potash is withheld from the mixture, the bread, when baked, resembles in hardness a piece of wood.

Treacle is alwars acidulous. Carbonate of magnesia and soda may be used as substitutes for the potashes. Dr. Colquhoun has found that carbonate of magnesia and tartaric acid may replace the potashes and the alum with great invantage, affording a gingerbread fully more agreeable to the taste, and much more wholesome than the common kind, which contains a notable quantity of potashes. His proportions are one pound of flour, a quarter of an ounce of carbonate of magnesia, and one eighth of an ounce of tartaric acid; in addition to the treacle, butter, and aromatics, as at present used. The acid and alkaline earth must be well diffused through the whole dough. The magnesia should, in fact, be first of all mixed with the flour. Pour the melted butter, the treacle, and the acid dissolved in a little water all at once among the flour, and linead into a consistent dough, which being set aside for half an hour or an hour will be reads for the oven, and should never be kept unbaked more than 2 or 3 hours. The following more complete recipe is given by Dr. Colquhoun, for making thin ginger. bread cakes :-

$\begin{array}{ll}\text { Flour } & 1 \mathrm{lb} . \\ \text { Treacle } & 0 \frac{1}{2} \\ \text { Raw sugar } & 0 \frac{1}{6}\end{array}$




$\begin{array}{ll}\text { Butter } & 2 \mathbf{o z} . \\ \text { Carbon. magnesia } & 0 \frac{1}{4} \\ \text { Tartaric acid } & 0 \frac{1}{8} \\ \text { Ginger } & 0 \frac{1}{8} \\ \text { Cinnamen } & 0 \frac{1}{8} \\ \text { Nutmeg } & 1\end{array}$

This compound has rather more butter than common thin gingerbread.

I shall here insert a passage from my Dictionary of Chemistry, as published in 1821; as it may prove interesting to many of my present readers.

"Under Process of Baking, in the Supplement to the Encyclopedia Britannica, we have the following statement:- 'An ounce of alum is then dissolved over the fire in tin pot, and the solution poured into a large tub, called by the bakers the seasoningtub. Four pounds and a half of salt are likewise put into the tub, and a pailful of hot water.'-Foot note on this passage.-' In London, where the goodness of bread is estimated enarely by its whiteness, it is usual with those bakers who employ flour of an in. ferior quality, to add as much alum as common salt to the dough; or, in other words, the quantity of salt added is dim.nished one half, and the deficiency supplied by an equal weight of alum. This improves the look of the bread very much, rendering it much whiter and firmer." "'

In a passage which we shall presently quote, our author represents the bakers of London in a conspiracy to supply the citizens with bad bread. We may hence infer that the full allowance he assigns of $2 \frac{1}{4}$ pounds of alum for every $2 \frac{1}{4}$ pounds of salt, will be adopted in converting the sack of flour into loaves. But as a sack of flour weighs 280 pounds, and furnishes on an average 80 quartern loaves, we have $2 \frac{1}{4}$ pounds divided by 80 , or $\frac{15750 \text { grains }}{80}=197$ grains, for the quantity present, by this writer, in a London quartern loaf. Yet in the very same page (39th of vol. ii.) we have the following passage: "Alum is not added by all baliers. The writer of this article has been assured by several bakers of respectability, both in Edinburgh and Glasgow, on whose testimony he relies, and who made excellent bread, that they never employed any alum. The reason for adding it given by the London bakers is, that it renders the bread whiter, and enables them to separate readily the loaves from each other. This addition has been alleged by medical men, and is considered by the community at large, as injurious to the health, by occasioning constipation. But if we consider the small quantity of this salt added by the baker, not quite $5 \frac{1}{2}$ grains to a quartern loaf, we will not readily admit these allegations. Suppose an individual to eat the seventh part of a quartern loaf a day, he would only swallow eight tenths of a grain of alum, or, in reality, not quite so much as half a grain; for one half of this salt consists of water. It seems absurd to suppose that half a grain of alum, swallowed at different times during the course of a day, should occasion constipation." Is it not more absurd to state $2 \frac{1}{4}$ pounds or 36 ounces, as the alum adulteration of a sack of flour by the London bakers, and within a few periods to reduce the adulteration to one ounce?

That this voluntary abstraction of $\frac{35}{36}$ of the alum, and substitution of superior and more expensive flour, is not expected by him from the London bakers, is sufficiently evident from the following story. It would appear that one of his friends had invented a new yeast for fermenting dough, by mixing a quart of beer barm with a paste made of ten pounds of flour and two gallons of boiling water, and keeping this mixture warm for six or eight hours.

"Yeast made in this way," says he, "answers the purposes of the baker much better than brewers' yeast, because it is clearer, and free from the lwp mixture which sometimes injures the yeast of the brewer. Some years ago the bakers of London, sensible of the superiority of this artificial yeast, invited a company of manufacturers from Glasgow to establish a manufactory of it in London, and promised to use no other. About $5,000 l$. accordingly was laid out on buildings and materials, and the manufactory was begun on a considerable scale. The ale-brewers, finding their yeast, for which they had drawn a good price, lie heavy on their hands, invited all the journeymen bakers to their cellars, gave them their full of ale, and promised to regale them in that manner every day, provided they would force their masters to take all their yeast from the ale-brewers. The journeymen accordingly declared, in a body, that they would work no more for their masters unless they gave up taking any more yeast from the manufactory. The masters were obliged to comply ; the new manufactory was stopped, and the inhabitants of London were obliged to continue to eat worse bread, because it was the interest of the alebrewers to sell the yeast. Such is the influence of journeymen bakers in the metropolis of England !"

This doleful diatribe seems rather extravagant; for surely beer yeast can derive nothing noxious to a porter drinking people, from a slight impregnation of hops; while it must form probably a more energetic ferment than the fermented paste of the new company, which at any rate could be prepared in six or eight hours by any baker who 
found it to answer his purpose of making a pleasant eating bread. But it is a very serious thing for a lady or gentleman of sedentary habits, or infirm constitution, to have their digestive process daily vitiated by damaged flour, whitened with 197 grains of alum pei quartern loaf. Acidity of stomach, indigestion, flatulence, headaches, palpitation, costiveness, and urinary calculi may be the probable consequences of the habitual introduction of so much acidulous and acescent matter.

I have made many experiments upon bread, and have found the proportion of alum very variable. Its quantity seems to be proportional to the badness of the flour; and hence when the best flour is used no alum need be introduced. That alum is not necessary for giving bread its utmost beauty, sponginess, and agreeableness of taste, is undoubted; since the bread baked at a very extensive establishment in Glasgow, in which about 20 tons of 1lour were regularly converted into loaves in the course of a week, united every quality of appearance with an absolute freedom from that acido-astringent drug. Six pounds of salt were used for every sack of flour; which, from its good quality, generally afforded 83 or 84 quartern loaves of the legal weight of four pounds five ounces and a half each. The loaves lost nine ounces in the oven.

Every baker ought to be able to analyze his fiour. He may proceed as follows :-A ductile paste is to be made with a pound of the flour and a sufficient quantity of water, and left at rest for an hour; then having tied across a bowl a piece of silken sieve-stuff, a little below the surface of the water in the bowl, the paste is to be laid upon the sieve on a level with the water, and kneaded tenderly with the hand, so as merely to wash the starchy particles out of it. This portion of the flour gets immediately diffused through the water, some of the other constituents dissolve, and the gluten alone remains upon the filter. The water must be several times renewed till it ceases to become milky. The last washings of the gluten are made out of the sieve.

The whole of the turbid washings are to be put into a tall conical glass or stoneware vessel, and allowed to remain at rest, in a cool place, till they deposite the starch. The elear supernatant liquor is then decanted off. The deposite consists of starch, with a litlle gluten. It must be washed till the water settles over it quite clear, and then it is to be dried.

The filtered waters being evaporated at a boiling heat, discover flocks floating through them, which have been supposed by some to be albumen, and by others gluten. At last, phosphate of lime precipitates. When the residuum has assumed a sirupy consistence in the cold, it is to be mixed with alcohol, in order to dissolve out its sugar. Cold water being added to what remains, effects a solution of the mucilage, and leaves the insoluble azotized matter with the phosphate of lime.

By this mode of analysis a minute portion of resin may remain in the gluten and in the washing water; the gluten retains also a small proportion of a fixed oil, and a volatile principle, which may be removed by alcohol. If we wish to procure the resin alone, we must first of all treat the flour, well dried, with alcohol.

When corn flour, poor in gluten, is to be analyzed, the dough must be enclosed in a linen bag, kneaded with water, and washed in that state.

In analyzing barley-meal by the above process, hordeine, mixed with common starch, is obtained: they may be separated by boiling water, which dissolves the starch, and leaves ihe hordeine under the aspect of saw-dust.

Fig. 170 is the plan of a London baker's oven, fired with coal fuel.

Fig. 171 is the longitudinal section.
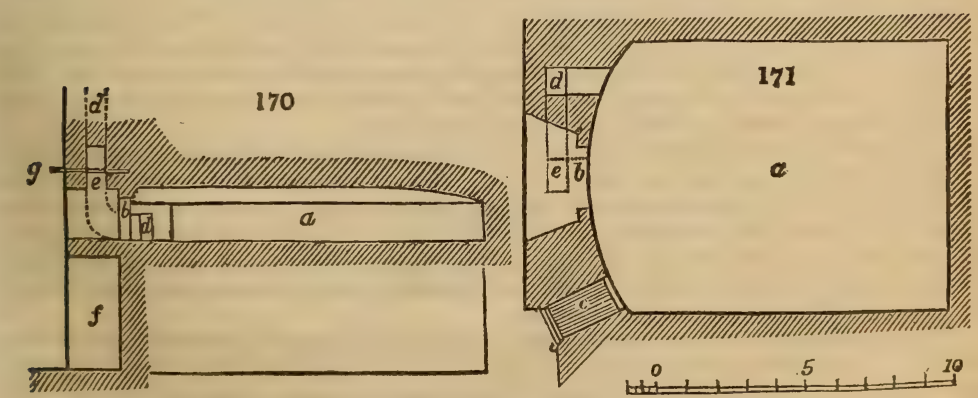

$a$, the body of the oven; $b$, the door; $c$, the fire-grate and furnace; $d$, the smoke flue; $e$, the flue above the door, to carry off the steam and hot air, when taking out the iread; $f$, recess below the door, for receiving the dust; $g$, damper plate to shut off the steam flue; $h$, damper plate to shut off smoke flue, after the oven has come to its proper 


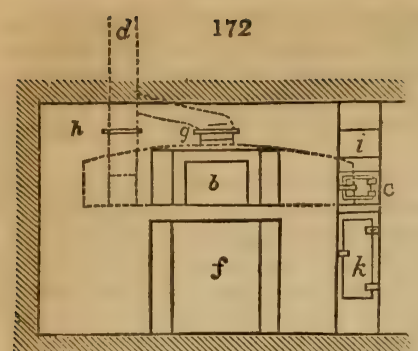

heat; $i$, a small ircn pan nver the fire-place $c$ : for heating water; $k$, ash-pit below the fur nace.

Fig. 172 is the front view; the same letters re. fer to the same objects in all the figures.

The flame and burnt air of the fire at $c$, sweep along the bottom of the oven by the right hand side, are reflected from the back to the left hand side, and thence escape by the flue $d$; (see plan fig. 171.) Whenever the oven has acquired the proper degree of heat, the fire is withdrawn, the flues are closed by the damper plates, and the lumps of fermented dough are introduced.

BRECCIA, an Italian term, used by mineralogists and architects to designate such compound stony masses, natural or artificial, as consist of hard rocky fragments of considerable size, united by a common cement. When these masses are formed of small rounded pebbles, the conglomerate is called a pudding-stone, from a fancied resemblance to plum pudding.

Concrete, now so much used for the foundations of large buildings, is a factitious breeeia, or pudding-stone. See Concrete.

BREWING. (Brasser, Fr.; Brauen, Germ.) The art of making beer, which see.

BRICK. (Brique, Fr.; Backsteine, ziegelsteine, Germ.) A solid, commonly rectangular, composed of clay, hardened by heat, and intended for building purposes. The natural mixture of clay and sand, called loam, as well as marl, which consists of lime and clay, with little or no sand, constitutes also a good material for making bricks. The poorer the marl is in lime, the worse adapted it is for agricultural purposes, and the better for the brick manufacturer, bsing less liable to fuse in his kiln. When a natural compound of silica and clay can be got nearly free from lime and magnesia, it forms a kind of bricks very refractory in the furnace, hence termed fire-bricks. Such a material is the slate-clay, schieferthon, of our coal measures, found abundantly, and of excellent quality, at Stourbridge, and in the neighborhood of Newcastle and Glasgow. The London brickmakers add to the clay about one third of coal ashes obtained from the kitchen dust-holes; so that when the bricks are put into the kiln, the quantity of coaly matter attached to their surface serves to economize fuel, and makes them less apt to shrink in the fire; though they are less compact, and probably less durable, than the bricks made in the coal districts of England.

The general process of brick-making consists in digging up the clay in autumn; exposing it, during the whole winter, to the frost, and the action of the air, turning it repeatedly, and working it with the spade; breaking down the clay lumps in spring, throwing them into shallow pits. to be watered and soaked for several days. The next step is to temper the clay, which is generally done by the treading of men or oxen. In the neighborhood of London, however, this process is performed in a horse-mill. The kneading of the clay is, in fact, the most laborious but indispensable part of the whole business; and that on which, in a great measure, the quality of the bricks depends. All the stones, particularly the ferruginous, calcareous, and pyritous kinds, should be removed, and the clay worked into a homogeneous paste, with as little water as possible.

The earth, being sufficiently kneaded, is brought to the bench of the moulder, who works the clay into a mould made of wood or iron, and strikes off the superfluous matter. The bricks are next delivered from the mould, and ranged on the ground; and when they have acquired sufficient firmness to bear handling, they are dressed with a knife, and stacked or built up in long dwarf walls, thatched over, and left to dry. An able workman will make, by hand, 5,000 bricks in a day.

The different kinds of bricks made in England are principally place bricks, gray and red stocks, marl facing bricks, and cutting bricks. The place bricks and stocks are used in common walling. The marls are made in the neighborhood of London, and used in the outside of buildings; they are very beautiful bricks, of a fine yellow color, hard, and well burnt, and, in every respect, superior to the stocks. The finest kind of marl and red bricks, called cutting bricks, are used in the arches over windows and doors, being rubbed to a centre, and gauged to a height.

In France attempts were long ago made to substitute animals and machines for the treading of men's feet in the clay-kneading-pit; but it was found that their sehemes could not replace, with advantage, human labor, where it is so cheap, particularly for separating the stones and heterogeneous matters from the loam. The more it is worked, the denser, more uniform, and more durable, the bricks which are made of it. A good French workman, in a day's labor of 12 or 13 hours, it has been said, is able to mould from 9,000 to 10,000 bricks, 9 inches long, $4 \frac{1}{2}$ inches broad, and $2 \frac{1}{4}$ thick; but he must 
have good assistants under him. In many brickworks near Paris, screw-presses are now used for consolidating the bricks and paving tiles in their mon!ds. M. Mollerat employed the hydraulic press for the purpose of condensing pulverized clay, which, after baling, formed beautiful bricks; but the process was too tedious and costly. An ingenious contrivance for moulding bricks mechanically, is said to be emploved near Washington, in America. This machine moulds 30,000 in a day's work of 12 hours, with the help of one horse, yoked to a gin wheel, and the bricks are so dry when discharged from their moulds, as to be ready for immediate burning. The machine is described, with figures, in the Bulletin de la Société d'Encouragement for 1819, p. 361. See further on, an account of our recent patents.

Brichs, in this country, are generally baked either in a clamp or in a kiln. The latter is the preferable method, as less waste arises, less fuel is consumed, and the bricks are souner burnt. The kiln is usially 13 feet long, by $10 \frac{1}{2}$ feet wide, and about 12 feet in height. The walls are one foot two inches thick, carried up a little out of the perpendicular, inclining towards each other at the top. The briclis are placed on flat arches, having holes left in them resembling lattice-work; the kiln is then covered with pieces of tiles and brichs, and some wood put in, to dry them with a gentle fire. This continues two or three days before they are ready for burning, which is linown by the smoke turning from a darkish color to transparent. The mouth or mouths of the kiln are now dammed up with a shinlog, which consists of pieces of bricks piled one upon another, and closed with wet brick earth, leaving above it just room sufficient to receir? ? fagot. The fagots are made of furze, heath, brake, fern, \&c., and the kiln is supplied with these until its arches look white, and the fire appears at the top; upon which the fire is slackened for an hour, and the kiln allowed gradually to cool. This heating and cooling is repeated until the bricks be thoroughly burnt, which is generally done in 48 hours. One of these kilns will hold about 20,000 bricks.

Clamps are also in common use. They are made of the bricks themselves, and generally of an oblong form. The foundation is laid with place brick, or the driest of those just made, and then the bricks to be burnt are built up, tier upon tier, as high as the clamp is meant to be, with two or three inches of breeze or cinders strewed between each layer of briclis, and the whole corered with a thick stratum of breeze. The fireplace is perpendicular, about three feet high, and generally placed at the west end; and the flues are formed by gathering or arching the bricks over, so as to leave a space between each of nearly a brick wide. The llues run straight through the clamp, and are filled with wood, coals, and breeze, pressed closely together. If the brichs are to be burnt off quiclily, which may be done in 20 or 30 days, according as the weather may suit, the flues should be only at about six feet distance; but if there be no immediate hurry, they may be placed nine feet asunder, and the clamp left to burn off slowly.

Floating bric's are a rery ancient invention : they are so light as to swim in water; and Pliny tells us, that they were made at Marseilles; at Colento, in Spain; and at Pittane, in Asia. This invention, however, was completely lost, until M. Fabbroni published a discovery of a method to imitate the floating brichs of the ancients. According to Posidonius, these bricks are made of a kind of argillaceous earth, which was employed to clean silver plate. But as it could not be our tripoli, which is too heavy to float in water, M. Fabbroni tried several experiments with mineral argaric, guhr, lac-lunæ, and fossil meal, which last was found to be the very substance of which he was in search. This earth is abundant in Tuscany, and is found near Casteldelpiano, in the territories of Sienna. According to the analysis of M. Fabbroni, it consists of 55 parts of silicious earth, 15 of magnesia, 14 of water, 12 of alumina, 3 of lime, and 1 of iron. It exhales an argillaceous odor, and, when sprinkled with water, throws out a light whitish smo'se. It is infusible in the fire; and, though it loses about an eighth part of its weight, its bulk is scarcely diminished. Bricks composed of this substance, either baked or unbaked, float in water; and a twentieth part of clay may be added to their composition without taking away their property of swimming. These hricks resist water, unite perfectly with lime, are subject to no alteration from heat or cold, and the baked differ from the unbaked only in the sonorous quality which they have acquired from the fire. Their strength is little inferior to that of common briclis, but much greater in proportion to their weight; for $\mathbf{M}$. Fabbroni found, that a floating brick, measuring 7 inches in length, $4 \frac{1}{2}$ in breadth, and one inch eight lines in thickness, weighed only $14 \frac{1}{4}$ onnces; whereas a common brick weighed 5 pounds $6 \frac{3}{4}$ ounces. The use of these bricks may be very important in the construction of powder magazines and reverberatory furnaces, as they are such bad conductors of heat, that one end may be made red hot while the other is held in the hand. They may also be employed for buildings that require to be light; such as cooking-places in ships, and floating batteries. the parapets of which would be proof against red-hot bullets.

The following plan of a furnace or kiln for burning tiles has been found very con. venient :- 
Fig. 173, front view, A A, B B, the solid walls of the furnace; $a \alpha a$, openings to the ash-pit, and the draught hole; $b \quad b \quad b$, openings for the supply of fuel, furnisher with a sheet-iron door. Fig. 174. Plan of the a-l! i!: and air channels c ic. The principal

178

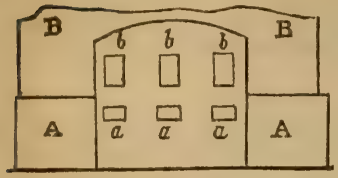

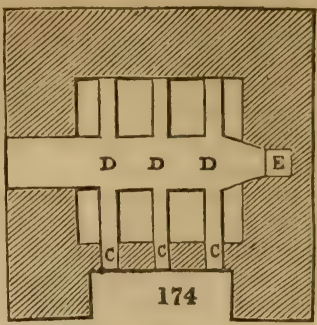

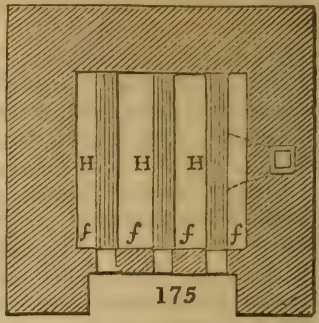

branch of the ash-pit $\mathrm{D} D \mathrm{D}$, is also the opening for taking out the tiles, after removing the grate; $\mathrm{E}$ the smoke flue. Fig. 175. Plan of the kiln seen from above. The grates H $\mathbf{H}$ H. The tiles to be fired are arranged upon the spaces $f f f f$.

Fig. 176 is the plan and section of one of the grates upon a much larger scale than in the preceding figures.

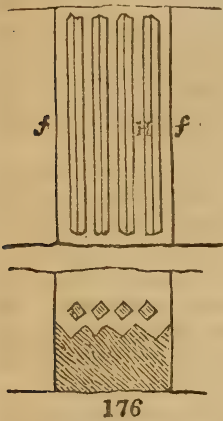

Mechanical brick moulding.-Messrs. Lyne and Stainford ob. tained, in August, 1825, a patent for a machine for making a considerable number of bricks at one operation. It consists, in the first place, of a cylindrical pug-mill of the kind usually employed for comminuting clay for bricks and tiles, furnished with rotatory knives, or cutters, for breaking the lumps and mixing the clay with the other materials of which bricks are commonly made. Secondly, of two moveable moulds, in each of which fifteen bricks are made at once; these moulds being made to travel to and fro in the machine for the purpose of being alternately brought under the pug-mill to be fitted with the clay, and then removed to situations where plungers are enabled to act upon them. Thirdly, in a contrivance by which the plungers are made to descend, for the purpose of compressing the material and discharging it from the mould in the form of bricks. Fourthly, in the meth $\mathrm{l}_{\mathrm{l}}$ of constructing and working trucks which carry the receiving boards, and conduct the bricks away as they are formed.

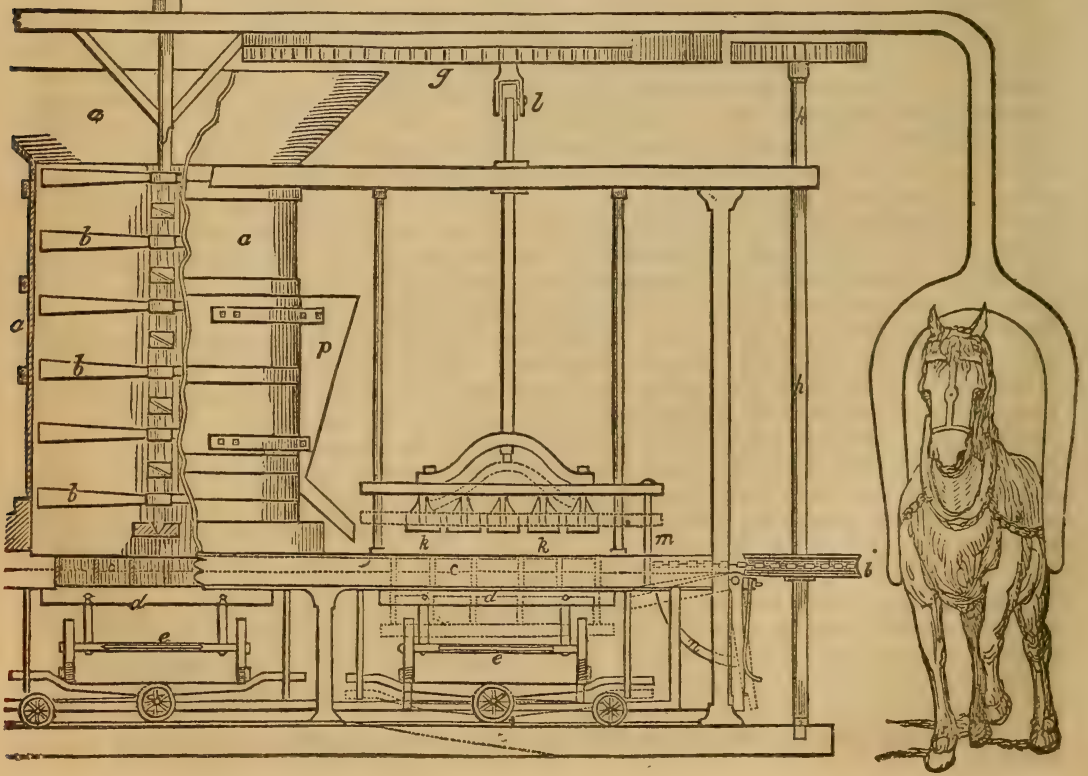


Fig. 177 exhihits the general construction of the apparatus; both ends of which being exactly sinilar, little more than half of the machine is represented. $a$ is the cylindrical pug-mill, shown partly in section, which is supplied with the clay and other materials from a hopper above; $b \quad b$, are the rotatory knives or cutters, which are attached to the vertical shaft, and being placed obliquely, press the clay down toward the bottoin of the cylinder, in the act of breaking and mixing it as the shaft revolves. The lower part of the cylincler is open; and immediately under it the mould is placed in which the bricks are to be formed. These moulds run to and fro upon ledges in the side frames of the machine; one of the moulds only can be shown by dots in the figure, the side rail intervening : they are situated at $c c$, and are formed of bars of iron crossing each other, and encompassed with a frame. The mould resembles an ordinary sash-window in its form, being dirided into rectangular compartments (fifteen are proposed in each) of the dimensions of the intended bricks, but sufficiently deep to allow the material, after being considerably pressed in the mould, to leave it, when discharged, of the usual thickness of a common brick.

The mould being open at top and bottom, the material is allowed to pass into it, when situater exactly under the cylinder; and the lower side of the mould, when so placed, is to be closed by a flat board $d$, supported by the truck $e$, which is raised by a lever and roller beneath, running upon a plane rail with inclined ends.

The central shaft $f$, is kept in continual rotatory motion by the revolution of the upper horizontal wheel $\mathrm{g}$, of which it is the axis; and this wheel may be turned by a horse yoked to a radiating arm, or by any other means. A part of the circumference of the wheel $g$, has teeth which are intended at certain periods of its revolution to take into a toothed pinion, fixed upon the top of a vertical shaft $h h$. At the lower part of this vertical shaft, there is a pulley $i$, over which a chain is passed that is connected to the two moulds $c$, and to the frame in which the trucks are supported; by the rotation of the rertical shaft, the pulley winds a chain, and draws the moulds and truck-frames along.

The clay and other material having been forced down from the cylinder into the mould, the teeth of the horizontal wheel g now come into gear with the pinion upon $h$, and turn it and the shaft and pulley $i$, by which the chain is wound, and the mould at the right hand of the machine brought into the situation shown in the figure; a scraper or edgebar under the pug-mill having levelled the upper face of the clay in the mould, and the board $d$, supported by the truck $e$, formed the flat under side.

The mould being brought into this position, it is now necessary to compress the materials, which is cione by the descent of the plungers $k k$. A friction-roller $l$, pendent from the under side of the horizontal wheel as that wheel revolves, comes in contact with an inclined plane, at the top of the shaft of the plungers; and, as the friction-roller passes over this inclined plane, the plungers are made to descend into the mould, and to compress the material; the resistance of the board beneath causing the clay to be squeezed into a compact state. When this has been effectually accomplished, the further descent of the plungers brings a pin $m$, against the upper end of a quadrant catch-lever $n$, and, by depressing this quadrant, causes the balance-lever upon which the truck is now supported to rise at that end, and to allow the truck with the board $d$ to descend, as shown by dots; the plingers at the same time forcing out the bricks from the moulds, whereby they are deposited upon the board $d$; when, by drawing the truck forward out of the machine, the board with the bricks may be removed, and replaced by another board. The truck may then be again introduced into the machine, ready to receive the next parcel of bricks.

By the time that the discharge of the bricks from this mould has been effected, the other mould under the pug-cylinder has become filled with the clay, when the teeth of the horizontal wheel coming round, take into a pinion upon the top of a vertical shaft exactly similar to that at $h$, but at the reverse end of the machine, and cause the moulds and the frame supporting the trucks to be slidden to the left end of the machine; the upper surface of the mould being scraped level in its progress, in the way already described. This movement brings the friction-wheel 0 , up the inclined plane, and thereby raises the truck with the board to the under side of the mould, ready to receive another supply of clay; and the mould at the left-hand side of the machine being now in its proper situation under the plungers, the clay becomes compressed, and the bricks discharged from the mould in the way described in the former instance; when this truck being drawn out, the bricks are removed to be dried and baked, and another board is placed in the same situation. There are boxes $p$, upon each side of the pug-cylinder containing sand, at the lower parts of which small sliders are to be opened (by contrivances not shown in the figure), as the mould passes under them, for the purpose of scattering sand upon the clay in the mould to prevent its adhering to the plungers. There is also a rack and toothed sector, with a balance-weight connected to the inclined plane at the top of the plunger-rods, for the purpose of raising the plunger after the 
friction-roller has passed over it. And there is a spring acting against the back of the quadrant-catch for the purpose of throwing it into its former situation, after the pin of the plunger has risen.

One of the latest, and apparently most effective machines for brick-making, is that patented by Mr. Eutward Jones, of Birmingham, in August, 1835. His improvements are described under four heads; the first applies to a machine for moulding the earth into briclss in a circular frarne-plate horizontally, containing a series of moulds or rectangular boxes, standing radially round the circumference of the circular frome, into which boxes successively the clay is expressed from a stationary hopper as the frame revolves, and after being so formed, the bricks are successively pushed out of their boxes, each by a piston, acted upon by an inclined plane below. The second head of the specification describes a rectangular horizontal frame, having a series of moulding boxes placed in a straight range, which are acted upon for pressing the clay by a corresponding range of pistons fixed in a horizontal frame, worked up and down by rods extending from a rotatory crank shaft, the moulding boxes being allowed to rise for the purpose of enabling the pistons to force out the bricks when moulded, and leave them upon the bed or board below. The third head applies particularly to the making of tiles, for the flooring of kilns in which malt or grain is to be dried. There is in this contrivance a rectangular mould, with pointed pieces standing up for the purpose of producing airholes through the tiles as they are moulded, which is done by pressing the clay into the moulds upon the points, and scraping off the superfluous matter at top by hand. The fourth or last head applies to moulding ehimney pots in donble moulds, which take to pieces for the purpose of withdrawing the pot when the edges of the slabs or sides are sufficiently brought into contact.

"The drawing which accompanies the specification very imperfectly represents some parts of the apparatus, and the description is still more defective; but as we are acquainted with the machinery, we will endeavor to give it an intelligible form, and quote those parts of the specification which point the particular features of novelty proposed to be claimed by the patentee as his invention, under the several heads." *

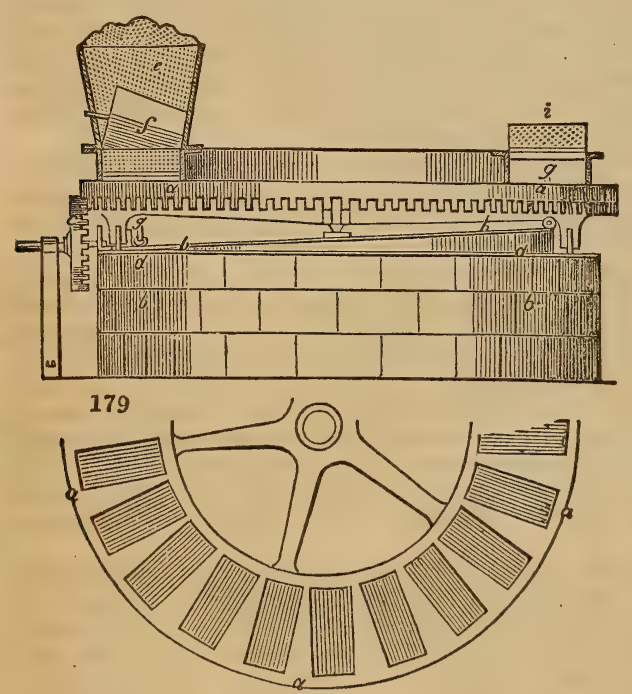

Fig. 178 represents, in elevation, the first-mentioned machine for moulding bricks. The moulds are formed in the face of a circular plate or wheel, $a a$, a portion of the upper surface of which is represented in the horizontal view, fig. 179 . Any convenient number of these moulds are set radially in the wheel, which is mounted upon a central pivot, supported by the masonry $b \quad b$. There is a rim of teeth round the outer edge of the wheel $a \quad a$, which take into a pinion $c$, on a shaft connected to the first mover; and by these means the wheel $a$, with the moulding boxes, is made to revolve horizontally, guided by arms with anti-friction rollers, which run round a horizontal plate $a a$, fixed upon the masonry.

A hopper, $e$, filled with the brick earth shown with one of the moulding boxes in section, is fixed

above the face of the wheel in such a way that the earth may descend from the hopper into the several moulding boxes as the wheel passes round under it; the earth being pressed into the moulds, and its surface scraped off smooth by a conical roller $f$, in the bottom of the hopper.

Through the bottom of each moulding box there is a hole for the passage of a piston rod $g$, the upper end of which rod carries a piston with a wooden pallet upon it, acting within the moulding box; and the lower end of this rod has a small anti-friction roller, which, as the wheel $a$ revolves, runs round upon the face of an cblique ring or inclined way, $h h$, fixed upon the masonry.

The clay is introduced into the moulding boxes from the hopper, fixed over the lowest * Mr. Newton, in his London Journal, February, 1837. 
part of the inclined way $h$, and it will be perceived that as the wheel revolves, the piston rods $g$, in passing up the inclined way, will cause the pistons to force the new-moulded bricks, with their pallet or board under them, severally up the mould, into the situalion shown at $i$, in fig. 178, from whence they are to be removed by hand. Fresh pallets being then placed upon the several pistons, they, with the moulds, will be rearly for moulding fresh bricks, when, by the rotation of the wheel $a$, they are severally brought under the hopper, the pistons having sunk to the bottoms of their boxes, as the piston rods passed down the other side of the inclined way $h$.

The patentee says, after having described the first head of his invention, he would have it understood that the same may be varied without departing from the main object of the invention; viz., that of arranging a series of moulds when worked by means of an inclined track, and in such manner that bricks, tiles, or other articles made of brick earth, may be capable of being formed in a mould with pallets or boards laid within the moulds, and constituting the bottom thereof, the bricks being removed from out of the moulds, with the pallets or boards under them, as above described. "I do not, therefore, confine myself to the precise arrangement of the machine here shown, though it is the best with which I am acquainted for the purpose."

The second head of the invention is another construction of apparatus ior moulding bricks, in this instance, in a rectangular frame. Fig. 180 is a front elevation of the ma-

180

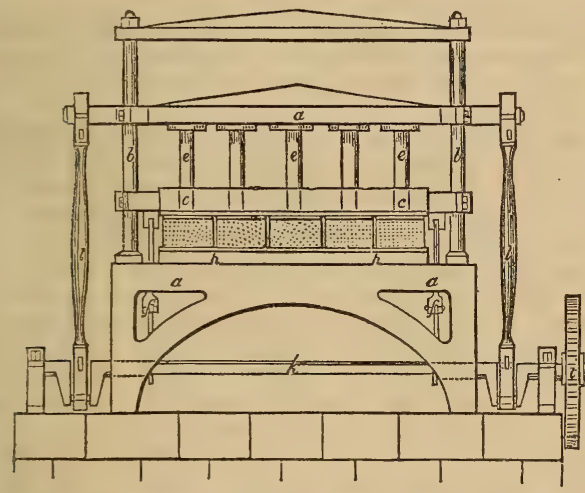

181

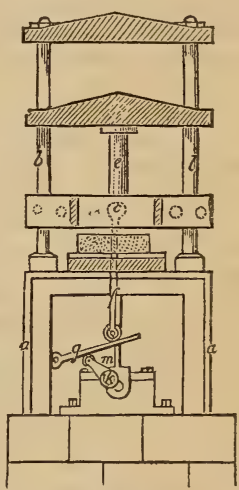

chine; fig. 181, a section of the same taken transversely. $a$ a is the standard frame-work and bed on which the bricks are to be moulded. Near the corners of this standard frame-work, four vertical pillars $b b$ are erected, upon which pillars the frame of the moulding boxes $c$, slides up and down, and also the bar $d$, carrying the rods of the pistons eee. These pistons are for the purpose of compressing the clay in the moulding box, and therefore must stand exactly over and correspond with the respective moulds in the frame $c$ beneath.

The sliding frame $c$, constituting the sides and ends of the moulding boxes, is supported at each end by an upright sliding rod $f$, which rods pass through guides fixed to the sides of the standard frame $a a$, and at the lower end of each there is a roller, bearing upon the levers $g$, on each side of the machine, but seen only in fig. 181, which levers, when depressed, allow the moulding boxes to descend, and rest upon the bed or table of the machine $h h$.

In this position of the machine resting upon the bed or table, the brick-earth is to be placed upon, and spread over, the top of the frame $c$, by the hands of workmen, when the descent of the plunger or pistons $e \varepsilon e$, will cause the earth to be forced into the moulds, and the bricks to be formed therein. To effect this, rotatory power is to be applied to the toothed wheel $i$, fixed on the end of the main driving crank shaft $k k$, which on revolving will, by means of the crank rods $l l$, bring down the bar $a$, with the pistons or plungers $e e e$, and compress the earth compactly into the moulds, and thereby form the bricks.

When this has been done, the bricks are to be released from the moulds by the moulding frame $c$ rising up from the bed, as shown in fig. 180, the pistons still remaining depressed, and bearing upon the upper surfaces of the bricks. The moulding frame is raised by means of cams $m$, upon the crank shaft, which at this part of the operation are brought under the levers $g$, for the purpose of raising the cams and the sliding rods $f$, into the position shown in $f$ ig. 181 .

The bricks having been thus formed and released from their moulds, they are to be removed from the bed of the machine by pushing forward, on the front side, fresh boards 
or pallets, which of course will drive the bricks out upon the other side, whence they are to be removed by hand.

There is to be a small hole in the centre of each pallet, and also in the bed, for the purpose of allowing any superfluous earth to be pressed through the moulding boxes when the pistons descend. And in order to cut off the projecting piece of clay which would be thus formed on the bottom of the brick, a knife-edge is in some way connected to the bed of the machine; and as the brick slides over it, the knife separates the protuberant lump : but the particular construction of this part of the apparatus is considered to be of little importance; and the manner of effecting the object is not clearly stated in the specification.

The patentee proposes a variation in this construction, which he describes in these words : "It will be evident that in place of having the moulds to rise, they may, by suitable arrangements, be made to descend below the bricks. In this case, in place of the boards, stationary blocks to receive the pallets must be fixed on the bed of the machine, and these blocks must be shaped in such a manner as to allow of the moulds passing orer them; and then it will be desirable to use the first part of my improvements, that of having the pallets within the moulds at the time of moulding the bricks; or in case of working with exceedingly stiff brick-earth, the paliets may be dispensed with." In 1835, 1,380,279,065 bricks paid duty in the United Kingdom; the revenue from which was $405,580 l$. $6 s$. $3 d$.

BRIMSTONE. (Soufre, Fr.; Schwefel, Germ.) Sulphur, which see.

BRITISH GUM. The trivial name given to starch, altered by a slight calcination in an oven, whereby it assumes the appearance and acquires the properties of gum, being soluble in cold water, and forming in that state a paste well adapted to thicken the colors of the calico printer. See STARch.

BROMINE, one of the archæal elements, which being developed from its combinations at the positive pole of the voltaic circuit, has been therefore deemed to be idio-electropositive, like oxygen and chlorine. It derives its name from its nauseous smell, Boringos, fator. It occurs in various saline springs on the continent of Europe, in those of Ashby de la Zouche, and some others in England; in the lake Asphaltites, in sponges, in some marine plants, in an ore of zinc, and in the cadmium of Silesia. At ordinary temperatures it is liquid, of a dark brown color in mass, but of a hyacinth-red in thin layers. Its smell is rank and disagreeable, somerwhat like that of chlorine. It has a very caustic taste. Its specific gravity is 2.966 . Applied to the skin it colors it deep yellow and corrodes it. One drop put within the bill of a bird suffices to kill it. It combines with oxygen with feeble affinity, forming bromic acid. Its attraction for hydrogen being far more energetic, it forms therewith a strong acid, the hydrobromic.

Bromine dissolves very sparingly in water, but it is very soluble in alcohol and ether. It combines with carbon, phosphorus, sulphur, and chlorine, as well as with most of the metals. From its scarcity it has not hitherto been applied to any purpose in the arts, but it is supposed to possess powerful discutient effeets upon scrofulous and other glandular tumors, whence the waters containing it are prescribed as an internal and external remedy in such forms of disease.

BRONZE. A compound metal consisting of copper and tin, to which sometimes a little zinc and lead are added. This alloy is much harder than copper, and was employed by the ancients to make swords, hatchets, \&c., hefore the method of working iron was generally understood. The art of easting bronze statues may be traced to the most remote antiquity, but it was first brought to a certain degree of refinement by Theodoros and Ræcus of Samos, about 700 years before the Christian era, to whom the invention of modelling is ascribed by Pliny. The ancients were well aware that by alloying copper with tin, a more fusible metal was obtained, that the process of casting was therefore rendered easier, and that the statue was harder and more durable; and yet they frequently made them of copper nearly pure, because they possessed no means of determining the proportions of their alloys, and because, by their mode of managing the fire, the copper became refined in the course of melting, as has happened to many founders in our own days. It was during the reign of Alexander that bronze statuary received its greatest extension, when the celebrated artist Lysippus succeeded by new processes of moulding and melting to multiply groups of statues to such a degree that Pliny called them the mob of Alexander. Soon afterwards enormous bronze colossuses were made, to the height of towers, of which the isle of Rhodes possessed no less than one hundred. The Roman consul Mutianus found 3,000 bronze statues at Athens, 3,000 at Rhodes, as many at Olympia and at Delphi, although a great number had been previously carried off from the last town.

In forming such statues, the alloy should be capable of flowing readily into all the parts of the mould, however minute; it should be hard, in order to resist accidental blows, be proof against the influence of the weather, and be of such a nature as to acquire that greenish oxydized coat upon the surface whith is so much admired in the 
antique bronzes, called patina antiqua. The chemical composition of the bronze alloy is a matter therefore of the first moment. The brothers Keller, celebrated founders in the time of Lonis XIV., whose chefs d'œurre are well known, directed 'their attention toward this point, to which too little importance is attached at the present day. The statue of Desaix in the Place Dauphine, and the column in the Place Vendome, are noted specimens of most defective worlimanship from mismanagement of the alloys of which they are composed. On analyzing separately specimens taken from the bass-reliefs of the pedestal of this column, from the shaft, and from the capital, it was found that the first contained only six per cent. of alloy, and 94 of copper, the second much less, and the third only 0.21. It was therefore obrious that the founder, unskilful in the melting of bronze, had gone on progressively refining his alloy, by the oxydizement of the tin, till he had exhansted the copper, and that he had then worked up the refuse scoriæ in the upper part of the column. The cannons which the government furnished him for casting the monument consisted of-

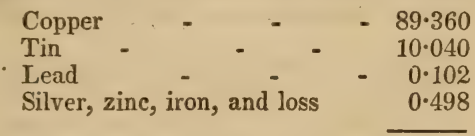

$100 \cdot 000$

The monlding of the several bass-reliefs was so ill executed, that the chisellers employed to repair the faults removed no less than 70 tons of bronze, which was given them, besides 300,000 franes for their work. The statues made by the Kellers at Versailles were found on chemical analysis to consist of-

\begin{tabular}{|c|c|c|c|c|}
\hline Copper & $\begin{array}{l}\text { No. } 1 . \\
91 \cdot 30\end{array}$ & $\begin{array}{l}\text { No. } 2 . \\
91 \cdot 68\end{array}$ & $\begin{array}{l}\text { No. } 3 . \\
91 \cdot 22\end{array}$ & $\begin{array}{c}\text { The mean } \\
91 \cdot 40\end{array}$ \\
\hline Tin & 1.00 & $2 \cdot 32$ & 1.78 & $1 \cdot 70$ \\
\hline Zine & 6.09 & $4 \cdot 93$ & $5 \cdot 57$ & $5 \cdot 53$ \\
\hline \multirow[t]{2}{*}{ Lead } & 1.61 & 1.07 & $1 \cdot 43$ & $1 \cdot 37$ \\
\hline & 1 & 100.00 & $100 \cdot 00$ & $100 \cdot 00$ \\
\hline
\end{tabular}

The analysis of the bronze of the statue of Louis XV. was as follows :-

$\begin{array}{lrl}\text { Copper } & 82 \cdot 45 & \text { Its specific gravity was } 8 \cdot 482 . \\ \text { Zine } & 10 \cdot 30 \\ \text { Tin } & 4 \cdot 10 \\ \text { Lead } & 3 \cdot 15 \\ & \frac{3.10}{100 \cdot 00}\end{array}$

The alloy most proper for bronze medals which are to be afterward struck, is composed of from 8 to 12 parts of tin, and from 92 to 88 of copper; to which if two or three parts in the hundred of zinc be added, they will make it assume a finer bronze tint. The alloy of the Kellers is famous for this effect. The medal should be subjected to three or four suecessire stamps of the press, and be softened between each blow by being heated and plunged into cold water.

The bronze of bells or bell metal is composed in 100 parts of copper $78, \operatorname{tin} 22$. This alloy has a fine compact grain, is very fusible and sonorous. The other metals sometimes added are rather prejudicial, and merely increase the profit of the founders. Some of the English bells consist of 80 copper, 10.1 tin, $5 \cdot 6$ zinc, and $4 \cdot 3$ lead; the latter metal when in such large quantity is apt to cause insolated drops, hurtful to the uniformity of the alloy.

The tam-tams and cymbals of bronze.-The Chinese make use of bronze instruments forged by the hammer, which are very thin, and raised up in the middle; they are called gongs, from the word ishoung, which signifies a bell. Klaproth has shown that they contain nothing but copper and tin; in the proportions of 78 of the former metal and 22 of the latter. Their specific gravity is 8.815 . This alloy when newly cast is as brittle as glass, but by being plunged at a cherry-red heat into cold water, and confined between two dises of iron to keep it in shape, it becomes tough and malleable. The cymbals consist of 80 parts copper and 20 tin.

Bronze vessels naturally brittle may be made tenacious by the same ingenious process, for which the world is indebted to M. Darcet. Bronze mortars for pounding have their lips tempered in the same way. Ancient warlike weapons of bronze were variously compounded; swords were formed of $87 \frac{1}{2}$ copper, and $12 \frac{1}{2}$ tin in 100 parts; the springs of balistæ consisted of 97 copper, and 3 tin.

Cannon metal consists of abont 90 or 91 copper, and 10 or 9 of tin. From the experiments of Papacino-d'Antony, made at Turin, in 1770, it appears that the most proper 
alloy for great guns is from 12 to 14 parts of tin to 100 of copper; but the Comte Lamar tilliere concluded from his experiments made at Douay, in 1786, that never less than 8 nor more than. 11 of tin should be employed in 100 parts of bronze.

Gilt ornaments of bronze.-This kind of bronze should be easy of fusion, and take perfectly the impression of the mould. The alloy of copper and zinc is when fused of a pasty consistence, does not make a sharn east, is apt to absorb too much amalgam, is liable to crack in cooling, and is too tough or too soft for the chaser or the turner. Were the quantity of zinc increased to make the metal harder, it would lose the yellow color suitable to the gilder. A fourfold combination of copper, zinc, tin, and lead is preferable for making such ornamental bronze articles; and the following proportions are probably the best, as they unite closeness of grain with the other good qualities. Copper 82 , zine 18 , tin 3 or 1 , lead $1 \frac{1}{2}$ or 3 . In the alloy which contains most lead, the tenacity is diminished and the density is increased, which is preferable for pieces of small dimensions. Another alloy, which is sail to require for its gilding only two thirds of the ordinary quantity of gold, has the following composition: copper, 82.257 ; zinc, $17 \cdot 481$; tin, 0.238 ; lead, 0.024 .

The antique bronze color is given to figures and other objects made from these alloys by the following process :-Two drachms of sal-ammoniac, and half a drachm of salt of snrrel (binoxalate of potash) are to be dissolved in fourteen ounce measures (English) of colorless vinegar. A hair pencil being dipped into this solution, and pressed gently bctween the fingers, is to be rubbed equally over the clean surface of the object, slightly warmed in the sun or at a stove; and the operation is to be repeated till the wished-for shade is obtained. (See GiLdING.)

The bronze founder ought to melt his metals rapidly, in order to prevent the loss of tin, zinc, and lead, by their oxydizement. Reverberatory furnaces have been long used for this operation; the best being of an elliptical form. The furnaces with dome tops are employed by the bell-founders, because their alloy being more fusible, they do not require so intense a heat; bat they also would find their adrantage in using the most rarid mode of fusion. The surface of the melting metals should be covered with small charcoal, or colie; and when the zinc is added, it should be dexterously thrust to the bottom of the melted copper. Immediately after stirring the melted mass so as to incorporate its ingredients, it should be poured out into the monlds. In general, the metals most easily altered by the fire, as the tin, should be put in last. The cooling should be as quick as possible in the moulds, to prevent the risk of the metals separating from each other in the order of their density, as they are very apt to do. The addition of a little iron, in the form of tin-plate, to bronze, is reckoned to be advantageous.

One part of tin, and two parts of copper (nearly one atom of tin and four of copper, or more exactly, 100 parts of tin, and 215 copper), form the ordinary speculum metal of reflecting telescopes, which is of all the alloys the whitest, the most brilliant, the hardest. and the most brittle. The alloy of 1 part of tin, and 10 of copper (or nearly one atom of the former to eighteen of the latter), is the strongest of the whole series.

Ornamental objects of bronze, after being cast, are commonly laid upon red-hot coals till they take a dull red heat, and are then exposed for some time to the air. The surface is thereby freed from any greasy matter, some portion of the zinc is dissipated, the alloy assumes more of a coppery hue, which prepares for the subsequent gilding. The black tinge which it sometimes gets from the fire may be removed by washing it with a weak acid. It may be made very clean by acting upon it with nitric acid, of specific gravity $1 \cdot 324$, to which a little common salt and soot have been added, the latter being of doubtful utility; after which it must be well washed in water, and dried with rags or saw-dust.

BronzING is the art of giving to objects of wood, plaster, \&c., such a surface as makes them appear as if made of bronze. The term is sometimes extended to signify the production of a metallic appearance of any kind upon such objects. They ought first to be smeared over smoothly with a coat of size or oil varnish, and when nearly dry, the metallic powder made from Dutch foil, gold leaf, mosaic gold, or precipitated copper, is to be applied with a dusting bag, and then rubbed over the surface with a linen pad; or the metallic powders may be mixed with the drying oil beforehand, and then applied with a brush. Sometimes fine copper, or brass filings, or mosaic gold, are mixed previously with some pulverized bone-ash, and then applied in either way. A mixture of these powders with mucilage of gum arabic is used to give paper or wood a bronze appearance. The surface must be afterward burnished. Copper powder precipitated by clean plates of iron, from a solution of nitrate of copper, after being well washed and dried, has been em. ployed in this way, either alone or mixed with pulverized bone-ash. A finish is given to works of this nature by a coat of spirit varnish.

A white metallic appearance is given to plaster figures by rubbing over them an amal. gam of equal parts of mercury, bismuth, and tin, and applying a coat of varnish over it. The iron-colored bronzing is given by black lead or plumbago, finely pulverized and 
washed. Busts and other objects made of cast iron acquire a bronze aspect by being well cleane and piunged in solution of sulphate of copper, whereby a thin film of this metal is left upon the iron.

Copper ac puires by a certain treatment a reddish or yellowish hue, in consequence of a little oxyile being formed upon its surface. Coins and meda!s may be handsnmely bronzed as follows; two parts of verdigris and 1 pa:t of sal ammuniac are to be dissolved in vinegar: the soluti on is to be boiled, skimm:d, and diluted with water till it has only a weak metallic taste, and upon further dilution lets fall no white recipitate. This solution is made to boil brislily, and is poured upon the objects to be bronzed, which are previously made quite clean, particularly fiee from grease, and set in arıother copper pan. This pan is to be put upon the fire, that the bniling may be renewed. The pieces under operation must be so laid that the solution has tree access to every point of their surface. The copper hereby acquires an agreeable reddist: brown hue, without losing its lustre. But if the process be too long continued, the coat of oxyde becomes thick, and makes the objects appear sealy and dull. Henee they must be inspected every 5 minutes, and be taken out of the solution the moment their color arrives at the desired shade. If the solution be too strong, the bronzing cumes off with friction, or the copper gets covered with a white powder, which becomes green by exposure to air, and the labor is consequently lost. The bronzed pieces are to be washed with many repeated waters, and carefully dried, otherwise they wonld infallibly turn green. To give fresh made bronze objects an antique appearanee, three quarters of an ounce of sal ammoniac, and a drachm and a half of binoxalate of potash (salt of sorrel) are to be dissolved in a quart of vinegar, and a sofi rag or brush moistened with this solution is to be rubbed over the clean bright metal, till its surface becomes entirely dry by the friction. This process must be repeated several times to produce the full effect; and the object should be kept a litle warm. Copper acquires very readily a brown color by rubbing it with a solution of the common liver of sulphur, or sulphuret of potash.

The Chinese are said to bronze their copper vessels by taking 2 ounces of verdigris, 2 ounces of cinnabar, 5 ounces of sal ammoniac, and 5 ounces of alum, all in powder, making them into a paste with vinegar, and spreading this pretty thick like a pigment on the surfaces previously brightened. The piece is then to be held a little while over a fire, till it becomes uniformly heated. It is next cooled, washed, and dried; after which it is treated in the same way once and again till the wished for color is obtained. An addition of sulphate of copper makes the color incline more to chestnut brown, and of borax more to yellow. It is obvious that the cinnabar produces a thin coat of sulphuret of copper upon the surface of the vessel, and might probably be used with advantage by itself.

To give the appearance of antique bronze to modern articles, we should dissolve 1 part of sal ammoniac, 3 parts of cream of tartar, and 6 parts of common salt in 12 parts of hot water, and mix with the solution 8 parts of a solution of nitrate of copper of specific gravity $1 \cdot 160$. This compound, when applied repeatedly in a moderately damp place to bronze, gives it in a short time a durable green coat, which becomes by degrees very beautiful. More salt gives it a yellowish tinge, less salt a blueish cast. A large addition of sal ammoniac accelerates the operation of the mordant.

Browning of gun-barrels and other arms.-By this process, the surface of several articles of iron acquires a shining brown color. This preparation, which protects the iron from rust, and also improves its appearance, is chiefly employed for the barrels of fowling-pieces and soldiers' rifles, to conceal the fire-arms from the game and the enemy. The finest kind of browning is the Damascus, in which dark and bright lines run through the brown ground.

This operation consists in producing a very thin uniform film of oxyde or rust upon the iron, and giving a gloss to its surface by rubbing wax over it, or coating it with a shellac varnish.

Several means may be employed to produce this rust speedily and well. The effect may be obtained by enclosing the barrels in a space filled with the vapor of muriatic acid. Moistening their surface with dilute muriatic or nitric acid, will answer the same purpose. But the most common material used for browning, is the butter or chloride of antimony, which, on account of its being subservient to this purpose, has been called bronzing sult. It is mixed uniformly with olive oil, and rubbed upon the iron slightly heated; which is afterwards exposed to the air, till the wished-for degree of browning is produced. A little aquafortis is rubbed on after the antimony, to quicken its operation. The brown barrel must be then carefully cleaned, washed with water, dried, and finally polished, either by the steel burnisher, or rubbed with white wax, or varnished with a solution of 2 ounces of shellac, and three drachms of dragon's blood, in 2 quarts of spirit of wine.

The following process may also be recommended: Make a solution with half an 
ounce of aquafortis, half an ounce of sweet spirit of nitre, 1 ounce of spirit of winc, 2 ounces of sulphate of copper, and 1 ounce of tincture of iron, in so much water as will fill altogether a quart measure. The gun barrel to be browned must first of all be filed and polished bright, and then rubbed with unslaked lime and water to clear away all the grease. Its two ends must now be stopped with wooden rods, which may serve as handles, and the touch-hole must be filled with wax. The barrel is then to be rubbed with that solution, applied to linen rags or a sponge, till the whole smface be equally moistened; it is allowed to stand 24 hours, and is then scrubbed with a stitf brush. The application of the liquid and the brushing may be repeated twice or oftener, till the iron acquires a fine brown color. After the last brushing, the barrel must be washed with plenty of boiling water, containing a little potash; then washed with clcan water, dried, rubbed with polishing hard wood, and coated with shellac varnish, for which purpose the barrel must be heated to the boiling point of water. It is finally polished with a piece of hard wood.

Storch recommends to make a browning solution with 1 part of sulphate of copper, one third of a part of sulphuric ether, and 4 parts of distilled water.

To give the damask appearance, the barrel must be rubbed over first with very dilute aquafortis and vinegar, mixed with a solution of blue vitriol; washed and dried, and rubbed with a hard brush to remove any seales of copper which may be precipitated uper. it from the sulphate.

Statues, rases, bass-reliefs, and other objects made of gypsum, may be durably bronzed, and bear exposure to the weather better than after the ordinary oil-varnish, by the following process:-Prepare a soap from linseed oil, boiled with caustic soda ley, to which add a solution of common salt, and concentrate it by boiling, till it becomes somewhat granular upon the surface. It is then thrown upon a piece of linen cloth, and strained with moderate pressure. What passes through is to be diluted with boiling water, and again filtesed. On the other hand, 4 parts of blue vitriol and 1 part of copperas are tc be dissolved separately in hot water. This solution is to be poured slowly into the solution of soap, as long as it occasions any precipitate. This flocculent matter is a mixture of cupreous soap and ferruginous soap, that is, a combination of the oxydes of copper and iron with the margaric acid of the soda soap. The copper soap is green, the iron soap is reddish brown, and both together resemble that green rust which is characteristic of the antique bronzes. When the precipitate is completely separated, a fresh portion of the vitriol solution is to be poured upon it in a copper pan, and is made to boil, in order to wash it. After some time, the liquid part must be decanted, and replaced by warm water for the purpose of washing the metallic soaps. They are finally treated with cold water, pressed in a linen bag, drained, and dried. In this state the compound is ready for use in the following way:-

Three pounds of pure linseed oil are to be boiled with twelve ounces of finelypowdered litharge, then strained through a coarse canvass cloth, and allowed to stand in a warm place till the soap turns clear. Fifteen ounces of this soap rarnish, mixed with 12 ounces of the above metallic soaps, and 5 ounces of fine white wax, are to be melted together at a gentle heat in a porcelain basin, by means of a water bath. The mixture must be kept for some time in a melted state, to expel any moisture which it mar contain. It must be then applied, by means of a painter's brush, to the surface of the gypsum previously heated to the temperature of about $200^{\circ} \mathrm{F}$. By skilful management of the heat, the color may be evenly and smoothly laid on without filling up the minute lineaments of the busts. When, after remaining in the cool air for a few days, the smell of the pigment has gone off, the surface is to be rubbed with cotton wool, or a fine linen rag, and variegated with a few streaks of metal powder or shell gold. Small objects may be dipped in the melted mixture, and then exposed to the heat of a fire till they are thoroughly penetrated and evenly coated with it.

BROWN DYE. Upon this subject some general views are given in the article DrEING, explanatory of the nature of this color, to which I may in the first place refer. This dye presents a vast variety of tints, from yellow and red to black brown, and is produced either by mixtures of red, yellow, and blue with each other, or of yellow or red with black, or by substantive colors, such as catechu or oxyde of manganese, alone. We shall here notice only the principal shades; leaving their modifications to the caprice or skill of the dyer.

1. Brown from mixture of other colors.

Wool and woollen cloths must be boiled with one eighth their weight of alum and sulpho-tartrate of iron (see this article); afterwards washed, and winced through the madder bath, which dyes the portion of the stuff imbued with the alum red, and that with the salt of iron black; the tint depending upon the proportion of each, and the duration of the madder bath.

A similar brown is produced by boiling every pound of the stuff with two ounces of alum, and one ounce of conmon sait, and then dyeing it in a bath of logwood contain- 
ing either sulphotartrate, acetate, or sulphate of iron. Or the stuff may be boiled with. alum and tartar, dyed up in a madder bath, and then run through a hlack batl: of jion moriant and salls or sumach. Here the black tint is added to the red till the proper hice be hit. The brown mas be produced also by adding sorne iron liquor to the madder bith, after the stuff has been dyed up in it with alum and tartar. A better brown of this ki $\mathrm{a}$ is obtained by boiling every pound of wool with 2 ounces of alum. ajyeing it up in coshineal, then changing the crimson thus given into brown, by turning the stuff throngh the bath after acetate of iron has been added to it. Instead of the cochineal, archil, or cutbear, with a little galls or sumach, may be used.

Wool or silli may also receive a light blue ground from the indigo vat, then be morlanted with alum, washed, and turned through a madder bath till the wished-for brown be brousht out. For the deeper shades, galls or sumach may be added to the paler Brazilwood, with more or less iron mordant. Instead of the indigo vat, Saxon blue may be employed to ground the stuff before dyeing it with madder, or 5 pounds of madder, with 1 pound of alum, a solution of one tenth of a pound of indigo in sulphuric acid, may be used with the proper quantity of water for 20 pounds of wool; for dark shades, some iron mordant may be added. Or we may combine a bath of cochineal or cutbear, fustic, and galls, and add to it sulphate of iron and sulphate of indigo, blunted with a little potash.

If we boil woollen cloth with alum and tartar, then pass it through a madder bath, and afterward through one of weld or fustic, containing more or less iron mordant, we obtain shades variable, according to the proportions of the materials, from mordoré and cinnamon to ehestnut brown.

After the same manner, bronze colors may be obtained from the union of olive dyes with red. For 25 pounds of cloth, we take 4 pounds of fustic chips, boil them for 2 hours, turn the cloth in this bath for an hour, and drain it; then add to the bath from 4 to 6 ounces of sulphate of iron, and 1 pound of ordinary madder, or 2 pounds of sandal-wood; put the cloth again in this compound bath, and turn it through, till the desired shade be obtained. By changing the proportions, and adding an iron mordant, other tints may be produced.

This mode of dyeing is suitable for silk, but with three different baths; one of logwood, one of Brazil-wood, and one of fustic. The silk, after being boiled with soap, is to be alumed, and then dyed up in a bath compounded of these three decoctions, mixed in the requisite proportions. By the addition of walnut peels, sulphate of copper, and a little sulphate of iron, or by passing the silk through a bath of annotto, a variety of brown shades may be had.

Or the silk may receive an annotto ground, and then be passed through a bath of logwood or Brazil-wood. For 10 pounds of silk, 6 ounces of annotto are to be taken, and dissolved with 18 ounces of potashes in boiling water. The silk must be winced through this solution for 2 hours, then wrung out, dried, next alumed, passed through a bath of Brazil-wood, and finally through a bath of logwood, containing some sulphate of iron. It is to be wrung out and dried.

Brown of different shades is imparted to cotton and linen, by impregnating them with a mixed mordant of acetates of alumina and iron, and then dyeing them up, either with madder alone, or with madder and fustic. When the aluminous mordant predominates, the madder gives an amaranth tint. For horse-chestnut brown, the cotton must be gallet, plunged into a black bath, then into a bath of sulphate of copper, next dyed up in a decoction of fustic, wrung out, passed through a strong madder bath, then through the sulphate of copper solution, and finished with a soap boil. Different shacles of cinnamon are obtained, when cottons first dyed up with madder get an olive cast with iron liquor in a fustic bath.

These cinnamon and mordoré shades are also produced by dyeing them first in a bath or weld and rerdigris, passing them through a solution of sulphate of iron, wringing and arying them; next putting them through a bath containing 1 pound of galls for 10 pounds of stuff, again drying, next aluming, and maddering. They must be brightened by a boil in soap water.

A supierior brown is produced by like means upon cotton goods, which have undergone the oiling process of the Turkey red dye. Such stuffs must be galled, mordanted with alum (see MADDER), sulphate of iron, and acetate of lead (equal to $\frac{2}{3}$ of the alum); after washing and drying, dyed in a madder bath, and cleared with a soap boil. The tint of brown varies with the proportion of alum and sulphate of iron.

We perceive from these examples, in how many ways the browning of dyes may be modified, upon what principles they are founded, and how we have it in cur power to turn the shade more or less toward red, black, yellow, blue, \&c.

Brown may be produced by direct dyes. The decoction of oak bark dyes wool a fast brown of different shades, according to the concentration of the bath. The color is more lively with the addition of alum. 
The decoction of bastard marjoram (Origanum vulgare) dyes cotton and linen a reddish brown, with acetate of alumina. Wool takes from it a dark brown.

The bark of the mangrove tree (Rizophora mangle) affords to wool boiled with alum and tartar a fine red brown color, which, with the addition of sulphate of iron, passes into a fast chocolate.

The Bablah, the pods of the East Indian Mimosa cineraria, and the African Mimosa nilotica, gives cotton a brown with acetate or sulphate of copper.

The root of the white sea rose (Nymphœa alba) gives to cotton and wool beautiful shades of brown. A mordant of sulphate of iron and zinc is first given, and then the wool is turned through the decoction of the root, till the wished-for shade is obtained. The cotton must be mordanted with a mixture of the acetates of iron and zinc.

Walnut peels (Juglans regia), when ripe, contain a dark brown dye stuff, which communicates a permanent color to wool. The older the infusion or decoction of the peels, the better dye does it make. The stuff is dyed in the lukewarm bath, and needs no mordant, though it becomes brighter with alum. Or this dye may be combined with the madder or fustic bath, to give varieties of shade. For dyeing silk, this bath should be hardly lukewarm, for fear of eausing inequality of color.

The peelings of horse-chestnuts may be used for the same purpose. With muriate of tin they give a bronze color, and with acetate of lead a reddish brown.

Catechu gives cotton a permanent brown dye, as also a bronze, and mordoré, when its solution in hot water is combined with acetate or sulphate of copper, or when the stuff is previously mordanted with the acetates of copper and alumina mixed, sometimes with a little iron liquor, rinsed, dried, and dyed up, the bath being at a boiling heat.

Ferrocyanate of copper gives a yellow brown or a bronze to cotton and silk.

The brown color called carmelite by the French is produced by one pound of eatechn to four ounces of verdigris, with five ounces of muriate of ammonia. The bronze (solitaire) is given by passing the stuff through a solution of muriate or sulphate of manganese, with a little tartaric acid, drying, passing through a potash ley at $4^{\circ}$ Baumé, brightening and fixing with solution of chloride of lime.

BRUSHES. (Brosses, Fr.; Bürsten, Germ.) Mr. T. Mason obtained a patent in October, 1830 , for an improvement in the manufacture of this article. It consists in a firmer mode of fixing the knots or small bundles of hair into the stock or the handle of the brush. This is done by forming grooves in the stocks of the brushes, for the purpose of receiving the ends of the knots of hair, instead of the holes drilled into the wood, as in brushes of the common constructions. These grooves are to be formed like a dovetail, or wider at the bottom than the top; and when the ends of the knots of hair have been dipped into cement, they are to be placed in the grooves and compressed into an oval form, by which the ends of the hair will be pressed outwards into the recess or wider part of the dovetailed groove, or the grooves may be formed with threads or teeth on the sides, instead of being dovetailed; and the cement and hairs being pressed into the teeth or threads, will eause them to adhere firmly to the stock or handle of the brush.

A metal ferrule may be placed on the outside of the stock of the brush, if necessary, and secured by pins or rirets, or in any other convenient manner, which ferrule may

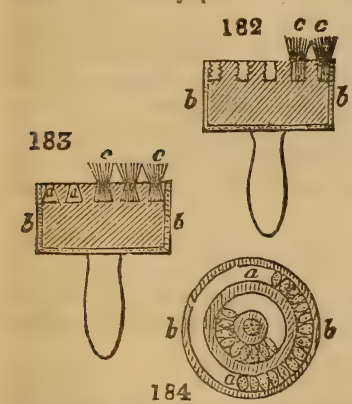
also form one side of the outer groove. Fig. 182 is a plan view of the stock of a round brush; fig. 183 is a section of the same; $a$ are the dovetailed grooves, which are turned out of the wood; $b$ is the metal ferrule; $c c$ are knots or small bundles of hair, to form the brush. After a number of the knots of hair are prepared, the ends are to be dipped into proper cement, and then placed into the grooves, when their ends are to be squeezed by a pair of pliers, or- other means, which will compress them into the oval shape, as shown in fig. 184, and cause the ends of the hairs to extend outward under the dovetailed part of the recess.

The knots of hair are to be successively placed in the grooves, and forced up by a tool against the last knot put in, and so on, until the grooves are filled; fig. 184 is a section taken through a brush with teeth or threads of a screw formed upon the sides of the groove; into these teeth or threads the cement and hairs will be forced by the compression, by which means they will be held firmly in the stock of the brush.

BUTTER. (Beurre, Fr.; Butter, Germ.) Milk contains a fatty matter of more or less consistency, modified very much according to the nature of the animals which afford it. This substance is butter, held suspended in the milk by means of the caserus matter and whey, with which it is intimateiy blended. Milk is a true emulsion resulting from the mixture of these three ingredients, owing its opacity and white solor to the diffusion through it of that butyraceous oil. When any circumstance 
dissolves this union, each component becomes insulated, and manifests its peciliar properties. Milk, even left to itself, at a temperature of from $50^{\circ}$ to $60^{\circ} \mathrm{F}$., separates spontaneously into several products. A layer of a fatter, more consistent, but lighter nature, floats upon its surface, while the subjacent liquid forms a white magma, which retains among its curdy flocks all the whey of the milk. The upper layer or cream contains nearly the whole of the butter; but a portion remains entangled with the curd and whey below.

It belongs to a work on husbandry or rural economy to treat fully of the operations of the dairy; one of the principal of which is the extraction of butter from milk.

The Tartars and French have been long in the habit of preserving butter, by melting it with a moderate heat, whereby are coagulated the albuminous and curdy matters remaining in it, which are very putrescible. This fusion should be made by a heat cf a water bath, about $176^{\circ} \mathrm{F}$., continued for some time, to effect the more complete purification of the butter. If in this settled liquefied state it be carefully decanted, strained through a tammy cloth, and slightly salted, it may be kept for a long time nearly fresh, without becoming in any degree rancid, more especially if it be put up in small jars closely covered.

Butter of Cacao. See Cacao and OrLs.

BUTTON MANUFACTURE. This art is divided into several branches, constituting so many distinct trades. Horn, leather, bone, and wood, are the substances frequently employed for buttons, which are either plain, or covered with silk, mohair, thread, or other ornamental materials. The most durable and ornamental buttons are made of various metals, polished, or covered with an exceedingly thin wash, as it is termed, of some more valuable metal, chiefly tin, silver, and gold.

Those buttons intended to be covered with silk, \&c. are termed, in general, moulds. They are small circles, perforated in the centre, and made from those refuse chips of bone which are too small for other purposes. These chips, which, for the large and coarser buttons, are pieces of hard wood, are sawn into thin flakes, of an equal thickness; from which, by a machine, the button moulds are cut out at two operations.

The shavings, sawdust, and more minute fragments, are used by manufacturers of cutlery and iron toys, in the operations of case-hardening; so that not the smallest waste takes place.

Metal buttons are formed of an inferior kind of brass, pewter, and other metallie compositions: the shanks are made of brass or iron wire, the formation of which is a distinct trade. The buttons are made by casting them round the shank. For this purpose the workman has a pattern of metal, consisting of a great number of circular buttons, connected together in one plane by very small bars from one to the next; and the pattern contains from four to twelve dozen of buttons of the same size. An impression from this pattern is taken in sand in the usual manner; and shanks are pressed into the sand in the centre of each impression, the part which is to enter the metal being left projecting above the surface of the sand. The buttons are now cast from a mixture of brass and tin; sometimes a small proportion of zinc is added, which is found useful in causing the metal to flow freely into the mould, and make a sharp casting. When the buttons are cast, they are cleaned from the sand by brushing; they are then broken asunder, and carried to a second workman at the lathe, who inserts the shank of a button into a chuck of a proper figure, in which it is retained by the back centre of the lathe being pressed against the button with a spring. The circumference is now, by filing it as it turns round, reduced to a true circle; and the button is instantly released by the workman's holding back the centre, and is replaced by another. A third workman now turns the back of the button smooth, in a chuck lathe, and makes the projecting part round the shank true; and a fourth renders the face of the button smooth, by placing it in a chuck, and applying the edge of a square bar of steel across its centre.

Gilt buttons are stamped out from copper, (having sometimes a small alloy of zinc,) laminated in the flatting mill to the proper thickness. The stamp is urged by a flypress, which cuts them out at one stroke. These circular pieces, called blanks, are annealed in a furnace to soften them; and the maker's name, \&c. is struck on the back by a monkey, which is a machine very similar to a pile-engine. This stamp also renders the face very slightly convex, that the buttons may not stick together in the gilding jrocess. The shanks are next soldered on. The burnishing is performed by a piece of hematites or blood-stone, fixed into a handle, and applied to the button as it revolves by the motion of the lathe.

A great number of the buttons, thus prepared for gilding, are put into an earthen pan, with the proper quantity of gold to cover them,* amalgamated with mercury in the fol-

* By ant of parliament 5 grains of gold are allotted for the purpose of gilding 144 buttons, though they may be tolerably well gilt by half that quantity. In this last case, the thickness would be about the $214,000 t h$ part of an inch. 
sowing manner: - The gold is put into an iron ladle, and a small quantity of mercuiry aidded to it; the ladle is held over the fire, till the gold and mercury are perfectiy united. This amalgam being put into the pan with the buttons, as much aquafurtis, diluted with water, as will wet them all over, is thrown in, and they are stirred up with a brush, till the acid, by its affinity to the copper, carries the amalgam to every part of its surface, covering it with the appearance of silver. When this is perfected, the acid is washed away with clean water. This process by the workman is called quiching.

The oid process in gilding buttons, called the drying off, was exceedingly pernicious to the operator, as he inhaled the vapor of the mercury, which is well known to be a violent poison. In order to obviate this, the following plan of apparatus has been employed with success. The vapor, as it rises from the pan of buttons heated by a charcoal fire, is conducted into an oblong iron flue or gallery, gently sloped downwards, having at its end a small vertical tube dipping into a water cistern, for condensing the mercury, and a large vertical pipe for promoting the draught of the products of the combustion.

Plated buttons are stamped by the fly-press, out of copper-plate, covered on one side with silver at the flatting-mill. The copper side is placed upwards in stamping, and the die or hole through which they are stamped is rather chamfered at its edge, to make the silver turn orer the edge of the button. The backs are stamped in the same manner as the gilt buttons. The shanks are soldered on with silver solder, and heated one by one in the flame of a lamp, with a blow-pipe urged by bellows. The edges are now filed smonth in the lathe, care being taken not to remove ang of the silver which is turned over the edge. They are next dipped in acid, to clean the backs, and boiled in cream of tartar and silver, to whiten them; after which they are burnished, the backs being first brushed clean by a brush held against them as they revolre in the lathe. The mode of burnishing is the same as for gilt buttons.

Button shanks are made by hand from brass or iron wire, bent and cut by the follow. ing means :-

The wire is lapped spirally round a piece of steel bar. The steel is turned round by screwing it into the end of the spindle of a lathe, and the wire by this means lapped close round it till it is corered. The coil of wire thus formed is slipped off, and a wire fork or staple with parallel legs put into it. It is now laid upon an anvil, and by a punch the coil of wire is struck down between the two prongs of the fork, so as to form a figure 8 , a little open in the middle. The punch has an edge which marks the middle of the 8 , and the coil being cut open by a pair of shears along this mark, divides each turn of the coil into two perfect button shanks or eyes.
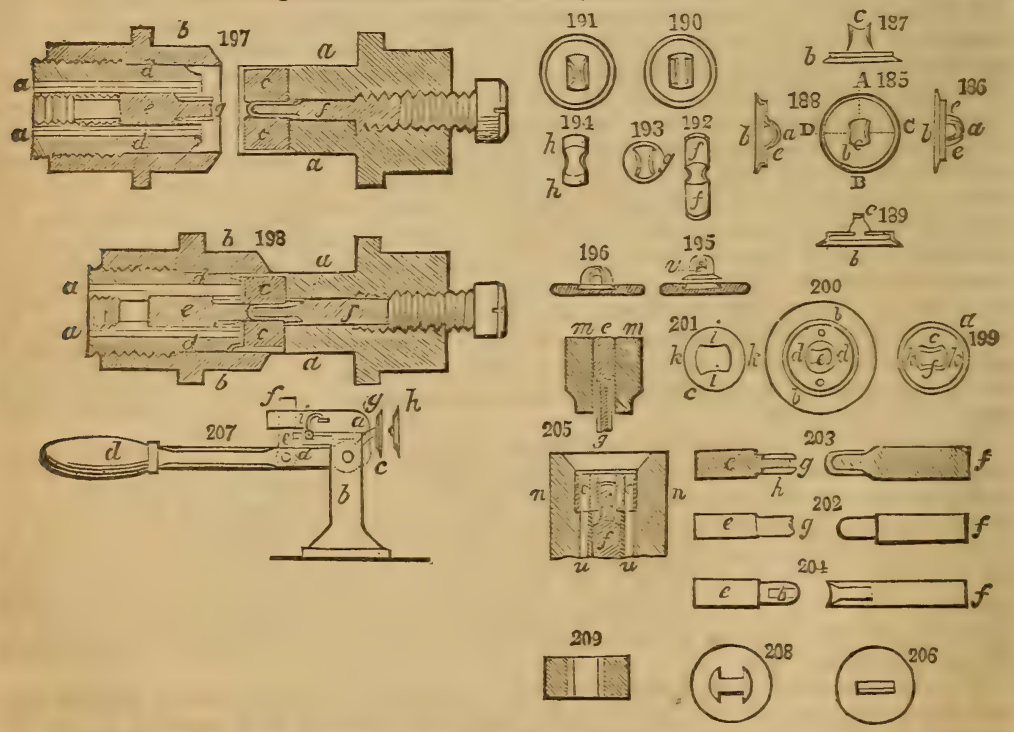

Mr. Holmes, of Birmingham, obtained in May, 1833, a patent for an improved construction of buttons. Fig. 185 represents the outside appearance of one of his improved shants, as raised or formed out of the dise of metal which is to constitute the 
back of the button; fig. 186 an edge view, looking through the shank or loop; fig. 187 is another edge view, looking at the raised shank or loop endways; fig. 188 is a section taken through the shank and disc in the direction of the dotted line A $\mathrm{B}$, in fig. 18.5 ; and $f i g .189$ another section taken in the direction of the dotted line $\mathrm{C} \mathrm{D}$, in fig. 185. All these figures of his improved shanks, as well as those hereinafter described, together with the tools used to form the same, are drawn at about half the real size, to show the parts nore distinctly. It will be seen that the shanks or loops $a$ a are formed by paitially cutting and raising, or forcing up a portion of the metal disc or back $b$, and are compressed or formed by the action of the tools, or punches and dies, so as to have a rounded figure on the inside of the top part of the shank, as at $c$, the edges of the metal being turned so as to prevent them cutting the threads by which the button is fastened to the cloth or garment. It will be observed that, there being but one passage or way through which the thread can be passed to sew on the button, and that opening being rounded on all edges, will cause the threads to keep in the centre of the shanks, the form of the shank allowing a much neater attachment to the garment, and keeping the threads from the edges of the metal. The ends of the shank, or portions $e$, which rise up from the dise or back $b$, are madt searly circular, in order to avoid presenting any edges of the metal to the sides of the button hole; and when the shank is sewed on the cloth, it forms, in conjunction with the threads, a round attachment, thereby preventing the shank from cutting or wearing the button-hole; the threads, when the shank is properly sewed to the garment, nearly filling up the opening through the shank, and completing that portion of the circle which has been taken out of the shank by the dies in forming the crescented parts of the loop. It will be therefore understood that the intention is, that the inside edges of the shank should be turned as much as possible away from the threads by which the button is sewed on the cloth, and that the outside of the shank should be formed so as to present rounded surfaces to the button-hole, and that the thread should fill up the opening through the shank, so as to produce a round attachment to the garment. It should here be observed, that the backs of the buttons shown in these figures are of the shape generally used for buttons covered with Florentine or other fabric, or faced with plates of thin metal, and are intended to have the edges of a disc, or what is termed a shell, forming the face, to be closed in upon the inclined or bevelled edges of the backs. Having now described the peculiar form of the improved shanks which he prefers, for buttons to be covered with Florentine or other fabric, or shells of thin metal plate, he proceeds to describe some of the different variations from the same.

Fig. 190 is a representation of a shank, the cut through the disc or back being effected by a parallel rib on the die, and corresponding groove in the shaping punch, instead of the semicircular or crescented cut shown in fig. $185 ; \mathrm{fig.} 191$ is a view of another shank, the separation of the sides of the loop being performed by straight edges in both punch and die. He prefers finishing this shaped shank (that is, giving it the rounded form, to prevent its cutting the threads) by detached punches, and dies, or pincers, as will be hereinafter described. Fig. 192 is a representation of one of the improved shanks, which has merely portions, $f f$, of the back of the button connected to its ends. This shank may be used for buttons which have a metal shell to be closed in upon the bevelled edges of the ends, or the shank piece may be otherwise connected to the face part of the button. Fig. 193 is a representation of a shank raised out of a small disc of metal $g$, intended to be sol. dered to the disc of metal forming the button, or it may be otherwise fixed to the back; fig. 194 is a representation of another shank for the same purpose, having only portions of metal $h h$, for soldering or otherwise attaching it to the back of the button, as by placing a ring or annular piece over it forming the back, which shall be confined to the face as before described; fig. 195 is a representation of a shank raised upon a dish or bevelled piece of metal, and is intended to be used for buttons made from pearl-shell, horn, wood, paper, or other substances. The back part of the button has a dovetailed recess formed in it to receive the dish-shaped back, which is pressed into the recess, the edges of the dish being expanded in the dovetailed parts of the recess by the ordinary muans, and thereby firmly fixing it to the button, as shown in fig. 196.

Haring now explained the peculiar forms of his improved shanks, he proceeds to describe tf e tou'.s, or punches and dies, by which he cuts the disc or back from out of a sheet of metal, and at the same operation produces and forms the shank complete. Fig. 197 is a longitudinal section taken through a pair of dies and punches when separated; fig. 198 is a similar section, taken when they are put together, and in the act of forming a shank after cutting out the disc or back of the button from a sheet of metal; fig. 199 is a face view of the punch; and fig. 200 is a similar representation of the counter die, with the tools complete; $a$ is the punch or cutter, and $b$ the counter bed, by the circular edges of which the disc of metal is cut out of the sheet; $c$ is a die, fixed in the cutter $a$ (upon which the name of the button-maker may be engraved). Fig. 201 is a face view of this die when removed out of the punch; $d$ is the counter die to the die $c$. It will be perceived that these dies $c$ and $d$, together with the punch and bed, compress the disc of metal into the form re- 
quired for the back of the button; that shown in the figures, as before stated, is of the shape used for buttons to be covered with Florentine or thin plate metal, in a round shell closed in upon the inclined or bevelled edge of the back; $e$ is the cutting and shaping punch of the shank, which is fixed within the counter die; this punch cuts through the metal of the disc, and forms the shank as the dies approach nearer together, by raising or forcing it up into the recess or opening in the die $c$, where it is met by the end of another shaping punch $f$, fixed in the punch $a$, which compresses the upper part of the shank into the recess $g$, in the end of the punch $e$, thereby giving the shank its rounded figure, and at the same time forming the other part of the shank into the required shape, as described at figs. 185 to 189 . The ends of these shaping punches fit into and over each other, as will be seen by the detached figures of the punches designed for form. ing the shank first described. Fig. 202 is a representation of the purches when apart and removed out of the dies; fig. 203 is a longitudinal section of the same; fig. 204 is another view of the punches as seen on the top. The sharp edge of the recess $h$, in the punch $e$, comes in contact with the cutting edges of the projecting rib $i$ of the die $c$, and thereby cuts through so much of the metal as is required. The edge $k$ of this die keeps the outside ends of the shank of a spherical figure, as before explained, while the punches force up the metal, and form the elevated loop or shank: $u u$ are holes made through the counter die $d$, for the passage of clearing pins, which force out the shank or back piece from the counter die when finished; the operation of which will be shown when describing the machinery hereafter. There are adjusting screws at the back of the punches and dies, by which they can be regulated and brought to their proper position one to the other.

Although he has shown the punches which form his improved shanks, fixed into and working in conjunction with the punch and dies which cut out and shape the discs of metal for the back of the button, yet he does not intend to confine himself to that mode of using them, as flat blanks or dises for the backs of buttons may be cut out in a separate stamping press, and afterwards shaped in the same press or in another, and then brought under the operation of the punches which form his improved shanks, fixed in any suitable press. This last mentioned mode of producing button shanks and backs he prefers when such metals are employed as require annealing between the operations of shaping the backs and forming the shank. Fig. 205 is a section taken through a pair of dies, in which the operation only of forming the shank is to be performed, the backs being previously shaped in another press. In this instance the punches $e$ and $f$ are mounted in guide-pieces $m$ and $n$, which keep them in the proper position towards each other, the die $c$ being mounted in the piece $n$, and acting against the face of the guide $m$. The blanks or backs of the buttons may be fed into these dies by hand or any other means; and after the shank is formed, the finished back can be pushed out of the lower die by clearing rods passed through the holes $u u$ and removed by hand, or in any convenient manner.

When his improved shanks are formed out of iron or other metal which is too brittle to allow of the shank being forced up and finished at one operation in the dies and punches, he prefers cutting out and shaping the blank or back of the button first, and after annealing it, to raise or force up the portion of metal to form the shank into the shape shown in fig. 206, that is, without the edges of the metal being turned to prevent their cutting the threads, and after again annealing it, to bend or turn the edges into the shape shown in fig. 191 by means of suitable punches in another press, or by a pair of pincers and punch as shown in fig. 207, which is a side view of a small apparatus to be used for turning the edges of the shank by hand, with a partly formed shank seen under operation. $a$, is the upper jaw of a pair of pincers, this jaw being fixed on to the head of the standard $b$; the under jaw $c$, is formed by the end of the lever or handle $d$, which has its fulcrum in the standard $b . \quad e$ is a small punch, passed through a guide hole in the head of the standard, one end projecting into the jaws of the pincers, the other against a piece $f$, attached by a joint to the lever $d$, and working through a slot in the head of the standard; this piece $f$, has an inclined plane on the side next the end of the punch, which, in its descent, projects the punch forward against the top of the loop of the shank, (placed at $g$,) as the pincers are closed by forcing down the lever $d$, and, in conjunction with the jaws of the pincers, compresses the shank into the required form, as shown at $h$, and in the enlarged fig. 191. A spring, $i$, acts against a pin fixed into the punch $e$, for the purpose of bringing it back as the jaws open after forming a shank. Figs. 208 and 209 represent the face and section of the dies mentioned before, for cutting the slits in the dises, as at fig. 190.

Having explained the peculiar forms of his improved metallic shanks for buttons, and the tools employed in making the same, he proceeds to describe the machinery or apparatus by which he intends to carry his invention into effect. He proposes to take a sheet of metal, say about 30 or 40 feet long, and of the proper width and thickness; which thin sheet is to be wound upcn a roller, and placed above the machine, so that it can be easily drawn down into the machine as required for feeding the punches and 
dies. Fig. 210 is a plan view of a machine, intended to work any convenient number of sets of punches and dies placed in rows. Eleven sets of punches and dies are re-

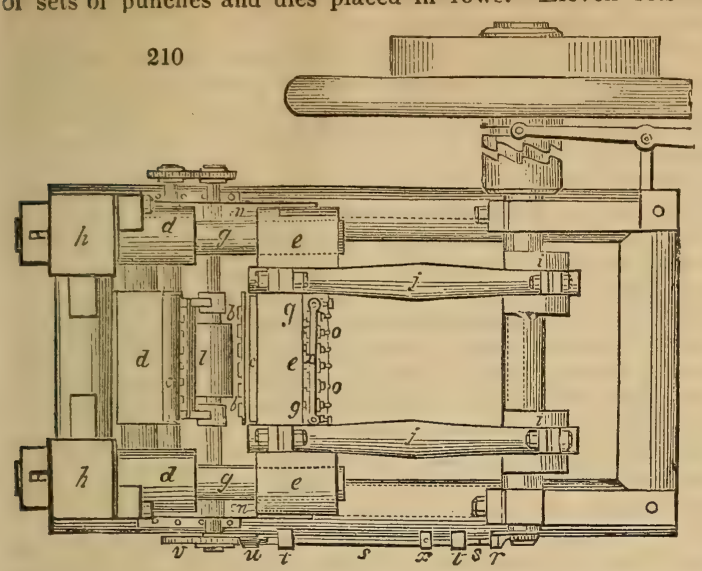
presented, each set being constructed as described under figs. 197 to 204 ; fig. 211 is a side view, and fig. 212 a longitudinal section, taken through the machine; figs. 213 and 214 are transverse sections taken through 'he machine between the punches and counter dies, fig. 213 representing its appearance at the face of the punches, and $f i g$. 214 the opposite view of the counter dies. $\quad \boldsymbol{a} \boldsymbol{a}$ are the punches; $b \quad b$ the counter dies; each being mounted in rows in the steel plates $c$, fixed upon two strong bars $d$ and $e$, by countersunk screws and nuts, the punches and dies being retained in their proper position by the plates, which are screwed on to the front of the steel plates, and press against the collars of the punches and dies. The bars $d$ and $e$ are both mounted on the guidepins $g$, fixed in the heads $h h$ of the frame, which guide pins pass through the bosses on the ends of the bars. The bar $d$ is stationary upon the guide pins, being fixed to the heads $h h$, by nuts and screws passed through ears cast on their bosses. The bar $e$ slides freely upon the guide pins $g^{\circ} g$, as it is moved backwards and forwards by the crank $i$, and connecting-rods $j j$, as the crank shaft revolves. The sheet of thin iron to be operated upon is placed, as before stated, above the machine; its end being brought down as at $a, a$, and passed between the guide rod
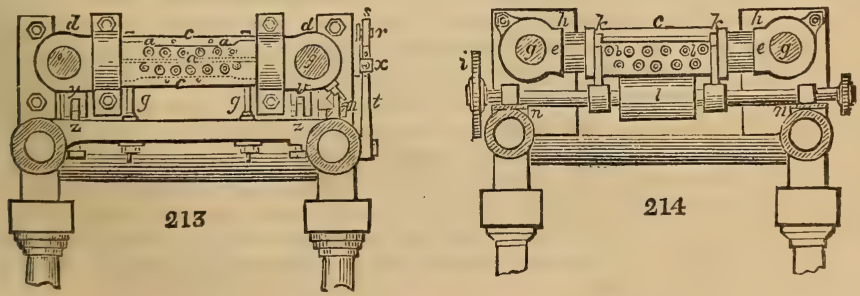

and clearing-plate $k$, and betwren the pair of feeding-rollers $l l$, which, by revolving, draw down a further purtion of the sheet of metal betweeil the punches and dies, after each operation of the purches. 
As the counter dies advance towards the punches, they first come in contact with the sheet of metal to be operated upon; and after having produced the pressure which cuts out the dises, the perforations of the sheet are pushed on to the ends of the punches by the counter dies; and in order that the sheet may be allowed to advance, the carriage which supports the axles of the feeding rollers, with the guide rod and clearine-plate, are made to slide by means of the pin $m$, which works in a slot in the sliding-piece $n$, bearing the axis of the feeding-roller $l l$, the slide $n$ being kept in its place on the framework by dovetailed guides shown in fig. 214.

When the counter dies have advanced near to the sheet of metal, the pin $m$ comes in contact with that end of the slot in the piece $n$ which is next to the punches, and forces the carriage with feed-rollers and clearing-plate, and also the sheet of metal, onwards, as the dies are advanced by the reaction of the cranks; and after they have cut out the dises, and raised the shanks, the sheet of metal will remain upon the punches; and when the bar $e$ returns, the finished backs and shanks are forced out of the counter dies, by the clearing-pins and rods 00 , which project through the bar $e$, and through the holes before mentioned in the counter dies; these clearing-pins being stationary between the bars $p p$, mounted upon the standard $q q$, on the cross bar of the frame, as shown in figs. $210,212,213$. Immediately after this is done, the pins $m$ come in contact with the other ends of the slots in the pieces $n$, and draw back the feeding-rollers $l l$, together with the clearing-plate $k$, and the sheet of metal, away from the punches into the position represented in the figures.

At this ime the feeding of the metal into the machine is effected by a cranli-pin, $r$, on the end of the crank-shafts coming in contact with the bent end of the sliding-bar $s$, supported in standards $t t$; and as the crank-shaft revolves, this pin $r$ forces the bar $s$ forward, and causes the tooth or pall $u$, on its reverse end, to drive the racket-wheel $v$, one or more teeth; and as the racket-wheel $v$ is fixed on to the end of the axle of one of the rollers 7 , it will cause that roller to revolve; and by means of the pair of spurpinions on the other ends of the axles of the feeding-rollers, they will hoth revolve simultaneously, and thereby draw down the sheet of metal into the machine. It will be perceived that the standards which support the clearing-plate and guide-bar are carried by the axles of the feeding-rollers, and partake of their sliding motion : also that the clearing-pins 0 , are made adjustable between the bars $p$, to correspond with the counter dies. There is an adjustable sliding stop $x$ upon the bai $s$, which comes in contact with the back standard $t$, and prevents the bar sliding back too far, and consequently regulates the quantity of sheet metal to be fed into the machine by the pall and ratchet-wheel, in order to suit different sizes of punches and dies. In case the weight of the bar $c$, carrying the counter dies, should wear upon its bearings, the guide pins $g$, have small friction-rollers $y y$, shown under the bosses of this bar, which frictionrollers run upon adjustable beds or planes $z z$, by which means the guide pins may be partially relieved from the weight of the bar $c$, and the friction consequently diminished.

\section{C.}

CABLE. (Cable, Fr.; Ankertau, Germ.) A strong rope or chain, connecting the ship with the anchor for the purpose of mooring it to the ground. The sheet anchor cable is the strongest, and is used at sea; the stream cable is more slender, being used chiefly in rivers. A cable's length is 120 fathoms. The greatest improvement in mooring vessels has been the introduction of the chain cable, which, when duly let out, affords in the weight of its long catenary curve, an elastic tension and play to the ship under the pressure of wind. The dead strain upon the anchor is thus greatly reduced, and the sudden pull by which the flukes or arms are readily snapped is in a great measure obviated. The best iron cables are chains made of links, bound and braced by rods across their middle. Experience has taught that the ends of these links wear out much sooner than the sides. To remedy this evil, Mr. Hawkes, iron manufacturer, obtained a patent in July, 1828, for constructing these anchor chains with links considerably stouter at the ends than in the middle. With this view, he forms the short rods of iron, of which the links are to be made, with swells or protuberances about one third of their length from each of their ends, so that when these are welded together, the slenderer parts are at the sides, and the thicker at the ends of the elliptic links. Such rods as the above are formed at once by rolling, swagging, or any other means. When the link is welded, it may be strengthened, by a brace or stretcher fixed across the middle.

The first avowed proposal to substitute iron cables for cordage in the sea service, was made by Mr. Slater, surgeon of the navy, who obtained a patent for the plan in 1808, though he does not seem to have had the means of carrying it into effect; a very general misfortune with ingenious projectors. It was Captain Brown of the West India 
mercliant service who, in 1811, first employed chain cables in the vessel Penelope, of 400 tous burden, of which he was captain. He made a vojage in this ship from England to Martinique and Guadaloupe and home again, in the csurse of four months, having anchured many times in every variety of ground without any accident. He multiplied his trials, and acquired certaiı proofs that iron might be substituted for hemp in making cables, not only for mooring vessels, but for the standing rigging. Since this period chain cables have been universally introduced into all the ships of the royal navy, but the twisted links employed at first by Brown have been replaced by straight ones, stayed in the middle with a cross rod, the contrivance of $\mathrm{Mr}$. Brunton, which was secured by patent in this country and in France; but the latter patent was suffered to fall from not being acted upon within the two years specified by law.

The first thing to be considered in the manufacture of iron cables is, to procure a material of the best quality, and, in using it, always to keep in view the direction of the strain, in order to oppose the maximum strength of the iron to it. The best form of the links may be deduced from the following investigation.

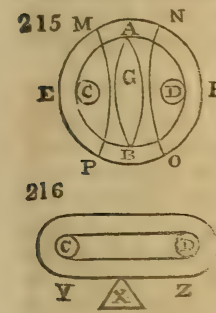

Let A B, fig. 215, be a circular link or ring, of one inch rod iron, the outer circumference of the ring being 15 inches, and the inner 9 . If equal opposite forces be applied to the two points of the link $F_{C}$, pulling $\mathrm{C}$ towards $\mathrm{E}$, and $\mathrm{D}$ towards $\mathrm{F}$, the result will be, when the forces are sufficiently intense, that the circular form of the link will be changed into another form with two round ends and two parallel sides, as seen in fig. 216. The ratio of the exterior to the interior periphery, which was originally as 15 to 9 , or 5 to 3 , is no longer the same in fig. 216. Hence there will be a derangement in the relative position of the component particles, and consequently their cohesion will be progressively impaired, and eventually destroyed. In fig. 215, the segment $\mathrm{M} \mathrm{N}$ of the outside periphery being ?qual to 3 inches, the corresponding inside segment will be $\frac{3}{5}$ of it, or $1 \frac{4}{5}$ inches. If this portion of the link, in consequence of the stretching force, comes to be extended into a straight line, as shown in fig. 216, the corresponding segments, interior and exterior, must both be reduced to an equal length. The matter contained in the 3 inches of the outside periphery must therefore be either compressed, that is, condensed into $1 \frac{4}{5}$ inches, or the inside periphery, which is only $1 \frac{4}{5}$ inches already, must be extended to 3 inches; that is to say, the exterior condensation and the interior expansion must take place in a reciprocal proportion. But, in every case, it is impossible to effect this contraction of one side of the rod, and extension of the other, without disrupture of the link.

Let us imagine the outside periphery divided into an infinity of points, upon each of which equal opposite forces act to straighten the curvature: they must undoubtedly occasion the rupture of the corresponding part of the internal periphery. This is not the sole injury which must result; others will occur, as we shall perceive in considering what passes in the portion of the link which surrounds $\mathrm{c} \mathrm{D}$, fig. 216 , whose length is $4 \frac{1}{2}$ inches outside, and $2 \frac{1}{1}$ inside. The segments $\mathrm{M} P$ and $\mathrm{N}, \mathrm{fig} .215$, are actually reduced to semi-circumferences, which are inside no more than half an inch, and outside as before. There is thus contraction in the interior, with a quicker curvature or one of shorter radius in the exterior. The derangement of the particles takes place here, in an order inverse to that of the preceding case, but it no less tends to diminish the strength of that portion of the link; whence we may certainly conclude that the circular form of cable links is an extremely faulty one.

Leaving matters as we have supposed in fig. 215, but suppose that $\mathrm{G}$ is a rod introdnced mto the mai-, hindering its two opposite points $\mathrm{A}$ B from approximating. This circum$21 \%$ stance makes a remarkable change in the results. The link pulled as above described, must assume the quadrilateral form shown in fig. 217. It offers more resistance to deformation than before; but as it may still suffer change of shape, it will lose strength in so doing, and cannot therefore be recommended for the construction of cables which are to be exposed to very severe strains.

Supposing still the link to be circular, if the ends of the stay comprehended a larger portion of the internal periphery, so as to leave merely the space necessary for the plan of the next link, there can be no doubt of its opposing more effectively the change of form, and thus rendering the chain stronger. But, notwithstanding, the circular portions which remain between the points of application of the strain and the stay, would tend always to be straightened, and of consequence to be destroyed. Besides, though we could construct circular links of sufficient strength to bear all strains, we ought still to reject them, because they would consume more materials than links of a more suitable form, as we shall presently see.

The effeci of two opposite forces applied to the links of a chain, is, as we have seen, 
to reduce to a straight line or a straight plane every curved part which is not stayed; whence it is obvious that twisted links, such as Brown first employed, even with a stay in their middle, must of necessity be straightened out, because there is no resistance in the direction opposed to the twist. A cable formed of twisted links, for a vessel of 400 tons, stretches 30 feet, when put to the trial strain, and draws backs only 10 feet. This elongation of 20 feet proceeds evidently from the straightening of the twist in each link, which can take place only by impairing the strength of the cable.

From the preceding remarks, it appears that the strongest links are such as present, in their original form, straight portions between the points of tension; whence it is clear that links with parallel sides and round ends would be prefersble to all others, did not a good cable require to be able to resist a lateral force, as well as one in the direction of its length.

Let us suppose that by some accident the link fig. 216 should have its two extremities

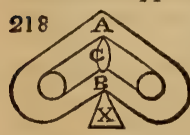
pulled towards $\mathrm{Y}$ and $\mathrm{z}$, whilst an obstacle $\mathrm{x}$, placed right opposite to its middle, resisted the effort. The side of the link which touches $x$ would be bent inwards; but if, as in $f i g .218$, there is a stay A G B, the two sides would be bent at the same time; the link would notwithstanding assume a faulty shape.

In thus rejecting all the vicious forms, we are naturally directed to that which deserves the preference. It is shown in fig. 219. This link has a cast-iron stay with large ends;

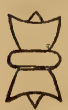

220

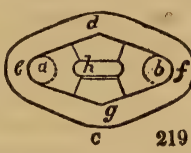

not get deformed or be broken without the whole link giving it presents in all directions a great resistance to every change of form; for let it be pulled in the direction $a b$, against an obstacle $c$, it is evident that the portions $d e$ and $d f$, which are supported by the parts $g e$ and $g f$, canway. As the matter composing $g e$ and $g f$ cannot be shortened, or that which composes $d e$ and $d f$ be lengthened, these four sides will remain necessarily in their relative positions, by virtue of the large-ended stay $h$, whose profile is shown in fig. 220 .

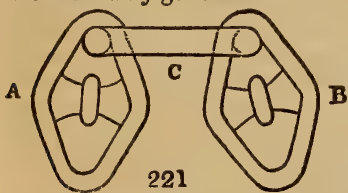

We have examined the strength of a link in every direction, except that perpendicular to its plane. Fig. 221 represents the assemblage of three links in the above

B predicament; but we ought to observe that the obstacle c, placed between the links $\mathrm{A}$ B, must be necessarily very small, and could not, therefore, resist the pressure or impact of the two lateral links.

Process of manufacturing iron cables.-The implements and operations are arranged in the following order:-

1. A reverberatory furnace (see IRoN), in which a number of rods or round bars of the best possible wrought-iron, and of proper dimensions, are heated to bright ignition.

2. The cutting by a machine of these bars, in equal lengths, but with opposite bevels, to allow of the requisite crossing and splicing of the ends in the act of welding.

3 . The bending of each of these pieces by a machine, so as to form the links; the last two operations are done rapidly while the iron is red-hot.

4. The welding of the links at small forge fires, fitted with tools for this express purpose, and the immediate introduction of the stay, by means of a compound lever press.

5. Proving the strength of the cables by an hydraulic press, worked by two men turning a winch furnished with a fly-wheel.

The furnace is like those used in the sheet-iron works, but somewhat larger, and needs no particular description here.

Figs. 222 and 223 are a plan and elevation of the shears with which the rods are cut

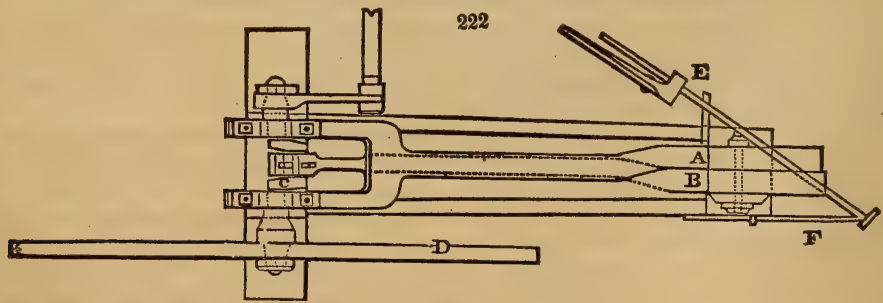

into equal pieces, for forming each a link. It is moved at Mr. Brunton's factory by a small stearn engine, but, for the sake of simplicity, it is here represented worked by fou or more laborers, as it may be in any establishment. These must be relieved, however, 
frequently by others, for I believe each shears' machine is calculated to require nearly one horse in steam power. It is portable, and must be placed in the neighborhood of both the furnace and bending machine.

$A$ and $\mathbf{B}$ are the two cast-iron limbs of the shears. The first is fixed, and the second is moveable by means of a crank shaft c, driven by a heavy fly-wheel weighing 7 or $8 \mathrm{cwt}$.

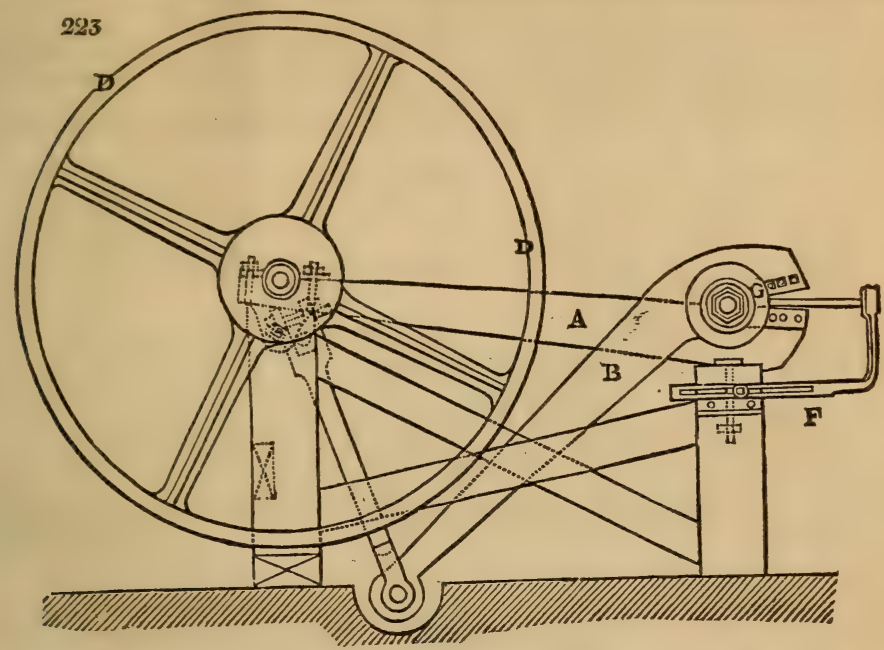

The cutting jaws G are mounted with pieces of steel which are made fast by bolts, and may be changed at pleasure.

$\mathbf{E}$, the bar of iron to be cut. It is subjected, immediately upon being taken out of the fire, to the shears, under a determinate uniform angle, care being taken not to let it turn round upon its axis, lest the planes of the successive incisions should become unequal.

F is a stop which serves to determine, for the same kind of chain, the equality of length in the link pieces.

Figs. 224, 225, 226, plan and elevations of the machine for bending the links into an elliptic form. It is represented at the moment when a link is getting bent upon it.
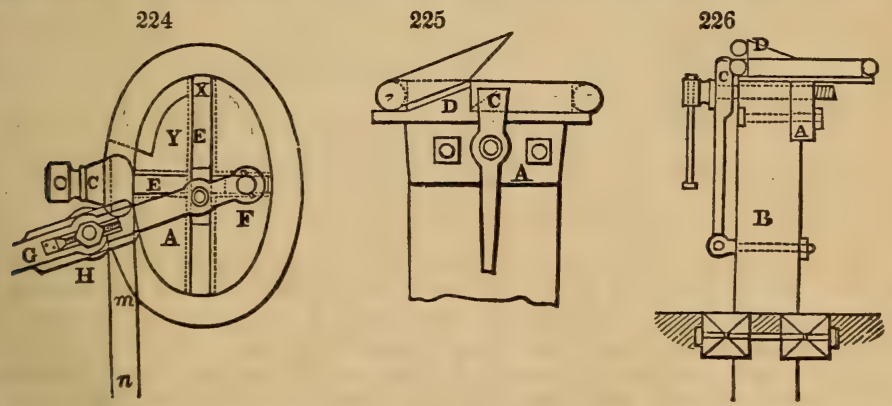

$A$ is an elliptic mandrel of cast-iron; it is fixed upon the top of a wooden pillar $B$, solidly supported in the ground. $\mathrm{c}$ is the jaw of the vice, pressed by a square-headed screw against the mandrel $A$.

$D$ part of the mandrel comprehended between $x$ and $\mathbf{y}$, formed as an inclined plane, so as to preserve an interval equal to the diameter of the rod between the two surfaces that are to be welded together.

$E$ rectangular slots (shears) passing through the centre of the nut of the mandrel, in which each of the pins $F$ may be freely slidden.

G horizontal lever of wrought-iron six feet long. It carries at $\mathbf{H}$ a pulley or friction. roller of steel, whose position may be altered according to the diameter of the links. If is obvious that as many mandrels are required as there are sizes and shapes of links. 
The piece of iron intended to form a link being cut, is carried, while red-hot, to the bending machine, where it is seized with the jaw of the vice $c$, by one of its ends, the slant of the cut being turned upwards; this piece of iron has now the horizontal direction $m n$; on pushing the lever $\mathrm{G}$ in the line of the arrow, the roller $\mathrm{H}$ will force $m n$ to be applied successively in the elliptic groove of the mandrel; thus finally the two faces that are to be welded together will be placed right opposite each other.

The length of the small diameter of the ellipse ought to exceed by a little the length of the stay-piece, to allow of this being readily introduced. The difference between the points $F E$, is equal to the difference of the radii vectores of the ellipse. Hence it will be always easy to find the eccentricity of the ellipse.

Fig. 227 is a lever press for squeezing the links upon their stays, after the links are

227

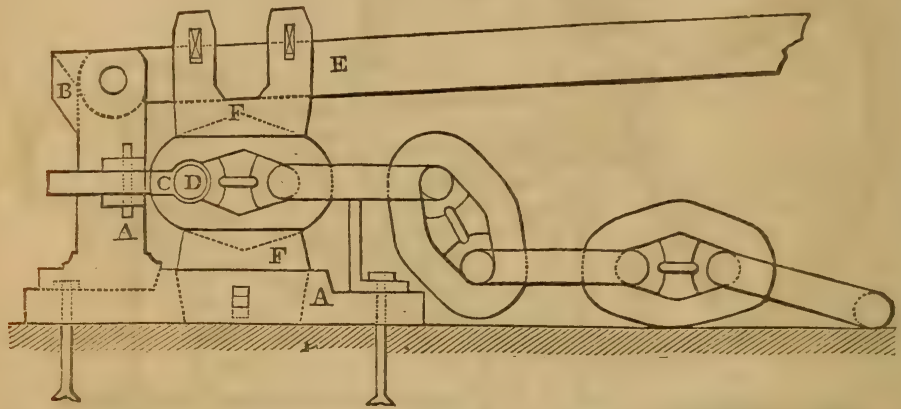

welded. Tris machine consists of a strong cast-iron piece $\mathrm{A}$, in the form of a square, of which one of the branches is laid horizontally, and fixed to a solid bed by means of bolts; the other branch, composed of two cheeks, leaving between them a space of two inches, stands upright. These two cheeks are united at top, and on the back of their plane by a cross piece $\mathrm{B}$. c, a rectangular staple, placed to the right and left of the cheeks through which is passed the mandrel $D$, which represents and keeps the place of the following link. $\mathrm{E}$, is a press lever, 6 feet long. $F$, clamp and counterclamp, between which the link is pressed at the moment when the stay is properly placed. There are other clamps, as well as staples $\mathrm{c}$, for changing with each changed dimension of links.

The links bent, as we hare seen, are carried to the forge hearth to be welded, and to receive their stay; two operations performed at one heating. Whenever the welding is finished, while the iron is still red-hot, the link is placed apright between the clamps $F$; then a rorkman introduces into the staple the mandrel $\mathrm{D}$, and now applies the stay with a pair of tongs or pincers, while another workman strikes down the lever $\mathrm{E}$ forcibly upon it. This mechanical compression first of all joins perfectly the sides of the link against the concare ends of the stay, and afterwards the retraction of the iron on cooling increases still more this compression.

If each link be made with the same care, the cable must be sound throughout. It is not delivered for use, however, till it be prosed by the hydraulic press, at a draw-bench made on purpose. The press is a horizontal one, haring the axis of its ram in the middle line of the draw-bench, which is about 60 feet long, and is secured to the body of the press by strong bolts.

The portion of chain under trial, being attached at the one end to the end of the ram of the press, and at the other to a cross-bar at the extremity of the draw-bench, two men put the press in action, by turning the winch, which works by a triple crank three forcing pumps alternately; the action being equalized by means of a heavy fly-wheel. As long as the resistance does not exceed the force of two men, the whole three pumps are kept in play. After a while one pump is thrown out of gear and next another, only one being worked towards the conclusion. The relocity of the ram being retarded first one third and next two thirds, gives the men a proportional increase of mechanical power.

The strength of two average men thus applied being compuied, enables us to know at every instant the resistance opposed by the chain to the pressure of the ram. The strain usually applied to the stronger cables is about 500 tons.

The side beams of the draw-bench are of east-iron, 6 inches in diameter; the different pieces composing it are adjusted to each other endwise by turned joints. Props also of cast-iron support the beams two feet asunder, and at the height of 30 inches above the ground. The space between them is filled with an oak plank on which the trial chain is laid. 
CABLE.

Strength of iron cables compared to hemp cables:-

\begin{tabular}{|c|c|c|}
\hline $\begin{array}{c}\text { Iron Cables. } \\
\text { Diameter of Iron Rod. }\end{array}$ & $\begin{array}{c}\text { Hemp Cables. } \\
\text { Circumference of Rope. }\end{array}$ & Resistance. \\
\cline { 1 - 3 } Inches. & Inches. & Tons. \\
$0 \frac{7}{8}$ & 10 & 12 \\
1 & 11 & 18 \\
$1 \frac{1}{8}$ & 12 & 26 \\
$1 \frac{2}{4}$ & 13 & 32 \\
$1 \frac{5}{16}$ & 14 to 15 & 35 \\
$11 \frac{3}{8}$ & 16 & 38 \\
$1 \frac{1}{2}$ & 17 & 44 \\
$1 \frac{5}{8}$ & 20 & 52 \\
$1 \frac{3}{4}$ & 22 to 24 & 60 \\
$1 \frac{\frac{3}{8}}{2}$ & & 80 \\
\hline
\end{tabular}

It would be imprudent to put hemp cables to severer strains than those indicated in. the preceding table, drawn up from Brunton's experiments; but the iron cables of the above sizes will support a double strain without breaking. They ought never in common cases, howerer, to be exposed to a greater stress. A cable destined for ships of a certain tonnage, should not be employed in those of greater burden. Thus treated it may be always trusted to do its duty, and will last longer than the ship to which it belongs. A considerable part of this decided superiority which iron cables have over hemp ones, is undoubtedly due to the admirable form contrived by Brunion. Repeated experiments have proved that his cables possess double the strength of the iron rods with which they are made - a fact which demonstrates that no stronger form can be devised or is in fact possible.

One of the most valuable qualities of iron cables is their resisting lateral as well as longitudinal strains, as explained under figs. 219 and 221.

Vessels furnished with such cables have been saved by them from the most imminent peril. The Henry, sent out with army stores during the peninsular war, was caught on the northern coast of Spain in a furious storm. She run for shelter into the Bay of Biscay among the rocks, where she was exposed for three days to the hurricane. She possessed furtunately one of Brunton's 70 fathom chain cables, which held good all the time, but it was found afterwards to have had the linlis of its lower portion polished bright by attrition against the rocky bottom. A hemp cable would have been speedily torn to pieces in such a predicament.

In the contracts of the Admiralty for chain cables for the British navy, it is stipulated that "the iron shall have been manufactured in the best manner from pig iron, smelted from iron-stone only, and selected of the best quality for the purpose, and shall not have received, in any process whatever subsequent to the smelting, the admixture of either the cinder or oxydes froduced in the manufacture of iron; and shall also have been puddled in the best manner upon iron bottoms, and at least three times sufficiently drawn out at three distinct welding heats, and at least twice properly fagoted."

The following is a table of the breaking proof of chain cables, and of the iron for the purpose of making them, also of the proofs required by her majesty's navy for chains.

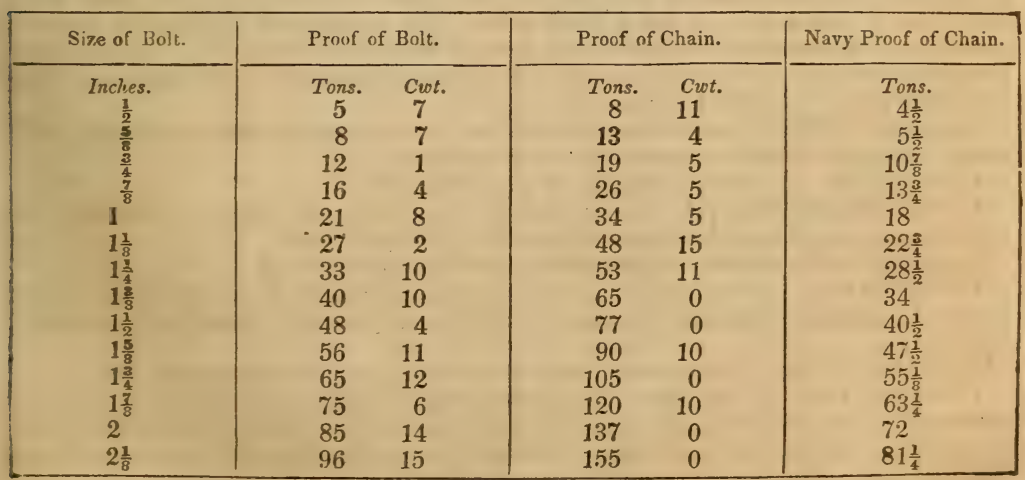

In Brunton's cable the matter in the link is thrown very much into one plane; the 
Jink being of an oval form, and provided with a stay. As there are emergencies in which the cable must be severed, this is accomplished in those of iron by means of a bolt and sheckle (shackle), at every fathom or two fathoms; so that by striking out this bolt or pin, this cable is parted with more ease than a hempen one can be cut.

CACAO, BUTTER OF. See CocoA, and OrLs, Unctuous.

CADMIUM is a metal discovered about the beginning of the year 1818. It occurs chiefly in Silesia in several ores of zinc; and may be readily recognised by means of the blowpipe; for at the first impression of the reducing or smoky part of the flame, the ores containing cadmium stain the charcoal all round them with a reddish yellow circle of oxyde of cadmium. The Silesian native oxyde of zinc contains from $1 \frac{1}{2}$ to 11 per cent. of cadmium.

The cadmium may be extracted by dissolving the ore in sulphuric acid, leaving the solution acidulous, and diluting it with water, then transmitting through it a stream of sulphureted hydrogen, till the yellow precipitate ceases to fall. This powder, which is sulphuret of cadmium, is to be dissolved in concentrated muriatic acid, the excess of which is to be expelled by evaporation; and the muriatic salt being dissolved in water, carbonate of ammonia is to be added in excess, whereby the cadmium separates as a carbonate, while the small portion of adhering copper or zinc is retained in solution by the ammonia. Herapath has shown, that in distilling zinc per descensum (see ZINC), the first portions of gaseous metal which are disengaged burn with a brown flame and deposite the brown oxyde of cadmium.

Cadmium has the color and lustre of tin, and is susceptible of a fine polish. Its fracture is fibrous; it crystallizes readily in regular octahedrons, and when it suddenly solidifies, its surface gets covered with fine mossy vegetations. It is soft, easily bent, filed, and cut, soils like lead any surface rubbed with it. It is harder and more tenacious than tin, and emits a creaking sound when bent, like that metal. It is very ductile, and may be drawn out into fine wire, and hammered into thin leaves without cracking at the edges. Its specific gravity, after being merely melted, is 8.604 ; and 8.6944 after it has been hammered. It is very fusible, melting at a heat much under redness; indeed, at a temperature little exceeding that of boiling mercury, it boils and distils over in drops. Its vapors have no smell. It is but slightly altered by exposure to air. When heated in the atmospnere, it readily takes fire, and burns with a brownish yellow smoke which is destitute of smell. In strong acids it dissolves with disengagement of hydrogen, and forms colorless solutions. Chromate of potash causes no precipitate in them, unless zinc or lead be present.

There is only one oxyde of cadmium, the brown above mentioned. Its specific gravity is 8.183 . It is neither fusible nor volatile at a very high temperature. When in the state of a hydrate it is white. The oxyde of cadmium consists of 87.45 parts of metal, and 12.55 oxygen in 100 parts. Berzelius states its atomic weight to be 55.833 to hydrogen $1 \cdot 000$. Its sulphuret has a fine orange yellow color, and would form a beautiful pigment, could the metal be found in sufficient quantity for the purposes of art. The sulphate is applied to the eyes by surgeons for removing specks of the cornea.

CAFEINE. A chemical principle discovered in eoffee, remarkable for containing much azote. See CoFres.

CAJEPUT OIL is obtained from the leaves of the tree called Melalenca Leucadendron by Linnæus, which grows upon the mountains of Amboyna, and in other of the Molucca islands. It is procured by distillation of the dried leaves along with water, is prepared in great quantities in the island of Banda, and sent to Holland in copper fiasks. Hence, as it comes to us, it has a green color. It is very limpid, lighter than water, of a strong smell resembling camphor, and pungent taste like cardamoms. When reetified the copper remains in the retort, and the oil comes over colorless. It is used in medicine as a stimulant. See Oils Etriereous.

CALAMANCO. A sort of woollen stuff of a shining appearance, checkered in the warp, so that the checks are seen only upon one side.

CALAMINE. A native carbonate of zinc. See Zinc.

CALCAREOUS EARTH, (Terre caicaire, Fr.; Kalkerde, Germ.), commonly denotes lime, in any form; but, properly speaking, it is pure lime.

CALCAREOUS SPAR. Crystallized native carbonate of lime.

CALCEDONY. A hard mineral of the silicious family, often cut into seals. Under it may be grouped common calcedony, heliotrope, chrysoprase, plasma, onyx, sardonyx, and sard.

CALCHANTUM. The ancient name of native copperas or sulphate of iron.

CALCINA'TION is the chemical process of subjecting metallic bodies to heat with access of air, whereby they are converted into a pulverulent matter, somewhat like lime in appearance, called calx in Latin. The term calcination, however, is now used when any substance whatever is exposed to a roasting heat.

CALCIUM. The metallic basis of lime. See Lime. 
CALC-SINTER. The incrustations of carbonate of lime upon the ground, or the pendulous conical picces called stalactites, attached to the roofs of caverns, arc so called. CALC-TUFF. A semi-hard, irregular deposite of carbonate of lime, formed from the waters of calcareous springs.

CALCULUS. The stony-looking morbid concretion, occasionally formed in the bladder of urine, gall-bladder, cystic duct, kidneys, and other parts of living animals, Its examination belongs to medical chemistry.

CALENDER (Calanlre, Fr.; Kalander, Germ.), a word derived from the Greek kalindros (cylinder), is the name of a machine, consisting of two or more cylinders, revolving so nearly in contact with each other that cloth passed through between them is smoothed, and even glazed, by their powerful pressure. It is employed either to finish goods for the market, or to prepare cotton and linen webs for the calico-printer, by rendering their surfaces level, compact, and uniform. This condensation and polish, or satinage, as the French call it, differ in degree according to the object in view, and may be arranged into three distinct series. 1. For goods which are to receive the first impression by the block, a very strong pressure is required; for, upon the uniformity of the polish, the neatness and regularity of the printing, and the correspondence of its members, depend. In many establishments the calico is passed twice through the calender before being sent to the tables. 2. The pieces already dyed up at the madder bath, or otherwise, and which remain to be filled in with other colors, or grounded-in, as it is technically styled, must receive a much less considerable gloss. This is a principle everywhere admitted and acted upon, because the outline of the figured design being deranged by the washing, and sometimes in consequence of the peculiar texture of the cloth, the printer, in order to apply his grounding blocks properly, and to fit them to the contours of the figures already impressed, is obliged to stretch the piece sometimes in the direction of the warp, and sometimes of the weft, which would be impossible if they had been hard glazed by the calender. 3. The degree of glazing given to finished goods depends upon the taste of purchasers, and the nature of the article; but it is, in general, much less than for the first course of block-printing.

The most complete calender probably in existence is that used by some of the eminent calico-printers of Alsace, as contrived by M. Charles Dollfus, and constructed by MM. Witz, Blech, and Co. 1. It passes two pieces at once, and thus does double the work of any ordinary machine. 2. It supersedes the necessity of having a workman to fold up the goods, as they emerge from the calender, with the aid of a self-acting folder. 3. It receives, at pleasure, the finished pieces upon a roller, instead of laying them in folds; and, by a very simple arrangement, it hinders the hands of the workmen from being caught by the rollers.

Calenders, in consequence of the irregular demand for foreign orders and shipments, are worked very irregularly, being sometimes overloaded with duty, and at others altogether unemployed. A machine which can, when required, turn out a double quantity of goods, must, therefore, be a desirable possession. For the first course of the printers, where high calendering is necessary, the goods are usually passed twice through between two paper cylinders, to give that equality of surface which could not be obtained by one passage, however strong the pressure ; and therefore the simplification of this calender will prove no economy. Besides, in order to increase the pressure to the requisite degree, the cylinders would need to be made bulging at their middle part, and with such cylinders common smoothing could not be given; for the pieces would be glazed in the central line, and rough towards the edges. For pieces already printed in part, and requiring only to be grounded-in for other colors, the system of double effect has fewer objections, as a single passage through the excellent calender described under BLEAcHING, page 140 , is found to answer very well.

The most remarkable feature of $M$. Dollfus's machine is its being managed by a single workman. Six or eight pieces are coiled upon the feed-roller, and they are neither pasted nor stitched together, but the ends are merely overlapped half a yard or so. The workman is careful not to enter the second piece till one third or one half of the first one has passed through on the other side, to prevent his being engrossed with two ends at a time. He must, no doubt, go sometimes to the one side, and sometimes to the other of the machine, to see that no folds or creases occur, and to be ready for supplying a fresh piece as the preceding one has gone through. The mechanism of the folder in the Alsace machine is truly ingenious: it performs extremely well, really saves the attendance of an extra workman, and is worthy the attention of manufacturers intent upon economizing hand labor. The lapping-roller works by friction, and does its duty fully better than similar machines guided by the hand.

The numerous accidents which have happened to the hands of workmen engaged in calenders should direct the attention towards its effective contrivance for preventing such misfortunes. These various improvements in the Alsace machine may be easily adapted "o the ordinary calenders of almost every construction. 
The folder is a kind of cage, in the shape of an inverted pyramid, shut on the four sides, and open at top and bottom; the top orifice is about five inches, the bottom one an inch and a half; the front and the back, which are about four feet broad, are made of tin-plate or smouth pasteboard, and the two sides are made of strong sheet-iron; the whole being boltel together by small bars of iron. Upon the sheet-iron of the sides, iron up. rights are fixed, perforated with holes, through which the whole cage is supjorted freely by means of studs that enter into them. One of the uprights is longer than the vther, and bears a slut with a small knob, which, by means of the iron piece, joins the guide to the crank of the cylinder, and thereby communicates to the cage a seesaw movement; at the bittom extremity of the great upright, there is a piece of iron in the shape of an anchor, which may be raised, or lowered, or made fast, by screws.

At the ends of this anchor are friction-rollers, which may be drawn out or pushed back and fixed by screws; these rollers lift alternately two levers made of wood, and fixed to a wooden shaft.

The paws are also made of wood: they serve to lay down alternately the plies of the cloth which passes upon the cage, and is folded zigzag upon the floor, or upon a board set below the cage; a motion imparted by the seesaw motion of the cage itself. See Stretching Machine.

To protect the fingers of the workmen, above the small plate of the spreading-board or bar, there is another bar, which forms with the former an angle of about $75^{\circ}$; they come sufficiently near together for the opening at the summit of the angle to allow the cloth to pass through, but not the fingers. See Bulletin de la Sociélé Industrièlle de Mulhausen, No. 18.

I shall now describe, more minutely, the structure of the powerful but less complicated calender mechanisms employed in the British manufactories.

A front elevation of a four-rollered calender (five rollers are often introduced) for giazing goods is given in fig. 228. $d l$ are two pasteboard or paper cylinders, each 20 inches

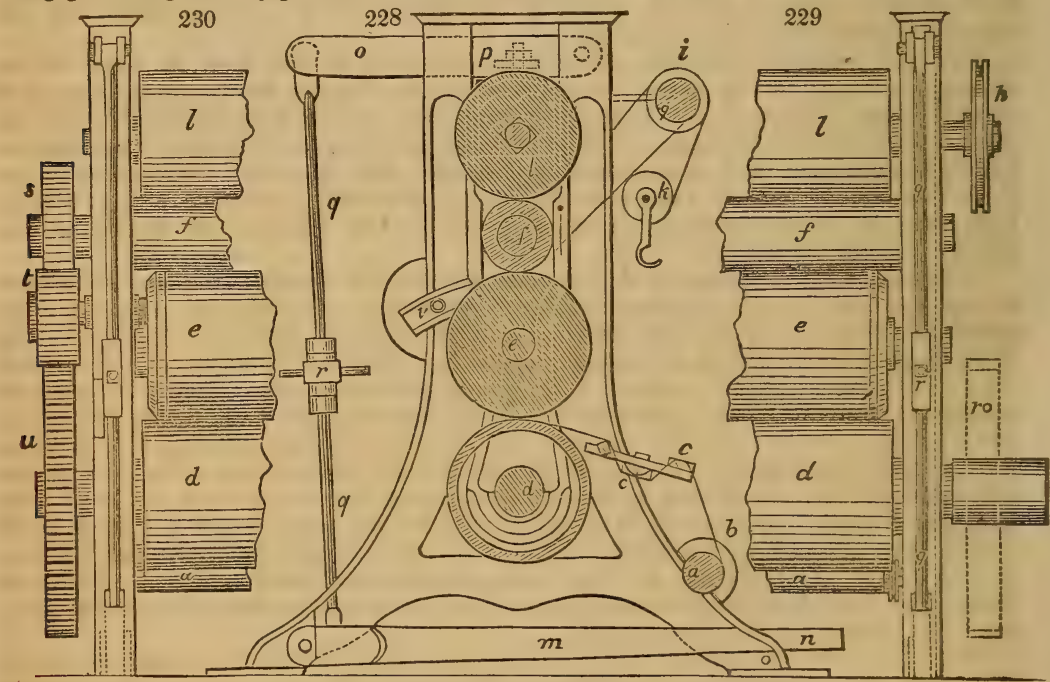

in diameter, whose structure will be presently described : $f$ is a cast-iron crlindel turned perfectly smooth (its fellow is often placed between $e$ and $d$ ): it is eight inches in diameter outside, four inches inside, with two inches thickness of metal. $e$ is another pasteboard crlinler, fourteen inches in diameter: the strong cast-iron fiame contains the bushes in which the journals of the rollers turn. o $p$, is one of the pair of levers for communicating a graduated pressure according to the quality of the goods. Figs. 2:29, 230, are end views of the same machine to show the working gear. The wheel $s$, on the end of the upper iron cylinder, is ten inches in diameter; that on the end of the fellow iron cylinder below (when it is present) is thirteen inches; both are connected by the larger carrier wheel $\%$. The lower wheel $u$ is one third larger than the upper wheel, and therefore receives from the carrier wheel $t$, a proportionally slower motion, which it imparts to the central pasteboard roller $e$, lying upon it, causing it to move one third more slowly than the upper pasteboard roller. Thus a sort of sliding motion is produced, which, by rubbing their surfaces, glazes the goods.

The iron rollers are made hollow for the purpose of admitting either a hot roller of 
Iron, or steam when hot calendering is required. The other cylinders used formerly to be made of wood, but it was liable to many defects. The advantage of the paner roller consists in its being deroid of any tendency to split, crack, or warp, especially when exposed to a considerable heat from the contact and pressure of the hot iron rollers. The paper, moreorer, takes a vastly finer polish, and, being of an elastic nature, presses into every pore of the cloth, and smooths its surface more effectually than any wooden cylinder, however truly turned, could possibly do.

The paper crlinder is constructed as follows:-The axis of the cylinder is a strong square bar of the best wrought iron, cut to the proper length. Upon this bar a strong round plate of cast iron is first put, somewhat less in diameter than the cylinder when finished A quantity of thick stout pasteboard is then procured, and cut into round pieces an inch larger in diameter than the iron plate. In the centre of the plates, and of every piece of the pasteboard, a square hole must be cut to receive the axis ; and, the circle being divided into six equal parts, a hole must also be cut at each of the divisions, an inch or two within the rim. These pieces of pasteboard being successively put upon the axis, a long bolt of malleable iron, with a head at one end, and serewed at the other, is also introduced through each of the holes near the rim; and this is continued until a sufficient number of pasteboards are thus placed to form a cylinder of the length required, proper allowance being made for the compression which the pasteboard is afterwards to undergo. Another round plate is then applied, and, nuts being put upon the screws, the whole are screwed tight, and a cylinder formed. This cylinder is now to be placed in a stove, exposed to a strong heat, and must be kept there for at leas? several days; and, as the pasteboard shrinks by exposure to the heat, the screws must be frequently tightened until the whole mass has been cempressed as much as possible. When the cylinder is thus brought to a sufficient degree of density, it is removed from the stove; and, when allowed to cool, the pasteboard forms a substance almost incon. ceirably dense and hard. Nothing now remains but to turn the cylinder; and this is an operation of no slight labor and patience. The motion in turning must be slow, not exceeding about forty revolutions in a minute; the substance being now so hard and tough that tools of a very small size must be used to cut, or rather scrape it, until it is true. Three men are generally employed for the turning, even when the motion of the cylinder is effected by mechanical power, two being necessary to sharpen tools for the third, who turns, as quickly as he blunts them.

Let us suppose it to be a five-rollered machine: when a person stands in front of the calender, the cloth coming from behind above the uppermost cylinder 1 , passes between 1 and 2 : proceeding behind 2, it again comes to the front between 2 and 3 : between 3 and 4 it is once more carried behind, and, lastly, brought in front between 4 and 5, where it is received, and smoothly folded on a clean board, or in a box, by a person placed there for the purpose. In folding the cloth at this time, care must be taken that it may be loosely done, so that no mark may appear until it be again folded in the precise length and form into which the piece is to be made up. The folding may be done either by two persons or by one, with the aid of two sharp polished spikes placed at a proper distance, to ascertain the length of the fold, and to make the whole equal. When folded into lengths, it is again folded across upon a smooth clean table, according to the shape intended, which varies with the different kinds of goods, or the particular market for which the goods are designed.

When the pieces have received the proper fold, the last operation previous to packing them is the pressing. This is commonly performed by placing a certain number of pieces, divided by thin smooth boards of wood, in a common screw press, similar to those used by printers for taking out the impression left by the types in the printing-press. Besides the wooden boards, a piece of glazed pasteboard is placed above and below every piece of cloth, that the outer folds may be as smooth and glossy as possible. The operation of the common screw press being found tedious and laborious, the hydraulic press is now in all well-mounted establishments had recourse to. See Hydraulic Press.

No improvements that have taken place in calendering can exceed the power and facility of the water press : one of these presses may be worked by two men, who can with great ease produce a pressure of 400 tons; but, in considerable establishments, the presses are worked by nower. See BANDANNA.

The appearance and finish of the goods, in consequence of such an immense weight acting on them, are materially improved.

The press is also used for the purpose of packing; whereby the bale is rendered much more compact than formerly. It is commonly roped, \&c., while in this compressed state; the dimensions are therefore greatly diminished from what they would otherwise be by any other method. For instance, the same quantity of goods packed in a ba!e are from one third to one half less bulky than if they were packed in a box with the utmost force of the hands. 
For lawns and muslins of a light texture, the operation of smoothing regaires a du. ferent process in some respects than close heavy fabrics. They only require to be slightly smoothed to remove any marks which they may have received at the bleaching; and as cheir beauty depends rather on their transparency than their closeness, the more the cy lyndrical form of the yarn is preserved the better. They are therefore put throngh a small machine, consisting of three rollers or cylinders; and as the power required to move this is small, the person who attends it generally drives it by a small winch; or - the same effect may be produced by passing the muslins between only two or three rollers of the above calender, lightly loaded.

In the thick fabries of cloth, including those kinds which are used for many parts of household furniture, as also those for female dress, the operation of glazing is used both to add to the original beauty of the cloth, and to render it more impervious to dust or smoke. The glazing operation is performed entirely by the friction of any smooth substance upon the cloth; and, to render the gloss brighter, a small quantity of bleached wax is previously rubbed over the surface. The operation of glazing by the common plan is very laborious, but the apparatus is of the most simple kind. A table is mounted with a thick stout cover of level and well-smoothed wood, forming an inclined plane; that side where the operator stands at work being the lowest. The table is generally placed near a wall, both for convenience in suspending the glazing apparatus, and for the sake of light. A long piece of wood is suspended in a groove formed between two longitudinal beams, placed parallel to the wall, and fixed to it. The groove resembles exactly the aperture between the shears of a common turning lathe. The lever, of which the groove may be supposed to be the centre or fulcrum, is faced at the bottom with a semi-cylindrical piece of finely polished flint, which gives the friction to the cloth stretched upon the table below. Above the flint are two cross handles, of which the operator lavs hold, and moves them backward and forward with his hands, keeping the flint pressing slightly upon the cloth. When he has glazed a portion equal to the breadth of the flint, he moves his lever between the shears sidewise, and glazes a fresh part: thus he proceeds from one side or selvage of the cloth to the other; and when all which is upon the table is sufficiently glazed, he draws it over, and exposes a new portion to the same operation. To preserve the cloth at a proper tension, it may be wound smoothly upon a roller or beam, which being set so as to revolve upon its own axis behind the table, another roller to receive the cloth may be placed before, both being secured by a catch, acting in a ratchet wheel. Of late years, however, a great part of the labor employed in glazing cloth has been saved, as the common four or five bowl calender has been altered to fit this purpose by direct presstire.

As a matter of accommodation, the different processes of packing, cording of boxes, sheeting of trunks, and, in general, all the arrangements preparatory to shipments, and also the intimations and surveys necessary for obtaining drawbacks, debentures, or bounties, according to the excise laws, are generally conducted at the calender houses where goods are finished. These operations sufficiently account for the general meaning attached to the word.

CALICO-PRINTING (Impression d'Indiennes, Fr.; Zeugdruckerei, Germ.) is the art of impressing cotton cloth with topical dyes of more or less permanence. Of late years, silk and woollen fabrics have been made the subjects of a similar style of dyeing. Linens were formerly stained with various colored designs, but since the modern improvements in the manufacture of cotton cloth, they are seldom printed, as they are both dearer, and produce less beautiful work, because flax possesses less affinity than cotton for coloring matters.

This art is of very ancient date in India, and takes its English name from Calicut, a district where it has been practised with great success from time immemorial. The Egyptians, also, appear from Pliny's testimony to have practised at a remote era some of the most refined processes of topical dyeing. "Robes and white veils," says he, "are painted in Egypt in a wonderful way. They are first imbued, not with dyes, but with dye-absorbing drugs, by which, though they seem to be unaltered, yet, when immersed for a little while in a caldron of the boiling dye-liquor, they are found to become painted. Yet, as there is only one color in the caldron, it is marvellous to see many colors imparted to the robe, in consequence of the influence of the excipient drug. Nor can the dye be washed out. A caldron, which would of itself merely confuse the colors of cloths previously dyed, is thus made to impart several pigments from a single dyestuff, painting as it boils." The last expression, pingitque dum coquit, is perfectly graphic and descriptive of calico-printing.

The cotton chints counterpanes of great size, called pallampoors, which have been manufactured in Madras from the earliest ages, have in like manner peculiar dye-absorbing drugs applied to them with the pencil, as also wax, to protect certain parts of the surface from the action of the dye, and are afterwards immersed in a staining liquor, which, when wax is applied, is usually the cold indigo-vat, but without the wax is a hot liquor similar to the Egyptian. M. Koechlin Roder, of Mulhouse, brought home lately from 
India a rich collection of cloths in this state of preparation, which $\mathbf{I}$ saw in the cabinet of the Société Indus/rielle of that interesting emporium of calico-printing. The native implements for applying the wax and coloring bases are placed alongside of the cloths, and form a curious picture of primeval art. There is among other samples an ancient pallampoor, five French yards long, and two and a half broad, said to be the labor of Hindoo princesses, which must have taken a lifetime to execute. The printing machinery of great Britain has begun to supersede, for these styles of work, the cheapest hand labor of India.

Calico-printing has been for several hundred years practised by the oriental methods in Asia Minor and the Levant; but it was unknown as an English art till 1696, when a small print-ground was formed upon the banks of the Thames, near Richmond, by a Frenchman-probably a refugee from his own country, in consequence of the revocation of the edict of Nantes. Some time afterwards, a considerable printing work was established at Bromley Hall, in Essex, and several others sprung up successively in Surrey, to supply the London shops with chintses, their import from India having been prohibited by act of parliament in 1700. The silk and woollen weavers, indeed, had all along manifested the keenest hostility to the use of printed calicoes, whether brought from the East or made at home. In the year 1680 they mobbed the India House in revenge for some large importations then made of the chintses of Malabar. They next induced the government, by incessant clamors, to exclude altogether the beautiful robes of Calicut from the British market. But the printed goods, imported by the English and Dutch East India companies, found their way into this country, in spite of the excessive penalties annexed to smuggling, and raised a new alarm among the manufacturing population of Spitalfields. The sapient legislators of that day, intimidated, as would appear, by the East London mobs, enacted in 1720 an absurd sumptuary law, prohibiting the wearing of all printed calicoes uhatsoever, either of foreign or domestic origin. This disgraceful enactment, worthy of the meridian of Cairo or Algiers, proved not only a death-blow to rising industry in this ingenious department of the arts, but prevented the British ladies from attiring themselves in the becoming drapery of Hindostan. After an oppressive operation of ten years, this act was repealed by a partially enlightened set of senators, who were then pleased to permit what they called British calicoes, if made of linen warp, with merely weft of the hated cotton, to be printed and worn, upon paying a duty of no less than sixpence the square yard. Under this burden, English calico-printing could not be expected to make a rapid progress. Accordingly, even so lately as the year 1750 , no more than 50,000 pieces of mixed stuff were printed in Great Britain, and that chiefly in the neighborhood of London; whereas a single manufacturer, Mr. Coates of Manchester, now-a-days will turn off nearly twenty times that quantity, and there are very many others who manufacture several hundred thousand pieces per annum. It was not till about 1766 that this art migrated into Lancashire, where it has since taken such extraordinary development; but it was only after 1774 that it began to be founded upon right principles, in consequence of the repeal of that part of the act of 1730 which required the warp to be made of linen yarn. Henceforth the printer, though still saddled with a heavy duty of $3 d$. the square yard, was allowed to apply his colors to a homogeneous web, instead of the mixed fabric of linen and cotton substances, which differ in their affinities for dyes.

France pursued for some time a similar false policy with regard to calico-printing, but she emerged sooner from the mists of manufacturing monopoly than England. Her avowed motive was to cherish the manufacture of flax, a native product, instead of that of cotton, a raw material, for which prejudice urged that money had to be exported. Her intelligent statesmen of that day, fully seventy years ago, replied that the money expended in the purchase of cotton was the produce of French industry, beneficially employed, and they therefore took immediate measures to put the cotton fabrics upon a footing of equality. Meanwhile the popular prejudices became irritated to such a degree, by the project of permitting the free manufacture and sale of printed cottons, that every French town possessed of a chamber of commerce made the strongest remonstrances against it. The Rouen deputies declared to the government, "that the intended measure would throw its inhabitants into despair, and make a desert of the surrounding country :" those of Lyons said, "the news had spread terror through all its workshops :" Tours "foresaw a commotion likely to convulse the body of the state :" Amiens said, "that the new law would be the grave of the manufacturing industry of France ;" and Paris declared that "her merchants came forward to bathe the throne with their tears upon that inauspicious occasion."

The government persisted in carrying its truly enlightened principles into effect, and with so manifest advantage to the nation, as to warrant the inspector-general of manufactures to make, soon afterwards, the following appeal to those prejudiced bodies:"Will any of you now deny that the fabrication of printed cottons has occasioned a vast extension of the industry of France, by giving profitable employment to a great many 
hands in spinning, weaving, bleaching, and printing the colors? Look only at the dyeing department, and say whether it has not done more good to France in a few years than many of your other manufactures have in a century ?"

The despair of Rouen has been replaced by the most signal prosperity in the cotton trade, and especially in printed calicoes, for the manufacture of which it possesses 70 different establishments, producing upwards of a million of pieces of greater average size and price than the English. In the district of the Lower Seine, round that town, there are 500 colton jactories of different kinds, which give employment to 118,000 operatives of all orders, and thus procure a comfortable livelihood to probably not less than half a million of people.

The repeal, in 1831, of the consolidated duty of $3 \frac{1}{2} d$. per square yard upon printed calicoes in Great Britain is one of the most judicious acts of modern legislation. By the improvements in calico-printing, due to the modern discoveries and inventions in chem:-try and mechanics, the trade had become so vast as to yield in 1830 a revenue of $2,280,000 l$. levied upon $8,596,000$ pieces, of which, however, about hree fourths were exported, with a drawback of $1,579,000 l$. 2,281,512 pieces were consumed in that year at home. When the expenses of collection were deducted, only 350,0007 . found their way into the exchequer, for which pitiful sum thousands of frauds and obstructions were committed against the honest manufacturer. This reduction of duty enables the consumer to get this extensive article of clothing from 50 to 80 per cent. cheaper than before, and thus places a becoming dress within the reach of thousands of handsome females in the humbler ranks of life. Printed goods, which in 1795 were sold for two shillings and three-pence the yard, may be bought at present for eight-pence. In fact, a woman may now purchase the materials of a pretty gown for two shillings. The repeal of the tax has been no less beneficial to the fair dealers, by putting an end to the contraband trade, formerly pursued to an extent equally injurious to them and the revenue. It has, moreover, emancipated a manufacture, eminently dependant upon taste, science, and dexterity, from the venal curiosity of petty excisemen, by whom private improvements, of great value to the inventor, were in perpetual jeopardy of being pirated and sold to any sordid rival. The manufacturer has now become a free agent, a master of his time, his workmen, and his apparatus; and can print at whatever hour he may receive an order; whereas he was formerly obliged to wait the convenience of the excise officer, whose province it was to measure and stamp the cloth before it could be packed, -an operation fraught with no little annoyance and delay. Under the patronage of parliament, it was easy for needy adventurers to buy printed calicoes, because they could raise such a sum by drawbacks upon the export of one lot as would go far to par for another, and thus carry on a fraudulent system of credit, which sooner or later merged in a disastrous bankruptey. Meanwhile the goods thus obtained were pushed of to some foreign markets, for which they were possibly not suited, or where they produced, by their forced sales, a depreciation of all similar merchandise, ruinous to the man who meant to pay for his wares.

The principles of calico-printing have been very profoundly studied by many of the French manufacturers, who generally keep a chemist, who has been educated in the Parisian schools of science, constantly at work, making experiments upon colors in a well-mounted laboratory. In that belonging to M. Daniel Kouchlin, of Mulhausen, there are upwards of 3000 labelled rials, filled with chemical reagents, and specimens subservient to dyeing. The great disadvantage under which the French printers labor is the higher price they pay for cotton fabries above that paid by the English printers. It is this circumstance alone which prevents them from becoming very formidable rivals to us in the markets of the world. M. Barbet, deputy and mayor of Rouen, in his replies to the ministerial commission of inquiry, rates the disadvantage proceeding from that cause at 2 franes per piece, or about 5 per cent. in value. In the annual report of the Sociéte Industrielle of Mulhausen, made in December, 1833, the number of pieces printed that year in Alsace is rated at 720,000 , to which if we add $1,000,000$ for the produce of the department of the Lower Seine, and 280,000 for that of St. Quentin, Lille, and the rest of France, we shall have for the total amount of this manufacture $2,000,000$ of pieces, equivalent to nearly 2,400,000 pieces English; for the French piece usually measures $33 \frac{1}{2}$ aunes, $=41$ yards nearly; and it is also considerably broader than the English pieces upon an average. It is therefore probable that the home consumption of France in printed goods is equal in quantity, and superior in value, to that of England. With regard tc the comparative shill of the workmen in the two countries, M. Nicholas Kœchlin, deputy of the Upper Rhine, say's, that one of his foremen, who worked for a year in a printfield in Lancashire, found little or no difference between them in that respect. The English wages are considerably higher than the French. The machines for multiplying production, which for some time gave us a decided advantage, are now getting into very general use among our neighbors. In my recent visit to Mulhausen, Rouen, and their environs, I had an opportunity of seeing many printing establishments mounted with all the resources of the most refined mechanisms. 
The calico-printing of this country still labors under the burden of considerable taxes upon madder and gallipoli oil, which have counteracted the prosperity of our Turliey red styles of work, and caused them to flourish at Elberfeldt, and some other places on the continent whither a good deal of the English yarns are sent to be dyed, then brought back, and manufactured into ginghams, checks, \&c., or fo:warded directly thence to our Russian customers. This fact places our fiscal laws in the same odious light as the facility of pirating printers' patterns with impunity does our chancery laws.

Befure cloth can receive good figured impressions its surface must be freed from fibrous down by Singeing, and be rendered smooth by the CALENDER. See these articles. They are next bleached, with the exception of those destined for Turkey red. See Bleaching and MADDER. After they are bleached, dried, singed, and calendered, they are lapped round in great lengths of several pieces, stitched endwise together, by means of an apparatus called in Manchester a candroy, which bears on its front edge a rounded iron bar, transrersely grooved to the right and left from the centre, so as to spread out the web as it is drawn over it by the rotation of the lapping roller. See a figure of this bar subservient to the cylinder printing-machine.

Four different methods are in use for imprinting figures upon calicoes: the first is by small wooden blocks, on whose face the design is cut, which are worked by hand; the second is by larger wood-cut blocks, placed in either two or three planes, standing at right angles to each other, called a Perrotine, from the name of its inventor; the third is by flat copper plates, a method now almost obsolete; and the fourth is by a system of copper cylinders, mounted in a frame of great elegance, but no little complexity, by which two, three, four, or even five colors may be printed on in rapid succession by the mere rotation of the machine driven by the agency of steam or water. The productive powers of this printing automaton are very great, amounting for some styles to a piece in the minute, or a mile of cloth in the hour. The fifth color is commonly communicated by means of what is called a surface cylinder, covered with wooden figures in bass-relief, which, by rotation, are applied to a plane of cloth imbued with the thickened mordants.

The hand blocks are made of sycamore or pear-tree wood, or of deal face 1 with these woods, and are from two to three inches thick, nine or ten inches long, and five broad, with a strong box handle on the back for seizing them by. The face of the block is either carved in relief into the desired design, like an ordinary wood-cut, or the figure is formed by the insertion edgewise into the wood of narrow slips of flattened copper wire. These tiny fillets, being filed level on the one edge, are cut or bent into the proper shape, and forced into the wood by the taps of a hammer at the traced lines of the configuration. Their upper surfaces are now filed flat, and polished into one horizorital plane, for the sake of equality of impression. As the slips are of equal thickness in th ir whole depth, from having been made by running the wire through between the steel cylinders of a flatting mill, the lines of the figure, however much they get worn by use, are always equally broad as at first; an advantage which does not belong to wood-cutting. The interstices between the ridges thus formed are filled up with felt-stuff. Sometimes a delicate part of the design is made by the wood-cutter, and the rest by the insertion of copper slins.

The coloring matter, properly thickened, is spread with a flat brush, by a child, upon fine woollen cloth, stretched in a frame over the wax cloth head of a wooden drum or sieve, which floats inverted in a tubful of old paste, to give it elastic buoyancy. The inverled sieve drum should fit the paste tub pretty closely. The printer presses the face of the block on the drum head, so as to take up the requisite quantity of color, applies it to the surface of the calico, extended upon a flat table covered with a blanket, and then strikes the back of the block with a wooden mallet, in order to transfer the impression fully to the cloth. This is a delicate operation, requiring equal dexterity and diligence. To print a piece of cloth 25 yards long, and 30 inches broad, no less than 672 applications of a block, 9 inches long and 5 inches broad, are requisite for each color; so that if there are three colors, or three hands as the French term it, no less than 2016 applications will be necessary. The blocks have pin-points fixed into their corners, by means of which they are adjusted to their positions upon the cloth, so as to join the different parts of the design with precision. Each printer has a color-tub placed within reach of his right hand; and for every different color he must have a separate sicre. Many manufacturers cause their blocks to be made of three layers of wood, two of them being deal with the grain crossed to prevent warping, and the third sycamore for engraving.

The printing shop is an oblong apartment, lighted with numerous windows at each side, and haring a solid table opposite to each window. The table $\mathrm{B}, \mathrm{fig}$. 231, is formed of a strong plank of well-seasoned hard wood, mahogany, or marble, with a surface truly plane. Its length is about 6 feet, its breadth 2 feet, and its thickness 3,4 , or 5 inches. It stands on strong fect, with its top about 36 inches above the floor. At one of its ends there are two brackets $c$ for supporting the axles of the roller $\mathrm{E}$, which carries the 
white calico to be printed. The hanging rollers $\mathrm{E}$ are laid across joists fixed near the roof of the apartment above the printing shop, the ceiling and floor between them being open bar work, at least in the middle of the room. Their use is to facilitate the exposure, and, consequently, the drying of the printed pieces, and to prevent one figure being daubed by another. Should they come to be all filled, the remainder of the goods must be folded lightly upon the stool $\mathrm{D}$.
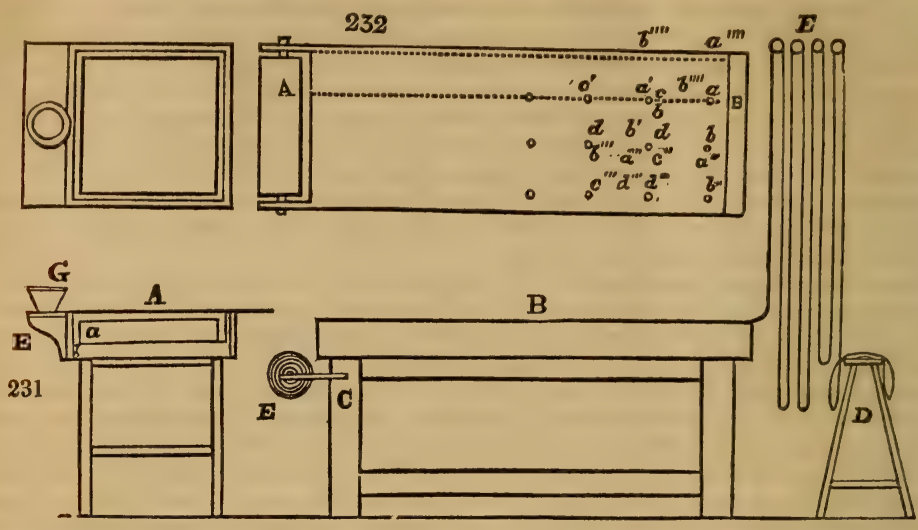

The printer stretches a length of the piece upon his table A B, taking care to place the selvage towards himself, and one inch from the edge. He presents the block towards the end, to determine the width of its impression, and marks this line A B, by means of his square and tracing point. The spreader now besmears the cloth with the color, at the commencement, upon both sides of the sieve head; because, if not uniformly applied, the block will take it up unequally. The printer seizes the block in his right hand, and daubs it twice in different directions upon the sieve cloth, then he transfers it to the calico in the line $\mathrm{A} \mathrm{B}$, as indicated by the four points $a b c d$, corresponding to the four pins in the corners of the block. Having done so, he takes another daub of the color, and makes the points $a b$ fall on $c d$, so as to have at the second stamp $a^{\prime} b^{\prime}$, covering $a b$ and $c^{\prime} d^{\prime}$; and so on, through the rest, as denoted by the accented letters. When one table length is finished, he draws the cloth along, so as to bring a new length in its place.

The grounding-in, or re-entering (rentrage), of the other colors is the next process. The blocks used for this purpose are furnished with pin-points, so adjusted that, when they are made to coincide with the pin-points of the former block, the design will be correet; that is to say, the new color will be applied in its due place upon the flower or other figure. The points should not be allowed to touch the white cloth, but should be made to fall upon the stem of a leaf, or some other dark spot. These rentrages are of four sorts :1. One for the mordants, as above; 2 . one for topical colors; 3 . one for the application of reds; and, 4. one for the application of resist pastes or reserves. These styles have superseded the old practice of pencilling.

The Perrotine is a machine for executing block-printing by mechanical power; and it performs as much work, it is said, as 20 expert hands. I have seen its operation, in many factories in France and Belgium, in a very satisfactory manner; but $I$ have reason to believe that there are none of them as yet in this country. Three wooden blocks, from $2 \frac{1}{2}$ to 3 feet long, according to the breadth of the cloth, and from 2 to 5 inches broad, faced with pear-tree wood, engraved in relief, are mounted in a powerful cast-iron frame work, with their planes at right angles to each other, so that each of them may, in succession, be brought to bear upon the face, top, and back of a square prism of iron covered with cloth, and fitted to revolve upon an axis between the said blocks. The calico passes between the prism and the engraved blocks, and receives successive impressions from them as it is successively drawn through by a winding cylinder. The blocks are pressed against the calico through the agency of springs, which imitate the elastic pressure of the workman's hand. Each block receives a coat of colored paste from a woollen surface, smeared after every contact with a mechanical brush. One man, with one or two children for superintending the colorgiving surfaces, can turn off about 30 pieces English per day, in three colors, which is the work of fully 20 men and 20 children in block printing by hand. It executes some styles of work to which the cylinder machine, without the surface roller, is inadequate. 
The copper-plate printing of calico is almost exactly the same as that used for printing engravings on paper from flat plates, and being nearly superseded by the next machine, need not be described.

The cylinder printing machine consists, as its name imports, of an engraved copper cylinder, so mounted as to revolve against another cylinder lapped in woollen cloth, and imbued with a colored paste, from which it derives the means of communicating

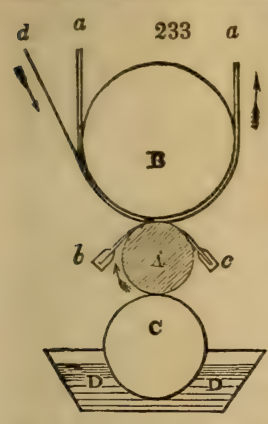
colored impressions to pieces of calico passed over it. Fig. 223 will give the reader a general idea of this elegant and expeditious plan of printing. The pattern is engraved upon the surface of a hollow cylinder of copper, or sometimes gunmetal, and the cylinder is forced by pressure upon a strong iron mandrel, which serves as its turning shaft. To facilitate the transfer of the impression from the engraving to the cotton cloth, the latter is lapped round another large cylinder, rendered elastic by rolls of woollen cloth, and the engraved cylinder presses the calico against this elastic cushion, and thereby prints it as it revolves. Let $\mathrm{A}$ be the engraved cylinder mounted upon its mandrel, which receives rotatory motion by wheels on its end, connected with the steam or water power of the factory. $\mathbf{B}$ is a large iron drum or roller, turning in bearings of the end frames of the machine. Against that drum the engraved cylin$\operatorname{der} \mathrm{A}$ is pressed by weights or screws; the weights acting steadily, by levers, upon its brass bearings. Round the drum B the endless web of felt or blanket stuff $a a$, travels in the direction of the arrow, being carried round along with the drum B, which again is turned by the friction of contact with the cylinder A. C represents a clothed wooden roller, partly plunged into the thickened color of the trough D D. That roller is also made to bear, with a moderate force, against $A$, and thus receives, by friction, in some cases, a movement of rotation. But it is preferable to drive the roller $\mathrm{c}$ from the cylinder A, by means of a system of toothed wheels attached to their ends, so that the surface speed of the wooden or paste roller shall be somewhat greater than that of the printing cylinder, whereby the color will be rubbed, as it were, into the engraved parts of the latter.

As the cylinder $\mathrm{A}$ is pressed upwards against $\mathrm{B}$, it is obvious that the bearers of the trough and its roller must be attached to the bearings of the cylinder $A$, in order to preserve its contact with the color-roller c. $\quad b$ is a sharp-edged ruler of gun-metal or steel, called the color doctor, screwed between two gun-metal stiffening bars; the edge of which wiper is slightly pressed as a tangent upon the engraved roller A. This ruler vibrates with a slow motion from side to side, or right to left, so as to exercise a delicate shaving action upon the engraved surface, as this revolves in the direction of the arrow. $c$ is another similar sharp-edged ruler, called the lint doctor, whose office it is to remove any fibres which may have come off the calico in the act of printing, and which, if left on the engraved cylinder, would be apt to occupy some of the lines, or at least to prevent the color from filling them all. This lint doctor is pressed very slightly upon the cylinder $A$, and has no transverse motion.

What was stated with regard to the bearers of the color trough $D$, namely, that they are connected, and moved up and down together with the bearings of the cylinder A, may also be said of the bearers of the two doctors.

The working of this beautiful mechanism may now be easily comprehended. The web of calico, indicated in the figure by the letter $d$, is introduced or carried in along with the blanket stuff $a a$, in the direction of the arrow, and is moved onward by the pressure of the revolving cylinder $A$, so as to receive the impression of the pattern engraved on that cylinder.

Before proceeding to describe the more complex calico-machine which prints upon eloth 3,4 , or 5 colors at one operation, by the rotation of so many cylinders, I shal] explain the modern methods of engraving the cylinder, which I am enabled to do by the courtesy of Mr. Locket, of Manchester, an artist of great ingenuity in this department, who politely allowed me to inspect the admirable apparatus and arrangements of his factory.

To engrave a copper cylinder 3 or 4 inches in diameter, and from 30 to 36 inches long, with the multitude of minute figures which exist in many patterns, would be a very aborious and expensive operation. The happy invention made by Mr. Jacob Perkins, in America, for transferring engravings from one surface to another by means of steel roller dies, was with great judgment applied by Mr. Locket to calico-printing, so long ago as the year 1808, before the first inventor came to Europe with the plan. The pattern is first drawn upon a scale of about 3 inches square, so that this size of figure being repeated a definite number of times, will cover the cylinder. This pattern is next engraved in intaglio upon a roller of softened steel, about 1 inch in diameter, and 3 inches long, so that it will exactly occupy its surface. The engraver aids his eye with 
lens, when employed at this delicate work. This roller is hardened by heating it to a cherry-red in an iron case containing pounded bone-ash, and then plunging it into cold water ; its surface being protected from oxydizement by a chally paste. This hardened roller is put into a press of a peculiar construction, where, by a rotatory pressure, it trans fers its design to a similar roller in the soft state; and as the former was in intaglio, tho latter must be in relievo. This second roller being hardened, and placed in an appro priate volutory press, is employed to engrave by indentation upon the full-siz d coppes cylinder the whole of its intended pattern. The first roller engraved by hand is calleo the die; the second, obtained from it by a process like that of a milling tool, is called the mill. By this indentation and multiplication system, an engraved crlinder may be had fol seven pounds, which engraved by hand would cost fifty or upwards. The restoration of a worn-ont cylinder becomes extremely easy in this way; the mill being preserved, need merely be properly rolled over the copper surface again.

At other times, the hard roller die is placed in the upper bed of a screw press, not unlike that for coining, while the horizontal bed below is made to move upon strong rollers mounted in a rectangular iron frame. In the middle of that bed a smooth calie or flat disc of very soft iron, about 1 inch thick, and 3 or 4 inches in diameter, is made fasi by four horizontal adjusting screws, that work in studs of the bed frame. The die being now brought down by a powerful screw, worked by toothed wheel-work, and made tc press with force upon the iron cake, the bed is moved backwards and forwards, causing the roller to revolve on its axles by friction, and to impart its design to the cake. This iron disc is now case-hardened by being ignited amidst horn sharings in a box. and then suddenly quenched in water, when it becomes itself a die in relievo. This disc die is fixed in the upper part of a screw press with its engrared face downwards, ret $s 0$ as to be moveable horizontally by traverse screws. Beneath this inverted bed, sustained at its upper surface by friction-rollers, a copper cylinder 30 inches long, or thereby, is mounted horizontally upon a strong iron mandrel, furnished with toothed wheels at one of its ends, to communicate to it a movement upon its axis through any aliquot arcs of the circle. The disc die being now brought down to bear upon the copper cylinder, this is turned round through an arc corresponding in length to the length of the die; and thus, by the steady downward pressure of the screw, combined with the revolution of the cylinder, the transfer of the engraving is made in intaglio. This is. I beliere, the most convenient process for engraving, by transfer, the copper of a one-crlinder machine. But when 2,3 , or 4 cylinders are to be engraved with the same pattern for a two, three, or four-colored machine, the die and the mill roller plan of transfer is adopted. In this case, the hardened roller die is mounted in the upper bed of the transfer press, in such a way as to be capable of rotation round its axis, and a similar roller of softened steel is similarly placed in the under bed. The rollers are now made to bear on each other by the action of the upper screw, and while in hard contact, the lower one is caused to revolve, which, carrying round the upper by friction, receives from it the figured impression in relief. When cylinders for a three-colored machine are wanted, three such mills are made fac-similes of each other; and the prominent parts of the figure which belong to the other two copper cylinders are filed off in each one respectively. Thus three differently figured mills are very readily formed, each adapted to engrave its particular figure upon a distinct copper eylinder.

Some copper cylinders for peculiar styles are not graved by indentation, as just described, but etched by a diamond point, which is moved by mechanism in the most curious variety of configurations, while the cylinder slowly revolves in a horizontal line beneath it. The result is extremely beautiful, but it would require a very elaborate set of drawings to represent the machinery by which $\mathrm{Mr}$. Locket produces it. The copper is covered by a resist varnish while being heated by the transmission of steam through its axis. After being etched, it is suspended horizontally by the ends, for about five minutes, in an oblong trough charged with dilute nitric acid.

With regard to the two and three-colored machines, we must observe, that as the calico in passing between the cylinders is stretched laterally from the central line of the web, the figures engraved upon the cylinders must be proportionally shortened, in their lateral dimensions especially, for the first and second cylinder.

Cylinder printing, though a Scotch invention, has received its wonderful development in England, and does the greatest honor to this country. The economy of labor introduced by these machines is truly marvellous; one of them, under the guidance of a man to regulate the rollers, and the service of a boy, to supply the color troughs, being capable of printing as many pieces as nearly 200 men and boys could do with blocks. The perfection of the engraving is most honorable to our artisans. The French, with all their ingenuity and neat-handedness, can produce nothing approaching in excellence to the engraved cylinders of Manchester, - a painful admission, universally made to me by every eminent manufacturer in Alsace, whom I visited in my late tour. 
Another modification of cylinder printing, is that with wooden rollers cut in relief; it is called surfuce printing, probably because the thickened color is applied to a tense sur face of woollen cloth, from which the roller takes it up by revolving in contact with the cloth. When the copper cylinders and the wooden ones are combined in one apparatus. it has got the appropriate name of the union printing machine.

In mounting three or more cylinders in one frame, many more adjustments become ne cessary than those described above. The first and most important is that which ensures the correspondence between the parts of the figures in the successive printing rollers, for umless those of the second and subsequent engraved cylinders be accurately inserted into their respective places, a confused pattern would be produced upon the cloth as it advances round the pressure cylinder B, figs. 233, 234.

Each cylinder must have a forward adjustment in the direction of rotation round its axis, so as to bring the patterns into correspondence with each other in the length of the piece; and also a lateral or traverse adjustment in the line of its axis, to effect the correspondence of the figures across the piece; and thus, by both together, each cylinder may be made to work symmetrically with its fellows.

Fig. 234 is a cross section of a four-color cylinder machine, by which the working parts are clearly illustrated.

A A $\mathrm{A}$ is a part of the two strong iron frames or cheeks, in which the various rollers are mounted. They are bound together by the rods and bolts $a \quad a \quad a \quad a$.

$\mathbf{B}$ is the large iron pressure cylinder, which rests with its gudgeons in bearings or bushes, which can be shifted up and down in slots of the side cheeks A A. These bushes are suspended from powerful screws $b$, which turn in brass nuts, made fast to the top of the frame $A$, as is plainly shown in the figure. These screws serve to counteract the strong pressure applied beneath that cylinder, by the engraved cylinders $\mathbf{D} \mathbf{E}$.

C D E F are the four printing cylinders, named in the order of their operation. They consist of strong tubes of copper or gun-metal, forcibly thrust by a screw press upon the iron mandrels, round which as shafts they revolve.

The first and last cylinder $\mathrm{c}$ and $\mathrm{F}$ are mounted in brass bearings, which may be shifted in horizontal slots of the frame A. The pressure roller B, against whose surface they bear with a rery little obliquity downwards, may be nicely adjusted to that pressure by its elevating and depressing screws. By this means $\mathrm{C}$ and $\mathbf{F}$ can be adjusted to $\mathrm{B}$ with geometrical precision, and made to press it in truly opposite directions.

The bearings of the cylinders $D$ and $E$ are lodged also in slots of the frame $A$, which point obliquely upwards, towards the centre of $\mathrm{B}$. The pressure of these two print cylinders $\mathrm{c}$ and $\mathrm{F}$ is produced by two screws $c$ and $d$, which work in brass nuts, made fast to the frame and very visible in the figure. The frame-work in which these bearings and screws are placed, has a curvilinear form, in order to permit the cylinders to be readily removed and replaced; and also to introduce a certain degree of elasticity. Hence the pressure applied to the cylinders $C$ and $F$, partakes of the nature of a spring; a circumstance essential to their working smoothly, on account of the occasional inequalities in the thickness of the felt web and the calico.

The pressure upon the other two print cylinders $\mathbf{D}$ and $\mathbf{E}$ is produced by weights acting with levers against the bearings. The bearings of $\mathrm{D}$ are, at each of their ends, acted upon by cylindrical rods, which slide in long tubular bosses of the frame, and press with their nuts $g$ at their under end upon the small arms of two strong levers $\mathrm{G}$, which lie on each side of the machine, and whose fulcrum is at $h$ (in the lower correr at the left hand). The long arms of these levers $\mathrm{G}$, are loaded with weights $\mathrm{H}$, whereby they are made to press up against the bearings of the roller $\mathrm{D}$, with any degree of force, by screwing up the nut $g$, and hanging on the requisite weights.

The manner in which the cylinder $\mathbf{E}$ is pressed up against $\mathbf{B}$, is by a similar construction to that just described. With each of its bearings, there is connected by the link $k$, a curved lever $\mathrm{I}$, whose fulcrum or centre of motion is at the bolt $l$. To the outer end of this lever, a screw, $m$, is attached, which presses downwards upon the link $n$, connected with the small arm of the strong lever $k$, whose centre of motion is at 0 . By turning therefore the screw $m$, the weight $\mathrm{L}$, laid upon the end of the long arm of the lever $\mathrm{k}$ (of which there is one upon each side of the machine), may be made to act or not at pleasure upon the bearings of the cylinder $\mathbf{E}$.

In tracing the operation of this exquisite printing machine, we shall begin with the first engraved cylinder c. Its bearings or bushes shift, as was already stated, in slots of the frame A. Each of them consists of a round piece of iron, to which the end of the screw $c$ is joined, in the same way as at $d$, in the opposite side. In each of these iron bearings, a concave brass is inserted to support the collar of the shaft, and in a dovetailed slit of this brass, a sliding piece is fitted, upon which a set or adjusting screw in the iron bearing acts, and which, being forced against the copper cylinder $\mathrm{c}$, serves to adjust the line of its axis, and to keep it steady between its bearings, and true in its rotatory motion. Upon the iren bearing a plate is screwed, provided with two fianges, 
which support the color trough $q$, and the color roller $\mathrm{M}$. This trough, as well as the others to be mentioned presently, is made of sheet copper in the sides and bottom, and 234

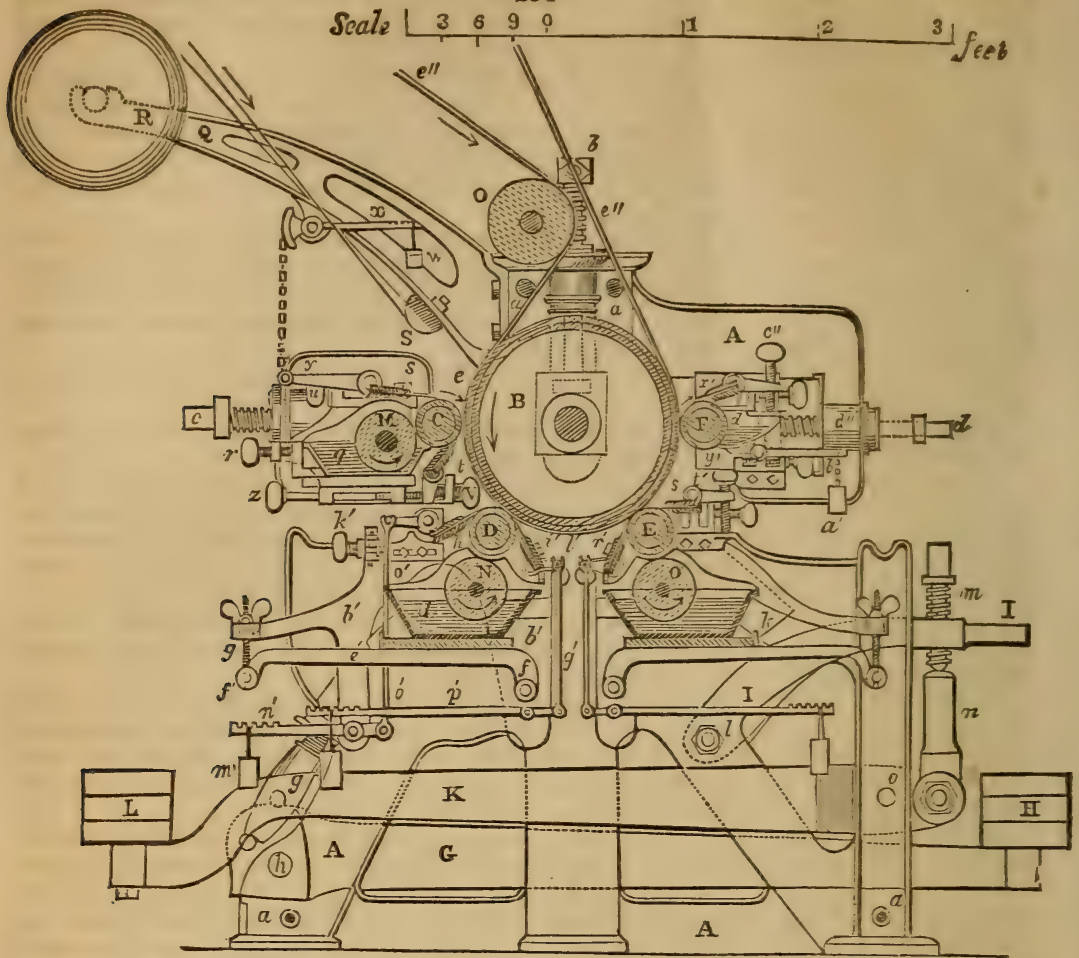

fixed upon a board; but its ends are made of plates of cast copper or gun-metal to serve as bearings to the color roller M. The trough and its roller may be shifted both together into contact with the printing cylinder $\mathrm{c}$, by means of the screw $r$. Near $s$, seen above the roller, c, and $t$ below it, are sections of the two doctors, which keep the engraved cylinders in sound working condition; the former being the colour doctor, and the latter the lint doctor. Their ends lie in brasses, which may be adjusted br the screws $u$ and $v$, working in the respectire brackets, which carry their brasses, and are made fast to the iron bearings of the cylinder.

The pressure of the color doctor is produced by two weights $w$, (see high up on the frame work, ) which act on a pair of small levers $x$, (one on each side of the machine,) and thus, by means of the chains, tend to lift the arms $y$, attached to the end axles of the doctor. The pressure of the lint doctor upon the cylinder c, is performed by the screw $z$, pressing upon an arm which projects downwards, and is attached to the axle of that doctor.

The bearings of the second printing cylinder $\mathrm{D}$, consist at each end of a mass of iron (removed in the drawing to show the mechanism below it), which shifts in the slanting slot of the frame A. In each of these masses there is another piece of iron, which slides in the transverse direction, and may be shifted by the adjusting screw $a^{\prime}$ fixed to it, and working in a nut cast upon the principal bearing above described. To the inner bearings, which carry the brasses in which the shaft lies, are screwed the two curved arms $b^{\prime} b^{\prime}$ to which are attached the bearings \&c., for the color trough and the doctors. In these brasses there are also doretailed pieces, which slide and are pressed by set screws furnished with square heads in the iron secondary bearings, which serve, as before said, to adjust the printing crlinder in the line of its axis, while other screws adjust the distance of the cloth upon which the second color is printed, and the line of contact with the cylinder $\mathrm{B}$.

$\mathrm{N}$, is the color roller of $\mathrm{D}$, and $d^{\prime}$ the color trough, which rests by its board upon the lever $e^{\prime}$; whose centres of motion $f^{\prime}$, are made fast to the curved arms $b^{\prime}$, fixed at the 
bearings of the cylinder, and whose ends are suspended by screws $g^{\prime}$; whereby the color roller $\mathrm{N}$, may be pressed with greater or less force to the cylinder $\mathbf{D} . h^{\prime}$ and $i^{\prime}$ are the two doctors of this cylinder; the former being the color, the latter the lint doctor. They rest, as was said of the cylinder $\mathrm{c}$, in brasses which are adjustable by means of screws, that work in the studs or brackets by which the brasses are supported. These brackets must of course be screwed to the secondary bearing-pieces, in order that they may keep their position, into whatever direction the bearings may be shifted. $k^{\prime}$ and $l^{\prime}$ are these set screws for the color and lint doctors. The pressure of the former upon the cylinder $\mathrm{D}$, is produced by weights $m^{\prime}$, acting upon levers $n^{\prime}$, and pressing by rods or links $o^{\prime}$, upon arms attached to each end of the axis of the doctor. (See the left hand side of the figure near the bottom.) The lint-doctor $i^{\prime}$ is pressed in a similar way at the other side upon the cylinder $\mathrm{D}$, by the weights acting upon levers $p^{\prime}$, and by rods $q^{\prime}$ upon arms fixed at each end of the axis of the doctor.

The bearings of the third printing cylinder $\mathrm{E}$, are of exactly the same construction as that above described, and therefore require no particular detail. The lint doctor $s$, is here pressed upon the engraved cylinder by screws $t^{\prime}$, working in the ends of studs or arms fixed upon each end of the axis of the doctor, and pressing upon flanges cast upon the brackets in which the brasses of the doctor's axis lie, which are made fast to the bearings of the cylinder $\mathbf{E}$.

The bearings of the fourth copper cylinder $\mathrm{F}$, are also constructed in a similar way, Each consists of a first bearing, to which is joined the end of the screw $d$, by which it is made to slide in a slot of the frame. Another bearing, which contains the brass for the shaft of the cylinder, can be shifted up and down in a transverse direction by a screw $z^{\prime}$, of the second bearing, working in a nut cast upon the first bearing. To this secondary bearing, plates are made fast by the screws $v^{\prime} v^{\prime}$ to the inside, to carry the studs or brackets of the doctors $x^{\prime}$ and $y^{\prime}$. In the brasses of the cylincier shaft, dovetailed pieces are made to slide, being pressed by set screws $w^{\prime}$, against the engraved cylinder $F$, similar to what has been described for adjusting the cylinders to one another. This cylinder has no separate color roller, nor trough, properly speaking, but the color doctor $y^{\prime}$ is made concave to serve the purpose of a trough in supplying the engraved lines of the cylinder with color. With this view the top plate of the doctor is curved to contain the colored paste, and it is shut up at the ends by pieces of wood made to fit the curvature of the doctor. Its pressure against the engraved surface is produced by weights $a^{\prime \prime}$, acting at the ends of arms $b^{\prime \prime}$, attached to the ends of the axis of the doctor. The pressure of the lint doctor $x^{\prime}$ is given by screws $c^{\prime \prime}$, working in arms attached to the ends of the axis of the doctor, and pressing upon the flanges $d^{\prime \prime}$, cast upon the brackets which carry the brasses for the axis of the doctor. These brasses are themselves adjustable, like those of all the other cylinders, by set screws in the brackets, which work in the nuts formed in the brasses.

$e^{\prime \prime} e^{\prime \prime}$, is the endless web of felt stuff which goes round the cylinder $\mathrm{B}$, and constitutes the soft elastic surface upon which the printing cylinders $c, D, E$, and $F$ exercise their pressure. This endless felt is passed over a set of rollers at a certain distance from the machine, to give opportunity for the drying up of any coloring paste which it may have imbibed from the calico in the course of the impressions. In its return to the machine in the direction of the arrow, it is led over a guide roller o, which is thereby made to revolve. Upon the two ends of this, and outside of the bearings which are fixed upon the tops of the frame $A$, are two eccentrics, one of which serves to give a vibratory traverse morement to the color doctors $s^{\prime}, h^{\prime}$, and $r^{\prime}$ of the three cylinders, $\mathrm{c}, \mathrm{D}$, and $\varepsilon$, whilst the other causes the color doctor $y^{\prime}$ of the cylinder $F$, to make lateral vibrations.

$\mathrm{Q}$ is one of a pair of cast-iron brackets, screwed on at the back of the side-frames or cheeks A A, to carry the roller filled with white calico $\mathrm{R}$, ready for the printing operations. Upon the end of the shaft whereon the calico is coiled, a pulley is fixed, over which a rope passes suspending a weight in order to produce friction, and thereby resistance to the action which tends to unwind the calico. In winding it upon that and similar rollers, the calico is smoothed and expanded in breadth by being passed over one or

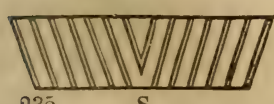

235 $\mathrm{S}$ more grooved rods, or over a wooden bar $s$, fig. 235 , the surface of which is covered with wire, so as to have the appearance of a united right and left-handed screw. By this device, the calico, folded or creased at any part, is stretched laterally from the centre, and made level. It then passes over the guide-roller 0 , where it comes upon the surface of the felt $e^{\prime \prime} e^{\prime \prime}$, and thence proceeds under its guidance to the series of printing cylinders.

Three and four-color machines, similar to the above, are now at work in many establishrnents in Lancashire, which will tarn off a piece of 28 yards per minute, each of the three or four cylinders applying its peculiar part of the pattern to the cloth as it passes along, by ceaseless rotation of the unwearied wheels. At this rate, the astonishing 
length of one mile of many-colored web is printed witn elegant flowers and other figures in an hour. When we call to mind how much knowledge and skill are involved in this process, we may fairly consider it as the greatest achievement of chemical and mechanical science.

Before entering upon the different styles of work which constitute calico-printing, 1 shall treat, in the first place, of what is common to them all, namely, the thickening of the mordants and colors. This is an operation of the greatest importance towards the successful practice of the art. Several circumstances may require the consistence of the thickening to be varied; such as the nature of the mordant, its density, and its acidity. A strong acid mordant cannot be easily thickened with starch; but it may be by roasted starch, vulgarly called British gum, and by gum arabic or senegal. Some mordants which seem sufficiently inspissated with starch, liquefy in the course of a few days, and, being apt to run in the printing-on, make blotted work. In France, this evil is readily obviated by adding one ounce of spirits of wine to half a gallon of color-a remedy which the English excise duties render too costly.

The very same mordant, when inspissated to different degrees, produces different tints in the dye-copper-a difference due to the increased bulk from the thickening substance; thus, the same mordant, thickened with starch, furnishes a darker shade than when thickened with gum. Yet there are circumstances in which the latter is preferred, because it communicates more transparency to the dyes, and because, in spite of the washing, more or less of the starch always sticks to the mordant. The gum has the inconvenience, however, of drying too speedily, and of also increasing too much the volume of the mordants; by both of which causes it obstructs their combination with the stuff, and the tints become thin or scratchy.

The substances generally employed as thickeners are the following :-
1. Wheat starch.
2. Flour.
3. Roasted starch.
4. Gum senegal.
5. Gum tragacanth.
6. Salep.
7. Pipe-clay, mixed with gum senegal.
8. Sulphate of lead.
9. Sugar.
10. Molasses.
11. Glue.

After thickening with gum, we ought to avoid adding metallic solutions in the liquid state; such as nitrate of iron, of copper, solutions of tin, of subacetate of lead, \&c.; as they possess the property of coagulating gum. I shall take care to specify the nature and proportion of thickening to be employed for each color; a most important matter, hitherto neglected by English writers upon calico-printing.

The atmosphere of the printing shops should never be allowed to cool under $65^{\circ}$ or $70^{\circ} \mathrm{F}$; and it should be heated by proper stoves in cold weather, but not rendered too dry. The temperature and moisture should therefore both be regulated with the aid of thermometers and hydrometers, as they exercise a great influence upon all the printing processes, and especially upon the combination of the mordant with the cloth. In the course of the desiccation, a portion of the acetic acid evaporates with the water, and subacetates are formed, which combine with the stuff in proportion as the solvent principle escapes; the water, as it evaporates, carries off acetic acid with it, and thereby aids the fixation of bases. These remarks are peculiarly appropriate to delicate impressions by the cylinder machine, where the printing and drying are both rapidly effected. In the lapis lazuli style, the strong mordants are apt to produce patches, being thickened with pipe-clay and gum, which obstruct the evaporation of the acids. They are therefore apt to remain, and to dissolve a portion of the mordants at their immersion in the blue vat, or at any rate in the dang bath. In such a case, a hot and humid air is indispensable, after the application of the mordants, and sometimes the stuffs so impregnated must be suspended in a damp chamber. To prevent the resist pastes becoming rapidly crusty, substances apparently useless are mixed with them, but which act beneficially by their hygrometric qualities, in retarding the desiccation. Oil also is sometimes added with that view.

It is often observed that goods printed upon the same day, and with the same mordant, exhibit inequalities in their tints. Sometimes the color is strong and decided in one part of the piece, while it is dull and meager in another. The latter has been printed in too dry an atmosphere. In such circumstances a neutral mordant answers best, espeeially if the goods be dried in a hot flue, through which humid vapors are in constant circulation.

In padding, where the whole surface of the calico is imbued with mordant, the drying 
apartment or flue, in which a great many pieces are exposed at once, should be so constructed as to afford a ready outlet to the aqueous and acid exhalations. The cloth ought to be introduced into it in a distended state; because the acetic acid may accumulate in the foldings, and dissolve out the earthy or metallic base of the mordant, causing white and gray spots in such parts of the printed goods. Fans may be employed with great advantage, combined with Hor Fuues. (See this article.)

In the color laboratory, all the decoctions requisite for the print work should be ready prepared. They are best made by a steam heat, by means of copper boilers of a cylin. dric form, rounded at the bottom, and incased within a cast-iron cylinder, the steam being supplied to the space between the two vessels, and the dye-stuff and water being introduced into the interior one, which for some delicate purposes may be made of tin, or copper tinned inside. A range of such steam apparatus should be placed either along one of the side walls, or in the middle line of the laboratory. Proper tables, diawers, vials, with chemical reagents, measures, balances, \&c., should also be provided. The most useful dye-extracts are the following:-

Decoction of logwood, of Brazil-wood, of Persian berries, of quercitron bark, of nutgalls, of old fustic, of archil or cutbear, of cochineal, of cochineal with ammonia, of eatechu.

The following mordants should also be kept ready prepared :-

1. Aluminous mordant.

Take 50 gallons of boiling water.

100 lbs. of alum.

$10 \mathrm{lbs}$. of soda crystals.

$75 \mathrm{lbs}$. of acetate of lead.

The soda should be added slowly to the solution of the alum in the water, snd when the effervescence is finished, the pulverized acetate of lead is put in and well stirred about till it be all dissolved and decomposed. During the cooling, the mixture should be raked up a few times, and then allowed to settle. The supernatant liquor is the mordant; it has a density of $11^{\circ}$ or $11 \frac{1}{2}^{\circ}$ Baumé. It serves for reds and pinks, and enters into the composition of puce and lilach.

2. Aluminous mordant.

Take 50 gallons of water.

100 lbs. of alum.

10 lbs. of soda crystals.

$100 \mathrm{lbs}$. of acetate of lead;-operate as above directed.

The supernatant liquor here has a density of $12^{\circ}$ Baumé; it is employed for lapis resists or reserves, and the cylinder printing of madder reds.

3. Aluminous mordant.

Take 50 gallons of water.

$100 \mathrm{lbs}$ of alum.

6 lbs. of soda crystals.

$50 \mathrm{lbs}$. of acetate of lead;-operate as above directed.

This mordant is employed for uniform yellow grounds.

4. Aluminous mordant.

This is made by adding potash to a solution of alum, till its earth begins to be separared, then boiling the mixture to precipitate the subsulphate of alnmina, which is to be strained upon a filter, and dissolved in acetic acid of moderate strength with the aid of heat. This mordant is very rich in alumina, and marks $20^{\circ} \mathrm{B}$.

5. Aluminous mordant.

Take $12 \frac{1}{2}$ gallons of water.

100 lbs. of alum.

$150 \mathrm{lbs}$. of liquid pyrolignite of lime at $11 \frac{1}{2}^{\circ}$ Baumé.

This mordant is made with heat like the first; after cooling, some alum crystallizes, and it marks only $12 \frac{10^{\circ}}{\mathrm{B}}$.

A mordant is made by solution of alum in potash, commonly called-

6. Aluminate of potash. The caustic ley is prepared by boiling together for an hour 100 gallons of water, $200 \mathrm{lbs}$. of potash, and $80 \mathrm{lbs}$. of quicklime; the mixture is then allowed to settle, the supernatant liquor is decanted, and evaporated till its density be $35^{\circ}$ B. In 30 gallons of that ley at a boiling heat, $100 \mathrm{lbs}$. of ground alum are to be dissolved. On cooling, crystals of sulphate of potash separate. The clear liquor is to be decanted off, and the crystals being washed with a little water, this is to be added to the ley. About 33 gallons of mordant should be obtained.

\section{Mordant for Black.}

The pyrolignite of iron, called iron liquor in this country, is the only mordant used in calico-printing for black, violet, puce, and brown colors. The acetate of alumina, prepared from pyroligneous acid, is much used by the calico-printers under the name of red or yellow liquor, being employed for these dyes. 
We may observe that a strong mordant, like No. 2, does not keep so well as one of mean density, such as No. 1. Too much mordant relatively to the demands of the works should therefore not be made at a time.

There are eight different styles of calico-printing, each requiring different methods of manipulation, and peculiar processes.

1. The madder style, to which the best chintses belong, in which the mordants are ap plied to the white cloth with many precautions, and the colors are afterwards brought up in the dye-bath. These constitute permanent prints.

2. The padding or plaquage style, in which the whole surface of the calico is imbued with a mordant, upon which afterwards different colored figures may be raised, by the topical application of other mordants joined to the actirn of the dye-bath.

3. The reserve style, where the white cloth is impressed with figures in resist paste, and is afterward subjected first to a cold dye, as the indigo vat, and then to a hot dyebath, with the effect of producing white or colored spots upon a blue ground.

4. The discharge or rongeant style, in which thickened acidulous matter, either pure or mixed with mordants, is impirinted in certain points upon the cloth, which is afterwards padded with a dark-colored mordant, and then dyed, with the effect of showing bright figures on a darkish ground.

5. China blues; a style resembling blue stone-ware, which requires very peculiar treatment.

6. The decoloring or enlevage style; by the topical application of chlorine or chromic acid to dyed goods. This is sometimes called a discharge.

7. Steam colors; a style in which a mixture of dye extracts and mordants is topical. ly applied to calico, while the chemical reaction which fixes the colors to the fibre is produced by steam.

8. Spirit colors; produced by a mixture of dye extracts, and solution of tin, vulgarly called spirit by dyers. These colors are brilliant but fugitive.

I. The madder style; called by some dip colors. The true chints patterns belong to it ; they have from 5 to 7 colors, several of which are grounded-in after the first dye has been given in the madder bath.

In dyeing with madder, sumach, fustic, or quercitron, is sometimes added to the bath, in order to produce a variety of tints with the various mordants at one operation.

1. Suppose we wish to produce flowers or figures of any kind containing red, purple, and black colors, we may apply the three mordants at once, by the three-color cylinder machine, putting into the first trough acetate of alumina thickened; into the second, acetate of iron; and into the third, a mixture of the two; then drying in the air for a few days to fix the iron, dunging and dyeing up in a bath of madder and sumach. If we wish to procure the finest madder reds and pinks, besides the purple and black, we must apply at first only the acetate of alumina of two densities, by two cylinders, dry, dung, and dye up, in a madder bath. The mordants of iron liquor for the black, and of iron liquor mixed with the aluminous for purple, must be now grounded-in by blocks, taking care to insert these mordants into their precise spots : the goods being then dried with airing for several days, and next dunged, are dyed up in a bath of madder and sumach. They must be afterwards cleared by branning. See Bran, Dunging, and MADDER.

2. Suppose we wish to produce yellow with red, pink, purple, and black; in this case the second dye-bath should contain quercitron or fustic, and the spots intended to be yellow should receive the acetate of alumina mordant.

3. The mordant for a full red may be acetate of alumina, of spec. grav. $1 \cdot 055$, thickened with starch, and tinged with Brazil-wood; that for a pale red or pink, the same at spec. gravity 1.014 , thickened with gum; that for a middling red, the same at spec. gravity 1.027, thicliened with British gum; and for distinction's sake, it may be tinged yellow with Persian berries. The mordant for black is a pyroligneous acetate of iron, of specific gravity 1.04 ; for purple the same, diluted with six times its volume of water; for chocolate, that iron liquor mixed with acetate of alumina, in various proportions according to the shade wanted. Sumach is mixed with the madder for all these colors except for the purple. The quantity of madder required varies according to the body of color to be put upon the cloth, being from one pound per piece to three or even four. The goods must be entered when the copper is cool, be gradually heated during two or three hours, up to ebullition, and sometimes boiled for a quarter of an hour; the pieces being all the while turned with a wince from the one side of the copper to the other. (See WincE.) They are then washed and boiled in bran and water for ten or fifteen minutes. When there is much white ground in the chints, they must be branned a second or even a third time, with alternate washing in the dash-wheel. To complete the purification of the white, they are spread upon the grass for a few days; or what is more expeditious, and equally good if delicately managed, they are winced for a few minutes in a weak solution of chloride of lime.

4. In the grounding-in for yellow, after madder reds, the aluminous mordant being 
applied, Sc., the piece is dyed, for about an hour, with one pound of quercitron bark, the infusion being gradually lieated to $150^{\circ}$ or $160^{\circ}$, but not higher.

5. A yelluw is sometimes applied in chints work after the other colors are dyed, by means of a decoction of Persian berries mixed with the aluminous mordant, thickened with flour or gum, and printed-on with the block; the piece, when dry, is passed through a weak carbonated alkaline water, or lime water, then washed and dried for the market.

6. Black mordant.-Take half a gallon of acetate of iron, of spec. grav. $1 \cdot 04,4$ ounces of starch, and 4 ounces of flour. The starch must first be moistened with the acetate, then the flour must be added, the rest of the acetate well mixed with both, and the whole made to boil over a brisk fire for five minutes, stirring meanwhile to prevent adhesion to the bottom of the pot. The color must be poured into an earthen pipkin, and well mixed with half an ounce of gallipoli oil. In general, all the mordants, thickened with starch and flour, must be boiled for a few minutes. With British gum or common gum, they must be heated to $160^{\circ} \mathrm{F}$., or thereby, for the purpose merely of dissolving them. The latter should be passed through a sieve to separate the impurities often present in common gum.

7. Puce mordant.-Take a quart of acetate of alumina and acetate of iron, each of spec. grav. 1.04, mixed and thickened like the black, No.6. To give the puce a reddish tinge, the acetate of alumina should have a specific gravity of $1 \cdot 048$, and the iron liquor only $1 \cdot 007$.

Red mordants are thickened with British gum, and are sufficiently colored with the addition of any tinging decoction.

8. Violet mordunts.-These consist either of a very weak solution of acetate of iron, of specific gravity 1.007 , for example; or of a little of the stronger acetate of $1 \cdot 04$, mixed with acetate of alumina, and a little acetate of copper, thickened with starch or British gum. The shades may be indefinitely varied by varying the proportions of the acetates.

When black is one of the colors wanted, its mordant is very commonly printed-on first, and the goods are then hung upon poles in the drying-room, where they are aired for a few days, in order to fix the iron by its peroxydizement; the mordants for red, violet, \&c., are then grounded in, and the pieces are dyed up, after dunging and washing, in the madder bath, into which, for certain shades, sumach, galls, or fustic is added. The goods are brightened with a boil in soap water; occasionally also in a bath, containing a small quantity of solution of tin or common salt. The following mode of brightening is much extolled by the French, who are famous for their reds and roses.

1. A soap boil of forty minutes, at the rate of 1 pound for every 2 pieces. Rinse in clear water.

2. Pass through chloride of soda solution of such strength that two parts of it decolor one part of Gay Lussac's test liquor. See CHLoride of Lime and Indigo. Wince the pieees through it for 40 minutes. Rinse again.

3. Pass it again through the soap bath, No. 1 .

4. Brighten it in a large bath of boiling water, containing 4 pounds of soap, and 1 pound of a cream-consistenced salt of tin, containing nearly half its weight of the muriate of tin, combined with as much nitric acid of spec. grav. 1·288. This strong nitro-muriate having been diluted with a little water, is to be slowly poured into the bath of soap water, and well mixed by stirring. The pieces are now put in, and winced through it for one half or three quarters of an hour.

5. Repeat the soap boil, No. 1. Rinse and dry.

9. Grounding-in of Indigo blue.

Take half a gallon of water of $120^{\circ} \mathrm{F} ., 8$ ounces of ground indigo, and 8 ounces of red sulphuret of arsenic (orpiment), 8 ounces of quicklime, mix together, and heat the mixture to the boiling point; withdraw from the fire, and add, when it is lukewarm, 6 ounces of carbonate of soda, stir and leave the whole at rest till the next day. Then decant the clear liquor, and thicken every quart of it with half a pound of gum. This color onght to be green, and be preserved in a close vessel. When used, it is put into a pot with a narrow orifice, the pencil is dipped into it, wiped on the edge of the pot, and immediately applied by hand. This plan is tedious, and is nearly superseded by the following grounding blue.

Take half a gallon of caustic soda ley of spec. grav. $1 \cdot 15$, heated to $120^{\circ} \mathrm{F}$.

12 ounces of hydrate of protoxyde of tin, obtained by precipitating it from the muriate of tin by solution of potash.

8 ounces of ground indigo; heat these mixed ingredients to the boiling point, then move the pot off and on the fire two or three times in succession, and finally thicken with 3 pounds of raw sugar. In order to apply this by the block, the following apparatus is employed, called the canvass frame; figs. 236, 237. It is formed of a copper 


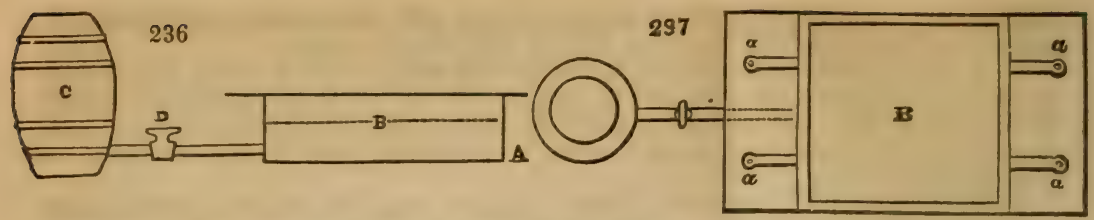

case or box A, in which is laid a frame $\mathrm{B}$, filled with pretty stout canvass The box communicates by a tube with the cistern c, mounted with a stop-cock D. Fig. 237 represents the apparatus in plan: A, the box; $\mathrm{B}$, the canvass, with its edges $a \quad a \quad a$, fixed by pin points to the sides. The color is teared (tiré), or spread even, with a wooden scraper as broad as the canvass. In working with this apparatus, the color being contained in the ressel $\mathrm{c}$ is drawn off into the case $\mathrm{A}$, by opening the stop-cock $\mathrm{D}$, till it rises to the level of the canvass. The instant before the printer daubs the block upon the canvass, the tearer (iveur), boy or girl, runs the scraper across it to renew its surface; and the printer immediately transfers the color to the cloth. In this kind of printing great skill is required to give evenly impressions. As the blue is usually applied to somewhat large designs, it is very apt to run; an inconvenience counteracted by dusting fine dry sand upon the cloth as soon as it is blocked. The goods must be washed within 24 hours after being printed.

10. Topical grounding blue for the cylinder press.

Take $3 \frac{1}{2}$ gallons of caustic soda ley of spec. grav. $1 \cdot 15$.

$3 \frac{1}{2}$ lbs. of ground indigo.

5 lbs. of precipitated protoxyde of tin (as above).

Boil the mixed ingredients for ten minutes, take them from the fire, and add, first, 3 lbs. of Venice turpentine; then 11 lbs. of gum.

Put this mixture into the color trough, print with it, and after two days wash in the dash-wheel; then pass it through a soap-bath, along with a little soda, to brighten the blue, and to take off its grayish tint.

The use of the turpentine is easily explained; it serves to exclude the atmospherical oxygen, and prevent the regeneration of the indigo blue, before it is spread upon the cloth.

After the application to white calico of a similar blue, into which a little acid muriate of tin has been put, the goods are dipped for ten minutes in thin milk of lime, shaling the frame all the time. They are then washed, and cleared with a soap boil. The following color remains long in the deoxydized state from its containing 8 ounces of indigo, 10 ounces of hydrated protoxyde of tin, and $1 \frac{1}{2}$ pounds of solution of muriate of tin, to 2 quarts of soda ley of $1 \cdot 15$, thickened with $2 \frac{1}{2}$ pounds of gum. This blue may be applied by either the block or the cylinder.

11. Topical Prussian blue for grounding.

2 quarts of water with 8 ounces of starch are to be mixed and boiled; add $2 \frac{1}{4}$ ounces of a liquid Prussian blue color, prepared by triturating three quarters of an ounce of that pigment with as much muriatic acid, leaving the ingredients to react upon each other for 24 hours, and then adding three quarters of an ounce of water.

Add 4 ounces of liquid perchloride of tin (oxymuriate).

Mix all together, and pass through a searce. This color is not very fast; cloth printed with it will bear only rinsing.

12. Prussian blue figures are impressed as follows:-

Dissolve 8 ounces of sulphate of iron, and as much acetate of lead, separately in 2 quarts of boiling water; mix well, and settle. Take one quart of this clear liquor reduced to spec. grav. 1.02, one quart of mucilage containing 3 pounds of gum, colored with a little prussiate of potash, mix into a mordant, and print it on with the cylinder. Two days afterwards wash in tepid water containing a little chalk, and then pass the cloth through a solution of prussiate of potash in water, sharpened with a little muriatic acid, till it takes the desired hue. Finally rinse.

III. The padding or plaquage style, called foulard also by the French. See Padding.

Any mordant whatever, such as the acetates of alumina, or of iron, or their mixture, may be applied to the piece by the padding machine, after which it is dried in the нот FLuE, washed, dunged, dyed, washed, and brightened.

Colors from metallic oxydes are very elegantly applied by the padding process. Thus

the iron buff, the manganese bronze, and the chrome yellows and greens are given.

1. Iron buff or chamois.

Take 50 gallons of boiling water;

150 pounds of sulphate of iron; dissolve along with

10 pounds of alum; which partly saturate by the gradual addition of

5 pounds of crystals of soda; and in this mixture dissolve 
50 pounds of pyroligneous acetate of lead. Allow the whole to settle, and draw of the clear supernatant liquid.

For furniture prints this bath should have the spec. grav. $1 \cdot 07$.

The calico being padded in it, is to be dried in the hot-flue; and after 48 hours suspension is to be washed in water at $170^{\circ}$ containing some chalk, by the wince apparatus. It is then washed, by the same apparatus, in hot water, containing a pailful of soda ley of sp?: gray. $1 \cdot 04$.

For light tirts the padding liquor should be reduced to the spec. grav. 1.01. The dye in either case may be brightened by wincing through a weak solution of chloride of lime.

Nitrate of iron diffused through a body of water may be also used for padding, with alternate washings in water, and a final wincing in a weak alkaline ley.

With a stronger solution, similar to the first, the boot-top color is given.

2. The bronze or solitaire.

The goods are to be padded in a solution of the sulphate or muriate of manganese, of a strength proportional to the shade desired, dried in the hot-flue, and then raised by wincing them in a boiling-hot caustic ley, of spec. grav. $1 \cdot 08$, and next through a weak solution of chloride of lime, or soda. They are afterwards rinsed. Instead of passing them through the chloride, they may be merely exposed to the air till , he manganese attracts oxygen, then rinsed and dried.

When the manganese solution has the density of 1.027 , it gives a light shade; at the density of $1 \cdot 06$, a shade of moderate depth, and at $1 \cdot 12$ a dark tint.

The texture of the stuff is apt to be injured during the oxydation of the manganese.

3. Carmeli'e is obtained by padding in a mixture of muriate or sulphate of manganese and acetate of iron, then proceeding as above.

4. Copper green is given by padding in a mixed solution of sulphate and acetate of copper with a little glue, drying in the hot-flue, and next day padding in a caustic ley of spec. grav. $1 \cdot 05$. The goods are then rinsed, and padded through a solution made with 8 ounces of arsenious acid combined with 4 ounces of potash diluted with 2 gallons of water. They are finally rinsed and dried.

5. Olive and cinnamon colors are given by padding through mixed solutions of the acetate of iron and sulphate of copper; drying, and padding in a caustic ley of spec. grav. 1:05.

6. Green and solitaire form a pleasing umber, or hellebore shade, which may be obtained by padding through a mixed solution of manganese and aceto-sulphate of copper, and raising the shades as above prescribed.

7. Chrome yellow.

$\mathrm{Pad}$ in a solution of bichromate of potash containing 8 ounces of it to the gallon of water; then dry with moderate heat, and pad in a solution of acetate or nitrate of lead, containing 6 or 8 ounces in the gallon of water; wash, and dry. Or we may pad first in a solution of acetate of lead containing a little glue; dry, and pad in solution of bichromate of potash. Then rinse. The last process is apt to occasion cloudiness. To obtain a light lemon tint, we must pad in a solution of acetate of lead of double the above strength, or 16 ounces to the gallon, then wince the pieces through weak milk of lime, rinse, pad through bichromate of potash, rinse and dry.

8. Chrome orange.

Pad through a mixed solution of the subacetate and acetate of lead, three times in succession, and dry in the hot-flue; then wince for ten minutes through weak milk of lime; rinse; wince for a quarter of an hour in a warm solution of bichromate of potash; and finally raise the color by wincing the goods through hot lime-water.

9. Prussian blue.

Pad in the preceding chamois liquor of the spec. grav. 1.007; dry in the hot-flue; wince well in chalky water at $160^{\circ} \mathrm{F}$., and then dye by wincing in the following liquor :-

Dissolve 5 ounces of prussiate of potash, in 25 gallons of water heated to $90^{\circ}$ or $100^{\circ}$, adding 2 ounces of sulphuric acid; afterwards rinse, and brighten in a very dilute sulphuric acid.

10. Green is given by padding goods, previously dyed in the indigo vat, in a solution of acetate of lead containing a little glue; and then padding them in a warm solution of bichromate of potash ; finally rinsing and drying.

III. Resist pastes or reserves; these are subservient to the cold indigo vat, and they may be distributed under four heads; 1 . fat reserves; 2 . reserves with bases of metallic salts; 3 . colored reserves capable of assuming different tints in the dyeing; 4 . reserves with mordants, for the cloth to be afterwards subjected to a dyeing bath, whereby variously colored figures are brought up on a blue ground, so as to resemble the mineral called lazulite; whence the name lupis or lapis lazuli.

1. The fatty resists are employed in the printing of silk; which see infra. 
2. With regard to reserves the following general observations may be made. After printing-on the paste, the goods must be hung up in a chamber, rather humid than toodry, and left there for a certain time, more or less, according to the nature of the reserve. In dipping them into the blue vat, if the reserve be too dry, it is apt to swell, scale off, and vitiate the pattern. This accident is liable to happen also when the vat is deficient in lime, especially with deep blues.

1. Simple whice resist paste for a full body of blue.

Take 1 gallon of water, in which are to be dissolved,

1 pound of binacetate of copper (distilled verdigris), and $3 \mathrm{lbs}$. of sulphate of copper.

This solution is to be thickened with

$2 \mathrm{lbs}$. of gum senegal, $1 \mathrm{lb}$. of British gum, and $4 \mathrm{lbs}$. of pipe-clay; adding after. wards, 2 ounces of nitrate of copper-as a deliquescent substance.

2. White reserve for light blues.

Take 1 gallon of water, in which dissolve

4 ounces of binacetate of copper,

$1 \mathrm{lb}$. of sulphate of copper; and thicken this solution with

$2 \mathrm{lbs}$. of gum senegal, $1 \mathrm{lb}$. of British gum, and $4 \mathrm{lbs}$. of pipe-clay.

3. White reserve for the cylinder machine.

Take $1 \frac{1}{2}$ gallons of water; in which dissolve

$2 \frac{1}{2} \mathrm{lbs}$. of binacetate of copper,

$10 \mathrm{lbs}$. of sulphate of copper; and add to the solution

$6 \mathrm{lbs}$. of acetate of lead; then thicken with

$10 \mathrm{lbs}$. of gum; adding afterwards $10 \mathrm{lbs}$. of sulphate of lead,

After printing-on this reserve, the goods are to be hung up for two days, then dipped till the proper blue tint be obtained. Finally they must be winced through dilute sulphuric acid to clear up the white, by removing the cupreous tinge.

3. Colored reserves.

1. Chamois reserve.

Take 1 gallon of the chamois bath (No. 1, page 232, at bottom); to which add

8 ounces of nitrate of copper,

24 ditto of muriate of zine; thicken with

6 pounds of pipe-clay, and 3 pounds of gum senegal.

After printing-on this paste, the goods must be hung up for five or six days in a some. what damp room. Then after having dipped them in the vat, they are to be steeped in water for half an hour, and slightly washed. Next wince for half an hour, through water at $100^{\circ} \mathrm{F}$. containing 2 pounds of soda crystals per 30 gallons. Rinse and dry.

2. Chrome yellow reserve.

Take 1 gallon of water; in which dissolve

3 lbs. of nitrate of lead,

$1 \mathrm{lb}$. of binacetate of copper; to the solution, add

$\frac{2}{2}$ lb. of subacetate of lead; and thicken the mixed solution with

3 lbs. of gum.

$6 \mathrm{lbs}$. of pipe-clay. Grind all the ingredients together, and pass through a searce.

After treating the goods as in No. 1, they must be winced for half an hour in a solution containing 5 ounces of bichromate of potash, per piece of calico, and also in a dilute muriatic bath, till the chrome yellow becomes sufficiently bright.

A chrome orange reserve may be made by introducing a larger proportion of subacetate of lead, and passing the reserve printed goods through weak milk of lime, as already prescribed for producing an orange by chrome.

The basis of the resist pastes used at Manchester is sometimes of more complex composition than the above; since, according to the private information I received from an extensive calico printer, they contain china clay (instead of pipe-clay, which often contains iron), strong solution of sulphate of copper, oil, tallow, and soap; the whole incorporated by trituration with heat.

In the Lancashire print-works, a little tartaric acid is added to the nitrate of lead, which prevents the color from taking a dingy cast.

4. Reserves with mordants, or the lazulite style.

1. Black upon a blue ground.

At Manchester the black pattern is printed-on with a mixture of iron liquor and extract of logwood, and the resist paste by the cylinder machine; in France the black is given by the following recipe:-

Take 1 gallon of decoction of galls of spec. grav. 1.04, mixed and boiled into a paste with

14 ounces of flour; into the paste, when nearly cold, there are added,

8 ounces of an acetated peroxyde of iron, made by adding $1 \mathrm{lb}$. acetate of lead to 3

lbs. of nitrate of iron, spec. grav. 1.56.

$\frac{1}{2}$ ounce of gallipoli oil. 
This topical black forms a fast color, and resists the fine blue vat, weak potash ley, bichromate of potash, boiling milk of lime, dunging, and maddering.

The preceding answers best for the block; the following for the cylinder,-

2. Take 1 gallon decoction of galls of spec. grav. $1 \cdot 056$.

18 ounces of flour, mix, boil into a paste, to which, when cool, add

8 ounces of the aceto-nitrate of "iron of the precediug formula, and

1 quart of iron liquor of spec. grav. 1·110.

In Lancashire a little prussiate of potash is sometimes added to nitrate of iron and decoction of logwood; and the goods are after washing, \&c. finished by passing through a weak solution of bichromate of potash. The chromic acid gives depth and permanence to the black dye, being supposed to impart oxygen to the iron, while it does not affeet any of the other colors that may happen to be impressed upon the cloth, as solution of chloride of lime would be apt to do. The solution of the bichromate deepens the spirit purples into blacks, and therefore with such delicate dyes becomes a very valuable application. This interesting fact was communicated to me by an eminent calico-printer in Lancashire.

Having premised the composition of the topical black dye, we are now prepared to apply it in the lazulite style.

1. Black resist.

Take 1 gallon of the above black without the flour,

2 ounces of sulphate of copper,

1 ounce of muriate of ammonia, dissolve and thicken with

4 pounds of pipe-clay and 2 pounds of gum.

Another good formula is the following :-

Take 1 gallon of iron liquor of 1.056 spec. grav.; dissolve in it, 2 ounces of binacetate of copper,

8 ounces of sulphate of copper; and thicken as just described.

2. Puce reserve paste, contains acetate of alumina mixed with the iron liquor.

3. Full red reserve.

Take 1 gallon of acetate of alumina, (made with 50 gallons water, $100 \mathrm{lbs}$. alum, $10 \mathrm{lbs}$. soda crystals, and $100 \mathrm{lbs}$. acetate of lead; the supernatant liquid being of spee. grav. 1.085 ;) dissolve in it

4 ounces of corrosive sublimate; thicken with

2 pounds of gum senegal,

4 pounds of pipe-clay, and mix in 8 ounces of gallipoli oil.

4. Reserve paste for a light red.

Take 1 gallon of the weaker sulpho-acetate of alumina formerly prescribed; dissolve in it 4 ounces of corrosive sublimate; and thicken with

4 pounds of pipe-clay, and 2 pounds of gum; adding to the mixture

8 ounces of oil.

5. Neutral resist paste.

Take 1 gallon of water; in which dissolve,

$3 \frac{1}{4}$ lbs. of binarseniate of potash, and

12 ounces of corrosive sublimate; thicken with

$3 \mathrm{lbs}$. of gum, and $6 \mathrm{lbs}$. of pipe-clay, adding to the paste 16 ounces of oil.

6. Carmelite reserve paste.

Take 1 half gallon of acetate of alumina, spec. grav. 1.014; (see second aluminous mordant, p. 230.)

1 half gallon iron liquor of spec. grav. $1 \cdot 027$; dissolve in them

4 ounces of sulphate of copper, 4 ounces of verdigris, and 1 ounce of nitrate of copper; thicken with

2 lbs. of gum,

4 lbs. of pipe-clay.

7. Neutral reserve paste.

Take 1 gallon of water; dissolve in it,

44 ounces of binarseniate of potash, and

12 ounces of corrosive sublimate; thicken with

3 lbs. of gum,

$6 \mathrm{lbs}$. of pipe-clay,

$16 \mathrm{oz}$. of oil.

To explain fully the manipulation of the lazulite style, we shall suppose that the calcoes are printed with the following reserves, taken in their order :-
1. Black reserve,
2. Full red reserve, No. 3 .
3. Light red reserve, No. 4.
4. Neutral reserve, No. 7.

No. 1. above.

Four days after printing-on these reserves, the goods must be twice dipped in the blue 
vat, ten minutes in and ten minutes out each time; but more dips may be given according to the desired depth of the shade. The cloth must be afterwards rinsed in running water for half an hour. The next process is to remove the paste; which is done by wincing the goods in a bran bath, lowered to $150^{\circ}$, during twenty minutes. They are then winced for five minutes in a bath of water slightly sharpened with vinegar. When well cleansed they are ready for the madder bath. The lapis goods are finally cleared in a bran bath, by exposure on the grass, and a soap boil.

The lazulite style is susceptible of many modifications.

8. Deep blue ground, with light blue, carmelite, and white figures.

1. Print-on the white reserve, No. 1.

2. Dip in the strongest blue vat; rinse and dry.

3. Ground-in with the block, the carmelite reserve (containing the mixed acetates of iron and alumina.)

4. Ground-in the neutral reserve.

э. Dip for the light blue; rinse.

6. Dung, dye, and clear, as above.

By varying the proportions of the reserve mordants, and the dye-stuffs, as madáct, quercitron, \&c. a great variety of effects may be produced.

9. Deep green ground, with buff and white figures.

1. Print-on the white reserve.

2. Dip in the blue vat; rinse and dry.

3. Pad in the buff liquor, as formerly prescribed.

4. Ground in upon the buff spots, the discharge No. 2, presently to be described.

5. Wash away the paste in chalky water.

6. Wince through a boiling alkaline ley, to raise the buff iron color.

IV. The Discharge style; first, of simple discharges.

1. Discharge for block printing.

Take 1 gallon of lemon or lime-juice, of spec. grav. 1.09, in which dissolve

1 pound of tartaric acid,

1 pound of oxalic acid, and thicken the solution with

4 pounds of pipe or china clay, and 2 pounds of pulverized gum; as soon as the gum is dissolved, the mixture must be put through a searce.

2. Another discharge is made of half the above acid strength.

3. A third with one half of the solid acids of the second.

4. Take 1 gallon of water, in which dissolve with heat

1 pound of cream of tartar, adding, to facilitate the solution,

1 pound of warm sulphuric acid of spec. grav. 1.7674; after 24 hours mix

$4 \mathrm{lbs}$. of pipe or China clay, and three lbs. of gum, with the decanted clear liquor.

In some cases British gum is used alone, as a thickener.

5. Discharge for the cylinder machine.

Take 1 gallon of lime-juice, of spec. grav. 1.085; dissolve in it

3 pounds of tartaric acid, and one pound of oxalic acid; thicken with

6 pounds of gum senegal, or 5 pounds of British gum.

6, 7. A stronger and weaker discharge is made of the same materials; and one is made without the tartaric acid.

Second; combination of discharges with mordants.

1. Black, red, lilach, and white figures upon an olive ground.

The olive being given in a madder bath, and the ground well whitened (see MADDER), the cloth is padded in a weak buff mordant; and upon the parts that are to remain white, the weakest simple discharge No. 3 is printed-on by the cylinder; (in some works the discharge paste is applied and made dry before padding through the iron liquor;) the goods are cleared of the paste in a tepid chalky water, then dyed in a quercitron bath, containing a little glue, and cleared in a bran bath.

Discharge mordants upon mordants may be regarded as a beautiful modification of the preceding style. Example.

$A$ violet ground or impression, with red and white.

1. Pad with an acetate of iron of 1.004 ; or print-on with the cylinder, iron liquor of 1.027 thickened with British gum.

2. Print-on a red mordant, strongly acidulated with lime-juice of $1 \cdot 226$.

3. Ground in the discharge No. 2; dry.

4. Clear off the paste in chalky water.

5. Dung, madder, and brighten.

6. Ground-in the topical colors at pleasure.

V. China blues.

Take 16 pounds of coarsely ground indigo, and

4 pounds of sulphuret of arsenic; dissolve 22 pounds of sulphate of iron in 6 gallons of water; introduce these three matters into the indigo mill, and grind them for 
three days. If it be wished to have a thickened blue, this mixture must have pourdeu gum added to it; but if not, 5 gallons of water are added. This color may be called blue No. 1.

The following table exhibits the different gradations of China blue:-

\begin{tabular}{|c|c|c|}
\hline Course. & $\begin{array}{c}\text { Quantity by measure of } \\
\text { No. 1. }\end{array}$ & $\begin{array}{c}\text { Quantity by measure of } \\
\text { water or muciage. }\end{array}$ \\
\cline { 1 - 3 } No. 1 & 1 & 0 \\
2 & 11 & 1 \\
3 & 10 & 2 \\
4 & 8 & 4 \\
5 & 6 & 6 \\
6 & 4 & 8 \\
7 & 2 & 10 \\
8 & 2 & 12 \\
9 & 2 & 14 \\
10 & 2 & 16 \\
11 & 2 & 18 \\
12 & 2 & 20 \\
\hline
\end{tabular}

I shall now give examples of working this style by the block and cylinder :Impression of a single blue with small dots.

For the block, blue No. 5, thickened with starch.

For the cylinder, No. 4, thickened with gum.

First blue, No. 4, with starch.

Impression of two different blues with the block.

Second blue, No. 9, with gum.

First blue, No. 5, with starch.

Impression of three blues with the block.

Second blue, No. 7, with starch.

Third blue, No. 10, with gum.

After printing-on the blues, the pieces are hung up for two days, in a dry and aury place, but not too dry ; then they are dipped as follows :-Three vats are mounted, which snay be distinguished by the numbers $1,2,3$.

No. 1. 300 pounds of lime to 1,800 gallons of water.

No. 2. Solution of sulphate of iron of spec. grav. 1.048.

3 . Solution of caustic soda of spec. grav. 1.055 ; made from soda crystals, quicklime, and water, as usual.

The pieces being suspended on the frames, are to be dipped in the first vat, and left in it ten minutes; then withdrawn, drained for five minutes; next plunged into the second vat for ten minutes, and drained also for five, \&c. These operations will be most intelligible when put into the form of a table :-

Dip in the 1st vat. During 10 minutes. Drain during 5 minutes.

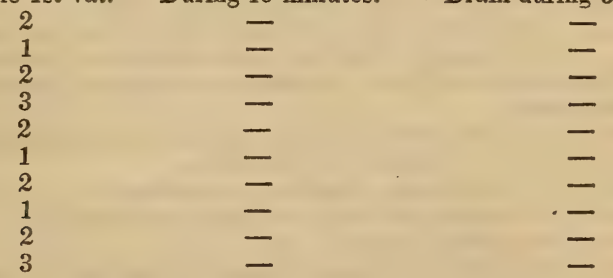

In the dipping of China blues, care should be taken to swing the frames during the operation; and when the last dip is given, the piece is to be plunged upon its frame into a fourth vat, containing dilute sulphuric acid of spec. grav. 1.027. This immersion is for the purpose of removing the oxyde of iron, deposited upon the calico in the alternate passages through the sulphate of iron and lime vats. They are then rinsed an hour in running water, and finally brightened in the above dilute sulphuric acid, slightly tepid. Sometimes they are subjected to a soap bath, at the temperature of $120^{\circ}$. By the addition of nitrate of lead to the indigo vat, the blue becomes more lively. Some use the roller dyeing apparatus for running the pieces through the respective baths instead of the square frames. (See Wincing.) But the frame-dip gives the most evenly dyes, and preserves the vats in good condition for a much longer time. 
The various phenomena which occur in the dipping of China blues are not difficult of explanation with the lights of modern chemistry. We have, on the one hand, indigo and sulphate of iron alternately applied to the cloth; by dipping it into the lime, the blue is deoxydized, because a film of the sulphate of iron is decomposed, and protoxyde of iron comes forth to seize the oxygen of the indigo, to make it yellow-green, and soluble, at the same time, in lime-water. Then, it penetrates into the heart of the fibres, and, on exposure to air, absorbs oxygen, so as to become insoluble and fixed within their pores. On dipping the calico into the second vat of sulphate of iron, a layer of oxyde is formed upon its whole surface, which oxyde exercises an action only upon those parts that are covered with indigo, and deoxydizes a portion of it; thus rendering a second dose soluble by the intervention of the second dip in the lime-bath. Hence we see that while these alternate transitions go on, the same series of deoxydizement, solution, and re-oxydizement recurs; causing a progressively increasing fixation of indigo within the fibres of the cotton. A deposite of sulphate of lime and oxyde of iron necessarily falls upon the cloth, for which reason the frame should be shaken in ti.e lime-water vat, to detach the sulphate; but, on the contrary, it should be held motionless in the copperas bath, to favor the deposition of as much protoxyde upon it as possible. These circumstances serve to account for the various accidents which sometimes befall the China blue process. Thus the blues sometimes scale off, which may proceed from one of two causes:-1. If the goods are too dry before being dipped, the color swells, and comes off in the vats, carrying along with it more or less of indigo. 2. If the quantity of sulphate of lime formed upon the cloth be considerable, the crust will fall off, and take with it more or less of the blue; whence arise inequalities in the impression. The influence of temperature is important; when it falls too low, the colors take a gray cast. In this case it should be raised with steam.

VI. The decoloring or enlevage style; not by the removal of the mordant, but the destruction of the dye. The acid, which is here mixed with the discharge paste, is intended to combine with the base of the chloride, and set the chlorine free to act upon the color. Among the topical colors for this style are the following :-

1. Black.-Take one gallon of iron liquor of spec. grav. 1·086.

One pound of starch; boil together, and while the paste is hot, dissolve in it

One pound of tartaric acid in powder; and when cold, add

Two pounds of Prussian blue, prepared with muriatic acid, see p. 232.

Two ounces of lamp black, with four ounces of oil.

2. White discharge.-Take one gallon of water, in which dissolve

One pound and a half of oxalic acid,

Three pounds of tartaric acid; add

One gallon of lime-juice of spec. grav. 1.22; and thicken with

Twelve pounds of pipe clay, and six pounds of gum.

\section{Chrome-green discharge.-}

Take one gallon of water, thicken with 18 ounces of starch; boil and dissolve in the hot paste;

Two pounds and a half of powdered nitrate of lead,

One pound and a half of tartaric acid,

Two pounds of Prussian blue, as above.

4. Blue discharge.-Take one gallon of water, thicken with

18 ounces of gum; while the boiled paste is hot, dissolve in it

Two pounds of tartaric acid, and mix one pound of Prussian blue.

5. Chrome-yellow discharge.-This is the same as the chrome-green given above, but without the Prussian blue.

6. A white discharge on a blue ground requires the above white discharge to be strengtr.

ened with 8 ounces of strong sulphuric acid, per gallon.

7. White discharge for Turkey red needs to be very strong.

Take one gallon of lime-juice of sp. grav. 1.086; dissolve in it

Five pounds of tartaric acid; thicken with

Eight pounds of pipe-clay, four pounds of gum; then dissolve in the mixture

Three pounds of muriate of tin in crystals; and add, finally,

Twenty-four ounces of sulphuric acid.

8. Yellow discharge for Turkey red.-

Take one gallon of lime-juice of spec. grav. 1.086 ; in which dissolve

Four pounds of tartaric acid,

Four pounds of nitrate of lead; thicken the solution with

Six pounds of pipe-clay, and three pounds of gum.

9. For green discharge, add to the preceding 24 ounces of Prussian hlue, as above.

The decoloring or chlo;ine bath is usualiy formed of wood lined with lead, and

has an area of ahout 5 feet square, with a dejth of 6 teet. A square iramc, mounted with a hurizontal scries of rullers at top and bottom, may be let down by cords, at 
pleasta ${ }^{\circ}$, into the cistern. The pieces are introduced and guided in a serpentine path, round the upper and lower rollers alternately, by a cord.

This bath is filled with a solution of chloride of lime, of the spec. grav. 1.045, whose decoloring strength is $65^{\circ}$ by Gay Lussac's indigo chlorometer. It ought to be made turbid by stirring before putting in the goods, which should occupy three minutes in their passage. The piece is drawn through by a pair of squeezer cylinders at the end of the trough, opposite to that at which the piece enters. With black, white, and blue impressions of all shades, the goods are floated in a stream of water for an hour; then rinsed and dried. When there is yellow or green, the pieces must be steeped in water, then merely washed by the wince, and passed through solution of bichromate of potash, containing from 3 to 5 ounces of the salt per piece. Here the pieces are winced during 15 or 20 minutes, rinsed, and next passed through dilute muriatic acid to clear the ground; then rinsed and dried.

Discharge by the intervention of the chromic acid.

After having dipped the pieces to the desired shade, they are padded in a solution of bichromate of potash; dried in the shade without heat; and then printed with the following mordant :-

Take 1 gallon of water; dissolve in it

2 pounds of oxalic and 1 pound of tartaric acid; thicken with

6 pounds of pipe-clay, and 3 pounds of gum; lastly, add

8 ounces of muriatic acid.

After the impression, the pieces are winced in chalky water, at $120^{\circ} \mathrm{F}$, then washed, and passed through a dilute sulphuric acid.

M. Daniel Kœchlin, of Mulhausen, the author of this very ingenious process, considers the action of the bichromate here as being analogous to that of the alkaline chlorides. At the moment that the block applies the preceding discharge to the bichromate dye, there is a sudden decoloration, and a production of a peculiar odor.

The pieces padded with the bichromate must be dried at a moderate temperature, and in the shade. Whenever watery solutions of chromate of potash and tartaric acid are mixed, an effervescence takes place, during which the mixture possesses the power of destroying vegetable colors. This property lasts no longer than the effervescence.

VII. Steam colors.-This style combines a degree of brilliancy with solidity of color, which can hardly be obtained in any other way except by the chints dyes. The steam apparatus employed for fixing colors upon goods, may be distributed under five heads:-1. the column; 2. the lantern; 3 . the cask; 4 . the steam-chest; and, 5. the chamber.

The column is what is most generally used in this country. It is a hollow cylinder of copper, from three to five inches in diameter, and about 44 inches long, perforated over its whole surface with holes of about one sixteenth of an inch, placed about a quarter of an inch asunder. A circular plate, about 9 inches diameter, is soldered to the lower end of the column, destined to prevent the coil of cloth from sliding down off the cylinder. The lower end of the column terminates in a pipe, mounted with a stop-cock for regulating the admission of steam from the main steamboiler of the factory. In some cases, the pipe fixed to the lower surface of the disc is made tapering, and fits into a conical socket, in a strong iron or copper box, fixed to a solid pedestal; the steam pipe enters into one side of that box, and is provided, of course, with a stop-cock. The condensed water of the column falls down into that chest, and may be let off by a descending tube and a stop-cock. In other forms of the column, the conical junction pipe is at its top, and fits there into an inverted socket connected with a steam chest, while the bottom has a very small tubular outlet, so that the steam may be exposed to a certain pressure in the column, when it is incased with cloth.

The pieces, after being printed with the topical colors presently to be described, and dried, are lapped round this column, but not in immediate contact with it ; for the copper cylinder is first enveloped in a few coils of blanket stuff; then with several coils of white calico; next with the severa] pieces of the printed goods, stitched endwise ; and lastly, with an outward mantle of white calico. In the course of the lapping and unlapping of such a length of webs, the cylinder is laid in a horizontal frame, in which it is made to revolve. In the act of steaming, however, it is fixed upright, by one or the methods above described. The steaming lasts for 20 or 30 minutes, according to the nature of the dyes; those which contain much solution of tin admit of less steaming. Whenever the steam is shut off, the goods must be immediately uncoiled, to prevent the chance of any aqueous condensation. I was much surprised, at first, on finding the unrolled pieces to be free from damp, and requiring only to be exposed for a few minutew in the air, to appear perfectly dry. Were water condensed during the process, it would be apt to make the colors run.

Steam ciars are ari toplcal, though, for many of them, the riuces ars previnisl: 
padded with mordants of various kinds. Some manufacturers run the goods before printing them through a weak solution of the perchloride of tin, with the view of brightening all the colors subseguently applied or raised upon them. I shall now illustrate steam calico-printing by some examples, kindly furnished me by a practical printer near Manchester, who conducts a great business with remarkable success.

Steam blue.-Prussiate of potash, tartaric acid, and a little sulphuric acid, are dissolved in water, and thickened with starch; then applied by the çlinder, dried at a moderate heat, and steamed for 25 minutes. They are rinsed and dried after the steaming. The tartaric acid, at a high temperature, decomposes here a portion of the ierrocyanic acid, and fixes the remaining ferrocyanate of iron (Prussian blue) in the fibre of the cloth. The ground may have been previously padded and dyed; the acids will remove the mordant from the points to which the above paste has been applied, and bring out a bright blue upon them.

Steam purple.-This topical color is made by digesting acetate of alumina upon ground logwood with heat; straining, thickening with gum senegal, and applying the paste by the cylinder machine.

Sleam pink.-A decoction of Brazil-wood with a small quantity of the solution of muriate of tin, called, at Manchester, new tin crystals, ${ }^{*}$ and a little nitrate of copper to assist in fixing the color; properly thickened, dried, and steamed for not more than 20 minutes, on account of the corrosive action of muriate of tin when the heat is too strong.

Cochineal pink.-Acetate of alumina is mixed with decoction of cochineal, a little tartaric acid and solution of tin; then thickened with starch, dried, and steamed.

Steam broun.-A mixed infusion of logwood, cochineal, and Persian berries, with cream of tartar, alum (or acetate of alumina), and a little tartaric acid, thickened, dried, and steamed.

Green, blue, chocolate, with white ground, by steam.-Prussiate of potash and tartaric acid, thickened, for the blue; the same mixture with berry-liquor and acetate of alumina, thickened, for the green; extract of logwood with acetate of alumina and cream of tartar, thickened, for the chocolate. These three topical colors are applied at once by the three-color cylinder machine; dried and steamed. Though greens are fixed by the steam, their color is much improved by passing the cloth through solution of bichromate of potash.

In France, solution of tin is much used for steam colors.

VIII. Spirit or fancy colors.- These all owe their vivacity, as well as the moderate degree of permanency they possess, to their tin mordant. After printing-on the topical color, the goods must be dried at a gentle heat, and passed merely through the rinsing machine. Purple, brown, or chocolate, red, green, yellow, hlue, and white discharge; any five of these are printed on at once by the five-color cylinder machine. See Rinsing MACHINE.

Chocolate is given by extract of Brazil-wood, extract of logwood, nitro-muriate of tin, with a little nitrate of copper : all mixed, thickened, and merely printed-on.

Red, by extract of Brazil-wood and tin, with a little nitrate of copper.

Green, by prussiate of potash, with muriate of tin and acetate of lead, dissolved, thickened, and printed-on.

The goods after rinsing must be passed through solution of bichromate of potash, to convert the Prussian blue color into green, by the formation of chrome yellow upon it.

Blue.-Prussian blue ground up with solution (nitromuriate) of tin; thickened, \&c.

Yellow. - Nitrate of lead dissolved in solution of tartaric acid, thickened, tenderly dried, passed through the bichromate vat or padding machine, washed and dried.

This yellow is pretty fast; though topical, it can hardly, therefore, be called a fancy color.

When purple is to be inserted instead of the above blue, extract of logwood with tin is used in the place of the Prussian blue. Tartaric acid is a useful addition to tin in brightening fancy colors.

Chocolate. - A good topical chocolate is made by digesting logwood with liquid acetate of alumina, adding a little cream of tartar to the infusion; thickening, applying by the cylinder, drying, washing, then passing through solution of bichromate of potash, which serves to darken and fix the color.

I shall conclude my account of the printing of cotton goods with some miscellaneous formulæ, which were given me by skilful calico-printers in Lancashire.

Prussian blue is prepared for topical printing by grinding it in a handmill, like that for grinding pepper or coffee, and triturating the powder with solution of muriate of tin.

Green.-The deoxydized indigo vat liquor is mixed with a little pearlash, and thickened with gum. This is applied by the cylinder or block to goods previousiy

* This preparation is made by addirg $3 \mathrm{lbs}$. of sal ammoniac to 1 gallon of solution of tin (see ScARLET DYE, and TIN), evaporating, and crystalizing. The sal ammoniac seems to counteract the separation 0 : the tin by peroxydizement. 
padded with nitrate of lead; the goods, after being dried, are passed through milky limewater, rinsed, and then winced or padded through the bichromate of potash bath.

Another green.-Nitrate of lead, prussiate of potash, and tartaric acid, dissolved, and mixed with a little sulphate, nitrate, and muriate of iron; this mixture is either thickened for cylinder printing, or used in its liquid state in the padding trough. The goods subjected to une of these two processes are dried, padded in weak solution of carbonate of potash, which serves to precipitate the oxyde of lead from the nitrate; they are finally padded with bichromate of potash, which induces a yellow upon the blue, constituting a green color of any desired tint, according to the proportion of the materials.

Chocolate and black. with white discharge; a fast color.-The cloth is padded with ace. tate of alumina, and dried in the hot-llue; it is then passed through a two-color machine, the one cylinder of which prints-on lime-juice discharge, thickened with gum senegal; the other a black topical dye (made with logwood extract and iron liquor). The cloths are now hung up to be aired during a week, after which they are dunged, and dyed up with madder, fustic, and quercitron bark, heated, with steam in the bath.

Blue, white, and olive or chocolale.-1. Pad with the aluminous mordant; 2. Apply thickened lemon-juice for discharge by the cylinder; 3 . Dung the goods after they are thoroughly dried; 4 . Pass them through the bath of madder, fustic, and quercitron, which dye a brown ground, and leave the discharge points white; then print-on a reserve paste of China clay and gum with sulphate of copper; dry, dip in the blue vat, which will communicate an olive tint to the brown ground; or a chocolate, if madder alone had been used.

When a black ground is desired, with white figures, the acid discharge paste should be printed-on by the cylinder, and dried before the piece is padded in the iron liquor. By following this plan the whites are much purer than when the iron is first applied.

Green, black, white. - The black is first printed-on by a mixture of iron liquor, and infusion (not decoction) of logwood; then resist or reserve paste is applied by the block, and dried; after which the goods are blued in the indigo vat, rinsed, dried, passed through solution of acetate of lead; next, through milky lime-water; lastly, through a very strong solution of bichromate of potash.

Turkey red, black, yellow.-Upon Turkey red cloth, print with a strong solution of tartaric acid, mixed with solution of nitrate of lead, thickened with gum; dry. The cloth is now passed through the chloride of lime bath, washed, and chromed. Lastly, the black is printed-on by the block as above, with iron liquor and logwood.

Black gronind dotted white, with red or pink and black figures.-1. Print-on the lime-juice discharge-paste by the cylinder; dry; 2. Then pad with iron liquor, containing a little acetate of alumina, and hang up the goods for a few days to fix the iron: 3. Dye in a logwood bath to which a little madder has been added; clear with bran. The red or pink is now put in by the block, with a mixture of extract of Brazil-wood, nitromuriate of tin, and nitrate of copper, as prescribed in a preceding formula.

Orange or brown; black; white; pink.-The black is topical, as above; it is printedon, as also the lemon-juice discharge and red mordant, with muriate of tin (both thickened), by the three-color machine. Then, after drying the cloth, a single-cylinder machine is made to apply in diagonal lines to it a mixture of acetate of iron and alumina. The cloth, being dried and dunged, is next dyed in a bath of quercitron, madder, and fustic.

Here the orange is the result of the mordant of tin and alumina; the brown, of the alumina and iron; white, of the citric acid discharge. The tin mordant, wherever it has been applied, resists the weaker mordant impressed in the diagonal lines. The pink is blocked-on at the end.

Orange brown, or aventurine; black and white.-The topical black (as above) and discharge lemon-juice, are printed-on by the two-color machine; then the cloth is subjected to the diagonal line cylinder, supplied with the alumino-iron mordant. The cloth is dried, dunged, and dyed in a bath of bark, madder, and fustic.

The manganese or solitaire ground admits of a great variety of figures being easily brought upon it, because almost every acidulous mordant will dissolve the oxyde of manganese from the spot to which it is applied, and insert its own base in its place; and of course, by dyeing such mordanted goods in various baths, any variety of colored designs may be produced. Thus, if the paste of nitrate of lead and tartaric acid solution be applied, and the goods after drying be passed first through lime-water, and then through a chrome bath, bright yellow spots will be made to appear upon the bronze ground.

Manganese bronze, buff and green; all metallic colors.-Pad-on the manganese solution, and dry; apply the aceto-sulphate of iron, of spec. grav. 1.02, and Scheele's green (both properly thickened), by the two-color machine. The goods are next to be dried, and padded through a cold caustic ley of spec. grav. $1 \cdot 086$. They are then 
rinsed, and passed through a weak solution of chloride of lime, to raise the bronze, again rinsed, and passed through a solution of arsenious acid to raise the green.

Scheele's green for the calico-printer is made as follows :-

Take 1 gallon of water, in which dissolve with heat,

5 pounds of sulphate of copper, and 1 pound of verdigris. When the two salts are dissolved, remove the kettle from the fire, and put into it 1 quart of solution of nitrate of copper, and 5 pounds of acetate of lead. Stir the mixture to facilitate the decomposition, and allow the pigment to subside.

It must be thickened with $2 \frac{1}{2} \mathrm{lbs}$. of gum per gallon, for pencilling; or $12 \mathrm{oz}$. of starch for the block. The goods printed with this paste are to be winced through a caustic ley, till a fine sky-blue be produced; then washed well and rinsed. They are now to be passed through water, containing from half an ounce to an ounce of white arsenic per piece; 4 turns are sufficient; if it be too long immersed it will take a yellow tint.

Catechu has been considerably employed by calico-printers of late years, as it affords a fine permanent substantive brown, of the shade called carmelite by the French. The following formula will exemplify its mode of application :-

Take 1 gallon of water;

1 pound of catechu in fine powder; reduce by boiling to half a gallon, pass the decoction through a fine sieve, and dissolve in it 4 ounces of verdigris; allow it then to cool, and thicken the solution with 5 ounces of starch; while the paste is hot, dissolve in it $\mathbf{5}$ ounces of pulverized muriate of ammonia.

Print-on this paste, dry, and wash. It is a fast color.

I shall subjoin the prescriptions for two fancy cochineal printing colors.

Amaranth by cochineal.-Pad the pieces in the aluminous mordant of spec. grav. 1.027, page 230.

Dry in the hot flue; and after hanging up the goods during 3 days, wince well through chalky water, and then dye, as follows:-

For each piece of 28 or 30 yards, 8 ounces of cochineal are to be made into a decoction of 2 gallons in bulk, which is to be poured into a kettle with a decoction of 3 ounces of galls, and with two ounces of bran. The pieces are to be entered and winced as in the madder bath, during two hours and a half; then washed in the dash wheel. On mixing with the amaranth bath a certain quantity of logwood, very beautiful lilachs and violets may be obtained.

Mixture of quercitron and cochineal.-Pad in the aluminous mordant, and dye with 2 lbs. of quercitron, and 4 ounces of cochineal, when a capuchin color will be obtained. If we pad with the following mordant, viz., 1 gallon of acetate of alumina of 1.056 spec. grav., and 1 of iron liquor of 1.02 spec. grav., and dye with 1 pound of quercitron, and 1 ounce of cochineal, we shall obtain a shade like boot-tops, of extreme vivacity.

Two ounces of cochineal will print a long piece of calico with rich pink figures, having acetate of alumina for a mordant. As the ground is hardly tinged by the dye, it neither needs nor admits of much clearing.

I have already mentioned that goods are sometimes padded with solution of perchloride of tin before printing-on them the steam colors, whereby they acquire both permanence and vivacity. I have also stated that the salts of tin at a high temperature are apt to corrode the fibre of the stuff, and therefore must be used with discretion. This danger is greatly lessened by adding to the perchloride of tin a sufficient quantity of caustic potash ley to form a stannate of potash. The goods are padded through this substance, diluted with water, dried with a moderate heat, and then immersed in very dilute sulphuric acid, which saturates the potash, and precipitates the tin oxyde within the pores of the cloth. Calico thus prepared affords brilliant and permanent colors by the steam pro cess, above described.

Printing of silks or woollen stuffs, such as merinoes and mousselin de laine, as also of mixed stuffs of silk and wool, such as chalys. - All these prints are applied, not by the cylinder but the block, and are fixed by the application of steam in one of four ways; 1. By the lantern; 2. By the cask; 3. By the chest; or, 4. By the chamber.

1. By the lantern.-In this mode of exposure to steam, the goods are stretched upon a frame; and therefore the apparatus may be described under two heads; the lantern and the frame. The former is made of copper, in the shape of a box A B C D E, fig. 238, open below, and with a sloping roof above, to facilitate the trickling down of the water condensed upon the walls. The sides в C D E are $4 \frac{1}{2}$ feet high, 6 feet long, and 4 feet wide. The distance of the point $\mathrm{A}$ from the line $\mathrm{E} \mathrm{B}$ is 2 feet. At $\mathrm{F}$ is a brass socket, which may be stopped with a cork; and there is a similar one at the other side. This kind of penthouse may be raised by means of a pulley with cords fixed to the four angles of the roof E B; and it rests upon the table G H, a little larger than the area of the box, which stands upon the four feet $\mathbf{I}$ K. Round the borders of the table there is a triangular groove $a b$, for receiving the lower edges of the box, and it is stuffed steamtight with lists of cloth. Through the centre of the table, the two-inch steam pipe $\boldsymbol{N}$ 
passes; it is surmounted with a hemispherical rose pierced with numerous holes for the equal distribution of the steam. Right above it, a disc $\mathrm{N}$ is placed upon fomr feet.

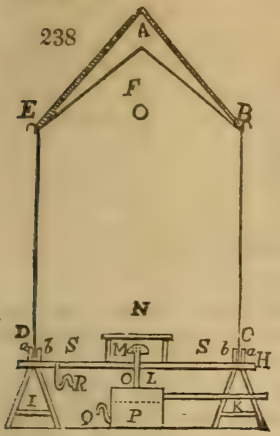

The tube $\mathrm{L}$ communicates with a box $\mathbf{P}$, which has a syphon $Q$ to let off the condensed water. At the upper part of this box the tube $\boldsymbol{L}$ terminates which brings the steam. The little table G $\mathbf{H}$ slopes towards the part $G$, where the syphon $R$ is placed for drawing off the water.

The frame has such dimensions, that it may stand in the four corners of the table at $\mathrm{s} s$, as pointed out by the dotted lines. The second part embraces an open square frame, which is formed by spars of wood 2 inches square, mortised together; and is 3 feet 8 inches wide, 5 feet 8 inches long, and 4 feet 3 inches high; it is strengthened with cross bars. Upon the two sides of its breadth, two rows of round brass hooks are placed, about half an inch apart; they are soldered to a copper plate fixed to uprights by means of screws.

Before hanging up the goods, a piece of cloth 3 feet 8 inches long, and 4 feet wide, is placed upon the row of hooks; and 3 feet of it are left hanging out.

One foot within, the hooks pass through the cloth. A similar one is fitted to the other side. This cloth is intended to cover the goods hung upon the hooks; and it is kept straight by resting upon strings. The pieces are altached zig-zag from one hook to another. When the frame is filled, the bag is put within the cloths; it has the same rectangular shape as the frame. The pieces are in this way all incased in the cloth; a bit of it being also put beneath to prevent moisture affecting that part.

When shawls are framed, they are attached with pins; and if they be too large, they are doubled back to back, with the fringes at top.

These arrangements being made, the frame is set upon the table, the penthouse is placed over it, and the steam is admitted during from 35 to 45 minutes, according to circumstances. The orifice $F$ is opened at first to let the air escape, and when it begins to discharge steam it is stopped. The frame is taken out at the proper time, the bag is removed, the cloths are lifted off, and the goods are spread out for airing. Three frames and six bags are required for a constant succession of work. The above apparatus is particularly suitable for silks.

2. The drum. - This is the most simple mode of steaming. The apparatus is a drum of white wood, 2 inches thick, fig. 239 ; the bottom is pierced with a hole which admits the steam-pipe $F$, terminating in a perforated rose. Four inches from the bottom there is a canvass partition $\mathbf{E}$, intended to stop any drops of water projected from the tube $\mathbf{F}$, and also to separate the condensed water from the body of the apparatus. The drum is covered in by a wooden head $\mathrm{H}$, under which the goods are placed. It is made fast either

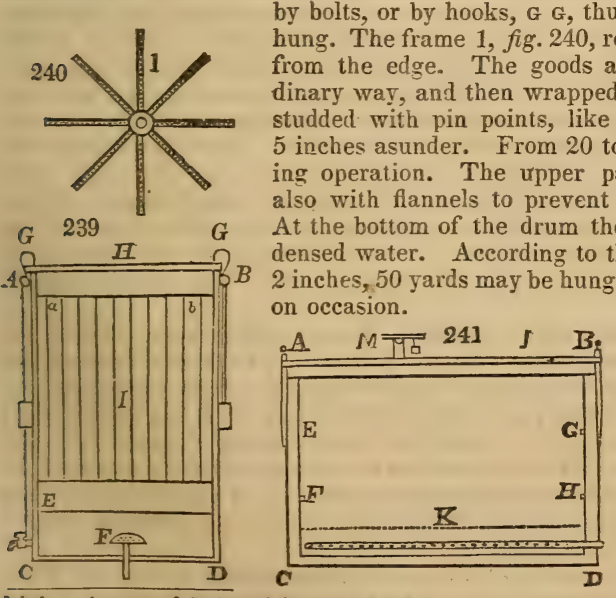
, thus $\mathbb{2}$, to which weighted cords are hung. The frame 1 , fig. 240, rests upon a hoop, $a$ a, a few inches from the edge. The goods are hung upon the frame in the ordinary way, and then wrapped round with flannel. The frame is studded with pin points, like that of the indigo vat, fixed about 5 inches asunder. From 20 to 30 minutes suffice for one steaming operation. The upper part of the frame must be covered also with flannels to prevent the deposition of moisture upon it. At the bottom of the drum there is a stopcock to let off the condensed water. According to the size of the figure, which is 3 feet 2 inches, 50 yards may be hung up single; but they may be doubled occasion.

3. The box.-This steaming apparatus is convenient from the large quantity of goods admissible at a time: it answers best for woollen stuffs. From 12 to 16 pieces, of 36 yards each, may be operated upon at once; and from 240 to 260 shawls. It is formed of a deal box, A B C D, fig. 241, 4 feet wide, 6 long, and 3

high; the wood being 4 inches thick. It is closed by a cover of the same substance, $I$, which is made steam-tight at the edges by a list of felt. The lid is fastened down by 5 cross bars of iron, $a$ a $a$ a $a$, which are secured by screws, $c c c c c$, fig. 242. The ends of these cross bars are let into the notches, $b b b b b$, on the edge of the box. The safety valve $M$, fig. 241, is placed upon the lid. For taking off the lid, there are rings at the four 
corners, $d d d d$, bearing cords, $\mathbf{F}$ F F F. These join at the centre into one, which passet over a pulley. Fight inches from the bottom of the box there is a horizontal canvass par
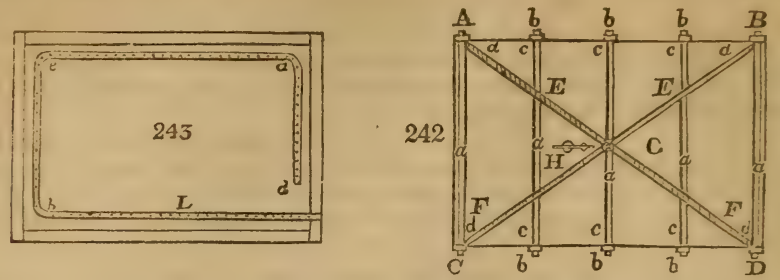

tition, beneath which the steam is discharged from the pipe $\mathbf{L}$, fig. 243. There are twq ledges, $E F G H$, at the sides for receiving the bobbins. Tis tube $L$ runs round the box, as

244
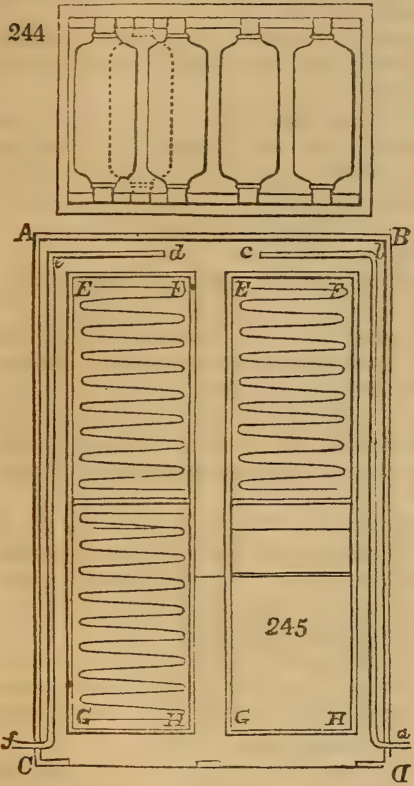
sincwn by the letters $d a e b$ : the end $d$ is shut; but the side and top are perforated with many holes in the direction towards the centre of the box. Fig. 244 shows the arrangement of the lower set of bobbins : that of the upper set is shown by the dotted lines: it is seen to be in an alternate position, one lying between two others. They are formed of pieces of deal 4 inches broad, 1 inch thick, and of a length equal to the width of the box. They are first wrapped round with 5 or 6 turns of doubled flannel or calico: the piece of goods is laid over it upon a table, and then wrapped round. At the end of the piece, several folds of the covering must be put, as also a roll of flannel. The two ends must be slightly tied with packthread. When these flat bobbins are arranged in a box, the steam is let on them, and continued about 45 minutes; it is then shut off, the lid is removed, and the pieces are unrolled.

4. The chamber.-The interior height of the chamber, A B C D, fig. 245, is nine feet, the length 12 feet, and the breadth 9 feet. The steam is introduced into it by two pipes, $a b c, d e f$. Their two ends, $d c$, are shut; but their sides are all along perforated with small holes. The frames E F G H, E F G H, are moveable, and run upon rollers : they are taken out by front doors, which are made of strong planks, shut by sliding in slots, and are secured by strong iron bars and pressure screws. The cross rods, $\mathbf{E}$ F G H, are provided with hooks for hanging up the pieces. There is a safety-valve in the top of this large chamber. The dimensions of the. frame are ten feet long, 3 feet wide, and 7 high. Three feet and a half from the upper part of the frame, a row of hooks is fixed for hanging on a double row of pieces, as show in the figure. Over the frame, woollen blankets are laid to protect it from drops of wa. ter that might fall from the roof of the chamber. When the hooks are two thirds of an inch apart, $2 t$ pieces, of 28 yards each, may be suspended at once. The period of steaming is from 45 to 60 minutes.

Muslins and sills do not require so high a temperature as woollen goods. When the stuffs are padded with color, like merinoes and chalys, they must not be folded together, for fear of stains, which are sometimes occasioned by the column in steam calico-printing, where the end which receives the first impression of the steam is seldom of the same shade as the rest of the roll of goods. The duration of the steaming depends upon the quantity of acid in the mordant, and of saline solution in the topical color; the more of which are present the shorter should be the steaming period. A dry vapor is requisite in all cases; for when it becomes moist, from a feeble supply or external condensation, the goods become streaky or stained by the spreading of the colors.

1. Black figures are given by decoction of logwood thickened with starch, to which a little oxalic acid is added while hot, and, after it is cold, neutralized solution of nitrate of iron.

2. Dark blue for a ground.-Decoction of logwood, and archil thickened with starch ; to which, while the paste is hot, a little soluble Prussian blue is added; and, when it is cold, neutralized nitrate of iron; see supra. 
3. Deep poppy or ponceau color.-Cochineal boiled in starch water, with oxalic acid (or tartaric), and perchloride of tin.

4. Rose.-Cochineal infusion; oxalic acid; perchloride of tin; thickened with gum.

5. Dark amaranth.-Decoctions of archil and cochineal, thickened with starch : to the paste, alum and perchloride of tin are added.

6. Capuchin color.-Quercitron and cochineal thickened with starch; to the paste add oxalic acid and perchloride of tin.

7. Amnotto orange.-Dissolve the annotto in soda ley, of spec. grav. 1.07, at a boiling heat; add aluminate of soda, and thicken with gum.

8. Golden yellow.-Decoction of Persian berries thickened with starch; to which some alum and muriate of tin are added, with a little perchloride of tin and oxalic acid.

9. Lemon yellow.--Persian berries; starch; alum.

10. An ammoniacal solution of cochineal is used for making many violet and mallow colors. It is prepared by infusing cochineal in water of ammonia for 24 hours; then diluting with water, heating to ebullition, and straining.

11. Fine violet is given by ammoniacal cochineal, with alum and oxalic acid; to which a jittle aceto-sulphate of indigo is adced, and gum for thickening. The following blue may be used instead of the solution of indigo. The mallow tint is given by adding a little perchloride of tin to the above formula, and leaving out the blue.

12. Dark blue.-Soluble Prussian blue; tartaric acid; alum; thicken with gum.

13. Emerald green.-One quart of decoction, equivalent to 1 pound of Persian berries; 1 quart of infusion of quercitron, of spec. grav. $1 \cdot 027$; in which dissolve 12 ounces of alum in powder; and add 6 ounces of the following blue bath for greens; thicken with 20 ounces of gum.

14. Blue bath for greens. Half a gallon of water at $140^{\circ} \mathrm{F}$., one pound of soluble Prussian blue, 3 ounces of tartaric acid, and 2 ounces of alum.

I. Prinling of Sills. - 1. Of the madder style. This is one of the most difficult to execute, requiring both much skill and experience. The first step is the removal of the gum. A copper being nearly filled with water, the pieces, tied up in a linen bag, are put into it, with a quarter of a pound of soap for every pound of silk, and are boiled for 3 hours. If the silk be Indian, half an ounce of soda crystals must be added. When the goods are taken out, they are rinsed in the river, then passed through water at $140^{\circ} \mathrm{F}$., holding 8 ounces of crystallized soda in solution, as a scourer. They are next rinsed in cold swater, and steeped in water very faintly acidulated with sulphuric acid, during 4 hours, then rinsed, and dried.

Preparation of Mordants.-1 gallon of boiling water ; 2 pounds of alum ; dissolve :

1 pound of acetate of lead; 4 ounces of sal-ammoniac; 1 of chalk; mix well together; after decomposition and subsidence, draw off clear.

1. Red.--1 gallon of the above mordant, thickened with 14 ounces of starch, and singed with decoction of Brazil-wood. If dark red be wanted, dissolve, in a gallon of the above red, 4 ounces of sulphate of copper.

2. Black. -1 gallon of iron liquor, of $1 \cdot 056$ spec. grav.; thicken with 14 ounces of starch; and dissolve in the hot paste 2 ounces of sulphate of copper.

3. Violet.-Take 1 gallon of iron liquor of 1.04 spec. grav.;

2 ounces of cream of tartar; 2 ounces of nitre; 2 ounces of copperas;

1 ounce of alum: dissolve, and mix the solution with

1 gallon of gum water, containing 6 lbs. of gum.

4. Puce.--Half a gallon of red mordant; half a gallon of iron liquor of 1.07 ;

7 ounces of starch for thickening; color with logwood.

Manipulation of the above colors.-Print-on the black, then the puce, next the violet, and lastiy the red. Dry in the hot flue, and 48 hours after the impression, wash away the paste. The copper employed for dyeing is of a square form: a boil is given with bran, at the rate of $4 \mathrm{lbs}$. per piece of the foulards: cold water is added to lower the temperature to $130^{\circ} \mathrm{F}$. The pieces must be entered with the printed surface undermost, and winced for half an hour, taking care to keep them expanded and well covered with the liquor: they are then taken ont and rinsed. When grounds are to be made on the foulards, 2 ounces of sumach must be added per piece.

Maddering.- - Suppose 48 pieces are to be grounded with madder. 12 pounds of madder must be put into the copper, 1 pound of sumach, and 6 pounds of bran; the bath must be tepid when the pieces are entered : it must be heated to $104^{\circ} \mathrm{F}$. in 20 minutes, and to the boiling point in an hour and a half. The goods must be briskly winced all the time, and finally turned out into cold water.

When they come out of the madder bath they are much loaded with color. They are cleared by a boil of half an hour in bran, then turned out into cold water, and rinsed. A copper must be now mounted with 3 pounds of soap, 1 ounce of solution of tin, and 2 pailsful of bran, in which the goods are to be boiled for half an hour, then rinsed, and passed through a very dilute sulphuric acid bath. Then rinse, and dry. By following this process, a light salmon ground is obtained. 
II. Steam colors upon silk.-The same plan of operations may be adopted here as deseribed for calico-printing; the main difference being in the method of mordanting the stuffs. After boiling in soap water, in the proportion of 4 ounces per pound of silk, the goods are washed in cold water, and then in hot water at $140^{\circ}$; they are next rinsed, passed through weak sulphuric acid, rinsed, squeezed between rollers, and afterwards steeped in a bath containing 8 ounces of alum per gallon, where they remain for four hours, with occasionally wincing. They are now rinsed and dried. The subsequent treatment resembles that of steam-color printed cottons.

Rlack.-Take a gallon of decoction, made with $4 \mathrm{lbs}$. of logwood, with which

14 ounces of starch are to be combinec $\operatorname{mix}$ in

2 ounces of powdered nut-galls : boil, and pour the color into a pipkin containing

2 ounces of tartaric acid; 2 ounces of oxalic, both in powder, and

2 ounces of olive oil. Stir the color till it is cold, and add

8 ounces of nitrate of iron, and 4 ounces of nitrate of copper.

The red, violet, lilach, yellow colors, \&c. are the same as for steam colors upon cotton. Topical colors are also applied without mordanting the silk beforehand. In this case a little muriate of tin is introduced. Thus, for

Yellow.-Take 1 gallon of a decoction, made with 4 lbs. of Persian berries : dissolve in it 8 ounces of salt of tin (muriate), and 4 ounces of the nitro-muriatic solution of tin. 'Thicken with 2 pounds of gum.

Printing of foulard pieces. The tables which serve for the impression of silk goods are so constructed as to receive them in their full breadth. Towards the part between the color or sieve tub and the table, the roller is mounted upon which the piece is wound.

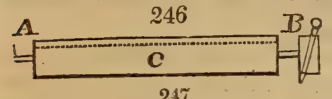

247

A

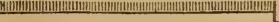

This roller, A B, fig. 216, has a groove, c, cut out parallel to its axis. Into this a bar is pressed, which fixes the end of the piece. The head, $\mathbf{B}$, of the roller is pierced with several holes, in which an iron pin passes for stopping its rotation at any point, as is shown at $\mathbf{B}$. At the other end $B$ of the table there is placed a comb, fig. 247 , which is supported by pivots A B at its ends. The teeth of the comb are on a level with the cloth.

The piece is arranged for printing as follows:-It is unwound, and its end is brought upon the teeth of the comb, and made to pass into them by slight taps with a brush. It is now stretched, by turning round the roller, and fixing it by the pin-handle. After tracing the outline, the printing blocks are applied. Care should be taken, in the course of printing, always to fix the teeth of the comb in the middle line between two handkerchiefs. The nperation of grounding-in is much facilitated by this plan of extension.

The pieces are washed in running water, and must be rapidly dried. The subsequent dressing is given by gum tragacanth: they are dried upon a stretching frame, and then folded up for the market.

III. Mandarining of silk stuffs and chalys.-This style of printing depends upon the property which nitric acid possesses of giving to silk and woollen stuffs a yellow color.

The first step is the scouring with a soap boil, as already described.

The designs are printed-on as also above described.

The swimming or color tub is usually double, and serves for two tables; instead of being placed, therefore, at the end of the table, it is put between two, and, conse-

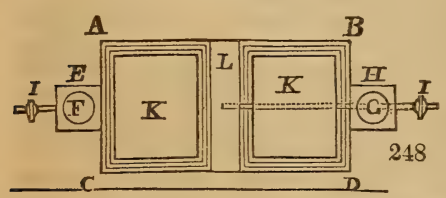
quently, behind the printer. It is formed of a copper chest, fig. 248 , A B C D, in which steam may circulate, introduced by the pipe $\mathbf{I}$; the excess being allowed to escape by the tube $J$, as also the water of condensation. The frame is placed in the hollow box $\mathrm{K} \mathrm{K}$. Between two such frames there is a plate of copper, $L$, which closes the box; it serves for laying the plates in order to keep them hot. At $\mathrm{E}$ and $\mathrm{H}$ are prolongations of the box, in which are set the vessels $\mathrm{F} \mathrm{G}$ for holding the reserve paste.

Preparation of the reserve or resist paste.-Melt in a kettle $2 \frac{1}{2}$ lbs. of rosin; 1 b. of suet; mix well, and put it into the basins $F^{\prime} G^{\prime}$. By means of steam the reserve is kept melted, as well as the false color upon which the sieve floats. The piece of silk being laid upon the table, and the reserve spread upon the frame, the printer heats his block, which should be mounted with lead, if the pattern will permit, upon the little table $\mathrm{L}$. He takes up the color from the frame, and transfers it instantly to the piece. He must strike the block lightly, and then lift it, lest, by its cooling, it might stick to the silk. When the table pattern is completed, he dusts it over with sand, and proceeds to another portion of the silk. The piece must not be 
taken out of the stretch till it is quite dry, which requires usually 6 hours. Let us con. sider first the most common case, that of a white upon an orange ground. We shal: afterwarls describe the other styles, which may be obtained by this process. The piece, being printed and dry, must next be subjected to the mandarining operation.

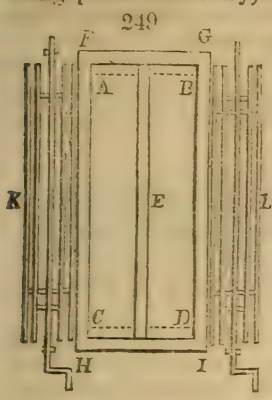

The apparatus here employed consists of a sandstone trough A B C D, fig. 249. Upon the two sides, A c, B D, of this trough are fixed two wooden planks, pierced with a hole an inch from the bottom to receive the roller $x$, under which the piece passes. In this trough the acid mixture is put. That trough is put into a wooden or copper trough, F G $\mathbf{H}$ I. Into the latter, water is put, which is heated by means of steam, or a convenient furnace. Before and behind are placed two winces, or reels, $\mathbf{K} \mathbf{L}$; one serves to guide the piece in entering into the trough, and the other in its leaving it. The piece falls immediately into a stream of cold water, or, failing that, into a large back, containing a mixture of chalk and water. The two winces are moved by handles: the velocity is proportioned to the action of the acid. The wince $L$ ought to be higher than $K$, to allow the aci I to drain off. Fig. 250 shows a section of the apparatus.

The temperature of the acid mixture ought to be maintained

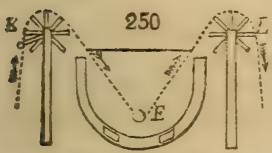
between $95^{\circ}$ and $100^{\circ} \mathrm{F}$.; for if it be raised higher, the resist would run the risk of melting, and the impression would become irregular and blotty.

The proportions of the acid mixture are the following :1 gallon of water; and 1 gallon of nitric acid, of spec. grav. i-288, which may be increased with the strength of the silk. It should be a little weaker for chalys. For the strong greens it may be 2 measures of acid of $1 \cdot 288$ to 1 measure of water. The duration of the passage through the acid should be 1 minute at most.

Mixture of orange color, and clearing away of the resist.-The goods, on coming out of the mandarining apparatus, are rinsed in running water; then boiled in soap water, quickened with a little soda, at the rate of $2 \mathrm{lbs}$. of the former and $4 \mathrm{oz}$. of the latter for a piece of 30 yards. They must be worked by the wince for half an hour. They are now rinsed in cold water, then passed through hot, again rinsed, and dried. I shall give some examples of the mode of manufacture, which is undoubtedly one of the most curious applications of chemical ingenuity.

1. Orange ground with white figures.

(1.) Print-on the fat reserve; (2.) mandarine; (3.) brighten the orange, and clear.

2. Orange ground with biue figures.

(1.) Dip in the indigo vat as for calico; (2.) print-on the fat resist to preserve the

blue; (3.) mandarine; (4.) clear, and brighten the orange by the boil.

3. Orange ground, with blue and white figures.

(1.) Print-on the resist to preserve the white; (2.) dip in the vat, rinse, and dry ; (3.) ground-in the fat resist to preserve the blue; (4.) mandarine; (5.) cleanse, and brighten.

4. Full green ground, and white figures.

(1.) Print-on the resist; (2.) mandarine, and rinse without drying; (3.) dip in the blue

vat; (4.) cleanse, and brighten.

5. Full green ground, and blue figures.

(1.) Dip a pale blue, rinse, and dry ; (2.) print-on the fat resist; (3.) mandarine, wash and dry; (4.) dip full blue; (5.) clean, and brighten.

6. Full green ground, with white and blue figures.

(1.) Print on the resist; (2.) dip a pale blue, and dry; (3.) ground-in the fat resist; (4.) mandarine and rinse; (5.) dip a full blue; (6.) clean, and brighten.

7. Full green ground, with white, blue, and orange figures.

(1.) Print-on the fat reserve; (2.) dip a pale blue, and dry; (3.) ground-in the reserve; (4.) mandarine, rinse, and dry; (5.) ground-in the reserve; (6.) dip a full blue; (\%.) clean, and brighten.

If blue grounds with white figures be wanted, the resist must be applied, and then the goods must be dipped in the blue vat : the resist is afterwards removed by a boil in soap-water.

The above processes are applicable to chalys.

The property which nitric acid possesses of staining animal matters yellow, such as the skin, wool, and silk, is here applied to a very elegant purpose.

of the bronze or solitaire style by mandarining.- The mandarining mixture is

1 gallon of nitric acid, of $1 \cdot 17$ spec. grav.; mixed with 3 pints of solution of nitrate of iron, of spec. grav. 1.65. If the quantity of nitrate of iron be increased, a darker tint wili be obtained. The temperature of the mixture should be $94^{\circ} \mathrm{F}$. The pieces, after mandarining, are let fall intc water, anc steeped for an hour. 
In order to raise the bronze, and clear away the fat resist, the goods must be boiled in a bath of soap and soda, as described for orange.

1. Bronze ground, with white figures.

(1.) Print-on the fat resist; (2.) dip in the blue vat, and dry; (3.) pad in a decoction of logwood, of $4 \mathrm{lbs}$. per gallon; dry, taking care to turn over the selvages; (4.) mandarine, and steep in water for an hour; (5.) cleanse, and pass through soap.

2. Bronze ground, with blue figures.

(1.) Dip in the blue vat, and dry; (2.) print-on the fat resist; (3.) pad in the above decoction of logwood, and dry; (4.) mandarine, and steep an hour; (5.) cle anse, and brighten.

3. Bronze ground, with white and blue.

(1.) Print-on the fat resist; (2.) dip in the blue vat, and dry ; $(3$.$) ground-in the fat re-$ sist; (4.) pad in the logwood liquor, and dry ; (5.) mandarine, and steep for an hour; (6.) cleanse, and give the brightening boil with soap.

This style of manufacture may be executea on chalys; and is capable of producing beautiful effects, which will in vain be sought for by other means.

With sillis, advantage may be derived from various metallic solutions which possess the property of staining animal substances; among which are nitrate of silver, nitrate of mercury, and muriate of iron. The solutions of these salts may be thickened with gum, and printed-on.

An orange upon an indigo vat ground.-After the blue ground has been dyed, orange figures may be produced by printing-on the following discharge paste:-

1 gallon of water, made into a paste with 1 pound of starch; when cold, add to it from 16 to 24 ounces of nitric acid, of spec. grav. 1.288. After fixing the color by steam, the orange is brightened with a soap boil.

An orange upon a Prussian-blue ground.-The dye is first given by Prussian blue in the ordinary way, and then the following discharge is printed-on:-

A caustic ley being prepared, of 1.086 specific gravity, dissolve in a gallon of it 2 pounds of annotto, and thicken with 3 pounds and a quarter of gum. Two dars after the impression of this paste, pass the goods through steam, and wash them in running water. With these two designs, the logwood and gall-black, formerly described, may be associated, to produce a rich effect.

To the preceding practical instructions for printing calicoes, silks, woollens, and mixed fabrics, made of the two latter, a few annotations may be added.

When a uniform color is to be applied to both sides of the cloth, the padding process is employed ; but, when only one side is to be thus colored, diagonal lines are cut very closely to each other upon the cylinder, which transfer so much color from the trough to the cloth passed under it as to make the surface appear uniformly stained. This process is called mattage by the French. Mordants or topical dyes, to be applied in this way, should not be much thickened.

The doubler is the piece of felt or blanket stuff placed between the cloth to be printed, and the block printing-table, or the cylinders. It should be kept very clean; because, were it soiled with acetate of iron, it would spoil all the light shades made with acetate of alumina.

Filters for the color shop of a print-house are best made of wool, formed into a substantial conical cap by felting. A filter ought to be set apart for each different dye-stuff.

When the goods after dyeing are washed, by being held by the selrage, dipped, and shaken in a stream of water, the process is called giving a lisi by the French (donner une lisière). The piece is transferred alternately from one hand to another.

Stains. When we obserre stains produced by mordants upon spots where no color is to come, we must, before dunging the goods, apply a little of the lime-juice, or tartarooxalic acid discharge paste, to the place. If, on the contrary, the stains are not perceived till after the maddering, we must then apply to it first a strong solution of chloride of lime with a pencil, next a solution of oxalic acid mixed with a little muriatic with another pencil, and immediately afterward wash with water. Every madder stain will be effaced by this means.

Rust stains are removeable by a mixture of oxalic and muriatic acids.

Indigo stains by the combined action of chloride of lime and muriatic acid.

Topical yellow stains, or yellow dyes, by the same combination.

Metallic greens and Scheele's green by the acid alone.

Chrome green, and Prussian blue. The blue may be taken out by a caustic alkali; after which the goods must be washed : the residuary rust stain may be removed by the mixture of oxalic and muriatic acids. The above methods refer to cotton and linen. The stains on silk and wonllen stuffs should be removed before fixing the colors by the soap boil; which may generally be done by scratching with the finger, with the aid of little water.

Fo: a direct calico green, see oxyde of Chrome. 
Mr. Hudson, of Gale, near Rochdale, obtained a patent, in December, 1834, for a me. chanism which furniches a continual and regular supply of color to the sieve or tear (tire, Fr.), into which the printer has to dip his block, for the purpose of recciving the color about to be trausterrel to the fabric in the operations of printing calicoes or paper hangings. The contrivance consists in a travelling endless web, moved by power, which, by passing provressively from the color vat over the diaphragm, brings forward continuously an equable supply of the colored paste for the workman's block.

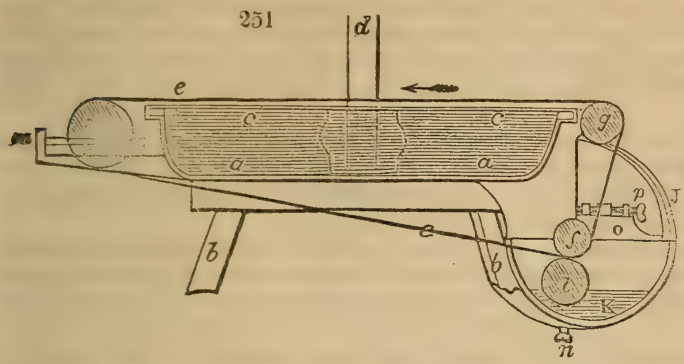

Fig. 251 represents the construction of this ingenious apparatus, shown partly in section. $a$ a is a vessel of iron, supported upon wooden standards $b b$ over the upper surface of which vessel a sheet or diaphragm, $c$ c, of oiled cloth, or other suitable elastic material, is distended and made fast at its edges by being bent over a flange, and packed or cemented to render the joints water-tight. A vertical pipe $d$ is intended to conduet water to the interior of the vessel $a$, and, by a small elevation of the column, to create such upward pressure as shall give to the diaphragm a slight bulge like the swimming tub.

An endless web, $e$ e e, passing over the surface of the diaphragm, is distended over three rollers, $f g h$, the lower of which, $f$, is in contact with the color-roller $i$ in the colortrough $\mathbf{K}$. On the axle of the roller $i$ a pulley wheel is fixed, which allows the roller to be turned by a band from any first mover; or the roller may receive rotatory motion by a winch fixed on its axle. On this said axle there is also a toothed wheel, taking into a another toothed wheel on the axle of the roller $f$; hence, the rotation of the color-roller $i$ in the one direction will cause the roller $f$ to revolve in the opposite, and to carry forward the endless web $e e e$, over the elastic diaphragm, the web taking with it a stratum of color received from the roller $i$, evenly distributed over its surface, and ready for the printer to dip his block into.

The axles of the rollers $f$ and $g$ turn in stationary bearings; but the axle of $h$ is mounted in sliding nuts, which may be moved by turning the screws $m$, for the purpose of tightening the endless web. The axle of the color-roller $i$ turns in mortises, and may be raised by screws $n$ in order to bring its surface into contact with the endless web. To prevent too great a quantity of color being taken up, the endless web passes through a long slit, or parallel aperture, in a frame 0 , which acts as a scraper or doctor, and is adjustable by a screw $p$, to regulate the quantity of color carried up. The contents of the vessel $a$, and of the color-trough $k$, may be discharged when required by a cock in the bottom of each. See PAPER HANGINGs, for the Fondu style.

CALOMEL. (Chlorure de Mercure, Fr.; Versüsstes Quecksilber, Germ.) The mila protochloride of mercury. The manufacture of this substance upon the great scale may be performed in two ways. The cheapest and most direct consists in mixing $1 \frac{1}{8}$ part of pure quicksilver with 1 part of pure nitric acid, of spec. grav. from 1.2 to 1.25 ; and in digesting the mixture till no more metal can be dissolved, or till the liquid has assumed a yellow color. At the same time, a solution of 1 part of common salt is made in 32 parts of distilled water, to which a little muriatic acid is added; and, when heated to nearly the boiling point, it is mixed with the mercurial solution. The two salts exchange bases, and a protochloride of mercury precipitates in a white powder, which, after being digested for some time in the acidulous supernatant liquor, is to be washed with the greatest care in boiling water. The circunstances which may injure the process are the following:-1. When less mercury is employed than the acid can dissolve, there is formed a deuto-nitrate of mercury, which forms some corrosive sublimate with the common salt, and causes a proportional defalcation of calomel. 2 . If the liquors are perfectly neutral at the moment of mixing them, some subnitrate of mercury is thrown down, which cannot be removed by washing, and which gives a noxious contamination to the bland calomel. The acid prescribed in the above formula obviates this danger.

The second manner of manufacturing calomel is to grind very carefully 4 parts of corrosive sublimate (bi-chloride of mercury) with 3 parts of quicksilver, adding a little water or spirits to repress the noxious dust during the trituration. The mass is then introduced into a glass globe, and sublimed at a temperature gradually raised. The quicksilver combines with the deutochloride, and converts it into the protochloride, or calomel. The 
following formula, upon the same principle, was recommended to the chemical manufaco turer in Brande's Journal for July, 1818 :-

"Prepare an oxysulphate of mercury, by boiling 25 pounds of mercury with 35 pounds of sulphuric acid to dryness. Triturate 31 pounds of this dry salt with 20 pounds 4 ounces of mercury, until the globules disappear, and then add 17 pounds of common salt. The whole is to be thoroughly mixed, and sublimed in earthen vessels. Between 46 and 48 pounds of pure calomel are thus produced : it is to be washed and levigated in the usual way." The above is the process used at Apothecaries' Hall, London. The oxysulphate is made in an iron pot; and the sublimation is performed in earthen vessels. The crystalline crust or cake of calomel should be separated from the accompanying gray powder, which is nearest the glass, and consists of mercury mixed with corrosive sublimate.

An ingenious modification of the latter process, for which a patent, now expired, was obtained by Mr. Jewell, consists in conducting the sublimed vapors over an extensive surface of water contained in a covered cistern. The calomel thus obtained is a superior article, in an impalpable powder, propitious to its medical efficacy.

The presence of corrosive sublimate in calomel is easily detected by digesting alcohol upon it, and testing the decanted alcohol with a drop of caustic potash, when the characteristic brick-colored precipitate will fall, if any of the poisonous salt be present. To detect subnitrate of mercury in calomel, digest dilute nitric acid on it, and test the acid with potash, when a precipitate will fall in case of that contamination. As it is a medicine so extensively administered to children at a very tender age, its purity ought to be scrupulously watched.

118 parts of calomel contain 100 of quicksilver.

CALORIC. The chemical name of the power or matter of heat.

CALORIFERE OF WATER. (Calorifère d'eau, Fr.; Wasser-Heitzung, Germ.) In the Diclionnaire Technologique, vol. iv., published in 1823, we find the following description of this apparatus, of late years so much employed in Great Britain for heating conservatories, \&c., by hot water circulating in pipes :-

"This mode of heating is analogous to that by stove-pipes : it is effected by the circuIation of water, which, like air, is a bad conductor, but may serve as a carrier of caloric by its mobility. We may readily form an idea of the apparatus which has been employed for this purpose. We adapt to the upper part of either a close kettle, or of an ordinary

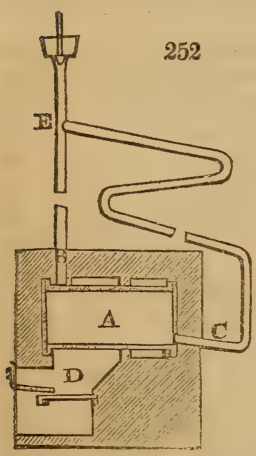
cylindric boiler A, fig. 252, a tube B, which rises to a certain height, then descends, making several sinuosities with a gentle slope till it reaches the level of the bottom of the boiler, to whose lowest part, as that which is least heated, it is fitted at c. At the highest point of the tube $F$ we adapt a vertical pipe, des. tined to serve as an outlet to the steam which may be formed if the temperature be too much raised: It serves also for the escape of the air expelled from the water by the heat: and it permits the boiler to be replenished from time to time as the water is dissipated by evaporation; lastly, it is a tube of safety.

"The apparatus being thus arranged, and all the tubes as well as the boiler filled with water, if we kindle fire in the grate $\mathrm{D}$, the first portions of water heated, having become specifically lighter, will tend to rise : they will actually mount into the upper part of the boiler, and, of course, enter the tube в $F$ : at the same time an equivalent quantity of water will re-enter the boiler by the other extremity $\mathrm{c}$ of the tube. We perceive that these simultaneous movements will determine a circulation in the whole mass of the liquid, which will continue as long as heat is generated in the fireplace; and if we suppose that the tubes, throughout their different windings, are applied against the walls of a chamber, or a stove-room, the air will get warmed by contact with the hot surfaces; and we may accelerate the warming by multiplying these contacts in the mode indicated.

"This calorifere cannot be employed so usefully as those with heated air, when it is wished to heat large apartments. In fact, the passage of heat through metallic plates is in the ratio of the difference of temperature and quantity of the heating surfaces. in the present case, the temperature of the water, without pressure, in the tubes, must be always under $100^{\circ} \mathrm{C}$. $\left(212^{\circ} \mathrm{F}\right.$.), even in those points where it is most heated, and less still in all the other points, while the temperature of the flues in air stoves, heated directly by the products of combustion, may be greatly higher. In these stoves, also, the pipes may without inconvenience have a Jarge diameter, and present, consequently, a large heating surface; whereas, with the water calorifere, the pressure exercised by the liquid upon the sides of the tubes being in the ratio of the surfaces, we are obliged, in order to avoid too great pressure, to employ a multitude of small tubes, which is expensive. Lastlv, if 
the hot-water circulation is to be carried high, as may be often necessary in lofty buildings, the pressure resulting from the great elevation would call for proportional thickiness in the tubes and the boiler : for these reasons, and others which we shall state in treating of heating by steam, it appears that water cannot be advantageously substituted for air or steam in the applications above stated; yet this mode of heating presents very decided advantages where it is useful to raise the temperature a small number of degrees in a uniform manner." See Incubation, ARTificial.

"M. Bonnemain applied, with much success, these ingenious processes of heating by the circulation of water, to maintain a very equal temperature in hot-houses (serreschaudes), in stoves adapted to artificial incubation, and in preserving or quickening vegetation within hot-houses, or outside of their walls, during seasons unpropitious to horticulture.

"Since the capacity of water for heat is very great, if the mass of it in a circulationapparatus be very considerable, and the circulation be accelerated by proper arrangements, as by cooling the descending tube exterior to the stove-room, we may easily obtain by such means a moderately high and uniform temperature, provided the heat generated in the fire-place be tolerably regular. We may easily secure this essential point by the aid of the fire-regulator, an instrument invented by $\mathbf{M}$. Bonnemain, and which is described under the article IncuBAtion, because there its use seems to be indispensable."

From the above quotation, and, more especially, from the evidence adduced in the article Incubation, we see how little claim the Marquis de Chabannes, or any of his followers, can have to invention in their arrangements for heating apartments by the calorific motions of the particles of water, enclosed in pipes of any kind.

CAMBRIC. (Batiste, Fr.; Kammertuch, Germ.) A sort of very fine and rather thin linen fabric, first made at Cambray. An excellent imitation of this fabric is made in Lancashire, woven from fine cotton yarn hard twisted. Linen cambric of a good quality is also now manufactured in the United Kingdom from power-spun flax.

CAMLET, or CAMBLET. A light stuff, much used for female apparel. It is made of long wool hard spun, sometimes mixed in the loom with cotton or linen yarn.

CAMPHOR, oR CAMIPHIRE. This immediate product of vegetation was known to the Arabs under the names of kamphur and kaphur, whence the Greek and Latin name camphora. It is found in a great many plants, and is secreted, in purity, by several laurels; it occurs combined with the essential oils of many of the labiacce ; but it is extracted, for manufacturing purposes only, from the Laurus camphora, which abounds in China and Japan, as well as from a tree which grows in Sumatra and Borneo, called, in the country, Kapour barros, from the name of the place where it is most common. The camphor exists, ready formed, in these vegetables, between the wood and the bark; but it does not exude spontaneously. On cleaving the tree Laurus sumatrensis, masses of pure camphor are found in the pith.

The wood of the laurus is cut into small pieces, and put, with plenty of water, into large iron boilers, which are covered with an earthen capital or dome, lined within with rice straw. As the water boils, the eamphor rises with the steam, and attaches itself as a sublimate to the stalks, under the form of granulations of a gray color. In this state, it is picked off the straw, and packed up for exportation to Europe.

Formerly Venice held the monopoly of refining camphor, but now France, England, Holland, and Germany refine it for their own markets. All the purifying processes proceed on the principle that camphor is volatile at the temperature of $400^{\circ} \mathrm{F}$. The substance is mixed, as intimately as possible, with 2 per cent. of quicklime, and the mixture is introduced into a large bottle made of thin uniform glass, sunk in a sand bath. The fire is slowly raised till the whole vessel becomes heated, and then its upper part is gradually laid bare in proportion as the sublimation goes on. Much attention and experience are required to make this operation succeed. If the temperature be raised too slowly, the neck of the bottle might be filled with camphor before the heat had acquired the proper subliming pitch; and, if too quickly, the whole contents might be exploded. If the operation be carried on languidly, and the heat of the upper part of the bottle be somewhat under the melting point of camphor, that is to say, a little under $350^{\circ} \mathrm{F}$., the condensed camphor would be snowy, and not sufficiently compact and transparent to be saleable. Occasionally, sudden alternations of temperature cause little jets to be thrown up out of the liquid camphor at the bottom upon the cake formed above, which soil it, and render its re-sublimation necessary.

If, to the mixture of 100 parts of crude camphor and 2 of quicklime, 2 parts of boneblack, in fine powder, be added, the small quantity of coloring matter in the camphor will be retained at the bottom, and whiter cakes will be produced. A spiral slip of platina foil immersed in the liquid may tend to equalize its ebullition.

By exposing some volatile oils to spontaneous evaporation, at the heat of about $70^{\circ} \mathbf{F}$.g Proust obtained a residuum of camphor; from oil of lavender, 25 per cent. of its weight; from oil of sage, $12 \frac{1}{2}$; from oil of marjoram, 10 . 
Refined camphor is a white translucid solid, possessing a peculiar taste and smell. It may be obtained, from the slow cooling of its alcoholic solution, in octahedral crystals. Ii may be seratched by the nail, is very flexible, and can be reduced into powder merely by mixing it with a few drops of alcohol. Its specific gravity varies from 0.985 to 0996. Mixed and distilled with six times its weight of clay, it is decomposed, and yields a golden yellow aromatic oil, which has a flavor analogous to that of a mixture of thyme and rosemary; along with a small quantity of acidulous water tinged with that oil, charcoal remains in the retort. In the air, camphor takes fire on contact of an ignited body, and burns all away with a bright fuliginous flame.

Camphor is little soluble in water; one part being capable of communicating smell and taste to 1000 of the fluid. 100 parts of alcohol, spec. grav. 0.806, dissolve 120 parts of camphor, at ordinary temperatures. It is separated in a pulverulent state, by water. Ether and oils, both expressed and volatile, also dissolve it.

When distilled with eight parts of aquafortis, camphor is converted into camphoric acid. Camphor absorbs 144 times its volume of muriatic acid gas, and is transformed into a colorless transparent liquid, which becomes solid in the air, because the acid attrants humidity, which precipitates the camphor. One part of strong acetic acid dissolies two parts of cainphor. By my analysis, camphor consists of 77.38 carbon, 11.14 hydrogen, and 11.48 oxygen. Berzelius's numbers are certainly erroneous.

CAMWOOD. An article imported from Sierra Leone, which seems to possess similar dyeing powers with Brazil or Nicaragua wood.

CANDLE. (Chandelle, Fr.; Kerze, Licht, Germ.) I shall first briefly describe the ordinary manufacture of candles. They are either dipped or moulded. But the first part of the process is the sorting of the tallow. Mutton suet with a proportion of oxtallow is selected for mould candles, because it gives them gloss and consistence. Coarser tallow is reserved for the dipped candles. After being sorted, it is cut into small pieces, preparatory to being melted or rendered; and the sooner this is done after the fat is taken from the carcass the better, because the fibrous and fleshy matters mixed with it promote its putrefaction. Tallow is too commonly melted by a naked fire applied to the bottom of the vessel, whereas it should be done either in a cold set pan, where the flame plays only round the sides a little way above the bottom, or in a steam-cased pan. After being fused a considerable time, the membrancous matters collect at the surface, constituting the cracklings used sometimes for feeding dogs, after the fat has been squeezed out of it by a press. The liquid tallow is strained through a sieve into another copper, where it is treated with water at a boiling temperature in order to wash it. After a while, when the foul water has settled to the botton, the purified tallow is lifted out, by means of tinned iron buckets, into tubs of a moderate size, where it concretes, and is ready for use.

It is a remarkable circumstance, that the wicks for the best candles are still cotton rovings imported from Turkey, notwithstanding the vast extension and perfection of cotton-spinning in this country. Four or more of these Turkey skeins, according to the intended thickness of the wick, are wound off at once into bottoms or clews, and afterward cut by a simple machine into lengths corresponding to those of the candles to be made. Mr. Colebank obtained a patent, in June, 182?, for a machine for cutting, twisting, and spreading wicks, which, though convenient, does not seem to have come into general use. The operations are performed upon a series of threads at once. The apparatus is placed in a box, in front of which the operator sits. A reel extends across the box, at the hinder part, upon which the cotton threads have been previously wound: from this reel they are drawn off in proper lengths, doubled, and cut by an ingenious mechanism. By dipping the wicks into the melted tallow, rubbing them between the palms of the hands, and allowing the tallow which adheres to harden, they may be arranged with facility upon the broaches for the purpose of dipping. The dipping-room is furnished with a boiler for melting the tallow, the dipning-mould, or cistern, and a large wheel for supporting the broaches. From the ceiling of the workshop a long balanceshaped beam is suspended, to one end of which a wooden frame is attached for holling the broaches with the wicks arranged at proper distances. The opposite arm is loaded with a weight to counterbalance the wooden frame, and to enable the workman to ascer. tain the proper size of the candles. The end of the lever which supports the frame is placed immediately above the dipping-cistern; and the whole machine is so balanced that by a gentle pressure of the hand, the wicks are let down into the melted tallow as ofter es may be required.

The fullowing convenient apparatus for dipping candles has been long in use at Edin burgh. In the centre of the dipping-room a strong upright post A A, fig. 253, is erected, with turning iron pivots at its two ends. Near its middle, six mortises are cut at small distances from one another, into each of which is inserted a long bar of wood B B, which moves vertically upon an iron pin, also passing through the middle of the shaft. The whole presents the appearance of a large horizontal wheel with twelve arms. A complete 
view uf two of them only is given in the figure. From the extremity of each arm is suspended a frame, or port, as the workmen call it, containing 6 rods, on each of which

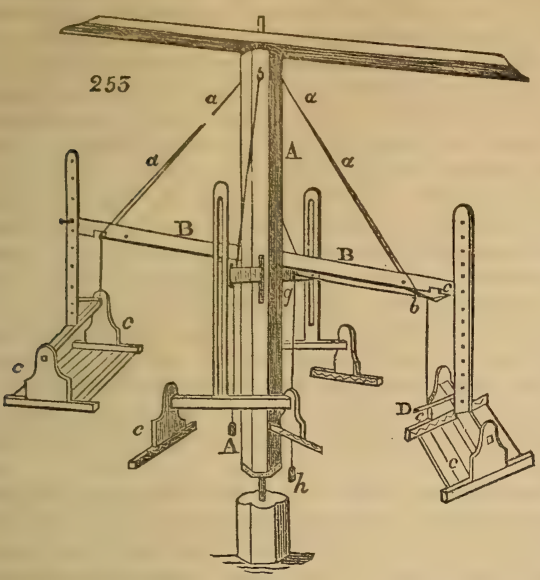
are hung 18 wicks, making the whole number of wicks upon the wheel 1296. The machine, though apparently heavy, turns round by the smallest effort of the workman; and each port, as it comes in succession over the dipping-mould, is gently pressed downwards, hy which means the wicks are regularly immersed in melted tallow. As the arms of the lever are all of the same length, and as each is loaded with nearly the same we vht, it is obvious that they will all naturally assume a horizontal position. In order, however, to prevent any oscillation of the machine in turning round, the levers are kept in a horizontal position by means of small chains $a a$, one end of which is fixed to the top of the upright shaft, and the other terminates in a small square piece of wood, $b$, which exactly fills the notch $c$ in the lever. As one end of the levers must be depressed at each dip, the square piece of wood is thrown out of the notch by the workman pressing down the handle $\mathrm{D}$, which communicates with the small lever $e$, inserted into a groove in the bar $\mathrm{B}$. In order that the square piece of wood, fixed in one extremity of the chain, mav recover its position upon the workman's raising the port, a small cord is attached to it, which passes over a pulley inserted in a groove near $c$, and communicates with another pulley and weight, which draws it forward to the notch. In this way the operation of dipping may be conducted by a single workman with perfect ease and regularity, and even despatch. No time is lost, and no -unnecessary labor expended, in removing the ports after each dip; and, besides, the process of cooling is much accelerated by the candles being kept in constant motion through the air. The number of revolutions which the wheel must make, in order to complete one operation, must obviously depend upon the state of the weather and the size of the candles; but it is said that, in moderately cold weather, not more than two hours are necessary for a single person to finish one wheel of candles of a common size. Upon the supposition, therefore, that six wheels are completed in one day, no less a number than 7776 candles will be manufactured in that space of time by one workman.

I shall next describe the process of moulding, which, if possible, is even less complicated in its details than that of dipping. The moulds are made of some metallic substance, usually pewter, and consist of two parts. The shaft or great body of the mould is a hollow cylinder, finely polished in the inside, and open at both extremities. The top of the mould is a small metallic cup, having a moulding within-side, and a hole to admit the wick. The two parts are soldered together, and when united, as will readily be imagined, have the shape of a moulded candle. A third piece, called the foot, is sometimes added: it is a kind of small funnel, through which the liquid tallow runs into the mould, and, being screwed to the opposite extremity of the shaft, is removeable at pleasure. This additional piece may certainly be useful in very mild weather, since, by removing it, the candles may be drawn more easily from the moulds; but, in general, it may be dispensed with.

Eight or twelve of these moulds, according to their size, are fixed in a frame, which bears a great resemblance to a wooden stool, the upper surface of which forms a kind of trough. The top of the moulds points downwards, and the other extremity, which is open, is inserted into the bottom trough or top of the stool, and made quite level with its upper surface. In order to introduce the wicks into the mould, the workman lays the frame upon its side on an adjoining table, and holding in his left hand a quantity of wicks, previously cut to the proper length, he introduces into the mould a long wire with a hooked point. As soon as the hook of the wire appears through the hole in the top of the mould, he attaches to it the looped end of the wick, and, immediately drawing back the wire, carries the wick along with it. In this manner each mould in succession is furnished with a wick. Another workman now follows, and passes a small wire through the loop of each wick. This wire is obviously intended to keep the wick stretched, and to prevent it from falling back into the mould upon the frame being placed in the proper position for filling. The frame is then handed to the person that fills the moulds, who 
previously arranges the small wires in such a manner that each wick may be exactly in the middle of the rnould.

The moulds are filled by running tallow into each of them, or into the trough, from a eistern furnished with a cock, and which is regularly supplied with tallow of the proper temperature from an adjoining boiler. When the workman observes that the moulds are nearly half filled he turns the cock, and, laying hold of that portion of the wick which hangs out of the mould, pulls it tight, and thus prevents any curling of the wick, which might injure the candles: he then opens the cock, and completes the process of filling. The frame is now set aside to cool; and when the tallow has acquired a proper consistence which the workman easily discovers by a snapping noise emitted br the candles upon pressing his thumb against the bottom of the moulds, he first withdraws the small wires which hept the wicks tense, and then, scraping off the loose tallow from the top of the frame with a small wooden spade, he introduces a bodkin into the loop of the wick, and thus draws each candle in succession from its mould. The candles are now laid upon a table for the inspection of the exciseman, and afterwards remored to the storehouse. Previous to storing them up, some candle-makers bleach their candles, by exposing them to the air and dews for several days. This additional labor can be necessary only when the dealer is obliged to hare early sales; for if the candles are kept for some months, as they ought to be, before they are brought to market, they become sufficiently whitened by age.

Wax candles.-Next to tallow, the substance most emplored in the manufacture of candles is wax. Wax candles are made either by the hand or with a ladle. In the former case, the wax, being lept soft in hot water, is applied bit by bit to the wick, which is hung from a hook in the wall; in the latter, the wicks are hung round an iron circle, placed immediately orer a large copper-tinned basin full of melted wax, which is poured upon their tops, one after another, by means of a large ladle. When the candles have by either process acquired the proper size, they are laken from the hooks, and rolled upon a table, usually of walnut-tree, with a long square instrument of box, smooth at the bottom.

A few years ago I made a set of experiments upen the relative intensities of light, and duration of different candles, the results of which are contained in the following table.

\begin{tabular}{|c|c|c|c|c|c|c|}
\hline Number in a pound. & $\begin{array}{l}\text { Duration of a } \\
\text { candle. }\end{array}$ & $\begin{array}{l}\text { Weight in } \\
\text { grains. }\end{array}$ & $\begin{array}{l}\text { Consumption } \\
\text { per hour in } \\
\text { graing. }\end{array}$ & $\begin{array}{l}\text { Proportion } \\
\text { of light. }\end{array}$ & $\begin{array}{l}\text { Economy } \\
\text { of light. }\end{array}$ & $\begin{array}{l}\text { Candles } \\
\text { equal one } \\
\text { Argand. }\end{array}$ \\
\hline $\begin{array}{l}10 \text { mould - - } \\
10 \text { dipped - } \\
8 \text { mould - } \\
6 \text { ditto - } \\
4 \text { ditto - } \\
\text { Argand oil fiame }\end{array}$ & $\begin{array}{cc}h . & m . \\
5 & 9 \\
4 & 36 \\
6 & 31 \\
7 & 2 \frac{1}{2} \\
9 & 3 \cdot 6 \\
& -\end{array}$ & $\begin{array}{r}682 \\
672 \\
856 \\
1160 \\
1707 \\
-\end{array}$ & $\begin{array}{l}132 \\
150 \\
132 \\
163 \\
186 \\
512\end{array}$ & $\begin{array}{l}12 \frac{1}{4} \\
13 \\
10 \frac{1}{2} \\
14 \frac{2}{3} \\
20 \frac{1}{4} \\
69 \cdot 4\end{array}$ & $\begin{array}{l}68 \\
65 \frac{1}{2} \\
59 \frac{1}{2} \\
66 \\
80 \\
100\end{array}$ & $\begin{array}{l}5 \cdot 7 \\
5 \cdot 25 \\
6 \cdot 6 \\
5 \cdot 0 \\
3 \cdot 5\end{array}$ \\
\hline
\end{tabular}

A Scotch mutchkin, or $\frac{1}{8}$ of a gallon of good seal oil, weighs 6010 gr., or $13 \frac{1}{10}$ oz., avoirdupois, and lasts in a bright Argand lamp 11 hours 44 minutes. The weight of oil it consumes per hour is equal to 4 times the weight of tallow in candles 8 to the pound, and $\frac{1}{7}$ the weight of tallow in candles 6 to the pound. But, its light being equal to that of 5 of the latter candles, it appears from the above table that 2 pounds weight of oil, ralue $9 d$. in an Argand, are equiralent in illuminating power to 3 pounds of tallow candles, which cost about two shillings. The larger the flame in the above candles the greater the economy of light.

In June, 1825, M. Gay Lussac obtained a patent in England for making candles from margaric and stearic acids, improperly called stearine, by converting tallow into the above fat acids br the following process :-Tallow consists, by Cherreul's researches, of stearine, a solid fat, and elaine, a liquid fat; the former being in much the larger proportion. When tallow is treated with an alkaline body, such as potash, soda, or lime, it is saponified; that is, its stearine and elaine become respectirely stearic and elaic acids, and, as such, form compounds with these bases. When by the action of an acid, such as the sulphuric or muriatic, these combinations are decomposed, the fats reappear in the altered form of stearic and elaic acids; the former body being harder than tallow, and of a texture somewhat like spermaceti, the latter body being fluid, like oil. "The decomposition of the soap should be made," says the patentee, "in a large quantity of water, liept well stirred during the operation, and warmed by steam introduced in any convenient way. When the mixture has been allowed to stand, the acid of the tallow or fat will rise to the surface, and the water being drawn off will carry the alkaline or saline matters with it, but if the acids of the tallow should retain any portion of the salts, fresh water may be 
thrown upon it, and the whole well agitated, until the acids have become perfectly free from the allialine matters; and, when allowed to cool, the acids will be formed into a solid mass. This mass is now to be submitted to considerable pressure ... such an apparatus as is employed in expressing oil from seeds; when the liquid acid will run off in the form of a substance resembling oil, leaving a solid matter, similar, in every respect, to spermaceti, which is fit for making candles.

The wick to be used in the manufacture of these improved candles, and which forms one of the features of this invention, is to be made of cotton yarn, twisted rather hard, and laid in the same manner as wire is sometimes coiled round the bass strings of musical instruments. For this purpose, straight rods or wires are to be procured, of suitable lengths and diameters, according to the intended size of the candles about to be made; and these wires, having been covered with cotton coiled round them as described, are to be inserted in the candle-moulds as the common wicks are; and when the candle is made, and perfectly hard, the wire is to be withdrawn, leaving a hollow cylindrical aperture entirely through the middle of the candle. See STLARINE.

CANE-MILL. See Mill and Sugar.

CANION. For the composition of these implements of destruction, see BronzE.

CANVASS. (Canevas, Fr.; Segeltuch, Germ.) It has been found that sails of ships made with the selvages and seams of the canvass running down parallel to their edges, are very apt to bag, and become torn in the middle, from the strain to which they are subjected by the pressure of the wind. To obviate this inconvenience, a mode of making sails, with the seams and selvages running diagonally, was proposed by Admiral Brooking, and a patent granted to him for the same on the 4 th of September, 1828. The invention of Messrs. Ramsay and Orr, which we are about to describe, has a similar object, viz., that of giving additional strength to sails by a peculiar manner of weaving the canvass of which they are made.

The improvement proposed under their patent of March, 1830, consists in weaving the canvass with diagonal threads; that is, placing the weft yarn, or shoot, in weaving, at an oblique angle to the warp yarns, instead of making the decussation of the warp, or weft threads, or yarns, at right angles to each other, as in the ordinary mode of weaving.

To accomplish this object, the loom must be peculiarly constructed; that is, its warp and worl beams must stand at an oblique angle with the sides of the loom, and the batten and slay must be hung in a peculiar manner, in order to beat up the weft, or shoot, in lines ranging diagonally with the warp. No drawing is shown of the method by which this arrangement of the loom is to be made, but it is presumed that any wearer would know how to accomplish it: the invention consisting solely in producing sail-cloth with the threads or yarns of the weft ranging diagonally at any desired angle with the direction of the warp thread.

CAOUTCHOUC, GUM-ELASTIC, on INDIAN-RUBBER (Federharz, Germ.), occurs as a milky juice in several plants, such as the siphonia cahuca, called also hevea guianensis, cautschuc, jatropha elastica, castilleja elastica, cecropia pellata, ficus religiosa. and undica, urceolaria elastica, \&c. It is, however, extracted chiefly from the first plant, which grows in South America and Java. The tree has incisions made into it through the bark in many places, and it discharges the milky juice, which is spread upon clay movlds, and dried in the sun, or with the smoke of a fire, which blackens it.

The juice itself has been of late years imported. It is of a pale yellow color, and has the consistence of cream. It becomes covered, in the bottles containing it, with a pellicle of concrete caoutchouc. Its spec. grav. is 1.012. When it is dried, it loses 55 per cent. of its weight: the residuary 45 is elastic gum. When the juice is heated, it immediately coagulates, in virtue of its albumen, and the elastic gum rises to the surface. It mixes with water in any proportion; and, when thus diluted, it coagulates with heat and alcohol as before.

The specific gravity of caoutchouc is 0.925 , and it is not permanently increased by any degree of pressure. By cold or long quiescence, it becomes hard and stiff. When the milky juice has become once coherent, no means hitherto known can restore it to the emulsive state. By long boiling in water it softens, swells, and becomes more readily soluble in its peculiar menstrua; but when exposed to the air, it speedily resumes its pristine consistence and volume. It is quite insoluble in alcohol; but in ether, de. prived of alcohol by washing with water, it readily dissolves, and affords a colorless solution. When the ether is evaporated, the caoutchouc becomes again solid, but is somewhat clammy for a while. When treated with hot naphtha, distilled from native petroleum, or from coal tar, it swells to 30 times its former bulk; and if then triturated with a pestle, and pressed through a sieve, it affords a homogeneous varnish, which being applied by a flat edge of metal or wood to cloth, prepares it for forming the patent water-proof cloth of Mackintosh. Two surfaces of cloth, to which several coats of the above varnish have been applied, are, when partially dried, hrought evenly in contact, and then passed between rollers, in order to condense and smooth them together. This double 
cloth is afterwards suspended in a stove-room to dry, and to discharge the disagreeable odor of the naptha.

Caoutchotic dissolves in the fixed oils, such as linseed oil, but the varnish has not the property of becoming concrete upon exposure to air.

It has been lately asserted that caoutehouc is soluble in the oils of lavender and sassafras.

It melts at $248^{\circ} \mathrm{F}$, and stands afterwards a much higher heat without undergoing any further change. When the melted caoutchouc is exposed to the air, it becomes hard on the surface in the course of a year. When kindled it burns with a bright flame and a great deal of smoke.

Neither chlorine, sulphurous acid gas, muriatic acid gas, ammonia, nor fluosilicic acid gas affects it, whence it forms very valuable flexible tubes for pneumatic chemistry. Cold sulphuric acid does not readily decompose it, nor does nitric acid, unless it be somewhat strong. The strongest caustic potash ley does not dissolve it even at a boiling heat.

Caoutchouc, according to my experiments, which hare been confirmed by those of $\mathrm{Mr}$. Faraday, contains no oxygen, as almost all other solid vegetable products do, but is a mere compound of carbon and hydrogen, in the proportion, by my results, of 90 carbnn to 10 hydrogen, being three atoms of the former to two of the latter. Mr. Faraday obtained only 87.2 carbon, from which I would infer that some of the carbon, which in this substance is difficult to acidify by peroxyde of copper, had escaped its action. It is obvious that too little carbonic acid gas may be obtained, but certainly not more than corresponds to the carbon in the body. No carbon can be created in the process of ultimate analysis by pure peroxyde of copper such as I employed; and I repeated the ignition after attrition of the mixture used in the experiment. Melted caoutchouc forms a very excellent chemical lute, as it adheres very readily to glass ressels, and withstands the corrosive action of acid vapors. This substance is much used for effacing the traces of plumbago pencils, whence it derived the name of Indian-rubber. It has been lately employed very extensively for making elastic bands or braces. The caoutchouc bottles are skilfully cut into long spiral slips, which are stretched, and kept extended till nearly deprived of their elasticity, and till they form a thread of moderate fineness. This thread is put into a braid machine, and covered with a sheath of cotton, silk, linen, or worsted. The clothed caoutchouc is then laid as warp in a loom, and woven into an elegant riband. When woven, it is exposed upon a table to the action of a hot smoothing iron, which restoring to the caoutchouc all its primitive elasticity, the riband retracts considerably in length, and the braiding corrugates equally upon the caoutchouc cores. Such bands possess a remarkable elasticity, combined with any desired degree of softness. Sometimes cloth is made of these braided strands of caoutchouc used both as warp and as weft, which is therefore elastic in all directions. When a light fabric is required, the strands of caoutchouc, either naked or braided, are alternated with common warp yarns. For this mixed fabric a patent has been obtained. The original manufacturer of these elastic webs is a major in the Austrian service, who has erected a great factory for them at St. Denys, near Paris. See Elastic Bands.

Mr. William Henry Barnard, in the course of some experiments upon the impregnation of ropes with caoutchouc, at the factory of Messrs. Enderby, at Greenwich, discorered that

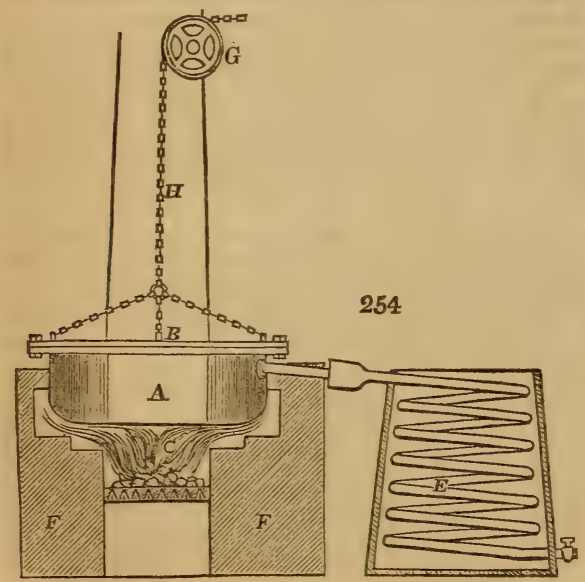
when this substance was exposed to a heat of about $600^{\circ} \mathrm{F}$. it resolved itself into a vapor, which, by proper refrigeratory methods, was condensable into a liquid possessing very remarkable properties, to which the name caoutchoucine has been given. For this invention " of a solvent not hitherto used in the arts" Mr. Barnard obtained a patent, in August, 1833. His process for preparing it is described in his specification as follows:-I take a mass of the said caoutchouc, or Indian rubber, as imported, and having cut it into small lumps, eontaining about two cubic inches each (which I prefer), I throw these lumps into a cast-iron still (which I find adapted for the purpose, and a diagram of which is annexed to, and forms part of, this my specification), with a worm attached; fig. 254, A is the still, B the cover ground to a metallic 
fit, to admit of a thermometer to take the temperature; $\mathbf{c}$ the fire-place, $\mathbf{D}$ the ash-pit, $\boldsymbol{E}$ the worm-tub and worm, $\boldsymbol{F}$ the brick-work of the still, $\mathrm{G}$ a roller and carriage, in conjunction with a crane, or other means, to raise the cover to take out the residue, and to charge the same; $\mathrm{H}$ the chain.

I then apply heat to the still in the usual manner, which heat is increased until the thermometer ranges at 600 degrees of Fahrenheit, or thereabouts. And as the thermometer ranges progressively upwards to 600 degrees of Fahrenheit, a dark-colored oil or liquid is distilled over, which I claim as my said invention, such liquid being ^ solvent of caoutchouc, and other resinous and oleaginous substances. When the thermometer reaches 600 degrees, or thereabouts, nothing is left in the still but dirt and charcoal.

I have found the operation of distillation to be facilitated by the addition of a portion of this oil, either previous or subsequent to rectification, as hereinafter mentioned, in the proportion of one thïrd of oil to two thirds of caoutchouc.

I afterwards subject the dark-colored liquid thus distilled to the ordinary process of rectification, and thereby obtain fluids varying in specific gravity, of which the lightest hitherto has not been under 670 , taking distilled water at 1000 , which fluids I also claim as my saic invention.

At each rectification the color of the liquid becomes more bright and transparent, until, at the specific gravity of 680 , or thereabouts, it is colorless and highly volatile.

In the process of rectification (for the purpose of obtaining a larger product of the oil colorless) I put about one third of water into the still. In each and every state the liquid is a solvent of caoutchouc, and several resinous and oleaginous substances, and also of other substances (such as copal), in combination with very strong alcohol.

Having experienced much difficulty in removing the dirt which adheres to the bottom of the still, I throw into the still lead and tin in a state of alloy (commonly called solder), to the depth of about half an inch, and, as this becomes fused, the dirt which lies on the surface of it is more easily removed.

Objections have been made to the smell of this liquid: I have found such smell removed by mixing and shaking up the liquid with nitro-muriatic acid, or chlorine, in the proportion of a quarter of a pint of the acid (of the usual commercial strength) to a gallon of the liquid.

The discovery of the chemical solvent, which forms the subject of the patent above described, has excited considerable interest in the philosophic world, not only from its probable usefulness as a new article of commerce, but also from two very extraordinary characteristics which it is found to possess, viz., that, in a liquid state, it has less specific gravity than any other liquid known to chemists, being considerably lighter than sulphuric ether, and, in a state of vapor, is heavier than the most ponderous of the gases.

Its elementary constituents are,

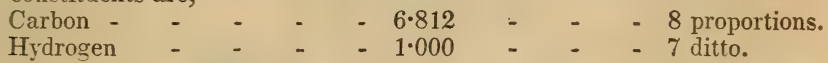

This new material (when mixed with alcohol) is a solvent of all the resins, and particularly of copal, which it dissolves, without artificial heat, at the ordinary temperature of the atmosphere; a property possessed by no other solvent known; and hence it is peculiarly useful for making varnishes in general. It also mixes readily with oils, and will be found to be a valuable and cheap menstruum for liquefying oil-paints ; and, without in the slightest degree affecting the most delicate colors, will, from its ready evaporation, cause the paint to dry almost instantly.

Cocoa-nut oil, at the common temperature of the atmosphere, always assumes a concrete form; but a portion of this caoutchoucine mixed with it will cause the oil to become fluid, and to retain sufficient fluidity to burn in a common lamp with extraordinary brilliancy.

Caoutchoucine is extremely volatile; and yet its vapor is so exceedingly heavy, that it may be poured, without the liquor, from one vessel into another like water.

CAPERS. The caper is a small prickly shrub, cultivated in Spain, Italy, and the southern provinces of France. The flowers are large roses of a pretty appearance, but the flower-buds alone are the objects of this cultivation.

They are plucked before they open, and thrown' into strong vinegar slightly salted, where they are pickled. The crop of each day is added to the same vinegar iub, so that, in the course of the six months during which the caper shrub flowers, the vessel gets filled, and is sold to persons who sort the capers (the smallest being most valued) by means of copper sieves. This metal is attacked by the acid, wherefrom the fruit acquires a green color, much admired by ignorant connoisseurs.

The capers, as found in the French market, are distinguished into five sorts; the nonpareille, the capucine, the capote, the second, and the third; this being the decreasing order of their quality, which depends upon the strength of the vinegar used in pickling them, as also the size and color of the buds. 
The caper shrub grows in the driest situations, even upon walls, and does not disdan any soil; but it loves a hot and sheltered exposure. It is multiplied by grafts made in autumn, as also by slips of the roots taken off in spring.

CAPSTAN. (Cabes/an, Fr.; Spille, Germ.) A machine wherenn the cable is wound successirely in weighing the anchor of a vessel. It is a species of wheel and axle; the axle being vertical, and pierced with holes near its top for the insertion of the ends of horizontal levers, called handspikes, which represent the wheel. These are turned by the force of men moving in a circle. The power applied to the lever is to the resistance to be overcome (the weight of the anchor, for example), when the forces are in equilibrio, as the radius of the cylinder round which the cable is coiled is to the circumference described by the power.

It is manifest that the radius of the axle must be augmented in this computation by half the diameter of the cable, which is supposed to lie always one coil thick upon it. The force of a man, thus applied, has been commonly estimated as equal to the traction of 27 pounds hanging over a pulley.

Friction being so variable a quantity in capstans, renders the exact calculation of it: mechanical effect somewhat uncertain.

A stout man, stationed near the bottom of the axle, holds fast the loose part of the cable, which has already made two or three turns; and, being aided by its friction upon the wood, he both prevents it from slipping backwards, and uncoils each turn as it is progressively made.

Mr. Hindmarsh, master mariner of Newcastle, obtained a patent, in February, 1827, for a contrivance to enable a capstan or windlass to be occasionally worked with increased mechanical advantage. With this riew, he placed toothed wheel-work, partly in the drum-head of the capstan, and partly in the upper part of the barrel, upon which the cable is coiled and uncoiled in successive portions.

The drum-head, and also the barrel, turn loosely upon a central spindle, independent of each other, and are connected together either by the toothed gear, or by bolts. On raising or withdrawing the connecting pinion from the toothed wheels, and then locking the drum-head and barrel together, the capstan works with a power equal only to that exerted by the men at the capstan-bars, as an ordinary capstan; but on lowering the pinion into gear with the wheel-work, and withdrawing the bolts which locked the drumhead to the barrel, the power exerted by the men becomes increased in proportion to the diameter and numbers of teeth in the wheels and pinions.

Fig. 255 is the external appearance of this capstan. Fig. 256 a horizontal view of the toothed gear at the top of the barrel. The barrel, with the whelps $a \quad a$, turns loosely 256

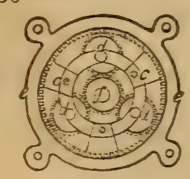

$25 \%$
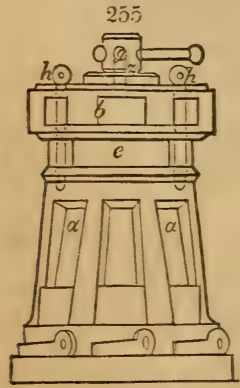

257

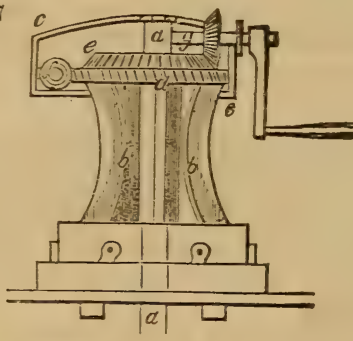

258

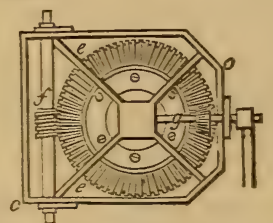
upon a vertical spindle fixed into the deck of the vessel. The drum-head $b$ also turns loosely upon the same spindle. The circular frame $c c$, in fig. 256 , in which the axes of the toothed wheels $d d d$ are mounted, is fixed to the central spindle. The rim $e e \varepsilon$, with internal teeth, is made fast to the top of the barrel; and the pinion $f$, which slides upon the spindle, is connected to the drum-head.

When it is intended to work the capstan with ordinary power, the pinion $f$ is raised up into the recess of the drum-head, by means of a screw $g$, fig. 255 , which throws it out of gear with the toothed wheels, and it is then locked up by a pin $z$ : the bolts $h h$ are now introduced, for the purpose of fastening the drum-head and barrel together, when it becomes an ordinary capstan.

But when it is required that the same number of men shall exert a greater power, the bolts $h$ are withdrawn, and the pinion $f$ lowered into gear with the toothed wheels. The rotation of the drum-head, then carrying the pinion round, causes it to drive the toothed wheels $d d d$; and these working into the toothed $\operatorname{rim} e e$, attached to the barrel, cause the barrel to revolve with an increased power.

Thus, under particular circumstances, a smaller number of men at the capstan or 
windilass (which is to be constructed upon the same principle) will be enabled to havl in the cable and anchor, or warp off the vessel, which is an important object to be effected.

In 1819, Captain Phillips obtained a patent for certain improvements in capstans, a part of which invention is precisely the same as this in principle, though slightly varied in its adaptation.

James Brown, ship-rigger, in his capstan, patented in 1833, instead of applying the moving power by handspikes, having fixed two rims of teeth round the top of the capstan, acts upon them by a rotatory worm, or pinions turned by a winch.

Fig. 257 is an elevation of this capstan, and fig. 258 is a horizontal top view : $a$ is an upright shaft, fixed firmly to the deck, serving as an axle round which the body of the capstan revolves. A frame $c$, fixed to the top of a stationary shaft $a$, above the body of the capstan, carries the driving apparatus.

The upper part of the body of the capstan has a ring of oblique teeth $d$ formed round its edge ; and above this, on the top of the capstan, is a ring of bevel teeth $e$. A horizontal shaft $f$, mounted in the top frame $c$, has a worm or endless screw, which takes into the teeth of the ring $d$; and a short axle $g$, having its bearings in the central shaft $a$, and in the frame $c$, carries a bevel pinion, which takes into the bevel teeth of the ring $e$.

The bearings of the shaft $f$, in the top frame, are in long slots, with angular returns, something like the fastening of a bayonet, which is for the purpose of enabling the shaft to be readily lifted in and out of gear with the teeth of the ring $d$ : the outer bearing of the axle $g$ of the bevel pinion is also supported in the frame $c$, in a similar way, in order to put it in and out of gear with the teeth of the bevel ring $e$. A mode of shifting these is essential; because the two toothed rings, and their driving worm and pinion, give different speeds, and, of course, cannot be both in operation at the same time.

The worm of the shaft $f$, being placed in gear with the teeth of the ring $d$, on applying rotatory power thereto, by means of winches attached to the ends of the shaft, the barrel or body of the capstan will be made to revolve with a slow motion, but with great power; and thus two men at the winches will do the same work as many men with capstan bars in the ordinary way.

If a quicker movement than that of the endless screw is desired, then the driving power may be applied by a winch to the axle $g$ of the bevel pinion, that pinion being put into gear with the bevel ring $e$, and the endless screw withdrawn. It should, however, be here remarked, that the patentee proposes to employ two short axles g, placed opposite to each other, with bevel pinions acting in the bevel-toothed ring, though only one is shown in the figure, to avoid confusion. He also contemplates a modification of the same contrivance, in which four short axles g, placed at right angles, with pinions taking into a bevel ring, may be employed, and made effective in giving rotatory motion to the barrel of a capstan by means of winches applied to the outer ends of the axle, and turned by the labor of four men.

CARAT, or CARACT, is a weight used by goldsmiths and jewellers. See Assax and DIAMOND.

CARBON (Carbone, Fr.; Kohlenstoff, Germ.), in a perfectly pure state, constitutes diamond. Carbonaceous substances are usually more or less compound, containing hydrogen, or sometimes oxygen, and azote, along with earthy and metallic matters. Carbon, tolerably pure, abounds in the mineral kingdom; and, in a combined state, it forms a main constituent of vegetable and animal bodies. Anthracite is a mineral charcoal, differing from common pit-coal in containing no bitumen, and therefore burning without flame or smoke. Coke is the carbonaceous mass which remains after pit-coal has been exposed to ignition for some time out of contact of air; its volatile parts having been dissipated by the heat. It is a spongy substance, of an iron-black color, a somewhat metallic lustre, and does not easily burn unless several pieces are kindled together. With a good draught, however, it produces a most intense heat. Wood charcoal is obtained by the calcination of wood in close vessels, as described under the article AcETIC AcrD, or in piles of various shapes, covered with loam, to screen it from the free action of the atmosphere, which would otherwise consume it entirely. See Charcoar. Such carbon is a solid, without smell or taste, and bears the strongest heats of our furnaces without suffering any change, provided air be excluded: it is a bad conductor of heat, but conducts electricity very well. When burned, it unites with oxygen, and forms carbonic acid, the fixed air of Dr. Black, the choke-damp of the miner. When this carbonic acid is made to traverse red hot charcoal it dissolves a portion of it, and becomes carbonic oxyde, which contains only one half of its volume of oxygen; whereas carbonic acid consists of one volume of oxygen combined with one volume of the vapor of carbon, the two being condensed into one volume. If the specific gravity of oxygen, $=1 \cdot 1025$, be deducted from that of carbonic acid, $=1 \cdot 5245$, the difference, $=0.422$, will be the specific gravity of the vapor of earbon; as well as the proportion present in that weight of the acid. 
Charcoal obtained by the action of a rapid fire in close vessels is not so solid and so good a fuel as that which is made in the ancient way by the slow calcination of pyramidal piles covered with earth. One of the most economical ovens for making wood charcoal is that invented by M. Foucauld, which he calls a shroud, or abri. To construct one of these, 30 feet in diameter at the base, 10 feet at its summit, and from 8 to 9 feet high, he forms, with wood 2 inches square, a frame 12 feet long, 3 feet broad at one end, and one foot at the other. The figure will explain the construction. The uprights, A B and $\mathrm{C} \mathrm{D}$, of this frame are furnished with three wooden handles $a a a$, and $a^{\prime} a^{\prime} a^{\prime}$, by means of which they can be joined together, by passing through two contiguous handles a wooden fork, the frame being previously provided with props, as shown in fig. 259, and covered with loam mixed with grass. A flat cover of 10 feet diameter, made of planks well joined, and secured by four cross bars, is mounted with two trap doors, $\mathbf{M} N$, fig. $2 € 1$, for giring egress to the smoke at the commencement of the operation; a triangular
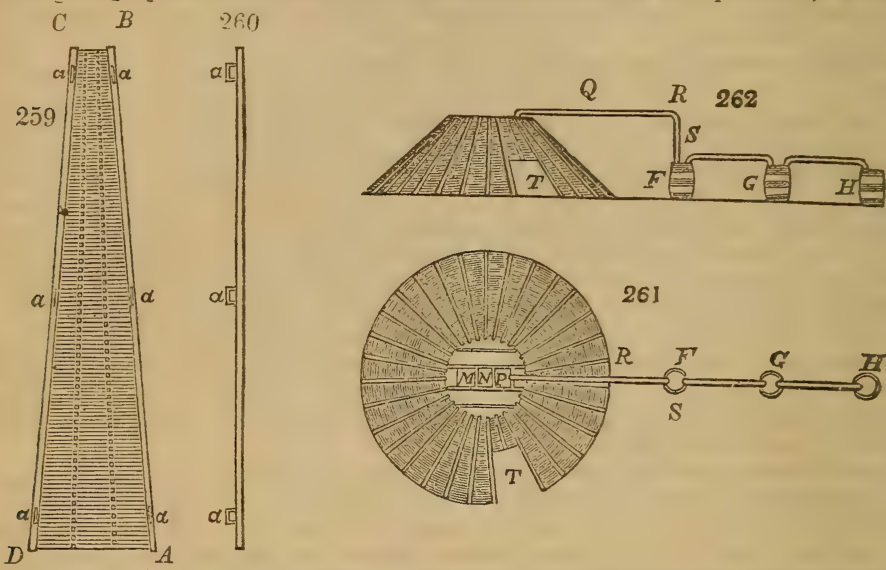

hole $\mathrm{P}$, cut out in the cover, receives the end of a conduit $\mathrm{Q} \mathrm{R} \mathrm{S}$, (figs. 262 and 261,) of wood formed of three deals, destined to convey the gases and condensed liquids into the casks F G H. Lastly, a door T, which may be opened and shut at pleasure, permits the operator to inspect the state of the fire. The charcoal calcined by this abri, has been found to be of superior quality.

When it is wished to change the place where the abri is erected, and to transport it to a store of new-felled timber, the frame is taken down, after beating off the clay which covers it, the joints are then cut by a saw, as well as the ends of the forks which fixed the frames to one another. This process is economical in use, simple and cheap in. construction; since all the pieces of the apparatus are easily moved about, and may be readily mounted in the forests. For obtaining a compact charcoal, for the use of artisans, this mixed process of Foucauld is said to be preferable to either the close iron cylinder or the pile.

For making gunpowder-charcoal the lighter woods, such as the willow, dogwood, and alder answer best; and in their carbonization care should be taken to let the vapors freely escape, especially towards the end of the operation, for when they are re-absorbed, they greatly impair the combustibility of the charcoal.

By the common process of the forests, about 18 per cent. of the weight of the wood is obtained; by the process of Foucauld about 24 per cent. are obtained, with 20 of crude pyroligneous acid of 10 degrees Baumé. By the process described under AcEtic Acid, 27 of charcoal, and 18 of acid at 6 degrees, are procured from 100 parts of wood, besides the tar. These quantities were the results of careful experimenting, and are greater than can be reckoned upon in ordinary hands.

Charcoal for chemical purposes may be extemporaneously prepared by calcining piece of wood covered with sand in a crucible, till no more volatile matter exhales.

The charcoal of some woods contains silica, and is therefore useful for polishing metals. Being a bad conductor of heat, charcoal is employed sometimes in powder to incase small furnaces and steam-pipes. It is not affected by water; and hence the extremities of stakes driven into moist ground are not liable to decomposition. In like manner casks when charred inside preserve water much better than common casks, because they furnish no soluble matter for fermentation or for food to animalcules.

Lowitz discovered that wood charcoal removes offensive smells from animal and vegetable substances, and counteracts their putrefaction. He found the odor of succinic 
and benzoic acids, of bugs, of empyreumatic oils, of infusions of valerian, essenve of wornwood, spirits distilled from bad grain, and sulphureous substances were all absorbable by freshly calcined charcoal properly applied. A very ingenious filter has been con. structed for purifying water, by passing it through strata of charcoal of different fineness.

When charcoal is burned, one third of the heat is discharged by radiaticn, and two thirds by conduction.

The following table of the quantity of charcoal yielded by different woods was published by Mr. Mushet, as the result of experiments carefully made upon the small scale. He says, the woods before being charred were thoroughly dried, and pieces of each lind were selected as nearly alike in every respect as possible. One hundred parts of each sort were taken, and they produced as under:-

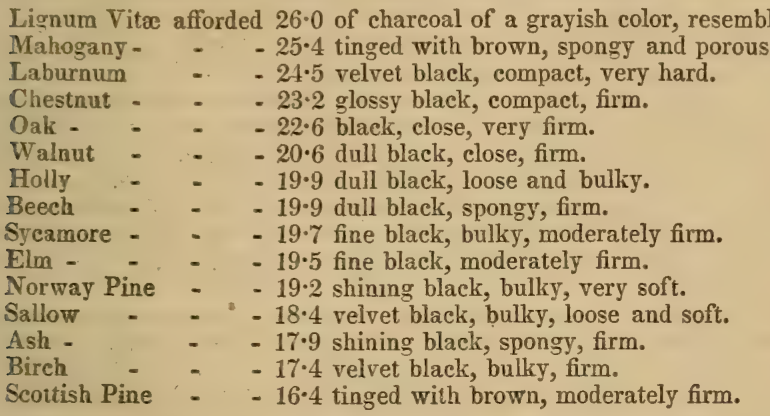

Messrs. Allen and Pepys, from 100 parts of the following woods, obtained the quanti. ues of charcoal as under:-

\begin{tabular}{lll|llll} 
Beech - & - & $-15 \cdot 00$ & Oak & - & - & $-17 \cdot 40$ \\
Mahogany & - & $-15 \cdot 75$ & Fir & - & - & $-18 \cdot 17$ \\
Lignum Vitæ & - & -17.25 & Box & - & - & -20.25
\end{tabular}

It is observable that the quantities obtained by Messrs. Allen and Pepys are in general less than those given by Mr. Mushet, which may be owing to Mr. Mushet not having applied sufficient heat, or operated long enough, to dissipate the aqueous matter of the gaseous products.

To those persons who buy charcoal by weight, it is important to purchase it as soon after it is made as possible, as it quickly absorbs a considerable portion of water from the atmospherc. Different woods, however, differ in this respect. Messrs. Allen and Pepys found, that by a week's exposure to the air, the charcoal of

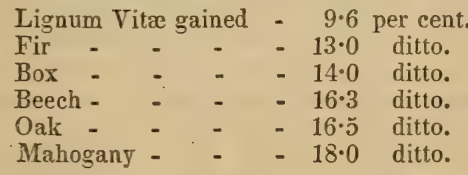

The following is a tabular view of the volumes of the different gases which were absorbed in the course of 24 hours, by one volume of charcoal, in the experiments of M. Theodore de Saussure, which were conducted in a way likely to produce correct results. Each portion of charcoal was heated afresh to a red heat, and allowed to cool under mercury. When taken from the mercury, it was instantly plunged into the vessel of gas:

\begin{tabular}{|c|c|c|c|}
\hline Ammoniacal gas & -90 & Bicarbureted hy & $35 \cdot 00$ \\
\hline Muriatic acid gas & - 85 & Carbonic oxyde & $9 \cdot 42$ \\
\hline Sulphurous acid & -65 & Oxygen gas - & $9 \cdot 25$ \\
\hline Sulphureted hydrogen & -55 & Nitrogen & $\cdot 5$ \\
\hline Nitrous oxyde - & -40 & Carbureted hyd & $\cdot 00$ \\
\hline Carbonic acid gas - & -35 & Hydrogen gas & $1 \cdot 75$ \\
\hline
\end{tabular}

Neumann, who made many experiments on charcoal, informs us, that for the reduction of the metallic oxydes, the charcoal of the heavier woods, as that of the oak and the beech, is preferable, and that, for common fuel, such charcoal gives the greatest heat, and requires the most plentiful supply of air to keep it burning; while those of the lighter woods preserre a glowing heat with a much less draught of air; and that for purposes where it is desirable to have a steady and a still fire, charcoal should be employel which 
has been made from wood previously divested of its bark, sinse it is the cortical par which crackles and flies off in sparks during combustion, while the coal of the wood itself seldom does.

For making crayons of charcoal, the willow is the best wood that can be employed, as the softness is uniform in all its parts. Its durability may be seen in several of our old churchyards, where the letters made with lamp-black are still perfect, though the white sead with which the body of the stones was painted is entirely destroyed.

This property of carbon is shown, however, in a more striking manner by the writings that were found in the ruins of Herculaneum, which have retained their original blackness for two thousand years. The ancients wrote with ink made from ground charcoal.

If it be required to purify any carbonaceous matter, to render it fitter for delicate pigments, this may be done by first calcining it in a close vessel, and then lixiviating it in water slightly acidulated by nitric acid.

The incorruptibility of charcoal was well known to the ancients, and they availed themselves of this property upon all important occasions.

About sixty years ago a quantity of oak stakes were found in the bed of the Thames, in the very spot where Tacitus say's that the Britons fixed a vast number of such stakes to prevent the passage of Julius Cæsar and his army. These stakes were charred to considerable depth, had retained their form completely, and were firm at the heart.

Most of the houses in Venice stand upon piles of wood, which have all been previonsly charred for their preservation. In this country, estates were formerly marked ont by charred stakes driven to a considerable depth into the ground. See BoNE-BLAcK, Charcoat, and Graphite.

CARBONATED WATER is water either pure, or holding various saline matters in solution, impregnated with carbonic acid gas. Fnr general sale in this country, the water usually contains a little soda, which being charged with the gas, is called Soda water ; see this article for a description of an excellent machine for the manufacture of this fashionable beverage.

CARBONATES. Saline compounds in definite proportions of carbonic acid, with alkalis, earths, and the ordinary metallic oxydes.

The carbonates principally used in the arts and manufactures are those of ammonia, copper, iron, lead, lime, magnesia, potash, soda. Native carbonate of copper is the beautiful green mineral called Malachite.

Carbonates are easily analyzed by estimating either by weight or measure the quantity of carbonic acid which they evolve under the decomposing action of somewhat dilute sulphuric, nitric, or muriatic acid; for as they are all compounds of acid, and base in equivalent proportions, the quantity of acid will indicate the quantity of base. Thus, as pure limestone consists of 56 of lime and 44 of acid, in 100 parts, if upon examining a sample of limestone we find it to gire out only 22 per cent. of carbonic aeid gas, during its slow solution in muriatic acid, we are sure that there are only 28 parts of lime present. I have described, in the Annals of Philosophy for October, 1817, a simple form of apparatus for analyzing the carbonates with equal readiness and precision. The simple rule by measure to which I was led, may be thus stated: From the bulk of exolved gas, expressed in cubic inches and tenths, deduct $\frac{1}{2}$, the remainder will express the proportion of real limestone present in the grains employed. Pure magnesian limestone yields very nearly a cubic inch of the gas for every grain in weight.

CARBONATE OF AMMONIA. A salt called in modern chemistry sesqui-carbonate, to denote its being composed of one and a half equivalent primes of carbonic acid, and one of ammonia. It consists by my analysis of 55.89 carbonic acid, 28.86 ammonia, and $15 \cdot 25$ water, in 100 parts. It is generally prepared by mixing from $1 \frac{1}{4}$ to $1 \frac{1}{2}$ parts of well-washed dry chalk, with 1 of sal-ammoniac, introducing the mixtare into an earthen or cast-iron retort, or subliming pot, and exposing it to a heat gradually raised to redness. By double decomposition, the ammonia is volatilized in combination with the caronnic acid of the chalk, and the vapors are recerved in a condensing receiver made either of glass, stone ware, or lead. The chlorine of the sal-ammoniac remains in the retort, associated with the basis of the chalk in the state of chloride of calcium. Some ammonia gas escapes during the process.

The saline mass thus sublimed is purified by a second sublimation in glass or saltglazed earthen ressels. The salt may be obtained, by the abore method carefully coizducted, in rhomboidal octahedrons, but it is generally made for the market in a comp $\varepsilon$ at semi-crystalline white cake. It has a pungent ammoniacal smell; a hot, pungent, alk :line taste; a strong alkaline reaction, and dissolves in two parts of cold water. It must be kept in well-closed vessels, as by exposure to the air a portion of its ammonia exhales, and it passes into the state of the scentless bi-carbonate. It is employed much in medicine, chemical analysis, and by the pastry-cooks to gire sponginess to their cakes, in consequence of its volatilization from their dough in the oven. See Sal-Ammoniac.

For the other carbonates used in the arts, see their respective bases; copper, lead, lime, \&c. 
CARBONIC ACID (Acile carbonique, Fr.; Kohlensaure, Germ.) consists of 1 prime equiralent of carbon $=6 \cdot 125-2$ of oxygen $=16.026$, whose joint sum $=22.151$, represents the atomic weight or combining ratio of this acid, in the neutral or protocarbonate salts. Its composition by volume is stated under CARBON. Its natural form is a gas, whose specific gravity is 1.5245 , compared to atmospheric air 1.000 ; and being so Jense, it may be poured out of one vessel into another. Hence it vas called at first aërial acill. From its existing copiously, in a solid state, in limestones and the mild alkalis, it was styled fixed air by its proper discoverer, Dr. Black. About one volume of it exists in 1000 volumes of common atmospheric air, which may be made manifest sy the erust of carbonate it occasions upon the surface of lime water. Carbonic acid gas is found accumulated in many caverns of volcanic districts, and particularly in the grotto dei cani at Pausilippo, near Puzzuoli; being disengaged in such circumstances by the action of subterranean fire, and, possibly, of certain acids, upon the limestone strata. It often issues from fountains in copious currents, as at Franzensbrunn, near Eger, in Polterbrunnen; near Trier; and Byrreshorn. This acid gas occurs also frecuently in mines and wells, being called choke dxmp, from its suffocating quality. Its presence may. at all times, be detected, by letting down a lighted candle, suspended from a string, into the places suspected of containing this mephitic air. It exists, in considerable quantities, in the water of every pump well, and gives it a fresh and pleasant taste. Water, exposed some time to the air, loses these aerial particles, and becomes vapil. Many springs are highly impregnated with carbonic acid gas, and form a sparkling beverage; such as the Sellerswasser, from Selters upon the Lahn, in the grand dutchy of Nassau; of which no less than two millions and a half of bottles are sold every year. A prodigious quantity of a similar water is also artificially prepared in Great Britain, and many other countries, under the name of aërated or soda water.

Carbonic acid occurs in nature, combined with many salifiable bases; as in the carbonates of soda, baryta, strontia, magnesia; the oxydes of iron, manganese, zinc, copper, lead, sc. From these substances it may be separated, generally spealing, by strong ignition, or, more readily, by the superior affinity of muriatic, sulphuric, or nitric acid, for the earth or metallic oxyde. It is formed whenever vegetable or animal substances are burned with free access of air, from the union of their carbonaceous principle with atmospheric oxygen. It is also formed in all cases of the spontaneous decomposition of organic substances, particularly in the process of fermentation; and constitutes the pungent, noxious, heavy gas thrown off, in vast volumes, from beer vats. See Distillatiox and Fermentation. Carbonic acid is also generated in the breathing of animals; from $t$ to 5 per cent., in volume, of the inhaled oxygen being converted, at each expiration, into this gas, which contaminates the air of crowded apartments, and renders rentilation essential to health, and even to life; witness the horrible catastrophe of the Black-hole at Calcutta.

Carbonic acid gas is destitute of color, has a sourish, suffocating smell, an acidulous pungent taste, imparts to moist, but not dry, litmus paper, a transient reddish tint, and weighs per 100 cubic inches, $46 \frac{1}{2}$ grains; and per cubic foot, $803 \frac{1}{2}$ grains; a little more than $3 \frac{3}{4}$ oz. avoirdupoise. A cubic foot of air weighs about two thirds of that quantity, or 527 grains. It may be condensed into the liquid state by a pressure of 40 atmospheres, and this liquid may be then solidified by its own sudden sporitaneous evaporation. If air contain more than 15 per cent. in bulk of this gas, it becomes unfit for respiration and combustion, animal life and candles being speedily extinguished by it.

Before a person ventures into a deep well, or vault containing fermenting materials, he should introduce a lighted candle into the space, and observe how it burns. Carbonic acid, being so much denser than common air, may be drawn out of cellars or fermenting tubs, by a pump furnished with a leather hose, which reaches to the bottom. Quichlime, mixed with water, may be used also to purify the air of a sunk apartment, by its affinity for, or power of, absorbing this aërial acid. See MrNeral Waters and Soda W ATER.

CARBONIC OXYDE. See the article CARBON.

CARBUNCLE. A gem highly prized by the ancients; most probably a variety of the noble garnet of modern mineralogists.

CARBURET OF SULPHUR, called also sulphuret of carbon, and alcohol of sulphur, is a limpid volatile liquid possessing a penetrating fetid smell, and an acrid burning taste. Its specific gravity is 1.265 ; and its boiling point is about $112^{\circ} \mathrm{Fahr}$. It evaporates so readily, and absorbs so much heat in the vaporous state, that if a tube con. taining quicksilter, surrounded with lint dipped in this liquid, be suspended in the re ceiver of an air-pump on making the vacuum, the quicksilver will be congealed. It con sists of 15.8 carbon and $84 \cdot 2$ sulphur, in 100 parts; being two equivalent primes of the latter to one of the former. 
CARBURETED HYDROGEN. A compound of carbon and hydrogen, of whice there are several species-such as oil-gas, coal-gas, olefiant gas, oil of len:ons, otto of roses, oil of turpentine, petroleum, naptha, napthaline, oil of wine, caoutchoucine, ana caoutchouc.

CARIS, PLAYING. (Cartes à jouer, Fr.; Karten, Germ.) Mr. de la Rue obtained, in Feluary, 1832, a patent for certain improvements in the manufacture of plarins cards, which he distributed under three heads; first, printing the pips, and also the picture or court cards, in oil colors by means of types or blocks; secondly, effecting the same i oil colors by means of lithography; and thirdly, gilding or silvering borders, and othe parts of the characters, by the printing process, either by types, blocks, or litho. graphy.

In the ordinary mode of manufacturing playing cards, their devices are partiy produced by copperplate printing, and they are filled up with water colors by the means called stencilling.

The patentee doles not propose any material alteration in the devices or forms $\mathrm{u}_{\mathrm{i}}$, on , he cards, but only to produce them with oil colors; and, to effect this, he follows precisely the same mode as that practised by calico printers.

A set of blocks or types properly devised, are produced for printing the different pips of hearts, diamonds, spades, and clubs, or they are drawn, as other subjects, in the usual way upon stone. The ink or color, whether black or red, is to be prepared from the best French lamp-black, or the best Chinese vermilion ground in oil, and laid on the types and blocks, or on the stone, in the same way as printers' ink, and the impressions taken-on to thick drawing paper by means of a suitable press in the ordinary manner of printing.

The picture or court-cards are to be produced by a series of impressions in different colors, fitting into each other exactly in the same way as in printing paper hangings, or silks and calicoes, observing that all the colors are to be prepared with oil.

For this purpose a series of blocks or types are to be provided for each suhrect, and which, when put together, will form the whole device. These blocks are to be used separately, that is, all the yellow parts of the picture, for instance, are to be printed at one impression, then all the red parts, next all the flesh color, then the blue portions, and so on, finishing with the black outlines, which complete the picture.

If the same is to be done by lithography, there must be as many stones as there are to be colors, each to print its portion only; and the impression, or part of the picture given by one stone, must be exactly fitted into by the impression given from the next stone, and so on until the whole subject is complete.

A superior kind of card is proposed to be made, with gold or silver devices in parts of the pictures, or gold or silver borders round the pips. This is to be effected by printing the lines which are to appear as gold or silver, with gilders' size, in place of ink or color; and immediately after the impression has been giren, the face of the card is to be powdered over with gold dust, silver, or bronze, by means of a soft cotton or wool dabber, by which the gold, silver, or bronze will be made to adhere to the picture, and the superfluous portions of the metal will wipe off by a very slight rubbing. When the prints are perfectly dry, the face of the card may be polished by means of a soft brush.

If it should be desirable to make these improved cards to resemble irory, that may be Jone by preparing the face of the paper in the first instance with a composition of size and fine French white, and a drying oil, mixed together to about the consistency of cream; this is to be washed over the paper, and dried before printing, and when the cards are finished they will exactly resemble ivory.

The only thing remaining to be deseribed, is the means by which the successive impressions of the types, blocks, or stones, forming the parts of the pictures, are to be brought exactly to join each other, so as to form a perfect whole design when complete; this is by printers called registering, and is to be effected much in the usual way, by points in the tympan of the press, or by marks upon the stones.

The parts of the subject having been all accurately cut or drawn to fit, small holes are to be made with a fine awl through a quire or more of the paper at once, by placing upon the paper a gauge-plate, having marks or guide-holes, and by observing these, the same sheet laid on several times, and always made to correspond with the points or marks, the several parts of the picture must inevitably register, and produce a perfect subject.

CARD CUTTING. Mr. Dickinson's patent machine for cutting cards, consists of a pair of rollers with circular revolving cutters, the edges of which are intended to act against each other as circular shears, and the pasteboards in passing between these rollers ane cut by the circular shears into cards of the desired dimensions. These rollers are mounted in suitable standards, with proper adjustments, and are made to revolve by 
- band and pulley connected to the axle of a crank, or by any other convenient means.

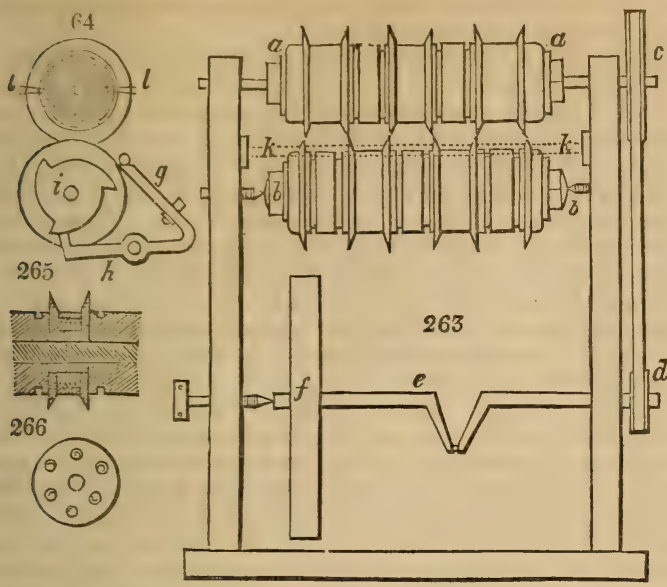

Fig. 263 is a front view of this machine; $a$ a and $b b$ are the two rollers, the upper one turning upon an extended axle, bearing in the standards, the lower one upon pivots. These rollers are formed by a series of circular blocks, between a series of eircular steel cutters, which are slidden on to iron shafts, and held togeiher upon their axle by nuts screwed up at their ends. The accurate adjustment of the cutters is of the first importance to their correct performance; it is therefore found necessary to introduce.spiral springs within the blocks, in order to press the entters up to

their proper bearings. A section of one of the blocks is shown at fig. 265, and an end view of the same at $f \mathrm{~g}$. 266, with the spiral springs inserted.

At the outer extremity of the axle of the roller $a$, a rigger $c$, is attached, whence a band passes to a pulley $d$, on the crank shaft $e$, to which a fly-wheel $f$, is affixed, for the purpose of rendering the action uniform. Rotatory motion being given to the crank shaft, the upper roller is turned, the lower roller moving at the same time by the friction against the edges of the cutters.

Fig. $26 t$ is an end view of the rollers, showing the manner in which the pasteboards are guided and conducted between the cutters. In the front of the machine a moveable frame $g$, is to be placed, for the purpose of receiving the pasteboards, preparatory to cutting them into cards, and a stop is screwed to this frame for the edge of the pasteboard to bear against, which stop is adjustable to suit different sizes. From the back part of this frame an arm $h$, extends, the extremity of which acts against the periphery of a ratchet wheel $i$, fixed at the end of the roller $b$, and hence, as the roller goes round, the frame is made to rise and fall upon its pivots, for the purpose of guiding the pasteboard up to the cutters; at the same time a rod $k$, hanging in arms from the sides of the standards (shown by dots in fig. 263), falling upon the pasteboard, confines it, while the cutters take hold, and racks corresponding with the indentations of the rollers are placed as at $l l$, by means of which the cards, when cut, are pushed out of the grooves.

As various widths of cards will require to be cut by this machine, the patentee proposes to have several pairs of rollers ready adjusted to act together, when mounted in the standards, in preference to shifting the circular cutters, and introducing blocks of greater or less width.

The second part of the invention is a machine for pasting the papers, and pressing the sheets together to make pasteboard. This machine consists of several reels (we suppose rollers are intended) on which the paper is to be wound, along with a paste trough, and rotatory brushes. The several parts of this machine, and their operations in making pasteboard, are described in the specification, but the patentee having omitted the letters of reference in the drawing which he has enrolled, it becomes difficult to explain it.

As far as we are enabled to understand the machine, it appears, that damped paper is to be wound upon two rollers, and conducted from thence over two other rollers; that two fluted rollers revolving in the paste trough are to supply paste to two circular brushes, and that by those brushes the papers are to be pasted upon one side, and then pressed together, to make the pasteboard; after this, the pasteboard is to be drawn on to a table, and to remain there until sufficiently dry to be wound upon other rollers. By comparing this description with the figure, perhaps the intended operations of the machine may be discovered : it is the best explanation we are enabled to give.

CARDS (Cardes, Fr.; Karden, Germ.) are instruments which serve to disentangle the fibres of wool, cotton, or other analogous bodies, to arrange them in an orderly lap or flece, and thereby prepare them for being spun into uniform threads. The fineness and the leveiness of the yarn, as well as the beauty of the cloth into which it enters. 
depend as much upon the regularity and perfection of the carding, as upon any subsequent opcrations of the factory. The quality of the carding depends more upon that of the cards than upon any attention or skill in the operative; since it is now nearly an automa tic process, conducted by young women called card-tenters.

Cards are formed of a sheet or fillet of leather pierced with a multitude of small holes, in which are implanted small staples of wire with bent projecting ends called teeth. Thus every piece of wire is double toothed. The leather is afterwards applied to a flat or cylindrical surface of wood or metal, and the co-operation of two or more such surfaces constitutes a card. The teeth of cards are made thicker or slenderer, according as tk a filaments to be carded are coarser or finer, stiffer or more pliant, more valuable or cheaper. It is obviously of great importance that the teeth should be all alike, equably distributed, and equally inclined over the surface of the leather, a degree of precision which is scarcely possible with handwork. To judge of the difficulty of this manipulation we need only inspect the annexed figures. The wire must first be bent at right angles in $c$ and $d, f i g .268$, then each branch must receive a second bend in $a$ and $b$ at a determinate obtuse angle, invariable for each system of cards. It is indispensable that the two angles $c a e$ and $d b f$ be mathematically equal, not only as to the twin teeth of one staple, but through the whole series; for it is easy to see that if one of the teeth be more or less sloped than its fellow, it will lay hold of more or less wool than it, and render the carding irregular. But though the perfect regularity of the teeth be important, it is not the sole condition towards making a good card. It must be always kept in view that these teeth are to be implanted by pairs in a piece of leather, and kept in it by the cross part $c d$. The leather must therefore be pierced with twin holes at the distance $c d$; and pierced in such a manner, that the slope of the holes, in reference to the plane of the leather, be invariably the same; for otherwise the length of the teeth would vary with this angle of inclination, and the card would be irregular.

A third condition essential towards producing perfect regularity, is that the leather ought to be of the same thickness throughout its whole surface, otherwise the teeth, though of the same length and fixed at the same angle, would be rendered unequal by the different thicknesses of the leather, and the operation of carding would be in consequence

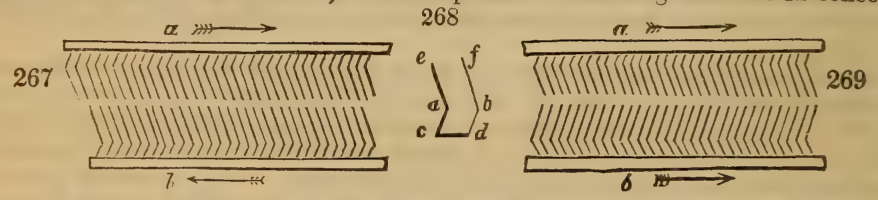

extremely defective. Fig. 267 shows the card teeth acting against each other, ฉs indicated by the arrows in two opposite directions; in fig. 269 they work one way.

of late years very complex but complete and well-acting machines have been constructed for splitting the leather or equalizing it by shaving, for bending and cutting the wires, and implanting them in the leather, into holes pierced with perfect regularity. Card machines which fashion the teeth with great precision and rapidity, and pierce the leather, have been for a considerable time in use at Halifax, in Yorkshire, a town famous for the excellence of its card-cloth, as also at Leeds, Glasgow, and several other places. The wires and the leather thus prepared are given out by the manufacturer to women and children, who put them together.

1. The simplest machine for equalizing the leather which can be employed, is that which I saw operating in MM. Scrive's automatic card factory at Lille, the most magnificent I believe in the world, where the leather was drawn forwards by a roller over a solid horizontal table, or bed, and passed under a nicely adjusted vertical blade, which shaved it by a scraping motion to a perfectly uniform thickness. About one half the weight of the leather is lost in this process, and in the subsequent squaring and trimming.

The machine for making cards, invented I believe by a Mr. Ellis of the United States, for which a first patent was obtained in this country by Joseph Cheeseborough Dyer, Esq. of Manchester, in 1811, and a second and third with further improvements in 1814, at 1 1824, is one of the most elegant automatons ever applied to productive industry. It is, however, necessarily so complicated with different mechanisms as to render its representation impracticable in such engravings as are compatible with the scope of this dictionary. I must therefore content myself with the following general description of its constituent parts.

The first thing to be done after having, as above, prepared the long sheets or fillets of leather of suitable length, breadth, and thickness, for making the cards, is to stretch the leather, and hold it firmly; which is accomplished by winding the fillet of leather upon the roller or dium, like the warp roller of a ioom, and then conducting it upwards between gurde rollers, to a receiving or work roller at top of the machine, where the fillet is held fast by a cramp, by which means the leather is kept stretched.

Secondly, the holes are pierced in the leather to receive the wire staples or teeth of th 
eard, by means of a sliding fork, the points of which are presented to the face of the leather; while the fork is made to advance and recede continually, by the agency of levers worked by rotatory cams upon a revolving main shaft.

The points of the fork being thus made to penetrate into the leather, the holes for raceiring the staples are pierced at regular distances, and in correct order, by shifting the leather fillet so as to bring different parts of its surface opposite to the points of the sliding fork. This is done by cams, or indented wheels and gear, which shift the guide rollers and confining drums laterally, as they revolve, and consequently move the fillet of leather at intervals a short distance, so as to present to the points of the fork or piercer, at every movement, a different part of the surface of the leather.

Thirdly, the wire of which the teeth or points of the card are to be made, is supplied from a coil on the side of the machine, and is brought forward at intervals by a pair of sliding pincers, which are slidden to and fro through the agency of levers actuated by rotatory cams upon the main shaft. The pincers having advanced a distance equal to the length of wire intended to form one staple or two points, this length of wire is pressed upon exactly in the middle by a square piece of steel, and being there confined, a cutter is brought forward, which cuts it off from that part of the wire held in the pincers.

The length of wire thus separated and confined is now, by a morement of the machine, bent up along the sides of the square steel holder, and shaped to three edges of the square, that is, formed as a staple; and in the same way, by the continued movements of the machine, a succession of pieces of wire are cut off, and bent into staples for making the teeth of the card as long as the mechanism is kept in action.

Fourthly, the wire staple thus formed is held with its points or ends outwards, closely contiguous to the forked piercer described above, and by another movement of the mechanism, the staple is protruded forward, its end entering into the two holes made previously in the leather by the sliding of the fork.

While the wire staple is being thus introduced into the leather, its legs or points are to be bent, that is, formed with a knee or angle, which is the fifth object to be effected. This is done by means of a small apparatus consisting of a bar or bed, which bears up against the under side of the wire staple when it has been passed half way into the holes in the leather, and another bar above it, which, being brought down behind the staple, bends it over the resisting bar to the angle required; that is, forms the knee in each leg. A pusher.now acts behind the staple, and drives it home into the leather, which completes the operation.

The leather being thus conducted, and its position shifted before the piercer progressively, a succession of the above described operations of cutting the wire, forming the staple, passing it into the leather, and bending its legs to the angular form, produces a sheet of card of the kind usually employed for carding or combing wool, cotton, and other fibrous materials. It may be necessary to add, that as these wire staples are re. quired to be set in the leathers sometimes in lines crossing the sheet, which is called ribbed, and at other times in oblique lines, called twilled, these rariations are produced by the positions of the notches or steps upon the edge or periphery of the cam or indented wheel, which shifts the guide rollers that hold the fillet or sheet of leather as already described.

CARMINE (Eng. and Fr.; Karminstoff, Germ.) is, according to Pelletier and Caventou, a triple compound of the coloring substance, and an animal matter contained in eochineal, combined with an acid added to effect the precipitation. The preparation of this article is still a mystery, because, upon the one hand, its consumption being very limited, few persons are engaged in its manufacture, and upon the other, the raw material being costly, extensive experiments on it cannot be conveniently made. Success in this business is said to depend not a little upon dexterity of manipulation, and upon knowing the instant for arresting the further action of heat upon the materials.

There is sold at the shops different kinds of carmine, distinguished by numbers, and possessed of a corresponding value. This difference depends upon two causes; either upon the proportion of alumina added in the precipitation, or of a certain quantity of vermilion put in to dilute the color. In the first case the shade is paler, in the second it has not the same lustre. It is always easy to discover the proportion of the adulteration. By availing ourselves of the property of pure carmine to dissolve in water of ammonia, the whole foreign matter remains untouched, and we may estimate its amount by drying the residuum.

Take 1 pound of cochineal in powder;

To make Ordinary Carmine.

3 drachms and a half of carbonate of potash;

8 drachms of alum in powder;

3 drachms and a half of fish-glue.

The cochineal must be boiled along with the potash in a copper containing five pailsful of water ( 60 pints); the ebullition being allayed with cold water. After boiling a 
few minutes the copper must be taken from the fire, and placed on a table at such an angle as that the liquor may be conveniently transvased. The pounded alum is then thrown in, and the decoction is stirred; it changes color immediately, and inclines to a more brilliant tint. At the end of fifteen minutes the cochineal is deposited at the bottom, and the bath becomes as clear as if it had been filtered. It contains the coloring matter, and probably a little alum in suspension. We decant it then into a copper of equal capacity, and place it orer the fire, adding the fish-glue dissolved in a great deal of water, and passed through a searce. At the moment of ebullition, the carmine is perceived to rise up to the surface of the bath, and a coagulum is formed, like what takes place in clarifications with white of egg. The copper must be immediately taken from the fire, and its contents be stirred with a spatula. In the course of fifteen or twenty minutes the carmine is deposited. The supernatant liquor is decanted, and the deposite must be drained upon a filter of fine canvass or linen. If the operation has been well conducted, the carmine, when dry, crushes readily under the fingers. What remains after the precipitation of the carmine is still much loaded with color, and mar be employed very advantageously for carminated lakes. See LAKE.

By the old German process, carmine is prepared by means of alum without any other addition. As soon as the water boils, the powdered cochineal is thrown into it, stirred well, and then boiled for six minutes; a little ground alum is added, and the boiling is continued for three minutes more; the vessel is remored from the fire, the liquor is filtered and left for three dass in porcelain vessels, in the course of which time a red matter falls down, which must be separated and dried in the shade. This is carmine, which is sometimes preriously purified by washing. The liquor after three dar's sore lets fall an inferior hind of carmine, but the residuary coloring matter may also be separated by the muriate of tin.

The proportions for the above process are 580 parts of clear river water, 16 parts of cochineal, and 1 part of alum; there is obtained from $1 \frac{1}{2}$ to 2 parts of carmine.

Another carmine wi!h tartar.- To the boiling water the cochineal is added, and after some time a little cream of tartar; in eight minutes more we add a little alum, and con tinue the boiling for a minute or two longer. Then take it from the fire and pour it into glass or porcelain ressels, filter, and let it repose quietly till the carmine falls down. We then decant and dry in the shade. The proportions are 8 pounds of water, $8 \mathrm{oz}$. of cochineal, $\frac{1}{2} \mathrm{oz}$. of cream of tartar, $\frac{3}{4} \mathrm{oz}$. of alum, and the product is an ounce of carmine.

The process of Alxon or Langlois.-Boil two pails and a half of river water (30 pints), throw into it, a little afterwards, a pound of cochineal, add a filtered solution of six drachms of earbonate of soda and a pound of water, and let the mixture boil fur half an hour; remore the copper from the fire, and let it cool, inclining it to one side. Add six drachms of pulrerized alum, stir with a brush to quichen the solution of the salt, and let the whole rest 20 minutes. The liquor, which has a fine scarlet color, is to be carefully decanted into another ressel, and there is to be put into it the whites of two eggs well beat up with half a pound of water. Stir again with a brush. The copper is replaced on the fire, the alumina becomes concrete, and carries down the coloring matter with it. The copper is to be taken from the fire, and left at rest for 25 or 30 minutes to allow the carmine to fall down. When the supernatant liquor is drawn off, the deposite is placed upon filter cloth stretched upon a frame to drain. When the carmine has the consistence of cream cheese, it is taken from the filter with a silver or irory knife and set to dry upon plates corered with paper, to screen it from dust. A pound of cochineal gives in this way an ounce and a half of carmine.

Process of Madame Cenette, of Amsterdam, with salt of sorrel.-Into six pails of rirer water boiling hot throw two pounds of the finest cochineal in powder, continue the ebullition for ito hours, and then add $3 \mathrm{oz}$. of refined saltpetre, and after a few minutes $4 \mathrm{oz}$. of salt of sorrel. In ten minutes more take the copper from the fire and let it settle for four hours; then draw off the liquor with a syphon into flat plates and leare it there for three weelis. Afterwards there is formed upon the surface a prettr thick mouldiness, which is to be removed dexterously in one pellicle by a slip of whalebone. Should the film tear and fragments of it fall down, they must be removed with the utmost care. Decant the supernatant water with a syphon, the end of which mas touch the botton of the ressel, because the lajer of carmine is very firm. Whaterer water remains must be sucked away by a pipette. The carmine is dried in the shade, and has an extraordinary lustre.

Curmine by the salt of tin, or the Carmine of China.-Boil the cochineal in river water, adding some Roman alum, then pass through a fine cloth to remore the cochineal, and set the liquor aside. It becomes brighter on keeping. After haring heated this liquor, pour into it, drop by drop, solution of tin till the carmine be precipitated. The proportions are one pailful of water, $20 \mathrm{oz}$. of cochineal, and 60 grains of alum, with a solu. ion of tin containing $4 \mathrm{oz}$. of the metal. 
To revive or brighten carmine. - We may brighten ordinary carmine, and obtain a very fine and clear pigment, by dissolving it in water of ammonia. For this purnose we leave ammonia upon carmine in the heat of the sun, till all its color be extracted, and the liquor has got a fine red tinge. It must be then drawn off and precipitated, by acetic acid and alchohol, next washed with alcohol, and dried. Carmine dissolved in ammonia has been long employed by painters, under the name of liquid carmine.

Carmine is the finest red color which the painter possesses. It is principally employed in miniature painting, water colors, and to tint artificial flowers, because it is more transparent than the other colors. For Carminium, see CochineaL.

CARPET. (Tapis, Fr.; Teppich, Germ.) A thick woollen fabric of variegated colors, for covering the floors of the better sort of apartments. This luxurious manufacture took its origin in Persia and Turkey, whence the most beautiful patterns were wont to come into Europe; but they have been for some time surpassed by the workmanship of France, Great Britain, and Belgium. To form a just conception of the elegant and ingenious processes by which carpets are made, we should visit the royal establishment of the Gobelins at Paris, where we would see the celebrated carpet manufactory of the Savounerie, which has been transported thither. A detailed set of engravings of this art is given by Roland de la Platière in the first and second volumes of the Encyclopédie Méthodique, to which I must refer my readers, as a due exposition of its machines and operations would far exceed the scope of the present volume.

The warp, says M. Roland, being the foundation of the fabric, ought to be of fine wool, equally but firmly spun, and consist of three yarns twisted into one thread. The yarns that are to form the velvety surface of the carpet, ought also to be of the best quality, but soft and downy in their texture, so that the dye may penetrate every filament. Hemp, or linen yarns, are likewise employed in this manufacture, as a woof, to bind the warp firmly together after each shoot of the velvety threads. Thus we see that good carpeting consists essentially of two distinct webs woven at the same time, and firmly decussated together by the woof threads. Hence the form of the pattern is the same upon the two sides of the cloth, only the colors are reversed, so that what was green upon one side becomes red or black upon the other, and vice versa. The smaller the figures the more frequent the decussations of the two planes, and the firmer and more durable the fabric.

The carpet manufacture, as now generally practised, may be distributed into two systems-that of double fabries, and that cut in imitation of velvet. Of late years the Jacquard loom has been much used in weaving carpets, the nature of which will be found fully explained under that title.

For the sake of.illustration, if we suppose the double carpets to be composed of only two colors, the principle of weaving will be easily understood; for it is only necessary to raise the warp of each web alternately for the passage of the shuttle, the upper web being entirely above when the under web is being woven, or decussated, and vice versa. In a Brussels carpet the worsted yarn raised to form the pile, and make the figure, is not cut ; in the Wilton the pile is cut to give it a velvety aspect and softness. In the imperial Brussels carpet the figure is raised above the ground, and its pile is cut, but the ground is uncut; and in the royal Wilton, the pile is both raised higher than in the common Wilton, and it is cut, whereby it has a rich cushion-like appearance. The cloth of all these superior carpets consists of woollen and linen, or hemp; the latter being put upon a beam, and brought, of course, through heddles and a reed; but as its only purpose is to bind together the worsted fabric, it should not be visible upon the upper face of the carpet. The worsted yarn is wound upon small bobbins or pirns, with a weight affixed to each, for giving proper tension to the threads. Their number varies, for one web, from 1300 to 1800 , according as the carpet is to be 27 or 36 inches wide; and they are placed, in frames, behind the loom, filled with differently colored yarn, to correspond with the figure. This worsted warp is then drawn through the harness, heddles, and reed, to be associated with the linen yarn in the compound fabric.

In Kidderminster carpeting, both warp and weft appear upon the face of the eloth, whereas, in the Brussels style, only the warp is seen, its binding weft being fine hempen or linen threads. The three-ply imperial carpet, called the Scotch, is coming very much into vogue, and is reckoned by many to be little inferior in texture, look, and wear to the Brussels. Kilmarnock has acquired merited distinction by this ingenious industry. In this fabric, as well as in the two-ply Kidderminster, the weft predominates, and displays the design; but in the French carpets, the worsted warp of the web shows the figure. Plain Venetian carpets, as used for stairs and passages, are woven in simple looms, provided merely with the common heddles and reed. The warp should be a substance of worsted yarn, so heavy as to cover in the weft completely from the view. Figured Venetian carpets are woven in the two-ply Kidderminster looms, and are 
provided with a mechanism to raise the pattern upon the worsted warp. The weft is an alternate shoot of worsted and linen yarn, and must be concealed.

'The following figure and description will explain the construction of the three-ply imperial Scotch and two-ply Kidderminster carpet-loom, which is merely a modification of the Jacquard métier. The Brussels carpet-loom, on the contrary, is a draw-Loy loom 270

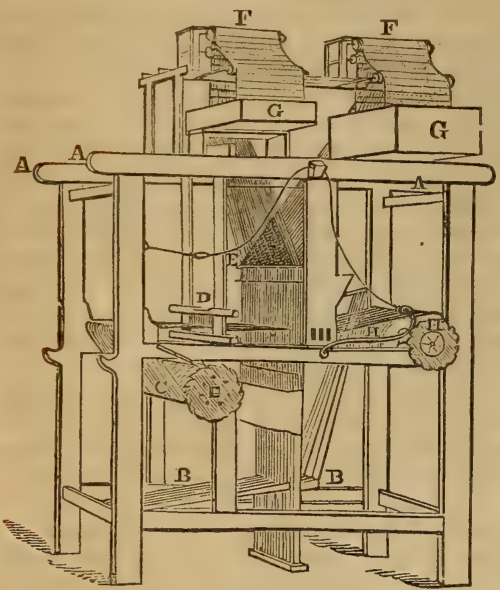

on the damask plan, and requires the weaver to have an assistant. Fig. 270, A A A, is the frame of the loom, consisting of four upright posts, with caps and cross rails to bind them together. The posts are about six feet high. c $c$. the cloth-beam, is a wooden cylinder, six inches or thereby in diameter, of sufficient length to traverse the loom, with iron gudgeons in the two ends, which work in bushes in the side frame. On one end of this beam is a ratchet wheel, with a tooth to keep it from turning round backwards by the tension of the wib. D, the lay, with its reed, its under and upper shell, its two lateral rulers or swords, and rocking-tree above. There are grooves in the upper and under shell, into which the reed is fitted. $\mathbf{E}$, the heddles, or harness, with a double neck attached to each of the tower or card mechanisms F F, of the Jacquard loom. The heddles are connected and work with

the treddles $\mathrm{B} \mathrm{B}$, by means of cords, as shown in the figure. $\mathrm{G} \mathrm{G}$ are wooden boxes for the cards. $\mathrm{H}$, the yarn or warp-beam.

In draw-looms of every kind, there is no sinking of any portion of the warp, as in plain cloth-weaving; but the plane of the warp is placed low, and the threads under which the shuttle is to pass are raised, while all the rest remains stationary. The harness part of this carpet-loom is moved by an assistant boy or girl, who thus allows the weft to be properly decussated, while the weaver attends to working the front

271

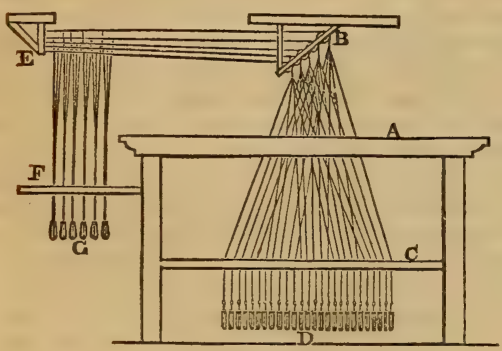

mounting or heddles. Fig. 271 , A represents the frame of a carpet draw-loom; B is a box or frame of pulleys, over which the cords of the harness pass, and are then made fast to a piece of wood, seen at $E$, which the weavers call a table. From the tail of the harness the simples descend, and to the end of each is attached a small handle $\mathrm{G}$, called a bob. These handles being disposed in pairs, and their regularity preserved by means of a perforated board $\mathrm{c}$, it is merely necessary to pull every handle in succession; the weaver, at the same time, working his treddles with his feet, as in any other loom. The treddles are four in number, the fabric being that of plain or alternate cloth, and two treddles allotted for each web. The harness part of the carpet draw-loom is furnished with mails, or metallic eyes, to save friction; two threads being drawn through each eye. The design or pattern of a carpet is drawn upon cross-rule paper, exactly in the same way as every other kind of fancy-loom work, and is transferred from the paper to the mounting by the rules for damask weaving. Suppose that a double web is so mounted that every alternate thread of the one may be raised, so as to form a sufficient shed-way for the shuttle, without depressing the other in the least. Then suppose another web placed above the former, at such a distance that it will exactly touch the convexity of those threads of the former which are raised. Then, if the threads of the latter web are sunk while the others are raised, the two would be entirely incorporated. But if this be only partially done, that is, at particular places, only those parts immediately operated upon will be affected by the action of the apparatus. If the carpet is a two-colored pattern, as black and red, and if upon the upper surface, as extended in the loom, red flowers are to be represented upon a black ground, then all those species of design paper which are colored may be supposed to represent 
the red, and those which are vacant the black. Then counting the spaces upon the paper, omit those which are vacant, and cord those which are colored, and the effect will be produced. But as the two webs are to be raised alternately, whatever is corded for the first handle must be passed by for the second, and vice versa; so that the one wil] form the flower, and the other the ground.

The board by which the simples are regulated appears at $F$. D shows the weights.

CARTHAMLS, or safflower (carthamus tinctorius), (Carthame, Fr.; $F$ rber distel, Germ.), the flower of which alone is used in dyeing, is an annual plant cultivated in Spain, Egypt, and the Levant. There are two varieties of it - one which has large leaves, and the other smaller ones. It is the last which is cultivated in Egypt, where it forms a considerable article of commerce.

Carthamus contains two coloring matters, one yellow and the other red. The first alone is soluble in water; its solution is always turbid: with re-agents it exhibits the characters usually remarlied in yellow coloring matters. The acids render it lighter, the alkalis deepen it, giring it more of an orange hue: both produce a small dur precipitate, in consequence of which it becomes clearer. Alum forms a precipitate of a deep yellow, in small quantity. The solution of tin and the other metallic solutions cause precipitates which have nothing remarkable in them.

The vellow matter of carthamus is not employed; but in order to extract this portion, the carthamus is put into a bag, which is trodden under water, till no more color can be pressed out. The flowers, which were yellow, become reddish, and lose in this opera tion nearly one half of their weight. In this state they are used.

For cxtracing the red part of carthamus, and thereafter applying it to stuff, the property which alkalis possess of dissolving it is had recourse to, and it is afterwards precipitated by an acid.

The process of dyeing consists, therefore, in extracting the coloring matter by means of an alkali, and precipitating it on the stuff by means of an acid. It is this fecula which serves for making the rouge employed by ladies.

As to this rouge, the solution of carthamus is prepared with crystallized carbonate of soda, and it is precipitated by lemon juice. It has been remarlied that lemons, beginning to spoil, were fitter for this operation than those which were less ripe, whose juice retained much mucilage. After squeezing out the lemon juice, it is left to settle for some days. The precipitate of carthamus is dried at a gentle heat upon plates of stone-ware ; from which it is detached and very carefully ground with talc, which has been reduced .0 a rery subtile powder, by means of the leaves of share-grass (presle), and successively passed through sieres of increasing fineness. It is the fineness of the talc, and the greater or less proportion which it bears to the carthamus precipitate, which constitute the difference between the high and low priced rouges.

Carthamus is used for dyeing silk, poppy, nacarat (a bright orange-red), cherry, rose color, and flesh color. The process differs according to the intensity of the color, and the greater or less tendency to flame color that is wanted. But the carthamus bath, whose application may be varied, is prepared as follows :

The carthamus, from which the yellow matter has been extracted, and whose lumps have been broken dow $\mathrm{n}$, is put into a trough. It is repeatedly sprinkled with cendres gravelées (crude pearlashes), or soda (barilla) well powdered and sifted at the rate of 6 pounds for $120 \mathrm{lbs}$. of carthamus; but soda is preferred, mixing carefully as the alkali is introduced. This operation is called amestrer. The amestred carthamus is put into a small trough with a grated bottom, first lining this trough with a closely woven cloth. When it is about half filled, it is placed over the large trough, and cold water is poured into the upper one, till the lower becomes full. The carthamus is then set over another trough, till the water comes from it almost colorless. A little more alkali is now mixed with 1 , and fresh water is passed through it. These operations are repeated till the carthamus be exhausted, when it turns yellow.

After distributing the silk in hanks upon the rods, lemon juice, brought in casks from Provence, is poured into the bath till it becomes of a fine cherry color; this is called turning the bath (virer le bain). It is well stirred, and the silk is immersed and turned round the slicin-sticks in the bath, as long as it is perceived to take up the color. For ponceau (poppy color), it is withdrawn, the liquor is run out of it upon the peg, and it is turned through a new bath, where it is treated as in the first. After this it is driea and passed through fresh baths, continuing to wash and dry it between each operation, till it has acquired the depth of color that is desired. When it has reached the proper point, a brightening is given it by turning it round the sticks seven or eight times in a bath of hot water, to which about half a pint of lemon juice for each pailful of water has been added.

When silk is to be dyed ponceau or flame color, it must be previously boiled as for white; it must then receive a slight foundation of annotto, as explained in treating of this substance. The silk should not be alumed. 
The nacarats, and the deep cherry colors, are given precisely like the poncerux, only they receive no annotto ground; and baths may be employed wbich have served for the poncea $u$, so as to complete their exhaustion. Fresh baths are not made for the latter zolors, unless there be no occasion for the poppy.

With regard to the lighter cherry-reds, rose color of all shades and flesh colors, they are made with the second and last runnings of the carthamus, which are weaker. The deepest shades are passed through first.

The lightest of all these shades, which is an extremely delicate flesh color, requires a little soap to be put into the bath. This soap lightens the color, and prevents it from taking too speedily, and becoming uneven. The silk is then washed, and a little brightening is given it, in a bath which has served for the deeper colors

All these baths are employed the moment they are made, or as speedily as possible, because they lose much of their color upon keeping, by which they are even entirely destroyed at the end of a certain time. They are, moreover, used cold, to prevent the color from being injured. It must have been remarked in the experiments just described, that the caustic alkalis attack the extremely delicate color of carthamus, making it pass to yellow. This is the reason why crystals of soda are preferred to the other alkaline matters.

In order to diminish the expense of the carthamus, it is the practice in preparing the deeper shades to mingle with the first and the second bath about one fifth of the bath of archil.

Dobereiner regards the red coloring matter of carthamus as an acid, and the yellow as a base. His carthamic acid forms, with the alkalis, colorless salts, decomposed by the tartaric and acetic acids, which precipitate the acid of a bright rose-red. Heat has a remarkable influence upon carthamus, rendering its red color yellow and dull. Hence, the colder the water is by which it is extracted, the finer is the color. Light destroys the color very rapidly, and hitherto no means have been found of counteracting this effect. For this reason this brilliant color must be dried in the shade, its dye must be given in a shady place, and the silk stuffs dyed with it must be preserved as much as possible from the light. Age is nearly as injurious as light, especially upon the dye in a damp state. The color is very dear, because a thousand parts of carthamus contain only five of it.

In preparing the finest rouge, the yellow coloring matter being separated by washing with water, the red is then dissolved by the aid of alkali, and is thrown down on linen or cotton rags by saturating the solution with vegetable acid. The color is rinsed out of these rags, dissolved anew in alkalis, and once more precipitated by lemon juice. The oest and freshest carthamus must be selected. It is put into linen bags, which are placed in a stream of water, and kneaded till the water runs off colorless. The bags are then put into water soured with a little vinegar, kneaded till the color is all expelled, and finally rinsed in running water. By this treatment the carthamus loses nearly half its weight. $6633 \mathrm{crvts}$. of safflower were imported into the United Kingdom in 1835, of which $2930 \mathrm{cwts}$. were retained for internal consumption.

CASE-HARDENING is the name of the process by which iron tools, keys, \&c., have their surfaces converted into steel.

Steel when very hard is brittle, and iron alone is for many purposes, as for fine keys, far too soft. It is therefore an important desideratum to combine the hardness of a steely surface with the toughness of an iron body. These requisites are united by the process of case-hardening, which does not differ from the making of steel, except in the shorter duration of the process. Tools, utensils, or ornaments, intended to be polished, are first manufactured in iron and nearly finished, after which they are put into an iron box, together with vegetable or animal charcoal in powder, and cemented for a certain time. This treatment converts the external part into a coating of steel, which is usually very thin, because the time allowed for the cementation is much shorter than when the whole substance is intended to be converted. Immersion of the heated pieces into water hardens the surface, which is afterwards polished by the usual methods. Moxon, in his Mechanic Exercises, p. 56, gives the following receipt for case-hardening:- "Cow's horn or hoof is to be baked or thoroughly dried and pulverized. To this add an equal quantity of bay salt; mix them with stale chamber-ley or white wine vinegar: cover the iron with this mixture, and bed it with the same in loam, or enclose it in an iron box; lay it on the hearth of the forge to dry and harden: then put it into the fire, and blow till the lump have a blood-red heat, and no higher, lest the mixture be burnt too much. Take the iron out, and immerse it in water to harden." I consider the vinegar to be quite superfluous.

I shall now describe the recent application of prussiate (ferrocyanate) of potash to this purpose. The piece of iron, after being polished, is to be made brightly red-hot, and then rubbed or sprinkled over with the above salt in fine powder, upon the part intended to be hardened. The prussiate being decomposed, and apparently dissipated, the iron is 
to be quenched in cold water. If the process has been well managed, the surface of the metal will have become so hard as to resist the file. Others propose to smear over the surface of the iron with loam made into a thin paste with a strong solution of the prussiate, to dry it slowly, then expose the whole to a nearly white heat, and finally plunge the iron into cold water, when the heat has fallen to dull redness. See STEE:I.

CASHMERE or CACHEMERE, a peculiar textile fabric first imported from the kingdom of Cashmere, and now well imitated in France and Great Britain. The material of the Cashmere shawls is the downy wool found about the roots of the hair of the Thibet goat. The year 1819 is remarkable in the history of French husbandry for the acquisition of this breed of goats, imported from the East under the auspices of their government, by the indefatigable courage and zeal of M. Jaubert, who encountered every fatique and dancer to enrich his country with these valuable animals, aided by the patriotism of M. Ternaux, who first planned this importation, and furnished funds for executing it at his own expense and responsibility. He placed a portion of the flock brought by M. Jaubert, at his villa of Saint Ouen, near Paris, where the climate seemed to be very farorable to them, since for several successive years after their introduction M. Ternaux was enabled to sell a great number of both male and female goats. The quantity of fine fleece or down afforded by each animal annually, is from a pound and a half to two pounds.

The wool imported into Europe comes by the way of Casan, the capital of a government of the Russian empire npon the eastern bank of the Wolga; it has naturally a grayish color, but is easily bleached. Its price a few years back at Paris was 17 franes per kilogramme; that is, about 6 shillings the pound avoirdupois. The waste in picking, carding, and spinning, amounts to about one third of its weight.

The mills for spinning Cachemere wool have multiplied very much of late years in France, as appears from the premiums distributed at the exposition of 1834, and the prices of the yarn have fallen from 25 to 30 per cent. notwithstanding their improved fineness and quality. There is a fabric made with a mixture of Cachemere down and spun silk, which is becoming very general. One of the manufacturers, M. Hindenlang, exhibited samples of Cachemere cloth woven with yarn so fine as No. 130 for warp, and No. 228 for weft.

Messrs. Pollino, brothers, of Paris, produced an assortment of Cachemere pieces from 22 to 100 francs the yard, dyed of every fancy shade. Their establishment at Ferté-Bernard oceupies 700 operatives, with an hydraulic wheel of 60 horse power.

The oriental Cashmere shawls are woven by processes extremely slow and consequently costly; whence their prices are very high. They are still sold in Paris at from 4,000 to 10,000 francs a piece; and from 100 to 400 pounds sterling in London. It became necessary, therefore, either to rest satisfied with work which should have merely a surface appearance, or contrive economical methods of weaving, to produce the real Cachemere style with much less labor. By the aid of the draw-loom, and still better of the Jacquard loom, M. Ternaux first succeeded in weaving Cachemere shawls perfectly similar to the oriental in external aspect, which secame fashionable under the name of French Cachemere. But to construct shaw's altogether identical on both sides with the eastern, was a more difficult task, which was accomplishea only at a later period by M. Bauson of Paris.

In both modes of manufacture, the piece is mounted by reeding-in the warp for the different leaves of the hedliles, as is commonly practised for warps in the Jacquard looms. The weaving of imitation shawls is executed, as usual, by as many shuttles as there are colors in the design, and which are thrown across the warp in the order established by the reeder. The greater number of these weft yarns being introduced only at intervals into the web, when the composition of the pattern requires it, they remain floating loose at the back of the piece, and are cut anterwards, without affecting in the least the quality of the texture; but there is a considerable waste of stuff in the weaving, which is worked up into earpets.

The weaving of the imitation of real Cachemere shawls is different from the above. The yarns intended to form the weft are not only equal in number to that of the colors of the pattern to be imitated, but besides this, as many little shuttles or pirns (like those used by embroiderers) are filled with these yarns, as there are to be colors repeated in the breadth of the piece; which renders their number considerable when the pattern is somewhat complicated and loaded with colors. Each of these small bobbins or shuttles passes through only that portion of the flower in which the color of its yarn is to appear, and stops at the one side and the other of the cloth exactly at its limit; it then returns upon itself after having crossed the thread of the adjoining shuttle. From this reciprocal intertexture of all the yarns of the shuttles, it results, that although the weft is composed of a great many different threads, they no less constitute a continuous line in the whole brearth of the web, upon which the lay or batten acts in the ordinary way 
We see, therefore, that the whole art of manufacturing this Cachemere cloth consists in avoiding the confusion of the shuttles, and in not striking up the lay till all have fulfilled their function. The labor does not exceed the strength of a woman, even though she has to direct the loom and work the treddles. Seated on her bench at the end opposite to the middle of the beam, she has for aids in weaving shawls from 45 to 52 inches wide, two girl apprentices, whom she directs and instructs in their tasks. About four hundred days of work are required for a Cachemere shawl of that breadth. For the construction of the loom, see JACQUARD.

In the oriental process, all the figures in relief are made simply with a slender pirn without the shuttle used in European weaving. By the Indians the flower and its ground are made with the pirn, by means of an intertwisting, which renders them in some measure independent of the warp. In the Lyons imitation of this style, the leaves of the heddles lift the yarns of the warp, the needles embroider as in lappet weaving, and the flower is united to the warp by the weft thrown across the piece. Thus a great deal of labor is saved, the eye is pleased with an illusion of the loom, and the shawls cost little more than those made by the common fly shuttle.

Considered in reference to their materials, the French shawls present three distinct classes, which characterize the three fabrics of Paris, Lyons, and Nimes.

Paris manufactures the French Cachemere, properly so called, of which both the warp and the weft are the yarn of pure Cachemere down. This web represents with fidelity the figures and the shades of color of the Indian shawl, which it copies; the deception would be complete if the reverse of the piece did not show the cut ends. The Hindoo shawl, also woven at Paris, has its warp in spun silk, which reduces its price without impairing its beauty much.

Lyons, however, has made the greatest progress in the manufacture of shawls. It excels particularly in the texture of its Thibet shawls, the weft of which is yarn spun with a mixture of wool and spun silk.

Nimes is remarkable for the low price of its shawls, in which spun silk, Thibet down, and cotton, are all worked up together.

The value of shawls exported from France in the following years was-

\begin{tabular}{|c|c|c|c|}
\hline & 1831. & 1832. & 1833. \\
\hline & Francs. & Francs. & Francs. \\
Woollen - . - . & $1,863,147$ & $2,070,926$ & $4,319,601$ \\
Cachemere down - & 433,410 & 655,200 & 609,900 \\
Spun silk - . - & 401,856 & 351,152 & 408,824 \\
\hline
\end{tabular}

It appears that M. J. Girard at Sèvres, near Paris, has succeeded best in producing Cachemere shawls equal in stuff and style of work to the oriental, and at a lower price. They have this advantage over the Indian shawls, that they are woven without seams, in a single piece, and exhibit all the variety and the raised effect of the eastern colors. Women and children alone are employed in his factory.

CASK (Tonneau, Fr.; Fass, Germ.), manufacture of by mechanical power. Mr. Samuel Brown ohtained a patent in November, 1825, for certain improvements in machinery for making casks, which seems to be ingenious and worthy of record. His mechanism consists in the first place of a circular saw attached to a bench, with a sliding rest, upon which rest each piece of wood intended to form a stave of a cask is fixed; and the rest being then slidden forward in a curved direction, by the assistance of an adjustable guide, brings the piece of wood against the edge of the rotatory saw, and causes it to be cut into the curved shape required for the edge of the stave. The second feature is an apparatus with cutters attached to a standard, and traversing round with their carrier upon a centre, by means of which the upper and lower edges of the cask are cuf: round and grooved, called chining, for the purpose of receiving the heads. Thirdly, an apparatus not very dissimilar to the last, by which the straight pieces of wood designed for the heads of the cask are held together, and cut to the circular figure required, and also the bevelled edges produced. And fourthly, a machine in which the cask is made to revolve upon an axis, and a cutting tool to traverse for the purpose of shaving the external part of the cask, and bringing it to a smooth surface.

The pieces of wood intended to form the staves of the cask, having been cut to their required length and breadth, are placed upon the slide-rest of the first mentioned machine, and confined by cramps; and the guide, which is a flexible bar, having been previously bent to the intended curve of the stave and fixed in that form, the rest is then slidden forward upon the bench by the hand of the workman, which as it advances (moving in a curved direction) brings the piece of wood against the edge of the revolving circular saw, by which it is cut to the curved shape desired.

The guide is a long bar held by a series of moveable blocks fitted to the bench by 
serews, and is bent to any desired curve by shifting the screws : the edge of the slide-rests which holds the piece of wood about to be cut, runs against the long guide bar, and of consequence is conducted in a corresponding curved course. The circular saw receives a rapid rotatory motion by means of a band or rigger from any first mover; and the piece of wood may be shifted laterally by means of racks and pinions on the side-rest, by the workman turning a handle, which is occasionally necessary in order to bring the piece of wood up to, or away from, the saw.

The necessary number of staves being provided, they are then set round within a confining hoop at bottom, and brought into the form of a cask in the usual way, anc braced by temporary hoops. The barrel part of the cask being thus prepared, in order to effect the chining, it is placed in a frame upon a platform, which is raised up by a treddle lever, that the end of the barrel may meet the cutters in a sort of lathe above : the cutters are then made to traverse round within the head of the barrel, and, as they proceed, occasionally to expand, by which means the bevels and grooves are cut on the upper edge of the barrel, which is called chining. The barrel being now reversed, the same apparatus is brought to act against the other end, which becomes chined in like manner.

The pieces of wood intended to form the heads of the cask are now to be cut straight by a circular saw in a machine, similar to the first described; but in the present instance the slide-rest is to move forward in a straight course. After their straight edges are thus produced, they are to be placed side by side, and confined, when a scribing cutter is made to traverse round, and cut the pieces collectively into the circular form desired for heading the cask.

The cask having now been made up, and headed by hand as usual, it is placed between centres, or upon an axle in a machine, and turned round by a rigger or band with a shaving cutter, sliding along a bar above it, which cutter, being made to advance and recede as it slides along, shaves the outer part of the cask to a smooth surface.

CASSAVA. Cassava bread, conaque, \&c., are different names given to the starch of the root of the Manioc (Jatropha Manitol, Linn.), prepared in the following manner in the West Indies, the tropical regions of America, and upon the African coast. The tree belongs to the natural family of the euphorbiacece.

The roots are washed, and reduced to a pulp by means of a rasp or grater. The pulp is put into coarse strong canvass bags, and thus submitted to the action of a powerful press, by which it parts with most of its noxious juice (used by the Indians for poisoning the barbs of their arrows.) As the active principle of this juice is volatile, it is easily dissipated by baking the squeezed cakes of pulp upon a plate of hot iron. Fifty pounds of the fresh juice, when distilled, afford, at first, three ounces of a poisonous water, possessing an intolerably offensive smell; of which, 35 drops being administered to a slave convicted of the crime of poisoning, caused his death in the course of six minutes, amid horrible convulsions.*

The pulp dried in the manner above described concretes into lumps, which become hard and friable as they cool. They are then broken into pieces, and laid out in the sun. to dry. In this state they afford a wholesome nutriment, and are habitually used as such by the negroes, as also by many white people. These cakes constitute the only provisions laid in by the natives, in their voyages upon the Amazons. Boiled in water with a little beef or mutton they form a kind of soup similar to that of rice.

The cassava cakes sent to Europe (which I have eaten with pleasure) are composed almost entirely of starch, along with a few fibres of the ligneous matter. It may be purified by diffusion through warm water, passing the milky mixture through a linen cloth, evaporating the strained liquid over the fire, with constant agitation. The starch dissolved by the heat, thickens as the water evaporates, but on being stirred, it becomes granulated, and must be finally dried in a proper stove. Its specific gravity is $1.530-$ that of the other species of starch.

The product obtained by this treatment is known in commerce under the name of $t a$. pioca; and being starch very nearly pure, is often prescribed by physicians as an aliment of easy digestion. A tolerably good imitation of it is made by heating, stirring, and drying potato starch in a similar way.

The expressed juice of the root of manioc contains in suspension a very fine fecula, which it deposites slowly upon the bottom of the vessels. When freed by decantation from the supernatant liquor, washed several times and dried, it forms a beautiful starch, which creaks on pressure with the fingers. It is called cipipa, in French Guyana; it is employed for many delicate articles of cookery, especially pastry, as also for hair powder, starching linen, \&c.

Cassava flour, as imported, may be distinguished from arrow-root and other kinds

* Memoir of Dr. Fermin, communicated to the Academy of Berlin concerning experiments made at Cayenne ugon the juice of the Manioc. 
of starch, by the appearance of its particles viewed in a microscope. They are spherical, all about 1-1000th of an inch in diameter, and associated in groups; those of potato starch are irregular ellipsoids, varying in size from 1-300th to $1-3000$ th of an inch; those of arrow-root have the same shape nearly, but vary in size from 1-500th to $1-800$ th of an inch; those of wheat are separate spheres 1-1000th of an inch.

CASSIS, the black currant (ribes nigra, Linn.), which was formerly celebrated for its medicinal properties, with very little reason.

The only technical use to which it is now applied is in preparing the agreeable liqueur called ratafia, by the following French recipe :- Stone, and crush three pounds of black currants, adding to the magma one drachm of cloves, two of cinnamon, four quarts of spirit of wine, at $18^{\circ}$ Baumé (see Areonftre of BAume), and $2 \frac{1}{2}$ pounds of sugar. Put the mixture into a bottle which is to be well corked; let it digest for a fortnight, shaking the bottle once daily during the first eight days; then strain through a linen cloth, and finally pass through filtering paper.

CASTING OF METALS. (See Founding.) Casis from elastic moulds. - Being much engaged in taking casts from anatomical preparations, Mr. Douglas Fox, Surgeon, Derby, found great difficulty, principally with hard bodies, which, when undercut, or having considerable overlaps, did not admit of the removal of moulds of the ordinary kind, except with injury. These difficulties suggested to him the use of elastie moulds, which, giving way as they were withdrawn from complicated parts, would return to their proper shape; and he ultimately succeeded in making such moulds of glue, which not only relieved him from all his difficulties, but were attended with great advantages, in consequence of the small number of pieces into which it was necessary to divide the mould.

The body to be moulded, previously oiled, must be secured one inch above the surface of a board, and then surrounded by a wall of clay, about an inch distant from its sides. The clay must also extend rather higher than the contained body : into this, warm melted glue, as thick as possible so that it will run, is to be poured, so as to completely cover the body to be moulded; the glue is to remain till cold, when it will have set into an elastic mass, just such as is required.

Having removed the clay, the glue is to be cut into as many pieces as may be necessary for its removal, either by a sharp-pointed knife, or by having placed threads in the requisite situations of the body to be moulded, which may be drawn away when the glue is set, so as to cut it out in any direction.

The portions of the glue mould having been removed from the original, are to be placed together and bound round by tape.

In some instances it is well to run small wooden pegs through the portions of glue, so as to keep them exactly in their proper positions. If the mould be of considerable size, it is better to let it be bound with moderate tightness upon a board to prevent it bending whilst in use; having done as above described, the plaster of Paris, as in common easting, is to be poured into the mould, and left to set.

In many instances wax may also be cast in glue, if it is not poured in whilst too hot; as the wax cools so rapidly when applied to the cold glue, that the sharpness of the impression is not injured.

Glue has been described as succeeding well where an elastic mould is alone applicable; but many modifications are admissible. When the moulds are not used soon after being made, treacle should be previonsly mixed with the glue (as employed by printers) to prevent it becoming hard.

The description thus given is with reference to moulding those bodies which cannot be so done by any other than an elastic mould; but glue moulds will be found greatly to facilitate casting in many departments, as a mould may be frequently taken by this method in two or three pieces, which would on any other principle require many.

CASTOR. (Eng. and Fr.; Biber, Germ.). The castor is an amphibious quadruped, inhabiting North America; also found in small numbers in the islands of the Rhone. In the arts, the skin of this animal is employed either as a fur or as affording the silky hair called beaver, with which the best hats are covered. Beaver skins, which form a very considerable article of trade, are divided into 3 sorts: 1 . The fresh beaver skins from castors, killed in winter before shedding their hair; these are most in request among the furriers, as being the most beautiful. 2. The dry or lean beavers are the skins of the animals killed during the moulting season; they are not much esteemed, as the shin is rather bare. 3. The fat castors: these are the skins of the first sort, which have been worn for some time upon the persons of the savages, and have got imbued with their sweat. The last are principally used in the hat manufacture. In France, the marine otter has been for many years substituted in the place of the castor of beaver.

CASTOR or CASTOREUM. This name is given to a secretion of the castors, 
contained in pear-shaped cellular organic sacs, placed near the genital organs of both the male and female animals. It is a substance analogous to civet and musk, of a consistence similar to thick honey. It has a bitter acrid taste; a powerful, penetrating, fetiủ, fnd very volatile smell; but, when dried, it becomes incdorous. Several chemists, and in particular Bouillon Lagrange, Laugier, and Hildebrandt, have examined castor, and found it to be composed of a resin, a fatty substance, a volatile oil, an extractive matter, benzoic acid, and some salts.

The mode of preparing it is very simple. The sacs are cut off from the eastors when they are killed, and are dried to prevent the skin being affected by the weather. In this state, the interior substance is solid, of a dark color, and a faint smell; it softens with heat, and becomes brittle by cold. Its fracture betrays fragments of membranes, indicating its organic structure. When chewed, it adheres to the teeth somewhat like wax; it has a bitter, slightly acrid, and nauseous taste.

The castor bags, as imported, are often joined in pairs by a kind of ligature. Sometimes the substance which constitutes their value is sophisticated; a portion of the castoreum being extracted, and replaced by lead, clay, gums, or some other foreign matters. This fraud may be easily detected, even when it exists in a small degree, by the absence of the membranous partitions in the interior of the bags, as well as by the altered smell and taste.

The use of castoreum in medicine is considerable, especially in nervous and spasmodic diseases, and it is often advantageously combined with opium.

CASTORINE. A chemical principle lately discovered to the amount of a few parts per cent. in Castoreum.

CASTOR OIL. The expressed oil of the seeds of the Palma Christı, or Ricinus commrnis, a native tree of the West Indies and South America; but which has been cultivated in France, Italy, and Spain. Bussy and Lecanu discovered in it 3 species of fatty matters, obtained partly by saponification, and partly by dry distillation-the margaritic, ricinic, and elaiodic acids. None of these has been separately applied to any use in the arts.

The quantity of eastor oil imported in 1835 into the United Kingdom was 1,109,307 Ibs. ; retained for home consumption, $670,205 \mathrm{lbs}$. See Orls.

CATECHU, absurdly called Terra Japonica, is an extract made from the wood of the tree mimosa catechu, which grows in Bombay, Bengal, and other parts of India. It is prepared bs boiling the chips of the interior of the trunk in water, evaporating the solution to the consistence of sirup over the fire, and then exposing it in the sun to harden. It occurs in flat rough cakes, and under two forms. The first, or the Bombay, is of uniform texture, of a dark red color, and of specific gravity $1 \cdot 39$. The second is more friable and less solid. It has a chocolate color, and is marked inside with red streaks. Its specifie gravity is $1 \cdot 28$.

According to Sir H. Davy, these two species are composed as follows :-

\begin{tabular}{|c|c|c|c|c|c|c|c|c|}
\hline \multirow{4}{*}{$\begin{array}{l}\text { Tannin - } \\
\text { Extractive } \\
\text { Mucilage }\end{array}$} & \multirow[b]{2}{*}{ - } & \multirow[b]{2}{*}{ - } & \multirow[b]{2}{*}{ - } & \multirow[b]{2}{*}{ - } & \multirow[b]{2}{*}{ - } & \multirow[b]{2}{*}{ - } & Bombay. & Bengal. \\
\hline & & & & & & & $54 \cdot 5$ & $48 \cdot 5$ \\
\hline & - & - & & - & - & - & $34 \cdot 0$ & $36 \cdot 5$ \\
\hline & - & - & - & - & - & - & $6 \cdot 5$ & 8 \\
\hline \multirow{2}{*}{\multicolumn{2}{|c|}{ Insoluble matters, }} & sand & and & lime & - & - & 5 & 7 \\
\hline & & & & & & & $100 \cdot 0$ & $100 \cdot 0$ \\
\hline
\end{tabular}

Areka nuts are also found to yield catechu; for which purpose they are cut into pieces watered in an earthen pot with solution of nitre, and have a little of the bark of a species of mimosa added to them. The liquor is then boiled with the nuts, and affords an inspissated decoction.

Good catechu is a brittle, compact solid, of a dull fracture. It has no smell, but a very astringent taste. Water dissolves the whole of it, except the earthy matter, which is probably added during its preparation. Alcohol dissolves its tannin and extractive. 'The latter may' be oxydized, and thus rendered insoluble in alcohol, by dissolving the catcchu in water, exposing it for some time to a boiling heat, and evaporating to dryness.

The tannin of catechu differs from that of galls, in being soluble in alcohol, and more soluble in water. It precipitates iron of an olive color, and gelatin in a mass which gradually becomes brown.

It has been long einployed in India for tanning skins, where it is said to effect this object in five days. I have seen a piece of sole leather completely tanned by it in this country in ten days, the ox-hide having been made into a bag, with the hair outside, and kept filled with the solution of catechu. In India it has also been used to give a brown dye to 
cotton goods, and of late years it has been extensively introduced into the calico print works of Europe. The salts of copper with sal ammoniac cause it to give a bronze color, which is very fast; the proto-muriate of tin, a brownish yellow; the per-chloride of tin, with the addition of nitrate of copper, a deep bronze hue; acetate of alumina alone, a reddish brown, and, with nitrate of copper, a reddish olive gray ; nitrate of iron, a dark brewn gray. For dyeing a golden coffee brown, it has entirely superseded mad. der; one pound of it being equivalent to six pounds of this root.

A solution of one part of catechu in ten parts of water, which is reddish brown, exhibits the following results : with-

\begin{tabular}{|c|c|c|c|}
\hline \\
\hline Acids & - & - & - A brightened shade. \\
\hline Alkalis & - & - & - A darkened shade. \\
\hline Proto-su & alphate of ir & con & - Olive brown precipitate. \\
\hline Per-sulp & hate of iro & & - Olive green \\
\hline Sulphate & e of copper & - & - Yellowish brown. \\
\hline Alum & - & - & A brightening of the liquor. \\
\hline Per-nitr: & ate of iron & - & Olive green precipitate. \\
\hline Nitrate & of copper & - & - Yellowish brown do. \\
\hline Nitrate & of lead & - & - Salmon \\
\hline Proto-ni & itrate of me & reury & - Milk-coffee \\
\hline Muriate & of alumin & & - Brown-yellow. \\
\hline Muriate & of tin & - & Do. \\
\hline Per-chlo & ride of tin & - & darker. \\
\hline Corrosiv & ve sublimat & & - Light chocolate do. \\
\hline Acetate & of alumina & - & - Brightening of the liquor. \\
\hline Acetate & of copper & - & - Copious brown precipitate \\
\hline Aceta & of lead & - & Salmon colored \\
\hline chro & nate of $\mathrm{po}$ & & Copious brown \\
\hline
\end{tabular}

Pure tannin may be obtained from catechu, by treating it with sulphuric acid and car. bonate of lead; but this process has no manufacturing application.

CATGUT (Corde à boyau, Fr.; Darmsaite, Germ.), the name absurdly enough giver to cords made of the twisted intestines of the sheep. The guts being taken while warm out of the body of the animal, are to be cleared of feculent matter, freed from any adhering fat, and washed in a tub of water. The small ends of all the intestines are next to be tied together, and laid on the edge of the tub, while the body of them is left to steep in some water, frequently changed, during two days, in order to loosen the peritoneal and mucous membranes. The bundle of intestines is then laid upon a sloping table which overhangs the tub, and their surface is scraped with the back of a knife, to try if the external membrane will come away freely in breadths of about half the circumference. This substance is called by the French manufacturers filandre, and the process filer. If we attempt to remove it hy beginning at the large end of the intestine, we shall not succeed. This filandre is employed as thread to sew intestines, and to make the cords of rackets and battledoors. The flayed guts are put again into fresh water, and, after steeping a night, are taken out and scraped elean next day, on the wooden bench with the rounded back of a knife. This is called curing the gut. The large ends are now cut off, and sold to the pork-butchers. The intestines are again steeped for a night in fresh water, and the following day in an alkaline lixivium made by adding 4 ounces of potash, and as much pearl-ash, to a pail of water containing about 3 or 4 imperial gallons. This ley is poured in successive quantities upon the intestines, and poured off again, after 2 or 3 hours, till they be purified. They are now drawn several times through an open brass thimble, and pressed against it with the nail, in order to smouth and equalize their surface. They are lastly sorted, according to their sizes, to suit different purposes.

Whip-cord is made from the above intestines, which are sewed together endwise by the filandre, each junction being cut aslant, so as to make it strong and smooth. The cord is put into the frame, and each end is twisted separately; for whip-cord is seldom made out of two guts twisted together. When twisted, it is to be sulphured (see SULPHURING) once or twice. It may also be dyed black with common ink, pink with red ink, which the sulphurous acid changes to pink, and green with a green dye which the color dealers sell for the purpose. The guts take the dyes readily. After being well smoothed, the cord is to be dried, and coiled up for sale.

Hatter's' cords for bews/rings. - The longest and largest intestines of sheep, after being properly treated with the potash, are to be twisted $4,6,8,10$, or 12 together, according to the intended size of the cord, which is usually made from 15 to 25 feet long. This cord must be free from seams and knots. When half dry, it must be exposed twice to the fumes of burning sulphur; and, after cach operation, it is to be well stretched and moothed: it should be finally dried in a state of tension.

Clockmaker's cord. - This cord should be extremely thin, and be therefore made from 
very small intestines, or from intestines slit up in their length by a knife fitted for the purpose; being a lind of lancet surmounted with a ball of lead or wood. The wet gut is strained wer the ball which guides the knife, and the two sections fall down into a vessel placel beneath. Each hand pulls a section. Clockmakers also make use of stronger cords made of 2 or more guts twisted together.

Fiddle and harp strings. - These require the greatest care and dexterity on the part of the worlimen. The treble strings are peculiarly difficult to make, and are best made at Naples, probably because therr sheep, from their small size and leanness, afford the best raw material.

The first scraping of the guts intended for fiddle-strings must be very carefully performed; and the alkaline less, being clarified with a little alum, are added, in a progressively stronger state from day to day, during 4 or 5 days, till the guts be well bleached and swollen. They must then be passed through the thimble, and again cleansed with the lixivium; after which they are washed, spun, or twisted and sulphured during two hours. They are finally polished by friction, and dried. Sometimes they are sulphured twice or thrice before being dried, and are polished between norse-hair cords.

It has been long a subject of complaint, as well as a serious inconvenience to musicians, that catgut strings cannot be made in England of the same goodness and strength as those imported from Italy. These are made of the peritoneal covering of the intestines of the sheep; and, in this country, they are manufactured at Whitechapel, and probably elsewhere in considerable quantity; the consumption of them for harps, as well as for the instruments of the violin family, being very great. Their chief fault is wealiness; whence it is difficult to bring the smaller ones, required for the higher notes, to concert pitch; maintaining at the same time, in their form and construction, that tenuity or smallness of diameter, which is required to produce a brilliant and clear tone.

The inconvenience arising from their breaking when in use, and the expense in the case of harps, where so many are required, are such as to render it highly desirable to improre a manufacture which, to many individuals may, however, appear sufficiently contemptible.

It is well known to physiologists, that the membranes of lean animals are far more tough than of those animals which are fat or in high condition; and there is no reason to doubt that the superiority of the Italian strings arises from the state of the sheep in that country. In London, where no lean animals are slaughtered, and where, indeed, an extravagant and useless degree of fattening, at least for the purpose of food, is given to sheep in particular, it is easy to comprehend why their membranes can never afford a material of the requisite tenacity. It is less easy to suggest an adequate remedy; but a linowledge of the general principle, should this notice meet the eyes of those interested in the subject, may at least serve the purpose of diminishing the evil and improving the manufacture, by inducing them to choose in the market the offal of such carcasses as appear least overburdened with fat. It is probable that such a manufacture might be advantageously established in those parts of the country where the fashion has not, as in London, led to the use of meat so much overfed; and it is equally likely, that in the choice of sheep for this purpose, advantage would arise from using the Welch, the Highland, or the Southdown breeds, in preference to those which, like the Lincoln, are prone to excessive accumulations of fat. It is equally probable, that sheep dying of some of the diseases accompanied by emaciation, would be peculiarly adapted to this pur, ose.

That these suggestions are not merely speculative is proved by comparing the strength of the membranes in question, or that of the other membranous parts, in the unfattened Highland sheep, with that of those found in the London markets.

CATHARTINE. The name proposed by MM. Feneulle and Lassaigne for a chemical principle, which they suppose to be the active constituent of senna.

CAUSTIC. Any chemical substance corrosive of the skin and flesh; as potash, called common caustic, and nitrate of silver, called lunar caustic, by surgeons.

CAVIAR. The salted roe of certain species of fish, especially the sturgeon. This pioduct forms a considerable article of trade, being exported annually from the town of Astrachan alone, upon the shores of the Caspian sea, to the amount of several hundred tons. The Ilalians first introduced it into Eastern Europe from Constantinople, under the name of caviale. Russia has now monopolized this branch of commerce. It is prepared in the following manner : -

The female sturgeon is gutted; the roe is separated from the other parts, and cleaned by passing it through a very fine searce, by rubbing it into a pulp between the hands; this is afterwards thrown into tubs, with the addition of a considerable quantity of salt; the whole is then well stirred, and set aside in a warm apartment. There is another sort of caviar, the compressed, in which the roe, after having been cured in strong brine, is dried in the sun, then put into a cask, and subjected to strong pressure.

CAWK. The English miner's name for sulphate of baryta, or heavy spar. 
CEDRA (Cedrat, Fr.) is the fruit of a species of orange, citron, or lemon, a tree which bears the same name. Its peel is rery thick, and covered with an epidermis which encloses a very fragrant and highly prized essential oil. The preserves flavored with it are very agreeable. The citrons are cut into quarters for the dry comfits, but are put whole into the liquid ones. The liquorist-perfumer makes with the peel of the cerlra an excellent liqueur; for which purpose, he plucks them before they are quite ripe; grates down the peel into a little brandy, or cuts them into slices, and infuses these in the spirits. This infusion is distilled for making perfume; but the flavor is better wher. the infusion itself is used. See Essences, Lrquorist, Perfumery.

CELESTINE. Native sulphate of strontia, found abundantly near Bristol, in the red marl formation. It is decomposed, by ignition with chareoal, into sulphuret of strontia, which is converted into nitrate by saturation with nitric acid, evaporation, and crystallization. This nitrate is employed for the production of the red light in theatrical fire-works.

CEMENTATION. A chemical process, which consists in imbeddino a solid body in a pulverulent matter, and exposing both to ignition in an earthen or metallic case. In this way, iron is cemented with charcoal to form steel, and bottle glass with gypsum powder, or sand, to form Reaumur's porcelain.

CEMENTS. (Ciments, Fr.; Cämente, Kille, Germ.) Substances eapable of taking the liquid form, and of being in that state applied between the surfaces of two bodies, so as to unite them by solidifying. They may be divided into two classes, those which are applieo through the agency of a liquid menstruum, such as water, alcohol, or oil, and those which are applied by fusion with heat.

The diamond cement for uniting broken pieces of china, glass, \&c., which is sold as a secret at an absurdly dear price, is composed of isinglass soaked in water till it becomes soft, and then dissolved in proof spirit, to which a little gum resin, ammoniac, or galbanum, and resin mastic are added, each previously dissolved in a minimum of alcohol. When to be applied, it must be gently heated to liquefy it; and it should be kept for use in a well-corked vial. A glass stopper would be apt to fix so as not to be removeable. This is the cement employed by the Armenian jewellers in Turkey for glueing the ornamental stones to trinkets of various kinds. When well made it resists moisture.

Shellac dissolved in alcohol, or in a solution of borax, forms a pretty good cement. White of egg alone, or mixed with finely sifted quicklime, will answer for uniting objects which are not exposed to moisture. The latter combination is very strong, and is much employed for joining pieces of spar and marble ornaments. A similar composition is used by copper-smiths to secure the edges and rivets of boilers; only bullock's blood is the albuminous matter used instead of white of egg. Another cement in which an analogous substance, the curd or caseum of milk is employed, is made by boiling slices of skim-milk cheeses into a gluey consistence in a great quantity of water, and then incorporating it with quicklime on a slab with a muller, or in a marble mortar. When this compound is applied warm to broken edges of stoneware, it unites them very firmly after it is cold.

A cement which gradually indurates to a stony consistence may be made by mixing 20 parts of clean river sand, two of litharge, and one of quicklime, into a thin putty with linseed oil. The quicklime may be replaced with litharge. When this eement is applied to mend broken pieces of stone, as steps of stairs, it acquires after some time a stony hardness. A similar composition has been applied to coat over brick walls, under the name of mastic.

The iron-rust cement is made of from 50 to 100 parts of iron borings, pounded and sifted, mixed with one part of sal-ammoniac, and when it is to be applied moistened with as much water as will give it a pasty consistency. Formerly flowers of sulphur were used, and much more sal-ammoniac in making this cement, but with decided disadrantage, as the union is effected by the oxydizement, consequent expansion and solidification of the iron powder, and any heterogeneous matter obstructs the effect. The best proportion of sal-ammoniac is, I believe, one per cent. of the iron borings. Another composition of the same kind is made by mixing 4 parts of fine borings or filings of iron, 2 parts of potter's clay, and 1 part of pounded potsherds, and making them into a paste with salt and water. When this cement is allowed to concrete slowly on iron joints, it becomes very nard.

For making architectural ornaments in relief, a moulding composition is formed of chalk, glue, and paper paste. Even statues have been made with it, the paper aiding the cohesion of the mass.

Mastics of a resinous or bituminous nature which must be softened or fused by heat are the following:-

Mr. S. Varley's consists of sixteen parts of whiting sifted and thoroughly dried by a red heat, adding when cold a melted mixture of 16 parts of black rosin and 1 of bees'-wax, and stirring well during the cooling. 
Mr. Singer's electrical and chemical apparatus cement consists of 5 lbs. of rosin 1 of bees'-wax, 1 of red ochre, and two table-spoonsful of Paris plaster, all melted torether. A cheaper one for cementing voltaic plates into wooden troughs is made with 6 pormuls of rosin, 1 pound of red ochre, $\frac{1}{2}$ of a pound of plaster of Paris, and $\frac{1}{4}$ of a pound of linseed oil. The ochre and the plaster of Paris should be calcined beforehand, and added to the other ingredients in their melted state. The thinner the stratum of cement that is inter rosed, the stronger, generally speaking, is the junction.

Boiled linseed oil and red lead mixed together into a putty are often used by coppersmiths and engineers, to secure joints. The washers of leather or cloth are sineared with this mixture in a pasty state.

The resin mastic alone is sometimes used by jewellers to cement by heat cameos of white enamel or colored glass to a real stone, as a ground to produce the appearance of an onix. Mastic is likewise used to cement false backs or doublets to stones, to alter their hue.

Yelted brimstone, either alone, or mixed with rosin and brick dust, forms a tolerably good and very cheap cement.

Plumber's cement consists of black rosin one part, brick dust two parts, well incorpo. rated by a melling heat.

The cement of dihl for coating the fronts of buildings consists of linseed oil, renderen dry by boiling with litharge, and mixed with porcelain clay in fine powder, to give it the consistence of stiff mortar. Pipe-clay would answer equally well if well dried, and any color might be given with ground bricks, or pottery. A little oil of turpentine to thin this cement aids its cohesion upon stone, brick, or wood. It has been applied to sheets of wire eloth, and in this state laid upon terraces, in order to make them water tight; but it is little less expensive than lead.

The bituminous or black cement for bottle-corks consists of pitch hardened by the ad. dition of rosin and brick-dust.

In certain localities where a limestone impregnated with bitumen occurs, it is dried, ground, siftel, and then mixed with about its own weight of melted pitch, either mineral, vegetable, or that of coal tar. When this mixture is getting semifluid, it may be moulded into large slabs or tiles in wooden frames lined with sheet iron, previously smeared over with common lime mortar, in order to prevent adhesion to the moulds, which, being in moveable pieces, are easily dismounted so as to turn out the cake of artificial bituminous stone. This cement is manufactured upon a great scale in many places, and used for making Italian terraces, covering the floors of balconies, flat roofs, water reservoirs, water conduits, \&c. When laid down, the joints must be well run together with hot irons. The floor of the terrace should be previously covered with a layer of Paris plaster or common mortar, nearly an inch thick, with a regular slope of one inch to the yard. Such bituminous cement weighs 144 pounds the cubic foot; or a foot of square surface, one inch thick, weighs 12 pounds. Sometimes a second layer of these slabs or tiles is applied over the first, with the precaution of making the seams or joints of the upper correspond with the middle of the under ones. Occasionally a bottom bed, of coarse cloth or gray paper, is applied. The larger the slabs are made, as far as they can be conveniently transpurted and laid down, so much the better. For hydraulic cements, see Mortar.

CERASIN. The name given by Dr. John to those gums which swell, but do not dissolve in water; such as gum tragacanth. It is synonymous with BAssorine, which see.

CERATE, from cera, wax. An unguent, of rather a stiff consistence, made of oil, or lard and wax, thickened occasionally with pulverulent matters.

CERINE. A substance which forms from 70 to 80 per cent. of bees'-wax. It may be obtained by digesting wax, for some time, in spirit of wine, at a boiling temperature. The myricine separates, while the cerine remains dissolved, and may be obtained from the decanted liquor by evaporation. Cerine is white, analogous to wax, fusible at $134^{\circ} \mathrm{F}$., hardly acted upon by hot nitric acid, but is readily carbonized by hot sulphuric acid. When treated with caustic alkaline ley, it is converted into margaric acid and ceraine.

CERIUM. A peculiar metal discovered in the rare mineral, called cerite, found only in the copper mine of Bastnaes, near Riddarhytta, in Sweden. Cerium, extracted from its chloride by potassium, appears as a dark red or chocolate powder, which assumes a metallic lustre by friction. It does not conduct electricity well, like other metals; it is infusible; its specific gravity is unknown. It has been applied to no use in the arts.

CERUSE. A name of white lead. See LEAD.

CETINE. The name given by Chevreul to spermaceti.

CHAINWORK is a peculiar style of textile fabric, to which hosiery and tambouring belong. See Hosiery.

CHALK. (Craie, Fr.; Kreide, Germ.) A friable carbonate of lime, white, opaque, 
soft, dull, or without any appearance of polish in its fracture. Its specific gravity varies from $2 \cdot 4$ to $2 \cdot 6$. It usually contains a little silica, alumina, and oxyde of iron. It may be purified by trituration and elutriation. The silicious and ferruginous matters subside first, and the finer chalky particles floating in the supernatant liquid, may be decanted with it, and obtained by subsidence. When thus purified, it is called whitening and Spanish white, in England; schlemmkreide, in Germany; blanc de Troyes, and blanc de Meudon, in France. Pure chalk should dissolve readily in dilute muriatic acid, and the solution should afford no precipitate with water of anc monia.

CHALK-Black. A mineral, called also drawing-slate.

CHALK-French. Steatite, or soap-stone; a soft magnesian mineral.

CHALK-Red. A clay colored with the peroxyde of iron, of which it contains about 17 per cent.

CHARCOAL. The fixed residuum of vegetables exposed to ignition out of contact of air. In the article CARBoN, I have described the general properties of charcoal and the simplest mode of making it. I shall here detail the best systems of manufacturing this product upon the continent of Europe.

To carbonize wood under a moveable covering, the plan of meiler, or heaps, is employed very much in Germany. The wood is arranged either in horizontal layers, or in nearly vertical ones, with a slight slope, so as to form conical rounded heaps of differ'nt sizes. The former are called lying meiler, fig. 272; the latter standing meiler, figs. 27:... and 274 .

272

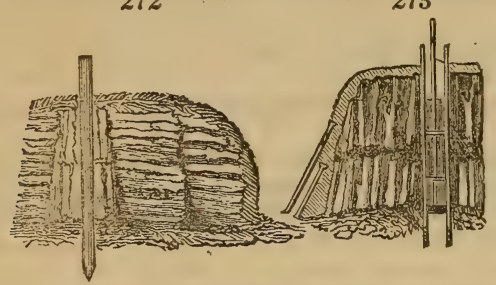

274

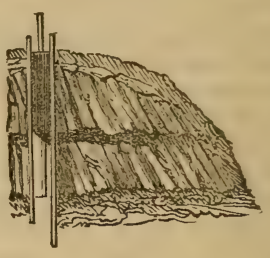

Both are distributed in much the same way.

In districts where the wood can be transported into one place by means of rivers, or mountain-slides, a dry flat space must be pitched upon, screened from storms and floods, which may be walled round, having a slight declivity made in the ground, toward the centre. See fig. 275 . Into this space the tarry acid will partially fall, and may be conducted outward, through a covered gutter beneath, into a covered tank. The mouth of the tank must be shut, during the coaking, with an iron or stone slab, luted with clay. A square iron plate is placed over the inner orifice of the gutter, to prevent

275

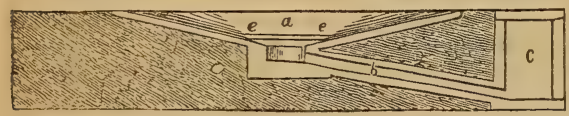

it being choked with cual ashes. Fig. $27 \overline{5}$ represents a walled meiler station; $a$, the station; $b$, the gutter ; $c$, the tank, which is covered with the slab $d ; e$, a slab which serves to keep the gutter clear of coals. The cover of the heaps is formed of earth, sand, ashes, or such other matter as may be most readily found in the woods. They should be kindled in the centre. From 6 days to 4 weeks may be required for charring a heap, according to its size ; hard wood requiring most time; and the slower the process, the better and greater is the product, generally speaking.

276
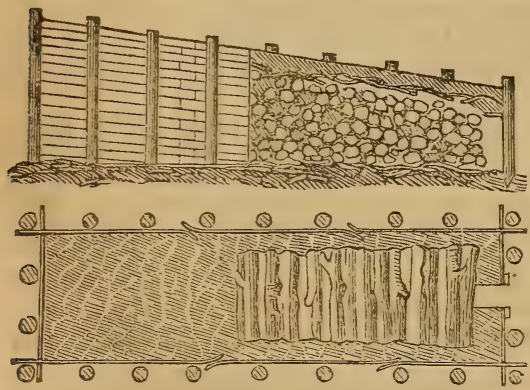

277
Charring of wood in mounds (Haufe or liegende werke), figs. 276 and 277 , differs from that in the meiler, because the wood in the haufe is successively charred, and the charcoal is raked out by little and little. The product is said to be greater in this way, and also better. Uncleft billets, 6 or 8 feet long, being laid over each other, are covered with ashes, and then carbonized. The station is sometimes horizontal, and sometimes made to slope. The length may be 24 feet, the breadth 8 feet; anc the wood is laid cross. 
wise. Piles are set perpendicularly to support the roof, made of boughs and leaves, corered with ashes. Pipes are occasionally laid within the upper part of the mounds, which serve to catch and carry off some of the liquid products into proper tanks.
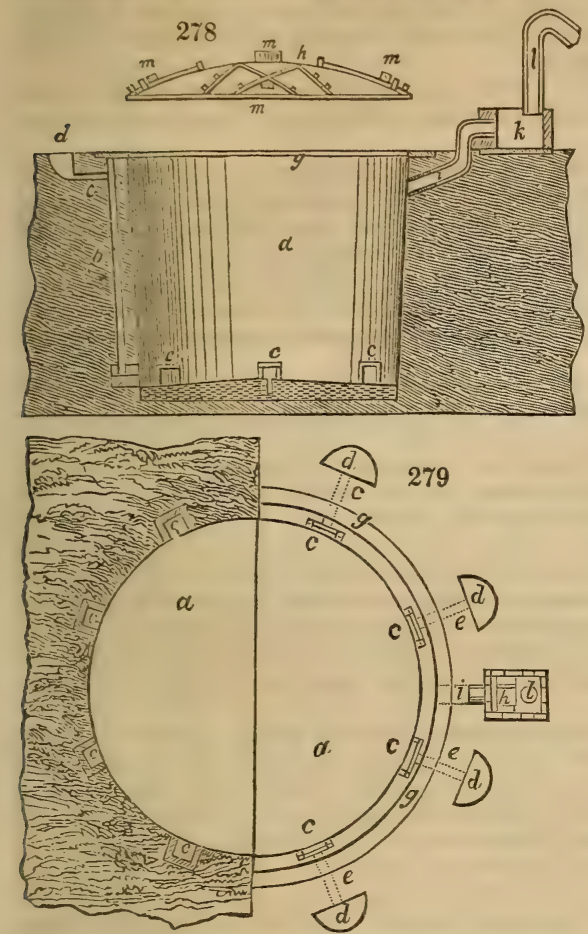

Fig. 278 is a vertical section, and fig. 279, a half bird's- eye view, and half cross section, at the height of the pit-bottom, of Chabeaussiere's kiln for making wood charcoal. $a$ is the oven; $b$, vertical air-pipes; $c c$, horizontal flues for admitting air to the kiln; $d d$, small pits which communicate by short horizontal pipes $e e$, with the vertical ones; $f$, the sole of the kiln, a circle of brickwork, upon which the cover or hood $h$ reposes; $i$, a pipe which leads to the cistern $k ; l$, the pipe destined for carrying off the gaseous matter; $m m$, holes in the iron cover or lid.

The distribution of the wood is like that in the horizontal meilers, or heaps ; it is kindled in the central vertical canal with burning fuel, and the lid is covered with a few inches of earth. At the beginning of the operation all the draught flues are left open, but they are progressively closed, as occasion requires. In eight kilns of this kind, 500 decasters of oak wood are earbonized, from which 16,000 hectolitres of chareoal are obtained, equal to 64,000 pounds French, being about 25 per cent. ; besides tar and 3000 velts of wood vinegar, of from $2^{\circ}$ to $3^{\circ}$ Baumé.

At Crouy upon the Ourcq, near Meaux, there is a well-constructed kiln for making turf-charcoal. It resembles most nearly a tar-kiln. In fig. 280, $a$ is the cylindrical

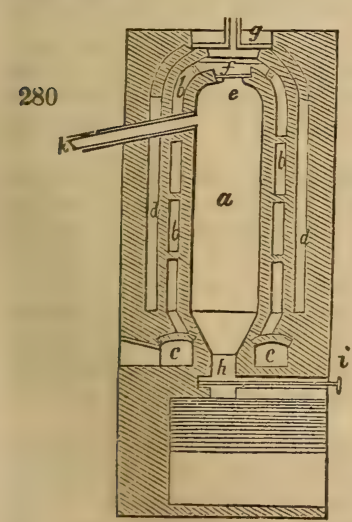
coaking place, whose surrounding walls are heated by the flame which passes through the intermediate space $b$. The place itself is divided by partitions of fire tiles into three stages, through the apertures in which the flames of the fire $c c$, rise, and heat the exterior of the coaking apartment. In order to confine the heat, there is in the enclosing walls of the outer kiln a cylindrical hollow space $d$, where the air is kept stagnant. Through the apertures left in the upper end at $e$, the turf is introduced; they are then shut with an iron plate $f$, which is covered with ashes or sand. The fire-place opens above this aperture, and its outlet is provided with a moveable iron cover $g$, in which there is a small hole for the issue of the gases. The sole of the kiln consists of a cast iron slab $h$, which may be raised by means of a hook $i$ upon it. This is drawn back after the carbonization is completed, whereby the charcoal falls from the coaking space into a subjacent vault. The volatile products are carried off by the pipe $k$, and led into the condensing cistern; the gases escaping to the fire-place where they are burned. The iron slab is protected from the corrosion of the acid vapors by a layer of coal ashes.

CHICA is a red coloring principle made use of in America by some Indian tribes to stain their skins. It is extracted from the bignonia chica by boiling its leaves in water, decanting the decoction, and allowing it to settle and cool, when a red matter falls down, which is formed into cakes and dried. This substance is not fusible, and 
when burned, diffuses the same odor as animal bodies do. $t$ is insoluble in cold water, very soluble in alcohol and ether, but after the evaporation of these liquids, it is recovered unchanged. Fats and unctuous oils both dissolve it. It is soluble in carbonated and caustic allaline leys, from which it is precipitated by the acids without alteration. An excess of alkali, huwever, speedily decomposes it. Nitric acid transforms it into oxalic acid, and a bitter matter. Chlorine makes it white.

The savages mix this pigment with the fat of the cayman or alligator, and rub their skins with the mixture. It may probably be turned to account in the arts of civilized nations.

CHIMNEY. (Cheminée, Fr.; Schornstein, Germ.) Chimney is a modern invention for promoting the draught of fires and carrying off the smoke, introduced into England so late as the age of Elizabeth, though it seems to have been employed in Italy 100 years before. The Romans, with all their luxurious refinements, must have had their Epicurean cookery placed in perpetual jeopardy from their kitchen fires, which, having no vent by a vertical tunnel in the walls, discharged their smoke and frequently their flames at the windows, to the no small alarm of their neighbors, and annoyance of even the street passengers.

Chimneys in dwelling houses serve also the valuable purpose of promoting salubrious circulation of air in the apartments, when not foolishly sealed with anti-ventilating stovechests.

The first person who sought to investigate the general principles of chimney draughts, in subserviency to manufacturing establishments, was the celebrated Montgolfier. As the ascent of heated air in a conduit depends upon the diminution of its specific gravity, or, in other words, upon the increase of its volume by the heat, the ascensional force may be deduced from the difference between the density of the elastic fluid in the interior of the chimney, and of the external air; that is, between the different heights of the internal and external columns of elastic fluid supposed to be reduced to the same density. In the latter case, the velocity of the gaseous products of combustion in the interior of the chimney is equal to that of a heavy body let fall from a height equal to the difference in height of the two aerial columns.

To illustrate this position by an example, let us consider the simple case of a chimney of ventilation for carrying off foul air from a factory of any kind; and suppose that the tunnel of iron be incased throughout with steam at 212 degrees Fahr. Suppose this tunnel to be 100 yards high, then the weight of the column of air in it will be to that of a column of external air 100 yards high, assumed at $32^{\circ} \mathrm{F}$., inversely as its expansion by $180^{\circ}$; that is, as 1000 is to 1.375 ; or as 72.727 is to 100 . The column of external air at $32^{\circ}$ being 100 yards, the internal column will be represented by 72.727 ; and the difference $=27 \cdot 27$, will be the amount of unbalanced weight or pressure, which is the effective cause of the ventilation. Calculating the velocity of current due to this difference of weight by the well-known formula for the fall of heavy bodies, that is to say, multiplying the above difference, which is $27 \cdot 27$, by the constant factor 19.62 , and extracting the square root of the product; thus $\sqrt{V 19 \cdot 62 \times 27 \cdot 27}=23 \cdot 13$ will be the velocity in yards per second, which, mnltiplied by 3 , gives $60^{\circ} 30$ feet. The quantity of air which passes in a second is obtained of course by multiplying the area or cross section of the tunnel by this velocity. If that section is half a yard, that is = a quadrangle $2 \frac{1}{4}$ feet by 2 , we shall have $23.13 \times 0.5=11.565$ cubic yards, $=312 \frac{1}{4}$ cubic feet.

The problem becomes a little more complicated in calculating the velocity of air which has served for combustion, because it has changed its nature, a variable proportion of its oxygen gas of specific gravity $1 \cdot 111$, being converted into carbonic acid gas of specific gravity 1.524 . The quantity of air passed through well constructed furnaces may, in general, be regarded as double of what is rigorously necessary for combustion, and the proportion of carbonic acid generated, therefore, not one half of what it would be were all the oxygen so combined. The increase of weight in such burned air of the temperature of $212^{\circ}$, over that of pure air equally heated, being taken into account in the preceding calculation, will give us about 19 yards or 57 feet per second for the velocity in a chimney 100 yards high incased in steam.

Such are the deductions of theory ; but they differ considerably from practical results, in consequence of the friction of the air upon the sides of the chimneys, which varies likewise with its form, length, and quality. The direction and force of the winds also exercise a variable influence upon chimney furnaces differently situated. In chimneys made of wrought iron, like those of steamboats, the refrigeration is considerable, and causes a diminution of velocity far greater than what occurs in a factory stalk of well built brick work. In comparing the numbers resulting from the trials made on chimneys of different materials and of different forms, it has been concluded that the obstruction to the draught of the air, or the deduction to be made from the theoretical velocity of efflux, is directly proportional to the length of the chimneys and to the square of the velocity, and inversely to their diameter. With an ordinary wrought-iron pipe, of from 4 inches 
io $y$ inches diameter, attached to an ordinary stove, burning good charcoal, the difference is prodigious between the velocity calculated by the above theoretical rule, and that observed by means of a stop-watch, and the ascent of a puff of smoke from a little tow, dipped in oil of turpentine thrust quickly into the fire. The chimney being 45 feet high, the temperature of the atmosphere $68^{\circ} \mathrm{Fahr}$, the velocity per second was, -

$\begin{array}{cc}\text { Trials. } & \text { By theory. } \\ 1 & 26.4 \text { feet } \\ 2 & 29 \cdot 4 \\ 3 & 34 \cdot 5\end{array}$

By experiment.
5 feet
$5 \cdot 76$
$6 \cdot 3$

Mean temperature of chimney. $190^{\circ} \mathrm{Fahr}$. 214

To obtain congruity between calculation and experiment, se $;$ eral circumstances must be introduced into our formulæ. In the first place, the theoretical velocity must be multiplied by a factor, which is different according as the chimney is made of bricks, pottery, sheet iron, or cast iron. This factor must be multiplied by the square root of the diameter of the chimney (supposed to be round), divided by its length, increased by four times its diameter. Thus, for pottery, its expression is $2.06 \sqrt{\mathrm{L}+\mathrm{D}}$; $\mathrm{D}$ being the diameter, and $\mathrm{L}$ the length of the chimney.

A pottery chimney, 33 feet high, and 7 inches in diameter, when the excess of its mean temperature above that of the atmosphere was $205^{\circ} \mathrm{Fahr}$., had a pressure of hot air equal to 11.7 feet, and a velocity of $7 \cdot 2$ feet per second. By calculating from the last formula, the same number very nearly is obtained. In none of the experiments did the velocity exceed 12 feet per second, when the difference of temperature was more than $410^{\circ} \mathrm{Fahr}$.

Every different form of chimney would require a special set of experiments to be made for determining the proper factor to be used.

This troublesome operation may be saved by the judicious application of a delicate differential barometer, such as that invented by Dr. Wollaston; though this instrument does not seem to have been applied by its very ingenious author in measuring the draughts or ventilating powers of furnaces.

If into one leg of this differential syphon water be put, and fine spermaceti oil into the other, we shall have two liquids, which are to each other in density as the numbers $S$ and 7 . If proof spirit be employed instead of water, we shall then have the relation of very nearly 20 to 19 . I have made experiments on furnace draughts with the instrument in each of these states, and find the water and oil syphon to be sufficiently sensible; for the weaker draughts of common fire-places the spirits and oil will be preferable barometric fluids.

To the lateral projecting tube of the instrument, as described by Dr. Wollaston, I found it necessary to attach a stop-cock, in order to cut off the action of the chimney, while placing the syphon, to allow of its being fixed in a proper state of adjustment, with its junction line of the oil and water at the zero of the scale. Since a slight deviation of the legs of the syphon from the perpendicular changes very considerably the line of the level, this adjustment should be made secure by fixing the horizontal pipe tightly into a round hole, bored into the chimney stalk, or drilled through the furnace door. On gently turning the stop-cock, the difference of atmospherical pressure corresponding to the chimney draught, will be immediately indicated by the ascent of the junction line of the liquids in the syphon. This modification of apparatus permits the experiment to be readily rectified by again shutting off the draught, when the air will slowly re-enter the syphon; because the projecting tube of the barometer is thrust into the stop-cock, but not hermetically joined; whereby its junction line is allowed to return to the zero of the scale in the course of a few seconds.

Out of many experiments made with this instrument, I shall content myself with describing a few, very carefully performed at the breweries of Messrs. Trueman, Hanbury, and Buxton, and of Sir H. Meux, Bart., and at the machine factory of Messrs. Braithwaite; in the latter of which I was assisted by Captain Ericsson. In the first trials at the breweries, the end of the stop-cock attached to the differential barometer was lapped round with hemp, and made fast into the circular peep-hole of the furnace door of a wort copper, communicating with two upright parallel chimneys, each 18 inches square and 50 feet high. The fire was burning with fully its average intensity at the time. The adjustment of the level being perfect, the stop-cock orifice was opened, and the junction level of the oil and water rose steadily, and stood at $1 \frac{1}{4}$ inches, corresponding to $\frac{1.25}{8}=0.156$ of 1 inch of water, or a column of air 10.7 feet high. This difference of pressure indicates a velocity of 26 feet per second. In a second set of experiments, the extremity of the stop-cock was inserted into a hole, bored through the chimney stalk of the boiler of a Boulton and Watt steam-engine of twenty-horse power. The area of this chimney was exactly 18 inches square at the level of the bored hole, and its summit rose 50 feet above it. The fire-grate was about 10 feet below that level. On 
opening the stop-cock, the junction line rose $2 \frac{1}{4}$ inches. This experiment was rerified hy repetition upon different days, with fires burning at their average intensity, and consuming fully 12 lbs. of the best coals hourly for each horse's power, or nearly one ton and a third in twelve hours. If we divide the number $2 \frac{1}{4}$ by 8 , the quotient 0.28 will represent the fractional part of 1 inch of water, supported in the syphon by the unbalanced pressure of the atmosphere in the said chimney; which corresponds to $19 \frac{1}{4}$ feet of air, and indicates a velocity in the chimney current of 35 feet per second. The consumption of fuel was much more considerable in the immense grate under the wort copper, than it was under the steam-engine boiler.

In my experiments at Messrs. Braithwaite's factory, the maximum displacement of the junction line was $1 \mathrm{inch}$, when the differential oil and water barometer was placed in direct communication with a chimney 15 inches square, belonging to a steam boiler, and when the fire was made to burn so fiercely, that on opening the safety-valve of the boiler, the excess of steam beyond the consumption of the engine rushed out with such violence as to fill the whole premises. The pressure of one eighth of an inch of water denotes a velocity of draught of 23.4 feet per second.

In building chimneys, we should be careful to make their area rather too large than too small; because we can readily reduce it to any desired size, by means of a sliding register plate near its bottom, or a damper plate applied to its top, adjustable by wires or chains, passing over pulleys. Wide chimneys are not so liable as narrow ones to have their draught affected by strong winds. In a factory, many furnace flues are often conducted into one vertical chimney stalk, with great economy in the first erection, and increased power of draught in the several fires.

Vast improvements have been made in this country, of late years, in building stalks for steam boilers and chemical furnaces. Instead of constructing an expansive, lofty scaffolding of timber round the chimney, for the bricklayers to stand upon, and to place their materials, pigeon-holes, or recesses, are left at regular intervals, a few feet apart, within the chimney, for receiving the ends of stout wooden bars, which are laid across, so as to form a species of temporary ladder in the interior of the tunnel. By means of these bars, with the aid of ropes and pulleys, everything may be progressively hoisted, for the building of the highest engine or other stalks. An expert bricklayer, with a handy laborer, can in this way raise, in a few weelis, a considerable chimney, 40 feet high, 5 feet 8 inches square outside, 2 feet 8 inches inside at the base, 28 inches outside, and 20 inches inside at the top. To facilitate the erection, and at the same time increase the solidity of an insulated stalk of this kind, it is built with three or more successive plinths, or recedures, as shown in fig. 281. It is necessary to make such chimneys thick and substantial near the base, in order that they may sustain the first violence of the fire, and prevent the sudden dissipation of the heat. When many flues are conducted into one chimney stalk, the area of the latter should be nearly equal to the sum of the areas of the former, or at least of as many of them as shall be going simultaneously. When the products of combustion from any furnace must be conducted downwards, in order to enter near the bottom of the main stalk, they will not flow off until the lowest part of the channel be heated by burning some wood shavings or straw in it, whereby the air syphon is set agoing. Immediately after hindling this transient fire at that spot, the orifice must be shut by which it was introduced; otherwise the draught of the furnace would be seriously impeded. But this precaution is seldom necessary in great factories, where a certain degree of heat is always maintained in the flues, or, at least, should be preserved, by shutting the damper plate of each separate flue, whenever its own furnace ceases to act. Sach chimneys are finished at top with a coping of stone-slabs, to secure their brickwork against the infiltration of rains, and they should be furnished with metallic conducting rods, to protect them from explosions of lightning.

When small domestic stoves are used, with very slow combustion, as has been recently proposed, upon the score of a misjudged economy, there is great danger of the inmates being suffocated or asphyxied, by the regurgitation of the noxious burned air. The smoke doctors who recommend such a vicious plan, from their ignorance of chemical science, are not aware that the carbonic acid gas, of coke or coal, must be heated $250^{\circ} \mathrm{F}$. above the atmospheric air, to acquire the same low specific gravity with it. In other words, unless so rarefied by heat, that gaseous poison will descend through the orifice of the ash-pit, and be replaced by the lighter air of the apartment. Drs. Priestley and Dalton have long ago shown the co-existence of these two-fold crossing currents of air, even through the substance of stone-ware tubes. True economy of heat, and salubrity, alike require vivid combustion of the fuel, with a somewhat brisk draught inside of the chimney, and a corresponding abstraction of air from the apartment. Wholesome continuous ventilation, under the ordinary circumstances of dwelling houses, cannot be secured in any other way. Were these mephitic stoves, which have been of late so ridiculously puffed in the public prints, generally introduced, the faculty would 
need to be immediately quadrupled to supply the demand for medical advice; for headaches. sicliness, nervous ailments, and apoplexy, would become the constant inmates of every inhabited mansion. The phenomeria of the grottc of Pausilippo might then be daily realized at home, among those who ventured to recline upon sofas in such carbonated apartments; only instead of a puppy being suffocated pro tempore, human beings would be sacrificed, to save two-penny worth of fuel per diem.

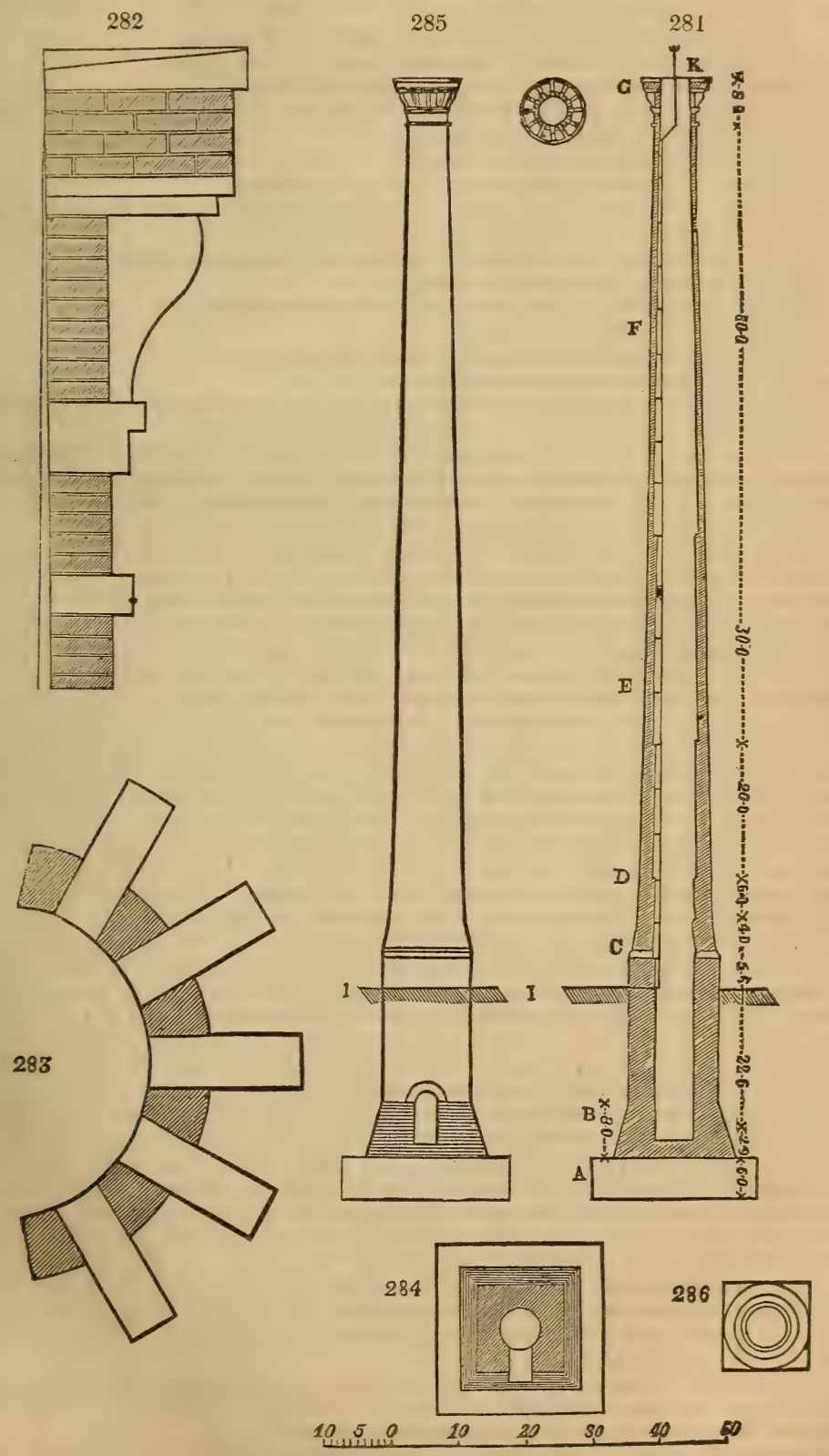


The figures upon the preceding page represent one of the two chimneys recently erect ed at the Camden Town station, for the stearn boilers of the two engines of 60 horsepower each, belonging to the London and Birmingham Railway Company. These en gines draw their train of carriages up the inclined plane of Hampstead Hill. The chim. neys were designed by. Robert Stephenson, Esq., engineer to the Company, executed by William Cubitt, Esq., of Gray's Inn Road,-and do equal honor to both gentlemen, being probably the most elegant and substantial specimens of this style of architecture in the world. In the section, fig. 281,

A represents a bed of concrete, 6 feet thick, and 24 feet square.

B, brick footings set in cement; the lower course 19 feet square.

c, Bramley-fall stone base, with a chain of wrought iron let into it.

$\mathrm{D}$, a portion, 15 feet high, curved to a radius of 113 feet, built entirely of Malm paviors, (a peculiarly good kind of bricks.)

$\mathbf{E}$, shaft built of Malm paviors in mortar.

F, ditto, built from the inside, without exterior scaffolding.

$\mathrm{G}$, the cap ornamented (as shown in the plan alongside) with Portland stone, the dressings being tied together with copper cramps and an iron bond.

Fig. 282 represents the mouldings of the top, upon an enlarged scale.

Fig. 283, a plan of the foundation, ditto.

Fig. 284, ditto, at the level of the entrance of the flue, as seen in

Fig. 285, the elevation of the chimney.

Fig. 286, plan at the ground level I, in figs. 281 and 285.

K, fig. 281, the lightning conducting rod.

CHINTS is a peculiar style of fast-printed calico, in which figures of at least five different colors are impressed upon a white or light colored ground.

CHLORATE OF POTASH, commonly called oxymuriate of potash. This interesting saline compound has become the object of a pretty extensive manufacture, in consequence of its application to make matches for procuring instantaneous light, and a detonating powder for fire-arms. It may be prepared both in the humid and dry way.

Having made a strong solution of purified potash, or carbonate of potash, with from two to three parts of water, we pass through it in a Woulfe's apparatus a current of chlorine gas, till it ceases to absorb any more. Chloride of potash and chloride of potassium alone are formed as long as there is an excess of alkali in the solution; but afterwards, in the further reaction of the materials, the chloride passes into the state of a chlorate, and, as such, precipitates from the solution. During the first half of the operation, that is, till the potash be about one half saturated with chlorine, as indicated by litmus paper ceasing to be darkened and beginning to be blanched, only the chloride of potassium or muriate of potash falls. The process should be interrupted at this point in order to remove the salt, to wash it, to add the washings to the liquor, and then to transmit the gas freely through the solution. As the operation advances, less muriate of potash is formed. and at length nothing but the pure chlorate is separated in crystals. When finally the bubbles of gas pass through without being sensibly absorbed, the process is known to be completed; the liquid may then be allowed to settle, and be poured off from the crystals of chlorate of potash, which are purified from the muriate by dissolving them in three times their weight of boiling water, and filtering the solution while hot. On its cooling, the chlorate will separate in pearly-looking crystalline plates. It may be rendered quite pure by a second crystallization, in which state it does not affect solution of nitrate of silver.

The above potash ley usually gets a reddish tint in the course of the process in consequence of a little manganesic acid coming over with the chlorine, but it gradually loses this color as the saturation becomes complete, when the solution turns yellow. The tubes for conveying the gas should be of large diameter, if they be plunged into the saline solution, because the crystallization which takes place in it is apt to choke them up. This inconvenience may however be obviated by attaching to the end of the glass tube, a tube of caoutchouc terminated in a small glass funnel, or simply the neck of a caoutchouc bottle with a part of its body, whose width will not be readily closed with a saline crust. The residuary lixivium may be used against another operation, or it may be evaporated down to half its bulk and set aside to crystallize, whereby sume more chlorate will be obtained, mixed indeed with muriate and carbonate, from which however it may be separated by a second crystallization. In general the pure chlorate obtained does not exceed one tenth the weight of the potash employed; because in thus treating potash with chlorine, five sixths of it are converted into muriate of potash and only one sixth into chlorate, and a part of the latter adheres to the muriate, or is lost in the mother waters of the crystallizations.

The chlorate of yotash may be more conveniently mannfactured, like that of lime, in the dry way. St. Romer patented at Vienna the following method for that purpose in 1821 : - Ten pounds of crystallized peroxyde of manganese are to be finely pulverized, mixed with ten pounds of plumbage, and thirty pounds of common salt, and put into the 
leaden retort represented in fig. 287, p. 293. From the middle of the helmet-sliaped lid of this vessel, a leat tube, two feet long, and two inches wide, conducts to the receiver, which is a square earthen pan, hard glazed both within and without, of the same capacity with the retort. The end of the tube must be made fast to a frame at the height of six inches above the bottom of the receiver. Upon its inner sides, four inches apart, brackets are to be fixed for supporting a series of laths or shelves of white wood, on which a number of little paper or pasteboard boxes are to be laid. In these boxes ten pounds of the purest carbonate of potash, prepared from tartar, are to be spread. The receiver must now be covered with a lid made tight by a water lute. Twenty pounds of concentrated sulphuric acid previously diluted with sixteen pounds of water, and then cooled, are to be poured upon the mixed materials in the retort, the lid immediately secured, with the tube adjusted in the receiver. The whole must be allowed to operate spontaneously without heat for twelve hours. At the end of this time the retort is to be surrounded with a water bath and steadily heated during twelve hours, and then left to cool for six hours. The apparatus must now be opened, the cakes of chlorate of potash removed, and freed from muriate by solution and crystallization.

M. Liebig proposes the following process for obtaining chlorate of potash :-

Heat chloride of lime in water till it ceases to destroy vegrtable colors. In this case a mixture of chloride of calcium and chlorate of potash is obtained. This is to be dissolved in hot water, and to the solution concentrated by evaporation, chloride of potassium is to be added, and then suffered to cool. After cooling, a quantity of crystals of chlorate of potash is obtained, which are to be redissolved and crystallized again to purify them. $M$. Liebig considers that this will be a cheap process for obtaining chlorate of potash. From 12 ounces of chloride of lime, of so bad a quality that it left 65 per cent. of insoluble matter, he obtained an ounce of chlorate of potash.

The only difficulty to overcome in this process is, from the chloride of lime not being so easily decomposed by heat as is generally supposed; a solution of it may be kept boiling for an hour without losing its bleaching power. The best method is to form a thin paste with chloride of lime and water, and then to evaporate it to dryness. If it be required to prepare it by passing chlorine into cream of lime, it is advantageous to keep it very hot.

The chlorate of potash which separates from the solution by crystallization, has not the form of scales which it usually possesses, but is prismatic : whether this is occasioned by some admixture has not been ascertained; but on re-crystallizing, it is obtained in the usual form.

The solution ought not merely to be left to cool, in order to procure crystals, for the crystallization is far from being terminated even after complete cooling; crystals continue to be deposited for 3 or 4 days.

The following modification of the process for making chlorate of potash is that of $\mathbf{M}$. Vée. A solution of chloride of lime, marking $18^{\circ}$ or $20^{\circ}$ Baumé, is to be set upon the fire in a lead or cast iron pot, and when it begins to get hot, there is to be dissolved in it a quantity of chloride of potassium sufficient to raise the hydrometer 3 or 4 degrees. It must be then concentrated as quickly as possible till it marks $30^{\circ}$ or $31^{\circ}$, taking care that it does not boil over by the sudden extrication of oxygen. The concentrated liquor is set aside to crystallize in a cool place; where a deposite of chlorate of potash forms, mixed with chloride of potassium. The mother waters being evaporated to the density of $36^{\circ}$, afford another crop of crystals, after which they may be thrown away.

The salts obtained at the first crystallization are to be re-dissolved, and the solution being brought to $15^{\circ}$ or $16^{\circ}$ is to be filtered, when it will afford upon cooling pure chlarate of potash.

Chlorate or oxymuriate of potash has a cooling, somewhat unpleasant and nitrous taste. It does not bleach. At $60^{\circ} \mathrm{F} .100$ parts of water dissolve six parts of it, and at its boiling point or $220^{\circ}$, sixty parts. When heated to dull ignition in a glass retort, it gives out $39 \cdot 15$ per cent. of its weight of oxygen, and becomes thereby chloride of potassium. When strongly triturated in a mortar it crackles, throws out sparks, and becomes luminous. It deflagrates upon red-hot cinders like nitre: when triturated along with sulphur, or phosphorus, it detonates with great violence, not without danger to the hands of the operator, if they be not protected by a thick glove. Similar detonations may be produced with cinnibar or vermilion, sulphuret of potassium, sugar, volatile oils, \&c., but they can be effected only by the smart blow of a heated hammer and anvil. A mixture of sugar or starch with chlorate of potash is readily inflamed by a drop of sulphuric acid, and this experiment is the basis of the preparation of the oxygenated matches, as they have been commonly called. The following formula forms a good paste for tipping the said matches, made of narrow slips of either wood or card. Thirty parts of the chlorate in fine powder are to be mixed gently with a spatula upon paper with ten parts of flowers of sulphur well levigated, eight of sugar, five of gum arabic, and enough of vermilion to give the whole a rose tint. We begin by mixing tenderly together 
the sugar, the gum, and the salt previously pulverized; we then add as mach water as shall reduce the mixture to a thin paste, and lastly introduce the sulphur; after which all must be well incorporated The points of the matches, either previously tipped with sulphur or not, are to be dipped in that paste, so as to get coated with a little of it, and are lastly laid in a warm place till they become thoroughly dry. To kindle one of them, it must be touched with strong sulphuric acid, which for this purpose is usually kept in a small well-stoppered vial, and thickened with amianthus. Aspen is reckoned the best wood for matches.

Of late years a detonating priming for fire-arms has been much used with the percussion locks. The simplest formula for making it is to take ten parts of gunpowder, to lixiviate it with water, and to mix the residuum, while moist, with five parts and a quarter of chlorate of potash, reduced to an extremely fine powder. The paste may be made pretty thin, for the salt is sparingly soluble in the cold water, and it mixes hest when tolerably fluid. This powder when dry is dangerous to handle, being very apt to explode. But this danger is guarded against by letting fall a drop of the paste into each copper percussion cap, and leaving it to dry there. In the detonation of this powder, besides muriate of potash, there are generated, a little sulphate of potash and chlorine gas. which rust the metal very fast. For which reason fulminate of mercury is now preferred by many sportsmen as a detonating powder. See FuLminate.

CHLORATES, compounds of chloric acid with the salifiable bases. The only acid belonging to this class of any manufacturing importance is the following :

CHLORIC ACID; the acid constituent of the preceding salt; it consists of one equivalent prime of chlorine $=35 \cdot 476,+5$ of oxygen, $=40 \cdot 065$; of which the sum $75 \cdot 535$ is the prime equivalent of the acid.

CHLORINE; the most energetic of the undecompounded bodies, or chemical elements as they are usually called, exists, under ordinary circumstances, as a greenish yellow gas, but, when exposed to a pressure of 4 atmospheres, it becomes a yellow transparent liquid. In the first state, its density compared to air, reckoned $1 \cdot 000$, is $2 \cdot 47$; in the second, its density compared to water, $1 \cdot 000$, is $1 \cdot 33$. No degree of cold hitherto tried, has liquefied the gas when dry. It is obtained by putting into a giass retort a mixture of 3 parts of common salt, with 2 parts of peroxyde of manganese, and pouring upon it 2 parts of sulphuric acid diluted with its own weight of water; or, more conveniently, by pouring moderately strong muriatic acid upon peroxyde of manganese in a retort; and in either case applying the gentle heat of a spirit lamp or a water bath, while the beak of the retort is plunged under brine upon the shelf of the pneumatic trough. The gas issues, and may be received in the usual way into inverted glass jars, or vials; but the first which comes over, being mixed with the air of the retort, must be rejected. It has a peculiar smell, and irritates the nostrils most violently when inhaled, as also the windpipe and lungs. It is eminentiy roxious to animal life, and, if breathed in its undiluted state, would prove instantly fatal. It supports the combustion of many bodies, and indeed spontaneously burns several without their being previously kindled. The resulting combinations are called chlorides, and act most important parts in many manufacturing processes.

Water absorbs, at the ordinary temperature of the atmosphere, about double its volume of chlorine, and acquires the color, smell, and taste of the gas, as well as its power of destroying or bleaching vegetable colors. When this aqueous chlorine is cooled to $36^{\circ} \mathrm{F}$. dark yellow crystalline plates appear in it of the hydrate of chlorine, which are composed in 100 parts of $27 \cdot \%$ chlorine, and 72.3 water. If these crystals be heated to about $45^{\circ}$ they liquefy, and the gas flies off.

Chlorine has a powerful affinity for hydrogen, not only combining with it rapidly in the gaseous, but seizing it in many of its liquid and solid combinations, as in volatile oils, which it inflames, and in yellow wax, cotton, and flax, which it whitens. The compound of chlorine and hydrogen gases is muriatic acid gas. Manganese, when mixed with liquid muriatic acid, as in the above process, abstracts the hydrogen, and lets the chlorine gas go free. When chlorine is passed into water, it decomposes some of it, seizes its hydrogen to form a little muriatic acid, and enables its oxygen to unite either with the chlorine, into chlorous acid, or with the remainin water, and to constitute oxygenated water. Hence, aqueous chlorine, exposed to the sunbeam, continually evolves oxygen, and ere long becomes muriatic acid.

This watery compound acts in a powerful way upon colored vegetable fibres, extracting their hydrogen or coloring element by the twofold affinities of the chlorine and oxyger for it.

Hence chlorine, as a bleaching agent, requires to be tempered by the quiescent affinity of some alkaline base, potash or lime. Malaria, or morbific and putrescent miasmata. consist chiefly of hydrogenous matter as their basis, and are best counteracted by chlorine, where it can be conveniently applied.

Chlorides of Potash, Soda, and Lime.-These are the most important preparation 
through winich chiorine exercises its peculiar powers upon the objects of manufuctures. When a weak solution of caustic potash or soda is saturated with chlorine, it afiords a bieaching liquor which is still used by some bleachers and calico-printers for their most delicate processes; but the price of the alkalis has led to the disuse of these chlorides as a general means, and has occasioned an extensive employment of chloride of lime. Upon the manufacture of this interesting compound I made an elaborate series of experiments, several years ago, and published the results in the 13th volume of Brande's Journal for A pril, 182.2. I have no reason to suppose, from any thing that has been published since, that the processes there described have been essentially improved, or that any errors, either theoretical or practical, of any moment, exist in that memoir. I shall therefore first present my readers with a brief abstract of it, and then make such observations as subsequent inquiries suggest.

In the researches which I made, at many different times, upon the nature of the chloride of lime, I generally sought to combine the information flowing from both synthesis and analysis; that is, I first converted a known portion of hydrate of lime into bleaching-powder, and then subjected this chloride to analysis.

Two hundred grains of the atomic proto-hydrate of pure lime were put into a glass globe, which was kept cold by immersion in a body of water at $50^{\circ}$. A stream of chlorine, after being washed in water of the same temperature in another glass globe, connected to the former by a long narrow glass tube, was passed over the calcareous hydrate. The globe with the lime was detached from the rest of the apparatus from time to time, that the process might be suspended as soon as the augmentation of weight ceased. This happened when the 200 grains of hydrate, containing 151.9 of lime, had absorbed 130 grains of chlorine. By one analytical experiment, it was found that dilute muriatic acid expelled from 50 grains of the chloride, 20 grains of chlorine, or 40 per cent. ; and by another, from 40 grains, 16.25 of gas, which is 40.6 per cert. From the residuum of the first 39.7 grain of carbonate of lime were obtained by carbonate of ammonia ; from that of the second, 36.6 of ignited muriate of lime. The whole results are therefore as follows :-

\begin{tabular}{|c|c|c|c|c|}
\hline \multirow{5}{*}{$\begin{array}{l}\text { Chlorine - - } \\
\text { Lime - - - } \\
\text { Water - - }\end{array}$} & Synthesis. & 1st Analysis. & 2d Analysis. & Mean. \\
\hline & $39 \cdot 39$ & $40 \cdot 00$ & $40 \cdot 62$ & $40 \cdot 31$ \\
\hline & $46 \cdot 00$ & $44 \cdot 74$ & $46 \cdot 07$ & $45 \cdot 40$ \\
\hline & $14 \cdot 60$ & $15 \cdot 26$ & $13 \cdot 31$ & $14 \cdot 28$ \\
\hline & $100 \cdot 00$ & $100 \cdot 00$ & $100 \cdot 00$ & $100 \cdot 00$ \\
\hline
\end{tabular}

Though the heat generated by the action of the dilute acid had carried off in the analytical experiments a small portion of moisture with the chlorine, yet their accordance with the synthetic experiment is sufficiently good to confirm the general results. The above powder appears to have been a pure chloride, without any mixture of muriate. But it exhibits no atomic constitution in its proportions.

To 200 grains of that hydrate of lime 30 grains of water being added, the powder was subjected to a stream of chlorine in the above way, till saturation took place. Its increase of weight was 150 grains.

It ought to be remarked, that in this and the preceding experiment there was no appreciable pneumatic pressure employed to aid the condensation of the chlorine. In the last case, we see that the addition of 30 grains of water has enabled the lime to absort 20 grains :-ore of chlorine, being altogether a quantity of gas nearly equal to that of the dry lime. Thus, an atom of lime seems associated with $\frac{7}{9}$ of an atom of chlorine. Analysis by muriatic acid confirmed this composition. It gave

$$
\begin{aligned}
& \text { Chlorine - }-39.5=51.8 \text { cubic inches. } \\
& \text { Lime - }-\frac{39.9}{100 \cdot 0}
\end{aligned}
$$

A great variety of apparatus has been at different times contrived for favoring the eombination of chlorine with the slaked lime for the purposes of commerce. One of the most ingenious forms is that of a cylinder, or barrel, furnished with narrow wooden shelves within, and suspended on a hollow axis by which the chlorine was admitted, and round which the barrel was made to revolve. By this mude of agitation, the lime-dust, being exposed on the most extensive surface, was speedily impregnated with the gas to the requisite degree. Such a mechanism I saw at MM. Oberkampf and Widmer's celebrated fabrique de toiles peintes, at Jouy, in 1816. But this is a cnstly refinement, inadmissible on the largest scale of British manufacture. The simplest, and, in my opinion, the best construction for subjecting lime-powder to chlorine, is a large chamber 
8 or 9 feet high, built of silicious sandstone, having the joints of the masonry secured with a cement composed of pitch, resin, and dry gypsum in equal parts. A door is fitted into it at one end, which can be made air-tight by strips of cloth and clay-lute. A window on each side enables the operator to judge how the inpregnation goes on by the color of the air, and also gives light for making the arrangements within at the commencement of the process. As water lutes are incomparably superior to all others, where the pneumatic pressure is small, I would recommend a large valve or door on this principle to be male in the roof, and two tunnels of considerable width at the bottom of each sile wall. The three covers could be simultaneously lifted off by cords passing over a pulley, without the necessity of the workman approaching the deleterious gas, when the apartment is to be opened. A great number of wooden shelves, or rather trays, 8 or 10 feet long, 2 feet broad, and 1 inch deep, are provided to receive the riddled slaked lime, containing generally about 2 atoms of lime to 3 of water. These shelves are piled one over another in the chamber, to the height of 5 or 6 feet, cross bars below each keeping them about an inch asunder, that the gas may have free room to circulate over the surface of the calcareous hydrate.

The alembics for generating the chlorine, which are usually nearly spherical, are in some cases made entirely of lead, in others of two hemispheres, joined together in the middle, the upper hemisphere being lead, the under one cast-iron. The first kind of alembic is enclosed, for two thirds from its bottom, in a leaden or iron case, the interval of two inches between the two being destined to receive steam from an adjoining boiler. Those which consist below of cast-iron have their bottom directly exposed to a very gentle fire; round the outer edge of the iron hemisphere a groore is cast, into which the under edge of the leaden hemisphere fits, the joint being rendered air-tight by Roman or patent cement. In this leaden dome there are four apertures, each secured by a waterlute. The first opening is about 10 or 12 inches square, and is shut with a leaden valve, with incurvated edges, that fit into the water channel at the margin of the hole. It is destined for the admission of a workman to rectify any derangement in the apparatus of rotation, or to detach hard concretions of salt from the bottom.

The second aperture is in the centre of the top. Here a tube of lead is fixed, which descends nearly to the bottom, and down through which the vertical axis passes. To its lower end the cross bars of iron, or of wood, sheathed with lead, are attached, by whose revolution the materials receive the proper agitation for mixing the dense manganese with the sulphuric acid and salt. The motion is communicated either by the hand of a workman applied from time to time to a winch at top, or it is given by connecting the axis with wheel work, impelled by a stream of water or a steam-engine. The third opening admits the syphon-formed funnel, through which the sulphuric acid is introduced; and the fourth is the orifice of the eduction-pipe.

Manufacturers differ much from each other in the proportion of their materials for generating chlorine. In general, $10 \mathrm{cwt}$. of salt are mixed with from 10 to $14 \mathrm{cwt}$. of manganese, to which mixture, after its introduction into the alembic, from 12 to $14 \mathrm{cwt}$. of sulphuric acid are added in successive portions. That quantity of oil of ritriol must, howerer, be previously diluted with water, till its specific grarity becomes about 1.6. But, indeed, this dilution is seldom actually made, for the manufacturer of bleachingpowder almust alwars prepares his own sulphuric acid for the purpose, and therefore carries its concentration no higher in the leaden boilers than the density of $1 \cdot 65$, which from my table of sulphuric acid, indicates $\frac{1}{4}$ of its weight of water, and therefore $\frac{1}{3}$ more of such acid must be used.

The fourth aperture, I have said, admits the eduction pipe. This pipe is afterwards conveyed into a leaden chest or cylinder, in which all the other eduction pipes also terminate. They are connected with it simply by water-lutes, having a hydrostatic pressure of 2 or 3 inches. In this general diversorium the chlorine is washed from adhering muriatic acid, by passing through a little water, in which each tube is immersed, and from this the gas is let off by a pretty large leaden tube, into the combination room. It usually enters in the top of the ceiling, whence it diffuses its heavy gas equally round.

Four days are required, at the ordinary rate of working, for making good marketable bleaching-powder. A more rapid formation would merely endanger an eleration of temperature, productive of muriate of lime, at the expense of the bleaching quality. But skilful manufacturers use here an alternating process. They pile up, first of all, the wooden trays only in alternate shelres in each column. At the end of two days the distillation is intermitted, and the chamber is laid open. After two hours the workman enters, to introduce the alternate trays covered with fresh hydrate of lime, and at the same time rakes up thoroughly the half-formed chloride in the others. The door is then secured, and the chamber, after being filled for two days more with chlorine, is again opened, to allow the first set of trays to be removed, and to be replaced by others, con taining fresh hydrate, as before. Thus the process is conducted in regular alternation: 
thus, to my lnowledge, very superior bleaching-powder is manufactured, and thus the chlorine may be stiflered to enter in a pretty uniform stream. But for this judicious pian, as the hydrate advances in impregnation, its faculty of absorption becoming diminshel, it would be requisite to diminish proportionately the erolution of chlorine, or to allow the excess to escape, to the great loss of the proprietor, and, what is of more consequence, to the great detriment of the health of the workmen.

The manufacturer generally reckions on obtaining from one ton of rock-salt, employed as above, a ton and a half of good bleaching-powder. But the following analysis of the operation will show that he ought to obtain two tons.

When a mixture of sulphuric acid, common salt, and black oxyde of manganese are the ingredients used, as by the manufacturer of bleaching-powder, the absolute proportions are, upon the oxygen scale of equivalents :-

\begin{tabular}{|c|c|c|c|c|}
\hline \multirow{3}{*}{1 atom muriate of soda - } & & \multirow{2}{*}{\multicolumn{2}{|c|}{$29 \cdot 70$}} \\
\hline & - & $7 \cdot 5$ & & \\
\hline & - & $5 \cdot 5$ & $21 \cdot 78$ & 7 \\
\hline & & $12 \cdot 25$ & $4 S \cdot 52$ & \\
\hline \multirow{2}{*}{\multicolumn{5}{|c|}{ And the products ought to be ; - }} \\
\hline & & & & \\
\hline Chlorine disengaged - & -1 & atom. & $4 \cdot 5$ & $17 \cdot 82$ \\
\hline Sulphate of soda & -1 & - & $9 \cdot 0$ & $35 \cdot 64$ \\
\hline Proto-sulphate of manganese & -1 & - & $9 \cdot 5$ & $37 \cdot 62$ \\
\hline \multirow[t]{2}{*}{ Water } & -2 & - & $2 \cdot 25$ & $8 \cdot 92$ \\
\hline & & & $25 \cdot 25$ & 100 \\
\hline
\end{tabular}

These proportions are, however, very different from those emplored by many, nay, I believe by all manufacturers; and they ought to be so, on account of the impurity of their oxyde of manganese. Let maling allowance for this, I am afraid that many of them commit great errors in the relative quantities of their materials.

From the preceding computation, it is erident that 1 ton of salt with 1 ton of the abore natire oxtde of manganese properly treated, would yield 0.59 of a ton of chlorine, which would impregnate 1.41 tons of slaked lime, producing 2 tons of bleaching-powder, stronger than the average of the commercial specimens; or allowing for a little loss, which is unaroidable, would afford 2 tons of ordinary powder, with a little more slaked lime.

$F_{i g}$. $25 \%$ represents a retort of lead, well adapted to the evolution of chlorine from the

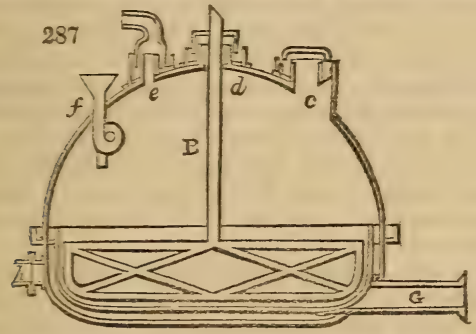

288

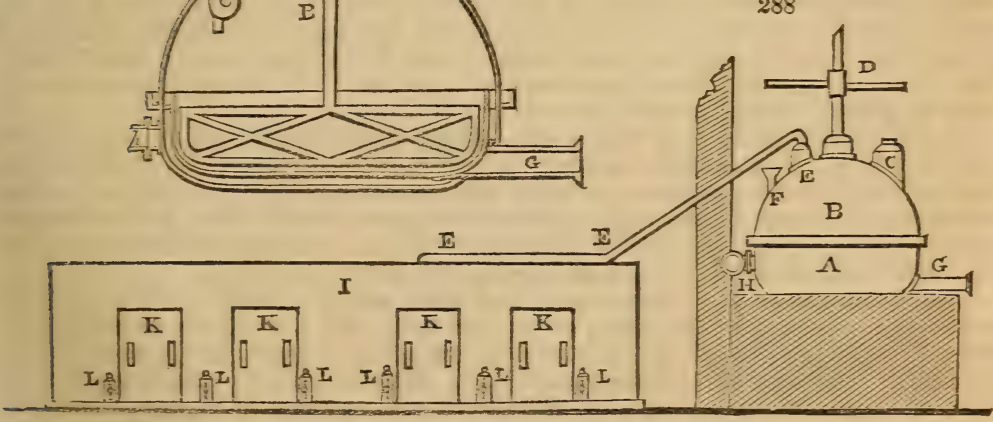

mirture of salt, manganese, and sulphuric acid, or from manganese and muriatic acid. The interior vessel is cast in lead, and it has round its bottom part a cast-iron steam cast. The salt and manganese are introduced by the aperture c, and the sulphuric acid by the syphon funnel $F$. The contact of these three substances is continually renewed by the agitator or stirrer $\mathrm{B}$, which consists of wrought or cast iron sheathed with lead. $e$ is the gas discharge pipe. The residuums are drawn off by the bottom discharge pipe $\mathrm{G}$. The heating case receives its steam by the pipe $h$.

The chlorine gas, fig. 288 , is conveyed from the retort $\mathrm{B}$ into the chamber $\mathrm{r}$, by the tube $\mathbf{E}$ E. This chamber is divided into four compartments, to receive the gas disengaged from four retorts, lite the abore. The bottom of it is corered with a stratum three or four inches thirk of quicklime, newly slaked and sifted, which is stirred about from 
tinse to time, by the rakes $\mathbf{L} \mathbf{L} \mathbf{L} \mathbf{L}$. When the saturation is sufficient, the chloride of lime is taken out by the doors $\mathrm{K} \mathrm{K} \mathrm{K} \mathrm{K}$. The size of this apparatus allows $2 \mathrm{cwt}$. of manganese, and its equivalent quantity of salt and sulphuric acid, or of muriatic acid, to be introduced at once into the retort. $D$ is the handle of the agitator.

The same form of retort will suit perfectly well to prepare chlorine for making liquid chloride of lime, which is preferred by many bleachers and calico-printers who have conveniences for preparing it themselves. The most concentrated solutions of the dry chloride of lime do not mark more than $6^{\circ} \mathrm{B}$. (sp. grar. 1.04), and discolor only 50 volumes of Gay Lussac's solution of indigo, whilst the chloride made in the humid way marks from $8^{\circ}$ to $9^{\circ}$ B. (about $1 \cdot 060$ ), and discolors 80 volumes of the same solution.

In the chloride of lime apparatus, most generally used by the skilful calico-printers of Mulhausen, the mixture of muriatic acid and manganese is put into glass globes, with long necks, heated upon a sand-bath. The chlorine is conveyed by glass tubes into a cylindrical stone cistern, containing milk of lime. The furnace of the sand baths is made of cast iron, and has brick partitions, to give each retort its own fire. The smoke of all these fires goes off by a flue into sheet iron pipes. The cistern is made of siliceous sandstone. Its cover is of wood. coated with a resinous cement; and it fits at its edges into grooves cut in the stone. A wheel serves to agitate the liquid continually; its paddles being liept at two inches distance from the sides of the cistern. The milk of lime is introduced by a funnel, and the chloride is drawn off by a discharge pipe. I think the lead retort and agitator used in this country greatly preferable to the experimental laboratory plan described above. In all such apparatus we should arnid giring any pressure to the tubes or vessels, and should not therefore dip the extremities of the gas pipes beneath the surface of the liquid, but rather facilitate the combination of the chlorine and the lime, by enlarging the surfaces of contact and by agitating. Intermediate vessels containing water, or the chemical cascade of $M$. Clement, are very useful for absorbing any muriatic acid which may be disengaged along with the chlorine, and thereby preventing the needless formation of muriate of lime in the chambers or cisterns of impregnation.

When the solution of the chloride of lime is mixed with hydrate of lime, it bears, without decomposing, a pretty high temperature, provided it be not too long continued; it may eren, in certain cases, be raised to near the boiling point without suffering a marked loss of its discoloring power; but when the chloride is deprived of that excess of lime, it is decomposed in a short time, even at a heat of $110^{\circ} \mathrm{F}$.

When chlorine is admitted to milk of lime, it infallibly produces some muriate of lime; but the quantity is kept at a minimum by constantly presenting an excess of lime to the gas with the agitator, and by keeping the temperature as low as possible. Hence the influx of gas should not be so rapid as to generate much heat. An automatic agitator, moved by steam or water power, is therefore much better than one driven by the hand of the operator, who is apt to intermit his labors. If the liquor becomes hot at the end of the process, it should be immediately drawn off into large stone bottles, and cooled. The rose-color, which sometimes supervenes, is due to a minute quantity of manganese. The strongest liquid chloride of lime that can be prepared will not discolor more than 80 times its volume of Gay Lussac's indigo test.

On acting upon cotton cloth with a concentrated solution of chloride of lime, at from $110^{\circ}$ to $120^{\circ} \mathrm{F}$., pure carbonic acid gas is disengaged, and the texture of the cloth is injured. Here the hrdrogen of the water and the cotton being seized by the chlorine, the liberated oxygen combines with the carbon to form carbonic acid. In the discharge troughs where printed calicoes are passed through strong solutions of chloride of lime, stalactitic crusts of carbonate of lime come to be formed in this way.

The chlorometre of Gay Lussac consists of a test solution of indigo and a graduated tube. One part of the best indigo, passed through a silk siere, is to be dissolved in nine parts of concentrated sulphuric acid, by the aid of a water-bath heat applied for six hours. The sulphate of indigo is now to be diffused through such a body of water that one volume of chlorine gas shall discolor exactly ten times its rolume of this dilute solution. The test liquor should be protected from the agency of light.

Mr. Crum, of Thorniebank, near Glasgow, has lately modified Dr. Dalton's copperas test for chloride of lime, and made it convenient to the practical man. The doctor justly considered that the more chlorine any bleaching powder contains, the more of the green sulphate of iron will it convert into the red sulphate, so that we hare on] to add successive portions of the chloride to a given weight of the dissolved copperas, and note the point at which all the iron gets peroxydized. See BlEAching.

Besides the method of analysis already quoted from my memoir on the manufacture of the chloride of lime, another occurred to me long ago, which I often practised as an easy and expeditious test. Chlorine decomposes ammonia. If therefore water of ammonia, faintly tinged with litmus, be added slowly to a solution of a given weight of 
chloride of lime, the color will continue to disappear till the chlorine be all neutralized by the reaction of the hydroren of the ammonia. The quantity of liquid ammonia of a certain stre thequisite to neutralize in this way a certain volume, say one cubic inch, or a thousand grain measures of chlorine gas, may be assumed as the standard of such a chlorumeter. As chlorine or chloride of lime, when mixed with water of ammonia, causes the disengagement of azote, the quantity of this gas evolved may also be made the foundation of an accurate and convenient chlorometer. The two substances should be mixed over mercury, in a graduated syphon tube. The shut end $\mathrm{A}$ and the open end $\mathbf{B}$ are butin sraduated to une scale; for example, to hundredths of a cubic inch, or to grain.

259 or 10 grain measures. The tube is to be filled with mercury, and then 10 $\Lambda \quad$ measures of it are to be displaced at the open end, by inserting a wooden plug. This space being filled with the solution of chloride of lime, is to be turned up into the shut end by covering the open end with the finger, and inverting the tube; a few drops of water may be sent through to wash the mercury. The ammonia being now let up, will cause a reaction, and evolve a quantity of azote, equivalent to the chlorine present. The action may be quickened by holding the sealed end of the tube obliquely over a lamp heat. The mercury is protected from the chlorine by the ammonia; and should any notion be entertained of such an action, the ammonia may be let up first. I have made innumerable researches over mercury with a detached apparatus of that kind, which combines precision with rapidity of result. It was by a similar mercurial syphon that I analyzed the carbonates, as described in the first edition of my Dictionary of Chemistry, twenty-one years ago.

M. Gay Lussac takes, as the basis of his indigo chlorometer, the fact, that one pound of pure crystallized peroxyde of manganese is capable of affording, with muriatic acid, 0.7964 parts of a pound of chlorine; or one kilogramme yields $251 \frac{1}{4}$ litres; that is, one pound yields $251 \frac{1}{4}$ pound measures. Hence 3.98 grammes of that manganese are capable of affording 1000 gramme measures, or 1 litre of chlorine; or, in round numbers, 4 grains will yield 1000 grain measures. This quantity of gas, being received into that volume of milk of lime, constitutes therefore Gay Lussac's primary standard. The small retort in which the manganese and muriatic acid are put, ought to be heated to ebullition, to discharge every particle of chlorine. To prevent the manganese, in this experiment, from sticking to the bottom in a cake, it has been proposed to mix it previously with a little plumbago. See Chroromerry.

For preparing the chlorides of potash and soda, the same apparatus may be employed as for the liquid chloride of lime. The alkaline solutions should be weak, containing not more than a pound to the gallon of water. Potash liquor saturated with chlorine, is much employed at Paris for whitening linen, under the name of the water of Javelle, the place where it was first made as a manufacture. One hundred parts of chlorine are said to saturate 133 parts of pure potash, and 195 of the carbonate; but the latter should not be used for preparing the bleaching fluid, as the carbonic acid resists the combination of the chlorine. A chloride of carbonate of soda has been lately recommended as a disinfecting substance against contagious miasmata or fomites. One hundred parts of chlorine will saturate 150 of the dry carbonate, and 405 of the crystallized. M. Payen prepares this medicinal chloride by adding 138 parts of carbonate of soda to a liquid, consisting of water 1800 , chloride of lime 100 , at $98^{\circ}$ of strength, by Gray Lussac's standard. The chloride of lime is to be dissolved, and the sediment well washed; the carbonate of soda, dissolved by heat, is to be poured into the solution, the precipitate allowed to subside, the clear fluid decanted, and the solid matter washed upon a filter. The collected solutions are neutral chloride of soda. Sixty-two parts of the carbonate of soda are then to be dissolved in the remainder of the water, and added to the preparation; the whole being thus filtered, a limpid liquor is obtained, indicating $5^{\circ}$ by the hydrometer of Baumé.

The chloride of magnesia was long ago proposed by Sir H. Davy for bleaching linen, as being preferable to chloride of lime, because the resulting muriate of magnesia was not injurious to the fibre of cloth, as muriate of lime may be, under certain circumstances. I prepared a quantily of chloride of magnesia, by exposing a iydrate of that earth in the chlorine chamber of a large manufactory of chloride of lime at Glasgow, and obtained a compound possessed of considerable discoloring powers; but $I$ found that the chlorine was so feebly saturated by the base, that it destroyed the colors of fast-dyed calicoes as readily as chlorine gas or chlorine water did, and was therefore dangerous for common bleaching, and destructive in clearing the grounds of printed goods, which is one of the most valuable applications of the calcareous and alkaline chlorides. The occasion of my making these experiments was the importation of a considerable quantity of magnesite, or native atomic carbonate of magnesia, from the district of Madras, by an enterprising friend of mine Encouraged by the encomiums bestowed on the chloride of magnesia by many chemical writers, he 
expected to have benefited both the country and himself, by bringing home the earthy base of that compound, at a moderate price; but was disappointed, to his cost.

Dr. Thomson is of opinion that the bleaching compound of lime and chlorine is not a shloride of lime, but a combination of chlorous acid with lime and of chlorine with calcium ; consisting in its most concentrated state of

3 atoms of chloride of calcium $=21$

1 atom of chlorite of lime - = 11

so that about one third of the weight is chlorite of lime, to which alone the bleaching powers of the substance are owing. He admits a fact, rather inconsisient with this opinion, that blcaching powder does not attract moisture from the atmosphere with nearly so much rapidity as inight be expected from a mixture containing two thirds of its weight of so deliquescent a salt as muriate of lime; un!ess this indeed be prevented by the chloride and chlurite being united into a double salt, which is a mere conjecture without either proof or analogy. And further, when dilute sulphuric or muriatic acid is poured upon bleaching powder, a profusion of chlorine is given out immediately, which he also admits to be inconsistent with the notion of its being a mixture of chloride of calcium and chlorite of lime, for no such evolution takes place when the above acids are mixed with solutions of chloride of calcium and chlorate of potash. Though I am of opinion that bleaching powder is simply a chloride of lime, in which the lime corresponds to the water in the aqueous chlorine, yet I cannot see the truth or appositeness of his last reason, because chlorine is certainly given out when chlorate of potash is acted upon by dilute muriatic acid, as any man may prove by adding to a mixture of these two substances a vegetable color; for it will be speedily blanched. Dr. Thomson considers the chloride which is at present made in $\mathrm{Mr}$. Tennant's great factory, as containing one atom of chlorine associated with one atom of lime, or, taking his numbers, as consisting of

\section{Hydrate of lime 4.625}

Chlorine - 4.5

Or nearly equal weights of the chlorine and the base; indicating a surprising degree of excellence in the preparation. The arerage commercial samples of bleaching powder from different factories which I examined some years ago, tid zot posst.s nearly that strength; but varied in their quantity of chlorine from 20 to 28 per cent. In my synthetic experiments related above, the greatest quantity of chlorine that would combine with the atomic hydrate of lime, was in the proportion of 130 to 200 ; but there is no doubt that if the lime contains additional water, it will condense more gas. I have never seen a chloride of lime of the strength mentioned by Dr. Thomson, and I should think there must be some fallacy in his statements. I have recorded in the paper above quoted an experiment which proves that with additional moisture, a chloride of lime may be obtained of the following composition:-

$$
\begin{aligned}
& \text { Chlorine } 39.5 \\
& \text { Lime }-39.9 \\
& \text { Water }-\frac{20.6}{100.0}
\end{aligned}
$$

In the article Bleaching, of the Encyclopædia Britannica, Dr. Thomson deduces from a test trial of Mr. Crum, that the best bleaching powder is a compound of 1 atom chlorite of lime $=11,3$ atoms chloride of calcium $=21$, and 8 atoms of water $=9$. "But," adds he, "in general the whole lime is not accurately saturated with chlorine. Accordingly, when the bleaching powder is dissolved in water a small residue almost alwars remains undissolved. Unless the powder be fresh made, a portion of chlorite is always converted into chloride of calcium. It is probable therefore that the best bleaching powder, as it comes into the hands of the bleachers, consists of

$$
\begin{aligned}
& 1 \text { atom chlorite of lime - } 11 \\
& 3 \text { atoms chloride of calcium } 21 \\
& 6 \text { atoms water - } \quad 6.75 \\
& \text { Impurity - - } 2.25
\end{aligned}
$$

"If we consider the bleaching powder as a compound of chlorine and lime, our mode of calculating will not be altered. Instead of 1 atom chlorite of lime, and 3 atoms chloride of calcium, we shall have 4 atoms chloride of lime, 6 atoms water, and $2 \cdot 25$ of impurity as before." In such ambiguity does this able chemist place this interesting compound, for theoretical reasons, of which I cannot see the value. Surely there is no difficulty in conceiving chlorine to exercise a direct attractive force towards the hydrate of lime, as it is known to do towards each of its elementary constituents, the oxygen and the calcium. buch refinements as the preceding tend merely to mystify a plain matter. Even the 
chiorous acid here brought into play to form the ideal chlorite, is by his own admission a hyputhetical being. "When chlorate of potash," says Dr. Thomson, "is mixed with sulphuric acid, and made into small balls the size of a pea, if we expose these balls to a heat somewhat lower than that of boiling water, a bright yellowish green gas separates, which may be received over mercury. Its smell is peculiar and aromatic. Water absorbs at least seven times its volume of it. It destroys vegetable blues. Its constituents are-

1 volume chlorine 2.5 or 4.5 .

2 volumes oxygen $2 \cdot 222$ or 4 .

Thus this compound consists in weight of chlorine $4 \cdot 5$, oxygen $4=8 \cdot 5$. It has been called quarteroxyde of chlorine, but it is more probably a teroxyde. It has been supposed by some to possess acid properties, and has therefore been called chlorous acid. But this is only as yet a hypothesis."

Surely this, by the doctor's own showing, is very slender authority for renouncing our long-received doctrines concerning the constitution of bleaching powder. I shall conclude by remarking that the ultra-atomists are now in a dilemma about this substance; M. Welter, and many French chemists calling it a sub-chloride, of 1 atom of chlorine to 2 atoms of lime, and Dr. Thomson showing that Mr. Tennant, the greatest and best manufacturer of it, has produced it in the state of a chloride, or 1 atom of each. The fact is, in chloride of lime, as in water of ammonia, alcohol, and muriatic acid, there is no sufficient reason for definite proportion in any term short of saturation, and therefore we shall find that chloride in every gradation of strength from one per cent. of chlorine up to 40 per cent.-the strongest which I succeeded in preparing, though I passed a constant stream of chlorine in great excess over a pure hydrate of lime for upwards of 24 hours, with frequent renewal of the surface; indeed, till it refused to absorb any more gas, as indicated by its remaining stationary in weight.

CHLOROMETRY; Chlorometrie, is the name given by the French to the process for testing the decoloring power of any combination of chlorine, but especially of the commercial articles, the chlorides of lime, potash, and soda. M. Gay Lussac proposed many years ago the following graduated method of applying indigo to this purpose. As indigo varies much in its dyeing quality, and of consequence in the proportion of chlorine required for its decoloration, he assumes as the unity of blanching power, one litre of chlorine gas, measured at the mean pressure of $29 \cdot 6$ inches, and at the temperature of melting ice. This volume of gas, when combined with a determinate quantity of water, is employed to test the standard solution of indigo. For this purpose a solution in sulphuric acid of any sample of indigo is taken, and diluted with water to such a degree that 10 measures of it, in a graduated tube, are decolored by that one measure of combined chlorine gas. Each measure of indigo solution so destroyed is called a degree, and this measure being divided into five parts, the real test of chlorine is given to fiftieths, which is sufficiently nice. For the standard of the assays, a chloride of lime as pure and fully saturated as possible is taken, and dissolved in such a quantity of water, that the solution shall contain, or be equivalent to, one volume of chlorine gas. Calculation proves that this condition is exactly fulfilled by dissolving 4938 grammes of the said chloride in half a litre of water ; or in English measures, $5 \mathrm{gr}$. very nearly in 500 grain measures of water. This solution, which serves for a type, indicates $10^{\circ}$ in the assay, or proof; that is to say, each single volume destroys the color of 10 volumes of the dilute indigo solution. It may be remarked that a greater degree of precision is in general attainable with a weak solution of chlorine or a chloride, for example at $4^{\circ}$ or $5^{\circ}$, than with one much stronger; consequently if, after a preliminary trial, the standard considerably exceeds $10^{\circ}$, a given volume of water must be added to the solution, and then the above proof must be taken. If the volume of water added was double, the number of degrees afterward found must be tripled, to obtain the true title of the chloride. It is, however, to be observed that the degree of decoloration varies with the time taken in making the mixture; the more slowly the chlorine is added to the indigo, the less of it escapes into the atmosphere, and the more effective it becomes in destroying the color. The best mode of obtaining comparahle results, is to pour suddenly into the test quantity of chlorine the whole volume of the indigo solution likely to be deeolored; but it is requisite to find approximately beforehand, what quantity of indigo-blue will probably be destroyed. When it comes to the verge of destruction, it is green; but yellowish-brown when entirely decomposed.

I have tried the indigo test in many ways, but never could confide in it. The sulphuric solution of indigo is very liable to change by keeping, and thus to lead to erroneous results. The method of testing the chlorides by green sulphate of iron, described under bleaching, is in my opinion preferable to the above.

M. Gay Lussac has recently proposed another proof of chlorine, founded on the same princinle as that by green vitriol, namely, the quantity of it requisite to raise a metallic substance from a lower to a higher stage of oxydizement. He now prescribes as the preferable plan of chlorometry, to pour very slowly from a graduated glass tube, a 
standard solution of the chloride, to be tested upon a determinate quantity of arsenious acid dissolved in muriatic acid, till the whole arsenious be converted into the arsenic acid. The value of the chloride is greater the less of it is required to produce this effect. It is easy to recognise, by a few drops of solution of indigo, the instant when all the arsenious acid has disappeared; for then the blue tint is immediately effaced, and cannot be restored by the addition of a fresh drop of the indigo solution.

In graduating the arsenical chlorometer, M. Gay Lussac takes for his unity the decoloring power of one volume of chlorine at $32^{\circ} \mathrm{Fahr}$, and divides it into 100 parts. Suppuse that we prepare a solution of chlorine containing its own volume of the gas, and an arsenious solution, such, that under a like volume, the two solutions shall reciprocally destroy each other. Let us call the first, the normal solution of chlorine, and the second, the normal arsenious solution. We shall fix at 10 grammes the weight of chloride of lime subjected to trial; and dissolve it in water, so that the total volume of the solution shall be a litre (1000 grammes measure), including the sediment. If we take a constant volume of this solution, 10 centimetres cube (10 gramme measures), for example, divided into 100 equal parts, and pour into it gradually the arsenious solution (measured by like portions), till the chlorine be destroyed, the bleaching power will be proportional to the number of portions of the arsenious solution, which the chloride shall have required. If the chloride has destroyed 100 portions of the arsenious solution, its title will be 100 ; if it has destroyed 80 portions, its title will be 80 , \&c., and so forth.

On pouring the acidulous arsenious solution into the chloride of lime, this will become very acid; the chlorine will be emitted abundantly, and the proof will be quite incorrect. If, on the contrary, we pour the solution of the chloride of lime into the arsenious solution, this evil will not occur, since the chlorine will always find plenty of arsenious acid to act upon, whatever be the dilution of the one or the other; but in this case, the standard of the chlorine is not given directly, as it is in the inverse ratio of the number of portions which are required to destroy the measures of the arsenious solution. If 50 portions of the chloride have been required, the proof will be $100 \times \frac{100}{50}=200^{\circ}$; if 200 have been required, the proof will be $100 \times \frac{100}{20}=50^{\circ}$, \&c. This evil is not, however, very serious, since we have merely to consult a table, in which we can find the proof corresponding to each volume of the chloride employed for destroying the constant measure of the arsenious solution. The arsenious solution should be slightly tinged with sulphate of indigo, so as to show, by the disappearance of the color, the precise point or instant of its saturation with chlorine, that is, its conversion into arsenic acid. If the arsenious acid be pure, the normal solution may be made directly by dissolving $4 \cdot 439$ grammes of it in muriatic acid (free from sulphurous acid), and diluting the solution till it occupies one litre, or 1000 grammes measure. Annales de Chimie et Physique, Lx. 225.

CHOCOLATE is an alimentary preparation of very ancient use in Mexico, from which country it was introduced into Europe by the Spaniards in the year 1520, and by them long kept a secret from the rest of the world. Linnæus was so fond of it, that he gave the specific name, theobroma, food of the gods, to the cacao-tree which produced it. The cacao-beans lie in a fruit somewhat like a cucumber, about 5 inches long and $3 \frac{1}{2}$ thick, which contains from 20 to 30 beans, arranged in 5 regular rows with partitions between, and which are surrounded with a rose-colored spongy substance, like that of water-melons. There are fruits, however, so large as to contain from 40 to 50 bearss. Those grown in the West India islands, Berbice and Demarara, are much smaller, and have only from 6 to 15 ; their development being less perfect than in South America. After the maturation of the fruit, when their green color has changed to a dark yellow, they are plucked, opened, their beans cleared of the marrowy substance, and spread out to dry in the air. Like almonds, they are covered with a thin skin or husk. In the West Indies they are immediately packed up for the market when they are dried; but in the Caraceas they are subjected to a species of slight fermentation, by putting them into tubs or chests, covering them with boards or stories, and turning them over every morning, to equalize the operation. They emit a good deal of moisture, lose the natural bitterness and acrimony of their taste by this process, as well as some of their weight. Instead of wooden tubs, pits or trenches dug in the ground are sometimes had recourse to for curing the beans; an operation ealled earthing (terrer). They are lastly exposed to the sun, and dried. The latter kind are reckoned the best; being larger, rougher, of a darker brown color, and, when roasted, throw off their husk readily, and split into several irregular fragments; they have an agreeable mild bitterish taste, without acrimony. The Guiana and West India sorts are smaller, flatter, smoother-skinned, lighter-colored, more sharp and bitter to the taste. They answer best for the extraction of the butter of cacao, but afford a less aromatic and agreeable chocolate. According to Lampadius, the kernels of the West India cacao beans contain, in 100 parts, besides water, $53 \cdot 1$ of fat or oil, 16.7 of an albuminous brown matter, which contains all the aroma of the bean, 10.91 of starch, $7 \frac{3}{4}$ of gurn or 
mucilage, 0.9 of lignine, and 2.01 of a reddish dye-stuff somewhat akin to the pigment of cochineal. The husks form 12 per cent. of the weight of the beans; they contain no fat, but, besides lignine, or woody fibre, which constitutes half their weight, they yield a light brown $m^{-c}$ cilaginous extract by boiling in water. The fatty matter is of the consistence of tallow, white, of a mild agreeable taste, called butter of cacao, and not apt to turn rancid by keeping. It melts only at $122^{\circ} \mathrm{Fahr}$., and should, therefore, make tolerable candles. It is soluble in bonling alcohol, but precipitates in the cold. It is obtained by exposing the beans to strong pressure in canvass bags, after they have been steamed or soaked in boiling water for some time. From 5 to 6 ounces of butter may be thus obtained from a pound of cacao. It has a reddish tinge when first expressed, but it becomes white by boiling with water.

The beans, being freed from all spoiled and mouldy portions, are to be gently roasted over a fire in an iron cylinder, with holes in its ends for allowing the vapors to escape; the apparatus being similar to a coffee-roaster. When the aroma begins to be well developed, the roasting is known to be finished; and the beans must be turned out, cooled, and freed by fanning and sifting from their husks. The kernels are then to be converted into a paste, either by trituration in a mortar heated to $130^{\circ} \mathrm{F}$., or by the following ingenious and powerful machine. The chocolate paste has usually in France a little vanilla incorporated with it, and a considerable quantity of sugar, which varies from one third of its weight to equal parts. For a pound and a half of cacao, one pod of vanilla is sufficient. Chocolate paste improves in its flavor by keeping, and should there. fore be made in large quantities at a time. But the roasted beans soon lose their aroma, if exposed to the air.

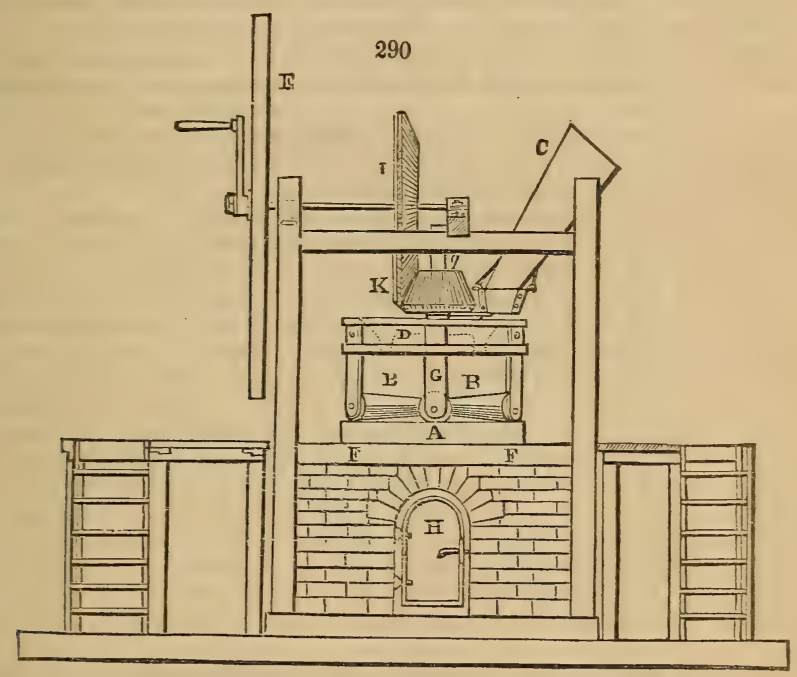

Fig. 290 represents the chocolate mill. Upon the sole A, made of marble, six conical rollers $\mathrm{B} B$, are made to, run by the rerolution of the upright axis or shaft $q$, driven by the agericy of the fly wheel $\mathrm{E}$ and bevel wheels $1 \mathrm{~K}$. The sole $\mathrm{A}$ rests upon a strong iron plate, which is heated by a small stove, introduced at the door $H$. The wooden frame work $F$, forms a ledge, a few inches high, round the marble slab, to confine the cocoa in the act of trituration. $c$ is the hopper of the mill through which the roasted beans are introduced to the action of the rollers, passing first into the flat vessel $n$, to be thence evenly distributed. After the cacao has received the first trituration, the paste is returned upon the slab, in order to be mixed with the proper quantity of sugar, and vanilla, previously sliced and ground up with a little hard sugar. When the chocolate is sufficiently worked, and while it is thin with the heat and trituration, it must be put carefully into the proper moulds. If introduced too warm, it will be apt to becoine damp and dull on the surface; and, if too cold, it will not take the proper form. It must be previously well kneaded with the hands, to ensure the expulsion of
every air bubble.

In Batcelnna, chocolate mills on this construction are very common, but they are turned by a horse-gin set to work in the under story, corresponding to $H$ in the above figure. The shaft $G$ is, in this case, extended down through the marble slab, and is 
surrounded at its centre with a hoop, to prevent the paste coming into contact with it Each of these horse-mills turns out about ten pounds of fine chocolate in the hour, from a slab two feet seven inches in diameter.

Chocolate is flarored with cinnamon and cloves, in several countries, instead of the more expensive vanilla. In roasting the beans, the heat should be at first very slow, to give time to the humidity to escape; a quick fire hardens the surface, and injures the process. In putting the paste into the tin plate, or other moulds, it must be well shaken down, to ensure its filling up all the cavities, and giving the sharp and polished impression so much admired by connoisseurs. Chocolate is sometimes adulterated with starch; in which case it will form a pasty consistenced mass when treated with boiling water. The harder the slab upon which the beans are triturated, the better; and hence perphyry is far preferable to marble. The grinding rollers of the mill should be made of iron, and kept very clean.

CHROMATES, saline compounds of chromic acid with the bases. See CHroMIUM.

CHROMIC ACID; see CHRomiom.

CHROMILM. The only ore of this metal, which occurs in sufficient abundance for the purposes of art, is the octohedral chrome-ore, commonly called chromate of iron, though it is rather a compound of the oxydes of chromium and iron. The fracture of this mineral is uneren; its lustre imperfect metallic; its color between iron-black and brownish-black, and its streak brown. Its specific gravity, in the purest state, rises to 4.5 ; but the usual chrome-ore found in the market varies from 3 to 4 . According to Klaproth, this ore consists of oxyde of chromium, 43 ; protoxyde of iron, $34 \cdot 7$; alumina, 20.3; and silica, 2; but Vauquelin's analysis of another specimen gave as above, respectively, $55.5,33,6$, and 2 . It is infusible before the blowpipe; but it acts upon the magnetic needle, after having been exposed to the reducing smoky flame. It is entirely soluble in borax, at a high blowpipe heat, and imparts to it a beautiful green color.

Chrome-ore is found at the Bare Hills, near Baltimore, in Maryland; in the Shetland Isles, Unst and Fetlar; the department of Var, in France, in small quantity; and near Portsoy, in Banffshire; as also in Silesia and Bohemia.

The chief application of this ore is to the production of chromate of potash, from which salt the rarious other preparations of this metal used in the arts are obtained. The ore, freed, as well as possible, from its gangue, is reduced to a fine puwder, by being ground in a mill under ponderous edge-wheels, and sifted. It is then mixed with one third or one half its weight of coarsely bruised nitre, and exposed to a powerful heat, for several hours, on a reverberatory hearth, where it is stirred about occasionally. In the large manufactories of this country, the ignition of the above mixture in pots is laid aside, as too operose and expensive. The calcined matter is raked out, and lixiriated with water. The bright yellow solution is then evaporated briskly, and the chromate of potash falls down in the form of a granular salt, which is lifted out from time to time from the bottom with a large ladle, perforated with small holes, and thrown into a draining-box. This saline powder may be formed into regular crystals of neutral chromate of potash, by solution in water and slow evaporation; or it may be converted into a more beautifu] crystalline body, the bichromate of potash, by treating its concentrated solution with nitric, muriatic, sulphuric, or acetic acid, or, indeed, any acid exercising a stronger affinity for the second atom of the potash than the chromic acid does.

Bichromate of potash, by evaporation of the above solution, and slow cooling, may be obtained in the form of square tables, with bevelled edges, or flat four-sided prisms. They are permanent in the air, have a metallic and bitter taste, and dissolve in about one tenth of their weight of water, at $60^{\circ} \mathrm{F}$; ; but in one haif of their weight of boiling water. They consist of chromic acid 13 , potash 6 ; or, in 100 parts, $68 \cdot 4+31.6$. This salt is much employed in calico-printing and in dyeing; which see.

Chromate of lead, the chrome-Jellow of the painter, is a rich pigment of various shades, from deep orange to the palest canary yellow. It is made by adding a limpid solution of the neutral chromate (the above granular salt) to a solution, equally limpid, of acelate or nitrate of lead. A precipitate falls, which must be well washed, and carefully dried out of the reach of any sulphureted vapors. A lighter shade of yellow is obtained by mixing some solution of alum, or sulphuric acid, with the chromate, before prouring it into the snlution of lead; and an orange tint is to be procured by the addition of subacetate of lead, in any desired proportion.

For the production of chromate of potash from chrome ore, varjous other processes have been recommended. The following formulæ, which have been verified in practice, will prove useful to the manufacturers of this important article:-

I. Two parts of chrome ore, containing about 50 per cent. of protoxyde of chromium: One part of saltpetre. 
II. Four parts of chrome ore, containing 34 per cent. of protoxyde of chromium. Two parts of potashes.

One part of saltpetre.

III. Four parts of chrome ore

Two of potashes.

Four tenths of a part of peroxyde of manganese.

IV. Three parts of chrome ore.

Four parts of saltpetre.

Two parts of argal.

Some manufacturers have contrived to effect the conversion of the oxyde into an acid and of cuurse to form the chromate of potash, by the agency of potash alone, in a calcining furnace, or in earthen pots fired in a pottery kiln.

After lixiviating the calcined mixtures with water, if the solution be a tolerably pure chromate of potash, its value may be inferred, from its specific gravity, by the following rable :-

At specific gravity $\mathbf{1} \cdot 28$ it contains about 50 per cent. of the salt.

$\begin{array}{ll}1 \cdot 21 & 33 \\ 1 \cdot 18 & 25 \\ 1 \cdot 15 & 20 \\ 1 \cdot 12 & 16 \\ 1 \cdot 11 & 14 \\ 1 \cdot 10 & 12\end{array}$

In making the red bichromate of potash from these solutions of the yellow salt, nitric acid was at first chiefly used; but in consequence of its relatively high price, sulphuric, muriatic, or acetic acid has been frequently substituted upon the great scale.

There is another application of chrome which merits some notice here; that of its green oxyde to dyeing and painting on porcelain. This oxyde may be prepared by decomposing, with heat, the chromate of mercury, a salt made by adding to nitrate of protoxyde of mercury, chromate of potash, in equivalent proportions. This chromate has a fine cinnabar red, when pure; and, at a dull red heat, parts with a portion of its oxygen and its mercurial oxyde. From M. Dulong's experiments it would appear, that the purest chromate of mercury is not the best adapted for preparing the oxyde of chrome to be used in porcelain painting. He thinks it ought to contain a little oxyde of manganese and chromate of potash, to afford a green color of a fine tint, especially for pieces that are to receive a powerful heat. Pure oxyde of chrome preserves its color well enough in a muffle furnace; but, under a stronger fire, it takes a dead-leaf color.

The green oxyde of chrome has come so extensively into use as an enamel color for porcelain, that a fuller account of the best modes of manufacturing it must prove acceptable to many of my readers.

That oxyde, in combination with water, called the hydrate, may be economically prepared by boiling chromate of potash, dissolved in water, with half its weight of flowers of sulphur, till the resulting green precipitate ceases to increase, which may be easily ascertained by filtering a little of the mixture. The addition of some potash accelerates the operation. This consists in combining the sulphur with the oxyger of the chromic acid, so as to form sulphuric acid, which unites with the potash of the chromate into sulphate of potash, while the chrome oxyde becomes a hydrate. An extra quantity of potash facilitates the deoxydizement of the chromic acid by the formation of hyposulphite and sulphuret of potash, both of which have a strong attraction for oxygen. For this purpose the clear lixivium of the chromate of potash is sufficiently pure, though it should hold some alumina and silica in solution, as it generally does. The hydrate may be freed from particles of sulphur by heating dilute sulphuric acid upon it, which dissolves it; after which it may be precipitated, in the state of a carbonate, by carbonate of potash, not added in excess.

By calcinins a mixture of bichromate of potash and sulphur in a crucible, chromic acid is also decomposed, and a hydrated oxyde may be obtained; the sulphur being partly converted into sulphuret of potassium, and partly into sulphuric acid (at the expense of the chromic acid), which combines with the rest of the potash into a sulphate. By careful lixiviation, these two new compounds may be washed away, and the chrome green may be freed from the remaining sulphur, by a slight heat.

Liebig and Wöhler have lately contrived a process for producing a subchromate of lead of a beautiful vermilion hue. Into saltpetre, brought to fusion in a crucible at a gentle heat, pure chrome yellow is to be thrown by small portions at a time. A strong ebullition takes place at each addition, and the mass becomes black, and continues so while it is hot. The chrome yellow is to be added till little of the saltpetre remains undecomposed, care being taken not to overheat the crucible, lest the color of the mixture should become brown. Having allowed it to settle for a few minutes, during which the dense basic salt falls to the bottom, the fluid part, consisting of 
chromate of potash and salipetre, is to be poured off, and it can be employed again in preparing chrome yellow. The mass remaining in the crucible is to be washed with water, and the chrome red being separated from the other matters, is to be dried after proper edulcoration. It is essential for the beauty of the color, that the saline solution should not stand long over the red powder, because the color is thus apt to become of a dull orange hue. The fine crystalline powder subsides so quickly to the bottom after every ablution, that the above precaution may be easily observed.

As Chromic Icid will probably ere long become an object of interest to the calico printer, I shall describe here the best method of preparing it. To 100 parts of yellow ehromate of potash, add 136 of nitrate of barytes, each in solution. A precipitate of the yellow chromate of barytes falls, which being washed and dried would amount to 130 parts. But while still moist it is 10 be dissolved in water by the intervention of a little nitric acid, and then decomposed by the addition of the requisite quantity of sulphuric acid, whereby the barytes is separated, and the chromic acid remains associated with the nitric acid, from which it can be freed by evaporation to dryness. On re-dissolving the chromic acid residuum in water, filtering and evaporating to a proper degree, 50 parts of chromic acid may be obtained in crystals.

This acid may also be obtained from chromate of lime, formed by mixing chromate of potash and muriate of lime; washing the insoluble chromate of lime which precipitates, and decomposing it by the equivalent quantity of oxalic acid, or for ordinary purposes even sulphuric acid may be employed.

Chromic acid is oblained in quadrangular crystals, of a deep red color; it has a very acrid and styptic taste. It reddens powerfully litmus paper. It is deliquescent in the air. When heated to redness it emits oxygen, and passes into the deutoxyde. When a little of it is fused along with vitreous borax, the compound assumes an emerald green color.

As chromic acid parts with its last dose of oxygen very easily, it is capable in certain styles of calico printing of becoming a valuable substitute for chlorine, where this more powerful substance would not from peculiar circumstances be admissible. For this ingenious application, the arts are indebted to that truly scientific manufacturer, M. Daniel Kachlin, of Mülhouse. He discovered that whenever chromate of potash has its acid set free by its being mixed with tartaric or oxalic acid, or a neutral vegetable substance, (starch or sugar for example,) and a mineral acid, a very lively action is produced, with disengagement of heat, and of several gases. The result of this decomposition is the active reagent, chromic acid, possessing valuable properties to the printer. Watery solutions of chromate of potash and tartaric acid being mixed, an effervescence is produced which has the power of destroying vegetable colors. But this power lasts no longer than the effervescence. The mineral acids react upon the chromate of potash only when regetable coloring matter, gum, starch, or a vegetable acid are present, to determine the disengagement of gas. During this curious change carbonic acid is evolved; and wher it takes place in a retort, there is condensed in the receiver a colorless liquid, slightly acid, exhaling somewhat of the smell of vinegar, and containing a little empyreumatic oil. This liquid heated with the nitrates of mercury or silver reduces these metals. On these principles M. Kœchlin discharged indigo blue by passing the cloth throngh a solution of chromate of potash, and printing nitric acid thickened with gum upon certain spots. It is probable that the employment of chromic acid would supersede the necessity of having recourse in many cases to the more corrosive chlorine.

The following directions have been given for the preparation of a blue oxyde of chrome. The concentrated alkaline solution of chromate of potash is to be saturated with weak sulphuric acid, and then to every $8 \mathrm{lbs}$. is to be added $1 \mathrm{lb}$. of common salt, and half $\mathbf{a}$ pound of concentrated sulphuric acid; the liquid will now acquire a green color. To be certain that the yellow color is totally destroyed, a small quantity of the liquor is to have potash added to it, and filtered; if the fluid is still yellow, a fresh portion of salt and of sulphuric acid is to be added; the fluid is then to be evaporated to dryness, redissolved, and filtered; the oxyde of chrome is finally to be precipitated by caustic potash. It will be of a greenish-blue color, and being washed, must be collected upon a filter.

Chromate of Polash, adulteration of, to detect. The chromate of potash has the power of combining with other salts up to a certain extent without any very sensible change in its form and appearance; and hence it has been sent into the market falsified by very considerable quantities of sulphate and muriate of potash, the presence of which has often escaped observation, to the great loss of the dyers who use it so extensively. The following test process has been devised by M. Zuber, of Mülhouse. Add a large excess of tartaric acid to the chromate in question, which will decompose it, and produce in a few minutes a deep amethyst color. The supernatant liquor will, if the chromate be pure, afford now no precipitate with the nitrates of barytes or silver; whence the absence of the sulphates and muriates may be inferred. We must, however, use dilute solutions of the chromate and acid, lest bitartrate of potash be precipitated, which will take place if less than 60 parts of water be employed. Nor must 
we test the liquid till the decomposition be complete, and till the color verge rather toward the green than the rellow. Eight parts of tartaric acid should be alded to one of chromate to obtain a sure and rapid result. If nitrate of potash (saltpetre) is the adulterating ingredient, it may be detected by throwing it on burning coals, when deflagration will ensue. The green color is a certain mark of the transformation of the chromie acid partially into the chrome oxyde; which is effected equally by the sulphurous acid and sulphureted hydrogen. Here this metallic acid is disoxygenated by the tartaric, as has been long known. The tests which I should prefer, are the nitrates of silver and baryta, having previonsly added so much nitric acid to the solution of the suspected chromate, as to prevent the precipitation of the chromate of silver or baryta. The smallest adulteration by sulphates or muriates will thus be detected.

CINIABAR; the native red sulphuret of mercury. It occurs sometimes crystallized in rhombuids; has a specific gravity varying from 6.7 to 8.2 ; a flat conchoidal fracture; is fine grained; opaque; has an adamantine lustre, and a color passing from cochineal to ruby red. The fibrous and earthy cinnabar has a scarlet hue. It is met with disseminated in smaller or larger lumps in veins, which are surrounded by a black clay, and is associated with native quicksilver, amalgam, with iron-ore, lead-glance, blende, copper-ore, gold, Sc. Its principal localities are Almaden in Spain, Idria in the Schiefergebirge, Kremnitz and Schemnitz in Hungary; in Saxony, Bavaria, Bohemia, Nassau, China, Japan, Mexico, Columbia, Peru. It consists of two primes of sulphur, $=32 \cdot 240$, combined with one of mercury, $=202,863$; or in 100 parts of $12 \cdot 7$ sulphur $+87 \cdot 3$ mercury. It is th 4 most prolific ore of this metal; and is easily smelted by exposing a mixture of it with iron or lime to a red heat in retorts. Factitious cinnabar is called in commerce Vermilion, which see, as also Mercury.

CINIAIION. (Cannelle, Fr.; Zimmt, Germ.) Is the inner bark of the laurus cinnamomum, a handsome-looking tree which grows naturally to the height of 18 or 20 feet, in Java, Sumatra, Ceylon, and other islands in the East Indian seas. It has been transplanted to the Antilles, particularly Guadaloupe and Martinique, as well as Cayenne, but there it produces a bark of very inferior value to the Oriental.

Cinnamon is gathered twice a year, but not till after the tree has attained to a certain age and maturity. The young twigs yield a bark of better quality than the larger branches. The first and chief harvest takes place from April to August; the second, from November to January. After having selected the proper trees, all the branches more than three years old are cut off; the epidermis is first removed with a two-edged pruning knife, then a longitudinal incision is made through the whole extent of the bark, and lastly, with the bluntest part of the knife, the true bark is carefully stripped off in one piece. All these pieces of bark are collected, the smaller ones are laid within the larger, and in this state they are exposed to the sun, whereby in the progress of drying, they become rolled into the shape of a quill. These convoluted pieces are formed into oblong bundles of 20 or $30 \mathrm{lbs}$. weight, which are placed in warehouses, sorted and covered with mats. Good cinnamon should be as thin as paper, have its peculiar aromatic taste, without burning the tongue, and leave a sweetish flavor in the mouth The broken bits of cinnamon are used in Ceylon for procuring the essential oil by distillation. 445,367 lbs. of cinnamon were imported into this kingdom in 1835, of which 16,604 only were retained for internal consumption.

CITRIC ACID. (Acide citrique, Fr.; Citronensaure, Germ.) Scheele first procured this acid in its pure state from lemon juice, by the following process. The juice put into a large tub, is to be saturated with dry chalk in fine powder, noting carefully the quantity employed. The citrate of lime which precipitates, being freed from the supernatant foul liquor, is to be well washed with repeated affusion and decantation of water. For every 10 pounds of chalk employed, nine and a half pounds of sulphuric acid, diluted with six times its weight of water, are to be poured while warm upon the citrate of lime, and well mixed with it. At the end of twelve hours, or even sooner, the citrate will be all decomposed, dilute citric acid will float above, and sulphate of lime will be found at the bottom. The acid being drawn off, the calcareous sulphate must be thrown on a canvass filter, drained, and then washed with water to abstract the whole acid.

The citric acid thus obtained may be evaporated in leaden pans, over a naked fire, till it acquires the specific gravity 1.13 ; after which it must be transferred into another vessel, evaporated by a steam or water bath till it assumes a sirupy aspect, when a pellicle appears first in patches and then over the whole surface. This point must be watched with great circumspection, for if it be passed, the whole acid runs a risk of being spoiled by carbonization. The stearn or hot water must be instantly withdrawn, and the concentrated acid put into a crystallizing vessel in a dry, but not very cold apartment. At the end of four days, the crystallization will be complete. The crystals must be drained, redissolved in a smali portion of water, the solution set aside to settle its impurities, then decanted, re-evaporated, and re-crystallized. A third or fourth crystallization may be necessary to obtain a colorless acid, 
If any citrate of lime be left undecomposed by the suiphuric acid, it will dissolve in the citric acid, and obstruct its crystallization, and hence it will be safer to use the slightest excess of sulphuric acid, than to leave any citrate undecomposed. There should not however be any great excess of sulphuric acid. If there be, it is easily detected by nitrate of barytes, but not by the acetate of lead as prescribed by some chemical authors; because the citrate of lead is not very soluble in the nitric acid, and might thus be confound. ed with the sulphate, whereas citrate of barytes is perfectly soluble in that test acid. Sometimes a little nitric acid is added with advantage to the solution of the colored crys. tals, with the effect of whitening them.

Twenty gallons of good lemon juice will afford fully ten pounds of white crystals of citric acid.

Attempts were made, both in the West Indies and Sicily, to convert the lime and lemon juice into citrate of lime, but they seem to have failed through the difficulty of drying the citrate for shipment.

The crystals of citric acid are oblique prisms with four faces, terminated by dihedral summits, inclined at acute angles. Their specific gravity is $1 \cdot 617$. They are unalterable in the air. When heated, they melt in their water of crystallization; and at a higher heat, they are decomposed. They contain 18 per cent. of water, of which one half may be separated in a dry atmosphere, at about $100^{\circ} \mathrm{F}$., when the crystals fall into a white powder.

Citric acid. in crystals is composed by my analysis of carbon $35 \cdot 8$, oxygen $59 \cdot 7$, and rydrogen 45 ; results which differ very little from those of Dr. Prout, subsequently obained. I found its atomic weight to be $8 \cdot 375$, compared to oxygen 1,000 . I cannot acsount for Berzelius's statements relative to the composition of this acid.

Citric acid in somewhat crude crystals is employed with much advantage in calicoprinting. If adulterated with tartaric acid, the fraud may be detected by adding potash to the solution of the acid, which will occasion a precipitate of cream of tartar.

CIVET. (Civette, Fr.; Zibelh, Germ.) This substance approaches in smell to musk and ambergris; it has a pale yellow color, a somewhat acrid taste, a consistence like that of honey, and a very strong aromatic odor. It is the product of two small quadrupeds of the genus viverra ( $v$. zibetha and $v$. civetta), of which the one inhabits Africa, the other Asia. They are reared with tenderness, especially in Abyssinia. The civet is contained in a sac, situated between the anus and the parts of generation in either sex. The animal frees itself from an excess of this secretion by a contractile movement which it exercises upon the sac, when the civet issues in a vermicular form, and is carefully collected. The negroes are accustomed to increase the secretion by irritating the animal; and likewise introduce a little butter, or other grease, by the natural slit in the bag, which mixes with the odoriferous substance, and increases its weight. It is employed only in perfumery.

According to M. Boutron-Chalard, it contains a volatile oil, to which it owes its smell, some free ammonia, resin, fat, an extractiform matter, and mucus. It affords, by calcination, an ash, in which there are some carbonate and sulphate of potash, phosphate of lime, and oxyde of iron.

CLAY (Argile, Fr.; Thon, Germ.) is a mixture of the two simple earths, alumina and silica, generally tinged with iron. Lime, magnesia, with some other coloring metallic oxydes, are occasionally present in small quantities in certain natural clays.

The different varieties of clay possess the following common characters:-

1. They are readily diffusible through water, and are capable of forming with it a plastuc ductile mass, which may be kneaded by hand into any shape. This plasticity exists, however, in very different degrees in the different clays.

2. They concrete into a hard mass upon being dried, and assume, upon exposure to the heat of ignition, a degree of hardness sometimes so great as to give sparks by collision with hardened steel. In this state they are no longer plastic with water, even when pulverized. Tolerably pure clays, though infusible in the furnace, become readily so by the admixture of lime, iron, manganese, \&e.

3. All clays, even when previously freed from moisture, shrink in the fire in virtue of the reciprocal affinity of their particles; they are very absorbent of water in their dry state, and adhere strongly to the tongue.

4. Ochrey, impure clays, emit a disagreeable earthy smell when breathed upon.

Brongniart distributes the clays into :-

1. Fire-clays, (argiles apyres, Fr.; feuerfeste, Germ.)

2. Fusible, (schmelzbare, Germ.)

3. Effervescing (brausende, Germ.), from the presence of chalk.

4. Ochrey (ocreuses, Fr.; ockrige, Germ.)

Fire-clay is found in the greatest abundance and perfection for manufacturing purposes in,

1. Slate-clay. (Thon-schiefer, Germ.) Its color is gray or grayish-yellow. Massir 
dull, or glimmering from admixture of particles of mica. Fraeture slaty, approaching sometimes to earthy. Fragments tabular. Soft, sectile, and easily broken. Sp.gr. $=2 \cdot 6$. Adheres to the tongue, and breaks down in water. It occurs along with pit coal; which see. Slate-clay is ground, and reduced into a paste with water, for making fire-bricks ; for which purpose it should be as free as possible from lime and iron.

2. Common clay or loam.-This is an impure coarse pottery clay, mixed with iron ochre, and occasionally with mica. It has many of the external characters of plastic clay. It is soft to the touch, and forms, with water, a somewhat tenacious paste; but is in general less compact, more friable, than the plastic clays, which are more readily diffusible in water. It does not possess the property of acquiring in water that commencement of translucency which the purer clays exhibit. Although soft to the touch, the common clay wants unctuosity, properly so called. The best example of this argillaceous substance is afforded in the London clay formation, which consists chiefly of bluish or blackish clay, mostly very tough. Those of its strata which effervesce with acids partake of the nature of marl. This clay is fusible at a strong heat, in consequence of the iron and lime which it contains. It is employed in the manufacture of bricks, tiles, and coarse pottery ware.

3. Potter's clay, or Plastic clay.-This species is compact, soft, or even unctuous to the touch, and polishes with the pressure of the finger; it forms, with water, a tenacious, very ductile, and somewhat translucent paste. It is infusible in a porcelain kiln, but assumes in it a great degree of hardness. Werner calls it pipe-clay. Good plastic clay remains white, or if gray before, becomes white in the porcelain kiln.

The geological position of the plastic clay is beneath the London clay, and above the sand which covers the chalk formation. The plastic clay of the Paris basin is described as consisting of two beds separated by a bed of sand. The lower bed is the proper plastic clay. The plastic clay of Abondant, near the forest of Dreux, analyzed by Vauquelin, gave-

Silica, 43.5 ; alumina, $33 \cdot 2$; lime, 0.35 ; iron, 1 ; water, 18.

This clay is employed as a fire clay for making the bungs or seggars, or coarse earthenware cases, in which china ware is fired.

The plastic clay of Dorsetshire and Devonshire supplies the great Staffordshire potteries. It is gray colored, less unctuous than that of Dreux, and consequently more friable. It becomes white in the pottery kiln, and is infusible at that heat. It causes no effervescence with nitric acid, but falls down quickly in it, and becomes higher colored. Its refractoriness allows of a harder glaze being applied to the ware formed from it without risk of the heat requisite for making the glaze flow, affecting the biscnit either in shape or color. "Most of the plastic clays of Franee," says M. Brongniart, "employed for the same ware, have the disadvantage of reddening a little in a somewhat strong heat ; and hence it becomes necessary to coat them with a soft glaze, fusible by means of excess of lead at a low heat, in order to preserve the white appearance of the biscuit. Such a glaze has a dull aspect, and cracks readily into innumerable fissures by alternations of hot and cold water." Hence one reason of the vast inferiority of the French stone-ware to the English.

4. Porcelain clay, or Kaolin earth.-The Kaolins possess very characteristic properties. They are friable in the hand, meager to the touch, and difficultly form a paste with water. When freed from the coarse and evidently foreign particles interspersed through them, they are absolutely infusible in the porcelain kiln, and retain their white color unaltered. They harden with heat like other clays, and perhaps in a greater degree; but they do not acquire an equal condensation or solidity, at least when they are perfectly pure. The Kaolins in general appear to consist of alumina and silica in nearly equal proportions. Most of the Kaolin clays contain some spangles of mica, which betray their origin from disintegrated granite.

This origin may be regarded as one of their most distinctive features. Almost all the porcelain clays are evidently derived from the decomposition of the feldspars, granites, and principally those rocks of feldspar and quartz, called graphic granite. Hence, they are to be found only in primitive mountain districts, among banks or blocks of granite, forming thin seams or partings between them. In the same partings, quartz and mica occur, being relics of the granite; while some seams of Kaolin retain the external form of feldspar.

The most valuable Kaolins have been found-

In China and Japan. The specimens imported from these countries appear pretty white; but are more unctuous to the tnuch, and more micaceous than the porcelain clays of France.

In Saxony. The Kaolin employed in the porcelain manufactories of that country has a slight yellow or flesh color, which disappears in the kiln, proving, as Wallerius observed, that this tint is not owing to any metallic matter.

In France, at Saint-Yriex-la-Perche, about 10 leagues from Limoges. The Kaolin 
occurs there in a bed, or perhaps a vein of beds of granite, or rather of that feldspar rock called Pe-tun-tse, which exists here in every stage of decomposition. This Kaolin is generally white, but sometimes a little yellowish, with hardly any mica. It is meager to the touch, and some beds include large grains of quartz, called pebbly by the China manufacturers. This variety, when ground, affords, without the addition of any fusible ingre. dient, a very transparent porcelain.

Near Bayonne. A Kaolin possessing the lamellated structure of feldspar, in many places. The rock containing it is a graphic granite in every stage of decomposition.

In England, in the county of Cornwall. This Kaolin or China clay is very white, and more unctuous to the touch than those upon the continent of Europe mentioned above. Like them it results from the decomposition of the feldspars and granites, occurring in the middle of these rocks. Mr. Wedgewood found it to contain 60 of alumina or pure clay, and 40 of silica, in 100 parts.

Pure clay, the alumina of the chemist, is absolutely infusible; but when subjected to the fire of a porcelain kiln, it contracts into about one half of its total bulk. It must, however, be heated very cautiously, otherwise it will decrepitate and fly in pieces, owing to the sudden expansion into steam of the water combined with its particles, which is retained with a considerable attractive force. It possesses little plasticity, and consequently affords a very short paste, which is apt to crack when kneaded into a cake.

It is not only infusible by itself, but it will not dissolve in the fusible glasses; making them merely opaque. If either lime or silica be added separately to pure clay, in any proportion, the mixture will not melt in the most violent furnace; but if alumina, lime, and silica be mixed together, the whole melts, and the more readily, the nearer the mixture approaches to the following proportions :- 1 of alumina, 1 of lime, and 3 of sand. If the sand be increased to five parts, the compound becomes infusible. These interesting facts show the reciprocal action of those earths which are mixed most commonly in nature with alumina.

Iron in small quantity, but in a state not precisely determined, though probably of protoxyde, does not color the clays till they are subjected to a powerful heat. There are very white clays, such as those of Montereau, which do not become red till calcined in the porcelain kiln; the oxyde of iron contained in them, which colors them in that case, was previously imperceptible. It appears from this circumstance, that the clays fit for making fine white stone ware, as also the Kaolins adapted to the manufacture of porcelain, are very rare.

Iron, in larger proportion, usually colors the clays green or slate-blue, before they have been heated. Such clays, exposed to the action of fire, become yellow or red according to the quantity of iron which they contain. When the iron is very abundant, it renders the clays fusible; but a little lime and silica must also be present for this effect. The earthenware made with these ferruginous clays can bear but a moderate baking heat; it is thick, porous, and possesses the advantage merely of cheapness, and of bearing considerable alternations of temperature without breaking.

Alumina and the very aluminous natural clays which possess most plasticity, are apt to crack in drying, or to lose their shape. This very serious defect for the purposes of pottery is rectified, in some measure, by adding to that earth a certain quantity of sand or silica. Thus, a compound is formed which possesses less attraction for water, and dries more equably from the openness of its body. The principal causes of the distortion of earthenware vessels, are the unequal thickness of their parts, and quicker desiccation upon one side than another. Hard burnt stone-ware ground to powder, and incorporated with clay, answers still better than sand for counteracting the great and irregular contraction which natural pottery paste is apt to experience. Such ground biscuit is called cement; and its grains interspersed through the ware, may be regarded as so many solutions of continuity, which arrest the fissures.

The preceding observations point out the principles of those arts which employ clay for moulding by the wheel, and baking in a kiln. See Porcelain and Pottery.

CLOTH, MANUFACTURE OF. See Trxtile Fabrics, Weaving, Wool.

CLOTH-BINDING. Nothing places in so striking a point of view the superior taste, judgment, and resources of London tradesmen over those of the rest of the world, as the extensive substitution which they have recently made of embossed sillss and calicoes for leather in the binding of books. In old libraries, cloth-covered boards indeed may occasionally be seen, but they have the meanest aspect, and are no more to be compared with our modern cloth-binding, than the jupon of a trull, with the ballette dress of Taglioni. The silk or calico may be dyed of any shade which use or fancy may require, impressed with gold or silver foil in every form, and variegated by ornaments in relief, copied from the most beautiful productions in nature. This new style of binding is distinguished not more for its durability, elegance, and variety, than for the economy and despatch with which it ushers the offspring of intellect into the world. 
For example, should a house eminent in this line, such as that of Westleys, Friarstreet, Doctors'-commons, receive 5000 volumes from Messrs. Longman \& Co. upon Monday morning, they can have them all ready for publication, within the incredibly short period of two days; being far sooner than they could have rudely boarded them upon the former plan. The reduction of price is not the least advantage incident to the new method, amounting to fully 50 per cent. upon that with leather.

The dyed cloth, being cut by a pattern to the size suited to the volume, is passed rapidly through a roller press, between engraved cylinders of hard steel, whereby it receives at once the impress characteristic of the back and the sides, along with embossed designs over the surface in sharp relief. The cover, thus rapidly fashioned, is as rapiàly applied by paste to the stitched and pressed volume; no time being lost in mutual adjustments; since the steel rollers turn off the former, of a shape precisely adapted to the latter. Hard glazed and varnished calico is moreover much less an object of depredation to moths, and other insects, than ordinary leather has been found to be.

COBALT. This metal, being difficult to reduce from its ores, is therefore very little known, and has not hitherto been employed in its simple state in any of the arts; but its oxyde has been extensively used on account of the rich blue color which it imparts to glass, and the glaze of porcelain and stone-ware. The principal ores of cobalt are those designated by mineralogists under the names of arsenical cobalt and gray cobalt. The first contains, in addition to cobalt, some arsenic, iron, nickel, and occasionally silver, \&c. The other is a compound of cobalt with iron, arsenic, sulphur, and nickel. Among the gray cobalts, the ore most esteemed for its purity is that of Tunaberg in Sweden. It is often in regular crystals, which possess the lustre and color of polished steel. The specific gravity of cobalt pyrites is 6.36 to $4 \cdot 66$. The Tunaberg variety afforded to Klaproth, cobalt, 44 ; arsenic, 55.5 ; sulphur, 0.5 ; so that it is an arseniuret. Others, however, contain much sulphur as well as iron. It imparts at the blowpipe a blue color to borax and other fluxes, and gives out arsenical fumes.

The ore being picked, to separate its concomitant stony matter, is pounded fine and passed through a sieve; and is also occasionally washed. The powder is then spread on the sole of a reverberatory furnace, the flue of which leads into a long horizontal chimney. Here it is exposed to calcination for several hours, to expel the sulphur and arsenic that may be present ; the former burning away in sulphurous acid gas, the latter being condensed into the white oxyde or arsenious acid, whence chiefly the market is supplied with this article. This calcining process can never disengage the whole of these volatile ingredients, and there is therefore a point beyond which it is useless to push it ; but the small quantities that remain are not injurious to the subsequent operations. The roasted ore is sifted anew; reduced to a very fine powder, and then mixed with 2 or 3 parts of very pure silicious sand, to be converted into what is called zaffre. With this product glasses are generally colored blue, as well as enamels and pottery glaze. In the works where cobalt ores are treated, a blue glass is prepared with the zaffre, which is well known under the name of smalt or azure blue. This azure is made by adding to the zaffe 2 or 3 parts of potash, according to its richness in cobalt, and melting the mixture in earthen crucibles. The fused mass is thrown out while hot into water; and is afterwards triturated and levigated in mills mounted for the purpose. There remains at the bottom of the earthen pot a metallic lump, which contains a little cobalt, much nickel, arsenic, iron, \&c. This is called speiss.

As it is the oxyde of cobalt which has the coloring quality, the calcination serves the purpose of oxydizement, as well as of expelling the foreign matters.

A finer cobalt oxyde is procured for painting upon hard porcelain, by boiling the cobalt ore in nitric acid, which converts the arsenic into an acid, and combines it with the different metals present in the mineral. These arseniates, being unequally soluble in nitric acid, may be separated in succession by a cautious addition of carbonate of soda or potash ; and the arspniate of cobalt as the most soluble remains unaffected. It has a rose color, and is easil; distinguishable, whence the precipitation may be stopped at the proper point. The above solution should be much diluted, and the alkali should be cautiously added, with frequent agitation.

The cobalt ores, rich in nickel, are exposed to slow oxydizement in the air, whereby the iron, cobalt, arsenic, and sulphur get oxygenated by the atmospheric moisture, but the nickel continues in the metallic state. This action of the weather must not be extended beyond a year, otherwise the nickel becomes affected, and injures the cobalt blue. The ore hereby increases in weight, from 8 to 10 per cent. Fig. 291 is a longitudinal section of the furnace : fig. 292, a horizontal section upon a level with the sole of the hearth. It is constructed for wood fuel, and the hearth is composed of are-bricks or tiles. The vapors and gases disengaged in the roasting pass off through the flues $a$, into the channels $b b$, and thence by $c$ into the common vent, or poison chamber. See the representation of the poison tower of Altenberg, under the 
article Arsfinic. The flues are cleared out by means of openings left at suitable situa tions in the brick-work of the chimneys.
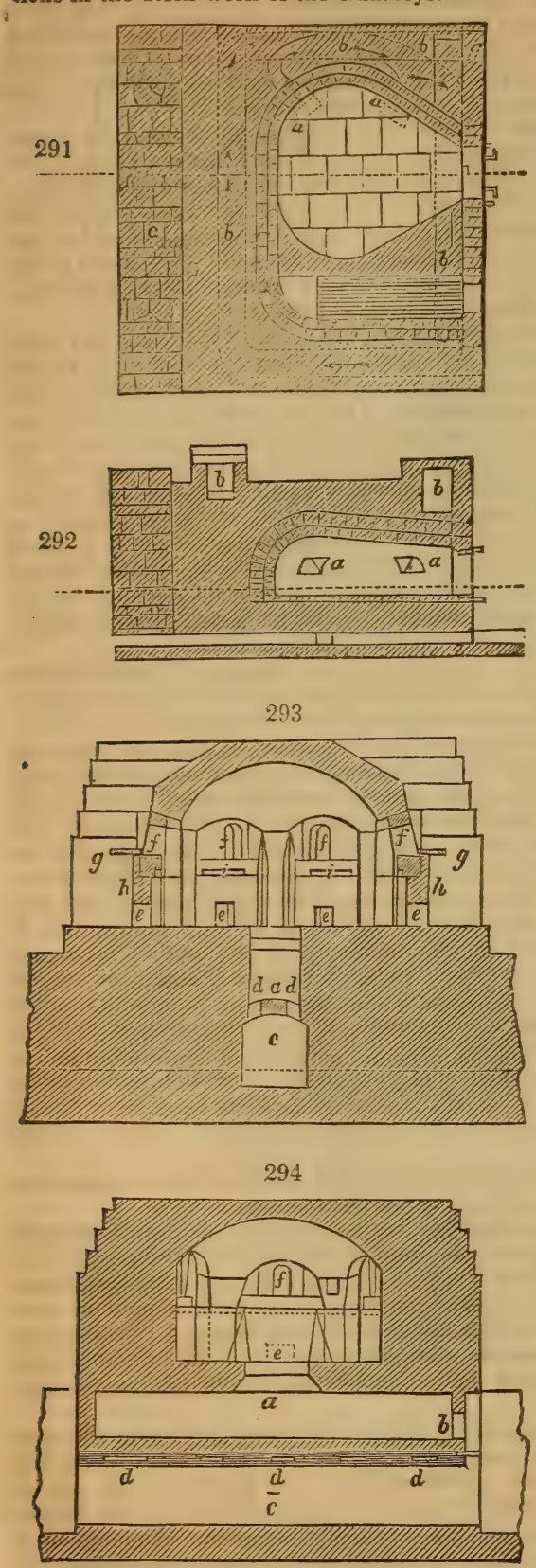

about several times in the earthen melting pots.

The azure manufacture is carried on chiefly in winter, in order that the external cold may favor the more complete condensation of the acids of arsenic. From 3 to $5 \mathrm{cwt}$. of Schlich (pasty ore) are roasted at one operation, and its bed is laid from 5 to 6 inches thick. After two hours, it must be turned over; and the stirring must be repeated every half hour, till no more arsenic is observed to exhale. The process being then finished, the ore must be raked out of the furnace, and another charge introduced.

The duration of the roasting is regulated partly by the proportion of sulphur and arsenic present, and partly by the amount of nickel; which must not be suffered to become oxydized, lest it should spoil the color of the smalt. The latter ores should be but slightly roasted, so as to convert the nickel into speiss. The roasted ore must be sifted in a safety apparatus. The loss of weight in the roasting amounts, upon the average, to 36 per cent. The roasted ore has a brownish gray hue, and is called safflor in German, and is distributed into different sorts. F F S is the finest safre; F S, fine; O S, ordinary; and M S, middling. These varieties proceed from various mixtures of the calcined ores. The roasted ore is ground up along with sand, elatriated, and, when dry, is called $z$ affre. It is then mixed with a sufficient quantity of potash for converting the mixture into a glass.

Figs. 293 and 294, represent a round smalt furnace, in two vertical sections, at right angles to each other. The fire-place is vaulted or arched; the flame orifice $a$, is in the middle of the furnace; $b$ is the feed hole ; $c$, a tunnel which serves as an ash-pit, and to supply air; $d$, openings through which the air arrives at the fuel, the wood being placed upon the vault; $e$, knee holes for taking out the scoriæ from the pot bottoms; $f$, working orifices, with cast-iron plates $g$, in front of them. Under these are the additional outlets $h$. The smoke and flame pass off through the orifices $i$, which terminate in expanded flues, where the sand may be calcined or the wood may be baked. Eight hours are sufficient for one vitrifying operation, during which the glass is stirred

The preparation of the different shades of blue glass is considered a secret in the smelting works; and marked with the following letters:-F F F C, the finest; F C, 
fine; M C, middling; O C, ordinary. A melting furnace, containing 8 pots of glass, produces in 24 hours, from 24 cwts. of the mixture, 19 cwts. of blue glass; and from to $3 \mathrm{cwt}$. of scoriæ or speiss (speise). The composition speise, according to Berthier, is-nickel, 49.0; arsenic, $37 \cdot 8$; sulphur, $7 \cdot 8$; copper, 1.6 ; cobalt, $3 \cdot 2$ in 100 . Nickel, arsenic, and sulphur, are its essential constituents; the rest are accidental, and often absent. The freer the cobalt ore is from foreign metals, the finer is the color, and the deeper is the shade; paler tints are easily obtained by dilution with more glass. The presence of nickel gives a violet tone.

The production of smalt in the Prussian states amounted, in 1830 , to $7452 \frac{1}{2}$ cwts.; and in Saxony to 9697 cwts.; in 1825 , to $12,310 \mathrm{cwts}$.

One process for making fine smalt has been given under the title Azure; I shall introduce another somewhat different here.

The ore of cobalt is to be reduced to very fine powder, and then roasted with much carc. One part, by weight, is next to be introduced, in successive small portions, into an iron vessel, in which three parts of acid sulphate of potassa have been previously fused, at a moderate temperature. The mixture, at first fluid, soon becomes thick and firm, when the fire is to be increased, until the mass is in perfect fusion, and all white vapors have ceased. It is then to be taken out of the crucible with an iron ladle, the crucible is to be recharged with acid sulphate of potash, and the operation continued as before, until the vessel is useless. The fused mass contains sulphate of cobalt, neutral sulphate of potassa, and arseniate of iron, with a little cobalt. It is to be pulverized, and boiled in an iron vessel, with water, as long as the powder continues rough to the touch. The white, or yellowish white residue, may be allowed to separate from the solution, either by deposition or filtration. Carbonate of potassa, free from silica, is then to be added to the solution, and the carbonate of cobalt thrown down is to be separated and well washed, if possible, with warm water; the same water may be used to wash other portions of the fused mass. The filtered liquid which first passes is a saturated solution of sulphate of potassa : being evaporated to dryness in an iron vessel, it may be reconverted into acid sulphate by fusing it with one half its weight of sulphuric acid : this salt is then as useful as at first.

The oxyde of cobalt thus obtained contains no nickel; so little oxyde of iron is present, that infusion of galls does not show its presence; it may contain a little copper, if that metal exists in the ore, but it is easily separated by the known methods. Sometimes sulphureted hydrogen will produce a yellow brown precipitate in the solution of the fused mass; this, however, contains no arsenic, but is either sulphuret of antimony or bismuth, or a mixture of both.

It has been found advantageous to add to the fused mass sulphate of iron, calcined to redness, and one tenth of nitre when the residue is arseniate of iron, and contains no arseniate of cobalt. There is then no occasion to act upon the residue a second time for the cobalt in it.

This process is founded on the circumstances that the sulphate of cobalt is not decomposed by a red heat, and that the arseniates of iron and cobalt are insoluble in all neutral liquids. It is quite evident, that, to obtain a perfect result, the excess of acid in the bisulphate of potassa must be completely driven off by the red heat applied.

$110,646 \mathrm{lbs}$. of smalts were imported into the United Kingdom in 1835, and 96,949 were retained for home consumption. In 1834, only 16,223 lbs. were retained.

In $1835,322,562 \mathrm{lbs}$. of zaffres were imported, and 336,824 are stated to have been retained, which is obviously an error. 284,000 lbs. were retained in 1834 .

COCCULUS INDICUS, or Indian berry, is the fruit of the Menispermum Cocculus, a large tree, which grows upon the coasts of Malabar, Ceylon, \&c. The fruit is blackish, and of the size of a large pea. It owes its narcotic and poisonous qualities to the vegeto-alkaline chemical principle called picrotoxia, of which it contains about one fiftieth part of its weight. It is sometimes thrown into waters to intoxicate or kill fishes; and it is said to have been employed to increase the inebriating qualities of ale or beer. Its use for this purpose is prohibited by act of parliament, under a penalty of $200 \mathrm{l}$. upon the brewer, and $500 l$. upon the seller of the drug.

COCHINFAL was taken in Europe at first for a seed, but was proved by the observations of Lewenhoeck to be an insect, being the female of that species of shield-louse, or coccus, discovered in Mexico, so long ago as 1518. It is brought to us from Mexico, where the animal lives upon the cactus opuntia or nopal. Two sorts of cochineal are gathered-the wild, from the woods, called by the Spanish name grana silvestra; and the cultivated, or the grana fina, termed also mesteque, from the name of a Mexican province. The first is smaller, and covered with a cottony down, which increases its bulk with a matter useless in dyeing; it yields, therefore, in equal weight, much less coior, and is of inferior price to that of the fine cochineal. But these disadvantages are cornpensated in some measure to the growers by its being reared more easily, and 
less expensively ; partly by the effect of its down, which enables it better to resist rains and storms.

The wild cochineal, when it is bred upon the field nopal, loses in part the tenacity and quantity of its cotton, and acquires a size double of what it has on the wild opuntias. It may, therefore, be hoped that it will be improved by persevering care in the rearing of it, when it will approach more and more to fine cochineal.

The fine cochineal, when well dried and well preserved, should have a gray color, bordering on purple. The gray is owing to the powder, which naturally covers it, and of which a little adheres; as also to a waxy fat. The purple shade arises from the color extracted by the water in which they were killed. It is wrinkled with parallel furrows across its back, which are intersected in the middle by a longitudinal one; hence, when viewed by a magnifier, or even a sharp naked eye, especially after being swollen by soaking for a little in water, it is easily distinguished from the factitious, smooth, glistening, black grains, of no value, called East India cochineal, with which it is often shamefully adulterated by certain London merchants. The genuine cochineal has the shape of an egg, bisected through its long axis, or of a tortoise, being rounded like a shield upon the back, flat upon the belly, and without wings.

These female insects are gathered off the leaves of the nopal plant, after it has ripened its fruit, a few only being left for brood, and are killed, either by a momentary immersion in boiling water, by drying upon heated plates, or in ovens : the last become of an ash-gray color, constituting the silver cochineal, or jaspeada; the second are blackish, called negra, and are most esteemed, being probably driest ; the first are reddish brown, and reckoned inferior to the other two. The dry cochineal being sifted, the dust, with the imperfect insects and fragments which pass through, are sold under the name of granillo.

Cochineal keeps for a long time in a dry place. Hellot says that he has tried some 130 years old, which produced the same effect as new cochineal.

We are indebted to MM. Pelletier and Caventou for a chemical investigation of cochi. neal, in which its coloring matter was skilfully eliminated.

Purified sulphuric ether acquired by digestion with it a golden yellow color, amounting by Dr. John to one tenth of the weight of the insect. This infusion left, on evaporation, a fatty wax of the same color.

Cochineal, exhausted by ether, was treated with alcohol at $40^{\circ} \mathrm{B}$. After 30 infusions in the digester of M. Chevreul, the cochineal continued to retain color, although the alcohol had ceased to have any effect on it. The first alcoholic liquors were of a red verging on yellow. On cooling, they let fall a granular matter. By spontaneous evaporation, this matter, of a fine red color, separated, assuming more of the crystalline appearance. These species of crystals dissolved entirely in water, which they tinged of a yellowish-red.

This matter has a very brilliant purple-red color; it adheres strongly to the sides of the vessels; it has a granular and somewhat crystalline aspect, very different, however, from those compound crystals alluded to above; it is not altered by the air, nor does it sensibly attract moisture. Exposed to the action of heat, it melts at about the fiftieth degree centigrade $\left(122^{\circ} \mathrm{Fahr}\right.$.). At a higher temperature it swells up, and is decomposed with the production of carbureted hydrogen, much oil, and a small quantity of water, very slightly acidulous. No trace of ammonia was found in these products.

The coloring principle of cochineal is very soluble in water. By evaporation, the liquid assumes the appearance of sirup, but never yields crystals. It requires of this matter a portion almost imponderable to give a perceptible tinge of bright purplish red to a large body of water. Alcohol dissolves this coloring substance, but, as we have already stated, the more highly it is rectified the less of it does it dissolve. Sulphuric ether does not dissolve the coloring principle of cochineal; but weak acids do, possibly owing to their water of dilution. No acid precipitates it in its pure state. This eoloring principle, however, appears to be precipitable by all the acids, when it is accompanied by the animal matter of the cochineal.

The affinity of alumina for the coloring matter is very remarkable. When that earth, newly precipitated, is put into a watery solution of the coloring principle, this is immediately seized by the alumina. The water becomes colorless, and a fine red lake is obtained, if we operate at the temperature of the atmosphere; but if the liquor has been hot, the color passes to crimson, and the shade becomes more and more violet, accord. ing to the elevation of the temperature, and the continuance of the ebullition.

The salts of tin exercise upon the coloring matter of cochineal a remarkable action. The muriatic protoxyde of tin forms a very abundant violet precipitate in the liquid. This precipitate verges on crimson, if the salt contains an excess of acid. The muriatic deutoxyde of tin produces no precipitate, but changes the color to scarlet-red. If gelatinous alumina be now added, we obtain a fine red precipitate, which does not pass to crimson by boiling. 
To this colnring principle the name carminium has been given, because it forms the basis of the pigment called carmine.

The procuss fullowed in Germany for making carmine, which consists in pouring a certain quantity of solution of alum into a decoction of cochineal, is the most simple of all, and affords an explanation of the formation of carmine, which is merely the carminium and the animal matter precipitated by the excess of acid in the salt, which has taken down with it a small quantity of alumina; though it appears that alumina ought not to be regarded as essential to the formation of carmine. In fact, by another process, called by the name of Madaine Cenette of Amsterdam, the carmine is thrown down, by pouring into the decoction of cochineal a certain quantity of the binoxalate of potash. When carbonate of soda is added, then carminated lake also falls down. That carmine is a triple compound of animal matter, carminium, and an acid, appears from the circumstance that liquors which have afforded their carmine, when a somewhat strong acid is poured into them, yield a new formation of carmine by the precipitation of the last portions of the animal matter. But whenever the whole animal matter is thrown down, the decoctions, although still much charged with the coloring principle, can afford no more carmine. Such decoctions may be usefully employed to make carminated lakes, saturating the acid with a slight excess of alkali, and adding gelatinous alumina. The precipitates obtained on adding acids to the alkaline decoctions of cochineal, are therefore true carmines, since they do not contain alumina; but the small quantity of alumina which is thrown down by alum in the manufacture of carmine, augments its bulk and weight. It gives, besides, a greater lustre to the color, even though diluting and weakening it a little.

The carmines found in the shops of Paris were analyzed, and yielded the same products. They are decomposed by the action of heat, with the diffusion at first of a very strong smell of burning animal matter, and then of sulphur. A white powder remained, amounting to about one tenth of the matter employed, and which was found to be alumina. Other quantities of carmine were treated with a solution of caustic potash, which completely dissolved them, with the exception of a beautiful red powder, not acted on by potash and concentrated acids, and which was recognised to be red sulphuret of mercury or vermilion. This matter, evidently foreign to the carmine, appears to have been ad. ded in order to increase its weight.

The preceding observations and experiments seem calculated to throw some light on the art of dyeing scarlet and crimson. The former is effected by employing a cochineal bath, to which there have been added, in determinate proportions, acidulous tartrate of potash, and nitro-muriatic deutoxyde of tin. The effect of these two salts is now well known. The former, in consequence of its excess of acid, tends to redden the color, and to precipitate it along with the animal matter; the latter acts in the same manner, at first by its excess of acid, then by the oxyde of tin which falls down also with the carmine and animal matter, and is fixed on the wool, with which it has of itself a strong tendency to combine. MM. Pelletier and Caventou remark, that " to abtain a beautiful shade, the muriate of tin ought to be entirely at the maximum of oxydizement; and it is in reality in this state that it must exist in the solution of tin prepared according to the proportions prescribed in M. Berthollet's treatise on dyeing."

We hence see why, in dyeing scarlet, the employment of alum is carefully avoided, as this salt tends to convert the shade to a crimson. The presence of an alkali would seem less to be feared. The alkali would occasion, no doubt, a crimson-colored bath; but it would be easy in this case to restore the color, by using a large quantity of tartar. We should, therefore, procure the advantage of having a bath better charged with coloring matter and animal substance. It is for experience on the large scale to determine this point. As to the earthy salts, they must be carefully avoided; and if the waters be selenitish, it would be a reason for adding a little alkali.

To obtain crimson, it is sufficient, as we know, to add alum to the cochineal bath, or to boil the scarlet cloth in alum water. It is also proper to diminish the dose of the salt of tin, since it is found to counteract the a tion of the alum.

The alkalis onght to be rejected as a means of changing scarlet to crimson. In fact, crimsons by this process eannot be permanent colors, as they pass into reds by the action of acids.

According to M. Von Grottluss, carmine may be deprived of its golden shade by ammonia, and subsequent treatment with acetic acid and alcohol. Since this fact was made known, M. Herschel, color maker at Halle, has prepared a most beautiful carmine.

The officers of Her Majesty's Customs have lately detected a system of adulterating cochineal, which has been practised for many years upon a prodigious scale by a mercan. tile house in London. I have analyzed about 100 samples of such cochineal, from which it appears that the genuine article is moistened with gum-water, agitated in a box or 
.eather bag, first, with sulphate of baryta in fine powder, afterwards with bone or ivory black, to give it the appearance of negra cochineal, and then dried. By this means about 12 per cent. of the worthless heavy spar is sold at the price of cochineal, to the enrichment of the sophisticators, and the disgrace and injury of British trade and manufactures.

The specific gravity of genuine cochineal is 1.5 ; that of the cochineal loaded with the barytic sulphate 1.35 . It was taken in oil of turpentine and reduced to water as unity, because the waxy fat of the insects prevents the intimate contact of the latter liquid with them, and the ready expulsion of air from their wrinkled surface. They are not at all acted upon by the oil, but are rapidly altered by water, especially when they have been gummed and barytified.

The quantities of cochineal imported into the United Kingdom in the following years were : -

$$
\begin{array}{lllllllll}
1827 . & 1828 . & 1829 . & 1830 . & 1831 . & 1832 . & 1833 . & 1834 . & 1835 .
\end{array}
$$

$\begin{array}{lllllllll}\text { Lbs. } 320,722 & 258,032 & 288,456 & 316,589 & 244,371 & 388,478 & 359,381 & 410,387 & 418,320\end{array}$

The quantities re-exported were:-

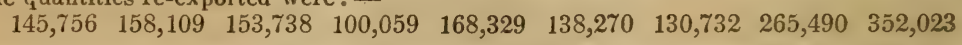

Humboldt states that so long ago as the year 1736, there was imported into Europe from South America cochineal to the value of 15 millions of francs. Its high price had for a long time induced dyers to look out for cheaper substitutes in dyeing red, and since science has introduced so many improvements in tinctorial processes, both madder and lac have been made to supersede cochineal to a very great extent. Its price has, in consequence of this substitution, as well as from more successful modes of cultivation, fallen very greatly of late years. At present it is only $7 s$. per $1 b$. in London. See SCARLET Dyerng.

COCOA, STEARINE, and ELAINE. Mr. Soames obtained a patent in September, 1829 , for making these useful articles, by the following process :-

He takes the substance called cocoa-nut oil, in the state of lard, in which it is imported into this country, and submits it to a strong hydraulic pressure, having made it up in small packages, 3 or 4 inches wide, 2 feet long, and 1 or $1 \frac{1}{2}$ inches thick. These packages are formed by first wrapping up the said substance in a strong linen cloth, of close texture, and then in an outward wrapper of strong sail cloth. The packages are to be placed side by side, in single rows, between the plates of the press, allowing a small space between the packages for the escape of the elaine.

The temperature at which the pressure is begun, should be from about 50 to 55 degrees, or in summer as nearly at this pitch as can be obtained, and the packages of the said substance intended for pressure, should be exposed for several hours previously to about the same temperature. When the packages will no longer yield their oil or elaine freely at this temperature, it is to be gradually raised; but it must at no time exceed 65 degrees, and the lower the temperature at which the separation can be effected, the better will be the quality of the oil expressed.

When the packages are sufficiently pressed, that is, when they will give out no more oil, or yield it only in drops at long intervals, the residuum in them is to be taken out and cleansed and purified, which is done by melting it in a well-tinned copper vessel, which is fixed in an outer vessel, having a vacant space between, closed at the top, into which steam is admitted, and the heat is kept up moderately for a sufficient time to allow the impurities to subside; but if a still higher degree of purity is required, it is necessary to pass it through filters of thick flannel lined with blotting paper.

Having been thus cleansed or purified, it is fit for the manufacture of candles, which are made by the ordinary process used in making mould tallow candles. Having thus disposed of the stearine, or what is called the first product, he proceeds with the elaine or oil expressed from it, and which he calls the second product, as follows: that is to say, he purifies it by an admixture, according to the degree of its apparent foulness, of from 1 to 2 per cent. by weight of the sulphuric acid of commerce, of about 1.80 specific gravity, diluted with six times its weight of water. The whole is then to be violently agitated by mechanical means, and he prefers for this purpose the use of a vessel constructed on the principle of a common barrel churn. When sufficiently agitated, it will have a dirty whitish appearance, and is then to be d awn off into another vessel, in which it is to be allowed to settle, and any scum that rises is to be carefully taken off. In a day or two the impurities will be deposited at the bottom of the oil, which will then become clear, or nearly so, and it is to be filtered through a thick woollen cloth, after which it will be fit for burning in ordinary lamps and for other uses.

The process of separating the elaine from the stearine, by pressure, in manner afore. said, had never before been applied to the substance called cocoa-nut oil, and consequentiy no product had heretofore seen obtained thereby from that substance, fit for being manufactured into candles in the ordinary way, or for being refined by any of the usual 
morles, so as to burn in ordinary lamps, both which objects are obtained by this method of preparing or manufacturing the said substance.

Candles welı made from the above material are a very superior article. The light produeed is more brilliant than from the same sized candle made of tallow; the flame is perfectly colorless, and the wick remains free from cinder, or any degree of foulness during combustion.

COFFEE. The coffee is the seel of a tree of the family rubiacea, and belongs to the Pentandria monogynia of Linnæus. There are several species of the genus, but the only one cultivated is the Coffea Arabica, a native of Upper Ethiopia and Arabia Felix. It rises to the height of 15 or 20 feet ; its trunk sends forth opposite branches in pairs above and at right angles to each other; the leaves resemble those of the common laurel, although not so dry and thick. From the angle of the leaf-stallss small groups of white flowers issue, which are like those of the Spanish jasmine. These flowers fade very soon, and are replaced by a kind of fruit not unlike a cherry, which contains a yellow glairy fluic, enveloping two small seeds or berries convex upon one side, flat and furrowed upon the other in the direction of the long axis. These seeds are of a horny or cartilaginous nature; they are glued together, each being surrounded with a peculiar coriaceans membrane. They constitute the coffee of commerce.

It was not till towards the end of the 15 th century that the coffee-tree began to be cultivated in Arabia. Historians usually ascribe the discovery of the use of coffee as a be. verage to the superior of a monastry there, who, desirous of preventing the monks from sleeping at their nocturnal services, made them drink the infusion of coffee upon the report of shepherds, who pretended that their flocks were more lively after browsing on the fruit of that plant. The use of coffee was soon rapidly spread, but it encountered much opposition on the part of the Turkish government, and became the occasion of public assemblies. Under the reign of Amurath III. the mufti procured a law to shut all the coffee-houses, and this act of suppression was renewed under the minority of Mahomet IV. It was not till 1554, under Solyman the Great, that the drinking of coffee was accredited in Constantinople; and a century elapsed before it was known in London and Paris. Solyman $\mathrm{A}$ ga introduced its use into the latter city in 1669, and in 1672 an Armenian established the first café at the fair of Saint Germain.

When coffee became somewhat of a necessary of life, from the influence of habit among the people, all the European powers who had colonies between the tropics, projected to form plantations of coffee-trees in them. The Dutch were the first who transported the coffee plant from Moka to Batavia, and from Batavia to Amsterdam. In 1714 the magistrates of that city sent a root to Louis XIV., which he caused to be planted in the Jardin du Roi. This became the parent stock of all the French coffee plantations in Martinique.

The most extensive culture of coffee is still in Arabia Felix, and principally in the kingdom of Yemen, towards the cantons of Aden and Moka. Although these countries are very hot in the plains, they possess mountains where the air is mild. The coffee is generally grown half way up on their slopes. When cultivated on the lower grounds it is alwavs surrounded by large trees which shelter it from the torrid sun, and prevent its fruit from withering before their maturity. The harvest is gathered at three periods; the most considerable occurs in May, when the reapers begin by spreading cloths under the trees, then shaking the branches strongly, so as to make the fruit drop, which they collect, and expose upon mats to dry. They then pass over the dried berries a very heavy roller, to break the envelops, which are afterwards winnowed away with a fan. The interior bean is again dried before being laid up in store.

In Demarara, Berbice, and some of our West India islands, where much good coffee is now raised, a different mode of treating the pulpy fruit and curing the beans is adopted. When the cherry-looking berry has assumed a deep-red color it is gathered, and immediately subjected to the operations of a mill composed of two wooden rollers, furnished with iron plates, which revolvt near a third fixed roller called the chops. The berries are fed into a hopper above the rollers, and falling down between them and the chops, they are stripped of their outer skin and pulp, while the twin beans are separated from each other. These beans then fall upon a sieve, which allows the skin and the pulp to pass through, while the hard beans accumulate and are progressively slid over the edge into baskets. They are next steeped for a night in water, thoroughly washed in the morning, and afterwards dried in the sun. They are now ready for the peeling mill, a wooden edge wheel turned vertically by a horse roked to the extremity of its horizontal axis. In travelling over the coffee, it bursts and detaches the coriaceous or parchment-like skin which surrounds each hemispherical bean. It is then freed from the membranes by a winnowing machine, in which four pieces of tin made fast to an axle are caused to revolve with great velocity. Corn fanners would answer better than this rude instrument of negro invention. The coffee is finally spread upon mats or tables, picked clean, and packed up for shipment. 
The most highly esteemed coffee is that of Moka. It ias a smaller and a rounder bean; a more agreeable taste and smell than any other. Its zolor is yellow. Next to it in European reputation are the Martinique and Bourbon coffees; the former is larger than the Arabian, and more oblong; it is rounded at the ends; its color is greensh, and it preserves almost always a silver gray pellicle, which comes off in the roasting. The Bourbon coffee approaches nearest to the Moka, from which it originally sprung. The Saint Domingo coffee has its two extremities pointed, and is much less esteemed than the preceding.

The coffee-tree flourishes in billy districts, where its root can be kept dry, while its leaves are refreshed with frequent showers. Rocky ground, with rich decomposed mould in the fissures, agrees best with it. Though it would grow, as we have said, to the height of 15 or 20 feet, yet it is usually kept down by pruning to that of five feet, for increasing the production of the fruit, as well as for the convenience of cropping. It begins to yield fruit the third year, but is not in full bearing till the fifth, does not thrive beyond the twenty-fifth, and is useless in general at the thirtieth. In the coffee husbandry, the plants should be placed eight feet apart, as the trces throw out extensive horizontal branches, and in holes ten or twelve feet deep, to secure a constant supply of moisture.

Coffee has been analyzed by a great many chemists, with considerable diversity of results. The best analysis perhaps is that of Schrader. He found that the raw beans distilled with water in a retort communicated to it their flavor and rendered it turbid, whence they seem to contain some volatile oil. On reboiling the beans, filtering, and evaporating the liquor to a sirup, adding a little alcohol till no more matter was precipitated, and then evaporating to dryness, he obtained $17 \cdot 58$ per cent. of a yellowishbrown transparent extract, which constitutes the characteristic part of coffee, though it is not in that state the pure proximate principle called cafeine. Its most remarkable reaction is its producing, with both the protoxyde and the peroxyde salts of iron, a fine grass green color, while a dark green precipitate falls, which re-dissolves when an acid is poured into the liquor. It produces on the solution of the salts of copper scarcely any effect, till an alkali be added, when a very beautiful green color is produced, which may be employed in painting. Coffee beans contain also a resin, and a fatty substance somewhat like suet. According to Robiquet, ether extracts from coffee beans nearly 10 per cent. of resin and fat, but he probably exaggerates the amount. The peculiar substance cafeine contained in the above extract is crystallizable. It is remarkable in regard to composition, that after urea and the uric acid, it is among organic products the richest in azote. It was discovered and described in 1820 by Runge. It does not possess alkaline properties. Pfaff obtained only 90 grains of cafeine from six pounds of coffee beans. There is also an acid in raw coffee, to which the name of cafeic acid has been given. When distilled to dryness and decomposed, it has the smell of roasted coffee.

Coffee undergoes important changes in the process of roasting. When it is roasted to a yellowish brown it loses, according to Cadet, $12 \frac{1}{2}$ per cent. of its weight, and is in this state difficult to grind. When roasted to a chestnut brown it loses 18 per cent., and when it becomes entirely black, though not at all carbonized, it has lost 23 per cent. Schrader has analyzed roasted coffee comparatively with raw coffee, and he found in the first $12 \frac{1}{2}$ per cent. of an extract of coffee, soluble in water and alcohol, which possesses nearly the properties of the extract of the raw coffee, although it has a deeper brown color, and softens more readily in the air. $\mathrm{He}$ found also 10.4 of a blackish brown gum; 5.7 of an oxygenated extract, or rather apothème, soluble in alcohol, insoluble in water ; 2 of a fatty substance and resin; 69 of burnt vegetable fibre, insoluble. On distilling roasted coffee with water, Schrader obtained a product which contained the aromatic principle of coffee; it reddened litmus paper, and exhaled a strong and agreeable odor of roasted coffee. If we roast coffee in a retort, the first portions of the aromatic principle of coffee condense into a yellow liquid in the receiver; and these may be added to the coffee roasted in the common way, from which this matter has been expelled and dissipated in the air.

Chenevix affirmed that by the roasting of coffee a certain quantity of tannin possessing the property of precipitating gelatin is generated. Cadet made the same observation, and found, moreover, that the tannin was most abundant in the lightly roasted coffee, and that there was nearly none of it in coffee highly roasted. Payssé and Schrader, on the contrary, state that solution of gelatin does not precipitate either the decoction of roasted coffee or the alcoholic extract of this coffee. Runge likewise asserts that he could obtain no precipitate with gelatin; but he says that albumen precipitates from the decoction of roasted coffee the same kind of tannin as is precipitated from raw cotfee by the acetate of lead, and set free from the lead by selphureted hydrogen. With these results my own experiments agree. Gelatin certainly joes not disturb clear infusion of roasted coffee, but the salts of iron blacken it. 
Schrader endeavored to roast separately the different principles of coffee, but none of them exhaled the aromatic odor of roasted coffee except the horny fibrous matter. He therefore concludes that this substance contributes mainly to the characteristic taste of roasted coffee, which cannot be imitated by any other vegetable matter, and which, as we have seen, should be ascribed chiefly to the altered cafeic acid. According to Garot, we may extract the cafeine without alteration from roasted coffee by precipitating its decoction by subacetate of lead, treating the washed precipitate with sulphureted hydrogen, and evaporating the liquid product to dryness.

Of late years, much ingenuity has been expended in contriving various forms of apparatus for making infusions of coffee for the table. I have tried most of them, and find, after all, none so good as a cafetière à la Belloy, the coffee biggin, with the perforated tinplate strainer, especially when the filtered liquor is kept simmering in a close vessel, set over a lamp or steam pan. The useful and agreeable matter-in coffee is very soluble : it comes off with the first waters of infusion, and needs no boiling.

Tc roast coffee rightly we should keep in view the proper objects of this process, which are to develop its aroma, and destroy its toughness, so that it may be readily ground to powder. Too much heat destroys those principles which we should wish to preserve, and substitutes new ones which have nothing in common with the first, but add a disagreeable empyreumatic taste and smell. If, on the other hand, the rawness or greenness is not removed by an adequate heat, it masks the flavor of the bean, and injures the beverage made with it. When well roasted in the sheet-iron cylinders set to revolve over a fire, it should have a uniform chocolate color, a point readily hit by experienced roasters, who now manage the business very well for the principal coffee-dealers both of London and Paris, so far as my judgment can determine. The development of the proper aroma is a criterion by which coffee-roasters frequently regulate their operations. When it loses more than 20 per cent. of its weight, coffee is sure to be injured. It should never be ground till immediately before infinsion.

COKE is carbonized pitcoal. See Charcoal; and Pitcoal at the end.

COLCOTHAR OF VITRIOL (Rouge d'Angleterre, Fr.; Rothes Eisenoxyd, Germ.) is the brown-red peroxyde of iron, produced by calcining sulphate of iron with a strong heat, levigating the resulting mass, and elutriating it into an impalpable powder. A better way of making it so as to complete the separation of the acid, is to mix 100 parts of the green sulphate of iron with 42 of common salt, to calcine the mixture, wash away the resulting sulphate of soda, and levigate the residuum. The sulphuric acid in this case expels the chlorine of the salt in the form of muriatic acid gas, and saturates its alkaline base produced by the chemical reaction; whence an oxyde will be obtained free from acid, much superior to what is commonly found in the shops. The best sort of polishing powder, called jewellers' red rouge or plate powder, is the precipitated oxyde of iron prepared by adding solution of soda to solution of copperas, washing, drying, and calcining the powder in shallow vessels with a gentle heat, till it assumes a deep brown red color. See IRON.

COLOPHANY, black rosin, the solid residuum of the distillation of turpentine, when all the oil has been worked off.

COLORING MATTER. (Matiere colorante, Fr.; Farbstoff, Germ.) See Dreing. the several dye-stuffs and pigments.

COLUMBIUM, a peculiar metal extracted from a rare mineral brought from Haddam, in Connecticut. It is also called Tantalium, from the mineral tantalite and yttro-tantalite, found in Sweden. It has hitherto no application to the arts. It combines with two successive doses of oxygen; by the second it becomes an acid.

COLZA is a variety of cabbage, the brassica oleracea, whose seeds afford, by pressure, an oil much employed in France and Belgium for burning in lamps, and for many other purposes. This plant requires a rich but light soil; it does not succeed upon either sandy or clayey lands. The ground for it must be deeply ploughed and well dunged. It should be sown in July, and be afterward replanted in a richly-manured field. In October it is to be planted out in beds, 15 or 18 inches apart. Colza may also be sowed in furrows 8 or 10 inches asunder.

Land which has been just cropped for wheat is that usually destined to colza ; it may be fresh dunged with advantage. The harvest takes place in July, with the sickle, little before the seeds are completely ripe, lest they should drnp off. As the seed is productive of oil, however, only in proportion to its ripeness, the cut plants are allowed to complete their maturation, by laying them in heaps under airy sheds, or placing them in a stack, and thatching it with straw.

The cabbage-stallis are thrashed with flails, the seeds are winnowed, sifted, spread out in the air to dry; then packed away in sacks, in order to be subjected to the oil-mill at the beginning of winter. The oil-cake is a very agreeable food to cattle, and serves to fatten them. It is reckoned to defray the cost of the mill.

Colza impoverishes the soil very much, as do, indeed, all the plants cultivated for the 
sake of their oleaginous seeds. It must not, therefore, be come back upon again for sir years, if fine crops be desired. The double ploughing which it requires effectually cleans the ground. See OrLs, Unctuous.

COMB, the name of an instrument made of a thin plate either plane or curved of wood, horn, tortoise-shell, ivory, bone, or metal, cut out upon one or both of its sides or edges, into a series of somewhat long teeth, not far apart; which is employed for disentangling, laying parallel and smooth the hairs of man, horses, or other animals.

A thin steel saw bow, mounted in an iron or wooden handle, is the implement used by the comb-maker to cut the bone, ivory, and wood into slices of from a twelfth to a quarter of an inch thick, and of a size suitable to that of the comb. The pieces of tortoise-shell as found in commerce are never flat, or, indeed, of any regular curvature, such as the comb must have. They are therefore steeped in boiling water sufficiently long to softer them, and set to cool in a press between iron or brass moulds, which impart to them the desired form which they preserve after cooling. After receiving their outline shape and curvature, by proper flat files or fine rasps, the place of the teeth is marked with a triangular file, and then the teeth themselves are cut out with a double saw, composed of two thin slips of tempered steel, such as the main-spring of a watch, notched with very fine sharp teeth. These slips are mounted in a wooden or iron stock or handle, in which they may be placed at different distances, to suit the width of the comb-teeth. A comb-maker, however, well provided in tools, has an assortment of double saws set at every ordinary with. The two slips of this saw have their teeth in different planes, so that when it begins to cut, the most prominent slip alone acts, and when the teeth of this one have fairly entered into the comb, the other parallel blade begins to saw. The workman, meanwhile, has fixed the plate of tortoise-shell or ivory between the flat jaws of two pieces of wood, Jike a vice made fast to a bench, so that the comb intended to be cut is placed at an angle of $45^{\circ}$ with the horizon. He now saws perpendicularly, forming two teeth at a time, proceeding truly in the direction of the first tracing.

A much better mode of making combs is to fix upon a shaft or arbor in a lathe a series of circular saws, with intervening brass washers or discs to keep them at suitable distances; to set in a frame like a vice, in front of these saws, the piece of ivory or horn to be eut; and to press it forward upon the saws at an angle of 45 degrees, by means of a regulated screw motion. When the teeth are thus cut, they are smoothed and polished with files, and by rubbing with pumice-stone and tripoli.

Mr. Bundy, of Camden Town, obtained a patent so long ago as 1796, for an apparatus of that kind, which had an additional arbor fitted with a series of circular saws, or rather files, for sharpening the points of the comb-teeth.

More recently, Mr. Lyne has invented a machine in which, by means of pressure, two combs are cut out at once with chisels from any tough material, such as horn or tortoiseshell, somewhat softened at the moment by the application of a heated iron to it. The piece of horn is made fast to a carriage, which is moved forward by means of a screw until it comes under the action of a ratchet-wheel, toothed upon a part of its circumference. The teeth of this wheel bring a lever into action, furnished with a chisei or knife, which cuts out a double comb from the flat piece, the teeth of which combs are opposite to each other. By this means, no part of the substance is lost, as in sawing out combs. The same carriage may be used, also, to bear a piece of ivory in the hard state toward a circular saw, on the principles above explained, with such precision, that from 80 to 100 teeth can be formed in the space of one inch by a proper disposition of the tool.

Bullocks' horns, after the tips are sawed off, are roasted in the flame of a wood fire, till they are sufficiently softened; when they are slit up, pressed in a machine between two iron plates, and then plunged into a trough of cold water, whereby they are hardened. A paste of quicklime, litharge, and water is used to stain the horn to resemble tortoise-shell. See HorN.

COMBINATION (Combinaison, Fr.; Verbindung, Germ.); a chemical term which denotes the intimate union of dissimilar particles of matter, into a homogeneous-looking compound, possessed of properties generally different from those of the separate constituents.

COMBUSTIBLE (Eng. and Fr.; Brennstoff, Germ.); any substance which, exposed in the air to a certain temperature, consumes spontaneously with the emission of heat and light. All such combustibles as are cheap enough for common use go under the name of Fuel; which see. Every combustible requires a peculiar pitch of temperature to be kindled, called its accendible point. Thus phosphorus, sulphur, hydrogen, carbureted hydrogen, earbon, each takes fire at successively higher heats.

COMBUSTION (Eng. and Fr.; Verbrennung, Germ.) results in common cases from the mutual chemical reaction of the combustible, and the oxygen of the atmosphere, 
whereby a new compound is formed; the heat and light evolved being most probably produced by the rapict motions of the particles during the progress of this combination.

COMPOUND COLORS. If the effects of the coloring particles did not vary according to the combinations which they form, and the actions exercised upon them by the different substances present in a dyeing bath, we might determine with precision the shade which ought to result from the mixture of any two colors, or of the ingredients aflording these colors separately. Though the chemical action of the mordants, and of the liquor in the dye-bath often changes the results, yet theory may always predict them within a certain degree. It is not the color appropriate to the dye-stuffs which is to be considered as the constituent part of compound colors, but that which they must assume with a certain mordant and dye-bath. Our attention ought therefore to be directed principally to the operation of the chemical agents employed.

1. The mixture of blue and yellow dyes produces green. D'Ambourney, indeed, says that he has extracted a fast green from the fermented juice of the berries of the buckthorn (rhamnus fragula), but no dyer would trust to such a color.

2. The mixture of red and blue produces violet, purple, columbine (dove-color), pansy, amaranth, lilach, mallow, and a great many other shades, determined by the nature and tone of the red and blue dye-stuffs, as well as their relative proportions in the bath.

3. The mixture of red and yellow produces orange, mordoré, cinnamon, coquelicot, brick, capuchin; with the addition of blue, olives of various shades; and with duns instead of yellows, chestnut, snuff, musk, and other tints.

4. Blacks of the lighter kinds constitute grays; and, mixed with other colors, produce marrone (marroons), coffees, damascenes. For further details upon this subect, see Calico Printing, Dreing, as also the individual colors in their alphabetical places.

CONCRETE. The name given by architects to a compact mass of pebbles, sand, and lime cemented together, in order to form the foundations of buildings. Semple says that the best proportions are 80 parts of pebbles, each about 7 or 8 ounces in weight, 40 parts sharp river sand, and 10 of good lime; the last is to be mixed with water to a thinnish consistence, and grouted in. It has been found that Thames ballast, as taken from the bed of the river, consists nearly of 2 parts of pebbles to 1 of sand, and therefore answers exceedingly well for making concrete; with from one seventh to one eighth part of lime. The best mode of making concrete, according to $\mathrm{Mr}$. Godwin, is to mix the lime, previously ground, with the ballast in a dry state; sufficient water is now thrown over it to effect a perfect mixture, after which it should be turned over at least twice with shovels, or oftener; then put into barrows, and wheeled away for use instantly. It is generally found advisable to employ two sets of men to perform this operation, with three in each set; one man to fetch the water, \&c., while the other two turn over the mixture to the second set, and they, repeating the process, turn over the concrete to the barrow-men. After being put into the barrows, it should at once be wheeled up planks, so raised as to give it a fall of some yards, and thrown into the foundation, by which means the particles are driven closer together, and greater solidity is given to the whole mass. Soon after being thrown in, the mixture is observed usually to be in commotion, and much heat is evolved with a copious emission of vapor. The barrow-load of ?oncrete in the fall, spreading over the ground, will form generally a stratum of from 7.09 inches thick, which should be allowed to set before throwing in a second.

Another method of making concrete, is first to cover the foundation with a certain quantity of water, and then to throw in the dry mixture of ballast and lime. It is next turned and levelled with shovels; after which more water is pumped in, and the operation is repeated. The former method is undoubtedly preferable.

In some cases it has been found necessary to mix the ingredients in a pug-mill, as in mixing clay, \&c. for bricks. For the preparation of a concrete foundation, as the hardening should be rapid, no more water should be used than is absolutely necessary to effect a perfect mixture of the ingredients. Hot water accelerates the induration. There is about one fifth of contraction in volume in the concrete, in reference to the bulk of its ingredients. To form a cubical yard of concrete, about 30 feet cube of ballast and $3 \frac{1}{2}$ feet cube of ground lime must be employed, with a sufficient quantity of water.

CONGELATION (Eng. and Fr.; Gefrierung, Germ.); the act of freezing liquids. Many means are supplied by chemistry of effecting or promoting this process, but they do not constitute any peculiar art or manufacture. See ICE-HousE.

COOLING OF FLUIDS. In Mr. Derosnes's method, the cooling agents employed are a current of atmospheric air, and warm water of the same or nearly the same temperature as that of the vapors which are to be operated upon.

Fig. 295 represents merely a diagram of the general features of an apparatus con- 
structed upon the principles proposed to be exployed, which will serve to explain the nature of this improvement.

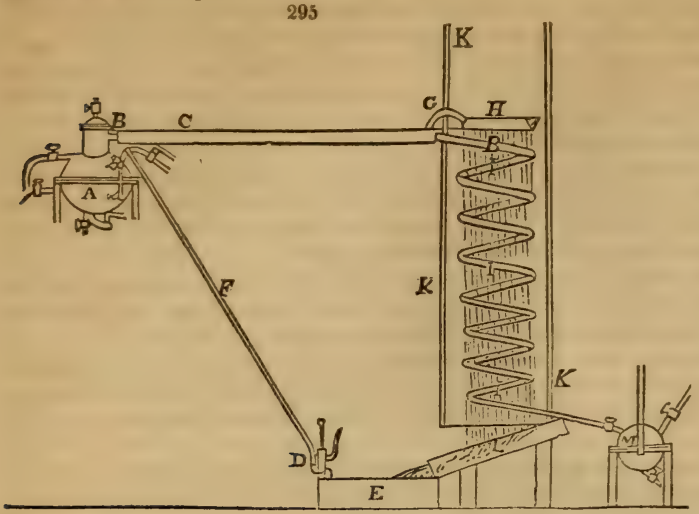

Let $\mathrm{A}$ be the source of the vapors, or the vessel, boiler, alembic, or closed pan that contains the liquid or sirup to be evaporated or concentrated. The pipe $\mathrm{B}$, through which the vapor passes as it rises in the boiler, is surrounded by another tube c, of larger diameter, closed at both ends. A pump D, draws from thereservoir $\mathbf{E}$, warm water, which water has been heated by its previous and continual passage through the apparatus in contact with the surface of the vapor pipes. This pump forces the water by the pipe $F$, into the annular space or chamber between the prpes $\mathrm{B}$ and $\mathrm{c}$, in which chamber, by its immediate contact with the pipe $\mathrm{B}$, it acquires the temperature of the vapors intended to be refrigerated. The pipe $\mathrm{G}$ convers the water from the pipe $\mathrm{c}$, into the annular colander or sieve $\mathrm{H}$, which has a multitude of small holes pierced through its under part, and whence the warm water descends in the form of a continued shower of rain. To the end of the pipe B, a distiller's worm I r, is connected, which is placed beneath the colander $\mathrm{H}$. The entire length of the worm-pipe should be bound round with linen or cotton cloth, as a conductor of the heat, which cloth will be continually moistened by the rain in its descent from the colander. As this water has been heated in passing along the tube $c$, the shower of rain descending from the colander will be at a higher temperature than that of the atmosphere, and, consequently, by heating the surrounding air as it descends, a considerable upward draft will be produced through the coils of the worm-pipe.

If the colander and the worm-pipe are enclosed within a chimney or upright tube, as $\mathbf{K} \mathbf{K}$, open at top and bottom, a current of ascending air will be produced within it by the descending shower of hot water, similar in effect to that which would be produced in a chimney communicating with a furnace, or to that of the burner of an argana lamp. Consequently, it will be perceived that in opposition to the descending rain, a strong upward current of air will blow through that part of the cylinder $\mathrm{K} \mathrm{K}$, which is beneath the colander. When the air first enters the lower aperture of the chimney or tube $\mathbf{K}$, it is of the same temperature and moisture as the external atmosphere; but in its passage up the tube it meets with a warmer and damper atmosphere, caused by the heat given out from the hot fluid continually passing through the plpes, and by the hot shower of rain, and also by the steam evolved from the surfaces of the coils of the worm, which are continually wetted by the descending rain, the evaporation being considerably augmented by the cloth bound round the worm-pipe, retaining the water as it descends in drops from coil t $\mathrm{c}$ coil.

The atmosphere within the tube being of a higher temperature than without, a current of air constantly ascends and escapes at the upper aperture $k$, and its place is supplied by fresh air from the surrounding atmosphere, entering the tube below. The fresh air thus admitted at the bottom of the tube, being cold and dry, will be suited to take up the heat and moisture within, because the water within the tube, being in a state of dispersion as rain, presents to the air many points, or a very extended surface, and also because it is of a higher temperature than the air; and, besides, cold dry air is cont inually renewed, and a source of warmth is furnished by the latent caloric to the steam as fast as it is evolved. Thus a portion of the descending rain, or water, is evaporated, and the effect of this evaporation is to subtract caloric not only from the water held in contact with the coils of the worm-pipe by the cloth enveloping it, but also from the hot vapors which pass through the worm. This process of evaporation nas, therefore, a cooling power, which is but slight in the lower part of the chimney or tube $\mathbf{z}$; because the temperature of the water, or rain, and of the worm, at this part, is of a lower temperature; but its refrigerating power increases as it rises towards the colander, and there it acquires its maximum of intensity, so that at any point between the lower aperture of the cylinder and the colander, the current of air is always a little cooler than the atmosphere of the region through which it passes (that is, at its maximum); and in passing this region of higher temperature, it is not only put in equili- 
brium of temperature, but also made to take up an additional quantity of aqueous rapors, which equalizes the new temperature it acquires with its capacity of saturation. The coling caused br the evaporation acts in an incessant and progressive manner from the lower aperture of the cylinder to the under side of the colander; and this cooling not only acts as an agent of the evaporation which the current of air cools, but it refrigerates also, because it becomes warmed in abstracting caloric from the rapors or liquids passing through the worm; and this refrigeration acts also incessantly and progressirely from the lower part of the tube or chimney to the colander.

The patentee states, in conclusion, that "the velocity or force of the current of air that passes through the chimne! or tube $\mathbf{K}$, can be accelerated by artificial means, either by conlucting the air and vapor passing from the upper aperture of the crlinder into the chimney or flues of a furnace, or by means of a revolving, forcing, or exhausting fan, or ventilator, or any other contrirance which will produce an increased current of air, but which is not necessary to be particularly described, as I only wish to explain the principles of a simple apparatus, constructed in an conrenient form : and I would remark, that the area of the lower aperture through which the air is introdaced into the chimney or tube $\mathrm{k}$, and also the area of the upper aperture, or that through which it passes to the atmosphere, should be in accordance with the effect intended to be obtained.

"It is further to be remarkel, that in order to obtain from this apparatus the best effect, the relocity of the current of air must be itself a maximum: and as the speed or velocity of the current of air is owing to and determined by the excess of the temperature of the descending water, or rain, and of the coils of the worm to that of the exteris: atmosphere, it ensues that the temperature of the water, or rain, must be a maximum. But this excess of temperature is a maximum only when the source of the rain is at the same temperature as the rapors to be condensed: if less warm, it would attract less air; or, if warmer, it would augment the temperature of the rapors intended to be condensed. Consequently, the shower of water emplosed in the tube $\mathrm{K}$, as the agent for cooling, bestows its maximum of effect when it is as warm as the raporz to be condensed; therefore, I may express this proposition, viz., "That in refrigerating with water, less of it mey be expended when it is warm than when it is cold, and that the least quantity of water will be evaporated when it is as warm as the aqueous or spirituous vapors upon which it is to operate."

"This proposition may appear strange, netertheless it is conformable to the laws of nature; and appears only strange, because until now warm water has not been employed with currents of air for refrigerating.

"Hence it is necessary to raise the temperature of the water in the colander to the temperature of the rapors to be condensed: therefore, I cause the lukewarm water, pumpel from the reservoir $E_{\text {, to }}$ circulate in the chamber $c$. In this circulation it also begins to act as a refrigerating medium, taking up a portion of heat from the rapors that pass through the pipe $B$, and afterwards it acts as a further condenser in the crlinder, in the way described. Finaliy, the portion of this water that is still in the fluid state, after haring fallen down from coil to coil, arrires lukewarm to the inclined surface $I$, which conducts it into the reservoir $E$, from whence it is pumped ap into the chamber $c$, as before described.

"The tube or chirnner $\mathrm{x}$ mar have more or less altitude; the higher it is the greater is the current produced. The force or relocity of the current of air can be gorerned by the areas of the introduction and exit apertures. If the crlinder rises onlr to the height of the sieve, the effect is much less than when it is prolonged berond this height. I would further remark. that if the cylinder was remored, a slight effect might be produced, provited that a current of air be preserved in the crlindrical space limited by the coils of the worm, and also if the current was produced between the coils; or a central passas: might be formed in an apparatus of another shape than that abore described.

"I hare only shown the application of the worm, because intending only to explain the principles of this method of condensing and refrigerating.

"The small quantity of water wasted in this manner of condensation, (that is, that portion passed off to the atmosphere in the form of vapors, at the upper aperture of the crinder $\mathrm{k}$, mas he replaced bs a small stream of cold water, which may be brought to the apparatus, and ferbaps most conreniently introduced into the reserroir $\mathrm{E}$, or into the chamber between the pipes $\mathrm{B}$ and $\mathrm{c}$. When operating upon aqueous rapors, the waste of water is always less in weight than that of the rapors liquefied. When this apparatus is applied to the purposes of distillation, the end of the worm should terminate in a ressel $\mathrm{sr}$, which is to receire the produce of the condensation. It will be seen that this improred process is applicable to various purposes, where condensation or refrigeration is required; for instance, in the boiling or concentration of sugar: to condensing and refrigerating distilled rapors, or steam, or saline liqui is, either in racwm or not; to cooling brewers: worts; and to the refrigeration of other liquors, or an: ctier processes, when it may be required."

I have inserted the specification of this patent verbatim. M. Derosne has busied 
himself during a long life with a prodigious number of ingenious little contrivances tor clarifying and boiling sirups, distillation, \&c., but he has in this invention taken a bolder fight, having secured the exclusive privilege of condensing vapors, and corling liquors, with hot water, in preference to cold. No man at all versant in the scientific doctrines, or the practical applications of caloric, will ever seek to meddle with his monopoly of such a scheme. He may find, perhaps, some needy coppersmith ready to espouse that or any other equally foolish project, provided a productive job can be made of it, against credulous customers.

For some rational methods of cooling liquors, and condensing vapors, see REFrigerATION, STILL, and SUgar.

COPAL, a resin which exudes spontaneously from two trees, the Rhus copallinum, and the Elcocarpus copalifer, the first of which grows in America, and the second in the East Indies. A third species of copal tree grows on the coasts of Guinea, especially on the banks of some rivers, among whose sands the resin is found. It occurs in lumps of various sizes and of various shades of color, from the palest greenish yellow to darkish brown. I found its specific gravity to vary in different specimens from 1.059 to 1.071 , being intermediate in density between its two kindred resins, animé and amber. Some rate its specific gravity so high as $1 \cdot 139$, which I should think one of the errors with which chemical compilations teem. Copal is too hard to be scratched by the nail, whence the excellence of its varnish. It has a conchoidal fracture, and is without smell or taste. When exposed to heat in a glass retort over a spirit lamp, it readily melts into a liquid, which being further heated boils with explosive jets. A viscid oily-looking matter then distils over. After continuing the process for some time, no succinic acid is found in the receiver, but the copal blackens in the retort. Anhydrous alcohol boiled upon it, causes it to swell, and transforms it by degrees into an elastic viscid substance. It is not soluble in alcohol of 0.825 at the boiling point, as I have ascertained. Copal dissolves in ether, and this ethereous solution may be mixed with alcohol without decomposition. Caoutchoucine acts very slightly upon it by my experiments, even at the boiling temperature of this very volatile fluid; but a mixture of it with alcohol of 0.825 in equal parts dissolves it very rapidly in the cold into a perfectly liquid varnish. Alcohol holding camphor in solution also dissolves it, but not nearly so well as the last solvent. According to Unverdorben, copal may be completely dissolved by digesting one part of it for 24 hours with one part and a half of alcohol (probably anhydrous), because that portion of copal which is insoluble in alcohol dissolves in a very concentrated solution of the soluble portion. Oil of petroleum and turpentine dissolve only 1 or 2 per cent. of raw copal. By particular management, indeed, oil of turpentine may be combined with copal, as we shall describe under the article VARNISH.

Fused copal possesses different properties from the substance in its solid state; for it then may be made to combine both with alcohol and oil of turpentine.

Unverdorben has extracted from the copal of Africa five different kinds of resin, none of which has however been applied to any use in the arts.

The ultimate constituents of copal by my analysis are, carbon $79 \cdot 87$, hydrogen $9 \cdot 00$, oxyger $11 \cdot 1$; being of hydrogen $7 \cdot 6$ in excess above the quantity necessary to form water with the oxygen. Of copal and animé, 551,166 lbs. were imported in 1835.

COPPER is one of the metals most anciently known. It was named from the island of Cyprus, where it was extensively mined and smelted by the Greeks. It has a reddish brown color inclining to yellow; a faint but nauseous and rather disagreeable taste; and when rubbed between the fingers it imparts a smell somewhat analogous to its taste. Its specific gravity is from 8.8 to 8.9 . It is much more malleable than it is ductile; so that far finer leaves may be obtained from it than wire. It melts at the 27 th degree of Wedgewood's pyrometer, and at a higher temperature it evaporates in fumes which tinge the fire of a bluish green. By exposure to heat with access of air, it is rapidly converted into black scales of peroxyde. In tenacity it yields to iron; but surpasses gold, silver, and platinum, considerably in this respect.

In mineralogy, the genus copper includes about 13 different species, and each of these contains a great many varieties. These ores do not possess any one general exterior character by which they can be recognised; but they are readily distinguished by chemical re-agents. Water of ammonia digested upon any of the cupreous ores in a pulverized state, after they have been calcined either alone or with nitre, assumes an intense blue color, indicative of copper. The richest of the ordinary ores appear under two aspects; the first class has a metallic lustre, a copper red, brass yellow, iron gray, or blackish gray color, sometimes inclining to blue; the second is without metallic appearance, has a red color, verging upon purple, blue, or green, the last tint being the most usual. Few copper ores are to be met with, indeed, which do not betray the presence of this metal by more or less of a greenish film.

1. Native copper occurs in crystals, branches, and filaments, its most common locality being in primitive rocks. It is found abundantly in Siberia, at the mines of Tourinski, in those of Hungary, of Fundo-Moldavi in Gallicia, of Fahlun in Sweden, 
of Cornwall, \&c. The gangues of native copper are granite, gneiss, mica-slate, clayslate, quartz, carbonate or fluate of lime, sulphate of barytes, \&c. The most remarkable masses of native copper hitherto observed were-first, one in Brazil, 14 leagues from Basa, which weighed 2616 pounds; and secondly, another which Dr. Francis-le-Baron discovered in America to the south of Lake Superior. It was nearly 15 feet in circumfernnce.

2. Sulphurct of Copper, the vitreous ore of Brochant. The texture of this ore is compact; its fracture, conchcidal, surface sometimes dull; color, iron black or lead gray, often bluish, iridiscent, or reddish from a mixture of protoxyde. It is easily melted even by the heat of a candle; but more difficult of reduction than protoxyde. This ore yields to the knife, assuming a metallic lustre when cut. Its density varies from $4 \cdot 8$ to $5 \cdot 34$. Its composition, according to Klaproth, is 78.5 copper, 18.5 sulphur, with a little iron and silica. Its equivalent constitution by theory is 80 copper +20 sulphur $=100$; whence 78.5 of metal should be associated with 19.6 of sulphur. This ore is therefore one of the richest ores, and forms very powerful veins, which likewise contain some orange protoxyde. It is to be found in all considerable copper districts; in Siberia, Saxony, Sweden, and especially Cornwall, where the finest crystals occur.

3. Copper Pyrites resembles in its metallic yellow hue, sulphuret of iron; but the latter is less pale, harder, and strikes fire more easily with steel. It presents the most lively rainbow colors. Its specific gravity is $4 \cdot 3$. It contains generally a good deal of iron, as the following analysis will show: copper 30, sulphur 37, iron 33, in 100 parts. According to Hisinger, the Swedish pyrites contains 63 of copper, 12 of iron, and 25 of sulphur. These ores occur in primitive and transition districts in vast masses and powerful veins; and are commonly accompanied with gray copper, sulphuret of iron, sparry iron, sulphurets of lead, and zinc.

4. Gray Copper has a steel gray color, more or less deep, either shining or dull; fracture uneven; a distinct metallic lustre; difficult of fusion at the blowpipe ; it communicates to glass of borax a yellowish-red color. Its density in crystals is 4.86. Its composition is very variable; consisting essentially of copper, iron, antimony, and sulphur. The exploration of this ore is profitable, in consequence of the silver which it frequently contains. It occurs in primitive mountains; and is often accompanied with red silver ore, copper pyrites, and crystallized quartz.

5. Protoxyde of Copper, or red oxyde of Copper : its color is a deep red, sometimes very lively, especially when bruised. It is friable, difficult of fusion at the blowpipe, reducible on burning charcoal, soluble with effervescence in nitric acid, forming a green liquid. Its constitution, when pure, is 88.9 copper $+11 \cdot 1$ oxygen $=100$.

6. Black oxyde of Copper is of a velvet black, inclining sometimes to brown or blue; and it acquires the metallic lustre on being rubbed. It is infusible at the blowpipe. Its composition is, copper $80+$ oxygen 20 ; being a true peroxyde.

7. Hydrosilicate of Copper consists essentially of oxyde of copper, silica, and water. Its color is green; and its fracture is conchoidal with a resinous lustre, like most minerals which contain water. Its specific gravity is $2 \cdot 73$. It is infusible at the blowpipe alone, but it melts easily with borax.

8. Dioptase Copper, or Emerald Malachite; a beautiful but rare cupreous mineral, consisting of oxyde of copper, carbonate of lime, silica, and water in varying proportions.

9. Carbonale of Copper, Malachite, is of a blue or green color. It occurs often in beautiful crystals.

10. Sulphate of Copper, Blue Vitriol, similar to the artificial salt of the laboratory The blue water which flows from certain copper mines is a solution of this salt. The copper is easily procured in the metallic state by plunging pieces of iron into it.

11. Phosphate of Copper is of an emerald green, or verdigris color, with some spots of black. It presents fibrous or tuberculous masses with a silky lustre in the fracture. It dissolves in nitric acid without effervescence, forming a blue liquid; melts at the blowpipe, and is reducible upon charcoal, with the aid of a little grease, into a metallic globule. Its powder does not color flame green, like the powder of muriate of copper.

12. Muriate of Copper is green of various shades; its powder imparts to flame a remarkable blue and green color. It dissolves in nitric acid without effervescence; and is easily reduced before the blowpipe. Its density is 3.5 . By Klaproth's analysis, it con. sists of oxyde of copper 73, muriatic acid 10, water 17.

13. Arseniate of Copper. It occurs in beautiful blue crystals. Before the blowpipe it melts, exhaling fumes of a garlic odor, and it affords metallic globules when in contact with charcoal. See more upon the ores at the end of this article.

In the article Metallurgy, I have described the mode of working certain coppex mines; and shall content myself here with giving a brief account of two cupreous forma. tions, interesting in a geological point of view; that of the copper slate of Mansfeldt, and of the copper veins of Cornwall.

The curious strata of bituminous schist in the first of these localities, are among the 
most ancient of any which contam the exuviæ of organized bodies not testaceous. From among their tabular slabs the vast multitudes of fossil fish were extracted, which have rendered the cantons of Mansfeldt, Eisleben, Ilmenau, and other places in Thuringia and Voigtland so celebrated. Many of the fish are transformed into copper pyrites. Here, also, have been found the fossil remains of the lizard family, called Monilors.

Such is the influence of a wise administration upon the prosperity of mines, that the thin layer of slate in this formation, of which 100 pounds commonly contain but one pound and a half of copper, occasionally argentiferous, has been for several centuries the object of smelting works of the greatest importance to the territory of Mansfeldt and the adjoining country.

The frequent derangements which this metallic deposite experiences, led skilful directors of the under-ground operations at an early period to study the order of superposition of the accompanying rocks. From their observations, there resulted a system of facts which have served to guide miners, not only in the country of Mansfeldt, but over a great por tion of Germany, and in several other countries where the same series of rocks, forming the immediate envelope of the cupreous schists, were found to occur in the same order of superposition.

Of the English copper worlss. - The deposites of copper in Cornwall occur always es veins in granite, or in the schistose rozks which surround and cover it; and hence, the Cornish miners work mostly in the granite or greenish clay slate; the former of which they call growan, the latter killas. But tin is sometimes disseminated in small veins in porphyry or elvan, which itself forms great veins in the above rocks. No stratification has been observed in Cornwall.

The copper veins are abundant in the killas and rare in the granite; but most numerous near the line of junction of the two rocks. The different kinds of mineral veins in Cornwall may be classed as follows :-

1. Veins of elvan; elvan courses, or elvan channels.

2. Tin veins, or tin lodes; the latter word being used by the Cornish miners to signify

a vein rich in ore, and the word course, to signify a barren vein.

3. Copper veins running east and west; east and west copper lodes.

4. Second system of copper veins, or contra copper lodes.

5. Crossing veins; cross courses.

6. Modern copper veins; more recent copper lodes.

7. Clay veins; of which there are two sets, the more ancient, called Cross-Fluckans ; and the more modern, called Slides.

There are therefore three systems of copper veins in Cornwall; of which the first is considered to be the most ancient, because it is always traversed by the two others, and because, on the contrary, it never cuts them off. The width of these veins does not exceed 6 feet, though occasional enlargements to the extent of 12 feet sometimes take place. Their length is unknown, but the one explored in the United Mines has been traced over an extent of seven miles. The gangue of these veins is generally quartz, either pure, or mixed with green particles analogous to chlorite. They contain iron pyrites, blende, sulphuret, and several other compounds of copper, such as the carbonate, phosphate, arseniate, muriate, \&c. The most part of the copper veins are accompanied with small argillaceous veins, called by the miners fluckan of the lode. These are often found upon both sides of the vein, so as to form cheeks or salebandes.

When two veins intersect each other, the direction of the one thrown out becomes an object of interest to the miner and geologist In Saxony it is regarded as a general fact that the rejected portion is always to the side of the obtuse angle; this also holds generally in Cornwall, and the more obtuse the angle of incidence, the more considerable the out-throw.

The great copper vein of Carharack, in the parish of Gwenap, is a most instructive example of intersection. The power of this vein is 8 feet; it runs nearly from east to west, and dips toward the north at an inclination of 2 feet in a fathom. Its upper part Is in the killas, its lower part in the granite. The vein has suffered two intersections; the first results from encountering the vein called Steven's fluckan, which runs from northeast to south-west, throwing it out several fathoms. The second has been caused by another vein, almost at right angles to the first, and which has driven it 20 fathoms out to the right side. The fall of the vein occurs, therefore, in one case to the right, and in the other to the left; but in both instances, it is to the side of the obtuse angle. This disposition is very singular; for one portion of the vein appears to have ascended, while another has sunk.

The mining works in the copper veins are carried on by reverse steps; see MrNes The grand shafts for drainage and extraction are vertical, and open upon the roof side of the vein, traversing it to a certain depth. These pits are sunk to the lowest point of the exploration; and, in proportion as the workings descend, by means of excavations in the vein, the pits are deepened and put into communication toward their bottom with 
each new gallery of elongation, by means of transverse galleries. At present, the main shafts are fully 160 fathoms deep. Their horizontal section is oblong, and is divided into two compartments; the one destined for extraction, the other for the pumps. Their timbering has nothing remarkable, but is executed with every attention to economy, the whole wood employed in these mines being brought from Norway.

The descent of the workmen is effected by inclined shafts scooped out of the vein; the ladders are slightly inclined; they are interrupted every 10 fathoms by floors; the steps are made of iron, and, to prevent them from turning under the foot, the form of a miner's punch or jumper has been given them, the one end being round, and the other being wedge-shaped.

The ore is raised either by means of horse-gins, or by steam-engine power, most frequently of high pressure. I shall take the Consolidated Mines as an example.

The draining, which is one of the most considerable sources of expense, both from the quantity of water, and from the depth of the mine, is executed by means of sucking and forcing pumps, the whole piston-rods of which, 120 feet long, are attached to a main-rod suspended at the extremity of the working beam of a steam-engine.

On this mine three steam-engines are erected of very great power, for the gurpose of Jrainage; the one called the Maria engine is of the first-rate force, and most improved construction. The cylinder is 90 inches in internal diameter, and the length of the stroke is 9 feet 11 inches. It works single stroke, and is incased in a coating of bricks to prevent dissipation of the heat. The vapor is admitted at the upper end of the cylinder during the commencement of the fall of the piston, at a pressure capable of forming an equilibrium with a column of 60 inches of mercury. The introduction of the steam ceases whenever the piston has descended through a certain space, which may be increased or diminished at pleasure. During the remainder of the descent the piston is pressed merely by this vapor in its progressive expansion, while the under side of the piston communicates with the condenser. It ascends by the counterweight at the pump end of the working beam. Hence, it is only during the descent of the piston that the effective stroke is exerted. Frequently the steam is admitted only during the sixth part of the course of the piston, or 18 inches. In this way the power of the engine is proportioned to the work to be done; that is, to the body of water to be raised. The maximum force of the above engine is about 310 horses; though it is often made to act with only one third of this power.

The copper mines of the Isle of Anglesey, those of North Wales, of Westmoreland, the adjacent parts of Lancashire and Cumberland, of the south west of Scotland, of the Isle of Man, and of the south east of Ireland, occur also in primitive or transition rocks. The ores lie sometimes in masses, but more frequently in veins. The mine of Ecton in Staffordshire, and that of Cross-gill burn, near Alston-moor in Cumberland, occur in Iransition or metalliferous limestone.

The copper ores extracted both from the granitic and schistose localities, as well as from the calcareous, are uniformly copper pyrites more or less mixed with iron pyrites; the red oxyde, carbonate, arseniate, phosphate, and muriate of copper, are very rare in these districts.

The working of copper in the Isle of Anglesey may be traced to a very remote era. It appears that the Romans were acquainted with the Hamlet mine near Holyhead; but it was worked with little activity till about 70 years ago. This metalliferous deposite lies in a greenish clay slate, passing into talc slate; a rock associated with serpentine and euphotide (gabbro of Von Buch). The veins of copper are from one to two yards thick, and they converge towards a point where their union forms a considerable mass of ore. On this mass the mine was first pierced by an open excavation, which is now upwards of 300 feet deep, and appears from above like a vast funnel. Galleries are formed at different levels upon the flank of the excavation to follow the several small veins, which run in all directions, and diverge from a common centre like so many radil. The ore receives in these galleries a kind of sorting, and is raised by means of hand. windlasses to the summit of a hill, where it is cleaned by breaking and riddling.

The water is so scanty in this mine that it is pumped up by a six-horse steam-engine. A great proportion of it is charged with sulphate of copper. It is conveyed into reserroirs containing pieces of old iron; the sulphate is thus decomposed into copper of cementation. The Anglesea ore is poor, yielding only from 2 to 3 per cent. of copper : a portion of its sulphur is collected in roasting the ore.

Mechanical preparation of the copper ores in Cornwall.-The ore receives a first sorting, either within the mine itself, or at its mouth, the object of which is to separate all the pieces larger than a walnut. These are then reduced by the hammer to a smaller size; after which the whole are sorted into four lots, according to their relative richness. The fragments of poor ore are pounded in the stamps so that the metallic portion may be separated by washing.

The rich ore is broken into small bits, of the size of a nut, with a flat beater, formed 
of a piece of tron 6 inches square and 1 inch thick, adapted to a wooden handle. The ore to be broken is placed upon plates of east-iron; each about 16 inches square and $1 \frac{1}{2}$ inch thick. These iron plates are set towards the edge of a small mound about a yard high, constructed with dry stones rammed with earth. The upper surface of this mound is a little inclined from behind forwards. The work is performed by women, each furnished with a beater; the ore is placed in front of them besond the plates; they break it, and strew it at their feet, whence it is lifted and disposed of to the smeltingnouses.

Inferior ores, containing a notable proportion of stony matters, are also broken with the beater, and the rich parts are separated by riddling and washing from the useless matters.

The smaller ore is washed on a sieve by shaking it in a stream of water, which carries away the lighter stony pieces, and leaves the denser metalliferous. They are then sorted by hand. Thus by beating, stamping, and riddling in water, the stony substances are in a great measure separated. The finer ground matter is washed on a plane table, over which a current of water is made to flow. Finally, the ore nearly fine is put into a large tub with water, and briskly stirred about with a shovel, after which it settles in the order of richness, the pure metallic ore being nearest the bottom. The stamps used for copper ore in Cornwall are the same as those used for tin ores, of which we shall speak in treating of the latter metal, as well as of the boxes for washing the fine powder or slime. These, in fact, do not differ essentially from the stamping mills and washing apparatus described in the article Metallurgy. Crushing rolls are of late years mueh employed. See LEAd and TiN.

Cornwall being destitute of coal, the whole copper ore which this county produces is sent for smelting to South Wales. Here are 15 copper works upon the Swansea and Neath, which pursue a nearly uniform and much improred process, consisting in a series of calcinations, fusions, and roastings, executed upon the ores and the matters resulting from them.

The furnaces are of the reverberatory construction; they vary in their dimensions and in the number of their openings, according to the operations for which they were in tended. There are 5 of them:-1. The calcining furnace or calciner; 2 . The melting furnace; 3 . The roasting furnace or roaster; 4 . The refining furnace; 5 . The heating or igniting furnace.

1. The calcining furnace rests npon a vault, $c$, into which the ore is raked down after being calcined; it is built of bricks, and bound with iron bars, as shown in the eleration, fig. 296. The hearth, в в, figs. 296 and 298 , is placed upon a level with the lower horizontal binding bar, and has nearly the form of an ellipse, truncated at the two extremities of its great axis. It is horizontal, bedded with fire-bricks set on edge, so that it may be removed and repaired without disturbing the arch upon which it reposes. Holes, not visible
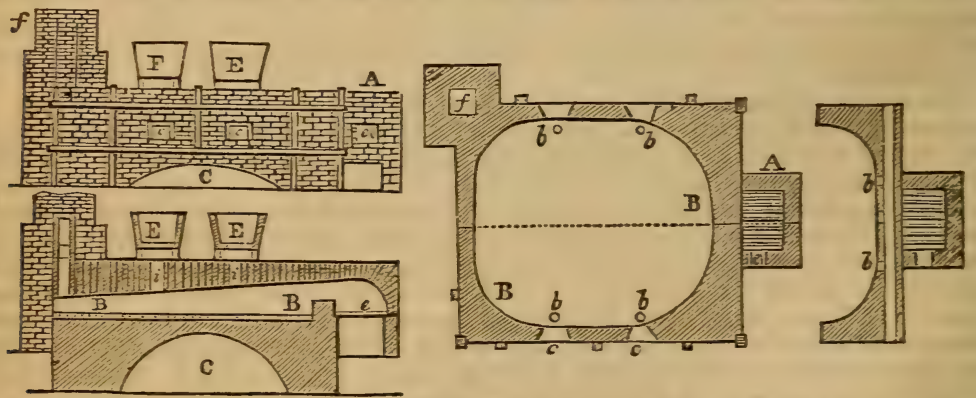

in the figure, are left in the shelves before each door, $c c$, through which the roasted ore is let fall into the subjacent vault. The dimensions of the hearth в В are immense, being from 17 to 19 feet in length, and from 14 to 16 in breadth. The fire-place, A, fig. 298, is from $4 \frac{1}{2}$ to 5 feet long, and 3 feet wide. The bridge or low wall, $b$, fig. 302 , which separates the fire-place from the hearth, is 2 feet thick; and in Mr. Vivian's smelting-works is hollow, as shown in the figure, and communicates at its two ends with the atmosphere, in order to conduct a supply of fresh air to the hearth of the furnace. This judicious contrivance will be described in explaining the roasting operation. The arched roof of the furnace slopes down from the bridge to the beginning of the chimney, f, figs. 297, 298 , its height above the hearth being at the first point about 26 inches, and from 8 to 12 at the second.

Such great calcining furnaces have 4 or 5 doors, c c c c, fig. 298, one for the fire-place, as shown at the right hand in fig. 297, and 3 or 4 others for working the ore upon the 
reverberatory hearth. If there be 3,2 of them are placed between the vertical binding bars upon one side, and a third upon the opposite side of the furnace; if there be 4,2 are placed upon each side, facing one another. These openings are 12 inches square, and are bound with iron frames. The chimney is about 22 feet high, and is placed at one angle of the hearth, as at $f, f i g .298$, being joined by an inclined flue to the furnace.

For charging it with ore there are usually placed above the upper part of the vault 2 hoppers, E E, in a line with the doors; they are formed of 4 plates of iron, supported in an iron frame. Beneath each of them there is an orifice for letting the ore down into the hearth.

These furnaces serve for calcining the ore, and the matts or crude coppers : for the latter purpose, indeed, furnaces of two stories are sometimes employed, as represented in fig. 301 . The dimensions of each floor in this case are a little less than the preceding. Two doors, $c c$, correspond to each hearth, and the workmen, while employed at the upper story, stand upon a raised moveable platform.

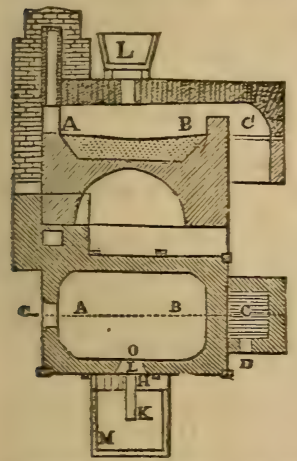

2. Melling furnace, figs. 299 and 300 . The form of the hearth is also elliptical, but the dimensions are smaller than in the calcining furnace. The length does not exceed 11 or $11 \frac{1}{2}$ feet, and the breadth varies from 7 to 8 . The fire-place is however larger in proportion, its length being from $3 \frac{1}{2}$ feet to 4 , and its breadth from 3 to $3 \frac{1}{2}$; this size being requisite to produce the higher temperature of this furnace. It has fewer openings, there being commonly three; one to the fire-place at $\mathrm{D}$, a second one, $\mathrm{O}$, in the side, kept generally shut, and used only when incrustations need to be scraped off the hearth, or when the furnace is to be entered for repairs; and the third or working-door, G, placed on the front of the furnace beneath the chimney. Through it the scoriæ are raked out, and the melted matters are stirred and puddled, \&c.

The hearth is bedded with infusible sand, and slopes slightly towards the side door, to facilitate the discharge of the metal. Above this door there is a hole in the wall of the chimney ( fig. 300) for letting the metal escape. An iron gutter, 0, leads it into a pit, $\mathbf{k}$, bottumed with an iron receiving-pot, which may be lifted out by a crane. The pit $\mathrm{M}$ is filled with water, and the metal becomes granulated as it falls into the receiver. The melting furnaces are surmounted by a hopper, L, as shown in fig. 299.

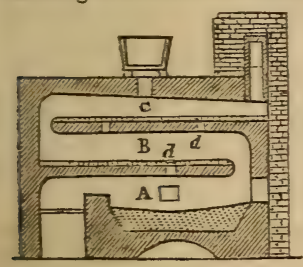

Melting furnaces are sometimes used also for calcination. There are some such near Swansea, which serve this double purpose; they are composed of 3 floors (fig. 301.) The floor $A$ is destined for melting the calcined ore; the other two, B c, serve for calcination. The heat being less powerful, upon the upper sole $\mathrm{c}$, the ore gets dried upon it, and begins to be calcined - a process completed on the next floor. Square holes, $d$, left in the hearths $\mathbf{B}$ and $\mathbf{c}$, put them in communication with each other, and with the lower one A; these perforations are shut during the operation by a sheet

of iron, removeable at pleasure.

The hearths $b$ and $c$ are made of bricks; they are horizonal at top and slightly vaulted beneath; they are 2 bricks thick, and their dimensions are larger than those of the inferior hearths, as they extend above the fire-place. On the floors destined for calcination the furnace has two doors on one of its sides : on the lower story there are also two; but they are differently collocated. The first, being in the front of the furnace, serves for drawing off the scoriæ, for working the metal, \&c. ; and the second, upon the side, admits workmen to make necessary repairs. Below this door the discharge or tap-hole $A$ is placed, which communicates by a cast-iron gutter with a pit filled with water. The dimensions of this furnace in length and breadth are nearly the same as those of the melting furnace above described; the total height is nearly 12 feet. It is charged by means of one or two hoppers.

3. Roasting furnace. - The furnaces employed for this purpose are in general analogous to the calcining ones; but in the smelting works of Hafod, the property of Messrs. Vivian, these furnaces, alluded to above, present a peculiar construction, for the purpose of introducing a continuous current of air upon the metal, in order to facilitate its oxydizement. This process was originally invented by Mr. Sheffield, who disposed of his patent right to Messrs. Vivian.

The air is admitted by a channel, $c c$, through the middle of the fire-bridge, fig. 302 , and extending all its length; it communicates with the atmosphere at its two ends $c c$; square holes, $b$, left at right angles to this channel, conduct the air into the fur- 
nace. This very simple construction produces a powerful effect in the rnastıng operation. It not only promotes the oxydizement of the metals, but burns the smoke, and assists in the vaporization of the sulphur ; while by keeping the bridge cool it preserves it from wasting, and secures uniformity of temperature to the hearth.

4. Refining furnace. - In this, as in the melting furnace, the sole slopes towards the door in front, instead of towards the side doors, because in the refining furnace the copper collects into a cavity formed in the hearth towards the front door, from which it is lifted out by ladles; whereas, in the melting furnaces, the metal is run out by a taphole in the side. The hearth sole is laid with sand; but the roof is higher than in the melting furnace, being from 32 to 36 inches. If the top arch were too much depressed, there might be produced upon the surface of the metal a layer of oxyde very prejudicial to the quality of the copper. When the metal in that case is run out, its surface solidifies and eracks, while the melted copper beneath breaks through and spreads irregularly orer the cake. This accident, called the rising of the copper, hinders it from being laminated, and requires it to be exposed to a fresh refining process, when lead must be added to dissolve the oxyde of copper. This is the only occasion upon which the addition of lead is proper in refining copper. When the metal to be refined is mixed with others, particularly with tin, as in extracting copper from old bells, then very wide furnaces must be employed, to expose the metallic bath upon a great surface, and in a thin stratum, to the oxydizing action of the air.

The door G, fig. 300, upon the side of the refining furnace, is very large, and is shut with a framed brick door, balanced by a counter-weight. This door being open during the refining process, the heat is stronger at B than at $\mathbf{A},(f i g s .299,300$.

5. Heating furnaces, being destined to heat the pigs or bars of copper to be laminated, as well as the copper sheets themselves, are made much longer in proportion to their breadth. Their hearth is horizontal, the vault not much depressed; they have only one door, placed upon the side, but which extends nearly the whole length of the furnace; this door may be raised by means of a counter-weight, in the same way as in the furnaces for the fabrication of sheet-iron and brass.

Series of operations to which the ore is subjected. - The ores which are smelted in the Swansea works are cupreous pjrites, more or less mingled with gangue (rein-stone). The pyrites is composed of neariy equal proportions of sulphuret of copper and sulphuret of iron.

The earthy matters which accompany the pyrites are usually silicious, though in some mines the metalliferous deposite is mixed with clay or fluate of lime. Along with these substances, pretty uniformly distributed, tin and arsenical pyrites occur occasionally with the copper; and though these two metals are not chemically combined, ret ther cannot be separated entirely in the mechanical preparations. The constituent parts of the ore prepared for smelting are, therefore, copper, iron, sulphur, with tin, arsenic, and earthy matters in some cases. The different ores are mixed in such proportions that the average metallic contents may amount to $8 \frac{1}{2}$ per cent. The smelting process consists in alternate roastings and fusions. The following description of it is chiefly taken from an excellent paper, published by John Virian, Esq., in the Annals of Philosophy for 1823.

In the roasting operation the rolatile substances are disengaged mostly in the gaseous state, while the metals that possess a strong affinity for oxrgen become oxrdized. In the fusion the earthy substances combine with these oxydes, and form glassy scoriæ or slags which float upon the surface of the melted metal.

These calcinations and fusions take place in the following order :-

1. Calcination of the ore. 2. Melting of the calcined ore. 3. Calcination of the soarse metal. 4. Melting of the calcined coarse metal. 5. Calcination of the fine metal (second matt). 6. Melting of the calcined fine metal. 7. Roasting of the coarse copper. In some smelting works, this roasting is repeated four times; in which case a calcination and a melting are omitted. In the Havod works, howerer, the same saring is made without increasing the number of roastings. 8. Refining or toughening the copper.

Besides these operations, which constitute the treatment of copper properly speaking, two others are sometimes performed, in which only the scoriæ are melted. These may be designated by the letters $a$ and $b . \quad a$ is the re-melting of the portion of the scoriæ of the second process, which contain some metallic granulations. $b$ is a particular melting of the scoriæ of the fourth operation. This fusion is intended to concentrate the particles of copper in the scoriæ, and is not practised in all smelting works.

First operation. Calcination of the ore. - The different cres, on arriving from Cornwall and other distıicts where they are mined, are discharged in continuous cargoes at the smelting works, in such a way, that by taking out a portion from several heaps at a time, a tolerably uniform mixture of ores is obtained; which is very essential in a foundry, because, the ores being different in qualities and contents, they act as 
fuxes upon cach other. The ore thus mixed is transported to the works in wonden measures lint hold a hunclred-weight. The workmen intrusted with the calcination corvey the rre into the hoppers of the calcining furnace, whence it falls into the hearth; other workn en spread it uniformly on the surface by iron rakes. The charge of a furnace i: fivi: three tons to three tons and a half. Fire is applied and gradually increased, till, lowards the end of the uperation, the temperature be as high as the ore can support without meluing or agglutinating. To prevent this running together, and to aid the extrication of the sulphur, the surfaces are renewed, by stirring up the ore at the end of every hour. The calcinaticn is usually completed at the end of 12 hours, when the ore is tumbled into the arch under the sole of the furnace. Whenever the ore is cold enough to be moved, it is taken out of the arch, and conveyed to the calcined heap.

The ore in this process hardly changes weight, having gained in oxydizement nearly as much as it has lost in sulphur and arsenic; and if the roasting has been rightly managed, the ore is in a black powder, owing to the oxyde of iron present.

Second operution. Fusion of the calcined ore.-The calcined ore is likewise given to the melters in measures containing a hundred weight. They toss it into hoppers, and after it has fallen on the hearth, they spread it uniformly. They then let down the door, and lute it tightly. In this fusion there are added about 2 cwt. of scoriæ proceeding from the melting of the calcined matt, to be afterwards described. The object of this addition is not only to extract the copper that these scoriæ may contain, but especially to increase the fusibility of the mixture. Sometimes also, when the composition of the ore requires it, lime, sand, or fluor spar is added; and particularly the last fluxıng article.

The furnace being charged, fire is applied, and the sole care of the founder is to keep up the heat so as to have a perfect fusion; the workman then opens the door, and stirs about the liquid mass to complete the separation of the metal (or rather of the matt) from the scoria, as well as to hinder the melted matter from sticking to the sole. The furnace being ready, that is, the fusion being perfect, the founder takes out the scoriæ by the front door, by means of a rake. When the matt is thus freed from the scoriæ, a second charge of calcined ore is then introduced to increase the metallic bath; which second fusion is executed like the first. In this way, new charges of roasted ore are put in till the matt collected on the hearth rises to a level with the door-way, which happens commonly after the third charge. The tap hole is now opened; the matt flows out into the pit filled with water, where it is granulated during its immersion; and it collects in the pan placed at the bottom. The granulated matt is next conveyed into the matt warehouse. The oxydation with which the grains get covered by the action of the water does not allow the proper color of the matt or coarse metal to be distinguished; but in the bits which stick in the gutter, it is seen to be of a steel gray. Its fracture is compact, and its lustre metallic. The scoriæ often contain metallic grains; they are broken and picked with care. All the portions which include some metallic particles are re-melted in an accessory process. The rejected scoriæ have been found to be composed of silicious matter 59, oxyde of copper 1, oxyde of $\operatorname{tin} 0 \cdot \%$.

In this operation, the copper is concentrated by the separation of a great part of the matters with which it was mixed or combined. The granulated matt produced, contains in general 33 per cent. of copper; it is therefore four times richer than the ore; and its mass is consequently diminished in that proportion. The constituent parts are principally copper, iron, and sulphur.

The most important point to hit in the fusion just described, is to make a fusible mixture of the earths and the oxydes, so that the matt of copper may, in virtue of its greater specific gravity, fall to the under-part, and separate exactly from the slag. This point is attained by means of the metallic oxydes contained in the scoriæ of the fourth operation, of which $\dot{z}$ cwt. were added to the charge. These consist almost entirely of black oxyde of iron. When the ores are very difficult to melt, a measure of about half a hundredweight of fluor spar is added; but this must be done with precaution, for fear of increasing the scoriæ too much.

The business goes on day and night. Five charges are commonly put through hands in the course of 24 hours; but when all circumstances are favorable-that is to say, when the ore is fusible, when the fuel is of the first quality, and when the furnace is in good condition, even six charges a day have been despatched.

The charge is a ton and a half of calcined ore, so that a melting furnace corresponds nearly to a calcining furnace; the latter turning out nearly 7 tons of calcined ore in 24 hours.

The workmen are paid by the ton.

Thirl operation. Calcination of the coarse metal, or the matt.-The object of this operation is principally to oxydize the iron, an oxydation easier to execute than in the first 
ealcining, because the metal is now disengaged from the earthy substances, which screened it from the action of the air.

This calcination is executed in the furnace already represented, figs. 296, 297, 298, page 324 , exactly in the same way as the ore was calcined. The metal must be perpetu. ally stirred about, to expose all its surfaces to the action of the hot air, and to hinder the clotting together. The operation lasts 24 hours; durng the first six, the fire should be very moderate, and thereafter gradually increased to the end of the calcination. The charge is, like that of the first, 3 tons and a half.

Fourth operation. Melting of the calcined coarse metal, or calcined matt.-In the fusion of this first calcined matt, some scoriæ of the latter operations must be added, which are very rich in oxyde of copper, and some crusts from the hearth, which are likewise impregnated with it. The proportion of these substances varies according to the quality of the calcined matt.

In this second fusion, the oxyde of copper contained in the scoriæ is reduced by the affinity of the sulphur, one portion of which passes to the state of acid, while the other forms a subsulphuret with the copper become free. The matt commonly contains a sufficient quantity of sulphur to reduce the oxyde of copper completely; but if not, which may happen if the calcination of the matt has been pushed too far, a small quantity of uncalcined matt must be introduced, which, by furnishing sulphur, diminishes the richness of the scoriæ, and facilitates the fusion.

The scoriæ are taken out by the front door, by drawing them forward with a rake. They have a great specific gravity; are brilliant with metallic lustre, very crystalline, and present, in the eavities, crystals like those of pyroxene; they break easily into very sharp-edged fragments. They contain no granulated metal in the interior; but it some times occurs, on account of the small thicknesses of the stratum of scoriæ, that these car ry off with them, when they are withdrawn, some metallic particles.

These scoriæ, as we have already stated, under the fusion of the roasted ore, are in general melted with it. In some cases, however, a special melting is assigned to them.

The matt obtained in this second fusion is either run out into water like the first, or moulded into pigs (ingots), according to the mode of treatment which it is to undergo. This matt, called by the smelters fine metal when it is granulated, and blue metal when it is in pigs, is of a light gray color, compact, and bluish at the surface. It is collected in the first form when it is to be calcined anew; and in the second, when it must immediately undergo the operation of roasting. Its contents in copper are 60 per cent. This operation, which is sometimes had recourse to, lasts 5 or 6 hours. The charge is 1 ton.

(b) Particular fusion of the scoria of the fourth operation.-In re-melting these scoria, the object is to procure the copper which they contain. To effect this fusion, the scoria are mixed with pulverized coal, or other carbonaceous matters. The copper and several other metals are deoxydized, and furnish a white and brittle alloy. The scoriæ resulting from this melting are in part employed in the first melting, and in part thrown away. They are crystalline, and present crystals often in the cavities, which appear to belong to bisilicate of iron. They have a metallic lustre, and break into very sharp-edged fragments. The white metal is melted again, and then united to the product of the second fusion.

Fifth operation. Calcination of the second matt, or fine metal of the smeller.-This is executed in precisely the same way as that of the first matt. It lasts 24 hours; and the charge is usually 3 tons.

Sixth operation. Melting of the calcined fine metal.-This fusion is conducted like that of the first matt. The black copper, or coarse copper, which it produces, contains from 70 to 80 per cent. of pure metal; it is run into ingots, in order to undergo the operation of roasting.

The scoriæ are rich in copper; they are added to the fusion of the calcined coarse metal of the fourth operation.

In the smelting houses of Messrs. Vivian, at Hafod, near Swansea, the fifth and sixth operations have been omitted of late years. The second matt is run into pigs, under the name of blue metal, to be immediately exposed to the roasting.

The disposition of the canal $a a^{\prime}$, fig. 302, which introduces a continuous current of alr to the hearth of the furnace, accelerates and facilitates the calcination of the matt; an advantage which has simplified the treatment, by diminishing the number of calculations.

Seventh operation. Roasting of the coarse copper, the product of the sixth operation.The chief object of this operation is oxydizement; it is performed either in an ordinary roasting furnace, or in the one belonging to fig. 302 , which admits a constant current of air. The pigs of metal derived from the preceding melting are exposed, on the hearth of the furnace, to the action of the air, which oxydizes the iron and other foreign metals with which the copper is still contaminated. The duration of the roasting varies from 
12 to 24 hours, according to the degree of purity of the crude copper. The temperature should be graduated, in order that the oxydizement may have time to complete, and that the volatile substances which the copper still retains may escape in the gaseous form. The fusion must take place only towards the end of the operation.

The charge varies from a ton and a quarter to a ton and a half. The metal obtained is run out into moulds of sand. It is covered with black blisters, like steel of cementation; whence it has got the name of blistered copper. In the interior of these pigs, the copper presents a porous texture, occasioned by the ebullition produced by the escape of the gases during the moulding. The copper being now almost entirely purged from the sulphur, iron, and the other substances with which it was combined, is in a fit state to be refined. This operation affords some scoriæ; they are very heavy, and contain a great deal of oxyde of copper, sometimes even metallic copper.

These scoriæ, as well as those of the third melting and of the refining, are added to the second fusion, as we have already stated, in describing the fourth operation.

In some works, the roasting is repeated several times upon the blue metul, in order to bring it to a state fit for refining. We shall afterwards notice this modification of the treatment.

Eighth operation. Refining or toughening. - The pigs of copper intended for refining are put upon the sole of the refining furnace through the door in the side. A slight heat is first given, to finish the roasting or oxydation, in case this operation has not already been pushed far enough. The fire is to be increased by slow degrees, so that, by the end of 6 hours, the copper may begin to flow. When all the metal is melted, and when the heat is considerable, the workman lifts up the door in the front, and withdraws with a rake the few scoriæ which may cover the copper bath. They are red, lamellated, very heavy, and closely resemble protoxyde of copper.

The refiner takes then an assay with a small ladle, and when it cools, breaks it in a vice, to see the state of the copper. From the appearance of the assay, the aspect of the bath, the state of the fire, \&cc., he judges if he may proceed to the toughening, and what quantity of wooden spars and wood charcoal he must add to render the metal malleable, or, in the language of the smelters, bring it to the proper pitch. When the operation of refining begins, the copper is brittle or dry, and of a deep red color approaching to purple. Its grain is coarse, open, and somewhat crystalline.

To execute the refining, the surface of the metal is covered over with wood charcoal, and stirred about with a spar or rod of birch wood. The gases which escape from the wood occasion a brisk effervescence. More wood charcoal is added from time to time, so that the surface of the metal may be always covered with it, and the stirring is continued with the rods, till the operation of refining be finished, a circumstance indicated by the assays taken in succession. The grain of the copper becomes finer and finer, and its color gradually brightens. When the grain is extremely fine, or closed, when the trial pieces, half cut through and then broken, present a silky fracture, and when the copper is of a fine light red, the refiner considers the operation to be completed; but he verifies still further the purity of the copper, by trying its malleability. For this purpose, he takes out a sample in his small ladle, and pours it into a mould. When the copper is solidified, but still red-hot, he forges it. If it is soft under the hammer, if it does not crack on the edges, the refiner is satisfied with its ductility, and he pronounces it to be in its proper state. He orders the workmen to mould it; who then lift the copper out of the furnace in large iron ladles lined with clay, and pour it into moulds of the size suitable to the demands of commerce. The ordinary dimensions of the ingots or pigs are 12 inches broad, 18 long, and from 2 to $2 \frac{1}{2}$ thick.

The period of the refining process is 20 hours. In the first six, the metal heats, and suffers a kindi of roasting; at the end of this time it melts. It takes four hours to reach the point at which the refining, properly speaking, begins; and this last part of the process lasts about 4 hours. Finally, 6 hours are required to arrange the moulds, cast the ingots, and let the furnace cool.

The charge of copper in the refining process depends upon the dimensions of the furnace. In the Hafod works, one of the most important in England, the charge varies from 3 to 5 tons; and the quantity of pure copper manufactured in a week is from 40 to 50 tons.

The consumption of fuel is from 15 to 18 parts of coal, for one part of refined copper in pigs.

When the copper offers difficulties in the refining, a few pounds of lead are added to it. This metal, by the facility with which it scorifies, acts as a purifier, aiding the oxydation of the iron and other metals that may be present in the copper. The lead ought to be added immediately after removing the door to skim the surface. The copper should be constantly stirred up, to expose the greatest possible surface to the action of the air, and to produce the complete oxydation of the lead; for the smallest quantity of this metal alloyed in copper, is difficult to clear up in the lamination; that is to say, the scale of oxyde does not come cleanly from the surface of the sheets. 
The operation of refining copper is delicate, and requires, upon the part of the wor men, great skill and attention to give the metal its due ductility. Its surface ought to be entirely covered with wood charcoal; without this precautior, the refinitg of the metal would go back, as the workmen say, during the long interval which elapses in the moulding; whenever this accident happens, the metal must be stirred up anew with the wooden pole.

Too long employment of the wooden rod gives birth to another remarkable accident, for the copper becomes more brittle than it was prior to the commencement of the refining; that is, when it was $d r y$. Its color is now of a very brilliant yellowish red, and its fracture is fibrous. When this circumstance occurs, when the refining, as the workmen say, has gone too far, the refiner removes the charcoal from the top of the melted metal; he opens the side door, to expose the copper to the action of the air, and it then resumes its malleable condition.

Mr. Vivian, to whom we owe the above very graphic account of the processes, has explained, in a very happy manner, the theory of refining. He conceives, we may conclude, that the copper in the dry state, before the refining, is combined with a small por tion of oxygen, or, in other words, that a small portion of oxyde of copper is diffused through the mass, or combined with it; and that this proportion of oxygen is expelled by the deoxydizing action of the wood and charcoal, whereby the metal becomes malleable. 2. That when the refining process is carried too far, the copper gets combined with a little charcoal. Thus copper, like iron, is brittle when combined with oxygen and charcoal; and becomes malleable only when freed entirely from these two substances.

It is remarkable, that copper, in the dry stale, has a very strong action upon iron; and that the tools employed in stirring the liquid metal become very glistening, like those used in a farrier's forge. The iron of the tools consumes more rapidly at that time, than when the copper has acquired its malleable state. The metal requires, also, when $d r y$, more time to become solid, or to cool, than when it is refined; a circumstance depending, probably, upon the difference in fusibility of the copper in the two states, and which seems to indicate, as in the case of iron, the presence of oxygen.

When the proper refining point has been passed, another very remarkable circumstance has been observed; namely, that the surface of the copper oxydizes more difficultly, and that it is uncommonly brilliant; reflecting clearly the bricks of the furnace vault. This fact is favorable to the idea suggested above, that the metal is in that case combined with a small quantity of carbon; which absorbs the oxygen of the air, and thus protects the metal from its action.

Copper is brought into the market in different forms, according to the purposes which it is to serve. What is to be employed in the manufacture of brass is granulated. In this condition it presents more surface to the action of zinc or calamine, and combines with it more readily. To produce this granulation, the metal is poured into a large ladle pierced with holes, and placed above a cistern filled with water, which must be hot or cold, according to the form wished in the grains. When it is hot, round grains are obtained analogous to lead shot; and the copper in this state is called bean shot. When the melted copper falls into cold water perpetually renewed, the granulations are irregular, thin, and ramified; constituting feathered shot. The bean shot is the form employed in brass making.

Copper is also made into small ingots, about 6 ounces in weight. These are intended for exportation to the East Indies, and are known in commerce by the name of Japan copper. Whenever these little pieces are solidified, they are thrown, while hot, into cold water. This immersion slightly oxydizes the surface of the copper, and gives it a fine red color.

Lastly, the copper is often reduced into sheets, for the sheathing of ships, and many other purposes. The Hafod works possess a powerful rolling mill, composed of four pairs of cylinders. It is moved by a steam engine, whose cylinder has 40 inches diamete: See the representation of the rolling mill of the Royal Mint, under GOLD.

The cyınders for rolling copper into sheets are usually 3 feet long, and 15 inches in diameter. They are uniform. "The upper roller may be approached to the under one, by a screw, so that the cylinders are brought closer, as the sheet is to be made thinner.

The ingots of copper are laid upon the sole of a reverberatory furnace to be heated; they are placed alongside each other, and they are formed into piles in a cross-like arrangement, so that the hot air may pass freely round them all. The door of the furnace is shut, and the workman looks in through a peep-hole from time to time, to see if they have taken the requisite temperature; namely, a dull red. The copper is now passed between the cylinders; but although this metal be very malleable, the ingots cannot be reduced to sheets without being several times heated; because the copper cools, and ac quires, by compression, a texture which stops the progress of the lamination.

These successive heatings are given in the furnace indicated above; though, when the 
sheets are to have a very great size, furnaces somewhat different are had recourse to. They are from 12 to 15 feet long, and 5 wide. See Brass.

The copper, by successive heating and lamination, gets covered with a coat of oxyde, which is removed by steeping the sheets for a few days in a pit filled with urine; they are then put upon the sole of the heating furnace. Ammonia is formed, which acts on the copper oxyde, and lays bare the metallic surface. The sheets are next rubbed with a piece of wood, then plunged, while still hot, into water, to make the oxyde scale off; and lastly, they are passed cold through the rolling press to smooth them. They are now eut square, and packed up for home sale or exportation.

The following estimate has been given by MM. Dufrenoy and Elie de Beaumont of the expense of manufacturing a ton of copper in South Wales.

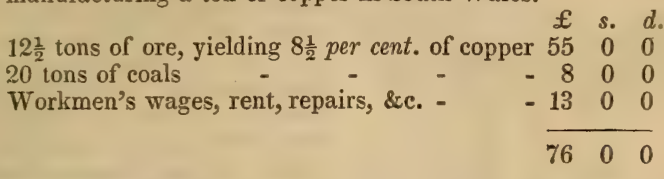

The exhalations from the copper smelting works are very detrimental to both vegetable and animal life. They consist of sulphurous acid, sulphuric acid, arsenic, and arsenious acids, various gases and fluoric vapors, with solid particles mechanically swept away into the air, besides the coal smoke. Mr. Vivian has invented a very ingenious method of passing the exhalations from the calcining ores and matts along horizontal flues, or rather galleries of great dimensions, with many crossings and windings of the current, and exposure during the greater part of the circuit to copious showers of cold water. By this simple and powerful system of condensation, the arsenic is deposited in the bottoms of the flues, the sulphurous acid is in a great measure absorbed, and the nuisance is remarkably abated.

The following figures represent certain modifications of the copper calcining and smelting copper furnaces of Swansea.

Fig. 304, is the section of the roasting furnace lengthwise; fig. 303, the ground plan; in which $a$ is the fire-door; $b$ the grate; $c$ the fore-bridge; $d$ the chimney; $e e$ working 303
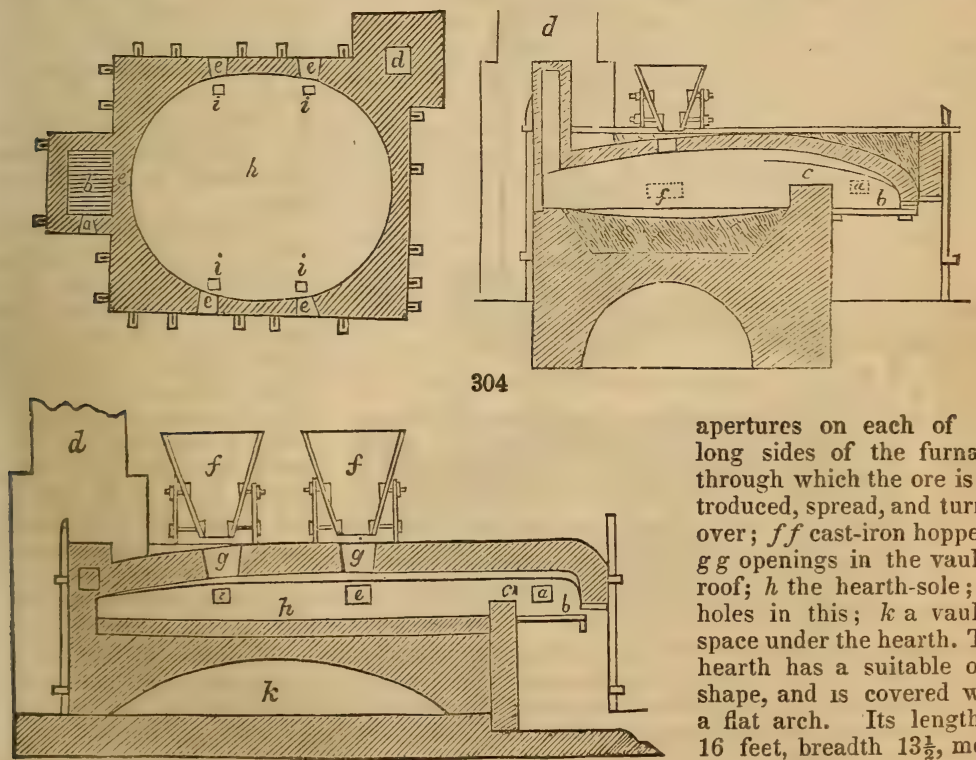

apertures on each of the long sides of the furnace, through which the ore is in. troduced, spread, and turned over; $f f$ cast-iron hoppers ; $g \mathrm{~g}$ openings in the vaulted roof; $h$ the hearth-sole; $i$ i holes in this; $k$ a vaulted space under the hearth. The hearth has a suitable oval shape, and is covered with a flat arch. Its length is 16 feet, breadth $13 \frac{1}{2}$, mean

height 2 feet.

Fig. $30 j$, is a longitudinal section of the melting furnace; fig. 306, the ground plan, in which $a$ is the fire door; $b$ the grate; $c$ the fire bridge; $d$ the chimney; $e$ the side openings; $f$ the working doors; $g$ the raking-out hole; $h$ iron spouts, which conduct the melted metal into pits filled with water.

The melting furnace is altogether smaller; but its firing hearth is considerably larger 
than in the roasting furnace. 'The long axis of the oval hearth is 14 feet; itg ghort axu 10 feet; its mean height 2 feet.

300

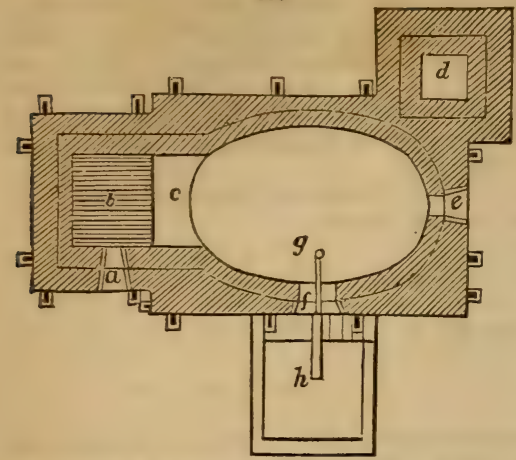

The principal ore smelted at Chessy is the azure copper, which was discovered by accident in 1812 . Red copper ore, also, has come into operation there since 1825 . The average metallic contents of the richest azure ore are from 33 to 36 per cent. ; of the poorer, from 20 to 24 . The red ore contains from 40 to 67 parts in 100 . The ore is sorted, so that the mean contents of metal may be 27 per cent., to which 20 per cent. of limestone are added; whence the cinder will amount to 50 per cent. of the ore. A few per cents. of red copper slag, with some quicklime and gahrslag, are added to each charge, which consists of 200 pounds of the above mixture, and 150 pounds of coke. When the furnace (fourneau à manche, see the Scotch smelting hearth, under LEAD) is in good action, from 10 to 14 such charges are worked in 12 hours. When the crucible is full of metal at the end of this period, during which the cinder has been frequently raked off, the blast is stopped, and the matte floating over the metal being sprinkled with water and taken off, leaves the black copper to be treated in a similar way, and converted into roseltes. The refining of this black copper is performed in a kind of reverberatory furnace.

The einders produced in this reduction process are either vitreous and light blue, which are most abundant; cellular, black, imperfectly fused from excess of lime; or, lastly, red, dense, blistery, from defect of lime, from too much heat, and the passage of protoxyde into the cinders. They consist of silicate of alumina, of lime, protoxyde of iron; the red contain some silicate of copper.

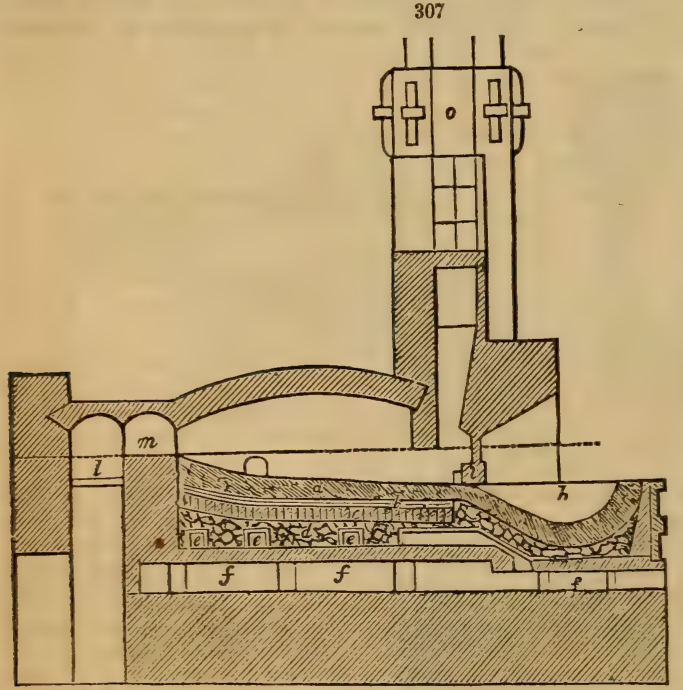

The copper-refining furnace at Chessy, near Ly. ons, is of the kind called Spleiss-ofen (split hearths) by the Germans. Fig. 307 is a section lengthwise in the dotted line $\mathbf{A}$ B of $f i g$. 308 , which is the ground plan.

The foundation-walls are made of gneiss; the arch, the fire-bridge, and the chimney, of fire-bricks. The hearth, $a$, is formed of a dense mixture of coal-dust, upon a bottom of well-beat clay, $b$, which reposes upon a bed of brickwork $c$. Beneath this there is a slag bottom $d ; e$ is the upper, and $f$ the under discharge hole. The hearth is eggshaped; the longer axis being 8 feet, the shorter $6 \frac{3}{2}$ feet; in the middle it is 10 inches deep, and furnished with the outlets $g \mathrm{~g}$, which lead to each of the Spleiss-hearths $h h, f i g .308$. These outlets are contracted with fire-bricks $i i$, till the proper period of the discharge. The two hearths are placed in communication by a canal $k$ : they are $3 \frac{1}{2}$ feet in diameter, 16 inches deep ; are floored with well-beat coal ashes, and receive about 27 ewt. for a charge.

$l$ is the grate; $m$, the fire-bridge; $n$, the boshes in which the tuyères lie; 0 , the chimney; $p$, the working door through which the slags may be drawn off. Above this is a small chimney, to carry off the flame and smoke whenever the door is opened.

The smelting post or charge, to be purified at once, consists of $60 \mathrm{cwt}$. of black copper, to which a little granular copper and copper of cementation is added; the 
consumption of pit-coal amounts to 36 cwt. As soon as the copper is melter, the beilows are set a-going, and the surface of the metal gets soon covered with a

\section{8}

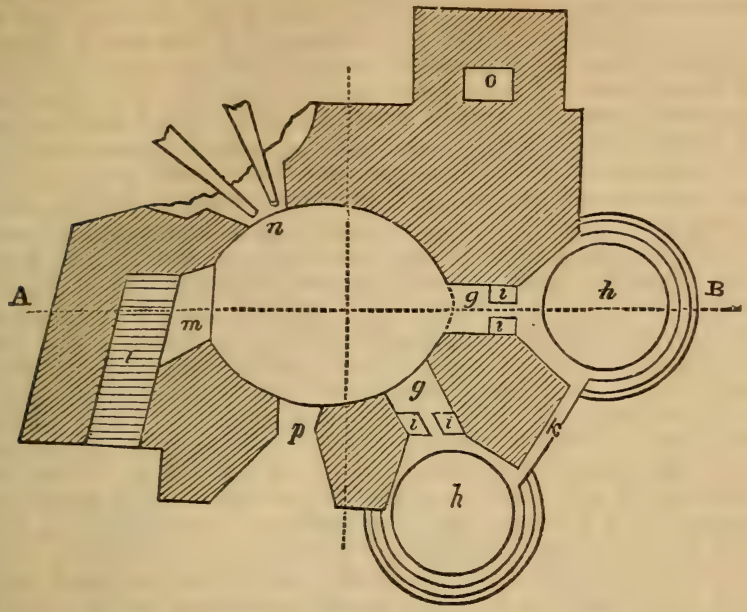

moderately thick layer of cinder, which is drawn off. This is the first skimming or decrassage. By and by, a second layer of cinder forms, which is in like manner removed; and this skimming is repeated, to allow the blast to act upon fresh metallic surfaces. After 4 or 5 hours, no more slag appears, and then the fire is increased. The melted mass now begins to boil or work (travailler), and continues so to do, for about $\frac{3}{4}$ of an hour, or an hour, after which the motion ceases, though the fire be kept up. The gahrproof is now taken; but the metal is seldom fine in less than $\frac{3}{4}$ of an hour after the boil is over. Whenever the metal is run off by the tap-hole into the iwo basins $i i$, called spLIT-HEARTHS, a reddish vapor or mist rises from its surface, composed of an infinite number of minute globules, which revolve with astonishing velocity upon their axes, constituting what the Germans called spratzen (crackling) of the copper. They are composed of a nucleus of metal, covered with a film of protoxyde, and are used as sand for strewing upon manuscript. The copper is separated, as usual, by sprinkling water upon the surface of the melted metal, in the state of rosettes, which are immediately immersed in a stream of water. This refining process lasts about 16 or 17 hours; the skimmings weigh about $50 \mathrm{cwt}$. ; the refuse is from 15 to 17 per cent. ; the ioss from 2 to 3 per cent. The gahrslag amounts to $11 \mathrm{ewt}$.

The refining of the eliquated copper (called darrlinge) from which the silver has been sweated out by the intervention of lead, can be performed only in small hearths. The following is the representation of such a furnace, called, in German, Kupfergahrheerd. Fig. 309 is the section lengthwise; fig. 310 is the section across; and fig. 311 is the
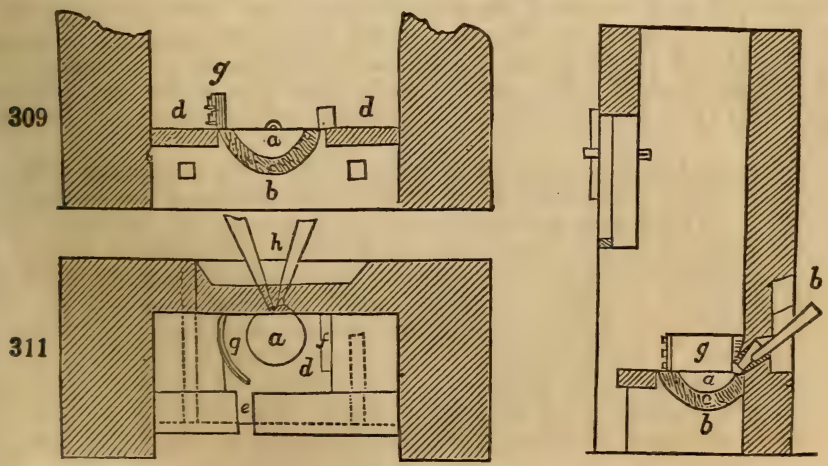

ground plan, in which $a$ is the hearth-hollow; $b$, a massive wall; $c$, the mass out $\boldsymbol{e f}$ wich the hearth is formed; $d$, cast-iron plates covering the hearth; $e$, opening 
running oft the liquid slag; $f$, a small wall; $g$, iron curb for keeping the coals together.

The hearth being heated with a bed of charcoal, $\frac{3}{4} \mathrm{cwt}$. of darrlinge are laid over it, and covered with more fuel : whenever this charge is melted, another layer of the coal and darrlinge is introduced, and thus in succession till the hearth become full, or contain from $2 \frac{1}{4}$ to $2 \frac{1}{2} \mathrm{cwt}$. In Neustadt $7 \frac{1}{2} \mathrm{cwt}$. of darrlinge have been refined in one furnace, from which $5 \mathrm{cwt}$. of gahrcopper has been obtained. The blast oxydizes the foreign metals, namely; the lead, nickel, cobalt, and iron, with a little copper, forming the gahrslag; which is, at first, rich in lead oxyde, and poor in copper oxyde; but, at the end, this order is reversed. The slag, at first blackish, assumes progressively a copper red tint. The slag flows off spontaneously along the channel $e$, from the surface of the hearth. The gahre is tested by means of a proof-rod of iron, called gahr-eisen, thrust though the iuyire into the melted copper, then drawn out and plunged in cold water. As soon as the gahrspan (scale of copper) appears brownish red on the outside, and copper red within, so thin that it seems like a net-work, and so deficient in tenacity that it cannot be bent without breaking, the refining is finished. The blast is then stopped; the coals covering the surface, as also the cinders, must be raked off the copper, after being left to cool a little; the surface is now cooled by sprinkling water upon it, and the thick cake of congealed metal (rondelle) is lifted off with tongs, a process called schleissen (slicing), or sheibenreissen (shaving), which is continued till the last convex cake at the bottom of the furnace, styled the kingspiece, is withdrawn. These rondelles are immediately immersed in cold water, to prevent the oxydation of the copper; whereupon the metal becomes of a cochineal red color, and gets covered with a thin film of protoxyde. Its under surface is studded over with points and hooks, the result of tearing the congealed disc from the liquid metal. Such cakes are called rosette copper. When the metal is very pure and free from protoxyde, these cakes may be obtained very thin, one 24th of an inch for example.

The refining of two cwts. and a lialf of darrlinge takes three quarters of an hour, and yields one cwt. and a half of gahrcopper in 36 rosettes, as also some gahrslag. Gahr. copper generally contains from $1 \frac{1}{2}$ to $2 \frac{1}{2}$ per cent. of lead, along with a little nickel, silver, iron, and aluminum.

Smeling of the Mannsfeld copper schist, or bituminous Mergelschiefer.-The cupreous ore is first roasted in large heaps, of 2000 cwts., interstratified with brush-wood, and with some slates rich in bituminous matter, mixed with the others. These heaps are 3 ells high, and go on burning 15 weeks in fair and 20 in rainy weather. The bitumen is decomposed; the sulphur is dissipated chiefly in the form of sulphurous acid; the metal gets partially oxydized, particularly the iron, which is a very desirable circumstance towards the production of a good smelting slag. The calcined ore is diminished one tenth in bulk, and one eighth in weight; becoming of a friable texture and a dirty yellow gray color. The smelting furnaces are cupolas (schachtofen), 14 to 18 feet high; the fuel is partly wood charcoal, partly coke from the Berlin gas-works, and Silesia. The blast is given by cylinder bellows, recently substituted for the old barbarous Blasebälgen, or wooden bellows of the household form.

The cupreous slate is sorted, according to its composition, into slate of lime, clay, iron, \&c., by a mixture of which the smelting is facilitated. For example, 1 post or charge may consist of $20 \mathrm{cwt}$. of the ferruginous slate, 14 of the calcareous, 6 of the argillaceous, with 3 of fluor spar, 3 of rich copper slags, and other refuse matters. The nozzle at the tuyere is lengthened 6 or 8 inches, to place the melting heat near the centre of the furnace. In 15 hours 1 fodder of 48 cwts. of the above mixture may be smelted, whereby 4 to 5 cwts. of matte (crude copper, called Kupferstein in Germany) and a large body of slags are obtained. The matte contains from 30 to 40 per cent. of copper, and from 2 to 4 loths ( 1 to $2 \mathrm{oz}$.) of silver. The slags contain at times one tenth their weight of copper.

The matte is composed of the sulphurets of copper, iron, silver, zinc, along with some arsenical cobalt and nicke. The slaty slag is raked off the surface of the melted matte from time to time. The former is either after being roasted six successive times, smelted into black copper; or it is subjerted to the following concentration process. It is broken to pieces, roasted by brushwood and coals three several times in brick-walled kilns, containing $60 \mathrm{cwts}$, and turned over after every calcination; a process of four weeks' duration. The thrice roasted mass, called spurrost, being melted in the cupola fig. 313, with ore-cinder, yields the spurstein, or concentrated matte. From 30 to 40 cwts. of spurrost are smelted in 24 hours; and from 48 to 60 per cent. of spursiern are obtained, the slag from the slate smelting being employed as a flux. The spurstein contains from 50 to 60 per cent. of copper, combined with the sulphurets of copper, of iron, and vilver.

'The spurstein is now mixed with dünnstein (a sulphuret of copper and iron produced in the original smeltings) roasted six successive times, in the quantity of $60 \mathrm{cwts}$,, with 
brushwood and charcoal; a process which requires from 7 to 8 weeks. The product of this six-fold calcination is the Gahrrost of the Germans (done and purified); it has a color like red copper ore, varying from blue gray into cochineal red; a granular fracture; it contains a little of the metal, and may be immediately reduced into metallic copper, called kupfermachen. But before smelting the mass, it is lixiviated with water, to extract from it the soluble sulphate, which is concentrated in lead pans, and crystallized.

The lixiviated gahröste mixed with from $\frac{x}{4}$ to $\frac{1}{5}$ of the lixiviated dünnsteinrost, and $\frac{1}{6}$ to $\frac{1}{10}$ of the copper slate slag, are smelted with charcoal or coke fuel in the course of 24 hours, in a mass of 60 or $80 \mathrm{cwts}$. The product is black copper, to the amount of about $\frac{1}{4}$ the weight, and $\frac{1}{6}$ of dïnnstein or thin matte. This black copper contains in the cwt. from 12 to 20 loths ( 6 to $10 \mathrm{oz}$.) of silver. The dünnstein consists of from 60 to 70 per cent. of copper combined with sulphur, sulphuret of iron, and arsenic; and when thrice roasted, yields a portion of metal. The black copper lies undermost in the crucible of the furnace; above it is the dünnstein, covered with the stone slag, or copper cinder, resulting from the slate-smelting. The slags being raked off, and the crucible sufficiently full, the eve or nozzle hole is shut, the dünnstein removed by cooling the surface and breaking the crust, which is about $\frac{1}{4}$ to $\frac{1}{2}$ inch thick. The same method is adopted for taking out the black copper in successive layers. For the de-silvering of this and similar black coppers, see Sitver.

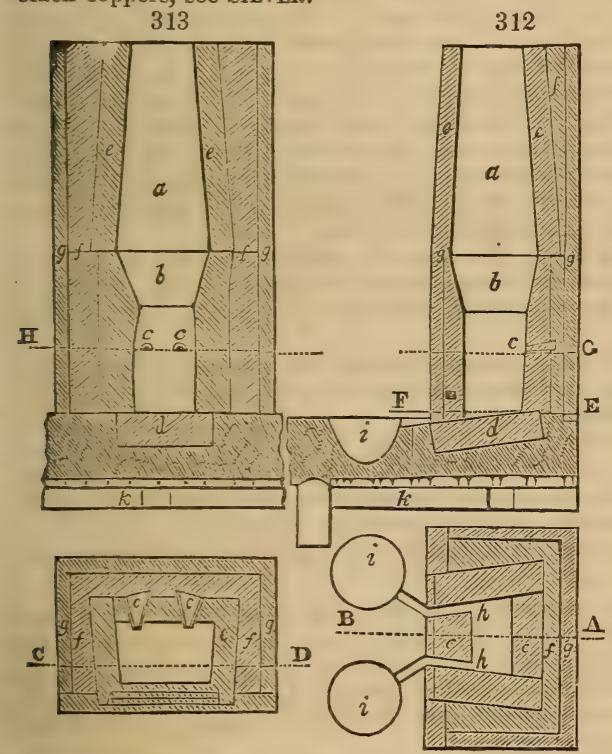

Fig. 312 is a vertical section through the form or tuyère in the dotted line A B of fig. 314. Fig. 313 is a vertical section in the dotted line c D of $f i g .315$. $a$ is the shaft of the furnace, $b$ the rest, $c c$ the forms; $d$ the sole or hearth-sione, which has a slope of 3 inches towards the front wall; $e e, \& c$. casing walls of fire bricks; $f f$, \&c. filling up walls built of rubbish stones; $g \mathrm{~g}$ a mass through which the heat is slowly conducted; $h h$ the two holes through one or other of which alternately the product of the smelting process is run, off into the fore-hearth. Beneath the hearth-sole there is a solid body of loam; and the forehearth is formed with a mixture of coal-dust and clay; $k$ is the discharge outlet. Fig. 314 is a horizontal section of the furnace through the hole or eye in the dotted line E F of fig. 312 ; fig. 315 , a horizontal section of the shaft of the furnace through the

form in the dotted line $\mathrm{G} \mathrm{H}$ of figs. 312 and 313 . The height of the shaft, from the line I $\mathbf{F}$ to the top, is 14 feet; from $\mathbf{E}$ to $\mathrm{G}, 25$ inches; from $c$ to the line below $b, 2$ feet; from that line to the line opposite $g \mathrm{~g}, 2$ feet. The width at the line $g \mathrm{~g}$ is 3 feet 3 inches, and at $c 26$ inches. The basins $i$, fig. 314, are 3 feet diameter, and 20 inches deep.

The refining of copper is said to be well executed at Seville, in Spain; and, therefore, some account of the mode of operating there may be acceptable to the reader.

The first object is to evaporate in a reverberatory furnace all the volatile substances, such as sulphur, arsenic, antimony, \&c., which may be associated with the sulphur; ant the second, to oxydize and to convert into scoriæ the fixed substances, such as iron, lead, \&c., with the least possible expense and waste. The minute quantities of gold and sil. ver which resist oxydation cannot be in any way injurious to the copper. The hearth is usually made of a refractory sand and clay with ground charcoal, each mixed in equal volumes, and worked up into a doughy consistence with water. This composition is beat firmly into the furnace bottom. But a quartzose hearth is found to answer better, and to be far more durable; such as a bed of fire-sandstone.

Before kindling the furnace, its inner surface is smeared over with a cream-consistenced mixture of fire-clay and water.

The cast pigs, or blocks of black or crude copper, are piled upon the hearth, each successive layer crossing at right angles the layer beneath it, in order that the flame may 
have access to play upon the surface of the hearth, and to heat it to a proper pitch for making the metal flow.

The weight of the charge should be proportional to the capacity of the furnace, and such that the level of the metallic bath may be about an inch above the nozzle of the bellows; for, were it higher, it would obstruct its operation, and were it too low, the stream of air wonld strike but imperfectly the surface of the metal, and would fail to effect, or would retard at least, the refining process, by leaving the oxydation and volatilization of the foreign metals incomplete.

As the scoriæ form upon the surface, they are drawn off with an iron rabble fixed to the end of a wooden rod.

Soon after the copper is melted, charcoal is to be kindled in three iron basins lined with loam, placed alongside the furnace, to prepare them for receiving their charge of copper, which is to be converted in them, into rosettes.

The bellows are not long in action before the evaporation of the mineral substances is so copious, as to give the bath a boiling appearance; some drops rise up to the roof of the reverberatory, others escape by the door, and fall in a shower of minute spherical globules. This phenomenon proves that the process is going on well; and, when it ceases, the operation is nearly completed. A small proof of copper, of the form of a watch-case, and therefore called montre, is taken out from time to time, upon the round end of a polished iron rod, previously heated. This rod is dipped two or three inches into the bath, then withdrawn and immersed in cold water. The copper cap is detached from the iron rod, by a few blows of a hammer; and a judgment is formed from its thickness, color, and polish, as to the degree of purity which the copper has acquired. But these watches need not be drawn till the small rain, above spoken of, has ceased to fall At the end of about 11 hours of firing, the numerous small holes observable in the first watch samples begin to disappear; the outer surface passes from a bright red to a darker hue, the inner one becomes of a more uniform color, and always less and less marked with yellowish spots. It will have acquired the greatest pitch of purity that the process can bestow, when the watches become of a dark crimson color.

Care must be taken to stop this refining process at the proper time; for, by prolonging it unduly, a small quantity of cupreous oxyde would be formed, which, finding no oxygen to reduce it, would render the whole body of copper hard, brittle, and incapable of lamination.

The basins must now be emptied of their burning charcoal, the opening of the tuyère must be closed, and the melted copper allowed to flow into them through the tap-hole, which is then closed with loam. Whenever the surface is covered with a solid crust, it is bedewed with water; and as soon as the crust is about $1 \frac{1}{2}$ inch thick it is raised upon hooks above the basin, to drain off any drops, and then carried away from the furnace. If these cakes, or rosettes, be suddenly cooled by plunging them immediately in water, they will assume a fine red color, from the formation of a film of oxyde.

Each refining operation produces, in about 12 hours, $1 \frac{7}{10}$ tons of copper, with the consumption of about $\frac{4}{5}$ of a ton of dry wood.

Care should be taken that the copper cake or rosette be all solidified before plunging it into water, otherwise a very dangerous explosion might ensue, in consequence of the sudden extrication of oxygen from the liquid metal, in the act of condensation. On the other hand, the cake should not be allowed to cool too long in the air, lest it get peroxydized upon the surface, and lose those fine red, purple, and yellow shades, due to a film of the protoxyde, which many dealers admire.

When a little oxyde of antimony and oxyde of copper are combined with copper, they occasion the appearance of micaceous scales in the fractured faces. Such metal is hard, brittle, yellowish within, and can be neither laminated nor wire-drawn. These defects are not owing to arsenic, as was formerly imagined; but, most probably, to antimony in the lead, which is sometimes used in refining copper. They are more easily prevented than remedied.

According to $M$. Frèrejean, proprietor of the great copper works of Vienne, in Dauphiny, too low a temperature, or too much charcoal, gives to the metal a cubical structure, or that of divergent rays; in either of which states it wants tenacity. Too high a temperature, or too rapid a supply of oxygen, gives it a brick red color, a radiated crystallization without lustre, or a very fine grain of indeterminate form; the last structure being unsuitable for copper that is to be worked under the hammer or in the rolling-press. The form which indicates most tenacity is radiated with minute fibres glistening in mass. Melted copper will sometimes pass successively through these three states in the space of ten minutes.

Fig. 316 represents a roasting mound of copper pyrites in the Lower Hartz, near Goslar, where a portion of the sulphur is collected. It is a vertical section of a truncated quadrangular pyramid. A layer of wooden billets is arranged at the base of the pyramid in the line $a$ a. 
c, a wooden chimney which stands in the centre of the mound with a small pile of eharcoal at its bottom, c ; $d \cdot d$ are large lumps of ore surrounded by smaller pieces; $f f$

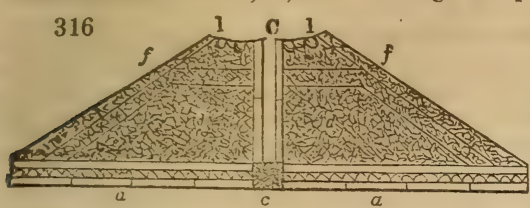

are rubbish and earth to form a covering. A current of air is admitted under the billets by an opening in the middle of each of the four sides of the base $a$, so that two principal currents of air cross under the vertical axis $\mathrm{c}$ of the truncated pyramid, as indicated in the figure.

The fire is applied through the chimney $\mathrm{c}$; the cirarcoal at its bottom $c$, and the piles $a d$ are kindled. The sulphureous ores, $d f$, are raised to such a high temperature as to expel the sulphur in the state of vapor.

In the Lower Hartz a roasting mound continues burning during four months. Some days after it is hindled the sulphur begins to exhale, and is condensed by the air at the upper surface of the pyramid. When this seems impregnated with it, small basins 11 are excavated, in which some liquid sulphur collects; it is removed from time to time with iron ladles, and thrown into water, where it solidifies. It is then refined and cast into roll brimstone.

A similar roasting mound contains, in the Lower Hartz, from 100 to 10 tons of ore and 730 cubic feet of wood. It yields in four months about one ton and a half of sulphur from copper pyrites. Lead ore is treated in the same way, but it furnishes less sulphur.

There are usually from 12 to 15 roasting heaps in action at once for three smelting works of the Lower Hartz. After the first roasting two heaps are united to form a third, which is calcined anew, but under a shed; the ores are then stirred up and roasted for the third time, whence a crude mixture is procured for the smelting-house.

The most favorable seasons for roasting in the open air are spring and autumn; the best weather is a light wind accompanied with gentle rain. When the wind or rain obstructs the operation, this inconvenience is remedied by planks distributed round the npper surface of the truncated pyramid over the sulphur basins.

Manufacturing assays of copper.-The first thing is to make such a sample as will represent the whole mass to be valued; with which view, fragments must be taken from different spots, mixed, weighed, and ground together. A portion of this mixture being tried by the blow-pipe, will show, by the garlic or sulphurous smell of its fumes, whether arsenic, sulphur, or both, be the mineralizers. In the latter case, which often occurs, $100 \mathrm{gr}$. or $1000 \mathrm{gr}$. of the ore are to be mixed with one half its weight of saw-dust, then imbued with oil, and heated moderately in a crucible till all the arsenical fumes be dissipated. The residuum, being cooled and triturated, is to be exposed in a shallow earthen cup to a slow roasting heat, till the sulphur and charcoal be burned away. What remains, being ground and mixed with half its weight of calcined borax, one twelfth its weight of lamp black, next made into a dough with a few drops of oil, is to be pressed down into a crucible, which is to be covered with a luted lid, and to be subjected, in a powerful air furnace, first to a dull red heat, and then to vivid ignition for 20 minutes. On cooling and brealing the crucible, a button of metallic copper will be obtained. Its color and malleability indicate pretty well the qua?ity, as does its weight the relative value of the ore. It should be cupelled with lead, to ascertain if it contains silver or gold. See Assay, and Silver.

If the blow-pipe trial showed no arsenic, the first calcination may be onitted; an? if neither sulphur nor arsenic, a portion of the ground ore should be dried, and treated directly with borax, lamp-black, and oil. It is very common to make a urv assay of copper ores, by one roasting and one fusion along with 3 parts of black flix : from the weight of the metallic button the richness of the ore is inferred.

The humid assay is more exact, but it requires more skill and time.

The sulphur and the silica are easily got rid of by the acids, which do not dissolve them, but only the metallic oxydes and the other earths. These oxydes may then be thrown down by their appropriate reagents, the copper being precipitated in the state of either the black oxyde or pure metal. 105 parts of black oxyde represent 100 of copper Before entering upon the complete analysis of an ore, preliminary trials should be made, to ascertain what are its chief constituents. If it be sulphuret of copper, or copper pyrites, without silver or lead, 100 grains exactly of its average powder may be weighed out, treated in a matrass with boiling muriatic acid for some time, gradually adding a few drops of nitric acir', till all action ceases, or till the ore be all dissolved. The insoluble matter found floating in the liquid contains most of the sulphur; it may be separated upon a filter, washed, dried, and weighed; then verified by burning away. The incombustible residuum, treated by muriatic acid, may leave an insoluble deposite, which is to be added to the former. To the whole of the filtered solutions carbonate of potash ig 
to be added; and the resulting precipitate, being washed, and digested repeatedly in water of ammonia, all its cupric oxyde will have been dissolved, whenever the ammonia is eo longer rendered blue.

Caustic potash, boiled with the ammoniacal solution, will separate the copper in the state of black oxyde; which is to be thrown upon a filter, washed. dried, and weighed. The matter left undissolved by the ammonia, consists of oxyde of iron, with probably a little alumina. The latter being separated by caustic potash, the iron oxyde may be also washed, dried, and weighed. The powder which originally resisted the muriatic acid, is silica.

Assay of copper ores, which contain iron, sulphur, silver, lead, and antimony.

100 grains of these ores, previously sampled, and pulverized, are to be boiled with nitric acid, adding fresh portions of it from time to time, till no more of the matte? be dissolved. The whole liquors which have been successively digested and decanted uff, are to be filtered and treated with common salt, to precipitate the silver in the state of a chloride.

The nitric acid, by its reaction upon the sulphur, having generated sulphuric acid, this will combine with the lead oxydized at the same time, constituting insoluble sulphate of lead, which will remain mixed with the gargue. Should a little nitrate of lead remain in the liquid, it may be thrown down by sulphate of soda, after the silver has been separated. The dilute liquid, being concentrated by evaporation, is to be mixed with ammonia in such excess as to dissolve all the cupric oxyde, while it throws down all the oxyde of iron and alumina; which two may be separated, as usual, by a little eaustic potash. The portion of ore insoluble in the nitric acid being digested in muriatic acid, everything will be dissolved except the sulphur and silica. These being collected upon a filter, and dried, the sulphur may be burned away, whereby the proportion of each is determined.

Ores of the oxyde of copper are easily analyzed by solution in nitric acid, the addition of ammonia, to separate the other metals, and precipitation by potash. The native carbonate is analyzed by calcining 100 grains; when the loss of weight will show the amount of Ivater and carbonic acid; then that of the latter may be found, by expelling it from another 100 grains, by digestion in a given weight of sulphuric acid. The copper is finally obtained in a metallic state by plunging bars of zinc into the solution of the sulphate.

The native arseniates of copper are analyzed by drying them first at a moderate heat; after which they are to be dissolved in nitric acid. To this solution, one of nitrate of lead is to be added, as long as it occasions a precipitate; the deposite is to be drained upon a filter, and the clear liquid which passes through, being evaporated nearly to driness, is to be digested in hot alcohol, which will dissolve everything except a little arseniate of lead. This being added to the arseniate first obtained, from the weight of the whole, the arsenic acid, constituting 35 per cent., is directly inferred. The alcoholic solution being now evaporated to dryness, the residue is to be digested in water of ammonia, when the cupric oxyde will be dissolved, and the oxyde of iron will remain. The copper is procured, in the state of black oxyde, by boiling the filtered ammoniacal solution with the proper quantity of potash.

The analysis of muriate of copper-atacamite-is an easy process. The ore being dissolved in nitric acid, a solution of nitrate silver is added, and from the weight of the chloride precipitated, the equivalent amount of muriate or chloride of copper is given ; for 100 of chloride of silver represent 93 of chloride of copper, and 43.8 of its metallic basis. This calculation may be verified by precipitating the copper of the muriate from its solution in dilute sulphuric acid, by plates of zirc.

The phosphate of copper may be analyzed either by solution in nitric acid, and precipstation by potash; or by precipitating the phosphoric acid present, by means of acetate of lead. The phosphate of lead thus obtained, after being washed, is to be decomposed by dilute sulphuric acid. The insoluble sulphate of lead, being washed, dried, and weighed, indicates by its equivalent the proportion of phosphate of lead, as also of phosphate of copper; for 100 of sulphate of lead correspond to 92.25 phosphate of lead, and 89.5 phos. phate of copper; and this again to 52.7 of the black oxyde.

Copper forms the baris of a greater number of important ALLOYs than any other metal. With zinc, it forms Brass in all its varieties; which see.

Bronze and Bell Metal are alloys of copper and tin. This compound is prepared in crucibles when only small juantities are required; but in reverberatory hearths, when statues, bells, or cannons are to be cast. The metals must be protected as much as possible during their combination from contact of air by a layer of pounded charcoal, otherwise two evils would result, waste of the copper by combustion, and a rapid oxydizement of the tin, so as to change the proportions and alter the properties of the alloy. The fused materials ought to be well mixed by stirring, to give uniformity to the compound. See Bronze. 
An alloy of 100 of copper and $4 \cdot 17$ of tin has been proposed by M. C naudet for tise ready manufacture of melals. After melting this alloy, he casts it in rnoulds rnade of such bone-ash as is used for cupels. The medals are afterward suiajected to the action of the coining press, not for striking them, for the mould furnishes perfect impressions, but for finishing and polishing them.

By a recent analysis of M. Berthier, the bells of the pendules, or ornamental clocks, made in Paris, are found to be composed-of copper $72 \cdot 00$, tin 26.56 , iron $1 \cdot 44$, in 100 parts.

An alloy of 100 of copper and 14 of tin is said by M. Dussauszy to furnish tools, which, hardened and sharpened in the manner of the ancients, afford an edge nearly equal to that of steel.

Cymbals, gongs, and the tamtam of the Chinese are made of an alloy of 100 of copper with about 25 of tin. To give this compound the sonorous property in the highest degree, it must be subjected to sudden refrigeration. M. D'Arcet, to whom this discovery is due, recommends to ignite the piece after it is cast, and to plunge it immediately into cold water. The sudden cooling gives the particles of the alloy such a disposition, that, with a regulated pressure by skilful hammering, they may be made to slide over each other, and remain permanently in their new position. When by this means the instrument has received its intended form, it is to be heated and allowed to cool slowly in the air. The particles now take a different arrangement from what they would have done by sudden refrigeration; for instead of being ductile, they possess such an elasticity, that on being displaced by a slight compression, they return to their primary position after a series of extremely rapid vibrations; whence a very powerful sound is emitted. Bronze, bell-metal, and probably all the other alloys of tin with copper, present the same peculiarities.

The alloy of 100 of copper with from 60 to 33 of tin forms common bell-metal. It is yellowish or whitish gray, brittle, and sonorous, but not so much so as the preceding. The metal of house-clock bells contains a little more tin than that of church-bells, and the bell of a repeater contains a little zinc in addition to the other ingredients.

The bronze-founder should study to obtain a rapid fusion, in order to avoid the causes of waste indicated above. Reverberatory furnaces have been long adopted for this operation; and among these, the elliptical are the best. The furnaces with spheroidal domes are used by the bell-founders, because their alloy being more fusible, a more moderate melting heat is required; however, as the rapidity of the process is always a matter of consequence, they also would find advantage in employing the elliptical hearths, (see the form of the melling furnace, as figured under Smelting of copper ores.) Coal is now universally preferred for fuel.

The alloy of 100 of copper with 50 of tin, or more exactly of 32 of the former with $14 \frac{1}{2}$ of the latter, constitutes speculum metal, for making mirrors of reflecting telescopes. This compound is nearly white, very brittle, and susceptible of a fine polish with a brilliant surface. The following compound is much esteemed in France for making specula. Melt 2 parts of pure copper and 1 of grain-tin in separate crucibles, incorporate thoroughly with a wooden spatula, and then run the metal into moulds. The lower surface is the one that should be worked into a mirror.

Mr. Edwards, in the Nautical Almanack for 1787, gave the following instructions for making speculum metal.

The quality of the copper is to be tried by making a series of alloys with tin, in the proportion of 100 of the former to 47 , to 48 , to 49 , and to 50 of the latter metal; whence the proportions of the whitest compound may be ascertained. Beyond the last proportion, the alloy begins to lose in brilliancy of fracture, and to take a bluish tint. Having determined this point, take 32 parts of the copper, melt, and add one part of brass and as much silver, covering the surface of the mixture with a little black flux; when the whole is melted, stir with a wooden rod, and pour in from 15 to 16 parts of melted tin (as indicated by the preparatory trials), stir the mixture again, and immediately pour it out into cold water. Then melt again at the lowest heat, adding for every 16 parts of the compound 1 part of white arsenic, wrapped in paper, so that it may be thrust down to the bottom of the crucible. Stir with a wooden rod as long as arsenical fumes rise, and then pour it into a sand mould. While still red hot, lay the metal in a pot-full of very hot embers, that it may cool very slowly, whereby the danger of its cracking or flying into splinters is prevented.

Having described the different alloys of copper and tin, I shall now treat of the method of separating these metals from each other as they exist in old cannons, damaged bells, scc. The process employed on a very great scale in France, during the Revolution, for obtaining copper from bells, was contrived by Fourcroy; founded upon the chemical fact that tin is more fusible and oxydizable than copper.

1. A certain quantity of bell-metal was completely oxydized by calcination in a reverberatory furnace; the oxyde was raked out, and reduced to a fine powder.

2. Into the same furnace a fresh quantity of the same metal was introduced; it was 
Lelted, and there was added to it one half of its weight of the oxyde formed in the first operation. The temperature was increased, and the mixture well incorporated; at the end of a few hours, there was ohtained on the one hand copper almost pure, which subsided in a liquid state, and spread itself upon the sole of the hearth, while a compound of oxyde of tin, oxyde of copper, with some of the earthy matters of the furnace, collected on the surface of the metallic bath in a pasty form. These scoriæ were removed with a rake, and as soon as the surface of the melted copper was laid bare, it was run out. The scoriæ were levigated, and the particles of metallic copper were obtained after elutriation. By this process, from 100 pounds of bell-metal, about 50 pounds of copper were extracted, containing only one per cent. of foreign matters.

3. The washed scoriæ were mixed with $\frac{1}{8}$ their weight of pulverized charcoal; the mixture was triturated to effect a more intimate distribution of the charcoal; and it was then put into a reverberatory hearth, in which, by aid of a high heat, a second reduction was effected, jielding a fluid alloy consisting of about 60 parts of copper and 20 of tin; while the surface of the bath got covered with new scoriæ, containing a larger proportion of tin than the first.

4. The alloy of 60 of copper with 40 of tin was next calcined in the same reverberatory furnace, but with stirring of the mass. The air, in stveeping across the surface of the bath, oxydized the tin more rapidly than the copper; whence proceeded crusts of oxyde that were skimmed off from time to time. This process was continued till the metallic alloy was brought to the same standard as bell-metal, when it was run out to be subjected to the same operations as the metal of No. 1.

The layers of oxyde successively removed in this way were mixed with charcoal, and reduced in a fourneau à manche, or Scotch lead smelting furnace.

I shall not prosecute any further the details of this complicated process of Fourcroy; because it has been superseded by a much better one contrived by M. Bréant. He employed a much larger quantity of charcoal to reduce the scoriæ rich in tin; and increased the fusibility by adding crushed oyster-shells, bottle glass, or even vitrified scoriæ, according to the nature of the substance to be reduced; and he treated them directly in a reverberatory furnace.

The metal, thus procured, was very rich in tin. He exposed it in masses on a sloping hearth of a reverberatory furnace, where, by a heat regulated according to the proportions of the two metals in the alloy, he occasioned an eliquation or sweating out of the tin. Metallic drops were seen to transpire round the alloyed blocks or pigs, and, falling like rain, flowed down the sloping floor of the furnace; on whose concave bottom the metal collected, and was ladled out into moulds. When the alloy, thus treated, contained lead, this metal was found in the first portions that sweated out. The purest tin next came forth, while the last portions held more or less copper in solution. By fractioning the products, therefore, there was procured-

\section{Tin with lead.}

2. Tin nearly pure.

3. Tin alloyed with a little copper.

A spongy mass remained, exhibiting sometimes beautiful crystallizations; this mass, commonly too rich in copper to afford tin by liquation, was treated by oxydizement. In this manner, M. Bréant diminished greatly the reductions and oxydations; and therefore incurred in a far less degree the enormous waste of tin, which flies off with the draught of air in high and long-continued heats. He also consumed less fuel as well as labor, and obtained purer products of known composition, ready to be applied directly in many arts.

He treated advantageously in this manner more than a million of kilogrammes (1000 tuns) of scoriæ, for every 2 cwts. of which he paid 40 centimes (four-pence), while several million kilogrammes of much richer scoriæ had been previously sold to other refiners at 5 centimes or one sous.

I have said that the ancients made their tools and military weapons of Bronze. Several of these have been analyzed, and the results are interesting.

An antique sword, found in 1799, in the peat moss of the Somme, consisted of copper $87 \cdot 47$; tin $12 \cdot 53$, in 100 parts.

The bronze springs for the balistæ, according to Philo of Byzantium, were made of copper $9 \%$, tin 3 .

Hard and brittle nails afforded by analysis, 92 of copper, and 8 of tin.

Of three antique swords found in the environs of Abbeville, one was found to consist of 85 of copper to 15 of tin. The nails of the handle of this sword were flexible; they were composed of copper 95 , tin 5 .

Another of the swords consisted of 90 of copper and 10 of tin; and the third, of 96 copper, with 4 tin.

A fragment of an ancient scythe afforded to analysis $92 \cdot 6$ copper, and $7 \cdot 4$ tin.

The process of coating copper with tin, exemplifies the strong affinity between the two metals. The copper surface to be tinned is first cleared up with a smooth sand- 
stone; then it is heated and rubbed over with a little sal ammoniac, till it be perfectly clean and bright: the tin, along with some pounded rosin, is now placed on the copper, which is made so hot as to melt the tin, and allow of its being spread over the suriace with a dossil or pad of tow. The layer thus fixed on the copper is exceedingly thin ; Bayen found that a copper pan, 9 inches in diameter and $3 \frac{1}{4}$ inches deep, being weighed immediately before and after tinning, became only 21 grains heavier. Now as the area tinned, including the bottom, amounted to 155 square inches, 1 grain of tin had been spread over nearly $7 \frac{2}{2}$ square inches; or only 20 grains over every square foot.

Copper and Arsenic form a white-colored alloy, sometimes used for the scales of thermometers and barometers; for dials, candlesticks, \&c. To form this compound, successive layers of copper clippings and white arsenic are put into an earthen crucible; which is then covered with sea salt, closed with a lid, and gradually heated to redness. If 2 parts of arsenic have been used with 5 of copper, the resulting compound commonly contairs one tenth of its weight of metallic arsenic. It is white, slightly ductile, denser, and more fusible than copper, and without action on oxygen at ordinary temperatures; but, at higher heats, it is decomposed with the exhalation of arsenious acid. The white copper of the Chinese consists of 40.4 copper; 31.6 nickel; 25.4 zine; and 2.6 iron. This alloy is nearly silver white; it is very sonorous, well polished, malleable at common temperatures, and even at a cherry red, but very brittle at a red-white heat. When heated with contact of air, it oxydizes, burning with a white flame. Its specific gravity was $8 \cdot 432$. When worked with great care, it mas be reduced to thin leaves, and to wires as small as a needle. See German Srlver, infra.

Tutenag, formerly confounded with white copper, is a different composition from the above. Keir says it is composed of copper, zinc, and iron; and Dick describes it as a short metal, of a grayish color, and scarcely sonorous. The Chinese export it, in large quartities, to India.

Copper, White, or German silver. M. Gersdorf, of Vienna, states, that the proportions or the metals in this alloy should vary according to the uses for which it is destined. When intended as a substitute for silver, it should be composed of 25 parts of nickel, 25 of zine, and 50 of copper. An alloy better adapted for rolling, consists of $25 \mathrm{of} \mathrm{nickel,}$ 20 of zine, and 60 of copper. Castings, such as candlesticks, bells, \&c., may be made of an alloy, consisting of 20 of nickel, 20 of zinc, and 60 of copper; to which 3 of lead are added. The addition of 2 or $2 \frac{1}{2}$ of iron (in the shape of tin plate?) renders the packfong much whiter, but, at the same time, harder and more brittle.

Keferstein has giren the following analysis of the genuine German silver, as made from the original ore found in Hildburghausen, near Suhl, in Henneberg:-

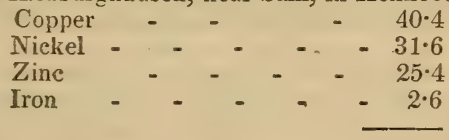

$100 \cdot 0$

Chinese packfong, according to the same authority, consists of 5 parts of copper, alloyed with 7 parts of nickel, and 7 parts of zinc.

The best alloy for making plummer blocks, bushes, and steps for the steel or iron gudgeons and pirots of machinery to run in, is said to consist of 90 parts of copper, 5 of zine, and 5 of antimony.

A factitious protoxyde of copper, of a fine red color, may be made by melting together, with a gentle heat, 100 parts of sulphate of copper, and 59 of carbonate of soda in crystals, and continuing the heat till the mass become solid. This being pulverized and mixed exactly with 15 parts of copper filings, the mixture is to be heated to whiteness, in a crucible, during the space of 20 minutes. The mass, when cold, is to be reduced to powder, and washed. A beautiful metallic pigment may be thus prepared, at the cost of $2 s$. a pound.

All the oxydes and salts of copper are poisonous; they are best counteracted by administering a large quantity of sugar, and sulphureted hydrogen water.

The following scientific summary of copper ores in alphabetical order may prove acceptable to many readers, amid the present perplexing distribution of the native metallic compounds in mineralogical systems.

1. Arseniate of Copper.

A. Erinite, rhomboidal arseniate of copper, micaceous copper, kupferglimmer. Emerald green: specific gravity $4 \cdot 043$; scratches calc-spar; yields water by heat; fusible at the blowpipe, and reducible into a white metallic globule. Soluble in nitric acid; the solution throws down copper by iron. It consists of arsenic acid $33 \cdot 78$; oxyde of copper 59.24; water 5 ; alumina $1.7 \%$. It is found in Coinwall, Ireland, Hungary.

B. Liroconite; octahedral arseniate of copper; lens ore, so called from the flatness 
of the crystal. Blue; specific gravity 2.88 ; scratches calc-spar. It consists of arsenie acid 14; oxycle of copper 49; water 35. It is found in Huel-Mutrel, Huel-Gorland, Huel-Unity, mines in Cornwall.

C. Olivenite; right prismatic arseniate of copper; olive-ore. Dull green; specific gravity $4 \cdot 28$; scratches Huor; yields no water by heat; fusible at the blowpipe into a glassy bead, enclosing a white metallic grain. It consists of arsenic acid 45 , oxyde of copper 50.62 . It affords indications of phosphoric acid, which the analysts seem to have overlooked. It occurs in the above and many other mines in Cornwall.

D. Aphanese. Trihedral arseniate of copper. Bluish green, becoming gray upon the surface; specific gravity $4 \cdot 28$; scarcely scratches calc-spar; yields water with heat; and traces of phosphoric acid.

The fibrous varieties called wood copper, contain water, and resemble the last species in composition.

2. Carbonate of Copper.

A. Azurite; kupferlazur. Blue. Crystallizes in oblique rhomboidal prisms; speente gravity 3 to 3.83 ; scratches calc-spar, is scratched by fluor; yields water with heat, and blackiens. Its constituents are, carbonic acid 25.5; oxyde of copper $69 \cdot 1$; water $\mathbf{5 \cdot 4}$. The Chessy and Banat azurite is most profitably employed to make sulphate of copper.

B. Mulachite; green carbonate or mountain green. Crystallizes in right rhomboidal prisms; specific gravity 3.5 ; affords water with heat, and blachens. It consists of carbonic acid $18 \cdot 5$; oxyde of copper $72 \cdot 2$; water $9 \cdot 3$.

C. Mysorine; anhydrous carbonate of copper. Dark brown generally stained green or red; conchoidal fracture; soft, sectile; specific gravity 2.62 . It consists of carbonic acid 16.7; oxyde of copper 60.75 ; peroxyde of iron 19.5 ; silica $2 \cdot 10$. This is a rare mineral found in the Mysore.

3. Chromate of Copper and Lead; vauquelinite. Green of various shades; speeitic gravity 6.8 to $7 \cdot 2$; brittle; scratched by fluor; fusible at the blowpine with froth and the production of a leaden bead. It consists of chromic acid 28.33 ; oxyde of lead 60.87 ; oxyde of copper $10 \cdot 8$. It occurs at Berezof in Siberia along with chromate of lead.

4. Dioptase; silicate of copper; emerald copper. Specific gravity 3.3 ; scratehes glass with difficulty; affords water with heat, and blackens; infusible at the blowpipe. It consists of silica $43 \cdot 18$; oxyde of copper $45 \cdot 46$; water $11 \cdot 36$. This rare substance comes from the government of Kirgis.

The silicate of Dillenberg is sinilar in composition.

5. Gray copper ore called Panabase, from the number of metallic bases which $\mathbf{n t}$ contains; and Fablerz. Steel gray; specific gravity $4 \cdot 79$ to $5 \cdot 10$; crystallizes in regular tetrahedrons; fusible at the blowpipe, with disengagement of fumes of antimony and occasionally of arsenic; swells up and scorifies, affording copper with soda flux. Is acted upon by nitric acid with precipitation of antimony; becomes blue with ammonia; yields a blue precipitate with ferrocyanide of potassum; as also indications frequently of zine, mercury, silver, \&c. Its composition which is very complex is as follows : sulphur $26 \cdot 83$; antimony $12 \cdot 46$; arsenic $10 \cdot 19$; copper $40 \cdot 60$; iron 4.66 ; zinc $3 \cdot 69$; silver 0.60 Some specimens contain from 5 to 31 per cent. of silver. The gray copper ores are rery common ; in Saxony; the Hartz; Cornwall; at Dillenberg; in Mexicos Peru, \&c. They are important on account both of their copper and silver. Tennantite is a variety of Fahlerz. It occurs in Cornwall. Its constituents are, sulphur 28.74; arsenic 11.84 ; copper $45 \cdot 32$; iron $9 \cdot 26$.

6. Hydrated silicate of Copper; or Chrysocolla. Green or bluish green; specifie gravity 2.03 to 2.16 ; scratched by steel; very brittle; affords water with heat, and blackens; is acted upon by acids, and leaves a silicious residuum. Solution becomes blue with ammonia. Its constituents are silica 26 ; oxyde of copper 50 ; water 17 ; carbonic acid 7.

7. Muriate of Copper. Gtakamite; green; crystallizes in prisms; specific gravity $4 \cdot 43$. Its constituents are, chlorine 15.90 ; copper 14.22 ; oxyde of copper $54 \cdot 22$; water $14 \cdot 16$; oxyde of iron 1.50 . The green sand of Peru, collected by the inhabitants of Atakama, is this substance in a decomposed state.

8. Oxyde of Copper.

A. Black, or Melaconise; a black earthy looking substance found at Chessy and other places. It is deatoxydc of copper.

B. Protoxyde or red oxyde of copper; ziegelerz. Crystallizes in the regular octahe dron; specific gravity 5.69; scratches calc-spar; fusible at the blowpipe into the blach oxyde; and reducible in the smolse of the flame to copper; acted upon by nitric acid with disengagement of nitrous gas; solution is rendered blue by ammonia. Its constituents are oxygen $11 \cdot 22$; copper $88 \cdot 78$. It occurs near Chessy, and upon the eastern slope of the Altai mountains.

9. Phosphale of Copper. Dark green; crystallizes in octahedrons; specific gravity 3.6 to 3.8 ; scratches calc-spar; yields water with heat; and affords metallic coppes 
with soda flux; acted on by nitric acid. Its constituents are, phosphoric acid 28.7; oxyde of copper 63.9 ; water $7 \cdot 4$. It occurs at the mines of Libethen in Hungary.

10. Pyri uns Copper; Kupferkies; a metallic looking substance, of a bronze-yellow color, crystallizing in octahedrons which pass into tetrahedrons; specific gravity $4 \cdot 16$; fusible at the blowpipe into beads attractable by the magnet, and which afterwards afford copper with a soda flux; soluble in nitric acid; solution is rendered blue by ammonia, an! affords an abundant precipitate of iron. Its composition is, sulphur 36; copper 34.5 ; iron 30.5 ; being a combined sulphuret of these two metals. This is the most important metallurgic species of copper ores. It occurs chiefly in primitive formations, as among gneiss and mica slate, in veins, or more frequently masses, in very many parts of the world-Cornwall, Anglesea, Wicklow, \&c. It is found among the early secondary rocks, in Shetland, Yorkshire, Mannsfeldt, \&c. The finest crystallized specimens come from Cornwall, Derbyshire, Freyberg, and Saint iMarie-aux-Mines in France.

11. Seleniale of Copper; Berzeline. Is of metallic aspect; silver white; ductile ; fusible at the blowpipe into a gray bead, somewhat malleable; is acted upon by nitric acid; consists of selenium 40 ; copper 64 .

12. Sulphate of Copper; Cyanose. Blue; soluble, \&c. like the artificial sulphates, which see.

Brochantite is a subsulphate of copper, observed in small crystals at Ekaterinenbourg in Siberia.

13. Sulphuret of Copper; Kupferglanz. Of a steel gray metallic aspect; crystallizes in rhomboids; specific gravity 5.69 ; somewhat sectile, yet brittle; fusible with intumescence at the blowpipe, and yields a copper bead with soda; soluble in nitric acid; becomes blue with ammonia, but lets fall scarcely any oxyde of iron. Its constituents are sulphur 19 ; copper 79.5 ; iron 0.75 ; silica 1.00 . It occurs in small quantities in Cornwall, \&c.

The chemical preparations of copper which constitute distinct manufactures are, Blue or Roman vitriol; for which see Sulphate of Copper; Scheele's green and Schweinurth green, Verditer, and Verdigris. See these articles in their alphabetical places.

COPPER, Statistics of.-Copper ores may be imported into Great Britain for smelting, from any country, and under any flag. On arrival of the cargo at Swansea or elsewhere, a bond is given at the Custom-house, which binds the party to return the quantity of copper which the lot of ores shall be ascertained to contain, into bond within a limited period, or pay thereon the duty as foreign copper, which is $27 l$. per ton. The cargo of ore is then weighed out by the custom-house officer, and samples are taken, which are sent to two assay-masters in Cornwall, the highest produce of the two being entered as that of the cargo. This fixes the quantity of copper that must be exported under the bond.

The copper produced from foreign ores must then find a market, as cake or pig copper, in France, Holland, Germany, Italy, the United States of America, \&c. At Calcutta, it is subject to a duty of 6 per cent.; and at Bombay, to a duty of 10 per cent. ad valorem.

The export of British unwrought copper to the continent of Europe, and to the United States of America, was formerly inconsiderable. These countries drew the bulk of their supplies either from the north of Europe, or direct from South America in pig copper. In point of fact, the copper derived from the import of foreign ores for smelting has produced for itself a new market, as the following table, taken from the official returns, will show.

Export of unwrought copper from Great Britain to all parts, except Asia :-

\begin{tabular}{ccc} 
Years ending January 5, & 1830 & 881 tons. \\
- & 1831 & $857-$ \\
- & 1832 & $1326-$ \\
- & 1833 & $2471-$ \\
- & 1834 & $2523-$ \\
- & 1835 & $3267-$ \\
- & 1836 & $4083-$ \\
\hline
\end{tabular}

In the last year, that ended with 5th January, 1838, the export of unwrought copper was about 5000 tons.

Let any candid and practical man consider attentively this table, and compare it with the import of foreign ores for the same period, and with the gradual advance in the value of copper; and then let him, if he can, avoid the conclusion that the admission of oreign ores for smelting was a great boon conferred upon the British copper mines, for it made this country what it now is, the regulator and distributor of the copper produce of the world-the country to which all others consuming and not producing copper, must look for a regular, certain, and economical supply. We want the admission merely under proper and safe regulations, of foreign copper for refining, to draw to this country 
he whole supply of copper for the world, by which prices would be regulated and main tained, and our copper-mining interests put beyond the reach of successful rivalry.

This country did not furnish any supply of unwrought copper to the continent of Europe, or to the United States of America, which was worthy of notice, before the yeas 1830 ; in fact, previous to that time, we imported considerable quantities of foreign copper for re-exportation to India. It is easy to explain how the produce of foreign ores, being prohibited from export in any other shape, has, in fact, opened for itself a new debouché, and this is illustrated by the table, showing the growth of the export of unwrought copper from 1830. To prove that this is not merely a simultaneous advance in the export of all sorts of copper, a corrected table is subjoined from the nfficial returns. comprising the whole export, and divided so as to illustrate the operation of the coppes produce of foreign ores upon our foreign. copper trade.

\section{Copper exported :-}

\begin{tabular}{|c|c|c|c|c|c|}
\hline \multirow{2}{*}{\multicolumn{2}{|c|}{ Years ending }} & \multirow{2}{*}{$\begin{array}{l}\text { Wrought. } \\
\text { To all parts. }\end{array}$} & \multicolumn{2}{|c|}{ Unwrought. } & \multirow{2}{*}{$\frac{\text { Total. }}{\text { To all parts. }}$} \\
\hline & & & To India. & To all parts. & \\
\hline $\begin{array}{r}\text { 5th January, } 1825 \\
1826 \\
1827 \\
1828 \\
1829 \\
1830 \\
1831 \\
1832 \\
1833 \\
1834 \\
1835 \\
1836 \\
1837\end{array}$ & 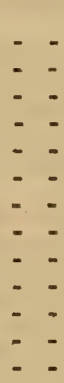 & $\begin{array}{c}\text { Tons. } \\
- \\
- \\
- \\
- \\
- \\
5327 \\
6172 \\
5171 \\
5855 \\
5417 \\
4787 \\
5948 \\
6105\end{array}$ & $\begin{array}{c}\text { Tons. } \\
- \\
- \\
- \\
- \\
- \\
1801 \\
2317 \\
2423 \\
2312 \\
1769 \\
2104 \\
1993 \\
1588\end{array}$ & $\begin{array}{c}\text { Tons. } \\
960 \\
{ }^{\frac{1}{2}} \\
130 \\
1329 \\
1079 \\
2682 \\
3150 \\
3714 \\
4569 \\
4019 \\
5283 \\
5935 \\
3909\end{array}$ & $\begin{array}{r}8,009 \\
9,322 \\
8,885 \\
10,424 \\
9,436 \\
10,072 \\
11,883 \\
10,014^{*}\end{array}$ \\
\hline
\end{tabular}

Production of Copper in Great Britain:

\begin{tabular}{|c|c|c|}
\hline Years. & Ores. & Metal. \\
\cline { 2 - 3 } & Tons. & Tons. \\
$1771-1781$ & 28,185 & 3380 \\
$1781-1791$ & 32,854 & 4123 \\
$1791-1801$ & 48,034 & 4083 \\
$1801-1811$ & 67,533 & 6060 \\
$1811-1816$ & 78,237 & 7181 \\
1816 & 83,058 & 7045 \\
1817 & 75,016 & 6608 \\
1818 & 80,525 & 8714 \\
1819 & 92,234 & 7214 \\
1820 & 92,672 & 7364 \\
1821 & 98,803 & 8163 \\
1822 & 106,723 & 9331 \\
1826 & 128,459 & - \\
1827 & - & 12,381 \\
1828 & 153,600 & 12,169 \\
1829 & - & 11,994 \\
1830 & - & 13,097 \\
1831 & - & 14,480 \\
1832 & - & $14,463 \dagger$ \\
\hline
\end{tabular}


COPPER.

Quantity of Copper produced in the several districts of Grear Britain and Ireland :-

\begin{tabular}{|c|c|c|c|c|c|}
\hline With Ores from- & 1828. & 1829. & 1830. & 1831. & 1832. \\
\hline Cornwall - & $\begin{array}{l}\text { T'ons. } \\
1966\end{array}$ & $\begin{array}{l}\text { Tons. } \\
9763\end{array}$ & $\begin{array}{r}\text { Tons. } \\
10,890\end{array}$ & $\begin{array}{r}\text { Tons. } \\
12,218\end{array}$ & $\begin{array}{r}\text { Tons. } \\
12,099\end{array}$ \\
\hline Devonshire - - & 434 & 318 & 368 & 312 & 249 \\
\hline Other parts of England - & 71 & 36 & 10 & 31 & 42 \\
\hline Island of Anglesea - - & 738 & 901 & 815 & 809 & 852 \\
\hline Other parts of Wales - & 259 & 172 & 237 & 123 & 237 \\
\hline Ireland - - - - & 706 & 790 & 768 & 972 & 974 \\
\hline Isle of Man - - & - & 4 & 9 & 15 & 12 \\
\hline $\begin{array}{l}\text { Total copper from the ores } \\
\text { of the United Kingdom - } \\
\text { Copper smelted from foreign }\end{array}$ & 12,169 & 11,994 & 13,097 & 14,480 & 14,465 \\
\hline ores - - - & - & 30 & 124 & 100 & 56 \\
\hline General total - & 12,169 & 12,024 & 13,221 & 14,580 & 14,521 \\
\hline
\end{tabular}

Statistics of Copper for Cornwall in 1837.-The total quantity of ore sold was 142,089 sons (of 21 civts.), yielding an average produce of eight per cent.; the quantity of fine eopper being 11,209 tons $1 \mathrm{cwt}$; and the average price of the ore $5 l .15 \mathrm{~s} .6 \mathrm{~d}$.; the sotal amount of the sales for the twelve months being 822,516l. The standard upon the 5 th of January was $127 \mathrm{l} .16 \mathrm{~s}$; this was the highest for the year. Upon the $22 \mathrm{~d}$ of June it was at the lowest, being only $93 l$. 18s. It went up again to $120 l$. 10s. upon the 5 th of October; but declined with some slight fluctuation to 107l. 18s. upon the 28th of December. The largest quantity sold at any one ticketing was 4670 tons, upon the 4th of May: and the smallest 1088, upon the 17th of August. The highest produce was nine and five eighths per cent. upon the 13th of July; and the lowest, seven, upon the 26th of January. The greatest weekly total was $25,887 \mathrm{l}$., upon the $2 \mathrm{~d}$ of November; and the least $5694 l$., upon the 17 th of August. The average sum per week wa.s $15,817 l . *$

Table of the produce of Copper Ores and fine Metal in Cornwall, from 1800 to 1830.

\begin{tabular}{|c|c|c|c|c|c|c|c|}
\hline Years. & Ores. & Metal. & Value of & f Ore & & Metal. & Average Standard. \\
\hline & Tons of $21 \mathrm{Cwts}$. & Tons. Cwt. & & & $d$ & Per Cent. of Ore. & Price per Ton. \\
\hline 1800 & 55,981 & $\begin{array}{ll}5187 & 0\end{array}$ & 550,925 & $\ddot{0}$ & 0 & $9 \frac{1}{4}$ & $\begin{array}{lll}133 & 3 & 6\end{array}$ \\
\hline 1801 & 56,611 & $5268 \quad 0$ & 476,331 & 0 & 0 & $9 \frac{1}{4}$ & $\begin{array}{lll}117 & 8 & 0\end{array}$ \\
\hline 1802 & 53,937 & $5228 \quad 15$ & 445,094 & 0 & 0 & $9 \frac{5}{8}$ & $110 \quad 18 \quad 0$ \\
\hline 1804 & 64,637 & $5374 \quad 18$ & 507,840 & 11 & 0 & $8 \frac{3}{8}$ & $\begin{array}{lll}136 & 5 & 0\end{array}$ \\
\hline 1805 & 79,269 & 686310 & 730,845 & 6 & 0 & $8 \frac{5}{8}$ & $\begin{array}{lll}138 & 5 & 0\end{array}$ \\
\hline 1308 & 67,867 & $6795 \quad 13$ & 495,303 & 10 & 0 & $10^{8}$ & 10070 \\
\hline 1810 & 66,048 & 568219 & 570,035 & 8 & 0 & $8 \frac{1}{2}$ & $\begin{array}{lll}132 & 5 & 0\end{array}$ \\
\hline 1812 & 71,547 & 67207 & 549,665 & 6 & 0 & $9 \frac{3}{8}$ & $\begin{array}{lll}111 & 0 & 0\end{array}$ \\
\hline 1814 & 74,322 & $6369 \quad 13$ & 627,501 & 10 & 0 & $8 \frac{1}{2}$ & $\begin{array}{lll}130 & 120\end{array}$ \\
\hline 1816 & 77,334 & $6697 \quad 4$ & 447,959 & 17 & 0 & $8 \frac{2}{\frac{5}{3}}$ & $\begin{array}{lll}98 & 13 & 0\end{array}$ \\
\hline 1818 & 86,174 & $6849 \quad 7$ & 686,005 & 4 & 0 & $7 \frac{8}{8}$ & $134 \quad 15 \quad 0$ \\
\hline 1820 & 91,473 & $7508 \quad 0$ & 602,441 & 12 & 0 & $8 \frac{1}{8}$ & 113150 \\
\hline 1822 & 104,523 & 91408 & 663,085 & 13 & 0 & $8 \frac{3}{4}$ & 10400 \\
\hline 1824 & 99,700 & $7823 \quad 15$ & 587,178 & 0 & 0 & $7 \frac{4}{8}$ & $110 \quad 0 \quad 0$ \\
\hline 1826 & 117,308 & $9026 \quad 12$ & 788,971 & 15 & 0 & $\begin{array}{l}18 \\
7 \frac{5}{8}\end{array}$ & $123 \quad 3 \quad 0$ \\
\hline 1828 & 130,366 & $9921 \quad 1$ & 756,174 & 16 & 0 & $7 \frac{8}{8}$ & 11270 \\
\hline 1829 & 124,502 & $\begin{array}{ll}9656 \quad 10\end{array}$ & 717,334 & 0 & 0 & $7 \frac{3}{4}$ & $10914 \quad 0$ \\
\hline 1830 & 143,296 & 11,22419 & 887,900 & 0 & 0 & $7 \frac{\frac{3}{4}}{4}$ & $114 \quad 4 \quad 0$ \\
\hline $\begin{array}{l}1834 \\
1835\end{array}$ & $\{150,617$ & $12,271 \quad 14$ & 893,402 & 15 & 0 & $8 \frac{1}{8}$ & 106110 \\
\hline
\end{tabular}

* Mining Review, Feb. 28, 1838. 
Produce of Copper Mines in Cornwall, (on the authority of John Taylor, Esq.,F.R.S.)

\begin{tabular}{|c|c|c|c|c|c|c|}
\hline Years. & Orè. & Metal. & Value. & & Produce. & Standard \\
\hline 1831 & $\begin{array}{l}\text { Tons. } \\
144,402\end{array}$ & $\begin{array}{c}\text { Tons. } \\
12,044\end{array}$ & $\stackrel{f}{f}{ }^{s}$ & $\begin{array}{l}d \\
6\end{array}$ & $\begin{array}{c}\text { Per Cwt } \\
8 \frac{1}{4}\end{array}$ & 100 \\
\hline 1832 & 137,357 & 11,948 & 825,612 & 0 & $8 \frac{5}{8}$ & 100 \\
\hline 1833 & 138,300 & 11,191 & 858,70810 & 0 & $8 \frac{1}{8}$ & 111 \\
\hline 1834 & 143,296 & 11,226 & 887,902 & 0 & $7 \frac{3}{4}$ & 114 \\
\hline 1835 & 150,617 & 12,270 & $893,402 \quad 1$ & 0 & $8 \frac{1}{8}$ & 106 \\
\hline 1836 & 140,981 & 11,647 & 957,752 & 6 & $8 \frac{1}{4}$ & 115 \\
\hline 1837 & 140,753 & 10,832 & 908,613 & 0 & $7 \frac{5}{8}$ & 120 \\
\hline
\end{tabular}

An account of the quantities of Foreign wrought and unwrought Copper, and Copper Ore imported and exported, and of British wrought and unwrought Copper exported from the United Kingdom; together with the quantities and value of Copper Ore smelted in Cornwall and Swansea, and the quantity of Copper produced in those places; and in the county of Devon; together with the market prices of sheet and cake Copper, in the year ending 5th January, 1835.

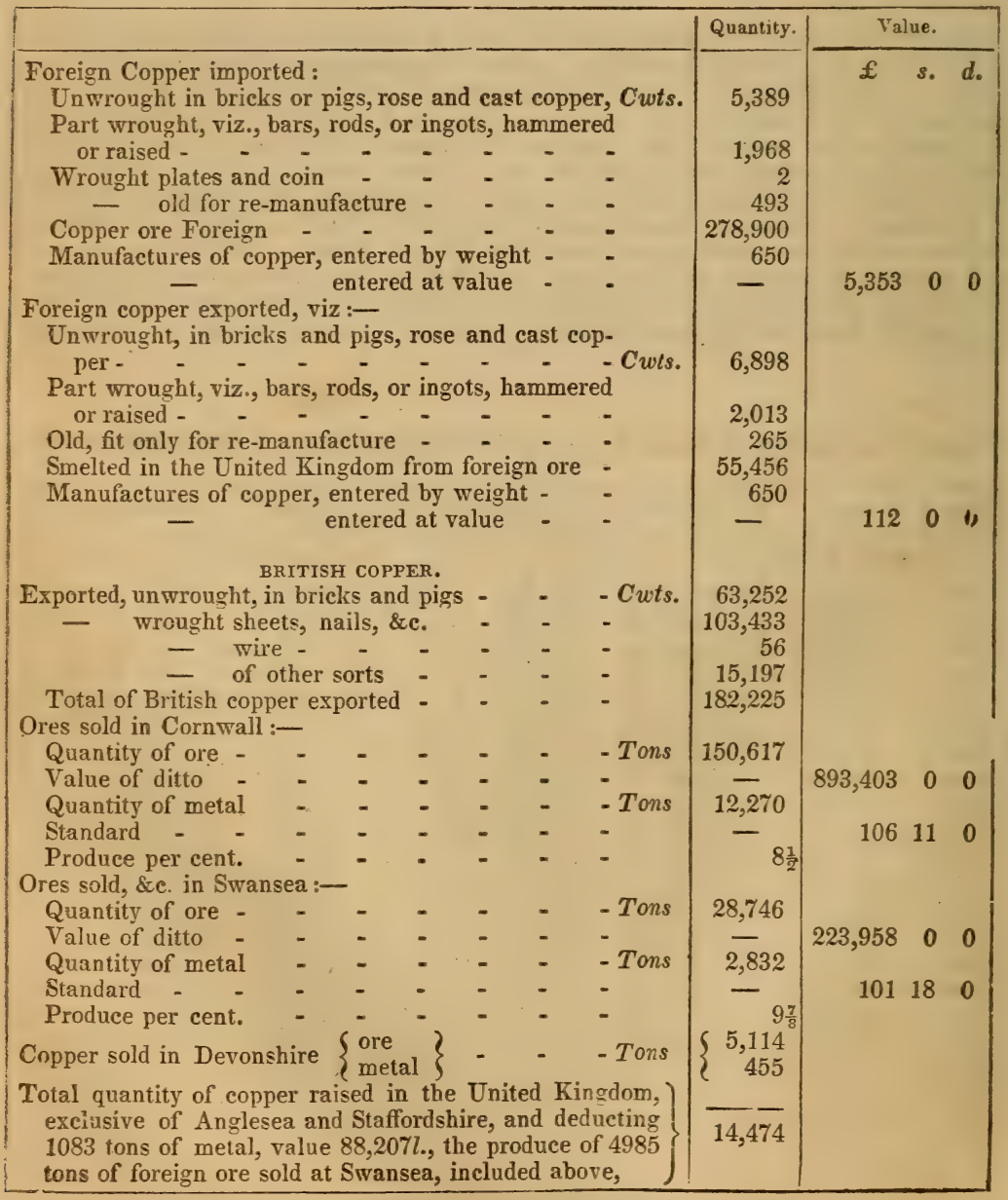


COPPERAS. (Couperose verte, Fr.; Eisenvitrol, Germ.) Sulphate of iron.

CORAL (Corail, Fr.; Koralle, Germ.) is a calcareous substance, formed iny a species of sea polypus, which constructs in concert immense ramified habitations, consisting of an assemblage of small cells, each the abode of an animal. The coral is therefore a real polypary, which resembles a tree stripped of its leaves. It has no roots, but a foot not unlike a hemispherical scull-cap, which applies closely to every point of the surface upon which it stands, and is therefore difficult to detach. It merely serves as a basis or support to the coral, but contributes in no manner to its growth, like the root of an ordinary tree; for detached pieces have been often found at the bottom of the sea in a state of increase and reproduction. From the above base a stem usually single proceeds, which seldom surpasses an inch in diameter, and from it a small number of branches ramify in very irregular directions, which are studded over with cells, each containing an insest. The polypi, when they extend their arms, feelers, or tentacula, resemble flowers, whence, as well as from the form of the coral, they were classed among vegetable productions. They are now styled zoophites by the writers upon Natural History.

The finest coral is found in the Mediterranean. It is fished for upon the coasts of Provence, and constitutes a cunsiderable branch of trade at Marseilles. The coral is attached to the submarine rocks, as a tree is by its roots, but the branches, instead of growing upwards, shoot downwards towards the bottom of the sea; a conformation favorable to breaking them off and bringing them up. For this kind of fishing, eight men, who are excellent divers, equip a felucca or small boat, called commonly a coralline. They carry with them a large wooden cross, with strong, equal, and long arms, each bearing a stou oag-net. They attach a strong rope to the middle of the cross, and let it down horizontally into the sea, having loaded its centre with a weight sufficient to sink it. The diver follows the cross, pushes one arm of it after another into the hollows of the rocks, so as to entangle the coral in the nets. Then his comrades in the boat pull up the cross and its accompaniments.

Coral fishing is nearly as dangerous as pearl fishing, on account of the number of sharks which frequent the seas where it is carried on. One would think the diring-bell in its now very practicable state might be emploved with great advantage for both purposes.

Coral is mostly of a fine red color, but occasionally it is flesh-colored, yellow, or white. The red is preferred for making necklaces, crosses, and other female ornaments. It is worked up like precious stones. See LAPIDARY.

CORK (Lige, Fr.; Kork, Germ.) is the bark of the quercus liber, Linn., a species of oak-tree, which grows abundantly in the southern provinces of France, Italy, and Spain. The bark is taken off by making coronal incisions above and below the portions to be removed; vertical incisions are then made from one of these circles to another, whereby the bark may be easily detached. It is steeped in water to soften it, in order to be flattened by pressure under heavy stones, and next dried at a fire which blackens its surface. The cakes are bound up in bales and sent into the market.

There are two sorts of cork, the white and the black; the former grows in France and the latter in Spain. The cakes of the white are usually more beautiful, more smooth, lighter, freer from knots and cracks, of a finer grain, of a yellowish gray color on both sides, and cut more smoothly than the black. When this cork is burned in close vessels it forms the pigment called Spanish black.

This substance is employed to fabricate not only bottle corks, but small architectural and geognostic models, which are very convenient from their lightness and solidity.

The cork-cutters divide the boards of cork first into narrow fillets, which they after wards subdivide into short parallelopipeds, and then round these into the proper conical or cylindrical shape. The bench before which they work is a square table, where 4 workmen are seated, one at every side, the table being furnished with a ledge to prevent the corks from falling over. The cork-cutter's knife is a broad blade, very thin, and fine edged. It is whetted from time to time upon a fine-grained dry whetstone. The workman ought not to draw his knife edge over the cork, for he would thus make misses, and might cut himself, but rather the cork over the knife edge. He should seize the knife with his left hand, rest the back of it upon the edge of the table, into one of the notches made to prevent it from slipping, and merely turns its edge sometimes upright and sometimes to one sile. Then holding the squared piece of cork by its two ends, between his finger and his thumb, he presents it in the direction of its length to the edge ; the cork is now smoothly cut into a rounded form by being dexterously turned in the hasd. He next cuts off the two ends, when the cork is finished and thrown into the proper basket alongside, to be afterwards sorted by women or boys.

Of late years a much thicker kind of cork boards have been imported from Catalonia, from which longer and better corks may be made. In the art of cork-cutting the French surpass the English, as any one may convince himself by comparing the corlis of their champagne bottles with those made in this country.

Cork, on account of its buoyancy in water, is extensively employed for making floats 
to fishermen's nets, and in the construction of life-boats. Its impermeability to water has led to its employment for inner soles to shoes.

When cork is rasped into powder, and subjected to chemical solvents, such as alcohol, \&c., it leaves 70 per cent. of an insoluble substance, called suberine. When it is treated with nitric acid, it yields the following remarkable products: - White fibrons matter $0 \cdot 18$, resin $14 \cdot 72$, oxalic acid $16 \cdot 00$, suberic acid (peculiar acid of cork) 14.4 in 100 parts.

Michine cork-culting. - A patent was obtained some years ago by Sarah Thomson for this purpose. The cutting of the cork into slips is effected by fixing it upon the sliding bed of an engine, and bringing it, by a progressive motion, under the action of a circular knife, by which it is cut into slips of equal widths. The nature or construction of a machine to be used for this purpose may be easily conceived, as it possesses no new mechanical feature, except in its application to cutting cork. The motion communicated to the knife by hand, steam, horse, or other power, moves at the same time the bed also. which earries the cork to be cut.

The second part of the invention, viz., that for separating the cork into square pieces, after it has been cut into slips as above, is effected by a moving bed as before, upon which the slips are to be placed and submitted to the action of a cutting lever, which may be regulated to chop the cork into pieces of any given length.

The third part of the invention, viz., that for rounding or finishing the corks, consists of an engine to which is attached a circular knife that turns vertically, and a carriage or frame upon its side that revolves upon an axle horizontally.

This carriage or frame contains several pairs of clamps, intended respectively to hold a piece of the square cut cork by pressing it at the ends, and carrying it lengthways perpendicularly; which clamps are contrived to have a spindle motion, by means of a pinion at the lower end of their axles, working into a spur-wheel.

The machinery, thus arranged, is put in motion by means of bands and drum-wheels, or any other contrivance which may be found most eligible; and at the same time that the circular knife revolves vertically, the frame containing the clamps with the pieces of eork, turns horizontally, bringing the corks, one by one, up to the edge of the knife, when, to render each piece of cork cylindrical, the clamps, as above described, revolve upon their axes, independently of their carriage, by which means the whole circumference of the cork is brought under the action of the knife, the superfluous parts are uniformly pared off, and the cork is finished smooth and cylindrical.

CORROSIVE SUBLIMATE; bichloride of mercury.

CORUNDUII ; or Telesie; a very hard genus of aluminous minerals, to which the gems, sapphire, ruby, salamstein, and adamantine spar belong.

COTTON DYEING. (Teinture de Coton, Fr.; Baumwollenfärberei, Germ.) Cotton and linen yarns and cloths have nearly the same affinity for dyes, and may therefore with propriety be treated, in this respect, together. After they have acquired the proper degree of whiteness (see BLEAcHING), they are still unfit to receive and retain the dyes in a permanent manner. It is necessary, before dipping them into the dye-bath, to give them a tendency to condense the coloring particles within their cavities or pores, and to communicate such chemical properties as will fix these particles so that they will not separate, to whatever ordinary trial they may be subjected. All the colors which it would be desirable to transfer to these stuffs unfortunately do not possess this permanence. Men of science engaged in this important art have constantly aimed at the discovery of some new processes which may transfer into the class of fast colors those dyes which are at present more or less fugitive. Almost all the goods manufactured of cotton, flax, or hemp, are intended to be washed, and ought, therefore, to be so dyed as to resist the alkaline and soapy solutions commonly used in the laundry. Vitalis distinguished dyed cottons into three classes; 1 . the frigitive, or fancy-colored (pe'it teint), which change their hue or are destroyed by one or two boils with soap ; 2. those which resist five or six careful washings with soap, are good dyes, (bon teint); and those which were still more durable, such as Turkey reds, may be called fast colors (grand tein'). The colors of Brazil-wood, logwood, annotto, safflower, \&c., are fugilive; those made with madder without an oily base, are good; and those of madder with an oily mordant, are fast. It is, however, possible to point out certain processes for giving these different orders of dyes a greater degree of fixity.

I shall describe, in the five following paragraphs, the operations conducive to the fixation of eolors upon cotton and linen.

1. Galling. Either gall-nuts alone, or sumach alone, or these two substances united, are employed to give to cotton the fast dye preparation. 2 or 3 ounces of galls for every pound of cotton, being coarsety pounded, are to be put into a copper containing about 30 gallons of water for every 100 pounds of cotton, and the bath is to be boiled till the bits of galls feel pasty between the fingers. The fire being withdrawn, when the bath becomes moderately cool, it is passed through a hair-cloth sieve. If during this operation the 
liquor should become cold, it must be made once more as hot as the hand can bear. $\Lambda$ portion of it is now transferred into another vessel, called a back, in which the cotton is worked till it be well penetrated with the decoction. It is then taken out, wrung at the peg or squeezed in a press, and straightway hung up in the drying-house. Some more of the fresh decoction being added to the partially exhausted liquor in the back, the pro cess is resumed upon fresh goods.

The manipulation is the same with sumach, but the bath is somewhat differently made; because the quantity of sumach must be double that of galls, and must be merely infused in very hot water, without boiling. When galls and sumach are both prescribed, their baths should be separately made and mixed together.

2. Aluming. Alum is a salt which serves to prepare cotton for receiving an indefinite variety of dyes. Its bath is made as follows: For 100 pounds of scoured cotton, about 30 gallons of water, being put into the copper, are heated to aboi $-22^{\circ} \mathrm{F}$., when 4 ounces of alum, coarsely pounded, are thrown in for every pound of cotton, and instantly dissolved. Whenever the heat of the bath has fallen to about $98^{\circ} \mathrm{F}$., the cotton is well worked in it, in order that the solution may thoroughly penetrate all its pores. It is then taken out, wrung at the peg or squeezed in the press, and dried in the shade. The solution of alum is of such constant employment in this kind of dyeing, that it should be made in large quantities at a time, kept in the alum tun, where it can suffer no deterioration, and drawn off by a spigot or stop-cock as wanted.

There are certain colors which require alum to be deprived of a portion of its acid excess, as a supersalt; which may be done by putting 1 ounce of crystals of soda into the tun for every pound of alum. But so much soda should never be used as to cause any permanent precipitation of alumina. When thus prepared, it is called saturated alum, though it is by no means neutral to litmus paper; but it crystallizes differently from ordinary alum.

Cotton does not take up at the first aluming a sufficient quantity of alum; but it must receive a second, or even a third immersion. In every case the stuff should be thoroughly dried, with an interval of one or two days between each application; and it may even be left for 10 or 12 hours moist with the alum bath before being hung in the air. When the cotton is finally dry, it must be washed before being plunged into the dye bath; otherwise, the portion of alum not intimately combined with the cotton, but adhering externally to its filaments, would come off by the heat, mix with the bath, alter the color by dissolving in it, and throw it down to the bottom of the copper, in the form of a lake, to the great loss of the dyer. Madder reds, weld yellows, and some other colors are more brilliant and faster when acetate of alumina, prepared with acetate of lead, alum, and a little potash, is used, than even saturated alum. This mordant is employed cold, and at $4^{\circ}$ Baumé.

3. Mordants. See this article in its alphabetical place.

4. Dye baths are distinguished into two classes; the coloring bath, and the dyeing bath. The former serves to extract the coloring matters of the different substances with the exception of madder, which is always used in substance, and never as vr extract, infusion, or decoction. In all these cases, when the color is extracted, that is when the dye bath is completed by the degree of heat suited to each substance, it is tnen allowed to cool down a certain way, and the cotton is worked or winced through ii, wo get the wishedfor tint. This is what is called the dye bath. Several coloring batns are made in the cold; and they serve to dye also in the cold; but the greater part require a heat of $90^{\circ}$ or $100^{\circ}$ to facilitate the penetration of the stuffs by the coloring particles. The description. of the several dye baths is given under the individual dyes.

5. Of the washing after the dyeing.-The washing of the cottons after they have received the dyes, is one of the most important operations in the business. If it is not carefully performed, the excess of color not combined with the fibres is apt to stain whatever it touches. This inconvenience would be of little consequence, if the friction carried off the color equally from all the points ; but it does not do so, and hence the surface appears mottled. A well-planned dye house should be an oblong gallery, with a stream of water flowing along in an open conduit in the middle line, a series of dash-wheels arranged against the wall, at one side, and of dyeing coppers, furnished with self-acting winces or reels against the other. In such a gallery, the washing may be done either by hand, by the rinsing machine, or by the dash-wheel, according to the quality of the dye, and the texture of the stuffs. And they may be stripped of the water either by the jack and pin, by the squeezing roller, or by the press. Wooden pins are placed in some dye houses on each side of the wash cistern or pool. They are somewhat conical, $1 \frac{1}{2}$ foot high, $3 \frac{1}{2}$ inches in diameter at the base, $1 \frac{1}{2}$ at the top, are fixed firmly upright, and at a level of about 3 feet above the bottom of the cistern, so as to be handy for the workmen. See Brazil-wood, Fustic, Madder, Black Dye, Brown Dye, \&c., as also Bleaching, Bran, Calico Printing, Dunging, Dyeing, \&c.

COTTON MANUFACTURE. (Filature de Coton, Fr.; Baumwollenspinnerie, 
Germ.) Cotton is a filamentous down, which invests tre seeds of the plant called gas. sypium by Linnæus, and placed by him in the class monadelphia, and order monanilria, but belonging to the natural family of mulvacee. It has a cup-shaped calyx, obtusely five-toothed, enclosed in a thiee-cleft exterior calyx; the leaflets are united at their base, of a heart shape, and toothed; stigmas three to five; capsule three to five celled and many seedted; seeds bearing a downy wool. Thirteen species are deseribed by Decandolle, but their characters are very uncertain, and no botanist can assign to a definite species of the plant, the very dissimilar staples of the cotton filaments found in commerce. The leaves are generally palmate and hairy; and the blossoms are large, and of a beautiful yellow. The gossypium religiosum of Tranquebar has white blossoms in some of its varieties, to which probably the white cotton of Rome, cultivated in the Jardin des Plantes at Paris, helongs. The filaments difter in length, flexibility, tenacity, and thick ness, in different cottons, whence the great differences of their value to the cotton-spinner, as the prices current in the market show. Thus, at Liverpool, on the 1st of December, 1835, the following values were assigned to the following cottons:-

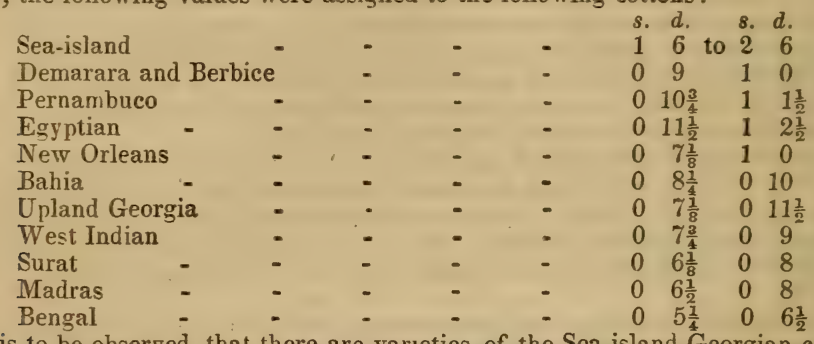

But it is to be observed, that there are varieties of the Sea-island Georgian cotton, so highly prized by the spinner of fine yarn, as to fetch $3 s ., 4 s$., or even $5 s$. per pound.

The filaments of cotton, when examined with a good microscope, are seen to be more or less riband-like, and twisted; having a breadth varying from $\frac{1}{80} 0$ of an inch in the strongest Smyrna or candle-wick cotton of the Levant, to $\frac{1}{2500}$ of an inch in the finest
Sea-island.

The main distinction between cottons in the pod, is that of the black seeded and the green seeded; for the former part with their downy wool very readily to a pair of simple rollers, made to revolve nearly in contact, by the power of the human arm; while the latter retain the wool with much force, and require to be ginned, as the operation is called, by a powverful revolving circular saw-mechanism, usually driven by horse or water power. After the cotton wool is thus separated from the seeds, it is packed in large canvass bags, commonly with the aid of a screw or hydraulic press, into a very dense bale, for the convenience of transport. Each of the American bags contains about 340 lbs. of cotton wool. When this cotton is delivered to the manufacturer, it is so foul and flocky, that he must clean and disentangle it with the utmost care before he can subject it to the carding operation.

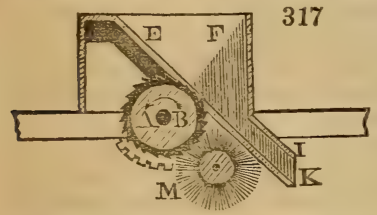

Fig. $317, \mathrm{~A} \mathrm{~B}$, is a roller, about 9 inches in diameter, which revolves in the direction of the arrow. This cylinder consists of a parallel series of oblique pointed circular saws made fast to one axis; and parted from each other by wooden rings nearly one inch and a half in thickness. Above the cylinder, is a kind of hopper E F, into which the ginner throws the seed cotton, which falls upon a grating, up through which small segments of the sarv-teeth project, so as to lay hold of the fibres in their revolution, and pull them through, while the seeds, being thus separated, roll down the slope of the grid, to be discharged from the spout $\mathbf{I} \mathbf{K}$. $M$ is a cylindrical brush placed below the grating, which revolves against the saw-teeth, so as to clear them of the adhering cotton filaments

The willow, which was originally a cylindrical willow basket, whence its name, but is now a box made of wood, with revolving iron spikes, is the first apparatus to which cotton wool is exposed, after it has been opened up, picked, and sorted by hand or a rake, in what is called a bing. The willow exercises a winnowing action, loosens the large flocks, and shakes out much of the dirt contained in them. The frame of the willow is about 2 feet wide, and turns with its spikes at the rapid rate of 600 revolutions per minute, whereby it tosses the cotton about with great violence. The heavy impurities fall down through the grid bottom. It is exposed, however, for only a few minutes to the action of this machine. For factories, which work up chiefly the coarser and fouler cottons of India, and Upland Georgia, the conical self-acting willow, as 
sonstructed by Mr. Lillie at Manchester, is much employed. In it, the cotton is put in at the narrow end of the truncated cone, which, being spiked, and revolving rapidly within a nearly concentric case upon a horizontal axis, wafts it on towards the wide end, while its impurities are partly shaken out through the grid or perforated bottom, and partly sucked up through revolving squirrel wire cages, by the centrifugal action of a fan. This is a powerful automatic engine, deserving the study of the curious, and is as safe as it is powerful. The cone of this huge machine makes from 400 to 600 turns per minute, and will clean 7200 pounds, or 24 bags, in a day.

After shaking out the grosser impurities by the willow, the cotton spinner proceeds to separate each individual filament of cotton wool from its fellow, so as to prepare it for carding, and to free it from every particle of foreign matter, whether lighter or heavier than itself. This second operation is performed by what are called batting (beating), scutching, and blowing machines, which are all now much the same, whatever difference of signification the name may have. Indeed, each machine not only

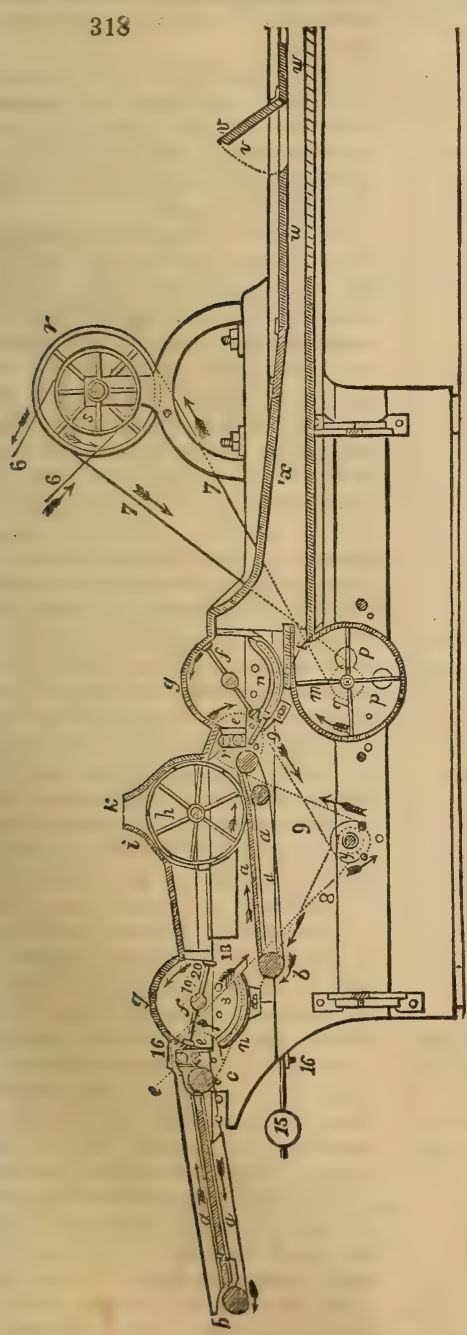
beats, scutches, bit blows. Fig. 318 exhibits a longitudinal section of a good blowing engine of modern construction. The machine is about 18 or 19 feet long, and three feet across within the case. The whole frame is made of cast-iron, lined with boards, forming a close box, which has merely.openings for introducing the raw cotton wool, for taking out the cleansed wool, and removing the dust as it collects at the bottom. These doors are shut during the operation of the machine, but may be opened at pleasure, to allow the interior to be inspected and repaired.

The introduction of the cotton is effected by means of an endless cloth or double apron, which moves in the direction of the arrow $a a$, at the left end of the figure, by passing round the continually revolving rollers at $b$ and $c$. The two rollers at $e$, being the ones which immediately introduce the cotton into the jaws, as it were, of the machine, are called the feed rollers. The batting arm, or revolving diameter, $f e$, turns in the direction of the arrow, and strikes the flocks violently as they enter, so as to throw down any heavy particles upon the iron grating or grid at $n$, while the light cotton filaments are wafted onwards with the wind, from the rotation of the scutcher in the direction of arrow $a^{\prime}$, along the second travelling apron, upon which the squirrel cage cylinder presses, and applies the cotton in the form of a lap. Above the cylindric cage $h$, which turns in the direction of its arrow, there is a pipe $k$, the continuation of the case $i$. This pipe, though broken off in the figure, communicates by a branch pipe with an airsucking fan ventilator, not seen in this figure, but explained under Foundry. The cage $h$, by its rotation, presses down, as we have said, the half-cleaned cotton upon the cloth $a^{\prime}$, which carries it forward to the second scutcher $f^{\prime}$, by the second set of feed rollers $e^{\prime}$. The second scutcher throws down the heavy dust upon the second grid $n^{\prime}$, through which it falls upon the bottom of the case. The first scutcher makes about 1280 strokes of each of its two arms in a minute; the second 1300 .

The feed rollers for each are fluted. The feed cloth is either sustained by a board, or is made of parallel spars of wood, to secure it against bagging, which would render the 
delivery of the cotton irregular, The feed rollers make 8 turns in the minute; and as their diameter is $1 \frac{1}{2}$ inches, they will introduce 8 times their circumference, or $37 \cdot 7$ inches of the cotton spread upon the apron in that time. Upon every $12 \mathrm{th}$ part of an inch of the cotton, therefore, nearly 3 blows of the scutcher arm will be applied. The second feed rollers move relatively with more slowness, so that for every $2 \cdot 4$ blows of the scutcher, only one twelfth of an inch of cotton wool is presented.

The fan is enclosed in a cylindrical case. The wings or vanes revolve from 120 to 150 times in the minute; and while they throw the air out with nearly this velocity at their sccentric outlet in the circumference, they cause it to enter, with equal velocity, at the centre. With this centre the squirrel cage is connected by a pipe, as above

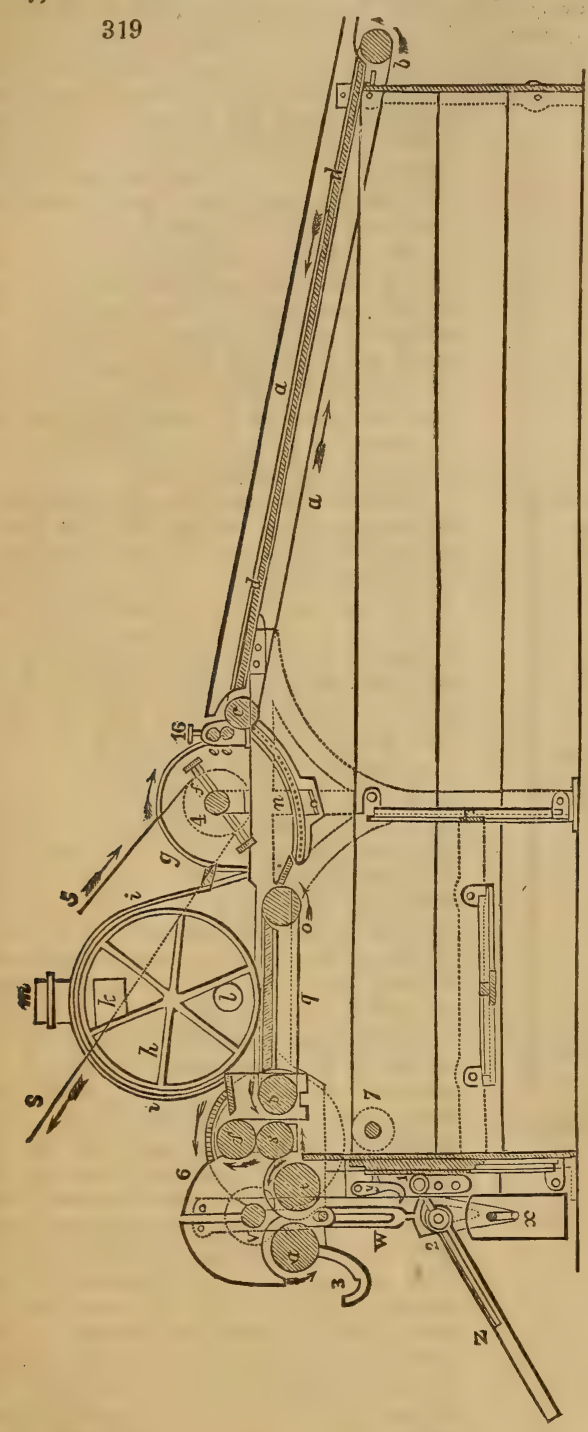

stated. The sound filaments of the cotton are arrested by the sieve surface of the cylindric cage, and nothing but the broken fragments and the light dust can pass through.

The cotton wool in the blowing machine is wafted by the second scutcher into the space $x, w, w$, provided with a fine grid bottom; or it is sometimes wound up there by rollers into a lap.

In fig. 318 an additional ventilator is introduced beneath at $m$, $o, o$, to aid the action of the scutchers in blowing the cotton onwards into the oblong trough $a$. The outlet of that fan is at $t$; and it draws in the air at its axis $q . u$ and $v$ are two doors or lids for removing the cleaned cotton wool. This last fan is suppressed in many blowing machines, as the scutching arms supply a sufficient stream of air. The dotted lines show how the motion is transmitted from the first mover at $s$, to the various parts of the machine. $6^{\prime} 6^{\prime}$ represent the bands leading to the main shafting of the mill. A machine of this kind can clean fully 600 pounds of short-stapled cotton wool in a day, with the superintendence of one operative, ustally a young woman, to distribute the cotton upon the first feed cloth.

The second Blowing machine is usually called a lap machine, because, after blowing and scutching the cotton, as above described, it eventually coils the fleece upon a wooden roller at the delivering end of the apparatus. It is sometimes, also, called a spreading machine. A section of it is shown in fig. 319 . The breadth of this machine is about 3 feet, as the lap formed is prepared for the usual breadth of the breaker cards, namely, 3 feet. Where the cards are only 18 inches broad, the lap machine is also made of

the same breadth. In the figure we see the feed-cloth, the scutching barrel, the squirrel suction, and spreading cage, and the rollers for coiling up the lap. The lever shown below is for removing the pressure weight from the axis of the lap 
rollers, when a full one is to be removed, and replaced by an empty one. $m$, at the top, is the commencement of the pipe which leads to the suction fan, or ventilator. The tnickiness of the lap in this machine must be nicely regulated, as it determines, in a great measure, the grist of the card-ends, and even the rovings. In 12 hours such a lap machine will prepare 650 pounds of cotton.

Fig. 320 is the first scutching machine, now never seen except in the oldest factories. $\mathbf{B}$ is the feed cloth; $\mathrm{G} \mathrm{H}$ and $\mathrm{M} \mathbf{N}$ are the two scutcher frames.

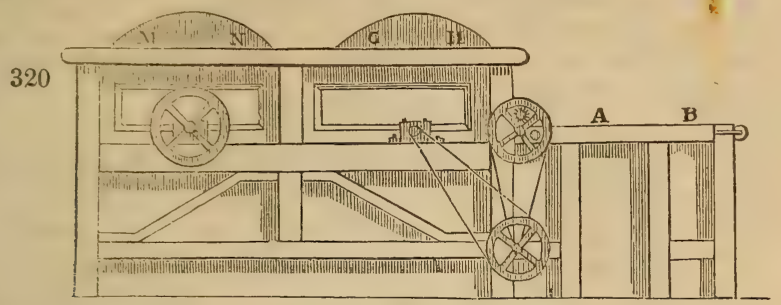

Carding is the next operation in a cotton factory. Cards are destined to disentangle the individual filaments from each other, and to lay them lengthwise, instead of being donbled up and convoluted, as they usually are in leaving the blowing and lap machines. Carding consists in the mutual action of two opposite surfaces, which are studded thick with oblique angled hooks. The wires of which these hooks are made must be very hard drawn in order to render them stiff and elastic. The middle part of the figures shows one of the staples or double teeth, the structure of which has been partly

321

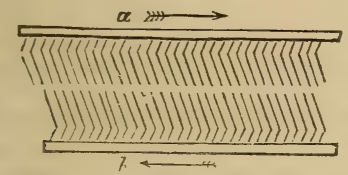

322

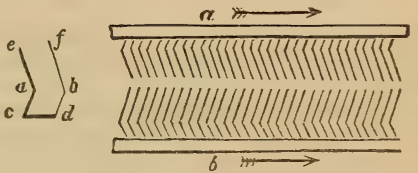

explaineà under CARD. Suppose $a$, fig. 321 , to be a piece of a card fillet, and $b$ to be another piece, each being made fast with pins to a board; the teeth of these two cards are set in opposite directions, but are very near together, and parallel. Now suppose a flock or tuft of cotton placed between two such bristling surfaces. Let $a$ be moved in the direction of its arrow, and let $b$ be moved in the opposite direction, or even let it remain at rest. Every filament of the cotton will be laid hold of by each set of teeth, when their surfaces are thus drawn over each other; the teeth of $a$ will pull them in a forward direction, while those of $b$ will tend to retain them, or to pull them backwards. The loops or doublings will, by both movements, be opened or drawn out, so that the flocks will be converted into rows of parallel filaments, lying alongside or before each other. Each tooth. will secure to itself one or more of them, and by the friction of its sides, as well as the hooks of its points, will draw them to their utmost elongation. Though one stroke of the opposite cards be inadequate to produce this equable arrangement, yet many repeated strokes must infallibly accomplish the end in view, of laying the fibres parallel.

Let us suppose this end effected, and that all the fibres have been transferred to the card $a$, a transterse stroke of $b$ will draw over to it a certain number of them, and indeed at each stroke there will be a new partition between the two cards, with increased parallelism, but still each card will retain a great deal of the cotton. To make one card strip another, the teeth of one of them must be placed in a reverse position, as shown in fig. 322 .

If $a$ be now drawn in the direction of its arrow along the face of $b$, it will inevitably comb out all, or almost all, the filaments from it, since the hooks of $b$ have, in this position, no power of retaining them. Even the doubled fibres or loops will slip over the sloping point of $b$, in obedience to the traction of $\alpha$. By considering these two relative positions of the cards, which take place in hand cards, simply by reversing one of them any person will be able to understand the play of a cylinder card against its flat top, or against another cylinder card, the respective teeth being in what we may call the teasing position of $f \mathrm{~g} .321$; and also the play of a cylinder card against the doffer cylinder, in what may be called the stripping position of fig. 322 .

Cylinder cards, so essential to the continuity and despatch of cotton factory labor, were the ingenious invention of Lewis Paul of Northampton, but were greatly improved and brought into nearly their present operative state by Sir Richard Arkwright. A 
carding engine consists of one or more cylinders, covered with card-leather (sometımes called card cluth), and a set of plane surfaces similarly covered, made to work against each other, but so that their points do not come into absolute contact. Some cards consist entirely of cylinders, the central main cylinder being surrounded by a series of smaller ones called urchins or squirrels. These are used solely for preparing the coarser stapled cotton, and sheep's wool for the wool spinner.

Fig. 323 represents a card of excellent construction, which may be called a breaker and finisher, as it is capable of working up the fleece roll of the lapping machine di rectly into a card-end or riband fit for the drawing machine. In fine spinning mills there are always, however, two cards; one coarser, called a breaker, which turns off

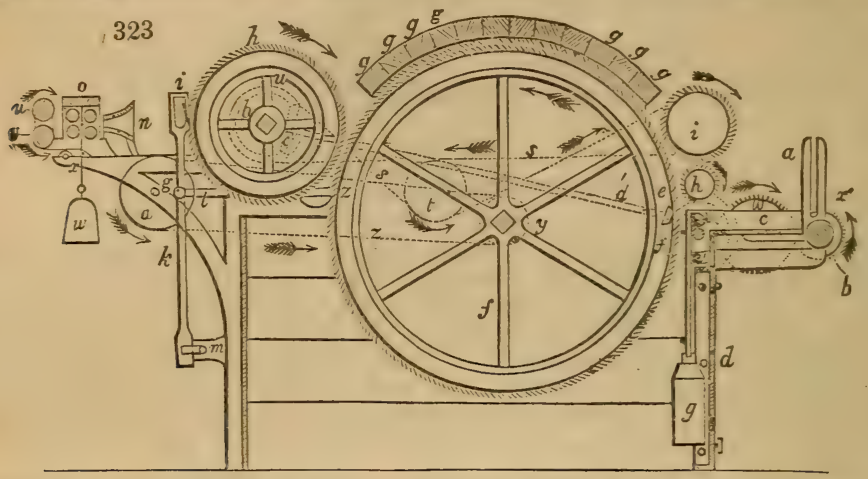

the cotton in a broad fleece of extreme thinness, which is lapped round a cylinder ; and constitutes the material presented to the finisher card, which has teeth of a fines construction.

$a$ is one of the two upright slots, which are fixed at each side of the engine for receiving theiron gudgeons of the wooden cylinders round which the fleece of the lapping machine is rolled. The circumference of this coil rests upon a roller $b$, which is made to turn slowly in such a direction as to aid the unfolding of the lap by the fluted cylinders e. The lap proceeds along the table seen beneath the letter $c$, in its progress to the fluted rollers, which are an inch and one sixth in diameter, and have 28 flutings in their circumference. $g$ is a weight which hangs upon the axis of the upper roller, and causes it to press upon the under one : $f$ is the main card drum; $g g g$, the arch formed by the flat top cards; $h$, the small card cylinder for stripping off the cotton, and therefore called the doffer, as we have said; $i$, the doffer-knife or comb for stripping the fleecy web from the doffer; $k l q \mathrm{~m}$, the lever mechanism for moving these parts. At $d$ there is a door for permitting the tenter to have access to the interior of the engine, and to remove whatever dirt, \&c. may happen to fall into it. In fig. 324 we see the manner of fixing the flat tops $g$ g over the drum; and for making the matter clearer, three of the tops are removed. Upon the arched cast-iron side of the frame, a row of strong iron pins $k$ are made fast in the middle line; and each top piece has, at each of its ends, a hole, which fits down upon two such opposite pins. $l l$ are screws whose heads serve as supports to the tops, by coming into contact with the bottom of the holes, which are not of course bored through the wood of the tops. By turning the heads of these screws a little the one way or the other, the pins may be lengthened or shortened in any degree, so as to set the tops rery truly in adjustment with the drum teeth revolving beneath them. $\quad h^{\prime}$ is the small runner or urchin, and $i^{\prime}$ the large runner; both of which are spirally covered from end to end with narrow card fillets in the same manner as the doffer. The main drum is on the contrary covered with card cloth, in strips laid on parallel to its axis, with interjacent parallel smooth leather borders. The teeth of these sereral cards are set as represented in the figure, and their cylinders revolve as the arrows indicate. The runners as well as the doffer cylinder may be set nearer to or farther from the $\operatorname{drum} f$; but the screws intended for this adjustment are omitted in the draw. ings, to avoid confusion of the lines.

The card-end or fleece taken off the doffer $h$ by the crank and comb mechanism $i k m$, passes through the tin plate or brass funnel $n$, fig. 323 , whereby it is hemmed in and contracted into a riband, which is then passed through between a pair of drawing rollers 0 . It is next received by the rollers $u v$, which carry it off with equable velocity, and let it fall into the tin cans placed below, or conduct it over a friction pulley, to be wound along with many other card-ends upon a lap roller or large bobbin. The latter mechanism is not shown in this figure. A sloping curved tin or brass plate, channelled or 
ridged along its surface, conducts the card ribands separately; there are two smooth iron rollers for condensing the several ribands, and a wooden pin round which the ribands are lapped, resting between two leather-covered rollers, onc of which receives motion

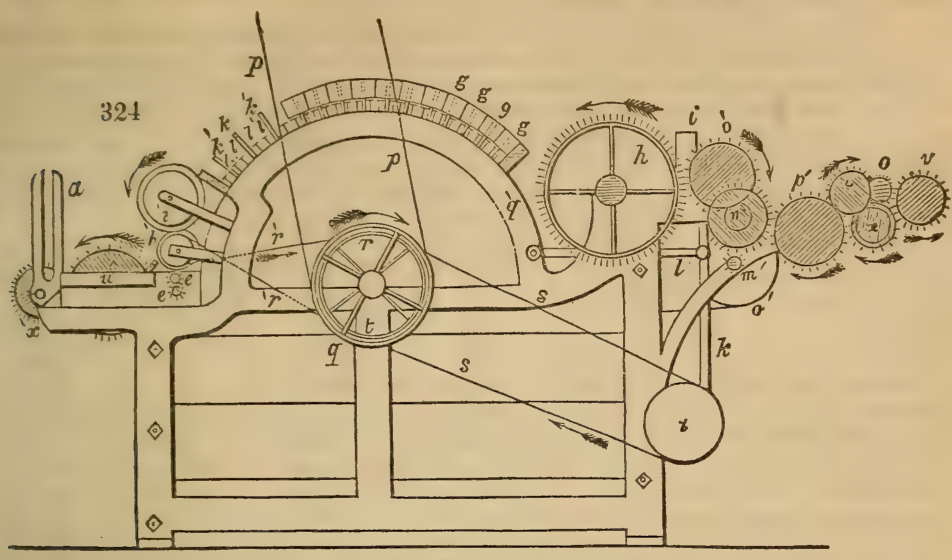

from mill gearing, and imparts it by friction to the lap roller over it. The iron ends of ihe lap roller lie in upright slots, which allow them freedom to rise as the roller gets slled with fleece.

The two pairs of rollers at 0 effect the extension of the card-end, and reduce its size. The under rollers are made of iron and fluted; the upper ones are also made of iron. but they are covered with a coat of leather, nicely glued on over a coat of flannel, which two coats render them both smooth and elastic. Two weights, $w$, press the upper cylinders steadily down upon the under ones. Between the first and second pair there is a certain interval, which should be proportioned to the length of the cotton staple. The second, or that furthest from the funnel, revolves with greater velocity than the first, and therefore turns out a greater length of riband than it receives from its fellow; the consequence is a corresponding extension of the riband in the interval between the two pairs of rollers.

The motions of the several parts of the engine are effected in the following way. The band, $p$, fig. 324, which comes down from the pulley upon the main shaft near the ceiling of the work-r $\approx 0 m$, drives, by means of the pulley $q$, the $\operatorname{drum} f$, fig. 323 , with a velocity of from 120 to 140 revolutions in a minute. From another pulley $r$, on the axis of the drum, the axis of $t$ is driven by the band $s$ working round the pulley $t$ on its end. This shaft drives the crank and lever mechanism of the stripper knife $i$. A third pulley of the same size as $r$ is fixed just within the frame to the other end of the drum, and from it a crossed or close band $r^{\prime}$ goes to a pulley upon the small runner $h^{\prime}$, to give this its rapid rotation. Upon the opposite end of the engine in fig. 323 , these wheels and pulleys are marked with dotted lines. Here we may observe, first, a pulley $y$ upon the drum, and a pulley $a^{\prime}$, which receives motion from it by means of the band $z$. The axis of $a^{\prime}$ carries in front a pinion $m^{\prime}$, which sets in motion the wheel $n^{\prime}$. The latter imparts motion, by means of a pinion and intermediate wheel $o^{\prime}$, to the wheel $h$ on the doffer cylinder, and consequently to that cylinder on the one hand; and it turns, by the carrier wheel $p^{\prime}$, a wheel $x$, whose axis is marked also with $x$ in fig. 323 , upon the other hand. The axis of $x^{\prime}$, fig. 323 , carries, towards the middle of the engine, a very broad wheel, which is represented by a small dotted circle. The toothed wheel $v$ of the smooth roller $v^{\prime}$, fig. 323, and the two toothed wheels 0 o, fig. 324, of the under rollers 00 , fig. 323 , work into that broad wheel. The wheel of the second or delivery fluted roller is seen to be smaller than that of the first, by which means the difference of their velocities is obtained. The large runner $i$ is driven from the main drum pulley, by means of the 'Jand 's', and the pulley $u^{\prime}, f i g .323$. The said band is crossed twice, and is kept in tension by the pulley $t^{\prime}$, round which it passes. The motion of the fluted rollers $e$, which feed in the cotton fleece, is effected by means of a bevel wheel $b^{\prime}$ on the end of the doffer, which works into a similar wheel $c^{\prime}$ on the oblique axis $d^{\prime}$ (dotted lines across the drum), of the pinion $e^{\prime}$ upon the lower end of the same axis which turns the wheel $f^{\prime}$, upon the ander feed roller.

Each of the feed rollers, fig. 324, bears a pinion $e$ at one end, so that the upper roller turns round with the under one. The roller $b, f i g .323$, is set in motion by means of 
its wheel $x^{\prime}$; which is driven by a wheel $v^{\prime}$ on the other end of the under feed roller, through the intervention of the large carrier wheel $w^{\prime}$. The original or first motion of $b$ must be as quick as that of the fluted feed rollers $e$, in order that the former may uncoil as much lap as the latter can pass on.

The annexed table exhibits the proper velocities of the different cylinders and rollers of the carding engine, which, however, are not invariable, but may be modified according to circumstances, by changing the pinions $e^{\prime}, f i g .323$, and $w^{\prime}$, according to the quality or length of the cotton staple. The velocities stated in the table will be obtained when the pulley $u^{\prime}$, fig. 323 , is made greater than $y$ in the proportion of 3 to 2 , and the wheels and pinions have the following number of teeth : $m^{\prime}, 18 ; n^{\prime}, 50$; its pinion, $18 ; h, 128$; $x, 24$; the broad wheel upon the shaft of $x, 37$ teeth; the wheel 0 of the first fluted roller, 35 ; that of the second, $21 ; v, 44 ; b^{\prime}$ and $e^{\prime}, 54 ; e^{\prime}, 10 ; f^{\prime}, 63$.

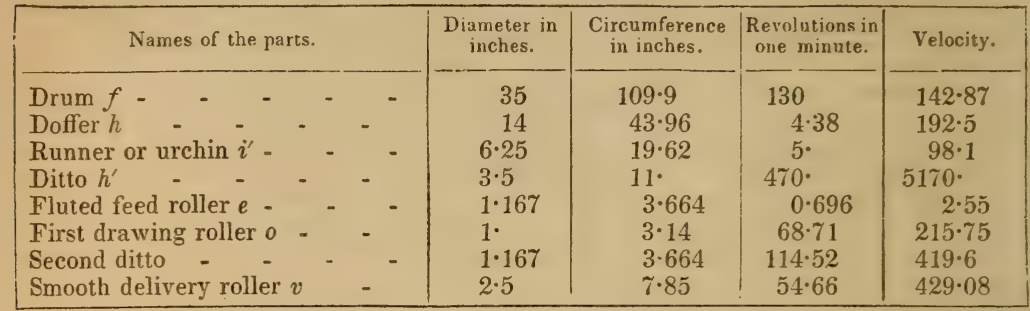

The operation of the runners, $h^{\prime}$ and $i^{\prime}$, becomes very plain on comparing their speed with one another and with that of the main-drum, and taking into account the direction of the card teeth. The cotton wool, taken off from the feed-rollers by the drum, is caught by the opposite teeth of the large runner $i^{\prime}$, which, on account of its slower surface rotation ( 98 inches per minute), may be considered to be at rest with reference to the drum, and therefore, by holding the cotton in its teeth, will commence its carding. The small runner $h^{\prime}$, in consequence of its greater surface velocity (5170 inches per minute), will comb the cotton-wool back out of the teeth of the large runner, but it will give it up in its turn to the swifter teeth of the dzum, which, in carrying it forwards, encounters the teeth of the top cards, and delivers up the filaments to their keeping for some time. We thus see how essential the runners are to the perfection as well as to the acceleration of the carding process for ordinary cotton wool, though for the slenderer and .onger filaments of the sea-island kind they are not so well adapted. In cleaning the carding-engines the little runner must be looked to every time that the drum is examined. The large runner and the doffer require to be cleaned together. The quantity of cotton spread upon the feed-cloth, the velocity of it, and of the drawing-rollers, must all be carefully adjusted to the grist of the yarn intended to be spun.

Suppose the sizes and velocities to be as represented in the preceding table, that the engine is a double card 36 inches broad, and that it is furnished with a lap from the lapmachine of which 30 feet in length weigh $5 \mathrm{lbs}$. In one minute the surface of the feedrollers, $e$, passes 2.55 inches of that lap onwards; in the same time the main-drum will work it off. To card the whole 30 feet, therefore, 141 minutes, or 2 hours and 21 minutes will be required. In this time the circumference of the rollers, $u v$, moves through a space of $141 \times 42,908 \mathrm{in} .=5042 \mathrm{ft}$., and delivers a card-end of that length, weighing 5 lbs., minus 6 per cent. for waste, that is, 4 lbs. $11 \frac{1}{2} \mathrm{oz}$. One pound will form a riband 1072 feet long, being, according to the English mode of counting, about number $\frac{1}{3}$, or 0.357. The extension of the cotton-fleece to this degree proceeds as follows:-In the 141 minutes which the feed-rollers take to introduce the 30 feet of lap, the doffer, $h$, makes 617.58 revolutions, and the comb, or doffer knife, $i$, detaches from the doffer teeth a thin fleecy web of 2262 feet in length. The first drawing pair of fluted rollers,

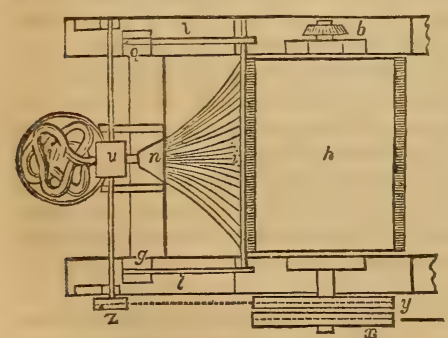

325

by its quick motion, with the aid of the funnel, $m$, converts this fleece into a riband 2535 feet long. The second pair of the fluted rollers extends this riband to 4390 feet, since their surface velocity is greater than the first pair in that proportion. The slight elongation (of only 112 feet, or about $\frac{1}{4 \frac{1}{4}}$ ) which takes place between the delivery fluted rollers and the smooth cylinders, $v, u$, serves merely to keep the card-end steadily upon the stretch without folding. Fig. 325 is a plan of the card and the fleece, where $h$ is the cylinder, $n$ is the funnel, $u$ the pressing rollers, and $h^{\prime}$ the card-ends in the can. 
Figs. 326, 327, represent skeletons of the old cards to facilitate the comprehenston of these complex machines. Fig. 326 is a plan; $F$ is the main drum; $M$ M is the doffes
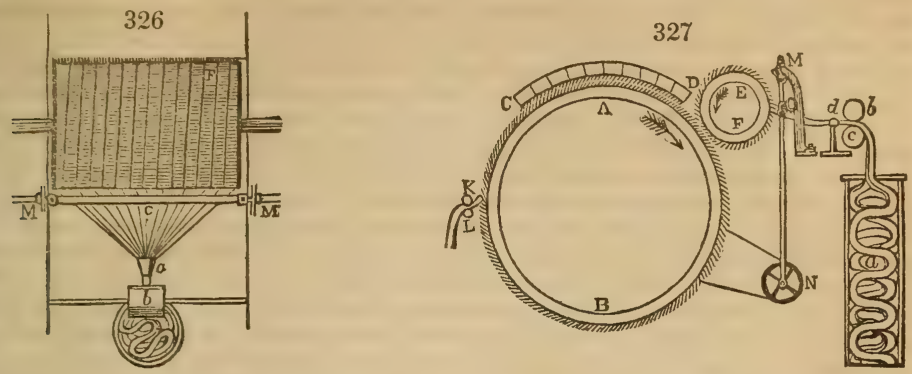

knife or comb; $\mathrm{G}$ the carded fleece hemmed in by the funnel $a$, pressed between the roll. ers $b$, and then falling in narrow fillets into its can. Fig. $327, \mathrm{~K} \mathrm{~L}$ are the feed rollers; $\mathrm{A} \mathrm{B}$ the card drum; $\mathrm{C}$ D the tops; $\mathrm{E}$ F the doffer card; $\mathrm{M} N$ the doffer knife; $d, b, c$, the card-end passing between compressing rollers into the can $a$.

The drawing and doubling are the next operation. The ends, as they come from the cards, are exceedingly tender and loose, but the filaments of the cotton are not as yet laid so parallel with each other as they need to be for machine spinning. Before any degree of torsion therefore be communicated, a previous process is required to give the filaments a level arrangement in the ribands. The drawing out and doubling accomplish this purpose, and in a manner equally simple and certain. The means employed are dratvingrollers, whose construction must here be fnlly explained, as it is employed in all the folLowing unachines; one example of their use occurred, indeed, in treating of the cards.

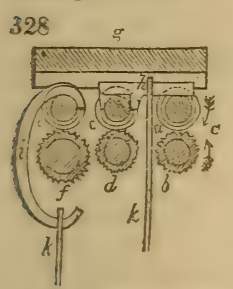

Let $a$ and $b$, fig. 328, represent the section of two rollers lying over each other, which touch with a regulated pressure, and turn in contact upon their axes, in the direction shown by the arrows. These rollers will lay hold of the fleecy riband presented to them at $a$, draw it through between them, and deliver it quite unchanged. The length of the piece passed through in a given time will be equal to the space which a point upon the circumference of the roller would have percured in the same time; that is, equal to the periphery of one of the rollers multiplied by the number of its entire revolutions. The same thing holds with regard to the transmission of the riband through between a second pair of rollers, $c, d$, and a third, $e, f$. Thus the said riband issues from the third pair exactly the same as it entered at $a$, provided the surface speed of all the rollers be the same. But if the surface speed of $c$ and $d$ be greater than that of $a$ and $b$, then the first-named pair will deliver a greater length of riband than the last receives and transmits to it. The consequence can be nothing else in these circumstances than a regulated drawing or elongation of the riband in the interval betwixt $a, b$, and $c, d$, and a condensation of the filaments as they glide over each other, to assume a straight parallel direction. In like manner the drawing may be repeated by giving the rollers, $e, f$, a greater surface speed than that of the rollers, $c$ and $d$. This increase of velocity may be produced, either by enlarging the diameter, or by increasing the number of turns in the same time, or finally by both methods conjoined. In general the drawing-machine is so adjusted, that the chief elongation takes place between the second and third pair of rollers, while that between the first and second is but slight and preparatory. It is obvious, besides, that the speed of the middle pair of rollers can have no influence upon the amount of the extension, provided the speed of the first and third pair remains inchanged. The rollers, $r, \delta$, and $c, d$, maintain towards each other continually the same position, but they may be removed with their frame-work, more or less, from the third pair, $e, f$, according as the length of the colton staple may require. The distance of the middle point from $b$ and $d$, or its line of contact with the upper roller, is, once for all, so calculated, that it shail exered the length of the cotton filaments, and thereby that these filaments are never in dar.ger of being torn asunder by the second pair pulling them while the first holds them fast. Between $d$ and $f$, where the greatest extension takes place, the distance must be as small as it can be without risk of tearing them in that way; for thus will the uniformity of the drawing be promoted. If the distance between $d$ and $f$ be very great, a riband passing through will become thinner, or perhaps break in the middle; whene we see that the drawing is more equable, tha shorter is the portion submitted to extension at n time, and the nearer the rollers are to each other, supposing .hem always distant enough not to tear the staple. 
The under rollers, $b d f$, are made of iron, and, to enable them to lay firmer hold of the filaments, their surfaces are fluted with triangular channels prarallel to their axes. The upper rollers, a $c$ e, are also made of iron, but they are smooth, and covered with a double coating, which gives them a certain degree of softness and elasticity. A coat of flannel is first applied by sewing or glueing the ends, and then a coat of leather in the same way. The junction edges of the leather are cut slanting, so that when joined by the glue (made of isinglass dissolved in ale) the surface of the roller may be smoothly cylindrical. The top rollers are sometimes called the pressers, because they press by means of weights upon the under ones. These weights are suspended to the slight rods $k k^{\prime}$; of which the former eperates on the roller $e$ alone, the latter on the two rollers $a$ and $e$ together. For this purpose the former is hung to a $\mathrm{c}$ shaped curve $i$, whose upper hook embraces the roller $e$; the latter to a brass saddle $h$, which rests upon $a$ and c. A bar of hard wood, g, whose under surface is covered with flannel, rests, with merely its own weight, upon the top rollers, and strips off all the loose hanging filaments. Similar bars with the same view are made to bear up under the fluted rollers $b d f$, and press against them by a weight acting through a cord passing over a pulley. Instead of the upper dust-covers, light wooden rollers covered with flannel are occasionally applied.

Were the drawing of a riband continued till all its fibres acquired the desired degree of parallelism, it would be apt, from excessive attenuation, to tear across, and thereb; to defeat the purpose of the spinner. This dilemma is got rid of in a very simple way, namely, by laying several ribands together at every repetition of the process, and incorporating them by the pressure of the rollers. This practice is called doubling. It is an exact imitation of what takes place when we draw a tuft of cotton wool between our fingers and thumb in order to ascertain the length of the staple, and replace the drawn filaments over each other, and thus draw them forth again and again, till they are all parallel and of nearly equal length. The doubling has another advantage, that of causing the inequalities of thickness in the ribands to disappear, by applying their thicker to their thinner portions, and thereby producing uniformity of substance.

329

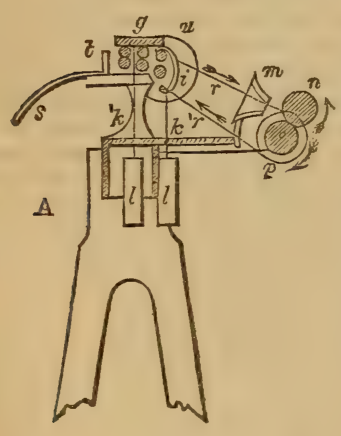

330

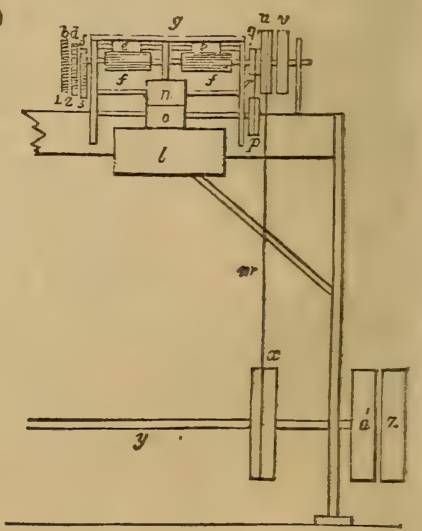

The drawing frame, as shown in section in figs. 328,330 , and in a back riew in fig. 329 , will require, after the above details, little further explanation. $l l$ are the weights which press down the top rollers upon the under ones, by means of the rods $k k^{\prime}$ and hook $i$. Each fluted roller is, as shown at $f$, fig. 329, provided in the middle of its length with a thinner smooth part called the neck, whereby it is really divided into two fluted portions, represented by $e$ e in the figure. Upon this middle neck in the pressure rollers, the hool $i$ and the saddle $h$ immediately bear, as shown in the former fig. 328. The card-ends, to the number probably of six, are introduced to the drawing frame either from tin cans, placed at e e, fig. 330, and at A, fig. 329, or from lap-bobbins; and, after passing through it, the ribands or slivers are received either into similar tin cans, as $g$, or upon other lapbobbins upon the other side. These appendages may be readily conceived, and are therefore not exhibited in all the drawings. Three of the slivers being laid together are again introduced to the one fluted portion $a b, f i g .328$, and three other slivers to the other portion. The sloping curved tin or brass plate $s$, fig. 329, with its guide pins $t$, serves to conduct the slivers to the rollers. When the two threefold slivers have passed through between the three pairs of rollers, and been thereby properly drawn, they run towards each other in an eblique direction, behind the last roller pair $e f, f i g .328$, and unite, on issuing through the 
conical funnel $m$, fig. 329 , into a single riband or spongy sliver; which is immediately carried oll " ith equable velc city by two smooth cast-iron rollers, $n$, figs. 329 and 330 , and either diopped into a can, or wound upon a large bobbin. The surface speed of these rullers is made a trifle sreater than that of the delivery drawing rollers, in order to leep the portion of sliver between them always in an extended state. Four fluted drawing portions are usually mounted in one drawing frame, which are set a-going or at rest together. To save all unnecessary carrying of the cans from the back to the front of the frame, the drawing heads are so placed, that the first and third discharge their slivers at the one side, and the second and fourth at the other. By this arrangement, the cans filled behind one head, are directly pushed aside in front of the next drawing head; by which alternate distribution the work goes on without interruption.

The fast pulley $u$, fig. 330 , by which the whole machine is driven, derives its motion from the main shaft of the mill by means of the band $w$. The similar pulley $z$, which sits loose upon the axis, and turns independently of it, is called the loose pulley; both together being technically styled riggers. When the operative desires to stop the machine, he transfers the band from the fast to the loose pulley by means of a lever, bearing a fork at its end, which embraces the band. Upon $y$, four pulleys such as $x$ are fixed, each of which sets in motion a drawing head, by means of a band like $w$ going round the pulleys $x$ and $u$. On account of the inverted position of the heads, which requires the motion of $u$ to be inverted, the bands of the first and third heads are open, but those of the second and fourth are crossed. Every head is provided with a loose pulley $v$, as well as the fast pulley $u$, in order to make the one stop or move without affecting the others. The shaft of the pulley $u$ is the prolonged shaft of the backmost fluted roller $f$. It carries besides a small pulley $q$, which, by means of the band $r$, and the pulley $p$, fig. 329 , sets in motion the undermost condensing roller 0 . The upper roller $n$ presses with its whole weight upon it, and therefore turns by friction. The toothed wheel-work, by which the motions are communicated from the backmost fluted roller to the middle and front ones, is seen in fig. 330 .

The wheel $f$, fig. 328 , of 20 teeth, works in a 44-toothed carrier-wheel, on whose axis there are two smaller wheels; 2 with 26 teeth, and 1 with 22 teeth. The wheel $d$, fig. 330 of the middle roller, and the wheel $b$ of the front roller, are set in motion by other carrier wheels; the first has 27 teeth, and the last 40 . For every revolution of $b$, the roller $d$ makes nearly $1 \frac{3}{4}$ turns, and the roller $f 4$ revolutions. The top rollers revolve, as we have stated, simply by the friction of contact with the lower ones. Now suppose the diameter of the rollers $b$ and $d$ to be 1 inch or 12 lines, that of $f 1 \frac{1}{4}$ inches or 15 lines, the surface velocities of the three pairs of rollers in the series will be as 1 , $1 \frac{3}{4}$, and 5. Every inch of the cotton sliver will be therefore extended between the first and second pairs of rollers into $1 \frac{3}{4}$ inches, and between the second and third or delivery pair into 5 inches; and after the sliver has passed through all the four drawing heads, its length will be increased 625 times $=5 \times 5 \times 5 \times 5$.

The further the drawing process is pushed, the more perfectly will its object be accomplished, namely, the parallelism of the filaments. The fineness of the appearance of the sliver after the last draught depends upon the number of doublings conjointly with the original fineness and number of drawings. The degree of extension may be increased or diminished, by changing the wheels in fig. 330 , for others with a different number of teeth. Thus the grist or fineness of the sliver may be modified in any desired degree; for, when the subsequent processes of the mill remain the same, the finer the drawings the finer will be the yarn. For spinning coarse numbers or low counts, for example, six card-ends are usually transmitted through the first drawing head, and converted into one riband. Six such ribands again form one in the second draught; six of these again go together into the third sliver; and this sliver passes five-fold through the last draught. By this combination 1080 of the original card-ends are united in the finished drawn sliver $=6 \times 6 \times 6 \times 5$. The fineness of the sliver is, however, in consequence of these doublings, not increased, but rather diminished. For, by the drawing, the card-end has been made 625 times longer, and so much smaller; by the doubling alone it would have become 1080 times thicker; therefore, the original grist is to the resent as 1 to the fraction $\frac{625}{1080}$; that is, supposing 1072 feet of the riband delivered by the card to weigh one pound, 625 feet, the sliver of the last drawing, will also weigh a pound, which corresponds in fineness to number $0 \cdot 24$, or nearly $\frac{1}{4}$.

The rearmost or last drawing roller has a circumference of nearly 4 inches and makes abrut 150 revolutions per minute; hence, each of these drawing heads may turn off 35,000 feet of sliver in 12 hours.

Some manufacturers have lately introduced a double roller beam, and a double draught at the same doubling, into their drawing frames. I have seen this contrivance working satisfactorily in mills where low counts were spun, and where the tube roving frame was emplored; but I was informed oy competent judges, that it was not advisable where a level yarn was required for good printing calicoes. 
The loss which the cotton suffers in the drawing frame is quite inconsiderable. It consists of those flaments which remain upon the drawing rollers, and collect, in a great measure, upon the flannel facing of the top and bottom cleaner bars. It is thrown among the top cleanings of the carding engine. When from some defect in the rollers, or negligence in piecing the running slivers, remarkably irregular portions occur in the ribands, these must be torn off, and returned to the lap machine to be carded anew.

The fifth operation may be called the first spinning process, as in it the cotton sliver receives a twist; whether the twist be permanent, as in the bobbin and fly frame, or be undone immediately, as in the tube-roving machine. In fact, the elongated slivers of parallel filaments could bear little further extension without breaking asunder, unless the precaution were taken to condense the filaments by a slight convolution, and at the same time to entwine them together. The twisting should positively go no further than to fulfil the purpose of giving cohesion, otherwise it would place an obstacle in the way of the future attenuation into level thread. The combination of drawing and twisting is what inainly characterizes the spinning processes, and with this fifth operation, therefore, commences the formation of yarn. As, however, a sulden extension to the wished-for fineness is not practicable, the draught is thrice repeated in machine spinning, and after each draught a new portion of torsion is given to the yarn, till at last it possesses the degree of fineness and twist proportioned to its use.

The prelimmary spinning process is called roving. At first the torsion is slight in proportion to the extension, since the solidity of the still coarse sliver needs that cohesive

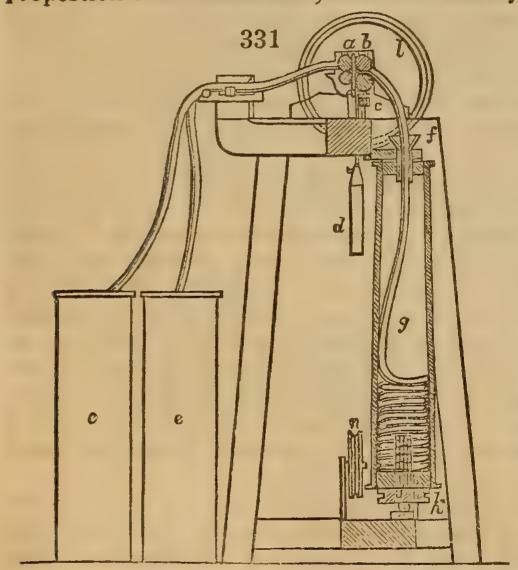
aid only in a small degree, and looseness of texture must be maintained to facilitate to the utmost the further elongation.

Fig. 331 is a section of the can roving frame, the ingenious invention of Ark wright, which, till within these 14 years, was the principal machine for communi cating the incipient torsion to the spongy cord furnished by the drawing heads. I differs from that frame in nothing bu the twisting mechanism; and eonsists of two pairs of drawing rollers, $a$ and $b$, be tween which the sliver is extended in the usual way; $c$ are brushes for cleaning the rollers; and $d$ is the weight which presses the upper set upon the lower. The wiping covers (not shown here) rest upon $a b$. The surface speed of the posterior or secone pair of rollers is 3,4 , or 5 times greates than that of the front or receiving pair, according to the desired degree of attenua. tion. Two drawn slivers were generally united into one by this machine, as is shown in the figure, where they are seen coming from the two cans $e$, to be brought together by the pressure rollers, before they reach the drawing rollers $a b$. The sliver, as it escapes from these rollers, is conducted into the revolving conical lantern $g$, through the funnel $f$ at its top. This lantern-can receives its motion by means of a cord passing over a pulley $k$, placed a little way above the step on which it turns. The motion is steadied by the collet of the funnel $f$, being embraced by a brass busk. Such a machine generally contained four drawing heads, each mounted with two lanterns; in whose side there was a door for taking out the conical coil of roving.

The motion imparted to the back roller by the band pulley or rigger $m$, was conveyed to the front one by toothed wheel work.

The vertical guide pulley at bottom, $n$, served to lead the driving band descending from the top of the frame round the horizontal whorl or pulley upon the under end of the lantern. The operation of this can-frame was pleasing to behold; as the centrifugal force served both to distribute the soft cord in a regular coil, and also to condense a great deal of it most gently within a moderate space. Whenever the lantern was filled, the tenter carried the roving to a simple machine, where it was wound upon bobbins by band. Notwithstanding every care in this transfer, the delicate texture was very apt to be seriously injured, so as to cause corresponding injuries in every subsequent operation, and in the finished yarn. Messrs. Cocker and Higgins, of Salford, had the singular merit, as I have said, of superseding that beautiful but defective mechanism, which had held a prominent place in all cotton mills from almost the infancy of the factory system, by the following apparatus.

The Bobbin and Fly frame is now the great roving machine of the cotton manufac- 
ture; to which may be added, for coarse spinning, the tube roving frame. Of such a complicated machine as the bobbin and fly frame, it is not possible to give an ade-

332

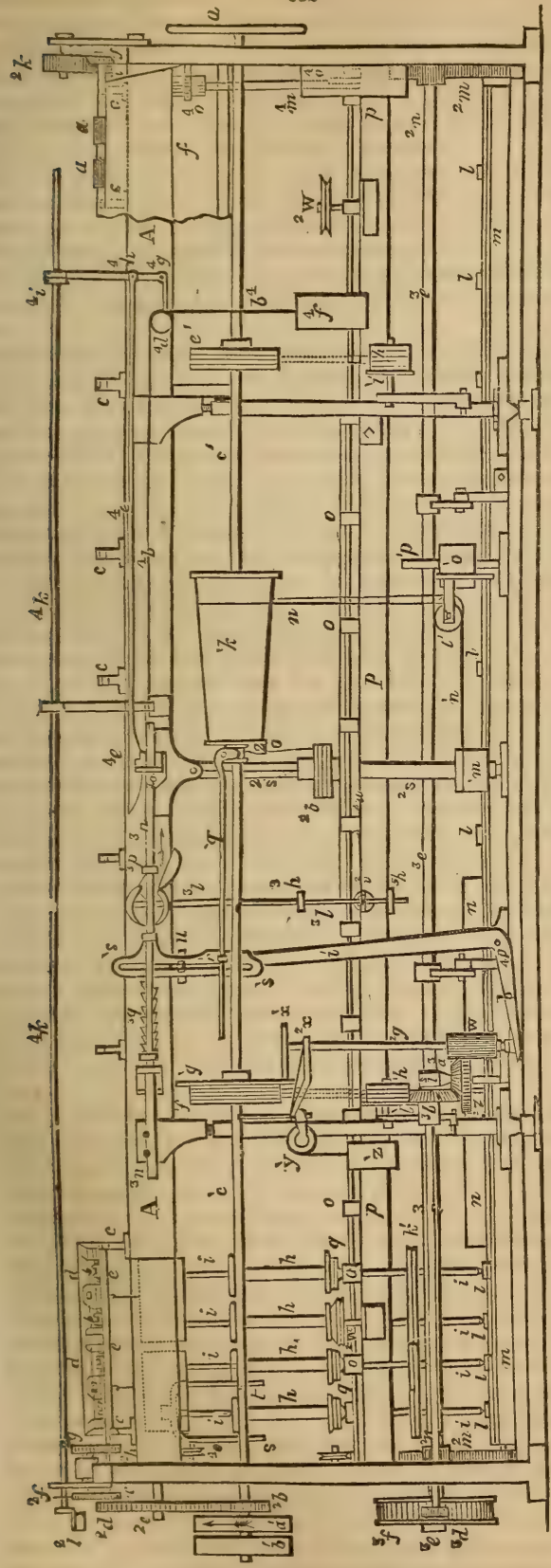
quately detailed description in the space due to the subject in this Dictionary. Its mechanical combinations are, however, so admirable as to require such an account as will make its functions intelligible by the general reader.

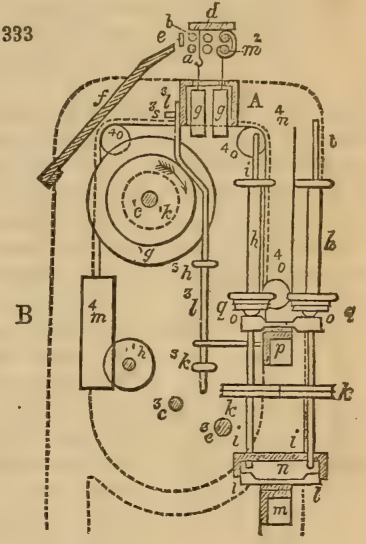

Fig. 332 exhibits a back view ol this machine ; and fig. 333 a section of some of the parts not very visible in the former figure. The back of the machine is the side at which the cotton is introduced between the drawing rollers.

The cans, or lap-bobbins filled with slivers at the drawing frame, are placed in the situation marked B, fig. 333, in rows parallel with the length of the machine. The sliver of each ean, or the united slivers of two contiguous cans, are conducted upwards along the sur face of a sloping board $f$, and through an iron staple or guide $e$, betwixt the usual triple pair of drawing rollers, the first of which is indicated by $a, b$. In fig. 332 , for the purpose of simplifying the figure, the greater part of these rollers and their subordinate parts are omitted. After the slivers have been sufficiently extended and attenuated between the rollers, they proceed forwards, towards the spindles $i i i$, where they receive the twist, and are wound upon the bobbins $h$. The machine deline ated contains thirty spindles, but many bobbin and fly frames contain double or even four times that number. Only a few of the spin dles are shown in fig. 332 , for fear of confusing the drawing.

With regard to the drawing functions of this machine, I have already given abundan: 
explanation, so far as the properties and operation of the rollers are concerned. The frame-work of this part of the machine, called the roller-beam, is a cast-iron bench, upon which nine bearers, $c$, are mounted for carrying the rollers. The fluted rollers $a$ a a, fig. 334 , are constructed in four pieces for the whole length, which are parted from each other by thinner smooth cylindric portions, $z$, called necks. Seven such partings for four rollers, and one parting for two rollers, constitute together the 30 fluted rollers of which the whole series consists. The coupling of these roller subdivisions into one cylinder, is secured by the square holes $x$, and square pins $y, f i g .334$, which fit into the 334

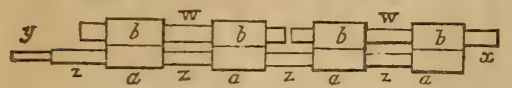

holes of the adjoining subdivision. The top or pressure rollers $b$, are two-fold over the whole set; and the weighted saddle presses upon the neck $w$, which connects every pair, as was already explained under fig. 329. These weights $g \mathrm{~g}, \mathrm{fig} .333$, are applied in this as in the drawing frame; $d$ are the bars faced with flannel for cleaning the top rollers. A similar bar is applied beneath the rollers, to keep the flutings clean.

The structure and operation of the spindles $i$ may be best understood by examining

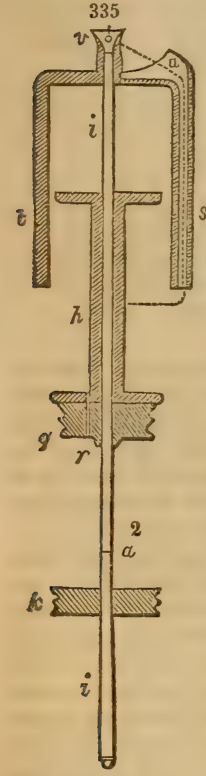
the section fig. 335. They are made of iron, are cylindrical from the top down to $a 2$, but from this part down to the steel tipped rounded points they are conical. Upon this conical portion there is a pulley $k$, furnished with two grooves in its circumference, in which the cord runs that causes the spindle to revolve. The wooden bobbin $h$ is slid upon the cylindrical part, which must move freely upon it, as will be presently explained. To the bobbin another two-grooved pulley or whorl $\mathrm{g}$ is made fast by means of a pin $r$, which passes through it; by removing this pin, the bobbin can be instantly taken off the spindle. The upper end of the spindle bears a fork $s t$, which may be taken off at pleasure by means of its left-handed screw; this fork, or flier, has a funnel-formed hole at $v$. One arm of the fork is a tube, $s, u$, open at top and bottom; the leg, $t$, is added merely as a counterpoise to the other. In fig. 333, for the sake of clearness, the forks or fliers of the two spindles here represented are left out; and in fig. 332, only one is portrayed for the same reason. It is likewise manifest from a comparison of these two figures that the spindles are alternately placed in two rows, so that each spindle of the back range stands opposite the interval between two in the front range. The object of this distribution is economy of space, as the machine would need to be greatly longer if the spindles stood all in one line. If we suppose the spindles and the bobbins (both of which have independent motions) to revolve simultaneously and in the same direction, their operation will be as follows: The sliver, properly drawn by the fluted rollers, enters the opening of the funnel $v$, proceeds thence downwards through the hole in the arm of the fork, runs along its tube $u, s$, and then winds round the bobbin. This path is marked, in fig. 335 , by a dotted line.

The revolution of the spindles in the above circumstances effects the twisting of the sliver into a soft cord; and the flier $s, t$, or particularly its tubular arm $s$, lays this cord upon the bobbin. Were the speed of the bobbins equal to that of the spindles, that is, did the bobbin and spindle make the same number of turns in the same time, the process would be limited to mere twisting. But the bobbin anticipates the fliers a little, that is, it makes in a given time a somewhat greater number of revolutions than the spindle, and thereby effects the continuous winding of the cord upon itself. Suppose the bobbin to make 40 revolutions, while the spindle completes only 30 ; 30 of these revolutions of the bobbin will be inoperative towards the winding-on, because the fliers follow at that rate, so that the cord or twisted sliver will only be coiled 10 times round the bobbin, and the result as to the winding-on will be the same as if the spindle had stood still, and the bobbin had made $40-30=10$ turns. The 30 turns of the spindles serve, therefore, merely the purpose of communicating twist.

The mounting and operation of the spindles are obvious.y the same as they are upon the household flax wheel. In the bobbin and fly frame there are some circumstances which render the construction and the winding-on somewhat difficult, and the mechanism not a little complicated. It may be remarked, in the first place, that as the cord is wound on, the diameter of the bobbin increases very rapidly, and therefore every turn made round it causes a greater length of roving to be taken up in succession. Were the motions of the bobbins to continue unchanged in this predicament, the increased velocity of the winding-on would require an increased degree of extension, or it would 
occasion the rupture of the cord, because the front fluted rollers move with uniform speed, and therefore deliver always the same length of sliver in the same time. It is therefore necessary to diminish the velocity of the bobbins, or the number of their turns, in the same proportion as their diameter increases, in order that the primary velocity may remain unchanged. Moreover, it is requisite for the proper distribution of the cord upon the bobbin, and the regular increase of its diameter, that two of its successive corjvolutions should not be applied orer each other, but that they should be laid close side by side. This object is attained by the up and down sliding motion of the bobbin upon the spindle, to the same extent as the length of the bobbin barrel. This up and down motion must become progressively slower, since it increases the diameter of the bobbin at each range, by a quantity equal to the diameter of the sliver. What has now been stated generally, will become more intelligible by an example.

Let it be assumed that the drawing rollers deliver, in 10 seconds, 45 inches of roving, and that this length receives 30 twists. The spindles must, in consequence, make 30 revolutions in 10 seconds, and the bobbins must turn with such speed, that they wind up the 45 inches in 10 seconds. The diameter of the bobbin barrels being $1 \frac{1}{2}$ inches, their circumference of course $4 \frac{1}{2}$ inches, they must make 10 revolutions more in the same tim than the spindles. The effective speed of the bobbins will be thus $30+10=40$ turns in 10 seconds. Should the bobbins increase to 3 inches diameter, by the winding-on of the sliver, they will take up 9 inches at each turn, and consequently 45 inches in 5 turns. Their speed should therefore be reduced to $30 \div 5=35$ turns in 10 seconds. In general, the excess in number of revolutions, which the bobbins must make over the spindles, is inversely as the diameter of the bobbins. The speed of the bobbins must remain uniform during the period of one ascent or descent upon the spindle, and must diminish at the instant of changing the direction of their up and down motion; because a fresh range of convolutions then begins with a greater diameter. When, for example, 30 coils of the sliver or roove are laid in one length of the bobbin barrel, the bobbin must complete its vertical movement up or down, within 30 seconds in the first case above mentioned, and within 60 seconds in the second case.

The motions of the drawing rollers, the spindles, and bobbins, are produced in the following manner:-A shaft $c^{\prime}$, figs. 332 and 333 , extending the whole length of the machine, and mounted with a fly wheel $d^{\prime}$, is set in motion by a band from the running pulley upon the shaft of the mill, which actuates the pulley $a^{\prime} . b^{\prime}$ is the loose pulley upon which the band is shifted when the machine is set at rest. Within the pulley $a^{\prime}$, but on the outside of the frame, the shaft $c^{\prime}$ carries a toothed wheel $b_{2}$ with 50 teeth, which by means of the intermediate wheel $c^{2}$ turns the wheel $d_{2}$ upon the prolonged shaft of the backmost fluted roller ( $m 2$, fig. 333.) This wheel d2 has usually 54 teeth; but it may be changed when the roove is to receive more or less twist; for as the spindles revolve with uniform velocity, they communicate the more torsion the less length of sliver is delivered by the rollers in a given time. Upon the same shaft with $d^{2}$, a pinion $e^{2}$ of 32 teeth is fixed, which works in a wheel $f_{2}$ of 72 teeth. Within the frame a change pinion $g^{2}$ is made fast to the shaft of $f 2$. This pinion, which has usually from 24 to 28 teeth, regulates the drawing, and thereby the fineness or number of the roving. It works in a 48-toothed wheel $h 2$ upon the end of the backmost fluted roller $\alpha$, fig. 333. The other extremity of the same roller, or, properly speaking, line of rollers, carries a pinion 72 , furnished with 26 teeth, which, by means of the broad intermediate wheel $k^{2}$, sets in motion the pinion $\imath^{\prime} 2$ of 22 teeth upon the middle roller. When the diameter of all the drawing rollers is the same, suppose 1 inch, their proportional relocities will be, with the above number of teeth in the wheel work, if g2 have 24 teeth, as $1: 1 \cdot 18: 4 \cdot 5$; and the drawn sliver will have $4 \frac{1}{2}$ times its original length. The front or delivery roller of the drawing frame is of late years usually made $1 \frac{1}{4}$ or $1 \frac{3}{8}$ inches in diameter. If 625 feet of the sliver from the drawing frame weighed one pound, $2 \tau 90$ feet of the roving will now go tn this weight, and the number will be $1 \cdot 12$; that is, 1 hank and 12 hundredths to the pound. The front pair of fluted rollers makes about 90 revolutions, and deliver; 282.6 inches of roving in the minute, when of one inch diameter.

The spindles $i$ (figs. 332 and 333), rest, with their lower ends, in steps $l$, which are fixed in an immoveable beam or bar $m$. To protect it from dust and cotton filaments, this beam is furnished with a wooden cover $n$, in which there are small holes for the passage of the spindles right over the steps. In fig. 332 , two of the eight covers $n$, which compose the whole range $m$, are removed to let the steps be seen. The crlindrical part of each spindle passes through a brass ring 0 ; and all these 30 rings, whose centres must be vertically over the steps $l$, are made fast to the copping beam $p$. This beam is su called, because it is destined not merely to keep the spindles upright by the rings attached to it, but, at the same time, to raise and lower along the spindles the bobbins 
which rest on these rings; for which purpose the two racks, or toothed bars $m^{2} m^{2}$, made fast to it, are designed, as will be presently explained. To effect the revolution of the spindles, there are attached to the main shaft $c^{\prime}$ two whorls or pulleys $e^{\prime} f^{\prime}$, each bearing four grooves of equal diameter. Each of these pulleys puts one half of the spindles in motion, by means of a cord, which, after going round the whorls $k$, turns four times about the pulleys of the shaft $c^{\prime}$. Two guide pulleys $h^{\prime}$, each four-grooved, and two others $i$, with a single groove, which turn independently of the others, upon the above shaft, serve to give the whorl cords the proper direction, as well as to keep them tight. 'The spindles revolve 200 times or thereby in the minute; and therefore impart two turns or twists to every three inches of the roving.

The revolution of the bobbins is independent of that of the spindles, although it likewise proceeds from the shaft $c^{\prime}$, and differs from it in being a continually retarded motion. The simplest method of effecting this motion, is by means of the wooden or tin plate cone $k^{\prime \prime}$, which revolves equally with the shaft $c^{\prime}$, and at the same time slides along it.

The manner in which this operates is shown in section in fig. 336 . Here we perceive the rod $q^{2}$, which extends from the base toward the narrow end of the truncated cone, and $p^{2}$ a forked bearer or carrier made fast to the shaft $c^{\prime}$ by, a screw, which compels the cone, by means of that rod, to obey the movements of $c^{\prime}$. In the large end

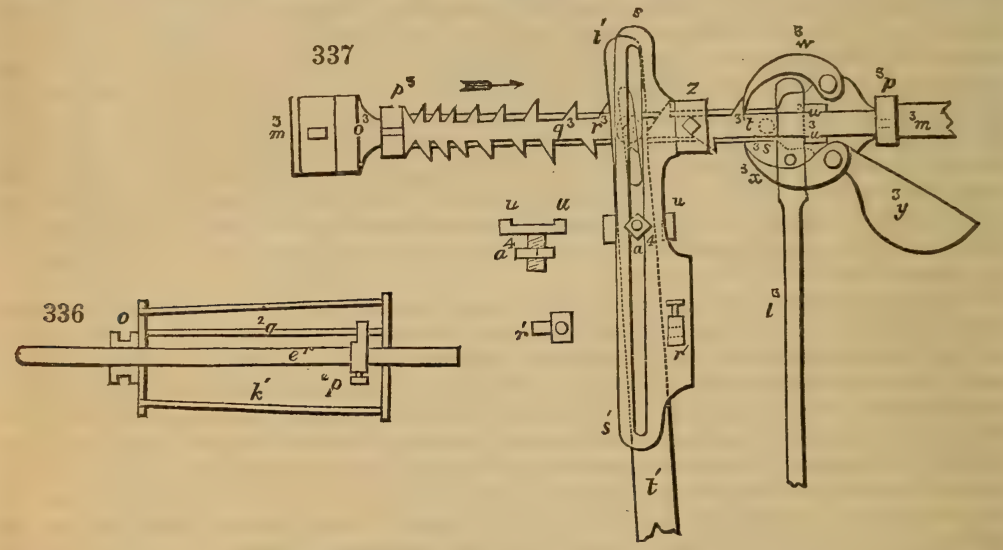

of the cone there is an aperture, through which the bearer can be got at. The smaller end carries outside a projection 02, provided with a groove, which is embraced by the forked end of a rod $q^{\prime}, f i g$. 337, that serves to shove the cone along upon the shaft $c^{\prime}$. Directly under the cone, there is an upright round pillar $p^{\prime}$, upon which the holder $\sigma$ of the two guide pulleys $l^{\prime}$ is adjustable. A bar $r^{2}$ placed along-side of the holder, prevents its turning round, but allows it to slide along $p^{\prime}$ by friction. The weight of the holder and the pulley is sufficient to distend the endless band $n^{\prime}$, which runs from the cone $k^{\prime}$, through under the prlley $l^{\prime}$, and round the small drum $m^{\prime}$ on the shaft $s 2$. A pulley or whorl $t 2$, with four grooves, is made fast by means of a tube to this shaft, and slides along it backwards and forwards, without ever ceasing to follow its revolutions. The shaft possesses for this purpose a long fork, and the interior of the tube a corresponuing tongue or catch. There is besides upon the tube beneath the pulley, at $u 2$, a groove that goes round it, in which the staple or forked end of an arm like v2, fig. 333, made fast to the copping beam $p$, catches. By the up and down movement of shat beam, the pulley $t 2$ takes along with it the arm that embraces the tube, which therefore rises and falis equally with the bobbins $h^{\prime}$, and their pulleys or whorls $q$. This is requisite, since the bobbins are made to revolve by the pulleys $t 2$, by means of two endless cords or bands.

The most intricate part of the mechanism is the adjustment, by which the revolution of the bobbins is continually retarded, and their up and down, or copping motion, along the spindles, is also retarded in like proportion. The vertical pulley $f^{\prime}$ (towards the left end of the shaft $c$ ) has at its right side a somewhat larger disc or sheave $g^{\prime}$, with a perfectly uniform, but not a very smooth surface. Upon this sheave, a smaller horizontal pulley $x^{\prime}$ rubs, whose upper face is covered with leather to increase the friction The under end of the shaft $y^{2}$ of the pulley $x^{\prime}$ turns in a step, which is so connected with the arm $v^{\prime}$ of the large bent lever $t^{\prime} v^{\prime}$, that it always stands horizontally, whatever direction the arms of that lever may assume. The shaft $y^{2}$ is steadied at top by an 
annular holder or bush, which embraces the fast arm $x^{2}$ with its forked end. Upon its opposite side, this arm carries a pulley $y^{2}$, upon which a cord goes, that is made fast to the holder of the shaft $y^{2}$, and loaded with the weight $z^{\prime}$. The weight presses the pulley $x^{\prime}$ against the surface of $g^{\prime}$, in such wise as to effect the degree of friction necessary in order that the revolution of $g^{\prime}$ may produce an uninterrupted revolution in $x^{\prime}$. A pinion $w^{\prime}$, whose length must be equal at least to the semi-diameter of the sheave $g^{\prime}$, is placed upon the under end of the shaft. $y^{2}$. It has 22 teeth, and takes into a 62-toothed horizontal wheel $z 2$. Upon the upper end of this wheel the conical pinion $a^{3}$ is made fast, which may be changed for changing the speed, but usually has from 28 to 30 teeth. By this pinion the conical wheel $b^{3}$ is turned, which has 30 teeth, and whose shaft is $c^{3}$ 。 This shaft carries upon its opposite end a six-leaved pinion, $d 3$, which takes into the calender wheel $f 3$, formed with cogs like a trundle, upon the long shaft $e^{3}$. In fig. 338 the wheel $f 3$ is exhibited with its pinion $d^{3}$. Here we may remark, that in the circumference of the wheel there is a vacant place, g3, void of teeth. When, by the motion of the wheel, the pinion comes opposite to this opening, it turns round about the last tooth of the wheel, falls into the inside of the toothed circle marked by the dotted lines, and thus gives now an inverse movement to the wheel $f 3$, while itself revolves always in the same direction. This reversed motion continues till the opening $g^{3}$ comes once more
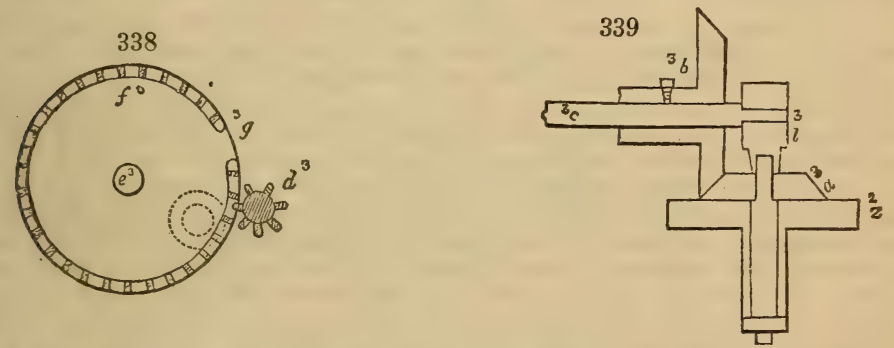

opposite to the pinion, when this turns round about the last tooth of that side, and begins again to work in the exterior teeth. Thus, by the uniform motion of $d 3$ and its dependant parts, the wheel $f 3$, with its shaft $e^{3}$, revolves alternately to the right hand and the left. That this result may ensue, the shaft $c^{3}$ of the pinion must be able to slide endwise, without losing its hold of $a^{3}$ and $b_{3}$. This adjustment is effected by placing the end of the said shaft, nearest $b 3$, in a box or holder $i 3$, in which it can turn, and which forms a vertical tube to this box, as a downward prolongation which is fixed to the tail of the conical pinion $\alpha^{3}$. Fig. 339 shows this construction in section upon an enlarged scale. The second bearer of the shaft nearest $d 3$, must possess likewise the means of lateral motion. When therefore the pinion $d 3$ shifts through the opening of the wheel $f^{3}$ outwards or inwards, its shaft $c^{3}$, makes a corresponding small angular motion upon the pivot of $a^{3}$, by means of the tube $i_{3} ; a^{3}$ and $b^{3}$ remain thereby completely in gear with one another.

The above-described alternate revolutions of the wheel $f^{3}$ serve to produce the up and down motions of the bobbins. The shaft $e^{3}$ has for this purpuse two pinions $n^{2} n^{2}$, which work in the rack teeth $m^{2} m^{2}$ of the copping rail $p$, and thus alternately raise and sink it with the bobbins which rest upon it. The weight of the copping beam and all its dependant parts, is poised by two counterweights $m 4$, whose cords run over the pulleys 0404 04, fig. 332, and have their ends made fast to the frame, so as to make the upward motion as easy as the downward. The two upper pulleys out of the three of each weight are fixed to the frame; the under one, round which the cord first runs, is attached to the coping beam, rising and falling along with it.

As long as the friction disc $x^{\prime}$ remains at the same height, the pulley $g^{\prime}$ derives its motion from the same circle of the said disc, and the up and down motion of the copping beam is also uniform. But when that disc ascends so as to describe with its edge a small circle upon the face of $\mathrm{g}^{\prime}$, its motion must become proportionally more slow. This is the method, or principle of retarding the copping motions of the bobbins. It has been shown, however, that the rotation of the bobbins should be also retarded in a progressive manner. This object is effected by means of the cone $k^{\prime}$, which, as the band $n$ progressively approaches towards its smaller diameter, drives the pulleys or whorls $q$ of the bobbins with decreasing speed, though itself moves uniformly quick with the shaft $c$. To effect this variation, the cone is shifted lengthwise along its shaft, while the band running upon it remains continually in the same vertical plane, and is kept distended by the weight of the pulley $o^{\prime}$. The following mechanism serves to shift the cone, which may be best understood by the aid of the figures 340,341, and 337. A long cast iron bar $m^{3}$, 
which bears two horizontal projecting puppets, $0_{3} 0_{3}$, is made fast to the front uprigh face of the copping beam A. Through the above puppets a cylindrical rod $n^{3}$ passes freely

340

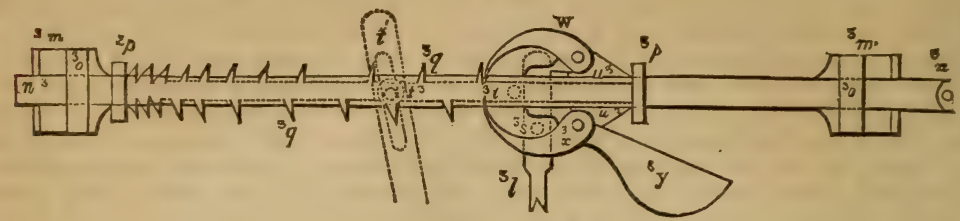

341

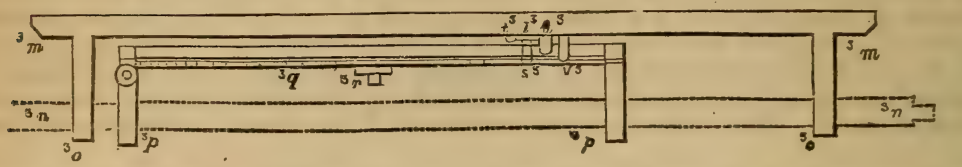

which is left out in fig. 337, that the parts lying behind it may be better seen. Upon this rod there is a kind of fork, $p^{3} p^{3}$, to which the alternating rack bars $q^{3}$ are made fast. The teeth of these racks are at unequal distances from. each other, and are so arranged, that each tooth of the under side corresponds to the space between two teeth in the upper side. Their number depends upon the number of coils of roving that may be required to fill a bobbin; and consists in the usual machines of from 20 to 22 . The rod $n^{3}$ may be shifted in the puppet $0^{3}$, like the fork $p^{3}$ of the rack-rod, upon the rod $n 3$, and along the surface of $m^{3}$, where two wings $u^{3} u^{3}$ are placed, to keep the fork in a straight direction. Upon the bar $m 3$, there are the pivots or fulcra of two stop catches $w^{3}, x^{3}$, of which the uppermost presses merely by its own weight, but the undermost by means of a counterweight $y^{3}$, against the rack, and causes them thus to fall in between the teeth. In fig. 341, $v^{3}$ shows the pivot of the eatch or detent $w^{3}$ by itself, the detent itself being omitted, to render the construction plainer. A pushing rod $l \beta$, upon which there is a pin above at $s^{3}$, that passes behind the rack rod, between this and the bar $m^{3}$, has for its object to remove at pleasure the one or the other of the two catches; the upper, when the upper end of the rod pushes against it; the under, by means of the above mentioned pin $s^{3}$. Both the catches are never raised at once, but either the under or the upper holds the rack bar fast, by pressing against one of the teeth. The vertical motion up or down, which the rod $l 3$ must take to effect the lifting of the catches, is given to it from the copping beam $p$; since upon it a horizontal arm $v 2$, fig. 341, is fixed, that lays hold of that rod. Upon the pushing rod are two rings, $h 3$ and $k 3$, each made fast by a screw. When the copping beam is in the act of going up, the $\operatorname{arm} v^{3}$ at the end of this movement, pushes against the ring $h 3$, raises up the rod $l 3$, and thus removes the eatch $w 3$, fig. 337, from the teeth of the rod $q^{3}$, before which it lies flat. At the descent of the copping rail, $v 2$ meets the ring $k 3$, when the motion in this direction is nearly completed, draws down the rod $l 3$ a little, by means of the same, and thereby effects the removal of the catch $x^{3}, f i g$. 337, from the rod $q^{3}$. Every time that one of the catches is lifted, the rack recovers its freedom to advance a little bit in the direction of the arrow; so far, namely, till the other catch lays hold upon the tooth that next meets it. The reason is thus manifest why the teeth of the upper and under sides of the bar $q^{3}$ are not right opposite to each other, but, in an alternate position.

From the rack-bar, the sliding of the cone $k^{\prime}$, and the raising of the shaft $y^{2}$, each by minute steps at a time, is produced as follows:-

A large rectangular lever $t 1, v^{1}$, whose centre of motion is at $p^{4}$, has at the upper end of its long arm $t 1$, a long slot through which a stud $r^{3}$ upon the rack $q^{3}$ goes, ( $f$ igs. 340, 341,337, ) so that the lever must follow the motions of the rack bar. The end of the short arm of the lever bears, as already mentioned, the step of the shaft $y^{2}$; hence the friction dise $x^{1}$ will be raised in proportion as the rack bar advances, and will come nearer to the middle point of $\mathrm{gl}^{1}$; consequently, its revolution and the shifting of the bobbins will become slower. Upon the cylindrical rod $n^{3}$, the piece $\delta^{1} s^{1}$ furnished with a long slot is made fast, by means of a tube $z 3$, ( fig. 337,) and a screw. A fork $u$, which by means of the screw nut $a^{4}$ is made fast in the slot, embraces the arm $t^{1}$ of the bent lever; and a tube $r^{1}$ riveted to the surface of $s 1$, is destined to take up the draw rod $q^{1}$ of the cone $k^{1}, f i g$. 337. A weight $f^{4}$, whose cord $b 4$ is made fast to the cylindrical rod $n^{3}$, endeavors to draw this rod continually in the direction of the arrow. In consequence of this arrangement, every time that the pushing bar ${ }^{3}$ lifts up one of the catches, the cone $k^{1}$, the lever $t^{1} v^{1}$, and by it the rack bar $q^{3}$, are set in motion. It is 
obvious, that the motion of the eone may be made greater or less, according as the fork $u v$ is fixed further up or down in the slot of $s^{1}$.

The number of the teeth upon the bar $q^{3}$ is so ordered, that the bobbins are quite full when the last tooth has reached the catch and is released by it. The rack bar, being restrained by nothing, immediately slides onwards, in consequence of the traction of the weight $f 4$, and brings the machine to repose by this very movement, for which purpose the tollowing construction is employed. A rectangular lever which has its centre of motion in $g_{4}$ is attached to the side face of the beam A, and has at the end of its horizontal arm a pulley $d t$, over which the cord $b^{4}$ of the counterweight $f t$ is passed. The end of the perpendicular arm is forked and embraces the long and thin rod $k$, to whose opposite end the fork $l \pm$ is made fast. Through this fork the band which puts the machine in motion passes down to the pulley $a 1$. With the bent lever another rod $c^{4}$ is connected at $h 4$, which lies upon the puppet $e^{3}$ with a slot at es, and hereby keeps the lever $g_{4}$ in its upright position notwithstanding the weight $f_{4}$. In the moment when, as above stated, the rack bar $q^{3}$ becomes free, the arm $p 3$ of its fork pushes in its rapid adrance against the under oblique side of $e^{4}$, raises this rod, and thereby sets the lever $g^{4}$ free, whose upright arm bends down by the traction of the weight, drives the rod $k 4$ before it into the ring it fastened to it, and thus, by means of the fork $l 4$, shifts the band upon the loose pulley $b^{l}$. But the machine may be brought to repose or put out of gear at any time merely by shifting the rod $k 4$ with the hand.

The operation of the bobbin and fly frame may be fully understood from the preceding description. A few observations remain to be made upon the cone $k^{1}$, the rack-bar $q^{3}$, and the speed of the work.

When we know the diameter of the empty bobbins, and how many turns they should make in a given time in order to wind-on the sliver delivered by the fluted rollers and the spindles; when we consider the diameters of the spindle pulleys $q$, and $t 2$, as also the drum $\mathrm{m}$, fig. 332, we may easily find the diameter which the cone must have for producing that number of turns. This is the diameter for the greatest periphery of the base. The diameter of the smaller is obtained in the same way, when the diameter of the bobbins before the last winding-on, as well as the number of turns necessary in a given time, are known.

A bobbin and fly frame of the construction just described delivers from each spindle in a day of twelve hours, from 6 to $8 \mathrm{lbs}$. of roving of the fineness of $1 \frac{1}{2}$ English counts. One person can superintend two frames, piece the broken slivers, and replace the full bobbins by empty ones. The loss of cotton wool in this machine consists in the portions carried off from the torn slivers, and must be returned to the lapping machine.

The fine bobbin and fly frame does not differ essentially from the preceding machine. The rovings from the coarse bobbin and fly frame are placed in their bobbins in a frame called the creel, behind and above the roller beam, two bobbins being allowed for one fluted portion of the rollers. These rovings are united into one, so as to increase the uniformity of the slivers.

The invention of the beautiful machine above described is due to Messrs. Cocker and Higgins, of Manchester, and as lately improved by Henry Houldsworth, jun., Esq., it may be considered the most ingeniously combined apparatus in the whole range of productive industry.

In the fine roving frame the sliver is twisted in the contrary direction to that of the coarse roving frame. For this reason the position of the cone is reversed, so as to present in succession to the band, or strap, diameters continually greater, in order that the rotation of the bobbins may be accelerated in proportion as their size is increased, because here the flier and the bobbin turn in the same direction, and the winding-on is effected by the precession of the bobbin; but if the winding-on took place by its falling behind, as in the coarse bobbin and fly frame, that is, if the flier turned less quickly than the bobbin, the rotatory speed of the bobbin would be uniformly retarded; in which case the cone would be disposed as in the coarse frame.

When, by any means whatever, a uniform length of thread is delivered by the rollers in a given time, the bobbin must wind it up as it is given out, and must therefore turn with a speed decreasing with the increase of its diameter by successive layers of thread. Hence proceeds the proposition, that the velocity of the bobbin must be in the inverse ratio of its diameter, as already explained.

With respect to the bobbin and fly frame, the twist is given to the sliver by means of a spindle, or flier, which turns in the same direction with the bobbin, but quicker or slower than it, which establishes two predicaments. The first case is where the flier turns faster than the bobbin. Here the winding-on goes in advance, as in the coarse roving frame, or as in throstle spinning, where the yarn is wound on merely in consequence of the friction of the lower disc or washer of the bobbin upon the copping rail, and of the drag of the yarn. The second case is where the flier revolves more slowly than the bobbin. Here the winding goes on in arrear, and as the bobbin 
turns faster, it must receive a peculiar motion, which is uniformiy retarded in the ratio of its increase of diameter. This is the case with the fine bobbin and fly frame. When the cone is placed as in fig. 332, the winding-on, in either the coarse or fine frame, results from the difference, whether greater or less, between the rotatory speed of the flier and bobbin.

The motion of the bobbin and spindle is simultaneous, and takes place in the same direction, with a difference varying more or less with the varying diameters of the bobbins. To render the matter still clearer, suppose for a moment the spindle to be motionless, then the bobbin must revolve with such a speed as to lap-on the roving as fast as the rollers deliver it. The sliver comes forward uniformly; but the bobbin, by its increase of diameter, must revolve with a speed progressively slower. Now, suppose the spindle set a-whirling, it is obvious that the bobbin must add to the movement requisite for winding-on the sliver, that of the spindle in the case of winding-on in arrear, or when it follows the fliers, and subtract its own motion from the twisting motion of the spindles, in the case of winding-on in adrance, that is, when the bobbin precedes or turns faster than the fliers; for the diameter of the bobbin being $1 \frac{1}{2}$ inches, 10 iurns will take up 45 inches. Deducting these 10 turns from the 30 made by the spindle in the same time, there will remain for the effective movement of the bobbin only 20 turns; or when the diameter of the bobbin becomes 3 inches, 5 turns will take up the 45 inches, if the spindle be at rest; but if it makes 30 turns in the time, the effective velocity of the bobbin will be 25 turns, $=30--5$. Hence in the fine bobbin and fly frame, the number of turns of the spindle, minus the number of turns made by the bobbin in equal times, is in the inverse ratio of the diameter of the bobbin. We thus perceive, that in the coarse frame the bobbin should more faster than the spindle, and that its speed should always diminish; whilst in the fine frame the bobbin should move slower than the spindle, but its speed should alwars increase. It is easy to conceive, therefore, why the cones are placed in reverse directions in the two machines. Not that this inversion is indispensably necessary; the cone of the fine roving frame might, in fact, be placed like that of the coarse roving frame; but as the torsion of the ruving becomes now considerable, and as on that account the bobbin would need to move still faster, which would consume a greater quantity of the moving power, it has been deemed more economical to give its movement an opposite direction.

We mentioned that the twist of the sliver in the fine roring frame was the reverse of that in the coarse; this is a habit of the spinners, for which no good reason has been given.

The divisions of the rack-bar, and the successive diameters of the cone, must be nicely adjusted to each other. The first thing to determine is, how much the rack should advance for every layer or range of roving applied to the bobbin, in order that the cone may occupy such a place that the strap which regulates the pulley barrel mar be at the proper diameter, and thus fulfil every condition. The extent of this progressive morement of the rack depends upon the greater or less taper of the cone, and the increase which the diameter of the bobbin receives with every traverse, that is, every layer of roving laid on. But care should be taken not to taper the cone too rapidly, especially in the fine roving frame, because in its progress towards the smaller end the strap would not slide with certainty and ease. We have already shown that the number of effective turns of the bobbin is inversely, as the diameter of the bobbin; or directly, as the successive diameters of the different points of the cone.

H. Houldsworth, jun. Esq. has introduced a capital improvement into the bobbin and fly frame, by his differential or equation-box mechanism, and by his spring fingers, which, by pressing the soft sliver upon the bobbin, cause at least a double quantity to be wound upon its barrel. With the description of his patent equation-bos, I shall conclude the description of the bobbin and fly-frame.

Fig. 342 represents a portion of a fly frame with Mr. Houldsworth's invention. a $a \quad a$ are the front drawing rollers, turning upon bearings in the top of the machine, and worked by a train of toothed wheels, in the way that drawing rollers are usually actuated.

From the drawing rollers, the filaments of cotton or other material, $b b$, are brought down to, and passed through the arms of the fliers $c$, mounted on the tops of the spindles $d d$, which spindles also carry the loose bobbins $e e$. In the ordinary mode of constructing such machines, the spindles are turned by cords or bands passing from a rotatory drum roind their respective pullers or whirls $f$, and the loose bobbins $e$, turn with them by the friction of their slight contact to the spindle, as before said; in the improved machine, however, the movements of the spindles and the bobbins are independent and distinct from each other, being actuated from different sources.

The main shaft of the engine $g$, turned by a band and rigger $\mathrm{A}$ as usual, communicates motion by a train of wheels $h$, through the shaft $i$, to the drawing rollers at the reverse end of the machine, and causes them to deliver the filaments to be twister. 
Upon the main shaft $g$, is mounted a cylindrical hollow box or drum-pulley, whence one cord passes to drive the whirls and spindles $f$ and $d$, and another to drive the bobbins $e$.

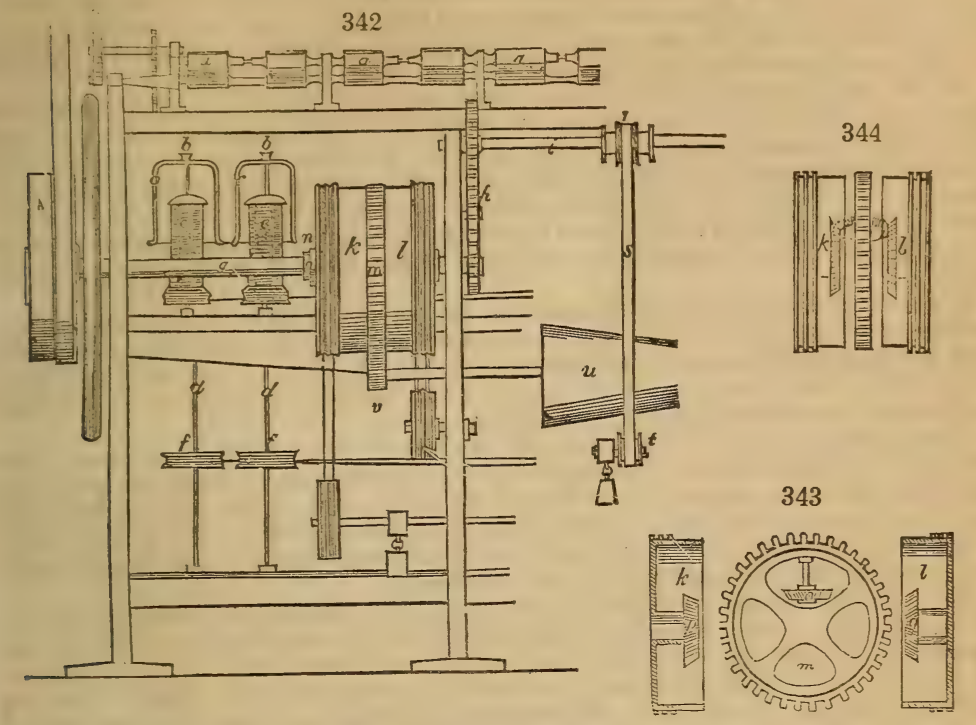

This cylindrical box pulley is made in two parts, $k$ and $l$, and slipped upon the axle with a toothed wheel $m$, intervening between them. The box and wheel are shown detached in fig. 343 , and partly in section at fig. 344. That portion of the box with its pulley marked l, is fixed to the shaft $g$; but the other part of the box and its pulley $k$, and the toothed wheel $m$, slide loosely round upon the shaft $g$, and when brought in contact and confined br a fixed collar $n$, as in the machine shown at fig. 342 , they constitute two distinct pulleys, one being intended to actuate the spindles, and the other the bobbins.

In the web of the wheel $m$, a small bevel pinion 0 , is mounted upon an axle standing at right angles to the shaft $\mathrm{g}$, which pinion is intended to take in to the two bevel pinions $p$ and $q$, respectively fixed upon bosses, embracing the shaft in the interior of the boxes $k$ and $l$. Now it being remembered that the pinion $q$, and its box $l$, are fixed to the shaft $g$, and turn with it, if the loose wheel $m$ be independently turned upon the shaft, with a different velocity; its pinion $o$, taking into $q$, will be made to revolve upon its axle, and to drive the pinion $p$, and pulley box $k$, in the same direction as the wheel $m$; and this rotatory morement of the box $k$ and wheel $m$, may be faster or slower than the shaft $g$, and box $l$, according to the velocity with which the wheel $m$ is turned.

Having explained the construction of the box pulleys $k$ and $l$, which are the peculiar features of novelty claimed under this patent, their office and advantage will be seen by describing the general movements of the machine.

The main shaft $g$, being turned by the band and rigger $A$, as above said, the train of wheels $h$, connected with it, drives the shaft $i$, which at its reverse end has a pinion (not seen in the figure) that actuates the whole series of drawing rollers $a$. Upon the shaft $i$ there is a sliding pulley $r$, carrying a band $s$, which passes down to a tension pulley $t$, and is kept distended by a weight. This band $s$, in its descent, comes in contact with the surface of the cone $u$, and causes the cone to revolve by the friction of the band running against it. The pulley $r$ is progressively slidden along the shaft $i$, by means of a rack and weight not shown, but well understood as common in these kind of machines, and which morement of the pulley is for the purpose of progressively shifting the band $s$ from the smaller to the larger diameter of the cone, in order that the speed of its rotation may gradually diminish as the bobbins fill by the winding-on of the yarns.

At the end of the axle of the cone $u$ a small pinion $v$ is fixed, which takes into the teeth of the loose wheel $m$, and, as the cone turns, drives the wheel $m$ round upon the shaft g, with a speed dependant always upon the rapidity of the rotation of the cone. Now the box pulley $l$, being fixed to the main shaft $g$, turns with one uniform speed, and by cords passing from it over guides to the whorls $f$, drires all the spindles and fliers, which twist the yarns with one continued uniform velocity; but the box pulley $k$, 
being loose upon the shaft, and actuated by the bevel pinions within, as described, is made to revolve by the rotation of the wheel $m$, independent of the shaft, and with a different speed from the pulley box $l$; cords passing from this pulley box $k$, over guides to small pulleys under the bobbins, communicate the motion, whatever it may be, of the pulley box $k$, to the bobbins, and cause them to turn, and to take up or wind tire yarn with a speed derived from this source, independent of, and different from, the speed of the spindle and flier which twist the yarn.

It will now be perceived, that these parts being all adjusted to accommodate the taking up movements to the twisting or spinning of any particular quality of yarn intended to be produced, any variations between the velocities of the spinning and taking up, which another quality of yarn may require, can easily be effected, by merely changing the pinion $v$, for one with a different number of teeth, which will cause the wheel $m$, and the pulley box $k$, to drive the bobbins faster or slower, as would be required in winding-on fine or coarse yarn, the speed of the twisting or spinning seing the same.

The rovings or spongy cords, of greater or less tenuity, made on the bobbin and fly, or tube roving frame, are either spun immediately into firm cohesive yarn, or receive a further preparation process in the stretching frame, which is, in fact, merely a mulejenny, without the second draught and second speed, and therefore need not be described at present, as it will be in its place afterwards.

The finishing machines of a cotton mill, which spin the cohesive yarn, are of two classes ; 1 . the water-twist or throstle, in which the twisting and winding are performed simultaneously upon progressive portions of the roving; and, 2 . the mule, in which the thread is drawn out and stretched, with little twist, till a certain length of about 5 feet is extended, then the torsion is completed, and the finished thread is immediately wound upon the spindles into double conical coils called cops.

The water-twist frame, so called by its inventor, Sir R. Arkwright, because it was first driven by water, is now generally superseded by the throstle frame, in which the mechanical spinning fingers, so to speak, are essentially the same, but the mode of communicating the motion of the mill-gearing to them is somewhat different. Fig. 345 exhibits a vertical section of the throstle. This machine is double, possessing upon each side of its frame a row of spindles with all their subsidiary parts. The bobbins, filled with rovings from the bobbin and fly, or the tube frame, are set up in the creel $a a$, in two ranges. $b, c, d$, are the three usual pairs of drawing rollers, through which the yarn is attenuated to the proper degree of fineness, upon the principles already explained. At its escape from the front rollers, every thread runs through a guide eyelet $e$ of wire, which gives it the vertical direction down towards the spindles $f, g$. The spindles which perform at once and uninterruptedly the twisting and winding-on of the thread delivered by the rollers, are usually made of steel, and tempered at their lower ends. They stand at $g$ in steps, pass at $v$ through a brass bush or collet which keeps them upright, and revolve with remarkable speed upon their axes. The bobbins $h$, destined to take up the yarn as it is spun, are stuck loosely upon the spindles, and rest independently of the rotation of the spindles upon the copping beam $l$, with a leather washer between. Upon the top of the spindles an iron-wire fork, called a fly or flier, $i, k$, is made fast by a left-hand screw, and has one of its forks turned round at the end into a little ring. The branch of the flier at $f$ is tubular, to allow the thread to pass through, and to escape by a little hole at its side, in order to reach the eyelet at the end of that fork. From this eyelet $i$, it proceeds directly to the bobbin. By the twirling of the spindle, the twisting of the portion of thread between the front roller $d$ and the nozzle $f$ is effected. The winding-on takes place in the following way:-Since the bobbin has no other connexion with the spindle than that of the thread, it would, but for it, remain entirely motionless, relatively to the spindle. But the bobbin is pulled after it by the thread, so that it must follow the rotation of the spindle and fly. When we consider that the thread is pinched by the front roller $d$, and is thereby kept fully upon the stretch, we perceive that the rotation of the bobbin must be the result. Suppose now the tension to be suspended for an instant, while the rollers $d$ deliver, for example, one inch of yarn. The inertia or weight of the bobbin, and its friction upon the copping beam $l$, by means of the leather washer, will, under this circumstance, cause the bobbin to hang back in a state of rest, till the said inch of yarn be wound on by the whirling of the fly $i$, and the former tension be restored. The delivery of the yarn by the drawing rollers, however, does not take place inch after inch, by starts, but at a certain continuous rate; from whence results a continuous retardation or loitering, so to speak, of the bobbins behind the spindles, just to such an amount that the delivered yarn is wound up at the same time during the rotation.

This process in spinning is essentially the same as what occurs in the fine bobbin and fly frame, but is here simplified, as the retardation regulates itself according to the diameter of the bobbin by the drag of the thread. In the fly frame the employment 
of this tension is impossible, because the roving has too little cohesion to bear the strain; and hence it is necessary to give the bobbins that independent movement of rotation which so complicates this machine.

The up and down mution of the bobbins along the spindles, which is required for the equal distribution of the yarn, and must have the same range as the length of the bobbin barrels, is performed by the following mechanism. Every copping rail, $l$, is made fast to a bar $m$, and this, which slides in a vertical groove or slot at the end of the frame, is connected by a $\operatorname{rod} n$, with an equal-armed, moveable lever $o$. The rod $p$ carries a weight $r$, suspended from this lever; another rod, $q$, connects the great lever 0 with a smaller one $s, t$, upon which a heart-shaped disc or pulley, $u$, works from below at $t$. By the rotation of the disc $u$, the arm $t$, being pressed constantly down upon it by the reaction, the weight $r$ must alternately rise and fall; and thus the copping rail $l$ must obviously move with the bobbins $h$ up and down; the bobbins upon one side of the frame rising, as those upon the other sink. Strictly considered, this copping motion should become slower as the winding-on proceeds, as in the fiy roving frame; but, on account of the smallness of the finished thread, this construction, which would render the machine complicated, is without inconvenience neglected, with the result merely that the coils of the yarn are successively more sparsely laid on, as the diameter of the bobbin increases.

The movement of the whole machine proceeds from the shaft of a horizontai drum, which drives the spindles by means of the endless bands $x x$. Each spindle is mounted with a small pulley or wharf, $w$, at its lower part, and a particular band, which goes round that wharf or whorl, and the drum $y$. The bands are not drawn tense, but hang down in a somewhat slanting direction, being kept distended only by their own weight. Thus every spindle, when its thread breaks, can readily be stopped alone, by applying a slight pressure with the hand or knee, the band meanwhile gliding loosely round the whorl.

The velocities of rotation of the three drawing rollers are, according to this arrangement, in the proportion of $1: 1 \frac{1}{2}: 8$; and as their diameters are the same, namely, one inch, the elongation of the yarn in spinning is eight-fold. If, for example, the roving was of the number $4 \frac{1}{2}$, the yarn would become No. 36 . The extension of the thread may be changed by changing the wheels of the drawing rollers. To perceive the power of this change, let us put, for example, in the place of the 18-toothed wheel of the back rollers, a wheel with 16 teeth; we shall find that the elongation will amount, in that case, only to $7 \frac{1}{2}$ times, whence the number of the yarn would come out $32=7 \frac{1}{2} \times 4 \frac{1}{2}$. The extension by the throstle is extremely various : it amounts, in some cases, to only 4 times; at others to 10,12 , or even 15 .

The copping motion of the bobbins is produced in consequence of a bevel pinion working in a small bevel wheel upon an upright shaft; while this wheel gives a slow motion by means of a worm screw to the wheel of the heart-shaped pulley $u, f i g .345$.

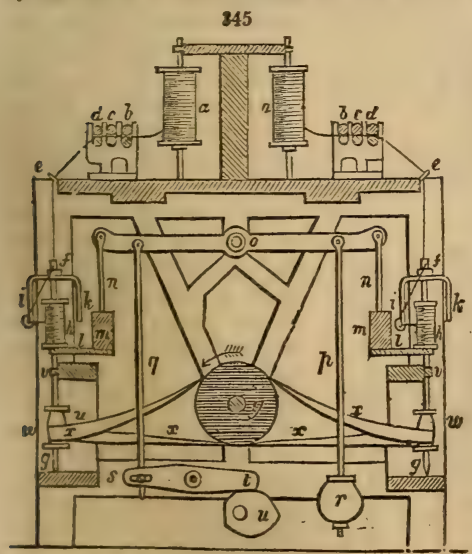

The driving pulley makes about 600 turns in a minute; and as the diameter of the drum $y$, fig. 345, is six times the diameter of the spindle wharves $w$, it will give 3600 turns to the spindle in that time. If the pulley be driven faster, for example, 700 times in a minute, it will increase the revolutions of the spindles to 4200 . The degree of twist which will be thereby imparted to the yarn, depends, with like speed of spindles, upon the rate at which the soft yarn is delivered by the drawing-rollers; for the quicker this delivery the quicker is the winding-on, and the less twist goes into a given length of yarn. If, for example, the front rollers $d$ turn 24 times in a minute, giving out of course 72 inches of yarn in this time, upon which the 3600 revolutions of the spindle are expended, there will be 50 twists to every inch of yarn. By changing the wheel-work of fig. 345 , or by sticking greater or smaller wharves upon the spindles, the proportion between their velocity and that of the drawing rollers, and thence the degree of twist, can be modified at pleasure.

The number of spindles in a throstle frame 12 feet long is about 60 on each side. The drawing rollers are coupled together as in the bobbin and fiy frame, so that each row forms one continuous cylinder. There is a complete roller beam on each side; each of the rollers of the front row is pressed bv its top rollers with a weight of ten a 
twelve pounds; but those of the middle and back rows bear weights of only one pound. In the throstles, there is a guide bar which traverses a small way horizontally to the lef and right, in front of the roller beam, to lead the thread along different points of the rollers, and thus prevent the leather of the top ones from being grooved by its constant pressure in one line.

For the service of 240 spindles, in two double frames, one young woman and an assistant piecer are sufficient. They mend the brokien ends, and replace the empty bobbins in the creel with full ones, and the full bobbins of the throstle by empty ones. The average quantity of yarn turned off in a week of 69 hours is about 24 hanks per spindle of 30 's twist. Throstle yarn is of a firm wiry quality, adapted to the warps of fustians and other strong stuffs, as well as to the manufacture of stockings and serving thread.

There are many modifications of the throstle syst $\in m$ besides the one above described; the most celebrated of which are Danforth's, called the American throstle, Montgomery's, and Gore's. I must refer for an account of them to my work entitled "The Cotton Manufacture of Great Britain," where they are minutely described and illustrated with accurate figures.

Mule-spinning.-The general principles of the mule have been already stated. This machine is so named because it is the offspring, so to speak, of two older machines, the jenny and the water-frame. A mule is mounted with from 240 to 1000 spindles, and pins, of course, as many threads.

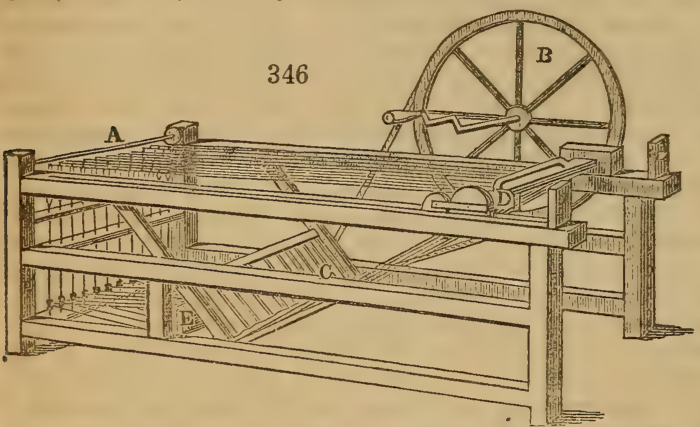

Fig. 346 represents the original jenny of Hargreaves, by which one person was enabled to spin from 16 to 40 threads at once. The soft cords of rovings wound in double conical cops upon skewers were placed in the inclined frame at $\mathrm{c}$; the spindles for first twisting and then winding-on the spun yarn were set upright in steps and bushes at $A$, being furnished near their lower ends with whorls, and endless cords, which were driven by passing round the longrevolving drum of tin plate $E$. $D$ is the clasp or clove, having a handle for lifting its upper jaw a little way, in order to allow a few inches of the soft roving to be 347

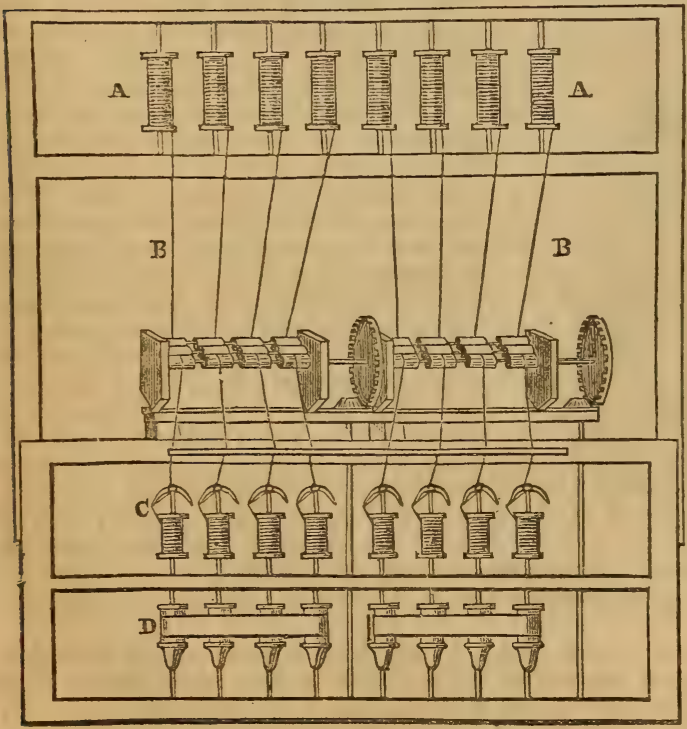

introduced. The compound clove $\mathrm{D}$ being now pushed forward upon its îiction wheels to $\mathrm{A}$, was next gradually drawn backward, while the spindles were made to revolve with proper speed by the right hand of the operative turning the fly-wheel B. Whenever one stretch was thereby spun, the clove frame was slid home towards $\mathrm{A}$; the spindles being simultaneously whirled slowly to take up the yarn, which was laid on in a conical cop by the du: depression of the faller wire at $A$ with the spinner's left hand.

Fig. 347 is a diagram of Arkwright's original water-frame spinning machine, called afterwards the water - twist 


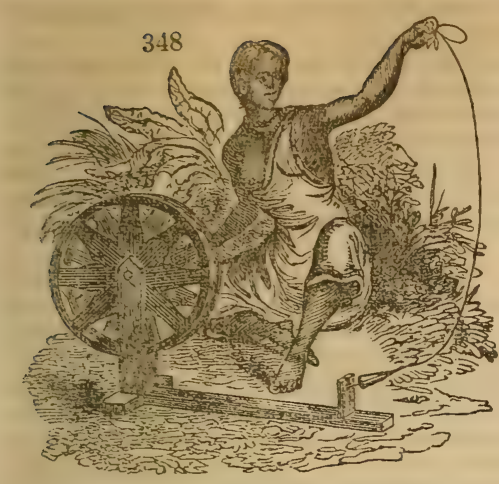

the most diligent spinsters of Hindostan.

Fig. 349 is a transverse section of the mule, in which its principal parts are shown.

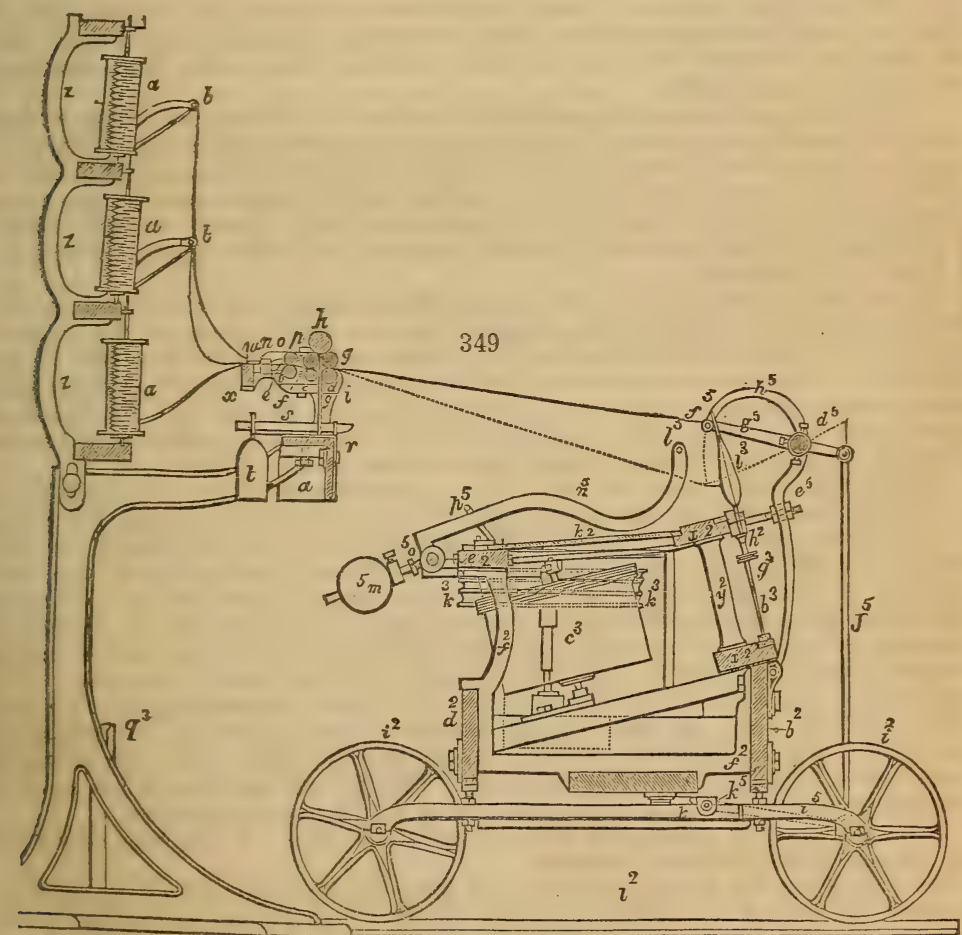

The machine consists of two main parts; a fixed one corresponding in some measure to the water-frame or throstle, and a moveable one corresponding to the jenny. The first contains in a suitable frame the drawing roller-beam and the chief moving machinery: the second is called the carriage, in which the remainder of the moving mechanism and the spindles are mounted.

The fiame of the fixed part consists of two upright sides, and two or more intermediate parallel bearings, unon which the horizontal roller beam $a$, the basis of the drawing rollers is supported. $b, c, d$, are the three ranges of fluted iron rollers; $e, f, g$, are the upper iron rollers covered with leather; $h$, the wooden wiper-rollers covered with flannel, which being occasionally rubbed with chalk, imparts some of it to the pressure rollers beneath, so as to prevent the cotton filaments adhering to them. The rollers are made through. 
out the whole length of the mule in portions containing six flutings, which are coupled together by squared ends fitted into square holes.

The skewers upon which the bobbins containing the rovings from the bobbin and fily or stretching frame are set up, are seen at $a^{1}, a^{1}, a^{1}$, arranged in three rows in the creel $z$. The soft threads unwound from these bobbins, in their way to the drawing zollers, pass first through eyelets in the ends of the wire arms $b^{1}$, then through the rings or eyes of the guide bar $w$, and enter between the back pair of rollers. The number of these bobbins is equal to the number of spindles in the mule, and twice as great as the number of fluted portions of the rollers; for two threads are assigned to each portion.

The carriage consists of two cast-iron side pieces, and several cast-iron intermediate similar pieces, such as $f 2$, which all together are made fast to the planks $b^{2}, c^{2}, d 2$. The top is covered in with the plank $k 2$. The carriage runs by means of its cast-iron grooved wheels, upon the cast-iron railway $l 2$, which is fixed level on the floor.

The spindles stand upon the carriage in a frame, which consists of two slant rails $x^{2}$, $x^{2}$, connected by two slender rods $y^{2}$, and which frame may be set more or less obliquely. The lower rail carries the brass steps for the points of the spindles bs; upon the upper rail brass slips are fixed pierced with holes through which the tops of the spindles play. The spindles are as usual made of steel, perfectly straight, turned truly round, and are all arranged in one plane. To each of them a small wooden or east-iron whorl $\mathrm{g}^{2}$ is made fast. They are distributed into groups of 24 , and the whorls are arranged at such different heights, that only two of them in each group are upon a level with each other. A small brass head $h 2$, which every spindle has beneath the upier slant rail of the frame $x^{2}$, prevents their sitting down into the step, during their rotation, or sliding off their cop of yarn.

$c^{3}$ are drums, mounted in the carriage in a plane at right angles to the plane in which the spindles are placed. At top they have a double groove for a cord to run in, and the motion which they receive from the great fly wheel, or rim of the mule (not visible in this view) they impart to the spindles. Such a drum is assigned to every 24 spindles; and therefore a mule of 480 spindles contains 20 drums. In the middle of the carriage is seen the horizontal pulley $k 3$, furnished with three grooves, which stands in a line with the drums $c^{3}$.

The motion is given to the drums $c 3$, upon the right hand half of the carriage, by a single endless band or cord which proceeds from the middle groove of the pulley $k$. The rotation of the spindles is produced by a slender cord, of which there are 12 upon each drum $c^{3}$; because every such cord goes round the drum, and also every two wharves which stand at the same level upon the spindles. It is obvious that the drums, and consequently the spindles, must continue to revolve as long as the main rim of the mule is turned, whether the carriage be at rest or in motion upon its railway.

If we suppose the carriage to be run in to its standing point, or to be pushed home to the spot from which it starts in spinning, its back plank $d^{2}$ will strike the post $q^{3}$ upon the fixed frame, and the points of the spindles will be close in front of the roller beam. The rollers now begin to turn and to deliver threads, which receive immediately a portion of their twist from the spindles; the carriage retires from the roller beam with somewhat greater speed than the surface speed of the front rollers, whereby the threads receive a certain degree of stretching, which affects most their thicker and less twisted portions, and thereby contributes greatly to the levelness of the yarn. When the carriage has run out to the end of its course, or has completed a stretch, the fluted rollers suddenly cease to revolve (and sometimes even beforehand, when a second stretch is to be made), but the spindles continue to whirl till the fully extended threads have received the proper second or after-twist. Then the carriage must be put up, or run back towards the rollers, and the threads must be wound upon the spindles.

This is the order of movements which belong to the mule. It has been shown how the rotation of the spindles is produced.

For winding-on the yarn the carriage has a pecnliar apparatus, which we shall now describe. In front of it, through the whole extent to the right hand as well as the left, a slender iron rod, $d 5$, runs horizontally along, in a line somewhat higher than the middle of the copping portion of the spindles, and is supported by several props, such as e5. Upon each end of the two rods, $d^{5}$, there is an arm, $g^{5}$; and betwixt these arms an iron wire, called the copping wire, $f 5$, is stretched, parallel with the rod $d 5$. For the support of this wire, there are several slender bent arms $h 5$ extended from the rod $d 5$ at several points betwixt the straight arms $g^{5}$. The rod $d 5$ has, besides, a wooden handle at the place opyosite to where the spinner stands, by which it can be readily grasped. This movement is applied at the left division of the machine, and it is communicated to the right by an apparatus which resembles a crane's bill. The two arms, $\mathrm{g}^{5}$, in the middle of the machine, project over the rods $d 5$, and are connected by hinges with two vertical rods $j 5$, which hang together downwards in like manner with two arms $i 5$, proceeding from a horizontal axis $k 5$. 
By means of that apparatus the yarn is wound upon the spindles in the following manner. As long as the stretching and twisting go on, the threads form an obtuse angle with the spindles, and thereby slide continually over their smooth rounded tips during theif revolution, without the possibility of coiling upon them. When, however, the spinnink process is completed, the spinner seizes the carriage with his left hand and pushes it back towards the roller beam, while with his right hand he turns round the handle of the rim or fly wheel, and eonsequently the spindles. At the same time, by means of the handle upon the rod $d 5$, he moves the copping-wire $f 5$, so that it presses down all the threads at once, and places them in a direction nearly perpendicular to the spindles; as shown by the dotted line $y 5$. That this movement of the copping wire, however, may take place without injury to the yarn, it is necessary to turn the rim beforehand a little in the opposite direction, so that the threads may get uncoiled from the upper part of the spindles, and become slack; an operation called in technical language the backing off. The range upon which the threads should be wound, in order to form a conical cop upon the spindle, is hit by depressing the copping wire to various angles, nicely graduated by an experienced eye. This faller wire alone is not, however, sufficient for the purpose of winding-on a seemly cop, as there are always some loose threads which it eannot reach without breaking others.

Another wire called the counter-faller, $l 5$, must be applied under the threads. It may be raised to an elevation limited by the angular piece $p 5$; and is counterpoised by a very light weight $m^{5}$, applied through the bent lever $n^{5}$, which turns upon the fulcrum 05 . This wire, which applies but a gentle pressure, gives tension to all the threads, and brings them regularly into the height and range of the faller $f 5$. This wire must be raised once more, whenever the carriage approaches the roller beam. At this instant a new stretch commences; the rollers begin again to revolve, and the carriage resumes its former course. These motions are performed by the automatic machinery.

There is a little eccentric pulley mechanism for moving the guide beam to and frc with the soft yarns, as they enter between the back rollers. On the right hand end of the back roller shaft, a worm screw is formed which works into the oblique teeth of a pinion attached to the end of the guide beam, in which there is a series of holes for the passage of the threads, two threads being assigned to each fluted roller. In the flat disc of the pinion, an eccentric pin stands up which takes into the jointed lever upon the end of the guide beam, and, as it revolves, pushes that beam alternately to the left and the right by a space equal to its eccentricity. This motion is exceedingly slow, since for each revolution of the back roller, the pinion advances only by one tooth out of the 33 which are ent in its circumference.

After counting the number of teeth in the different wheels and pinions of the mule, or measuring their relative diameters, it is easy to compute the extension and twist of the yarns; and when the last fineness is given to ascertain their marketable value. Let the ratio of speed between the three drawing rollers be $1: 1 \frac{3}{22}: 7 \frac{1}{2}$; and the diameter of the back and middle roller three quarters of an inch : that of the front roller one inch; in which case the draving is thereby increased $1 \frac{1}{3}$ times, and $7 \frac{1}{2} \times 1 \frac{1}{2}=10$. If the rovings in the creel bobbins have been No. 4, the yarn, after passing through the rollers, will be No. 40. By altering the change pinion (not visible in this view) the fineness may be changed within certain limits, by altering the relative speed of the rollers. For one revolution of the great rim or fly wheel of the mule, the front roller makes about 6 tenths of a turn, and delivers therefore $22 \cdot 6$ lines or 12 ths of an inch of yarn, which, in consequence of the tenfold draught through the rollers, corresponds to 2.26 lines of roving fed in at the back rollers. The spindles or their whorls make about 66 revolutions for one turn of the rim. The pulleys or grooved wheels on which the carriage runs, perform 0.107 part of a turn while the rim makes one revolution, and move the carriage $24 \cdot 1$ lines upon its rails, the wheels being 6 inches in diameter.

The 22.6 lines of soft yarn delivered by the front rollers will be stretched $1 \frac{1}{2}$ lines by the carriage advancing $24 \cdot 1$ lines in the same time. Let the length of the railway, or of each stretch, be 5 feet, the carriage will complete its course after 30 revolutions of the rim wheel, and the 5 feet length of yarn (of which $56 \frac{1}{2}$ inches issue from the drawing rollers, and $3 \frac{1}{2}$ inches proceed from the stretching) is, by the simultaneous whirling of the spindles, twisted 1980 times, being at the rate of 33 twists for every inch. The second twist, which the threads receive after the carriage has come to rcpose, is regulated according to the quality of the cotton wool, and the purpose for which the yarn is spun. For warp yarn of No. 40 or 50 , for example, 6 or 8 turns of the rim wheel, that is, from 396 to 528 whirls of the spindles for the whole stretch, therefore from 7 to 9 twists per inch will be sufficient. The finished yarn thus receives from 40 to 42 twists per inch.

One spinner attends to two mules, which face each other, so that he needs merely turn round in the spot where he stands, to find himself in the proper position for the other mule. For this reason the rim wheel and handle, by which he operates, are not 
placed in the middle of the length of the machine, but about two fifths of the spindles are to the righ: hand and three fifths to the left; the rim wheel being towards his right hand. The carriage of the one mule is in the act of going out and spinning, while that of the other is finishing its twist, and being put up by the spinner.

The quantity of Jarn manufactured by a mule in a given time, depends directly upon the number of the spindles, and upon the time taken to complete every stretch of the carriage. Many circumstances have an indirect influence upon that quantity; and particularly the degree of skill possessed by the spinner. The better the machine, the steadier and softer all its parts revolve, the better and more abundant is its production. When the toothed wheels do not work iruly into their pinions, when the spindles shake in their bushes, or are not accurately made, many threads break, and the work is much injured and retarded. The better the staple of the cotton wool, and the more careful has been its preparation in the carding, drawing, and roving processes, the more easy and excellent the spinning will become: warmth, dryness, cold, and moisture have great influence on the ductility, so to speak, of cotton. A temperature of $65^{\circ} \mathrm{F}$., with an atmosphere not too arid, is found most suitable to the operations of a spinning mill. The finer the yarn, the slower is the spinning. For numbers from 20 to 36 , from 2 to 3 stretches of warp may be made in a minute, and nearly 3 stretches of weft; for numbers above 50 up to 100, about 2 stretches; and for numbers from 100 to 150 , one stretch in the minute. Still finer yarns are spun more slowly, which is not wonderful, since, in the fine spinning mills of England, the mules usually contain upwards of 500 spindles each, in order that one operative may manage a great number of them, and thereby earn such high wages as shall fully remunerate his assiduity and skill.

In spinning fine numbers, the second speed is given before the carriage is run out to the end of its railway; during which course of about six inches, it is made to move very slowly. This is called the second stretch, and is of use in making the yarn level by drawing down the thicker parts of it, which take on the twist less readily than the thinner, and therefore remain softer and more extensible. The stretch may therefore be divided into three stages. The carriage first moves steadily out for about 4 feet, while the drawing rollers and spindles are in full play; now the rollers stop, but the spindles go on whirling with accelerated speed, and the carriage advances slowly about 6 inches more; then it also comes to rest, while the spindles continue to revolve for a little longer, to give the final degree of twist. The acceleration of the spindles in the second and third stages, which has no other object but to save time, is effected by a mechanism called the counter, which shifts the driving band, at the proper time, upon the loose pulley, and, moreover, a second band, which had, till now, lain upon its loose pulley, upon a small driving pulley of the rim-shaft. At length, both bands are shifted upon their loose pulleys, and the mule comes to a state of quiescence.

The SELF-ACTOR MULE, or the IRON MAN, as it has been called in Lancashire, is an invention to which the combinations among the operative spinners obliged the masters to have recourse. It now spins good yarn up to $40 \mathrm{~s}$ with great uniformity and promptitude, and requires only juvenile hands to conduct it, to piece the broken yarns, to replace the bobbins of rovings in the creel, and to remove the finished cops from the spindies.

The self-acting mules were first constructed, I believe, by Messrs. Eaton, formerly of Manchester, who mounted ten or twelve of them in that town, four at Wiln, in Derbyshire, and a few in France. From their great complexity and small productiveness, the whole were soon relinquished, except those at Wiln. M. de Jong obtainel two patents for self-acting mules, and put twelve of them in operation in a mill at Warrington, of which he was part proprietor; but with an unsuccessful result. I saw the débris of one of M. de Jong's self-actors in the factory of M. Nicholas Schlumberger, at Guebwiller, in Alsace, where the machine had been worked for three months, without advantage, nnder the care of the inventor, who is a native of that valley.

The first approximation to a successful accomplishment of the objects in view, was an invention of a self-acting mule, by Mr. Roberts, of Manchester; one of the principa] points of which was the mode of governing the winding-on of the yarn into the form of a cop; the entire novelty and great ingenuity of which invention was universally admitted, and proved the main step to the final accornplishment of what had so long been a desideratum. For that invention a patent was obtained in 1825 , and several headstocks upon the principle were made, which are still working successfully.

In $1830, \mathrm{Mr}$. Roberts obtained a patent for the invention of certain improvements; and by a combination of both his inventions, he produced a self-acting mule, which is generally admitted to have exceeded the most sanguine expectations, and which has been extensively adopted. There are probably, at present, upwards of half a million of spin. dles of Messrs. Sharp, Roberts, and Co.'s construction, at work in the United Kingdom, and giving great satisfaction to their possessors. T'he advantages of these self-actors are the following:- 
The saving of a spinner's wages to each pair of mules, piecers only being required, as one overlooker is sufficient to manage six or eight pairs of mules. The produciinn of a greater quantity of yarn, in the ratio of from 15 to 20 per cent. The yarn possesses a more uniform degree of twist, and is not liable to be strained during the spinning, or in winding-on, to form the cop; consequently, fewer threads are broken in these processes, and the yarn, from having fewer piecings, is more regular.

The cops are made firmer, of better shape, and with undeviating uniformity; and, from being more regularly and firmly wound, contain from one third to one half more yarn than cops of equal bulk wound by hand; they are consequently less liable to injury in packing or in carriage, and the expense of packages and freight (when charged by measurement) is considerably reduced.

From the cops being more regularly and firmly wound, combined with their superior formation, the yarn intended for warps less frequently breaks in winding or reeling, consequently there is a considerable saving of waste in those processes.

Secondly, the advantages connected with weaving.

The cops being more regularly and firmly wound, the yarn, when used as weft, seldom breaks in weaving; and as the cops also contain a greater quantity of weft, there are fewer bottoms, consequently there is a very material saving of waste in the process of weaving.

From those combined circumstances, the quality of the cloth is improved, by being more free from defects caused by the breakage of the warp or weft, as well as the selvages being more regular.

The looms can also be worked at greater speed; and, from there being fewer stoppages, a greater quantity of cloth may be produced.

That the advantages thus enumerated, as derivable from the use of self-acting mules, have not been overrated, but, in many instances, have been considerably exceeded, I have, by extensive personal inquiry and observation, had ample opportunity of ascertaining.

Statement of the quantity of yarn produced on Messrs. Sharp, Roberts, and Co.'s selfacting mules, in twelve working hours, including the usual stoppages connected with spinning, estimated on the average of upwards of twenty mills :-

No. of Yarn.

$\begin{array}{llll}16 & - & - & 4 \frac{1}{2} \text { hanks } \\ 24 & - & - & 4 \frac{1}{4}- \\ 32 & - & - & 4 \\ 40 & - & - & 3 \frac{2}{4}\end{array}$

No, of Weft.

$4 \frac{7}{8}$ hanks per spindle.

$4 \frac{5}{8}$

$4 \frac{3}{8}$

$4 \frac{1}{8}$

Of the intermediate numbers the quantities are proportionate.

Results of trials made by Messrs. Sharp, Roberts, and Co., at various mills, to ascertain the comparative power required to work self-acting mules, in reference to handmules, during the spinning, up to the period of backing off.

Particulars of the trials referred to, and their results:-

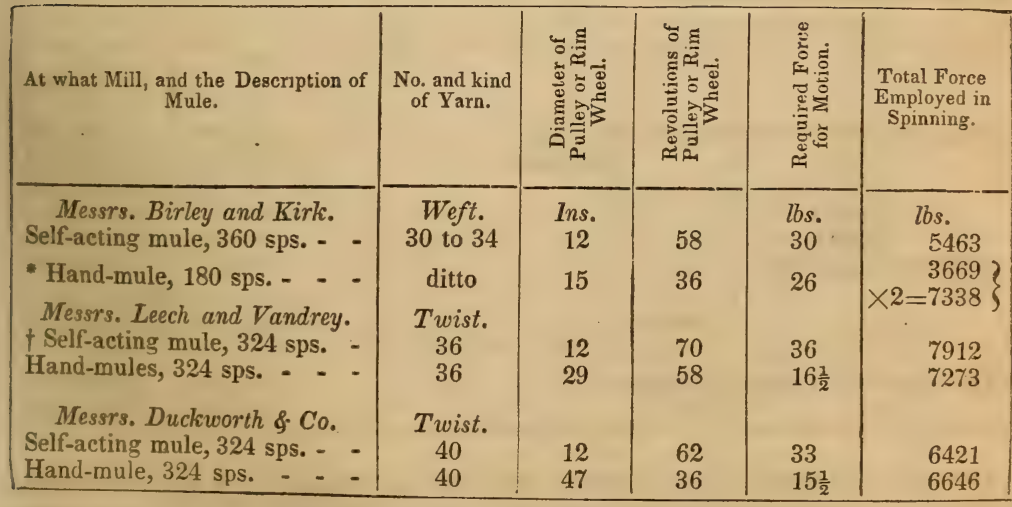

The mode adopted to make the trials was as follows, viz. :

A force, indicated by weight in pounds, was applied to the strap working upon the

* The trial was disadvantageous for the hand-mules, being two for 360 spindles.

$t$ The trial was clisadvantageous for the self-acting mules, being driven by a very short and light vertical otran, the hand-mul having a long horizontal strap. 
driving-pulley of the respective mules, sufficient to maintain the motion of the inule whilst spunning, which weight, being multiplied by the length of strap delivered by each rerolution of the puller, and again by the number of revolutions made by the pulley whilst spinning, gare the total force in pounds, applied to the respectire mules whilst spinning; for instance, suppose a mule to be driven by a pulley 12 inches diameter $(3 \cdot 14$ feet in circumference), such pulley making 58 revolutions during the spinning as above, and that it required a force equal to $30 \mathrm{lbs}$. weight to maintain the motion of the mule, then $30 \mathrm{lbs}: \times 3 \cdot 14$ feet circumference of pulley $\times 58$ revolutions in spinning $=5,463 \mathrm{lbs}$. of force employed during the spinning, to the period of backing off.

Mr. James Smith, of Deanstone cotton works in Scolland, obtained a patent for the invention of a self-actor, in Februart, 1834. He does not perform the backing-off by reversing the rotation of the spindle, as in common mules, or as in Mr. Roberts', but by elerating the counterfaller wire, which, being below the ends of the yarn or thread, along the whole extent of the carriage, thereby pulls off or strips the spiral coils at the point of the spindle, instead of unwinding them, as of old. This morement he considers to be of great importance towards simplifying the machiners for rendering the mule self-acting; and the particular way in which he brings the stripper into action is no doubt ingenious, but it has been supposed by many to strain the rarn. He claims as his invention the application and adaptation of a mangle wheel or mangle rack to the mule, for effecting certain successive movements, either separately or in conjunction; he claims that arrangement of the carriages of a pair of mules, by which the streteh is caused to take place orer part of the same ground by both carriages, and therebr the space required for the working of the pair of mules is greatly diminished; and he claims the application of a weight, spring, or friction, for balancing the tension of the ends of the threads.

A patent was granted, in April, 1835, to Mr. Joseph Whitworth, engineer in Manchester, for some ingenious modifications of the mechanism of the mule, subservient to atomatic purposes. His machinerr is designed, first, to traverse the carriage in and out, by means of screws or worm-shafts, which are placed so as to keep the carriage parallel to the drawing rollers, and prevent the necessity of squaring bands, hitherto unirersally emplored : secondly, his invention consists in an improved manner of working the drums of a self-acting mule br gear; thirdly, in the means of effecting the backing off; fourthly, in the mechanism for working the faller-wire in building the cops; and fifthly, in the apparatus for effecting the winding of the rarns upon the spindles. As regards the throstles and doubling frames, his improvements apply, first, to the peculiar method of constructing and adapting the flers and spindles, and producing the drag; and, secondly, to the arrangement of the other parts of the doubling machinery.

See Lace-Making, Singeing, Textile Fabric, Thread Manufactere, and WEATING.

The Imports of Cotton Wool for home consumption into the United Kingdom were, in the year ending 5 th January -

\begin{tabular}{|c|c|c|}
\hline \multirow{3}{*}{$\begin{array}{l}\text { From the British possessions in America } \\
\text { do. do. } \\
\text { United States of America Indies } \\
\text { Brazil } \\
\text { Egrpt }\end{array}$} & 1836. & 1837. \\
\hline & $\begin{array}{r}\text { lbs. } \\
1,346,220 \\
43,404,058 \\
287,346, \pi 21 \\
26,879,779 \\
5,184, \pi 43 \\
6, \pi 89,603\end{array}$ & $\begin{array}{r}\text { lb8. } \\
1,041,434 \\
34,060,055 \\
309,027,306 \\
20,822,509 \\
7,465,774 \\
5,602,602\end{array}$ \\
\hline & $370,951,124$ & $378,019,680$ \\
\hline $\begin{array}{l}\text { The Exports of Cotton Manufactures _. } \\
\text { Yarn }\end{array}$ & \begin{tabular}{r}
\multicolumn{1}{c}{} \\
$18,511,692$ \\
$6,120,366$
\end{tabular} & \begin{tabular}{r}
\multicolumn{1}{c}{} \\
$13,625,464$ \\
$6,953,467$
\end{tabular} \\
\hline
\end{tabular}

COURT PLASTER is a considerable object of manufacture. It is made as follows :

Black silk is strained and brushed orer ten or twelve times with the following preparation:-Dissolve $\frac{1}{2}$ an ounce of balsam of benzoin in 6 ounces of rectified spirits of wine; and in a separate ressel dissolve 1 ounce of isinglass in as little water as mar be. Strain each solution, mix them, and let the mixture rest, so that any undissolred parts may sobside; when the clear liquid is cold it will form a jelly, which must be warmed befure it is applied to the silk. When the silk coated with it is quite dry, it must bo 
finished off with a coat of a solution of 4 ounces of Chian turpentine in 6 ounces of tincture of benzoin, to prevent its cracking.*

CRAPE. (Crepe, Fr.; Krepp, Germ.) A transparent textile fabric, somewhat like gauze, made of raw silk, gummed and twisted at the mill. It is woven with any cressing or tweel. When dyed black, it is much worn by ladies as a mourning dress. Crapes are crisped (crepés) or smooth; the former being double, are used in close mourning, the latter in less deep. White crape is appropriate to young unmarried females, and to virgins on taking the veil in nunneries. The silk destined for the first is spun harder than for the second; since the degree of twist, particularly of the warp, determines the degree of erisping which it assumes after being taken from the loom. It is for this purpose steeped in clear water, and rubbed with prepared wax. Crapes are all woven and dyed with the silk in the raw state. They are finished with a stiffening of gum water.

Crape is a Bolognese invention, but has been long manufactured with superior excellence at Lyons in France, and Norwich in England. There is now a magnificent fabric of it at Yarmouth, hy power-loom machinery.

There is another kind of stuff, called crepon, made either of fine wool, or of wool and silk, of which the warp is twisted much harder than the weft. The crepons of Naples consist altogether of silk.

CRAYONS. (Eng. and Fr.; Pastelstifte, Germ.) Slender, soft, and somewhat friable cylinders, variously colored for delineating figures upon paper, usually ealled chalk drawings. Red, green, brown, and other colored crayons, are made with fine pipe or china clay paste, intimately mixed with earthy or metallic pigments, or in general with body or surface colors, then moulded and dried. The brothers Joel, in Paris, employ as crayon cement the following composition : 6 parts of shellac, 4 parts of spirit of wine, 2 parts of turpentine, 12 parts of a coloring powder, such as Prussianblue, orpiment, white lead, vermilion, \&c., and 12 parts of blue clay. The clay being elutriated, passed through a hair sieve, and dried, is to be well incorporated by trituration with the solution of the shellac in the spirit of wine, the turpentine, and the pigment; and the doughy mass is to be pressed in proper moulds, so as to acquire the desired shape. They are then dried by a stove heat.

In order to make cylindrical crayons, a copper cylinder is employed, about 2 inches in diameter, and $1 \frac{1}{2}$ inches long, open at one end, and closed at the other with a perforated plate, containing holes corresponding to the sizes of the crayons. The paste is introduced into the open end, and forced through the holes of the bottom by a piston moved by a strong press. The vermicular pieces that pass through are cut to the proper lengths, and dried. As the quality of the crayons depends entirely upon the fineness of the paste, mechanical means must be resorted to for effecting this object in the best manner. The following machine has been found to answer the purpose exceed ingly well.

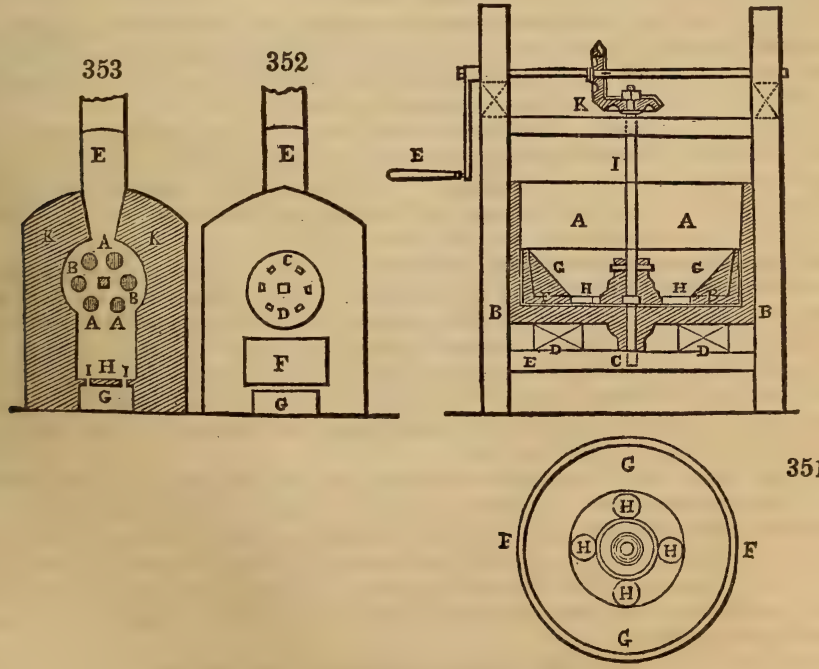


Fig. 350 is a vertical section through the centre of the crayon mill. Fig. 351 is a view of the mill from above. A, the nill tub, whose bottom $B$ must be a hard flat plate of cast-iron; the sides A being of wood or iron at pleasure. In the centre of the bottom there is a pivot c, screwed into a socket cast upon the bottom, and which may be strengthened by two cross bars $D$, made fast to the frame $E$. $F$, the millstone of castiron, concare, whose diameter is considerably smaller than that of the ressel $A$; it is furnished within with a circular basin of wood $G$, which receives the materials to be ground, and directs them to the holes $\mathrm{H}$, which allow them to pass down between the uncer part of the muller, and the bottom of the tub, to undergo trituration.

By the centrifugal motion, the paste is driven towards the sides of the ressel, rises over the sides of the muller, and comes again through the holes $H$, so as to be repeatedly subjected to the grinding operation. This millstone is mounted upon an upright shaft $\mathrm{I}$, which receives rotatory motion from the bevel wheel work $\mathrm{K}$, driven by the winch $\mathrm{L}$.

The furnace in which some kinds of crayons, and especially the factitious blacklead pencils are baked, is represented in fig. 352 in a front elevation; and in fig. 353 , which is a vertical section through the middle of the chimney.

A A, six tubes of greater or less size, according as the substance of the crayons is a better or worse conductor of heat. These tubes, into which the crayons intended for baking are to be put, traverse horizontally the laboratory в of the furvace, and are supported by two plates $\mathrm{c}$, pierced with six square holes for corering the axes of the tubes A. These two plates are hung upon a common axis D; one of them, with a ledge, shuts the cylindrical part of the furnace, as is shown in the figure. At the extremity of the bottom, the axis $D$ is supported by an iron fork fixed in the brickwork; at the frort it crosses the plate c, and lets through an end about four inches square to receive a key, by means of which the axis $\mathrm{D}$ may be turned round at pleasure, and thereby the two plates c, and the six tubes $A$, are thus exposed in succession to the action of the fire in an equal manner upon each of their sides. At the two extremities of the furnace are two chimneys $\mathrm{E}$, for the purpose of diffusing the heat more equably orer the body of the crayons. F, fig. 352, is the door of the fire-place, by which the fuel is introduced; G, fig. 353 , the ash-pit; $\mathrm{H}$, the fire-place; $\mathrm{I}$, holes of the grate which separate the fireplace from the ash-pit; $\kappa$, brickwork exterior to the furnace.

General Lomet proposes the following composition for red crayons. He takes the softest hematite, grinds it upon a porphyry slab; and then carefully elutriates it. He makes it into a plastic paste with gum arabic and a little white soap, which he forms by moulding, as above, through a syringe, and drying, into crayons. The proportions of the ingredients require to be carefully studied.

CRAYONS, lithographic. Various formulæ have been given for the formation of these crayons. One of these prescribes, white wax, 4 parts ; hard tallow-soap, shellac, of each 2 parts; lamp black, 1 part. Another is, dried tallow soap and white wax, each 6 parts; lamp black, 1 part. This mixture, being fused with a gentle heat, is to be cast into moulds for forming crayons of a proper size.

CREOSOTE, or the flesh-preserver, from kpras and $\sigma \omega \zeta \omega$, is the most important of the five new chemical products obtained from wood tar by Dr. Reichenbach. The other four, paraffine, eupione, picamar, and pittacal, have hitherto been applied to no use in the arts, and may be regarded at present as mere analytical curiosities.

Creosote may be prepared either from tar or from crude pyroligneous acid. The tar must be distilled till it acquires the consistence of pitch, and at the utmost till it begins to exhale the white vapors of paraffine. The liquor which passes into the receiver divides itself into 3 strata, a watery one in the middle, placed between a heavy and a light oil. The lower stratum alone is adapted to the preparation of creosote.

1. The liquor, being saturated with carbonate of potash, is to be allowed to settle, and the oily matter which floats at top is to be decanted off. When this oil is distilled, it affords, at first, products lighter than water, which are to be rejected, but the heavier oil which follows is to be separated, washed repeatedly by agitation, with fresh portions of dilute phosphoric acid, to free it from ammonia, then left some time at rest, after which it must be washed by water from all traces of acidity, and finally distilled along with new portion of dilute phosphoric acid, taking care to cohobate, or pour back the distilled product repeatedly into the retort.

2. The oily liquid thus rectified is colorless; it contains much creosole, but at the same time some eupione, \&c. It must therefore be mixed with potash ley at $1 \cdot 12 \mathrm{sp}$. grav., which dissolves the creosote. The upione floats upon the surface of that solution, and may be decanted off. The alkaline solution is to be exposed to the air, till it நlackens by decomposition of some foreign matter. The potash being then saturated with dilute sulphuric acid, the creosote becomes free, when it may be decanted or syphoned off and distilled.

3. The treatment by potash, sulphuric acid, \&c., is to be repeated upon the brownish 
sreosote till it remains colorless, or nearly so, even upon exposure to air. It must be now dissolved in the strongest potash ley, subjected to distillation anew, and, lastly, re distilled with the rejection of the first products which contain much water, retaining only the following, but taking care not to push the process too far.

In operating upon prroligneous acid, if we dissolve effloresced sulphate of soda in it to saturation, at the temperature of $167^{\circ} \mathrm{F}$., the creosote oil will separate, and float upon the surface. It is to be decanted, left in repose for some days, during which it will part with a fresh portion of the vinegar and salt. Being now saturated while hot, with carbonate of potash, and distilled with water, an oily liquor is obtained, of a pale yellow color. This is to be rectified by phosphoric acid, \&c., like the crude product of creosote from tar.

Creosote is apparently composed of 76.2 carbon, $7 \cdot 8$ hydrogen, and 16.0 oxygen, in 100 narts. It is an oily looking liquid, slightly greasy to the touch, void of color, having an acrid burning taste, and capable of corroding the epidermis in a short time. It possesses a penetrating disagreeable smell, like that of highly smoked hams, and, when inhaled up the nostrils, causes a flow of tears. Its specific gravity is 1.037 , at $58^{\circ} \mathrm{F}$. Its consistence is similar to that of oil of almonds. It has no action upon the colors of litmus or turmeric, but communicates to white paper a stain which disappears spontaneously in a few hours, and rapidly by the application of heat.

It boils without decomposition at $398^{\circ} \mathrm{F}$, under the average barometric pressure, remains fluid at $16^{\circ} \mathrm{F}$, is a non-conductor of electricity, refracts light powerfully, and burns in a lamp with a ruddy smoky flame.

When mixed with water at $58^{\circ} \mathrm{F}$. it forms two different combinations, the first being a solution of 1 part of creosote in 400 of water; the second, a combination of 1 part of water with 10 parts of creosote. It unites in all proportions with alcohol, hydric ether, acetic ether, naptha, eupione, carburet of sulphur, \&c.

Creosote dissolves a laroe quantity of iodine and phosphorus, as also of sulphur with the aid of heat, but it deposites the greater part of them in crystals, on cooling. It combines with potash, soda, ammonia, lime, baryta, and oxyde of copper. Oxyde of mercury converts creosote into a resinous matter, while itself is reduced to the metallic state. Strong sulphuric and nitric acids decompose it.

Creosote dissolves several salts, particularly the acetates, and the chlorides of calcium and in; it reduces the nitrate and acetate of silver. It also dissolves indigo blue; a remarkable circumstance. Its action upon animal matters is very interesting. It coagulates albumen, and prevents the putrefaction of butchers' meat and fish. For this purpose these substances must be steeped a quarter of an hour in a weak watery solution of creosote, then drained and hung up in the air to dry. Hence Reichenbach has inferred that it is owing to the presence of creosote that meat is cured by smoking; but he is not correct in ascribing the effect to the mere coagulation of the albumen, since fibrine aione, without creosote, will putrefy in the course of 24 hours, during the heats of summer. I kills plants and smail animals. It preserves flour paste unchanged for a long time.

Creosote exists in the tar of beech-wood, to the amount of from 20 to 25 per cent., and in crude pyroligneous acid, to that of $1 \frac{1}{2}$.

It ought to be kept in well-stoppered bottles, because when left open it becomes progressively yellow, brown, and thick.

Creosote has considerable power upon the nervous system, and has been applied to the teeth with adrantage in odontalgia, as well as to the skin in recent scalds. But its medicinal and surgical virtues have been much exaggerated. Its flesh-preserving quality is rendered of little use, from the difficulty of removing the rank flavor which it imparts.

CRUCIBLES (Creusets, Fr.; Schmelzticgel, Germ.) are small conical vessels, narrower at the bottom than the mouth, for reducing ores in docimasy by the dry analysis, for fusing mixtures of earthy and other substances, for melting metals, and compounding metallic alloys. They ought to be refractory in the strongest heats, not readily acted upon by the substances ignited in them, not porous to liquids, and capable of bearing considerable alternations of temperature without cracking; on which account they should not be made too thick. The best crucibles are formed from a pure fire clay, mixed with finely ground cement of old crucibles, and a portion of black-lead or graphite. Some pounded coke may be mixed with the plumbago. The clay should be prepared in a similar way as for making pottery ware; the vessels after being formed must be slowly dried, and then properly baked in the kiln. Crucibles formed of a mixture of 8 parts in bulk of Stourbridge clay and cement, 5 of coke, and 4 of graphite, have been found to stand 23 meltings of 76 pounds of iron each, in the Royal Berlin foundry. Such crucibles resisted the greatest possible heat that could be produced, in which even wrought iron was melted, cqual to $150^{\circ}$ or $155^{\circ}$ Wedgewood; and bore sudlen cooling without cracking. Another composition for brass-founding crucibles is the following:- $\frac{1}{2}$ Stourbridge clay; $\frac{1}{4}$ burned clay cement; $\frac{1}{8}$ coke powder; $\frac{1}{8}$ pipe 
clay. The pasty mass must be compressed in moulds. The Hessian crucibles from ireat Almerode and Epterode are made from a fire clay which contains a little iron, but . no lime; it is incorporated with silicious sand. The dough is compressed in a mould, dried, and strongly kilned. They stand saline and leaden fluxes in docimastic operations very well; are rather porous on account of the coarseness of the sand, but are thereby less apt to crack from sudden heating or cooling. They melt under the fusing point of bar iron. Beaufay in Paris has lately succeeded in making a tolerable imitation of the Hessian crucibles with a fire clay found near Namur in the Ardennes.

Berthier has published the following elaborate analyses of several kinds of crusibles :-

\begin{tabular}{|c|c|c|c|c|c|c|c|}
\hline & Hessian. & Beaufay. & $\begin{array}{l}\text { English for } \\
\text { Cast Steel. }\end{array}$ & $\begin{array}{c}\text { St. Etienne } \\
\text { for } \\
\text { Cast Steel. }\end{array}$ & $\begin{array}{l}\text { Glass Pots } \\
\text { at Nemour }\end{array}$ & $\begin{array}{l}\text { Bohemian } \\
\text { Glass Pots. }\end{array}$ & $\begin{array}{l}\text { Glass Pots } \\
\text { of Creusot }\end{array}$ \\
\hline Silica & $70 \cdot 9$ & $64 \cdot 6$ & $63 \cdot 7$ & $65 \cdot 2$ & $67 \cdot 4$ & $68 \cdot 0$ & $68 \cdot 0$ \\
\hline Alumina - & $24 \cdot 8$ & $34 \cdot 4$ & $20 \cdot 7$ & $25 \cdot 0$ & $32 \cdot 0$ & $29 \cdot 0$ & $28 \cdot 0$ \\
\hline Oxyde of Iron & $3 \cdot 8$ & $1 \cdot 0$ & $4 \cdot 0$ & $7 \cdot 2$ & $0 \cdot 8$ & $2 \cdot 2$ & $2 \cdot 0$ \\
\hline Magnesia - & trace & - & - & trace & trace & 0.5 & trace \\
\hline Water - - & - & - & $10 \cdot 3 *$ & - & - & - & $1 \cdot 0$ \\
\hline
\end{tabular}

Wurzer states the composition of the sand and clay in the Hessian crucibles as follows :-

Clay; silica 10.1 ; alumina 65.4 ; oxydes of iron and manganese $1 \cdot 2$; lime 0.3 ; water 23 Sand; $\quad 95 \cdot 6 \quad 2.1 \quad 1.5 \quad 0.8$

Black lead crucibles are made of two parts of graphite and one of fire clay; mixed with water into a paste, pressed in moulds, and well dried; but not baked hard in the kiln. They bear a higher heat than the Hessian crucibles, as well as sudden changes of temperature; have a smooth surface, and are therefore preferred by the melters of gold and silver. This compound forms excellent small or portable furnaces.

$\mathrm{Mr}$. Anstey describes his patent process for making crucibles, as follows: Take two parts of fine ground raw Stourbridge clay, and one part of the hardest gas coke, previously pulverized, and sifted through a sieve of one eighth of an inch mesh (if the coke is ground too fine the pots are very apt to crack). Mix the ingredients together with the proper quantity of water, and tread the mass well. The pot is moulded by hand upon a wooden block, supported on a spindle which turns in a hole in the bench; there is a gauge to regulate the thickness of the melting pot, and a cap of linen or cotton placed wet upon the core before the clay is applied, to prevent the clay from sticking partially to the core, in the taking off; the cap adheres to the pot only while wet, and may be removed without trouble or hazard when dry. He employs a wooden bat to assist in moulding the pot; when moulded, it is carefully dried at a gentle heat. A pot dried as above, when wanted for use, is first warmed by the fire-side, and is then laid in the furnace with the mouth downwards (the red cokes being previously damped with cold ones in order to lessen the heat); more coke is then thrown in till the pot is covered, and it is now brought up gradually to a red heat. The pot is next turned and fixed in a proper position in the furnace, without being allowed to cool, and is then charged with cold iron, so that the metal, when melted, shall have its surface a little below the mouth of the pot. The iron is melted in about an hour and a half, and no flux or addition of any kind is made use of. A pot will last for fourteen or even eighteen successive meltings, provided it is not allowed to cool in the intervals; but if it cool, it will probably crack. These pots, it is said, can bear a greater heat than others without softening, and will, consequently, deliver the metal in a more fluid state than the best Birmingham pots will. See a figure of the crucible mould under STEEL.

CRYSTAL is the geometrical form possessed by a vast number of mineral and saline substances; as also by many vegetable and animal products. The integrant particles of matter have undoubtedly determinate forms, and combine with one another, by the attraction of cohesion, according to certain laws, and points of polarity, whereby they assume a vast variety of secondary crystalline forms. The investigation of these laws belongs to crystallography, and is foreign to the practical purpose of this volume. Instructions are given under each object of manufacture which requires crystallization, how to conduct this process; see Borax, Salt, \&c.

CUDBEAR was first made an article of trade in this country, by Dr. Cuthbert Gordon, from whom it derived its name, and was originally manufactured on a great scale by Mr. G. Mackintosh at Glasgow, nearly 60 years ago. Cudbear or persio is 
powder of a vivlet red color, difficult to moisten with water, and of a peculiar but not disagreeable odor. It is partially soluble in boiling water, becomes red with acids, and violet blue with alkalis. It is prepared in the same way as archil, only towards the end the substance is dried in the air, and is then ground to a fine powder, taking care to avoid decomposition, which renders if glutinous. In Scotland they use the lichen tartareus, more rarely the lichen calcareus, and omphalodes; most of which lichens are imported from Sweden and Norway, under the name of rock moss. The lichen is suffered to ferment for a month, and is then stirred about to allow any stones which may be present to fall to the bottom. The red mass is next poured into a flat vessel, and left to evaporate till its urinous smell has disappeared, and till it has assumed an agreeable color verging upon violet. It is then ground to fine powder. During the fermentation of the lichen, it is watered with stale urine, oz with an equivalent ammoniacal liquor of any kind, as in making archil.

CUPELLATION is a mode of analyzing gold, silver, palladium, and platinum, by adding to small portions of alloys, containing these metals, a bit of lead, fusing the mixture in a little cup of bone earth called a cupel, then by the joint action of heat and air, oxydizing the copper, tin, \&c., present in the precious metals. The oxydes thus produced are dissolved and carried down into the porous cupel in a liquid state, by the vitrified oxyde of lead. See Assay, Gold, and Silver.

CURRYING OF LEATHER (Corroyer, Fr.; Zurichten, Germ.) is the art of dressing skins after they are tanned, for the purpose of the shoe-maker, coach and harness maker, \&c., or of giving them the necessary smoothness, lustre, color, and suppleness. The currier's shop has no resemblance to the tanner's premises, having a quite different set of tools and manipulations.

The currier employs a strong hurdle about a yard square, made either of basket twigs, or of wooden spars, fixed rectangularly like trellis work, with holes 3 inches square, upon which he treads the leather, or beats it with a mallet or hammer, in order to soften it, and render it flexible.

The head knife, called in French couteau a revers, on account of the form of its edge, which is much turned over, is a tool 5 or 6 inches broad, and 15 or 16 long; with two handles, one in the direction of the blade, and the other perpendicular to it, for the

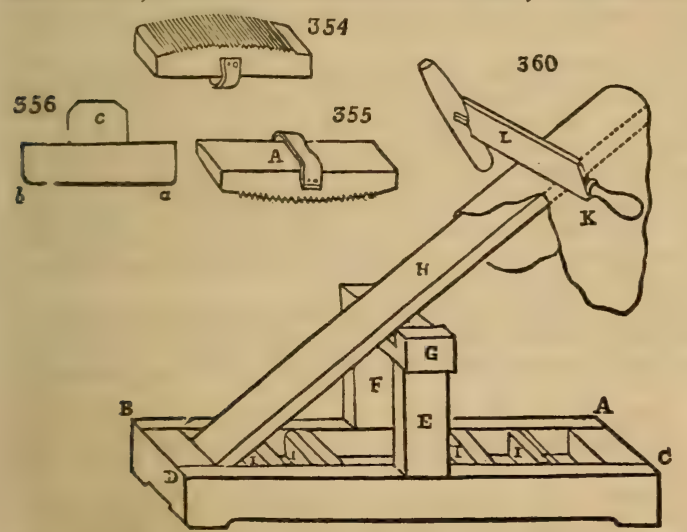
purpose of guiding the edge more truly upon the skin. The pommel (paumelle) is so called because it clothes the palm of the hand, and performs its functions. It is made of hard wood, and is of a rectangular shape, 1 foot long, 5 inches broad, flat above and rounded below. It is furrowed over the rounded surface with transverse parallel straight grooves. These grooves are in section sharp-edged isosceles triangles. Figs. 354 and 355 , represent the pommel in an uppes and under view. The flat surface is provided with a leather strap for securing at to the hand of the workman. Pommels are made of different sizes, and witl. grooves of various degrees of fineness. Cork pommels are also used, but they are rot grooved. Pommels serve to give grain and pliancy to the skins.

The stretching iron, fig. 356, is a flat plate of iron or copper, fully a fourth of an inch thick at top, and thinning off at bottom in a blunt edge, shaped like the are of a circle of large diameter, having the angles $a$ and $b$ rounded, lest in working they should penetrate the leather. The top $c$ is mounted with leather to prevent it from hurting the hands. A copper stretching knife is used for delicate skins. The workman holds this tool nearly perpendicular, and scrapes the thick places powerfully with his two hands, especially those where some tan or flesh remains. He thus equalizes the thickness of the skin, and renders it at the same time more dense and uniform in texture. This tool is of very general use in currying.

The round knife, figs. 357 and 358 (lunette in French), is a circular knife from 10 to 12 inches in diameter, with a round 4 or 5 inch hole in its centre, for introducing the hands and working it. It is concave, as shown in the section fig. 358 , presenting the 
form of a spherical zone. The concave part is that applied to he kin Its edge is nor perfectly straight; but is a little turned over on the side opposite to the skin, to preven

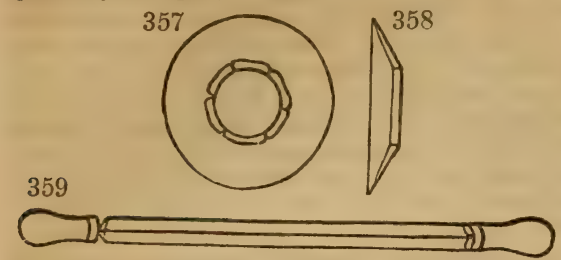
it from entering too far into the leather. The urrier first -lopes off with the head knife from the edges, a portion equal to what he afterwards removes with the round ons. By this division the work is done sooner and more exactly. All the cileo or greased skins are dressed with the round knife.

The cleaner is a straight two-handled knife two inches broad, of which there are two kinds, a sharp-edged and a blunt one. Fig. 359.

The mace is made of wood, having a handle 30 inches long, with a cubical head or mallet; upon the two faces of which, parallel to the line of the handle, there are 4 pegs of hard wood turned of an egg-shape, and well polished, so as not to tear the moistened leather when it is strongly beat and softened with the mace.

The horse or trestle, fig. 360 , consists of a strong wooden frame, A B C D, which serves as a leg or foot. Upon the middle of this frame there are two uprights, $E F$, and a strong cross beam, G, for supporting the thick plank $\mathrm{H}$, upon which the skins are worked. This plank may be set at a greater or less slope, according as its lower end is engaged in one or other of the cross bars, I I I I, of the frame. In the figure, a skin $\mathrm{k}$ is represented upon the plank with the head knife upon it, in the act of being pared.

A cylindrical bar fixed horizontally at its ends to two buttresses projecting from the wall, serves by means of a parallel stretched cord, to fix a skin by a coil or two in order to dress it. This is accordingly called the dresser. The tallow cloth is merely a mop made of stout rags, without the long handle; of which there are several, one for wax, another for oil, \&c. Strong-toothed pincers with hook-end handles, drawn together by an endless cord, are employed to stretch the leather in any direction, while it is being dressed. The currier uses clamps like the letter $U$, to fix the edges of the leather to his table. His polisher is a round piece of hard wood, slightly convex below, with a handle standing upright in its upper surface, for seizing it firmly. He first rubs with sour beer, and finishes with barberry juice.

Every kind of tanned leather not intended for soles or such coarse purposes, is generally curried before being delivered to the workmen who fashion it, such as shoemakers, coachmakers, saddlers, \&c. The chief operations of the currier are four :-

1. Dipping the leather, which consists in moistening it with water, and beating it with the mace or a mallet upon the hurdle. He next applies the cleaners, both blunt and sharp, as well as the head knife, to remove or thin down all inequalities. After the leather is shaved, it is thrown once more into water, and well scoured by rubbing the grain side with pumice stone, or a piece of slaty grit, whereby it parts with the bloom, a whitish matter, derived from the oak bark in the tan pit.

2. Applying the pommel to give the leather a granular appearance, and correspondent flexibility. The leather is first folded with its grain side in contact, and rubbed strongly with the pommel, then rubbed simply upon its grain side; whereby it becomes extremely flexible.

3. Scraping the leather. This makes it of uniform thickness. The workman holds the tool nearly perpendicular upon the leather, and forcibly scrapes the thick places with both his hands.

4. Dressing it by the round knife. For this purpose he stretches the leather upon the wooden cylinder, lays hold of the pendent under edge with the pincers attached to his girdle, and then with both hands applies the edge of the knife to the surface of the leather, slantingly from above downwards, and thus pares off the coarser fleshy parts of the skin. This operation requires great experience and dexterity; and when well performed improves greatly the look of the leather.

The hide or skin, being rendered flexible and uniform, is conveyed to the shed or drying house, where the greasy substances are applied, which is called dubbing (daubing) or stuffing. The oil used for this purpose is prepared by boiling sheep-skins or doe-skins, in cod oil. This application of grease is often made before the graining board or pommel is employed.

Before waxing, the leather is commonly colored by rubbing it with a brush dipped into a composition of oil and lamp black on the flesh side, till it be thoroughly black; it is then black-sized with a brush or sponge, dried, tallowed with the proper cloth, and slicked upon the flesh with a broad smooth lump of glass; sized again with a sponge; and when dry, again curried as above described.

Currying leather on the hair or grain side, termed black on the grain, is the same in the first operation with that dressed on the flesh, till it is scoured. Then the first black 
is applied to it while wet, by a solution of copperas put upon the grain, after this has been rubbed with a stone; a brush dipped in stale urine is next rubbed on, then an iron sliclier is used to make the grain come out as fine as possible. It is now stuffed with oil. When dry, it is seasoned; that is, rubbed over with a brush dipped in copperas water, on the grain, till it be perfectly black. It is next slicked with a good grit-stone, to take out the wrinkles, and smooth the coarse grain. The grain is finally raised with the pommel or graining board, by applying it to the leather in different directions. When thoroughly dry, it is grained again in two or three ways.

Hides intencled for covering coaches are shaved nearly as thin as shoe hides, and blacked upon the grain.

CUTLERY. (Coutellerie, Fr.; Messerschmidwaare, Germ.) Three kinds of steel are made use of in the manufacture of different articles of cutlery, viz., common steel, shear steel, and cast steel. Shear steel is exceedingly plastic and tough. All the edge tools which require great tenacity without great hardness are made of it, such as table knives, scythes, plane-irons, \&c.

Cast steel is formed by melting blistered steel in covered crucibles, with bottic glass, and pouring it into cast-iron moulds, so as to form it into ingots; these ingots are then taken to the tilt, and drawn into rods of suitable dimensions. No other than cast steel can assume a very fine polish, and hence all the finer articles of cutlery are made of it, such as the best scissors, penknives, razors, \&c.

Formerly cast steel could be worked only at a very low heat; it can now be made so as to be welded to iron with the greatest ease. Its use is consequently extended to making very superior kinds of chisels, plane-irons, \&c.

Forging of table knives. - Two men are generally employed in the forging of table knives; one called the foreman or maker, and the other the striker.

The steel called common steel is employed in making the very common articles; but for the greatest part of table knives which require a surface free from flaws, shear steel is generally preferred. That part of the knife termed the blade, is first rudely formed and cut off. It is next welded to a rod of iron about $\frac{1}{2}$ inch square, in such a manner as to leave as little of the iron part of the blade exposed as possible. A sufficient quantity of the iron now attached to the blade, is taken off from the rod to form the bolster or shoulder, and the tang.

In order to make the bolster of a given size, and to give it at the same time shape and neatness, it is introduced into a die, and a swage placed upon it; the swage has a few smart blows given it by the striker. This die and swage are, by the workman, called prints.

After the tangs and bolster are finished, the blade is heated a second time, and the foreman gives it its proper anvil finish; this operation is termed smithing. The blade is now heated red-hot, and plunged perpendicularly into cold water. By this means it becomes harlened. It requires to be tempered regularly down to a blue color : in which state it is ready for the grinder.

Mr. Brownill's method of securing the handles upon table-knives and forks, is, by lengthening the tangs, so as to pass them completely through the handle, the ends of which are to be tinned after the ordinary mode of tinning iron; and, when passed through the handle. the end of the tang is to be spread by beating, or a small hole drilled through it, and a pin passed to hold it upon the handle. After this, caps of metal, either copper plated or silver, are to be soldered on to the projecting end of the tang. and while the solder is in a fluid state, the cap is to be pressed upon the end of the handle and held there until the solder is fixed, when the whole is to be cooled by being immersed in cold water.

Mr. Thomason's patent improvements consist in the adaptation of steel edges to the blades of gold and silver knives. These steel edges are to be attached to the other metal, of whaterer quality it may be, of which the knife, \&c. is made, by means of solder, in the ordinary mode of effecting that process. After the edge of steel is thus attached to the gold, silver, \&c., it is to be ground, polished, and tempered by immersion in cold water or oil after being heated. This process being finished, the other parts of the knife are then wrought and ornamented by the engraver or chaser, as usual.

A patent was obtained in 1827 , by Mr. Smith of Sheffield, for rolling out knives at one operation.

In the ordinary mode of making knives, a sheet of steel being provided, the blades are cut out of the sheet, and the backs, shoulders, and tangs, of wrought iron, are attached to the steel blades by welding at the forge. The knife is then ground to the proper shape, and the blade polished and hardened.

Instead of this welding process, the patentee proposes to make the knives entirely of steel, and to form them by rolling in a heated state between massive rollers; the shoulders or bolsters, and the tangs for the handles, being produced by suitable recesses in the peripheries of the rollers; just as rail-way rails are formed. When the knife is to 
be made with what is called a scale tang, that is, a broad flat tang, to which the handle is to be attached in two pieces, riveted on the sides of the tang, the rollers are then only to have recesses cut in them, in a direction parallel to the axis for forming the bolster.

The plate of steel, having been heated, is to be pressed between the two rollers, by which the blades and the parts for the scale tangs will be pressed out flat and thin, and those parts which pass between the grooves or recess will be left thick or protuberant, forming the bolster for the shoulder of the blade. But if the tangs are to be round in order to be fixed into single handles, then it will be necessary also to form transverse grooves in the rollers, that is, at right angles to those which give shape to the bolsters, the transverse grooves corresponding in length to the length of the intended tang. When the plates of steel have been thus rolled, forming three or more knives in a breadth, the several knives are to be cut out by the ordinary mode of what is called slitting, and the blades and shoulders ground, hardened, and polished in the usual way.

Forks are generally a distinct branch of manufacture from that of knives, and are purchased of the fork makers by the manufacturers of table knives, in a state fit for receiving the handles.

The rods of steel from which the forks are made, are about $\frac{3}{8}$ ths of an inch square. The tang and shank of the fork are first roughly formed. The fork is then cut off, leaving at one end about 1 inch of the square part of the steel. This part is afterwards drawn out flat to about the length of the prongs. The shank and tang are now heated, and a proper form given to them by means of a die and swage. The prongs are afterwards formed at one blow by means of the stamp; this machine is very similar to that used in driving piles, but it is worked by one man. It consists of a large anvil fixed in a block of stone nearly on a level with the ground. To this anvil are attached two rods of iron of considerable thickness, fixed twelve inches asunder, perpendicularly to the anvil, and diagonally to each other. These are fastened to the ceiling. The hammer or stamp, about $100 \mathrm{lbs}$. in weight, having a groove upon either side corresponding to the angles of the upright rods, is made to slide freely through its limited range, being conducted by its two iron supporters. A rope is attached to the hammer, which goes over a pulley on the floor of the room above, and comes down to the person who works the stamp: two corresponding dies are attached, one to the hammer, and the other to the anvil. That part of the fork intended to form the prongs, is heated to a pretty white heat and placed in the lower die, and the hammer containing the other die is made to fall upon it from a height of about 7 or 8 feet. This forms the prongs and the middle part of the fork, leaving a very thin substance of steel between each prong, which is afterwards cut out with an appropriate instrument called a fly-press. The forks are now annealed by surrounding a large mass of them with hot coals, so that the whole shall become red-hot. The fire is suffered gradually to die out, and the forks to cool without being disturbed. This process is intended to soften, and by that means to prepare them for filing. The inside of the prongs is then filed, after which they are bent into their proper form and hardened. When hardened, which is effected by heating them red-hot and plunging them into cold water, they are tempered by exposing them to the degree of heat at which grease inflames. See STAMPs.

Penknives are generally forged by a single hand, with the hammer and the anvil sim. ply. The hammer in this trade is generally light, not exceeding $3 \frac{1}{2} \mathrm{lbs}$. The breadth of the face, or the striking part, is about one inch; if broader, it would not be convenient for striking so small an object. The principal anvil is about 5 inches, and 10 upon the face, and is provided with a groove into which a smaller anvil is wedged. The smaller anvil is about 2 inches square upon the face. The blade of the knife is first drawn out at the end of the rod of steel, and as much more is cut off along with it as is thought necessary to form the joint. The blade is then taken in a pair of tongs, and heated a second time to finish the joint part, and at the same time to form a temporary tang for the purpose of driving into a small haft used by the grinder. Another heat is taken to give the blade a proper finish. The small recess called the nail-hole, used in opening the knife, is made while it is still hot by means of a chisel, which is round on one side, and flat upon the other.

Penknives are hardened by heating the blade red-hot, and dipping them into water up to the shoulder. They are tempered by setting them side by side, with the back downwards upon a flat iron plate laid upon the fire, where they are allowed to remain till they are of a brown or purple color.

The blades of pocket knives, and all that come under the denomination of spring knives, are made in the same way.

The forging of razors is performed by a foreman and striker, as in making table nives.

They are generally made of cast steel. The rods, as they come from the tilt, are about 1 inch broad, and of a thickness sufficient for the back of the razor.

There is nothing peculiar in the tools made use of in forging razors: the anvil is a 
.uttle rounded at the sides. which affords the opportunity of making the eilge thinner, and sares an immense labor to the srinter.

Racurs are bardenct and tempered in a similar manner to penknives. Thes are, however, ieft harker, beinz only let down to jellow or brown color.

The forzinz of scissors is wholly nerformed by the hammer, and all the sizes are made by a single hand. The antil of the scissor-maker weizhs about $1 \frac{1}{2} \mathrm{ewt}$; it measures, on the lace, about $\div$ by 11 inches. It is provided with two grates or groores for the reception of various litile inderted towls termed by the workman bosses: one of these bossts is employed to sire proper frure to the shank of the scissors : anather for form. ing that part which has to make the joint: and a thind is made use of for giring a proper fizure to the upper sile of the biate. There is also another anvil places on the same block. containing two or three tocls called beah-irons, each consisting of an ypriaht stem about is inches Lizh, at the ton of which a Lorizontal beak projeets; one of these beaks is conical, and is used for extending the bow of the scissors; the other is a segment of a cylin ier with the round side upwards, containing a recess for giring a proper simpe and smoothness to the inside of the bow.

The shank of the scissors is Irst formed by means of one of the bosses, abore described. learing as much steel at the end as will form the blade. A bole is then punched about $\frac{2}{4}$ inch in with, a little abore the shank. The blate is drawn out and finished, and the scissors separated from the rod a lirtle above the hole. It is teated a third time, and the small hole abore mentioned is extended upon the beak-irons so as to form the bow. This finishes the furging of scissors. They are promiscuonsly trade in this war, without anj other guibe than the ere, haring no regand to their being in pairs. They are nest annealed for the purpose of filing such parts of them as cannot be ground, and afterwards paired.

The rery larze scissors are cade partly of iron. the blades being of steel.

titer tice forzinz, the bow and joints, and such shanks as cannot be ground, are ile. The riret tole is then bored, through which the are to be scretrel or riveted together. Ttis common kind of scissurs is only handened up to the joint. They are terpered down to a purple or blue color. In this state ther are taken to the grinjer.

Grining and poilsing of cutlery. - The rarious processes which come under this denmination are periomed by machiner, moting in general by the power of the steamengine or water-wheel.

Grindiog wheels or grinding mills are dirided into a number of separate rooms : every roum contains sir places calied troughs : pach trongh consists of a convenience for running a grinistone and a poisher at the same time, which is generally occupied by a man and a bos.

The business of the grinder is generall! dirided into three stages, riz., grinding, glazing, and polishing.

The grindirg is periormed upon stones of rarious qualities and sizes, depending on the articles to be ground. Those exposing much lat surface, such as sars, fenders, kc., require stones of great diameter, while razors, whose surface is concare, require to be ground upon stones of reT small dimensions. Those articles which require a certain temper, which is the case with most cutting instruments, are mostly fround on a wet sione: for which purpose the stone bangs within the iron trongh, illed with water to such a height that its surface may just touch the face of the stone.

Glazing is a process following that of crinding: it consists in giving that degree of lustre and scoothness to an article which can be effected by means of ererg of the rarious degrees of fineness. The tool cm which the glazing is performed, is termed a giazes. It consists of a circular piece of wood. formed of a number of pieces in such a Eanner that its eige or face may always present the endwas of the wood. Were it made otherwise, the contraction of the parts would destroy its circular fgure. It is fred upon an iron axis similar to that of the stone. Some glazers are corered on the face with leather, others with metal, consisting of an alloy of lead asd tin; the latter are termed caps. In others, the woolen suriace abote is made use of. Soze of the leatier-faced clazers, such as are used for forhs, table knires, edge tocls, and all the coarser polished articles, are first coated with a solution of slue, and then corered with exery. The suriacs of the others are prepared for use by first turning the face rery truc, then fillic 3 it with small notches by means of a sharp-ended hammer, and lastly fuling up the intersices with a compound of tallow and emery.

The puller of the glazer is so much less than that of the stone, that its relocity is wore than doutle, haring in general a surface speed of 1500 feet in a second.

The process of poishing consists in giving the most perfect polish to the different articles. Fothing is subjected to this operation but $\pi$ hat is made of cast steel, and has been previonsly hardened and tempered.

The polisher consists of a circular piece of wood covered with buff leather, the surface 
of which is covered from time to time, while in use, with the crocus of iron, called also colcothar of vitriol.

The polisher requires to run at a speed much short of that of the stone, or the glazer. Whatever may be its diameter, the surface must not move at a rate exceeding 70 or 80 feet in a second.

CYANATES; saline compounds of cyanic acid with the bases potash, soda, ammonia, baryta, \&c. The first is prepared by calcining, at a dull red heat, a mixture of ferro. cyanide of potassium (prussiate of potash) and black oxyde of manganese. The cyanates have not hitherto been applied to any use in the arts.

CYANHYDRIC Acid; another name for the hydrocyanic or prussic acid. See Prusstan Blue and Prussic Acid.

CYANIDES; compounds of cyanogen with the metals; as cyanide of potassium, sodium, barium, calcium, iron, mercury. The last is the only one of importance in a manufacturing point of view, since from it prussic acid is made.

CYANIDES, FERRO. Double compounds of cyanogen with iron, and of cyanogen with another metal, such as potassium, sodium, barium, \&c. The ordinary yellow prussiate of potash has this constitution, and is called the ferro-cyanide.

CYANOGEN. A gaseous compound of two prime equivalents of charcoal = 12, and one of azote $=14=26$; hydrogen being the radix, or 1 . It consists of two volumes of vapor of carbon, and one volume of azote, condensed into one volume; and has therefore a density equal to the sum of the weights of these three gaseous volumes $=1.815$. Cyanogen is readily procured by exposing the cyanide of mercury to a dull red heat in a retort; the gas is evolved and may be collected over mercury. Its smell is very sharp and penetrating; it perceptibly reddens tincture of litmus; it is condensable by pressure at a low temperature into a liquid; and by a still greater degree of cold, it is solidified. When a lighted taper is applied to a mixture of cyanogen and oxygen, an explosion takes place; carbonic acid is formed, and the azote is set at liberty.

For a connected view of the various compounds of cyanogen employed in the arts, see Prussian Blue.

CIDER (Cidre, Fr.; Apfelwein, Germ.); the vinous fermented juice of the apple. The ancients were acquainted with cider and perry, as we learn from the following passage of Pliny the naturalist: "Wine is made from the Syrian pod, from pears and apples of every kind." Book xiv. chap. 19. The term cider or cidre in French, at first written sidre, is derived from the Latin word sicera, which denoted all other fermented liquors except grape wine. Cider seems to have been brought into Normandy by the Moors of Biscay, who had preserved the use of it after coming into that country from Africa. It was afterwards spread through some other provinces of France, whence it was introduced into England, Germany, and Russia. It is supposed that the first growths of Normandy afford still the best specimens of cider. Devonshire and Herefordshire are the counties of England most famons for this beverage.

Strong and somewhat elevated ground, rather dry, and not exposed to the air of the sea, or to high winds, are the best situations for the growth of the cider apple. The fruit should be gathered in dry weather. The juice of apples is composed of a great deal of water; a little sugar analogous to that of the grape; a matter capable of causing fermentation with contact of air; a pretty large proportion of mucilage, with malic acid, acetic acid, and an azotized matter in a very small quantity. The seeds contain a bitter substance and a little essential oil; the pure parenchyma or cellular membrane constitutes not more than two per cent. of the whole. After the apples are gathered, they are left in the barn-loft for fifteen days or upwards to mellow; some of them in this case, bowever, become soft and brown. This degree of maturation diminishes their mucilage, and develops alcohol and carbonic acid; in consequence of which the cider suffers nc injury. There is aiways, however, a little loss; and if this ripening goes a little further it is very apt to do harm, notwithstanding the vulgar prejudice of the country people to the contrary. Too much care, indeed, cannot be taken to separate the sound from the spoiled apples; for the latter merely furnish an acid leaven, give a disagreeable taste to the juice, and hinder the cider from fining, by leaving in it a certain portion of the parenchyma, which the gelatinous matter or the fermentation has diffused through it. Unripe apples should be separated from the ripe also, for they possess too little saccharum to be properly susceptible of the vinous fermentation.

In France, where cider making is most scientifically practised, it is prepared by crushing the apples in a mill with revolving edge-stones, turned in a circular stone cis. tern by one or two horses. When the fruit is half mashed, about one fifth of its weight of river water is added, or the water of lakes. The latter have been found by experience to be preferable to other water.

In some places a mill composed of two cast-iron fluted cylinders placed parallel to each other under the bottom of a hopper, is employed for crushing the apples. One of the cylinders is turned by a winch, and communicates its motion in the opposite direc- 
tion by means of the flutings working into each other. Each portion of the fruit must be passed thrice through this rude mill in order to be sufficiently mashed; and the same quantity of water must be added as in the edge stone mill.

After the apples are crushed they are usually put into a large tub or tun for 12 or 24 hours. This steeping aids the separation of the juice, because the fermentative motion which takes place in the mass breaks down the cellular membranes; but there is always a loss of alcohol carried off by the carbonic acid disengaged, while the skins and seeds develop a disagreeable taste in the liquid. The vatting might be suppressed if the apples were so comminuted as to give out their juice more readily. With slight modifications, the process employed in rasping and squeezing the beet-roots might in my opinion be applied with great advantage to the cider manufacture. See Sugar.

After the vatting, the mashed fruit is carried to the press and put unon a square wicker frame or into a hair bag, sometimes between layers of straw, and exposed stratum super stratum to strong pressure till what is called a cheese or cake is formed. The mass is to be allowed to drain for some time before applying pressure, which ought to be very gradually increased. The juice which exudes with the least fressure afiords the best cider; that which flows towards the end acquires a disagreeable taste from the seeds and the skins. The must is put into casks with large bungholes, where it soon begins to exhibit a tumultuous fermentation. The cask must be completely flied, in order that all the light bodies suspended in the liquid when floated to the top by the carbonic acid may flow over with the froth; this means of clearing eider is particularly necessary with the weak kinds, because it cannot be expected that these matters in suspension will fall to the bottom of the casks after the motion has ceased. In almost every circumstance besides, when no saccharine matter has been added to the must, that kind of yeast which rises to the top must be separated, lest by precipitation it may excite an acid fermentation in the cider. The casks are raised upon gavntrees or stillions, in order to place fiat tubs below them to receive the liquor which flows over with the froth. At the end of two or three days, for weak ciders which are to be drunk somewhat sweet, of 6 or 10 days or more for stronger ciders, with rariations for the state of the weather, the fermentation will be sufficiently advanced, and the cider may be racked off into other casks. Spirit puncheons preserve cider better than any other, but in all cases the casks should be well seasoned and washed. Sometimes a sulphur match is burned in them before introducing the cider, a precaution to be gencrally recommended, as it suspends the activity of the fermentation, and prevents the formation of vinegar.

The cider procured by the first expression is called cider without water. The cake remaining in the press is taken out, divided into small pieces, and mashed anew, adding gbont half the weight of water, when the whole is carried back to the press and treated as above described. The liquor thus obtained furnishes a weaker cider which will not keep, and therefore must be drunk soon.

The cake is once more mashed up with water, and squeezed, when it yields a liquor which may be used instead of water for moistening fresh ground apples.

The processes above described, although they have been long practised, and have therefore the stamp of ancestral wisdom, are extremely defective. Were the apples ground with a proper rotatory rasp which would tear all their cells asunder, and the mash put through the hydraulic press in bags between hurdles of wicker-work, the juice would be ohtained in a state of perfection fit to make a cider superior to many wines. An experimental process of this kind has been actually executed in France upon a considerable scale; with the best results. The juice had the fine flavor of the apple, was fermented by itself without any previous fermentation in the mash, and afforded an excellont strong cider which kept well.

When the must of the apples is weak or sour, good cider cannot be made from it without the addition of some saccharine matter. The sirup into which potato farina is convertible by diastase (saccharine ferment), see STARcH and SUGAR, would answer well for enriching poor apple juice.

\section{D.}

DAHLINE, the same as Inuline, the fecula obtained from elecampane, analogous in many respects to starch. It is not employed in the arts.

DAMLSCUS BLADES, are swords or cimeters, presenting upon their surface a variegated appearance of watering, as white, silvery, or black veins, in fine lines, or fillets ; fibrous, crossed, interlaced, or parallel, \&c. They are brought from the East, being fabricated chiefly at Damascus, whence their name. Their excellent quality has become proverbial; for which reason these blades are much sought after by military 
men, and are high priced. The oriental processes have never been satisfactorily de. scribed; but of late years methods have been devised in Europe to imitate the fabric very well.

Clouet and Hachette pointed out the three following processes for producing $\mathrm{Da}$. mascus blades: 1 , that of paraliel fillels; 2 , that by torsion; 3 , the mosuic. The first, which is still pursued by some French cutlers, consists in scooping out with a graving tool the faces of a piece of stuff composed of thin plates of different kinds of steel. These hollows are by a subsequent operation filled up, and brought to a level with the external faces, upon which they subsequently form tress-like figures. 2. The method of torsion which is more generally employed at present, consists in forming a bundle of rods or slips of steel, which are welded together into a well-wrought bar, twisted several times round its axis. It is repeatedly forged, and twisted alternately; after which it is slit in the line of its axis, and the two halves are welded with their outsides in contact; by which means their faces will exhibit very various configurations. 3. The mosaic method consists in preparing a bar, as by the torsion plan, and cutting this bar into short pices of nearly equal length, with which a fagot is formed and welded together; taling care to preserve the sections of each piece at the surface of the blade. In this way, all the variety of the design is displajed, corresponding to each fragment of the cut bar.

The blades of Clouet, independently of their excellent quality, their flexibility, and extreme elasticity, have this advantage over the oriental blades, that they exhibit in the very substance of the metal, designs, letters, inscriptions, and, generally speaking, all kinds of figures which had been delineated beforehand.

Notwithstanding these successful results of Clouet, it was pretty clear that the watered designs of the true Damascus cimeter were essentially different. M. Bréant has at last completely solved this problem. He has demonstrated that the substance of the oriental blades is a cast-steel more highly charged with carbon than our European steels, and in which, by means of a cooling suitably conducted, a crystallization takes place of two distinct combinations of carbon and iron. This separation is the essential condition; for if the melted steel be suddenly cooled in a small erucible or ingot, there is no damascene appearance.

If an excess of carbon be mixed with iron, the whole of the metal will be converted into steel; and the residuary carbon will combine in a new proportion with a portion of the steel so formed. There will be two distinct compounds; namely, pure steel, and carbureted steel or cast-iron. These at first being imperfeetly mixed will tend to separate, if while still fluid they be left in a state of repose; and form a erystallization in which the particles of the two compounds will place themselves in the crucible in an order determined by their affinity and density conjoined. If a blade forged out of steel so prepared be immersed in acidulous water, it will display a very distinet Damascus appearance; the portions of pure steel becoming black, and those of earbureted stee? remaining white, because the acids with difficulty disengage its carbon. The slower such a compound is cooled, the larger the Damascus veins will be. Travernier relates that the steel crucible ingots, like those of wootz, for making the true oriental Damascus, come from Golconda, that they are of the size of a halfpenny roll, and when cut in two, form two swords.

Steel combined with manganese forges easily, but it is brittle when cold; it displays however the Damascus appearance very strongly.

A mixture of 100 parts of soft iron, and 2 of lamp black, melts as readily as ordinary steel. Several of the best blades which M. Bréant presented to the Société d'Eneouragement are the product of this combination. This is an easy way of making cast-steel without previous cementation of the iron. 100 parts of filings of very gray cast-iron, and 100 parts of like filings previously oxydized, produced, by their fusion together, a beautiful damascene steel, fit for forging into white arms, sabres, swords, \&c. This compound is remarkable for its elasticity, an essenial quality, not possessed by the old Indian steel. The greater the proportion of the oxydized cast-iron, the tougher is the steel. Care should be taken to stir the materials during their fusion, before it is allowed to cool ; otherwise they will not afford a homogeneous damasc. If the steel contains much earbon it is difficult to forge, and cannot be drawn out except within a narrow range of temperature. When heated to a red-white it crumbles under the hammer; at a cherry-red it becomes hard and brittle; and as it progressively cools it becomes still more unmalleable. It resembles completely Indian steel, which European blacksmiths cannot forge, because they are ignorant of the suitable temperature for working it. M. Bréant, by studying this point, succeeded in forging fine blades.

Experience has proved that the orbicular veins, called by the workmen knots or thorns-(ronces), which are seen upon the finest Eastern cimeters, are the result of the manner of forging them, as well as the method of twisting the Damascus bars. If these be drawn in length, the veins will be longitudinal; if they be spread equally in all 
lirections, the stiuf will have a crystalline aspect; if they be made wavy in the two directions, lin.lulated veins will be produced like those in the oriental Damascus.

DAMASK is a variegated textile fabric, richly ornamented with figures of flowers, fruits, landscapes, animals, scc., woven in the loom, and is by far the most rich, elegant, and expensive species of ornamental weaving, tapestry alone excepted. The name is said to be derived from Damascus, where it was anciently made.

Damask belongs to that species of texture which is distinguished by practical men by the name of tweeling, of which it is the richest pattern. The tweel of damask is usually half that of full satin, and consequently consists of eight leaves moved either in regular succession or by regular intervals, eight leaves being the smallest number which will admit of alternate tweeling at equal intervals. loon:

In the article CARPET, two representations have been given of the damask draw-

The generic difference of tweeling, when compared with common cloth, consists in the intersections, although uniform and equidistant, being at determinate intervals, and not between the alternate threads. Hence we have specimens of tweeled cloth, where the intersections take place at the third, fourth, fifth, sixth, seventh, eighth, or sixteenth interval only. The threads thus deflecting only from a straight line at intervals, preserve more of their original direction, and a much greater quantity of materials can be combined in an equal space, than in the alternate intersection, where the tortuous deflection, at every interval, lieeps them more asunder. On this principle tweeled cloths of three and four leaves are woren for facility of combination alone. The coarser species of ornamented cloths, known by the names of dornock and diaper, usually intersect at the fifth, or half satin interval. The sixth and seventh are rarely used, and the intersection at the eighth is distinguished by the name of satin in common, and of damask in ornamental tweeling. It will further be very obvious, that where the warp and woof cross only at evers eighth interval, the two sides of the cloth will present a diversity of appearance; for on one side the longitudinal or warp threads will run parallel from one end of a web to the other, and, on the other, the threads of woof will run also parallel, but in a transverse direction across the cloth, or at right angles to the former. The points of intersection being only at every eighth interval, appear only like points; and in regular tweeling these form the appearance of diagonal lines, inclined at an angle of $45^{\circ}$ (or nearly so) to each of the former.

The appearance, therefore, of a piece of common tweeled cloth is very similar to that of two thin boards glued together, with the grain of the upper piece at right angles to that of the under one. That of an ornamental piece of damask may, in the same manner, be rery properly assimilated to a piece of veneering, where all the wood is of the same substauce and color, and where the figures assume a diversity of appearance from the ground, merely by the grain of the one being disposed perpendicularly to that of the other. See Textrle FaBric.

From this statement of the principle, it results that the most unlimited variety of figures will be produced, by constructing a loom by which every individual thread of warp may be placed either above or below the woof at every intersection; and to effect this, in boundless variety, is the object of the Jacquard mounting; which see.

The chief seat of this manufacture is probably the town and neighborhood of Dunfermline, in Fifeshire, and Lisburn and Ardoyne, near Belfast, where it is considered as the staple, having proved a very profitable branch of traffic to the manufacturer, and given employment to many industrious people.

The material used there is chiefly linen; but many have been recently woven of cotton, since the introduction of that article into the manufacture of cloth has become so prevalent. The cotton damasks are considerably cheaper than those of linen; but are not considered either so elegant or durable. The cotton, also, unless frequently bleached, does not preserve the purity of the white color nearly so well as the linen.

DA.MASKEE.ING; the art of ornamenting iron, steel, \&e., by making incisions upon its surface, and filling them up with gold or silver wire; chiefly used in enriching sword blades, guards, and gripes, locks of pistols, \&c.

Its name shows the place of its origin, or, at least, the place where it has been practised in the greatest perfection; viz., the city of Damascus, in Syria; though M. Felibien attributes the perfection of the art to his countryman, Cursinet, who wrought under the reign of Henry $\mathrm{IV}$.

Damaskeening is partly mosaic work, partly engraving, and partly carving. As mosaic work it consists of pieces inlaid; as engraving, the metal is indented, or cut in intaglio; and as carving, gold and silver are wrought into it in relievo.

There are two ways of damaskeening; in the first, which is the most beautiful, the artists cut into the metal with a graver, and other tools proper for engraving upon steel, and afterwards fill up the incisions, or notches, with a pretty thick silver or gold sire. In the other, which is only superficial, they content themselves to make hatches, 
or strokes across the iron, \&c. with a cutting knife, such as is used in making of sman files. As to the first, it is necessary for the gravings or incisions to be made in the dovetail form; that the gold or silver wire, which is thrust forcibly into them, may adhere the more strongly. As to the second, which is the more usual, the method is this :- Having heated the steel till it changes to a violet, or blue color, they hatch it over and acros with the knife; then draw the ensign or ornament intended, upon this hatching, with a fine brass point or bodkin. This done, they take fine gold wire, and conducting or chasing it according to the figures already designed, they sink it carefully into the hatches of thr metal with a copper tool.

DAMASSIN is a kind of damask, with gold and silver flowers, woven in the warp anc woof; or occasionally with silk organzine.

DAMPS, in mining, are noxious exhalations, or rather gases, so called from the German dampf, vapor. There are two principal kinds of mine gases, the fire-damp, or carbureter hydrogen, and the choke-damp, or carbonic acid gas. See Mines.

DAPHNINE; the bitter principle of the Daphne Alpina.

DATOLITE. A mineral composed of silica, lime, and boracic acid.

DECANTATION (Eng. and Fr.; Abgiessen, Germ.) is the act of pouring off the clear supernatant fluid from any sediment or deposite. It is much employed in the chemical arts; and is most conveniently effected by a syphon.

DECOCTION (Eng. and Fr.; Ablochung, Germ.) means either the act of boiling a liquid along with some organic substance, or the liquid compound resulting from that act.

DECOMPOSITION (Eng. and Fr.; Zersetzung, Germ.) is the separation of the constituent principles of any compound body. The following table, the result of important researches recently made by M. Persoz, Professor of Chemistry at Strasburgh, shows the order in which decompositions take place among the successive substances.

\begin{tabular}{|c|c|c|c|}
\hline & Nitric Acid. & & Muriatic Acid. \\
\hline Oxyde of & Magnesium & Oxyde of & f Magnesium \\
\hline- & Silver & - & Cobalt \\
\hline - & Cobalt & - & Nickel \\
\hline & Nickel & Protox. of & f Mercury \\
\hline Protox. of & Cerium & - & Cerium \\
\hline Oxyde of & Zinc & Oxyde of & Zine \\
\hline Protox. of & Manganese & Protox. of & Manganese \\
\hline Oxyde of & Lead & - & Iron \\
\hline - & Cadmium & - & Uranium \\
\hline - & Copper & - & Copper \\
\hline - & Glucinum & - & Tin \\
\hline - & Alumium & Oxyde of & Glucinum \\
\hline - & Uranium & - & Alumium \\
\hline - & Chromium & - & Uranium \\
\hline Protox. of & Mercury & - & Chromium \\
\hline Oxyde of & Mereury & - & Iron \\
\hline - & Iron & - & Tin \\
\hline - & Bismuth & - & Bismuth \\
\hline & & - & Antimony \\
\hline
\end{tabular}

By means of the cupric oxyde we may separate, 1 , the ferric oxyde from the manganous oxyde; 2 , the cobaltic, nickelic, zincic and cerous oxydes from the uranic, ferric, chromic, and aluminic oxydes; 3 , the ferrous oxyde from the chromic oxyde, when dissolved in the muriatic acid.

In boiling a muriatic solution of the cobaltic, nickelic, and manganous oxydes, with the mercuric oxyde, the first two oxydes alone are precipitated. Alumina separates the cadmic oxyde from the bismuthic oxyde, the stannous oxyde from the stannic oxyde, and the stannous oxyde from the antimonic acid. The cupric oxyde separates also by precipitation, the aluminic, uranic, chromic, titanic, and vanadic oxydes from all the oxydes which are precipitable in the state of sulphuret, by hydrosulphuret of ammonia.

As an example of this mode of analysis-

Dissolve pech-blende in aqua regia, precipitate its copper by sulphureted hydrogen, boil the liquid along with nitric acid, in order to transform all the uranium into uranic acid. Next boil it along with cupric oxyde, which precipitates only the uranic and ferric oxydes. Redissolve the precipitate in nitric acid, and boil the solution with mercuric oxyde, which does not precipitate the ferric oxyde. Finally, separate the copper and the mercury from the uranium, by means of sulphureted hydrogen. In this process we may substitute plumbic oxyde for the cupric oxyde, and succeed equally well.

Knowledge, like the above, of the elective affinities and habitudes of chemical bodies, simple and compound, imparts to its possessor an irresistible power over the unions and 
disunions of the elements, which he can exercise with certainty in effecting innumerable transformations in the arts.

DECREPIT ATION (Eng. and Fr.; Verknistern, Germ.) is the crackling noise, attended with the flying asunder of their parts, made by several salts and minerals, when heated. It is caused by the unequal sudden expansion of their substance by the heat. Silphate of barrta, chloride of sodium, calcareous spar, nitrate of baryta, and many more budies which contain no water, decrepitate most violently, separating at the natural joints of their crystalline structure. Some chemists have preposterously enough ascribed the phenomenon to the expansion of the combined water into steam. What a specimen of inductive philosophy !

DEFECATION (Eng. and Fr.; Klaren, Germ.), the freeing from dregs or impurities.

DEFI.AGR.ATION (Eng. and Fr.; Verpuffung, Germ.), the sudden blazing up of a combustible; as of a charcoal or sulphur when thrown into melted nitre.

DELPHINIA. The regeto-alkaline principle of the Delphinium staphysagria, or stavesacre. It is poisonous.

DELIQUESCENT (Zerfliessen, Germ.) is said of a solid which attrants so much moisture from the air as to become spontaneously soft or liquid; such as potash and muriate of lime.

DEPHLEGMATION is the process by which liquids are deprived of theil watery particles. It is applied chiefly to spirituous liquors, and is now nearly obsolete, as involving the alchemistical notion of a peculiar principle called phlegm.

DEPHLOGISTICATED; deprived of phlogiston, - formerly supposed to be the common combustible principle. It is nearly synonymous with oxygenated. The idea originally attached to the word having proceeded from false logic, the word itself should never be used either in science or manufactures.

DEPILATORY (Depilatoire, Fr.; Enthaarensmittel, Germ.) is the name of any substance capable of removing hairs from the human skin without injuring its texture. They act either mechanically or chemically. The first are commonly glutinous plasters formed of pitch and rosin, which stick so closely to the part of the skin where they are applied, that when removed, they tear away the hairs with them. This method is more painful, but less dangerous than the other, which consists in the solvent action of a menstruum, so energetic as to penetrate the pores of the skin, and destroy the bulbous roots of the hairs. This is composed either of caustic allalis, sulphuret of barvta, or arsenical preparations. Certain vegetable juices have also been recommended for the same purpose; as spurge and acacia. The bruised eggs of ants hare likewise been prescribed. But the oriental rusma yields to nothing in depilatory power. Gadet de Gassincourt has published in the Dictionnaire des Sciences Medicales, the following recipe for preparing it.

Mix two ounces of quicklime with half an ounce of orpiment or realgar, (sulphuret of arsenic;) boil that mixture in one pound of strong alkaline ley, then try its strength by dipping a feather into it, and when the flue falls off, the rusmu is quite strong enough. It is applied to the human skin by a momentary friction, followed by washing with warm water. Such a caustic liquid should be used with the greatest circumspection, beginning with it somewhat diluted. A soap is sometimes made with lard and the above ingredients; or soft soap is combined with them; in either case to form a depilatory pommade. Occasionally onc ounce of orpiment is taken to eight ounces of quicklime, or two to twelre, or three to fifteen; the last mixture being of course the most active. Its causticity may be tempered by the addition of one eighth of starch or rye flour, so as to form a soft paste, which being laid upon the hairy spot for a few minutes, usually carries away the hairs with it.

The rusma should never be applied but to a small surface at a time, for independently of the risk of corroding the skin, dangerous consequences might ensue from absorption of the arsenic.

DETONATION. See Funminating, for the mode of preparing detonating powder for the percussion caps of fire-arms.

DEUTOXYDE literally means the second oxyde, but is usually employed to denote a compound containing two atoms or two prime equivalents of oxygen to one or more of a metal. Thus we say deutoxyde of copper, and deutoxyde of mercury. Berzelins has abbreriated this expression by adopting the principles of the French nomenclature of 1787 ; according to which the higher stage of oxydizement is characterized by the termination $i c$, and the lower by ous, and he writes accordingly cupric and mercuric, to designate the dentoxydes of these two metals; cuprous and mercurous to designate their protoxydes. I have adopted this nomenclature in the article Decomposition, and in some other parts of this Dictionary, as being short and sufficiently precise.

DEXTRINE is a matter of a gummy appearance into which the interior substance of the molecules of starch is converted, through the influence of diastase or acids. It derives its name from the circumstance that it turns, more than any other body, the 
plane of polarization to the right hand. It is white, insipid, withont smell, transparent in thin plates, friable, with a glassy fracture when well dried. It is not altered by the heat of boiling water, but at $280^{\circ} \mathrm{F}$. it becomes brown, and acquires the flavor of toasted bread. It is not colored by iodine, like starch, it does not form muric acid with the nitric, as common gum does, and it is transformed into grape sugar, when heated along with dilute sulphuric acid or diastase.

Dextrine is much employed by the French pastry-cooks and confectioners; it is a good substitute for gum arabic in medicine. For the conversion of potato or other starch into dextrine by the action of diastase, see this article.

DIAMOND. Since this body is merely a condensed form of carbon, it cannot in a chemical classification be ranked among stones; but as it forms in commerce the most precious of the gems, it claims our first attention in a practical treatise on the arts. Diamonds are distinguishable by a great many peculiar properties, very remarkable and easily recognised, both in their rough state, and when cut and polished. Their most absolute and constant character is a degree of hardness superior to that of every mineral, whence diamonds scratch all other bodies, and are scratched by none. Their peculiar adamantine lustre, not easy to define, but realily distinguishable by the eye from that of every other gem, is their most obvious feature. Their specific gravity is 3.55 . Whether rough or polished, diamonds acquire by friction positive electricity, but do not retain it for more than half an hour. The natural form of diamonds is derivable from an octahedron, and they never present crystals having one axis longer than the other. Their strueture is very perceptibly lamellar, and therefore, notwithstanding their great hardness, they are brittle and give way in the line of their cleavage, affording a direct means of arriving at their primitive form, the regular octahedron.

The diamond possesses either single or double refraction, according to its different crystalline forms; its refractive power on light is far greater than it ought to be in the ratio of its density; the index of refraction being $2 \cdot 44$, whence Newton long ago supposed it to consist of inflammable matter. Its various forms in nature present a circumstance peculiar to this body; its faces are rarely terminated by planes, like most other native crystals, but they are often rounded off, and the edges between them are curved. When these secondary faces are attentively examined with a lens, we remark that they are marked with striæ, sometimes very fine and almost imperceptible, but at others well defined; and that these striæ are parallel to the edges of the octahedron, and consequently to those of the plates that are applied on the primitive faces of this figure.

Diamonds are usually colorless and transparent; when colored, their ordinary tint تerges upon yellow, or smoke-yellow, approaching sometimes to ilackish-brown. Green diamonds are next to yellow the most common; the blue possess rarely a lively hue, but they are much esteemed in Scotland. The rose or pink diamonds are the most valued of the colored kind, and exceed sometimes in price the most limpid; though generally speaking the latter are the most highly prized.

The geological locality of the diamond seems to be in diluvial gravel, and among conglomerate rocks; consisting principally of fragments of quartz, or rolled pebbles of quartz mixed with ferruginous sand, which compose sometimes hard aggregated masses. This kind of formation is called cascalho in Brazil. Its accompanying minerals are few in number, being merely black oxyde of iron, micaceous iron ore, pisiform iron ore, fragments of slaty jasper, several varieties of quartz, principally amethyst. In Mr. Heuland's splendid collection there was a Brazilian diamond imbedded in brown iron ore; another in the same, belonging to M. Schuch, librarian to the Crown Princess of Portugal; and in the cabinet of M. Eschwege there is a mass of brown iron ore, containing a diamond in the drusy cavity of a green mineral, conjectured to be arseniate of iron. From these facts it may be inferred with much probability that the matrix or original repository of the diamond of Brazil is brown iron ore, which occurs in beds of slaty quartzose micaceous iron ore, or in beds composed of iron-glance and magnetic iron ore, both of which are apparently subordinate in that country to primitive clay slate.

The loose earth containing diamonds lies always a little way beneath the surface of the soil, towards the lower outlet of broad valleys, rather than upon the ridges of the adjoining hills.

Only two places on the earth can be adduced with certainty as diamond mines, or rather districts; a portion of the Indian peninsula, and of Brazil.

India has been celebrated from the most remote antiquity as the country of diamonds. Its principal mines are in the kingdoms of Golconda and Visapour, extending from Cape Comorin to Bencal, at the foot of a chain of mountains called the Oruxu, which appear to belong to the trap rock formation. In all the Indian diamond soils, these gems are so dispersed, that they are rarely found directly, even in searching the richest spots, because they are enveloped in an earthy crust, which must be removed before they can be seen. The stony matter is therefore broken into pieces, and is then, as well as the looser earth, washed in basins scooped out on purpose. The gravel thus washed is 
collected, spread out on a smooth piece of ground, and left to dry. The diamonds are now recognised by their sparkling in the sun, and are picked out from the stones.

The diamond mines of Brazil were discovered in 1728, in the district of Serro-doFrio. The ground in which they are imbedded has the most perfect resemblance to that of the East Indies, where the diamonds occur. It is a solid or friable conglomerate, consisting chiefly of a ferruginous sand, which encloses fragments of various magnitude of yellow and bluish quartz, of schistose jasper, and grains of gold disseminated with oligist iron ore; all mineral matters different from those that constitute the neighboring mountains; this conglomerate, or species of pudding-stone, almost always superficial, occurs sometimes at a considerable height on the mountainous table-land. The most celebrated diamond mine is that of Mandarga, on the Jigitonhonha, in the district of Serro-do-Frio to the north of Rio Janeiro. The river Jigitonhonha, three times broader than the Seine at Paris, and from 3 to 9 feet deep, is made nearly dry, by drawing the waters off with sluices at a certain season; and the cascalho or diamond-gravel is removed from the channel by various mechanical means, to be washed elsewhere at leisure. This cascalho, the same as the matrix of the gold mines, is collected in the dry season, to be searched into during the rainy; for which purpose it is formed into little mounds of 15 or 16 tons weight each. The washing is carried on beneath an oblong shed, by means of a stream of water admitted in determinate quantities into boxes containing the cascalho. A negro washer is attached to each box; inspectors are placed at regular dis. tances on elevated stools, and whenever a negro has found a diamond, he rises up and exhibits it. If it weighs $17 \frac{1}{2}$ carats, he receives his liberty. Many precautions are taken to prevent the negroes from secreting the diamonds. Each squad of workmen consists of 200 negroes, with a surgeon and an almoner or priest.

The flat lands on either side of the river are equally rich in diamonds over their whole surface, so that it becomes very easy to estimate what a piece of ground not yet washed may produce.

It is said that the diamonds surronnded with a greenish crust are of the first water, or are the most limpid when cut. The diamonds received in the different mines of the district are deposited once a month in the treasury of Tejuco; and the amount of what was thus delivered from 1801 to 1806 , may be estimated at about 18 or 19 thousand carats per anuum.

On the banks of the torrent called Rio Pardo, there is another mine or diamonds. The ground presents a great many friable rocks of pudding-stone, distributed in irregujar strata. It is chiefly in the bed of this stream that masses of cascalho occur, peculiarly rich in diamonds. They are much esteemed, particularly those of a greenish-blue color. The ores that accompany the diamond at Rio Pardo differ somewhat from those of the washing grounds of Mandanga, for they contain no pisiform iron ore; but a great many pebbles of slaty jasper. This table land seems to be very high, probably not less than 5500 feet above the level of the sea.

Tocaya, a principal village of Minas Novas, is 34 leagues to the northeast of Tejuco, in an acute angle of the confluence of the Jigitonhonha and the Rio Grande. In the bed of the streamlets which fall westward into the Jigitonhonha, those rolled white topazes are found which are known under the name of minas novas with blue topazes, and aquamarine beryls. In the same country are found the beautiful cymophanes or erysoberyls so much prized in Brazil. And it is from the cantons of Indaia and Abaite that the largest diamonds of Brazil come; yet they have not so pure a water as those of the district of Serro-do-Frio, but incline a little to the lemon yellow.

Diamonds are said to come also from the interior of the island of Borneo, on the banks. of the river Succadan, and from the peninsula of Malacca.

It is known that many minerals become phosphorescent by heat, or exposure to the sun's light. Diamonds possess this property, but all not in equal degree, and certain precautions must be observed to make it manifest. Diamonds need to be exposed to the sunbeam for a certain time, in order to become self-luminous; or to the blue rays of the prismatic spectrum, which augment still more the faculty of shining in the dark. Diamonds susceptible of phosphorescence exhibit it either after a heat not raised to redness, or the electric discharge. They possess not only a great refractive power in the mean ray of light, but a high dispersive agency, which enables them to throw out the most varied and vivid colors in multiplied directions.

Louis de Berquem discovered, in 1476, the art of cutting diamonds by rubbing them against one another, and of polishing them with their own powder. These operations may be abridged by two methods: 1 . By availing ourselves of the direction of the laminæ of the diamond to split them in that direction, and thus to produce several facets. This process is called cleaving the diamond. Some, which appear to be macle crystals, resist this mechanical division, and are called diamonds of nature. 2. By sawing the diamonds by means of a very delicate wire, coated with diamond powder.

Diamonds take precedence of every gem for the purposes of dress and decoration; and 
hence the price attached to those of a pure water increases in so rapid a proportion, that beyond a certain term there is no rule of commercial valuation. The largest diamond that is known seems to be that of the Rajah of Mattan, in the East Indies. It is of the purest water, and weighs 367 carats, or at the rate of 4 grains to a carat, upwards of 3 ounces troy. It is shaped like an egg, with an indented hollow near the smaller end; it was discovered at Landak about 100 years ago; and though the possession of it has cost several wars, it has remained in the Mattan family for 90 years. A governor of Batavia, after ascertaining the qualities of the gem, wished to be the purchaser, and offered 150,000 dollars for it, besides two war brigs with their guns and ammunition, together with a certain number of great guns, and a quantity of powder and shot. But this diamond possessed such celebrity in India, and was regarded as a talisman involving the fortunes of the Rajah and his family, that he refused to part with it at any price.

The diamond possessed, in the time of the traveller Tavernier, by the emperor of Mogul, a kingdom now no more, weighed 279 carats, and was reckoned worth upwards of $400,000 l$. sterling. It was said to have lost the half of its original weight in the cutting. After these prodigious gems, the next are:-1. That of the emperor of Russia, bought by the late empress Catharine, which weighs 193 carats. It is said to be of the size of a pigeon's egg, and to have been bought for $90,000 l$., besides an annuity to the Greek merchant of $4000 l$. It is reported thai he above diamond formed one of the eyes of the famous statue of Sheringan, in the temple of Brama, and that a French grenadier, who had deserted into the Malabar service, found the means of robbing the pagoda of this precious gem; and escaped with it to Madras, where he disposed of it to a ship captain for 2,000l., who resold it to a Jew for 12,000l. From him it was transferred for a large sum to the Greek merchant. 2. That of the emperor of Austria, which weighs 139 carats, and has a slightly yellowish hue. It has, however, been valued at 100,000l. 3. That of the king of France, called the Regent or Pitt diamond, remarkable for its form and its perfect limpidity. Although it weighs only 136 carats, its fine qualities have caused it to be valued at $160,000 l$., though it cost only $100,000 l$.

The largest diamond furnished by Brazil, now in possession of the crown of Portugal, weighs, according to the highest estimates, 120 carats. It was found in the streamlet of Abaïte, in a clay-slate district.

The diamonds possessed of no extraordinary magnitude, but of a good form and a pure water, may be valued by a certain standard rule. In a brilliant, or rose-diamond of regular proportions, so much is cut away that the weight of the polished gem does not exceed one half the weight of the diamond in the rough state; whence the value of a cut diamond is esteemed equal to that of a similar rough diamond of double weight, exclusive of the cost of workmanship. The weight and value of diamonds are reckoned by carats of 4 grains each; and the comparative value of two diamonds of equal quality but different weights, is as the squares of these weights respectively. The arerage price of rough diamonds that are worth working is about $2 l$. for one of a single carat; but as a polished diamond of one carat must have taken one of 2 carats, its price in the rough state is double the square of $2 l$., or $8 l$. Therefore, to estimate the value of a wrought diamond, ascertain its weight in carats, double that weight, and multiply the square of this product by $2 l$.

Hence, a wrought diamond of 1 carat is worth $£ 8$

$\begin{array}{rrr}2 & - & 32 \\ 3 & - & 72 \\ 4 & - & 128 \\ 5 & - & 200 \\ 6 & - & 288 \\ 7 & - & 392 \\ 8 & - & 512 \\ 9 & - & 612 \\ 10 & - & 800 \\ 20 & - & 3200\end{array}$

$20 \quad 3200$, beyond which weight the prices can no longer rise in this geometrical progression, from the small number of purchasers of such expensive toys. A very trifling spot or flaw of any kind lowers exceedingly the commercial value of a diamond.

Diamonds are used not only as decorative gems, but for more useful purposes, as for cutting giass by the glazier, and all kinds of hard stones by the lapidary.

On the structure of the glazier's diamond, we possess some very interesting observations and reflections by Dr. Wollaston. He remarks, that the hardest substances brought to a sharp point scratch glass, indeed, but do not cut it, and that diamond alone possesscd that property; which he ascribes to the peculiarity of its crystallization in rounded faces, and curvilinear edges. For glass-cutting, those rough diamonds are always selected which are sharply crystallized, hence called diamond sparks; but cut 
diamonds are never used. The inclination to be given to a set diamond in cutting glass is comprised within very narrow limits; and it ought, moreover, to be inoved in the direction of one of its angles. The curvilinear edge adjoining the curved faces, entering as a wedge into the furrow opened up by itself, thus tends to separate the parts of the glass; and in order that the crack which causes the separation of the ritreous particles may take place, the diamond must be held almost perpendicular to the surface of the glass. The Doctor proved this theory by an experiment. If, by suitable cutting with the wheel, we malie the edges of a spinel ruby, or corundum-telesie (sapphire) curvilinear, and the adjacent faces curved, these stones will cut glass as well as a glazier's diamond, but being less hard than it, they will not preserve this property so long. He found that upon giving the surface of even a fragment of flint the same shape as that of the cutting diamond, it ac luired the same property; but, from its relative softness, was of little duration. The depth to which the fissure caused by the glazier's diamond penetrates, does not seem to exceed the two-hundredth of an inch.

I shall here introduce Mr. Milburn's valuable observations on the choice of rough diamonds, as published in his work on Oriental Commerce.

The color should be perfectly crystalline, resembling a drop of clear spring water, in the middle of which you will perceive a strong light, playing with a great deal of spirit. If the coat be smooth and bright, with a little tincture of green in it, it is not the worse, and seldom proves bad, but if there is a mixture of yellow with green, then beware of it; it is a soft greasy stone, and will prove bad.

If the stone has a rough coal, so that you can hardly see through it, and the coat be white and look as if it were rough by art, and clear of flaws or veins, and no blemish cast in the body of the stone (which may be discovered by holding it against the light), the stone will prove good.

It often happens that a stone will appear of a reddish hue on the outward coat, not unlilie the color of rusty iron, yet by looking through it against the light, you may observe the heart of the stone to be white (and if there be any black spots, or flaws, or veins in it, they may be discovered by a true eye, although the coat of the stone be the same), and such stones are generally good and clear.

If a diamond appears of a greenish bright coat, resembling a piece of green glass, inclining to black, it generally proves hard, and seldom bad; such stones have been linown to have been of the first water, and seldom worse than the second; but if any tincture of yellow seems to be mixed with it, you may depend on its being a very bad stone.

All stones of a milky cast, whether the coat be bright or dull, if ever so little inclining to a bluish cast, are naturally soft, and in danger of being flawed in the cutting; and though they should have the good fortune to escape, yet they will prove dead and milky, and turn to no account.

All diamonds of cinnamon color are dubious; but if of a bright coat mixed with a little green, then they are certainly bad, and are accounted among the worst of colors. You will meet with a great many diamonds of a rough cinnamon-colored coat, opaque; this sort is generally very hard, and, when cut, contain a great deal of life and spirit; but the color is very uncertain; it is sometimes white, sometimes brown, and sometimes of a fine yellow. Rough diamonds are frequently beamy, that is, look fair to the eye, yet are so full of veins to the centre, that no art or labor can polish them. A good diamond should never contain small spots of a white or gray color of a nebulous form; it should be free from small reddish and brownish grains, that sometimes occur on their surface, or in their interior. A good diamond should split readily in the direction of the cleavage; it sometimes happens, however, that the folia are curved, as is the case in twin crystals. When this happens, the stone does not readily cut and polish, and is therefore of inferior value.

In the cut and polished gem, the thickness must always bear a certain proportion to the breadth. It must not be too thin nor thick; for, when too thin, it loses much of its fire, and appears not unlike glass.

The term carat is said to be derived from the name of a bean, the produce of a species of erythina, a native of the district of Shangallas, in Africa; a famous mart of gold-dust. The tree is called kuara, a word signifying sun in the language of the country; because it bears flowers and fruit of a flame color. As the dry seeds of this pod are always of nearly uniform weight, the savages have used them from time immemorial to weigh gold. The beans were transported into India, at an ancient period, and have been long employed there for weighing diamonds. The carat of the civilized world is, in fact, an imaginary weight, consisting of 4 nominal grains, a little lighter than 4 grains troy ( $p o i d s$ de marc) ; it requires 74 carat grains and $\frac{1}{16}$ to equipose 72 of the other.

In valuing a cut diamond, we must reckon that one half of its weight has been lost in the lapidary's hands; whence its weight in this state should be doubled before we calculate its price by the general rule for estimating diamonds. The French multiply 
by 48 the square of this weight, and they call the product in francs the value of the diamond. Thus, for example, a cut diamond of 10 carats would be worth $(10 \times 2)$ s $\times 48=19,200$ francs, or 7681 , allowing only 25 francs to the pound sterling.

The diamond mines of Brazil have brought to its government, from the year 1730 till $1814,3,023,000$ carats; being at the average rate annually of 36,000 carats, or a little more than 16 lbs. weight. They have not been so productive in the later years of that period; for, according to Mr. Mawe, between 1801 and 1806, only $115,6 \% \bar{j}$ carats were obtained, being 19,279 a year. The actual expenses incurred by the government, during this interval, was $4,419,700$ francs; and, deducting the production in gold from the washings of the diamond gravel, or cascalho, it is found that the rough diamonds cost in exploration, per carat, 38 franes 20 c., or nearly 31 s. British money. The contraband is supposed to amount to one third of the above legitimate trade. Brazil is alınost the only country where diamonds are mined at the present day; it sends annually to Europe from 25 to 30 thousand carats, or from 10 to $16 \frac{1}{2}$ lbs.

DIAMONDS, culting of. Although the diamond is the hardest of all known substances, yet it may be split by a steel tool, provided a blow be applied; but this requires a perfect knowledge of the structure, because it will only yield to such means in certain directions. This circumstance prevents the workman from forming facettes or planes generally, by the process of splitting; he is therefore obliged to resort to the process of abrasion, which is technically called cutting. The process of cutting is effected by fixing the diamond to be cut on the end of a stick, or handle, in a small ball of cement, that part which is to be reduced being left to project. Another diamond is also fixed in a similar manner; and the two stones being rubbed against each other with considerable force, they are mutually abraded, flat surfaces, or facettes, being thereby produced. Other facettes are formed by shifting the diamonds into fresh positions in the cement, and when a sufficient number are produced, they are fit for polishing. The stones, when cut, are fixed for this purpose, by imbedding them in soft solder, contained in a small copper cup, the part, or facette, to be polished, being left to protrude.

A flat circular plate of cast-iron is then charged with the powder produced during the abrasion of the diamonds; and by this means a tool is formed which is capable of producing the exquisite lustre so much admired on a finely-polished gem. Those diamonds that are unfit for working, on account of the imperfection of their lustre or color, are sold, for various purposes, under the technical name of Bort. Stones of this kind are frequently broken in a steel mortar, by repeated blows, until they are reduced to a fine powder, which is used to charge metal plates, of various kinds, for the use of jewellers, lapidaries, and others. Bort, in this state of preparation, is incapable of polishing any gems; but it is used to produce flat surfaces on rubies and other precious stones.

Fine drills are made of small splinters of bort, which are used for drilling small holes in rubies, and other hard stones, for the use of watch-jewellers, gold and silrer wiredrawers, and others, who require very fine holes drilled in such substances. These drills are also used to pierce holes in china, where rivets are to be inserted; also for piercing holes in artificial enamel teeth, or any vitreous substances, however hard.

DIAMOND MICROSCOPES were first suggested by Dr. Goring, and have been well executed by Mr. Pritchard. Previous to grinding a diamond into a spherical figure, it should be ground flat and parallel upon both sides, that by looking through it, as opticians try flint glass, we may see whether it has a double or triple refractive power, as many have, which would render it useless as a lens. Among the 14 different crystalline forms of the diamond, probably the octahedron and the cube are the only ones that will give single vision. It will, in many cases, be advisable to grind diamond lenses, plano-convex, both because this figure gives a low spherical aberration, and because it saves the trouble of grinding one side of the gem. A concave tool of cast iron, paved with diamond powder, hammered into it by a hardened steel punch, was empluyed by Mr. Pritchard. This ingenious artist succeeded in completing a double convex of equal radii, of about $\frac{1}{25}$ of an inch focus, bearing an aperture of $\frac{1}{30}$ of an inch with distinctness upon opaque objects, and its entire diameter upon transparent ones. This lens gives vision with a trifling chromatic aberration; in other respects, like Dr. Goring's Amician reflector, but without its darkness, its light is said to be superior to that of any compound microscope whatever, acting with the same power, and the same angle of aperture. The advantage of seeing an object without aberration by the interposition of only a single magnifier, instead of looking at a picture of it with an eyeglass, is evident. We thus have a simple direct view, whereby we shall see more accurately and minutely the real texture of objects.

DIAPER is the name of a kind of eloth, used chiefly for table linen. It is known among the French by the name of toile fourré, and is ornamented with the most extensive 
figures of any kind of tweeled cloth, excepting damask. The mounting of a loom for working diayer is, in principle, much the same as a draw-loom, but the figures being less extensive, the mounting is more simple, and is wrought entirely by the wearer, without the aid of any other person. As tweeled cloths, of any number of leaves, are only interwoven at those intervals when one of the leaves is raised, the woof above, and the warp below, is kept floating or flushed, until the intersection takes place. Of consequence, the floating yarn above appears across the fabric, and that below longitudinally. This property of tweeled cloths is applied to form the ornamental figures of all kinds of tweeled goods, merely by reversing the floating yarn when necessary. In the simpler patterns, this is effected by a few additional leaves of treddles; but when the range of pattern becomes too great to render this convenient, an apparatus called a back harness is employed, and the cloth woven with this mounting is called diaper. Diapers are generally five-leaf tweels; that is to say, every warp floats under four threads of woof, and is raised, and of course interwoven with the fifth. This is done either successively, forming diagonals at $45^{\circ}$ upon the cloth, or by intervals of two threads, which is called the broken tweel. The latter is generally, if not universally, adopted in the manufacture of diaper. The reason of preferring the broken to the regular tweel, where ornaments are to be formed, is very obvious. The whole depending upon reversed flushing to give the appearance of oblique or diagonal lines, through either, would destroy much of the effect, and materially injure the beauty of the fabric. The broken tweel, on the contrary, restores to the tweeled cloth a great similarity of appearance to plain, or alternately interwoven fabrics, and at the same time preserves the facility of producing ornaments by reversing the flushing. The simplest kinds of reversed tweels will be found described under TExTILE FABRICS.

DIASTASE. This curious substance, extracted by water from crushed malt, and precipitated from that infusion by alcohol, as is described under FERnientation, has been made the subject of new researches by M. Guerin Varry. The conclusions deducible from his interesting experiments are the following:-

1. One part of diastase, dissolved in 30 parts of cold water, put with 408 parts of potato starch out of contact of air, did not exercise the slightest action upon this substance in the course of 63 days, under a temperature varying from $68^{\circ}$ to $79^{\circ} \mathrm{Fahr}$.

2. Two parts of diastase do not in the course of an hour cause the globules of three parts of starch to burst, at a temperature approaching very nearly to that of the hot water which bursts them into a paste. It follows that diastase acts no part in the process of germination, towards eliminating the teguments of the starch, or transforming its interior portion into sugar, and a gummy matter assimilated by plants.

3. Diastase liquefies and saccharifies the paste of starch without absorption or disengagement of gas; a reaction which takes place equally in vacuo as in the open air.

4. 100 parts of starch made into a paste with 39 times their weight of water, mixed with 6.13 parts of diastase dissolved in 40 parts of water, and kept for an hour between $140^{\circ}$ and $149^{\circ} \mathrm{Fahr}$., afforded 86.91 parts of sugar.

5. A paste containing 100 parts of starch, and 1393 parts of water, put in contact with 12.25 parts of diastase dissolved in 367 parts of cold water, having been maintained at $68^{\circ}$ Fahr. during 24 hours, produced $77 \cdot 64$ parts of sugar.

6. The preceding experiment, repeated at the temperature of melting ice, afforded at the end of 2 hours, 11.82 parts of sugar.

7. The most favorable proportions and circumstances for the production of a great quantity of sugar, are a slight excess of diastase or barley malt (at least 25 per cent. of the latter), about 50 parts of water to one of starch, and a temperature between $140^{\circ}$ and $149^{\circ} \mathrm{Fahr}$. It is of the greatest consequence for the saccharification to take place as speedily as possible, so that the sugar produced may not be left in contact with much gummy matter (dextrine), in which case the diastase will not convert the latter into sugar. In fact, the liquefaction and saccharification should proceed simultaneously.

8. The sugar of starch, prepared either with diastase or sulphuric acid, crystallizes in cauliflowers, or in prisms with rhomboidal facets. It has the same composition as sugar of grapes.

9. Diastase even in excess does not saccharify the gummy matter dissolved in the water along with the starch sugar, but when the gum is insulated, it is convertible almost entirely into sugar.

10. Gum arabic, cane sugar, and beer yeast, suffer no change from diastase.

11. A watery solution of diastase readily decomposes on keeping, either in contact or out of contact of air.

12. When starch-sugar, whether obtained by means of diastase or sulphuric acid, is submitted to the spirituous fermentation, the sum of the weights of the alcohol, carbonic acid, and water of erystallization of the sugar, is less than the weight of the sugar by about $3 \frac{1}{2}$ per cent. This difference proceeds in a great measure from the form- 
ation of some acetic acid, lactic acid, volatile oil, and probably some other unknown pre ducts in the act of fermentation.

DIMITY is a kind of cotton cloth originally imported from Ind:a, and now manufac tured in great quantities in various parts of Britain, especially in Lancashire. Dr Johnson calls it dimmity, and describes it as a kind of fustian. The distinction between fustian and dimity seems to be, that the former designates a common tweeled cotton cloth of a stout fabric, which receives no ornament in the loom, but is most frequently dyed after being woven. Dimity is also a stout cotton cloth, but not usually of so thick a tex. ture; and is ornamented in the loom, either with raised stripes or fancr figures; is seldom dyed, but usually worn white, as for bed and bed-room furniture. The striped dimities are the most common; they require less labor in weaving than the others; and the mounting of the loom being more simple, and consequently less expensive, they can be sold at much lower rates. See Textile Fabrics, for particular details of the plan of mounting them.

DIES FOR STAMPING. (Coins, Fr.; Münzstampeln, Germ.) The first circumstance that claims particular attention in the manufacture of dies, is the selection of the best kind of steel for the purpose, and this must in some measure be left to the experience of the die-forger, who, if well skilled in his art, will be able to form a tolerably correct judgment of the fitness of the metal for the purpose, by the manner in which it works upon the anvil. It should be rather fine-grained than otherwise, and above all things perfectly even and uniform in its texture, and free from spots and patches finer or coarser than the general mass. But the very fine and uniform steel with a silky fracture, which is so much esteemed for some of the purposes of cutlery, is unfit for our present purpose, from the extreme facility with which it acquires great hardness by pressure, and its liability to cracks and flaws. The very coarse-grained or highly crystalline steel is also equally objectionable; it acquires fissures under the die-press, and seldom admits of being equally and properly hardened. The object, therefore, is to select a steel of a medium quality as to fineness of texture, not easily acted upon by dilute sulphuric acid, and exhibiting a uniform texture when its surface is washed over with a little aqua-fortis, by which its freedom from pins of iron, and other irregularities of composition, is sufficiently indicated.

The best kind of steel being thus selected, and properly forged at a high heat into the rough die, it is softened by very careful annealing, and in that state, having been smoothed externally, and brought to a table in the turning lathe, it is delivered to the engraver.

The process of annealing the die consists in heating it to a bright cherry red, and suffering it to cool gradually, which is best effected by bedding it in a crucible or iron pot of coarsely-powdered charcoal, that of animal substances being generally preferred. In this operation it is sometimes supposed that the die, or at least its superficial parts, becomes super-carbonized, or highly-converted steel, as it is sometimes called; but experience does not justify such an opinion, and I believe the composition of the die is scarcely, certainly not materially, affected by the process, for it does not remain long enough in the fire for the purpose.

The engraver usually commences his labors by working out the device with small steel tools, in intaglio; he rarely begins in relief (though this is sometimes done); and having ultimately completed his design, and satisfied himself of its general effect and correctness, by impressions in clay, and dabs, or casts in type metal, the die is ready for the important operation of hardening, which, from various causes, a few of which I shall enumerate, is a process of much risk and difficulty ; for should any accident now occur, the labor of many months may be seriously injured, or even rendered quite useless.

The process of hardening soft steel is in itself very simple, though not very easily explained upon mechanical or chemical principles. We know by experience that it is a property of this highly valuable substance to become excessively hard, if heated and suddenly cooled; if, therefore, we heat a bar of soft malleable and ductile steel red hot, and then suddenly quench it in a large quantity of cold water, it not only becomes hard, but fragile and brittle. But as a die is a mass of steel of considerable dimensions, this hardening is an operation attended by many and peculiar difficulties, more especially as we have at the same time to attend to the careful preservation of the engraving. This is effected by covering the engraved face of the die with a protecting face, composed of fixed oil of any kind, thickened with powdered charcoal : some persons add pipe-clay, others use a pulp of garlic, but pure lamp-black and linseed oil answer the purpose perfectly. This is thinly spread upon the work of the die, which, if requisite, may be further defended by an iron ring; the die is then placed with its face downwards in a crucible, and completely surrounded by powdered charcoal. It is heated to a suitable temperature, that is, about cherry red, and in that state is taken out with proper tongs, and plunged into a body of cold water, of such magnitude as not 
to become materially increased in temper iture; here it is rapidly moved about, until all noise ceases, and then lell in the water till quite cool. In this process it should produce a bubbling and hissing noise; if it pipes and sings, we may generally apprehend a crack or fissure.

No process has been found to answer better than the above simple and common mode of hardening dies, though others have had repeated and fair trials. It has been proposed to leep up currents and eddies of cold water in the hardening cistern, by means of delivery-pipes, coming from a height; and to subject the hot die, with its face uppermost, to a suduen and copious current of water, let upon it from a large pipe, supplied from a high reservoir; but these means have not in any way proved more successfu, either in saving the die or in giving it any good qualities. It will be recollected, from the form of the die, that it is necessarily only, as it were, case-hardened; the hardest strata being outside, and the softer ones within, which envelop a core, something in the manner of the successive coats of an onion; an arrangement which we sometimes hare an opportunity of seeing displayed in dies which have been smashed by a violent blow.

The hardening having been effected, and the die being for the time safe, some further steps may be taken for its protection; one of these consists in a very mild kind of tempering, produced by putting it into water, gradually raised to the boiling point, till heated throughout, and the 1 suffering it gradually to cool. This operation renders the die less apt to crack in very cold weather. A great safeguard is also obtaineu by thrusting the cold die into a red-hot iron ring, which just fits it in that state, and which, by contracting as it cools, keeps its parts together under considerable pressure, preventing the spreading of external cracks and fissures, and often enabling us to employ a split or die for obtaining punches, which would break to pieces without the protecting ring.

If the die has been successfully hardened, and the protecting paste has done its duty, by preserving the face from all injury and oxydizement, or burning, as it is usually called, it is now to be cleaned and polished, and in this state constitutes what is technically called a MATRIX; it may, of course, be used as a multiplier of medals, coins, or impressions, but it is not generally thus employed, for fear of accidents happening to it in the coining press, and because the artist has seldom perfected his work upon it in this state. It is, therefore, resorted to for the purpose of finishing a $\mathrm{PCNCH}$, or steel impression for relief. For this purpose a proper block of steel is selected, of the same quality, and with the same precautions as before, and being carefully annealed, or softened, is turned like the matrix, perfectly true and flat at the bottom, and obtusely conical at top. In this state, its conical surface is carefully compressed by powerful and proper machinery upon the matrix, which, being very hard, soon allows it to receive the commencement of an impression; but in thus receiving the impression, it becomes itself so hard by condensation of texture as to require, during the operation, to be repeatedly annealed, or softened; otherwise it would split into small superficial fissures, or would injure the matrix; much practical skill is therefore required in taking this impression, and the punch, at each annealing, must be carefully protected, so that the work may not be injured.

Thus, after repeated blows in the die-press, and frequent annealing, the impression from the matrix is at length perfected, or brought completely up, and having been retouched by the engraver, is turned, hardened, and collared, like the matrix, of which it is now a complete impression in relief, and, as we have before said, is called a punch.

This punch becomes an inexhaustible parent of dies, without further reference to the original matrix; for now by impressing upon it plugs of soft steel, and by pursuing with them an exactly similar operation to that by which the punch itself was obtained, we procure impressions from it to any amount, which of course are fac-similes of the matrix, and these dies being turned, hardened, polished, and, if necessary, tempered, are employed for the purposes of coinage.

The distinction between striking medals and common coin is very essential, and the work upon the dies is accordingly adjusted to each. Medals are usually in very high relief, and the effect is produced by a succession of blows; and as the metal in which they are struck, be it gold, silver, or copper, acquires considerable hardness at each stroke of the press, they are repeatedly annealed during the process of bringing them up. In a beautiful medal, which Mr. Wyon some time since completed for the Rayal Navy College, the obverse represents a head of the King, in very bold relief; it required thirty blows of a very powerful press to complete the impression, and it was necessary to anneal each medal after every third blow, so that they went ten times into the fire for that purpose. In striking a coin or medal, the lateral spread of the metal, which otherwise would ooze out as it were from between the dies, is prerented by the application of a steel collar, accurately turned to the dimensions of the dies, and which, when left plain, gives to the edge of the piece a finished and polished appearance; it is 
sometimes grooved, or milled, or otherwise ornamented, and occasionally lettered, in which case it is made in three separate and moveable pieces, confined by a ring, into which they are most accurately fitted, and so adjusted that the metal may be furced intc the letters by its lateral spread, at the same time that the coin receives the blow of the screw-press.

Coins are generally completed by one blow of the coining-press. These presses are worked in the Royal Mint by machinery, so contrived that they shall strike, upon an average, sixty blows in a minute; the blank piece, previously properly prepared and annealed, being placed between the dies by part of the same mechanism.

The number of pieces which may be struck by a single die of good steel, properly hardened and duly tempered, not unfrequently amounts at the Mint to between three and four liundred thousand, but the average consumption of dies is of course much greater, owing to the rariable qualities of steel, and to the casualties to which the dies are liable: thus, the upper and lower die are often violently struck together, owing to an error in the layer-on, or in that part of the machinery which ought to put the blank into its place, but which now and then fails so to do. This accident very commonly arises from the boy who superintends the press neglecting to feed the hopper of the layer-on with blank pieces. If a die is too hard, it is apt to break or split, and is especially subject to fissures, which run from letter to letter upon the edge. If too soft, it swells, and the collar will not rise and fall upon it, or it sinks in the centre, and the work becomes distorted and faulty. He, therefore, who supplies the dies for an extensire coinage has many accidents and difficulties to encounter. There are eight presses at the Mint, frequently at work for ten hours each day, and the destruction of eight pair of dies per day (one pair for each press) may be considered a fair average result, though they much more frequently fall short of, than exceed this proportion. It must be remembered that each press produces 3600 pieces per hour, but, making allowance for occasional stoppages, we may rechion the daily produce of each press at 30,000 pieces; the eight presses, therefore, will furnish a diurnal average of 240,000 pieces.

DIGESTER is the name of a strong liettle or pot of small dimensions, made very strong, and mounted with a safety valve in its top. Papin, the contriver of this apparatus, used it for subjecting bones, cartilages, \&c. to the solrent action of high-pressure steam, or highly heated water, whereby he proposed to facilitate their digestion in the stomach. This contrivance is the origin of the French cookery pans, called autoclares, because the lid is self-keyed, or becomes steam-light by turning it round under clamps or ears at the sides, having been previously ground with emery to fit the edge of the pot exactly. In some autoclaves the lid is merely laid on with a fillet of linen as a lute, and then secured in its place by means of a screw bearing down upon its centre from an arched bar above. The safety valve is loaded either by a weight placed vertically upon it, or by a lever of the second lind pressing near its fulcrum, and acted upon by a weight which may be made to bear upon any point of its graduated arm.

Cherreul has made a useful application of the digester to vegetable analysis. His instrument consists of a strong copper cylinder, into which enters a tight crlinder of silver, having its edge turned over at right angles to the axis of the cylinder, so as to form the rim of the dicester. A segment of a copper sphere, also lined with silver, stops the aperture of the silter cylinder, being applied closely to its rim. It has a conical ralve pressed with a spiral spring, of an is enclosed within a brass box perforated with four holes; which may be screwed into a tapped orifice in the top of the digester. A tube screwed into another hole serves to conduct away the condensable vapors at pleasure into a Woulfe's apparatus.

DISTILLATION (Eng. and Fr.; Branntweinbrennerei, Germ.) means, in the commercial language of this country, the manufacture of intoxicating spirits; under which are comprehended the four processes, of mushing the regetable materials, cooling the worts, exciting the vinous fermentation, and separating by a peculiar vessel, called a still, the alcohol combined with more or less water. This art of eroking the fiery demon of drunkenness from his attempered state in wine and beer, was unknown to the ancient Greeks and Romans. It seems to have been inrented by the barbarians of the north of Europe, as a solace to their cold and humid clime; and was first made known to the southern nations in the writings of Arnoldus de Villa Nova, and his pupil, Rarmond Lully of Majorca, who declares this admirable essence of wine to be an emanation of the Divinity, an element newly revealed to man, but hid from antiquity, because the hnman race were then too young to need this beverage, destined to revive the energies of modern decrepitude. He further imagined that the discovery of this aqua vita, as it was called, indicated the approaching consummation of all things-the end of this world. Howerer much he erred as to the value of this remarkable essence, he truly predicted its vast influence upon humanity, since to both cirilized and savage nations it has realized greater ills than were threatened in the fabled box of Pandora. 
I shall consider in this place the first three of these subjects, reserving for the article SriLl an account of the construction and use of that apparatus.

Whiskey, from the Irish word Usquebaugh, is the British name of the spirituous liquor manufactured by our distillers, and corresponds to the Eau de vie of the French, and the Branntwein of the Germans. It is generated by that intestine change which grape juice and other glutino-saccharine liquids spontaneously undergo when exposed to the atmosphere at common temperatures; the theory of which will be expounded under the article Fermentation. The production of whiskey depends upon the simple fact, that when any vinous fluid is boiled, the alcohol, being very volatile, evaporates first, and may thereby be separated from the aqueous vegetable infusion in which it took its birth. Sugar is the only substance which can be transformed into alcohol. Whatsoever fruits, seeds, or roots afford juices or extracts capable of conversion into vinous liquor, either coniain sugar ready formed, or starch susceptible of acquiring the saccharine state by proper treatment. In common language, the intoxicating liquor obtained from the sweet juices of fruits is called wine; and that from the infusions of farinaceous seeds, beer; though there is no real difference between them in chemical constitution. A similar bererage, though probably less palatable, is procurable from the juices and infusions of many roots, by the process of fermentation. Wine, cider, beer, and fermented wash of every kind, when distilled, yields an identical intoxicating spirit, which differs in these different cases merely in Hlavor, in consequence of the presence of a minute quantity of volatile oils of different odors.

I. The juices of sweet fruits contain a glutinous ingredient which acts as a ferment in causing their spontaneous change into a vinous condition; but the infusions of seeds, eren in their germinated or malted state, require the addition of a glutinous substance called yeast, to excite the best fermentation. In the fabrication of wine or beer for drinking, the fermentative action should be arrested before all the fruity saccharum is decomposed; nor should it on any account be suffered to pass into the acetous stage; whereas for making distillery wash, that action should be promoted as long as the proportion of alcohol is increased, because the formation of a little acetic acid is not injurious to the quality of the distilled spirit, but rather improves its flavor by the addition of acetic ether, while all the undecomposed sugar is lost. Distillers operate upon the saccharine matter from corn of various kinds in two methods; in the first they draw off a pure watery extract from the grain, and subject this species of wort to fermentation; in the second they ferment and distil the infused mass of grains. The former is the practice of the distillers in the United Kingdom, and is preferable on many accounts; the latter, which is adopted in Germany, Holland, and the north of Europe, is less economical, more uncertain in the product, and affords a cruder spirit, in consequence of the fetid volatile oil evolved from the husks in the still. The substances employed by the distillers may be distributed into the following classes :-

1. Saccharine juices. At the head of these stands cane-juice, which fresh from the mill contains from 12 to 16 per cent. of raw sugar, and like the must of the grape enters into the vinous fermentation without the addition of yeast, affording the species of spirit called Rum, which is possessed of a peculiar aroma derired from an essential oil in the cane. An inferior sort of rum is fabricated from molasses, mixed with the skimmings and washings of the sugar pans. When molasses or treacle is diluted with twenty times its weight of warm water, and when the mixture has cooled to $78^{\circ} \mathrm{F}$., if one twelfth of its weight of yeast be added, fermentation will speedily ensue, and an ardent spirit will be generated, which when distilled has none of the aroma of rum; proring this to reside in the immediate juice or substance of the cane, and to be dissipated at the high temperature employed in the production of molasses. Though the cane juice will spontaneously undergo the vinous fermentation, it does so more slowly and irregularly than the routine of business requires, and therefore is quickened by the addition of the lees of a preceding distillation. So sensible are the rum distillers of the adrantage of such a plan, that they soak woollen cloths in the yeast of the fermenting rats, in order to preserve a ferment from one sugar season to another. In Jamaica ard some other of our colonies, 50 gallons of srent wash or lees are mixed with 6 gallons of molasses, 36 gallons of sugar-pan skimmings (a substance rich in aroma), and 8 gallons of water; in which mixture there is about one twelfth part of solid saccharum. Those who attend more to the quality than the quantity of their rum, will use a smaller proportion of the spent wash, which is always empyreumatic, and imparts more or less of its odor to the spirit distilled from it. The fermentation is seldom complete in less than 9 days, and most commonly it requires from 12 to 15 ; the period being dependant upon the capacity of the fermenting tun, and the quality of its contents. The liquid now becomes clear, the froth having fallen to the bottom, and few bubbles of gas are extricated from it, while its specific gravity is reduced from 1.050 down to 0.992. The sooner it is subjected to distillation after this period, the better, to prevent 
the loss of alcohol by the supervention of the acetous stage of fermentation, an accident very liable to happen in the sugar colonies. The crude spirit obtained from the large single sill at the first operation, is rectified in a smaller still. About 114 gallons of rum, proof strenoth, specific gravity 0.920 , are obtained from 1200 gallons of wash. Now these 1200 gallons weigh $12,600 \mathrm{lbs}$, and contain nearly one eighth of their weight of sugar $=1575$ Jbs.; which should yield nearly its own weight of proof spirit, whose bulk is $=\frac{1}{0} 575=1712$ pound measures $=171.2$ gallons; whereas only 114 are obtained; proving the processes to be conducted in a manner far from economical, even with every reasonable allowance.

Mr. Edwards gives the following estimate: "The total amount of sweets from an estate in Jamaica which makes 200 hogsheads of sugar, is 16,666 gallons. The wash set at the rate of 12 per cent. sweets should return 34,720 gallons of low wines, which should give 14,412 gallons of rum, or 131 puncheons of 110 gallons each."

By my own experiments on the quantity of proof spirit obtainable from molasses by fermentation (afterwards to be detailed), one gallon of sweets should yield one gallon of spirit; and hence the above 16,666 gallons should have afforded the same bulk of rum. But here we are left somewhat in the dark, by not knowing the specific gravity of the rum spoken of by Mr. Edwards. The only light let in upon us is when he mentions rum oilproof, that is, a spirit in which olive oil will sink; indicating a density nearly the same with our actual excise proof, for olive oil at $60^{\circ} \mathrm{F}$. has the specific gravity 0.919 . When a solution of sugar of the proper strength is mixed with wine lees, and fermented, it affords a spirit by distillation not of the rum, but of the brandy flavor.

The sweet juices of palm trees and cocoa nuts, as also of the maple, and ash, birch, \&c., when treated like cane juice afford vinous liquors from which ardent spirits, under various names, are obtained; as arrack, \&c.; the quantity being about 50 pounds of alcohol of 0.825 for every 100 pounds of solid saccharine extract present. Honey similarly treated affords the metheglin so much prized by our ancestors. Good whey, freed from curd by boiling, will yield 4 per cent. of spirit of wine, when fermented with the addition of a little yeast.

2. The juices of apples, pears, currants, and such fruits, afford by fermentation quan. tities of alcohol proportional to the sugar they contain. But the quality of the spirit is much better when it is distilled from vinous liquids of a certain age, than from recently fermented must. Cherries are employed in Germany, and other parts of the Continent, for making a high-flavored spirit called Kirsch-wasser, or cherry water. The fully ripe fruit is crushed by a roller press, or an edge-stone mill, along with the kernels; the pulp is fermented in a mass, the liquid part is then drawn off, and distilled. More or iess prussic acid enters from the kernels into this spirit, which renders it very injurious, as a liquor, to many constitutions. I was once nearly poisoned by swallowing a wine glass of it in the valley of Chamouni. The ripened red fruit of the mountain ash constitutes a good material for vinous fermentation. The juice being mixed with some water and a little yeast, affords when well fermented, according to Hermstaedt, 12 pounds, or $1 \frac{1}{2}$ gallons, of alcohol from 2 bushels of the ripe berries.

3. Many roots contain sugar, particularly beet, from which no less than 7 per cent. of it may be extracted by judicious means. Hermstaedt recommends to mash the steam boiled clean roots, and add to the paste two thirds of its weight of boiling water, and a thirtieth of its weight of ground malt, mixing the materials well, and then leaving them three hours in a covered vessel. The mixture must now be passed through a wire sieve, with meshes of one third of an inch square each; the residuum is washed with a little cold water, and, when the temperature has fallen to $77^{\circ} \mathrm{F}$., the proper quantity of yeast must be added, and the fermentation suffered to proceed in a covered tun. In 5 or 6 days it will be complete, and will afford by distillation, from 100 pounds of beet root, about 10 or 12 pounds of proof spirits. Carrots and parsnips, when similarly treated, yield a considerable quantity of alcohol.

II. Ardent spirits or whiskey from fecula or starchy materials.

I have already pointed out, in the article BEER, how the starch is transformed into a saccharine condition, by malting and mashing; and how a fermentable wort may be obtained from starchy meal. By like operations may all vegetable substances, which consist chiefly of starch, become materials for a whiskey distillery. To this class belong all the farinaceous grains, potatoes, and the pods of shell fruits, as beans, vetches, horsechestnuts, acorns, \&c.

1. Whisliey from corn. All those species of corn which are employed in breweries answer for distilleries; as wheat, rye, barley, and oats; as well as buckwheat, and maize or Indian corn. The product of spirits which these different grains afford, depends upon the proportion of starch they contain, including the small quantity of uncrystallizable sugar present in them. Hermstaedt, who has made exact experiments upon the subject, reckons a quart (Prussian or British) of spirits, containing 30 per cent. of the absolute alcohol of Richter, fer 2 pounds of starch. Hence 100 pounds of starch should yield 
35 pounds of alcohol; or 4.375 gallons imperial, equal to $7 \cdot 8$ gallons of spirits, excise proof:

100) pounds of the following grains afford in spirits of specific gravity 0.9427 , containing 45 per cent. of absolute alcohol, ( $=\frac{9}{11}$ of British proof, ) the following quantities :-

Wheat, 40 to 45 pounds of spirits; rye, 36 to 42 ; barley, 40 ; oats, 36 ; buckwheat, 40 ; maize, 40 . The mean of the whole may be taken at 40 pounds, equal to $4 \frac{1}{4}$ gallons imperial, of 0.9427 specific gravity $=3.47$ gallons, at excise proof. The chief difference in these several hinds of corn consists in their different bulks under the same weight; a matter of considerable importance; for since a bushel of oats weighs little more than the half of a bushel of wheat, the former becomes for some purposes less convenient in use than the latter, though it affords a good spirit.

Barley and ryc are the species of grain most commonly employed in the European distilleries for making whiskey. Barley is mostly taken either partly or altogether in the malted state; while the other corns are not malted, but merely mixed with a certain proportion of barley malt to favor the saccharine fermentation in the mashing. It is deemed preferable to use a mixture of several sorts of grain, instead of a single one; for example, wheat with barley and oats; or barley with rye and wheat; for the husks of the oats diffused through the wheat flour and rye meal keep it open or porous when mashed, and thus favor the abstraction of the wort; while the gluten of the wheat tends to convert the starch of the barley and oats into sugar. When the whole of the grain, however, is malted, a much more limpid wort is obtained than from a mixture of malt with raw grain; hence the pure malt is preferable for the ale and porter brewer, while the mixture affords a larger product, at the same cost of materials, to the distiller. When barley is the only grain employed, from one third to one sixth of malt is usually mixed with it; but when wheat and rye are also taken, the addition of from one eighth to one sixteenth of barley malt is sufficient. Oats are peculiarly proper to be mixed with wheat, to keep the meal open in the mashing.

The following are the proportions used by some experienced Scotch distillers.

250 bolls, containing 6 bushels each, being used for a mashing, consist of,

\begin{tabular}{llcc}
25 & bolls of & oats, weighing & 284 lbs. per boll, or $47 \frac{1}{2}$ lbs. per bushel; \\
42 & malt & 240 & 40 \\
25 & rye & 320 & $53 \frac{1}{3}$ \\
158 & barley & 320 & $53 \frac{1}{3}$ \\
\hline 250 & & & mean $\frac{48 \frac{1}{2}}{2}$
\end{tabular}

From each boll, weighing $291 \mathrm{lbs}$, 14 imperial gallons of proof whiskey are obtained on an average; equivalent to 11.2 gallons at 25 over proof.

The malting for the distilleries is to be conducted on the same principles as for the breweries, but the malt ought to be lightly kiln-dried, and that preferably at a steam heat, instead of a fire, which is apt to give an empyreumatic smell to the grain that passes into the spirits. For cuch persons, indeed, as relish the smell of burned turf, called leat-reek in Seotland, the malt should be dried by a turf fire, whereby the whiskey will acquire that peeuliar odor.

But this smell, which was originally prized as a criterion of whiskey made from pure malt, moderately fermented and distilled with peculiar care, has of late years lost its value, since the artifice of impregnating bad raw grain whiskey with peat-smoke has been extensively practised.

Dr. Kolle, in his treatise on making spirits, describes a malting kiln with a copper plate heated with steam, 18 feet long, and 12 feet broad, on which a quantity of malt being spread thin, is changed every 3 or 4 hours, so that in 24 hours he turns ont upwards of 28 cwt. of an excellent and well kilned article. The malt of the distiller should be as pale as possible, because with the deepening of the color an empyreumatic principle is generated.

When Indian corn is the subject of distillation, it must be malted in the same way as deseribed in the article BEER. According to Hermstaedt, its flour may be advantageously mixed with the crushed malt in the mash tun. But its more complete dissolution may be accomplished by Siemen's mode of operating upon potatoes, presently to be deseribed.

1. Mrashing. Barley and raw grain are ground to meal by millstones, but malt is merely crushed between rollers. If only one tenth or one eighth of malt be used with nine tenths or seren eighths of barley, some husks of oats are added, to render the mash mixture more drainable.

When 40 bushels of barley and 20 of malt form one mashing, from 600 to 700 gallons of water, heated to $150^{\circ} \mathrm{F}$, are mixed with these 60 bushels in the mash tun, 
and carefully incorporated by much manual labor with wooden oars, or in great concerne by the mechanical apparatus used in the breweries. This agitation must be continued for 2 or 3 hours, with the admission from time to time of about 400 additional gallons of water, at a temperature of $190^{\circ}$, to counteract the cooling of the materials. But since the discovery of diastase, as the best heat for saccharifying starch is shown to be not higher than $160^{\circ} \mathrm{F}$., it would be far better to mash in a tun, partially, at least, steam incased, whereby we could preserve the temperature at the appropriate degree for generating the greatest quantity of sugar.

If the wort be examined every half-hour of the mashing period, it will be found to become progressively sweeter to the taste, thinner in appearance, but denser in reality.

The wort must be drawn off from the grains whenever it has attained its maximum density, which seldom exceeds 150 lbs. per barrel; that is, $\frac{360+150}{360}=1 \cdot 42$, or 42 per cent. As the corn of the distiller of raw grain has not the same porosity as the brewer's, the wort cannot be drawn off from the bottom of the tun, but through a series of holes at the level of the liquor, bored in a pipe stuck in at the corner of the vessel. About one third only of the water of infusion can thus be drawn off from the pasty mass. More water is therefore poured on at the temperature of $190^{\circ}$, well mixed by agitation for half an hour, then quietly infused for an hour and a half, and finally drawi off as before. Fully 400 gallons of water are used upon this occasion, and nearly as much liquor may be drawn off. Lastly, to extract from the grains everything soluble, about 700 gallons of boiling hot water are turned in upon them, thoroughly ineorporated, then left quietly to infuse, and drawn off as above. This weak wort is commonly reserved for the first liquor of the next mashing operation upon a fresh quantity of meal and malt.

The English distiller is bound by law to make his mixed worts to be let down into the fermenting tun of a specific gravity not less than 1.050, nor more than 1.090; the Scoteh and Irish distillers not less than 1.030 , nor more than 1.080 ; which numbers are called, gravity $50,90,30$, and 80 , respectively.

With the proportion of malt, raw grain, and water, above prescribed, the infusion first drawn off may have a strength $=20$ per cent. $=$ spec. grav. 1.082 , or 73 lbs. per barrel; the second of $50 \mathrm{lbs}$. per barrel, or 14 per cent.; and the two together would have a strength of 61.2 lbs. per barrel $=17$ per cent., or spec. grav. 1.070. From experiments carefully made upon a considerable scale, it appears that no more than four fifths of the soluble saccharo-starchy matter of the worts is decomposed in the best regulated fermentations of the distiller from raw grain. For every.2 lbs. so decomposed, $1 \mathrm{lb}$. of alcohol, spec. grav. 0.825 , is generated; and as every gallon of spirits of the spec. grav. 0.909 contains $4.6 \mathrm{lbs}$. of such alcohol, it will take twice 4.6 or $9.2 \mathrm{lbs}$. of saccharine matter to produce the said gallon. To these $9 \cdot 2 \mathrm{lbs}$., truly transmuted in the process, we must add one fifth, or $1.84 \mathrm{lbs}$, which will raise to $11: 04$ the amount of solid matter employed in producing a gallon of the above spirits.

Some distillers mash a fourth time; and always use the feeble wort so obtained in mashing fresh grain.

2. As the imperfect saccharine infusion obtained from raw grain is much more acescent than the rich sugary solution got from malt in the breweries, the distiller must use every precaution to cool his worts as quickly as possible, and to keep them clear from any acetous taint. The different schemes of cooling worts are considered under BE.ER and ReFrigeration. As the worts cool, a quantity of starchy matter is precipitated, but it is all carefully swept along into the fermenting tun, and undoubtedly contributes to increase the production of alcohol. During the winter and temperate months, when the distilleries are most actively at work, the temperature at which the worts are set is usually about $70^{\circ} \mathrm{F}$. When much farinaceous deposite is present, the heat may be only $65^{\circ}$, because, in this case, a slow fermentation seems to favor the conversion of that starch into sugar. In some German distilleries a little chalk is mixed with the worts, to check acidity.

3. The fermentation.

The yeast added to the worts as a ferment, ought to be the best top barm of the London porter breweries. About 1 gallon of it is requisite for every 2 bushels of meal and malt worked up in the mashing process; and of this quantity only a certain proportion is introduced at the beginning; the remainder being added by degrees, on the second and third days.

Should the fermentation flag, a little more may be added on the fourth or fifth day, and the contents of the tun may be roused by an agitator. About 8 or 9 gallons mav be introduced four days in succession to the quantity of worts extracted from 60 bushels of the farinaceous materials; or the third day's dose may be intermitted, and joined to the fourth on the subsequent day. 
Great diversity and no little caprice prevail among distillers in respect of the periods of administering the yeast; but they should be governed very much by the appearance of the fermentalion. This process continues from nine to twelve or even fourteen days, according to circumstances; the tuns being left quite open during the first five days, but being covered moderately close afterwards to favour the full impregnation of the liquor with carbunic acid, as a fermenting agent. In consequence of the great attenuation of the wort by the generation of so much alcohol, no good body of yeast continues to float on the surface, and what is formed is beat down into the liquor on purpose to promote the fermentation. The temierature of the wash gradually increases till towards the end of the forrth day, when it attains its maximum height of about $25^{\circ}$ above the pitch of $55^{\circ}$ or $60^{\circ}$ at which it may have been set. The time of the greatest elevation of temperature, as well as its amount, depends conjointly upon the quality of the yeast, the nature of the saccharo-starchy matter, and the state of the weather. It is highly probable that the electrical condition of the atmosphere exercises a considerable influence upon fermentation. We linow the power of a thunder-storm to sour vinous fluids. An experimental inquirv into the relation between electricity and fermentation, could not fail to prove both curious and profitable.

The dimunition of the density of the wort is carefully watched by the distiller, as the true criterion of the success of his process. This attenuation, as he calls it, is owing part? $y$ to the decomposition of the sugar, which communicated its gravity to the solution, and partly to the introduction of the lighter alcoholic particles. Were all the saccharostarchy matter resolved into gaseous compounds, the wort would become water; but since a part of it remains undecomposed, and a portion of alcohol is produced at the expense of the decomposed part, the degree of attenuation becomes a somewhat complicated problem in a theoretical point of view ; the density due to the residuary sugar being masked and counteracted by the spirit evolved. Could the alcohol be drawn off as it is formed, the attenuation would probably become greater, because the alcohol checks the fermentative action, and eventually stops it, before all the saccharum is decomposed. After the wash has taken its highest degree of temperature, not much more spirit is found to be generated; were this therefore removed by proper means, the remaining vegetable matter would undoubtedly yield a further product of alcohol.

In the attenuation of raw-grain wash, the specific gravity seldom arrives at 1.000 ; but most commonly stops short at $1 \cdot 002$ or $1 \cdot 004$. When the vinous fermentation comes to an end, the acetous is apt to commence, and to convert a portion of the alcohol into vinegar; a result which is easily ascertained by the increasing specific gravity, sour smell, and acidulous reaction of the wash upon litmus paper, which remains after the paper is heated, showing that the red color is not caused by carbonic acid.

Fermentation proceeds with more uniformity and success in the large tuns of the distiller, than in the experimental apparatus of the chemist; because the body of heat generated in the former case maintains the action. But I have succeeded in obviating this inconvenience in operating upon 80 or 90 gallons, by keeping up the temperature, when it begins to flag, by transmitting hot water through a recurved pipe plunged into the tun.

We have already mentioned that one gallon of spirits, one in ten over-proof, is upon the average generated from $11.04 \mathrm{lbs}$. of starch sugar; hence we conclude that one pound water-measure of spirits at proof $\left(=\frac{1}{1^{10}}\right.$ imperial gallon $)$ is produced from one pound of the saccharum.

1 Mult whiskey. - The treatment and produce of malt distilleries are in some respects different from those of raw grain. Having been professionally employed by the proprietors of both, I am prepared to state the peculiarities of the latter, by an example. 500 bushels of ground malt are first mashed with 9000 gallons of water, heated to the temperature of $160^{\circ} \mathrm{F}$.: 6000 gallons of worts are drawn off into the coolers, and let down into the fermenting tun at $68^{\circ}$. From 3 to 4 per cent. of a mixture of London porter yeast with quick Scotch barm are added, and well stirred through the mass. At the end of two or three days, in general, the fermentation is finished. On the residuary grains of the malt, from 4500 to 5000 gallons of water at $180^{\circ}$ are run, which after proper mashing as before, are drawn off; then 4500 more are poured on, the drainage of which is added to the second. Both of these together, constituting 9000 gallons, are heated next day, and employed for the mashing of 500 bushels of fiesh malt. During the fermentation, the wash which was set at the spec. grav. 1.065, comes down to water $=1 \cdot 000$.

The wash is distilled in two stills, appropriated to it, of about 800 gallons capacity each, provided with a rotatory chain apparatus for preventing the lees from adhering to the britom of the still. Into about 800 gallons of wash 8 lbs. of soap are put. The liquor obtained at this first distillation is called low wines. These low wines are redis. tilled in the spirit stills; the first and last portions of liquid being more or less blue or milky in color, and rank in flavor, are run into a separate receiv called the faints-back; 
while the middle portion, constituting in a well-managed distillery, from three fourths to four fifths of the whole, are received into the spirit-back. The faints are mixed with a 'arze quantity of water, and redistilled, in order to free them from the fetid oil derived from the husks of the grain. The interception of this noxious oil may be best effected by $\mathbf{a}$ self-regulating bath, between the capital of the still and the refrigeratory, as will be explained in treating of STrLls. The capitals of the common Senteh stills are marle from 15 to 20 feet high, in order to prevent the chance of the wash boiling over into the worm; and they are, towards the beginning of the process, struck from time to time with a rod, and by the sound emitted it is known whether they be empty, partially filled, or in danger of an overflow; in which case the fire is damped, by a spout near the furnace door, connected by a leather pipe with an elevated reservoir of wa*or. When very pure spirits are wished for, a third or even a fourth distillation is hal recourse to; there being a quantity of water mixed each time with the spirit in the still, to prevent its acquiring a harsh alcoholic flavor.

According to some experienced distillers from raw grain, the mashing temperature of the first liquor should not exceed $140^{\circ} \mathrm{F}$.; whereas with malt it may be safely and beneficially $165^{\circ}$ or $170^{\circ}$. When rye is used instead of malt, 90 bushels of it are mixed with 190 bushels of raw grain, constituting 280 bushels in whole, for the mashing of which 5200 gallons of water are required. An hour and a half more time is necessary for settling the mashing of the above mixture, than of grain alone. Gin is made in this way.

The distiller of malt whiskey calculates on obtaining two gallons of proof spirits from one bushel of malt, in average years. The highest yield is 20 gallons per quarter of 8 bushels; and the lowest is 16, when the malt and fermentation are indifferent. The best temperature to set the fermenting tun with malt wash is about $70^{\circ}$ or $72^{\circ} \mathrm{F}$.

When malt is $5 \mathrm{~s}$. the bushel, 6 bushels at $30 \mathrm{~s}$. will yield 12 gallons of proof spirits. These cost therefore $2 s .6 d$. per gallon for the malt; to which must be added $3 d$. per bushel for the amount of malt duty not returned, or $1 \frac{1}{2} d$. on the gallon; this added to the Scotch duty of $3 s$. $4 d$. the gallon, makes the price altogether $5 \mathrm{~s} .11 \frac{1}{2} d$.; besides the expenses in fuel, yeast, labor, and rent, which may be estimated at $8 \frac{1}{2} d$. per gallon. But $3 d$. may be deducted for what is paid by the dairymen for the spent wash and grains. The total cost, therefore, exclusive of use of capital, is $6 \mathrm{s.} 5 \mathrm{~d}$. per gallon in Scotland.

The following is the work of a Scotch distillery, where good malt whiskey was made.

One bushel of the malt weighed $35 \mathrm{lbs}$, or the boll, $=6$ bushels, 210 lbs. In mashing each boll of malt, 110 gallons of water were run on it at $160^{\circ} \mathrm{F}$. As soon as the fermenting tun of 3000 gallons capacity was charged with the wash at from $64^{\circ}$ to $74^{\circ} \mathrm{F}$., 2 gallons per cent. of barm were added. When the wash had become attenuated from $1 \cdot 060$ to $1 \cdot 040$, another gallon of barm was introduced.

The temperature of the fermenting wash sometimes rises to $96^{\circ}$, which is, however, an extreme case, and not desirable. When the bubbles of carbonic acid mount in rapid succession, it is reckoned an excellent sign. If the tun be small, and stand in a cool apartment, it should be started at a higher temperature than in the reverse predicament. Should the fermentation be suffered to flag, it is in seneral a hopeless task to sestore vigorous action. Some try the addition of $b u b s$, that is, of some wort brought into a state of rapid fermentation in a tub, by a large proportion of yeast, but seldom with much success. Indeed, the law prohibits the addition of any wort to the tun at a later period than 24 hours after it is set; so that if bubs are used afterwards, the distiller is apt to incur a penalty.

The maximum quantity of proof spirits obtained on the great scale at any time from raw grain mixed with from one fourth to one eighth of malt, seems to be 22 gallons per quarter.

By the British laws a distiller is not allowed to brew and distil at the same time; but he must work alternately, one week, for instance, at fermentation, and next week at distillation.

In fermenting solutions of sugar mixed with good yeast, the attenuation has been earried down to 0.984 , and even 0.982 , that is, in the language of the excise, 16 and 18 degrees below water, from $1 \cdot 060$, the density at which it was originally set in the tun. This was excellent work done on the sca!e of a great distillery nearly 30 years ago, when distillation from sugar was encouraged, in consequence of bad corn harvests.

In an experiment which I made in 1831 , for the information of a committee of the House of Commons, on the use of molasses in the breweries and distilieries, I dissolved $1 \mathrm{cwt}$. of raw sugar in water, so as to form $74 \frac{1}{2}$ gallons, inclusive of 2 gallons of yeast. The specific gravity of the mixture was 1.0593 on the 31 st of March. By the 6 th of April, that is, in 6 days, the gravity had sunk to 0.992 , or 8 degrees under water, which was reckoned a good attenuation, considering the circumstances and the small quantity operated upon. By diftillation it afforded at the rate of 14.875 gallons of proof spirits for 100 gallons of the wash. 
When the distillers first worked from sugar, they only obtained upon an average from 1 cwt. 10.09 galluns imp. of proof spirit; but they afterwards got no less than 11.92 imp. Qallons.

The following experiment, which I made upon the fermentation of West India molasses into spirits, for the information of the said committee, may prove not uninteresting to my readers. 150 lbs. were dissolved in water and mixed with 2 gallons of yeast, weighing exactly $20 \mathrm{lbs}$. The wash measured 70 gallons, and had a spec. gravity of 1.0647 at $60^{\circ} \mathrm{F}$. In two days the gravity had fallen to 1.0055 ; in three days to $1.002 \%$; and in five days to 1.001 . The temperature was kept up at from $80^{\circ}$ to $90^{\circ} \mathrm{F}$. during the last two days, by means of a steam pipe, to favor the fermentation. The product of spirits was 11 gallons, and $\frac{35}{100}$ of a gallon. Now $150 \mathrm{lbs}$. of the above molasses were found to contain of solid matter, chiefly uncrystallizable, $112 \mathrm{lbs}$. And as $112 \mathrm{lbs}$. of sugar are estimated by the revenue laws to afford by fermentation $11 \frac{1}{2}$ gallons imp. of proof spirit, the result of that experiment upon molasses must be considered satisfactory, bearing in mind that the saccharine substance in molasses has been not only partially decornposed by heat, but is mixed with some of the glutinous or extractive matter of the cane.

Since the alteration of the excise laws relative to distillation in 1825 and 1826 , when permission was given to set the wort at lower gravities, the quantity of spirits produced from 1 quarter of corn has been much increased, even up to fully 20 gallons; and the proportion of malt has been much diminished. The latter was soon reduced from three sevenths malt, and four sevenths barley, or two fifths malt and three fifths barley, to one fifth of malt and now to one tenth or even one sixteenth.

A discussion having lately taken place in Ireland between certain persons connecied with the distilleries and the officers of the excise, whether, and to what extent, raw grain worts would pass spontaneously into the vinous fermentation, the Board in London requested me to superintend a series of researches in a laboratory fitted up at their office, to settle this important point. I shall content myself here with giving the result of one experiment, out of several, which seems to me quite decisive. Three bushels of mixed grains were taken, consisting of two of barley, one half of oats, and one half of malt, which, being coarsely ground by a hand-mill, were mashed in a new tun with 24 gallons of water at $155^{\circ}$. The mash liquor drawn off amounted to 18 gallons, at the density of 1.0465 ; and temperature of $82^{\circ} \mathrm{F}$. Being set in a new tun, it began to ferment in the course of 12 hours, and in 4 days it was attenuated down to gravity 1.012. This yielded, upon distillation in low wines, $3 \cdot 22$ gallons, and by rectification, in spirits, $3 \cdot 05$; while the quantity equivalent to the attenuation by the tables was 3.31 , being an excellent accordance in such circumstances.

The inquisitorial régime imposed by law upon our distilleries, might lead a stranger to imagine that our legislators were desirous of repressing by every species of annoyance the fabrication of the fiery liquid which infuriates and demoralizes the lower population of these islands. But alas! credit can be given them for no such moral or philanthropic motive. The necessity of the exchequer to raise a great revenue, created by the wastefu? expenditure of the state, on the one hand, and the efforts of fraudulent ingen'xity on the ocher, to evade the payment of the high duties imposed, are the true origin of that régime: Examinations in distilleries are constantly making by the officers of excise. There is a survey at 6 o'clock in the morning, when the officers take their accounts and gauges, and make calculations which occupy several hours. At 10 o'clock they again survey, going over the whole premises, where they continue a considerable time, frequently till the succeeding officer comes on duty; at 2 in the afternoon another survey takes place, but not by the same people; at 6 in the evening the survey. is repeated; at 10 there comes another survey by an officer who had not been engaged in any of the previous surveys of that day. He is not relieved till 6 o'clock next morning. In addition to these regular inspections, the distilleries are subject to frequent and uncertain visits of the surveyor and general surveyor. "We are never," says Mr. Smith, the eminent distiller of Whitechapel, "out of their hands."**

Before the fermented wort goes into the still, a calculation is made of the quantity of wash drawn from the wash back, and which is first pumped into what is called the wash charger. If the quantity in the wash charger exceeds the quantity in the wash back, the distiller is charged upon the higher quantity; if it contains less, he must pay according to the wash back, as being the larger quantity. When the quantity of wash is all transferred to the charger, the discharge cock of the wash charger is unlocked, and the wash is allowed to be drawn off from the charger into the still, the charging and discharging cock of the still being locked by the officer. There can be no transfer of wash but through the pumps, which are locked also. The first distillation from the wash is worked into the low-wine receiver, which is also a locked up vessel; then of

* Report of Committee on Molasses, 2198. 
those low wines, the strength and quantity are ascertained by the excise. The account of them affords a comparison with the quantity which the contents of the wash-inck had been estimated to produce; they are then pumped from the low wine receiver, through pumps previously locked into the low wine charger, which is also a locked up vessel; from the locked up charger, after the officer has done his duty regarding it, they are allowed to be drawn off into the low wine still, which is a distillation of the second extraction; then that lo:v wine still works into another locked up cask, called the spirit receiver, for the receiving of raw spirits; when that distillation is finished, the officer, attending again on regular notice for that purpose, takes the quantity and strength of the spirits therein, and upon the quantity so ascertained he charges the duty. In distilling low wines, one portion of them goes into the spirit receiver, and a portion into what is called the faint receiver, which is another locked up vessel. These faints are in the next distillation united with the low wines, from the succeeding wash-back on their second distillation, and are worked together; the united produce of these goes partly into the spirit cask, and partly back again into the faint cask. The operation is thus continued till all the backs in the house are emptied.*

There is a kind of ardent spirits manufactured in Holland, vulgarly called Dutch gin, Hollands, and sometimes geneva, from genievre, the French for juniper, a plant with the essential oil of whose berries it is flavored. One cwt. of ground malt mixed with two cwts. of rye meal are mashed for two hours, with about 450 gallons of water at the temperature of $160^{\circ} \mathrm{F}$. The mash drawn off is reduced with cold water till the liquid part has the density of $45 \mathrm{lbs}$. per barrel, = specific oravity 1.047 ; and is then put all together into the fermenting back at the temperature of $80^{\circ} \mathrm{F}$. One or two gallons of yeast are added. The fermentation soon becomes so vigorous as to raise the heat to $90^{\circ}$ and upwards, but it is not pushed far, being generally over in two days, when the gravity of the wash still indicates 12 pounds of saccharum per barrel. By this moderate attenuation, like that practised by the contraband distillers of the Highlands of Scotland, it is supposed that the fetid oil of the husks is not evolved, or at least in very small quantity. The grains are put into the alembic along with the liquid wash, and distilled into low wines, which are rectified twice over, some juniper berries and hops being added at the last distillation. But the junipers are sometimes bruised and put into the mash. The produce of worts so imperfectly fermented, is probably little more than one half of what the British distiller draws from the same quantily of grain. But the cheapness of labor and of grain, as well as the superior flavor of the Skiedam spirits, enables the Dutch distiller to carry on his business with a respectable profit. In opposition to the above facts, Dubrunfaut says that about one third more spirits is obtained in Holland from grain than in France, because a very calcareous spring water is employed in the mashing operation. Were this account well founded, all that the distillers of other countries would have to do would be merely to introduce a portion of chalk into their mash tuns, in order to be on a par with the Dutch. But the statement is altogether a mistake.

In the vine countries, the inferior wines or those damaged by keeping, as also a fermented mash of the pressed grapes, mixed with water, are distilled to form the eau de vie de Cognac of the French, called Brandy in this country. It contains less essential oil, and that of a more agreeable flavor, than corn spirits. See BRANDY.

Berzelius says that there are distillers who are guilty of putting a little arsenious acid into the still; that the spirits contain pretty frequently traces of arsenic, which may be detected by adding to them a little muriatic acid, then evaporating off the alcohol, and passing a current of sulphureted hydrogen gas through the residuary liquid, which will give it the characteristic orpiment yellow tinge, arsenic being present. Copper, which is sometimes introduced into distilled grain, or even malt spirits, in consequence of the soap employed in the process of distillation, may be deterted best by the brown precipitate which it occasions with ferroprussiate of potash. No arsca $c$ is ever used in this country.

When damaged grain has been mashed in making whiskey, a peculiar oily substance makes its appearance in it. On approaching the nostrils to such whiskey slightly heated, this rolatile matter irritates the pituitary membrane and the eyes very powerfully. These opirits have exactly the smell of an alcoholic solution of cyanogene; they intoxicate more powerfully than pure alcohol of equal strength, and produce even temporary phrensy, with subsequent sicliness and disordered functions. This rolatile body is not cyanogene, though it be so like it, for it forms no such combinations as cyanogene does. It may be extracted from diluted alcohol by agitating it with an unctuous oil, and then distilling the oil along with water. At the end of 3 or 4 months, this volatile matter disappears in a great measure, even when the spirits impregnated with it are enclosed in well-corked bottles; obviously from its undergoing a spontaneous decomposition. It may be preserved much longer in the state of a watery solution.

When acetic ether is added to well purified or clean spirits, such as the distillers call

* Thomas Smith, Esq., of Whitechapel Road, in Report of Molasses Committee, Part II. p. 149. 
silent whiskey, it gives it somewhat of the flavor of brandy. For this purpose, also, the spirits are rectified from bruised prunes, or the lees of the cognac distilleries, whereby they acquire additional flavor. The astringent taste of old brandy is imitated by the introduction of a little catechu into the British spirits. Burned sugar is employed as a coloring in these imitations.

IV. Of making whiskey from potatoes.-This root, in certain localities where it abounds at a moderate price, is an excellent material for fermenting into alcohol. When sound, it possesses from 20 to 25 per cent. of solid substance, of which starch constitutes at least three fourths; hence 100 pounds contain from 16 to 22 pounds of starch susceptible of being saccharified. In the expressed juice there is a small quantity of tartaric acid.

Previously to mashing, potatoes must be first well washed in a horizontal cylindrical cage revolving partially in a trough of water, as will be described in treating of the manufactare of sugar from beet root. They must be then boiled in a close vessel with steam, provided with a perforated bottom a few inches above the real one. The top has an opening with a cover fitted tightly to it; through that the potatoes are introduced; and inmediately above the false bottom there is a similar aperture through which the boiled potatoes are taken out. The steam-pipe enters at the top, runs down the side a little way, and terminates in a widened mouth. The large lids are secured by cross bars, the small hole by folds of linen. In the lower valve there are two small holes closed with pins, for inserting a wire to feel whether the potatoes be sufficiently boiled. If so, the steam is immediately stopped off, the lower lid is removed, and the potatoes

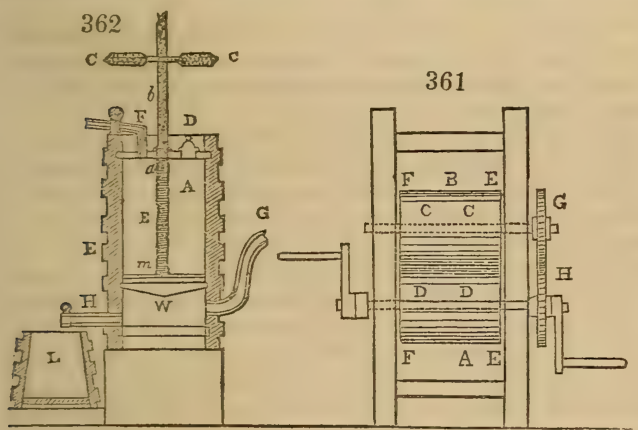
pulled out.with a hook into a tub. They must be immediately made into a homogeneous paste before they get cold. Fig. 361 represents, in plan, or horizontal section, the apparatus used in France for this purpose. A B are two cylinders covered with wire cloth, but open at the ends; $\mathrm{C} C$ and $\mathrm{D} D$ are two pieces of wood fixed on the two axes, in the form of two cones, with the adjoining surfaces truncated; upon which, as also upon iron rings $\mathbf{E} F$, of the same diameter, made fast to the axes, the wire cylinder rests. Of the two wheels $\mathrm{G} H$, the smaller has 18 , the greater has 21 teeth. The diameter of each cylinder is 14 inches, the length 18. Abore and between the two cylinders, there is a hopper for the reception of the boiled potaloes. This machine triturates 1200 pounds of potatoes per hour. Their paste must be forthwith mashed with some ground wheat or barley, and a proportion of malt; then be set a fermenting.

As in the above mode of trituration, the potatoes are apt to cool to such a degree as to obstruct their ready admixture with water, it is better to make them into a paste in the vessel in which they are steamed. The apparatus contrived by Siemens fully answers this end. It consists essentially of a tub A, represented in fig. 362, in section. It is cylindrical, and made of planks from 3 to 4 inches thick, joined firmly and steam-tight; the upper and under ends being well secured with iron hoops. The lower part is about $\mathbf{2}$ inches more in diameter than the upper. About a foot from the bottom, in a circular groove, a cast iron partition w, or disc full of holes, is made fast, which serves the purpose of a searce, the apertures being an inch asunder; above, from $\frac{1}{8}$ to $\frac{1}{10}$ of an inch in diameter, and below, scooped out to half an inch. This disc is half an inch thick in the elges, and five fourths of an inch in the middle.

Through the female screw $a$ in the top of the cylinder, there passes the screwed rod $b$, one and a half inches thick, provided at top with a strong cross bar $\mathrm{c} c$, for turning it round. The under end of this rod has a square piece terminating in a short screw, upon which a wrought iron cross is secured by means of a screw nut, so as to stand at right angles to the rod. This cross is composed of two distinct arms; of which one of them is mcunted on the upper side with little knives an inch and a half long; the other, upon the under side, with a wire brush, that may be made to rub against the perforated cast iron disc. On the side of the cylinder at $\mathrm{E}$, fig. 362, there is a narrow aperture provided with a bung secured by a cross bar, and near the bottom at $H$ there is another like it. Both openings serve for taking out the residuary matter. Through the opening $\mathrm{k}$, the above two arms are introduced; and secured to the square of the rod by the screw nut. In the top there is an opening, $D$, 
for putting in the potatoes which may be shut in the same way. From the lid thero likewise issues a lateral tube $F$, which terminates in a tubful of water, for condensing the waste steam. G is the tube connected with the steam boiler, for conducting the steam into the space under the iron dise $\mathrm{w}$.

With this apparatus the potatues are prepared as follows: when the screw rod is so fixed that the cross touches the disc, the cylinder is to be filled with washed potatoes to within one foot of the top, leaving them some space to expand. The orifice $\mathrm{D}$ is to be then closed, and the steam admitted. When the potatoes are boiled enough, two laborers lay hold of the lever handles $c \mathrm{c}$, of the screw rod $b$, and turn it round with the effect of screwing up the spitied cross, and of triturating the potatoes; an operation which may be still more efiectually done by screwing it down again. The potato paste is now let off by the plug hole $\mathrm{H}$, into the tub $\mathrm{L}$, where it is mixed with about 30 per cent. of boiling water, and one thousandth part of potash, made caustic with quicklime, in order to dissolve the albuminous matter coagulated by the heat, and give complete fluidity to the mass. The alliali also neutralizes the tartaric acid present. The mashed matter must now be mixed with the crushed malt diffused through 40 or 50 pounds of cold water for every 100 pounds of potatoes, which lowers the temperature to $16 \%^{\circ}$. The wort must be then diligently stirred during two hours; mixed with 40 or 50 pounds of cold water for 100 pounds of potatoes, and, when reduced to the temperature of $77^{\circ}$, put into the fermenting tun along with the proper quantity ( 3 or 4 per cent.) of yeast. As potatoes readily pass into the acetous fermentation, the admixture of the malt, the mashing, and the cooling should be rapidly performed, while the utmost cleanliness must be observed.

The fermentation is brisk, probably from the agency of the albumen, and furnishes a good head of barm, which answers well for the bakers; 100 pounds of potatoes yield from 18 to 20 pounds measure of spirits, nine elevenths of our excise proof; or about 16 pounds measure of proof, $=$ about $1 \frac{2}{3}$ gallons.

It has been observed that after the month of December potatoes begin to yield a smaller product of fermented spirits; and when they have once sprouted or germinated, they afford very little indeed. From the difficulty of keeping and transporting potatoes, distillation from them, even though our laws now permit it, can never become general till some plan be adopted for overcoming these disadvantages. A scheme of this kind, however, has been successfully practised in Vienna, which consists in subjecting the washed potatoes to strong pressure in a perforated chest by a hydraulic or screw press, whereby they lose about three fourths of their weight, and may then be readily dried into a white flour, that may be kept for several years without injury, and transported to considerable distances with comparative ease. This flour, mixed with a moderate quantity of ground malt, and saccharified by mashing with water, at the temperature of $167^{\circ} \mathrm{F}$, becomes capable of affording a sweet wort convertible by fermentation either into beer or whiskey.

Horse-chestnuts, according to Hermstaedt, are an eligible material for producing alcohol, as 128 pounds of them afford 100 pounds of meal; which 100 pounds yield, by proper treatment, 34 pounds of spirits, containing 36 per cent. of absolute alcohol, by Richter's tables. Barley to the extent of 10 pounds per 100 should be ground up with them, after they have been boiled in a steam apparatus, not only for the purpose of softening them, but freeing them from their bitter astringent matter. Acorns are pro ductive of alcohol by similar treatment.

The best means hitherto discovered for depriving bad whiskey of its nauseous smell and taste is to pass it through well burned and coarsely pulverized charcoal, distributed as follows in a series of cylindrical casks. Each vessel must have a double bottom, the false one being perforated with conical holes, and placed a few inches above the true. Upon this perforated board a layer of chopped clean straw, one inch thick, is laid; and over the straw, a stratum of small river gravel, the size of large peas. This is to be covered with a pretty thick stratum of the charcoal, previously freed from dirt and dust by washing; upon which a piece of close canvass is to be spread, and pressed down by a thin bed of river sand. The cylinder or cask should be filled with these successive layers to within two inches of its top, and it is then to be closed air-tight. Immediately below the head, a round orifice is pierced in the side, for receiving an orerflow tube, which is either screwed rectangularly to another elbow pipe, or is bent (when of block tin) so as to enter tight into an orifice beneath the false bottom of the second crlinder or cask. In this way, the series may be continued to any desired number of vessels; the last discharging the purified spirit into the store-back. The foul spirit must be made to flow into the bottom space of the first cylinder down through a pipe in communication with a charging-back placed upon such an elevated level as to give sufficient pressure to force the spirits up through the series of filters; the supply-pipe being provided with a regulating stop-cock. The spirit may be filtered dounwards through sand and cloth in 
its final passage to the receiver. It has been found, with very crude spirits, that eight successire cylinders were required to deprive them entirely of the rank flavor.

In the year 1831,23,000,000 gallons of spirits were made in the United Kingrom, equivalent to the consumption of $1,500,000$ quarters of grain, and for that year and the four preceding years, there were imported annually 2,000,000 of quarters of foreign barley.

In $1832,20,778,521$ gallons paid excise duty.

$1834,23,397,806$.

$1836,27,137,000$; of which $14,000,000$ were Irish.

We may add to the last quantity, 3 millions of gallons at least on the score of smuggling, in licensed and illicit distilleries; making 30 millions to be the frightful amount of whisliey consumed by the British people, independent of other intoxicating liquors.

DOCIMACY, from the Greek $\Delta_{о \kappa \iota \mu} \zeta \omega$, I prove (Docimasie, Fr.; Probierkunst, Germ.); is the art by which the nature and proportions of an ore are determined. This analytical examination was originally conducted in the dry way, the metal being extracted from its mineralizers, by means of heat and certain fluxes. But this method wes eventually found to be insufficient and even fallacious, especially when volatile metals were in question, or when the fluxes could absorb them. The latter circumstance became a very serious evil, whenever the object was to appreciate an ore that was to be worked at great expense. Bergmann first demonstrated, in an elaborate dissertation, that the humid analysis was much to be preferred; and since his time the dry way has been consecrated chiefly to the direction of metallurgic operations, or, at least, it has been employed merely in concert with the humid, in trials upon the small scale.

After discovering an ore of some valuable metal, it is essential to ascertain if its quantity and state of combination will justify an adventurer in working the mine, and smelting its products. The metal is rarely found in a condition approaching to purity; it is often disseminated in a mineralizing gangue far more bulky than itself; and more frequently still it is combined with simple non-metallic substances, such as sulphur, carbon, chlorine, oxygen, and acids, more or less difficult to get rid of. In these compound states its distinctive characters are so altered, that it is not an easy task either to recognise its nature, or to decide if it can be smelted with advantage. The assayer, without neglecting any of the external characters of the ore, seelis to penetrate, so to speak, into its interior; he triturates it to an impalpable powder, and then subjects it to the decomposing action of powerful chemical reagents; sometimes with the aid of allialis or salts appropriate to its nature, he employs the dry way by fire alone; at others, he calls in the solvent power of acids with a digesting heat; happy, if after a series of labors, long, varied, and intricate, he shall finally succeed in separating a notable proportion of one or more metals either in a pure state, or in a form of combination such, that from the amount of this known compound, he can infer, with precision, the quantity of fine metal, and thereby the probable value of the mine. The blow-pipe, skilfully applied, affords ready indications of the nature of the metallic constituents, and is therefore usually the preliminary test. The separation of the several constituents of the ore can be effected, however, only by a chemist, who joins to the most extensive knowledge of the habitudes of mineral substances, much experience, sagacity, and precision, in the conduct of analytical operations. Under the individual metals, as also in the articles Metallurgy, Mines, and Ores, I have endeavored to present such a copious and correct detail of docimastic processes, as will serve to guide the intelligent student through this most mysterious labyrinth of nature and art.

DORNOCK is a species of figured linen of stout fabric, which derives its name from a town in Scotland, where it was first manufactured for table-cloths. It is the most simple in pattern of all the varieties of the diaper or damask style, and therefore the goods are usually of coarse quality for common household wear. It receives the figure by reversing the flushing of the warp and woof at certain intervals, so as to form squares, or oblong rectangles upon the cloth. The most simple of these is a succession of alternate squares, forming an imitation of a checker board or mosaic work. The coarsest kinds are generally woven as tweels of three leaves, where every thread floats over two, and is intersected by the third in succession. Some of the finer are tweels of four or five leaves, but few of more; for the six and seven leaf tweels are seldom or never used, and the eight leaf tweel is confined almost exclusively to damask. See TeXtile Fabric.

DRAGON'S BLOOD (Sang dracon, Fr.; Drachenblut, Germ.) is a resinous substance, which comes to us sometimes in small balls of the size of a pigeon's egg, sometimes in rods, like the finger, and sometimes in irregular cakes. Its color, in lump, is dark brown red; in powder, bright red; friable; of a shining fracture, sp. grav. 1·196. It contains a little benzoic acid, is insoluble in water, but dissolves 
readily in alcohol, ether, and oils. It is brought from the East Indies, Africa, South America, as the produce of several trees, the Draccena Draco, the Pterocarpus santalinus, the Pterocarpus Draco, and the Calamus Rolang.

Dragon's blood is used chiefly for tingeing spirit and turpentine varn shes, for preparing gold lacker, for tooth tinctures and powders, for staining marble, \&c. According to Herbenger, it consists of 9.07 parts of red resin, 2 of fat oil, 3 of benzoic acid, 1.6 of oxalate, and 3.7 of phosphate of lime.

DRUGGET is a coarse, but rather slight, woollen fabric, used for covering carpets, and as an article of clothing by females of the poorer classes. It is now-a-days nearly superseded by coarse cotton goods.

DRYING HOUSE. An apartment fitted up in a peculiar manner for drying calicoes, and other textile fabrics. Mr. Southworth, of Sharples, a Lancashire bleacher, obtained a patent, in 1823, for the following ingenious arrangement, which has been since generally adopted, with certain modifications, in most of our extensive bleaching and printing works. Fig. 363 is a section of the drying-house, where $a$ is a furnace and

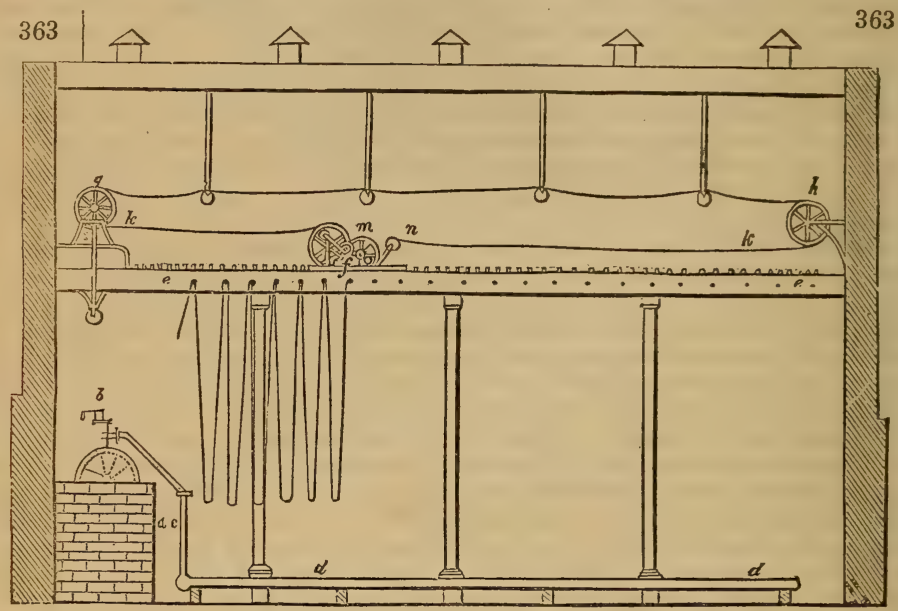

boiler for the purpose of generating steam; it is furnished with a safety valve in the tube $b$, at top, and from this tube the steam main $c$ passes down to the floor of the basement story. From this main, a series of steam-pipes, as $d d$, extend over the surface of the floor, and from them heat is intended to be diffused for the purpose of warming the drying-house.

Along the middle of the building a strong beam of timber $e e$, extends, and is supported by cast-iron pillars; from this beam, to bearings on the side walls, a series of rails are carried in a cross direction, over which rails the wet cloth is to be hung in folds, and the steam or evaporation emitted in drying is allowed to escape through apertures or ventilators in the roof.

The mode in which the cloth is delivered on to the rails, on either side of the beam, will be best understood by reference to the delivering carriage, which is shown, with its rollers partly in section.

The wet cloth is first to be coiled upon a roller, and then placed in the carriage, as at $f$, with its pivots bearing upon inclined planes. The carriage is to be placed at the commencement of the rails, running upon the middle beam, and also upon the sidebearings or railways extending along the side walls of the building, parallel to and upon a level with the same beam. It is made to travel by means of an endless hand passing over two riggers, $g$ and $h$, in $f i g .363$, and over pulleys and a band-wheel attached to the carriage, as will be explained. The rigger $g$, which moves this endless band, is actuated by bevel gear, seen at $i$, which is put in motion by a pinion at the end of a revolving shaft leading from a steam engine.

In the same fig., $k k$ is the endless band passing over a pulley under the band-wheel, and over the pulley $n$, by which it will be perceived that the traversing of the band, as described, would cause these pulleys and wheels to revolve. On the axle of the bandwheel $m$, there is a drum against which the roll of wet cloth $f$ presses, and as this drum revolves, the roll of wet cloth is, by its friction, made to turn in a contrary direction, and to deliver off the cloth on to the periphery of the drum, whence it passes over a roller and descends to the rails. Upon the end of the axle of the 
band-wheel $m$, there is a pinion which takes into the teeth of the large wheel, and upon the axle of this large wheel there is a pinion that actuates the intermediate wheel, which turns another toothed wheel. This last-mentioned toothed wheel takes into cogs upon the side railway, and hence, as the train of wheels moves round, the carriage to which the wheels are attached is slowly impelled forward.

As soon as the wheels begin to move, and the carriage to advance, the wet cloth begins to uncoil, and to pass down over the first roller; a small roller attached to the carriage, as it passes over the rails in succession, holds the cloth against each rail for a short space of time, and prevents it from slipping, by which means the cloth descends in folds or loops between the rails, and is thereby made to hang in a series of folds or loops, as shown in the figure.

It will be perceived that as the pivots of the cloth roller $f$ bear upon inclined planes, the roller will continually slide down as the cloth diminishes in bulk, keeping in contact with the drum, and delivering the cloth from the roller on to the several rails, as described.

In order to stop the carriage in any part of its course, or to adjust any of the folds of the cluth, a man is usually placed upon the platform travelling with the carriage, over which he has perfect command. This apparatus may be also employed for taking the cloth when dried off the rails; in which case the carriage must be made to travel backwards, and by first guiding the end of the cloth on to the roller $f$, and then putting the wheels in a retrograde motion, the cloth will be progressively coiled upon the roller $f$, in a similin way to that by which it was uncoiled.

DCCIILITY (Streckbarkeit, Germ.) is the property of being drawn out in length without brealing, possessed in a pre-eminent degree by gold and silver, as also by many other metals, by glass in the liquid state, and by many semifluid resinous and gummy substances. The spider and the silk-worm exhibit the finest natural exercise of ductility upon the peculiar viscid secretions from which they spin their threads. When a body can be readily extended in all directions under the hammer, it is said to be malleable, and when into fillets under the rolling press, it is said to be laminable.

\section{Table of the ductility and malleability of Metals.}

\begin{tabular}{|c|c|c|c|}
\hline $\begin{array}{c}\text { Metals ductile and } \\
\text { malleable in alphabetical } \\
\text { order. }\end{array}$ & $\begin{array}{l}\text { Brittle metals } \\
\text { in } \\
\text { alphabetical order. }\end{array}$ & $\begin{array}{l}\text { Metals in the order } \\
\text { of their wire-drawing } \\
\text { ductility. }\end{array}$ & $\begin{array}{c}\text { Metals in the order of } \\
\text { their laminable } \\
\text { ductility. }\end{array}$ \\
\hline $\begin{array}{l}\text { Cadmium. } \\
\text { Copper. } \\
\text { Gold. } \\
\text { Iron. } \\
\text { Iridium. } \\
\text { Lead. } \\
\text { Magnesium. } \\
\text { Mercury. } \\
\text { Nickel. } \\
\text { Osmium. } \\
\text { Palladium. } \\
\text { Platinum. } \\
\text { Potassium. } \\
\text { Silver. } \\
\text { Sodium. } \\
\text { Tin. } \\
\text { Zinc. }\end{array}$ & $\begin{array}{l}\text { Antimony. } \\
\text { Arsenic. } \\
\text { Bismuth. } \\
\text { Cerium. ? } \\
\text { Chromium. } \\
\text { Cobalt. } \\
\text { Columbium. } \\
\text { Iridium. } \\
\text { Manganese. } \\
\text { Molybdenum. } \\
\text { Osmium. } \\
\text { Rhodium. } \\
\text { Tellurium. } \\
\text { Titanium. } \\
\text { Tungsten. } \\
\text { Uranium. }\end{array}$ & $\begin{array}{l}\text { Gold. } \\
\text { Silver. } \\
\text { Platinum. } \\
\text { Iron. } \\
\text { Copper. } \\
\text { Zinc. } \\
\text { Tin. } \\
\text { Lead. } \\
\text { Nickel. } \\
\text { Palladium. ? } \\
\text { Cadmium. ? }\end{array}$ & $\begin{array}{l}\text { Gold. } \\
\text { Silver. } \\
\text { Copper. } \\
\text { Tin. } \\
\text { Platinum. } \\
\text { Lead. } \\
\text { Zinc. } \\
\text { Iron. } \\
\text { Nickel. } \\
\text { Palladium.? } \\
\text { Cadmium. ? }\end{array}$ \\
\hline
\end{tabular}

There appears to be, therefore, a real difference between ductility and malleability; for the metals which draw into the finest wire are not those which afford the thinnest leaves under the hammer or in the rolling press. Of this fact iron affords a good illustration. Among the metals permanent in the air, 17 are ductile and 16 are brittle. But the most ductile cannot be wire-drawn or laminated to any considerable extent without being annealed from time to time during the progress of the extension, or rather the sliding of the particles alongside of each other, so as to loosen their lateral cohesion.

DUTGING, in calico-printing, is the application of a bath of corvdung, diffused through hot water, to cotton goods in a particular stage of the manufacture. Dunging and scouring are commonly alternated, and are two of the most important steps in the process. The operation of dunging has for its objects :-

1. To determine the entire combination of the aluminous sub-salts with the stuffs, by 
separating almost all the acetic acid which was not volatilized in the stove-drying of the mordant.

2. To dissolve and earry off from the cloth a portion of the thickening matters.

3. To separate from the cloth the part of the mordant that is uncombined, and merely mixed mechanically with the gum or starch.

4. To prevent, by the peculiar action of the dung, the uncombined mordant, as well as the acetic acid with which the bath is apt to get loaded, from affecting the blank parts of the cloth, or being injurious to the mordant.

The aluminous base or mordant on the cloth, more or less neutralized by the dunging, is next subjected to the dash-wheel or fulling mill, where by the stream of water the remainder of the thickening and other impurities are washed away.

No very exact analysis has been made of cowdung. Morin's, which is the most recent and elaborate, is as follows:-

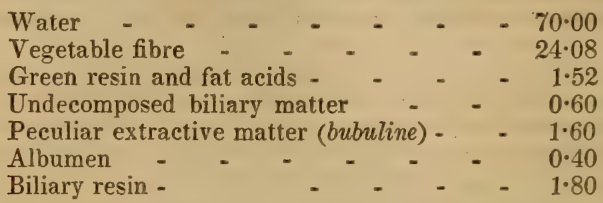

According to M. Kœchlin's practical knowledge on the great scale, it consists of a moist fibrous vegetable substance, which is animalized, and forms about one tenth of its weight; 2. of albumen; 3 . of animal mucus; 4 . of a substance similar to bile; 5 . of muriate of soda, muriate and acetate of ammonia, phosphate of lime and other salts; 6 . of benzoin or musk.

Probably the hot water in which the calico-printer diffuses the dung exerts a powerful solvent action, and in proportion as the uncombined mordant floats in the bath it is precipitated by the albumen, the animal mucus, and the ammoniacal salts; but there is reason to think that the fibrous matter in part animalized or covered with animal matter, plays here the principal part; for the great affinity of this substance for the aluminous salts is well known.

All practical men are aware that the affinity of cotton for alumina is increased by its combination with oil or animal substances, to such a degree as to take it from the dung bath; which would not be possible without this combination. It would therefore appear that the principal function of dunging is to hinder the nncombined mordant, diffused in the dung bath, from attaching itself to the unmordanted portion of the cloth, as already observed; for if we merely wished to abstract the thickening stuffs, or to complete by the removal of acetic acid the combination of the aluminous base with the goods, dung would not be required, for hot water would suffice. In fact, we may observe, that in such cases the first pieces passed through the boiler are fit for dyeing; but when a certain number have been passed through, the mordant now dissolved in the water is attracted to the white portions of the cloth, while the free acid impoverishes the mordanted parts, so that they cannot afford good dyes, and the blank spaces are tarnished.

The cowdung may be in some measure replaced by bran, but not with perfect suecess. The former both answers the purpose better and is cheaper. The bran is only preferred for the most delicate yellows, for cochineal pinks and lilachs, to which the dung may sometimes impart a greenish cast. It is to be presumed that the action of the bran in this process has much analogy with that of the dung, and that the ligneous fibre is the most active constituent; with which the gluten and mucilage co-operate, no doubt, in zeizing the aluminous salts.

It seems to be ascertained that the mordant applied to the cloth does not combine entırely with it during the drying; that this combination is more or less perfect according to the strength of the mordants, and the circumstances of the drying; that the operation of dunging, or passing through hot water, completes the combination of the cloth with the aluminous base now insoluble in water; that this base may still contain a very minute quantity of acetic acid or sulphate of alumina; that a long ebullition in water impoverishes the mordant but a little; and that even then the liquid does not contain any perceptible quantity of acetate or subsulphate of alumina.

The manner of immersing the goods, or passing them through the dung bath, is an important circumstance. They should be properly extended and free from folds, which is secured by a series of cylinders.

The cistern is from 10 to 12 feet long, $4 \frac{1}{2}$ feet wide, and 6 or 8 feet deep. The piece passes alternately over the upper rollers and under rollers near the bottom. There are two main squeezing rollers at one end, which draw the cloth through between them. Whenever the goods come out of the bath they are put into the dash-wheel. 
The immersion should take place as fast as possible, for the moment the hot water penetrates the mordanted cloth, the acetic acid quits it; and, therefore, if the immersion was made slowly or one ply after another, the acid as well as the uncombined mordant become free, would spreal their influence, and would have time to dissolve the aluminous sub. salts now combined with the cloth; whence inequalities and impoverishment of the colors would ensue.

It is difficult to determine the number of pieces which may be passed through a given quantity of dung and water. This depends upon the state of the mordants, whether they are strong or acid, and on the quantity of the surface covered with the figures. The number varies usually from 20 to 60 pieces, for from 240 to 300 gallons of water and 6 gallons of dung. The time of the immersion varies with the concentration of the mordants, and the nature of their thickening. The temperature must be regulated by the same circumstances; for starch or flour paste a much warmer bath is needed than for gum. The heat varies usually from $130^{\circ}$ to $212^{\circ} \mathrm{F}$. When the printing is heavy and the thickening is starch or flour, the goods are usually twice dunged, with two washings between the two dungs. A strong acid mordant is more aifficult to dung and to wash than a neutral mordant, especially when it is to receive the madder dye. Sometimes a little chalk is added to the bath, when the goods have been padded in an acid mordant. Too much dung is injurious to weak mordants, as well as to pinks. It has also been semarked that a mordant when neutralized does not produce as brilliant tints, especially yellows. The latter are obtained of a finer shade when, instead of dunging, they are exposed for an hour in a stream of water, provided its temperature is not too low. In winter they are passed through a slightly chalky water, then washed at the wheel, and dyed in quercitron or weld.

A very able and learned memoir upon this subject, by M. Penot, Professor of Chemistry, appeared in the Bulletin of the Society of Mulhausen, in October, 1834, with an ingenious commentary upon it, under the title of a Report by M. Camille Kœchlin, in March, 1835.

Experience has proved that dunging is one of the most important steps in the process of calico printing, and that if it be not well performed the dyeing is good for nothing. Before we can assign its peculiar function to the dung in this case, we must know its composition. Fresh cow's dung is commonly neutral when tested by litmus paper; but sometimes it is slightly alkaline, owing, probably, to some peculiarity in the food of the animal.

The total constituents of 100 parts of cow dung are as follows : Water, $69 \cdot 58$; bitter matter, 0.74 ; sweet substance, 0.93 ; chlorophylle, 0.28 ; albumine, 0.63 ; muriate of soda, 0.08 ; sulphate of potash, 0.05 ; sulphate of lime, 0.25 ; carbonate of lime, 0.24 ; phosphate of lime, 0.46 ; carbonate of iron, 0.09 ; woody fibre, 26.39 ; silica, 0.14 ; loss, $0 \cdot 14$.

In dunging calicoes the excess of uncombined mordant is in part attracted by the soluble matters of the cow's dung, and forms an insoluble precipitate, which has no affinity for the cloth, especially in presence of the insoluble part of the dung, which strongly attracts alumina. The most important part which that insoluble matter plays, is to seize the excess of the mordants, in proportion as they are dissolved by the water of the bath, and thus to render their reaction upon the cloth impossible. It is only in the deposite, therefore, that the matters carried off from the cloth by the dung are to be found.

M. Camille Kœchlin ascribes the action of cow dung chiefly to its albuminous constituent, combining with the alumina and iron, of the acetates of these bases dissolved by the hot water of the bath. The acids consequently set free, soon become evident by the test of litmus paper, after a few pieces are passed through, and require to be got rid of either by a fiesh bath or by adding chalk to the old one. The dung thus serves also to fix the bases on the cloth, when used in moderation. It exercises likewise a disoxydating power on the iron mordant, and restores it to a state more fit to combine with coloring matter.

DYEING, (Teinture, Fr.; Färberei, Germ.) is the art of impregnating wool, silk, cotton, linen, hair, and skins, with colors not removable by washing, or the ordinary usage to which these fibrous bodies are exposed when worked up into articles of furniture or raiment. I shall here consider the general principles of the art, referring for the particular dyes, and peculiar treatment of the stuffs to be dyed, to the different tinctorial substances in their alphabetical places; such as cochineal, indigo, madder, \&c.

Dyeing is altogether a chemical process, and requires for its due explanation and practice an acquaintance with the properties of the elementary bodies, and the laws which regulate their combinations. It is true that many operations of this, as of other chemical arts, have been practised from the most ancient times, long before any just views were entertained of the nature of the changes that took place. Mankind, equally in the rudest and most refined state, have always sought to gratify the love of distinction 
by staining their dress, sometimes even their skin, with gaudy colors. Moses speaks of raiment dyed blue, and purple, and scarlet, and of sheep stins dyed red; circumstances which indicate no small degree of tinctorial skill. He enjoins purple stuff's for the works of the tabernacle and the vestments of the high priest.

In the article Calico Printing, I have shown from Pliny that the ancient Egyptians cultivated that art with some degree of scientific precision, since they knew the use of mordants, or those substances which, though they may impart no color themselves, yet enable white robes (candidu vela) to absorb coloring drugs (colorem sorbendibus medicamentis). Tyre, however, was the nation of antiquity which made dying its chief occupation and the staple of its commerce. There is little doubt that purple, the sacred symbol of roval and sacerdotal dignity, was a color discovered in that city, and that it contributed to its opulence and grandeur. Homer marks no less the value than the antiquity of this dye, by describing his heroes as arrayed in purple robes. Purple habits are mentioned among the presents made to Gideon by the Israelites from the spoils of the kings of Midian.

The juice emploved for communicating this dye was obtained from two different kinds of shell-fish, described by Pliny under the names of purpura and buccinum; and was extracted from a small ressel, or sac, in their throats, to the amount of only one drop from each animal. A darker and inferior color was also procured by crushing the whole substance of the buccinum. A certain quantity of the juice collected from a vast number of shells being treated with sea-salt was allowed to ripen for three days; after which it was diluted with five times its bulk of water, kept at a moderate heat for six days more, occasionally skimmed to separate the animal membranes, and when thus clarified was applied directly as a dye to white wool, previously prepared for this purpose by the action of lime-water, or of a species of lichen called fucus. Two operations were requisite to communicate the finest Tyrian purple; the first consisted in plunging the wool into the juice of the purpura : the second, into that of the buccinum. Fifty drachms of wool required one hundred of the former liquor, and two hundred of the latter. Sometimes a preliminary tint was given with coccus, the kermes of the present day, and the cloth receired merely a finish from the precious animal juice. The colors, though probably not nearly so brilliant as those producible by our cochineal, seem to have been very durable, for Plutarch says, in his Life of Alexander, (chap. 36, ) that the Greeks found in the treasury of the King of Persia a large quantity of purple cloth, which was as beautiful as at first, though it was 190 years old.*

The difficulty of collecting the purple juice, and the tedious complication of the dyeing process, made the purple wool of Tyre so expensive at Rome, that in the time of Augustus a pound of it cost nearly 30l. of our money. Notwithstanding this enormous price, such was the wealth accumulated in that capital, that many of the leading citizens decorated themselves in purple attire, till the emperors arrogated to themselves the privilege of wearing purple, and prohibited its use to every other person. This prohibition operated so much to discourage this curious art as eventually to occasion its extinction, first in the western and then in the eastern empire, where, however, it existed in certain imperial manufacturies till the eleventh century.

Dyeing was little cultivated in ancient Greece; the people of Athens wore generally woollendresses of the natural color. But the Romans must have bestowed some pains upon this art. In the games of the circus parties were distinguished by colors. Four of these are described by Pliny, the green, the orange, the gray, and the white. The foliowing ingredients were used by their dyers. A crude native alum mixed with copperas, copperas itself, blue vitriol, alkanet, lichen rocellus, or archil, broom, madder, woad, nutgalls, the seed of pomegranate, and of an Egyptian acacia.

Gage, Cole, Plumier, Reaumur, and Duhamel hare severally made researches concerning the coloring juices of shell-fish caught on various shores of the ocean, and have succeeded in forming a purple dye, but they found it much inferior to that furnished by. other means. The juice of the buccinum is at first white; it becomes by exposure to air of a yellowish green bordering on blue; it afterwards reddens, and finally changes to a deep purple of considerable vivacity. These circumstances coincide with the minute descrip. tion of the manner of catching the purple-dye shell-fish which we possess in the work of an eye-witness, Eudocia Macrembolitissa, daughter of the Emperor Constantine VIII., who lived in the eleventh century.

The moderns have obtained from the New World several dye-drugs unknown to the ancients; such as cochineal, quercitron, Brazil wood, logwood, annatto; and they have

* "A mong other things, there was purple of Hermione (?) to the amount of five thousand talents." (Plutarch's Lives, translated by Langhorne, Wrangham's edition, vol. v. p. 240.) Horace celebrates the Laconian dye in the following lines:-

Ner Laconicas mihi

Trahunt honestæ purpuras clientæ.

(Carm. lib. ii., Ode 18.)

I Fli ny says that a pound of the double-dipped Tyrian purple was sold in Rome for a hundred crowna 
discovered the art of using indigo as a dye, which the Romans knew only as a pigment. But the vast superiority of our dyes over those of former times must be ascribed principally to the employment of pure alum and solution of tin as mordants, either alone or mixed with other bases; substances which give to our common dye-stuffs remarkable depth, durability, and lustre. Another improvement in dyeing of more recent date is the application to textile substances of metallic compounds, such as Prussian blue, chrome yellow, manganese brown, \&c.

Indigo, the innoxious and beautiful product of an interesting tribe of tropical plants, which is adapted to form the most useful and substantial of all dyes, was actually denounced as a dangerous drug, and forbidden to be used, by our parliament in the reign of Queen Elizabeth. An act was passed authorizing searchers to burn both it and $\log$-wood in every dye-house where they could be found. This act remained in full force till the time of Charles II.; that is, for a great part of a century. A foreigner $\mathrm{I}$ ight have supposed that the legislators of England entertained such an affection for their native woad, with which their naked sires used to dye their skins in the old times, that they would alluw no outlandish drug to come in competition with it. A most instructive book might be written illustrative of the evils inflicted upon arts, manufactures, and commerce, in consequence of the ignorance of the legislature.*

Colors are not, properly speaking, material; they are impressions which we receive from the rays of light reflected, in a decomposed state, by the surfaces of bodies. It is well known that a white sunbeam consists of an indeterminate number of differently colored rays, which being separated by the refractive force of a glass prism, form the solar spectrum, an image distinguishable into seven sorts of rays; the red, orange, yellow, green, blue, indigo, and violet. Hence, when an opaque body appears colored, for example, red, we say that it reflects the red rays only, or in greatest abundance, mixed with more or less of the white beam, which has escaped decomposition. According to this manner of viewing the coloring principle, the art of dyeing consists in fixing upon stuffs, by means of corpuscular attraction, substances which act upon light in a different manner from the surfaces of the stuffs themselves. The dyer ought, therefore, to be familiar with two principles of optics; the first relative to the mixture of colors, and the second to their simultaneous contrast.

Whenever the different colored rays, which have been separated by the prism, are totally reunited, they reproduce white light. It is evident, that in this composition of light, if some rays were left out, or if the colored rays be not in a certain proportion, we should not have white light, but light of a certain color. For example; if we separate the red rays from the light decomposed by a prism, the remaining colored rays will form by their combination a peculiar bluish green. If we separate in like manner the orange rays, the remaining colored rays will form by their combination a blue color. If we separate from the decomposed prismatic light the rays of greenish yellow, the remaining colored rays will form a violet. And if we separate the rays of yellow bordering on orange, the remaining colored rays will form by their union an indigo color.

Thus we see that every colored light has such a relation with another colored light that, by uniting the first with the second, we reproduce white light; a relation which we express by saying that the one is the complement of the other. In this sense, red is the complementary colur of bluish green; orange, of blue; greenish yellow, of violet; and orange yellow, of indigo. If we mix the yellow ray with the red, we produce orange; the blite ray with the yellow, we produce green; and the blue with the red, we produce violet or indigo, according as there is more or less red relatively to the blue. But these tints are distinguishable from the orange, green, indigo, and violet of the solar spectrum, because when viewed through the prism they are reduced to their elementary compound colors.

If the dyer tries to realize the preceding results by the mixture of dyes, he will succeed only with a certain number of them. Thus, with red and yellow he can make orange; with blue and yellow, green; with blue and red, indigo or violet. These facts, the results of practice, have led him to conclude that there are only three primitive colors; the red, yellow, and blue. If he attempts to make a white, by applying red, yellow, and blue dyes in certain quantities to a white stuff, in imitation of the philosopher's experiment on the synthesis of the sunbeam, far from succeeding, he will deviate still further from his purpose, since the stuff will by these dyes become so dark colored as to appear black.

The fact must not, however, lead us to suppose that in every case where red, yellow, and blue are applied to white eloth, black is produced. In reality, when a little ultramarine, cobalt blue, Prussian blue, or indigo, is applied to goods with the view of giving them the best possible white, if only a certain proportion be used, the goods will appear whiter after this addition than before it. What happens in this case? The violet blue 
Eorms, with the brown yellow of the goods, a mixture tending to white, or less colored than the yellow of the goods and the blue logether were. For the same reason, a mix ture of Prussian blue and cochineal pirk has been of late years used in the whitening or the azuring of silks, in preference to a pure blue; for on examining closely the color $\dot{x}$ the silk to be nentralized, it was found by the relations of the complementary colors, that the violet was more suitable than the indigo blue formerly used. The dyer should linow, that when he applies several different coloring matters to stuffs, as yellow and blue, for example, if they appear green, it is because the eye cannot distinguish the points which reflect the yellow from those which reflect the blue; and that, consequently, it is only where the distinction is not possible, that a mixture or combination appears. When we examine certain gray substances, such as hairs, feathers, \&c., with the microscope, we see that the gray color results from black points disseminated over a colorless or slightly colored surface. In reference to compound colors, this instrument might be used with advantage by the dyer.

The dyer should be acquainted also with the law of the simultaneous contrast of colors. When the eye views two colors close alongside of each other, it sees them differing most in their optical composition, and in the height of their tone, when the two are not equally pale or full-bodied. They appear most different as to their optical composition, when the complementary of the one of them is added to the color of the other. Thus, put a green zone alongside of an orange zone; the red color complementary of green, being added to the orange, will make it appear redder; and in like manner the blue, complementary of orange, being added to the green, will make it appear more intensely blue. In order to appreciate these differences, let us take two green stripes and two orange stripes, placing one of the green stripes near one of the orange: then place the two others so that the green stripe may be at a distance fiom the other green stripe, but on the same side, and the orange at a distance from the other orange, also on the same side.

As to the contrast in the height of the tone, we may satisfy ourselves by taking the tones No. 1, No. 2, No. 15, and No. 16, from a graduated pallet of reds : for example, by placing No. 2 and No. 15 close alongside, putting No. 1 at a distance from No. 2 on the same side, and No. 16 at a distance from No. 15 on the same side, - we shall see (if the pallet is sufficiently lowered in tone) No. 2 equal to No. 1, and No. 15 equal to No. 16; whence it follows that No. 2, by the "vicinity of No. 15, will appear to have lost some of its color; while No. 15 will appear to have acquired color. When black or gray figures are printed upon colored grounds, these figures are of the color complementary of the ground. Consequently, in order to judge of their color, we must cut ont spaces in a piece of gray or white paper, so as to allow the eye to see nothing but the figures; and if we wish to compare figures of the same color, applied upon grounds of different colors, we can judge rightly of the figures only by insulating them from the grounds.

The relations of dyeing with the principles of chemistry, constitute the theory of the art, properly speaking; this theory has for its basis, the knowledge-1. of the species of bodies which dyeing processes bring into contact; 2 . of the circumstances in which these species act; 3 . of the phenomena which appear during their action; and 4 . of the properties of the colored combinations which are produced. These generalities may be specified under the ten following heads :-

1. The preparation of the stuffs to be dyed, whether fibres, yarn, or cloth; under the heads of ligneous matter, cotton, hemp, flax; and of the animal matters, silk and wool.

2. The mutual action of these stuffs, and simple bodies.

3. The mutual action of these stuffs, and acids.

4. The mutual action of these stuffs, and salifiable bases, as alumina, \&c.

5. The mutual action of these stuffs, and salts.

6. The mutual action of these stuffs, and neutral compounds not saline.

7. The mutual action of these stuffs, and of one or more definite compounds.

8. Of dyed stuffs considered in reference to the fastness of their color, under the influence of heat, light, water, oxygen, air, boilings with soap, and reagents.

9. Of dyeing, considered in its connexions with chemistry.

10. Of dyeing, considered in its relations with caloric, mechanies, hydraulics, and optics.

1. The preparation of stuffs.

The operations to which stuffs are subjected before dyeing, are intended-1. to separate from them any foreign matters; 2 . to render them more apt to unite with the coloring tinctures which the dyer proposes to fix upon them, in order to give them a more agreeable, or more brilliant aspect, or to lessen their tendency to assume a soiled appearance by use, which white surfaces so readily do. The foreign matters are either naturally inherent in the stuffs, or added to them in the spinning, weaving, or other 
manipulation of manufacture. The ligneous fibres must be freed from the colored azof.ze. V varnish on their surface, from a yellow coloring matter in their substance, from sonue lime and iron, from chlorophylle or leaf-green, and fiom pectic acid; all natura! combinations. Some of these principles require to be oxygenized before alkaline leys can cleanse them, as I have stated in the article BLEAching, which may be consulted in reference to this subject. See also Sirk and Wool. A weak bath of soda has the property of preparing wool for taking on a uniform dye, but it must be well rinsed and aired before being put into the dye-vat.

2. Mutual action of stuffs and simple bodies.

Stuff: chemically consilered being composed of three or four elements, already in a state of reciprocal saturation, have but a feeble attraction for simple substances. Te know in fact, that the latter combine only with each other, or with binary compounds, and that in the greater number of cases where ther exert an action upon more complete compunds, it is by disturbing the arrangement of their elements, and not by a resulting affinity with the whole together.

3. 4. Although stuffs may in a general point of view be considered as neutral in relatiun to coloring reagents, yet experience shows that they are more disposed to combine with acid than with alkaline compounds; and that consequently their nature seems to be more alkaline than acid. By steeping dry wool or other stuff in a clean state in an allisline or acid solution of known strength, and by testing the liquor after the stuff is taken out, we shall ascertain whether there be any real affinity between them, by the colution being rendered more dilute in consequence of the abstraction of alkaline or acid particles from it. Wool and silk thus immersed, abstract a portion of both sulphuric and muriatic acids; but cotton and flax imbibe the water, with the rejection of a portion of the acid. The acid may be again taken from the stuffs by washing them with a sufficient quantity of water.

5. The affinity between saline bodies and stuffs may be ascertained in the same way as that of acids, by plunging the dry stuffs into solutions of the salts, and determining the density of the solution before the immersion, and after withdrawing the stuffs. Woul abstracts alum from its solution, but it gives it all out again to boiling water. The sulphates of protoxyde of iron, of copper, and zinc, rescmble alum in this respect. When silk is steeped for some time in solution of protosulphate of iton, it abstracts the oxyd?, gets thereby dred, and leaves the solution acidulous. Wool put in contact $\pi$ ith crean of tartar decomposes a portion of it; it absorbs the acid into its pores, and leaves a nemtral salt in the liquor. The study of the action of salts upon stuffs is at the present lis the foundation of the theory of dyeing; and some of them are employed immediately as dye-drugs.

6. Mutual action of stuffs, and neutral compounds not saline.

Several sulphurets, such as those of arsenic, lead, copper, antimony, tin, are susceptible if being applied to stuffs, and of dyeing them in a more or less fast manner. Indigo, hematine, breziline, carmine, and the peculiar coloring principles of many dyes belong to this dirision.

7. Intual action of goods with one or more definite compounds, and dye-stuffs.

I sha'l consider here in a theoretical point of view, the most general results which a certain number of organic coloring matters present, when applied upon stuffs by tine dyer.

Indigo. This dye-drug, when tolerably good, contains half its weight of indigotine. The cold rat is prepared commonly with water, copperas, indigo, lime, or sometimes carbonate of soda, and is used almost exclusively for cotton and linen; immersion in acidulatel water is occasionally had recourse to for remoring a little oxyde of iron which attaches itself to the cloth dyed in this vat.

The indigo vat for wool and silk is mounted exclusively with indigo, good potashes of commerce, madder, and bran. In this vat, the immediate principles with base of carbon and hylrozen, such as the extracts of madder and bran, perform the disorrdizing functirn of the copperas in the cold vat. The pastel vats require most skill and experience, in consequence of their complexity. The greatest difficulty occurs in keeping them in a goxt condition, becanse they vary progressively as the dyeing goes on, by the abstraction of the in ligotine, and the modification of the fermentable matter employed to disoxrgenate the indizo. The alkaline matter also changes br the action of the air. Br the successive additions of indigo, alkali, \&c., this vat becomes very difficult to manage with profit and success. The great affair of the dyer is the proper addition of lime; too much or too little being equally injurious.

Sulphate of indigo or Saxon blue is used also to dre silk and wool. If the wools be Ill sorted, it will show their differences by the inequalities of the dye. Wool dyed in this bath put into water satırated with sulphureted hydrogen, becomes soon colorless, owing to the disoxygenation of the indigo. The woollen cloth, when exposed to the air for some time resumes its blue color, but not so intensely as before. 
The properties of hematine explain the mode of using logwood. When stuffs are dyed in the infusion or decoction of this wood, under the influence of a base which acts upon the hematine in the manner of an alkali, a blue dye, bordering upon violet, is obtained. Such is the process for dyeing cotton and wool a logwood blue by means of verdigris, crystallized acetate of copper, and acetate of alumina.

When we dye a stuff yellow, red, or orange, we have always bright tints; with blue, we may have a very dark shade, but somewhat violet; the proper black can be obtained only by using the three colors, blue, red, and yellow, in proper proportions. Hence we can explain how the tints of yellow, red, orange, blue, green, and violet, may be browned, by applying to them one or two colors which along with themselves would produce black; and also we may explain the nature of that variety of blacks and grays which seems to be indefinitc. Nutgalls and sulphate of iron, so frequently employed for the black dye, give only a violet or bluish gray. The pyrolignite of iron, which contains a brown empyreumatic matter, gives to stuffs a brown tint, bordering upon greenish yellow in the pale hues, and to chestnut brown in the dark ones. By galling cotton and silk, and giving them a bath of pyrolignite of iron, we may, after some alternations, dye them black. Galls, logwood, and a salt of iron, produce merely a very deep violet blue; but by boiling and exposure to air, the hematate of iron is changed, becoming red-brown, and favors the production of black. Galls and salts of copper dye stufts an olive drab, logwood and salts of copper, a violet blue; hence their combination should produce a black. In using sumach as a substitute for galls, we should take into account the proportion of yellow matter it contains. When the best possible black is wanted upon wool, we must give the stuff a foundation of indigo, then pass it into a bath of lig. wood, sumach, and proto-sulphate of iron. The sumach may be replaced by one third of its weight of nutgalls.

8. Of dyed stuffs considered in reference to the fastness of their colors, when exposed to water, light, heat, air, oxygen, boiling, and reagents.

Pure water without air has no action upon any properly dyed stuff.

Heat favors the action of certain oxygenized bodies upon the carbonaceous and hydrogenous constituents of the stuff; as is seen with regard to chromic acid, and peroxyde of manganese upon cotton goods. It promotes the solvent action of water, and it even affects some colors. Thus Prussian blue applied to silk, is reduced to peroxyde of iron by long boiling.

Light without contact of air affects very few dyes.

Oxygen, especially in the nascent state, is very powerful upon dyes. See BLEAching.

The atmosphere in a somewhat moist state affects many dyes, at an elevated temperature. Silk dyed pink, with safflower, when heated to $400^{\circ} \mathrm{F}$, becomes of a dirty white hue in the course of an hour. The violet of logwood upon alumed wool becomes of a dull brown at the same temperature in the same time. But both stand a heat of $300^{\circ} \mathrm{F}$. Brazil red dye, turmeric, and weld yellow dyes, display the same phenomena. These facts show the great fixity of colors commonly deemed tender. The stuffs become affected to a certain degree, under the same circumstances as the dyes. The alterability even of indigo in the air is shown in the wearing of pale blue clothes; in the dark blue cloth there is such a body of color, that it resists proportionally longer; but the seams of coats exhibit the effect very distinctly. In silk window curtains, which have been long exposed to the air and light, the stuff is found to be decomposed, as well as the color.

Boiling was formerly prescribed in France as a test of fast dyes. It consisted in putting a sample of the dyed goods in boiling water, holding in solution a determinate quantity of alum, tartar, soap, and vinegar, \&c. Dufay improved that barbarous test. He considered that fast-dyed cloth could be recognised by resisting an exposure of twelve hours to the sunshine of summer, and to the midnight dews; or of sixteen days in winter.

In trying the stability of dyes, we may offer the following rules :-

That every stuff should be exposed to the light and air ; if it be intended to be worn abroad, it should be exposed also to the wind and rain; that carpets, moreover, should be subjected to friction and pulling, to prove their tenacity; and that cloths to be washed should be exposed to the action of hot water and soap.

In examining a piece of dyed cotton goods, we may proceed as follows :-

Suppose its color to be orange-brown. We find first that it imparts no color to boiling water; that protochloride of tin takes out its color; that plunged into a solution of ferroprussiate of potash it becomes blue; and that a piece of it being burned, leares a residuum of peroxyde of iron; we may thence conclude that the dyeing matter is peroxyde of iron.

Suppose we have a blue stuff which may hare been dyed either with indigo or with Prussian blue, and we wish to know what it will become in use. We inquire first into the nature of the blue. Hot water slightly alkaline will be colored blue by it, if 
it has been dyed with sulphate of indigo; it will not be colored if it was dyed in the indigo vat, but it will become velluw by nitric acid. Boiling water, without becoming colored itself, will de-troy the Prussian blue dye; an alkaline water will convert its color into an iron rust tint; nitric acid, which makes the indigo dye yellow, makes that of Prussias bluc green. The liquor resulting from boiling alkaline water on the Prussian blue cloth, will convert sulnhate of iron into Prussian blue.

9. Division. Of dyeing viewed in its relation to chemistry.

The phenomena of dyeing have been ascribed to very different causes; by some they were snp:osed to depend upon mechanical causes, and by others upon the forces from which chemical effects flow. Hellot, in conformity with the first mode of explanation, thought that the art of dreing consisted essentially in opening the pores in order to admit coloring matters into them, and to fix them there by cooling, or by means of a mordant imagined to act like a cement.

Dufay in $173 \pi$, Bergmann in 1776 , Macquer in 1778 , and Berthollet in 1790 , had recourse to chemical affinities, to explain the fixation of the coloring principles upon stuffs, either without an intermedium, like indigo, walnut peels, annotto; or by the intervention of an acid, a salifiable base, or a salt, which were called mordants. When bodies present phenomena which we refer to an attraction liniting particles of the same nature, whether simple or compound, to form an aggregate, or to an affinity which unites the particles of different natures to form them into a chemical compound, these bodies are in apparent contact. This happens precisely in all the cases of the mutual action of bodies in an operation of dyeing; if their particles were not in apparent contact, there would be absolutely no change in their respective condition. When we see stuffs and metallic oxydes in apparent contact, form a mutual union of greater or less force, we cannot therefore help referring it to affinity. We do not know how many dyes mar be fixed upon the same piece of cloth; but in the operations of the dye-house sufficiently complex compounds are formed, since they are always stuffs, composed of three or four elements, which are combined with at least binary acid or basic compounds; with simple salts compounded themselves of two immediate principles at least binary; with double salts composed of two simple salts; and finally with organic dyestuffs containing three or four elements. We may add that different species belonging to one of these classes, and different species belonging to different classes, may unite simultaneously with one stuff. The union of stuffs with coloring matters appears, in general, not to talie place in definite proportions; though there are probably some exceptions.

We may conclude this head by remarking, that, besides the stuff an 1 the coloring matter, it is not necessary, in dyeing, to distinguish a third body, under the name of mordant; for the idea of mordant does not rest upon any definite fact; the body to which this name has been given being essentially only one of the immediate principles of the colored combination which we wish to fix upon the stuff.

10. Division. Of dyeing in its relation with caloric, mechanics, hydraulics, pneumatics, and opties.

Dyeing baths, or coppers, are heated directly by a furnace, or by means of steam conducted in a pipe from a boiler at a certain distance from the bath. In the first case, the vessels are almost always made of copper; only, in special cases, for the scarlet and some delicate silk dyes, of tin; in the second case, they are of copper, iron, or wood. A direct fire is more economical than heating steam pipes, where there is only one or two baths to heat, or where the labors are often suspended. Madder and indigo vats, when heated by steam, have it either admitted directly into the liquor, or made to circulate through pipes plunged into it, or between the copper and an exterior iron or wood case. See the end of this article.

Everythinz else being equal, dyeing with heat presents fewer difficulties towards obtaining an evenly color, than dyeing in the cold; the reason of which may be found in the following facts:-The air adhering to the surface of stuffs, and that interposed between the fibres of their constituent yarns, is more easily extricated in a hot bath than a cold one, and thus allows the dye liquor to penetrate more easily into their interior : in the secund place, the currents which take place in a hot bath, and which tend incessantly to renler its contents uniform, by renewing continually the strata of liquid in contact with the stuff, contribute mainly to render the dyeing evenly. In cold dyeing, it is necessary to stir up the bath from time to time: and when goods are first put in, they must be carefully dipperl, then taken out, pressed, and wrung, several times in succession till they be uniformly moistened.

The mechanical relations are to be found in the apparatus employed for wincing, siring, and pressing the goods, as we have deseribed under CALICo Printing and BANidNa. The hydranlic relations refer to the wash-wherls and other similar apparatus, of which an account is given under the same articles. The optical relations 
have been already considered. In the sequel of this article an automatic dyeing vat will

be described.

I he exiracts of solutions of native dye-stuffs may be divided into two classes, in reference to theil habitudes with the oxygen of the atmosphere; such as continue essentiaily unaltered in the air, and such as suffer oxydation, and thereby precipitate a determinate coloring malter. The dyes contained in the watery infusions of the different vezelable and animal substances which do not belong to the second class, are feebly attaclied to their solvents, and quit them readily for any other bodies that possess an attraction for them. On this principle, a decoction of cochineal, logwood, brazil wrot, or a solutior. of sulphate of indigo, by digestion with powdered bone black, lose their color, in consequence of the coloring particles combining by a kind of capillary attraction with the porous carbon, without undergoing any change. The same thing happens when wellscoured wool is steeped in such colored liquids; and the color which the wool assumes by its attraction for the dye, is, with regard to most of the above colored solutions, but feeble and fugitire, since the dye may be again abstracted by copious washing trith sitnple water, whese attractive force therefore prercomes that of the wool. The aiu of a high temperature, indeed, is requisite for the austraction of the color from the wool and the Bone-black, probably by enlarging the size of the pores, and increasing the solvent power of the water.

Those dire-baths, on the contrary, whose coloring matter is of the nature of extractive cr aputheme, form a faster combination with stuffs. Thus the yelluw, fawn, ard brown dyes, which contain tannin and extractive, become oxygenated by contact of air, and insoluble in water; br which means they can impart a durable dre. When woul is impregnated with decections of that lind, its pores get charged by capillarity, and when the liquid becomes orygenated, they remain filled with a color now become insoluble in water. A similar change to insolubility ensues when the yellow liquor of the indigo rat gets oxydized in the pores of cotton and wool, into which it har been introduced in a fluid state. The same change occurs when protosulphate of iron is converted into persulphate, with the deposition of an insoluble peroxyde in the substance of the stuff. The change here effected by oxydation can, in other circumstances, be produced by acids which hare the power of precipitating the dye-stuff in an insoluble state, as happens with decoction of fustic.

Hence we perceive that the dreing of fast colors rests upon the principle, that the colors dissolred in the vat, during their union with the stuff, should suffer such a change as to become insoluble in their former menstruum. The more this dye, as altered in its union with the stuff, can resist other menstrua or agents, the faster it will be. This is the essential difference between dreing and painting; or applying a coat of pigment devoid of any true affinity for the surface.

If we mix a clear infusion of a dye with a small quantity of a solution of an earthy or metallic salt, both in water, the limpid liquids soon become turbid, and there gradually subsides sooner or later, according to the nature of the mixture, a colored precipitate, consisting of the altered dỵe united with a basic or subsalt. In this compound the coloring matter seems to act the part of an acid, which is saturated br a small quantity of the basis, or in its acid relationship is feeble, so that it c in also combine with acids, being in reference to them a base. The decomposition of a salt, as alum, by dres, is effected principally through the formation of an insoluble subsalt, with which the color combines, while a supersalt remains in the bath, and modifies, by its solvent reaction, the shade of the dyed stuff. Dyed stuffs may be considered as composed of the fibrous body intimately associated with the coloring matter, the oxrde, and acid, all three constituting a compound salt. Many persons hare erroneously imaginel, that dred goods contained none of the acid emplored in the dre bath; but they forget that even potash added to alum does not throw duwn the pure earthy basis, but a subsalt; and ther should not ascribe to coloring matter a power of decomposition at all approaching to that of an alkali. Salts, containing strong acids, saturate a rery large quantity of coloring matter, in proportion to their place in the scale of chemical equivalents. Mere bases, such as pure alumina, and pure oxyde of tin, have no power of precipitating coloring matter; when they seem to do so, they alwars contain some acid.

Such salts, therefore, as have a tendency to pass readily into the basic state, are peculiarly adapted to act as mordants in dreing, and to form colored lakes. Magnesia affords as fine a white powder as alumina, and answers equally well to dilute lakes, but its soluble salts cannot be emplored to form lakes, because ther do not pass into the basic state. This illustration is calculated to throw much light upon dyeing processes in general.

The color of the lake deperals verr much upon the nature of the acid, and the basis of the precipitating salt. If it be white, like alumina and oxyde of tin, the lake will have, more or less, the color of the dje, but brightened by the reflection of white 
Ingt fiom the basis; while the difference of the acid occasions a difference in the hue. The colored base's impart more or less of their color to the lakes, not merely in virtue of thei own tints, but of their chemical action upon the dye.

Upun these principles a crimson precipitate is obtained from infusions of cochineal by alum and sali of tin, which becomes scarlet by the addition of tartar; by acetate of lead, a viviet bite precipitate is obtained, which is durable in the air; by muriate of lime, a pink bruwn precipitate falls, which soon becomes black, and at last dirty green; by the solution of a ferruginous salt, the precipitates are dark violet and black; and, in lilie manner, all other salts with earthy or metallic bases, afford diversities of shade with cochineal. If this dye stuff be dissolved in weal water of ammonia, and be precipitalcl with acetate of lead, a green lake is obtained, which, after some time, will become reen on the surface by contact of air, but violet and blue beneath. Hence it appears, that the shade of color of a lake depends upon the degree of oxydation or change of the culor caused by the acid of the precipitating salt, upon the degree of oxydation or color of the oxyde which enters into union with the dye, and upon its quantity in terince to that of the coloring principle.

Such lakes are the difficulty soluble salts which constitute the dyeing materials of stufts. Their particles, however, for the purposes of dyeing, must exist in a slate of extremely fine division in the bath liquor, in order that they mar penetrate along with it intu the ininute pores of textile fibres, and fill the cavities observed by means of the microscope in the filaments of wool, silk, cotton, and flax. I have examined these stuffs with an achromalic microscope, and find that when they are properly dyed with fast colors, the interior of their tubular texture is filled, or lined at least, with coloring matter. When the bath contains the coloring particles, so finely divided that they can pass through filtering paper, it is capable of dyeing; but if the infusion mixed with its mordant be flocculent and ready to subside, it is unfit for the purpose. In the latter case, the ingredients of the dye bave already become aggregated into compounds too coherent and too gross for entering into combination with fibrous stuffs. Extractive matter and tannin are particularly liable to a change of this kind, by the prolonged action of heat in the bath. Hence, also, an alkaline solution of a coloring matter affords no useful dye bath, when mixed with the solution of a salt having an earthy or metallic basis.

These circumstances, which are of frequent occurrence in the dye-house, render it necessary alwars to have the laky matter in a somewhat soluble condition, and to effect its precipitation within the pores of the stuff, by previously impregnating them with the saline solutions by the aid of heat, which facilitates their introduction.

When a mordant is applied to any stuff, the portion of it remaining upon the suxface of the fibres should be remored; since, by its combination with the coloring matter, it would be apt to form an external crust of mere pigment, which would block up the pores, obstruct the entrance of the dye into the interior, and also exhaust to no purpose the dyeing power of the bath. For this reason the stuffs, after the application of the moidant, are drained, squeezed, washed, and sometimes (particularly with cotton and linen, in calico printing) even hard dried in a hot stove.

The saline mordants, moreover, should not in general possess the crystallizing property in any considerable degree, as this opposes their affnity of composition for the cloth. On this account the deliquescent acetates of iron and alumina are more ready to aid the dyeing of cotton than copperas and alum.

Alum is the great mordant employed in wool dyeing. It is frequently dissolved in waler, holding tartar equal to one fourth the weight of the alum in solution; by which addition its tendency to crystallize is diminished, and the resulting color is brightened. The alum and tartar combine with the stuff without suffering any change, and are decomposed only by the action of the coloring matters in the dre bath. The alum operates solely in virtue of its sulphuric acid and earthy basis; the sulphate of potash present in that salt being rather injurious. Hence, if a sulphate of alumina free from iron could be readily obtained, it would prove a preferable mordant to alum. It is also probable, for the reasons above assigned, that soda alum, a salt much less apt to crystallize than potash or arnmonia alum, would suit the dyer very well. In order to counteract the tendency of common alum to crystallize, and to promote its tendency to pass into a basic salt, one eighth part of its weight of potash is added to its solution, or the equivalent in chalk or soda.

We shall conclude this account of the general principles of dyeing, with Mr. Delaval's observations on the nature of dyes, and a list of the different substances used in dyeing, in reference to the colors produced by them.

Sir I-aac Tewton supposed colored matters to reflect the rays of light; some bodies reflecting the more, others the less, refrangible rays most copiously; and this he conceived to be the true, and the only reason of their colors. Mr. Delaval, however, proved, in the $2 \mathrm{~d}$ vol. of the "Memoirs of the Philosophical and Literary Society of Manchester," that, "in transparent colored substances, the coloring substance does not refiect any 
light; aic that when, by intercepting the light which was transmitted, it is hindered from passing through substances, they do not vary from their former color to any orher color, but become entirely black;" and he instances a considerable nurnber of colored liquors, none of them endued with reflective powers, which, when seen by transmitted light, appeared severally in their true colors; but all of them, when seen by incident light, appeared black; which is also the case of black cherries, black currants, black berries, \&c., the juices of which appeared red when spread on a white ground, or otherwise viewed by transmitted instead of incident light; and he concludes, that bleached linen, \&c., "when dyed or painted with vegelable colors, do not differ in their manner of acting on the rays of light, from natural vegetable bodies; both yielding their colors by transmitting through the transparent colored matter the light which is reflected from the white ground:" it being apparent, from different experiments, "that no reflecting power resides in any of their components, except in their white matter only," and that "transparent colored substances, placed in situations by which transmission of light through them is intercepted, exhibit no color, but become entirely black."

The art of dyeing, therefore, (according to Mr. Delaval,) "consists principally in covering white substances, from which light is strongly reflected, with transparent colored media, which, according to their several colors, transmit more or less copiously the rays reflected from the white," since "the transparent media themselves reflect no light; and it is evident that if they yielded their colors by reflecting, instead of transmitting the rays, the whiteness or color of the ground on which they are applied, would not in anywise alter or affect the colors which they exhibit."

But when any opaque basis is interposed, the reflection is doubtless made by it, rather than by the substance of the dyed wool, silk, \&c., and more especially when such basis consists of the white earth of alum, or the white oxyde of tin ; which, by their strong reflective powers, greatly augment the lustre of colors. There are, moreover, some opaque coloring matters, particularly the acetous, and other solutions of iron, used to stain linen, cotton, \&c., which must necessarily themselves reflect, instead of transmitting the light by which their colors are made perceptible.

The compound or mixed colors, are such as result from the combination of two differently colored dye stuffs, or from dyeing stuffs with one color, and then with another. The simple colors of the dyer are red, yellow, blue, and black, with which, when skil. fully blended, he can produce every variety of tint. Perhaps the dun or fawn color might be added to the above, as it is directiy obtained from a great many vegetable sub. stances.

1. Red with yellow, produces orange; a color which, upon wool, is given usually with the spent scarlet bath. To this shade may be referred flame color, pomegranate, capuchin, prawn, jonquil, cassis, chamois, café au laii, aurora, marigold, orange peel, mordorés, cinnamon, gold, \&c. Snuff, chestnut, musk, and other shades are produced by substituting walnut peels or sumach for bright yellow. If a little blue be added to orange, an olive is obtained. "The only direct orange dyes are annotto, and subchromate of lead; see Silk and Wool Dyeing.

2. Red with blue produces purple, violet, lilach, pigeon's neck, mallow, peach-blossom, bleu de roi, lint-blossom, amaranth.

3. Red with black; brown, chocolate, marone, \&c.

4. Yellow with blue; green of a great variety of shades, such as nascent green, gay green, grass green, spring green, laurel green, sea green, celadon green, parrot green, cabbage green, apple green, duck green.

5. Mixtures of colors, three and three, and four and four, produce an indefinite diversity of tints; thus red, yellow, and blue, form brown olives, and greenish grays; in which the blue dye ought always to be first given, lest the indigo vat should be soiled by other colors. Red, yellow, and gray, (which is a gradation of black, give the dead-leaf tint, as well as dark orange, snuff color, \&c. Red, blue, and gray, give a vast variety of shades; as lead gray, slate gray, wood-pigeon gray, and other colors, too numerous to specify. See Brown Dye.

The following list of dyes, and the coloring substances which produce them, may prove useful.

Red. Cochineal, kermes, lac, madder, archil, carthamus or safflower, brazil wood logwood, periodide of mercury, alkanet.

Yellow. Quereitron, weld, fustic, (yellow wood, annotto, sawwort, dyer's broom, tur. meric, fustet, (rhus cotinus,) Persian and Avignon berries, (rhamnus infectorius, ) willow, peroxyde of iron; chromate of lead, (chrome yellow,) sulphuret of arsenic, hydrasulphuret of antimony; nitric acid on silk.

Blue. Indigo, woad or pastel, Prussian blue, turnsole or litmus, logwood with a salt of copper. 
Black. Galls, sumach, logwood, walnut peels, and other vegetables which contain tannin and gallic acid, along with ferruginous mordants. The anacardium of India.

Green. These are produced by the blue and yellow dyes skilfully combined; with the exception of the chrome green, and perhaps the copper green of Schweinfurt.

Orange. Annotto, and mixtures of red and yellow dyes; subchromate of lead.

Broun. See the remarks at the beginning of this article; Brown in its alphabetical place; Calico Printing, Catechu, and Manganese.

Faun, Dun, or Root. Walnut peels, sumach, birch-tree, henna, sandal wood. See Calico Printing, for a great variety of these dyes.

Figs. 364 and 365 represent in a cross and longitudinal section the automatic dyeing ateam copper, si generally employed in the well-appointed factories of Lancashire.

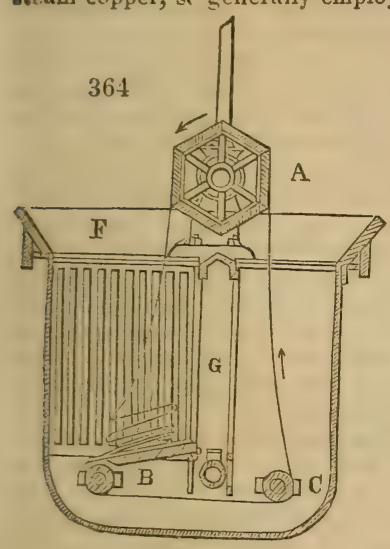

$A$ is the long reel, composed at each end of six radial iron arms or spokes, bound at their outer extremities with a six-sided wooden frame; these two terminal hexagons are connected by long wooden laths, seen above and below A in fig. 365. is shows the sloping border or ledge of the copper. $B$ and $c$ are rollers laid horizontally, for facilitating the continuous motion of the series of pieces of goods stitched together into an endless web, which are made to travel by the incessant rotations of the reel. Immediately above the roller $\mathrm{B}$ in $f i g .364$, all the spare foldings of the web are seen resting upon the sloping wooden grating, which guides them onwards in the direction indicated by the arrow. The dye stuffs are put within the middle grating, like a hencoop, marked G. Each copper is 6 feet long, $3 \frac{1}{2}$ feet wide, $3 \frac{1}{2}$ feet deep, exclusive of the top ledge, 9 inches high. Such steam coppers are usually erected in pairs, and moved by a common horizontal bevel wheel seen at $\mathrm{D}$ in $f i g$. 365 , fixed upon a vertical shafts

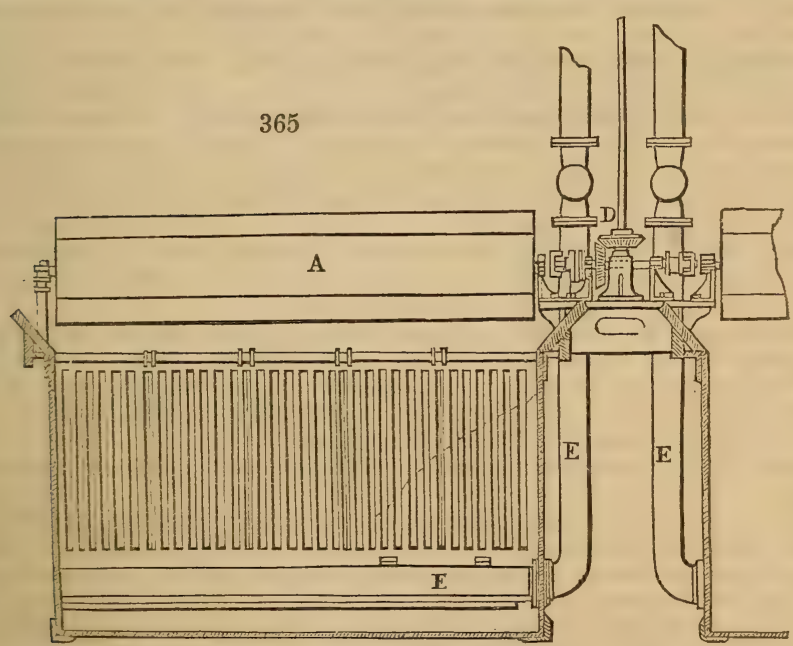

shifted into gear by a wheel at its top, with one of the driving shafts of the factory. Upon each side of $\mathrm{D}$, the two steam pipes for supplying the right and left hand coppers are seen ; each provided with a stop cock for acimitting, regulating, or cutting off the steam. These steam pipes descend at $\mathrm{E}_{\mathrm{E}}$, the horizontal branch having several orifices in its upper surface. The horizontal shaft in a line with the axes of the reels, and which turns them, is furnished upon each side with a clutch for putting either of the reels into or out of cear, that is to say, setting it a going, or at rest, in a moment by the touch of a forked lever.

The steam pipe of distribution $\mathrm{E}$ lies horizontally near the bottom of the middle coop, as shown under $\mathrm{G}$ in fig. 364, and sends up the steam through its numerous orifices, among the dye-stuffs and water by which it is covered. Thus the infusion or decoc- 
lion is continually advancing in the copper, during the incessant loco-motion of the end. less web. The horizontal pipe traverses the copper from end to end, and is not stopped short in the inidule. Each of these coppers can receive two, three, or more parallel pieces of goods at a time, the reel and copper being divided into so many compartments by transverse wooden spars.

E.

EARTHS. (Terres, Fr.; Erden, Germ.) Modern science has demonstrated that the substances called primitive earths, and which prior to the great electro-chemical career of Sir H. Davy, were deemed to be elementary matter, are all compounds of certain metallic bases and oxygen, with the exception of silica, whose base, silicon, being analn gous to boron, has led that compound to be regarded as an acid; a title characteristie of the part it extensively performs in neutralizing alkaline bodies, in mineral nature, and in the processes of art. Four of the earths, when pure, possess decided alkaline properties, being more or less soluble in water, having (at least three of them) an acrid alkaline taste, changing the purple infusion of red cabbage to green, most readily saturatins ne acids, and affording thereby neutro-saline crystals. These four are hary'u, strontia, line (calcia), and magnesia. The earths proper are five in number; alumina, glucina, yttria, zirconia, and thorina. These do not change the color of infusion o! cabbage or tincture of litmus, do not readily neutralize acidity, and are zuite insoluble in water. The alkalis are soluble in water, even when earbonated; a property which distinguishes them from the alkaline earths. Lithia must for this reason be considered to be an alkali. See the above substances in their alphabetical places.

EAU DE COLOGNE. This preparation has long possessed great celebrity, in consequence chiefly of the numerous virtues ascribed to it by its venders; and is resorted to by many votaries of fashion as a panacea against ailments of every kind. It is however nothing more than aromatized alcohol, and as such, an agreeable companion of the toilet. Numerous fictitious recipes have been offered for preparing eau de Cologne; the following may be reckoned authentic, having been imparted by Farina himself to a friend.

Take 60 gallons of silent brandy; sage, and thyme, each 6 drachms; balm-mint and spearmint, each 12 ounces; calamus aromaticus, 4 drachms; root of angelica, 2 drachms; camphor, 1 drachm; petals of roses and violets, each 4 ounces; flowers of lavender, 2 ounees; flowers of orange, 4 drachms; wormwood, 1 ounce; nutmegs, cloves, cassia lignea, mace, each 4 drachms. Two oranges and two lemons, cut in pieces. Allow the whole to macerate in the spirit during 24 hours, then distil off 40 gallons by the heat of a water bath. Add to the product :

Essence of lemons, of cedrat, of balm-mint, of lavender, each 1 ounce 4 drachms; neroli and essence of the seed of anthos, each 4 drachms; essence of jasmin, 1 ounce; of bergamot, 12 ounces. Filter and preserve for use.

Cadet Gassincourt has proposed to prepare eau de Cologne by the following recipe: Take alcohol at $32^{\circ}$ B., 2 quarts ; neroli, essence of cedrat, of orange, of lemon, of bergamot, of rosemary, each 24 drops; add 2 drachms of the seeds of lesser cardamoms, distil by the heat of a water bath a pint and a half. When prepared as thus by simple mixture of essences without distillation, it is never so good.

EAU DE LUCE is a compound formed of the distilled oil of amber and water of ammonia.

ELEMI is a resin which exudes from incisions made during dry weather through the bark of the anyris elemifera, a tree which grows in South America and Brazil. It comes to us in yellow, tender, transparent lumps, which readily soften by the heat of the hand. They have a strong aromatic odor, a hot spicy taste, and contain $12 \frac{1}{2}$ per celit. of ethereous oil. The crystalline resin of elemi has been called Elemine. It is used in making lacker, to give toughness to the varnish.

EBULLITION. (Eng. and Fr.; Kochen, Germ.) When the bottom of an open vessel containing water is exposed to heat, the lowest stratum of fluid immediately expands, becomes therefore specifically lighter, and is forced upwards by the superior gravity of the superineumbent colder and heavier particles. The heat is in this way diffused through the whole liquid mass, not by simple communication of that power from particle to particle as in solids, called the conduction of caloric, but by a translation of the several particles from the bottom to the top, and the top to the bottom, in alternate succession. This is denominated the carrying power of fluids, being common to both liquid and gaseous bodies. These internal movements may be rendered very conspicuous and instructive, by mingling a little powdered amber with water, contained in a 
tall glass cylinder, standing upon a sand-bath. A column of the heated and lighter particles will be seen ascending near the axis of the cylinder, surrounded by a hollow column of the cooler ones descending near the sides. That this molecular translation or locomotion is almost the sole mode in which fluids get heated, may be demonstrated by placing the middle of a pretty long glass tube, nearly filled with water, ubliquely over an argand flame. The upper half of the kquid will soon boil, but the portion under the midlle will contiuue cool, sn that a lump of ice may remain for a considerable time at the bottom. When the heat is rapidly applied, the liquid is thrown into agitation, in consequence of elastic vapor being suddenly generated at the bottom of the vessel, and being as suddenly condensed at a little distance above it by the surrounding cold columns. These alternate expansions and contractions of volume become more manifest as the liquid becomes hotter, and constitute the simmering vibratory sound which is the prelude of ebullition. The whole mass being now heated to a pitch compatible with its permanent elasticity, becomes turbulent and explosive under the continued influence of fire, and emitting more or less copious volumes of vapor, is said to boil. The further elevation of temperature, by the influence of caloric, becomes impossible in these circumstances with almost all liquids, because the vapor carries off from them as much heat in a latent state as they are capable of receiving from the fire.

The temperature at which liquids boil in the open air varies with the degree of atmospheric pressure, being higher as that is increased, and lower as it is diminished. Hence boiling water is colder by some degrees in bad weather, or in an elevated situation, with a depressed barometer, than in fine weather, or at the bottom of a coal-pit, when the barometer is elevated. A high column of liquid, also, by resisting the discharge of the steam, raises the boiling point. In vacuo, all liquids boil at a temperature about $124^{\circ} \mathrm{F}$. lower than under the average atmospheric pressure. For a table of elasticities, see VAPOR. Gay Lussac has shown that liquids are converted into vapors more readily, or with less turbulence, when they are in contact with angular or irregular, than with smooth surfaces; that they therefore boil at a heat $2^{\circ} \mathrm{F}$. lower in metallic than in glass ressels, probably owing to the greater polish of the latter. For example, if into water about to boil in a glass matrass, iron filings, ground glass, or any other insoluble powder be thrown, such a brisk ebullition will be instantly determined as will sometimes throw the water out of the vessel; the temperature at the same time sinking two degrees F. It would thence appear that the power of caloric, like that of electricity, becomes concentrated by points.

The following table exhibits the boiling heats, by Fahrenheit's scale, of the most important liquids :-

Ether, specific gravity 0.7365 at $48^{\circ}$

Carburet of sulphur

Alcohol, sp. grav. $0 \cdot 813$.

Nitric acid, $1 \cdot 500-$

Water

Saturated solution of Glauber salt

$\begin{array}{lll}\text { do. do. } & \text { Acetate of lead } \\ \text { do. do. } & \text { Sea salt } \\ \text { do. } & \text { do. } & \text { Muriate of lime, }\end{array}$

do. do.
do. do.
do. do.

do.

do. do.

Muriatic acid, sp. gr. 1.094 do.

do. $1 \cdot 127$

Nitric acid, do. 1.420 do. do. 1.30

Rectified petroleum

Oil of turpentine

Sulphuric acid, sp. gr. $\mathbf{1} \cdot 848$ do.

do.

do.

do.

do.

do.

do.

Phosphorus

Sulphur -

Linseed oil do. $1 \cdot 810$

do. $1 \cdot 780$

do. $1 \cdot 700$

do. $1 \cdot 650$

do. $1 \cdot 520$

do. 1.408

do. $1 \cdot 300+$
$1+$ water 2, do.

\section{$35.5+$ do. 64.5 , do.}

$40.5+$

do. $59 \cdot 5$, do.

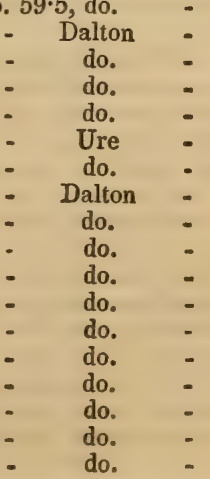

$\begin{array}{llr}- & - & 100^{\circ} \\ - & - & 113 \\ - & - & 173 \cdot 5 \\ - & - & 210 \\ - & - & 212 \\ - & - & 213 \frac{1}{3} \\ - & - & 2153 \\ - & - & 224 \frac{1}{3} \\ - & - & 285 \\ - & - & 230 \\ - & - & 235 \\ - & - & 240 \\ - & - & 232 \\ - & - & 222 \\ - & - & 248 \\ - & - & 236 \\ - & - & 306 \\ - & - & 316 \\ - & - & 600 \\ - & - & 473 \\ - & - & 435 \\ - & - & 374 \\ - & - & 350 \\ - & - & 290 \\ - & - & 260 \\ - & - & 240 \\ - & - & 554 \\ - & - & 570 \\ - & & 640\end{array}$




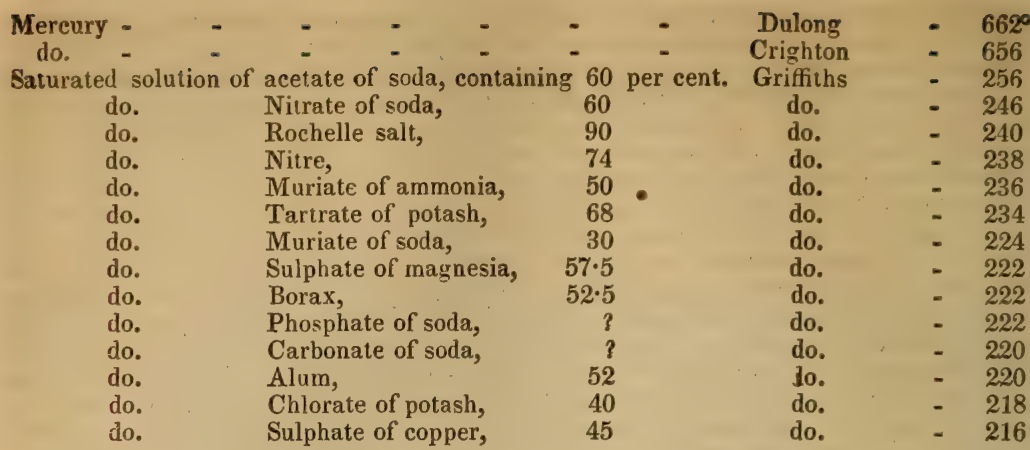

EDULCORATE (Edulcorer, Fr.; Aussiissen, Germ.) is a word introduced by the alchemists to signify the sweetening, or rather rendering insipid, of acrimonious pulverulent substances, by copious ablutions with water. It means, in modern language, the washing away of all particles soluble in water, by agitation or trituration with this fluid, and subsequent decantation or filtration.

EFFER VESCENCE. (Eng. and Fr.; Aufbrausen, Germ.) When gaseous matter is suddenly extricated with a hissing sound during a chemical mixture, or by the application of a chemical solvent to a solid, the phenomenon, from its resemblance to that of simmering or boiling water, is called effervescence. The most familiar example is afforded in the solution of sodaic powders; in which the carbonic acid gas of sesqui-carbonate of soda is extricated by the action of citric or tartaric acid.

EFFLORESCENCE (Eng. and Fr.; Verwittern, Germ.) is the spontaneous conversion of a solid, usually crystalline, into a powder, in consequence either of the abstraction of the combined water by the air, as happens to the crystals of sulphate and carbonate of soda; or by the absorption of oxygen and the formation of a saline compound, as in the case of alum schist, and iron pyrites. Saltpetre appears as an efflorescence upon the ground and walls in many situations.

EDGE-TOOIS. See Cutrery and Steel.

EGgS, Hatching. See Incubation, Artificial.

EIDER-DOWN is a kind of precious down, so called because it is obtained from the Eider-duck. These birds build their nests among precipitous rocks, and the female lines them with fine feathers plucked from her breast, among which she lays her five eggs. The natives of the districts frequented by the eider-ducks let themselves down by cords among the dangerous cliffs, to collect the down from the nests. It is used to fill coverlets, pillows, cushions, \&c.

ELAINE is the name given by Chevreul to the thin oil which may be expelled from tallow, and other fats, solid or fluid, by pressure either in their natural state, or after being saponified, so as to harden the stearine. It may be extracted also by digesting the fat in 7 or 8 times its weight of boiling alcohol, spec. grav. 0.798 , till it dissolves the whole. Upon cooling the solution, the slearine falls to the bottom, while the elaine collects in a layer like olive oil, upon the surface of the supernatant solution, reduced by evaporation to one eighth of its bulk. If this elaine be now exposed to a cold temperature, it will deposite its remaining stearine, and become pure. See FAT, OILs, and Stearine.

ELASTIC BANDS. (Tissus Elastiques, Fr.; Federharz-zeige, Germ.) The manufacture of braces and garters, with threads of caoutchouc, either naked or covered, seems to have originated, some time ago, in Vienna, whence it was a few years since imported into Paris, and thence into this country. At first the pear-shaped bottle of Indian rubber was cut into long narrow strips by the scissors; a single operative turning off only about 100 yards in a day, by cutting the pear in a spiral direction. He succeeded next in separating with a pair of pincers the several layers of which the bottle was composed. Another mode of obtaining fine threads was to cut them out of a bottle which had been rendered thin by inflation with a forcing pump. All these operations are facilitated by previously steeping the caoutchoue in boiling water, in its moderately inflated state. More recently, machines have been successfully employed for cutting out these filaments, but for this purpose the bottle of caoutchouc is transformed into a dise of equal thickness in all its parts, and perfectly circular. This preliminary operation is executed as follows: 1 . the bottle, softened in hot water, is squeezed between the two plates of a press, the neck having been removed beforehand, as useless in this point of view; 2 . the bottle is then cut into two equal parts, and is allowed to con- 
solidate by cooling before subjecting it to the cutting instrument. When the bottle is strong enough, and of variable thickness in its different points, each half is submitted to powerful pressure in a very strong cylindrical mould of metal, into which a metallic plunger descends, which forces the caoutchouc to take the form of a flat cylinder with a circular base. The mould is plunged into hot water during the compression. A stem or rod of iron, which goes across the hollow mould and piston, retains the latter in its place, notwithstanding the resilience of the caoutchouc, when the mould is taken from the press. The mould being then cooled in water, the caoutchouc is withdrawn.

The transformation of the disc of caoutchouc into fine threads is performed by two machines; the first of which cuts it into a riband of equal thickness in its whole extent, running in a spiral direction from the circumference to the centre; the second subdivides this riband lengthwise into several parallel filaments much narrower but equally thick.

The following figs. $366,367,368$, represent the machine for cutting the spiral riband. The disc $\mathrm{D}$, placed horizontally, turns round its vertical axis, so as to present its

366

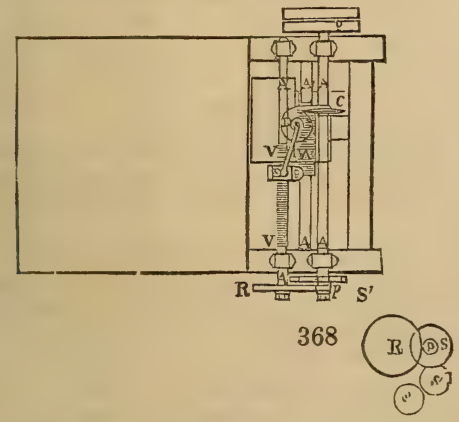

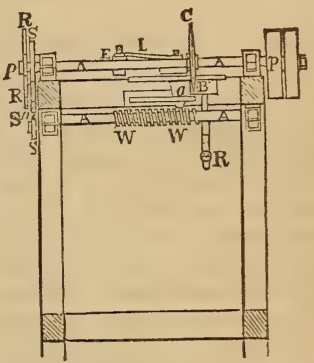

367

periphery to the edge of a knife c, formed like a circular blade, whose plane is perpendicular to that of the bases of the disc. This knife turns round its centre, which is fixed. The rotatory motion of the dise forces the knife to penetrate further and further into its mass, and the motion of the knife itself makes it cut the riband more easily. It is obvious, that if the disc alone revolve, the motionless knife could act only by pressure, and would meet with an enormous resistance. A third movement becomes necessary. In proportion as the disc is diminished by the removal of the spiral band, the centre of this disc must advance upon the knife, in order that the riband may have always the same breadth. The inspection of fig. 368 will make the accordance of the three motions intelligible.

The $\mathrm{knife} \mathrm{c}$ is placed upon a shaft or axis $\mathrm{A}$, which carries a pulley, round which a belt or cord runs which drives the whole machine. This knife is six inches in diameter. In order that by being kept cool it may cut the caoutchouc better, it is plunged at its lower part into a trough $\mathrm{B}$, full of water; a stopcock $\mathrm{R}$, serves to empty this trough.

The shaft $\mathrm{A}$ bears a pinion $\mathrm{p}$, which takes into a wheel $\mathrm{R}$, placed upon the shaft $\mathrm{A}^{\prime}$; upon which there is cut a worm or endless screw, $\nabla, \nabla$. This worm bears a nut E, which advances as the screw turns, and carries with it a tie $L$, which in its turn pushes the disc D, carried upon a shoulder constantly towards the knife. This shoulder is guided by two ears which slide in two grooves cut in the thickness of the table. The diameter of the pinion $p$ is about one fifth of that of the wheel $\mathrm{R}$; so that the arbor A turns five times less quickly than the arbor $A^{\prime}$; and the fineness of the screw $v$ contributes further to slacken the movement of translation of the disc.

When the disc is all cut down, the shoulder, the tie, and the nut, are brought back to their original position by lifting the nut, which is hinged on. The disc is fixed upon the shoulder by means of sharp points, and an upper washer. The shoulder and the washer have a very small diameter, in order that the knife may, in cutting down the disc, advance as near as possible to the centre.

The rotatory movement of the dise and its shoulder, is given by an endless screw w, w, which governs a pinion $p^{\prime}$, provided with 10 teeth, and carried by the shaft $A$, upon which the shoulder is mounted. The arbor $A^{\prime}$ of this endless screw receives its motion from the first shaft $A$, by mears of the wheels $s$ and $s^{\prime}$ mountea upon these shafts, and of an intermediate wheel $\mathrm{s}^{\prime \prime}$. This wheel, of a diameter equal to that of the shaft $A^{\prime \prime}$, is intended merely to allow this shaft to recede from the shaft $A$. The diameter of the wheel of this last shaft is to that of the two others in the ratio of 10 so 8 . 
Second machine for subdividing the ribands. Fig. 369.-The riband is engaged between the circular knives, $\mathrm{c}$, $\mathrm{c}$, which are mounted upon the rollers $\mathrm{R}, \mathrm{R}$; thin brass washers keep these knives apart at a distance which may be varied, and two extreme washers mounted with screws on each roller maintain the whole system. The axes of these rollers traverse two uprights $\mathrm{M}$, $\mathrm{M}$, furnished with brasses, and with adjusting screws to approximate them at pleasure. The axis of the lower roller carries a wheel

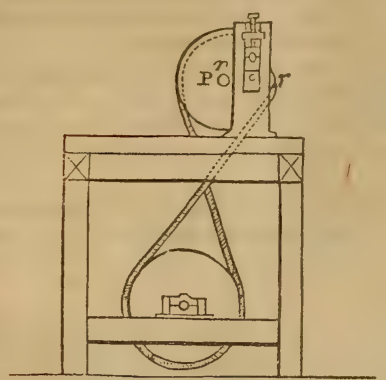

369

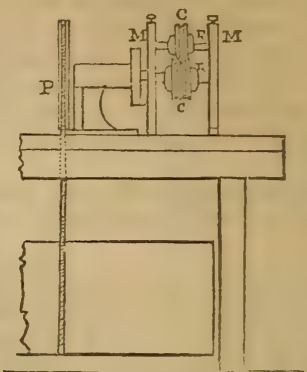

', which takes into another smaller wheel $r$, placed upon the same shaft as the pulley $\mathbf{P}$, which is driven by a cord. The diameter of the wheel $r$ is three times greater than the wheel $r^{\prime}$. The pulley $\mathrm{P}$ is twice the size of the wheel $r^{\prime}$; and its cord passes round a drum $B$, which drives the rest of the machine.

The threads, when brought to this state of slenderness, are put successively into tubs filled with cold water; they are next softened in hot water, and elongated as much as possible in the following manner:- They are wound upon a reel turned quickly, while the operative stretches the caoutchouc thread with his hand. In this way it is rendered 8 or 10 times longer. 'The reels when thus filled are placed during some days in a cold apartment, where the threads become firm, and seem to change their nature.

This state of stiffness is essential for the success of the subsequent operations. The threads are commonly covered with a sheath of silk, cotton, or linen, by a braiding machine, and are then placed as warp in a loom, in order to form a narrow web for braces, garters, \&c. If the gum were to exercise its elasticity during this operation, the different threads would be lengthened and shortened in an irregular manner, so as to firm a puckered tissue. It is requisite therefore to weave the threads in their rigid and inextensible, or at least incontractile condition, and after the fabric is woven to restore to the threads of caoutchouc their appropriate elasticity. This restoration is easily effected by passing a hot smoothing iron over the tissue laid smoothly upon a table covered with blanket stuff. See Braiding Machine.

ELECTIVE AFFINITY (Wahlverwandtschaft, Germ.) denotes the order of preference, so to speak, in which the several chemical substances choose to combine; or really, the gradation of attractive force infused by Almighty Wisdom among the different objects of nature, which determines perfect uniformity and identity in their compounds amidst indefinite variety of combination. The discussion of this interesting subject belongs to pure chemistry. See Decomposition.

ELEMENTS, (Eng. and Fr.; Grundstoffe, Germ.) The ancients considered fire, air, water, and earth, as simple substances, essential to the constitution of all terrestrial bcings. This hypothesis, evidently incompatible with modern chemical discovery, may be supposed to correspond, however, to the four states in which matter seems to exist; namely, 1 . the unconfinable powers of fluids-caloric, light, electricity; 2. ponderable gases, or elastic fluids; 3 . liquids; 4 . solids. The three elements of the alchemists, salt, earth, mercury, were, in their sense of the word, mere phantasms.

In modern science, the term Element signifies merely a substance which has not yet been resolved by analysis into any simple form of matter; and it is therefore synonymous with undecompounded. This class cumprehends 54 different bodies, of which no less than 41 are metallic. Five may be styled Archcal, from the intensity and universality of their affinities for the other bodies, which they penetrate, corrode, and apparently consume, with the phenomena of light and heat. These $\bar{j}$ are chlorine, oxygen, iodine, bromine, fluorine. Eight elements are eminently inflammable when acted upon by any of the preceding five, and are thereby converted into incombustible compounds. The simple non-metallic inflammables are hydrogen, azote, sulphur, phosphorus, selenium, carbon, boron, silicon.

The following table exhibits all the undecompounded bodies in alphabetical order, with their prime equivalent numbers, atomic weights, or reciprocal combining and saturating proportions, as given by Berzelius, in reference to oxygen, reckoned $100,000:-$ 
Table of undecompounded Bodies, or modern Chemical Elements.

A signifies Archæal : I, Inflimmable: M, Metal.

\begin{tabular}{|c|c|c|c|c|c|c|c|c|c|}
\hline Alumium & M. & $\cdot$ & $\bullet$ & 171,167 & Mercury & M. & • & & 1265,822 \\
\hline Antimony & - & • & & 806,542 & Molybdenum & - & . & . & 598,525 \\
\hline Arsenic & - & . & . & 470,012 & Nickel & - & . & . & 369,675 \\
\hline Azote & I. & ${ }^{\circ}$ & $\bullet$ & 88,518 & Osmium & - & . & . & 1244,210 \\
\hline Barium & M. & 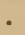 & . & 856,880 & Oxygen & A. & - & - & 100,000 \\
\hline Bismuth & - & . & . & 886,000 & Palladium & M. & . & - & 665,840 \\
\hline Boron & I. & . & • & 135,983 & Phosphorus & I. & - & - & 196,155 \\
\hline Bromine & A. & . & . & 489,150 & Platinum & M. & . & . & 1233,260 \\
\hline Cadmium & M. & . & • & 696,970 & Rhodium & - & . & - & 651,400 \\
\hline Calcium & - & . & . & 256,019 & Selenium & I. & . & . & 494,582 \\
\hline Carbon & I. & . & . & 76,437 & Silicon & - & . & . & 277,478 \\
\hline Cerium & M. & . & . & 574,718 & Silver & M. & . & . & 675,804 \\
\hline Chlorine & A. & . & . & 221,325 & Strontium & - & . & - & 547,285 \\
\hline Chromium & M. & . & . & 351,819 & Sulphur & I. & . & . & 201,165 \\
\hline Cohalt & - & . & . & 369,991 & Tantalum & M. & - & . & 153,715 \\
\hline Copper & - & . & - & 395,695 & Tellurium & - & . & . & 801,760 \\
\hline Fluorine & I. & . & . & 116,900 & Thorinum & - & . & . & 744,900 \\
\hline Gold & M. & . & . & 1243,013 & Tin & - & . & . & 735,294 \\
\hline Hydrogen & I. & - & - & 62,398 & Titanium & - & . & . & 803,686 \\
\hline Iodine & A. & . & . & 789,145 & Tungsten & - & - & . & 1183,000 \\
\hline Iridium & M. & . & . & 1233,260 & Uranium & - & . & . & 2711,360 \\
\hline Iron & - & . & . & 339,213 & Vanadium & - & . & . & $85 \check{5}, 84$ \\
\hline Lead & - & $\theta^{\circ}$ & . & 1294,489 & Yttrium & - & - & . & 401,840 \\
\hline Lithium & - & . & . & 81,320 & Zine & - & . & • & 403,2 \\
\hline Màgnesium & - & . & . & 158,353 & Zirconium & - & . & . & 420,23 \\
\hline Manganesium & - & . & . & 345,900 & & & & & \\
\hline
\end{tabular}

ELUTRIATE. (Soutirer, Fr.; Schlemmen, Germ.) When an insoluble pulverulent matter, like whitening or ground flints, is diffused through a large body of water, and the mixture is allowed to settle for a little, the larger particles will subside. If the supernatant liquid be now carefully decanted, or run off, with a syphon, it will contain an impalpable powder, which on repose will collect at the bottom, and may be taken out to dry. This process is called elutriation.

E\BALIIING (Embaument, Fr.; Einbalsamen, Germ.) is an operation in which balsams (baumes, $\mathrm{Fr}$.) were employed to preserve human corpses from putrefaction; whence the name.

The ancient Eqyptians had recourse to this process for preserving the bodies of numerous families, and eren of the animals which they loved or worshipped. An excellent account of their methods is giren in Mr. Pettigrew's work upon Mummies. Modern chemistry has made us acquainted with many means of counteracting putrefaction more simple and efficacious than the Egyptian system of salting, smoking, spicing, and bituminizing. See Putrefaction.

EMBOSSING WOOD. (Bossage, Fr.; Erhabenes Arbeit, Germ.) Raised figures upon wood, such as are employed in picture frames and other articles of ornamental cabinet work, are usually produced by means of carving, or by casting the pattern in plaster of Paris, or other composition, and cementing, or otherwise fixing it on the surface of the wood. The former mode is expensive; the latter is inapplicable on many occasiors. The invention of Mr. Streaker may be used either by itself, or in aid of carving ; and depends on the fact, that if a depression be made by a blunt instrument on the surface of the wood, such depressed part will again rise to its original level by subsequent immersion in the water.

The wood to be ornamented having been first worked out to its proposed shape, is in a state to receive the drawing of the pattern; this being put on, a blunt steel tool, or burnislier, or die, is to be applied successively to all those parts of the pattern intended to be in relief, and, at the same time, is to be driven very cautiously, without breaking the grain of the wood, till the depth of the depression is equal to the intended prominence of the figures. The ground is then to be reduced by planing or filing to the level of the depressed part; after which, the piece of wood being placed in water, either hot or cold, the part previously depressed will rise to its former height, and will then form an embossed pattern, which may be finished by the usual operations of carving.

For this invention the Society of Arts voted to Mr. Streaker their silver Isis medal and ten guineas.

EMBOSSING CLOTH. Mr. Thnmas Greig, of Rose Bank, near Bury, patented an invention, in November, 1835, which consists in an ingenious construction of machinery 
for both embossing and printing silk, cotton, woollen cloth, paper, and other fabrics, in one or more colors, at one operation.

Figs. $370,370^{*}$ represent three distinct printing cylinders of copper, or other suitable

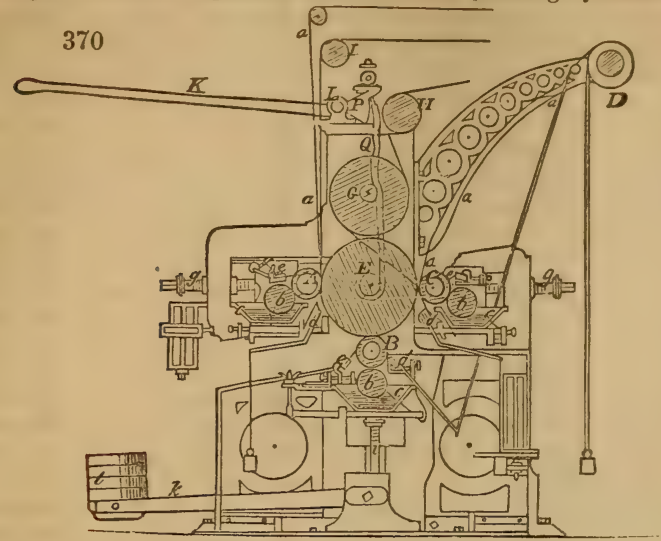
material, A, B, c, with their necessary appendanges for printing three different colors upon the fabric as it passes through the machine: either of these cylinders A, B, or C, may be employed as an embossing cylinder, without performing the printing process, or may be made to effect both operations at the same time.

The fabric or goods to be operated upon being first wound tightly upon a roller, that roller is to be mounted upon an axle or pivot, bearing in arms or brackets at the back of the machine, as shown at D. From this roller the fabric

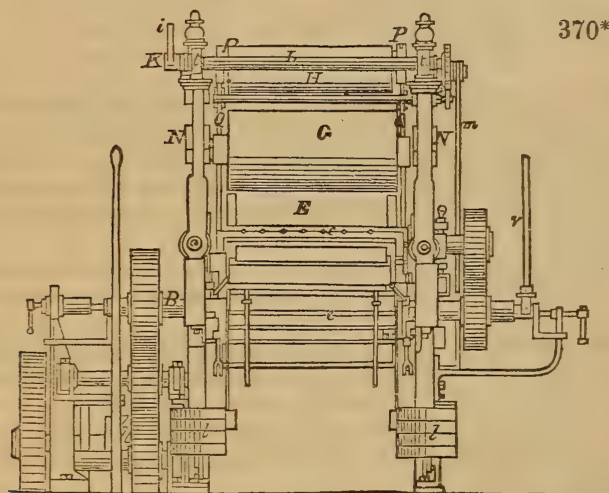
a $a$ a $a$ is conducted between tension rails, and passed under the bed cylinder or paper bowl $\mathbf{E}$, and from thence proceeds over a carrier roller $\boldsymbol{F}$, and over steam boxes not shown in the drawing, or it may be conducted into a hot room, for the purpose of drying the colors.

The cylinders A, B, and $\mathrm{c}$, having neither engraved or raised surfaces, are connected to feeding rollers $b \quad b \quad b$, revolving in the ink or colored troughs $c c c$; or endless ts, called sieves, may be employed, as in ordinary printing machines, for supplying the color, when the device on the surface of the cylinders is raised: these cylinders may be furnished with doctors or scrapers when required, or the same may be applied to the endless felts.

The blocks have adjustable screws $g \mathrm{~g}$, for the purpose of bringing the cylinders up against the paper bowl, with any required degree of pressure : the cylinder в is supported by its gudgeons running in blocks, which blocks slide in the lower parts of the side frames, and are connected to perpendicular rods $i$, having adjustable screw nuts.

The lower parts of these rods bear upon weighted levers $k k$, extending in front of the machine; and by increasing the weights $l l$, any degree of upward pressure may be given to the cylinder $\mathbf{B}$.

The color boxes or troughs $c c c$, carrying the feeding rollers $b b b$, are fixed on boards which slide in grooves in the side frames, and the rollers are adjusted and brought into contact with the surface of the printing cylinders by serews.

If a back cloth should be required to be introduced between the cylindrical bed or paper bowl $\mathrm{E}$, and the fabric $a a a$, as the ordinary felt or blanket, it may, for printing and embossing cotton, silk, or paper, be of linen or cotton; but if woollen goods are to be operated upon, a cap of felt, or some such material, must be bound round the paper bowl, and the felt or blanket must be used for the back cloth, which is to be conducted over the rollers $\mathrm{H}$ and $\mathrm{l}$.

For the purpose of embossing the fabric, either of the rollers $A, B$, or $c$, may be 
employed, observing that the surface of the roller must be cut, so as to leave the pattern or device elevated lor embossing relvets, plain cloths, and papers; but for woollens the levice must be excavated, that is, cut in recess.

The pattern of the embossing cylinder will, by the operation, be partially marked throush the fabric on to the surface of the paper bowl $E$; to obliterate which marks from the surface of the bowl, as it revolves, the iron cylinder roller $\mathrm{G}$ is employed; but as in the embossing of the same patterns on paper, a counter roller is required to produce the pattern perfectly, the iron roller is in that case dispensed with, the impression given to the paper bowl being required to be retained on its surface until the operation is finished.

In : 1 is case the relative circumferences of the embossing cylinder, and of the paper bowl, must be exactly proportioned to each other; that is, the circumference of the bowl must be equal, exactly, to a given number of circumferences of the embossing cylinder, very accurately measured, in order to preserve a perfect register or coincidence, as they continue revolving between the pattern on the surface of the embossing cylinder, and that indented into the surface of the paper bowl.

The axle of the paper bowl $E$, turns in brasses fitted into slots in the side frames, and it may be raised by hand fiom its bearings when required, by a lever $k$, extending in front. This lever is atfixed to the end of a horizontal shaft $x, L$, crossing the machine seen in the figures, at the bac.i of which shaft there are two segment levers $\mathrm{P}, \mathrm{P}$, to which bent rods $Q, Q$, are attached, having hooks at their lower ends, passed under the axle of the buwl. At the reverse end of the shaft $L$, a ratchet-wheel $r$, is affixed, and a pall or click mounted on the side of the frame takes into the teeth of the wheel $r$, and thereby holds up the paper bowl when required.

When the iron roller $\mathrm{G}$, is to be brought into operation, the vertical screws $t$, $t$, mounted in the upper parts of the side frames, are turned, in order to bring down the brasses $\mathrm{N}$, which carry the axle of that roller and slide in slots in the side frames.

The cylinders $\mathrm{A}, \mathrm{B}$, and $\mathrm{C}$, are represented hollow, and may be kept at any desired temperature during the operation of printing, by introducing steam into them; and under the color boxes $c, c, c$, hollow chambers are also made for the same purpose. The degree of temperature required to be given to these must depend upon the nature of the coloring material, and of the goods operated upon. For the purpose of conducting steam to these hollow cylinders and color boxes, pipes, as shown at $v, v, v$, are attached, which lead from a steam boiler. But when either of these cylinders is employed for embossing alone, or for embossing and printing at the same time, and particularly for some kinds of goods where a higher temperature may be required, a red-hot heater is then introduced into the hollow cylinder in place of steam.

If the cylinder $\mathbf{B}$ is employed as the embossing cylinder, and it is not intended to print the fabric by that cylinder simultaneously with the operation of embossing, the feeding roller $b$, must be removed, and also the color box $c$, belonging to that cylinder; and the cylinders $\mathrm{A}$ and $\mathrm{c}$, are to be employed for printing the fabric, the one applying the color before the embossing is effected, the other after it. It is however to be remarked, that if $\mathrm{A}$, and $\mathrm{C}$, are to print colors on the fabric, and $\mathrm{B}$ to emboss it, in that case it is preferred, where the pattern would allow it. A and c, are wooden rollers having the pattern upon their surfaces, and not metal, as the embossing cylinders must of necessity be.

It will be perceived that this machine will print one, two, or three colors at the same time, and that the operation of embossing may be performed simultaneously with the printing, by eithe: of the cylinders $\mathrm{A}, \mathrm{B}$, or $\mathrm{C}$, or the operation may be performed consecutively by the cylinlers, either preceding or succeeding each other.

The situations of the doctors, when required to be used for removing any superfluous color from the surface of the printing cylinder, are shown at $d, d, d$; those for removing any lint which may attach itself, at $e, e, e$. They are kept in their bearings by weighted levers and screws, and receive a slight lateral movement to and fro, by means of the vertical rod $m$, which is connected at top to an eccentric, on the end of the axle of the roller $\mathbf{E}$, and at its lower end to a horizontal rod mounted at the side of the frame; to this horizontal rod, arms are attached, which are connected to the respective doctors; and thus, by the rotation of the eccentric, the doctors are made to slide laterally.

When the cylinders $\mathrm{A}, \mathrm{B}$, or $\mathrm{c}$, are employed for embossing only, those doctors will not be required. The driving power is communicated to the machine from any first mover through the agency of the toothed gear, which gives rotatory motion to the cylinder $B$, and from thence to the other cylinders $\mathrm{A}$, and $\mathrm{c}$, by toothed gear shown in fig. 370 .

E.MBROIDERING MACHINE. (Machine à broder, Fr.; Steckmaschine, Germ.) This art has been till of late merely a handicraft employment, cultivated on account of its elegance by ladies of rank. But a few years ago M. Heilmann of Mulhause invented a machine of a most ingenious kind, which enables a female to embroider any design with 80 or 140 needles as accurately and expeditionsly as she formerly could do with one. A brief 
account of this remarkable invention will therefore be acceptable to many readers. It was displayed at the national exposition of the products of industry in Paris for 1834, and was unquestionably the object which stood highest in public esteem; for whether at rest or in notion, it was always surrounded with a crowd of curious visiters, admiring the figures which it had formed, or inspecting its movements and investigating its mechanism. 130 neelles were occupied in copring the same pattern with perfect regularity, all set in motion by one person.

Several of these machines are now mounted in France, Germanr, and Switzerland. I have seen one factory in Manchester, where a great many of them are doing beantifui work.

The price of a machine having 130 needles, and of consequence 260 pincers or fingers and thumbs to lay hold of them, is 5000 francs, or 200l. sterling; and it is estimated to do daily the work of 15 expert hand embroiderers, employed upon the ordinary frame. It requires mereiy the labor of one grown-up person, and two assistant chsldren. The operative must be well taught to use the machine, for he has many things to attend to; with the one hand he traces out, or rather follows the design with the point of the pantograph: with the other he turns a handle to plant and pull all the needles, which are seized by pincers and mored along by carriages, approaching to and receding from the web, roliing all the time along an iron railway; lastly, by means of two pedals, upon which he presses alternately with the one foot and the other, he opens the 130 pincers of the first carriage, which ought to give up the needles after planting them in the stuff, and he shuts with the same pressure the 130 pincers of the second carriage, which is to receire the needles, to draw them from the other side, and to bring them back again. The children hare nothing else to do than to change the needles when all their threads are used, and to see that no needle misses its pincers.

This machine deserves particular attention, because it is no less remarkable for the happr arrangement of its parts, than for the effects which it produces. It may be described under four heads: 1 . the structure of the frame: 2. the disposition of the web; 3 . the arrangement of the carriages; and 4. the construction of the pincers.

1. The structure of the frame. It is composed of cast-iron, and is very massive. Fig. $3 i 1$ exibits a front elevation of it. The length of the machine denends upon the number of pincers to be morked. The model at the exposition had 260 pincers, and was 2 metres and a half (about 100 inches or 8 feet 4 inches English) Jong. The figure here given has been shortened considerably, but the other proportions are not disturbed. The breadth of the frame ought to be the same for erery machine, whether it be long or short, for it is the breadth which determines the length of the thread to be put into the needles, and there is an adrantage in giring it the full breadth of the model machine, fully 100 inches, so that the needles mat carry a thread at least $t 0$ inches long.

Disposition of the piece to be embroidered. - We have already stated that the pincers which hold the needles always present themselres opposite to the same point, and that in consequence they would continually pass oachwards and forwards through the same hole, but the piece is displaced with sufficient precision to bring opposite the tips progressively of the needles. erery point upon which thes are to mork a design, such as a flower.

The piece is strained perpendicularly upon a large rectangular frame, whose four sides are visible in $f i g .3 \% 1$; namely, the two rertical sides at $F \bar{F}$, and the two horizontal sides, the upper and lower at $F^{\prime} F^{\prime \prime}$. Te see also in the figure two long wooden rollers G and G, whose ends, mounted with iron studs, are supported upon the sides $F$ of the frame, so as to turn freely. These form a system of beams upon which the piece destined to receive the embroidery, is wound and hept rerticalls stretched to a proper degree, for each of these beams k:ars upon its end a small ratchet wheel $g, g$ : the teeth of one of them being inclined in the opposite direction to those of the other. Besides this srstem of lower beams, there is anotiner of two upper beams, which is howerer but imperfectly seen in the figure, on account of the interference of other parts in this riem of the machine. One of these sistems presents the web to the inferior needles, and the other to the upper needles. As the two beams are not in the same rertical plane, the plane of the web would be presented obliquely to the needles were it not for a straight bar of iron, round whose edge the cloth passes, and which renders it rertical. The piece is lept in tension crosswise by small brass templets, to which the strings $g^{\prime \prime}$ are attached, and by which it is pulled towards the sides of the frame $F$. It remains to show by what ingenious means this frame may be shifted in erery possible direction. M. Heilmann has em. ploted for this purpose the pantograph which draughtsmen use for reducing or enlarging their plans in determinate proportions.

$b b^{\prime} f^{\prime \prime} b^{\prime \prime}\left(f i g .3 \sim 1\right.$ ) represents a parallelogram of which the four angles $b b^{\prime}, f b^{\prime \prime}$, are jointed in such a way that they may become very acute or very obtuse at pleasure, while the sides of course continue of the same length; the sides $\dot{b}, b^{\prime}$ and $b, b^{\prime \prime}$ are prolonged, the one to the point $d$, and the other to the point $c$, and these points $c$ and $d$, 
are chosen under the condition that in one of the positions of the parallelogram, the line $c d$ which joins them passes through the point $f$; this condition may be fulfilled in

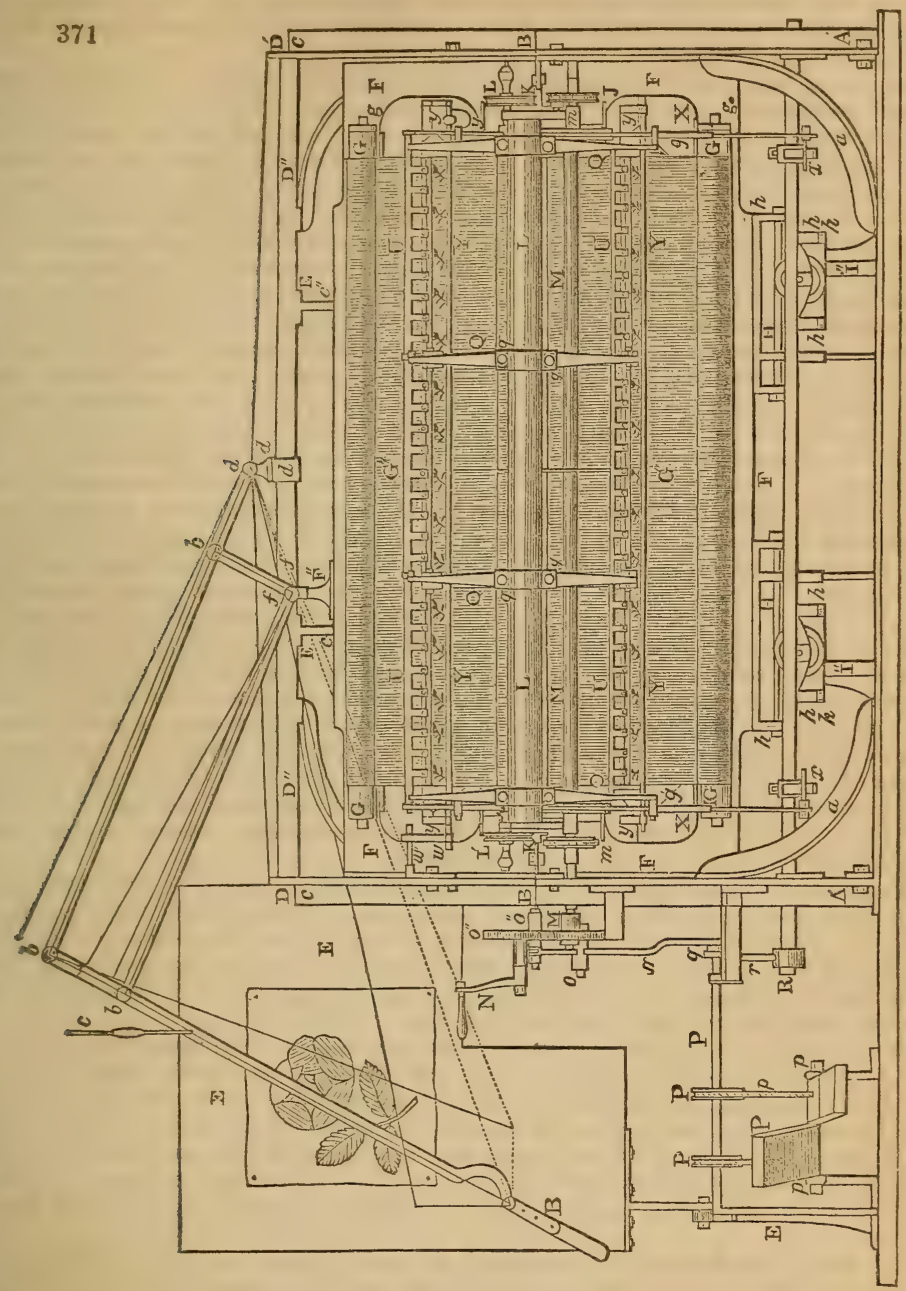

an infinite number of manners, since the position of the parallelogram remaining the same, we sce that if we wished to shift the point $d$ further from the point $b^{\prime}$, it would be sufficient to bring the point $c$ near enough to $b^{\prime \prime}$, or vice versa; but when we have once fixed upon the distance $b^{\prime} d$, it is evident that the distance $b^{\prime \prime} c$ is its necessary consequence. Now the principle upon which the construction of the pantograph rests is this; it is sufficient that the three points $d, f$, and $c$ be in a straight line, in one only of the positions of the parallelogram, in order that they shall remain always in a straight line in every position which can possibly be given to it.

We see in the figure that the side $b c$, has a handle $\mathbf{B}^{\prime \prime}$ with which the workman puts the machine in action. To obtain more precision and solidity in work, the sides of the pantograph are joined, so that the middle of their thickness lies exactly in the vertical plane of the piece of goods, and that the axes of the joints are truly perpendicular to this plane, in which consequently all the displacements are effected. We arrive at this result by making fast to the superior great cross bar $\mathrm{D}^{\prime \prime}$ an elbow piece $d^{\prime \prime}$, having a suitable projection, and to which is adapted in its turn the piece $d^{\prime}$, which receives in a 
socket the extremity of the side $\delta, a$; this piece $d^{\prime}$ is made fast to $d^{\prime \prime}$ by a bolt, but it carries an oblong hole, and before screwing up the nut, we make the piece advance or recele, till the fulcrum point comes exactly into the plane of the web. This condition being fulfilled, we have mereiy to attach the frame to the angle $f$ of the parallelogram, which is done by means of the piece $F^{\prime \prime}$.

It is now obvious that if the embroiderer takes the handle $\mathbf{B}^{\prime \prime}$ in his hand and makes the pantograph move in any direction whatever, the point $f$ will describe a figure similar to the figure described by the point $c$, and six times smaller, but the point $f$ cannot move without the frame, and whatever is upon it moving also. Thus, in the movement of the pantograph, every point of the web describes a figure equal to that described by the point $f$, and consequently similar to that described by the point $c$, but six times smaller; the embroidered object being produced upon the cloth in the position of that of the pattern. It is sufficient therefore to give the embroidering operative who holds the handle $\mathrm{B}^{\prime \prime}$, a design six times greater than that to be executed by the machine, and to afford him at the same time a sure and easy means of tracing over with the point $c$, all the outlines of the pattern. For this purpose he adapts to $c$, perpendicularly to the plane of the parallelogram, a small style terminated by a point $c^{\prime}$, and he fixes the pattern upon a vertical tablet $E$, parallel to the plane of the stuff and the parallelogram, and distant from it only by the length of the style $c \mathrm{c}^{\prime \prime}$; this tablet is carried by the iron rod $e^{\prime}$, which is secured to a cast iron foot $\mathrm{E}^{\prime}$, serving also for other purposes, as we shall presently see. The frame loaded, with its beams and its cloth forms a pretty heavy mass, and as it must not swerve from its plane, it needs to be lightened in order that the operative may cause the point of the pantograph to pass along the tablet without straining or uncertainty in its movemenis. M. Heilmann has accomplished these objects in the following way. A cord $e$ attached to the side $b c$ of the pantograph passes over a return pulley, and carries at its extremity, a weight which may be graduated at pleasure; this weight equipoises the pantograph, and tends slightly to raise the frame. The lower sile of the frame carries two rods $\mathrm{H}$ and $\mathrm{H}$, each attached by two arms $h h$, a little bent to the left; both of these are engaged in the grooves of a pulley. Through this mechanism a pressure can be exercised upon the frame from below upwards, which may be regulated at pleasure, and without preventing the frame from moving in all directions, it hinders it from deviating from the primitive plane to which the pantograph was adjusted. The length of the rods $H$ ought to be equal to the amount of the lateral movement of the frame. Two guides $i i$ carried by two legs of cast iron, present vertical slits in which the lower part of the frame $F^{\prime}$ is engaged.

Disposition of the carriages.-The two carriages, which are similar, are placed the one to the right, and the other to the left of the frame. The carriage itself is composed merely of a long hollow cylinder of cast iron $L$, carrying at either end a system of two grooved castors or pulleys $L^{\prime}$, which roll upon the horizontal rails $\mathrm{K}$; the pulleys are mounted upon a forked piece $l^{\prime}$, with two ends to receive the axes of the pulleys, and the piece $l$ is itself bolted to a projecting ear $l$ cast upon the cylinder.

This assemblage constitutes properly speaking the carriage, resting in a perfectly stable equilibrium upon the rails $\mathrm{k}$, upon which it may be most easily moved backwards and forwards, carrying its train of needles to be passed or drawn through the eloth.

M. Heilmann has contrived a mechanism by which the operative without budging from his place may conduct the carriages, and regulate as he pleases the extent of their course, as well as the rapidity of their movements. By turning the axes $\mathrm{s}^{\prime \prime}$ in the one direction or the other, the carriage may be made to approach to, or recede from the web.

When one of the carriages has advanced to prick the needles into the stuff, the other is there to receive them; it lays hold of them with its pincers, pulls them through, performs its course by withdrawing to stretch the thread, and close the stitch, then it goes back with the needles to make its pricks in return. During these movements the first carriage remains at its post waiting the return of the second. Thus the two chariots make in succession an advance and a return, but they never move together.

To effect these movements M. Heilmann has attached to the piece $o^{\prime}$ made fast to the two uprights A $\mathrm{C}$ and A D of the frame, a bent lever $n 0 n^{\prime} n^{\prime \prime}$ moveable round the point 0 ; the bend $n^{\prime}$ carries a toothed wheel $o^{\prime}$, and the extremity $n^{\prime \prime}$ a tonthed wheel $o^{\prime \prime}$; the four wheels $M \mathrm{M}^{\prime} \mathrm{o}^{\prime}$ and $\mathrm{o}^{\prime \prime}$ have the same number of teeth and the same diameter; the two wheels $o^{\prime}$ and $o^{\prime \prime}$ are fixed in reference to each other, so that it is sufficient to turn the handle $N$ to make the wheel $\sigma^{\prime \prime}$ revolve, and consequently the wheel $o^{\prime}$; when the lever $n o$ is vertical, the wheel $o^{\prime}$ touches neither the wheel $M$ nor the wheel $\mathrm{m}^{\prime}$; but if it be inclined to the one side or the other, it brings the wheel o' aiternately into gear with the wheel $\mathrm{m}$ or the wheel $\mathrm{sI}^{\prime}$. As the operative has his two hands occupied, the one with the pantograph and the other with the handle of impulsion, he has merely his feet for acting upon the lever $n o$, and as he has many other things to do, M. Heilmann has adapted before him a system of two pedals, by which he executes 
with his feet a series of operations no less delicate than those which he executes with his hands.

The pedals $\mathrm{P}$ are moveable round the axis $p$, and carry cords $p^{\prime}$ wound in an opposite direction "1pon the pulleys $\mathrm{P}^{\prime}$; these pulleys are fixed upon a moveable shaft $\mathrm{P}^{\prime \prime}$, supported upon one side by the prop $\mathrm{E}^{\prime}$, and on the other in a piece $\mathbf{K}^{\prime}$ attached to the two great uprights of the frame. In depressing the pedal $\mathbf{P}$ (now raised in the figure), the upper part of the shaft $\mathrm{P}^{\prime \prime}$ will turn from the left to the right, and the lever $n o$ will become inclined so as to carry the wheel $o^{\prime}$ upon the wheel $\mathrm{M}^{\prime}$, but at the same time the pedal which is now depressed will be raised, because its cord will be forced to wind itself upon its pulley, as much as the other cord has unwound itself; and thus the apparatus will be ready to act in the opposite direction, when wanted.

Disposition of the pincers.-The shaft $\mathrm{L}^{\prime}$ carries, at regular intervals of a semi-diameter, the appendages $q q$ cast upon it, upon which are fixed, by two bolts, the curved branches $Q$ destined to bear the whole mechanism of the pincers. When the pincers are opened by their appropriate leverage, and the half of the needle, which is pointed at each end, with the eye in the middle, enters the opening of its plate, it gets lodged in an angular groove, which is less deep than the needle is thick, so that when the pincers are closed, the upper jaw presses it into the groove. In this way the needle is firmly held, allhough touched in only three points of its circumference.

Suppose, now, that all the pincers are mounted and adjusted at their proper distances upon their prismatic bar, forming the upper range of the right carriage. For opening all the pincers there is a long plate of iron, $U$, capable of turning upon its axis, and which extends from the one end of the carriage to the other. This axis is carried by a kind of forks which are bolted to the extremity of the branches Q. By turning that axis the workman can open the pincers at pleasure, and they are again closed by springs. This movement is performed by his feet acting upon the pedals.

The threads get stretched in proportion as the carriage is run out, but as this tension has no elastic play, inconveniences might ensue which are prevented by adapting to the carriage a mechanism by means of which all the threads are pressed at the same time by a weight susceptible of graduation. A little beneath the prismatic bar, which carries the pincers, we see in the figure a shaft, $\mathbf{y}$, going from one end of the carriage to the other, and even a little beyond it ; this shaft is carried by pieces $y$ which are fixed to the arms $Q$, and in which it can turn. At its left end it carries two small bars $y^{\prime}$ and $w^{\prime}$, and at its right a single bar $y^{\prime}$, and a counterweight (not visible in this view); the ends of the two bars $y^{\prime}$ are joined by an iron wire somewhat stout and perfectly straight. When the carriage approaches the web, and before the iron wire can touch it, the little bar $w$ presses against a pin, $w^{\prime}$, which rests upon it, and tends to raise it more and more. In what has preceded we have kept in view only the upper range of pincers and needles, but there is an inferior range quite similar, as the figure shows, at the lower ends of the arms Q. In conclusion, it should be stated, that the operative does not follow slidingly with the pantograph the trace of the design which is upon the tablet or the picture, but he must stop the point of the style upon the point of the pattern into which the needle should enter, then remove it, and put it down again upon the point by which the needle ought to re-enter in coming from the other side of the piece, and so on in succession. To facilitate this kind of reading off, the pattern upon the tablet is composed of right lines terminated by the points for the entrance and return of the needle, so that the operative (usually a child) has continually under her eyes the series of broken lines which must be followed by the pantograph; if she happens to quit this path an instant, without having left a mark of the point at which she had arrived, she is under the necessity of looking at the piece to see what has been already embroidered, and to find by this comparison the point at which she must resume her work, so as not to leave a blank, or to repeat the same stitch.

\section{Explanation of figure.}

$\Lambda$, lower cross bars, which unite the legs of the two ends of the frame.

$a$, the six feet of the front end of the frame.

$a^{\prime}$, the six feet of the posterior end of the frame.

$a^{\prime \prime}$, curved pieces which unite the cross bars $\mathrm{A}^{\prime \prime}$ to the uprights.

$\mathbf{B}^{\prime \prime}$, handle of the pantograph.

$b b^{\prime} b^{\prime \prime}$, three of the angles of the pantograph.

$c$, point of the side $b b^{\prime \prime}$ on which the point is fixed.

$c^{\prime \prime}$, point of the pantograph.

$D^{\prime \prime}$, cross bar in form of a gutter, which unites the upper parts of the frame.

$d$, fixed point, round which the pantograph turns.

$\boldsymbol{E}$, tablet upon which the pattern to be embroidered is put.

$\boldsymbol{E}^{\prime}$, support of that tablet.

$e$, cord attached at one end to the side $b c$ of the pantograph passing over a guide pulley, and carrying a weight at the other end. 
$\mathbf{e}^{\prime}$, iron rod by which the table $\mathbf{E}$ is joined to its support $\mathbf{\Sigma}^{\prime}$.

$F$ F, uprights of the cloth-carrying frame.

$F^{\prime} F^{\prime}$, horizontal sides of the same frame.

$\mathrm{G}$, four roll beams.

$\mathbf{G}^{\prime \prime}$, the piece of cloth.

$\mathrm{g}^{\prime \prime}$, the strings, which serve to stretch the cloth laterally.

EMERALD (Emeraude, Fr.; Smaragd, Germ.) is a precious stone of a beautiful green color; valued next to diamond, and in the same rank as oriental ruby and sapphire. It occurs in prisms with a regular hexagonal base; sp. grav. 2.7; scratches quartz with difficulty; is scratched by topaz; fusible at the blowpipe into a frothy bead; the precipitate affurded by ammonia, frum its solution, is soluble, in a great measure, in carbonate of ammonia. Its analysis is given very variously by different chemists. It contains about 14 per cent. of glucina, which is its characteristic constituent; along with 68 of silica, 16 of alumina, a very little lime and iron. The beautiful emerald of Peru is found in a clay schist mixed with some calcareous matter. A stone of 4 grains weight is said to be worth from $4 l$. to $5 l$.; one of 8 grains, $10 l$. ; one of 15 grains, being fine, is worth $60 l$; one of 24 grains fetched, at the sale of M. de Drée's cabinet, 2400 franes, or nearly $100 l$.

The beryl is analogous in composition to the emerald, and is employed (when of the common opaque kind, found near Limoges) by chemists for procuring the earth glucina.

EMERY. This mineral was long regarded as an ore of iron; and was called by Haüy fer oxidé quartzifêre. It is very abundant in the island of Naxos, at cape Emeri, whence it is imported in large quantities. It occurs also in the islands of Jersey and Guernsey, at Almaden, in Poland, Saxony, Sweden, Persia, \&c. Its color varies from red brown to dark brown; its specific eravity is about 4.000 ; it is so hard as to scratch quartz and many precious stones. By Mr. Tenant's analysis it consists of alumina, 80 ; silica, 3 ; iron, 4 . Another inferior kind yielded 32 of iron, and only 50 of alumina.

The alumina of emery is believed to be aggregated to the same degree of hardness as in corundum or adamantine spar; which is one of the hardest minerals linown. Emery is extensively employed for grinding metals, glass, \&c.; for which purpose it is reduced to powders of different degrees of fineness, by grinding and elutriation. When so treated, it is sold under the name of flour of emery, or washed emery.

EMPYREUMA, means the offensive smell produced by fire applied to organic matters, chiefty vegetable, in close vessels. Thus, empyreumatic rinegar is oblained by distilling wood at a red heat, and empyreumatic oil from many animal substances in the same way.

ENAMELS (Emaux, Fr.; Schmelzglas, Germ.) are varieties of glass, generally opaque and colored, always formed by the combination of different metallic oxydes, to which certain fixed fusible salts are added, such as the borates, fluates, and phosphates.

The simplest enamel, and the one which serves as a basis to most of the others, is obtained by calcining first of all a mixture of lead and tin, in proportions varying from 15 to 50 parts of tin for 100 of lead. The middle term appears to be the most suitable for the greater number of enamels; and this alloy has such an affinity for oxygen, that it may be calcined with the greatest ease in a flat cast-iron pot, and at a temperature not above a cherry red, provided the dose of tin is not too great. The oxyde is drawn off to the sides of the melted metal according as it is generated, new pieces of the alloy being thrown in from time to time, till enough of the powder be obtained. Great care ought to be taken that no metallic particles be left in the oxyde. and that the calcining heat be is low as is barely sufficient; for a strong fire frits the powder, and obstructs its subsexuent comminution. The powder when cold is ground in a proper mill, levigated with water, and elutriated, as will be described under Red lead. In this state of fineness and purity, it is called calcine, or flux, and it is mixed with silicious sand and some alkaline matter or sea-salt. The most ordinary proportions are, 4 of sand, 1 of sea-salt, and 4 of calcine. Chaptal states that he has obtained a very fine product from 100 parts of calcine, made by calcining equal parts of lead and tin, 100 parts of ground flint, and 200 parts of pure subcarbonate of potash. In either case, the mixture is put into a crucible, or laid simply on a stratum of sand, quicklime spontaneously slaked, or wood-ashes, placed under a pottery or porcelain kiln. This mass undergoes a semi-vitrification; or even a complete fusion on its surface. It is this kind of frit which serves as a radical to almost every enamel; and by varying the proportions of the ingredient, more fusible, more opaque, or whiter enamels are obtained. The first of these qualities depends on the quantity of sand or flux, and the other two on that of the tin.

The sea-salt employed as a flux may be replaced either by salt of tartar, by pure potash, or by soda; but each of these fluxes gives peculiar qualities to the enamel.

Most authors who have written on the preparation of enamels, insist a great deal on 
the necessity of selecting carefully the particular sand that should enter into the composi tion of the firit, and they even affirm that the purest is not the most suilable. Clouet states, in the 34 th volume of the Innales de Chimie, that the sand ought to contain at least 1 part of tale for 3 of silicious matter, otherwise the enamel obtained is never very glassy, and that some wrinkled spots from imperfect fusion are seeen on its surface; and jet we fiml prescribed in sume old treatises, to make use of ground flints, frilted by ineans of salt of tartar or some other flux. It would thence appear that the presence of talc is of no use towards the fusibility of the silica, and that its absence may be supplied by increasing the duse of the flux. In all cases, however, we ought to beware of metallic oxyles in the sand, particularly those of iron and manganese, which most frequently occur, and always injure the whiteness of the frit.

The aticients arried the art of enamelling to a very high perfection, and we occasionally find beautiful specimens of their work, of which we know neither the composition, nor the manner of applying it. Then, as at present, each artist made a mystery of the mean: that strcceeded best with ?im, and thus a multitude of curious processes have been buried with their authors. Another cause contributes powerfully to this sort of declension in the arts. Among the vast number of recipes which have been published for the formation of enamels, there are several in which substances are mentioned that can no longer be procured. whether owing to a change of denomination, or becanse the substances cannot now be found in commerce, or becanse they are not of the same nature as of old. Hence, in many cases, we find it impossible to obtain satisfactory results. What we have now said renders it desirable that the operations should be resumed anew, or upon new bases, and availing ourselves of all the known chemical facts, we should employ in the proluction of enamels, raw materials of the purest kind.

The Tenetians are still in possession of the best enamel processes, and they supply the French and other nations with the best kinds of enamel, of every colored shade.

Enamels are distinguished into transparent and opaque; in the former all the elements have experienced an equal degree of liguefaction, and are thus run into crystal glass, whilst in the others, some of their elements have resisted the action of heat more, so that their particles retain sufficient aggregation to prevent the transmission of light. This eflect is produced, particularly by the oxyde of tin, as we shall perceive in treating of white enamel.

The frits for enamels that are to be applied to metallic surfaces require greater fusibility, an $l$ should therefore contain more flux; and the sand used for these should be calcined beforehan 1 with one fourth its weight of sea-salt; sometimes, indeed, metallic fluxes are added, as minium or litharge. For some metallic colors, the oxydes of lead are very injurious, and in this case recourse must be had to other fluxes. Clouet states that he had derivel adrantage from the following mixtures, as bases for purples, blues, and some other delicate colors :-

Three parts of silicious sand, one of chalk, and three of calcined borax; or, three of glass (of broken crystal goblets), one of calcined borax, one fourth of a part of nitre, and one part of well washed diaphoretic antimony. These compositions afford a very white enamel, which accords perfectly well with blue.

It is obvious that the composition of this primary matter may be greatly varied; but we should never lose sight of the essential quality of a good enamel; which is, to acquire, at a molerate heat, sufficient fluidity, to take a shining surface, without running 100 thin. It is not complete fusion which is wanted; but a pasty state, of such a degree as may give it, after cooling, the aspect of having suffered complete liquefaction.

Deat-whit. Enamel.-This requires greater nicety in the choice of its materials than any other enamel, as it must be free from every species of tint, and be perfectly white; hence the frit employed in this case should be itself composed of perfectly pure ingredients. But a frit should not be rejected hastily because it may be somewhat discolored, since this may depend on two causes; either on some metallic oxydes, or on fuliginous particles proceeding from vegetable or animal substances. Now the latter impurities may be easily removed by means of a small quantity of peroxyde of manganese, which has the property of readily parting with a portion of its oxygen, and of thus facilitating the combustion, that is to say, the destruction of the coloring carbonaceous matter. Manga. nese indeed possesses a coloring power itself on glass, but only in its highest state of oxydizement, and when reduced to the lower state, as is done by incombustible matters, it no lunger communicates color to the enamel combinations. Hence the proportion of manganese should never exceed what is just; for the surplus would cause color. Sometimes, inleed, it becomes necessary to give a little manganese cclor, in order to obtain a more agreeable shaile of white; as a little azure blue is added to linens, to brighten or counteract the dulness of their yellow tint.

A white enamel may be conveniently prepared also with a culcine composed of two parts of tin and one of lead calcined together; of this combined oxyde, one part is melted with two parts of fine crystal and a very little manganese, all previously ground together. 
When the fusion is complete, the vitreous matter is to be poured into clear water, and the frit is then dried, and melted anew. The pouring into water and fusion are sometimes repeated four times, in order to secure a very uniform combination. The crucible must be carefully screened from smoke and flame. The smallest portions of oxyde of iron or copper admitted into this enamel will destroy its value.

Some practitioners recommend the use of washed diaphoretic antimony (antimoniate of potash, from metallic antimony and nitre deflagrated together) for white enamel; but this product cannot be added to any preparation of lead or other metallic oxydes; for it would tend rather to tarnish the color than to clear it up; and it car be userl therefore only with ordinary glass, or with saline fluxes. For three parts of white glass (without lead) one part of washed diaphoretic antimony is to be taken; the substances are well ground together, and fused in the common way.

Blue enamel. - This fine color is almost always obtained from the oxyde of cobalt or some of its combinations, and it produces it with such intensity that only a very little can be used, lest the shade should pass into black. The cobalt blue is so rich and lively that it predominates in some measure over every other color, and masks many so that they can hardly be perceived; it is also most easily obtained. To bring it out, however, in all its beauty, the other colors must be removed as much as possible, and the cobalt itself should be tolerably pure. This metal is associated in the best known ores with a considerable number of foreign substances, as iron, arsenic, copper, nickel, and sulphur, and it is difficult to separate them completely; but for enamel blues, the oxyde of cobalt does not require to be perfectly free from all foreign metals; the iron, nickel, and copper, being most prejudicial, should be carefully eliminated. This object may be most easily attained by dissolving the ore in nitric acid, evaporating the solution to a sirupy consistence, to expel the excess of acid, and separate a portion of arsenic. It is now diluted with water, and solution of carbonate of soda is dropped slowly into it with brisk agitation, till the precipitate, which is at first of a whitish gray, begins to turn of a rose-red. Whenever this color appears, the whole must be thrown on a filter, and the liquid which passes through must be treated with more of the carbonate of soda, in order to obtain the arseniate of cobalt, which is nearly pure. Since arsenic acid and its derivatives are not capable of communicating color themselves, and as they moreover are volatile, they cannot impair the beaity of the blue, and hence this preparation affords it in great perfection.

Metallic fluxes are not the most suitable for this color; because they always communicate a tint of greater or less force, which never fails to injure the purity of the blue. Nitre is a useful addition, as it keeps the oxyde at the maximum of oxydation, in which state it produces the richest color.

Yellow Enamel.-There are many processes for making this color in enamel; but it is somewhat difficult to fix, and it is rarely obtained of a uniform and fine tint. It may be produced directly with some preparations of silver, as the phosphate or sulphate; but this method does not always succeed, for too strong a heat or powerful fluxes readily destroy it, and nitre is particularly prejudicial. This uncertainty of success with the salts of silver causes them to be seldom employed; and oxydes of lead and antimony are therefore preferred, which afford a fine yellow when combined with some oxydes that are refractory enough to prevent their complete vitrification. One part of white oxyde of antimony may be taken with from one to three parts of white lead, one of alum, and one of sal-ammoniac. Each of these substances is to be pulverized, and then all are to be exactly mixed, and exposed to a heat adequate to decompose the sal-ammoniac. This operation is judged to be finished when the yellow color is well brought out. There is produced here a combination quite analogous to that known under the name of Naples yellow.

Other shades of yellow may be procured either with the oxyde of lead alone, or by adding to it a little red oxyde of iron; the tints varying with the proportion of the latter.

Clonet says, in his memoir on enamels, that a fine yellow is obtained with pure oxyde of silver, and that it is merely necessary to spread a thin coat of it on the spot to be colored. The piece is then exposed to a moderate heat, and withdrawn as soon as this has reached the proper point. The thin film of metallic silver revived on the surface being removed, the place under it will be found tinged of a fine yellow, of hardiy any thickness. As the pellicle of silver has to be removed which covers the color, it is requisite to avoid fixing this film with fluxes; and it ought therefore to be applied after the fusion of the rest. The yellows require in general little flux, and they answer better with one of a metallic nature.

Green Enamel.-It is known that a green color may be produced by a mixture of yellow and blue; but recourse is seldom had to this practice for enamels, as they can be obtain. ed almost always directly with the oxyde of copper; or still better with the oxyde of ehrome, which has the advantage of resisting a strong heat. 
Chemists describe two oxydes of copper, the protoxyde, of an orange red color, which communicates its color to enamels, but it is difficult to fix; the deutoxyde is blue in the state of hydrate, but blackish-brown when dry, and it colors green all the vitreous combinations into which it enters. This oxyde requires, at most, one or two proportions of flux, either saline or metallic, to enter into complete fusion; but a much smaller dose is commonly taken, and a little oxyde of iron is introduced. To four pounds of frit, for instance, two ounces of oxyde of copper and 48 grains of red oxyde of iron are used; and the ordinary measures are pursued for making very homogeneous enamel.

The green produced by the oxyde of chrome is much more solid; it is not affected by a powerful fire, but it is not always of a fine shade. It generally inclines too much to the deail-leaf yellow, which depends on the degree of oxygenation of the chrome.

Rel Enumel.-We have just stated that protoxyde of copper afforded a fine color when it could be fixed, a result difficult to obtain on account of the fugitive nature of this oxyde; slight variations of temperature enabling it to absorb more oxygen. The proper point of fusion must be seized, for taking it from the fire whenever the desired color is brought out. Indeed, when a high temperature has produced peroxydizement, this may be corrected by adding some combustible matter, as charcoal, tallow, tartar, \&cc. The copper then returns to its minimum of oxydizement, and the red color which had vanished, reappears. It is possible, in this way, and by pushing the heat a little, to accomplish the complete reduction of a part of the oxyde; and the particles of metallic copper thereby disseminated in a reddish ground, give this enamel the aspect of the stone called avanturine. The surest and easiest method of procuring protoxyde of copper is to boil a solution of equal parts of sugar, and sulphate or rather acetate of copper, in four parts of water. The sugar takes possession of a portion of the oxygen of the cupreous oxyde, and reduces it to the protoxyde; when it may be precipitated in the form of a granular powder of a brilliant red. After about two hours of moderate ebullition, the liquid is set aside to settle, decanted off the precipitate, which is washed and dried.

This pure oxyde, properly employed by itseif, furnishes a red which vies with the finest carmine, and by its means every tint may be obtained from red to orange, by adding a greater or smaller quantity of peroxyde of iron.

The preparations of gold, and particularly the oxyde and purple of Cassius, are likewise employel, with advantage, to color enamel red, and this composition resists a powerful fire tolerably well. For some time back, solutions of gold, silver, and platinum have been usel with success instead of their oxydes; and, in this way, a more intimate mixture may be procured, and, consequently, more homogeneous tints.

Blick Enamel.-Black enamels are made with peroxyde of manganese or protoxyde of iron; to which more depth of color is given with a little cobalt. Clay alone, melted with about a third of its weight of protoxyde of iron, gives, according to Clouet, a fine black enamel.

Violet Enamel.-The peroxyde of manganese in small quantity by itself furnishes, with saline or alkaline fluxes, an enamel of a very fine violet hue; and variations of shade are easily had by modifying the proportions of the elements of the colored frit. The great point is to maintain the manganese in a state of peroxydation, and consequently to beware of placing the enamel in contact with any substance attractive of oxygen.

Such are the principal colored enamels hitherto obtained by means of metallic oxydes; but since the number of these oxydes is increasing every day, it is to be wished that new trials be made with such as have not yet been employed. From such researches some interesting results would unquestionably be derived.

Of paining on Enamel.-Enamelling is only done on gold and copper; for silver swells up, and causes blisters and holes in the coat of enamel. All enamel paintings are, in fact, done on copper or gold.

The gollsmith prepares the plate that is to be painted upon. The gold should be 22 carats fine: if purer, it would not be sufficiently stiff; if coarser, it would be subject to melt; and its alloy should be half white and half red, that is, half silver and half corper; whereby the enamel with which it is covered will be less disposed to turn green, than if the alloy were entirely copper.

The workman must reserve for the edge of the plates small fillet, which he calls the borler. This ledge serves to retain the enamel, and hinders it from falling off when applied an.l pressed on with a spatula. When the plate is not to be counter-enamelled, it shoul.1 be charged with less enamel, as, when exposed to heat, the enamel draws up the gold (1) it-self, and makes the piece convex. When the enamel is not to cover the whole plate, it becomes necessary to prepare a lodgment for it. With this view, all the outlines of the figure are traced on the plate with a black-lead pencil, after which recourse is had to the graver.

The whole space enclosed by the outlines must be hollowed out in bas-relief, of a 
depth equal to the height of the fillet, had the plate been ertirely enamelled. This sinking of the surface must be done with a flat graver as equally as possible; for if there be an eminence, the enamel would be weaker at that point, and the green would appear. Some artists hatch the bottom of the hollow with close lines, which cross each other in all directions; and others make lines or scratches with the end of a file broken off square. The hatchings or scratches lay hold of the enamel, which might otherwise separate from the plate. After this operation, the plate is cleansed by boiling it in an alkaline ley, and it is washed first with a little weak vinegar, and then with clear water.

The plate thus prepared is to be covered with a coat of white enamel, which is done by bruising a piece of enamel in an agate or porcelain mortar to a coarse powder like sand, washing it well with water, and applying it in the hollow part in its moist state. The plate may meanwhile be held in an ordinary forceps. The enamel powder is spread with a spatula. For condensing the enamel powder, the edges of the plate are struck with this spatula.
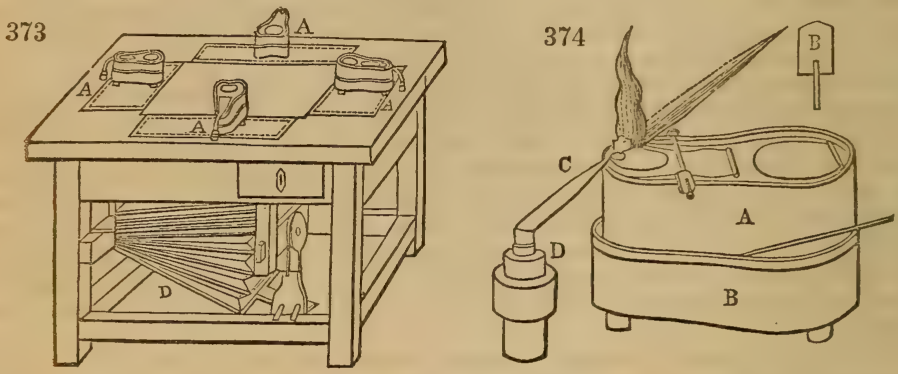

Whenever the piece is dry, it is placed on a slip of sheet iron perforated with several small holes, see fig. 375. which is laid on hot cinders; and it is left there until it ceases to steam. It must be kept hot till it goes to the fire; for were it allowed to cool it would become necessary to heat it again very gradually at the mouth of the furnace of fusion, to prevent the enamel from decrepitating and flying off.
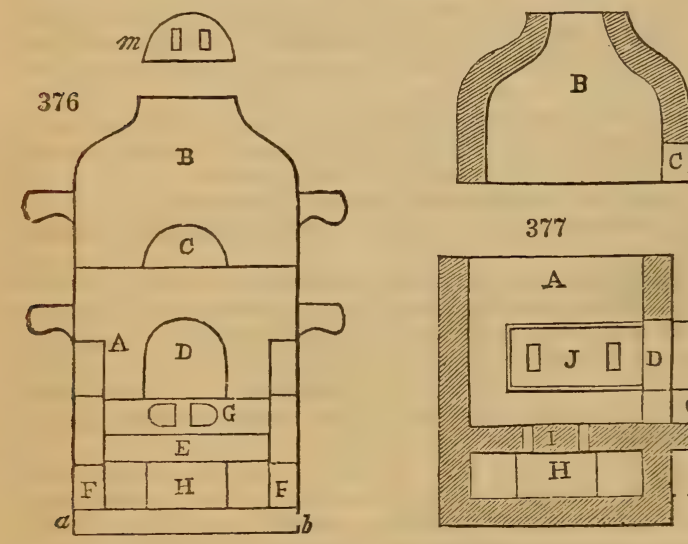

375
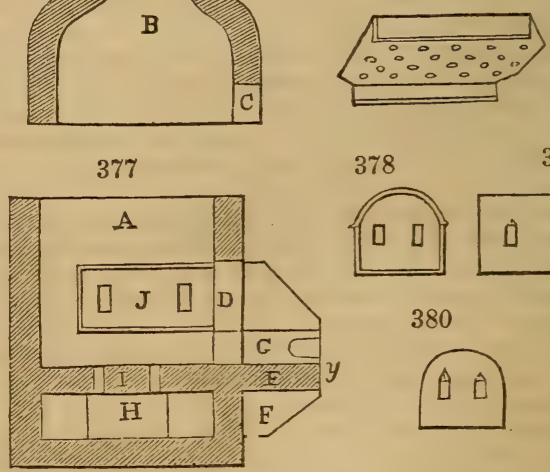

378

379

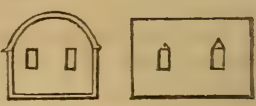

380

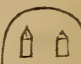

Before describing the manner of exposing the piece to the fire we must explain the construetion of the furnace. It is square, and is shown in front elevation in fig. 376. It consists of two pieces, the lower part A, or the body of the furnace, and the upper part $\mathbf{B}$, or the capital, which is laid on the lower part as is shown in fig. 377 , where these two parts are separately represented. The furnace is made of good fire-clay, moderately baked, and resembles very closely the assay or cupellation furnace. Its inside dimensions are 9 inches in width; 13 inches in height in the body, and 9 in the capital. Its general thickness is 2 inches.

The capital has an aperture or door c, fig. 376, which is closed by a fire-brick stopper $\boldsymbol{m}$, when the fire is to be made active. By this door fuel is supplied. 
The boty of the furnace has likewise a door $\mathrm{D}$, which reaches down to the projecting shelf $\mathrm{E}$, called the bib (mentomnicre), whose prominence is seen at E, fig. 376 . This shelf is supported and secured by the two brackets F, F; the whole being earthenware. The height of the door $\mathrm{D}$ is abridged by a peculiar fire-brick $\mathrm{G}$, which not only covers the whole projection of the shelf $\mathrm{E}$, but enters within the opening of the door $\mathrm{D}$, filling its breadth, and advancine into the same plane with the inner surface of the furnace. This plate is cilled the hearth; its purpose will appear presently; it may be taken out and replaced at pleasure, by laying hold of the handle in its front.

Below the shelf $\mathrm{E}$, a square hole, $\mathrm{H}$, is seen, which serves for admitting air, and for extracting the ashes. Similar holes are left upon each side of the furnace, as is shown in the ground plan of the base, fig. 377 , at $\mathbf{~ H ~} \mathbf{~ H .}$

On a level with the shelf, in the interior of the furnace, a thin fire-tile I rests, perforated wilh numerous small holes. This is the grate represented in a ground view in fig. 375. Fiqs. 3 8 8, 379, 380, represent, under different aspects, the muffle. Fig. 377 shows the elevation of its further end; fig. 379 its sides; and fig. 380 its front part. At $\mathrm{J}$, fig. $3 \% \tau$, the muffe is seen in its place in the furnace, resting on two bars of iron, or, still better, on ledges of fire-clay, supported on brackets attached to the lateral sides of the firnace. The muffle is made of earthenware, and as thin as possible. The fuel consists of diy beech-wood, or oaken branches, about an inch in diameter, cut to the length of 9 inches, in order to be laid in horizontal strata within the furnace, one row only being placed above the muffle. When the muffle has attained to a white-red heat, the sheet iron tray, bearing its enamel plate, is to be introduced with a pair of pincers into the front of the muffle, and gradually advanced towards its further end. The mouth of the muffle is to be then closed with two pieces of charcoal only, between which the artist may see the progress of the operation. Whenever the enamel begins to flow, the tray must be turned round on its base to ensure equality of temperature; and as soon as the whole surface is melted, the tray must be withdrawn with its plate, but slowly, lest the vitreous matter be cracked by sudden refrigeration.

The enamel plate, when cold, is to be washed in very dilute nitric acid, and afterwards in cold water, and a second coat of granular enamel paste is to be applied, with the requisite precautions. This, being passed through the fire, is to be treated in the same way a third time, when the process will be found complete. Should any chinks happen to the enamel coat, they must be-widened with a graver, and the space being filled with ground enamel, is to be repaired in the muffle. The plate, covered with a ptre white enamel, requires always to be polished and smoothed with sandstone and water, particularly if the article have a plane surface; and it is then finally glazed at the fire.

The painting operation now follows. The artist prepares his enamel colors by pounding them in an agate mortar, with a pestle of agate, and grinding them on an agate slab, with oil of lavender, rendered viscid by exposure to the sun in a shallow vessel, loosely corered with gauze or glass. The grinding of two drachms of enamel pigment into an impalpable powder, will occupy a laborer a whole day. The painter should have alongside of him a stove in which a moderate fire is kept up, for drying his work whenever the figures are finished. It is then passed through the muffle.

Enamelling at the Lamp.-The art of the lamp enameller is one of the most agreeable and amusing that we know. There is hardly a subject in enamel which may o be executed by the lamp-flame in very little time, and more or less perfectly, according to the dexterity of the artist, and his acquaintance with the principles of modelling.

In working at the lamp, tubes and rods of glass and enamel must be provided, of all sizes and colors.

The enamelling table is represented in fig. 373, round which several workmen, with their lamps, may be placed, while the large double bellows $\mathrm{D}$ below is set a-blowing by a treadle moved with the foot. The flame of the lamp, when thus impelled by a powerful jet of air, acquires surprising intensity. The bent nozzles or tubes, A A A A, are made 0. glass, and are drawn to points modified to the purpose of the enameller.

Fig. 374 shows, in perspective, the lamp A of the enameller standing in its cistern $\mathbf{B}$; the blowpipe $\mathrm{c}$ is seen projecting its flame obliquely upwards. The blowpipe is adiustable in an elastic cork $\mathrm{D}$, which fills up exactly the hole of the table into which it enters. When only one person is to work at a table provided with several lamps, he sits down at the same side with the pedal of the bellows; he takes out the other blowpipes, and plugs the holes in the table with solid corks.

The lamp is made of copper or tin-plate, the wick of cotton threads, and either tallow or oil may be used. Between the lamp and the workman a small board or sheet:of white iron $\mathrm{B}$, called the screen, is interposed to protect his eyes from the glare of light. The screen is fastened to the table by a wooden stem, and it throws its shadow on his face. 
The enamelling workshop ought to admit little or no daylioht, otherwise the artist, not perceiving his fiame distinctly, would be apt to commit mistakes.

It is impossible to describe all the manipulations of this ingenious art, over which taste and dexterity so entirely preside. But we may give an example. Suppose the enameller wishes to make a swan. He takes a tube of white enamel, seals one of its ends hermetically at his lamp, and while the matter is sufficiently hot, he blows on it a minikin flask, resembling the body of the bird; he draws out, and gracefully bends the neck; he shapes the head, the beak, and the tail; then, with slender enamel rods of a proper color, he makes the eyes; he next opens up the beak with pointed scissors; he forms the wings and the legs; finally attaching the toes, the bird stands complete.

The enameller also makes artificial eyes for human beings, imitating so perfectly the colors of the sound eye of any individual, as to render it difficult to discover that he has a blind and a seeing one.

It is difficult to make large articles at the blowpipe; those which surpass 5 or 6 inches become nearly unmanageable by the most expert workman.

EPSOM SALTS. Sulphate of Magnesia.

EQUIVALENTS, CHEMICAL. (Stöchiometrie, Germ.) This expression was first employed by Dr. Wollaston, to denote the primary proportions in which the various chemical bodies reciprocally combine; the numbers representing these proportions being referred to one standard substance of general interest, such as oxygen or hydrogen reckoned unity, or 1,000 . Dr. Dalton, who is the true author of the grand discovery of definite and multiple chemical ratios, calls these equivalent numbers atomic weights, when reduced to their lowest terms, either hydrogen or oxygen being the radix of the scale. Though it belongs to a chemical work to discuss the principles and develop the applications of the Atomic Theory, I shall be careful, upon all proper occasions, to point out the vast advantages which the chemical manufacturer may derive from it, and to show how much he may economize and improve his actual processes by its means. See Element.

ESSENCES are either ethereous oils, in which all the fragrance of vegetable products reside; or the same combined and diluted with alcohol. See OrLs, Ethereous.

ESSENCE D'ORIENT, the name of a pearly looking matter procured from the blay or bleak, a fish of the genus cyprinus. This substance, which is found principally at the base of the seales, is used in the manufacture of artificial pearls. A large quantity of the scales being scraped into water in a tub, are there rubbed between the hands to separate the shining stuff, which subsides on repose. The first water being decanted, more is added with agitation till the essence is thoroughly washed from all impurities; when the whole is thrown upon a sieve; the substance passes through, but the scales are retained. The water being decanted off, the essence is procured in a viscid state, of a bluish white color, and a pearly aspect. The intestines of the same fish are also covered with this beautiful glistening matter. Several other fish yield it, but in smaller proportion. When well prepared, it presents exactly the appearance and reflections of the real pearls, or the finest mother of pearl; properties which are probably owing to the interposition of some portions of this same substance, between the laminæ of these shelly concretions. Its chemical nature has not been investigated; it putrefies readily when kept moist, an accident which may, however, bo counteracted by water of ammonia. See Pearls.

ETCHING Varnish. (Aelzgrund-Deckfirniss, Germ.) Though the practice of this eleogant art does not come within the scope of our Dictionary, the preparation of the varnishes, and of the biting menstrua which it employs, legitimately does.

The varnish of Mr. Lawrence, an English artist resident in Paris, is made as follows: Take of virgin wax and asphaltum, each two ounces, of black pitch and burgundy-pitch each half an ounce. Melt the wax and pitch in a new earthenware glazed pot, and add to them, by degrees, the asphaltum, finely powdered. Let the whole boil till such time as that, taking a drop upon a plate, it will break when it is cold, on bending it double two or three times betwixt the fingers. The varnish, being then enough boiled, must be taken off the fire, and after it cools a little, must be poured into warm water that it may work the more easily with the hands, so as to be formed into balls, which must be kneaded, and put into a piece of taffety for use.

Care must be taken, first, that the fire be not too violent, for fear of burning the ingredients, a slight simmering being sufficient; secondly, that whilst the asphaltum is putting in, and even after it is mixed with the ingredients, they should be stirred continually with the spatula; and thirdly, that the water into which this composition is thrown should be nearly of the same degree of warmth with it, in order to prevent a kind of eracking that happens when the water is too cold.

The varnish ought always to be made harder in summer than in winter, and it will become so if it be suffered to boil longer, or if a greater proportion of the asphaltum or 
brown rosin be used. The experiment above mentioned, of the drop suffered to cool, will determine the degree of hardness or softness that may be suitable to the season when it is used.

Preparation of the hard varnish used by Callot, commonly called the Florence Varnish :-Take four ounces of fat oil very clear, and made of good linseed oil, like that usel by painters; heat it in a clean pot of glazed earthenware, and afterwards put to it four ounces of mastick well powdered, and stit the mixture briskly till the whole be well melted, then pass the mass through a piece of fine linen into a glass bottle with a long neck, that can be stopped very securely; and keep it for the use that will be explained below.

Method of applying the soft varnish to the plate, and of blackening it:-The plate being well polished and burnished, as also cleansed from all greasiness by chalk or Spanish white, fix a hand-vice on the edge of the plate where no work is intended to be, to serve as a handle for managing it when waim'; then put it upon a chafing dish, in which there is a moderate fire, and cover the whole plate equally with a thin coat of the varnish; and whilst the plate is warm, and the varnish upon it in a fluid state, beat every part of the varnish gently with a small ball or dauber nade of cotton tied up in taffety, which operation smooths and distributes the varnish equally over the plate.

When the plate is thus uniformly and thinly covered with the varnish, it must be blackened iy a piece of flambeau, or of a large candle which affords a copious smoke; sometimes two or even four such candles are used together for the sake of despatch, that the rarnish nay nor grow cold, which if it does during the operation, the plate must be heate? uyain, that it may be in a melted state when that operation is performed; but great care must be taken not to burn it, which, when it happens, may be easily perceived by the varnish appearing burnt, and losing its gloss.

The menstruum used and recommended by Turrell, an eminent London artist, for etching upon steel, was prepared as follows:-

\section{Take Pyroligneous acid 4 purts by measure, Alcohol 1 part, mix, and add Nitric acid 1 part.}

This mixed liquor is to be applied from $1 \frac{1}{2}$ to 15 minutes, according to the depth desired. The nitric acid was employed of the strength of $1 \cdot 28$-the double aquafortis of the shops.

The eau forle or menstruum for copper, used by Callot, as also by Piranesi, with a slight modification, is prepared with 8 parts of strong French vinegar,

$$
\begin{aligned}
& 4 \text { parts of verdigris, } \\
& 4 \text { ditto sea salt, } \\
& 4 \text { ditto sal ammoniac, } \\
& 1 \text { ditto alum, } \\
& 16 \text { ditto water. }
\end{aligned}
$$

The solid substances are to be well ground, dissolved in the vinegar, and diluted with the water; the mixture is now to be boiled for a moment, and then set aside to cool. This menstruum is applied to the washed, dried, and varnished plate, after it has suffered the ordinary action of aquaf rtis, in order to deepen and finish the delicate touches. It is at present called the eau forte à passer.

ETHER is the name of a class of very light, volatile, inflammable, and fragrant spirituous liquids, obtained by distilling, in a gjlass retort, a mixture of alcohol with almost any strong acid. Every acid modifies the result, in a certain degree, whence several varieties of ether are produced. The only one of commercial importance is sulphuric ether, which was first made known under the name of sweet oil of vitriol, in 1540 , by the receipt of Walterus Cordus. Froberus, 190 years after that date, directed the attention of chemists afresh to this substance, under the new denomination of ether.

There are two methods of preparing it; by the first, the whole quantity of acid and alcohol are mixed at once, and directly subjected to distillation; by the second, the alcohol is admitted, in a slender streamlet, into a body of acid previously mixed with a little alcohol, and heated to $220^{\circ} \mathrm{Fahr}$.

1. Mix equal weights of alcohol at spec. grav. $0 \cdot 830$, and sulphuric acid at $1 \cdot 842$, by introducing the former into a large tubulated retort, giving it a whirling motion, so that the alcohol may revolve round a central conical cavity. Into this species of whirlpool the acid is to be slowly poured. The rixture, which beccmes warm, is to be forthwith distilled by attaching a spacious receiver to the retort, and applying the heat of a sand-bath. The formation of ether takes place orly at a certain temperature. If the contents of the retort be allowed to coct, and be then slowly heated in a water-bath, alcohol alone will come over for some time without ether, till the mixture acquires the 
proper degree of heat. The first receiver should be a globe, with a tube proceeding frum its bottom, into second receiver, of a cylindric shape, surrounded with ice-cold water. The joints must be well secured by lutes, after the expanded air has been allowed to escape. The liquid in the retort should be kept in a steady state of ebullition. The ether, as long as it is produced, condenses in the balloon and neck of the receiver in striæ; when these disappear the process is completed. The retort inust now be removed from the sand; therwise it would become filled with white fumes containing sulphurous acid, and denser striæ would flow over, which would contaminate the light product with a liquid called sweet oil of wine.

The theory of etherification demonstrates that when strong sulphuric acid is mixed with alcohol, there is formed, on the one hand, a more aqueous sulphuric acid, and, on the other, sulphovinic acid. When this mixture is made to boil, the sulphorinic acid is decomposed, its dihydrate of carbon combines with the alcohol, and constitutes ether; while the proportion of sulphovinic acid progressively diminishes. Mr. Hennell, of the Apothecaries' Hall, first explained these phenomena, and he was confirmed in his views by the interesting researches of Serullas. The acid left in the retort is usually of a black color, and may be employed to convert into ether half as much alcohol aoain; an experiment which may be repeated several times in succession.

The most profitable way of manufacturing ether has been pointed out by Boullay. It consists in letting the alcohol drop in a slender stream into the acid, previously heated to the etherifying temperature. If the acid in this case were concentrated to 1.846 , the reaction would be too violent, and the ether would be transformed into bicarbureted hydrogen (dihydrate of carbon). It is therefore necessary to dilute the acid down to the density of 1.780 ; but this dilution may be preferably effected with alcohol, instead of water, by mixing three parts of the strongest acid with 2 of alcohol, specific gravity 0.830 , and distilling off a portion of the ether thereby generated; after which the st:eam of alcohol is to be introduced into the tubulure of the retort through a small glass tube plunged into the mixture; this tube being the prolongation of a metallic syphun, whose shorter leg dips into a bottle filled with the alcohol. The longer leg is furnished with a stop-cock, for regulating at pleasure the alcoholic streamlet. The distilled vapurs should be transmitted through a worm of pure tin, surrounded by cold water, and the condensed fluid received in a glass bottle. The quantity of alcohol which can be thus converted into ether by a given weight of sulphuric acid, has not hitherto been accurately determined; but it is at least double. In operating in this way, neither sulphurous acid nor sweet oil of wine is generated, while the residuary liquid in the retort continues limpid and of a merely brownish yellow color. No sulphovinic acid is formed, and according to the experiments of Geiger, the proportion of ether approaches to what theory shows to be the maximum amount. In fact, 57 parts of alcohol of $0.83 \mathrm{sp.} \mathrm{grav.} \mathrm{being} \mathrm{equiva-}$ lent to 46.8 parts of anhydrous alcohol, yield, according to Geiger, $33 \frac{1}{2}$ parts of ether; and by calculation they should yield $37 \frac{1}{4}$.

The ether of the first distillation is never pure, but always contains a certain quantity of alcohol. The density of that product is usually 0.78 , and if prepared by the first of the above methods, contains, besides alcohol, pretty frequently sulphurous acid, and sweet oil of wine; impurities from which it must be freed. Being agitated with its bulk of milk of lime, both the acid and the alcohol are removed at the same time; and if it be then decanted and agitated, first with its bulk of water, next decanted into a retort containing chloride of calcium in coarse powder, and distilled, one third of perfectly pure ether may be drawn over. Gay Lussac recommends to agitate the ether, first with twice its volume of water, to mix it, and leave it in contact with powdered unslaked lime for 12 or 14 hours, and then to distil off one third of pure ether. The remaining two thirds consist of ether containing a little alcohol. If in preparing ether by Boullay's method, the alcohol be too rapidly introduced, much of this liquid will come over unchanged. If in this state the ether be shaken with water, a notable quantity of it will be absorbed, because weak alcohol dissolves it very copiously. The abuve product should therefore be re-distilled, and the first half that comes over may be considered as ether, and treated with water and lime. The other half must be exposel afiesh to the action of sulphuric acid.

Pure ether possesses the following properties. It is limpid, of spec. grav. $0 \cdot 713$, or 0.715 at $60^{\circ}$; has a peculiar penetrating strong smell; a taste at first acria, burning, sweetish, and finally cooling. It has neither an acid nor alkaline reaction; is a nonconductor of electricity, and refracts light strongly. It is very volatile, boiling at $96^{\circ}$ or $97^{\circ} \mathrm{F}$., and produces by its evaporation a great degree of cold. At the temperature of 62.4 , the vapor of ether balances a column of mercury 15 inches high, or half the weight of the atmosphere. When ether is cooled to $-24^{\circ} \mathrm{F}$. it begins to crystallize in brilliant white plates, and at $-47^{\circ}$ it becomes a white crystalline solid. When vapor of ether is made to traverse a red hot porcelain tube, it deposites within it one half per cent. of charcoal, and there are condensed in the receiver one and two thirds 
per cent. of a brown oil, partly in crystalline scales, and partly viscid. The crystalline portion is suluble in alcuhol, but the viscid only in ether. The remainder of the decom. posed ether consists of bi-carburetel hydrogen gas, tetrahydric carburet, carbonic oxyde gas, and one per cent. at most of gaseous carbonic acid.

Ether talies fire readily, even at sone distance from a flame, and it should not there. fore be poured fiom one vessel to another in the neighborhood of a lighted candle. It may be likewise set on fire by the electric spark. It burns all away with a bright fuliginous Hame. When the vapor of ether is mixed with 10 times its volume of oxygen, it burns with a violent explosion, absorbs 6 times its bulk of oxygen, and produces 4 times its volume of carbonic acid gas.

Etlier alters gradually with contact of air ; absorbing oxygen, and progressively changing into acetic acid and water. This conversion takes place very rapidly when the ether is boiled in an open vessel, while the acid enters into a new combination forming acetic ether. Ether should be preserved in bottles perfectly full and well corked, and kept in a cool place, otherwise it becomes sour, and is destroyed. In contains in this state 15 per cent. of its bulli of azote, but no oxygen gas, as this has combined with its elements. Ether is composed of oxygen 21.24 ; hydrogen 13.85 ; carbon 65.05 . This composition may be represented by 1 prime equivalent of water, and 4 primes of bi-carburetted hydrogen gas; in other words, ether contains for 1 prime of water, once as much olefiant gas as alcohol, and its prime equivalent is therefore $468 \cdot 15$ to oxygen 100 . By my analysis, as published in the Phil. Trans. for 1822 , ether is composed of oxygen $27 \cdot 10$; hydrogen 13.3 ; and carbon 59.6 in 100 parts. The density of my ether was 0.700 . One volume of vapor of ether consists of one volume of aqueous vapor and two volumes of olefiant gas (bi-carbureted hydrogen), while alcohol consists of two volumes of each.

ETHER, ACETIC, is used to flavor silent corn spirits in making imitation brandy. It may be prepared by mixing 20 parts of acetate of lead, 10 parts of alcohol, and $11 \frac{1}{2}$ of concentrated sulphuric acid; or 16 of the anhydrous acetate, 5 of the acid, and $4 \frac{1}{2}$ of absolute alcohol; distilling the mixture in a glass retort into a very cold receiver, agitating along with weak potash ley the liquor which comes over, decanting the supernatant ether, and rectifying it by re-distillation over magnesia and ground charcoal.

Acetic ether is a colorless liquid of a fragrant smell and pungent taste, of spec. grav. 0.866 at $15^{\circ} \mathrm{F}$, boiling at $166^{\circ} \mathrm{F}$, burning with a yellowish flame, and disengaging fumes of acetic acid. It is soluble in 8 parts of water.

Acetic ether may be economically made with 3 parts of acetate of potash, 3 of very strong alcohol, and 2 of the strongest sulphuric acid, distilled together. The first product must be re-distilled along with one fifth of its weight of sulphuric acid; as much ether will be obtained as there was alcohol employed.

ETHIOPS is the absurd name given by the alchemists to certain black metallic preparations. VIartial ethiops was the black oxyde of iron; mineral ethiops, the black sulphuret of mercury; and ethiops per se, the black oxyde of mercury.

EVAPORATION (Eng. and Fr.; Abicimpfen; Abdunsten, Germ.) is the process by which any substance is converted into, and carried off in, vapor. Though ice, camphor, and many other solids evaporate readily in dry air, I shall consider, at present, merely the vaporization of water by heat artificially applied.

The rapor of water is an elastic fluid, whose tension and density depend upon the temperature of the water with which it is in contact. Thus the vapor rising from water heated to $165^{\circ} \mathrm{F}$. possesses an elastic force capable of supporting a column of mercury 10.8 high; and its density is such that 80 cubic feet of such vapor contain one pound weight of water; whereas $32 \frac{1}{2}$ cubic feet of steam of the density corresponding to a temperature of $212^{\circ}$ and a pressure of 30 inches of mercury, weigh one pound. When the temperature of the water is given, the elasticity and specific gravity of the vapor emitted by it may be found.

Since the vapor rises from the water only in virtue of the elasticity due to its gaseous nature, it is obvious that no more can be produced, unless what is already incumbent upon the liquid have its tension abated, or be withdrawn by some means. Suppose the temperature of the water to be midway between freezing and boiling, viz., $122^{\circ} \mathrm{Fahr}$., as also that of the air in contact with it, to be the same but replete with moisture, so that its interstitial spaces are filled with vapor of corresponding elasticity and specific gravity with that given off by the water, it is certain that no fresh formation of vapor can take place in these circumstances. But the moment a portion of vapor is allowed to escape, or is drawn off by condensation to another vessel, an equivalent portion of vapor will be immediately exhaled from the water.

The pressure of the air and of other vapors upon the surface of water in an open vessel, does not prevent evaporation of the liquid; it merely retards its progress. Experience shows that the space filled with an elastic fluid, as air or other gaseous body, is capable of receiving as much aqueous vapor as if it were vacuous, only the repletion of that 
space with the vapor proceeds mare slowly in the former predicament than in the lat. ter, but in both cases it arrives eventually at the same pitch. Dr. Dalton has very in. geniously proved, that the particles of aeriform bodies present no permanent obstacle to the introduction of a gaseous atmosphere of another kind among them, but merely obstruct its diflusion momentarily, as if by a species of friction. Hence, exhalation at atmospheric temperatures is promoted by the mechanical diffusion of the vapors through the air with ventilating fans or chimney draughts; though under brisk ebullition, the force of the steam readily overcomes that mechanical obstruction.

The quantities of water evaporated under different temperatures in like times, are proportional to the elasticities of the steam corresponding to these temperatures. A vessel of boiling water exposing a square foot of surface to the fire, evaporates 725 grains in the minute; the elasticity of the vapor is equivalent to 30 inches of mercury. To find the quantity that would be evaporated from the same surface per minute at a heat of $88^{\circ} \mathrm{F}$. At this temperature the steam incumbent upon water is capable of supporting 1.28 inch of mercury; whence the rule of proportion is $30: 1.28:: 725: 30.93$; showing that about 31 grains of water would be evaporated in the minute. If the air contains already some aqueous vapor, as it commonly does, then the quantity of evaporation will be proportional to the difference between the elastic force of that vapor, and what rises from the water.

Suppose the air to be in the hygrometric state denoted by 0.38 of an inch of mercury, then the above formula will become: $30: 1.28-0.38:: 725: 21.11$; showing that not more than $21 \frac{1}{2}$ grains would be evaporated per minute under these circumstances.

The elastic tension of the atmospheric vapor is readily ascertained by the old experiment of Le Roi, which consists in filling a glass cylinder (a narrow tumbler for example) with cool spring water, and noting its temperature at the instant it becomes so warm that dew ceases to be deposited upon it. This temperature is that which corresponds to the elastic tension of the atmospheric vapor. See VAPor, Table of.

Whenever the elasticity of the vapor, corresponding to the temperature of the water, is greater than the atmospheric pressure, the evaporation will take place not only from its surface, but from every point in its interior; the liquid particles throughout the mass assuming the gaseous form, as rapidly as they are actuated by the caloric, which subverts the hydrostatic equilibrium among them, to constitute the phenomena of ebullition. This turbulent vaporization takes place at any temperature, even down to the freezing point, provided the pneumatic pressure be removed from the liquid by the air pump, or any other means. Ebullition always accelerates evaporation, as it serves to carry off the aqueous particles not simply from the surface, but from the whole body of the water.

The vapors exhaled from a liquid at any temperature, contain more heat than the fluid from which they spring; and they cease to form whenever the supply of heat into the liquid is stopped. Any volume of water requires for its conversion into vapor five and $a$ hulf times as much heat as is sufficient to heat it from the freezing to the boiling temperature. The heat, in the former case, seems to be absorbed, being inappreciable by the thermometer; for steam is no hotter than the boiling water from which it rises. It has been therefore called latent heat; in contradistinction to that perceived by the touch and measured by the thermometer, which is called sensible heat. The quantity of heat absorbed by one volume of water in its conversion into steam, is about $1000^{\circ} \mathrm{Fahr}$.; it would be adequate to heat 1000 volumes of water, one degree of the same scale; or to raise one volume of boiling water, confined in a non-conductirig vessel, to $1180^{\circ}$. Were the vessel charged with water so heated, opened, it would be instantaneously emptied by vaporization, since the whole caloric equivalent to its constitution as steam, is present. When, upon he other hand, steam is condensed by contact with cold substances, so much heat is set free as is capable of heating five and a half times its weight of water, from $32^{\circ}$ to $212^{\circ} \mathrm{F}$. If the supply of heat to a copper be uniform, five hours and a half will be required to drive off its water in steam, provided one hour was taken in heating the water, from the freezing to the boiling pitch, under the atmospherical pressure.

Equal weights of vapor of any temperature contain equal quantities of heat; for example, the vapor exhaled from one pound of water, at $77^{\circ} \mathrm{F}$, absorbs during its formation, and will give out in its condensation, as much heat as the steam produced by one pound of water, at $212^{\circ} \mathrm{F}$. The first portion of vapor with a tension $=30$ inches, occupies a space of 27.31 cubic feet; the second, with a tension of 0.92 inch, occupies a space of 890 cubic feet.* Suppose that these 890 volumes were to be compressed into 27.31 in a cylinder capable of confining the heat, the temperature of the vapor would rise from $77^{\circ}$ to $212^{\circ}$, in virtue of the condensation, as air becomes so hot by com-

* One pound avoirdupois of water contains $27 \cdot 72$ cubic inches ; one cubic inch of water forms 1696 cubio inches of steam at $212^{\circ} \mathrm{F}$ : therefore one pound of water will form $27 \cdot 31$ cubic feet of such steam : and 0.92 $30:: 27 \cdot 31: 890$ cubic feet. 
pression in a syringe, as to ignite amadou. The latent heat of steam at $212^{\circ} \mathrm{F}$. is $1180^{\circ}-180=1000$; that of vapor, at $77^{\circ}$, is $1180-45=1135^{\circ}$; so that, in fact, the lower the temperature at which the vapor is exhaled, the greater is its latent heat, as Joseph Black and James Watt long ago proved by experiments upon distillation and the steam engine.

From the preceding researches it follows, that evaporation may be effected upon two different plans :-

1. Under the ordinary pressure of the atmosphere; and that either,

$A$, by external application of heat to boilers, with $a$, an open fire; $b$, steam; $c$, hot liquid media.

$B$, by evaporation with air; $a$, at the ordinary temperature of the atmosphere; $b$, by currents of warm air.

2. Under plogressively lower degrees of pressure than the atmospheric, down to evaporation in as perfect a vacuum as can be made.

It is generally affirmed, that a thick metallic boiler obstructs the passage of the heat through it so much more than a thin one, as to make a considerable difference in their relative powers of evaporating liquids. Many years ago, I made a series of experiments upon this subject. Two cylindrical copper pans, of equal dimensions, were provided : but the metal of the one was twelve times thicker than that of the other. Each being charged with an equal volume of water, and placed either upon the same hot plate of iron, or immersed, to a certain depth, in a hot solution of muriate of lime, I found that the ebullition was greatly more vigorous in the thick than in the thin vessel, which I ascribed to the conducting substance up the sides, above the contact of the source of heat, being 12 times greater in the former case than in the latter.

If the bottom of a pan, and the portions of the sides, immersed in a hot fluid medium, solution of caustic potash or muriate of lime, for example, be corrugated, so as to contain a double expanse of metallic surface, that pan will evaporate exactly double the quantity of water, in a given time, which a like pan, with smooth bottom and sides, will do immersed equally deep in the same bath. If the corrugations contain three times the quantity of metallic surface, the evaporation will be threefold in the above circumstances. But if the pan, with the same corrugated bottom and sides, be set over a fire, or in an oblong flue, so that the current of flame may sweep along the corrugations, it will evaporate no more water from its interior than a smooth pan of like shape and dimensions placed alongside in the same flue, or over the same fire. This curious fact I have verified upon models constructed with many modifications. Among others, I caused a cylindrical pan, 10 inches diameter, and 6 inches deep, to be made of tinplate, with a vertical plate soldered across its diameter; dividing it into two equal semi-cylindrical compartments. One of these was smooth at the bottom, the other corrugated; the former afforded as rapid an evaporation over the naked fire as the latter, but it was far outstripped by its neighbor when plunged into the heated liquid medium.

If a shallow pan of extensive surface be heated by a subjacent fire, by a liquid medium or a series of steam pipes upon its bottom; it will give off less vapor in the same time when it is left open, than when partially covered. In the former case, the cool incumbent air precipitates by condensation a portion of the steam, and also opposes considerable mechanical resistance to the diffusion of the vaporous particles. In the latter case, as the steam issues with concentrated force and velocity from the contracted orifice, the air must offer less proportional resistance, upon the known hydrostatic principle of the pressure being as the areas of the respective bases, in communicating vessels.

In evaporating by surfaces heated with ordinary steam, it must be borne in mind that a surface of 10 square feet will evaporate fully one pound of water per minute, or $725 \times 10=7250 \mathrm{gr}$, the same as over a naked fire; consequently the condensing surface must be equally extensive. Suppose that the vessel is to receive of water 2500 lbs., which corresponds to a boiler 5 feet long, 4 broad, and 2 deep, being 40 . cubic feet by measure, and let there be laid over the bottom of this vessel 8 connected tubes each 5 inches in diameter and 5 feet long, possessing therefore a surface of 5 feet square. If charged with steam, they will cause the evaporation of half a pound of water per minute. The boiler to supply the steam for this purpose must expose a surface of 5 square feet to the fire. It has been proved experimentally that 10 square feet surface of thin copper can condense $3 \mathrm{lbs}$. of steam per minute, with a difference of temperature of 90 degrees Fahr. In the above example, 10 square feet evaporate $1 \mathrm{lb}$. of water per minute; the temperature of the evaporating fluid being $212^{\circ} \mathrm{F}$, consequently $3: 1:: 90: \frac{90}{3}$. During this evaporation the difference of the temperature is therefore $=30^{\circ} .{ }^{3}$ Consequently the heat of the steam placed in connexion with the interior of the boiler, to produce the calculated evaporation, should be, $212+30=242^{\circ}$, corresponding to an elastic force of 53.6 inches of mercury. Were the temperature of 
the steam only 224 , the same boiler in the same time would produce a diminished quan. tity of steam, in the proportion of 12 to 30 ; or to produce the same quantity the boiler or tubular surface should be enlarged in the proportion of 30 to 12. In general, however, steam boilers employed for this mode of evaporation are of such capacity as to give an unfailing supply of steam.

I shall now illustrate by some peculiar forms of apparatus, different systems of eva. poration. Fig. 381 explains the principles of evaporating in vacuo. A B represents

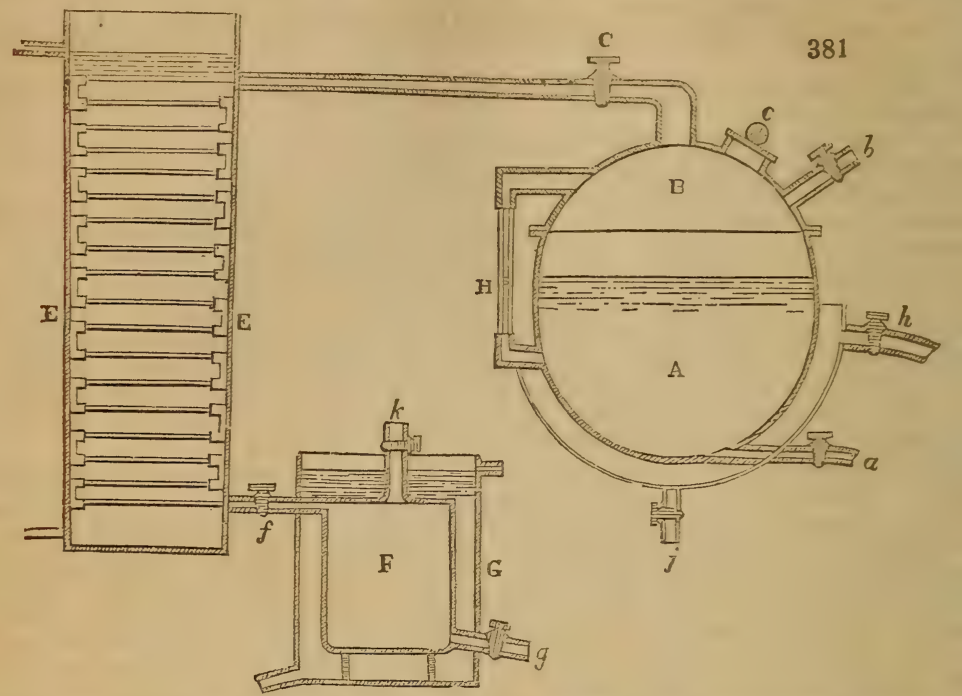

a pan or kettle charged with the liquor to be evaporated. The somewhat wide ornfice $c$, secured with a screw-plug, serves to admit the hand for the purpose of cleaning it thoroughly out when the operation is finished; $h$ is the pipe of communication with the steam boiler; $b$ is a tube prolonged and then bent down with its end plunged into the liquor to be evaporated, contained in the charging back, (not shown in the figure). $H$ is a glass tube communicating with the vacuum pan at the top and bottom, to show by the height of the column the quantity of liquid within. The eduction eraporating pipe c is provided with a stop-cock to cut off the communication when required. $i$ is a tube for the discharge of the air and the water from the steam-case or jachet; the refrigerator $\mathbf{E}$ is best formed of thin copper tubes about 1 inch in diameter, arranged zig-zag or spirally Jike the worm of a still in a cylinder. The small air-tight condenser $F$, connected with the efflux pipe $f$ of the refrigerator, is furnished below with a discharge cock $g$, and surrounded by a cooling case, for the collection of the water co.densed by the refrigerator. In its upper part there is a tube $k$, also furnished with a cock, which communicates with the steam boiler, and through which the pan A $\mathrm{B}$ is heated.

The operation of this apparatus is as follows: after opening the cocks c, $f, g$; and before admitting the cold water into the condenser $\mathrm{E}$, the cock of the pipe $k$ is opened, in order that by injecting steam it may expel the included air; after which the cocks $k$ and $g$ are to be shut. The water must now be introduced into the condenser, and the cock $b$ opened, whereon the liquid to be evaporated rises from the charging back, through the tube $b$, and replenishes the racuum pan to the proper height, as shown by the register glass tube $\mathrm{H}$. Whenever the desired eraporation or concentration is effected, the cock c must be closed, the pipe $k$ opened, so as to fill the pan with steam, and then the effux cock $a$ is opened to discharge the residuary liquor. By shutting the cocks $a$ and $k$, and opening the cock $b$, the pan will charge itself afresh with liquor, and the operation will be begun ancw, after $b$ has been shut and $c$ opened.

The contents of the close water cistern F, may be drawn off during each operation. For this purpose, the cock $f$ must first be shut, the cold water is to be then run out of the condenser $\mathrm{G}$, and $k$ and $g$ are to be opened. The steam entering by $k$ makes the water flow, but whenever the steam itself issues from the cock $g$, this orifice must be immediately shut, the cock $f$ opened, and the cold water again introduced, whereupon the condensed water that had meanwhile collected in the under part of the refrigerator, flows off into the condenser vessel $F$. Since some air alwars enters with the liquor 
sucked into the pan, it must be removed at the time of drawing off the water from the two condensers, by driving steam through the apparatus. This necessity will be less urgent if the liquor be made to boil before being introduced into the vacuum pan.

Such an apparatus may be modified in size and arrangement to suit the peculiar object in view, when it will be perfectly adapted for the concentration of extracts of every kind, as well as saline solutions containing vegetable acids or alkalis. The interior vessel of A B should be made of tinned or plated copper. For an account of Howard's vacuum pan, made upon the same principle, see Sugar.

When a boiler is set over a fire, its bottom should not be placed too near the grate, lest it refrigerate the flame, and prevent that vivid combustion of the fuel essential to the maximum production of heat by its means. The evil influence of leaving too little room between the grate and the copper may be illustrated by a very simple experiment. If a small copper or porcelain capsule containing water be held over the flame of a candle a little way above its apex, the flame will suffer no abatement of brightness or size, but will continue to keep the water briskly boiling. If the capsule be now lowered into the middle of the flame, this will immediately lose its brightness, becoming dull and smoky, corering the bottom of the capsule with soot; and owing to the imperfect combustion, though the water is now surrounded by the flame, its ebullition will cease.

Fig. 382 is a section of two evaporating coppers en suite, so mounted as to favor the

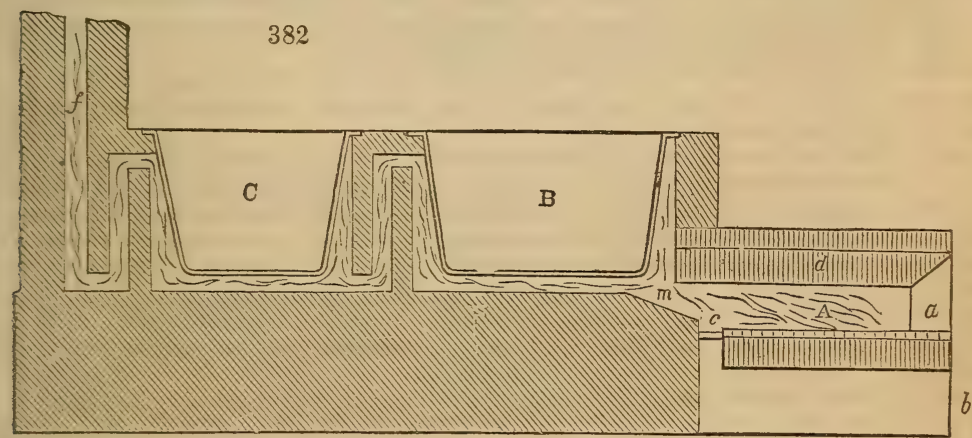

full combustion of the fuel. A is the hearth, in which wood or coal may be burned. For coal, the grate should be set higher and be somewhat smaller. $a$ is the door for feeding the fire; $d$, an arch of fire-bricks over the hearth; $c$, a grate through which the ashes fall into the pit beneath, capable of being closed in front to any extent by a sliding door b. $B$ and $c$ are two coppers incased in brickwork; $f$ the flue. At the end of the hearth near $m$, where the fire plays first upon the copper, the sole is made somewhat lower and wider, to promote the spreading of the flame under the vessel. The second copper, c, receives the benefit of the waste heat; it may be placed upon a higher level, so as 10 discharge its concentrated liquor by a slop-cock or syphon into the first. When coals are burned for heating such boilers, the grate should be constructed as shown in the figure of the brewing copper, page 122 .

Fig. 383 represents a pan for evaporating liquids, which are apt, during concentra-

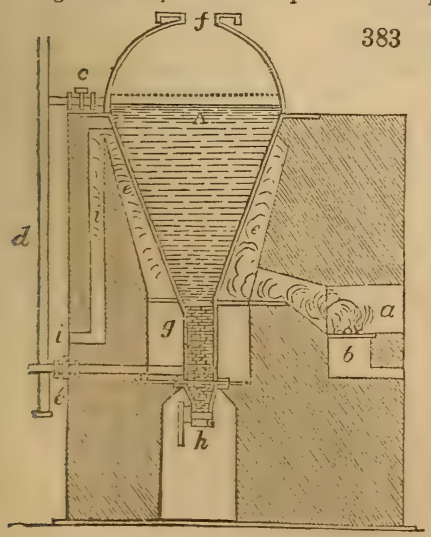
tion, to let fall crystals or other sediment. These would be injured either by the fire play ing upon the bottom of the pan, or, by adhesion to it, they would allow the metal to get red hot, and in that state run every risk of being burnt or rent on the sudden intrusion of a little liquor through the incrustation. When large coppers have their bottoms planted in loam, so that the flame circulates in flues round their sides, they are said to be cold-set.

A is a pear-shaped pan, charged with the liquid to be evaporated; it is furnished with a dome cover, in which there is an opening with a flange $f$, for attaching a tube, to conduct the steam wherever it may be required. $a$ is the fire-place; $b$ the ash-pit. The conical part terminates below in the tube $g$, furnished with a stop-cock at its nozzle $h$. Through the tube $c d c^{\prime}$, furnished above and below with the stopcocks $c$ and $c^{\prime}$, the liquid is run from the 
charging back or reservoir. During the operation, the upper cock $c$ is kept partially open, to replace the fluid as it evaporates; but the under cock $c^{\prime}$ is shut. The flane from the fire-place plays round the liettle in the space $e$, and the smoke escapes driwnwards through the flue $i$ into the chimney. The lower cylindrical part $g$ remains thus comparatively cool, and collects the crystalline or other solid matter. After some time, the under stop-cock c', upon the supply-pipe, is to be opened to admit some of the cold liquor into the cylindrical neck. That cock being again shut, the sediment settled, and the large stop-cock (a horizontal slide-valve would be preferable) $h$ opened, the crystals are suffered to descend into the subjacent receiver; after which the stop-cock $h$ is shut, and the operation is continued. A construction upon this principle is well adapted for heating dyeing coppers, in which the sediment should not be disturbed, or exposed to the action of the fire. The fire-place should be built as for the brewing copper.

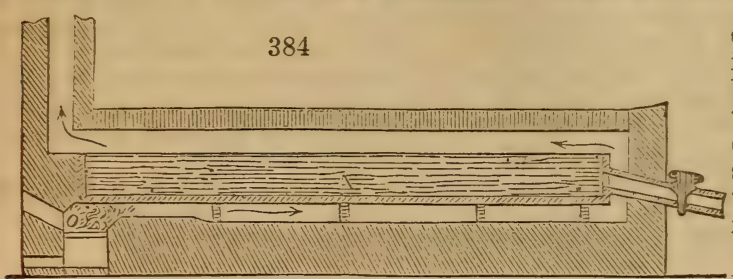

Fig. 384 represents an oblong evaporating pan, in which the flame, after beating along its bottom, turns up at its further end, plays back along its surface, and passes off in. to the chimney. A is a rectangular vessel, from 10 to 15 feet long, 4 to 6 feet broad, and 1 or $1 \frac{1}{2}$

feet deep. The fire-bricks, upon which the pan rests, are so arranged as to distribute the flame equably along its bottom.

EUDIOMETER is the name of any apparatus subservient to the chemical examination of the atmospheric air. It means a measure of purity, but it is employed merely to determine the proportion of oxygen which it may contain. The explosive eudiometer, in which about two measures of hydrogen are introduced into a graduated glass tube, containing five measures of atmospheric air, and an electric spark is passed across the mixture, is the best of all eudiometers; and of these the syphon form, proposed by me in a paper published by the Royal Society of Edinburgh in 1819 , is probably the surest and most convenient. Volta's explosive eudiometer, as made in Paris, costs 3 guineas; mine may be had nicely graduated for 6 or 8 shillings.

EXPANSION (Eng. and Fr.; Ausdehnung, Germ.) is the increase of bulk experienced by all bodies when heated, unless a change of chemical texture takes place, as in the case of clays in the potter's kiln. Table $\mathbf{1}$. exhibits the linear expansion of several solids by an increase of temperature from $32^{\circ}$ to $212^{\circ}$ Fahr.; Table II. exhibits the expansion in bulk of certain liquids.

TABLE I.-Linear Dilatation of Solids by Heat.

Dimensious which a bar takes at $212^{\circ}$, whose length at $32^{\circ}$ is 1.000000 .

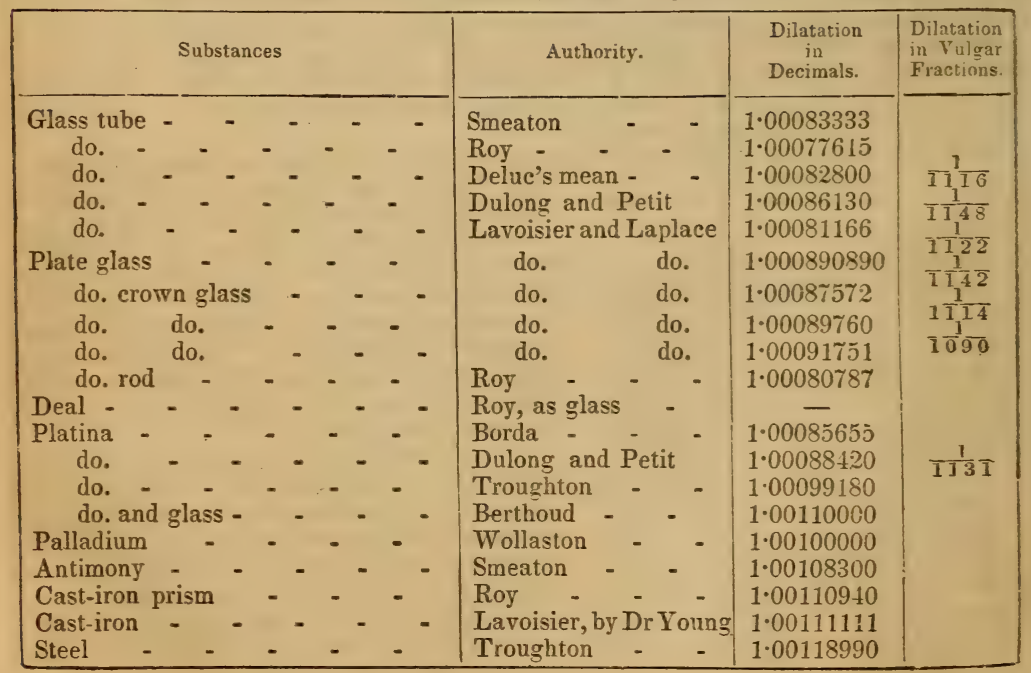




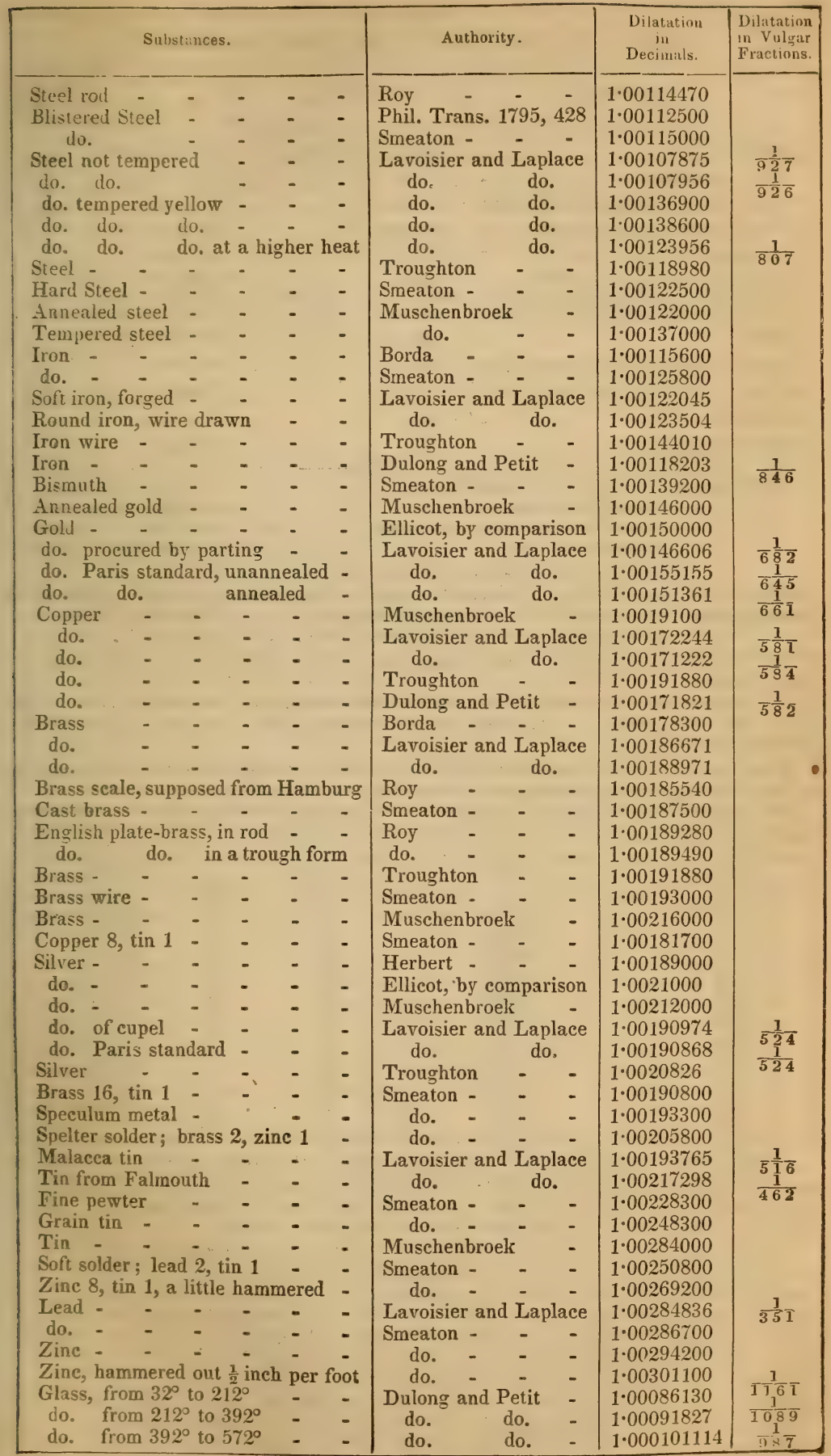

The last two measurements be an air thermometer. 
TABLE II.

Expansion of certain Liçaidy $t y$ being Heated from 320 to 2120 .

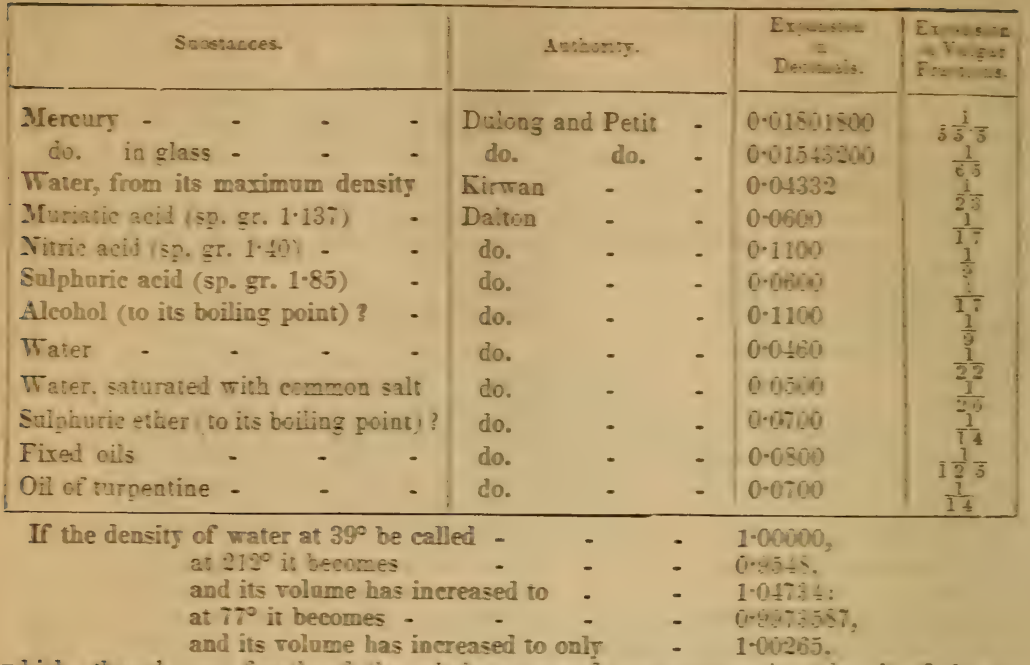

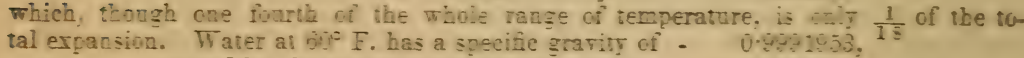
and has inereased in rolnme from $39^{\circ}$ to - 1-00008,

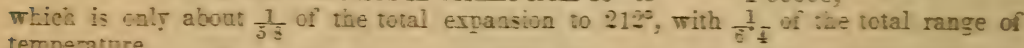
temperature.

All gaves expand the same guentity br the same increase of temperatre, which from 320 to $212^{2}$ Faht $=\frac{1}{\frac{1}{4}}=\frac{2}{3}$, or 100 rolumes become 1.375 . For each Legree of Fahr. the expansion is $\frac{1}{1-5}$.

- When dre air is saturated with meisture, its buth increases, and is specifie grarity diminishes, beeacse auge ns vapor is less dense than air, at lihe temperatires.

The following table gires the maltiphiers to be emploged for conrerting cne volume of moist gas at the several temperatures into a rolume of dry gas.

\begin{tabular}{|c|c|c|c|}
\hline Temperature. & Uriugher. & Temperature. & Wulpoter. \\
\hline $\begin{array}{l}55^{\circ} F . \\
51 \\
55 \\
503 \\
57 \\
59 \\
59 \\
60 \\
61 \\
62 \\
63\end{array}$ & $\begin{array}{l}0.3570 \\
0.9504 \\
0.9858 \\
0.9552 \\
0.9546 \\
0.9539 \\
0.033 \\
0.0527 \\
0.9527 \\
0.9513 \\
0.9985\end{array}$ & $\begin{array}{l}61^{\circ} \\
65 \\
65 \\
67 \\
65 \\
59 \\
70 \\
71 \\
72 \\
73\end{array}$ & $\begin{array}{l}0.9750 \\
0.9793 \\
0-9785 \\
0 \cdot 97: 9 \\
0.972 \\
19 \cdot 9705 \\
0.9758 \\
0.9751 \\
0-9713 \\
0.9735\end{array}$ \\
\hline
\end{tabular}

ETTRACTS. (Extraits. Fr. : Extrano, Germ.) The clder apothenaries csed th is term to designate the proluct of the eraporation of anj regetable juise. infusinn, or deontion: whether the latter two mere made with water. alectol, or ether: whence arose the distinction of aqueous, aleoholie, and ethereous ertracts.

Fourcory made many cesearches upon these preparations, and supponsed that they had

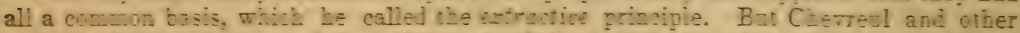
chemists have sicce proved that this pretenied priciple is a heterozenecos and rer rariable compound. By the term extract, therebire, is now meant menely the ritule of the soluble matrers chtained from rezetables, reluced by careful erapuration to either a

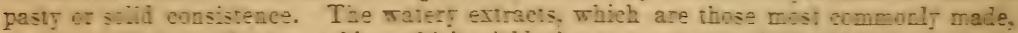
are as rarives as the rezetables which yill them: some containing ehiefly suger or gom in great abondance, and are therefore innocent or inert: whlle onters con. tain rert energetie imprezuations. The conduct of the eraporating beat is the capital point in the greparation of extracts. Ttey storid be almays prepared, if possible, from the juice of the fresh plant, by subjecting its leares or oither suceslen 
part, to the action of a powerful serew or hrdraulic press; and the evaporation should be elfictel b; the warmth of a water-bath, heated not beyond $100^{\circ}$ or $120^{\circ} \mathrm{F}$. Sieam heat may perinaps be applied advantageously in some cases, where it is not litiely to decomine any of the principles of the plant. But by far the best process for making extract is in vacuo, upon the principles explained in the article Evaporation. It is much vasier to fit up a proper apparatus of this kind, than most practical men inagine. The vacuum may either be made through the agency of steam, as there pointed out, or by means of an air-pump. One powerful air-pump may form and maintain a good racuun under several receivers, placed upon the flat-ground flanges of so many basins, each provided with a stop-cock at its side for exhaustion. The airless basin containing the ju:ce b-in set on the shelf of a water-bath, and exposed to a proper temperature, will furnish, in a short time, a large quantity of medicinal extract, possessing the properties of the plant unimpaired.

Fur exceedingly deicate purposes, the concentration may be performed in the cold, by placin! saucers illed with the expressed juice orer a basin containing sulphuric acid, putting a glass receiver over them and exhausting its air.

F.

FAHLERZ. Gray copper-ore, called also Panabase, from the many oxtđes it contains.

FAINTS is the name of the impure spirit, which comes orer first and last in the distillation of whisier; the former being called the strong, and the latter, which is much more abundant. the weak faints. This crude spirit is much impregnated with felid essential oil, is therefure rery unwholesome, and must be puritied by rectificatin.

F-til (Eventail, Fr.; Fücher, Germ.) is usually a semi-circular piece of silt or paper, pasted double, enclosing slender slips of wood, ivory, tortoise-shell, whale-bone, \&c., arranged like the tail of a peacock, in a radiating form, and susceptible of being folted together, and expanded at pleasure. This well-known hand ornament is use $i$ by lailies to cool their faces br agitating the air. Fans made of feathers, like the wing of a bird, have been employed from time immemorial by the natires of tropical countries.

Fum is also the name of the apparatus for winnowing corn. For an account of the powertul bluwing and ventilating fan machine, see Fotwdrs and Textrator.

FARIVA (Farine, Fr.; Mehl, Germ.) is the flour of any species of corn, or starchy root, such as potato, arrow root, \&c. See BREAD and STsRcH.

FATS (Grauses, Fr.; Fette, Germ.) occur in a great number of the animal tissues, being abundant under the shin in what is called the cellular membrane, round the kilners, in the folds of the omentum, at the base of the heart, in the mediastinum, the mesenteric webb, as well as upon the surface of the intestines, and among many of the muscies. They rary in consistence, color, and smell, according to the animals from which they are obtained; thus, they are generally fluid in the cetaceous tribes, soft and rank-flarored in the carnirorous, solid and nearly scentless in the ruminants, usually white and copious in well-fed young animals; yellowish and more scanty in the old. Their consistence raries also according to the orcan of their production; being firmer under the slin, and in the neighborhood of the kidness, than among rine moreable viscera. Fat forms about one twentieth of the weight of a healthr animal. But as taken out br the butcher it is not pure, for being of a resicular structure, it is alwars enclosed in membranes, mixed with blood, blood-ressels, lỵphatics, \&e. These foreign matters must first be separated in some measure mechanically, after the fat is minced small, and then wore completely by meling it along with hot water, passing it through a sieve, and letting the whole cool vert slowly. Br this means a cake of cleansed iat will be obtained. Many plans of purifying fats have been proposed; one of the best is to mix two per cent. of strong sulphuric acid with a quantity of water, in which the tallow is heated for some time with much stirring; to allow the materials to cool, ?o take off the supernatant fat, and re-melt it with abundance of hot water. More tallow will thus be obtained, and that considerably whiter and harder than is usually procured by the melters.

I have found that chlorine and chloride of lime do not improre, but rather deteriorate, the appearance of oils and other fatty bodies. According to Appert, minced suet subjected to the action of high-pressure steam in a digester, at $250^{\circ}$ or $260^{\circ} \mathrm{F}$, becomes so hard as to be sonorous when struck, whiter, and capable, when made into candles, of giring a superior light. A conrenient mode of rendering minced tallow, or melting it, is to put it in a tub, and drive steam through it from numerous orifices in ramifing pipes placed near the bottom. Mr. Watt assures me that his plan of purifying fats, patented in March, 1536, has been quite successful. He emplors dilute sulphintic acil, to which he adds a little nitric acid, with a very small quantity of bichromate of potash, " to sup- 
ply oxygen," and some oxalic acid. These are mixed with the fat in the steaming tub. When the lumps of it are nearly dissolved, he takes for every ton of fat, one pound of strong nitric acid, diluted with one quart of water; to which he adds two ounces of alcolol, naptha, sulphuric ether, or spirits of turpentine; and after introducing this mixture, he continues the boiling for half an hour. The fat is finally washed.-As I do not comprehend the modus operandi of these ingredients, I shall abstain from any comment upon the recipe.

Others have proposed to use vegetable or animal charcoal first, especially for rancid oils, then to heat them with a solution of sulphate of copper and common salt, which is supposed to precipitate the fetid albuminous matter. Milk of lime has been also prescribed; but it is, I believe, always detrimental.

Davidson treats whale oil with infusion of tan, in order to separate the gelatine and albumine in flocks; next with water and chloride of lime, to destroy the smell; and lastly, with dilute sulphuric acid, to precipitate all the lime in the state of a sulphate. This is certainly one of the cheapest and most effective methods of purifying that substance.

Braconnot and Raspail have shown that solid animal fats are composed of very small, microscopic, partly polygonal, partly reniform particles, which are connected together by very thin membranes. These may be ruptured by mechanical means, then separated by triturating the fresh fats with cold water, and passing the unctuous matter through a siere. The particles float in the water, but eventually collect in a white granular crystalline appearance, like starch. Each of them consists of a vesicular integument, of the nature of stearine, and an interior fluid like elaine, which afterwards exudes. The granules float in the water, but subside in spirits of wine. When digested in strong alcohol, the liquid part dissolves, but the solid remains. These particles differ in shape and size, as obtained from different animals; those of the calf, ox, sheep, are polygonal, from $\frac{1}{50}$ to $\frac{1}{30}$ of an inch in diameter; those of the sow are kidney-shaped, and from $\frac{1}{50}$ to $\frac{1}{10} \overline{0}$; those of man are polygonal, and from $\frac{1}{50}$ to $\frac{1}{600}$; those of insects are spherical, and at most $\frac{1}{500}$ of an inch.

Fats all melt at a temperature much under $212^{\circ} \mathrm{F}$. When strongly heated with contact of air, they diffuse white pungent fumes, then blacken, and take fire. When subjected to distillation, they afford a changed fluid oil, carbureted hydrogen, and the other products of oily bodies. Exposed for a certain time to the atmosphere, ther become rancid, and generate the same fat acids as they do by saponification. In their fresh state they are all composed principally of stearine, margarine, and oleine, with a little coloring and odorous matter; and, in some species, hircine, from the goat ; phocenine, from the dolphin; and butyrine, from butter. By subjecting them to a great degree of cold, and compressing them between folds of blotting paper, a residuum is obtained, consisting chiefly of stearine and margarine; the latter of which may be dissolved out by oil of turpentine.

Beef and Mutton Suet.-When fresh, this is an insipid, nearly inodorous fat, of a firm consistence, almost insoluble in alcohol, entirely so if taken from the kidners and mesenteric web of the ox, the sheep, the goat, and the stag. It varies in its whileness, consistence, and combustibility, with the species and health of the animals. That of the sheep is very white and very solid. They may all be purified in the manner above described. Strong sulphuric acid developes readily the acid fats by stirring it through melted suet. Alkalis, br saponification, give rise at once to the three acids, - the stearic, margaric, and oleic. Beef suet consists of stearine, margarine, and oleine; mutton and goat suet contain a little hircine. The specific grarity of the tallow of which common candles are made is, by my experiments, 0.936. The melting point of suet is from $98^{\circ}$ to $104^{\circ} \mathrm{F}$. The proportion of solid and fluid fat in it is somewhat variable, but the former is in much larger proportion. Mutton suet is soluble in 44 parts of boiling alcohol, of 0.820 ; beef suet in 44 parts. Marrow fat consists of 76 of stearine, and 24 of oleine; it melts at $115^{\circ} \mathrm{F}$.

- Hog s'lard is soft, fusible at $81^{\circ} \mathrm{F}$., convertible, by an alkaline solution, into a stearate, margarate, oleate, and glycerine. Its sp. grav. is 0.938 , at $50^{\circ} \mathrm{F}$. It consists of 62 of oleine, and 38 of stearine, in 100 parts.

Goose-fat consists of 68 oleine and 32 stearine.

Butter, in summer, consists of 60 of oleine and 40 of stearine; in winter, of 35 of oleine, and 65 of stearine; the former substance being yellow, and the latter white It differs, howewer, as produced from the milk of different cows, and also according to their pasture.

The ultimate constituents of stearine, according to Chevreul, are 79 carbon, $11 \cdot 7$ hydrogen, and $9 \cdot 3$ oxygen, in 100 parts.

$1,294,009$ crvts. of the tallow imported in 1837 were retained for internal consumption. See Margarine, Oleine, Soap, Stearine.

FAULTS (Failles, Fr.), in mining, are disturbances of the strata which interrupt 
the miner's operations, and put him at fault, to discover where the vein of ore or bed of coal has been thrown by the convulsions of nature. Many examples of faults are exhib. ited under Prtcoal.

FEATHERS (Plumes, Fr.; Federn, Germ.) constitute the subject of the manufacture of the Plumassier, a name given by the French (and also the English) to the artisan who prepares the feathers of certain birds forornaments to the toilet of ladies and for military men, and to him also who combines the feathers in various forms. We shall content ourselves with describing the method of preparing ostrich feathers, as most others are prepared in the same way.

Several qualities are distinguished in the feathers of the ostrich; those of the male, in particular, are whiter and more beautiful. Those upon the back and above the wings are preferred; next, those of the wings, and lastly, of the tail. The down is merely the feathers of the other parts of the body, which vary in length from 4 to 14 inches. This down is black in the males, and gray in the females. The finest white feathers of the female have always their ends a little grayish, which lessens their lustre, and lowers their price. These feathers are imported from Algiers, Tunis, Alexandria, Madagascar, and Senegal; this being the order of their value.

The scouring process is thus performed:-4 ounces of white soap, cut small, are dissolved in 4 pounds of water, moderately hot, in a large basin; and the solution is made into a lather by beating with rods. Two bundles of the feathers, tied with packthread, are then introduced, and are rubbed well with the hands for five or six minutes. After this soaping they are washed in clear water, as hot as the hand can bear.

The whitening or bleaching is performed by three successive operations.

1. They are îmmersed in hot water mixed with Spanish white, and well agitated in it; after which they are washed in three waters in succession.

2. The feathers are azured in cold water containing a little indigotied up in a fine cloth. They should be passed quickly through this bath.

3. They are sulphured in the same way as straw hats are (See Sur.PHuRING); they are then dried by hanging upon cords, when they must be well shaken from time to time to open the fibres.

The ribs are scraped with a bit of glass cut circularly, in order to render them very pliant. By drawing the edge of a blunt knife over the filaments they assume the curly form so much admired. The hairs of a dingy color are dyed black. For 20 pounds of feathers, a strong decoction is made of 25 pouncs of $l o g$ wood in a proper quantity of water. After boiling it for 6 hours, the wood is taken out, 3 pounds of copperas are thrown in; and, after continuing the ebuilition for 15 or 20 minutes, the copper is taken from the file. The feathers are then immersed by handfuls, thoroughly soaked, and worked about; and left in for two or three days. They are next cleansed in a very weak alkaline ley, and soaped three several times. When they feel very soft to the touch, they must be rinsed in cold water, and afterwards dried. White feachers are very difficult to dye a beautiful black. The acetate of iron is said to answer better than the sulphate, as a mordant.

For dying other colors, the feathers should be previously well bleached by the action oî the sun and the dew; the end of the tube being cut sharp like a toothpick, and the feathers being planted singly in the grass. After fifteen days' exposure, they are cleared with soap as above described.

Rose color or pink, is given with safflower and lemon juice.

Deep red, by a boiling hot bath of Brazil wood, after aluming.

Crimson. The above deep red feathers are passed through a bath of cudbear.

Prune de Monsieur. The deep red is passed through an alkaline bath.

Blues of every shade, are dyed with the indigo vat.

Yellow; after aluming, with a bath of turmeric or weld.

Other tints may be obtained by a mixture of the above dyes.

Feathers have some more useful employments than the decoration of the heads of women and soldiers. In one case, they supply us with a soft elastic down on which we can repose our wearied frames, and enjoy sweet slumbers. Such are called bed feathers. Others are employed for writing, and these are called quills.

Goose feathers are most esteemed for beds, and they are best when plucked from the living bird, which is done thrice a year, in spring, midsummer, ans the beginning of harvest. The qualities sought for in bed feathers, are softness, elasticity, lightness, and warmth. Their only preparation when cleanly gathered is a slight beating to clear away the loose matter, but for this purpose they must be first well dried either by the sun or a stove. Bleaching with lime water is a bad thing, as they never can be freed from white dust afterwards.

The feathers of the eider duck, anas mollissima, called eider down, possess in a superior degree all the good qualities of goose down. It is used only as a covering to beds, and never should be slept upon, as it thereby loses its elasticity. 
Quills for writing. These consist usually of the feathers plucked out of the wings of geese. Dutch quills have been highly esteemed, as the Dutch were the first who hit upon the art of preparing them well, by clearing them both inside and outside from a fatty humor with which they are naturally impregnated, and which prevents the ink from flowing freely along the pens made with them. The Dutch for a long time employed hot cinders or ashes to attain this end; and their secret was preserved very carefully, but it at length transpired, and the process was then improved. A bath of very fine sand must be kept constantly at a suitable temperature, which is about $140^{\circ} \mathrm{F}$; into this, the quill end of the feather must be plunged, and left in it a few instants. On taking them out they must be strongly rubbed with a piece of flannel, after which they are found to be white and transparent. Both carbonate of potash in solution and dilute sulphuric acid have been tried to effect the same end, but without success. The yellow tint which gives quills the air of age, is produced by dipping them for a little in dilute muriatic acid, and then making them perfectly dry. But this process must be preceded by the sand-bath operation. The above is the French process.

Quills are dressed by the London dealers in two ways; by the one, they remain of treir natural color; by the other, they acquire a yellow tint. The former is called the Dutch method, and the principal workman is called a Dutcher. He sits before a small store fire, into which he thrusts the barrel of the quill for about a second, then lays its root quickly below his blunt-edged knife called a hook, and, pressing this firmly with the left hand, draws the quill briskly through with his right. The bed on which the quill is laid to receive this pressure is called the plate. It is a rectangular smooth lump of iron, about 3 inches long, $1 \frac{1}{2}$ broad, and $2 \frac{1}{2}$ thick, which is heated on his stove to about the 350 th degree Fahr. The hook is a ruler of about 15 inches in length, somewhat lilie the pattenmaker's knife, its fulcrum being formed at the one end by a hooli and staple, and the power of pressure being applied by the hand at the other end. The quill, rendered soft and elastic by the heat, endures the strong scraping action of the tool, and thus gets stripped of its opaque outer membrane, without hazard of being split. A skilful workman can pass 2000 quills through his hands in a day of 10 hours.

They are next cleaned by being scrubbed by a woman with a piece of rough dog-fish skin, and finally tied up by a man in one quarter of a hundred bundles.

In another mode of dressing quills, they are steeped a night in decoction of turmeric, to stain them yellow; taken out and dried in warm sand contained in a pot, then scraped by the Dutcher as above described. The first are reclioned to make the best pens, though the second may appear more beautiful.

Crow quills for draughtsmen, as well as swan quills, are prepared in the same way. The quills plucked from well-fed living birds have most elasticity, and are least subject to be moth-eaten. The best are those plucked, or which are spontaneously cast in the month of May or June, because they are then fully ripe. In the goose's wing the five exterior feathers only are valuable for writing. The first is the hardest and roundest of all, but the shortest. The next two are the best of the five. They are sorted into those of the right and the left wing, which are differently bent. The heaviest quills are, generally speaking, the best. Lately, steaming for four hours has been proposed as a good preparation.

FECULA (Fecule, Fr.; Slärkemehl, Germ.) sometimes signifies corn flour, sometimes starch from whatever source obtained.

FELDSPAR (Or/hose, Fr.; Feldspath, Germ.) is a mineral crystallizing in oblique rhomboidal prisms, susceptible of two clivages; lustre more pearly than vitreous; spec. grav. $2 \cdot 39$ to $2 \cdot 58$; scratches glass; yields no water when calcined; fusible at the blowpipe into a white enamel; not affected by acids. The liquid left from its analytical treatment with nitrate of baryta, nitric acid, and carbonate of ammonia, affords on evaporation an alkaline residuum which precipitates platina from its chloride, and appears from this, as well as other tests, to be potash. Feldspar consists of - silica, 66.75 ; alumina, 17.50 ; potash, 12 ; lime, 1.25 ; oxyde of iron, 0.75 . Rose: This mineral is a leading constituent of granite; and in its decomposed state furnishes the petuntse or Cornish stone, so much used in the porcelain and best pottery manufactures.

FELTING (Feutrage, Fr.; Filzen, Germ.) is the process by which loose flocks of wool, and hairs of various animals, as the beaver, rabbit, hare, \&c., are mutually interlaced into a compact textile fabric. The first step towards making felt is to mix, in the proper proportions, the different kinds of fibres intended to form the stuff; and then, by the vibratory strokes of the bowstring, to toss them up in the air, and to cause them to fall as irregularly as possible, upon the table, opened, spread, and scattered. The workman corers this layer of loose flocks with a piece of thick blanket stuff slightly moistened; he presses it with his hands, moving the hairs backwards and forwards in all directions. Thus the difierent fibres get interlaced, by their ends pursuing ever tortuous paths; their vermicular motion being always, however, root foremost. As the 
matting gets denser, the hand pressure should be increased in order to overcome the increasing resistance to the decussation.

A first thin sheet of soft spongy felt being now formed, a second is condensed upon it in like manner, and then a third, till the requisite strength and thicliness be obtained. These ditlerent pieces are successively brought together disposed in a way suitable to the wished-for article, and united by continued dexterous pressure. The stuff must be next subjected to the fulling-mill. See Hat Manufacture.

FERMENT (Eng. and Fr.; Hefe, Germ.) is the substance which, when added in a small quantity to vegetable or animal fluids, tends to excite those intestine motions and changes which accompany fermentation. It seems to be the result of an alteration which $\mathbf{v} \epsilon$, etable albumen and gluten undergo with contact of air amidst a fermenting mass. The precipitate or lees which fall down when fermentation is finished consist of a mixcure of the fermenting principle with the insoluble matters contained in the ferzented liquor, some of which, like hordeine, existed in the worts, and others are probably generated at the time.

To prepare a pure ferment, or at least a compound rich in that principle, the precipitate separated during the fermentation of a clear infusion of malt, commonly called yeast or barm, is made use of. This pasty matter must be washed in cold distilled water, drained and squeezed between the folds of blotting paper. By this treatment it becomes a pulverulent mass, composed of small transparent grains, yellowish gray when viewed in the coinpound microscope. It contains much water, and is therefore soft, like moist gluten and albumen. When dried, it becomes like these bodies, translucid, yellowish brown, horny, hard, and brittle. In the soft humid state it is insipid, inodorous, insoluble in water and alcohol. If, in this state, the ferment be left to itself at a temperature of from $60^{\circ}$ to $70^{\circ} \mathrm{F}$., but not in too dry a situation, it putrefies with the same phenomena as vegetable gluten and albumen, and leaves, like them, a residuum resembling old cheese.

At the beginning of this change, particularly if the ferment be enclosed in a limited portion of air, there is an absorption of oxygen gas with a fivefold disengagement of carbonic acid gas; while acetic acid makes its appearance in the substance. When distilled by itself it affords the same products as gluten. Dilute acids dissolve it very readily; and so does potash with the production of ammonia, a peculiar circumstance, for in dissolving gluten the alkali causes no such evolution.

The property possessed by yeast of determining the fermentation of a properly diluted solution of sugar is very fleeting, and is lost by very trifling alterations. It is destroyed by complete desiccation, and cannot be restored by moistening it again. The attempts made in London to squeeze out the liquid part of yeast in bags placed in a powerful press, and to obtain a solid cake, in order to transport ferment to India, have had but a very partial success; for its virtue is so impaired that it will rarely excite a perfect fermentation in the best prepared worts. The same method is adopted in Germany, to send yeast to only moderate distances; and therefore with more advantage.

If yeast be boiled for ten minutes, it loses the greater part of its fermenting power, and by longer boiling it becomes inert.

When alcohol is poured upon yeast, it immediately destroys its fermenting faculties, though, on filtering it off, it seems to carry no remarkable principle with it. One thousandth part of sulphuric acid equally deprives yeast of its peculiar property, and so does a little strong acetic acid. All the acids and the salts, especially those which part readily with their oxygen, produce the same effect. A very small quantity of sulphurous acid, or sulphites, mustard powder, particularly the volatile oil of mustard, and in general the volatile oils that contain sulphur, as well as the vegetables which yield them, such as horse-radish and garlic, all kill the fermenting agent. Lastly, fermentation is completely stopped by a moderate depression of temperature.

During fermentation the yeast undergoes a change; it loses the property of causing another wort to ferment. This change probably depends upon the chemical reaction between the ferment and the sugar that is decomposed; for a certain quantity of yeast can effect the fermentation of only a certain quantity of sugar, and all the sugar exceeding this quantity remains unaltered in the liquor. It has been concluded from some rather loose experiments, that one part and a half of yeast (supposed to be in the dry state) is adequate to the fermentation of a solution of 100 parts of pure sugar. When such a solution is fermented by the precise proportion of yeast, the fermenting principle is exhausted, for no new yeast is formed in it. There is a deposite indeed to about half the weight of the yeast employed, of a white matter insoluble in water, which affords no ammonia by dry distillation, and is incapable of acting as a ferment upon a fresh saccharine solution.

Of all the bodies convertible into yeast during fermentation, vegetable gluten and albumen possess the most rapid and energetic powers. But ordinary glue, isingglass, animal fibrine, curd or caseum, albumine, urine, and other azotized substances, all 
enjoy the property of causing a solution of sugar to ferment; with this difference, that whilst yeast can establish a complete fermentation in less than an hour, at a temperature of about $68^{\circ}$, the above substances require several days, with a heat of from $77^{\circ}$ to $87^{\circ}$ F., for becoming ferments, and for occasioning fermentation. Substances devoid of nitrogen do not produce a ferment.

FERMENTATION. (Eng. and Fr.; Gahrung, Germ.) When organic substances, under the influence of water, air, and warmth, are abandoned to the reciprocal operation of their proximate principles (sugar, starch, gluten, \&c.), they are entirely changed and decomposed, so that their ultimate principles (oxygen, hydrogen, carbon, and in some cases azote) combine in new proportions, and thus gire birth to various new compounds. To this process, the general name of fermentation has been given. These operations and their products differ according to the differences of the substances, and of the circumstances in which they are placed. The following may be enumerated as sufficiently distinct species of fermentation. 1. The saccharine fermentation, in which starch and gum are changed into sugar. 2. The rinous fermentation, in which sugar is converted into alcohol. 3. The mucilaginous fermentation, in which sugar is conrerted into slime, instead of alcohol. 4. The acetous fermentation, in which alcohol and other substances are converted into vinegar. 5. The putrid fermentation or putrefaction, which characterizes particularly the decomposition of azotized organic substances.

1. The saccharine fermentation. When a paste made by boiling one part of starch with twelre parts of water is left entirely to itself, water merely being stirred in as it evaporates, at the end of a month or two in summer weather it is changed into sugar, equal in weight to from one third to one half of the starch, and into gum, equal to from one fifth to one tenth, with a residuum of starch paste somewhat altered. This saccharifying process advances much quicker through the co-operation of vegetable albumine or gluten, acting as a ferment. If we boil two parts of potato starch into a paste with twenty parts of water, mix this paste with one part of the gluten of wheat flour, and set the mixture for 8 hours in a temperature of from $122^{\circ}$ to $167^{\circ} \mathrm{F}$, the mixture soon loses its pasty character, and becomes by degrees limpid, transparent, and sweet, passing at the same time first into gum, and then into sugar. The remainder consists of the unchanged starch with the altered gluten, which has become sour, and has lost the faculty of acting upon fresh portions of starch. It is probable, howerer, that the sugar formation in the first case, when the starch undergoes a spontaneous change, may be due to the action of a small portion of gluten and albumine left in the starch, since a putrefactive smell is eventually evolved indicative of that azotized matter. The gum into which during this process the starch is first converted, and which becomes afterwards sugar, is of the same nature as British gum, formed by the roasting of starch.

This production of sugar takes place in the germination and kiln-drying of malt; and the mashing of the brewer as well as the sweetening of bread in baking, rests upon the same principles. In many cases the vinous fermentation precedes the saccharification, or accompanies it; the starchy parts of the fermenting mass changing into sugar, while the previously formed sugar becomes wine or beer. In the sweetening of fruits by keeping, a similar process occurs; the gummy and starchy fibres become sugar from the action of the glutinous ferment which they contain; as happens also to the juices of many fruits which sweeten for a little while after they have been expressed.

The nature of this sugar formation through the influence of gluten upon starch, is undoubtedly the same as the conversion of starch into sugar, by boiling it with sulphuric acid; though the whole theory of this change is not entirely developed.

The most energetic substance for the conversion of starch into sugar, is the malt of barley. According to the researches of Payen and Persoz, the gum which br this process is first formed, may be prevented from going into sugar, by merely exposing it to a boiling heat, and hence we have it in our power either to make sugar or gum at pleasure. Of finely ground malt from 10 to 25 parts must be taken for 100 parts of starch. Into a pan placed in a water bath, 400 parts of water being warmed to from $77^{\circ}$ to $86^{\circ} \mathrm{F}$., the ground malt must be stirred in, and the temperature must be raised to $140^{\circ}$. The 100 parts of starch must now be added, and well mixed. The heat is then to be increased to $158^{\circ} \mathrm{F}$.; and be so regulated that it shall not fall below $149^{\circ}$, nor rise above $16 \tau^{\circ}$. In the course of 20 or 30 minutes the originally milky and pasty liquid will become gradually more attenuated, and eventually it will turn as fluid nearly as water. This is the point of time in which the starch has passed into gum, or into the substance lately denominated dextrine by the chemists. Should this mucilaginous matter, which appears to be a mixture of gum and a little starchy sugar, be wished for in that state, the temperature of the liquid must be suddenly raised to the boiling point, whereby the further action of the malt upon it is stopped. But on the other hand if sugar be desired, then the temperature must be steadily maintained at from $158^{\circ}$ to $167^{\circ}$ for threc quarters of an hour, in which time the greater part of the starch will have become 
sugar, and from the evaloration of the fluid a starchy sirup will be obtained, entirely similar to that procurable by the action of very dilute sulphuric acid upon starch.

The substance which operates this saccharine change, or the appropriate yeast of the sugar fermentation, which had been previously imagined to be a residuum of gluten or vegetable albumen in the germinated grain, has been traced by Payen and Persoz to a peculiar proximate vegetable principle called by them diastase. This substance is generated during the germination of barley, oats, and wheat, and may be obtained separately by infusing the ground malt in a small quantity of cold water, straining off the liquor, then filtering it, and heating the clear solution in a water-bath to the temperature of $158^{\circ} \mathrm{F}$. The greater part of the vegetable albumen is thus coagulated, and must be separated by a fresh filtration; the liquid is afterwards treated with alcohol as long as the flocculent precipitate of diastase falls. In order to purify it still more completely from the azotized matter, it may be once more dissolved in water, and again precipitated by alcohol. When dried at a low temperature, it appears as a white solid, which contains no azote, is insoluble in strong alcohol, but dissolves in weak alcohol and water. lits solution is neutral and tasteless; and if left to itself, it changes spontaneously, sooner or later, according to the degree of warmih, and becomes sour. At the temperature of from $149^{\circ}$ to $168^{\circ}$, it has the property of converting starch into gum or dextrine, and sugar; and, when sufficiently pure, it does this with such energy, that one part of it is capable of saccharifying 2000 parts of dry starch. It acts the more rapidly the larger its proportion. Whenever the solution of diastase with starch or dextrine has been heated in the boiling point, it loses the property of transforming these substances. One hundred parts of well malted barley appear to contain about one part of this new body.

2. The Vinous Fermentation.-In this fermentation the sugar existing in watery solution is, by the operation of the ferment or yeast, converted into alcohol, with disengagement of carbonic acid gas. If we dissolve one part of pure sugar in ten parts of water, and leave the solution in a temperature of from $68^{\circ}$ to $77^{\circ} \mathrm{F}$., which is that most favorable to fermentation, it will remain unaltered. But if we stir into that solution some beer yeast, the phenomena of fermentation soon appear in the above circumstances; for carbonic acid gas is evolved, with intestine movements of the liquid, and an increase of its temperature. A body of yeast rises to the surface, and exhibits a continual formation and rupture of air bubbles. At length the sugar being in a great measure decomposed, the motions cease, the liquor becomes clear, and instead of being a sirup, it is now a dilute alcohol. The yeast has by this time fallen to the bottom in a somewhat compact form, and of a whitish color, deprived of the property of exciting fermentation in fresh sirup, provided no undue excess of it was added at first, for that alone would remain effective. Experience shows that for the conversion of a determinate quantity of sugar by fermentation, a determinate quantity of yeast is necessary, which has been estimated at about $1 \frac{1}{2}$ per cent. in the dry state. When the yeast has been decomposed by fermenting its definite proportion of sugar, it loses its fermentable property, and leaves the excess of sugar unaffected, forming a sweet vinous solution. The same thing happens if the yeast be separated from the wort by a filter in the progress of the fermentation, for then all intestine motion speedily stops, although much saccharine matter remains.

In the juices of sweet fruits, of grapes, for example, the ferment is intimately associated with the sugar. It is at first soluble and inactive, till it absorbs oxygen from the atmosphere, whereby it becomes an operative ferment, but, at the same time, insoluble, so as to precipitate at the end of the process. When the expressed juice of the grape, or must, is enclosed in a vessel out of contact of air, and there subjected to the heat of boiling water, the small portion of oxygen present is rendered inactive, and the liquor experiences no fermentative change. If the grapes be squeezed in an atmosphere deprived of oxygen, and confined in the same, the juice will also remain unaltered. Recently expressed grape juice is limpid, and manifests the commencement of fermentation by the separation of the yeasty substance, which can take place only with access of air. The sclution becomes turbid after a certain time, gas begins to be evolved, and the separated ferment decomposes the sugar. At the end of the process the yeast collects at the bottom of the vessel, usually in larger quantity than was sufficient to complete the fermentation; and hence a considerable portion of it possesses still the fermentative faculty. The fermentation itself, when once begun, that is, whenever the yeasty particles are erolved and float in the liquid, for which evolution a very minute quantity of oxygen is sufficient, is thenceforth independent of the contact of air, and goes on as well in close as in open vessels; so that the production of alcohol and carbonic acid depends solely upon the mutual reaction of the ferment and the sugar.

The yeast, which may be obtained tolerably pure from a fine infusion of malt in a state of fermentation, after being washed with cold water to separate the soluble, gummy, and saccharine matter, and after being pressed between folds of blotting paper, constitutes a pulverulent, grayish yellow, granular substance, destitute of both taste and sineli, insoluble both in water and alcohol. Cold water dissolves, indeed, only $\frac{1}{400}$, and boiling water very little more. 
The essentially operative constituent of yeast is a peculiar azotized matter, which in the wine rat is mixed with some tartar and other salts, and in the beer tun with gum, starch, \&c. This animalized substance may be obtained in a separate state, according to Braconnot, by acting upon the washed yeast powder with a weak ley of carbonate of potash, and by decomposing the solution with vinegar, whereby the matter is thrown down in a gelatinous form. The substance thus obtained is insoluble in cold water and alcohol, but dissolves readily in very dilute alkaline leys, and even in lime water When diflised thiough water, it assumes a homogeneous aspect, as if it were really dissolved; but when this mixture is heated, the animalized matter coagulates, and separates in thick flocks. In this state it has lost its former properties, bein no longer soluble in allialine leys, even when concentrated. Acids exercise no solvent prower over this peculiar matter; they precipitate it from its solutions, as do also the earthy and metallic salts, which, moreover, combine with it. This is also the case with tannin. The combination of the ferment stuff with acids increases the stability of its constitution, and counteracts its tendency to influence solutions of sugar. These properties of the operative principle of yeast explain many of the phenomena of fermentation, as we shall presently see.

The animalized matter of yeast resembles gluten, albumen, caseum, and other azotized substances; if any one of these be put into a saccharine solution ready for fermentation, it will begin to operate a change, when aided by warmth and time, if it be previously decomposed in some measure to facilitate its influence; or if these substances be brought into a slightly putrescent state beforehand, they will cause more speedy fermentation. Thus white of egg, when added to saccharine liquors, requires a period of three weeks, with a temperature of $96^{\circ} \mathrm{F}$., before it will excite fermentation; afterwards the excess of the albumen forms a precipitate which may be used instead of yeast upon other sweet worts. The rapidity with which such azotized substances are capable of being converted into ferments of more or less purity and power is very variable; vegetable gluten and albumen being best fitted for this purpose. This conversion is accelerated when the sweet liquor in which the substance is diffused or dissolved has already begun to ferment; whence it appears that the presence of carbonic acid gas, combined with the liquor, is here of singular influence. Upon it, in fact, the formation and elimination of the yeast in fermenting liquors depend.

A solution of pure sugar, which has been made to ferment by the addition of yeast, furnishes no new yeast; but there remains after the process a portion of the yeast originally mixed, in an altered inoperative condition, should its quantity have been exactly adequate to the decomposition of the sugar, or in an operative state, should the quantity have been originally excessive.

But if the fermentable liquor contains vegetable albumen and gluten, as is commonly the case with the sweet juices of fruits and beer worts, these substances become changed into ferments in the course of the fermentation induced by the yeast, and, being superfluous, so to speak, for that particular process, they remain entire at the end, and may be collected for use in other operations.

Upon this principle is founded the increased production of yeast, and the manufacture of what has been called arificial barm, in which the fermentation is conducted chiefly with a view to the formation of yeast. To the fermenting mass, those kinds of meal are added, which abound in albumen and gluten, as barley, beans, or wheat, for instance; and the process is similar to the production of a great lump of learen, from the action of a small piece of it upon dough. The following prescription will illustrate this subject. Take three ounces of bean flour, add to it five quarts of boiling water, and boil the mixture for half an hour. Pour the decoction into a vessel, and stir into it, while hot, 56 ounces of wheaten flour. After the mixture cools to the temperature of $54^{\circ} \mathrm{F}$., add to it about two quarts of beer barm, stirring the whole well together. About 24 hours after the commencement of the fermentation, incorporate with the mixture 112 ounces of barley or bean flour, till it becomes a uniform dough, which must be thoroughly kneaded, rolled out into cakes about an inch thick, and cut into pieces of the size of a dollar. These cakelets must be dried upon laths in the sun in favorable weather, and then put up in a dry situation. For use, one of these dises is to be broken into pieces, laid in warm water, and set in a warm place during 12 hours. The soft mass will then serve the purpose of beer yeast.

Or we may mix equal parts of barley malt, wheat malt, and crushed rye, pour water at the temperature of $122^{\circ} \mathrm{F}$. over them into a tub till it stand a span above their surface; then stir well together, and allow the whole to remain at rest for a few hours, till it cools to about $65^{\circ} \mathrm{F}$. We must now add for each pound of the mingled meals, a quarter of an ounce of beer barm. The tub must be then covered, and preserved at a temperature of $63^{\circ} \mathrm{F}$. The husks, as they begin to rise to the surface, in consequence of the fermentation, must be taken off and squeezed through a cloth over the vessel. When the meal comes afterwards to subside to the bottom, the whole must be strained 
through a canvass bag, and freed from the superfluous moisture by squeezing. The bag with its doughy mass must next be surrounded with dry ashes, to remove the remaining humidity, and to arrest any further fermentation. This consistent ferment may be used instead of beer yeast.

It is dificult to prepare an artificial yeast without barm. The best process for this purpose is the following. Take five parts of honey, one part of powdered tartar, and sixteen parts of wheat or barley malt, stir the whole in water of the temperature of $122^{\circ}$ F., and place in a fermenting heat; when the yeast will, as usual, be eliminated.

The change which gluten or vegetable albumen undergoes in the different kinds of meal, when it becomes a ferment, consists apparently in an oxydation, since analysis shows that this ferment contains more oxygen than gluten does.

It has been already stated that yeast in its liquid condition readily putrefies, and becomes altogether useless for the process of fermentation. In order to preserve it for some time, it must be dried to such a degree as to resist spontaneous decomposition without losing its fermentative faculty; but completely dried yeast loses that property, and does not recover it by being again moistened. Beer barm may be dried after heing washed several times with cold water, till the last quantity comes off clear; but the insoluble portion must be allowed to settle fully before the water is poured away from it. The residuum being freed as much as possible from water, by drainage and pressure between flannel cloths, is to be dried in the shade by a current of warm air as quickly as possible, with the aid of frequent turning over. It must be afterwards kept in dry earthen vessels. Yeast may also be preserved a short time in activity by being kneaded with as much barley or wheat flour as it can take up without losing the doughy consistence. Dried yeast has, however, always an impaired activity. The easiest and most certain method of preserving yeast in its primitive power, is by mixing it, after pressure in flannel, with as much pulverized sugar as will render it dry, and putting up the mixture in air-tight vessels. The fermentative power of yeast is destroyed by the following means : 1. as already stated, by making it completely dry either by the evaporation of the water, or its abstraction by alcohol; 2 . by boiling, which if continued for ten minutes renders yeast quite inoperative; 3 . by the action of such substances as dissolve out its essential constituents; by alkalis, for instance, since the particles of yeast seem to be operative only in their insoluble granular state; 4 . by such substances as form combinations with it, and thereby either alter its nature, or at least increase the cohesion of its constituent parts, so that they can no longer operate upon sweet liquors by the decomposing affinity of its ultimate particles. Such bodies are the acids, especially the mineral ones, tannin and most salts, particularly the metallic, which unite with the yeast into new compounds. The volatile oils which contain sulphur exercise the same paralyzing influence upon yeast.

The circumstances which promote, and are necessary to, the vinous fermentation are, conformably to the above views, the following:-1. The presence of the proper quantity of active yeast, and its proper distribution through the worts. If in the course of a slack fermentation the yeast subsides to the bottom, the intestine motions cease entirely, but they may be excited anew by stirring up the ingredients, or rousing the tun, as the brewers say. 2. A certain degree of warmth, which should never be less than $51^{\circ} \mathrm{F}$, nor more than $86^{\circ}$; the temperature of from $68^{\circ}$ to $77^{\circ}$ being the most propitious for the commencement and progress of fermentation. When other circumstances are the same, the rapidity of the fermentation is proportional to the temperature within certain limits, so that by lowering it, the action may be moderated at pleasure. 3. The fermentation proceeds the better and more equably the greater the mass of fermenting liquor, probably on account of the uniformly high temperature, as well as the uniform distribution of the active particles of the yeast by the greater energy of the intestine movements. 4. The saccharine solution must be sufficiently diluted with water; when too much concentrated it will not ferment. Hence very sweet musts furnish wines containing much undecomposed sugar. For a complete fermentative action, one part of sugar should be dissolved in ten parts of water.

Fermentation may be tempered or stopped : 1 . by those means which render the yeast inoperative, particularly by the oils that contain sulphur, as oil of mustard; as also by the sulphurous and sulphuric acids. The operation of the sulphurous acid in obstructing the fermentation of must consists partly, no doubt, in its absorbing oxygen, whereby the elimination of the yeasty particles is prevented. The sulphurous acid, moreover, acts more powerfully upon fermenting liquors that contain tartar, as grape juice, than sul phuric acid. This acid decomposes the tartaric salts, and, combining with their bases, sets the vegetable acid free, which does not interfere with the fermentation; but the sulphurous acid operates directly upon the yeast : 2 . by the separation of the yeast, either with the filter or by subsidence: 3 . by lowering the temperature to $45^{\circ} \mathrm{F}$. If the fermenting mass become clear at this temperature, and be drawn off from the subsided yeast, it will not ferment again, though it should be heated to the proper pitch. 
The products of vinous fermentation are carbonic acid gas, and alcohol; of which the former escapes during the process, except in the case of the sparkling wines, like champaign, that are partially fermented in close vessels. The alcohol remains in the fermented liquor. 100 parts of sugar afford by complete decomposition nearly 50 parts of alcohol. According to Thenard, 100 parts of sugar are converted into 46.8 parts of carbonic acid, and 49.38 of alcohol ; besides 3.82 parts of carbon otherwise employed, which the sugar contained, above what is present in the former two products. This chemist found in the fermented liquor 4 per cent. of an extractive matter, soluble in water, and having an acidulous reaction, to whose formation, probably, that excess of carbon may be necessary. In what way the action of the yeasty particles upon the saccharine substance is carried on in the vinous fermentation, or what may be the interior working of this process, is not accurately understood. The quantitative relation of the carbonic acid and alcohol to the sugar is pretty well made out; but the determination of the ultimate principles of the ferment itself, before and after the vinous change, and of the residuum dissolved in the fermented liquor, has not been well ascertained. It is probable that the yeast undergoes in the process a similar decomposition to that of the putrefactive, and that its elementary constituents enter into new combinations, and abstract so much carbon and hydrogen from the sugar, that the remainder, amounting to 96 per cent. of the whole, may constitute one atom of alcohol and one of carbonic acid.

3. The slimy or glutinous fermentation.-This process takes place in weak solutions of sugar, at ordinary fermenting temperatures, where, from defect of good yeast, the vinous fermentation cannot proceed. In such circumstances, from one part of sugar, one third part of gum is formed. According to Desfosses, however, 100 parts of sugar afford 109.48 of gum or slime. This is formed when one part of sugar is dissolved in twenty parts of water, which had been previously boiled with washed barm or gluten, and then filtered. The process proceeds slowly and quietly, equally well in close vessels, as with contact of air, and continues at ordinary temperatures abcut 12 days; but it goes on more rapidly and completely at the heat of from $77^{\circ}$ to $86^{\circ} \mathrm{F}$. A small quantity of hydrogen and carbonic acid gas is disengaged, in the proportion of two to one by volume. The fermented liquor becomes turbid, and assumes a tough thready appearance, like a decoction of linseed. A small addition of sulphuric or sulphurous acid, of muriatic acid and alum, or of tannin, impedes this species of fermentation; because these substances combine, as in the vinous fermentation, with the ferment into an insoluble precipitate, unsusceptible of further change. In many wines, especially when bottled, this slimy fermentation occurs, and occasions their ropiness, which may be best remedied or prevented by the addition of as much tannin as will precipitate the dissolved mucous matter. This species of fermentation attacks very rapidly the rinsing waters of the sugar refiner, which always contain some fermentative gluten. A little alum is the best preventive in this case, because it precipitates the dissolved ferment.

4. The acetous or sour fermentation.-In this process, alcohol, more or less dilute, is resolved into water and vinegar, in consequence of the operation of the ferment; oxydizement of the alcohol being effected by the oxygen of the atmospherical air. The requisites of this process have been already detailed under the article AcETIC AcrD. They are the presence of atmospherical air; alcohol diluted to a certain degree with water ferment or yeast, and a temperature above $66^{\circ} \mathrm{F}$. The most active ferments are such substances as have already passed into the acetous state; hence vinegar, especially when it contains some yeasty particles, or is combined with porous and spongy bodies, so as to multiply its points of contact with the vinous liquor, is particularly powerful. Common yeast may also be employed for vinegar ferments, if it be imbued with a little vinegar, with leaven, crusts of bread soaked in vinegar, the stalks and husks of grapes, sawdust and shavings of beech or oak impregnated with vinegar, or the slimy sediment of vinegar casks called mother; all of which operate as ferments chiefly in consequence of the vinegar which they contain. The inside shavings of the staves of vinegar tuns act on the same principle.

The acetous fermentation may, moreover, go on along with the vinous in the same liquor, when this contains sugar as well as alcohol. Whilst the acidification of the alcohol is effected by the absorption of oxygen from the atmosphere, the sugar becomes alcohol with disengagement of carbonic acid, and then passes into vinegar. Since most liquors intended for making vinegar, such as wine, juices of fruits, ales, \&c., contain still a little sugar, they disengage always a little carbonic acid. Besides spirits, some other substances, such as gum, the mucilage of plants, and starch paste, directly ferment into vinegar. Sugar also seems to be convertible into vinegar without any vinous change. The albuminous matter of potato juice, precipitated by vinegar, serves as a proper ferment for that purpose, when added in its moist state to weak sirup. 5. See Putrefaction.

Mr. William Black, in his treatise on Brewing, has, with much ingenuity and apparent truth, endeavored to show that the process of fermentation is strongly influenced by electricity, not only that of the atmosphere, as has been long known from the circumstance 
of beer and wine becoming speedily sour after thunderstorms, but the voltaic, produced by electric combinations of metals in the fermenting tuns. He therefore recommends these tuns to be made with as little metallic work as possible, and to be insulated from the floor of the brewhouse. For the propriety of this advice he adduces some suriking examples. Wort which had become stationary in its fermentation, on being pumped out of square gyles imbedded in the floor, into casks placed upon wooden stillions, began immediately to work very well, and gained about 6 degrees of attenuation while throwing off its yeast. From the stagnation of the process in the gyles, he had in the morning predicted an approaching thunderstorm, which accordingly supervened in the course of the evening. In further support of his views he instances the fact, that, in dairies where the milk is put into porcelain vessels, and placed upon wooden shelves, it is seldom injured by lightning; but when contained in wooden or leaden vessels, and placed upon the ground, it almost invariably turns sour in thunders weather. His general conclusion is, "that the preservation or destruction of beer depends upon electricity; and the most certain mode of preservation is to insulate as much as possible, both the squares and all other utensils or vessels connected with the brewing or storing of beer."

Mr. Black further considers that unsoundness of worts is often the result of electricity excited between the mash tun and the copper.

Why is beer liable to get spoiled in thunder-storms, though apparently well insulated in glass bottles?

I shall conclude this article with Mr. Black's description of the phenomena of beer fermentation. In every regular process there are five distinct stages. In the first we see a substance like cream forming all round the edges of the gyle tun; which extends towards the centre until the whole is creamed over, constituting the first change. Next a fine curl appears like caulillower, which also spreads over the square surface, and according to the strength and appearance of this curl, the quality of the fermentation may be predicated. This he calls the second stage. What is technically called the stomach or vinous vapor now begins to be smelt, and continues to gain strength till the process is concluded. From the vinous energy of this odor, and the progressive attenuation of the wort, the vigor of the fermentation may be inferred. The experienced brewer is much guided in his operations by the peculiarity of this effluvium. The third change is when the cauliflower or curling top rises to a fine rocky or light yeasty head; and when this falls down, the fourth stage has arrived. Finally the head should rise to what is called close reasty, having the appearance of yeast all over. About this period the gas becomes so powerful as to puff up occasionally in little bells or bladders about the size of a walnut, which immediately break. The bells should appear bright and clear. If they be opaque or whey colored, there is some unsoundness in the wort. The great point is to add just. so much yeast as to carry the fermentation completely through these five changes at the regular periods.

FERROCYANATE, or, more correctly, FERROCYANIDE. (Ferrocyanure, Fr.; Eisencyanid, Germ.) Several compounds of cyanogen and metals possess the property of uniting together into double cyanides; of which there are none so remarkable in this respect, as the protocyanide of iron. This appears to be capable of combining with several simple cyanides, such as that of potassium, sodium, barium, s1 contium, calcium, and ammonium. The only one of these double cyanides of any importance in manufactures is the first, which is described under its commercial name, Prussiate of Potash.

FERROPRUSSIATES; another name for Ferrocyanides.

FIBRE, VEGETABLE, called also Lignire (Ligneux, Fr.; Pfanzen-faserstoff, Germ.), is the most abundant and general ingredient of plants, existing in all their parts, the root, the leaves, the stem, the flowers, and the fruit; amounting in the compact wood to 97 or 98 per cent. It is obtained in a pure state by treating saw-dust successively with hot alcohol, water, dilute muriatic acid, and weak potash ley, which dissolve, first, the resinous; second, the extractive and saline matters; third, the carbonate and phosphate of lime; and, lastly, any residuary substances. Ligneous fibres, such as saw-dust, powdered barks, straw, hemp, flax, linen, and cotton cloth, are convertible by the action of strong sulphuric acid into a gummy substance analogous to dex/rine, and a sugar resembling that of the grape.

If we put into a glass mortar 24 parts, by weight, of dry old cordage, chopped small, and sprinkle over it 34 parts of sulphuric acid, by degrees, so as to avoid heating the mixture, while we constantly stir it; and if, in a quarter of an hour, we triturate the mass with a glass pestle, the fibres will disappear without the disengagement of gas. A tenacious mucilage will be produced, almost entirely soluble in water. The gum being thus formed, may be separated from the acid ivy dilution with water, and addition of the requisite quantity of chalk; then straining the saturated liquid through linen cloth, concentrating it by evaporation, throwing down any remaining lime by oxalie 
acid, filtering anew, and mixing the mucilage with alcohol in great excess, which will take up the free acid, and throw down the gum. From 24 parts of hemp fibres thus treated, fully 24 parts of a gummy mass may be obtained, containing, bowever, probably some water.

When, instead of saturating the diluted acid paste with chalk, we boil it for 10 hours, the gummy matter disappears, and is replaced by sugar, which may be purified without any difficulty, by saturation with chalk, filtration, and evaporation to the consistence of sirup. In 24 hours crystallization begins, and, in 2 or 3 days, a concrete mass of grape sugar is formed; which needs merely to be pressed strongly between old linen cluths doubled, and then crystallized a second time. If this sirup be treated with bone black, a brilliant white sugar will be procured. 20 parts of linen rags yield 23 of good sugar. Bracomot. Guerin got $87 \frac{1}{2}$ of dry sugar from 100 parts of rags, treated with 250 of sulphuric acid. See Woon.

FIBRINE (Eng. and Fr.; Thierischer Fasersloff, Germ.) constitutes the principal part of animal muscle; it exists in the chyle, the blood, and may be regarded as the most abundant constituent of animal bodies. It may be obtained in a pure state by agitating or beating new drawn blood with a bundle of twigs, when it will attach itself to them in long reddish filaments, which may be deprived of color by working them with the hands under a streamlet of cold water, and afterwards freed from any adhering grease by digestion in alcohol or ether.

Fibrine, thus obtained, is solid, white, flexible, slightly elastic, insipid, inodorous, denser than water, but containing four fifths of its weight of it, and without action on litmus. When dried, it becomes semi-transparent, yellowish, stiff, and brittle: water restores its softness and flexibility. 100 parts of fibrine consist of 53.36 carbon, $19 \cdot 68$ oxygen, $7 \cdot 02$ hydrogen, and $19 \cdot 31$ azote. As the basis of flesh, it is a very nutritious substance, and is essential to the sustenance of carnivorous animals.

FILE (Lime, Fr.; Feile, Germ.) is a well known steel instrument, having teeth upor the surface for cutting and abrading metal, ivory, wood, \&c.

When the teeth of these instruments are formed by a straight sharp-edged chisel, extending across the surface, they are properly called files; but when by a sharp-pointed tool, in the form of a triangular pyramid, they are termed rasps. The former are used for all the metals, as well as ivory, bone, horn, and wood; the latter for wood and horn.

Files are divided into two varieties, from the form of their teeth. When the teeth are a series of sharp edges, raised by the flat chisel, appearing like parallel furrows, either at right angles to the length of the file, or in an oblique direction, they are termed single cut. But when these teeth are crossed by a second series of similar teeth, they are said to be double cut. The first are fitted for brass and copper, and are found to answer better when the teeth run in an oblique direction. The laiter are suited for the harder metals, such as cast and wrought iron and steel. Such teeth present sharp angles to the substance, which penetrate it, while single cut files would slip over the surface of these metals. The double cut file is less fit for filing brass and copper, because its teeth would be very liable to become clogged with the filings.

Files are also called by different names according to their various degrees of fineness. Those of extreme roughness are called rough; the next to this is the bastard cut; the third is the second cut; the fourth, the smooth; and the finest of all, the dead smooth. The very heavy square files used for heavy smith-work, are sometimes a little coarser than the rough; they are known by the name of rubbers.

Files are also distinguished from their shape, as flat, half-round, three-square, foursquare, and round. The first are sometimes of uniform breadth and thickness throughout, and sometimes tapering. The cross section is a parallelogram. The half-round is generally tapering, one side being flat, and the other rounded. The cross section is a segment of a circle, varying a little for different purposes, but seldom equal to a semi-circle. The three-square generally consists of three equal sides, being equilateral prisms, mostly tapering; those which are not tapering are used for sharpening the teeth of saws. The four-square has four equal sides, the section being a square. These files are generally thickest in the middle, as is the case with the smith's rubber. In the round file, the sec. tion is a circle, and the file generally conical.

The heavier and coarser kinds of files are made from the inferior marks of blistered steel. Those made from the Russian iron, known by the name of old sable, called from its mark CCND, are excellent. The steel made from the best Swedish iron, called hoop L or Dannemora, makes the finest Lancashire files, for watch and clock makers; a manufacture for which the house of Stubbs in Warrington is celebrated.

The steel intended for files is more highly converted than for other purposes, to give them proper hardness. It should however be recollected, that if the hardness be not accompanied with a certain degree of tenacity, the teeth of the file break, and do but little service.

Small files are mostly made of cast steel, which would be the best for all others, if 
it were not for its higher price. It is much harder than the blistered steel, and from having been in the fluid state, is entirely free from those seams and loose parts so common to blistered steel, which is no sounder than as it comes from the iron forge before conversion.

The smith's rubbers are generally forged in the common smith's forge, from the converted bars, which are, for convenience, made square in the iron before they come into this country. The files of lesser size are made from bars or rods, drawn down from the blistered bars, and the cast ingots, and known by the name of tilted steel.

The file-maker's forge consists of large bellows, with coke as fuel. The anvil-block, particularly at Sheffielu, is one large mass of mill stone girt. The anvil is of considerable size, set into and wedged fast into the stone; and has a projection at one end, with a hole to contain a sharp-edged tool for cutting the files from the rods. It alsc contains a deep groove for containing dies or bosses, for giving particular forms to the files.

The flat and square files are formed entirely by the hammer. One man holds the hot bar, and strikes with a small hammer. Another stands before the anvil with a two-handed hammer. The latter is generally very heavy, with a broad face for the large files. They both strilie with such truth as to make the surface smooth and flat, without what is cailed hand-hammering. This arises from their great experience in the same kind of work. The expedition arising from the same cause is not less remarkable.

The hali-round files are made in a boss fastened into the groove above mentioned. The steel being drawn out, is laid upon the rounded recess, and hammered till it fills the die.

The three-sided files are formed similarly in a boss, the recess of which consists of two sides, with the angle downwards. The steel is first drawn out square, and then placed in a boss with an angle downwards, so that the hammer forms one side, and the boss two. The round files are formed by a swage similar to those used by common smiths, but a little conieal.

The n̂le-cutter requires an anvil of a size greater or less, proportioned to the size of his fles, with a face as even and flat as possible. The hammers weigh from one to five or six pounds. The chisels are a little broader than the file, sharpened to an angle of about 20 degrees. The length is just sufficient for them to be held fast between the finger and thumb, and so strong as not to bend with the strokes of the hammer, the intensity of which may be best conceired by the depth of the impression. The antil is placed in the face of a strong wooden post, to which a wooden seat is attached, at a small distance befow the level of the anril's face. The file is first laid upon the bare anvil, one end projecting over the front, and the other over the back edge of the same. A leather strap now goes over each end of the file, and passes down upon each side of the block to the workman's feet, which, being put into the strap on each side, like a stirrup, holds the file firmly upon the anvil as it is cut. While the point of the file is cutting, the strap passes over one part of the file only, the point resting upon the anvil, and the tang upon a prop on the other side of the strap. When one side of the file is single cut, a fine file is run slightlr orer the teeth, to tabe away the roughness; when they are to be double cut, another set of teeth is cut, crossing the former nearly at right angles. The file is now finished upon one side, and it is erident that the cut side cannot be laid upon the bare anvil to cut the other. A flat piece of an alloy of lead and tin is interposed between the toothed surface and the anvil, while the other side is cut, which completely preserves the side already formed. Similar pieces of lead and tin, with angular and rounded grooves, are used for cutting triangular and half-round files.

Rasps are cut precisely in the same way, by using a triangular punch instead of a flat chisel. The great art in cutting a rasp is to place every new tooth as much as possible opposite to a vacancy.

\any abortive attempts have been made to cut the teeth of files by machinery. The folluwing plan, for which a patent was obtained by Mr. William Shilton, of Birmingham, in April, 1833, is replete with ingenious mechanical resources, and deserves to sueceed.

The blantis of steel for making the files and rasps, are held in a pair of clamps in connexion with a slide, and are moved forward at intervals under the head of the tilt hammer which carries the tool; the distance which the blank is to be adranced at every morement being dependant upon the required fineness or coarseness of the cut of the file, which movernent is effected and regulated by a rack and pinion, actuated by a pall and ratchet wheel, or the movement may be produced by any other convenient means.

When the machine is employed for cutting or indenting the teeth of rasps, the cutting tooi being pointed and only producing one tooth at a blow, the tilt hammer carrying the tool must be made to traverse at intervals across the width of the blank piece of steel 
from one edge to the other and back again; the blank being advanced in rength only when the hammer has produced the last cut or tooth toward either edge of the rasp.

In order to render this invention better understood, two views of the apparatus for prom ducing the cross-cut or teeth of the files are given.

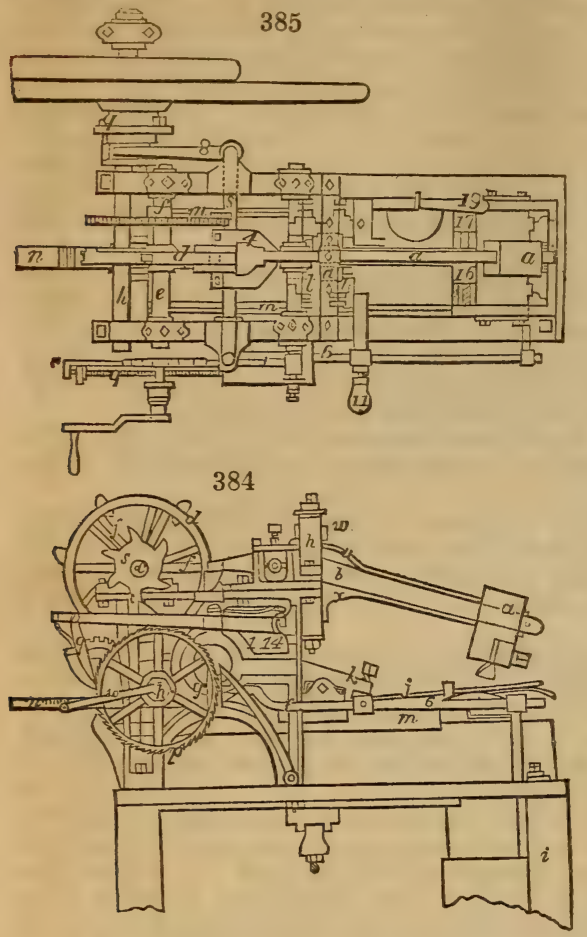

Fig. 384 is an elevation of the upper part of the file-cutting machine, as seen on one side; fig. 385 is a plan or horizontal view, as the machine appears on the top.

$a$, is the head of the tilt hammer placed in the end of the lever $b$, which is mounted on an axle $c_{3}$ turning in proper bearings in the frame work of the machine; $d$, is the tilt wheel mounted on another axle $s$, also turning in bearings on the frame work of the machine, and having any required number of projections or tappets upon it for depressing the tail or shorter end of the hammer or tilt lever $b$.

The tilt wheel $d$, receives its rotatory motion from the toothed wheel $f$, mounted upon the same axle, and it takes into gear with a pinion $g$, upon the main shaft $h$, which is actuated by a band passed from any first mover to the rigger on its end, or in any other convenient manner. The bed upon which the blank piece of steel bears is marked $i$. This bed is firmly supported upon masonry placed upon proper sleepers; $j$, is one of the blank pieces of steel under operation, and is shown secured in the pair of jaws or holding elamps $k$, mounted on centre pins in the slide $l$, fig. 385 ; which slide is held down by a spring and slide beneath, and is moved backwards and forwards in the machine upon the (v) edges $m, m$, of the frame, by means of the rack $n$, and its pinion; the latter being mounted upon the axle of the ratchet wheel $p$, and which ratchet wheel is made to turn at intervals by means of the pall $q$, upon the end of the lever $r, f i g .385 . \quad T h i s ~ l e v e r$ is depressed, after every cut has been effected upon the blank by means of the teeth or tappets of the wheel $s$, coming in contact with the inclined plane $t$, upon the lever $r$. The tappet wheel $s$, is mounted upon the end of the axle $e$, of the tilt wheel, and consequently revolves with it, and by depressing the lever $r$, every time that a tooth passes the inclined plane $t$, the eliek $q$, is made to drive the ratchet wheel $p$, and thereby the advancing movement of the blank is effected after each blow of the tilt hammer.

There is a strong spring $u$, attached to the upper side of the tilt hammer, its end being confined under an adjustable inclined plane $v$, mounted in the frame $w$, which inclined plane can be raised or lowered by its adjusting screws as required, to produce more or less tension of the spring.

A similar spring is placed on the under side of the tilt hammer, to raise and sustain the cutter or tool clear of the bed after every blow, and in conjunction with safety holders or eatchers, to counteract any vibration or tendency the spring $u$ may have to cause the hammer to reiterate the blow.

The end of the lower spring acts on an inclined plane, mounted in the frame $w$, which has an adjusting serew similar to $v$, to regulate the tension of the spring.

In case the under spring should raise, that is, return the hammer, with sufficient force or velocity to cause the top spring $u$, to reiterate the blow, the ends of the safety holders or catchers are made to move under and catch the tail of the lever $b$, immediately on its being raised by the under springs, which is effected by the following means :-The holders are mounted upon a plate or carriage 1 , fig. $38 \dot{t}$, which turns upon a small pin or axle mounted in the ears of a cross bar; the upper ends of the holders are kept inclined towards the tail of the tilt hammer by means of a spring fixed to the crcss bar, and which acts upon one end of the plate or carriage 1 . 
In order that the holders may be removed out of the way of the tail of the hammer $b$, when the tilt wheel is about to effect a blow, the tooth of the tilt wheel which last acted upon the han:mer comes in contact with an inclined plane fixed on the plate or carriage 1 , and by depressing that end of the plate, causes the upper ends of the holders to be withulrawn fiom under the tail of the hammer $b$. The tilt wheel continuing to revolve, the next torth advances, and depresses the tail of the hammer, but before it leaves the tail of the lranmer, the tooth last in operation will have quitted the inclined plane and allowed the spring to return the holders into their former position. After the tooth has eseaped from the tail of $b$, the hammer will immediately descend and effect the blow or cut on the blank, and as the tail of the hammer rises, it will come in contact with the inclined planes at the upper ends of the holders, and force them backwards; and as soon as the tail of the hammer has passed the top of the holders, the spring will immediately force the holders forward under the tail of the hammer, and prevent the hammer rising again until the next tooth of the tilt wheel is about to depress the end of the hammer, when the same movements of the parts will be repeated, and the machine will continue in operation until a sufficient length of the blank of steel (progressively advanced under the hammer) has been operated upon, when it will be thrown out of gear by the following means :-

Upon the sliding bar 6 there is placed an adjustable stop, against which the foremost end of the slide $l \vec{l}$, fig. $38 \mathrm{j}$, comes in contact as it is moved forward by the rack $n$, and its pinion. The sliding bar 6 is connected at its left end to the bent lever 8 , the other end of this lever being formed into a forked arm, which embraces a clutch upon the main shaft, and as the slide $l$ continues to advance, it will come in contact with a stop; and when it has brought a sufficient length of the blank pieces of steel under the operation of the cutting tool, the slide $l$, in its progress, will have moved that stop and the bar 6 forward, and that bar, by means of the bent lever 8 , will withdraw the clutch on the main shaft, from locking into the boss of the fly-wheel, and consequently stop the further progress of the machine; the rigger and fly-wheel turning loosely upon the main shaft.

The cut file can now be removed from out of the clamps, and reversed to cut the other side, or another blank piece put in its place; and after throwing back the pall $q$ of the ratchet wheel $p$, the slide $l$, and with it the fresh blank may be moved back into the machine by turning the winch handle, on the axle of the ratchet wheel $p$, the reverse way, which will turn the pinion backwards, and draw back the rack $n$, without affecting any other parts of the machine; and on moving back the bar 6 , by the handle 11 , placed on the stop, the clutches will be thrown into gear again, and the machine proceed to cut the next blank.

When the blanks have been thus cut on one side, and are reversed in the machine to form the tecth upon the other side, there should be a piece of lead placed between the blank and the bed to protect the fresh cut teeth.

It will be seen that the position of the stop upon the bar 6 will determine the length or extent of the blank piece of steel which shall be cut or operated upon; and in order that the progressive movement of the blanks under the cutting tool may be made to suit different degrees of fineness or coarseness of the teeth (that is, the distance between the cuts), there is an adjusting screw upon the lever $r$, the head of which screw stops against the under side of an ear projecting from the frame-work, and thereby determines the extent of the motion of the lever $r$, when depressed by the tappets of the wheel $s$, acting upon the inclined plane $t$, consequently determining the number of teeth the ratchet wheel $p$ shall be moved round by the pall $q$; and hence the extent of motion communicated by the rack and pinion to the slide $l$, and the blank $j$, which regulates the distance that the teeth of the file are apart, and the lever $r$ is forced upwards by a spring pressing against its under side.

It will be perceived that the velocity of the descent of the hammer, and consequently the force of the blow, may be regulated by raising or lowering the inclined plane $v$ of the spring $u$; and in order to accommodate the bed upon which the blanks rest to the different inclinations they may be placed at, that part of the bed is formed of a semi-globular piece of hardened steel, which fits loosely into a similar concavity in the bed $r$, and is therefore capable of adjusting itself so that the blanks shall be properly presented to the cutting tool, and receive the blow or cut in an equal and even manner; or the piece of steel may be of a conical shape, and fit loosely in a similar shaped concavity.

There are guides, 16 , placed on the top of the bed $i$, for the purpose of keeping the blanks in their proper position towards the cutting tool, and these can be regulated to suit blanks of any width, by turning the right and left handed screw 17 . There is also another adjustable stop on the jaws or clamps $k$, which serves as a guide when placing the blanks within the jaws; and 19 is a handle or lever for raising the clamps when required, which has a weight suspended from it for the purpose of keeping down the blanks with sufficient pressure upon the bed.

The cutting tool in the face of the hammer, can be placed at any required angle or . 
inclination with the blank, it being secured in the head of the hammer by clamps and screws. In cutting fine files a screw is employed in preference to the rack and pinion, for advancing the slide $l$, and the blank piece of steel in the machine.

Hardening of files. - This is the last and most important part of file making. Whatever may be the quality of the steel, or however excellent the workmanship, if it is not well hardened all the labor is lost.

Three things are strictly to be observed in hardening; first, to prepare the file on the surface, so as to prevent it from being oxrdated by the atmosphere when the file is red hot, which effect would not only take off the sharpness of the tooth, but render the whole surface so rough that the file would, in a little time, become clogged with the substance it had to work. Secondly, the heat ought to be very uniformly red throughout, and the water in which it is quenched, fresh and cold, for the purpose of giring it the proper degree of hardness. Lastly, the manner of immersion is of great importance, to prevent the files from warping, which in long thin files is very difficult.

The first object is accomplished by laring a substance upon the file, which, when it fuses, forms, as it were, a rarnish upon the surface, defending the metal from the action of the oxrgen of the air. Formerly the process consisted in first coating the surface of the file with ale grounds, and then corering it orer with pulverized common salt (muriate of soda). After this coating became dry, the files were heated red hot, and hardened; after this, the surface was lightly brushed orer with the dust of cokes, when it apprared white and metallic, as if it had not been heated. This process has lately been improred, at least so far as relates to the economy of the salt, which, from the quantity used, anc the increased thichness, had become a serious object. Those who use the improred method are now consuming about one fourth the quantity of salt used in the old method. The process consists in dissolving the salt in water to saturation, which is about three pounds to the gallon, and stiffening it with ale grounds, or with the cheapest kind of flour, such as that of beans, to about the consistence of thick cream. The files require to be dipped only into this substance, and immediately heated and hardened. The grounds or the flour are of no other use than to give the mass consistence, and br that means to allow a larger quantity of salt to be laid upon the surface. In this method the salt forms immediately a firm coating. As soon as the water is evaporated, the whole of it becomes fused upon the file. In the old method the dry salt was so loosely attached to the file, that the greatest part of it was rubbed off into the fire, and was sublimed up the chimney, without producing any effect.

The carbonaceous matter of the ale grounds is supposed to have some effect in giving hardness to the file, br combining with the steel, and rendering it more highly carbonated. It will be found, however, upon experiment, that vegetable carbon does not combine with iron, with sufficient facility to produce any effect, in the short space of time a file is heating for the purpose of hardening. Some file makers are in the habit of using the conl of burnt leather, which doubtless produces some effect; but the earbon is generally so ill prepared for the purpose, and the time of its operation so short, as to render the result inconsiderable. Animal carbon, when proper]r prepared and mixed with the above hardening composition, is capable of giving hardness to the surface eren of an iron file.

This carbonaceous matter mar be readily obtained from any of the soft parts of animals, or from blood. For this purpose, however, the refuse of shoemaliers and eurriers is the most convenient. After the volatile parts have been distilled orer, from an iron still, a bright shining coal is left behind, which, when reduced to powder, is fit to mix with the salt. Let about equal parts, br bulk, of this powder, and muriate of soda be ground together, and brought to the consistence of cream, by the addition of water. Or mix the powdered carbon with a saturated solution of the salt, till it become of the abore consistence. Files which are intended to be very hard should be corered with this composition previous to hardening. All files intended to file iron or steel, particularly saw files, should be hardened with the aid of this mixture, in preference to that with the flour or grounds. Indeed, it is probable that the carbonaceous powder might be used br itself, in point of economr, since the ammonia or hartshorn, obtained br distillation, would be of such ralue as to render the coal of no expense. Br means of this method the files made of iron, whioh, in itself, is unsusceptible of hardening, acquire a superficial hardness sufficient for any file whatever. Such files mar, at the same time, be bent into ant form; and, in consequence, are particularly useful for sculptors and die-sinkers.

The next point to be considered is the best method of heating the file for hardening. For this purpose a fire, similar to the common smith's fire, is generally emplored. The file is held in a pair of tongs by the tang, and introduced into the fire, consisting of very small cokes, pushing it more or less into the fire for the purpose of heating it regularly. It must frequently be withdrawn with the view of observing that it is not too hot in any part. When it is uniformly heated, from the tang to the point, of a 
eherry red color, it is fit to quench in the water. At present an oven formed of firebricks is used for the larger files, into which the blast of the bellows is directed, being open at one end, for the purpose of introducing the files and the fuel. Near to the top of the oven are placed two cross bars, on which a few files are placed, to be partially heating. In the hardening of heavy files this contrivance afiords a considerable saving, in point of time, while it permits them also to be more uniformly and thoroughly heated.

After the file is properly heated for the purpose of hardening, in order to produce the greatest possible hardness, it should be cooled as soon as possible. The most common method of effecting this is by quenching it in the coldest water. Some file-makers have been in the habit of putting different substances in their water, with a view to increase its hardening property. The addition of sulphuric acid to the water was long held a great secret in the hardening of saw files. After all, however, it will be.found that clear spring water, free from animal and vegetable matter, and as cold as possible, is the best calculated for hardening files of every description.

In quenching the files in water, some caution must be observed. All files, except the half-round, should be immersed perpendicularly, as quickly as possible, so that the upper part shall not cool. This management prevents the file from warping. The half-round file must be quenched in the same steady manner; but, at the same time that it is kept perpendicular to the surface of the water, it must be moved a little horizontally, in the direction of the round side, otherwise it will become crooked backwards.

After the files are hardened, they are brushed over with water and powdered cokes, when the surface becomes perfectly clean and metallic. They ought also to be washed well in two or three clean waters, for the purpose of carrying off all the salt, which, if allowed to remain, will be liable to rust the file. They should moreover be dipped into lime-water, and rapidly dried before the fire, after being oiled with olive oil, containing a little oil of turpentine, while still warm. They are then finished.

FILLIGREE (Filigrane, Fr.; Filigran, or Feine Drahtgeflecht, Germ.) is, as the last term justly expresses it, intertwisted fine wire, used for ornamenting gold and silver trinkets. The wire is seldom drawn round, but generally flat or angular, and soldered by gold or silver solder with borax and the blowpipe. The Italian word, filigrana, is compounded of filum and granum, or granular net-work; because the Italians, who first introduced this style of work, placed small beads upon it.

FILTRATION (Eng. and Fr.; Filtriren, Germ.) is a process, purely mechanical, for separating a liquid from the undissolved particles floating in it, which liquid may be either the useful part, as in vegetable infusions, or of no use, as the washings of mineral precipitates. The filtering substance may consist of any porous matter in a solid, foliated, or pulverulent form; as porous earthenware, unsized paper, cloth of many kinds, or sand. The white blotting paper sold by the stationers, answers extremely well for filters in chemical experiments, provided it be previously washed with dilute muriatic acid, to remove some lime and iron that are generally present in it. Filter papers are first cut square, and then folded twice diagonally into the shape of a cornet, having the angular parts rounded off. Or the piece of paper being cut into a circle, may be folded fan-like from the centre, with the folds placed exteriorly, and turned out sharp by the pressure of the finger and thumb, to keep intervals between the paper and the funnel into which it is fitted, to favor the percolation. The diameter of the funnel should be about three fourths of its height, measured from the neck to the edge. If it be more divergent, the slope will be too small for the ready efflux of the fluid. A filter covered with the sediment is most conveniently washed by spouting water upon it with a little syringe. A small camel's-hair paint brush is much employed for collecting and turning over the contents in their soft state. Agitation or vibration is of singular efficacy in quickening percolation, as it displaces the particles of the moistened powders, and opens up the pores which had become closed. Instead of a funnel, a cylindrical vessel may be employed, having its perforated bottom covered with a disc of filtering powder folded up at the edges, and made tight there by a wire ring. Linen or calico is used for weak alkaline liquors; and flannels, twilled woollen cloth, or felt-stuff, for weak acid ones. These filter bags are often made conical like a fool's cap, and have their mouths supported by a wooden or metallic hoop. Cotton wool put loose into the neck of a funnel answers well for filtering oils upon the small scale. In the large way, oil is filtered in conical woollen bags, or in a cask with many conical tubes in its bottom, filled with tow or cotton wool. Stronger acid and alkaline liquors must be filtered through a layer of pounded glass, quartz, clean sand, or bruised charcoal. The alcarrhazas are a porous biscuit of stone ware made in Spain, which are convenient f r filtering water, as also the porous filtering stone of Teneriffe, largely imported into England at one time, but now superseded in a great measur eby the artificial filters patented under many forms, consisting essentially of strata of gravel, sand, and charcoal powder.

It is convenient to render the filter self-acting, by accommodating the supply of liquid 
to the rate of percolation, so that the pressure upon the porous surface may be always equally great. Upon the small scale, the lamp-fountain or bird's-glass form, so generally used for lamps, will be found to answer.

Fig. 386 represents a glass bottle, A, partly filled with the flnid to be filtered, supported in the ring of a chemical stand, and having its mouth inverted into the same liquor in the filter funnel. It is obvious that whenever this liquor by filtration falls below the lip of the bottle, air will enter into it, let down a fresh supply to feed the filter, and keep the funnel regularly charged. If larger quantities are to be operated upon, the

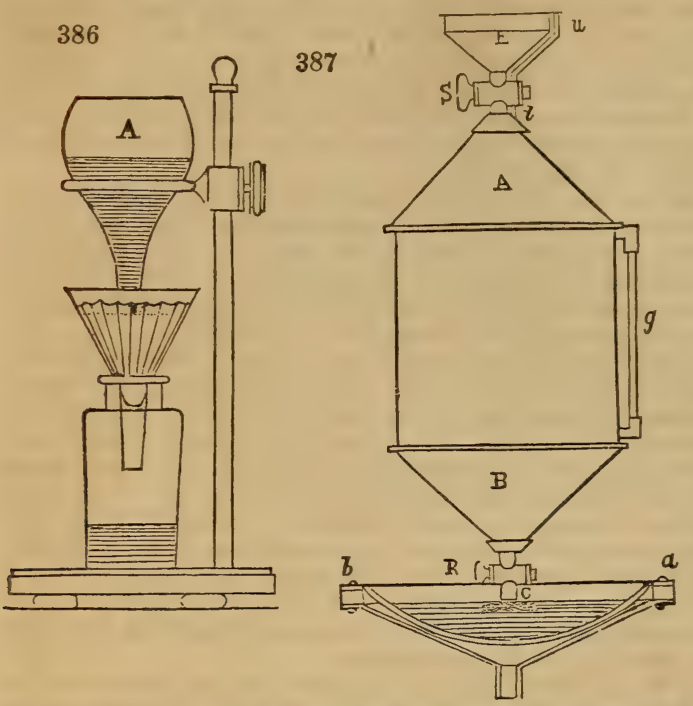

following apparatus may be employed. Fig. 387, $\mathrm{A} \mathrm{B}$, is a metallic vessel which may be made airtight ; $\mathrm{c}$ is the under pipe, provided with a stopcock, $\mathrm{R}$, for letting down the liquor into the filter $a b$. The upper pipe $t$, through which the fluid is poured by means of the funnel $E$, has also a stopeock which opens or shuts, at the same time, the small side tube $u t$, through which, during the entrance of the fluid, the air is let off from the receiver. A glass tube, $g$, shows the level of the liquor in the body of the apparatus. In using it, the cock $\mathbf{R}$ must be first closed, and the cock 8 must be opened to fill the receiver. Then the filter is set a going, by re-opening the cock $\mathrm{R}$, so as to keep the fluid in the filter upon a level with the opening of the tube c. Both these pieces of apparatus are essentially the same.

In many manufactures, self-acting filters are fed by the plumber's common con390 388

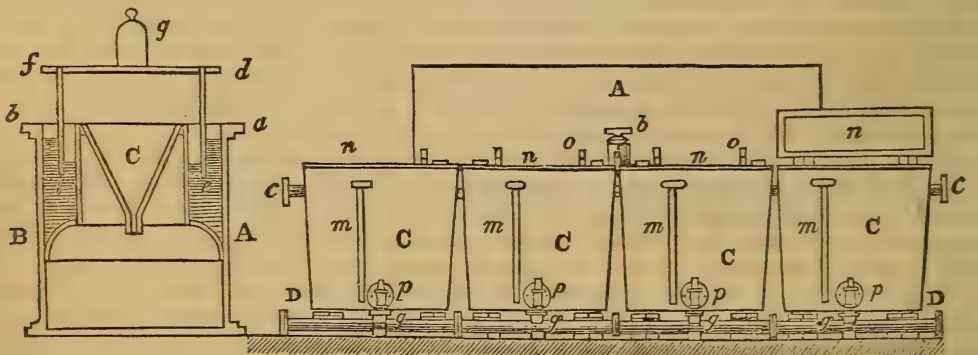

trivance of a ball-cock, in which the sinking and rising of the ball, within certain

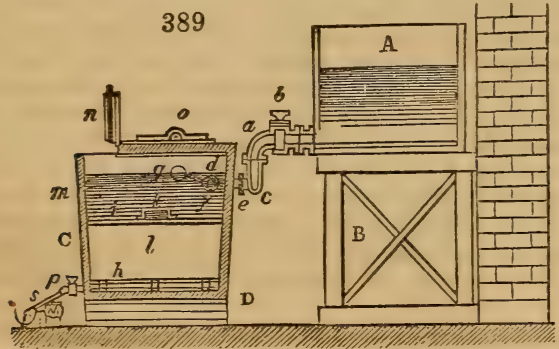

limits, serves to open or shut off the supply of liquor, as it may be required or not. Dumont has adopted this expedient for his system of filtering sirup through a stratum of granularly ground animal charcoal । or bone-black. Fig. 388 is a front view of this apparatus with 4 filters, c; and fig. 389 is a cross section. The framework B supports the eiszern $A$, in which the sirup is contained. From it the liquor flows through the stopcock 
$b$, and the connexion-tube $a$, into the common pipe $c$, which communicates, by the short branch tubes $e$, with each of the four filters. The end of the branch tube, which is inside of the filter tub, is provided with a stop-cock $d f$, whose opening, and thereby the efflux of the liquor from the cistern through the tube $a$, is regulated by means of the float-ball $g$. Upon the brickwork $\mathrm{D}$ the filter tub stands, furnished at $h$ with a false bottom of zinc or copper pierced with fine holes; besides which, higher up at $i$ there is another such plate of metal furnished with a strong handle $k$, by which it may be removed, when the bone black needs to be changed. In the intervening space $l$, the granular coal is placed. $o$ is the cover of the filter tub, with a handle also for lifting it. One portion of it may be raised by a hinge, when it is desired to inspect the progress of the filtration within. $m m$ is a slender vertical tube, forming a communication between the bottom part $h$, and the upper portion of the filter, to admit of the easy escape of the air from that space, and from among the bone black as the sirup descends; otherwise the filtration could not go on. $p$ is the stopeock through which the fluid collected in the space under $h$, is let off from time to time inte the common pipe $q, f i g .388 . \quad r$ is a trickling channel or groove lying parallel to the tube $q$, and in which, by means of a tube $s$, inserted at pleasure, the sirup is drawn off in case of its flowing in a turbid state, when it must be returned over the surface of the charcoal.

The celerity with which any fluid passes through the filter depends, 1 . upon the porosity of the filtering substance; 2 . upon the pressure exercised upon it; and 3 . upon the extent of the filtering surface. Fine powders in a liquor somewhat glutinous, or closely compacted, admit of much slower filtration than those which are coarse and free; and the former ought, therefore, to be spread in a thinner stratum and over a more extensive surface than the latter, for equal effect; a principle well exemplified in the working of Dumont's apparatus, just described.

In many cases filtration may be accelerated by the increase of hydrostatic or pneumatic pressure. This happens when we close the top of a filtering cylinder, and connect it by a pine with a cistern of fluid placed upon a higher level. The pressure of the air may be rendered operative also either by withdrawing it partially from a close vessel, into which the bottom of the filter enters, or by increasing its density over the top of the liquor to be filtered. Either the air pump or steam may be employed to create a partial void in the receiver beneath the filter. In like manner, a forcing pump or steam may be employed to exert pressure upon the surface of the filtering liquor. A common syphon may, on the same principle, be made a good pressure filter, by making its upper leg trumpet-shaped, covering the orifice with filter paper or cloth, and filling the whole with liquor, the lower ley being of such length so as to create considerable pressure by the difference of hydrostatic level. This apparatus is very convenient either on the small or great scale, for filtering off a clear fluid from a light muddy sediment. The pressure of the atmosphere may be elegantly applied to common filters, by the apparatus represented in $f i g .390$, which is merely a funnel enclosed within a gasometer. The case A $B$ bears an annular hollow vessel $a b$, filled with water, in which receiver the cylindrical gasometer $d, e, f, i$, is immersed. The filter funnel is secured at its upper edge to the inner surface of the annular vessel $a b$. In consequence of the pressure of the gasometer regulated by the weight $g$, upon the air enclosed within it, the liquid is equally pressed, and the water in the annular space rises to a corresponding height on the outer surface of the gasometer, as shown in the figure. Were the apparatus made of heet iron, the annular space might be charged with mercury.

In general, relatively to the application of pressure to filters, it may be remarked, that it cannot be pushed very far, without the chance of deranging the apparatus, or rendering the filtered liquor muddy. The enlargement of the surface is, generally speaking, the safest and most efficacious plan of increasing the rapidity of filtration, especially for liquids of a glutinous nature. This expedient is well illustrated in the creased bag filter now in use in most of the sugar refineries of London. See Sugar.

In many cases it is convenient so to construct the filtering apparatus, as that the liquid shall not descend, but mount by hydrostatic pressure. This method has two advantages : 1. that without much expensive apparatus, any desired degree of hydrostatic pressure may be given, as also that the liquid may be forced up through several filtering surfaces placed alongside of each other; 2 . that the object of filtering, which is to separate the particles floating in the fluid without disturbing the sediment, may be perfectly attained, and thus very foul liquids be cleared without greatly soiling the filtering surface.

Such a construction is peculiarly applicable to the purification of water, either alone, or combined with the downwards plan of filtration. Of the former variety an example is shown in fig. 391. The wooden or zinc conical vessel is provided with two perforated bottoms or sieves $e e$, betwixt which the filtering substance is packed. Over this, for the formation of the space $h h$, there is a third shelf, with a hole in its middle, through which the tube $d b$ is passed, so as to be water tight. This places the upper 
open part of the apparatus in communication with the lowest space $\alpha$. From the compartment $h h$ a small air tube $l$ runs upwards. The filtering substance consists at bottom of pebbles, in the middle of gravel, and at the top of fine sand, which may be mixed with coarsely ground bone black, or covered with a layer of the same. The water to be filtered being poured into the cistern at top, fills through the tube $b d$ the inferior compartment $a$, from which the hydrostatic pressure forces the water upward through the perforated shelf, and the filtering materials. The pure water collects in the space $h h$, while the air escapes by the small tube $l$, as the liquid enters. The stopcock $i$ serves to draw off the filtered water. As the motion of the fluid in the filter is slow, the particles suspended in it have time to subside by their own gravity; hence there collects over the upper shelf at $d$, as well as over the under one at $a$, a precipitate or deposite which may be washed out of the latter cavity by means of the stopcock $m$.

As an example of an upwards and downwards filter, fig. 392 may be exhibited. A B C D

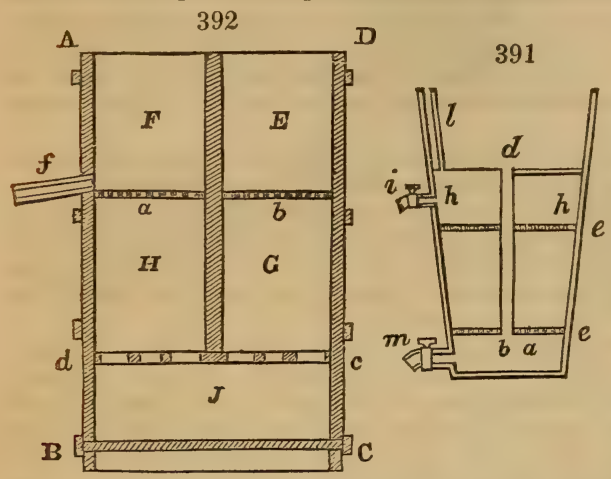
is a wooden or metallic cistern furnished with the perforated shelf $c d$ near its under part, upon which a vertical partition is fixed through the axis of the vessel. A semicircular perforated shelf is places at $\alpha$, and a second similar one at $b$. These horizontal shelves rest upon brackets in the sides of the cisterns, so that they may be readily lifted out. The space $\mathrm{G}$ is filled with coarse sand, J with moderately fine, and $\mathbf{H}$ with very fine. The foul water is poured into the chamber $\mathbf{E}$, and presses through $\mathbf{G} \mathbf{J} \mathbf{H}$ and into the space $\mathbf{F}$; whence it may be drawn by the stop$\operatorname{cock} f$.

Fig. 393 represents in section a filtering apparatus consisting of two concentric chambers; the interior being destined for downwards filtration, and the exterior for upwards. Within the larger cistern A, a smaller one B is placed concentrically, with its

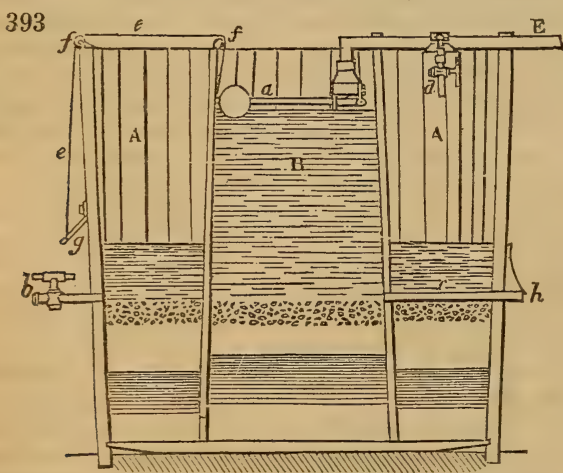
under part, and is left open from distance to distance, to make a communication between the interior cavity and the exterior annular space. These cavities are filled to the marked height with sand and gravel. The inner cylindrical space has fine sand below, then sharper sand with granular charcoal, next coarse sand, and lastly gravel. The annular space has in like manner fine sand below. The foul water is introduced by the pipe $\mathbf{E}$, the orifice at whose end is acted upon by a ball-cock with its lever $a$; whereby the water is kept always at the same

level in the inner vessel. The water sinks through the sand strata of the middle vessel, passes outwards at its bottom into the annular space, thence up through the sand in it, and collecting above it, is let off by the stopcock on the pipe $b$. When a muddy deposite forms after some time, it may be easily cleared out. The cord $e$, running over the pulleys $f f$, heing drawn tight, the ball lever will shut up the valve. The stopcock $d$ made fast to the conducting tube $\mathrm{E}$ must then be opened, so that the water now overflows into the annular space at $\mathrm{A}$; the tube $c$, in communication with the inner space $\mathrm{B}$, being opened by taking out the stopper $h$. The water thereby percolates through the sand strata in the reverse direction of its usual course, so as to clear away the impurities in the space $B$, and to discharge them by the pipe $c h$. An apparatus of this kind of moderate size is capable of filtering a great body of water. It should be constructed for that purpose of masonry; but upon a small scale it may be made of stone-ware.

A convenient apparatus for filtering oil upwards is represented in fig. $394 . \mathrm{g}$ is an bil cask, in which the impure parts of the oil have accumulated over the bottom. Immediately above this, a pipe $a$ is let in, which communicates with an elevated water cistern 
n. $f$ is the filter (placed on the lid of the cask), furnished with two perforated shelves, one at $e$ and another at $d$; which divide the interior of the filter into three com-

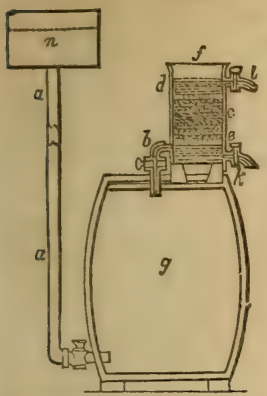
partments. Into the lower space immediately over the shelf $e$, the tube $b$, furnished with a stopcok, enters, to establish a communication with the cask; the middle cavity $e$ is filled with coarsely ground charcoal or other filtering materials; and the upper one has an eduction pipe, $l$. When the stopcocks of the tubes $a$ and $b$ are opened, the water passes from the cistern into the oil cask, occupies from its density always the lowest place, and presses the oil upwards, without mixing the two liquids; whereby first the upper and purer portion of the oil is forced through the tube $b$ into the filter, and thence out through the pipe $l$. When the fouler oil follows, it deposites its impurities in the space under the partition $c$, which may from time to time be drawn off through the stopeock $k$, while the purer oil is pressed upwards through the filter. In this way the different strata of oil in the cask may be filtered off in succession, and kept separate, if found necessary, for sale or use, without running any risk of mixing up the muddy matter with what is clear. According to the height of the water cistern $n$, will be the pressure, and, of course, the filtering force. When the filter gets choked with dirt, it may be easily recharged with fresh materials.

In filtering caustic alkaline leys through linen or quartz, it is proper to exclnde the free contact of air; which is done by enclosing the upper vessel, and attaching a pipe of communication between its cover and the shoulder of the lower vessel or recipient of the leys. In proportion as these flow down, they will displace their bulk of air, and drive it into the top of the upper vessel above the foul leys.

Many modifications of the above described apparatus are now on sale in this country; but certainly the neatest, most economical, and effective means of transforming the water of a stagnant muddy pool into that of a crystalline fountain, is afforded by the Royal Patent Filters of George Robins.

FIRE ARMS, MANUfacture of. This art is divided into two branches, that of the metallic and of the wooden work. The first includes the barrel, the lock, and the mounting, as also the bayonet and ramrod, with military arms. The second comprises the stock, and in fowling pieces, likewise the ramrod.

1. The Barrel. Its interior is called the bore; its diameter, the calibre; the back end, the breech; the front end, the muzzle; and the closing of the back end, the breech pin or plug. The barrel is generally made of iron. Most military muskets and lowpriced guns are fashioned out of a long slip of sheet-iron, folded together edgewise round a skewer into a cylinder, are then lapped over at the seam, and welded at a white heat. The most ductile and tenacious soft iron, free from all blemishes, must be selected for this slip. It is frequently welded at the common forge, but a proper airfurnace answers better, not being so apt to burn it. It should be covered with ashes or cinders. The shape of the bore is given by hammering the cylinder upon a steel mandril, in a groove of the anvil. Six inches of the barrel at either end are left open for forming the breech and the muzzle by a subsequent welding operation; the extremity put into the fire being stopped with clay, to prevent the introduction of cinders. For every length of two inches there are from two to three welding operations, divided into alternating high and low heats; the latter being intended to correct the defects of the former. The breech and muzzle are not welded upon the mandril, but upon the horn of the anvil; the breech being thicker in the metal, is more highly heated, and is made somewhat wider to save labor to the borer. The barrel is finally hammered in the groove of the anvil without the mandril, during which process it receives a heat every two minutes. In welding, the barrel extends about one third in length; and for muskets, is eventually left from 3 to $3 \frac{1}{2}$ feet long; but for cavalry pistols, only 9 inches.

The best iron plates for gun-barrels are those made of stub iron, that is, of old horse-shoe nails welded together, and forged into thin bars, or rather narrow ribands. At one time damascus barrels were much in vogue; they were fashioned either as above described, from plates made of bars of iron and steel laid parallel, and welded together, or from ribands of the same damascus stuff coiled into a cylinder at a red heat, and then welded together at the seams. The best modern barrels for fowling pieces are constructed of stub-nail iron in this manner. The slip or fillet is only half an inch broad, or sometimes less, and is left thicker at the end which is to form the breech, and thinner at the end which is to form the muzzle, than in the intermediate portion. This fillet being moderately heated to increase its pliancy, is then lapped round the mandril in a spiral direstion till a proper length of cylinder is formed; the edges being made to overlap a little in order to give them a better hold in the welding process. The coil 
being taken of the mandril and again heated, is struck down vertically with its muzzlo end upon the anvil, whereby the spiral junctions are made closer and more uniform. It is now welded at several successive heats, hammered by horizontal strokes, called jumping, and brought into proper shape on the mandril. The finer barrels are made of still narrower stub-iron slips, whence they get the name of wire twist. On the Continent, barrels are made of steel wire, welded together lengthwise, then coiled spirally into a cylinder. Barrels that are to be rifled require to be made of thicker iron, and that of the very best quality, for they would be spoiled by the least portion of scale upon their inside. Soldiers' muskets are thickened a little at the muzzle, to give a stout holding to the bayonet.

The barrels thus made are annealed with a gentle heat in a proper furnace, and slowly cooled. They are now ready for the borer, which is an oblong square bit of steel, pressed in its rotation against the barrel, by a slip of wood applied to one of its flat sides, and held in its place by a ring of metal. The boring bench works horizontally, and has a very shaky appearance, in respect at least of the bit. In some cases, however, it has been attempted to work the barrels and bits at an inclination to the horizon of $30^{\circ}$, in order to facilitate the discharge of the borings. The barrel is held in a slot by only one point, to allow it to humor the movements of the borer, which 395

396
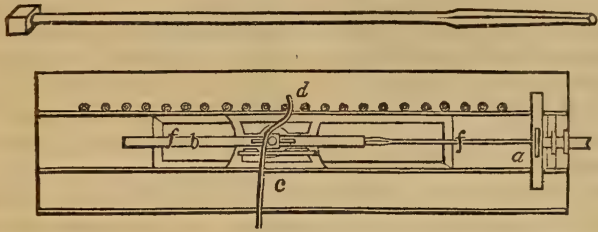
would otherwise be infallibly broken. The bit, as represented in fig. 395, has mereiy its square head inserted into a clamp-chuck of the lathe, and plays freely through the rest of its length.

Fig. 396 represents in plan the boring bench for musket barrels; $f f$ is the sledge or carriage frame in which the barrel is supported; $a$ is the revolving chuck of the lathe, into which the square end of the bit, fig. 395, is inserted; $b$ is the barrel, clamped at its middle to the carriage, and capable of being pressed onwards against the tapering bit of the borer, by the bent lever $c$, worked by the left hand of the operative against fulcrum knobs at $d$, which stand about two inches asunder. Whenever the barrel has been thereby advanced a certain space to the right, the bent end of the lever is shifted against another knob or pin. The borer appears to a stranger to be a very awkward and unsteady mechanism, but its perpetual vibrations do not affect the accuracy of the bore. The opening broach may be of a square or pentagonal form, and either gradually tapered from its thickest part, or of uniform diameter till within two inches of the end, whence it is suddenly tapered to a point.

A series of bits may be used for boring a barrel, beginning with the smallest and ending with the largest. But this multiplication of tools becomes unnecessary, by laying against the cutting part of the bit slips of wood, called spales, of gradually increasing thickness, so that the edge is pressed by them progressively further from the axis. The bore is next polished. This is done by a bit with a very smooth edge, which is mounted as above, with a wedge of wood besmeared with a mixture of oil and emery. The inside is finished by working a cylindrical steel file quickly backwards and forwards within it, while it is revolving slowly.

In boring, the bit must be well oiled or greased, and the barrel must be kept cool by letting water trickle on it; for the bit, revolving at the rate of 120 or 140 times a minute, generates a great deal of heat. If a flaw be detected in the barrel during the boring, that part is hammered in, and then the bit is employed to turn it out.

Many sportsmen are of opinion that a barrel with a bore somewhat narrowed towards the muzzle serves to keep shot better together; and that roughening its inside with pounded glass has a good effect, with the same view. For this purpose, also, fine spiral lines have been made in their interior surface. The justness of its calibre is tried by means of a truly turned cylinder of steel, 3 or 4 inches long, which ought to move without friction, but with uniform contact from end to end of the barrel. Whatever irregularities appear must be immediately removed.

The outer surface of the barrel is commonly polished upon a dry grindstone, but it is better finished, and less dangerously to the workman, at a turning lathe with a slide rest. If a stone be used, it should be made to revolve at the mouth of a tunnel of some kind, into which there is a good draught to carry off the ferruginous particles. A piece of moist cloth or leather should be suspended before the orifice.

Rifle barrels have parallel grooves of a square or angular form cut within them, each groove being drawn in succession. These grooves run spirally, and form each an aliquot part of a revolution from the chamber to the muzzle. Rifles should not be too deeply indented; only so much as to prevent the ball turning round within the barrel, 
and the spires should be truly parallel, that the ball may glide along with a regular pace. See infra.

The Parisian gun-makers, who are reckoned very expert, draw out the iron for the barrels at hand forges, in fillets only one ninth of an inch thick, one inch and a half broad, and four feet long. Twenty-five of these ribands are laid upon each other, between two similar ones of double thickness, and the bundle, weighing 60 pounds, bound with wire at two places, serves to make two barrels. The thicker plates are intended to protect the thinner from the violence of the fire in the numerous successive heats necessary to complete the welding, and to form the bundle into a bar two thirds of an inch broad, by half an inch thick; the direction of the individual plates relatively to the breadth being preserved. This bar, folded flat upon itself, is again wrought at the forge, till it is only half an inch broad, and a quarter of an inch thick, while the plates of the primitive ribands are now set perpendicular to the breadth of the narrow fillet; the length of which must be 15 or 16 feet French (16 or 17 English), to form a fowling piece from 28 to 30 inches long. This fillet, heated to a cherry red in successive portions, is coiled into as close a spiral as possible, upon a mandril about two fifths of an inch in diameter. The mandril has at one end a stout head for drawing $;$ out, by means of the hammer and the grooves of the anvil, previous to every heating. The welding is performed upon a mandril introduced after each heat; the middle of the barrel being first worked, while the fillets are forced back against each other, along the surface of the mandril, to secure their perfect union. The original plates having in the formation of the ultimate long riband become very thin, appear upon the surface of the barrel like threads of a fine screw, with blackish tints to mark the junctions. In making a double-barrelled gun, the two are formed from the same bundle of slips, the coils of the one finished fillet being turned to the right hand, and those of the other to the left.

The Damascus barrels forged as above described, from a bundle of steel and iron plates laid alternately together, are twisted at the forge several times, then coiled and welded as usual. Fifteen Parisian workmen concur in one operation: six at the forge; two at the boring mill; seven at filing, turning, and adjusting; yet all together make only six pairs of barrels per week, which are sold at from 100 to 300 francs the pair, ready for putting into the stock.

The breeching is of three kinds : the common; the chamber, plug, or mortar, fig. 397; 398 and the patent, fig. 398. The common

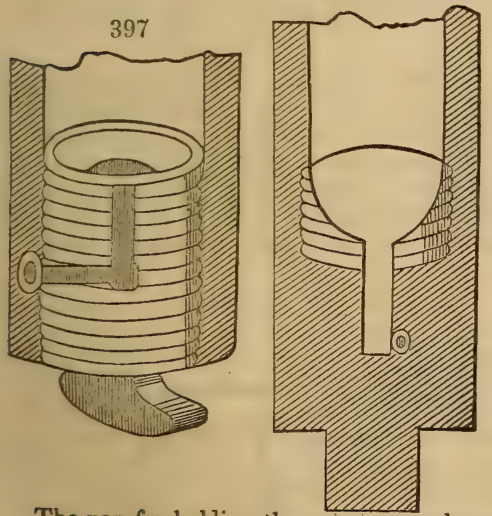
was formerly used for soldiers' muskets and inferior pieces. The second is a trifling improvement upon it. In the patent breeching, the screws do not interfere with the touch-hole, and the ignition is quicker in the main chamber.

The only locks which it is worth while to describe are those upon the percussion principle, as flint locks will certainly soon cease to be employed even in "military muskets. Forsyth's lock ( fig. 399) was an ingenious contrivance. It has a magazine $a$, for containing the detonating powder, which revolves round a roller $b$, whose end is screwed into the breech of the barrel. The priming powder passes through a small hole in the roller, which leads to a channel in communication with the chamber of the gun.

The pan for holding the priming is placed immediately over the litt.e hole in the roller. There is a steel punch $c$, in the magazine, whose under end stands above the pan, ready

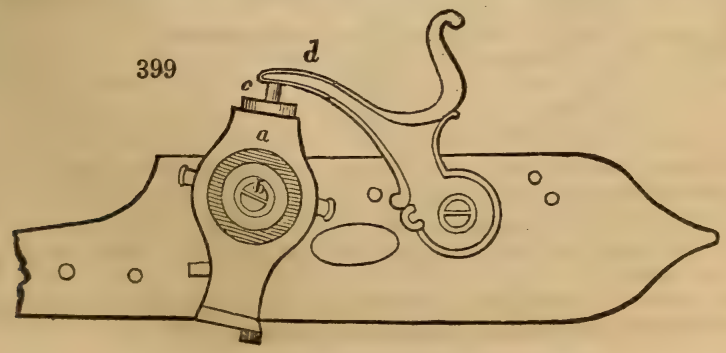


to ignite the priming when struck upon the top by the cock $d$, whenever the trigger is drawn. The punch immediately after being driven down into the pan is raised by the action of a spiral spring. For each explosion, the magazine must be turned so far round as to let fall a portion of the percussion powder into the pan; after which it is turned back, and the steel punch recovers its proper position for striking another blow into the pan.

The invention of the copper percussion cap was another great improvement upon the detonating plan. Fig. 400 represents the ordinary percussion lock, which is happily divested of three awkward projections upon the flint lock, namely, the hammer, hammer spring, and the pan. Nothing now appears upon the plate of the lock, but the cock or striking hammer, which inflicts the proper blow upon the percussion cap. It is concave, with a small metallic ring or border, called a shield or fence, for the purpose of enclosing the cap, as it were, and preventing its splinters doing injury to the sportsman, as also protecting against the line of flame which may issue from the touch-hole in the cap nipple. This is screwed into the patent breech, and is perforated with a small hole.

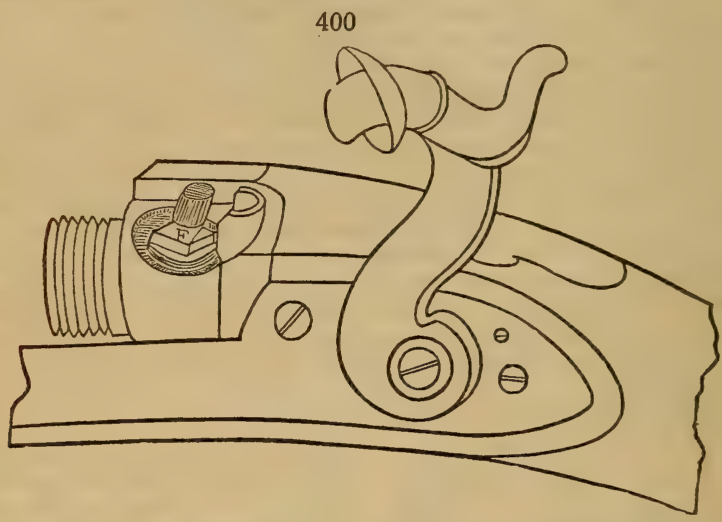

The safety lock of Dr. Somerville is a truly humane invention. Its essential feature is a slide stop or catch, placed under the trigger A, fig. 401 . It is pulled forward into a notch in the trigger, by means of a spring $B$, upon the front of the guard, which is worked by a key c, pressing upon the spring when the piece is discharged. In another safety plan there is a small moveable curved piece of iron, A, which rises through an opening $\mathbf{B}$, in the lock-plate $\mathbf{c}$, and prevents the cock from reaching the nipple, as represented in the figure, until it is drawn back within the plate of the lock when the piece is fired.

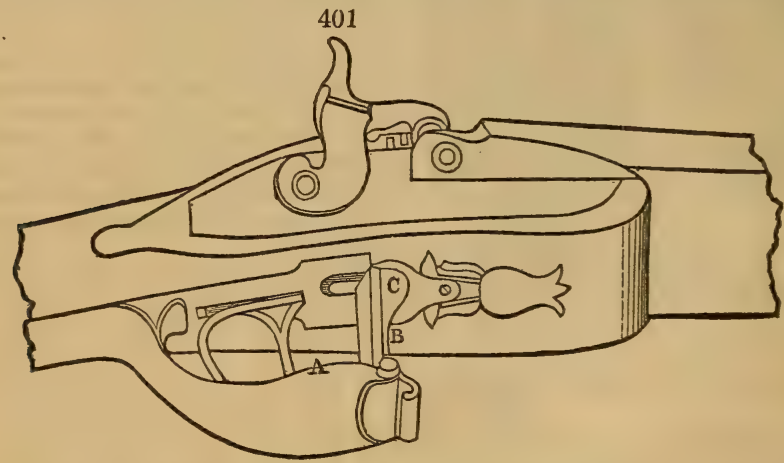

To fire this gun, two different points must be pressed at the same time. If by accident the key which works the safety be touched, nothing happens, because the trigger is not drawn; and the trigger touched alone can produce no effect, because it is locked. The pressure must be applied to the trigger and the key at the same instant, otherwise the lock will not work.

The French musket is longer than the British, in the proportion of 44.72 inches to 48 but the French bayonet is 15 inches, whereas the British is 17. 
Diameter of Dimensions.

of the bore - - $\quad-0.75$ in

Diameter of the ball - $\quad$ - $\quad$ - 0.676

Weight of the ball in oz. - _ _ - 1.06

Weight of the firelock and bayonet in lbs. - 12.25

Length of the barrel and bayonet -
- $59 \cdot 00$
Fr. Dimensions.

0.69 in.

0.65

0.958

$10 \cdot 980$

$59 \cdot 72$

Within these few years a great many contrivances have been brought forward, and several have been patented for fire arms. The first I shall notice is that of Charles Random, Baron de Berenger. Fig. 402 shows the lock and breech of a fowling piece, with a sliding protector on one of the improved plans; $a$ is the hammer, $b$ the nipple of the touch-hole, $c$ a bent lever, turning upon a pin, fixed into the lock-plate at $d$. The upper end of this bent lever stands partly under the nose of the hammer, and while in that situation stops it from striking the nipple. A slider $g f h$, connected with the under part of the gun-stock, is attached to the tail of the bent lever at $i$; and when the piece is brought to the shoulder for firing, the hand of the sportsman pressing against the bent part of the slider at $g$, forces this back, and thereby moves the end of the lever $c$ forward

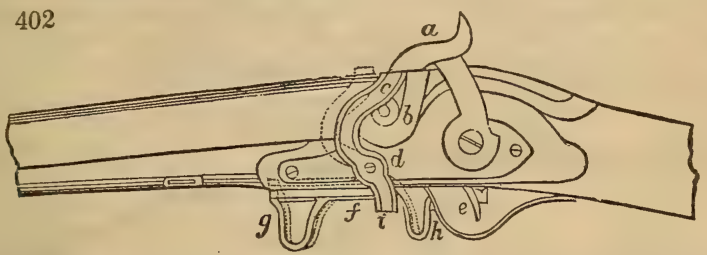

from under the nose of the cock or hammer, as shown by the dotted lines. The trigger being now drawn, the piece will be discharged; and on removing the hand from the end $g$, of the slider $f$, the spring at $h$ acting against the guard, will force the slider forward, and the lever into the position first described.

Mr. Redford, gun-maker of Birminghan, proposes a modification of the lock for small fire-arms, in which the application of pressure to the sear spring for discharging the piece is made by means of a plug, depressed by the thumb, instead of the force of the finger exerted against the trigger. Fig. 403 represents a fowling piece partly in

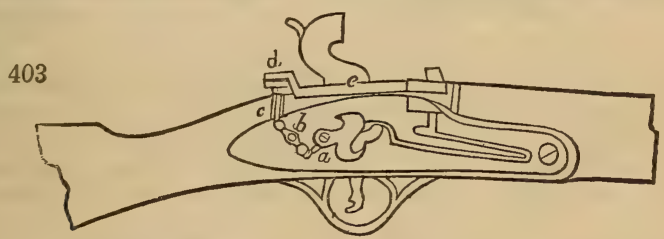

section. The sear spring is shown at $\alpha$. It is not here connected with the irigger as in other locks; but is attached by a double-jointed piece to a lever $b$, which turns upon a fulcrum pin in its centre. At the reverse end of this lever an arm extends forwards, like $t$ at of an ordinary sear spring, upon which arm the lower end of the plug $c$ is intended to bear; and when this plug is depressed by the thumb bearing upon it, that end of the lever $b$ will be forced downwards, and the reverse end will be raised, so as to draw up the end of the sear spring, and set off the piece. For the sake of protection, the head of the plug $c$ is covered by a moveable cap $d$, forming part of a slider $e$, which moves to and fro in a groove in the stock, behind the breech end of the barrel; this slider $e$ is acted upon by the trigger through levers, which might be attached to the other side of the lock-plate; but are not shown in this figure, to avoid confusion. When the piece is brought to the shoulder for firing, the fore-finger must be applied as usual to the trigger, but merely for the purpose of drawing back the slider $e$, and uncovering the head of the plug; when this is done, the thumb is to be pressed upon the head of the plug, and will thus discharge the piece. A spring bearing against the lever of the slider $e$, will, when the finger is withdrawn from the trigger, send the slider forward again, and cover the head of the plug, as shown.

It is with pleasure I again advert to the humane ingenuity of the Rev. John Somerville, of Currie. In April, 1835, he obtained a patent for a further invention to prevent the accidental discharge of fire arms. It consists in hindering the hammer from reaching the nipple of a percussion lock, or the flint reaching the steel of an ordinary one, by the interposition of moveable safety studs or pins, which protrude from under the false breech before the hammers of the locks, and prevent them from descending to strike. 
These safety studs or pins are moved ont of the way by the pressure of the right hand of the person using the gun only when in the act of firing, that is, when the force of the right hand and arm is exerted to press the butt end of the stock of the gun against the shuulder while the aim is taken and the trigger pulled. In carrying the gun at rest, the proper parts of the thumb or hand do not come over Mr. Somerville's moveable buttons or studs.

Fig. 404 is a side view of part of a double percussion gun; and fig. 405 is a top or plan view, which will serve to explain these improvements, and show one, out of many, methods of carrying them into effect. A is the stock of the gun; B the barrels; $\mathrm{c}$ the breech; $D$ the nipples; $E$ the false breech, on the under side of which the levers which work the safety studs or pins are placed; $F$ is the shield of the false breech; $G$, triggers; $\boldsymbol{H}$ the locli-plate; and $\mathrm{r}$ the hammers : all of which are constructed as usual: $u a$ are the safety studs or pins, which protrude before the shield $F$, and work through guide pieces on the under side of the false breech. The button piece is placed in the position for the thumb of the right hand to act upon it; but when the pressure of the ball of the right thumb is to produce the movement of the safety studs, it must be placed

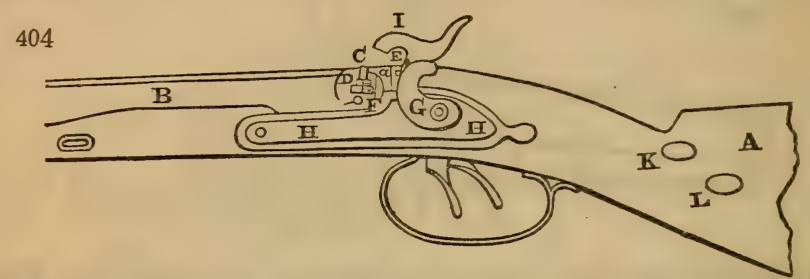

in or near the position $\mathrm{K}$; and when the heel of the right hand is to effect the movements of the safety studs, the button piece must be placed at $L$, or nearly so.
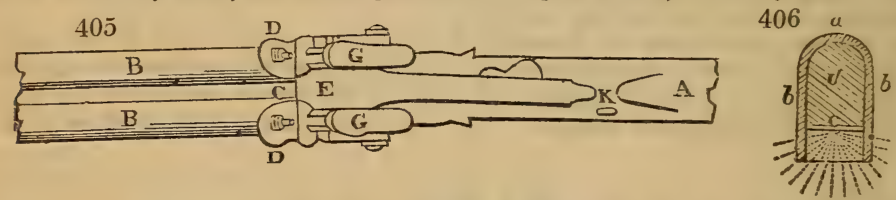

In these last two positions, the lever (which is acted upon by the button piece to work the safety studs through a slide) would require to be of a different shape and differently mounted. When the hammers are down upon the nipples after discharging the gun, the ends of the safety pins press against the inner sides of the hammers. When this invention is adapted to single-barrelled guns, only one pin, $a$, one lever and button piece will be required.

Mr. Richards, gun-maker, Birmingham, patented, in March, 1836, a modification of the copper cap for holding the percussion powder, as represented fig. 406 ; in which the powder is removed from the top of the cap, and brought nearer the mouth; $a$ being the top, $b$ the sides, and $c$ the position of the priming. The dotted lines show the direction of the explosion, whereby it is seen that the metal case is opened or distended only in a small degree, and not likely to burst to pieces, as in the commion caps, the space between $a$ and $c$ being occupied by a piece of any kind of hard metal $d$, soldered or otherwise fastened in the cap.

George Lovell, Esq., director of the Royal Manufactory of Arms at Enfield, has recently made a great improvement upon the priming chamber. He forms it into a vertical double cone, joined in the middle by the common apex; the base of the upper cone being in contact with the percussion cap, presents the most extensive surface to the fulminate upon the one hand, while the base of the under one being in a line with the interior surface of the barrel, presents the largest surface to the gunpowder charge, upon the other. In the old nipple the apex of the cone being at its top, afforded very injudiciously the minimum surface to the exploding force.

Guns, Rifling of the Barrels. - The outside of rifle barrels is, in general, octagonal, After the barrel is bored, and rendered truly cylindrical, it is fixed upon the rifling machine. This instrument is formed upon a square plank of wood 7 feet long, to which is fitted a tube about an inch in diameter, with spiral grooves deeply cut internally through its whole length; and to this a circular plate is attached, about 5 inches diameter, accurately divided in concentric circles, into from 5 to 16 equal parts, and supported by two rings made fast to the plank, in which rings it revolves. An arm eonnected with the dividing graduated plate, and pierced with holes, through which a 
pin is passed, regulates the change of the tube in giving the desired number of grooves to the barrel. An iron rod, with a moveable handle at the one end, and a steel cutter in the other, passes through the above rifling tube. This rod is covered with a core of lead one foot long. The barrel is firmly fixed by two rings on the plank, standing in a straight line on the tube. The rod is now drawn repeatedly through the barrel, from end to end, until the cutter has formed one groove of the proper depth. The pin is then shifted to annther hole in the dividing plate, and the operation of grooving is repeated till the whole number of riflings is completed. The barrel is next taken out of the machine, and finished. This is done by casting upon the end of a small iron rod a core of lead, which, when besmeared with a mixture of fine emery and oil, is drawn, for a considerable time, by the workmen, from the one end of the barrel to the other, till the inner surface has become finely polished. The best degree of spirality is found to be from a quarter to half a revolution in a length of three feet.

Mililary Rifles.-An essential improvement in this destructive arm has lately been introduced into the British service at the suggestion of $\mathrm{Mr}$. Lovell.

The intention in all rifles is to impart to the ball a rotatory or spinning motion round its axis, as it passes out through the barrel. This object was attained, to a certain degree, in the rifles of the old pattern, by cutting seven spiral grooves into the inside of the barrel, in the manner shown by fig. 407 , the spherical ball, fig. 408 , being a little

410

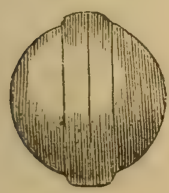

409

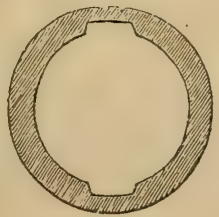

407

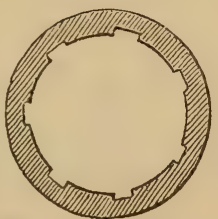

408

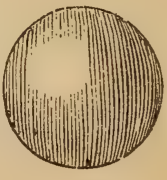

larger than the bore, was driven down with a mallet, by which the projecting ribs were forced into the surface of the ball, so as to keep it in contact with their curvatures, during its expulsion. Instead of this laborious and insecure process, the barrel being now cut with only two opposite grooves, fig. 409, and the ball being formed with a projecting belt, or zone, round its equator, of the same form as the two grooves, fig. 410, it enters so readily into these hollows, that little or no force is required to press it down upon the powder. So much more hold of the barrel is at the same time obtained, that instead of one quarter of a turn, which was the utmost that could be safely given in the old way, without danger of stripping the ball, a whole turn round the barrel, in its length, can be given to the two gronved rifles; whereby a far more certain and complete rotatory motion is imparted to the ball. The grand practical result is, that better practice has been performed by several companies of the Rifle Corps, at 300 yards, than could be produced with the best old military rifles at 150 yards; the soldier being meanwhile enabled to load with much greater ease and despatch. The belt is bevelled to its middle line, and not so flat as shown in the figure.

This mode of rifling is not, however, new in England. In fact, it is one of the oldest upon record; and appears to have fallen into disuse from faults in the execution. The ideg was revived within the last few years in Brunswick, and it was tried in

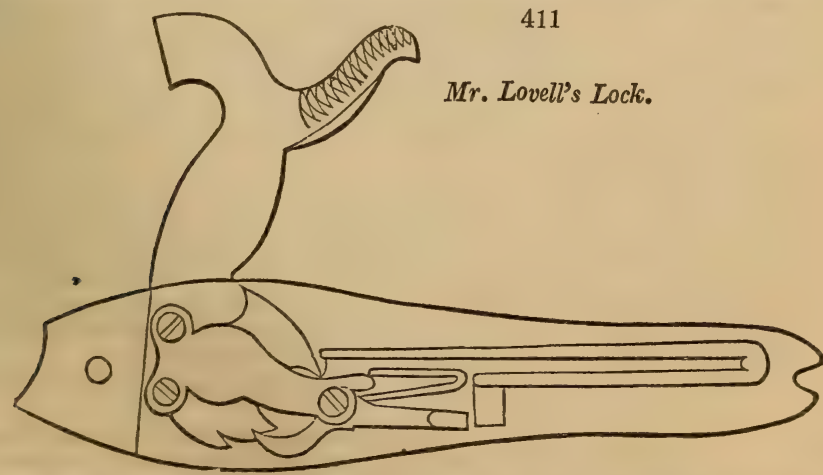

Hanover also, but with a lens-shaped (Linsenförmig) ball. The judicious modifications and improvements it has finally received in $\mathrm{Mr}$. Lovell's hands, have brought out all its 
advantages, and rendered it, when skilfully used, a weapon of unerring aim, even at the prodigious distance of 700 yauds.

The locks, also, for the military service generally, are now receiving an important im. provement by means of his labors, having been simplified in a remarkable manner. The action of the main spring is reversed, as shown by fig. 411 ; thus rendering the whole mechanism more solid, compact, and convenient; while the ignition of the charge being eflected by percussion powders in a copper cap, the fire of the British line will, in future, be more murderous than ever, as a mis-fire is hardly ever experienced with the fire-arms made at the Royal manufactory, under Mr. Lovell's skilful superintendence.

FIRE-DAMP ; the explosive carbureted hydrogen of coal mines. See Pitcoal.

FIRE-WORKS. (Feux d'artifice, Fr.; Feuerwerke, Germ.) The composition of luminous devices with explosive combustibles, is a modern art resulting from the discovery of gunpowder. The finest inventions of this kind are due to the celebrated Ruggieri, father and son, who executed in Rome and Paris, and the principal capitals of Europe, the most brilliant and beautiful fireworks that were ever seen. The following description of their processes will probably prove interesting to many of my readers.

The three prime materials of this art are, nitre, sulphur, and charcoal, along with filings of iron, steel, copper, zine, and resin, camphor, lycopodium, \&c. Gunpowder is used either in grain, half crushed, or finely ground, for different purposes. The longer the iron filings, the brighter red and white sparks they give; those being preferred which are made with a very coarse file, and quite free from rust. Steel filings and cast-iron borings contain carbon, and afford a more brilliant fire, with wavy radiations. Copper filings give a greenish tint to flame; those of zinc, a fine blue color; the sulphuret of antimony gives a less greenish blue than zine, but with much smoke; amber affords a yellow fire, as well as colophony, and common salt; but the last must be very dry. Lampblack produces a very red color with gunpowder, and a pink with nitre in excess. It serves for making golden showers. The yellow sand or glistening mica, communicates to fire-works golden radiations. Verdigris imparts a pale green; sulphate of copper and sal-ammoniac, a palm-tree green. Camphor yields a very white flame and aromatic fumes, which mask the bad smell of other substances. Benzoin and storax are used also on account of their agreeable odor. Lycopodium burns with a rose color and a magnificent flame; but it is principally employed in theatres to represent lightning, or to charge the torch of a fury.

Fire-works are divided into three classes: 1 . those to be set off upon the ground; 2. those which are shot up into the air; and 3 . those which act upon or under water.

Composition for jets of fire; the common preparation for rockets not more than $\frac{3}{4}$ of an inch in diameter, is; gunpowder, 16 parts; charcoal, 3 parts. For those of larger diameter; gunpowder, 16 ; steel filings, 4 .

Brilliant revolving wheel; for a tube less than $\frac{3}{4}$ of an inch; gunpowder, 16 ; steel filings, 3 . When more than $\frac{3}{4}$ : gunpowder, 16 ; filings, 4 .

Chinese or Jasmine fire; when less than $\frac{3}{4}$ of an inch : gunpowder, 16 ; nitre, 8 ; charcoal (fine), 3 ; sulphur, 3 ; pounded cast-iron borings (small), 10 . When wider than $\frac{3}{4}$ : gunpowder, 16 ; nitre, 12 ; charcoal, 3 ; sulphur, 3 ; coarse borings, 12 .

A fixed brilliant; less than $\frac{3}{4}$ in diameter : gunpowder, 16 ; steel filings, 4 ; or, gunpowder, 16 ; and finely pounded borings, 6 .

Fixed suns are composed of a certain number of jets of fire distributed circularly, like the spokes of a wheel. All the fusees take fire at once through channels charged with quick matches. Glories are large suns with several rows of fusees. Fans are portions of a sun, being sectors of a circle. The Palte d'oie is a fan with only three jets.

The mosaic represents a surface covered with diamond shaped compartments, formed by two series of parallel lines crossing each other. This effect is produced by placing at each point of intersection, four jets of fire, which run into the adjoining ones. The intervals between the jets must be associated with the discharge of others, so as to keep ap a succession of fires in the spaces.

Palm trees. Ruggieri contrived a new kind of fire, adapted to represent all sorts of trees, and especially the palm. The following is the composition of this magnificent green fire-work : crystallized verdigris, 4 parts; sulphate of copper, 2 ; sal-ammoniac, 1 . These ingredients are to be ground and moistened with alcohol. An artificial tree of any kind being erected, coarse cotton rovings about 2 inches in diameter, impregnated with that composition, are to be festooned round the trunk, branches, and among the leaves; and immediately kindled before the spirits have had time to evaporate.

Cascades imitate sheets or jets of water. The Chinese fire is best adapted to such decorations.

Fixed stars. The bottom of a rocket is to be stuffed with clay, and one diameter in height of the first preparation being introduced, the vacant space is to be filled with the following composition, and the mouth tied up. The pasteboard must be pierced into the preparation, with five holes, for the escape of the luminous rays, which represent a star. 


\section{Composition of fixed stars :-}

\begin{tabular}{|c|c|c|c|}
\hline Nitre - & $\begin{array}{c}\text { Ordinary. } \\
-\quad 16\end{array}$ & $\begin{array}{c}\text { Brighter. } \\
12\end{array}$ & $\begin{array}{c}\text { Colored. } \\
0\end{array}$ \\
\hline Sulphur & 4 & 6 & 6 \\
\hline Gunpowder mea & 4 & 12 & 16 \\
\hline Antimony & 2 & 1 & 2 \\
\hline
\end{tabular}

Lances are long rockets of small diameter, made with cartridge paper. Those which burn quickest should be the longest. They are charged by hand without any mould, with rods of different lengths, and are not strangled at the mouth, but merely stuffed with a quick match of tow. These lances form the figures of great decorations; they are fixed with sprigs upon large wooden frame works, representing temples, palaces, pagodas, \&c. The whole are placed in communication by conduits, or small paper cartridges like the lances, but somewhat conical, that they may fit endwise into one another to any extent that may be desired. Each is furnished with a match thread fully $1 \frac{1}{2}$ inches long, at its two ends.

Composition for the while lances: nitre, 16 ; sulphur, 8 ; gunpowder, 4 or 3 . For a bluish-white : nitre, 16 ; sulphur, 8 ; antimony, 4 . For blue lances : nitre, 16 ; antimony, 8. For yellow: nitre, 16; gunpowder, 16; sulphur, 8; amber, 8. For yellower ones: nitre, 16 ; gunpowder, 16 ; sulphur, 4 ; colophony, 3 ; amber, 4 . For greenish ones: nitre, 16 ; sulphur, 6 ; antimony, 6 ; verdigris, 6 . For pink lances : nitre, 16; gunpowder, 3 ; lampblack, 1 . Others less vivid are made with nitre, 16 ; colophony, 3 ; amber, 3 ; lycopodium, 3.

Cordage is represented in fire-works, by imbuing soft ropes with a mixture of nitre, 2; sulphur, 16 ; antimony, 1 ; resin of juniper, 1.

The Bengal flames rival the light of day. They consist of, nitre, 7; sulphur, 2; antimony, 1. This mixture is pressed strongly into earthen porringers, with some bits of quick match strewed over the surface. These flames have a fine theatrical effect for conflagrations.

Revolving suns are wheels upon whose circumference rockets of different styles are fixed, and which communicate by conduits, so that one is lighted up in succession after another. The composition of their common fire is, for sizes below $\frac{3}{4}$ of an inch: gunpowder meal, 16; chareoal, not too fine, 3. For larger sizes: gunpowder, 20; charcoal, not too fine, 4. For fiery radiations: gunpowder, 16 ; yellow micaceous sand, 2 or 3 . For mixed radiations: gunpowder, 16 ; pitcoal, 1 ; yellow sand, 1 or 2.

The waving or double Catharine wheels are two suns turning about the same axis in opposite directions. The fusees are fixed obliquely and not tangentially to their peripheries. The wheel spokes are charged with a great number of fusees; two of the four wings revolve in the one direction, and the other two in the opposite; but always in a vertical plane.

The girandoles, caprices, spirals, and some others, have, on the contrary, a horizontal rotation. The fire-worker may diversify their effects greatly by the arrangement and color of the jets of flame. Let us take for an example the globe of light. Imagine a large sphere turning freely upon its axis, along with a hollow hemisphere, which revolves slso upon a vertical axis passing through its under pole. If the two pieces be covered with colored lances or cordage, a fixed luminous globe will be formed; but if horizontal fusees be added upon the hemisphere, and vertical fusees upon the sphere, the first will have a relative horizontal movement, the second a vertical movement, which being combined with the first, will cause it to describe a species of curve, whose effect will be an agreeable contrast with the regular movement of the hemisphere. Upon the surface of a revolving sun, smaller suns might be placed, to revolve like satellites round their primaries.

Ruggieri exhibited a luminous serpent, pursuing, with a rapid winding pace, a butterfly which flew continually before it. This extraordinary effect was produced in the following way. Upon the summits of an octagon he fixed eight equal wheels turning freely upon their axles, in the vertical plane of the octagon. An endless chain passed round their circumference, going from the interior to the exterior, covering the outside semi-circumference of the first, the inside of the second, and so in succession; whence arose the appearance of a great festooned circular line. The chain, like that of a watch, carried upon a portion of its length a sort of scales pierced with holes for receiving colored lances, in order to represent a fiery serpent. At a little distance there was a butterfly constructed with white lances. The piece was kindled commonly by other fireworks, which seemed to end their play, by projecting the serpent from the bosom of the flames. The motion was communicated to the chain by one of the wheels, which received it like a clock from the action of a weight. This rumarkably curious mechanism was called by the artists a salamander.

The rockets which rise into the air with a prodigious velocity are among the mos: 
common, but not least interesting fire-works. When employed profusely, they form those rich volleys of fire which are the crowning ornaments of a public fete. The cartridge is similar to that of the other jets, except in regard to its length, and the necessity of pasting it strongly, and planing it well; but it is charged in a different manner. As the sky-rockets must fly off with rapidity, their composition should be such as to kindle instantly throughout their length, and extricate a vast volume of elastic fluids. To effect this purpose, a small cylindric space is left vacant round the axis; that is, the central line is tubular. The fire-workers call this space the soul of the rocket (a me de la fusée). On account of its somewhat conical form, hollow rods, adjustable to different sizes of broaches or skewers, are required in packing the charge; which must be done while the cartridge is sustained by its outside mould, or copper cylinder. The composition of skyrockets is as follows :-

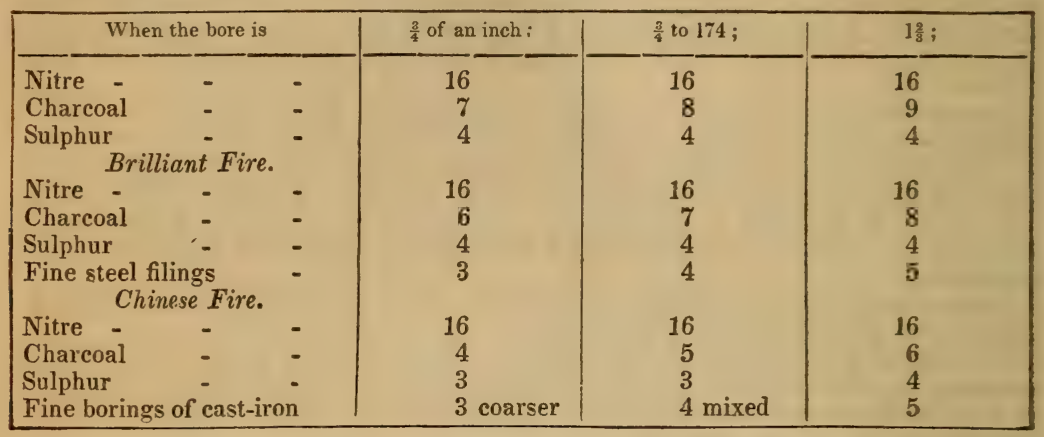

The cartridge being charged as above described, the pot must be adjusted to it, with the garniture; that is, the serpents, the crackers, the stars, the showers of fire, \&ce. The pot is a tube of pasteboard wider than the body of the rocket, and about one third of its length. After being strangled at the bottom like the mouth of a vial, it is attached to the end of the fusee by means of twine and paste. These are afterwards covered with paper. The garniture is introduced by the neck, and a paper plug is laid over it. The whole is enclosed within a tube of pasteboard terminating in a cone, which is firmly pasted to the pot. The quick-match is now finally inserted into the soul of the rocket. The rod attached to the end of the sky-rockets to direct their flight is made of willow or any other light wood. M. Ruggieri replaced the rod by conical wings containing explosive materials, and thereby made them fly further and straighter.

The garnitures of the sky-rocket pots are the following:-

1. Stars are small, round, or cubic solids, made with one of the following compositions, and soaked in spirits. White stars, nitre, 16 ; sulphur, 8 ; gunpowder, 3 . Others more vivid consist of nitre, 16 ; sulphur, 7 ; gunpowder, 4 .

Stars for golden showers, nitre, 16 ; sulphur, 10 ; charcoal, 4 ; gunpowder, 16 ; lampblack, 2. Others yellower are made with nitre, 16; sulphur, 8; charcoal, 2; lampblack, 2 ; gunpowder, 8.

The serpents are small fusees made with one or two playing cards; their bore being less than haif an inch. The lardons are a little larger, and have three cards; the vetilles are smaller. Their composition is, nitre, 16 ; charcoal, not too fine, 2; gunpowder, 4 ; sulphur, 4 ; fine steel filings, 6 .

The petards are cartridges filled with gunpowder and strangled.

The saxons are cartridges clayed at each end, charged with the brilliant turning fire, and perforated with one or two holes at the extremity of the same diameter.

The cracker is a round or square box of pasteboard, filled with granulated gunpowder, and hooped all round with twine.

Roman candles are fusees which throw out very bright stars in succession. With the composition (as under) imbued with spirits and gum-water, small cylindric masses are made, pierced with a hole in their centre. These bodies, when kindled and projected into the air, form the stars. There is first put into the cartridge a charge of fine gunpowder of the size of the star; above this charge a star is placed; then a charge of composition for the Roman candles.

The stars, when less than $\frac{3}{4}$ of an inch, consist of nitre, 16 ; sulphur, 7 ; gunpowder, $\supset$. When larger, of nitre, 16 ; sulphur, 8 ; gunpowder, 8 .

Roman candles, nitre, 16 ; charcoal, 6 ; sulphur, 3 . When above $\frac{3}{4}$ of an inch, nitre, 16 ; charcoal, 8 ; sulphur, 6 .

The girandes, or bouquets, are those beautiful pieces which usually conclude a fire- 
work exhihilinn; when a multitude of jets seem to emblazon the sky in every direction, and then fill in golden showers. This effect is produced by distributing a number of eases open it top, each containing 140 sky-rockets, communicating with one another by quicli-match strings planted among them. The several cases communicate with each other by cominits, whereby they take fire simultaneously, and produce a volcanic display.

The water fire-uorks are prepared like the rest; but they must be floated either by wooden bowls, or by dises and hollow cartridges fitted to them.

Blue fire for lances may be made with nitre, 16 ; antimony, 8 ; very fine zinc filings, 4 . Chinese paste for the stars of Roman candles, bombs, \&c. :-Sulphur, 16; nitre, 4 ; gunpowder meal, 12; camphor, 1; linseeu oil, 1; the mixture being moistened with spirits.

The feu grégois of Ruggieri, the son:-Nitre, 4; sulphur, 2; naptha, 1. See PrroTECHNY and Rockets.

The red fire composition is made by mixing 40 parts of nitrate of strontia, 13 of flowers of sulphur, 5 of chlorate of potash, and 4 of sulphuret of antimony.

White fire is produced by igniting a mixture of 48 parts nitre; $13 \frac{1}{4}$ sulphur; $7 \frac{1}{4}$ sulphuret of antimony; or, 24 nitre, 7 sulphur, 2 realgar; or, 75 nitre, 24 sulphur, 1 charcoal; or, finally, 100 of gunpowder meal, and 25 of cast-iron fine borings.

The blue fire composition is 4 parts of gunpowder meal; 2 of nitre; sulphur and zinc, each 3 parts.

FISH-HOOKS (Hamecons, Fr.; Fishangeln, Germ.) are constructed with simple tools, but require great manual dexterity in the workmen. The iron wire of which they are made should be of the best quality, smooth, and sound. A bundle of such wire is cut in lengths, either by shears or by laying it down upon an angular wedge of hard steel fixed horizontally in a block or anvil, and striking off the proper lengths by the blows of a hammer. In fashioning the barbs of the hooks the straight piece of wire is laid down in the groove of an iron block made on purpose, and is dexterously struck by the chisel in a slanting direction, across so much of the wire as may be deemed necessart. A sharp-pointed little wedge is thus formed, whose base graduates into the substance of the metal.

The end of the wire where the line is to be attached is now flattened or screw-tapped; the other end is sharp pointed, and the proper twisted curvature is given. The soft iron hooks are next case-hardened, to give them the steely stiffness and elasticity, by imbedding them in animal charcoal contained in an earthen or iron box; see CASE-HARDENING; after which they are brightened by heating and agitating them with bran, and finally tempered by exposure to a regulated temperature upon a hot iron plate. Hooks for salt-water fishing are frequently tinned, to prevent them wearing rapidly away in rust. See Try Plate.

FLAKE WHITE is the name sometimes given to pure white-lead.

FLAME (Flumme, Fr. and Germ.) is the combustion of an explosive mixture of an inflammable gas or vapor with air. That it is not, as many suppose, combustion merely at the exterior surface, is proved by plunging a fragment of burning phosphorus or sulphur into the centre of a large flame of alcohol. Either of these bodies will continue to burn there with its peculiar light; thus proving that oxygen is mixed with the whole of the burning rapor. If we mix good coal gas with as much atmospheric air as can conrert all its carbon into carbonic acid, the mixture will explode with a feeble blue light; but if we mix the same gas with a small quantity of air, it will burn with a rich white flame. In the latter case the carbonaceous particles are precipitated, as Sir H. Davy first showed, in the interior of the flame, become incandescent, and constitute white light : for from the ignition of solid matter alone can the prismatic rays be emitted in that concentrated union. Towards the interior of the flame of a candle, a lamp, or a gas jet, where the air is scanty, there is a deposition of solid charcoal, which first by its ignition, and afterwards by its combustion, increases in a high degree the intensity of the light. If we hold a piece of fine wire ganze over a jet of coal gas close to the orifice, and if we then kindle the gas, it will burn above the wire with its natural brilliancy; but if we elevate the gauze progressively higher, so as to $\mathrm{mix}$ more and more air with it before it reaches the burning point, its flame will become fainter and less white. At a certain distance it becomes blue, like that of the above explosive mixture. Since the combustion of all the constituents is in this case direct and complete, the heat becomes greatest in proportion nearly as the light is diminished. If a few platina wires be held in that dim flame they will grow instantly white hot, and illuminate the apartment. On reversing the order of this experiment, by lowering progressively a flat piece of wire gauze from the summit towards the base of a gas flame. we shall find no charcoal deposited at its top, beca!nse plenty of air has been introduced there to convert all the carbon of the gas into carbonic acid, and therefore the apex is blue; but as we descend, more and more charcoal will appear upon the meshes. At the very bottom, indeed, where the 
atmospheric air impinges upon the gauze, the flame is again blue, and no charcoal can therefore be deposited.

The fact of the increase of the brilliancy and whiteness of flame by the developmen and ignition of solid matter in its bosom illustrates many curious phenomena. We can thus explain why olefiant gas afiords the most vivid illumination of all the gases; because. being surcharged with charcoal, its hydrogen lets it go in the middle of the flame, as it does in an ignited porcelain tube, whereby its solid particles first get ignited to whiteness, and then burn away. When phosphorus is inflamed it always yields a pure white light, from the ignition of the solid particles of the snowy acid thus produced.

In the blowpipe the inner blue flame has the greatest heat, because there the combustion of the whole fatty vapor is complete. The feeble light of burning hydrogen, carbonic oxyde, and sulphur, may, upon the principles now expounded, be increased by simply placing in them a few particles of oxyde of zinc, slender filamants of amianthus, or fine platina wire. Upwards of twenty years ago I demonstrated, in my public lectures in Glasgow, that by narrowing the top of a long glass chimney over an argand flame either from oil or coal gas, the light could be doubled at the same cost of material. The very tall chimneys used by the Parisian lampists are very wasteful. I find that with a narrow chimney of half the length of theirs, I can have as good a light, and sare 30 per cent. of the oil. Thus the light of a flame may be increased by diminishing its heat, or the intensity of its combustion; and conversely the heat of a flame may be increased by diminishing its light.

FLANNEL; a plain woollen stuff, of a rather open and slight fabric.

FLAX. By this term we understand the bast or inner bark of the Linum usitatissimum, which is spun into yarn for weaving linen webs. This plant blossoms in June or July, and commonly ripens its seed in September. As varieties, we distinguish the spring flax, with short knotty stems, whose seed capsules, at the period of maturity, spring open with a perceptible sound; and the close flax, with longer smoother stems, whose eapsules give out their seeds only when thrashed. The Germans, who have bestowed much attention upon the culture of flax, call the former Klanglein or Springlein, and the latter Dreschlein. This is the kind most commonly grown, but from the difference of climate, soil, and culture, it affords flax of rery different qualities. The best ground for this plant is an open, somewhat friable clay, mingled with sand and mould. The early flax is usually sown in the end of April or beginning of May, the late, in June. The seeds ought to be sown thick, whereby the stalks are forced to grow more slender, and the fibres of the bast or harl are not only smoother and finer, but more uniform in length. If the raising of seed be the principal object, the flax must be more thinly sown, whereby it will produce stronger stalks, but more knotty, with shorter fibres, and more productive of tow.

Whenever the flax is ripe, which is shown by the bottom of the stalk becoming yellow and the leaves beginning to drop off, it must be immediately reaped by pulling it up by the roots. The seeds are still immature, fit merely for the oil press, and not for sowing When the seed crop is the object, the plant must be suffered to acquire its full maturity; in which case the fibres are less fine and soft.

The flax is carried off the field in bundles to be rippled, or stripped of its seeds, which is done by drawing it by handfuls through an iron comb with teeth eight inches long, fixed upright in a horizontal beam. When the seeds are more fully ripened, they may be separated by the thrashing mill.

The operations next performed upon the flax will be understood by attending to the structure of the stem. In it two principal parts are to be distinguished; the woody heart or boon, and the harl (covered outwardly witk a fine cuticle), which encloses the former like a tube, consisting of parallel lines. In the natural state, the fibres of the harl are attached firmly, not only to the boon, but to each other, by means of a green or yellowish substance. The rough stems of the flax, after being stripped of their seeds, lose in moisture, by drying in warm air, from 55 to 65 per cent. of their weight; but somewhat less when they are quite ripe and woody. In this dry state they consist in 100 parts of from 20 to 23 per cent. of harl, and from 80 to 77 per cent. of boon. The latter is composed upon the average of 69 per cent. of a peculiar woody substance, 12 per cent. of a matter soluble in water, and 19 per cent. of a body not soluble in water, but in alkaline leys. The harl contains at a mean 58 per cent. of pure flaxen fibres, 25 parts soluble in water (apparently extractive and albumen), and 17 parts insoluble in water, being chiefly gluten. By treating the harl with either cold or hot water, the latter substance is dyed brown by the soluble matter, while the fibres retain their coherence to one another Alkaline leys, and also, though less readily, soap water, dissolve the gluten, which seems to be the cement of the textile fibres, and thus set them free.

The cohesion of the fibres in the rough harl is so considerable that by mechanical means, as by beating, rubbing, \&c., a complete separation of them cannot be effected, unless with great loss of time and rupture of the filaments. This circumstance 
shows the necessity of having recourse to some chemical method of decomposing the gluten. The process employed with this view is a species of fermentation, to which the flax stalks are exposed; it is called retting, a corruption of rotting, since a certain degree of putrefaction talies place. The German term is rusting. This is the first important step in the preparation of flax. After the retting is completed, the boon of the stalks must be removed by the second operation called breaking, and other subordinate processes. The harl freed from the woody parts contains still a multitude of fibres, more or less coherent, or entangled, and of variable lengths, so as to be ill adapted for spinning. These are removed by the heckle, which separates the connected fibres into their finest filaments, removes those that are too short, and disentangles the longer ones.

1. Of relting.-The fermentation of this process may be either rendered rapid by steeping the flax in water, or slow by using merely the ordinary influence of the atmospheric damp, dews, and rain. Hence the distinction of water-retting and dew-retting. Both may also be combined.

Prior to being retted, the flax should be sorted according to the length and thickness of its stalks, and its state of maturity : the riper the plant, the lorger must the retting last. The due length of the process is a point too little studied.

Waler-retting.-When flax stalks are macerated in water, at a temperature not too low, fermentation soon begins, evinced in the dingy infusiun, by disengagement of carbonic acid gas, and the production of vinegar. If the flax be taken out at the end of a few days, dried, and rubbed, the textile filaments are found to be easily separable from each other. By longer continuance of the steep, the water ceases to be acid, it becomes to a certain degree alkaline, from the production of ammonia, diffuses a fetid odor, from the disengagement of sulphureted hydrogen gas, along with the carbonic acid; the acetous fermentation being in fact now changed into the pütrid. The filaments become yellowish brown, afterwards dark brown, and lose much of their tenacity, if the process be carried further.

When the operation is conducted with discernment, the water-retting may be completed by the acetous fermentation alone, as the putrefaction should never be suffered to proceed to any length; because when over-retted, flax is partially rotten, gets a bad color, and yields a large proportion of tow.

For water-retting, the flax must be bound up in sheaves, placed in layers over each other in the water, or sometimes upright, with the roots undermost. Straw may be put below to keep it from touching the ground, and boards may be laid upon the top, with weights to hold it immersed about a foot beneath the surface, especially when the fermentative gases make it buoyant. As soon as it sinks at the end of the fermentation, it must be inspected at least twice a day, and samples must be taken out to see that no over-retting ensues. A single day too long often injures the flax not a little. We may judge that the retting is sufficient when the harl separates easily from the boon by the fingers, when the boon breaks across without bending, and when several stalks knotted together sink to the bottom upon being thrown into the water. For this completion, a shorter or longer time is required according to the quality of the flax, the temperature, \&c., so that the term may vary from five to fourteen days. It may be done either in running or in stagnant water. For the latter purpose, tanks five feet deep are dug in the ground. In stagnant water, the process is sooner finished, but it is more hazardous, and gives a deeper stain to the fibres, than in a stream, which carries off much of the color. The best place for steeping flax is a pond with springs of water at its bottom; or a tank into which a rivulet of water can be occasionally admitted, while the foul water is let off. For every fresh quantity of flax, the pond should be emptied, and supplied with clear water. Water impregnated with iron, stains flax a permanent color, and should therefore never be used. After retting, the flax should be taken out without delay, rinsed in clean water, and exposed in an airy situation to dry by the sun.

Rough rippled flax stalks, well seasoned before being retted, and dried afterwards, show a loss of weight, amounting to 20 or 30 per cent., affecting both the boon and the harl. This loss is greater the finer the stems, and the longer the retting. The harl contains, beside the textile filaments, a certain portion of a glutinous cement; but nothing soluble in water. The destruction of the gluten cannot be pushed to the last point by steeping, without doing an essential injury to the filaments.

Dew-retting. - The fetid and noxious exhalations which the water-retting diffuses over an extensive district of country, and the danger of over-retting in that way, especially with stagnant water, are far from recommending that process to general adoption. Dew-retting accomplishes the same purpose, by the agency of the air, dews, and rain, in a much more convenient, though far slower manner. The flax, with this view, should be spread out thin upon meadow or grass lands, but never upon the bare ground, and turned over, from time to time, till the stems, on being rubbed between the fingers, show that the harl and the boon are ready to part. The duration of dewretting is, of course, very various, from 2 to 6 , or 8 weeks, as it depends upon the state 
of the weather; a moist air being favorable, and dry sunshine the reverse. The loss of weight by dew-retting is somewhat less than by water-retting; and the textile fibres are of a brighter color, softer and more delicate to the touch.

Mixed reting.-This may be fairly regarded as the preferable plan, the retting being begun in the water, and finished in the air. The flax should be taken out of the steep whenever the acetous fermentation is complete, before the putrid begins, and exposed, for 2 or 3 weeks, on the grass.

II. The breaking is performed by an instrument called a brake. In order to give the wood or boon such a degree of brittleness as to make it part readily from the harl, whereby the execution of this process is rendered easy, the flax should be well dried in the sun or what is more suitable to the late period of the year, in a stove. Such is often attached to the bakers' ovens in Germany, and other flax-growing countries. The drying temperature should never exceed $120^{\circ} \mathrm{F}$., for a higher heat makes it brittle, easy to tear, and apt to run into tow. Before subjecting the flax to the brake, the stems should be equalized and laid parallel by the hand, and the entangled portions should be straightened with a coarse heckle. The brake has one general construction, and consists of two principal parts, the frame or case, and the sword or beater. In the simplest brakes, the frame $e$, fig. 412, is a piece of wood cleft lengthwise in the middle, supported by the legs $a$ and $c$. The sword $f$, also of hard wood, is formed with an edge beneath, and turns round the centre of motion at $q$, when seized by the handle $h$, and moved up and down. As it descends, the sword enters the cleft of the frame, and breaks the flax stalks laid transversely upon it, scattering the boon in fragments.

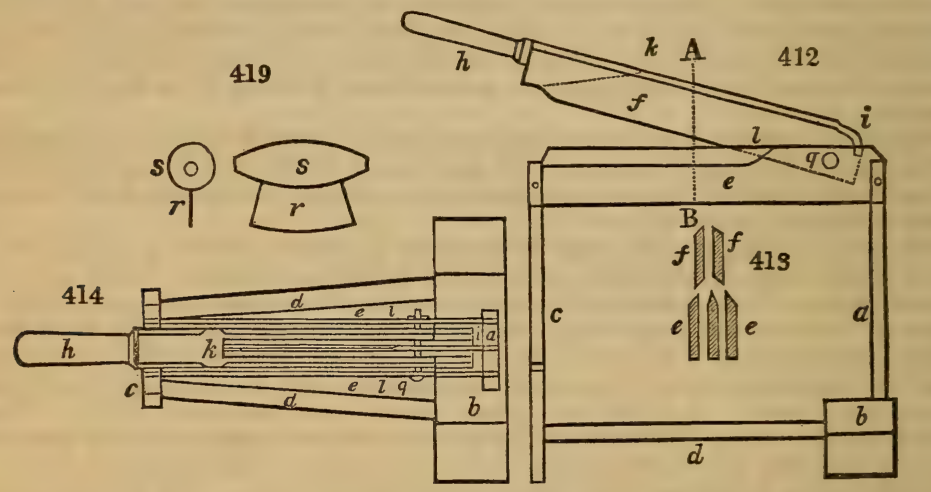

But those hand brakes are more convenient which are provided with a double cleft, or triple row of oblong teeth, with a double sword. This construction will be understood by inspecting figs. $412,413,414$. Fig. 412 is the section of that side at which the operative sits; fig. 413 is a section in the line A, B, of fig. 412 ; and fig. 414 the ground plan. The whole machine is made of hard wood, commonly red beech. Two planks $a$ and $e$, form the legs of the implement. $a$ is mortised in a heavy block, to give the brake a solid bearing; two stretchers $d$, bind $a$ and $c$, firmly together. The frame $\ell$ consists of three thin boards, which are placed edgewise, and have their ends secured in $a$ and $c$. The sword $f$ is a piece of wood, so chamfered from $i$ to $k$, that it appears forklike, and embraces the middle piece of the frame; its centre of motion is the wooden pin $q$; in front is the handle $h$, which the operative seizes with the right hand. Both the lathes of the frame and those of the sword are sharpened, from $l$ to the front end, as is best shown in fig. 413; but the edges must not be too sharp, for fear of injuring the flax; and, for the same reason, the sword should not sink too far between the lathes of the frame. Such hand-brakes are laborious in use, and often tear the harl into tow. The operative, usually a female, in working the brake, seizes with her left hand a bundle of flax, lays it transversely across the frame, and strikes it smartly with repeated blows of the sword, pushing forwards continually new portions of the flax into the machine. She begins with the roots, turns next round the tips, then goes on through the length of the stalks. Flax is frequently exposed twice to the brake, with a stove drying between the two applications.

The brake machines afford a far preferable means of cleaning flax than the above hand tools. The essential part of such a machine, consists in several deeply fluted rollers of wood or iron, whose teeth work into each other, and while they stretch out the flaxen stalks betwixt them, they break the wood or boon, without doing that violence to the harl which hand mechanisms are apt to do. The following may be regarded as 
one of the best constructions hitherto contrived for breaking flax. Fig. 415 is a view of the right side of this machine. Fig. 416 the view from behind, where the broken

416

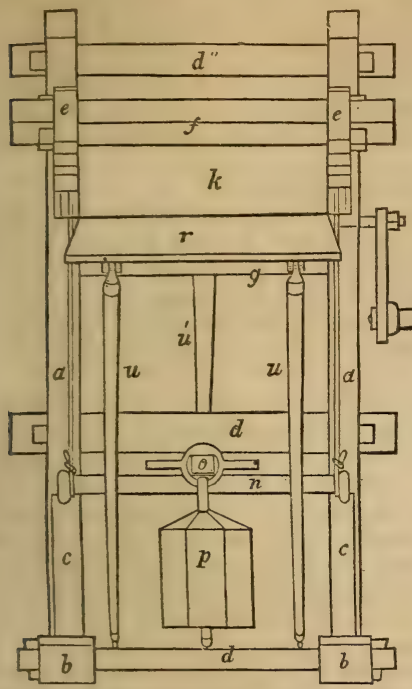

flax issues from between the rollers. walls $a, a$, which are mortised into the bottom $b, b$; and are firmly fixed to it by braces. Two transverse rods $d, d$, secure the base, two others $d^{\prime} d^{\prime \prime}$, the sides. In each of these a lateral $\operatorname{arm} e$, is mortised in an oblique direction; a cross bar $f$,

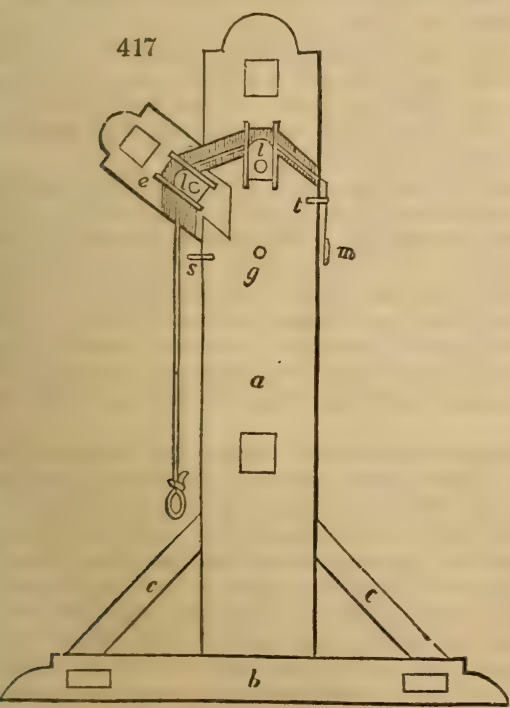
unites both arms. Fig. 417 shows the inside of the left side of the frame, with the subsidiary parts. The three rollers $g, i, k$, may be made of red beech, with iron gudgeons, and fluted in their length, each of the flutes being $\frac{5}{12}$ ths of an inch broad, and $\frac{4}{12}$ ths deep. The large roller $g$, bears upon the right side, a handle $h$, which on being turned, sets the whole train in motion. The side partitions $a, a$, are furnished with brasses in whose round holes $l, \mathrm{~g}$, fig. 417, the gudgeons $g$ work For the extremities of the two smaller rollers, there are at $\alpha$ and $e$, slots in brasses, as may be seen in fig. 415 . Within the partition $\alpha$, there are moveable brasses $l$, for the pivots of $i$ and $k$, shown in fig. 417. Each brass slides in a groove, between two ledges. A strong cord made fast at $m$ to the partition $a$, runs over the brass of $i$, next over that of $k$, then descends perpendicularly, and passes over the cross bar n, figs. 415 and 416 . This construction being repeated at both ends of the rollers, the rod $n$, binds both cords. Against the cross bar $d^{\prime}$ of the frame, a lever $o$ is sustained, which lies upon the rod $n$, and carries a weight $p$. The farther or nearer this weight hangs towards the end of the lever, it stretches the cord more or less, and presses by means of the brasses $l$, the rollers $i, i$, , towards the main roller $g$. A table $q$, serves for spreading out the flax to be broken, and a second one $r$, for the reception of the stalks at their issuing from between the rollers. Both tables hang by means of iron hooks to rings of the frame $s$, $t$, figs. 415 and 417 , and are supported by the moveable legs $u, u, u$, figs. 415 and 416. 
In using the machine the operative lays an evenly spread handful of flax upon the table, $q$, intruduces their root ends with his left hand between the rollers $g$ and $i$, and turns round the handle $h$, with the right. The stems are first broken betwixt $g$ and $i$, then between $g$ and $k$, and come out upon the table $r$. The handle is mored alternately forwards and backwards, in order that the flax may be rolled alternately in the same directions, and be more perfectly broken. The boon falls down in very small pieces, and the harl remains expanded in parallel bands. This should be drawn orer the points of a heckle, then laid for a couple of days in a cellar to absorb some moisture, and afterwards worked once more through the machine, whereby the flax acquires a peculiar softness.

The advantages of this brake machine are chiefly the following :-

It takes up little room, and from its simplicity is easily and cheaply constructed; it requires no more power to work, than the ordinary hand-bralie; it tears none of the filaments, and grinds nothing except the boon, in consequence of the flutings of the rollers going much less deep into each other, than the sword of the hand-bralie; it prevents all entanglements of the flax, whence in the subsequent heckling the quantity of short fibres or tow is diminished; and it accomplishes the cleaning of even the shortest flax, which cannot be well done by hand machines.

The comminution of the boon of the stems, which is the object of the breaking process, can however be performed by thrashing or beating, although in this way the separation of the woody matter from the textile fibres is much less completely effected.

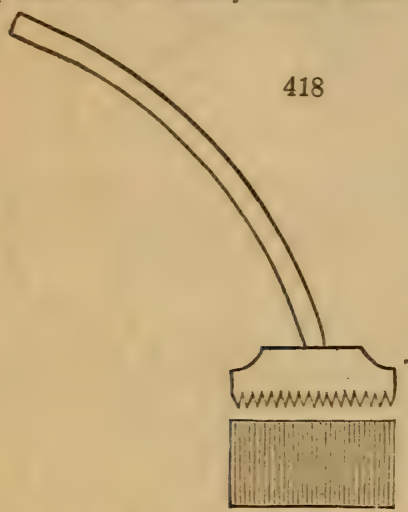

It is the practice in Great Britain, instead of breaking, to employ a water-driven wooden mallet, between which and a smooth stone the flax is laid. In that part of Belgium where the preparation of flax las been studied, the brake is not used, but beating by means of the Bott-hammer, to the great improvement, it is said, of the flax. The Bott-hammer, fig. 418 , is a wooden block, having on its under face channels or flutings, 5 or 6 lines deep, and it is fixed to a long bent helve or handle. In using .it, a bundle of the dried flax stalks is spread evenly upon the floor, then powerfully beaten with the hammer, first at the roots, next at the points, and lastly in the middle. When the upper surface has been well beat in this way, it is turned over, that the under surface may get its turn. The flax is then removed, and well shaken to free it from the boon.

$\mathrm{By}$ the brake or the hammer the whole wood is never separated from the textile fibres, but a certain quantity of chaff stuff adheres to them, which is removed by another operation. This consists either in rubbing or shaking. The rubbing is much practised in Westphalia, and the neighboring districts. In this process, the operative lars the rubbing apron on a piece of dressed leather, one foot square, upon her linee; then seizes a bundle of flar in the middle with her left hand, and scrapes it strongl 5 with the Ribbeknife held in her right, fig. 419. This tool, which consists of a wooden handle $s$, and a thin iron blade $r$, with a blunt and somewhat bent edge, acts admirably in cleaning and also in parting the filaments, without causing needless waste in flax previously well broken.

The winnorring, which has the very same object as the rubbing, is, however, much more generally adopted than the latter. Two distinct pieces of apparatus belong to it, namelr, the suing-stock and the suing-knife. The first consists of an upright board with a groove in its side, into which a handful of flax is so placed that it hangs down orer half the surface of the board. While the left hand holds the flax fast above, the right carries the swing-knife, a sabre-shaped piece of wood from $1 \frac{1}{2}$ to 2 feet long, planed to an edge on the conrex side, and provided with a handle. With this knife the flax is struck parallel to the board, with perpendicular blows, so as to scrape off its woody asperities. The breadth of the swing-knife is an important circumstance; when too narrow it easily causes the fiax to twist round it, and thereby tears away a portion of the fibres. When $\varepsilon$ or 10 inches broad, it is found to act best. Knives made of iron will not answer, for they injure the filaments.

Figs. 420, 421 show the best construction of the swing-stock. The board $a$ has for its base a heary block of wood $b$, upon which two upright pins $e e$, are fixed. The band $f$, which is stretched between the pins, serves to guide the swing-knife in its movements, and prevent the operative from wounding his feet. The under edge of the groove $c$, upon which the flax comes to be laid, is cut obliquely and rounded off (see $d$ 
in $f$ g. 420$)$; thus we perceive that the swing-knife can never strike against that edge, 80 as to injure the flax.

Fig. 422 exhibits the form of a very convenient implement which is employed in 421

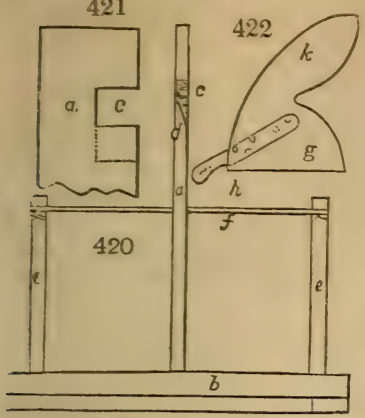
Belgium instead of the swing-knife. It is a sort of wooden hatchet, which is not above two lines thick, and at the edge $g h$ is reduced to the thickness of the back of a knife. The fly $k$ gives force to the blow, and preserves the tool in an upright position. The short flat-pressed helve $i$ is glued to that side of the leaf which in working is turned from the swing-stock; and is, moreover, fastened with a wooden pin.

The rubbing and swinging throw off the coarsest sort of tow, by separating and shaking out the shortest fibres and those that happen to get torn. That tow is used for the inferior qualities of sacking, being mixed with many woody fibres.

We may in general estimate that 100 pounds of the stalks of retted flax, taken in the dry state, afford from 45 to 48 pounds of broken flax, of which, in the stringing or scutching, about 24 pounds of flax, with 9 or 10 pounds of senteh tow are obtained. The rest is boon-waste. The breaking of 100 pounds of stalks requires, in the ordinary routine of a double process by hand, about 20 hours; and with the above described machine, from 17 to 18 hours. To scutch 100 pounds of broken flax clean, 130 hours of labor are required by the German swinging method.

Mr. Bundy obtained a patent in 1819, for certain machinery for breaking and preparing flax, which merits description here. Fig. 423, A A A A, is the frame, made either of

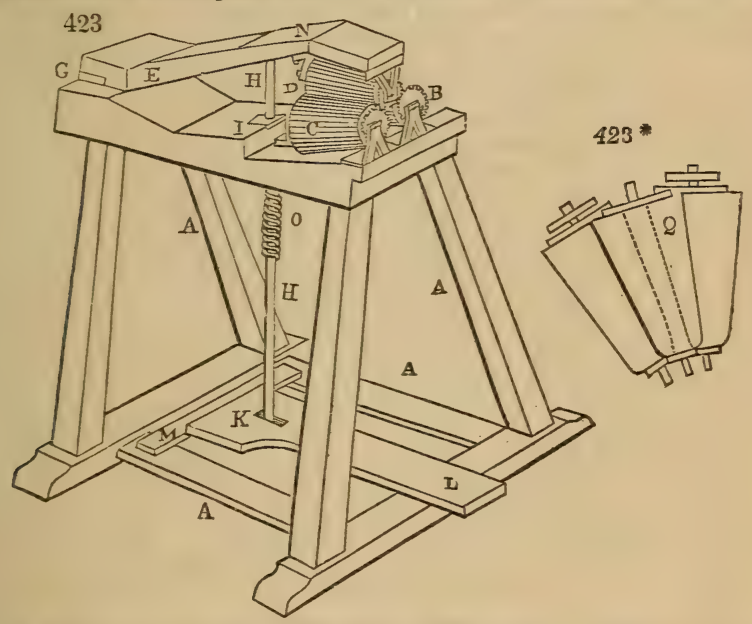

wood or metal, which supports the two conical rollers в and c. These revolve independently of each other in proper brass bearings. A third conical roller $\mathrm{D}$ is similarly supported under the top piece $\mathrm{E}$ of the machine. All these rollers are frusta of cones, made of cast iron. Whatever form of tooth be adopted, they must be so shaped and disposed with regard to each other as to have considerable play between them, in order to admit the quantity of flax stem which is intended to be broken and prepared. The upper piece $\mathrm{E}$ of the machine which carries the upper conical roller $\mathrm{D}$, is fixed or attached to the main frame A A A A by strong hinges or any other moveable joint at G, and rods of iron or other sufficiently strong material; $\mathrm{H} \mathrm{H}$ is attached at its upper end by a joint to the top piece $E$, through a hole near $I$, and is fixed at its lower end by another joint $\mathrm{K}$ to the treadle or lever $\mathrm{K} \mathrm{L}$, which turns upon the joint or hinges $\mathrm{m}$. A spring or weight (but the former is preferable for many reasons) is applied to the machine in such manner, that its action will always keep the upper piece $E$, and consequently the upper roller $\mathrm{D}$, in an elerated or raised position above the rollers $\mathrm{B}$ and $\mathrm{c}$, when the machine is not in action; and of course the end $L$ of the treadle will also be raised, which admits of the flax to be worked being introduced between the rollers, viz., over the two lower rollers $B, C$, and under the upper roller $D$; such a spring may be applied in a variety of ways, as between the top piece $\mathrm{\Sigma}$, and the top or platform of the machine at $\mathrm{N}$; or it 
may be a strong spiral wire spring, having its upper end fastened to the platform while its lower extremity is lixed to the rod $\mathrm{H} \mathrm{H}$, round which it coils as shown at o, or it may be placed under the end $\tau$ of the treadle; but in every case its strength must be no more than will be just sufficient to raise the upper roller $\mathrm{D}$ about $t$ wo inches from the lower rollers, otherwise it will occasion unnecessary fatigue to the person working the machine.

The manner of using it is as follows: the upper and lower rollers being separated as aforesaid, a small handful of dried flax or hemp stems is to be introduced between them, and held extended by the two hands, while the rollers are brought together by the pressure of the foot upon the treadle $\mathbf{L}$. This pressure being continued, the flax or hemp is to be drawn backwards and forwards by the hards between the rollers, in a direction at right angles to their axes, and eventually withdrawn by pulling with one hand only. The foot is now to be removed until the flax or hemp is again replaced, and each end is this way to be drawn several times through the machine, until such ends are respectively finished.

By a succession of these operations, using the pressure of the foot upon $\mathrm{L}$, each time that the flax or hemp is introduced between the rollers, and regulating such pressure according to the progress of the work, the flax or hemp will soon be sufficiently worked, and the fibre brought into a clean and divided state fit for bleaching; or if it be required to spin it in the yellow state, it may be made sufficiently fine by a longer continuation of the same process, particularly if worked between the smaller ends of the rollers.

Indeed, the operation may be commenced and continued for some time, with the larger part of the rollers, and finished with their smaller ends; and, in this point of view, the invention of conical rollers will be found both convenient and useful; for as the flutes, grooves, or teeth, vary in their distance from each other at all points between the large and small ends, so it becomes almost impossible for the workman to draw the flax or hemp through such rollers in the same track; and thus the breaking of the boon must be much more irregular, and the fibre will be much more effectually cleansed than it can be by the flutes, grooves, or teeth of cylinders, or other such contrivances formerly employed; because they would probably fall frequently upon the same points of the fibres. If it is intended that the flax shall be bleached before it is spun, then the second part of Mr. Bundy's invention may be had recourse to, which consists in moving certain trays or cradles in the water, or other fluid used for bleaching the flax or hemp, in the manner following, viz. : The flax or hemp, after having been broken and worked in the machine, should be divided into small quantities of about one ounce each, and these should be tied loosely in the middle with a string, and in this state laid in the trays or cradles, and then be soaked in cold soft water for a day or two, when each parcel should be worked separately, while wet, through a machine, precisely similar to that already described, except only that the rollers should be cylindrical, and made entirely of wood with metal axles, and the teeth, which will be parallel, should be similar in form to those shown in section at $\mathrm{Q}, \mathrm{fig}$. $423^{*}$. Such operation will loosen the gluten and coloring matter, for the rinsing and wringing which must follow. The flax must then be again disposed in a flat and smooth manner, in such trays or cradles, and once more set to soak in sufficient soft water to cover it, in which a small quantity of soap, in the proportion of about seven pounds of soap to each hundred weight of flax, has been previously dissolved, and in this state it should remain for two or three days longer, and then be finally worked through the machine, rinsed with clear water, and wrung; which will render it sufficiently white for most purposes.

III. The Heckling.-We have already stated that, by the operation of heckling, a three-fold object is proposed: 1 . the parting of the filaments into their finest fibrils; 2. the separation of the short fibres which are unfit for spinning; 3 . the equable and parallel arrangement of the long filaments. The instrument of accomplishing these objects is a comb-fashioned tool, called the heckle or hackle; a surface studded more or less

$+424$
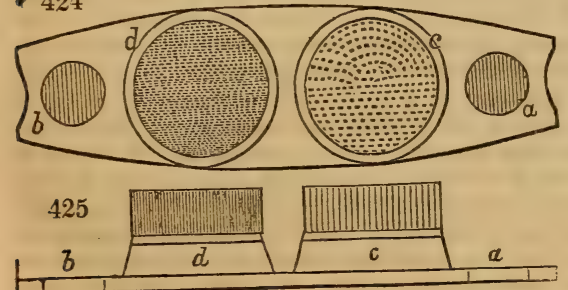

thickly with metal points, called heckle teeth; over which the flax is drawn in such a way that the above three required operations may be properly accomplished.

The common construction of the heckle is the following: (see fig. 424.) Fig. 424 is the ground plan, and fig. 425 is the section. Upon an oblong plank $a b$, two circular or square blocks of wood $c$ and $d$ are fixed, in which the heckle teeth stand

urright. To give these a firmer hold they are stuck into holes in a brass cr iron plate, 
with which the upper surface of $c$ and $d$ is covered. Both heckles may be either associated upun one board or separated; and of different finenesses; that is, the teeth of the one may be thinner, and stand closer together; because the complete preparation of the flax requires, for its proper treatment, a twofold heckling; one upun the coarse, and one upon the fine heckle; nay, sometimes 3 or 4 heckles are employed of progressive fineness. The heckle teeth are usually made of iron, occasionally of steel, and from 1 to 2 inches long. Their points must be very sharp and smooth, all at an equal level, and must all graduate very evenly into a cylindrical stem, like that of a sewing needle, without any irregularity. The face of the heckle block must be uniformly beset with teeth, which is done by different arrangements, some persons setting them in a circle, anu others in parallel rows; the former being practised in Germany, the latter in England. The coarse heckle is furnished with teeth about one tenth of an inch thick, one and a quarter of an inch long, and tapering from the middle into a very fine point. In the centre of the circular heckle is a tooth planted; the rest are regularly set in 12 similar concentric circles, of which the outermost is $5 \frac{3}{4}$ inches in diameter. The fine heckles contain no fewer than 1109 teeth. Instead of making the points of the teeth round, it is better to make them quadrangular, in a rhombus form, in which case the edges serve to separate or dissect the fibres.

The operation of heckling is simple in principle, although it requires much experience to acquire dexterity. The operative seizes a flock of flax by the middle with the right hand, throws it upon the points of the coarse heckle, and draws it towards him, while he hold = the left hand upon the other side of the heckle, in order to spread the flax, and to prevent it from sinking too deeply among the teeth. From time to time the short fibres or tow sticking to the teeth are removed. Whenever one half of the length of the strake of flax is heckled, it is turned round to heckle the other half. This process is repeated upon the fine heckle. From 100 pounds of well-cleaned flax, about 45 or 50 pounds of heckled flax may be obtained by the hand labor of 50 hours; the rest being tow, with a small waste in boony particles and dust. The process is continued, till by careful handling little more tow is formed.

Many contrivances have been made to heckle by machinery, but it may be doubted whether any of them as yet make such good work with so little loss as hand labor. In heckling by the hand, the operative feels at once the degree of resistance, and can accommodate the traction to it, or throw the flax more or less deep among the teeth, according to circumstances, and draw it with suitable force and velocity. To aid the heckle in splitting the filaments, three methods have been had recourse to; beating, brushing, and boiling with soap-water, or an alkaline ley.

Beating flax, either after it is completely heckled, or between the first and second heckling, is practised in Bohemia and Silesia. Each heckled tress of flax is folded in the middle, twisted once round, its ends being wound about with flaxen threads; and this head, as it is called, is then beat by a wooden mallet upon a block, and repeatedly turned round till it has become hot. It is next loosened out, and rubbed well between the hands. The brushing is no less a very proper operation for parting the flax into fine filaments, softening and strengthening it without risk of tearing the fibres. This process requires in tools, merely a stiff brush made of swine's bristles, and a smooth board, 3 feet long and one foot broad, in which a wooden pin is made fast. The end of the flax is twisted two or three times round this pin to hold it, and then brushed through its whole length. Well heckled flax suffers no loss in this operation; unheckled only a little tow; which is of no consequence, as the waste is thereby diminished in the following process. A cylindrical brush turned by machinery might be employed bere to advantage.

The boiling of flax with potash ley alone, or with ley and soap, dissolves that portion of the glutinous cement which had resisted the retting, completes the separation of the fibres, and is therefore a good practical means of improving flax. When it is performed upon the heckled fibres, a supplementary brushing is requisite to free it from the dust, soapy particles, \&c.

Can flax be prepared without retting? -The waste of time and labor in the steeping of flax; the dyeing of the fibres consequent thereon, which must be undone by bleaching; the danger of injuring the staple by the action of putrescent water; and, lastly, the diminished value of flax which is much water-retted, are all circumstances which have of late years suggested the propriety of superseding that process entirely by mechanical operations. It was long hoped, that by the employment of breaking machines, the flax merely dried could be freed from its woody pariicles, while the textile filaments might be sufficiently separated by a subsequent heckling. Experience has, however, proved the contrary. The machines, which consisted for the most part of fluted rollers of iron or wood, though expensive, might hrve been expected to separate the ligneous matter from the fibres; but, in the further working of the flax, no advantage was gained over the water-retting process. 
1. Unretted flax requires a considerably longer time for breaking than retted, under the employment of the same manipulations.

2. Unretted stalks deliver in the breaking and heckling a somewhat greater product than the same weight of flax which has been retted; but there is no real advantage in this, as the greater weight of the unretted flax consists in the remainder of ligneous or glutinous matter, which being foreign to the real fibre, must be eventually removed. In the bleaching process, the water and the alkaline leys take away that matter, so that the weight of the bleached fibre is not greater from the unretted than the retted flax.

3 . The parting of the fibres in the unretted stalks is imperfectly effected by the neckling, the flax either remains coarser as compared with the retted article, and affords a coarser thread, or if it be made to receive greater attenuation by a long continued heckling, it yields incomparably more torn filaments and tow.

4. The yarn of unretted flax feels harder, less glossy, and rougher; and, on account of these qualities, turns out worse in the weaving than the retted flax. Nor is the yarn of unretted flax, whether unbleached or bleached, in any degree stouter than the yarn of the retted flax.

5. Fabrics of unretted flax require for complete bleaching about a sixth less time and materials than those of the retted. This is the sole advantage, but it is more than counterbalanced by the other drawbacks above specified.

In Mr. Wordsworth's improved apparatus for heckling flax and hemp, a succession of stricks is subjected to the operation of several series of revolving heckles of different degrees of fineness, for the purpose of gradually separating or combing the long fibres, and dressing them smooth; while at the same time, the tow or entangled refuse portions of the material taken off from the stricks by the heckle points are removed from the heckles by rotatory brushes and rollers covered with wire cards, and discharged into suitable receivers, whence it may be taken to a carding engine, to be worked in the ordinary way.

The accompanying figures represent in plan and section the heckling machine, which is made double, for the purpose of allowing two series of stricks of flax to be acted upon at one time. Fig. 426 is a horizontal view of the machine; fig. 427 is an end view, the

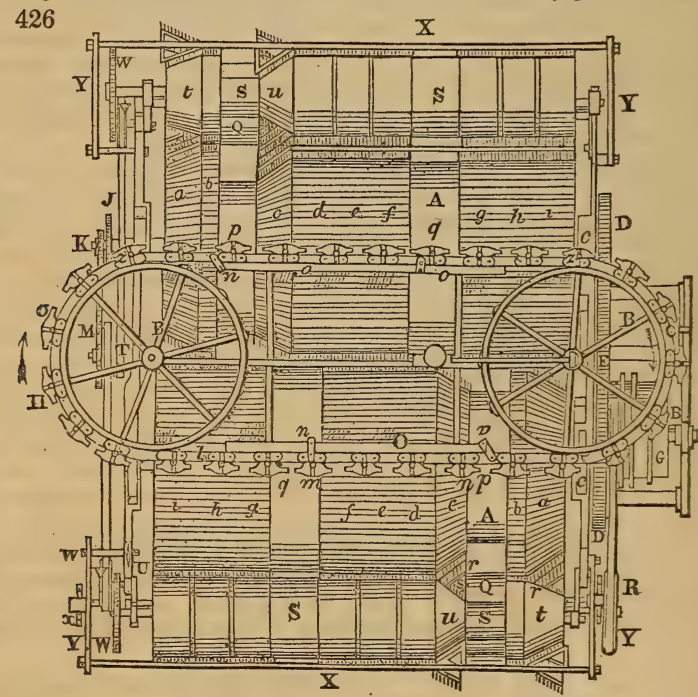

whole being represented in working order, and the respective letters of reference pointing out corresponding parts of the machine.

A A are two large barrels or drums, upon the surfaces of which are fixed longitudinally several series of brass ribs $a, b, c, d, e, f, g, h, i$, holding heckle points. These ribs are placed at small distances apart round the barrels, all the heckle points standing radially from the axes, and the barrels are mounted upon axles supported by pedestals, with plummer blocks bearing on the rails of the end frames. B B, are two horizontal wheels or pulleys turning upon vertical shafts, which pulleys conduct an endless chain c c c c, carrying the holders, whereon the stricks of flax or other material intended to be heckled are suspended.

At one end of the axle of each of the barrels a tonthed wheel D D is made fast, and these 
are connected by a similar wheel $\mathrm{s}$, and a pinion $\mathrm{F}$, fig. 427 , the latter being fixed upon the axle of the driving rigger $\mathrm{G}$.

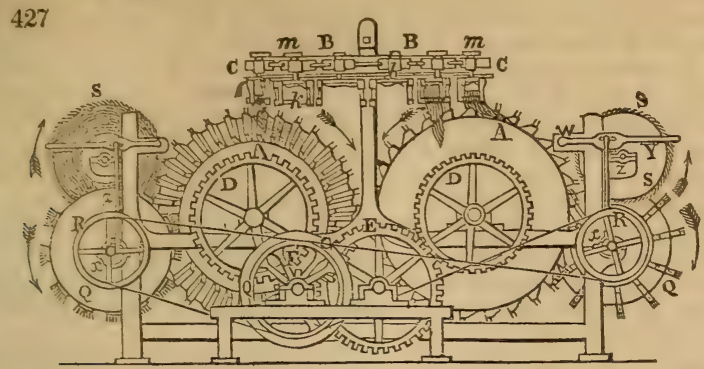

The power of a steam engine, or any other first mover, being applied by a band and rigger, or otherwise to the axle of $\mathrm{G}$, the pinion $\mathrm{F}$ is driven round, which, being in gearing with the toothed wheels $\mathrm{E}$ and $\mathrm{D} \mathrm{D}$, causes the heckle barrels $\mathrm{A} A$ to revolve simultaneously in opposite directions, as shown by the arrows in fig. 427.

The stricks of flax intended to be operated upon are severally confined between pairs of clamps $k$, fastened together, which clamps, with the stricks, are then suspended in their respective holders $\mathrm{H} \mathrm{H}$, attached to the endless chain $\mathrm{c}$ : the lower portion of the flax hanging down for the purpose of being acted upon by the rotatory heckles, while the upper portions are turned up in loops and confined by spring levers attached to each carrier.

The respective holders of the clamps consist of a forked frame, with hooks at the lower parts of their arms, which receive the ends of the clamps $k$, that confine the strick of flax. From the upper part of each forked frame, a perpendicular pin extends, which pins, when inserted into the sockets $l l l$, in front of the chain, form axles for the frames to turn upon at certain periods of the operation.

On the upper end of each pin a small arm or tappet piece $m, f i g .427$, is fixed, standing at right angles to the face of the forked frame of the holder $\mathrm{H}$. Those tappets, as the endless chain conducts the holders along at certain periods, come in contact with stationary pins or wipers $n n$, fixed to the guide rails 0 , on which the chain c slides; and these wipers acting against the tappets as they pass, cause the holders to be turned round at those periods for the purpose of bringing the reverse side of the strick of flax on to the heckle points.

Let it now be supposed, that all the holders connected to the endless chain have been furnished with stricks of flax, or other material to be heckled, and that the barrels A A are put in motion in the way described, revolving in the direction of the arrows shown in fig. 427. A pinion on the end of the axles of one of the barrels $\mathrm{A}$ will drive a train of toothed gear, J K L M and N, on the axle of the latter, of which there is a bevelled pinion taking into a bevelled wheel, turning horizontally at the lower end of the perpendicular shaft of one of the chain pulleys. It will hence be perceived, that as the barrels go round, such rotatory motion will be communicated to the pulley $\mathrm{B}$, as will cause it to drive the chain c forward, and by that means conduct the several stricks of flax progressively along the barrel.

When each successive holder, with its strick of flax or other material, is brought to the part $z$, fig. 426, the fibres come in contact with the rotatory barrel, and first strike upon the series of coarse heckles $a a$, placed upon an inclined or conical surface of the barrel, by which means the lower ends of the flax in each strick are first acted upon; and as it advances, the upper part, and ultimately the whole length of the long fibres of the suspended strick, are gradually brought on to the heckles, which progressive operation prevents the long fibres from being broken, and causes a smaller quantity of tow to be produced than is usually taken off in any of the ordinary modes of heckling.

After the strick of flax or other material has been carried by the travelling chain past the first inclined or conical surface $\alpha$, of the heckling barrel, it then comes upon the cylindrical part, $b$, of the barrel, which is also furnished with coarse heckles that penetrate and comb down the whole pendent lengths of the fibres. But in order that both sides of the strick of flax may be equally operated upon, the holder is now to be turned round upon its pin or pivot, which movement is effected by one arm of the lever or tappet $m$, (as the carrying chain moves onward), coming against the stationary pin or wiper $n$, which changes the position of the holder, as shown at $p$, in the horizontal view fig. 426 .

The under part of the guide rail $o$, upon which the chain slides, is at this part cut away, for the purpose of allowing the holder to turn round horizontally; and a pin or projection at the under side of the guide rail, as the chain continues moving, acts against 
the side of the carrier frame, and forces it into a position parallel with the chain. The other side of the strick of flax is by these means brought on to the heckles of the second inclined or conical surface of the barrel at $c$; and the travelling chain proceeding onward, the fibres of the material are in succession passed over and combed by the heckles of increasing fineness, $d, e$, and $f$, on the cylindrical part of the revolving barrel, until the strick having arrived at the second wiper $n$, the frame or holder is at $q$, turned round as before, and the reverse side of the strick, or that first operated upon by the heckles $a$ and $b$, is brought progressively on to the heckles of increasing fineness, $g, h$, and $i$; and having passed the last series of rotatory heckles, the holders are in succession to be removed from the machine, the material having been sufficiently dressed.

The clamps of the holders are now opened by the aitendant, and the stricks of flax or other material are taken out, and again placed between the clamps in reversed positions, in order that the other ends of the fibres may be operated upon. The clamps, with the stricks, are then suspended again in the holders, the uncombed ends of the fibres hanging dowu upon the heckle barrel.

In order to avoid interrupting the continual operation of the machine, it is proposed that the strick, on its second introduction, shall be placed in the holders on the opposite side at $y$, which is one of the reasons for constructing a double machine, and the strick being thence carried along by the travelling endless chain in the way already described, the fibres will be first brought under the operation of the coarse heckles on the inclined or conical surface of the second revolving barrel, and then of the other heckles increasing in fineness on the cylindrical part of the barrel, until having reached the end, as in the former instance, the fibres of the flax may be considered to be sufficiently dressed, and may then be withdrawn.

It may be necessary here to remark, that as different kinds and qualities of material will require different degrees of working by the heckles, this can be effected by varying the comparative speeds of the travelling holders and the heckle barrels. These comparative speeds, it will be perceived, depend upon the diameters of the wheels and pinions by which the pulley $\mathrm{B}$ is driven from the rotation of the heckle barrel. These wheels and pinions are therefore intended to be removed and changed for others of differcnt diameters, as circumstances may require. It will be perceived that the faster the stricks travel through the machine compared to the rotatory speed of the heckle barrels, so much the less will the material be acted upon by the rotatory heckles; but as different qualities of material must be differently operated upon, according to circumstances, it is impossible to set out any definite speeds or proportions of speed: that will, however, be readily perceived by competent workmen when working at the machine.

In the process of opening the fibres of the material by the rotatory heckles, a quantity of short or loose fibres, as tow, will be taken off the stricks by the heckle points, and will remain adhering to the barrel between the points of the heckles: in order, therefore, to remove this tow or other loose entangled materials from the heckles, several series of brushes, or blocks, with bristles, are affixed longitudinally tø rotatory barrels Q Q.

These brush barrels are mounted parallel to the heckle barrels upon axles, supported in plummer blocks affixed to brackets extending from the end frames of the machine. Those parts of the brush barrels which are opposite to the cylindrical portions of the heckle barrel are cylindrical, and those parts which are opposite to the bevels are contrabevelled, or made as frustrums of cones reversed, or in an opposite angle, as $r, s$, so as to run parallel to the inclined surfaces of the heckle barrels $a$ and $c$.

Upon the periphery of these barrels $Q \mathbf{Q}$, ribs or blocks, with bristles or brushes, are fixed longitudinally, at suitable distances apart, the bristles all standing radially from the axle, and taking into the points of the heckles.

Rotatory motions are given to the brush barrels $Q Q$, by bands passing from the riggers at $\mathbf{G}$, over pulleys $\mathbf{R}$, fixed at the end of each of the axles of the brush barrels. Hence, it will be perceived that the barrels $Q Q$ will revolve in opposite directions to the heckle barrels, and with sufficient speed to enable the brushes to pass through between the points of the heckles, and, in so doing, to remove the tow or other loose matter therefrom.

The tow or other loose fibrous material collected upon the brushes is transferred thence on to wire cards placed round the periphery of the barrels $s$, , which barrels are mounted upon axles parallel to the brush rollers, and turn in plummer blocks upon brackets, extending from the end frames of the machine.

These barrels are cylindrical, and covered with sheets of wire eards at those parts which are opposite to the cylindrical portions of the brush barrels, but those portions of the barrel $s$ which are opposite to the bevelled points, $r$ and $s$, of the brush barrels, are bevelled or made conical at $t u$, to fit or correspond with the inclined surfaces $r$ and $s$; these are covered with sheets of wire card also.

Rotatory motions are communicated to the card barrels s $\mathrm{s}$, by bands from the pulley 
r, fixed on to the sxic of the toothed wheel in (see fig. 427), which band drives similar pulleys $\mathrm{v} v$, mounted upon studs fixed in the end frame. Upon the side of each of these pulleys $\mathrm{v} v$, a pinion $t$ is fixed, which pinion takes into the teeth of the wheel $\mathrm{w}$, on the end of the axle of each of the card barrels s s; by which means such slow motions are given to the barrels $\mathrm{s}$, as will allow the brushes of the barrels $\mathrm{Q}$ to comb off and deposite the tow or other fibrous material upon the wire cards as they revolve, and from whence it is to be removed by a doffing comb, and let fall into any convenient receptacle below, in the same way as in ordinary carding engines.

The doffing combs, $\mathrm{x} x \mathrm{x}$, are formed to the shape of the card barrels, and are attached to straight bars extending along the machine on both sides, which are supported at their extremities by levers $\mathbf{y} \mathbf{y}$, vibrating upon fulcrum pivots at $w w$. To these levers perpendicular rods, $z \mathrm{z}$, are connected by joints, and the lower end of each of these rods is attached to an eccentric disc, roller, or crank, $x x$, on the axle of the brush barrel; whence it will be perceived that by the rotation of the eccentrics $x$, the levers $\mathrm{y}$ will be made to vibrate and strike off, or doff the tow or other material from the card barrels, in a similar manner to the operations of the doffing comb of an ordinary carding engine,

Mr. Evans's patent improvements in machinery for preparing and dressing flax and hemp apply, first, to the operation of scutching, swingling, or beating away the boom or woody particles of the rind which covers the flax, or hemp, in its rough state; and, secondly, to the subsequent operation of heckling, combing, or opening of the fibres of the material preparatory to spinning it into yarns.

Fig. 428 represents the scutching or swingling machine, in different positions.

429

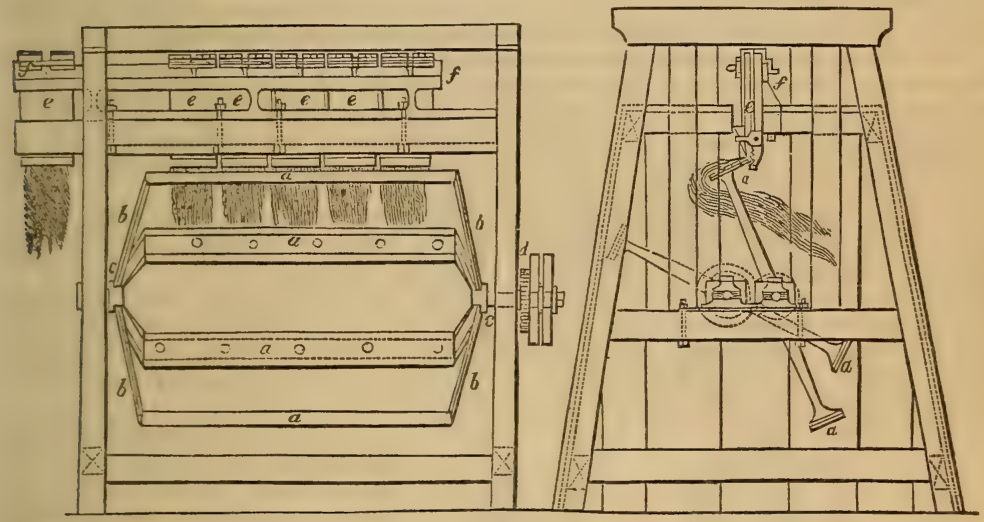

Fig. 428 is an end view of the machine in operation; fig. 429 is a front view of the same. The essential parts of the machine, and those in which the invention especially consists, are two pairs of revolving beaters or scutchers, each formed by long ribs or blades mounted upon arms. The blades of the beaters, $a$ a, may be made of ribs of hard wood, or other suitable material, broad but thin, and slightly rounded on their edges, to pre zent their cutting the fibres of the flax or hemp when they strike it. The two blades are placed parallel to each other, and mounted upon a hexagonal frame, the arms $b b$ inclining or forming obtuse angles with the blades, and from the middle of the arms short axles, $c c$, extend, upon which the beaters revolve.

The axles of both pairs of beaters are mounted in plummer boxes, bearing upon horizontal rails at the ends of the machine, as shown in fig. 428, and are at such distance apart as will allow of the arms and the beaters of each pair passing alternately within those of the other pair as they revolve in opposite directions, which they are enabled to do without coming in contact, in consequence of the inclination of the arms.

On the axle at one end of each pair of beaters a toothed wheel, $d$, is affixed, and these wheels being of similar diameters, and taking into each other, cause the beaters to revolve with smilar speed in opposite directions, rotatory motion being given to them by a band and rigger fixed upon one of the axles; and in order that the beaters in revolving may not come in contact as they pass, the positions of the two pairs are so arranged that the blades of one shall be in a perpendicular situation, while those of the other are horizontal.

The rind of the flax or hemp having been previously broken by any of the ordinary modes of performing that operation, small bunches or stricks of the material are spreas out, and their ends confined between the jaws of clamps or holders. 
These clar:ns or holders differ considerably from the clamps which are commonly used. I shall therefore particularly describe their construction, before showing them in operation. Figs. 430 and 431 are views of the clamp in two different positions; $a$ and $b$

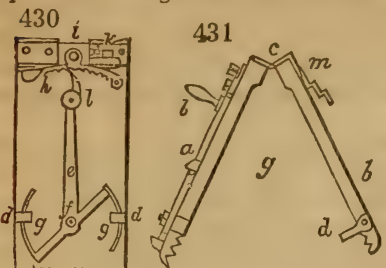
are two boards united together by a hinge, $c$, at top, which of course allows them to shut and open. The lower parts, forming the jaws of the clamps, are made with teeth or indentations, between which parts the ends of the flax or hemp are securely held when the clamps are brought together; $d d$ are two pieces projecting from the board $b$, at the end of each of which is an eye shown by dots, and at the back of the board $a$ (see fig. 430), there is a double armed lever, $e$, turning upon a fixed pin, $f$, which lever carries two circular wedges, $g$. These wedges pass into the eyes of the pieces $d d$, when the clamps are closed and hold them fast. There is a segment ratchet $h$, at the upper part of the board $a$, which turns upon a stud, $i$, and is pressed downward by a spring, $k$. This ratchet receives the end of the lever $e$, and consequently keeps the circular wedges firm in the eyes, which hold the clamps securely together, and prevent their opening by the shaking of the machine.

When it is required to open the clamps, the ratchet $h$ must be raised, and the lever $e$ pushed aside by its handle $l$, which draws the circular wedges $f$ from the eyes of the pieces $d d$, and the boards of the clamps immediately separale. For the convenience of suspending the holders in the machines, a piece of sheet iron, $m$, is bent at right angles, and fastened to the back of the board $b$, as seen in fig. 431, forming a groove by means of which the holders are enabled to slide into the machine and hang there.

These clamps or holders are, when charged with the material, placed in the scutching machine, as shown at $e e e$, in figs. 428 and 429, bearing upon the edge-rail or bar $f$. The beaters are now made to revolve in the manner already described, by which the edges of the blades will strike against the pendent stricks of flax or hemp alternately on each side, and beat off, scutch, or swingle the boom from the material, and render it fit for the operation of heckling which is to follow.

The whole machine is incased with boards, to prevent the inconvenience arising from dust, and an apparatus might be adapted with a blower to conduct away the dust created by the machine, and to discharge it out of the building.

In introducing these stricks of flax or hemp into the machine, the holder is placed upon the projecting end of the bar or edge-rail $f$, and is thence slidden into the machine; and after the material has been sufficiently scutched or swingled, the holders, with the stricks, are removed through the top of the machine, and others successively introduced at the end, and pushed along the rail.

432

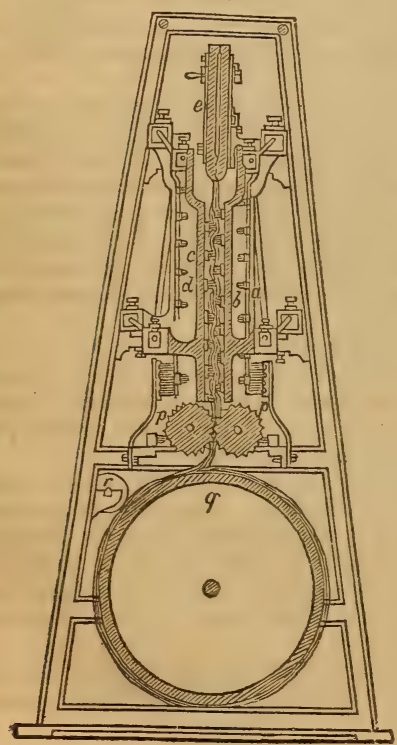

433

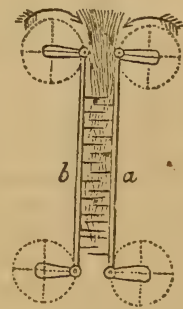

434

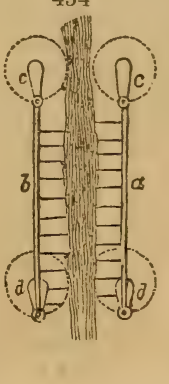

If, however, it should be thought desirable, the stricks may be progressively carried through the scutching machine, and delivered into a similar edge-rail in the heckling machine, there to be operated upon in the way about to be described, by which means the whole process of scutching and heckling may go on without interruption.

Fig. 432 represents the heckling or combing machine by which the fibres of the material are to be opened, and the tow removed. It is a transverse section, taken nearly through the middle, in a vertical direction. Perpendicular standards form the ends of the machine, which are connected together by longitudinal rods or bars secured by nuts. The heckle points intended to act upon the flax are mounted in the frames $a, b, c$, and $d$, and the stricks of flax held in the clamps $e e e$, as described, are suspended from the bar or edge-rail, extending through the machine. 
In order to render the principles of this machine and its mode of working evident, it may be desirable to show in an abstract form the manner in which the heckles are brought into operation upon the flax, and for this purpose two diagrams are delineated in figs. $433,434$.

Suppose two sets of combs or heckle points be mounted upon frames $a$ and $b$, as in these figures, each frame being moveable by means of cranks $c, c$, and $d$, $d$, connected in such manner that they both turn with the same speed in opposite directions, it is evident that every part of the frames and combs will move in circles corresponding to those described by the cranks; the points of the combs travelling in the directions of the arrows, and in circles represented by dots.

During this movement, whilst performing the first descending quarter of the circle, the cranlis bring the frames together as in fig. 433 . They begin after this to separate in describing the second descending quarter, and come to the position fig. 434, when, continuing to revolve, they move further from each other in describing the first ascending quarter of the circle, and arrive at the position where the distance is the greatest; lastly, they deseribe the second ascending quarter returning to the third position. If, therefore, a strich of flax be suspended between the two sets of combs as in fig. 433, and the rotatory motion be continued for a sufficient length of time, the flax will be combed in the whole length which is submitted to the actions of the combs, although the points severally have only operated in a very small space.

Such a system of combs or heckles would make a very good and simple heckling engine, if it were not for the inconvenience experienced by the points dragging some of the fibres with them when withdrawing from the flax, which would produce a great waste of material; and to obviate this it would be necessary to introduce sume contrivance for clearing the points, which must be attended with considerable complication. The plan, however, of the present improved engine, affords the means of producing the same effect by more simple and efficient means.

There are two series of combs, see fig. 435, attached to two moveable frames repre-

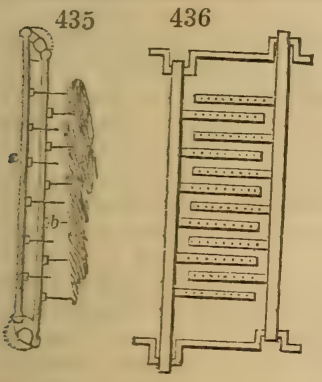
437

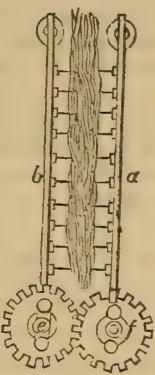
sented at $a$ and $b$. Each frame is formed by vertical bars $a b$, with lateral, branches or arms, which carry the heckle points. The branches or arms are parallel, and at equal distances apart, but fixed in such positions in each frame that they may occupy the intervening space when the frames are brought together, as fig. 436 . The frames are put in motion by means of revolving cranks to which they are attached, as shown in $f \mathrm{~g} .436$, and when the cranks turn upon their axes, the branches of one frame pass between those of the other. without touching. This forms what may be called a set of combs; but one of the improved machines contains two such sets, the points of the combs of one set being opposed to the points of the combs in the other set.

The way in which the series of combs that compose one set act upon the flax, is shown in the side view fig. 435. When the cranks are nearly vertical, the points of both frames are away from the flax, but as the cranks move round in the direction of the arrows, the rrames come into another position, and it is then that the points or heckles of one of the frames $a$, begin to penetrate the flax, and descending they comb or divide its fibres. The rotation of the cranks continuing, the two frames $a$ and $b$ come into the position shown at fig. 435 , the points of the frame $a$, withdrawing from the flax, and those of the frame $b$, approaching and pushing the fibres off from the former, which are now combed by the descending stroke of the points.

It will hence be perceived that as the combs of the frame $a$ and $b$, respectively advance, they will push forward the whole of the strick of flax, and render it impossible for the fibres to be raised and entangled, as each frame in advancing clears the fibres from the points which preceded it.

A single set, however, of such combs or heckles acting only on one side of the flax, would but imperfectly perform the operation of opening its fibres; it is therefore necessary, in order to accomplish the desired object in the most effectual way, that two such sets of combs or heckles should be brought to act on opposite sides of the strick of flax, which may be done in the manner shown in the figures. The cranks of the two opposite sets of comb-frames or heckles $a b$, and $c d$, are connected by a pair of toothed wheels e $f$, as fig. 437, or by four toothed wheels, by which the heckles are actuated at once, the two sets moving in opposite directions, but with similar speeds, and the combing or heckling of the material will go on in the way shown in the figure last andicated. 
Thus far I have considered only two frames of combs or heckles constituting a set, as acting upon each side of the strick of flax ; but in order to perform a greater quantity of work, several sets may be mounted in one machine, working alongside of each other, extending over the breadth of the machine. The combs may then be supported upon three frames, of which the middle one may have branches or arms extending upon both sides, and the other two frames branches extending inwards only. To drive the frames so ar ranged they must be connected to treble cranks.

Such is the principle of the improved machine for combing or heckling, exhibited in the several figures of which I now proceed to describe the particular construction. The machine or engine, fig. 432, has four sets of combs, acting both at the back and front of the flax; $a b$ are the front set of combs, and $c d$ the back set of combs; $e e e$, are the clamps holding the stricks of flax previously scutched, which clamps hang upon the edge-raii. The comb frames are attached at top and bottom to the cranks $g \mathrm{~g}$, which are all connected by toothed gear, and driven by a band and rigger.

The combs or heckles being put in motion in the way described, act upon the suspended stricks of flax, and upon their fibres, os explained; which stricks are progressively conducted through the machine by their elamps sliding upon the edge-rail through the agency of the endless chain, to which the clamps are severally attached, by a hook falling into one of the links. The chain is driven by a spur wheel upon the axle of a bevel wheel, which receives a slow rotatory motion through a bevel pinion on the axis of a simiiar wheel, actuated by another pinion on the end of the upper crank axle. By these means, clamps, with the stricks of flax placed on the edge-rail, are slowly carried through the machine, when the flax will be gradually acted upon first by heckle points of a coarse kind, set wide apart, and ultimately by finer points set near together; after which, the clamp with the strick of flax is discharged from the machine, at the reverse end of the edgerail. But should the workman neglect to remove the holder or clamp, when it arrives at the end of the rail, the machine would be stopped by means of a jointed lever, having a fork at its end, which pushes the band from the fast rigger on to the loose one, and throws off the driving power.

As the combs or heckles, in acting upon the flax to divide its fibres, tear parts of the fibres, and reduce them into tow, the downward motion of the heekles brings the tow with them out of the flax, which is deposited between two fluted rollers $p$, fig. 432, and is by them conducted down to the large drum $q$, where it becomes lapped in two endless sheets round the periphery of the drum; the one of coarse tow, the other of fine, the adhesion being assisted by a pressing roller $r$; and when a quantity of the tow has been thus accumulated round the periphery of the drum, it may be removed thence by cutting it off in sheets. The fluted rollers, and also the large drum, are driven by gear bands.

After the strick of flax has been thus carried through the scutching machine or the heckling machine, the jaws of the clamps are to be opened, the ends of the flax reversed, - and the strick again confined in the clamps, so that the other end of the striek may be operated upon in a similar way. In order to prevent any part of the flax from attaching itself to the branches of the moveable frames, each frame is furnished with a shield or guard of polished iron or brass plate, which covers a part of the combs and the heads of the screws by which they are fixed to the branches. When the plate metal is bent into the form of a shield, it is slipped on to the branches of the heckle frames, and is sufficiently elastic to hold fast.

But it is to be observed, that the edges of the shields are to vary in the extent of their projection according to the situation in which they are to be placed; those which are to shield the upper branches of heckles are to project but little, so as to leave the points uncovered and free to enter the strick of flax; but the shields of the lower heckles are to project considerably over the points, to prevent them from penetrating ton far into the fibres, which is so contrived for the purpose of facilitating the falling of the tow, which would otherwise be with difficulty removed from the lower combs, were it thrust upon the whole length of the points.

It being advantageous that each strick of flax should be combed near the lower extremities before the middle is acted upon, it is necessary, in order to obtain this effect, to remove sume of the points of the combs in the upper branches. By these means, the operation of the heckles upon the flax begins and proceeds gradually, and ceases at the opposite extremity of the machine in the same gradual way, which is very advantageous in clearing completely the flax from the tow.

IV. Flax-spinning. - If we compare flax with other spinning materials, such as wool and cotton, we shall find it to possess several characteristic properties. While cotton and wool are presented by nature in the form of insulated fibres, the former requiring merely to be separated from its seeds, and the latter to be purified from dirt and grease before being delivered to the spinner, flax must have its filaments separated from each 
other by tedinus and painful treatment. In reference to the spinning and the subsequent operations, the following properties of flax are influential and important :-

1. The cunsiderable length of the fibres, which renders it difficult, on the one hand, to form a fine, level, regular thread, on the other, gives the yarn a considerably greater tenacity, so that it cannot be broken by pulling out the threads from each other, but by tearing them across.

2. The smooth and slim structure of the filaments, which gives to linen its peculiar polished aspect, and feel so different from cotton, and especially from woollen stuffs, unless when disquised by dressing. The fibres of flax have no mutual entanglement, whereby one can draw out another as with wool, and they must therefore be made adhesive by mois. ture. This wetting of the fibres renders them more pliant and easier to twist together.

3. The small degree of elasticity, by which the simple fibres can be stretched only one twenty-fifth of their natural length before they break, while sheep's wool will stretch from one fourth to one half before it gives way.

Good flax should have a bright silver gray or yellowish color (inclining neither to green nor black); it should be long, fine, soft, and glistening, somewhat like silk, and contain no broad tape-like portions, from undissevered filaments. Tow differs from flax in having shorter fibres, of very unequal length, and more or less entangled. Hemp agrees in its properties essentially with flax, and must be similarly treated in the spinning processes.

The manufacture of linen and hemp yarn, and the tow of either, may be effected by different processes; by the distaff, the hand-wheel, and spinning machinery. It will be unnecessary to occupy the pages of this volume with a description of the first two well known domestic employments. I shall therefore proceed directly to describe the last method, or

Spiming of Flax by Machinery. - This branch of manufacture has been much more recently brought to a practical state than the spinning of cotton and wool by machines, of which the cause must be sought for in the nature of flax as above described. The first attempts at the machine spinning of flax, went upon the principle of cutting the filaments into short fragments before beginning the operation. But in this way the most valuable property of linen yarn, its cohesive force, was greatly impaired; or these attempts were restricted to the spinning of tow, which on account of its short and somewhat tortuous fibres, could be treated like cotton, especially after it had been further torn by the carding engine. The first tolerably good results with machinery seem to have been obtained by the brothers Girard at Paris, about the year 1810. But the French have never carried the apparatus to any great practical perfection. The towns of Leeds in Yorkshire, of Dundee in Scotland, and Belfast in Ireland, have the merit of bringing the spinning of flax by machines into a state of perfection little short of that for which the coiton trade has been so long celebrated.

For machine spinning, the flax is sometimes heckled by hand, and sometimes by machinery. The series of operations is the following :-

1. The heckling.

2. The conversion of the flax into a band of parallel rectilinear filaments, which forms the foundation of the future yarn.

3. The formation of a sliver from the riband, by drawing it out into a narrower range of filaments.

4. The coarse spinning, by twisting the sliver into a coarse and loose thread.

5. The fine spinning, by the simultaneous extension and twisting of that coarse thread.

The spinning of tow requires a different treatment: we shall first treat of the heckling of flax by machines; and secondly, of the mechanical spinning of flax. The mechanical carding and spinning of tow are very similar to those of cotton; which see. Though machine heckling be far from perfect, yet the tow it throws off can be spun into very good yarn by machines, while it would afford very indifferent yarn to the hand spinner.

All heckle machines have this common property, that the flax is not drawn through them, as in working by hand, but, on the contrary, the system of heckles is moved through the flax properly suspended or laid. Differences exist in the shape, arrangement, and movements of the heckles, as also in regard to the means by which the adhering tow is removed from them. The simplest and most common construction is to place the heckles upon the surface of a horizontal cylinder, while the flax is held either by mechanical means or by the hand during its exposure to the heckle points. Many machines have been made upon this principle. It is proper in this case to set the heckle teeth obliquely in the direction in which the cylinder turns, whereby they penetrate the fibres in a more parallel line, effect their separation more easily, and cause less waste in torn filaments. To conduct the flax upon the cylinders, two horizontal fluted rollers of iron are employed, which can be so modified in a moment by a lever as to present the flax more or less to the heckling mechanism. The operator seizes a tress lock of 
flax with her hand and introduces it between the fluted rolers, so that the tips on which the operation must begin, reach the heckles first, and by degrees the advancing flax gets heckled through two thirds or three fourths of its length, after which the tress or strick is turned, and its other end is subjected to the same process. By its somewhat rapid revo lution the heckle cylinder creates a current of air which not only carries away the boomy particles, but also spreads out the Hax like a sheaf of corn upon the spikes, effecting the same object as is done by the dexterous swing of the hand. The tow collects betwixt the teeth of the heckle, and may, wijen its quantity has become considerable, be removed in the form of a flock of parallel kayers.

The essential parts of such a construction will be understood from fig. 438 , though the futed rollers are absent. The flax $a, b$, is held by the hand, or in a kind of clamp. The cylinder is partly covered with a curvilinear plate of iron $c$, $d$, which serves to sustain the flax, and to guide it in circular tresses round the periphery of the heckle. At the beginning it is placed near $b$, when the tips of the flax are only presented to the heckles; during the working the shield is continually drawn back in the direction from $d$ to $c$, and thus lets the uperation be performed upon the remaining part of the flax.

438
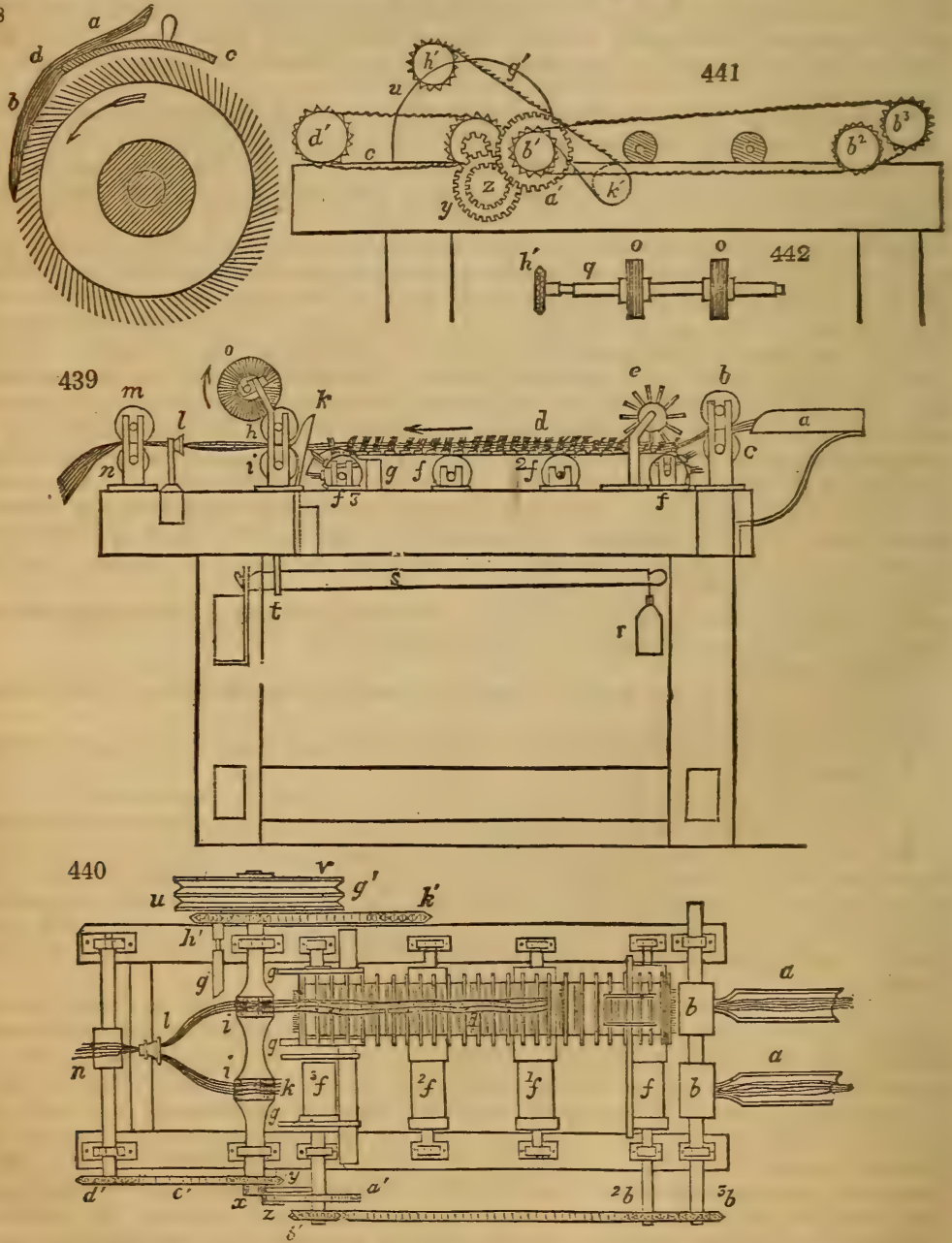

First operation; the conversion of flax into ribands or slivers.-This is effected by sub jecting the flax to a series of advancing gills or heckle-teeth, and at the same time drawing out its fibres by means of rollers. Figs. 439, 440,441, show the outline of the con- 
struction of a machine for this purpose. Here two rows of heckles are placed alongside of each other, though only one of them be shown in the ground plan, fig. 440 , in order to allow the parts beneath the other to be seen. The flax is placed in the sheet iron channels $a$ a by laying down one handful after another, so that the points of the second strick reach to only the middle of the first, and thus preserve a uniformity of thiclincss in the feeding. This process is necessary, since, as every one knows, the heckled stricks are always thick in the middle, and thin at the ends. The flax being introduced between the rollers $b$ and $c$, is drawn out by their agency, and at the same time subdivided by the heckles $d$, between whose teeth the pins of the roller $e$ press it down. At the rollers $f^{3}$ it is loosened from the heckles by the transverse bars which rise from the springs $g$, after which it is seized by the rollers $h i$, and drawn again. A little beyond these rollers, it runs through a funnel $l$, in order to gather the fibres together; in front of these rollers the slivers from both rows of heckles are united, and proceed in one riband throngh that polished brass funnel; the rollers $m n$ extend this riband, pressing it gently together, and then let it fall into a tin can. The union of the two slivers contributes to the uniformity, since the irregular thicknesses are thereby compensated. The diameter of the roller $c$, is equal to that of each of the cylinders $f, f_{1}, f_{2}, f_{3}$; and the whole five move with equal velocity. The same correspondence exists between the rollers $n$ and $i$. Thus the sliver of flax is not stretched either by its passage from $e$, upon the heckles, nor between $i$ and $n$, but solely in passing from the heckles to the rollers $i h$. The heckle teeth of this machine do not stand perpendicularly, but are bent somewhat backwards; so as to retain the flax more firmly. The revolving cylindrical brush $o$, is placed over and a little in front of the pressing roller $h$, in order to take off all the filaments of flax adhering to their circumference, and to toss them onwards where they may again unite with the slivers. For the sake of perspicuity, the rollers $h$, and those brushes are left out in fig. 440, but the latter are particularly shown in fig. 442, while a portion of their axis $q$, is however shown in fig. 440. The pressure of the cylinder $h$, upon the cylinder $i$, is produced by the weight $r$, fig. 439, which hangs upon the lever $s$; the lever pulls down at $t$, a vertical rod, whose upper hook-shaped end embraces the axis of $h$ in the middle of its length.

Second principal operation; the formation of rovings.-Mr. Wordsworth's improvements in machinery for preparing, drawing, and roving flax, hemp, wool, and other fibrous substances, consists in a novel contrivance or mechanism to be adapted to the machine commonly called the gill, employed for preparing, drawing, and roving flax and hemp, and for combing and spinning long wool ; which improvements allow the points of the travelling heckles to continue longer in operation than in the ordinary construction of gill, and cause the heckle points to be withdrawn from the fibres at the end of the stroke without the possibility of their drawing the fibres down with them.

The manner of effecting this object will be seen by reference to the several figures

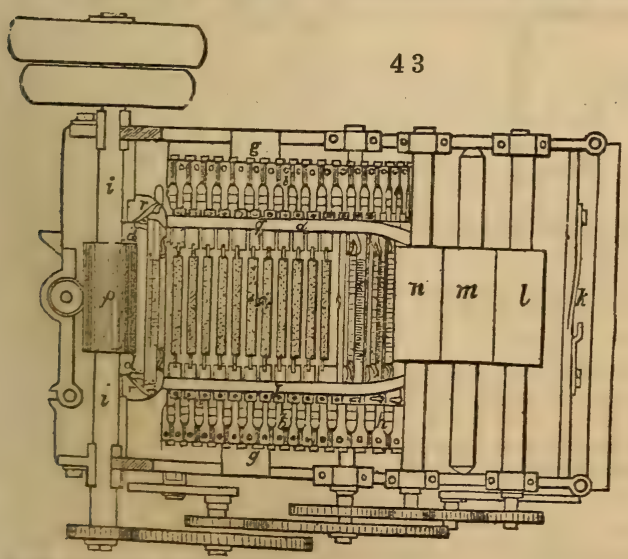
which exhibit a gill on this improved plan in different views. Fig. 443 is a plan or horizontal view, exhibiting the upper surface of the machine; and fig. 444 is a longitudinal section taken through the middle of the machine: fig. 445 is a representation of the front of the machine, but in which several parts have been removed to show the action of the heckles more perfectly.

The several heckles $a$ a $a$ are formed by a series of needles or heckle points set into a metal bar, as represented on an enlarged scale in figs. 446 and 447. These bars are each of them sus. pended in a frame or carriage $b b b$ (shown in two views at figs. 448 and 449), by means of double jointed levers $c c$, seen in two positions, at figs. 450 and 451 ; the heckle bar, its levers and carriage or frame, being shown put together in figs. 452 and 453.

When the heckles are in operation, the points are raised, as in fig. 445 ; but when they are withdrawn from the fibres, then the points are sunk down into the carrying frames, as fig. 453 .

These two positions of the heckles are produced by the knobs or parts $d$, that project 
from the jointed levers $c$, acting against the edges of guide bars, which will be explainea

in describing the operations of the machine.

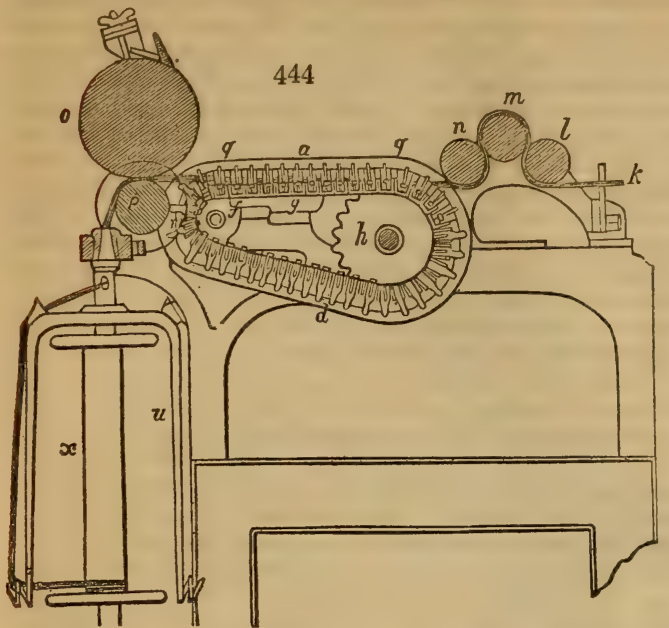

445
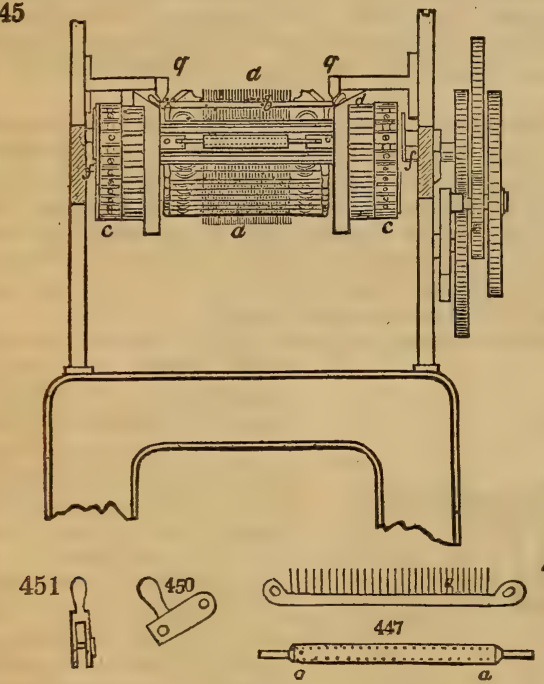

446

448

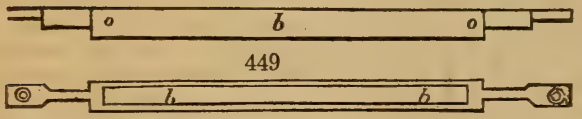

452

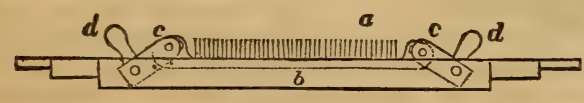

453

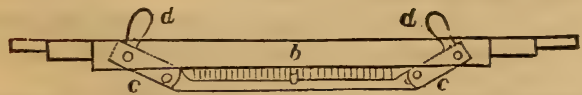

The several heckles are adapted and made to work in the machine by attaching the ends of the respective frames or carriages $b$, to travelling endless chains $e, e$, seen in figs. 443, 444, and 445. These endless chains pass over fluted guide rollers $f f$, seen best in figs. 444 and 445 , and over horizontal bars $g \mathrm{~g}$, seen best in figs. 443 and 444 . The chains with the heckles are driven through the machine by rotatory spur wheels $h h$; see figs. 443 and 444 , the teeth of which take into the spaces between the cylindrical parts of the several heckle carriages $b \quad b$, and consequently drive the heckles forward; and these spur wheels are actuated by a train of toothed gear from the first driving shaft $i$, which gives motion to all the operative parts of the machine.

If flax, hemp, long wool, or other fibrous material, be passed into the machine at the back part by a feeding cloth or creeper through a guide $k$, best seen in figs. 443 and 444 , and be conducted under and over the feeding rollers $l, m$, and $n$, and over the heckles $a a a$ to the drawing rollers 0 and $p$, and thence to the flier and bobbin, or to 2 receiving can, the fibres will be opened in their progress, and combed by the points of the heckles entering into and separating the fibres, the material being drawn by a different speed to that with which the heckles travel.

This operation of preparing, drawing, and roving flax and hemp, and the general construction of a machine of this kind being well understood, it is not necessary to explain its details, excepting as respects those parts which constitute the present improvements.

It will be perceived, by reference to figs. 443 and 444 , that the knobs $d$, which project from the jointed levers $c$, as they travel along the machine, bear against the outer

edges of the two fixed guide bars $q q$ that extend along the top of the machine above 
the heckles, which keep the heckle points raised, as in $f g .451$. This will also be very evidently seen in the front view of the machine, fig. 445 , where the upper heckle bar $a$ is raised in its carriage $b$, by the knobs $d d$ bearing against the outer edges of these guide bars, $q q$. But when the endless chains $e e$, which support and conduct the frames or carriages of the heckles, have advanced the heckle points to within a very little distance of the drawing rollers (see fig. 444), then the knob $d$ of the jointed levers at each end of the heckle bar passes the ends of the guide bars $q q$, and they immediately come in contact with two inclined planes, $r r$, seen in figs. 443 and 444, which instantly depres6 the levers $c$, and consequently cause the heckle bar $a$, with its points, to descend in the frame or carriage $b$, withdrawing the points from the fibres of the material almost in 2 perpendicular direction.

The heckles that have become thus depressed pass with their carriages by the traversing of the endless chains along the under part of the machine, and when they arrive at the back, and begin to rise, the guide bars $q q$, being at their commencement slightly bent, conduct the knobs $b$ of the levers $c$ until they are forced back into the positions first described, whereby the heckle points are raised, as they come to the upper part of the machine into effective operation. The fibres of material operated upon, after passing through the drawing process between the rollers, may be roved, twisted, or spun, by the employment of a bobbin and flier, as shown fig. 444, or may be delivered into a can, to be roved, twisted, or spun, by other machinery, by substituting a pair of conducting rollers instead of the bobbin and flier, which shall conduct the sliver of material into a tin can below.

The descent of the heckles $a$ into their frames $b$, by the falling of the levers $c c$, precludes the possibility of the fibres of the material operated upon being carried down under the machine by the points, as frequently happens in gill machines of the ordinary construction; and this mode of mounting the heckles and traversing them with the assistance of the guide bars $q q$, and inclined planes $r r$, allows the heckle points to be brought much nearer to the drawing rollers $o p$, by means of the metal bars in which the heckle points or needles are set, falling below the centre of the endless chain $e e$, as shown in figs. 443 and 444, and thereby affords the means of preparing, drawing, and roving various qualities of flax, hemp, wool, and other fibrous materials, particularly such as have a much shorter staple than any fibrous materials hitherto operated upon in gill machinery.

Another most ingenious and effective improvement made of late years in the flax spinning machinery, is that patented by Messrs. Westley and Lawson, in August, 1833, and since then introduced into practice with great advantage. It applies to the gill or mechanism employed for opening, straightening, and separating the fibres of flax, hemp, and long wool, in the operation of slivering. The peculiar feature here is a method of driving the heckle bars through the gill machine by means of perpetual screws or worm shafts, instead of by chains and spur wheels, as in the former constructions.

The heckle bars which lie across the machine are, by the present patentees, supported at their ends by fixed horizontal guide rails, on which they slide, while the extremities of the heckle bars are inserted in the helical grooves of the worm shafts, which are placed in horizontal positions at the sides of the machine; and hence the rotatory motions given to these screw shafts cause the heckle bars to be driven along the guide rails with a uniform simultaneous movement.

The heckle bars having performed their usual office, that is, having combed and separated the fibres of the material as they move onward, are at the front part of the machine depressed and put out of operation by means of rotatory cams; and by the assistance of guide levers, each heckle bar, when it arrives at the end of the upper horizontal guide rail, is conducted down to the lower horizontal guide rails, where the extremities of the comb-bars falling into the helical grooves of a lower pair of worm shafts, revolving in an opposite direction to the former, thereby give the heckle bars a retrograde movement. When they arrive at the back end of their horizontal guide rails, they are, by similar rotatory cams, raised again to the upper horizontal guide rails, which coming into gear with the upper worm shafts, are moved onwards as at first.

By this means a succession of heckles is continually advancing upon the upper guide rails, having their points in constant operation between the fibres of the textile materials, while their vertical position is secured during their whole course.

Fig. 454 is a horizontal representation of a gill machine, showing the present improvernents; but sume of the upper portions of the machine are removed, to let the working parts be seen more clearly. Fig. 455 is a side view of the gill; and fig. 456 a vertical section taken longitudinally. The driving rigger or pulley $a$ is fixed upon the front roller $b$, cornmonly called the drawing roller, because when pressed upon by the upper wooden roller $c$, it draws out the fibres between them. The rollars $d, e, f$, are the ordinary back or holding rollers, for retaining the fibres, while they suffer powerful traction 
by the rollers $b, c$, over the needles or points of the heckle bars. The upper guide rail above mentioned, upon which the heckle bars slide, is shown at $g$, in fig. 456 , and the

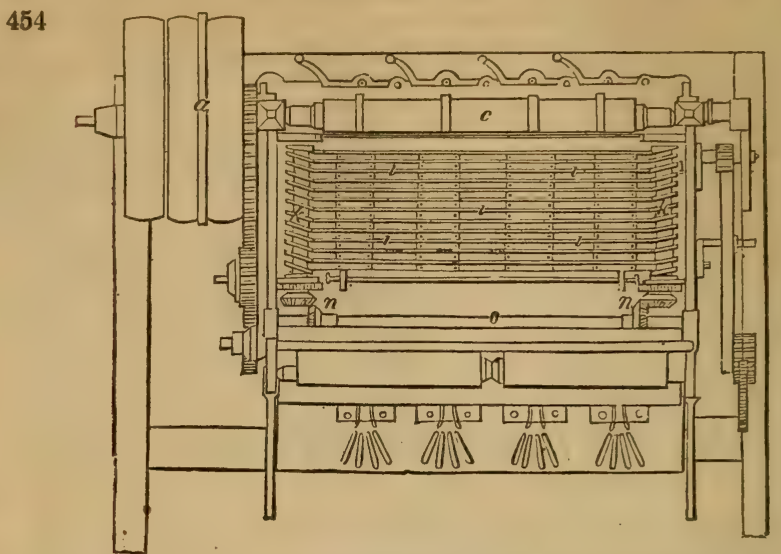

lower guide rail at $h$; the series of heckle bars with their needles are represented at $i, i, i, i, i, i$; the upper worm shafts $k, k$, are mounted in brackets made fast to the sides

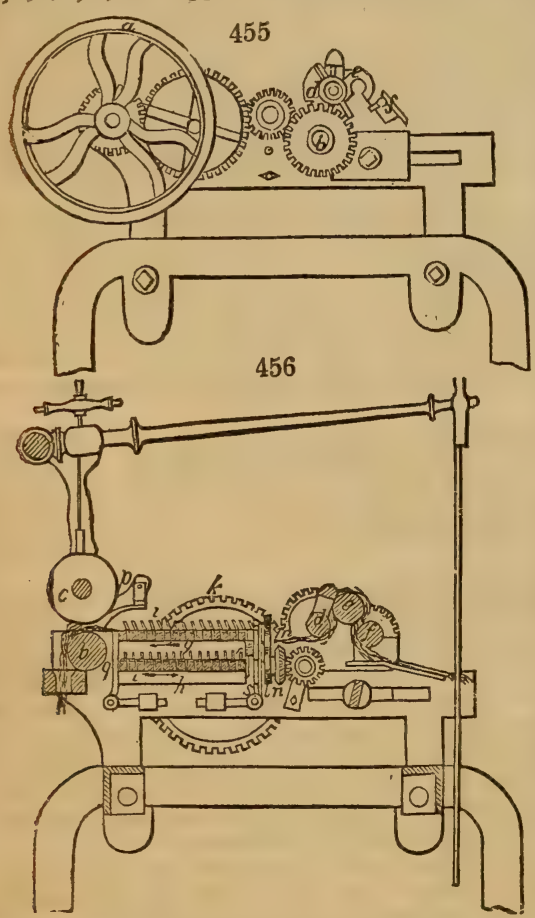
of the frame; a similar pair of worm shafts $l$, being mounted in like manner below. These worm shafts $k$ and $l$, on each side are connected together by toothed wheels $m$, and upon the axles of the lower worm shafts, bevelled pinions $n$ are fixed, which take into corresponding bevel pinions on the transverse shaft or axle $o$. This shaft $o_{\text {, }}$ heing connected by a train of toothed wheel work with the axle of the drawing roller $b$, as shown in figs. 454 and 455 , the rotation of the roller $b$, causes the shaft 0 to turn also, and the bevel gear $n$ and 0 , produces the rotatory motion of the worm shafts $k$ and $l$, which turn in contrary directions.

It will be seen, from fig. 454, that the ends of the heckle bars $i$, have nibs or projections which fall into the grooves of the screw or worm shaft, and that being supported below, upon their guide rails, as the worm-shafts $k k$ revolve, the upper range of heckle bars will be progressively advanced towards the front part of the machine. By referring to fig. 456 it will be perceived, that as each heckle bar arrives at the front end of the guide rail $g$, a finger $p$ called a tappet or cam, on the shaft $k$, strikes it down to the lower guide rails $h$; and, in order that its descent may be truly vertical, weighted levers $q q$, in front, are made to press against the face of the heckle bar as it descends. This

bar having now arrived at the lower guide rails $h$, lets fall its nibs into the grooves of the lower worm shafts $l$, by whose rotation the heckle bar is made to retrogade, or return towards the back of the machine. When the heckle bar has reached the hinder end of the guide rail $h$, a finger or tappet, $r$, on the lower worm-shaft, comes under it and raises the heckle bar, guided by the back-weighted levers $s$, as shown in fig. 456, till it is elevated to the level of the upper guide rail $\mathrm{g}$; when the threads of the upper worm. shafts take hold of its nibs as before, and conduct it forward upon the guide rail in ths 
way already described. Thus the continued rotation of the worm shafts $k k$ and $l l$, causes the whole series of heckle bars to travel along the guide rails, and the tappets $p$ and $r$, by alternately depressing and raising them at the ends of the said rails, cause them to move in a regular circuit, yet so as to preserve their verticality.

The claim made under this patent is for every mode in which screw or worm shafts may be adapted to conduct the bars carrying the needles or heckle-teeth through a machine for preparing, drawing, or roving textile fibres.

In December, 1835, Messrs. Hope and Dewhurst obtained a patent for improvements in the manufacture of flax, which deserve notice. These are of both a chemical and mechanical nature. The first consists in steeping the flax in dilute sulphuric acid, of a certain strength, and for a certain time, proportioned to the quality of the fibres, the coarser requiring the stronger application. By this means the gummy matter and the outer shell will be loosened and easily detached. It is then to be passed between squeezing rollers, afterwards well washed, boiled in a solution of soap and water for a few hours, and finally passed again through the rollers. These processes may be repeated till the flax acquires the desired glossiness and separation of fibres. It is next to be beaten, and passed once or twice over an ordinary heckle or stiff brush. 459

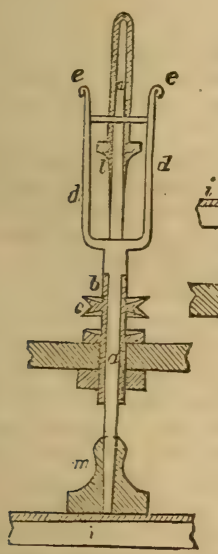

457

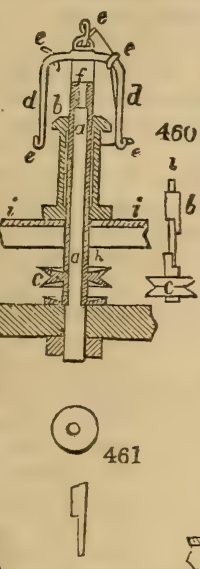

458

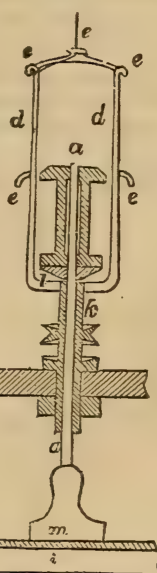

The second part, or the mechanical, is represented by the figures $457,458,459,460$, and 461. Fig. 457 is a sectional elevation in part of the construction of the spindle, bobbin, and flier, proposed for spinning all kinds of flax or hemp. Fig. 458 answers for spinning coarser yarns; fig. 459 shows how yarns are to be spun for weft, and wound upon what is called a "pin cop bobbin."

$a \quad a \quad a$ is the stationary or fixed spindle of the ordinary throstle frame, which is surrounded by the tube $b b$, and connected to the wharve or pulley $c$, by which the flier $d$ is driven. The flier is furnished with guides or conductors, $e$, which lead the yarn immediately to the bobbin; this flier is also provided with a small central shaft which supports it, and runs in the small cup or recess at the top of the stationary spindle $a$, and is fixed with the flier to the tube $b b$, which is altogether carried round or driven by the wharve $c$.

It will be seen by fig. 460 that the wharve $c$ and tube $b$ are connected at bottom by a half-lap coupling joint or clutch; this is for the purpose of allowing the tube $b$ to be slidden up the spindle, and more readily removing the bobbin when it is full of yarn, without stopping the frame, or removing the band from the wharve $c$, the tube of which runs in the step or cup $h$, fixed upon the bolster rail near the bottom of the throstle frame. The traversing of the bobbin or the copping motion is effected exactly in the same manner as in ordinary throstles, that is, by the lifting and lowering of the copping rail $i$, which in this instance supports the bobbin. In fig. 458 the flier is constructed of twice the length of the bobbin, to allow this to rise and fall freely within it, and is connected at top by a slight cross piece, for the purpose of preventing the arms of the flier from expanding by the centrifugal force, when turning with great velocity. The flier for spinning coarse numbers requires to have an inner tube, $k$, to support the spindle. The bobbins are supported upon a washer, $l l$. The spindle is allowed to revolve in a slight degree by the friction of the drag-weight $m \mathrm{~m}$. This weight has a hole formed in it with a flat side, as shown in fig. 461 .

Flax has been for a long period spun wet in the mills; a method no doubt copied from the practice of housewives moistening their yarn with their saliva at the domestic wheel. Within a few years the important improvement has been introduced of substituting hot for cold water, in the troughs through which the fibres in the act of spinning pass. By this means a much finer, smoother, and more uniform thread can be spun than in the old way. The flax formerly spun to twelve pounds a bundle is, with hot water, spun to six. The inconvenience of the spray thrown from the yarn on the fliers remains, aggravated by increased heat and dampness of the room where this hot process goes on. Being a new expedient, it receives daity changes and ameliorations. When first employed, the troughs of hot water were quite open; they are now 
usually covered in, so as almost entirely to obviate the objections to which they were previously liable. With the covers has been also introduced a new method of piecening or joining on any end, which may have been run down, namely, by splicing it to the adjoining roving, whereby it is carried through the water without imposing a necessity on the spinner to put her hand into the water at all. In some places she uses a wire, for the purpose of drawing through the end of the roving to mend a broken yarn.

This may be considered the inherent evil of flax-spinning,- the spray thrown off by the wet yarn, as it whirls about with the fier of the spindles. A working dress, indeed, is generally worn by the spinners; but, unless it be made of stuff impermeable to water, like Mackintosh's cloth, it will soon become uncomfortable, and cause injury to health by keeping the body continually in a hot bath. In some mills, water-proof cloth and leather aprons have actually been introduced, which are the only practicable remedy; for the free space which must be left round the spindles for the spinner to see them play, is incompatible with any kind of fixed guard or parapluie.

There was, before the late Factory Bill passed, a class of very young children employed in the flax mills, under the name of little doffers, forming generally a troop of from four to ten in each spinning-room, who, the moment they perceived the bobbins of any frame or side of a frame exhausted of roving, ran together, and furnished it with full ones as quickly as possible. They were not numerous in all, but they had an occupation requiring a great activity and attention. It was practised also in the fine spinning-rooms, which are perfectly free from dust; and, as it involved a kneeling and stooping position, seemed peculiarly appropriate to children, and is still done by them at a somewhat more advanced age.

The adjoining fig. 462 will serve to explain the mechanism by which the fine 462

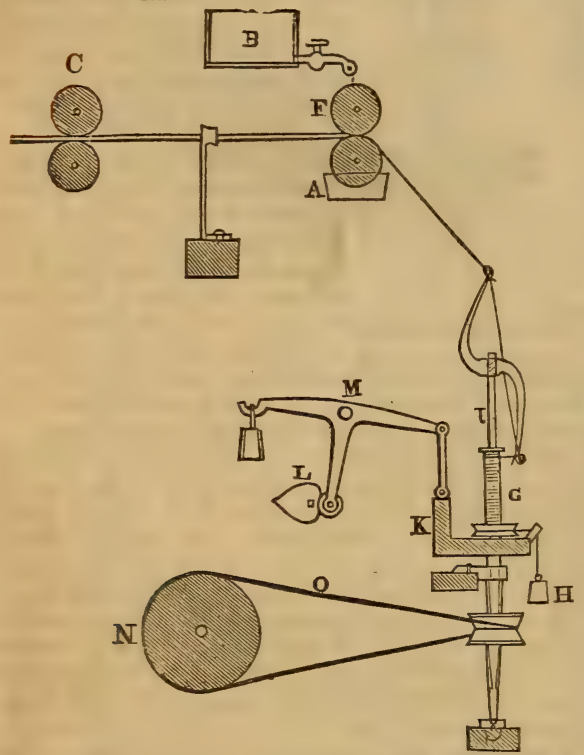
spinning of flax is performed. The front pair of drawing rollers represented at $\mathbf{F}$ was at one time moistened by letting water trickle upon it, from a vessel B, furnished with a stopcock placed a little above, or by immersing one half of the under-roller in the water-trough as at $A$. The roller pair $c$, which receives the fine rovings from bobbins placed on skewers or upright pins in the creel behind, is so mounted as to be fixed at any desired distance from the front rollers $\boldsymbol{F}$. This distance should be always a little more than the average length of the filaments of the line; for if it were equal to it, they would be seized at both ends by the two pairs of rollers, which move with different velocities, and would be torn asunder, instead of being drawn out alongside of each other. The front rollers, indeed, move in many such machines four times faster than the back pair. The rest of this flax-spinning apparatus resembles in every respect the throstle frame of the cotton-spinner. The

thread, as it escapes from the front rollers, gets twisted by the spindle and flier, and wound up in constant progression on the bobbin, the motion of the latter being retarded either by a washer of leather beneath its lower end, or sometimes, as shown in the figure, by a weighted lever $\mathrm{H}$, suspended from a cord, which embraces the pulley-groove turned on the lower end of the bobbin. This friction of this cord on the pulley, which may be varied by changing the length of leverage at which the weight acts, gives the bobbin the requisite retardation for winding up the yarn.

The bobbin G, at the same time that it has this retarded movement of revolution on its axis, has another motion up and down on the spindle $I$, to present itself at different points to the thread, and to cause the equal distribution of this over the surface of the bobbin-barrel. This latter motion is given by a double eccentric $\mathbf{L}$, which, by turning slowly on its axis, makes the balance-lever $\mathrm{m}$ oscillate, and thereby raises or depresses the bobbin-rail with its row of spindles. $\mathrm{N}$ is a section of the long tin drum, which 
extends the whole breadth of the frame, and communicates its rotatory motion, derived from the steam-pulley, to the spindles, by the intervention of the endless cotton cords 0 , as also to the fluted rollers $c, F$, and to the axis of the heart-shaped or eccentric wheel 1 . working in an endless screw.

The ratio of the velocity of the rollers of supply $c$, with the front or delivering rollers , and with the spindles, is proportional to the fineness of the yarn. For low numbers, the draught is usually fourfold. The speed of the spindles also varies with the quality of the yarn, according as it is intended for warp or weft; the former requiring more twist than the latter; but never so much as to cause it to snarl into a knot, when left free to turn on itself.

One of the most important improvements hitherto made in the spinning of flax is that for which James Kay, of Preston, obtained a patent in July, 1825. Its peculiar feature is the maceration in warm water of the slivers or rovings, previously to spinning them, by conducting them into tin cans, with open bottoms, fitted into circular boxes having holes like a cullender, and immersed into a trough of warm water. The slivers as they pass from the rollers are let fall through the cans into these boxes, when they are to be repeatedly pressed and beaten down by a plunger, or the action of rollers, as may be most convenient. The material must be thoroughly freed from air, and macerated. After five or six hours it is to be removed from the water, and placed in its compressed state at the back part of a drawing and spinning machine. The cake being now turned over, the end of the roving first deposited in the can is drawn out with care, then raised up and passed over a tension roller to the drawing apparatus. The first pair of rollers for the drawing process merely retains the filaments; while at a distance of two inches and a half the drawing rollers are placed. Both are fluted, for the purpose of taking firm hold of the material; and the drawing pair is made to move eight times quicker than the retaining. As the flax fibres have in this state little or no elasticity, and as they adhere loosely in their macerated condition, the drawing rollers must be placed thus close to the retaining rollers, and being made to move at a proper speed, produce an extremely attenuated thread.

The adjoining table represents, in three compartments, the most important rooms in a flax-mill, viz.:-

I. The tow preparing room.

II. The line preparing room for the long flax.

III. One room of spinning machines as a pattern for the rest.

463 TOW PREPARING ROOM.

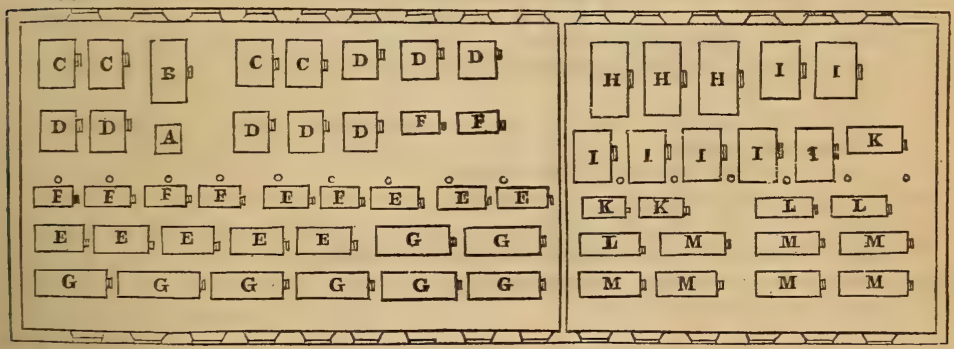

A, lap machine; B, 4-feet breaker card; c, 3 feet 6 inches ditto; D, 3-feet finisher card, 3 workers; E, cut tow, second drawing, 5 heads; F, cut tow, first drawing, 4 heads ; G, cut tow, reg. roving, 32 spindles; $\mathbf{H}, 4$-feet breaker card; I, 4-feet finisher ditto; $\mathbf{K}$, long tow, first drawing, 3 heads; $\mathbf{L}$, long tow, second drawing, 4 heads; $M$, long tow roving 4 spindles.

LINE PREPARING ROOM.

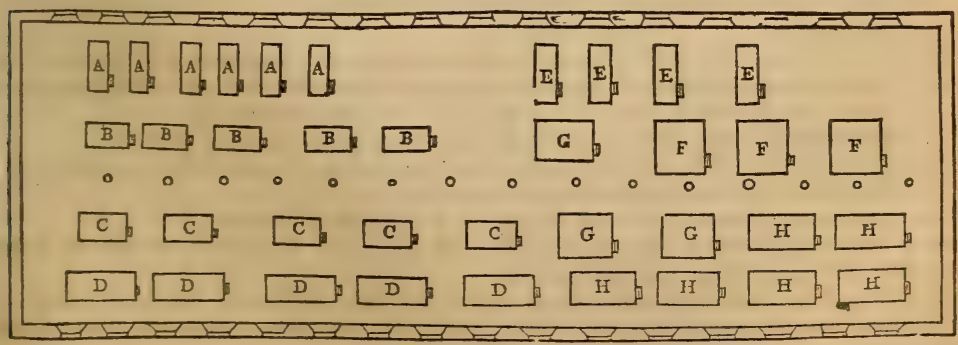


A, cut line, first drawing; B, cut line, second drawing, 4 heals; c, cut line, thi-a drawing, 5 heads ; D, cut line, reg. roving 32 spindles each; $\mathbf{E}$, long line, first drawing, $\mathbf{F}$, long line, second drawing, 3 heads each; $G$, long line, third drawing, 4 heads each; $\mathbf{H}$, long line, roving 16 spindles.

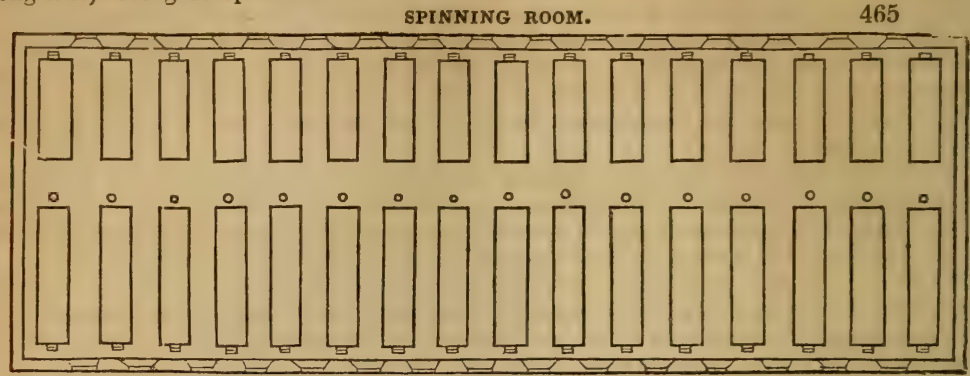

I. The line preparing room comprehends :-

1. Heckling machines with heckles.

2. Line spreaders, or first drawing slivers.

3. Frames for the second drawing, of 3 heads each.

4. Frames for the third drawing, of 4 heads each.

5. Roving frames of 16 spindles each.

6. Spare fallers for first drawing with gills.

7. Ditto ditto for second and third drawing with ditto.

8. Ditto ditto for roving.

II. The cut flax line preparing room :-

1. Sets of heckling frames (eccentric.)

2. Cutting or breaking machine.

3. Line spreaders or drawing ditto.

4. Frames for second drawing, 4 heads each.

5. Ditto third ditto, 5 ditto.

6. Ditto, regulator roving, 32 spindles each.

7. Spare fallers with gills for first drawing.

8. Ditto, ditto for second and third ditto.

9. Ditto, ditto, with gills for roving.

III. Long or uncut flax tow preparation :-

1. Lap machine.

2. Breaker cards, 4 feet diameter.

3. Finisher ditto, ditto.

4. Frames for first drawing, 3 heads each.

5. Ditto for second drawing, 3 heads each.

6. Ditto for roving, 16 spindles each.

7. Spare fallers, with gills for first and second drawing.

8. Ditto, ditto, ditio, for roving.

IV. Cut flax tow preparation :-

1. Lap machine.

2. First breaker cards, 4 feet diameter.

3. Second ditto, ditto, 3 feet 6 inches ditto.

4. Finisher cards with 8 workers.

5. First drawing frames, of 4 heads each.

6. Second ditto, ditto, of 5 ditto.

7. Frames for regulator roving, 32 spindles each frame.

8. Spare fallers with gills for first and second drawing.

9. Ditto, ditto, for roving.

V. Spinning rooms for both lines and tows:-spindles in frames in a number proportional to the number of the above preparation machines; and consequently to the quantity and quality of the flax yarn intended to be spun.

VI. Utensils and tools; such as cards clothing with needle pointed filleting.

Observations upon the above statement of the series of machinery requisite in a modern flax mill of the most improved construction :-

The long or uncut flax to be spun into yarns averaging 30 leas per $1 \mathrm{~b}$.

Each heckling machine will produce about $4 \frac{1}{2} \mathrm{cwts}$. per day, which would be distributed into $200 \mathrm{lbs}$. of line, and $266 \frac{2}{3}$ of tow.

The total with 3 machines would be therefore $600 \mathrm{lbs}$. of line, and $800 \mathrm{lbs}$. of tow.

The preceding statement contains three systems of line preparing, each system being composed of - 
1 line spreader, or first drawing;

1 st frame of 3 heads; 2 ditto, 2 slivers each;

1 ditto of 4 ditto ; 3 d ditto, ditto ditto;

2 ditto rovings, of 32 spindles, which are capable of supplying about 640 spinning do.;

1 line spreader being allowed for contingencies.

The above statement contains 3 systems of tow (uncut) preparation, each system being composed of -

1 breaker card;

2 finisher ditto;

1 frame of first drawing, 3 heads of 4 slivers each;

1 ditto second ditto, 4 ditto, 4 ditto ditto;

$2 \frac{1}{3}$ ditto rovings, or 37 spindles, which are capable of supplying about 660 spinning do.;

1 lap machine being sufficient for 2 or 3 systems;

1 extra finisher is deemed desirable.

The statement contains 2 systems of heckling machines for cut flax, a system consisting of either 8 or ten machines; for the coarser work, 8 machines in succession, finer and finer, are sufficient; but for the finest, 10 or 12 are required. Each system will produce between 2 and 300 lbs. per diem of raw flax, heckled, divided on the average into 170 Ibs. line, $280 \mathrm{lbs}$. tow, which will about equal the supply of the 5th system contained in the statement, each consisting of-

1 line spreader or 1st drawing;

1 frame 2 d drawing; 4 heads, 4 slivers each;

1 ditto 3 d ditto, 5 ditto, 4 ditto ditto;

1 ditto roving, 32 spindles;

and are capable of supplying about 480 ditto, of spinning.

The statement contains 2 systems of tow (cut flax) preparings, each system being composed of-

2 second breaker cards :

4 finishers ditto;

4 frames 1st drawing, 4 heads, each 4 slivers;

4 ditto $2 d$ ditto, 5 ditto ditto 4 ditto;

4 regulator rovings, 128 spindles;

and are capable to supply about 1800 spinning ditto.

1 first-breaker card and lap frame are sufficient to 2 or 3 systems.

Summary view :-

$$
\begin{aligned}
& \text { Long or uncut line } 3 \text { systems of } 640 \text { spindles }=1920 \\
& \text { Ditto - tow } 3 \text { ditto } 660 \text { ditto } 1980 \quad 3900 \\
& \begin{array}{llrrr}
\text { Cut } & \text { - line } 5 \text { ditto } & 480 \text { ditto } & \overline{2400} & \\
\text { Ditto } & \text { tow } 2 \text { ditto } & 1800 \text { ditto } & 3600 & 6000
\end{array}
\end{aligned}
$$

3900 spindles, at an average of 30 leas yarn per lb., would turn off 9 leas per spindle per diem, with waste circa 1400 lbs.

6000 spindles, at an average of 100 leas yarn per lb., would turn off 6 leas per spindle per diem, with waste circa $450 \mathrm{lbs}$.

Yarns produced:

Of average 30 leas per lb. per week circa 1050 boles at $9 s$.

Of ditto 100 ditto

1080

f. s. $d$.

472100

$486 \quad 00$

Total weekly produce, $\overline{2130}$

958100

$$
\text { f. s. } d \text {. }
$$

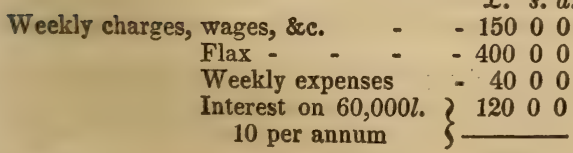

\section{0}

Measures of flax yarn, and statistics of the linen trade for the United Kingdom.

One lea of flax yarn at Leeds is $=300$ yards.

One spindle Seotch

One rand

$=38$ leas $=11400$ yards.

One dozen is 12 rands

$$
\begin{aligned}
& =6 \text { ditto }=1800 \text { ditto. } \\
& =72 \text { ditto }=21600 \text { ditto. }
\end{aligned}
$$

When yarn is estimated in Nos. it implies the number of leas in one pound weight; as in cotton, it means the number of hanks of 840 yards each in one pound. 
Imports of flax and tow, or codilla of hemp and flax, at a duty of 1 d. per cwt., in

$\begin{array}{lllll}\frac{1834 .}{\text { lbs. }} & \frac{1835 .}{\text { lbs. }} & & \frac{1837 .}{\text { lbs. }} & \frac{1838 .}{\text { lbs. }} \\ 811,722 & 740,814 & & 1,529,116 & 1,002,256 \\ 794,272 & 728,143 & & 1,532,059 & 1,002,408\end{array}$

Linen yarn exported - $\quad-\quad 2,611,215$

Linen manufactures exported, including

flax yarn, declared value $\quad-\quad-£ 3,208,139$

FLINT. (Pierre à fusil, Fr.; Feuerstein, Germ.) The fracture of this fossil is perfectly conchoidal, sometimes glossy, and sometimes dull, on the surface. It is very hard, but breaks easily, and affords very sharp-edged splintery fragments; whence it is a stone which strikes most copious sparks with steel. It is feebly translucid, has so fine and homogeneous a texture as to bear polishing, but possesses little lustre. Its colors are very various, but never vivid. The blackish-brown flint is that usually found in the white chalk. It is nearly black and opaque, loses its color in the fire, and becomes grayish-white and perfectly opaque. Flints occur almost always in nodules or tubercular concretions of various and very irregular forms. These nodules distributed in strata among the chalk, alongside of one another and almost in contact, form extensive beds; interrupted, indeed, by a multitude of void spaces, so as to present, if freed from the earthy matter in which they are imbedded, a species of network with meshes, very irregular both in form and dimension.

The nodules of silex, especially those found in the chalk, are not always homogeneous and sulid. Sometimes there is remarked an organic form towards their centre, as a madrepore or a shell, which seems to have served as their nucleus; occasionally the centre is hollow, and its sides are studded over with crystals of quartz, carbonate of iron, pyrites, concretionary silex or calcedony, filled with pulverulent silica, nearly pure, or silex mixed with sulphur; a very singular circumstance.

Flints are observed to be generally humid when broken immediately after being dug out of the ground; a property which disappears after a short exposure to the air. When dried they become more brittle and more splintery, and sometimes their surfaces get covered at old fractures with a thin film or crust of opaque silex.

Flints calcined and ground to a powder enter into the composition of all sorts of fine pottery ware.

The next important application of this silicious substance is in the formation of gunflints, for which purpose it must be cut in a peculiar manner. The following characters distinguish good flint nodules from such as are less fit for being manufactured. The best are somewhat convex, approaching to globular; those which are very irregular, knobbed, branched, and tuberose, are generally full of imperfections. Good nodules seldom weigh more than 20 pounds; when less than 2 , they are not worth the working. They should have a greasy lustre, and be particularly smooth and fine grained. The color may vary from honey-yellow to blackish-brown, but it should be uniform throughout the lump, and the translucency should be so great as to render letters legible through a slice about one fiftieth of an inch thick, laid down upon the paper. The fracture should be perfectly wmooth, uniform, and slightly conchoidal; the last property being essential to the cutting out of perfect gun flints.

Four tools are employed by the gun-flint makers.

First, a hammer or mace of iron with a square head, from 1 to 2 pounds weight, with a handle 7 or 8 inches long. This tool is not made of steel, because so hard a metal would render the strokes too harsh, or dry, as the workmen say, and would shatter the nodules irregularly, instead of cutting them with a clean conchoidal fracture.

Second, a hammer witu 2 points, made of good steel well hardened, and weighing from 10 to 16 ounces, with a handle 7 inches long passing through it in such a way that the points of the hammcr are nearer the hand of the workman than the centre of gravity of the mass.

Third, the disc hammer or roller, a small solid wheel, or flat segment of a cylinder, parallel to its base, only two inches and a third in diameter, and not more than 12 ounces in weight. It is formed of steel not hardened, and is fixed upon a handle 6 inches long, which passes through a square hole in its centre.

Fourth, a chisel tapering and bevelled at both cxtremities, 7 or 8 inches long, and 2 inches broad, made of steel not hardened; this is set on a block of wood, which serves also for a bench to the workmen. To these 4 tools a file must be added, for the purpose of restoring the edge of the chisel from time to time.

After selecting a good mass of flint, the workman executes the following four operations on it.

1. He breaks the block. Being seated upon the ground, he places the nodule of flint on 
his left thigh, and applies slight strokes with the square hammer to divide it into smaller pieces of about a pound and a half each, with broad surfaces and almost even fractures. The blows should be moderate, lest the lump crack and split in the wrong direction.

2. He cleaves or chips the flint. The principal point is to split the flint well, or to chip off scales of the length, thickness, and shape adapted for the subsequent formation of gun flints. Here the greatest dexterity and steadiness of manipulation are necessary ; but the fracture of the flint is not restricted to any particular direction, for it may be chipped in all parts with equal facility.

The workman holds the lump of flint in his left hand, and strikes with the pointed : ammer upon the edges of the great planes produced by the first breaking, whereby the white coating of the flint is removed in small scales, and the interior body of the flint s laid bare; after which he continues to detach similar scaly portions from the clean mass.

These scaly portions are nearly an inch and a half broad, two inches and a half long, and about one sixth of an inch thick in the middle. They are slightly convex below, and consequently leave in the part of the lump from which they were separated a space slightly concave, longitudinally bordered by two somewhat projecting straight lines or ridges. The ridges produced by the separation of the first scales must naturally constitute nearly the middle of the subsequent pieces; and such scales alone as have their ridges thus placed in the middle are fit to be made into gun-flints. In this manner the workman continues to split or chip the mass of flint in various directions, until the defects usually found in the interior render it impossible to make the requisite fractures, or until the piece is too much reduced to sustain the smart blows by which the flint is divided.

3. He fashions the gun-fiints. Five different parts may be distinguished in a gun-flint. 1. The sloping facet or bevel part, which is impelled against the hammer of the lock. Its ihichess should be from two to three twelfths of an inch; for if it were thicker it would be too liable to break; and if more obtuse, the scintillations would be less vivid. 2. The sides, or lateral edges, which are always somewhat irregular. 3. The back or thick part opposite the tapering edge. 4. The under surface, which is smooth and rather concave. And 5. The upper face, which has a small square plane between the tapering edge and the back, for entering into the upper claw of the cock.

In order to fashion the flint, those scales are selected which have at least one of the above mentioned longitudinal ridges; the workman fixes on one of the two tapering borders to form the striking edge, after which the two sides of the stone that are to form the lateral edges, as well as the part that is to form the back, are successively placed on the edge of the chisel in such a manner that the convex surface of the flint, which rests on the forefinger of the left hand, is turned towards that tool. Then with the disc hammer he applies some slight strokes to the flint just opposite the edge of the chisel underneath, and thereby breaks it exactly along the edge of the chisel.

4. The finishing operation is the trimming, or the process of giving the flint a smooth and equal edge; this is done by turning up the stone and placing the edge of its tapering end upon the chisel, in which position it is completed by 5 or 6 slight strokes of the dise hammer. The whole operation of making a gun-flint, which I have used so many words to describe, is performed in less than one minute. A good workman is able to manufacture 1000 good chips or scales in a day (if the flint-balls be of good quality), or 500 gunflints. Hence, in the space of 3 days, he can easily cleave and finish 1000 gun-flints without any assistance.

A great quantity of refuse matter is left, for scarcely more than half the scales are good, and nearly half the mass in the best flints is incapable of being chipped out; so that it seldom happens that the largest nodules furnish more than 50 gun-flints.

Flints form excellent building materials; because they give a firm hold to the mortar by their irregularly rough surfaces, and resist, by their nature, every vicissitude of weather. The counties of Kent, Essex, Suffolk, and Norfolk, contain many substantial specimens of flint-masonry.

FLOSS of the puddling furnace, is the fluid glass floating upon the iron produced by the vitrification of the oxydes and earths which are present.

FLOSS-SILK (Filoselle, Bourre de soie, or fleuret, Fr.) is the name given to the portions of ravelled silk broken off in the filature of the cocoons, which is carded like cotton or wool, and spun into a soft coarse yarn or thread, for making bands, shawls, socks, and other common silk fabrics. The floss or fleuret, as first obtained, must be steeped in water, and then subjected to pressure, in order to extract the gummy matter, which renders it ton harsh and short for the spinning-wheel. After being dried it is made still more pliant by working a little oil into it with the hands. It is now ready to be submitted to the carding engine. See CotTon Mandfacture. It is spun upon the flax wheel.

The female peasants of Lombardy generally wear clothes of homespun floss-silk. Of late years, by improved processes, pretty fine fabrics of this material have been pro- 
duced both in E.ngland and France. M. Ajac, of Lyons, presented at one of the Frencd national exhibitions of the objects of industry, a great variety of scarfs and square shawls, of bourre de soie, closely resernbling those of cacnemire.

FLOUR; the finely ground meal of wheat, and of any other corns or cerealia. See BREAD.

FLOJR OF WHEAT, Adullerations of, to detect.

The first method is by specific gravity. If potato flour be added, which is frequently done in France, since a vessel which contains one pound of wheat flour will contain one pound and a half of the fecula, the proportion of this adulteration may be easily estinated. If gypsum or ground bones be mixed with the flour, they will not only increase its density still more, but they will remain after burning away the meal.

The second method is by ascertaining the quantity of gluten which the suspected sample will afford, by the process prescribed under the article BREAD. The two following chemical criteria may also be employed.

1st. Nitric acid has the property of coloring wheat flour of a fine orange yellow, whereas it affects the color neither of fecula nor starch.

2d. Pure muriatic acid colors good wheat flour of a deep violet, but dissolves fecula or starch, and forms with it a light, colorless, viscous fluid, decomposable by alkalis. It may also be abserved, that as fecula absorbs less water than flour, this affords a ready means of detection.

The adulteration with bean or pea flour may be detected by pouring boiling water upon it, which developes the peculiar smell of these two substances.

FLOWERS (Fleurs, Fr.; Blumen, Germ.) of benzoin, of sulphur, of zinc, \&c., is the appellation given by the older chemists to such substances as were obtained in a pulverulent or rather minutely erystalline form by the process of sublimation.

FLOWERS, ARTIFICIAL, MANUFACTURE OF. The art of representing by flowers, leaves, plants, \&c., vegetable nature in her ornamental productions, constitutes the business of the artificial florist. The Italians appear to have been the first people in Europe who excelled in the art of making artificial flowers; but of late years the French have been most ingenious in this branch of industry.

Ribands folded in different forms and of different colors were originally employed for imitating flowers, by being attached to wire stems. This imitation soon gave way to that by feathers, which are more delicate in texture, and more capable of assurning a variety of flower-like figures. But a great difficulty was encountered in dyeing them with due vivacity. The savages of South America manufacture perfect feather flowers, derived from the brilliant plumage of their birds, which closely resemble the products of vegetation. The blossoms and leaves are admirable, while the colors never fade.

The Italians employ frequently the cocoons of the silkworm for this purpose; these take a brilliant dye, preserve their color, and possess a transparent velvety appearance, suitable for petals. Of late years, the French have adopted the finest cambric for making petals, and the taffeta of Florence for the leaves. M. de Bernardiere employs whalebone in very thin leaves for artificial flowers; and by bleaching and dyeing them of various hues, he has succeeded in making his imitations of nature to be very remarkable.

The coloring matters used in flower dyeing are the following :-

For red; carmine dissolved in a solution of salt of tartar.

For blue; indigo dissolved in sulphuric acid, diluted and neutralized in part by Spanish whitening.

For bright yellow; a solution of turmeric in spirit of wine. Cream of tartar brightens sll these colors.

For violet; archil, and a blue bath.

For lilach; archil.

Some petals are made of velvet, and are colored merely by the application of the finger dipped in the dye.

FLUATES, more properly fluorides (Eng. and Fr.; Flusssüure, Germ.); compounds of fluorine and the metals; as fluor spar, for example, which consists of fluorine and calcium.

FLUOR SPAR. (Chaux fluatée Fr.; Spath fluor, Germ.) This mineral often exhibits a variety of vivid colors. It crystallizes in the cubic system: with regular octahedral and tetrahedral cleavages; spec. grav. $3 \cdot 1$ to $3 \cdot 2$; scratches calc spar, but is scratched by a steel point; usually phosphorescent with heat; fusible at the blowpipe into an opaque bead; acted on by the acids, with disengasement of a vapor which corrodes glass; its solution affords precipitates with the oxalates, but not with ammonia. Its constituents are, fluorine, 48.13 ; calcium, 51.87 in 100.

Fluor spar occurs subordinate to metallic veins as to those of lead, in Derbyshire; of tin, in Saxony and Bohemia; but it is found also in masses or veins, either in crystalline rocks, associated with quartz, heavy spar, \&c., as in Auvergne, Forez, Vosges, Norberg in Sweden; Norway; Petersburg; near Hall; Gourock, in Scotland, \&c.; or 
among secondary limestones, slates, and sandstones, in Derbyshire, Cumberland, Corn wall, and New Jersey. It exists also in the amygdaloids of Scotland, and in the volcanic products of Monte Somma at Vesuvius. The variously colored specimens, called Derbyshire spar, are worked upon the turning-lathe into vases and other ornamental objects.

FLUX (Eng. and Fr.; Fluss, Germ.) signifies any substance capable of promoting the fusion of earths or metallic ores by heat. White flux is the residuum of the deflagration in a red-hot crucible, of a mixture of two parts of nitre, and one of cream of tartar. It is in lact merely a carbonate of potash. Black flux is obtained when equal parts of nitre and tartar are deflagrated. It owes its color to the carbonaceous matter of the tartaric acid, which remains unconsumed; the quantity of nitre being too small for that purpose. The presence of the charcoal renders this preparation a convenient flux for reducing calcined or oxydized ores to the metallic state. Limestone, fluor-spar, borax, and several earthy or metallic oxydes are employed as fluxes in metallurgy.

FLY POWDER; the black colored powder obtained by the spontaneous oxydizement of metallic arsenic in the air.

FODDER is the name of a weight by which lead and some other metals are sold in this country. It varies in its amount in different parts of the kingdom; being in Northumberland estimated at $21 \mathrm{cwts.}$, and in other counties 22,23 , or even more cwts.

FONDUS is the name given by the French to a particular style of calico-printing resembling the rainbow, in which the colors are graduated or melted (fondus) into one another, as in the prismatic spectrum. See PAPER HANGings, for a description of the proeess.

FORGE (Eng. and Fr.; Feuer, Germ.) is the name either of the furnace, where wrought-iron is hammered and fashioned with the aid of heat, or the great workshop where iron is made malleable. The former is called a smith's forge, the latter a shingling mill. See Iron. 466

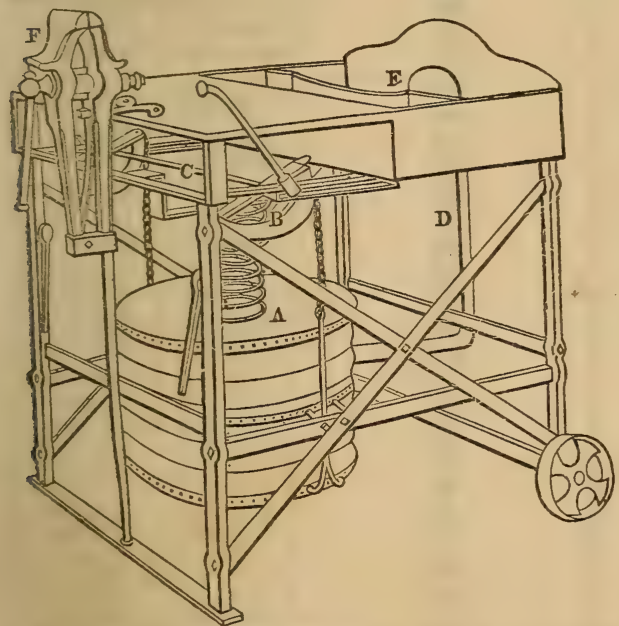

Fig. 466 represents a portable truck forge of a very commodious construction. A is the cylindric leather bellows, pressed down by a helical spring, and worked by means of the handle at $\mathbf{B}$, which moves the horizontal shaft $\mathrm{c}$, with its two attached semicircular levers and chains. D is the pipe which conducts the blast to the nozzle at E. The hearth may be covered with a thin fire-tile or with cinders. $F$ is a vice fixed to the strong rectangular frame. This apparatus answers all the ordinary purposes of a smith's forge; and is peculiarly adapted to ships, and to the execution of engineering jobs upon railways, or in the country. The height is 2 feet 6 inches; the length is 2 feet 9 inches; the width 2 feet. Weight about 2 cwts.

FOR MIATES are compounds of formic acid, with the salifiabie bases. Many of them are susceptible of erystallization.

FORMIC ACID (Acide Formique, Fr ; Ameisansaure, Germ.) exists in the bodies of wood ants, associated with the malic or acid of apples. The artificial formation of this animal secretion is one of the most remarkable triumphs of modern chemistry. If 10 parts of tartaric acid, 14 of black oxyde of manganese, 15 of concentrated sulphuric acid, and from 20 to 30 of water be mixed and distilled in a retort, formic acid will be the liquid product; while carbonic acid will be disengaged. It may also be generated from other mixtures. This acid is transparent and colorless, of a pungent sour smell, a strongly acil taste, of specific gravity 1.1168 at $60^{\circ} \mathrm{F}$., and may be re-distilled without suffering any change. It contains in its most concentrated form $19 \frac{3}{4}$ per cent. of water. The dry acid, as it exists in the formiates, is composed of 32.54 carbon, 2.68 hydrogen, and 61.78 oxygen; or of two volumes carbonic oxyde gas, and one volume of vapor of water. It reduces the oxydes of mercury and silver to the metallic state. It has not hitherto been applied to any use in the arts.

FORMULA, CHEMICAL, are symbols representing the different substances, simple and compound. 


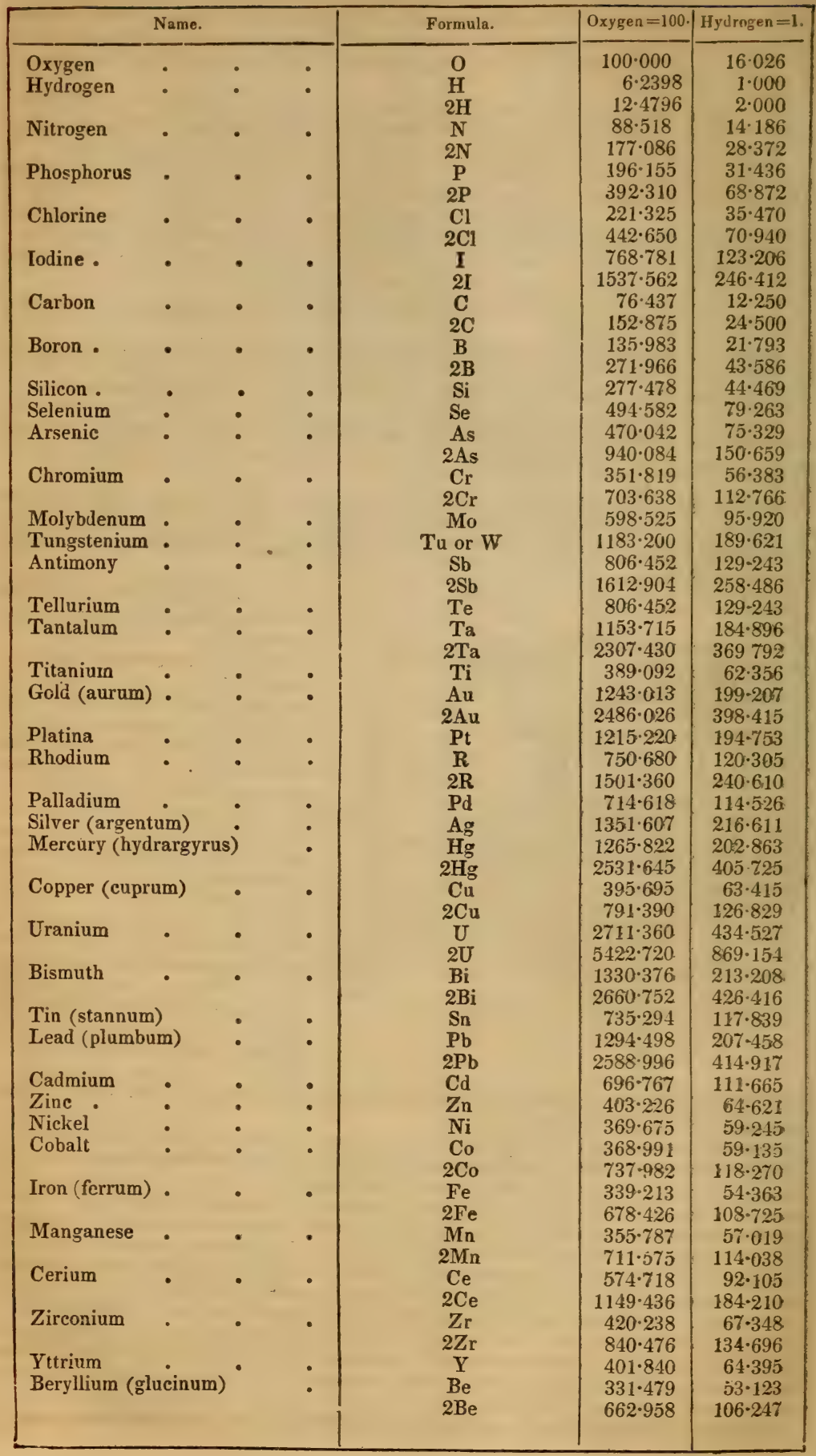




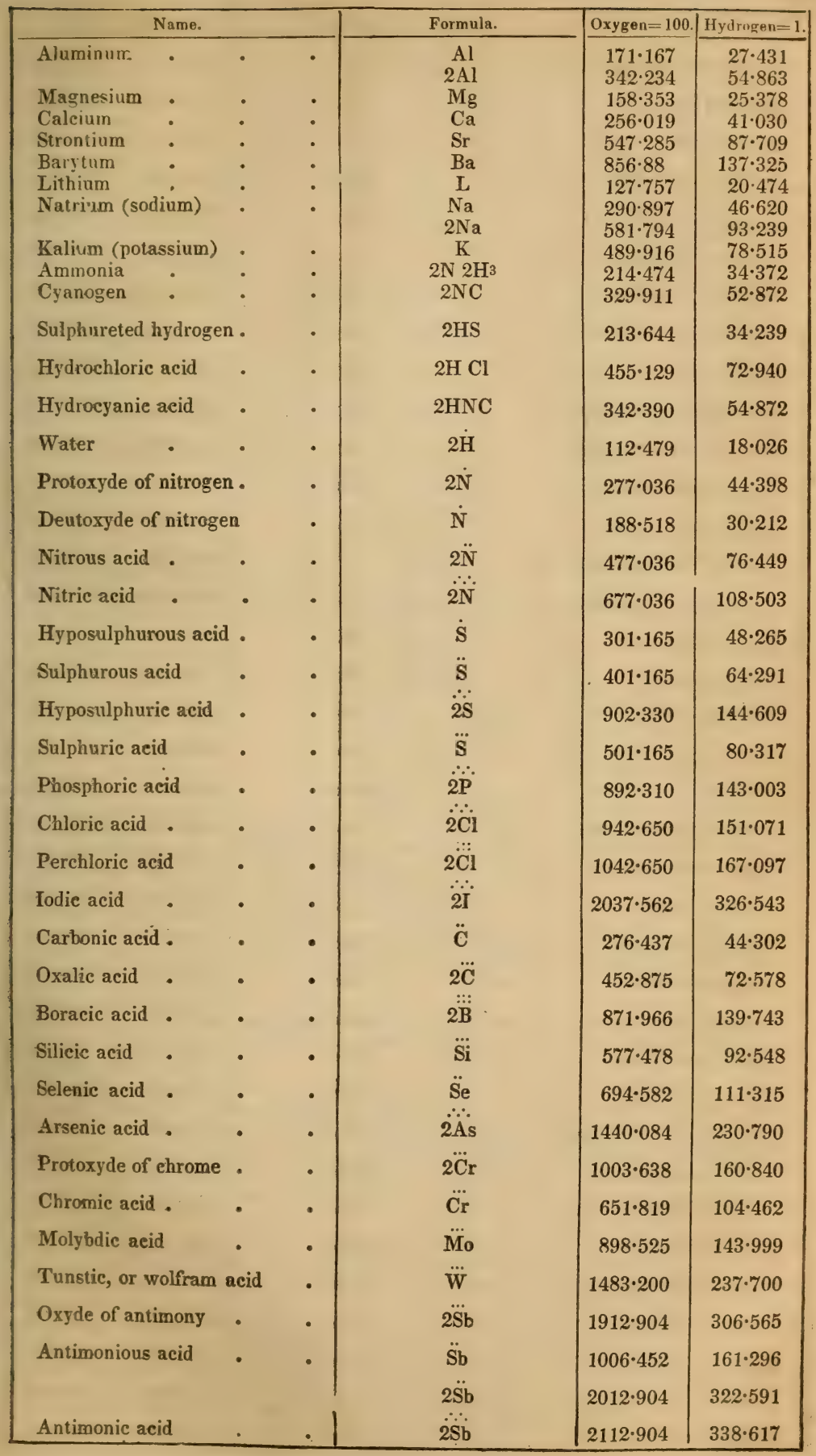




\begin{tabular}{|c|c|c|c|c|c|}
\hline \multicolumn{3}{|l|}{ Name. } & Formula. & Oxygen $=100$ & Hýdrogen $=1$ \\
\hline Oxyde of tellurium & - & - & $\ddot{\mathrm{Te}}$ & $1006 \cdot 452$ & $161 \cdot 296$ \\
\hline Tantalic acid & - & $\cdot$ & $2 \mathrm{Ta}$ & $2607 \cdot 430$ & $417 \cdot 871$ \\
\hline Titanic acid . & - & • & Ti & $589 \cdot 092$ & $94 \cdot 409$ \\
\hline Protoxyde of gold & - & - & $2 \mathrm{Au}$ & $2586 \cdot 026$ & $414 \cdot 441$ \\
\hline Peroxyde of gold & - & - & $2 \mathrm{Au}$ & $2786 \cdot 026$ & $446 \cdot 493$ \\
\hline Oxyde of platina & - & - & $\mathbf{P t}$ & $1415 \cdot 220$ & $226 \cdot 086$ \\
\hline Oxyde of rhodium & - & $\cdot$ & $2 \mathrm{R}$ & $1801 \cdot 360$ & $228 \cdot 689$ \\
\hline Oxyde of palladium & - & - & $\mathbf{P d}$ & $814 \cdot 618$ & $130 \cdot 552$ \\
\hline Oxyde of silver & · & - & $\mathrm{Ag}$ & $1451 \cdot 607$ & $232 \cdot 637$ \\
\hline Protoxyde of mercury & & $\cdot$ & $2 \mathrm{Hg}$ & $2631 \cdot 645$ & $421 \cdot 752$ \\
\hline Peroxyde of mercury & $\cdot$ & - & $\mathrm{Hg}$ & $1365 \cdot 822$ & $218 \cdot 889$ \\
\hline Protoxyde of copper & - & - & $2 \mathrm{Cu}$ & $801 \cdot 390$ & $142 \cdot 856$ \\
\hline Peroxyde of copper & - & $\cdot$ & $\mathrm{Cu}$ & $495 \cdot 695$ & $79 \cdot 441$ \\
\hline Protoxyde of uranium & a. & - & $\mathrm{U}$ & $2811 \cdot 360$ & $450 \cdot 553$ \\
\hline Peroxyde of uranium & . & - & $2 \ddot{U}$ & $5722 \cdot 720$ & $917 \cdot 132$ \\
\hline Oxyde of bismuth & - & - & $2 \mathrm{Bi}$ & $2960 \cdot 752$ & $474 \cdot 49$ \\
\hline Protoxyde of tin & - & - & $\dot{S} n$ & $835 \cdot 294$ & $133 \cdot 866$ \\
\hline Peroxyde of tin & - & - & $\ddot{\mathrm{S} n}$ & $935 \cdot 294$ & $149 \cdot 892$ \\
\hline Oxyde of lead. & - & - & $\dot{\mathrm{Pb}}$ & $1394 \cdot 498$ & $223 \cdot 484$ \\
\hline Minium & • & $\cdot$ & $2 \mathrm{~Pb}$ & 2888.996 & $462 \cdot 995$ \\
\hline Brown oxyde of lead & - & - & $\mathrm{Pb}$ & $1494 \cdot 498$ & $239 \cdot 511$ \\
\hline Oxyde of cadmium & • & - & $\mathrm{Cd}$ & $796 \cdot 767$ & $127 \cdot 691$ \\
\hline Oxyde of zinc & - & - & $\mathrm{Zn}$ & $503 \cdot 226$ & $80 \cdot 649$ \\
\hline Oxyde of nickel & - & - & $\mathrm{Ni}$ & $469 \cdot 675$ & $75 \cdot 271$ \\
\hline Oxyde of cobalt & - & - & Co & $468 \cdot 991$ & $75 \cdot 161$ \\
\hline Peroxyde of cobalt & - & $\cdot$ & $2 \mathrm{CB}$ & $1037 \cdot 982$ & $166 \cdot 349$ \\
\hline Protoxyde of iron & • & - & Fe & $439 \cdot 213$ & $70 \cdot 389$ \\
\hline Peroxyde of iron & - & - & $2 \mathrm{~F}$ & $978 \cdot 426$ & $156 \cdot 804$ \\
\hline Protoxyde of mangane & ese & - & Mn & $455 \cdot 787$ & $73 \cdot 045$ \\
\hline Oxyde of manganese & - & - & $2 \mathrm{Mn}$ & 1011.575 & $162 \cdot 117$ \\
\hline Peroxyde of mangane & & - & Mn & $555 \cdot 787$ & $89 \cdot 071$ \\
\hline Manganesic acid & - & $\cdot$ & $\dot{2} \dot{M n}$ & $1211 \cdot 575$ & $194 \cdot 169$ \\
\hline Protoxyde of cerium & - & - & $\mathrm{Ce}$ & $674 \cdot 718$ & $108 \cdot 132$ \\
\hline Oxyde of cerium & - & $\cdot$ & $2 \mathrm{Ce}$ & $1449 \cdot 436$ & $232 \cdot 289$ \\
\hline Zirconia & - & $\cdot$ & $2 \mathrm{Zr}$ & $1140 \cdot 476$ & $182 \cdot 775$ \\
\hline Yttria & - & $\cdot$ & $\dot{Y}$ & $501 \cdot 840$ & $80 \cdot 425$ \\
\hline Glucina, or berryllia & $\dot{.}$ & $\dot{\circ}$ & $2 \mathrm{Be}$ & 962.958 & $154 \cdot 325$ \\
\hline
\end{tabular}




\begin{tabular}{|c|c|c|c|c|c|c|}
\hline \multicolumn{4}{|c|}{ Name } & Formula. & Oxygen $=100$. & $\longdiv { \text { IIydrogen } = 1 }$ \\
\hline Alumina & - & . & . & $2 \ddot{\mathrm{Al}}$ & $642 \cdot 334$ & $109 \cdot 942$ \\
\hline Magnesia & - & • & • & Mg & $258 \cdot 353$ & $41 \cdot 404$ \\
\hline Lime . & . & • & - & $\mathrm{Ca}$ & 356.019 & $57 \cdot 056$ \\
\hline Strontia & - & • & - & sir & $647 \cdot 285$ & $103 \cdot 735$ \\
\hline Baryta & - & • & - & $\dot{\mathrm{Ba}}$ & $956 \cdot 880$ & $153 \cdot 351$ \\
\hline Lithia & - & • & • & $\mathbf{L}$ & 227.757 & $36 \cdot 501$ \\
\hline Natron, or $\mathrm{s}$ & oda & • & • & $\mathrm{Na}$ & 390.897 & $62 \cdot 646$ \\
\hline Peroxyde of & sodium . & • & - & $2 \mathrm{Na}$ & $881 \cdot 794$ & $141 \cdot 318$ \\
\hline Kali, or potz & sssa & • & - & K & $589 \cdot 916$ & $94 \cdot 541$ \\
\hline Peroxyde of & potassium & & • & $\dddot{\mathbf{K}}$ & $789 \cdot 916$ & $126 \cdot 593$ \\
\hline Sulphate of & ootassa . & • & - & $\dot{\mathrm{K}} \ddot{\mathrm{S}}$ & $1091 \cdot 081$ & $174 \cdot 859$ \\
\hline Protosulphat & e of iron. & • & - & $\dot{\mathrm{Fe}} \ddot{\mathrm{s}}$ & $940 \cdot 378$ & $150 \cdot 706$ \\
\hline Persulphate & of iron. & & - & $2 \ddot{\mathrm{Fe}} \dddot{\mathrm{S}} 3$ & $2481 \cdot 906$ & $397 \cdot 754$ \\
\hline Protochlorid & e of iron. & • & - & $\mathrm{Fe} 2 \mathrm{Cl}$ & $781 \cdot 863$ & $125 \cdot 303$ \\
\hline Perchloride & of iron . & • & - & $2 \mathrm{Fe} 2 \mathrm{Cl} 3$ & $2006 \cdot 376$ & $321 \cdot 545$ \\
\hline Protochlorid & of mercur & & - & $2 \mathrm{Hg} 2 \mathrm{Cl}$ & $2974 \cdot 295$ & $476 \cdot 666$ \\
\hline Perchloride & f mercury & & - & $\mathrm{Hg} 2 \mathrm{Cl}$ & $1708 \cdot 472$ & $273 \cdot 803$ \\
\hline Ferrocyanide & of iron . & & • & $\mathrm{Fe} 2 \mathrm{NC}+2 \mathrm{~K} 2 \mathrm{NC}$ & $2308 \cdot 778$ & $370 \cdot 008$ \\
\hline Alum . & • & . & • & $\dot{\mathrm{K}} \ddot{\mathrm{S}}+2 \ddot{\mathrm{Al}} \ddot{\mathrm{S}} 3+242 \dot{\mathrm{H}}$ & $5936 \cdot 406$ & $951 \cdot 378$ \\
\hline Feldspar & - & - & $\cdot$ & $\dot{\mathrm{K}} \ddot{\mathrm{Si}}+2 \ddot{\mathrm{Al}} \mathrm{i} \mathrm{Si} 3$ & $3542 \cdot 162$ & $567 \cdot 673$ \\
\hline
\end{tabular}

FOUNDING of metals, chiefly of Iron. The operations of an iron foundry consist in re-melting the pig-iron of the blast furnaces, and giving it an endless variety of forms by casting it in moulds of different kinds, prepared in appropriate manners. Coke is the only kind of fuel emplcyed to effect the fusion of the cast-iron.

The essential parts of a well-mounted iron foundry are,

1. Magazines for pig-irons of different qualities, which are to be mixed in certain proportions, for producing castings of peculiar qualities; as also for coal, coke, sands, clay, powdered charcoal, and cow-hair for giving tenacity to the loam mouldings.

2. One or more coke ovens.

3. A workshop for preparing the patterns and materials of the moulds. It should contain small edge millstones for grinding and mixing the loam, and another mill for grinding coal and charcoal.

4. A vast area, called properly the foundry, in which the moulds are made and filled with the meited metal. These moulds are in general very heavy, consisting of two parts at least, which must be separated, turned upside down several times, and replaced very exactly upon one another. The casting is generally effected by means of large ladles or pots, in which the melted iron is transported from the cupola, where it is fused. Hence, the foundry ought to be provided with cranes, having jibs moveable in every direction.

5. A stove in which such moulds may be readily introduced as require to be entirely deprived of humidity, and where a strong heat may be uniformly maintained.

6. Both blast and air furnaces, capable of melting speedily the quantity of cast-iron to be employed each day.

7. A blowing machine to urge the fusion in the furnaces.

Fig. 467 represents the general plan of a well-mounted foundry.

$a$ is a cupola furnace, of which the section and view will be afterwards given; it is capable of containing 5 tons of cast-iron.

$\grave{a}$ is a similar furnace, but of smaller dimensions, for bringing down $1 \frac{3}{4}$ tons.

$a^{\prime \prime}$ is a furnace like the first, in reserve for great castings. 
$b, b, b, b$, a vast foundry apartment, whose floor, to a yard in depth, is formed of sand and charcoal powder, which have already been used for castings, and are ready for heap467

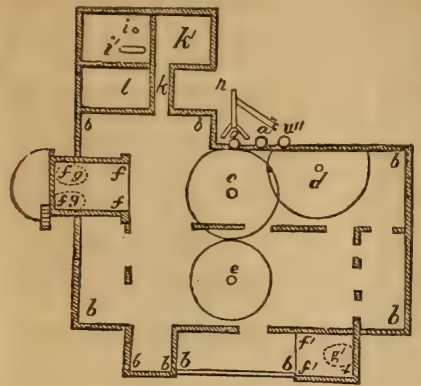

ing up into a substratum, or to be scooped out when depth is wanted for the moulds. There are besides several cylindrical pits, from five to seven yards in depth, placed near the furnaces. They are lined with brick work, and are usually left full of moulding sand. They are emptied in order to receive large moulds, care being had that their top is always below the orifice from which the melted metal is tapped.

These moulds, and the ladles full of melted metal, are lifted and transported by the arm of one or more men, when their weight is moderate; but if it be considerable, they are moved about by cranes whose vertical shafts are placed at $c, d, e$, in correspondence, so that they may upon occasion transfer the load from one to another. Each crane is composed principally of an upright shaft, embraced at top by a collet, and turning below upon a pivot in a step; next of a horizontal beam, stretched out from nearly the top of the former, with an oblique stay running downwards, like that of a gallows. The horizontal beam supports a moveable carriage, to which the tackle is suspended for raising the weights. This carriage is made to glide backwards or forwards along the beam by means of a simple rack and pinion mechanism, whose long handle descends within reach of the workman's hand.

By these arrangements in the play of the three cranes, masses weighing five tons may be transported and laid down with the greatest precision upon any point whatever in the interior of the three circles traced upon fig. 467 with the points $c, d, e$, as centres.

$c, d, e$, are the steps, upon which the upright shafts of the three cranes rest and turn. Each shaft is 16 feet hioh.

$f, f$, is the drying stove, having its floor upon a level with that of the foundry.

$f^{\prime}, f^{\prime}$, is a supplementary stove for small articles.

$g, g, g$, are the coking ovens.

$h$, is the blowing machine or fan.

$i$, is the steam-engine, for driving the fan, the loam-edge stones,

$\boldsymbol{k}$, and the charcoal mill.

$i^{\prime \prime}$, are the boiler and the furnace of the engine.

$\boldsymbol{k}^{\prime}$, workshop for preparing the loam and other materials of moulding.

$l$, is the apartment for the patterns.

- The pig-iron, coals, \&c. are placed either under sheds or in the open air, round the above buildings; where are also a smith's forge, a carpenter's shop, and an apartment mounted with vices for chipping and rough cleaning the castings by chisels and files.

Such a foundry may be erected upon a square surface of about 80 yards in each side, and will be capable, by casting in the afternoon and evening of each day, partly in large and partly in small pieces, of turning out from 700 to 800 tons per annum, with an establishment of 100 operatives, including some moulding boys.

Of making the moulds.-1. Each mould ought to present the exact form of its object.

2. It should have such solidity that the melted metal may be poured into it, and fill it entirely without altering its shape in any point.

3 . The air which occupies the vacant spaces in it, as well as the carbureted gases generated by the heat, must have a ready vent; for if they are but partially confined, they expand by the heat, and may crack, even blow up the moulds, or at any rate become dispersed through the metal, making it vesicular and unsound.

There are three distinct methods of making the moulds :-

1. In green sand; 2 . In baked sand; 3 . In loam.

To enumerate the different means employed to make every sort of mould exceeds the limits prescribed to this work. I shall merely indicate for each species of moulding, what is common to all the operations : and I shall then describe the fabrication of a few such moulds as appear most proper to give general views of this peculiar art.

Moulding in green sand.- The name green is given to a mixture of the sand as it comes from its native bed, with about one twelfth its bulk of coal reduced to powder, and damped in such a manner as to form a porous compound, capable of preserving the forms of the objects impressed upon it. This sand ought to be slightly argillaceous, with particles not exceeding a pin's head in size. When this mixture has once served for a mould, and been filled with metal, it cannot be employed again except for the coarsest castings, and is generally used for filling up the bottoms of fresh moulds.

For moulding any piece in green sand, an exact pattern of the object must be pre- 
pared in wood or metal; the latter being preferable, as not liable to warping, swelling, or shrinkage.

A couple of iron frames form a case or box, which serves as an envelope to the mould. Such boxes constitute an essential and very expensive part of the furniture of a foundry. It is a rectangular frame, without bottom or lid, whose two largest sides are united by $\mathbf{2}$ series of cross bars, parallel to each other, and placed from 6 to 8 inches apart.

The two halves of the box carry ears corresponding exactly with one another; of which one set is pierced with holes, but the other has points which enter truly into these holes, and may be made fast in them by cross pins or wedges, so that the pair becomes one solid body. Within this frame there is abundance of room for containing the pattern of the piece to be moulded with its incasing sand, which being rammed into the frame, is retained by friction against the lateral faces and cross bars of the monld.

When a mould is to be formed, a box of suitable dimensions is taken asunder, and each half, No. 1 and No. 2, is laid upon the floor of the foundry. Green sand is thrown with a shovel into No. 1, so as to fill it; when it is gently pressed in with a rammer. The object of this operation is to form a plain surface upon which to lay in the pattern with a slight degree of pressure, varying with its shape. No. 1 being covered with sand, the frame No. 2 is laid upon it, so as to form the box. No. 2 being now filled carsfully with the green sand, the box is inverted, so as to place No. 1 uppermost, which is then detached and lifted off in a truly vertical position; carrying with it the body of sand formed at the commencement of the operation. The pattern remains imbedded in the sand of No. 2, which has been exactly moulded upon a great portion of its surface. The moulder condenses the sand in the parts nearest to the pattern, by sprinkling a little water upon it, and trimming the ill-shaped parts with small iron trowels of different kinds. He then dusts a little well-dried finely-sifted sand over all the visible surface of the pattern, and of the sand surrounding it; this is done to prevent adhesion when he replaces the frame No. 1.

He next destroys the preparatory smooth bed or area formed in this frame, covers the pattern with green sand, replaces the frame 1 upon 2, to reproduce the box, and proceeds to fill and ram No. 1 , as he had previously done No.2. The object of this operation is to obtain very exactly a concavity in the frame No. 1, having the shape of the part of the model impressed coarsely upon the surface formed at the beginning, and which was meant merely to support the pattern and the sand sprinkjed over it, till it got imbedded in No. 2.

The two frames in their last position, along with their sand, may be compared to a box of which No. 1 is the lid, and whose interior is adjusted exactly upon the enclosed pettern.

If we open this bor, and after taking out the pattern, close its two halves again, then pour in melted metal till it fill every void space, and become solid, we shall obviously attain the wished-for end, and produce a piece of cast iron similar to the pattern. But many precautions must still be taken before we can hit this point. We must first lead through the mass of sand in the frame No. 1 one or more channels for the introduction of the selted metal; and though one may suffice for this purpose, another must be made for letting the air escape. The metal is run in by several orifices at once, when the piece has considerable surface, but little thickness, so that it may reach the remotest points sufficiently hot and liquid.

The parts of the mould near the pattern must likewise be pierced with small holes, by means of wires traversing the whole body of the sand, in order to render the mould more porous, and to facilitate the escape of the air and the gases. Then, before lifting off the frame No. 1, we must tap the pattern slightly, otherwise the sand enclosing it would stick to it in several points, and the operation would not succeed. These gentle jolts are given by means of one or more pieces of iron wire which have been screwed vertically into the pattern before finally ramming the sand into the frame No. 1, or which enter merely into holes in the pattern. These pieces are sufficiently long to pass out through the sand when the box is filled; and it is upon their upper ends that the horizontal blows of the hammer are given; their force being regulated by the weight and magniiude of the pattern. These rods are then removed by drawing them straight out; after which the frame No. 1 may be lifted off smoothly from the pattern.

The pattern itself is taken out, by lifing it in all its parts at once, by means of screw pins adjusted at the moment. This mancuvre is executed, for large pieces, almost always by several men, who, while they lift the pattern with one hand, strike it with the other with small repeated blows to detach the sand entirely, in which it is generally more engaged than it was in that of the frame No. 1. But in spite of all these precautions, there are always some degradations in one or other of the two parts of the mould; which are immediately repaired by the workman with damp sand, which he applies and pressea gently with his trowel, so as to restore the injured forms.

Hitherto I have supposed all the sand rammed into the box to be of one kind; but 
from economy, the green sand is used only to form the portion of the mould next the pattern, in a stratum of about an inch thick; the rest of the surrounding space is filled with the sand of the floor which has been used in former castings. The interior layer round the pattern is called, in this case, new sand.

It may happen that the pattern is too complex to be taken out without damaging the mould, by two frames alone; then 3 or more are mutually adjusted to form the box.

When the mould, taken asunder into two or more parts, has been properly repaired, its interior surface must be dusted over with wood charcoal reduced to a very fine powder, and tied up in a small linen bag, which is shaken by hand. The charcoal is thus sifted at the moment of application, and sticks to the whole surface, which has been previously damped a little. It is afterwards polished with a fine trowel. Sometimes, in order to avoid using too much charcoal, the surfaces are finally dusted over with sand, very finely pulverized, from a bag like the charcoal. The two frames are now replaced with great exactness, made fast together by the ears, with wedged bolts laid truly level, or at the requisite slope, and loaded with considerable weights. When the casting is large, the charcoal dusting, as well as that of fine sand, is suppressed. Everything is now ready for the introduction of the fused metal.

Moulding in baked or used sand.-The mechanical part of this process is the same as of the preceding. But when the castings are large, and especially if they are tall, the hydrostatic pressure of the melted metal upon the sides of the mould cannot be counteracted by the force of cohesion which the sand acquires by ramming. We must in that case adapt to each of these frames a solid side, pierced with numerous small holes to give issue to the gases. This does not form one body with the rest of the frame, but is attached extemporaneously to it by bars and wedged bolts. In general, no ground coal is mixed with this sand. Whenever the mould is finished, it is transferred to the drying stove, where it may remain from 12 to 24 hours at most, till it be deprived of all its humidity. The sand is then said to be baked or annealed. The experienced moulder knows how to mix the different sands placed at his disposal, so that the mass of the mould as it comes out of the stove may preserve its form, and be sufficiently porous. Such moulds allow the gases to pass through them much more readily than those made of green sand; and in general the castings they turn out are less vesicular, and smoother upon the surface. Sometimes in a large piece, the three kinds of moulding, that in green sand, in baked sand, and in loam, are combined to produce the best result.

Moulding in loam.-This kind of work is executed from drawings of the pieces to be moulded, without being at the expense of making patterns. The mould is formed of a pasty mixture of clay, water, sand, and cow's-hair, or other cheap filamentous matter, kneaded together in what is called the loam mill. The proportions of the ingredients are varied to suit the nature of the casting. When the paste requires to be made very light, horse dung or chopped straw is added to it.

I shall illustrate the mode of fabricating loam moulds, by a simple case, such as that of a sugar pan. Fig. 468 is the pan. There is laid upon the floor of the foundry an annular plaiform of cast-iron, $a b$, fig. 469 ; and upon its centre $c$, rests the lower extremity of a vertical shaft, adjusted so as to turn freely upon itself, while it makes a wooden pattern, $e f, f i g .470$, describe a surface of revolution identical with the interr al surface reversed of the boiler intended to be made. The outline, $e \mathrm{~g}$, of the pattern is rashioned so as to describe the surface of the edge of the vessel. Upon the part $a d b d$, fig. 469, of th: flat cast-iron ring, there must next be constructed, with bricks laid either flat or on their edge, and clay, a kind of dome, $h i k$, fig. 470, from two to four inches thick, 469
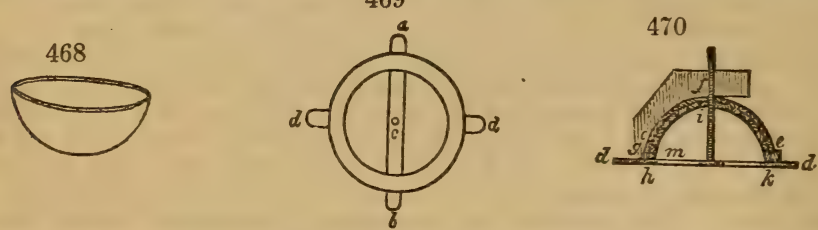

according to the size and weight of the piece to be moulded. The external surface of the brick dome ought to be every where two inches distant, at least, from the surface described by the arc $e f$. Before building up the dome to the point $i$, coals are to be placed in its inside upon the floor, which may be afterwards kindled for drying the mould. The top is then formed, leaving at $i$, round the upright shaft of revolution, only a very small outlet. This aperture, as also some others left under the edges of the iron ring, enable the moulder to light the fire when it becomes necessary, and to graduate it so as to make it last long enongh without needing more fuel, till the mould be quite finished and dry. The combustion should be always extremely slow.

Over the brick dome a pasty layer of loam is applied, and rounded with the mould 
$g$ e $f$; this surface is then coated with a much smoother loam, by means of the concave edge of the same mould. Upon the latter surface, the inside of the sugar pan is cast; the linf $g$ having traced, in its revolution, a ledge $m$. The fire is now kindled, and as the surface of the mould becomes dry, it is painted over by a brush, with a mixture of water, charcoal powder, and a little clay, in order to prevent adhesion between the surface already dried and the coats of clay ahout to be applied to it. The board $g e f$ is now removed, and replaced by another, $g^{\prime} e^{\prime} f^{\prime}$, fig. 471, whose edge $e^{\prime} f^{\prime}$ describes the outer surface of the pan. Over the surface $e, f$, a layer of loam is applied, which is turned and polished so as to produce the surface of revolution $e^{\prime} f^{\prime}$, as was done for the surface $e f$; only in the latter case, the line $e^{\prime} g^{\prime}$ of the board does not form a new shoulder, but rubs lightly against $m$.

The layer of loam included between the two surfaces $e f, e^{\prime} f^{\prime}$, is an exact representation of the sugar pan. When this layer is well dried by the heat of the interior fire, it must be painted like the former. The upright shaft is now removed, leaving the small vent hole through which it passed to promote the complete combustion of the coal. There must be now laid horizontally upon the ears of the platform $d d$, fig. 469, another annular platform $p q$, like the former, but a little larger, and without any cross-bar.

471

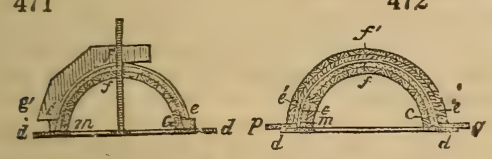

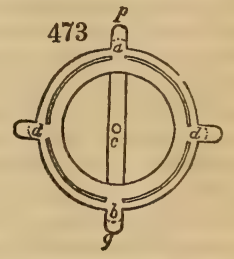

The relative position of these two platforms is shown in fig. 473. Upon the surface $e^{\prime} f^{\prime}$, fig. 472, a new layer of loam is laid, two inches thick, of which the surface is smoothed by hand. Then upon the platform $p q$, fig. 473, a brick vault is constructed, whose inner surface is applied to the layer of loam. This contracts a strong adherence with the bricks which absorb a part of its moisture, while the coat of paint spread over the surface $e^{\prime} f^{\prime}$, prevents it from sticking to the preceding layers of loam. The brick dome ought to be built solidly.

The whole mass is now to be thoroughly dried by the continuance of the fire, the draught of which is supported by a small vent left in the upper part of the new dome; and when all is properly dry, the two iron platforms are adjusted to each other by pin points, and $p q$ is lifted off, taking care to keep it in a horizontal position. Uprn this platform are removed the last brick dome, and the layer of loam which had been applied next to it ; the latter of which represents exactly by its inside the mould of the surface $e^{\prime} f^{\prime}$, that is, of the outside of the pan. The crust contained between $e f$ and $e^{\prime} f^{\prime}$ is broken away, an operation easily done without injury to the surface $e f$, which represents exactly the inner surface of the pan; or only to the shoulder $m$, corresponding to the edge of the ressel. The top aperture through which the upright shaft passed must be now closed; only the one is kept open in the portion of the mould lifted off upon $p q$; because through this opening the melted metal is to be poured in the process of casting. The two platforms being replaced above each other very exactly, by means of the adjusting pin points, the mould is completely formed, and ready for the reception of the metal.

When the object to be moulded presents more complicated forms than the one now chosen for the sake of illustration, it is always by analogous processes that the workman constructs his loam moulds, but his sagacity must hit upon modes of executing many things which at first sight appear to be scarcely possible. Thus, when the forms of the interior and exterior do not permit the mould to be separated in two pieces, it is divided into several, which are nicely fitted with adjusting pins. More than two cast-iron rings or platforms are sometimes necessary. When ovals or angular surfaces must be traced instead of those of revolution, no upright shaft is used, but wooden or cast-iron guides made on purpose, along which the pattern cut-out board is slid according to the drawing of the piece. Iron wires and claws are often interspersed through the brick work to give it cohesion. The core, kernel, or inner mould of a hollow casting is frequently fitted in when the outer shell is moulded. I shall illustrate this matter in the case of a gas-light retort, fig. 474. The core of the retort ought to have the form $e e e$, and be very solid, since it cannot be fixed in the outer mould, for the casting, except in the part standing out of the retort towards $m \mathrm{~m}$. It must be modelled in loam, upon a piece of cast-iron called a lantern, made expressly for this purpose. The lantern is a cylinder or a truncated hollow cone of cast-iron, about half an inch thick; and differently shaped for every different core. The surface is perforated with holes of about half an inch in diameter. It is mounted by means of iron cross-bars, upon an iron axis, 
which traverses it in the direction of its length. Fig. 475 represents a horizonta section through the axis of the core; $g h$ is the axis of the lantern, figured itself at $i k$

474

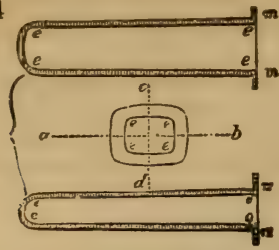

475

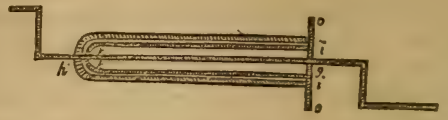

$k i$; $o i i o$ is a kind of disc or dish, perpendicular to the axis, open at $i i$, forming one piece with the lantern, whose circumference $o o$ presents a curve similar to the section of the core, made at right angles to its axis. We shall see presently the two uses for which this dish is intended. The axis $\mathrm{g} h$ is laid upon two gudgeons, and handles are placed at each of its extremities, to facilitate the operation in making the core. Upon the whole surface of the lantern, from the point $h$ to the collet formed by the dish, a hay cord as thick as the finger is wound. Even two or more coils may be applied, as occasion requires, over which loam is spread to the exact form of the core, by applying with the hand a board, against the dish 00 , with its edge cut out to the desired shape; as also against another dish, adjusted at the time towards $h$; while by means of the handles a rotatory movement is given to the whole epparatus.

The hay interposed between the lantern and the loam, which represents the crust of the core, aids the adhesion of the clay with the cast-iron of the lantern, and gives passage to the holes in its surface, for the air to escape through in the casting.

When the core is finished, and has been put into the drying stove, the axis $g$ is taken out, then the small opening which it leaves at the point $h$, is plugged with clay. This is done by supporting the core by the edges of the dish, in a vertical position. It is now ready to be introduced into the hollow mould of the piece.

This mould executed in baked sand consists of three pieces, two of which, absolutely

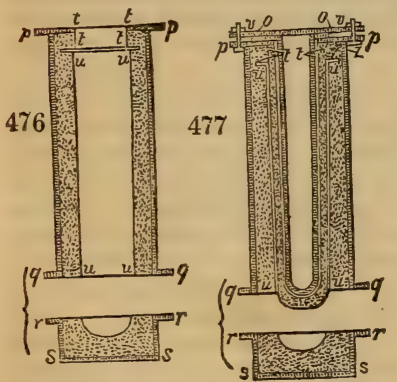
similar, are represented, fig. 476 , at $p q$, the third is shown at $r s$. The two similar parts $p q$, present each the longitudinal half of the nearly cylindrical portion of the outer surface of the gas retort; so that when they are brought together, the cylinder is formed; $r s$ contains in its cavity the kind of hemisphere which forms the bottom of the retort. Hence, by adding this part of the mould to the end of the two others, the resulting apparatus presents in its interior, the exact mould of the outside of the retort; an empty cylindrical portion $t t$, whose axis is the same as that of the cylinder $u u$, and whose surface, if prolonged, would be everywhere distant from the surface $u v$, by a quantity equal to the desired thickness of the retort. The diameter of the cylinder $t t$ is precisely equal to that of the core, which is slightly conical, in order that it may enter easily into this aperture $t t$, and close it very exactly when it is introduced to the collet or neck.

The three parts of the mould and the core being prepared, the two pieces $p q$, must first be united, and supported in an upright position; then the core must be let down into the opening $t t$, fig. 477. When the plate or disc 00 of the core is supported upon the mould, we must see that the end of the core is everywhere equally distant from the edge of the external surface $u \quad u$, and that it does not go too far beyond the line $q q$. Should there be an inaccuracy, we must correct it by slender iron slips placed under the edge of the disc 00 ; then by means of a cast-iron cross, and screw bolts $v v$, we fix the core immoveably. The whole apparatus is now set down upon $r s$, and we fix with screw bolts the plane surface $q q$ upon $r r$; then introduce the melted metal by an aperture $z$, which has been left at the upper part of the mould.

When, instead of the example now selected, the core of the piece to be cast must go beyond the mould of the external surface, as is the case with a pipe open at each end, the thing is more simple, because we may easily adjust and fix the core by its two ends.

In casting a retort, the metal is poured into the mould set upright. It is important to maintain this position in the two last examples of casting; for all the foreign matters which may soil the metal during its flow, as the sand, the charcoal, gases, scoriæ, being less dense than it, rise constantly to the surface. The hydrostatic pressure produced by a high gate, or filling-in aperture, contributes much to secure the soundness and solidity of the casting. This gate-piece being superfluous, is knocked off almost immediately 
after, or even before the casting cools. Very long, somewhat slender pieces, are usually cast in moulds set up obliquely to the horizon. As the metal shrinks in cooling, the mould should always be somewhat larger than the object intended to be cast. The iron founder reckons in general upon a linear shrinkage of a ninety-sixth part; that is, one eighth of an inch per foot.

Melting of the cast iron. - The metal is usually melted in a cupola furnace, of which the dimensions are very various. Fig. 478 represents in plan, section, and elevation, one of these furnaces of the largest size; being capable of founding 5 tons of cast iron at a time. It is kindled by laying a few chips of wood upon its bottom, leaving the orifice $c$ open, and it is then filled up to the throat with coke. The fire is lit at $c$, and in a quarter or half an hour, when the body of fuel is sufficiently kindled, the tuyère blast is set in action. The flame issues then by the mouth as well as the orifice $c$, which has been lefi open on purpose to consolidate it by the heat. Without this precaution, the sides, which are made up in argillaceous sand after each day's work, would not present the necessary resistance. A quarter of an hour afterwards, the orifice $c$ is closed with a lump of moist clay, and sometimes, when the furnace is to contain a great body of melted metal, the clay is supported by means of a small plate of cast iron fixed against the furnace. Before the blowing machine is set a going, the openings $g \mathrm{~g} g$ had been kept shut. Those of them wanted for the tuyères are opened in succession, beginning at the lowest, the tuyères being raised according as the level of the fused iron stands higher in the furnace. The same cupola may receive at a time from one to six tuyères, through which the wind is propelled by the centrifugal action of an eccentric fan or ventilator. It does not appear to be ascertained whether there be any advantage in placing more than two tuyères facing each other upon opposite sides of the furnace. Their diameter at the nozzle varies from 3 to 5 inches. They are either cylindrical or slightly conical. A few minutes after the tuyères have begun to blow, when the coke sinks in the furnace, alternate charges of coke and pig iron must be thrown in. The metal begins to melt in about 20 minutes after its introduction; and successive charges are then made every 10 minutes nearly ; each charge containing from $2 \mathrm{cwts}$. to $5 \mathrm{cwts}$. of iron, and a quantity proportional to the estimate given below. The amount of the charges varies of course with the size of the furnace, and the speed required for the operation. The pigs must be previously broken into pieces weighing at most 14 or 16 pounds. The vanes of the blowing fan make from 625 to 650 turns per minute. The two cupolas represented fig. 478 , and another alongside in the plan, may easily melt $6 \frac{1}{2}$ tons of metal in $2 \frac{3}{4}$ hours;
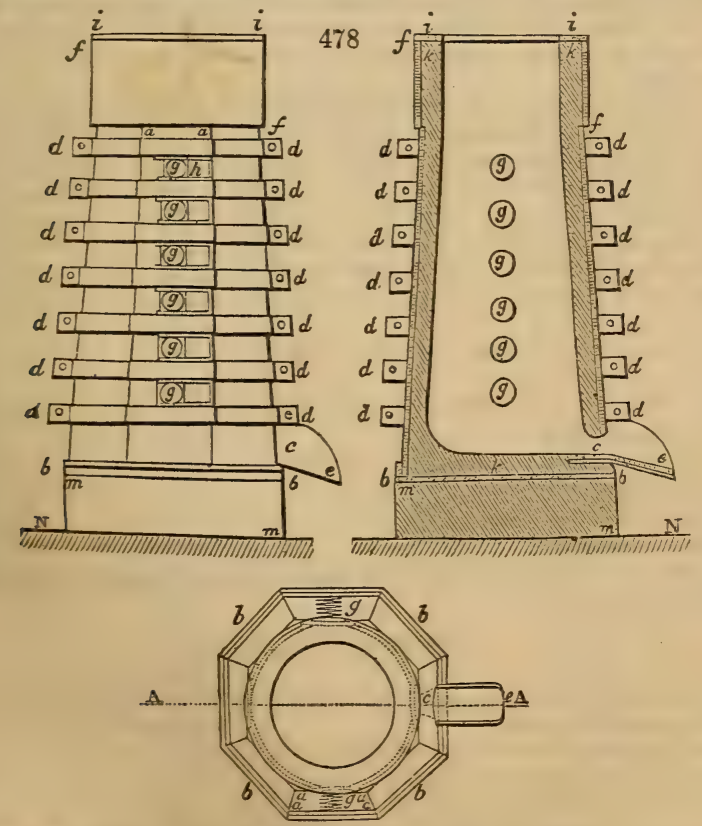

that is, $2 \frac{2}{3}$ tons per hour. This result is three or four times greater than what was 
formerly obtained in similar cupolas, when the blast was thrown in from sn.all nozzles with cylinder bellows, moved by a steam engine of 10 horse power.

In the course of a year, a considerable foundry like that represented in the plan, fig. $46 \%$, will consume about 300 tons of coke in melting 1240 tons of cast iron; consisting of 940 tons of pigs of different qualities, and 300 tons of broken castings, gate-pieces, \&c. Thus, it appears that 48 pounds of coke are consumed for melting every $2 \mathrm{cwt}$. of metal.

Somewhat less coke is consumed when the fusion is pushed more rapidly to collect a great body of melted metal, for casting heavy articles; and more is consumed when, as in making many small castings, the progress of the founding has to be slackened from time to time; otherwise, the metal would remain too long in a state of fusion, and probably become too cold to afford sharp impressions of the moulds.

It sometimes happens that in the same day, with the same furnace, pieces are to be cast containing several proportions of different kinds of iron; in which case, to prevent an intermixture with the preceding or following charges, a considerable bed of coke is interposed. Though there be thus a little waste of fuel, it is compensated by the improved adaptation of the castings to their specific objects. The founding generally begins at about $3 o^{\text {'} c l o c k, ~ P . ~ M ., ~ a n d ~ g o e s ~ o n ~ t i l l ~} 6$ or 8 o'clock. One founder, aided by four laborers for charging, \&c., can manage two furnaces.

The following is the work of a well-managed foundry in Derby.

$200 \mathrm{lbs}$. of coke are requisite to melt, or bring down (in the language of the founders), 1 ton of cast-iron, after the cupola has been brought to its proper heat, by the combustion in it of 9 baskets of coke, weighing, by my trials, 40 pounds each, $=360 \mathrm{lbs}$.

The chief talent of the founder consists in discovering the most economical mixtures, and so compounding them as to produce the desired properties in the castings. One piece, for example, may be required to have great strength and tenacity to bear heavy weights or strains; another must yield readily to the chisel or the file; a third must resist sudden alternations of temperature; and a fourth must be pretty hard.

The filling in of the melted metal is managed in two ways. For strong pieces, whose moulds can be buried in the ground at 7 or 8 yards distance from the furnace, the metal may be run in gutters, formed in the sand of the floor, sustained by plates or stones. The clay plug is pierced with an iron rod, when all is ready.

When from the smaller size, or greater distance of the moulds, the melted metal cannot be run along the floor from the furnace, it is received in cast-iron pots or ladles, lined with a coat of loam. These are either carried by the hands of two or more men, or transported by the crane. Between the successive castings, the discharge hole of the furnace is closed with a lump of clay, applied by means of a stick, having a small disc of iron fixed at its end.

After the metal is somewhat cooled, the moulds are taken asunder, and the excrescences upon the edges of the castings are broken off with a hammer. They are afterwards more carefully trimmed or chipped by a chisel when quite cold. The loss of weight in founding is about $6 \frac{1}{2}$ per cent. upon the pig iron employed. Each casting always requires the melting of considerably more than its own weight of iron. This excess forms the gates, false seams, \&c.; the whole of which being deducted, shows that $1 \mathrm{cwt}$. of coke is consumed for every 3 cwts. of iron put into the furnace; for every $138 \mathrm{cwts}$. of crude metal, there will be $100 \mathrm{cwts}$. of castings, 32 of refuse pieces, and 6 of waste.

Explanation of the plates.

\section{Manner of constructing the Mould of a Sugar-pan.}

Fig. 468. View of the pan.

- 469. Flat ring of cast-iron for supporting the inner mould.

- 470. Construction of the inner mould.

- 471. Formation of the outer surface of the pan.

- 472. Finished mould.

- 473. Position of the two flat cast-iron rings, destined to sustain the moulds of the inner and the outer surface.

\section{Gas-retort Moulding.}

- 474. Vertical projection, perpendicular to the axis of the retort; and two sectrons, the one upright, the other horizontal.

- 475. Construction of the core of the retort.

- 476. Disposition of the outer mould.

- 477. Adjustment of the core in the mould.

- 478. Cupola furnace. It is 3 feet wide within, and $13 \frac{1}{2} \mathrm{~h} / \mathrm{gh}$.

$m \boldsymbol{m}$, solid body of masonry, as a basis to the furnace. 
$b b$, oetagonal platform of cast iron, with a ledge in which the plates $a \quad a \quad a \quad a$ are engaged.

a $a$, eight plates of cast iron, 1 inch thick, absolutely similar; only one of them is notched at its lower part in $c$, to allow the melted metal to run out, and two of the others have six apertures $g g g$, \&c. to admit the tuyères.

$c$, orifice for letting the metal flow out. A kind of cast iron gutter, $e$, lined with loam, is fitted to the orifice.

$d$, hoops of hammered iron, $4 \frac{1}{4}$ inches broad; one half of an inch thick for the bottom ones, and a quarter of an inch for the upper ones. The intermediate hoops decrease in thickness from below upwards between these limits.

$e$, cast iron gutter or spout, lined with loam, for running off the metal.

$f f$, cylindrical piece of cast iron, for increasing the height and draught of the furnace.

$g$, side openings for receiving the tuyères, of which there are six upon each side of the furnace. Each of them may be shut at pleasure, by means of a small cast iron plate $h$, made to slide horizontally in grooves sunk in the main plate, pierced with the holes g g.

$k k$, interior lining of the surface, made of sand, somewhat argillaceous, in the following

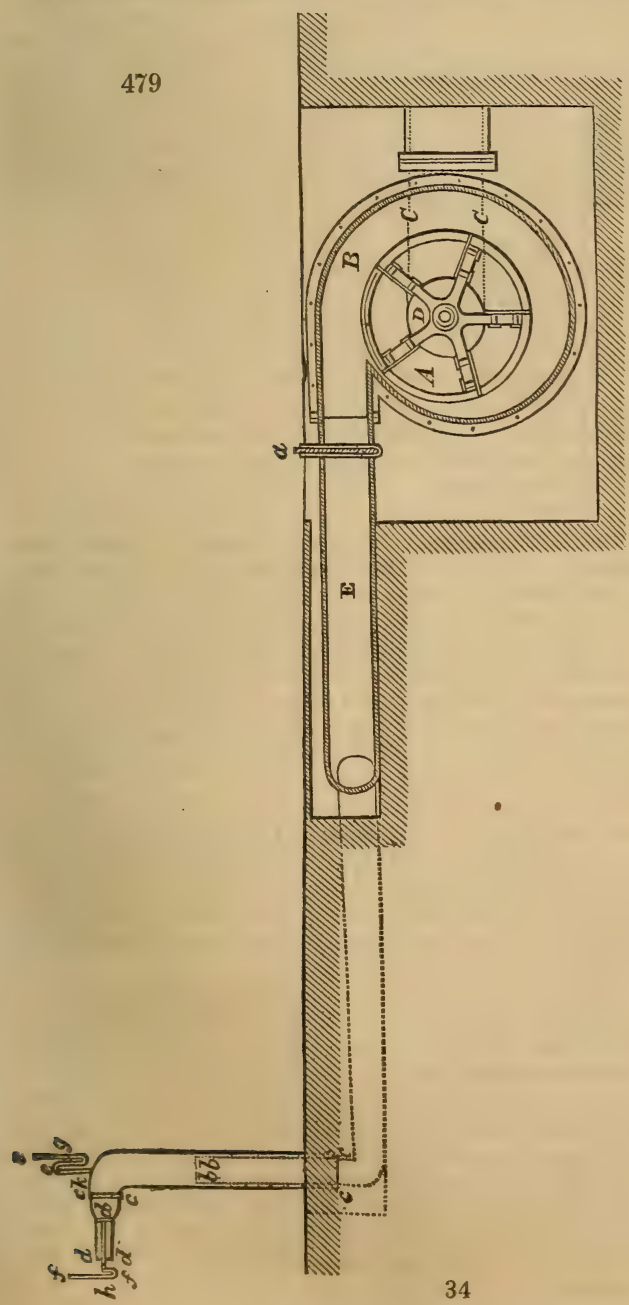
way. After having laid at the bottom of the furnace a bed of sand a few inches thick, slightly sloped towards the orifice of discharge, there is set upright, in the axis of the cupola, a wooden cylinder of its whole height, and of a diameter a little less than that of the vacant space belonging to the top of the furnace. Sand is to be then rammed in so as to fill the whole of the furnace; after which the wooden cylinder is withdrawn, and the lining of sand is cut or shaved away, till it has received the proper form.

This lining lasts generally 5 or 6 weeks, when there are 6 meltings weekly.

$i i$, cast iron circular plate, through which the mouth of the furnace passes, for protecting the lining in $k$ during the introduction of the charges.

$N \mathrm{~N}$, level of the floor of the foundry. The portion of it below the running out orifice consists of sand, so that it may be readily sunk when it is wished to receive the melted metal in ladles or pots of large dimensions.

The fan distributes the blast from the main pipe to three principal points, by three branch tubes of distribution. A register, consisting of a cast-iren plete sliding witk. friction in 2 frame, serves to intercept the blast at any moment, when it is not desirable to stop the moving power. A large main pipe of zine or sheet iron is fitted to the orifice of the slide valve. It is square at the beginning, of only rounded at the angles 
but at a little distance it becomes cylindrical, and conducts the blast to the divaricating points. There, each of the branches turns up vertically, and terminates at $b b$, fig. 479 , where it presents a circular urifice of $7 \frac{1}{2}$ inches. Upon each of the upright pipes $b$, the one end of an elbow-tube of zinc $c c c c$, fig. 479, is adjusted rather loosely, and the other end receives a tuyère of wrought iron $d d$, through the intervention of a shifting hose or collar of leather $c c d$, hooped with iron wire to both the tube and the tuyère. The portion $c c c c$ may be raised or lowered, by sliding upon the pipe $b$, in order to bring the nozzle of the tuyère $d d$, to the requisite point of the furnace. The portion c $c$ c $c$ may be made also of wrought iron. A power of 4 horses is adequate to drive this fan, for supplying blast to 3 furnaces.

The founders have observed the efflux of air was not the same when blown into the atmosphere, as it was when blown into the furnaces; the velocity of the fan, with the same impulsive power, being considerably increased in the latter case. Ther imagine that this circumstance arises from the blast being sucked in, so to speak, by the dranght of the furnace, and that the fan then supplied a greater quantity of air.

The following experimental researches show the fallacy of this opinion. Two water syphons, e e e, $f f f$, made of glass tubes, one fifth of an inch in the bore, were inserted into the tuyère, containing water in the portions, $g g g, h h h$. The one of these manometers for measuring the pressure of the air was inserted at $k$, the other in the centre of the nozzle. The size of this glass tube was too small to obstruct in any sensible degree the outlet of the air. It was found that when the tuyères of the fan discharged into the open air, the expenditure by a nozzle of a constant diameter was proportional to the number of the revolutions of the vanes. It was further found, that when the speed of the vanes was constant, the expenditure by one or by two nozzles was proportional to the total area of these nozzles. The following formulæ give the volume of air furnished bv the fan, when the number of turns and the area of the nozzles are known.

$$
\begin{aligned}
& \text { Volume }=\frac{25 \cdot 32 \mathrm{~S} \mathrm{n}}{1 \cdot 000,000} \\
& \text { Volume }=\frac{0 \cdot 86^{\prime} 6^{\prime} 7 \mathrm{~S} \mathrm{n}}{1,000,000}
\end{aligned}
$$

The volume is measured at $32^{\circ} \mathrm{Fahr}$., under a pressure of $29 \cdot 6$ inches barom.

$\mathbf{S}=$ is the total area of the orifices of the tuyères in square inches.

$n=$ the number of turns of the vanes in a minute.

After measuring the speed of the vanes blowing into the atmosphere, if we introduce the nozzle of discharge into the orifice of the furnace, we shall find that their speed immediately augments in a notable degree. We might, therefore, naturally suppose that the fan furnishes more air in the second case than in the first; but a little reflection will show that it is not so. In fact, the air which issues in a cold state from the tuyère encounters instantly in the furnace a very high temperature, which expands it, and contributes, along with the solid matters with which the furnace is filled, to diminish the facility of the discharge, and consequently to retard the efflux by the nozzles. The oxygen gas consumed is replaced by a like volume of carbonic acid gas, equally expansible by heat. Reason leads us to conclude that less air flows from the nozzles into the furnace than into the oper atmosphere.

The increase in the velocity of the vanes takes place precisely in the same manner, when after having made the nozzles blow into the atmosphere, we substitute for these nozzles others of a smaller diameter, instead of directing the larger ones into the furnace. Hence we may conceire that the proximity of the charged furnace acts upon the blast iike the contraction of the nozzles. When the moring power is uniform, and the velocity of the vanes remains the same, the quantity of air discharged must also be the same in the two cases.

Two tuyères, one 5 inches in diameter, the other $4 \frac{1}{2}$, and which, consequently, presented a total area of $35 \frac{1}{2}$ square inches, discharged air into one of the furnaces, from a fan whose vanes performed 654 turns in the minute. These two nozzles being briskly withdrawn from the furnace, and turned round to the free air, while a truncated pasteboard cone of $3 \frac{1}{2}$ inches diameter was substituted for the nozzle of $4 \frac{1}{2}$ inches, whereby the area of efflux was reduced to 29.3 square inches, the velocity of the vanes continued exactly the same. The inverse operation having been performed, that is to say, the two original nozzles having been smartly replaced in the furnace, to discover whether or not the moving power had changed in the interval of the experiment, they betrayed no perceptible alteration of speed. From the measures taken to count the speed, the error could not exceed 3 revolutions per minute, which is altogether unimportant upon the number 654 . 
It follows, therefore, that when the vanes of the fan have the velocity of 654 turns per minute, the expenditure by two nozzles, whose joint area is $35 \frac{1}{2}$ square inches, both blowing into a furnace, is to the expenditure which takes place, when the same nozzles blow into the air, as 35.5 is to 29.3 ; that is, a little more than four fifths.

If this be, as is probable, a general rule for areas and speeds cunsiderably different from the above, to find the quantity of air blown into one or more furnaces by the fan, we should calculate the volume by one of the above formulæ (1) or (2), and take four fifths of the result as the true quantity.

The fan A c here represented is of the best eccentric form, as constructed by Messrs. Braithwaite and Ericsson. D is the circular orifice round the axis by which the air is admitted; and $\mathrm{c} \mathrm{CB}$ is the eccentric channel through which the air is wafted towards the main discharge pipe $\mathbf{E}$.

FOUNTAIN; a stream of water rising up through the superficial strata of the earth. See Artesian Wells.

FOXING is a term employed by brewers to characterize the souring of beer, in the process of its fermentation or ripening.

FRANKFORT BLACK is made by calcining vine branches, and the other refuse lees of the vinegar vats in Germany. They must be previously washed.

FREEZING. (Congelation, Fr.; Gefrierung, Germ.) The three general forms, solid, liquid, and gaseous, under one or other of which all kinds of matter exist, seem to be immediately referable to the influence of heat; modifying, balancing, or subduing the attraction of cohesion. Every solid may be liquefied, and every liquid may be vaporized, by a certain infusion of caloric, whether this be regarded as a moving power or an elastic essence. The converse of this proposition is equally true ; for many gases, till lately styled permanent, may be liquefied, nay, even solidified, by diminution of their temperature, either alone, or aided by a condensing force, to bring their particles within the sphere of aggregative attraction. When a solid is transformed into a liquid, and a liquid into a gas or vapor, a quantity more or less considerable of heat is absorbed, or becomes latent, to use the term of Dr. Black, the celebrated discoverer of this great law of nature. When the opposite transformation takes place, the heat absorbed is again emitted, or what was latent becomes sensible caloric. Upon the first principle, or the absorption of heat, are founded the various artificial methods of producing cold and congelation.

Tables exhibiting a collective view of all the Frigorific Mixtures contained in Mr. Walker's publication, 1808.

I.-Table consisting of Frigorific Mixtures, composed of ice, with chemical salts and acids.

Frigorific Mixtures with Ice.

\begin{tabular}{|c|c|c|c|}
\hline \multicolumn{2}{|c|}{ MIXTURES. } & Thermometer sinks. & Degree of cold \\
\hline $\begin{array}{l}\text { Snow, or pounded ice } \\
\text { Muriate of soda }\end{array}$ & $\begin{array}{l}-2 \text { parts } \\
-1\end{array}$ & to $-5^{\circ}$ & $*$ \\
\hline $\begin{array}{l}\text { Snow, or pounded ice } \\
\text { Muriate of soda } \\
\text { Muriate of ammonia - }\end{array}$ & $\begin{array}{l}-5 \text { parts } \\
-\quad 2 \\
-\quad 1\end{array}$ & 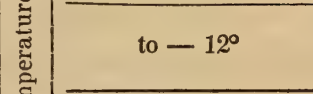 & * \\
\hline $\begin{array}{l}\text { Snow, or pounded ice } \\
\text { Muriate of soda } \\
\text { Muriate of ammonia } \\
\text { Nitrate of potash }\end{array}$ & $\begin{array}{l}-24 \text { parts } \\
=10 \\
=5 \\
-5\end{array}$ & 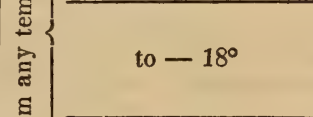 & * \\
\hline $\begin{array}{l}\text { Snow, or pounded ice } \\
\text { Muriate of soda } \\
\text { Nitrate of ammonia - }\end{array}$ & $\begin{array}{l}-12 \text { parts } \\
-5 \\
-\quad 5\end{array}$ & to $-25^{\circ}$ & $\cdot$ \\
\hline $\begin{array}{l}\text { Snow } \\
\text { Diluted sulphuric acid }\end{array}$ & $\begin{array}{l}-3 \text { parts } \\
-2\end{array}$ & From $+32^{\circ}$ to $-23^{\circ}$ & 55 \\
\hline $\begin{array}{l}\text { Snow - } \\
\text { Muriatic acid }\end{array}$ & $\begin{array}{l}-8 \text { parts } \\
-5\end{array}$ & From $+32^{\circ}$ to $-27^{\circ}$ & 59 \\
\hline $\begin{array}{l}\text { Snow - } \\
\text { Diluted nitric acid - }\end{array}$ & $\begin{array}{l}-7 \text { parts } \\
-4\end{array}$ & From $+32^{\circ}$ to $-30^{\circ}$ & 62 \\
\hline $\begin{array}{l}\text { Snow - } \\
\text { Muriate of lime - }\end{array}$ & $\begin{array}{l}-4 \text { parts } \\
-5\end{array}$ & From $+32^{\circ}$ to $-40^{\circ}$ & 72 \\
\hline $\begin{array}{l}\text { Snow } \\
\text { Cryst. muriate of lime }\end{array}$ & $\begin{array}{l}-2 \text { parts } \\
-3\end{array}$ & From $+32^{\circ}$ to $-50^{\circ}$ & 82 \\
\hline $\begin{array}{l}\text { Snow } \\
\text { Potash }\end{array}$ & $\begin{array}{l}-3 \text { parts } \\
-4\end{array}$ & From $+32^{\circ}$ to $-51^{\circ}$ & 83 \\
\hline
\end{tabular}


N. B. - The reason for the omissions in the last column of the preceding table is, the thermometer sinking in these mixtures to the degree mentioned in the preceding column, and never lower, whatever may be the temperature of the materials at mixing.

II.-Table, consisting of Frigorific Mixtures, having the power of generating or creating cold, without the aid of ice, sufficient for all useful and philosophical purposes, in any part of the world at any season.

Frigorific Mixtures without Ice.

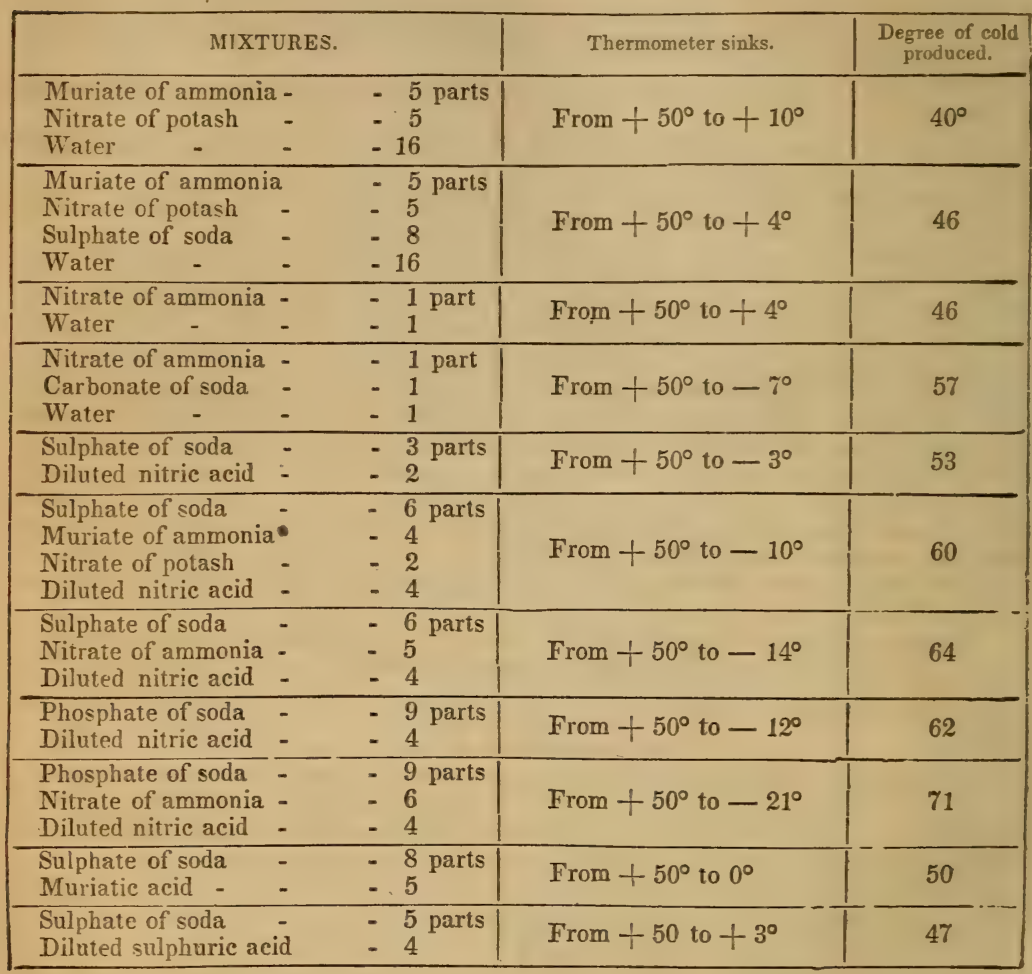

N. B.-If the materials are mixed at a warmer temperature than that expressed in the table, the effect will be proportionably greater; thus, if the most powerful of these mixtures be made when the air is $+85^{\circ}$, it will sink the thermometer to $+2 \circ$.

III.-Table consisting of Frigorific Mixtures selected from the foregoing Tables, and combined so as to increase or extend cold to the extremest degrees.

Combinations of Frigorific Mixtures.

\begin{tabular}{|c|c|c|c|}
\hline \multicolumn{2}{|c|}{ MIXTURES. } & Thermometer sinks. & $\begin{array}{l}\text { Degree of cold } \\
\text { produced. }\end{array}$ \\
\hline $\begin{array}{l}\text { Phosphate of soda } \\
\text { Nitrate of ammonia } \\
\text { Diluted nitric acid }\end{array}$ & $\begin{array}{l}-5 \text { parts } \\
-3 \\
-4\end{array}$ & From $0^{\circ}$ to $-34^{\circ}$ & $34^{\circ}$ \\
\hline $\begin{array}{l}\text { Phosphate of soda } \\
\text { Nitrate of ammonia } \\
\text { Diluted mixed acids - }\end{array}$ & $\begin{array}{l}-3 \text { parts } \\
-\quad 2 \\
-\quad 4 \\
\end{array}$ & From $-34^{\circ}$ to $-50^{\circ}$ & 16 \\
\hline $\begin{array}{l}\text { Snow } \\
\text { Diluted nitric acid }\end{array}$ & $\begin{array}{l}3 \text { parts } \\
-2\end{array}$ & From $0^{\circ}$ to $-46^{\circ}$ & 46 \\
\hline
\end{tabular}


FUEL.

TABLE III.-continued.

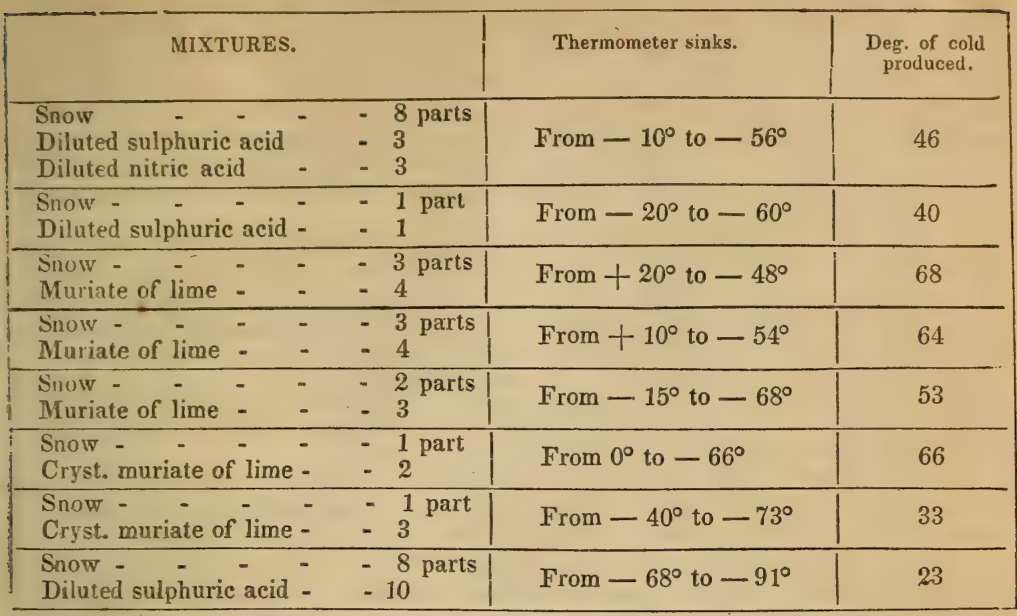

N. B.-The materials in the first column are to be cooled, previously to mixing, to the semperature required, by mixtures taken from either of the preceding tables.

Water absorbs 1000 degrees of heat in becoming vapor; whence, if placed in a saucer within an exhausted receiver, over a basin contanning strong sulphuric acid, it will freeze by the rapid absorption of its heat into the vapor so copiously formed under these circumstanees.

But the most powerful means of artificial refrigeration is afforded by the evaporation of liquefied carbonic acid gas; for the frozen carbonic acid thus obtained has probably a temperature $100^{\circ}$ under zero; so that when a piece of it is laid upon quicksilver, it instantly congeals this metal. The more copious discussion of this subject belongs to chemical science.

FRENCH BERRIES; Berries of Avignon.

FRICTION, counteraction of ; see LUBRICATION.

FRIT; see Enamel and Grass.

FUEL (Combustible, Fr.; Brennstoff, Germ.)

Such combustibles as are used for fires or furnaces are called fuel, as wood, turf, pitcoal. These differ in their nature and in their power of giving heat.

I. Wood, which is divided into hard and soft. To the former belong the oak, the beech, the alder, the birch, and the elm; to the latter, the fir, the pine of different sorts, the larch, the linden, the willow, and the poplar.

Under like dryness and weight different woods are found to afford equal degrees of 。 heat in combustion. Moisture diminishes the heating power in three ways; by diminishing the relative weight of the ligneous matter, by wasting heat in its evaporation, and by causing slow and imperfect combustion. If a piece of wood contain, for example, 25 per cent. of water, then it contains only 75 per cent. of fuel, and the evaporation of that wvater will require $\frac{\lambda}{28}$ part of the weight of the wood. Hence the damp wood is of less value in combustion by $\frac{8}{28}$ or $\frac{2}{7}$ than the dry. The quantity of moisture in newly felled wood amounts to from 20 to 50 per cent.; birch contains 30 , oak 35 , beech and pine 39 , alder 41 , fir 45 . According to their different natures, woods which have been felled and cleft for 12 months contain still from 20 to 25 per cent. of water. There is never less than 10 per cent. present, even when it has been kept long in a dry place, and though it be dried in a strong heat, it will afterwards absorb 10 or 12 per cent. of water. If it be too strongly kiln dried, its heating powers are impaired by the commencement of carbonization, as if some of its hydrogen were destroyed. It may be assumed as a mean of many experimental results, that 1 pound of artificially dried wood will heat 35 pounds of water from the freezing to the boiling point; and that a pound of such wood as contains from 20 to 25 per cent. of water will heat 26 pounds of ice-cold water to the same degree. It is better to buy wood by measure than by weight, as the bulk is very little increased by moisture. The value of different woods for fuel is inversely as their moisture, and this may easily be ascertained by taking their shavings, drying them in a heat of $140^{\circ} \mathrm{F}$., and seeing how much weight they lose.

From every combustible the heat is diffused either by radiation or by direct communication to bodies in contact with the flame. In a wood fire the quantity of radiating heat is to that diffused by the air as 1 to 3 ; or it is one fourth of the whole heating power 
II. Charcoal. The different charcoals afford, under equal weights, equal quantities of heat. We may reckon, upon an average, that a pound of dry charcoal is capable of heating 73 pounds of water from the freezing to the boiling point; but when it has been for some time exposed to the air, it contains at least 10 per cent. of water, which is partially decomposed in the combustion into carbureted hydrogen, which causes flame, whereas pure dry charcoal emits none.

A cubic foot of charcoal from soft wood weighs, upon an average, from 8 to 9 pounds, and from hard wood 12 to 13 pounds; and hence the latter are best adapted to maintain a high heat in a small compass. The radiating heat from charcoal fires constitutes one third of the whole emitted.

III. Pitcoal. The varieties of this coal are almost indefinite, and give out very various quantities of heat in their combustion. The carbon is the heat-giving constituent, and it amounts, in different coals, to from 75 to 95 per cent. One pound of good pitcoal will, upon an average, heat 60 pounds of water from the freezing to the boiling point. Small coal gives out three fourths of the heat of the larger lumps. The radiating heat emitted by burning pitcoal is greater than that by charcoal.

IV. The coke of pitcoal.-The heating power of good coke is to that of pitcoal as 75 to 69 . One pound of the former will heat 65 pounds of water from $32^{\circ}$ to $212^{\circ}$; so that its power is equal to nine tenths of that of wood charcoal.

V. Turf or peat.-One pound of this fuel will heat from 25 to 30 pounds of water from freezing to boiling. Its value depends upon its compactness and freedom from earthy particles; and its radiating power is to the whole heat it emits in burning as 1 to 3.

VI. Carbureted hydrogen or coal gas.-One pound of this gas, equal to about 24 cubic feet, disengages, in burning, as much heat as will raise 76 pounds of water from the freezing to the boiling temperature.

In the following table the fourth column contains the weight of atmospherical air, whose oxygen is required for the complete combustion of a pound of each particular substance.

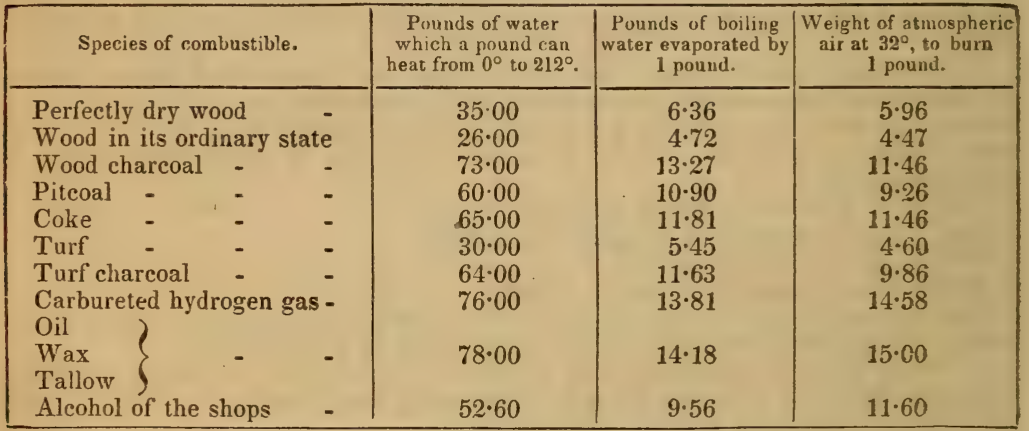

The quantity of air stated in the fourth column is the smallest possible required to burn the combustible, and is greatly less than would be necessary in practice, where much of the air never comes into contact with the burning body, and where it consequently never has its whole oxygen consumed. The heating power stated in the second column is also the maximum effect, and can seldom be realized with ordinary boilers. The draught of air usually carries off at least $\frac{1}{7}$ of the heat, and more if its temperature be very high when it leaves the vessel. ${ }^{7}$ In this case it may amount to one half of the whole heat or more; without reckoning the loss by radiation and conduction, which, however, may be rendered very small by enclosing the fire and flues within proper non-conducting and non-radiating materials.

It appears that, in practice, the quantity of heat which may be obtained from any combustible in a properly mounted apparatus must vary with the nature of the object to be heated. In heating chambers by stoves, and water boilers by furnaces, the effluent heat in the chimney which constitutes the principal waste may be reduced to a very moderate quantity, in comparison of that which escapes from the best constructed reverberatory hearth. In heating the boilers of steam engines, one pound of coal is reckoned adequate to convert $7 \frac{1}{2}$ pounds of boiling water into vapor; or to heat $41 \frac{1}{4}$ pounds of water from the freezing to the boiling point. One pound of fir of the usual dryness will evaporate 4 pounds of water, or heat 22 pounds to the boiling temperature; which is about two thirds of the maximum effect of this combustible. According to Watt's experiments upon the great scale, one pound of coal can boil off, with the best built boiler, 9 pounds of water; the deficiency from the maximum effect being here $\frac{1}{5} \frac{0}{7}$, or nearly one sixth.

In many cases, the hot air which passes into the flues or chimneys may be bene- 
ficially applird to the heatins, drying, or roasting of objects; but care ought to be taken that the draught of the fire be not thereby impaired, and an imperfect combustion of the fuel producul. For at a low smothering temperature both carbonic oxyde and carburet. ed hydrogen may be generited from coal, without the production of much heat in the fre-place.

To determine exactly the quantity of heat disengaged by any combustible in the act of burning, three different systems of apparatus have been employed: 1 . the calorimeter of Lavuisier and Laplace, in which the substance is burned in the centre of a vessel, whose walls are lined with ice; and the amount of ice melted, measures the heat evolved; 2 . the calorimeter of Watt and Rumford, in which the degree of heat communicated to a given body of water affords the measure of temperature; and 3 . by the quantity of water evaporated by different kinds of fuel in similar circumstances.

If our object be to ascertain the relative heating powers of different kinds of fuel, we need not care so much about the total waste of heat in the experiments, provided it be the same in all; and therefore they should be burned in the same furnace, and in the same way. But the more economically the heat is applied, the greater certainty will

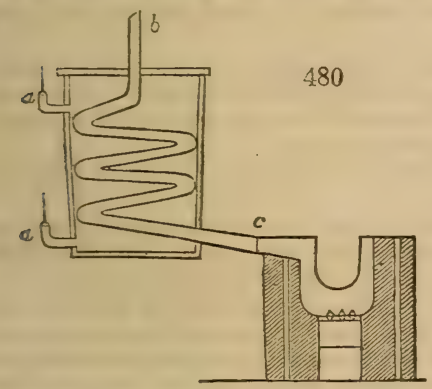
there be in the results. The apparatus fig. 480, is simple and well adapted to make such comparative trials of fuel. The little furnace is covered at top, and transmits its burned air by $c$, through a spiral tube immersed in a cistern of water, having a thermometer inserted near its top, and another near its bottom, into little side orifices $a a$, while the effluent air escapes from the upright end of the tube $b$. Here also a thermometer bulb may be placed. The average indication of the two thermometers gives the mean temperature of the water. As the water evaporates from the cistern, it is supplied from a vessel placed alongside of it. The experiment should be begun when the furnace has acquired an equability of temperature. A throttle valve at $c$ serves to regulate the draught, and to equalize it in the different experiments by means of the temperature of the effluent air. When the water has been heated the given number of degrees, which should be the same in the different experiments, the fire may be extinguished, the remaining fuel weighed, and compared with the original quantity. Care should be taken to make the combustion as vivid and free from smoke as possible.

FULGURATION designates the sudden brightening of the melted gold and silver in the cupel of the assayer, when the last film of vitreous lead and copper leaves their surface.

FULLER'S EARTH (Terre à foulon, Argile Smectique, Fr.; Walkererde, Germ.) Is a soft, friable, coarse or fine grained mass of lithomarge clay. Its color is greenish, or yellowish gray; it is dull, but assumes a fatty lustre upon pressure with the fingers, feels unetuous, does not adhere to the tongue, and has a specific gravity varying from 1.82 to $2 \cdot 19$. It falls down readily in water, into a fine powder, with extrication of air bubbles, and forms a non-plastic paste. It melts at a high heat into a brown slag. Its constituents are 53.0 silica; 10.0 alumina; 9.75 red oxyde of iron; 1.25 magnesia ; 0.5 lime; 24 water, with a trace of potash. Its cleansing action upon woollen stuffs depends upon its power of absorbing greasy matters. It should be neither tenacious nor sandy; for in the first case, it would not diffuse itself well through water, and in the second it would abrade the cloth too much. The finely divided silica is one of its useful ingredients.

Fuller's earth is found in several counties of England; but in greatest abundance in Bedfordshire, Berkshire, Hampshire, and Surry.

In the county of Surry there are great quantities of fuller's earth found about Nutfield, Ryegate, and Blechingley, to the south of the Downs, and some, but of inferior quality, near Sutton and Croydon, to the north of them. The most considerable pits are near Nutfield, between which place and Ryegate, particularly on Redhill, about a mile to the east of Ryegate, it lies so near the surface as frequently to be turned up by the wheels of the wagons. The fuller's earth to the north of the road between Redhill and Nutfield, and about a quarter of a mile from the latter place, is very thin; the seam in general is thickest on the swell of the hill to the south of the road. It is not known how long this earth has been dug in Surry; the oldest pit now wrought is said to have lasted between 50 and 60 years, but it is fast wearing out. The seam of fuller's earth dips in different directions. In one, if not in more cases, it inclines to the west with a considerable angle. There are two kinds of it, the blue and the yellow; the former, on the eastern side of the pit, is frequently within a yard of the surface, being 
covered merely with the soil-a tough, wet, clayey loam. A few yards to the west, the blue kind appears with an irony sand-stone, of nearly two yards in thickness, between it and the soil. The blue earth in this pit is nearly 16 feet deep. In some places the yellow kind is found lying upon the blue; there seems, indeed, to be no regularity either in the position or inclination of the strata where the fuller's earth is found, nor any mark by which its presence could be detected. It seems rather thrown in patches than laid in any coninued or regular vein. In the midst of the fuller's earth are often found large pieces of stone of a yellow color, translucent and remarkably heavy, which have been found to be sulphate of barytes, encrusted with quartzose crystals. These are carefully remored from the fuller's earth, as the workmen say they often spoil many tons of it which lie about them. There is also found with the yellow fuller's earth a dark brown crust, which the workmen consider as injurious also. In Surry the price of fuller's earth seems to have varied very little, at least for these last 80 years. In 1730 , the price at the pit was $6 l$. a sack, and $6 s$. per load or ton. In 1744 , it was nearly the same. It is carried in wagons, each drawing from three to four tons, to the beginning of the iron railway near Westham, along which it is taken to the banks of the Thames, where it is sold at the different wharves for about 25s. or 26s. per ton. It is then shipped off either to the north or west of England.

The next characteristic stratum, owing to its forming a ridge of censpicuous hills through the country, is the Woburn land, a thick ferruginous stratum, which below its middle contains a stratum of fuller's earth. This is thicker and more pure in Aspley and Hogstyeend, two miles north-west of Woburn, than in any known place.

Fuller's earth is found at Tillington, and consumed in the neighboring fulling mills.

Mode of preparing fuller's earth :-

After baking it is thrown into cold water, where it falls into powder, and the separation of the coarse from the fine is effectually accomplished, by a simple method used in the dry color manufactories, called washing over. It is done in the following manner: Three or four tubs are connected on a line by spouts from their tops; in the first the earth is beat and stirred, and the water, which is continually running from the first to the last through intermediate ones, carries with it and deposites the fine whilst the coarse settles in the first. The advantages to be derived from this operation are, that the two kinds will be much fitter for their respective purposes of cleansing coarse or fine cloth; for without baking the earth they would be unfit, as before noticed, to incorporate so minutely with the water in its native state; it would neither so readily fall down, nor so easily be divided into different qualities, without the process of washing over. When fuel is scarce for baking the earth, it is broken into pieces of the same size, as mentioned above, and then exposed to the heat of the sun.

The various uses of fuller's earth may be shortly explained. According to the above method, the cuarse and fine of one pit being separated, the first is used for cloths of an inferior, and the second for those of a superior quality. The yellow and the blue earths of Surry are of different qualities naturally, and are, like the above, obtained artificially, and used for different purposes. The former, which is deemed the best, is employed in fulling the kerseymeres and finer cloths of Wiltshire and Gloucestershire, whilst the blue is principally sent into Yorkshire for the coarser cloths. Its effect on these cloths is owing to the affinity which alumine has for greasy substances; it unites readily with them, and forms combinations which easily attach themselves to different stuffs, and thereby serve the purpose of mordants in some measure. The fullers generally apply it before they use the soap.

FULLING; for the theory of the process, see FELTING and Woor.

FULLING MILL. Willan and Ogle obtained a patent in 1825 for improved ful$480^{*}$

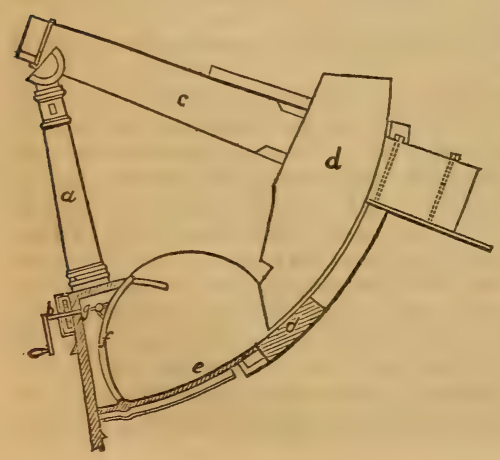
ling machinery, designed to act in a similar way to the ordinary stocks, in which cloths are beaten, for the purpose of washing and thickening them; but the standard and the bed of the stocks are made of iron instead of wood as heretofore; and a steam vessel is placed under the bed, for heating the cloths during the operation of fulling; whereby their appearance is said to be greatly improved.

Fig. $480^{*}$ is a section of the fulling machine or stocks; $a$ is a cast-iron pillar, made hollow for the sake of lightness; $b$ is the bed of the stocks, made also of iron, and polished smooth, the side of the stock being removed to show the interior; $c$ is the lever that carries the beater $d$. The cloths are to be placed on the bed $b$, a 
bottom, and water allowed to pass through the stock, when by the repeated blows of the beater 1 , which is raised and let fall in the usual way, the cloths are beaten, and become cleansed and fulled.

A part of the bed at $e$, is made hollow, for the purpose of forming a steam box, into which steam from a boiler is introduced by a pipe with a stop-cock. This steam heats the bed of the stock, and greatly facilitates, as well as improves the process of cleansing and fulling the cloths.

The smoothness of the surface of the polishor metal, of which the bed of the stock is constituted, is said to be very much preferable to the roughness of the surface of wood of which ordinary fulling stocks are male, as by these iron stocks less of the nap or felt of the cloth is removed, and its appearance when finished is very much superior to cloths fulled in ordinary stocks.

In the operation of fulling, the cloths are turned over on the bed, by the falling of the beaters, but this turning over of the cloths will depend in a great measure upon the form of the firont or breast of the stock. In these improved stocks, therefore, there is a contrivance by which the form of the front may be varied at pleasure, in order to suit cloths of different qualities; $f$, is a moveable curved plate, constituting the front of the stock; its lower part is a cylindrical rod, extending along the entire width of the bed, and being fitted into a recess, forms a hinge joint upon which the curved plate moves; $g$, is a rod attached to the back of the curved plate $f$, with a screw thread upon it; this rod passes through a nut $h$, and by turning this nut, the rod is moved backward or forward, and consequently the position of the curved plate altered.

The nut $h$, is a wheel with teeth, taking into two other similar toothed wheels, one on each side of it, which are likewise the nuts of similar rods jointed to the back of the curved plate $f$; by turning the central wheel, therefore, which may be done by a winch, the other two wheels are turned also, and the curved plate moved backward or forward. At the upper part of the plate there are pins passing through curved slots, which act as guides when the plate is moved.

The patentees state in conclusion, that steam has been employed before for heating cloths while fulling them, they therefore do not exclusively claim its use, except in the particular way described; the advantages arising from the construction of iron stocks, with polished surfaces in place of wooden ones, together with the moveable curved plates described, are in their opinion "sufficiently important to constitute a patent right."

FULIINATES, or fulminating powders. Of these explosive compounds, there are sereral species; such as fulminating gold, mercury, platinum, silver; besides the old fusible mixture of nitre, sulphur, and potash. The only kind at all interesting in a manufacturing point of view is the fulminate of mercury, now so extensively used as a priming to the caps of percussion locks. Having published a paper in the Journal of the Royal Institution for 1831, upon gunpowder (see GuNPowder), the result of an elaborate suite of experiments, I was soon afterivards requested by the Hon. the Board of Ordnance to make such researches as would enable me to answer, in a satisfactory practical manner, a series of questions upon fulminating powders, subservient to the future introduction of percussion muskets into the British army. The following is a verbatim copy of my report upon the subject :-

\section{To the Secretary of the Board of Ordnance.}

"Sir, -I have the honor of informing you, for the instruction of the Honorable the Master General and the Board of Ordnance, that the researches on fulminating inercury, which I undertook by their desire, have been brought to a satisfactory conclusion, after a numerous, diversified, and somewhat hazardous series of experiments. The following are the questions submitted to me, with their respective answers :-

Question 1. What proportions of mercury, with nitric acid and alcohol of certain strengths, will yield the greatest quantity of pure fulminate of mercury?

Answer. One hundred parts, by weight, of mercury, must be dissolved with a gentle heat, in 1000 parts (also by weight) of nitric acid, spec. gx. $1 \cdot 4$; and this solution, at the temperature of about $130^{\circ} \mathrm{Fahr}$., must be poured into 830 parts by weight of alcohol, spec. gr. 0.330 . - Note. 830 parts of such alcohol, by weight, constitute 1000 by measure; and 1000 parts of such nitric acid, by weight, constitute 740 by measure. Hence, in round numbers, one ounce weight of quicksilver must be dissolved in $7 \frac{1}{2} \mathrm{oz}$. measures of the above designated nitric acid, and the resulting solution must be poured into $10 \mathrm{oz}$. measures of the said alcohol.

Question 2. What is the most economical and safe process for conducting the manipulation, either as regards the loss of nitrous gas and residuum, or as respects danger to the operator; also, what is the readiest and safest mode of mixing the fulminate intimately with its due proportions of common gunpowder.

Answer. The mercury should be dissolved in the acid in a glass re:ort, the beak of which is loosely inserted into a large balloon or bottle of glass or eartl:? nware, whereby 
the offensive fumes of the nitrous gas disengaged during the solution, are, in a consider able measure, condensed into liquid acid, which should be returned into the retort. As soon as the mercury is all dissolved, and the solution has acquired the prescribed temperature of about $130^{\circ}$, it should be slowly poured, through a glass or porcelain funne? into the alcohol contained in a glass matrass or bottle capable of holding fully 6 times the bulk of the mixed liquids. In a few minutes bubbles of gas will proceed from the bottom of the liquid; these will gradually increase in number and magnitude till a general fermentative commotion, of a very active kind, is generated, and the mixture assumes a somewhat frothy appearance. A white voluminous gas now issues from the orifice of the matrass, which is very combustible, and must be suffered to escape freely into the air, at a distance from any flame. These fumes consist of an ethereous gas, holding mercury in suspension or combination. I have made many experiments with the view of condensing this gas, or, at least, the mercury, but with manifest disadvantage to the perfection of the process of producing fulminate. When the said gas is transmitted, through a glass tube, into a watery solution of carbonate of soda, a little oxyde of mercury is, no doubt, recovered; but the pressure on the fermentative mixture, though slight, necessary to the displacement of the soda solution, seems to obstruct or impair the generation of the fulminate; this effect is chiefly injurious towards the end of the operation, when the gaseous fumes are strongly impregnated with nitrous gas. When this is not allowed freely to come off, a portion of subnitrate or nitrate of mercury is apt to be formed, to the injury of the general process and the product.

As soon as the effervescence and concomitant emission of gas are observed to cease, the contents of the matrass should be turned out upon a paper double filter, fitted into a glass or porcelain funnel, and washed by the affusion of cold water till the drainings no longer redden litmus paper. The powder adhering to the matrass should be washed out and thrown on the filter by the help of a little water. Whenever the filter is thoroughly drained, it is to be lifted out of the funnel, and opened out on plated copper or stone ware, heated to $212^{\circ} \mathrm{Fahr}$. by steam or hot water. The fulminate, being thus dried, is to be put up in paper parcels of about 100 grains each; the whole of which may be afterwards packed away in a light box, or a bottle with a cork stopper. The excellence of the fulminate may be ascertained by the following characters. It consists of brownishgray small crystals which sparkle in the sun, are transparent when applied to a slip of glass with a drop of water, and viewed by transmitted light. These minute spangles are entirely soluble in 130 times their weight of boiling water; that is to say, an imperial pint of boiling water will dissolve $67 \mathrm{grs}$. of pure fulminate. Whatever remains indicates impurity. From that solution beautiful pearly spangles of fulminate fall down as the liquid cools.

It may now be proper to show within what nice and narrow limits the best proportions of the ingredients used in making the fulminate of mercury lie. The following are selected from among many experiments instituted to determine that point, as well as the most economical process.

1. According to the formula given by the celebrated chemist Berzelius, in the 4 th vol. of his "Traité de Chimie," recently published (p.383), the mercury should be dissolved in 12 times its weight of nitric acid, sp. gr. $1 \cdot 375$; and alcohol of sp. gr. 0.850 , amounting to $16 \cdot 3$ times the weight of the mercury, should be poured at intervals into the nitric solution. The mixture is then to be heated till effervescence with the characteristic cloud of gas appears. On the action becoming violent, alcohol is to be poured in from time to time to repress it, till additional 16.3 parts have been employed.

On this process I may remark, that it is expensive, troublesome, dangerous, and unproductive of genuine pure fulminate. One fifth more nitric acid is expended very nearly than what is necessary, and almost four times the weight of alcohol which is beneficial. Of alcohol at $0.83,8.3$ parts by weight are sufficient; whereas Berzelius prescribes nearly 4 times this quantity in weight, though the alcohol is somewhat weaker, being of sp. gr. $0 \cdot 850$. By using such an excess of alcohol, much of the fulminate is apt to be revived into globules of quicksilver at the end of the process, as I showed in my paper on this subject published in the Journal of the Royal Institution two years ago. There is no litle hazard in pouring the alcohol into the nitric solution; for at each affusion an explosive blast takes place, whereas by pouring the solution into the alcohol, as originally enjoined by the Hon. Mr. Howard, the inventor of the process, no danger whatever is incurred. 100 parts of mercury treated in the way recommended by Berzelius afforded me only 112 parts of fulminate, instead of the 130 obtained by my much more economical and safe proportions and process from the same weight of juicksilver.

2. If 10 parts of nitric acid of sp. gr. $1 \cdot 375$ be used for dissolving 1 of quicksilver, and if 14 parts of alcohol of sp. gr. 0.85 be thereafter mixed with the solution, the product of such proportions will either be not granular, and therefore not fulminating, or 
it will be partially gianular and partially pulverulent, being a mixture of fulminate and subnitrate of mercury ill adapted for priming detonating caps. Instead of 130 parts of genuine fulminate, as I do obtain, probably not more than 10 parts of potvder will be produced, and that of indifferent quality. In fact, whenever the ethereous fermentation is defective, or not vigorous, little true fulminate is generated; but much of the mercury remains in the acidulated alcoholic liquid.

3. If the alcohol be poured in successive portions, and of proper strength (sp. gr. 0.83 ), into a proper nitric solution of mercury, the explosive action which accompanies each affusion dissipates much of the alcohol, and probably impairs the acid, so that the subsequent ethereous fermentation is defective, and little good fulminate is formed. From 100 parts of mercury submitted to this treatment, I obtained in one experiment, carefully made, only 51 parts of a powder, which was impalpable, had a cream color, and was not explosive either by heat or percussion.

4. When, with 100 parts of mercury, 800 of nitric acid of sp. gr. $1 \cdot 375$, are employed with 650 of alcohol of sp. gr. $\cdot 846$, no fulminate whatever is generated.

5 . When, with the proper proportions of mercury, acid, and alcohol, the process is advanced into a proper energy of fermentative commotion, if the matrass be immersed in cold water so as materially to repress that action, the process will be impaired, and will turn out ultimately defective both as to the quantity and quality of the fulminate. It is therefore evident that a certain energy or vivacity of etherization is essential to the full success of this curious process, and that anything which checks it, or obstructs its taking place, is injurious and to be avoided.

When my proportions are observed in making fulminating mercury, somewhat less than one fourth of the nitric acid used in making the solution remains in the alcoholie mixture along with the fulminate. When other proportions are taken, much more acid remains. This acid is not recoverable to any useful or economical purpose, nor is the alcohol that is associated with it. Many distillations, with various reagents, have led me to this practical conclusion. In fact, when the process is most complete, as described in the first paragraph, the alcohol is entirely and profitably employed in etherization, an generating fulminic acid.

I have made a series of analytical experiments on the pure fulminate of mercury, with the view of determining its composition, the quantity of quicksilver present in it, and consequently the loss of mercury in the operation. I have stated that my maximum product of fulminate from 100 grs. of quicksilver is 130 grs. Occasionally, from slight differences in the temperature of the mixture, or the ambient atmosphere, 2 grs. less may be obtained.

A. I dissolved $130 \mathrm{grs}$. with a gentle heat in muriatic acid contained in a small matrass, adding a few drops of the nitric to quicken the solution. On evaporating it to dryness, with much care to avoid volatilization of the salt, I obtained 125 grs. of corrosive sublimate or bi-chloride of mercury. But 125 grs. of this bi-chloride contain only $\mathbf{9 1 \cdot 1}$ grs. of quicksilver. Therefore, by this experiment, $130 \mathrm{grs}$. of fulminate contain no more than 91.1 of mercury, indicating an exhalation of 8.9 parts in the form of fumes, or a retention in the residuary liquid of some of these 8.9 parts, out of the 100 originally employed.

B. In another experiment for analysis, 130 grs. dissolved as above, were thrown down by carbonate of soda. 95 grs. of black oxyde of mercury were obtained, which are equivalent to 91.2 grs. of quicksilver; affording a confirmation of the preceding result.

C. 130 grs. of fulminate were dissolved in strong muriatic acid, and the solution was decomposed by crystals of proto-muriate of tin at a boiling temperature. The mercury was precipitated in globules to such amount as to verify the two preceding experiments.

Regarding fulminate of mercury as a bi cyanate, that is, as a compound of one atom or one equivalent prime of deutoxyde of mercciry. $n$ ind two primes of cyanic acid, we shall find its theoretical composition to be as follows, hyarogen being the radix, or 1 .

$$
\begin{aligned}
& 2 \text { Primes of Cyanic or fulminic Acid }=34 \times 9=68 \\
& \text { Deutoxyde of Mercury }=\quad 216 \\
& \frac{218}{284} \quad \frac{76}{100}
\end{aligned}
$$

As these 284 parts of fulminate contain 200 of quicksılver, .0 142 parts of fulminate will contain 100 of quicksilver. Whence it appears, that when only 130 parts of fulminate can be obtained in practiee from 100 of quicksilver, $8 \frac{1}{2}$ iarts of quicksilver out of the 100 are unproductive, that is, are expended in the etherisea pas, or left in the residuary acidulous liquid. By the above experimental and theortical aralvgig 91.5 parts of quicksilver enter into the composition of 130 parts of true crysta'line fulminate. The complete accordance here exhibited between theory and practice remuves arary 
shadow of doubt as to the accuracy of the statements. 100 parts of fulminate eon. sist of -

$$
\begin{aligned}
& \left.\begin{array}{l}
\text { Mercury } \\
\text { Oxygen }
\end{array} \begin{array}{r}
70 \cdot 4 \\
5.6
\end{array}\right\} \text { Peroxyde } 76 \cdot 0 \\
& \text { Fulminic acid - - } \quad 24 \\
& 100 \cdot 0
\end{aligned}
$$

Question 3. May the gas or vapor produced by the inflammation of the fulminate of mercury, when combined with a portion of gunpowder, be considered in its nalure corrosive of iron or brass?

Answer. I have suggested to Mr. Lovell, of Waltham Abbey works, that the fulminate may be probably diluted most advantageously with spirit varnish made of a proper consistence by dissolving sandarach in alcohol. When well mixed with this varnish, a small drop of the mixture will suffice for priming each copper cap or disc; and as the spirit evaporates immediately, the fulminate will be fixed to the copper beyond the risk of shaking or washing away. On the Continent, tincture of benjamin is used for the same purpose; but as that balsamic resin leaves in combustion a voluminous coal, which sandarach does not, the latter, which is the main constituent of spirit varnish, seems better adapted for this purpose. It is sufficiently combustible, and may be yet made, by a due proportion, to soften the violence of the explosive mercury on the nipple of the touch-hole. Fulminate prepared by my formula has no corrosive influence whatsoever on iron or steel ; and, therefore, if such a medium of applying it, as I have now taken leave to suggest, should be found to answer, all fears on the score of corrosion may for ever be set at rest.

Quesition 4. How far is the mixture (of fulminate and gunpowder) liable to be affected by the moisture of the atmosphere, or by the intrusion of water; and will such an accident affect its inflammability when dried again?

Answer. Well made fulminate, mixed with gunpowder and moistened, undergoes no change, nor is it apt to get deteriorated by keeping any length of time in a damp climate or a hazy atmosphere. Immersion in water would be apt to wash the nitre out of the pulverine; but this result would be prevented if the match or priming mixture were liquefied or brought to the pasty consistence, not with water, but spirit varnish. Such detonating caps would be indestructible, and might be alternately moistened and dried without injury.

Question 5. Is it at all probable that the composition would be rendered more inflam. mable or dangerous of use by the heat of tropical climates?

Answer. No elevation of temperature of an atmospheric kind, compatible with human existence, could cause spontaneous combustion of the fulminating mercury, or the detonating matches made with it. In fact, its explosive temperature is so high as $367^{\circ}$ of Fahrenheit's scale, and no inferior heat will cause its detonation.

Question 6. Is the mercurial vapor or gas arising from the ignition of a great number of primers, and combined with the smoke of gunpowder in a confined space (as in the ease of troops in close bodies, squares, casemates, \&c.), likely in its nature to be found prejudicial to human health?

Answer. I have exploded in rapid succession of portions, 100 grains of fulminate of mercury (equivalent to 300 or 400 primers), in a close chamber of small dimensions, without experiencing the slightest inconvenience at the period, or afterwards, though my head was surrounded by the vapors all the time of the operation. These vapors are, in fact, so heavy that they subside almost immediately. When the fulminate mixed with pulverine is exploded in the primers by condensed masses of troops, the mercury will cause no injury to their health, nor 100th part of the deleterious impression on weak lungs which the gases of exploded gunpowder might by possibility inflict. These gases are all, theoretically speaking, noxious to respiration; such as carbonic acid gas, azote, carbureted hydrogen, and sulphureted hydrogen, a deadly gas. Yet the soldier who should betray any fcar of gunpowder smoke would be an object of just ridicule."

In the following September, I executed for the Board of Ordnance a set of experiments, complementary to those of the memoir, with the view of ascertaining the best manner of protecting the fulminate when applied to the copper caps, from being detached by carriage, or altered by keeping. The following were my results and conclusions.

"1. Fulminate of mercury moistened upon copper is speedily decomposed by the superior affinity of the copper over mercury, for oxygen and fulminic acid. Dryness is, therefore, essential to the preservation of the fulminate; and hence charcoal, which is apt to become moist, should not be introduced into percussion caps destined for distant service.

2. An alcoholic solution of sandarach, commonly called spirit varnish, acts powerfully on copper, with the production of a green eflorescence, which decomposes ful- 
minate of mercury. Indeed, sandarach can decompose the salte of copper. It is therefore ill adapted for attaching the fulminate to copper caps.

3. An alcoholic solution of shellac acts on copper, though more feebly than the sandarach.

4. A solution of mastic in spirits of turpentine, whether alone or mixed with fulminate, has no action whaterer on bright copper, but protects it from being tarnished. Such a varnish is very cheap, dries readily, adheres strongly, screens the fulminate from damp, and does not impair or counteract its detonating powers. This, therefore, is, in my opinion, the fittest medium for attaching the fulminate, and for softening the force of its impulsion in any degree preportional to the thickness of the varnish."

Fulminate of mercury is obtained in white grains, or short needles, of a silky lustre, which become gray upon exposure to light, and detonate either by a blow or at a heat unfer $3 \pi()^{\circ} \mathrm{F}$.; with the disengagement of azote, carbonic acid, as also of aqueous and mercurial vapors; to the sudden formation of which gaseous products the report is due. It detunates even in a moist condition; and when dry it explodes readily when struck betreen two pieces of iron, less so between iron and bronze, with more difficulty between marble and glass, or between two surfaces of marble or glass. It is hardly possible to explode it by a blow with iron upon lead; and impossible by striking it with iron upon wood. It fulminates easily when rubbed between two wooden surfaces; less so between two of marble, two of iron, or one of iron against one of wood or marble. The larger its erystals, the more apt they are to explode. By damping it with 5 per cent. of water, it becomes less fulminating; the part of it struck still explodes with a proper blow, but will not kindle the adjoining portion. Though moistened with 30 per cent. of water, it will occasionally explode by trituration between a wooden muller and a marble slab, but only to a small extent, and never with any danger to the operator. When an ounce of it, laid upon the bottom of a cask, is kindled, it strikes a round hole down through it, as if it had been exposed to a four-pound shot, without splintering the wood. If a train of fulminate of mercury be spread upon a piece of paper, covered with some loose gunpowder, in exploding the former the latter will not be kindled, but merely scattered. When gunpowder, however, is packed in a cartridge, or otherwise, it may be certainly kindled by a percussion cap of the fulminate, and more completely than by a priming of gunpowder. $8 \frac{1}{2}$ parts of gunpowder exploded by a percussion cap, have an equal projectile force as 10 exploded by a flint lock. If we add to this economy in the charge of the barrel, the saving of the powder for priming, the advantage in military service of the percussion system will become conspicuous.

The French calculate that 1 kilogramme of mercury will furnish $1 \frac{1}{4} \mathrm{kil}$. ( $2 \frac{1}{2} \mathrm{lbs}$. nearly) of fulminate, which will be sufficient to charge 40,000 percussion caps. For this purpose they grind the crystalline salt along with 30 per cent. of water upon a marble table with a wooden muller; mixing with every 10 parts of the fulminate 6 of gunpowder. A consistent dough is thus obtained, which, being dried in the air, is ready for introducing into the bottoms of the copper caps. One quarter of a grain of the fulminate is said to be fully sufficient for one priming.

Mr. Lovell, of the Royal Manufactory of Arms, has lately executed a series of experiments upon priming powders. His trials, which occupied nearly 18 months, were made for the purpose of ascertaining what is the advantage in point of force obtained by using percussion primes. He had anticipated some extra energy would be imparted to the charge of powder in the barrel, because he had repeatedly proved that a good strong cap, exploded by itself on the nipple of the musket (without any charge of gunpowder), will exert sufficient force upon the air within the barrel to blow a candle out at a distance of 12 feet from the muzzle. He concluded also that stopping the escape of fluid from the vent, as is done by the cap, would have some effect, but he attributed most to the quickness and energy with which the powder of the charge is ignited by the vivid stream of flame, generated by the percussion prime. The trials were made from one and the same barrel, having a percussion lock on one side and a flint lock on the other. The balls were fired against Austen's recoiling target, a very delicate plegometer, beginning with a charge of 1.50 grains (the present musket charge), and descending by 10 grains at a time (niring 30 rounds with each weight), down to 50 grains. The machine marked the decrease of force at each reduction in the charge very satisfactorily, and the result of the whole average was, that 8.84 parts of gunpowder fired by percussion are equal to 10 parts fired by the flint.

To find out what sort of liberties might be taken with fulminate of mercury in handling it, he placed 3 grains on an anvil, putting the end of a steel punch gently on the top of it, and while so placed he covered the fulminate over with a drachm of dry gunpowder. He then ignited the fulminate by a blow on the punch with the hammer, but not a grain of the gunpowder was lighted, though it was blown about in all directions. He then placed a train of fulminate as thick as a quill, and about 3 feet long, on a table, and covered it over entirely with gunpowder except about an inch at one 
end; this he lighted with a hot iron, when the whole train went off without blazing a grain of the gunpowder, which he swept together and blew up afterwards with a match. He then took a tin box containing 500 copper caps, made a hole in the top of the box, and through this hole ignited one of the caps in the middle, by means of the punch and hammer on the outside; only two other caps besides the one struck exploded; no injury was sustained by the remainder, except being discolored. This he tried repeatedly, and alway's with the same kind of result, never more than 3 or 4 caps exploding. He then made a steel rammer red hot, and passed it through the hole in the box right in amongst the caps, but it only ignited them where the hot iron came in actual contact with the priming composition; when, however, he placed a few grains of gunpowder loose among the caps, the hot iron lighted this, and produced a flame that blew off the whole of them.

The same thing has been tried at Woolwich, where large packages of percussoion caps (some thousands) have been fired at with musket balls, and only a few of the caps actually hit by the ball exploded; but when any cartridges were connected with the packages, the whole, caps and all, were blown up. The flame of the fulminate is therefore hazardous, but being so very ethereal, it requires for making primes, an admixture of some combustible matter, as a little gunpowder, to condense or modify the flame.

FULMINIC ACID (Acid fulminique, Fr.; Knallsaure, Germ.) is the explosive constituent of the fulminating mercury of Howard, and the fulminating silver of Brugnatelli, being generated by the reaction of alcohol and the acid nitrates of these metals. It is a remarkable chemical fact, that fulminic acid has exactly the same composition as cyanic acid; though the salts of the latter possess no detonating property, and afford, in their decomposition by an oxygen acid, ammonia with carbonic acid; while those of the former afford ammonia and prussic acid. All attempts to insulate fulminic acid have proved unsuccessful, as it explodes with the slightest decomposing force. It consists, by weight, of 2 primes of carbon, 1 of azote, and 1 of oxygen; or of two volumes of carbonic acid, and one of azote. When two different bodies, like the above, have the same composition, they are said to be isomeric.

FUMIGATION, is the employment of fumes or vapors to purify articles of apparel, and goods or apartments supposed to be imbued with some infectious or contagious poison or fumes. The vapors of vinegar, the fumes of burning sulphur, explosion of gunpowder, have been long preseribed and practised, but they have in all probability little or no efficacy. The diffusion of such powerful agents as chlorine gas, muriatic acid gas, or nitric acid vapor, should alone be trusted to for the destruction of morbific effluvia.

FUR; See Peltry.

FURNACE OF ASSAY. Under AsSAY, I have referred to a furnace constructed by Messrs. Anfrye and d'Arcet, which gives some peculiar facilities and economy to the ancient process by fire. It had originally a small pair of bellows attached to it, for raising the heat rapidly to the proper vitrifying pitch. The furnace, $17 \frac{1}{2}$ inches high, and $7 \frac{1}{2}$ inches wide, made of pottery or fine clay, is represented fig. 481, supported upon a table, having a pair of bellows beneath it. The laboratory is at $b$, the blow-pipe of the bellows at $d$, with a stop-cock, and the dome is surmounted by a chimney $a c$, in whose lower part there is an opening with a sliding door, for the introduction of the charcoal fuel. The furnace is formed in three pieces; a dome, a body, and an ash-pit. A pair of tongs, a stoking hook, and cupel, are seen to the right hand, and the plan of the stone-ware grate, pierced with conical holes, and a poker, are seen to the left. This grate suits the furnace represented under
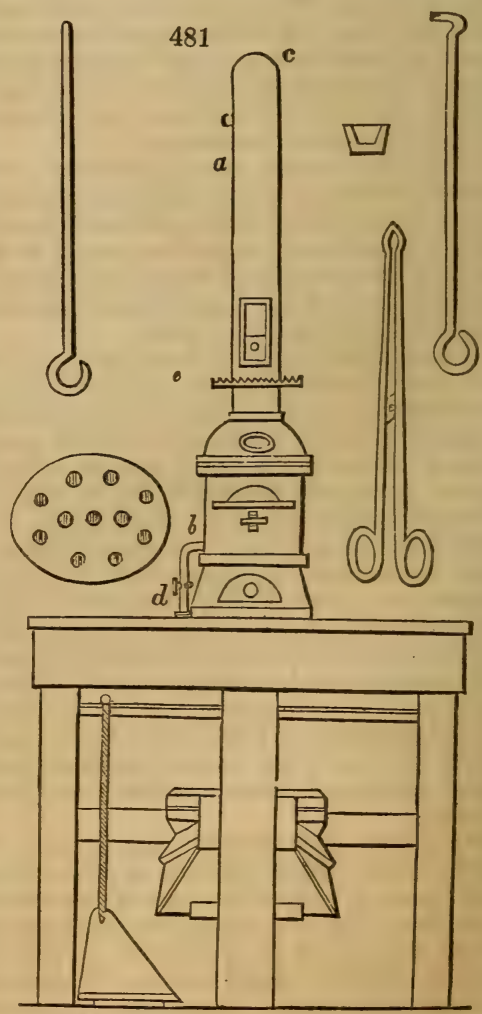

Assay. The following are comparative experiments made by means of this furnace. 


\begin{tabular}{|c|c|c|c|c|c|}
\hline Numbers. & Silver employed. & Lead employed. & Time of assay. & Stundards. & Charcoal used. \\
\hline 1 & 1 Grain. & 4 Grains. & 12 minutes. & 947 millièmes. & 173 Grains. \\
\hline 2 & - & - & 11 & 950 & 86 \\
\hline 3 & - & - & 13 & 949 & 93 \\
\hline 4 & 一 & - & 10 & 949 & 60 \\
\hline
\end{tabular}

Each assay was therefore performed at an average in $11 \frac{1}{2}$ minutes, and not much more than a quarter of a pound of charcoal was used. An experiment of verification in the ordinary assay furnace showed the standard to be 949 thousandths.

This furnace becomes a very convenient one for melting small quantities of metals in analyses, by removing the muffle, and closing the several apertures with their appropriate stoppers. A small pedestal may be then set in the middle of the grate, to support a crucible, which may be introduced throngh the opening $h$. Coke may also be used as fuel, either by itself or mixed with charcoal. For descriptions of various furnaces, see Assay ; Beer; Copper; Evaforation; Iron; Metallurgy; Ores ; Silver, Tin, \&c.

FUSIBILITY. That property by which solids assume the fluid state.

Some chemists have asserted that fusion is simply a solution in caloric; but this opinion includes too many yet undecided questions, to be hastily adopted.

\section{Fusibility of Metals, as given by M. Thenard.}

\section{Fusible below a red heat.}

\section{Infusible below a red heat.}

Mercury
Potassium
Sodium
Tin
Bismuth
Lead
Tellurium
Arsenic
Zinc
Antimony
Cadmium

Centigr.

$-39^{\circ}$

$\left.+58^{\circ}\right\}$

$90\}$

$210 ?$

256

260

Gay Lussac and Thenard.

Newton.

A little less fusible than lead.-Klaproth.

Undetermined.

$370^{\circ} \quad$ Brongniart.

A little below a read heat.

Stromeyer.

Silver
Copper
Gold
Cobalt

Iron

Manganese

Nickel

Palladium

Molybdenum

Uranium

Tungsten

Chromium

Titanium

Cerium

Osmium

Iridium

Rhodium

Platinum

Columbium

yrometer of Wedgewood.
20 Kennedy.

\section{7 ? Kennedy.}

$32\}$ Wedgewood.

A little less difficult to melt than iron.

$\{130$ Wedgewood.

$\{158$ Sir G. M`Kenzie.

160 Guyton.

As manganese.-Richter.

Nearly infusible; and to be obtained at forge heat only in small buttons.

Infusible at the forge furnace. Fusible at the oxyhydrogen blowpipe. See BrowPIPE.

\section{FUSTBLE METAL. See ArLoy.}

FLSTET. (Fuslec, Fr.) The wood of the rhus cotinus, a fugitive yellow dye.

FUSTIAN is a species of coarse thick tweeled cotton, and is generally dyed of an olive, leaden, or other dark color. Besides the common fustian, which is known by the name of pillow (probably pilaw), the cotton stuffs called corduroy, velverett, velveteen, thicksett, used for men's wearing apparel, belong to the same fabric. The commonest kind is merely a tweel of four, or sometimes five leaves, of a very close stout texture, and very narrow, seldom exceeding 17 or 18 inches in breadth. It is cut from the loom in half pieces, or ends, as they are usually termed, about 35 yards long, and after undergoing the subsequent operations of dyeing, dressing, and folding, is ready for the market. 
The draught and cording of common fustian is very simple, being generally a regular or unbroken tweel of four or five leaves. Below are specimens of a few different kinds, selected from those most general in Lancashire.

The number of leaves of heddles are represented by the lines across the paper, and the cording by the ciphers in the little squares, those which raise every leaf being distinguished by these marks, and those which sink them left blank, as more particularly explained in the article Textile Fabric.

Oi velvet, there are properly only two kinds, that with a plain, and that with a tweeled, or, as it is here called, a Genoa ground, or back. When the material is silk, it is called velvet, when cotton, velveteen; and this is the sole difference. In the same way a common twceled cloth, when composed of silk is called satin; when of cotton, fustian or jean - of wonllen, plaiding, serge, or kerseymere; and in the linen trade is distinguished by a variety of names according to the quality or fineness, or the place where the article is manufactured.

No. 1.-Pillow Fustian.

No. 2.-Plain Velveret.

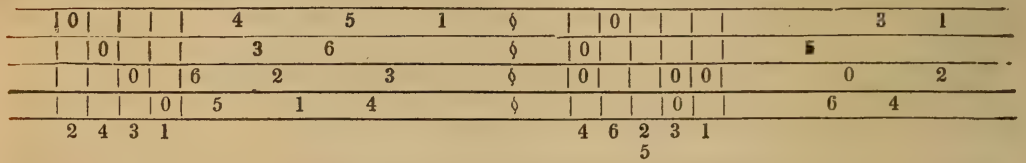

Of the above, each contains four leaves of heddles or healds; that represented by No. 1 is wrought by four treddles, and that which is distinguished by No. 2 by five; the succession of inserting the threads of warp into the heddles will be discovered by the figures between the lines, and the order in which the treddles are to be successively pressed down by the figures below.

No. 3.-Double Jean.

No. 4.-Plain Thickset.

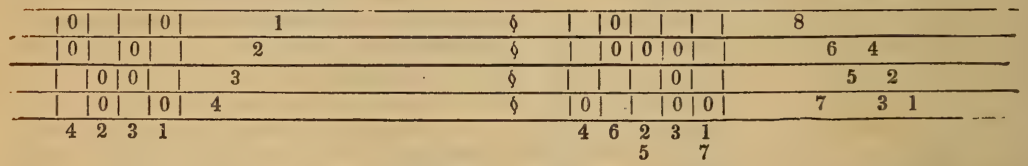

These, like the former, are wrought with leaves. No. 3 requires four, and No. 4 five treddles. The succession of inserting the threads of warp, and of working the treddles, are marked by the respective numbers between and under the lines, as in the former example. Both are fabries of cloth in very general use and estimation as low priced articles.

No. 5.-Best Thickset.

No. 6.-Velvet Tuft.

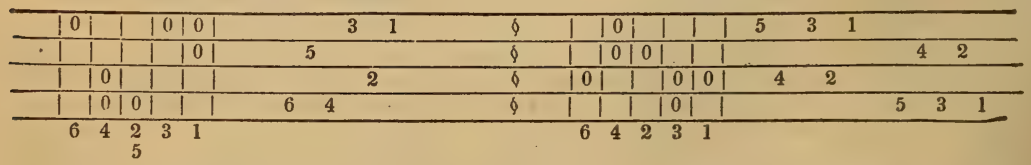

These are further specimens of what may be, and is, executed with four leaves, and in both examples five treddles are used. With two other specimens we shall conclude our examples of this description of work, and shall then add a very few specimens of the more extensive kinds.

No. 7.-Cord and Velveret.

No. 8.-Thickset Cord.

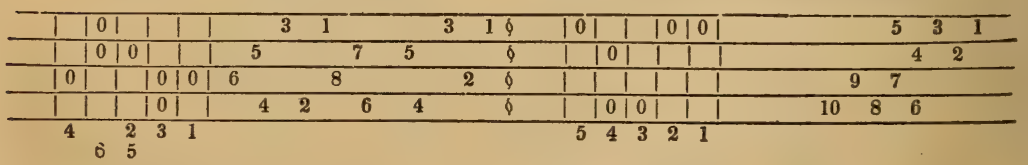

In these the succession of drawing and working are marked like the former. The next are examples of patterns wrought with six leaves. No. 9 has eight, and No. 10 five heddles. 


\section{FUSTIAN.}

No. 9.-Double Corduror.

No. 10.-Genoa Thickset.

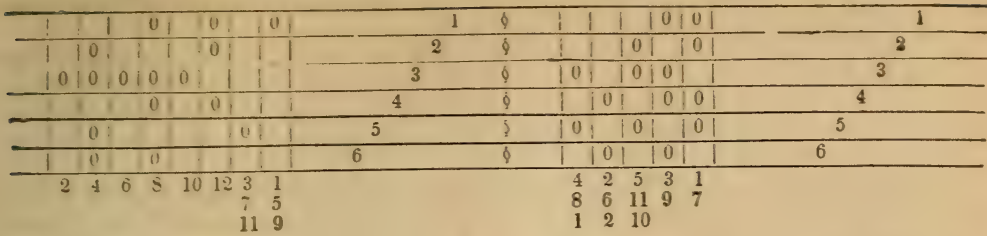

In both these the warp is inserted into the heddles the same way. The difference is entireir in the application of the cords, and in the succession of pressing uawn the treddles. We now give four specimens of the flusbed and cut work, known by the name of relreteen. Thes are also upon six leaves, and the difference is solely in the cording and in the treading.

ำ. 11.

Queen's Velreteens.

No. 12.

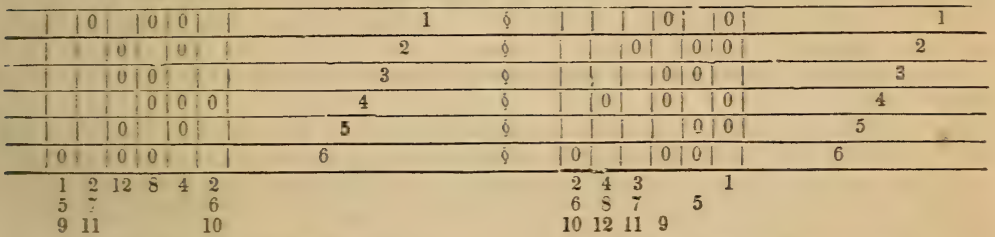

No. 13.-Plain Velveteen.

No. 14.-Genoa Velveteen.

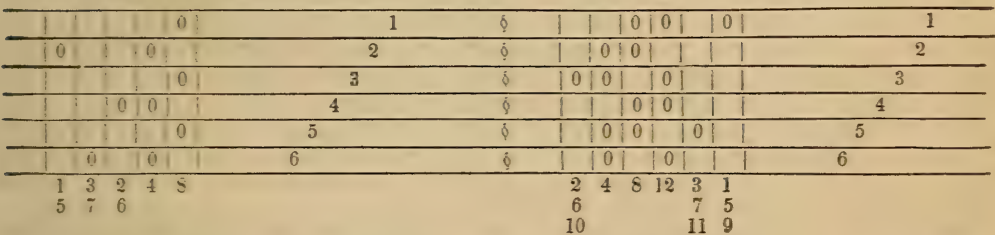

The additional rarieties of figure which might be given are almost endless, but the limits of this article will not admit a further detail. Those already given are the articles in most general use. The rarieties of fancr may be indulged to a great extent; but it is unirersally found, that the most simple patterns in every department of ornamental weaving are those which attract attention and command purchasers. We shall therefore only add two examples of king's cord or corduror, two of Genoa and common velvet, and two more of jean. These will be found below.

No. 15.-King's Cord.

No. 16.-Dutch Cord.

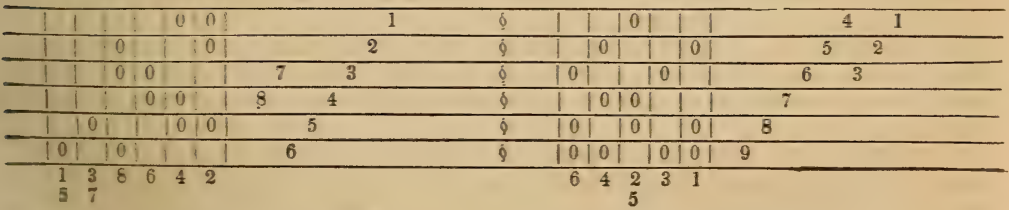

No. 17.-Genoa Velvet.

No. 18.-Plain Velvet.

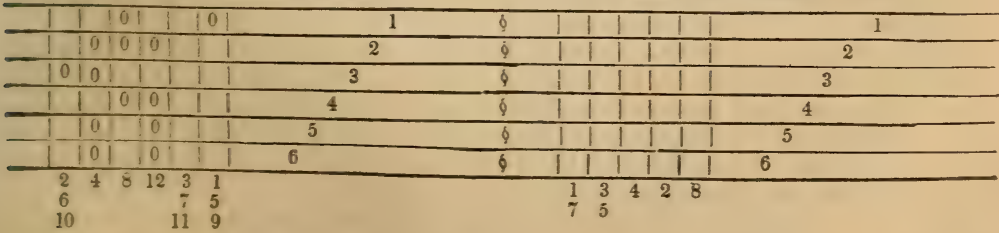

After the fustian cloth is taken from the loom-beam, it is carried to the cutter, who nps up the surface-threads of weft, and produces thereby a hairy-looking stuff. 
Preparatory to its being cut, the cloth is spread evenly upon a table about six feet long, upon each end of which a roller mounted with a ratchet-wheel is fixed; the one to give off, and the other to wind up the piece, in the above six-feet lengths.

The knife is a steel rod about two feet long, and three eighths of an inch square, having a square handle at the one end; the other end is tapered away to a blade, as thin as paper. To prevent this point from turning downwards and injuring the cloth, its under side is covered by a guide which serves to stiffen it, as well as to prevent its lower edge from cutting the fustian.

The operative (male or female) grasps the handle in the right hand, and insinuating the projecting point of the guide under the weft, pushes the knife smaitly forward through the whole length of six feet, with a certain dexterous movement of the shoulder and right side, balancing the body meanwhile, like a fencer, upon the left foot. This process is repeated upon every adhesive line of the weft.

The next process to which fustians are exposed is steeping in hot water, to take out the dressing paste. They are then dried, reeled, and brushed by a machine, \&ce. From twenty to thirty pieces, each eighty yards long, may be brushed in an hour. The breadth of the cloth is twenty inches. The maceration is performed by immersing the bundled pieces in tanks of water, heated by waste steam; and the washing by means of a reel or winch, kept revolving rapidly under the action of a stream of cold water, for an hour or longer.

After being thus ripped up, it is taken to the brushing or teazling machine, to make it shaggy.

This consists of a series of wooden rollers, turning freely upon iron axles, and covered with tin-plate, rough with the burs of punched holes; and blocks of wood, whose concave under surfaces are covered with card-cloth or card-brushes, and which are made to traverse backwards an! forwards in the direction of the axes of the revolving rollers, during the passage of the cloth over them.

After they are brushed in the machine, the goods are singed by passing their cut surface over a cylinder of iron, laid in a horizontal direction, and kept red hot by a flue. See Singeing. They are now brushed again by the machine, and once more passed over the singeing surface. The brushing and singeing are repeated a third, or even occasionally a fourth time, till the cord acquires a smooth polished appearance.

The goods are next steeped, washed, and bleached, by immersion in solution of chloride of lime. They are then dyed by appropriate chemical means. After which they are padded (imbued by the padding machine of the calico printers) with a solution of glue, and passed over steam cylinders to stiffen them.

Smooth fustians, when cropped or shorn before dyeing, are called moleskins; but when shorn after being dyed, are called beaverteen : they are both tweeled fabrics. Cantoon is a fustian with a fine cord risible upon the one side, and a satiny surface of yarns running at right angles to the cords upon the other side. The satiny side is sometimes smoothed by singeing. The stuff is strong, and has a very fine aspect. Its price is one shilling and sixpence a yard.

Common plain fustian, of a brown or drab color, with satin top, is sold as low as sevenpence a yard.

A fustian, with a small cord running in an oblique direction, has a very agreeable appearance. It is called diagonal. Moleskin shorn, of a very strong texture, and a drab dyed tint, is sold at $20 d$. per yard.

The weight of 90 yards of the narrow velveteen, in the green or undressed state, is about 24 pounds. The goods made for the German, Italian, and Russian markets are lighter, on account of the peculiarity in the mode of levying the import duty in these countries.

Velveteens as they come from the loom, are sold wholesale by weight, and average a price of $20 \mathrm{~d}$. per pound. They are usually woven with yarns of Upland and Brazil cotton wool, spun together for the warp; or, sometimes, New Orleans alone. The weft is usualiy Upland, sometimes mixed with East India cotton wools.

Trouser velveteens are woven 19 inches wide, if they are to be cut up; if not, they are woven 30 inches, and called beaverteen.

Cutting or cropping fustians by hand is a very laborious and delicate operation. The invention of an improved apparatus for effecting the same end with automatic precision and despatch, was therefore an object of no little interest to this peculiar manufacture of Manchester. An ingenious machine, apparently well calculated for this purpose, was made the subject of a patent by Messrs. William Wells and George Scholefield, of Salford, in November, 1834 .

FUSTIC. (Bois jaune, Fr.; Gelbholz, Germ.) The old fustic of the English dyer, as the article fustet is their yellow fustic. It is the wood of the Morus tinctoria. It is light, not hard, and pale yellow with orange veins; it contains two coloring matters, one resinous, and another soluble in water. The latter resembles weld, but it has more of an orange cast, and is not so lively. 
Its decoctions in water are brightened by the addition of a little glue, and more by curdled milk. This wood is rich in color, and imparts permanent dyes to woollen stuffs, when aided by proper mordants. It unites well with the blue of the indigo vat, and Saxon blue, in producing green of various shades. Alum, tartar, and solution of tin, render its color more vivid; sea salt and sulphate of iron deepen its hue. From 5 to 6 parts of old fustic are sufficient to give a lemon color 1016 parts of cloth. The color of weld is however purer and less inclining to orange; but that of fustic is less affected by acids than any other yellow dye. This wood is often employed with sulphate of iron in producing olive and brownish tints, which agree well with its dull yellow. For the same reason it is much used for dark greens.

\section{G.}

GABRONITE is a yellowish stony substance, of a greasy lustre and spec. $\mathrm{gr} .=2 \cdot 74$; affording no water by calcination; fusible at the blowpipe into an opaque glass; soluble in muriatic acid; solution affords hardly any precipitate by oxalate of ammonia. This mineral is distinguished by the large quantity of soda which it contains; its constituents being-silica, 54 ; alumina, 24 ; soda, $17 \cdot 25$; magnesia, 1.5 ; exyde of iron, $1 \cdot 25$; water, 2. It belongs to the species Nepheline.

GADOLINITE, called also Yttrite and Ytterbite, is a mineral of a black, brownish, or yellowish color, granular, or compactly vitreous, and conchoidal fracture; of spec. grav. 4.23 ; readily scratching glass; fusibie at the blowpipe into an opaque glass, sometimes with intumescerice. It affords, with acids, a solution that lets fall, with caustic soda, a precipitate partly re-soluble in carbonate of ammonia. It is remarkable for containing from 45 to 55 per cent. of the earth Yttria ; its remaining constituents being silica, $25 \cdot 8$; oxyde of cerium, $17 \cdot 92$; oxyde of iron, $11 \cdot 43$. This mineral is very rare, having been hitherto found only in the neighborhood of Fahlon and Ytterby, in Sweden; its peculiar constituent was discovered by Professor Gadolin.

GALACTOMETER, or LACTOMETER, is an instrument to ascertain the quality of milk; an article often sophisticated in various ways. Fresh milk, rich in cream, has a less specific gravity than the same milk after it has been skimmed; and milk diluted with water becomes proportionably lighter. Hence, when our purpose is to determine the quantity of cream, the galactometer may consist merely of a long graduated glass tube standing upright upon a sole. Having filled 100 measures with the recent milk, we shall see, by the measures of cream thrown up, its value in this respect. A delicate longranged glass hydrometer, graduated from 1.000 up to $1 \cdot 060$, affords the most convenient means of detecting the degree of watery dilution, provided the absence of thickening materials has been previously ascertained by filtration. Good fresh milk indicates from $1 \cdot 030$ to 1.032 ; when the cream is removed, 1.035 to 1.037 . When its density is less than 1.028 , we may infer it has been thinned with water.

GALBANUM is a gum-resin, which occurs sometimes in yellow, shining tears, easily agglutinated ; of a strong durable smell; an acrid and bitter taste; at other times in lumps. It exudes either spontaneously or from incisions made into the stem of the bubon galbanum, a plant of the family of umbelliferce, which grows in Africa, particularly in Ethiopia. It contains 67 of resin; 19.3 of gum; 6.4 of volatile oil and water; $7 \cdot 5$ of woody fibres and other impurities; with traces of acid malate of lime.

GALENA, (Plomb sulfuré, Fr.; Bieiglanz, Germ.;) is a metallic looking substance of a lead-gray color, which crystallizes in the cubical system, and is susceptible of cleavages parallel to the faces of the cube; spec. gr. $7 \cdot 7592$; cannot be cut; fusible at the blowpipe with exhalation of sulphureous vapors; is easily reduced to metallic lead. Nitric acid first dissolves it, and then throws down sulphate of lead in a white precipitate; the solution affording, with plates of zinc, brilliant laminæ of lead (arbor Saturni.) It consists of sulphur, 13 ; lead, 85 ; with a little iron, and sometimes a minute quantity of silver. This is the richest ore of lead, and it occurs in almost every geological formation, in rein s, in masses, or in beds. It is almost always accompanied by sulphuret of zinc, dirferent salts of lead, heavy spar, fluor spar, \&c. Galena in powder, ealled Alquifoux, is employed as a glaze for coarse stoneware.

G-ALIPOT is a name of a white semi-solid viscid rosin found on fir-trees; or an inferior sort of turpentine, poor in oil.

GALLATES; salts consisting of gallic acid combined with bases; the most important being that with oxyde of iron, constituting a principal part of the black dye.

F. ALILIC ACID is the peculiar acid extracted from gall-nuts; which see.

G.1LLIPOLI OIL is a coarse olive oil, containing more or less mucilage; imported from a seaport so named, of the province of Otranto, in the kingdom of Naples.

G.ALL-NUTS, or GALLS, (Noix de Galle, Fr.; Galläpfel, Germ.;) are excrescences found upon the leaves and leaf-stalks of a species of oak, called Quercus infectoric, which grows in the Levant. They are produced in consequence of the puncture of the female of the gall wasp (Cynips folii quercus), made in order to deposite her 
eggs; round which the juice of the tree exudes, and dries in concentric portions. When the insect gets fully formed, it eats through the nut, and flies off.

The Levant galls are of two different appeaiances and qualities; the first are heavy cumpact, imperforated, the insect not having been sufficiently advanced to eat its way through the shell; prickly on the surface; of a blackish or bluish green hue; about the size of a musket-ball. These are called black, blue, or Aleppo galls. The second are light, spongy, pierced with one or more holes; smooth upon the surface, of a pale grarish or reddish yellow color, generally larger than the first, and are called white galls. Be. sides the galls of the Levant, others come from Dalmatia, Illyria, Calabria, \&c. ; but they are of inferior quality, being found upon the Quercus Cerris; they are smaller, of a brownsh color, and of inferior value. The further south the galls are grown, they are reckoned the better.

Galls consist principally of three substances; tannin or tannic acid; yellow extractive; and gallic acid. Their decoction has a very astringent and unpleasant bitter taste. The ollowing are their habitudes with various reagents:-

Litmus paper is powerfully reddened.

Stannous chloride (protomuriate of $t i n$ ) produces an isabel yellow precipitate.

Alum; a yellowish gray precipitate.

Acetate of lead; a thick yellowish white precipitate.

Acetate of copper; a chocolate brown precipitate.

Ferric sulphate (red sulphate of iron); a blue precipitate.

Sulphuric acid; a dirty yellowish precipitate.

Acetic acid brightens the muddy decoction.

The galls of the Quercus Cerris and common oak (Galles à l'épine, Fr.; Knoppern, Germ.) are of a dark brown color, prickly on the surface, and irregular in shape and size. They are used chiefly for tanning in Hungary, Dalmatia, and the southern provinces of the Austrian states, where they abound.

Tannin or tannic acid is prepared as follows: Into a long narrow glass adopter tube, shut at its lower orifice with a cotton wick, a quantity of pounded galls are put, and slightly pressed down. The tapering end of the tube being inserted into a matrass or bottle, the vacant upper half of the tube is filled with sulphuric ether, and then closed with a ground-glass stopper. Next day there will be found in the bottle a liquid in two distinct strata; of which the more limpid occupies the upper part, and the other, of a sirupy consistence and amber color, the lower. More ether must be filtered through the galls, ill the thicker liquid cuases to augment. Both are now poured into a funnel, closed with he finger, and after the dense liquor is settled at the bottom, it is steadily run off into a zapsule. This, after being washed repeatedly with ether, is to be transferred into a stove shamber, or placed under the receiver of an air pump, to be evaporated. The residuary natter swells up in a spongy crystalline form of considerable brilliancy, sometimes color-ess, but more frequently of a faintly yellowish hue.

This is pure tannin, which exists in galls to the amount of from 40 to 45 per cent. It . $s$ indispensable that the ether employed in the preceding process be previously agitated with rater, or that it contain some water, because by using anhydrous etber, not a particle of tannin will be obtained.

Tannic acil is a white or yellowish solid, inodorous, extremely astringent, very soluble in water and alcohol, much less so in sulphuric ether, and uncrystallizable. Its watery solution, out of contact of air, undergoes no change; but if, in a very dilute state, t $t$ be leit exposed to the atmosphere, it loses gradually its transparency, and lets fall a slightly grayish crystalline matter, consisting almost entirely of gallic acid. For procuring this acid in a perfectly pure state, it is merely necessary to treat that solution thus shanged with animal charcoal, and to filter it, in a boiling state, through paper previously washed with dilute muriatic acid. The gallic acid will fall down in crystals as the liquid cools.

If the preceding experiment be made in a graduated glass tube containing oxygen over mercury, this gas will be absorbed, and a corresponding volume of carbonic acid gas will be disengaged. In this case the liquor will appear in the course of a few weeks as if traversed with numerous crystalline colorless needles of gallic acid.

Tannin or tannic acid consists of carbon $51 \cdot 56$; hydrogen $4 \cdot 20$; oxygen $44 \cdot 24$.

From the above facts it is obvious that gallic acid does not exist ready formed in gallnuts, but that it is produced by the reaction of atmospheric oxygen upon the tannin of these concretions.

Gallic acid is a solid, feebly acidulous and styptic to the taste; inodorous, crystallizing in silky needles of the greatest whiteness; soluble in about 100 times its weight of cold, and in a much smaller quantity of boiling water; more soluble in alcohol than in water, Dut little so in sulphuric ether.

Gallic acid does not decompose the salts of protoxyde of iron, but it forms, with the sulphate of the peroxyde, a dark blue precipitate, much less insoluble than the tannate of iron. Gallic acid takes the oxyde from the acetate and nitrate of lead, and throws 
down a white gallate unchangeable in the air, when it is mixed with that acetate and nitrate. It occasions no precipitate in solutions of gelatine (isinglass or glue), by which eriterion its freedom from tannin is verified.

Gallic acid occurs but seldom in nature; and always united to brucine, veratrine, or lime. Its constituents are carbon 49.89; hydrogen 3.49 ; oxygen 46.62. In the crystal. line state it contains one atom of water, which it loses by drying.

Scheele obtained gallic acid by infusing pounded galls for 3 or 4 days in 8 times their weight of water, and exposing the infusion to the air, in a vessel covered loosely with paper. At the end of two months, the liquor had almost all evaporated, leaving some mouldiness mixed with a crystalline precipitate. The former being removed, the deposite was squeezed in a linen cloth, and then treated with boiling water. The solution, being gradually evaporated, yielded crystals of gallic acid, granular or star-like, of a grayish color. "These crystals might be whitened by boiling their solution along with a little animal charcoal. About one fifth of gallic acid may be obtained by Scheele's process from good gall-nuts.

From a decoction of 500 parts of galls, Sir H. Davy obtained 185 parts of solid extract; which consisted of 130 parts of tannin; 31 parts of gallic acid with extractive; 13 parts of mucilage; 12 parts of lime and salts. Hence gall-nuts would seem to contain, by this statement, more than two thirds of their weight of tannin. This result is now seen, from the above experiments of Pelouze, to have been incorrect, in consequence of the admixture of yellow extractive in Davy's tannin.

The use of galls in many processes of dyeing, and in making black ink, is detailed under their respective heads.

GALL OF ANIMALS, or OX-GALL, purification of. Painters in water colors, scourers of elothes, and many others, employ ox-gall or bile; but when it is not purified, it is apt to do harm from the greenness of its own tint. It becomes therefore an important object to clarify it, and to make it limpid and transparent like water. The following process has been given for that purpose. Take the gall of newly killed oxen, and after having allowed it to settle for 12 or 15 hours in a basin, pour the supernatant liquor off the sediment into an evaporating dish of stone ware, and expose it to a boiling heat in a water bath, till it is somewhat thick. Then spread it upon a dish, and place it before a fire till it becomes nearly dry. In this state it may be kept for years in jelly pots covered with paper, without undergoing any alteration. When it is to be used, a piece of it of the size of a pea is to be dissolved in a table spoonful of water.

Another and probably a better mode of purifying ox-gall is the following. To a pint of the gall boiled and slimmed, add one ounce of fine alum in powder, and leave the mixture on the fire till the alum be dissolved. When cooled, pour into a bottle, which is to be loosely corked. Now take a like quantity of gall, also boiled and skimmed, add an ounce of common salt to it, and dissolve with heat; put it when cold into a bottle, which is likewise to be loosely corlied. Either of these preparations may be liept for several years without their emitting a bad smell. After remaining three months, at a moderate temperature, they deposite a thick sediment, and become clearer, and fit for ordinary uses, but not for artists in water colors and miniatures, on account of their yelluwish-green color. To obviate this inconvenience, each of the above liquors is to be decanted apart, after they have become perfectly settled, and the clear portion of both mixed together in equal parts. The yellow coloring matter still retained by the mixture coagulates immediately and precipitates, leaving the ox-gall perfectly purified and colorless. If wished to be still finer, it inay be passed through filtering paper; but it becomes clearer with age, and never acquires a disagreeable smell, nor loses any of its good qualities.

Clarified ox-gall combines readily with coloring matters or pigments, and gives them solidicy either by being mixed with or passed over them upon paper. It increases the brilliancy and the durability of ultramarine, carmine, green, and in general of all delicate colors, whilst it contributes to make them spread more cvenly upon the paper, ivory, \&c. When mixed with gum-arabic, it thickens the colors without communicating to them a disagreeable glistering appearance; it prevents the gum from cracking, and fixes the colors so well that others may be applied over them without derradation. Along with lamp black and gum, it forms a good imitation of China ink. When a coat of ox-sall is put upon drawings made with black lead or crayons, the lines can no longer be effaced, but may be painted over safely with a variety of colors previously mixed uf with the same ox-gall.

Miniature painters find a great advantage in employing it; by passing it over ivory, it removes completely the unctuous matter from its surface; and when ground with the colors, it makes them spread with the greatest ease, and renders them fast.

It serves also for transparencies. It is first passed over the varnished or oiled paper, and is allowed to dry. The colors mixed with the gall are then applied, and cannot afterwards be removed by any means.

It is adapted finally for taking out spots of grease and oil. 
GALL OF GLASS, called also sandiver, is the neutral salt skimmed off the surface of melted crown glass; which, if allowed to remain too long, is apt to be re-absorbed in part, and to injure the quality of the metal, as the workmen call it.

GALVANIZED IRON is the somewhat fantastic name newly given in France to iron tinned by a peculiar patent process, whereby it resists the rustins influence of damp air, and even moisture, much longer than ordinary tin plate. The following is the prescribed process. Clean the surface of the iron perfectly by the joint action of dilute acid and friction, plunge it into a bath of melted zinc, and stir it about till it be alloyed super ficially with this mils ; then take it out, and immerse it in a bath of tin, such as is used for making tin plate. The tin forms an exterior coat of alloy. When the metal thus prepared is exposed to humidity, the zinc is said to oxydize slowly by a galvanic action, and to protect the iron from rusting within it, whereby the outer tinned surface remains for a long period perfectly white, in circumstances under which iron tinned in the usual way would have been superficially browned and corroded with rust.

GAMBOGE (Gomme Gutte, Fr.; Gutti, Germ.) is a gum resin, concreted in the air, from the milky juice which exudes from several trees. The gambogia gutta, a tree which grows wild upon the coasts of Ceylon and Malabar, produces the coarsest kind of gamboge; the guttaefera vera (Stalagmites cambogioides) of Ceylon and Siam affords the best. It comes to us in cylindrical lumps, which are outwardly brown yellow, but reddish yellow within, as also in cakes; it is opaque, easily reducible to powder, of specine gravity $1 \cdot 207$, scentless, and nearly devoid of taste, but leaves an acrid feeling in the throat. Its powder and watery emulsion are yellow. It consists of 80 parts of a hyacinth red resin, soluble in alcohol; and 20 parts of gum; but by another analysis, of 89 of resin, and 10.5 of gum. Gamboge is used as a pigment, and in miniature painting, to tinge gold varnish; in medicine as a powerful purge. It should never be employed by confectioners to color their liquors, as they sometimes do.

GANGUE. A word derived from the German gang, a vein or channel. It signifies the mineral substance which either encloses or usually accompanies any metallic ore in the vein. Quartz, lamellar carbonate of lime, sulphate of baryta, sulphate and fluate of lime, generally form the gangues; but a great many other substances become such when they predominate in a vein. In metallurgic works the first thing is to break the mixed ore into small pieces, in order to separate the valuable from the useless parts, by processes called stamping, picking, sorting. See Metallurgy and Mines.

GARNET (Grenat, Fr.; Granat, Germ.) is a vitreous mineral of the cubie system, of which the predominating forms are the rhomboidal dodecahedron and the trapœzohedron; specific gravity varying from $3 \cdot 35$ to $4 \cdot 24$; fusible at the blowpipe. Its constituents are, silica, 42 ; alumina, $20^{\circ} 0$; lime, $34 \cdot 0$; protoxyde of iron, 4 . Garnets are usually disseminated, and occur in all the primitive strata from gneiss to clay slate. The finer varieties, noble garnet or Almandine, and the reddish varieties of Grossulaire (Essonite), are employed in jewellery; the first are called the Syrian or oriental; the others, hyacinth, In some parts of Germany, garnets are so abundant as to be used as fluxes to some iron ores; in others, the garnet gravel is washed, pounded, and employed as a substitute for emery. The garnets of Pegu are most highly valued. Factitious garnets may be made by the following composition:-Purest white glass, 2 ounces; glass of antimony, 1 ounce: powder of eassius, 1 grain, manganese, 1 grain.

GAS (Eng. and Fr.; Gaz, Germ.) is the seneric name of all those elastic fluids which are permanent under a considerable pressure, and at the temperature of zero of Fahrenheit. In many of them, however, by the joint influence of excessive cold and pressure, the repulsive state of the particles may be balanced or subverted, so as to transform the elastic gas into a liquid or a solid. For this most interesting discovery, we are indebted to the fine genius of Mr. Faraday.

The following table exhibits the temperatures and pressures at which certain gases are liquefied.

\begin{tabular}{|c|c|c|c|c|c|}
\hline \multirow{2}{*}{\multicolumn{3}{|c|}{ Name of the gas. }} & \multicolumn{2}{|c|}{ Becomes liquid } & \multirow{2}{*}{$\begin{array}{l}\text { Calculated boiling print; } \\
\text { Barom. }=30 \text { inches. }\end{array}$} \\
\hline & & & \multirow{3}{*}{\begin{tabular}{l}
\multicolumn{1}{c}{ At } \\
$59^{\circ} \mathrm{F}$. \\
60
\end{tabular}} & Under a pressure of & \\
\hline Sulphurous acid & - & - & & 3 atmospheres. & $4^{\circ}$ Fahr. \\
\hline Chlorine & - & - & & & -22 \\
\hline Ammonia & - & - & 50 & $6 \cdot 5$ & -64 \\
\hline Sulphureted hydrogen & - & - & 50 & 17 & -142 \\
\hline Carbonic acid - & - & - & 32 & 36 & -229 \\
\hline Hydrochloric or muriat & tic acid & - & 50 & 50 & -249 \\
\hline Deutoxyde of azote & - & - & 45 & 50 & -254 \\
\hline
\end{tabular}


Liquid earhonic acid becomes solidified, into a snowy-looking substance, by its own rapid evapuration. Oxygen, hydrogen, and azote, have hitherto resisted all attempts to divest them it their elastic furm. For this purpose, it is probable that a condensing force equal to that of 650 atmospheres, will be required.

The voluu!e of any gas is, generally speaking, inversely as the pressure to which it is exposed: thus, under a double pressure its bulk becomes one half; under a triple pressure, one third; and so on. For the change of volume in gaseous bodies by heat, see Expansion.

Ammonia, carbonic acid, carbureted hydrogen, chlorine, muriatic acid, sulphurous acid, sulphureted hydrogen, are the gases of most direct interest in the arts and manu. factures. Their detailed examination belongs to a work on chemistry.

GAS-LIGHT. (Eclairage par gas, Fr.; Gaslicht, Germ.) Dr. Clayton demonstrated, by numerous experiments in 1737 and 1738 , that bituminous pit-coal subjected to a red heat in close vessels, afforded a great deal of an air similar to the fire-damp of mine's, but which burned with a brighter flame. It does not appear that this species of factitious air was ever produced from pit-coal for the purpose of artificial illumination till 1792, when Mr. William Murdoch, engineer to Messrs. Bolton and Watt, employed coal gas for lighting his hoise and offices, at Redruth in Cornwall. The gas was generated in an iron retort, whence it was received in a gasometer, distributed in different situations by pipes, and finally burned at small apertures which could be opened and stopped at pleasure. He moreover made this light moveable, by confining the gas in portable tin-plate vessels, and burning it wherever he pleased. Between this period and 1802, Mr. Murdoch continued at intervals to make similar experiments; and upon occasion of the national illumination in the spring of the latter year, at the peace of Amiens, he lighted up part of the Soho manufactory with a public display of gaslights.

The earliest application of this artificial light, on a large systematic scale, was made at Manchester; where an apparatus for lighting the great cotton mills of Messrs. Philips and Lee, was fitted up in 1804 and 1805, under the direction of Mr. Murdoch. A quantity of light, nearly equal to 3000 candles, was produced and distributed in this building. This splendid pattern has been since followed very generally in Great Britain, and more or less in many parts of the continents of Europe and America. By the year 1822, gas-lighting in London had become the business of many public companies. At the Peter street station, for example, 300 retorts had been erected, supplying 15 gasometers, having each an average capacity of 20.626 cubic feet, but, being never quite filled, their tolal contents in gas might be estimated at 309,385 cubic feet. The extent of main pipes of distribution belonging to this station was then about 57 miles, with two separate mains in some of the streets. The product of gas was from 10,000 to 12,000 cubic feet from a chaluron of coals. The annual consumption of coals was therefore altogether 9282 chaldrons, affording $11,384,000$ cubic feet of gas, allowing 153 retorts to be in constant daily action, upon an average of the year; and illuminating 10,660 private lamps, 2248 street lamps, and 3894 theatre lamps.

At the Brick-lane works, 371 retorts were fixed in 1822, 133 being worked on an aver age of summer and winter. There were 12 gasometers, charged with an average quantity of gas amounting to 197,214 cubic feet. Of coals, 8060 chaldrons were annually consumed; $96,720,000$ cubic feet of gas were generated; for the supply of 1978 public lamps, and 7366 private ones, connected with main pipes 40 miles long.

At the Curtain-road gas establishment, there were 240 retorts; but the greatest number worked in 1821 was only 80 , and the lowest 21 . The six gasometers had an average contents of 90,467 cubic feet. Of coals, 3336 chaldrons were annually consumed, yielding $40,010,000$ cubic feet of gas, that supplied 3860 private lamps, and 629 public ones, by means of mains 25 miles long. The above three stations belonged to the London GasLight and Coke Company.

The City of London Gas-Light Company, Dorset street, had built up 230 retorts, and 6 gasometers, while two were preparing; having a total capacity of 181,282 cubic feet. Of private lamps 5123 were lighted, and 2413 public ones, from mains extending 50 miles. The quantily of coals carbonized amounted to 8840 chaldrons; producing $106,080,000$ cubic feet of gas.

The Sinth London Gas-Light and Coke Company had mounted at Bankside 143 retorts, with 3 gasometers; the contents of the whole being 41,110 cubic feet, connected with mains frum 30 to 40 miles long. At their other station, in Wellington street, 9 large gasometers were then erecting, with a capacity of 73,565 cubic feet, which were to be aupplied with gas from Bankside, till retorts were mounted for them.

The Imperial Gas-Light and Coke Company had at that time 6 gasometers in progress at their Hackney station.

In 1822 there were thus four great companies, having in all 47 gasometers at work, eapable of containing 917,940 cubic feet of gas, supplied by 1315 retorts, which generated 
per annum upwards of $397,000,000$ cubic feet of gas, by which 61,203 private lamps, and 7268 public or street lamps, were linhted in the metropolis. Besides these public companies, there were likewise several private ones.

1. Of the generation of illuminating gases.-Pure hydrogen gas burns with too feeble a flaine to be employed for illumination. But carbureted hydrogen having the property of precipitating its carbon in the act of burning, its solid particles become incandescent, and diffuse a vivid light. The more carbon it contains, the more brightly does it burn. This gas exists in two distinct states of combination. In the first, tivo measures of hydrogen gas are combined with one measure of the vapor of carbon, forming together one measure whose specific gravity is of course the sum of the weights of the constituents, or 0.559 ; atmospherical air being 1.000 . This is the gas which is found in mines, and is also evolved in ditches from decomposing vegetable matter. In the second, two measures of hydrogen gas are combined with two of gaseous carbon, forming also one volume or measure whose weight or specific gravity is 0.985 . This was at one time called the olefiant gas, because when mixed with chlorine an oily looking compound was produced. It may be called as well oil gas, because it is generated in considerable quantities by the igneous decomposition of oil. Thus the olefiant gas contains in the same volume double the quantity of carbon of common carbureted hydrogen, and it burns with a proportionably brighter flame. The gaseous oxyde of carbon, as well as sulphureted hydrogen gas, burns with a feeble blue light, but the latter produces in combustion sulphurous acid, an offensive and noxious gas.

By dry distillation or carbonization in close vessels, all bodies of vegetable and animal origin disengage carbureted hydrogen gas; even charcoal, when placed in ignition in contact with steam, by decomposing the water, produces abundance of earbonic acid, carbureted hydrogen, hydrogen, and carbonic oxyde. After separating the carbonic acid with lime water, that mixed gas contains in 100 measures, 20 of carbureted hydrogen; the rest being hydrogen and carbonic oxyde, so that the gaseous mixture cannot be used for illumination. The best substances for furnishing a gas rich in luminiferous materials are, pitcoal, especially the cannel coal, resin, oil, fats of all kinds, tar, wax, \&c. In some cases the gases evolved during the igneous decomposition of bones and other animal matters for the production of ammonia, may be employed for procuring light, but they are apt to emit a fetid odor.

When coals are heated in a cast-iron retort to ignition, the progress of decomposition is as follows. First, and before the retort becomes red hot, steam issues along with the atmospheric air. When the retort begins to redden, tar distils in considerable quantity with some combustible gas, of which hydrogen mixed with ammoniacal gas forms a part. The evolution of gas increases as the retort becomes hotter, with a continual production of tar and ammoniacal liquor as well as sulphurous acid from the pyrites of the coal, which unites with the ammonia. When the retort has come to a bright cherry red heat, the disengagement of gas is most active. By and by the gaseous production diminishes, and eventually ceases entirely, although the heat be increased. In the retort a quantity of carbonized coal or coke remains, while tar is found at the bottom of the receiver, covered with the ammoniacal liquor, and combined with carbonic and sulphurous acids, and sulphureted hydrogen.

If, during this distillation, the combustible gas be collected and examined at the several stages of the process, it is found to differ extremely in its luminiferous powers. That which comes off before the retort has acquired its proper temperature, gives a feeble light, and resembles the gas obtained by the ignition of moist charcoal, consisting chiefly of hydrogen. That evolved when the retort has just acquired throughout a vivid red heat, is the best of all, consisting chiefly of bi-carbureted hydrogen or olefiant gas. From good coal, it consists, for example, in 100 measures, of 13 of olefiant gas, 82.5 of carbureted hydrogen, 3.2 carbonic oxyde, 1.3 azote; the mixture having a specific gravity of 0.650 . At a later period, as after 5 hours, it contains 7 measures of olefiant gas, 56 of carbureted hydrogen, 11 of carbonic oxyde, 21.3 of hydrogen, 4.7 of azote; the specific gravity of the whole being 0.500 . Towards the end of the operation, as after 10 hours, it contains twenty measures of carbureted hydrogen, 10 of carbonic oxyde, 60 of hydrogen, 10 of azote, with a specific gravity of only $0 \cdot 345$. The hydrogen becomes sulphureted hydrogen, if there be much pyritous matter in the coal. The larger proportion of the gas is disengaged during the first hour, amounting to about one fifth of the whole; in the three following hours the disengagement is tolerably uniform, constituting in all fiftyfour hundredths; in the sixth hour, it is one tenth; in the seventh and eighth hours. :sixteen hundredths.

From these observations are derived the rules for the production of a good light gas from coals. They show that the distillation should commence with a retort previously heated to a cherry red, since thereby good gas is immediately produced, and a portion of the tar is also converted into gas, instead of being simply distilled over into the condenser pit; that this heat should be steadily continued during the whole operation, from 5 to 8 hours; that it should not be increased, especially towards the end, for fear 
of generating carbonic oxyde and hydrogen gases, as well as of injuring the retort when the cuning ayency of gasefication has become feeble; and that the operation should be stopped some time before gas ceases to come over, lest gases with feeble illuminating powet should impoverish the contents of the gasometer. Upon the average, a pound of avod coas atlurds fuur cubic feet of gas, or a chaldıon $=26 \mathrm{cwts}$. London measure, affords from 12,000 to 15,000 eubic feet, according to the form of the retort, and the manner of firing it.

When oil, fats, rosin, tar, \&c. are employed for the production of a light gas, it is not sufficient to introunce these substances into the retorts, and to heat thein, as is done with cuals. In this case, the greater part of them would distil over in the state of volatile vils, and very little gas be generated, only as much as corresponded to the quantity of fat, Sc. in immediate contact with the retort. It becomes therefore necessary to fill the returts with pieces of brick $\mathrm{c}$ : colse; and to keep them in ignition, while the oil, Sc. is slowly introduced into their interior. The fats instantly assume the vaporous state, and thus coming into contact upon an extensive surface with the ignited bricks, are decumpused into combustible gases. A small portion of carbonaceous matter remains in the retort, while much olefiant gas is formed, possessing a superior illuninating power to common coal gas, and entirely free from sulphureous impregnation. The best oil gas is generated at a dull red, a heat much below what is requisite for the decomposition of coal: A more intense heat would indeed produce a greater volume of gas, but of a poorer quality, because the olefiant gas thereby deposites one half of its carbon, and is conrerted into common carbureted hrdrogen. Oil affords at a lively red heat, gases which contain in 100 measures, 19 of olefiant gas, 32.1 of carbureted hydrogen, 12.2 of carbonic oxyde gas, 32.4 of hydrogen, and 4 of azote; the mean specific gravity being only 0.590 . At a more moderate temperature it yields 22.5 of the olefiant, 50.3 carbureted hydrogen, 15.5 carbonic oxyde, $7 \cdot 7$ hydrogen, and 4 azote, with a specific grarity of $0 \cdot 758$. It contains when generated by dull ignition, as is usual in works on the manufacturing scale, in 100 parts from 38 to 10 of olefiant gas, and besides the carbureted hydrogen, a few per cent. of carbonic oxrde and azote, with a specific gravity of 0.900 , and even upwards. One pound of oil or fluid fat affords 15 cubic feet of gas; of tar affords about 12 cubic feet; of rosin or pitch, 10 cubic feet.

When the oil gas is compressed by a force of from 15 to 20 atmospheres, as was the practice of the Portable Gas Company, about one fifth of the volume of the gas becomes liquefied into an oily, very volatile fluid, having the specific gravity 0.821 . It is a mixture of three fluids (consisting of carbureted hydrogen), of different degrees of volatility. The most volatile of these boils even under $32^{\circ} \mathrm{F}$. Some of the vapor of this gas-oil is mixed with the olefiant gas in the general products of decomposition; in consequence of which they are sometimes richer in carbon than even olefiant ras, and have a higher illuminating power. Oil gas contains about 22 per cent. and coal gas about $3 \frac{1}{4}$ per cent. of this oily vapor. In the estimations of the composition of the gases given above, this rapor is included under olefiant gas. This rapor combines readily with sulphuric acid, and is thus precipitated from the gaseous mixture. The amount of olefiant gas is shown, by adding to the gas, contained over water, one half of its rolume of chlorine, which, in the course of an hour or two, condenses the olefiant gas into an oily looking liquid (chloride of hydrocarbon.) After the mixture, the gases must be screened from the light, otherwise the common carbureted hydrogen would also combine with the chlorine, while water and carbonic acid would make their appearance.

The oil employed for affording gas is the crudest and cheapest that can be bought; even the blubber and sediment of whale oil are employed with advantage. After all, however, coal is so much cheaper, and the gas produced from it is now so well purified, that oil and rosin are very little used in gas apparatus.

-Appuralus for Coal Gas._-Coal gas, as it issues from the retort, cannot be directly employel for illumination; for it contains vapors of tar and coal oil, as also steam impregnated with the carbonate, sulphite, and hydrosulphuret of ammonia. These rapors would readily condense in the pipes through which the gas must be dis. tributed, and would produce obstructions; they must therefore be so far remored by previous cuoling, as to be liable to occasion no troublesome condensation at ordinary temperatures. The crude coal gas contains moreover sulphureted hrdrogen, whose combustion for light would exhale an offensive sulphureous odor, that ought to be got rid of as much as possible. Carbonic acid and carbonic oxyde gases, generated at first from the decomposition of the steam by the ignited coal, enfeeble the illuminating power of the gas, and should be removed. The disengagement of gas in the retorts is never uniform, but varies with the degree of heat to which they are exposed; for which reason the gas must be receired in a gasometer, where it may experience uniform pressure, and be discharged uniformly into the pipes of distribution, in order to ensure a steady discharge of gas, and uniform intensity of light in the burners. A coal gas apparatus ought therefore to be su constructed as not only to generate the gas itself, but to fulfi] the above conditions. 
In $f i g .482$, such an apparatus is represented, where the various parts are shown con. nected with each other, in section.

$A$ is the furnace with its set of cylindrical or elliptical retorts, five in number. From each of these retorts, a tube $b$ proceeds perpendicularly upwards, and then by a curve 482

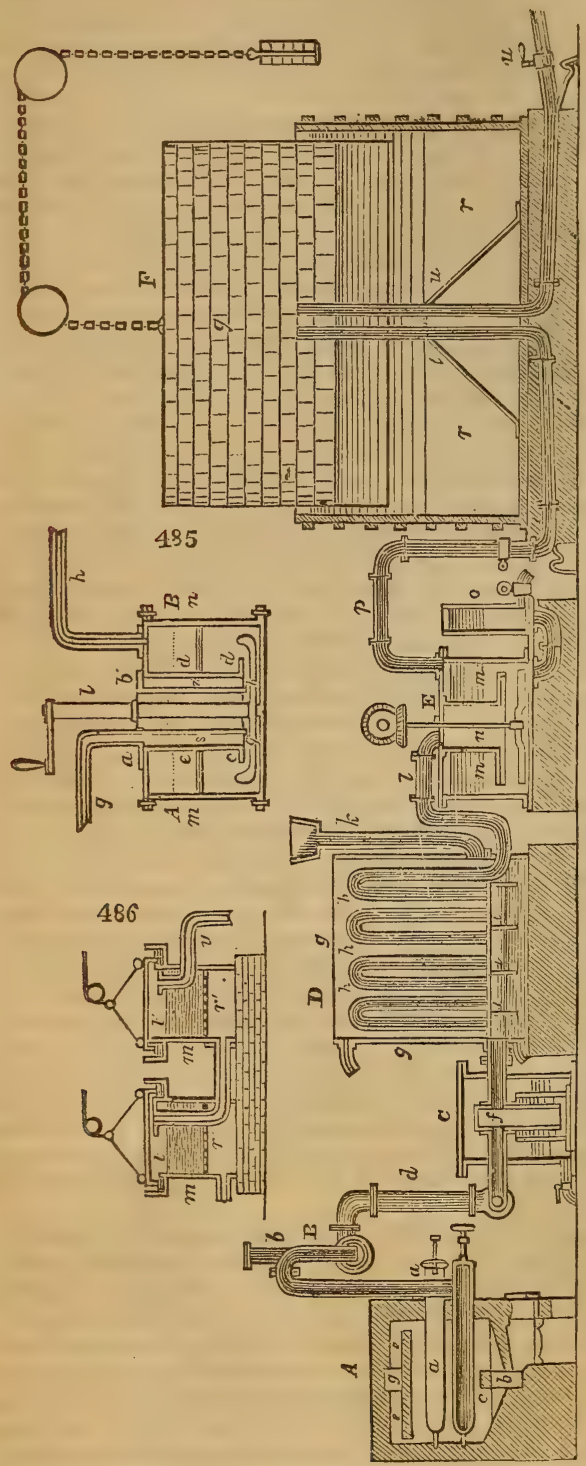
or saddle-tube, it turns down wards, where it enters a long horizontal cylinder under $\mathbf{B}$, shut at each end with a screw cap, and descends to beneath its middle, so as to dip about an inch into the water contained in it. From one end of this cylinder the tube $d$ passes downward, to connect itself with a horizontal tube which enters into the tar pit or cistern $\mathrm{c}$, by means of the verti cal branch $f$. This branch reaches to near the bottom of the cylindrical vessel, which sits on the sole of the tar cistern. From the other side of the vertical branch $f$, the main pipe proceeds to the condenser $\mathrm{D}$, and thence by the pipe $l$, into the purifier $\mathbf{E}$; from which the gas is immediately transmitted by the pipe $p$ into the gasometer $\mathbf{F}$.

The operation proceeds in the following way :-As soon as gas begins to be disengaged from the ignited retort, tar and ammoniacal liquor are deposited in the cylindrical receiver $\mathrm{B}$, and fill it up till the superfluity runs over by the pipe $d$, the level being constantly preseryed at the line shown in the figure. By the same tarry liquid, the orifices of the several pipes $b$, issuing from the retorts, are closed; whereby the gas in the pipe $d$ has its com. munication cut off with the gas in the retorts. Hence if one of the retorts be opened and emp. tied, it remains shut off from the rest of the apparatus. This insulation of the several retorts is the function of the pipe under $\mathbf{B}$, and therefore the recurved tube $b$ must be dipped as far under the surface of the tarry liquid, as to be in equilibrio with the pressure of the gas upon the water in the purifier. The tube $b$ is closed at top with a screw cap, which can be taken off at pleasure, to permit the interior to be cleansed.

Both by the overflow from the receiver-pipe $B$, and by subse-

quent condensation in the tube $d, \operatorname{tar}$ and ammoniacal liquor collect progressively in the cistern or pit under $\mathrm{c}$, by which mingled liquids the lower orifice of the vertical tube $f$ is closed, so that the gas cannot escape in to the empty space of this cistern. These liquids flow over the edges of the inner vessel when it is full, and may from time to time be drawn off by the stopcock at the bottom of the cistern.

Though the gas has, in its progress hitherto, deposited a good deal of its tarry and ammoniacal vapors, yet, in consequence of its high temperature, it still retains a considerable portion of them, which must be immediately abstracted, otherwise the tar 
would pollute the lime in the vessel $\mathbf{E}$, and interfere with its purification. On this account the gas should, at this period of the process, be cooled as much as possible, in order to condense these vapors, and to favor the action of the lime in the purifier $\mathrm{F}$, upon the sulphureted hydrogen, which is more energetic the lower the temperature of the gas. The cual gas passes, therefore, from the tube $f$ into the tube $h$ of the condenser $\mathbf{D}$, which is placed in an iron chest $g$ filled with water, and it deposites more tar and ammoniacal liquor in the under part of the cistern at $t, t$. When these liquids have risen to a certain level, they overflow into the tar-pit, as shown in the figure, to be drawn off by the stopcock as occasion may require.

The refrigerated gas is now conducted into the purifier $\mathrm{E}$, which is filled with milk of lime, made by mixing one part of slaked lime with 25 parts of water. The gas, as it enters by the pipe $l$, depresses the water in the wide cylinder $n$, thence passes under the perlorated disc in the under part of that cylinder, and rising up through innumerable small holes is distributed throughout the lime liquid in the vessel $m$. By contact with the lime on this extended surface, the gas is stripped of its sulphureted hydrogen and carbonic acid, which are condensed into the hydro-sulphuret and carbonate of lime; it now enters the gasumeter $F$ in a purified state, through the pipe $p t$, and occupies the space $q$. The gasometer, pressing with a small unbalanced force over the counterweight $s$, expels it through the main $u u$, in communication with the pipes of distribution through the buildings or streets to be illuminated.

The parts A B C D E and F, of which this apparatus consists, are essential constituents of every good coal-gas work. Their construction rests upon peculiar principles, is susceptible of certain modifications, and therefore deserves to be considered in detail.

The Retorls. - These are generally made of cast iron, though they have occasionally been made of baked clay, like common earthentware retorts. The original form was : cylinder, which was changed to an ellipse, with the long axis in a horizontal direction, then into the shape of the letter $\mathrm{D}$ with the straight line undermost, and lastly into a semi-cylinder, with its horizontai diameter 22 inches, and its vertical varying from 9 to 12. The kidney form was at one time preferred, but it has been little used of late.

The form of retort represented in fig. 483 has been found to yield the largest quantity

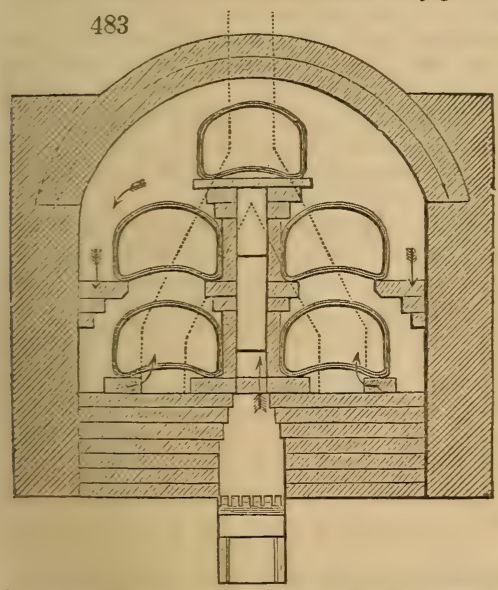
of good gas in the shortest time, and with the least quantity of firing. The length is $7 \frac{1}{2}$, and the transverse area, from one foot to a foot and a half square. The arrows show the direction of the flame and draught in this excellent bench of retorts, as mounted by Messrs. Barlow.

The charge of coals is most conveniently introduced in a tray of sheet iron, made somewhat like a grocer's scoop, adapted to the size of the retort, which is pushed home to its further end, inverted so as to turn out the contents, and then immediately withdrawn.

The duration of the process, or the time of completing a distillation, depends upon the nature of the coal and the form of the retort. With eylindrical retorts it cannot be finished in less than 6 hours, but with elliptical and semi-cylindrical retorts, it may be completed in 4 or 5 hours. If the distillation be continued in the formet for 8 hours, and in the latter for 6 , gas will continue to be obtained, but during the latter period of the operation, of indifferent quality.

The Receiver. - If the furnace contains only 2 or 3 retorts, a simple cylindrical vessel standing on the ground half filled with water, may serve as a receiver; into which the tube fiom the retort may be plunged. It should be provided with an overflow pipe for the tar and ammoniacal liquor. For a range of several retorts, a long horizontal cylinder is preferable, like that represented at $\mathrm{B}$ in fig. 484 . Its diameter is from 10 to 15 inches. This cylinder may be so constructed as to separate the tar from the ammoniacal liquor, by means of a syphon attached to one of its ends.

The Condenser. - The condenser, represented in fig. 482 , consists of a square chest $g$, made of wrought iron plates open at top, but having its bottom pierced with a row of holes, to receive a series of tubes. To these holes the upright four-inch tubes $h h$ are secured by flanges and screws, and they are connected in pairs at top by the curved or saddle tubes. The said bottom forms the cover of the chest $t, t$, which is divided by vertical iron partitions, into half as many compartments as there are tubes. 
These partition plates are left open at bottom, so as to place the liquids of each cumpartment in communication. Thereby the gas passes up and down the series of tubes, in proceeding from one compartment to another. The condensed liquids descend in:o the

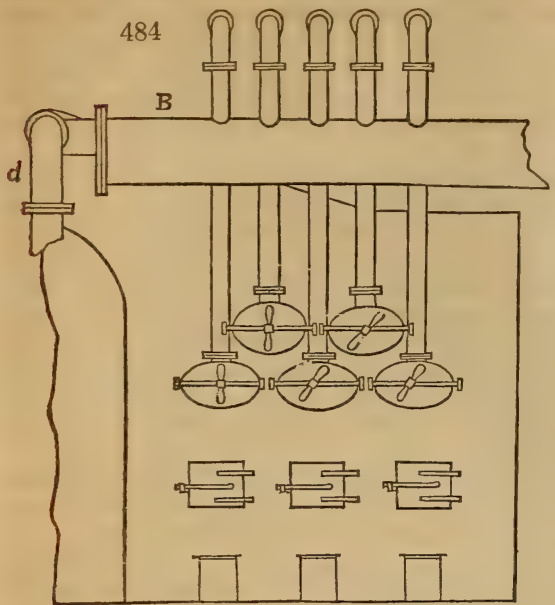
box $t, t$, and flow over into the tar cistern, when they rise above the level $t, t$. The tar may be drawn off from time to time by the stopcock. Through the tube $k$, cold water flows into the condenser chest, and the warm water passes away by a pipe at its upper edge.

The extent of surface which the gas requires for its refrigeration before it is admitted into the washing-lime apparatus, depends upon the emperature of the milk of lime, and the quantity of gas generated in a certain time.

It may be assumed as a determination sufficiently exact, that 10 square feet of surface of the condenser can cool a cubic foot of gas per minute to the temperature of the cooling water. For example, suppose a furnace or arch with 5 retorts of 150 pounds of coal each, to produce in 5 hours 3000 cubic feet of gas, or 10 cubic feet per minute, there would be required, for the cooling surface of the condenser, 100 square feet $=10 \times 10$. Suppose 100,000 cubic feet of gas to be produced in 24 hours, for which 8 or 9 such arches must be employed, the condensing surface must contain from 800 to 900 square feet.

The Purifier. - The apparatus represented in the preceding figure is composed of a cylindrical iron vessel, with an air-tight cover screwed upon it, through which the cylinder $n$ is also fixed air-tight. The bottom of this cylinder spreads out like the biim of a hat, forming a horizontal circular partition, which is pierced with holes. Through a stuffing box, in the cover of this interior cylinder, the vertical axis of the agitator passes, which is turned by wheel and pinion work, in order to stir up the lime from the buttom of the water in the purifier. The vessel o serves for introducing fresh milk of lime, as also for letting it off by a stopcock when it has become too foul for further use.

The quantity of lime should be proportioned to the quantity of sulphureted hydrogen and carbonic acid contained in the gas. Supposing that in good coal gas there is 5 per cent. of these gases, about one pound and a half of lime will be requisite for every hundred cubic feet of coal gas generated, which amounts to nearly one sixteenth of the weight of coal subjected to decomposition. This quantity of lime mixed with the proper quantity of water will form about a cubic foot of milk of lime. Consequently, the capacity of the purifier, that is, of the interior space filled with liquid, may be talien at four sevenths of a cubic foot for every hundred cubic feet of gas passing throngh it in one operation; or for 175 cubic feet of gas, one cubic foot of liquor. After every operation, that is, after every five or six hours, the purifier must be filled afresh. Suppose that in the course of one operation 20,000 cubic feet of gas pass through the machine, this should be able to contain $\frac{20,000}{175}=114$ cubic feet of milk of lime; whence its diareter should be seven feet, and the height of the liquid three feet. If the capacity of the vessel be less, the lime milk must be more frequently changed.

In some of the large gas works of London the pririfier has the following construction, whereby an uninterrupted influx and efflux of milk of lime takes place. Three single purifiers are so connected together, that the second vessel stands higher than the first, and the third than the second; so that the discharge tube of the superior vessel, placed somewhat below its cover, enters into the upper part of the next lower vessel; consequently, should the milk of lime in the third and uppermost vessel rise above its ordinary level, it will flow over into the second, and thence in the same way into the first; from which it is let off by the eduction pipe. A tube introduces the gas from the condenser into the first vessel, another tube does the same thing for the second vessel, \&c.; and the tube of the third vessel conducts the gas into the gasometer. Into the third vessel, milk of lime is constantly made to flow from a cistern upon a higher level. By this arrangement, the gas passing through the several vessels in proportion as it is purified, comes progressively into contact with purer milk of lime, whereby its purification becomes more complete. The agitator $c$, provided with two stirring paddles, is 
kept in continual rotation. The pressure which the gas has here to overcome is naturally three times as great as with a single purifier of like depth.

F Ł. 485 is a simple form of purifier, which has been found to answer well in practice. Through the cover of the vessel A $B$, the wide cylinder $e d$ is inserted, having its lower end pierced with numerous holes. Concentric with that cylinder is the narrower one $s z$, bound ahove with the flange $a b$, but open at top and bottom. The under edge $g h$ of this crlinder descends a few inches below the end $c d$ of the outer one. About the iniddle of the ressel the perforated shelf $m n$ is placed. The shaft of the agitator $l$, passes through a stuffing box upon the top of the vessel. The gas-pipe g, proceeding from the condenser, enters through the flange $a b$ in the outer cylinder, while the gas-pipe $h$ goes from the cover to the gasometer. A stopcock upon the side, whose orifice of discharge is somewhat higher than the under edge of the outer cylinder, serves to draw off the millk of lime. As the gas enters through the pipe $g$ into the space between the two cylinders, it displaces the liquor till it arrives at the holes in the under edge of the outer cylinder, through which, as well as under the edge, it flows, and then passes up through the apertures of the shelf $m n$ into the milk of lime chamber; the level of which is shown by the dotted line. The stirrer, $l$, should be turned by wheel work, though it is here shown as put in motion by a winch handle.

In order to judge of the degree of purity of the gas aftel its transmission through the lime machine, a slender syphon tube provided with a stopcock may have the one end inserted in its cover, and the other dipped into a vessel containing a solution of acetate of lead. Whenever the solution has been rendered turbid by the precipitation of sulphuret of lead, it should be renewed. The saturated and fetid milk of lime is evaporated in oblong cast-iron troughs placed in the ash-pit of the furnaces, and the dried lime is partly employed for luting the apparatus, and partly disposed of for a mortar or manure.

By this purifier, and others of similar construction, the gas in the preceding parts of the apparatus, as in the retorts and the condenser, suffers a pressure equal to a column of water about two feet high; and in the last described purifier even a greater pressure. This pressure is not disadrantageous, but is of use in two respects; 1 . it shows by a brisk jet of gas when the apparatus is not air-tight, and it prevents common air from entering into the retorts; 2. this compression of the gas favors the conclensation of the tar and ammoniacal liquor. The effect of such a degree of pressure in expanding the metal of the ignited retorts is quite inconsiderable, and may be neglected. Two contrivances have, however, been proposed for taking off this pressure in the purifier.

In fig. 486, $m \mathrm{~m}$ are two similar vessels of a round or rectangular form, furnished at their upper border with a groove filled with water, into which the under edge of the cover fits, so as to make the vessel air-tight. The cover is suspended by a cord or chain, which goes over a pulley, and may be raised or lowered at pleasure. The vessels themselves have perforated bottoms, $r r^{\prime}$, covered with wetted moss or hay sprinkled over with slaked and sifted quicklime. The gas passes through the loosely compacted matter of the first vessel, by entering between its two bottoms, rises into the upper space $t$, thence it proceeds to the second vessel, and, lastly, through the pipe $u$, into the gasometer.

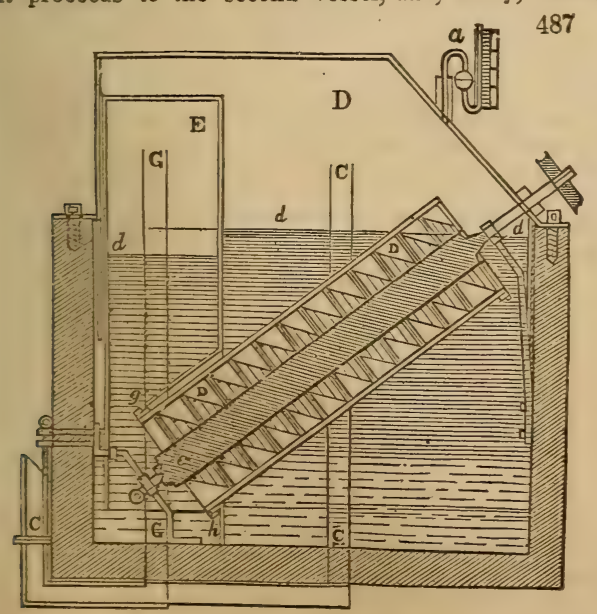

water level $d$; the upper cells of the warm scoop
This method, however, requires twice as much lime as the former, without increasing the purity of the gas.

The second method consists in compressing the gas by the action of an Archimedes serew, to such a degree, before it is admitted into the purifier, as that it may overcome the pressure of the column of water in that vessel. Fig. 487 exhibits this apparatus in section. $\mathrm{D} D$ is the Archimedes worm, the axis of which revolves at bottom upon the gudgeon $e$; it possesses a three-fold spiral, and is turned in the opposite direction to that in which it scoops the water. The cistern which contains it has an air-tight cover. The gas to be purified passes through the pipe $c$ into the space $D$, over the in the gas at this point, and 
carry it downwards, where it enters at $g$ into the cavity $\mathrm{E}$ of a second cistern. In order that the gas, after it escapes from the bottom of the worm, may not partially return through $g$ into the carity $\mathrm{D}$, an annular plate $g h$ is attached to its under edge, so as tc turn over it. The compressed gas is conducted from the cavity $\mathrm{E}$ through the pipe $\mathrm{G}$ into the purifying machine; $a$ is a manometer, to indicate the elastic tension of the gas in D. On the top of the worm a mechanism is fitted for keeping it in constant rotation.

A perfect purification of light-gas from sulphureted hydrogen, either by milk of lime or a solution of the green sulphate of iron, is attended with some difficulty, when carried so far as to cause no precipitation of sulphuret in acetate of lead, because such a degree of washing is required as is apt to diminish its illuminating power, by abstracting the vapor of the rich oily hrdrocarburet which it contains. Moreover, the coal gas obtained towards the end of the distillation contains some sulphuret of carbon, which affords sulphurous acid on being burned, and can be removed by no easy method hitherto known. The lime in the purifier disengages from the carbonate and hydro-sulphuret of ammonia carried over with the gas, especially when it has been imperfectly cooled in the condenser, a portion of ammoniacal gas, which, howeyer, is not injurious to its illuminating power. The best agent for purifying gas would be the pyrolignite of lead, were it not rather ex pensive, because it would save the trouble of stirring, and require a smaller and simpler apparatus.

The Gasometer.-The gasometer serves not merely as a magazine for receiring the gas when it is purified, and keeping it in store for use, but also for communicating to the gas in the act of burning such a uniform pressure as may secure a steady unflickering flame. It consists of two essential parts; 1 . of an under cistern, open at top and filled with water; and 2. of the upper floating eylinder or chest, which is a similar cistern inverted, and of somewhat smaller dimensions, called the gas-holder; see $\mathbf{F}$, fig. 482. The best form of this ressel is the round or crlindrical; both because under equal capacity it requires least surface of metal, and it is least liable to be warped by its own weight or accidents. Since a cylindrical body has the greatest capaciy with a given surface when its height is equal to its semi-diameter, its dimensions ought to be such that when elevated to the highest point in the water, the height may be equal to the radius of the base. For example, let the capacity of the gas-holder in cubic feet be $k$, the semi-diameter of its base be $x$, the height out of the water be $h$; $h$ is $=x=\sqrt[3]{\frac{k}{3 \cdot 14}}$. This height may be increased by one or two feet, according to its magnitude, to prevent the chance of any gas escaping beneath its under edge, when it is raised to its highest elevation in the water.

The size of the gasometer should be proportional to the quantity of gas to be con sumed in a certain time. If 120,000 cubic feet be required, for instance, in 10 hours for street illumination, and if the gas retorts be charged four times in $2 \frac{1}{4}$ hours, 30,000 feet of gas will be generated in 6 hours. Hence the gasometer should have a cafracity of at least 70,000 cubic feet, supposing the remaining 50,000 cubic feet to be produced during the period of consumption. If the gasometer has a smaller capacity, it must be supplied from a greater number of retorts during the lighting period, which is not advantageous, as the first heating of the supernumerary retorts is wasteful of fuel. Some engineers consider that a capacity of 30,000 cubic feet is the largest which can with propriety be given to a gasometer; in which case they make its diameter 42 feet, and its height 23. When the dimensions are greater, the sheet iron must be thicker and more expensive; and the hollow cylinder must be fortified by strong internal cross braces.

The water cistern is usually constructed in this country with cast-iron plates bolted together, and made tight with rust-cement.

In cases where the weight of water required to fill such a cistern might be inconvenient to sustain, it may be made in the form represented in fig. 188 ; which, however, will cost nearly $t$ wice as much. Parallel with the side of the cistern, a second cylinder $c$, of the same shape, but somewhat smaller, is fixed in an inverted position to the bottom of the first, so as to leave an annular space в в between them, which is filled with water, and in which the floating gasometer A plays up and down. The water must stand above the cover of the inverted cylinder. $\quad a$ and $b$ are the pipes for leading the gas in and out. Through an opening in the masonry upon which the gasometer apparatus rests, the space $\mathrm{c}$ may be entered, in order to make any requisite repairs.

The water cistern may also be sunk in the ground, and the sides made tight with hydraulic mortar, as is shown in fig. 488, and to make it answer with less water, a concentric cylindrical mass of masonry may be built at a distance of 2 or 3 inches within it.

Every large gasometer must be strengthened interiorly with cross iron rods, to stiffen both its top and bottom. The top is supported by rods stretching obliquely down to 
the sides, and to the under edge an iron ring is attached, consisting of curved cast iron bars bolted together; with which the oblique rods are connected by perpendicular ones.

489

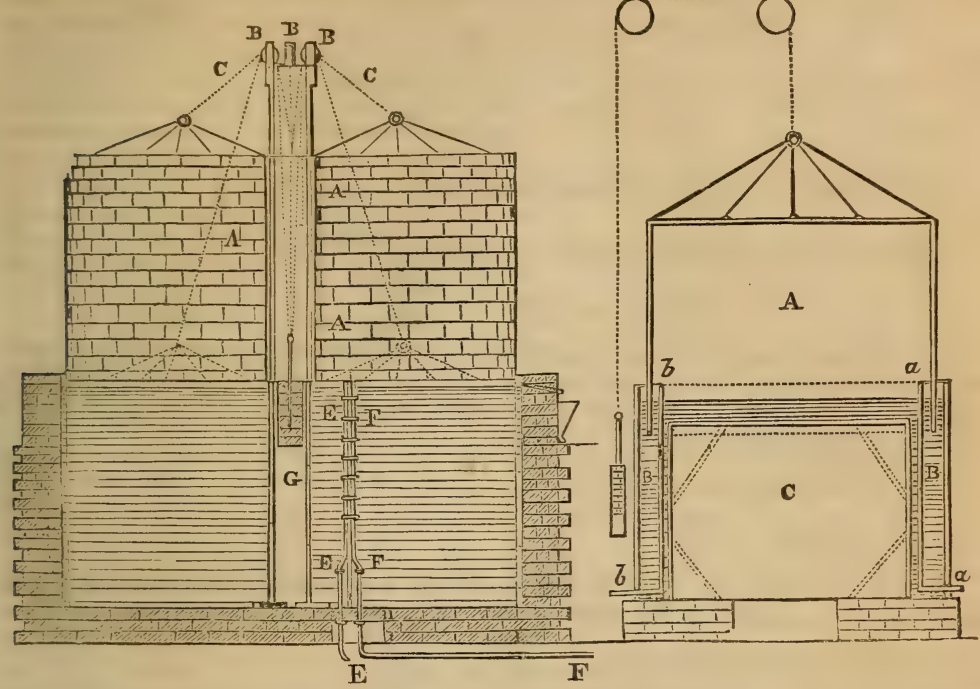

Other vertical rods stretch directly from the top to the bottom edge. Upon the periphery of the top, at the end of the rods, several rings are made fast, to which the gas-holder is suspended, by means of a common chain which runs over a pulley at the centre. Upon the other end of the chain there is a counterpoise, which takes off the greater part of the weight of the gas-holder, leaving only so much as is requisite for the expulsion of the gas. The inner and outer surfaces of the gas-holder should be a few times rubbed orer with hot tar, at a few days' interval between each application. The pulley must be made fast to a strong frame.

If the water cistern be formed with masonry, the suspension of the gas-holder may be made in the fullowing way. A A, fig. 489, is a hollow cylinder of cast iron, standing up through the inidule of the gasometer, and which is provided at either end with another small hollow cylinder G, open at both ends and passing through the top, with its axis placed in the axis of the gas-holder. In the hollow cylinder $\mathrm{G}$, the counterweight moves up and down, with its chain passing over the three pulleys B, B, B, as shown in fig. 489. E $F$ are the gas pipes made fast to a vertical iron rod. Should the gasometer be made to work without a counterweight, as we shall presently see, the central cylinder A A, serves as a vertical guide.

In proportici as the gas-holder sinks in the water of the cistern, it loses so much of its weight, as is equal to the weight of the water displaced by the sides of the sinking ressel; so that the gas-holder when entirely immersed, exercises the least pressure upon the gas, and when entirely out of the water, it exercises the greatest pressure. In order to counteract this inequality of pressure, which would occasion an unequal velocity in the effux of the gas, and of course an unequal intensity of light in its flame, the weight of the chain upon which the gas-holder hangs is so adjusted as to be equal, throughout the length of its motion, to one half of the weight which the gas holder loses by immersion. In this case, the weight which it loses by sinking into the water, is replaced by the portion of the chain which, passing the pulley, and hanging over, balances so much of the chain upon the side of the counterweight; and the weight which it gains by rising out of the water, is counterpoised by the links of the chain which, passing over the pulley, add to the amount of the counterweight. The pressure which the gas-holder exercises upon the gas, or that with which it forees it through the first main pipe, is usuaily so regulated as to sustain a column of from one to two inches of water; so that the water will stand in the cistern from one to two inches higher within, than without the gas-holder. The following computation will place these particulars in a clear light.

Let the semi-diameter of the gas-holder, equal tc the vertical extent of its motion into and ont of the water, $=x$; let the weight of a foot square of the side of the gas-holder, including that of the strengthening bars and ring, which remain plunged under the water, be $=p_{\text {; }}$ then 
1. ihe weight of the gas-holder in its highest position $=3 p \pi x$;

2. the weight of the sides of the gas-holder which play in the water $=2 p \pi x$;

3. the cubic contents of the immersed portion of the gas-holder $=\frac{2 p \pi x}{400}$;

4. its loss of weight in water $=\frac{112}{400} p \pi x^{2}$;

5. the weight of the gas-holder in its lowest position $=$

$$
p \pi x^{2}\left(3-\frac{112}{400}\right)=2 \cdot 72 p \pi x^{2} ;
$$

6. the weight of $n$ inches, height of water $=\frac{56}{12} n \pi x^{2}$;

7. the amount of the counterweight $\left.=\pi x 2) 3 p-\frac{56 n}{12}\right)$;

8. the weight of the chain for the length $x=\frac{112}{800} p \pi x$.

If we reduce the weight of the gas-holder in its highest and lowest positions to the height of a stratum of water equal to the surface of its top, this height is that of the column of water which would press the gas within the gasometer, were no counterweight employed ; it consists as follows :-

9. for the highest position $=\frac{3 p}{56}$;

10. for the lowest $=\frac{2 \cdot 72 p}{50}$;

For the case, when the height of the gas-holder is different from its semi-diameter, let this height $=m x$; then the height of the water level is-

11. for the highest position $=p\left(\frac{1+2 m}{56}\right)$;

12. for the lowest $=p\left(\frac{1+1.72 m}{6}\right)$;

13. the counterweight $=\pi x 2\left(p(1+2 m)-\frac{56 n}{12}\right)$;

14. the weight of the equalizing chain $=\frac{112}{800} p \pi m \times 2$.

For example, let the diameter of the gas-holder be 30 feet, the height 15 (the contents in cubic feet will be 10,597$),=4$ pounds; then the counterweight for a height of an inch and a half of water pressure $=3532$ pounds; the weight of the chain for a length of 15 feet $=395$ pounds. Were no counterweight employed, so that the gas-holder pressed with its whole weight upon the gas, then the height of the equivalent column of water in its highest position $=2.56$ inches; and in its lowest, $2 \cdot 33$. The counterweight may hence be lessened at pleasure, if the height of the pressing water-column $n$ be increased. The weight of the equalizing or compensating portion of the chain remains the same. When $n=2$ inches, for instance, the counterweight $=1886$ pounds.

The velocity with which the gas passes along the mains for supplying the various jets of light, may be further regulated by opening the main-cock or slide-valve in a greater or less degree. Gasometers whose height is greater than their semi-diameter, are not only more costly in the construction, but require heavier counterweights and equilibration chains.

The above estimate is made on the supposition of the gas in the gas-holder being of the same specific gravity as the atmospherical air, which would be nearly true with regard to oil gas under the ordinary pressure. But coal gas, whose specific gravity may be taken on an average at about 0.5 , exercises a buoyancy upon the top of the gas-holder, which of course diminishes its absolute weight. Supposing the cubic foot of gas to be $=0.0364$ pounds, the buoyancy will be $=0.0364 \pi x^{3}$ pounds; a quantity which deserves to be taken into account for large gasometers. Hence,

15. the weight of the gas-holder in its highest position $=3 p \pi x^{2}-0 \cdot 1143 x^{3}$;

16. the counterweight $=\pi x 2\left(3 p-\frac{56 n}{12}\right)-0.1143 x 2$;

17. the weight of the chain for the length $x=\frac{112}{800} p \pi x^{2} \frac{0 \cdot 1143 x^{3}}{2}$;

18. The height of the water pressure for the highest position, without the counterweight $=\frac{3 p \pi-0 \cdot 1143 x}{56 \pi}$;

19. the same for the lowest position $=\frac{2 \cdot 72 p}{56}$ in feet 
The preceding values of $p$ and $x$ are,

$(16)=3147 ;(17)=203 ;(18)=2.44$ inches; $(19)=2.33$ inches.

The water columns in the highest and lowest situations of the gas-holder here differ about 0.1 of an inch, and this difference becomes still less when $p$ has a smaller value, for example, 3 pounds, or when the diameter of the gas-holder is still greater.

It would thus appear that for coal-gas gasometers, in which the height of the gas-holder does not exceed its semi-diameter, and especially when it has a considerable size, neither a compensation chain nor a counterweight is necessary. The only thing requisite, is to preserve the vertical motion of the gas-holder by a sufficient number of guide rods or pillars, placed either within the water cistern, or round about it. Should the pressure of the gas in the pipe proceeding from the gasometer, be less than in the gasometer itself, this may be regulated by the main valve. or by water valves of various kinds. Or a smal! intermediate regulating gasometer may be introduced between the great gas-holder, and the main pipe of distribution. With a diameter of 61 feet in the gas-holder, the pressure in the highest and lowest positions is the same.

The gasometers employed in storing up gas until required for use, occupy, upon the old plan, much space, and are attended with considerable expense in erecting. The water tank, whether sunk in the ground or raised, must be of equal dimensions with the gasometer, both in breadth and depth. The improved construction which we are about to describe, affords a means of reducing the depth of the tank, dispensing with the bridge of suspension, and of increasing at pleasure the capacity of the gasometer, upon a given base; thus rendering a small apparatus capable, if required, of holding a large quantity of gas, the first cost of which will be considerably less than even a small gasometer constructed upon the ordinary plan.

Mr. Tait, of Mile-End Road, the inventor, has, we believe, been for some years connected with gas establishments, and is therefore fully aware of the practical defects

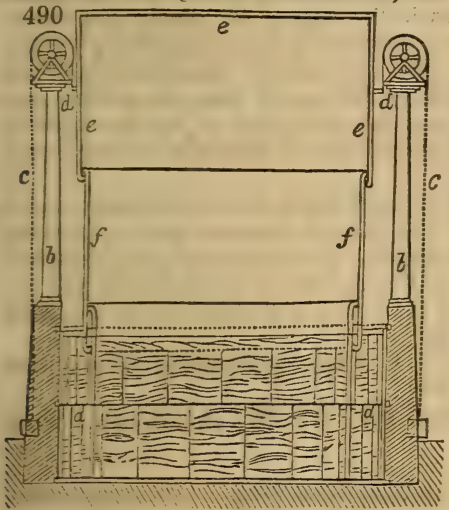
or advantages of the different constructions of gasometers now in use. Fig. 490 is a section of Mr. Tait's improved contrivance; $a \boldsymbol{a}$ is the tank, occupied with water, $b \quad b$ two iron columns, with pulley-wheels on the top, $c c$, chains attached to a ring of iron, $d d$, extending round the gasometer, which chains pass over the pulley-wheels, and are loaded at their extremities, for the purpose of balancing the weight of the materials of which the gasometer is composed.

The gasometer is formed by 2 or 3 cylinders, sliding one within the other, like the tubes of a telescope; $e, e, e$, is the first or outer cylinder, closed at the top, and having the ring of iron $d$, passing round it, by which the whole is suspended; $f f$, is the second cylinder, sliding freely within the first, and there may be a third and fourth within these if necessary.

When there is no gas in the apparatus, all the cylinders are slidden down, and remain one within the other jmmersed in the tank of water; but when the gas rises through the water pressing against the top of the gasometer, its buoyancy causes the cylinder $e$ to ascend. Round the lower edge of this cylinder a groove is formed by the turning in of the plate of iron, and as it rises, the edge takes hold of the top rim of the cylinder $f$, which is jverlapped for that purpose. The groove at the bottom of the cylinder fills itself with water as it ascends, and by the rim of the second cylinder falling into it, an air-tight hydraulic joint is produced.

Thus, several cylinders may be adapted to act in a small tank of water, by sliding one within the other, with lapped edges forming hydraulic joints, and by supporting the apparatus in the way shown, the centre of gravity will always be below the points of suspension. A gasometer may be made upon this plan of any diameter, as there will be no need of frame-work, or a bridge to support it; and the increasing weight of the apparatus, as the cylinders are raised one after the other, may be counterpoised by loading the ends of the chains $c c$.

The water in the gasometer need not be renewed; but merely so much of it as evaporates or leaks out, is to be replaced. Indeed, the surface of the water in the cistern gets covered with a stratum of coal oil, a few inches deep, which prevents its evaporation, and allows the gas to be saturated with this volatile substance, so as to increase its illaminating powers.

The gasometer may be separated from the purifier by an intermediate vessel, such os is represented fig. 491, with which the two gas pipes are connected. A is the 
cylndrical vessel of cast iron $a$, the end of the gas pipe which comes from the purifier,

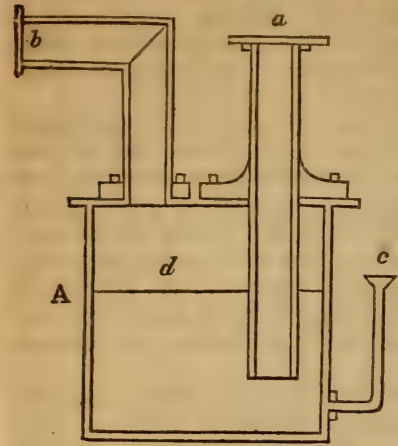
immersed a few inches deep into the liquid with which the vessel is about two thirds filled; $b$ is the gas-pipe which leads into the gasometer; $c$ is a perpendicular tube, piaced over the bottom of the vessel, and reaching to within one third of the top, through which the liquid is introdueed

491 into the vessel, and through which it escapes when it overflows the level $d$. In this tube the liquid stands towards the inner level higher, in pruportion to the pressure of the gas in the gasometer. The fluid which is condensed in the gas-pipe $b$, and in its prolongation from the gasometer, runs off into the vessel $A$; and therefore the latter must be laid so low that the said tube may have the requisite declivity. A straight stopeock may also be attached to the side over the bottom, to draw off any sediment.

II. Application of Light-Gas.

1. Distribution of the pipes.-The pressure by which the motion of the gas is maintained in the pipes, corresponds to a certain height of water in the cstern of the gasometer. From the magnitude of this pressure, and the quantity of gas which in a given time, as an hour, must be transmitted through a certain length of pipes, depends the width or the diameter that they should have, in order that the motion may not be retarded by the friction which the gas, like all other fluids, experiences in tubes, and thereby the gas might be prevented from issuing with the velocity required for the jets of flame. The velocity of the gas in the main pipe increases in the ratio of the square root of the pressing column of water upon the gasometer, and therefore by increasing this pressure, the gas may be forced more rapidly along the remoter and smaller ramifications of the pipes. Thus it happens, however, that the gas will be discharged from the orifices near the gasometer, with superfluous velocity. It is therefore advisable to lay the pipes in such a manner, that in every point of their length the velocity of discharge may be nearly equal. This may be nearly effected as follows :-

From experiment it appears that the magnitude of the friction, or the resistance which the air suffers in moving along the pipes, under a like primary pressure, that is, for equal initial velocity, varies with the square root of the length. The volume of gas discharged from the end of a pipe is directly proportional to the square of its diameter, and inversely as the square root of its length; or, calling the length $\mathrm{L}$, the diameter $\mathrm{D}$, the cubic feet of gas discharged in an hour $k$; then $k=\frac{D_{2}}{\sqrt{\mathrm{L}}} \cdot$ Experience likewise shows, that for a pipe 250 feet long, which transmits in an hour 200 cubic feet of gas, one inch is a sufficient diameter.

$$
\text { Consequently, } 200: k:: \frac{1}{144 \sqrt{250}}: \frac{\mathrm{D} 2}{\sqrt{\mathrm{L}}} ; \text { and } \mathrm{D}=\frac{\overline{V^{k}}}{\frac{\sqrt{\mathrm{L}}}{455,000}}
$$

From this formula the following table of proportions is calculated.

\begin{tabular}{|c|c|c|}
\hline Number of cubic feet per hour. & Length of pipe, in feet. & Diameter, in inches \\
\hline 50 & 100 & $0 \cdot 40$ \\
250 & 200 & $1 \cdot 00$ \\
500 & 600 & $1 \cdot 97$ \\
700 & 1000 & $2 \cdot 65$ \\
1000 & 1000 & $3 \cdot 16$ \\
1500 & 1000 & $3 \cdot 87$ \\
2000 & 1000 & $4 \cdot 47$ \\
2000 & 2000 & $5 \cdot 32$ \\
2000 & 4000 & $6 \cdot 33$ \\
2000 & 6000 & $7 \cdot 00$ \\
6000 & 1000 & $7 \cdot 75$ \\
6000 & 2000 & $9 \cdot 21$ \\
8000 & 1000 & $8 \cdot 95$ \\
8000 & 2000 & $16 \cdot 65$ \\
\hline
\end{tabular}

These dimensions are applicable to the case where the body of gas is transmitted through 
pipes without being let off in its way by burners, that is, to the mains which conduct the gas to the places where it is to be used. If the main sends off branches for burners, then for the same length the diameter may be reduced, or for like diameter the length may be greater. For example, if a pipe of 5.32 inches, which transmits 2000 cubic feet through a length of 2000 feet, gives off, in this space, 1000 cubic feet of gas; then the remainder of the pipe, having the same diameter, can continue to transmit the gas through a length of 2450 feet $=\left(\frac{450,000}{k}\right)^{2}$ with undiminished pressure for the purposes of lighting. Inversely, the diameter should be progressively reduced in proportion to the number of jets sent off in the length of the pipe.

Suppose, for instance, the gasometer to discharge 2000 cubic feet per hour, and the last point of the jets to be at a distance of 4000 feet. Suppose also that from the gasometer to the first point of lighting, the gas proceeds through 1000 feet of close pipe, the diameter of the pipe will be here 4.47 inches; in the second 1000 feet of length, suppose the pipe to give off, at equal distances, 1000 cubic feet of gas, the diameter in this length (calculated at 1500 cubic feet for 1000 feet long) $=3.87$ inches; in the third extent of 1000 feet, 600 cubic feet of gas will be given off, and the diameter (reckoning 700 cubic feet for 1000 feet long) will be 2.65 inches; in the fourth and last space (for 200 cubic feet in 1000 feet long) the pipe has a diameter of only an inch and a half, for which, in practice, a two-inch cast iron pipe is substituted; this being the smallest used in mains, into which branch pipes can be conveniently inserted.

The same relations hold with regard to branch pipes through which the gas is transmitted into buildings and other places to be illuminated. If such pipes : ake frequent angular turnings, whereby they retard the motion of the gas, they must be a third or a half larger in diameter. The smallest tubes of distribution are never less than one fourth of an inch in the bore.

Where, from one central gas work, a very great quantity of light is required in particular localities, there ought to be placed near these spots gasometers of distribution, which, being filled during the slack hours of the day, are ready to supply the burners at night, without making any considerable demand upon the original main pipe. Suppose the first main be required to supply 8000 cubic feet in the hour, for an illumination of 8 hours, at the distance of 2000 feet, a pipe $10 \frac{2}{3}$ inches in diameter would be necessary ; but if two or three gasometers of distribution, or station gasometers, be had recourse to, into which the gas during the course of 24 hours would flow through the same distance continuously from the central gas works, the quantity required per hour from them would be only one third of $8,000=2666^{\circ} 6$ cubic feet; consequently the diameter for such a pipe is only $6 \cdot 15$ inches.

All the principal as well as branch pipes, whose interior diameter exceeds an inch and a half, are made of cast iron from 6 to 8 feet long, with elbow pipes cast in them where it is necessary. These pipe lengths are shown in fig. 492, having at one end a wide socket $a$, and at the other a nozzle $b$, which fits the former. After inserting the one in the other in their proper horizontal position, a coil of hemp soaked with tar is driven home at the junction; then a luting of clay is applied at the mouth, within which a ring of lead is cast into the socket, which is driven tight home with a mallet and blunt chisel.
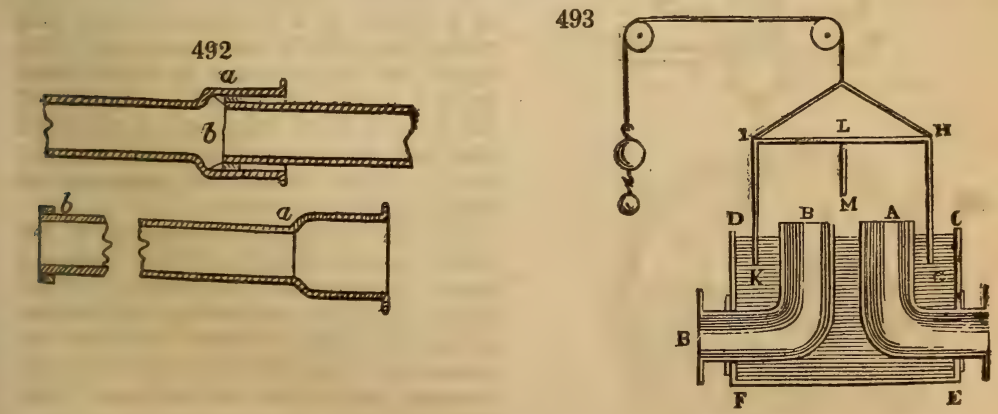

The pipes should be proved by a force pump before being received into the gas works: two or three lengths of them should be joined before laying them down, and they should be placed at least two feet below the surface, to prevent their being affected by changes of temperature, which would loosen the joints. The tubes for internal distribution, when of small size, are made of lead, copper, wrought iron, or tin. 
Instead of a stopcock for letting off the gas in regulated quantities from the gasometer, a pecuiarly formed water or mercurial valve is usually employed. Fig. 493 shows the mode of construction for a water trap or lute, and is, in fact, merely a gasometer in miniature. C D E F is a square cast iron vessel, in the one side of which a pipe $A$ is placed in communication with the gasometer, and in the other, one with the nain $\mathbf{B}$. The moveable cover or lid $\mathbf{H} \mathbf{G} \mathbf{I} \mathbf{K}$ has a partition, $\mathrm{I}, \mathrm{M}$, in its middle. If this cover be raised by its counterweight, the gas can pass without impediment from $\mathrm{A}$ to $\mathrm{B}$; but if the cunterweight be diminished so as to let the partition plate $\mathrm{L}$ M sink into the water, the communication of the two pipes is thereby interrupted. In this case the water-level stands in the compartment A so much lower than outside of it, and in the compartment $\mathbf{B}$, as is equivalent to the pressure in the gasometer; therefore the pipes $\mathbf{A}$ and $\mathbf{B}$ must project thus far above the water. In order to keep the water always at the same height, and to prevent it from flowing into the mouths of these pipes, the rim $\mathrm{c} D$ of the outer vessel stands somewhat lower than the orifices $A B$; and thence the vessel may be kept always full of water.

If a quicksilver valve be preferred, it may be constructed as shown in fig. 494. A B are

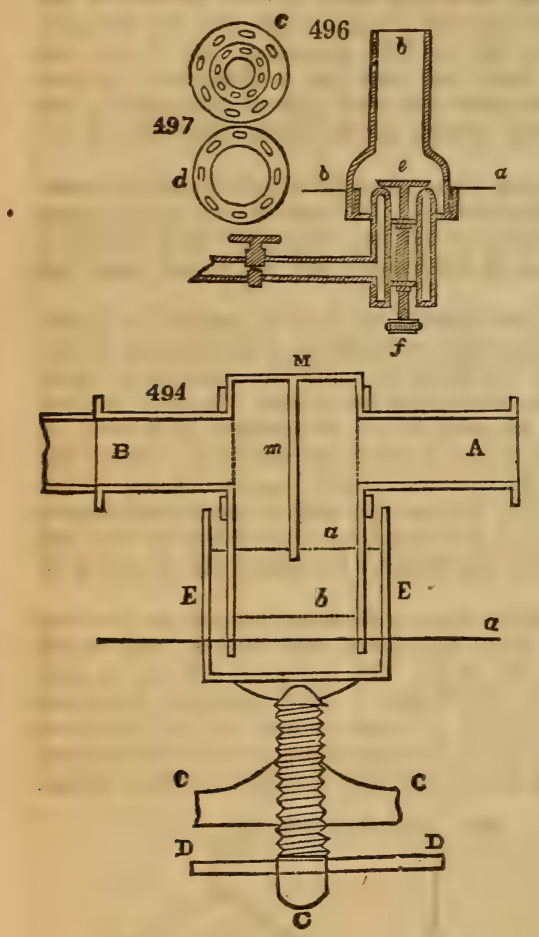
the terminations of the two gas pipes, which are made fast in the rectangular iron vessel M. E is an iron vessel of the same form, which is filled with quicksilvet up to the level $a$, and which, by means of the screw G, which presses against its bottom, and works in the fixed female screw c c, may be moved up or down, so that the vessel M may be immersed more or less into the quicksilver. The vessel $\mathrm{M}$ is furnished with a vertical partition $m$; the passage of the gas from $A$ to $B$ is therefore obstructed when this partition dips into the quicksilver, and from the gradual depression of the vessel $\mathbf{E}$ by its serew, the interval be tween the quicksilver and the lower edge of the partition, through which the gas must enter, may be enlarged at pleasure, whereby the pressure of the gas in B may be regulated to any degree. The transverse section of that interval is equal to the area of the pipe or rather greater; the breadth of the vessel $M$ from $A$ to $B$ amounts to the double of that space, and its length to the mere diameter of $\mathrm{A}$ or $\mathrm{B}$. The greatest height to which the partition $m$ can rise out of the quicksilver, is also equal to the above diameter, and in this case the line $a$ comes to the place of $b$. The vertical movement of the outer vessel $\mathfrak{E}$, is secured by a rectangular rim or hoop which surrounds it, and is made fast to the upper part of the vessel $\mathbf{m}$, within which guide it moves up and down. Instead of the lever D D, an index with a graduated plate may be employed to turn the screw, and to indicate exactly the magnitude in the opening of the valve.

In order to measure the quantity of gas which passes through a pipe for lightirg a factory, theatre, \&c., the gas-meter is em. ployed, of whose construction a safficiently precise idea may be formed from the consideration of fig. 495, which shows the instrument in a section perpendicular to its axis.

Within the cylindrical case $a$, there is a shorter cylinder $b \quad b$, shut at both ends, and moveable round an axis, which is

divided into four compartments, that communicate by the opening $d$, with the interval between this cylinder and the outer case The mode in which thi 
eylinder turns round its axis is as follows :-The end of the tube $c$, which is made fast to the side of the case, and by which the gas enters, carries a pivot or gudgeon, upon which the centre of its prop turns; the other end of the axis runs in the cover, which here forms the side of a superior open vessel, in which, upon the same axis, there is a toothed wheel. The vessel is so far filled with water, that the tube $c$ just rises above it, which position is secured by the level of the side vessel. When the gas enters through the tube $c$, by its pressure upon the partition $e$ ( fig. 495), it turns the cylinder from right to left upon its axis, till the exterior opening $d$ rises above the water, and the gas expands itself in the exterior space, whence il passes off through a tube at top. At every revolution, a certain volume of gas thus uoes through the cylinder, proportional to its known capaeity. The wheel on the axis works in other toothed wheels, whence, by means of an index upon a graduated disc or dial, placed at the top or in front of the gas-meter, the number of cubic feet of gas, which pass through this apparatus in a given time, is registered.

B. Employment of the gas for lighting.-The illuminating power of different gases burned in the same circumstances, is proportional, generally speaking, to their specific gravity, as this is to the quantity of carbon they hold in combination. The following table exhibits the different qualities of gases in respect to illumination.

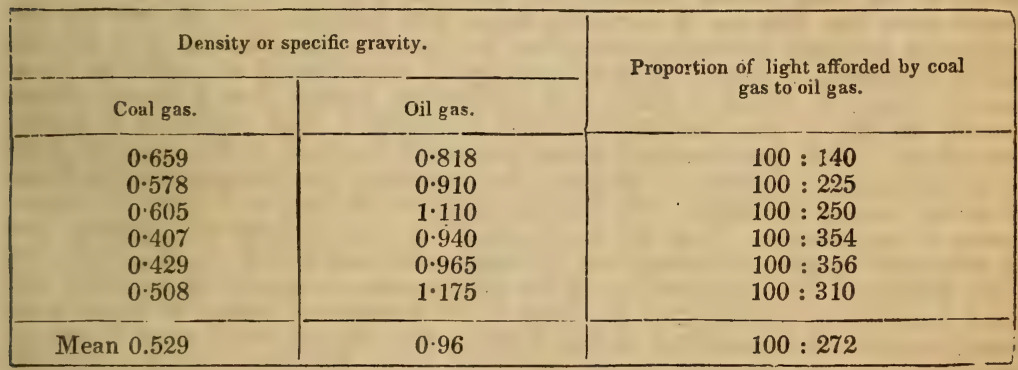

In the last three proportions, the coal gas was produced from coals of middle quality ; in the first three proportions, from coals of good quality; and therefore the middle proportion of 100 io 270 may be raken to represent the fair average upon the great scale. On comparing the gas from had coals, with good oil gas, the proportion may become 100 to 300 . Nay, coal gas of specific gravity $0 \cdot 4$, compared to oil gas of $1 \cdot 1$, gives the proportion of 1 to 4 . A mould tallow candle, of 6 in the pound, burning for an hour, is equivalent to half a cubic foot of ordinary coal gas, and to four tenths of a foot of good gas. The flame of the best argand lamp of Carcel, in which a steady supply of oil is maintained by pump-work, consuming 42 grammes $=649$ grains English in an hour, and equal in light to 9.38 such candles, is equivalent to 3.75 cubic feet of coal gas per hour. The sinumbra lamp, which consumes 50 grammes $=772$ grains English, of oil per hour, and gives the light of 8 of the above candles, is equivalent to the light emitted by 3.2 cubic feet of coal gas burning for an hour. A common argand lamp, equal to 4 candles, which consumes 30 grammes $=463$ grains English per hour, is represented by 1.6 cubic feet of gas burning during the same time. A common lamp, with a flat wick and glass chimney, whose light is equal to 1.13 tallow candles, and which consumes 11 grammes $=169.8$ grains English per hour, is represented by 0.452 of a cubic foot of gas burning for the same time.

Construclion of the Burners.-The mode of burning the gas as it issues from the jets has a great influence upon the quantity and quality of its light. When carbureted hydrogen gas is transmitted through ignited porcelain tubes, it is partially decomposed with a precipitation of some of its carbon, while the resulting gas burns with a feebler flame. Coal gas, when kındled at a small orifice in a tube, undergoes a like decomposition and precipitation. Its hydrogen, with a little of its carbon, burns whenever it cornes into contact with the atmospherical air, with a bluish colored flame; but the carhonaceous part not being so accendible, takes fire only when mixed with more air; therefure at a greater distance from the beak, and with a white light from the vivid ignition of its solid particles. Upon this principle pure hydrogen gas may be made to burn with a white instead of its usual blue flame, by dusting into it particles of lamp black, or by kindling it at the extremity of a tube containing finely pulverized rinc. The metallic particles become ignited, and impart their bright light to the pale blue flame. Even platinum wire and asbestos, when placed in the flame of hydrogen gas, eerve to whiten it. Hence it has been concluded, that the intensity of light which a gas is capable of affording is proportional to the quantity of solid particles which it 
contains, and can precipitate in the act of burning. Carbonic oxyde gas burns witt the feeblest light next to hydrogen, because it deposites no carbon in the act of burning Phosphureted hydrogen gives a brilliant light, because the phosphoric acid, into which its base is converted during the combustion, is a solid substance, capable of being ignited in the flame. Olefiant gas, as also the vapor of hydro-carbon oil, emits a more vivid light than common coal gas; for the first is composc 3 of two measures of hydrogen and two measures of the vapor of carbon condensed into one volume; while the last contains only one measure of the vaper of carbon in the same bulk, and eombined with the same proportion of hydrogen. Olefiant gas may therefore be expected to evolve a double quantity of carbon in its flame, which should emit a double light.

The illuminating power of the flame of coal gas is, on the contrary, impaired, when, by admixture with other species of gas which precipitate no carbon, its own ignited particles are diffused over a greater surface. This happens when it is mixed with hydrogen, earbonic oxyde, carbonic acid, and nitrogen gases, and the diminution of the light is proportional to the dilution of the coal gas.

In like manner the illuminating power of eoal gas is impaired, when it is consumed too rapidly to allow time for the separation and ignition of its carbonaceous matter; it burns, in this ease, without decomposition, and with a feeble blue flame. 1. This occurs when the light-gas is previously mixed with atmospherical air, because the combustion is thereby accelerated throughout the interior of the flame, so as to prevent the due separation of carbon. A large admixture of atmospherical air makes the flame entirely blue. 2. When it issues, with considerable velocity, from a minute orifice, whereby the gas, by expansion, gets intimately mixed with a large proportion of atmospherical air. If the jet be vertical, the bottom part of the flame is blue, and the more so the less carbon is contained in the gas. The same thing may be observed in the flame of tallow, wax, or oil lights. The burning wick acts the part of a retort, in decomposing the fatty matter. From the lower part of the wick tne gases and vapors of the fat issue with the greatest velocity, and are most freely mixed with the air; while the gases disengaged from the upper part of the wick compose the interior of the flame, and being momentarily protected from the action of the atmosphere, acquire the proper high temperature for the deposition of carbon, which is then diffused on the outer surface in an ignited state, and causes its characteristic white light. Henee with coal gas, the light increases in a certain ratio with the size of the flame as it issues from a larger orifice, because the intermixture of air becomes proportionately less. 3. If by any means too great a draught be given to the flame, its light becomes feebler by the rapidity and completeness with which the gas is burned, as when too tall a chimney is placed over an argand burner, see fig. 496. Fig. 497, $c$, is a view of the upper plate, upon which the glass chimney $b$ rests. The gas issues through the smaller openings of the inner ring, and forms a hollow cylindrical flame, upon the outside as well as the inside of which the atmospherical air acts. The illuminating power of this flame may be diminished at pleasure, according as more or less air is allowed to enter through the orifices beneath. With a very full draught the light almost vanishes, leaving only a dull blue flame of great heating power, like that of the blowpipe, corresponding to the perfect combustion of the gas without precipitation of its carbon. 4. On the other hand, too small a draught of air is equally prejudicial; not merely because a portion of the carbon thus escapes unconsumed in smoke, but also because the highest illuminating power of the flame is obtained only when the precipitated charcoal is heated to whiteness; a circumstance which requires a considerable draught of air. Hence the flame of dense oil gas, or of oil in a wick, burns with a yellow light without a chimney; but when it is increased in intensity by a chimney draught, it burns with a brilliant white flame.

From the consideration of the preceding facts, it is possible to give to coal gas its highest illuminating power. The burners are either simple beaks perforated with a smali round hole, or circles with a series of holes to form an argand flame, as shown in fig. 497, or two holes drilled obliquely, to make the flame cross, like a swallow's tail, or with a slit constituting the sheet of flame called a bat's wing, like most of the lamps in the streets of London. These burners are mounted with a stopcock for regulating the quantity of gas.

The height of the flame, which with like pressure depends upon the size of the orifice, and with like orifice upon the amount of pressure, the latter being modified by the stopcock, is, for simple jets in the open air, as follows :-

\begin{tabular}{|c|c|c|c|c|c|}
\hline In & $\stackrel{2}{55 \cdot 6}$ & $\begin{array}{c}3 \\
100\end{array}$ & $\begin{array}{c}4 \\
150\end{array}$ & $\begin{array}{c}5 \\
197 \cdot 8\end{array}$ & $\begin{array}{l}6 \text { inch } \\
247.4\end{array}$ \\
\hline umed & $60 \cdot 5$ & $101 \cdot 4$ & $126 \cdot 3$ & $143 \cdot 7$ & $182 \cdot 2$ \\
\hline nsumpti & 100 & & 121 & 150 & 150 \\
\hline
\end{tabular}

When the length exceeds five inches, nothing is gained in respect to light. For of 
gas the came statements will serve, only on account of its superior richness in carbon, is does nut bear so long a flame without smoke. Thus:

\begin{tabular}{|c|c|c|c|c|c|}
\hline Length of the flame & 1 & 2 & 3 & 4 & 5 inches. \\
\hline Intensity of the light & 22 & $63 \cdot 7$ & 96.5 & 141 & 178 \\
\hline Gas consumed & $-\quad 33 \cdot 1$ & $78 \cdot 5$ & 90 & 118 & 153 \\
\hline Light with equal consump & $\operatorname{tion} 100$ & 122 & 159 & 181 & 174 \\
\hline
\end{tabular}

The diameter of the orifice for single jets, or for several jets from the same beak, is one twenty-eighth of an inch for coal gas, and one forty-fifth for oil gas.

When several jets issue from the same burner, the light is improved by making all the flames unite into one. In this case the heat becomes greater, for the combined flame presents a smaller surface to be cooled, than the sum of the smaller flames. The advantage gained in this way may be in the ratio of 3 to 2 , or 50 per cent. In an argand burner the distances of the orifices for coal gas should be from $\frac{16}{100}$ to $\frac{18}{160}$ of an inch, and for oil gas $\frac{12}{100}$. If the argand ring has 10 orifices, the diameter of the central opening should be $=\frac{4}{1}$ of an inch; if 25 orifices, it should be one inch for coal gas; but for oil gas with 10 orifices, the central opening should have a diameter of half an inch, and for 20 orifices, one inch. The pin holes should be of equal size, otherwise the larger ones will cause smoke, as in an argand flame with an uneven wick. The glass chimney is not necessary to promote the combustion of an argand coal gas flame, but only to prevent it from flickering with the wind, and therefore it should be made so wide as to exercise little or no influence upon the draught. A narrc $\mathbb{x}$ chimney is necessary merely to prevent smoke, when a very strong light with a profusion of cas is desired. Oil gas burned in an argand beak requires a draught chimney, like a common argand lamp, on account of the large quantity of carbon to be consumed. The most suitable mode of regulating the degree of draught can be determined only by experiment, and the best construction hitherto ascertained is that represented in fig. 498 . Fig. 499 exhibits the

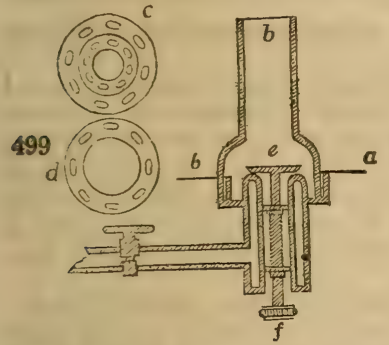
view from above, of the rim or ring $c$, upon which the chimney $b$ stands, and which surrounds the perforated beak. The ring is made of open fretwork, to permit the free passage of air upwards to strike the outside of the flame. The thin annular dsic $d$, 498 which is laid over its fellow disc $c$, in the bottom of the ehimney-holder, being turned a little one way or other, will allow more or less air to pass through for promoting, more or less, the draught or ventilation. The draught in the central tube of the burner may be regulated by the small dise $e$, whose diameter is somewhat smaller than that of the ring of the burner, and which, by turning the milled head $f$, of the screw, may be adjusted with the greatest nicety, so as to admit a greater or smaller body of air into the centre of the cylindrical flame.

In mounting gas-lights, and in estimating beforehand their illuminating effects, we must keep in mind the optical proposition, that the quantity of light is inversely as the square of the distance from the luminous body, and we must distribute the burners accordingly. When, for example, a gas-light placed at a distance of ten feet, is required for reading or writing to afford the same light as a candle placed at a distance of twe feet; squaring each distance, we have 100.4 ; therefore $1 \frac{00}{4}=25$, shows us that 25 such lights will be necessary at the distance of 10 feet.

Concerning portable gas-light, with the means of eondensing it, and carrying it from the gas works to the places where it is to be consumed, we need say nothing, as by the improvements lately made in the purification and distribution of coal gas, the former system has been superseded.

It is well known that light gas deteriorates very considerably by keeping, especially when exposed to water over an extensive surface; but even to a certain degree over oil, or in close vessels. An oil gas which when newly prepared has the specific gravity of 1.054, will give the light of a candle for an hour, by consuming 200 cubic inches; will, afte? two days, give the same light by consuming 215 cubic inches per hour; and after four- lays, by consuming 240 cubic inches in the like time. With coal gas the deterioration appears to be more rapid. When newly prepared, if it affords the light of a candle with a consumption of 400 cubic inches per hour, it will not give the same light after being kept two days, except with a consumption of 430 inches; and after four days, of 460. Oil gas three weeks old has become so much impaired in quality that 600 inches of it were required per hour to furnish the light of a candle. All light gas should be used therefore as soon as possible after it is properly purified.

Economical considerations. - The cost of gas-light depends upon so many local cir cumstances, that no estimate of it can be made of general application; only a few 
leading points may be stated. The coals required for heating the retorts used to const.ute one half of the quantity required for charging the retorts themselves. When five retorts are heated by one fire, the expenditure for fuel is only one third of that when each retort has a fire. The coke which remains in the retorts constitutes about 60 per cent. of the weight of the original coal; but the volume is increased by the coking in the proportion of 100 to 75. When the coke is used for heating the retorts, about one half of the whole is required. If we estimate the coke by its comparative heating power, it represents 65 per cent. of the coals consumed. One hundred pounds of good coal yield in distillation 10 pounds of ammoniacal liquor, from which sulphate or muriate of ammonia may be made, by saturation with sulphuric or muriatic acid, and evaporation. The liquor contains likewise some cyanide of ammonia, which may be converted into Prussian blue by the addition of sulphate of iron, after saturation with muriatic acid.

Two hundred pounds of coal afford about 17 pounds of tar. This contains in 100 pounds 26 pounds of coal oil, and 48 pounds of pitch. The tar is sometimes employed as a paint to preserve wood and walls from the influence of moisture, but its disagreeable smell limits its use. The coal oil, when rectified by distillation, is extensively employed for dissolving caoutchouc in making the varnish of waterproof cloth, and also for burning in a peculiar kind of lamps under the name of naptha. Oil of turpentine, however, is often sold and used for this purpose, by the same name. If the coal oil be mixed with its volume of water, and the mixture be made to boil in a kettle, the mingled vapors when passed through a perforated nozzle may be kindled, and employed as a powerful means of artificial heat. The water is not decomposed, but it serves by its vapor to expand the bulk of the volatile oil, and to make it thereby come into contact with a larger volume of atmospherical air, so as to burn without smoke, under a boiler or any other vessel. The pitch may be decomposed into a light-gas.

The relative cost of light from coal gas and oil gas may be estimated as one to six at least. Rosin gas is cheaper than oil gas. See Rosin.

I shall conclude this article with a summary of the comparative expense of different modes of illumination, and some statistical tables.

One pound of tallow will last 40 hours in six mould candles burned in succession, and costs $8 d$.; a gallon of oil, capable of affording the light of 15 candles, for 40 hours $\operatorname{costs}^{5} 5 \mathrm{~s}$; ; being therefore $\frac{1}{2}$ of the price of mould candles, and $\frac{6}{15}$ of the price of dips. The cost of wax is about $3 \frac{1}{2}$ times that of tallow; and coal gas, as sold at the rate of $9 \mathrm{~s}$. for 1000 cubic feet, will be one sixth the price of mould candles; for 500 cubic incher of coal gas give a light equal to the above candle for an hour; therefore $40 \times 500$. 20,000 cubic inches $=1157$ cubic feet, worth $1 \frac{1}{4} d$., which multiplied by 6 gives $7 \frac{1}{2} d$, the average price of mould candles per pound.

The author of the article Gas-light in the Encyclopædia Britannica, observes, in refes ence to the economy of this mode of illumination, that while the price of coal, in conss quence of the abundant and regular supply of that article, is liable to little fluctuation, the cost of wax, tallow, and oil, on account of the more precarious nature of the source from which they are obtained, varies exceedingly in different seasons. "Assuming that a pound of tallow candles, which last when burned in succession forty hours, costs nincpence" (seven-pence halfpenny is the average price), "that a gallon of oil, yielding the light of 600 candles for an hour, costs two shillings" (five shillings is the lowest price of a gallon of such oil as a gentleman would choose to burn in his lamp), " that the expense of the light from wax is three times as great as from tallow, and that a thousand cubic feet of coal gas cost nine shillings;" he concludes the relative cost to be for the same quantity of light,-from wax, 100; tallow, 25; oil, 5; and coal-gas, 3 . I conceive the estimate given above to be much nearer the truth; when referred to wax called 100 , it becomes, for tallow, $28 \cdot 6$; oil, $14 \cdot 3$; coal-gas, $4 \cdot 76$.

Gas-lighting has received a marvellous development in London. In the year 1834, the number of gas lamps in this city was 168,000 , which consumed daily about 4,200,000 cubic feet of gas. For the purpose of generating this gas, more than 200,000 chaldrons, or $10,800,000$ cubic feet of coals were required.

For the following valuable statistical details upon gas-light, my readers are indebted to Joseph Hedley, Esq., engineer, of the Alliance Gas Works, Dublin; a gentleman who to a sound knowledge of chemistry, joins such mechanical talent and indefatigable diligence, as qualify him to conduct with success any great undertakıng committed to his care. He has long endeavored to induce the directors of the London gas-works to employ a better coal, and generate a more richly carbureted gas, which in much smaller quantity would give as brilliant a light, without heating the apartments unpleasantly, as their highly hydrogenated gas now does. Were his judicious views adopted, coal gas would soon supersede oil, and even wax candles, for illuminating pri. vate mansions. 
Copy of a paper laid before a Committee of the House of Commons, showing not only the relative values of the Gases produced at the undermentioned places, but showing in like manner the relative economy of Gas, as produced at the different places, over candles. By Joseph Hedley, Esq.

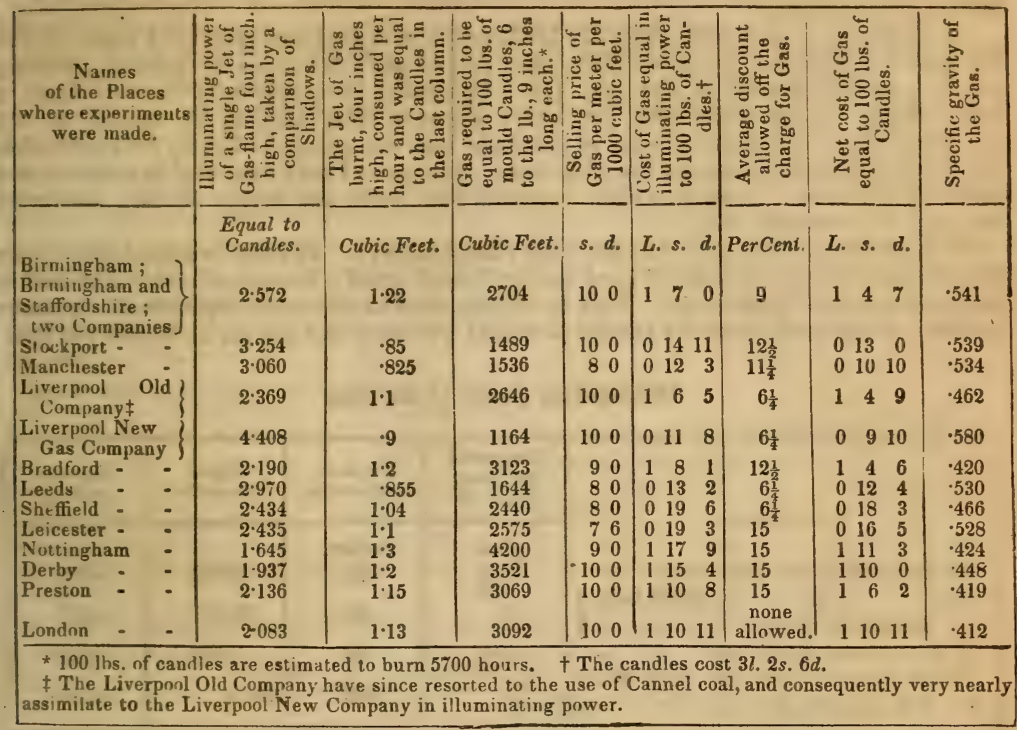

MEMORANDUM.-It will not fail to be observed that in deducing the comparative value between candles and gas by these experiments, the single jet (and in every instance, of course, it was the same) has been the medium. This, however, though decidedly the most correct way of making the comparative estimate of the illuminating power of the several gases, is highly disadvantageous in the economical comparison, inasmuch as gas burnt in a properly regulated argand burner, with its proper sized glass, air aperture, and sufficient number of holes, gives an advantage in favor of gas consumed in an argand, over a jet burner, of from 30 to 40 per cent. At the same time it must not be overlooked, that in many situations where great light is not required, it will be found far more economical to adopt the use of single jets, which, by means of swing brackets and light elegant shades, become splendid substitutes for candles, in banking establishments, offices, libraries, \&c. \&c.

Note.-In Glasgow, Edinburgh, Dindee, Perth, and the Scotch towns generally, the Parrot or Scotch Cannel coal is used; in illuminating power and specific gravity the gas produceu is equal to that from the best fescription of Cannel coal in England. The price per $10 \mathrm{vi}^{\mathrm{c}}$ cubic feet ranges about $9 \mathrm{~s}$., with from 5 to 30 per cent. off for discounts, leaving the net frice about $9 \mathrm{~s}$. to bis equal in the above table to $100 \mathrm{lbs}$. of candles.

Epitome of Experiments made in Gas produser from different qualities of Coal, and consumed in different kinds of Burners:

Tried at the Sheffield Gas Light Company's Works, and laid before a Committee of the House of Commons. By Joseph Hedley, Esq.

\begin{tabular}{|c|c|c|c|c|c|c|c|c|c|c|}
\hline $\begin{array}{l}\text { Date } \\
1835 .\end{array}$ & $\begin{array}{l}\text { Description } \\
\text { of Burner. }\end{array}$ & $\begin{array}{l}\text { Species of } \\
\text { Coal. }\end{array}$ & 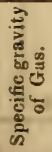 & 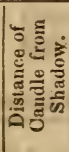 & 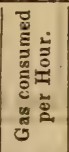 & 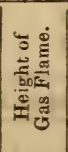 & 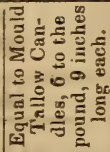 & 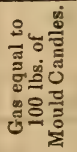 & 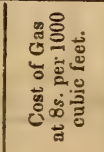 & 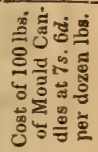 \\
\hline $\begin{array}{c}\text { May } \\
8 \\
9 \\
9\end{array}$ & $\begin{array}{l}\text { Single Jet } \\
\text { Ditto } \\
\text { Ditto }\end{array}$ & $\begin{array}{l}\text { Deep Pit } \\
\text { Murtormley } \\
\text { Canuel }\end{array}$ & $\begin{array}{l}.410 \\
.450 \\
.660\end{array}$ & $\begin{array}{c}\text { Inches } \\
75 \\
74 \\
614\end{array}$ & $\begin{array}{l}\text { Cubic } \\
\text { Feet. } \\
1 . \\
\cdot 95 \\
\cdot 7\end{array}$ & $\begin{array}{c}\text { Inches } \\
4 \\
4 \\
4\end{array}$ & $\begin{array}{l}\text { Candles. } \\
2 \cdot 36 \\
2 \cdot 434 \\
3 \cdot 54\end{array}$ & $\begin{array}{l}\text { Cubic } \\
\text { Feet. } \\
2415 \\
2224 \\
1127\end{array}$ & $\left(\begin{array}{ccc}L . & s . & d . \\
0 & 19 & 3 \frac{1}{2} \\
0 & 17 & 9 \frac{1}{2} \\
0 & 9 & 0\end{array}\right)$ & L. s. d. \\
\hline 8 & $\left\{\begin{array}{l}\text { A rgand } \\
14 \text { holes }\end{array}\right\}$ & Deep Pit & .410 & 34 & $3 \cdot 3$ & $3 \frac{1}{2}$ & $11 \cdot 53$ & 1631 & $\begin{array}{lll}0 & 13 & 0 \frac{1}{2}\end{array}$ & \\
\hline$\frac{9}{9}$ & \begin{tabular}{|l|} 
Ditto \\
Ditto
\end{tabular} & $\begin{array}{l}\text { Mortormley } \\
\text { Canuel }\end{array}$ & $\begin{array}{r}.450 \\
.660\end{array}$ & $\begin{array}{l}33 \\
29\end{array}$ & $\begin{array}{l}3 \cdot 1 \\
2 \cdot 6\end{array}$ & $\begin{array}{l}3 \frac{1}{2} \\
3 \frac{1}{2}\end{array}$ & $\begin{array}{l}12 \cdot 24 \\
15 \cdot 85\end{array}$ & $\begin{array}{r}1443 \\
935\end{array}$ & {$\left[\begin{array}{rrr}0 & 11 & 6 \frac{1}{2} \\
0 & 7 & 5 \frac{3}{4}\end{array}\right.$} & \\
\hline
\end{tabular}


Copy of Experiments made at the Alliance Gas Company's Works in Dublin, durng the past year 1837. By Joseph Hedley, Esq.

Results of experiments on the qualities of various coals for the production of gas; its value in illuminating power; produce of coke, and quality; and other particulars important in gas-making:-

1st Experiment, Saturday, May 27, 1837.-Deane coal (Cumberland), 2 cwts. of 112 lbs. each (or 224 lbs.) produced 970 cubic feet of gas; 4 bushels of coke of middling quality; specific gravity of the gas, 475 . Consumed in a single-jet burner, flame 4 inches high, $1 \frac{4}{10}$ ths cubic feet per hour; distance from shadow 76 inches, or 2.3 mould candles. ${ }^{10}$ Average quantity of gas made from the charge ( 6 hours) 4.33 cubic feet per $\mathrm{lb}$., or 9,700 cubic feet per ton of $20 \mathrm{cwts}$. Increase of coke over coal in measure, not quite 30 per cent. Lose in weight between coal, coke, and breize 56 lbs., converted into gas, tar, ammonia, \&ce.

$2 d$ Experiment, May 28.-Carlisle coal (Blenkinsopp). $224 \mathrm{lbs}$. produced 1010 cubic feet of gas, 4 bushels of coke of good quality though small; increase of coke over coal in measure not quite 30 per cent. Loss in weight, same as foregoing experiment. Average quantity of gas made from the charge ( 6 hours) 4.5 cubic feet per lb. or 10,080 per ton.

Illuminating power of the Gas.

\begin{tabular}{|c|c|c|c|c|}
\hline & $\begin{array}{l}\text { Consumed } \\
\text { per hour, } \\
\text { single jet. }\end{array}$ & $\begin{array}{l}\text { Distance } \\
\text { from candle. }\end{array}$ & $\begin{array}{l}\text { Equal } \\
\text { to candles. }\end{array}$ & $\begin{array}{l}\text { Specific } \\
\text { gravity. }\end{array}$ \\
\hline At the end of the first hour & feet. & $\begin{array}{l}\text { inches. } \\
\quad 70\end{array}$ & $2 \cdot 72$ & •475 \\
\hline $\begin{array}{c}\text { Ditto ditto } \\
\text { argand burner }\end{array}$ & 5 & 25 & $21 \cdot 33$ & $\cdot 475$ \\
\hline When charge nearly off & $1 \frac{4}{10}$ & 85 & 1.84 & $\cdot 442$ \\
\hline $\begin{array}{l}\text { When charge quite off, with } 20- \\
\text { hole argand burner }\end{array}$ & 9 & 100 & not 1 & $\cdot 266$ \\
\hline
\end{tabular}

$3 d$ Experiment, May 29.-Carlisle coal (Blenkinsopp). $112 \mathrm{lbs}$. produced 556 cubic feet of gas. Other products, loss of weight, \&c., same proportion as foregoing experiment. Average quantity of gas made from the charge ( 6 hours) 4.96 cubic feet per lb., or 11,120 per ton.

In this experiment the quantity of gas generated every hour was ascertained; the illuminating power, the specific gravity, and the quantity of gas consumed by the single jet with a flame 4 inches high, was tried at the end of each hour, with the respective gases generated at each hour; and the following is a table of results.

RESULTS.

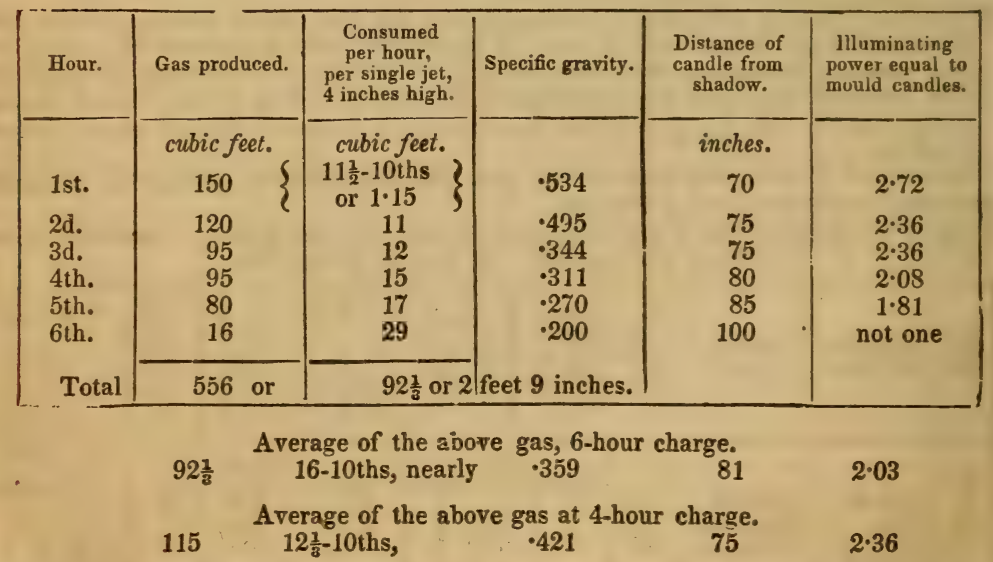

Production of gas in 6 hours 556 feet, or at the rate of 11,120 cubic feet per ton. Ditto in 4 hours 460 feet, or at the rate of $9,200 \ldots$ ditto 
The relative value of these productions of gas is as follows, viz. :

11,120 at $16-10$ ths per hour nearly (or 1.5916 accurately), and equal to 203 candles; the 11,120 feet would be equal to and last as long as 1597 candles, or $266 \frac{1}{6} \mathrm{lbs}$. of candles.

9200 at $12 \frac{1}{8}-10$ ths per hour (or 1.2375 accurately), and equal to 236 candles; the 9200 feet would be equal to 1949 candles, or $324 \frac{5}{6} \mathrm{lbs}$. candles.

Now $266 \frac{1}{6}$ lbs. of mould candles, at $7 s .6 d$. per ${ }^{6}$ dozen lbs., will cost $8 l .6 s .4 \frac{1}{2} d$., whilst $3245 \mathrm{lbs}$ of do. do. at 7 s. $6 d$. per do. do. 10l. 3 s.

Showing ${ }^{6}$ the value of 4 -hour charges over 6 -hour charges; and of 9,200 cubic feet over 11,120 cubic feet.

Note.-9500 cubi. feet of Wigan cannel coal gas are equal in illuminating power to 859 1-6th Ibs of candles, which at $7 s .6 d$. per dozen lbs. will cost $25 l$. $10 s .5 \frac{1}{2} d$. It is also found that any burner with superior gas will consume only about half the quantity it would do with common gas.

4th Experiment, May 30th.-Cannel and Cardift coai mixed $\frac{-}{2}$ and $\frac{1}{2}$, together $112 \mathrm{lbs}$., produced 460 feet of gas; 2 bushels of coke of good quality; increase of coke over coal in measure, about 30 per cent.; loss in weight, $41 \mathrm{lbs}$. ; coke weighed $71 \mathrm{lbs}$., no breize. Average quantity of gas made from the charge ( 4 hours), $4 \cdot 1$ cubic feet per lb., or $9 \cdot 200$ per ton.

Illuminating power.-At the end of the first hour.

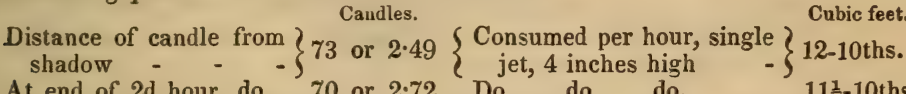

At end of $2 \mathrm{~d}$ hour, do. 70 or 2.72 Do. do. do. $11 \frac{1}{2}$-10ths.

At end of $3 \mathrm{~d}$ hour. This gas very indifferent.

Average of the three -70 or 2.72 Do. do. do. $11 \frac{1}{2}-10$ ths.

Specific gravity $3 \cdot 44$; 5 feet per hour, with a 20 -hole argand burner, equal to $14 \cdot 66$ candles.

5th Experiment, May 31st.-Carlisle coal, 112 lbs. produced 410 feet of gas; other products, same as in former experiments with this coal, but heat very low.

Illuminating power and produce of gas.

$410 \mathrm{ft} . \begin{cases}1 \text { st } & \text { hour } 120 \text { cubic feet } \\ 2 \mathrm{~d} & 100 \\ 3 \mathrm{~d} & 90 \\ 4 \text { th } & 100\end{cases}$

Average of this gas: specific gravity, 540 ; distance of candle from shadow, 55 inches, or 4.4 candles consumed per single jet, 9-10ths of a cubic foot per hour. 20-hole argand burner, 4 feet per hour, equal to $21 \cdot 33$ candles.

It is possible, from the superior quality of this gas, that a little of the cannel gas made for a particular purpose, may have got intermixed with it in the experimental gasholder and apparatus.

A variety of other experiments were tried on different qualities of coal, and mixtures of ditto, too tedious to insert here, though extremely valuable, and all tending to show the superior value of gas produced at short over long charges; and also showing the importance and value of coal producing gas of the highest illuminating power; among which the cannel coal procured in Lancashire, Yorkshire, and some other counties of England and Wales, and the Parrot or splent coal of Scotland, stand pre-eminent.

Note.-In all the foregoing experiments the same single-jet burner was used; its flame in all instances exactly 4 inches high.

The coal when drawn from the retort was slaked with water, and after allowing some short time for drying, was weighed.

A TABLE of the number of hours Gas is burnt in each month, quarter, and year.

\begin{tabular}{|c|c|c|c|c|c|c|c|c|c|c|c|c|c|c|c|c|c|c|}
\hline Time & $\frac{2}{3}$ & 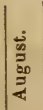 & ले & סूँ & 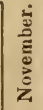 & 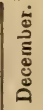 & 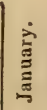 & 节 & & $\frac{\bar{a}}{4}$ & $\stackrel{\vec{E}}{\Sigma}$ & $\stackrel{\Xi}{\Xi}$ & 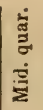 & 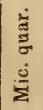 & 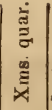 & 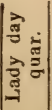 & 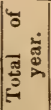 & \\
\hline & & & & & & & & & & & & & & & & & & \\
\hline m dus & - & - & 2 & 31 & 62 & 80 & 65 & 33 & 4 & - & - & - & - & 2 & 173 & 102 & 277 & $\ddot{0}$ \\
\hline 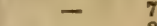 & - & 14 & 22 & 62 & 92 & 111 & 96 & 61 & 31 & 4 & - & _ & 4 & 36 & 265 & 188 & 493 & \\
\hline & $\overline{10}$ & 40 & 52 & 93 & 122 & 12 & 127 & 81 & 62 & 28 & 4 & & 32 & 92 & 357 & 278 & 759 & \\
\hline & 13 & 311 & 82 & 124 & 152 & 173 & 158 & 117 & 0 & & 2 & 8 & 9 & 166 & 449 & 368 & & \\
\hline & 44 & 102 & 112 & 155 & 182 & 04 & 39 & 145 & 124 & 8 & 6 & 38 & 180 & $1+$ & 541 & 458 & 14 & \\
\hline 11 & 75 & 133 & 142 & 186 & 212 & 235 & 220 & 173 & & 11 & & 0 & & & 633 & 548 & & \\
\hline- & 106 & 164 & 172 & 217 & 242 & 266 & 251 & 201 & 6 & 148 & 122 & 98 & 36 & 2 & 725 & 638 & 2173 & \\
\hline All night & $21 \%$ & 307 & 345 & 421 & 473 & 52 & 512 & 411 & 382 & & 248 & 195 & 73 & 869 & 1421 & 1305 & & \\
\hline om 4 & - & 16 & 48 & 80 & 110 & 137 & 1 & 98 & 71 & 28 & 2 & - & 30 & 64 & 327 & $\begin{array}{r}306 \\
306\end{array}$ & 727 & Iี \\
\hline- & 一 & - & 18 & 49 & 80 & 106 & 10 & 7 & 4 & & - & - & 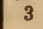 & 18 & 235 & 216 & 472 & \\
\hline 6 & 一 & - & - & 18 & 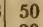 & 7 & $\pi$ & 42 & 9 & & - & - & & & 143 & 126 & 269 & \\
\hline & - & - & - & - & 20 & 44 & 44 & 14 & - & - & - & - & - & - & 64 & 58 & 122 & \& \\
\hline
\end{tabular}


Copy of a Paper submitted to a Cummittee of the flouse of Commons in the Session of 1857, of Kingland; and procured by actual Survey and

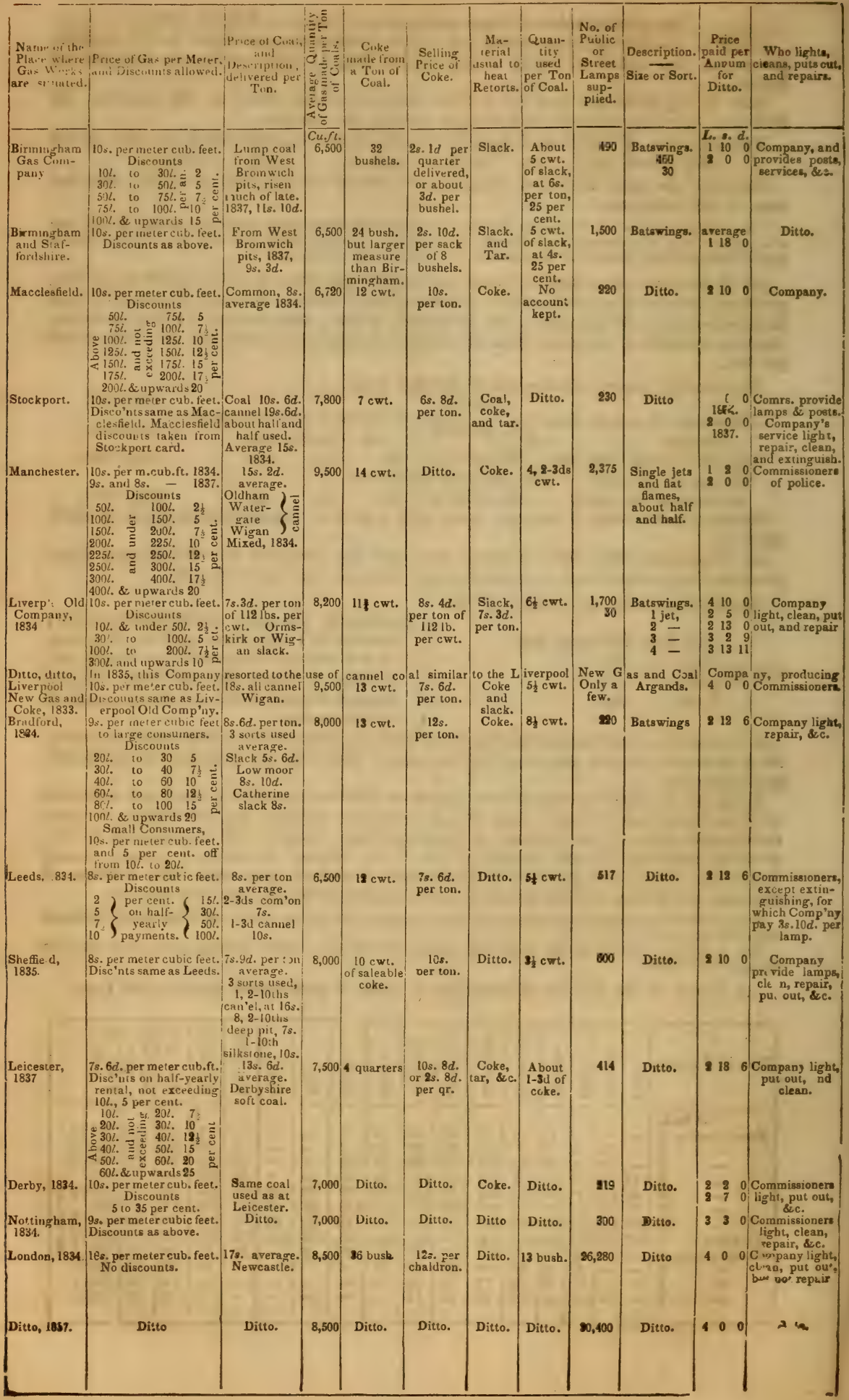


being a Synopsis of the proceedings of the undermentioned principal (ias-Light Establishments Experiments between the Years 1831 and 1837. By Joseph Hedley, Esq.

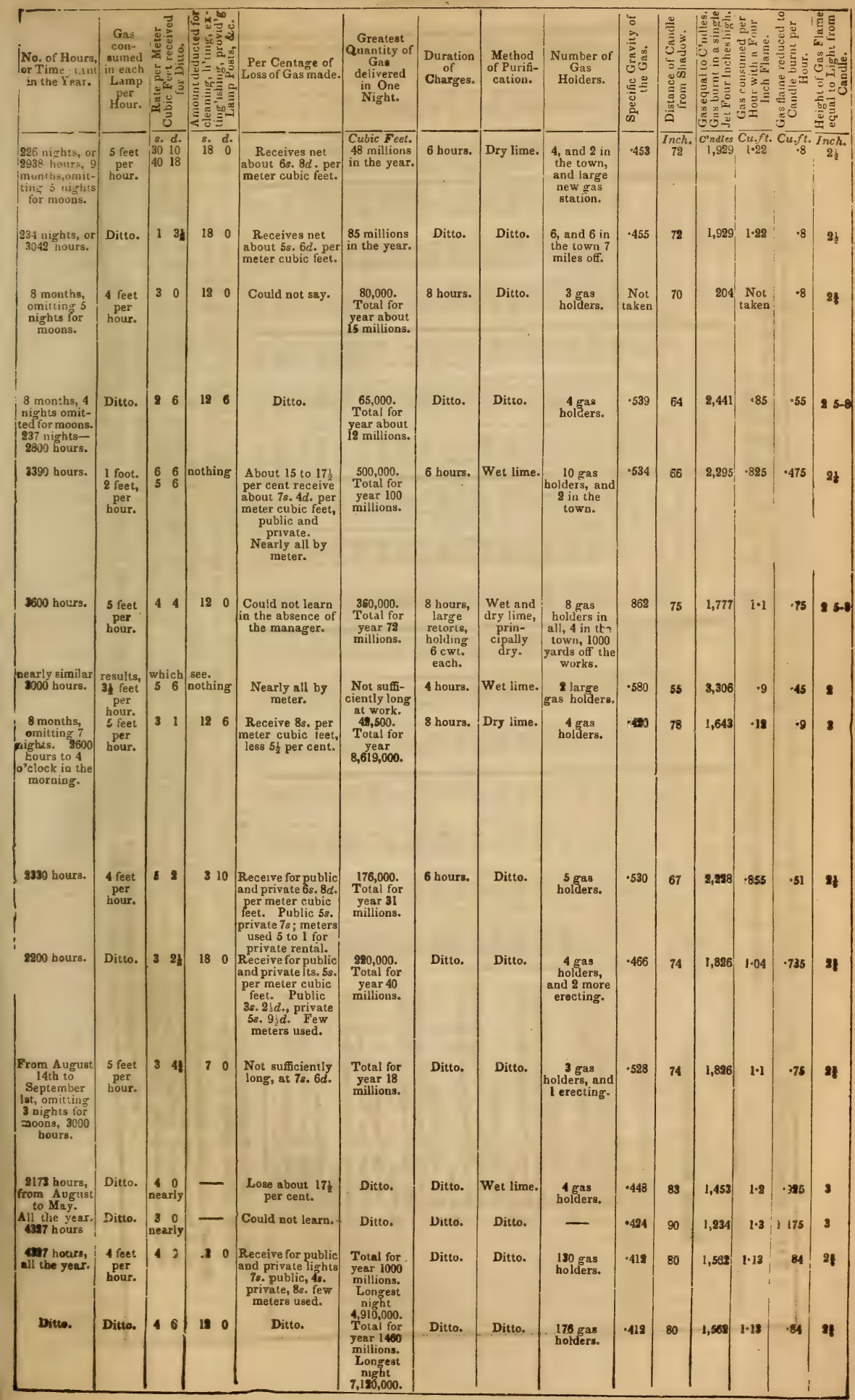




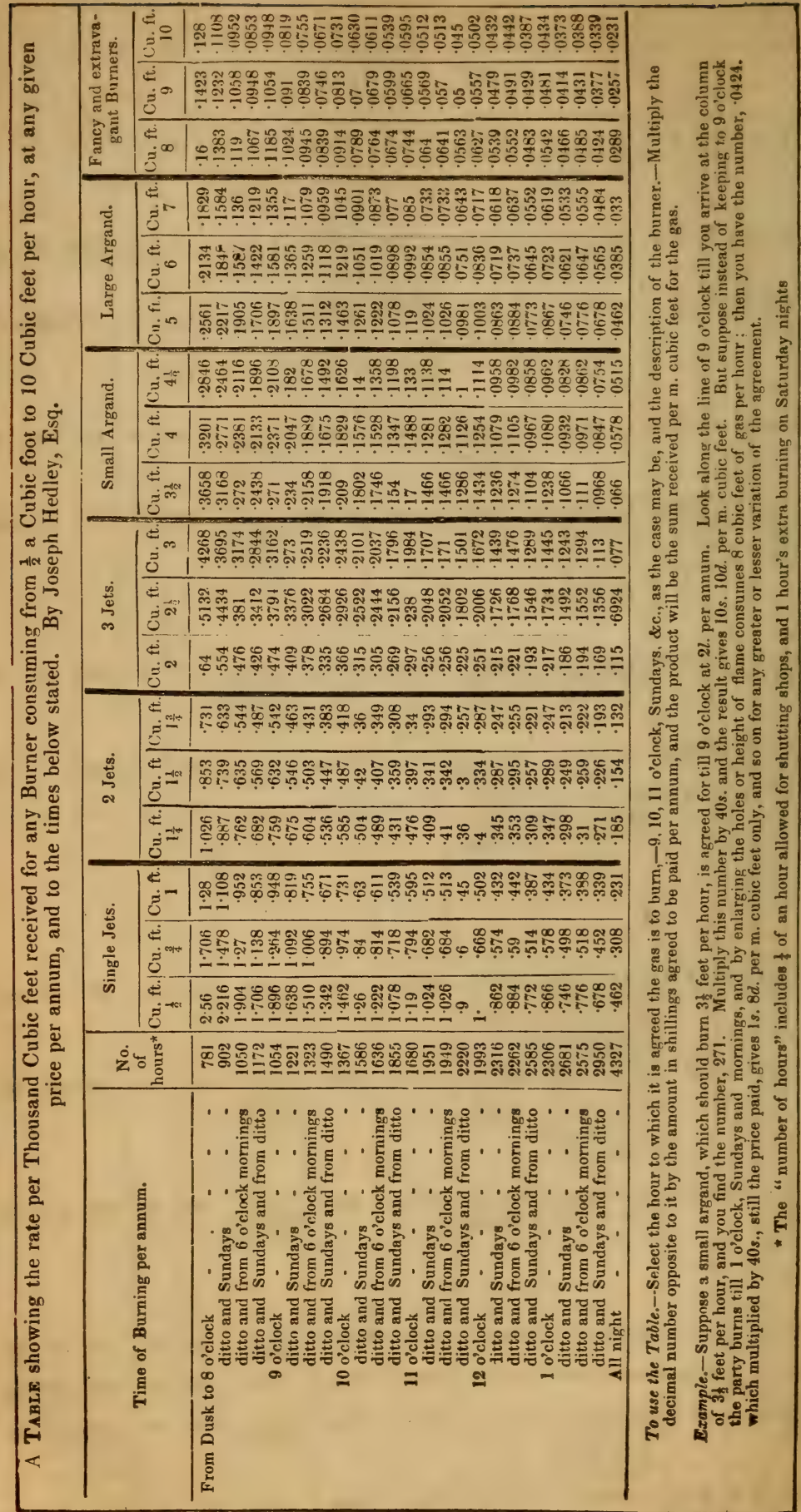


GENERAX SUMMARY.

For lighting London and its suburbs with gas, there are-

18 public gas works.

12 do. companies.

$2,800,000 l$. capital employed in works, pipes, tanks, gas-holders, apparatus.

$450,000 l$. yearly revenue derived.

180.000 tons of coals used in the year for making gas.

$1,460,000,000$ cubic feet of gas made in the year.

134,300 private burners supplied to about 40,000 consumers.

30,400 public or street do. N. B. about 2650 of these are in the city of London.

380 lamplighters employed.

176 gas-holders; several of them double ones, capable of storing 5,500,000 cubic feet.

890 tons of coals used in the retorts on the shortest day, in 24 hours.

$7,120,000$ cubic feet of gas used in longest night, say 24th December.

About 2500 persons are employed in the metropolis alone, in this branch of manufacture.

Between 1822 and 1827 the quantity nearly doubled itself, and that in 5 years.

Between 1827 and 1837 it doubled itself again.

Mr. Kirkham, engineer, obtained a patent, in June, 1837, for an improved mode of removing the carborflceous incrustation from the internal surfaces of gas retorts. He employs a jet or jets of heated atmospheric air, or other gases containing oxygen, which he impels with force into the interior of such retorts as have become incrusted in consequence of the decomposition of the coal. The retort is to be kept thoroughly red hot during the application of the proposed jets. An iron pipe, constructed with several flexi ble joints, leading from a blowing machine, is bent in such a way as to allow its nozzle end to be introduced within the retort, and directed to any point of its surface.

I should suppose that air, even at common temperatures, applied to a retort ignited to the pitch of making gas, would burn away the incrustations; but hot air will, no doubt, be more powerful.

GAS.HOLDER; a vessel for containing and preserving gas, of which various forms are described by chemical writers.

GASOMETER means properly a measurer of gas, though it is employed often to denote a recipient of gas of any kind. See the article GAS-LIGHT.

GAUZE WIRE CLOTH is a textile fabric, either plane or tweeled, made of brass, iron, or copper wire, of very various degrees of fineness and openness of texture. Its chief uses are for sieves, and safety-lamps.

GAY-LUSSITE is a white mineral of a vitreous fracture, which crystallizes in oblique rhomboidal prisms; specific gravity from 1.93 to 1.95 ; scratches gypsum, but is scratched by calespar; affords water by calcination; it consists of carbonic acid, 28.66 ; soda, 20.44 ; lime, $17 \cdot 70$; water, $32 \cdot 20$; clay, 1.00 . It is, in fact, by my analysis, a hydrated soda-carbonate of lime in atomic proportions. This mineral occurs abundantly in insulated crystals, disseminated through the bed of clay which covers the urao, or native sesquicarbonate of soda, at Lagunilla in Colombia.

GELATINE (Eng. and Fr.; Gallert, Leim, Germ.) is an animal product which is never found in the humors, but it may be obtained by boiling with water the soft and solid parts; as the muscles, the skin, the cartilages, bones, ligaments, tendons, and membranes. Isinglass consists almost entirely of gelatine. This substance is very soluble in boiling water; the solution forms a tremulous mass of jelly when it cools. Cold water has litle action upon gelatine. Alcohol and tannin (tannic acid, see GaLLNUTS) precipitate gelatine from its solution; the former by abstracting the water, the latter by combining with the substance itself into an insoluble compound, of the nature of leather. No other acid, except the tannic, and no alkali possesses the property of precipitating gelatine. But chlorine and certain salts render its solution more or less turbid ; as the nitrate and bi-chloride of mercury, the proto-chlorile of tin, and a few others. Sulphuric acid converts a solution of gelatine at a boiling heat into sugar. See LIGNEous Frbre. Gelatine consists of carbon, $47 \cdot 88$; hydrogen, $7 \cdot 91$; oxygen, $27 \cdot 21$. See Glue and Isinglass.

GEMS are precious stones, which, by their color, limpidity, lustre, brilliant polish, purity, and rarity, are sought after as objects of dress and decoration. They form the principal part of the crown jewels of kings, not only from their beauty, but because they are suppused to comprise the greatest value in the smallest bulk; for a diamond, no larger than a nut or an acorn, may be the representative sign of the territorial value of a whole country, the equivalent in commercial exchange of a hundred fortunes, acquired by severe toils and privations.

Among these beautiful minerals mankind have agreed in forming a select class, to which the title of gems or jewels has been appropriated; while the term precious stone is more particularly given to substances which often occur under a more considerable volume than fine stones ever do. 
Diamonds, sapphires, emeralds, rubies, topazes, hyacinths, and chrysoberyls, are reckoned the most valuable gems.

Cyrstalline quartz, pellucid, opalescent, or of various hues, amethyst, lapis lazuli, malachite, jasper, agate, \&c., are ranked in the much more numerous and inferior class of ornamental stones. These distinctions are not founded upon any strict philosophical principle, but are regulated by a conventional agreement, not very well defined; for it is impossible to subject these creatures of fashion and taste to the rigid subdivisions of science. We have only to consider the value currently attached to them, and take care not io confound two stones of the same color, but which may be very differently prized by the virtuoso.

Since it usually happens that the true gems are in a clit and polished state, or even set in gold or silver, we are thereby unable to apply to them the criteria of mineralogical and chemical science. The cutting of the stone has removed or masked its crystalline character, and circumstances rarely permit the phenomena of double or single refraction to be observed; while the test by the blowpipe is inadmissible. Hence the only scientific resources that remain are the trial by electricity, which is often inconclusive; the degree of hardness, a criterion requiring great experience in the person who employs it; and, lastly, the proof by specific gravity, unquestionably one of the surest means of distinguishing the really fine gems from ornamental stones of similar color. This proof can be applied only to a stone that is not set; but the richer gems are usually dismounted, when offered for sale.

This character of specific gravity may be applied by any person of common intelligence, with the aid of a small hydrostatic balance. If, for example, a stone of a fine crimson-red color be offered for sale, as an oriental ruby; the purchaser must ascertain If it be not a Siberian tourmaline, or ruby spinel. Supposing its weight in air to be 100 grains, if he finds it reduced to 69 grains, when weighed in water, he concludes that its bulk is equal to that of 31 grains of water, which is its loss of weight. Now, a real sapphire which weighs 100 grains in air, would have weighed 76.6 in water; a spinel ruby of 100 grains would have weighed 72.2 in water, and a Siberian tourmaline of 100 grains would have weighed only 69 grains in water. The quality of the stone in question is, therefore, determined beyond all dispute, and the purchaser may be thus protected from fraud.

The sard of the English jewellers (Sardoine, French) is a stone of the nature of agate, having an orange color more or less deep, and passing by insensible shades into yellow, reddish, and brown; whence it has been agreed to unite under this denomination all the agates whose color verges upon brown. It should be remarked, however, that the sard presents, in its interior and in the middle of its ground, concentric zones, or small nebulosities, which are not to be seen in the red cornelian, properly so called. The ancients certainly knew our sard, since they have left us a great many of them engraved, but they seem to have associated under the title sarda both the sardoine of the French, and our cornelians and calcedonies. Pliny says that the sarda came from the neighborhood of a city of that name in Lydia, and from the environs of Babylon. Among the engraved sards which exist in the collection of antiques in the Bibliothèque Royale of Paris, there is an Apollo remarkable for its fine color and great size. When the stone forms a part of the agate-onyx, it is called sardonyx. For further details upon Gems, and the art of cutting and engraving them, See LAPIDARY.

GEOGNOSY, means a knowledge of the structure of the earth; GEoLOGY, a description of the same. The discussion of this subject does not come within the province of this Dictionary.

GERM AN SILVER. See the latter end of the article_CoPPER.

GERMINATION (Eng. and Fr.; Das Keimen, Germ.) is the first sprouting of a seed after it is sown, or when, after steeping, it is spread upon the malt floor. See BEvR.

GIG MACHINES are rotatory drums, mounted with thistles or wire teeth for teazling eloth. See Woollen Mandfacture.

GILDING (Dorure, Fr.; Vergoldung, Germ.) is the art of coating surfaces with a thin film of gold. For a full discussion of this subject, See GoLD. Mr. Elkington, gilt toy maker, obtained a patent in June, 1836, for gilding copper, brass, \&c., by means of potash or soda combined with carbonic acid, and with a solution of gold. Dissolve, says he, $5 \mathrm{oz}$. troy of fine gold in $52 \mathrm{oz}$. avoirdupois of nitro-muriatic acid of the following proportions : viz. $21 \mathrm{oz}$. of pure nitric acid, of spec. grav. $1.45,17 \mathrm{oz}$. of pure muriatic acid, of spec. grav. $1 \cdot 15$; with $14 \mathrm{oz}$. of distilled water.

The gold being put into the mixture of acids and water, they are to be heated in a glass or other convenient vessel till the gold is dissolved; and it is usual to continue the application of heat after this is effected, until a reddish or yellowish vapor ceases to rise.

The clear liquid is to be carefully poured off from any sediment which generally appears and results from a small portion of silver, which is generally found in alloy with gold. The clear liquid is to be placed in a suitable vessel of stone, pottery ware is preferred. Add to the solution of gold 4 gallons of distilled water, and 20 pounds of bicar- 
bonate of potash of the best quality ; let the whole boil moderately for two hours, the mixture will then be ready for use.

The articles to be vilued having been first perfectly cleaned from scale or grease, they are to be suspended on wires, conveniently for a workman to dip them in the liquid, which is liept boiling. The time required for gilding any particular article will depend on circumstances, partly on the quantity of gold remaining in the liquid, and partly on the size and weight of the article; but a little practice will readily give sufficient guidance to the workman.

Supposing the artieles desired to be gilded be brass or copper buttons, or small articles for wilt toys, or crnaments of dress, $s^{*}$ h as ear-rings or bracelets, a considerable number of which may be strung on a hoop, - I bended piece of copper or brass wire, and dipyed into the ressel containing the boiling liqu: $d$ above described, and moved therein, the requisite giluing will be generally obtained in from a few seconds to a minute; this is when the liquid is in the condition above described, and depending on the quality of the gilaing desired; but if the liquid has been used some time, the quantity of gold will be lessened, which will vary the time of operating to produce a given effect, or the color required, all which will quickly be observed by the workman; and by noting the appearance of the articles from time to time, he will know when the desired object is obtained, though it is desirable to avoid as much as possible taking the articles out of the liquid.

When the operation is completed, the workman perfectly washes the articles so gilded with clean water; they may then be submitted to the usual process of coloring.

If the articles be cast figures of animals, or otherwise of considerable weight, compared with the articles above mentioned, the time required to perform the process will be greater.

In case it is desired to produce what is called a dead appearance, it may be performed by several processes: the one usually employed is to dead the articles in the process of cleaning, as practised by brass-founders and other trades; it is produced by an acid, prepared for that purpose, sold by the makers under the term "deading aquafortis," which is well understood.

It may also be produced by a weak solution of nitrate of mercury, applied to the articles previous to the gilding process, as is practised in the process of gilding with mercury, previous to spreading the amalgam, but generally a much weaker solution; or the articles having been gilded may be dipped in a solution of nitrate of mercury, and submitted to heat to expel the same, as is practised in the usual process of gilding.

It is desirable to remark, that much of the beauty of the result depends on the well cleaning of the articles, and it is better to clean them by the ordinary processes, and at once pass them into the liquid to be gilded. See GoIn, towards the end.

GIN, or Geneva, from Genievre (juniper), is a kind of ardent spirits manufactured in Holland, and hence called Hollands gin in this country, to distinguish it from British gin. The materials employed in the distilleries of Schiedam, are two parts of unmalted rye from Riga, weighing about 54 lbs. per bushel, and one part of malted bigg, weighing about 37 lbs. per bushel. The mash tun, which serves also as the fermenting tun, has a capacity of nearly 700 gallons, being about five feet in diameter at the mouth, rather narrower at the bottom, and $4 \frac{1}{2}$ feet deep; the stirring apparatus is an oblong rectangular iron grid, made fast to the end of a wooden pole. About a barnel, $=36$ gallons of water, at a temperature of from $162^{\circ}$ to $168^{\circ}$ (the former heat being best for the most highly dried rye), are put into the mash tun for every $1 \frac{1}{2} \mathrm{cwt}$. of meal, after which the malt is introduced and stirred, and lastly the rye is added. Powerful agitation is given to the magma till it becomes quite uniform; a process which a vigorous workman piques himself upon executing in the course of a few minutes. The mouth of the tun is immediately covered over with canvass, and further secured by a close wooden lid, to confine the heat; it is left in this state for two hours. The contents being then stirred up once more, the transparent spent wash of a preceding mashing is first added, and next as much cold water as will reduce the temperature of the whole to about $85^{\circ} \mathrm{F}$. The best Flanders yeast, which had been brought, for the sake of carriage, to a doughy consistence by pressure, is now introduced to the amount of one pound for every 100 gallons of the mashed materials.

The gravity of the fresh wort is usually from 33 to 38 lbs. per Dicas' hydrometer; and the fermentation is carried on from 48 to 60 hours, at the end of which time the attenuation is from 7 to $4 \mathrm{lbs}$., that is, the specific gravity of the supernatant wash is from 1.007 to 1.004 .

The distillers are induced by the scarcity of beer-barm in Holland, to skim off a quantity of the yeast from the fermenting tuns, and to sell it to the bakers, whereby they obstruct materially the production of spirit, though they probably improve its quality, by preventing its impregnation with yeasty particles; an unpleasant result which seldom 1.ils to take place in the whiskey distilleries of the United Kingdom. 
On the third day after the fermenting tun is set, the wash containing the grains is trane ferred to the still, and converted into low wines. To every 100 gallons of this liquor, two pounds of juniper berries, from 3 to 5 years old, being added along with about one quarter of a pound of salt, the whole are put into the low wine still, and the fine Hollands spurit is drawn off by a gentle and well-regulated heat, till the magma becomes exhausted; the first and the last products being mixed together; whereby a spirit, 2 to 3 per cent. above our hydrometer proof, is obtained, possessing the peculiar fine aroma of gin. The quantity of spirit varies from 18 to 21 gallons per quarter of grain; this large product being partly due to the employment of the spent wash of the preceding fermentation; an addition which contributes at the same time to "mprove the flavor.

For the above instructive details of he manufacture of genuine Hollands, I am indebted to Robert More, Esq., formerly of Underwood, distiller, who, after studying the ait at Schiedam, tried to introduce that spirit into general consumption in this country, but found the palates of our gin-drinkers too much corrupted to relish so pure a beverage.

GINNING, is the name of the operation by which the filaments of cotton are separated from the seeds. See Cotron Mandfacture.

GI.ANCE COAL, or anthracite, of which there are two varieties, the slaty and the conchoidal. See ANTHracite.

GLASS (Verre, Fr.; Glas, Germ.) is a transparent solid formed by the fusion of silicious and alkaline matter. It was known to the Phenicians, and constituted for a long time an exclusive manufacture of that people, in consequence of its ingredients, natron, sand, and fuel, abounding upon their coasts. It is probable that the more ancieni Egyptians were unacquainted with glass, for we find no mention of it in the writings of Moses. But according to Pliny and Strabo, the glass works of Sidon and Alexandria were famous in their times, and produced beautiful articles; which were cut, engraved, gilt, and stained of the most brilliant colors, in imitation of precious stones. The Romans employed glass for various purposes; and have left specimens in Herculaneum of window-glass, which must have been blown by methods analogous to the modern. The Phenician processes seem to have been learned by the Crusaders, and transferred to Venice in the 13th century, where they were long held secret, and formed a lucrative commercial monopoly. Soon after the middle of the 17 th century, Colbert enriched France with the blown mirror glass manufacture.

Chance undoubtedly had a principal share in the invention of this curious fabrication, but there were circumstances in the most ancient arts likely to lead to it; such as the fusing and vitrifying heats required for the formation of pottery, and for the extraction of metals from their ores. Pliny ascribes the origin of glass to the following accident. A merchant-ship laden with natron being driven upon the coast at the month of the river Belus, in tempestuous weather, the crew were compelled to cook their victuals ashore, and having placed lumps of the natron upon the sand, as supports to the kettles, found to their surprise masses of transparent stone among the cinders. The sand of this small stream of Galilee, which runs from the foot of Mount Carmel, was in consequence sup, posed to possess a peculiar virtue for making glass, and continued for ages to be sought after and exported to distant countries for this purpose.

Agricola, the oldest author, who has written technically upon glass, describes furnaces and processes closely resembling those employed at the present day. Neri, Kunckel, Henckel, Pott, Achard, and some other chemists, have since then composed treatises upon the subject; but Neri, Bosc, Antic, Loysel, and Allut, in the Encyclopedie Methodique, are the best of the elder authorities.

The window-glass manufacture was first begun in England in 1557, in Crutched Friars, London; and fine articles of flint-glass were scon afterwards made in the Savoy House, Strand. In 1635 the art received a great improvement from Sir Robert Mansell, by the use of coal fuel instead of wood. The first sheets of blown glass for looking glasses and coach windows were made in 1673 at Lambeth, by Venetian artisans employed under the patronage of the Duke of Buckingham.

The casting of mirror-plates was commenced in France about the year 1688, by Abraham Thevart; invention which gave rise soon afterwards to the establishment of the celebrated works of St. Gobin, which continued for nearly a century the sole place where this highly prized object of luxury was well made. In excellence and cheapness, the French mirror-plate has been, however, for some time rivalled by the English.

The analysis of modern chemists, which will be detailed in the course of this article, and the light thrown upon the manufacture of glass in general by the accurate means now possessed of purifying its several ingredients, would have brought the art to the highest state of perfection in this country, but for the vexatious interference and obstructions of our excise laws.

The researches of Berzelius having removed all dowbts concerning the acid character of silica, the general composition of glass presents now no difficulty of conception. This substance consists of one or more salts, which are silicates with bases of potash 
soda, lime, oxyde of iron, alumina, or oxyde of lead; in any of which compounds we can substitute one of these bases for another, provided that one alkaline base be left. Silica in its turn may be replaced by the boracic acid, without causing the glass to lose its principal characters.

Under the title glass are therefore comprehended various substances fusible at a high temperature, solid at ordinary temperatures, brilliant, generally more or less transparent, and always brittle. The following chemical distribution of glasses has been proposed.

1. Soluble glass; a simple silicate of potash or soda; or of both these alkalis.

2. Bohemian or crown glass; silicate of potash and lime.

3. Common window and mirror glass; silicate of soda and lime; sometimes also of potash.

4. Bottle glass; silicate of soda, lime, alumina, and iron.

5. Ordinary crystal glass; silicate of potash and lead.

6. Flint glass; silicate of potash and lead; richer in lead than the preceding.

7. Strass; silicate of potash and lead; still richer in lead.

8. Enamel; silicate and stannate or antimoniate of potash or soda and lead.

The glasses which contain several bases are liable to suffer different changes when they are melted or cooled slowly. The silica is divided among these bases, forming new compounds in definite proportions, which by crystallizing, separate from each other, so that the general mixture of the ingredients which constituted glass is destroyed. It becomes then very hard, fibrous, opaque, much less fusible, a better conductor of electricity and of heat; forming what Reaumur styled devitrified glass; and what is called after him, Reaumur's porcelain.

This altered glass can always be produced in a more or less perfect state, by melting the glass and allowing it to cnol very slowly; or merely by heating it to the softening pitch, and keeping it at this heat for some time. The process succeeds best with the most complex vitreous compounds, such as bottle glass; next with ordinary window glass ; and lastly with glass of potash and lead.

This property ought to be kept constantly in view in manufacturing glass. It shows why in making bottles we should fashion them as quickly as possible with the aid of a mould, and reheat them as seldom as may be absolutely necessary. If it be often heated and cooled, the glass loses its ductility, becomes refractory, and exhibits a multitude of stony granulations throughout its substance. When coarse glass is worked at the enameller's lamp, it is apt to change its nature in the same way, if the workman be not quick and expert at his business.

From these facis we perceive the importance of making a careful choice of the glass intended to be worked in considerable masses, such as the large object glasses of telescopes; as their annealing requires a very slow process of refrigeration, which is apt to cause devitrified specks and clouds. For such purposes, therefore, no other species of glass is well adapted except that with basis of potash and lead; or that with basis of potash and lime. These two form the best flint glass and crown glass; and they should be exclusively employed for the construction of the object glasses of achromatic telescopes.

GLASS-MAKING, general principles of. Glass may be defined in technical phrase. ology, to be a transparent homogeneous compound formed by the fusion of silica with oxydes of the alkaline, earthy, or common metals. It is usually colorless, and then resembles rock crystal, but is occasionally stained by accident or design with colored metallic oxydes. At common temperatures it is hard and brittle, in thick pieces; in thin plates or threads, flexible and elastic; sonorous when struck; fracture conchoidal, and of that peculiar lustre called vitreous; at a red heat, becoming soft, ductile, and plastic. Besides glass properly so called, other bodies are capable of entering into vitreous fusion, as phosphoric acid, boracic acid, arsenic acid, as also certain metallic oxydes, as of lead and antimony, and several chlorides; some of which are denominated glasses. Impure and opaque vitriform masses are called slags; such are the productions of blast iron furnaces and many metallurgic operations.

Silica, formerly styled the earth of flints, which constitutes the basis of all commercial glass, is infusible by itself in the strongest fire of our furnaces; but its vitreous fusion is easily effected by a competent addition of potash or soda, either alone or mixed with lime or litharge. The silica, which may be regarded as belonging to the class of acids, combines at the heat of fusion with these bases, into saline compounds; and hence glass may be viewed as a silicate of certain oxydes, in which the acid and the bases exist in equivalent proportions. Were these proportions, or the quantities of the bases which silica requires for its saturation at the melting point, exactly ascertained, we might readily determine beforehand the best proportions of materials for the glass manufacture. But as this is far from being the ease, and as it is, noreover, not improbable that the capacity of saturation of the silica varies with the temperature, and that the properties of glass also vary with the bases, we must, in the present state of our knowledge, regulate the proportions rather by practice than by theory, though the latter may throw an 
indirect light upon the subject. For example, a good colorless glass has been found by analysis to consist of 72 parts of silica, 13 parts of potash, and 10 parts of lime, in 95 parts. If we reduce these numbers to the equivalent ratios, we shall have the following results; taking the atomic weights as given by Berzelius.

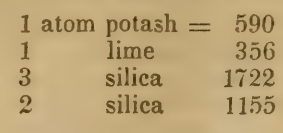

3823

$\left.\begin{array}{r}14.67 \\ 8 \cdot 84 \\ 42 \cdot 79 \\ 28 \cdot 70\end{array}\right\} 71 \cdot 49$

$95 \cdot 00$

This glass would therefore have been probably better compounded with the just atomic proportions, to which it nearly approaches, viz. 71.49 silica, 14.67 potash, and 8.84 lime, instead of those given above as its actual constituents.

The proportions in which silica unites with the alkaline and other oxydes are modified by the temperature as above stated; the lower the heat, the less silica will enter into the glass, and the more of the base will in general be required. If a glass which contains an excess of alkali be exposed to a much higher temperature than that of its formation, a portion of the base will be set free to act upon the materials of the earthen pot, or to be dissipated in fumes, until such a silicate remains as to constitute a permanent glass corresponding to that temperature. Hence the same mixture of vitrifiable materials will vield very different results, according to the heats in which it is fused and worked in the glass-house; and therefore the composition should a]ways be referrible to the going of the furnace. When a species of glass which at a high temperature formed a transparent combination with a considerable quantity of lime, is kept for some time in fusion at a lower temperature, a portion of the lime unites with the silica into another combination of a semi-vitreous or even of a stony aspect, so as to spoil the transparency of the glass altogether. There is probably a supersilicate and a sub-silicate formed in such cases; the latter being much the more fusible of the two compounds. The Reaumur's porcelain produced by exposing bottle glass to a red heat for 24 hours, is an example of this species of vitreous change, in which new affinities are exercised at a lower temperature. An excess of silica, caused by the volatilization of alkaline matter with too strong firing, will bring on similar appearances.

The specific gravity of glass varies from $2 \cdot 3$ to $3 \cdot 6$. That of least specific gravity consists of merely silica and potash fused together; that with lime is somewhat denser, and with oxyde of lead denser still. Plate glass made from silica, soda, and lime, has a specific gravity which varies from $2 \cdot 50$ to $2 \cdot 6$; erystal or flint glass from $3 \cdot 0$ to $3 \cdot 6$.

The power of glass to resist the action of water, alkalis, acids, air, and light, is in general the greater, the higher the temperature employed in its manufacture, the smaller the proportion of its fluxes, and the more exact the equiralent ratios of its constituents. When glass contains too much alkali, it is partially soluble in water. Most crystal glass is affected br haring water boiled in it for a considerable time; but crown glass being poorer in alliali, and containing no lead, resists that action much longer, and is therefore better adapted to chemical operations. The affinity of glass for water, or its hygrometric attraction, is also proportional to the quantity of alkali which it contains. In general also potash glass is more apt to become damp than soda glass, agreeably to the respective hygrometric properties of these two alkalis, and also to the smaller proportion of soda than of potash requisite to form glass.

Air and light operate upon glass probably by their oxydizing property. Bluish or greenish colored glasses become by exposure colorless, in consequence undoubtedly of the peroxydizement of the iron, to whose protoxyde they owe their tint; other glasses become purple red from the peroxydizement of the manganese. The glasses which contain lead, suffer another kind of change in the air, if sulphureted hydrogen be present; the oxyde of lead is converted into a sulphuret, with the effect of rendering the surface of the glass opaque and iridescent. The more lead is in the glass, the quicker does this iridescence supervene. By boiling concentrated sulphuric aeid in a glass vessel, or upon glass, we can ascertain its power of resisting ordinary menstrua. Good glass will remain smooth and transparent; bad glass will become rough and dim.

The brittleness of unannealed glass by change of temperature is sometimes very great. I have known a thick ressel to fly by vicissitudes of the atmosphere alone. This defect may be corrected by slowly heating the vessel in salt water or oil to the highest pitch consistent with the nature of these liquids, and letting it cool very slowly. Within the limits of that range of heat, it will, in consequence of this treatment, bear alternations of temperature without cracking as before.

It has been said that glass made from silica and alkalis alone will not resist the action of water, but that the addition of a little lime is necessary for this effect. In general 
100 parts of quartzose sand require 33 parts of dry carbonate of soda for their vitrification, and 45 parts of dry carbonate of potash. But to make unchangeable alkaline glass, especially with potash, a smaller quantity of this than the above should be used, with a very violent heat. A small proportion of lime increases the density, hardness, and lustre of glass; and it aids in decomposing the alkaline sulphates and muriates always present in the pearlash of commerce. From 7 to 20 parts of dry slaked lime have been added for 100 of silica, with advantge, it is said, in some German glass manufactories, where the alkaline matter is soda; for potash does not assimilate well with the calcareous earth.

In many glass works on the Continent, sulphate of soda is the form under which alkaline matter is introduced into glass. This salt requires the addition of 8 per cent. of charcoal to decompose and dissipate its acid; $\bullet$ a result which takes place at a high heat, without the addition of any lime. 88 pounds of quartz-sand, 44 pounds of dry glauber salt, and 3 pounds of charcoal, properly mixed and fused, afford a limpid, fluent, and workable glass; with the addition of 17 pounds of lime, these materials fuse more readily into a plastic mass. If less carbon be added, the fusion becomes more tedious. The two following formulæ afford good glauber salt glass.

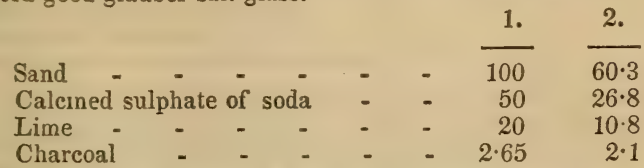

The first mixture has been proved in the looking-glass manufactory of Neuhaus near Vienna, and the second by the experiments of Kirn. The fusion of the first requires 19, of the second 21 hours. The bluish green tinge which these otherwise beautiful and brilliant glasses possess, is not removeable by the ordinary means, such as manganese or arsenic, which decolor alkaline glass. When the sulphate of soda and charcoal are used in smaller proportions, the glass becomes more colorless. The tinge is no doubt owing to the sulphur combining with the oxyde of sodium, in some such way as in the pigment ultramarine.

By a proper addition of galena (the native sulphuret of lead), to glauber salt and quartz sand, without charcoal, it is said a tolerably good crystal glass may be formed. The sulphuric acid of the salt is probably converted by the reaction of the sulphuret of lead into sulphurous acid gas, which is disengaged.

One atom of sulphuret of lead $=1495 \cdot 67$, is requisite to decompose 3 atoms of sulphate of soda $=2676$. It is stated, on good authority, that a good colorless glass may be obtained by using glauber salt without charcoal, as by the following formula.

$\begin{array}{llll}\text { Quartz-sand - } & - & - & 100 \text { pounds } \\ \text { Calcined glauber salt } & - & - & 24 \\ \text { Lime - - - } & - & - & 20 \\ \text { Cullet of soda glass } & - & - & 12\end{array}$

The melting heat must be continued for $26 \frac{1}{2}$ hours. A small quantity of the sand is reserved to be thrown in towards the conclusion of the process, in order to facilitate the expulsion of air bubbles. The above mixture will bear to be blanched by the addition of manganese and arsenic. The decomposition of the salt is in this case effected by the lime, with which the sulphuric acid first combines, is then converted into sulphurous acid, and dissipated. Glass made in this way was found by analysis to consist of 79 parts of silica, 12 lime, and $9 \cdot 6$ soda, without any trace of gypsum or sulphuric acid.

Glauber salt is partially volatilized by the heat of the furnace, and acts upon the arch of the oven and the tops of the pots. This is best prevented by introducing at first into the pots the whole of the salt mixed with the charcoal, the lime, and one fourth part of the sand; fusing this mixture at a moderate heat, and adding gradually afterwards the remainder of the sand, increasing the temperature at the same time. If we put in the whole ingredients together, as is done with potash glass, the sand and lime soon fall to the bottoin, while the salt rises to the surface, and the combination becomes difficult and unequal.

Sulphate of potash acts in the same way as sulphate of soda.

Muriate of soda also, according to Kirn, may be used as a glass flux with advantage. The most suitable proportions are 4 parts of potash, 2 of common sali, and 3 of lime, agreeably to the following compositions :-

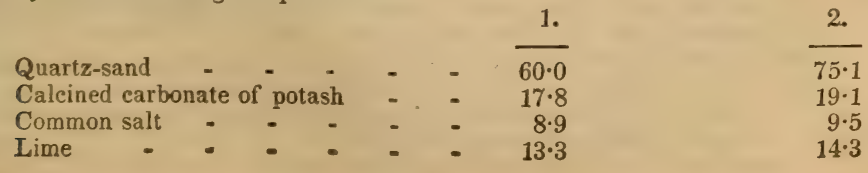


For No. 1, the melting heat mist be 10 hours, which turns out a very pure, solid, good glass; for No. 2, 23 hours of the furnace are required. Instead of the potash, glauber salt may be substituted; the p:oportions being then 19.1 glauber salt, 9.5 muriate of soda, $14 \cdot 3$ lime, $75 \cdot 1$ sand, and 1.3 charcoal.

The oxyde of lead is an essential constituent of the denser glasses, and may be regarded as replacing the lime, so as to form with the quartz-sand a silicate of lead. It assimilates best with purified pearlash, on account of the freedom of this alkali from iron, which is present in most sodas.

Its atomac constitution may be represented as follows :-

\begin{tabular}{|c|c|c|c|c|c|c|}
\hline \multirow{5}{*}{\multicolumn{2}{|c|}{$\begin{array}{llc}\text { Silicic acid } & - & - \\
\text { Oxyde of Lead - } & - & - \\
\text { Potash } & - & - \\
\text { Oxydes of iron and manganese. }\end{array}$}} & \multicolumn{3}{|c|}{ • } & \multirow{5}{*}{$\begin{array}{c}\text { Computation. } \\
59 \cdot 19 \\
28 \cdot 68 \\
12 \cdot 13 \\
-\end{array}$} & \multirow{2}{*}{$\frac{\text { Analysis. }}{59 \cdot 20}$} \\
\hline & & \multirow{5}{*}{$\begin{array}{l}5 \text { atoms } \\
1 \\
1\end{array}$} & $=$ & 2877. & & \\
\hline & & & $=$ & 1394.5 & & $28 \cdot 20$ \\
\hline & & & $=$ & 590.0 & & $9 \cdot 00$ \\
\hline & & & & - & & $1 \cdot 40$ \\
\hline & & & & $4861 \cdot 5$ & $100 \cdot 00$ & $100 \cdot 00$ \\
\hline
\end{tabular}

The above analysis by Berthier relates to a specimen of the best English erystal glass, perfectly colorless and free from air-bubbles. This kind of glass may however take several different proportions of potash and silica to the oxyde of lead.

The composition of mirror plate, as made on the Continent, is as follows :-

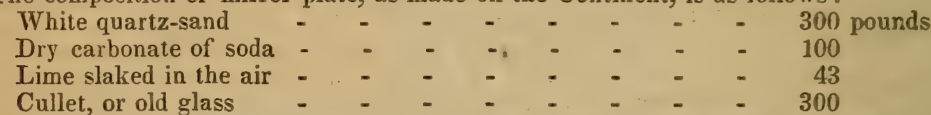

The manganese should not exceed one half per cent. of the weight of the soda.

Optical glass requires to be made with very peculiar care. It is of two different kinds; namely, crown glass and fint glass. The latter contains a considerable proportion of lead, in order to give it an increased dispersive power upon the rays of light, in proportion to its mean refractive power.

Optical crown glass should be perfectly limpid, and have so little color, that a pretty thick piece of it may give no appreciable tinge to the rays of light. It should be exempt from striæ or veins as well as air-bubbles, and have not the slightest degree of milkiness. It should moreover preserve these qualities when worked in considerable quantities. Potash is preferable to soda for making optical crown glass, because the latter alkali is apt to make a glass which devitrifies and becomes opalescent, by long exposure to heat in the annealing process. A simple potash silicate would be free from this defect, but it would be too attractive of moisture, and apt to decompose eventually by the humidity of the atmosphere. It should therefore contain a small quantity of lime, and as little potash as suffices for making a perfect glass at a pretty high temperature. It is probably owing to the high heats used in the English crown glass works, and the moderate quantity of alkali (soda) which is employed, that our crown glass has been found to answer so well for optical purposes.

\section{Practical Details of the Manufacture of Glass.}

The Venetians were the first in modern times who attained to any degree of excellence in the art of working glass, but the French became eventually so zealous of rivalling them, particularly in the construction of mirrors, that a decree was issued by the court of France, declaring not only that the manufacture of glass should not derogate from the dignity of a nobleman, but that nobles alone should be masters of glass-works. Within the last 30 or forty years, Great Britain has made rapid advances in this important art, and at the present day her pre-eminence in every department hardly admits of dispute.

There are five different species of glass, each requiring a peculiar mode of fabrication, and peculiar materials : 1 . The coarsest and simplest form of this manufacture is bottle glass. 2. Next to it in cheapness of material may be ranked broad or spread window glass. An improved article of this kind is now made near Birmingham, under the name of British or German plate. 3. Crown glass comes next, or window glass, formed in large circular plates or dises. This glass is peculiar to Great Britain. 4. Flint glass, crystal glass, or glass of lead. 5. Plate or fine mirror glass.

The materials of every kind of glass are vitrified in pots made of a pure refractory clay; the best kind of which is a species of shale or slate clay dug out of the coal-formation near Stourbridge. It contains hardly any lime or iron, and consists of silica and alumina in nearly equal proportions. The masses are carefully picked, brushed, and ground under edge iron wheels of considerable weight, and sifted through sieves 
having 20 mrshes in the square inch. This powder is molstened with water (best hot), and kneaded by the feet or a loam-mill into a uniform smooth paste. A large body of this dough shou'd be made up at a time, and laid by in a damp cellar to ripen. Previously to working it into shapes, it should be mixed with about a fourth of its weight of cement of whl pots, ground to powder. This mixture is sufficiently plastic, and being lcss contraciile by heat, forms more solid and durable vessels. Glass-house pots have the figure of a truncated cone, with the narrow end undermost; those for bottle and window-slass being open at top, about 30 inches diameter at bottom, 40 inches at the mouth, and 40 inches deep; but the flint-glass pots are covered in at top with a dome-cap, having a mouth at the side, by which the materials are introduced, and the glass is extracted. Bottle and crown-house pots are from 3 to 4 inches thick; those for flint-houses are an inch thinner, and of proportionally smaller capacity.

The well-mixed and kneaded dough is first worked upon a board into a cake for the bottom; orer this the sides are raised, by laying on its edges rolls of clay above each other with much manual labor, and careful condensation. The clay is made into lumps, is equalized, and slapped much in the same way as for making Pottery. The pots thus fashioned must be dried very prudently, first in the atmospheric temperature, and finally in a stove floor, which usually borrows its heat directly from the glass-house. Before setting the pots in the furnace, they are annealed during 4 or 5 days, at a red heat, in a small reverberatory vault, made on purpose. When completely annealed, they are transferred with the utmost expedition into their seat in the fire, by means of powerful tongs supported on the axle of an iron-wheel carriage frame, and terminating in a long lever for raising them and swinging them round. The pot-setting is a desperate service, and when unskilfully conducted without due raechanical aids, is the forlorn hope of the glass-founder.-Quaque ipse miserrima vidi. The celebrated chemist, Dr. Irvine, caught his last illness by assisting imprudently at this formidable operation. The working breast of the hot furnace must be laid bart so as to open a breach for the extraction of the faulty pot, and the insertion of the fresh one, both in a state of bright incandescence. It is frightful to witness the eyes ane fuming visages of the workmen, with the blackening and smoking of their scorched woollen clothes, exposed so long to the direct radiations of the flame. A light mask and sack dress conted with tinfoil, would protect both their faces and persons from any annoyance, at a very cheap rate.

The glass-houses are usually built in the form of a cone, from 60 to 100 feet high, and from 50 to 80 feet in diameter at the base. The furnace is constructed in the centre of the area, above an arched or groined gallery which extends across the whole space, and terminates without the walls, in large folding doors. This cavern must be sufficiently high to allow laborers to wheel out the cinders in their barrows. The middle of the vaulted top is left open in the building, and is covered over with the grate-bars of the furnace.

1. Bo'tle glass. - The bottle-house and its furnace resemble nearly fig. 505. The furnace is usually an oblong square chamber, built of larçe fire-bricks, and arched over with fre-stone, a silicious grit of excellent quality extracted from the coal measures of Newcastle. This furnace stands in the middle of the area; and has its base divided into three compartments. The central space is occupied by the grate-bars; and on either side is the platform or fire-brick siege (seat), raised about 12 inches above the level of the ribs upon which the pots rest. Each siege is about 3 feet broad.

In the sides of the furnace, semi-circular holes of about a foot diameter are left opposite to, and a little above the top of, each pot, called working holes, by which the workmen shovel in the materials, and take out the plastic glass. At each angle of the furnace there is likewise a hole of about the same size, which communicates with the calcining furnace of a cylindrical form, dome-shaped at top. The flame that escapes from the founding or pot-furnace is thus economically brought to reverberate on the raw materials of the bottle-glass, so as to dissipate their carbonaceous or volatile impurities, and convert them into a frit. A bottle-house has generally eight other furnaces or fire-arches; of which six are used for annealing the bottles after they are blown, and two for annealing the pots, before setting them in the furnace.

The laws of this country till lately prohibited the use for making common bottles of any fine materials. Nothing but the common river sand, and soap-boilers' waste, was allowed. About 3 parts of waste, consisting of the insoluble residuum of kelp, mixed with lime and a little saline substance, were used for 1 part of sand. This waste was first of all calcined in two of the fire arches or reverberatories reserved for that purpose, called the coarse arches, where it was kept at a red heat, with occasional stirring, from 24 to 30 hours, being the period of a journey or journée, in which the materials could be melted and worked into bottles. The roasted soap-waste was then withdrawn, under the name of ashes, from its arch, coarsely ground, and mixed with its proper proportion of sand. This mixture was now put into the fine arch, and calcined during the working jour. ney, which extended to 10 or $1 \hat{\xi}$ hours. Whenever the pots were worked out, that frit 
was immediately transferred into them in its ignited state, and the founding process proceeded with such despatch that this first charge of materials was completely melted down in 6 hours, so that the pots might admit to be filled up again with the second charge of frit, which was founded in 4 hours more. The heat was briskly continued, and in the course of from 12 to 18 hours, according to the size of the pots, the quality of the fuel, and the draught of the furnace, the vitrification was complete. Before blowing the bottles, however, the glass must be left to settle, and to cool down to the blowing consistency, by shutting the cave doors and feeding holes, so as to exclurle the air from the fire-grate and the bottom of the hearth. The glass or metal becories more diense, and by its subsidence throws up the foreign lighter earthy and saline matters in the form of a scum on the surface, which is removed with skimming irons. The furnace is now charged with coal, to enable it to afford a working heat for 4 or 5 hours, at the erd of which time more fuel is cautiously added, to preserve adequate heat for finishing the journey.

It is hardly possible to convey in words alone a correct idea of the manipulations necessary to the formation of a wine bottle; but as the manufacturers make no mystery of this matter, any person may have an opportunity of inspecting the operation. Six people are employed at this task; one, called a gatherer, dips the end of an iron tube, about five feet long, previously made red-hot, into the pot of melted metal, turns the rod round so as to surround it with glass, lifts it out to cool a little, and then dips and turns it round again; and so in succession till a ball is formed on its end sufficient to make the required bottle. He then hands it to the blower, who rolls the plastic lump of glass on a smouth stone or cast-iron plate, till he brings it to the very end of the tube; he next introduces the pear-shaped ball into an open brass or cast-iron mould, shuts this together by pressing a pedal with his foot, and holding his tube vertically, blows through it, so as to expand the cooling glass into the form of the mould. Whenever he takes his foot from the pedal-lever, the mould spontaneously opens out into two halves, and falls asunder by its bottom hinge. He then lifts the bottle up at the end of the rod, and transfers it to the finisher, who, touching the glass tube at the end of the pipe with a cold iron, cracks off the bottle smoothly at its mouth-ring. The finished bottles are immediately piled up in the hot annealing arch, where they are afterwards allowed to cool slowly for 24 hours at least. See Bottle Mould.

2. Broad or spread window-glass.-This kind of glass is called inferior window-glass, in this country, because coarse in texture, of a wavy wrinkled surface, and very cheap, but on the Continent spread window-glass, being made with more eare, is much better than ours, though still far inferior in transparency and polish to crown olass, which has, therefore, nearly superseded its use among us. But Messrs. Chance and Hartley, of West Bromwich near Birmingham, have of late years mounted a spread-glass work, where they make British sheet glass, upon the best principles, and turn out an article quite equal, if not superior, to anything of the kind made either in France or Belgium. Their materials are those used in the crown-glass manufacture. The vitrifying mixture is fritted for 20 or 30 hours in a reverberatory arch, with considerable stirring and puddling with long-handled shovels and rakes; and the frit is then transferred by shovels, while red hot, to the melting pots to be founded. When the glass is rightly vitrified, settled, and brought to a working heat, it is lifted out by iron tnbes, as will be described under the article Crown Glass, blown into pears, which being elongated into cylinders, are cracked up along one side, parallel to the axis, by touching them with a cold iron dipped in water, and are then opened out into sheets. Glass cylinders are spread in France, and at West Bromwich, on a bed of smooth stone Paris-plaster, or laid on the bottom of a reverberatory arch; the cylinder being placed on its side horizontally, with the cracked line uppermost, gradually opens out, and flattens on the hearth. At one time, thick plates were thus prepared for subsequent polishing into mirrors; but the glass was never of very good quality ; and this mode of making mirror-plate has accordingly been generally abandoned.

The spreading furnace or oven is that in which cylinders are expanded into takles or plates. It ought to be maintained at a brisk red heat, to facilitate the softening of the glass. The oven is placed in immediate connexion with the annealing areh, so that the tables may be readily and safely transferred from the former to the latter. Sometimes the cylinders are spread in a large mufle furnace, in order to protect them from being tarnished by sulphureous and carbonaceous fumes.

Fig. 500 represents a ground plan of both the spreading and annealing furnace; fig. 501 is an oblong profile in the direction of the dotted line $x \mathrm{x}$, fig. 500 .

$a$ is the fire-place; $b \quad b$ the canals or flues through which the flame rises into both furnaces; $c$ the spreading furnace, upon whose sole is the spreading slab. $d$ is the cooling and annealing oven; $e e$ iron bars which extend obliquely across the annealing arch, and serve for resting the glass tables against, during the cooling. $f f$ the channel along which the previously cracked cylinders are slid, so as to be gradually warmed; 
8 the opening $n$ the spreading furnace, for enabling the workmen to regulate the pro.

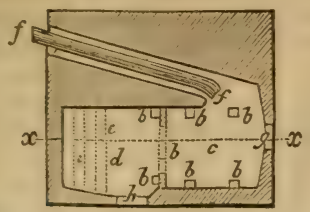

501

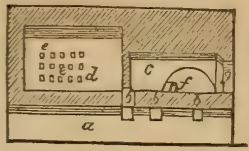

cess; $h$ a door in the annealing arch, for introducing the tools requisite for raising up and removing the tables.

In forming glass-plates by the extension of a cylinder into a plane, the workman first blows the lump of glass into the shape of an oblong pear, the length of which must be nearly equal to the length of the intended plate, and its diameter such, that the circumference, when developed, will be equal to the breadth of the plate. He now rests the blowing iron on a stool or iron bar, while an assistant, with a pointed iron, pierces a hole into the extreme end of the pear, in the line of the blowing-pipe. This opening is then enlarged, by introducing the blade of a pair of spring-tongs, while the glass is turned round; and by skilful management, the end of the pear is eventually opened out into a cylindrical mouth. The workman next mounts upon a stool, and holds the blowing-iron perpendicularly. The blown cylinder is now cracked off, a punto rod of iron having been previously stuck to its one end, to form a spindle for working the other by. This rod has a flat disc on its end, or three prongs, which being dipped in melted glass, are applied to the mouth of the cylinder. By this as a handle, the glass cone is carried to the fire, and the narrow end being heated, is next opened by spring tongs, and formed into a cylinder of the same size as the other end. The cylinder, thus equalized, is next cracked or slit down in its side with a pair of shears, laid on a smooth copper plate, detached from the iron rod, spread out by heat into a plane surface, and finally annealed. This series of transformations is represented in fig. 502 , at $\mathrm{A}, \mathrm{B}, \mathrm{C}, \mathrm{D}, \mathrm{E}, \mathrm{F}, \mathrm{G}, \mathrm{H}$.

Figs. 503 and 504 represent a Bohemian furnace in which excellent white window glass is founded. Fig. 503 is a longitudinal section of the glass und annealing furnace. Fig. 504 is the ground plan. $a$ is the ash-pit vaulted under the sole of the furnace; the fire-place itself is divided into three compartments ; with a middle slab at $d$, which is hollowed in the centre, for collecting any split glass, and two hearth tiles or slabs $b \quad b . \quad c c$

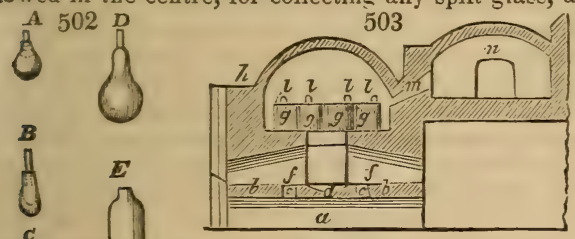

504
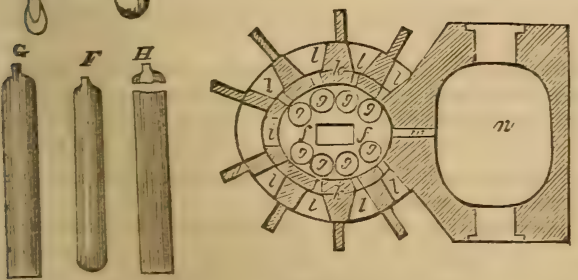
are the draught or air holes; $e$ e are arches upon which the bearing slabs $f f$ partly rest. In the middle between these arches, the flame strikes upwards upon the pots $g \mathrm{~g}$, placed as closely together as possible, for economy of room. $h$ is the breast wall of the furnace; $i$, fig. 504, the opening through which the pots are introduced; it is bricked up as soon as they are set. $k k$, is the base of the cone or dome of the furnace; $l \quad l \quad l$, the working orifices, which are made larger or smaller according to the size of the glass articles to be made. $m$ is the flue which leads to the annealing stove $n$, with an arched door. Exterior to this, there is usually a drying kiln not shown in the figure; and there are adjoining stoves called arches, for

drying and annealing the new pots before they are set.

The cooling or annealing arch, or leer, is often built independent of the glass-house furnace, is then heated by a separate fire-place, and constructed like a very long reverberatory furnace. See Copper.

The leer pans or trays of sheet-iron, are laid upon its bottom in an oblong series, and hooked to each other.

3. Crown-glass.-The crown-glass house with its furnace is represented in fig. 505, where the blowing operation is shown on the one side of the figure, and the flashing on the other. The furnace is usually constructed to receive 4 or 6 pots, of such dimen. sions as to make about a ton of glass each at a time. There are, however, several sub- 
sidiary furnaces to a crown-house. 1. A reverberatory furnace or calcar, ior calcining or

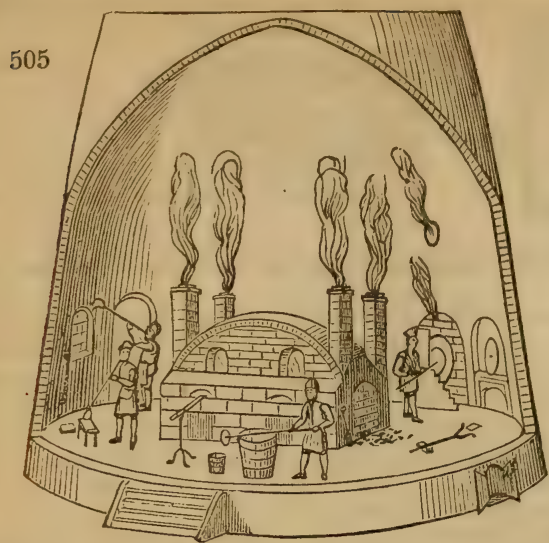
fritting the materials; 2. a biow ing furnace, for blowing the pearshaped balls, made at the potholes, into large globes; $\mathbf{3}$. a flashing furnace, and bottoming hole for communicating a softening heat, in expanding the globe into a circular plate; 4 . the annealing arch for the finished tables; 5 . the reverberatory oven for annealing the pots prior to their being set upon the founding siege.

The materials of crown glass used to be, fine sand, by measure 5 parts, or by weight 10 ; ground kelp by measure 11 parts, or by weight $16^{\circ} \frac{1}{2}$; but instead of kelp, soda ash is now generally employed. From 6 to $8 \mathrm{cwts}$. of sand, lime, and soda-ash, mixed together in wooden boxes with a shovel, are thrown on the sole of a large reverberatory, such as is represented in the article Copper. Here the mixture is well worked tugether, with iron paddles, flat shovels, and rakes with long handles; the area of this furnace being about 6 feet square, and the height 2 feet. The heat soon brings the materials to a pasty consistence, when they must be diligently turned over, to favor the dissipation of the carbon, sulphur, and other volatile matters of the kelp or soda ash, and to incorporate the fixed ingredients uniformly with the sand. Towards the end of three hours, the fire is considerably raised, and when the fourth hour has expired, the fritting operation is finished. The mass is now shovelled or raked out into shallow cast-iron square cases, smoothed down, and divided, before it hardens by cooling, into square lumps, by cross sections with the spade. These frit-bricks are afterwards piled up in a large apartment for use; and have been supposed to improve with age, by the efflorescence of their saline constituents into carbonate of soda on their surface.

The founding-pots are filled up with these blocks of frit, and the furnace is powerfuliy urged by opening all the subterranean passages to its grate, and closing all the doors and windows of the glass-house itself. After 8 or 10 hours the vitrification has made such progress, and the blocks first introduced are so far melted down, that another charge of frit can be thrown in, and thus the pot is fed with frit till the proper quantity is used. In about 16 hours the vitrification of the frit has taken place, and a considerable quantity, amounting often to the ewt. of liquid saline matter, fluats over the glass. This salt is carefully skimmed off into iron pots with long ladles. It is called Sandiver or Glass-gall, and consists usually of muriate of soda, with a little sulphate. The pot is now ready for receiving the topping of cullet, which is broken pieces of window glass, to the amount of 3 or 4 cwts. This is shovelled in at short intervals; and as its pressure forces up the residuary saline matter, this is removed; for were it allowed to remain, the body of the glass would be materially deteriorated.

The heat is still continued for several hours till the glass is perfect, and the extrication of gas called the boil, which accompanies the fusion of crown glass, has nearly terminated, when the fire is abated, by shuiting up the lower vault doors and every avenue to the grate, in order that the glass may settle fine. At the end of about 40 hours altogether, the fire being slightly raised by adding some coals, and opening the doors, the glass is carefully skimmed, and the working of the pots commences.

Before describing it, however, we may state that the marginal figure 506 shows the 506

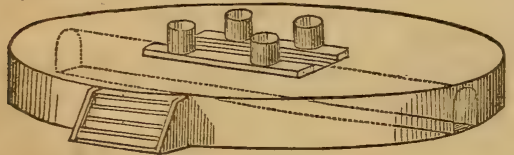
base of the crown-house cone, with the four open pots in two ranges on opposite sides of the furnace, sitting on their raised sieges, at each side of the grate. At one side of the base the door of the vault is shown, and its course is marked by the dotted lines.

Detailed description of the crown-glass furnace, figs. 507, 508.-It is an oblong square, built in the centre of a brick cone, large enough to contain within it, two or three pots at each side of the grate room, which is either divided as shown in the plan, or runs the whole length of the furnace, as the manufacturer chooses. Fig. 507 is a ground plan, and fig. 508 a front elevation, of a six-pot furnace. 1, 2, 3, fig. 507, are the working hole: 
for the purposes of ventilation, of putting in the materials, and of taking out the metal to be wrought. $4,5,6,7$, are pipe holes for warming the pipes before beginning to work with them. $8,9,10$, are foot holes for mending the pots and sieges. 11 is a bar of iron for binding the furnace, and keeping it from swelling.

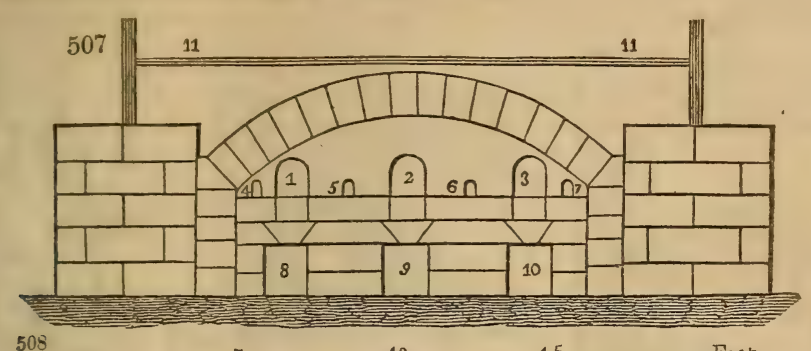

508 51 10 $1,5,1,1$, Eeet,

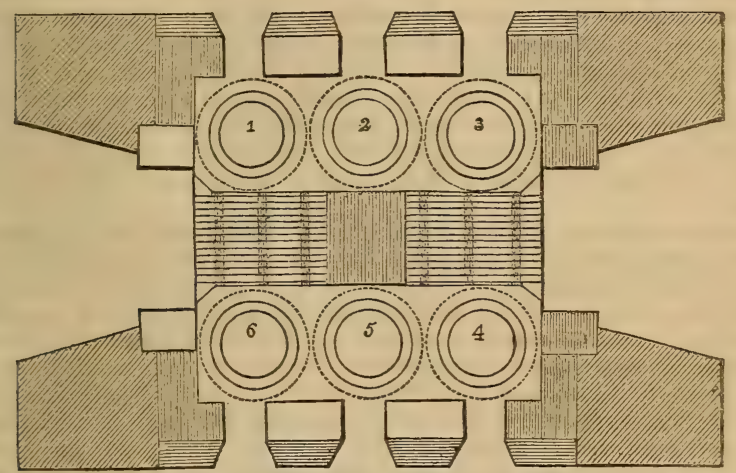

The arch is of an elliptic form; though a barrel areh, that is, an arch shaped like the half of a barrel cut longwise through the centre, is sometimes used. But this soon gives way when used in the manufacture of crown glass, although it does very well in the elayfurnace used for bottle houses.

The best stone for building furnaces is fire-stone, from Coxgreen in the neighborhood of Newcastle. Its quality is a close grit, and it contains a greater quantity of talc than the common fire-stone, which seems to be the chief reason of its resisting the fire better. The great danger in building furnaces is, lest the cement at the top should gire way with the excessive heat, and by dropping into the pots, spoil the metal. The top should therefore be built with stones only, as loose as they can hold together after the centres are removed, and without any cement whatever. The stones expand and come quite close together when annealing; an operation which takes from eight to fourteen days at most. There is thus less risk of any thing dropping from the roof of the furnace.

The inside of the square of the furnace is built either of Stourbridge fire-clay annealed, or the Newcastle fire-stone, to the thickness of sixteen inches. The outside is built of common brick about nine inches in thickness.

The furnace is thrown over an ash-pit, or cave, as it is called, which admits the atmospheric air, and promotes the combustion of the furnace. This cave is built of stone until it comes beneath the grate room, when it is formed of fire-brick. The abutments are useful for binding and keeping the furnace together, and are built of masonry. The furnaces are stoutly clasped with iron all round, to keep them tight. In four-pot furnaces this is unnecessary, provided there be four good abutments.

Fig. 509 is an elevation of the flashing furnace. The outside is built of common brick, the inside of fire-brick, and the mouth or nose of Stourbridge fire-clay.

Fig. 510 is the annealing kiln. It is built of common brick, except round the grate room, where fire-brick is used.

Few tools are needed for blowing and flashing crown-glass. The requisite ball of plastic glass is gathered, in successive layers as for bottles, on the end of an iron tube, and rolled into a pear-shape, on a cast-iron plate; the workman taking care that the air blown into its cavity is surrounded with an equal body of glass, and if he perceives any side to be thicker than another, he corrects the inequality by rolling it on the sloping iron table called marver, (marbre). He now heats the bulb in the fire, and rolls it so a. 
to form the grass upon the end of the tube, and by a dexterous swing or two he lengthens it, as shown in $\mathrm{r}$, fig. 511. To extend the neck of that pear, he next rolls it over a smooth iron rod, turned round in a horizontal direction, into the shape $\mathrm{K}$, fig. 511. By fut ther expansion at the blowing-furnace, he now brings it to the shape $L$, represented in fig. 511 .

510

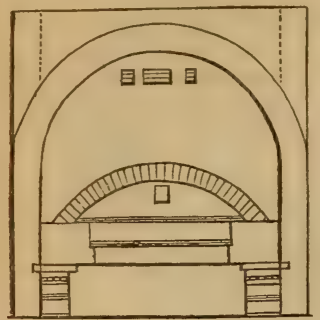

509

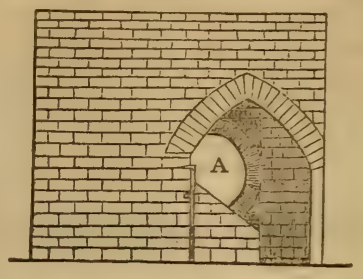

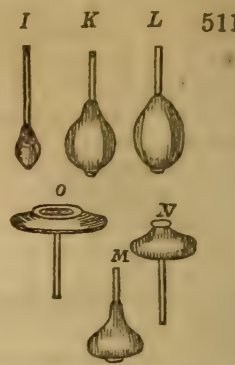

This spheroid having become cool and somewhat stiff, is next carried to the bottoming hole (like fig. 509), to be exposed to the action of flame. A slight wall erected before one half of this hole, screens the workman from the heat, but leaves room for the globe to pass between it and the posterior wall. The blowing-pipe is made to rest a little way froro the neck of the globe, on a hook fixed in the front wall; and thus may be made easily to revolve on its axis, and by giving centrifugal force to the globe, while the bottom of it, or part opposite to the pipe, is softened by the heat, it soon assumes the form exhibited in M, fig. 511 .

In this state the flattened globe is removed from the fire, and its rod being rested on the casher box covered with coal cinders, another workman now applies the end of a solid iron rod tipped with melted glass, called a punto, to the nipple or prominence in the middle; and thus attaches it to the centre of the globe, while the first workman cracks off the globe by touching its tubular neck with an iron chisel dipped in cold water. The workman having thereby taken possession of the globe by its bottom or knobbed pole attached to his punty rod, he now carries it to another circular opening, where he exposes it to the action of moderate flame with regular rotation, and thus slowly heats the thick projecting remains of the former neck, and opens it slightly out, as shown at $\mathrm{N}$, in fig. 511. He next hands it to the flasher, who, resting the iron rod in a hook placed near the side of the orifice A, fig. 509, wheels it rapidly round opposite to a powerful flame, till it assumes first the figure 0 , and finally that of a flat circular table.

The flasher then walks off with the table, keeping up a slight rotation as he moves along, and when it is sufficiently cool, he turns down his rod into a vertical position, and lays the table flat on a dry block of fire-clay, or bed of sand, when an assistant nips it off from the punto with a pair of long iron shears, or cracks it off with a touch of cold iron. The loose table or plate is lastly lifted up horizontally on a double pronged iron fork, introduced into the annealing arch fig. 510 and raised on edge; an assistant with a long-kneed fork preventing it from falling too rapidly backwards. In this arch a great many tables of glass are piled up in iron frames, and slowly cooled from a heat of about $600^{\circ}$ to $100^{\circ} \mathrm{F}$., which takes about 24 hours; when they are removed. A circular plate or table of about 5 feet diameter weighs on an average 9 pounds.

4. Flint glass.-This kind of glass is so called because originally made with calcined flints, as the silicious ingredient. The materials at present employed in this country for the finest flint glass or crystal, are first, Lynn sand, calcined, sifted, and washed; second, an oxyde of lead, either red lead or litharge; and third, pearlash. The pearlash of commerce must however be purified by digesting it in a very little hot water, which dissolves the carbonate of potash, and leares the foreion salts, chiefly sulphate of potash, muriate of potash, and muriate of soda. The solution of the carbonate being allowed to cool and become clear in lead pans, is then run off into a shallow iron boiler, and evaporated to dryness. Nitre is generally added as a fourth ingredient of the body of the glass; and it serves to correct any imperfections which might arise from accidental combustible particles, or from the lead being not duly oxydized. The above four substances constitute the main articles; to which we may add arsenic and manganese, introduced in very small quantities, to purify the color and clear up the transparency of the glass. The black oxyde of manganese, when used in such quantity only as to peroxydize the iron of the sand, simply removes the green tinge caused by the iron; but if more mangan sse be added than accomplishes that purpose, it will give a purple tinge to the glass; and in fact, most manufacturers prefer to have an excess rather than a defect of manganese, since cut glass has its brilliancy increased by a faint lilach hue. The arsenic is supposed to counteract the injury arising from excess of manganese, but is itself very apt on the other hand to communicate some degree of opalescence, 
or at least, 10 impair the lustre of the glass. When too much manganese has been added, the purple tinge may indeed be removed by any carbonaceous matter, as by thrusting a wooden rod down into the liquid glass; but this cannot be done with good effect in practice, since the final purple tinge is not decided till the glass is perfectly formed, and then the introduction of charcoal would destroy the uniformity of the whole contents of the pot.

The raw materials of flint glass are always mixed with about a third or a fourth of their weight of broken erystal of like quality; this mixture is thrown into the pot with a shovel; and more is added whenever the preceding portions by melting subside; the object being to obtain a pot full of glass, to facilitate the skimming off the impurities

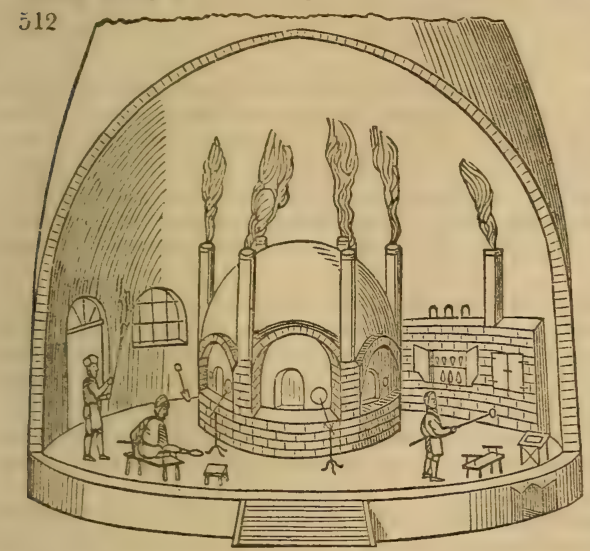
and sandiver. The mouth of the pot is now shut, by applying clay-lute round the stopper, with the exception of a small orifice below, for the escape of the liquid saline matter. Flint glass requires about 48 hours for its complete vitrification, though the materials be more fusible than those of crown glass ; in consequence of the contents of the pot being partially screened by its cover from the action of the fire, as also from the lower intensity of the heat.

Fig. 512 represents a flint glass house for 6 pots, with the arch or leer on one side for annealing the crystal ware. In fig. 513, the base of the cone is seen, and the glass pots in situ on their platform ranged ronnd the central fire grate. The dotted line denotes the contour of the furnace, fig. 512.

Whenever the glass appears fine, and is freed from its air bubbles, which it usually is in about 36 hours, the heat is suffered to fall a little by closing the bottom valves, \&c., that the pot may settle; but prior to working the metal the heat is somewhat raised again.

It would be useless to describe the manual operations of fashioning the various articles of the flint-glass manufacture, because they are indefinitely varied to suit the conveniences and caprices of human society.

Every different flint-house has a peculiar proportion of glass materials. The following have been offered as good practical mixtures.

1. Fine white sand - - -

- $\quad$ - $\quad 300$ parts.

Refined pearlashes

Nitre

$-\quad-$

Arsenic and manganese, a minute quantity.

In my opinion, the proportion of lead is too great in the above recipe, which is given on the authority of Mr. James Geddes, of Leith. The glass made with it would be probably yellowish and dull.
2. Fine sand
Litharge -
Nitre
$-$

To these quantities from 30 to 50 parts of broken glass or cullet are added; with about a two-thousandth part of manganese, and a three-thousandth part of arsenic. But manganese varies so extremely in its purity, and contains often so much oxyde of iron, that nothing can be predicated as to its quantity previously to trial.

M. Payen, an eminent manufacturing chemist in France, says that the composition of erystal does not deviate much from the following proportions :-

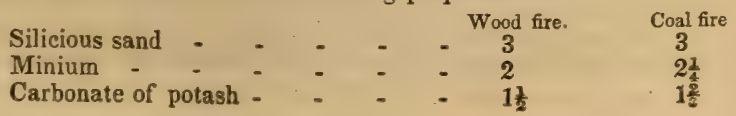


I conceive that this glass contains too much lead and potash. Such a mixture will pre. duce a dull metal, very attractive of moisture; defects to which the French crown-glass also is subject.

The flint-glass leer for annealing glass, is an arched gallery or large flue, about 36 feet long, 3 feet high, 4 wide; having its floor raised above 2 feet above the ground of the glass-house. The hot air and smoke of a fire-place at one end pass alnng this gallery, and are discharged by a chimney 8 or 10 feet short of the other end. On the floor of the vanlt, large iron trays are laid and hooked to each other in a series, which are drawn from the fire end towards the other by a chain, wound about a cylinder by a winch-handle projecting through the side. The flint-glass articles are placed in their hot state into the tray next the fire, which is moved on wards to a cooler station whenever it is filled, and an empty tray is set in its place. Thus, in the course of about 20 hours, the glass advances to the cool end thoroughly annealed.

Besides colorless transparent glass, which forms the most important part of this manufacture, various colored glasses are made to suit the taste of the public. The taste at Paris was lately for opaline crystal; which may be prepared by adding to the above composition (No. 2) phosphate of lime, or well burnt bone ash in fine powder, washed and dried. The article must be as uniform in thickness as possible, and speedily worked into shape, with a moderate heat. Oxyde of tin, putty, was formerly used for making opalescent glass, but the lustre of the body was always impaired by its means.

Crystal vessels have been made recently of which the inner surface is colorless, and all the external facets colored. Such works are easily executed. The end of the blowingrod must be dipped first in the pot containing colorless glass, to form a bulb of a certain size, which being cooled a little is then dipped for an instant into the pot of colored glass. The two layers are associated without intermixture; and when the article is finished in its form, it is white within and colored without. Fluted lines, somewhat deeply cut, pass through the colored coat, and enter the colorless one; so that when they cross, their ends alone are colored.

For some time past, likewise, various crystal articles have been exhibited in the market with colored enamel-figures on their surface, or with white incrustations of a silvery lustre in their interior. The former are prepared by placing the enamel object in the brass mould, at the place where it is sought to be attached. The bulb of glass being put into the mould, and blown while very hot, the small plate of enamel gets cemented to the surface. For making the white argentine incrustations, small figures are prepared with an impalpable powder of dry porcelain paste, cemented into a solid by means of a little gypsum plaster. When these pieces are thoroughly dried, they are laid on the glass while it is red hot, and a large patch of very liquid glass is placed above it, so as to encase it and form one body with the whole. In this way the incrustation is completely enclosed; and the polished surface of the crystal, which scarcely touches it, gives a brilliant aspect, pleasing to the eye.

A uniform flint-glass, free from striæ, or wreath, is much in demand for the optician. It would appear that such an article was much more commonly made by the English manufacturers many years ago, than at present; and that in improving the brilliancy of crystal-glass they have injured its fitness for constructing optical lenses, which depends not so niuch on its whiteness and lustre as on the layers of different densities being parallel to each other. The oxyde of lead existing in certain parts of a potful of glass in greater proportion than in other parts, increases the density uriequally in the same mass, so that the adjoining strata are often very different in this respect. Even a potful of pretty uniform glass, when it stands some time liquid, becomes eventually unequable by the subsidence of the denser portions; so that striæ and gelatinous appearances begin to manifest themselves, and the glass becomes of little value. Glass allowed to cool slowly in mass in the pot is particularly full of wreath; and if quickly refrigerated, that is, in two or three hours, it is apt to split into a multitude of minute splinters, of which no use can be made. For optical purposes, the glass must be taken out in its liquid state, being gathered on the end of the iron rod from the central portion of a recently skimmed pot, after the upper layers have been worked off in general articles.

M. Guinand, of Brennets near Geneva, appears to have hit upon processes that fur. nished almost certainly pieces of flint-glass capable of forming good lenses of remarkable dimensions, even of 11 inches diameter; of adequate density and transparency, and nearly free from strice. M. Cauchoix, the eminent French optician, says, that out of ten object glasses, 4 inches in diameter, made with M. Guinand's flint glass, eight or nine turned out very good, while out of an equal number of object glasses made of the flint glass of the English and French manufactories, only one, or two at most, were found serviceable. The means by which M. Guinand arrived at these results have not been published. He has lately died, and it is not known whether his son be in possession of his secret. 
An achromatic object glass for telescopes and microscopes consists of at least two lenses; the one made with glass of lead, or flint-glass, and the other with crown-glass; the former possessing a power of dispersing the colored rays relatively to its mean refractive power, much greater than the latter; upon which principle the achromatism of the image is produced, by reuniting the different colored rays into one focus. Flint-glass to be fit for this delicate purpose must be perfectly homogeneous, or of uniform density throughout its substance, and free from wavy veins or wreaths; for every such inequality would occasion a corresponding inequality in the refraction and dispersion of the light; like what is perceived in looking through a thick and thin solution of gum-arabic imperfectly mixed. Three plans have been prescribed for obtaining homogeneous pieces of optical glass: 1. to lift a mass of it in large ladles, and let it cool in them; 2 . to pour it out from the pots into moulds; 3 . to allow it to cool in the pots, and afterwards to cut it off in horizontal strata. The last method, which is the most plausible, seldom affords pieces of uniform density, unless peculiar precautions have been adopted to settle the flint glass in uniform strata; because its materials are of such unequal density, the oxyde of lead having a specific gravity of 8 , and silica of $2 \cdot 7$, that they are apt to stand at irregular heights in the pots.

One main cause of these inequalities lies in the construction of the furnace, whereby the bottom of the pot is usually much less heated than the upper part. In a plate glass furnace the temperature of the top of the pot has been found to be $130^{\circ} \mathrm{W}$ edgew., while that of the bottom was only $110^{\circ}$, constituting a difference of no less than $2610^{\circ} \mathrm{F}$. The neressary consequence is that the denser particles which subside to the bottom, during the fusion of the materials, and after the first extrication of the gases, must remain there, not being duly agitated by the expansive force of caloric, acting from below upwards.

The preparation of the best optical glass is now made a great mystery by one or two proficients. The following suggestions, deduced from a consideration of principles, may probably lead to some improvements, if judiciously applied. The great object is to counteract the tendency of the glass of lead to distribute itself into strata of different densities; which may be effected either by mechanical agitation or by applying the greatest heat to the bottom of the pot. But however homogeneous the glass may be thereby made, its subsequent separation into strata of different densities must be prevented by rapid cooling and solidification. As the deeper the pots, the greater is the chance of unequal specific gravity in their contents, it would be advisable to make them wider and shallower than those in use for making ordinary glass. The intermixture may be effected either by lading the glass out of one pot into another in the furnace, and back again, with copper ladles, or by stirring it up with a rouser, then allowing it to settle for a short time, till it becomes clear and free from air bubbles. The pot may now be removed from the furnace, in order to solidify its contents in their homogeneous state; after which the glass may be broken in pieces, and be perfected by subjecting it to a second fusion; or, what is easier and quicker, we may form suitable discs of glass without breaking down the potful, by lifting it out in flat copper ladles with iron shanks, and transferring the lumps after a little while into the annealing leer.

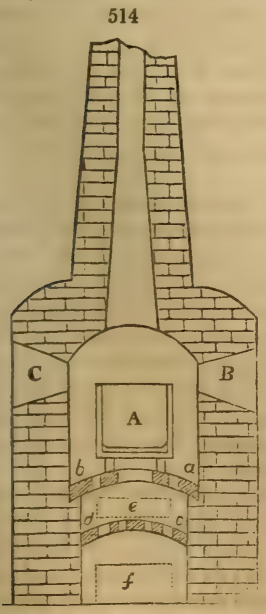

To render a potful of glass homogeneous by agitation, is a more difficult task, as an iron rod would discolor it, and a copper rod would be apt to melt. An iron rod sheathed in laminated platinum would answer well, but for its expense. A stone-ware tube supported within by a rod of iron, might also be employed for the purpose in careful hands; the stirring being repeated several times, till at last the glass is suffered to stiffen a little by decrease of temperature. It must then be allowed to settle and cool, after which the pot, being of small dimensions, may be drawn out of the fire.

2 . The second method of producing the desired uniformity of mixture, consists in applying a greater heat to the bottom than to the upper part of the melting pot. Fig. 514 represents in section a furnace contrived to effect this object. It is cylindrical, and of a diameter no greater than to allow the flames to play round the pot, containing from three to four cwts. of vitreous materials. $\Lambda$ is the pot, resting upon the arched grid $b a$, built of fire-bricks, whose apertures are wide enough to let the flames rise freely, and strike the bottom and sides of the vessel. From $1 \frac{1}{2}$ to 2 feet under that arch, the fuel grate $c d$ is placed. B c are the two working openings for introducing the materials, and inspecting the progress of the fusion; they must be closed with fire-tiles and luted with fire-clay at the beginning of the process. At the back of the furnace, opposite the mouth 
of the fire-place there is a door-way, which is bricked up, except upon occasion of puttıng in and taking out the pot. The draught is regulated by means of a slide-plate upon the mouth of the ash-pit $f$. The pot being heated to the proper pitch, some purified pearlash, mixed with fully twice its weight of colorless quartz sand, is to be thrown into it, and after the complete fusion of this mixture, the remaining part of the sand along with the oxyde of lead (fine litharge) is to be strown upon the surface. These silicious particles in their lescent serve to extricate the air from the mass. Whenever the whole is fused, the heat must be strongly urged, to ensure a complete uniformity of combination by the internal motions of the particles. As soon as the glass has been found, by making test vials, to be perfectly fine, the fire must be withdrawn, the two working holes must be opened, as well as the mouths of the fire-place and ash-pit, to admit free ingress to cooling currents of air, so as to congeal the liquid mass as quickly as possible; a condition essential to the uniformity of the glass. It may be worth while to stir it a little with the pottery rod at the commencement of the cooling process. The solidified glass may be afterwards detached by a hammer in conchoidal discs, which, after chipping off their edges, are to be placed in proper porcelain or stone-ware dishes, and exposed to a softening heat, in order to give them a lenticular shape. Great care must be taken that the heat thus applied by the muffle furnace be very equable, for utherwise wreaths might be very readily reproduced in the dises. A small oven, upon the plan of a baker's, is best fitted for this purpose, which being heated to dull redness, and then extinguished, is ready to soften and afterwards anneal the conchoidal pieces.

Guinand's dense optical flint glass, of specific gravity $3 \cdot 616$, consists, by analysis, of oxyde of lead, 43.05 ; silica, 44.3 ; and potash, 11.75 ; but requires for its formation the following ingredients: 100 pounds of ground quartz; 100 pounds of fine red lead; 35 pounds of purified potash; and from 2 to 4 pounds of saltpetre. As this species of glass is injured by an excess of potash, it should be compounded with rather a defect of it, and melted by a proportionably higher or longer heat. A good optical glass has been made in Germany with 7 parts of pure red lead, 3 parts of finely ground quartz, and 2 parts of calcined borax.

\section{Plate glass.}

This, like English crown-glass, has a soda flux; whereas flint-glass requires potash, and is never of good quality when made with soda. We shall distribute our account of this manufacture under two heads.

1. The different furnaces and principal machines, without whose knowledge it would be impossible to understand the several processes of a plate-glass factory.

2. The materials which enter into the composition of this kind of glass, and the series of operations which they undergo; devoting our chief attention to the changes and improvements which long experience, enlightened by modern chemistry, has introduced into the great manufactory of Saint-Gobin, in France, under the direction of M. Tassaert. It may however be remarked, that the English plate-glass manufacture derives peculiar advantages from the excellence of its grinding and polishing machinery.

The clay for making the bricks and pots should be free from lime and iron, and very refractory. It is mixed with the powder of old pots passed through a silk sieve. If the clay be very plastic it will bear its own weight of the powder, but if shorter in quality, it will take only three fifths. But before mingling it with the cement of old pots, it must be dried, bruised, then picked, ground, and finally elutriated by agitation with water, decantation through a hair sieve, and subsidence. The clay fluid after passing the sieve is called slip (coulis).

The furnace is built of dry bricks, cemented with slip, and has at each of its four angles a peculiar annealing arch, which communicates with the furnace interiorly, and thence derives sufficient heat to effect in part, if not wholly, the annealing of the pots, which are always deposited there a long time before they are used. Three of these arches, exclusively appropriated to this purpose, are called pot-arches. The fourth is called the arch of the materials, because it serves for drying them before they are founded. Each arch has, moreover, a principal opening called the throat, another called bonnard, by the French workmen, through which fire may be kindled in the arch itself, when it was thought to be necessary for the annealing of the pots; a practice now abandoned. The duration of a furnace is commonly a year, or at most 14 months; that of the arches is 30 years or upwards, as they are not exposed to so strong a heat.

In the manufacture of plate-glass two sorts of crucibles are employed, called the pots and the basins (cuveties). The first serve for containing the materials to be founded, and for keeping them a long time in the melted state. The cuvetles receive the melted glass after it is refined, and decant it out on the table to be rolled into a plate. Three pots hold liquid glass for six small basins, or for three large ones, the latter being employed for making mirrors of great dimensions, that is, 100 inches long and up- 
wards. Furnaces have been lately constructed with 6 pots, and 12 cuvettes, 8 of which are smail, and 4 large; and cuvettes of three sizes are made, called small, middling, and large. The small are perfect cubes, the middling and the large ones are oblıng paralle! opipeds. Towards the middle of their height, a notch or groove, two or three inches broad, and an inch deep, is left, called the girdle of the cuvette, by which part they are grasped with the tongs, or rather are clamped in the iron frame. This frame goes round the four sides of the small cuvettes, and may be placed indifferently upon all their sides; in the other cuvettes, the girdle extends only over the two large sides, because they can. not be turned up. See $m$ T, fig. 515 , p. 596 .

The pot is an inverted truncated cone, like a crown glass pot. It is about 30 inches high, and from 30 to 32 inches wide, including its thickness. There are only a few inches of difference between the diameter of the top and that of the bottom. The bottom is 3 inches thick, and the body turns gradually thinner till it is an inch at the mouth of the pot.

The large building or factory, of which the melting furnace occupies the middle space, is called the halle in French. At Ravenhead in Lancashire it is called the foundry, and is of magnificent dimensions, being probably the largest apartment under one roof in Great Britain, since its length is 339 feet, and its breadth 155 . The famous halle of St. Gobin is $17+$ feet by 120 . Along the two side walls of the halle, which are solidly constructed of hewn stone, there are openings like those of common ovens. These ovens, destined for the annealing of the newly cast plates, bear the name of carquaises. Their soles are raised two feet and a half above the level of the ground, in order to bring them into the same horizontal plane with the casting tables. Their length, amounting sometimes to $30 \mathrm{feet}$, and their breadth to 20 , are required in order to accommodate 6,8 , or even 10 plates of glass, alongside of each other. The front aperture is called the throat, and the back door the little throat (gueulette). The carquaise is heated by means of a fire-place of a square form called a tisar, which extends along its side.

The founding or melting furnace is a square brick building laid on solid foundations, being from 8 to 10 feet in each of its fronts, and rising inside into a vault or crown about 10 feet high. At each angle of this square, a small oven or arch is constructed, likewise vaulted within, and communicating with the melting furnace by square flues, called $l u$ nettes, through which it receives a powerful heat, though much inferior to that round the pots. The arches are so distributed as that two of the exterior sides of the furnace stand wholly free, while the two other sides, on which the arches encroach, offer a free spaze of only three feet. In this interjacent space, two principal openings of the furnace, of equal size in each side, are left in the building. These are called tunnels. They are destined for the introduction of the pots and the fuel.

On looking through the tunnels into the inside of the furnace, we perceive to the right hand and the left, along the two free sides, two low platforms or sieges, at least 30 inches in height and breadth. See figs. 506, 508.

These- sieges (seats) being intended to support the pots and the cuvettes filled with heary materials, are terminated by a slope, which ensures the solidity of the fire-clay mound. The slopes of the two sieges extend towards the middle of the furnace so near as to leave a space of only from 6 to 10 inches between them for the hearth. The end of this is perforated with a hole sufficiently large to give passage to the liquid glass of a broken pot, while the rest is preserved by lading it from the mouth into the adjoining cuvette.

In the two large parallel sides of the furnace, other apertures are left much smaller than the tunnels, which are called ouvreaux (peep holes). The lower ones, or the ouvreaux en bas, called curette openings, because, being allotted to the admission of these vessels, they are exactly on a level with the surface of the sieges, and with the floor of the hall:Plates of cast-iron form the thresholds of these openings, and facilitate the ingmess and egress of the cuvettes. The apertures are arched at top, with hewn stone iike the tunnels, and are 18 inches wide when the cuvettes are 16 inches broad.

The upper and smaller apertures, or the higher ouvreaux called the lading holes, because they serve for transvasing the liquid glass, are three in number, and are placed 31 or 32 inches above the surface of the sieges. As the pots are only 30 inches high, it becomes easy to work through these openings either in the pots or the curettes. The pots stand opposite to the two pillars which separate the openings, so that a space is left between them for one or more cuvettes according to the size of the latter. It is obvious that if the tunnels and ouvreaux were left open, the furnace would not draw or take the requisite founding heat. Hence the openings are shut by means of fire-tiles. These are put in their places, and removed by means of two holes left in them, in correspondence with the two prongs of a large iron fork supported by an axle and two iron wheels, and terminated by two handles which the workmen lay hold of when they wish to move the tile.

The closing of the tunnel is more complex. When it is shut or ready for the iiring, 
the aperture appears built up with bricks and mortar from the top of the arch to the middle of the tunnel. The remainder of the door-way is closed; 1 . on the two sides down to the bottom, by a small upright wall, likewise of bricks, and 8 inches broad, called walls of the glaye; 2 . by an assemblage of pieces called pieces of the glaye, because the whole of the closure of the tunnel bears the name of glaye. The upper hole, 4 inches square, is called the tisar, through which billets of wood are tossed into the fire. Fuel is also introduced into the posterior openings. The fire is always kept up on the hearth of the tunnel, which is, on this account, 4 inches higher than the furnacehearth, in order that the glass which may accidentally fall down on it, and which does not flow off by the bottom hole, may not impede the combustion. Should a body of glass, however, at any time obstruct the grate, it must be removed with rakes, by opening the tunnel and dismounting the fire-tile stoppers of the glaye.

Formerly wood fuel alone was employed for heating the melting-furnaces of the mirror-plate manufactory of Saint Gobin; but within these few years, the Director of the works makes use with nearly equal advantage of pit-coal. In the same establishment, two melting furnaces may be seen, one of which is fixed with wood, and the other with coals, without any difference being perceptible in the quality of the glass furnished by either. It is not true, as has been stated, that the introduction of pit-coal has made it necessary to work with covered pots in order to avoid the discoloration of the materials, or that more alkali was required to compensate for the diminished heat in the covered pots. They are not now covered when pit-coal is used, and the same success is obtained as heretofore by leaving the materials two or three hours longer in the pots and the cuvettes. The construction of the furnaces in which coal is burned, is the same as that with wood, with slight modifications. Instead of the close bottomed hearth of the wood furnace, there is an iron grate in the coal-hearth through which the air enters, and the waste ashes descend.

When billets of wood were used as fuel, they were well dried beforehand, by being placed a few days on a frame-work of wood called the wheel, placed two feet above the furnace and its arches, and supported on four pillars at some distance from the angles of the building.

Composition of plate-glass. - This is not made now, as formerly, by random trials. The progress of chemistry, the discovery of a good process for the manufacture of soda from sea salt, which furnishes a pure alkali of uniform power, and the certain methods of ascertaining its purity, have rendered this department of glass-making almost entirely new, in France. At Saint Gobin no alkali is employed at present except artificial crystals of soda, prepared at the manufactory of Chauny, subsidiary to that establishment. Leaden chambers are also erected there for the production of sulphuric acid from sulphur. The first crop of soda crystals is reserved for the plate-glass manufacture, the other crystals and the mother-water salts are sold to the makers of inferior glass.

At the mirror-plate works of Ravenhead, near St. Helen's in Lancashire, soda crystals, from the decomposition of the sulphate of soda by chalk and coal, have been also tried, but without equal success as at Saint Gobin; the failure being unquestionably due to the impurity of the alkali. Hence, in the English establishment the soda is obtained by treating sea-salt with pearl-ash, whence carbonate of soda and muriate of potash result. The latter salt is crystallized out of the mingled solution, by evaporation at a moderate heat, for the carbonate of soda does not readily crystallize till the temperature of the solution falls below $60^{\circ} \mathrm{Fahr}$. When the muriate of potash is thus removed, the alkaline carbonate is evaporated to dryness.

Long experience at Saint Gobin has proved that one part of dry carbonate of soda is adequate to vitrify perfectly three parts of fine silicious sand, as that of the mound of Aumont near Senlis, of Alum Bay in the Isle of Wight, or of Lynn in Norfolk. It is also known that the degree of heat has a great influence upon the vitrification, and that increase of temperature will compensate for a certain deficiency of alkali; for it is certain that a very strong fire always dissipates a good deal of the soda, and yet the glass is not less beautiful. The most perfect mirror-plate has constantly afforded to M. Vauquelin in analysis, a portion of soda inferior to what had been employed in its formation. Hence, it has become the practice to add for every 100 parts of cullet or broken plate that is mixed with the glass composition, one part of alkali, to make up for the loss that the old glass must have experienced.

To the above mentioned proportions of sand and alkali independently of the cullet which may be used, dry slaked lime carefully sifted is to be added to the amount of one seventh of the sand; or the proportion will be, sand $7 \mathrm{cwts}$.; quicklime $1 \mathrm{cwt}$. ; dry carbonate of soda 2 cwts. and 37 lbs.; besides cullet. The lime improves the quality of the glass; rendering it less brittle and less liable to change. The preceding quantities of materials, suitably blended, have been uniformly found to afford most advantageous results. The practice formerly was to dry that mixture as soon as it was made, in the 
arch for the materials, but it has been ascertained that this step may be dispensed with, and the small portion of humidity present is dissipated almost instantly after they are thrown into the furnace. The coat of glaze previously applied to the inside of the pot, prevents the moisture from doing them any harm. For this reason, when the demand for glass at Saint-Gobin is very great, the materials are neither fritted nor even dried, but shovelled directly into the pot; this is called founding raw. Six workmen are employed in shovelling-in the materials either fritted or otherwise, for the sake of expedition, and to prevent the furnace getting cooled. One third of the mixture is introduced at first; whencver this is melted, the second third is thrown in, and then the last. These three stages are called the first, second, and third fusion or founding.

According to the ancient practice, the founding and refining were both executed in the pots, and it was not till the glass was refined, that it was laded into the cuvettes, where it remained only 3 hours, the time necessary for the disengagement of the air bubtles introduced by the transvasion, and for giving the metal the proper consistence for casting. At present, the period requisite for founding and refining, is equally divided between the pots and the cuvettes. The materials are left 16 hours in the pots, and as many in the curettes; so that in 32 hours the glass is ready to be cast. During the last two or three hours, the fireman or tiseur ceases to add fuel; all the openings are shut, and the glass is allowed to assume the rcquisite fluidity; an operation called stopping the glass, or performing the ceremony.

The transfer of the glass into the cuvettes, is called lading, (tréjetage). Before this is done, the cuvettes are cleared out, that is, the glass remaining on their bottom is removed, and the ashes of the firing. They are lifted red hot out of the furnace by the method presently to be described, and placed on an iron plate, near a tub filled with water. The workmen, by means of iron paddles 6 feet long, flattened at one end and hammered to an edge, scoop out the fluid glass expeditiously, and throw it into water; the curettes are now returned to the furnace, and a few minutes afterwards the lading begins.

In this operation, ladles of wrought iron are employed, furnished with long handles, which are plunged into the pots through the upper openings or lading holes, and immediately transfer their charge of glass into the buckets. Each workman dips his ladle only three times, and empties its contents into the cuvette. By these three immersions (whence the term (réjeler is derived), the large iron spoon is heated so much that when plunged into a tub full of water, it makes a noise like the roaring of a lion, which may be heard to a very great cistance.

The founding, refining, and ceremony, being finished, they next try whether the glass be ready for casting. With this view, the end of a rod is dipped into the bucket, which is called drawing the glass; the portion taken up being allowed to run off, naturally assumes a pear-shape, from the appearance of which, they can judge if the consistence be proper, and if any air bubbles remain. If all be right, the cuvettes are taken out of the furnace, and conveyed to the part of the halle where their contents are to be poured out. This process requires peculiar instruments and manipulations.

Casting.-While the glass is refining, that is, coming to its highest point of perfection, preparation is made for the most important process, the casting of the plate, whose success crowns all the preliminary labors and cares. The oven or carquaise destined to receive and anneal the plate is now heated by its small fire or tisar, to such a pitch that its sole may have the same temperature as that of the plates, being nearly red-hot at the moment of their being introduced. An unequal degree of heat in the carquaise would cause breakage of the glass. The casting table is then rolled towards the front door or throat, by means of levers, and its surface is brought exactly to the level of the sole of the oven.

The table T, fig. 515, is a mass of bronze, or now preferably cast iron, about 10 feet long, 5 feet broad, and from 6 to 7 inches thick, supported by a frame of carpentry, which rests on three cast iron wheels. At the end of the table opposite to that next to the front of the oven, is a very strong frame of timber-work, called the puppet or standard, upon which the bronze roller which spreads the glass is laid, before and after the casting. This is 5 feet long by 1 foot in diameter; it is thick in the metal but hollow in the axis. The same roller can serve only for two plates at one casting, when another is put in its place, and the first is laid aside to cool ; for otherwise the hot roller would at a third casting make the plate expand unequally, and cause it to crack. When the rollers are not in action, they are laid aside in strong wooden trestles, like those employed by sawyers. On the two sides of the table in the line of its length, are two parallel bars of bronze, $t, t$, destined to support the roller during its passage from end to end; the thickness of these bars determines that of the plate. The table being thus arranged, a crane is had recourse to for lifting the cuvette, and keeping it suspended, till it be emptied upon the table. This raising and suspension are effected by means of an iron gib, furnished with pulleys, beld horizontally, and which turns with them. 
The tongs $T$, fig. 515, are made of four iron bars, bent into a square frame in their middle, for embracing the bucket. Four chains proceeding from the corners of the frame $\mathbf{v}$ are united at their other ends into a ring which fits into the hook of the crane.

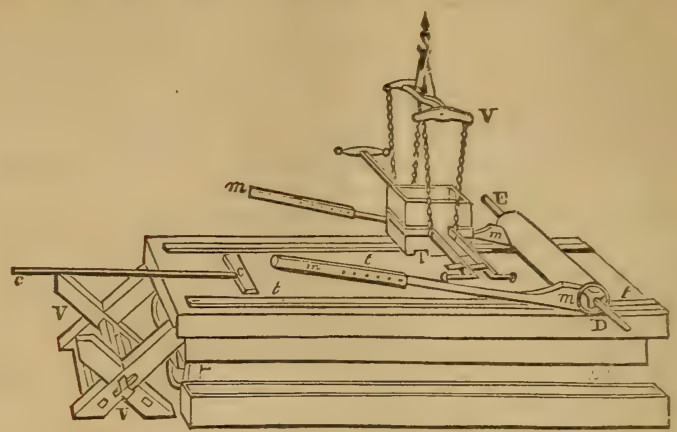

Things being thus arranged, all the workmen of the foundry co-operate in the manipulations of the casting. Two of them fetch, and place quickly in frunt of one of the lower openings, the small cuvette-carriage, which bears a forked bar of iron, having two prongs corresponding to the two holes left in the fire-tile door. This fork, mounted on the axle of two cast-iron wheels, extends at its other end into two branches terminated by handles, by which the workmen move the fork, lift out the tile stopper, and set it down against the outer wall of the furnace.

The instant these men retire, two others push forward into the opening the extremity of the tongs-carriage, so as to seize the bucket by the girdle, or rather to clamp it. At the same time, a third workman is busy with an iron pinch or long chisel, detaching the bucket from its seat, to which it often adheres by some spilt glass; whenever it is free, he withdraws it from the furnace. Two powerful branches of iron united by a bolt, like two scissor blades, which open, come together, and join by a quadrant near the other end, form the tongs-carriage, which is mounted upon two wheels like a truck.

The same description will apply almost wholly to the iron-plate carriage, on which the bucket is laid the moment it is taken out of the furnace; the only difference in its construction is, that on the bent iron bars which form the tail or lower steps of this car. riage (in place of the tongs) is permanently fastened an iron plate, on which the bucket is placed and carried for the casting.

Whenever the cuvette is set upon its carriage, it must be rapidly wheeled to its station near the crane. The tongs $T$ above described are now applied to the girdle, and are then hooked upon the crane by the suspension chains. In this position the bucket is skimmed by means of a copper tool called a sabre, because it has nearly the shape of that weapon. Every portion of the matter removed by the sabre is thrown into a copper ladle (poche de gamin), which is emptied from time to time into a cistern of water. After being skimmed, the bucket is lifted up, and brushed very clean on its sides and bottom; then by the double handles of the suspension-tongs it is swung round to the table, where it is seized by the workmen appointed to turn it orer; the roller having been previously laid on its ruler-bars, near the end of the table which is in contact with the annealing oven. The cuvette-men begin to pour out towards the right extremity $\mathrm{E}$ of the roller, and terminate when it has arrived at the left extremity D. While preparing to do so, and at the instant of casting, two men place within the ruler-bar on each side, that is, between the bar and the liquid glass, two iron instruments called hands, $m, m, m, m$, which prevent the glass from spreading beyond the rulers, while another draws along the table the wiping bar $c, c$, wrapped in linen, to remove dust, or any small objects which may inter: pose between the table and the liquid glass.

Whenever the melted glass is poured out, two men spread it over the table, guiding the roller slowly and steadily along, beyond the limits of the glass, and then run it smartly into the wooden standard prepared for its reception, in place of the trestles $\mathrm{v}, \mathrm{v}$.

The empty bucket, while still red-hot, is hung again upon the crane, set on its plateiron earriage, freed from its tongs, and replaced in the furnace, to be speedily cleared out anew, and charged with fresh fluid from the pots. If while the roller glides along, the two workmen who stand by with picking tools, perceive tears in the matter in advance of the roller, and can dexterously snatch them out, they are suitably rewarded, according to the spot where the blemish lay, whether in the centre, where it would have proved most detrimental, or near the edge. These tears proceed usually from 
small portions of semi-vitrified matter which fall from the vault of the furnace, and from their density occupy the bottom of the cuvettes.

While the plate is still red-hot and ductile, about 2 inches of its end opposite to the carquaise door is turned up with a tool; this portion is called the head of the mirror; against the outside of this head, the shovel, in the shape of a rake without teeth, is applied, with which the plate is eventually pushed into the oven, while two other workmen press upon the upper part of the head with a wooden pole, eight feet long, to preserve the plate in its horizontal position, and prevent its being warped. The plate is now left for a few moments near the throat of the carquaise, to give it solidity ; after which it is pushed farther in by means of a very long iron tool, whose extremity is forked like the letter $y$, and hence bears that name; and is thereby arranged in the most suitable spot for allowing other plates to be introduced.

However numerous the manipulations executed from the moment of withdrawing the curette from the furnace, till the cast-plate is pushed into the annealing oven, I have seen them all performed in less than five minutes; such silence, order, regularity, and despatch prevail in the establishment of Saint-Gobin.

When all the plates of the same casting have been placed in the carquaise, it is sealed up, that is to say, all its orifices are closed with sheets of iron, surrounded and made tight with plastic loam. With this precaution, the cooling goes on slowly and equably in every part, for no cooling current can have access to the interior of the oven.

After they are perfectly cooled, the plates are carefully withdrawn one after another, keeping them all the while in a horizontal position, till they are entirely out of the carquaise. As soon as each plate is taken out, one set of workmen lower quickly and steadily the edge which they hold, while another set raise the opposite edge, till the glass be placed upright on two cushions stuffed with straw, and covered with canvass. In chis vertical position they pass through, beneath the lower edge of the plate, three girths or straps each four feet long, thickened with leather in their middle, and ending in wooden handles; so that one embraces the middle of the plate, and the other two, the ends. The workmen, six in number, now seize the handles of the straps, lift up the glass closely to their bodies, and convey it with a regular step to the warehouse. Here the head of the plate is first cut off with a diamond square, and then the whole is attentively examined, in reference to its defects and imperfections, to determine the sections which must be made of it, and the eventual size of the pieces. The pairings and small cuttings detached are set aside, in order to be ground and mixed with the raw materials of " another glass-pot.

The apartment in which the roughing-down and smoothing of the plates is performed, is furnished with a considerable number of stone tables truly hewn and placed apart like billiard tables, in a horizontal position, about 2 feet above the ground. They are rectangular, and of different sizes proportional to the dimensions of the plates, which they ought always to exeeed a little. These tables are supported either on stone pillars or wooden frames, and are surrounded with a wooden board whose upper edge stands somewhat below their level, and leaves in the space between it and the stone all round an interval of 3 or 4 inches, of which we shall presently see the use.

A cast plate, unless formed on a table quite new, has always one of its faces, the one next the table, rougher than the other; and with this face the roughing-down begins. With this view, the smoother face is cemented on the stone table with Paris-plaster. But often, instead of one plate, several are cemented alongside of each other, those of the same thickness being carefully selected. They then take one or more crude plates of about one third or one fourth the surface of the plate fixed to the table, and fix it on them with liquid gypsum to the large base of a quadrangular truncated pyramid of stone, of a weight proportioned to its extent, or about a pound to the square inch. This pyramidal muller, if small sized, bears at each of its angles of the upper face a peg or ball, which the grinders lay hold of in working it; but when of greater dimension, there is adapted to it horizontally a wheel of slight construction, 8 or 10 feet in diameter, whose circumference is made of wood rounded so as to be seized with the hand. The upper plate is now rubbed over the lower ones, with moistened sand applied between.

Tlis operation is however performed by machinery. The under plate being fixed or imbedded in stucco, on a solid table, the upper one likewise imbedded by the same cernent in a cast-iron frame, has a motion of circumrotation given to it closely resembling that communicated by the human hand and arm, moist sand being supplied between them. While an eccentric mechanism imparts this double rotatory movement to the upper plate round its own centre, and of that centre round a point in the lower plate, this plate, placed on a moveable platform, changes its position by a slow horizontal motion, both in the direction of its length and its breadth. By this ingenious contrivance, which pervades the whole of the grinding and polishing machinery, a remarkable regularity of friction and truth of surface is produced. When the plates are sufficiently worked on one face, they are reversed in the frames, and worked together on 
the other. The Paris plaster is usually colored red, in order to show any defects in the glass.

The smoothing of the plates is effected on the same principles by the use of moist emery washed to successive degrees of fineness, for the successive stages of the operation; and the polishing process is performed by rubbers of hat-felt and a thin paste of coleothar and water. The colcothar, called also crocus, is red oxyde of iron prepared by the ignition of copperas, with grinding and elutriation of the residuum.

The last part or the polishing process is performed by hand. This is managed by females, who slide one plate over another, while a little moistened putty of tin finely levigated is thrown between.

Large mirror-plates are now the indispensable ornaments of every large and sumptrous apartment; they diffuse lustre and gayety round them, by reflecting the rays of light in a thousand lines, and by multiplying indefinitely the images of objects placed between op. posite parallel planes.

The silvering of plane mirrors consists in applying a layer of tin-foil alloyed with mercury to their posterior surface. The workshop for executing this operation is provided with a great many smooth tables of fine freestone or marble, truly levelled, having round their contour a rising ledge, within which there is a gutter or groove which terminates by a slight slope in a spout at one of the corners. These tables rest upon an axis of wood or iron which runs along the middle of their length; so that they may be inclined easily into an angle with the horizon of 12 o: 13 degrees, by means of a hand-screw fixed below. They are also furnished with brushes, with glass rules, with rolls of woollen stuff, several pieces of flannel, and a great many weights of stone or cast-iron.

The glass-tinner, standing towards one angle of his table, sweeps and wipes its surface with the greatest care, along the whole surface to be occupied by the mirror-plate; then taking a sheet of tin-foil adapted to his purpose, he spreads it on the table, and applies it closely with a brush, which removes any folds or wrinkles. The table being horizontal, he pours over the tin a small quantity of quicksilver, and spreads it with a roll of woollen stuff; so that the tin-foil is penetrated and apparently dissolved by the mercury. Placing now two rules, to the right and to the left, on the borders of the sheet, he pours on the middle a quantity of mercury sufficient to form everywhere a layer about the thickness of a crown piece; then removing with a linen rag the oxyde or other impurities, he applies to it the edge of a sheet of paper, and advances it about half an inch. Meanwhile another workman is occupied in drying very nicely the surface of the glass that is to be silvered, and then hands it to the master workman, who, laying it flat, places its anterior edge first on the table, and then on the slip of paper; now pushing the glass forwards, he takes care to slide it along so that neither air nor any coat of oxyde on the mercury can remain beneath the plate. When this has reached its position, he fixes it there by a weight applied on its side, and gives the table a gentle slope, to run off all the loose quicksilver by the gutter and spout. At the end of five minutes he covers the mirror with a piece of flannel, and loads it with a great many weights which are left upon it for twenty-four hours, under a gradually increased inclination of the table. By this time the plate is ready to be taken off the marble table, and laid on a wooden one sloped like a reading desk, with its under edge resting on the ground, while the upper is raised successively to different elevations by means of a cord passing over a pulley in the ceiling of the room. Thus the mirror has its slope graduated from day to day, till it finally arrives at a vertical position. About a month is required for draining out the superfluous mercury from large mirrors; and from 18 to 20 days from those of moderate size. The sheets of tin-foil being always somewhat larger than the glass. plate, their edges must be paired smooth off, before the plate is lifted off the marble table.

Process for silvering concave mirrors.-Having prepared some very fine Paris plaster by passing it through a silk sieve, and some a little coarser passed through hair-cloth, the first is to be made into a creamy liquor with water, and after smearing the concave surface of the glass with a film of olive oil, the fine plaster is to be poured into it, and spread by turning about, till a layer of plaster be formed about a tenth of an inch thick. The second or coarse plaster, being now made into a thin paste, poured over the first, and moved about, readily incorporates with it, in its imperfectly hardened state. Thus an exact mould is obtained of the concave surface of the glass, which lies about three quarters of an inch thick upon it, but is not allowed to rise above its outer edge.

The mould, being perfectly dried, must be marked with a point of coincidence on the glass, in order to permit of its being exactly replaced in the same position, after it has been lifted out. The mould is now removed, and a round sheet of tin-foil is applied to it, so large that an inch of its edge may project beyond the plaster all round; this border being necessary for fixing the tin to the contour of the mould by pellets of white wax softened a little with some Venice turpentine. Before fixing the tin-foil, however, it must be properly spread over the mould, so as to remove every wrinkle; which the 
pliancy of the foil easily admits of, by uniform and well-directed pressure with the fingers.

The glass being placed in the hollow bed of a tight sack filled with fine sand, set in a well-jointed box, capable of retaining quicksilver, its concave surface must be dusted with sifted wood-ashes, or Spanish white contained in a small cotton bag, and then well wiped with clean linen rags, to free it from all adhering impurity, and particularly the moisture of the breath. The concavity must be now filled with quicksilver to the very lip, and the mould, being dipped a little way into it, is withdrawn, and the adhering mercury is spread over the tin with a soft flannel roll, so as to amalgamate and brighten its whole surface, taking every precaution against breathing on it. Whenever this brightening scems complete, the mould is to be immersed, not vertically, but one edge at first, and thus obliquely downwards till the centres coincide; the mercury meanwhile being slowly displaced, and the mark on the mould being brought finally into coincidence with the mark on the glass. The mould is now left to operate by its own weight, in expelling the superfluous mercury, which runs out upon the sand-bag and thence into a groove in the bottom of the box, whence it overflows by a spout into a leather bag of reception. After half an hour's repose, the whole is cautiously inverted, to drain off the quicksilver more completely. For this purpose, a box like the first is provided with a central support rising an inch above its edges; the upper surface of the support being nearly equal in ciarreter to that of the mould. Two workmen are required to execute the following operation. Each steadies the mould with the one hand, and raises the box with the other, taking eare not to let the mould be deranged, which they rest on the (convex) support of the second box. Before inverting the first apparatus, however, the reception bag must be removed, for fear of spilling its mercury. The redundant quicksilver now drains off; and if the weight of the sandbag is not thought sufficient, supplementary weights are added at pleasure. The whole is left in this position for two or three days. Before separating the mirror from its mould, the border of tinfoil, fixed to it with wax, must be pared off with a knife. Then the weight and sandbag being removed, the glass is lifted up with its interior coating of tinamalgam.

For silvering a convex surface.-A concave plaster mould is made on the convex glass, and their points of coincidence are defined by marks. This mould is to be lined with tinfoil, with the precautions above described; and the tin surface being first brightened with a little mercury, the mould is then filled with the liquid metal. The glass is to be well cleaned, and immersed in the quicksilver bath, which will expel the greater part of the metal. A sandbag is now to be laid on the glass, and the whole is to be inverted as in the former case on a support; when weights are to be applied to the mould, and the mercury is left to drain off for several days.

If the glass be of large dimensions, 30 or 40 inches, for example, another method is adopted. A circular frame or hollow ring of wood or iron is prepared, of twice the diameter of the mirror, supported on three feet. A circular piece of new linen cloth of close texture is cut out, of equal diameter to the ring, which is hemmed stoutly at the border, and furnished round the edge with a row of small holes, for lacing the cloth to the ring, so as to leave no folds in it, but without bracing it so tightly as to deprive it of the elasticity necessary for making it into a mould. This apparatus being set horizontally, a leaf of tinfoil is spread over it, of sufficient size to cover the surface of the glass; the tin is first brightened with mercury, and then as much of the liquid metal is poured on as a plane mirror requires. The convex glass, wel, cleaned, is now set down on the cloth, and its own weight, joined to some additional weights, gradually presses down the cloth, and causes it to assume the form of the glass which thus comes into close contact witr the tin submersed under the quicksilver. The redundant quicksilver is afterwards drained off by inversion, as in common cases.

The follnwing recipe has been given for silvering the inside of glass globes. Melt in an irnn ladle or a crucible, equal parts of tin and lead, adding to the fused alloy one part of bruised bismuth. Stir the mixture well, and pour into it as it cools, two parts of dry mercury; agitating anew and skimming off the drossy film from the surface of the amalgam. The inside of the glass globe, being freel from all adhering dust and humidity, is to be gently heated, while a little of the semi-fluid amalgam is introduced. The liquidity being increased by the slight degree of heat, the metallic coating is applied to all the points of the glass, by turning round the globe in every direction, but so slowly as to favor the adhesion of the alloy. This silvering is not so substantial as that of plane mirrors : but the form of the vessel, whether a globe, an ovoid, or a cylinder, conceals or palliates the defects by counter reflection from the opposite surfaces.

Colored Glasses und Artificial Gems. - The general vitreous body preferred by Fontanieu in his treatise on this subject, which he calls the Mayence base, is prepared in the following manner. Eight ounces of pure rock-crystal or flint in powder, mixed with 24 ounces of salt of tartar, are baked and left to cool. This is afterwards poured 
into a basin of hot water, and treated with dilute nitric acid till it ceases to effervesce, when the frit is to be washed till the water comes off tasteless. 'The frit is now dried and mixed with 12 ounces of fine white lead, and the mixture is to be levigated and elutriated with a little distilled water. An ounce of calcined borax is to be added to abour 12 ounces of the preceding mixture in a dry state, the whole rubbed together in a porce lain mortar, then melted in a clean crucible, and poured out into cold water. This vitreous matter must be dried, and melted a second and a third time, always in a new crucible, and after each melting poured into cold water as at first, taking care to separate the lead that may be revired. To the last glass ground to powder, five drachms of nitre are to be added, and the mixture being melted for the last time, a mass of crystal will be found in the crucible with a beautiful lustre. The diamond is well imitated by this Mayence base. Another very fine white crystal may be obtained, according to $\mathbf{M}$. Fontanieu, from eight ounces of white lead, two ounces of powdered borax, half a grain of manganese, and three ounces of rock-crystal, treated as above.

The colors of artificial gems are obtained from metallic oxydes. The oriental topaz is prepared by adding oxyde of antimony to the base; the amethyst from manganese, with a little purple precipitate of Cassius; the beryl from antimony and a very little cobalt; yellow artificial diamond and opal from horn-silver (chloride of silver); blue stone from cobalt. See Pastes and Pigmente Vitrifiable.

The following are recipes for making the different kinds of glass.

1. Bottle glass. -11 pounds of dry glauber salts; 12 pounds of soaper salts; a half bushel of waste soap ashes; 56 pounds of sand; 22 pounds of glass skimmings; 1 cwt. of green broken glass; 25 pounds of basalt. This mixture affords a dark green glass.

2. Yellow or white sand, 100 parts; kelp, 30 to 40 ; lixiviated wood ashes, from 160 to 170 parts ; fresh wood ashes, 30 to 40 parts; potter's clay, 80 to 100 parts; cullet or broken glass, 100 . If basalt be used, the proportion of kelp may be diminished.

In two bottle-glass houses in the neighborhood of Valenciennes, an unknown ingredient, sold by a Belgian, was employed, which he called spar. This was discovered by chemical analysis to be sulphate of baryta. The glass-makers observed that the bottles which contained some of this substance were denser, more homogeneous, more fusible, and worked more kindly, than those formed of the common materials. When one prime equivalent of the silicate of baryta $=123$, is mixed with three primes of the silicate of soda $=(3 \times 77 \cdot 6) 232 \cdot 8$, and exposed in a proper furnace, vitrification readily ensues, and the glass may be worked a little under a cherry red heat, with as much ease as a glass of lead, and has nearly the same lustre. Since the ordinary run of glass-makers are not familiar with atomic proportions, they should have recourse to a scientific chemist, to guide them in using such a proportion of sulphate of baryta as may suit their other vitreous ingredients; for an excess or defect of any of them will injure the quality of the glass.

3. Green window glass, or broad glass. -11 pounds of dry glauber salts; 10 pounds of soaper salts; half a bushel of lixiviated soap waste; 50 pounds of sand; 22 pounds of glass pot skimmings; 1 ewt. of broken green glass.

4. Crown glass. -300 parts of fine sand; 200 of good soda ash; 33 of lime; from 250 to 300 of broken glass; 60 of white sand; 30 of purified potash; 15 of saltpetre ( 1 of borax); $\frac{1}{2}$ of arsenious acid.

5. Nearly white table glass. -20 pounds of potashes; 11 pounds of dry glauber salts; 16 of soaper salt; 55 of sand; 140 of cullet of the same kind. Another.-100 of sand; 235 of kelp; 60 of wood ashes; $1 \frac{1}{3}$ of manganese; 100 of broken glass.

6. Whire table glass. -40 pounds of potashes; 11 of chalk; 76 of sand; $\frac{1}{2}$ of manganese; 95 of white cullet.

Another.-50 of purified potashes; 100 of sand ; 20 of chalk; and 2 of saltpetre.

Bohemian table or plate glass is made with 63 parts of quartz; 26 of purified potashes; 11 of sifted slaked lime, and some cullet.

7. Crystral glass. -60 parts of purified potashes; 120 of sand ; 24 of chalk; 2 of saltpetre; 2 of arsenious acid; $\frac{1}{16}$ of manganese.

Another. - 70 of purified pearlashes; 120 of white sand; 10 of saltpetre; $\frac{1}{2}$ of arsenious acid; $\frac{1}{3}$ of manganese.

A third. -67 of sand; 23 of purified pearlashes; 10 of sifted slaked lime; $\frac{1}{4}$ of manganese; ( 5 to 8 of red lead).

A fourth. -120 of white sand; 50 of red lead; 40 of purified pearlash; 20 of saltpetre; $\frac{3}{3}$ of manganese.

A fifth.-120 of white sand; 40 of pearlash purified; 35 of red lead; 13 of saltpetre; $\frac{1}{1} \frac{1}{2}$ of manganese.

A sixth. -30 of the finest sand; 20 of red lead; 8 of pearlash purified; 2 of salt. petre; a little arsenious acid and manganese. 
GLASS-CUTTING.

A seventh. -100 of sand; 45 of red lead; 35 of purified pearlashes; $\frac{1}{7}$ of manganese; of arsenious acid.

8. Plaie gıass.-Very white sand 300 parts; dry purified soda 100 parts; carbonate of lime 43 parts; manganese 1 ; cullet 300 .

Another.-Finest sand 720 ; purified soda 450 ; quicklime 80 parts; saltpetrre 25 parts ; cullet 425 .

A little borax has also been prescribed; much of it communicates an exfoliating property to glass.

Tabular view of the composition of several kinds of Glass.

\begin{tabular}{|c|c|c|c|c|c|c|c|c|c|}
\hline & No. 1. & No.2. & No. 3. & No. 4 . & No. 5. & No. 6 . & No. 7 . & No. 8. & No. 9. \\
\hline Silica - & $71 \cdot 7$ & $69 \cdot 2$ & $62 \cdot 8$ & $69 \cdot 2$ & $60 \cdot 4$ & $53 \cdot 55$ & $59 \cdot 2$ & $51 \cdot 93$ & $42 \cdot 5$ \\
\hline Potash & $12 \cdot 7$ & $15 \cdot 8$ & $22 \cdot 1$ & $8 \cdot 0$ & $3 \cdot 2$ & $5 \cdot 48$ & $9 \cdot 0$ & $13 \cdot 77$ & $11 \cdot 7$ \\
\hline Soda - & $2 \cdot 5$ & $3 \cdot 0$ & & $3 \cdot 0$ & S. pot. & & & & \\
\hline Lime - & $10 \cdot 3$ & $7 \cdot 6$ & 12.5 & $13 \cdot 0$ & $20 \cdot 7$ & $29 \cdot 22$ & & & 0.5 \\
\hline Alumina & 0.4 & $1 \cdot 2$ & & $3 \cdot 6$ & $10 \cdot 4$ & $6 \cdot 01$ & & & $1 \cdot 8$ \\
\hline Magnesia - & & $2 \cdot 0$ & & $0 \cdot 6$ & $0 \cdot 6$ & & & & \\
\hline $\begin{array}{l}\text { Oxyde of iron } \\
\text { - manganese }\end{array}$ & $\begin{array}{l}0 \cdot 3 \\
0 \cdot 2\end{array}$ & 0.5 & $\{2.6$ & $1 \cdot 6$ & $3 \cdot 8$ & $5 \cdot 74$ & $\begin{array}{l}0 \cdot 4 \\
1 \cdot 0\end{array}$ & & \\
\hline -lead - & & & & & 0 & & $28 \cdot 2$ & $33 \cdot 28$ & $43 \cdot 5$ \\
\hline Daryta & & & & & 0.9 & & & & \\
\hline
\end{tabular}

No. 1 is a very beautiful white wine glass of Neuwelt in Bohemia.

No. 2. Glass tubes, much more fusible than common wine glasses.

No. 3. Crown glass of Bohemia.

No. 4. Green glass, for medicinal vials and retorts.

No. 5. Flask glass of St. Etienne, for which some heavy spar is used.

No. 6. Glass of Sevres.

No. 7. London glass employed for chemical and physical purposes.

No. 8. English flint glass.

No. 9. Guinand's flint glass.

The manufacture of Glass beads at Murano near Venice, is most ingeniously simple, Tubes of glass of every color are drawn out to great lengths in a gallery adjoining the glass-house pots, in the same way as the more moderate lengths of thermometer and barometer tubes are drawn in our glass-houses. These tubes are chopped into very small pieces of nearly uniform length on the upright edge of a fixed chisel. These elementary cylinders, being then put in a heap into a mixture of fine sand and wood ashes, are stirred about with an iron spatula till their cavities get filled. This curious mixture is now transferred to an iron pan suspended over a moderate fire and continually stirred about as before, whereby the cylindrical bits assume a smooth rounded form; so that when removed from the fire and cleared ont in the bore, they constitute beads, which are packed in casks, and exported in prodigious quantities to almost every country, especially to Africa and Spain.

GLASS CUTTING AND GRINDING, for common and optical purposes. By this mechanical process the surface of glass may be modified into almost any ornamental or useful form.

1. The grinding of crystal ware. This kind of glass is best adapted to receive polished facets, both on account of its relative softness, and its higher refractive power, which gives lustre to its surface. The cutting shop should be a spacious long apartment, furnished with numerous sky-lights, having the grinding and polishing lathes arranged right under them, which are set in motion by a steam-engine or water-wheel at

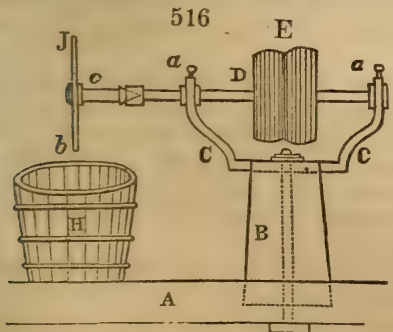
one end of the building. A shaft is fixed as usual in sallowses along the ceiling; and from the pulleys of the shaft, bands descend to turn the different lathes, by passing round the driving pulleys near their ends.

The turning lathe is of the simplest construction. Fig. 516, $\mathrm{D}$ is an iron spindle with two well-turned prolongations, running in the iron puppets $a a$, between two concave bushes of tin or type metal, which may be pressed more or less together by the thumb-screws shown in the figure. These two puppets are made fast to the wooden support $\mathbf{B}$, which is attached by a strong screw and bolt to the

longitudinal beam of the workshop A. $\quad \mathbf{E}$ is the fast and loose pulley for putting the 
lathe into and out of gear with the driving shaft. The projecting end of the spindle is furnished with a hollow head-piece, into which the rod $c$ is pushed tight. This rod car. ries the cutting or grinding disc plate. For heavy work, this rod is fixed into the head by a screw. When a conical fit is preferred, the cone is covered with lead to increase the friction.

Upon projecting rods or spindles of that kind the different dises for cutting the glass are made fast. Some of these are made of fine sandstone or polishing slate, from 8 to 10 inches in diameter, and from $\frac{3}{4}$ to $\frac{1}{2}$ inch thick. They must be carefully turned and polished at the lathe, not only upon their rounded but upon their flat face, in order to grind and polish in their turn the flat and curved surfaces of glass vessels. Other discs of the same diameter, but only $\frac{3}{4}$ of an inch thick, are made of cast tin truly turned, and serve for polishing the vessels previously ground; a third set consist of sheet iron from $\frac{1}{6}$ to $\frac{1}{2}$ an inch thick, and 12 inches in diameter, and are destined to cut grooves in glass by the aid of sand and water. Small dises of well-hammered copper from $\frac{1}{2}$ to 3 inches in diameter, whose circumference is sometimes flat, and sometimes concave or convex, serve to make all sorts of delineations upon glass by means of emery and oil. Lastly, there are rods of copper or brass furnished with small hemispheres from $\frac{1}{21}$ to $\frac{1}{4}$ of an inch in diameter, to excavate round hollows in glass. Wooden discs are also employed for polishing, made of white wood cut across the grain, as also of cork.

The cutting of deep indentations, and of grooves, is usually performed by the iron disc, with sand and water, which are allowed constantly to trickle down from a wooden hopper placed right over it, and furnished with a wooden stopple or plug at the apex, to regulate by its greater or less looseness the flow of the grinding materials. The same effect may be produced by using buckets as shown in fig. 517. The

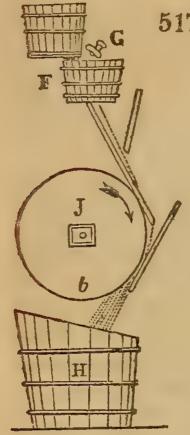
sand which is contained in the bucket $r$, above the lathe, has a spigot and faucet inserted near its bottom, and is supplied with a stream of water from the stopeock in the vessel $\mathrm{G}$, which, together running down the inclined board, are conducted to the periphery of the dise as shown in the figure, to whose lowest point the glass vessel is applied with pressure by the hand. The sand and water are afterwards collected in the tub H. Finer markings, which are to remain without lustre, are made with the small copper discs, emery, and oil. The polishing is effected by the edge of the tin disc, which is from time to time moistened with putty (white oxyde of tin) and water. The wooden disc is also employed for this purpose with putty, colcothar, or washed tripoli. For fine delineations, the glass is first traced over with some colored varnish, to guide the hand of the cutter.

In grinding and facetting crystal glass, the deep grooves are first cut, for example the cross lines, with the iron disc and rounded edge, by means of sand and water. That disc is one sixth of an inch thick and 12 inches in diameter. With another iron disc about half an inch thick, and more or less in diameter, according to the curvature of the surface, the grooves may be widened. These roughly' cut parts must be next smoothed down with the sandstone disc and water, and then polished with the wooden disc about half an inch thick, to whose edge the workman applies, from time to time, a bag of fine linen containing some ground pumice moistened with water. When the cork or wooden disc edged with hat felt is used for polishing, putty or colcothar is applied to it. The above several processes in a large manufactory, are usually committed to several workmen on the principle of the division of labor, so that each may become expert in his department.

2. The grinding of optical glasses. - The glasses intended for optical purposes, being spherically ground, are called lenses; and are used either as simple magnifiers and spectacles, or for telescopes and microscopes. The curvature is always a portion of a sphere, and either convex or concave. This form ensures the convergence or divergence of the rays of light that pass through them, as the polishing does the brightness of the image.

The grinding of the lenses is performed in brass moulds, either concave or convex, form: $\in$ d to the same curvature as that desired in the lenses; and may be worked either by hand or by machinery. A gauge is first cut out out of brass or copper plate to suit the curvature of the lens, the circular are being traced by a pair of compasses. In this way both a convex and concave circular gauge are obtained. To these gauges the brass moulds are turned. Sometimes, also, lead moulds are used. After the two moulds are made, they are ground face to face with fine emery.

The piece of glass is now roughed into a circular form by a pair of pincers, learing It a little larger than the finished lens ought to be, and then smoothed round upon the stone disc, or in an old mould with emery and water, and is next made fast to a holdfast. This consists of a round brass plate having a screw in its back; and is somewhat smaller in diameter than the lens, and two thirds as thick. This as turned concave upon 
the lathe, and then attached to the piece of glass by drops of pitch applied to several points of its surface, taking care, while the pitch is warm, that the centre of the glass coincides with the centre of the brass plate. This serves not merely as a holdfast, by enabling a person to seize its edge with the fingers, but it prevents the glass from bending by the necessary pressure in grinding.

The glass must now be ground with coarse emery upon its appropriate mould, whether convex or concave, the emery being all the time kept moist with water. To prevent the heat of the hand from affecting the glass, a rod for holding the brass plate is screwed to its back. For every six turns of circular motion, it must receive tyo or three rubs across the diameter in different directions, and so on alternately. The middle point of the glass must never pass beyond the edge of the mould; nor should strong pressure be at any time applied. Whenever the glass has assumed the shape of the mould, and touches it in every point, the coarse emery must be washed away, finer be substituted in its place, and the grinding be continued as before, till all the scratches disappear, and a uniforis dead surface be produced. A commencement of polishing is now to be given with pumice stone powder. During all this time the convex mould should be occasionally worked in the concave, in order that both may preserve their correspondence of shape between them. After the one surface has been thus finished, the glass must be turned over, and treated in the same way upon the other side.

Both surfaces are now to be polished. With this view equal parts of pitch and rosin must be melted together, and strained through a cloth to separate all impurities. The coricave mould is next to be heated, and covered with that mixture in a fluid state to the thichness uniformly of one quarter of an inch. The cold convex mould is now to be pressed down into the yielding pitch, its surface being quite clean and dry, in order to give the pitch the exact form of the ground lens; and both are to be plunged into cold water till they be chilled. This pitch impression is now the mould upon which the glass is to be polished, according to the methods above described, with finely washed colcothar and water, till the surface become perfectly clear and brilliant. To prevent the pitch from changing its figure by the friction, cross lines must be cut in it about $\frac{1}{2}$ an inch asunder, and 1-12th of an inch broad and deep. These grooves remove all the superfluous parts of the polishing powder, and tend to preserve the polishing surface of the pitch clean and unaltered. No additional colcothar after the first is required in this par: of the process; but only a drop of water from time to time. The pitch gets warm as the polishing advances, and renders the friction more laborious from the adhesion between the surfaces. No interruption must now be suffered in the work, nor must either water or colcothar be added; but should the pitch become too adhesive, it must be merely breathed upon, till the polish be complete. The nearer the lens is brought to a true and fine surface in the first grinding, the better and more easy does the polishing become. It should never be submitted to this process with any scratches perceptible in it, even when examined by a magnifier.

As to small lenses and spectacle eyes, several are ground and polished together in a mould about 6 inches in diameter, made fast to a stiffening plate of brass or iron of $a$ shape corresponding with the mould. The pieces of glass are affixed by means of drops of pitch, as above described, to the mould, close to each other, and are then all treated as if they formed but one large lens. Plane glasses are ground upon a surface of pitch rendered plane by the pressure of a piece of plate glass upon it in its softened state.

Lenses are also ground and polished by means of machinery, into the details of which the limits of this work will not allow me to enter.

A Return to an Order of the Honorable the House of Commons, dated 1st March, 1838 , of the Amount of Duty charged on Glass; distinguishing the Amount charged on Flint, Plate, Broad, Crown, Bottle, and German Sheet, in the Year ending the 5 th day of January, 1838; together with the Amount of Drawback on each description of Glass; the produce of the Duties in England, Scotland, and Ireland, stated separately.

\begin{tabular}{|c|c|c|c|c|c|c|c|}
\hline \multicolumn{7}{|c|}{ Amount of Duty charged on } & \multirow[b]{2}{*}{ Total. } \\
\hline- & Flint Glass. & Plate. & Broad. & Crown. & Bottle. & $\begin{array}{c}\text { German } \\
\text { Sheet. }\end{array}$ & \\
\hline $\begin{array}{l}\text { Enaland } \\
\text { Scotland } \\
\text { Ireland }\end{array}$ & $\left|\begin{array}{ccr}f & s . & d \\
76.052 & 1 & 0 \\
7.53 n & 9 & 4 \\
6,736 & 12 & 11\end{array}\right|$ & $\begin{array}{cc}\mathcal{E} & 8 \\
68,902 & 10\end{array}$ & 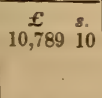 & \begin{tabular}{cccc}
$£$ & s. & $d$. \\
533,404 & 6 & 7 \\
16,423 & 11 & 6 \\
\multicolumn{2}{c}{}
\end{tabular} & \begin{tabular}{rrr}
\multicolumn{1}{c}{} & $s$. & $d$. \\
122,617 & 10 & 2 \\
32,246 & 4 & 1 \\
3,642 & 0 & 3
\end{tabular} & $\begin{array}{c} \pm \\
25,511 \\
-\end{array}$ & $\begin{array}{rrr}f & s . & d . \\
837.277 & 14 & 9 \\
56,200 & 4 & 11 \\
10,378 & 13 & 2\end{array}$ \\
\hline Total & $\begin{array}{lll}90.319 & 3 & 3\end{array}$ & $68,902 \quad 10$ & 10,78910 & $\longdiv { 5 4 9 , 8 2 7 \quad 1 8 \quad 1 }$ & $\mid \overline{158,505} \overline{14} 6$ & $25,511 \overline{1 \pi}$ & $903,856 \quad 12 \quad 10$ \\
\hline
\end{tabular}




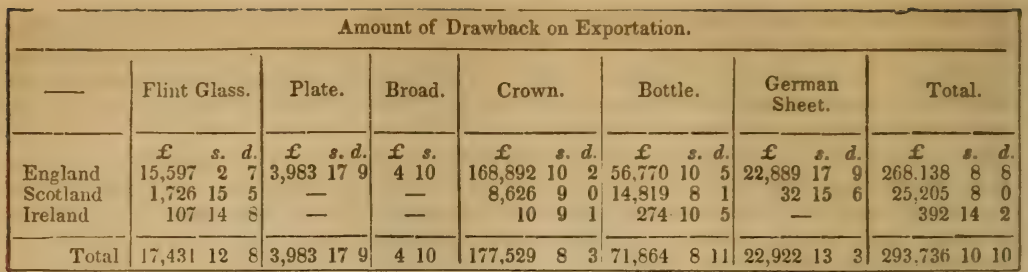

The duties payable in the United Kingdom upon the different descriptions of glass are, for-

Flint glass, the finished article

British plate or German sheet, and crown glass, ditto

Broad glass,

Bottles,

Plate glass, the fused material in pot
$£$ s. $d$.

- $0 \quad 02$ per lb.

- 3136 per cwt.

- 1100 -

$-0770-$

GLAZES. See PotTery.

GLAZIER is the workman who cuts plates or panes of glass, with the diamond, and fastens them by means of putty in frames or window casements. See Diamond, for an explanation of its glass-cutting property.

GLAUBER SALT is the old name of sulphate of soda.

GLOVE MANUFACTURE. In February, 1822, Mr. James Winter, of Stokeunder-Hambdon, in the county of Somerset, obtained a patent for an improvement upon

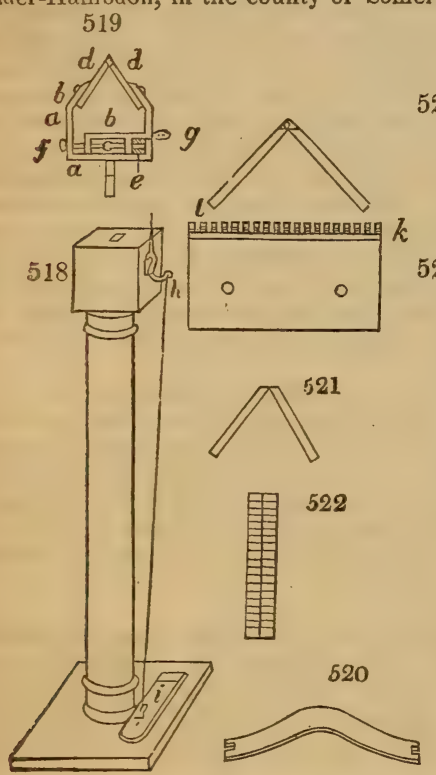
a former patent machine of his for sewing and pointing leather gloves. Fig. 518 represents a pedestal, upon which the instrument called 523 the jaws is to be placed. Fig. 519 shows the jaws, which instead of opening and closing by a circular movement upon a joint as described in the former specification, are now made to open and shut by a parallel horizontal movement, effected by a slide and serew; $a \boldsymbol{a}$ is the 524 fixed jaw, made of one piece, on the under side of which is a tenon, to be inserted into the top of the pedestal. By means of this tenon the jaws may be readily removed, and another similar pair of jaws placed in their stead, which affords the advantage of expediting the operation by enabling one person. to prepare the work whilst another is sewing $; b b$ is the moveable jaw, made of one piece. The two jaws being placed together in the manner shown at fig. 519, the moveable jaw traverses backwards and forwards upon two guide-bars, $c$, which are made to pass through holes exactly fitted to them, in the lower parts of the jaws. At the upper parts of the jaws are what are called the indexes, $d d$, which are pressed tightly together by a spring, shown at fig. 520, and intended to be introduced between the perpendicular ribs of the jaws at e. At $f$ is a thumbscrew, passing through the ribs for the purpose of tightening the jaws, and holding the leather fast between the indexes while being sewn; this screw, however, will seldom, if ever, be necessary if the spring is sufficiently strong; $g$ is an eye or ring fixed to the moveable jaw, through which the end of a lever, $h$, in fig. 518 passes; this lever is connected by a spring to a treadle, $i$, at the base of the pedestal, and by the pressure of the right foot upon this treadle, the moveable jaw is withdrawn; so that the person employed in sewing may shift the leather, and place another part of the glove between the jaws. The pieces called indexes are connected to the upper part of the jaws, by screws passing through elongated holes which render them capable of adjustment.

The patentee states, that in addition to the index described in his former patent, which is applicable to what is called round-seam sewing only, and which permits the leather to expand but in one direction when the needle is passed through it, namely, upwards, he 
now makes two indexes of different construction, one of which he calls the receding index, and the other the longitudinally grooved index. Fig. 521 represents an end view, and $f i g .522$ a top view of the receding index, which is particularly adapted for what are called "drawn sewing, and prick-seam sewing;" this index, instead of biting to the top, is so rounded off in the inside ftom the bottom of the cross grooves, as to permit the needles, by being passed backwards and forwards, to carry the silk thread on each side of the leather without passing over it. Fig. 523 represents an end view of the longitulinally grooved index, partly open, to show the section of the grooves more distinctly; and fig. 524 represents an inside view of one side of the same index, in which the longitudinal groove is shown passing from $k$ to $l$. This index is more particularly adapted to round seam sewing, and permits the leather to expand in every direction when the needle is passed through it, by which the leather is less strained, and the sewing consequently rendered much stronger.

It is obrious that the parallel horizontal movement may be effected by other mechanical means besides those adopted here, and the chief novelty claimed with respert to that movement, is its application to the purpose of carrying the index used in sewing and pointing leather gloves.

\section{Importation of leather gloves for home consumption; and amount of duty in

\begin{tabular}{ccc|cc}
1836. & 1837. & 1836. & 1837. \\
$1,461,769$. & $\mid$ & $1,221,350$. & $£ 27,558$. & $£ 22,923$.
\end{tabular}

GLOVE-SEWING. The following simple and ingenious apparatus, invented by an Englishman, has been employed extensively in Paris, and has enabled its proprietors to realize a handsome fortune. The French complain that "it has inundated the world with gloves, male of excellent quality, at 30 per cent. under their former wholesale

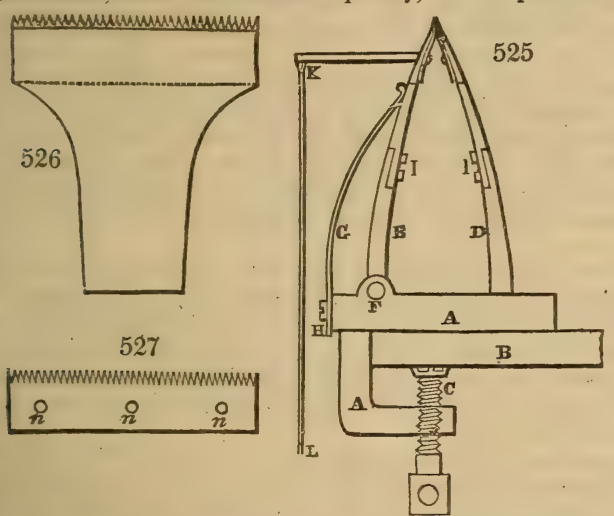

prices." The instrument is shown in profile ready for action in fig. 525. It resembles an iron vice, having the upper portion of each jaw made of brass, and tipped with a kind of comb of the same metal. The teeth of this comb, only one twelfth of an inch long, are perfectly regular and equal. Change combs are provided for different styles of work. The vice: $\mathbf{A} \mathbf{A}$ is made fast to the edge of the bench or table $B_{\text {, }}$ of the proper height, by a thumb-screw c, armed with a cramp which lays hold of the wood. Of the two jaws composing the machine, the one $D$ is made fast to the foot $\mathbf{A} A$, but the other $\mathrm{x}$ is moveable upon the solid base of the machine, by means of a hinge at the point $\mathrm{F}$. At I I is shown how the upper brass portion is adjusted to the lower part made of iron; the two being secured to each other by two stout serews. The comb, seen separately in fig. 527, is made fast to the upper end of each jaw, by the three screws $n n n$. Fig. 526 is a front view of the jaw mounted with its comb, to illustrate its construction.

The lever $\mathbf{k}$ corresponds by the stout iron wire $\mathbf{L}$, with a pedal pressed by the needlewoman's foot, whenever she wishes to separate the two jaws, in order to insert between them the parallel edges of leather to be sewed. The instant she lifts her foot, the two jaws join by the force of the spring $\mathrm{G}$, which pushes the moveable jaw $\mathrm{E}$ against the stationary one $\mathrm{D}$. The spring is made fast to the frame of the vice by the screw $\mathrm{H}$.

After putting the double edge to be sewed in its place, the woman passes her needle successively through all the teeth of the comb, and is sure of making a regular seam in every direction, provided she is careful to makie the needle graze along the bottom of the notches. As soon as this piece is sewed, she presses down the pedal with her toes, whereby the jaws start asunder, allowing her to introduce a new seam, and so in quick succession.

The comb may have any desired shape, straight or curved; and the teeth may be larger or smaller, according to the kind of work to be done. With this view, the combs might be changed as occasion requires; but it is more economical to have sets of vices ready monnted with combs of every requisite size and form.

GLUCINA (Glucine, Fr.; Berryllerde, Germ.) is one of the primitive earths, 
originally discovered by Vauquelin, in the beryl and emerald. It may be extracted from either of these minerals, by treating their powder successively with potash, with water, and with muriatic acid. The solution by the latter, being evaporated to dryness, is to be digested with water, and filtered. On pouring carbonate of ammonia in excess into the liquid, we form soluble muriate of ammonia, with insoluble carbonates of lime, chrome, and iron, as also carbonate of glucina, which may be dissolved out from the rest by an excess of carbonate of ammonia. When the liquid is filtered anew, the glucina passes through, and may be precipitated in the state of a carbonate by boiling the liquid, which expels the excess of ammonia. By washing, drying, and calcining the carbonate, pure glncina is obtained. It is a white insipid powder, infusible in the heat of a smith's forge, insoluble in water, but soluble in caustic potash and soda; as also, especially when it is a hydrate, in carbonate of ammonia. It has a metallic base called glucinum, of which 100 parts combine with $45 \cdot 252$ of oxygen to form the earth. It is too rare to be susceptible of application in manufactures.

GLUE (Colle forte, Fr.; Leim, Tischlerleim, Germ.) is the chemical substance gelatine in a dry state. The preparation and preservation of the skin and other animal matters employed in the manufacture of glue, constitute a peculiar branch of industry. Those who exercise it should study to prevent the fermentation if the substances, and to diminish the cost of carriage by depriving them of as much water as can conveniently be done. They may then be put in preparation by macerating them in milk of lime, renewed three or four times in the course of a fortnight or three weeks. This process is performed in large tanks of masonry. They are next taken out with all the adhering lime, and laid in a layer, 2 or 3 inches thick, to drain and dry, upon a sloping parement, where they are turned over by prongs, two or three times a day. The action of the lime dissolves the blood and certain soft parts, attacks the epidermis, and disposes the gelatinous matter to dissolve more readily. When the cleansed matters are dried, they may be packed in sacks or hogsheads, and transported to the glue manufactory at any distance. The principal substances of which glue is made are the parings of ox and other thick hides, which form the strongest article; the refuse of the leather dresser; both afford from 45 to 55 per cent. of glue. The tendons, and many other offals of slaughter houses, also afford materials, though of an inferior quality, for the purpose. The refuse of tanneries, such as the ears of oxen, calves, sheep, \&c., are better articles; but parings of parchment, old gloves, and, in fact, animal skin, in every form, uncombined with tannin, may be made into glue.

The manufacturer who receives these materials, is generally careful to ensure their purification by subjecting them to a weak lime steep, and rinsing them by exposure in baskets to a stream of water. They are lastly drained upon a sloping surface, as above described, and well turned over till the quicklime gets mild by absorption of carbonic acid; for, in its caustic state, it would damage the glue at the heat of boiling water. It is not necessary, however, to dry them before they are put into the boiler, because they dissolve faster in their soft and tumefied state.

The boiler is made of copper, rather shallow in proportion to its area, with a uniform frat bottom, equably exposed all over to the flame of the fire. Above the true bottom there is a false one of copper or iron, pierced with holes, and standing upon feet 3 or 4 inches high; which serves to sustain the animal matters, and prevent them from being injured by the fire. The copper being filled to two thirds of its height with soft water, is then heaped up with the bulky animal substances, so high as to surmount its brim. But soon after the ebullition begins they sink down, and, in a few hours, get entirely immersed in the liquid. They should be stirred about from time to time, and well pressed down toward the false bottom, while a steady but gentle boil is maintained.

The solution must be drawn off in successive portions; a method which fractions the products, or subdivides them into articles of various value, gradually decreasing from the first portion drawn off to the last. It has been ascertained by careful experiments that gelatine gets altered over the fire very soon after it is dissolved, and it ought therefore to be drawn off whenever it is sufficiently fluid and strong for forming a clear gelatinous mass on cooling, capable of being cut into moderately firm slices by the wire. This point is commonly determined by filling haif an egg-shell with the liquor, and exposing it to the air to cool. The jelly ought to get very consistent in the course of a few minutes; if not so, the boiling must be persisted in a little longer. When this term is attained, the fire is smothered up, and the contents of the boiler are left to settle for a quarter of an hour. The stop-cock being partially turned, all the thin gelatinous liquor is run off into a deep boiler, immersed in a warm water bath, so that it may continue hot and fluid for several hours. At the end of this time, the supernatant clear liquid is to be drawn off into congealing boxes, as will be presently explained.

The grounds, or undissolved matters in the boiler, are to be again supplied with a quantity of boiling water from an adjoining copper, and are to be once more subjected to 
the action of the fire, till the contents assume the appearance of dissolved jelly, and afford a fresh quantity of strong glue liquor, by the stop-cock. The grounds should be subjected a third time to this operation, after which they may be put into a bag, and squeezed in a press to leave nothing unextracted. The latter solutions are usually too weak to form glue directly, but they may be strengthened by boiling with a portion of fresh skin-parings.

Fig. 528 represents a convenient apparatus for the boiling of skins into glue, in which

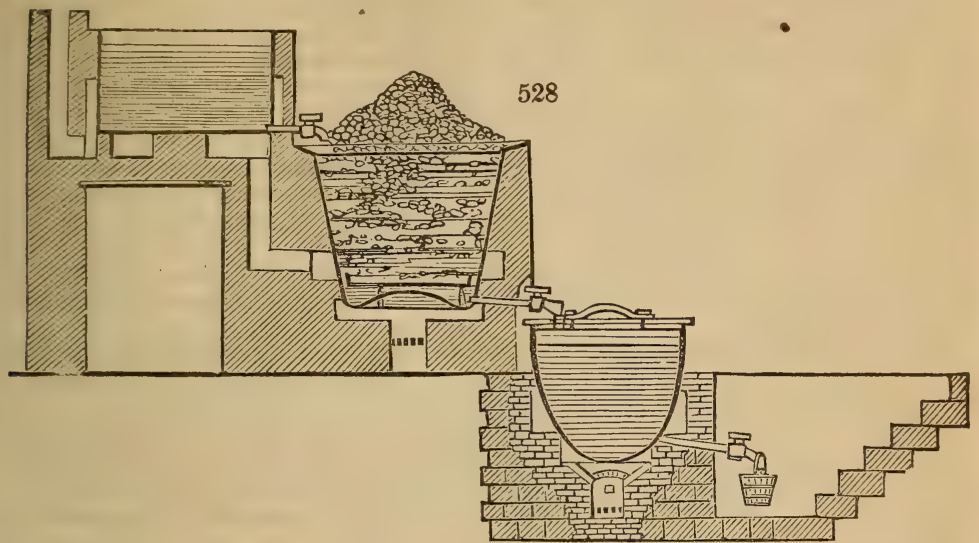

there are three coppers upon three different levels; the uppermost, being acted upon by the waste heat of the chimney, provides warm water in the most economical way; the second contains the crude materials, with water for dissolving them; and the third receives the solution to be settled. The last vessel is double, with water contained between the outer and inner one; and discharges its contents by a stop-cock into buckets for filling the gelatinizing wooden boxes. The last made solution has about one five-hundredth part of alum in powder usually added to it, with proper agitation, after which it is left to settle for several hours.

The three successive boils furnish three different qualities of glue.

Flanders or Dutch glue, long much esteemed on the Continent, was made in the manner above described, but at two boils, from animal offals well washed and soaked, so as to need less boiling. The liquor, being drawn off thinner, was therefore less colored, and being made into thinner plates, was very transparent. The above two boils gave two qualities of glue.

By the English practice, the whole of the animal matter is brought into solution at once, ind the liquor being drawn off, hot water is poured on the residuum, and made to boil on it for some time, when the liquor thus obtained is merely used instead of water upon a fresh quantity of glue materials. The first drawn off liquor is kept hot in a settling copper for five hours, and then the clear solution is drawn off into the boxes.

These boxes are made of deal, of a square form, but a little narrower at bottom than at top. When very regular cakes of glue are wished for, cross grooves of the desired square form are cut in the bottom of the box. The liquid glue is poured into the boxes placed very level, through funnels furnished with filter cloths, till it stands at the brim of each. The apartment in which this is done ought to be as cool and dry as possible, to favor the solidification of the glue, and should be floored with stone flags kept very clean, so that if any glue run through the seams, it may be recovered. At the end of 12 or 18 hours, or usually in the morning if the boxes have been filled overnight, the glue is sufficiently firm for the nets, and they are at this time removed to an upper story, mounted with ventilating windows to admit the air from all quarters. Here the boxes are inverted upon a moistened table, so that the gelatinous cake thus turned out will not adhere to its surface; usually the moist blade of a long knife is insinuated round the sides of the boxes beforehand, to loosen the glue. The mass is first divided into horizontal layers by a brass wire stretched in a frame, like that of a bowsaw, and guided by rulers which are placed at distances corresponding to the desired thichness of the cake of glue. The lines formed by the grooves in the bottom of the box define the superficial area of each cake, where it is to be cut with a moist knife. The gelatinous layers thus formed must be dexterously lifted, and immediately laid upon nets stretched in wooden frames, till each frame be filled. These frames are set over each other at distances of about three inches, being supported by small wooden 
pegs, stuck into mortise holes in an upright, fixed round the room; so that the au may have perfectly free access on every side. The cakes must moreover be turned upside down upon the nets twice or thrice every day, which is readily managed, as each frame may be slid out like a drawer, opon the pegs at its two sides.

The drying of the glue is the most precarious part of the manufacture. The least disturbance of the weather may injure the glue during the two or three first days of its exposure; should the temperature of the air rise considerably, the gelatine may turn so soft as to become unshapely, and even to run through the meshes upon the pieces below, or it may get atached to the strings and surround them, so as not to be separable without plunging the net into boiling water. If frost supervene, the water may Ireeze and form numerous cracks in the cakes. Such pieces must be immediately re-melted and re-formed. A slight fog even produces upon glue newly exposed a serious deterioration; the damp condensed upon its surface occasioning a general mouldiness. A thunderstorm sometimes destroys the coagulating power in the whole laminæ at once; or causes the glue to turn on the nets, in the language of the manufacturer. A wind too dry or too hot may cause it to dry so quickly, as to prevent it from contracting to its proper size without numerous cracks and fissures. In this predicament, the closing of all the flaps of the windows is the only means of abating the mischief. On these accounts it is of importance to select the most temperate season of the year, such as spring and autumn, for the glue manufacture.

After the glue is dried upon the nets it may still preserve too much flexibility, or softness at least, to be saleable; in which case it must be dried in a stove by artificial heat. This aid is peculiarly requisite in a humid climate, like that of Great Britain.

When sufficiently dry it next receives a gloss, by being dipped cake by cake in hot water, and then rubbed with a brush also moistened in hot water; after which the glue is arranged upon a hurdle, and transferred to the stove room, if the weather be not sufficiently hot. One day of proper drought will make it ready for being packed up in casks.

The pale-colored, hard, and solid article, possessing a brilliant fracture, which is made from the parings of ox-hides by the first process, is the best and most cohesive, and is most suitable for joiners, cabinet-makers, painters, \&c. But many workmen are influenced by such ignorant prejudices, that they still prefer a dark-colored article, with somewhat of a fetid odor, indicative of its impurity and bad preparation, the result of bad materials and too long exposure to the boiling heat.

There is a good deal of glue made in France from bones, freed from the phosphate of lime by muriatic acid. This is a poor article, possessing little cohesive force. It dissolves almost entirely in cold water, which is the best criterion of its imperfection. Glue should merely soften in cold water, and the more considerably it swells, the better, generally speaking, it is.

Some manufacturers prefer a brass to a copper pan for boiling glue, and insist much on skimming it as it boils; but the apparatus I have represented renders skimming of little consequence. For use, glue should be broken into small pieces, put along with some water into a vessel, allowed to soak for some hours, and subjected to the heat of a boiling-water bath, but not boiled itself. The surrounding hot water keeps it long in a fit state for joiners, cabinet-makers, \&c.

Water containing only one hundredth part of good glue, forms a tremulous solid. When the solution, however, is heated and cooled several times, it loses the property of gelatinizing, even though it be enclosed in a vessel hermetically sealed. Isinglass or fishglue undergoes the same change. Common glue is not soluble in alcohol, but is pre. cipitated in a white, coherent, elastic mass, when its watery solution is treated with that fluid. By transmitting chlorine gas through a warm solution of glue a combination is very readily effected, and a viscid mass is obtained like that thrown down by alcohol. A little chlorine suffices to precipitate the whole of the glue. Concentrated sulphuric acid makes glue undergo remarkable changes; during which are produced, sugar of gelatine, leucine, an animal matter, \&c. Nitric acid, with the aid of heat, converts glue into malic acid, oxalic acid, a fat analogous to suet, and into tannin; so that, in this way, one piece of skin may be made to tan another. When the mixture of glue and nitric acid is much evaporated, a detonation at Jast takes place. Strong acetic acid renders glue first soft and transparent, and then dissolves it. Though the solution does not gelatinize, it preserves the property of gluing surfaces together when it dries. Liquid glue dissolves a considerable quantity of lime, and also of the phosphate of lime recently precipitated. Accordingly glue is sometimes contaminated with that salt. Tannin both natural and artificial combines with glue; and with such effect, that one part of glue dissolved in 5000 parts of water affords a sensible precipitate with the infusion of nutgalls. Tannin unites with glue in several proportions, which are to each other as the numbers $1,1 \frac{1}{2}$, and 2 ; one compound consists of 100 glue and 89 tannin; another of 100 glue and 60 tannin; and a third of 100 glue and 120 tannin. These two sub- 
sfances cannot be afterwards separated from each other by any known chemical pricess.

Glue may be freed from the foreign animal matters generally present in it, by softening it in cold water, washing it with the same several times till it no longer gives out any color, then bruising it with the hand, and suspending it in a linen bag beneath the surfice of a large quantity of water at $60^{\circ} \mathrm{F}$. In this case, the water loaded with the soluble impurities of the glue gradually sinks to the bottom of the vessel, while the pure glue remains in the bag surrounded with water. If this softened glue be heated to $92^{\circ}$ withont adding water, it will liquefy; and if we heat it to $122^{\circ}$, and filter it, some albuminous and other impurities will remain on the filter, while a colorless solution of glue will pass through.

Experiments have not yet explained how gelatine is formed from skin by ebullition. It is a change somewhat analogous to that of starch into gum and sugar, and takes place without any appreciable disengingement of gas, and even in close vessels. Gelatine, says Berzelius, does not exist in the living body, but several animal tissues, such as skin, cartilages, hartshorn, tendons, the serous membranes, and bones, are susceptible of being converted into it.

GLUTEN (Colle Vegetale, Fr.; Kleber, Germ.) was first extracted by Becearia from wheat flour, and was long regarded as a proximate principle of plants, till Einhof, Taddei, and Berzelius succeeded in showing that it may be resolved by means of alcohol into three different substances, one of which resembles closely animal albumine, and has been called Zymome, or vegetable albumine; another has been called Gliadine; and a third, Mucine. The mode of separating gluten from the other constituents of wheat flour has been described towards the end of the article BrEAD.

Gluten, when dried in the air or a stove, diminishes greatly in size, becomes hard, brittle, glistening, and of a deep yellow color. It is insoluble in ether, in fat, and essential oils, and nearly so in water. Alcohol and acetic acid cause gluten to swell and make a sort of milky solution. Dilute acids and alkaline leys dissolve gluten. Its ultimate constituents are not determined, but azote is one of them, and accordingly when moist gluten is left to ferment, it exhales the smell of old cheese.

GLYCERINE is a sweet substance which may be extracted from fatty substances. If we take equal parts of olive oil and finely-ground litharge, put them into a basin with a little water, set this on a sand bath moderately heated, and stir the mixture constantly, with the occasionzl addition of hot water to replace what is lost by evaporation, we shall obtain in a short time a soap or plaster of lead. After having added more water to this, we remove the vessel from the fire, decant the liquor, filter it, pass sulphureted hydrogen throcgh it to separate the lead, then filter afresh, and concentrate the liquor as much as is possible without burning upon the sand bath. What remains must be finally evaporated within the receiver of the air-pump. Glycerine thus prepared is a transparent liquid, whthout color or smell, and of a sirupy consistence. It has a very sweet taste. Its specific gravity is 1.27 at the temperature of $60^{\circ}$. When thrown upon burning coals, it takes fire and burns like an oil. Water combines with it in almost all proportions; alcohol dissolves it readily; nitric acid converts it into oxalic acid; and according to Vogel, sulphuric acid transforms it into sugar, in the same way as it does starch. Ferment or yeast does not affect it in any degree.

Its constituents are, carbon, 40 ; hydrogen, 9 ; oxygen, 51 ; in 100.

GNEISS is the name of one of the great mountain formations, being reckoned the oldest of the stratified rocks. It is composed of the same substances as granite, viz.: quartz, mica, and feldspar. In gneiss, however, they are not in granular crystals, but in scales, so as to give the mass a slaty structure. It abounds in metallic treasures.

GOLD (Eng. and Germ.; Or, Fr.). This metal is distinguished by its splendid yellow color; its great density $=19 \cdot 3$, compared to water 1.0 ; its fusibility at the $32 \mathrm{~d}$ degree of Wedgewood's pyrometer; its pre-eminent ductility and malleability, whence it can be beat into leaves only one 282,000th of an inch thick; and its insolubility in any acid menstruum, except the mixture of muriatic and nitric acids, styled by the alchymists aqua regia, because gold was deemed by them to be the king of metals.

Gold is found only in the metallic state, sometimes erystallized in the cube, and its de rivative forms. It occurs also in threads of various size, twisted and interlaced into a chain of minute octahedral crystals; as also in spangles or roundish grains, which, when of a certain magnitude, are called pepitas. The small grains are not fragments broken from a greater mass; but they show by their flattened ovoid shape, and their rounded outline, that this is their original state. The spec. grav. of native gold varies from $13 \cdot 3$ to $17 \cdot 7$. Humboldt states that the largest pepita known was one found in Peru, weighing about 12 kilogrammes ( $26 \frac{1}{2}$ lbs. avoird.); but masses have been quoted in the province of Quito which weighed nearly four times as much.

Another ore of gold is the alloy with silver, or argental gold, the electrum of Pliny 
so called from its amber shade. It seems to be a definite compound, containing in 100 parts, 64 of gold and 36 of silver.

The mineral formations in which this metal occurs, are the crystalline primitive rocks, the compact transition rocks, the trachytic and trap rocks, and alluvial grounds.

It never predominates to such a degree as to constitute veins by itself. It is either disseminated, and as it were impasted in stony masses, or spread out in thin plates or grains on their surface, or, lastly, implanted in their cavities, under the shape of filaments or crystallized twigs. The minerals composing the veins are either quartz, calcspar, or sulphate of baryta. The ores that accompany the gold in these veins are chiefly iron pyrites, copper pyrites, galena, blende, and mispickel (arsenical pyrites).

In the ores called auriferous pyrites, this metal occurs either in a visible or invisible form, and though invisible in the fresh pyrites, becomes visible by its decomposition; as the hydrated oxyde of iron allows the native gold particles to shine forth on their reddishbrown ground, even when the precious metal may constitute only the five n.. llionth part of its weight, as at Rammelsberg in the Hartz. In that state it has been extracted with profit; most frequently by amalgamation with mercury, proving that the gold was in the native state, and not in that of a sulphuret.

Gold exists among the primitive strata, disseminated in small grains, spangles, and crystals. Brazil affords a remarkable example of this species of gold mine. Beds of granular quartz, or micaceous specular iron, in the Sierra of Cocäes, 12 leagues beyond Villa Rica, which form a portion of a mica-slate district, include a great quantity of native gold in spangles, which in this ferruginous rock replace mica.

Gold has never been observed in any secondary formation, but pretty abundantly in its true and primary locality among the trap rocks of igneous origin; implanted on the sides of the fissures, or disseminated in the veins.

The auriferous ores of Hungary and Transylvania, composed of tellurium, silver pyrites or sulphuret of silver, and native gold, lie in masses or powerful veins in a rock of trachyte, or in a decomposed feldspar subordinate to it. Such is the locality of the gold ore of Königsberg, of Telkebanya, between Eperies and Tokay in Hungary, anc probably that of the gold ores of Kapnick, Felsobanya, \&c., in Transylvania ; an arrange. menent nearly the same with what occurs in Equatorial America. The auriferous veins of Guanaxuato, of Real del Monte, of Villalpando, are similar to those of Schemnitz in Hungary, as to magnitude, relative position, the nature of the ores they include, and of the rocks they traverse. These districts have impressed all mineralogists with the evidences of the action of volcanic fire. Breislak and Hacquet have described the gold mines of Transylvania as situated in the crater of an ancient volcano. It is certain that the trachytes which form the principal portions of the rocks including gold, are now almost universally regarded as of igneous or volcanic origin.

It would seem, however, that the primary source of the gold is not in these rocks, but rather in the sienites and greenstone porphyries below them, which in Hungary and Transylvania are rich in great auriferous deposites; for gold has never been found in the trachyte of the Euganean mountains, of the mountains of the Vicentin, of those of Auvergne; all of which are superposed upon granite rocks, barren in metal.

Finally, if it be true that the ancients worked mines of gold in the island of Ischia, it would be another example, and a very remarkable one, of the presence of this metal in trachytes of an origin evidently voleanic.

Gold is, however, much more common in the alluvial grounds than among the primitive and pyrogenous rocks just described. It is found disseminated under the form of spangles, in the silicious, argillaceous, and ferruginous sands of certain plains and rivers, especially in their re-entering angles, at the season of low water, and after storms anc temporary floods.

It has been supposed that the gold found in the beds of rivers had been torn out by the waters from the veins and primitive rocks, which they traverse. Some have even searched, but in vain, at the source of auriferous streams, for the native bed of this precious metal. The gold in them belongs, however, to the grounds washed by the waters as they glide along. This opinion, suggested at first by Delius, and supported by Deborn, Guettard, Robitant, Balbo, \&c., is founded upon just observations. 1. The soil of these plains contains, frequently, at a certain depth and in several spots, spangles of gold, separable by washing. 2. The beds of the auriferous rivers and streamlets contain more gold after storms of rain upon the plains than in any other circumstances. 3 . It happens almost always that gold is found among the sands of rivers only in a very eircumscribed space; on ascending these rivers their sands cease to afford gold; though did this metal come from the rocks above, it should be found more abundantly near the source of the rivers. Thus it is known that the Orco contains no gold except from Pont to its junction with the Po. The Ticino affords gold only below the Lago Maggiore, and consequently far from the primitive mountains, after traversing a lake, where its course is slackened, and into which whatsoever it carried down from these mountains 
must have been deposited. The Rhine gives more gold near Strasburg than near Basle, though the latter be much closer to the mountains. The sands of the Danube do not contain a grain of gold, while this river runs in a mountainous region; that is, from the frontiers of the bishopric of Passau to Efferding; but its sands become auriferous in the plains below. The same thing is true of the Ems; the sands of the upper portion of this river, as it flows among the mountains of Styria, include no gold; but from its entrance into the plain at Steyer, till its embouchure in the Danube, its sands become auriferous, and are even rich enough to be washed with profit.

The greater part of the auriferous sands, in Europe, Asia, Africa, and America, are black or red, and consequently ferruginous; a remarkable circumstance in the geological position of alluvial gold. M. Napione supposes that the gold of these ferruginous grounds is due to the decomposition of auriferous pyrites. The auriferous sand occurring in Hungary almost always in the neighborhood of the beds of lignites, and the petrified wood covered with gold grains, found buried at a depth of 55 yards in clay, in the mine of Vorospatak near Abrabanya in Transylvania, might lead us to presume that the epoch of the formation of the auriferous allnvia is not remote from that of the lignites. The same association of gold ore and fossil wood occurs in South America, at Moco. Near the village of Lloro, have been discovered at a depth of 20 feet, large trunks of petrified trees, surrounded with fragments of trap rocks interspersed with spangles of gold and platinum. But the alluvial soil affords likewise all the characters of the basaltic rocks; thus in France, the Cèze and the Gardon, auriferous ricers, where they afford most gold, flow over ground apparently derived from the destruction of the trap rocks, which occur in situ higher up the country. This fact had struck Reaumur, and this celebrated observer had remarked that the sand which more immediately accompanies the gold spangles in most rivers, and particularly in the Rhone and the Rhine, is composed, like that of Ceylon and Expailly, of black protoxyde of iron and small grains of rubies, corindon, hyacinth, \&c. Titanium has been observed more recently. It has, lastly, been remarked that the gold of alluvial formations is purer than that extracted from rocks.

\section{Principal Gold Mines.}

Spain anciently possessed mines of gold in regular veins, especially in the province of Asturias; but the richness of the American mines has made them to be neglected. The Tagus, and some other streams of that country, were said to roll over golden sands. France contains no workable gold mines; but it presents in several of its rivers auriferous sands. There are some gold mines in Piedmont; particularly the veins of auriferous pyrites of Macugnagna, at the foot of Monte Rosa, lying in a mountain of gneiss; and although they do not contain 10 or 11 grains of gold in a hundred weight, they have long defrayed the expense of working them. On the southern slope of the Pennine Alps, from the Simplon and Monte Rosa to the valley of Aoste, several auriferous districts and rivers occur. Such are the torrent Evenson, which has afforded much gold by washing; the Orco, in its passage from the Pont to the $\mathrm{Po}$; the reddish grounds over which this little river runs for several miles, and the hills in the neighborhood of Chivasso, contain gold spangles in considerable quantity.

In the county of Wicklow, in Ireland, a quartzose and ferruginous sand was discovered not long ago, containing many particles of gold, with pepitas or solid pieces, one of which weighed 22 ounces. No less than 1000 ounces of gold were collected.

There are auriferous sands in some rivers of Switzerland, as the Reuss and the Aar. In Germany no mine of gold is worked, except in the territory of Salzburg, amid the chain of mountains which separates the Tyrol and Carinthia.

The mines of Hungary and Transylvania are the only gold mines of any importance in Europe; they are remarkable for their position, the peculiar metals that accompany them, and their product, estimated at about 1430 pounds avoird. annually. The principal ones are in Hungary. 1. Those of Konigsberg. The native gold is disseminated in ores of sulphuret of silver, which occur in small masses and in veins in a decomposing feldspar rock, amid a conglomerate of pumice, constituting a portion of the trachytic formation. 2. Those of Borson, Schemnitz. And, 3. of Felsobanya; ores also of auriferous sulphuret of silver, occur in veins of sienite and greenstone porphyry. 4. Those of Telkebanya, to the south of Kaschau, are in a deposite of auriferous pyrites amid trap rocks of the most recent formation.

In Transylvania the gold mines occur in veins often of great magnitude, 6,8 , and sometımes 40 yards thick. These veins have no side plates or wall stones, but abut without intermediate gangues at the primitive rock. They consist of carious quartz, ferriferous limestone, heary spar, fluor spar, and sulphuret of silver. The mine of Kapnik deserves notice, where the gold is associated with orpiment, and that of Vcrospatak in granite rocks; those of Offenbanya, Zalatna, and Nagy-Ag, where it is associated with tellu rium. The last is in a sienitic rock on the limits of the trachyte. 
In Sweden, the mine of Edelfors in Smoland may be mentioned, where the gold occurs native and in auriferous pyrites; the veins are a brown quart $z$, in a mountain of foliated hornstone.

In Siberia, native gold occurs in a hornstone at Schlangenberg or Zmeof, and at Zmeino-garsk in the Altai mountains, accompanied with many other ores.

The gold mine of Berezof in the Oural mountains has been long known, consisting of partially decomposed auriferous pyrites, disseminated in a vein of greasy quartz. A bout 1820 , a very rich deposite of native gold was discovered upon the eastern side of the Oural mountains, disseminated at some yards depth, in an argillaceous loam, and accompanied with the déhris of rocks which usually compose the auriferous alluvial soils, as greenstone, serpentine, protoxyde of iron, corundum, \&c. The rivers of this district possess auriferous sands. The annual product of the gold mines of Siberia is 3710 pounds avoirdupois.

In Asia, and especially in its southern districts, there are many mines, streams, rivers, and wastes, which contain this metal. The Pactolus, a small river of Lydia, rolled over such golden sands, that it was supposed to constitute the origin of the wealth of Crœsus. But these deposites are now poor and forgotten. Japan, Formosa, Ceylon, Java, Sumatra, Borneo, the Philippines, and some other islands of the Indian Archipelago, are supposed to be very rich in gold mines. Those of Borneo are worked by the Chinese in an alluvial soil on the western coast, at the foot of a chain of volcanic mountains.

Little or no gold comes into Europe from Asia, because its servile inhabitants place their fortune in treasure, and love to hoard up that precious metal.

Numerous gold mines occur on the two slopes of the chain of the Cailas mountains in the Oundès, a province of Little Thibet. The gold lies in quartz veins which traverse a very crumbling reddish granite.

Africa was, with Spain, the source of the greater portion of the gold possessed by the ancients. The gold which Africa still brings into the market in abundance is always in dust, showing that the metal is obtained by washing the alluvial soils. None of it is collected in the north of that continent; three or four districts only are remarkable for the quantity of gold they produce.

The first mines are those of Kordofan, between Darfour and Abrssinia. The negroes transport the gold in quills of the ostrich or vulture. These mines seem to have been known to the ancients, who considered Ethiopia to abound in gold. Herodotus relates that the king of that country exhibited to the ambassadors of Cambyses, all their prisoners bound with golden chains.

The second and chief exploitation of gold dust is to the south of the great desert of Zaara, in the western part of Africa, from the mouth of the Senegal to the Cape of Palms. The gold occurs in spangles, chiefly near the surface of the earth, in the bed of rivulets, and always in a ferruginous earth. In some places the negroes dig wells in th soil to a depth of about 40 feet, unsupported by any props. They do not follow any vein; nor do they construct a gallery. By repeated washings they separate the gold from the earthy maiters.

The same district furnishes also the greater part of what is carried to Morocco, Fez, and Algiers, by the caravans which go from Timbuctoo on the Niger, across the great desert of Zaara. The gold which arrives by Senna at Cairo and Alexandria, comes from the same quarter. From Mungo Park's deseription, it appears that the gold spangles are found usually in a ferruginous small gravel, buried under rolled pebbles.

The third spot in Africa where gold is collected, is on the south-east coast, between the twenty-fifth and the twenty-second degree of south latitude, opposite to Madagascar, in the country of Sofala. Some persons think that this was the kingdom of Ophir, whence Solomon obtained his gold.

In modern times, the richest gold mines are found in America, from which there is exported annually, 3700 or 4000 pounds avoirdupois of this metal. It occurs there principally in spangles among the alluvial earths, and in the beds of rivers; more rarely in veins.

There is little gold in the northern part of America. The United States have hitherto produced but a slight quantity of alluvial gold, collected in the gravel pits of the creeks of Rockhole, district of Lebanon, in North Carolina. In 1810, a mass was found there, weighing 28 pounds. This district has furnished the mint of the United States with about $100 \mathrm{lbs}$. avoirdupois of gold.

Sonth America, especially Brazil, Choco, and Chili, are the regions which furnish most gold.

The gold of Mexico is in a great measure contained in the argentiferous veins, so numerous in that country, whose principal localities are mentioned under the article Silver. The silver of the argentiferous ores of Guanaxato, contains one 360 th of its weight of gold; the annual product of the mines being valued at from 2640 to 3300 pounds avoirdupois. 
Oaxaco contains the only auriferous veins exploited as gold mines in Mexico; they traverse rocks of gneiss and mica slate.

All the rivers of the province of Caraccas, to ten degrees north of the line, flow over golden sands.

Peru is not rich in gold ores. In the provinces of Huailas and Pataz, this metal is mined in veins of greasy quartz, variegated with red ferruginous spots, which traverse primitive rochs. The mines called pacos de oro, consist of ores of iron and copper oxydes, containing a great quantity of gold.

All the gold furnished by New Grenada (New Colombia) is the product of washings, established in alluvial grounds. The gold exists in spangles and in grains, disseminated among fragments of greenstone and porphyry. At Choco, along with the gold and platinum, hyacinths, zircons, and titanium occur. There have been found, as already stated, in the auriferous localities, large trunks of petrified trees. The gold of Antioquia is 20 earats fine, that of Choco 21, and the largest lump or pepita of gold weighed about $27 \frac{1}{2}$ pounds avoirdupois. The gold of Chili also occurs in alluvial formations.

Brazil furnishes the greatest part of the gold now brought into the market. Yet there is not in this country any gold mine properly so called; for the veins containing the metal are seldom worked.

It is in the sands of the Mandi, a branch of the Rio-Dolce, at Catapreta, that the auriferous ferruginous sands were first discovered in 1682. Since then, they have been found almost everywhere at the foot of the immense chain of mountains, which runs nearly parallel with the coast, from the 5th degree south to the 30 th. It is particularly near Villa Rica, in the environs of the village Cocäes, that the numerous washings for gold are established. The pepitas occur in different forms, often adhering to micaceous specular iron. But in the province of Minas Geräes, the gold occurs also in veins, in beds, and in grains, disseminated among the alluvial loams. It has been estimated in annual product, by several authors, at about 2800 pounds avoirdupois of fine metal; worth nearly a million sterling.

We thus see that almost all the gold brought into the market, comes from alluvial lands, and is extracted by washing.

The gold coin of the ancients was made chiefly out of alluvial gold, for in these early times the metallurgic arts were not sufficiently advanced to enable them to purify it. The gold dust from Bambouk in Africa, is of $22 \frac{1}{4}$ carats fine, and some from Morocco is even 23.

The gold of Giron, in New Grenada, is of $23 \frac{3}{4}$ carats ; being the purest from America. "For those who traffic in gold," says Humboldt, "it is sufficient to learn the place where the metal has been collected, to know its title."

Metellurgic treatment of gold. - The gold found in the sands of rivers, or in auriferous soils, needs not be subjected to any metallurgic process, properly speaking. The Orpaillers separate it from the sands, by washing them first upon inclined tables, sometimes covered with a cloth, and then by hand in wooden bowls of a particular form. Amalgamation is employed to carry off from the sand the minuter particles of gold they may contain. The people called Bohemians, Cigans, or Tehinganes, who wash the aurirerous sands in Hungary, employ a plank with 24 transverse grooves cut in its surface. They hold this plank in an inclined position, and put the sand to be washed in the first groove; they then throw water on it, when the gold mixed with a little sand collects usually towards the lowest furrow. They remove this mixture into a flat wooden basin, and by a peculiar sleight of hand, separate the gold entirely from the sand. The richest of the auriferous ores consist of the native gold quite visible, disseminated in a gangue, but the veins are seldom continuous for any length. The other ores are auriferous metallic sulphurets, such as sulphurets of copper, silver, arsenic, \&c., and particularly iron.

The stony ores are first ground in the stamping mill, and then washed in hand-basins, or on wooden tables.

The auriferous sulphurets are much more common, but much poorer than the former ores; some contain only one 200,000th part of gold, and yet they may be worked with advantage, when treated with skill and economy.

The gold of these ores is separated by two different processes; namely, by fusion and amalgamation.

The auriferous metallic sulphurets are first roasted; then melted into mattes, which are ruasted anew; next fused with lead, whence an auriferous lead is obtained, which may be refined by the process of cupellation.

When the gold ores are very rich, they are melted directly with lead, without preliminary calcination or fusion. These processes are, however, little practised, because they are less economical and certain than amalgamation, especially when the gold ores are very poor.

If these ores consist of copper pyrites, and if their treatment has been pushed to the point 
of obtaining auriferous rose copper, or even black copper including gold, the precious metal cannot be separated by the process of liquation, because the gold, having more affinity for copper than for lead, can be but partially run off by the latter metal. For these reasons the process of amalgamation is far preferable.

This process being the same for silver, I shall reserve its description for this metal. The rich ores in which the native gold is apparent, and merely disseminated in a stony gangue, are directly triturated with quicksilver, without any preparatory operation. As to the poor ores, in which the gold seems lost amid a great mass of iron, sulphuret of copper, \&c., they are subjected to a roasting before being amalgamated. This process seems requisite to lay bare the gold enveloped in the sulphurets. The quicksilver with which the ore is now ground, seizes the whole of its gold, in however small quantity this metal may be present.

The gold procured by the refining process with lead, is free from copper and lead, but it may contain iron, tin, or silver. It cannot be separated from iron and tin without great difficulty and expense, if the proportion of gold be too small to admit of the employment of muriatic acid.

By cupellation with lead, gold may be deprived of any antimony united with it.

Tin gives gold a remarkable hardness and brittleness; a piece of gold, exposed for some time over a bath of red hot tin, becomes brittle. The same thing happens more readily over antimony, from the volatility of this metal. A two thousandth part of antimony, bismuth, or lead, destroys the ductility of gold. The tin may be got rid of by throwing some corrosive sublimate or nitre into a crucible, containing the melted alloy. By the first agent, perchloride of tin is volatilized; by the second, stannale of potash forms, which is carried off in the resulting alkaline scoriæ.

Gold treated by the process of amalgamation, contains commonly nothing but a little silver. The silver is dissolved out by nitric acid, which leaves the gold untouched; but to make this parting with success and economy on the great scale, several preeautions must be observed.

If the gold do not contain fully two thirds of its weight of silver, this metal, being thoroughly enveloped by the gold, is partially screened from the action of the acid. Whenever, therefore, it is known by a trial on a small scale, that the silver is much below this proportion, we must bring the alloy of gold and silver to that standard by adding the requisite quantity of the latter metal. This process is called quartation.

This alloy is then granulated or laminated; and from twice to thrice its weight of sulphuric or nitric acid is to be boiled upon it; and when it is judged that the solution has been pushed as far as possible by this first acid, it is decanted, and new acid is poured on. Lastly, after having washed the gold, some sulphuric acid is to be boiled over it, which carries off a two or three thousandth part of silver, which nitrie acid alone could net dissolve. Thus perfectly pure gold is obtained.

The silver held in solution by the sulphuric or nitric acid is precipitated in the metallic state by copper, or in the state of chloride by sea-salt. See PArTing.

Not only has the ratio between the value of gold and silver varied much in different ages of the world; but the ratio between these metals and the commodities they represent has undergone variations, owing to the circumstances in which their mines have been successively placed; since they have always poired a greater quantity of the metals into the market than has been absorbed by use. This quantity has greatly increased since the discovery of America, a period of little more than 300 years. The mines of that continent, rich, numerous, and easily worked, by augmenting the mass of gold and silver, necessarily lessened the value of these metals compared with that of the objects of commerce represented by them, so that everything else being equal, there is now required for purchasing the same quantity of commodities, much more gold or silver than was necessary in the reign of Henry VII., before the discovery of America. This productiveness of the American mines has had an influence on those of the ancient continent; many of whose silver and gold mines have been abandoned, not because the veins or auriferous sands are less rich than they were, but because their product no longer represents the value of human labor, and of the goods to be furnished in return for their exploitation.

In the $3 \mathrm{~d}$ vol. of the Mining Journal, p. 331, we have the following statement as to the produce of the precious metals.-" In 40 years, from 1790 to 1830 , Mexico produced 6,436,4537. worth of gold, and 139,818,032l. of silver. Chili, 2,768,488l. of gold, and 1,822,924l. of silver. Buenos Ayres, 4,024,895l. of gold, and 27,182,673l. of silver. Russia, 3,703,743l. of gold, and 1,502,981l. of silver. Total, 1880 millions sterling, or 47 millions per annum.

The following table shows what proportion the product of the mines of Ametica bears to that of the mines of the ancient continent. 
Table of the Quantities of Gold which may be considered as having been brought into the European Marliet, every Year on an Average, from 1790 to 1802.

\begin{tabular}{|c|c|c|c|c|c|c|}
\hline \multicolumn{6}{|c|}{ Continents. } & \multirow{2}{*}{$\begin{array}{c}\text { Gold. } \\
\text { lbs. Avoir. }\end{array}$} \\
\hline \multicolumn{6}{|c|}{ Ancient Continent. } & \\
\hline Asia : $\quad-$ & - & - & - & - & - & \\
\hline Siberia & - & - & - & - & - & 3740 \\
\hline Africa - & - & - & - & - & - & 3300 \\
\hline Europe: - & - & - & - & - & - & \\
\hline Hungary & - & - & & $=$ & - & 1430 \\
\hline Salzbourg & $=$ & - & - & - & - & 165 \\
\hline Austrian s & tates - & - & - & - & -3 & \\
\hline Hartz and & Hessia & - & - & - & - & \\
\hline Saxony & - & - & - & - & - & \\
\hline Norway & - & - & - & - & - & 165 \\
\hline Sweden & - & - & - & - & - & \\
\hline France & - & - & - & - & - & \\
\hline Spain, \&c. & - & - & 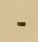 & - & $-j$ & \\
\hline \multicolumn{4}{|c|}{ Total of the Ancient Continent } & - & - & 8800 \\
\hline \multicolumn{6}{|c|}{ New Continent. } & \\
\hline North Americ & - & - & - & - & - & 2860 \\
\hline \multicolumn{2}{|c|}{ South America - } & - & - & - & - & \\
\hline \multicolumn{2}{|c|}{ Spanish dominion } & - & - & - & - & 22,000 \\
\hline Brazil & $-\quad-$ & - & - & - & - & 15,400 \\
\hline \multicolumn{3}{|c|}{ Total of the New Continent } & - & - & - & 40,260 \\
\hline
\end{tabular}

The mines of America have sent into Europe three and a half times more gold, and twelve times more silver, than those of the ancient continent. The total quantity of silver was to that of gold in the ratio of 55 to 1 ; a very different ratio from that which holds really in the value of these two metals, which is in Europe as 1 to 15 . This difference depends upon several causes, which cannot be investigated here at length; but it may be stated that gold, by its rarity and price, being much less employed in the arts than silver, the demand for it is also much less; and this cause is sufficient to lower its price much beneath what it would have been, if it had followed the ratio of its quantity compared to that of silver. Thus also bismuth, tin, \&c., though much rarer than silver, are, nevertheless, very inferior in price to it. Before the discovery of America, the value of gold was not so distant from that of silver, because since that era silver has been distributed in Europe in a far greater proportion than gold. In Asia the proportion is now actually only 1 to 11 or 12 ; the product of the gold mines in that quarter, being not so much below that of the silver mines as in the rest of the world.

The total annual production of Gold at present has been estimated as follows.

From the ancient Spanish colonies of America - - - 10,400 kilogrammes

Brazil -
Europe and Asiatic Russia
The Indian Archipelago :
Africa

without taking into account the quantity of gold now extracted from silver.

600

6,200

4,700

14,000 ?

$35,900=36$ tons nearly

Gold has less affinity for oxygen than any other metal. When alone, it cannot be oxydized by any degree of heat with contact of air, although in combination with other oxydized bodies, it may pass into the state of an oxyde, and be even vitrified. 'The purple smoke into which gold leaf is converted by an electric discharge is not an oxyde, for it is equally formed when the discharge is made through it in hydrogen gas. There are two oxydes of gold; the first or protoxyde is a green powder, which may be obtained by pouring, in the cold, a solution of potash into a solution of the metallic chloride. It is not durable, but soon changes in the menstruum into metallic gold, 
and peroxyde. Its constituents are 96.13 metal, and 3.87 oxygen. The peroxyde is best prepared by adding magnesia to a solution of the metallic chloride; washing the precipitate with water till this no longer takes a yellow tint from muriatic acid; then digesting strong nitric acid upon the residuum, which removes the magnesia, and leaves the peroxyde in the form of a black or dark brown powder, which seems to partake more of the properties of a metallic acid than a base. It contains 10.77 per cent. of oxygen. For the curious combination of gold and tin, called the Purple Precipitate of Cassius, see this article, and Pigments Vitrifiable.

Gold bealing.-This is the art of reducing gold to extremely thin leaves, by beating with a hammer. The processes employed for this purpose may be applied to other metals, as silver, platinum, and copper. Under tin, zinc, \&c., we shall mention such modifications of the processes as these metals require to reduce them to thin leaves. The Romans used to gild the ceilings and walls of their apartments; and Pliny tells us, that from an ounce of gold forming a plate of 4 fingers square, about 600 leaves of the same area were hammered. At the present day, a piece of gold is extended so as to cover a space 651,590 times greater than its primary surface when cast.

The gold employed in this art ought to be of the finest standard. Alloy hardens gold, and renders it less malleable; so that the fraudulent tradesman who should attempt to debase the gold, would expose himself to much greater loss in the operations, than he could derive of profit from the alloy.

Four principal operations constitute the art of gold beating. 1. The casting of the gold ingots. 2. The hammering. 3. The lamination; and 4 , the beating.

1. The gold is melted in a crucible along with a little borax. When it has vecome squid enough, it is poured out into the ingot-moulds previously heated, and greased on the inside. The ingot is taken out and annealed in hot ashes, which both soften it and free it from grease. The moulds are made of cast iron, with a somewhat concave internal surface, to compensate for the greater contraction of the central parts of the metal in cooling than the edges. The ingots weigh about 2 ounces each, and are $\frac{3}{4}$ of an inch broad.

2. The forging.-When the ingot is cold, the French gold-beaters hammer it out on a mass of steel 4 inches long and 3 broad. The hammer for this purpose is called the forging hammer. It weighs about 3 pounds, with a head at one end and a wedge at the other, the head presenting a square face of $1 \frac{1}{2}$ inches. Its handle is 6 inches long. The workman reduces the ingot to the thickness of $\frac{1}{6}$ of an inch at most ; and during this operation he anneals it whenever its substance becomes hard and apt to crack. The English gold-beaters omit this process of hammering.

3. The lamination. - The rollers employed for this purpose should be of a most perfectly cylindrical figure, a polished surface, and so powerful as not to bend or yield in the operation. The ultimate excellence of the gold leaf depends very much on the precision with which the riband is extended in the rolling press. The laminating machine represented under the article MrNT, is an excellent pattern for this purpose. The gold-beater desires to have a riband of such thinness that a square inch of it will weigh $6 \frac{1}{2}$ grains. Frequent annealings are requisite during the lamination.

4. Beating.- - The riband of gold being thus prepared uniform, the gold-beater cuts it with shears into small squares of an inch each, having previously diviled it with compasses, so that the pieces may be of as equal weight as possible. These squares are piled over each other in parcels of 150 , with a piece of fine calf-skin vellum interposed between each, and about 20 extra vellums at the top and bottom. These vellum leaves are about 4 inches square, on whose centre lie the gold laminæ of an inch square. This packet is kept together by being thrust into a case of strong parchment open at the ends, so as to form a belt or band, whose open sides are covered in by a second case drawn over the packet at right angles to the first. Thus the packet becomes sufficiently compact to bear beating with a hammer of 15 or 16 pounds weight, having a circular face nearly 4 inches diameter, and somewhat convex, whereby it strikes the centre of the packet most forcibly, and thus squeezes out the plates laterally.

The beating is performed on a very strong bench or stool framed to receive a heavy block of marble, about 9 inches square on the surface, enclosed upon every side by wodwork, except the front where a leather apron is attached, which the workman lays before him to preserve any fragments of gold that inay fall out of the packet. The hammer is short-handled, and is managed by the workman with one hand; who strikes fairly on the middle of the packet, frequently turning it over to beat both sides alike; a feat dexierously done in the interval of two strokes, so as not to lose a blow. The packet is occasionally bent or rolled between the hands, to loosen the leaves and secure the ready extension of the gold; or it is taken to pieces to examine the gold, and to shift the central leaves to the outside, and vice versa, that every thing may be equalized. Whenever the gold plates have extended, under this treatment, to nearly the size of the vellum, they are removed from the packet, and cut into four equal squares by a knife. Ther 
are thus reduced to nearly the same size as at first, and are again made up into packets and enclosed as before, with this difference, that skins prepared from ox-gut are now interposed between each gold leaf instead of vellum. The second course of beating is performed will a smaller hammer, about 10 pounds in weight, and is continued till the leares are extended to the size of the skins. During this period the packet must be often folled, to render the gold as loose as possible between the membranes; otherwise the leaves are easily chafed and broken. They are once more spread on a cushion, and subdivided intu four square pieces by means of two pieces of cane cut to very sharp edges, and fixed down transversely on a board. This rectangular cross being applied on each leal, with slioht pressure, divides it into four equal portions. These are next made up into a thirl packet of convenient thickness, and finally hammered out to the area of fine gold leaf, whose arerage size is from 3 to $3 \frac{1}{2}$ inches square. The leaves will now have obtained an area 192 times greater than the plates before the hammering begun. As these were originally an inch square, and 75 of them weighed an ounce $\left(=6 \frac{1}{2} \times 75=487 \frac{1}{2}\right)$, the surface of the finished leaves will be $192 \times 75=14,400$ square inches, or 100 square feet per ounce troy. This is by no means the ultimate degree of attenuation, for an ounce may be hammered so as to cover 160 square feet; but the waste incident in this case, from the number of broken leaves, and the increase and nicety of the labor, make this an unprofitable refinement; while the gilder finds such thin leaves to make less durable and satisfactory work.

The finished leaves of gold are put up in small books made of single leaves of soft paper, rubbed over with red chalk to prevent adhesion between them. Before putting the leave' in these books, however, they are lifted one by one with a delicate rair of pincers out of the finishing packet, and spread out on a leather cushion by bluwing them flat down. They are then cut to one size, by a sharp-edge square moulding of cane, glued on a flat board. When this square-framed edge is pressed upon the gold, it cuts it to the desired sizie and shape. Each book commonly contains 25 gold leaves.

I shall now describe some peculiarities of the French practice of gold beating. The workman cuts the laminated ribands of an inch broad into portions an inch and a half long. These are called quartiers. He takes 24 of them, which he places exactly over each other, so as 10 form a thickness of about an inch, the riband being $\frac{1}{2}$ of a line, or $\frac{1}{24}$ of an inch thick; and he beats them logether on the steel slab with the round face (panne) of the hammer, so as to stretch them truly out into the square form. He begins by extending the substance towards the edges, thereafter advancing towards the middle; he then does as much on the other side, and finally hammers the centre. By repeating this mode of beating as often as necessary, he reduces at once all the quartiers (squares) of the same packet, till none of them is thicker than a leaf of gray paper, and of the size of a square of 2 inches each side.

When the quartiers are brought to this state, the workman takes 56 of them, which he piles over each other, and with which he forms the first packet (caucher) in the manner already described; only two leaves of vellum are interposed between each gold leaf. The empty leaves of vellum at the top and bottom of the packet are called emplures. They are 4 inches square, as well as the parchment pieces.

The packet thus prepared forms a rectangular parallelopiped; it is enclosed in two sheaths, composed each of several leaves of parchment applitd to each, and glued at the two sides, forming a bag open at either end.

The block of black marble is a foot square at top, and 18 inches deep, and is framed as above described. The hammer used for beating the first packet is called the flat, or the enlarging hammer; its head is round, about 5 inches in diameter, and very slightly convex. It is 6 inches high, and tapers gradually from its head to the other extremity, which gives it the form of a hexagonal truncated pyramid. It weighs 14 or 15 pounds.

The French gold-beaters employ, besides this hammer, three others of the same form; namely, 1. The commencing hammer, which weighs 6 or 7 pounds, has a head 4 inches in diameter, and is more convex than the former. 2. The spreading hammer (marleau à chasser); its head is two inches diameter, more convex than the last, and weighs only 4 or 5 pounds. 3. The finishing hammer; it weighs 12 or 13 pounds, has a head four inches diameter, and is the most convex of all.

The heating processes do not differ essentially from the English described above. The vellum is rubbed over with fine calcined Paris plaster, with a hare's foot. The skin of the gold-beater is a pellicle separated from the outer surface of ox-gut; but before being employed for this purpose, it must undergo two preparations. 1. It is sweated, in order to expel any grease it may contain. With this view, each piece of membrane is placed between two leaves of white paper; several of these pairs are piled over each other, and struck strongiy with a hammer, which drives the grease from the gut into the paper.

2. A body is given to the pieces of gut; that is, they are moistened with an infusion 
of cinnamon, nutmeg, and other warm and aromatic ingredients, in order to preserve them; an operation repeated after they have been dried in the air. When the leaves of skin are dry, the $y$ are put in a press, and are now ready for use. After the parchment, vellum, and gut membrane have been a good deal hammered, they become unfit for work, till they are restored to proper flexibility, by being placed leaf by leaf between leaves of white paper, moistened sometimes with vinegar, at others with white wine. They are left in this predicament for 3 or 4 hours, under compression of a plank loaded with weights. When they have imbibed the proper humidity, they are put between leaves of parchment 12 inches square, and beat in that situation for a whole day. They are then rubbed over with fine calcined gypsum, as the vellum was originally. The gut-skin is apt to contract damp in standing, and is therefore dried before being used.

The average thickness of common gold leaf is $\frac{1}{282000}$ of an inch.

The art of Gilding.-This art consists in covering bodies with a thin coat of gold; which may be done either by mechanical or chemical means. The mechanical mode is the application of gold leaf or gold powder to various surfaces, and their fixation by various means. Thus gold may be applied to wood, plaster, pasteboard, leather; and to metals, such as silver, copper, iron, tin, and bronze; so that gilding, generally speaking, includes several arts, exercised by very different classes of tradesmen.

I. Mechanical Gilding.-Oil gilding is the first method under this head, as oil is the fluid most generally used in the operation of this mechanical art. The following process has been much extolled at Paris.

1. A coat of impression is to be given first of all, namely, a coat of white lead paint, made with drying linseed oil, containing very little oil of turpentine.

2. Calcined ceruse is to be ground very well with unboiled linseed oil, and tempered with essence of turpentine, in proportion as it is laid on. Three or four coats of this hard tint are to be applied evenly and dryly on the ornaments and the parts which are to be most carefully gilded.

3. The Gold color is then to be smoothly applied. This is merely the dregs of the solors, ground and tempered with oil, which remain in the little dish in which painters zlean their brushes. This substance is extremely rich and gluey; after being ground up, and passed through fine linen cloth, it forms the ground for gold leaf.

4. When the gold color is dry enough to catch hold of the leaf gold, this is spread on the cushion, cut into pieces, and carefully applied with the palette knife, pressed down with cotton, and in the small ornaments with a fine brush.

5. If the gildings be for outside exposure, as balconies, gratings, statues, \&c., they must not be varnished, as simple oil gilding stands better; for when it is varnished, a bright sun-beam, acting after heavy rain, gives the gilding a jagged appearance. When the objects are inside ones, a coat of spirit varnish may be passed over the gold leaf, then a glow from the gilder's chafing dish may be given, and finally a coat of oil varnish. The workman who causes the chafing dish to glide in front of the varnished surface, must avoid stopping for an instant opposite any point, otherwise he would cause the varnish to boil and blister. This heat brings out the whole transparency of the varnish and lustre of the gold.

Oil Gilding is employed, with varnish polish, upon equipages, mirror-frames, and other furniture. The following method is employed by eminent gilders at Paris.

1. White lead, with half its weight of yellow ochre, and a little litharge, are separately ground very fine; and the whole is then tempered with linseed oil, thinned with essence of turpentine, and applied in an evenly coat, called impression.

2. When this coat is quite dry, several coats of the hard tint are given, even so many as 10 or 12 , should the surface require it, for smoothing and filling up the pores. These coats are given daily, leaving them to dry in the interval in a warm sunny exposure.

3. When the work is perfectly dry, it is first softened down with pumice stone and water, afterwards with worsted cloth and very finely powdered pumice, till the hard tint give no reflection, and be smooth as glass.

4. With a camel's hair brush, there must be given lightly and with a gentle heat, from 4 to 5 coats at least, and even sometimes double that number, of fine lac varnish.

"5. When these are dry, the grounds of the panels and the sculptures must be first polished with shave-grass (de la prìle); and next with putty of tin and tripoli, tempered with water, applied with woollen cloth; by which the varnish is polished till it shines like a mirror.

6. The work thus polished is carried into a hot place, free from dust, where it receives very lightly and smoothly a thin coat of gold color, mush softened down. This coat is passed over it with a clean soft brush, and the thinner it is the better.

7. Whenever the gold color is dry enough to take the gold, which is known by laying the back of the hand on a corner of the frame work, the gilding is begun and finished as usual.

8. The gold is smoothed off with a very soft brush, one of camel's hair, for example, of three fingers' breadth; after which it is left to dry for several days. 
9. It is then varnished with a spirit of wine varnish; which is treated with the chafing dish as above described.

10. When this varnish is dry, two or three coats of copal or oil varnish are applied, at intervals of two days.

11. Finally, the panels are polished with a worsted cloth, imbued with tripoli and water, and lustre is given by friction with the palm of the hand, previously softened with a little olive oil, taking care not to rub off the gold.

In this country, burnished gilding is practised by first giving a ground of size whiting, in several successive coats; next applying gilding size; and then the gold leaf, which is burnished down with agate, or a dog's tooth.

Gilding in distemper of the French, is the same as our burnished gilding. Their process seems to be very elaborate, and the best consists of 17 operations; each of them said to be essential.

1. Encollage, or the glue coat. To a decoction of wormwood and garlic in water, strained through a cloth, a little common salt and some vinegar are added. This composition, as being destructive of worms in wood, is mixed with as much good glue; and the mixture is spread in a hot state, with a brush of boar's hair. When plaster or marble is to be gilded, the salt must be left out of the above composition, as it is apt to attract humidity in damp places, and to come out as a white powder on the gilding. But the salt is indispensable for wood. The first glue coating is made thinner than the second.

2. While preparation. This consists in covering the above surface with 8,10 , or 12 coats of Spanish white, mixed up with strong size, each well worked on with the brush, and in some measure incorporated with the preceding coat, to prevent their peeling off in scales.

3. Stopping up the pores, with thick whiting and glue, and smoothing the surface with dog-skin.

4. Polishing the surface with pumice-stone and very cold water.

5. Reparation; in which a skilful artist retouches the whole.

6. Cleansing; with a damp linen rag, and then a soft sponge.

7. Prêler. This is rubbing with horse's tail (shave-grass) the parts to be yellowed, in order to make them softer.

8. Yellowing. With this view yellow ochre is carefully ground in water, and mixed with transparent colorless size. The thinner part of this mixture is applied hot over the white surface with a fine brush, which gives it a fine yellow hue.

9. Ungraining consists in rubbing the whole work with shave-grass, to remove any granular appearance.

10. Coat of assiette; trencher coat. This is the composition on which the gold is to be laid. It is composed of Armenian bole, 1 pound; bloodstone (hematite), 2 ounces; and as much galena; each separately ground in water. The whole are then mixed together, and ground up with about a spoonful of olive oil. The assiette well made and applied gives beauty to the gilding. The assiette is tempered with a white sheep-skin glue, very clear and well strained. This mixture is heated and applied in three successive coats, with a very fine long-haired brush.

11. Rubbing, with a piece of dry, clean linen cloth; except the parts to be burnished, which are to receive other two coats of assiette tempered with glue.

12. Gilding. The surface, being damped with cold water (iced in summer), has then the gold leaf applied to it. The hollow grounds must alivays be gilded before the prominent parts. Water is dexterously applied by a soft brush, immediately behind the gold leaf, before laying it down, which makes it lie smoother. Any excess of water is then removed with a dry brush.

\section{Burnishing with bloodstone.}

14. Deadening. This consists in passing a thin coat of glue, slightly warmed, over the parts that are not to be burnished.

15. Mending; that is, moistening any broken points with a brush, and applying bits of gold leaf to them.

16. The vermeil coat. Vermeil is a liquid which gives lustre and fire to the gold; and makes it resemble or moulu. It is composed as follows: 2 ounces of annotto, 1 ounce of gamboge, 1 ounce of vermilion, half an ounce of dragon's blood, 2 ounces of salt of tartar, and 18 grains of saffron, are boiled in a litre ( 2 pints English) of water, over a slow fire, till the liquid be reduced to a fourth. The whole is then passed through a silk or muslin sieve. A little of this is made to glide lightly over the gold, with a very soft brush.

17. Repassage is passing over the dead surfaces a second coat of deadening glue, which must be hotter than the first. This finishes the work, and gives it strength.

Leaf gilding, on paper or vellum, is done by giving them a coat of gum water or fine size, applying the gold leaf ere the surfaces be hard dry, and burnishing with agate. 
Gold lettering, on bound books, is given without size, by laying the gold leaf on the seather, and imprinting it with hot brass types.

The edges of the leaves of books are gilded while they are in the press, where they have been cut smouth, by applying a solution of isinglass in spirits, and laying on the gold when the edges are in a proper state of dryness. The French workmen employ a ground of Armenian bole, mixed with powdered sugar-candy, by means of white of egg. This ground is laid very thin upon the edges, after fine size or gum water has been applied; and when the ground is dry it is rubbed smooth with a wet rag, which'moistens it sufficiently to take the gold.

Japanner's gilding is done by sprinkling or daubing with wash leather, some gold pow. Ler, over an oil sized surface, mixed with oil of turpentine. This gives the appearance of frosted gold. The gold powder may be obtained, either by precipitating gold from its solution in aqua regia by a solution of pure sulphate of iron, or by evaporating away the mercury from some gold amalgam.

faces.

II. Chermical gilding, or the application of gold by chemical affinity to metallic sur

A compound of copper with one seventh of brass is the best metal for gilding on ; copper by itself being too soft and dark colored. Ordinary brass, however, answers very well. We shall describe the process of wash gilding, with M. D'Arcet's late improvements, now generally adopted in Paris.

Wash gilding consists in applying evenly an amalgam of gold to the surface of a copper alloy, and dissipating the mercury with heat, so as to leave the gold film fixed. The surface is afterwards burnished or deadened at pleasure. The gold ought to be quite pure, and laminated to facilitate its combination with the mercury; which should also be pure.

Preparation of the amalgam. After weighing the fine gold, the workman puts it in a crucible, and as soon as this becomes faintly red, he pours in the requisite quantity of mercury; which is about 8 to 1 of gold. He stirs up the mixture with an iron rod, bent hookwise at the end, leaving the crucible on the fire till he perceives that all the gold is dissolved. He then pours the amalgam into a small earthen dish containing water, washes it with care, and squeezes out of it with his fingers all the running mercury that he can. The amalgam that now remains on the sloping sides of the vessel is so pasty as to preserve the impression of the fingers. When this is squeezed in a shamoy leather bag, it gives up much mercury; and remains an amalgam, consisting of about 33 of mercury, and 57 of gold, in 100 parts. The mercury which passes through the bag, under the pressure of the fingers, holds a good deal of gold in solution; and is employed in making fresh amalgam.

Preparation of the mercurial solution. The amalgam of gold is applied to brass, through the intervention of pure nitric acid, holding in solution a little mercury.

100 parts of mercury, and 110 parts by weight of pure nitric acid, specific gravity $1 \cdot 33$, are to be put into a glass matrass. On the application of a gentle heat the mercury dissolves with the disengagement of fumes of nitrous gas, which must be allowed to escape into the chimney. This solution is to be diluted with about 25 times its weight of pure water, and bottled up for use.

1. Annealing.-The workman anneals the piece of bronze after it has come out of the hands of the turner and engraver. He sets it among burning charcoal, or rather peats, which have a more equal and lively flame; covering it quite up, so that it may be oxydized as little as possible, and taking care that the thin parts of the piece do not become hotter than the thicker. This operation is done in a dark room, and when he sees the piece of a cherry red color, he removes the fuel from about it, lifts it out with long tongs, and sets it to cool slowly in the air.

2. The decapage.-The object of this process is to clear the surface from the coat of oxyde which may have formed upon it. The piece is plunged into a bucket filled with extremely dilute sulphuric acid; it is left there long enough to allow the coat of oxyde to be dissolved, or at least loosened; and it is then rubbed with a hard brush. When the piece becomes perfectly bright, it is washed and dried. Its surface may howerer be still a little variegated; and the piece is therefore dipped in nitric acid, specific gravity $1 \cdot 33$, and afterwards rubbed with a long-haired brush. The addition of a little common salt to the dilute sulphuric acid would probably save the use of nitric acid, which is so apt to produce a new coat of oxyde. It is finally made quite dry (after washing in pure water), by being rubbed well with tanners' dry bark, saw-dust, or bran. The surface should now appear somewhat de-polished; for when it is very smooth, the gold does not adhere so well.

Application of the amalgam.-The gilder's scratch-brush or pencil, made with fine brass wire, is to be dipped into the solution of nitrate of mercury, and is then to be drawn over a lump of gold amalgam, laid on the sloping side of an earthen ressel, after which it is to be applied to the surface of the brass. This process is to be 
repeated, dipping the brush into the solution, and drawing it over the amalgam, till the whole surface to be gilued is coated with its just proportion of gold. The piece is then washed in a body of water, dried, and put to the fire to volatilize the mercury. If one coat of gilding be insufficient, the piece is washed over anew with amalgam, and the operation recommenced till the work prove satisfactory.

4. Folatilization of the mercury.-Whenerer the piece is well coated with amalgam, the gilder exposes it to glowing charcoal, turning it about, and heating it by degrees to the proper point; he then withdraws it from the fire, lifts it with long pincers, and, seizing it in his left hand, protected by a stuffed glove, he turns it over in every direction, rubbing and striking it all the while with a long-haired brush, in order to equalize the amalgam. He now restores the piece to the fire, and treats it in the same way till the mercury be entirely volatilized, which he recognises by the hissing sound of a drop of water let fall on it. During this time he repairs the defective spots, taking care to rolatilize the mercury very slowly. The piece, when thoroughly coated with gold, is washed, and scrubbed well with a brush in water acidulated with vinegar.

If the piece is to have some parts burnished, and others dead, the parts to be burnished are covered with a mixture of Spanish white, bruised sugar-candy, and gum dissolved in water. This operation is called in French epargner (protecting). When the gilder has prolected the burnished points, he dries the piece, and carries the heat high enough to expel the little mercury which might still remain on it. He then plunges it, while still a little hot, in water acidulated with sulphuric acid, washes it, dries it, and gives it the burnish.

5. The burnish is given by rubbing the piece with burnishers of bematite (bloodstone). The workman dips his burnisher in water sharpened with vinegar, and rubs the piece alwars in the same direction backwards and forwards, till it exhibits a fine polish, and a complete metallic lustre. He then washes it in cold water, dries it with fine linen cloth, and concludes the operation by drying it slowly on a grating placed above a chafing dish of burning charcoal.

6. The deadening is given as follows. The piece, covered with the protection on those parts that are to be burnished, is attached with an iron wire to the end of an iron rod, and is heated strongly so as to give a brown hue to the epargne by its partial carbonization. The gilded piece assumes thus a fine tint of gold; and is next coated over with a mixture of sea salt, nitre, and alum, fused in the water of crystallization of the latter salt. The piece is now restored to the fire, and heated till the saline crust which covers it becomes homogeneous, nearly transparent, and enters into true fusion. It is then taken from the fire and suddenly plunged into cold water, which separates the saline crust, carrying away even the coat of epargne. The piece is lastly passed through very weak nitric acid, washed in a great body of water, and dried by exposure either to the air, over a drying stove, or with clean linen cloths.

7. Of or-moulu color. - When it is desired to put a piece of gilded bronze into or moulu color, it must be less scrubbed with the scratch-brush than usual, and made to come back again by heating it more strongly than if it were to be deadened, and allowing it then to cool a little. The or-moulu coloring is a mixture of hematite, alum, and sea salt. This mixture is to be thinned with vinegar, and applied with a brush so as to cover the gilded brass, with reserve of the burnished parts. The piece is then put on glowing coals, urged a little by the bellows, and allowed to heat till the color begins to blacken. The piece ought to be so hot that water sprinkled on it may cause a hissing noise. It is then taken from the fire, plunged into cold water, washed, and next rubbed with a brush dipped in vinegar, if the piece be smooth, but if it be chased, weak nitric acid must be used. In either case, it must be finally washed in a body of pure water, and dried over a gentle fire.

8. Of red gold color.-To give this hue, the piece, after being coated with amalgam and heated, is in this hot state to be suspended by an iron wire, and tempered with the composition known under the name of gilder's wax; made with yellow wax, red ochre, verdigris, and alum. In this state it is presented to the flame of a wood fire, is heated strongly, and the combustion of its coating is favored by throwing some drops of the wax mixture into the burning fuel. It is now turned round and round over the fire, so that the flame may act equally. When all the wax of the coloring is burned away, and when the flame is extinguished, the piece is to be plunged in water, washed, and scrubbed with the scratch-brush and pure vinegar. If the color is not beautiful, and quite equal in shade, the piece is coated with verdigris dissolved in vinegar, dried over a gentle fire, plunged in water, and scrubbed with pure vinegar, or even with a little weak nitric acid if the piece exhibit too dark a hue. It is now washed, burnished, washed anew, wiped with linen cloth, and finally dried over a gentle fire.

The fullowing is the outline of a complete gilding factory, as now fitted up at Paris.

Fig. 529. Front elevation and plan of a complete gilding workshop. 
P. Furnace of appel, or draught, serving at the same time to heat the deadening pan (poêlon au mat).
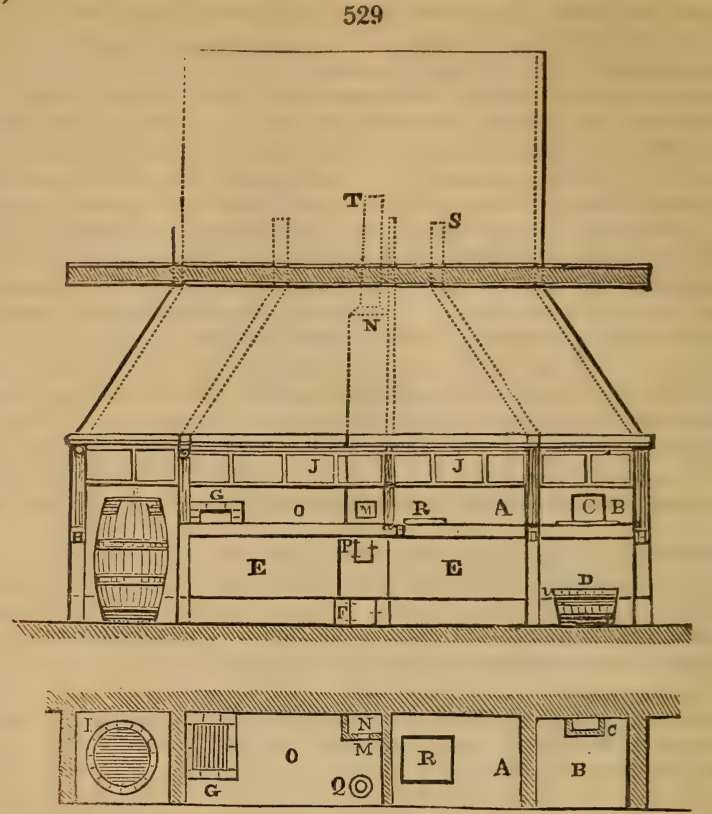

F. Ashpit of this furnace.

N. Chimney of this furnace constructed of bricks, as far as the contraction of the great chimney s of the forge, and which is terminated by a summit pipe rising 2 or 3 yards above this contraction.

в. Forge for annealing the pieces of bronze; for drying the gilded pieces, \&c.

c. Chimney of communication between the annealing forge $\mathrm{B}$, and the space $\mathrm{D}$ below the forge. This chimney serves to carry the noxious fumes into the great vent of the factory.

v. Bucket for the brightening operation.

A. Forge for passing the amalgam over the piece.

n. Shelf for the brushing operations.

E E. Coal cellarets.

o. Forge for the deadening process.

G. Furnace for the same.

M. An opening into the furnace of appel, by which vapors may be let off from any operation by taking out the plug at $\mathrm{M}$.

r. Cask in which the pieces of gilded brass are plunged for the deadening process. The vapors rising thence are carried up the general chimney.

J J. Casement with glass panes, which serves to contract the opening of the hearths, without obstructing the view. The casement may be rendered moveable to admit larger objects.

H H. Curtains of coarse cotton cloth, for closing at pleasure, in whole or part, one or several of the forges or hearths, and for quickening the current of air in the places where the curtains are not drawn.

Q. Opening above the draught furnace, which serves for the heating of the poélon au mat (deadening pan).

Gilding on polished tron and steel. - If a nearly neutral solution of gold in muriatic acid be mixed with sulphuric ether, and agitated, the ether will take up the gold, and float above the denser acid. When this auriferous ether is applied by a hair pencil to brightly polished iron or steel, the ether flies off, and the gold adheres. It must be fixed by polishing with the burnisher. This gilding is not very rich or durable. In fact, the affinity vetween gold and iron is feeble, compared to that between gold and copper or silver. But polished iron, steel, and copper, may be gilded with heat, by gold leaf. They are first heated till the iron takes a bluish tint, and till the copper has attained to a like temperature; a first coat of gold leaf is now applied, which is pressed geritly 
down with a burnisher, and then exposed to a gentle heat. Several leaves either single or double are thus applied in succession, and the last is burnished down cold.

Cold gilding.- Sixty grains of fine gold and 12 of rose copper are to be dissolved in two ounces of aqua regia. When the solution is completed, it is to be dropped on clean linen rags, of such bulk as to absorb all the liquid. They are then dried, and burned into ashes. These ashes contain the gold in powder.

When a piece is to be gilded, after subjecting it to the preliminary operations of softening or annealing and brightening, it is rubbed with a moistened cork, dipped in the above powder, till the surface seems to be sufficiently gilded. Large works are thereafter burnished with pieces of hematite, and small ones with steel burnishers, along with soap nater.

In gilding small articles, as buttons, with amalgam, a portion of this is taken equivalent to the work to be done, and some nitrate of mercury solution is added to it in a wooden trough: the whole articles are now put in, and well worked about with a hard brush, till their surfaces are equably coated. They are then washed, dried, and put altogether into an iron frying-pan, and heated till the mercury begins to fly off, when they are turned out into a cap, in which they are tossed and well stirred about with a painter's brush. The operation must be repeated several times for a strong gilding. The surfaces are funally brightened by brushing them along with small beer or ale grounds.

Gold wire is formed by drawing a cylindrical rod of the metal, as pure as may be, through a series of holes punched in an iron plate, diminishing progressively in size. The gold, $s=$ it is drawn through, becomes hardened by the operation, and requires frequent annealing.

Gold thread, or spun gold, is a flatted silver-gilt wire, wrapped or laid over a thread of yellow silk, by twisting with a wheel and iron bobbins. By the aid of a mechanism like the Braiding Machine, a number of threads may thus be twisted at once by one master wheel. The principal nicety consists in so regulating the movements that the successive volutions of the flatled wire on each thread roay just touch one another, and form a continuous covering. The French silver for gilding is said to be alloyed with 5 or 6 pennyweights, and ours with 12 pennyweights of copper in the pound troy. The gold is applied in leaves of greater or less thickness, according to the quality of the gilt wire. The smallest proportion formerly allowed in this country by act of parliament, was 100 grains of gold 10 one pound, or 5760 grains of silver; but more or less may now be used. The silver rod is encased in the gold leaf, and the compound cylinder is then drawn into round wire down to a certain size, which is afterwards flatted in a rolling mill such as is described under Mint.

The liquor employed by goldsmiths to bring out a rich color upon the surface of their trinkets, is made by dissolving 1 part of sea salt, 1 part of alum, 2 parts of nitre, in 3 or 4 of water. This pickle or sauce, as it is called, takes up not only the copper alloy, but a notable quantity of gold; the total amount of which in the Austrian empire, has been estimated annually at 47,000 franes. To recover this gold, the liquor is diluted with at least twice its bulk of boiling water; and a solution of very pure green sulphate of iron is poured into it. The precipitate of gold is washed upon a filter, dried, and purified by melting in a crucible along with a mixture of equal parts of nitre and borax.

GONG-GONG, or tam-tam of the Chinese; a kind of cymbal made of a copper alloy, described towards the end of the article CopPER.

GONIOMETER is the name of a little instrument made either on mechanical or optical principles, for measuring the angles of crystals. It is indispensable to the mineralogist.

GRADUATOR, called by its contriver, M. Wagenmann, Essigbilder, which means

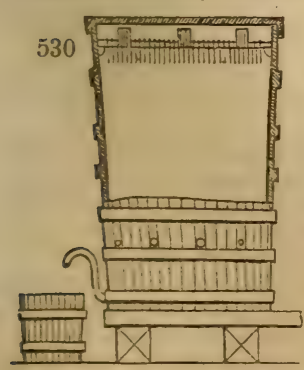
in German, vinegar-maker, is represented in fig. 530 . It is an oaken tub, $5 \frac{1}{2}$ feet high, $3 \frac{1}{2}$ feet wide at top, and 3 at bottom; set upon wooden beams, which raise its bottom about 14 inches from the fioor. At a distance of 15 inches above the bottom, the tub is pierced with a horizontal row of 8 equidistant round holes, of an inch in diameter. At 5 inches beneath the mouth of the tub, a thick beech-wood hoop is made fast to the inner surface, which supports a circular oaken shelf, leaving a space round its edge of $1 \frac{1}{4}$ inches, which is stuffed water tight with hemp or tow. In this shelf, 400 holes at least must be bored, about $\frac{1}{8}$ of an inch in diameter and $1 \frac{1}{2}$ inches apart; and each of these must be loosely filled with a piece of packthread, or cotton wick, which serves to filter the liquid slowly downwards. In the same shelf there are likewise four larger holes of $1 \frac{1}{2}$ inches diameter, and 18 inches apart, each of which receives air-tight a glass tube 3 or 4 inches long, hring its ends projecting above and below the shelf. These tubes serve to allow the air that 
enters by the 8 circumferential holes, to circulate freely through the graduator. The mouth of the tube is covered with a wooden lid, in whose middle is a hole for the insertion of a funnel, when the liquor of acetification requires to be introdnced. One inch abuve the bottum, a hole is bored for receiving a syphon-formed discharge pipe, whose upper curvature stands one inch below the level of the holes in the side of the tub, to prevent the liquor from rising so high as to overflow through them. The syphon is so bent as to retain a body of liquor 12 inches deep above the bottom of the tub, and to allow the ex cess only to escape into the subjacent receiver. In the upper part of the graduator, but under the shelf, the bulb of a thermometer is inserted through the side, some way into the interior, having a scale exteriorly. The whole capacity of the cask from the bottom up to within one inch of the perforated shelf, is to be filled with thin shavings of beech wood, grape stalks, or birch twigs, previously imbued with vinegar. The manner of using this simple apparatus is deseribed under Acetic AcrD.

GRANITE is a compound rock, essentially composed of quartz, feldspar, and mica, each in granular crystals. It constitutes the lowest of the geological formations, and therefore has been supposed to serve as a base to all the rest. It is the most durable material for building, as many of the ancient Egyptian monuments testify.

The obelisk in the place of Saint Jean de Lateran at Rome, which was quarried at Syene, under the reign of Zetus, king of Thebes, 1300 years before the Christian sra; and the one in the place of Saint Pierre, also at Rome, consecrated to the Sun by a son of Sesostris, have resisted the weather for fully 3000 years. On the other hand there are many granites, especially those in which feldspar predominates, which crack and crumble down in the course of a few years. In the same mountain, or even in the same quarry, granites of very different qualities as to soundness and durability occur. Some of the granites of Cornwall and Limousin readily resolve themselves into a white kaolin or argillaceous matter, from which pottery and porcelain are made.

Granite, when some time dug out of the quarry, becomes refractory, and difficult to cut. When this rock is intended to be worked it should be kept under water ; and that variety ought to be selected which contains least feldspar, and in which the quartz or gray crystals predominate.

GRANULATION is the process by which metals are reduced to minute grains. It is effected by pouring them, in a melted state, through an iron cullender pierced with small holes, into a body of water; or directly upon a bundle of twigs immersed in water. In this way copper is granulated into bean shot, and silver alloys are granulated preparatory to PARTING; which see.

GRAPHITE (Plombagine,Fr.; Reissblei, Germ.) is a mineral substance of a lead or iron gray color, a metallic lustre, soft to the touch, and staining the fingers with a lead gray hue. Spec. grav. 2.08 to 2.45 . It is easily scratched, or cut with a steel edge, and displays the metallic lustre in its interior. Burns with great difficulty in the outward flame of the blow-pipe. It consists of carbon in a peculiar state of aggregation, with an extremely minute and apparently accidental impregnation of iron. Graphite, called also plumbago and black lead, occurs in gneiss, mica slate, and their subordinate clay slates and lime stones; in the form of masses, veins, and kidney-shaped disseminated pieces; as also in the transition slate, as at Borrodale in Cumberland, where the most precious deposite exists, both in reference to extent and quality, for making pencils. It has been found also among the coal strata, as near Cumnock in Ayrshire. This substance is employed for counteracting friction between rubbing surfaces of wood or metal, for making crucibles and portable furnaces, for giving a gloss to the surface of cast iron, \&c. See Plumbago, for some remarks concerning the Cumberland mine.

GRAUWACKE, or GREYWACKE, is a rock formation, composed of pieces of quartz, flinty slate, feldspar, and clay slate, cemented by a clay-slate basis; the pieces varying in size from small grains to a hen's egg.

GRAY DYE. (Teinture grise, Fr.; Graufärbé, Germ.) The gray dyes, in their numerous shades, are merely various tints of black, in a more or less diluted state, from the deepest to the lightest hue.

The dyeing materials are essentially the tannic and gallic acid of galls or other astringents, along with the sulphate or acetate of iron, and occasionally wine stone. Ash gray is given for 30 pounds of woollen stuff, by one pound of gall-nuts, $\frac{1}{2} \mathrm{lb}$. of wine stone (crude tartar), and $2 \frac{1}{2}$ lbs. of sulphate of iron. The galls and the wine stone being boiled with from 70 to 80 pounds of water, the stuff is to be turned through the decoction at a boiling heat for half an hour, then taken out, when the bath being refreshed with cold water, the copperas is to be added, and, as soon as it is dissolved, the stuff is to be put in and fully dyed. Or, for 36 pounds of wool; 2 pounds of tartar, $\frac{2}{2}$ pound of galls, 3 pounds of sumach, and 2 pounds of sulphate of iron are to be taken. The tartar being dissolved in 80 pounds of boiling water, the wool is to be turned through the solution for half an hour, and then taken out. The copper being filled up to its former level with fresh water, the decoction of the galls and sumach is to 
be poured in, and the wool boiled for half an hour in the bath. The wool is then taken out. while the copperas is being added and dissolved; after which it is replaced in the bath, and dyed gray with a gentle heat.

If the gray is to have a yellow cast, instead of the tartar, its own weight of alum is to be taken; instead of the galls, one pound of old fustic; instead of the copperas, $\frac{3}{4}$ of a pound of Salıburg vitriol, which consists, in $22 \frac{3}{8}$ parts, of 17 of sulphate of iron, and $5 \frac{3}{8}$ of stilphate of copper; then proceed as above directed. Or the stuff may be first stained in a bath of fustic, next in a weak bath of galls with a little alum; then the wool being taken out, a little vitriol (common or Saltzburg) is to be put in, previously dissolved in a decoction of logwood; and in this bath the dye is completed.

Pearl gray is produced by passing the stuff first through a decoction of sumach and logwood ( $2 \mathrm{lbs}$. of the former to one of the latter), afterwards through a dilute solution of sulphate or acetate of iron; and finishing it in a weak bath of weld containing a little alum. Mouse-gray is obtained, when with the same proportions as for ash-gray, a small quantity of alum is introduced.

For several other shades, as tawny-gray, iron-gray, and slate-gray, the stuff must receire a previous blue ground by dipping it in the indigo vat; then it is passed first through a boiling bath of sumach with galls, and lastly through the same bath at a lower temperature after it has received the proper quantity of solution of iron.

For dyeing silk gray, fustet, logwood, sumach, and elder-tree bark, are employed instead of galls. Archil and annotto are frequently used to soften and beautify the tint.

The mode of producing gray dyes upon cotton has been sufficiently explained in the articles Calrco Printing and Dyeing.

GREEN DYE is produced by the mixture of a blue and yellow dye, the blue being first applied. See Dyeing; as also Blue and Yellow Dyes, and Calico Printing.

GREEN PAINTS. (Couleurs vertes, Fr.; Grüne pigmente, Germ.) Green, which is so common a color in the vegetable kingdom, is very rare in the mineral. There is only one metal, copper, which affords in its combinations the various shades of green in general use. The other metals capable of producing this color are, chromium in its protoxyde, nickel in its hydrated oxyde, as well as its salts, the seleniate, arseniate, and sulphate; and titanium in its prussiate.

Green pigments are prepared also by the mixture of yellows and blues; as, for example, the green of Rinman and of Gellert, obtained by the mixture of cobalt blue, and floirers of zinc; that of Barth, made with yellow lake, Prussian blue, and clay; but these paints seldom appear in the market, because the greens are generally extemporaneous preparations of the artists.

Mountain green consists of the hydrate, oxyde, or carbonate of copper, either factitious, or as found in nature.

Bremen or Brunswick green is a mixture of carbonate of copper with chalk or lime, and sometimes a little magnesia or ammonia. It is improved by an admixture of white lead. It may be prepared by adding ammonia to a mixed solution of sulphate of copper and alum.

Frise green is prepared with sulphate of copper and sal ammoniac.

Mittis green is an arseniate of copper; made by mixing a solution of acetate or sul. phate of copper with arsenite of potash. It is in fact Scheele's green.

Sap green is the inspissated juice of buckthorn berries. These are allowed to ferment for 8 days in a tub, then put in a press, adding a little alum to the juice, and concentrated by gentle evaporation. It is lastly put up in pigs' bladders, where it becomes dry and hard.

Schweinfurt green; see SchwEINFuRT.

Veronc green is merely a variety of the mineral called green earth.

GREEN VITRIOL is sulphate of iron in green crystals.

GUAIAC (Gaiac, Fr.; Guajaharz, Germ.) is a resin which exudes from the trunk of the Guaiacum officinale, a tree which grows in the West India islands. It comes to us in large greenish-brown, semi-transparent lumps, having a conchoidal or splintery fracture, brittle, and easy to pulverize. It has an aromatic smell, a bitterish, acrid taste, melts with heat, and has a spec. grav. of from 1.20 to $1 \cdot 22$. It consists of 67.88 carbon; 7.05 hydrogen; and 25.07 oxygen; and contains two different resins, the one of which is soluble in all proportions in ammonia, and the other forms, with water of ammonia, a tarry consistenced mixture. It is soluble in alkaline leys, in alcohol, incompletely in ether, still less so in oil of turpentine, and not at all in fat oils. Its chief use is in medicine.

GUANO is a substance of a dark yellow color; of a strong mbrosial smell; which blackens in the fire, with the exhalation of an ammoniacal ndor; soluble with effervescence in hot nitric acid. When this solution is evaporated to dryness, it assumes 
a fine red color, evincing the presence of uric acid. Guano is found upon the coasts of Peru, in the islands of Chinche, near Pisco, and several other places more to the south. It forms a deposite 50 or 60 feet thick, and of considerable extent; and appears to be the accumulation of the excrements of innumerable flocks of birds, especially herons and flamands, which inhabit these islands. It is an excellent manure, and forms the object of a most extensive and profitable trade.

GUM (Gomme, Fr.; Gummi, Pflanzenschleim, Germ.) is the name of a proximate vegetable product, which forms with water a slimy solution, but is insoluble in alcohol, ether, and oils; it is converted by strong sulphuric acid into oxalic and mucic acids.

There are six varieties of gum: 1. gum arabic;2. gum senegal; 3 gum of the cherry and other stone fivit trees: 4. gum tragacanth; 5. gum of Bassora; 0 . the gum of seeds and roots. The first five spontaneously flow from the branches and trunks of their trees, and sometimes from the fruits, in the form of a mucilage which dries and hardens in the air. The sixth kind is extracted by boiling water.

Gum arabic and gum senegal consist almost wholly of the purest gum : rlled arabine by the French chemists; our native fruit trees contain some cercsine, along with arabine; the gum of Bassora and gum tragacanth consist of arabine and bassorine.

Gum arabic flows from the acacia arabica, and the acacia vera, which grow upon the banks of the Nile and in Arabia. It occurs in commerce in the form of small pieces, rounded upon one side and hollow upon the other. It is transparent, without smell, brittle, easy to pulverize, sometimes colorless, sometimes with a yellow or brownish tint. It may be bleached by exposure to the air and the sun-beams, at the temperature of boiling water. Its specific gravity is $1 \cdot 355$. Moistened gum arabic reddens litmus paper, owing to the presence of a little supermalate of lime, which may be removed by boiling alcoho]; it shows also traces of the chlorides of potassium and calcium, and the acetate of potash. 100 parts of good gum contain 70.40 of arabine, 17.60 of water, with a few per cents. of saline and earthy matters. Gum arabic is used in medicine, as also to give lustre to crapes and other silk stuffs,

Gum senegal is collected by the negroes during the month of November, from the acacia senegal, a tree 18 or 20 feet high. It comes to us in pieces about the size of a partridge ego, but sometimes larger, with a hollow centre. Its specific gravity is 1.436. It consists of $81 \cdot 10$ arabine; $16 \cdot 10$ water; and from 2 to 3 of saline matters. The chemical properties and uses of this gum are the same as those of gum arabic. It is much employed in calico-printing.

Cherry-tree gum consists of 52.10 arabine; 54.90 cerasine; 12 water; and 1 saline matter.

Gum tragacanth is gathered about the end of June, from the astragalus tragacantha of Crete and the surrounding islands. It has the appearance of $t$ wisted ribands; is white or reddish; nearly opaque, and a little ductile. It is difficult to pulverize, without heating the mortar. Its specific gravity is $1 \cdot 384$. When plunged in water, it dissolves in part, swells considerably, and forms a very thick mucilage. 100 parts of it consist of 53.30 arabine; 33.30 bassorine and starch; 11.0 water; and from 2 to 3 parts of saline matters. It is employed in calico printing, and by shoemakers.

Gum of Bassora; see BAssonine.

Gum of seeds, as linseed, consists of 52.70 arabine; 28.9 of an insoluble matter; 10.3 water; and $7 \cdot 11$ saline matter. Neither bassorine nor cerasine seems to be present in seeds and roots. For British Gum, see STARcH.

GUM RESINS. (Gomme-résines, Fr.; Schleimharze, Germ.) When incisions are made in the stems, branches, and roots of certain plants, a milky juice exudes, which gradually hardens in the air; and appears to be formed of resin and essential oil, held suspended in water charged with gum, and sometimes with other vegetable matters, such as caoutchouc, bassorine, starch, wax, and several saline matters. The said concrete juice is called a gum-resin; an improper name, as it gives a false idea of the nature of the substance. They are all solid; heavier than water; in general opaque and brittle; many have an acrid taste, and a strong smell; their color is very variable. They are partially soluble in water, and also in alcohol; and the solution in the former liquid seldom becomes transparent. Almost all the gum resins are medicinal substances, and little employed in the arts and manufactures. The following is a list of them: Asafœtida; gum ammoniac; bdellium; euphorbium; galbanum; gamboge ; myrrh; olibanum or frankincense; opoponax; and scammony. Some of these are described in this work under their peculiar names.

GUNPOWDER. The following memoir upon this subject was published by me in the Journal of the Royal Institution for October, 1830. It contains the results of several careful analytical experiments, as also of observations made at the Royal Gunpowder works of Waltham Abbey, and at some similar establishments in the neighborhood of London.

GUNPOWDER is a mechanical combination of nitre, sulphur, and charcoal; deriving 
the intensity of its explosiveness from the purity of its constituents, the proportion in which they are mixed, and the intimacy of the atmixture.

1. On the nitre.-Nitre may be readily purified, by solution in water and crystallization, from the muddy particles and foreign salts with which it is usually contaminated. In a saturated aqueous solution of nitre, boiling hot, the temperature is $240^{\circ} \mathrm{F}$; and the relation of the salt to its solvent is in weight as three to one, by my experiments : not five to one, as MMI. Bottée and Riffault have stated. We must not, however, adopt the general language of chemists, and say that three parts of nitre are soluble in one of boiling water, since the liquid has a much higher heat and greater solvent power than this expression implies.

Water at $60^{\circ}$ dissolves only one fourth of its weight of nitre; or, more exactly, this saturated solution contains 21 per cent. of salt. Its specific gravity is $1.1415 ; 100$ parts in volume of the two constituents occupy now $97 \cdot 91$ parts. From these data we may perceive that little advantage could be gained in refining crude nitre, by making a boilinghot saturated solution of it; since on cooling, the whole would concrete into a moist saline mass, consisting by weight of $2 \frac{3}{4}$ parts of salt, mixed with 1 part of water, holding $\frac{1}{4}$ of salt in solution, and in bulk of $1 \frac{7}{8}$ of salt, with about 1 of liquid; for the specific gravity of nitre is 2.005 , or very nearly the double of water. It is better, therefore, to use equal weights of saltpetre and water in making the boiling-hot solution. When the filtered liquid is allowed to cool slowly, somewhat less than three fourths of the nitre will separate in regular crystals; while the foreign salts that were present will remain with fully one fourth of nitre in the mother liquor. On redissolving these crystals with heat, in about two thirds of their weight of water, a solution will result, from which crystalline nitre, fit for every purpose, will concrete on cooling.

As the principal saline impurity of saltpetre is muriate of soda (a substance scarcely more soluble in hot than in cold water), a ready mode thence arises of separating that salt from the nitre in mother waters that contain them in nearly equal proportion. Place an iron ladle or basin, perforated with small holes, on the bottom of the boiler in which the solution is concentrating. The muriate, as it separates by the evaporation of the water, will fall down and fill the basin, and may be removed from time to time. When small needles of nitre begin to appear, the solution must be run off in to the crystallizing cooler, in which moderately pure nitre will be obtained, to be refined by another similar operation.

At the Waltham Abbey gunpowder works the nitre is rendered so pure by successive solutions and crystallizations, that it causes no opalescence in a solution of nitrate of silver. Such crystals are dried, fused in an iron pot at a temperature of from $500^{\circ}$ to $600^{\circ} \mathrm{F}$., and cast into moulds. The cakes are preserved in casks.

About the period of 1794 and 1795, under the pressure of the first wars of their revolution, the French chemists employed by the government contrived an expeditious, economical, and sufficiently effective mode of purifying their nitre. It must be observed that this salt, as brought to the gunpowder-works in France, is in general a much cruder article than that imported into this country from India. It is extracted from the nitrous salts contained in the mortar-rubbish of old buildings, especially those of the lowest and filthiest descriptions. By their former methods, the French could not refine their nitre in less time than eight or ten days; and the salt was obtained in great lumps, very difficult to dry and divide; whereas the new process was so easy and so quick, that in less than twenty-four hours, at one period of pressure, the cride saltpetre was converted into a pure salt, brought to perfect dryness, and in such a state of extreme division as to supersede the operations of grinding and sifting, whence also considerable waste was avoided.

The following is a brief outline of this method, with certain improvements, as now practised in the establishment of the Administration des poudres et saltpêtres, in France.

The refining boiler is charged over night with 600 kilogrammes of water, and 1200 kilogrammes of saltpetre, as delivered by the salpetriers. No more fire is applied than is adequate to effect the solution of this first charge of saltpetre. It may here be observed, that such an article contains several deliquescent salts, and is much more soluble than pure nitre. On the morrow morning the fire is increased, and the boiler is charged at different intervals with fresh doses of saltpetre, till the whole amounts to 3000 kilogrammes. During these additions, care is taken to stir the liquid very diligently, and to skim off the froth as it rises. When it has been for some time in ebullition, and when it may be presumed that the solution of the nitrous salts is effected, the muriate of soda is scooped out from the bottom of the boiler, and certain affusions or inspersions of cold water are made into the pot, to quicken the precipitation of that portion which the boiling motion may have kept afloat. When no more is found to fall, one kilogramme of Flanders glue, dissolved in a sufficient quantity of hot water, is poured into the boiler; the mixture is thoroughly worked together, the froth being shimmed off, with several successive inspersions of cold water, till 400 additional kilogrammes have been introduced, constituting altogether 1000 kilogrammes. 
When the refining liquor affords no more froth, and is grown perfectly clear, all manipulation must cease. The fire is withdrawn, with the exception of a mere kindling, so ag to maintain the temperature till the next morning at about $88^{\circ} \mathrm{C} .=190.4 \mathrm{~F}$.

This liquor is now transferred by hand-basins into the crystallizing reservoirs, taking care to disturb the solution as little as possible, and to leave untouched the impure matter at the bottom. The contents of the long crystallizing cisterns are stirred backwards and furwards with wooden paddles, in order to quicken the cooling, and the consequent precipitation of the nitre in minute crystals. These are raked, as soon as they fall, to the upper end of the doubly-inclined bottom of the crystallizer, and thence removed to the washing chests or boxes. By the incessant agitation of the liquor, no large crystals of nitre can possibly form. When the temperature has fallen to within $7^{\circ}$ or $8^{\circ}$ F., of the apartment, that is, after seven or eight hours, all the saltpetre that it can yield will have been obtained. By means of the double inward slope given to the crystallizer, the supernatant liquid is collected in the middle of the breadth, and may be easily laded out.

The saltpetre is shovelled out of the crystallizer into the washing chests, and heaped up in them so as to stand about six or seven inches above their upper edges, in order to allow for the subsidence which it must experience in the washing process. Each of these chests being thus filled, and their bottom holes being closed with plugs, the salt is besprinkled from the rose of a watering-can, with successive quantities of water saturated with saltpetre, and also with pure water, till the liquor, when allowed to run off, indicates by the hydrometer, a saturated solution. The water of each sprinkling ought to remain on the salt for two or three hours; and then it may be suffered to drain off through the plug-holes below, for about an hour.

All the liquor of drainage from the first watering, as well as a portion of the second, is set aside, as being considerably loaded with the foreign salts of the nitre, in order to be evaporated in the sequel with the mother waters. The last portions are preserved, because they contain almost nothing but nitre, and may therefore serve to wash another dose of that salt. It has been proved by experience, that the quantity of water employed in washing need never exceed thirty-six sprinklings in the whole, composed of three waterings, of which the first two consist of fifteen, and the last of six pots $=3$ gallons E.; or in other words, of fifteen sprinklings of water saturated with saltpetre, and twentyone of pure water.

The saltpetre, after remaining five or six days in the washing chests, is transported into the drying reservoirs, heated by the flue of the nearest boiler; here it is stirred up from time to time with wooden shovels, to prevent its adhering to the bottom, or running into lumps, as well as to quicken the drying process. In the course of about four hours, it gets completely dry, in which state it no longer sticks to the shovel, but falls down into a soft powder by pressure in the hand, and is perfectly white and pulverulent. It is now passed through a brass sieve, to separate any small lumps or foreign particles accidentally present, and is then packed up in bags or barrels. Even in the shortest winter days, the drying basin may be twice charged, so as to dry 700 or 800 kilogrammes. By this operation, the nett produce of 3000 kilogrammes ( 3 tons) thus refined, amounts to from 1750 to 1800 kilogrammes of very pure nitre, quite ready for the manufacture of gunpowder.

The mother waters are next concentrated; but into their management it is needless to enter in this memoir.

On reviewing the above process as ractised at present, it is obvious that, to meet the revolutionary crisis, its conductors must have shortened it greatly, and have been content with a brief period of drainage.

2. On the sulphur.-The sulphur now imported into this country, from the voleanic districts of Sicily and Italy, for our manufactories of sulphuric acid, is much purer than the sulphur obtained by artificial heat from any varieties of pyrites, and may, therefore, by simple processes, be rendered a fit constituent of the best gunpowder. As it is not my purpose here to repeat what may be found in common chemical compilations, I shall say nothing of the sublimation of sulphur; a process, moreover, much too wasteful for the gunpowder-maker.

Sulphur may be most easily analyzed, even by the manufacturer himself ; for I find it to be soluble in one tenth of its weight of boiling oil of turpentine, at $316^{\circ}$ Fahrenheit, forming a solution which remains clear at $180^{\circ}$. As it cools to the atmospheric temperature, beautiful crystalline needles form, which may be washed sufficiently with cold alcohol, or even tepid water. The usual impurities of the sulphur, which are carbonate and sulphate of zinc, oxyde and sulphuret of iron, sulphuret of arsenic and silica, will remain unaffected by the volatile oil, and may be separately eliminated by the curious, though such separation is of little practical importance.

Two modes of ren̂ning sulphur for the gunpowder works have been employed; the first is by fusion, the second by distillation. Since the combustible solid becomes as 
limpid as water, at the temperature of about $230^{\circ}$ Fahrenheit, a ready mode offers of removing at once its denser and lighter impurities, by subsidence and slimming. But I may take the liberty of observing, that the French melting pot, as described in the elaborate work of MMI. Bottee and Riffault, is singularly ill-contrived, for the fire is bindled right under it, and plays on its bottom. Now a pot for subsidence ought to be cold set; that is, should have its bottom part imbedded in clay or mortar for four or six inches up the side, and be exposed to the circulating flame of the fire only round its middle zone. This arrangement is adopted in many of our great chemical works, and is found to be very adrantageous. With such a boiler, judiciously heated, I believe that crude sulphur might be made remarkably pure; whereas by directing the heat against the bottom of the vessel, the crudities are tossed up, and incorporated with the mass. See Evaporation.

The sulphur of commerce occurs in three prevailing colors; lemon yellow verging on green, dark yellow, and brown yellow. As these different shades result from the different degrees of heat to which it has been exposed in its original extraction on the great scale, we may thereby judge to what point it may still be heated anew in the refinery melting. Whatever be the actual shade of the crude article, the art of the refiner consists in regulating the heat, so that after the operation it may possess a brilliant yellow hue, inclining somewhat to green.

In seeking to accomplish this purpose, the sulphur should first be sorted according to its shades; and if a greenish variety is to be purified, since this kind has been but little beated in its extraction, the fusion may be urged pretty smartly, or the fire may be kept up till everything is melted but the uppermost layer.

Sulphur of a strong yellow tinge cannot bear so great a heat, and therefore the fire must be withdrawn whenever three fourths of the whole mass have been melted.

Brown-colored brimstone, haring been already somewhat scorched, should be heated as little as possible, and the fire may be removed as soon as one half of the mass is fused.

Instead of melting, separately, sulphurs of different shades, we shall obtain a better result by first filling up the pot to half its capacity, with the greenish-colored article, putting over this layer one quarter volume of the deep yellow, and filling it to the brim with the brown-colored. The fire must be extinguished as soon as the yellow is fused. The pot must then be closely covered for some time; after which the lighter impurities will be found on the surface in a black froth, which is skimmed off, and the heavier ones sink to the bottom. The sulphur itself must be left in the pot for ten or twelve hours, after which it is laded out into the crystallizing boxes or casks.

Distillation affords a more complete and very economical means of purifying sulphur, which was first introduced into the French gunpowder establishments, when their importation of the best Italian and Sicilian sulphur was obstructed by the British navy. Here the sulphur need not come over slowly in a rare vapor, and be deposited in a pulverulent form called flowers; for the only object of the refiner is to bring over the whole of the pure sulphur into his condensing chamber, and to leave all its crudities in the body of the still. Hence a strong fire is applied to elevate a denser mass of vapors, of a yellowish color, which passing over into the condenser, are deposited in a liquid state on its bottom, whilst only a few lighter particles attach themselves to the upper and lateral surfaces. The refiner must therefore give to the heat in this operation rery considerable intensity; and, at some height above the edge of the boiler, he should provide an inclined plane, which may let the first ebullition of the sulphur overflow into a safety recipient. The condensing chamber should be hot enough to maintain the distilled sulphur in a fluid state-an object most readily procured by leading the pipes of several distilling pots into it; while the continuity of the operations is secured, by charging each of the stills alternately, or in succession. The heat of the receiver must be never so high as to bring the sulphur to a sirupy consistence, whereby its color is darkened.

In the sublimation of sulphur, a pot containing about $4 \mathrm{cwts}$. can be worked off only once in twenty-four hours, from the requisite moderation of its temperature, and the precaution of an inclined plane, which restores to it the accidental ebullitions. But, by distillation, a potcontaining fully ten cwts. may complete one process in nine hours at most, with a very considerable saving of fuel. In the former plan of procedure, an interval must elapse between the successive charges; but in the latter, the operation must be continurus to prevent the apparatus from getting cooled; in sublimation, moreover, where communication of atmospheric air to the condensing chamber is indispensable, explosive combustions of the sulphurous vapors frequently occur, with a copious production of sulphurous acid, and correspondent waste of the sulphur; disadvantages from which the distillatory process is in a great measure exempt.

I shall here describe briefly the form and dimensions of the distilling apparatus einployed at Marseilles in purifying sulphur for the national gunpowder worlis, whicb 
was found adequate to supply the wants of Napoleon's great empire. This apparatus consists of only two still-pots of cast iron, formed like the large end of an egg, each about three feet in diameter, two feet deep, and nearly half an inch thick at the bottom, but much thinner above, with a horizontal ledge four inches broad. A pot of good cast iron is capable of distilling 1000 tons of sulphur before it is rendered unserviceable, by the action of the brimstone on its substance, aided by a strong red heat. The pot is covered in with a sloping roof of masonry, the upper end of which abuts on the brickwork of the vaulted dome of condensation. A large door is formed in the masonry in front of the mouth of the pot, through which it is charged and cleared ont; and between the roof-space over the pot, and the cavity of the vault, a large passage is opened. At the back of the pot a stone step is raised to prevent the sulphur boiling over into the condenser. The vault is about ten feet wide within, and fourteen feet from the bottom up to the middle of the dome, which is perforated, and carries a chimney about twelve feet high, and twelve feet diameter inside.

As the dome is exposed to the expansive force of a strong heat, and to a very considerable pressure of gases and vapors, it must possess great solidity, and be therefore bound with iron straps. Between the still and the contiguous wall of the condensing chamber, a space must be left for the circulation of air ; a precaution found by experience indispensable; for the contact of the furnaces would produce on the wall of the chamber such a heat as to make it crack and form crevices for the liquid sulphur to eseape. The sides of the chamber are constructed of solid masonry, forty inches thick, surmounted by a brick dome, covered with a layer of stones. The floor is paved with tiles, and the walls are lined with them up to the springing of the dome; a square hole being left in one side, furnished with a strong iron door, at which the liquid sulphur is drawn off at proper intervals. In the roof of the vault are two valve-holes covered with light plates of sheet-iron, which turn freely on hinges at one end, so as to give way readily to any sudden expansion from within, and thus prevent dangerous explosions.

As the chamber of condensation is an oblong square, terminating upwards in an oblong vault, it consists of a parallelopiped below, and semi-cylinder above, having the following dimensions :-

Length of the parallelopiped
Width
Height
Radius of the cylinder
Height or length of semi-cylinder

Whenever the workman has introduced into each pot its charge of ten or twelve hundred weight of crude sulphur, he closes the charging doors carefully with their iron plates and cross-bars, and lutes them tight with loam. He then kindles his fire, and makes the sulphur boil. One of his first duties (and the least neglect in its discharge may oceasion serious accidents) is to inspect the roof-valves and to clean them, so that they may play freely and give way to any expulsive force from within. By means of a cord and chain, connected with a crank attached to the valves, he can, from time to time, ascertain their state, without mounting on the roof. It is found proper to work one of the pots a certain time before fire is applied to the other. The more steadily vapors of sulphur are seen to issue from the valves, the less atmospherical air can exist in the chamber, and therefore the less danger there is of combustion. But if the air be cold, with a sharp north wind, and if no vapors be escaping, the operator should stand on his guard, for in such circumstances a serious explosion may ensue.

As soon as both the boilers are in full work the air is expelled, the fumes cease, and every hazard is at an end. He should bend his whole attention to the cutting off all communication with the atmosphere, securing simply the mobility of the valves, and a steady vigor of distillation. The conclusion of the process is ascertained by introducing his sounding-rod into the pot, through a small orifice made for its passage in the wall. new charge must then be given.

By the above process, well conducted, sulphurs are brought to the most perfect state of purity that the arts can require; while not above four parts in the hundred of the sulphur itself are consumed; the cpude, incombustible residuum varying from five tc eight parts, according to the nature of the raw material. But in the sublimation of sulphur, the frequent combustions inseparable from this operation carry the loss of weight in flowers to about twenty per cent. See SULPHUR, for a figure of the subliming apparatus.

The process by fusion, performed at some of the public works in this country, does not afford a return at all comparable with that of the above French process, though a much better article is operated upon in England. After two meltings of rough sulphur (as imported from Sicily or Italy), eighty-four per cent. is the maximum amount 
obtained, the average being probably under eighty ; while the product is certainly inferior in yuality to that by distillation.

3. On the tarcoal.-Tender and light woods, capable of affording a friable and porous charenal, which burns rapidly away, leaving the smallest residuum of ashes, and containing therefure the largest proportion of carbon, ought to be preferred for charring in gunpowder works.

After many trials made long ago, black dogwood came to be preferred to every plant for this purpose; but modern experiments have proved that many other woods afford an equally suitable charcoal. The woods of black alder, poplar, lime-tree, horse-chestnut, and chestnut-tree, were carbonized in exactly similar circumstances, and a similar gunpowder was made with each, which was proved by the same proof-mortar. The following results were obtained :-

\begin{tabular}{|c|c|c|c|c|c|c|}
\hline \multirow{2}{*}{\multicolumn{2}{|c|}{ Poplar-mean range }} & \multirow[b]{2}{*}{-} & \multirow[b]{2}{*}{ - } & \multirow[b]{2}{*}{ - } & Toises. & \multirow{2}{*}{$\begin{array}{r}\text { Feet. } \\
2\end{array}$} \\
\hline & & & & & 113 & \\
\hline Black alder - & - & - & - & - & 110 & 4 \\
\hline Lime - & - & - & - & - & 110 & 3 \\
\hline Horse-chestnut & - & - & - & - & 110 & 3 \\
\hline Chestnut-tree & - & - & - & - & 109 & \\
\hline
\end{tabular}

By subsequent experiments, confirmatory of the above, it has been further found that the willow presents the same advantages as the poplar, and that several shrubs, such as the hazel-nut, the spindle-treee, the dogberry, the elder-tree, the common sallow, and some others, may be as advantageously employed. But whichever wood be used, we should always cut it when full of sap, and never after it is dead; we should choose branches not more than five or six years old, and strip them carefully, because the old branches and the bark contain a larger proportion of earthy constituents. The branches ought not to exceed three quarters of an inch in thickness, and the larger ones should be divided lengthwise into four, so that their pith may be readily burned away.

Wood is commonly carbonized in this country into gunpowder-charcoal in cast-iron cylinders, with their axes laid horizontally, and built in brick-work, so that the flame of a furnace may circulate round them. One end of the cylinder is furnished with a door, for the introduction of the wood and the removal of the charcoal; the other end terminates in a pipe, connected with a worm-tub for condensing the pyroligneous acid, and giving vent to the carbureted hydrogen gases that are disengaged. Towards the end of the operation, the connexion of the cylinder with the pyroligneous acid, cistern ought to be cut off, and a very free egress opened for the volatile matter, otherwise the charcoal is apt to get coated with a fuliginous varnish, and to be even penetrated with condensable matter, which materially injure its qualities.

In France, the wood is carbonized for the gunpowder works either in oblong vaulted ovens, or in pits, lined with brick-work or cylinders of strong sheet-iron. In either case, the heat is derived from the imperfect combustion of the wood itself to be charred. In general, the product in charcoal by the latter method is from 16 to 17 part by weight, from 100 of wood. The pit-process is supposed to afford a more productive return, and a better article; since the body of wood is much greater, and the fuliginous vapors are allowed a freer escape. The surface of a good charcoal should be smooth, but not glistening. See Charcoal.

The charecal is considered by the scientific manufacturers to be the ingredient most influential, by its fluctuating qualities, upon the composition of gunpowder; and, therefure, it ought alwars to be prepared under the vigilant and skilful eye of the directer of the powder establishment. If it has been kept for some time, or quenched at first with water, it is unsuitable for the present purpose. Charcoal extinguished in a close vessel by exclusion of air, and afterwards exposed to the atmosphere, absorbs only from three to fuur per cent. of moisture, while red-hot charcoal quenched with water may lose by drying twenty-nine per cent. When the latter sort of charcoal is used for gunpowder, a deduction of weight must be made for the water present. But charcoal which has remained long impregnated with moisture, constitutes a most detrimental ingredient of gunpowder.

\section{On mixing the Constituents and forming the Powder.}

The three ingredients thus prepared are ready for manufacturing into gunpowder. They are, 1. Separately ground to a fine powder, which is passed through sorted silk sieves or bolting machines. 2. They are mfxed together in the proper proportions, which we shall afterwards discuss. 3. The composition is then sent to the gunpowder 
mill, which consists of two edge-stones of a calcareous kind, turning by means of a hortzontal shaft, on a bed-stone of the same nature; incapable of affording sparks by collision with steel, as sand-stones would do. On this bed-stone the composition is spread, and moistened with as small a quantity of water as will, in conjunction with the weight of the revulving stones, bring it into a proper body of cake, but by no means into a pasty state. The line of contact of the rolling edge-stone is constantly preceded by a hard copper scraper, which goes round with the wheel, regularly collecting the caking inass, and bringing it into the track of the stone. From 50 to 60 pounds of cake are isually worked at one operation, under each millstone. When the mass has been thoroughly kneaded and incorporated, it is sent to the corning-house, where a separate mill is employed to form the cake into grains or corns. Here it is first pressed into a hard firm mass, then broken into small lumps; after which the corning process is performed, by placing these lumps in sieves, on each of which is laid a disc or fiat cake of lignum vitæ. The sieves are made of parchment skins, or of copper, perforated with a multitude of round holes. Several such sieves are fixed in a frame, which, by proper machinery, has such a motion given to it as to make the lignum vitæ runner in each sieve move about with considerable velocity, so as to break down the lumps of the cake, and force its substance through the holes, in grains of certain sizes. These granular particles are afterwards separated from the finer dust by proper sieves and reels.

The corned powder must now be hardened, and its rougher angles removed, by causing it to revolve in a close reel or cask turning rapidly round its axis. This ressel resembles somewhat a barrel-churn, and is frequently furnished inside with square bars parallel to its axis, to aid the polish by attrition.

The gunpowder is finally dried, which is now done generally with a steam heat, or in some places by transmitting a current of air, previously heated in another chamber, over canvass shelves, covered with the damp grains.

\section{On the proportion of the Constituents.}

A very extensive suite of experiments, to determine the proportions of the constituents for producing the best gunpowder, was made at the Essonne works, by a commission of French chemists and artillerists, in 1794.

Powders in the five following proportions were prepared :-

\begin{tabular}{|l|l|l|cl|}
\hline & Nitre. & Charcoal. & \multicolumn{2}{|c|}{ Sulphur. } \\
\cline { 2 - 5 } 1 & 76 & 14 & 10 & Gunpowder of Bâle. \\
2 & 76 & 12 & 12 & Gunpowder works of Grenelle. \\
3 & 76 & 15 & 9 & M. Guyton de Morveau. \\
4 & $77 \cdot 32$ & $13 \cdot 44$ & $9 \cdot 24$ & Idem. \\
5 & $77 \cdot 5$ & 15 & $7 \cdot 5$ & M. Riffault. \\
\hline
\end{tabular}

The result of more than two hundred discharges with the proof-mortar showed that the first and third gunpowders were the strongest; and the commissioners in consequence recommended the adoption of the third proportions. But a few years thereafter it was thought proper to substitute the first set of proportions, which had been found equal in force to the other, as they would have a better keeping quality, from containing a little more sulphur and less charcoal. More recently still, so strongly impressed have the French government been with the high value of durability in gunpowders, that they have returned to their ancient dosage of 75 nitre, $12 \frac{1}{2}$ charcoal, and $12 \frac{1}{2}$ sulphur. In this mixture, the proportion of the substance powerfully absorbent of moisture, riz., the charcoal, is still further reduced, and replaced by the sulphur, or the conservative ingredient.

If we inquire how the maximum gaseous volume is to be produced from the chemical reaction of the elements of nitre on charcoal and sulphur, we shall find it to be by the generation of carbonic oxyde and sulphurous acid, with the disengagement of nitrogen. This will lead us to the following proportions of these constituents.

\begin{tabular}{|c|c|c|c|}
\hline \multirow{3}{*}{$\begin{array}{ll}1 \text { prime equivalent of nitre - } \\
1 & \text { sulphur } \\
3 & \text { charcoal }\end{array}$} & \multirow{3}{*}{$\div \quad:$} & Hydrogen $=1$. & Per cent \\
\hline & & $\begin{array}{r}102 \\
16 \\
18\end{array}$ & $\begin{array}{l}75 \cdot 00 \\
11 \cdot 77 \\
13 \cdot 23\end{array}$ \\
\hline & & 136 & $100 \cdot 00$ \\
\hline
\end{tabular}


The nitre contains five primes of oxygen, of which three, combining with the three of eliarcual, will furnish three of carbonic oxyde gas, while the remaining two will convert the one prime of sulphur into sulphurous acid gas. The single prime of nitrogen is, therefore, in this view, disengaged alone.

The gaseous volume, on this supposition, evolved from 136 grains of gunpowder, equi valent in bulk to $75 \frac{1}{2}$ grains of water, or to three tenths of a cubic inch, will be, at the atmospheric temperature, as follows :-

\begin{tabular}{|c|c|c|c|c|c|c|}
\hline \multirow{3}{*}{$\begin{array}{l}\text { Carbonic oxyde } \\
\text { Sulphurous acid - } \\
\text { Nitrogen - }\end{array}$} & \multirow{3}{*}{-} & \multirow{3}{*}{$\cdot$} & \multirow{3}{*}{$\cdot \cdot$} & \multirow{2}{*}{\multicolumn{3}{|c|}{$\begin{array}{c}\text { Grains. Cubic Inches } \\
42=141 \cdot 6 \\
32=47 \cdot 2 \\
14=47 \cdot 4\end{array}$}} \\
\hline & & & & & & \\
\hline & & & & & & $236 \cdot 2$ \\
\hline
\end{tabular}

being an expansion of one volume into $78 \% \cdot 3$. But as the temperature of the gases as the instant of their combustive formation must be incandescent, this volume may be safely estimated at three times the above amount, or considerably upwards of two thousand times the bulk of the explosive solid.

But this theoretical account of the gases developed does not well accord with the experimental products usually assigned, though these are probably not altogether exact. Much carbonic acid is said to be disengaged, a large quantity of nitrogen, a little oxyde of carbon, sleam of water, with carbureted and sulphureted hydrogen. From experiments to be presently detailed, I am convinced that the amount of these latter products printed in italies must be very inconsiderable indeed, and unworthy of ranking in the calculation; for, in fact, fresh gunpowder does not contain above one per cent. of water, and can therefore yield little hydrogenated matter. Nor is the hydrogen in the carbon of any consequence.

It is obvious that the more sulphur is present, the more of the dense sulphurous acid will be generated, and the less forcibly explosive will be the gunpowder. This is sufficiently confirmed by the trials at Essonne, where the gunpowder that contained 12 of sulphur and 12 of charcoal in 100 parts, did not throw the proof-shell so far as that which contained only 9 of sulphur and 15 of charcoal. The conservative property is, however, so capital, especially for the supply of our remote colonies and for humid climates, that it justifies a slight sacrifice of strength, which at any rate may be compen. sated by a small addition of charge.

Table of Composition of different Gunpowders.

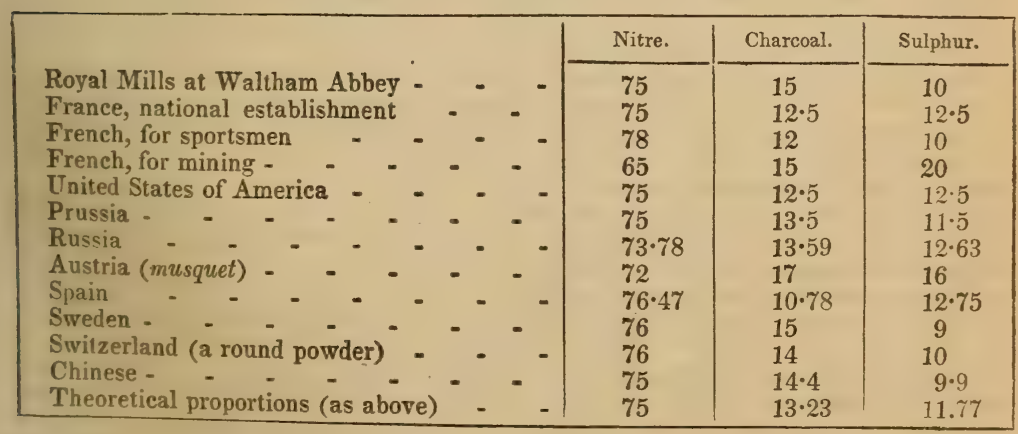

\section{On the Chemical Examination of Gunpowders.}

I have treated five different samples: 1 . The government powder made at Waltham Abbey; 2. Glass gunpowder made by John Hall, Dartford; 3. The treble strong gunpowder of Charles Lawrence and Son; 4. The Dartford gunpowder of Pigou and Wilks; 5. Superfine treble strong sporting gunpowder of Curtis and Harvey. The first is coarse-grained, the others are all of considerable fineness. The specific gravity of each was taken in oil of turpentine: that of the first and last three was exactly the same, being 1.80 ; that of the second was 1.793 , all being reduced to water as unity. 
The above density for specimen first, may be calculated thus :-

75 parts of nitre, specific gravity $=2 \cdot 000$

15 parts of charcoal, specific gr. $=1 \cdot 154$

10 parts of sulphur, specific gr. $=2 \cdot 000$

The volume of these constituents is 55.5 (the volume of their weight of water being 100 ); by which if their weight 100 be divided, the quotient is 1.80 .

The specific gravity of the first and second of the above powders, including the interstices of their grains, after being well shaken down in a vial, is 1.02. This is a curious result, as the size of the grains is extremely different. That of Pigou and Wilks, similarly tried, is only 0.99 ; that of the Battle powder is 1.03 ; and that of Curtis and Harvey is nearly 1.05 . Gunpowders thus appear to have nearly the same weight as water, under an equal bulk; so that an imperial gallon will hold from 10 pounds to 10 pounds and a half, as above shown.

The quantities of water which 100 grains of each part with on a steam bath, and absorb when placed for 24 hours under a moistened receiver standing in water, are as follows :-

100 grains of Waltham Abbey, lose 1.1 by steam heat, gain 0.8 over water.

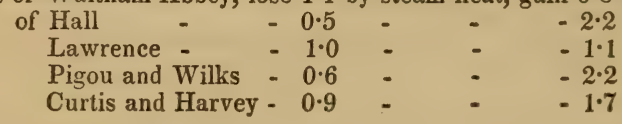

I'hus we perceive that the large grained government powder resists the hygrometric influence better than the others; among which, however, Lawrence's ranks nearly as high. These two are therefore relatively the best keeping gunpowders of the series.

The process most commonly practised in the analysis of gunpowder, seems to be tolerably exact. The nitre is first separated by hot distilled water, evaporated and weighed. A minute loss of salt may be counted on, from its known volatility with boiling water. I have evaporated always on a steam bath. It is probable that a small portion of the lighter and looser constituent of gunpowder, the carbon, flies off in the operations of corning and dusting. Hence, analysis may show a small deficit of charcoal below the synthetic proportions originally mixed. The residuum of charcoal and sulphur left on the double filter-paper, being well dried by the heat of ordinary steam, was estimated, as usual, by the difference of weight of the inner and outer papers. This residuum was cleared off into a platina capsule with a tooth-brush, and digested in a dilute solution of potash at a boiling temperature. Three parts of potash are fully sufficient to dissolve out one of sulphur. When the above solution is thrown on a filter, and washed first with a very dilute solution of potash boiling hot, then with boiling water, and afterwards dried, the carbon will remain; the weight of which deducted from that of the mixed powder, will show the amount of sulphur.

I have tried many other modes of estimating the sulphur in gunpowder more directly, but with little satisfaction in the results. When a platina capsule, containing gunpowder spread on its bottom, is floated in oil, heated to $400^{\circ}$ Fahrenheit, a brisk exhalation of sulphur fumes rises, but, at the end of several hours, the loss does not amount to more than one half of the sulphur present.

The mixed residuum of charcoal and sulphur digested in hot oil of turpentine gives up the sulphur readily; but to separate again the last portions of the oil from the charcoal or sulphur, requires the aid of alcohol.

When gunpowder is digested with chlorate of potash and dilute muriatic acid, at a moderate heat in a retort, the sulphur is acidified; but this process is disagreeable and slow, and consumes much chlorate. The resulting sulphuric acid being tested by nitrate of baryta, indicates of course the quantity of sulphur in the gunpowder. A curious fact occurred to me in this experiment. After the sulphur and charcoal of the gunpowder had been quite acidified, I poured some solution of the baryta salt into the mixture, but no cloud of sulphate ensued. On evaporating to dryness, however, and redissolving, the nitrate of baryta became effective, and enabled me to estimate the sulphuric acid generated; which was of course 10 for every 4 of the sulphur.

The acidification of the sulphur by nitric or nitro-muriatic acid is likewise a slow and unpleasant operation.

By digesting gunpowder with potash water, so as to convert its sulphur into a sulphuret, mixing this with nitre in great excess, drying and igniting, I had hoped to convert the sulphur readily into sulphuric acid. But on treating the fused mass with dilute nitric acid, more or less sulphurous acid was exhaled. This occurred even though chlorate of potash had been mixed with the nitre to aid the oxygenation. 
The following are the results of my analyses, conducted by the first described method :

\begin{tabular}{|c|c|c|c|c|c|}
\hline 100 grains afford, o & & Nitre. & Charcoal. & Sulphur. & Water. \\
\hline Waltham Abbey & - & $74 \cdot 5$ & $14 \cdot 4$ & $10 \cdot C$ & $1 \cdot 1$ \\
\hline Hall, Dartford - & - & $76 \cdot 2$ & $14 \cdot 0$ & $9 \cdot 0$ & $0.5 \operatorname{loss} 0.3$ \\
\hline Pigou and Wilks & - & $77 \cdot 4$ & $13 \cdot 5$ & $8 \cdot 5$ & $0 \cdot 6$ \\
\hline Curtis and Harvey & - & $76 \cdot 7$ & $12 \cdot 5$ & $9 \cdot 0$ & $1 \cdot 1$ loss $0 \cdot 7$ \\
\hline Battle gunpowder & - & $77 \cdot 0$ & $13 \cdot 5$ & $8 \cdot 0$ & $0.8 \operatorname{loss} 0.7$ \\
\hline
\end{tabular}

It is probable, for reasons already assigned, that the proportions mixed by the manufacturers may differ slightly from the above.

The English sporting gunpowders have long been an object of desire and emulation in France. Their great superiority for fowling pieces over the product of the French national manufactories, is indisputable. Unwilling to ascribe this superiority to any genuine cause, M. Vergnaud, captain of French artillery, in a little work on fulminating powders lately published, asserts positively, that the English manufacturers of 'poudre de chasse' are guilty of the 'charlatanisme' of mixing fulminating mercury with it. To determine what truth was in this allegation, with regard at least to the above five celebrated gunpowders, I made the following experiments.

One grain of fulminating mercury, in crystalline particles, was mixed in water witk. 200 grains of the Waltham Abbey gunpowder, and the mixture was digested over a samp with a very little muriatic acid. The filtered liquid gave manifest indications of the corrosive sublimate, into which fulminating mercury is instantly convertible by mu riatsc acid; for copper was quicksilvered by it ; potash caused a white cloud in it that Decame rellow, and sulphureted hydrogen gas separated a dirty yellow white precipl. tate of bisulphuret of mercury. When the Waltham Abbey powder was treated alone with dilute muriatic acid, no effect whatever was produced upon the filtered liquid by the sulphureted hydrogen gas.

200 grains of each of the above sporting gunpowders were treated precisely in the same way, but no trace of mercury was obtained by the severest tests. Since by this process there is no doubt but one 10,000 th part of fulminating mercury could be detected, we may conclude that Captain Vergnand's charge is groundless. The supcriority of our sporting gunpowders is due to the same cause as the superiority of our cotton fabrics - the care of our manufacturers in selecting the best materials, and their skill in combining them.

I shall subjoin here some miscellaneous observations upon gunpowder.

In Bengal, mixing is performed by shutting up the ingredients in barrels, which are turned either by hand or machinery; each containing $50 \mathrm{lbs}$. weight, or more, of small brass balls. They have ledges on the inside, which occasion the balls and composition to tumble about and mingle together, so that the intermixture of the ingredients, after tne process has been gone through, cannot fail to be complete. The operation is coninued two or three hours; and I think it would be an improvement in Her Majesty's system of manufacture if this method of mixing were adopted.

In England two or three pints of water are used for a $42 \mathrm{lb}$. charge : but the quantity is variable; both the temperature and the humidity of the atmosphere influence it.

Bramah's hydrostatic press, or a very strong wooden press working with a powerfu? screw, lever, and windlass, constitutes the description of mechanism by which density is imparted to gunpowder. The incorporated or mill-cake powder is laid on the bed or follower of the press, and separated, at equal distances, by sheets of copper, so that when the operation is over, it comes out in large thin solid cakes, or strata, distinguished by the term press-cake. The mill-cake powder at Waltham Abbey, is submitted to a mean theoretic pressure of 70 to 75 tons per superficial foot.

Gunpowder should be thoroughly dried, but not by too high a degree of heat; that of $140^{\circ}$ or $150^{\circ}$ of Fahrenheit's thermometer is sufficient. It appears to be of no consequence whether it be dried by solar heat, by radiation from red-hot iron, as in the gloom stove, or by a temperature raised by means of steam. Her Majesty's gunpowder is dried by the last two methods. The grain should not be suddenly exposed to the highest degree of heat, but gradually.

The method of trial best adapted to show the real inherent strength and goodness of gunpowder, appears to be an eight or ten inch iron or brass mortar, with a truly spherical solid shot, having not more than one tenth of an inch windage, and fired with a low charge. The eight-inch mortar, fired with two ounces of powder, is one of the established methods of proof at Her Majesty's works. Gunpowders that range equally in this mode of trial, may be depended on as being equally strong.

Another proof is by four drachms of powder laid in a small neat heap, on a clean, polished 
copper plate; which heap is fired at the apex, by a red hot iron. The explosion should be sharp and quick; not tardy, nor lingering; it should produce a sudden concussion in the air ; and the force and power of that concussion ought to be judged of by comparison with that produced by powder of known good quality. No sparks should fly off, nor should beads, or globules of alkaline residuum, be left on the copper. If the copper be left clean, i. e. without gross foulness, and no lights, i. e. sparks, be seen, the ingredients may be considered to have been carefully prepared, and the powder to have been well manipulated, particularly if pressed and glazed; but if the contrarv be the result, there has been a want of skill or of carefulness manifested in the manufacture.

"Gunpowder," says Captain Bishop, "explodes exactly at the $600^{\circ}$ of heat by Fahrenheit's thermometer; when gunpowder is exposed to $500^{\circ}$ it alters its nature altogether; not only the whole of the moisture is driven off, but the saltpetre and sulphur are actually reduced to fusion, both of which liquefy under the above degree. The powder, on cooling, is found to have changed its color from a gray to a deep black ; the grain has necome extremely indurated, and by exposure even to very moist air, it then suffers no alteration by imbibing moisture."

The mill for grinding the gunpowder cake may be understood from the following 531

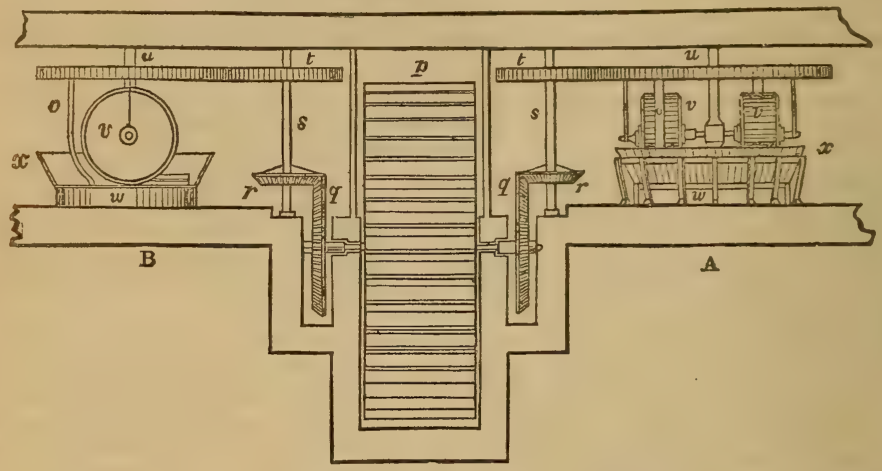

representation ( $f g .531$ ) ; $p$, is the water wheel, which may drive several pairs of stones ; $q, q$, two vertical bevel wheels, fixed upon the axis of the great wheel; $r,{ }^{\prime}, t$ two horizontal bevel wheels working in $q, q$, and turning the shafts $s, s ; t, t$, two horizontal spur wheels fixed to the upper part of the vertical shafts, and driving the large wheels $u, u$. To the shafts of these latter wheels are fixed the runners $v, v$, which traverse upon the bed stone $w, w ; x, x$, are the curbs surrounding the bed stone to prevent the powder from fallung off; $O$ is the scraper. Mill A represents a view, and mill в a section of the bed stone and curb.

GYPSUM, Sulphate of lime, Alabaster, or Paris Plaster. This substance is found in three geological positions in the crust of the earth; among transition rocks; in the red marl formation; and above the chalk, in the tertiary beds.

1. The alpine gypsums are ranged by $\mathbf{M}$. Brochant among the transition class, and are characterized by the presence of anthracite or stone coal; some of them are white and pure, others gray or yellowish, and mixed with mica, talc, steatite, black oxyde of iron, prrites, compact carbonate of lime, sulphur, and common salt. Examples of such localities are found in the gypsum of Val-Canaria at the foot of Saint Gothard, that of Brigg in the upper Valais; of the Grilla in the valley of Chamouni, and of Saint Gervais-les-Bains, near Sallenches in Savoy.

2. The secondary gypsum, or that of the salt mine districts, belongs to the red ground, immediately beneath the lias in the order of stratification, and therefore a rock relatively ancient. Near Northwick, the red marl beds above the great deposite of rock salt, are irregularly intersected with gypsum, in numerous laminæ or plates. At Newbiogin in Cumberland, the gypsum lies in red argillaceous marl, between two strata of sandstone; and a mile south of Whitehaven, the subterraneous workings for the alabaster extend 30 yards in a direct line; with two or three lateral branches extending about 10 yards, at whose extremities are large spaces where the gypsum is blasted with gunpowder. It is generally compact, forming a regular and conformable bed, with crystals of selenite (crystallized gypsum) in drusy cavities. Gypsum occurs in the red marl in the isle of Axholme, and various other places in Nottinghamshire. In Derbyshire some considerable deposites have been found in the same red sandstone, several of which are mined, as at Chellaston hill, which would exhibit a naked and water-worn rock of gypsum, 
were it not for a corering of alluvial clay. It appears in general to present itself chiefly in particular patches, occasioning a sudden rise, or an insulated hill, by the additional thickness which it gives to the stratum of the ren ground in these places. The principal demand for the pure white gypsum, or that faintly streaked with red, is by the potters in Staffurdshire, who form their monlds with the calcined powder which it affords; only particularly fine blocks are selected for making alabaster ornaments on the turning lathe. In one of the salt pits near Droitwich, the strata sunk through, were vegetable mould. 3 feet ; red marl, 35 feet; gypsum, 40 feet; a river of brine, 22 inches; gipsum, 75 feet; a rock of salt, bored into only 5 feet, but probably extending much deeper. On the Welsh side of the Bristol channel, gypsum occurs in the red marl cliffs of Glamorganshire, from Pennarth to Lavernock. No organic remains or metallic minerals have hitherto been found in the gypsum of this formation.

3. The most interesting gypsums in a general point of view, are certainly the tertiary, or those of the plains, or hills of comparatively modern formation. They are characterized $b_{y}$ the presence of fossil bones of extinct animals, both mammifera and birds, by shells, and a large proportion of carbonate of lime, which gives them the property of effervescing wi h acids, and the title of limestone gypsums. Such are the gypsums of the environs of Paris, as at the heights of Montmartre, which contain crystallized sulphate of lime in many forms, but most commonly the lenticular and lance-shaped.

Sulphate of lime occurs either as a dense compound without water, and is called anhydri:e from that circumstance; or with combined water, which is its most ordinary state. Of the latter there are 6 sub-species; sparry gypsum or selenite in a variety of crystalline forms; the foliated granular; ihe compact; the fibrous; the scaly foliated; the earthy.

The prevailing color is white, with various shades of gray, blue, red, and yellow. Mure or less translucent. Soft, sectile, yielding to the nail. Specific gravity $2 \cdot 2$. Water dissolves about one five-hundredth part of its weight of gypsum, and acquires the quality of hardness, with the characteristic selenitic taste. When exposed on red hot coals, it decrepitates, becomes white, and splits into a great many brittle plates. At the heat of a baker's oven, or about $400^{\circ}$ Fahr., the combined water of gypsum escapes with a species of ebullition. At a higher temperature the particles get indurated. When rightly calcined and pulverized, g!psum is mixed with water to the consistence of cream, and poured into moulds by the manufacturers of stucco ornaments and statues. A species of rapid crrstallization ensues, and the thin paste soon acquires a solid consistence, which is increased by drying tho figure in proper stoves. During the consolidation of the plaster, its volume expands into the finost lines of the mould, so as to give a sharp and faithful impression.

The plaster stone of the Paris basin contains about 12 per cent. of carbonate of lime. This body, ground and mixed with water, forms an adhesive mortar much used in building, as it fixes rery speedily. Works executed with pure gypsum never become so hard as those made with the calcareous kind; and hence it might be proper to add a certain portion of white slaked lime to our calcined gypsum, in order to give the stucco this valuable property. Colored stuccos of great solidity are made by adding to a clear solution of glue, any desired coloring tincture, and mixing-in the proper quantity of the calcined calcareous gypsum.

The compact, fine-grained, gypseous alabaster is often cut into various ornamental figures, such as vases, statuary groups, \&c., which take a high polish and look beautiful, but from their softness are easily injured, and require to be kept enclosed within a glass shade.

In America and France, the virtues of gypsum in fertilizing land have been highly extolled, but they have not been realized in the trials made in this kingdom.

Pure gypsum consists of lime 28 ; sulphuric acid 40 ; water 18 ; which are the respective weights of its prime equivalent parts.

M. Gay Lussac, in a short notice, in the Annales de Chimie for April, 1829, on the setting of gypsum, says that the purest plasters are those which harden least, and that the addition of lime is of no use toward promoting their solidity, nor can the heat proper for boiling gypsum ever expel the carbonic acid gas from the calcareous carbonate present in the gypsum of Montmartre. He conceives that a hard plaster-stone having lost its water, will resume more solidity in returning to its first state, than a plaster-stone naturally tender or soft; and that it is the primitive molecular arrangement which is regene. rated. See Alabaster. 
H.

HADE signifies, among English miners, the inclination, or deviation from the vertical of any mineral vein.

HAIR (Cheveu, Crin, Fr.; Haar, Germ.) is of all animal products, the one least liable to suontaneous change. It can be dissolved in water only at a temperature somewhat above $230^{\circ} \mathrm{F}$., in a Papin's digester, but it appears to be partially decomposed by this heat, since some sulphureted hydrogen is disengaged. By dry distillation, hair gives off several sulphureted gases, while the residuum contains sulphate of lime, common salt, much silica, with some oxyde of iron and manganese. It is a remarkable fact that fair hair affords magnesia, instead of these latter two oxydes. Horse-hair yields about 12 per cent. of phosphate of lime.

Hairs are tubular, their cavities being filled with a fat oil, having the same color with themselves. Hair plunged in chlorine gas, is immediately decomposed and converted into a viscid mass; but when immersed in weak aqueous chlorine, it undergoes no change, except a little bleaching. The application of nitrate of mercury to hairy skins in the process of secretage, is explained under Peltry.

For the dyeing of horse-hair, see the next article.

Living hairs are rendered black by applying to them, for a short time, a paste made by mixing litharge, slaked lime, and bicarbonate of potash, in various proportions, according to the shade of color desired.

We have no recent analysis of hair. Vauquelin found nine different substances in black hair ; in red hair, a red oil instead of a greenish-black one.

The salts of mercury, lead, bismuth, as well as their oxydes, blacken hair, or make it of a dark violet, by the formation, most probably, of metallic sulphurets.

Hair as an object of manufactures is of two kinds, the curly and the straight. The former, which is short, is spun into a cord, and boiled in this state, to give it the tortuons springy form. The long straight hair is woven into cloth for sieves, and also for ornamental purposes, as in the damask-hair cloth of chair bottoms. For this purpose the hair may be dyed in the following way.

Forty pounds of tail hair about 26 inches long are steeped in lime water during twelve hours. Then a bath is made with a decoction of 20 pounds of logwood, kept boiling for three hours, after which time the fire is withdrawn from the boiler, and ten ounces of copperas are introduced, stirred about, and the hair is immersed, having been washed from the lime in river water. The hair should remain in this cooling bath for 24 hours, when the operation will be finished. For other colors, see the respective dyes.

The looms for weaving hair differ from the common ones, only in the templet and the shuttle. Two templets of iron must be used to keep the stuff equably, but lightly stretched. These templets, of which one is represented in fig. 532, are constructed in the shape of flat pincers; the jaws c c being furnished with teeth inside. A screw D,

532

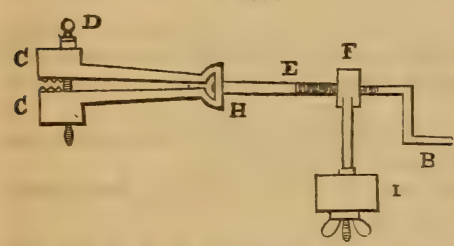
binds the jaws together, and hinders the selvage from going inwards. Upon the side cross beam of the loom, seen in section at $\mathrm{r}$, a bolt is fixed which carries a nut $F$ at its end, into which a screwed iron rod $\mathbf{E}$ enters, on one of whose ends is the handle $\mathbf{B}$. The other extremity of the screw $E$ is adapted by a washer and pin to the back of the pincers at the point $\mathrm{H}$, so that by turning the handle to the right or the left, we draw onwards or push backwards the pincers and the stuff at pleasure. The warp of the web is made of black linen yarn. The weft is of hair, and it is thrown with a long hooked shuttle; or a long rod, having a catch hook at its end. The length of this shuttle is about 3 feet; its breadth half an inch, and its thickness one sixth. It is made of box-wood. The reed is of polished steel; the thread warns are conducted through it in the usual way. The workman passes this shuttle between the hairs of the warp with one hand, when the shed or shuttle way is opened by the treddles; a child placed on one side of the loom presents a hair to the weaver near the selvage, who catches it with the hook of his shuttle, and by drawing it out passes it through the warp. The hairs are placed in a bundle on the side where the child stands, in a chest filled with water to keep them moist, for otherwise they would not have the suppleness requisite to form a web. Each time that a hair is thrown across, the batten is driven home twice. The warp is dressed with paste in the usual way. The hair cloth, after it is woven, is hot calendered to give it lustre. 
HAIR PENCILS OR BRUSHES for painting. Two sorts are made; those with coarse nair, as that of the swine, the wild boar, the dog, \&c., which are attached usually to short wouden rods as handles; these are commonly called brushes; and hair pencils, properly so called, which are composed of very fine hairs, as of the minever, the marten, the badger, the polecat, \&c. These are mounted in a quill when they are small or of moderate size, but when larger than a quill, they are mounted in white-iron tubes.

The most essential quality of a good pencil is to form a fine point, so that all the hairs without exception may be united when they are moistened by laying them upon the tongue, or drawing them through the lips. When hairs present the form of an elongated cone in a pencil, their point only can be used. The whole difficulty consists after the hairs are cleansed, in arranging them together so that all their points may lie in the same horizontal plane. We must wash the tails of the animals whose hairs are to be used, by scouring them in a solution of alum till they be quite free from grease, and then steeping them for 24 hours in luke-warm water. We next squeeze out the water by pressing them strengly from the root to the tip, in order to lay the hairs as smooth as possible. They are to be dried with pressure in linen cloths, combed in the longitudinal direction, with a very fine-toothed comb, finally wrapped up in fine linen, and dried. When perfectly dry, the hairs are seized with pincers, cut across close to the skin, and arranged in separate heaps, according to their respective lengths.

Each of these little heaps is placed separately, one after the other, in small tin pans with flat bottoms, with the tips of the hair upwards. On striking the bottom of the pan slightly upon a table, the hairs get arranged parallel to each other, and their delicate points rise more or less according to their lengths. The longer ones are to be picked out and made into so many separate parcels, whereby each parcel may be composed of equally long hairs. The perfection of the pencil depends upon this equality; the tapering point being produced simply by the attenuation of the tips.

A pinch of one of these parcels is then taken, of a thickness corresponding to the intended size of the pencil; it is set in a little tin pan, with its tips undermost, and is shaken bv striking the pan on the table as before. The root end of the hairs being tied by the fisnerman's or seaman's knot, with a fine thread, it is taken out of the pan, and then hooped with stronger thread or twine; the knots being drawn very tight by means of two little sticks. The distance from the tips at which these ligatures are placed, is of course relative to the nature of the hair, and the desired length of the pencil. The base of the pencil must be trimmed flat with a pair of scissors.

Nothing now remains to be done but to mount the pencils in quill or tin-plate tubes as above described. The quills are those of swans, geese, ducks, lapwings, pigeons, or larks, according to the size of the pencil. They are steeped during 24 hours in water, to swell and soften them, and to prevent the chance of their splitting when the hair brush is pressed into them. The brush of hair is introduced by its tips into the large end of the cut quill, having previously drawn them to a point with the lips, when it is pushed forwards with a wire of the same diameter, till it comes out at the other and narrower end of the quill.

The smaller the pencils, the finer ought the hairs to be. In this respect, the manufacture requires much delicacy of tact and experience. It is said that there are only four first-rate hands among all the dexterous pencil-makers of Paris, and that these are principally women.

HALOGENE is a term employed by Berzelius to designate those substances which form compounds of a saline nature, by their union with metals; such are Bromine, Chlorine, Cyanogene, Fluorine, Iodine. Haloid is his name of the salt thereby formed.

HANDSPIKE is a strong wooden bar, used as a lever to move the windlass and capstan in heaving up the anchor, or raising any heavy weignts on board a ship. The handle is smooth, round, and somewhat taper; the other end is squared to fit the holes in the head of the capstan or barrel of the windlass.

HARDNESS (Dureté, Fr.; Härle, Festigkeit, Germ.) is that modification of cohesive attraction which enables bodies to resist any effort made to abrade their surfaces. Its relative intensity is measured by the power they possess of cutung or scratching other substances. The following table exhibits pretty nearly the successive hardnesses of the several bodies in the list:- 


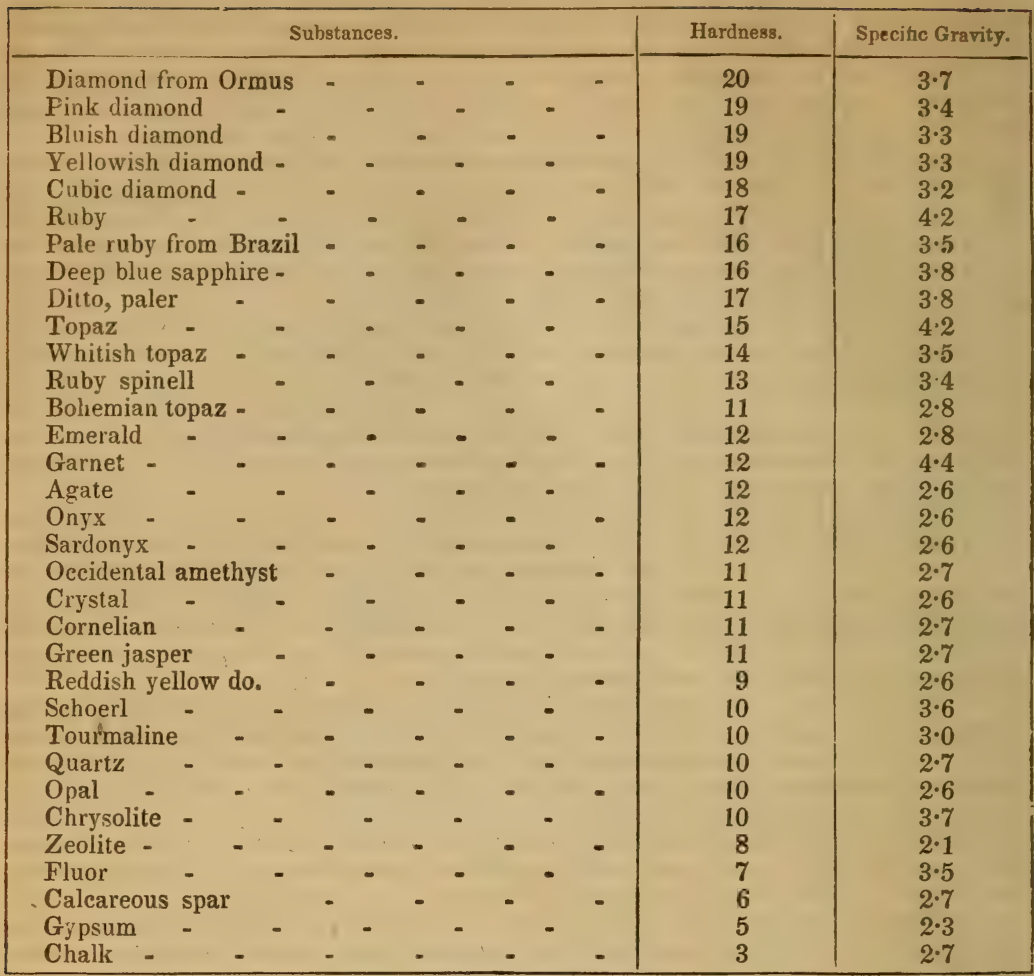

HARTSHORN, SPIRIT OF, is the old name for water of ammonia.

hatCHing OF CHiCKENS. See Incubation, Artificial.

HAT MANUFACTURE. (L'art de Chapelier, Fr.; Hutmacherkunst, Germ.) Hat is the name of a piece of dress worn upon the head by both sexes, but principally by the men, and seems to have been first introduced as a distinction among the ecclesiastics in the 12 th century, though it was not till the year 1400 that it was generally adopted by respectable laymen.

As the art of making common hats does not involve the description of any curious machinery, or any interesting processes, we shall not enter into very minute details upon the subject. It will be sufficient to convey to the reader a general idea of the methods employed in this manufacture.

The materials used in making stuff hats are the furs of hares and rabbits freed from the long hair, together with wool and beaver. The beaver is reserved for the finer hats. The fur is first laid upon a hurdle made of wood or wire, with longitudinal openings; and the operator, by means of an instrument called the bow (which is a piece of elastic asn, six or seven feet long, with a catgut stretched between its two extremities, and made so vibrate by a bowstick), causes the vibrating string to strike and play upon the fur, so as to scatter the fibres in all directions, while the dust and filth descend through the grids of the hurdle.

After the fur is thus driven by the bow from the one end of the hurdle to the other, it forms a mass called a bat, which is only half the quantity sufficient for a hat. The bat or capade thus formed is rendered compact by pressing it down with the hardening skin (a piece of half-tanned leather), and the union of the fibres is increased by covering them with a cloth, while the workman presses them together repeatedly with his hands. The cloth being taken off, a piece of paper, with its corners doubled in, so as to give it a triangular outline, is laid above the bat. The opposite edges of the bat are then folded over the paper, and being brought together and pressed again with the hands, they form a conica. cap. This cap is next laid upon another bat, ready hardened, so that the joined 
edges of the first bat rest upon the new one. This new bat is folded over the other, and its edices joined by pressure as before; so that the joining of the first conical cap is opposite to that of the second. This compound bat is now wrought with the hands for a consitlerable time upon the hurdle between folds of linen cloth, being occasionally sprinkled with clear water, till the hat is basined or rendered tolerably firm.

The cap is now taken to a wooden receiver, like a very flat mill-hopper, consisting of eight wooden planes, sloping gently to the centre, which contains a kettle filled with

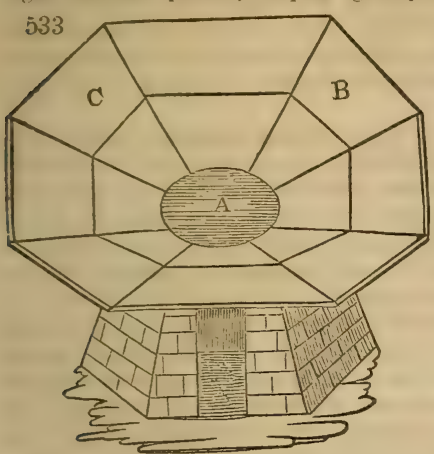
water acidulated with sulphuric acid. The technical name of this vessel is the battery. It consists of a kettle A; and of the planks, B c, which are sloping planes, usually eight in number, one being allotted to each workman. The half of each plank next the kettle is made of lead, the upper half of mahogany. In this liquor the hat is occasionally dipped, and wrought by the hands, or sometimes with a roller, upon the sloping planks. It is thus fulled or thickened during four or five hours; the knots or hard substances are picked out by the workman, and fresh felt is added by means of a wet brush to those parts that require it. The beaver is applied at the end of this operation. In the manufacture of beaver hats, the grounds of beer are added to the liquor in the kettle.

Stopping, or thickening the thin spots, seen by looking through the body, is performed by daubing on additional stuff with successive applications of the hot acidulous liquor from a brush dipped into the kettle, until the body be sufficiently shrunk and made uniform. After drying, it is stiffened with varnish composition rubbed in with a brush; the inside surface being more copiously imbued with it than the outer; while the brim is peculiarly charged with the stiffening.

When once more dried, the body is ready to be covered, which is done at the battery. The first cover of beaver or napping, which has been previously boued, is strewed equably over the body, and patted on with a brush moistened with the hot liquor, until it gets incorporated; the cut ends towards the root, being the points which spontaneously intrude. The body is now put into a coarse hair cloth, then dipped and rolled in the hot liquor, until the root ends of the beaver are thoroughly worked in. This is technically called rolling off, or roughing. A strip for the brim, round the edge of the inside, is treated in the same way; whereby every thing is ready for the second cover (of beaver), which is incorporated in like manner; the rolling, \&c. being continued, till a uniform, elose, and well-felted hood is formed.

The hat is now ready to receive its proper shape. For this purpose the workman turns up the edge or brim to the depth of about $1 \frac{1}{2}$ inch, and then returns the point of the cone back again through the axis of the cap, so as to preduce another inner fold of the same depth. A third fold is produced by returning the point of the cone, and so on till the point resembles a flat circular piece having a number of concentric folds. In this state it is laid upon the plank, and wetted with the liquor. The workman pulls out the point with his fingers, and presses it down with his hand, turning it at the same time round on its centre upon the plank, till a flat portion, equal to the crown of the hat, is rubbed nut. This flat crown is now placed upon a block, and, by pressing a string called a commander, down the sides of the block, he forces the parts adjacent to the crown, to assume a cylindrical figure. The brim now appears like a puckered appendage round the cylindrical cone; but the proper figure is next given to it, by working and rubbing it. The body is rendered waterproof and stiff by being imbued with a varnish composed of shellac, sandarach, mastic, and other resins dissolved in alcohol or naptha.

The hat being dried, its nap is raised or loosened with a wire brush or card, and sometimes it is previously pounced or rubbed with pumice, to take off the coarser parts, and afterwards rubbed over with seal-skin. The hat is now tied with pack-thread upon its rock, and is afterwards dyed. See HAT-DYerNG, infra.

The dyed hats are now removed to the stiffening shop. Beer grounds are next applied on the inside of the crown, for the purpose of preventing the glue from coming through; and when the beer grounds are dried, glue (gum Senegal is sometimes used) a little thinner than that used by carpenters, is laid with a brush on the inside of the crown, and the lower surface of the brim.

The hat is then softened by exposure to steam, on the steaming basin, and is brushed and ironed $t i l$ it receives the proper gloss. It is lastly cut round at the brim by a knife fixed at the end of a gauge, which rests against the crown. The brim, however, is not 
cut entirely through, but is torn off so as to leave an edging of beaver round the external rim of the hat. The crown being tied up in a gauze paper, which is neatly ironed down, is then ready for the last operations of lining and binding.

The furs and wools of which hats are manufactured contain, in their early stage of preparation, hemps and hairs, which must be removed in order to produce a material for the better description of hats. This separation is effected by a sort of winnowing machine, which wafts away the finer and lighter parts of the furs and wools from the coarser. Messrs. Parker and Harris obtained a patent in 1822 for the invention and use of such an apparatus, whose structure and functions may be perfectly understood, from its analogy to the blowing and scutching machine of the cotton manufacture; to which I therefore refer my readèrs.

I shall now proceed to describe some of the recent improvements proposed in the manufacture of hats, but their introduction is scarcely possible, on account of the perfectly organized combination which exists among journeyman hatters throughout the kingdom, by which the masters are held in a state of complete servitude, having no power to take a single apprentice into their works beyond the number specified by the Union, nor any sort of machine which is likely to supersede hand labor in any remarkable degree. Hence the hat trade is, generally speaking, unproductive to the capitalist, and incapable of receiving any considerable development. The public of a free country like this, ought to counteract this disgraceful state of things, by renouncing the wear of stuff hats, a branch of the business entirely under the control of this despotic Union, and betake themselves to the use of silk hats, which, from recent improvements in their fabric and dyeing, are not a whit inferior to the beaver hats, in comfort, appearance, or durability, while they may be had of the best quality for one fourth part of their price.

The annexed figures represent Mr. Ollerenshaw's machine, now generally employed or ironing hats. Fig. 534 is the frame-work or standard upon which three of these

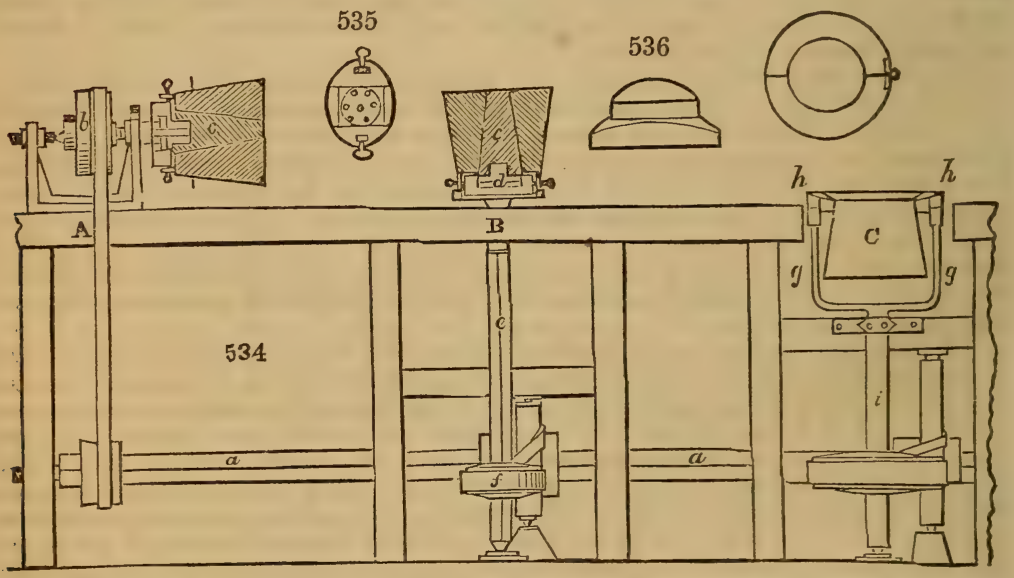

lathes are mounted, as $A, B, C$. The lathe $A$ is intended to be employed when the crown of the hat is to be ironed. The lathe $B$, when the flat top, and the upper side of the brim is ironed, and lathe $c$, when its under side is ironed; motion being given to the whole by means of a band passing from any first mover (as a steam-engine, water-wheel, $\& c$.$) to the drum on the main shaft a a$. From this drum a strap passes over the rigger $\mathrm{b}$,which actuates the axle of the lathe $\mathrm{A}$. On to this lathe a sort of chuck is screwed, and to the chuck the block $c$ is made fast by screws, bolts, or pins. This block is represented in section, in order to show the manner in which it is made, of several pieces held fast. by the centre wedge-piece, as seen at fig. 535 .

The hat-block being made to turn round with the chuck, at the rate of about twenty turns per minute, but in the opposite direction to the revolution of an ordinary turning lathe, the workman applies his hot iron to the surface of the hat, and thereby smooths it, giving a beautiful glossy appearance to the beaver; he then applies a plush cushion, and rubs round the surface of the hat while it is still revolving. The hat, with its block, is now removed to the lathe $\mathrm{B}$, where it is placed upon the chuck $d$, and made to turn in a horizontal direction, at the rate of about twenty revolutions per minute, for the purpose of ironing the flat-top of the crown. This lathe $\mathrm{B}$ moves upon an upright shaft $e$, and is actuated by a twisted band passing from the main shaft, round the 
ngger $f$. In order to iron the upper surface of the brim, the block $c$ is removed from the lathe, and taken out of the hat, when the block fig. 536, is nounted upon the chuck $d$, and made to turn under the hand of the workman, as before.

The hat is now to be removed to the lathe $c$, where it is introduced in an inverted position, between the arms $g \mathrm{~g}$ supporting the $\operatorname{rim} h h$, the top surface of which is shown at fig. 537. The spindle $i$ of the lathe turns by similar means to the last, but slower; only ten turns per minute will be sufficient. The workman now smooths the under side of the brim, by drawing the iron across it, that is, from the centre outwards. The hat is then carefully examined, and all the burs and coarse hairs picked out, after which the smonthing process is performed as before, and the dressing of the hat is complete.

Messrs. Gillman and Wilson, of Manchester, obtained a patent in 1823, for a peculiar kind of fabric to be made of cotton, or a mixture of cotton and silk, for the covering of hats and bonnets, in imitation of beaver. The foundation of the hat may be of felt, hemp, wool, which is to be covered by the patent fabric. This debased article does not seem to have got into use; cotton, from its want of the felting property and inelasticity, being very ill-adapted for making hat-stuff.

A more ingenious invention of John Gibson, hatter, in Glasgow, consisting of an elastic fabric of whalebone, was made the subject of a patent, in June, 1824. The whalebone, being separated into threads no larger than hay stalks, is to be boiled in some alkaline liquid for removing the oil from it, and rendering it more elastic. The longest threads are to be employed for warp, the shorter for weft; and are to be woven in a hair-cloth loom. This fabric is to be passed between rollers, after which it is fit to be cut out into forms for making hats and bonnets, to be sewed together at the joints, and stiffened with a preparation of resinous varnishes, to prevent its being acted upon by perspiration or rain. A very considerable improvement in the lightness and elasticity of silk hats has been the result of this invention.

The foundation of men's hats, upon whose outside the beaver, down, or other fine fur is laid to produce a nap, is, as I have described, usually made of wool felted together by hand, and formed first into conical caps, which are afterwards stretched and moulded upon blocks to the desired shape. Mr. Borradaile, of Bucklersbury, obtained a patent in November, 1825, for a machine, invented by a foreigner, for setting up hat bodies, which seems to be ingeniously contrived; but I shall decline describing it, as it has probably not been suffered by the Union to come into practical operation, and as I shall presently give the details of another later invention for the same purpose.

Silk hats, for several years after they were manufactured, were liable to two objections; first, the body or shell over which the silk covering is laid, was, from its hardness, apt to hurt the head; second, the edge of the crown being much exposed to blows, the silk nap soon got abraded, so as to lay bare the cotton foundation, which is not capable of taking so fine a black dye as the silk; whence the hat assumed a shabby appearance. Messrs. Mayhew and White of London, hat-manufacturers, proposed in their patent of February, 1826, to remedy these defects, by making the hat body of stuff or wonl, and relieving the stiffness of the inner part round the brim, by attaching a coating of beaver upon the under side of the brim, so as to render the hat pliable. Round the edge of the tip or crown, a quantity of what is called stop wool is to be attached by the ordinary operation of bowing, which will render the edge soft and elastic. The hat is to be afterwards dyed of a good black color, both outside and inside; and being then properly stiffened and blocked, is ready for the covering of silk.

The plush employed for covering silk hats, is a raised nap or pile woven usually upon a cotton foundation; and the cotton, being incapable of receiving the same brilliant black dye as the silk, renders the hat apt to turn brown whenever the silk nap is partially worn off. The patentees proposed to counteract this evil, by making the foundation of the plush entirely of silk. To these two improvements, now pretty generally introduced, the present excellence of the silk hats may be, in a good measure, ascribed.

The apparatus above alluded to, for making the foundations of hats by the aid of mechanism, was rendered the subject of a patent, by $\mathrm{Mr}$. Williams, in September, 1826 ; but I fear it has never obtained a footing, nor even a fair trial in our manufactures, on ac count of the hostility of the operatives to all labor-saving machines.

Fig. 538 is a side view of the carding engine, with a horizontal or plan view of the lower part of the carding machine, showing the operative parts of the winding apparatus, as connected to the carding engine. The doffer cylinder is covered with fillets of wire cards, such as are usually employed in carding engines, and these fillets are divicied into two, three, or more spaces extending round the periphery of the cylinder; the object of which division is to separate the sliver into two, three, or more breadths; which are to be conducted to, and wound upon distinct blocks, for making so many sep. arate hats or caps. 
The principal cylinder of the carding engine is made to revolve by a rigger upon it axie, actuated by a band from any first mover as usual, and the subordinate rollers or

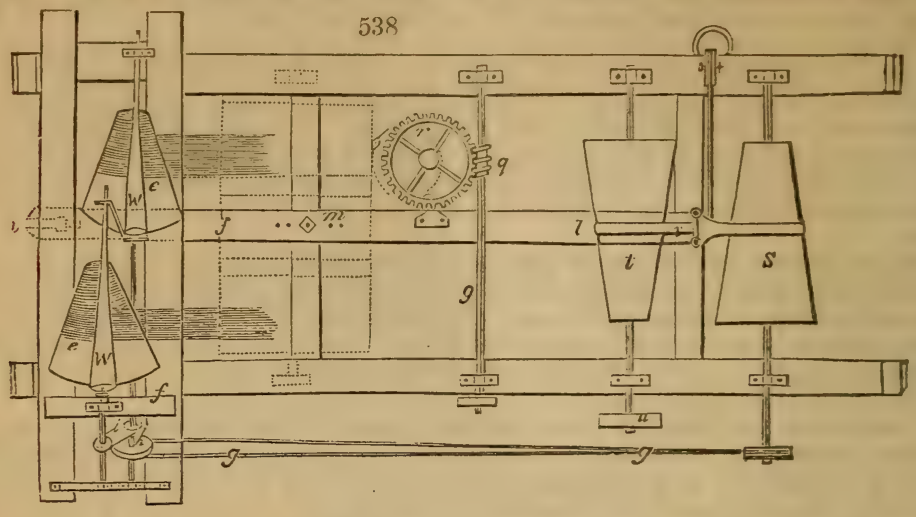

539

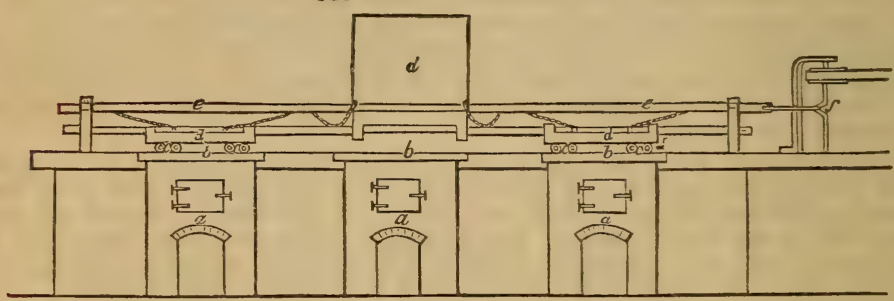

cylinders belonging to the carding engine, are all turned by pulleys, and bands, and gear, as in the ordinary construction.

The wool or other material is supplied to the feeding cloth, and carried through the engine to the doffer cylinder, as in other carding engines; the doffer comb is actuated by a revolving crank in the common way, and by means of it the slivers are taken from the doffer cylinder, and thence received on to the surfaces of the blocks $e e$. These blocks, of which two only are shown to prevent confusion, are mounted upon axles, supported by suitable bearings in a carriage $f f$, and are made to revolve by means of a band $g \mathrm{~g}$, leading from a pulley on the axle of a conical drum beneath. The band $g$ passes over a pulley $h$, affixed to the axle of one of the blocks, while another pulley $i$, upon the same axle, gives motion, by means of a band, to as many other blocks as are adapted to the machine.

As it is necessary, in winding the slivers on to the blocks, to cross them in different directions, and also to pass the sliver over the hemispherical ends of the blocks, in order that the wool or other material may be uniformly spread over the surface in forming the cap or hood for the shell or foundation of the intended hat, the carriage $f$, with the blocks, is made to traverse to and fro in lateral directions upon rollers at each end.

This alternating motion of the carriage is caused by a horizontal lever $l l$ (seen in the horizontal view fig. 538), moving upon a fulcrum pin at $m$, which lever is attached to the carriage at one extremity $n$, and at the other end has a weighted cord which draws the side of this lever against a cam wheel $o$. This cam is made to revolve by means of a band and pulley, which turns the shaft and endless screw $q$, and this endless screw taking into a toothed wheel $r$, on the axle of the cam 0 , causes the cam to revolve, the periphery of which cam running against a friction roller on the side of the lever $l$, causes the lever to vibrate, and the carriage $f f$ attached to it, to traverse to and fro upon the supporting rollers, as described. By these means the slivers are laid in oblique directions (varying as the carriage traverses), over the surface of the blocks.

The blocks being conically formed, or of other irregular figures, it is necessary, in order to wind the slivers with uniform tension, to vary their speed according to the diameter of that part of the block which is receiving the sliver. This is effected by giving different velocities to the pulley on the axle of the conical drum $s$, corresponding with $e$. There is a similar eonical drum $t$, placed in a reverse position in the lowe? 
part of the frame, which is actuated by a band from any convenient part of the machine passing over a pulley $u$, upon the axle of $t$. From the drum $t$, to the drum $s$, there is a band $t$, which is made to slide along the drums by the guidance of two rollers at the ena of the lever $l$.

It will now be seen that when the larger diameter of the cam wheel $o$ forces the lever outwards, the band $v$ will be guided on to the smaller part of the conical drum $t$ and the larger part of $s$, consequently the drum $s$ will at this time receive its slowest motion, and the band $g$ will turn the blocks slower also; the reverse end of the lever $l$ having, by the same movement, slidden the carriage into that position which causes the sivers to wind upon the larger diameter of the blocks.

When the smaller diameter of the cam is acting against the side of the lever, the weighted cord draws the end of the lever to the opposite side, and the band $v$ will bc guided on to the larger part of the cord $t$, and the smaller part of the cone $s$; consequently, the quicker movement of the band $g$ will now cause the blocks $e e$ to revolve with a corresponding speed. The carriage $f$ will also be moved upon its rollers, to the reverse side, and the sliver of wool or other material be now wound upon the smaller parts and ends of the blocks, at which tıme the quicker rotation of the blocks is required. It may be here observed, that the cam wheel $o$ should be differently formed according to the different shaped blocks employed, so as to produce the requisite movements of the lever and carriage suited thereto.

It only remains to state, that there are two heavy conical rollers $w w$, bearing upon the peripheries of the blocks $e e$, which turn loosely upon their axles by the friction of contact, for the purpose of pressing the slivers of wool or other material on the blocts as it comes from the doffer cylinder of the carding engine, and when the blocks have been eoated with a sufficient quantity of the sliver, the smaller end of the pressing rollers is to be raised, while the cap is withdrawn from the block. The process being continued as before, the formation of other bodies or caps is effected in the manner above described.

After the caps or bodies of hats, \&c. are formed in the above described machine, they are folded in wet cloths, and placed upon heated plates, where they are rolled under pressure, for the purpose of being hardened. Fig. 539 represents the front of three furnaces $a$ a $a$, the tops of which are covered with iron plates $b b b$. Upon these plates, which are heated by the furnace below, or by steam, the bodies wrapped in the wet cloths $c$ c c are placed, and pressed upon by the flaps or corers $d d d$, sliding upon guide rods, to which flaps a traversing motion is given, by means of chains attached to an alternating bar $e$. This bar is moved by a rotatory crank $f$, which has its motion by pulleys from any actuating power. When any one of the flaps is turned up to remove the bodies from beneath, the chains hang loosely, and the flap remains stationary.

These caps or hat bodies, after having been hardened in the manner above described, may be felted in the usual way by hand, or they are felted in a fulling mill, by the usual process employed for milling cloths, except that the hat bodies are occasionally taken out of the fulling mill, and passed between rollers, for the purpose of rendering the felt more perfect.

540

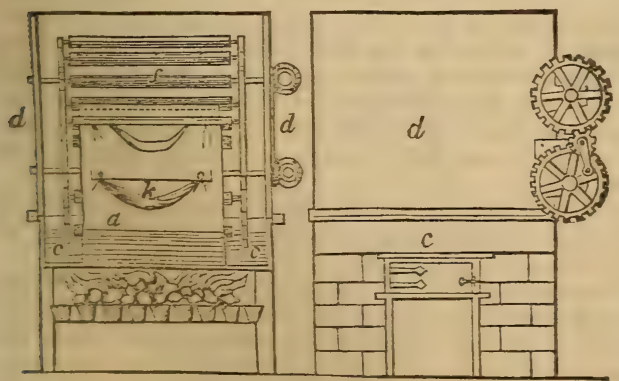

Mr. Carey, of Basford, obtained a patent in October, $1834_{3}$ for an invention of certain machinery to be employed in the manufacture of hats, which is ingenious, and seems to be worthy of notice in this place. It consists in the adaptation of a system of rollers, forming a machine, by means of which the operation of roughing or plaiting of hats may be performed; that is, the beaver or other fur may be made to attach itself, and work into the felt or hat body, without the necessity of the ordinary manual operations.

The accompanying drawings represent the machine in several views, for the purpose of showing the construction of all its parts. Fig. $5 \pm 0$ is a front elevation of the machine; fig. 541 is a side elevation of the same; fig. 542 is a longitudinal section of the machine; and fig. 543 is a transverse section; the similar letters indicating the same parts in all the figures. 
Upon a brick or other suitable base, a furnace or fire-place $a$, is made, having a descend 541

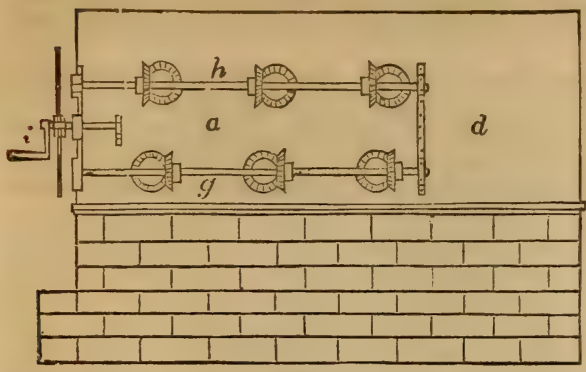

542

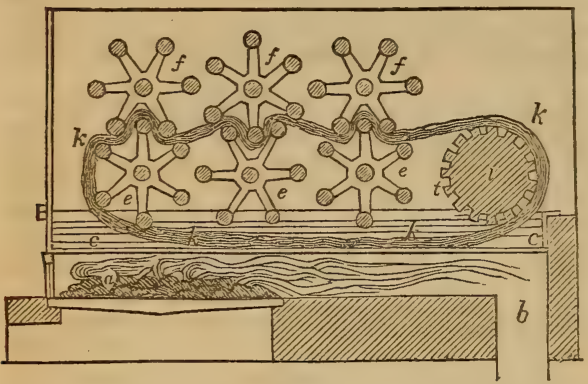
ing flue $b$, for the purpose of carry. ing away the smoke. A pan or shallow vessel $c$, formed of lead, is placed over the furnace; which vessel is intended to contain a sour liquor, as a solution of vitriolic acid and water. On the edge of this pan is erected a wooden casing $d d d$, which encloses three sides, leaving the fourth open for the purpose of obtaining access to the working apparatus within. A series of what may be termed lantern rollers, $e e e$, is mounted on axles turning in the side casings; and another series of similar lantern rollers, $f f f$, is in like manner mounted above. These lantern rollers are made to revoive by means of bevel pinions, fixed on the ends of their axles, which are turned by similar bevel wheels on the lateral shafts $g$ and $h$, driven by $a$ winch $i$, and gear, as shown in figs. 540 and 541.

Having prepared the bodies of the hats, and laid upon their surfaces the usual coatings of beaver, or other fur, when so prepared they are to be placed between hair cloths, and these hair cloths folded within a canvass or other suitable wrapper. Three or more hats being thus enclosed in each wrapper, the packages are severally put into bags or pockets in an endless band of sackcloth, or other suitable material; which endless band is extended over the lantern rollers in the machine.

In the first instance, for the purpose of merely attaching the furs to the felts (which is called slicking, when performed by hand), Mr. Carey prefers to pass the endless band $k k k$, with the covered hat bodies, over the upper series $f f f$, of the lantern rollers, in ordes to avoid the inconvenience of disturbing the fur, which might occur from subjecting them to immersion in the solution contained in the pan, before the fur had become attached to the bodies.

After this operation of slicking has been effected, he distends the endless band $k k k$, over the lower series of lantern rollers $e e e$, and round a carrier roller $l$, as shown in $f i g$. 542 ; and having withdrawn the hat bodies for the purpose of examining them, and chang. ing their folds, he packs them again in a similar way in flannel, or other suitable cloths. and introduces them into the pockets or bags of the endless bands, as before.

On putting the machinery in rotatory motion in the way described, the hats will be carried along through the apparatus, and subjected to the scalding solution in the pan, as also to the pressure, and to a tortuous action between the ribs of the lantern rollers, as they revolve, which will cause the ends of the fur to work into the felted bodies of the hats, and by that means permanently to attach the nap to the body; an operation which, wher performed by hand, is called rolling off.

The improved stiffening for hat bodies proposed by Mr. Blades, under his patent of January, 1828, consists in making his solution of shellac in an alkaline ley, instead of spirits of wine, or pyroxylic spirit, vulgarly called naptha.

He prepares his water-proof stiffening by mixing 18 pounds of shellac with $1 \frac{1}{2}$ pounds of salt of tartar (carbonate of potash), and $5 \frac{1}{2}$ gallons of water. These materials are to be put into a kettle, and made to boil gradually until the lac is dissolved; when the liquor will become as clear as water, without any scum upon the top, and if left to cool, will have a thin crust upon its surface of a whitish cast, mixed with the light impurities of the gum. When this skin is taken off, the hat body is to be dipped into the mixture in a cold state, so as to absorb as much as possible of it; or it may be applied with a brush or sponge. The hat body, being thus stiffened, may stand till it become dry, or nearly so; and after it has been brushed, it must be immersed in very dilute sulphuric or acetic acid, in order to neutralize the potash, and cause the shellac to 
set. If the hats are not to be napped immediately, they may be thrown into a cistern of pure water, and taken out as wanted.

Should thr hat bodies have been worked at first with sulphuric acid (as usual), they must be sualied in hot water to extract the acid, and dried before the stiffening is applied: care being taken that no water falls upon the stiffened body, before it has been immersed in the acid.

This ingenious chemical process has not been, to the best of my knowledge, introduced into the hat manufacture. A varnish made by dissolving shellac, mastic, sandarach, and other resins in alcohol, or the naptha of wood vinegar, is generally employed as the stiffening and water-proof ingredient of hat bodies. A solution of caoutchouc is oflen applied to whalebone and horse-hair hat bodies.

The following recipe has been prescribed as a good composition for stiffening hats; four parts of shellac, one part of mastic, one half of a part of turpentine, dissolved in fire narts of alcohol, by agitation and subsequent repose, without the aid of heat. This stiffening varnish should be applied quickly to the body or foundation with a soft oblong brush, in a dry and rather warm workshop; the hat being previously fitted with its inside turned outwards upon a block. The body must be immediately afterwards taken off, to prevent adhesion.

Hat-Dyeing.-The ordinary bath for dyeing hats, employed by the London manufacturers, consists, for 12 dozen, of-

\section{4 pounds of $\log w o o d$;}

12 pounds of green sulphate of iron, or copperas;

$7 \frac{1}{2}$ pounds of verdigris.

The copper is usually made of a semi-cylindrical shape, and should be surrounded with an iron jacket or case, into which steam may be admitted, so as to raise the temperature of the interior bath to $190^{\circ} \mathrm{F}$., but no higher, otherwise the heat is apt to affect the stiffening varnish, called the gum, with which the body of the hat has been imbued. The logwood having been introduced and digested for some time, the copperas and verdigris are added in successive quantities, and in the above proportions, along with every successive two or three dozens of hats, suspended upon the dipping machine. Each set of hats, after being exposed to the bath with occasional airings during 40 minutes, is taken off the pegs, and laid out upon the ground to be more completely blackened by the peroxydizement of the iron with the atmospheric oxygen. In 3 or 4 hours the dyeing is completed. When fully dyed, the hats are well washed in running water.

Mr. Buffum states that there are four principal objects accomplished by his patent invention for dyeing hats.

1. in the operation;

2. the production of a better color;

3. the prevention of any of the damages to which hats are liable in the dyeing;

4. the accomplishment of the dyeing process in a much shorter time than by the usual methods, and consequently lessening the injurious effects of the dye-bath upon the texture of the hat.

Fig. 544 shows one method of constructing the apparatus. $\quad a \quad a$ is a semi-cylindrical

544

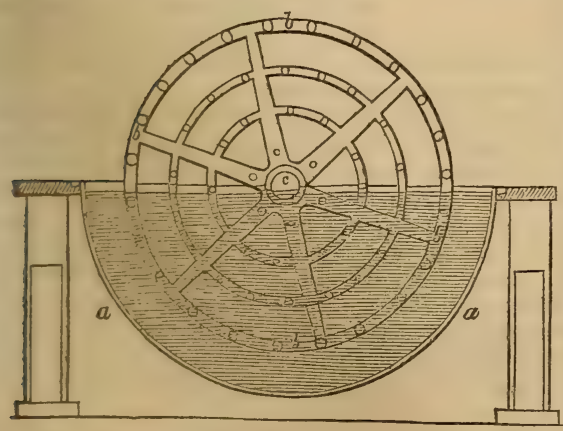

545

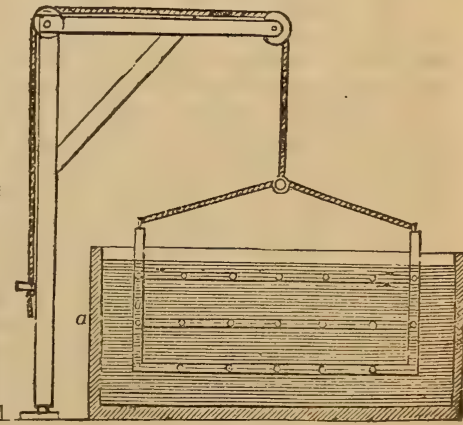

shaped copper vessel, with flat ends, in which the dyeing process is carried on. $b b b$ is a wheel with several circular rims mounted upon arms, which revolve upon an axle $c$. In the face of these rims a number of pegs or blocks are set at nearly equal distances apart, upon each of which pegs or blocks it is intended to place a hat, and as the whee! 
revolves, to pass it into and out of the dyeing liquor in the vat or copper. This wree, may be kept revolving with a very slow motion, either by gear connecting its axle, $c_{\text {s }}$ with any moving power, or it may be turned round by hand, at intervals of ten minutes; whereby the hats hung upon the pegs, will be alternately immersed for the space of ten minutes in the dyeing liquor, and then for the same space exposed to the atmospherie air. In this way, the process of dyeing, it is supposed, may be greatly facilitated and improved, as the occasional transition from the dye vat into the air, and from the as again in to the bath, will enable the oxygen of the atmosphere to strike the dye more per fectly and expeditiously into the materials of which the hat is composed, than by a continued immersion in the bath for a much longer time.

A variation in the mode of performing this process is suggested, and the apparatus fig. 545 is proposed to be employed. $a$ a is a square vat or vessel containing the dyeing liquor; $b b$ is a frame or rack having a number of pegs placed in it for hanging the hats upon, which are about to be dyed, in a manner similar to the wheel above described. This frame or rack is suspended by cords from a crane, and may in that way be lowered down with the hats into the vat, or drawn up and exposed in the air; changes which may be made every 10 or 20 minutes.

I have seen apparatus of this kind doing good work in the hat-dyeing manufactories of London, that being a department of the business with which the Union has not thought it worth their while to interfere.

Mr. William Hodge's patent improvements in hat dyeing, partly founded upon an invention of $\mathrm{Mr}$. Bowler, consist, first in causing every alternate frame to which the suspenders or blocks are to be attached, to slide in and out of grooves, for the purpose $\begin{array}{llll}548 & 546 & 547 & \text { of more easily removing the said }\end{array}$

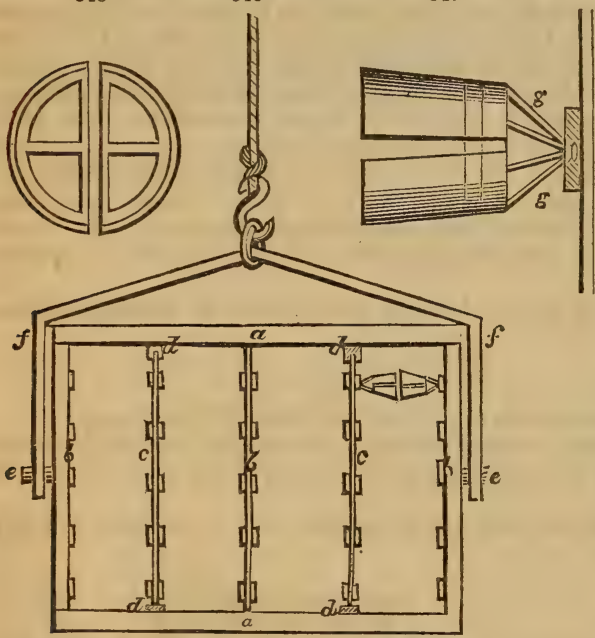
suspenders when required. Fig. 546 represents the improved dye. ing frame, consisting of two circular rims, $a$, which are connected together at top and bottom, by three fixed perpendicular bars or the frame-work $b \quad b \quad b$. Two other perpendicular frames $c c$, similar to the former, slide in grooves, $d d d d$, fixed to the upper and lower rims. These grooves have anti-friction rollers in them, for the purpose of making the frames $c c$, to slide in and out more freely. The suspenders or substitutes for blocks, by these means, may be more easily got at by drawing out the frames $c c$, about half way, when the suspenders, which are attached to the frames with the hats upon them, may be easily reached, and either removed or altered in position; and when it is done on one side, the sliding frame may be brought out on the other, and the remaining quantity of "suspenders" undergo the same operation.

The patentee remarks, that it is well known to all hat dyers, that after the hats have been in the dyeing liquor some time, they ought to be taken out and exposed to the action of the atmospheric air, when they are again immersed in the copper, that part of the hat which was uppermost in the first immersion, being placed downwards in the second. This is done for the purpose of obtaining a uniform and regular dye. The patentee's mode of carrying this operation into effect, is shown in the figure: $e e$ are pivots for the dyeing-frame to turn upon, which is supported by the arms $f$, from a erane above. The whole apparatus may be raised up or lowered into the copper by means of the crane or other mechanism. When the dyeing-frame is raised out of the copper, the whole of the suspenders or blocks are reversed, by turning the apparatus over upon the pivots $e e$, and thus the whole surfaces of the hats are equally acted upon by the dyeing material.

It should be observed, that when the dyeing-frame is raised up out of the copper, it should be tilted on one side, so as to make all the liquor run out of the hats, as also to eause the rims of the hats to hang down, and not stick to the body of the hat, or leave bad place or uneven dye upon it. The second improvement described by the patentee. is the construction of "suspenders," to be substituted instead of the ordinary blocks. 
These "suspenders" are composed of thin plates of copper, bent into the renuired form, that is, nearly resembling that of a hat block, and made in such a manner as to be capable of contraction and expansion to suit different sized hats, and keep them distended, which may be altered by the workmen at pleasure, when it is required to place the hats upon them, or remove them therefrom. The dyeing-frame at fig. 546 is shown with only two of these "suspenders," in order to prevent confusion. One of these suspenders is represented detached at fig. 547, which exhibits a side view; and $f i g .548$ a front view of the same. It will be seen by reference to the figure, that the suspenders consist of two distinct parts, which may be enlarged or collapsed by a variety of means, and which means may be suggested by any competent mechanic. The two parts of the suspenders are proposed to be connected together by arms $g \mathrm{~g}$, and at the junction of these arms a key is connected for turning them round when required. It will be seen on reference to the front view, fig. 548, that the "suspenders" or substitutes for blocks, are open at the top or crown part of the hat; this is for the purpose of allowing the dyeing liquor to penetrate.

From the mixture of copperas and verdigris employed in the hat-dye, a vast quantity of an ochreous muddy precipitate results, amounting to no less than 25 per cent. of the weight of the copperas. This iron mud forms a deposite upon the hats, which not only corrodes the fine filaments of the beaver, but causes both them and the felt stuff to turn speedily of a rusty brown. There is no process in the whole circle of our manufactures so barbarous as that of dyeing stuff hats. No ray of chemical science seems hitherto to have penetrated the dark recesses of their dye shops. Some hatters have tried to remove this corrosive brown ochre by a bath of dilute sulphuric acid, and then counteract the evil effect of the acid upon the black dye by an alkaline bath; but with a most unhappy effect. Hats so treated are most deceptious and unprofitable; as they turn of a dirty brown hue, when exposed for a few weeks to sunshine and air.

HEALDS, is the harness for guiding the warp threads in a loom; that is, for lifting a certain number of them alternately to open the shed, and afford passage to the decussating weft threads of the shuttle. See Weaving.

HEARTH (Foyer, Fr.; Heerde, Germ.) is the flat or hollow space in a smelting furnace upon which the ore and fluxes are subjected to the influence of flame. See CoPPER, Iron, Metallurgy, \&ec.

HEAT, is that power or essence called caloric, the discussion of whose habitudes with the different kinds of matter belongs to the science of chemistry.

HEAT-REGULATOR. The name given by M. Bonnemain to an ingenious apparatus for regulating the temperature of his incubating stove rooms. See Incubation, ArtiFICraL, for the manner of applying the Heat-Regulator.

The construction of the regulator is founded upon the unequal dilatation of different metals by the same degree of heat. A rod of iron $x$, fig. 549, is tapped at its lower 549

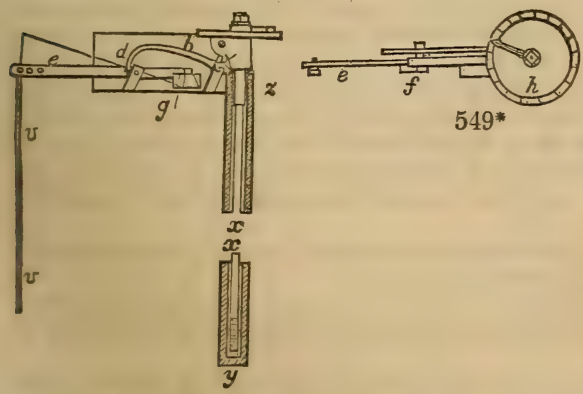
end into a brass nut $y$, enclosed in a leaden box or tube, terminated above by a brass collet $z$. This tube is plunged into the water of the boiler, alongside of the smokepipe. (Fig. 549* is a bird's-eye view of the dial, \&c.) The expansion of the lead being more than the iron for a like degree of temperature, and the rod enclosed within the tube being less easily warmed, whenever the heat rises to the desired pitch, the elongation of the tube puts the collet $z$ in contact with the heel $a$, of the bent lever $a, b, d$; thence the slightest increase of heat lengthens the tube anew, and the collet lifting the heel of the lever depresses its other end $d$ through a much greater space, on account of the relative lengths of its legs. This movement operates near the axis of a balance-bar $e$, sinks one end of this, and thereby increases the extent of the movement which is transmitted directly to the iron skewer $v$. This pushing down a swing register diminishes or cuts off the access of air to the fire-place. The combustion is thereby obstructed, and the temperature falling by degrees, the tube shrinks and disengages the heel of the lever. The counterpoise $g$, fixed to the balance-beam $e$, raises the other extremity of this beam, by raising the end $d$ of the lever as much as is necessary to make the heel bear upon the collet of the tube. The swing register acted upon by this means, presents a greatcr section to the passage of the air; whence the combustion is increased. To counter. 
balance the effect of atmospheric changes, the iron stem which supports the regulator is terminated by a dial disc, round the shaft of the needle above $h$, fig. 549*; on turning this needle the stem below it turns, as well as a screw at its under end, which raises or lowers the leaden tube. In the first case, the heel falls, and opens the swing register, whence a higher temperature is required to shut it, by the expansion of the tube. We may thus obtain a regularly higher temperature. If, on the contrary, we raise the tube by turning the needle in the other direction, the register presents a smaller opening, and shuts at a lower temperature; in this case, we obtain a regularly lower temperature. It is therefore easy, says M. Bonnemain, to determine $\grave{a}$ priori the degree of temperature to be given to the water circulating in the stove pipes. In order to facilitate the regulation of the apparatus, he graduated the dise dial, and inscribed upon its top and bottom the words, Strong and Weak heat. See Thermostat, for another Heat-REgulator.

HEAVY SPAR, sulphale of Baryta, or Cawk (Spath pesant, Fr.; Schwerspath, Germ.), is an abundant mineral, which accompanies veins of lead, silver, mercury, \&c., but is often found, also, in large masses. Its color is usually white, or flesh-colored. It occurs in many crystalline forms, of which the cleavage is a right rhomboidal prism. It is met with also of a fibrous, radiated, and granular structure. Its spec. grav. varies from $4 \cdot 1$ to $4 \cdot 6$. It has a strong lnstre, between the fatty and the vitreous. It melts at $35^{\circ}$ Wedgew. into a white opaque enamel. Its constituents are 65.63 baryta, and 34.37 sulphuric acid. It is decomposed by calcination in contact with charcoal at a white heat, into sulphuret of baryta; from which all the baryta salts may be readily formed. Its chief employment in commerce is for adulterating white lead; a purpose which it readily serves on account of its density. Its presenee here is easily detected by dilute nitric acid, which dissolves the carbonate of lead, and leaves the heavy spar. It is also a useful ingredient in some kinds of pottery and glass.

HECKLE (Seran, Fr.; Hechel, Germ.) is an implement for dissevering the filaments of flax, and laying them in parallel stricks or tresses. See Flax.

HELIOTROPE is a variety of jaspar, mixed with chlorite, green earth, and diallage; oceasionally marked with blood-red points; whence its vulgar name of bloodstone.

HEMATINE is the name given by its discoverer Chevreul to a crystalline substance, of a pale pink color, and brilliant lustre when viewed in a lens, which he extracted from logwood, the hamatoxylon Campechianum of botanists. It is, in fact, the characteristic principle of this dye-wood. To procure hematine, digest during a few hours ground logwood in water heated to a temperature of about $130^{\circ} \mathbf{F}$.; filter the liquor, evaporate it to dryness by a steam bath, and put the extract in alcohol of 0.835 for a day. Then filter anew, and after having inspissated the alcoholic solution by evaporation, pour into it a little water, evaporate gently again, and then leave it to itself in a cool place. In this way a considerable quantity of crystals of hematine will be obtained, which may be readily purified by washing with alcohol and drying.

When subjected to dry distillation in a retort, hematine affords all the usual products of vegetable bodies, along with a little ammonia; which proves the presence of azote. Boiling water dissolves it abundantly, and assumes an orange-red color, which passes into yellow by cooling, but becomes red again with heat. Sulphurous acid destroys the color of solution of hematine. Potash and ammonia convert into a dark purple-red tint, the pale solution of hematine; when these alkalis are added in large quantity, they make the color violet blue, then brown-red, and lastly brown-yellow. By this time, the hematine has become decomposed, and cannot be restored to its pristine state by neutralizing the alkalis with acids.

The waters of baryta, strontia, and lime exercise an analogous power of decomposition; but they eventually precipitate the changed coloring matter.

A red solution of hematine subjected to a current of sulphureted hydrogen becomes yellow; but it resumes its original hue when the sulphureted hydrogen is removed by a little potash.

The protoxyde of lead, the protoxyde of tin, the hydrate of peroxyde of iron, the hydrate of oxydes of copper and nickel, oxyde of bismuth, combine with hematine, and color it blue with more or less of a violet cast.

Hematine precipitates glue from its solution in reddish flocks. This substance has not hitherto been employed in its pure state; but as it constitutes the active principle of logwood, it enters as an ingredient into all the colors made with that dye stuff.

These colors are principally violet and black. Chevreul has proposed hematine as an excellent test of acidity.

HEMATITE (Fer Oligiste, Fr.; Rotheisenstein, Germ.) is a native reddish-brown peroxyde of iron, consisting of oxygen 30.66 ; iron 60.34 . It is the kidney ore of Cumberand, which is smelted at Ulverstone with charcoal, into excellent steel iron.

HEMP (Chanvre, Fr.; Hanf, Germ.) is the fibrous rind of the bark of the canna. 
bis sativa, which is spun into strands or yarn for making ropes, sail-cloth, \&c. It is prepared for spinning in the same way as flax, which see. Hemp-seed contains an oil which is employed for making soft soap, for painting, and for burning in lamps. See OrLS.

Importation of undressed hemp for home consumption ; and amount of duty, in

\begin{tabular}{|c|c|}
\hline $\begin{array}{c}1837 . \\
\text { Cwts. } 596,994 \cdot 3 .\end{array}$ & $\begin{array}{c}1838 \\
667,017 .\end{array}$ \\
\hline
\end{tabular}

HEPAR, which signifies liver in Latin, was a name given by the older chemists to some of those compounds of sulphur with the metals which had a liver-brown color. Thus the sulphuret of potassium was called liver of sulphur.

HEPATIC AIR; sulphureted hydrogen gas.

HERMETICAL SEAL, is an expression derived from Hermes, the fabulous parent of Egyptian chemistry, to designate the perfect stoppage of a hollow vessel, by the cementing or melting of the lips of its orifice; as in the case of a glass thermometer, or matrass.

HIDE (Peau, Fr.; Haut, Germ.), the strong skin of an ox, horse, or other large animal. Sec Leather.

Importation of untanned hides for home consumption ; and amount of duty, in

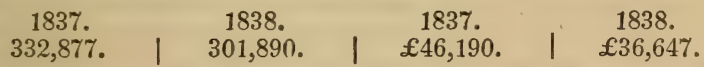

HIRCINE; from hircus, a ram; is the name given by Chevreul to a liquid fatty substance, which is mixed with the oleine of mutton suet, and gives it its peculiar rank smell. Hircine is much more soluble in alcohol than oleine. It produces hircic acid by saponification.

HOG'S LARD; see FATS.

HONEY ( Mel, Fr.; Honig, Germ.) is a sweet viscid liquor, elaborated by bees from the sweet juices of the nectaries of flowers, and deposited by them in the waxen cells of their combs. Virgin honey is that which spontaneously flows with a very gentle heat from the comb, and common honey is that which is procured by the joint agency of pressure and heat. The former is whitish or pale yellow, of a granular texture, a fragrant smell, and a sweet slightly pungent taste; the latter is darker colored, thicker, and not so agreeable either in taste or smell. Honey would seem to be merely collected by the bees, for it consists of merely the vegetable products; such as the sugars of grape, gum, and manna ; along with mucilage, extractive matter, a little wax, and acid.

HONEY-STONE (Mellite, Fr.; Honigstein, Germ.) is a mineral of a yellowish or reddish color, and a resinous aspect, crystallizing in octahedrons with a square base; specific gravity 1.58 . It is harder than gypsum, but not so hard as calc-spar; it is deeply scratched by a steel point; very brittle; affords water by calcination; blackens, then burns at the flame of the blowpipe, and leaves a white residuum which becomes blue, when it is calcined after having been moistened with a drop of nitrate of cobalt. It is a mellate of alumina, and consists of :

\begin{tabular}{|c|c|c|c|c|c|}
\hline $\begin{array}{l}\text { Mellitic acio } \\
\text { Alumina } \\
\text { Water }\end{array}$ & - & $\cdot$ & - & $\begin{array}{c}\text { Klaproth. } \\
46 \\
16 \\
38\end{array}$ & $\begin{array}{c}\text { Wohler. } \\
44 \cdot 4 \\
14.5 \\
41 \cdot 1\end{array}$ \\
\hline & & & & 100 & $100 \cdot 0$ \\
\hline
\end{tabular}

The honey-stone, like amber, belongs to the geological formation of lignites. It has been hitherto found clearly in only one locality, at Artern in Thuringia.

HOP (Houblon, Fr.; Hopfen, Germ.) is the name of a well-known plant of the natural family of Urticæ, and of the dioecia pentandria of Linnæus. The female flowers, placed upon different plants from the male, grow in ovoid cones formed of oval leafy scales, concave, imbricated, containing each at the base an ovary furnished with two tubular open styles, and shurp pointed stigmata. The fruit of the hop is a small rounded seed, slightly compressed, brownish colored, enveloped in a scaly calyx, thin but solid, which contains, spread at its base, a granular yellow substance, appearing te the cye like a fine dust, but in the microscope seen to be round, yellow, transparent grains; deeper colored, the older the fruit. This secretion, which constitutes the useful portion of the hop, has been examined in succession by Ives, Planche, Payen, and Cheva. lier. I have given a pretty full account of the results of their researches in treating of the hop, under the article BEER. 
HORDEINE is the name given by Proust to the peculiar starchy matter of barley. If seems to be a mixture of the starch, lignine, and husks, which constitutes barley meal. See BeEr.

HORN (Eng. and Germ.; Corne, Fr.), particularly of oxen, cows, goats, and sheep, is a substance soft, tough, semi-transparent, and susceptible of being cut and pressed into a varitly of forms; it is this property that distinguishes it from bone. Turtle or tortoise sheli seems to be of a nature similar to horn, but instead of being of a uniform color, it is variegated with spots.

These valuable properties render horn susceptible of being employed in a variety of works fit for the turner, snuff-box, and comb maker. The means of softening the horn need not be described, as it is well known to be by heat; but those of cutting, polishing, and soldering it, so as to make the plates of large dimensions, suitable to form a variety of articles, may be detailed. The kind of horn to be preferred is that of goats and sheep, from its being whiter and more transparent than the horn of any other animals. When horn is wanted in sheets or plates, it must be steeped in water, in order to separate the pith from the kernel, for about fifteen days in sum mer, and a month in winter; and after it is soaked, it must be taken out by one end, well shaken and rubbed, in order to get off the pith; after which it must be put for half an hour into boiling water, then taken out, and the surface sawed even, lengthways; it must again be put into the boiling water to soften it, so as to render it capable of separating ; then, with the help of a small iron chisel, it can be divided into sheets or leaves. The thick pieces will form three leaves, those which are thin will form only two, whilst young horn, which is only one quarter of an inch thick, will form only one. These plates or leaves must again be put into boiling water, and when they \&.e surficiently soft, they must be scraped with a sharp cutting instrument, to render those parts that are thick even and uniform; they must be put once more into the boiling water, and finally carried to the press.

At the bottom of the press employed, there must be a strong block, in which is formed a cavity, of nine inches square and of a proportionate depth; the sheets of horn are to be laid within this cavity, in the following manner at the bottom : first a sheet of hot iron, upon this a sheet of horn, next again a sheet of hot iron, and so on, taking care to place at the top a plate of iron even with the last. The press must then be screwed down tight.

There is a more expeditious process, at least in part, for reducing the horn into sheets, when it is wanted very even. After having sarved it with a very fine and sharp saw, the pieces must be put into a copper made on purpose, and there boiled, until sufficiently soft, so as to be able to split with pincers; the sheets of horn must then be put in the press, where they are to be placed in a strong vice, the chaps of which are of iron and larger than the sheets of horn, and the vice must be screwed as quick and tight as possible; let them cool in the press or vice, or it is as well to plunge the whole into cold water. The last mode is preferable, because the horn does not shrink in cooling. Now draw out the leaves of horn, and introduce other horn to undergo the same process. The horn so enlarged in pressing, is to be submitted to the action of the saw, which ought to be set in an iron frame, if the horn is wanted to be cut with advantage, in sheets of any desired thickness, which cannot be done without adopting this mode. The thin sheets thus produced must be kept constantly very warm between plates of hot iron to preserve their softness; every leaf being loaded with a weight heavy enough to prevent its warping. To join the edges of these pieces of horn together, it is necessary to provide strong iron moulds suited to the shape of the article wanted, and to place the pieces in contact with copper-plates or with polished metal surfaces against them; when this is done, the whole is to be put into a vice and screwed up tight, then plunged into boiling water, and after some time it is to be removed from thence and immersed in cold water. The edges of the horn will be thus made to cement together and become perfect$1 y$ united.

To complete the polish of the horn, the surface must be rubbed with the subnitrate of bismuth by the palm of the hand. The process is short, and has this advantage, that it makes the horn dry promptly.

When it is wished to spot the horn in imitation of tortoise-shell, metallic solutions must be employed as follows :-To spot it red, a solution of gold, in aqua regia, must be employed; to spot it black, a solution of silver in nitric acid must be used; and for brown, a hot solution of mercury in nitric acid. The right side of the horn must be impregnated with these solutions, and they will assume the colors intended. The brown spots can be produced on the horn by means of a paste made of red lead, with a solution of potash, which must be put in patches on the horn, and subjected some time to the action of heat. The deepness of the brown shades depends upon the quantity of potash used in the paste, and the length of time the mixture lies on the horn. A desoction of Brazil wood, or a solution of indigo, in sulphuric acid, or a deoction of saffron, 
and Berbary wood may also be used. After having employed these materials, the horn may be left for half a day in a strong solution of vinegar and alum.

In France, Holland, and Austria, the comb-makers and horn-turners use the clippings of horn, which are of a whitish yellow, and tortoise-shell skins, out of which they make snufl-buxes, powder-horns, and many curious and handsome things. They first soften the horn and shell in boiling water, so as to be able to submit them to the press in iron moulds, and by means of heat form them into one mass. The degree of heat necessary to join the horn-clippings must be stronger than that for shell skins, and it can only be found out by experience. The heat must not however be too great, for fear of scorching the horn or shell. Considerable care is required in these operations, not to touch the horn with the fingers, nor with any greasy body, because the grease will prevent the perfect joining. Wooden instruments should be used to move them while they are at the fire, and for carrying them to the moulds.

In making a ring of horn for bell-pulls, \&c., the required piece is to be first cut out in the flat of its proper dimensions, and nearly in the shape of a horse-shoe; it is then pressed in a pair of dies to give its surface the desired pattern; but previous to the pressure, both the piece of horn and the dies are to be heated; the niece of horn is to be introduced between the dies, squeezed in a vice, and when cold, the impression or pattern will be fixed upon the horn. One particular condition, however, is to be observed in the construction of the dies for forming a ring. They are to be so made, that the open ends of the horse-shoe piece of horn, after being pressed, shall have at one end a nib, and at the other a recess of a dovetailed form, corresponding to each other; and the second operation in forming this ring of horn is to heat it, and place it in another pair of dies, which shall bring its open ends together, and cause the dovetailed joints to be locked fast into each other, which completes the ring, and leaves no appearance of the junction.

In forming the handles of table knives and forks, or other things which require to be made of two pieces, each of the two pieces or sides of the handle is formed in a separate pair of dies; the one piece is made with a counter-sunk groove along each side, and the other piece with corresponding leaves or projecting edges. When these two pieces are formed, by first being cut out of the flat horn, then pressed in the dies in a heated state, for the purpose of giving the pattern, the two pieces are again heated and put together, the leaves or edges of the one piece dropping into the counter-sunk grooves of the other piece, and being introduced between another pair of heated dies, the joints are pressed together and the two pieces formed into one handle.

In making the knobs for drawers which have metal stems or pins to fasten them into the furniture, the face of the knob is to be first made in a die, as above described, and then the back part of the knob with a hole in it; a metal-disc of plate-iron is next provided, in which the metal stem or screw pin is fixed, and the stem being passed through the aperture in the back piece, and the two, that is, the back and front pieces of horn put together, they are then heated and pressed in dies as above described; the edge of the back piece falling into the counter-sunk groove of the front piece, while by the heat they are perfectly cemented together.

HORNSILVER (Argent Corné, or Kerargyre, Fr. ; Hornsilber, Germ.) is a white or brownish mineral, sectile like wax or horn, and crystallizing in the cubic system. Its specific gravity varies from $4 \cdot 75$ to $5 \cdot 55$. Insoluble in water; not volatile; fusible at the blowpipe, but difficult of reduction by it. It deposites metallic silver when rubbed with water upon a piece of clean copper or iron. It consists of 24.67 chlorine, and $75^{\prime} 32$ silver.

Hornsilver is rare in the European mines, but it occurs in great quantity in the districts of Zacatecas, Fresnillo, and Catarce, in Mexico; and in Huantajaya, Yauricocha, \&c., in Peru; where it is abundantly mixed with the ores of hydrate of iron, called Pacos and Colorados, interspersed with veins of metallic silver, which form considerable deposites in the pencean limestones. There it is profitably mined as an ore of silver.

HORNSTONE is a variety of rhomboidal quartz. Being both hard and tough, it is well adapted to form the stones of pottery mills for grinding flints; it is called chert in Derbyshire, where it abounds.

Hornstone occurs under three modifications; splintery hornstone, conchoidal hornstone, and woodstone. The colors of the first two are gray, white, and red; they are all massive; dull, or of a glimmering lustre. Translucent only on the thin edges. Difficult to break. Hornstone is less brittle than flint; and by its infusibility before the blowpipe it may be distinguished from petrosilex, which it resembles in external appearance. The geological locality of hornstone is remarkable; for it occurs in both ancient and recent formations. It is found frequently in the veins that traverse primitive crystalline rocks, filling up the interstices, and enveloping their metallic ores. In the lead mine of Huelgoët in Brittany it is whitish; but its prevailing color is gray. It occurs likewise in the middle beds of the coarse limestone (calcaire grossier) in the Paris basin, which is a 
comparatively modern formation, as well as in the sand beds of the upper parts of this district, near Saint Clond, Neuilly, \&c. The hornstone which occurs in secondary limestone is called chert by the English miners. It is valuable for forming the grinding blocks of flint mills in the pottery manufacture.

HORSE POWER, in steam engines, is estimated by Mr. Watt at 32,000 pounds avoirdupois lifted one foot high per minute, for one horse. M. D'Aubuisson, from an examination of the work done by horses in the whims, or gigs (machines à molettes) for raising ore from the mines at Freyberg, the horses being of average size and strength, has concluded that the useful effect of a horse yoked during eight hours, by two relays of four hours each, in a manege or mill course, may be estimated at 40 kilogrammes raised 1 mètre per second; which is nearly 16,440 pounds raised one foot per minute; being very nearly one half of Mr. Watt's liberal estimate for the work of his steam engines.

HOSIERY (Bonnèterie, Fr.; Strumpfueberei, Germ.). The stocking frame, which is the great implement of this business, though it appears at first sight to be a complicated machine, consists merely of a repetition of parts easily understood, with a moderate degree of attention, provided an accurate conception is first formed of the nature of the hosiery fabric. This texture is totally different from the rectangular decussation which constitutes cloth, as the slightest inspection of a stocking will show; ir this, insiead of having two distinct systems of thread, like the warp and the weft, which are woven together, by crossing each other at right angles, the whole piece is composed of a single thread united or looped together in a peculiar manner, which is called stocking-stitch, and sometimes chain-work.

This is best explained by the view in fig. 550. A single thread is formed into

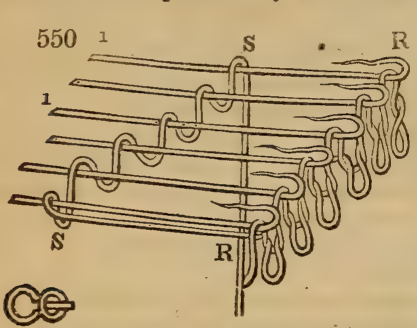
a number of loops or waves, by arranging it over a number of parallel needles, as shown at $\mathbf{R}$ : these are retained or kept in the form of loops or waves, by being drawn or looped through similar loops or waves formed by the thread of the preceding course of the work, $s$. The fabric thus formed by the union of a num ber of loops is easily unravelled, because the stability of the whole piece depends upon the ultimate fastening of the first end of the thread; and if this is undone, the loops formed by that end will open, and release the subsequent loops, one at a time, until the whole is unravelled, and drawn out into the single thread from which it was made. In the same manner, if a thread in a stocking-piece fails, or breaks at any part, or drops a stitch, as it is called, it immediately produces a hole, and the extension of the rest can only be prevented by fastening the end. It should be observed that there are many different fabrics of stocking-stitch for various kinds of ornamental hosiery, and as each requires a different kind of frame or machine to produce it, we should greatly exceed our limits to enter into a detailed description of them all. That species which we have represented in fig. 550 is the common stocking-stitch used for plain hosiery, and is formed by the machine called the common stocking-frame, which is the groundwork of all the others. The operation, as we see, consists in drawing the loop of a thread successively through a series of other loops, so long as the work is continued, as is very plainly shown for one stitch in fig. 551 .

There is a great variety of different frames in use for producing various ornamental kinds of hosiery. The first, which forms the foundation of the whole, is that for knitting plain hosiery, or the common stocking-frame.

Of this valuable machine, the invention of Mr. Lee, of Cambridge, a side elevation is given in fig. 552, with the essential parts. The framing is supported by four upright posts, generally of oak, ash, or other hard wood. Two of these posts appear at A A, and the connecting cross rails are at c c. At B is a small additional piece of framing, which supports the hosier's seat. The iron-work of the machine is bolted or screwed to the upper rails of the frame-work, and consists of two parts. The first rests upon a sole of polished iron, which appears at $\mathrm{D}$, and to which a great part of the machinery is attached. The other part, which is generally called the carriage, runs upon the iron sole at $\mathrm{D}$, and is supported by four small wheels, or trucks, as they are called by the workmen. At the upper part of the back standard of iron are joints, one of which appears at $Q$; and to these is fitted a frame, one side of which is seen extending to $H$. By means of these joints, the end at $\mathrm{H}$ may be depressed by the hosier's hand, and it returns, when relieved, by the operation of a strong spring of tempered steel, acting between a cross bar in the frame and another below. The action of this spring is very apparent in fig. 553. In the front of the frame, immediately opposite to where the hosier sits, are placed the needles which form the loops. These needles, or rather 
hooks, are more or less numerous, according to the coarseness or fineness of the atocking; and this, although unavoidable, proves a very considerable abatement of

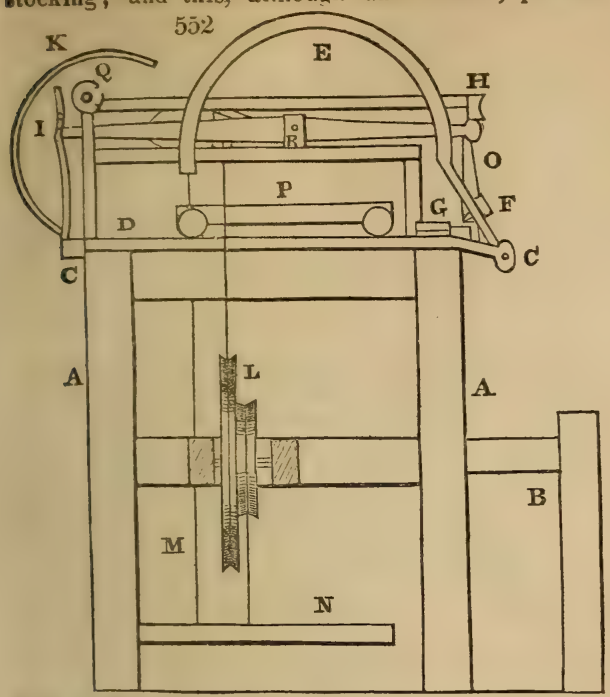
the value of a stocking-frame. In almost every other machine (for example, those employed in spinning or weaving), it is easy to adapt any one either to work coarser or finer work, as it may be wanted. But in the manufacture of hosiery, a frame once finished, is limited for ever in its operation to the same quality of work, with this exception, that by changing the stuff, the work may be made a little more dense or flimsy; but no alteration in the size or quantity of loops ran take place. Hence where the manufacture is extensively prosecuted, many frames may be thrown idle by every vicissitude of demand; and where a poor mechanic does purchase his own frame, he is for ever limited to the same kind of work. The gauge, as it is called, of a stocking-frame is regulated by the number of loops contained in three inches of breadth, and varies very much; the coarsest frames in common use being about what are termed Fourteens, and the finest employed in great extent about Forties. The needles are of iron wire, the manufacture of which is very simple; but long practice in the art is found necessary before a needle-maker acquires the dexterity which will enable him both to execute his work well, and in sufficient quantity to render his labor productive.

The process of making the needles is as follows:-Good sound iron wire, of a proper fineness, is to be selected; that which is liable to split or splinter, either in filing, punching, or bending, being totally unfit for the purpose. The wire is first to be cut into proper lengths, according to the fineness of the frame for which the needles are designed, coarse needles being considerably longer than fine ones. When a sufficient number (generally some thousands) have been cut, the wire must be softened as much as possible. This is done by laying them in rows in a flat iron box, about an inch deep, with a close cover; the box being filled with charcoal between the strata of wires. This box, being placed upon a moderate fire, is gradually heated until both the wires and charcoal have received a moderate red heat, because, were the heat increased to what smiths term the white heat, the wire would be rendered totally unfit for the subsequent processes which it has to undergo, both in finishing and working. When the box has been sufficiently heated, it may be taken from the fire, and placed among hot ashes, until both ashes and box have gradually cooled; for the slower the wires cool, the softer and easier wrought they will be. When perfectly cool, the next process is to punch a longitudinal groove in the stem of every needle, which receives the point or barb, when depressed. This is done by means of a small engine worked by the power of a screw and lever. The construction of these engines is various; but a profile

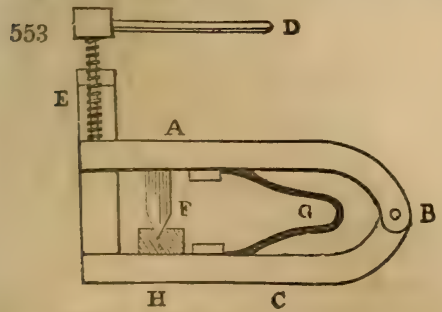
elevation of one of the most simple and commonly used will be found in fig. 553. It consists of two very strong pieces of malleable iron, represented at $A$ and $c$, and these two pieces are connected by a strong wellfitted joint at $\mathrm{B}$. The lower piece, or sole of the engine at $c$, is screwed down by bolts to a strong board or table, and the upper piece $\Lambda$ will then rise or sink at pleasure, upon the joint B. In order that A may be very steady in rising and sinking, which is indispensable to its correct operation, a strong bridle of iron, which is shown in section at $\mathrm{E}$, is added to confine it, and direct its motion. In the upper part of this bridle is a female screw, through which the furcing screw passes, which is turned by the handle or lever $D$. To the sole of the engine $c$ is fixed a bolster 
of tempered steel, with a small groove to receive the wire, which is to be punched; anc in the upper or moving part $\mathrm{A}$, is a sharp chisel, which descends exactly into the groove, when $\mathrm{A}$ is depressed by the screw. These are represented at $\mathrm{F}$, and above $\mathrm{H}$. At $\mathrm{G}$ is a strong spring, which forces up the chisel when the pressure of the screw is removed. The appearance of the groove, when the punching is finished, will be rendered familiar by inspecting fig. 554, p. 657. When the punching is finished, the wires are to be brought to a fine smooth point by filing and burnishing, the latter of which should be very completely done, as, besides polishing the wire, it tends greatly to restore that spring and elasticity which had been removed by the previous operation of softening. The wire is next to be bent, in order to form the hook or barb; and this is done with a small piece of tin plate bent double, which receives the point of the wire, and by its breadth regulates the length of the barb. The stem of the needle is now flattened with a small hammer, to prevent it from turning in the tin socket in which it is afterwards to be cast; and the point of the barb being a little curved by a pair of small pliers, the needle is completed.

In order to fit the needles for the frame, they are now cast into the tin sockets, or leads, as they are called by the workmen; and this is done by placing the needles in an iron mould, which opens and shuts by means of a joint, and pouring in the tin while in a state of fusion. In common operations, two needles are cast into the same socket. The form of the needle, when complete and fitted to its place in the frame, will be seen in fig. 555, which is a profile section of the needle-bar, exhibiting one needle. In this figure a section of the pressure is represented at $F$; the needle appears at $G$, and the socket or level at $\mathrm{K}$. At $\mathbf{H}$, is a section of the needle-bar, on the fore part of which is a small plate of iron called a verge, to regulate the position of the needles. When placed upon the bar resting against the verge, another plate of iron, generally lined with soft leather, is screwed down upon the sockets or leads, in order to keep them all fast. This plate and the screw appear at $I$. When the presser at $F$ is forced down upon the barb, this sinks into the groove of the stem, and the needle is shut ; when the presser rises, the barb opens again by its own elasticity.

The needles or hooks being all properly fitted, the next part of the stocking-frame to which attention ought to be paid, is the machinery for forming the loops; and this consists of two parts. The first of these, which sinks between every second or alternate needle, is represented at o, fig. 552, and is one of the most important parts of the whole machine. It consists of two moving parts; the first being a succession of horizontal levers moving upon a common centre, and called jacks, a term applied to vibrating levers in various kinds of machinery as well as the stocking-frame. One only of these jacks can be represented in the profile fig. 552 ; but the whole are distinctly shown in a horizontal position in fig. 556 ; and a profile upon a very enlarged scale is given in fig. 557 .

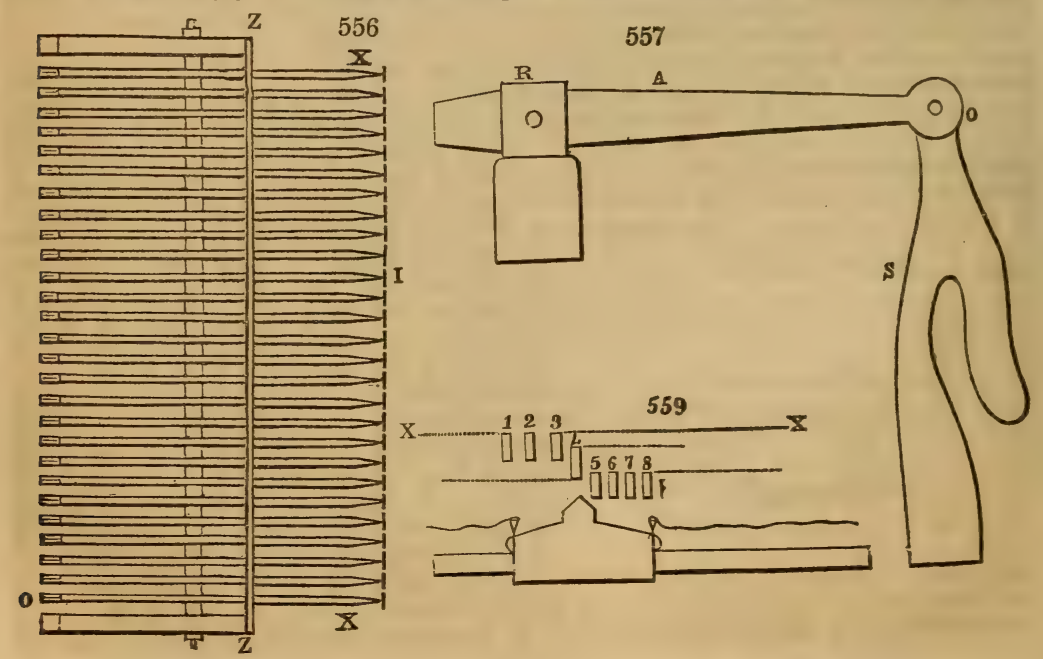


The jack shown in fig. 552, extends horizontally from $\mathrm{o}$ to $\mathrm{x}$, and the centre of motion is at $R$. On the front, or right hand part of the jack at 0 , is a joint suspending a very thin plate of polished iron, which is termed a sinker. One of these jacks and sinkers is allotted for every second or alternate needle. The form of the sinker will appear at $\mathrm{s}$, fig. 557; and in order that all may be exactly uniform in shape, they are cut out and finished between two stout pieces of iron, which serve as moulds or gauges to direct the frame-smith. The other end of the jack at $\mathrm{r}$, is tapered to a point ; and when the jacks are in their horizontal position, they are secured by small iron springs, one of which is represented at I, fig. 552 , each spring having a small obtuse angled notch to receive the point of the jack, against which it presses by its own elasticity. In fig. 557 the centre is at $R$; the pointed tail is omitted for want of room, the joint is at 0 , and the throat of the sinker, which forms the loop, is at $\mathrm{s}$. The standards at $\mathrm{R}$, upon which the jack noves, are called combs, and consist of pieces of flat smooth brass, parallel to, and equidistant from each other. The cross-bar $\mathrm{k}$, which contains the whole, is of iron, with a perpendicular edge or rim on each side, leaving a vacancy between them, or a space to receive the bottom part or tails of the combs. The combs are then placed in the bar, with a flat piece of brass called a countercomb, between each, to ascertain and preserve their distances from each other. These countercombs are exactly of the same shape as the combs, bui have no tails. When both combs and countercombs are placed in the bar, it is luted with clay so as to form a mould, into which is poured a sufficient quantity of melted tin. When the tin has had time to cool, the countercombs having no tails are easily taken out, and the combs remain well fastened and secured by the tin, which has been fused entirely round them. Thus they form a succession of standards for the jacks; and a hole being drilled through each jack and each comb, one polished wire put through, serves as a com mon centre for the whole.

The jack sinkers being only used for every alternate or second needle, in order to complete this part of the apparatus, a second set of sinkers is employed. These are, in form and shape, every way the same as the jack sinkers, but they are jointed at the top into pieces of tin, all of which are screwed to the sinker bar H, fig. 552; and thus a sinker of each kind descends between the needles alternately. By these sinkers the loops are formed upon all the needles, and the reason of two sets different in operation being employed, will be assigned in describing the mode of working the frame. The presser of the operation, of which something has already been said, appears at F; and of the two arms which support and give motion to it, one appears very plainly at $\mathrm{E}$, its centre of motion being at c. The circular bend given to these arms, besides having an

\section{8}
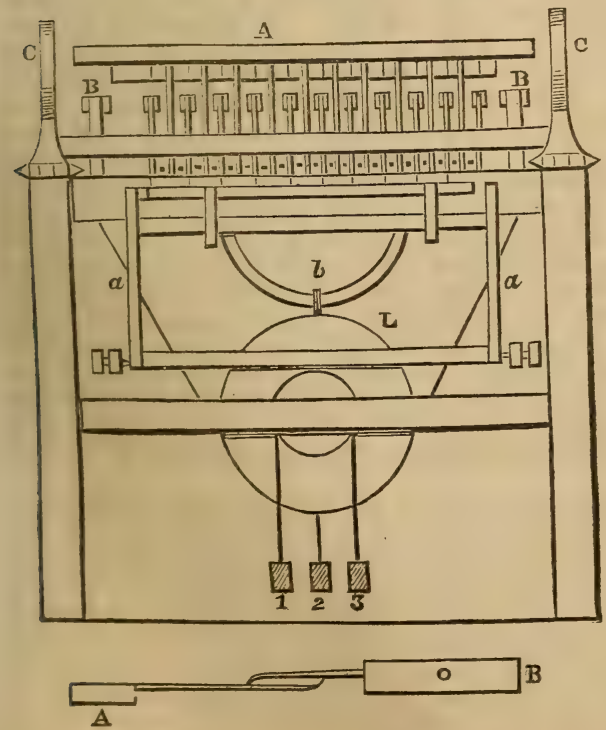

554 ornamental effect, is very useful, in order to prevent any part from interfering with the other parts which are behind, by elevating them entirely above them. The extremities of these arms at the termination of the bends behind, are connected by a cross bar, which has also a circular bend in the middle, projecting downwards, for a reason similar to that already assigned. This bend is concealed in fig. 552 , but visible in the front elevation, fig. 558. From the middle of the bend, the presser is connected with the middle treadle by a depending wire appearing at $\mathrm{M}$, fig. 552, and thus, by the pressure of that treadle, the presser is forced down to close the barbs of the needle. The re-ascent of the presser is sometimes effected by means of a counterpoising weight passing over a pulley behind; and sometimes by the reaction of a wooden spring, formed of a strong hoop like that represented at $\mathrm{k}$. The latter of these is preferred, especially by the Nottingham hosiers, because, as they assert, it makes the presser spring up with greater 
rapidity, and consequently saves time in working. How far this may be practically the case, it would be superfluous here to investigate; but it is obvious that the wooden suring, if very stiff, must add much to the hosier's exertion of his foot, already exercised against the united spring of all his barbs; and this inconvenience is much complained of by those who have been accustomed to work with the counterpoise.

At $\mathrm{L}$ are two pulleys or wheels, of different diameters, moving upon a common centre, by which the jack sinkers are relieved from the back springs, and thrown downwards to form the loops upon the needles. About the larger wheel is a band of whipcord, passing twice round, the extremities of which are attached to what is called the slur, which disengages the jacks from the back sfrings. The smaller pulley, by another band, communicates with the right and left treadle; so that these treadles, when pressed alternately, turn the pulleys about in an inverted order. The directions of these bands also appear more plainly in the front elevation, fig. 558. The construction of the slur, and its effect upon the jacks, will also be rendered apparent by fig. 559. In this figure, eight jacks are represented in section, the tail part of three of which, 1, 2, 3, are thrown up by the slur in its progress from left to right; the fourth is in the act of rising, and the remaining four, $5,6,7$, and 8 , are still unacted upon, the slur not yet having reached them. As the slur acts in the direction of the dotted line $\mathrm{x}, \mathrm{x}, \mathrm{fig}$. 556 , behind the centres of the jacks, it is hardly necessary to remark, that this forcing up of the tails must of course depress the joints by which the sinkers in front are suspended. The jack sinkers falling successively from the loops on every alternate needle, in the way

$$
\text { A }
$$

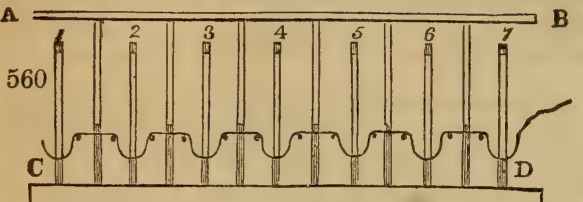

represented at fig. 560 , where both kinds of sinkers appear in section, the light part expressing what is above the point at which the throat of the sinker operates upon the thread, and the dark part what is below. The second set, or, as they are called, the lead sinkers, from the manner of jointing them, and suspending them from the bar above, appear still elevated; the position of the bar being represented by the line A, B. But when these are pulled down to the level of the former by the operator's hands, the whole looping will be completed, and the thread c, D, which is still slack, will be brought to its full and proper degree of tension, which is regulated by stop screws, so as to be tempered or altered at pleasure.

561

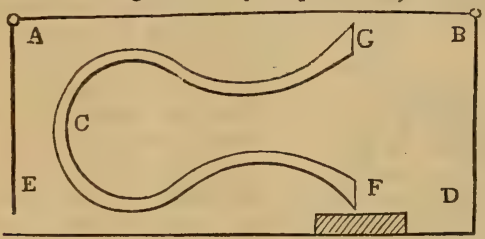
The sinking of this second set of sinkers, may be easily explained by fig. 561. The direction of the sinkers is expressed by the line $\mathrm{E}$; the bar from which they are suspended will be at $A$; the top frame is in the direction from $A$ to $B$; the back standards at $\mathrm{D}$, and the joint at $\mathrm{B}$, is the centre of motion. If $\mathbf{E}$ is pulled perpendicularly downwards, the spring $\mathrm{c}$ will be contracted, and its upper extreme point G, will be brought nearer to its lower extreme point F, which is fixed. Again, when the force which has depressed $\mathrm{E}$ is removed, the spring $\mathrm{c}$ will revert to its former state, and the sinkers will rise. The raising of the jack sinkers and jacks takes place at the same time, by the hosier raising his hands; and for the cause of this we must revert to fig. 556. The lead sinkers in rising lay hold of notches, which raise the extreme parts of the set of jacks z, z, which are called half-jacks. Between the extremities of these at $z, z$, is a cross bar, which, in descending, presses all the intermediate jacks behind the common centre, and restores them to their original posture, where they are secured by the back springs, until they are again relieved by the operation of the slur recrossing at the next course.

Working of the frame.-In order to work a frame, the whole apparatus being previously put into complete order, the hosier places himself on the seat $\mathrm{B}$ in front, and pro- vides himself with a bobbin of yarn or stuff. This bobbin he places loosely on a vertical pin of wire, driven into one side of the frame contiguous to the needles, so that it may turn freely as the stuff is unwound from it. Taking the thread in his hand, he draws it loosely along the needles, behind the barbs, and under the throats of the sinkers. He then presses down one of the treadles to pass the slur along, and unlock the jacks from the back springs, that they may fall in succession. When this is done, the number of loops thus formed is doubled by bringing down the lead sinkers, and the new formed loops are lodged under the barbs of the needles by bringing forward the sinkers. The preceding course, and former fabric, being then again pushed back, the barbs are shut by depressing the middle treadle, and forcing down the presser upon the needles. The former work is now easily brcught over the shut needles, after which, by raising the 
hands, both sets of sinkers are raised; the jacks are locked by the back springs, and the hosier goes on to another course.

From this it will be apparent, that the remark made in the outset is well founded, that there are, in reality, no complicated or difficult movements in the stocking-frame. Almost the whole are merely those of levers moving upon their respective fulcra, excepting that of the carriage which gives the horizontal motion to the sinkers, and that is merely an alternate motion on four wheels. Yet the frame is a machine which requires considerable experience and care, both to work it to advantage, and also to keep it in good order. This circumstance arises greatly from the small compass in which a number of moving parts must be included. Owing to this, the needles, unless cautiously and delicately handled, are easily bent or injured. The same circumstance applies with equal or greater force to the sinkers, which must be so very thin as to be easily injured. But as these must work freely, both in a perpendicular and horizontal direction between the needles, in a very confined and limited space, the slightest variation in either, from being truly and squarely placed, unavoidably injures the others. When a hosier, either ignorant of the mechanical laws, of their relation to each other, or too impatient to wait for the assistance of another, attempts to rectify defects, he in most cases increases them tenfold, and renders the machine incapable of working at all, until repaired by some more experienced person. This circumstance has given rise to a set of men employed in this trade, and distinguished by the name of upsetters; and these people, besides setting new frames to work, have frequently more employment in repairing old ones injured by want of care or skill, than many country apothecaries, who live in unhealthy parishes, find in tampering with the disorders of mankind.

It seems unnecessary to go further into detail respecting a machine so well known, and which requires practical attention even more than most others. It may, therefore, be sufficient to describe shortly some of its varieties, the most simple and common of which is the rib stocking-frame.

Rib slocking-frame. - This frame, which, next to the common frame, is most extensively in use, is employed for working those striped or ribbed stockings, which are very common in all the different materials of which hosiery is formed. In principle it does not differ from the common frame, and not greatly in construction. The preceding general description will nearly apply to this machine with equal propriety as to the former; that part, however, by which the ribs or stripes are formed, is entirely an addition, and to the application of this additional machinery it may be proper to pay the chief attention, referring chiefly to fig. 558, which is a front elevation.

This figure has been already referred to for the illustration of those parts of the machinery which are common to both, and those parts therefore require no recapitulation. The principle of weaving ribbed hosiery possesses considerable affinity to that which subsists in the weaving of that kind of cloth which is distinguished by the name of tweeling, for the formation of stripes, with some variation arising merely from the different nature of the fabric. In cloth weaving, two different kinds of yarn intersecting each other at right angles, are employed; in hosiery only one is used. In the tweeling of cloth, striped as dimity, in the cotton or kerseymere, and in the woollen manufacture, the stripes are produced by reversing these yarns. In hosiery, where only one kind of yarn is used, a similar effect is produced by reversing the loops. To effect this reversing of the loops, a second set of needles is placed upon a vertical frame, so that the bends of the hooks may be nearly under those of the common needles. These

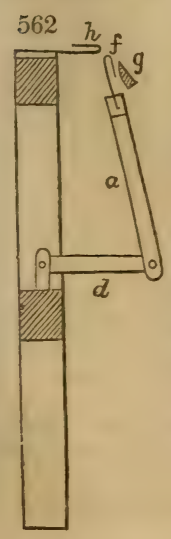
needles are cast into tin moulds, pretty similar to the former, but more oblique or bevelled towards the point, so as to prevent obstructions in working them. They are also screwed to a bar of iron, generally lighter than the other, and secured by means of plates : this bar is not fixed, but has a pivot in each end, by means of which the bar may have a kind of oscillatory motion on these pivots. The two frames of iron support this bar; that in which it oscillates being nearly vertical, but inclined a little towards the other needles. Fig. 562, which is a profile elevation, will serve to illustrate the relative position of each bar to the other. The lower or horizontal frame, the ends only of which can be seen in fig. 558 under $a a$, appears in profile in fig. 562, where it is distinguished by $d$. The vertical frame at $a$ is attached to this by two centre screws, which serve as joints for it to move in. On the top of this frame is the rib-needle bar at $f$, in figs. 552 and 562 , and one needle is represented in $f i g .562$ at $f$. At $g$ is a small presser, to shut the barbs of the ribneedles, in the same manner as the large one does those of the frame. At $h$ is one of the frame needles, to show the relative position of the one set to the other. The whole of the rib-bar is not fitted with needles like the other; for here needles are only placed where ribs or stripes are to be formed, the intervals being filled up with blank leads, that is 
to say, with sockets of the same shape as the others, but without needles; being merely designed to fill the bar and preserve the intervals. Two small handles depend from the needle bar, by which the oscillatory motion upon the upper centres is given. The rising and sinking motion is communicated to this machine by chains which are attached to iron sliders below, and which are wrought by the hosier's heel when necessary. The pressure takes place partly by the action of the small presser, and partly by the motion of the needles in descending. A small iron slider is placed behind the rib-needles, which rises as they descend, and serves to free the loops perfectly from each other.

In the weaving of ribbed hosiery, the plain and rib courses are wrought alternately. When the plain are finished, the rib-needles are raised between the others, but no additional stuff is supplied. The rib-needles, intersecting the plain ones, merely lay hold of the last thread, and, by again bringing it through that which was on the rib-needle before, give it an additional looping, which reverses the line of chaining, and raises the rits above the plain intervals, which have only received a single knitting.

HOT-FLLE is the name given in England to an apartment heated by stoves or steam pipes, in which padded and printed calicoes are dried hard. Fig. 563 represents

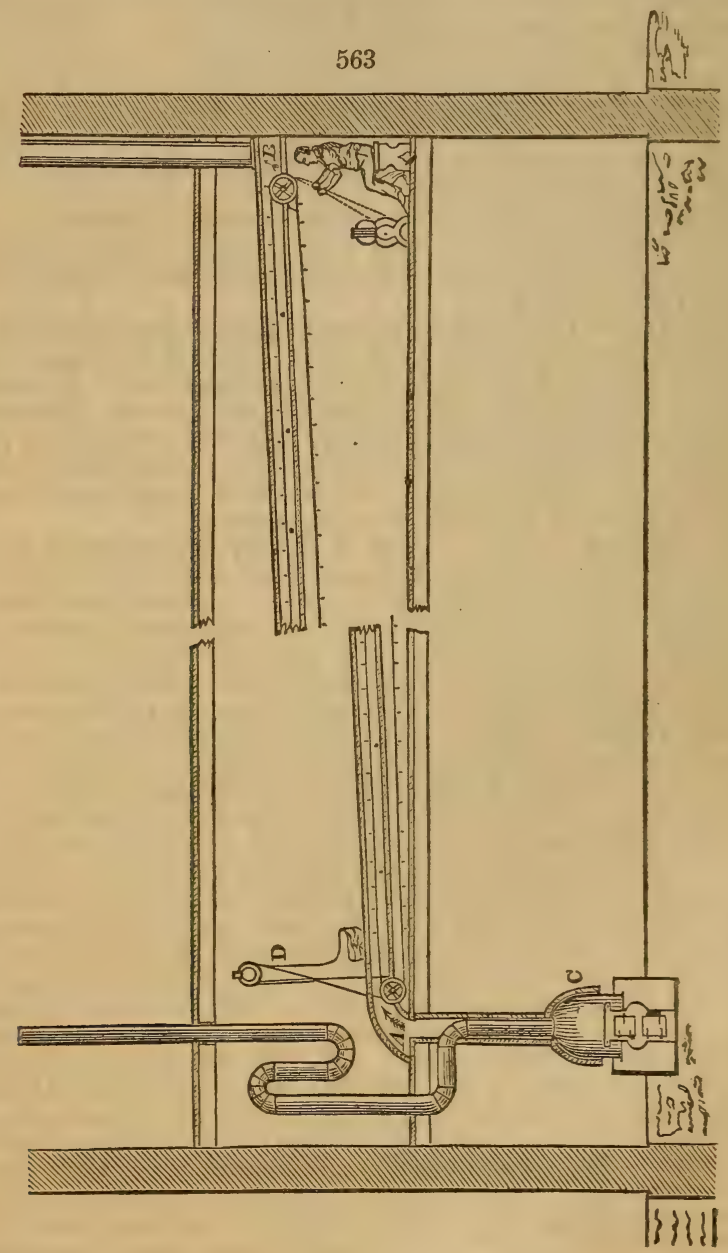

the simplest form of such a flue, heated by the vertical round iron stove $c$, from whose top a wide square pipe proceeds upwards in a slightly inclined direction, which receives the current of air heated by the body and capital of the stove. In this wide channe. there are pulleys, with cords or bands which suspend by hooks, and conduct the web of 
ealico, from the entrarice at $\mathrm{B}$, where the operative sits, to near the point $\mathrm{A}$, and back again. This circuit may be repeated once or oftener till the goods are perfectly dried. At D the driving pulley connected with the main shaft is shown. Near the feet of the operative is the candroy or reel upon which the moist goods are rolled in an endless web; so that their circulation in the hot-air channel can be continued without interruption, as long as may be necessary.

Fig. 564 is a cross section of the apparatus of the regular hot-flue, as it is mounted 564

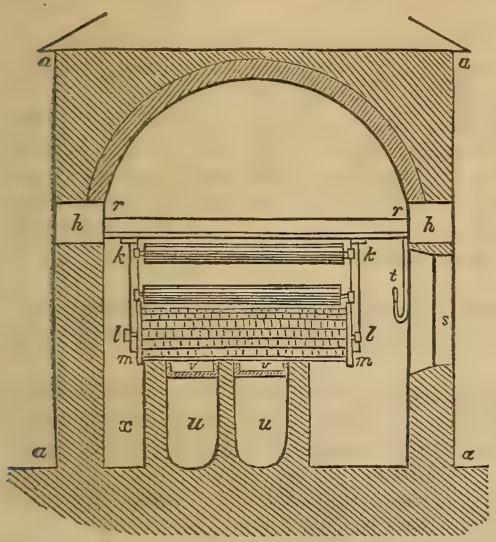
in the most scientific calico works of England, those of James Thomson, Esq., of Primrose, near Clitheroe, Lancashire. a $a$ a $\alpha$, is an arched apartment, nearly 30 yards long, by 13 feet high, and 10 feet wide. Through about one half of this gallery there is a horizontal floor supported on arches, above which is the driest space, through which the goods are finally passed before they escape from the hot-flue, after they have been previously exposed to the hot but somewhat moist air of the lower compartment. A large scuare flue covered with cast-iron plates runs along the whole bottom of the gallery. It is divided into two long parallel vaults, whose sections are seen at $u, u, f i g .564$, covered with the cast-iron plates $v v$, grooved at their ends into one another. The thickness of these plates is increased progressively as they come nearer to the fireplace or furnace. There are dampers which regulate the draught, and of course the heat of the stove. $h h$ are the air-passages or vent-holes, left in the side walls, and which by means of a long iron rod, mounted with iron plates, may be opened or closed together to any degree. $k k$ are the cast-iron supports of the tinned brass rollers which guide the goods along, and which are fixed to the cross pieces represented by $r$ r, fig. 564 . l $l$ are iron bars for supporting the ventilators or fans (see the fan under FouNDRY). These fans are here enclosed within a wire grating. They make about 300 turns per minute, and expel the moist air with perfect effect. $s$ indicates the position of the windows, which extend throughout the length of the building. $t$ is a gas-light jet, placed at the side of each window to supply illumination for night work.

The piece is stretched along the whole extent of the gallery, and runs through it in the course of one minute and a half; being exposed during its passage to the heat of $-212^{\circ} \mathrm{Fahr}$.

In fig. $565, \mathrm{~A}$ is the iron door of entrance to the hot-flue gallery; at $b$ is the padding machine, where the goods are imbued with the general mordant. The speed of

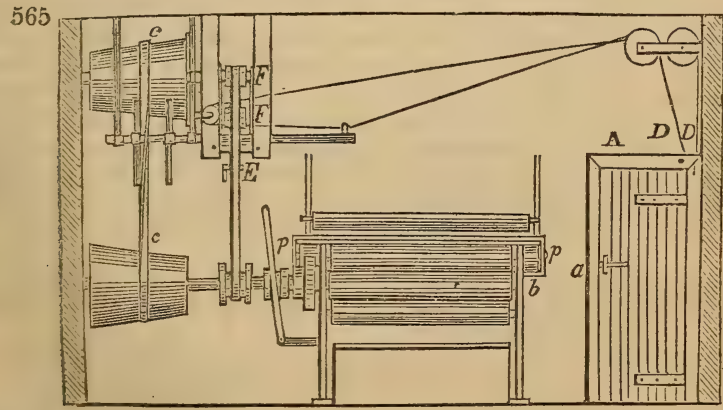

this machine may be varied by means of the two conical drums $c c$, which drive it; since when the band $c c$ is brought, by its forks and adjusting screws, nearer to the narrow end of the lower drum, the cylinder upon the same shaft with the latter is driven quicker; and vice versa. Over D D the cords are shown for drawing the drum mechanism into gear with the main shaft band, F F E; or for throwing it out of gear. The pulleys $F F$ carry the bands which transmit the motion to the padding machine. A cylindrical drum exterior to the hot-flue, covered with flannel, serves to receive the end of the series of pieces. and to draw them through the apartment. This mode of drying 
the padded calicoes requires for each piece of 28 yards, 3 pounds of coals for the furnace when a fan is employed, and 4 pounds without it.

HYDRATES are compounds of the oxydes, salts, \&c. with water in definite or equivalent proportions. Thus slaked lime consists of one atom of quick-lime $=28,+$ one atom of water $=9$, of which the sum is 37 on the hydrogen scale.

HYDRAUliC PRESS. See Oil, Press, and Stearine.

HYDRIODIC ACID (Acide Hydriodique, Fr.; Hydriodsïure, Germ.) is an acid formed by the combination of 99.21 parts of iodine, and $0 \cdot 79$ hydrogen. When pure, it occurs in the gaseous state, but it combines with water like the hydrochloric or muriatic acid gas into a liquid acid.

HYDROCHLORIC ACID; the new chemical name of muriatic acid, which see.

HYDROGEN (Eng. and Fr.; Wasserstoff, Germ.), an undecompounded gaseous body ; the lightest of all ponderable matter, whose examination belongs to chemistry.

HYDROMETER; an instrument for ascertaining the specific gravities of liquids. Baumés hydrometer, which is much used in France, and other countries of the continent of Europe, when plunged in pure water, at the temperature of $58^{\circ} \mathrm{Fahr}$., marks 0 upon its scale; in a solution containing 15 per cent. of common salt (chloride of sodium) and 85 of water by weight, it marks $15^{\circ}$; so that each degree is meant to indicate a density corresponding to one per cent. of that salt. See AREOMETER, for comparative tables of hydrometers.

HYDROSULPHURETS ; chemical compounds of bases with sulphureted hydrogen.

HYMENCEA COURBARIL; a tree growing in South America, from which the resin animé exudes.

HYOSCIAMUS NIGER. Henbane is a plant used in medicine, from which modern chemistry has extracted a new crystalline vegetable principle called hyosciamine, which is very poisonous, and when applied in solution to the eye, determines a remarkable dilatation of the pupil; as belladonna also does.

HYPOSULPHATES; HyPoSUlPHITES; saline compounds of the hyposulphuric $\alpha$ hyposulphurous acid with bases.

HYPEROXYMURIATES; the old and incorrect name of ChLORATES.

\section{I. \& J.}

JACK, called also jack in a box, and hand jack, is a portable mechanical instrument, consisting of a rack and pinion, or a pair of claws and ratchet bar, moved by a winch handle, for raising heavy weights a little way off the ground.

JACK and JACK-SINKERS, are parts of a stocking frame; see HosIERY.

JACK-BACK, is the largest jack of the brewer.

JACQUARD. A peculiar and most ingenious mechanism, invented by M. Jacquart of Lyons, to be adapted to a silk or muslin loom for superseding the employment of draw-boys, in weaving figured goods. Independently of the ordinary play of the warp threads for the formation of the ground of such a web, all those threads which should rise simultaneously to produce the figure, have their appropriate healds, which a child formerly raised by means of cords, that grouped them together into a system, in the order, and at the time desired by the weaver. This plan evidently occasioned no little complication in the machine, when the design was richly figured; but the apparatus of Jacquart, which subjects this manœuvre to a regular mechanical operation, and derives its motion from a simple pedal put in action by the weaver's feet, was generally adopted soon after its invention in 1800. Every common loom is susceptible of receiving this beautiful appendage. It costs in France, 200 francs, or $8 l$. sterling; and a little more in this country.

Fig. 566 is a front elevation of this mechanism, supposed to be let down. Fig. 567 is a cross section, shown in its highest position. Fig. 568 the same section as the preceding, but seen in its lower position.

$\Lambda$, is the fixed part of the frame, supposed to form a part of the ordinary loom; there are two uprights of wood, with two cross bars uniting them at their upper ends, and leaving an interval $x y$, between them, to place and work the moveable frame $B$, vibrating round two fixed points $a$, placed laterally opposite each other, in the middle of the space $x y$, fig. 566 .

c, is a piece of iron with a peculiar curvature, seen in front, $f i g .566$, and in profile, figs. 567 and 568. It is fixed on one side upon the upper cross-bar of the frame B, and on the other, to the intermediate cross-bar $b$ of the same frame, where it shows an inclined curvilinear space $c$, terminated below by a semi-circle.

$\mathrm{D}$, is a square wooden axis, moveable upon itself round two iron pivots, fixed into its two 
encis; which axis occupies the bottom of the moveable frame $B$. The four faces of this square axis are pierced with three round, equal, truly-bored holes, arranged in a quin-
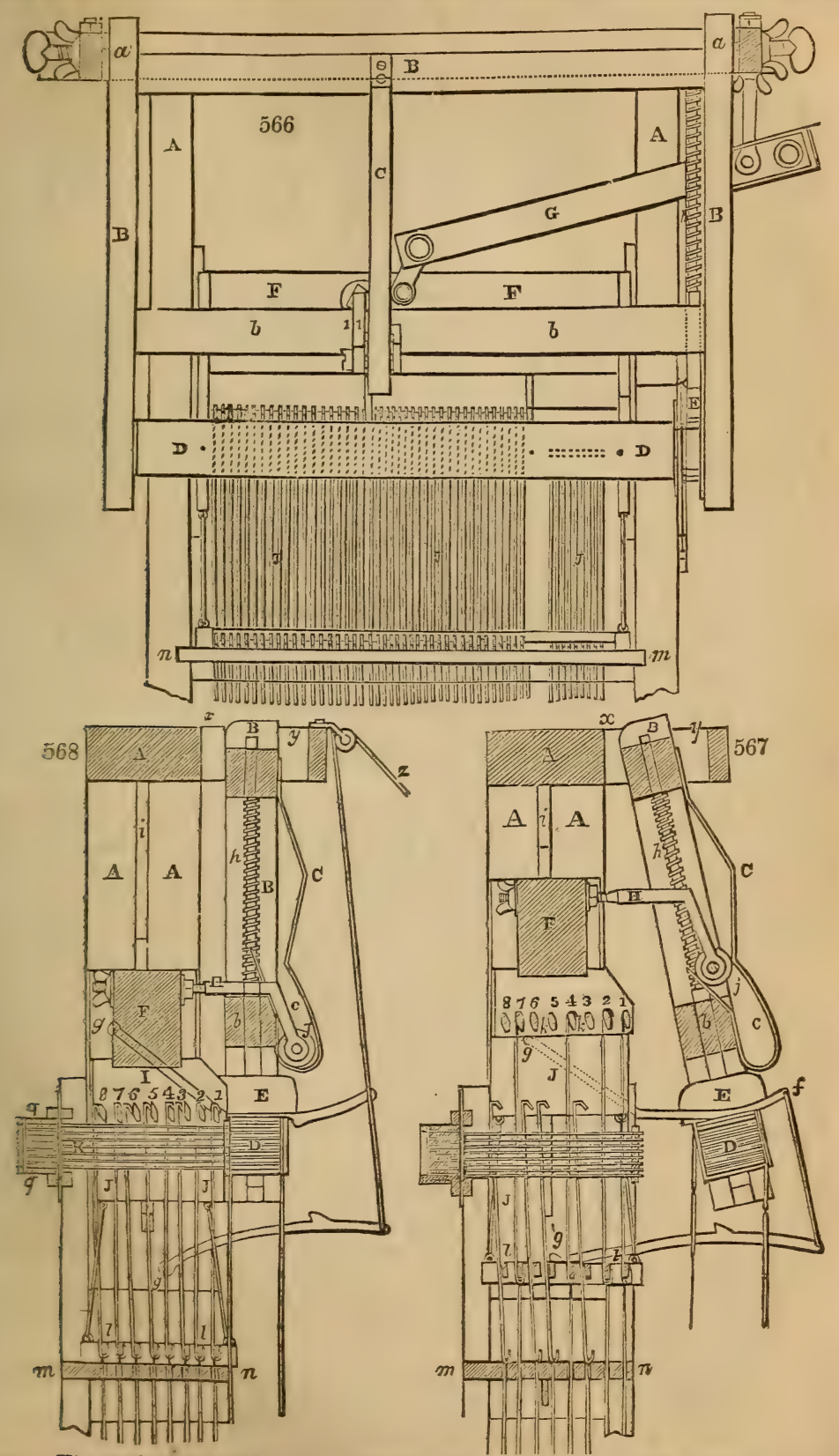

eunx. The teeth $a, f i g .570$, are stuck into each face, and correspond to holes $a, f i g .573$ made in the cards which constitute the endless chain for the healds; so that in the sue cessive application of the cards to each face of the square axis, the holes pierced in one card may always fall opposite to those pierced in the other. 
The right-hand end of the square axis, of which a section is shown in double size, fig. 569, carries two square plates of sheet iron $d$, kept paral el to each other and

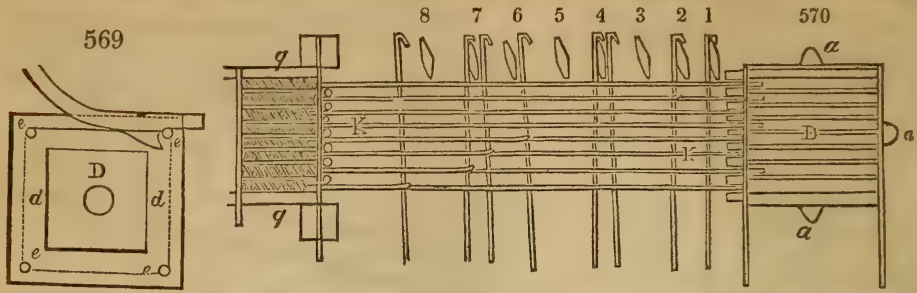

a little apart, by four spindles $e$, passed opposite to the corners. This is a kind of lantern, in whose spindles the hooks of the levers $f f^{\prime}$, turning round fixed points $g g^{\prime}$ beyond the right hand upright $\mathrm{A}$, eatch hold, either above or below, at the pleasure of the weaver, according as he merely pulls or lets go the cord $z$, during the vibratory movement of the frame $B$.

$\mathbf{E}$ is a piece of wood shaped like a $\mathbf{T}$, the stem of which, prolonged upwards, passes freely through the cross-bar $b$, and through the upper cross-bar of the frame $\mathrm{B}$, which serve as guides to it. The head of the $T$ piece being applied successively against the two spindles $e$, placed above in a horizontal position, first by its weight, and then by the spiral spring $h$, acting from above downwards, keeps the square axis in its position, while it permits it to turn upon itself in the two directions. The name press is given to the assemblage of all the pieces which compose the moveable frame в в.

$F$ is a cross-bar made to move in a vertical direction by means of the lever $G$, in the notehes or grooves $i$, formed within the fixed uprights $A$.

$\mathrm{H}$ is a piece of bent iron, fixed by one of its ends with a nut and screw, upon the crossbar $F$, out of the vertical plane of the piece $C$. Its other end carries a friction roller $J$, which working in the curvilinear space $c$ of the piece $c$, forces this, and consequently the frame $B$, to recede from the perpendicular, or to return to it, according as the cross-bar $F$ is in the top or bottom of its course, as shown in figs. 56 ? and 568.

I, cheeks of sheet iron attached on either side to the cress-isar $F$, which serve as a safe to a kind of claw $\mathrm{x}$, composed here of eight small metallie bars, seen in section fig. 567 and 568 , and on a greater seale in fig. 570 .

$\mathrm{J}$, upright skewers of iron wire, whose tops, bent down hook-wise, naturally place themselves over the little bars $\mathrm{K}$. The bottom of these spıniles, likewise hooked in the same direction as the upper ones, embraces small wooden bars $l$, whose office is to keep them in their respective places, and to prevent them from twirling round, so that the uppermost hooks may be always directed towards the small metallic bars upon which they impend. To these hooks from below are attached strings, which, after having crossed a fixed board $m n$, pierced with corresponding holes for this purpose, proceed next to be attached to the threads of the loops destined to lift the warp threads. $\mathbf{K} \mathbf{K}$, horizontal spindles or needles, arranged here in eight several rows, so that each spindle corresponds both horizontally and vertically to each of the holes pierced in the four faces of the square axis $\mathrm{D}$. There are therefore as many of these spindles as there are holes in one of the faces of the square.

Fig. 571 represents one of these horizontal spindles. $n$ is an eyelet through which the

\section{1}

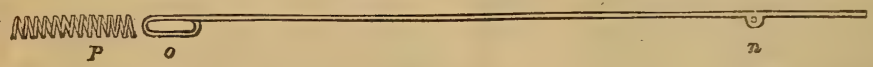

corresponding vertical skewer passes. o another elongated eyelet, through which a small fixed spindle passes to serve as a guide, but which does not hinder it from moving lengthwise, within the limits of the length of the eyelet. $p$, small spiral springs placed in each hole of the case $q q$, fig. 570. They serve the purpose of bringing back to its primitive position, every corresponding needle, as soon as it ceases to press upon it.

Fig. 572 represents the plan of the upper row of horizontal needles. Fig. 573 is a fragment of the endless chain, formed with perforated eards, which are made to cireulate

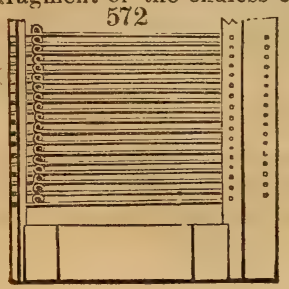
573

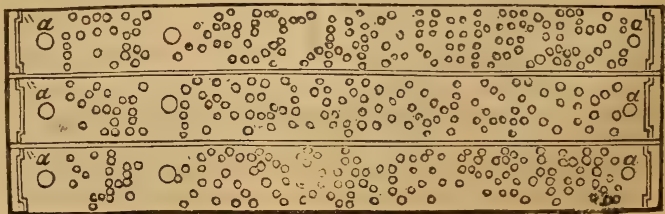


or travel by the rotation of the shaft $\mathrm{D}$. In this movement, each of the perforated cards, whose position, form, and number, are determined by the operation of tying-up of the warp, comes to be applied in succession against the four faces of the square axis or drum, leaving open the corresponding holes, and covering those upon the face of the axis, which have no corresponding holes upon the card.

Now let us suppose that the press в is let down into the vertical position shown in fig. 568 ; then the card applied against the left face of the axis, leaves at rest or untouched the whole of the horizontal spindles (skewers), whose ends correspond to these holes, but pushes back those which are opposite to the unpierced part of the card; thereby the corresponding upright skewers, $3,5,6$, and 8 , for example, pushed out of the perpendicular, unhook themselves from above the bars of the claw, and remain in their place, when this claw comes to be raised by means of the lever $\mathrm{G}$; and the skewers $1,2,4$, and 7, which have remained hooked on, are raised along with the warp threads attached to them. Then by the passage across of a shot of the color, as well as a shot of the common weft, and a stroke of the lay after shedding the warp and lowering the press $\mathrm{B}$, an element or point in the pattern is completed.

The following card, brought round by a quarter revolution of the axis, finds all the needles in their first position, and as it is necessarily perforated differently from the preceding card, it will lift another series of warp threads; and thus in succession for all the other cards, which compose a complete system of a figured pattern.

This machine, complicated in appearance, and which requires some pains to be understood, acts however in a very simple manner. Its whole play is dependant upon the movement of the lever $\mathrm{G}$, which the weaver himself causes to rise and fall, by means of a peculiar pedal; so that without the aid of any person, after the piece is properly read in and mounted, he can execute the most complex patterns, as easily as he could weave plain goods ; only attending to the order of his weft yarns, when these happen to be of different colors.

If some warp yarns should happen to break without the weaver observing them, or should he mistake his colored shuttle yarns, which would so far disfigure the pattern, he must undo his work. For this purpose, he makes use of the lower hooked lever $f^{\prime}$, whose purpose is to make the chain of the card go backwards, while working the loom as usual, withdrawing at each stroke the shot both of the ground and of the figure. The weaver is the more subject to make mistakes, as the figured side of the web is downwards, and it is only with the aid of a bit of looking-glass that he takes a peep of his work from time to time. The upper surface exhibits merely loose threads in different points, according as the pattern requires them to lie upon the one side or the other.

Thus it must be evident, that such a number of paste-boards are to be provided and mounted as equal the number of throws of the shuttle between the beginning and end of any figure or design which is to be woven; the piercing of each paste-board individually, will depend upon the arrangement of the lifting rods, and their connexion with the warp, which is according to the design and option of the workman; great care must be taken that the holes come exactly opposite to the ends of the needles; for this purpose two large holes are made at the ends of the paste-boards, which fall upon conical points, by which means they are made to register correctly.

It will be hence seen, that, according to the length of the figure, so must be the number of paste-boards, which may be readily displaced so as to remount and produce the figure in a few minutes, or remove it, or replace it, or preserve the figure for future use. The machine, of course, will be understood to consist of many sets of the lifting rods and needles, shown in the diagram, as will be perceived by observing the disposition of the holes in the paste-board; those holes, in order that they may be accurately distributed, are to be pierced from a gauge, so that not the slightest variation shall take place.

To form these card-slips, an ingenious apparatus is employed, by which the proper steel punches required for the piercing of each distinct card, are placed in their relative situations preparatory to the operation of piercing, and also by its means a card may be punched with any number of holes at one operation. This disposition of the punches is effected by means of rods connected to cords disposed in a frame, in the nature of a false simple, on which the pattern of the work to be performed is first read in.

These improved pierced cards, slips, or paste-boards, apply to a weaving apparatus, which is so arranged that a figure to be wrought can be extended to any distance along the loom, and by that means the loom is rendered capable of producing broad figured works; having the long lever $\mathrm{G}$ placed in such a situation that it affords power to the foot of the weaver, and by this means enables him to draw the heaviest morintures and figured works, without the assistance of a draw-boy.

The machinery for arranging the punches, consists of a frame with four upright standards and cross-pieces, which contains a series of endless cords passing under a wooden roller at bottom, and over pulleys at the top. These pulleys are mounted on 
axles in two frames, placed obliquely over the top of the standard frame, which pulleyframes constitute the table commonly used by weavers.

In order better to explain these endless cords, fig. 574 represents a single endleas cord 1,1 , which is here shown in operation, and part of another endless cord 2 , 2, shown

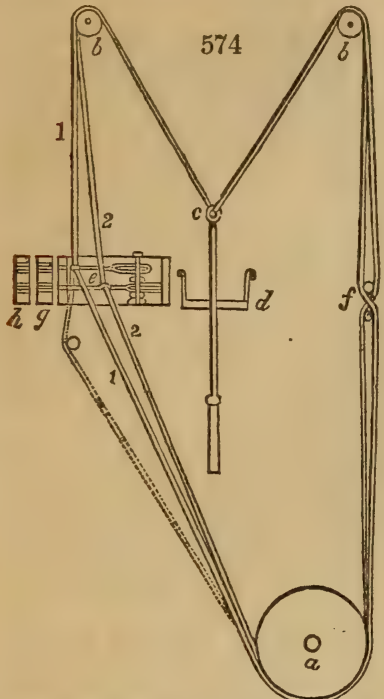
stationary. There must be as many endless cords in this frame as needles in the weaving-loom. $a$ is the wooden cylinder, revolving upon its axis at the lower part of the standards; $b b$, the two pulleys of the pulley-frames above, over which the individual endless cord passes; $c$ is a small traverse ring. To each of these rings a weight is suspended by a single thread, for the purpose of giving tension to the endless cord. $d$ is a board resembling a common comber-bar, which is supported by the cross-bars of the standard frame, and is pierced with holes, in situation an ${ }^{3}$ number, corresponding with the perpendicular threads that pass through them; which board keeps the threads distinct from each other.

At $e$, the endless cord passes through the eyes of wires resembling needles, which are contained in a wooden box placed in front of the machine, and shown in this figure in section only. These wires are called the punch-projectors; they are guided and supported by horizontal rods and vertical pins, the latter of which pass through loops formed at the hinder part of the respective wires. At $f$ are two horizontal rods extending the whole width of the machine, for the purpose of producing the cross in the cords; $g$ is a thick brass plate, extending along in front of the machine, and lying close to the box which holds the

punch-projectors; this plate g, shown also in section, is called the punch-holder; it contains the same number of apertures as there are punch-projectors, and disposed so as to correspond with each other. In each of these apertures, there is a punch for the purpose of piercing the cards, slips, or pasteboards with holes; $h$ is a thick steel plate of the same size as $g$, and shown likewise in section, corresponding also in its number of apertures, and their disposition, with the punch-projectors and the puseh-holder. This plate $h$, is called the punch-receiver.

The object of this machine is to transfer such of the punches as may be required for piercing any individual card from the punch-holder $g$, into the punch-receiver $h$; when they will be properly situated, and ready for piercing the individual card or slip, with such holes as have been read in upon the machine, and are required for permitting the warp threads to be withdrawn in the loom, when this card is broughit against the ends of the needles. The process of transferring the patterns to the punches will be effected in the following manner.

The pattern is to be read in, according to the ordinary mode, as in a false simple, upon the endless cords below the rods $f$, and passed under the revolving wooden cylinder $a$, to a sufficient height for a person in front of the machine to reach conveniently. He there takes the upper threads of the pattern, called the beard, and draws them forward so as to introduce a stick behind the cords thus advanced, as shown by dots, for the purpose of keeping them separate from the cords which are not intended to be operated upon. All the punch-projectors which are connected with the cords brought forward, will be thus made to pass through the corresponding apertures of the punch-holder $g$, and by this means will project the punches out of these apertures, into corresponding apertures of the punch-receiver $h$. The punches will now be properly arranged for piercing the required holes on a card or slip, which is to be effected in the following manner.

Remove the punch-receivers from the front of the machine; and having placed one of the slips of card or pasteboard between the two folding plates of metal, completely pierced with holes corresponding to the needles of the loom, lay the punch-receiver upon those perforated plates; to which it must be made to fit by mortises and blocks, the cutting parts of the punches being downwards. Upon the back of the punch-receiver is then to be placed a plate or block, studded with perpendicular pins corresponding to the above described holes, into which the pins will fall. The plates and the blocks thus laid together, are to be placed under a press, by which means the pins of the block will be made to pass through the apertures of the punch-receiver; and wherever the punch has been deposited in the receiver by the above process, the said punches will be forced through the slip of pasteboard, and pierced with such holes as are required for producing the figured design in the loom. 
Each card being thus pierced, the punch recaiver is returned to its place in front of the machine, and all the punches forced back again into the apertures of the punch holder as at first. The next set of cords is now drawn forward by the next beard, as above described, which sends out the punch-projectors as before, and disposes the punches in the punch-receiver, ready for the operation of piercing the next card. The process being thus repeated, the whole pattern is, by a number of operations, transferred to the punches, and afterwards to the cards or slips, as above described.

JADE, axe-stone (Nephrite, Ceraunile, Fr.), is a mineral commonly of a greenish color, compact, and of a fatty lustre. Spec. Grav. 2.95 ; scratches glass, is very tough; fuses into a white enamel. Its constituents are, silica, 50.5 ; alumina, 10 ; magnesia, 31 ; oxyde of iron, $5 \cdot 50$; oxyde of chrome, 0.05 ; water, $2 \cdot 75$. It comes from China, is used among rude nations for making hatchets; and is susceptible of being cut into any form.

JAPANNING, is a kind of varnishing or lackering, practised with excellence by the Japanese, whence the name. See Varnish.

JASPER (Jaspe calcedoine, Fr.; Jaspis, Germ.) is a sub species of calcedony quartz, of which there are five varieties. 1. The Egyptian red and brown, with ring or tendrilshaped delineations. 2. Striped jasper. 3. Porcelain jasper. 4. Common jasper. 5. Agate jasper. The prettiest specimens are cut for seals, and for the inferior kinds of jewellery ornaments. See LAPIDARY.

ICE-HOUSE; (Glacière, Fr.; Eishaus, Germ.) Under the article FreEzING I have enumerated the different artificial methods of producing cold. But for the uses of common life, in these climates, the most economical and convenient means of refrigeration in hot weather may be procured by laying up a store of ice in winter, in such circumstances as will preserve it solid during summer.

An ice-house should not be regarded as an object of mere luxury, for pleasing the palates of gourmands with iced creams and orgeats. In the southern countries of Europe it is considered among people in easy circumstances as an indispensable appendage to a country mansion. During the Dog-days, especially at those periods and in those districts where the sirocco blows, a lassitude and torpor of mind and body supervene, with indigestion or total loss of appetite, and sometimes dysenteries, frach are obviously oc. casioned by the excessive heat, and are to be prevented or counteracted chiefly by the use of cold beverages. By giving tone to the stomach, iced drinks immediately restore the functions of the nervous and muscular systems when they are languid; while they enable persons in health to endure without much inconvenience an atmosphere so close and sultry as would be intolerable without this remedy. Ice-houses, moreover, afford to country gentlemen a great advantage in enabling them to preserve their fish, butcher meat, dead poultry, and game, which would otherwise, in particular states of the weather, immediately spoil. Considering at how little expense and trouble an ice-house can be constructed, it is surprising that any respectable habitation in the country should not have one attached to it. The simplest and most scientific form is a double cone, that is, two cones joined base to base; the one being of stones or brickwork, sunk under ground with its apex at the bottom, into which the ice is rammed; the other being a conical roof of carpentry covered with thatch, and pointed at top. The entrance should be placed always on the north side; it should consist of a corridor or porch with double doors, and be screened from the sunbeams by a small shrubbery. Such are, in general, the principles upon which an ice-house should be formed; but they will be better understood by the following explanation and figure.

A dry sandy soil should be selected, and, if possible, a spot sheltered by a cliff or other natural barrier from the direct rays of the sun. Here a cavity is to be dug about 16 feet in diameter, terminating below like the point of a sugar loaf. Its ordinary depth, for a moderate family, may be about 24 feet; but the larger its dimensions are, the longer will it preserve the ice, provided it be filled. In digging, the workman should slope the ground progressively towards the axis of the cone, to prevent the earth falling in. This conical slope should be faced with brick or stone work about one foot thick, and jointed with Roman cement so as to be air and water tight. A well is to be excavated at the bottom two feet wide and four deep, covered at top with an iron grating for supporting the ice, and letting the water drain away.

The upper cone may likewise be built of brickwork, and covered with thatch; such a roof would prove the most durable. This is the construction shown in fig. 575. Whatever kind of roof be preferred, there must be left in it an oblong passage into the interior. This porch should face the north, and be at least 8 feet long by $2 \frac{1}{2}$ feet wide; and perfeetly closed by a well-fitted door at each end. All round the bottom of this conical cover, a gutter should be placed to carry off the rain to a distance from the ice-house, and prevent the circumjacent ground from getting soaked with moisture.

Fig. 575 shows the section of a well-constructed ice-house. Under the ice-ehamber $\mathrm{A}$ the ice is rammed into the space B. $\mathrm{C}$ is the grate of tha drain-sink $\mathrm{D}$. The 
portion $\mathrm{E} \mathrm{E}$ is built in brick or stone; the base $\mathrm{L}$ of the ice-chamber slopes inwards towards the centre at $\mathrm{c}$. The upper part of the brickwork $\mathbf{E} \mathbf{E}$ is a little way below the

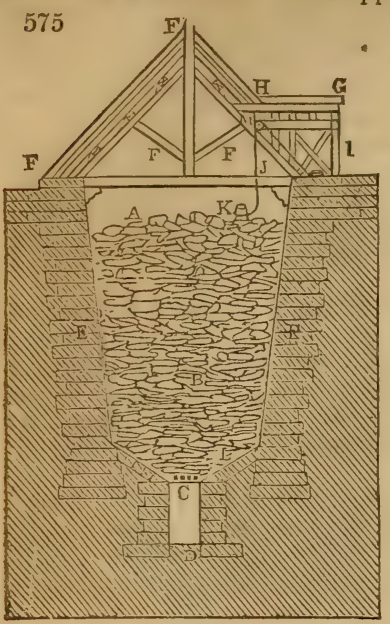
level of the ground. The wooden frame work F F F F forms the roof, and is covered with thick thatch. G $\mathrm{H}$ is the wooden work of the door $\mathrm{x}$. At $\boldsymbol{K}$ the bucket is seen for lifting up a charge of ice, by means of the cord $\mathrm{J}$ passing over the pulley $\mathrm{B}_{3}$ which enables the servant to raise it easily.

The icehouse should have no window to admit light; but be, so to speak, hermetically sealed in every point, except at its cess-pool, which may terminate in a water trap to prevent circulation of air.

A clear day should be selected for charging the icehouse; but before beginning to fill, a quantity of long dry straw should be laid on the bottom crosswise; and as the ice is progressively introduced, straw is to be spread against the conical sides, to prevent the ice from coming into contact with the brick or stone work. The more firmly compacted the ict is, the better does it keep; with which view it should be broken into pieces with mallets before being thrown in. No layers of straw should be stratified among the ice, for they would make its body porous. Some persons recommend to pour in a little water with the successive layers of ice, in order to fill up its small crevices, and convert the whole into one mass.

Over the top layer a thick bed of straw should be spread, which is to be covered with boards surmounted with heavy stones, to close up the interstices in the straw. The inner and outer doors should never be opened at once; but the one should alway's be shut before the other is opened.

Dry snow well rammed keeps equally well with hard ice, if care be taken to leare no cavities in the mass, and to secure its compactness by sprinlling a little water upon the successive charges.

To facilitate the extraction of the ice, a ladder is set up against its sloping wall at one side of the door, and left there during the season.

JELI,Y, VEGETABLE, of ripe currants and other berries, is a compound of mucilage and acid, which loses its power of gelatinizing by prolonged ebullition.

JELLY, ANIMAL; see Gelatine, Glue, and Isinglass.

JET (Jaiet or jais, Fr.), a species of pitch-coal or glance-coal, which, being found abundantly in a beautiful compact form, in the valley of Hers, arrondissement of Pamiers, department of the Arriège, has been worked up extensively there from time immemorial, into a multitude of ornamental articles. With this black lignite, buttons, crosses, rosaries, necklaces, ear-drops, bracelets, waist-buckles, \&c. are made, which were at one time much worn by ladies for mourning dresses. The greater number of these ornaments are fashioned upon grind-stones which turn in a horizontal direction, and are kept continually wet; others are turned at the lathe, or shaped by files.

About 40 years ago this manufacture employed from 1000 to 1200 operatives ; at present it gives bread to only 60 . This falling off may be ascribed to the successful imitation of the jet articles by those of black glass, which are equally beautiful, and not nearly so apt to lose their polish by use.

IMPERMEABLE, is the epithet given to any kind of textile fabric, rendered water proof by one or other of the following substances;-

1. Linseed oil to which a drying quality has been communicated by boiling with litharge or sugar of lead, \&c.

2. The same oil holding in solution a little caoutchouc.

3. A varnish made by dissolving caoutchouc in rectified petroleum or naptha, applied between two surfaces of cloth, as described under Mackintosh's patent. See CAOUTchouc.

4. Vegetable or mineral pitch, applied hot with a brush, as in making tarpawling for covering goods in ships.

5. A solution of soap worked into cloth, and decomposed in it by the action of a solution of alum; whence results a mixture of acid fats and alumina, which insinuates itself among all the woolly filaments, fills their interstices, and prevents the passage of water.

6. A solution of glue or : singlass, intro luced into a stuff, and then acted upon by \& 
clear infusion of galls, whereby the fibres get impregnated with an insoluble, impermeable, pulverulent leather.

7. Plaster work is rendered impermeable by mixing artificial or natural asphaltum with it.

JEWELLERY, Art of. See GEM and LAPIDARY.

INCOMBUSTIBLE CLOTH is a tissue of the fibrous mineral called amianthus or asbestos. This is too rare to form the object of any considerable manufacture. Cotton and linen cloth may be best rendered incapable of taking fire, or burning with flame, by being imbued with a solution of sal ammoniac.

INCUBATION, ARTIFICIAL. The Egyptians have from time immemorial been accustomed to hatch egos by artificial warmth, without the aid of hens, in peculiar stoves, called Mammals. The inhabitants of the village Bermé still travel through the most distant provinces of Egypt at certain seasons of the year, with a portable furnace, heated by a lamp, and either hatch chickens for sale, or undertake to hatch the eggs belonging to the natives at a certain rate per dozen. M. de Reaumur published in France, about a century ago, some ingenious observations upon this subject; but $M$. Bonnemain was the first person who studied with due attention all the circumstances of artificial incubetion, and mounted the process successfully upon the commercial scale. So far back as 1777 he communicated to the Academy of Sciences an interesting fact, which he had noticed, upon the mechanism employed by chicks to break their shells; and for some time prior to the French revolution he furnished the Parisian market with excellent poultry at a period of the year when farmers had ceased to supply it. His establishment was ruined at that disastrous era, and no other has ever since been constructed or conducted with similar care. As there can be no doubt however of the practicability and profitableness of the scheme, when judiciously managed, I shall insert a brief account of his ingenious arrangements. I had the pleasure of making the acquaintance of this amiable old man at my first visit to Paris, many years ago, and believe all his statements to be worthy of credit. Some imitations of his plans have been made in this country, but how far they have succeeded, in an economical point of view, it is difficult to determine. His apparatus derives peculiar interest from the fact that it was founded upon the principle of the circulation of hot water, by the intestine motions of its particles, in a returning series of connected pipes; a subject afterwards illustrated in the experimental researches of Count Rumford. It has of late years been introduced as a novelty into this country, and applied to warm the apartments of many public and private buildings. The following details will prove that the theory and practice of hot water circulation were as perfectly understood by M. Bonnemain, fifty years ago, as they are by any of our stove-doctors at the present day. They were then publicly exhibited at his residence in Paris, and were afterwards communicated to the world at large in the interesting article of the Dictionnaire Technologique, entitled Incubation Artificielle.

The apparatus of M. Bonnemain consisted: 1. of a boiler and pipes for the circula tion of water; 2 . of a regulator calculated to maintain an equable temperature; 3 . of a stove-apartment, heated constantly to the degree best fitted for incubation, which he called the hatching pitch. He attached to one side a poussinière or chick-room, for cherishing the chickens during a few days after incubation.

The boiler is represented in vertical section and ground plan, in figs. 576 and $57 \%$. It is composed of a double cylinder of copper or cast-iron, $l l$, having a grate, $b$ (see

576

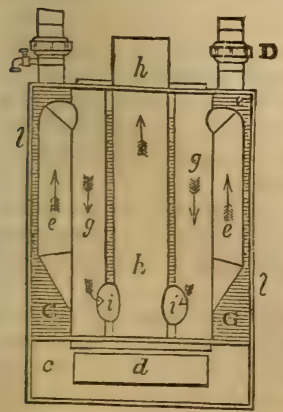
plan), an ashpit at $d$ (section). The water occupies the shaded space $\mathrm{c}, \mathrm{c}$. $h, g, g, e, e$, are five vertical flues, for conducting the burnt air and smoke, which first rise in the two exterior flues $e, e$, then descend in the two adjoining flues $g$, $g$, and finally re-mount through the passages $i, i$, in the central flue $h$. During this upwards and downwards circulation, as shown by the arrows in the section, the products of combustion are made to impart nearly the whole of their heat to the water by which they are surrounded. At the commencement, some burning paper or wood shavings are inserted at the orifice $m$, to establish a draught in this circuitous chimney. The air is admitted into the ashpit at the side, in regulated quantities, through a small square door, moveable round a rod which runs horizontally along its middle line. This swing valve is acted upon by an expanding bar (see HEAT-REGULATOR), which opens it more or less, according to the temperature of the stove apartment in which the eggs are placed.

D is the upper orifice of the boiler, by which the hotter and consequently lighter particles of the water continually ascend, and are replaced by the cooled particles, which enter the boiler near its bottom, as shown in fig. 578, at $\mathbf{R}$. Into further details relative to the boiler it is needless to enter; for though its form, as designed by $\mathbf{M}$. Bonnemain, 
is excellent and most economical of heat for a charcoal fire, it would not suii one of pitcoal. on account of the obstruction to the pipes which would soon be occasioned oy it coot.

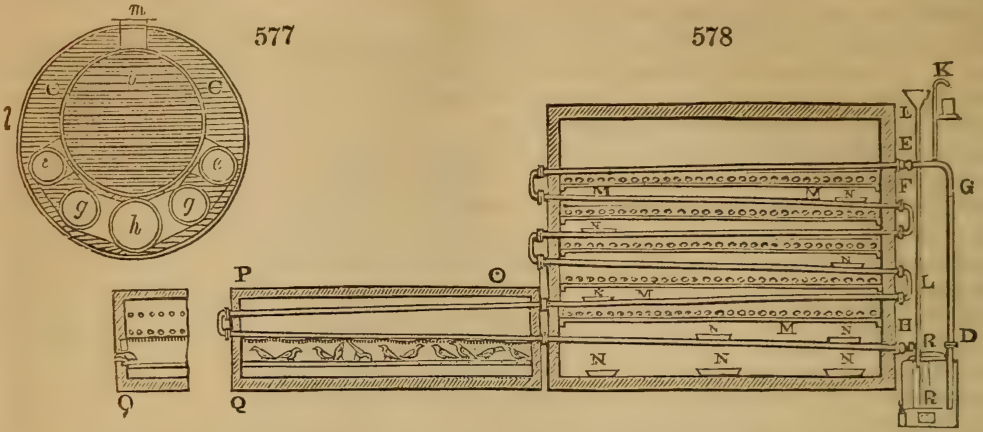

In fig. 578 the boiler is shown at $\mathbf{R}$, with the rod which regulates the air door of the ashpit. $\mathrm{D}$ is a stopcock for modifying the opening by which the hotter particles of water ascend; $\mathrm{G}$ is the water-pipe of communication, having the heating pipe of distrbution attached between E F, which thence passes backwards and forwards with a very slight slope from the horizontal direction, till it reaches the poussinière o $F \mathrm{Q}$. It traverses this apartment, and returns by $\mathrm{N} \mathrm{N}$ to the orifice of the boiler $\mathrm{H}$, where it turns vertically downwards, and descends to nearly the bottom of the boiler, discharging at that point the cooled and therefore denser particles of water, to replace those which continually issue upwards at $D$. L $\mathbf{R}$ is a tube surmounted with a funnel for keeping the range of pipes always full of water; and $\mathrm{K}$ is a syphon orifice for permitting the escape of the disengaged air, which would otherwise be apt to occupy partially the pipes and obstruct the aqueous circulation.

The faster the water gets cooled in the serpentine tubes, the quicker its circulation will be, because the difference of density between the water at the top and bottom of the boiler, which is the sole cause of its movement, will be greater. N represents small saucers filled with water, to supply the requisite moisture to the heated air, and to place the eggs, arranged along the trays $\mathrm{M} M$, in an atmosphere analogous to that under the body of the hen.

When we wish to hatch eggs with this apparatus, the fire is to be kindled in the boiler, and as soon as the temperature has risen to about $100^{\circ} \mathrm{F}$., the eggs are introduced; but only one twentieth of the whole number intended, upon the first day; next day, a like number is laid upon the trays, and thus in succession for twenty days, so that upon the twenty-first day the eggs first placed may be hatched, for the most part, and we may obtain daily afterwards an equal number of chicks. In this way, regularity of care is established in the rearing of them.

During the first days of incubation, natural as well as artificial, a small portion of the water contained in the egg evaporates by the heat, through the shell, and is replaced by a like quantity of air, which is afterwards useful for the respiration of the animal. If the warm atmosphere surrounding the eggs were very dry, such a portion of the aqueous part of the eggs would evaporate through the pores of the shells as would eildanger the future life of the chick in ovo. The transpiration from the body of the hen, as she sits upon her eggs, counteracts this desiccation in general; yet, in very dry weather, many hatching eggs fail from that cause, unless they be placed in moist decomposing straw. The water saucers $\mathrm{N} \mathrm{N}$ are therefore essential to success in artificial incubation.

After the chickens are hatched they are transferred into the nursery, $o$, on the front side of which there is a small grated trough filled with millet seed. Small divisions are made between the broods of successive days, to enable the superintendent to vary their feeding to their age.

In order to supply an establishment of the common kind, where 100 eggs are to be hatched daily, a dozen of hens would be needed, and 150 eggs must be placed under them, as only two thirds in general succeed. At this rate, 4300 mothers would be required to sit. Now supposing we should collect ten times as many hens, or 43,000 , we should not be able to command the above number of chickens, as there is seldom a tenth part of hens in a brooding state. Besides, there would be in this case no fewer than 720 hens every day coming out with a fresh brood of chickens, which would require a regiment of superintendents.

Artificial Incubation by means of Hot Mineral Waters.-This curious process is 
sescribed very briefly in a letter by M. D'Arcet. The following are extracts from this letter :-

'. In June, 1825, I obtained chickens and pigeons at Vichy, by artificial incubation, effected through the means of the thermal waters of that place. In 1827 I went to the baths of Chandes-Aigues, principally for the purpose of doing the same thing there. Finding the proprietor a zealous man, I succeeded in making a useful application of this source of heat to the production of poultry.

"The advantage of this process may be comprehended, when it is known that the invalids who arrive at Vichy, for instance in the month of May, find chickens only the size of quails; whereas, by this means, they may be readily supplied six months old.

"The good which may be done by establishing artificial incubation in places where hot springs exist is incalculable; it may be introduced into these establishments without at all interfering with the medical treatment of patients, since the hatching would go on in winter, at a time when the baths for other purposes are out of use.

"There is no other trouble required in breeding chickens, by means of hot baths, than to break the eggs at the proper time; for, when the apartments are closed, the whole of the interior will readily acquire a sufficiently elevated and very constant temperature."

In addition to these details by M. D'Arcet, a letter was received from M. Felgeris, the proprietor of the baths at Chaudes-Aigues (Cantal), in which he describes the success he had in following M. D'Arcet's process. This consists in putting the eggs into a small basket, suspending it in one of the stove-rooms heated by the hot mineral water, and turning round the eggs every day. The very first trial was attended with success, and no failure was experienced in four repetitions of it.

INDIGO. This invaluable blue dye-stuff, for which no tolerable substitute has been found, was known to the ancients as a pigment under the name of indicum, whence its present denomination. In modern Europe, it first came into extensive use in Italy, but, about the middle of the 16th century, the Dutch began to import and employ it in considerable quantities. Its general introduction into the dye-houses of both England and France was kept back by absurd laws, fuunded upon an opinion that it was a fugitive substance, and even prejudicial to the fibre of wool. See Dyeing, p. 419.

The plants which afford this dye-drug grow in the East and West Indies, in the middle regions of America, in Africa, and Europe. They are all species of the genera Indigofera, Isatis, and Nerium.

The following are cultivated:-Indigofera tinctoria affords in Bengal, Malabar, Madagascar, the Isle of France, and St. Domingo, an article of middling quality, but in large quantity. The indigofera disperma, a plant cultivated in the East Indies and America, grows higher than the preceding, is woody, and furnishes a superior dye stuff. The Guatimala indigo comes from this species. Indigofera Anil grows in the same countries, and also in the West Indies. The Indigofera Argentea, which grows also in Africa; it yields little indigo, but of an excellent quality. Indigofera Pseudotinctoria, which is cultivated in the East Indies, furnishes the best of all : the Indigofera Glauca is the Egyptian and Arabian species. There are also the carulea, cinerea erecta, hirsuta, glabra, and several others. The Nerium tinctorium of the East Indies affords some indigo; as does the Isatis tinctoria, or Woad, in Europe; and the Polygonum tinctorium.

The districts of Kishenagar, Jessore, and Moorshedabad, in Bengal, ranging from $88^{\circ}$ to $90^{\circ} \mathrm{E}$. L. and $22 \frac{1}{2}^{\circ}$ to $24^{\circ} \mathrm{N}$. L., produce the finest indigo. That from the districts about Burdwan and Benares is of a coarser or harsher grain. Tyroot, in lat. $26^{\circ}$, yields a tolerably good article. The portion of Bengal most propitious to the cultivation of indigo lies between the river Hoogly and the main stream of the Ganges.

In the East Indies, after having ploughed the ground in October, November, and the beginning of December, they sow the seed of the indigo plant in the last half of March and the beginning of April, while the soil, being neither too hot nor too dry, is most propitious to its germination. A light mould answers best; and sunshine, with occasional light showers, are most favorable to its growth. Twelve pounds of seeds are sufficient for sowing an acre of land. The plants grow rapidly, and will bear to be cut for the first time at the beginning of Ju'y, nay, in some districts, so early as the middle of June. The indications of maturity are the bursting forth of the flower buds, and the expansion of the blossoms; at which period the plant abounds most in the dyeing principle. Another indication is taken from the leaves; which, if they break across, when doubled flat, denote a state of maturity. But this character is somewhat fallacious, and depends upon the poverty or richness of the soil. When much rain falls, the plants grow too rapidly, and do not sufficiently elaborate the blue pigment. Bright sunshine is most advantageous to its production.

The first cropping of the plants is the best; after two months a second is made; after another interval, a third, and even a fourth; but each of these is of diminished value, There are only two croppings in America. 
Twn methods are pursued to extract the indigo from the plant ; the first effects it by fermentation of the fresh leaves and stems; the second, by maceration of the dried 'eaves; the latter process being most advantageous.

1. Frcm the recent leaves.-In the indigo factories of Bengal there are two large stone-built cisterns, the bottom of the first being nearly upon a level with the top of the second, in order to allow the liquid contents to be run out of the one into the other. The uppermost is called the fermenting vat, or the steeper; its area is 20 feet square, and its depth 3 feet; the lowermost, called the beater or beating vat, is as broad as the other, but one third longer. The cuttings of the plant, as they come from the field, are stratified in the steeper, till this be filled within 5 or 6 inches of its brim. In order that the plant, during its fermentation, may not swell and rise out of the vat, beams of wood and twigs of bamboo are braced tight over the surface of the plants, after which water is pumped upon them till it stands within three or four inches of the edge of the vessel. An active fermentation speedily commences, which is completed within 14 or 15 hours; a little longer or shorter, according to the temperature of the air, the prevailing winds, the quality of the water, and the ripeness of the plants. Nine or ten lours after the immersion of the plant, the condition of the vat must be examined; frothy bubbles appear, which rise like little pyramids, are at first of a white color, but soon become gray-blue, and then deep purple-red. The fermentation is at this time violent, the fluid is in constant commotion, apparently boiling, innumerable bubbles mount to the surface, and a copper-colored dense scum covers the whole. As long as the liquor is agitated, the fermentation must not be disturbed; but when it becomes more tranquil, the liquor is to be drawn off into the lower cistern. It is of the utmost consequence not to push the fermentation too far, because the quality of the whole indigo is deteriorated; but rather to cut it short, in which case there is, indeed, a loss of weight, but the article is better. The liquor possesses now a glistening yellow culor, which, when the indigo precipitates, changes to green. The average temperature of the liquor is commonly $85^{\circ} \mathrm{Fahr}$; its specific gravity at the surface is 1.0015 ; and at the bottom, $1 \cdot 003$.

As soon as the liquor has been run into the lower cistern, ten men are set to work to beat it with oars, or shovels 4 feet long, called busquets. Paddle wheels have also been employed for the same purpose. Meanwhile two other laborers clear away the compressing beams and bamboos from the surface of the upper vat, remove the exhausted plant, set it to dry for fuel, clean out the vessel, and stratify fresh plants in it. The fermented plant appears still green, but it has lost three fourths of its bulk in the process, or from 12 to 14 per cent. of its weight, chiefly water and extractive matter.

The liquor in the lower vat must be strongly beaten for an hour and a half, when the indigo begins to agglomerate in flocks, and to precipitate. This is the mument for judging whether there has been any error committed in the fermentation; which must be corrected by the operation of beating. If the fermentation has been defective, much froth rises in the beating, which must be allayed with a little oil, and then a reddish tinge appears. If large round granulations are formed, the beating is continued, in order to see if they will grow smaller. If they become as small as fine sand, and if the water clears up, the indigo is allowed quietly to subside. Should the vat have been over fermented, a thick fat-looking crust covers the liquor, which does not disappear by the introduction of a flask of oil. In such a case the beating must be moderated. Whenever the granulations become round, and begin to subside, and the liquor clears up, the beating must be discontinued. The froth or scum diffuses itself spontaneously into separate minute particles, that move about the surface of the liquor; which are marks of an excessive fermentation. On the other hand, a rightly fermented vat is easy to work; the froth, though abundant, vanishes whenever the granulations make their appearance. The color of the liquor, when drawn out of the steeper into the beater, is bright green; but as soon as the agglomerations of the indigo commence, it assumes the color of Madeira wine; and speedily afterwards, in the course of beating, a small round grain is formed, which, on separating, makes the water transparent, and falls down, when all the turbidity and froth vanish.

The object of the beating is three-fold: first, it tends to disengage a great quantity of carbonic acid present in the fermented liquor; secondly, to give the newly developed indigo its requisite dose of oxygen by the most extensive exposure of its particles to the atmosphere; thirdly, to agglomerate the indigo in distinct flocks or granulations. In order to hasten the precipitation, lime-water is occasionally added to the fermented liquor in the progress of beating, but it is not indispensable, and has been supposed eapable of deteriorating the indigo. In the front of the beater a beam is fixed upright, in which three or more holes are pierced a few inches in diameter. These are closed with plugs during the beating, but, two or three hours after it, as the indigo subsides, the upper plug is withdrawn to run off the supernatant liquor, and then the lower ylugs in succession. The state of this liquor being examined, affords an indication of 
the success of both the processes. When the whole liquor is run off, laborer enters the rat, sweeps all the precipitate into one corner, and empties the thinner part into a spont which leads into a cistern, alongside of a boiler, 20 feet long, 3 feet wide, and 3 deep. When all this liquor is once collected, it is pumped through a bag for retaining the impurities, into the boiler, and heated to ebullition. The froth soon subsides, and shows an vily looking film upon the liquor. The indigo is by this process not only freed from the "ellow extractive matter, but is enriched in the intensity of its color, and increased in weignt. From the boiler the mixture is run, after two or three hours, into a general receiver called the dripping vat, or table, which, for a factory of twelve pairs of preparation rats, is 20 feet long, 10 feet wide, and 3 feet deep; having a false bottom, 2 feet under the top edge. This cistern stands in a basin of masonry (made water tight with Chunam hrdraulic cement), the bottom of which slopes to one end, in order to facilitate the drainage. A thick woollen web is stretched along the bottom of the inner vessel, to act as a filter. As long as the liquor passes through turbid, it is pumped back into the receiver. Whenever it runs clear, the receiver is covered with another piece of cloth to exclude the dust, and allowed to drain at its leisure. Next morning the drained magma is put into a strong bag, and squeezed in a press. The indigo is then carefully takien out of the bag, and cut with a brass wire into bits, about 3 inches cube, which are dried, in an airy house, upon shelves of wicker work. During the drying, a whitish efflorescence comes upon the pieces, which must be carefully remored with a brush. In some places, particularly on the coast of Coromandel, the dried indigo lumps are allowed to effloresce in a cask for some time, and when they become hard they are wiped and packed for exportation.

From some experiments it would appear that the gas disengaged during the middle period of the fermentation is composed in 100 parts of $27 \cdot 5$ carbonic acid, $5 \cdot 8$ oxrgen, and $66 . \tau$ azote; and towards its end, of 40.5 carbonic acid, 4.5 oxygen, and 55.0 azote. The fermenting leaves apparently convert the oxygen of the atmosphere into carbonic acid gas, and leave its azote; besides the quantity of carbonic acid which they spontaneously evolve. Carbureted hydrogen does not seem to be disengaged. That the liquor in the beating vat absorbs oxygen from the air in proportion as the indigo becomes flocculent and granular, has been ascertained by experiment, as well as that sunshine accelerates the separation of the indigo blue. Out of 1000 parts of the fermented liquor of specific gravity 1.003, the blue precipitate may constitute 0.75 of a part. Such a proportion upon the great scale is however above the average, which is not more than 0.5 . When lime water is added, an extractive matter is thrown down, which amounts to from 20 to $4 \tau$ parts in 1000 of the liquor. It has a dark brown tint, a viscid appearance, an unpleasant smell, and a bitter taste. It becomes moist in damp air, and dissolves in water without decomposition. It is precipitated by lime, alkalis, infusion of galls, and acetate of lead. All indigo contains a little lime derived from the plant, even though none has been used in its preparation.

2. Indigo from dried leares.-The ripe plant being cropped, is to be dried in sunshine from 9 o'clock in the morning till 4 in the afternoon, during two dars, and thrashed to separate the stems from the leaves, which are then stored up in magazines till a sufficient quantity be collected for manufacturing operations. The newly dried leaves must be free from spots, and friable between the fingers. When kept dry, the leaves undergo, in the course of 4 weeks, a material change, their beautiful green tint turning into a pale bluegray, previous to which the leaves afford no indigo by maceration in water, but subsequently a large quantity. Afterwards the product becomes less considerable.

The following process is pursued to extract indigo from the dried leaves. They are infused in the steeping vat with six times their bulk of water, and allowed to macerate for two hours with continual stirring till all the floating leaves sink. The fine green liquor is then drawn off into the beater vat, for if it stood longer in the steeper, some of the indigo would settle among the leaves and be lost. Hot water, as emplored br some manufacturers, is not necessary. The process with dry leaves possesses this advantage, that a provision of the plant may be made at the most suitable times, independently of the vicissitudes of the weather, and the indigo may be uniformly made; and moreover, that the fermentation of the fresh leaves, often capricious in its course, is superseded by 8 much shorter period of simple maceration.

The process for obtaining indigo from the Nerium is altogether the same, but hot water has been generally applied to the dried leares. For woad, hot water must be employed, and also lime water as a precipitant, on account of the small proportion of indigo in the plant. Dilute muriatic acid is digested upon the woad indigo to remove the lime, without which no dye could be precipitated. According to the warmth of the summer and the ripeness of the plant, from 2 to 5 ounces of indigo may be obtained from 100 pounds of the dried woad, or upon an average 4 ounces to the hundred weight.

The indigo found in European commerce is imported from Bengal, Coromandel, Madras, the Mauritius, Manilla, and Jara in the Eastern hemisphere; from Senegal, 
Caraccas, Guatimala, Brazil (South Carolina and Louisiana in small quantity), and for. merly from the West India islands, especially St. Domingo. Its quality depends upon the species of the plant, its ripeness, the soil and climate of its growth, and mode of manufacture. The East Indian and Brazilian indigo comes packed in chests, the Guatimala in ox-hides, called surons.

The organ which affords the indigo is confined entirely to the pellicle of the leaves, and exists in largest quantity at the commencement of maturation while the plant is in flower. The indigofera is remarkable for giving a blue tinge to the urine of cows that feed upon its leaves.

According to some manufacturers, the plants should be cut down in dry weather, an hour or two before sunset, carried off the field in bundles, and immediately spread upon a dry floor. Next morning the reaping is resumed for an hour and a half, before the sun acts too powerfully upon vegetation; and the plants are treated in the same way. Both cuttings become sufficiently dry by 3 o'clock in the afternoon, so as to permit the leaves to be separated from the stems by thrashing. They are now thoroughly dried in the sunshine, then coarsely bruised, or sometimes ground to powder in a mill, and packed up for the operations of manufacture.

In the spring of $1830 \mathrm{I}$ subjected a variety of specimens of indigo to comparative analyses, by dissolving a few grains of each in strong sulphuric acid, diluting the solutions with an equal volume of water, and determining the resulting shade of color in a hollow prism of plate glass, furnished with a graduated scale. The following are the results, compared to the shade produced by a like weight of absolute indigo.

I. East India Indigos; prices as at the last October sales.

\begin{tabular}{|c|c|c|c|}
\hline No. & Price. & $\begin{array}{l}\text { Real Indigo } \\
\text { in } 100 \text { parts. }\end{array}$ & Characters by the Brokers. \\
\hline & s. $d$. & & \\
\hline $\begin{array}{l}1 \\
2\end{array}$ & $\begin{array}{ll}3 & 9 \\
3 & 6\end{array}$ & $\begin{array}{l}42 \\
56 \cdot 5\end{array}$ & $\begin{array}{l}\text { Broken, middling violet, and coppery violet spotted. } \\
\text { Ditto, a little being coppery violet and coner. }\end{array}$ \\
\hline 3 & $3 \quad 3$ & $46 \cdot 0$ & Ditto, middling red violet, dull violet and lean. \\
\hline 4 & 43 & $54 \cdot 5$ & Large broken, and square, even middling red violet. \\
\hline 5 & & $75 \cdot 0$ & $\begin{array}{l}\text { Much broken and very small, very crumbly and limy, soft, } \\
\text { good violet. }\end{array}$ \\
\hline 6 & 49 & $60 \cdot 0$ & $\begin{array}{l}\text { Square and large broken, } \frac{1}{2} \text { middling violet, and } \frac{1}{2} \text { good } \\
\text { coppery violet. }\end{array}$ \\
\hline 7 & 53 & $70 \cdot 0$ & Large broken, very good; paste a little limy, good violet. \\
\hline 8 & 66 & $60 \cdot 0$ & Square and large broken, soft, fine paste, fine violet. \\
\hline 9 & 60 & 66 롱 & Square, ditto, good red violet. \\
\hline 10 & 70 & 75 & Square, ditto, fine purple and blue. \\
\hline 11 & 23 & $37 \cdot 5$ & Middling ordinary Madras. \\
\hline 12 & 36 & $60 \cdot 0$ & Good Madras. \\
\hline 13 & 43 & $58 \cdot 0$ & Very fine ditto. \\
\hline 14 & 20 & & Low, pale Oude. \\
\hline 15 & 24 & $27 \frac{3}{4}$ & Middling, ordinary Oude. \\
\hline 16 & 33 & 54 & Good Oude. \\
\hline 17 & 19 & 29 & Lundy, very low quality. \\
\hline
\end{tabular}

II. American Indigos ; wholesale prices at present. (March, 1830.)

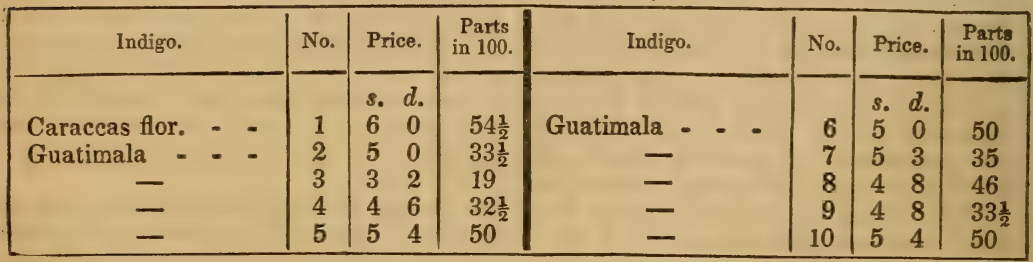

Properties of Indigo.-It possesses a dark blue color, passing into violet-purple, is void of taste and smell, dull, but by rubbing with a smooth hard body, it assumes the lustre and hue of copper. It occurs sometimes less and sometimes more dense apparently than water, which circumstance depends upon its freedom from foreign impurities, as well as upon the treatment of its paste in the boiling, pressing, and drying operations. It is insoluble in water, cold alcohol, ether, muriatic acid, dilute sulphuric acid, cold 
ethereous and fat oils; but boiling alcohol and oils dissolve a little of it, which they deposite on cooling. Creosote has the property of dissolving indigo.

Indigo is a mixture of several dye-stuffs, and other substances. Berzelius found in it a matter resembling vegetable gluten or: gliadine, a brown, red, and blue pigment, besides oxyde of iron, clay, lime, magnesia, and silica.

1. Indigo gluten or gliadine is dissolved along with the calcareous and magnesian salts by acids. If the powder be treated with cilute sulphuric acid, if the solution be saturated with carbonate of lime, evaporated to dryness, and its residuum treated with alcohol ; the solution thus formed leaves, after being evaporated, a yellow transparent extract, easily soluble in water, more difficultly in acid liquids; showing that acids extract only a portion of the gliadine from the indigo. It yields, by dry distillation, much ammonia, a fetid oil, and comports itself in other respects like vegetable gluten.

2. Indigo-brown occurs in combination with lime, as also with vegetable acid in considerable quantity, and more abundantly in the coarser sorts of indigo than in the finer. Indigo purified by acids is to be treated with hot strong canstic ley, which dissolves the indigo-brown; the liquid part of the mixture passes with difficulty through the filter, is black-brown, opaque, and holds some indigo-blue in solution, or diffused in fine powder. The alkali being neutralized with acetic acid, the liquor is to be evaporated, and alcohol poured on the residuum, whereby the alkaline acetate is dissolved out from the brown.

This pigment is a dark brown, almost black, but is not yet entirely ?eprived of the other constituents of indigo. It is nearly tasteless, is combustible, affords, by dry distillation, ammonia and fetid oil, forms with acids combinations hardly soluble in water, with alkalis soluble ones, but with earths hardly soluble. Lime possesses the property of precipitating the indigo-brown completely from its alkaline solution. Chlorine occasions a pale yellow brownish precipitate, which consists of indigo brown and muriatic acid, but causes no further change. By drying, it becomes again dark colored. Indigo-brown seems to exist also in woad.

3. Indigo-red, or more properly red resin of indigo. This may be obtained by boiling alcohol of sp. grav. $0 \cdot 830$ upon some indigo which has been previously treated with acids and alkalis; for the red substance is hardly soluble in cold alcohol. The solution is dark red, opaque, and leaves, by distillation, the indigo-red in the form of a black-brown powder, or a glistening varnish, slightly soluble in alcohol and ether. Alkalis do not dissolve it, but concentrated sulphuric acid forms with it a dark yellow dye, from which water causes no precipitation; wool extracts the color from the acid solution, and becomes of a dirty brown hue. Chlorine does not seem capable of destroying the color, for though it makes it yellow, it becomes as dark as ever on being dried. Indigo-red melts with heat, burns with a bright flame, affords, when heated in vacuo, first a white crystalline sublimate, and then unchanged indigo-red. That white matter is changed by nitric acid into indigo-red.

4. Indigo-blue, or pure indigo, remains, after treating the indigo of commerce with dilute acid, alkalis, and alcohol; it retains, however, still traces of the matters thereby extracted, along with some earthy substances. In order to procure indigo-blue in its jtmost purity, we must deoxydize the above blue residuum, thus form colorless indigo, which again acquires a blue color from the air, and constitutes the pure pigment. For this purpose the above moist indigo is to be mixed with slaked lime, green sulphate of iron, and hot water in an air-tight matrass. The indigo when deoxydized by protoxyde of iron being soluble in lime-wwater, the clear yellow solution is to be poured off, and exposed to the air. The indigo absorbs oxygen, and becomes again blue. By digestion with dilute muriatic acid the foreign matters are dissolved, and may then be washed away with distilled water, from the absolute indigo.

The indigo-blue obtained in this manner has a cast of purple red, displaying the characteristic copper lustre in a high degree, but in powder, it is blue. It is void of taste and smell, is by my experiments of specific gravity 1.50 , affords at $554^{\circ}$ Fahr. a purple vapor, and sublimes in shining purple scales, or slender needles in an apparatus open to the air, whereby, however, much of it is destroyed. Some carbon remains after the sublimation. A quick heat produces most sublimate. These needles contain a brown oily matter, which may be dissolved out by means of hot alco. hol. Their specific gravity is 1.35 , according to Mr. Crum. The sublimate from common indigo does not contain any oil, but some indigo-red and the above white crystalline matter. According to Mr. Crum, indigo-blue consists of carbon, 73.22; oxygen, 12.60 ; azute, 11.26 ; hydrogen, 2.92 ; while according to Dumas, crystallized indigo consists of carbon, 73.26 ; oxygen, 10.43 ; azote, 13.81 ; and hydrogen, 2.50 ; precipitated indigo consists of carbon, $74 \cdot 81$; oxygen, $7 \cdot 88$; azote, 13.98 ; and hydrogen, 3.33 ; sublimed indigo, of carbon, 71.71 ; oxygen, 12.18 ; azote, $13 \cdot 45$; hydrogen, 2.66 . My own analysis afforded-carbon, 71.37 ; oxygen, $14 \cdot 25$; azote, 10.00 ; hydrogen, 4.38 . In another analysis of Dumas, 3.93 parts of hydrogen were obtained. Hence we must infer that considerable differences exist in the composition of indigo in its purest state. Reagents 
act upon it much as upon common indigo. Chlorine, iodine, and bromine convert it into a reddish brown soluble substance. Concentrated sulphuric acid, especially the smoking or anhydrous of Nordhausen, dissolves indigo-blue with the disengagement of heat, but it makes it suffer some modification; for though it retains an interse dark blue color, it has become soluble in water, and may be blanched by light, which does not happen with indigo itself. Nitric acid destroys indigo-blue, forms indigotic (carbazotic) acid, carbonic acid, artificial resin, and bitter principle.

Indigo-blue may be reduced by substances oxydized, with the co-operation of alkalis or alkaline earths; for example, by such substances as have a strong affinity for oxygen, and are imperfeetly saturated with this principle, as the sulphurous and phosphorous acids and their salts, the protoxydes of iron and manganese, the protoxyde salts of $i$, and the corresponding compounds of chlorine, as the proto-chlorides of tin and iron; and the solution of the former in potash. When in these circumstances, in the presence of alkali, a deoxydation or reduction of the indigo-blue takes place, the other bodies get oxydized by absorption of the oxygen of the indigo-blue; the protoxydes become peroxydes, and the acids in ous become acids in $i c$, \&c. Several metallic sulphurets also reduce the indigo-blue in the same predicament, as the sulphurets of potassium, of calcium, of antimony, and of arsenic (orpiment.) A similar influence is exercised by fermenting vegetable substances, such as woad, madder, bran, raw sugar (molasses), starch, sirup, in consequence of the formation of carbonic and acetic acids, by absorption of the oxygen of the indigo-blue, for acetic acid and acetic salts are found in the liquor of the warm blue vat, in which indigo has been reduced by means of woad, madder, and bran.

Formation of colorless reduced indigo-blue, or indigotine.-Purified indigo-blie is to be treated with copperas and slaked lime, as above described; or the clear wine-yellow supernatant liquor of the cold blue-vat mixture is to be taken, run by a syphon into a matrass, a few drops of concentrated acetic or sulphuric acid, deprived of air, are to be poured into it, and the vessel, being made quite full, is to be well corked. The reduced indigo soon falls in white flocks, or crystalline scales. They must be edulcorated upon a filter with water deprived of its air by boiling, then pressed between folds of blottingpaper, and dried under the receiver in vacuo. Indigo-blue may likewise be reduced and dissolved by solution of hydro-sulphuret of ammonia; and the colorless indigotine may be precipitated by muriatic acid.

The reduced indigo is sometimes white at the instant of its elimination, sometimes grayish, of a silky lustre, but becomes very readily greenish, blue green, and blue, in the air ; in which case it absorbs, according to Berzelius, 4.2 per cent. of oxygen; but aecording to Liebeg, 11.5 per cent. It is void of taste and smell, is insoluble in water; well boiled water free from air is not affected by it, but is turned blue by common water. It dissolves in alcohol and ether into a yellow dye; not in dilute acids, but in concentrated sulphuric acid, whereby probably a portion of this is decomposed, and some hyposulphurous acid formed; the color of this solution is blue. Solutions of the caustic and carbonated alkalis, even the alkaline earths, readily dissolve reduced indigo into a wineyellow liquid; but in contact with air, oxygen is absorbed, and indigo-blue falls, while a purple-colored froth, passing into copper-red, appears upon the surface, just as in the indigo vats of the dyer.

The reduced indigo may be combined, by means of complex affinity, with other bases, with the exception of the oxydes of copper, zinc, and mercury, which oxydize it. These combinations are white, in part crystallizable, become speedily blue in the air, and afford by sublimation indigo-blue. Berzelius formed with lime a two-fold combination; one easily soluble in water, and another difficultly soluble, of a lemon color, which contained an excess of lime; this is formed both in the hot and the cold blue vat; in the latter it is occasioned by an overdose of lime.

When pure indigo-blue is treated with concentrated sulphuric acid, and particularly with six times its weight of the smoking $d r y$ acid, it dissolves completely, and several different compounds are produced in the solution. There is first a blue sulphate of indigo; secondly, a similar compound with the resulting hyposulphurous acid; thirdly, a combination of sulphuric acid with the purple of indigo (called Phænicin by Crum), a peculiar substance, generated from indigo-blue. These three compounds are here dissolved in an excess of sulphuric acid. The more concentrated the sulphuric acid is, the more blue hyposulphite is formed. The solution in smoking acid, when diluted with water and filtered, affords a considerable precipitate of indigo purple, which that in oil of vitriol does not. The vapor of anhydrous sulphuric acid combines with indigo-blue into a purple fluid.

In order to obtain from the dark blue solution each of these blue acids in a pure state, we must dilute it with forty times its weight of water, and immerse in the filtered liquor, well washed wool or flannel, with which the blue acids combine, while most of the sulphuric acid and some other foreign substances remain free in the liquor. The wool must be then scoured with water containing about a half per cent. of carbonate of ammonia, or potash, which neutralizes both of the blue acids, and produces a blue compound, 
This being evaporated to dryness at the temperature of $140^{\circ} \mathrm{F}$., alcohol of 0.833 is to be poured upon the residuum, which dissolves the blue hyposulphite, but leaves the blue sulphate undissolved. From either salt, by precipitating with acetate of lead, by acting upon the precipitate with sulphureted hydrogen water, and evaporation, either of the two blue acids may be obtained. They may be both evaporated to dryness, especially the blue sulphate of indigo; they both become somewhat moist in the air, they are very soluble in water, and the blue sulphate also in alcohol; they have a not unpleasant smell, and an acid astringent taste.

From these habitudes, particularly in reference to the bases, it appears that indigo-blue does not comport itself like a saline base towards the acids, but rather like an acid, since it enters into the salts, just as the empyreumatic oil of vinegar and oil of turpentine do into resin soaps. The blue pigment of both acids is reduced by zinc or iron without the disengagement of hydrogen gas; as also by sulphureted hydrogen, tepid protochloride of tin, while the liquor becomes yellow.

Indigo-blue sulphate of potash, or ceruleo-sulphate of potash, may be obtained by extracting the blue color from the wool by water containing 1 per cent. of carbonate of potash, evaporating nearly to dryness, treating the extract with alcohol to remove the indigo-blue hyposulphile, then with acetic acid and alcohol to remove any excess of carbonate of potash. It is found in commerce under the name of precipitated indigo, indıgo paste, blue carmine, and soluble indigo. To prepare it economically, indigo is to be dissolved in ten times its weight of concentrated sulphuric aeid; the solution after twenty-four hours is to be diluted with ten times its weight of water, filtered, and imperfectly saturated with carbonate of potash; whereby a blue powder falls down; for the resulting sulphate of potash throws down the ceruleo-sulphate, while the hyposulphite of potash remains dissolved. It is a dark blue copper shining powder, soluble in 140 parts of cold water, and in much less of boiling water. It is made use of as a dye, and to give starch a blue tint. When mixed with starch into cakes, it is sold under the name of blue for washerwomen.

Ceruleo-sulphate of ammonia may be formed in the same way. It is much more soluble in water. Ceruleo-sulphate of lime is obtained by saturating the above dilute acid with chalk, filtering to separate the undyed gypsum, and washing with water till the purple color be extracted. This liquor, evaporated and decomposed by alcohol, affords a bluish flocky precipitate, which is more soluble in water than common gypsum, and dries up in a purple-blue film. Ceruleo-sulphate of alumina may be obtained by double affinity; it is dark blue while moist, but becomes black-blue by drying, and is soluble in water.

The blue present in all these salts of ceruline is destroyed by sunshine, becomes greenishgray by caustic alkalis; and turns immediately yellow-brown by alkaline earths. But when the solution is very dilute, the color becomes first green, then yellow. The carbonates of alkalis do not produce these changes. Nitric acid decomposes the color quickly. $\mathrm{Mr}$. Crum considers ceruline to be a combination of indigo-blue with water.

Phenicine, or indigo-purple combined with sulphuric acid, is obtained when the solution of indigo-blue in concentrated sulphuric acid has been diluted for a few hours with water, and then filtered. It seems to be an intermediate body into which the indigo-blue passes, before it becomes soluble ceruline. Hence it occurs in greater quantity soon after digesting the indigo with the acid, than afterwards. It is dark blue, dissolves gradually in water, affords after evaporation a blue residuum, of the same appearance as the above blue acids. When a salt is added to it a purple precipitate ensues, which is a compound of indigo-purple, sulphuric acid, and the base of the salt. Indigo-purple is reduced by bodies having a strong attraction for oxygen, if a free alkali or alkaline earth be present, and its solution is yellow, but it becomes blue in the atmosphere. According to Mr. Crum, Phenicine contains half as much combined water as ceruline.

The table which I published in 1830 (as given above) shows very clearly how much the real quality and value of indigo differ from its reputed value and price, as estimated from external characters by the brokers. Various test or proof processes of this drug have been proposed. That with chlorine water is performed as follows. It is known that chlorine destroys the blue of indigo, but not the indigo-red or indigo-brown, which by the resulting muriatic acid is thrown down from the sulphuric solution in flocks, and the chlorine acts in the same way on the gliadine or gluten of the indigo. Pure indigo-blue is to be dissolved in 10 or 12 parts of concentrated sulphuric acid, and the solution is to be diluted with a given weight of water, as, for example, 1000 parts for 1 of indigo-blue. If we then put that volume of liquor into a graduated glass tube, and add to it chlorine water of a certain strength till its blue color be destroyed by becoming first green and then red-brown, we can infer the quantity of color from the quantity of chlorine water expended to produce the effect. The quantity of real indigo-blue cannot, however, be estimated with any accuracy in this way, because the other coloring matters in the drug act also upon the chlorine; and, indeed, the indigo itself soon changes when dissolved in 
sulphuric acid even out of access of light, while the chlorine water itself is very susceptible of alteration. Perhaps a better appreciation might be made by avoiding the sulphuric acid altogether, and adding the finely powdered indigo to a definite volume of the chlorine water till its color ceased to be destroyed, just as Prussian-blue is decolored by solution of potash in making the ferro-cyanide.

Another mode, and one susceptible of great precision, is to convert 10 or 100 grains of indigo finely powdered into its deoxydized state, as in the blue vat by the proper quantity of slaked lime and solution of green sulphate; then to precipitate the indigo. collect and weigh it. The indigo should be ground upon a muller along with the quick: lime, the levigated mixture should be diluted with water, and added to the solution of the copperas. This exact analytical process requires much nicety in the operator, and can hardly be practised by the broker, merchant, or manufacturer.

Employment of indigo in dyeing. - As indigo is insoluble in water, and as it can penetrate the fibres of wool, cotton, silk, and flax, only when in a state of solution, the dyer must study to bring it into this condition in the most complete and economical manner. This is effected either by exposing it to the action of bodies which have an affinity for oxygen superior to its own, such as certain metals and metallic oxydes, or by mixing it with fermenting matters, or, finally, by dissolving it in a strong acid, such as the sulphurie. The second of the above methods is called the warm blue, or pastel vat; and being the most intricate, we shall begin with it.

Before the substance indigo was known in Europe, woad haring been used for dyeine blue, gave the name of woad vats to the apparatus. The vats are sometimes made of copper, at other times of iron or wood, the last alone being well adapted for the employ. ment of steam. The dimensions are very variable; but the following may be considered as the average size : depth, $7 \frac{1}{2}$ feet; width below, 4 feet, above, 5 feet. The vats are built in such a way that the fire does not affect their bottom, but merely their sides half way up; and they are sunk so much under the floor of the dyehouse, that their upper half only is above it, and is surrounded with a mass of masonry to prevent the dissipatior of the heat. About 3 or $3 \frac{1}{2}$ feet under the top edge an iron ring is fixed, called the champagne by the French, to which a net is attached in order to suspend the stuffs out of contact of the sediment near the bottom.

In mounting the vat the following articles are required: 1 . woad prepared by fermen. tation, or woad merely dried, which is better, because it may be made to ferment in the vat, without the risk of becoming putrid, as the former is apt to do; 2 . indigo, previousi? ground in a proper mill; 3 . madder; 4 . potash; 5 . slaked quicklime; 6 . bran. Ir France, weld is commonly used instead of potash.

The indigo mill is represented in figs. 579 and 580. $a$ is a four-sided iron cister
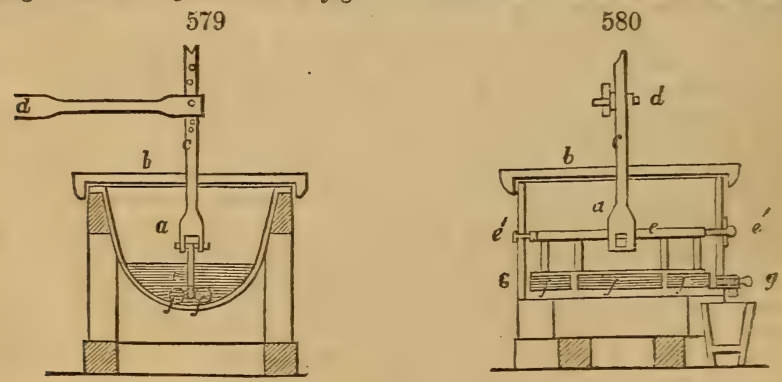

cylindrical or rounded in the bottom, which rests upon gudgeons in a wooden frame; it has an iron lid $b$, consisting of two leaves, between which the rod $c$ moves to and fro, receiving a vibratory motion from the crank $d$. By this construction, a frame $e$, which is made fast in the cistern by two points $e$ é, is caused to vibrate, and to impart its swing movement to six iron rollers $f f f$, three being on each side of the frame, which triturate the indigo mixed with water into a fine paste. Whenever the paste is uniformly ground, it is drawn off by the stopcock $\mathrm{g}$, which had been previously filled up by a serewed plug, to prevent any of the indigo from lodging in the orifice of the cock, and thereby escaping the action of the rollers. The cistern is nearly three feet long.

The vat being filled with clear river water, the fire is to be kindled, the ingredients introduced, and if fermented woad be employed, less lime is needed than with the merely dried plant. Meanwhile the water is to be heated to the temperature of $160^{\circ} \mathrm{Fahr}$, and maintained at this pitch till the deoxydizement and solution of the indigo begin to show themselves, which, according to the state of the constituents, may happen in 12 hours, or not till after several days. The first characters of incipient solution are blue 
bubbles, called the flowers, which rise upon the surface, and remain like a head of soapsuds for a considerable time before they fall; then blue coppery shining veins appear with a like colored froth. The hue of the liquor now passes from blue to green, and an ammoniacal odor begins to be exhaled. Whenever the indigo is completely dissolved, an acetic smelling acid may be recognised in the vat, which neutralizes all the alkali, and may occasion even an acid excess, which should be saturated with quicklime. The time for doing this cannot be in general very exactly defined. When quicklime has been added at the beginning in sufficient quantity, the liquor appears of a pale wine-yellow color, but if not, it acquires this tint on the subsequent introduction of the lime. Experience has not hitherto decided in favor of the one practice or the other.

As soon as this yellow color is formed in the liquor, and its surface becomes blue, the vat is ready for the dyer, and the more lime it takes up without being alkaline, the better is its condition. The dyeing power of the vat may be kept up during six months, or more, according to the fermentable property of the woad. From time to time, madder and bran must be added to it, to revive the fermentation of the sediment, along with some indigo and potash, to replace what may have been abstracted in the progress of dyeing. The quantity of indigo must be proportional, of course, to the depth or lightness of the tints required.

During the operation of this blue vat two accidents are apt to occur; the first, which is the more common one, is called the throwing back, in French, the cuve rebute, and in German, the Scharf or Schwartzwerden (the becoming sharp or black); the second is the putrefaction of the ingredients. Each is discoverable by its peculiar smell, which it is impossible to describe. The first is occasioned by the employment of too much quicklime, whereby the liquor becomes neutral or even alkaline. This fault may be recognised by the fading of the green, or by the dark green, or nearly black appearance of the liquor; and by a dull blue froth, owing to a film of lime. The remedy for a slight degree of this vicious condition, is to suspend in the liquor a quantity of bran tied up in a bag, and to leave it there till the healthy state be restored. Should the evil be more inveterate, a decoction of woad, madder, and bran must be introduced. Strong acids are rather Jetrimental. Sulphate of iron has been recommended, because its acid precipitates the anne, while its oxyde reduces the indigo to the soluble state.

The decomposition or putrefaction of the blue vat is an accident the reverse of the preceding, arising from the transition of the acetous into the putrid fermentation, whereby the dyeing faculty is destroyed. Such a misfortune can happen only towards the commencement of working the vat, whilst the woad is still powerful, and very little indigo has been dissolved. Whenever the vat is well charged with indigo, that accident cannot easily supervene. In both of these distemperatures the elevation of the temperature of the vat aggravates the evil.

Dyeing in the blue vat is performed as follows :-

Wool is put into a net, and pressed down into the liquor with rods; but cloth is smoothly stretched and suspended by hooks upon frames, which are steadily dipped into the vat, with slight motions through the liquor; yarn-hanks must be dipped and turned about by hand. All unnecessary stirring of the liquor must however be avoided, lest the oxygen of the atmosphere be brought too extensively into contact with the reduced indigo, for which reason mechanical agitation with rollers in the vat is inadmissible. The stuffs to be dyed take at the first dip only a feeble color, though the vat be strong, but they must be deepened to the desired shade by successive immersions of fifteen minutes or more each time, with intervals of exposure to the air, for absorption of its oxygen.

After the lapse of a certain time, if the fermentative power be impaired, which is recognised by the dye stuffs losing more color in a weak alkaline test ley than they ought, the vat should be used up as far as it will go, and then the liquor should be poured away, for the indigo present is not in a reduced state, but merely mixed mechanically, and therefore incapable of forming a chemical combination with textile fibres. If cotton goods preriously treated with an alkaline ley are to be dyed blue, the vat should contain very little lime.

Theory of the Indigo vat.-The large quantity of extractive matter in woad and madder, as also the sugar, starch, and gluten, in the bran and woad, when dissolved in warm water, soon occasion a fermentation, with an absorption of oxygen from the air, but especially from the indigo of the woad, and from that introduced in a finely ground state. When thus disoxygenated, it becomes soluble in alkaline menstrua; the red-brown of the indigo being dissolved at the same time. When lime is added, the indigo-blue dissolves, and still more readily if a little potash is conjoined with it; but whatever indigo-brown may have been dissolved by the potash, is thrown down by the lime. Lime in too large a quantity, however, forms an insoluble combination with the redaced indigo, and thus makes a portion of the dye ineffective; at the same time it combines with the extractive. In consequence of the fermentative action, carbonic acid, acetic acid, and ammonia are disengaged; the first two of which neutralize a portion of the lime, 
and require small quantities of this earth to be added in succession; hence also a con siderable quantity of the carbonate of lime is found as a deposite on the sides and bottom of the rat. In the sound condition of the indigo rat, no free lime should be perceived, but on the contrary a free acid. Yet when the disengaged carbonic and acetic acids saturate the lime completely, no indigo can remain at solution; therefore a sufficient supply of lime must always be left to dissolve the dye, otherwise the indizo would fall down and mix with the extractive matter at the bottom. Goods dyed in the blue vat are oceasionally brightened by a boil in a logwood bath, with a mordant of sulpho-muriate of tin, or in a bath of cudbear.

Another mode of mounting the indigo vat without woad and lime, is br means of madder, bran, and putash. The water of the rat is to be heated to the temperature of $122^{c}$ F.; and for 120 cubic feet of it, 12 pounds of indigo, 8 pounds of madder, and as much bran, are to be added, with 24 pounds of good potashes; at the end of 36 honrs, 12 pounds more of potash are introduced, and a third 12 pounds in other 12 hours. In the course of 72 hours, all the characters of the reduction and solution of the indigo become apparent; at which time the fermentation must be checked by the addition of quicklime. The liquor has a bright full color, with a beautiful rich froth. In feeding the vat with indigo, an equal weight of madder, and a double weight of potash, should be added. The odor of this vat, in its mild but actire state, is necessarily different from that of the woad rat, as no ammonia is exhaled in the present case, and the sediment is much smaller. The reduced indigo is held in solution br the carbonated potash, while the small addition of quicklime merely serres to precipitate the indigo-brown.

A potash vat dyes in about half the time of the ordinary warm vat, and penetrates fine cloth much better; while the goods thus dyed lose less color in allialine and soap solutions. This rat mar moreorer be kept with ease in good condition for severa] months; is more readily mounted; and from the minute proportion of lime present, it cannot impair the softness of the woollen fibres. It is merely a little more expensive. It is said that cloth dyed in the potash indigo rat, requires one third less soap in the washing at the fulling mill, and does not soil the hands after being dressed. At Elbeuf and Louviers, in France, such vats are much emplojed. Wool, silk, cotton, and linen, may all be dyed in them.

Cold rats. - The copperas or common blue vat of this country is so named because the indigo is reduced by means of the protoxyde of iron. This salt should therefore be as free as possible from the red oxyde, and especially from any sulphate of copper, which would re-oxydize the indigo. The necessary ingredients are: copperas green sulphate of iron), newly slaked quicklime, finely ground indigo, and water; to which sometimes a little potash or soda is added, with a proportional diminution of the lime. The operation is conducted in the following way : the indigo, well triturated with water or an alkaline les, must be mixed with hot water in the preparation rat, then the requisite quantity of lime is added, after which the solution of copperas must be poured in with stirring. Of this preparation rat, such a portion as may be wanted is laded into the dreing rat. For one pound of indigo three pounds of copperas are taken, and four pounds of lime (or 1 of indigo, $2 \frac{1}{2}$ of copperas, and 3 of lime). If the copperas be partially peroxidized, somewhat more of it must be used.

A rat containing a considerable excess of lime is called a sharp vat, and is not wel] adapted for dyeing. A soft rat, on the contrary, is that which contains too much copperas. In this case the precipitate is apt to rise, and to prevent uniformity of tint in the dyed goods. The sediment of the copperas rat consists of sulphate of lime, nxscde of iron, lime with indigo brown, and lime with indigo blue, when too much quicklime has been employed. The clear, dark wine sellow fluid contains indigo blue in a reduced state, and indigo red, both combined with lime and with the gluten of indigo dissolved. After using it for some time the vat should be refreshed or fed with copperas and lime, upon which occasion the sediment must first be stirred up, and then allowed time to settle again and become clear. For obtaining a series of blue tints, a series of vats of different strengths is required.

Linen and cotton yarn, before being dyed, should be boiled with a weak alkaline ley, then put upon frames or tied up in hanks, and after remoring the froth from the rat, plunged into and moved gently through it. For pale blues, an old, nearly exhausted vat is used; but for deep ones, a fresh, nearly saturated rat. Cloth is stretched upon a proper square dipping frame made of wood, or preferably of iron, furnished with sharp hooks or points of attachment. These frames are suspended by cords orer a pulley, and thus immersed and lifted out alternately at proper intervals. In the course of 8 or 10 minutes, the cluth is sufficiently saturated with the solution of indigo, after which it is raised and suspended so as to drain into the rat. The number of dippings determines the depth of the shade; after the last, the goods are allowed to dry, taken off the frame, plunged into a sour bath of very dilute sulphuric or muriatic acid, to remore the adhering lime, and then well rinsed in running water. Instead of the dipping frames, some 
dyers vse a peculiar roller apparaius, called gallopers, similar to what has been described under Calico Printing; particularly for pale blues. This cold vat is applicable to cotton, linen, and silk goods.

When white spots are to appear upon a blue ground, resist pastes are to be used, as described under Calico Printing.

The urine rat is prepared by digestion of the ground indigo in warmed stale urine, which first disoxygenates the indigo, and then dissolves it by means of its ammonia. Madder and alum are likewise added, the latter being of use to moderate the fermentation. This rat was employed more commonly of old than at present, for the purpose of dyeing woollen and linen goods.

The mode of making the China blue dye has been described under Calico PRINTING; as well as the pencil blue, or blue of application.

A blue dre may likewise be given by a solution of indigo in sulphuric acid. This process was discovered by Barth, at Grossenhayn, in Saxony, about the year 1740 , and is hence called the Saxon blue dye. The chemical nature of this process has been already fully explained. If the smoking sulphuric acid be employed, from 4 to 5 parts are sufficient for 1 of indigo; but if oil of vitriol, from 7 to 8 parts. The acid is to be poured into an earthenware pan, which in summer must be jaced in a tub of cold water, to prevent it getting hot, and the indigo, in fine powder, is to be added, with careful stirring, in small successire portions. If it becomes heated, a part of the indigo is decomposed, with the disengagement of sulphurous acid gas, and indigo green is produced. Whenever all the indigo has been dissolved, the vessel must be covered up, allowed to stand for 48 hours, and then diluted with twice its weight of clear river water.

The undiluted mass has a black blue color, is opaque, thick, attracts water from the air, and is called indigo composition, or chemic blue. It must be prepared beforehand, and kept in store. In this solution, besides the cerulin, there are also indigo-red, indigobrown, and gluten, by which admixture the pure blue of the dye is rendered foul, assuming a brown or a green cast. To remove these contaminations, wool is had recourse to. This is plunged into the indigo previously diffused through a considerable body of water, brought to a boiling heat in a copper kettle, and then allowed to macerate as it cools for 24 hours. The wool takes a dark blue dye by absorbing the indigo-blue sulphate and hyposulphite, while at the same time the liquor becomes greenish blue; and if the wool be left longer immersed, it becomes of a dirty yellow. It must therefore be taken out, drained, washed in running water till this runs off colorless, and without an acid taste. It must next be put into a copper full of water, containing one or two per cent. of carbonate of potash, soda, or ammonia (to about one third the weight of the indigo), and subjected to a boiling heat for a quarter of an hour. The blue salts forsake the wool, learing it of a dirty red-brown, and dye the water blue. The wool is in fact dyed with the indigo-red, which is hardly soluble in alkali. The blue liquor may now be employed as a fine dye, possessed of superior tone and lustre. It is called distilled blue and soluble blue. Sulphuric acid throws down from it the small quantity of indigo-red which had been held in solution by the alkali.

When wool is to be dyed with this sulphate of indigo-blue, it must be first boiled in alum, then treated with the blue liquor, and thus several times alternately, in order to produce a uniform blue color. Too long continuance of boiling is injurious to the beauty of the dye. In this operation the woollen fibres get impregnated with the indigoblue sulphate of alumina.

With sulphate of indigo, not only blues of every shade are dyed, but also green, olive, gray, as also a fast ground to logwood blues; for the latter purpose the preparatory boil is giren with alum, tartar, sulphates of copper and iron, and the blue solution; after which the goods are dyed up with a logwood bath containing a little potash.

\section{Statistical Tables of INdigo; per favor of James Wilkinson, Esq.,} of Leadenhall Street.

EAst India Indigo.

\begin{tabular}{|c|c|c|c|c|c|c|}
\hline Years. & Produce in India. & \begin{tabular}{|c} 
Consumption of \\
the World ; ave- \\
rage, 4 years.
\end{tabular} & $\begin{array}{l}\text { Stock in England } \\
\text { 31st December. }\end{array}$ & Highest Price. & $\underset{V}{\text { Good }}$ & $\begin{array}{l}\text { ddling } \\
\text { et. }\end{array}$ \\
\hline $\begin{array}{l}1811 \\
1812 \\
1813 \\
1814 \\
.1815 \\
1816\end{array}$ & $\begin{array}{l}\text { Chests. } \\
21,000 \\
23,500 \\
22,800 \\
28,500 \\
30,500 \\
25,000\end{array}$ & $\begin{array}{l}\text { Chests. } \\
22,200 \\
22,500 \\
22,800 \\
23,000 \\
23,200 \\
26,900\end{array}$ & $\begin{array}{l}\text { Chests. } \\
26,900 \\
29,500 \\
24,500 \\
24,900 \\
30,400 \\
25,700\end{array}$ & $\begin{array}{cc}\text { Per lb. } & \\
s . & d . \\
10 & 6 \\
11 & 6 \\
15 & 5 \\
13 & 0 \\
11 & 0 \\
10 & 0\end{array}$ & $\begin{array}{ll}\text { s. } & d . \\
5 & 6 \\
6 & 9 \\
9 & 0 \\
7 & 9 \\
6 & 9 \\
5 & 0\end{array}$ & $\begin{array}{ll}s . & d \\
6 & 0 \\
7 & 3 \\
9 & 6 \\
8 & 3 \\
7 & 0 \\
5 & 6\end{array}$ \\
\hline
\end{tabular}


East India Indigo.-Continued.

\begin{tabular}{|c|c|c|c|c|c|c|c|c|c|c|}
\hline \multirow{2}{*}{$\begin{array}{l}\text { Years. } \\
1817\end{array}$} & \multirow{2}{*}{$\frac{\text { Produce in India. }}{20,500}$} & \multirow{2}{*}{$\left|\begin{array}{c}\text { Consumption of } \\
\text { the World; ave- } \\
\text { rage, } 4 \text { years. }\end{array}\right|$} & \multirow{2}{*}{$\begin{array}{c}\text { Stock in England } \\
\text { slst December. }\end{array}$} & \multicolumn{3}{|c|}{ Highest Price. } & \multicolumn{4}{|c|}{$\begin{array}{l}\text { Good middling } \\
\text { Violet. }\end{array}$} \\
\hline & & & & & 10 & 0 & 7 & 3 & 7 & 9 \\
\hline 1818 & 19,100 & 26,500 & 24,000 & & 9 & 3 & 6 & 9 & 7 & 3 \\
\hline 1819 & 20,700 & 26,400 & 19,700 & & 8 & 6 & 5 & 6 & 6 & 0 \\
\hline 1820 & 27,200 & 24,200 & 14,500 & & 9 & 0 & 6 & 3 & 6 & 9 \\
\hline 1821 & 21,100 & 25,300 & 9,800 & & 11 & 6 & 8 & 6 & 9 & 0 \\
\hline 1822 & 25,700 & 26,000 & 8,200 & & 12 & 0 & 9 & 0 & 9 & 6 \\
\hline 1823 & 29,800 & 25,300 & 13,100 & & 10 & 0 & 7 & 3 & 7 & 9 \\
\hline 1824 & 24,100 & 26,500 & 12,200 & & 15 & 0 & 12 & 0 & 12 & 6 \\
\hline 1825 & 43,500 & 23,500 & 16,400 & & 15 & 6 & 12 & 0 & 12 & 6 \\
\hline 1826 & 28,000 & 27,300 & 22,300 & & 11 & 3 & 7 & 6 & 7 & 9 \\
\hline 1827 & 45,300 & 28,900 & 22,800 & & 12 & 6 & 8 & 0 & 8 & 6 \\
\hline 1828 & 30,000 & 31,000 & 31,100 & & 10 & 0 & 6 & 3 & 6 & 6 \\
\hline 1829 & 43,200 & 33,000 & 31,200 & Years & 8 & 9 & 5 & 3 & $\grave{5}$ & 9 \\
\hline $1830-1$ & 32,100 & 32,800 & 37,600 & 1831 & 7 & 9 & 4 & 3 & 4 & 9 \\
\hline 1831-2 & 32,500 & 34,500 & 35,700 & 1832 & 6 & 3 & 4 & 3 & 4 & 6 \\
\hline $1832-3$ & 35,200 & 35,500 & 32,500 & 1833 & 6 & 0 & 4 & 2 & 4 & 4 \\
\hline $1833-4$ & 27,100 & 34,600 & 35,800 & 1834 & 8 & 0 & 6 & 3 & 6 & 6 \\
\hline 1834-5 & 30,500 & 33,800 & 29,319 & 1835 & 7 & 0 & 5 & 3 & 5 & 6 \\
\hline $1835-6$ & 32,600 & 34,700 & 21,449 & 1836 & 6 & 3 & 4 & 9 & 5 & 0 \\
\hline $1836-7$ & - & 32,600 & 26,219 & $183 \tau$ & 8 & 9 & 6 & 9 & 7 & 0 \\
\hline
\end{tabular}

East India and Spanish, \&c. Indigo.

\begin{tabular}{|c|c|c|c|c|}
\hline \multirow{2}{*}{ Years. } & \multicolumn{2}{|c|}{ Importations. } & \multirow{2}{*}{ Exported. } & \multirow{2}{*}{ Home Consumption } \\
\hline & East India. & Spanish, \&c. & & \\
\hline & lbs. & lbs. & lbs. & lbs. \\
\hline 1785 & 154,291 & $1,539,218$ & $584,88 \tilde{5}$ & \\
\hline 1786 & 253,345 & $1,724,945$ & 466,696 & \\
\hline 1787 & 364,046 & $1,514,784$ & 502,800 & \\
\hline 1788 & 622,691 & $1,473,920$ & 445,857 & \\
\hline 1789 & 371,469 & $1,594,618$ & 673,630 & \\
\hline 1790 & 531,619 & $1,307,088$ & 821,131 & \\
\hline 1791 & 465,198 & $1,141,589$ & 870,185 & \\
\hline 1792 & 581,827 & $1,274,538$ & 880,951 & \\
\hline 1793 & 990,766 & $1,066,817$ & 929,707 & \\
\hline 1794 & $1,403,650$ & $1,487,642$ & $1,623,908$ & \\
\hline 1795 & $2,862,684$ & $1,424,941$ & $1,387,171$ & \\
\hline 1796 & $3,897,120$ & 680,915 & $1,883,320$ & \\
\hline 1797 & $1,754,233$ & 535,845 & $3,105,610$ & \\
\hline 1798 & $3,862,188$ & 192,060 & $1,718,624$ & \\
\hline 1799 & $2,529,377$ & 512,459 & $2,585,755$ & \\
\hline 1800 & $2,674,317$ & $1,076,417$ & $2,586,833$ & \\
\hline 1801 & $2,123,637$ & 827,696 & $2,281,812$ & \\
\hline 1802 & $2,264,199$ & 669,679 & $1,961,346$ & \\
\hline 1803 & $2,632,110$ & 522,825 & $1,130,194$ & \\
\hline 1804 & $2,765,871$ & 395,258 & $1,523,095$ & \\
\hline 1805 & $4,666,292$ & 687,319 & $1,845,035$ & \\
\hline 1806 & $2,612,181$ & 319,394 & $2,904,614$ & \\
\hline 1807 & $5,326,032$ & 715,809 & $2,006,463$ & \\
\hline 1808 & $5,314,860$ & 477,625 & $1,568,351$ & \\
\hline 1809 & $2,179,083$ & 674,048 & $3,179,861$ & • \\
\hline 1810 & $5,243,613$ & 883,061 & $2,485,679$ & \\
\hline 1811 & $4,453,932$ & 658.577 & $1,566,056$ & \\
\hline 1812 & $4,461,793$ & 354,171 & $1,853,916$ & \\
\hline 1813 & Accounts destr & oyed by Fire at & Custom House. & \\
\hline 1814 & $6,803,064$ & 328,881 & $5,501,851$ & $3,406,282$ \\
\hline 1815 & $5,543,852$ & 79,253 & $4,278,674$ & $2,774,091$ \\
\hline 1816 & $7,247,227$ & 39,275 & $4,214,454$ & $1,899,819$ \\
\hline 1817 & $5,001,280$ & 134,313 & $2,427,443$ & $2,377,659$ \\
\hline 1818 & $5,497,768$ & 187,257 & $2,963,462$ & $2,302,163$ \\
\hline
\end{tabular}


East India and Spanish, \&c. Indigo.-Continued.

\begin{tabular}{|c|c|c|c|c|}
\hline & \multicolumn{2}{|c|}{ Importations. } & Exported. & Home Consumption. \\
\cline { 2 - 3 } Years. & East India. & Spanish, \&c. & & \\
\cline { 2 - 3 } & $3,689,052$ & 129,682 & $3,126,739$ & $2,033,601$ \\
1819 & $4,924,222$ & 161,164 & $4,378,857$ & $2,288,196$ \\
1820 & $3,943,592$ & 119,517 & $2,985,364$ & $1,959,509$ \\
1822 & $2,549,284$ & 374,230 & $2,378,948$ & $2,004,062$ \\
1823 & $6,557,296$ & 664,408 & $2,783,504$ & $2,322,221$ \\
1824 & $4,595,707$ & 485,110 & $2,795,740$ & $2,493,350$ \\
1825 & $6,233,335$ & 560,296 & $3,870,929$ & $2,381,233$ \\
1826 & $7,699,439$ & 386,312 & $4,365,163$ & $1,901,047$ \\
1827 & $5,404,811$ & 662,936 & $3,315,675$ & $2,399,365$ \\
1828 & $9,683,626$ & 229,384 & $4,588,658$ & $3,064,915$ \\
1829 & $5,978,527$ & 769,757 & $4,286,605$ & $2,113,830$ \\
1830 & $7,920,924$ & 295,516 & $4,686,784$ & $2,676,945$ \\
1831 & $7,004,510$ & 290,089 & $4,374,241$ & $2,490,134$ \\
1832 & $6,221,725$ & 131,340 & $5,346,725$ & $2,395,653$ \\
1833 & $6,304,016$ & 331,016 & $3,664,814$ & $2,323,300$ \\
1834 & $3,798,144$ & 357,152 & $3,928,226$ & $2,447,827$ \\
1835 & $3,986,233$ & 183,480 & $4,074,598$ & $2,606,772$ \\
1836 & $6,753,898$ & 418,800 & $3,691,951$ & $2,864,274$ \\
1837 & $5,872,601$ & 673,270 & $3,587,561$ & $2,240,451$ \\
\hline
\end{tabular}

INDIAN RUBBER is the vulgar name of caoutchouc in this country.

INK (Encre, Fr. ; Tinte, Germ.) is a colored liquid for writing on paper, parchment, linen, \&c. with a pen.

Black ink.-Nutgalls, sulphate of iron, and gum, are the only substances truly useful in the preparation of ordinary ink; the other things often added merely modify the shade, and considerably diminish the cost to the manufacturer upon the great scale. Many of these inks contain little gallic aeid, or tannin, and are therefore of inferior quality. To make 12 gallons of ink, we may take-

12 pounds of nutgalls,

5 pounds of green sulphate of iron,

5 pounds of gum senegal,

12 gallons of water.

The bruised nutgalls are to be put into a cylindrical copper, of a depth equal to its diameter, and boiled, during three hours, with three fourths of the above quantity of water, taking care to add fresh water to replace what is lost by evaporation. The decoction is to be emptied into a tub, allowed to settle, and the clear liquor being drawn off, the lees are to be drained. Some recommend the addition of a little bullock's blood or white of egg, to remove a part of the tannin. But this abstraction tends to lessen the product, and will seldom be practised by the manufacturer intent upon a large return for his capital. The gum is to be dissolved in a small quantity of hot water, and the mucilage thus formed, being filtered, is added to the clear decoction. The sulphate of iron must likewise be separately dissolved, and well mixed with the above. The color darkens by degrees, in consequence of the peroxydizement of the iron, on exposing the ink to the action of the air. But ink affords a more durable writing when used in the pale state, because its particles are then finer, and penetrate the paper more intimately. When ink consists chiefly of tannate of peroxyde of iron, however black, it is merely superficial, and is easily erased or effaced. Therefore, whenever the liquid made by the above prescription has acquired a moderately deep tint, it should be drawn off clear into bottles, and well corked up. Some ink-makers allow it to mould a little in the casks before bottling, and suppose that it will thereby be not so liable to become mouldy in the bottles. A few bruised cloves, or other aromatic perfume, added to ink, is said to prevent the formation of mouldiness, which is produced by the ova of infusoria animalcules. I prefer digesting the galls to boiling them.

The operation may be abridged, by peroxydizing the copperas beforehand, by moderate calcination in an open vessel; but, for the reasons above assigned, ink made with such a sulphate of iron, however agreeable to the ignorant, when made to shine with gum and sugar, under the name of japan ink, is neither the most durable nor the most pleasant to write with.

From the comparatively high price of gall-nuts, sumach, logwood, and even 
oak bark, are too frequently substituted, to a considerable degree, in the manufacture of ink.

The ink made by the prescription given above, is much more rich and powerful than many of the inlis commonly sold. To bring it to their standard, a half more water may safely be added, or even 20 gallons of tolerable ink may be made from that weight of materials, as I have ascertained.

Sumach and logwood admit of only about one half of the copperas that galls will take to bring out the maximum amount of black dye.

Chaptal gives a prescription in his Chimie appliquée aux arts, which, like many other things in that book, are published with rery little knowledge and discrimination. He uses $\log$ wood and sulphate of copper, in addition to the galls and sulphate of iron; a pernicious combination, productive of a spurious fugitive black, and a liquor corrosive of pens. It is, in fact, a modification of the vile dye of the hatters.

Lewis, who made exact experiments on inks, assigned the proportion of 3 parts of galls to 1 of sulphate of iron, which, with average galls, will answer rery well; but good galls will admit of more copperas.

Gold ink is made by grinding upon a porphyry slab, with a mnller, gold leares along with white honer, till they be reduced to the finest possible division. The paste is then sollected upon the edge of a knife or spatula, put into a large glass, and diffused thrc agh water. The gold by gravity soon falls to the bottom, while the honer dissolres in the water, wnich must be decanted off. The sediment is to be repeatedly washed till entirely freed from the honey. The powder, when dried, is very brilliant, and when to be used as an ink, may be mixed up with a little gum water. After the writing becomes dry, it should be burnished with a wolf's tooth.

Silver ink is prepared in the same manner.

Indelible ink. - A rery good ink, capable of resisting chlorine, oxalic acid, and ablution with a hair pencil or sponge, may be made by mixing some of the inl made by the preceding prescription, with a little genuine China ink. It writes well. Many other formulæ have been given for indelible inks, but they are all inferior in simplicity and usefulness to the one now prescribed. Solution of nitrate of silver thickened with gum, and written with upon linen or cotton cloth, previously imbued with a solution of soda, and dried, is the ordinary permanent ink of the shops. Before the cloths are washed, the writing should be exposed to the sun-beam, or to bright daylight, which blacliens and fixes the oxyde of silver. It is easily discharged by chlorine and ammonia.

Red ink.-This ink may be made by infusing, for 3 or 4 dars in weak vinegar, Brazil wood chipped into small pieces; the infusion may be then boiled upon the wood for an hour, strained, and thickened slightly with gum arabic and sugar. A little alum improves the color. A decoction of cochineal with a little water of ammonia, forms a more beautiful red ink, but it is fugitive. An extemporaneous red ink of the same kind may be made by dissolving carmine in weak water of ammonia, and adding a little mucilage.

Green ink.-According to Klaproth, a fine ink of this color may be prepared by boiling a mixture of two parts of verdigris in eight parts of water, with one of cream of tartar till the total bulk be reduced one half. The solution must be then passed through : cloth, cooled, and bottled for use.

Yellow ink is made by dissolving 3 parts of alum in 100 of water, adding 25 parts of Persian or Arignon berries bruised, boiling the mixture for an hour, straining the liquor, and dissolving in it 4 parts of gum arabic. A solution of gamboge in water forms a convenient yellow ink.

By examining the different dye-stuffs, and considering the processes used in dyeing with them, a variety of colored inks may be made.

China ink.-Proust says, that lamp-black purified by potash ley, when mixed with a solution of glue, and dried, formed an ink which was preferred by artists to that of China. M. Merimée, in his interesting treatise, entitled, De la peinture à l'huile, says, that the Chinese do not use glue in the fabrication of their ink, but that they add vegetable juices, which render it more brilliant and more indelible upon paper. When the best lamp-black is levigated with the purest gelatine or solution of glue, it forms, no doubt, an ink of a good color, but wants the shining fracture, and is not so permanent on paper as good China ink; and it stiffens in cold weather into a tremulous jelly. Glue may be deprired of the gelatinizing property by boiling it for a long time, or subjecting it to a high heat in a Papin's digester; but as ammonia is apt to be generated in this way, M. Merimée recommends starch gum made by sulphuric acid (British gum) to be used in preference to glue. He gires, however, the following directions for preparing this ink with glue. Into a solution of glue he pours a concentrated solution of gall-nuts, which occasions an elastic resinous-looking precipitate. He washes this matter with hot water, and dissolves it in a spare s. ution of clarified glue. He filters 
anew, and concentrates it to the proper degree for being incorporated with the purified amp-black. The astringent principle in vegetables does not precipitate gelatine when its acid is saturated, as is done by boiling the nutgalls with limewater or magnesia. The first mode of making the ink is to be preferred. The lamp-black is said to be made in China, by collecting the smoke of the oil of sesame. A little camphor (about 2 per cent.) has been detected in the ink of China, and is supposed to improve it. Infusion of galls renders the ink permanent on paper.

Sympathetic ink. The best is a solution of muriate of cobalt.

Printer's ink. See this article.

By decomposing vanadate of ammonia with infusion of galls, a liquid is obtained of a perfectly black hue, which flows freely from the pen, is rendered blue by acids, is insoluble in dilute alkalis, and resists the action of chlorine. Whenever the metal vanadium shall become more abundant, as it probably may ere long, we shall possess the means of making an ink, at a moderate price, much superior to the tannate and gallate of iron.

To prepare the above vanadic salt cheaply, the cinder or hammerschlag obtained from the iron made at Ekersholm, in Sweden, or other iron which contains vanadium, being reduced to a fine powder, is to be mixed with two thirds of its weight of nitre, and one third of effloresced soda. The mixture is to be ignited in a crucible; cooled and lixiviated, whereby solutions of the vanadates of potash and soda are obtained, not pure, indeed, but sufficiently so for being decomposed, by means of sal ammoniac, into a vanadate of ammonia. This being rendered nearly neutral with any acid, constitutes an excellent indelible ink.

INULINE (Eng. and Fr.) is a substance first extracted from the root of the Inula-Hellenium, or Elecampane. It is white and pulverulent like starch; and differs from this substance chiefly because its solution, when it cools, lets fall the inuline unchanged in powder, whereas starch remains dissolved in the cold, as a jelly or paste.

Inuline is obtained by boiling the root sliced in 3 or 4 times its weight of water, and setting the strained decoction aside till it cools, when the pulverulent inuline precipitates. It exists also in the roots of colchicum, and pellitory.

IODINE (Iode, Fr.; Iod, Germ.) is one of the archæal undecompounded chemical bodies, which was discovered accidentally in 1812 by M. Courtois, a manufacturer of saltpetre, in the mother-waters of that salt. Its affinities for other substances are so powerful as to prevent it from existing in an insulated state. It occurs combined with potassium and sodium in many mineral waters, such as the brine spring of Ashby-de-laZouche, and other strongly saline springs. This combination exists sparingly in seawater, abundantly in many species of fucus or sea-weed, and in the kelp made from them; in sponges; in several marine molluscre, such as the doris, the venus, oysters, \&c.; in several polyparies and sea plants, as the gorgonia, the zostera marina, \&c.; particularly in the mother-waters of the salt-works upon the Mediterranean sea; and it has been found in combination with silver, in some ores brought from the neighborhood of Mexico.

Iodine is most economically procured from the mother-water of kelp, as furnished by those manufacturers of soap in Scotland and elsewhere who employ this crude alkaline matter. By pouring an excess of sulphuric acid upon that liquid, and exposing the mixture to heat in a retort, iodine rises in violet vapors (whence its name), and condenses in the receiver into black, brilliant, soft, scaly crystals, resembling graphite or plumbago. An addition of the peroxyde of manganese to the above mixture, favors the production of iodine. Souberain has proposed as a means of extracting it in greater abundance from a given quantity of the said mother-waters, to transform the iodide of potash or soda, present, into an insoluble iodide of copper, by pouring into them solution of sulphate of copper, which precipitates first of all one half of the iodine. He then decants the supernatant liquor, and adds to it a fresh quantity of the sulphate along with some iron filings. The latter metal seizes the oxygen and sulphuric acid of the cupreous salt, sets the copper free, which then seizes the other half of the iodine. To separate this iodide from the remaining iron filings, he agitates the whole with water, and decants the liquor. The filings immediately subside, but the iodide of copper remains for some time in a state of suspension. This compound, separated by a filter cloth, is to be mixed with twice its weight of the black peroxyde of manganese, and as much sulphuric acid as will make the mixture into a paste; which mixture being introduced into a retort, and distilled, the iodine comes over in its characteristic violet vapors, which are condensed into the glistening black substance in the receiver.

Iodine is always solid at atmospheric temperatures, though it slowly flies off with a peculiar offensive penetrating odor somewhat like chlorine. Its specific gravity is 4.946 at the temperature of $58^{\circ} \mathrm{Fahr}$. Its prime equivalent, according to Berzelius, is 
63.283 , one volume of hydrogen being 1.000 ; but 126.566 , if two volumes of hydrogen be reckoned unity, as most British chemists estimate it, from the composition of water. It possesses in a high degree electro-negative properties, like oxygen and chlorine; and therefore makes its appearance at the positive pole, when its compounds are placed in the voltaic circuit. It stains the skin yellow; and if applied for some time to it, is apt to produce painful ulcerations.

Iodine melts only at about $390^{\circ} \mathrm{Fahr}$.; but with the vapor of water it volatilizes at $212^{\circ}$ It has a great affinity for hydrogen, and constitutes by that union hydriodic acid; a compound resembling in some respects muriatic or hydrochloric acid. It also can be combined with oxygen, and forms thereby iodic acid. Its compounds with carbon, phosphorus, sulphur, chlorine, azote, and many metals, have not been applied to any manufacturing purpose, and therefore need not be described here.

The chief application of iodine in the arts, is for the detection of starch, which its watery solution, though containing only one part in 5000, does readily, by the production of a deep purple color; this vanishes by exposing the starch to the air for some time, or more quickly by heating it.

As a medicine, iodine and its compounds, such as the iodides of potassium and iron, are supposed to possess great powers in resolving glandular swellings. The periodide of mercury is a brilliant red pigment, but somewhat evanescent.

Chlorine, bromine, and iodine, are frequently associated; and it has hitherto been reckoned a difficult problem to separate them from one another. The following -lan is proposed by Mr. Lövig.

Heat the mixture of the dried chloride and bromide (or chloride and iodide) while a current of chlorine is made to pass over it, till no more bromine is carried off by the chlorine. Receive the gases in a solution of potash; saturate this fluid mixture of the chloride of potassium, and the chlorate and bromate of potash with nitric acid, adding afterwards nitrate of silver. A mixture of bromate and chloride of silver will precipitate. Dry the precipitate, calcine it, and calculate the proportion of bromine from the volume of oxygen gas now disengaged. It would be preferable to digest in a vial, the precipitate while moist, along with water of baryta, which decomposes the bromate of silver without acting upon the chloride. The excess of baryta being thrown down by carbonic acid, and the liquid being evaporated, a bromate of baryta is obtained, which may be washed with alcohol of 0.840 . The solution of bromate of baryta may also be neutralized by nitric acid, and the bromic acid may be precipitated by nitrate of silver. The same method is applicable to the separation of iodine from chlorine.

After throwing down the solution of the mixed salts by nitrate of silver, Berzelius digests the washed precipitate in a closed bottle of water of baryta; whence results bromate of baryta without any chloride of barium. On evaporating the liquor we obtain crystallized bromate of baryta, which may be freed from a small accidental quantity of chloride, by washing with alcohol at 0.840 . By calcination we then obtain bromide of barium, which, being distilled with sulphuric acid and peroxyde of manganese, affords bromine.

IRIDIUM is a metal discovered by Descotils in 1803, as also by Tenant in 1804 ; and is so called because its different solutions exhibit all the colors of the rainbow. It occurs only in the ore of platinum, being found there in two states; 1 . united to that metal, and 2. as alloy of osmium and iridium, in the form of small, insulated, hard grains. Iridium is the most refractory of all the metals; and appears as a gray metallic powder. It is not fused by the flame of the hydroxygen lamp.

IRON (Fer, Fr.; Eisen, Germ.) is a metal of a bluish-gray color, and a dull fibrous fracture, but it is capable of acquiring a brilliant surface by polishing. Its specific gravity is $7 \cdot 78$. It is the most tenacious of metals, and the hardest of all those which are malleable and ductile. It is singularly susceptible of the magnetic virtue, but in its pure state soon loses it. When rubbed it has a slight smell, and it imparts to the tongue a peculiar astringent taste, called chalybeate. In a moist atmosphere, iron speedily oxydizes, and becomes covered with a brown coating, called rust.

Every person knows the manifold uses of this truly precious metal; it is capable of being cast in moulds of any form; of being drawn out into wires of any desired strencth or fineness; of being extended into plates or sheets; of being bent in every direction; of being sharpened, hardened, and softened at pleasure. Iron accommodates itself to all our wants, our desires, and even our eaprices; it is equally serviceable to the arts, the sciences, to agriculture, and war; the same ore furnishes the sword, the ploughshare, the scythe, the pruning hook, the needle, the graver, the spring of a watch or of a carriage, the chisel, the chain, the anchor, the compass, the cannon, and the bomb. It is a medicine of much virtue, and the only metal friendly to the human frame.

The ores of iron are scattered over the crust of the globe with a beneficent profusion. groportioned to the utility of the metal; they are found under every latitude, and every 
zone ; in every mineral formation, and are disseminated in every soil. Considered in a purely mineralogical point of view, without reference to their importanee for reduction, they may be reckoned to be 19 in number; namely, 1. native iron of three kinds: pure, nickeliferous, and steely; 2 . arsenical iron; 3 . yellow sulphuret of iron; 4 . white sulphuret of iron; 5 . magnetic sulphuret of iron; 6 . black oxyde of iron, either the loadstone, or susceptible of magnetism, and titaniferous; 7. compact fer oligiste, specular iron ore, as of Elba, and scaly fer oligiste; 8 . hematite, affording a red powder; 9 . hematite or hydrate of iron, affording a yellow powder, of which there are several varieties; 10. pitchy iron ore; 11 . siliceo-calcareous iron, or yenite; 12 . sparry carbonate of iron, and the compact clay iron-stone of the coal formation; 13 . phosphate of iron; 14 . sulphate of iron, native copperas; 15 . chromate of iron; 16 . arseniate of iron; 17 . muriate of iron; 18. oxalate of iron; 19. titanate of iron.

Among all these different species, ten are worked by the miner, either for the sake of the iron which they contain; for use in their native state; or for extracting some principles from them advantageous to the arts and manufactures; such are arsenical iron, sulphate of iron, sulphuret of iron, and chromate of iron.

1. Native iron A. Pure.-This species is very rare, and its existence was long matter of dispute; though it has been undoubtedly found not only in volcanic formations, but in veins properly so called. It is not entirely like our malleable iron; but is whiter, more ductile, more permanent or less oxydizable in the air, and somewhat less dense. Among the best attested examples of pure native iron is that observed by M. Schreber, in the mountain of Oulle near Grenoble. The metal was entangled in a vein running through gneiss, and appeared in ramifying stalactites, enveloped in fibrous brown-oxyde of iron mixed with quartz and clay.

B. The native nickeliferous or meteoric iron is very malleable, often cellular, but sometimes compact, and in parallel plates, which pass into rhomboids or octahedrons. It is naturally magnetic, and by its nickel is distinguishable from terrestrial native iron. Macquart, in describing the famous mass found at mount Kemir in Siberia, says that the iron is perfectly flexible, and fit for making small instruments at a moderate heat; but in too strong a fire, the metal becomes short, brittle, and falls into grains under the hammer. Meteoric iron is covered with a sort of varnish which preserves its surface from the rusting action of the air; but this preservative property does not extend to the interior. Chladni has given a list of masses of meteoric iron, which have been known to fall at different times from the atmosphere, and of many specimens which indicate their atmospheric origin, by their aspect and composition. A portion of the mass of meteoric iron found at Santa-Rosa near Santa-Fe-de-Bogota, was made into a sword and presented to Bolivar.

c. Native steel-iron.-This substance has all the characters of cast-steel; it occurs in a kind of small button ingots, with a finely striated surface, and a fracture exceedingly fine grained. It is hardly to be touched by the file, and will scarcely flatten under the hammer. M. Mossier found this native steel at the village of Bouiche, near Nery, department of the Allier, in a spot where there had existed a seam of burning coal. A mass of 16 pounds and 6 ounces of native steel was discovered in that place, besides a great many small globules.

2. Arsenical iron, Arsenikkies, or Mispickel, is a tin-white mineral, which emits a garlic smell at the blowpipe, or even when sparks are struck from it by steel, accompanied with a small train of white smoke. It contains generally more or less sulphur, and sometimes a little silver, associated with metallic arsenic and iron.

3. Yellow sulphuret of iron, commonly called Marcasite, or Martial pyrites. The bronze or brass yellow color enables us to recognise this mineral. At the blowpipe it gives off its sulphur, and is converted into a globule attractable by the blowpipe. It is a bisulphuret of iron containing 32 of sulphur and 28 of metal.

Copper pyrites may be distinguished from it by its golden yellow color, which is frequently iridescent, and by its inferior hardness; for it does not strike fire with steel, like the preceding persulphuret. There is no vein, stratum, or mass of metallic ore which does not contain some iron pyrites; and it is often the sole mineral that fills the veins in quartz. It sometimes contains gold, and at other times silver.

4. White sulphuret of iron.-This is distinguishable from the preceding species only by is color and form of crystallization, and was hence till lately confounded with it by mineralogists. Its surface is often radiated.

5. Magnelic sulphuret of iron, the Magnetkies of the Germans.-This ore is attractable by the magnet like common iron. Its color is reddish-yellow, passing into brown; its fracture is rough. It consists of 16 of sulphur and 28 of iron.

6. Black oxyde of iron, magnet ore, or nalive loadstone.-One variety of this species has two poles in each specimen, which manifest a repulsive action against the corresponding poles of a magnetic needle. All the varieties furnish a black powder. Its external color is a gray approaching to that of metalic iron, but somewhat duller; 
with occasional iridescence of surface. Neither nitric acid nor the blowpipe has any action upon it. Its specific gravity varies from $4 \cdot 24$ to $5 \cdot 4$; and its constituents are $\mathbf{7 1} .86$ peroxyde, and 28.14 protoxyde, according to Berzelius; or in 100 parts, 71.74 of metallic iron, and 28.26 of oxygen. Assuming the prime equivalent of iron to be 28 , with the British chemists, then an ore consisting, like the above, of two prime proportions of peroxyde, and one of protoxyde, would be represented by the number $116=80$ +36 ; and would consist in 100 parts, of iron $72 \cdot 4$, oxygen $27 \cdot 6$.

Magnetic iron-ore belongs to primitive rock formations, and occurs abundantly in Sweden, Dalecarlia, Norway, Siberia, China, Siam, and the Philippine isles; but it is rare in England and France. It is worked extensively in Sweden, and furnishes an excellent iron.

The titaniferous oxyde of iron, or iron sand, is also attractable by the magnet. Its color is a deep black, with some metallic lustre; it is perfectly opaque; its fracture is conchoidal; it is hard and difficult to grind under the pestle into a dull black powder, which stains the fingers when it is very fine; it melts at a high heat into a black enamel without lustre. All volcanic rocks contain a greater or less quantity of titanic iron-ore, disseminated through them, which may be recognised by its brilliant metallic lustre, anit its perfect conchoidal fracture.

7. Fer oligiste, iron-glance, specular iron, and red iron ore.-This ore has the color of polished steel; and the light transmitted through the thin edges of its crystals appears of a beautiful red. Its powder is always of a well marked brown-red hue, passing into cherry-red, which distinguishes it from the black-oxyde ore. Its fracture is rongh, or vitreous in certain varieties; it breaks easily ; but it is hard enough to scratch glass. It usually contains from 60 to 70 of metallic iron in 100 parts; the equivalent proportion of oxygen in the pure red oxyde of iron being 30 parts combined with 70 of metal. It is a mistake to suppose any specular iron ore capable of yielding 85 per cent. of iron, for 100 parts of even protoxyde of iron contain only $77 \cdot 77$ parts of metal.

The compact variety comprises the crystals of the island of Elba, and of Framont in the Vosges, which have a rough-grained fracture. It exists in very great masses, constituting even entire mountains; in the cavities and fissures of these masses, the beautiful crystals so much prized by collectors of minerals, occur.

The island of Elba is equally celebrated for its in exhaustible abundance of rich specular iron ore, and for the immemorial antiquity of its mining operations. Fig. 581 is a vertical

581

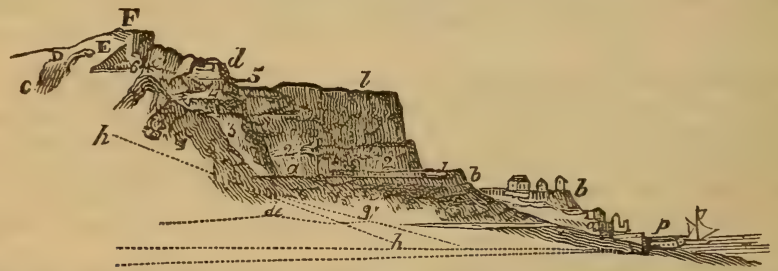

section passing through the three workings, called Pietamonte (D), Sanguinaccio (E), Antenna ( $\mathrm{F})$, through an ancient excavation $a$, through the coast 0 , and the mole $p$, ending at the canal of Piombino. The total height of the metalliferous mountain above the level of the sea, is no more than 180 metres, or 600 feet.

The rock which constitutes the body of this little mountain $d, l$, is called bianchetta by the workmen. It is a white slaty talc, slightly ochreous, or yellowish, consisting chiefly of silica and alumina, with some magnesia.

The ore of Antenna $(\mathrm{F})$ is a very hard compact fer oligiste, of a brilliant metallic aspect. The workable bed has a height of 66 feet, and consists of metalliferous blocks mixed confusedly with sterile masses of the rock; the whole covered with a rocky detritus, under a brownish mould. From its metallic appearance and toughness, this bed is called vena ferrata, the iron vein. In Pietamonte the workable bed is composed entirely of micaceous specular iron ore ( fer oligiste), with its fissures filled with yellow ochre. This bed rests upon the rock called bianchetta; the brilliant aspect of ore in this place has gained for it the name of vena lucciola.

The metalliferous hill $d l$, extends to the north-east, about a mile beyond the workings D E F. The ore contains about 65 per cent. of iron, and is smelted in Catalan forges.

The following description of the figure will make the structure of this extraordinary mine well understood. $a$, is a great excavation, the result of ancient workings.

1,$1 ; 2,2 ; 3,3,4,4,5,6$, and 7 , are roads for carrying off the rubbish, in correspond ence with the several working levels. 
$b, b, b$, masses of old rubbish (deblais).

$c$, $c$, ditto, from the present workings $\mathrm{D}, \mathrm{E}, \mathrm{F}$.

$d$, the rocky mass calied bianchetta, against which the ore extracted from $a$ abuts.

$e$, the surface of a bed of ore, near the streamlet $g$.

$f$, $f$, indication of beds of iron pyrites and fer oligiste.

$g$, a small rivulet proceeding from the infiltration of rains, and which is impregnated

with acidulous sulphate of iron.

$h, h$, ravine which separates the metalliferous hill $d l$ from the barren hill $i$.

$k$, masses of slags from ancient smelting operations; such are very common in this island. None of any consequence now exist; nearly the whole of the ore being exported to Tuscany, the Romagna, the Genoese territories, Piedmont, Naples, and Corsica.

$l$, a considerable body of rubbish from ancient workings, towards the summit of the metalliferous hill $d, l$.

$m, m$, part of this hill covered with rubbish, the result of old workings.

$n$, the site called Vigneria.

o, houses upon the shore called Marine de Rio, where the workpeople live, and the mineral is kept in store.

$p$, wooden pier (mole) whence the ore is shipped; terminated by a small tower, $q$.

Compact fer oligiste occurs also in the Vosges, in Corsica, at Altenberg and Freyburg, in Saxony, Presnitz, in Bohemia, Norberg and Bisberg, in Sweden, \&c.

The varieties called specular fer oligiste, and scaly fer oligiste, or iron-glance, do not differ essentially from the compact. None of them affects the magnetic needle, and their powder is a red of greater or less vivacity.

8. Red oxyde of iron.-The varieties included under this species afford a red powder, do not affect the magnetic needle, and are destitute of metallic lustre. At the blowpipe they all become black, or deep brown; and then they act on the needle. The crystallized rariety consists of 70 iron and 30 oxygen in 100 parts. The concretionary kind, or hematite, has a brown-red color; is solid, compact, and sometimes very hard; its surface may be filed and polished so as to acquire a lustre almost metallic; its internal structure is fibrous, and it exhibits sometimes a resemblance to splinters of wood. Its outer surface is constantly concretionary, mammelated, and presents occasionally sections of a sphere, or cylinders attached to each other. This is the blood-stone of the burnisher of metals. It is a very common mineral. The ochry variety or red-iron-ochre is distinguished from the solid hematite by the brightness of its color. It is used as a pigment.

9. Brown oxyde of iron, brown iron-stone.-This affords always a yellow powder, without any shade of red, which passes sometimes into the bistre brown, or velvet black. At the blowpipe this oxyde becomes brown, and very attractable by the magnet; but after calcination and cooling, the ore yields a red powder, which stains paper nearly as red as hematite does, and which is much employed in polishing metals. All the yellow or brown oxydes contain a large proportion of water, in chemical combination; and hence this species has been called hydrate of iron. There are several varieties which assume globular, reniform, stalactitic, and fruticose shapes. As impure varieties of the species we must consider some of the clay-iron ores, such as the granular, the common, the pisifurm, and the reniform clay-iron ore According to D'Aubuisson, the present specie: consists of peroxyde of iron, from 82 to 84 per cent.; water, 14 to 11 ; oxyde of manganese, 2 ; silica, 1 to 2 . It is therefore a hydrated peroxyde of iron; and ought by theory to consist, in its absolute state, of 81.63 peroxyde, and 18.37 water. It occurs both in beds and veins. The cetites or eagle-stones form a particular variety of this ore. On breaking the balls, so named, they are observed to be composed of concentric coats, the outside ones being very hard, but the interior becoming progressively softer towards the centre, which is usually earthy and of a bright yellow color; sometimes, however, the centre is quite empty, or contains only a few drops of water. Etites occur in abundance, often even in continuous beds in secondary mountains, and in certain argillaceous strata. These stones are still considered by the French shepherds as amulets or talismans, and may be found in the small bags which they suspend to the necks of their favorite rams; and they are in such general use, that a large quantity is annually imported into France from the frontiers of Germany, for this superstitious purpose. When smelted, they yield a good iron.

The variety called granular brown oxyds, or bone ore, is merely a modification of the preceding. It occurs in grains nearly round, varying in size from a millet seed to a pea, each being composed of concentric coats, hard outside and soft within. They are generally agglutinated by a calcareous or argillaceous paste; but are occasionally quite loose. This ore occurs in calcareous formations, and is sometimes accompanied with shells, such as terebratula. The brittle quality of the iron afforded by it has been ascribed to the phosphorus derived from the large quantity of organic bodies, with 
which the ore is frequently mixed. The bog-iron ore and swamp-iron ore belong to this species.

10. Pitchy hydrate of iron.-This is a rare mineral of a resinous aspect, found in a vein in the mine of Braunsdorf, two leagues from Freyberg, and seems to consist of red oxyde of iron and water. lime.

11. Yenite is a mineral species, rather rare, composed of red oxyde of iron, silica, and

12. Carbonate of iron, sparry iron, or brown-spar.-This important species has been divided into two varieties; spathose iron and the compact carbonate. The first has a sparry and lamellar fracture; with a color varying from yellowish-gray to isabella yellow, or even to brownish-red. It turns brown without melting at the blowpipe, and becomes attractable by the magnet after being slightly roasted in the flame of a candle. Even by a short exposure to the air, after its extraction from the mine, it also assumes the same brown tint, but without acquiring the magnetic quality. It affords but a slight effervescence with nitric acid, changing merely to a red-brown color. Its specific gravity varies from 3.00 to 3.67 . Its primitive form is like that of carbonate of lime, an obtuse rhomboid. Without changing this form, its crystals are susceptible of containing variable quantities of carbonate of lime, till it passes wholly into this mineral. Manganese and magnesia enter also occasionally into its composition.

Sparry carbonate of iron belongs to primitive formations; forming powerful veins in mountains of gneiss, and is associated in these veins with quartz, copper pyrites, gray copper, fibrous brown oxyde of iron, and a variety of ramose carbonate of lime, vulgarly called flos ferri. Thus it is found at Allevard and Vizille, near Grenoble, at SaintGeorge d'Huretière, in the Alps of Savoy ; at Baigorry, in the Lower Pyrenees ; at Eisenerz, in Styria; at Huttenberg, in Carinthia; at Schwartz, in the Tyrol; in Saxony, Hungary, other places in Germany, as also in Spain, Sweden, Norway, and Siberia. It also occurs, along with galena and other ores of lead, in the mines of Lead-Hills and Wanlockhead, in Scotland; and in the mines of Cumberland, Northumberland, and Derbyshire; likewise with tin-ore, at Wheal Maudlin, Saint-Just, and other places in Cornwall.

This ore, viewed as a metallurgic object, is one of the most interesting and valuable that is known; it affords natural steel with the greatest facility, and accommodates itself best to the Catalan smelting forge. It was owing in a great measure to the peculiar quality of the iron which it produces, that the excellence long remarked in the cutlery of the Tyrol, Styria, and Carinthia, was due. It was called by the older mineralogists steel ore.

The carbonate of iron of the coal formation, is the principal ore from which iron is smelted in England and Scotland, and it yields usually from 30 to 33 per cent. of cast metal. We are indebted to Dr. Colquhoun for several elaborate analyses of the sparryirons of the Glasgow coal field; ores which afford the best qualities of iron made in that district. The richest specimen, out of the nine which he tried, came from the neighborhood of Airdrie; it had a specific gravity of 3.0533 , and afforded in 100 parts, carbonic acid, $35 \cdot 17$; protoxyde of iron, $53 \cdot 03$; lime, 3.33 ; magnesia, 1.77 ; silica, $1 \cdot 4$; alumina, 0.63 ; peroxyde of iron, 0.23 ; carbonaceous or bituminous matter, 3.03 ; moisture and loss, $1 \cdot 41$. Its contents in metallic iron are $41 \cdot 25$.

The compact carbonate of iron has no relation xternally with the sparry variety. It comprehends most of the clay-iron stones, and particularly that which occurs in flattened spheroidal masses of various size, among the coal measures. The color of this ore is often a yellowish-brown, reddish-gray, or a dirty brick-red. Its fracture is close grained; it is easily scratched, and gives a yellowish-brown powder. It adheres to the tongue, has an odor slightly argillaceous when breathed upon, makes no effervescence with any acid, blackens at the blow-pipe without melting, and becomes attractable by the magnet with the slightest calcination.

This ore affords from 30 to 40 per cent. of iron of excellent quality; and it is the object of most extensive workings in Great Britain. It occurs in the slaty clay which serves as a roof or floor to the strata of coal; and also in continuous beds, from 2 to 18 inches thick, among the coal measures, as in Staffordshire, Shropshire, and Wales. It is remarkable, that the coal-basin of Newcastle contains little clay iron-stone, while the coal-basin of Dudley is replete with it.

13. Phosphate of iron.-A dull blue color is the most remarkable external character of this species, which occurs in small masses composed of aggregated plates, sometimes in an excessively fine powder, or giving other bodies a blue tinge. It assumes at the blowpipe a rusty hue, and is then reduced to a button of a metallic aspect. It dissolves completely in dilute nitric acid, as well as in ammonia, but it dues not communicale its color to them, and oil turns it black; characters which distinguish it readily from blue carbonate of copper, whose color is not altered by ammonia. It is of no use as a smelting ore. 
14. Sulphate of iron, native green vitriol.-This is formed by the oxygenation of sul phuret of iron, and is unimportant in a metallurgic point of view.

15. Chromate of iron.-For the treatment and use of this ore, see Снrome.

16. Arseniate of iron, Wurfelerz.

17. Muriate of iron.

18. Oxalate of iron; Humboldtite, found by M. Breithaupt in the lignite of Kolaw. It consists of protoxyde of iron, $53 \cdot 86$; oxalic acid, $46 \cdot 14$; in 100 .

19. Titanate of iron consists of protoxyde and peroxyde of iron, 86 ; titanic acid, 8; oxyde of manganese, 2 ; gangue, $1=97$. See Black Oxyde of iron.

Of the assay of iron-ores by fusion.-In the assays by the dry way, the object is to separate exactly all the iron which the ore may contain, with the view of comparing the result with the product of smelting on the great scale. In order to succeed in this operation, we must deoxydize the iron, and produce at the same time such a temperature as will melt the metal and the earths associated with it in the ore, and obtain the former in a dense button at the bottom of a crucible, and the latter in a lighter glass or slag, above it. Sometimes the gangue of the ores, consisting mostly of a single earth, as quartz, alumina, or lime, is of itself very refractory, and hence some flux must be added to bring about the fusion. The substance most commonly employed for this purpose is borax ; but ordinary flint glass may be substituted for it. Sometimes, also, instead of adding borax, which always succeeds, lime or clay may be added to the ore, according to the nature of its mineralizer; that is, lime for a clay iron-stone, and clay for a calcareous carbonate of iron; and both, when the gangue is silicious, as occurs with the black oxyde.

The ore, pulverized and passed through a silk sieve, is to be well mixed with the flux, and the mixture introduced into the smooth concavity made in the centre of a crucible lined with hard-rammed damp charcoal dust. Were the mixture diffused throngh the charcoal, the reduced iron would be apt to remain scattered in little globules through the crucible, and no metallic button would be formed at its bottom. The mingled ore and flux must be covered with charcoal. The crucible thus filled must be shut with an earthen lid luted on with fire-clay; and it is then set on its base, either in an air furnace, or on the hearth of a forge urged with a smith's bellows. The heat should be very slowly raised, not employing the bellows till three quarters of an hour have expired. In this way, the water of the damp charcoal (brasque) is allowed to exhale slowly, and the deoxydation is completed before the fusion begins; for by acting otherwise, the slags formed would dissolve some oxyde of iron, and the assay would not indicate the whole of the iron to be obtained from the ore. At the end of the above period, the fire must be raised progressively to a white heat, at which pitch it must be maintained for a quarter of an hour, after which the crucible should be withdrawn. Whenever it has cooled, it is to be opened, the brasque must be carefully removed or put aside, and the button of cast-iron taken out and weighed. The brasque may sometimes contain a few globules, which must be collected by washing in water, or the application of a magnetic bar. The quantity of iron denotes, of course, the richness of the ore. These assays furnish always a gray cast-iron; and, therefore, the quality of the products can hardly be judged of, except by an experiment on the large scale. The temperature necessary for the success of an assay is about $150^{\circ}$ of Wedgewood.

In the assays by the humid way, we may expect to find manganese, silica, alumina, lime, magnesia, and sometimes carbonic acid, associated with the iron. 100 grains of the ore in fine powder are to be digested with nitro-muriatic-acid; which will leave only the silica with perhaps a very little alumina. If an effervescence takes place in the cold with a dilute acid, the loss of weight will indicate the amount of carbonic acid gas expelled. The muriatic solution contains the iron, the manganese, the lime, magnesia, and most of the alumina, with a little silica. On evaporating to dryness, and digesting in water, all the silica will remain in an insoluble state. If the solution somewhat acidulated be treated with oxalate of ammonia, the lime will fall down in the form of an oxalate; ammonia will now precipitate the alumina and the oxyde of iron together, while the manganese and magnesia will continue dissolved in the state of triple salts (ammoniamuriates). The alumina may be separated from the ferric oxyde by potash-ley. The manganese may be thrown down by hydrosulphuret of potash; and, finally, the magnesia may be precipitated by carbonate of soda. 100 parts of the red oxyde of iron contain $69 \cdot 34$ of metal, and $30 \cdot 66$ of oxygen.

If phosphorus be present in the ore, the nitro-muriatic solution, being rendered nearly neutral, will afford with muriate of lime a precipitate of phosphate of lime, soluble in an excess of muriatic acid.

When the sole object is to learn readily the per-centage of iron, the ore may be treated with hot nitro-muriatic, the acid solution filtered and supersaturated with ammonia, which will throw down only the iron oxyde and alumina ; because the lime is not preeipitable by that alkali, nor is magnesia and manganese, when in the state of ammonia- 
muriates. The red precipitate, being digested with some potash-ley, will lose its alumina. and will leave the ferric oxyde nearly pure. The presence of sulphur, phosplioras, or arsenic, in iron ores, may always be detected by the blowpipe, or ustulation in the assay muffle, as described under FurNacE.

Of the smelting of iron ores.-We shall describe, in the first place, the methods practised in Great Britain, and shall afterwards consider those pursued in other countries, in the treatment of their peculiar ores.

Iron is divided into three kinds, according to the different metallic states in which it may be obtained; and these are called crude or cast iron; steel; and bar or mal'eable iron. These states are determined essentially by the different proportions of charcoal or carbon held in chemical combination; cast iron containing more than steel, and steel more than malleable iron; which last, indeed, ought to be the pure metal, a point of perfection, however, rarely if ever attained. It is impossible to assign the limits between these three forms of iron, or their relative proportions of carbon, with ultimate precision ; for bar iron passes into steel by insensible gradations, and steel and cast iron malie such mutual transitions as to render it difficult to define where the former commences, and the latter ceases, to exist. In fact, some steels may be called crude iron, and some cast irons may be reckoned among steels.

Towards the conclusion of the last century the manufacture of iron underwent a very important rerolution in Great Britain, by the substitution of pitcoal for charcoal of wood, the only combustible preriously used in smelting the ores of this metal. This improvement served not merely to diminish the cost of reduction, but it furnished a softer cast iron, fit for many new purposes in the arts. From this era, iron works have assumed an immense importance in our national industry, and have given birth to many ingenions and powerful machines for fashioning the metal into bars of every form, with almost incredible economy and expedition.

The profusion of excellent coal, and its association in many localities with iron-stone, have procured hitherto for our country a marked superiority over all otliers in the iron trade; though now every possible effort is making by foreign policy to rival or to limit our future operations. In 1802, M. de Bonnard, now divisionary inspector in the royal corps of mines of France, and secretary of the general council, made a tour in England, in order to study our new processes of manufacturing iron, and published, on his return, in the Journal des Mines, tom. 17, a memoir descriptive of them. Since the peace, many French engineers and iron-masters have exerted themselves in naturalizing in France this species of industry ; and M. de Gallois, in particular, after a long residence in Great Britain, where he was admitted to see deliberately and minutely every department of the iron trade, returned with ample details, and erected at Saint-Etieune a large establish. ment entirely on the English model. More recently, MM. Dufrénoy and Elie de Beaumont, and MIM. Coste and Perdonnet, have published two very copious accounts of their respectire metallurgic tours in Great Britain, illustrated with plans and sections of our furnaces, for the instruction of the French nation.

The argillaceous carbonate of iron, or clay iron-stone of the coal measures, is the chief ore smelted in England. Some red hematite is used as an auxiliary in certain works in Cumberland and Lancashire; but nowhere is the iron-sand, or other ferruginous matters of the secondary strata, employed at present for procuring the metal.

Among the numerous coal-basins of England there are two, in particular, which furnish more than three fourths of the whole cast iron produced in the kingdom; namely, the coal field of Dudley, in the south of Staffordshire; and the coal fields of Monmouthshire, in South Wales, along with those of Gloucestershire and Somersetshire.

Dudley is peculiarly farored by nature. There are found associated the coal, the iron ore, the limestone for flux, and the refractory fire-clay for constructing the interior brick-work of the furnaces. This famous clay is mined at Stourbridge, and exported to every part of the kingdom for making cast steel crucibles and glass-house melting pots.

At Merthyr-Tydvil, the centre of the iron-works of Wales, the iron-stone is extremely plentiful, forming 16 beds, or rather constituting an integrant portion of 16 beds of slate-clay. Sometimes it occurs in pretty long tables adjoining each other, so as to resemble a continuous stratum; but more frequently it forms nodules of various size and abundance, placed in planes both above and below the coal seam. Eight varieties of ore, belonging to different beds, have been distinguished by the following barbarous names : black balls, black pins, six-inch-wide vein, six-inch jack, blue vein, blue pins, gray pins, seven pins. The bed containing the first quality of iron-stone is analogous to the black ore of Staffordshire, called gubbin; it is often cleft within like septaria, and its cavities are sometimes besprinkled with crystals of carbonate of lime or quartz. In the superior beds there are nodules decomposing into concentric coats, of which the middle is clay. Crystals of oxyde of titanium are occasionally found in the middle of 
the balls of clay iron-stone; to which the metallic titanium observed in the inside of the dome of blast furnaces, may be traced. Both at Dudley and South Wales, casts of shells, belonging to the genus unio, are observed on the iron-stone.

The average richness of the iron-stones of South Wales is somewhat greater than that of those of Staffordshire. The former is estimated at 33 parts of cast iron, while the latter rarely exceeds 30 parts in 100 of ore ; and this richness, joined to the superior quality or cheapness of the coals, and the proximity of the sea, gives South Wales a decided advantage as a manufacturing district.

The number of blast furnaces in the parish of Merthrr-Tydril amounts to upwards of 30. The cast iron produced is, however, seldom brought into the market, but is almost entirely converted into bar iron, of which, at Mr. Crawshay's works, 600 tons are manufactured in a week. Numerous iron railways, extending through a length of 220 miles, facilitate the transport of the materials and the exportation of the products. That concurrence of farorable circumstances, which we have noticed as occurring at Dudley, prevails in an equal degree in South Wales.

The same economy which the use of coal has introduced into the smelting of cast iron from the ore, also extends to its refinery into bars. And this process would supersede in every iron work the use of wood charcoal, were not the iron produced by the latter combustible better for many purposes, particularly the manufaiture of steel. In some English smelting works, indeed, where sheet iron is prepared for making tin plate, a mixed refining process is employed, where the cast iron is made into bar iron by wood charcoal, and laminated by the aid of a coal fire.

Till 1740, the smelting of iron ores in England was executed entirely with wood charcoal; and the ores emplosed were principally brown and red hematites. Earthy iron ores were also smelted; but it does not appear that the clay iron-stones of the coal-basins were then used, though they constitute almost the sole smelting material at the present day. At that era, there were 59 blast furnaces, whose annual product was 17,350 tons of cast iron; that is, for each furnace, 294 tons per annum, and $5 \frac{1}{8}$ tons per week. By the year 1788 , several attempts had been made to reduce iron ore with colied coal; and there remained only 24 charcoal blast furnaces, which produced altogether 13,000 tons of east iron in the year; being at the rate of 546 tons for each per annum, or nearly 11 tons per week. This remarliable increase of 11 tons for $5 \frac{1}{8}$, was due chiefly to the substitution of cylinder blowing machines worled with pistons, for the common wooden bellows. Already 53 blast furnaces fired with coke were in actirity; which furnished in toto 48,800 tons of iron in a ycar; which raises the annual product of each furnace to $90 \tau$ tons, and the weelily product to about $17 \frac{1}{2}$ tons. The quantity of cast iron produced that year (1788) by means of coal, was and that by wood eharcoal, was

Constituting a total quantity of

$$
\div \quad-\quad-\quad-\quad=\quad-48,800 \text { tons, }
$$

61,900 tons.

In 1796 , the wood charcoal process was almost entirely given up; when the returns of the iron trade made by desire of Mr. Pitt, for establishing taxes on the manufacture afforded the following results :-

121 blast furnaces, furnishing in whole per annum 121,879 tons, constituting an arerage amount for eaeh furnace of 1032 tons.

In 1802, Great Britain possessed 168 blast furnaces, yielding a product of about 170,000 tons; and this product amounted, in 1806 , to 250,000 tons, derived from $22 \tau$ coke furnaces, of which only 159 were in activity at once. These blast furnaces were distributed 2. follows.

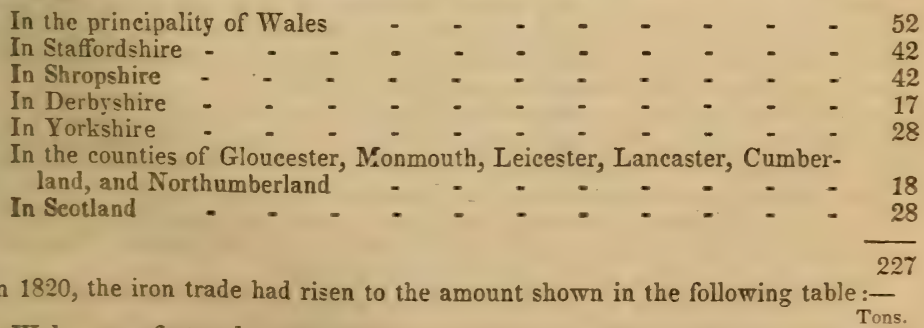

-Wales manufactured, per annum -

Shropshire and Staffordshire

Yorkshire and Derbyshire

Scotland, with some places in England - 
In a statistical view given by $M$. de Villefosse, of the French and English iron works he assigns to the latter, in 1826, 305 blast furnaces, distributed as follows :-

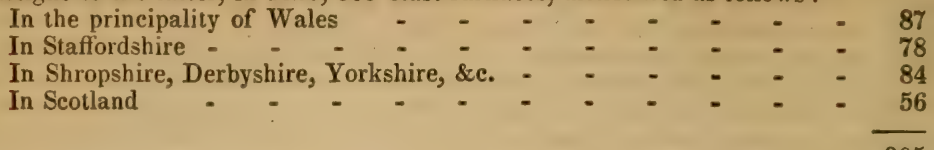

Out of these, 280 were in activity at the same time; and if we suppose their mean product to have been 50 tons a week, the total product would have been, in $1826,728,001$ ) tons. But this estimate seems to be somewhat above the truth; for, from the information communicated by Mr. Philip Taylor to M. Achille Chaper, a considerable French ironmaster, who, in the summer of 1826, inspected two thirds of the blast furnaces of Great Britain, their product during this year was about 600,000 tons.

The preceding details show the successive increments which the manufacture of cast iron has received; and a similar progression has taken place in its refinery into wrought iron. This operation was formerly effected by the agency of wood charcoal in refineries analogous to those still made use of in France. But when that kind of fuel began to be scarce in this island, it came to be mixed with coke in various proportions. The bar iron thus produced was usually hard, and required much time to convert, so that an establishment which could produce 20 tons of bar iron in a week, was deemed considerable. At that time, England imported annually from Sweden and Russia the enormous quantity of 70,000 tons of iron.

Mr. Cort, to whom Great Britain is indebted for the methods now pursued in this country, succeeded about that time, after many unsuccessful experiments, in converting cast iron into bar iron, by exposing it on the hearth of a reverberatory furnace to the flame of pitcoal. This method, which possessed the advantage of employing this species of combustible alone, likewise simplified the treatment, because it required no blast apparatus. But this mode of refinery, consisting in the use of a reverberatory furnace alone, did not produce altogether the desired result. It was irregular; sometimes the loss of iron was small, but at others it was very considerable; and there were great variations in the quality of the iron, as well as in the quantity of fuel consumed. Mr. Cort succeeded in removing this uncertainty of result, by causing the puddling in the reverberatory furnace to be preceded by a kind of refinery with coke. The intent of this operation was to decarburate the iron, and to prepare it for becoming malleable. The metal took in that case the name of finery metal, called, for the sake of brevity, fine-metal.

He also substituted the drawing cylinders for the extension under the hammer, an improvement which accelerated greatly the manufacture of bar iron. The iron then yielded by the operation of puddling was of a very inferior quality, and could not be directly employed in the arts. In order to give it more consistence, it was subjected to a secoud heating in a reverberatory furnace; and whenever this method had arrived at a high enough degree of perfection to afford products fit for the market, it became exclusively employed in Great Britain. This new method of transforming cast iron into malleable iron speedily gained such an extension, that of late years, a single iron-work, Cyfartha in Wales, manufactured annually more than twice as much as was made annually from 1740 to 1750 , in the whole kingdom.

In surveying the improvements which the iron manufacture has received in England in the space of the last 60 years, they are seen to be resolvable into two ; the first set relating to the smelting of the ores; the other, to the conversion of the pigs into bar iron; hence naturally arise two heads under which the subject of iron must be treated.

1. Manufaclure of cast-iron by coke and coal.-The cast-iron produced by the English and Scoteh blast furnaces is in general black and very soft; but yet may be distinguished into several qualities, of which three are particularly noticed.

No. 1. Very black cast-iron, in large rounded grains, obtained commonly near the commencement of the casting, when an excess of carbon is present; in flowing, it appears pasty, and throws out blue scintillations. It exhibits a surface where crystalline vegetations develop themselves rapidly in very fine branches; it congeals or fixes very slowly; its surface when cold is smooth, concave, and often charged with plumbago ; it has but a moderate tenacity, is tender under the file, and susceptible of a dull polish. When melted over again, it passes into No. 2 , and forms the best castings.

No. 2. Black cast-iron has a somewhat lighter shade than the preceding, and may therefore on comparison be called blackish-gray. It presents less large granulations than No. 1 ; is tenacious, easily turned, filed, and polished; excellent for casting when it ap. proaches to No. 1 , and for the manufacture of bar iron when it has on the contrary a shade somewhat lighter. If repeatedly melted, it passes into the next quality, or

No. 3. White cast iron; this is brittle, and indicates always some derangement in the 
working of the furnace; it flows imperfectly, and darts out, in casting, abundance of brilliant white scintillations; it fixes very quickly; and on cooling, exhibits on its surface irregular asperities, which make it extremely rough. It is easily broken, and presents a lamellar and radiated fracture; and is so hard that tempered steel cannot act upon it. It is cast only into weights, bullets, or bombs, but never into pieces of machinery. When exposed to the refinery processes, it affords a bad bar iron. It is owing probably to the different nature of the cast iron obtained in different counties in England, that Staffordshire and Shropshire furnish the greater part of the great iron castings, while Wales manufactures almost exclusively malleable iron. The lower price of coals in Wales is perhaps the cause to a certain extent of this difference in the results of these two iron districts. It will be interesting, at any rate, to describe separately the processes employed in Staffurulshire and Wales.

The blast furnaces of Staffordshire, in the neighborhood of Dudley, Bilston, and Wednesbury, are constructed almost wholly of bricks. Their outer form is frequently a cone, often also a pyramid with a square base. They are bound about with a great many iron hoops, or with iron bars placed at different heights. This powerful armor allows the furnaces to be built much less massively than they formerly were; and admits lighter and more elegant external forms. They are seldom insulated; but are usually associated to the number of two or three in the same line. A narrow passage is left between them, which leads to the lateral openings where the tuyères are placed. At the

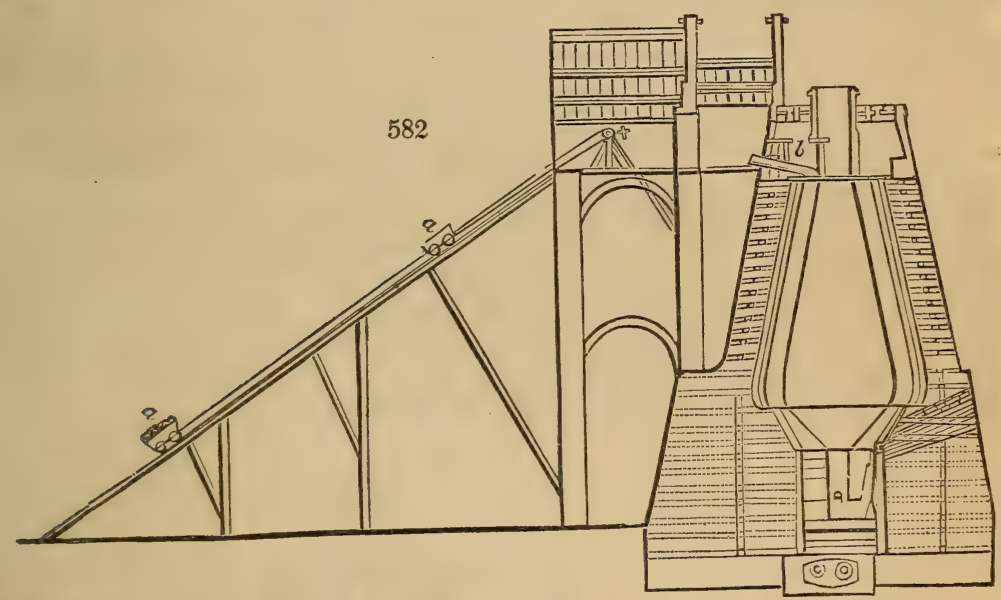

front of the furnace, a large shed is always raised. The roofs of these sheds present in general circular profiles, and being made of cast or bar iron, they display a remarkable lightness of construction. The east iron columns likewise, which support the joists and girders, give additional elegance.

In the Dudley field, the furnaces are almost always in the middle of the plain, and an inclined rail-way must be formed to reach their platform. These inclined planes, composed of beams or rails placed alongside of each other, and sustained by props and crossbars, as indicated in fig. 582, are set up mostly against the posterior face of the furnace. Two chains or ropes, passing over the drums of gins, moved by a steam engine (commonly the same that drives the bellows), draw up the wagons of wood or sheet iron $a$ a, which contain the various materials for supplying the furnace. To facilitate this service, the platform round the furnace is sometimes enlarged behind by a floor; while a balustrade, which opens when the wagons arrive at the platform, prevents accidents. This projection is occasionally covered by a roof. For a furnace of the largest size, the force expended by this lifting apparatus is not more than a two-horse power.

Fig. 582 is a vertical section through the furnace from front to rear, or at right angles to the line of the lateral tuyeres. The erection of a pair of blast furnaces, of 40 feet high each, costs, in the Dudley district, 1800 pounds sterling; and requires for building each, 160,000 common bricks for the outside work, 3900 fire-bricks for the lining or shirt of the furnace, and 825 for the boshes. The dimensions of the fire-bricks are various; 5 kinds are employed for the lining, and 9 kinds for the boshes. They are all 6 inches thick, and are curved to suit the voussoirs.

The number of charges given in 12 hours is different in different furnaces; being sometimes 20, 25, and even so high as 40 ; but 20 is a fair average. Each charge is 
composed of from 5 to $6 \mathrm{cwts}$. of coke, (or now of 3 to $4 \mathrm{cwts}$. of coal with the hot blast); 3 , 4 , and sometimes $6 \mathrm{cwts}$. of the roasted mine, according to its richnass and the quality of cast iron wanted; the limestone flux is usually one third of the weight of the roasted iron stone. There are 2 casts in 24 hours; one at 6 in the morning, and another at 6 in the evening.

The height of the blast furnaces is very variable; some being only 36 feet high including the chimney, while others have an elevation of 60 feet. These extreme limits are very rare: so that the greater part of the furnaces are from 45 to 50 feet high. They are all terminated by a cylindrical chimney of from 8 to 12 feet long; being about one fifth of the total height of the furnace. The inside diameter of this chimney is the same as that of the throat or mouth; and varies from 4 to 6 feet. The chimney is frequently formed of a single course of bricks, and acciuires solidity from its hoops of iron, so thickly placed that one half of the surface is often covered with them. At its lower end, the mouth presents one or two rectangular openings, through which the charge is given. It is built on a basement circle of cast-iron, which forms the circumference of the throat; and a sloping plate of cast-iron $b$ is so placed as to make the materials slide over into the furnace, as shown in the figure.

The insile of the blast furnaces of Staffordshire is most frequently of a circular form, except the hearth and working area. The inner space is divided into four portions, different in their forms, and the functions which they fulfil in the smelting of the ore.

The undermost, called the hearth, or crucible, in which the cast-iron collects, is a right rectangular prism, elongated in a line prependicular to the axes of the turères. The sides of the hearth consist in general of refractory sandstone (fire-stone), obtained mostly from the bed of the coal basin, called millstone grit ; and the bottom of the hearth is formad of a large block of the same nature, laid on a cast-iron plate.

The second portion is also made of the same refractory grit stone. It has the form of quadrangular pyramidal, approaching considerably to a prism, from the smallness of the angle included between the sides and the axis.

The third portion or lower body of the furnace is conical, but here the interior space suddenly expands; the slope outwards at this part seems to have a great influence on the quality of the cast-iron obtained from the furnace. When No. 2 of the blackest kind is wanted for castings, the inclination of this cavity of the furnace is in general less considerable than when No. 2 cast iron for conversion into bar iron is required. The inclination of this conical chamber, called the boshes, varies from 55 to 60 degrees with the zorizon. The diameter of this part is equal to that of the belly, and is from 11 to 13 feet. The boshes are built of masonry, as shown in figs. 583, 584 .

583

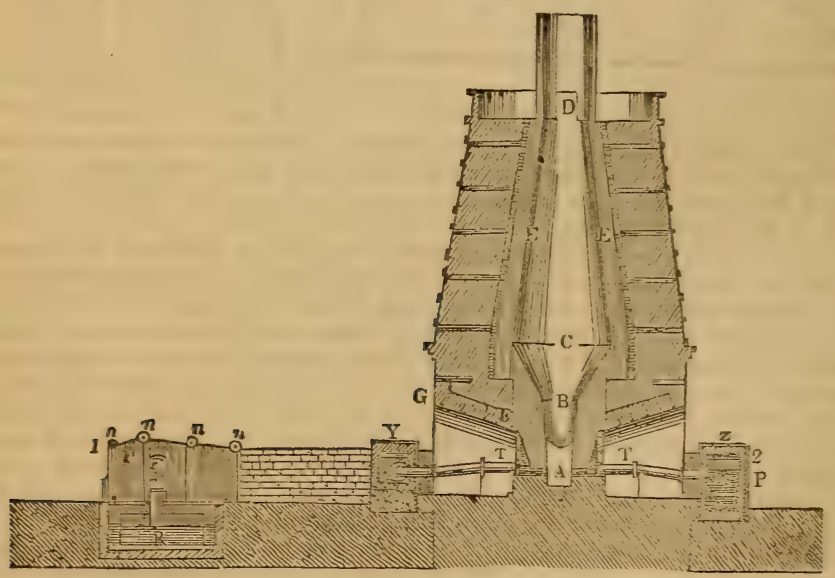

The fourth part, which constitutes about two thirds of the height of the furnace from the base of the hearth up to the throat, presents the figure of a surface of revolution, generated by a curve whose concavity is turned towards the axis of the furnace, and vhose last tangent towards the bottom is almost vertical. This surface is sloped off with that of the boshes (étalages in French), so that no sharp angle may exist at the belly. In some furnaces of considerable dimensions, as in that with three tuyères, this portion of the furnace is cylindrical for a certain height. 
The following measurements represent the interior structure of two well-going furnaces.

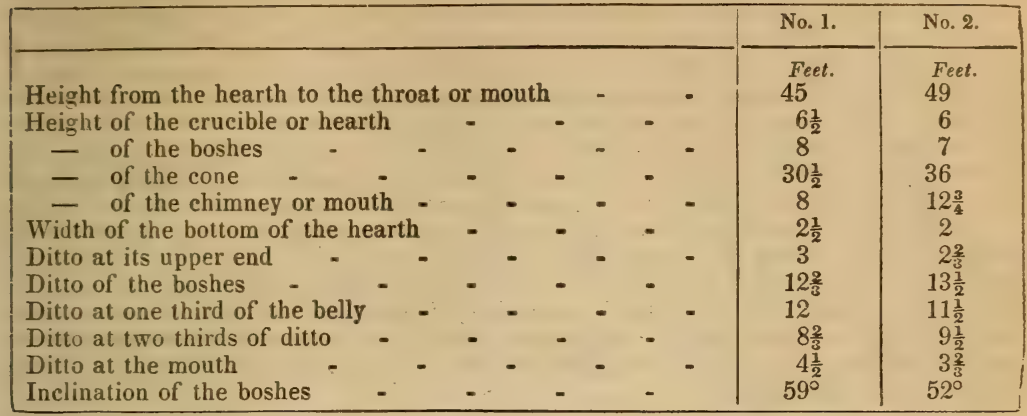

The conical orifice called the tuyère, in which the tapered pipes are placed, for imparting the blast, is seen near the bottom of the furnace, fig. 583, at A. Nose tubes of various sizes, from 2 to 4 inches in diameter, are applied to the extremity of the main blast-pipe. Under $\mathrm{A}$ is the bottom of the hearth, which, in large furnaces, may be two feet square. B is the top of the hearth, about two feet six inches square. A $B$ is the height of the hearth, about six feet six inches. B shows the round bottom of the conical or funnel part, called in this country the boshes, standing upon the square area of the hearth. $\quad \mathrm{c}$ is the top of the boshes, which may be about 12 feet in diameter, and 8 feet in perpendicular height. D is the furnace top or mouth (gueulard in French), at which the materials are charged. It may be $4 \frac{1}{2}$ feet in diameter. The line between $\mathrm{c}, \mathrm{D}$, is the height of the internal cavity of the furnace, from the top of the boshes upwards, supposed to be 30 feet. A, D, is the total height of the interior of the furnace, reckoned at $44 \frac{1}{2}$ feet. $\quad \mathrm{E} E$ is the lining, which is built in the nicest manner with the best fire-bricks, from 12 to 14 inches long, 3 inches thick, and curved to suit the circle of the cone. A vacancy of 3 inches wide is left all round the outside of the first lining by the builder; which is sometimes filled with coke dust, but more generally with sand firmly rammed. This void space in the brick-work is for the purpose of allowing for any expansion which might occur, either by an increase in the bulk of the building, or by the pressure and weight of the materials when descending to the bottom of the furnace. Exterior to $\mathrm{E} E$ is a second lining of fire-bricks similar to the first. At $F$, on either side, is a cast-iron lintel, $8 \frac{1}{2}$ feet long, by 10 inches square, upon which the bottom of the arches is supported. F, G, is the rise of the tuyère arch, which may be 14 feet high upon the outside, and 18 feet wide. The extreme size of the bottom or sole of the hearth, upon each side of $\mathrm{A}$, may be 10 feet square. This part and the boshing stones are preferably made from a coarse sandstone grit, containing large rounded grains of quartz, united by a siliceo-argillaceous cement.

The bottom of the hearth consists, first, of a course of the said gritstone; beneath

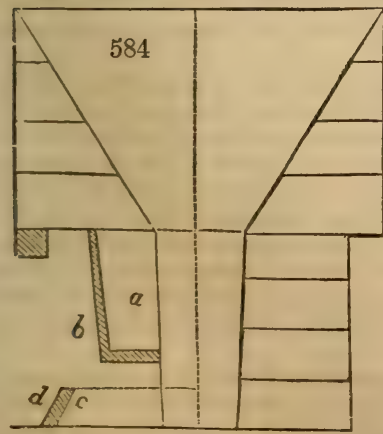
which is a layer of bedding sand, having, in its under part, passages for the escape of the vapors generated by damps; the whole being supported upon pillars of brick.

Fig. 584 represents the hearth and boshes, in a vertical side section. $a$ is the tymp stone, and $b$ the tymp plate for confining the liquid metal in the hearth. The latter is wedged firmly into the side-walls of the hearth; $c$ is the dam-stone, which occupies the whole breadth at the bottom of the hearth, excepting about 6 inches, which space, when the furnace is at work, is filled, before every cast, with a strong binding sand. This stone is faced outside by a cast-iron plate $d$, called the dam-plate, of considerable thickness, and peculiar shape. The top of the dam-stone, or rather the notch of the dam-plate, lies from 4 to 8 inches under the level of the tuyère hole. The space under the tymp plate, for 5 or 6 inches down, is rammed full, for every cast, with strong loamy earth, or even fine clay; a process called the tymp stopping. The area of the base of this furnace being 38 feet, its extreme height is 55 leet.

The blast furnaces of Staffordshire have always two tuyères, at least, placed on oppo- 
site sides, but so pointed thut the blast may not pursue directly opposite lines. In a furnacs acting well in the neighborhond of Dudley, the one of the tuyeres was 10 inches distant from the posterior wall of the hearth, and the other only four inches. In other furnaces with 3 tuyères, the side ones are placed, the one $16 \frac{1}{2}$ inches, and the other $6 \frac{1}{2}$ inches from the back. Three tuyères are seldom made to blow simultaneously. The third is brought into action only when the furnace seems to be choked up, and when it becomes necessary to clear it up by a powerful concussion. Too much pains cannot be bestowed on the masonry and brickwork of a blast furnace, and on the solidity of its foundation. In a soft ground it should rest on piles, so driven that the channel left beneath for the drainage of the building may be above any water level. Small passages should likewise be left throughout the body of the work, for the transpiration of moisture.

The blowing machines employed in Staffordshire are generally cast-iron cylinders, in which a metallic piston is exactly fitted as for a steam engine, and made in the same way. Towards the top and bottom of the blowing cylinders orifices are left covered with valves, which open inside when the vacuum is made with the cylinders, and afterwards shut by their own weight. Adjutages conduct into the iron globe or chest, the air expelled by the piston, both in its ascent and descent; because these blowing machines have al ways a double stroke.

The pressure of the air is made to vary through a very considerable range, according to the nature of the fuel and season of the year; for as in summer the atmosphere is more rarefied, it must be expelled with a compensating force. The limits are from $1 \frac{1}{2}$ pounds to $3 \frac{1}{2}$ pounds on the inch; but these numbers represent extreme proportions, the average amount in Staffordshire being 3 pounds. With this pressure a furnace usually works, which affords 60 tons of cast-iron in the week; and the pressure may be $2 \frac{1}{2}$ pounds on an average. The orifices, or nose-pipes, through which the air issues, also vary with the nature of the coke and the ore. In Staffordshire they are generally from 2 inches and 5 tenths to 2 inches and 8 tenths in diameter.

The blowing machines of Staffordshire are always impelled by steam engines. At Mr. Bagnall's works, two blast furnaces, 40 feet high, exclusive of the chimney or top, and two finery furnaces, are worked by a steam engine of 40 horses power; and therefore the power of one horse corresponds to the production of $2 \frac{1}{2}$ tons of cast iron per week, independently of the finery.

In South Wales, especially at Pontypool, there are slighter blast furnaces, whose upper portion is composed of a single range of bricks, each of which is 20 inches long, 4 thick, and 9 broad. The interior of the chimney represents an inverted cone. These furnaces derive solidity, and power to resist the expansions and contractions from change of temperature, by being eased, as it were, in horizontal hoops, placed 3 feet, or, even in some cases, only 6 inches asunder. These flat rings consist of four pieces, which are joined by means of vertical bars, that carry a species of ears or rings, into which the hoops enter, and are retained by bolts or keys. Instead of these ears, screw nuts are also employed for the junction. Each hoop is alternately connected to each of the eight vertical bars. The interior of these furnaces is the same as of the others; being generally from 12 to 14 feet diameter at the belly, and from 50 to 55 feet high. Though slight, they last as long as those composed of an outer body of masonry and a double lining of bricks; and have continued constantly at work for three years. In Wales also the blast furnaces are generally somewhat larger than in Staffordshire; because there the object being to refine the cast iron, they wish to procure as large a smelting product as possible. But in Staffordshire, a fine quality of casting iron is chiefly sought after, and hence their furnaces have less height, but nearly the same width.

In a blast apparatus employed at the Cyfartha works, moved by a 90 horse steam power, the piston rod of the blowing cylinder is connected by a parallelogram mechanism with the opposite end of the working beam of the steam engine. The cylinder is 9 feet 4 inches diameter, and 8 feet 4 inches high. The piston has a stroke 8 feet long, and it rises 13 times in the minute. By calculating the sum of the spaces percurred by the piston in a minute, and supposing that the volume of the air expelled is equal to only 96 per cent. of that sum, which must be admitted to hold with machines executed with so much precision, we find that 12,588 cubic feet of air are propelled every minute. Hence a horse power applied to blowing machines of this nature gives, on an average, $137 \mathrm{cu}$ bic feet of air per minute. The pressure on the air, as it issues, rarely exceeds two pounds on the square inch in the Welsh works.

At the establishment of Cyfartha, for blowing seven smelting furnaces, and the seven corresponding fineries, three steam engines are employed, one of 90 horse power, another of 80 , and a third of 40 ; which constitutes in the whole a force of 210 horses, or 26 horses and $\frac{1}{5}$ per furnace, supposing the fineries to consume one eighth of the blast. In the whole of the works of Messrs. Crawshay, the proprietors of Cyfartha, the power of about 350 horses is expended in blowing 12 smelting furnaces, and their subordinate fineries; which gives from 25 to 26 horses for each, allowing as before one eighth for the fineries. As these furnaces produce each about 60 tons of cast iron weekly, we find 
What a horse power corresponds to 2 tons and a tenth in that time. Each of the furnaces consumes about 3567 cubic feet of air per minute. These works have becn greatly increased of late years.

The following analyses of the English coal ronstones have been made by M. Berthier, at the school of mines in Paris.

\begin{tabular}{|c|c|c|c|c|}
\hline & & Rich Welsh Ore. & Poor Welsh Ore. & \begin{tabular}{|c|} 
Rich Ore of Dudley, \\
or gubbin.
\end{tabular} \\
\hline Loss by ignition - & - & & & \\
\hline Inso & - & & & \\
\hline Lime & - & 0 . & 6 & $2 \cdot 66$ \\
\hline Peroxyde of iron - & - & $60 \cdot 00$ & $42 \cdot 66$ & $58 \cdot 33$ \\
\hline \multicolumn{5}{|c|}{$\begin{array}{l}\text { On calculating the quantities of carbonate of iron, and metallic iron, to which the } \\
\text { above peroxyde corresponds, we have:- }\end{array}$} \\
\hline Carbon & $-\quad-$ & $88 \cdot 77$ & & $85 \cdot 20$ \\
\hline Metallic iron & - & $42 \cdot 15$ & $31 \cdot 38$ & $40 \cdot 45$ \\
\hline
\end{tabular}

The mean richness of the ores of carbonate of iron of these coal basins is not far from 33 per cent. About 28 per cent. is dissipated on an average, in the roasting of the ores.

Every ferruginous clay-stone is regarded as an iron ore, when it contains more than 20 per cent. of metal; and it is paid for according to its quality, being on an average at 12 shillings per ton in Staffordshire. The gubbin, however, fetches so high a price as $\mathbf{1 6}$ or 17 shillings. The ore must be roasted before it is fit for the blast furnace, a process carried on in the open air. A heap of ore mingled with small coal (if necessary) is piled up over a stratum of larger pieces of coal; and this heap may be 6 or 7 feet high, by 15 or 20 broad. The fire is applied at the windward end, and after it has burned a certain way, the heap is prolonged at the other extremity, as far as the nature of the ground or convenience of the work requires. The quantity of coal requisite for roasting the ore varies from one to four hundred weight per ton, according to the proportion of bituminous matter associated with the iron-stone. The ore loses in this operation from 25 to 30 per cent. of its weight. Three and a quarter tons of crude ore, or two and a quarter tons of roasted ore, are required to produce a ton of cast-iron; that is to say, the crude material yields on an average 30.7 per cent., and the roasted ore 44.4 of pig metal. In most smelting works in Staffordshire, about equal weights of the rich ore in round nodules called gubbin, and the poorer ore in cakes called blue flat, are employed together in their roasted state; but the proportions are varied, in order to have a uniform mixture, capable of yielding from 30 to 33 per cent. of metal.

The transition or carboniferous limestone of Dudley is used as the flux; it is compact and contains little clay. The bulk of the flux is made nearly equal to that of the ore. To treat two tons and a quarter of roasted ore, which furnish one ton of pig iron, 19 hundred weight of limestone are employed; constituting nearly 1 of limestone for 3 of unroasted ore. The limestone costs 6 shillings the ton.

Carbonized pitcoal or coke was, till within these few years, the sole combustible used in the blast furnaces of Staffordshire.

The coal is distributed in circular heaps, about 5 feet diameter, by 4 feet high; and the middle is occupied by a low brick chimney, piled with loose bricks, so open as to leave interstices between them, especially near the ground. The larger lumps of coal are arranged round this chimney, and the smaller towards the circumference of the heap. When every thing is adjusted, a kindling of coals is introduced into the bottom of the brick chimney; and to render the combustion slow, the whole is covered over with a coat of coal dross, the chimney being loosely closed with a slab of any kind. Openings are occasionally made in the crust and afterwards shut up, to quicken and retard the ignition at pleasure, during its continuance of 24 hours. Whenever the carbonization has reached the proper point for forming good coke, the covering of coal dross is removed, and water is thrown on the heap to extinguish the combustion; a circumstance deemed useful to the quality of the coke. In this operation the Staffordshire coal loses the half of its weight, or two tons of coal produce one of coke.

As soon as the blast furnace gets into a regular heat, which happens about 15 days or three weeks after fires have been put in it, the working consists simply in charging it, at the opening in the throat, whenever there is a sufficient empty space; the only rule being to keep the furnace always full. The coke is measured in a basket, thirteen of which go to the ton. The ore and the flux (limestone) are brought forwards in wheelbarrows of sheet iron. In 24 hours, there are thrown into a furnace such as fig. $582,14 \frac{2}{3}$ tons of coke, 16 tons of roasted ore, and $6 \frac{3}{4}$ tons of limestone; from which about 7 tons of pig iron are procured. This is run off every 12 hours; in some works the blast is suspended during the discharge. The metal intended to be converted into 
bar iron, or to be cast again into moulds, is ran into small pigs 3 feet long, and 4 inches diameter; weighing each about 2 hundred weight and a half.

The disorders to which blast furnaces are liable have a tendency always to produce white cast-iron. The color of the slag or scoriæ is the surest test of these derangements, as it indicates the quality of the products. If the furnace is yielding an iron proper for casting into moulds, the slag has a uniform vitrification, and is slightly translucid. When the dose of ore is increased in order to obtain a gray pig iron, fit for fabrication into bars, the slag is opaque, dull, and of a greenish-yellow tint, with blue enamelled zones. Lastly, when the furnace is producing a white metal, the slags are black, glassy, full of bubbles, and emit an odor of sulphureted hydrogen. The scoriæ from a coke are much more loaded with lime than those from a charcoal blast furnace. This excess of lime appears adapted to absorb and carry off the sulphur, which would otherwise injure the quality of the iron. The slags, when breathed on, emit an argillaceous odor.

A blast furnace of 50 or 60 feet in height gives commonly from 60 to 70 tons of castiron per week; one from 50 to 55 feet high, gives 60 tons; two united of 45 feet produce together 100 tons; and one of 36 feet furnishes from 30 to 40 . A blast furnace should go for four or five years without needing restoration. From $3 \frac{1}{2}$ to 4 tons of coal, inclusive of the coal of calcination, are required in Staffordshire to obtain one ton of cast-iron; and the expense in workmen's wages is about 15 shillings on that quantity.

At the Cyfartha works of Messrs. Crawshay in South Wales, the average price of the lithoid carbonate of iron, ready for roasting, is only $7 s .6 d$. a ton, and its richness is about 33 per cent. The furnaces for roasting the ore in that country are made in the form of cylinders, placed above an inverted cone. The cylindrical part is 6 feet high and wide, and the cone is about 4 feet high, with a base equal to that of the cylinder; towards the bottom or narrowest part of the inverted cone, there is an aperture which terminates in an outlet on a level with the bottom of the terrace in which the furnace is built. Sometimes, however, all the roasting furnaces are in a manner combined into one, which resembles a long pit about 6 feet in width and depth, and whose bottom presents a series of inverted hollow quadrangular pyramids, $6 \mathrm{feet}$ in each side, and 4 deep. The bottom or apex of each of these pyramids communicates with a mouth or door-way that opens on a lower terrace, through which the ore falls in proportion as it is roasted; and whence it is wheeled and tumbled into the throat of an adjoining blast furnace, on the same level with the terrace; for in Wales the blast furnace is generally built up against the face of a hill, which makes one of its fronts. The above roasting furnaces, which closely resemble lime-kilns, after being filled with alternate strata of small coal and ore, are set on fire; and the roasted ore is progressively withdrawn below, as already mentioned.

The product of coke from a certain weight of coal is greater in Wales than in Staffordshire, though the mode of manufacture is the same. At Pen-y-Darran, for example, 5 of coal furnish $3 \frac{1}{2}$ of coke; or 100 give 70 ; at Dowlais 100 of coal afford 71 of coke, and the product would be still greater if more pains were bestowed upon the process. At Dowlais, coal costs only 2 shillings a ton; at Cyfartha, it is worth from $2 s .6 d$. to 5 shillings. About 2 tons of coke are employed in obtaining 1 ton of cast-iron.

According to M. Berthier's analysis, the slag or cinder of Dowlais consists of silica, 40.4 ; lime, 38.4 ; magnesia, $5 \cdot 2$; alumina, $11 \cdot 2$; protoxyde of iron, 3.8 ; and a trace of sulphur. He says that the silica contains as much oxygen as all the other bases united; or is equivalent to them in saturating power; and to the excess of lime he ascribes the freedom from sulphur, and the good quality of the iron produced. The specimen examined was from a furnace at Merthyr-Tydvil. Other slags from the same furnace, and one from Dudley, furnished upwards of 2 per cent. of manganese. Those which he analyzed from Saint Etienne, in France, afforded about 1 per cent. of sulphur.

The consumption of coal in the Welsh smelting furnaces may be estimated, on an average, at 3 tons per ton of cast-iron; corresponding to 2.1 of their coke. From this economy in the quantity of fuel, as well as from its cheapness and that of the iron ore, the iron of South Wales can be brought into the market at a much lower rate than that of any other district. These blast furnaces remain in action from 5 to 10 years; at the end of which time, only their interior surface has to be repaired. The lining of the upper part lasts much longer; for examples are not wanting of its holding good for nearly 40 years.

One of the greatest improvements ever made by simple means in any manufacture is the employment of hot air, instead of the ordinary cold air of the atmosphere, in supplying the blast of furnaces for smelting and founding iron. The discovery of the superior power of a hot over a cold blast in fusing refractory lumps of cast-iron was accidentally observed by my pupil, Mr. James Beaumont Neilson, engineer to the Glasgow gas works, about the year 1827, at a smith's forge in that city, and it was made the subject of a patent in the month of September of the following year. No particular construction of apparatus was described by the inventor by which the air was to be 
heated, and conveyed to the furnace; but it was merely stated that the air may be heated in a chamber or closed vessel, having a fire under it, or in a vessel connected in any convenient manner with the forge or furnace. From this versel the air is to be forced by means of bellows into the furnace. The quantity of surface which a heating furnace is sequired to have for a forge, is about 1260 cubic inches; for a cupola furnace, about 10,000 subic inches. The vessel may be enclosed in brickwork, or fixed in any other manner that may be found desirable, the application of heated air in any way to furnaces or iorges, for the purposes of working iron, being the subject claimed as constituting the invention.

Wherever a forced stream of air is employed for combustion, the resulting temperature must evidently be impaired by the coldness of the air injected upon the fuel. The heat developed in combustion is distributed into three portions; one is communicated to the remaining fuel, another is communicated to the azote of the atmosphere, and to the volatile products of combustion, and a third to the iron and fluxes, or other surrounding matter to be afterwards dissipated by wider diffusion. This inevitable distribution takes place in such a way, that there is a nearly equal temperature over the whole extent of a fire-place, in which an equal degree of combustion exists.

We thus perceive that if the air and the coal be very cold, the portions of heat absorbed by them might be very considerable, and sufficient to prevent the resulting temperature from rising to a proper pitch; but if they were very hot they would absorb less caloric, and would leave more to elevate the common temperature. Let us suppose two furnaces charged with burning fuel, into one of which cold air is blown, and into the other hot air, in the same quantity. In the same time, nearly equal quantities of fuel $w_{i}^{*}$, be consumed with a nearly equal production of heat; but notwithstanding of this, there will not be the same degree of heat in the two furnaces, for the one which receives the hot air will be hotter by all the excess of heat in its air above that of the other, since the former air adds to the heat while the latter abstracts from it. Nor are we to imagine that by injecting a little more cold air into the one furnace, we can raise its temperature to that of the other. With more air indeed we should burn more coals in the same time, and we should produce a greater quantity of heat, but this heat being diffused proportionally among more considerable masses of matter, would not produce a greater temperature; we should have a larger space heated, but not a greater intensity of heat in the same space.

Thus, according to the physical principles of the production and distribution of heat, fires fed with hot air should, with the same fuel, rise to a higher pitch of temperature than fires fed with common cold air. This consequence is independent of the masses, being as true for a small stove which burns only an ounce of charcoal in a minute, as for a furnace which burns a hundred weight; but the excess of temperature produced by hot air cannot be the same in small fires as in great; because the waste of heat is usually less the more fuel is burned.

This principle may be rendered still more evident by a numerical illustration. Let us take, for example, a blast furnace, into which 600 cubic feet of air are blown per minute; suppose it to contain no ore, but merely coal or coke, and that it has been burn: ing long enough to have arrived at the equilibrium of temperature, and let us see what excess of temperature it would have if blown with air of $300^{\circ} \mathrm{C}$. $\left(572^{\circ} \mathrm{F}\right.$ ) $)$, instead of being blown with air at $0^{\circ} \mathrm{C}$.

600 cubic feet of air under the mean temperature and pressure, weigh a little more than 45 pounds avoirdupois; they contain 10.4 pounds of oxygen, which would burn very nearly 4 pounds of carbon, and disengage 16,000 times as much heat as would raise by one degree Cent. the temperature of two pounds of water. These 16,000 portions of heat, produced every minute, will replace 16,000 other portions of heat, dissipated by the sides of the furnace, and employed in heating the gases which escape from its mouth. This must take place in order to establish the assumed equilibrium of caloric.

If the 45 pounds of air be heated beforehand up to $300^{\circ} \mathbf{C}$., they will contain about the eighth part of the heat of the 16,000 disengaged by the combustion, and there will be therefore in the same space one eighth of heat more, which will be ready to operate upon any bodies within its range, and to heat them one eighth more. Thus the blast of $300^{\circ} \mathrm{C}$. gives a temperature which is nine eighths of the blast at zero C., or at even the ordinary atmospheric temperature; and as we may reckon at from $2200^{\circ}$ to $2700^{\circ} \mathrm{F}$. (from $1200^{\circ}$ to $1500^{\circ} \mathrm{C}$.), the temperature of blast furnaces worked in the common way, we perceive that the hot air blast produces an increase of temperature equal to from $270^{\circ}$ to $360^{\circ} \mathrm{F}$.

Now in order to appreciate the immense effects which this excess of temperature may produce in metallurgic operations, we must consider that often only a few degrees more temperature are required to modify the state of a fusible body, or to determine the play of affinities dormant at lower degrees of heat. Water is solid at $1^{\circ}$ under $32^{\circ} \mathrm{F}$.; it is liquid at $1^{\circ}$ above. Every fusible body has a determinate melting point, a very few 
degrees above which it is quite fluid, though it may be pasty below it. The same ob servation applies to ordinary chemical affinities; charcoal, for example, which reduces the greater part of metallic oxydes, begins to do so only at a determinate pitch of temperature, under which it is inoperative, but a few degrees above, it is in general lively and complete. It is unnecessary, in this article, to enter into any more details to show the influence of a few degrees of heat, more or less, in a furnace, upon chemical operations, or merely upon physical changes of state.

These consequences might have been deduced long ago, and industry might thus have been enriched with a new application of science; but philosophers have been and still

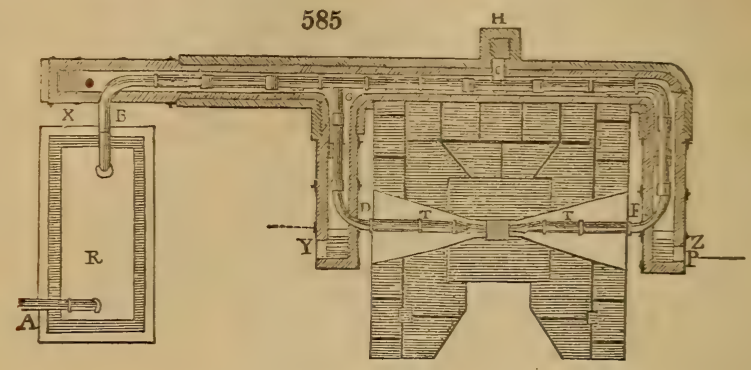

are too much estranged from the study of the useful arts, and content themselves too much with the minutiæ of the laboratory or theoretic abstractions. Within the space of 7 years, the use of the hot blast has been so much extended in Great Britain, as to have enabled many proprietors of iron works to add 50 per cent. to their weekly production of metal, to diminish the expenses of smelting by 50 per cent., and, in many cases, to produce a better sort of east iron from indifferent materials.
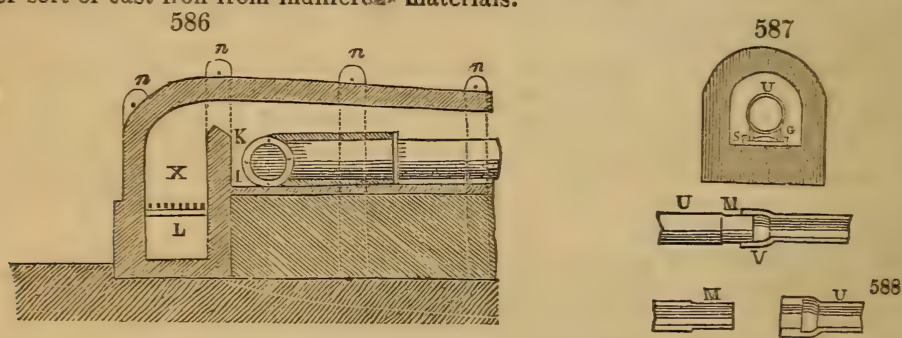

The figures here given represent the blast furnace, and all the details of the air heating, 589 at one view. Fig. 583 is a vertical section of the furnace and the apparatus; fig. 585 represents the plan at the height of the line 1,2 , of fig. 583. The blowing machine, which is not shown in this view, injects the air through the pipe $\mathrm{A}$, into the regulator chamber $\mathrm{R}$, fig. 585 ; the air thence issues by the pipe $B$, proceeds to c, where it is subdivided into two portions; the one passes along the pipe $\mathrm{C} D$ to get to the tuyère $T$, the other passes behind the furnace, and arrives at the tuyère $T^{\prime}$ by the pipe $C E F$.

These pipes are distributed in a long furnace or flue, whose bottom, sides, and top are formed with fire-brick, where they are exposed to the action of the flame of the three fires $x, x, z$. The flame of the fire $\mathrm{x}$ plays round the pipe $\mathrm{B}$ at its entrance into the flue, and quits it only to go into the chimney $\mathrm{H}$; that of the fire $\mathrm{Y}$ acts from the point $\mathrm{D}$ to the same chimney, passing by the elbow $\mathrm{c}$; that of the fire $\mathrm{z}$ acts equally upon $\mathrm{F}$ and $\mathrm{H}$, in passing by the elbow $\mathrm{E}$.

Disposition of the fires and furnace.-Fig. 586 represents, upon a scale three times larger than fig. 585, the section of the 
fire $\mathbf{x}$, of which the plan is seen in fig. 585, and the elevation in fig. 583 ; as also in the outside view of the blast furnace, fig. 589 .

The grate is at $\mathrm{L}$; the fuel is introduced by the door $\mathrm{P}$, fig. 583 ; the flame rises above the bridge I $\mathrm{K}$, and proceeds along the vaulted flue towards the chimney $\mathrm{H}$. Through a length of about $13 \mathrm{feet}$, including the grate, the furnace is on each side supported by oblung plates of cast-iron, which are bound together by 4 upright ribbed or feathered bars, also on each side; these bars, $n$, being bound together by iron rods furnished with screw nuts at their ends ( $f$ igs. 583, 585, 586). Beyond this distance, the outside of the furnace is mere brickwork.

The fires $\mathrm{y}$ and $\mathrm{z}$ have exactly a like disposition with the above.

Fig. 586 indicates the dimensions and the curvature of the arch above the grate, near the bridge ; fig. 587 represents the section of the furnace and of the pipe beyond the castiron casing.

I find that the furnace is only about 3 feet wide at the bottom, and that the elevation of the arch above the bottom is no more than 30 inches. Perhaps it might be made a little wider with advantage; the combustion would be more vigorous and effective; and if the sides also were a little thicker, the heat would be better confined.

The distance from the fire-place $\mathrm{x}$ to the chimney $\mathrm{H}$, is $43 \frac{1}{2}$ feet.

$$
\text { - } \quad \text { - } \quad \mathrm{z} \text { to the chimney, is } 29 \text { - including the }
$$

Dis'r:3ution of the pipes.-At в the pipe is 18 inches diameter outside, and one inch thick of metal, and it tapers to $\mathrm{C}$; from $\mathrm{c}$ to $\mathrm{D}$ and from $\mathrm{D}$ to $\mathrm{c}$ the pipes are only 11 inches in external diameter, and three fourths of an inch thick; they are 5 feet long, and are united by two kinds of joints; the ordinary ones, and those of compensation, to give play for the expansion and contraction. One of these is seen between $B$ and $c$, one between $\mathrm{c}$ and $\mathrm{D}$, one between $\mathrm{C}$ and $\mathrm{E}$, and $\mathrm{a}$ fourth between $\mathrm{E}$ and $\mathrm{F}$. These pipes and their adjustment are seen more at large in fig. $588 ; \mathrm{u} v$ is one of these pipes, its widened mouth receives the extremity M of the preceding pipe. These pieces are truly bored and turned to fit each other, and slide out and in like telescope tubes, by the effect of dilatation and contraction of the pipes with changes of temperature.

At certain distances castors or friction-rollers of cast-iron are placed to carry the pipes, which roll upon oblong plates of cast-iron laid upon the floor of the flues. These castors are shown at $a, b, c, d, e, f, g, f i g .585$; one of them is shown separate upon a larger scale at $\mathrm{G}$, in fig. 587, as also the plate or rail s, on which it runs.

The tuyerres $\mathrm{T}^{\mathrm{T}} \mathrm{T}^{\prime}$ are adjusted into the pipe behind them; this is truly bored, so as to allow the thick end of the tuyère to slide tightly backwards and forwards in it, like a piston in the barrel of a pump; a diaphragm moreover prevents the tuyère from being drawn or forced entirely out of its tube. At the side of this tube there is a small orifice, which may be shut or opened at pleasure with a stopcock or screw-plug : it serves to try the degree of heat of the air-blast; if a lead wire does not melt when held at this hole, the temperature is reckoned too low; being under the 612th degree of Fahrenheit. The nozzles are 2 inches in diameter.

Near the fire-places of the air-heating furnaces the pipes are at a cherry-red heat ; and lest they should be burned, they are there coated with a lute of fire-clay, as shown near $\mathbf{K}$, in fig. 586. By this means the air is kept up at the heat of $350^{\circ} \mathrm{C}$., or $662^{\circ} \mathrm{F}$, a little above the boiling point of quicksilver.

Quantity of air and pressure.-The blowing-machine belonging to the above blastfurnace is moved by a water wheel of 22 horse power; the pistons are 4 feet in diameter, have a $3 \frac{1}{2}$-feet stroke, work double, and expel 1200 cubic feet of air in the minute; or 600 cubic feet for each nozzle. The pressure of the air is equivalent to no more than 2 or $2 \frac{1}{4}$ inches of mercury ; formerly with cold air it amounted to $3 \frac{1}{2}$ inches. This furnace yields, upon an average, $5 \frac{1}{4}$ tons of cast-iron daily, and consumes $1 \frac{1}{3} \mathrm{cwt}$. of coke for each ewt. of cast-iron produced; being 7 tons of coke per diem.

The consumption of the three flue fires is 30 pounds of small coal, for 100 pounds of cast-iron produced, which may be reckoned equivalent to 15 pounds of coke; hence altogether each ton of cast-iron requires for its production $1 \frac{1}{2}$ tons of coke.

The same furnace worked with the cold blast, the same pressure, and the same ores, produced only $3 \frac{1}{2}$ tons of cast-iron daily, with an expenditure of 2.55 of coke for 1 of cast-iron; in which case the coke amounted to 9 tons daily.

The returns by the hot blast compared with those by the cold, are therefore as the numbers 3 and 2 , which shows an advantage by the former plan of 50 per cent. The consumption of fuel in the two cases is as 8 to 9, being a saving in this article of about 11 per cent. Coke is used on account of sulphur in the coal.

Hot-blast heated by the flame of the furnace mouth. - This system is mounted in Staffordshire. The heating apparatus is there set immediately upon the mouth of the fur. nace; and is composed of two large cast-iron cylinders of the same length, the one within 
the other, leaving a space between them. This annular interval amounts to 16 inches and it is closed at top and bottom: but the innermost cylinder is open at both ends, and forms, indeed, the vent of the chimney or furnace. It carries nine rows of pipes, three in each row, which cross its interior, and open into the annular space.

The flame of the furnace passes between the intervals of the cross pipes, heating them, and also the two upright cylinders with which they are connected. The air of the blowing machine arrives by a vertical pipe, which is placed at the back of the furnace; it enters into the above annular space, and thence circulates, with more or less velocity, through the 27 cross tubes, upon which the flame is continually playing; lasily, it is drawn through to the bottom of the annuiar space; the two tubes which conduct it to the two tuyères, pass down within the brickwork of the furnace, and thus prevent the dissipation of its heat.

Below this heating apparatus there is a door for putting the charges into the furnace.

The above arrangement does not seem to be the best for obtaining the greatest possible heat for the blast, nor for favoring the free action of the furnace; but it illustrates perfectly well the principle of this application. A serpentine movement in a long bent hot channel would be much better adapted for communicating heat to so bad a conductor as air is known to be.

In the month of July, 1836, I paid a visit to Codner Park and Butterly works, in Derbyshire, belonging to the eminent iron-masters, Messrs. Jessop \& Co., where I was kindly permitted not only to study the various processes of the manufacture of cast and wrought iron, but to inspect the registers of the products of cast iron in their blast furnaces for several years back. It appeared that in the year 1829, only 29 tons of cast-iron were made weekly in each of the blast furnaces at Codner Park. They were then worked with coke, and blown with cold air. Each ton of iron required for its production, at that time, 6.82 tons of coals, made into coke for smelting; with 2.64 of roasted iron ore (carbonate), called mine; and 0.87 of limestone, the castine of the French.

In 1835 and 1836 , the same furnaces turned out weekly 49 tons of cast-iron each; an every ton of iron required for its production only 3 tons of coal (not made into coke); 2.72 tons of mine; and 0.77 of lime.

In 1829, and for many years before, as well as one or two after, each ton of coals is said to have cost for coking the sum of $6 s$., whence the 6.82 tons of coals then converted into coke for smelting one ton of iron, cost fully 40 s. in coking alone, in addition to their prime cost. The saving in this respect, therefore, is $40 s$. upon each ton of iron, besides the saving of fully half the coal, and the increased produce of nearly 60 per cent. of metal per week. The iron-master pays the patentee $1 s$. upon every ton of iron which he makes, and, at the prices of 1836 , he lessened his expenses by at least 30 s. or 40 s. per ton by the patent improvement.

The following tabular view of the progression in the management and results of the hot blast, is given by $M$. Dufrènoy, after visiting the various iron works in this country where it had been introduced.

"At the Clyde iron works, near Glasgow ; in 1829, when the combustion was effected by the cold air blast,-

There were consumed, for smelting, 3 tons of coke, equivalent to

$$
\text { - for the blowing engine - . - }
$$

Total coal per ton of iron

\section{Limestone}

- 6130

- 107

In 1831 , with the hot blast at $450^{\circ} \mathrm{F}$., coke being still used in smelting,-

- 7137

There were consumed, for smelting, $1 \mathrm{ton}, 18 \mathrm{cwt}$. of coke, equiva-

lent to

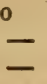

for

for heating the air, $5 \mathrm{cwt}$. for the blowing engine, $7 \mathrm{cwt} .4 \mathrm{lbs}$.

Total coal per ton of iron

Limestone
- 460

- $\begin{array}{lll}0 & 12 & 4\end{array}$

In July, 1833 , with the hot blast at $612^{\circ} \mathrm{F}$., raw coal alone being used for smelting, -

There wern consumed : for smelting

for heating the air for the blowing engine -

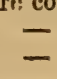

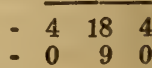

Total coal per ton of iron

Limestone

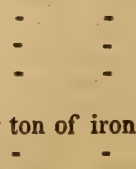

- 200

- 080

- 0112

- $\overline{2192}$ 
"At the last period the use of hot air had increased the make of the furnaces by more than one third, and had consequently produced a great saving of expense in the article of labor. The quantity of blast necessary for the furnaces was also sensibly diminished; for a blowing engine of seventy-horse power, which, in 1829 , served only for three blast furnaces, was now sufficient for the supply of four.

"On comparing these sereral results, we find that the economy of fuel is in proportion to the temperature to which the air is raised. As for the actual saring, it raries in every work, according to the nature of the coal, and the care with which the operation is conducted.

"This process, though it has been four rears in use in the works near Glasgow (which it has rescued from certain ruin), has scarcely passed the borders of Scotland; the marvellous adrantages, howerer, which it has produced, are beginning to triumph over prejudice, and gradually to extend its use into the different English iron districts. There are one-and-twenty works, containing altogether sixty-seren blast furnaces, in which hot air is used. The pig iron run out of these furnaces is generally No. 1 , and is fit for making the most delicate castings. This process is equally applicalle to forge pigs for the manufacture of bar iron; since in order to obtain this quality of iron, it is only necessart to alter the proportion of fuel and mineral. In the forges of the Trne ironworks, near Newcastle, and of Codner Park, near Derby, pigs made in furnaces blown by hot air, are alone used in the manufacture of bar iron.

"In the side of the turère pipe a small hole is made, by means of which the heat of the air mar be ascertained at any moment. This precaution is indispensable, it being of importance to the beneficial use of hot air, that it he kept at a uniformly high temperature. With a proper apparatus the air is raised to 612 degrees Fahr., which is a greater heat, by several degrees, than is necessary for the fusion of lead."

"At Calder works the consumption of fuel has diminished in the proportion of 7 tons $17 \mathrm{cwts}$. to 2 tons $2 \mathrm{cwts}$. There has also been a great diminution of expense in limestone, of which only $5 \frac{1}{2}$ cwts. are now used, instead of $13 \mathrm{cwts}$, which were used in 1828 . This decrease results, as I have already said, from the high temperature which the furnace has acquired since the introduction of hot air.

"The quantity of blast has been reduced from 3500 cubic feet per minu e, to 2627 cubic feet; the pressure also has been reduced from $3 \frac{1}{4}$ to $2 \frac{z}{4}$ lbs."

Of the refinery of cast-iron, or its conversion into bar-iron, in England.-This . operation is naturally divisible into three distinct parts. The first, or the finery properly s yeaking, is executed in peculiar furnaces called runing out fires; the second operation ccupletes the first, and is called puddling; and the third consists in welding several iron $\mathrm{l}$ ars together, and working them under forge hammers, and between rolls.

1. The finery furnaces are composed of a body of brickwork, about 9 feet square; rising but little abore the surface of the ground. The hearth, placed in the middle, is two feet and a half deep; it is rectangular, being in general, 3 feet by 2 , with its greatest side parallel to the face of the turères; and it is made of cast iron in four plates. On the side of the turères there is a single brick wall. On the three other sides, sheet iron doors are placed, to prerent the external air from cooling the metal, which is almost alwars worked under an open shed, or in the open air, but never in a space surrounded by walls. The chimner, from 15 to 18 feet high, is supported upon four columns of cast iron; its lintel is four feet abore the level of the hearth, in order that the laborers may work with. out restraint.

The number of turères is from two to three; they are placed at the height of the lip of the crucible or hearth, and distributed so as to divide its length into equal parts; their axes being inclined towards the bottom, at an angle of from $25^{\circ}$ to $30^{\circ}$, so as to point upon the bath of melted metal as it flows. The cast-iron nose-pipe is incased, and water is made to circulate in the hollow space by means of crlindrical tubes; being introduced by one tube, and let off by another, so as to prevent the tuyères from getting burned in the process.

Two nozzles are usually placed in each tuyère, to render the blast constant and uni. form; and for the same end, the air impelled by the bellows, is sometimes receired at first in a regulator. The quantity of air blown into the fineries is considerable; being nearly. 400 cubic feet per minute for each finery ; or about the eighth part of the consumption of a blast furnace.

The finery furnace, or running out fire, is represented in figs. 590 and 591 . It is a smelting hearth, in which by first fusing and then cooling gray cast iron in a peculiar way, it is converted into white cast iron, called fine iron, or fine metal, of the quality of forge pig, for making malleable iron by the puddling process. The furnace resembles the forge hearth employed in Germany and France for converting forge pig into wrought iron; but it differs, particularly in this, that the fused iron is run out into an oblong iron trough, for sudden congelation.

$a$ is the air-chest, in communication with the uluwing cylinder, or bellows; the air 
Deing conducted through at least two blast pipes to the fire, and sometimes throug even 4 or 6 pipes. $b$ is the side of the furnace, corresponding to the tuyère plates, in
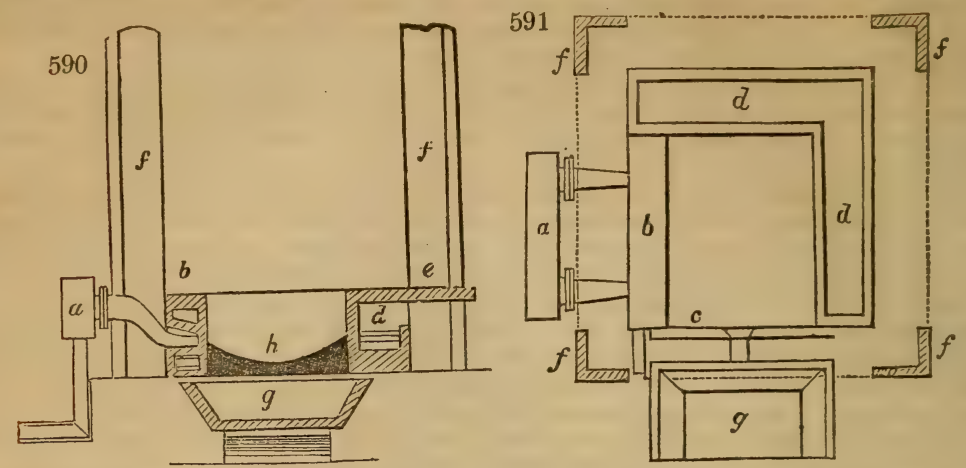

which are the openings for the.blast pipes. All the sides of the furnace are hollow, and are kept cool by the circulation of water through the cavity between them. $c$ is the front wall of the furnace, having a strong cast iron plate containing the tap holes for running off the melted metal. $d \dot{d}$ is the exterior wall of the furnace, which corresponds to the contre-vent and ash-hearth of the French refining forge. $e$, is the top plate upon which the coke is piled up in store. $f f, f f$, iron props of the chimney, (not shown in this view). $g$, cast iron trough into which the fine iron is run off in fusion; which is sometimes made in one piece, but more usually in separate plates joined together. Beneath this mould a stream of water is made to flow. $h$ is the bottom of the hearth, covered with sand.

In the finery process, the hearth or crucible of the furnace is filled with coke; then six pigs of cast iron are laid horizontally on the hearth, namely, four of them parallel to the four sides, and two in the middle above; and the whole is covered up in a domeform, with a heap of coke. The fire is now lighted, and in a quarter of an hour the :blast is applied. The cast iron flows down gradually, and collects in the crucible; more coke being added as the first quantity burns away. This operation proceeds by itself; the melted metal is not stirred about, as in some modes of refinery, and the temperature is always kept high enough to preserve the metal liquid. During this stage the coals -are observed continually lieaving up, a movement due in part to the action of the blast, and in part to an expansion caused in the metal by the discharge of gaseous oxyde of carbon. When all the pig iron is collected at the bottom of the hearth, which happens commonly at the end of two hours, or two and a half, the tap hole is opened, and the fine metal flows out with the slag, into the loam-coated pit, on a plate 10 feet long, 3 broad, and from 2 inches to $2 \frac{1}{2}$ thick. A portion of the slag forms a small crust on the surface of the metal; but most part of it collects in a basin scooped out at the bottom of the pit, into which the fine metal is run.

A large quantity of water is thrown on the fine metal, with the view of rendering it brittle, and perhaps of partially oxydizing it. This metal suddenly cooled, is very white, and possesses in general a fibrous radiated texture; or sometimes a cellular, including a considerable number of small spherical cavities, like a decomposed amygdaloid rock. If the cast iron be of bad quality, a little limestone is occasionally used in the above operation.

Three samples of cinder, analyzed by Berthier, gave.

Silica $0 \cdot 276$; protox. of iron, 0.612 ; alumina, $0 \cdot 040$; phosp. acid, $0 \cdot 072$, Dudley.

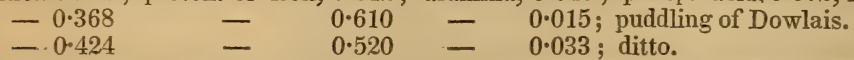

The remarkable fact of the presence of phosphoric acid, shows how important this operation is to the purification of the iron. The charge varies from a ton and a quarter to a ton and a half of pigs; and the loss by the process varies from 12 to 17 per cent.

The fine metal is broken into fragments, and sent to the puddling furnace after the product of each operation has been weighed. The coal consumed in the fine metal process is from 4 to 5 hundred weight for the ton of cast iron. About 10 tons may be refined per diem, a quantity somewhat greater than the supply from a blast furnace; but the fineries are not worked on the Sundays; and therefore a smelting furnace just keeps one of them in play. Whatever care be taken in this process, the bar iron finally re.ulting is never so goo! as if wood charcoal had been used in the refinery; and hence in making sheet iron for the tin plate manufacture, wood charcoal is substituted for coke in one Welsh establishment. The east iron treated with charcoal, gets into clots 
Dr lumps in the finery furnace, which are lifted out, set under the hammer, and flattened into thin cakes.

The main effect of the finery process, is probably the separation of the plumbaginous part of the charcoal, which is disseminated through the gray cast iron in a state of imperfect chemical combination. When that is removed the metal becomes more homogeneous, haring no crystalline carbon present to counteract its transition into pure iron; much of the silica and manganese are also vitrified together, and run off in the finery cinder.

2 . The puddling furnace is of the reverberatory form. It is bound generally with iron, as represented in the side view, fig. 592, by means of horizontal and vertical bars, which

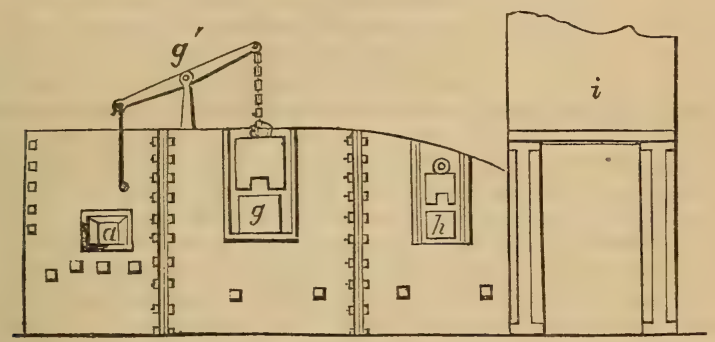

are joinea together and fixed by wedges, to prevent them from starting asunder. Very frequently, indeed, the reverberatory furnaces are armed with cast-iron plates over their whole surface. These are retained by upright bars of cast iron applied to the side walls, and by horizontal bars of iron, placed across the arch or roof. The furnace itself is divided interiorly into three parts; the fire-place, the hearth, and the flue. The fire-place varies from $3 \frac{1}{2}$ to $4 \frac{1}{2}$ feet long, by from 2 feet 8 inches to 3 feet 4 inches wide. The door way by which the coke is charged, is 8 inches square, and is bevelled off towards the outside of the furnace. This opening consists entirely of cast iron, and has a quantity of coal gathered round it. The bars of the fire grate are moveable, to admit of more readily clearing them from ashes.

Fig. 593 is a longitudinal section referring to the elevation; fig. 592, and fig. 594,

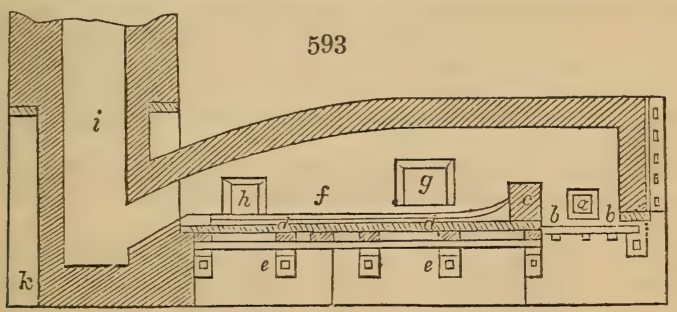

594

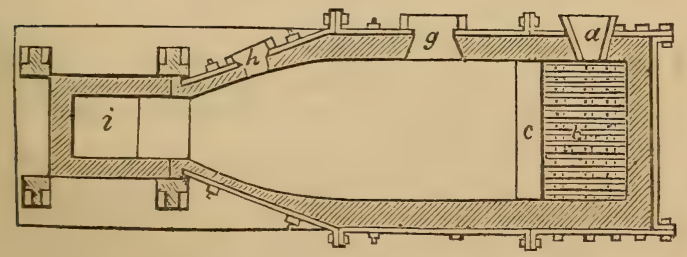

is a ground plan. When the furnace is a single one, a square hole is left in the side of the fire-place opposite to the door, through which the rakes are introduced, in order to be heated.

$a$ is the fire door; $b$, the grate ; $c$, the fire bridge ; $d d$, cast-iron hearth plates, resting upon cast-iron beams $e e$, which are bolted upon both sides to the cast-iron binding plates of the furnace. $f$ is the hearth covered with cinders or sand; $g$, is the main working door, which may be opered and shut by means of a lever $g^{\prime}$, and chain to move it up and down. In this large door there is a hole 5 inches square, through which the iron may be worked with the paddles or rakes; it may also be closed air. 
tight. There is a second working door $h$, near the flue, for introducing the cast ron, so that it may soften slowly, till it be ready for drawing towards the bridge. $i$, is the chimney, from 30 to 50 feet high, which receives commonly the flues of two furnaces, each 595

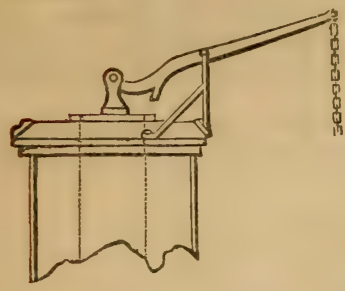
provided with a damper plate or register. Fig. 595, shows the main damper for the top of the common chimney, which may be opened or shut to any degree by means of the lever and chain. $k$, fig. 593, is the tap or floss hole for running off the slag or cinder.

The sole is sometimes made of bricks, sometimes of cast iron. In the first case it is composed of fire-bricks set on edge, forming a species of flat vault. It rests immediately on a body of brickwork either solid or arched below. When it is made of cast iron, which is now beginning to be the general practice, it may be made either of one piece or of several. It is commonly in a single piece, which, howerer, causes the inconvenience of reconstructing the furnace entirely when the sole is to be changed. In this case it is a little hollow, as is shown in the preceding vertical section; but if it consists of sereral pieces, it is usually made flat.

The hearths of cast iron rest upon cast iron pillars, to the number of four or five; which are supported on pedestals of cast iron placed on large blocks of stone. Such an arrangement is shown in the figure, where also the square hole $a, f i g .592$, for heating the rake irons, may be observed. The length of the hearth is usually six feet; and its breadth varies from one part to another. Its greatest breadth, which is opposite the door, is four feet. In the furnace, whose horizontal plan is given above, and which produces good results, the sole exhibits, in this part, a species of ear, which enters into the mouth of the door. At its origin towards the fireplace, it is 2 feet 10 inches wide; from the fire it is separated, moreover, by a low wall of bricks (the fire-bridge) 10 inches thick, and from 3 inches to 5 high. At the other extremity its breadth is 2 feet. The curvature presented by the sides of the sole or hearth is not symmetrical; for sometimes it makes an advancement, as is observable in the plan. At the extremity of the sole furthest from the fire, there is a low rising in the bricks of $2 \frac{1}{2}$ inches, called the altar, for preventing the metal from running out at the floss-hole when it begins to fuse. Beyond this shelf the sole terminates in an inclined plane, which leads to the floss, or outlet of the slag from the furnace. This floss is a little below the level of the sole, and is hollowed out of the basement of the chimney. The slag is prevented from concreting here, by the flame being made to pass over it, in its way to the sunk entry of the chimney; and there is also a plate of cast iron near this opening, on which a moderate fire is kept ap to preserve the fluidity of the scoriæ, and to burn the gases that escape from the furnace, as also to quicken the draught, and to keep the remote end of the furnace warm. On the top of this iron plate, and at the bottom of the inclined plane, the cinder accumulates in a small carity, whence it afterwards flows away; whenever it tends to congeal, the workman must clear it out with his rake.

The door is a cast iron frame filled up inside with fire-bricks; through a small hole in its bottom the workmen can observe the state of the furnace. This hole is at other times shut with a stopper. The chimney has an area of from 14 to 16 inches.

The hearth stands 3 feet above the ground. Its arched roof, only one brick thick, is raised 2 feet above the fire-bridge, and above the level of the sole, taken at the middle of the furnace. At its extreme point near the chimney, its elevation is only 8 inches; and the same height is given to the opening of the chimney.

In most iron works the sole is covered with a lajer of refractory sand from $2 \frac{1}{2}$ to 3 inches thick, which is lightly beat down with a shovel. At each operation a portion of the sand is carried away; and is replaced before another. Within these few years, there has been substituted for the sand a body of pounded slags; a substitution which has oceasioned, it is said, a great economy of iron and fuel.

The fine metal obtained by the cole is puddled by a continuous operation, which calls for much care and skill on the part of the workmen. To charge the puddling furnace, pieces of fine metal are successively introduced with a shovel, and laid one over another on the sides of the hearth, in the form of piles rising to the roof; the middle being left open for puddling the metal, as it is successively fused. Indeed, the whole are kept as far separate as possible, to give free circulation to the air round the piles. The working door of the furnace is now closed, fuel is laid on the grate, and the mouth of the fireplace, as well as the side opening of the grate, are both filled up with coal, at the same time that the damper is entirely opened.

The fine mctal in about twenty minutes comes to a white-red heat, and its thin-edged fragments begin to melt and fall in drops on the sole of the furnace. At this period the workman opens the small hole of the furnace door, detaches with a rake the pieces of fine metal that begin to melt, tries to expose new surfaces to the action of the heat, and 
in order to prevent the metal from runnirg together as it softens, he removes it from the vicinity of the fire-bridge. When the whole of the fine metal has thus got reduced to a pasty condition, he must lower the temperature of the furnace, to prevent it from becoming more fluid. He closes the damper, takes out a portion of the fire, and the ribs of the grate, and also throws a little water sometimes on the semi-fused mass. $\mathrm{He}$ then works about with his paddie the clotty metal, which swells up, with the discharge of gaseous oxyde of carbon, burning with a blue flame, as if the bath were on fire. The metal becomes finer by degrees, and less fusible; or in the language of the workmen, it begins to get dry. The disengagement of the oxyde of carbon diminishes, and soon stops. The workmen continue meanwhile to puddle the metal till the whole charge be reduced to the state of incoherent sand; and at that time, the ribs of the grate are replaced, the fire is restored, and the register is progressively opened up. With the return of the heat, the particles of metal begin to agglutinate, the charge becomes more difficult to raise, or in the lahorers' language, it works heavy. The refining is now finished, and nothing remains but to gather the iron into balls. The founder with his paddle takes now a little lump of metal, as a nucleus, and makes it roll about on the surface of the furnace, so as to collect more metal, and form a ball of about 60 or 70 pounds weight. With a kind of rake, called in England a dolly, and which he heats beforehand, the workman sets this ball on that side of the furnace most exposed to the action of the heat, in order to unite its different particles; which he then squeezes together to force out the scoriæ. When all the balls are fashioned, (they take about 20 minutes work, ) the small opening of the working door is closed with a brick, to cause the heat to rise, and to facilitate the welding. Each ball is then lifted out, either with tongs, if roughing rollers are to be used, as in Wales, or with an iron rod welded to the lump as a handle, if the hammer is to be employed, as in Staffordshire. Thus we see that the operation lasts in whole from 2 hours to $2 \frac{1}{2}$; in a quarter of an hour, the fine metal melts at its edges, when the puddling begins, in order to effect its division; at the end of as hour or an hour and a half, the metal is entirely reduced to a sand; a state shat is kept up for half an hour by continual stirring; and finally, the balling operation takes nearly the same time.

The charge for each operation is from $3 \frac{1}{2}$ to 4 hundred weight; and sometimes the cuttings of bar-ends are introduced, which are puddled apart. The loss of iron is here zery variable, according to the degree of skill in the workman, who by negligence may sufier a considerable body of iron to scorify or to flow into the hearth and raise the bottom. In good working, the loss is from 8 to 10 per cent. In Wales, the consumption of coal is estimated at one ton for every ton of fine metal. About five puddling furnaces are required for the service of one smelting furnace and one finery. The hearth of the pudding furnace should be exposed to heat for 12 hours before the work begins on the Mondays; and on the Saturdays, the old sole must be cleared out, by melting it off, and running it out by the floss-hole.

III. Sehafthault obtained, in May, 1835, a patent for the conversion of cast into wrought iron, by adding a mixture of black oxyde of manganese, common salt, and potter's clay, in certain small portions, successively, to the melting iron in the puddling furnace.

The reheating furnaces, balling furnaces, or mill furnaces, are analogous to the puddling furnaces, but only of larger dimensions.

The wood charcoal forge hearth is employed for working up scrap iron into boiler plate, \&c. Herc 22 bushels of charcoal are consumed in making one ton of iron of that description, from boiler plate parings.

Machines for forging and condensing the iron.-In England there are employed for the forging and drawing out of the iron, cast-iron hammers of great weight, and cylinders of different dimensions, for beating out the balls, or extending the iron into bars, as also porrerful shears. These several mechanisms are moved either by a steam engine, as in Staffordshire, and in almost all the other counties of England, or by water-wheels when the localities are favorable, as in many establishments in South Wales. We shall here offer some details concerning these machines.

The main driving shaft usually carries at either end a large toothed wheel, which communicates motion to the different machines through smaller toothed wheels. Of these, there are commonly six, four of which drive four different systems of cylinders, and the two others work the hammer and the shears. The different cylinders of an iron work should never be placed on the same arbor, because they are not to move together, and they must have different velocities, according to their diameter. In order to economize time and facilitate labor, care is taken to associate on one side of the motive machine the hammer, the shears, and the reducing cylinders; and on the other side to place the several systems of crlinders for drawing out the iron into bars. For the same reason the puddling furnaces ought to be grouped on the side of the hammer; and the reheating furnaces on the other side of the works. 
The hammers, fig. 596, are made entirely of cast iron; they are nearly 10 feet long, and consist usually of two parts, the helve $c$, and the head or pane $d$. The latter enter 596

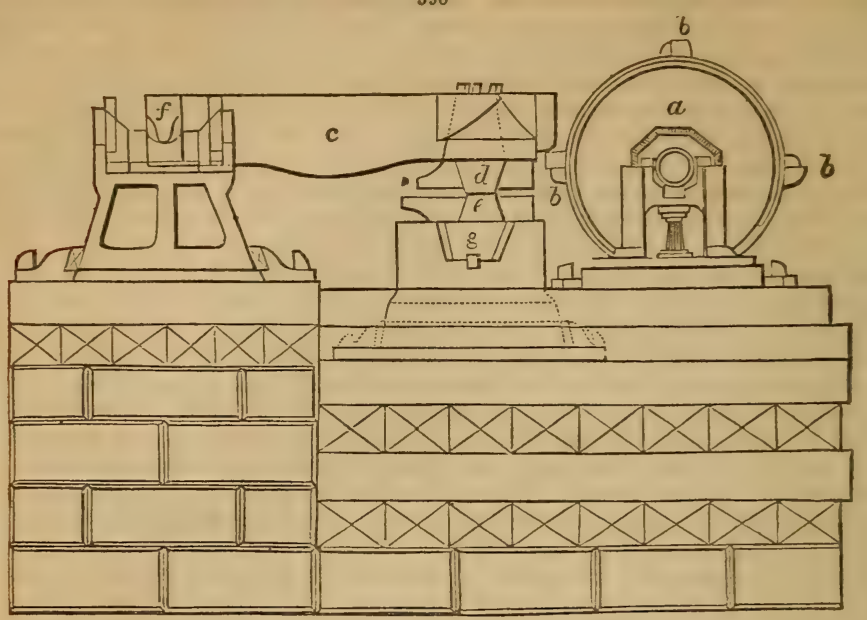

with friction into the former, and is retained in its place by wedges of iron or wood. The head consists of several faces or planes receding from each other; for the purpose of giving different forms to the ball lumps. A ring of cast-iron $\alpha$, called the cam-ring bag, bearing moveable cams $b b$, drives the hammer $d$, by lifting it up round its fulcrum $f$, and then letting it fall alternately. In one iron work, this ring was found to be 3 feet in diameter, 18 inches thick, and to weigh 4 tons. The weight of the helve (handle) of the corresponding hammer was 3 tons and a half, and that of the head of the hammer, 8 hundred weight.

The anvil $e$ consists also of two parts ; the one called the pane of the anvil, is the counterpart of the pane of the hammer; it likewise weighs 8 hundred weight. The second, $g$, named the stock of the anril, weighs 4 tons. Its form is a parallelopiped, with the edges rounded. The bloom or rough ball, from the puddle furnace, is laid and turned about upon it, by means of a rod of iron welded to each of them, called a porter. Since the weight of these preces is very great, and the shocks very considerable, the utmost precautions should be taken in setting the hammer and its anril upon a substantial mass of masonry, as shown in the figure, over which is laid a double, or eren quadruple flooring of wood, formed of beams placed in transverse layers close to each other. Such beams possess an elastic force, and thereby partially destroy the injurous reaction of the shock. In some works, a six-feet cube of cast iron is placed as a pedestal to the anvil.

Forge hammers are very frequently mounted as levers of the first kind, with the centre of motion about one third or one fourth of the length of the helve from the cam wheel. The principle of this construction will be understood by inspection of fig. 605. The short end of the lerer which is struck down by the tappet $c$, is driven against the end of an elastic beam $\alpha$, and immediately rebounds, causing the long end to strike a harder blow upon the anvil s.

The shears are composed of two branches, the one fixed and the other moveable, each formed of two pieces. The fixed branch is a cast-iron plate, which forms one mass with a horizontal base fixed to a piece of wood or cast iron buried in the ground. A sharpened chisel is fastened to its upper part by screws and nuts. The moveable branch is likewise of cast iron; it bears an axis round which it turns, and this axis passes through the fixed part. It is also furnished with a cutting chisel, fixed on by nuts and screws. An eccentric or an ellipse, mored directly by a toothed wheel, lifts the moreable branch of the shears, and forces it to cut the iron bars presented to it. The pressure exerted by these scissors is such, that they can cut without difficulty, iron bars, one half or two thirds of an inch thick.

Cylinders. - The compression between crlinders now effects, in a few seconds, that condensation and distribution of the fibres, which, 40 years ago, could not be aczomplished till after many heats in the furnace, and many blows of the hammer. $-2 \cdot 8$ cylinders mar be distinguished into two kinds; 1 . those which serve to draw out the ball, called puddling rolls, or roughing rolls, and which are, in fact, reducing cylinders; 2. the cylinders of extension, called rollers, for drawing inte bars the massire iron after it bas received a welding, to make it more malleable. This second kind of cylinders is 
subdirided into several varieties, according to the patterns of bar iron that are required. These may va:y from 2 inches square to less than one sirth of an inch.

Beneath 11: : cylinders there is usually formed an oblong fosse, into which the scoriæ and the scal: fall when the iron is compressed. The sides of this fosse, constructed of stone, are fomded on a body of solid masonry, capable of supporting the enormous load of the eylimlers. Beams of wood form in some measure the sides of this pit, to which crlinders may be made fast, by securing them with screws and bolts. Massive bars of cast iron are found, however, to answer still better, not only because the uprights and bearers may be more solidly fixed to them, but because the basement of heavy metal is more dificult to shatter or displace, an accident which happens frequently to the wooden beams. A rill of water is supplied by a pipe to each pair of cylinders, to hinder them from getting hot; as also to prevent the hot iron from adhering to the cylinder, by cooling its surface, and perhaps producing on it a slight degree of oxydizement.

The shafts are one foot in diameter for the hammer and the roughing rolls; and six inches where they communicate motion to the cylinders destined to draw the iron into bars.

The roughing rolls are employed either to work out the lump or ball immediately after it leaves the puddling furnace, as in the Welsh forges, or only to draw out the piece, after it has been shaped under the hammer, as is practised in most of the Staffordshire establishments. These roughing cylinders are generally 7 feet long, including the trunnions, or 5 feet between the bearers, and 18 inches diameter; and weigh in the whole from 4 to $4 \frac{1}{2}$ tons. They contain from 5 to 7 grooves, commonly of an elliptical form, one smaller than another in regular progression, as is seen in fig. 597. The small axis of each ellipse, as formed by the union of the upper and under grooves, is always placed in the vertical direction, and is equal to the great axis, or horizontal axis of the succeeding groove; so that in transferring the bar from one oroove to another, it must receive a quarter of a revolution, whereby the iron gets elongated in every direction. Sometimes the roughing rolls serve as preparatory cylinders, in which case they bear towards one extremity rectangular grooves, as the figure exhibits. Several of these large groores are bestudded with small asperities analogous to the teeth of files, for biting the lump of iron, and preventing its sliding. On a level with the under side of the grooves of the lower cylinder, there is a plate of cast iron with notches in its edge adanted to the grooves. This piece, called the apron, rests on iron rods, and serves to support the balls and bars exposed to the action of the rollers, and to receive the fragments of ill-welded metal, which fall off during the drawing. The housing frames in which the rollers are supported and revolve, are made of great strength. Their height is 5 feet; their thickness is 1 foot in the side perpendicular to the axis of the cylinders, and 10 inches in the other. Each pair of bearers is connected at their upper ends by two iron rods, on which the workmen rest their tongs or pincers for passing the lump or bar from one side of the cylinders to the other.

The cods or bushes are each composed of two pieces; the one of hard brass, which presents a cylindrical notch, is framed into the other which is made of cast iron, as is clearly seen in fig. 597.

The iron bar delivered from the square grooves, is cut by the shears into short lengths, which are collected in a bundle in order to be welded together. When this bundle of bars has become hot enough in the furnace, it is conveyed to the rollers; which differ in their arrangement according as they are meant to draw iron from a large or small piece. The first, fig. 597, possess both elliptical and rectangular grooves; are 1 foot in diameter and 3 feet long between the bearers. The bar is not finished under these cylinters, but is transferred to another pair, whose grooves have the dimensions proper for the bar, with a round, triangular, rectangular, or fillet form. The trianguiar grooves made use of for square iron, have for their profile an isosceles triangle, slightly obtuse, so that the space left by the two grooves together may be a rhombus, differing little from a square, and whose smaller diagonal is rertical. When the bar is to be passed successively through several grooves of this kind, the larger or horizontal diagonal of each following groore is made equal to the smaller or upright of the preceding one, whereby the iron must be turned one fourth round at each successive draught, and thus receive pressure in opposite directions. Indeed, the bar is often turned in succession through the triangular and rectangular grooves, that its fibres mas be more accurately worked together. The decrement in the capacity of the grooves follows the proportion of 15 to 11 .

When it is intended to reduce the iron to a small rod, the cylinders have such a diameter, that three may be set in the same housing frame. The lower and middle cylinders are employed as roughing rollers, while the upper and middle ones are made to draw out the rod. When a rod or har is to be drawn with a channel or gutter in its face, the grooves of the rollers are suitably formed. 
To draw out square rods of a very small size, as nail-rods, a system of small rollers w employed, called slitters. Their ridges are sharp-edged, and enter into the opposite

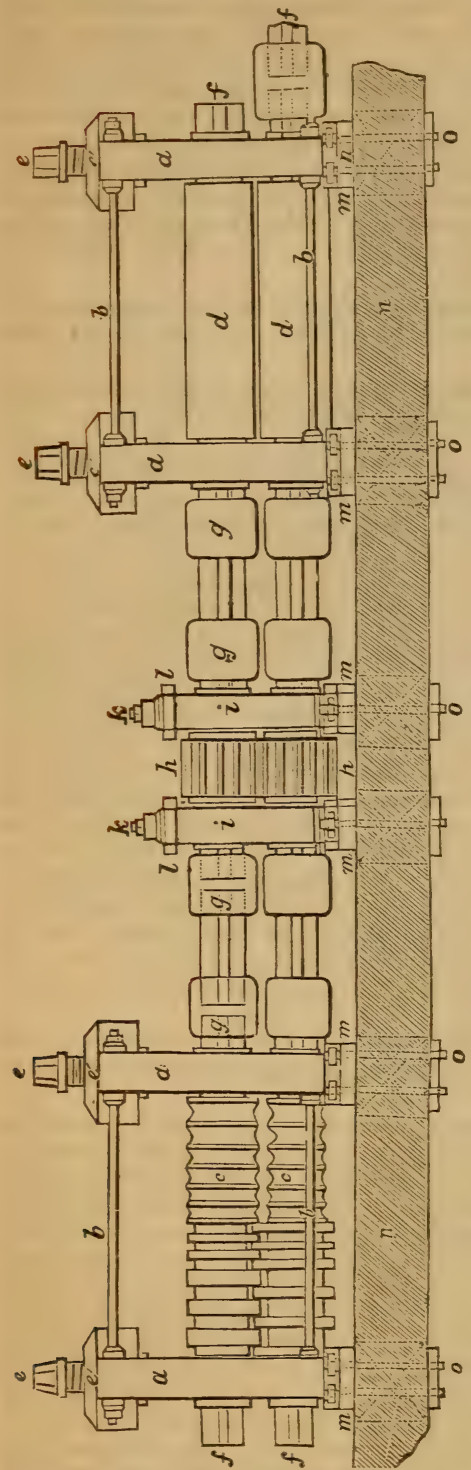
grooves $2 \frac{1}{2}$ inches deep; so that the flat bar in passing between such rollers is instantaneously divided into several slips. For this purpose the rollers represented in fig. 598 may be

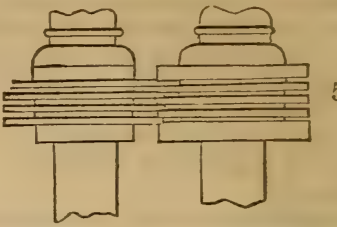

put on and removed from the shaft at plea sure.

The velocity of the cylinders varies with their dimensions. In one work, cylinders for drawing out iron of from one third to two thirds of an inch thick, make 140 revolutions per minute; while those for iron of from two thirds of an inch to 3 inches, make only 65 . In another work, the cylinders for two inch iron, make 95 revolutions per minute; those for iron from two thirds of an inch to an ineh and a third, make 128; and those for bars from one third to two thirds of an inch, 150. The roughing rollers move with only one third the velocity of the drawing cylinders.

The shingling and plate-rolling mill is represented in fig. 597. The shingling mill, for converting the blooms from the balling furnace into bars, consists of two sets of grooved cylinders, the first being called puddling rolls or roughing rolls; the second are for reducing or drawing the iron into mill-bars, and are called simply rolls.

$a, a, a, a$, are the powerful uprights or standards called housing frames, of cast iron, in which the gudgeons of the rolls are set to revolve; $b, b, b, b$, are bolt rods for binding these frames together at top and bottom; $c$, are the roughing rolls, having each a series of triangular grooves, such that between those of the upper and under cylinder, rectangular concavities are formed in the circumference with slightly sloping sides. The end groove to the right of $c$, should be channelled like a rough file, in order to take the better hold of the blooms, or to bite the metal, as the workmen say; and give it the preparatory elongation for entering into and passing through the remaining grooves till it comes to the square ones, where it becomes a mill-bar. $d, d$, are the smooth cylinders, hardened upon the surface, or chilled as it is called, by being cast in iron moulds, for rolling iron into plates or hoops. $e, e, e, e$, are strong screws with rectangular threads, which work by means of a wrench or key, into the nuts $e^{\prime} e^{\prime} e^{\prime} e^{\prime}$, fixed in the standards; they serve to regulate the height of the plummer blocks or bearers of the gudgeons, and thereby the distance between the upper and under cylinders. $f$ is a junction shaft; $g$ $g$, $g$, are solid coupling hoxes, which emurace the two separate ends of the shafts, and make them turn together. $h, h$, are junction pinions, whereby motion is communicated from the driving shaft $f$, through the under pinion to the upper one, and thus to both upper and under rolls at once. $i, i$, are the pinion standards in which their shafts run; they are smaller than the uprights of the rolls. $k, k$, are screws for fastening the head pieces $l$ tc the top of the pinion standards. All the standards are provided with sole plates $m_{1}$ 
whereby they are screwed to the foundation beams $n$, of wood or preferab.y iron, as shown by dotted lines; 00 are the binding screw bolts. Each pair of rolls at work is kept cool by a small stream of water let down upon it from a pipe and stop-cock.

In the crlinder drawing, the workman who holds the ball in tongs, passes it into the first of the elliptical grooves; and a second workman on the other side of the cylinders, receires this lump, and hands it over to the first, who re-passes it between the rollers, after bringing them somewhat closer to each other, by giving a turn to the adjusting pressure screws. After the lump has passed five or six times through the same groove, it has got an elliptical form, and is called in England a bloom. It is next passed through a second groore of less size, which stretches the iron bar. In this state it is subjected to a second pair of cylinders, by which the iron is drawn into flat bars, 4 inches broad and half an inch thick. Fragments of the ball or bloom fall round about the cylinders; which are afterwards added to the puddling charge. In a minute and a half, the rude lump is transformed into bars, with a neatness and rapidity which the inexperienced eve can hardly follow. A steam engine of thirty-horse power can rough down in a week, 200 tons of coarse iron.

This iron, called mill-bar iron, is however of too inferior a quality to be employed in any machinery; and it is subjected to another operation, which consists in welding several pieces together, and working them into a mass of the desired quality. The iron bars, while still hot, are cut by the shears into a length proportional to the size of iron bar that is wanted; and four rows of these are usually laid over each other into a heap or pile, which is placed in the re-heating furnace above described, and exposed to a free circulation of heat; one pile being set crosswise over another. In a half or three quarters of an hour, the iron is hot enough, and the pieces now sticking together, are carried in successive piles to the bar-drawng cylinders, to be converted into strong bars, which are reckoned of middle quality. When a very tough iron is wanted, as for anchors, another welding and rolling must be given. In the re-heating ovens, the loss is from 8 to 10 per cent. on the large bar iron, and from 10 to 12 in smaller work. A ton of iron consumes in this process about $150 \mathrm{lbs}$. of coals.

It is thought by many that a purer iron is obtained by subjecting the balls as they come out of the puddling furnace, to the action of the hammer at first, than to the roughing rollers; and that by the latter process vitrified specks remain in the metal, which the hammer expels. Hence, in some works, the balls are first worked under the forgehammer; and these stampings being afterwards heated in the form of pies or cakes piled over each other, are passed through the roughing rollers.

Having given ample details concerning the manufacturing processes used in England for making cast iron, it may be proper to subjoin a few observations upon its chemical constitution. It has been generally believed and taught that the dark gray cast iron, No. 1 or No. 2, contains more carbon than the white cast iron; and that the superior quality of the former in tenacity and softness, is to be ascribed to that excess. But the distinguished German metallurgist, M. Karsten, in his instructive volume, "Handbuch der Eisenhüttenkunde," or manual of the art of smelting iron ores, has proved, on the contrary, that the white cast iron contains most charcoal; that this substance exists in it in a state of combination with the whole body of the iron; that the foliated or lamellar white cast iron contains as much carbon as iron can absorb in the liquid state; and that this constitutes a compound of 4 atoms of iron combined with 1 of charcoal, or $112+6$; or $5 \frac{1}{3}$ per cent.; whereas the dark gray cast iron contains generally from 3 to 4 per cent., in the state of plumbago merely dispersed through the metal. He has further confirmed his opinion, by causing the white variety to pass into the gray, and reciprocally. Thus, dark gray cast metal melted and suddenly cooled, gives a silvery white metal, hard and brittle. On the other hand, when the white cast iron is cooled very slowly after fusion, the condition of the carbon in it changes, and a dark gray cast iron is obtained. These phenomena show that the graphite or plumbago, which requires a high temperature for its formation, cannot be produced but by a slow cooling, which allows the carbon to agglomerate itself in the iron in the state of graphite; while under a rapid congelation, the carbon remains dissolved in the mass, and produces a white metal. Hence we may understand how each successive fusion of dark gray iron hardens and whitens it, though in contact with coke, by completing that chemical dissolution of the carbon on which the white state depends.

In the manufacture of the blackest No. 1 cast iron, it sometimes happens that a considerable quantity of a glistening carburet of iron appears, floating on the top of the metal as it is run out into the sand-moulds. This substance is called kish by the English workmen; and it affords a sure test of the good state of the furnace and quality of the iron.

The most remarkable fact relative to the smelting of cast iron, is the difference of pro. duct between the workings of the summer and the winter season, though all the materials and machinery be the same. In fact, no cold-blast furnace will carry so great a burden 
in summer as in winter, that is, afford so great a product of metal, or bear so great a charce of ore with the same quantity of colie. This difference is undoubtedly due to the dilated and humid state of the atmosphere in the warm season. A very competent judge of this matter, states the diminution in summer at from one fifth to one seventh, independently of deterioration of quality.

Some of the forcign irons, particularly certain Swedish and Russian bars, are imported into Great Britain in large quantities, and at prices much greater than those of the English bars, and therefore the modes of manufacturing such excellent metal deserve examination. All the best English cast steel, indeed, is made from the hoop L iron from Dannemora, in Sweden.

The processes pursued in the smelting works of the Continent have frequently in view to obtain fiom the ore malleable iron directly, in a pure or nearly pure state. The furnaces used for this purpose are of two linds, called in French, 1. Feux de Loupes, or Forges Catalanes; and 2. Fourneaux à pièce, or Forges Allemañdes.

In the Catalan, or French method, the ore previously roasied in a kiln is afterwards strongly torrefied in the forge before the smelting begins; operations which follow in immediate succession. Ores treated in this way should be very fusible and very rich; such as black oxyde of iron, hematites, and certain spathose iron ores. From 100 parts of ore, 50 of metallic iron have been procured, but the average product is 35 . The furnaces employed are rectangular hearths, figs. 599, and 600, the water-blowing

599

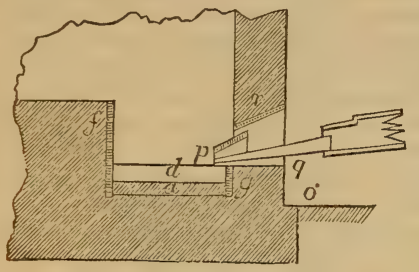

600

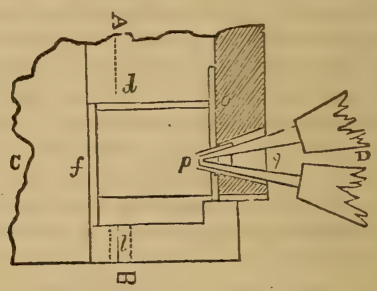

machine being employed to give the blast. See Metallorgy. There are three rarieties of this forge; the Catalan, the Navarrese, and the Biscayan. The dimensions of the first, the one most generally employed, are as follows: 21 inches long, in the lirection $p$ f, fig. 600 ; $18 \frac{1}{2}$ broad, at the bottom of the hearth or creuset, in the line A B ; and 17 inches deep, fig. 599. The tuyère, $q$, is placed $9 \frac{1}{2}$ inches above the bottom, so that its axis is directed towards the opposite side, about 2 inches above the bottom. But it must be moveable, as its inclination needs to be changed, according to the stage of the operation, or the quantity of the ores. It is often raised or lowered with pellets of clay; and even with a graduated circle, for the workmen make a great mystery of this matter. The hearth is lined with a layer of brasque (loam and charcoal dust worked together), and the ore after being roasted is sifted; the small powder being set aside to be used in the course of the operation. The ore is piled up on the side opposite to the blast in a sharp saddle ridge, and it occupies one third of the furnace. In the remaining space of two thirds, the charcoal is put. To solidify the small ore on the hearth, it is covered with moist cinders mixed with clay.

The fire is urged with moderation during the first two hours, the workman being continually employed in pressing down more charcoal as the former supply burns away, so as to keep the space full, and prevent the ore from crumbling down. By a blast so tempered at the beginning, the ore gets well calcined, and partially reduced in the way of cementation. But after two hours, the full force of the air is given; at which period the fusion ought to commence. It is easy to see whether the torrefaction be sufficiently advanced, by the aspect of the flame, as well as of the ore, which becomes spongy or cavernous; and the workman now completes the fusion, by detaching the pieces of ore from the bottom, and placing them in front of the tuyère. When the fine siftings are afterwards thrown upon the top, they must be watered, to prevent their being blown away, and to keep them evenly spread over the whole surface of the light fuel. They increase the quantity of the products, and give a propel fusibility to the scoriæ. When the scoriæ are viscid, the quantity of siftings must be diminished; but if thin, they must be increased. The excess of slag is allowed to run off by the chio or floss hole. The process lasts from five to six hours, after which the pasty mass is taken out, and placed under a hammer to be cut into lumps, which are afterwards forged into bars.

Each mass presents a mixed variety of iron and steel; in proportions which may be modified at pleasure; for by using much of the siftings, and making the tuyère dip towards the sole of the hearth, iron is the chief product; but if the operation be cor 
ducted slowly, with a small quantity of siftings, and an upraised tuycre, the quantity ct steel is more considerable. This primitive process is farorably spoken of by $M$. Brongniart. The weight of the lump of metal varies from 200 to 400 pounds. As the consumption of charcoal is very great, amounting in the Palatinate or Kheinkreis to seven times the weight of iron obtained, though in the Pyrenees it is only thrice, the Catalan forge can be profitably employed only where wood is exceedingly cheap and abundant.

The Fourneanx à pièce of the French, or Stuck-ofen of the Germans, resembles fig. 313 (COPPER); the tuyère (not shown there) having a dip towards the bottom of the hearth, where the smclted matter collects. When the operation is finished, that is, at least once in every 24 hours, one of the sides of the hearth must be demolished, to take out the pasty mass of iron, more or less pure. This furnace holds a middle place in the treatment of iron, between the Catalan forge and the cast-iron floss-ofen, or high-blast furnaces. The stuck-ojen are from 10 to 15 feet high, and about 3 feet in diameter at the hearth. Most usually there is only one aperture for the tuyère and for working; with a small one for the escape of the slag; on which account, the bellows are removed to make way for the lifting out of the lump of metal, which is done through an opening left on a level with the sole, temporarily closed with brickis and potters' clay, while the furnace is in action.

This outlet being closed, and the furnace filled with charcoal, fire is kindled at the bottom. Whenever the whole is in combustion, the roasted ore is introduced at the top in alternate charges with charcoal, till the proper quantity has been introduced. The ore falls down; and whenever it comes opposite to the tuyère the slag begins to flow, and the iron drops down and collects at the bottom of the hearth into the mass or sluck; and in proportion as this mass increases, the floss-hole for the slag and the tuyère is raised higher. When the quantity of iron accumulated in the hearth is judged to be sufficient, the bellows are stopped, the scoriæ are raked off, the little brick wall is taken down, and the mass of iron is remored hy rakes and tongs. This mass is then flattened under the hammer, into a cake from 3 to 4 inches thick, and is cut into two lumps, which are submitted to a new operation; where it is treated in a peculiar refinery, lined with charcoal brasque, and exposed to a nearly horizontal blast. The above mass, seized in the jaws of a powerful tongs, is heated before the tuyère; a portion of the metal flows down to the bottom of the hearth, loses its carbon in a bath of rich slags or fused oxydes, and forms thereby a mass of iron thoroughly refined. The portion that remains in the tongs furnishes steel, which is drawn out into bars.

This process is employed in Carniola for smelting a granular oxyde of iron. The mass or sincli amounts to from 15 to 20 hundred weigh1, after each operation of 24 hours. Eight strong men are required to lift it out, and to carry it under a large hammer, where it is cut into pieces of about 1 cwt. each. These are afterwards refined and drawn into bars as above described. These furnaces are now almost generally abandoned on the Continent, in favor of charcoal high or blast furnaces.

Fig. 313 represents a shachtofen (but without the tuyère, which may be supposed to be in the usual place), and is, like all the continental Hauls Fourneaux, remarkable for the excessirt thickness of its masonry. The charge is put in at the throat, near the summit of the octaconal or square concarity, for they are made of both forms. At the bottom of the hearth there is a dam-stone with its plate, for permitting the overflow of the slag, while it confines the subjacent fluid metal; as well as a tymp-stone with its plate, which forms the liey to the front of the hearth; the boshes are a wide funnel, almost flat, to obstruct the easy descent of the charges, whereby the smelting with charcoal would proceed tor rapidly. The bottom of the hearth is constructed of two large stones, and the hinue part of one great stone, called in German, riicksiein (black stone), which the French have corrupted into rustine. In other countries of the Continent, the boshes are frequently a good deal more tapered downwards, and the hearth is larger than here representel. The refractory nature of the $\mathrm{Hartz}$ iron ores is the reason assigned for this peculiarity.

In Swclen there are blast-furnaces, schachtofen, 35 feet in height, measured from the boshes above the line of the hearth, or creuset. Their cavity has the form of an elonga ted ellipse, whose small diameter is 8 feet across, at a height of 14 feet above the bottom of the hearth; hence, at this part, the interior space constitutes a belly, corresponding with the upper part of the boshes. In other respects, the details of the construction of the Siredish furnaces resemble the one figured above. Marcher relates that a furnace of that lind, whose height was only 30 feet, in which brown hydrate of iron (hematite) was smelted, yielded 47 per cent. in cast-iron, at the rate of 5 hundred weight a day, or 36 hundred weight one week after another; and that in the production of 100 pounds of cast-iron, 130 pounds of charcoal were consumed. That furnace was worked with forge bellows, mounted with leather. 
The decarburation of cast-iron is merely a restoration of the carbon to the surlace, in tracing inversely the same progressire steps as had carried it into the interior during the smelting of the ore. The oxygen of the air, acting first at the surface of the cast metal, upon the carbon which it finds there, burns it: fresh charcoal, oozing from the interior, comes then to occupy the place of what had been dissipated; till, finally, the whole carbon is transferred from the centre to the surface, and is there converted into either carbonic acid gas, or oxyde of carbon; for no direct experiment has hitherto proved which of these is the precise product of this combustion.

This diffusibility of carbon through the whole mass of iron constitutes a movement by means of which cast-iron may be refined even without undergoing fusion, as is proved by a multitude of phenomena. Every workman has observed that steel loses a portion of its steely properties every time it is heated in contact with air.

On the above principle, cast-iron may be refined at one operation. Three kinds of iron are susceptible of this continuous process:-1. The speckled cast-iron, which contains such a proportion of oxygen and carbon as with the oxygen of the air and the carbon of the fuel may produce sufficient and complete saturation, but nothing in exces 2 . The dark gray cast-iron. 3. The white cast-iron. The nature of the crude metri requires variations both in the forms of the furnaces, and in the manipulations.

Indeed, malleable iron may be obtained directly from the ores by one fusion. This mode of working is practised in the Pyrenees to a considerable extent. All the ores of iron are not adapted for this operation. Those in which the metallic oxyde is mixed with much earthy matter, do not answer well; but those composed of the pure black oxyde, red oxyde, and carbonate, succeed much better. To extract the metal from such ores, it is sufficient to expose them to a high temperature, in contact either with charcoal, or with carbonaceous gases; the metallic oxyde is speedily reduced. But when several earths are present, these tend continually, during the vitrification which they suffer, to retain in their vitreous mass the unreduced oxyde of iron. Were such earthy ores, as our ironstones, to be put into the low furnaces called Catalan, through which the charges pass with great rapidity, and in which the contact with the fuel is merely momentary, there would be found in the crucible or hearth merely a rich metallic glass, instead of a lump of metal.

In smelting and refining by a continuous operation, three different stages may be distinguished: 1. The roasting of the ore to expel the sulphur, which would be less easily separated afterwards. The roasting dissipates likewise the water, the carbonic acid, and any other volatile substances which the minerals may contain. 2. The deoxydizement and reduction to metal by exposure to charcoal or carbureted vapors. 3. The melting, agglutination, and refining of the metal to fit it for the heary hammers where it gets nerre. There are several forges in which these three operations seem to be con founded into a single one, because, although still successive, they are practised at one single heating without interruption. In other forges, the processes are performed separately, or an interval elapses between each stage of the work. Three systems of this kind are known to exist:-1. The Corsican method; 2. The Catalan with wood charcoal; and 3. The Catalan with coke.

The furnaces of Corsica are a kind of semicircular basins, 18 inches in diameter, and 6 inches deep. These are excavated in an area, or a small elevation of masonry, 8 or 10 feet long by 5 or 6 broad, and covered in with a chimney. This area is quite similar to that of the ordinary hearths of our blast-furnaces.

The tuyère stands 5 or 6 inches above the basin, and has a slight inclination downwards. In Corsica, and the whole portion of Italy adjoining the Mediterranean shores, the iron ore is an oxydde similar to the specular ore of the Isle of Elba. This ore contains a little water, some carbonic acid, occasionally pyrites, but in small quantity. Before deorydizing the ore, it is requisite to expel the water and carbonic acid combined with the oxyde, as well as the sulphur of the pyrites.

The operations of roasting, reduction, fusion, and agglutination, are executed in the same furnace. These are indeed dirided into two stages, but the one is a continuation of the other. In the first, the two primary operations are performed at once; - the reduction of a portion of the roasted ore is begun at the same time that a portion of the raw ore is roasted: these two substances are afterwards separated. In the second stage, the deoxydizement of the metal is continued, which had begun in the preceding stage; it is then melted and agglutinated, so as to form a ball to bs submitted to the forge-hammer.

The roasted pieces are broken down to the size of nuts, to make the reduction of the metal easier. In executing the first step, the basin and area of the furnace must be lined with a brasque of charcoal dust, 3,4 , or even 5 inches thick: over this brasque a mound is raised with lumps of charcoal, very hard, and 4 or 5 inches high. A semicircle is formed round the tuyère, the inner radius of which is 5 or 6 inches. This mass of charcoal is next surrounded with another pile of the roasted and broken ores, which 
must be core red with charcoal dust. The whole is sustained with large blocks of the raw ore, which form externally a third wall.

These three piles of charcoal, with roasted and unroasted ore, are raised in three sucsessire beds, each 7 inches thick : they are separated from each other by a layer of char:oal dust of about an inch, which makes the whole 24 inches high. This is afterwards sovered over with a thick coat of pounded charcoal.

The blocks of raw ore which compose the outward wall form a slope; the larger and stronger preces are at the bottom, and the smaller in the upper part. The large blocks are sunk very firmly into the charcoal dust, to enable them better to resist the pressure from within.

On the bottom of the semicircular well formed within the charcoal lumps, kindled pieces are thrown, and over these, pieces of black charcoal; after which the blast of a waterblowing machine (trompe) is given. The fire is kept up by constantly throwing charcoal into the central well. At the beginning of the operation it is thrust down with wooden rods, lest it should affect the building; but when the heat becomes 100 intense for the workmen to come so near the hearth, a long iron rake is employed for the purpose. At the end of about 3 hours, the two processes of roasting and reduction are commonly finished: then the raw ore no longer exhales any fumes, and the roasted ore, being softened, unites into lumps more or less coherent.

The workman now removes the blocks of roasted ore which form the outer casing, rolls them to the spot where they are to be broken into small pieces, and pulls down the brasque (small charcoal) which surrounds the mass of reduced ore.

The second operation is executed by cleaning the basin, removing the slags, covering the basin anew with 2 or 3 brasques (coats of pounded charcoal), and piling up to the right and the left, two heaps of charcoal dust. Into the interval between these conical piles two or three baskets of charcoal are cast, and on its top some cakes of the reduced crude metal being laid, the blast is resumed. The cakes, as they heat, undergo a sort of liquation, or sweating, by the action of the earthy glasses on the unreduced black oxyde present. Very fusible slags flow down through the mass; and the iron, reduced and melted, passes finally through the coals, and falls into the slag basin below. To the first parcel of cakes, others are added in succession. In proportion as the slags proceeding from these run down, and the melted iron falls to the bottom, the thin slag is run off by an upper overflow or chio hole, and the reduced iron kept by the heat in the pasty condition, remains in the basin : all its parts get agglutinated, forming a soft mass, which is remored by means of a hooked pole in order to be forged. Each lump or bloom of malleable iron requires 3 hours and a half for its production.

The iron obtained by this process is in general soft, very malleable, and but little steely. In Corsica four workmen are employed at one forge. The produce of their labor is only about $4 \mathrm{cwts}$. of iron from $10 \mathrm{cwts}$. of ore and 20 of charcoal, mingled with wood of beech and chestnut. Though their ore contains on an average 65 per cent. of iron, only about 40 parts are extracted; evincing a prodigious waste, which remains in the slags.

The difference between the Corsican and the Catalonian methods consists in the latter roasting the ore at a distinct operation, and employing a second one in the reduction, agglutination, and refining of the metal. In the Catalonian forges, 100 pounds of iron are obtained from 300 pounds of ore and 310 pounds of charcoal; being a produce of only 33 per cent. It may be concluded that there is a notable loss, since the sparry iron ores, which are those principally smelted, contain on an average from 54 to 56 per cent. of iron. The same ores, smelted in the ordinary blast furnace, produce about 45 per cent. of cast iron.

On the Continent, iron is frequently refined from the cast metal of the blast furnaces by three operations, in three different ways. In one, the pig being melted, with aspersion of water, a cake is obtained, which is again melted in order to form a second cake. This being treated in the refinery fire, is then worked into a bloom. In another system, the pig iron is melted and cast into plates: these are melted anew in order to obtain crude balls, which are finally worked into blooms. In a third mode of manufacture, the pig-iron is melted and cast into plates, which are roasted, and then strongly heated, to form a bloom.

The French fusible ores, such as the silicates of iron, are very apt to smelt into white cast iron. An excess of fluxes, light charcoals, too strong a blast, produce the same resuits. A surcharge of ores which deranges the furnace and affords impure slags mixed with much iron, too rapid a slope in the boshes, too low a degree of heat, and too great condensation of the materials in the upper part of the furnace; all tend also to produce a white cast iron. In its state of perfection, white cast iron has a silver color, and a bright metallic lustre. It is employed frequently in Germany for the manufacture of steel, and is then called steel floss, or lamellar floss, a title which it still retains, though it be hardly silver white, and have ceased to be foliated. When its color takes a bluish- 
gray tinge, and its fracture appears striated or splintery, or when it exhibits gray spots, it is then styled flower floss. In a third species of white cast iron we observe still much lustre, but its color verges upon gray, and its texture is variable. Its fracture has been sometimes compared to that of a broken cheese. This variety occurs very frequently. It is a white cast iron, made by a surcharge of ore in the furnace. If the white color becomes less clear and turns bluish, if its fracture be contorted, and contains a great many empty soaces or air-cells, the metal takes the name of cavernous-finss, or tender-floss. The whitest metal cannot be employed for casting. When the white is mixed with the gray cast iron, it becomes riband or trout cast iron.

The German refining forge.-Figs. 601, 602 represent one of the numerous refinery
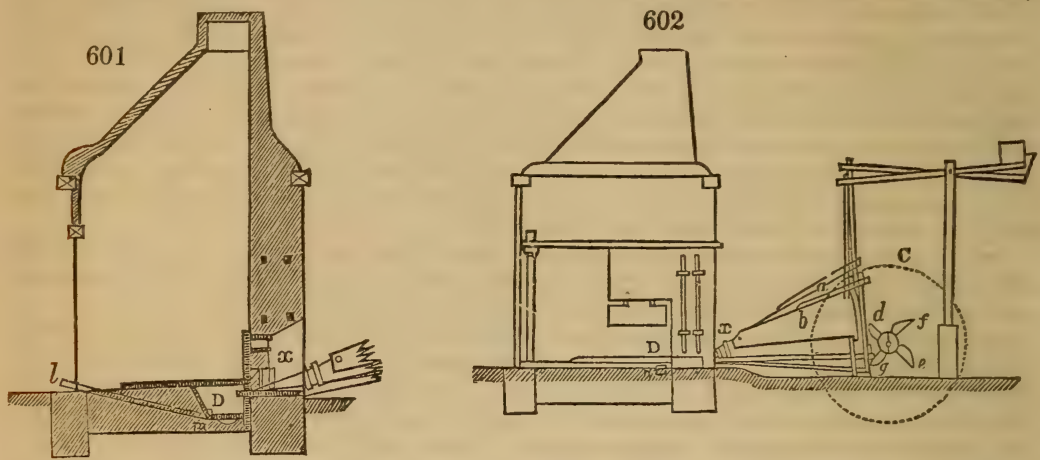

furnaces so common in the Hartz. The example is taken from the Mandelholz works, in the neighborhood of Elbingerode. Fig. 602 is an elevation of this forge. $\mathrm{D}$ is the refinery hearth, provided with two pairs of bellows. Fig. 601 is a vertical section, showing particularly the construction of the crucible or hearth in the refinery forge $\mathbf{D}$. $\mathrm{c}$ is an overshot water wheel, which gives an alternate impulsion to the two bellows $a b$ by means of the revolving shaft $c$, and the cams or tappets $d f e g$.

$\mathrm{D}$, the hearth, is lined with cast iron plates. Through the pipe $l$, cold water may be introduced, under the bottom plate $m$, in order to keep down, when necessary, the temperature of the crucible, and facilitate the solidification of the loupe or bloom. An orifice $n$, figs. 601, 602, called the chio (floss hole), allows the melted slag or cinder to flow off from the surface of the melted metal. The copper pipe or nose piece $p$, fig. 600, conducts the blast of both bellows into the hearth, as shown at $b x, f i g .602$, and $\mathbf{D} g p$, fig. 600 .

The substance subjected to this mode of refinery, is a gray carbonaceous cast iron, from the works of Rothehütte. The hearth $D$, being filled and heaped over with live charcoal, upon the side opposite to the tuyère $x$, figs. 601, 602, long pigs of cast iron are laid with their ends sloping downwards, and are drawn forwards successively into the hearth by a hooked poker, so that the extremity of each may be plunged into the middle of the fire, at a distance of 6 or 8 inches from the mouth of the tuyère. The workman proceeds in this way, till he has melted enough of metal to form a loupe. The cast iron, on melting, falls down in drops to the bottom of the hearth ; being covered by the fused slags, or vitreous matters more or less loaded with oxyde of iron. After running them off by the orifice $n$, he then works the cast iron by powerful stirring with an iron rake (ringard), till it is converted into a mass of a pasty consistence.

During this operation, a portion of the carbon contained in the cast iron combines with the atmospherical oxygen supplied by the bellows, and passes off in the form of carbonic oxyde and carbonic acid. When the lump is coagulated sufficiently, the workman turns it over in the hearth, then increases the heat so as to melt it afresh, meanwhile exposing it all round to the blast, in order to consume the remainder of the carbon, that is, till the iron has become ductile, or refined. If one fusion should prove inadequate to this effect, two are given. Before the conclusion, the workman runs off a second stratum of vitreous slag, but at a higher level, so that some of it may remain upon the metal.

The weight of such a loupe or bloom is about 2 cwts., being the product of $2 \mathrm{cwts}$. and $\frac{7}{10}$ of pig iron; the loss of weight is therefore about 26 per cent. 149 pounds of charcoal are consumed for every 100 pounds of bar iron obtained. The whole operation lasts about 5 hours. The bellows are stopped as soon as the bloom is ready; this is immediatcly transferred to a forge hammer, such as is represented fig. 605 ; the cast iron head of which weighs 8 o: 9 cwts. The bloom is greatly condensed thereby, and discharges a considerable quantity of semi-fluid cinder. The lump is then divided by the hammer 
ana a chisel into 4 or 6 pieces, which are reheated, one after another, in the same refinery n̂re, in order to be forged into bars, while another pig of cast iron is laid in its place, to prepare for the formation of a new bloom. The above process is called by the Germans klump-frischen, or lump-refining. It differs from the durch-brech-frischen, because in the latter, the lump is not turned over in mass, but is broken, and exposed in separate pieces successively to the refining power of the blast near the tuyère. The French call this $a f$ finage par portions; it is much lighter work than the other.

The quality of the iron is tried in various ways; as first, by raising a bar by one end, with the two hands over one's head, and bringing it forcibly down to strike across a narrow an ril at its centre of percussion, or one third from the other extremity of the bar; after which it may be bent backwards and forwards at the place of percussion several times; 2. a heavy bar may be laid obliquely over props near its end, and struck strongly with a hammer with a narrow pane, so as to curve it in opposite directions; or while heated to redness, they may be kneed backwards and forwards at the same spot, on the edge of the anvil. This is a severe trial, which the hoop L, Swedish iron, bears surprisingly, emitting as it is hammered, a phosphoric odor, peculiar to it and to the bar ir. $\mathrm{n}$ of Ulverstone, which also resembles it, in furnishing a good steel. The forging of a horseshoe is reckoned a good criterion of the quality of iron. Its freedom from flaws is detected by the above modes; and its linear strength may be determined by suspending a scale to the lower end of a hard-drawn wire, of a given size, and adding weights till the wire breaks. The treatises of Barlow and Tredgold may be consulted with advantage on the methods of proving the strength of different kinds of iron, in a great variety of circumstances.

Steel of cementation, or blistered steel and cast steel, are treated under the article STEEL. But since in the conversion of cast iron into wrought iron, by a very slight difference in the manipulations, a species of steel may be produced called natural steel, I shall describe this process here.

Fig. 603 is a view of the celebrated steel iron works, called Königshütte, (kingss-forge), in Upper Silesia, being one of the best arranged in Germany, for smelting iron ore by

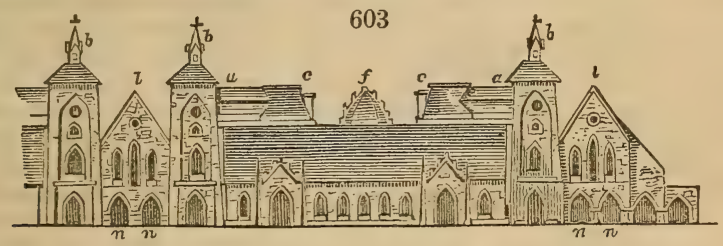

means of coke. The front shown here is about 400 English feet long. $a a$ are two blast furnaces. A third blast furnace, all like the English, is situated to the left of one of the towers $b$. $b \quad b$ are the charging towers, into which the ore is raised by machinery from the level of the store-houses $l l$, up to the mouth of the furnaces $a a ; c c$ point to the positions of the boilers of the two steam engines, which drive two cylinder bellows at $f$. $n n n n$ are arched cellars placed below the store-houses $l l$, for containing materials and tools necessary for the establishment.

Figs. 599, 604, are vertical sections of the forge of Königshütte, for making natural

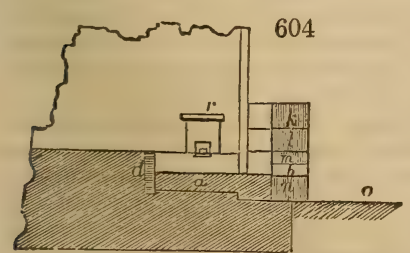
steel; fig. 599 being drawn in the line $\mathbf{A}$ B of the plan, fig. 600. $a$ is the bottom of the hearth, consisting of a fire-proof gritstone; $b$ is a space filled with small charcoal, damped with water, under which, at $n$, in fig. 604, is a bed of well rammed clay; $d$ is a plate of cast iron, which lines the side of the hearth called ruckstein (backstone) in German, and corrupted by the French into rustine; $f$ is the plate of the counter-blast; $g$ the plate of the side of the tuyère; behind, upon the face $d$, the fire-place or hearth is only $5 \frac{1}{2}$ inches deep; in front as well as upon the lateral faces, it is 18 inches deep. By means of a mound made of dry charcoal, the posterior face $d$ is raised to the height of the face $f . i$, fig. 600, is the floss-hole, by which the slags are run off from the hearth during the working, and through which, by removing some bricks, the lump of steel is taken out when finished.

$k l m$ are pieces of cast iron, for confining the fire in front, that is, towards the side where the workman stands; 0 is the level of the floor of the works; $p$ a copper tuyere; it is situated $4 \frac{1}{2}$ inches above the bottom $a$, slopes 5 degrees towards it, and advances 4 inches into the hearth or fire-place, where it presents an orifice, one half inch in horizontal length, and one inch up and down; $q$ the nose pipes of two bellows, like those represented 
in fig. 602, and under SrLver; the round orifice of each of them within the tuyère being one inch in diameter. $r$ is the lintel or top arch of the tuyère, beneath which is seen the cross section of the pig of cast iron under operation.

For the production of natural steel, a white cast iron is preferred, which contains little carbon, which does not flow thin, and which being cemented over or above the wind, falls down at once through the blast to the bottom of the hearth in the state of steel. With this view, a very flat fire is used; and should the metal run too fluid, some malleable lumps are introduced to give the mass a thicker pasty consistence.

If the natural steel be supposed to contain too little carbon, which is ₹ very rare case, the metal bath covered with its cinder slag, is diligently stirred with a wo nden pole, or it may receive a little of the more highly carbureted iron. If it contains the right dose of carbon, the earthy and other foreign matters are made progressively to sweat out, into the supernatant slag. When the mass is found by the trial of a sample to be completely converted, and has acquired the requisite stiffness, it is lifted out of the furnace, by the opening in front, subjected to the forge hammer, and drawn into bars. In Sweden, the cast iron pigs are heated to a cherry-red, and in this state broken to pieces under the hammer, before they are exposed in the steel furnace. These natural steels are much employed on the Continent in making agricultural implements, on account of their cheapness. The natural steel of Styria is regarded as a very good article.

Wootz is a natural steel prepared from a black ore of iron in Hindostan, by a process analogous to that of the Catalan hearth, but still simpler. It seems to contain a minute portion of the combustible bases of alumina and silica, to which its peculiar hardness when tempered may possibly be ascribed. It is remarkable for the property of assuming a damask surface, by the action of dilute sulphuric acid, after it has been forged and polished. See Damascus and Steel.

Fig. 605 is the German forge-hammer; to the left of 1 , is the axis of the rotatory

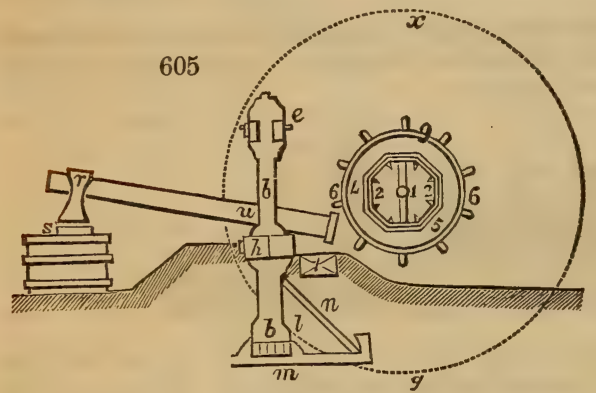
cam, 2,3 , consisting of 8 sides, each formed of a strong broad bar of cast iron, which are joined together to make the octagon wheel. $4,5,6$, are cast iron binding rings or hoops, made fast by wooden wedges. $b, b$, are standards of the frame-work $e, l, m$, in which the helve of the forge-hammer has its fulcrum near $u$. $h$, the sole part of the frame. Another cast iron base or sole is seen at $m$. $n$ is a strong stay, to strengthen the frame-work. At $r$ two parallel hammers are placed, with eastiron heads and wooden helves. $s$ is the anvil, a very massive piece of cast iron. $t$ is the end of a vibrating beam, for throwing back the hammer from it forcibly by recoil. $x y$ is the outline of the water-wheel which drives the whole. The cams or tappets are shown mounted upon the wheel $6, \mathrm{~g}, 6$.

Analysis of irons.--Oxydized substances cannot exist in metallic iron, and the foreign substances it does contain are present in such small quantities, that it is somewhat difficult to determine their amount. The most intricate point is, the proportion of carbon. The free carbon, which is present only in gray cast iron, may, indeed, be determined nearly, for most of it remains after solution of the metal in acids. The combined charcoal, however, changes by the action of muriatic acid into gas and oil ; sulphuric acid also occasions a great loss of carbon, and nitric acid dissipates it almost entirely. Either nitre or chloride of silver may be employed to ascertain the amount of carbon; but when the iron contains chromium and much phosphorus, the determination of the carbon is attended with many difficulties.

The quantity of sulphur is always so small, that it can scarcely be ascertained by the weight of the precipitate of sulphate of barytes from the solution of the iron in nitromuriatic acid. The iron should be dissolved in muriatic acid; and the hydrogen, as it escapes charged with the sulphur, should be passed through an acidulous solution of acetate of lead. The weight of the precipitated sulphuret shows the amount of sulphur, allowing 13.45 of the latter for 100 of the former. In this experiment the metal should be slowly acted upon by the acid. Cast iron takes from 10 to 15 days to dissolve, steel from 8 to 10 , and malleable iron 4 days. The residuum of a black color does not contain a trace of sulphur.

Phosphorus and chromium are determined in the following way. The iron must be dissolved in nitro-muriatic acid, to oxygenate those two bodies. The solution must be evaporated cautiously to dryness in porcelain capsules, and the saline residuum heated 
to redness. A little chloride of iron is volatilized, and the remainder resembles the red-bruwn oxyde. This must be mixed with thrice its weight of carbonate of potash, and fused in a platinum crucible; the quantity of iron being from 40 to 50 grains at most.

The mixture, after being acted upon by boiling water, is to be left to settle, to allow the oxrde to be deposited, for it is so fine as to pass through a filter. If the iron containei manganese, this would be found at first in the alkaline solution; but manganes: spontaneously separates by exposure to the air. The allialine liquor must be supersaturated with muriatic acid, and evaporated to dryness. The liquor acidulated, and deprived of its silica by filtration, is to be supersaturated with ammonia; when the alumina will precipitate in the state of a subphosphate. When the liquor is now supersaturated with acetic acid, and then treated with acetate of lead, a precipitate of phosphate of lead almost always falls. There is hardly a bit of iron to be found which does not contain phosphorus. The slightest trace of chrome is detected by the yellow color of the lead precipitate; if this be white there is none of the coloring metal present.

100 parts of the precipitated phosphate of lead contain, after calcination, $19 \cdot 4$ parts of phosphoric acid. The precipitate should be previously washed with acetic acid, and then with water. These $19 \cdot 4$ parts contain 8.525 parts of phosphorus.

Cast iron sometimes contains calcium and barium, which may be detected by their well-known reagents, oxalate of ammonia. and sulphuric acid. In malleable iron they are seldom or never present.

The charcoal found in the residuum of the nitro-muriatic solution is to be burned away under a muffle. The solution itself contains along with the oxyde of iron, protoxyde of manganese, and other oxydes; as well as the earths, and the phosphoric and arsenic acids. Tartaric acid is to be added to it, till no precipitate be formed by supersaturation with caustic ammonia. The ammoniacal liquor must be treated with hydrosulphuret of ammonia as long as it is clouded, then thrown upon a filter. The precipitate is usually very voluminous, and must be well washed. The liquor which passes through is to be saturated with muriatic acid, to decompose all the sulphurets.

The solution still contains all the earths and the oxyde of titanium, besides the phosphoric acid. It is to be eraporated to dryness, whereby the ammonia is expelled, and the carbonaceous residuum must be burned under a muffle. If the iron contains much phosphorus, the ashes are strongly agglutinated. They are to be fused as already described along with carbonate of potash, and the mass is to be treated with boiling water. The residuum may be examined for silica, lime, barytes, and oxyde of titanium. Muriatic acid being digested on it, then evaporated to dryness, and the residuum treated with water, will leave the silica. Caustic ammonia, poured into the solution, will separate the alumina, if any be present, and the oxyde of titanium; but the former almost never occurs.

Manganese is best sought for by a distinct operation. The iron must be dissolved at the heat of boiling water, in nitro-muriatic acid; and the solution, when very cold, is to be treated with small successive doses of solution of carbonate of ammonia. If the iron has been oxrdized to a maximum, and if the liquor has been sufficiently acid, and diluted with water, it will retain the whole of the manganese. This process is as good as that by succinate of ammonia, which requires many precautions.

The liquor is often tinged yellow by carbon, after it has ceased to contain a single trace of iron oxyde. As soon as litmus paper begins to be blued by carbonate of ammonia, we should stop adding it; immediately throw the whole upon a filter, and wash continuously with cold water. What passes through is to be neutralized with muriatic acid, anci concentrated by evaporation. It may contain, besides manganese, some lime or barytes. It should therefore be precipitated with hydro-sulphuret of ammonia, the hydrosulphuret of manganese should be collected, dissolved in strong muriatic acid, filtered, and treated, at a boiling heat, with carbonate of potash. The precipitate, well washed and calcined, contains, in 100 parts, 72.75 parts of metallic manganese.

The copper, arsenic, lead, tin, bismuth, antimony, or silver, are best separated by a stream of sulphureted hydrogen gas passed through the solution in nitro-muriatic acid, after it is largely diluted with water. The precipitate must be cautiously roasted in a porcelain test, to burn away the large quantity of sulphur which is deposited in consequence of the conversion of the peroxyde of iron into the protoxyde. If nothing remains upon the test, none of these metals is present. If a residuum be obtained, it must be dissolved in nitro-muriatic acid, and subjected to examination. But, in fact, carbon, sulphur, phosphorus, silicon, and manganese, are the chief contaminators of iron.

Chloride of silver affords the means of determining the proportion of carbon contained in iron, and of ascertaining the state in which that substance exists in the metal. Fused 
chloride of a pale yellow color must be employed. The operation is to be performed in c'ose ressels, with the addition of a great deal of water, and a few drops of muriatic acid. The carbonaceous residuum is occasionally slightly acted upon. We may judge of this circumstance by the gases disengaged, as well as by the appearance of the charcoal.

Ductile iron and soft steel, as well as white cast-iron which has been rendered gray by roasting, when decomposed by chloride of silver, afford a blackish-brown unmagnetic charcoal, and a plumbaginous substance perfectly similar to what is extracted from the same kinds of iron, by solution in acids. A portion of this plumbago is also converted into charcoal of a blackish brown color, by the action of the chloride. Hence this agent does not afford the means of obtaining what has been called the poly-carburet, till it has produced a previous decomposition. But we obtain it, in this manner, purer and in greater quantity than we could by dissolving the metal in the acids. The only subject of regret is, that we possess no good criterion for judging of the progress of this analytical operation.

Gray cast iron leaves, besides the poly-carburet, a residuum of plumbago, and carbon which was not chemically combined with the iron; while tempered steel and white cast iron afford merely a blackish brown charcoal; but the operation is extremely slow with the latter two bodies, because a layer of charcoal forms upon the surface, which obstructs their oxydizement. For this reason the white cast iron ought to be previously changed into gray by fusion in a crucible lined with charcoal, before being subjected to the chloride of silver; if this process be emrloyed for tempered steel, the combined carbon becomes merely a poly-carburet. It world not be possible to operate upon more than 15 grains, which require from 60 to 80 times that quantity of the chloride, and a period of 15 days for the experiment.

The residuum, which is separable from the silver only by mechanical means, should be dried a long time at the heat of boiling water. It contains almost always iron and silica. After its weight is ascertained, it is to be burned in a crucible of platinum till the ashes no longer change their color, and are not attractable by the magnet. The difference between the weights of the dried and calcined residuum is the weight of the charcoal. The oxyde of iron is afterwards separated from the silica by muriatic acid.

In operating upon gray cast iron, we should ascertain separately the proportion of graphite or plumbago, and that of the combined charcoal. To determine the former, we dissolve a second quantity of the cast iron in nitric acid, with a little muriatic; the residuum, which is graphite, is separated from the silica and the combined carbon by the action of caustic potash. After being washed and dried, it must be weighed. The weight of the graphite obtained being deducted from the quantity of carbon resulting from the de. composition effected by the chloride of silver, the remainder is the amount of the chemi cally combined carbon.

By employing muriatic acid, we could dissipate at once the combined carbon; but thi method would be inexact, because the hydrogen disengaged would carry off a portion of the graphite.

According to Karsten, Mushet's table of the quantities of carbon contained in different steels and cast irons is altogether erroneous. It gives no explanation why, with equal proportions of charcoal, cast iron constitutes at one time a gray, soft, granular metal, and at another, a white, hard, brittle metal in lamellar facets. The incorrectness of Mushet's statement becomes most manifest when we see the white lamellar cast iron melted in a - crucible lined with charcoal, take no increase of weight, while the gray cast iron treated in the same way becomes considerably heavier.

Analysis has nerer detected a trace of carbon unaltered or of graphite in white cast iron, if it did not proceed from small quantities of the gray mixed with it; while perfect gray - cast iron affords always a much smaller quantity of carbon altered by combination, and a much greater quantity of graphite. Neither kind of cast iron, however, betrays the presence of any oxygen. Steel affords merely altered carbon, without graphite; the same thing holds true of malleable iron; while the iron obtained by fusion with 25 per cent. of scales of iron contains no carbon at all.

The graphite of cast iron is obtained in scales of a metallic aspect, whereas the combined carbon is obtained in a fine powder. When the white cast iron has been rossted, and become gray, and is as malleable as the softest gray cast iron, it still affords no graphite as the latter does, though in appearance both are alike. Yet in their properties they are still essentially dissimilar.

With $4 \frac{1}{4}$ per cent. of carbon, the white cast iron preserves its lamellar texture; but with less carbon, it becomes granular and of a gray color, growing paler as the dose of carbon is diminished, while the metal, after passing through an indefinite number of gradations, becomes steely cast iron, very hard steel, soft steel, and steely wrought iron.

The steels of the forge and the cast steels examined by Karsten, afforded him from 
$2 . ?$ to $1 \frac{1}{4}$ per cent. of carbon; in the steel of cementation (blistered steel), he never found above $1 \frac{3}{4}$ of carbon. Some wrought irons which ought to contain no charcoal, hold as much as $\frac{1}{2}$ per cent. and they then approach to steel in nature. The softest and purest irons contain still 0.2 per cent. of carbon.

The quantity of graphite which gray cast-iron contains, varies, according to Karsten's experiments, from 2.57 to $3 \cdot 75$ per cent. ; but it contains, besides, some carbon in a state of alteration. The total contents in carbon varied from $3 \cdot 15$ to 4.65 per cent. When the congelation of melted iron is very slow, the carbon separates, probably in consequence of its crystallizing force, so as to form a gray cast-iron replete with plumbago. If the gray do not contain more charcoal than the white from which it has been formed, and if it contain the charcoal in the state of mechanical mixture, then it can have little or none in a state of combination, even much less than what some steels contain. Hence we can account for some of its peculiarities in reference to white cast-iron; such as its granular texture, its moderate hardness, the length of time it requires to receive annealing colors, the modifications it experiences by contact of air at elevated temperatures, the high degree of heat requisite to fuse it, its liquidity, and finally its tendency to rust by porosity, much faster than the white cast-iron.

We thus see that carbon may combine with iron in several masszers; that the gray cast-iron is a mixture of steely iron and plumbago; that the white, rendered gray and soft by roasting, is a compound of steely iron and a carburet of iron, in which the carbon predominates; and that untempered steel is in the same predicament.

For the following analyses of cast-irons, we are indebted to MM. Gay Lussac and Wilson.

TABLE.-In 100 parts.

\begin{tabular}{|c|c|c|c|c|c|c|c|}
\hline \multicolumn{2}{|r|}{ Cast-iron. } & Iron. & Carbon. & Silica. & $\begin{array}{l}\text { Phos- } \\
\text { phorus. }\end{array}$ & $\left|\begin{array}{c}\text { Manga- } \\
\text { nese. }\end{array}\right|$ & Remarks. \\
\hline White & cast from Siegen & $94 \cdot 338$ & $2 \cdot 690$ & $0 \cdot 230$ & $0 \cdot 162$ & 2.590 & By wood charcoal. \\
\hline Do. & Coblentz - & $94 \cdot 654$ & $2 \cdot 441$ & $0 \cdot 230$ & $0 \cdot 185$ & $2 \cdot 490$ & do \\
\hline Do. & a. d. Champ & $96 \cdot 133$ & $2 \cdot 324$ & $0 \cdot 840$ & 0.703 & a trace & do \\
\hline Do. & Isère & $94 \cdot 687$ & $2 \cdot 636$ & $0 \cdot 260$ & 0.280 & $2 \cdot 137$ & do \\
\hline Gray & Nivernais - & $95 \cdot 673$ & $2 \cdot 254$ & $1 \cdot 030$ & $1 \cdot 043$ & a trace & do \\
\hline Do. & Berry - - & $95 \cdot 573$ & $2 \cdot 319$ & $1 \cdot 920$ & $0 \cdot 188$ & do. & Mixt'e of coke \& do. \\
\hline Do. & a. d. Champ & $95 \cdot 971$ & $2 \cdot 100$ & $1 \cdot 060$ & 0.869 & do. & Charcoal. \\
\hline Do. & Creusot - & $93 \cdot 385$ & $2 \cdot 021$ & $3 \cdot 490$ & $0 \cdot 604$ & do. & Coke. \\
\hline Do & $\begin{array}{l}\text { a.d. Franche } \\
\text { Comté - }\end{array}$ & $95 \cdot 6$ & $2 \cdot 800$ & $1 \cdot 160$ & 1 & $d$ & \\
\hline Do & Wales - - & $94 \cdot 8$ & $1 \cdot 666$ & $3 \cdot 000$ & $0 \cdot 492$ & $d$ & \\
\hline Do. & Do. - & 9 & $2 \cdot 550$ & $1 \cdot 200$ & $0 \cdot 440$ & do & \\
\hline Do. & Do. - - & $95 \cdot 150$ & $2 \cdot 450$ & $1 \cdot 620$ & 0.780 & do. & do. \\
\hline
\end{tabular}

Karsten has given the following results as to carbon, in 100 parts of gray cast-iron.

\begin{tabular}{|c|c|c|c|c|}
\hline Gray cast-iron. & $\begin{array}{c}\text { Combined } \\
\text { carbon. }\end{array}$ & $\begin{array}{c}\text { Free } \\
\text { carbon. }\end{array}$ & $\begin{array}{c}\text { Total. } \\
\text { carbon. }\end{array}$ & Remarks. \\
\cline { 5 - 5 } & 0.89 & $3 \cdot 71$ & 4.60 & By wood charcoal. \\
\hline $\begin{array}{c}\text { Siegen, from brown ron stone - } \\
\text { Sicgen (Widderstein), from brown }\end{array}$ & & & & \\
and sparry iron - - - - & 1.03 & 3.62 & 4.65 & do. \\
Malapane, from spherosiderite - & 0.75 & $3 \cdot 15$ & 3.90 & do. \\
Königshütte, from brown ore - & 0.58 & 2.57 & $3 \cdot 15$ & coke. \\
Do. at a lower smelting heat - - & 0.95 & $2 \cdot 70$ & $3 \cdot 65$ & do. \\
\hline
\end{tabular}

Fig. 607 represents in section, and fig. 606 in plan, the famous cupola furnace for casting iron employerl at the Royal Foundry in Berlin. It rests upon a foundation $\alpha$, from 18 to 24 inches high, which supports the basement plate of cast-iron, furnished with ledges, for binding the lower ends of the upright side plates or cylinder, $e$. Near the mouth there is a top-plate $d$, made in several pieces, which serves to bind the sides at their upper end, as also to cover in the walls of the shaft. These plates are most readily secured in their places by screws and bolts. Within this iron case, at a little distance from it, the proper furnace-shaft $e$ is built with fire-bricks, and the space between this and the iron is filled up with ashes. The sole of the hearth $f$, over the basement-plate, is composed of a mixture of fire-clay and quartz-sand firmly beat down to the thickness of 6 or 9 inches, with a slight slope towards the discharge-hole for running off the metal. .g 
is the form or the tuyère (there are sometimes one on each side); $h$ the nose pipe : the discharge aperture $i$ is 12 inches wide and 15 inches high; across which the sole of the hearth is rammed down. During the melting operation, this opening is filled up with fire-clay; when it is completed, a small hole merely is pierced through it at the lowest point, for running off the liquid metal. The hollow shaft should be somewhat
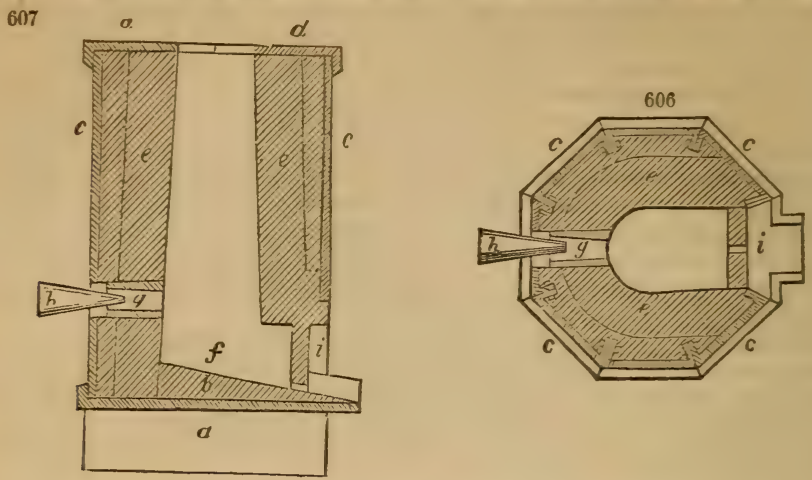

wider at bottom than at top. Its dimensions rary with the magnitude of the foundry. When $\check{5}$ feet high, its width at the level of the turère or blast-hole may be from 201022 inches. From 250 to 300 cubic feet of air per minute are required for the working of such a cupola. For running down 100 pounds of iron, after the furnace has been brought to its heat, 48 pounds of ordinary coke are used; but with the hot blast much less will suffice. The furnace requires feeding with alternate charges of coke and iron every 8 or 10 minutes. The waste of iron, by oxydization and slag, amounts in most foundries to fully 5 per cent. For carrying off the burnt air, a chimney-hood is commonly erected over the cupola. See FoundRY.

The double-arched air or wind-furnace used in the foundries of Staffordshire for melting cast-iron has been found advantageous in saring fuel, and preventing waste by slag. It requires fire-bricks of great size and the best composition.

The main central hey-stone is constructed of large fire-bricks made on parpose; against that ker-stone the two arches press, haring their abutments at the sides against the walls. The highest point of the roof is only 8 inches above the melted metal. The sole of the hearth is composed of a layer of sand 8 inches thick, resting upon a bed of iron or of brickwork. The edge of the fire-bridge is only 3 inches abore the fluid iron.

In from 2 to 4 hours from 1 to 3 tons of metal may be founded in such a furnace, according to its size; but it ought alwars to be heated to whiteness before the iron is introduced. 100 pounds of cast-iron require from 1 to $1 \frac{1}{2}$ cubic foot of coal to melt them. The waste varies from 5 to 9 per cent.

I shall conclude the subject of iron with a few miscellaneous observations and statistical tables. Previously to the discorery by Mr. Cort, in 1785, of the methods of puddling and rolling or shingling iron, this country imported 70,000 tons of this metal from Russia and Sweden; an enormous quantity for the time, if we consider that the cotton and other automatic manufactures, which now consume so vast a quantity of iron, were then in their infancy; and that two years ago, the whole of our importation from these countries did not exceed 40,000 tons. From the following table of the prices of bar iron in successive years, we may infer the successive rates of improvement and economy, with slight vicissitudes.

\begin{tabular}{|c|c|c|c|c|c|c|c|c|}
\hline Years & \multicolumn{4}{|c|}{ Per Ton. } & Years. & \multicolumn{3}{|c|}{ Per Ton. } \\
\hline & $£ s$. & & $\stackrel{£}{£}$ & s. & 1830 & $£ s$. & 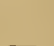 & $£ s$. \\
\hline $\begin{array}{l}1824 \\
1825\end{array}$ & $\begin{array}{rl}9 & 0 \\
10 & 0\end{array}$ & 10 & $\begin{array}{l}10 \\
14\end{array}$ & $\begin{array}{l}0 \\
0\end{array}$ & 1831 & & $\overline{-}$ & 510 \\
\hline 1826 & $\begin{array}{ll}8 & 10\end{array}$ & - & 10 & 0 & 1832 & & - & 510 \\
\hline 1827 & 80 & - & 9 & 0 & 1833 & 510 & - & 60 \\
\hline 1828 & $7 \quad 10$ & - & 8 & 0 & 1834 & 60 & - & $\begin{array}{ll}6 & 10\end{array}$ \\
\hline 1829 & 510 & - & 7 & 0 & 1835 & 510 & - & 70 \\
\hline
\end{tabular}

I have been informed upon good authority that the total production of iron in Great Britain, in the year 1836, was almost exactly ONE MrLIION OF TONs! 
The export of iron that rear, in bars, rods, pigs, castings, wire, anchors, hoops, nails, and old iron, amounted to 189,390 tons; in unwrought steel to 3,014 , and in cutlery, to 21,072 ; in whele to 213,478 : learing apparently for internal consumption, 776,522 tons, from which however one tenth probably should be deducted for waste, in the conversion of the bar iron. Hence 700,000 tons may be taken as the approximate quantity of inn made use of in the United Kingdom, in the year 1836.

The years $1 \$ 35$ and $1 \$ 36$ being those of the railway mania orer the world, produced a considerable temporary rise in the price of bar iron; but as this increased demand caused the construction of a great many more smelting and refining furnaces, it has tended erentually to lower the prices; an effect also to be ascribed to the more general use of the hot blast.

The relatire cost of making cast iron at Merthyr Tydril in South Wales, and at Glasgow, was as follows, eight or nine years ago.

- At Merthyr.

s.

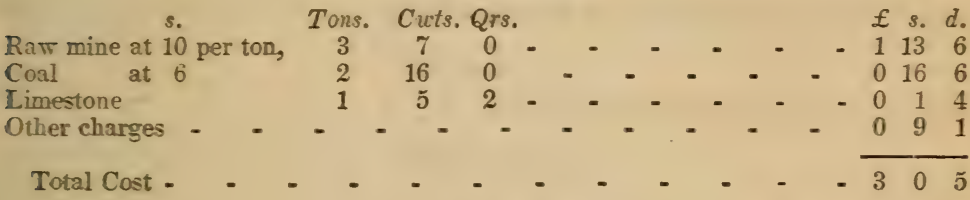

At Glasgore.

s. d. Tons. Cwts. $\quad$ f. s. a.

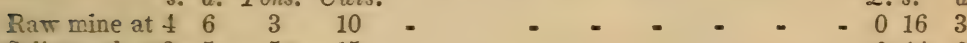

Splint coal at $2 \quad 5 \quad 5 \quad 15-\quad-\quad-\quad-\quad-\quad-00140$

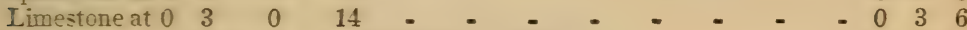

Coals for the engine 110 - 1 - - - - - 030

Other charges

Total cost

The cost is still nearly the same at Merthyr, but it has been greatly decreased at Glasgow.

The saring of fuel br the hot-blast is said to be in fact so great, that blowing crlinders, which were adequate merely to work three furnaces at the first period, were competent to work four furnaces at the last period. The saring of materials has moreorer been aecompanied by an increase of one fourth in the quantity of iron, in the same time; as a funace which turned out onlt 60 tons a week with the cold blast, now turns out no less than $\$ 0$ tons. That the iron so made is no worse, but probably better, when judiciously smelted, would appear from the following statement. A considerable order was not long since giren to four iron-work companies in England, to supply pipes to one of the London mater-companies. Three of these supplied pipes made from the cold-blast iron; the fourth, it is said, supplied pipes made with the hot-blast iron. On subjecting these sereral sets of pipes to the requisite trials br hrdraulic pressure, the last lot was found to stand the proof far better than any of the former three.-That iron was made with raw coal.

I have been since told br eminent iron-masters of Merthrr, that this statement stands in need of confirmation, or is probably altogether apocryphal, and that as they find the hot-blast weakens the iron, they will not adopt it.

Between the east irons made in different parts of Great Britain, there are characteristic differences. The Staffordshire metal runs remarkably fluid, and makes fine sharp castings. The Welsh is strong, less fluent, but produces bar iron of superior qualiț. The Derbyshure iron also forms excellent castings, and mar be worked with care into rery good bar iron. The Sectch iron is rery raluable for casting into hollow wares, as it affords a beautiful smooth slin from the moulds, so remarkable in the castings of the Carron compant, in Stirlingshire, and of the Phœnix foundrr, at Glasgow. The Shropshire iron resembles the Staffordshire in its good qualities.

The arerage quantity of fine metal obtainable from the forge-pigs at Merthrr Trdril, from the finery furnace, is one ton for $22 \frac{1}{2} \mathrm{cwts}$. of cast iron, with a consumption of about $9 \frac{1}{2}$ civts. of coal per ton. 
Foundations BUILDING LXPENSES.

Masony of hewn grit-stones

Common bricklayers' work

Lining of the furnace, hearth, \&c., in fure-bricks

Fire-clay for building

Lime and sand

CAST IRON.

Cast iron pieces, such as dam-plates, tymp-plates, beams, tuyère-plates, \&c., weighing about 24 tons for each furnace; -in whole

WROUGHT IRON.

For the binding-hoops, keys, \&c.; 5 tons for each

COST OF LABOR.

Bricklayers, masons, and laborers in building - . _ . . . 1080

VARIOUS EXPENSES.

Scaffolding

Tools

Shed in front of each furnace

Terracing, cost of ground, \&c. -

Total cost of erecting the furnaces

\section{INCIDENTAL CHARGES.}

Blowing machinery, and steam engine of 80 horse power - _ _ _ 6400

Inclined railway for mounting the charges - _ - _ _ _ - 120

Gallery for charging

Steam engine house

Chimneys, boilers, \&c.

Roasting liilns

Cuke kilns

Dwelling-houses for workmen

Total cost of three furnaces complete

Estimate from the Neath Abbey Works in S. Wales, of the cost of machines requisite for a forge and shingling-mill, capable of turning out 120 tons of bar iron per week.

1. Steam engine upon Bolton and Watt's construction; of 40 inches diameter in the cylinder, and 8 feet stroke; with boilers, pipes, grate, bars, firedoors, \&c., \&c., complete

2. System of great-gearing for transmitting the crank-motion of the engine to

the mill work, with fly-wheels, \&c. - - - - $-\overline{-}-$
3. A system of roughing rolls, with pinions, uprights, and every thing else necessary

4. Two pairs of finisher-rolls, with all their accessories

5. Two pairs of shear machines, at $170 l$. apiece

5. One pair of rolls of 10 inches diameter, for making small bar iron, with all their accessories

$\therefore$ Forge hammer, including the anvil, the cam-shafts, and all the other

\section{requisites}

8. A complete turning lathe

9. To the above must be added, spare cylinders weighing about 60 tons

10. Duplicate articles for the steam-engine

11. 150 tons of cast-iron plates, to cover the floor of the mill

12. Eight tons of cast-iron pieces for a reverberatory furnace

13. Tools of malleable iron; rakes, oars, \&c.

14. Castings for mounting a cupola furnace

:5. Blowing machine for the cupola 
Estimate from Neath Abbey Works.-continued.

16. Pieces of iron for a small forge, with two fires, two bellows, two anvils, iron tools faeed with steel, and common iron tools, \&c. -

17. Fight tons of cast-iron pieces, and wrought-iron pieces for 14 puddling furnaces

18. Seren tons of cast-iron pieces, and wrought-iron for 4 re-heating furnaces

19. Tools for the puddlers and other workmen - - - -

20. Iron mountings for two cranes, partly made of wood - _ _ -

Total cost of machines and pieces of iron -

- $£ 8165$

To the abore, the cost of the steam engine house is to be added, that of another forge hammer, and incidental expenses.

\section{In Staffordshire, the following estimate has been given :}

A steam engine of 60 -horse power

Rolls, with the iron work of the furnaces, \&c., to make 120 tons of bar iron weekly

The Neath Abbey estimate is greater, but that company has a high character for making substantial, well-finished machinery.

Bar iron, made entirely from ore without admixture of cinder, or vitrified oxyde, is alwars reckoned worth 10 s. a ton more than the average irun in the market, which is frequently made by smelting 25 per cent. of cinder with 75 of ore or mine, as it is called, Importation of iron in bars or unwrought, for home consumptiun; and amount of duty, in 1836. 1837. 1836. 1837

18,978 tons 18 cwts. | 13,470 tons 4 cwts. | $£ 28,450$ | 220,065

M. Virlet's Statistical Table of the produce of Iron in Europe.

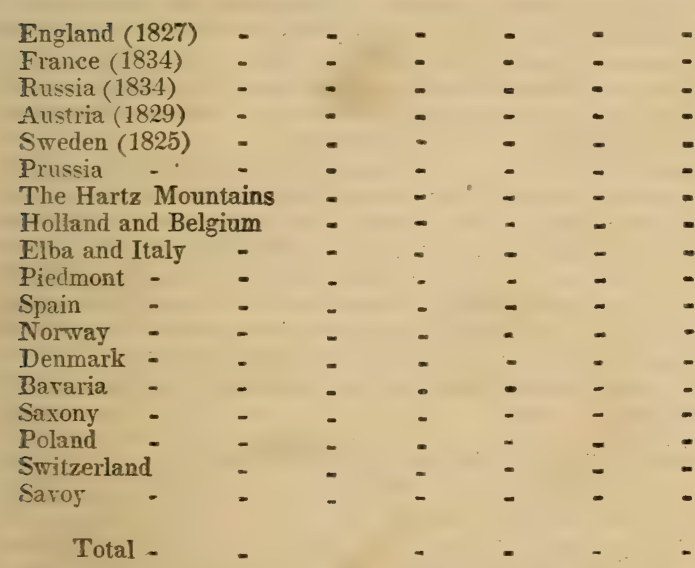

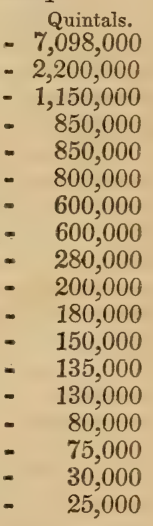

$-\overline{13,433,000}$ (equal to about 672,000 tons).

For additional statistics of iron, see Prtcoar, at the end.

Bronzing of polished iron.-The barrels of fowling-pieces and rifles are occasionally bronzed and varnished, to relieve the eye of the sportsman from the glare of a polished metal, and to protect the surface from rusting. The liquid used for browning the barrels is made by mixing nitric acid of specific gravity $1 \cdot 2$, with its own weight of spirit of nitric ether, of alcohol, and tineture of muriate of iron; and adding to that mixture a quantity of suiphate of copper equal in weight to the nitric acid and ethereous spirit taken together. The sulphate must be dissolved in water before being added; and the whole being diluted with about 10 times its weight of water, is to be bottled up for use. This liquid must be applied by friction with a rag to the clear barrel, which must then be rubbed with a hard brush; processes to be alternated two or three times. The barrel should be afterwards fijped in boiling water, rendered feebly allialine with carbonate of potash or soda, well dried, burnished, and heated slightly for receiving several coats of tin-smith's lacker, cunsisting of a sclution of shellac in alcohol, colored with dragon's blood.

ISINGLASS, or Fish-glue, called in Latin ichthyocolla, is a whitish, dry, tough, semi. 
transvarent substance, twiste 1 into different shapes, often in the formo of a lire, and consisting of membranes rolled together. Guod isinglass is unchangeable in the air, has a leathery aspect, and a mawhish taste nearly insipid; when steeped in cold water, it swells, sctiens, and separates in membranous laminæ. At the boiling heat it dissolves in water, ant the sulution, on cooling, forms a white jellr, which is semi-trans, arent, solubie in weak acits, but is precipitated frorn them by alhalis. It is gelatine nearly pure; and if not brittle, like other glue, this depeads on its fibrous and elastic texture. The whitest and finest is preferred in commerce. Isinglass is prepared from the airblatlers of sturgeons, and especially the great sturgeon, the accivenser huso; which is fishel on the sheres of the Caspian sea, and in the rirers flowing into it, for the sake chiefly of its swim bladder.

The preparation of isinglass in this part of Russia, and particularly at Astracan, consists in steeping these bladiers in water, remoring carefully their external ccat, and the blood which often covers them, putting them in a hempen bag, squeezing them, suttening them between the hands, and twisting them into small cylinders, which are afterwards bent into the shape of a lrre. Ther are ready for the market immediately after being dried in the sun, and whitened with the fumes of buraing sulphur.

In some districts of Moliavia, another process is follorred. The skin, the stomach, the intestines, and the swim bladder of the sturgeon are cut in small pieces, steened in cold water, and then gently boiled. The jelly thus ootained is spread in thin layers to drr, when it assumes the appearance of parchment. This being softened in a little water, then rolied into erlinders, or extended into plates, constitutes an inferior articie.

The swim bladider of the cod and many other tishes also furnishes a species of isinglass, but it is much more membranous, and less soluble, than that of the sturgeon.

The properties of isinglass are the same as those of gelatine or pure glue: and its uses are ter numerous. It is emplored in considerable quantities to clarif ale, wine, lizueurs, and colfee. As an article of food to the luxurious in the preparation of creams and jellies, it is in great request. Four parts of it convert 100 of water into a tremulous jellr, which is emplored to enrich mant soups and sances. It is used along with gum as a dressing to give lustre to ribands and other sill articles. The makers of artificial pearls employ it to fix the essence d Orient on the glass globules which form these pearls, and the Turhs set their precious stones or jewellery by means of isinglass dissolved in alcohol aiong with gum ammoniac; a combination which is also emplored in this country to join broken riees of China and glass, under the name of diamond cement. That setting preserves its transparency after it solidifies, if it be well made.

It is by corering taffety or thin silk with a coat of isinglass that court plaster is made. A solution of isinglass colored with carmine forms an excellent injection liquor to the anatumist. X. Rochen has made another pretty application of isinglass. He plunges into a limpid solution of it, made by means of a water bath, sheets of wire ganze set in window or lamp frames, which, when cold, hare the annearance of glass, and answer instead of it for shades and other purposes. If one din be not sufficient to make a proper trans?arent plate of isinglass, sereral mat he given in succession, allowing each film to harden in the interval betreen the dips. The outer surface should be varnished to protect it tiom damp air. These nanes of gelatine are now generally used for lamps instead of horn, in the maritime arsenals of France.

$$
\begin{aligned}
& \text { Isinglass imported for home consumption, and duties paid, in } \\
& \text { 1\$35. 1836. } 1835.1836 . \\
& \text { 1,814 cwts. | 1,735 cwts. | } £ 4,290 \text { | } £ 4,125
\end{aligned}
$$

ISLAND MOSS (Lichen d'Istande, Fr.; Flechte Isl., Germ.) is a lichen, the Cetraria islandiea, which contains a substance soluble in het water, but forming a jell when it cools, strled lichomine t.y . . Guerin. Lichenine has a rellowish tint in the dry state, is transparent in thin plates, insioid, inolorous, and dirkeult to pulverize. Cold water makes it swell, but does not dissolre it. It is precinitated in white flocks by aleohol and ether. Ialine tinges it of a brownish-green. Sulphuric acil conrerts it into sugar; and the nitric iato oxalie acil. Lichenine is prenaced br extractinz first of all from the plant a bitter coloring matter, by digesting 1 pound of it in 16 pornds of cold water, containing 1 ounee of pearlasi: then Graining the lichen, elulcorating with cold water, and boiling it in pounds of boiling water till 3 pounds be eraporated. The jells which forms, upon cooling the filtered solution, is dark colored, but, being dried and redissolred in hot water, it becomes elear and colorless. Lichenine consists of $39 \cdot 33$ carbon, $7 \cdot 24$ hrdrogen, and 55.43 orygen. With potash, lime, orgde of lead, and tincture of galls, the habitudes of lichenine and starch are the same. The mueilage of island moss is preferred in Germany to common paste for dressing the warp of webs in the loom, because it remains soft, from its bygrometric quality. It is also mired with the pulp for sizing paper in the rat.

IVORY (Iroire, Fr.; Elfenbein, Germ.) is the osseous matter of the tushs and 
teeth of the elephant, the hippopotamus, or morse, wild boar, sereral species of phocæ, as woll as the horn or tooth of the narwhal. Irory is a white, fine-grainel, dense substance, of consilerable elasticity, in thin plates, and more transparent than paper of equal thichness. The outside of the tusk is corered by the cortical part, which is softer and less compact than the interior substance, with the exception of the brown plate that sometimes lines the interior caritr. The hardest, toughest, whitest, and most translucent irory, has the preference in the market; and the tushs of the sea-horse-are considered to ationl the best. In these, a rough glassy enamel corers the cortical part, of such harlness, as to strike sparks with steel. The horn of the narwhal is sometimes ten feet lon $r$, an consists of an irory of the finest description, as hard as that of the elephant, an 1 susceptible of a better polish; but it is not in general so much esteemed as the latter.

Irory has the same constituents as the teeth of animals three fourths being phosphate, with a little carbonate of lime; one fourth cartilage. See Bones.

It is extensirely emplored br miniature painters for their tablets; br turners, in makine numberless useful and ornamental objects; by cutlers, for the handles of knives ant forks: by comb-makers; as also by philosophical instrument makers, for constructing the scales of thermometers, \&c. The irory of the sea-horse is preferred br dentists for making artificial teeth; that of the East India elephant is better than of the African. When it shows cracks or fissures in its substance, and when a splinter brolien off has a dull aspect, it is reckoned of inferior ralue. Irory is distinguishable from bone br its peculiar semi-transparent rhombohedral net-work, which mar be readily seen in slips of ivory cut transversely.

Iror! is very apt to take a rellow-brown tint by exposure to air. It mar be whitened or bleachet, br rubbing it first with pounded pumice-stone and water, then placing it moist unler a glass shade luted to the sole at the bottom, and exposing it to sunshine. The sunbeams without the shade would be apt to occasion fissures in the ivory The moist rubbing and exposure.may be repeated several times.

For etching irorr, a ground made by the following recipe is to be applied to the polished surface:-Take of pure white wax, and transparent tears of mastic, each one ounce; asphalt, half an ounce. The mastic and asphalt haring been separately reduced to fine powdor, and the wax being melted in an earthenware ressel orer the fire, the mastic is to be first slowly strewed in and dissolred br stirring; and then the asphalt in like manner. This compound is to be poured out into lukewarm water, well kneaded, as iteotis, br the hand, into rolls or balls about one inch in diameter. These should be kept wrappel round with taffetr. If white rosin be substituted for the mastic, a cheaper composition will be obtained, which answers nearly as well; 2 oz. asphalt, 1 oz. rosin, $\frac{1}{2}$ oz. white wax, being good proportions. Callot's etching ground for copper plates, is made by dissolving with heat $4 \mathrm{oz}$. of mastic in $4 \mathrm{oz}$. of very fine linseed oil ; fltering the rarnish through a rag, and bottling it for use.

Either of the two first grounds being applied to the ivory, the figured desion is to be traced through it in the usual way, a ledge of wax is to be applied, and the surface is to be then corered with strong sulphuric acid. The effect comes better out with the aid of a little heat; and br replacing the acid, as it becomes dilute by absorption of moisture, with concentrated oil of ritriol. Simple wax mar be emplored instead of the copperplate engrarer's ground; and strong muriatic acid instead of sulphuric. If an acid solution of silver or gold be used for etching, the design will become purple or black, on exposure to sunshine. The wax may be washed away with oil of turpentine. Acid nitrate of silver affords the easiest means of tracing permanent black lines upon ivory.

Ivory may be dyed by using the following prescriptions :-

1. Black dye. - If the ivory be laid for sereral hours in a dilute solution of nentral nitrate of pure silrer, with access of light, it will assume a black color, haring a slightly green cast. A still finer and deeper black may be obtained by boiling the irory for some time in a strained decoction of logwood, and then steeping it in a solution of red sulphate or red acetate of iron.

2. Blue dye. - When ivory is kept immersed for a longer or shorter time in a dilute solution of sulphate of indigo (partly saturated with potash), it assumes a blue tint of greater or less intensity.

3. Green dye. -This is giren by dipping blued irory for a little while in solution of nitro-muriate of tin, and then in a hot decoction of fustic.

4. Yellow dye is giren by impregnating the irory first with the abore tin mordant, and then diresting it with heat in a strained decoction of fustic. The color passes into oranre, if some Brazil wood has been mixed with the fustic. A very fine unchangeable yellow tnay be communicated to irorr br steeping it 18 or 24 hours in a strong solution of the neutral chromate of potash, and then plunging it for some time in a boiling hot solution of acetate of lead. 
5. Red dye-may be given by imbuing the ivory first with the tin mordant, then plunging it in a bath of Brazil wood, cochineal, or a mixture of the two. Lac-dye may be used with still more advantage, to produce a scarlet tint. If the scarlet ivory be plunged for a little in a solution of potash, it will become cherry red.

6. Violet dye-is given in the logwood bath to ivory previously mordanted for a short time with solution of tin. When the bath becomes exhausted, it imparts a lilach hue. Violet ivory is changed to purple-red by steeping it a little while in water containing a few drops of nitro-muriatic acid.

With regard to dyeing ivory, it may in general be observed, that the colors penetrate hetter before the surface is polished than afterwards. Should any dark spots appear, they may be cleared up by rubbing them with chalk; after which the ivory should be dyed once more to produce perfect uniformity of shade. On taking it out of the boiling hot dye bath, it ought to be immediately plunged into cold water, to prevent the chance of fissures being caused by the heat.

If the borings and chips of the ivory-turner, called ivory dust, be boiled in water, a kind of fine size is obtained.

The importation of elephants' teeth for home consumption was, in 1834, 4,282 cwts.; in $1835,3,698$; and in $1836,4,584$ cwts. ; duty, 1 . per cwt.

IVORY BLACK (Noir d'ivoire, Fr.; Kohle von Elfenbein, Germ.) is prepared from ivory dust, by calcination, in the very same way as is described under BonE BLAcK.

The calcined matter, being ground and levigated on a porphyry slab, affords a beautifil velvety black, much used in copperplate printing. Ivory black may be pred $d$ upon the small scale, by a well regulated ignition of the ivory dust in a covered ible.

K.

nALI. The Arabs gave this name to an annual plant which grows near the seashore; now known under the rame of salsola soda, and from whose ashes they extracted a substance, which they called alkali, for making soap. The term kali is used by German chemists to denote caustic potash; and kalium, its metallic basis; instead of our potassa and potassium, of preposterous pedigree, being derived from the words pot ashes, that is, ashes prepared in a pot.

KAOLIN (Terre à porcelaine, Fr.; Porzellanerde, Germ.) is the name given by the Chinese to the fine white clay with which they fabricate the biscuit of their porcelains. See ClAy. Berthier's analyses of two porcelain earths are as follows :-

\begin{tabular}{|c|c|c|c|}
\hline Analyses. & & From Passau. & From Saint Yriex. \\
\hline $\begin{array}{l}\text { Silica } \\
\text { Alumina } \\
\text { Lime } \\
\text { Oxyde of iron } \\
\text { Potass · } \\
\text { Water }\end{array}$ & 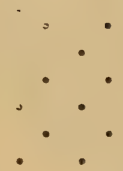 & $\begin{array}{r}45 \cdot 06 \\
32.00 \\
0.74 \\
0.90 \\
\frac{18 \cdot 0}{}\end{array}$ & $\begin{array}{c}46 \cdot 8 \\
37 \cdot 3 \\
\frac{-}{2 \cdot 5} \\
13 \cdot 0\end{array}$ \\
\hline & & $96 \cdot 7$ & $99 \cdot 6$ \\
\hline
\end{tabular}

KARABE', a name of amber, of Arabic origin, $\rightarrow$ use upon the Continent.

KELP (Varec, Fr.; Wareck, Germ.) is the crude alkaline matter produced ky ncinerating various species of fuci, or sea-weed. They are cut with sickles from the rocks in the summer season, dried and then burned, with much stirring of the pasty ash. I have analyzed many specimens of kelp, and found the quantity of soluble matter in 100 parts of the best to be from 53 to 62 , while the insoluble was from 47 to 38 . The soluble consisted of-

Sulphate of Soda -
Soda in carbonate and sulphuret -

Soda in carbonate and sulphuret :
Muriate of soda and potash

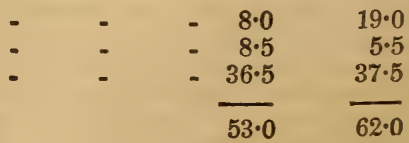




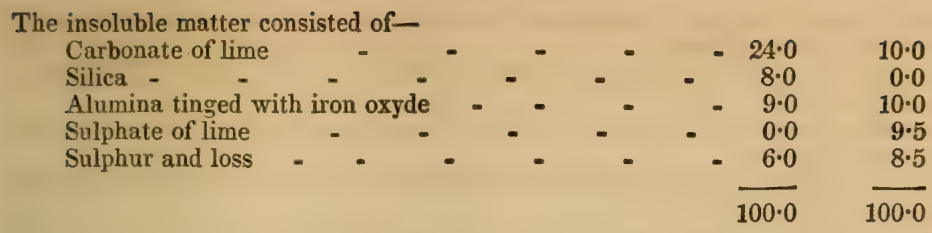

The first of these specimens was from Heisker, the second from Rona, both in the isle of Skye, upon the property of Lord Macdonald. From these, and many other analyses which I have made, it appears that kelp is a substance of very variable composition, and hence it was very apt to produce anomalous results, when employed as the chief alkaline flux of crown glass, which it was for a very long period. The fucus vesiculosus and fucus nodosus are reckoned to afford the best kelp by incineration; but all the species yield a better product when they are of two or three years growth, than when cut younger. The varec, made on the shores of Normandy, contains almost no carbonate of soda, but much sulphate of soda and potash, some hyposulphate of potash, chloride of sodium, iodide of potassium, and chloride of potassium ; the average composition of the soluble salts being, according to M. Gay Lussac, 56 of chloride of sodium, 25 of chloride of potassium, and a little sulphate of potash. The very low price at which soda ash, the dry erude carbonate from the decomposition of sea salt, is now sold, has nearly superseded the use of kelp, and rendered its manufacture utterly unprofitable-a great misfortune to the Highlands and Islands of Scotland.

KERMES. There are two substances so called, of totally different natures. Kermes mineral is merely a factitious sulphuret of antimony in a state of impalpable comminution, prepared in the moist way. Its minute examination belongs to pharmaceutical chemistry. It may be obtained perfectly pure, by diluting the proto-chloride of antimony with solution of tartaric acid, and precipitating the metal with sulphureted hydrogen; or by exposing the finely levigated native sulphuret to a boiling solution of carbonate of potash for some time, and filtering the liquor while boiling hot. The kermes falls down in a brown-red powder, as the liquor cools.

Kermes-grains, alkermes, are the dried bodies of the female insects of the species coccus ilicis, which lives upon the leaves of the quercus ilex (prickly oak). The word kermes is Arabic, signifies little worm. In the middle ages, this dye stuff was therefore called vermiculus in Latin, and vermilion in French. It is curious to consider how the name vermilion has been since transferred to red sulphuret of mercury.

Kermes has been known in the East since the days of Moses; it has been employed from time immemorial in India to dye silk; and was used also by the ancient Greek and Roman dyers. Pliny speaks of it under the name of coccigranum, and says that there grew upon the oak in Africa, Sicily, \&c. a small excrescence like a bud, called cusculium; that the Spaniards paid with these grains, half of their tribute to the Romans; that those produced in Sicily were the worst; that they served to dye purple; and that those from the neighborhood of Emerita in Lusitania (Portugal) were the best.

In Germany, during the ninth, twelfth, thirteenth, and fourteenth centuries, the rural serfs were bound to deliver annually to the convents, a certain quantity of kermes, the coccus polonicus, among the other products of husbandry. It was collected from the trees upon Saint John's day, between eleven o'clock and noon, with religious ceremonies, and was therefore called Johannisblut (Saint John's blood), as also German cochineal. At the abore period, a great deal of the German kermes was consumed in Venice, for dyeing the scarlet to which that city gives its name. After the discovery of America, cochineal having been introduced, began to supersede kermes for all brilliant red dyes.

The principal varieties of kermes are the coccus quercus, the coccus polonicus, the coccus fragaria, and the coccus uva ursi.

The coccus quercus insect lives in the south of Europe upon the kermes oak. The female has no wings, is of the size of a small pea, of a brownish-red color, and is covered with a whitish dust. From the middle of May to the middle of June the eggs are collected, and exposed to the vapor of vinegar, to prevent their incubation. A portion of eggs is left upon the tree for the mantenance of the brood. In the department of the Bouchesdu-Rhone, one half of the kermes crop is dried. It amounts annually to about 60 quintals or cwts., and is warehoused at Avignon.

The kermes of Poland, or coccus polonicus, is found upon the roots of the scleranthus perennis and the scleranthus annuus, in sandy soils of that country and the Ukraine. This species has the same properties as the preceding; one pound of it, according to Wolfe, being capable of dyeing 10 pounds of wool; but Hermstaedt could not obtain a fine color, although he employed 5 times as much of it as of cochineal. The Turks, Arme. 
nians, and Cossacks, dye with kermes their morocco leather, cloth, silk, as well as the manes and tails of their horses.

The kermes called coccus fragarice, is found principally in Siberia, upon the root of the common strawberry.

The coccus uva ursi is twice the size of the Polish kermes, and dyes with alum a fies red. It occurs in Russia.

Kermes is found not only upon the lycopodium complanatum in the Ukraine, but upon a great many other plants.

Good kermes is plump, of a deep red color, of an agreeable smell, and a rough and pungent taste. Its coloring matter is soluble in water and alcohol; it becomes yellowish or brownish with acids, and violet or crimson with alkalis. Sulphate of iron blackens it. With alum it dyes a blood-red; with copperas an agate sray; with copperas and tartar a lively gray; with sulphate of copper and tartar, an olive green; with tartar and salt of tin, a lively cinnamon yellow; with more alum and tartar, a lilach; with sulphate of zinc and tartar, a violet. Scarlet and crimson dyed with kermes, were called gruin colors ; and they are reckoned to be more durable than those of cochineal, as is proved by the brilliancy of the old Brussels tapestry.

Hellot says that previous to dyeing in the kermes bath, he threw a handful of wool into it, in order to extract a blackish matter, which would have tarnished the color. The red caps for the Levant are dyed at Orleans with equal parts of kermes and madder; and oc. casionally with the addition of some Brazil wood.

Cochineal and lac-dye have now nearly superseded the use of kermes as a tinctorial substance, in Fngland.

KILLAS is the name by which clay-slate is known among the Cornish miners.

KILN (Four, Fr.; Ofen, Germ.) is the name given to various forms of furnaces and stoves, by which an attempered heat may be applied to bodies; thus there are brickkilns, hop-kilns, lime-kilns, malt-kins, and pottery-kilns. Hop and malt-kilns, being designed merely to expel the moisture of the vegetable matter, may be constructed in the same way. See Brick, Limestone, Malt, and Pottery, for a description of their respective kilns.

KINIC ACID; a peculiar acid extracted by Vauquelin from cinchona.

KINO is an extractive matter obtained from the nauclea gambir, a shrub which grows at Bancoul and Sumatra, but principally in Prince of Wales' island. It is of a reddish-brown color, has a bitter styptic taste, and consists chiefly of tannin. It is used only as an astringent in medicine. Kino is often called a gum, but most improperly.

KIRSCHWASSER is an alcoholic liquor obtained by fermenting and distilling bruised cherries, called kirschen in German. The cherry usually employed in Switzerland and Germany is a kind of morello, which on maturation becomes black, and has a kernel very large in proportion to its pulp. When ripe, the fruit being made to fall by switching the trees, is gathered by children, thrown promiscuously, unripe, ripe, and rotten into tubs, and crushed either by hand, or with a wooden beater. The mashed materials are set to ferment, and whenever this process is complete, the whole is transferred to an old still corered with verdigris, and the spirit is run off in the rudest manner possible, by placing the pot over the common fire-place.

The fermented mash is usually mouldy before it is put into the alembic, the capital of which is luted on with a mixture of mud and dung. The liquor has accordingly, for the most part, a rank smell, and is most dangerous to health, not only from its own crude essential oil, but from the prussic acid, derived from the distillation of the cherry stones.

There is a superior kind of kirschwasser made in the Black Forest, prepared with fewer kernels, from choice fruit, properly pressed, fermented, and distilled.

KNOPPERN are excrescences produced by the puncture of an insect upon the flower-cups of several species of oak. They are compressed or flat, irregularly pointed, generally prickly and hard; brown when ripe. They abound in Styria, Croatia, Sclavonia, and Natolia; those from the latter country being the best. They contain a great deal of tannin, are much employed in Austria for tanning, and in Germany for dyeing fawn, gray, and black. Wool, with a mordant of sulphate of zinc, takes a grayish nankeen color. See GaLls.

KOUMISS is the name of a liquor which the Calmucs make by fermenting mare's milk, and from which they distil a favorite intoxicating spirit, called rack or racky. Cow's milk is said to produce only one third as much spirit, from its containing probably less saccharine matter.

The milk is kept in bottles made of hides, till it becomes sour, is shaken till it easts up its cream, and is then set aside in earthen vessels in a warm place to ferment, no yeast being required, though sometimes a little old koumiss is added. 21 pounds of milk put into the still afford 14 ounces of low wines, from which 6 ounces of pretty strong alcohol, of an unpleasant flavor, are obtained by rectification. 
L.

LABDANUM, or LADANUM, is an unctuous resin, of an agreeable odor, found besmearing the leaves and twigs of the cystus creticus, a plant which grows in the isiand of Candia, and in Syria. It is naturally a dark-brown soft substance, but it hardens on keeping. Its specific gravity is $1 \cdot 186$. It has a bitter taste. Its chief use is in surgery for maling plasters.

LABRADORITE, opaline, or Labradore feldspar, is a beautiful mineral, with brilliant changing colors, blue, red, and green, \&c. Spec. grav. 2.70 to 2.75. Scratches glass; affords no water by calcination; fusible at the blowpipe into a frothy bead; soluble in muriatic acid; solution affords a copious precipitate with oxalate of ammonia. Cleavages of $93 \frac{10}{2}$ and $86 \frac{1}{2}^{\circ}$; one of which is brilliant and pearly. Its constituents are silica, $55 \cdot 75$; alumina, $26 \cdot 5$; lime, 11 ; soda, 4 ; oxyde of iron, $1 \cdot 25$; water, 0.5 .

LABYRINT'H, in metallurgy, means a series of canals distributed in the sequel of a stamping-mill; through which canals a stream of water is transmitted for suspending, carrying off, and depositing, at different distances, the ground ores. See METaLLURGY.

LAC, LAC-DYE (Laque, Fr.; Lack, Lackfarben, Germ.). Stick-lac is produce oy the puncture of a peculiar female insect, called coccus lacca or ficus, upon the brancies of sereral plants; as the ficus religiosa, the ficus indica, the rhamnus jujuba, the croton lacciferum, and the butea frondosa, which grow in Siam, Assam, Pegu, Bengal, and Malabar. The twig becomes thereby incrusted with a reddish mammelated resin, having a crystalline-looking fracture.

The female lac insect is of the size of a louse ; red, round, flat, with 12 abdominal circles, a bifurcated tail, antennæ, and 6 claws, half the length of the body. The male is twice the above size, and has 4 wings; there is one of them to 5000 females. In November or December the young brood makes its escape from the eggs, lying beneath the dead body of the mother; they crawl about a little way, and fasten themselves to the bark of the shrubs. About this period the branches often swarm to such a degree with this vermin, that they seem covered with a red dust; in this case, they are apt to dry up, by being exhausted of their juices. Many of these insects, however, become the prey of others, or are carried off by the feet of birds, to which they attach themselves, and are transplanted to other trees. They soon produce small nipple-like incrustations upon the twigs, their bodies being apparently glued, by means of a transparent liquor, which goes on increasing to the end of March, so as to form a cellular texture. At this time, the animal resembles a small oval bag, without life, of the size of cochineal. At the commencement, a beautiful red liquor only is perceived, afterwards eggs make their apnearance; and in October or November, when the red liquor gets exhausted, 20 or 30 young ones bore a hole through the back of their mother, and come forth. The empty cells remain upon the branches. These are composed of the milky juice of the plant, which serves as nourishment to the insects, and which is afterwards transformed or elaborated into the red coloring matter that is found mixed with the resin, but in greater quantity in the bodies of the insects, in their eggs, and still more copiously in the red liquor secreted for feeding the young. After the brood escapes, the cells contain much less coloring matter. On this account, the branches should be broken off before this happens, and dried in the sun. In the East Indies this operation is performed twice $\mathrm{n}$ the year; the furst time in March, the second in October. The twigs incrusted with the radiated cellular substance constitute the stick-lac of commerce. It is of a red culor, inore or less deep, nearly transparent, and hard, with a brilliant conchoidal fracture. The stick-lac of Siam is the best; a piece of it presented to me by Mr. Rennie, of Fenchurch-street, having an incrustation fully one quarter of an inch thick all round the twig. The stick-lac of Assam ranks next; and last, that of Bengal, in which the resinous coat is scanty, thin, and irregular. According to the analysis of Dr. John, stick-lac consists, in 120 parts, of -

\begin{tabular}{|c|c|c|c|c|c|c|c|}
\hline An odorous common resin & - & - & - & - & - & - & $80 \cdot 00$ \\
\hline A resin insoluble in ether & - & - & - & - & 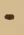 & - & $20 \cdot 00$ \\
\hline Coloring matter analogous & to that & f co & leal & - & - & - & $4 \cdot 50$ \\
\hline Bitter balsamic matter & - & - & - & - & - & - & $3 \cdot 00$ \\
\hline Dun yellow extract - & - & . & - & 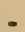 & - & - & 0.50 \\
\hline Acid of the stick-lac (lacci & ( acid) & - & - & 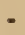 & - & - & 0.75 \\
\hline Fatty matter, like wax & - & - & - & & 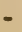 & 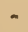 & $3 \cdot 00$ \\
\hline Skin of the insects and colo & ring $\mathrm{l}$ & & - & - & - & - & $2 \cdot 50$ \\
\hline
\end{tabular}




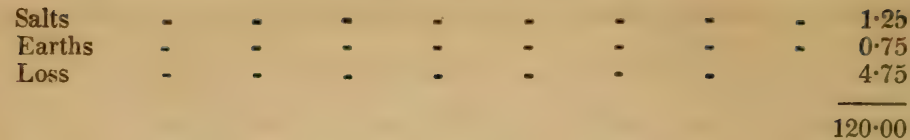

According to Franke, the constituents of stick-lac are, resin, 65.7 ; substance of the la:, $28 \cdot 3$; coloring matter, $0 \cdot 6$.

Seel-lac. - When the resinous concretion is taken off the twigs, coarsely pounded, and triturated with water in a mortar, the greater part of the coloring matter is dissolved, and the granular portion which remains, being dried in the sun, constitutes seed-lac. It contains of course less coloring matter than the stick-lac, nd is much less soluble. John found in 100 parts of it, resin, 66.7 ; wax, 1.7 ; matter of the lac, 16.7 ; bitter balsamic matter, 2.5 ; coloring matter, 3.9 ; dun yellow extract, $0 \cdot 4$; envelopes of insects, $2 \cdot 1$; laccic acid, $0 \cdot 0$; salts of potash and lime, $1 \cdot 0$; earths, $6 \cdot 6$; loss, $4 \cdot 2$.

In India the seed-lac is put into oblong bags of cotton cloth, which are held over a charcoal fire by a man at each end, and, as soon as it begins to melt, the bag is twisted so as to strain the liquefied resin through its substance, and to make it drop upon smooth stems of the banyan tree (musa paradisa). In this way, the resin spreads into thin plates, and constitutes the substance known in commerce by the name of shellac.

The Pegu stick-lac, being very dark-colored, furnishes a shellac of a corresponding deep hue, and therefore of inferior value. The palest and finest shellac is brought from the northern Circar. It contains very little celoring matter. A stick-lac of an intermediate kind comes from the Mysore country, which yields a brilliant lac-dye and a good shellac.

Lac-dye is the watery infusion of the ground stick-lac, evaporated to dryness, and formed into cakes about two inches square and half an inch thick. Dr. John found it to consist of coloring matter, 50 ; resin, 25 ; and solid matter, composed of alumina, plaster, chalk, and sand, 22.

Dr. Macleod, of Madras, informs me that he prepared a very superior lac-dye from stick-lack, by digesting it in the cold in a slightly alkaline decoction of the dried leaves of the Memecylon tinctorium (perhaps the $M$. capitellalum, from which the natives of Malabar and Ceylon obtain a saffron-yellow dye). This solution being used along with a mordant, consisting of a saturated solution of tin in muriatic acid, was found to dye woollen cloth of a very brilliant scarlet hue.

The cakes of lac-dye imported from India, stamped with peculiar marks to designate their different manufacturers, are now employed exclusively in England for dyeing scarlet cloth, and are found to yield an equally brilliant color, and one less easily affected by perspiration than that produced by cochineal. When the lac-dye was first introduced, sulphuric acid was the solvent applied to the pulverized cakes, but as muriatic acid has been found to answer so much better, it has entirely supplanted it. A good solvent (No. 1) for this dye-stuff may be prepared by dissolving three pounds of tin in 60 pounds of muriatic acid, of specific gravity $1 \cdot 19$. The proper mordant for the cloth is made by mixing 27 pounds of muriatic acid of sp. grav. $1 \cdot 17$, with $1 \frac{1}{2}$ pounds of nitric acid of $1 \cdot 19$; putting this mixture into a salt-glazed stone-bottle, and adding to it, in small bits at a time, grain tin, till 4 pounds be dissolred. This solution (No. 2) may be used within twelve hours after it is made, provided it has become cold and clear. For dyeing, three quarters of a pint of the solvent No. 1 is to be poured upon each pound of the pulverized lac-dye, and allowed to digest upon it for six hours. The cloth, before being subjected to the dye bath, must be scoured in the mill with fullers' earth. To dye 100 pounds of pelisse cloth, a tin boiler of 300 gallons capacity should he filled nearly brimful with water, and a fire kindled under it. Whenever the temperature rises to $150^{\circ} \mathrm{Fahr}$, a handful of bran and half a pint of the solution of tin (No. 2) are to be introduced. The froth, which rises as it approaches ebullition, must be skimmed off; and when the liquor boils, $10 \frac{1}{2}$ pounds of lack-dye, previously mixed with 7 pints of the solvent No. 1 , and $3 \frac{1}{2}$ pounds of solution of tin No. 2, must be poured in. An instant afterwards, $10 \frac{1}{2}$ pounds of tartar, and 4 pounds of ground sumach, both tied up in a linen bag, are to be suspended in the boiling bath for five minutes. The fire being now withdrawn, 20 gallons of cold water, with $10 \frac{1}{2}$ pints of solution of tin, being poured into the bath, the cloth is to be immersed in it, moved about rapidly during ten minutes; the fire is to be then rekindled, and the cloth winced more slowly through the bath, which must be made to boil as quickly as possible, and maintained at that pitch for an hour. The cloth is to be next washed in the river; and lastly, with water only, in the fulling mill. The above proportions of the ingredients produce a brilliant scarlet tint, with a slightly purple cast. If a more orange hue be wanted, white Florence argal may be used, instead of tartar, and some more sumach. Lac-dye may be substituted for cochineal in the orange-scarlets; but for the more delicate pink shades, it does not 
answer so well, as the lustre is apt to be impaired by the large quantity of acid neccssary to dissolve the coloring matter of the lac.

Shellac, by Mr. Hatchett's analysis, consists of resin, 90.5 ; coloring matter, 0.5 ; wax, $4 \cdot 0$; gluten, $2 \cdot 8$; loss, $1 \cdot 8$; in 100 parts.

The resin may be obtained pure by treating shellac with cold alcohol, and filtering the sulution in order to separate a yellow gray pulverulent matter. When the alcohol is again distilled off, a brown, translucent, hard, and brittle resin, of specific gravity $1 \cdot 139$, remains. It melts into a viscid mass with heat, and diffuses an aromatic odor. Anhydrous alcohol dissolves it in all proportions. According to John, it consists of two resins, one of which dissolves readily in alcohol, ether, the volatile and fat oils; while the other is little soluble in cold alcohol, and is insoluble in ether and the volatile oils. Unverlorben, however, has detected no less than four different resins, and some other substances, in shellac. Shellac dissolves with ease in dilute muriatic and acetic acids; but not in concentrated sulphuric acid. The resis? of shellac has a great tendency to combine with salifiable bases; as with caustic potash, which it deprives of its alkaline taste.

This solution, which is of a dark red color, dries into a brilliant, transparent, reddish brown mass; which may be re-dissolved in both water and alcohol. By passing chlorine in excess through the dark-colored alkaline solution, the lac-resin is precipitated in a colorless state. When this precipitate is washed and dried, it forms, with alcohol, an excellent pale-yellow varnish, especially with the addition of a little turpentine and mastic.

With the aid of heat, shellac dissolves readily in a solution of borax.

The substances which Unverdorben found in shellac are the following:

1. A resin, soluble in alcohol and ether;

2. A resin, soluble in alcohol, insoluble in ether;

3. A resinous body, little soluble in cold alcohol;

4. A crystallizable resin;

5. A resin, soluble in alcohol and ether, but insoluble in petroleum, and uncrvstallizable.

6. The unsaponified fat of the coccus insect, as well as oleic and margaric acids.

7. Wax.

8. The laccine of Dr. John.

9. An extractive coloring matter.

Statistical Table of Lac-Dye and Lac-Lake, per favor of James Wilkinson, Esq., of Leadenhall street.

\begin{tabular}{|c|c|c|c|c|c|}
\hline & Import. & Export. & $\begin{array}{c}\text { Home } \\
\text { Consumption. }\end{array}$ & Prices. & Stocks. \\
\hline 1802 & $\begin{array}{l}l b s . \\
253\end{array}$ & lbs. & $\begin{array}{l}\text { lbs. } \\
\text { none }\end{array}$ & & \\
\hline 1803 & 1,735 & none & account burned & & \\
\hline 1804 & 531 & - & & & \\
\hline 1805 & 1,987 & & & & \\
\hline 1806 & none & & & & \\
\hline 1807 & 25,350 & & & & \\
\hline $\begin{array}{l}1808 \\
1809\end{array}$ & $\begin{array}{r}5,731 \\
40,632\end{array}$ & & & & \\
\hline 1810 & 235,154 & & & & \\
\hline 1811 & 378,325 & & & & \\
\hline 1812 & 198,250 & & & & \\
\hline 1813 & 289,654 & & & & \\
\hline 1814 & 278,899 & 5,071 & 133,935 & & \\
\hline 1815 & 598,592 & 8,441 & 137,915 & & \\
\hline 1816 & 269,373 & 27,412 & 162,894 & & \\
\hline 1817 & 384,909 & 23,091 & 234,763 & & \\
\hline 1818 & 242,572 & 32,079 & 323,169 & & \\
\hline 1819 & 179,511 & 21,707 & $20 ; 063$ & & \\
\hline 1820 & 441,486 & 49,519 & $9=2,514$ & & \\
\hline 1821 & 641,755 & 91,925 & 322,$83 ;$ & & \\
\hline 1822 & 872,967 & 29,578 & 349,351 & & \\
\hline 1823 & 534,220 & 13,050 & 414,714 & & \\
\hline 1824 & 604,269 & 53,843 & 483,339 & & \\
\hline 1825 & 541,443 & 61.908 & 385,734 & & \\
\hline 1826 & 760,729 & 68,603 & 395,609 & & \\
\hline
\end{tabular}


Statistical Table of Lac-Dye and Lac-Lake.-continued.

\begin{tabular}{|c|c|c|c|c|c|c|c|c|}
\hline \multirow[b]{2}{*}{1827} & \multirow{2}{*}{$\begin{array}{c}\text { Import. } \\
\frac{1 b s .}{756,315}\end{array}$} & \multirow{2}{*}{$\begin{array}{c}\text { Export. } \\
\begin{array}{c}\text { lbs. } \\
76,875\end{array}\end{array}$} & \multirow{2}{*}{$\begin{array}{c}\begin{array}{c}\text { Home } \\
\text { Consumption. }\end{array} \\
\begin{array}{c}l b s \\
448,270\end{array}\end{array}$} & \multicolumn{4}{|c|}{ Prices. } & \multirow{2}{*}{$\begin{array}{c}\text { Stocks. } \\
\text { Chests. } \\
11,53 \text { r }\end{array}$} \\
\hline & & & & s. & $\begin{array}{c}d \\
9\end{array}$ & & $\begin{array}{c}a . \\
0\end{array}$ & \\
\hline 1828 & 512,874 & 54,999 & 397,867 & 1 & 3 & 3 & 9 & 11,085 \\
\hline 1829 & 475,632 & 39,344 & 433,851 & 1 & 3 & 3 & 6 & 11,976 \\
\hline 1830 & 534,341 & 78,099 & 548,865 & 0 & 9 & 3 & 3 & 11,834 \\
\hline 1831 & 913,562 & 175,717 & 597,568 & 0 & -4 & 2 & 6 & 12,5559 \\
\hline 1832 & 378,843 & 69,842 & วั94,155 & 0 & 4 & 2 & 3 & 11,420 \\
\hline 1833 & 326,894 & 66,447 & 426,460 & 0 & 9 & 2 & 4 & $11,45 \%$ \\
\hline 1834 & 708,959 & 89,229 & 398,832 & 0 & 11 & 2 & 4 & 11,928 \\
\hline 1835 & 528,564 & 203,840 & 573,288 & 0 & 11 & 3 & 0 & 10,454 \\
\hline 1836 & 642,436 & 200,975 & 642,615 & 1 & 0 & 4 & 0 & 9,492 \\
\hline 1837 & $1,011,674$ & 133,959 & 427,890 & 1 & 0 & 3 & 9 & 8,780 \\
\hline
\end{tabular}

LACCIC ACID crystallizes, has a wine-yellow color, a sour taste, is soluble in water, alcohol, and ether. It was extracted from sticklac by Dr. John.

LACCINE is the portion of shellac which is insoluble in boiling alcohol. It is brown, brittle, translucid, consisting of agglomerated pellicles, more like a resin than any thing else. It is insoluble in ether and oils. It has not been applied to any use.

LACE MANUFACTURE. The pillow-made, or bone-lace, which formerly gare occupation to multitudes of women in their own houses, has, in the progress of mech?nical invention, been nearly superseded by the bobbin-net lace, man:ufactured at first by hand-machines, as stockings are knit upon frames, but recently by the fower of water or steam. This elegant texture possesses all the strength and regularity of the old Buckingham lace, and is far superior in these respects to the point-net and warp lace, which had preceded, and in some measure pared the way for it. Bobbin-ner may be said to surpass every other branch of human industry in the complex ingenuity of its machinerr; one of Fisher's spotting frames being as much berond the most curious chronometer, in multiplicity of mechanical device, as that is bejond a common roastingjack.

The threads in bobbin-net lace form, by their intertwisting and deccussation, regular hexagonal holes or meshes, of which the two opposite sides, the upper and under, are directed along the breadth of the piece, or at right angles to the selvage or horder.

608

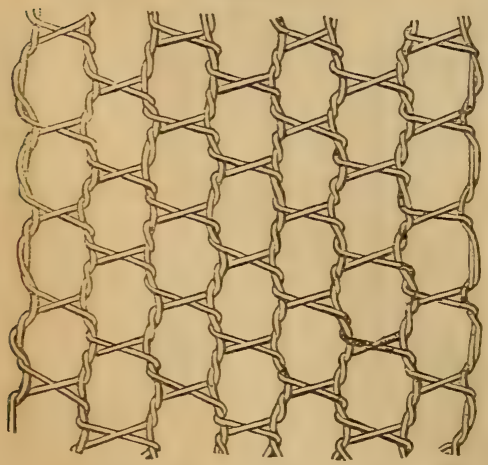

Fig. 608 shows how, by the crossing and twisting of the threads, the regular sixsided mesh is produced, and that the texture results from the union of three separate sets of threads, of which one set proceeds downwards in serpentine lines, a second set proceeds from the left to the right, and a third from the right to the left, both in slanting directions. These oblique threads twist themselves round the vertical ones, and also cross each other betwixt them, in a peculiar manner, which may be readily understood by examining the representation. In comparing bobbinnet with a common web, the perpendicular threads in the figure, which are parallel to the border, may be regarded as the warp, and the two sets of slanting threads, as the weft.

These warp threads are extended up and down, in the original mounting of the piece, between a top and bottom horizontal roller or beam, of which one is called the warp beam, and the other the lace beam, because the warp and finished lace are wound upon them respectirely. These straight warp threads receive their contortion from the tension of the weft threads twisted obliquely round them alternately to the right and the left hand. Were the warp threads so tightly drawn that they became inflexible, like fiddle-strings, then the lace would assume the appearance shown in fig $f n^{n} ;$ and although this condition does not reallv exist. it mav serre to illustraite inte structure of 
the web. The warp threads stand in the positions $a a, a^{\prime} a^{\prime}$, and $a^{\prime \prime} a^{\prime \prime}$; the one half of the weft proceeds in the direction $b b, b^{\prime} b^{\prime}$, and $b^{\prime \prime} b^{\prime \prime}$; and the second crosses the 609

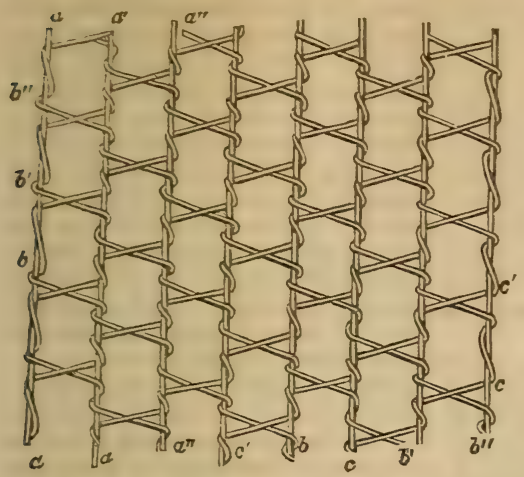
firsi bi running in the direction $c c$, or $c^{\prime} c^{\prime}$, towards the opposite side of the fab. ric. If we pursue the path of a wefi thread, we find it goes on till it reaches the outermost or last warp thread, which it twists about; not once, as with the others, but twice; and then returning towards the other border, proceeds in a reverse direction. It is by this double twist, and by the return of the weft threads, that the selvage is made.

The ordinary material of bobbin-net is two cotton yarns, of from No. 180 to No. 250 , twisted into one thread; but sometimes strongly twisted single yarn has been used. The beauty of the fabric depends upon the quality of the material, as well as the regularity and smallness of the meshes. The number of warp threads in a yard in breadth is from 600 to 900 ; which is equivalent to from 20 to 30 in an inch. The size of the holes cannot be exactly inferred from that circumstance, as it depends partly upon the oblique iraction of the threads. The breadth of the pieces of bobbin-net varies from edgings of a quarter of an inch, to webs 12 , or even 20 quarters, that is, 5 yards wide.

Bobbin-net lace is manufactured by means of very costly and complicatea machines, called frames. The limits of this Dictionary will admit of an explanation of no more than the general principles of the manufacture. The threads for crossing and twisting round the warp, being previously gassed, that is, freed from loose fibres by singeing with gas, are wound round small pulleys, called bobbins, which are, with this view, deeply grooved in their periphery. Figs. 610,611 exhibit the bobbin alone, and with its carriage. 611
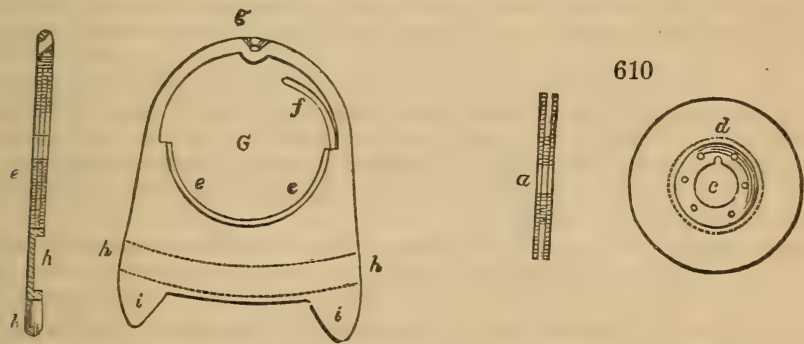

In the section of the bobbin $a, f i g .610$, the deep groove is shown in which the thread is wound. The bobbin consists of two thin discs of brass, cut out in a stamp-press, in the middle of each of which there is a hollow space $c$. These discs are riveted together, leaving an interval between their edge all round, in which the thread is coiled. The round hole in the centre, with the little notch at top, serves for spitting them upon a feathered rod, in order to be filled with thread by the rotation of that rod in a species of reel, called the bobbin-filling machine. Each of these bobbins (about double the size of the figure), is inserted into the vacant space G, of the carriage, fig. 611. This is a small iron frame (also double the size of the figure), which, at $e \quad e$, embraces the grooved border of the bobbin, and by the pressure of the spring at $f$, prevents it from falling out. This spring serves likewise to apply sufficient friction to the bobbin, so as to prevent it from giving off its thread at $\mathrm{g}$ by its rotation, unless a certain small force of traction be employed upon the thread. The curvilinear groove $h h$, sunk in each face or side of the carriage, has the depth shown in the section at $h$. This groove corresponds to the interval between the teeth of the comb, or bars of the bolt, in which each carriage is placed, and has its movement. A portion of that bolt or comb is shown at $a$, fig. 612, in plan, and one bar of a circular bolt machine at $b$, in section. If we suppose twc such combs or bolts placed with the ends of the teeth opposite each other, but a lintle apart, to let the warp threads be stretched, in one vertical plane, between their ends or tips, we shall have an idea of the skeleton of a bobbin-net machine. One of these two 
combs, in the double bolt machine, has an occasional lateral movement called shogging, equal to the interval of one tooth or bolt, by which, after it has received the bobbins,
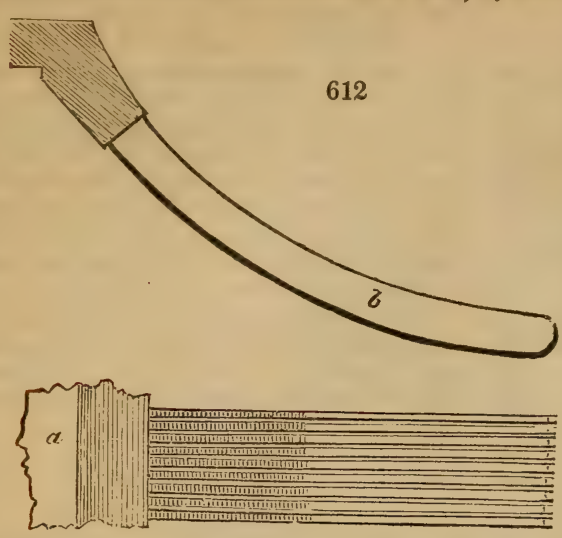
with their carriages, into its teeth, it can shift that interval to the one side, and thereby get into a position to return the bobbins, with their carriages, into the next series of interstices or gates, in the other bolt. By this means the whole series of carriages receives successive side steps to the right in one bolt, and to the left in the other, so as to perform a species of countermarch, in the course of which they are made to cross and twist round about the vertical warp threads, and thus to form the meshes of the net.

The number of movements required to form a row of meshes in the double tier machine, that is, in a frame with two combs or bars, and 2 rows of bobbins, is six; that is, he whole of the carriages (with their bobbins) pass from one bar or comb to the other six times, during which passages the different divisions of bobbin and warp threads change their relative positions 12 times.

This interchange or traversing of the carriages with their bobbins, which is the most difficult thing to explain, but at the same time the most essential principle of the lacemachine, may be tolerably well understood by a careful study of $f i g .613$, in which the

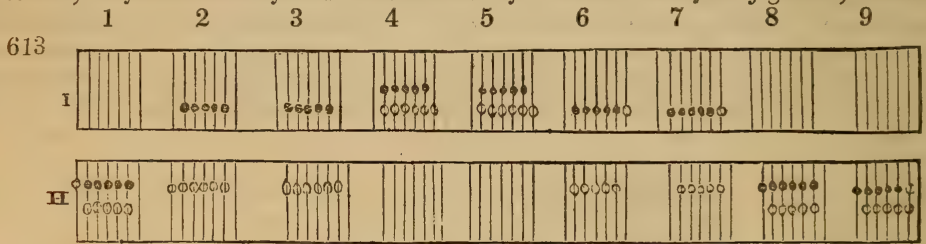

simple line / represents the boits or teeth, the sign o the back line of carriages, and the sign $\phi$ the front line of carriages. $H$ is the front comb or bolt bar, and $\mathrm{I}$ the back bolt har. The former remains always fixed or stationary, to receive the carriages as they may be presented to it by the shogging of the latter. There must be always one odd carriage at the end ; the rest being in pairs.

No. 1 represents the carriages in the front comb or bar, the odd carriage being at the left end. The back line of carriages is first moved on to the back bar 1 , the odd carriage, as seen in No. 1, having been left behind, there being no carriage opposite to drive it over to the other comb or bar. The carriages then stand as in No. 2. The bar I now shifts to the left, as shown in No. 3; the front carriages then go over into the back bar or comb, as is represented by No. 4. The bar I now shifts to the right, and gives the position No. 5. The front carriages are then driven over to the front bar, and leave the odd carriage on the back bar at the right end, for the same reason as before described, and the carriages stand as shown in No. 6. The bar I next shifts to the left, and the carriages stand as in No. 7 (the odd carriage being thereby on the back bar to the left.) The back carriages now come over to the front bar, and stand as in No. 8 . The back bar or comb I shifts to the right as seen in No. 9, which completes the traverse. The whole carriages with their bobbins have now changed their position, as will be seen by comparing No. 9 with No. 1 . The odd carriage, No. $1 \phi$ has advanced one step to the right, and has become one of the front tier; one of the back tier or line $\phi$ has advanced one step to the left, and has become the odd carriage; and one of the front ones ; has gone over to the back line. The bobbins and carriages throughout the whole width of the machine have thus crossed each other's course, and completed the mesh of net.

The carriages with their bobbins are driven a certain way from the one comb to the other, by the pressure of two long bars (one for each) placed above the level of the comb, until they come into such a position that their projecting heels or catches $i i, f i g .611$, are moved off by two other long flat bars below, called the locker plates, and thereby carried completely over the interval between the two combs.

There are six different systems of bobbin-net machines. 1. Heathcoate's patent 
machine. 2. Brown's traverse warp. 3. Morley's straight bolt. 4. Clarke's pusher prunciple, single tier. 5. Learer's machine, single tier. 6. Morley's circular bolt. All the others are mere variations in the construction of some of their parts. It is a remarkable fact, highly honorable to the mechanical judgment of Mr. Morley of Derby, that no machines except those upon his circular bolt principle, have been found capable of working successfully by mechanical power.

The circular bolt machine (comb with curved teeth) was used by Mr. Morley, for making narrow breadths or edgings of lace immediately after its first invention, and it has been regularly used by the trade for that purpose ever since, in consequence of the inventor having declined to secure the monopoly of it to himself by patent. At that time the locker bars for driving across the carriages had only one plate or blade. A machine so mounted is now called "the single locker circular bolt." In the year 1824, $\mathrm{Mr}$. Morley added another plate to each of the locker bars, which was a great improvement on the machines for making plain net, but an obstruction to the making of narrow breadths upon them. This machine is now distinguished from the former by the term " double locker.".*

A rack of lace, is a certain length of work counted perpendicularly, and contains 240 meshes or holes. Well-made lace has the meshes a little elongated in the direction of the selvage.

The term gauge, in the lace manufacture, means the number of gates, slits, or interstices, in one inch of the bolt-bar or comb; and corresponds therefore to the number of bobbins in an inch length of the double tier. Thus, when we say "gauge nine points," we mean that there are nine gates with nine bobbins in one inch of the comb or bolt-bar. Each of such bobbins with its carriage is therefore no more than one ninth of an inch thick. The common proportion or gauge up and down the machine is 16 holes in the inch for ten bobbins transversely, Circular bolt double tier machines can turn off by steam power fully 360 racks each day of 18 hours, with a relay of superintendents.

The number of new mechanical contrivances to which this branch of manufacture has given rise, is altogether unparalleled in any other department of the arts. Since Mr. Heathcote's first successful patent, in 1809, a great many other patents have been granted for making lace. In the year 1811, Mr. Morley, then of Nottingham, invented his straight bolt frame, more simple in construction, better combined, and more easy in its morements, than the preceding machines; but the modest inventor did not secure it, as he might have done, by patent. The pusher machine was invented in the same year, by Samuel Mart and James Clark, also of Nottingham. The following year is remarkable in the history of the lace trade, for the invention of the circular bolt machine, by $\mathbf{M r}$. Morley-a mechanism possessing all the advantages of his straight bolt machine, without its disadvantages.

Nearly at the same time Mr. John Leaver brought forward the lever machine, conjointly with one Turton, both of New Radford, near Nottingham. About the year 1817 or $1818, \mathrm{Mr}$. Heathcote applied the rotatory movemement to the circular bolt machine, and mounted a manufactory on that plan, by mechanical power, at Tiverton, after he and his partner, Mr. Boden, had been driven from Loughborough, in 1816, by the atrocious violence of the frame-destroying Luddites.

Such has been the progress of improvement and economy in this manufacture, that the cost of labor in making a rack, which was, twenty years ago, $3 s .6 d$., or 42 pence, is now not more than one penny. The prices of this beautiful fabric have fallen in an equally remarkable manner. At the former period, a 24 rack piece, five quarters broad, fetched 1\%l. sterling, in the wholesale market; the same is now sold for $7 \mathrm{~s}$.! The consequence is, that in lace decoration, the maid servant may be now more sumptuously arrayed than her mistress could afford to be twenty years ago.

LACKER, is a varnish, consisting chiefly of a solution of pale shellac in alcohol tinged with saffion, annotto, or other coloring matter. See VARNISH.

LACTIC ACID. (Acide Lactique, Fr.; Milchsaüre, Germ.) This acid was discovered by Scheele in buttermilk, where it exists most abundantly; but it is present also in fresh milk in small quantity, and communicates to it the property of reddening litmus. Lactic acil may be detected in all the fluids of the animal body; either free or saturated with alkaline matter.

Scheele obtained this acid by evaporating the sour whey of clotted milk to an eighth part of its bulk, saturating this remainder with slaked lime, in order to throw down the subpliosphate of lime held in solution, filtering the liquor, diluting it with thrice its weight of water, and precipitating the lime circumspectly, by the gradual addition of oxalic acid.

* $\mathrm{R}_{\mathrm{y}}$ leading the above brief account of Bobbin-net, in connexion with the more detailed description of it

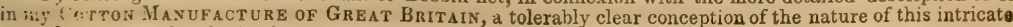
uakufictu: nay be obtained. 
He next filtered, evaporated to dryness on a water bath, and digested the residuum is strong alcohol, which dissolved the lactic acid, and left the sugar of milk. On evapora. ting off the alcohol, the acid was obtained. As thus procured, it requires to be purified by saturation with carbonate of lead (pure white lead), and precipitating the solution of this iactate with sulphate of zinc, not added in excess. Sulphate of lead falls, and the supernatant lactate of zinc being evaporated afiords crystals, at first brown, but which become colorless on being dissolved and re-crystallized twice or thrice. If the sulphurie acid of the dissulved salt be thrown down by water of baryta, the liquid when filtered and evaporated yields a pure lactic acid, of a sirupy consistence, colorless and void of smell. It has a puncent acid taste, which it loses almost entirely when moderately diluted with water. It does not mystallize. Its salts, with the exception of thos of magnesia an zinc, have a gummy appearance, and are very soluble in alcohol, unless they hold an excess of base. Lactic acid consists of 44.92 carbon; 6.55 hydrogen ; 48.53 oxrgen. It contains 9.92 per cent. of water. It has not bitherto been applied to any use in the arts, exceyt by the Dutch in their old process of bleaching linen with sour milk.

LACTOJETER is the name of an instrument for estimating the quality of mill;, called also a Gulactometer, which see. The most convenient form of apparatus would be a series of glass tubes each about 1 inch in diameter, and 12 inches long, graduated through a space of 10 inches, to tenths of an inch, haring a stop-coch at the bottom, and suspended upright in a frame. The arerage milk of the cow being poured in to the height of 10 inches, as soon as the cream has all separated at top, the thichness of its body may be measured by the scale; and then the slim milk may be run off below into a hridrometer glass, in order to determine its densiț, or relatire richness in caseous matter.

LAKES. Tnder this title are comprised all those colors which consist of a regetable dre, combined by precipitation with a white earthy basis, which is usually alumina. The general method of preparation is to add to the colored infusion a.solution of common alum, or rather a solution of alum saturated with potash, especiall ț when the infusion has been made with the aid of acids. At first only a slight precipitate falls, consisting of alumina and the coloring matter; but on adding potash, a copious precipitation ensues, of the alumina asscciated with the dye. When the dyes are not injured, but are rather brightened by allialis, the abore process is reversed; a decoction of the dye-stuff is made with an alkaline liquor, and when it is filtered, a solution of alum is poured into it. The third methol is practicable only with substances haring a great affinity for subsulphate of alumina; it consists in agitating recently precipitated alumina with the decoction of the dye.

Fellou lakes are made with a decoction of Persian or French berries, to which some potash or soda is added; into the mirture a solution of alum is to be poured as long as ant precipitate falls. The precipitate must be filtered, washed, and formed into calies, and dried. A lake mar be made in the same way with quercitron, taking the precaution to purify the decoction of the dye-stuff with buttermilk or glue. After filtering the lake it mar be brightened with a solution of tin. Annotto lake is formed br dissolring the dre-stuff in a weak alkaline les, and adding alum water to the solution. Solution of tin gives this lake a lemon rellow cast; acids a reddish tint.

Red lakes. - The finest of these is carmine.

This beautiful pigment mas accidentally discorered br a Franciscan monk at Pisa. He formed an extract of cochineal with salt of tartar, in order to emplor it as a medicine, and obtained, on the addition of an acid to it, a fine red precipitate. Homberg published a process for preparing it, in 1656. Carmine is the coloring matter of cochineal, prepared by precipitation from a decction of the drug. Its composition raries according to the mode of making it. The ordinary carmine is prepared with alum, and consists of carminium (see CochivesL), a little animal matter, alumina, and sulphuric acid. See Carmine.

Carminated lake, called lake of Florence, Paris, or Tienna. For making this pigment, the liquor is usually employed which is decanted from the carmine process. Into this, newly precipitated alumina is put; the mixture is stirred, and heated a little, but not too much. Whenerer the alumina has absorbed the color, the mixture is allowed to settle, and the liquor is drawn off.

Sometımes alum is dissolred in the decoction of cochineal, and potash is then added, to throw down the alumina in combination with the coloring matter; but in this way an indifferent pigment is obtained. Occasionally, solution of tin is added, to brighten the Uje.

A lake may be obtained from kermes, in the same way as from cochineal; but now it is seldom had recourse to.

Brazil-wood lakes.-Brazil wood is to be boiled in a proper quantity of water for 15 minutes; then, alum and solution of tin being added, the liquor is to be filtered, and a solution of potash poured in as long as it occasions a precipitate. This is separatea by 
the filter, washed in pure water, mixed with a little gum water, and made into cakes Or, the Brazil wood may be boiled along with a little vinegar, the decoction filtered, alum and salt of tin added, and then potash-ley poured in to precipitate the lake. For 1 pound of Brazil wood, 30 to 40 pounds of water, and from $1 \frac{1}{2}$ to 2 pounds of alum, may be talien, in producing a deep red lalie; or the same proportions with half a pound of solution of tin. If the potash be added in excess, the tint will become violet. Cream of tartar occasions a brownish cast.

Madder lake.-A fine lake may ae obtained from madder, by washing it in cold water as long as it gives out color; then sprinkling some solution of tin over it, and setting $\mathrm{i}_{i}$ aside for some days. A gentle heat may also be applied. The red liquor must be then separated by the filter, and decomposed by the addition of carbonate of soda, when a fine red precipitate will be obtained. Or, the reddish brown coloring matter of a decoction of madder may be first separated by acetate of lead, and then the rose-red color with alum. Or, madder tied up in a bag is boiled in water; to the decoction, alum is added, and then potash. The precipitate should be washed with boi ing water, till it ceases to tinge it yellow; and it is then to be dried.

The following process merits a preference:

Diffuse 2 pounds of ground madder in 4 quarts of water, and after a maceration of 10 minutes, strain and squeeze the grounds in a press. Repeat this maceration, \&c. twice upon the same portion of madder. It will now have a fine rose co.or. It must then be mixed with 5 or 6 pounds of water and half a pound of bruised alum, and heated upon a water bath for 3 or 4 hours, with the addition of water, as it evaporates, after which the whole must be thrown upon a filter cloth. The liquor which passes is to be filtered througin paper, and then precipitated by carbonate of potash. If the potash be added in three successive doses, three different lakes will be obtained, of successirely diminishing beauty. The precipitates must be washed till the water comes off colorless.

Blue lakes are hardly ever prepared, as indigo, Prussian blue, cobalt blue, and ultramarine, answer every purpose of blhe pigments.

Green lakes are made by a mixture of yellow lakes with blue pigments; but chrome vellows mixed with blues produce almost all the requisite shades of green.

LAMINABLE is said of a metal which may be extended by passing between steel or hardened (chilled) cast-iron rollers.

For a description of metal rolling presses, see Iron and Mint ; and

For a table of the relative laminability of metals, see Ductility.

LAMIUM ALBUM, or the dead nettle, is said by Leuchs to afford in its leares a greenish-rellow dye. The L. purpureum dyes a reddish-gray with salt of tin, and a greenish tint with iron liquor.

LAMPS differ so much in principle, form, and construction, as to render their description impossible, as a general subject of manufacture. In fact, the operations of the lampist, like those of the blachsmith, cabinet-maker, cooper, coppersmith, tinman, turner, \&c., belong to a treatise upon handicraft trades. I shall here, howerer, introduce a tabular view of the relative light and econorny of the lamps most generally kaown.

\begin{tabular}{|c|c|c|c|c|c|c|c|c|c|}
\hline \multirow[b]{2}{*}{ Kind of Lamps. } & \multicolumn{6}{|c|}{ Intensity of light during } & \multirow{2}{*}{$\begin{array}{l}\text { Mean of } \\
7 \text { hours. }\end{array}$} & \multirow{2}{*}{$\begin{array}{l}\text { Consump- } \\
\text { tion per } \\
\text { hour in } \\
\text { grammes. }\end{array}$} & \multirow{2}{*}{$\begin{array}{l}\text { Light from } \\
100 \text { parts } \\
\text { of oil. }\end{array}$} \\
\hline & $\begin{array}{c}1 \\
\text { hour }\end{array}$ & $\mid \begin{array}{c}2 \\
\text { hours }\end{array}$ & \begin{tabular}{|c|}
3 \\
hours
\end{tabular} & $\begin{array}{c}4 \\
\text { hours }\end{array}$ & \begin{tabular}{|c|}
5 \\
hours
\end{tabular} & $\begin{array}{c}6 \\
\text { hours }\end{array}$ & & & \\
\hline $\begin{array}{l}\text { 1. Mechanical lamp of } \\
\text { Carcel - }\end{array}$ & & & & & & & 100 & 42 & 238 \\
\hline $\left.\begin{array}{l}\text { 2. Fountain lamp, } \\
\text { and a chimney } \\
\text { with flat wick }\end{array}\right\}$ & 100 & 98 & 98 & 97 & 96 & 96 & 125 & 11 & 113 \\
\hline 3. Dome argand - & 103 & 90 & 72 & 61 & 42 & 34 & 31 & $26 \cdot 714$ & 116 \\
\hline $\begin{array}{l}\text { 4. Sinumbra lamp - } \\
\text { 5. Do. with fountain }\end{array}$ & 102 & 95 & 83 & 81 & 78 & 66 & 56 & $37 \cdot 145$ & 150 \\
\hline $\begin{array}{l}\text { above - } \\
\text { 6. Do. with another }\end{array}$ & 100 & 90 & 70 & 52 & 41 & 32 & 85 & 43 & 197 \\
\hline $\begin{array}{l}\text { beak - - - } \\
\text { 7. Girard's hydrostatic }\end{array}$ & 100 & 97 & 95 & 92 & 89 & 86 & 41 & 18 & 227 \\
\hline 8. Thilorier's or Par- & 101 & 96 & 84 & 81 & 76 & 70 & $63 \cdot 66$ & $34 \cdot 714$ & 182 \\
\hline $\begin{array}{l}\text { ker's hydrosta- } \\
\text { tie lamp - }\end{array}$ & 106 & 103 & 100 & 94 & 92 & 90 & $107 \cdot 66$ & $51 \cdot 143$ & 215 \\
\hline
\end{tabular}


In the above table, for the purpose of comparing the successive degrees of intensity 100 represents the mean intensity of light during the first hour. The quantity of oil con sumed per hour is given in grammes, of $15 \frac{1}{2}$ grains each. The last column expresses the quantity of light produced with a like consumption of oil, which was in all cases $10 \mathrm{C}$ grammes. See Candles.

The following table of M. Peclet is perhaps more instructive :-

\begin{tabular}{|c|c|c|c|c|c|c|}
\hline \multirow[b]{2}{*}{ Nature of the light. } & \multirow[b]{2}{*}{ Intensity. } & \multirow{2}{*}{$\begin{array}{l}\text { Consump- } \\
\text { tion per } \\
\text { hour in } \\
\text { grammes. }\end{array}$} & \multicolumn{2}{|c|}{ Cost } & \multirow{2}{*}{$\begin{array}{l}\text { Fat pro- } \\
\text { ducing the } \\
\text { same light. }\end{array}$} & \multirow{2}{*}{$\begin{array}{c}\text { Cost } \\
\text { per hour. }\end{array}$} \\
\hline & & & $\begin{array}{l}\text { per kilo- } \\
\text { gramme. }\end{array}$ & $\begin{array}{l}\text { of light } \\
\text { per hour. }\end{array}$ & & \\
\hline 1. Mechanical lamp & 100 & 42 & $\begin{array}{l}\text { francs. } \\
1 \cdot 40\end{array}$ & $\begin{array}{l}\text { cents. } \\
5 \cdot 8\end{array}$ & $\begin{array}{l}\text { grammes. } \\
42\end{array}$ & $\begin{array}{l}\text { cents. } \\
5 \cdot 8\end{array}$ \\
\hline $\begin{array}{l}\text { 2. Flat-wick mechan. do. } \\
\text { 3. Hemispherical dome }\end{array}$ & $12 \cdot 05$ & 11 & $1 \cdot 40$ & 1.5 & 88 & $12 \cdot 3$ \\
\hline lamp - - & $31 \cdot 0$ & $26 \cdot 714$ & $1 \cdot 40$ & $3 \cdot 7$ & $86 \cdot 16$ & $12 \cdot 0$ \\
\hline 4. Sinumbra lamp & 85 & 43 & $1 \cdot 40$ & $6 \cdot 0$ & $50 \cdot 58$ & $7 \cdot 0$ \\
\hline $\begin{array}{l}\text { 5. Do. with a lateral foun- } \\
\text { tain or vase - } \\
\text { 6. Do. with a fountain }\end{array}$ & 41 & 18 & $1 \cdot 40$ & $2 \cdot 5$ & $43 \cdot 90$ & $6 \cdot 1$ \\
\hline 7. Girard's hydrostatic - & 90 & 43 & $1 \cdot 40$ & $6 \cdot 0$ & $47 \cdot 77$ & $6 \cdot 6$ \\
\hline 8. Thilorier's or Parker's & $63 \cdot 66$ & $34 \cdot 71$ & $1 \cdot 40$ & $4 \cdot 8$ & $54 \cdot 52$ & $7 \cdot 6$ \\
\hline lamp - - - & $107 \cdot 66$ & $51 \cdot 143$ & $1 \cdot 40$ & $7 \cdot 1$ & $47 \cdot 5$ & $6 \cdot 6$ \\
\hline 9. Candle, 6 in lb. & $10 \cdot 66$ & $8 \cdot 51$ & $1 \cdot 40$ & $1 \cdot 2$ & $70 \cdot 35$ & $9 \cdot 8$ \\
\hline $\begin{array}{ll}\text { 10. Do. } 8 \text { in lb. } \\
\text { 11. } & \text { Do. } 6 \text { with smaller }\end{array}$ & $8 \cdot 74$ & $7 \cdot 51$ & $1 \cdot 40$ & $1 \cdot 0$ & $85 \cdot 92$ & $12 \cdot 0$ \\
\hline wick - - - & $7 \cdot 50$ & $7 \cdot 42$ & $2 \cdot 40$ & $1 \cdot 7$ & $98 \cdot 93$ & $23 \cdot 7$ \\
\hline 12. Wax candle, 5 in $\mathrm{lb}$. & $13 \cdot 61$ & $8 \cdot 71$ & $7 \cdot 60$ & $5 \cdot 7$ & $64 \cdot 04$ & 486 \\
\hline 13. Sperm candle, do. & $14 \cdot 40$ & $8 \cdot 92$ & $7 \cdot 60$ & $5 \cdot 8$ & $61 \cdot 94$ & $47 \cdot 8$ \\
\hline 14. Stearine candle, do. & $14 \cdot 30$ & $9 \cdot 35$ & 6.00 & $5 \cdot 5$ & $65 \cdot 24$ & $37 \cdot 1$ \\
\hline 15. Coal gas - - & 127 & 136 litres & & $5 \cdot 0$ & 107 litres & $3 \cdot 9$ \\
\hline 16. Oil gas - - & 127 & 136 do. & & $5 \cdot 0$ & 30 & $3 \cdot 9$ \\
\hline
\end{tabular}

The light of the mechanical lamp is greatly over-rated relatively to that of gas. The cost of the former is at least 5 times greater than that of the latter, in London.

LAMP OF DAVY consists of a common oil lamp, surmounted with a covered

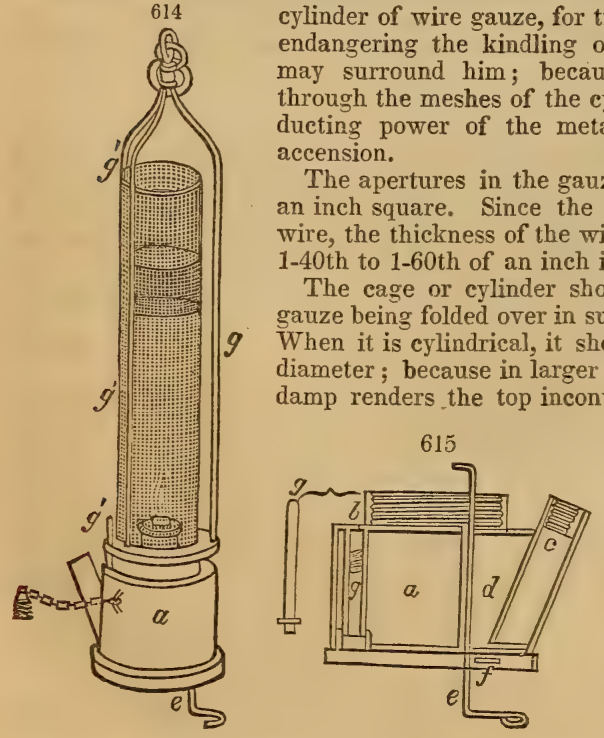
cylinder of wire gauze, for transmitting light to the miner without angering the kindling of the atmosphere of fire-damp which him; because carbureted hydrogen, in passing hrough the meshes of the cylindric cover, gets cooled by the conucting power of the metallic gauze, below the point of its

The apertures in the gauze should not be more than 1-20th of an inch square. Since the fire damp is not inflamed by ignited wire, the thickness of the wire is not of importance, but wire from -40 th to 1-60th of an inch in diameter is the most convenient.

The cage or cylinder should be made by double joinings, the over in such a manner as to leave no apertures. diameter; because in larger cylinders, the combustion of the firedamp renders the top inconveniently hot; a double top is always a proper precaution, fixed $\frac{3}{2}$ or $\frac{3}{4}$ of an inch above the first top. See fig. 614 .

The gauze cylinder should be fastened to the lamp by a screw $b$, fig. 615 , of four or five turns, and fitted to the screw by a tight ring. All joinings in the lamp should be made with haid solder; as the secu.. rity depends upon the circumstance that no aperture exists in the apparatus larger than in the wire-gauze. 
The parts of the lamp are,

1. The bra-s cistern $a, d, f i g .615$, which contains the oil. It is pierced at one side of the centre with a rertical narrow tube, nearly filled with a wire which is recurved above, at the level of the burner, to trim the wick, by acting on the lower end of the wire $e$ with the fingers. It is called the safety-trimmer.

2. The rim $b$ is the screw neck for fixing on the gauze cylinder, in which the wiregauze cover is fixed, and which is fastened to the cistern by a screw fitted to $b$.

3. An aperture $c$ for supplying oil. It is fitted with a screw or a cork, and communicates with the bottom of the cistern by a tube at $f$. A central aperture for the wick.

4. The wire-gauze cylinder, fig. 614 , which should not have less than 625 apertares to the square inch.

5. The second top, $\frac{3}{4}$ of an inch above the first, surmounted by a brass or copper plate, to which the ring of suspension may be fixed. It is covered with a wire cap in the figure.

6. Four or six thick vertical wires, $g^{\prime} g^{\prime} g^{\prime} g^{\prime}$, joining the cistern below with the top plate, and serving as protecting pillars round the cage. $g$ is a screw-pin to fix the cover, so that it shall not become loosened by accident or carelessness. The oil-cistern fig. 615 is drs wn upon a larger scale than fig. 614, to show its minuter parts.

When the wire-gauze safe-lamp is lighted and introduced into an atmosphere gradually mixed with fire-damp, the first effect of the fire-damp is to increase the length and size of the flame. When the inflammable gas forms so much as 1-12th of the volume of the air, the cylinder becomes filled with a feeble blue flame, while the flame of the wick apnears burning brightly within the blue flame. The light of the wick augments till the fire-damp increases to 1-6th or 1-5th, when it is lost in the flame of the fire-damp, which in this case fills the cylinder with a pretty strong light. As long as any explosive mixture of gas exists in contact with the lamp, so long it will give light; and when it is extinguished, which happens whenever the foul air constitutes so much as 1-3d of the volume of the atmosphere, the air is no longer proper for respiration; for though animal life will continue where flame is extinguished, yet it is always with suffering. By fixing a coil of platinum wire above the wick, ignition may be maintained in the metal when the lamp itself is extinguished; and from this ignited wire the wick may be again rekindled, on carrying it into a less inflammable atmosphere.

"We hare frequently used the lamps where the explosive mixture was so high as to heat the wire-gauze red-hot; but on examining a lamp which has been in constant use for three months, and accasionally subjected to this degree of heat, I cannot perceive that the gauze cylinder of iron wire is at all impaired. I have not, however, thought it prudent, in our present state of experience, to persist in using the lamps under such circumstances, because I have observed, that in such situations the particles of coal dust floating in the air, fire at the gas burning within the cylinder, and fly off in small luminous sparks. This appearance, I must confess, alarmed me in the first instance, but experience soon proved that it was not dangerous.

"Besides the facilities afforded by this invention to the working of coal-mines abound ing in fire-damp, it has enabled the directors and superintendents to ascertain, with the utmost precision and expedition, both the presence, the quantity, and correct situation of the gas. Instead of creeping inch by inch with a candle, as is usual, along the galleries of a mine suspected to contain fire-damp, in order to ascertain its presence, we walk firmly on with the safe-lamps, and, with the utmost confidence, prove the actual state of the mine. By observing attentively the several appearances upon the flame of the lamp, in an examination of this kind, the cause of accidents which happened to the most experienced and cautious miners is completely developed; and this has hitherto been in a great measure matter of mere conjecture.

"It is not necessary that I should enlarge upon the national advantages which must necessarily result from an invention calculated to prolong our supply of mineral coal, because I think them obvious to every reflecting mind; but I cannot conclude without expressing my highest sentiments of admiration for those talents which have developed the properties, and controlled the power, of one of the most dangerous elements which human enterprise has hitherto had to encounter."--See Letter to Sir H. Davy, in Journal of Science, vol. i. p. 302, by John Buddle, Esq., generally and justly esteemed one of the most scientific coal-miners in the kingdom.

Mr. Buddle, in a letter dated 21st August, 1835, which is published in Dr. Davy's life of his brother Sir Humphrey, says :-

"In the evidence given in my last examination before a committee of the House of Commons, I stated that after nearly twenty years' experience of ' the Davy' with from 1000 to 1500 lamps in daily use, in all the variety of circumstances incidental to coal mining, without a single accident having happened which could he attributed to 
a defect in its principle, or even in the rules for its practical application, as laid down by Sir Humphrey--I maintained that 'the Davy' approximated perfection, as nearly as anj instrument of human invention could be expected to do. We have ascertained distinctly that the late explosion did not happen in that part of the mine where the Darys were used. They were all found in a perfect state after the accident-many of them in the hands of the dead bodies of the sufferers."

LAMP-BLACK. See BLACK.

LAMPATES and LAMPIC ACID. When a spirit of wine lamp has its cotton wick surmounted with a spiral coil of platinum wire, after lighting it for a little, it may be blown out, without ceasing to burn the alcohol ; for the coil continues ignited, and a current of hot vapor continues to rise, as long as the spirit lasts. This vapor was first condensed and examined by Professor Daniell, who called it lampic acid. It has a peculiar, strongly acid, burning taste, and a spec. grav. of $\% \cdot 015$. It possesses in an eminent degree the property of reducing certain metallic solutions; such as those of platinum, gold, and silver. The lampates may be prepared by saturating the above acid with the alkaline and earthy carbonates.

LAPIDARY, Art of. The art of the lapidary, or that of cutting, polishing, and engraving gems, was known to the ancients, many of whom have left admirable specimens of their skill. The Greeks were passionate lovers of rings and engraved stones; and the most parsimonious among the higher classes of the Cyrenians are said to have worn rings of the value of ten minæ (about 30l. of our money.) By far the greater part of the antique gems that have reached modern times, may be considered as so many models for forming the taste of the student of the fine arts, and for inspiring his mind with correct ideas of what is truly beautiful. With the cutting of the diamond, however, the ancients were unacquainted, and hence they wore it in its natural state. Even in the middle ages, this art was still unknown; for the four large diamonds which enrich the clasp of the imperial mantle of Charlemagne, as now preserved in Paris, are uncut, octahedral crystals. But the art of working diamonds was probably known in Hindostan and China, in very remote periods. After Louis de Berghen's discovery, in 1476, of polishing two diamonds by their mutual attrition, all the finest diamonds were sent to Holland to be cut and polished by the Dutch artists, who long retained a superiority, now no longer admitted by the lapidaries of London and Paris.

The operation of gem cutting is abridged by two methods; 1 . by cleavage; 2 . by cutting off slices with a fine wire, coated with diamond powder, and fixed in the stock of a hand-saw. Diamond is the only precious stone which is cut and polished with diamond powder, soaked with olive oil, upon a mill plate of very soft steel.

Oriental rubies, sapphires, and topazes, are cut with diamond powder soaked with olive oil, on a copper wheel. The facets thus formed are afterwards polished on another copper wheel, with tripoli, tempered with water.

Emeralds, hyacinths, amethysts, garnets, agates, and other softer stones, are eut at a lead wheel, with emery and water; and are polished on a tin wheel with tripoli and water, or, still better, on a zinc wheel, with putty of tin and water.

The more tender precious stones, and even the pastes, are cut on a mill-wheel of hard wood, with emery and water; and are polished with tripoli and water, on another whee] of hard wood.

Since the lapidary employs always the same tools, whatever be the stone which he cuts or polishes, and since the wheel discs alone vary, as also the substance he uses with them, we shall describe, first of all, his apparatus, and then the manipulations for diamond-cutting, which are applicable to every species of stone.

The lapidary's mill, or wheel, is shown in perspective in fig. 616. It consists of

616

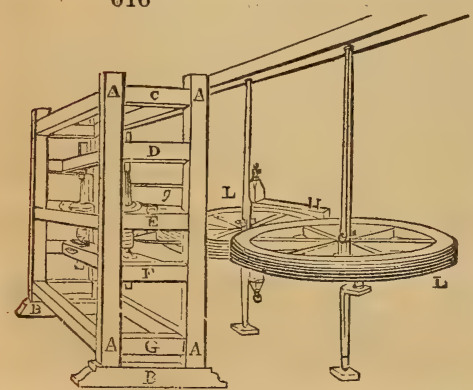
a strong frame made of oak carpentry, with tenon and mortised joints, bound together with strong bolts and screw nuts. Its form is a parallelopiped of from 8 to 9 feet long, by from 6 to 7 high; and about 2 feet broad. These dimensions are large enough to contain two cutting wheels alongside of each other, as represented in the figure.

Besides the two sole bars $\mathrm{B} \mathrm{B}$, we perceive in the breadth, 5 cross bars, $\mathrm{C}, \mathrm{D}, \mathrm{E}, \mathrm{F}, \mathrm{G}$. The two extreme bars $\mathrm{c}$ and $\mathrm{G}$, are a part of the frame-work, and serve to bind it. The two cross-bars $D$ and F, earry each in the middle of their length, a piece of wood as thick as themselves, but only $4 \frac{1}{2}$ inches long (see fig. 617), joined solidly by mortises and tenons with that cross bar, as well as 
with the one placed opposite on the other parallel face. These two pieces are called summers (lintels); the one placed at $\mathrm{D}$ is the upper; the one at $\mathrm{F}$, the lower.

Iir fig. 61\% this face is shown inside, in order to explain how the mill wheel is placed anil supported. The same letters point out the same objects, both in the preceding and the following figures.

In cach of these summers a square hole is cut out, exactly opposite to the other; in

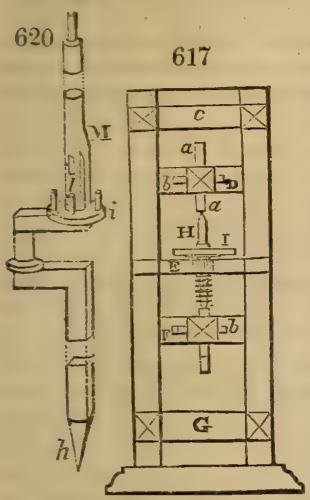
which are adjusted by friction, a square piece of oak $a, a, f i g$. 617 , whose extremities are perforated with a conical hole, which receives the two ends of the arbor $H$ of the wheel $r$, and forms its socket. The square bar is adjusted at a convenient height, by a double wooden wedge $b b$.

The cross bar in the middle $\mathbf{E}$ supports the table $: c$, a strong plank of oak. It is pierced with two large holes whose centres coincide with the centre of the conical holes hollowed out at the end of the square pins. These holes; of about 6 inches diameter each, are intended to let the arbor pass freely through, bearing its respective wheel. (See one of these holes at $\mathbf{I}$, in fig. 621 below.)

Each wheel is composed of an iron arbor $\mathrm{H}, \mathrm{fig} .618$, of a grinding-wheel $\mathrm{I}$, which differs in substance according to circumstances, as already stated, and of the pulley $\mathrm{J}$, furnished with several grooves (see fig. 619), which has a square fit upon the arbor. The arbor carries a collet $d$, on which are 4 iron pegs or pins that enter into the wheel to fasten it.

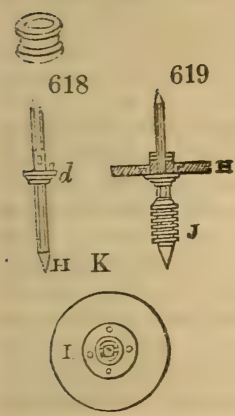

The wheel plate, of which the ground plan is shown at $\mathbf{K}$, is hollowed out towards its centre to half its thickness; when it is in its position on the arbor, as indicated in fig. 619 , a washer or ferrule of wrought iron is put over it, and secured in its place by a double wedge. In fig. 619 the wheel-plate is represented in section, that the connexion of the whole parts may be seen.

A board $g$ (see fig. 616 and fig. 624), about $7 \frac{1}{2}$ inches high, is fixed to the part of the frame opposite to the side at which the lapidary works, and it prevents the substances made use of in the cutting and polishing, from being thrown to a distance by the centrifugal force of the wheel-plate.

Behind this apparatus is mounted for each grinding-plate, a large wheel L (see fig. 616), similar to a cutler's, but placed horizontally. This wheel is grooved round its circumference to receive an endless cord or band, which passes round one of the grooves of the pulley $\mathrm{J}$, fixed below the wheel-plate. Hence, on turning the fly-wheel $\mathrm{L}$, the plate revolves with a velocity relative to the velocity communicated to the wheel $\mathrm{L}$, and to the difference of diameter of the wheel $\mathrm{L}$ and the pulley J. Each wheel $\mathrm{L}$, is mounted on an iron arbor, with a crank (see M, fig. 620.)

The lower pivot of that arbor $h$ is conical, and turns in a socket fixed in the floor. The great wheel $\mathrm{L}$ rests on the collet $i$, furnished with its 4 iron pins, for securing the connexion. Above the wheel an iron washer is laid, and the whole is fixed by a double wedge. which enters into the mortise $l$, fig. 620 .

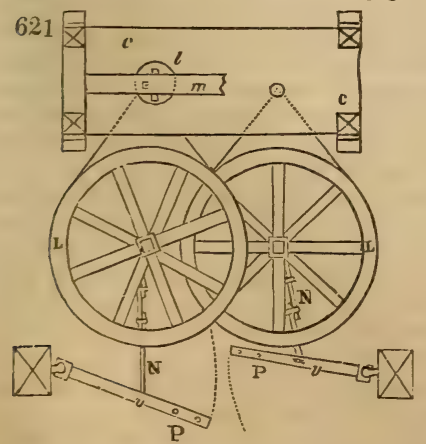

Fig. 621 exhibits a ground-plan view of all this assemblage of parts, to explain the structure of the machine. Every thing that stands above the upper summer-bar has been suppressed in this representation. Here we see the table $c c$; the upper summer $m$; the one wheel-plate $l$, the other having been removed to show that the endless cord does not cross; the two large wheels $L \mathrm{~L}$, present in each machine, the crank bar $\mathrm{N}$, seen separate in fig. 622, which serves for turning the wheel $\mathrm{L}$.

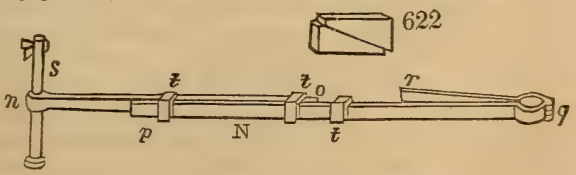

This bar is formed of 3 iron plates, $n, o ; p, q$; and $q, r ;(f i g .622$.$) The firs 1$ is 
bent round at the point $n$, to embrace the stud $s$; the second, $p q$, is of the same breadtn and thickness as the first; and the third is adjusted to the latter with a hinge joint, at the point $q$, where they are both turned into a circular form, to embrace the crank $\mathrm{M}_{\text {. }}$ When all these pieces are connected, they are fixed at the proper lengths by the buckles or square rings $t t t$, which embrace these pieces as is shown in fig. 621 .

The stud $s$, seen in fig. 622 , is fixed to the point $v$ by a wedge key upon the arm $\mathrm{P}$, represented separately, and in perspective in fig. 623. The laborer seizing the two upright pegs or handles $x x$, by the alternate forward and backward motion of his arm, he communicates the same motion to the crank rod, which transmits it to the crank of the arbor $\mathrm{M}$, and impresses on that arbor, and the wheel which it bears, a rotatory move ment.
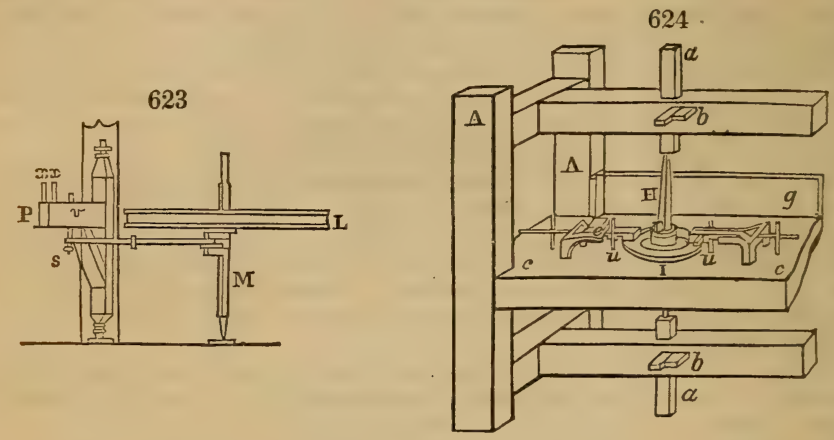

Fig. 624 shows piece-meal and in perspective, a part of the lapidary's wheel-mill There we see the table $c c$, the grind-plate $\mathrm{I}$, whose axis is kept in a vertical position by the two square plugs $a a$, fixed into the two summers by the wedges $b b$. On the two sides of the wheel-plate we perceive an important instrument called.a dial, which serves to hold the stone during the cutting and polishing. This instrument has received lately important ameliorations, to be described in fig. 625. The lapidary holds this instrument in his hand, he rests it upon the iron pins $u$ fixed in the table, lest he should be affected by the relocity of the revolving wheel-plate. He loads it sometimes with weights $e, e$, to make it take better hold of the grinding plate.

One of the most expert lapidaries of Geneva works by means of the following improved mechanism, of his own invention, whereby he cuts and polishes the facets with extreme regularity, converting it into a true dial.

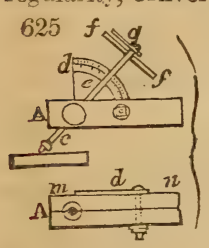

Fig. 625 shows this improvement. Each of the two jaws bears a large conchoidal cavity, into which is fitted a brass ball, which carries on its upper part a tube $e$, to whose extremity is fixed a dial-plate $f f$, engraved with several concentric circles, divided into equal parts, like the toothed-wheel cutting engine-plate, according to the number of facets to be placed in each cutting range. The tube receives with moderate friction the handle of the eement rod, which is fixed at the proper point by a thumb-screw, not shown in the figure, being concealed by the vertical limb $d$, about to be described.

A needle or index $g$, placed with a square fit on the tail of the cement rod, marks by its point the divisions on the dial plate $f f$. On the side $m n$ of the jaw $\mathrm{A}$, there is fixed by two screws, a limb $d$, forming a quadrant whose centre is supposed to be at the centre of the ball. This quadrant is divided as usual into 90 degrees, whose highest point is marked 0 , and the lowest would mark about 70 ; for the remainder of the arc down to 90 is concealed by the jaw. The two graduated plates are used as follows :-

When the cement rod conceals zero or 0 of the limb, it is then vertical, and serves to cut the table of the brilliant; or the point opposite to it, and parallel to the table. On making it slope a little, 5 degrees for example, all the facets will now lie in the same zone, provided that the inclination be not allowed to vary. On turning round the cement rod the index g marks the divisions, so that by operating on the circle with 16 dirisions, stopping for some time at each, 16 facets will have been formed, of perfect equality, and at equal distances, as soon as the revolution is completed.

Diamonds are cut at the present day in only two modes; into a rose diamond, and a brilliant. We shall therefore confine our attention to these two forms.

The rose diamond is flat beneath, like all weak stones, while the upper face rises intc a dome, and is cut into facets. Most usually six facets are put on the central region, 
which are in the form of triangles, and unite at their summits; their bases abut upon another range of triangles, which being set in an inverse position to the preceding, present their bases to them, while their summits terminate at the sharp margin of the stone. The latter triangles leave spaces between them which are likewise cut each into two facets. $\mathrm{By}$ this distribution the rose diamond is cut into 24 facets; the surface of the diamond being divided into two portions, of which the upper is called the crown, and that forming the contour, beneath the former, is called dentelle (lace) by the French artists.

According to Mr. Jeffries, in his Treatise on Diamonds, the regular rose diamond is formed br inscribing a regular octagon in the centre of the table side of the stone, and bordering it by eight right-angled triangles, the bases of which correspond with the sides of the octagon; beyond these is a chain of 8 trapeziums, and another of 16 triangles. The collet side also consists of a minute central octagon, from every angle of which proceeds a ray to the edge of the girdle, forming the whole surface into 8 trapeziums, each of which is again subdirided by a salient angle (whose apex touches the girdle) into one irregular pentagon and two triangles.

To fashion a rough diamond into a brilliant, the first step is to modify the faces of the original octahedron, so that the plane formed by the junction of the two pyramids shall be an exact square, and the axis of the crystal precisely twice the length of one of the sides of the square. The octahedron being thus rectified, a section is to be made parallel to he common base or girdle, so as to cut off 5 eighteenths of the whole height from the upper pyramid, and 1 eighteenth from the lower one. The superior and larger plane thus produced is called the table, and the inferior and smaller one is called the collet; in this state it is termed a complete square table diamond. To convert it into a brilliavis two trianoular facets are placed on each side of the table, thus changing it from a square to an octagon; a lozenge-shaped facet is also placed at each of the four corners of the table, and another lozenge extending lengthwise along the whole of each side of the original square of the table, which with two triangular facets set on the base of each lozenge, completes the whole number of facets on the table side of the diamond; viz., 8 lozenges, and 24 triangles. On the collet side are formed 4 irregular pentagons, alternating with as many irregular lozenges radiating from the collet as a centre, and bordered by 16 triangular facets adjoining the girdle. The brilliant being thus completed, is set with the table side uppermost, and the collet side implanted in the carity made to receire the diamond. The brilliant is always three times as thick as the rose diamond. In France, the thickness of the brilliant is set off into two unequal portions; one third is reserred for the upper part or table of the diamond, and the remaining two thirds for the lower part or collet (culasse). The table has eight planes, and its circumference is cut into facets, of which some are triangles, and others lozenges. The collet is also cut into facets called parillons. It is of consequence that the parillons lie in the same order as the upper facets, and that they correspond to each other, so that the symmetry be perfect, for otherwise the play of the light would be false.

Although the rose-diamond projects bright beams of light in more extensive proportion often than the brilliant, yet the latter shows an incomparably sreater play, from the difference of its cutting. In executing this, there are formed 32 faces of different figures, and inclined at different angles all round the table, on the upper side of the stone. On the collet (culasse) 24 other faces are made round a small table, which converts the culasse into a truncated prramid. These 24 facets, like the 32 above, are differently inclined and present different figures. It is essential that the faces of the top and the bottom correspond together in sufficiently exact proportions to multiply the reflections and refractions, so as to produce the colors of the prismatic spectrum.

The other precious stones, as well as their artificial imitations, called pastes, are cut in the same fashion as the brilliant; the only difference consists in the matter consticuting 626

627

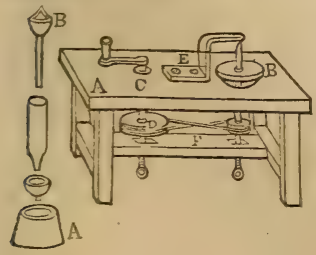
the wheel plates, and the grinding and polishing powcers, as already stated.

In cutting the stones, they are mounted on the cementrod $\mathrm{B}$, fig. 626 , whose stem is set upright in a socket placed in the middle of a sole piece at $\mathrm{A}$, which receives the stem of the cement-rod. The head of the rod fills the cup of A. A melted alloy of tin and lead is poured into the head of the cement-rod, into the middle of which the stone is immediately plunged; and whenever the solder has become solid, a portion of it is pared off from the top of the diamond, to give the pyramidal form shown in the figure at $\mathrm{B}$.

There is an instrument employed by the steel polishers for pieces of clock work, and br the manufacturers of watch-glasses for polishing their edges. It consists of a solid oaken table, fig. 627. The top is perforated with two holes, one for passing through the pulley and the arbor of the wheel-plate $\mathrm{B}$, made either of lead or of hard 
wood, according to circumstances; and the other $\mathrm{c}$ for receiving the upper part of the arbo: of the large pulley $\mathrm{D}$. The upper pulley of the wheel plate is supported by an iron prop k, fixed to the table by two wooden screws. The inferior pivots of the two pieces are supported by screw-sockets, working in an iron screw-nut sunk into the summer-bar F. The legs of the table are made longer or shorter, according as the workman chooses to stand or sit at his employment. Emery with oil is used for grinding down, and tinputty or colcothar for polishing. The workman lays the piece on the flat of the wheelplate with one hand, and presses it down with a lump of cork, while he turns round the handle with the other hand.

The Sapphire, Ruby, Oriental Amethyst, Oriental Emerald, and Oriental Topuz, are gems next in value and hardness to diamond; and they all consist of nearly pure alumina or clay, with a minute portion of iron as the coloring matter. The following analyses show the affinity in composition of the most precious bodies with others in little relative estimation.

\begin{tabular}{|c|c|c|c|c|}
\hline & & Sapphire. & Corundum Stone. & Emery. \\
\hline $\begin{array}{l}\text { Alumina or clay } \\
\text { Silica } \\
\text { Oxyde of iron } \\
\text { Lime }\end{array}$ & $\begin{array}{ll}- & - \\
- & -\end{array}$ & $\begin{array}{r}98 \cdot 5 \\
0 \cdot 0 \\
1.0 \\
0.5\end{array}$ & $\begin{array}{r}89 \cdot 50 \\
5 \cdot 50 \\
1 \cdot 25 \\
0 \cdot 00\end{array}$ & $\begin{array}{r}86 \cdot 0 \\
3 \cdot 0 \\
4 \cdot 0 \\
0 \cdot 0\end{array}$ \\
\hline & & $100 \cdot 0$ & $96 \cdot 25$ & $93 \cdot 0$ \\
\hline
\end{tabular}

Salamstone is a variety which consists of small transparent crystals, generally six-sided prisms, of pale reddish and bluish colors. The corundum of Battagammana is frequently found in large six-sided prisms : it is commonly of a brown color, whence it is called by the natives curundu gallé, cinnamon stone. The hair-brown and reddish-brown crystals are called adamantine spar. Sapphire and salamstone are chiefly met with in seconclary repositories, as in the sand of rivers, \&c., accompanied by crystals and grains of octahedral iron-ore and of several species of gems. Corundum is found in imbedded crystals in a rock, consisting of indianite. Adamantine spar occurs in a sort of granite.

The finest varieties of sapphire come from Pegu, where they occur in the Capelar. mountains near Syrian. Some have been found also at Hohenstein in Saxony, Bilin in Eohemia, Puy in France, and in several other countries. The red variety, the ruby, is most highly valued. Its color is between a bright scarlet and crimson. A perfect ruby above $3 \frac{1}{2}$ carats is more valuable than a diamond of the same weight. If it weigh 1 carat, it is worth 10 guineas; 2 carats, 40 guineas; 3 carats, 150 guineas; 6 carats, above 1000 guineas. A deep colored ruby, exceeding 20 carats in weight, is generally called a carbuncle; of which 108 were said to be in the throne of the Great Mogul, weighing from 100 to 200 carats each; but this statement is probably incorrect. The largest oriental ruby known to be in the world was brought from China to Prince Gargarin, governor of Siberia. It came afterwards into the possession of Prince Menzikoff, and constitutes now a jewel in the imperial crown of Russia.

A good blue sapphire of 10 carats is valued at 50 guineas. If it weighs 20 carats, its value is 200 guineas; but under 10 carats, the price may be estimated by multiplying the square of its weight in carats into half a guinea; thus, one of 4 carats would be worth $42 \times \frac{1}{2} G_{0}=8$ guineas. It has been said that the blue sapphire is superior in hardness to the red, but this is probably a mistake arising from confounding the corundum ruby with the spinelle ruby. A sapphire of a barbel blue color, weighing 6 carats, was disposed of in Paris by public sale for 70l. sterling; and another of an indigo blue, weighing 6 carats and 3 grains, brought $60 l$.; both of which sums much exceed what the preceding rule assigns, from which we may perceive how far fancy may go in such matters. The sapphice of Brazil is merely a blue tourmaline, as its specific gravity and inferior hardness show. White sapphires are sometimes so pure, that when properly cut and polished they have been passed for diamonds.

The yellow and green sapphires are much prized under the names of Oriental topaz and emerald. The specimens which exhibit all these colors associated in one stone are highly valued, as they prove the mineralogical identity of these varieties.

Besides these shades of color, sapphires often emit a beautiful play of colors, or chatoiement, when held in different positions relative to the eye or incident light; and some likewise present star-like radiations, whence they are called star-stones or asterias; sen ling forth 6 or even 12 rays, that change their place with the position of the stone. This property, so remarkable in certain blue sapphires, is not, however, peculiar to these gems. It seems to belong to transparent minerals which have a rhomboid for their 
nucleus, and arises from the combination of certain circumstances in their cutting and structure. Lapidaries often expose the light-blue variety of sapphire to the action of fire, in order to render it white and more brilliant; but with regard to those found at Expailly, in France, fire deepens their color.

3. Chrysoberyl, called by Haüy, Cymophane, and by others, Prismatic corundum, ranks next in hardness to sapphire, being 8.5 on the same scale of estimation. Its specific gravity is $3 . \% 5$. It usually occurs in rounded pieces about the size of a pea, but it is also found crystallized in many forms, of which 8-sided prisms with 8-sided summits are per haps the most frequent. Lustre vitreous, color asparagus green, passing into greenish white and olire-green. It shows a bluish opalescence, a light undulating, as it were, in the stone, when riewed in certain directions; which property constitutes its chief attraction to the jeweller. When polished, it has been sometimes mistaken for a yellow diamond; and from its hardness and lustre is considerably valued. Good specimens of it are rery rare. It has been found only in the alluvial deposites of rivers, along with other species of gems. Thus it occurs in Brazil, along with diamonds and prismatic topaz; also in Ceylon. Its constituents are alumina, 68.66 ; glucina, 16.00 ; silica, 6.00 ; protoxyde of iron, $4 \cdot 7$; oxyde of titanium, $2 \cdot 66$; moisture, $0 \cdot 66$; according to Seybert's analysis of a specimen from Brazil. It is difficultly but perfectly fusible before the blowpipe, with borax and salt of phosphorus. In composition it differs entirely from sapphire, or the rhombohedral corundum.

4. Spinelle Ruby, called Dodecahedral corundum, by some mineralogists, and Balas ruby, by lapidaries. Its hardness is 8 . Specific gravity, 3.523 . Its fundamental form is the hexahedron, but it occurs crystallized in many secondary forms : octahedrons, tetrahedrons, and rhombohedrons. Fracture, conchoidal; lustre, vitreous ; color, red, passing into blue and green, yellow, brown, and black; and sometimes it is nearly white. Red spinelle consists of alumina, 74.5 ; silica, 15.5 ; magnesia, 8.25 ; oxyde of iron, 1.5 ; lime, 0.75 . Tauquelin discovered 6.18 per cent. of chromic acid in the red spinelle. The red rarieties exposed to heat become black and opaque; on cooling, they appear first green, then almost colorless, but at last resume their red color. Pleonaste is a variety which yields a deep green globule with borax.

Crystals of spinelle from Ceylon have been observed imbedded in limestone, mixed with mica, or in rocks containing adularia, which seem to have belonged to a primitive district. Other varieties like the pleonaste occur in the drusy cavities of rocks ejected by Tesuvius. Crystals of it are often found in diluvial and alluvial sand and gravel, along with true sapphires, pyramidal zircon, and other gems; as also with octahedral iron ore, in Cerlon. Blue and pearl-gray varieties occur in Südermannland, in Sweden, imbedded in granular limestone. Pleonaste is met with also in the diluvial sands of CeyIon. Clear and finely colored specimens of spinelle are highly prized as ornamental stones. When the weight of a good spinelle exceeds 4 carats, it is said to be valued at half the price of a diamond of the same weight. M. Brard has seen one at Paris which weighed 215 grains.

5. Zircon or Hyacinth. Its fundamental form is an isosceles 4-sided pyramid; and the secondary forms have all a pyramidal character. Fracture, conchoidal, uneven; lustre, more or less perfectly adamantine; colors, red, brown, yellow, gray, green, white; which, with the exception of some red tints, are not bright. Hardness, $7 \cdot 5$. Specific grarity, 4.5. Zircon and hyacinth consist, according to Klaproth, of almost exactly the same constituents; namely, zirconia, 70 ; silica, 25 ; oxyde of iron, 5 . In the white zirconia there is less iron and more silica. Before the blowpipe the hyacinth loses its color, but does not melt. The brighter zircons are often worked up into a brilliant form, for ornamenting watch cases. As a gem, hyacinth has no high value. It has been often confounded with other stones, but its very great specific gravity makes it to be readily recognised.

6. Topaz. The fundamental form is a scalene 4-sided pyramid; but the secondary forms have a prismatic character; and are frequently observed in oblique 4-sided prisms, acuminated by 4 planes. The lateral planes of the prism are longitudinally striated. Fracture, conchoidal, uneven ; lustre, vitreous ; colors, white, yellow, green, blup; generally of pale shades. Hardness, 8 ; specific gravity, 3.5 . Prismatic topaz consists, according to Berzelius, of alumina, $57 \cdot 45$; silica, $34 \cdot 24$; fluoric acid, $7 \cdot 75$. In a strong heat the faces of crystallization, but not those of cleavage, are covered with small blisters, which however immediately crack. With borax, it melts slowly into a transparent glass. Its powder colors the tincture of violets green. Those crystals which possess different faces of crystallization on opposite ends, acquire the opposite electricities on being heated. By friction, it acquires positive electricity.

Most perfect crystals of topaz have been found in Siberia, of green, blue, and white colors, along with beryl, in the Uralian and Altai mountains, as also in Kamschatka; in Brazil, where they generally occur in loose crystals, and pebble forms of bright yel- 
low colors; and in Mucla, in A sia Minor, in pale straw-yellow regular crystals. They are also met with in the granitic detritus of Cairngorm, in Aberdeenshire. The blue varieties are ahsurdly called oriental aquamarine, by lapidaries. If exposed to heat, the Saxon topaz loses its color and becomes white; the deep yellow Brazilian varieties assume a pale pink hue; and are then sometimes mistaken for spinelle, to which, however, they are somewhat inferior in hardness. Topaz is also distinguishable by its double refiactive property. Tavernier mentions a topaz, in the possession of the Great Mogul, which weighed $15 \%$ carats, and cost $20,000 l$. sterling. There is a specimen in the museum of natural history at Paris which weighs 4 ounces 2 gros.

Topazes are not scarce enough to be much valued by the lapidary.

7. Emerald and Beryl are described in their alphabetical places. Emerald loses its lustre by candle-light ; but as it appears to most advantage when in the company of diamonds, it is frequently surrounded with brilliants, and occasionally with pearls. Beryl is the aquamarine of the jewellers, and has very little estimation among lapidaries.

8. Garnet. See this stone in its alphabetical place.

9. Chrysolite, called Peridot, by Haüy; probably the topaz of the ancients, as our topaz was their chrysolite. It is the softest of the precious stones, being scratched by quartz and the file. It refracts double.

10. Quartz, including, as sub-species, Amethyst, Rock-crystal, Rose-quarid, Prase, or Chrysoprase, and sereral varieties of calcedony, as Cat's-eye, Plasma, Chrysoprase, Onyx, Sardonyx, \&c. Lustre, vitreous, inclining sometimes to resinous; colors, very various; fracture, conchoidal; hardness, 7 ; specific gravity, $2 \cdot 69$.

11. Opal, or uncleavable quartz. Fracture, conchoidal; lustre, vitreous or resinous; colors, white, yellow, red, brown, green, gray. Lively play of light; hardness, 5.5 to 6.5 ; specific gravity, $2 \cdot 091$. It occurs in small kidney-shaped and stalactitic shapes, and large tuberose concretions. The phenomena of the play of colors in precious opal has not been satisfactorily explained. It seems to be connected with the regular structure of the mineral. Hydrophane, or oculis mundi, is a variety of opal without transparency, but acquiring it when immersed in water, or in any transparent fluid. Precious opal was found by Klaproth to consist of silica, 90 ; water, 10 ; which is a very curious combination. Hungary has been long the only locality of precious opal, where it occurs near Caschau, along with common and semi-opal, in a kind of porphyry. Fine varieties have, however, been lately discovered in the Faroe islands; and most beautiful ones, sometimes quite transparent, near Gracias a Dios, in the province of Honduras, America. The red and yellow bright colored varieties of fire-opal are found near Zimapan, in Mexico. Precious opal, when fashioned for a gem, is generally cut with a convex surface; and if large, pure, and exhibiting a bright play of colors, is of considerable value. In modern times, fine opals of moderate bulk have been frequently sold at the price of diamonds of equal size : the Turks being particularly fond of them. The estimation in which opal was held by the ancients is hardly credible. They called it Paideros, or Child beautiful as love. Nonius, the Roman senator, preferred banishment to parting with his farorite opal, which was coveted by Mark Antony. Opal which appears quite red when held against the light, is called girasol by the French; a name also given to the sapphire or corundum asterias or star-stone.

12. Turquois or Calaite. Mineral turquois occurs massive; fine-grained, impalpable; fracture, conchoidal; color, between a blue and a green, soft, and rather bright ; opaque; hardness, 6 ; spec. grav., $2 \cdot 83$ to $3 \cdot 0$. Its constituents are alumina, 73 ; oxyde of copper, 4.5 ; oxyde of iron, 4 ; water, 18 ; according to Dr. John. But by Berzelius, it consists of phosphate of alumina and lime, silica, oxydes of copper, and iron, with a little water. It has been found only in the neighborhood of Nichabour in the Khorassan, in Persia; and is very highly prized as an ornamental stone in that country. There is a totally different kind of turquois, called bone turquois, which seems to be phosphate of lime colored with oxyde of copper. When the oriental stone is cut and polished, it forms a pleasing gem of inferior value. Malachite, or mountain green, a compact carbonate of copper, has been substituted sometimes for turquois, but their shades are different. Malachite yields a green streak, and turquois a white one.

13. Lapis lazuli is of little value, on account of its softness.

LEAD (Plomb, Fr.; Blei, Germ.). This is one of the metals most anciently known, being mentioned in the books of Moses. It has a gray-blue color, with a bright metallic lustre when newly cut, but it becomes soon tarnished and earthy looking in the air. Its texture is close, without perceptible cleavage or appearance of structure; the specific gravity of common lead is 11.352 ; but of the pure metal, from 11.38 to 11.44. It is very malleable and ductile, but soft and destitute of elasticity; fusible at $612^{\circ}$ Fahr., by Crighton, at $634^{\circ}$ by Kupfer, and crystallizable on cooling, into octahedrons implanted into each other so as to form an assemblage of four-sided pyramids. 
There are four oxydes of lead. 1. The suboxyde, of a grayish-blue color, which forms a lind of crust upon a plate of lead long exposed to the air. It is procured in a perfect state by calcining oxalate of lead in a retort; the dark gray powder which remains, is the pure suboxyde. 2. The protoxyde is obtained by exposing melted lead to ihe atmosphere, or, more readily, by expelling the acid from the nitrate of lead by heat in a platinum crucible. It is yellow, and was at one time prepared as a pigment by calcining lead; but is now superseded by the chromate of this metal. Litharge is merely this uxvle in the form of small spangles, from having undergone fusion; it is more or less contaminated with iron, copper, and sometimes a little silver. It contains likewise some carbonic acid. The above oxyde consists of 104 of metal, and 8 of oxygen, its prime equiralent being 112, upon the hydrogen scale; and it is the base of all the salts of lear. 3. The plumbeous suroxyde of Berzelius, the sesquioxyde of some British chemists, is the well-linown pigment called RED LEAD or minium. It consists of 100 parts of metal anil 10 of oxygen. 4. The plumbic suroxyde of Berzelius, or the peroxyde of the British chemists, is obtained by putting red lead in chlorine water, or in dilute nitric acid. It is of a dark brown, almost black color, which gives out oxygen when heated, and besomes rellow oxyde. It kindles sulphur when triturated with it. This oxyde is used by the analytical chemist to separate, by condensation, the sulphurous acid existing in a gaseous mixture.

Among the ores of lead some have a metallic aspect; are black in substance, as well as when pulverized; others have a stony appearance, and are variously colored, with vially a vitreous or greasy lustre. The specific gravity of the latier ores is always less than 5. The whole of them, excepting the chloride, become more or less speedily black, with sulphureted hydrogen or with hydrosulphurets; and are easily reduceil to the metallic state upon charcoal, with a flux of carbonate of soda, after they have been properly roasted. They diffuse a whitish or yellowish powder over the charcoal, which, according to the manner in which the flame of the blowpipe is directed unon it, becomes yellow or red; thus indicating the two characteristic colors of the oxydes of lead.

ife shall not enter here into the controversy concerning the existence of native lead, which has been handled at length by M. Brongniart in the Dictionnaire des Sciences Naturelles, article Plomb, Mineralogie.

The lead ores most interesting to the arts are :-

1. Grilena, sulphuret of lead. This ore has the metallic lustre of lead with a crystalline structure derirable from the cube. When heated cautiously at the blowpipe it is decomposed, the sulphur flies off, and the lead is left alone in fusion; but if the heat be continued, the colored surface of the charcoal indicates the conversion of the lead into its orrdes. Galena is a compound of lead and sulphur, in equiralent proportions, and therefore consists, in 100 parts, of $86 \frac{2}{3}$ of metal, and $13 \frac{1}{3}$ of sulphur, with which numbers the analysis of the galena of Clausthal by Westrumb exactly agrees. Its specific gravity, when pure, is $7 \cdot 56$. Its color is blackish gray, without any shade of red, and its powder is black, characters which distinguish it from blende or sulphuret of zinc. Its structure in mass is lamellar, passing sometimes into the fibrous or granular, and even compact. It is brittle. The specular galena, so called from its brightly polished aspect, is remarkable for formang the slickensides of Derbyshire-thin seams, which explode with a loud noise when accidentally scratched in the mine.

The argentiferous galena has in general all the external characters of pure galena. The proportions of silver vary from one fifth part of the whole, as at Tarnowitz, in Silesia, 10 three parts in ten thousand, as in the ore called by the German miners Weisgültigerz; but it must be observed, that whenerer this lead ore contains above 5 per cent. of silver, several other metals are associated with it. The mean proportion of silver in galena, or that which makes it be considered practically as an argentiferous ore, because the silver may be profitably extracted, is about two parts in the thousand. See Silver. The above rich silver ores were first observed in the Freyberg mines, called Himmelsfurst and Beschertgluck, combined with sulphuret of antimony; but they have been no$t:$.ed since in the Hartz, in Mexico, and several other places.

The antimonial galena (Bournonite) exhales at the blowpipe the odor peculiar to antimony, and coats the charcoal with a powder partly white and partly red. It usually contains some arsenic.

2. The Seieniuret of lead resembles galena, but its tint is bluer. Its chemical characters are the only ones which can be depended on for distinguishing it. At the blowpipe it exhales a very perceptible smell of putrid radishes. Nitric acid liberates the sflenium. When heated in a tube, oxyde of selenium of a carmine red rises along with selenic acid, white and deliquescent. The specific gravity of this ore varies from $6 \cdot 8$ to $7 \cdot 69$.

3. Tutive minium or red lead has an earthy aspect, of a lively and nearly pure red colnr, but sometimes inclining to orange. It occurs pulverulent, and also compact, 
with a fracture somewhat lamellar. When heated at the blowpipe upon charcoal, it is realily reituced to metallic lead. Its specific gravity varies frcm $4 \cdot 6$ to $8 \cdot 9$. 'This ore is rare.

4. Plomb-gomme.-This lead ore, as singular in appearance as in composition, is of a dinty bruwnish or orange-yellow, and occurs under the form of s!cbular or gurm-like eoncretions. It has also the lustre and translucency of gum; with semewlat of a penily asprect at tires. It is havder than fuor spar. It consists of oxyde of lead, 40; alutima, 37: water, $1 \varsigma \cdot 8$; foreign matters and loss, 4.06 ; in 100 . Hitherto it has been fiund only at Huelgoet, near Poullaouen, in Brittany, corering with its tears $c^{-}$ snall concretions the ores of white lead and galena which compose the veins of that lead mine.

5. Wite lead, cartonate of lead.-This ore, in its purest state, is colorless and trans. parent the glass, with an adamantine lustre. It mas be recognised by the following characters :-

Its specific gravity is from 6 to $6 \cdot \tau$; it dissolres with more or less ease, and with efierrescence, in nitric acid; becomes immediately black br the action of sul hureted hydrucen, and melts on charcoal before the blowripe into a bution of lead. According to Klarroth, the carbonate of Leadhills contains 82 parts of oxyde of lead, and is of carbonic acid, in 19 parts. This mineral is tender, scarcely scratches cale-sjar, and breaks easily with a wared conchoidal fracture. It possesses the double refracting rroy erty in a very hith cegree: the double image being very risible on looking through the flat faces of the rrismatic crrstals. Its crrstalline forms are vert numerous, and are referrible to the octahedron, and the prramidal prism.

6. Titreous lead, or sulphate of lead.-This mineral closely resembles earbenate of lead: so that the external characters are inadequate to distinguish the two. But the foll wing are surficient. When pure, it has the same transparency and lustre. It does not effervesce mith nitric acid; it is but feebly blackened by sulphureted hrdrocen; it first lecrevitates and then melts before the blowripe into a transrarent glass, which becomes milit $\mathrm{Y}$ as it cools. Br the combined action of heat and charenal, it rasses first into a red pulrerulent oxte, and then into metallic lead. It consists, according to Klarreth, of 71 exrde of lead, 25 sulphuric acid, 2 water, and 1 iron. That srecimen was from Ansiesea; the Wanlochhead mineral is free from iron. The prerailing form of cristallization is the rectangular octahedron, whose angles and edges are rariouslr modified. The sulrhatc-carbonate, and sulnhato tri-carbonate of lead, now called Leudhillite, are rare minerals which belong to this head.

7. Phosphate of lead.-This, like all the combinations of lead with an acid, exhibits no metallic instre, but a rarietr of colors. Before the blowripe uron charcoal, it relts into a clobule externally crystalline, which, br a continuance of the heat, with the addition of ircn and boracic acid, affords metallic lead. Its constituents are $\$ 0$ oride of lead, 18 rhosphoric acid, and 1.6 muriatic acid, according to Klaproth's analrsis of the mineral from $W$ anlcelihead. The constant presence of muriatic acid in the rarious srecimens examined is a remarkable circumstance. The crystalline forms are derired from an obtuse rhomboid. Phosphate of lead is a little harder than white lead: it is easily scratehed, and its nowder is alwars grar. Its specific gravity is $6 \cdot 9$. It has a ritreous lustre, somewhat adamantine. Its lamellar texture is not rerr distinct; its fracture is wary, and it is easily frangible. The phosphoric and arsenic acids being, according to M. Mitscherlich, isomorr bous bodies, may replace each other in chemical combinations in evert proportion, so that the phosphate of lead mar include any proportion, from the smallest fracticn of arsenic acid to the smallest fraction of phosphoric acid, thus graduating indefnitely into arseniate of lead. The vellowish variety indicates, for the most part, the presence of arscnic acid.

\$. Pruricie of lead. Horn-lead, or murio-carbonate.-This ore has a pale rellow color, is reducible to metallic lead br the agencr of soda, and is not altered by the hydrosulrhurets. At the blowpipe it melts first into a pale rellow transparent globule, with salt of yosphorus and nxyde of copper; and it manifests the presence of muriatic acid by a bluish fiame. It is fragile, tender, softer than carbonate of lead, and is sometimes almost colorless, with an adamantine lustre. Srec. grav., 6016. Its constituents, according to Berzelius, are lead, 25.84 ; oxrde of lead, $5 \% \cdot 07$; carbonate of lead, $6 \cdot 25$; chlorine, 8.84 ; silica, $1 \cdot \frac{15}{5}$ mater, $0 \cdot 54 ;$ in 100 parts. The carbonate is an accidental ingredient, not being in equiralent proportion. Klaproth found chlorine, $13.6 \%$; lead, 39.98 ; oxrde of lead, 22.57 ; carbonate of lead, $23 \cdot 78$.

9. Arseniate of lead.-Its color of a prettr nure rellow, bordering slightly on the greenish, and its property of exhaling by the joint action of fire and charcoal a rert distinet arsenical odor, are the onlt characters which distinguish this ore from the rhosphate of lead. The form of the arseniate of lead, when it is crystallized, is a prism with six faces, of the same dimensions as that of phosrhate of lead. When pure, it is :tducible upon charcoal, before the blowpipe, into metallic lead, with the copious exhalation 
of arsettical fumes; but only in part, and learing a crystalline globule, wien it contains any this hate of lead. The arseniate of lead is tender, friable, scmetimes even pulveruleet, and of specific gravity 5.04. That of Johann-Georgenstadt consists, according to Ruse, of oxyde of lead $7 \% 5$; arsenic acid 12.5 ; phosphoric acid $7 \cdot 5$, and muriatic acid $1 \cdot 5$.

10. Red lead, or Chromate of lead.-This mineral is too rare to require consideration in the present work.

11. Plomb rauquelinite. Chromate of lead and copper.

12. Fellow lead. Molybdate of lead.

13. Tungstate of lead.

Haring thus enumerated the several species of lead ore, we mar remark, that galena is the only one which oceurs in sufficiently great masses to become the object of mining and metallurg. This mineral is found in small quantity among the crystalline primitire rocks, as granite. It is howerer among the oldest talc-schists and clay slates, that it usuall pecurs. But galena is much more abundant among the transition rochs, being its princinal locality, where it exists in interrupted beds, masses, and more rare in reins. The blachish transition limestone is of all rochs that which contains most galena; as at Pierrerilie in Normandy : at Clausthal, Z ellerfeldt, and most mines of the Harz; at Fahlur, in Swerlen; in Derbrshire and Northumberland, sc. In the transition graywacke of te south of Scotland, the galena mines of Leadhills occur. The galena of the primitive formations contains more silver than that of the calcareous.

The principal lead mines at present worked in the world, are the following: 1. Poulläouen and Huelgöet near Carhaix in France, department of Finisterre, being reins of galena, which traverse a clay slate resting upon granite. Ther have been lnown for rpwards of three centuries; the morkings penetrate to a depth of uptrards of 300 yards, and in 1815 furnished 500 tons of lead per annum, out of which $103 \frac{1}{4}$ pounds aroirdupois of silver were extracted. 2. At Villeforte and Viallaz, department of the Lozère, are galena mines said to produce 100 tons of lead per annum, with 400 kilogrammes of silver (5so lbs, aroird.). 3. At Pezer and Macot, to the east of Moutiers in Saror, a galena mine exists in tale-schist, which has produced annually 200 tons of lead, and about 600 hilogramrues of silver (1320 lbs. aroird.). 4. The mine of Tedrin, near Namur in the Low Countries, is opened upen a tein of galena, tratersing compact limestnne of a transition district; it has furnished 200 tons of lead, from which 355 pounds aroird. of silrer were extracted. 5. In Sarcn the galena mines are so rich in silter as to make the lead be almost orerlooked. They are enumerated under silter ores. 6 . The leal mines of the Harz hare been likewise considered as siltrer ores. T. Those of Blerberg in the Eifel are in the same predicament. 8. The galena mines of Blerberg and Villach in Carinthia, in compact limestone. 9. In Bohemia, to the south-west of Prague. 10. The mines of Joachimsthal, and Blerstadt, on the southern slope of the Erzgebirge, produce argentiferons galena. 11. There are numerous lead mines in Spain, the rost impurtant being in the granite hills of Linarès, upon the southern slope of the Sierra Morena, and in the district of the small torn of Canjagar. Sometimes enormous masses of galena are extracted from the mines of Linarès. There are also mines of galena in Catalonia, Grenada, Murcia, and Almeria, the ore of the last localit; being generalls poor in silver. 12. The lead mines of Sweden are rert argentiferous, and worked chiefly with a riem to the silver. 13. The lead mines of Daouria are numerous and rich, lring in a transition limestone, which rests on primitive rocks; their lead is neglected on account of the silver.

14. Of all the countries in the world, Great Britain is that which annually produces the greatest quantity of lead. Accord $1 \mathrm{~g}$ to M. Tillefosse, in his Richesse Minerale, published in 1810, we had furnished every . p2r 12,500 tons of lead, whilst all the rest of Europe taken together, did not produce so much; but from more recent documents, that estimate seems to hare been too low. Mr. Tarlor has rated the total product of the Cnited King. dom per anum at 31,900 tons, a quantity fulls $2 \frac{1}{2}$ times greater than the estimate of Villefosse (see Conybeare and Phillips's Geology, p. 354). Mr. Taylor distributes this product among the different distriets as follows :-

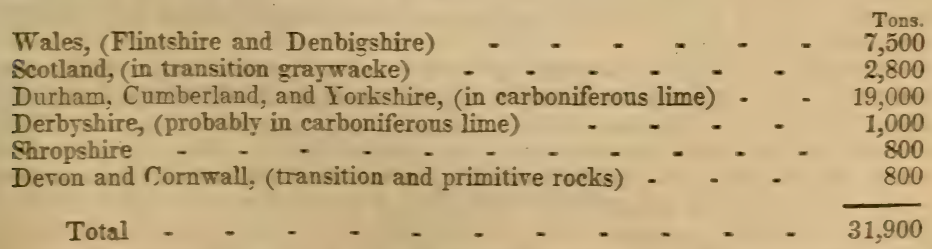

We thus see that Cumberland, and the adjacent parts of the counties of Durham and 
York, furnish of themselves nearly three fifths of the total product. Derbyshire was formerly much more productive. In Cornwall and Devonshire, the lead ore is found in veins in killes, a clay-slate passing into graywacke. In North Wales and the adjacent counties, as well as in Cumberland and Derbyshire, the lead occurs in the carboniferous limestone.

The English lead-miners distinguish three different kinds of deposites of lead ore; rake-vein.s, pipe-veins, and flat-veins. The English word vein corresponds to the Frerch term filon; but miners make use of it indifferently in England and France, to indicate all the deposites of this ore, adding an epithet to distinguish the different forms; thus, ralse veins are true veins in the geological acceptation of the word vein; pipe-veins are masses usually very narrow, and of oblong shape, most frequently parallel to the plane of the rocky strata; and flat-veins are small beds of ores interposed in the middle of these strata.

Rake-veins are the most common form in which lead ore occurs in Cumberland. They are in general narrower in the sandstone which covers the limestone, than in the calcareous beds. A thickness of less than a foot in the former, becomes suddenly 3 or 4 feet in the latter; in the rich vein of Hudgillburn, the thickness is 17 feet in the Great limestone, while it does not exceed 3 feet in the overlying Watersill or sandstone. This influence exercised on the veins by the nature of the enclosing rock, is instructive; it determines at the same time almost uniformly their richness in lead ore, an observation similar to what has been made in other countries, especially in the.veins of Kongsburg in Norway. The Cumberland veins are constantly richer, the more powerful they are, in the portions which traverse the calcareous rocks, than in the beds of sandstone, and more particularly the schistose rocks. It is rare in the rock called plate (a solid slaty clay) for the vein to include any ore; it is commonly filled with a species of potter's earth. The upper calcareous beds are also in general more productive than the lower ones. In most of these mines, the veins were not worked till lately below the fifth calcareous bed (the four-fathom limestone), which is 307 yards beneath the mill-stone grit; and as the first limestone stratum is 108 yards beneath it, it follows that the thickness of the part of the ground where the veins are rich in lead does not in general exceed 200 yards. It appears however that veins have been mined in the neighborhood of Alston Moor, downwards to the eleventh calcareous stratum, or Tyne bottom limestone, which is 418 yards under the milistone-grit of the coal formation, immediately above the whin-sill; and that they have been followed above the first limestone stratum, as high as the grindstone sill, which is only 83 yards below the same stratum of mill-stone grit; so that in the total thickness of the plumbiferous formation there is more than 336 yards. It has been asserted that lead veins have been traced even further down, into the Memerby scar limestone; but they have not been mined.

The greatest enrichment of a vein takes place commonly in the points where its two sides, being not far asunder, belong to the same rock; and its impoverishment occurs when one side is calcareous and the other a schistose clay. The minerals which most frequently accompany the galena, are carbonate of lime, fluate of lime, sulphate of baryta, quartz, and pyrites.

The pipe-veins (arnas in French) are seldom of great length; but some have a considerable width; their composition being somewhat similar to that of the rake-veins. 'They meet commonly in the neighborhood of the two systems, sometimes being in evident communication together; they are occasionally barren; but when a wide pipe-vein is metalliferous, it is said to be very productive.

The flat veins, or struta veins, seem to be nothing else than expansions of the matter of the rein between the planes of the strata; and contain the same ores as the veins in their vicinity. When they are metalliferous, they are worked along with the adjacent rake vein; and are proluctive to only a certain distance from that vein, unless they get enriched by crossing a rake vein. Some examples have been adduced of advantageous workings in flat veins in the great limestone of Cumberland, particularly in the mines of Coalcleugh and Nenthead. The rake veins, however, furnish the greater part of the lead which Cumberland and the adjacent counties send every year into the market. Mr. Forster gives a list of 165 lead mines, which have been formerly, or are now, worked in that district of the kingdom.

The metalliferous limestone occupies, in Derbyshire, a length of about 25 miles from north-west to south-east, under a very variable breadth, which towards the south, amounts to 25 miles. Castleton to the north, Buxton to the north-west, and Matlock to the south-east, lie nearly upon its limits. It is surrounded on almost all sides by the mill-stone grit which covers it, and which is, in its turn, covered by the coal strata. The nature of the rocks beneath the limestone is not known. In Cumberland the metalliferous limestone includes a bed of trap, designated under the name of whinsill. In Derbyshire the trap is much more abundant, and it is thrice interposed between the limestone. These two rocks constitute of themselves the whole mineral mass, through a 
:hickness of about 550 yards, measuring from the millstone grit; only in the upper portion, that is, near the millstone grit, there is a pretty considerable thickness of argillo-calcareous schists.

Four great bodies or beds of limestone are distinguishable, which alternate with three masses of trap, called toadstone. The lead veins exist in the calcareous strata, but disappear at the limits of the toadstone. It has now been ascertained, however, that they recur in the limestone underneath.

\section{Treatment of the Ores of Lead.}

The mechanical operations performed upon the lead ores in Great Britain, to bring them to the degree of purity necessary for their metallurgic treatment, may be divided into three classes, whose objects are,-

1. The sorting and cleansing of the ores;

2. The grinding;

3. The washing, properly so called.

The apparatus subservient to the first objects are sieves, running bucidies, and gratings. The large sieves employed in Derbyshire for sorting the ore at the mouth of the mine, into coarse and fine pieces, is a wire gauze of iron; its meshes are square, and an inch long in each side. There is a lighter sieve of wire gauze, similar to the preceding, for washing the mud from the ore, by agitating the fragments in a tub filled with water. But in Derbyshire, instead of using this siere, the pieces of ore are sometimes merely stirred about with a shovel, in a trough filled with water. This is called a standing buddle; a most defective plan.

The running buddle serves at once to sort and cleanse the ore. It consists of a plane surface made of slabs or planks, very slightly inclined forwards, and provided behind and on the sides with upright ledges, the back one having a notch to admit a stream of water. The ore is merely stirred about with a shovel, and exposed on the slope to the stream. For this apparatus, formerly the only one used at the mines of Alston Moor, the following has been substituted, called the grate. It is a grid, composed of square bars of iron, an inch thick, by from 24 to 32 inches long, placed horizontally, and parallelly to each other, an inch apart. There is a wooden canal above the grate, which conducts a stream of water over its middle; and an inclined plane is set beneath it, which leads to a hemispherical basin, about 24 inches in diameter, for collecting the metallic powder washed out of the ore.

The apparatus subservient to grinding the ore are,-

1. The bucker, or beater, formed of a cast-iron plate, 3 inches square, with a socket in its upper surface, for receiving a wooden handle. In the neighborhood of AlstonMIoor, crushing cylinders have been substituted for the beating bucker; but even now, in Derbyshire, buckers are generally employed for breaking the pieces of mixed ore, called knock-stone-stuff.

At the mines of this county, the knocker's workshop, or striking floor, is provided either with a strong stool, or a wall 3 feet high, beyond which there is a flat area 4 feet broad, and a little raised behind. On this area, bounded, except in front, by small walls, the ore to be bruised is placed. On the stool, or wall, a very hard stone slab, or cast-iron plate is laid, 7 feet long, 7 inches broad, and $1 \frac{1}{2}$ inches thick, called a knocks'one. The workmen seated before it, break the pieces of mixed ore, called bowse in Derbyshire, with the bucker.

Crushing machines are in general use at Alston Moor, to break the mingled ores, which they perform with great economy of time and labor. They have been employed there for nearly forty years.

This machine is composed of one pair of fluted cylinders, $x x, f i g .628$, and of two pairs of smooth cylinders $z z, z^{\prime} z^{\prime}$, which serve altogether for crushing the ore. The two cylinders of each of the three pairs turn simultaneously in an inverse direction, by means of two toothed wheels, as at $m$, fig. 629 , upon the shaft of every cylinder, which work by pairs in one another. The motion is given by a single water wheel, of which the circle $a$ a $a$ represents the outer circumference. One of the fluted cylinders is placed in the prolongation of the shaft of this wheel, which carries besides a cast-iron toothed wheei, geared with the toothed wheels $e e$, fixed upon the ends of two of the sinooth cylinders. Above the fluted cylinders, there is a hopper, which discharges down between them, by means of a particular mechanism, the ore brought forwards by the wagons $A$. These wagons advance upon a railway, stop above the hopper, and empty their contents into it through a trap-hole, which opens outwardly in the mirdle of their bottom. Below the hopper there is a small bucket called a shoe, into which the ore is shaken down, and which throws it without ceasing upon the cylinders, in consequence of the constant jolts given it by a crank-rod $i$ ( $f$ g. 629) attached to it, and moved by the teeth of the wheel $m$. The shoe is so regulated, that ton much ore can never fall upon the cylinders, and obstruct their movement. A small 
stream of water is likewise le. into the shoe, which spreads over the cylinders, and prevents them from growing hot. The ore, after passing between the fluted rollers 628

629

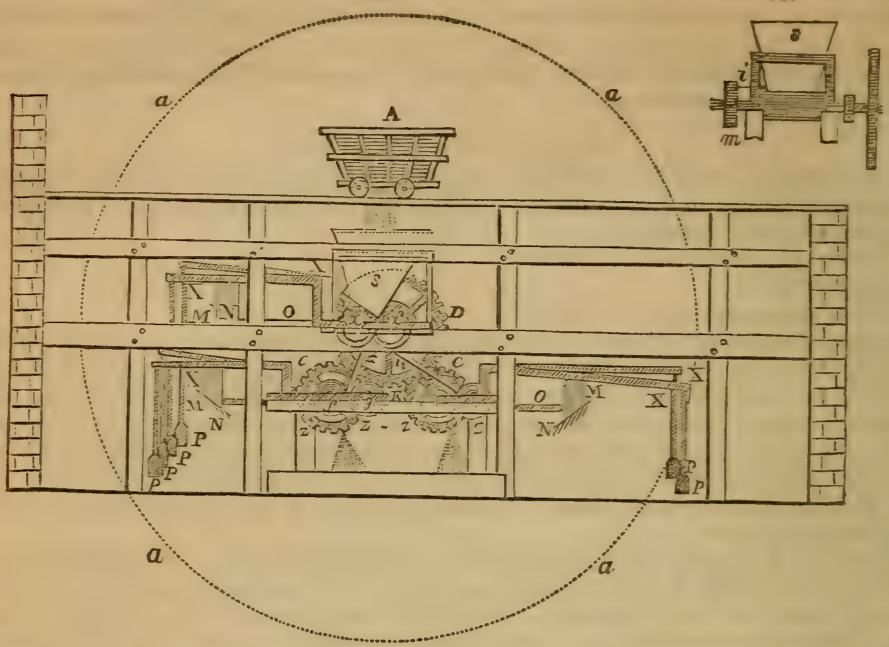

falls upon the inclined planes $\mathrm{N}, \mathrm{N}$, which tarn it over to one $\mathrm{cr}$ other of the pairs of smooth rolls.

These are the essential parts of this machine; they are made of iron, and the smooth ones are case-hardened, or chilled, by being cast in iron moulds. The gudgeons of both kinds more in brass bushes fixed upon iron supports $k$, made fast by bolts to the strong wood-work basis of the whole machine. Each of the horizontal bars has an oblong slot, at one of whose ends is solidly fixed one of the plummer-blocks or bearers of one of the crlinders $f$, and in the rest of the slot the plummer-block of the other crlinder $g$ slides; a construction which permits the two crlinders to come into contact, or to recede to such a distance from each other, as circumstances may require. The moreable cylinder is approximated to the fixed one by means of the iron levers $\mathrm{x} \mathrm{x}$, which carry at their ends the weights $\mathrm{P}$, and rest upon wedges $\mathrm{M}$, which may be slidden upon the inclined plane $\mathrm{N}$. These wedges then press the iron bar $\mathrm{o}$, and make it approach the moreable crlinder by adrancing the plummer block which supports its axis. When matters are so arranged, should a very large or hard piece present itself to one of the pairs of crlinders, one of the rollers would move awar, and let the piece pass withou: doing injury to the mechanism.

Besides the three pairs of crlinders which constitute essentially each crushing machne. there is sometimes a fourth, which serres to crush the ore when not in large fragments. for example, the chats and cuttings (the moderately rich and poorer pieces), produced by the first sifting with the brake siere, to be presently described. The crlinders composing that accessory piece, which, on account of their ordinary use, are called chats-rollers, are smooth, and similar to the rollers $z z$, and $z^{\prime} z^{\prime}$. The one of them is usually placed upon the prolongation of the shaft of the water-wheel, of the side opposite to the principal machine; and the other, which is placed alongside, receires its motion from the first, by means of toothed wheel-work.

The stamp mill is employed in concurrence with the crushing cylinders. It serves par. ticularly to pulterize those ores whose gangue is too hard to yield readily to the rollers. and also those which being already pulverized to a certain degree, require to be ground atill more finely. The stamps employed in the neighborhood of Alston Moor are moved by water wheels. They are similar to those described under TrN.

Proper sifting or jigging apparatus. - The hand sieve made of iron wire meshes, of various sizes, is shaken with the two hands in a tub of water, the ore vat, being held sometimes horizontally, and at others in an inclined position. This sieve is now in general use only for the cuttings that have passed through the grating, and which though not poor enough to require finer grinding, are too poor for the brake sieve. When the workman has collected a sufficient quantity of these smaller pieces, he puts them in his round hand sieve, shakes it in the ore vat with much rapidity and a dexterous toss, till he has separated the very poor portions called cuttings, from the mingled parts called chats, as well as from the pure ore. He then removes the first two qualities, with a 
sheet-iron scraper called a limp, and he finds beneath them a certain portion of ore which he reckons to be pure.

The brinte sieve is rectangular, as well as the cistern in which it is agitated. The meshes are made of strong iron wire, three eighths of an inch square. This siere is suspended at the extremity of a forked lever, or brake, turning upon an axis br means of two uprirht arms about $\zeta$ feet lon, , which are pierced with holes for connecting them with bolts or pins, both to the sieve-frame and to the ends of the two branches of the lever. These two arms are made of wrought iron, but the lever is made of wood; as it receires the jolt. A child placed near its end, by the action of leaping, jerks it smartly up and down, so as to shake effectually the siere suspended at the other extremity. Each jolt not only makes the fine parts pass through the meshes, but changes the relatire position of those which remain on the wires, bringing the purer and hearier pieces eventually to the bottom. The mingled fragments of galena, and the stony substances called chits lie abore them; while the poor and light pieces called cuttings, are at top. These are first scraped off br the limp, next the mixed lumps, or chats, and lastly the pure ore, which is carried to the bing heap. The cuttings are handed to a particular class of workmen, who br a new sifting, divide them into mere stones, or second cuttings, and into mixed ore analogous to chats.

The poor ore, called chats, is carried to a crushing machine, where it is bruised bettreen two crlinders appropriated to this purpose under the name of chats rollers; after which it is sifted afresh. During the sifting many parcels of small ore and stony substances pass through the siere, and accumulate at the bottom of the cistern. When it is two thirds filled, water is run slowir over it, and the sediment called smitham is taken out, and piled up in heaps. More being put into the tub, a child lifts up the smitham, an. l lars it on the siere, which retains still on its meshes the laver of fine ore. The sifter now aritates in the water nearly as at first, from time to time remoring with the limp the ligliter matters as they come to the surface; which being fit for washing only in boxes, are called buddler's offul, and are thrown into the buddle hole.

Mr. Petherich, the manager of Lanescot and the Fower Consol mines, has contrived an ingenious jigging machine, in which a series of 8 sieves are fixed in a stationary circular frame, connected with a plunger or piston working in a hollow cylinder, whereby a body of water is alternately forced up through the crushed ore in the sieves, and then left to descend. In this way of operating, the indiscriminate or premature passage of the finer pulrerulent matter through the meshes is aroided, because a regulated stream of water is made to trarerse the particles up and down. This mode has proved profitable in washing the copper ores of the above mentioned copper mines.

Proper washing apparatus.-For washing the ore after sifting it, the running buddle alreads described is employed, along with sereral chests or buddles of other linds.

1. The trink buddle is a species of German chest (see Metallergy and Tin) composel of two parts; of a cistern or box into which a stream of water flows, and of a large tank with a smooth lerel bottom. The ore to be trunked being placed in the box, the workman furnished with a shorel bent up at its sides, agitates it, and remores from time to time the coarser portions; while the smaller are swept off by the water and deposited apon the lerel area.

2. The stirring buddle, or chest for freeing the schlamms or slimy stuff from clar, is analogous to the German chests, and consists of two parts; namely; 1. a trough which reccives a stream of water through a plug hole, which is tempered at pleasure, to admit a greater or less current; 2. a settling tank with a horizontal bottom. The metallic slime being first floated in the water of the trough, then flows out and is deposited in the tank; the purest parts falling first near the beginning of the run.

3. The iniching buidle is analogous to the tables called dormantes or jumelles by the French miners. See Metallurgy. They have at their upper end a cross canal or epout, equal in length to the breadth of the table, with a plug hole in its middle for admitting the water. Alongside of this channel there is a slightly inclined plank, called aricking barrl, corresponding to the head of the twin table, and there is a nearly level plane below. The operation consists in spreading a thin laser of the slime upon the nickinz bourd, and in running over its surface a slender sheet of water, which in its progress is subdirided into rills, which gradually carry off the muddy matters, and strew them over the lower flat surface of the tank, in the order of their density.

690

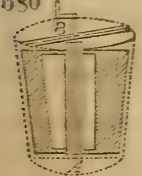

4. The dolly $t u b$ or rinsing bucket, fig. 630 , has an upright shaft which bears the vane or dolly $\mathrm{A}$ B, turned by the winch handle. This apparatus serves to bring into a state of suspension in water, the fine ore, already nearly pure; the separation of the metallic particles from the earthy ones by repose, being promoted by the sides of the tub being struck frequently during the subsidence.

5. Slime pits.-In the several operations of cleansing ores from mud, in grinding, and washing, where a stream of water is used, it is impossible to prevent 
some of the finely attenuated portions of the galena called sludge, floating in the water, from being carried off with it. Slime pits or labyrinths, called buddle holes in J)erbyshire, are employed to collect that matter, by receiving the water to settle, at a little distance from the place of agitation.

These hasins or reservoirs are about 20 feet in diameter, and from 24 to 40 inches deep. Here the suspended ore is deposited, and nothing but clear water is allowed to escape.

The workmen employed in the mechanical preparation of the ores, are paid, in Cumberland, by the piece, and not by day's wages. A certain quantity of crude ore is delivered to them, and their work is valued by the bing, a measure containing $14 \mathrm{cwts}$. of ore ready for smelting. The price varies according to the richness of the ore. Certain qualities are washed at the rate of two and sixpence, or three shillings the bing; while others are worth at least ten shillings. The richness of the ore varies from 2 to 20 bings of galena per shift of ore; the shift corresponding to 8 wagon loads.

1. The cleansing and sorting of the ores are well performed in Cumberland. These operations seem however to be inferior to the cleansing on the grid steps, grilles à gradin, of Saxony (See Metallurgy), an apparatus which in cleaning the ores, has the advantage of grouping them in lots of different qualities and dimensions.

2. The breaking or bruising by means of the crushing machine, is much more expeditious than the Derbyshire process by buckers; for the machine introduces not only great economy into the breaking operation, but it likewise diminishes considerably the loss of galena; for stamped ores may be often subjected to the action of the cylinders without waste, while a portion of them would have been lost with the water that runs from the stamp mill. The use of these rollers may therefore be considered as one of the happiest innovations hitherto made in the mechanical preparation of ores.

3 . The brake sieves appear to be preferable to the hand ones.

4. The system of washing used in Cumberland differs essentially from that of Brittany. The slime pits are constructed with much less care than in France and Germany. They never present, as in these countries, those long windings backwards and forwards, whence they have been called labyrinths; probably because the last deposites, which are washed with profit in France and Germany; could not be so in Cumberland. There is reason to believe, however, that the introduction of brake tables (tables à secousses, see Metallurgy) would enable deposites to be saved, which at present run to waste in England.

5. From what we have now said about the system of washing, and the basins of deposite or settling cisterns, it may be inferred that the operation followed in Cumberland is more expeditious than that used in Brittany, but it furnishes less pure ores, and oceasions more considerable waste; a fact sufficiently obvious, since the refuse stuff at Poulläouen is often resumed, and profitably subjected to a new preparation. We cannot howerer venture to blame this method, because in England, fuel being cheap, and labor dear, there may possibly be more advantage in smelting an ore somewhat impure, and in losing a little galena, than in multiplying the number of washing processes.

6. Lastly, the dolly tub ought to be adopted in all the establishments where the galena is mixed with much blende (sulphuret of zinc); for schlich (metallic slime) which appears very clean to the eye, gives off a considerable quantity of blende by means of the dolly $t u b$. While the vane is rapidly whirled, the sludge is gradually let down into the revolving water, till the quantity is sufficiently great. Whenever the ore is thoroughly disseminated in the liquid, the dolly is withdrawn. The workmen then strike on the sides of the tub for a considerable time, with mallets or wooden billets, to make the slime fall fast to the bottom. The lighter portions, consisting almost entirely of refuse matter, fall only after the knocking has ceased: the water is now run away; then the very poor slime upon the top of the deposite is skimmed off, while the pure ore found at the bottom of the tub is lifted out, and laid on the bingstead. In this way the blende, which always accompanies galena in a greater or smaller quantity, is well separated.

Smelling of lead ores.-The lead ores of Derbyshire and the north of England were anciently smelted in very rude furnaces, or boles, urged by the natural force of the wind, and were therefore placed on the summits or western slopes of the highest hills. More recently these furnaces were replaced by blast hearths, resembling smiths' forges, but iarger, and were blown by strong bellows, moved by men or water-wheels. The principal operation of smelting is at present always executed in Derbyshire in reverberatory furnaces, and at Alston Moor in furnaces similar to those known in France by the name of Scotch furnaces. Before entering into the detail of the founding processes, we shall give a description of the furnaces essential for both the smelting and accessory operations.

1. The reverberatory furnace called cupola, now exclusively used in Derbyshire for the smelting of lead ores, was imported thither from Wales, about the year 1747, by a company of Quakers. The first establishment in this country was built at Kalstedge, in the dist 1 ict of Ashover. 
In the wolks where the construction of these furnaces is most improved, they are interiorly 8 fet long by 6 wide in the middle, and two feet high at the centre. The fire, placed at one of the extremities, is separated from the body of the furnace by a borly of masunry, called the fire-bridge, which is two feet thick, leaving oniy from 14 to 18 inches betwee: its upper surface and the vault. From this, the highest point, the vault gradually sinks towards the further end, where it stands only 6 inches above the sole. At this extremity of the furnace, there are two openings separated by a triangular prism of fire-stone, which lead to a flue, a foot and a half wide, and 10 feet long, which is recurved towards the top, and runs into an upright chimney 55 feet high. The above flue is covered with stone slabs, carefully jointed with fire-clay, which may be removed when the deposite formed under them (which is apt to melt) requires to be cleaned out. One of the sides of the furnace is called the laborers' side. It has a door for throwing coal upon the fire-grate, besides three small apertures each about 6 inches square: These are closed with moveable plates of cast iron, which are taken off when the working requires a freer circulation of air, or for the stirring up of the materials upon the hearth. On the opposite side, called the working side, there are five apertures; namely, three equal and opposite to those just described, shuttiug in like manner with cast iron plates, and beneath them two other openings, ore of which is for running out the lead, and another for the scoriæ. The ash pit is also on this side, covered with a little water, and so disposed as that the gratc-bars may be easily cleared from the cinder slag.

The hearth of the furnace is composed of the reverberatory furnace slags, to which a proper shape has been given by beating them with a strong iron rake, before their entire solidification. On the laborers' side, this hearth rises nearly to the surface of the three openings, and falls towards the working side, so as to be 18 inches below the middle aperture. In this point, the lowest of the furnace, there is a tap-hole, through which the lead is run off into a large iron boiler (lea-pan), placed in a recess left outside in the masonry. From that lowest point, the sole gradually rises in all directions, forming thus an inside basin, into which the lead runs down as it is smelted. At the usual level of the metal bath, there is on the working side, at the end furthest from the fire, an aperture for letting off the slag

In the middle of the arched roof there is a small aperture, called the crown-hole, which is covered up during the working with a thick cast iron plate. Above this aperture a large wooden or iron hopper stands, leading beneath into an iron cylinder, through which the contents of the hopper may fall into the furnace when a trap or valve is opened.

2. The roasting furnace. - This was introduced abuut 30 years ago, in the neighborhood of Alston Moor, for roasting the ore intended to pass through the Scotch furnace, a process which greatly facilitates that operation. Since its first establishment it has successively received considerable improvements.

Figs. 631, 632, 633, represent the cupola furnace at the Marquess of Westminster's 631

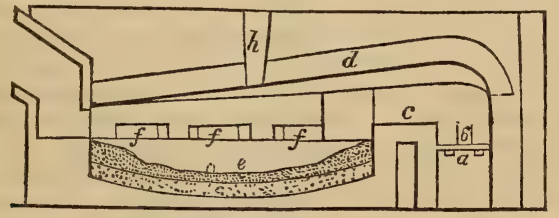

632
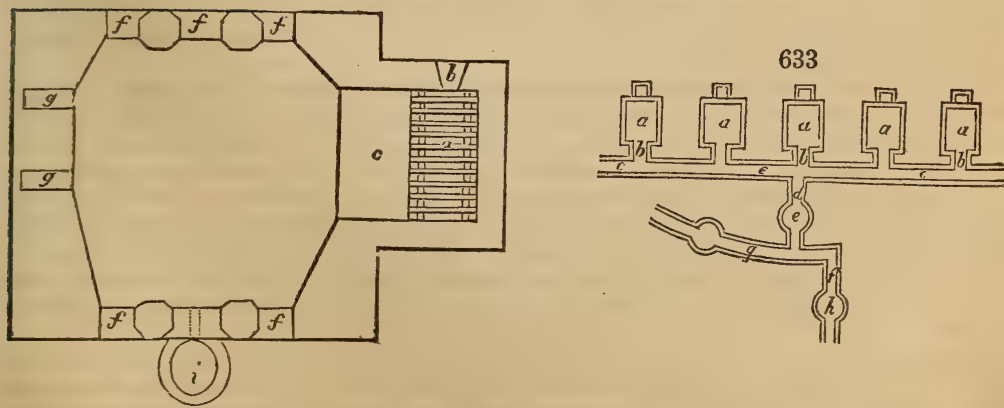

lead smelting works, two miles from Holywell. The hearth is hollowed out below the 
middle door of the furnace; it slopes from the back and onds towards this basin. The distance from the lowest point of this concavity up to the sill of the door, is usually 24 inches, but it is sometimes a little less, according to the quality of the ores to be smelted. This furnace has no hole for running off the slag, above the level of the top hole for the lead $i$, like the smelting furnace of Lea, near Matlock. A single chimney stalk serves for all the establishments; and receives all the flues of the various roasting and reducing furnaces. Fig. 633 gives an idea of the distribution of these flues. $a$ a $a$, scc. are the furnaces; $b$, the flucs, 18 inches square; these lead from each furnace to the principal conduit $c$, which is 5 feet deep by $2 \frac{1}{2}$ wide; $d$ is 6 feet deep by 3 wide; $e$ is a round chamber 15 feet in diameter $; f$ is a conduit 7 feet high by 5 wide; $g$ another, 6 feet. high $\mathrm{br} 3$ wide. The chimney at $h$ has a diameter at bottom of 30 feet, a top of 12 feet, including the thickness of its sides, forming a truncated cone 100 feet high; whose base stands upon a hill a little way from the furnaces, and 62 feet above their level.

$a, f i g s .631,632$, is the grate; $b$, the door of the fire-place; $c$, the fire-bridge; $d$, the arched roof; $e$, the hearth; $f f f$, \&c., the working doors; $g$, flues running into one conduit, which leads to the subterranean condensing chamber, $e$, and thence to the general chimney; $h$, a hopper-shaped opening in the top of the furnace, for supplying it with materials.

This magnificent structure is not destined solely for the reduction of the ores, but for dissipating all the vapors which might prove noxious to the health of the work-people and to vegetation.

The ores smelted at Holywell are very refractory galenas, mixed with blende, calamine, pyrites, carbonate of lime, \&c., but without any fluate of lime. They serve mutually as fluxes to one another. The coal is of inferior quality. The sole of each furnace is formed of slags obtained in the smelting, and they are all of one kind. In constructing it, 7 or 8 tons of these slags are first of all thrown upon the brick area of the hearth; are made to melt by a brisk fire, and in their stiffening state, as they cool, they permit the bottom to be sloped and hollowed into the desired shape. Four workmen, two at each side of the furnace, perform this task.

The ordinary charge of ore for one smelting operation is $20 \mathrm{cwts}$., and it is introduced through the hopper; see COPPER, fig. 304. An assistant placed at the back doors spreads it equally over the whole hearth with a rake; the furnace being meanwhile heated only with the declining fire of the preceding operation. No regular fire is made during the first two hours, but a gentle heat merely is kept up by throwing one or two shovelfuls of small coal upon the grate from time to time. All the doors are closed, and the register-plate of the chimney is lowered.

The outer basin in front of the furnace is at this time filled with the lead derived from a former process, the metal being covered with slags. A rectangular slit above the tap hole is left open, and remains so during the whole time of the operation, unless the lead should rise in the interior basin above the level of that orifice; in which case a little mound must be raised before it.

The two doors in front furthest from the fire being soon opened, the head-smelter throws in through them, upon the sole of the furnace, the slags swimming upon the bath of lead, and a little while afterwards he opens the tap-hole, and runs off the metallic lead reduced from these slags. At the same time his assistant turns over the ore with his paddle, through the back doors. These being again closed, while the abore two front doors are open, the smelter throws a shovelful of small coal or coke cinder upon the lead bath, and works the whole together, turning over the ore with the paddle or iron oar. About three quarters of an hour after the commencement of the operation, he throws back upon the sole of the hearth the fresh slags which then float upon the bath of the outer basin, and which are mixed with coaly matter. He next turns over these slags, as well as the ore with the paddle, and shuts all the doors. At this time the smelter runs off the lead into the pig-moulds.

The assistant now turns over the ore once more through the back doors. A little more than an hour after the operation began, a quantity of lead proceeding from the slag last remelted, is run off by the tap; being usually in such quantity as to fill one half of the outer basin. Both the workmen then turn over the ore with the paddles, at the several doors of the furnace. Its interior is at this time of a dull red heat; the roasting being carried on rather by the combustion of the sulphurous ingredients, than by the action of the small quantity of coal in the grate. The smelter, after shutting the front doors, with the exception of that next the fire-bridge, lifts off the fresh slags lying upon the surface of the outside bath, drains them, and throws them back into the furnace.

An hour and a half after the commencement, the lead begins to ooze out in small quantities from the ore; but little should be suffered to flow before two hours have expired. About this time the two workmen open all the doors, and turn over the ore, each at his own side of the furnace. An hour and three quarters after the beginning, 
there are few rapors in the furnace, its temperature being very moderate. No more lead is then seen to flow upon the sloping hearth. A little coal being thrown into the grate to raise the heat slightly, the workmen turn over the ore, and then close all the doors.

At the end of two hours, the first fire or roasting being completed, and the doors shut, the register is to be lifted a little, and coal thrown upon the grate to give the second fire, which lasts during 25 minutes. When the doors are now opened, the inside of the furnace is of a pretty vivid red, and the lead flows down from every side towards the inner basin. The smelter with his rake or paddle pushes the slags upon that basin bacli towards the upper part of the sole, and his assistant spreads them uniformly over the surface through the back doors. The smelter next throws in by his middle door, a few shorelfuls of quicklime upon the lead bath. The assistant meanwhile, for a quarter of an hour, works the ore and the slags together through the three back doors, and then spreads them out, while the smelter pushes the slags from the surface of the inner basin back to the upper parts of the sole. The doors being nuw left open for a little, while the interior remains in repose, the metallic lead, which had been pushed back with the slags, flows down into the basin. This occasional cooling of the furnace is thought to be necessary for the better separation of the products, especially of the slags, from the lead bath.

In a short time the workmen resume their rakes, and turn over the slags along with the ore. Three hours after the commencement, a little more fuel is put into the grate, merely to keep up a moderate heat of the furnace during the paddling. After three hours and ten minutes, the grate being charged with fuel for the third fire, the register is completely opened, the doors are all shut, and the furnace is left in this state for three quarters of an hour. In nearly four hours from the commencement, all the doors being opened, the assistant levels the surfaces with his rake, in orrler to favor the descent of any drops of lead; and then spreads the slags, which are pushed back towards him by the smelter. The latter now throws in a fresh quantity of lime, with the view not merely of covering the lead bath and preventing its oxydizement, but of rendering the slags less fluid.

Ten minutes after the third fire is completed, the smelter puts a new charge of fue? in the grate, and shuts the doors of the furnace to give it the fourth fire. In four hours and forty minutes from the commencement, this fire being finished, the doors are opened, the smelter pierces the tap hole to discharge the lead into the outer basin, and throws some quicklime upon the slags in the inner basin. He then pushes the slags thus dried up towards the upper part of the hearth, and his assistant rakes them out by the back doors.

The whole operation of a smelting shift takes about four hours and a half, or at mos? five hours, in which four periods may be distinguished.

1. The first fire for roasting the ores, requires very moderate firing and lasts two hours.

2. The second fire, or the smelting, requires a higher heat, with shut doors; at the end the slags are dried up with lime, and the furnace is also allowed to cool a little.

3,4 . The last two periods, or the third and fourth fires, are likewise two smeltings or foundings, and differ from the first only in requiring a higher temperature. The heat is greatest in the last. The form and dimensions of the furnace are calculated to cause a uniform distribution of heat over the whole surface of the hearth. Sometimes billets of green wood are plunged into the metallic lead of the outer basin, causing an ebullition which favors the separation of the slags, and consequently the production of a purer lead; but no more metallic metal is obtained.

Ten cwts. of coal are consumed at Holywell in smelting one ton of the lead-ore schlich or sludge; but at Grassington, near Skipton in Yorkshire, with a similar furnace worked with a slower heat, the operation taking from seven hours to seven hours and a half, instead of five, only $7 \frac{1}{2} \mathrm{cwts}$. of coal are consumed. But here the ores are less refractory, have the benefit of fluor spar as a flux, and are more exhausted of their metal, being smelted upon a less sloping hearth.

Theory of the above operation. - At Holywell, Grassington, and in Cornwall, the result of the first graduated roasting heat, is a mixture of undecomposed sulphuret of lead, with sulphate and oxyde of lead, in proportions which vary with the degree of care bestowed upon the process. After the roasting, the heat is raised to convert the sludge into a pasty mass; in which the oxyde and sulphate react upon the sulphuret, so as to produce a sub-sulphuret, which parts with the metal by liquation. The cooling of the furnace facilitates the liquation every time that the sub-sulphuret is formed, and the ore has passed by increase of temperature from the pasty into the liquid state. Cooling brings back the sludge to the pasty condition, and is therefore necessary for the due separation of the different bodies. The drying up of the thin slags by lime is intended to liberate the oxyde of lead, and allow it to react upon any sulphuret which may have 
resisted roasting or decomposition. It is also useful as a thickener, in a mechanical point of view. 'The iron of the tools, which wear away very fast, is also serviceable in redacing the sulphuret of lead. The small coal added along with the ime at Grassington, and also sometimes at Holywell, aids in reducing the oxyde of lead, and in transforming the sulphate into sulphuret.

3. The smelting furnace or ore hearth.-This furnace, called by the French écossais, is from 22 to 24 inches in height and 1 foot by $1 \frac{1}{2}$ in area inside; but its horizontal section, always rectangular, varies much in its dimensions at different levels, as shown in fig. 634.

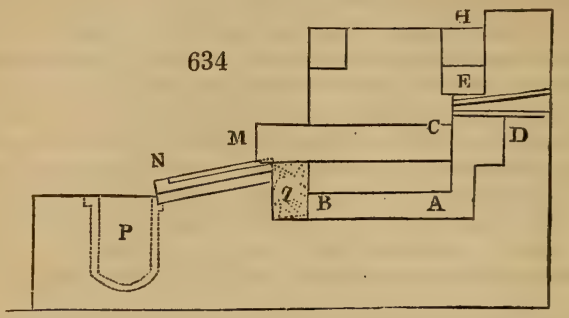

The hearth and the sides are of cast iron; the sole-plate A B is also of cast iron, $2 \frac{1}{2}$ inches thick, having on its back and two sides an upright ledge, A c, $2 \frac{1}{2}$ inches thick and $4 \frac{1}{4}$ high. In front of the hearth there is another cast iron plate $\mathrm{M} \mathrm{N}$, called the work stone, surrounded on every side excepting towards the sole of the furnace, by a ledge one inch in thickness and height. 'The plate slopes from behind forwards, and its posterior ledge, which is about $4 \frac{1}{2}$ inches above the surface of the hearth, is separated from it by a void space $q$, which is filled with a mixture of bone ash and galena, both in fine powder, moistened and pressed down together. The melted lead cannot penetrate into this body, but after filling the basin at the bottom of the furnace, flows naturally out by the gutter (nearly an inch deep) through a groove in the work-stone; and then passes into a caldron of reception $\mathrm{p}$, styled the melting-pot, placed below the front edge of the uork-stone.

The posterior ledge of the sole is surmounted by a piece of cast iron $\mathrm{c} D$, called the back-stone, 28 inches long, and $6 \frac{1}{2}$ high; on which the tuyère or blast-pipe is placed. It supports another piece of cast iron $\mathrm{E}$, called pipe-stone, scooped out at its under 'part, in the middle of its length, for the passage of the tuyere. This piece advances 2 inches into the interior of the furnace, the back wall of which is finally crowned by another piece of cast iron $\mathrm{E} \mathrm{H}$, called also back-stone.

On. the ledges of the two sides of the sole, are placed two pieces of cast iron, called bearers, each of which is 5 inches in breadth and height, and 26 inches long. They advance an inch or two above the posterior and highest edge of the work-stone, and contribute effectually to fix it solidly in its place. These bearers support, through the in tervention of several ranges of fire-bricks, a piece of cast iron called a fore-stone, which has the same dimensions as the piece called the back-stone, on which the base of the blowing-machine rests. This piece is in contact, at each of its extremities, with another mass of cast iron, 6 inches cube, called the key-stone, supported on the masonry. Lastly, the void spaces left between the two key-stones and the back part of the furnace are filled up with two masses of cast iron exactly like the key-stones.

The front of the furnace is open for about 12 inches from the lower part of the front cross-piece called fore-stone, up to the superior part of the work-stone. It is through this opening that the smelter operates.

The gaseous products of the combustion, on escaping from this ore-hearth, are frequently made to pass through a long flue, sloped very slightly upwards, in which they deposite all the particles of ore that they may have swept along; these flues, whose length is sometimes more than 100 yards, are usually 5 feet high and 3 feet wide in the inside, and always terminate in the chimney stalk. The matters deposited near the commencement of the flue require to be washed; but not the other dusty deposites. The whole may then be carried back to the roasting furnace, to be calcined and reagglutinated, or introduced without any preparation into the slag-hearth.

4. Figs. 635, 636 represent a slag-hearth, the fourneau à manche (elbow furnace) of the French, and the krummofen (crooked furnace) of the Germans ; such as is used at Alston Moor, in Cumberland, for the reduction of the lead-slag. It resembles the Scotch furnace. The shaft is a parallelopiped, whose base is 26 inches by 22 in area inside, and whose height is 3 feet; the sole-plate $a$, of cast iron, slopes slightly down to the basin of reception, or the fore-hearth $b$. Upon both of the long sides of the sole-plate there are east iron beams, called bearer's $\mathrm{c}$, of great strength, which support the side walls built of a coarse grained sand-stone, as well as the cast iron plate $d$ (fore stone), which forms 
the front of the shaft. This stands 7 inches off from the sole-plate, leaving an empry space between them. The back side is made of cast iron, from the sole-plate to the hurizontal tuyère in its midale; but above this foint it is made of sand-stone. 'The

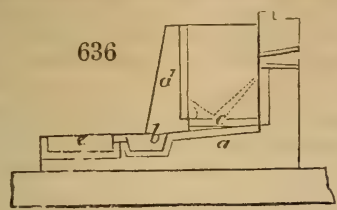

635

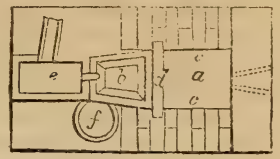

tuyere is from $1 \frac{1}{2}$ to 2 inches in diameter. In front of the fore-hearth $b$, a cistern $e$ is placed, through which water continually flows, so that the slags which spontaneously overflow the fore-hearth may become inflated and shattered, whereby the lead aisseminated through them may be readily separated by washing. The lead itself flows firom the fore-hearth $b$, through an orifice, into an iron pot $\hat{f}$, which is sept hot over a fire. The metal obtained from this slag-hearth is much less pure than that extracted directly from the ore.

The whole ou itom of the furnace is filled to a height of 17 inches, that is, to within 2 or 3 inches of the tuyere, with the rubbish of coke reduced to coarse powder and beat strongly down. At each smelting shift, this bel must be made anew, and the interior of the furnace above the tuyere repaired, with the exception of the front, consisting of east iron. In advance of the furnace there is a basin of reception, which is also filled with colie rubbish. Farther off is a pit, full of water, replenished by a cold stream, which incessantly runs in through a pipe. The scoria, in flowing out of the furnace, pass orer the coke bed in the basin of reception, and then fall into the water, whose coolness makes them fly into small pieces, after which they are easily washed, so as to serarate the lead that may be entangled among them.

These furnaces are urged, in general, by wooden bellows; fig. 63\%. But at the smelting

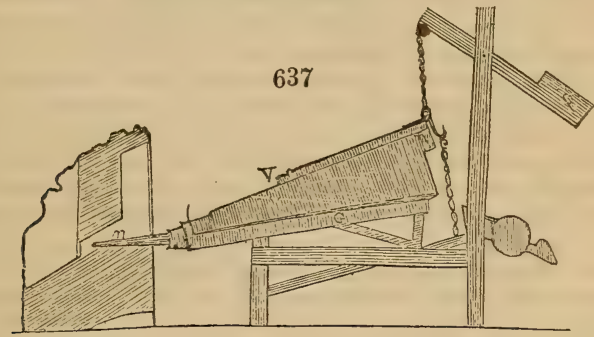

woris of Lea, near Matlock, the blowing-machine consists of two casks, which move upon horizontal axes. Each of these casks is divided into two equal parts by a fixed plane that passes through its axis, and is filled with water to a certain height. The water of one side communicates with that of the other by an opening in the lower part of the division. Each cask possesses a movement of oscillation, produced by a rod attached to a crank of a bucket-wheel. At each demi-oscillation, one of the compartments, being in communication with the external air, is filled; while the other, on the contrary, communicates with the nozzle, and supplies wind to the furnace.

5. Refining or cupellation furnacb. See Silver.

6. Sinelting by the reverberatory furnace is adopted exclusively in Derbyshire, and in some works at Alston Moor. The charge in the hopper consists commonly of 16 ewts.g each weighing $120 \mathrm{lbs}$. avoirdupois, composed of an intimate mixture of 5, 6, 7, or even 8 kinds of ore, derived from different mines, and prepared in different ways. The proportirns of the mixture are determined by experience, and are of great consequence to the success of the work.

The ore is rather in the form of grains than of a fine schlich ; it is sometimes very pure, and aflords 75 per cent.; but usually it is mixed up with a large proportion of carbonate and fluate of lime; and its product varies from 65 to 23 per cent.

After scraping the slaggy matters, out of the furnace, a fresh smelting shift is introduced at an interval of a few minutes; and thus, by means of two alternate workmen, who relieve each other tevery seven or eight hours, the weekly operations continue without interruption. The average product in lead of the reverberatory furnaces in Derbyshire, during several years, has been 66 per cent. of the ore. Very fine ore has, howerer, ifforded $\tau 6$.

7. Smelting of the drawn slag, on the slag-mill hearth. - The black slag of the reverbe. 
ratory furnace is broken by hammers into small pieces, and mixed in proper propurtinns with the coal cinders that fall through the grate of the reverberatory fire. The leaden matts that fluat on the surface of the bath, and the dust deposited in the chimney; are added, along with some poor ore containing a gangue of fluor spar and limestone, which had been put aside during the machanical preparation. W'ith such a mixture, the slag-hearth, already described figs. 635, 636, is charged. By the action of heat and cual, the lead is revired, the earthy matters flow into very liquid scoriæ, and the whole is made to pass across the body of fire into a basin of reception placed bencath. The scoriæ are thickened by throwing quicklime upon them, and they are then ralied away. At the end of the operation the lead is cast into pigs or ingots of a peculiar form. This is called slag-lead. It is harder, more sonorous than the lead obtained from the reverberatory furnace, and is preferred for the manufacture of minium, lead shot, and some other purposes.

8. Treatment of lead ores by the Scotch furnace, or ore-hearth.-This furnace is generally employed in the counties of Northumberland, Cumberland, and Durham, for the smelting of lead ores, which were formerly carried to them without any preparation, but now they are exposed to a preliminary calcination. The roasted ore yields in the Scotch furnace a more considerable product than the crude ore, because it forms in the furnace $i$ more porous mass, and at the same time it works drier, to use the founder's expression; that is, it allows the stream of air impelled by the bellows to diffuse itself more completely across the matters contained in the furnace.

The charge of the roasting furnace, figs. $631,632,633$, is from 9 to $11 \mathrm{cwts}$. of ore, put into the furnace without any addition. Three such shifts are usually passed thrcugh in eight hours. The fire should be urged in such a manner as to produce constantly a dense smoke, without letting any part of the ore melt and form a slag; an accident which would obstruct the principal end of the process, which is to burn off the sulpliur and antimony, and to expel the carbonic acid of the carbonate of lead. The ore must be frequently turned over, by moving it from the bridge to the other end and back again. To prevent the ore from running into masses as it cools, it is made to fall out of the furnace into a pit full of water, placed below one of the lateral doors.

Smelting of the lead ores in the Scotch furnace.-When a smelting shift has been finished in the Scotch furnace, a portion of the ore, called browse, remains in a semireduced state, mixed with coke and cinders. It is found of more advantage to preserve the browse for beginning the following operation, than to take raw or even roasted ore. To se the furnace in action, the interior of it is filled with peats, cut into the form of bricks. The peats towards the posterior part are heaped up without order, but those near the front are piled up with care in the form of a wall. A kindled peat is now placed before the nozzle of the bellows, which are made to blow, and the blast spreads the combustion rapidly through the whole mass. To increase the heat, and to render the fire more steady and durable, a few shovelfuls of coals are thrown over the turf. A certain quantity of the browse is to be next introduced; and then (or sometimes before all the browse is put in) the greater part of the matters contained in the furnace is drawn over on the work-stone, by means of a large rake called a govelock; the refuse of the ore called gray slag, which a skilful smelter knows by its shining more than the browse, is taken off with a shovel, and thrown to the right hand into a corner outside of the furnace. The browse left on the work-stone is to be now thrown back into the furnace, with the addition of a little coal, if necessary. If the browse be not well cleaned from the slag, which is perceived by the whole mass being in a soft state, and showing a tendency to fuse, quicklime must be added, which by its affinity for the argillaceous, silicious, and ferruginous substances, dries up the materials, as the smelters say, and gives to the earthy parts the property of concreting into lumpe or balls; but if, on the other hand, the silicious, argillaceous, or ferruginous parts contained in the ore be too refractory, lime is also to be added, but in smaller quantity, which, by rendering them more fusible, communicates the property of concreting into balls. These lumps, called gray slag, contain from one tenth to one fifteenth of the lead which was present in the ore. They must be smelted afterwards at a higher temperature in the slag hearth, to extract their lead. After the browse has been thrown back into the furnace, as has been said, a few shovelfuls of ore are to be strewed over it; but before doing this, and after removing the scoriæ, there must be always paced before the tuyère half a peat, a substance which being extremely porous and combustible, not only hinders any thing from entering the nozzle of the bellows, but spreads the blast through all the vacant parts of the furnace. After an interval of from 10 to 15 minutes, according to circumstances, the materials in the furnace are drawn afresh upon the workstone, and the gray slag is removed by the rake. Another peat being placed before the tuyère, and coal and quicklime being introduced in suitable proportions, the browse is thrown back into the furnace, a fresh portion of or is charged above it, and left in the furnace for the abøve-mentioned time. 
This mode of working, continued for 14 or 15 hours, forms what is called a smelting shift; in which time from 20 to $40 \mathrm{cwts}$. of lead, and even more, are produced.

By this process the purest part of the lead, as well as the silver, are sweated our, as it were, from the materials with which they are mixed, without anything entering into fusion except these two metals in the state of alloy. It is probable that the moderate temperature employed in the Scotch furnace is the main cause of the purity of the lead which it yields.

9. Smelting of the scoria of the Scotch furnace on the slag hearth.-Before putting fire to the slag hearth already described, figs. 635, 636, its empty space is to be filled with peats, and a lighted one being placed before the tuyère, the bellows are made to play. A layer of colie is to be now thrown upon the burning peats, and as soon as the heat is sufficiently high, a layer of the gray slag is to be introduced, or of any other scoriæ that are to be reduced. From time to time, as the fit moment arrives, alternate strata of coke and slag are to be added. In this operation, though the slag and the lead are brought to a state of perfect fluidity, the metal gets separated by filtering down through the bed of peat cinders, which the slag cannot do on account of its viscidity. Whenever that coke bed becomes covered with fluid slag, the workman makes a hole in it, of about an inch diameter, by means of a kneed poker; and runs it off by this orifice, as it cannot sink down into the hard rammed cinders, which fill the basin of reception. The slag flows over it in a glowing stream into the pit filled with water, where it gets granulated and ready for washing.

When lead is obtained from galena without the addition of combustible matter, we have : $n$ example, on the great scale, of the mutual decomposition of the oxydes and suljhates formed during the roasting heat, by the still undecomposed galena, especially when this action is facilitated by working up and skilfully mingling the various matters, as happens in the reverberatory and Scotch furnaces. It is therefore the sulphuret of lead itself which serres as the agent of reduction in regard to the oxyde and sulphate, when little or no charcoal has been added. Sometimes, however, towards the end of the operation in the reverberatory hearth, it becomes necessary to throw in some wood or charcoal, because the oxydizement having become too complete, there does not remain a sufficient body of sulphuret of lead to effect the decompositions and reductions just mentioned, and therefore it is requisite to regenerate some galena by means of carbonaceous matter, which immediately converts the sulphate of lead into the sulphuret. The sulphur and oxygen are eventually all separated in the form of sulphurous acid. Roasted galena contains sometimes no less than $\mathbf{7 7}$ per cent. of sulphate of lead.

At Ticonago, in the Valais, the process of smelting lead ore in the reverberatory furnace, with the addition of iron, as practised at Vienne, on the Isère, was introduced; but the difficulty of procuring a sufficient supply of old iron has led to an interesting modification.

On the hearth of the reverberatory furnace, 10 quintals of moderately rich ore are spread; these are heated temperately for some time, and stirred about to promote the sublimation of the sulphur. After three or four houns, when the ore seems to be sufficiently desulphureted, the heat is raised so as to melt the whole materials, and whenever they flux into a metallic glass, a few shovelfuls of bruised charcoal or cinders are thrown in, which soon thicken the liquid, and cause metallic lead to appear. By this means three fourths of the lead contained in the ore are usually extracted; but at length the substance, becoming less and less fluid, yields no more metal. Stamped and washed carbonate of iron (sparry iron ore) is now added, in the proportion of about 10 per cent. of the lead ore primarily introduced.

On stirring and working together this mixture, it assumes the consistence of a stiff paste, which is raked out of the furnace. When this has become cold, it is broken into pieces, and thereafter smelted in a slag-hearth, without the addition of flux. By this operation almost the whole lead present is obtained. 100 quintals of schlich yield 45 of argentiferous lead; and in the production of 100 quintals (cwts.) of marketable lead, 140 cubic feet of beech-wood, and $357 \frac{1}{2}$ quintals of charcoal are consumed.

This process is remarkable for the use of iron-ore in smelting galena.

10. Reduction in the reverberatory furnace of the litharge obtained in the refining of lead.- The litharge of Alston Moor is seldom sold as such, but is usually converted into lead, in a reverberatory furnace.

In commencing this reduction, a bed of coal about 2 inches thick is first of all laid on the hearth; which is soon kindled by the flame of the fire-place, and in a little while is reduced to red hot cinders. Upon these a certain quantity of a mixture of litharge and small coal is uniformly spread; the heat of the fire-place being meanwhile so managed as to maintain in the furnace a suitahle temperature for enabling the combustible to deprive the litharge of its oxygen, and to convert it into lead. The metal is run out by the tap-hole into an iron pot; and being cast into pigs of haif a hundred weight, is sold under the name of refined lead at a superior price. 
The quantity of small coal mixed with the litharge should be somewhat less than what may be necessary to effect the reduction, because if in the course of the process a deficiency of it is perceived in any part of the furnace, more can always be added; whereas a redundancy of coal necessarily increases the quantity of slag, which, at the end of the shift, must be removed from the furnace hefore a new operation is begun, whereby lead is lost. In the reverberatory furnace, six fodders of lead may be revived in nine or ten hours; during the first six of which the mixture of litharge and coal is added at short intervals. A fodder is from 21 to 24 cwts.

It deserves to be remarked that the work does not go on so well nor so quick when the coal and litharge are in a pulverulent form; because the reduction in this case takes place only at the surface, the air not being able to penetrate into the body, and to keep up its combustion, and the mutual action of the litharge and carbon in the interior. But, on the other hand, when the litharge is in porous pieces, as large as a hen's egg, the action pervades the whole body, and the sooty fumes of the coal effect the reduction even in the centre of the fragments of the litharge, penetrating into every fissure and carrying off the oxygen. The heat ought never to be urged so far as to melt the litharge.

The grounds of the cupel, and the slag of the reduction furnace, being a mixture of small coke, coal ash, and oxyde of iron, more or less impregnated with lead, are smelted upon the slag hearth, along with coke, and, by way of flux, with a certain quantity of the black scoriæ obtained from the same furnace, prepared for this purpose, by running it out in thin plates, and breaking it into small pieces. The lead thus obtained is usually very white, very hard, and not susceptible of refinement.

MM. Dufrènoy and Beaumont consider the smelting of lead ore by the reverberatory furnace, as practised in Derbyshire, as probably preferable to that with the slag hearth as carried on in Brittany; a process which seldom gives uniform products, while it occasions a more considerable waste of lead and consumption of fuel.

The mixed process employed in Cumberland of roasting the ore, and afterwards smelting it in a small furnace resembling that called the Scotch, apparently yields a little less lead than if both operations were executed in the reverberatory furnace; but according to Mr. Forster (see his Treatise on a Section of the Strata from Newcastle upon Tyne, \&c.), this slight loss is more than compensated by the smaller consumption of fuel, the increased rapidity of the operation, and especially by the much greater purity of the lead obtained from the Scotch furnace. When it comes to be refined, the loss is only about one twelfth or one thirteenth, whereas the lead revived in the reverberatory furnace loses frequently a ninth. Moreover, the lead furnished by the first method admits of being refined with profit, when it yields only 5 ounces of silver per fodder of 20 quintals, poids de marc, while that produced by the reverberatory furnace cannot be cupelled unless it gives 10 ounces per fodder; and as in the English cupellation lead is constantly added anew without skimming, the litharge obtained in the second case can never be brought into the market, whereas the litharge of the leads from the Scotch furnace is of good quality. See the new method of enriching lead for cupellation, under SiLver.

As the smelting of galena, the principal ore of lead, is not a little complex, the follow. ing tabular view of the different processes may prove acceptable to the metallurgist :-

Treatment of

1. Pure ores.

Ores mixed with ? England, in gensaline gangues.

3. Ores mixed with $\int_{\text {Vicenago, in }}^{\text {Italy, and Red- }}$ earthy gangues.

\section{A}

Desulphuration by roast-

I. Class. ing.

Treated in re-

verberatory furnaces.

4

4. Ores mixed with ruth, in Corn-
wall. several sulphu- Combined with rets.

5. Ores with earthy, saline, and sulphurous gan-
B

Desulphuration by iron. gues.

6. Ores with mattes, Ores with mattes, $\}$ Vienne, Poulläas at Vienne, in ouen, and TarDauphiny. nowitz. 


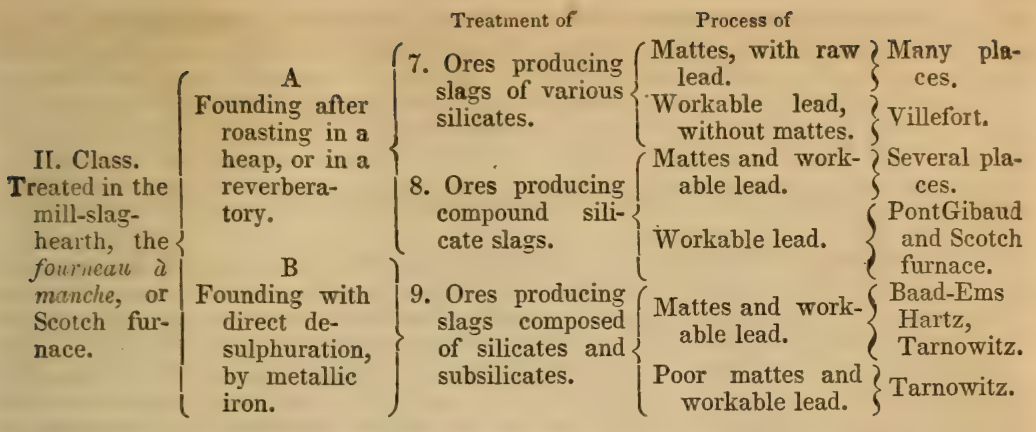

The annual production of lead in Europe may be estimated at about 80,000 tons; of which four sevenths are produced in England, two sevenths in Spain, the remainder in Gerinany and Russia. France does not produce more than one five-hundredth part of the whole; and only one fiftieth of its consumption.

See Litharge, Minium, or Red Lead, Solder, Sugar or Acetate of Leaz, Type Metat, and White Lead.

LEAD-SHOT (Plomb de chasse, Fr.; Schrot, Flintenschrot, Germ.). The origin of most of the imperfections in the manufacture of lead-shot is the too rapid cooling of the spherules by their being dropped too hot into the water, whereby their surfaces form a solid crust, while their interior remains fluid, and, in its subsequent concretion, shrinks, so as to produce the irregularities of the shot.

The patent shot towers origmally constructed in England obviate this evil by exposing the fused spherules after they pass through the cullender, to a large body of air during their descent into the water tub placed on the ground. The greatest erection of this kind is probably at Villach, in Carinthia, being 240 Vienna, or 249 English feet high.

The quantity of arsenic added to the mass of melted lead, varies according to the quality of this metal; the harder and less ductile the lead is, the more arsenic must be added. About 3 pounds of either white arsenic or orpiment is enough for one thousand parts of soft lead, and about 8 for the coarser kinds. The latter are employed preferably for shot, as they are cheaper and answer sufficiently well. The arsenical alloy is made either by introducing some of this substance at each melting, or by making a quantity of the compound considerably stronger at once, and adding a certain portion of this to each charge of lead. If the particles of the shot appear lens-shaped, it is a proof that the proportion of arsenic has been too great; but if they are flattened upon one side, if they are hollowed in their middle, called cupping by the workman, or drag with a tail behind them, the proportion of arsenic is too small.

The following is the process prescribed by the patentees, Ackerman and Martin. Melt a ton of soft lead, and sprinkle round its sides, in the iron pot, about two shovelfuls of wood ashes, taking care to leave the centre clear; then put into the middle about 40 pounds of arsenic to form a rich alloy with the lead. Cover the pot with an iron lid, and lute the joints quickly with loam or mortar, to confine the arsenical vapors, keeping up a moderate fire to maintain the mixture fluid for three or four hours; after which skim carefully, and run the alloy into moulds to form ingots or pigs. The composition thus made is to be put in the proportion of one pig or ingot into 1000 pounds of melted ordinary lead. When the whole is well combined, take a perforated skummer and let a few drops of it fall from some height into a tub of water. If they do not appear globular, some more arsenical alloy must be added.

Lead which contains a good deal of pewter or tin must be rejected, because it tends to produce elongated drops or tails.

From two to three tons are usuaily meited at once in the large establishments. The surface of the lead gets covered with a crust of oxyde of a white spongy nature, sometimes called cream by the workmen, which is of use to coat over the bottom of the cullender, because without such a bed the heavy melted lead would run too rapidly through the holes for the granulating process, and would form oblong spheroids. The mounting of this filter, or lining of the cullender, is reckoned to be a nice operation by the workmen, and is regarded usually as a valuable secret.

The cullenders are hollow hemispheres of sheet iron, about 10 inches in diameter, perforated with holes, which should be perfectly round and free from burs. These inust be of a uniform size in each cullender; but of course a series of different cullenders, with sorted holes for every different size of lead shot, must be prepared. The holes have nearly the following diameters for the annexed numbers of shot. 


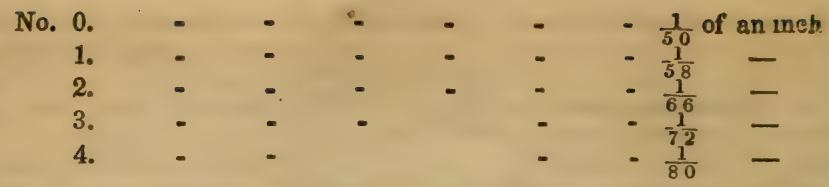

From No. 5 to No. 9 the diameter decreases by regular gradations, the latter being only $\frac{1}{36}=$ of an inch.

The operation is always carried on with three cullenders at a time; which are supported upon projecting grates of a kind of chafing dish made of sheet iron somewhat like a triangle. This chafing dish should be placed immeriately above the fall; while at its bottom there must be a tub half filled with water for receiving the granulated lead. The cullenders are not in contact, but must be parted by burning charcoal, in order to keep the lead constantly at the proper temperature, and to prevent its solidifying in the filter. The temperature of the lead bath should vary with the size of the shot; for the largest, it should be such that a bit of straw plunged into it will be scarcely browned, but for all it should be nicely regulated. The height from which the particles should be let fall raries likewise with the size of the shot; as the congelation is the more rapid, the smaller they are. With a fall of 33 yards or 100 feet, from No. 4 to No. 9 may be made; but for larger sizes, 150 feet of height will be required.

Every thing being arranged as above described, the workman puts the filter-stuff into the cullender, pressing it well against the sides. He next pours lead into it with an iron ladle, but not in too great quantity at a time, lest it should run through too fast. The shot thereby formed and found in the tub are not all equal.

The centre of the cullender being less hot affords larger shot than the sides, which are constantly surrounded with burning charcoal. Occasionally, also, the three cullenders employed together may have holes of different sizes, in which case the tub may contain shot of very various magnitudes. These are separated from each other by square sieres of different fineness, 10 inches broad and 16 inches long, their bottoms being of sheet iron, pierced with holes of the same diameters as those of the cullenders. These sieves are suspended by means of two bands above boxes for receiving the shot; one sieve being usually set above another in consecutive numbers, for instance, 1 and 2 . The shot being put into the upper sieve, No. 0 will remain in it, No. 1 will remain in the lower sieve, and No. 2 will, with all the others, pass through it into the chest below. It is obvious that by substituting sieves of successive fineness, shot of any dimension may be sorted.

In the preceding process the shot has been sorted to size; it must next be sorted to form, so as to separate all the spheroids which are not truly round, or are defectire in any respect. For this purpose a board is made use of about 27 inches long and 16 broad, furnished partially with upright ledges; upon this tray a handful or two of the shot to be sorted being laid, it is inclined very slightly, and gently shaken in the horizontal direction, when the globular particles run down by one edge, into a chest set to receive them, while those of irregular forms remain on the sides of the tray, and are reserved to be remelted.

After being sorted in this way, the shot requires still to be smoothed and polished bright. This object is effected by putting it into a small octagonal cask, through a door in its side, turning upon a horizontal iron axis, which rests in plummer boxes at its ends, and is made to revolve by any mechanical power. A certain quantity of plumbago or black lead is put in along with the shot.

LAZLLITE (Eng. and Fr.; Lazulith, Germ.) is a blue vitreous mineral, crystallizing in rhomboidal dodecahedrons; spec. grav., $2 \cdot 76$ to 2.94 ; scratches glass; affords a little water by calcination; fusible into a white glass; dissolves in acids with loss of color; solution leaves an alkaline residuum, after being treated with carbonate of ammonia, filtered, evaporated, and calcined. It consists of silica, $35 \cdot 8$; alumina, 34.8 ; so''a, $23 \cdot 2$; sulphur, $3 \cdot 1$; carbonate of lime, $3 \cdot 1$. This beautiful stone affords the native ultramarime pigment, which was very costly till a mode of making it artificially was lately discovered. See Ultramarine.

LEATHER (Cuir, Fr.; Germ., Leder) is the skin of animals, so modified by chemical means as to have become unalterable by the external agents which tend to decompose it in its natural state. The preparation in a rude manner of this valuable substance, has been known from the most ancient times, but it was not till the end of the last, and the beginning of the present century, that it began to be manufactured upon right principles, in consequence of the researches of Macbride, Deyeux, Seguin, and Davy. There are several varieties of leather, such as sole leather, boot or upper leather, shamoy leather, kid or glove leather, \&c. Skins may be converted into leather either with or without their hairy coat.

We shall treat first of sole and upper leathers, being the most important. and most 
costly and difficult to prepare in a proper manner. These kinds consist of organized fibrous gelatine or skin, combined with the proximate regetable principle, tannin, and probably also some vegetable extractive. Under the articles GaLLs and TANin, will be found an account of the properties of this substance, and the means of obtaining it in a state of purity. Calf leather quickly tanned by an infusion of galls, consists of 61 parts of shin, and 39 of regetable matter in 100 by weight; by solution of catechu, it consists of $\$ 0$ of skin, and 20 of regetable matter; by infusion of Leicester willow, of $74 \cdot 5$ skin, and 25.5 regetable matter; and by infusion of oak bark, of $73 \cdot 2$ shin, and 26.8 regetable matter. By the slow process of tanning, continued for three months, the increase of weight upon the skin in its conversion into leather, is greatly less; the vegetable constituents being from Leicester willow only 13 per cent. of the leather, and from oah bark 15 per cent. Sole leather, however, generally contains no less than 40 per cent. of vegetable matter. In evert astringent bark, the inner white part next to the albuman, contains the largest quantity of tannin, and the middle colored part contains most extractive matter. The outer surface or epidermis seldom furnishes either tannin or extractive matter. Young trees abound most in the white cortical layers, and are hence more productive of tannin under equal weights, than the barks of old trees. In no case is there any reason to believe that the gallic acid of astringent vegetables is absorbed in the process of making leather; hence Seguin's theory of the agency of that substance in disoxygenating slin, falls to the ground. The different qualities of leather made with the same kind of skin, seem to depend very much upon the different quantities of extractive matter it may have absorbed. The leather made with infusion of galls, is generally harder and more liable to crack than the leather obtained from infusions of barks; and It always contains a much larger proportion of tannin, and a smaller proportion of extractive matter.

When calf skin is slowly tanned in weak solutions of the bark, or of catechu, it combines with a good deal of extractive matter, and though the increase of the weight of the skin be comparatively small, yet it has become perfectly insoluble in water, forming a soft, but at the same time a strong leather. The saturated infusions of astringent barks contain much less extractive matter in proportion to their tannin, than the weak infusions; and when skin is quickly tanned in the former, it produces a worse and less durable leather than when slowly tanned in the latter. In quick tanning, a considerable quantity of regetable extractive matter is thus lost to the manufacturer, which might hare been made to enter as a useful constituent into the leather. These observations show that there is sufficient foundation for the opinion of the common workmen, concerning what is technically called feeding of leather, in the slow method of tanning; and though the processes of this art have been unnecessarily protracted by defective methods of steeping, and want of progressive infiltration of the astringent liquor through the skins, ret in general they appear to have arrived, in consequence of old experience, at a degree of perfection in the quality of the leather, which cannot be far exceeded by means of any theoretical suggestions which have been advanced.

On the first view it may appear surprising, that in those cases of quick ta ning, where extractive matter forms a certain portion of the leather, the increase of weight is less than when the skin is combined with the pure tannin; but the fact is easily accounted for, when we consider that the attraction of skin for tannin must be probably weakened by its union with extractive matter; and whether we suppose that the tannin and extractive matter enter together into combination with the matter of skin, or unite with separate portions of it, still, in either case, the primary attraction of skin for tan must be to a certain extent diminished.

In examining astringent regetables in relation to their power of making leather, it is necessary to take into account not only the quantity they may contain of the substance precipitable by gelatine, but likewise the quantity and the nature of the extractive matter; and in cases of comparison, it is essential to employ infusions of the same degree of concentration.

Of all astringent substances hitherto examined, catechu is that which contains the largest proportion of tannin; and in supposing, according to the usual estimation, that from four to five pounds of common oak bark are required to produce one pound of leather, it appears, from the various synthetical experiments, that about half a pound of catechu would answer the same purpose. Mr. Purkis found, by the results of different accurate experiments, that 1 pound of catechu was equivalent to 7 or 8 of oak bark. For the common purposes of the tanner, 1 pound of it would be equivalent also to $2 \frac{1}{4}$ pounds of galls, to $7 \frac{1}{2}$ of the Leicester willow, to 11 of the bark of the Spanish chestnut, to 18 of the bark of the common elm, to 21 of the bark of the common willow, and to 3 pounds of sumach.

Various menstrua have been proposed for the purpose of expediting and improving the process of tanning, among others, lime water, and solution of pearl-ash; but as these two substances form compounds with tannin which are not decomposable by gelatine, 't 
follows that their effects must be prejudicial. There is very little reason to suppose that any bodies will be found which, at the same time that they increase the solubility of tannin in water, will not likewise diminish its attraction for skin.

In this country all tanned leather is distinguished into two kinds, called hides and skins; the former term being appropriated to that made from the larger animals, as bulls, buffaloes, oxen, and cows, into thick strong sole leather; and the latter to that made from calves, seals, \&c., into thinner and more flexible upper leather. Sometimes the hides are brought into the market merely dried, as from Buenos Ayres; or dried and salted, as from Bahia and Pernambuco; but the greater part are fresh from recently slaughtered animals. The heaviest ox-hides are preferred for forming butts or backs, which are manufactured as follows:-

The washing process must be more or less elaborate, according to the state of the skins. Those that are salted and dry require to be steeped, beaten, and rubbed several times alternately, to bring them to the fresh condition.

After remoring the horns, the softened or recent hides are laid in a heap for two us three days, after which they are suspended on poles in a close room called a smokehouse, heated somewhat above the common temperature by a smouldering fire. In these circumstances, a slight putrefaction supervenes, which loosens the epidermis, and renders the hair easily deiachable by the fleshing knife; a large two handled implement, with a blunt edge, and bent to suit the curvature of the rounded beam of the wooden horse upon which the hide is scraped. See Currying.

The next step is immersion in a pit containing water impregnated with about a 1000 th part of sulphuric acid. This process is called raising, because it distends the pores, and makes the fibres swell, so as to render the skins more susceptible of the action of the tanning infusions. Forty-eight hours in general suffice for this operation, but more time may be safely taken.

When the hides are found to be sufficiently raised, they are transferred to a pit, in which they are stratified with oak bark, ground by a proper mill into a coarse powder. The pit is then filled up with an infusion of oak bark called ooze, and the hides are allowed to remain in it for about a month or six weeks. By this time the tannin and extractive matter of the bark having combined intimately with the animal fibre, the pit is exhausted of its virtue, and must be renewed, by taking out the spent bark, and subjecting the skins to a fresh dose of oak bark and ooze. The hides which were placed near the top of the first pit, must be placed near the bottom of the next. In this mixture they remain, upon the old practice, about three months. The last process being repeated twice or thrice, perfectly tanned leather is the result. The hides are now remored from the pit, and hung up in a shed. In the progress of drying, which should be slow, they are compressed with a steel tool, and beaten smooth, to render them more firm and dense.

Some manufacturers place on the bottom of the pit 5 or 6 inches of spent bark, over it 2 inches of fresh bark, then a skin ; and so, alternately, a layer of new bark and a skin, till the pit is nearly full, reserving a small space at top for a thicker layer of bark, over which weighted boards are laid, to condense the whole down into the tanning infusion.

The operation of tanning sole leather in the above way, lasts a year or a year and a half, according to the quality wanted, and the nature of the hides.

A perfect leather is recognised by its section, which should have a glistening marbled appearance, without any white streaks in the middle.

Crop hides are manufactured by immersion, during three or four days, in pits containing milk of lime; in which they are occasionally moved up and down in order to expose them equally to the action of this menstruum. They are then removed, and cleared from hair and impurities, by using the fleshing knife upon the horse; after which they must be completely freed from the lime by a thorough washing. They are next plunged in pits containing a weak ooze or infusion of oak bark, from which they are successively transferred into other pits with stronger ooze; all the while being daily handled, that is, moved up and down in the infusion. This practice is continued for about a month or six weeks. They are now ready to be subjected to a mixture of ground oak bark and stronger ooze in other pits, to a series of which they are progressively sub. jected during two or three months.

The hides are next put into large vats, called layers, in which they are smoothly stratified with more oak bark, and a stronger infusion of it. After six weeks they are taken out of these vats, and subjected to a new charge of the same materials for two months. This simple process is repeated twice or thrice, at the option of the manufacturer, till the hides are thoroughly tanned. They are then slowly dried, and condensed in the manner abore described. These crop hides form the principal part of the sole leather used for home consumption in England.

The process of tanning skins (as of calves, seals, \&c.) is in some respects peculiar. They are left in the lime pits for about twelve days, when they are stripped of their 
hair, washed in water, then immersed in a lixivium of pigeons' dung, called a grainer, of an allialine nature. Here they remain from eight to ten days, according to the state of the atmosphere, during which time they are frequently handled, and scraped on both sides upon a convex wooden beam. This scraping or working, as it is termed, joined to the action of the grainer, serves to separate the lime, oil, and glutinous matter, and to render the skin pliant, soft, and ready to imbibe the tanning principle. They are with this riew transferred into pits containing a weak solution of bark, in which they undergo nearly the same treatment as described above for crop hides; but they are not commonly stratified in the layers. The time occupied in tanning them is usually limited to three months. They are then dried, and disposed of to the currier, who dresses and blackens them for the upper leathers of boots and shoes, for harness, and other

- purposes. The light and thin sorts of cow and horse hides are often treated like calf skins.

In all the abore processes, as the animal fibres on the surface of the skin absorb most readily the tanning principles, and thereby obstruct, in a certain degree, their passage into the interior fibres, especially of thick hides, it becomes an object of importance to contrive some method of orercoming that obstacle, and promoting the penetration of the tan. The first manufacturer who appears to have employed efficacious mechanical means of faroring the chemical action was Francis G. Spilsbury, who in April, 1823, obtained a patent for the following operation:-After the hides are freed from the hairs, \&c. in the usual way, they are minutely inspected as to their soundness, and if any holes be found, they are carefully sewed up, so as to be water tight. Three frames of wood are provided of equal dimensions, fitted to each other, with the edges of the frames held together by screw bolts. A skin about to be tanned is now laid upon the frame, and stretched over its edges, then the second frame is to be placed upon it, so that the edges of the two frames may pinch the skin all round and hold it securely; another such skin is then stretched over the upper surface of the second frame, in like manner, and a third frame heing set upon this, confines the second skin. The three frames are then pinched tightly together by a series of screw bolts, passing through ears set round their outer edges, which fix the skin in a proper manner for being operated upon by the tanning liquor.

A space has been thus formed between the two skins, into which, when the frames are set upright, the infusion is introduced by means of a pipe from the cistern above, while the air is permitted to escape by a stopcock below. This cock must of course be shut whenerer the bag is filled, but the one above is left open to maintain a communication with the liquor cistern, and to allow the hydrostatic pressure to force the liquor through the cutaneous pores by a slow infiltration, and thus to bring the tannin into contact with all the fibres indiscriminately. The action of this pressure is evinced by a constant perspiration on the outer surfaces of the skins.

When the tanning is completed, the upper stopcock is closed, and the under is opened to run off the liquor. The fiames are now removed, the bolts are unscrewed, and the pinched edges of the skins pared off; after which they are to be dried and finished in the usual manner.

A modification of this ingenious and effectual process was made the subject of a patent, by William Drake, of Bedminster, tanner, in October, 1831. The hides, after the usual preparatory processes, are immersed in a weak tan liquor, and by frequent handling or turning over, receive an incipient tanning before being submitted to the infiltration plan. Two hides, as nearly of the same size and shape as possible, are placed grain to grain, when their corresponding edges are sewed firmly together all round by shoemakers' waxed thread, so as to form a bag sufficiently tight to hold tan liquor. This bag must then be suspended by means of loops sewed to its shoulder end, upon pegs, in such a manner that it may hang within a wooden-barred rack, and be confined laterally into a book form. About an inch of the bag is left unsewed at the upper end, for the purpose of introducing a funnel through which the cold tan liquor is poured into the bag till it be full. After a certain interval which varies with the quality of the hides, the outer surface becomes moist, and drops begin to form at the bottom of the bag. These are reccived in a proper vessel, and when they accumulate sufficiently may be poured hack into the funnel; the bag being thus, as well as by a fresh supply from above, kept constantly distended.

When the hides are observed to feel hard and firm, while every part of them feels equally damp, the air of the tanning apartment having been always well ventilated, is now to be heated by proper means to a temperature gradually increasing firom $70^{\circ}$ to $150^{\circ}$ of Fahrenheit's scale. This heat is to be maintained till the hides become firmer and harder in all parts. When they begin to assume a black appearance in some parts, and wnen the ian liquor undergoes little diminution, the hides may be considered to be tannec, and the hag may bo emptied by cutting a few stitches at its bottom. The outer e'iges being pared off, the hides are to be finished in the usual way. During 
their suspension within the racks, the hides should be shifted a little sideways, to prevent the formation of furrows by the bars, and to facilitate the equable action of the liquor.

By this process the patentee says, that a hide may be tanned as completely in ten days as it could be in ten months by the usual method. I have seen a piece of sole leather thus rapidly tanned, and it seemed to be perfect. How it may wear, compared with that made in the old way, I cannot pretend to determine.

Messrs. Knowlys and Duesbury obtained a patent in August, 1826, for accelerating the impregnation of shins with tannin, by suspending them in a close ressel, from which the air is to be extracted by an air pump, and then the tanning infusion is to be admitted. In this way, it is supposed to penetrate the hide so effectually as to tan it uniformly in a short time.

About 32 years ago, a similar vacuum scheme was employed to impregnate with wearers' paste or starch, the cops of cotton weft, for the dandy looms of Messrs. Radcliff and Ross, of Stockport.

Danish leather is made by tanning lamb and kid skins with willow bark, whence it de. rives an agreeable smell. It is chiefly worked up into gloves.

\section{Of the tawing or dressing of skins for gloves, and white sheep leather.}

The operations of this art are: 1 . washing the skins; 2. properly treating them with lime; 3. taking off the fleece; 4 . treatment in the leather steep.

A shed erected upon the side of a stream, with a cistern of water for washing the skins; wooden horses for cleaning them with the back of the fleshing knife; pincers for remoring the fibres of damaged wool; a plunger for depressing the slins in the pits; a lime pit; a pole with a bag tied to the end of it; a two-handed fleshing hnife; a rolling pin, from 15 to 18 inches long, thickened in the middle; such are some of the utensils of a taming establishment. There must be prorided also a table for applying the oil to the skins; a fulling mill, worked by a water-wheel or other power; a dressing peg; a press for squeezing out the fatty filth; a stove; planks mounted upon legs, for stretching the skins, \&c.

Fresh skins must be worked immediately after being washed, and then dried, otherwise they ferment, and contract either indelible spots, or get tender in certain points, so as to open up and tear under the tools. When receired in the dry state ther should be steeped in water for two days, and then treated as fresh skins. They are next strongly rubed on the convex horse-beam with a round-edged knife, in order to make them pliant. The rough parts are remored by the fleshing knife. One workman can in this way prepare 200 skins in a day.

The flesh side of each being rubbed with a cold cream of lime, the shins are piled together with the woolly side of each pair outermost, and the flesh sides in contact. They are left in this state for a few days, till it is found that the wool may be easils removed by plucking.

They are next washed in running water, to separate the greater part of the line. stripped of the wool by small spring tweezers, and then fleeced smooth br means of the rolling-pin, or sometimes by rubbing with a rhetstone. Unless they be fleeced soon after the treatment with lime, they do not well admit of this operation subsequently, as they are apt to get hard.

They are now steeped in the millk of lime-pit, in order to swell, soften, and cleanse them; afterwards in a weak pit of old lime-rrater, from which they are taken out and drained. This steeping and draining upon inclined tables, are repeated frequently during the space of 3 weeks. Only the slins of young animals, or those of inferior value, are tawed. Sometimes the wool is left on, as for housings, \&c.

The skins, after haring been well softened in the steeps, are rubbed on the outside with a whetstone set in a wooden case with two handles, in order to smooth them completely by remoring any remaining filaments of wool. Lamb skins are rubbed with the pin in the direction of their breadth, to gire them suppleness; but sheep skins are fulled with water a_one. They are now ready for the branning, which is done by mixing $40 \mathrm{lbs}$. of bran with 20 gallons of water, and keeping them in this fermentable mixture for three weeks-with the addition, if possible, of some old bran water. Here they must ie frequently turned over, and carefully watched, as it is a delicate operation. In the course of two days in summer, and eight in winter, the skins are said to be raised, when they sink in the water. On coming out of the bran, they are ready for the whije stuff; which is a bath composed of alum and sea-salt. Twelve, fourteen, and sometimes eighteen pounds of alim for 100 skins, form the basis of the bath; to which two and a half pounds of salt are added in winter, and three in summer. These ingredients are introduced into a copper with twelre gallons of water. The salt aids in the whitening action. When the solution is about to boil, three gallons of it are 
passed through the cullender into a basin ; in this 26 skins are worked one after another, and, after draining, they are put together into the bath, and left in it for ten minutes to imbibe the salts. They are now ready to receive the paste. For 100 skins, from 13 to 15 pounds of wheat flour are used along with the yolks of 50 eggs. After having warmed the alum bath through which the skins have been passed, the flower is dusted into it, with careful stirring. The paste is well kneaded by the gradual addition of the solution, and passed through the cullender, wherehy it becomes as cleir as honey. To this the yollis being added, the whole is incorporated with much manual labor. The skins are worked one after another in this paste; and afterwards the whole together are left immersed in it for a day. They are now stretched and dried upon poles, in a proper apartment, during from 8 to 15 days, according to the season.

The effects of the paste are to whiten the skins, to soften them, and to protect them from the hardening influence of the atmosphere, which would naturally render them brittle. They would not bear working upon the softening iron, but for the emulsion which has been introduced into their substance. With this view they are dipped in a tub of clear water during five or six minutes, and then spread and worked upon the board. They are increased by this means in length, in the proportion of 5 to 3 . No hard points must le left in them. The whiteness is also better brought out by this operation, which is performed upon the flesh side. The softening tool is an iron plate, about one foot broad, rounded over above, mounted upon an upright beam, 30 inches high, which is fixed to the end of a strong horizontal plank, $3 \frac{1}{2}$ feet long, and 1 broad. This plank is hearily loaded, to make it immoveable upon the floor. Sometimes the skins are next spread orer an undressed clean skin upon the horse, and worked well with the two-handled linife, for the purpose of removing the first and second epidermis, called the fleur and arritre-fleur by the French megissiers. They are then dried while stretched by hooks and strings. When dry they are worked on the streiching iron, or they are occasionally polished $\pi$ ith pumice stone. A delicate yellow tint is given by a composition made of two parts of whitening, and one of ochre, applied in a moistened state, and well worked in upon the grain side. After being polished with pumice, they are smoothed with a hot iron, as the laundresses do linen, whereby they acquire a degree of lustre, and are ready to be delivered to the glover.

For housings, the best sheepskins are selected, and such as are covered with the longest and most beautiful fleece. They are steeped in water, in order to be cleansed and softened; after which they are thinned inside by the fleshing knife. They are now steeped in an old bren pit for 3 or 4 days, when they are taken out and washed. They are next subjected to the white or alum bath, the wool being carefully folded within; about 18 pounds of alum being used for 100 skins. The paste is made as for the fleeced skins, but it is merely spread upon their flesh sile, and left upon them for 18 hours, so as to stiffen. They are then hung up to dry. They are next moistened by sprinkling cold water upon them, folded up, piled in a heap, and covered with boards weighted with heavy stones; in which state they remain for two days. They are next opened with a round iron upon the horse, and subjected to the stretching iron, being worked broadwise. Ther are dried with the fieece outermost, in the sun if possible; and are finished upon ife stretcher.

Calf and lamb skins with their hair and wool are worked nearly in the same manner ; only the thicker the skin, the stronger the alum bath ought to be. One pound of alum and one of salt are required for a single calf skin. It is left four days in this bath, after which it is worked upon the stretcher, then fulled; when half dry the skins are opened von the horse. In eight days of ordinary weather, they may be completely dressed. Lamb shins are sometimes steeped during eight days in a bath prepared with unbolted rye flour and cold water, in which they are daily moved about two or three times. They are then dried, stretched upon the iron, and switched upon the fleecy side.

Charnois ( $\mathrm{r}$ Shamoy leather.-The skins are first washed, limed, fleeced, and branned as ahore described. They are next efflowered, that is, deprived of their epidermis by a concsre knife, blunt in its middle part, upon the convex horse-beam. The cutting part se rves to remove all excrescences, and to equalize the thickness, while the blunt part softens and smooths. The skins of goats, does, and chamois, are always treated in this way. They are next subjected to the fermenting bran steep for one or two days, in ordir ary weather; but in hot weather for a much shorter time, sometimes only moving them in the sour bran liquor for a few minutes. They are lastly wrung at the peg, and subjected to the tulling mill.

When the skins have been sufficiently swelled and suppled by the branning, they may receive the first oil as follows: a dozen skins being stretched upon the table, the fingers are dipped in the oil, and shaken over the skins in different places, so as to impart enough of it to imbue the whole surface slightly, by friction with the palms of the hands. It is to the outside or grain that the oil is applied. The skins are folded four together, so as to form balls of the size of a hog's bladder, and thrown into the trough 
of the fulling mill, to the number of twelve dozen at once. Here they remam exposed to the beater for two, three, or four hours, according to their nature and the state of the weather. They are taken out, aired, oilerl, and again fulled. The airing and fulling are repeated several times, with more or less frequent oilings. Any cheap animal oil is employed.

After these operations, the shins require to be subjected to a fermenting process, to dilate their pores, and to facilitate their combination with the oil. This is performed in a cliamber only 6 feet high, and 10 or 12 feet square. Poles are suspended horizontally a few inches from the ceiling, with hooks fixed in them to which the skins are attached. A somewhat elevated temperature is maintained, and by a stove if need be. This operation requires great skill and experience.

The remainder of the epidermis is next removed by a blunt concave knife and the horse; whereby the surface is not cut, but rather forcibly scraped.

The skins are now scoured to carry off the redundant oil; which is effected by a pot-. ash ley, at two degrees Baumé, heated no hotter than the hand can bear. In this they are stirred briskly, steeped for an hour, and lastly wrung at the peg. The soapy liquor thus expelled is used for inferior purposes. The clean skins, after being dried, are finished first on the stretcher-iron, and then on the herse or stretching frame.

Leather of Hungary.-This is manufactured by impregnating strong hides with alnm, common salt, and suet; by a rapid process which is usually completed in the space of two months. The workshop is divided into two parts: 1 . A shed on the side of a stream, furnished with wooden horses, fleshing knires, and other small tools. In one corner is a furnace with a boiler for dissolving the alum, a vat for immersing the hides in the solution, and several subsidiary tubs. 2. A chamber, 6 feet high, by 15 feet square, capable of being made very tight, for preserting the heat. In one corner is a copper boiler, of sufficient size to contain 170 pounds of tallow. In the middle of the store is a square stone slab, upon which an iron grate is placed about a yard square. This is covered with charcoal. At each side of the stove are large tables, which oceupy its whole length, and on which the leather is spread to receive the grease. The upper part below the ceiling is filled with poles for hanging the leather upon to be heated. The door is made to shut perfectly close.

The first operations are analogous to those of tanning and tawing; the skins being washed, cut in halves, shared, and steeped for 24 hours in the river. They are then cleaned with 5 or 6 pounds of alum, and $3 \frac{1}{2}$ pounds of salt, for a piece of hide which weighs from 70 to 80 pounds. The common salt softens the effect of the alum, attraets the moisture of the air, and preserves the suppleness of the skin. When the alum and salt are dissolved, hot water is poured upon the hides placed in a rat, and they are tramped upon by a workman walking repeatedly from one end of the rat to the other. Ther are then transferred into a similar vat containing some hot water, and similarly tramped upon. They are next steeped for eight days in alum water. The same round of operations is repeated a second time.

The skins are now dried either in the air, or a stove room; but before being quite dry, they are doubled together, well stretched to take out the wrinkles, and piled up. Then dry, they are again tramped to open the pores as well as to render the skin pliant, after which they are whitened by exposure to the sun.

Tallow of inferior quality is employed for greasing the leather. With this riew the hides are hung upon the poles in the close stove room, then laid upon the table, and besmeared with the tallow melted till it begins to crackle. This piece is laid on another table, is there corered with a second, similarly greased, and so forth. Three pounds of iat are commonly employed for one piece of leather.

When the thirty strips, or fifteen hides passed through the grease in one operation are completed, two workmen take the first piece in their hands, and stretch it over the inurning charcoal on the grate for a minute, with the flesh side to the fire. The rest are passed orer the flame in like manner. After flaming, the pieces are successirely laid on an inclined table exposed to the fire, where they are corered with a cloth. They are finally hung upon poles in the air to dry; and if the weather be warm, they are suspended only during the night, so as to faror the hardening of the grease. Instead of the alum bath, M. Curaudau has employed with advantage a steep of dilute sulphuric acid.

Morocco leather.- The true morocco leather is goat skin tanned and then dyed on the side of the grain. Sheep skins are treated in the same way. The skins are steeped first in a fermenting mixture of bran water for a few days, they are then worked upon the horse, steeped in fresh water for 12 hours, and rinsed in the same. They are next drained, steeped in weak lime pits for a proper time, till the hairs can be readily detached. They are now subjected to the action of a blunt knife upon the horse-beam, in order to strip off their hair, after which they are cleansed in running water. Any excrescences must be carefully removed with the fleshing knife, and their edges neatly pared. The next 
process is rubing them strcngly with a piece of hard schist, set in a wooden frame, in order to expel by the pressure any lime which may still adhere, and to soften the grain. They are now worked upon the horse-beam with the blunt knife, and subjected to a siecies of fulling, by being agitated by pegs in a revolving cask along with water. Many manufacturers prefer a weak alkaline ley, or putrefied urine, to the lime bath.

The skins are immersed for a night and a day, in a bran bath, in a certain state of fermentation, then worked on the horse, and salted, to preserve them till they are to be dyed.

Preparatory to being dyed, each skin is sewed together edgewise, with the grain on the outside, and it is then mordanted either with a solution of tin, or with alum water. The color is siven by cochineal, of which from 10 to 12 ounces are required for a dozen of shin. The cochineal being boiled in water along with a little tartar or alum for a few minutes, forms a red liquor, which is filtered through a linen cloth, and put into a clean cask. The skins are imriersed in this bath, and agitated in it for about half an hour; they are taken out and beaten, and then subjected to a second immersion in the cochineal bath. After being thus dyed, they are rinsed and tanned with Sicilian sumach, at the rate of two pounds for a skin of moderate size. This process is performed in a large tub made of white wood, in the liquor of which the skins are floated like so many bladders, and moved about by manual labor during four hours. They are then taken out, drained, and again subjected to the tanning liquor; the whole process requiring a space of twenty-four hours. The skins are now unstitched, rinsed, fulled with beetles, drained, rubbed hard with a copper biade, and lastly hung up to dry.

Some manufacturers brighten the color by applying to the surface of the skins, in a damp staie, a solution of carmine in ammonia with a sponge; others apply a decoction of saffion to enliven the scarlet tint. At Paris the morocco leather is tanned by agitation with a decoction of sumach in large casks made to revolve upon a horizontal axis, like a barrel churn. White galls are sometimes substituted for sumach; a pound being used for a skin. The skins must be finally cleaned with the utmost care.

The black dye is given by applying with the brush a solution of red acetate of iron to the grain side. Blue is communicated by the common cold indigo vat; violets, with a light blue followed by cochineal red; green, by Saxon blue followed by a yellow dye, usually made with the ehopped roots of the barberry. This plant serves also for yellows. To dre olive, the skins are first passed through a weak solution of green vitriol, and then through the decoction of barberry root, containing a little Saxon blue. Puce color is communicated by logwood with a little alum; which may be modified by the addition of a little Brazil wood. In all these cases, whenever the skins are dyed, they should be rinsed, wrung or rather drained, stretched upon a table, then besmeared on the grain side with a film of linseed oil applied by means of a sponge, in order to promote their glossiness when curried, and to prevent them becoming horny by too rapid drying.

The last process in preparing morocco leather is the currying, which brings out the lustre, and restores the original suppleness. This operation is practised in different manners, according to the purpose the skins are to serve. For pocket-books, portfolios, and ease making in general, they must be thinned as much as possible upon the flesh side, moistened slightly, then stretched upon the table, to smooth them; dried again, moistened, and lastly passed two or three times through the cylinder press in different directions, to produce the erossing of the grain. The skins intended for the shoemaker, the saddler, the bookbinder, \&c., require more pliancy, and must be differently curried. After being thinned, they are glazed with a polisher while still moist, and a grain is formed upon the flesh side with the roughened lead plate or grainer of the curriers, called in French pornmelle; they are glazed anew to remove the roughness produced by the pommel, and finally grained on the flesh side with a surface of cork applied under a pommel of white wood.

Pussia leather.-The Russians have long been possessed of a method of making a peculiar leather, called by them jucten, dyed red with the aromatic saunders wood. This artiele has been much sought after, on account of not being subject to mould in damp situations, being proof against insects, and even repelling them from the vicinity of its odor. The skins are freed from the hair or fleece, by steeping in an ash-ley too weak to act upon the animal fibres. They are then rinsed, fulled for a longer or shorter time according to their nature, and fermented in a proper steep, after having been washed in hot water. They are taken out at the end of a week, but they may be steeped a second time if deemed necessary, to open their pores. They are now cleaned by working them at the horse on both the flesh and grain sides.

A paste is next composed, for 200 slins, of 38 pounds of rye flour, which is set to 
ferment with leaven. This dough is worked up with a sufficient quantity of water to form a bath for the skins, in which they are soaked for 48 hours; they are then trans. ferred into small tubs, where they remain during fifteen days, after which they are washed at the river. These operations serve to prepare the skins for absorbing the astringent juices with uniformity. A decoction of willow bark (salix cinerea and salix caprea) being made, the skins are immersed in the boiler whenever the temperature of the liquor is sutficiently lowered not to injure the animal fibres, and handled and pressed for half an hour. This manipulation is repeated twice daily during the period of a week. The tanning infusion is then renewed, and applied to the same skins for another week; after which, being exposed to the air to dry, they are ready for being dyed, and then curried with the empyreumatic oil of the bark of the birch tree. To this substance the Russia leather owes its peculiarities. Many modes have been prescribed for preparing it; but the following is the one practised in Russia.

The whitish membranous epidermis of the birch, stripped of all woody parts, is introduced into an iron boiler, whirin, when stuffed full, is covered tight with a vaulted iron lid, having a pipe rising from its centre. A second boiler into which this pipe passes without reaching its hottom, is set over the first, and is luted 10 it at the edges, after the two are bolted together. They are then inverted, so that the upper one contains the birch bark. The under half of this apparatus is sunk in the earth, the surface of the upper boiler is coated over with a clay lute, then surrounded with a fire of wood, and exposed to a red heat, till the distillation be completed. This operation, though rude in appearance, and wasteful of wood, answers its purpose perfectly well. The iron cylinder apparatus used in Britain for distilling wood vinegar, would, however, be much more convenient and productive. When the above boilers are unluted, there is found in the upper one a very light powder of charcoal, and in the under one which served as a receiver, there is an oily, brown, empyreumatic fluid, of a very strong smell, which is mixed with the tar, and which floats over a small quantity of crude vinegar. The former matter is the oil employed to impregnate the skins, by working it into the flesh side with the currier's tools. It is difficult to make this oil penetrate with uniformity; and the Russians do not always succeed in this process, for they turn out many skins in a spotted state. This oil is at present obtained in France by distilling the birch bark in copper stills, and condensing the products by means of a pipe plunged in cold water. About 60 per cent. of the weight of the bark is extracted.

The skins imbibe this oil most equally before they are fully dry. Care must be taken not to apply too much of it, for fear of its passing through and staining the grain side of the leather. Chevreul has investigated the chemical nature of this odoriferous substance, and finding it to be a peculiar compound, has called it betuline.

LEUUM PALUSTRE. This plant is employed in Russia to tan the skins of goats, calves, and sheep, into a reddish leather of an agreeable smeil; as also in the preparation of the oil of birch, for making what is commonly called Russia leather.

LEGUMINE is the name of a vegeto-alkali supposed to exist in leguminous plants.

LEMONS. See Crtric Acrd, and Orls, Essentral.

LEVIGATION is the mechanical process whereby hard substances are reduced to a very fine powder.

LEUCITE is a hard Vesuvian mineral, consisting of silica, 54 ; alumina, 23 ; potash, 23.

LEUCINE is a white crystalline substance produced by acting upon flesh with sulphuric acid.

LEWIS is the name of one kind of shears used in cropping woollen cloth.

LIAS is a fine-grained argillaceous limestone, whose geological position is under the oolite; it is the proper lithographic stone.

LIBAVIUS, LIQUOR oF, is the bichloride of tin, prepared by dissolving that metal, with the aid of heat, in aqua regia, or by passing chlorine gas through a solution of muriate of tin till no more gas be absorbed, evaporating the solution, and setting it aside to crystallize. The anhydrous bichloride is best prepared by mixing four parts of corrosive sublimate with one part of tin, previously amalgamated with just so much mercury as to render it pulverizable; and by distilling this mixture with a gentle heat. A colorless fluid, the dry bichloride of tin, or the proper fuming liquor of Libavius, comes over. When it is mixed with one third of its weight of water it becomes solid. The first bichloride of tin is used in ealico-printing.

LICHEN. See ArchiL.

LIGNEOUS MATTER is vegetable fibre. See Fibrous Matrer.

LIGNITE is one of the most recent geological formations, being the carbonaceous remains of forest trees. From this substance, as found in the neighborhood of Cologne the brown colors, called umber and earth of Cologne, are prepared.

LILACH DYE. See Calico-PRinting and Dyeing. 
LIMESTONE (Calcaire, Fr.; Kalskitein,Germ.), may be classed under the following heads :-

1. Calcareous spar occurs in colorless crystals or crystalline masses; dissolves with effervescence in muriatic acid; is scratched by soft iron, but not by the nitil; specific gravity $2 \cdot 7$; loses 46 per cent. by the expulsion of carbonic acid, and calcines into quicklime.

2. Calcsinter, or stalactitic carbonate of lime, called also concretionary limestone, because formed of zones more or less undulated, and nearly parallel. These zones have a fibr:)us structure, arising from the successive deposites of the crystalline limestone from its solvent water. The long conical pieces called stalactites, show fibres converging to the axis. The tubercular consists of irregular lumps often sprinkled over with small crystals, and associated so as to exhibit the appearance of cauliflower. The stratiform, commonly called stalagmite, or alabaster limestone, represents zones not «ncentric, but spread out, waring; and parallel; its texture is sometimes lamellar, and sometimes fibrous. These waving strata are distinguishable from one another by their different densities, and by their degrees of translucency. This stalagmitic mass bears the name of oriental alabaster, when it is reddish-yellow with distinct zones, and is susceptible of a fine polish. Stalactites are formed in the large excavations of calcareous rocks. The water percolating down through them, and dropping from the roofs of the caverns, is usually sharged with carbonate of lime held in suspension by an excess of carbonic acid. The exposure to air, the motion, and the consequent diminution of pressure, cause the precipitation of the carbonate of lime in the solid state. Each drop of water, on falling through the rault, abandons a small film of limestone, which enlarges by degrees, and forms either a cylinder or solid mass. This alabaster differs from marble in its parallel and waving layers, and its faint degree of transparency.

This alabaster serves for the decoration of public buildings, and is occasionally introduced into certain pieces of furniture. The fine Egyptian alabaster was anciently brought from the mountains of the Thebaid, between the Nile and the Red Sea, near a town called Alabastron, whence probably the name. Very fine red alabaster, of great hardness, was found at one time in the quarries of Montmartre, but the stock was soon exhausted.

The incrusting concretionary limestone differs little from the precedins except in the rapidity of its formation, and in being moulded upon some body whose shape it assumes. These deposites from calcareous springs, form equally on regetable bodies, on stones, metals, within pipes of cast iron, wood, or lead. The incrustations on vegetable and animal substances are vulgarly called petrifactions, as the organic fibres are replaced by stone. One of the most curious springs of this nature is at the baths of Saint Philip, in Tuscany, where the water flows in almost a boiling state, over an enormous mass of alabaster which it has produced. The carbonate of lime seems to be held in solution here by sulphureted hydrogen, which flies off when the water issues to the day. Dr. Vegny has taken advantage of this property of the spring, to obtain basso-relievo figures of great whiteness and solidity. He makes use of sulphur moulds.

Calcareous tuf consists of similar incrustations made by petrifying rivulets running over mud, sand, vegetable remains, \&c. It is porous, even cellular, somewhat soft, impure, and of a dirty gray color. Its surface is wavy, rough, and irregular. These incrustations or deposites are, however, sometimes so abundant, and the resulting stony matters so hard that buildings may be constructed with them. The stone with which the town of Pasti, in Italy, is built has been called pipe-stone by the Italians; and it has apparently derived its origin from incrustations upon large reeds.

The travertino, which served to construct all the monuments of Rome, appears to have been formed by the deposites of the Anio and the solfatara of Tivoli. The temples of Pæstum, which are of extreme antiquity, have been built with a travertino formed by the sediment of the waters which still flow in this territory. All these stones acquire great hardness in the air, and M. de Breislak thinks that it is to the happy union of travertino and pouzzolana in the same spot, that the monuments of Rome owe their great solidity.

Spongy limestone, usually called Agaric mineral, stone marrow, \&c., belongs to this kind of formation. It has a very white color, a very fine grain, is soft to the touch, very tender, and light enough to float for an instant on water. It occurs in rather thin layers, in the crevices of calcareous rocks, and is so common in Switzerland as to be employed for whitening houses.

3. Compact limestone, is of a grain more or less fine, does not polish, nor afford large blocks free from fissures, has a conchoidal, or uneven scaly fracture. Colors rery various. Its varieties are; $a$, The sub-lamellar, compact, with some appearance of a foliated texture. b, Compuct fine-grained limestone, the zechstein of the Germans, to which M. Brongniart refers the lithographic stone in his classification of rocks (Diction. 
naire des Sciences Naturelles), but the English geologists place the locality of the tamous lithographic quarry $0^{\circ}$ Solenhofen much higher in the plane of secondary superjosition. Its fracture is conchoidal; color from gray to whitish; $c$, Compact common limeslone. Grain of .middle-size; earthy aspect; uneven fracture; perfectly opaque; color, whitish to pale gray, yellow, or reddish. The limestones of the Jura formation are referred to this head, as well as most of those interspersed among the coal strata. $d$, The coarse compuct, or Cornbrash ; texture somewhat open, earthy aspect, rough to the touch, ragged fracture, color yellow, gray, or dirty red. e, Compact cellular, the Rauchekalk and Holekalk of the Germans, on account of the numerous holes or caverns distributed through it.

4. Oolite or roe-stone.-It consists of spherical grains of various size, from a millet seed, to a pea, or even an egg; texture compact ; fracture even ; colors, whitish, yellow, gray, reddish, brownish. The larger balls have almost always a foreign body for their centre or nucleus.

5. Chalk; texture earthy; grains fine, tender, friable; colors white, grayish, or pale yellowish.

6. Coarse-grained limestone; an earthy texture, in large particles, often loose; fracture foliated, uneven; color pale and dirty yellow. Coarse lias has been referred to this head.

7. Marly limestone; lake and fresh water limestone formation; texture fine-grained, more or less dense; apt to crumble down in the air; color white or pale yellow; fracture rough-grained, sometimes conchoidal; somewhat tenacious. Texture occasionally carern ous; with cylindrical winding cavities. This true limestone must not be confounded with the lime-marl, composed of calcareous matter and clay.

8. Silicious limestone; of a compact texture; scratching steel, and scratched by it; leaves a silicious residuum after the action of muriatic acid.

9. Calp; texture compact; fine-grained; schistose structure; hard, as the preceding; not burning into quicklime, affording to dilute muriatic acid a copious residuum of clay and silica; color blackish; found in beds in the transition district near Dublin.

10. Lucullite or stinkstone; texture compact or sub-lamellar, color grayish; emits the smell of sulphureted hydrogen by friction or a blow. It occurs at Assynt, in Sutherlandshire; in Derbyshire ; counties of Kilkenny, Cork, and Galway.

11. Bituminous limestone; black or blackish color; diffusing by the action of fire a bituminous odor, and becoming white.

Of all common limestones the purity may most readily be determined by the quantity of carbonic acid which is evolved during their solution in dilute nitric or muriatic acid. Perfect carbonate of lime loses in this way 46 per cent.; and if any particular limestone loses only 23 per cent., we may infer that it contains only one half its weight of calcareous carbonate. This method is equally applicable to marls, which are mixtures in various proportions of carbonate of lime, clay, and sand, and may all be recognised by their effervescing with acids.

The chief use of calcareous stones is for procuring quicklime by calcination in proper furnaces; and they are all adapted to this purpose provided they are not mixed with too large a proportion of sand and ferruginous clay, whereby they acquire a vitrescent texture in a high heat, and will not burn into lime. Limestone used to be calcined in a very rude kiln, formed by enclosing a circular space of 10 or 15 feet diameter, by rude stone walls 4 or 5 feet high, and filling the cylindrical cavity with alternate layers of turf or coal and limestone broken into moderate pieces. A bed of brushwood was usually placed at the bottom, to facilitate the kindling of the kiln. Whenever the combustion was fairly commenced, the top, piled into a conical form, was covered in with sorls, to render the calcination slow and regular. This method being found relatively inconvenient and ineffectual, was succeeded by a permanent kiln built of stones or brickwork, in the shape of a truncated cone with the narrow end undermost, and closed at bottom by an iron grate. Into this kiln, the fuel and limestone were introduced at the top in alternate layers, beginning of course with the former; and the charge was either allowed to burn out, when the lime was altogether removed at a door near the bottom, or the kiln was successively fed with fresh materials, in alternate beds, as the former supply sunk down by the calcination, while the thoroughly burnt lime at the bottom was successively raked out by a side door immediately above the grate. The interior of the lime kiln has been changed of late years from the conical to the elliptical form; and probably the best is that of an egg placed with its narrow end undermost, and truncated both above and below ; the ground plot or bottom of the kiln being compressed so as to give an elliptical section, with an eye or draft-hole towards each end of that ellipse. A kiln thus arched in above gives a reverberatory heat to the upper materials, anu also favors their falling freely down in proportion as the finished lime is raked out below; advan 
tages which the conical form does not afford. The size of the draft-notes for extracting the quicklime, should be proportionate to the size of the kiln, in order to admit a sufficient current of air to ascend with the smoke and flame, which is found to facilitate the extrication of the carbonic acid. The kilns are called perpetual, because the operation is carried on continuously as long as the building lasts; and draw-kilns, from the mode of discharging them by raking out the lime into carts placed against the draft-holes. Three bushels of calcined limestone, or lime-shells, are produced on an arerage for every bushel of coals consumed. Such kilns should be built up against the face of a cliff, so that easy access mar be gained to the mouth for charging, by making a sloping cart road to the top of the bank.

638

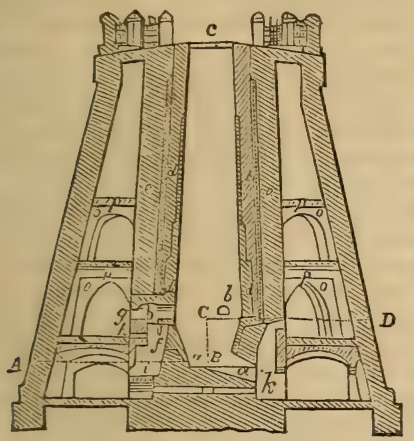

640

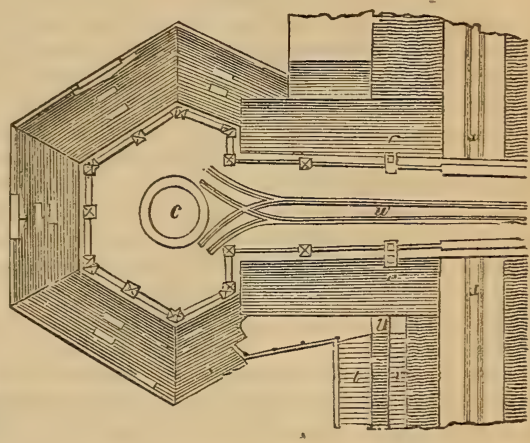

641
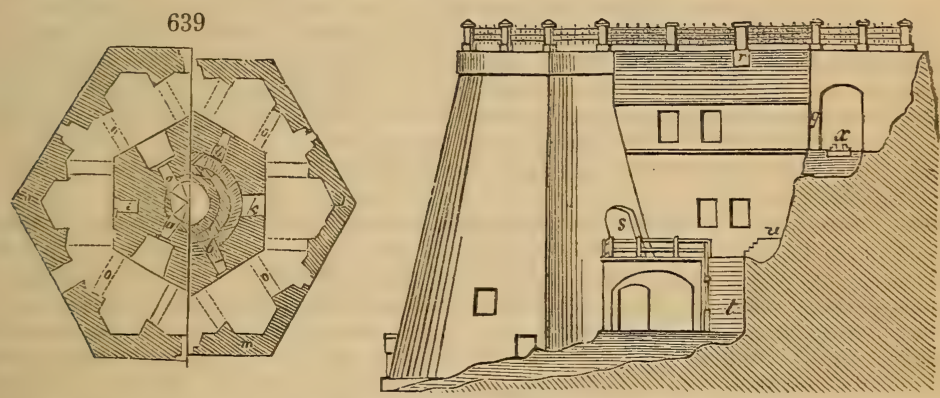

Figs. 638, 639, 640,641, represent the lime-kiln of Rüdersdorf near Berlin, upon the continuous plan, excellently constructed for economizing fuel. It is triple, and rields a threefold product. Fig. 640 , is a view of it as seen from above; fig. 641, the elevation and general appearance of one side; fig. 638, a vertical section, and fig. 639 , the ground plan in the line A B c D of fig. 638. The inner shaft fig. 638, has the form of two truncated cones, with their larger circular ends applied to each other; it has the greatest width at the level of the fire-door $b$, where it is 8 feet in diameter; it is narrower below at the discharge door, and at the top orifice, where it is about 6 feet in diameter. The interior wall $d$, of the upper shaft is built with hewn stones, to the height of 38 feet, and below that for 25 feet, with fire-bricks $d^{\prime} d^{\prime}$, laid stepwise. This inner wall is surrounded with a inantle $e$, of limestones, but between the two there is a small vacant space of a few inches filled with ashes, in order to allow of the expansion of the interior with heat taking place without shattering the mass of the building.

The fire-grate $b$, consists of fire-tiles, which at the middle, where the single pieces press together, lie upon an arched support $f$. The fire-door is also arched, and is secured by fire-tiles. $g$ is the iron door in front of that orifice. The tiles which form the grate have 3 or 4 slits of an inch wide for admitting the air, which enters through the canal $h$. The under part of the shaft from the fire to the hearth, is 7 feet, and the outer enclosing wall is constructed of limestone, the lining being of fire-bricks. Here are the ash-pit $i$, the discharge outlet $a$, and the canal $k$, in front of the outlet. Each ash-pit is shut with an iron dour, which is opened only when the space $i$ becomes filled with ashes. These 
indeed are a lowed to remain till they get cool enough to be removed without incon. venience.

The discharge outlets are also furnished with iron doors, which are opened only for taking out the lime, and are carefully luted with loam during the burning. The outer walls $l m n$ of the kiln, are not essentially necessary, but convenient, because they afford room for the lime to lie in the lower floor, and the fuel in the second. The several stories are formed of groined arches $o$, and platforms $p$, covered over with limestone slabs. In the third and fourth stories the workmen lodge at night. See fig. 641. Some enter their apartments by the upper door $q$; others by the lower doox s. $r$ is one of the chimneys for the several fire-places of the workmen. $t u v$ are stairs.

As the limestone is introduced at top, the mouth of the kiln is surrounded with a strong iron balustrade to prevent the danger of the people tumbling in. The platform is laid with rails $w$, for the wagons of limestone, drawn by horses, to run upon. $x$ is another rail-way, leading to another kiln. Such kilns are named after the number of their fire-doors, single, twofold, threefold, fourfold, \&c.; from three to five being the most usual. The outer form of the kiln also is determined by the number of the furnaces; being a truncated pyramid of equal sides; and in the middle of each altcrnate side there is a fireplace, and a discharge outlet. A cubic foot of limestone requires for burning, one and five twelfths of a cubic foot of wood, and one and a half of turf.

When the kiln is to be set in action, it is filled with rough limestones, to the height c i or to the level of the firing; a wood fire is kindled in $a$, and kept up till the lime is calcined. Upon this mass of quicklime, a fresh quantity of limestones is introduced, not thrown in at the mouth, but let down in buckets, till the kiln be quite full; while orer the top a cone of limestones is piled up, about 4 feet high. A turf-fire is now lindled in the furnaces $b$. Whenever the upper stones are well calcined, the lime under the fire-level is taken out, the superior column falls in, a new cone is piled up, and the process goes on thus without interruption, and without the necessity of once putting a fire into $a$; for in the space $\mathrm{c} \mathrm{s}$, the lime must be always well calcined. The discharge of lime takes place every 12 hours, and it amounts at each time in a threefold kiln, to from 20 to 24 Prussian tonnes of 6 imperial bushels each; or to 130 bushels imperial upon the average. It is found by experience, that fresh-broken limestone which contains a little moisture, calcines more readily than what has been dried by exposure for some time to the air; in consequence of the vapor of water promoting the escape of the carbonic acid gas; a fact well exemplified in distilling essential oils, as oil of turpentine and naptha, which come over with the steam of water, at upwards of 100 degrees F. below their natural term of ebullition. Six bushels of Rudersdorf quicklime weigh from 280 to 306 pounds.

When coals are used for fuel in a well-constructed perpetual, or draw kiln, about 1 measure of them should suffice for 4 or 5 of limestone.

The most extensive employment of quicklime is in agriculture, on which subject instructive details are given in Loudon's Encyclopædias of Agriculture and Gardening.

Quicklime is employed in a multitude of preparations subservient to the arts; for clarifying the juice of the sugar-cane and the beet-root; for purifying coal gas; for rendering the potash and soda of commerce caustic in the soap manufacture, and in the bleaching of linen and cotton; for purifying animal matters before dissolving out their gelatine; for clearing hides of their hair in tanneries; for extracting the pure volatile alkali from muriate or sulphate of ammonia ; for rendering confined portions of air very dry ; for stopping the leakage of stone reservoirs, when mixed with clay and thrown into the water; for making a powerful lute with white of egg or serum of blood; for preparing a depilatory pommade with sulphuret of arsenic, \&c. Lime water is used in medicine, and quicklime is of general use in chemical researches. Next to agriculture the most extensive application of quicklime is to MorTar-CEMENTs, which see.

LINEN. See Flax, and Textile Fabrics.

LINSEED (Graine de lin, Fr.; Leinsame, Germ.) contains, in its dry state, 11·265 of oil; 0.146 of wax; 2.488 of a soft resin; 0.550 of a coloring resinous matter; 0.926 of a yellowish substance analogous to tannin; 6.154 of gum; $15 \cdot 12$ of vegetable mucilage; 1.48 of starch; 2.932 of gluten; 2.782 of albumine; 10.884 of saccharine extractive; 44.382 of envelopes, including some vegetable mucilage. It contains also free acetic acid; some acetate, sulphate, and muriate of potash, phosphate and sulphate of lime; phosphate of magnesia ; and silica. See Orls, Unctuous.

LIQUATION (Eng. and Fr.; Saigerung, Germ.) is the process of sweating out, by a regulated heat, from an alloy, an easily fusible metal from the interstices of a metal difficuli of fusion. Lead and antimony are the metals most commonly subjected to liquation; the former for the purpose of carrying off by a superior affinity the silver present in any complex alloy, a subject discussed under SILver; the latter will be considered here, as referred to from the article ANтimony. 
Figs. 612, 643, 644, represent the celebrated antimonial liquation furnaces of Malbosc,
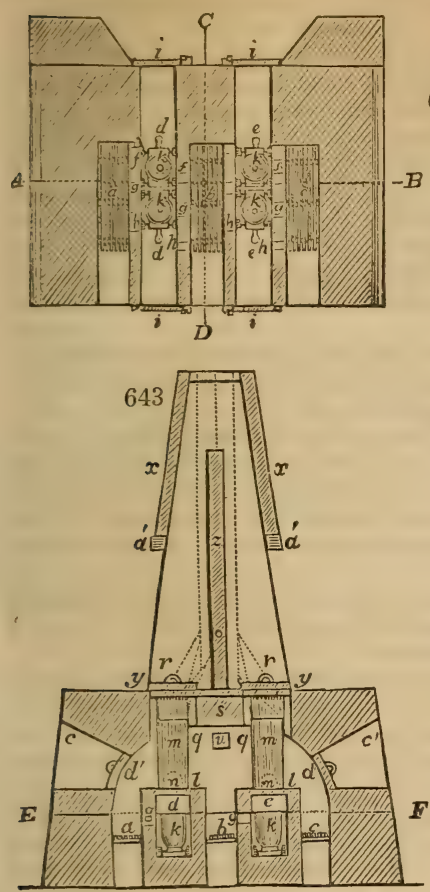
in the department of Ardèche, in France. Fig. 642 is a ground plan taken at the level of the draught holes $g \mathrm{~g}$, fig. 643 , and of the dotted line 642 E F ; fig. 643 is a vertical section through the

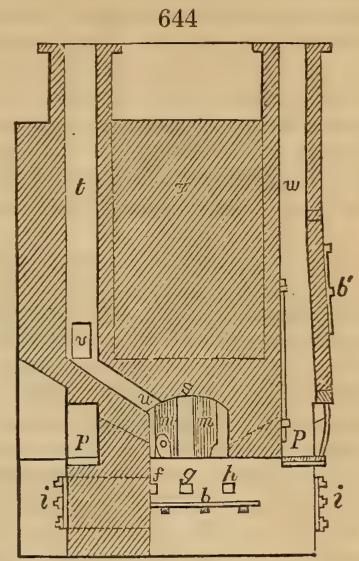

dotted line A B, of fig. 642 ; and fig. 644 is a vertical section through the dotted line $\boldsymbol{c}$ D of fig. 642. In the three figures, the same letters denote like objects. $a b c$ are three grates upon the same level above the floor of the works, $4 \frac{1}{2}$ feet long, by $10 \frac{1}{2}$ inches broad; between which are two rectangular galleries, $d e$, which pass transversely through the whole furnace, and lie at a level of 12 inches above the ground. They are separated by two walls from the three fire-places. The walls have three openings, $f \mathrm{~g} h$, alternately placed for the flames to play through. The ends of these galleries are shut in with iron doors $i$, containing peep holes. In each gallery are two conical cast-iron crucibles $k k$, into which the eliquating sulphuret of antimony drops. Their height is from 12 to 14 inches, the width of the mouth is 10 inches, that of the bottom is 6 , and the thickness four tenths of an inch. They are coated over with fire-clay, to prevent the sulphuret from acting upon them; and they stand upon cast-ron pedestals with projecting ears, to facilitate their removal from the gallery or platform. Both of these galleries are lined with tiles of fire-clay $l l$, which also serve as supports to the rertical liquation tubes $m m$, made of the same clay. The tiles are somewhat curved towards the middle, for the purpose of receiving the lower ends of these tubes, and have a small hole at $n$, through which the liquid sulphuret flows down into the crucible.

The liquation tubes are conical, the internal diameter at top being 10 inches, at bottom 8 ; the length fully 40 inches, and the thickness six tenths of an inch. They have at their lower ends notches or slits 0 , fig. 644, from 3 to 5 inches long, which look outwards, to make them accessible from the front and back part of the furnaces through small conical openings $p p$, in the walls. These are closed during the operation with clay stoppers, and are opened only when the gangue, rubbish, and cinders are to be raked out. The liquation tubes pass across the arch of the furnace $q q$, the space of the arch being wider than the tubes; they are shut in at top with fire-covers $r r . s s$, the middle part of the arch, immediately under the middle grate, is barrel-shaped, so that both arches are abutted together. The flames, after playing round about the sides of the liquation tubes, pass off through three openings and flues into the chimney $t$, about 13 feet high; $u$ being the one opening, and $v$ the two others, which are provided with register plates. In front of the furnace is a smoke flue $w$, to carry off the sulphureous vapors exhaled during the clearing out of the rubbish and slag; another, $x$, begins over $y y$, at the top of the tubes; a wall $z$, separates the smoke flue into halves, so that the workmen upon the one side may not be incommoded by the fumes of the other. This wall connects at the same time the front flue $w$ with the chimney $t . a^{\prime} a^{\prime}$ and $b^{\prime} b^{\prime}$ are iron and wooden bearer beams and rods for strengthening the smoke-flue. $c^{\prime} c^{\prime}$ are arches upon both sides of the furnace, which become narrower from without inwards, 
and are closed with well-fitted plates $d^{\prime} d^{\prime}$. They serve, in particular circumstances, to allow the interior to be inspected, and to see if either of the liquation furnaces be out of order.

Each tube being charged with about $500 \mathrm{lbs}$. of the antimonial ore, previously warmed upon the roof of the furnace, in a short time the sulphuret of a blue color begins to flow out. Whenever the liquation ceases, the cinders are raked out by the side openings, and the tubes are charged afiesh. The luted iron crucibles are suffered to become three fourths full, are then drawn out from the galleries, left to cool, and emptied. The ingots weigh about 85 pounds. The charging is renewed every three hours, and, when the process is in good train, $100 \mathrm{lbs}$. of sulphuret of antimony are obtained every hour. The average duration of the tubes is 3 weeks, though in some cases it may be 40 days. The product from the ore is from 40 to 50 per cent. The above plan of operation is remarkable for the small consumption of fuel, the economy of labor, and the complete exhaustion of the ore.

LIQUEURS, LIQUORISTE; names given by the French to liquors compounded of alcobol, water, sugar, and different aromatic substances; and to the person who compounds them. I shall insert here a few of their most approved recipes.

Infusion of the peels of fruits.-The outcr skin, pared off with a sharp knife, is to be dropped into a hard glazed jar, containing alcohol of $34^{\circ} \mathrm{B}$., diluted with half its bulk of water, and the whole is to be transferred into well-corked carboys. After an infusion of six weeks, with occasional agitation, the aromatized spirit is to be distilled off. In this way are prepared the liquors of cedrat, lemons, oranges, limettes (a sort of sweet lemon), poncires (the large citron), bergamots, \&c.

Infusion of aromatic seeds. - These must be pounded, put into a carboy, along with alcohol diluted as above, infused with agitation for six weeks, and then distilled.

Infusions of aromatic woods are made in the same way.

The liquorist should not bring his infusions and tinctures into the market till six months after their distillation.

Liqueurs have different titles, according to their mode of fabrication.

Thus waters are liquors apparently devoid of viscidity; creams and oils possess it in a high degree.

Water of cedrat, is made by dissolving six pounds of sugar in seven quarts of water; adding two quarts of spirit of cedrat, and one of spirit of citron. Boil the whole for a minute, and filter hot through a proper bag. Set it for a considerable time aside in a corked carboy, before it be bottled.

Oil or cream of cedrat.-Take eight quarts of river water, two of spirit of cedrat, one of spirit of citron, and as much rich sirup as is necessary to give the mixture an oily consistence. Stir it well and set it aside in carboys. Should it be at all clouded, it must be filtered till it be perfectly pellucid.

Balm of Molucca, is made by infusing for ten days, in a carboy capable of holding fully four gallons, 10 pounds of spirits of $18^{\circ} \mathrm{B} ., 4$ pounds of white sugar, 4 pounds of river water, 4 drachms of pounded cloves, and 48 grains of pounded mace. The mixture is to be shaken 3 or 4 times daily, colored with caramel (burnt sugar), filtered at the end of ten days, and set aside in bottles.

Tears of the widow of Malabar, are compounded with the preceding quantity of spirits, sugar, and water, adding 4 drachms of ground cinnamon, 48 grains of clores, and a like quantity of mace, both in powder. It may be slightly colored with caramel.

The delight of the Mandarins.-Take spirit, sugar, and water, as above, adding 4 drachms of anisum China, (Gingi), as much ambrette (seeds of the hibiscus abelmoschus, Lin.), all in powder; 2 drachms of saftlower.

The sighs of love.-Take spirits, water, and sugar, as above. Perfume with essence (otto) of roses; give a very pale pink hue with tincture of cochineal, filter and bottle up.

Crême de macarons. - Add to the spirit, sugar, and water as above, half a pound of bitter almonds, blanched and pounded; cloves, cinnamon, and mace in powder, of each 48 grains. A violet tint is given by the tinctures of turnsole and cochineal.

Curacoa.-Put into a large bottle nearly full of alcohol of trente-six (34 Baumé), the peels of six smooth Portugal oranges, (Seville?) and let them infuse for 15 days; then put into a carboy 10 pounds of spirits of $18^{\circ}$ B., 4 pounds of white sugar, and 4 pounds of river water. When the sugar is dissolved, add a sufficient quantity of the orange zesles to give flavor, then spice the whole with 48 grains of cinnamon, and as much mace, both in powder. Lastly introduce an ounce of ground Brazil wood, and infuse during 10 days, agitating 3 or 4 times daily. A pretty deep hue ought to be giren with caramel.

Swiss extract of wormwood is compounded as follows :-

'Tops of the absinthium majus 4 pounds ;

Ditto, absinthium minus 2 pounds; 
Roots of angelica,

Calamus aromaticus,

$\left.\begin{array}{l}\text { Ceeds of the anisum Chince, } \\ \text { Leaves of the dittany of Crete, }\end{array}\right\}$ of each a few grains at pleasure;

Alcohol of $20^{\circ}$ B., four gallons Imp.

Macerate these substances during eight days, then distil by a gentle fire; draw off two gallons of spirits, and add to it 2 drachms of essential oil of anise-seed. The two gallons left in the still serve for preparing the vulnerary spirituous water.

Of coloring the liqueurs.

Yellow is given with the yellow coloring matter of safflower (carthamus), which is readily extracted by water.

Fo un is given by caramel, made by heating ground white sugar in an iron spoon over a charcoal fire, till it assumes the desired tint, and then pouring it into a little cold water.

Red is given by cochineal alone, or with a little alum.

Violet is given by good litmus (turnsole).

Blue and green.--Sulphate of indigo gives the first. After saturating it nearly with chalk, alcohol being digested upon it, becomes blue. This tincture mixed with that of carthamus forms a good green.

LIQUID AMBER is obtained from the liquidambar styraciflua, a tree which grows in Mexico, Louisiana, and Virginia. Some specimens are thin, like oil, and others are thickish, likie turpentine. It is transparent, amber colored, has an agreeable and powerfu] smell, and an aromatic taste, which feels pungent in the throat. Boiling alcohol dissolves it almost entirely. It contains a good deal of benzoic acid, some of which effloresces whenever the liquid amber hardens with keeping.

LITHARGE (Eng. and Fr.; Glätte, Germ.) is the fused yellow protoxyde of lead, which on cooling passes into a mass consisting of small six-sided plates, of a reddish yellow color, and semi-transparent. It generally contains more or less red lead, whence the rariations of its color; and carbonic acid, especially when it has been exposed to the air for some time. See LEAD and Silver, for its mode of preparation.

IITHIA is a simple earthy or alkaline substance, discovered not many years ago, in the minerals called petalite and triphane. It is white, very caustic, reddens litmus and red cabbage, and saturates acids with great facility. When exposed to the air it attracts humidity and carbonic acid. It is more soluble in water than baryta; and has such a strong affinity for it, as to be obtained only in the state of a hydrate. It forms neutral salts with all the acids. It is most remarkable for its power of acting upon, or corroding platinum.

LITHICM is the metallic basis of Lithia; the latter substance consists of 100 of metal, and 123 of oxygen.

LITHOGRAPHY. Though this subject belongs rather to the arts of taste and design than to productive manufactures, its chemical principles fall within the province of this Dictionary.

The term lithography is derived from $\lambda_{\imath} \theta_{0} s, a$ stone, and yoa $\phi \eta$, writing, and designates the art of throwing off impressions, upon paper, of figures and writing previously traced upon stone. The processes of this ari are founded :-

1. Upon the adhesion to a smoothly polished limestone of an encaustic fat which forms the lines or traces.

2. Upon the power, acquired by the parts penetrated by this encaustic, of attracting to themselres, and becoming covered with a printer's ink, having linseed oil for its basis.

3. Upon the interposition of a film of water, which prevents the adhesion of the ink in all the parts of the surface of the stone not impregnated with the encaustic.

4. Lastly, upon a pressure applied by the stone, such as to transfer to paper the greater part of the ink which covers the greasy tracings of the encaustic.

The lithographic stones of the best quality are still procured from the quarry of Solenhofen, a village at no great distance from Munich, where this mode of printing had its birth. They resemble in their aspect the yellowish white lias of Bath, but their geological place is much higher than the lias. Abundant quarries of these fine-grained limestones occur in the county of Pappenheim, along the banks of the Danube, presenting slabs of every required degree of thickness, parted by regular seams, and ready for removal with very little violence. The good quality of a lithographic stone is generally denoted by the following characters: its hue is of a yellowish gray, and uniform throughout; it is free from veins, fibres, and spots ; a steel point makes an impression on it with difficulty; and the splinters broken off from it by the hammer display a conchoidal fracture.

The Munich stones are retailed on the spot in slabs or layers of equal thickness; they are quarried with the aid of a saw, so as to sacrifice as little as possible of the 
irregular edges of the rectangular tables or plates. One of the broad faces is then dress 3 d] and coursely smoothed. The thickness of these stones is nearly proportional to their other dimensions; and varies from an inch and two thirds to 3 inches.

In each lithographic establishment, the stones receive their finishing, dressing, and polishing; which are performed like the grinding and polishing of mirror plate. The work is done by hand, by rubbing circularly a moveable slab over another cemented in a horizontal position, with fine sifted sand and water interposed between the two. 'The style of work that the stone is intended to produce determines the lind of polis/l that it should get. For crayon drawing the stone should be merely grained more or less jine. according to the fancy of the draughtsman. The higher the finish of the surface, the softer are the drawings; but the printing process becomes sooner pasty, and a suialler number of impressions can be taken. Works in ink require the stone to be more softened down, and finally polished with pumice and a little water. The stones thus prepared are packed for use with white paper interposed between their faces.

Lithographic crayons.-Fine lithographic prints cannot be obtained unless the crayons possess every requisite quality. The ingredients composing them ought to be of such a nature as to adhere strongly to the stone, both after the drawing has undergone the preparation of the acid, and during the press-work. They should be hard enough to admit of a fine point, and trace delicate lines without risk of breaking. The following composition has been successfully employed for crayons by MM. Bernard and Delarue. at Paris :-

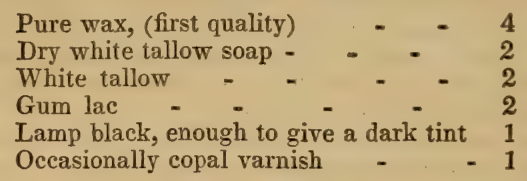

The wax is to be melted over a gentle fire, and the lac broken to bits is then to be added by degrees, stirring all the while with a spatula; the soap is next introduced in fine shavings; and when the mixture of these substances is very intimately accomplished, the copal-varnish, incorporated with the lamp black, is poured in. The heat and agitation are continued tili the paste has acquired a suitable consistence; which mar be recognised by taking out a little of it, letting it cool on a plate, and trying its quality with a penknife. This composition, on being cut, should afford brittle slices. The boiling may be quickened by setting the rising vapors on fire, which increases the temperature, and renders the exhalations less offensive. When ready, it is to be poured into a brass mould, made of two semi-cylinders joined together by clasps or rings, forming between them a cylindric tube of the crayon size. The mould should be preriously smeared with a greasy cloth.

M. Lasteyrie prescribes a more simple composition, said to be equally fit for the lithographer's use :-

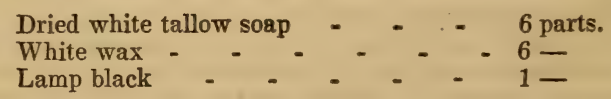

The soap and tallow are to be put into a small goblet and covered up. When the whole is thoroughly fused by heat, and no clots remain, the black is gradually sprinkled in with careful stirring.

Lithographic ink is prepared nearly on the same principles :-

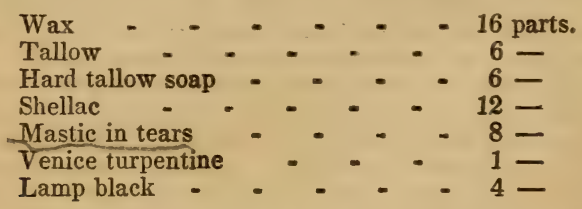

The mastic and lac, previously ground together, are to be heated with care in the turpentine; the wax and tallow are to be added after they are taken off the fire, and when their solution is effected, the soap shavings are to be thrown in. Lastly, the lamp black is to be well intermixed. Whenever the union is accomplished by heat, the operation is finished; the liquor is left to cool a ittle, then poured out on tables, and, when cold, cut into square rods.

Lithographic ink of good quality ought to be susceptible of forming an emulsion so attenuated, that it may appear to be dissolved when rubbed upon a hard body in dis 
tilled or river water. It should be flowing in the pen, not spreading on the stone; capa. ble of forming delicate traces, and rery black to show its delineations. The most essential quality of the ink is to sink well into the stone, so as to re-produce the most delicate outlines of the drawing, and to afford a great many impressions. It must therefore be able to resist the acid with which the stone is moistened in the preparation, without leting any of its greasy matter escape.

M. de Lasteyrie states that after having tried a great many combinations, he gives the oreference to the following :-

Tallow soap, dried -
Mastic, in tears -

The soap is first put into the goblet and melted over the fire, to which the lac being added fuses immediately; the soda is then introduced, and next the mastic, stirring all the while with a spatula. A brisk fire is applied till all these materials be melted completely, when the whole is poured out into the mould.

The inks now prescribed may be employed equally with the pen and the hair pencil, for writings, black-lead drawings, aqua tinta, mixed drawings, those which represent engravings on wood (wood cuts), \&c. When the ink is to be used it is to be rubbed down with water, in the manner of China ink, till the shade be of the requisite depth. The temperature of the place ought to be from $84^{\circ}$ to $90^{\circ} \mathrm{Fahr}$., or the saucer in which the ink-stick is rubbed should be set in a heated plate. No more ink should be dissolved than is to be used at the time, for it rarely keeps in the liquid state for 24 hours; and it should be covered or corked up.

Autographic paper.-Autography, or the operation by which a writing or a drawing is transferred from paper to stone, presents not merely a means of abridging labor, but also that of reverting the writings or drawings into the direction in which they were traced, whilst, if executed directly upon the stone, the impression given by it is inverted. Hence, a writing upon stone must be inverted from right to left to obtain direct impressions. But the art of writing thus is tedious and difficult to acquire, while, by means of the autographic paper and the transfer, proofs are obtained in the same direction with the writing and drawing.

Autographic ink.- It must be fatter and softer than that applied directly to the stone, so that though dry upon the paper, it may still preserve sufficient viscidity to stick to the stone by mere pressure.

To compose this ink, we take-

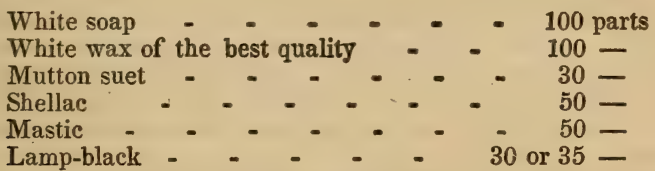

These materials are to be melted as above described for the lithographic ink.

Lithographic ink and paper.-The following recipes have been much commended :-

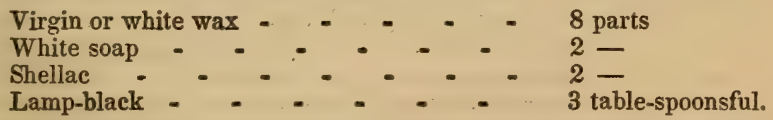

Preparation.-The wax and soap are to be melted together, and before they become so hot as to take fire, the lamp-black is to be well stirred in with a spatula, and then the mixture is to be allowed to burn for 30 seconds; the flame being extinguished, the lac is to be added by degrees, carefully stirring all the time; the vessel is to be put upon the fire once more in order to complete the combination, and till the materials are either kindled or nearly so. After the flame is extinguished, the ink must be suffered to cool a little, and then put into the moulds.

With the ink crayons thus made, lines may be drawn as fine as with the point of the graver, and as full as can be desired, without risk of its spreading in the carriage. Its traces will remain unchanged on paper for years before being transferred.

Some may think it strange that there is no suet in the above composition, but it has been found that ink containing it is only good when used soon after it is made, and when 
immediately transferred to the stone, while traces drawn on paper with the suet ink be come defective after 4 or $\mathbf{5}$ days.

I.ithographic paper.-Lay on the paper, 3 successive coats of sheep-feet jelly,

1 layer of white starch,

1 layer of gamboge.

The first layer is applied with a sponge dipped in the solution of the hot jelly, very equally over the whole surface, but thin; and if the leaf be stretched upon a cord, the gelatine will be more uniform. The next two coats are to be laid on, until each is dry. The layer of starch is then to be applied with a sponge, and it will also be very thin and equal. The coat of gamboge is lastly to be applied in the same way. When the paper is dry, it must be smoothed by passing it through the lithographic press; and the more polished it is, the better does it take on the ink in fine lines.

Transfer.-When the paper is moistened, the transfer of the ink from the gamboge is perfect and infallible. The starch separates from the gelatine, and if, after taking the paper off the stone, we place it on a white slab of stone, and pour hot water over it, it will resume its primitive state.

The coat of gamboge ought to be laid on the same day it is dissolved, as by keeping it becomes of an oily nature; in this state it does not obstruct the transfer, but it gives a gloss to the paper which renders the drawing or tracing more difficult, especially to persons little habituated to lithography.

The starch paste can be employed only when cold, the day after it is made, and after having the skin removed from its surface.

A leaf of such lithographic paper may be made in two minutes.

In transferring a writing, an ink drawing, or a lithographic crayon, even the impression of a copper-plate, to the stone, it is necessary, 1 . that the impressions be made unon a thin and slender body like common paper; 2 . that they may be detached and fixed totally on the stone by means of pressure; but as the ink of a drawing sinks to a certain depth in paper, and adheres pretty strongly, it would be difficult to detach all its parts, were there not previously put between the paper and the traces, a body capable of being separated from the paper, and of losing its adhesion to it by means of the water with which it is damped. In order to produce this effect, the paper gets a certain preparation, which consists in coating it over with a kind of paste ready to receive every delineation without suffering it to penetrate into the paper. There are different modes of communicating this property to paper. Besides the above, the following may be tried. Take an unsized paper, rather strong, and cover it with a varnish composed of :-

\begin{tabular}{|c|c|c|c|c|c|c|}
\hline Starch & - & - & - & - & - & 120 parts \\
\hline Gum arabic & - & - & - & - & - & $40-$ \\
\hline Alum & - & - & - & - & - & $20-$ \\
\hline
\end{tabular}

A paste of moderate consistence must be made with the starch and some water, with the aid of heat, into which the gum and alum are to be thrown, each previously dissolved in separate vessels. When the whole is well mixed, it is to be applied, still hot, on the leaves of paper, with a flat smooth brush. . A tint of yellow color may be given to the varnish, with a decoction of the berries of Avignon, commonly called. French berries by our dyers. The paper is to be dried, and smoothed by passing under the scraper of the Tithographic press.

Steel pens are employed for writing and drawing with ink on the lithographic stones.

LITMUS (Tournesol, Fr.; Lackmus, Germ.) is prepared in Holland from the species of lichen called Lecanora tartarea, Roccella tartarea, by a process which has been kept secret, but which is undoubtedly analogous to that for making archil and cudbear. The ground lichens are first treated with urine containing a little potash, and allowed to ferment, whereby they produce a purple-red; the colored liquor, treated with quicklime and some more urine, is set again to ferment during two or three weeks, then it is mixed with chalk or gypsum into a paste, which is formed into small cubical pieces, and dried in the shade. Litmus has a violet-blue color, is easy to pulverize, is partially soluble in water and dilute alcohol, leaving a residuum consisting of carbonate of lime, of clay, silica, gypsum, and oxyde of iron combined with the dye. The color of litmus is not altered by alkalis, but is reddened by acids; and is therefore used in chemistry as a delicate test of acidity, either in the state of solution or of unsized paper stained with it. It is employed to dye marble blue.

IIXIVIATION (Lessivage, Fr.; Auslagen, Germ.) signifies the abstraction by water of the soluble alkaline or saline matters present in any earthy admixture; as from that of quicklime and potashes to make potash ley, from that of effloresced alum schist to make aluminous liquors, \&c.

LOADSTONE, MAGNETIC IRON-STONE (Fer oxydulé, Fr.; Magnetersen. 
stein, (lerm.) ; an iron ore consisting of the protoxyde and peroxyde of iron in a state of combination

LOAM (Terre-limoneuse, Fr.; Lehm, Germ.); a native clay mixed with quartz sand and iron ochre, and occasionally with some carbonate of lime.

LODE, is the name given by the Cornish miners to a vein, whether it be filled with metallic or earthy matter.

LOGWOOD (Bois de Campèche, Bois bleu, Fr.; Blauholz, Germ.) is the wood of the Hamatoxylon Campechianum, a native tree of central America, grown in Jamaica since 1715. It was first introduced into England in the reign of Elizabeth, but as it afforded to the unskilful dyers of her time a fugitive color, it was not only prohibited from being used, under severe penalties, but was ordered to be burned wherever found, by a law passed in the $23 \mathrm{~d}$ year of her reign. The same prejudice existed, and the same law was enacted against indigo. At length, after a century of absurd prohibition, these two most valuable tinctorial matters, by which all our hats, and the greater part of our woollen cloths, are dyed, were allowed to be used.

Old wood, with black bark and with little of the white alburnum, is preferred. Logwood is denser than water, very hard, of a fine compact grain, and almost indestructible by the atmospheric elements; it has a sweet and astringent taste, and a peculiar not inoffensive smell.

For its chemical composition, see Hematin.

When chipped logwood is for some time exposed to the air, it loses a portion of its dyeing power. Its decoction absorbs the oxygen of the atmosphere, and then acquires the property of precipitating with gelatine, which it had not before. The dry extraet of logwood, made from an old decoction, affords only a fugitive color.

For its applications in dyeing, see Black Dye; Brown Dye; Calico Printing; Dyeing ; Hat Dyeing, \&c.

The imports of logwood for home use were, in 1836, 12,880 tons, 13 cwts.; in 1837, 14,677 tons, 13 cwts. And the amount of duty received was, in 1836, 2,480l.; in 1837, $2,552 l$.

LOOM (Metier à tisser, Fr.; Weberstuhl, Germ.) is the ancient and well-known machine for weaving cloth by the decussation of a series of parallel threads, which run lengthwise, called the warp or chain, with other threads thrown transversely with the shuttle, called the woof or weft. See JACQUARD LOOM and WEAVING.

LUBRICATION. The following simple and efficacious plan of lubricating the joints and bearings of machinery by capillary attraction, has been lindly communicated to me by its ingenious inventor, Edward Woolsey, Esq.:-

Fig. 645 , represents a tin cup, which has a small tin tube A, which passes through the bottom, as shown by the dotted lines. It may have a tin cover to keep out the dust.

Fig. 646, is a plan of the same.

Fig. 647, is a section of the same. Oil is poured into the cup, and one end of a worsted or cotton thread is dipped into the oil, and the other end passed through the tube. The capillary attraction causes the oil to ascend and pass over the orifice of the tube, whence it gradually descends, and drops slower or quicker, according to the length of the thread, or its thickness, until erery particle of oil is drawn over by this capillary syphon. The tube is intended to be put into the bearings of shafts, \&c., and is made of any size that may be wished. If oil, or other liquids, is desired to be dropped upon a grindstone or other surface, this cup can have a handle to it, or be hung from the ceiling.

Fig. 648 . It is frequently required to stop the capillary action when the machinery is not going; and this has been effected by means of a tightening screw, which passes through a screw boss in the corer of the cup, and presses against the internal orifice of the tube, preventing the oil from passing.

Fig. 619. As I find when these screw cups ( $f$ g. 648) are used upon beams of engines and moving bearings, that the screw is apt to be tightened by the motion; and also, as 1 think the action of the screw is uncertain, from the workman neglecting to screw it down sufficiently, it answers best to take out the capillary thread when the lubrication is not required; and to effect this easily, I have a tin top to the cup, with a round pipe soldered to it: this pipe has a slit in it, like a pencil case, and allows a bolt $\mathbf{B}$ to sline easily in it. In fig. 650 , the bolt is down; in fig. 651 , the bolt, which is a piece of brass wire, is drawn up, and there is no capillary action between the thread and the oil. In fig. 651 , it will be observed, that the bolt is kept in its place by its head c, resting in a lateral slit in the pipe, and it cannot be drawn out on account of the pin $\mathbf{E}$. One end of the thread is fastened to the eye-hole at the bottom of the bolt, and the other end is tierl to a small wire which crosses the lower orifice of the tube at $\mathrm{D}$, and which is shown in plan fig. 652 .

By this simple contrivance the capillary action can be stopped or renewed in a second, without removing the top of the lubricator. 
The saving by this plan, instead of pouring oil into the bearings, is 2 gallons out of $\mathbf{3}$, while the bearings are better oiled.

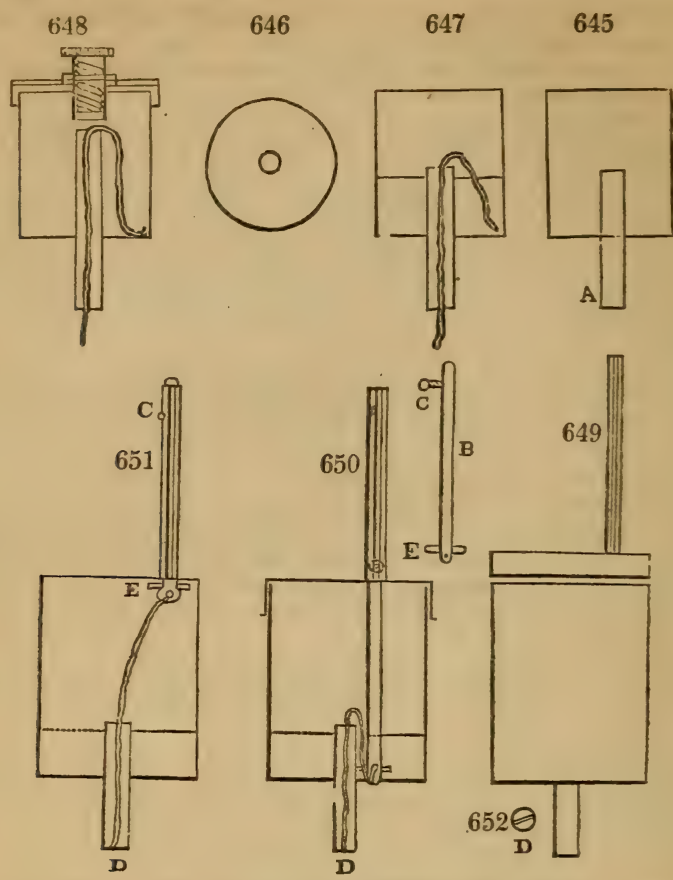

"I send you the drawings of the lubricators, with a detailed explanation. I have omitted to state, that the saving in labor is considerable where there are many joints to keep oiled three or four times a day; and that the workman does not with this apparatus, run the risk of being caught by the machinery. Perhaps your friends may be at a loss how to tie on the cotton or worsted thread. I pass a long thread through the eye-hole $\mathrm{E}$ of the bolt, and then draw the two ends through the tube by a fine wire with a hook to it, one end on one side of the cross wire $\mathrm{D}$, and the other end on the other side. I then put the cover on, and the bolt in the position sho rn in fig. 651 ; when by drawing the two ends of the thread, and tying them across the wire $\mathrm{D}$, Fou have the exact length required. When you wish to see the quantity of oil remaining in the lubricator, the bolt must be dropped as in fig. 650, and you can then lift the cover a little way off, without breaking the thread, and replenish with oil. The cost of fig. 650 , in tin plate, is $9 d$. The figures in the wood cuts are one third of the full size.

"Believe me to be yours sincerely,

"E. J. WoolsEY."

LUPININE, is a substance of a gummy appearance, so named by M. Cussola, because it was obtained from Lupines.

LUPULINE, from Humulus Lupulus; is the peculiar bitter aromatic principle of the hop. See BEER.

LUTE (from lutum, clay ; Lut, Fr. ; Kitte, Beschläge, Germ.) is a pasty or loamy matter employed to close the joints of chemical apparatus, or to coat their surfaces, and protect them from the direct action of flame. Lutes differ according to the nature of the vapors which they are destined to confine, and the degree of heat which they are to be exposed to.

1. Lute of linseed meal, made into a soft plastic dough with water, and immediately applied pretty thick to junctions of glass, or stone-ware, makes them perfectly tight, hardens speedily, resists acid and ammoniacal vapors, as also a moderate degree of heat. It becomes stronger when the meal is kneaded with milk, lime-water, or solution of glue.

2. Lute of thick gum-water, kneaded with clay, and iron filings, serves well for per. manent junctions, as it becomes extremely solid.

3. By softening in water a piece of thick brown paper, kneading it first with rye 
frnur paste, and then with some potter's clay, till it acquire the proper consistence, a lute is formed which does not readily crack or scale off.

4. Lute, consisting of a strong solution of glue lineaded into a dough with riew slaked lime, is a powerful cement, and with the addition of white of egg, forms the lut d'ane ; a composition adapted to mend broken vessels of porcelain and stone-ware.

5. Slim-milk cheese, boiled for some time in water, and then triturated into paste with fresh-slaked lime, forms also a good lute.

6. Calcined gypsum, diffused through milk, solution of glue or 'starch, is a valuable lute, in many cases.

7. A lute made with linseed, melted caoutchouc, and pipe-clay, incorporated into a smooth dough, may be kept long soft when covered in a cellar, and serves admirably to confine acid rapors. As it does not harden, it may therefore be applied and taken off as often as we please.

8. Caoutchouc itself, after being melted in a spoon, may be advantageously used for securing joints against chlorine and acid vapors, in emergencies when nothing else would be effectual. It bears the heat at which sulphuric acid boils.

9. The best lute for joining crucibles inverted into each other, is a dough made with a mixture of fresh fire-clay, and ground fire-bricks, worked with water. That cement if made with solution of borax answers still better, upon some occasions, as it becomes a compact vitreous mass in the fire. See Cements.

LUTEOLINE, is a yellow coloring matter discovered by Chevreul in weld. When sublimed, it crrstallizes in needles.

LYCOPODIUM CLAVATUM. The seeds of the lycopodium ripen in September. Thes are emplosed, on account of their great combustibility, in theatres, to imitate the sudden flash of lightning, by throwing a quantity of them from a powder puff, or bellows, aeross the flame of a candle.

LYDIAN STONE is flint-slate.

\section{M.}

MACARONI is a dough of fine wheat flour, made into a tubular or pipe form, of tilt: thichness of goose-quills, which was first prepared in Italy, and introduced into commerce under the name of Italian or Genoese paste. The wheat for this purpose must be ground into a coarse flour, ealled gruau or semoule, by the French, by means of a pair of light mill-stones, placed at a somewhat greater distance than usual. This semoule is the substance employed for making the dough. For the mode of manufacturing it into pipes, see VERMICELLI.

MACE is a somewhat thick, tough, unctuous membrane, reticulated or chapt, of a 5ellowish-brown or orange color. It forms the enrelop of the shell of the fruit of the myristica moschata, which contains the nutmeg. It is dried in the sun, after being dipped in brine; sometimes it is sprinkled over with a little hrine, before packing, to prevent the risk of moulding. Mace has a more agreeable flaror than nutmeg; with a warm and pungent taste. It contains two kinds of oil; the one of which is unctuous, bland, and of the consistence of butter; the other is volatile, aromatic, and thinner. The membrane is used as a condiment in cookery, and the aromatic oil in medicine.

MACERATION (Eng. and Fr.; Einweichen, Germ.), is a preparatory steep to which certain vegetable and animal substances are submitted, with the view of distending their fibres or pores, and causing them to be penetrated by such menstrua as are best adapted to extract their soluble parts. Water alone, or mixed with acids, alkalis, or salts; alcohol and ether, are the liquids usually employed for that purpose.

MACLE is the name of certain diagonal black spots in minerals, like the ace of diamonds in cards, supposed to proceed from some disturbance of the particles in the act of crystallization.

MADDER (Garance, Fr.; Farberröthe, Germ.), a substance very extensively used in dreing, is the root of the Rubia tinctorum, a plant, of which two species are distinguish. ed by Linnæeus.

The best roots are those which have the size of a writing quill, or, at most, of the little finger. They are semi-transparent, and reddish; have a strong odor, and a smooth bark. They should be of two or three years' growth.

The madder, taken from the ground and picked, must be dried in order to be ground and preserved. In warm climates it is dried in the open air; but, elsewhere, stoves must be employed. 
The stringy filaments and epidermis are to be removed, called mulle; as also the pith, so as to leave nothing but the ligneous fibres.

The preparation of madders is carried on in the department of the Rhone, in the fol. lowing manner.

The roots are dried in a stove heated by means of a furnace, from which the air is allowed to issue only at intervals, at the moment when it is judged to be saturated with moisture. The furnace-flue occupies a great portion of the floor; abore are three close gratings, on which the roots are distributed in layers of about two decimetres (nearly 8 inches). At the end of 24 hours, those which are on the first grated floor directly above the stove are dry, when they are taken away and replaced by those of the superior floors. This operation is repeated whenerer the roots over the stove are dry. The dry roots are thrashed with a flail, passed through fanners similar to those employed for corn, and then shaken upon a very coarse sieve. What passes through is farther winnowed and sifted through a finer sieve than the first. These operations are repeated fire times, proceeding successively to sieves still finer and finer, and setting aside every time what remains on the sieve. What passes through the fifth sieve is rejected as sand and dust. After these operations, the whole fibrous matters remaining on the sieve are cleaned with common fanners, and women separate all the foreign matters which had not been remored before. For dividing the roots, afterwards, into different qualities, a brass sieve is made use of, whose meshes are from six to three millimetres in diameter (from $\frac{1}{4}$ th to $\frac{1}{8}$ th inch E.) What passes through the finest is rejected; and what passes through the coarsest is regarded as of the best quality. These roots, thus separated, are carried into a store, of a construction somewhat different from the first. They are spread out in lasers of about a decimetre in thickness (nearly 4 inches E.), on large lattice-work frames, and the drying is known to be complete, when on taking up a handful and squeezing it, the roots break easily. On quitting the stove, the madder is carried, still hot, into a machine, where it is minced small, and a sieve separates the portion of the bark reduced to powder. This operation is repeated three or four times, and then the bolter is had recoarse to. What passes through the sieve, or the brass meshes of the bolter, is regarded as eommon madder; and what issues at the extremity of the bolter is called the flour. Lastly, the madder which passes through the bolter is ground in a mill with rertical stones, and then passed through sieves of different sizes. What remains above is always better than what goes through.

The madder of Alsace is reduced to a very fine powder, and its coloring matter is extracted by a much longer ebullition than is necessary for the lizari of the Lerant. The prepared madders ought to be carefully preserved from humidity, because they easily imbibe moisture, in which case fermentation spoils their color.

D'Ambourney and Beckman have asserted, that it is more advantageous to employ the fresh root of madder than what has been submitted to desiccation, especially by means of stoves. But in its states of freshness, its volume becomes troublesome in the dyeing bath, and uniform observation seems to prove that it ameliorates by age. Besides, it must be rendered suceptible of keeping and carrying easily.

It appears that madder may be considered as composed of two coloring substances, one of which is dun (tawny), and the other is red. Both of these substances may combine with the stuff. It is of consequence, however, to fix only the red part. The dun portion appears to be more soluble, but its fixity on stuffs may possibly be increased by the affinity which it has for the red portion.

The different additions made to madder, and the multiplied processes to which it is sometimes exposed, have probably this separation for their chief object.

The red portion of madder is soluble, but in small quantity, in water. Hence but a limited concentration can be given to its solution. If the portion of this substance be too much increased, so far from obtaining a greater effect, we merely augment the proportion of the dun part, which is the more soluble of the two.

In consequence of the Société Industrielle of Mulhausen having offered in the year 1826 large premiums to the authors of the best analrtical investigation of madder, eight memoirs were transmitted to it in the year 1827. They were examined with the greatest care by a committee consisting of able scientific and practical men. None of the competitors however fulfilled the conditions of the programme issued by the society; but four of them received a tribute of esteem and gratitude from it; MM. Robiquet and Colin at Paris, Kuhlmann at Iille, and Houton-Libillardière. Fresh premiums were offered for next year, to the amount of 2000 francs.

Every real discovery made concerning this precious root, would be of vast consequence to dyers and calico-printers. Both M. Kuhlmann, and Robiquet and Colin, conceired that they had discovered a new principle in madder, to which they gave the name alizarine. The latter two chemists treated the powdered madder with sulphuric acid, taking care to let it heat as littie as possible. By this action the whole is carbonized, except perhaps the red matter. The charcoal thus obtained is pulverized, mixed with 
water, thrown upon a filter, and well washed in the cold. It is next dried, ground, and diffused thron h fifty parts of water, containing six parts. of alum. This mixture is then boiled for on luarter of an hour, and thrown upon a filter cloth while boiling hot. The resiluum is unce more treated with a little warm alum water. The two liquors are to be mixel, and one part of sulphuric acid poured into them; when they are allowed to cool with occasional agitation. Flocks now make their appearance; the clear liquid is decanted, and the grounds are thrown upon a filter. The precipitate is to be washed, first with acidulated water, then with pure water, and dried, when the coloring matter is obtained in a red or purple state. This purple substance, when heated dry, gives out alizarine, and an empyreumatic oil, having an odor of animal matter ; while a charcoally matter remains.

M. Dan. Kinchlin, the justly celebrated calico-printer of Mulhausen, has no faith in alizarine as the dyeing principle of madder; and thinks moreover that, were it of value, it could not be extracted on the great scale, on account of the destructive heat which would result from the acid acting upon a considerable body of the ground madder. Their alizarine is not a uniform substance, as it ought to be, if a proximate principle ; for samples of it obtained in different repetitions of the process have produced very variable effects in dyeing. The madders of Avignon, though richer in color than those of Alsace, afford however little or no alizarine. In fact, purpurine, the crude substance from which they profess to extract alizarine, is a richer dye than this pure substance itself.

Madder contains so beautiful and so fast a color, that it has become of almost universal employment in dyeing; but that color is accompanied with so many other substances which mask and degrade it, that it can be brought out and fixed only after a series of operations more or less difficult and precarious. This dye is besides so little soluble, that much of it is thrown away in the dye-house; the portion supposed to be exhausted being often as rich as other fresh madder; hence it would be a most raluable improrement in this elegant art to insulate this tinctorial body, and make it a new product of manufacture.

Before the time of Haussmann, an apothecary at Colmar, the madder bath was subject to many risks, which that skilful chemist taught dyers how to guard against, by introducing a certain quantity of chalk into the bath. A change of residence led Haussman to this fortunate result. After having made very fine reds at Rouen, he encountered the greatest obstacles in dyeing the same reds at Logelbach near Colmar, where he went to lire. Numerous trials, undertaken with the view of obtaining the same success in his new establishment, proved that the cause of his favorable results at Rouen existed in the water, which contained carbonate of lime in solution, whilst the water of Logelbach was nearly pure. He then tried a factitious calcarcous water, by adding chalk to his dye bath. Having obtained the most satisfactory results, he was not long of producing here as beautiful and as solid reds as he had done at Rouen. This practice became soon general among the calico-printers of Alsace, though in many dye-works the chalk is now replaced by lime, potash, or soda. But when the madder of Avignon is used, all these antacid correctives become unnecessary, because it contains a sufficient quantity of carbonate of lime; an important fact first analytically demonstrated by that accurate chemist M. Henri Schlumberger of Mulhausen. Avignon madder indicates the presence of carbonate of lime in it, by effervescing with dilute acids, which Alsace madder does not.

M. Kuhlman found a free acid resembling the malic, in his analysis of madders. But his experiments were confined to those of Alsace. The madders of Avignon are on the contrary alkaline, as may be inferred from the violet tint of the froth of their infusions; whereas that of the Alsace madders is yellowish, and it strongly reddens litmus paper. This important difference between the plants of these two districts, depends entirely upon the soil; for madders grown in a calcareous shelly soil in Alsace, have been found to be possessed of the properties of the Avignon madder.

The useful action of the carbonate and the phosphate of lime in the madder of Avignon, explains why madders treated with acids which remove their calcareous salts, without taking away their coloring matter, lose the property of forming fast dyes. Many manufacturers are in the habit of mixing together, and with advantage, different sorts of madder. That of Avignon contains so much calcareous matter that, when mixed with the madder of Alsace, it can compensate for its deficiency. Some of the latter is so deficient as to afford colors nearly as fugitive as those of Brazil wood and quercitron. The Alsace madders by the addition of chalk to their baths, become as fit for dyeing Turkey reds as those of Avignon. When the water is very pure, one part of chalk ought to be used to fire of Alsace madder, but when the waters are calcareous, the chalk should be omitted. Lime, the neutral phosphate of lime, the carbonate of magnesia, oxyde and carbonate of zinc, and several other substances, have the property of causing madder to form a fast : dye, in like manner as the carbonate of lime. 
The temperature of from $50^{\circ}$ to $60^{\circ} \mathrm{R}$. $\left(145^{\circ}\right.$ to $167^{\circ} \mathrm{F}$.), is the best adapted to the solution of the coloring matter, and to its combination with the mordant; ; and thus a boiling heat may be replaced advantageously by the long continuance of a lower temperature. A large excess of the dye-stuff in the bath is unfavorable in two points of view; it causes a waste of coloring matter, and renders the tint dull. It is injurious to allow the bath to cool, and to heat it again.

In a memoir published by the Society of Mulhausen, in September, 1835, some interesting experiments upon the growth of madders in factitious soils are related by MM. Kœchlin, Persoz, and Schlumberger. A patch of ground was prepared containing from 50 to 80 per cent. of chalky matter, and nearly one fifth of its bulk of good horse-dung. Slips of Alsace and Avignon madders were planted in March, 1834, and a part of the roots were reaped in November following. These roots, though of only six months growth, produced tolerably fast dyes, nor was any difference observable between the Alsace and the Avignon species; whilst similar slips or cuttings, planted in a natural non-calcareous soil, alongside of the others, yielded roots which gave fugitive dyes. Others were planted in the soil of Palud, transported from Avignon, which contained more than 90 per cent. of carbonate of lime, and they produced roots that gave still faster dyes than the preceding. Three years are requisite to give the full calcareous impregnation to the indigenous madders of Avignon.

As to the function of the chalk, valuable observations, made long ago by M. Daniel Kœchlin, have convinced him, that the combination of two different bases with a coloring matter, gave much more solidity to the dye, in consequence, undoubtedly, of a greater insolubility in the compound. Experiments recently made by him and his colleagues above named, prove that in all cases of madder dyeing under the influence of chalk, a certain quantity of lime becomes added to the aluminous mordant. In the subsequent clearing with a soap bath, some of the alumine is removed, and. there remains upon the fibre of the cloth a combination of these two earths in atomic proportions. Thus the chalk is not for the purpose of saturating the acid, as had been supposed, but of forming a definite compound with alumina, and probably also with the fatty bodies, and the coloring matter itself.

The red mordants are prepared commonly in Alsace, as follows:-The crushed alum and acetate of lead being weighed, the former is put into a deep tub, and dissolved by adding a proper quantity of hot water, when about one tenth of its weight of soda crystals is introduced to saturate the excess of acid in the alum. The acetate of lead is now mixed in; and as this salt dissolves very quickly, the reaction takes place almost instantly. Care must be taken to stir for an hour. The vessel should not be covered, lest its contents should cool too slowly.

The different mordants most generally employed for madder, are detailed under Colors, in Calico-Printing and Mordant.

Much mordant should not be prepared at once, for sooner or later it will deposite some sub-acetate of alumina. This decomposition takes place even in corked vials in the cold; and the precipitate does not readily dissolve again in acetic acid. All practical men know that certain aluminous mordants are decomposed by heating them, and restored on cooling, as Gay Lussac has pointed out. He observed, that by adding to pure acetate of alumina, some alum or sulphate of potash, the mixture acquires the property of forming a precipitate with a heat approaching the boiling point, and of re-dissolving on cooling. The precipitate is alumina nearly pure, according to M. Gay Lussac; but, by M. Kœchlin's more recent researches, it is shown to be sub-sulphate of alumina, containing eight times as much base as the neutral sulphate.

Madder dye.-On account of the feeble solubility of its coloring matter in water, we cannot dye with its decoction; but we must boil the dye-stuff along with the goods to be dyed; whereby the water dissolves fresh portions of the dye, and imparts it in succession to the textile fibres. In dyeing with madder, we must endeavor to fix as little of the dun matter as possible upon the cloth.

Dyeing on wool.-Alumed wool takes, in the madder bath, a red color, which is not so bright as cochineal red, but it is faster; and as it is far cheaper, it is much used in England to dye soldiers' cloth. A mordant of alum and tartar is employed; the bath of madder, at the rate of from 8 to 16 ounces for the pound of cloth, is heated to such a degree that we can just hold our hand in it, and the goods are then dyed by the wince, without heating the bath more till the coloring matter be fixed. Vitalis prescribes as a mordant, one fourth of alum, and one sixteenth of tartar; and for dyeing, one third of madder, with the addition of a 24th of solution of tin diluted with its weight of water. He raises the temperature in the space of an hour to $200^{\circ}$, and afterwards he boils for 3 or $4 \mathrm{~min}$ utes; a circumstance which is believed to contribute to the fixation of the color. The bath, after dyeing, appears much loaded with yellow matter, because this ha: less aftinity for the alum mordant than the red. Sometimes a little archil is added to the madder, to give the dye a pink tinge; but this is fugitive. 
Silk is seldom dyed with madder, because cochineal affords brighter tints.

Dyeing on cotton and linen.-The most brilliant and fastest madder red is the Turkey or Adrianople. The common madder reds are given in the following way :-The yarn or cloth is boiled in a weak alkaline bath, washed, dried, and galled, by steeping the cotton in a decoction of bruised galls or of sumach. After drying, it is twice alumed; for which purpose, for every 4 parts of the goods, one part of alum is taken, mixed with 1-16th of its weight of chalk. The goods are dipped into a warm solution of the alum, wrung out, dried, and alumed afresh, with half the quantity. The acetate of alumina mordant, described above, answers much better than common alum for cotton. After the goods are dried and rinsed, they are passed through the dye-bath, which is formed of $\frac{3}{4} \mathrm{lb}$. of good madder for every pound of cotton; and it is raised to the boiling point by degrees, in the space of 50 or 60 minutes. Whenever the ebullition has continued a few minutes, the goods must be removed, washed slightly, and dyed a second time in the same way, with as much madder. They are then washed and passed through a warm soap bath, which removes the dun coloring matter.

Holterhoff prescribes for ordinary madder red the following proportions :-20 pounds of cotton yarn; 14 pounds of Dutch madder; 3 pounds of nut-galls; 5 pounds of alum; to which $\frac{1}{2} \mathrm{lb}$. of acetate of lead has been first added, and then a quarter of a pound of chalk.

In the calico-print works the madder goods are passed through a bran bath first, immediately after dyeing; next, after several days exposure to the air, when the dun dye has become oxydized, and is more easily removed. An addition of chalk, on the principles explained above, is sometimes useful in the madder bath. If bran be added to the madder bath, the color becomes much lighter, and of an agreeable shade. Sometimes bran-water is added to the madder bath, instead of bran.

Adrianople or Turkey red.-This is the most complicated and tedious operation in the art of dyeing; but it produces the fastest color which is known. This dye was discovered in India, and remained long a process peculiar to that country. It was afterwards practised in other parts of Asia and in Greece. In 1747, Ferquet and Goudard brought Greek dyers into France, and mounted near Rouen, and in Languedoc, Turkey-red dye works. In 1765, the French government, convinced of the importance of this business, caused the processes to be published. In 1808, Reber, at Mariakirch, furnished the finest yarn of this dye, and M. Kochlin became celebrated for his Turkey-red cloth.

Process for Turkey-red.-The first step consists in clearing the yarn or cloth in alkaline haths, and dipping them in oily liquors, to which sheep's dung was formerly added. This operation is repeated several times, the goods being dried after each immersion. There next follows the cleansing with alkaline liquors to remove the excess of oil, the galling, the aluming, the maddering, the brightening or removing the dun part of the dye by boiling, at a high temperature, with alkaline liquid, and the rosing by boiling in a bath of salt of tin. We shall give some details concerning this tedious manipulation, and the differences which exist in it in the principal dye-works.

At Rouen, where the process was first brought to perfection, two methods are pursued, called the gray and the yellow course or march. In the gray, the dye is given immediately after the cotton has received the oily mordant, the gall, and the alum, as it has then a gray color. In the yellow course, it is passed through fresh oils, alum, and galls before the maddering, the cotton having then a yellow tint.

Different views have been taken of the principles of the Turkey-red dye, and the object and utility of the various steps. The most ancient notion is that of animalizing the cotton by dung and blood, but experience has proved that without any animal matter the finest color may be obtained. According to Dingler, the cotton is imbued with oil by steeping it in combinations of oil and soda ; the oil is altered by repeated dryings at a high temperature; it attracts oxygen from the air, and thereby combines intimately with the cotton fibre, so as to increase the weight of the stuff. The dung, by a kind of fermentation, accelerates the oxydizement, and hence crude oil is preferable to pure. In England, the mucilaginous oils of Gallipoli are preferred, and in Malabar, oils more or less rancid. The drying oils do not answer. The subsequent treatment with the alkaline liquors removes the excess of oil, which has not been oxydized and combined; a hard drying completely changes that which remains in the fibres; the aluming which follows combines alumina with the cotton; the galling tans the fibres, producing a triple compound of oil and alum, which fixes the coloring matter. The object of the other steps is obvious.

According to Wuttich, the treatment with oil opens the cotton so as to admit the mordant and the coloring matter, but the oil and soap do not combine with the fibres. In the alkaline baths which follow, the oil is transformed into soap and removed: whence the cotton should not increase in weight in the galling and aluming; the coston 
suffers a kind of tanning, and the saline parts of the blood assist in fixing the madder dye.

The German process improved, according to Dingler, consists of the following operations : mordant of an oily soap or a soapy liniment, hard drying; alkaline bath, drying, steeping, rinsing away of the uncombined mordant, drying; galling, drying; aluming, drying, steeping in water containing chalk, rinsing; maddlering, airing, rinsing; brightening with an alkaline boil, and afterwards in a bath containing salt of tin; then washing and drying.

The varn or the cloth must be first well worked in a bath of sheep's dung and oil, compounded as follows:-25 pounds of sheep's dung are to be bruised in a solution of pure caustic potash of hydrometer strength $3^{\circ}$, and the mixed liquor is to be passed through a sieve. Two pounds of fine oil are now to be poured into 16 pounds of his ley, after which 30 pounds of coarse oil are to be added, with agitation for $\frac{1}{4}$ of an hour. Other 4 pounds of hot ley are to be well stirred in, till the whole is homogeneous. This proportion of mordant is sufficient for 100 pounds of cotton yarn, for 90 pounds of unbleached or 100 pounds of bleached cotton goods. The cotton stuff, after being well wrung out, is to be laid in a chest and covered with a lid loaded with weights, in which state it should remain for five days. At the end of 24 hours, the cotton becomes hot with fermentation, gets imbued with the mordant, and the oil becomes rapidly altered. The goods are next exposed freely to the air during the day, and in the evening they are dried in a hot chamber, exposed to a temperature of $158^{\circ} \mathrm{F}$., for 6 or 8 hours, which promotes the oxydizement of the oil.

The goods are now passed the second time through a soapy-oil mordant similar to the first, then dried in the air by day, and in the hot stove by night. The third and fourth oil-soap steeps are given in the same way, but without the dung. The fifth steep is composed of a ley at $2^{\circ}$, after which the goods must also be dried. Indeed, from the first to the fourth steep, the cotton stuff should be put each time into a chamber heated to $145^{\circ}$ F. for 12 or 15 hours, and during 18 hours after the fifth steep.

The uncombined oil must, in the next place, be withdrawn by the degraissage, which consists in steeping the goods for 6 hours in a very weak alkaline ley. After rinsing and wringing, they are dried in the air, and then put into the hot stove.

The goods are now galled in a bath formed of 36 pounds of Sicilian sumach, boiled for 3 hours in 260 pounds of water, and filtered. The residuum is treated with 190 fresh pounds of water. This decoction is heated with 12 pounds of pounded nut-galls to the boiling point, allowed to cool during the night, and used next morning as hot as the hand can bear; the goods being well worked through it. They are again dried in the air, and afterwards placed in a stove moderately heated. They are next passed through a tepid alum bath, containing a little chalk ; left afterwards in a heap during the night, dried in the air, and next in the stove. The dry goods are finally passed through hot water containing a little chalk, wrung out, rinsed, and then maddered.

For dyeing, the copper is filled with water, the fire is kindled, and an ounce and a half of chalk is added for every pound of madder; a pound and a quarter of madder being taken for every pound of cotton yarn. The goods are now passed through the bath, so that they penetrate to near its bottom. The fire must be so regulated, that the copper will begin to boil in the course of from $2 \frac{1}{2}$ to 3 hours; and the ebullition must be continued for an hour; after which the yarn is aired and rinsed. Cloth should be put into the dye-bath when its temperature is $77^{\circ}$ and winced at a heat of from $100^{\circ}$ to $122^{\circ}$ during the first hour; at $167^{\circ}$ during the second; and at the boiling point when the third hour begins. It is to be kept boiling for half an hour; so that the maddering lasts four hours. Dingler does not add sumach or galls to the madder bath, because their effect is destroyed in the subsequent brightening, and he has no faith in the utility of blood.

After being dyed, the goods are washed, pressed, and subjected to a soapy alkaline bath at a high heat, in a close boiler, by which the dun parts of the galls and the madder are dissolved away, and the red color remains in all its lustre. This operation is called brightening. It is repeated in a similar liquor, to which some muriate of tin is added for the purpose of enlivening the color and giving it a rosy tint. Last of all, the goods are rinsed, and dried in the shade.

The Elberfeld process consists for $100 \mathrm{lbs}$. of the following steps :-

1. Cleaning the cotton by boiling it for four hours in a weak alkaline bath, cooling and rinsing.

2. Working it thoroughly four times over in a steep, consisting of 300 pounds of water 15 pounds of potash, 1 pailful of sheep's dung, and $12 \frac{1}{2}$ pounds of olive oil, in which it should remain during the night. Next day it is drained for an hour, wrung out and dried. This treatment with the dung steep, and drying, is repeated 3 times.

3 It is now worked in a bath containing 120 quarts of water, 18 pounds of potash 
and 6 quarts of olive oil; then wrung out and dried. This steep is also repeated 4 times.

4. Steeping for a night in the river is the next process; a slight rinsing without wring. ing, and drying in the air.

5. Bath made of a warm decoction $\left(100^{\circ} \mathrm{F}\right.$.) of sumach and nut-galls, in which the goods remain during the night; they are then strongly wrung, and dried in the air.

6. Aluming with addition of potash and chalk; wringing; working it well through this bath, where it is left during the night.

7. Draining, and strong rinsing the following day; piling up in a water cistern.

8. Rinsing repeated next day, and steeping in water to remove any excess of alum from the fibres; the goods continue in the water till they are taken to the dyeing-bath.

9. The maddering is made with the addition of blood, sumach, and nut-galls; the bath is brought to the boil in 1 hour and $\frac{3}{4}$, and kept boiling for half an hour.

10. The yarn is rinsed, dried, boiled from 24 to 36 hours in a covered copper, with an oily alkaline liquid; then rinsed twice, laid for two days in clear water, and dried.

11. Finally, the greatest brightness is obtained by boiling for three or four hours in a soap bath, containing muriate of tin; after which the yarn is rinsed twice over, steeped in water, and dried.

Process of Hanssmann.-He treats cotton twice or 4 times in a solution of aluminated potash, mixed with one thirty-eighth part of linseed oil. The solution is made by adding caustic potash to alum. He dries and rinses each time, and dries after the last operation. He then rinses and proceeds to the madder bath. For the rose color, he takes one pound of madder for one pound of cotton; for carmine red, he takes from 2 to 3 pounds; and for the deepest red, no less than 4 pounds. It is said that the color thus obtained surpasses Turkey red.

The French process, by Vitalis of Rouen.-First operation. Scouring with a soda ley, of $1^{\circ}$ Baumé, to which there is usually added the remainder of the white preparation bath, which consists of oil and soda with water. It is then washed, wrung out, and dried.

In the second operation, he states that from 25 to 30 pounds of sheep's dung are commonly used for 100 pounds of cotton yarn. The dung is first steeped for some days in a ley of soda, of $8^{\circ}$ to $10^{\circ} \mathrm{B}$. This is afterwards diluted with about 500 pints of a weaker ley, and at the same time bruised with the hand in a copper basin whose bottom is pierced with small holes. The liquor is then poured into a vat containing 5 or 6 pounds of fat oil (Gallipoli), and the whole are well mixed. The cotton is washed in this, and the hanks of yarn are then stretched on perches in the open air, and turned from time to time, so as to make it dry equably. After receiving thus a certain degree of desiccation, it is carried into the drying house, which is heated to $50^{\circ}$ Reaumur $\left(144^{\circ}\right.$ Fahrenheit), where it loses the remainder of its moisture, which would have prevented it from combining with the other mordants which it is afterwards to receive. What is left of the bath is called avances, and is added to the following bath. Two, or even three dung baths are given to the cotton, when it is wished to have very rich colors. When the cotton has received the dung baths, care must be taken not to leave it lying in heaps for any length of time, lest it should take fire; an accident which has occasionally happened.

The white bath is prepared by pouring 6 pounds of fat oil into 50 pints of soda water, at $1^{\circ}$ or sometimes less, according as, by a preliminary trial, the oil requires. This bath ought to be repeated two, three, or even a greater number of times, as more or less body is to be given to the color.

To what remains of the white bath, and which is also styled avances, about 100 pints of soda ley of two or three degrees are added. Through this the cotton is passed as usual. Formerly it was the practice to give two, or three, or even four oils. Now, tw, are found to be sufficient.

The cotton is steeped for five or six hours in a tepid solution of soda, of $1^{\circ}$ at most ; it is set to drain, is then sprinkled with water, and at the end of an hour is washed, hank by hank, to purge it entirely from the oil. What remains of the water of degraissage, serves for the scouring or first operation.

For 100 pounds of cotton, from 20 to 25 pounds of galls in sorts must be taken, which are bruised and boiled in about 100 pints of water, till they crumble easily between the fingers. The galling may be done at two operations, dividing the above quantity of galls between them, which is thought to give a richer and more uniform color.

The aluming of 100 pounds of cotton requires from twenty-five to thirty pounds of pure alum, that is, alum entirely free from ierruginous salts. The alum should be dissolved without boiling, in about 100 pints of river or rain water. When the alum is dissolved, there is to be poured in a solution of soda, made with the sixteenth part of the weight of the alum. A second portion of the alkaline solution must not be poured in till the effervescence caused by the first portion has entirely ceased-and 
60 in succession. The bath of saturated alum being merely tepid, the cotton is passed through it, as in the gall bath, so as to impregnate it well, and it is dried with the precautions recommended above. The dyers who gall at two times, alum also twice, for like reasons.

For 25 pounds of cotton, 25 pints of blood are prescribed, and 400 pints of water. Whenever the bath begins to warm, 50 pounds of madder are diffused through the bath; though sometimes the maddering is given at two operations, by dividing the madder into two portions.

The brightening bath is prepared always for 100 pounds of cotton, with from four to five pounds of rich oil, six pounds of Marseilles white soap, and 600 litres of soda water of $2^{\circ} \mathrm{B}$.

The rosing is given with solution of tin, mixed with soap water.

The Turkey-red dye of Messrs. Monteith and Co., of Glasgow, is celebrated all over the world, and merits a brief description here.

The calico is taken as it comes from the loom without bleaching, for the natural color of the cotton wool harmonizes well with the dye about to be given; it is subjected to fermentative steep for 24 hours, like that preliminary to bleaching, after which it is wash . ed at the dash wheel. It is then boiled in a ley, containing about 1 pound of soda crys. tals for 12 pounds of cloth. The oiling process now begins. A bath is made with 10 gallons of Gallipoli oil, 15 gallon measures of sheep's dung not indurated ; 40 gallons of solution of soda crystals, of 1.06 specific gravity; 10 gallons of solution of pearl-ash of spec. grav. $1 \cdot 04$; and 140 gallons of water; constituting a milk-white, soapy solution of about spec. grav. $1 \cdot 022$. This liquor is put into a large cylindrical vat, and constantly agitated by the rotation of wooden vanes, which are best constructed on the plan of the mashing apparatus of a brewery, but far slighter. This saponaceous compound is let off as wanted by a stopcock into the trough of a padding machine, in order to imbue every fibre of the cloth in its passage. This impregnation is still more fully ensured by laying the padded cloth aside in wooden troughs during 16 or 18 days. The sheep's dung has been of late years disused by many Turkey-red dyers, both in England and France, but it is found to be advantageous in producing the very superior color of the Glasgow establishment. It is supposed, also, to promote the subsequent bleaching during the exposure on the green; which is the next process in favorable weather, but in bad weather the goods are dried over a hot-flue.

The cloth is padded again with the saponaceous liquor; and again spread on the grass, or dried hard in the stove. This alternation is repeated a third time, and occasionally, even a fourth.

The cloth by this time is varnished as it were with oil, and must be cleansed in a certain degree by being passed through a weak solution of pearl-ash, at the temperature of about $122^{\circ} \mathrm{F}$. It is then squeezed by the rollers and dried.

A second system of oiling now commences, with the following liquor :-10 gallons of Gallipoli oil; 30 gallons of soda crystals ley, of spec. grav. 1.06; and 10 gallons of caustic potash ley, of specific gravity $1 \cdot 04$, thoroughly diffused through 170 gallons of water. With this saponaceous liquor the cloth is padded as before, and then passed be. tween squeezing-rollers, which return the superfiuous iiquor into the padding-trough. The eloth may be now laid on the grass if convenient; but at any rate it must be hard dried in the stove.

These saponifying, grassing, and drying processes, are repeated three times; whereby the cloth becomes once more very oleaginous, and must be cleansed again by sieeping in a compound ley of soda crystals and pearl-ash of the spec. grav. 1.012, at the temperature of $122^{\circ}$. The eloth is taken out, squeezed between rollers to save the liquor, and washed. A considerable portion of the mingled alkalis disappear in this operation, as if they entered into combination with the oil in the interior of the cotton filaments. The cloth is now hard dried.

Galling is the next great step in the Turkey-red preparation; and for its success all the oil should have been perfectly saponified.

From 18 to 20 pounds of Aleppo galls (for each $100 \mathrm{lbs}$. of cloth) are to be bruised and boiler for 3 or 4 hours, in 25 gallons of water, till 5 gallons be evaporated; and the decoction is to be then passed through a searce. Two pounds of sumach may be substituted for every pound of galls. The goods must be well padded with this decoction, kept at $90^{\circ} \mathrm{F}$., passed through squeezing-rollers, and dried. They are then passed through a solution of alum of the spec. grav. 1.04 , to which a certain portion of chalk is added to saturate the acid excess of that supersalt; and in this cretaceous mixture, heated to $110^{\circ}$, the cloth is winced and steeped for 12 hours. It is then passed between squeezing-rollers, and dried in the stove.

The maddering comes next.

From two to three pounds of madder, ground to powder in a proper mill, are taken for every pound of cloth. The cloth, as usual in maddering, is entered into the cold 
bath, and winced by the automatic reel during one hour that the bath takes to boil, and during an ebullition of two hours afterwards. One gallon of bullock's blood is added to the cold bath for every 25 pounds of cloth; being the quantity operated upon in one bath. 'The utility of the blood in improving the color has been ascribed to its coloring particles; but it is more probably owing to its albuminous matter combining with the margarates of soda and potash condensed in the fibres.

As madder contains a dingy brown coloring matter associated with the fine red, the goods must be subjected to a clearing process to remove the former tinge, which is more fugitive than the latter. Every hundred pounds of cloth are therefore boiled during 12 hours at least, with water containing 5 pounds of soda crystals, 8 pounds of soap, and 16 gallons of the residual pearl-ash and soda ley of the last cleansing operation. By this powerful means the dun matter is well nigh removed; but it is completely so by a second boil, at a heat of $250^{\circ} \mathrm{F}$., in a tight globular copper, along with 5 pounds of soap, and 1 pound of muriate of tin crystals, dissolved in a sufficient body of water for 100 pounds of cloth. The muriate of tin serves to raise the madder red to a scarlet hue. A margarate of tin is probably fixed upon the cloth in this operation.

When the weather permits, the goods should be now laid out for a few days on the grass. Some manufacturers give them a final brightening with a weak bath of a chloride of lime; but it is apt to impoverish the color.

According to the latest improvements of the French dyers, each of the four processes of oiling, mordanting, dyeing, and brightening differs, in some respects, from the above.

1. Their first step is boiling the cloth for four hours, in water containing one pound of soap for every four pieces. Their saponaceous bath of a creamy aspect is used at a temperature of $75^{\circ} \mathrm{F}$.; and it is applied by the padding machine 6 times, with the grassing and drying alternations. In winter, when the goods cannot be exposed on the grass, no less than 12 alternations of the saponaceous or white bath are employed, and 8 in spring. They consider the action of the sun-beams to aid greatly in brightening this dye; but at midsummer, if it be continued more than 4 hours, the scarlet color produced begins to be impaired.

They conceive that the oiling operation impregnates the fibres with super-margarate of potash or soda, insoluble salts which attract and condense the alumina, and the red coloring particles of the madder, so firmly that they can resist the clearing boil.

2. Their second step, the mordanting, consists first in padding the pieces through a decoction of galls mixed with a solution of an equal weight of alum; and after drying in the hot-flue, \&c., again padding them in a solution of an acetate of alumina, made by decomposing a solution of $16 \mathrm{lbs}$. of alum with $16 \mathrm{lbs}$. of acetate of lead, for 6 pieces of cloth, each 32 aunes long.

3. The maddering is given at two successive operations; with 4 pounds of Avignon madder per piece at each time.

4. The brightening is performed by a 12 hours' boil in water with soda crystals, soap, and salt of tin; and the rosing by a 10 hours' boil with soap and salt of tin. Occasionally, the goods are passed through a weak solution of chloride of potash. When the red has too much of a crimson cast, the pieces are exposed for two days on the grass, which gives them a bright scarlet tint.

Process of M. Werdet to dye broadeloth and wool by madder :-

" Preparation for 24 pounds of scoured wool:

"Take $4 \frac{1}{4}$ pounds of cream of tartar, $4 \frac{1}{4}$ pounds of pure alum; boil the wool gently for 2 hours, transfer it into a cool place, and wash it next day in clear water.

" Dyeing.-12 pounds of Avignon madder, infused half an hour at $30^{\circ}$ R. (100 F.) Put into the bath 1 pound of muriate of tin, let the color rose for three quarters of an hour at the same heat, and drain or squeeze the madder through canvass. The whole of the red dye will remain upon the filter, but the water wlich has passed through will be as deep a yellow as a weld bath. The boiler with the Jye must now be filled up with ciear river water, and heated to $100^{\circ} \mathrm{F}$. Two ounces of the solution of the tartar and alum must be poured into it, and the wool must be turned over in it for an hour and a half, while the heat is gradually raised to the boiling point. The wool is then removed and washed. It must be rosed the following day.

"Rosing.-Dissolve in hot water 1 pound of white Marseilles soap ; let the bath cool, and pass the wool through it till it has acquired the desired shade; 15 or 20 minutes are sufficient. On coming out of this bath it should be washed.

"Solution of deuto-muriate of tin:-

" 2 ounces of pure muriatic acid; 4 drachms of pure nitric acid; 1 ounce of distilled water. Dissolve in it, by small portions at a time, 2 drachms of grain tin, in a large bottle of white glass, shutting it after putting in the tin. This solution may be preserved for years, without losing its virtue."

I have inserted this process, as recently recommended by the French minister cf com- 
merce, and published by M. Pouillet in vol. i. of his Portefeuille Industriel, to show what official importance is sometimes given by our neighbors to the most frivolous things.
Madders imported for home consumption.
Gross amount of Duty paid in 1836. 1837.
Cwts. 106,172
ewts. 79,228 1836.
$£ 10,810$ 1837. $£ 8.081$

MADREPORES are calcareous incrustations produced by polypi contained in cells of greater or less depth, placed at the surface of calcareous ramifications, which are fixed at their base, and perforated with a great many pores. The mode of the increase, reproduction, and death of these animals is still unknown to naturalists. Living madrepores are now-a-days to be observed only in the South American, the Indian, and the Red seas; but although their polypi are not found in our climate at present, there can be no doubt of their having existed in these northern latitudes in former times, since fossil madrepores occur in both the older and newer secondary strata of Europe.

MAGISTERY is an old chemical term to designate white pulverulent substances, spontaneously precipitated in making certain metallic solutions; as magistery of bismuth.

MAGISTRAL, in the language of the Spanish smelters of Mexico and South America, is the roasted and pulverized copper pyrites, which is added to the ground ores of silver in their patio, or amalgamation magma, for the purpose of decomposing the horn silver present. See SILver, for an account of this curious process of reduction.

MAGMA is the generic name of any crude mixture of mineral or organic matters, in a thin pasty state.

MAGNANIER is the name given in the southern departments of France to the proprietor of a nursery in which silk-worms are reared upon the great scale, or to the manager of the establishment. The word is derived from magnans, which signifies silk-worms in the language of the country people. See Sink.

MAGNESIA (Eng. and Fr.; Bittererde, Talkerde, Germ.) is one of the primitive earths, first proved by Sir $\mathrm{H}$. Davy to be the oxyde of a metal, which he called magnesium. It is a fine, light, white powder, without taste or smell, which requires 5150 parts of cold water and no less than 36,000 parts of boiling water for its solution. Its specific gravity is $2 \cdot 3$. It is fusible only by the heat of the hydroxygen blowpipe. A natural hydrate is said to exist which contains 30 per cent. of water. Magnesia changes the purple infusion of red cabbage to a bright green. It attracts carbonic acid from the air, but much more slowly than quicklime. It consists of 61.21 parts of metallic basis, and 38.79 of oxygen; and has, etherefore, 20 for its prime equivalent upon the hydrogen scale. Its only employment in the arts is for the purification of fine oil, in the preparation of varnish.

Magnesia mas be obtained by precipitation with potash or soda, from its sulphate, commonly called Epsom salt; but it is usually procured by calcining the artificial o: natural carbonate. The former is, properly speaking, a subcarbonate, consisting of 44.69 magnesia, $35 \cdot 86$ carbonic acid, and $19 \cdot 45$ water. It is prepared by adding to the solution $e^{*}$ the sulphate, or the muriate (the bittern of sea-salt evaporation works), a solution of carbonate of soda, or of carbonate of ammonia distilled from bones in iron cylinders. The sulphate of magnesia is generally made by acting upon magnesian limestone with somewhat dilute sulphuric acid. The sulphate of lime precipitates, while the sulphate of magnesia remains in solution, and may be made to crystallize in quadrangular prisms, by suitable evaporation and slow cooling. Where muriatic acid may be had in profusion for the trouble of collecting it, as in the soda works in which sea salt is decomposed by sulphuric acid, the magnesian limestone should be first acted upon with as much of the former acid as will dissolve out the lime, and then, the residuum being treated with the latter acid, will afford a sulphate at the cheapest possible rate; from which magnesia and all its other preparations may be readily made. Or, if the equivalent quantity of calcined magnesian limestone be boiled for some time in bittern, the lime of the former will displace the magnesia from the muriatic acid of the latter. This is the most economical process for manufacturing magnesia. The subcarbonate, or magnesia alba of the apothecary, has been proposed by Mr. E. Davy to be added by the baker to damaged flour, to counteract its acescency.

MAGNESIAN LIMESTONE (Dolomie, Fr.; Bittertalk, Talkspath, Germ.) is a min. eral which crystallizes in the rhombohedral system. Spec. grav. 2.86 ; scratches calcspar; does not fall spontaneously into powder, when calcined, as common limestone does It consists of 1 prime equivalent of carbonate of lime $=50$, associated with 1 of carbonate of magnesia $=42$.

Massive magnesiun limestone, is yellowish-brown, cream yellow, and yellowish-gray ; brittle. It dissolves slowly and with feeble effervescence in dilute muriatic acid; whenc 
At is called Calcaire lent dolomie by the French mineralogists. Specific gravity $2 \cdot 6$ to $2 \cdot 7$.

Near Sunderland, it is found in flexible slabs. The principal range of hills composing this geological formation in England extends from Sunderland on the northeast coast to Nottingham, and its beds are described as being about 300 feet thick on the east of the coal field in Derbyshire, which is near its southern extremity. On the western side of the Cumberland mountains magnesian limestone orerlies the coal measures near Whitehaven. The stratification of this rock is very distinct, the individual courses of stone not exceeding in general the thickness of a common brick.

The lime resulting from the calcination of magnesian limestone appears to hare an injurious action on vegetation, unless applied in quantities considerably less than common lime, when it is found to fertilize the soil. After two years, its hurtful influence on the ground seems to become exhausted, even when used in undue quantity. Great quantities of it are annually brought from Sunderland to Scotland by the Fifeshire farmers, and employed beneficially by them as a manure, in preference to other linds of lime. It has been unfairly denounced, by Mr. Tennent and Sir H. Davy, as a sterdizer.

This rock is used in many places for building; indeed, our most splendid monument of Gothic architecture, York Minster, is constructed of magnesian limestone.

MAGNESIA, NATIVE (Brucite; Guhr-magnesien, Fr.; Wassertalk, Germ.), is a white, lamellar, pearly-looking mineral, soft to the touch. Spec. grar. 2.336; tender; scratched by calc-spar; affording water by calcination; leaving a white suistance which browrs turmeric paper; and, by calcination with nitrate of cobalt, becoming of a lilach hue. It consists of $69 \cdot 75$ magnesia, and 30.25 water. It occurs in reins in the serpentine at Hoboken, in New Jersey, as also at Swinaness, in the island of Lnst, Shetland.

MAGNESITE, Gijbertite; native carbonate of magnesia occurs in white, hard, stony masses, in the presidency of Madras, and in a few other localities. It dissolves rery slowly in muriatic acid, and gives out carbonic acid in the proportion of 22 parts by weight to 42 of the mineral, according to my experiments, and is therefore an atomic carbonate. It forms an excellent and beautiful mortar cement for terraces; a purpose to which it has been beneficially applied in India by Dr. Macleod.

MAGNET, NATIVE, is a mineral consisting of the protoxyde and peroxyde of iron combined in equivalent proportions. See IRON.

MAHALEB. The fruit of this shrub affords a violet dye, as well as a fermented liquor like Kirschwasser. It is a species of cherry cultivated in our gardens.

MALACHITE, or mountain green, is natire carbonate of copper of a beautiful green color, with rariegated radiations and zones; spec. grav. $3 \cdot 5$; it scratches calc-spar, but not fluor; by calcination it affords water and turns black. Its solution in the acids deposites copper upon a plate of iron plunged into it. It consists of carbonic acid 18.5 ; deutoxyce of copper $72 \cdot 2$; water $9 \cdot 3$.

MALATES are saline compounds of the bases, with

MALIC ACID. (Acide malique, Fr.; Aepfelsaure, Germ.) This acid exists in the jusces of many fruits and plants, alone, or associated with the citric, tartaric, and oxalic acids; and occasionally combined with potash or lime. Unripe apples, sloes, barberries, the berries of the mountain ash, elder berries, currants, gooseberries, strawberries, raspberries, bilberries, brambleberries, whortleberries, cherries, ananas, afford malic acid; the house-leek and purslane contain the malate of lime.

The acid may be obtained most conveniently from the juice of the berries of the mountain ash, or barberries. This must be clarified, by mixing with white of egg, and heating the mixture to ebullition; then filtering, digesting the clear liquor with carbonate of lead, till it becomes neutral; and evaporating the saline solution, till crystals of malate of lead be obtained. These are to be washed with cold water, and purified by re-crystallization. On dissolving the white salt in water, and passing a stream of sulphureted $h$ y̦droger. through the solution, the lead will be all separated in the form of a sulphuret, and the liquor, after filtration and evaporation, will yield yellow granular crystals, or cauliflower concretions, of malic acid, which may be blanched by re-dissolution and digestion with bone-black, and re-crystallization.

Malic acid has no smell, but a very sour taste, deliquesces by absorption of moisture from the air, is soluble in alcohol, fuses at $150^{\circ}$ Fahr., is decomposed at a heat of $348^{\circ}$, and affords by distillation a peculiar acid, the pyromalic. It consists in 100 parts, of 41.47 carbon; 3.51 hydrogen; and 55.02 oxrgen; having nearly the same composition as citric acid. A crude malic acid might be economically extracted from the fruit of the mountain ash, applicable to many purposes; but it has not hitherto been manufactured upon the great scale.

MALLEABILITY is the property belonging to certair metals, of being extended un. der the ammer. A table of maeleability is given in the arlicle Ducrility. 
MALT (Eng. and Fr.; Malz, Germ.) is barley-corn, which has been subjected to an artificial process of germination. See BEER.

Table of the Quantity of Malt consumed by the undermentioned Brewers of London and Vicinity, from October 10, 1836, to October 10, 1837.

\begin{tabular}{|c|c|c|c|c|c|c|c|c|}
\hline \multicolumn{3}{|l|}{ Brewers. } & Qrs. & \multicolumn{4}{|c|}{ Brewers. } & \multirow{2}{*}{$\frac{\text { Qrs. }}{956}$} \\
\hline Barclay and Co. & - & - & 100005 & Jones & - & - & - & \\
\hline Hanbury and Co. & - & - & 82798 & Ufford and & Oldersha & aw & - & 953 \\
\hline Whitbread and Co. & - & - & 47012 & Blogg, B. & - & - & - & 943 \\
\hline Reid and Co. - & - & - & 13945 & Ing - & - & - & - & 900 \\
\hline Combe and Co. & - & - & 40366 & Keep & - & - & - & 886 \\
\hline Hoare and Co. & - & - & 32347 & Soulby & - & - & - & 861 \\
\hline Calvert and Co. & - & - & 32335 & Clarke, R. & - & - & - & 834 \\
\hline Meux and Co. & - & - & 30575 & Jenner & - & - & - & 833 \\
\hline Elliot and Co. & - & - & 24154 & Manvell & - & - & - & 824 \\
\hline Taylor and Co. & - & - & 23556 & M'Leods. & - & - & - & 820 \\
\hline Charrington and Co. & - & - & 18842 & Braithwaite & & - & - & 799 \\
\hline Thorne and Son & - & - & 16404 & Addison & - & - & - & 768 \\
\hline Gardner & - & - & 15256 & Turner & - & - & - & 766 \\
\hline Ramsbottom and Co. & - & - & 15227 & Holt & - & - & - & 756 \\
\hline J. \& C. Goding (11 m & honths) & - & 14023 & Church & - & - & - & 742 \\
\hline Bricheno - & - & - & 9863 & Clarke, S. & - & - & - & 741 \\
\hline Courage and Co. & - & - & 9284 & Mann, Joel & 1 - & - & - & 733 \\
\hline Wood and Co. & - & - & 7834 & Turner & - & - & - & 712 \\
\hline Goding, Thos. & - & - & 7095 & Mantell & - & - & - & 693 \\
\hline Hazard - & - & - & 6674 & Lock & - & - & - & 651 \\
\hline Mann, Jas. - & - & 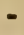 & 6588 & Hood & - & - & - & 649 \\
\hline Harris, Thos. - & - & & 6042 & Pink, A. & - & - & - & 636 \\
\hline More - - & - & - & 6025 & Collins & - & - & - & 598 \\
\hline M'Leod, B. - & - & - & 4960 & Wright & - & - & - & 588 \\
\hline Farren and Till & - & - & 4783 & West & - & - & - & 565 \\
\hline Manners and Co. & - & - & 4552 & Abbott & & - & - & 560 \\
\hline Hale, George - & - & 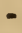 & 4547 & Hett $(6 \mathrm{mor}$ & nths) & - & - & 552 \\
\hline Halford and Topham & - & - & 3786 & Wells & - & - & - & 520 \\
\hline Stains and Fox & - & - & 5783 & Higgs & - & - & - & 475 \\
\hline Lamont and Co. & - & - & 3600 & Harris, Rob & bt. & - & - & 470 \\
\hline Laxton & - & - & 3583 & Woodward & - & - & - & 462 \\
\hline Richmond & - & - & 3174 & Wicks & - & - & - & 441 \\
\hline Maynard & - & - & 3133 & Bell - & - & - & - & 440 \\
\hline M'Leod and Thompsol & & - & 2834 & Thompson & - & - & - & 406 \\
\hline Tubb - & - & - & 2826 & Mattam & - & - & - & 400 \\
\hline Johnson and Wyatt & - & - & 2809 & M'Intosh & $\because$ & - & - & 397 \\
\hline Duggan and Co. & - & & 2665 & Thurlby & - & - & - & 392 \\
\hline Hodoson = & - & - & 2400 & Griffiths & - & - & - & 391 \\
\hline Sherborn and Co. & - & - & 2347 & Kay & - & - & - & 360 \\
\hline Griffith & - & - & 2221 & Tidman & - & - & - & 332 \\
\hline Cox, John & - & - & 2151 & Lindsay & - & - & - & 326 \\
\hline Masterman - & - & - & 1914 & Cooper & - & - & - & 315 \\
\hline Hill and Rice - & - & - & 1853 & West & - & - & - & 306 \\
\hline Gray and Dacre & - & . & 1760 & Carpenter & - & - & - & 299 \\
\hline Plimmer & - & 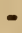 & 1747 & Green & - & - & - & 292 \\
\hline Hayward & 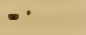 & - & 1737 & Chapman & - & - & - & 286 \\
\hline Verey, W. and C. & - & - & 1573 & Brace & - & - & - & 266 \\
\hline Williamson and Co. & - &. & 1566 & Clark & - & - & - & 248 \\
\hline Honeyball _- & - & 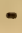 & 1512 & Allen & - & - & - & 245 \\
\hline Satchell and Son & - & & 1441 & Powditch & - & - & - & 238 \\
\hline Clarke, C. - & - & - & 1330 & Garnett & - & - & - & 232 \\
\hline Colyer & - & & 1299 & Hill & - & - & - & 222 \\
\hline Filmer and Wall & - & & 1298 & Olley & - & - & - & 214 \\
\hline Nicholls and Co. & - & & 1240 & Ward & - & - & - & 206 \\
\hline Hagan & - & 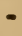 & 1143 & Bye & - & - & - & 201 \\
\hline Hume & - & & 1126 & Newton & - & - & - & 175 \\
\hline Buckley and Co. & - & - & 1025 & Chadwick & - & - & - & 169 \\
\hline Verey, J. - & - & - & 1017 & Prosser & - & - & - & 166 \\
\hline Collins, J. & $=$ & $=$ & 966 & Smith & - & - & - & 164 \\
\hline
\end{tabular}


Table of the Consumption of Malt.-Continued.

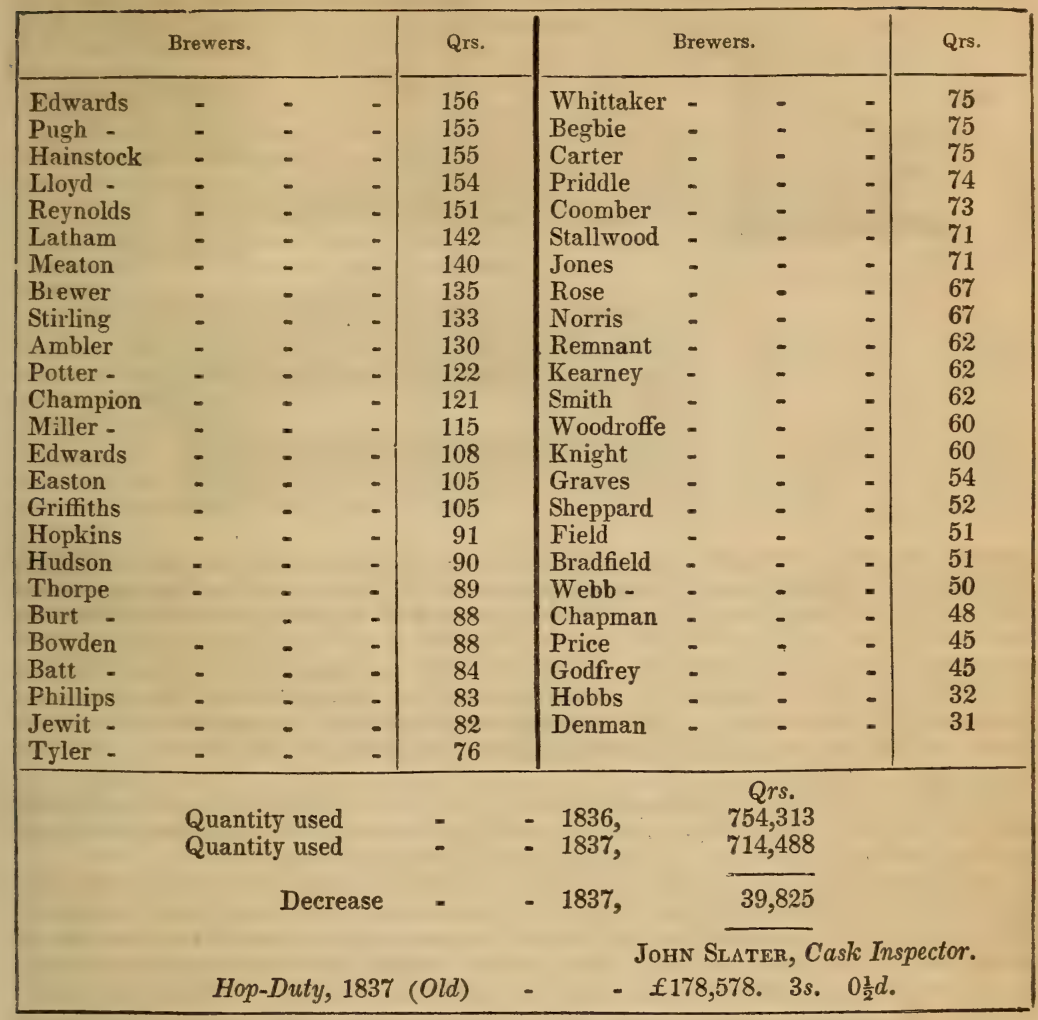

Table of the Quantity of Malt from Barley, which paid Duty in

\begin{tabular}{|c|c|c|c|}
\hline Years. & England. & Scotland. & Ireland. \\
\hline $\begin{array}{l}1834 . \\
1835 . \\
1836 .\end{array}$ & $\begin{array}{c}\text { Bushels. } \\
34,949,646 \\
36,078,855 \\
37,196,998\end{array}$ & $\begin{array}{c}\text { Bushels. } \\
3,580,758 \\
3,604,816 \\
4,168,854\end{array}$ & $\begin{array}{c}\text { Bushels. } \\
1,776,883 \\
1,825,300 \\
1,872,104\end{array}$ \\
\hline $\begin{array}{l}1834 . \\
1835 . \\
1836 .\end{array}$ & $\begin{array}{r}f^{\mathrm{A}} \\
4,449,745 \\
4,660,185 \\
4,804,612\end{array}$ & $\begin{array}{c}\text { outies paid } \\
£ \\
462,514 \\
465,622 \\
538,477\end{array}$ & $\begin{array}{c}£ \\
229,514 \\
235,767 \\
241,813\end{array}$ \\
\hline
\end{tabular}

MALT KILN (Darre, Germ.) The improved malt kiln of Pistorius is represented, fig. 653 , in a top view ; fig. 654, in a longitudinal view and section; and fig. 655 , in transverse section. $a a$ are two quadrangular smoke flues, constructed of fire-tiles, or fire-stones, and covered with iron plates, over which a pent-house roof is laid; the whole bound by the cross-pieces $b$ (figs. 654,655.) These flues are built above a grating $c c$, which commences at $c^{\prime}$; in front of $c^{\prime}$ there is a bridge of bricks. Instead of such a brick flue covered with plates, iron pipes may be used, covered with semi-cjlindrical tiles, to prevent the malt that may happen to fall from being burned. $d d$ are the breast walls of the kiln, 3 feet high, furnished with two apertures shut with iron doors, through which the malt that drops down may be removed from time to time. $e$ is a beam of wood lying on the breast wall, against which the hurdles are laid down 
slantingly towards the back wall of the kiln; $f f$ are two vertical flues left in the substance of the walls, through which the hot air, discharged by open pipes laid in a
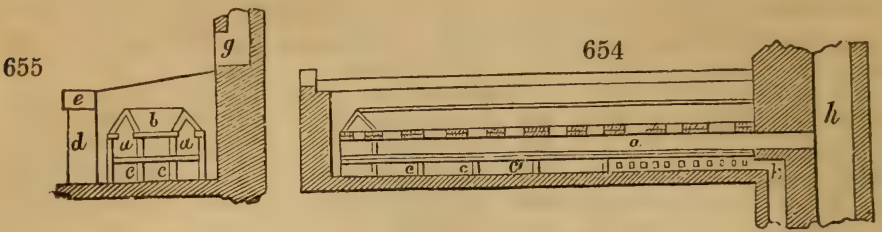

653

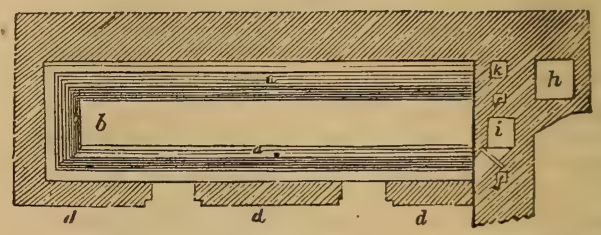

subjacent furnace, rises into the space between the pent-house roof and the iron plates, and is thence allowed to issue through apertures in the sides. $\mathrm{g}$ is the discharge flue in the back wall of the kiln for the air now saturated with moisture; $h$ is the smoke-pipe, from which the smoke passes into the anterior flue $a$, provided with a slide-plate, for modifying the draught; the smoke thence flows off through a flue fitted also with a damper-plate into the chimney $i . \quad k$ is the smoke-pipe of a subsidiary fire, in case no smoke should pass through $h$. The iron pipes are 11 inches in diameter, the air-flues $f^{\prime}, 5$ inches, and the smoke-pipe $h, 10$ inches square; the brick flues 10 inches wide, and the usual height of bricks.

MALTHA ; Bitume Glutineux, or mineral pitch. It is a soft glutinous substance, with the smell of pitch. It dissolves in alcohol, but leaves a bituminous residuum; as also in naptha, and oil of turpentine. It seems to be inspissated petroleum.

MANGANESE (Eng. and Fr.; Mangan, Braunsteinmetal, Germ.) is a grayish-white metal, of a fine-grained fracture, very hard, very brittle, with considerable lustre, of spec. grav. 8.013, and requiring for fusion the extreme heat of $160^{\circ}$ Wedgewood. It should be kept in closely stoppered bottles, under naptha, like potassium, because with contact of air it speedily gets oxydized, and falls into powder. It decomposes water slowly at common temperatures, and rapidly at a red heat. Pure oxyde of manganese can be reduced to the metallic state only in small quantities, by mixing it with lamp black and oil into a dough, and exposing the mixture to the intense heat of a smith's forge, in a luted crucible; which must be shaken occasionally to favor the agglomeration of the particles into a button. Thus procured, it contains, however, a little carbon.

Manganese is susceptible of five degrees of oxygenation.

1. The protoxyde may be obtained from a solution of the sulphate by precipitation with carbonate of potash, and expelling the carbonic acid from the washed and dried carbonate, by calcination in a close vessel filled with hydrogen gas, taking care that no air have access during the cooling. It is a pale green powder, which slowly attracts oxygen from the air, and becomes brown; on which account it should be kept in glass tubes, containing hydrogen, and hermetically sealed. It consists of 77.57 metal and 22.43 oxygen. It forms with 24 per cent. of water a white hydrate; and with acids, saline compounds; which are white, pink, or amethyst colored. They have a bitter, acerb taste, and afford with hydrogenated sulphuret of ammonia a flesh-red precipitate, but with caustic alkalis, one which soon turns brown-red, and eventually black.

2. The deutoxyde of munganese exists native in the mineral called Braunite; but it may be procured either by calcining, at a red heat, the proto-nitrate, or by spontaneous oxydizement of the protoxyde in the air. It is black; when finely pulverized, dark brown, and is convertible, on being heated in acids, into protoxyde, with disengagement of oxygen gas. It consists of 69.75 metal, and 30.25 oxygen. It forms, with 10 per cent. of water, a liverbrown hydrate, which occurs native under the name of Manganite. It dissolves readily in tartaric and citric acids, but in few others. This oxyde constitutes a bronze ground in calico-printing.

3. Peroxyde of manganese; Braunstein, occurs abundantly in nature. It gives ou* oxygen freely when heated, and becomes an oxydulated deutoxyde. It consists of 63.36 metal and 36.64 oxygen. 
4. Manganesic acid forms green-colored salts, but has not hitherto been insulated from the bases. It consists of 53.55 metal, and 46.45 oxygen.

5. Hypermanganesic ucid consists of $49 \cdot 70$ metal, and 50.30 oxygen.

Ores of manganese.-There are two principal ores of this metal which occur in great masses; the peroxyde and the hydrated oxyde; the first of which.is frequently found in primitive formations.

1. Metalloide oxyde of manganese; pyrolusite, or gray manganese ore; has a metallic lustre, a steel gray color, and affords a black powder. Spec. grav. $4 \cdot 85$. Seratches calcspar. It effervesces briskly with borax at the blow-pipe, in consequence of the disengagement of oxygen gas. This is the most common ore of manganese, and a very valuable one, being the substance mostly employed in the manufacture of chloride of lime and of flint-glass. It is the peroxyde. Great quantities are found near Tavistock, in Devonshire, and Launceston, in Cornwall.

2. Braunite, is a dark brown substance, of a glassy metallic lustre, affording a brown powder. Spec. grav. 4.8. It scratches feldspar; but is scratched by quartz. Infusible at the blow-pipe, and effervesces but slightly when fused with glass of borax. It is the deutoxyde. It gives out at a red heat only 3 per cent. of oxygen.

3. Manganite, or hydroxyde of manganese; is brownish-black or iron-black, powder brown, with somewhat of a metallic lustre. Spec. grav. 4.3. Scratches fluor spar; affords water by calcination in a glass tube; infusible at the blow-pipe; and effervesces slightly when fused with glass of borax. It consists of about 90 of deutoxyde, and 10 of water.

4. Haussmanite, black braunstein; is brownish-black, affords a reddish-brown powder. Spec. grav. $4 \cdot 7$; scratches fluor spar; infusible at the blow-pipe; does not effervesce when fused with borax. It is a deutoxyde. This is a rare mineral, and of no value to the arts.

5. Barytic oxyde of manganese; fibrous wad. It is a combination of deutoxyde and peroxyde, with some baryta.

6. Manganese blende, or sulphuret of manganese; has a metallic aspect; is black, or dark steel gray; spec. grav. 3.95; has no cleavage; cannot be cut ; infusible, but affords after being roasted distinct evidence of manganese, by giving a violet tinge to soda at the blow-pipe. Soluble in nitric acid ; solution yields a white precipitate with the ferro-cyanide of potassium. It consists of sulphur 53.65 ; manganese $66 \cdot 35$.

7. Carbonate of manganese; dialogite. Spec. grav. $3 \cdot 4$; affords a green frit by fusion with carbonate of soda; is soluble with some effervescence in nitric acid; solution when freed from iron by succinate of ammonia, gives a white precipitate, with ferrocyanide of potassium. It consists of 28 carbonic acid, 56 protoxyde of manganese, $5 \cdot 4$ of lime, 4.5 protoxyde of iron, and 0.8 magnesia.

8. Hydrosilicate of manganese is a black metallic looking substance, which yields a yellowish-brown powder, and water by calcination; is acted upon by muriatic acid, but affords no chlorine. It consists of silica 25 ; protoxyde of manganese 60 ; water 13.

9. Ferriferous phosphate of manganese is brown or black. Spec. grav. 3.6 ; scratches fluor; affords by calcination a very little of an acil water which corrodes glass; very fusible at the blow-pipe into a black metalloid magnetic bead; is acted upon by nitric acid; solution lets fall a blue precipitate with ferrocyanide of potassium; which tested by soda is shown to be manganese. It consists of phosphoric acid $32 \cdot 78$; protoxyde of iron 31.90 ; protoxyde of manganese 32.60 ; phosphate of lime $3 \cdot 2$. Another phosphate called hureaulite, contains 38 of phosphoric acid; $11 \cdot 10$ of protoxyde of iron; 32.85 of protoxyde of manganese, and 18 of water.

Bluck wad, is the old English name of the hydrated peroxyde of manganese. It occurs in various imitative shapes, in froth-like coatings upon other minerals, as alsc massive. Some varieties possess imperfect metallic lustre. The external color is brown of various shades, and similar in the streak, only shining. It is opaque, very sectile, soils and writes. Its specific gravity is about 3.7. Mixed with linseed oil into a dough, black wad forms a mass that spontaneously inflames. A variety from the Hartz, analyzed by Klaproth, afforded peroxyde of manganese 68 ; oxyde of iron 6.5 ; water 17.5 ; carbon 1 ; barytes and silica 9 . The localities of black wad are particularly Cornwall and Devonshire, the Hartz, and Piedmont. I have analyzed many varieties of the black wad sold to the manufacturers of bleaching salt and flint glass, and have found few of them so rich in peroxyde of manganese as the above. Very generally they contained no less than 25 per cent. of oxyde of iron, 8 or 9 of silica, about 7 of water, and the remainder amounting to only 60 per cent. of the peroxyde.

M. Gay Lussac has proposed to determine the commercial value of manganese ore, by the quantity of chlorine which it affords when treated with liquid muriatic acid. He places the manganese powder in a small retort or matrass, pours over it the acid, and the chlorine being disengaged with the aid of a gentle heat, is transmitted into a vessel containing milk of lime or potash water. This liquor is thereafter poured into a d lute 
- solution of sulphate of indigo ; and the quantity of chlorine is inferred from the quantity of the blue solution which is decolored. I pass the chlorine into test solution of indigo.

The manufacturer of flint glass uses a small proportion of the black manganese ore, to correct the green tinge which his olass is apt to derive from the iron present in the sand he employs. To him it is of great consequence to get a vative manganese contannig as little iron oxyde as possible; since in fact the color or lhipidity of his product will depend altogether upon that circumstance.

Sulphate of manganese has been of late years introduced into calico-printing, to give a chocolate or bronze impression. It is easily formed by heating the black oxyde, mixed with a little ground coal, with sulphuric acid. See CALico-Printing.

The peroxyde of manganese is used also in the formation of glass pastes, and in making the black enamel of pottery. See Oxasic Acid.

MANGLE. (Calandre, Fr; Mangel, Germ.) This is a well known machine for smoothing table cloths, table napkins, as well as linen and cotton furniture. As usually made, it consists of an oblong rectangular wooden chest, filled with stones, which load it to the degree of pressure that it should exercise upon the two cylinders on which it rests, and which, by rolling backwards and forwards over the linen spread upon a polished table underneath, render it smooth and level. The moving wheel, being furnished with teeth upon both surfaces of its periphery, and having a notch cut out at one part, allows a pinion, uniformly griven in one direction, to act alternately upon its outside and inside, so as to cause the reciprocating motion of the chest. This elegant and much admired En. glish invention, called the mangle-wheel, has been introduced with great advantage into the machinery of the textile manufactures.

Mr. Warcup, of Dartford, obtained a patent several years ago for a mangle, in which the linen, being rolled round a cylinder revolving in stationary bearings, is pressed downwards by heavy weights hung upon its axes, against a curved bed, made to slide to and fro, or twaverse from right to left, and left to right, alternately.

Mr. Hubie, of York, patented in June, 1832, another form of mangle, consisting of three rollers, placed one above another in a vertical frame, the axle of the upper roller being pressed downwards by a powerful spring. The articles intended to be smoothed are introduced into the machine by passing them under the middle roller, which is made to revolve by means of a fly wheel; the pinion upon whose axis works in a large toothed wheel fixed to the shaft of the same roller. The linen, \&c. is lapped as usual in protecting cloths. This machine is merely a small CALENDER.

MANIOC is the Indian name of the nutritious matter of the shrub jatropha manihot, from which cassava and tapioca are made in the West Indies.

MANNA is the concrete saccharine juice of the Fraxinus ornus, a tree much culti. vated in Sicily and Calabria. It is now little used, and that only in medicine.

MARBLE. This title embraces such of the primitive, transition, and purer compact limestones of secondary formation, as may be quarried in solid blocks without fissures, and are susceptible of a fine polished surface. The finer the white, or more beautifully rariegated the colors of the stone, the more valuable, ceteris paribus, is the marble. Its general characters are the following :-

Marble effervesces with acids; affords quicklime by calcination; has a conchoidal scaly fracture; is translucent only on the rery edges; is easily scratehed by the linife; has a spec. grav. of $2 \cdot 7$; admits of being sawn into slabs, and receires a brilliant polish. These qualities occur united in only three principal varieties of limestone; in the saccharoid limestone, so called from its fine granular texture resembling that of loaf sugar, and which constitutes modern statuary marble; like that of Carrara; 2 . in the foliated limestone, consisting of a multitude of small facets formed of little plates applied to one another in every possible direction, constituting the antique stataary marble, like that of Paros; 3. in many of the transition and carboniferous, or encrinitic limestones, subordiuate to the coal formation.

The saccharoid and lamellar, or statuary marbles, belong entirely to primitive and transition districts. The greater part of the close-grained colored marbles belong also to the same geological localities; and become so rare in the secondary limestone formations, that immense tracts of these occur without a single bed sufficiently entire and compact to constitute a workable marble. The limestone lying between the calcareo-silicious sands and gritstone of the under oolite, and which is called Forest marble in England, being susceptible of a tolerable polish, and variegated with imbedded shells, has sometimes been worked into ornamental slabs in Oxfordshire, where it occurs in the neighborhood of Whichwood forest; but this case can hardly be considered as an exception to the general rule. To constitute a profitable marble-quarry. there must be a large extent of homogeneous limestone, and a facility of transporting the blocks after they are dug. On examining these natural advantages of the beds of Carrara marble, we may readily understand how the statuary marbles discovered in the Pyrenees, Savoy, Corsica, \&c. have never been able to come into competition with it in 
the market. In fact, the two sides of the valley of Canara may be regarded as mountains of statuary marble of the finest quaity.

Gypseous alabaster may be readily distinguished from marbles, because it does not efferresce with acids, and is soft enough to be scratched by the nail; stalagmitic alabaster is somewhat harder than marble, translucent, and variegated with regular stripes or undulations.

Some granular marbles are flexible in thin slabs, or, at least, become so by being dried at the fire; which shows, as Dolomieu suspected, that this property arises from a diminution of the attractive force among the particles, by the loss of the moisture.

The various tints of ornamental marbles generally proceed from oxydes of iron; but the blue and green tints are sometimes caused by minute particles of hornblende, as in the slate-blue variety called Turchino, and in some green marbles of Germany. The black marbles are colored by charcoal, mixed occasionally with sulphur and bitumen; when they constitute stinkstone.

Brard dirides marbles, according to their localities, into classes, each of which contains eight subdivisions :-

1. Uni-colored marbles; including only the white and the black.

2. Variegated marbles; those with irregular spots or veins.

3. Madreporic marbles, presenting animal remains in the shape of white or gray spots, with regularly disposed dots and stars in the centre.

4. Shell marbles; with only a few shells interspersed in the calcareous base.

5. Lumachella marbles, entirely composed of shells.

6. Cipolin marbles, containing veins of greenish talc.

7. Breccia marbles, formed of a number of angular fragments of different marbles, united by a common cement.

8. Puddingstone marbles; a conglomerate of rounded pieces.

Antique marbles.-The most remarkable of these are the following:-Parian marble, called lychnites by the ancients, because its quarries were worked by lamps; it has a yellowish-white color; and a texture composed of fine shining scales, lying in all directions. The celebrated Arundelian tabies at Oxford consist of Parian marble, as well as the Medieean Venus. Pentelic marble, from Mount Penteles, near Athens, resembles the Parian, but is somewhat denser and firer grained, with occasional greenish zones, produced by greensh tale, whence it is called by the Italians Cipolino statuario. The Parthenon, Proprleum, the Hippodrome, and other principal monuments of Athens, were of Pentelic marble; of which fine specimens may be seen among the Elgin collection, in the British Museum. Marmo Greco, or Greek white marble, is of a very lirely snow white color, rather harder than the preceding, and susceptible of a very fine polish. It was obtained from several islands of the Archipelago, as Scio, Samos, Lesbos, \&ce. Translucent white marble, Marmo statuario of the Italians, is very much like the Parian, only not so opaque. Columns and altars of this marble exist in Venice, and sereral towns of Lombardy; but the quarries are quite unknown. Flexible white marble, of which fire or six tables are preserved in the house of Prince Borghese, at Rome. The white marble of Luni, on the coast of Tuscanj, was preferred by the Greek sculptors to both the Parian and Pentelic. White marble of Carrara, between Specia and Lucca, is of a fine white color, but often traversed by gray veins, so that it is difficult to procure moderately large pieces free from them. It is not so apt to turn yellow as the Parian marble. This quarry was worked by the ancients, having been opened in the time of Julius Cæsar. Many antique statues remain of this marble: Its two principal quarries at the present day are those of Pianello and Polvazzo. In the centre of its blocks very limpid rock-crystals are sometimes found, which are called Carrara diamonds. As the finest qualities are becoming excessively rare, it has risen in price to about 3 guineas the cubic foot. The White marble of Mount Hymettus, in Greece, was not of a very pure white, but inclined a little to gray. The statue of Meleager, in the French Museum, is of this marble.

Black antique marble, the Nero antico of the Italians. This is more intensely black than any of our modern marbles; it is extremely scarce, occurring only in sculptured pieces. The red antique marble, Egyptum of the ancients, and Rosso antico of the Italians, is a beautiful marble of a deep blood-red color, interspersed with white veins and with very minute white dots, as if strewed over with grains of sand. There is in the Gr:mani palace at Venice, a colossal statue of Marcus Agrippa in rosso antico, which was formerly preserved in the Pantheon at Rome. Green antique marble, verde antico, is a kind of breccia, whose paste is a mixture of talc and limestone, while the dark green fragments consist of serpentine. Very beautiful specimens of it are preserved at Parma. The best quality has a grass-green paste, with black spots of noble serpentine, but is never mingled with red spots. Red spotted green anlique marble, has a dark green ground marked with small red and black spots, with fragments of entrochi changed into white marble. It is known only in small tablets. Leck marble; a rare variety of that color, 
of which there is a table in the Mint at Paris. Marmo verde pagliocco is of a yellowish green color, and is found only in the ruins of ancient Rome. Cervelas marble of a deep red, with numerous gray and white veins, is said to be found in Africa, and highly esteemed in commerce. Yellow antique marble, giallo antico of the Italians; color of the yolk of an egg, either uniform or marked with black or deep yellow rings. It is rare, but may be replaced by Sienna marble. Red and white antique marbles, found only among the ruins of ancient Rome. Grand antique, a breccia martle, containing shells, consists of large fragments of a black marble, traversed by reins or lines of a shining white. There are four columns of it in the Museum at Paris. Antique Cipolino marble. Cipolin is a name given to all such marbles as have greenish zones produced by green talc; their fracture is granular and shining, and displays here and there plates of talc. Purple antique breccia marble, is very variable in the color and size of its spots. Antique African breccia has a black ground, variegated with large fragments of a grayish-white, deep red, or purplish wine color; and is one of the most beautiful marbles. Rose-colored antique breccia marble is very scarce, occurring only in small tablets. There are various other kinds of ancient breccias, which it would be tedious to particularize.

Modern marbles.-1. British. Black marble is found at Ashford, Matlock, and Monsaldale in Derbyshire; black and white in the north part of Devonshire; the rariegated marbles of Devonshire are generally reddish, brownish, and gravish, variously veined with white and yellow, or the colors are often intimately blended; the marbles from Torbay and Babbacombe, display a great variety in the mixture of their colors; the Plymouth marble is either ash-colored with black veins, or blackish-gray and white, shaded with black veins; the cliffs near Marychurch exhibit marble quarries not only of great extent, but of superior beauty to any other in Deronshire, being either of a dove-colored ground with reddish-purple and yellow reins, or of a black ground mottled with purplish globules. The green marble of Anglesea is not unlike the verde antico; its colors being greenish-black, leek-green, and sometimes dull purplish, irregularly blended with white. The white part is limestone, the green shades proceed from serpentine and asbestos. There are several fine varieties of marble in Derbyshire; the mottled-gray in the neighborhood of Moneyash, the light gray being rendered extremely beautiful by the number of purple reins which spread upon its polished surface in elegant irregular branches; but its chief ornament is the multitude of entrochi, with which this transition limestone-marble abounds. Much of the transition and earboniferous limestone of Wales and Westmoreland is capable of being worked up into agreeable dark marbles.

In Scotland, a particularly fine variety of white marble is found in immense berls, at Assynt in Sutherlandshire. A beautiful ash-gray marble of a very uniform grain, and susceptible of a fine polish, occurs on the north side of the ferry of Ballachulish in Invernesshire. One of the most beautiful varieties is that from the hill of Belephetrich in Tiree, one of the Hebrides. Its colors are pale blood-red, lisht flesh-red, and reddishwhite, with dark green particles of hornblende, or rather sahlite, diffused through the general base. The compact marble of Iona is of a fine grain, a dull white color, somewhat resembling pure compact feldspar. It is said by Bournon, to consist of an intimate mixture of tremolite and carbonate of lime, sometimes with yellowish or greenish-rellow spots. The carboniferous limestone of many of the coal basins in the lowlands of Scotland may be worked into a tolerably good marble for chimney-pieces.

In Ireland, the Kilkenny marble is the one best known, having a black ground more or less varied with white marks produced by petrifactions. The spar which occupies the place of the shells, sometimes assumes a greenish-rellow color. An exceedingly fine black marble has also heen raised at Crayleath in the county of Down. At Louthlougher, in the county of Tipperary, a fine purple marble is found, which when polished looks very beautiful. The county of Kerry affords several rariegated marbles, not unlike the Kilkenny.

France possesses a great many marble quarries which have been deseribed br Brard, and of which a copious abstract is given under the article marble-Rees' Cyclopedia.

The territory of Genc a furnishes several beautiful varieties of marble, the most remarkable of which is the polzevera di Genoa, called in French the vert d'Egypte and vert de mer. It is a mixture of granular limestone with a talcose and serpentine substance disposed in veins; and it is sometimes mixed with a reddish body. This marble was formerly much employed in Italy, France, and England, for chimney-pieces, but its sombre appearance has put it out of fashion.

Corsica possesses a good statuary marble of a fine close grain, and pure milky white. ness, quarried at Ornofrio; it will bear comparison with that of Carrara; also a gray marble (bardiglio), a cipolin, and some other varieties. The island of Elba has immense quarries of a white marble with blackish-green veins. 
Among the innumerable varieties of Italian marbles, the following deserve especial notice.

The rovigin, a white marble found at Padua; the white marble of St. Julien, at Pisa, of which the cathedral and celebrated slanting tower are built; the Biancone marble, white with a tinge of gray, quarried at Magurega for altars and tombs. Near Mergozza the white saline marble with gray veins is found; with which the cathedral of Milan 13 built. The black marble of Bergamo is called paragone, from its black color, like touchstone; it has a pure intense tint, and is susceptible of a fine polish. The pure black marble of Como is also much esteemed. The polveroso of Pistoya, is a black marble sprinkled with dots; and the beautiful white marble with. black spots, from the Lago Maggiore, has been einployed for decorating the interior of many churches in the Milanese. The Margorre marble found in several parts of the Milanese, is bluish veined with brown, and composes part of the dome of the cathedral of Milan. The green marble of Florence owes its color to a copious admixture of steatite. Another green marble, called verde di Prado, occurs in Tuscany, near the little town of Prado. It is marked with spots of a deeper green than the rest, passing even into blackish-blue. The beautiful Sienna marble, or brocatello di Sienu, has a yellow color like the yolk of an egg, which is disposed in large irregular spots, surrounded with veins of bluish-red, passing sometimes into purple. At Montarenti, two leagues from Sienna, another yellow marble is met with, which is traversed by black and purplish-black veins. The Brema marble is yellow with white spots. The maidelato of the Italians is a light red marble with yellowishwhite spots, found at Luggezzana, in the Veronese. The red marble of Verona is of a red rather inclining to yellow or hyacinth; a second variety of a dark red, composes the vast amphitheatre of Verona. Another marble is found near Verona, with large white spots in a reddish and greenish paste. Very fine columns have been made of it. The occhio di pavone is an Italian shell marble, in which the shells form large orbicular spots. red, white, and bluish. A madreporic marble known under the name of pietra slellaria, much employed in Italy, is entirely composed of star madrepores, converted into a gray and white substance, and is susceptible of an excellent polish. The village of Bretonico, in the Veronese, furnishes a splendid breccia marble, composed of yellow, steel-gray, and rose-colored spots. That of Bergamo consists of black and gray fragments in a greenish cement. Florence marble, called also ruin and landscape marble, is an indurated calcareous marl.

Sicily abounds in marbles, the most valuable of which is that called by the English stone-cutters, Sicilian jasper; it is red with large stripes like ribands, white, red, and sometimes green, which run zigzag with pretty acute angles.

Among the Genoese marbles we may notice the highly esteemed variety called portor, on account of the brilliant yellow veins in a deep black ground. The most beautiful kind comes from Porto-Venese, and Louis XIV. caused a great deal of it to be worked up for the decoration of Versailles. It costs now two pounds per cubic foot.

Of cutting and polishing marble.-The marble saw is a thin plate of soft iron, continually supplied during its sawing motion, with water and the sharpest sand. The sawing of moderate pieces is performed by hand, but that of large slabs is most economically done by a proper mill.

The first substance used in the polishing process is the sharpest sand, which must be worked with till the surface becomes perfectly flat. Then a second, and even a third sand of increasing fineness is to be applied. The next substance is emery of progressive degrees of fineness, after which tripoli is employed; and the last polish is given with tin-putty. The body with which the sand is rubbed upon the marble, is usually a plate of iron : but for the subsequent process, a plate of lead is used with fine sand and emery. The polishing rubbers are coarse linen cloths, or bagging, wedged tight into an iron planing tool. In every step of the operation, a constant trickling supply of water is required.

Visiters of Derby may have an opportunity of inspecting Brown's extensive machinery for eutting marble into many ornamental forms, which has been well described in Rees' Cyclopedia.

Sir James Jelf patented, in 1822, a combination of machinery for cutting any description of parallel mouldings upon marble slabs, for ornamental purposes; in which tools, supplied with sand and water, are made to traverse to and fro.

Mr. Tullock obtained a patent, in 1824, for improvements in machinery for sawing and grooring marble; the power being applied by means of toothed wheels bearing cranks, which gave the see-saw motion to the cutting iron plates.

In November, 1829, Mr. Gibbs secured, by patent, an invention for working ornamental derices in marble, by means of a travelling drill, guided by a mould of wood, \&c., in couster relief; and in April, 1833, Mr. G. W. Wilds obtained a patent for machinery, which consists of a series of circular cutters, for separating slabs from a block of marble; the block being advanced slowly to meet the cutters, by the progressive 
movement of a platform upon wheels, driven by the agency of a rack and pinion, as in the cylinder boring machine of the steam-engine manufacturer. Sand and water must be supplied, of course, from a hopper, to these smooth cutting dises of iron or copper. See Glass-Cutting. He proposes also to mould and polish marble, by applying a rotatory wheel or cylinder of any shape to it, in its carrying frame.

MARCASITE is a variety of iron pyrites, containing generally a little arsenic.

MARGARATES are saline compounds of margaric acid with the bases.

MARGARIC ACID is one of the acid fats, produced by saponifying tallow with alkaline matter, and decomposing the soap with dilute acid. The term Margaric signifies Pearly-looking.

The physical properties of the margaric and stearic acids are very similar; the chief difference is that the former is more fusible, melting at $140^{\circ} \mathrm{F}$. The readiest mode of obtaining pure margaric acid, is to dissolve olive oil soap in water, to pour into the solution a solution of neutral acetate of lead, to wash and dry the precipitate, and then to remove its oleate of lead by ether, which does not affect its margarate of lead. The residuum being decomposed by boiling hot muriatic acid, affords margaric acid. When heated in a retort this acid boils. It is insoluble in water, very soluble in aleohol and ether; it reddens litmus paper, and decomposes, with the aid of heat, the carbonates of soda and potash.

MARINE ACID. See MURIATIC AcID.

MARINE SALT. See SALT.

MARL (Marne, Fr.; Mergel, Germ.) is a mixed earthy substance, consisting of carbonate of lime, clay, and silicious sand, in very variable proportions; it is sometimes compact, sometimes pulverulent. According to the predominance of one or other of these three main ingredients, marls may be distributed into calcareous, clayey, and sandy. See LIMESTONE.

MARQUETRY is a peculiar kind of cabinet work, in which the surface of wood is ornamented with inlaid pieces of various colors and forms. The marqueteur puts gold, silver, copper, tortoise-shell, mother-of-pearl, ivory, horn, \&c. under contribution. These substances, being reduced to laminæ of proper thinness, are cut out into the desired forms by punches, which produce at once the full pattern or mould, and the empty one, which enclosed it; and both serve their separate purposes in marquetry, For the methods of dyeing the woods, \&c. see Ivory.

MARTIAL signifies, belonging to iron; from Mars, the mythological name of this metal.

MASSICOT is the yellow oxyde of lead.

MASTIC (Eng. and Fr.; Mastix, Germ.) is a resin produced by making incisions in the Pistacia Lentiscus, a tree cultivated in the Levant, and chiefly in the island of Chios. It comes to us in yellow, brittle, transparent, rounded tears; which soften between the teeth; with bitterish taste and aromatic smell, and a specific gravity of 1.07. Mastic consists of two resins; one soluble in dilute alcohol; but both dissolve in strong alcohol. Its solution in spirit of wine constitutes a good varnish. It dissolves also in oil of turpentine. See VARNISH.

MATRASS is a bottle with a thin egg-shaped bottom, much used for digestions in chemical researches.

MATTE is a crude black copper reduced, but not refined from sulphur and other heterogeneous substances.

MEADOW ORE is conchoidal bog iron ore.

MEDALS. For their composition, see BroNzE and CoPPER.

MEERSCHAUM (Germ.; sea-froth, Eng.; Ecume de Mer, Magnêsie carbonutêe silicifère, Fr.) is a white mineral, of a somewhat earthy appearance, always soft, but dry to the touch, and adhering to the tongue. Specific gravity, 2.6 to 3.4 ; affords water by calcination; fuses with difficulty at the blowpipe into a white enamel; and is acted upon by acid̀s. It consists, according to Klaproth, of silica, 41.5 ; magnesia, 18.25 ; water and carbonic acid, 39. Other analysts give, silica 50 , magnesia 25 , water 25 . It occurs in veins or kidney-shaped nodules, among rocks of serpentine, at Egribos, in the island of Negropont, Eski-Schehir in Anatolia, Brussa at the foot of Mount Olympus, at Baldissero in Piedmont, in the serpentine veins of Cornwall, \&c.

When first dug up, it is soft, greasy, and lathers like soap; and is on that account used by the Tartars in washing their linen. The well-known Turkey tobacco-pipes are made from it, by a process analogous to that for making pottery ware. The bowls of the pipes, when imported into Germany, are prepared for sale by soaking them first in tallow, then in wax, and finally by polishing them with shave-grass.

\section{MELLITE. (Eng. and Fr.; Honigstein, Germ.) See Honeystone.}

MELLITIC ACID, which is associated with alumina in the preceding mineral, crystallizes in small colorless needles, is without smell, of a strongly acid taste, permanent in the air, soluble in water and alcohol, as also in boiling hot concentrated sulphuric acid, but is decomposed by hot nitric acid, and consists of 50.21 carbon, ani 
49.79 oxygen. It is carbonized at a red heat, without the production of any inflammable oil.

MELLON is a new compound of carbon and azote, discovered by M. Liebig, by heating bi-sulpho-cyanide of mercury. The mellon remains at the bottom of the retort under the form of a yellow powder.

MEIACHANITE, an ore of titanium, found in the bed of a rivulet which flows into the valley Menacan, in Cornwall.

MERCURY or QUICKSILVER. This metal is distingurshed by its fluidity at com. mon temperatures ; its density $=13.6$; its silver blue lustre; and its extreme mobility. A cold of $39^{\circ}$ below zero of Fahrenheit, or $-40^{\circ}$ Cent., is required for its congelation, in which state its density is increased in the proportion of 10 to 9 , or it becomes of spec. grav. 15.0 . At a temperature of $656^{\circ} \mathrm{F}$. it boils and distils off in an elastic vapor; which, being condensed by cold, forms purified mercury.

Mercury combines with great readiness with certain metals, as gold, silver, zinc, tin, and bismuth, forming, in certain proportions, fluid solutions of these metals. Such mercurial alloys are called amalgams. This property is extensively employed in many arts; as in extracting gold and silver from their ores; in gilding, plating, making looking-glasses, \&c. Humboldt estimates at 16,000 quintals, of $100 \mathrm{lbs}$. each, the quantity of mercury annually employed at his visit to America, in the treatment of the mines of New Spain; three fourths of which came from European mines.

The mercurial ores may be divided into four species :-

1. Native quicksilver.-It occurs in most of the mines of the other mercurial ores, in the form of small drops attached to the rocks, or lodged in the crevices of other ores.

2. Argental mercury, or native silver amalgam.-It has a silver white color, and is more or less soft, according to the proportion which the mercury bears to the silver. Its density is sometimes so high as 14. A moderate heat dissipates the mercury, and leaves the silver. Klaproth states its constituents at silver 36, and mercury 64, in 100 ; but Cordier makes them to be, $27 \frac{1}{2}$ silver, and $72 \frac{1}{2}$ mercury. It occurs crystallized in a variety of forms. It has been found in the territory of Deux-Ponts, at Rozenau and Niderstana, in Hungary, in a canton of Tyrol, at Sahlberg in Sweden, at Kolyvan in Siberia, and at Allemont in Dauphiny; in small quantity at Almaden in Spain, and at Idria in Carniola. By the chemical union of the mercury with the silver, the amalgam, which should by calculation have a spec. grav. of only $12 \cdot 5$, acquires that of $14 \cdot 11$, according to M. Cordier.

3. Sulphuret of mercury, commonly called Cinnabar, is a red mineral of various shades; burning at the blowpipe with a blue flame, volatilizing entirely with the smell of burning sulphur, and giving a quicksilver coating to a plate of copper held in the fumes. Even the powder of cinnahar rubbed on copper whitens it. Its density varies from 6.9 to $10 \cdot 2$. It becomes negatively electrical by friction. Analyzed by Klaproth, it was found to consist of mercury $84 \cdot 5$, sulphur $14 \cdot 75$. Its composition, viewed as a bisulphuret of mercury, is, mercury $86 \cdot 2$, sulphur $13 \cdot 8$. The finest crystals of sulphuret of mercury come from China, and Almaden in Spain. These contain, according to Klaproth, 85 per cent. of mercury.

A bituminous sulphwret of mercury appears to be the base of the great exploration of Idria; it is of a dark liver-red hue; and of a slaty texture, with straight or twisted plates. It exists in large masses in the bituminous schists of Hiria. M. Beurard mentions also the locality of Munster-Appel, in the dutchy of Deux-Ponts, where the ore includes impressions of fishes, curiously spotted with cinnabar.

The compact variety of the Idria ore seems very complex in composition, according to the following analysis of Klaproth:-Mercury, 81.8 ; sulphur, 13.75 ; carbon, 2.3 ; silica, 0.65 ; alumina, 0.55 ; oxyde of iron, 0.20 ; copper, 0.02 ; water, 0.73 ; in 100 parts. M. Beurard mentions another variety from the Palatinate, which yields a large quantity of bitumen by distillation; and it was present in all the specimens of these ores analyzed by me for the German Mines Company. At Idria and Almaden the sulphurets are extremely rich in mercury:

4. Muriated mercury, or the Chloride of mercury, commonly called Horn mercury. This ore occurs in very small crystals of a pearl-gray or greenish-gray color, or in small nipples which stud, like crystals, the cavities, fissures, or geodes among the ferruginous gangues of the other ores of mercury. It is brittle, and entirely volatile at the blow-pipe, characters which distinguish it from horn silver.

The geological position of the mercurial ores, in all parts of the world, is in the strata which commence the series of secondary formations. Sometimes they are found in the red sandstone above the coal, as at Menildot, in the old dutchy of Deux-Ponts, at Durasno in Mexico, at Cuença in New Granada, at Cerros de Gauzan and Upar in Peru; in the subordinate porphyries, as at Deux-Ponts, San Juan de la Chica in Peru, and at Cerro-del-Fraile, near the town of San Felipe; they occur also among the strata below, or subordinate to the calcareous formation, called zechstein, in Germany, or 
among the accompanying bituminous schists, as at Idria in Carniola and, lastly, they form masses in the zechstein itself. Thus, it appears that the mercurial deposites are confined within very narrow geological limits, between the calcareous beds of zechstein, and the red sandstone. They occur at times in carbonaceous nodules, derived from the decomposition of mosses of various kinds; and the whole mercurial deposite is occasionally covered with beds of charcoal, as at Durasno.

They are even sometimes accompanied with the remains of organic bodies, such as casts of fishes, fossil shells, silicified wood, and true coal. The last fact has been observed at Potzberg, in the works of Drey-Koenigszug, by M. Brongniart. These sandstones, bituminous schists, and indurated clays, contain mercury both in the state of sulphuret and in the native form. They are more or less penetrated with the ore, forming sometimes numerous beds of rery great thickness; while, in the more ancient or the primitire formations, these ores exist only in very small quantity associated with tin. Mercury is, generally speaking, a metal sparingly distributed in nature, and its mines are very rare.

The great exploitations of Idria in Friuli, in the county of Goritz, were discovered in 1497, and the principal ore mined there is the bituminous sulphuret. The workings of this mine have been pushed to the depth of 280 yards. The product in quicksilver might easily amount annually to 6000 metric quintals $=600$ tons British; but, in order to uphold the price of the metal, the Austrian gorernment has restricted the production to 150 tons. The memorable fire of 1803 was most disastrous to these mines. It was extinguished only br drowning all the underground workings. The sublimed mercurr in this cat strophe occasioned diseases and nervous tremblings to more than 900 persons in the neighbornood.

Plint has recorded two interesting facts: 1 , that the Greeks imported red cinnabar from Almaden 700 years before the Christian era; and 2. that Rome, in his time, annually received 700,000 pounds from the same mines. Since 1827 , they have produced 22,000 ewts. of mercury every year, with a corps of 700 miners and 200 smelters; and, indeed, the reins are so extremely rich, that though they have been worked pretty constantly during so many centuries, the mines hare hardly reached the depth of 330 rards, or scmething less than 1000 feet. The lode actually under exploration is from 14 to 16 yards thich; and it becomes thicker still at the crossing of the reins. The totality of the ore is extracted. It rields in their smelting works only 10 per cent. upon an arerage, but there is no doubt, from the analysis of the ores, that nearly one half of the quicksilver is lost, and dispersed in the air, to the great injury of the workmen's health, in consequence of the barbarous apparatus of aludels employed in its sublimation; an apparatus which has remained without any material change for the better since the days of the Moorish dominion in Spain. M. Le Plar, the eminent Ingenieur des Mines, who published, in a recent volume of the Annales des Mines, his Itinéruire to Almaden, says, that the mercurial contents of the ores are notablement plus elevées than the product.

These reins extend all the way from the lown of Chillon to Almadenejos. Upon the borders of the streamlet Balde Azogues, a black slate is also mined which is abundantly impregnated with metallic mercury. The ores are treated in 13 double furnaces, which I shall presently describe. "Le mercure," sars M. Le Plar, "a sur la sante des ourriers la plus funeste influence, et l' on ne peut se déferdre d' un sentiment pénible en rorant l' empressement arec lequel des jeunes gens, pleins de force et de sante, se disputent la fareur d' aller chercher dans les mines, des maladies cruelies, et sourent une mort prématurée. La population des mineurs d'Almaden méritent le plus haut interét." These victims of a deplorable mismanagement are described as being a laborious, simple-minded, virtuous race of beings, who are thus condemned to breathe an atmosphere impregnated far and near with the fumes of a rolatile poison, which the lessons of science, as I shall presently demonstrate, might readily repress, with the effect of not only protecting the health of the population, but of rastly augmenting the revenues of the state.

These celebrated mines, near to which lie those of Las Cuebas and of Almadenejos, were known to the Romans. After haring been the property of the religious knights of Calatrara, who had assisted in expelling the Moors, ther were farmed off to the celebrated Fugger merchants of Augsbourg; and afterwards explored on account of the gorernment, from the date of 1645 till the present time. Their produce was, till very lately, entirely appropriated to the treatment of the gold and silver ores of the new world.

The mines of the Palatinate, situated on the left bank of the Rhine, though they do not approach in richness and importance to those of Idria and Almaden, merit, howerer, all the attention of the gorernment that farms them out. Ther are numerous, and raried in geological nosition. Those of Drey-Koenigszug, at Potzberg, near Kussel, deserre particular notice. The workings hare reached a depth of more than 220 yards ; the ore being a sandstone strongly impregnated with sulphuret of mercury. The produce of these mines is estimated at about 30 tons per annum. 
There are also in Hungary, Bohemia, and several other parts of Germany, some inconsiderable exploitations of mercury, the total produce of which is valued at about 30 or 40 tons on an average of several years.

The mines of Guancavelica, in Peru, are the more interesting, as their products are directly employed in treating the ores of gold and silver, which abound in that portion of America. These quicksilver mines, explored since 1570 , produced, up to $1800,53,700$ tons of that metal; but the actual produce of the explorations of these countries was, according to Helms, about the beginning of this century, from 170 to 180 tons per annum.

In 1782, recourse was had by the South American miners to the mercury extracted in the province of Yun-nan, in China.

The metallurgic treatment of the quicksilver ores is tolerably simple. In general, when the sulphuret of mercury, the most common ore, has been pulverized, and sometimes washed, it is introduced into retorts of cast-iron, sheet-iron, or even stoneware, in mixture with an equal weight of quicklime. These retorts are arranged in various ways.

Prior to the 17th century, the method called per descensum was the only one in use for distilling mercury; and it was effected by means of two earthen pots adjusted over each other. The upper pot, filled with ore, and closed at the top, was covered over with burning fuel; and the mercurial vapors expelled by the heat, passed down through small holes in the bot:om of the pot, to be condensed in another vessel placed below. However convenient this apparatus might be, on account of the facility of transporting it, whe ever the ore was found, its inefficiency and the losses it occasioned were eventsally recognised. Hence, before 1635, some smelting works of the Palatinate had given up the method per descensum, which was, however, still retained in Idria ; and they substituted for it the furnaces called galleries. At first, earthenware retorts were employed in these furnaces; but they were soon succeeded by iron retorts. In the Palatinate this mode of operating is still in use. At Idria, in the year 1750, a great distillatory apparatus was established for the treatment of the mercurial ores, in imitation of those which previously existed at Almaden, in Spain, and called aludel-furnaces. But, since 1794, these aludels have been suppressed, and new distillatory apparatus have been constructed at Idria, remarkable only for their magnitude; exceeding, in this respect, every other metallurgic erection.

There exist, therefore, three kinds of apparatus for the distillation of mercury : 1. the furnace called a gullery; 2 . the furnace with aludels; and 3 . the large apparatus of Idria. I shall describe each of these briefly, in succession.

1. Furnace called Gallery of the Palatinate.-The construction of this furnace is disposed so as to contain four ranges, $a a^{\prime}, b b^{\prime}$, of large retorts, styled cucurbits, of cast-iron, in which the ore of mercury is subjected to distillation. This arrangement is shown in fig. 656 , which presents a vertical section in the line $a b$ of the ground plan, fig. 657 . In the ground plan, the roof $e e$ of the furnace (fig. 656) is supposed to be lifted off, in order to show the disposition of the four ranges of cucurbits upon the grate $\mathrm{c} f$, figs. $656,65 \%$, which receives the pit-coal employed as fuel. Unde: this grate extends an ash-pit $d$. Fig. 658, which exhibits an elevation of the furnace, points ont this ash-pit, as well as one of the two doors $c$, by which the fuel is thrown apon the grate

656

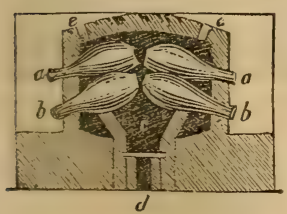

658

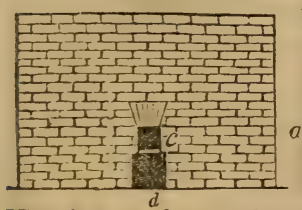
$65 \overline{7}$

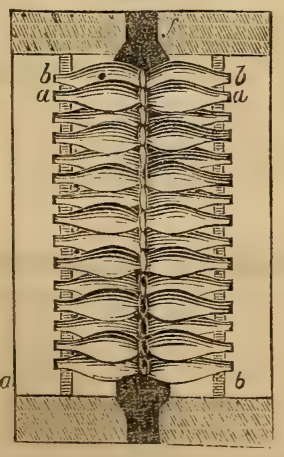
c $f$. Openings $e$ e, (fig. 656, ) are left over the top arch of the furnace, whereby the draught of air may receive a suitable direction. The grate of the fireplace extends over the whole length of the furnace, fig. 657, from the door $c$ to the door $f$, situated at the opposite extremity. The furnace called gallery includes commonly 30 cucurbits, and in some establishments even 52. Into each are introduced from 56 to 70 pounds of ore, and 15 to 18 pounds of quicklime, a mixture which fills no more than two thirds of the cucurbit; to the neck a stoneware receiver is adapted, containing water to half its height. The fire, at first moderate, is eventually pushed till the cucurbits are red hot. The operation being conclurled, the contents of the receivers are poured out into a wooden bowl placed upon a plank above a bucket; the quicksilver falls to the bottom of the bowl, and the water draws over the black mercury, for so the substance that coats 
the inside of the receivers is called. This is considered to be a mixture of sulphuret and oxyde of mercury. The black mercury, taken out of the tub and dried, is distilled anew with excess of lime; after which the residuum in the retorts is thrown away, as useless.

Aludel furnaces of Almaden.-Figs. 659 and 660 represent the great furnaces with aludels in use at Almaden, and anciently in Idria ; for between the two establishments there was in fact little difference before the year 1794. Figs. 659 and 662 present two vertical sections; figs. 660 and 661 are two plans of two similar furnaces, conjoined in

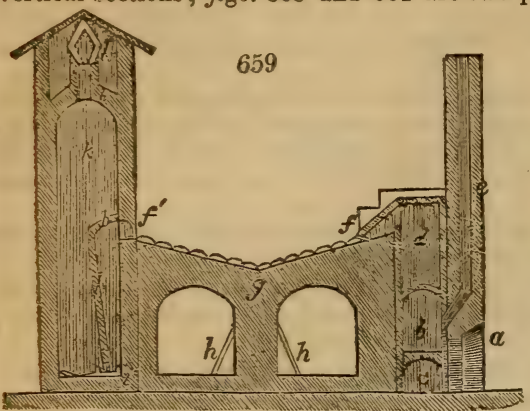

660

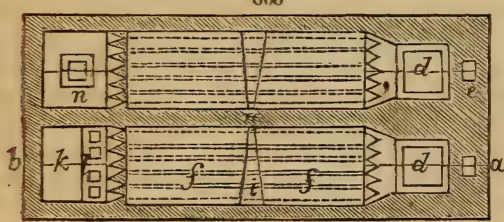
one body of brickwork. In the four figures the following objects are to be remarked: a door $a$, by which the wood is introduced into the fire-place $b$. This is perforated with holes for the passage of air; the ash-pit $c$ is seen beneath. An upper chamber, $d$, contains the mercurial ores distributed upon open arches, which form the perforated sole of this chamber. Immediately over these arches, there are piled up, in a dome form, large blocks of a limestone, very poor in quicksilver ore; above these are laid blocks of a smaller size, then ores of rather inferior quality, and stamped ores mixed with richer minerals. Lastly, the whole is covered up with soft bricks, formed of elay kneaded with schlich, and with small piees of sulphuret of mercury. Six ranges of aludels or stoneware tubes $f f$, of a pear shape, luted together with elay, are mounted in front of each of the two furnaces, on a double sloping terrace, having in its lowest middle line two gutters $t v$,

a little inclined towards the intermediate wall $m$. In each range the aludel placed at the line $t m$ v of fig. 660, that is to say, at the lowest point, $g$, figs. 659,662, is pierced with

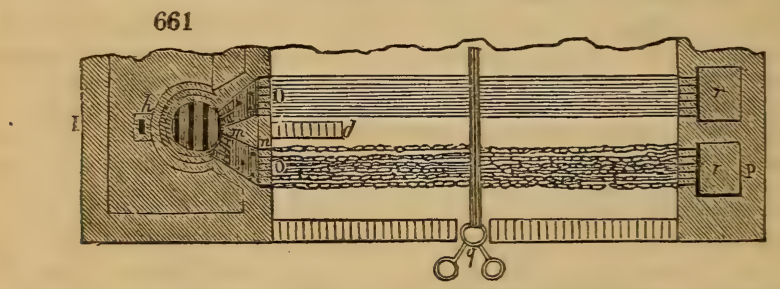

a hole. Thereby the mercury which had been volatilized in $d$, if it be already condensed by the cooling in the series of aludels $f g$, may pass into the corresponding gutter, next

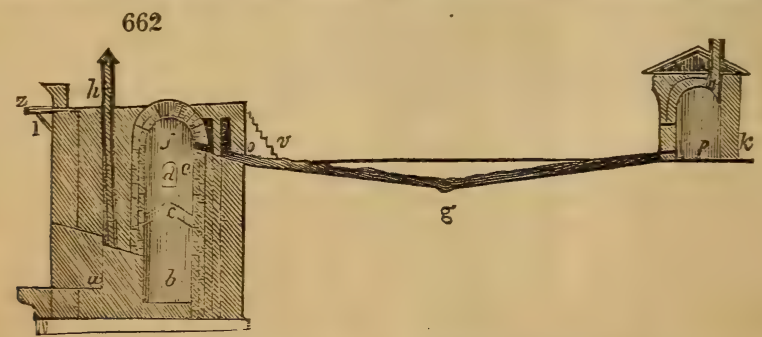

into the hole $m$, fig. 660 , and after that into the wooden pipes $h h^{\prime}, f i g .659$, which con duct it across the masonry of the terrace into cisterns filled with water; see $q, f \mathrm{~g} .661$. which is the plan of fig. 662 .

The portion of mercury not condensed in the range of aludels, $f g$, which is the most 
monsiderable, goes in the state of vapor, into a chamber $k$; but in passing under a partition $l l$, a certain portion is deposited in a cistern $i$, filled with water. The greater yart of the vapors diffused in the chamber $k^{\prime}$ is thereby condensed, and the mercury falls down upon the two inclined planes which form its bottom. What may still exist as rapor passes into an upper chamber $k^{\prime}$, by a small chimney $n$. On one of the sides of this chamber there is a shutter which may be opened at pleasure from below upwards, and beneath this shutter, there is a gutter into which a notable quantity of mercury collects. Much of it is also found condensed in the aludels. These facts prove that this process has inconveniences which have been tried to be rewiedied by the more extensive but rather unchemical grand apparatus of Idria.

Details of the aludel apparatus : 25 are set in each of the 12 ranges, seen in fig. 661 , constituting 300 pear-shaped stoneware vessels, open at both ends, being merely thrust into one another, and luted with loam. What a multitude of joints, of which a great many. must be continually giving way by the shrinkage of the luting, whereby the mercurial fumes will escape with great loss of product, to poison the air !

$a$, is the door of the fire-place; $c$, the perforated arches upon which the ore is piled in the chamber $e$, through the door $d$, and an orifice at top; the latter being closed durng the distillation; $f f$ are vents for conducting the mercurial vapors into two chambers $i$, separated by a triangular body of masonry $m n ; h$ is the smoke chimney of the fire-place; $O D$, are the ranges of aludels, in connexion with the chamber $i$, which are laid slantingly towards the gutter $q$, upon the double inclined plane terrace, and terminate in the chamber $h q$; this being surmounted by two chimneys $t$. The mercury is collected in these aludels and in the basins at $q$ and $p$, fig. 661. $r$ is a thin stone partition set up between the two principal walls of each of the furnaces. $v$ is the stair of the aludel terrace, leading to the platform which surmounts the furnace; $z$ is a gutter for conducting away the rains which may fall upon the buildings.

Great apparatus of Idria.-Before entering into details of this laboratory, it will not be useless to recapitulate the metallurgic classification of the ores treated in it. 1. the ores in large blocks, fragments, or shivers, whose size varies from a cubic foot to that of a nut. 2. The smaller ores, from the size of a nut to that of grains of dust.

The first class of large ores comprises three subdivisions, namely ; $a$, blocks of metalliferous rocks, which is the most abundant and the poorest species of ore, affording only one per cent. of mercury; $b$, the massive sulphuret of mercury, the richest and rarest ore, yielding 80 per cent. when it is picked; $c$, the fragments or splinters proceeding from the breaking and sorting, and which vary in value, from 1 to 40 per cent.

The second class of small ores comprises : $d$, the fragments or shivers extracted from the mine in the state of little pieces, affording from 10 to 12 per cent.; $e$, the kernels of orc separated on the sieve, yielding 32 per cent. ; $f$ the sands and paste called schlich, obtained in the treatment of the poorest ores, by means of the stamps and washing tables; 100 parts of this schlich give at least 8 of quicksilver.

The general aspect of the apparatus is indicated by figs. 663, 664, and 665. Fig. 665,

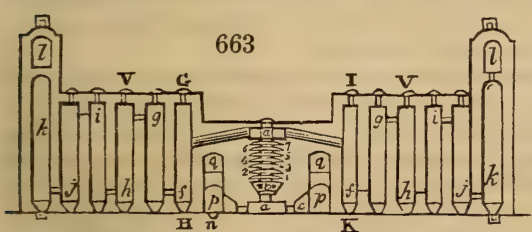
represents the exterior, but only one half, which is enough, as it resembles exactly the other, which is not shown. In these three figures the following objects may be distinguished; figs. 663, $664, a$, door of the fire-place; $b$, the furnace in which beech-wood is burned mixed with a little fir-wood; $c$, door of the ash-pit, extended beneath; $d$, a space in which the ores are deposited upon the seven arches, 1 to 7 , as indicated in figs. 663 , and 666 ; $e$, brick tunnels, by which the smoke of the fuel and the vapors of mercury pass, on the one side, into successive chambers $f k$.

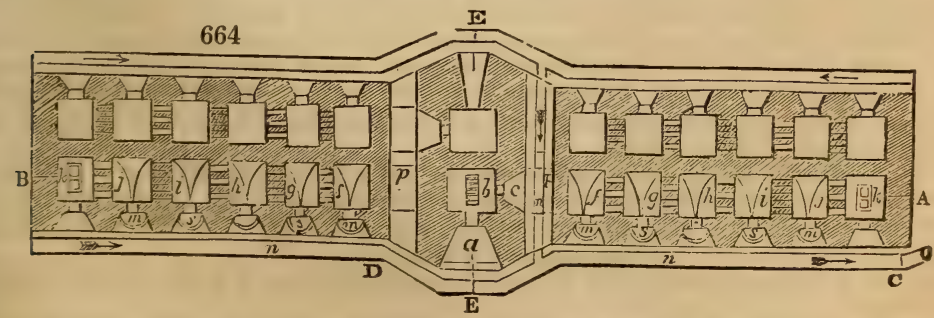

$f \mathrm{~g} h i j k l$ are passages which permit the circulation of the vapors from the furnace $a b c d$, to the chimneys $l l$. Figs. 663 and 664 exhibit clearlv the distribution of these 
openings on each side of the same furnace, and in each half of the apparatus, which is double, as fig. 664 shows; the spaces without letters being in every respect similar to the spaces mentioned below. Fig. 664 is double the scale of fig. 663 .

$m m^{\prime}$, fig. 664, are basins of reception, distributed before the doors of each of the chambers $f k f^{\prime} k^{\prime}$. The condensed mercury which flows out of the chambers is conveyed thither. $n n^{\prime}$ is a trench into which the mercury, after being lifted into the basins $m$, is poured, so that it may run towards a common chamber $o$, in the sloping direction indicated by the arrows. o leads to the chamber where the mercury is received into a porphyry trough; out of which it is laded and packed up in portions of 50 or $100 \mathrm{lbs}$. in sheep-skins prepared with alum. $p p^{\prime}, f i g .663$, are vaulted arches, through which a circulation may go on round the furnace $a b c d$, on the ground level. $q q^{\prime}$ are the vaults of the upper stories. $r r^{\prime}, f i g$. 665, vaults which permit access to the tunnels $e^{\prime} e^{\prime \prime}$, -fig. 663.

$s s^{\prime}$ and $t t^{\prime}$, fig. 665, are the doors of the chambers $f k$ and $f^{\prime} k^{\prime}$. These openings are shut during the distillation by wooden doors faced with iron, and luted with a mortar of clav and lime. $u u^{\prime}$ is the door of the vaults 1 to 7 of the furnace represeuted in fig. 663. These openings are hermetically shut, like the preceding. $\mathbf{v ~ v}^{\prime}$, fig. 663, are superior openings of the chambers, closed duing the operation by luted plugs; they are opened afterwards to facilitate the cooling of the apparatus, and to collect the mercurial soot. $x y z$, fig. 666, are floors which correspond to the doors $u u^{\prime}$ of the vaults 1 to 7 ,

$66 b$

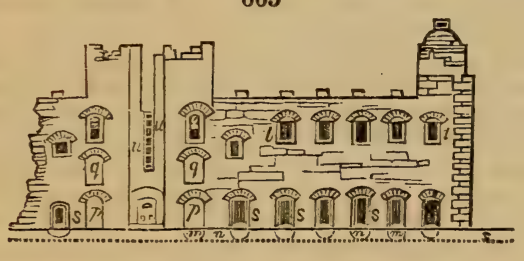

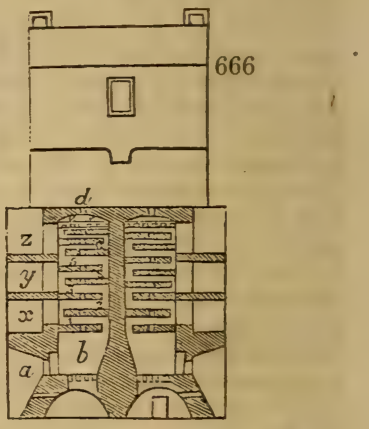

fig. 665. These floors are reached by stairs set up in the different parts of the building, which contains the whole apparatus.

On the lower arches the largest blecks of metalliferous rock are laid; orer these the less bulky fragments are arranged, which are covered with the shivers and pieces of less dimension. On the middle vaults, the small ore is placed, distributed into cylindrical pipkins of earthenware, of 10 inches diameter and 5 inches depth. The upper vaults receive likewise pipkins filled with the sands and pastes called schlich.

In 3 hours, by the labor of 40 men, the two double sets of apparatus are charged, and all the apertures are closed. A quick fire of beech-wood is then kindled; and when the whole mass has become sufficiently heated, the sulphuret of mercury begins to vapor. ize; coming into contact with the portion of oxygen which had not been carbonated, by combustion, its sulphur burns into sulphurous acid, while the mercury becomes free, passes with the other vapors into the chambers for condensing it, and precipitates in the liquid form at a greater or less distance from the fire-place. The walls of the chambers and the floors, with which their lower portion is covered, are soon coated over with a black mercurial soot, which, being treated anew, furnishes 50 per cent. of mercury. The distillation lasts from 10 to 12 hours; during which time the whole furnace is kept at a cherry-red heat. A complete charge for the two double apparatus, consists of from 1000 to 1300 quintals of ore, which produce from 80 to 90 quintals of running mercury. The furnace takes from 5 to 6 days ta cool, according to the state of the weather; and if to that period be added the time requisite for withdrawing the residuums, and attending to such repairs as the furnace may need, it is obvious that only one distillation can be performed in the course of a week.

In the works of Idria, in $1812,56,686$ quintals and a half of quicksilver ores were distilled, after undergoing a very careful mechanical preparation. They afforded 4832 quin. tals of running mercury ; a quantity corresponding to about $8 \frac{1}{2}$ per cent. of the ore. These smelting works are about 180 feet long and 30 feet high.

Upon the preceding three systems of smelting mercurial ores, I shall now make some observations.

It has been long well known, that quicksilver may be most readily extracted from cinnabar, by heating it in contact with quicklime. The sulphur of the cinnabar com- 
bines, by virtue of a superior affinity with the lime, to the exclusion of the quicksilver, to form sulphurets of lime and calcium, both of which being fixed hepars, remain in the retort while the mercury is volatilized by the heat. In a few places, hammerschlag, or the iron cinder, driven off from the blooms by the tilting hammer, has been used instead of lime in the reduction of this mercurial ore, whereby sulphurous acid and sulphuret of iron are formed.

The annual production of the Bavarian Rhine provinces has been estimated at from 400 to 550 quintals; that of Almaden, in the year 1827, was 22,000 quintals; and of Idria, at present, is not more than 1500 quintals.

All the plans hitherto prescribed for distilling the ore along with quicklime, are remarkiably rude. In that practised at Landsberg by Obermoschel, there is a great waste of labol, in charging the numerous small cucurbits; there is a great waste of fuel in the mode of heating them; a great waste of mercury by the imperfect luting of the retorts to the receivers, as well as the imperfect condensation of the mercurial vapors; and probably a considerable loss by pilfering.

The modes practised at Almaden and Idria are, in the greatest degree, barbarous ; the ores being heated upon open arches, and the vapors attempted to be condensed by enclosing them within brick or stone and mortar walls, which can never be rendered either sufficiently tight or cool.

To obviate all these inconveniences and sources of loss, the proper chemical arrangements suited to the present improved state of the arts ought to be adopted, by which labor, f:el, and mercury, might all be economized to the utmost extent. The only apparatus fit to be employed is a series of cast-iron cylinder retorts, somewhat like those employed in the coal gas works, but with peculiarities suited to the condensation of the mercurial vapors. Into each of these retorts, supposed to be at least one foot square in area, and 7 feet long, 6 or 7 cwts. of a mixture of the ground ore with the quicklime, may be easily introduced, from a measured heap, by means of a shovel. The specific gravity of the cinnabar being more than 6 times that of water, a cubic foot of it will weigh more than $3 \frac{1}{2}$ cwts.; but supposing the mixture of it with quicklime (when the ore does not contain the calcareous matter itself) to be only thrice the density of water, then four cubic feet might be put into each of the above retorts, and still leave $1 \frac{1}{2}$ cubic feet of empty space for the expansion of volume which may take place in the decomposition. The ore should certainly be ground to a moderately fine powder, by stamps, iron cylinders, or an edge wheel, so that when mixed with quicklime, the cinnabar may be brought into intimate contact with its decomposer, otherwise much of it will be dissipated unproductively in fumes, for it is extremely volatile.

Figs. 667, 668, 669, represent a cheap and powerful apparatus which I contrived at the request of the German Mines Company of London, and which is now mounted at Landsberg, near Obermoschel, in the Bavarian Rhein-Kreis.

Fig. 667 , is a section parallel to the front elevation of three arched benches of retorts,

667

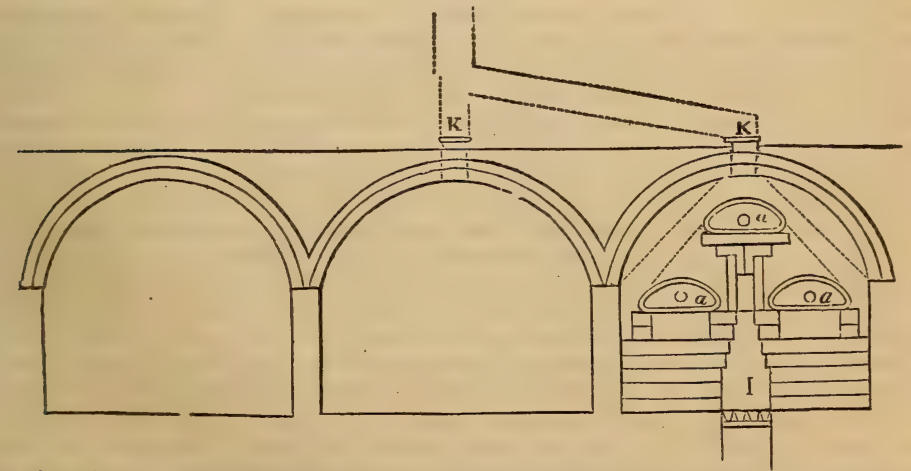

of the sizc above specified. Each bench contains 3 retorts, of the form represented by $\begin{array}{lll}a & a & a\end{array} \mathrm{I}$, is the single fire-place or furnace, capable of giving adequate ignition by coal or wood, to the three retorts. The retorts were built up in an excellont manner, by an English mason perfectly acquainted with the best modes of erecting coal-gas retorts, who was sent over on purpose. The path of the flame and smoke is precisely similar to that represented in fig. 483, page 555, whereby the uppermost retort is immersed in a bath of uniformly ignited air, whlle the currents reverberated from the top, play round the two undermost retorts, in their way to the vent-flues beneath 
them. The bottom of the uppermost retort is protected from the direct impulse of the flame by fire-tiles. The dotted lines $\mathrm{K} \mathrm{K}$, show the paths of the chimneys which rise at the back ends of the retorts.

In the section, fig. $668, a$ is the body of the retort; its mouth at the right hand end 668

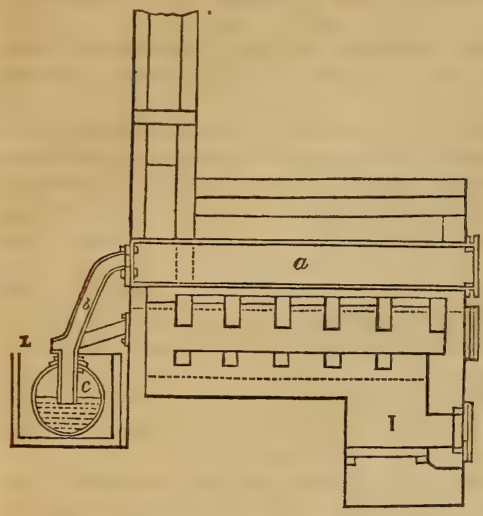

is shut, as usual, by a luted iron lid, secured with a cross-bar and screw-bolts; its other end is prolonged by a sloping pipe of cast iron, 4 inches in diameter, furnished with a nozzle hole at $L$, closed with a serew plug. Through this hole a wire rammer may be introduced, to ascertain that the tube is pervious, and to cleanse it from the mercurial soot, when thought necessary. $c$, is a cross section of the main condenser, shown in a longitudinal section at c c, fig. 669 . This pipe is 18 inches in diameter, and about 20 feet long. At $a$ a, \&c., the back ends of the retorts are seen, with the slanting tubes $b b$, \&c., descending through orifices in the upper surface of the condenser pipe, and dipping their ends just below the water-line $h i$. g, is the cap of a water valve, which removes all risk from sudden expansion or condensation. The condenser is placed within a rectangular trough, made either of wood or stone, through which a sufficient stream of water passes to keep it perfectly cool, and repress every trace of mercurial vapor, and it is laid with a slight inclination from $i$ to $h$, so that the condensed quicksilver may spontaneously flow along its bottom, and pass through the

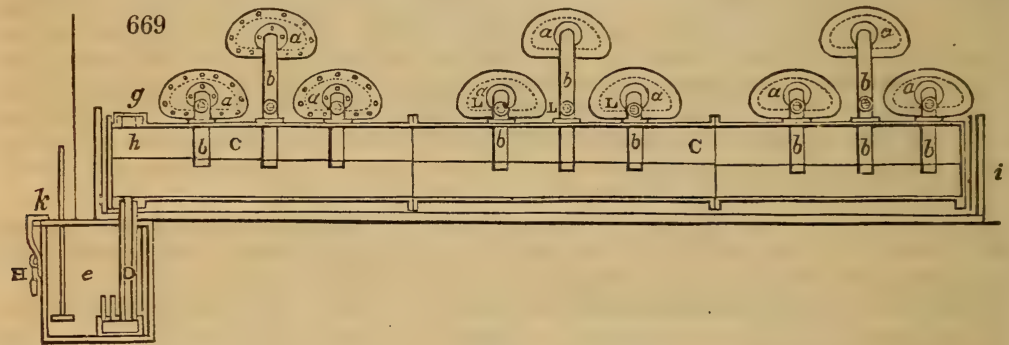

vertical tube $\mathrm{D}$ into the locked up iron chest, or magazine $e$. This tube $\mathrm{D}$ is from the beginning closed at bottom, by immersion in a shallow iron cup, always filled with mercury. $k$ is a graduated gauge rod, to indicate the progressive accumulation of quicksilver in the chest, without being under the necessity of unlocking it.

This air-tight apparatus was erected about a year ago, and has been found to act perfectly well ; İ regret, however, that my professional engagements at home have not. hitherto permitted me to conduct its operations personally for some days. The average samples of cinnabar ore from Obermoschel are ten times poorer than those of Almaden. Were such an apparatus as the above, with some slight modifications which have lately occurred to me, mounted for the Spanish mines, I am confident that their produce in quicksilver might be nearly doubled, with a vast economy of fuel, labor, and human life. The whole cost of the 9 large retorts, with their condensing apparatus, iron magazine, \&c., was very little more than tuso hundred pounds! As the retorts are kept in a state of nearly uniform ignition, like those of the gas works, neither they nor the furnaces are liable to be injured in their joints by the alternate contractions and expansions, which they would inevitably suffer if allowed to cool; and being always ready heated to the proper pitch for decomposing the mercurial ores, they are capable of working off a charge, under skilful management, in the course of 3 hours. Thus, in 24 hours, with a relay of laborers, 8 charges of at least 5 cwts. of ore each, might be smelted $=2$ tons, with 3 retorts, and 6 tons with 9 retorts; with a daily product from the rich ores of Almaden, or even Idria, of from $12 \mathrm{cwts}$. to $20 \mathrm{cwts}$. Instead of 3 benches of 3 retorts each, I would recommend 15 benches, containing 45 retorts, to be erected for either the Almaden or Idria mines; which, while they would smelt all their ores, could be got for a sum not much exceeding $1000 l$., an outlay whicb they would reimburse within a month or two.

Quicksilver is a substance of paramount value to science. Its great density and its reguar rate of expansion and contraction by increase and diminution of temperature, 
give it the preference over all liquids for filling barometric and thermometric tubes. In chemistry it furnishes the only means of collecting and manipulating, in the pneumatic trough, such gaseous bodies as are condensable over water. To its aid, in this respect, the modern advancement of chemical discovery is pre-eminently due.

This metal alloyed with tin-foil forms the reflecting surface of looking glasses, and by its ready solution of gold or silver, and subsequent dissipation by a moderate heat, it becornes the great instrument of the arts of gilding and silvering copper and brass. The same property makes it so available in extracting the e precious metals from their ores. The anatomist applies it elegantly to distend and display the minuter vessels of the lyc.phatic system, and secretory systems, by injecting it with a syringe through all their convolutions. It is the basis of many very powerful medicines, at present probably too indiscriminately used, to the great detriment of English society; for it is far more sparingly prescribed by practitioners upon the continent of Europe, not otherwise superior in skill or science to those of Great Britain.

The nitrate of mercury is employed for the secretage of rabbit and hare-skins, that is, for communicating to the fur of these and other quadrupeds the faculty of felting, which they do not naturally possess. With this view the solution of that salt is applied to them lightly in one direction with a sponge. A compound amalgam of zinc and tin is probably the best exciter which can be applied to the cushions of electrical machines. Mercury imported for home consumption in 1836, 286,808 lbs.; in 1837, 314,036 lbs.

The only mercurial compounds which are extensively used in the arts, are factitious cinnabar or VERMrION, and corrosive sublimate.

MERCURY, BICHLORIDE OF ; Corrosive sublimate (Deutochlorure de mercure, Fr. ; Aetzendes quecksilber sublimat, Germ.), is made by subliming a mixture of equal parts of persulphate of mercury, prepared as above described, and sea-salt, in a stoneware cucurbit. The sublimate rises in vapor, and incrusts the globular glass capital with a white mass of small prismatic needles. Its specific gravity is $5 \cdot 14$. Its taste is acrid, styptometallic, and exceedingly unpleasant. It is soluble in 20 parts of water, at the ordinary temperature, and in its own weight of boiling water. It dissolves in $2 \frac{1}{2}$ times its weight of cold alcohol. It is a very deadly poison. Raw white of egg swallowed in profusion, is the best antidote. A solution of corrosive sublimate has been long employed for preserving soft anatomical preparations. By this means the corpse of Colonel Morland was embalmed in order to be brought from the seat of war to Paris. His features remained unaltered, only his skin was brown, and his body was so hard as to sound like a piece of wood when struck with a hammer.

In the valuable work upon the dry rot, published by $\mathrm{Mr}$. Knowles, secretary of the committee of inspectors of the navy, in 1821, corrosive sublimate is enumerated among the chemical substances which had been prescribed for preventing the dry rot in timber; and it is well known that Sir H. Davy had, several years before that date, used and recommended to the Admiralty and Navy Board, corrosive sublimate as an anti-dry rot application. It has been since extensively employed by a joint-stock company for the same purpose, under the title of Kyan's patent.

MERCURY, PROTOCHLORIDE OF; Calomel; (Protochlorure de mercure, Fr.; Versiisstes quecksilber, Germ.) This compound, so much used and abused by medical practitioners, is commonly prepared by triturating four parts of corrosive sublimate along with three parts of running quicksilver in a marble mortar, till the metallic globules entirely disappear, with the production of a black powder, which is to be put into a glass balloon, and exposed to a subliming heat in a sand bath. The calomel, which rises in vapor, and attaches itself in a crystalline crust to the upper hemisphere of the balloon, is to be detached, reduced to a fine powder, or levigated and elutriated. $200 \mathrm{lbs}$. of mercury yield 236 of calomel and 272 of corrosive sublimate.

The following more economical process is that adopted at the Apothecaries' Hall, London. 140 pounds of concentrated sulphuric acid are boiled in a cast iron pot upon 100 pounds of mercury, till a dry persulphate is obtained. Of this sait, 124 pounds are triturated with 81 pounds of mercury, till the globules disappear, and till a protosulphate be formed. This is to be intimately mixed with 68 pounds of sea-salt, and the mixture, being put into a large stone-ware cucurbit, is to be submitted to a subliming heat. See Calomel.

From 190 to 200 pounds of ealomel rise in a crystalline cake, as in the former process, into the capital; while sulphate of soda remains at the bottom of the alembic. The ealomel must be ground to an impalpable powder, and elutriated. The vapors, instead of being condensed into a cake within the top of the globe or in a capital, may be allowed to diffuse themselves into a close vessel, containing water in a state of ebullition, whereby the calomel is obtained at once in the form of a washed impalpable powder. Calomel is tasteless and insoluble in water. Its specific gravity is $\mathbf{7 \cdot 1 7 6 .}$

For the compound of mercury with fulminic acid, see Fulmrnate. Periodide of nercury is a bright but fugitive red pigment. It is easily prepared by dropping a solution 
of iodide of potassrum into a solution of corrosive sublimate, as long as any precipitation takes place, decanting off the supernatant muriate of potash, washing and drying the precipitate.

METALLURGY (Erzkunde, Germ.) is the art of extracting metals from their ores. This art, which supplies industry with the instruments most essential to its wants, is alike dependant upon the sciences of chemistry and mechanics; upon the former, as directing the smelting processes, best adapted to disentangle each metal from its mineral izer; upon the latter, as furnishing the means of grinding the ores, and separating the light stony parts from the rich metallic matter.

Notwithstanding the striking analogy which exists between common chemical and metallurgic operations, since both are employed to insulate certain bodies from others, there are essential differences which should be carefully noted. In the first place, the quantity of materials being always very great in metallurgy, requires corresponding adaptations of apparatus, and often produces peculiar phenomena; in the second place, the agents to be employed for treating great masses, must be selected with a view to economy, as well as to chemical action. In analytical chemistry, the main object being exactness of result, and purity of product, little attention is bestowed upon the value of the reagents, on account of the small quantity required for any particular process. But in smelting metals upon the great seale, profit being the sole object, cheap materials and easy operations alone are admissible.

The metallic ores as presented by nature, are almost always mixed with a considerable number of foreign substances; and could not therefore be advantageously submitted to metallurgic operations, till they are purified and concentrated to a certain degree bs various methods.

OF THE PREPARATION OF ORES FOR THE SMELTING HOUSE.

There are two kinds of preparation; the one termed mechanical, from the means em. ployed, and the results obtained, consists in processes for breaking and grinding the ores, and for washing them so as to separate the vein-stones, gangues, or other mixed earthy matters, in order to insulate or concentrate the metallic parts.

Another kind of preparation, called chemical, has for its object to separate, by means of fire, various volatile substances combined in the ores, and which it is requisite to clear away, at least in a certain degree, before trying to extract the metals they may contain.

Lastly, an indispensable operation in several circumstances, is to discover, by simple and cheap methods, called assays, the quantity of metal contained in the different species of ores to be treated.

This head of our subject, therefore, falls under three subdivisions:-

$\S 1$. The mechanical preparation of ores, including picking, stamping, and different modes of washing.

$\S 2$. The chemical preparation, consisting especially in the roasting or calcination of the ores.

$\S 3$. The assay of ores, comprehending the mechanical part: that is, by washing; the chemical part, or assays by the dry way; and the assays by the moist way.

Section 1. Of the mechanical preparation or dressing of ores.-I. The first picking or sorting takes place in the interior, or underground workings, and consists in separating the fragments of rocks, that apparently contain no metallic matter, from those that contain more or less of it. The external aspect guides this separation; as also the feeling of density in the hand.

The substances, when turned out to the day, undergo another sorting, with greater or less care, according to the value of the included metal. This operation consists in breaking the lumps of ore with the hammer, into fragments of greater or less size, usually as large as the fist, whereby all the pieces may be picked out and thrown away that contain no metal, and even such as contain too little to be smelted with advantage. There is, for the most part, a building erected near the output of the mine, in which the breaking and picking of the ores are performed. In a covered gallery, or under a shed, bankis of earth are thrown up, and divided into separate beds, on each of which a thick plate of cast iron is lald. On this plate, elderly workmen, women, and children, break the ores with hand hammers, then pick and sort them piece by piece. The matters so treated, are usually separated into three parts; 1 . the rock or sterile gangue, which is thrown away; 2 . the ore for the stamping mill, which presents too intimate a mixture of rock and metallic substance to admit of separation by breaking and picking; and 3. the pure ore, or at least the very rich portion, called the sorted mine or the fat ore. On the sorting floors there remains much small rubbish, which might form a fourth subdivision of ore, since it is treated in a peculiar manner, by sifting, as will be presently sentioned.

The distribution of fragments more or less rich, in one class or another, is relative to the value of the included metal, taking into account the expenses necessary for its extraction. 
Thus in certain lead mines, pieces of the gangues are thrown away, which judged by the eye may contain 3 per cent. of galena, because it is known that the greater portion of this would be lost in the washings required for separating the 97 parts of the gangue, and that the remainder would not pay the expenses.

II. The very simple operations of picking are common to almost all ores; but there are other operations requiring more skill, care, and expense, which are employed in their final state of perfection only upon ores of metals possessing a certain value, as those of lead, silver, \&c. We allude to the washing of ores.

The most simple and economical washings are those that certain iron ores, particularly the alluvial, are subjected to, as they are found near the surface of the ground agglutinated in great or little pieces. It is often useful to clean these pieces, in order to pick out the earthy lumps, which would be altogether injurious in the furnaces.

This crude washing is performed sometimes by men stirring in the midst of a stream of water, with iron rakes or shovels, the lumps of ore placed in large chests, or basins of wood or iron.

In other situations, this washing is executed more economically by a machine called a buddle or dolly-tub by our miners. A trough of wood or iron, with a concave bottom, is filled with the ore to be washed. Within the tub or trough, arms or iron handles are moved round about, being attached to the arbor of a hydraulic wheel. The trough is kept always full of water, which as it is renewed carries off the earthy matters, . 'iffused through it by the motion of the machine, and the friction among the pieces of the ore. When the washing is finished, a door in one of the sides of the trough is opened, and the current removes the ore into a more spacious basin, where it is subjected to a kind of picking. It is frequently indeed passed through sieves in different modes. See LEAD and Tin, for figures of buddles and dollies.

III. Stamping. Before describing the refined methods of washing the more valuable ores of copper, silver, lead, \&c., it is proper to point out the means of reducing them into a powder of greater or less fineness, by stamping, so called from the name stamps of the pestles employed for that purpose. Its usefulness is not restricted to preparing the ores; for it is employed in almost every smelting house for pounding clays, eharcoal, scoriæ, \&c. A stamping mill or pounding machine, fig. 670 , consists of several

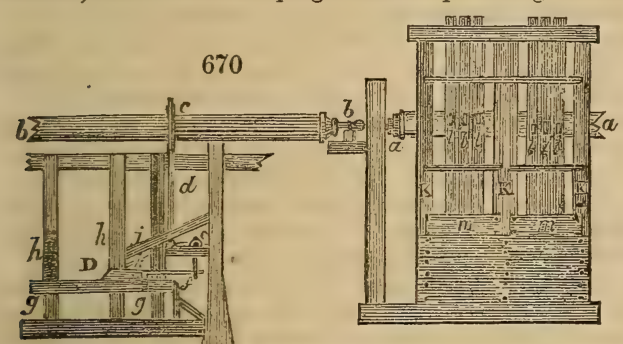
moveable pillars of wood $l l l$, placed vertically, and supported in this position between frames of carpentry $\mathbf{k} \mathbf{k} \mathbf{~}$. 'These pieces are each armed at their under end with a mass of iron $m$. An arbor or axle $a a$, moved by water, and turning horizontally, tosses up these wooden pestles, by means of wipers or cams, which lay hold of the shoulders of the pestles at $l l l$. These are raised in succession, and fall into an oblong trough below $m m$, scooped out in the ground, having its bottom covered either with plates of iron or hard stones. In this trough, beneath these pestles, the ore to be stamped is allowed to fall from a hopper above, which is kept constantly full.

The trough is closed in at the sides by two partitions, and includes three or four pestles; which the French miners call a battery. They are so disposed that their ascent and descent take place at equal intervals of time.

Usually a stamping machine is composed of several batteries (two, three, or four), and the arrangement of the wipers on the arbor of the hydraulic wheel is such that there is constantly a like number of pestles lifted at a time; a circumstance important for maintaining the uniform going of the machine.

The matters that are not to be exposed to subsequent washing are stamped dry, that is, without leading water into the trough; and the same thing is sometimes done with the rich ores, whose lighter parts might otherwise be lost.

Most usually, especially for ores of lead, silver, copper, \&c., the trough of the stamper is placed in the middle of a current of water, of greater or less force; which, sweeping off the pounded substances, deposites them at a greater or less distance onwards, in the order of the size and richness of the grain; constituting a first washing, as they escape from beneath the pestles.

In the dry stamping, the fineness of the powder depends on the weight of the pestles, the height of their fall, and the period of their action upon the ore; but in the stampers exposed to a stream of water, the retention of the matters in the trough is longer or shorte; according to the facility given for their escape. Sometimes these matters flow out of the chest orer its edges, and the height of the line they must surmount has an influence on the size of the grain; at other mes, the water and the pounded matter 
which it carries off, are inade to pass through a grating, causing a kind of sifting at the same time. There are, however, some differences in the results of these two methods. Lastly, the quantity of water that traverses the trough, as well as its velocity, has an influence on the discharge of the pounded matters, and consequently on the products of the stampers.

The size of the particles of the pounded ore being different, according to the variable hardness of the matters which compose them, suggests the means of classing them, and distributing them nearly in the order of their size and specific gravity, by making the water, as it escapes from the stamping trough, circulate in a system of canals called a labyrinth, where it deposites successively, in proportion as it loses its velocity, the earthy and metallic matters it had floated along. These metalliferous portions, especially when they have a great specific gravity like galena, would be deposited in the first passages, were it not that from their hardness being inferior to that of the gangue, they are reduced to a much finer powder, or into thin plates, which seem to adhere to both the watery and earthy particles; whence they have to be sought for among the finest portions of the pulverized gangue, called slime, schlich, or schlamme.

There are several methods of conducting the stamps; in reference to the size of the grains wished to be obtained, and which is previously determined agreeably to the nature of the ore, and of the gangue; its richness, \&c. The height of the slit that lets the pounded matters escape, or the diameters of the holes in the grating, their distance, the quantity of water flowing in, its velocity, \&c., modify the result of the stamping operation.

When it is requisite to obtain powder of an extreme fineness, as for ores that are to be subjected to the process of amalgamation, they are passed under millstones, as in common corn mills; and after grinding, they are bolted so as to form a species of flour; or they are crushed between rolls. See LEAD and TIN.

\section{Washing of ores.}

IV. The ores pounded under the stamps are next exposed to very delicate operations, both tedious and costly, which are called the washings. Their purpose is to separate mechanically the earthy matters from the metallic portion, which must therefore have a much higher specific gravity; for otherwise, the washing would be impracticable.

The medium employed to diminish the difference of specific gravity, and to move along the lightest matters, is water; which is made to flow with greater or less velocity and abundance over the schlich or pasty mud spread on a table of various inclination.

But as this operation always occasions, not only considerable expense, but a certain loss of metal, it is right to calculate what is the degree of richness below which washing is unprofitable; and on the other hand, what is the degree of purification of the schlich at which it is proper to stop, because too much metal would be lost comparatively with the expense of fusing a small additional quantity of gangue. There cannot, indeed, be any fixed rule in this respect, since the elements of these calculations vary for every work.

Before describing the different modes of washing, we must treat of the sifting or riddling, whose purpose, like that of the labyrinth succeeding the stamps, is to distribute and to separate the ores (which have not passed through the water stamps) in the order of the coarseness of grain. This operation is practised particularly upon the debris of the mine, and the rubbish produced in breaking the ores. These substances are put into a riddle, or species of round or square sieve, whose bottom is formed of a grating instead of a plate of metal pierced with holes. This riddle is plunged suddenly and repeatedly into a tub or cistern filled with water. This liquid enters through the bottom, raises up the mineral particles, separates them and keeps them suspended for an instant, after which they fall down in nearly the order of their specific gravities, and are thus classed with a certain degree of regularity. The sieve is sometimes dipped by the immediate effort of the washer; sometimes it is suspended to a swing which the workman moves; in order that the riduling may be rightly done, the sieve should receive bat a single movement from below upwards; in this case the ore is separated from the gangue, and if there be different specific gravities, there are formed in the sieve as many distinct strata, which the workman can easily take out with a spatula, throwing the upper part away when it is too poor to be re-sifted. This operation by the hand-sieve, is called riddling in the $t u b$, or riddling by deposite.

We may observe, that during the sifting, the particles which can pass across the holes of the bottom, fall into the tub and settle down there; whence they are afterwards gathered out, and exposed to washing when they are worth the trouble.

Sometimes, as at Poulläouen, the sieves are conical, and held by means of two handles by a workman; and instead of receiving a single movement, as in the preceaing method, the sifter himself gives them a variety of dexterous movements in succession. His object is to separate the poor portions of the ore from the richer; in order to subject the former to the stamp mill.

Among the siftings and washings which ores are made to undergo, we must notice a 
among the most useful and ingenious, those practised by iron gratings, called on the Continent grilles anglaises, and the step-washings of Hungary, laveries à gradins. These methods of freeing the ores from the pulverulent earthy matters, consist in placing them, at their ont-put from the mine, upon gratings, and bringing over them a stream of water, which merely takes down through the bars the small fragments, but carries off the pulverulent portions. The latter are receired in cisterns, where they are allowed to rest long enough to settle to the bottom. The washing by steps is an extension of the preceding plan. To form an idea, let us imagine a series of grates placed successively at different levels, so that the water, arriving on the highest, where the ore for washing lies, carries off a portion of it, through this first grate upon a second closer in its bars, thence to a third, \&cc., and finally into labyrinths or cisterns of deposition.

The grilles anglaises are similar to the sleeping tables used at Idria. The system of these en gradins is represented in fig. 671 . There are 5 such systems in the works at Idria, for

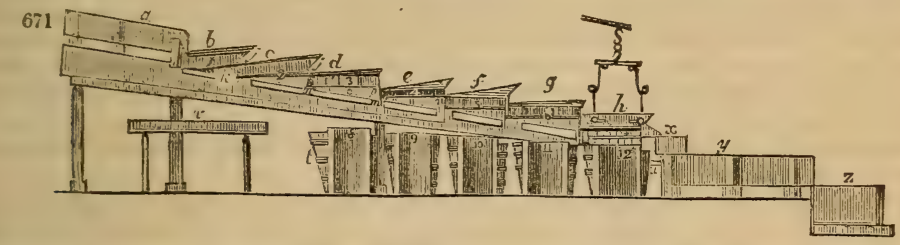

the sorting of the small morsels of quicksilver ore, intended for the stamping mill. These fragments are but moderately rich in metal, and are picked up at random, of various sizes, from that of the fist to a grain of dust.

These ores are placed in the chest $a$, below the level of which 7 grates are distributed, so that the fragments which pass through the first $b$, proceed by an inclined conduit on to the second grate $c$, and so in succession. (See the conduits $l, o, p)$. In front, and on a lerel with each of the grates $b, c, d, \&$ c., a child is stationed on one of the floors, 1,23 , to 7.

A current of water, which falls into the chest $a$, carries the fragments of ore upon the grates. The pieces which remain upon the two grates $b$ and $c$, are thrown on the adjoining table $v$, where they undergo a sorting by hand; there the pieces are classified, 1 . into gangue to be thrown away; 2 . into ore for the stamp mill; 3 . into ore to be sent directly to the furnace. The pieces which remain on each of the succeeding grates, $d, e, f, g, h$, are deposited on those of the floors 3 to 7 , in front of each. Before every one of these shelres a deposite-sieve is established, (see $t, u$, ) and the workmen in charge of it stand in one of the corresponding boxes, marked 8 to 12. The sieve is represented only in front of the chest $h$, for the sake of clearness.

Each of the workmen placed in $8,9,10,11,12$, operates on the heap before him; the upper layer of the deposite formed in his sieve, is sent to the stamping house, and the inferior layer directly to the furnace.

As to the grains which, after traversing the five grates, have arrived at the chest $x$, they are washed in the two chests $y$, which are analogous to the German chests to be presently described. The upper layer of what is deposited in $y$ is sent to the furnace; the rest is treated anew on three tables of percussion, similar to the English brake-sieves, also to be presently deseribed.

After sereral successire manipulations on these tables, an upper stratum of schlich is obtained fit for the furnace; an intermediate stratum, which is washed anew by the same process; and an inferior stratum, that is sent to the system of stamps, fig. 672.

$6 \% 2$

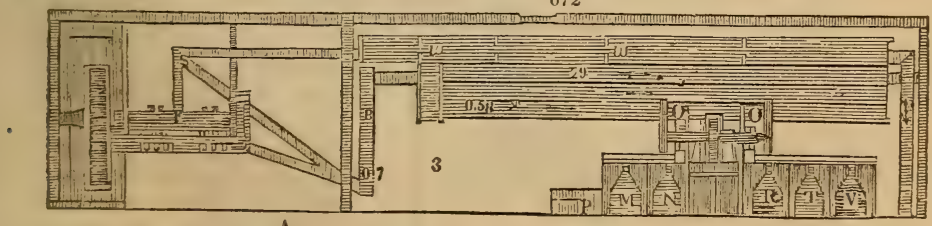

A

This figure represents the general ground plan of a stamping and washing mill. The stamps $\mathrm{F}$ are composed of two batteries similar to fig. 670. The ore passes in succession under three pestles of cast iron, each of which is heavier the nearer it is to the sieve through which the sand or pounded matter escapes.

In the upper part of the figure we see issuing from the stamps, two conduits destined to receive the water and the metalliferous sand with which it is loaded. The first, marked $\boldsymbol{F} \mathrm{s}, w$, is used only when a certain quality of ore is stamped, richer in metal than i 
usually treated by means of the second conduit, the first being closed. The second con. duit, or that employed for ordinary manipulation when the other is shut, is indicated by $F, 0.7, \mathbf{B}$; then by 0.58 and 0.29 . These numbers express the depth of the corresponding portions of this conduit. From $\mathrm{F}$ to $\mathrm{B}$, the conduit or water-course is divided into three portions much shallower, called the rich conduit, the middle conduit, and the inferior. Beyond the basin $\mathrm{B}$, the conduit takes the name of labyrinth. There the muddy sediments of ore are deposited; being the finer the further they are from the stamps $\boldsymbol{s}$. Darts indicate the direction of the stream in the labyrinth. On the German chests, placed at 3 , the sand derived from the rich and middle conduits is treated, in order to obtain three distinct qualities of schlich, as already mentioned. P is a cloth-covered table, for treating the deposite of the German chests at 3. M N are two sweep tables ( $\dot{a}$ balui), for treating the ore collected in the lower conduit, which precedes the midmost of the three German chests. Upon the three similar tables $\mathrm{R} \mathrm{T} \mathrm{v}$, are treated in like manner the muddy deposites of the labyrinth, which forms suite to three parallel German chests situated at 3, not shown for want of room in the figure, but connected in three rectangular zigzags with each other, as well as by a transverse branch to the points 0.7 and $\mathbf{P}$. At the upper part of these five sweep tables, the materials which are to undergo washing are agitated in two boxes 00 , by small paddle-wheels.

We shall now describe the percussion-tables used in the Hartz, for treating the sand of ore obtained from the conduits represented above.

Figs. 673, 674, and 675, exhibit a plan, a vertical section, and elevation, of one 673

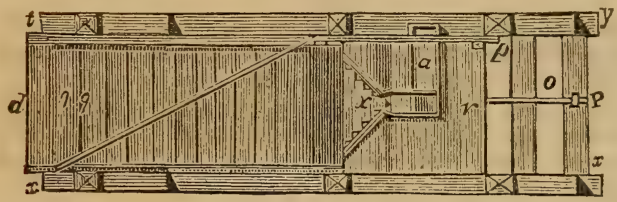

674
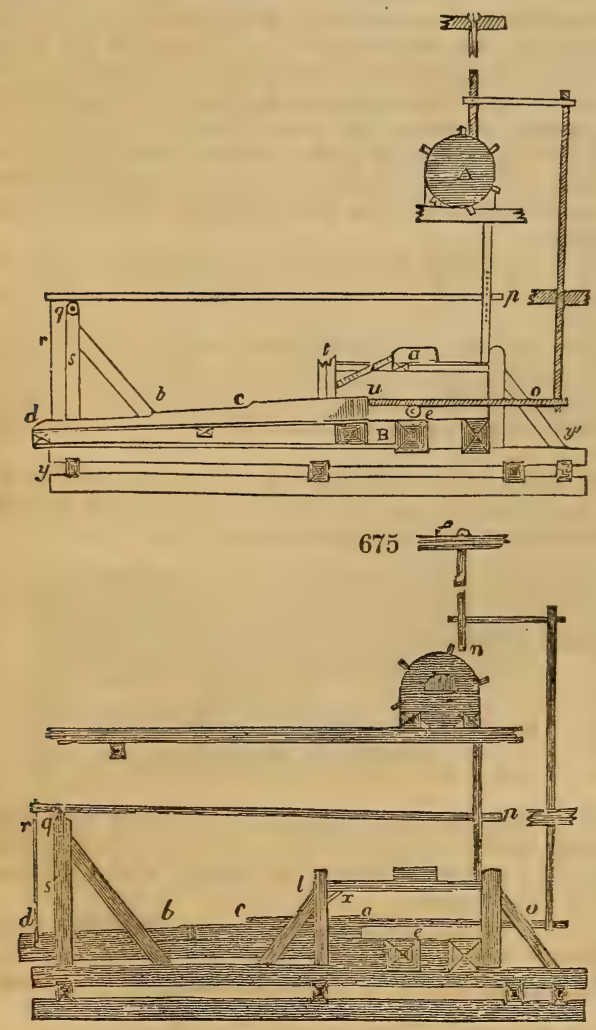
of these tables, taken in the direction of its length. The arbor or great shaft in prolongation from the stamps mill, is shown in section perpendicularly to its axis, at $\mathrm{A}$. The cams or wipers are shown round its eircumference, one of them having just acted on $n$.

These eams, by the revolntion of the arbor, cause the alternating movements of a horizontal bar of wood $o, u$, which strikes at the point $u$ against a table $d, b, c, w$. This table is suspended by two ehains $t$, at its superior end, and by two rods at its lower end. After having been pushed by the piece $o, u$, it rebounds to strike against a block or bracket B. A lever $p, q$, serves to adjust the inclination of the moveable table, the pivots $q$ being points of suspension.

The ore-sand to be washed, is placed in the chest $a$, into which a current of water runs. The ore floated onwards by the water, is carried through a sieve on a sloping small table $x$, under which is concealed the higher end of the moveable table $d, b, c, u$; and it thence falls on this table, diffusing itself uniformly over its surface. The particles deposited on this table form an oblong talus (slope) upon it; the successive percussions that it receives, deter mine the weightier matters, and consequently those richest in metal, to accumulate towards its upper end at $u$. Now the workman by means of the lever $p$, raises the lower end $d$ a little in order to preserve the same 
degree of inclination to the surface on which the deposite is strewed. According as the substances are swept along by the water, he is careful to remove them from the middle of the table ti:ivards the top, by means of a wooden roller. With this intent, he walks on the table $d b ; u$, where the sandy sediment has sufficient consistence to bear him. When the trable is abundantly charged with the washed ore, the deposite is divided into three bands or sexments $d b, b c, c u$. Each of these bands is removed separately and thrown into the particular heap assigned to it. Every one of the heaps thus formed becomes afterwaids the object of a separate manipulation on a percussion table, but always according to the same procedure. It is sufficient in general to pass twice over this table the matters contained in the heap, proceeding from the superior band $c u$, in order to obtain a pure schlich; but the heap proceeding from the intermediate belt $b c$, requires always a greater number of manipulations, and the lower band $d b$ still more. These successive manipulations are so associated that eventually each heap furnishes pure schlich, which is obtained from the superior band $c u$. As to the lightest particles which the water sweeps away beyond the lower end of the percussion table, they fall into conduits; whence they are lifted to undergo a new manipulation.

Fig. 676 is a profile of a plan which has been advantageously substituted, in the Hartz, for that part of the preceding apparatus which causes the jolt of the piece $o u$ against the table $d b c u$. By means of this plan, it is easy to vary, according to the circumstances of a manipulation always delicate, the force of percussion which a bar $x y$, ought to communicate by its extremity $y$. With this view, a slender piece of wood $u$ is made to slide in an upright piece, $v x$, adjusted upon an axis at $v$. To the piece $u$ a rod of iron is connected, by means of a hinge $z$; this rod is capable of entering more or less into a case or sheath in the middle of the piece $v x$, and of being stopped at the proper point, by a thumb-serew which presses against this piece. If it be wished to increase the force of percussion, we must lower the point $z$; if to diminish it, we must raise it. In the first case, the extremity of the piece $u$, advances so much further under

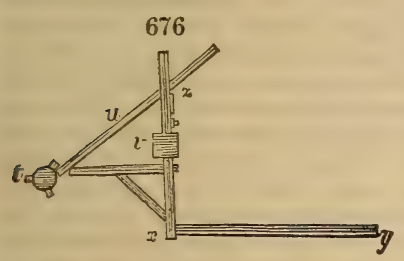

677

the eam of the driving shaft $t$; in the second, it goes so much less forwards; whereby the adjustment is produced.

Figs. $67 \%$ and 678 represent a complete system of sleeping tables, tables dormantes: 678

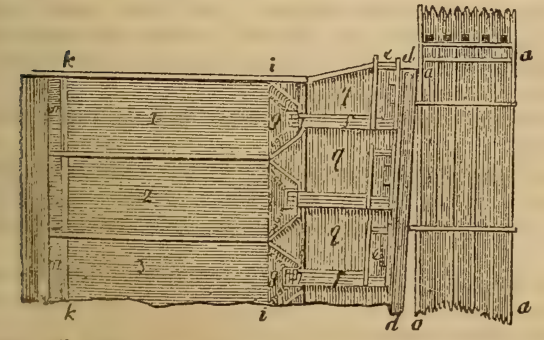
such as are mounted in Idria. Fig. 678 is the plan, and fig. 677 a vertical section. The mercurial ores, reduced to a sand by stamps like those of fig. 672 , pass into a series of conduits $a \cdot a, b b, c c$, which form three successive floors below the level of the floor of the works. The sand taken out of these conduits is thrown into the cells $q$; whence they are transferred into the trough $e$, and water is run upon them by turning two stopcocks for each trough. The sand thus diffused upon each table, runs off with the water by a groove $f$, comes upon a sieve $h$, spreads itself upon the board $s$, and thence falls into the slanting chest, or sleeping table $i k$. The under surface $k$ of this chest is pierced with holes, which may be stopped at pleasure with wooden plugs. There is a conduit $m$, at the lower end of each table, to catch the light particles carried off by the water out of the chest $i k$, through the holes properly opened, while the denser parts are deposited upon the bottom of this chest. A general conduit $\Omega$ passes across at the foot of all the chests $i k$; it receives the refuse of the washing operations.

Fig. 679 is a set of stamping and washing works for the ores of argentiferous galena, as mounted at Bockwiese, in the district of Zellerfeldt, in the Hartz.

$A$ is the stamp mill and its subsidiary parts; among which are $a$, the driving o 
main shaft; $b$, the overshot water-wheel; $c c$, six strong rings or hoops of cast iron, for receiving each a cam or tappet; $g$, the brake of the machine; $k, k, k$, the three 679

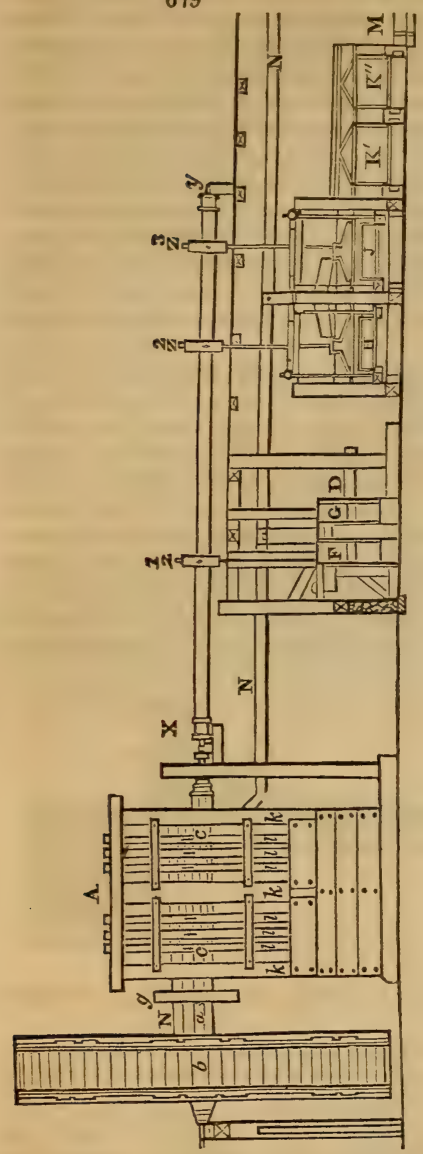
standards of the stamps; $l l$, \&c. six pestles of pine wood, shod with lumps of cast iron. There are two chests, out of which the ore to be ground falls spontaneously into the two troughs of the stamps. Of late years, however, the ore is mostly supplied by hand; the water-course terminates a short distance above the middle of the wheel $b$. There is a stream of water for the service of the stamps, and conduits proceeding from it, to lead the water into the two stamp troughs; the conduit of discharge is common to the two batteries or sets of stamps through which the water carries off the sand or stamped ore. There is a moveable table of separation, mounted with two sieves. The sands pass immediately into the conduit placed upon a level with the floor, and separated into two compartments, the first of which empties its water into the second. There are two boards of separation, or tables, laid upon the ground, with a very slight slope of only 15 inches from their top to their bottom. Each of these boards is divided into four cases with edges; the whole being arranged so that it is possible, by means of a flood-gate or sluice, to cause the superfluous water of the case to pass into the following ones. Thus the work ean go on without interruption, and alternately upon the two boards. There are winding eanals in the labyrinth, $\mathrm{N}, \mathrm{N}, \mathrm{N}$, in which are deposited the particles carried along by the water which has passed upon the boards. The depth of these canals gradually increases from 12 to 20 inches, to give a suitable descent for maintaining the water-flow. At $\mathrm{D}$, two percussion tables are placed. F.G are two German chests. H J are two percussion tables, which are driven by the eams $z z$, fixed upon the main shaft $x y$. $\quad \mathbf{K} \mathbf{K}^{\prime}$ are two sloping sweep tables (à balai).

The German chests are rectangular, being about 3 yards long, half a yard broad, with edges half a yard high; and their inclination is such that the lower end is about 15 inches beneath the level of the upper. At their upper end, usually called the bolster, a kind of trough or box, without any edge at the side next the chest, is placed, containing the ore to be washed. The water is allowed to fall upon the bolster in a thin sheet.

The sleeping tables have upright edges; they are from 4 to 5 yards long, nearly 2 yards wide, and have fully a yard of inclination.

The preceding tables are sometimes covered with cloth, particularly in treating ores that contain gold, on a supposition that the woollen or linen fibres would retain more surely the metallic particles; but this method appears on trial to merit no confidence, for it produces a very impure schlich.

Fig. 680 is a swing-sieve employed in the Hartz, for sifting the small fragments of the ore of argentiferous lead. Such an apparatus is usually set up in the ontside of

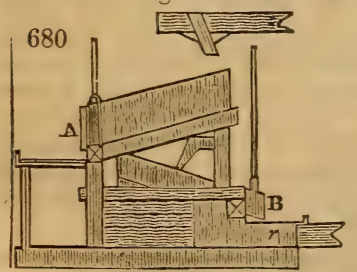
a stamp and washing mill; its place being denoted by the letter $\mathrm{A}$, in fig. 672. The two moveable ehests or boxes A B, of the sieve, are connected together, at their lower ends, with an upright rod, which terminates at one of the arms of a small balance beam, mounted between the driving shaft of the stamps and the sieve, perpendicularly to the length of both. The opposite arm of this beam carries another upright rod, which ears (cams or mentonnets), placed on purpose upon the driving shaft, may push down. During this movement the two lower ends A, B, are raised; and when the peg-cam of the shaft quits the irod which it had depressed, the swing chests fall by their own weight. Thus thev 
are made to vibrate alternately upon their axes. The small ore is put into the upper part of the chest $A$, over which a stream of water falls from an adjoining conduit. The framents which cannot pass through a cast iron grid in the bottom of that chest, are sorted by hand upon a table in front of $\mathrm{A}$, and they are classed by the workman, either among the ores to be stamped, whether dry or wet, or among the rubbish to be thrown away, or among the copper ores to be smelted by themselves. As to the small particles which fall through the grid upon the chest $B$, supplied also with a stream of water, they descend successively upon two other brass wire sieves, and also through the iron wire $r$, in the bottom of $\mathbf{B}$.

In certain mines of the Hartz, tables called a balais, or sweeping tables, are employed. The whole of the process consists in letting flow, over the sloping table, in successive currents, water charged with the ore, which is deposited at a less or greater distance, as also pure water for the purpose of washing the deposited ore, afterwards carried off by means of this sweeping operation.

At the upper end of these sweep-tables, the matters for washing are agitated in a chest, by a small wheel with vanes, or flap-boards. The conduit of the muddy waters opens above a little table or shelf; the conduit of pure water, which adjoins the preceding, opens below it. At the lower part of each of these tables, there is a transverse slit, covered by a small door with hinges, opening outwardly, by falling back towards the foot of the table. The water spreading over the table, may at pleasure be let into this slit, by raising a bit of leather which is nailed to the table, so as to corer the small door when it is in the shut position; but when this is opened, the piece of leather then hangs down into it. Otherwise the water may be allowed to pass freely above the leather, when the door is shut. The same thing mas be done with a similar opening placed abore the conduit. By means of these two slits, two distinct qualities of schlich may be obtained, which are deposited into two distinct conduits or canals. The refuse of the operation is turned into another conduit, and afterwards into ulterior reservoirs, whence it is lifted out to undergo a new washing.

In the percussion tables, the water for washing the ores is sometimes spread in slender streamlets, sometimes in a full body, so as to let two cubic feet escape per minute. The number of shochs communicated per minute, varies from 15 to 36 ; and the table may be pushed out of its settled position at one time, three quarters of an inch, at another nearly 8 inches. The coarse ore-sand requires in general less water, and less slope of table, than the fine and pasty sand.

The mechanical operations which ores undergo, take place commonly at their out-put from the mine, and without any intermediate operation. Sometimes, however, the hardness of certain gangues (vein-stones), and of certain iron ores, is diminished by subjecting them to calcination preriously to the breaking and stamping processes.

When it is intended to wash certain ores, an operation founded on the difference of their specific gravities, it may happen that by slightly changing the chemical state of the substances that compose the ore, the earthy parts may become more easily separable, as also the other foreign matters. With this view, the ores of tin are subjected to a roasting, which by separating the arsenic, and oxydizing the copper which are intermixed, furnishes the means of obtaining, by the subsequent washing, an oxyde of tin much purer than could be otherwise procured. In general, however, these are rare cases; so that the washing almost always immediately succeeds the picking and stamping; and the roasting comes next, when it needs to be employed.

The operation of roasting is in general executed by various processes, relatively to the nature of the ores, the quality of the fuel, and to the object in view. The greatest economy ought to be studied in the fuel, as well as the labor; two most important circumstances, on account of the great masses operated upon.

Three principal methods may be distinguished; 1 . the roasting in a heap in the open air, the most simple of the whole; 2 . the roasting executed between little walls, and which may be called case-roasting (rost-stadeln, in German); and 3. roasting in furnaces.

We may remark, as to the description about to be given of these different processes, that in the first two, the fuel is alwars in immediate contact with the ore to be roasted, whilst in furnaces, this contact may or may not take place.

1. The roasting in the open air, and in heaps more or less considerable, is practised upon iron ores, and such as are pyritous or bituminous. The operation consists in general in spreading orer a plane area, often bottomed with beaten clay, billets of wood arranged like the bars of a gridiron, and sometimes laid crosswise over one another, so as to form a uniform flat bed. Sometimes wood charcoal is scattered in, so as to fill up the interstices, and to prevent the ore from falling between the other pieces of the fuel. Coal is also employed in moderately small lumps; and even occasionally turf. The ore, either simply broken into pieces, or eren sometimes under the form of schlich, is piled up over the fuel; most usually alternate beds of fuel and ore are formed. 
The fire, kindled in general at the lower part, but sometimes, however, at the middle ehimney, spreads from spot to spot, putting the operation in train. The combustion must be so conducted as to be slow and suffocated, to prolong the ustulation, and let the whole mass be equably penetrated with heat. The means employed to direct the fire, are to cover outwardly with earth the portions where too much activity is displayed, and to pierce with holes or to give air to those where it is imperfectly developed. Rains, winds, variable seasons, and especially good primary arrangements of a calcination, have much influence on this process, which requires, besides, an almost incessant inspection at the beginning.

Nothing in general can be said as to the consumption of fuel, because it varies with its quality, as well as with the ores and the purpose in view. But it may be laid down as a good rule, to employ no more fuel than is strictly necessary for the kind of calcination in hand, and for supporting the combustion; for an excess of fuel would produce, besides an expense uselessly incurred, the inconvenience, at times very serious, of such a heat as may melt or vitrify the ores; a result entirely the reverse of a well-conducted ustulation.

Figs. 681, 682, 683, represent the roasting in mounds, as practised near Goslar in the 681

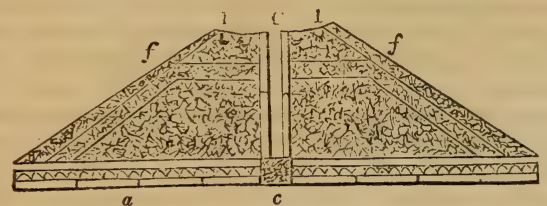
682

Hartz, and at Chessy in the department of the Rhone. Fig. 681 is a vertical section in the line $h c$ of figs. 682 and 683 . In fig. 682 there is shown in plan, only a little more than one half of the quadrangular truncated pyramid, which constitutes the heap. Fig. 683 shows a little more than one fourth of a bed of wood, arranged at the bottom of the pyramid, as shown by $a$, , fig. 681, and $c g h, f i g .683$. c is a wooden chimney,

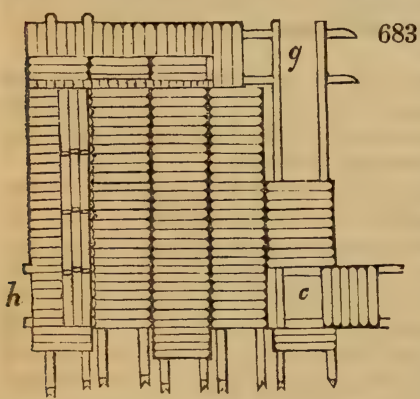
formed within the heap of ore, at whose bottom $c$ there is a little parcel of charcoal; $d d$ are large lumps of ore distributed upon the wooden pile $a a$; $e e$ are smaller fragments, to cover the larger; $f f$ is rubbish and clay laid smoothly in a slope over the whole. " $g$, fig. 683, a passage for air left under the bed of billets; of which there is a similar one in each of the four sides of the base $a a$, so that two principal currents of air cross under the upright axis $\mathrm{c} c$, of the truncated pyramid indicated in fig. 681 .

The kindling is thrown in by the chimney c. The charcoal $c$, and the wood $a \quad a$, take fire; the sulphureous ores $d$ ef are heated to such a high temperature as to vaporize the sulphur. In the Lower Hartz, a heap of this kind continues roasting during four months.

2. The second method. The difficulty of managing the fire in the roasting of substances containing little sulphur, with the greater difficulty of arranging and supporting in their place the schlichs to be roasted, and last of all, the necessity of giving successive fires to the same ores, or to inconsiderable quantities at a time, have led to the contrivance of surrounding the area on which the roasting takes place with three little walls, or with four, leaving a door in the one in front. This is what is called a walled area, and sometimes, improperly enough, a roasting furnace. Inside of these little walls, about 3 feet high, there are often vertical conduits or chimneys made to correspond with an opening on the ground level, in order to excite a draught of air in the adjacent parts. When the roasting is once set agoing, these chimneys can be opened or shut at their upper ends, according to the necessities of the process.

Several such furnaces are usually erected in connexion with each other by their lateral walls, and all terminated by a common wall, which forms their posterior part ; sometimes they are covered with a shed supported partly by the back wall, huilt sufficienily high for this purpose. These dispositions are suitable for the roasting of schlichs, and in general of all matters which are to have several fires; a circumstance often indispensable to a due separation of the sulphur, arsenic, \&c.

3 The furnaces employed for roasting the ores and the mattes differ much, according 
to the rature of the ores, and the size of the lumps. We shall content ourselves with referring to the principal forms.

When iron ores are to be roasted, which require but a simple calcination to disengage the combined water and carbonic acid, egg-shaped furnaces, similar to those in which limestone is burned in contact with fuel, may be conveniently employed; and they present the advantage of an operation which is continuous with a never-cooling apparatus. The analogy in the effects to be produced is so perfect, that the same furnace may be used for either object. Greater dimensions may, however, be given to those destined for the calcination of iron ores. But it must be remembered that this process is applicable only to ores broken into lumps, and not to ores in grains or powder.

It has been attempted to employ the same method a little modified, for the roasting of ores of sulphuret of copper and pyrites, with the view of extracting a part of the sulphur. More or less success has ensued, but without ever surmounting all the obstacles arising from the great fusibility of the sulphuret of iron. For sometimes it runs into one mass, or at least into lumps agglutinated together in certain parts of the furnace, and the operation is either stopped altogether, or becomes more or less languid; the air not being able to penetrate into all the parts, the roasting becomes consequently imperfect. This inconvenience is eren more serious than might at first sight appear; for, as the ill-roasted ores now contain too little sulphur to support their combustion, and as they sometimes fall into small fragments in the cooling, they cannot be passed again through the same furnace, and it becomes necessary to finish the roasting in a reverberatory hearth, which is much more expensive.

In the Pyrenees, the roasting of iron ores is executed in a circular furnace, so disposed that the fuel is contained and burned in a kind of interior oven, above which lie the pieces of ore to be calcined. Sometimes the vault of this oren, which sustains lis jre, is formed of bricks, leaving between them openings for the passage of the flame and the smoke, and the apparatus then resembles certain pottery kilns; at other times the vault is formed of large lumps of ore, carefully arranged as to the intervals requisite to be left for draught over the arch. The broken ore is then distributed above this arch, eare being taken to place the larger pieces undermost. This process is simple in the construction of the furnace, and economical, as branches of trees, without value in the forest, may be employed in the roasting. See Lime-kiln figures.

In some other countries, the ores are roasted in furnaces very like those in which porcelain is baked; that is to say, the fuel is placed exteriorly to the body of the furnace in a kind of brick shafts, and the flame traverses the broken ore with which the furnace is filled. In such an apparatus the calcination is continuous.

When it is proposed to extract the sulphur from the iren pyrites, or from pyritous min erals, different furnaces may be employed, anong which that used in Hungary deserves notice. It is a rectangular parallelopiped of four walls, each of them being perforated with holes and rertical conduits which lead into chambers of condensation, where the sulphur is collected. The ore placed between the four walls on hillets of wood arranged as in figs. $681,682,683$, for the great roastings in the open air, is calcined with the dersengagement of much sulphur, which finds more facility in escaping by the lateral conduits in the walls, than up through the whole mass, or across the upper surface esrered over with earth ; whence it passes into the chambers of condensation. In this way upwards of a thousand tons of pyrites may be roasted at once, and a large quantity of sulphur obtained.-See Copper.

Roasting of Pyrites.-Figs. 684, 685, represent a furnace which has been long em-

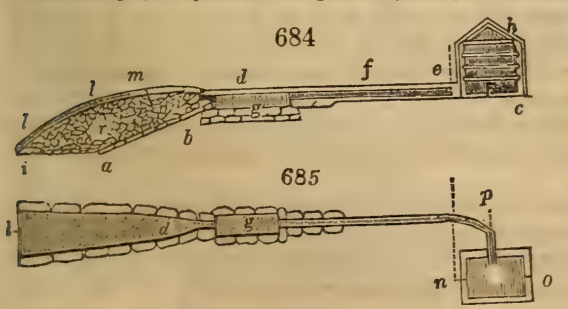
ployed at Fahlun in Sweden, and several other parts of that kingdom, for roasting iron pyrites in order to obtain sulphur. This apparatus was constructed by the celebrated Gahn. Fig. 684 is a vertical section, in the line $k d n o$ of fig. 685 , which is a plan of the furnace; the top being supposed to be taken off. In both figures the conduit may be imagined to be broken off at $e$; its entire length in a straight line is 43 feet berond the dotted line, $e n$, before the bend, which is an extension of this conduit. Upon the slope $a b$ of a hillock $a b c$, lumps $r$ of iron pyrites are piled upon the pieces of wood $i i$ for roasting. A conduit $d f e$ forms the continuation of the space denoted by $r$, which is coverel hy stone slabs so far as $f$; and from this point to the chamber $h$ it is constructed in briards. At the heginning of this conduit, there is a recipient $g$. The chamber $h$ is divided into five chambers by horizontal partitions, which permit the circulation of the 
vapors from one compartment to another. The ores $r$ being distributed npon the billets of wood $i i$, whenever these are fairly kindled, they are covered with small ore, and then with rammed earth $l l$. Towards the point $m$, for a space of a foot square, the ores are covered with moveable stone slabs, by means of which the fire may be regulated, by the displacement of one or more, as may be deemed necessary. The liquid sulphur runs into the recipient $g$, whence it is laded out from time to time. The sublimed sulphur passes into the conduit $f e$ and the chamber $h$, from which it is taken out, and washed with water, to free it from sulphuric acid, with which it is somewhat impregnated; it is afterwards distilled in cast-iron retorts. The residuum of the pyrites is turned to account in Sweden, for the preparation of a common red color much used as a pigment for wooden buildings.

The reverberatory furnace affords one of the best means of ustulation, where it is requisite to employ the simultaneous action of heat and atmospherical air to destroy certain combinations, and to decompose the sulphurets, arseniurets, \&c. It is likewise evident that the facility thus offered of stirring the matters spread out on the sole, in order to renew the surfaces, of observing their appearances, of augmenting or diminishing the degree of heat, \&c., promise a success much surer, a roasting far better executed, than by any other process. It is known, besides, that flame mingled with much undecomposed air issuing from the furnace, is highly oxydizing, and is very fit for burning away the sulphur, and oxydizing the metals. Finally, this is almost the only method of rightly roasting ores which are in a very fine powder. If it be not employed constantly and for every kind of ore, it is just because more economy is found in practising calcination in heaps, or on areas enclosed by walls; besides, in certain mines, a very great number of these furnaces, and many workmen, would be required to roast the considerable body of ores that must be daily smelted. Hence there would result from the construction of such apparatus and its maintenance a very notable outlay, which is saved in the other processes.

But in every case where it is desired to have a very perfect roasting, as for blende from which zinc is to be extracted, for sulphuret of antimony, \&c., or even for ores reduced to a very fine powder, and destined for amalgamation, it is proper to perform the operation in a reverberatory furnace. When very fusible sulphurous ores are treated, the workman charged with the calcination must employ much care and experience, chiefly in the management of the fire. It will sometimes, indeed, happen, that the ore partially fuses; when it becomes necessary to withdraw the materials from the furnace, to let them cool and grind them anew, in order to recommence the operation. The construction of these furnaces demands no other attention than to give to the sole or laboratory the suitable size, and so to proportion to this the grate and the chimney that the heating may be effected with the greatest economy.

The reverberatory furnace is always employed to roast the ores of precious metals, and especially those for amalgamation; as the latter often contain arsenic, antimony, and other volatile substances, they must be disposed of in a peculiar manner.

The sole, usually very spacious, is divided into two parts, of which the one farthest off from the furnace is a little higher than the other. Above the vault there is a space or chamber in which the ore is deposited, and which communicates with the laboratory by a vertical passage; which serves to allow the ore to be pushed down, when it is dried and a little heated. The flame and the smoke which escape from the sole or laboratory pass into condensing chambers, before entering into the chimney of draught, so as to deposite in them the oxyde of arsenic and other substances. When the ore on the part of the sole farthest from the grate has suffered so much heat as to begin to be roasted, has become less fusible, and when the roasting of that in the nearer part of the sole is completed, the former is raked towards the fire-bridge, and its ustulation is finished by stirring it over frequently with a paddle, skilfully worked, through one of the doors left in the side for this purpose. The operation is considered to be finished when the varets and the smell have alnost wholiy ceased; its duration depending obviously on the nat ure of the ores.

When this furnace is employed to roast very arsenical ores, as the tin ores of Schlackenwald in Bohemia, and at Ehrenfriedensdorf in Saxony, the arsenical pyrites of Geyer (in Saxony), \&c., the chambers of condensation for the arsenious acid are much more extensive than in the furnaces commonly used for roasting galena, copper, or even silver ores.

Figs. 686, 687, 688 represent a reverberatory furnace employed in the smelting works of Lautenthal, in the Hartz, for roasting the schlichs of lead ores, which contain much. blende or sulphuret of zinc. In fig. 686 we see that the two parts A B, B C, are absolutely like, the two furnaces being built in one body of brickwork. Fig. 687 is the plan of the furnace B $\mathbf{C}$, taken at the level $\mathbf{E} \mathbf{F}$ of fig. 686 . Fig. 688 is a vertical section of the similar furnace $A$, taken in the prolongation of the line $G \quad H$ in fig. 687 . 
$a$ is the fire-place of the furnace, its grate and ash-pit. $b$ is the conduit of vaporization, which communicates with the chambers $c$; $c$, chambers into which the vaporized
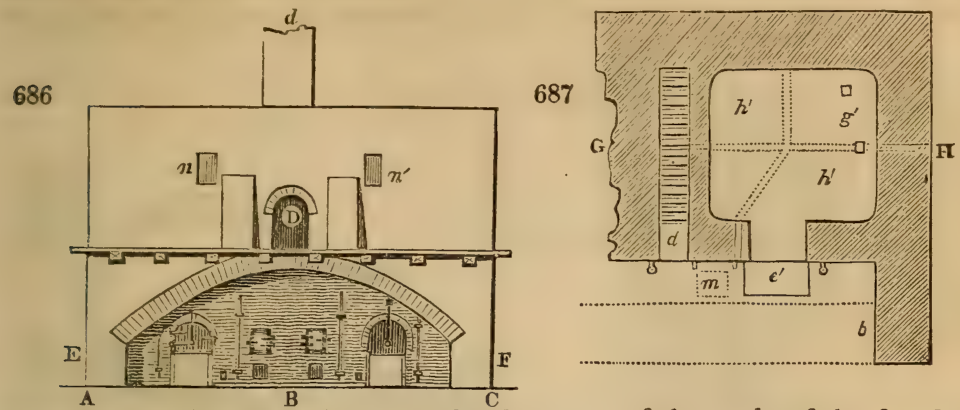

substances are deposited; $d$, chimney for the escape of the smoke of the fire place $a$, after it has gone through the space $b c c ; e^{\prime}$, is the charging door, with a hook hanging

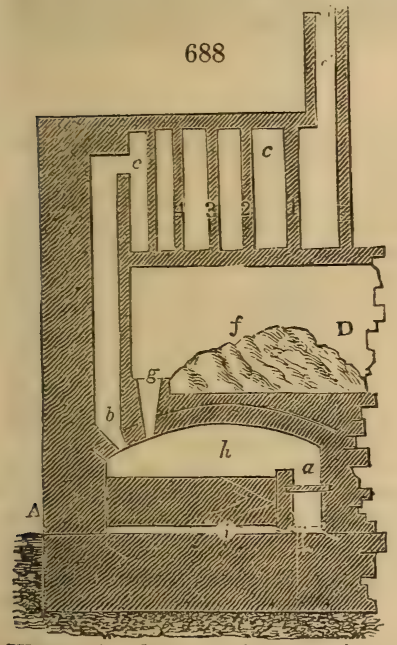
in front to rest the long iron rake upon, with which the materials are turned over; $f$, chamber containing a quantity of schlich destined for roasting; this chamber communicates with the vaulted corridor (gallery) D, seen in fig. $686 ; \mathrm{g}$, orifice through which the schlich is thrown into the furnace; $h$, area or hearth of the reverberatory furnace, of which the roof is certainly much too high; $i$, channels for the escape of the watery vapors; $k, l$, front arcade, between which and the furnace, properly speaking, are the two orifices of the conduits, which terminate at the channels $m, m^{\prime}$. $m$ is the channel for carrying towards the chimney $d$, the vapors which escape by the door $e^{\prime}$. $\quad n$ is a walled-up door, which is opened from time to time, to take out of the chambers $c, c$, the substances that may be deposited in them.

At the smelting works of Lautenthal, in such a roasting furnace, from 6 to 9 quintals (cwts.) of schlich are treated at a time, and it is stirred frequently with an iron rake upon the altar $h$. The period of this operation is from 6 to 12 hours, according as the schlich may be more or less dry, more or less rich in lead, or more or less charged with blende. When the latter substance is abundant, the process requires 12 hours, with about 60 cubic feet of cleft billets for fuel.

In such furnaces are roasted the cobalt ores of Schneeberg in Saxony, the tin ores of Schlackenwald in Bohemia, of Ehrenfriedersdorf in Saxony, and elsewhere; as also the arsenical pyrites at Geyer in Saxony. But there are poison towers and extensive condensing chambers attached in the latter case. See Arsenic.

Figs. 689, 690, 691 represent the reverberatory furnace generally employed in the

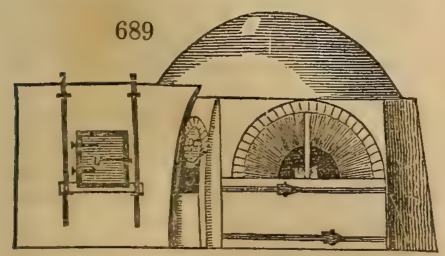
Hartz, in the district of Mansfeldt, Saxony, Hungary, \&c., for the treatment of black copper, and for refining rose copper upon the great scale. An analogous furnace is used at Andreasberg for the liquefaction or purification of the mattes, and for workable lead when it is much loaded with arsenic.

Fig. 689 presents the elevation of the furnace parallel to the line $\mathbf{I}$, of the plan fig. 690 ; which plan is taken at the level of the tuyère $n$, of $\mathrm{fig} .691$; fig. 691 is a vertical section in the line L M, fig. 690 . $k$ represents one of two basins of reception, brasqued with clay and charcoal; $n, n$, two tuyères, through which enters the blast of two pairs of bellows, like those shown at Cupellation of SiLvER; $q$, door by which the matter to be melted is laid upon the sole of the furnace; $v, v$, two points where the sole is perforated, when necessary to run off the melted matter into either of the basins $h$; $x$, door through which the slags or cinders floating upon the sur- 
face of the melted metal are raked out; $y$, door of the fire-place. The fuel is laid upon a grate above an ash-pit, and below the arch of a reverberatory which is contiguous to the dome or cap of the furnace properly so called. In the section, fig. 691, the following parts may be noted; $1,2,3$, mason-work of the foundation; 4 , vapor channels or con
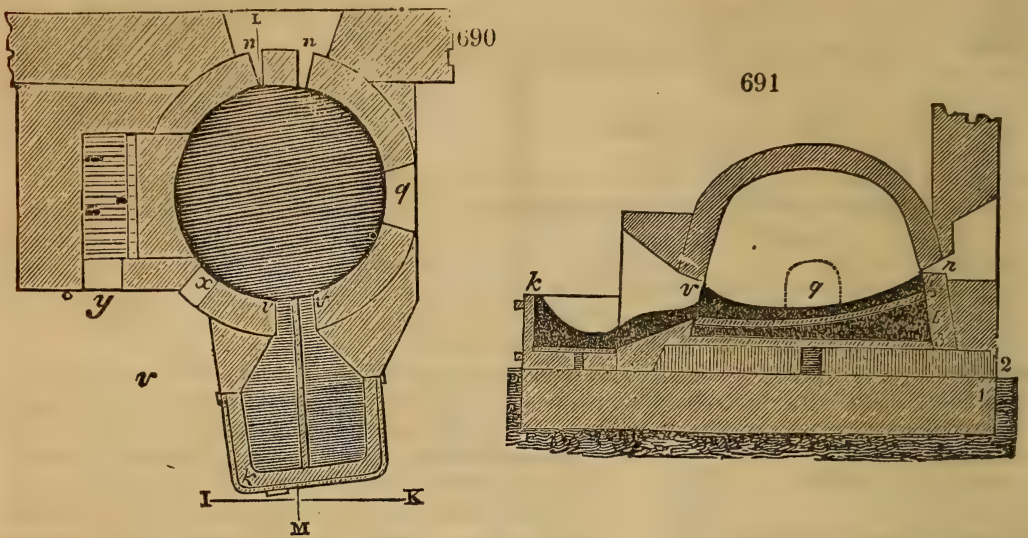

duits, for the escape of the humidity; 5 , bed of clay; 6 , brasque composed of clay and charcoal, which forms the concavity of the hearth.

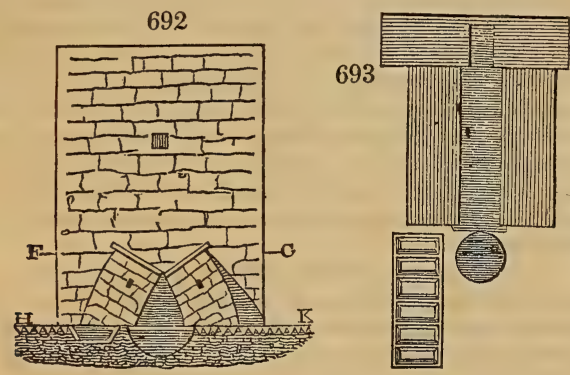

Figs. 692, 693, 694, show the furnace employed for liquation in one of the principal smelting works of the Hartz. Fig. 694 exhibits the working area charged with the liquation cakes and charcoal, supported by sheets of wrought iron; being an image of the process in action. Fig. 693 is the plan, in the line $\mathrm{F} \mathrm{G}$, of fig. 692 . of -

A liquation cake is composed

Black copper holding at least 5 or 6 loths ( $2 \frac{1}{2}$ or $3 \mathrm{oz}$.) of silver per cwt., and weighing 90 to $96 \mathrm{lbs}$.

Lead obtained from litharge, 2. cwts. Litharge, $\frac{1}{2} \mathrm{cwt}$.

From 30 to 32 cakes are successively worked in one operation, which lasts about 5 hours; the furnace is brought into action, as usual, with the aid of slags; then a little litharge is added; when the lead begins to flow, the copper is introduced, and when the copper flows, lead is added, so that the mixture of the metals may be effected in the hest way possible.

From 8 to 16 of these cakes (pains) are usually placed in the liquation furnace, figs. $692,693,694$. The operation lasts 3 or 4 hours, in which time about $1 \frac{1}{2}$ quintals of charcoal are consumed. The cakes are covered with burning charcoal, supported, as I have said, by the iron plates. The workable lead obtained flows off towards the basin in front of the furnace; whence it is laded out into moulds set alongside. See fig. 693. If the lead thus obtained be not sufficiently rich in silver to be worth cupellation, it is employed to form new liquation cakes. When it contains from 5 to 6 loths of silver per cwt., it is submitted to cupellation in the said smelting works. See SiLver.

The trompe, or water blowing engine, figs. 695, 696, 697. Fig. 695 is the elevation; fig. 696 is a vertical section, made at right angles to the elevation. The machine is formed of two cylindrical pipes, the bodies of the trompe $b$, set upright, called the funnels, which terminate above in a water cistern $a$, and below in a close basin under $c$, called the $t u b$ or 
drum. The conical part $p$, of the funnel has been called etranguillon, being strangled, as it were, in order that the water discharged into the body of the trompe shall not fill the

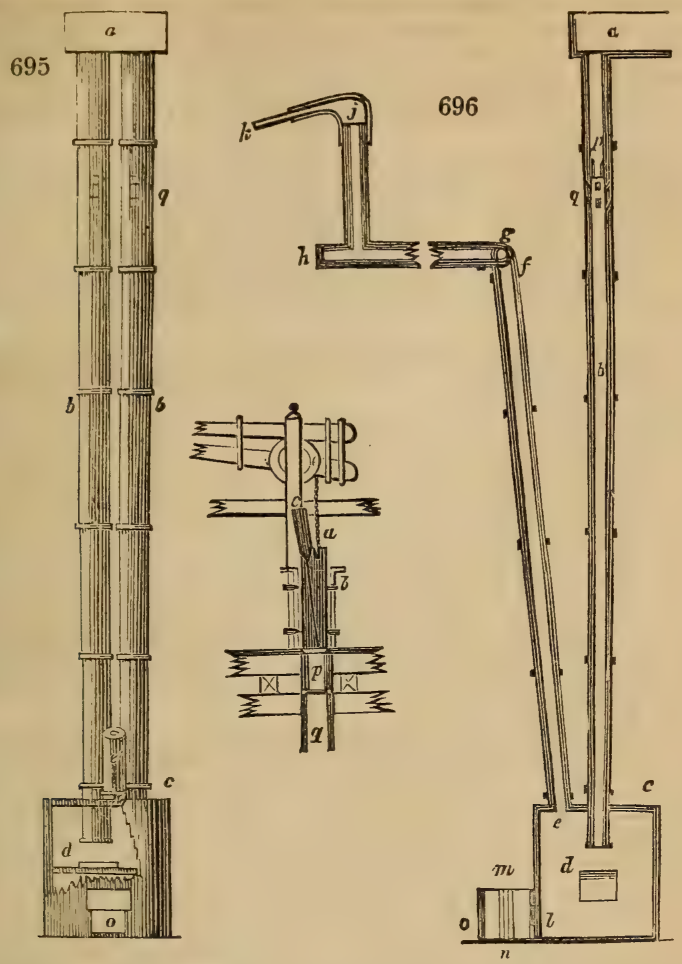

pipe in falling, but be divided into many streamlets. Below this narrow part, eight holes, $q q$, are perforated obliquely through the substance of the trompe, called the vent-holes or nostrils, for admitting the air, which the water carries with it in its descent. The air afterwards parts from the water, by dashing upon a cast-iron slab, placed in the drum upon the pedestal $d$. An aperture $l$, at the bottom of the drum, allows the water to flow away after its fall; but, to prevent the air from escaping along with it, the water as it issues is received in a chest $l m n o$, divided into two parts by a vertical slide-plate between $m n$. By raising or lowering this plate, the water may be maintained at any desired level within the drum, so as to give the included air any determinate degree of pressure. The superfluous water then flows off by the hole 0 .

The air-pipe e, fig. 696, is fitted to the upper part of the drum; it is divided, at the point $f$, into three tubes, of which the principal one is destined for the furnace of cupelation, while the other two $g \mathrm{~g}$, serve for different melting furnaces. Each of these tubes ends in a leather pocket, and an iron nose-pipe $k$, adjusted in the tuyère of the furnace. At Pesey, and in the whole of Savoy, a floodgate is fitted into the upper cistern $a$, to regulate the admission of water into the trompe; but in Carniola, the funnel $p$ is closed with a wooden plug, suspended to a cord, which goes round a pulley mounted upon a horizontal axis, as shown in fig. 697. By the plug $a$ being raised more or less, merely the quantity of water required for the operation is admitted. The plug is pierced lengthwise with an oblique hole $c c$, in which the small tube $c$ is inserted, with its top some way above the water level, through which air may be admitted into the heart of the column descending into the trompe $p q$.

The ordinary height of the trompe apparatus is about 26 or 27 feet to the upper level of the water cistern; its total length is 11 metres ( 36 feet 6 inches), and its width 2 feet, to give room for the drums. It is situated 10 mètres $\left(33 \frac{1}{3}\right.$ feet) from the melting furnace. This is the case at the smelting works of Jauerberg, in Upper Carniola.

OF THE ASSAY OF ORES.

Assays ought to occupy an important place in metallurgic instruetions, and theie is 
$r$ ason to believe that the knowledge of assaying is not sufficiently diffused, since rts practice is so offen neglected in smelting houses. Not only ought the assays of the ores under treatment to be frequently repeated, because their nature is subject (1) vary ; but the different products of the furraces should be subjected to reiterated assays, at the several periods of the operations. When silver or gold ores are in question, the il cimastic operations, then indispensable, exercise a salutary control orer the metall,:-aic processes, and aftord a clear indication of the quantities of precious metal which they ought to produce.

By the tille.tssays, in a metallurgic point of view, is meant the method of ascertaining for any substance whatever, not only the presence and the nature of a metal, but its proportional quantity. Hence the operations which do not lead to a precise determination of the metal in question, are not to be arranged among the assars now under consideration. Experiments made with the blow-pipe, although capable of yielding n.ost useful indications, are like the touchstone in regard to gold, and do not constitule genuine assars.

Three kinds of assays may be practised in different circumstances, and with more or less adrantage upon different ores. 1. The mechanical assay ; 2 . the assay by the dry way; 3 . the assay by the humid way.

1. Of mechanical assays.-These kinds of assays consist in the separation of the substances mechanically mixed in the ores, and are performed br a hand-washing, in a small trough of an oblong shape, called a sebilla. After pulrerizing with more or less pains the matters to be assayed by this process, a determinate weight of them is put into this wooden howl with a little water; and by means of certain morements and some precautions, to be learned only by practice, the lightest substances may be pretty exactly separated, namely, the earthy gangues from the denser matter or metallic particles, without losing any sensible portion of them. Thus a schlich of greater or less furity will be obtained, which may afford the means of judging by its quality of the richness of the as sayed ores, and which may thereafter be subjected to assays of another hind, whereby the whole metal may be insulated.

Washing, as an assay, is practised on auriferous sands; on all ores from the stumps, and even on schlichs already washed upon the great scale, to appreciate more nicely the degree of purity they have acquired. The ores of tin in which the oxyde is often disseminated in much earthy gangue, are well adapted to this species of assay, because the tin oxyde is very dense. The mechanical assay may also be employed in reference to the ores whose metallic portion presents a uniform composition, provided it also possesses con siderable specific gravity. Thus the ores of sulphuret of lead (galena) being susceptible of becoming almost pure sulphurets (within 1 or 2 per cent.) by mere washing skilfully conducted, the richness of that ore in pure galena, and consequently in lead, ray be at once concluded; since 120 of galena contain 104 of lead, and 16 of sulphur. The sulphuret of antimony mingled with its gangue may be subjected to the same mcde of assay, and the result will be still more direct, since the crude antimony is brought into the market after being freed from its gangue by a sim- e fusion.

The assay by washing is also had recourse to for ascertaining if the scorice or other products of the furnaces contain some metallic grains which might be extracted from them by stamping and washing on the great scale; a process employed considerably with the scorice of tin and copper works.

Of assays by the dry way.- The assay by the dry way has for its object, to show the nature and proportion of the metals contained in a mineral substance. To make a good assay, however, it is indispensably necessary to linow what is the metal associated with it, and even within certain limits, the quantity of the foreign bodies. Only one metal is commonly looked after; unless in the case of certain argentiferous ores. The mineralogical examination of the substances under treatment, is most commonly sufficient to afford data in these respects; but the assays may always be varied with different riews, before stopping at a definite result; and in erery instance, only such assays can be confided in, as have been verified by a double operation.

This mode of assaying requires only a little experience, with a simple apparatus; and is of such a nature as to be practised currently in the smelting works. The air furnace and crucibles employed are described in all good elementary chemical books. These assays are usually performed with the addition of a flux to the ore, or some agent for separating the earthy from the metallic substances; and they possess a peculiar adrantage relative to the smelting operations, because they offer many analogies between results on the great scale and experiments on the small. This may even enable us often to deduce, from the manner in which the assay has succeeded with a certain flux, and at a certain degree of heat, raluable indications as to the treatment of the ore in the great way. See FURNAcE.

In the smelting houses which purchase the ore, as in Germany, it is necessary to bestow much attention upon the assays, because they serve to regulate the qualitv and 
the price of the schlichs to be delivered. These assays are not by any means free from difficulties, especially when ores containing several useful metals are treated, and which are to be dosed or proportioned; ores, for example, including a notable quantity of iead, copper, and silver, mixed together.

In the central works of the Hartz, as well as in those of Saxony, the schlichs as de. livered are subjected to docimastic assays, which are verified three times, and by three different persons, one of whom is engaged for the interests of the mining partners, another for that of the smelting house, and a third as arbiter in case of a difference. If the first two results of assaying differ by $\frac{1}{2}$ loth (or $\frac{1}{4}$ ounce) of silver per cwt. of schlich, the operations must be resumed; but this rarely happens. When out of the three assays, the one differs from the two others by no more than $\frac{x}{4}$ loth of silver per cwt., but by more in one, and by less in another, the mean result is adopted. As to the contents of the schlich in lead, the mean results of the assays must be taken. The differences allowed are three pounds for the schlich, when it contains from 12 to 30 per cent. of lead, increasing to six pounds for schlich, when it contains less than 55 per cent. of that metal.

Assaying forms, in great establishments, an important object in reference to time and expense. Thus, in the single work of Franckenscharn, in the Hartz, no less thas 300 assays have to be made in a threefold way, every Monday, without taking into account the several assays of the smelting products which take place every Thursday. Formerly fluxes more or less compound were employed for these purposes, and every assay cost about fifteen pence. At present all these assays are made more simply, by much cheaper methods, and cost a penny farthing each upon an average.

of the assays by the humid way.-The assays by the humid way, not reducible to very simple processes, are true chemical analyses, which may in fact be applied with much advantage, either to ores, or to the products of the furnace; but which cannot be expected to be practised in smelting-houses, on account of the complication of apparatus and reagents they require. Moreover, an expert chemist is necessary to obtain results that can be depended on. The directors of smelting-houses, however, should never neglect any opportunities that may occur of submitting the materials operated upon, as well as their products, to a more thorough examination than the dry way alone can effect. One of the great advantages of similar researches is to discover and appreciate the minute quantities of injurious substances which impair the malleability of the metals, which give them several bad qualities, about whose nature and cause more or less error and uncertainty prevail. Chemical analysis, rightly applied to metallurgy, cannot fail to introduce remarkablt improvements into the processes. See the different metals, in their alphabetical places.

For assays in the dry way, both of stony and metallic minerals, the process of Dr. Abich deserves recommendation. It consists in mixing the pulverized mineral with 4 or 6 times its weight of carbonate of baryta in powder, fusing the mixture at a white heat, and then dissolving it, after it cools, in dilute muriatic acid. The most refractory minerals, even corundum, cyanate, staurolite, zircon, and feldspar, yield readily to this treatment. This process may be employed with advantage upon poor refraetory ores. The platinum crucible, into which the mixed materials are put for fusion, should be placed ip a Hessian crucible, and surrounded with good coke.

The following tabular view of the metallic produce of the British mines is given by two very skilful observers, in a work published in 1827 , entitled Voyage Metallurgique en Angleterre, par MM. Dufrénoy et Elie de Beaumont:-

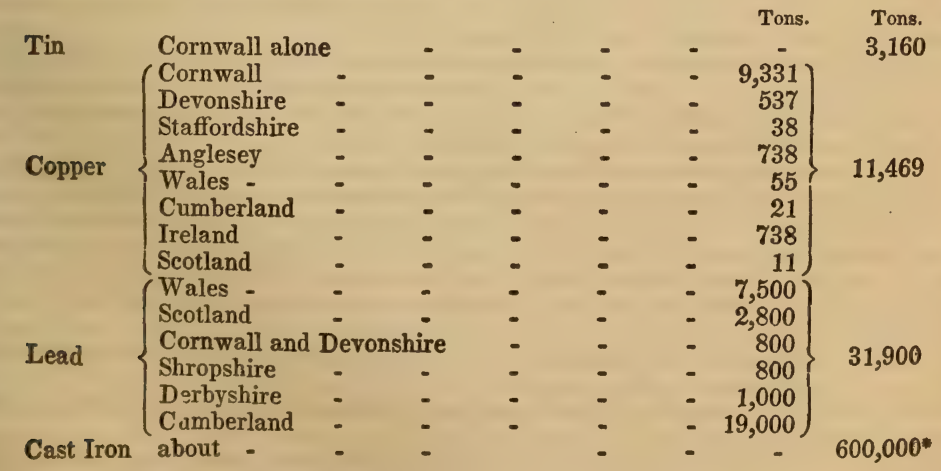

* I have converted the weights of lead and cast-iron, given in kilogrammes, into tons, at the rate of 1000 kilogrammes per ton; which is sufficiently near. 
The manganese raised in England exceeds 2000 tons.

M. Heron de Villefosse inserted in the last number of the Annales des Mines for 1827, the following statistical view of the metallic products of France:-

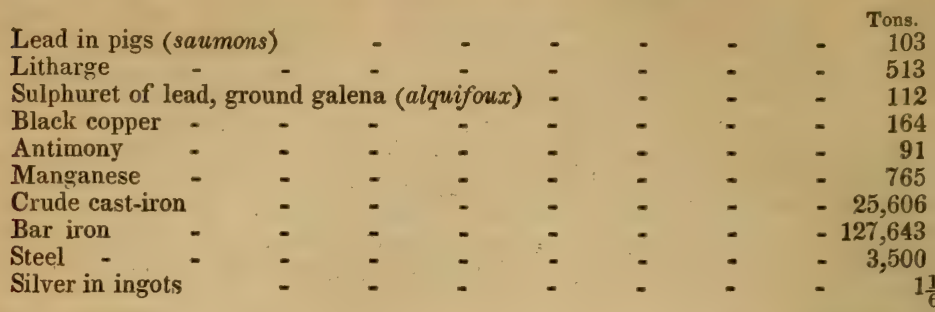

The total value of which is estimated at 80 millions of francs; or about $3,400,000$ pounds sterling.

METALS (Metaux, Fr.; Metalle, Germ.) are by far the most numerous class of undecompounded bodies in chemical arrangements. They amount to 41 ; of whic $"$ form, with oxygen, bodies possessed of alkaline properties: these are, 1. potassium; 2 . sodium; 3. lithium; 4. barytium, or barium; 5 . strontium; 6. calcium; 7. magnesium; for even magnesia, the last and feeblest base, tinges turmeric brown, and red cabbage green. The next 5 metals form, with oxygen, the earths proper; they are, 8 . yttrium; 9. glucinum; 10. alumium; 11. zirconium; 12. thorinum. The remaining 29 may be enumerated in alphabetical order, as they hardly admit of being grouped into subdivisions with any advantage. They are as follows : 13. antimony; 14. arsenic; 15 . bismuth; 16. cadmium ; 17. cerium; 18. chromium; 19 . cobalt ; 20. copper; 21. gold; 22. iridium; 23. iron; 24. lead; 25. manganese; 26. mercury; 27. molydenum; 28. nickel; 29. osmium; 30. palladium; 31 . platinum; 32 . rhodium; 33 . silver; 34 . tantalum; 35. tellurium; 36. tin; 37. titanium; 38. tungstenium; 39. vanadium; 40. uranium; 41. zinc.

1. They are all, more or less, remarkable for a peculiar lustre, called the metallic. This property of strongly reflecting light is connected with a certain state of aggregation of their particles, but is possessed, superficially at least, by mica, animal charcoal, selenium, polished indigo;-bodies not at all metallic.

2. The metals are excellent conductors of caloric, and most of them also of electricity, though probably not all. According to Despretz, they possess the power of conducting heat according to the following numbers :-Gold, 1000 ; platinum, 981 ; silver, 973 ; copper, 898 ; iron, 374 ; zinc, 363 ; tin, 304 ; lead, $179 \cdot 6$.

Beqquerel gives the following table of metals, as to electrical conduction :-

Copper, 100 ; gold, $93 \cdot 6$; silver, $73 \cdot 6$; zinc, 28.5 ; platina, $16 \cdot 4$; iron, $15 \cdot 8$; tin, 15.5 ; lead, $8 \cdot 3$; mercury, $3 \cdot 5$; potassium, $1 \cdot 33$.

The metals which hardly, if at all, conduct electricity, are zirconium, alumium, tantaIum in powder, and tellurium.

3. Metals are probably opaque ; yet gold leaf, as observed by Newton, seems to transmit the green rays, for objects placed behind it in the sunbeam appear green. This phenomena has, however, been ascribed to the rays of light passing through an infinite number of minute fissures in the thinly hammered gold.

4. All metals are capable of combining with oxygen, but with affinities and in quantities extremely different. Potassium and sodium have the strongest affinity for it; arsenic and chromium, the feeblest. Many metals become acids by a sufficient dose of oxygen, while with a smaller dose they constitute salifiable bases.

5. Metals combine with each other, forming a class of bodies called alloys, except when one of them is mercury, in which case the compound is styled an amalgam.

6. They combine with hydrogen into hydrurets ; with carbon, into carburets ; with sulphur, into sulphurets; with phosphorus, into phosphurets ; with selenium, into seleniurets ; with boron, into borurets (borides?); with chlorine, into chlorides; with iodine, into iodides; with cyanogen, into cyanides; with silicon, into silicides; and with fluorine, into fluorides.

7. Metallic salts are definite compounds, mostly crystalline, of the metallic oxydes with the acids. See HaLord.

METEORITES (Aerolithes, Fr.) are stones of a peculiar aspect and composition, which have fallen from the air.

METHYLE'NE, a peculiar liquid compound of carbon and hydrogen, extracted from pyroxylic spirit, which is reckoned to be a bi-hydrate of methylène.

MICA is a finely foliated mineral, of a pearly metallic lustre. It is harder than gypsum, but not so hard as calc-spar; flexible and elastic; spec. grav. 2.65 . It is 
an angredient of granite and gneiss. The large sheets of mica exposed for sale in Condon, are mostly brought from Siberia. They are used, instead or glass, to enclose the fire, without concealing the flame, in certain stoves.

The mica of Fahlun, analyzed by Rose, afforded, silica, 46.22 ; alumina, 34.52 ; peroxyde? of iron, $6 \cdot 04$; potash, $8 \cdot 22$; magnesia, with oxyde of manganese, $2 \cdot 11$; fluoric acid, $1 \cdot 09$; water, 0.98 .

MICROCOSMIC SALT; a term given to a salt extracted from human urine, because man was regarded by the alchemists as a miniature of the world, or the microcosm. It is a phosphate of soda and ammonia; and is now prepared by mixing equivalent proportions of phosphate of soda and phosphate of ammonia, each in solution, evaporating and crystallizing the mixture. A small excess of ammonia aids the crystallization.

MILK (Lait, Fr. ; Milche, Germ.) owes its whiteness and opacity to an emulsion composed of the caseous matter and butter, with sugar of milk, extractive matters, salts, and free lactic acid; the latter of which causes fresh milk to redden litmus paper. Milk, in general, contains from 10 to 12 per cent. of solid matter, on being evaporated to dryness by a steam heat. The mean specific gravity of cows' milk is 1.030 , but it is less if the milk be rich in cream. The specific gravity of the shimmed millk is 1.035 ; and of the cream is 1.0244 . 100 parts of creamed milk contain-

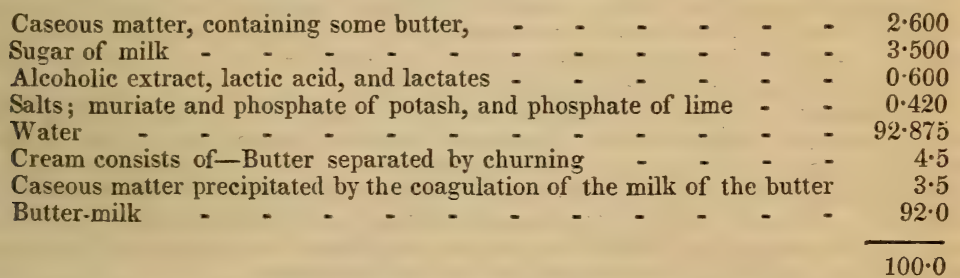

When milk contained in wire-corked bottles, is heated to the boiling point in a water bath, the oxygen of the included small portion of air under the cork seems to be carbonated, and the milk will afterwards keep fresh, it is said, for a year or two; as green gooseberries and peas do by the same treatment.

MILL-STONE, or BUHR-STONE. This interesting form of silica, which occurs in great masses, has a texture essentially cellular, the cells being irregular in number, shape, and size, and are often crossed by thin plates, or coarse fibres of silex. The Buhr-stone has a straight fracture, but it is not so brittle as flint, though its hardness is nearly the same. It is feebly translucent; its colors are pale and dead, of a whitish, grayish, or yellowish cast, sometimes with a tinge of blue.

The Buhr-stones usually occur in beds, which are sometimes continuous, and at others interrupted. These beds are placed amid deposites of sand, or argillaceous and ferruginous marls, which penetrate between them, filling their fissures and honeycomb cavities. Buhr-stones constitute a very rare geological formation, being found in abundance only in the mineral basin of Paris, and a few adjoining districts. Its place of superposition is well ascertained: it forms a part of the lacustrine, or fresh-water formation, which, in the locality alluded to, lies above the fossil-bone gypsum, and the stratum of sand and marine sandstone which covers it. Buhr-stone constitutes, therefore, the uppermost solid stratum of the crust of the globe; for above it there is nothing but alluvial soil, or diluvial gravel, sand, and loam.

Buhr-stones sometimes contain no organic forms, at others they seem as if stuffed full of fresh-water shells, or land shells and vegetables of inland growth. There is no exception known to this arrangement; but the shells have assumed a silicious nature, and their cavities are often bedecked with crystals of quartz. The best Buhr-stones for grinding corn, have about an equal proportion of solid matter, and of vacant space. The finest quarry of them is upon the high ground, near $L a$ F'erte-sous-Jouarre. The stones are quarried in the open air, and are cut out in cylinders, from one to two yards in diameter, by a series of iron and wooden wedges, gradually but equally inserted. The pieces of buhr-stones are afterwards cut in parallelopipeds, called panes, which are bound with iron hoops into large millstones. These pieces are exported chiefly to England and America. Good millstones of a bluish white color, with a regular proportion of cells, when six feet and a half in diameter, fetch 1200 francs a-piece, or $48 l$. sterling. A coarse conglom. erate sandstone or breccia is, in some cases, used as a substitute for buhr-stones; but it is a poor one.

MiNERAL WATERS. See Soda Water, and Waters, Mineral.

MINES, (Bergwerke, Germ.) Amidst the variety of bodies apparently infinite, which compose the crust of the globe, geologists have demonstrated the prevalence of a few 
general systems of roclis, to which they have given the name of formations or deposites. A large proportion of these mineral systems consists of parallel planes, whose length and breadth greatly exceed their thickness; on which account they are called stratified rocks; others occur in very thick blocks, without any parallel stratification, or horizontal seauns of considerable extent.

The stratiform deposites are subdivided into two great classes; the primary and the secondary. The former seem to have been called into existence before the creation of organic matter, because they contain no exuviæ of vegetable or animal beings; while the latter are more or less interspersed, and sometimes replete with organic rernair.s. The primary strata are characterized, moreover, by the nearly vertical or highly inclined position of their planes; the secondary lie for the most part in a nearly horizontal position.

Where the primitive mountains graduate down into the plains, rocks of an intermediate character appear, which, though possessing a nearly vertical position, contain a few vestiges of animal beings, especially shells. These have been called transition, to indicate their being the passing links between the first and second systems of ancient deposites; they are distinguished by the fractured and cemented texture of their planes, for which reason they are sometimes called conglomerate.

Between these and the truly secondary rocks, another very valuable series is interposed in certain districts of the globe; namely, the coal-measures, the paramount formation of Great Britain. The coal strata are disposed in a basin-form, and alternate with parallel beds of sandstone, slate-clay, iron-stone, and occasionally limestone. Some geologists have called the coal-measures the medial formation.

In every mineral plane, the inclination and direction are to be noted; the former being the angle which it forms with the horizon, the latter the point of the azimuth or horizon, towards which it dips, as west, north-east, south, \&c. The direction of the bed is that of a horizontal line drawn in its plane; and which is also denoted by the point of the compass. Since the lines of direction and inclination are at right angles to each other, the first may always be inferred fiom the second; for when a stratum is said to dip to the east or west, this implies that its direction is north and south.

The smaller sinuosities of the bed are not taken into account, just as the windings of a river are neglected in stating the line of its course.

Masses are mineral deposites, not extensively spread in parallel planes, but irregular heaps, rounded or oval, enveloped in whole or in a great measure by rocks of a different kind. Lenticular masses being frequently placed between two horizontal or inelined strata, have been sometimes supposed to be stratiform themselves, and have been accordingly denominated by the Germans liegende stocke, lying heaps or blocks.

The orbicular masses often occur in the interior of unstratified mountains, or in the bosom of one bed.

Nests, concretions, nodules, are small masses found in the middle of strata; the first being commonly in a friable state; the second often kidney-shaped, or tuberous; the third nearly round, and incrusted, like the kernel of an almond.

Lodes, or large veins, are flattened masses, with their opposite surfaces not parallel, which consequently terminate iike a wedge, at a greater or less distance, and do not run parallel with the rocky strata in which they lie, but cross them in a direction not far from the perpendicular; often traversing several different mineral planes. The lodes are sometimes deranged in their course, so as to pursue for a little way the space between two contiguous strata; at other times they divide into several branches. The matter which fills the lodes is for the most part entirely different from the rocks they pass through, or at least it possesses peculiar features.

This mode of existence, exhibited by several mineral substances, but which has been long known with regard to metallic ores, suggests the idea of clefts or rents having been made in the stratum posterior to its consolidation, and of the vacuities laving been filled with foreign matter, either immediately or after a certain interval. There can be no doubt as to the justness of the first part of the proposition, for thers may be observed round many lodes undeniable proofs of the movement or dislocation of the rock; for example, upon each side of the rent, the same strata are no longer situated in the same plane as before, but make greater or smaller angles with it; or the stratum upon one side of the lode is raised considerably above, or depressed considerably below, its counterpart upon the other side. With regard to the manner in which the rent has been filled, different opinions may be entertained. In the lodes which are widest near the surface of the ground, and graduate into a thin wedge below, the foreign matter would seem to have been introduced as into a funnel at the top, and to have carried along with it in its fluid state portions of rounded gravel and organic remains. In other cases, other conceptions seem to be more probable; since many lodes are largest at their under part, and become progressively narrower as they approach the surface; from which circumstance it has been inferred that the rent has been caused by an 
expansive force acting from within the earth, and that the foreign matter, having been injected in a fluid state, has afterwards slowly crystallized. This hypothesis accounts much better than the other for most of the phenomena observable in mineral veins, for the alterations of the rock at their sides, for the crystallization of the different substances interspersed in them, for the cavities bestudded with little crystals, and for many minute peculiarities. Thus, the large crystals of certain substances which line the walls of hollow veins, have sometimes their under surfaces besprinkled with small crystals of sulphurets, arseniurets, \&c., while their upper surfaces are quite smooth; suggesting the idea of a slow sublimation of these volatile matters from below, by the residual heat, and their condensation upon the under faces of the crystalline bodies, already cooled. This phenomenon affords a strong indication of the igneous origin of metalliferous veins.

In the lodes, the principal matters which fill them are to be distinguished from the accessory substances; the latter being distributed irregularly, amidst the mass of the first, in crystals, nodules, grains, seams, \&c. The non-metalliferous exterior purtion, which is often the largest, is called gangue, from the German gang, veth. The position of a vein is denoted, like that of the strata, by the angle of inclination, and the point of the horizon towards which they dip, whence the direction is deduced.

Veins, are merely small lodes, which sometimes traverse the great ones, ramifying in various directions, and in different degrees of tenuity.

A metalliferous substance is said to be disseminated, when it is dispersed in crystals, spangles, seales, globules, \&c., through a large mineral mass.

Certain ores which contain the metals most indispensable to human necessities, have been treasured up by the Creator in very bountiful deposites; constituting either great masses in rocks of different kinds, or distributed in lodes, veins, nests, concretions, or beds with stony and earthy admixtures; the whole of which become the objects of mineral exploration. These precious stores occur in different stages of the geological formations; hut their main portion, after having existed abundantiy in the several orders of the primary strata, suddenly cease to be found towards the middle of the secondary. Iron ores are the only ones which continue among the more modern deposites, even so high as the beds immediately beneath the chalk, when they also disappear, or exist merely as coloring matters of the tertiary earthy beds.

The strata of gneiss and mica-slate constitute in Europe the grand metallic domain. There is hardly any lind of ore which does not occur there in sufficient abundance to become the object of mining operations, and many are found nowhere else. The tran sition rocks, and the lower part of the secondary ones, are not so rich, neither do they contain the same variety of ores. But this order of things, which is presented by Great Britain, Germany, France, Sweden, and Norway, is far from forming a general law ; since in equinoetial America the gneiss is but little metalliferous; while the superior strata, such as the clay-schists, the sienitic porphyries, the limestones, which complete the transition series, as also several secondary deposites, include the greater portion of the immense mineral wealth of that region of the globe.

All the substances of which the ordinary metals form the basis, are not equally abundant in nature; a great proportion of the numerous mineral species which figure in our classifications, are mere varieties scattered up and down in the cavities of the great masses or lodes. The workable ores are few in number, being mostly sulphurets, some oxydes, and carbonates. These occasionally form of themselves rery large masses, but more frequently they are blended with lumps of quartz, feldspar, and carbonate of lime, which form the main body of the deposite; as happens always in proper lodes. The ores in that case are arranged in small layers parallel to the strata of the formation, or in small veins which araverse the rock in all directions, or in nests or concretions stationed irregularly, or finally disseminated in hardly visible particles. These deposites sometimes contain apparently only one species of ore, sometimes several, which must be mined together, as they seem to be of conternporaneous formation; whilst, in other cases, they are separable, having been probably formed at different epochs. In treating of the several metals in their alphabetical order, I have taken care to describe their peculiar geological positions, and the rocks which accompany or mineralize them.

In mining, as in architecture, the best method of imparting instruction is to display the master-pieces of the respective arts, which speak clearly to the mind through the medium of the eye. It is not so easy, however, to represent at once the general effect of a mine, as it is of an edifice; because there is no point of sight from which the former can be sketched at once, like the latter. The subterraneous structures certainly afford some of the finest examples of the useful labors of man, continued for ages, under the guidance of science and ingenuity; but, however curious, beautiful, and grand in themselves, they cannot become objects of a panoramic view. It is only by the lights of ge ometry and geology that mines can be contemplated and surveyed, either as a whole or in their details; and, therefore, these marvellous subterranean regions, in which roads are cut 
many hundred miles long, are altogether unknown or disregarded by men of the world. Should any of them, perchance, from curiosity or interest, descend into these dark recesses of the earth, they are prepared to discover only a few insulated objects which they may think strange or possibly hideous; but they cannot recognise either the symmetrical disposition of mineral bodies, or the laws which govern geological phenomena, and serve as sure guides to the skilful miner in his adventurous search. It is by exact plans and sections of subterraneous workings, that a knowledge of the nature, extent, and distribution of mine-al wealth can be acquired.

698. A general view of mining operations.

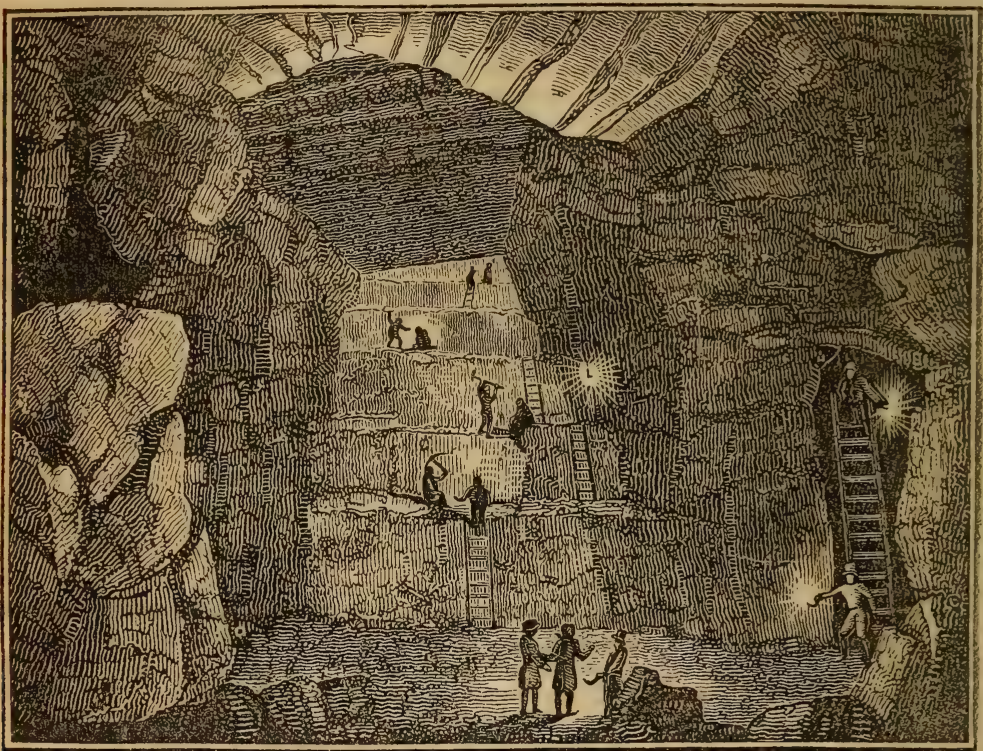

As there is no country in the world so truly rich and powersul, by virtue of its mineral stores, as Great Britain, so there are no people who ought to take a deeper interest in their scientific illustration. I have endeavored in the present artiele to eolleet from the most authentic sources the most interesting and instruetive examples of mining operations.

To the magnificent work of Ville-Fosse, Sur la Richesse Mineraie, no Bonger on sele, I have to acknowledge weighty obligations; many of the figures being copied from his great Atlas.

Lodes or mineral veins are usually distinguished by English miners into at least four species. 1. The rake vein. 2. The pipe vein. 3. The flat or dilated vein; and 4. The interlaced mass (stock-werke), indicating the union of a multitude of smoll veins mixed in every possible direction with each other, and with the roek.

1. The rake vein is a perpendicular mineral fissure; and is the form best known among practical miners. It commonly runs in a straight line, ficies of the strata, and cutting them downwards, generally further than can be reached. This vein sometimes stands quite perpendicular; but it more usually inclines or hangs over at a greater or smaller angle, or slope, which is ealled by the miners the hade or hading of the vein. The line of direction in which the fissure runs, is called the becrong of the vein.

2. The pipe vein resembles in many respects a huge irregular cavern, pmshing forward into the body of the earth in a sloping direction, under various inclinations, from an angle of a few degrees to the horizon, to a dip of $45^{\circ}$, or more. The pipe coes rot in general cut the strata across like the rake vein, but insinuates itself between them; so that if the plane of the strata be nearly horizontal, the bearing of the pipe vein will be conformable; but if the strata stand up at a high angle, the pipe shoots down nearly headlong-like a shaft. Some pipes are very wide and high, others are very low and narrow, sometimes not large than a common mine or drift.

3. The flat or dilated vein, is a space or opening between two strata or beds of stone, the one of which lies above, and the other below this vein, like a stratum of coai 
between its roof and pavement; so that the vein and the strata are placed in the same plane of inclitiation. These veins are subject, like coal, to be interrupted, broken, and thrown up or lown by slips, dikes, or other interruptions of the regular strata. In the case of a met.llic vein, a slip often increases the chance of finding more treasure. Such veins do not preserve the parallelism of their beds, characteristic of coal seams; but vary excessircly in thickness within a moderate space. Flat veins occur frequently in limestone, either in a horizontal or declining direction. The flat or strata veins open and close, as the rake veins also do.

4. The interlaced mass has been already defined.

To these may be added the accumulated vein, or irregular mass (butzenwerke), a great deposite placed without any order in the bosom of the rocks, apparently filling up cavern. ous spaces.

The interlaced masses are more frequent in primitive formations, than in the others; and tin is the ore which most commonly affects this locality. See figure of TrN mine.

The study of the mineral substances, called gangues or vein-stones, which usually accompany the different ores, is indispensable in the investigation and working of mines. These gangucs, such as quartz, calcareous spar, fluor spar, heavy spar, \&c., and a great number of other substances, although of little or no value in themselves, become of great consequence to the miner, either by pointing out by their presence that of certain useful minerals, or by characterizing in their several associations, different deposites of ores of which it may be possible to follow the traces, and to discriminate the relations, often of a complicated kind, provided we observe assiduously the accompanying gangues.

Mineral reins are subject to derangements in their course, which are called shifts or faults. Thus, when a transverse vein throws out, or intercepts, a longitudinal one, we must commonly look for the rejected vein on the side of the obtuse angle which the direction of the latter makes with that of the former. When a bed of ore is deranged by a fault, we must observe whether the slip of the strata be upwards or downwards; for in either circumstance, it is only by pursuing the direction of the fault that we can recover the ore; in the former case by mounting, in the latter by descending beyond the dislocation.

When two veins intersect each other, the direction of the offcast is a subject of interest, both to the miner and the geologist. In Saxony it is considered as a general fact that the portion thrown out is alwars upon the side of the obtuse angle, a circumstance which holds also in Cornwall; and the more obtuse the angle, the out-throw is the more considerable. A rein may be thrown out on meeting another vein, in a line which approaches either towards its inclination or its direction. The Cornish miners use two different terms to denote these two modes of rejection; for the first case, they say the vein is heaved; for the second, it is started.

The great copper lode of Carharack, $d, f i g .699$, in the parish of Gwenap, is one of the 699

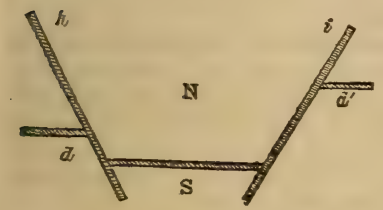
most instructive examples of intersection. The power or thickness of this vein is 8 feet; its direction is nearly due east and west, and it dips towards the north at an inclination of two feet per fathom; its upper part being in the killas (a greenish clay-slate); its lower part in the granite. The lode has suffered two intersections; the first produced by meeting the vein $h$, called Steven's fluckan, which runs from northeast to south-west, and which throws the lode several fathoms out; the second is produc a by another vein $i$, almost at right angles with the first, and which occasions another outthrow of 20 fathoms to the right side. The fall of the vein occurs therefore in the one case to the right, and in the other to the left; but in both it is towards the side of the obtuse angle. This distribution is very singular; for one part of the vein appears to have mounted while the other has descended. N. s. denotes North and South. $d$ is the copper lode running east and west. $h, i$, are systems of clay-slate veins called fluckans; the line over s, represents the down shift, and $d^{\prime}$ the up-shift.

\section{General observations on the localities of ores, and on the indications of metallic mines.}

1. Tin exists principally in primitive rocks, appearing either in interlaced masses, in beds, or as a constituent part of the rock itself, and more rarely in distinct veins. Tin ore is found indeed sometimes in alluvial land, filling up low situations between lofty mountains.

2. Gold occurs either in beds or in veins, frequently in primitive rocks; though in other formations, and particularly in alluvial earth, it is also found. When this metal exists in the bosom of primitive rocks, it is particularly in schists; it is not found in serpentine, but it is met with in graywacke in Transylvania. The gold of alluvial districts, 
called gold of washing or transport, occurs, as well as alluvial tin, among the debris of the more ancient rocks.

3. Silver is found particularly in veins and beds, in primitive and transition formations; though some veins of this metal occur in secondary strata. The rocks richest in it are, gneiss, mica-slate, clay-slate, graywacke, and old alpine limestone. Localities of silverore itself are not numerous, at least in Europe, among secondary formations; but it occurs in combination with the ores of copper or of lead.

4. Copper exists in the three mineral epochas; 1 . in primitive rocks, principally in the state of pyritous copper, in beds, in masses, or in vcins; 2 . in transition districts, sometimes in masses, sometimes in veins of copper pyrites; 3 . in secondary strata, especially in beds of cupreous schist.

5. Lead occurs also in each of the three mineral epochas; abounding particularly in primitive and transition grounds, where it usually constitutes veins, and occasionally beds of sulphureted lead (galena). The same ore is found in strata or in veins among secondary rocks, associated now and then with ochreous iron-oxyde and calamine (carbonate of zinc); and it is sometimes disseminated in grains through more recent strata.

6. Iron is met with in four different mineral eras, but in different ores. Among primitive rocks, magnetic iron ore and specular iron ore occur chiefly in beds, sometimes of enormous size; the ores of red or brown oxyde of iron (hematite) are found generally in veins, or occasionally in masses with sparry iron, both in primitive and transition rocks; as also sometimes in secondary strata ; but more frequently in the coal-measure strata, as beds of clay-ironstone, of globular iron oxyde, and carbonate of iron. In alluvial distriets we find ores of clay-ironstone, granular iron-ore, bog-ore, swamp-ore, and meadow-ore. The iron ores which belong to the primitive period have almost always the metallic aspect, with a richness amounting even to 80 per cent. of iron, while the ores in the posterior formations become in general more and more earthy, down to those in alluvial soils, some of which present the appearance of a common stone, and afford not more than 20 per cent. of metal, though its quality is often excellent.

7. Mercury occurs principally among secondary strata, in disseminated masses, along with combustible substances; though the metal is met with occasionally in primitive countries.

8. Cobalt belongs to the three mineral epochas; its most abundant deposites are veins in primitive rocks; small veins containing this metal are found, however, in secondary strata.

9. Antimony occurs in veins or beds among primitive and transition rocks.

10, 11. Bismuth and nickel do not appear to constitute the predominating sabstance of any mineral deposites; but they often accompany cobalt.

12. Zinc occurs in the three several formations : namely, as sulphuret or blende, particularly in primitive and transition rocks; as calamine, in secondary strata, usually along with oxyde of iron, and sometimes with sulphuret of lead.

An acquaintance with the general results collected and elassified by geology must be our first guide in the investigation of mines. This enables the observer to judge whether any particular district should, from the nature and arrangement of its rocks, be susceptible of including within its bosom, beds of workable ores; it indicates also, to a certain degree, what substances may probably be met with in a given series of rocks, and what locality these substances will preferably affect. For want of a knowledge of these facts, many persons have gone blindly into researches equally absurd and ruinous.

Formerly, indications of mines were taken from very unimportant circumstances; from thermal waters, the heat of which was gratuitously referred to the decomposition of pyrites; from mineral waters, whose course is however often from a far distant source; from vapors incumbent over particular mountain groups; from the snows melting faster in one mineral district than another; from the different species of forest trees, and from the greater or less vigor of vegetation, \&c. In general, all such indications are equally fallacious with the divining rod, and the compass made of a lump of pyrites suspended by a thread.

Geognostic observation has substituted more rational characters of metallic deposites, some of which may be called negative and others positive.

The negative indications are derived from that peculiar geological constitution, which from experience or general principles excludes certain metallic matters; for example, granite, and in general every primitive formation, forbids the hope of finding within them combustible fossils (pit-coal,) unless it be beds of anthracite; there also it would be vain to seek for sal gem. It is very seldom that granite rocks include silver; or limestones, ores of tin. Volcanic territories never afford any metallic ores worth the working; nor do extensive veins usually run into secondary and alluvial formations. The richer ores of iron do not occur in secondary strata; and the ores of this inetal peculiar to these localities, do not exist among primary rocks. 
Among positive indications, some are proximate and others remote. The proximate are, an efflorescence, so to speak, of the subjacent metallic masses; magnetic attraction for iron ores; bituminous stone, or inflammable gas for pit-coal ; the frequent occurrence of fragments of particular ores, \&c. The remote indications consist in the geological epocha, and nature of the rocks. From the examples previously adduced, marks of this kind acquire new importance when in a district susceptible of including deposites of workable ores, the gangues or vein-stones are met with which usually accompany any particular metal. The general aspect of mountains whose flanks present gentle and continuous slopes, the frequency of sterile veins, the presence of metalliferous sands, the neighborhood of some known locality of an ore, for instance, that of iron-stone in reference to coal, lastly, the existence of salt springs and mineral waters, may furnish some indications; but when ferruginous or cupreous waters issue from sands or clays, such characters merit in general little attention, because the waters may flow from a great distance. No greater importance can be attached to metalliferous sands and saline springs.

In speaking of remote indications, we may remark that in several places, and particularly near Clausthal in the Hartz, a certain ore of red oxyde of iron occurs above the most abundant deposites of the ores of lead and silver; whence it has been named by the Germans the iron-hat. It appears that the iron ore rich in silver, which is worked in America under the name of pacos, has some analogy with this substance; but iron ore is in general so plentifully diffused on the surface of the soil, that its presence can be regarded as only a remote indication, relative to other mineral substances, except in the case of clay iron-stone with coal.

Of the instruments and operations of subterranean operutions.-It is by the aid of geometry in the first place that the miner studies the situation of the mineral deposites, on the surface and in the interior of the ground; determines the sereral relations of the veins and the rocks; and becomes capable of directing the perforations towards a suitable end.

The instruments are, 1 . the magnetic compass, which is employed to measure the direction of a metallic ore, wherever the neighborhood of iron does not interfere with its functions; 2 . the graduated semi-circle, which serves to measure the inclination, which is also called the clinometer.

3. The chain or cord for measuring the distance of one point from another.

4. When the neighborhood of iron renders the use of the magnet uncertain, a plate or plane table is employed.

The dials of the compasses generally used in the most celebrated mines, are graduated. into hours; most commonly into twice 12 hours. Thus the whole limb is divided into 24 spaces, each of which contains $15^{\circ}=1$ hour. Each hour is subdivided into 8 parts.

Means of penetrating into the interior of the earth.-In order to penetrate into the interior of the earth, and to extract from it the objects of his toils, the miner has at his disposal sereral means, which may be divided into three classes; 1 . manual tools, 2 . gunpowder, and 3. fire.

The tools used by the miners of Cornwall and Devonshire are the following:

Fig. 700. The pick. It is a light tool, and somewhat varied in shape according to cir-

703

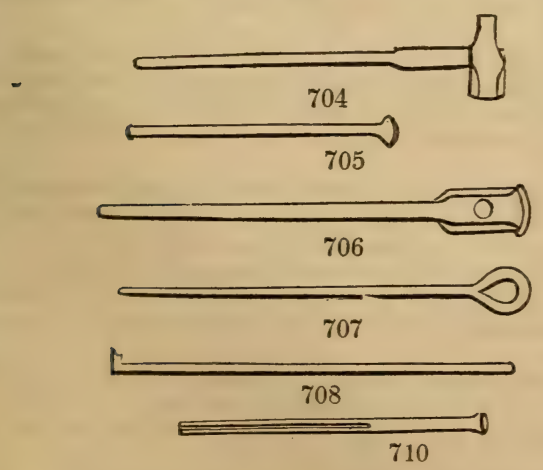

$702 \quad 700$

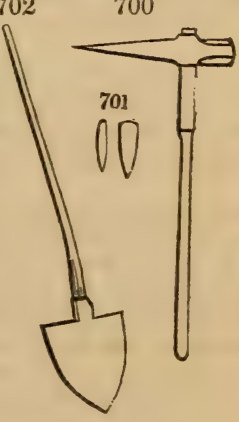

cumstances. One side used as a hammer is called the poll, and is emplored to drive in the gads, or to loosen and detach prominences. The point is of steel, carefully tempered, and drawn ander the hammer to the proper form, The French call it pointerolle. 
Fig. 701. The gad. It is a wedge of steel, driven into crevices of rocks, or into small openings made with the point of the pick.

Fig. 702. The miner's shovel. It has a pointed form, to enable it to penetrate among the coarse and hard fragments of the mine rubbish. Its handle being somewhat bent, a man's power may be conveniently applied without bending his body.

The blasting or shooting tools are:-

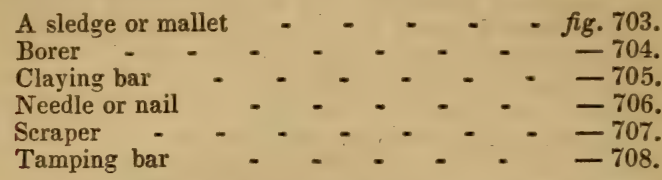

Besides these tools the miner requires a powder-horn, rushes to be filled with gunpowder, tin cartridges for occasional use in wet ground, and paper rubbed over with gunpowder or grease, for the smifts or fuses.

The borer, fig. 704, is an iron bar tipped with steel, formed like a thick chisel, and is used by one man holding it straight in the hole with constant rotation on its axis, while another strikes the head of it with the iron sledge or mallet, fig. 703. The hole is cleared out from time to time by the scraper, fig. 707 , which is a flat iron rod turned up at one end. If the ground be very wet, and the hole gets full of mud, it is cleaned out by a stick bent at the end into a fibrous brush, called a swab-stick.

Fig. 709 represents the plan of blasting the rock, and a section of a hole ready for

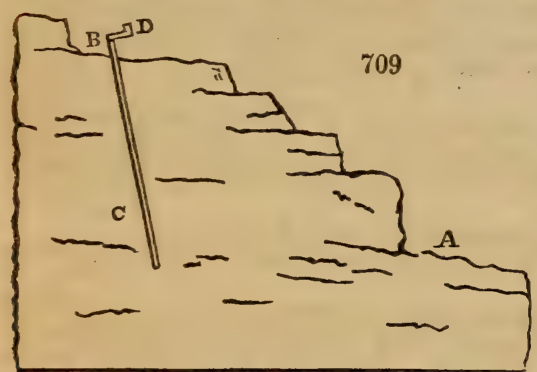
firing. The hole must be rendered as dry as possible, which is effected very simply by filling it partly with tenacious clay, and then driving into it a tapering iron rod, which nearly fills its calibre, called the claying bar. This being forced in with great violence, condenses the clay into all the crevices of the rock, and secures the dryness of the hole. Should this plan fail, recourse is had to tin cartridges furnished with a stem or tube, (see fig. 710 ,) through which the powder may be inflamed. When the hole is dry, and the charge of powder introduced, the nail, a small taper rod of copper, is inserted so as to reach the bottom of the hole, which is now ready for tamping. By this difficult and dangerous process, the gunpowder is confined, and the disruptive effect produced. Different substances are employed for tamping, or cramming the hole, the most usual one being any soft species of rock free from silicious or flinty particles. Small quantities of it only are introduced at a time, and rammed very hard by the tamping-bar, which is held steadily by one man, and struck with a sledge by another. The hole being thus filled, the nail is withdrawn by putting a bar through its eye, and striking it upwards. Thus a small perforation or vent is left for the rush which communicates the fire.

Besides the improved tamping-bar faced with hard copper, other contrivances have been resorted to for diminishing the risk of those dreadful accidents that frequently occur in this operation. Dry sand is sometimes used as a tamping material, but there are many rocks for the blasting of which it is ineffective. Tough clay will answer better in several situations.

For conveying the fire, the large and long green rushes which grow in marshy ground are selected. A slit is made in one side of the rush, along which the sharp end of a bit of stick is drawn, so as to extract the pith, when the skin of the rush closes again by its own elasticity. This tube is filled up with gunpowder, dropped into the vent-hole, and made steady with a bit of clay. A paper smift, adjusted to burn a proper time, is then fixed to the top of the rush-tube, and kindled, when the men of the mine retire to a safe distance.

In fig. 709 the portion of the rock which would be dislodged by the explosion, is that included between $\mathrm{A}$ and $\mathbf{B}$. The charge of powder is represented by the white part which fills the hole up to $\mathrm{c}$; from which point to the top, the hole is filled with tamping. The smift is shown at $D$.

Fig. 711 is an iron bucket, or as it is called in Cornwall, a kibble, in which the ore is raised in the shafts, by machines called whims, worked by horses. The best kibbles 
are made of sheet-iron, and hold each about three hundred weight of ore : 120 kibbles are supposed to clear a cubic fathom of rock.

712

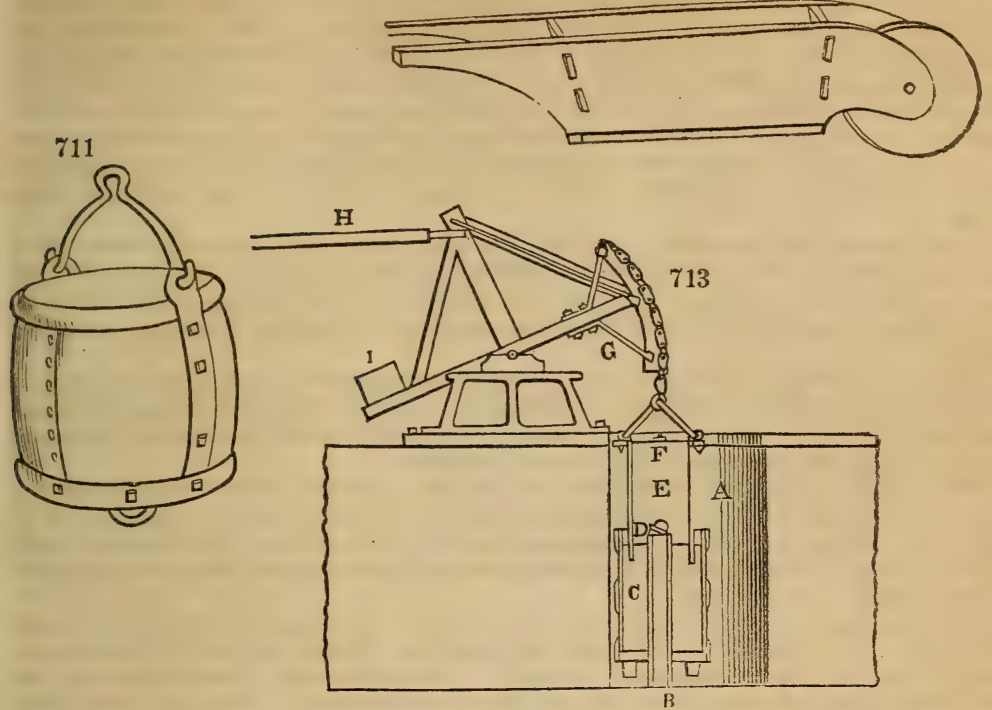

Fig. 712 represents the wheelbarrow used under ground for conveying ore and waste to the font of the shafts. It is made of light deal, except the wheel, which has a narrow rim of iron.

Fig. 713 represents $\mathrm{Mr}$. Taylor's ingenious ventilator, or machine for renewing fresh air in mines. It is so simple in construction, so complete in its operation, requires so little power to work it, and is so little liable to injury from wear, that nothing further of the kind can be desired in ordinary metallic mines. The shaft of the mine is represented at A; at either the top or bottom of which the machine may be placed, as is found most convenient, but the foul air must be discharged into a floor, furnished with a valve-door to prevent its return into the mine. в is the air-pipe from the mine, passing through the bottom of the fixed vessel or cylinder $c$, which is formed of timber, and bound with iron hoops. It is filled with water nearly to the top of the pipe $B$, on which is fixed a valve opening upwards at D. E, the air, or exhausting cylinder of cast-iron, open at bottom, and suspended over the air-pipe, but immersed some way in the wrater. It is furnished with a wooden top, having an aperture fitted with a valve likewise opening upwards at $\boldsymbol{F}$. This exhausting cylinder is moved up and down by the $b o b, \mathrm{G}$, broaght into connexion with any engine, by the horizontal rod $\mathrm{H}$; the weight of the cylinder being balanced, if necessary, by the counterpoise $\mathrm{I}$. The action is as follows : - When the cylinder rises, the air from the mine rushes up through the pipe and valve $\mathrm{D}$; and when it descends, this valve shuts, and prevents the return of the air, which is expelled through the valve $\mathbf{F}$. With a cylinder two feet in diameter and six feet long, working from two to three strokes per minute, 200 gallons of air may be discharged in the same time.

Gunpowder is the most valuable agent of excavation; possessing a power which has no iimit, and which can act everywhere, even under water. Its introduction, in 1615 , caused a great revolution in the mining art.

It is employed in mines in different manners, and in different quantities, according to circumstances. In all cases, however, the process resolves itself into boring a hole, and enclosing a cartridge in it, which is afterwards made to explode. The hole is always cylindrical, and is usually made by means of the borer, fig. 704, a stem of iron, terminated by a blunt-edged chisel. It sometimes ends in a cross, formed by two chisels set transversely. The workman holds the stem in his left hand, and strikes it with an iron mallet held in his right. He is careful to turn the punch a very little round at every stroke. Several punches are employed in succession, to bore one hole; the first shorter, the latter ones longer, and somewhat thinner. The rubbish is withdrawn as it accumulates, at the bottom of the hole, by means of a picker, which is a small spoon or dise of iron fixed at the end of a slender iron rod. When holes of a large size are to be 
made, scveral men must be employed; one to hold the punch, and one or more to wiela the iron mallet. The perforations are seldom less than an inch in diameter, and 18 inches deep; but they are sometimes two inches wide, with a depth of 50 inches.

The gunpowder, when used, is most commonly put up in paper cartridges. Into the side of the cartridge, a small cylindrical spindle or piercer is pushed. In this state the cartridge is forced down to the bottom of the hole, which is then stuffed, by means of the tamping bar, fig. 708, with bits of dry clay, or friable stones coarsely pounded.* The piercer is now withdrawn, which leaves in its place a channel through which fire may be conveyed to the charge. This is executed either by pouring gunpowder into that passage, or by inserting into it reeds, straw stems, quills, or tubes of paper filled with gunpowder. This is exploded by a long match, which the workmen kindle, and then retire to a place of safety.

As the piercer must not only be slender, but stiff, so as to be easily withdrawn when the hole is tamped, iron spindles are usually employed, though they occasionally give rise to sparks, and consequently to dangerous accidents, by their friction against the sides of the hole. Brass piercers have been sometimes tried; but they twist and break too readily.

Each hole bored in a mine, should be so placed in reference to the schistose structure of the rock, and to its natural fissures, as to attack and blow up the least resisting masses. Sometimes the rock is prepared beforehand for splitting in a certain direction, by means of a narrow channel excavated with the small hammer.

The quantity of gunpowder should be proportional to the depth of the hole, and the resistance of the rock, and merely sufficient to split it. Anything additional would serve no other purpose than to throw the fragments about the mine, without increasing the useful effect. Into the holes of about an inch and a quarter diameter, and 18 inches deep, only two ounces of gunpowder are put.

It appears that the effect of the gunpowder may be augmented by leaving an empty space above, in the middle of, or beneath the cartridge. In the mines of Silesia, the consumption of gunpowder has been eventually reduced, without diminishing the product of the blasts, by mixing sawdust with it, in certain proportions. The hole has also been filled up with sand in some cases, according to Mr. Jessop's plan, instead of being packed with stones, which has removed the danger of the tamping operation. The experiments made in this way have given results very advantageous in quarry blasts with great charges of gunpowder; but less favorable in the small sharges employed in mines.

Water does not oppose an insurmountable obstacle to the employment of gunpowder; but when the hole cannot be made dry, a cartridge bag impermeable to water must be had recourse to, provided with a tube also impermeable, in which the piercer is placed.

After the explosion of each mining charge, wedges and levers are employed, to drag away and break down what has been shattered.

Wherever the rock is tolerably hard, the use of gunpowder is more economical and more rapid than any tool-work, and is therefore always preferred. A gallery, for example, a yard and a half high, and a yard wide, the piercing of which by the hammer formerly cost from five to ten pounds sterling the running yard, in Germany, is executed at the present day by gunpowder at from two to three pounds. When, however, a precious mass of ore is to be detached, when the rock is cavernous, which nearly vullifies the action of gunpowder, or when there is reason to apprehend that the shock caused by the explosion may produce an injurious fall of rubbish, hand-tools alone must be employed.

In certain rocks and ores of extreme hardness, the use both of tools and gunpowder becomes very tedious and costly. Examples to this effect are seen, in the mass of quartz mingled with copper pyrites, worked at Rammelsberg, in the Hartz, in the masses of stanniferous granite of Geyer and Altenberg in the Erzgebirge of Saxony, sc. In these circumstances, fortunately very rare, the action of fire is used with advantage to diminish the cohesion of the rocks and the ores. The employment of this agent is not necessarily restricted to these difficult cases. It was formerly applied very often to the working of hard substances; but the introductioni of gunpowder into the mining art, and the increase in the price of wood, ocession fire to be little used as an ordinary means of excavation, except in places where tine scantiness of the poulation has

\footnotetext{
* Sir Rose Price invented a cap of bronze alloy, to tip the lower end of the iron rod; a contrivance now generally used in Cornwall. Before the Geological Society of that county introduced this invention into practice, scarcely a month elapsed without some dreadful sxplosion sending the miner to an untimely grave, or so injuring him by blowing out his eyes, or shatturing his limbs, as to render him a miserable object of charity for the rest of his days. Scarcely has any accident happened since the em ployment of the new tamping-bar. When the whole bar was made of the tin and copper alloy it was expensive, and apt to bend : but the iron rod tipp ?d with the bronze is both cheap and effectual. An ingenious instrument, called the shifting cartridge, was invented by $\mathrm{Mr}$ Chinalls, and is described in the Transactions of the a'sove society
} 
left a great extent of forest timber, as happens at Kongsberg in Norway, at Dannemora in Sweden, at Felsobanya in Transylvania, \&c.

The action of fire may be applied to the piercing of a gallery, or to the arlvancement of a horizontal cut, or to the crumbling down of a mass of ore, by the successive ujuraising of the roof of a gallery already pierced. In any of these cases, the process cunsists in forming bonfires, the flame of which is made to play upon the parts to be attacked. All the workmen must be removed from the mine, during, and even for some time after, the combustion. When the excavations have become sufficiently cool to allow them to enter, they break down with levers and wedges, or even by means of gunpowder, the masses which have been rent and altered by the fire.

To complete our account of the manner in which man may penetrate into the interior of the earth, we must point out the form of the excavations that he should make in it.

In mines, three principal species of excavations may be distinguished; viz., shafts, galleries, and the cavities of greater or less magnitude which remain in the room of the old workings.

A shaft or pit is a prismatic or cylindrical hollow space, the axis of which is eisher vertical or much inclined to the horizon. The dimension of the pit, which is never less than 32 inches in its narrowest diameter, amounts sometimes to several yards. Its depth may extend to 1000 feet, and more. Whenever a shaft is opened, means must be provided to extract the rubbish which continually tends to accumulate at its bottom, as well as the waters which may percolate down into it; as also to facilitate the descent and ascent of the workmen. For some time a wheel and axle erected over the mouth of the opening, which serve to elevate one or two buckets of proper dimensions, may be sufficient for most of these purposes. But such a machine becomes ere long inadequate. Horse-whims, or powerful steam-engines, must then be had recourse to ; and effectual methods of support must be employed to prevent the sides of the shaft from crumbling and falling down.

A Gallery is a prismatic space, the straight or winding axis of which does not usually deriate much from the horizontal line. Two principal species are distinguished; the galleries of elongation, which follow the direction of a bed or a vein; and the transverse galleries, which intersect this direction under an angle not much different from $90^{\circ}$. The most ordinary dimensions of galleries are a yard wide, and two yards high; but many still larger may be seen traversing thick deposites of ore. There are few whose width is less than 24 inches, and height less than 40 ; such small drifts serve merely as temporary expedients in workings. Some galleries are several leagues in length. We shall describe in the sequel the means which are for the most part necessary to support the roof and the walls. The rubbish is removed by wagons or wheelbarrows of various kinds. See fig. 712 .

It is impossible to advance the boring of a shaft or gallery beyond a certain rate, because only a limited set of workmen can be made to bear upon it. There are some galleries which have taken more than 30 years to perforate. The only expedient for accelerating the adrance of a gallery, is to commence, at several points of the line to be pursued, portions of galleries which may be joined together on their completion.

Whether tools or gunpowder be used in making the excavations, they should be so applied as to render the labor as easy and quick as possible, by disengaging the mass out of the rock at two or three of its faces. The effect of gunpowder, wedges, or picks, is then much more powerful. The greater the excavation, the more important is it to observe this rule. With this intent, the working is disposed in the form of steps, (gradins), placed like those of a stair; each step being removed in successive portions, the whole of which, except the last, are disengaged on three sides, at the instant of their being attacked.

The substances to be mined occur in the bosom of the earth, under the form of alluvial deposites, beds, pipe-veins, or masses, threads or small veins, and rake-veins.

When the existence of a deposite of ore is merely suspected, without positive proofs, recourse must be had to labors of research, in order to ascertain the richness, nature, and disposition of a supposed mine. These are divided into three kinds ; open workings, subterranean workings, and boring operations.

1. The working by an open trench, has for its object to discover the outcropping or basset edges of strata or veins. It consists in opening a fosse of greater or less width, which, after removing the vegetable mould, the alluvial deposites, and the matters disintegrated by the atmosphere, discloses the native rocks, and enables us to distinguish the beds which are interposed, as well as the veins that traverse them. The trench ought always to be opened in a direction perpendicular to the line of the supposed deposite. This mode of investigation costs little, but it seldom gives much insight. It is chiefly employed for verifying the existence of a supposed bed or vein.

The subterranean workings afford much more satisfactory knowledge. They are executed by different kinds of perforations; viz.. by longitudinal galleries hollowed out 
of the mass of the beds or veins themselves, in following their course; by transverse galleries, pushed at right angles to the direction of the veins; by inclined shafts, which pursue the slope of the deposites, and are excavated in their mass; or, lastly, by perpen. dicular pits.

If a vein or bed unveils itself on the flank of a mountain, it may be explored, according to the greater or less slope of its inclination, either by a longitudinal gallery opened in its mass, from the outcropping surface, or by a transverse gallery falling upon it in a certain point, from which either an oblong gallery or a sloping shaft may be opened.

If our object be to reconnoitre a highly inclined stratum, or a vein in a level country, we shall obtain it with sufficient precision, by means of shafts, 8 or 10 yards deep, dug at 30 yards distance from one another ; excavated in the mass of ore, in the direction of its deposite. If the bed is not very much inclined, only $45^{\circ}$, for example, vertical shafts must be opened in the direction of its roof, or of the superjacent rocky stratum, and zalleries must be driven from the points in which they meet the ore, in the line of its direction.

When the rocks which cover valuable minerals are not of very great hardness, as happens generally with the coal formation, with pyritous and aluminous slates, sal gem, and some other minerals of the secondary strata, the borer is employed with advantage to ascertain their nature. This mode of investigation is economical, and gives, in such cases, a tolerably exact insight into the riches of the interior. The method of using the borer has been described under Artesian Wells.

\section{OF MINING IN PARTICULAR.}

The mode of working mines is two-fold; by open excavations, and subterranean.

Workings in the open air present few difficulties, and occasion little expense, unless when pushed to a great depth. They are always preferred for working deposites little distant from the surface; where, in fact, other methods cannot be resorted to, if the substance to be raised be covered with incoherent matters. The only rules to be observed are, to arrange the workings in terraces, so as to facilitate the cutting down of the earth; to transport the ores and the rubbish to their destination at the least possible expense; and to guard against the crumbling down of the sides. With the latter view, they ought to have a suitable slope, or to be propped by timbers whenever they are not quite solid.

Open workings are employed for valuble clays, sands, as also for the alluvial soils of diamonds, gold, and oxyde of tin, bog iron ores, \&c., limestones, gypsums, building stones, roofing slates, masses of rock salt in some situations, and certain deposites of ores, particularly the specular iron of the island of Elba; the masses of stanniferous granite of Gayer, Altenberg, and Seyffen, in the Ertzgeberge, a chain of mountains between Saxony and Bohemia; the thick veins or masses of black oxyde of iron of Nordmarch, Dannemora, \&c., in Sweden; the mass of cupreous pyrites of Ræraas, near Drontheim in Norway; several mines of iron, copper, and gold in the Ural mountains, \&c.

Subterranean workings may be conveniently divided into five classes, viz. :-

1. Veins, or beds, much inclined to the horizon, having a thickness of at least two yards.

2. Beds of slight inclination, or nearly horizontal, the power or thickness of which does not exceed two yards.

3. Beds of great thickness, but slightly inclined.

4. Veins, or beds highly inclined, of great thickness.

5. Masses of considerable magnitude in all their dimensions.

Subterranean mining requires two very distinct classes of workings; the preparatory, and those for extraction.

The preparatory consist in galleries, or in pits and galleries destined to conduct the miner to the point most proper for attacking the deposite of ore, for tracing it all round this point, for preparing chambers of excavation, and for concerting neasures with a view to the circulation of air, the discharge of waters, and the transport of the extracted minerals.

If the vein or bed in question be placed in a mountain, and if its direction forms a very obtuse angle with the line of the slope, the miner begins by opening in its side, at the lowest possible level, a gallery of elongation, which serves at once to give issue to the waters, to explore the deposite through a considerable extent, and then to follow it in another direction; but to commence the real mining operations, he pierces either shafts or galleries, according to the slope of the deposite, across the first gallery.

For a stratum little inclined to the horizon, placed beneath a plain, the first thing is to nierce two vertical shafts, which are usually made to arrive at two points in the same line of slope, and a gallery is driven to unite them. It is, in the first place, for the sake of circulation of air that these two pits are sunk; one of them, which is also destined for the drainage of the waters, should reach the lowest point of the intended workings. 
If a vein is intersected by transverse ones, the shafts are placed so as fo follow, or, at least, to cut through the intersections. When the mineral ores lie in nearly vertical masses, it is right to avoid, as far as possible, sinking pits into their interior. These should rather be perforated at one side of their floor, even at some considerable distance, to avoid all risk of crumbling the ores into a heap of rubbish, and overwhelming the workmen.

With a vein of less than two vards thick, as soon as the preparatory labors have brought the miners to the point of the vein from which the ulterior workings are to ramify, whenever a circulation of air has been secured, and an outlet to the water and the matters mined, the first object is to divide the mass of ore into large parallelopipeds, by means of oblong galleries, pierced 20 or 25 yards below one another, with pits of communication opened up, 30,40 , or 50 yards asunder, which follow the slope of the vein. These galleries and shafts are usually of the same breadth as the vein, unless when it is very narrow, in which case it is requisite to cut out a portion of the roof or the floor. Such workings serve at once the purposes of mining; by affording a portion of ore, and the complete investigation of the nature and riches of the rein, a certain extent of which is thus prepared before removing the cubical masses. It is proper to adrance first of all, in this manner, to the greatest distance from the central point which can be mined with economy, and afterwards to remove the parallelopiped blocks, in working back to that point.

This latter operation may be carried on in two different ways; of which one consists in attacking the ore from above, and another from below. In either case, the excava tions a:e disposed in steps similar to a stair upon their upper or under side. The first is strled a working in direct or descending steps; and the second a working in reverse, or ascending steps.

1. Suppose, for example, that the post $\mathrm{N}$, fig. 714 , included between the horizontal

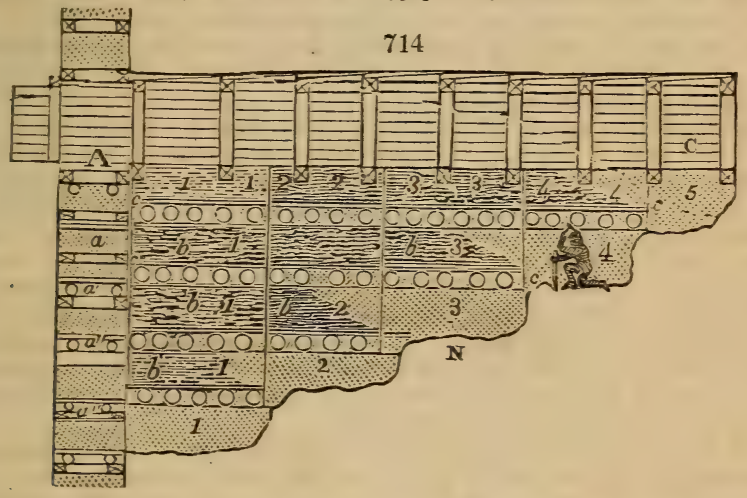

B

gallery $\mathrm{A} \mathrm{C}$ and the shaft $\mathrm{A} B$, is to be excavated by direct steps, a workman stationed upon a scaffold at the point $a$, which forms the angle between the shaft and the elongated drift, attacks the rock in front of him and beneath his feet. Whenever he has cut out a parallelopiped (a rectangular mass), of from four to six yards broad, and two tards high, a second miner is set to work upon a scaffold at $a^{\prime}$, two yards beneath the first, who, in like manner, excavates the rock under his feet and before him. As soon as the second miner has remored a post of four or six yards in width, by two in height, a third begins upon a scaffold at $a^{\prime \prime}$ to work out a third step. Thus, as many workmen are employed as there are steps to be made between the two oblong horizontal galleries which extend above and below the mass to be excavated; and since they all proceed simultaneousl, , they continue working in similar positions, in floors, over each other, as upon a stair with very long wide steps. As they advance, the miners construct before them wooden floors $c c c c$, for the purpose of sunnorting the rubbish which each Torkman extracts from his own step. This floor, which should be very solid, serves also for wheeling out his barrow filled with ore. The round billets which support the planks sustain the roof or the wall of the mineral vein or bed under operation. If the rubbish be very considerable, as is commonly the case, the floor planks are lost. However strongly they may be made, as they sannot be repaired, they sooner or later give way under the enormous pressure of the rubbish; and as all the weight is borne by the roof of the oblong gallery underneath, this must be suffi eiently timbered. By this ingenious plan, a great many miners may go to work together upon a vein without mutual interference; as the portions which ther detach have always two faces at least free, they are consequently more easily separable, either 
with gunpowder or with the pick. Should the vein be more than a yard thick, or if its substance betvery refractory, two miners are set upon each step. $b \quad b \quad b \quad b$ indicate the quadrangular masses that are cut out successively downwards; and $11,22,33$, forwards; the lines of small circles are the sections of the ends of the billets which support the floors.

2. To attack a mass $\mathrm{x}$, fig. 715 , a scaffold $m$ is erected in one of its terminal pits

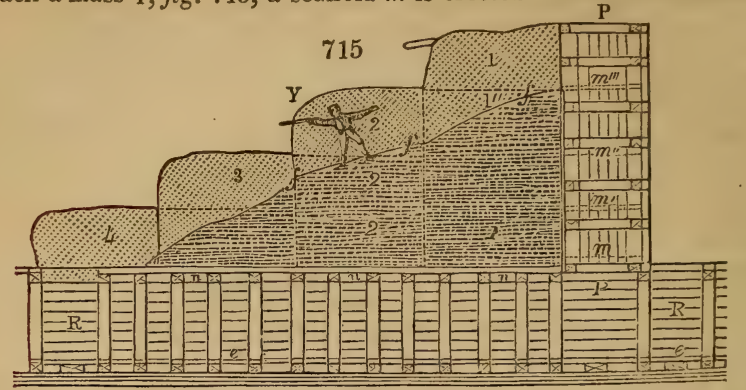

at the level of the ceiling of the gallery $\mathrm{R} \mathrm{R}^{\prime}$, where it terminates below. A miner placed on this scaffold, cuts off at the angle of this mass a parallelopiped 1, from one to two yards high, by six or eight long. When he has advanced thus far, there is placed in the same pit, upon another scaffold $m^{\prime}$, a second miner, who attacks the rein above the roof of the first cutting, and hews down, ahove the parallelopiped 1, a parallelopiped of the same dimensions $1^{\prime}$, while the first is taking out another 2 , in advance of 1. When the second miner has gone forward 6 or 8 yards, a third is placed also in the same pit. He commences the third step, while the first two miners are pushing forwarls theirs, and so in succession.

In this mode of working, as well as in the preceding, it is requisite to support the rubbish and the walls of the vein. For the first object, a single floor $n n n$, may be sufficient, constructed above the lower gallery, substantial enough to bear all the rubbish, as well as the miners. In certain cases, an arched roof may be substituted; and in others, several floors are laid at different heights. The sides of the vein are supported by means of pieces of wood fixed between them perpendicularly to their planes. Sometimes, in the middle of the rubbish, small pits are left at regular distances apart, through which the workmen throw the ore coarsely. picked, down into the lower gallery. The rubbish occasionally forms a slope $f f f$, so high that miners placed upon it can work conveniently. When the rich portions are so abundant as to leave too little rubbish to make such a sloping platform, the miners plant themselves upon moveable floors, which they carry forward along with the excavations.

These two modes of working in the step-form, have peculiar advantages and disadvantages; and each is preferred to the other according to circumstances.

In the descending workings, or in direct steps, fig. 714, the miner is placed on the very mass or substance of the vein; he works commodiously before him; he is not exposed to the splinters which may fly off from the roof; but by this plan he is obliged to employ a great deal of timber to sustain the rubbish; and the wood is fixed for ever.

In the ascending workings, or in reversed steps, fig. 715, the miner is compelled to work in the re-entering angle formed between the roof and the front wall of his excavation, a posture sometimes oppressive; but the weight of the ore conspires with his efforts to make it fall. He employs less timber than in the workings with direct steps. The sorting of the ore is more difficult than in the descending working, because the rich ore is sometimes confounded with the heap of rubbish on which it falls.

When seams of diluvium or gravel-mud occur on one of the sides of the vein, or on both, they render the quarrying of the ore more easy, by affording the means of uncovering the mass to be cut down, upon an additional face.

Should the vein be very narrow, it is necessary to remove a portion of the sterile rock which encloses it, in order to give the work a sufficient width to enable the miner to advance. If, in this case, the vein be quite distinct from the rock, the labor may be facilitated, as well as the separation of the ore, by disengaging the vein, on one of its faces, through a certain extent, the rock being attacked separately. This operation is called stripping the vein. When it is thus uncovered, a shot of gunpowder is sufficient to detach a great mass of it, unmixed with sterile stones.

By the methods now described, only those parallelopipeds are cut out, either in whole or in part, which present indications of richness adequate to yield a prospect of benefit. In other cases, it is enough to follow out the threads of ore which occur, by workings made in their direction. 
The miner, in searching within the crust of the earth for the riches which it conceals, is exposed to many dangers. The rocks amidst which he digs are seldom or never entire, but are almost always traversed by clefts in various directions, so that impending frag. ments threaten to fall and crush him at every instant. He is even obliged at times to cut through rotten friable rocks or alluvial loams. Fresh atmospheric air follows him with difficulty in the narrow channels which he lays open before him; and the waters which circulate in the subterranean seams and fissures filter incessantly into his excaration, anc tend to fill it. Let us now take a view of the means he employs to escape from these three classes of dangers.

1. Of the timbering of excavations.-The excavations of mines are divisible into three principal species; shafts, galleries, and chambers. When the width of these excavations is inconsiderable, as is commonly the case with shafts and galleries, their sides can sometimes stand upright of themselves; but more frequently they require to be propped or stayed by billets of wood, or by walls built with bricks or stones; or even by stufting the space with rubbish. These three kinds of support are called timbering, walling, and filling up.

Timbering is most used. It varies in form for the three species of excavations, according to the solidity of the walls which it is destined to sustain.

In a gallery, for example, it may be sufficient to support merely the roof, by means of joists placed across, bearing at their two ends in the rock; or the roof and the two walls by means of an upper joist s, fig. 716, which is then called a cap or conice beam,

716

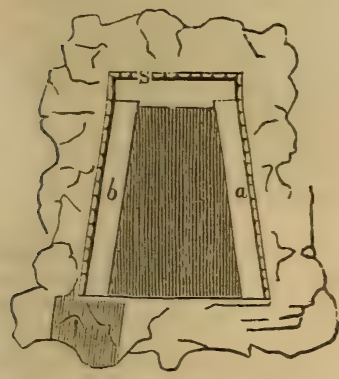
resting on two lateral upright posts or stanchions, $a b$, to which a slight inclination towards each other is given, so that they approach a little at the top, and rest entirely upon the floor. At times, only one of the walls and the roof need support. This case is of frequent occurrence in pipe veins. Pillars are then set up only on one side, and on the other the joists rest in holes of the rock. It. may happen that the floor of the gallery shall not be sufficiently firm to afford a sure foundation to the standards; and it may be necessary to make them rest on a horizontal piece called the sole. This is timbering with complete frames. The upright posts are usually set directly on the sole; but the extremities of the cap or ceiling, and the upper ends of the standards, are mortised in such a manner that these cannot come nearer, whereby the cap shall possess its whole force of resistance. In friable and shivery rocks there is put behind these beams, both upon the ceiling and the sides, facing boards, which are planks placed horizontally, or spars of cleft wood, set so close togther as to leave no interval. They are called fascines in French. In ordinary ground, the miner puts up these planks in proportion as he goes forwards ; but in a loose soil, such as sand or gravel, he must mount them a little in advance. He then drives into the mass behind the wooden frame-work, thick but sharp-pointed planks or stakes, and which, in fact, form the sides of the cavity, which be proceeds to excavate. Their one extremity is thus supported by the earth in which it is thrust, and their other end by the last framing. Whenever the miner gets sufficiently on, he sustains the walls by a new frame. The size of the timber, as well as the distance between the frames or stan. chions, depends on the degree of pressure to be resisted.

When a gallery is to serve at once for several distinct purposes, a greater height is given to it ; and a flooring is laid on it at a certain level. If, for example, a gallery is to be employed, both for the transport of the ores and the discharge of the waters, $\mathbf{a}$. floor $e$ e, fig. 715, is constructed above the bottom, over which the carriages are wheeled, and under which the waters are discharged.

The timbering of shafts varies in form, as well as that of galleries, according to the nature and the locality of the ground which they traverse, and the purposes which they are meant to serve. The shafts intended to be stayed with timber are usually square or rectangular, because this form, in itself more convenient for the miner, renders the execution of the timbering more easy. The wood-work consists generally of rectangular frames, the spars of which are about eight inches in diameter, and placed at a distance asunder of from a yard to a yard and a half. The spars are never placed in contact, except when the pressure of the earth and the waters is very great. The pieces composing the frames are commonly united by a half-check, and the longer of the two pieces extends often beyond the angles, to be rested in the rock. Whether the shaft is vertical or inclined, the frame-work is always placed so that its plane may be perpendicular to the axis of the pit. It happens sometimes in inclined shafts that there are only two sides, or even a single one, which needs to be propped. These are stayed by means of cross beams, which rest at their two ends in the rock. When the frames 
do not touch one another, strong planks or stakes are fastened behind them to sustain the ground. To these planks the frames are firmly connected, so that they cannot slide. In this case the whole timbering will be supported, when the lower frame is solidly fixed, or when the pieces from above pass by its angles to be abutted upon the ground.

In the large rectangular shafts, which serve at once for extracting the ores, for the discharge of the waters, and the descent of the workmen, the spaces destined for these several purposes are in general separated by partitions, which also serve to increase the strength of the timberings, by acting as buttresses to the planks in the long sides of the frame-work. Occasionally a partition separates the ascending from the descending bas ket, to prevent their jostling.-Lastly, particular passages are left for ventillation.

As it is desirable that the wood shall retain its whole force, only those pieces are squared which absolutely require it. The spars of the frames in shafts and galleries are deprived merely of their bark, which by holding moisture, would accelerate the decomposition of the wood. The alburnum of oak is also removed.

Resinous woods, like the pine, last much shorter than the oak, the beech, and the cherry-tree; though the larch is used with advantage. The oak has been known to last upwards of 40 years; while the resinous woods decay frequently in 10 . The fresher the air in mines, the more durable is the timbering.

The marginal figs. 717,718 represent two vertical sections of a shaft, the one at right
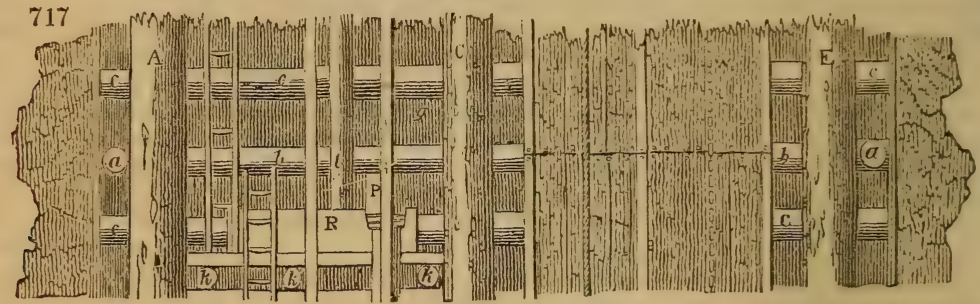

angles to the other, with the view of showing the mode of sustaining the walls of the excavation by timbering. It is copied from an actual mine in the Hartz. There we may 718 observe the spaces allotted to the descent of the miners by

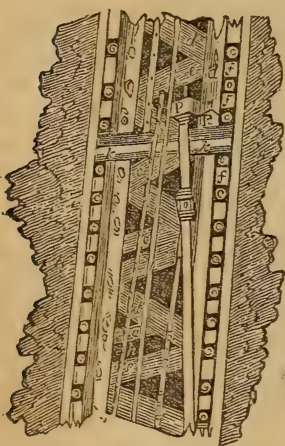
ladders, to the drainage of the waters by pumps $P$, and rods $t$, and to the extraction of the mineral substances by the baskets B. $a, b, c, f, h, k$, various cross timbers ; A, c, E, upright do.; $\mathrm{R}$, pump cistern; $\mathrm{v}, \mathrm{w}$, corve-ways. The shafts here shown, are excavated in the line of the vein itself, - - the rock enclosing it being seen in the second figure.

\%9 In a great many mines it is found advantageous to support the excavations by brick or stone buildings, constructed either with or without mortar. These constructions are often more costly than wooden ones, but they last much longer, and need fewer repairs. They are employed instead of timberings, to support the walls and roof of galleries, to line the sides of shafts, and to bear up the roofs of excavations.

Sometimes the two sides of a gallery are lined with vertical walls, and its roof is supported by an ogee vault, or an arch. If the sides of the mine are solid, a simple arch is sufficient to sustain the roof, and at other times the whole surface of a gallery is formed of a single elliptic vault, the great axis of which is vertical; and the bottom is surmounted by a wooden plank, under which the waters run off; see fig. 719 .

Walled shafts also are sometimes constructed in a circular or elliptic form, which is better adapted to resist the pressure of the earth and waters. Rectangular shafts of all dimensions, however, are frequently walled.

The sides of an excavation may also be supported by filling it completely with rubbish. Wherever the sides need to be supported for some time without the necessity of passing along them, it is often more economical to stuff them up with rubbish, than to keep up their supports. In the territory of Liege, for example, there have been shafts thus filled up for sereral centuries; and which are found to be quite entire when they are emptied. The rubbish is also useful for forming roads among steep strata, for closing air-holes, and forming canals of ventilation.

Figs. $719,720,721$ represent the principal kinds of mason-work employed in the galleries and shafts of mines. Fig. 722 exhibits the walling in of the cage of an over- shot water wheel, as mounted within a mine. Before beginning to build, an exca- 
vation large enough must be made in the gallery to leave a space three feet and a half high for the workmen to stand in, after the brick-work is completed. Between the two opposite sides, cross beams of wood must be fixed at certain distances, as chords of the vault, over which the rock must be hollowed out to receive the arch-stones, and the centring must then be placed, covered with deals to receive the voussoirs, beginning at the flanks and ending with the key-stone. When the vault is finished through a certain extent, the interval between the arch and the rock must be rammed ruli of rubbish, leaving passages, if necessary, through it and the arch, for currents of water.

In walling galleries, attention must be paid to the direction of the pressure, and to build vertically or with a slope accordingly. Should the pressure be equal in all directions, a closed vault, like fig. 719, should be formed. For walls not far from the vertical, salient or buttressed arches are employed, as shown in fig. 720, called in German überspringende bogen; for other cases, twin-arches are preferred, with an upright wall between.

Fig. 721 is a transverse section of a walled drain-gallery, from the grand gallery of the Hartz; see also fig. 722. $a$ is the rock, which needs to be supported only at the sides

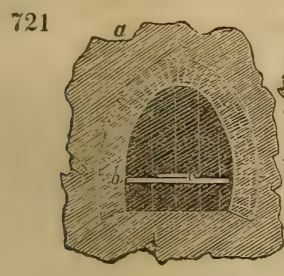

720
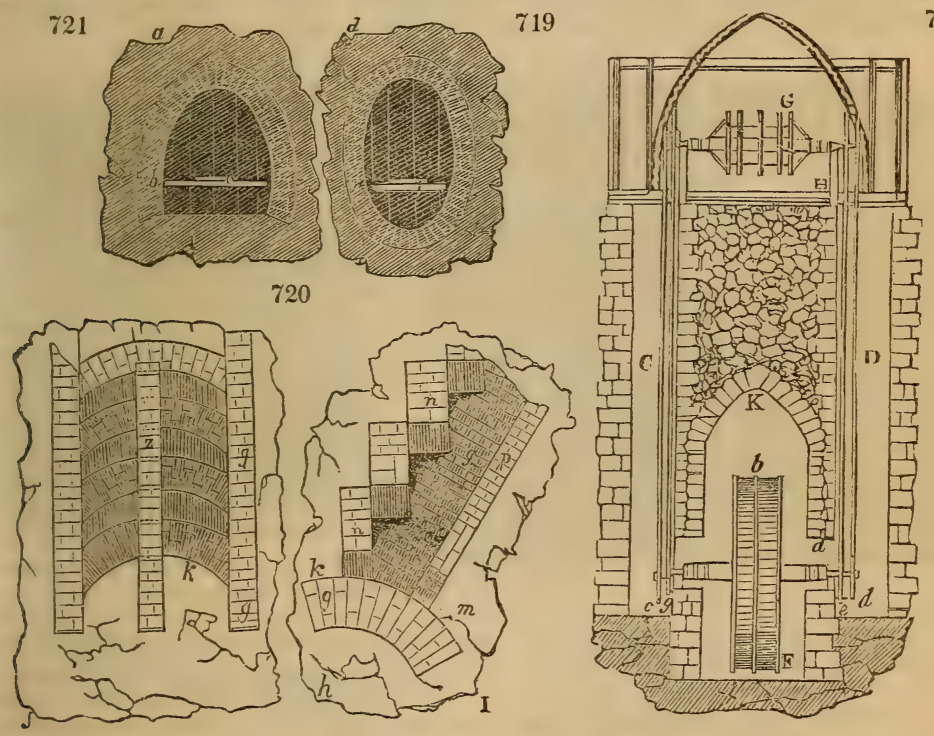

722

and top; $b$, the masonwork, a curve formed of the. three circular arcs upon one level; c, the floor for the water-course. Fig. 719 is a cross section of a walled gallery, as at Schneeberg, Rothenburg, Idria, \&c.; $d$, is the rock, which is not solid either at the flanks, roof, or floor; $e$, the elliptic masonwork; $f$, the wooden floor for the wagons, which is sometimes, however, arched in brick to allow of a water-course beneath it.

Fig. 720 shows two vertical projections of a portion of a walled shaft with buttresses, as built at the mine Vater Abraham, near Marienberg. $\mathrm{J}$ is a section in the direction of the rein $g h$, to show the roof of the shaft. I, a section exhibiting the slope of the vein $g h$, into which the shaft is sunk; $m$ is the wall of the vein; $k$ is the roof of the same vein; $n$, buttresses resting upon the flanks of the shaft; g, great ares on which the buttresses bear; $y$, vertical masonwork; $z$, a wall which divides the shaft into two compartments, of which the larger, $p$, is that for extracting the ore, and the smaller for the draining and descent of the miners.

Fig. 722, C D is the shaft in which the vertical crank-rods $c g, e d$, move up and down. F, is a double hydraulic wheel, which can be stopped at pleasure by a brake mounted upon the machine of extraction. G, is the drum of the gig or whim for raising the corves or tubs (tonnes); $\mathrm{H}$, is the level of the ground, with the carpentry which supports the whim and its roof. $k$, is the key-stone of the ogee arch which covers the wrer-wheel; $a$, is the opening or window, traversed by the extremity of the driving $\mathrm{sb}$ eft, upon each side of the water-wheel, through which a workman may enter to adjust or repair it ; $c b$, line of conduits for the streams of water which fall upon the hydraulic 
wheel ; $c, g$, double crank with rods, whose monion is ıaken off the left side of the wheel $e, d$, the same upon the right side. The distance from $\mathrm{H}$ to $\mathrm{F}$ is about 22 yards.

Figs. 723,724 present two vertical sections of the shaft of a mine walled, like the roof of a carern, communicating with the galleries of the roof and the wall of the vein, and well arranged for both the extraction of the ore, and the descent of the miners. The vertical partition of the shaft for separating the passage for the corves or tubs from the ladders is omitted in the figure, for the sake of clearness.

In $f i g .723, \mathrm{~A}$, B are the side walls supported upon the buttresses $\mathrm{c}$ and $\mathrm{v}$; in fig. $^{2} \mathbf{7 2 4}$, $E$ is the masonry of the wall, borne upon the arch $F$ at the entrance to a gallery; the continuation being at $\mathrm{G}$, which is sustained by a similar arch built lower.

$L$, is the vault arch of the roof, supported upon another vault $M$, which presents a double curvature, at the entrance of a gallery; at $\mathrm{H}$ is the continuation of the arch or vault $\mathbf{L}$, which underneath is supported in like manner at the entrance of a lower gallery.

$a b, c d$, fig. 723, are small upright guide-bars or rods for one of the corves, or kibbles. ef, $g h$, are similar guide-bars for the other corf.

$i i$, are cross-bars of wood, which support the stays of the ladders of descent.

$k k$, are also cross-bars by which the guide-rods are secured.

$t$, a corf, or extraction kibble, furnished with friction rollers; the other corf is supposed to be drawn up to a higher level, in the other vertical passage.

723

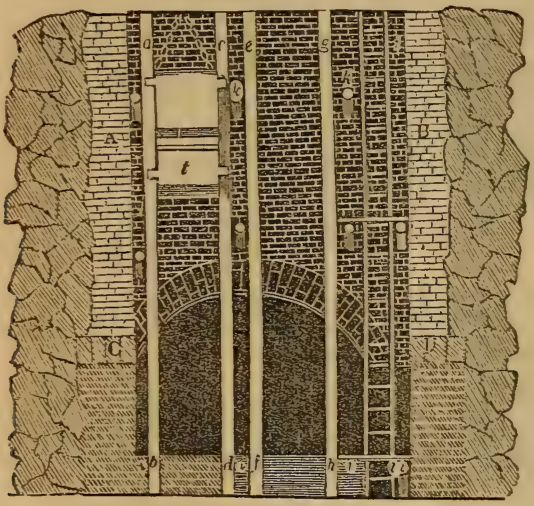

725
724

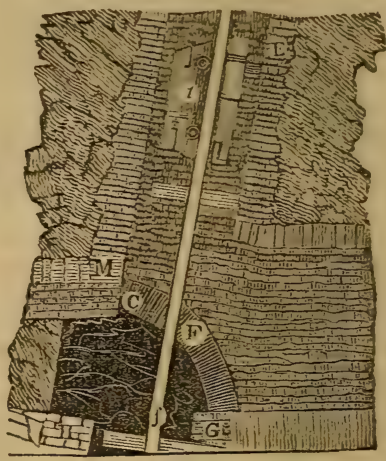

726
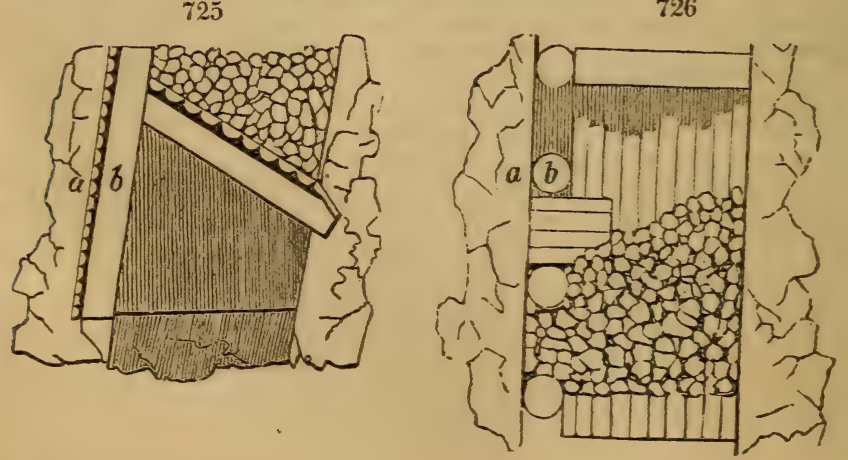

Figs. 725,726 represent in a vertical section the mode of timbering the galleries of the silver and lead mines at Andreasberg in the Hartz. Fig. 725 shows the plan viewed from above. Upon the roof of the timbering, the workman throws the waste rubbish, and in the empty space below, which is shaded black, he transports in his wagons or wheelbarrows the ores towards the mouth of the mine. Fig. 726 is the cross section of the gallery. In the two figures, $a$ represents the rock, and $b$ the timbering; round which there is a garniture of small spars or lathes for the purpose of drainage and ventilation, with the view of promoting the durability of the wood-work. 
The working of minerals by the mass is well exemplified a few leagues to the north of Siegen, near the village of Müsen, in a mine of iron and other metals, called Stahlberg, which forms the main wealth of the country. The plan of working is termed the excavation of a direct or transverse mass. It shows in its upper part the danger of bad mining, and in its inferior portion, the regular workings, by whose means art has eventually prevented the destruction of a precious mineral deposite.

Fig. 727 is a vertical section of the bed of ore, which is a direct mass of spathose 727

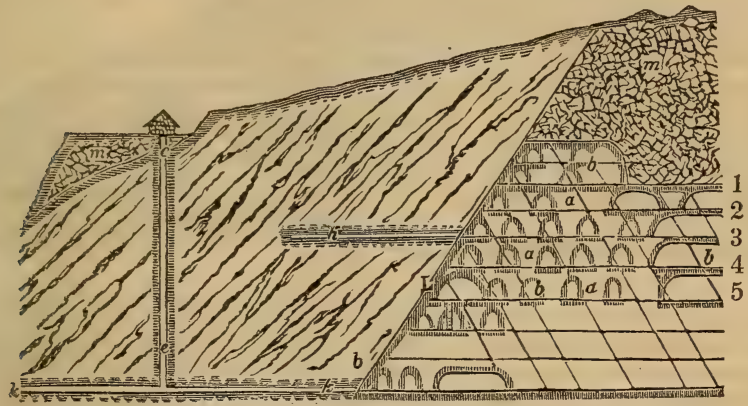

iron, contained in transition rock (graywacke). $a, a, a$, are pillars of the sparry ore, reserved to support the successive stages or floors, which are numbered $1,2,3$, \&c.; $b, b, b$, are excavations worked in the ore; which exhibit at the present day several floors of arehes, of greater or less magnitude, according to the localities. It may be remarked, that where the metallic deposite forms one entire mass, rich in spathose iron ore of good quality, there is generally given to the vaults a height of three fathoms; leaving a thickness over the roof of two fathoms, on account of the numerous fissures which pervade the mass. But where this mass is divided into three principal branches, the roof of the vaults has only a fathom and a half of thickness, while the excavation is three fathoms and a half high. In the actual state of the workings, it may be estimated that from all this direct mass, there is obtained no more out of every floor than one third of the mineral. Two thirds remain as labors of reserve, which may be resumed at some future day, in consequence of the regularity and the continuation of the subterranean workings. $e$ is a shaft for extraction, communicating below with the gallery of effux $k ; h$ is an upper gallery of drainage, which runs in different directions (one only being visible in this section) over a length of 400 fathoms. The lower gallery $k$ runs 646 fathoms in a straight line. The mine of Stahlberg has furnished annually on an average since 1760 about 25,000 cubic feet (French) of an excellent spathose ore of iron. in $m$, represents the mass of sparry iron.

Figs. 728, 729, 730 represent the cross system of mining, which consists in forming

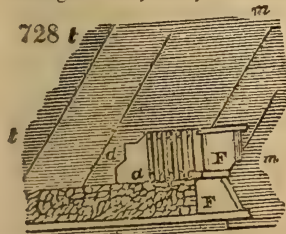
galleries through a mineral deposite, from its wall or floor towards its roof, and not, as usual, in the direction of its length. This mode was contrived towards the middle of the 18th century, for working the very thick veins of the Schemnitz mine in Hungary, and it is now employed with advantage in many places, particularly at Idria in Carniola. In the two sections figs. 728, 730, as well as in the ground plan fig. 729, the wall is denoted by $m m$, and the roof by $t$. A first gallery of prolongation E F, fig. 730, being formed to the wall, transverse cuts, $a, a$, are next established at right angles to this gallery, so that between every two there may be room enough to place three others, $b, c, b$, fig. 729. From cach of the euts $a$, ore is procured by advancing with the help of timbering, till the roof $t$ be reached. When this is done, these first cuts $a$, are filled up with rubbish, laid upon pieces of timber with which the ground is covered, so that if eventually it should be wished to mine underneath, no downfali of detritus is to be feared. These heaps of rubbish rise only to within a few inches of the top of the cuts $a$, in order that the working of the upper story may be easier, the bed of ore being there already laid open apon its lower face.

In proportion as the cuts $a$, of the first story $\mathbf{E} \mathbf{F}$, are thus filled up, the greater part of the timbering is withdrawn, and made use of elsewhere. The intermediate cuts $b, c, b$, are next mined in like manner, either beginning with the cuts $c$, or the cuts $b$, according to the localities. From fig. 729 it appears that the working may be so arranged, that in case of necessity, there may be always between two cuts in activity tho 
distance of three cuts, either not made, or filled up with rubbish. Hence, all the portion of the bed of ore may be removed, which corresponds to a first story E F, fig. 730 , and the portion is replaced by rubbish.

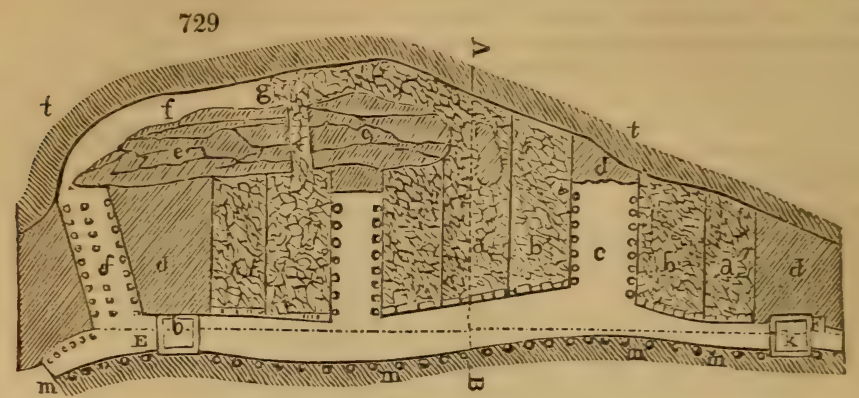

The exploration of the upper stories $\mathbf{E}^{\prime} \mathrm{F}^{\prime}, \mathrm{E}^{2} \mathrm{~F}^{2}, \mathrm{E}^{3} \mathrm{~F}^{3}$, is now prepared in a similar manner; with which view shafts $h h 3, k k 3$, are formed from below upwards in the wall $m$ of the deposite, and from these shafts oblong galleries proceed, established successirely on a level with the stories thus raised over one another. See fig. 730 . The following objects may be specified in the figures:-

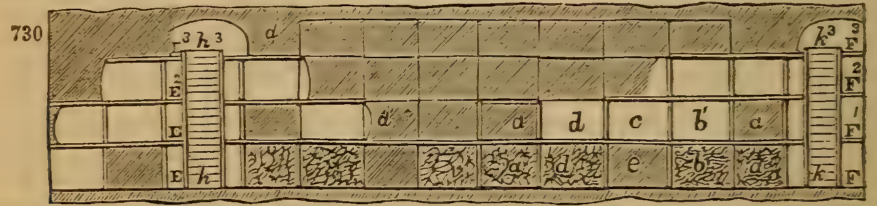

$a a$, the first cuts filled up with rubbish, upon the first story $x \mathrm{~F}$, fig. 729 .

$b$, other cuts subsequently filled up, upon the same story.

$c$, the cut actually working.

$d$, the front of the cut, or place of actual excavation of the mineral deposite.

$e$, masses of the barren rock, reserved in the cutting, as pillars of safety.

$f$, galleries, by means of which the workmen may turn round the mass $e$, in order to form, in the roof $t$, an excavation in the direction of the deposite.

$g$, rubbish behind the mass $e$.

$k k$, two shafts leading from the first story $\mathrm{E} \mathrm{F}$, to the upper stories of the workings, as already stated.

$m$, the wall, and $t$ the roof of the mineral bed.

In the second story $E^{\prime} F^{\prime}$, the gallery of prolongation $F^{\prime}$, figs. 728 and 730 , is not entirely perforated; but it is further advanced than that of the third story, which, in its turn, is more than the gallery of the fourth.

From this arrangement there is produced upon fig. 730 the general aspect of a working by reversed steps.

Whenerer the workings of the cuts $c$ in the first story are finished, those of the second, $a^{\prime} a^{\prime}$, mar be begun in the second; and thus by mounting from story to story, the whole deposite of ore may be taken out and replaced with rubbish. One great adrantage of this method is, that nothing is lost ; but it is not the only one. The facilities offered br the system of cross workings for disposing of the rubbish, most frequently a nuisance to the miner, and expensire to get rid of, the solidity which it procures br the banking up, the consequent economy of timbering, and saring of expense in the excaration of the rock, reckoning from the second story, are so many important circumstances which recommend this mode of mining. Sometimes, indeed, rubbish mar be wanted to fill up, but this may always be procured by a few accessory perforations; it being easy to establish in the vicinity of the workings a vast excaration in the form of a rault, or kind of subterraneous quarry, which may be allowed to fall in with proper precautions and where rubbish will thus accumulate in a short time, at little cost.

Fig. 731 represents a section of the celebrated lead mines of Blerberg in Carinthia, not far from Villach.

$b, c$, is the ridge of the mountains of compact limestone, in whose bosom the workings are carried on.

$e$ is the metalliferous valley, running from east to west, between the two parellel 
valleys of the Gail and the Drave, but at a level considerably above the waters of these rivers.

$f \mathrm{~g}$ is the lirection of a great many vertical beds of metalliferous limestone.

On considering the direction and dip of the marly schist, and metalliferous limestone,

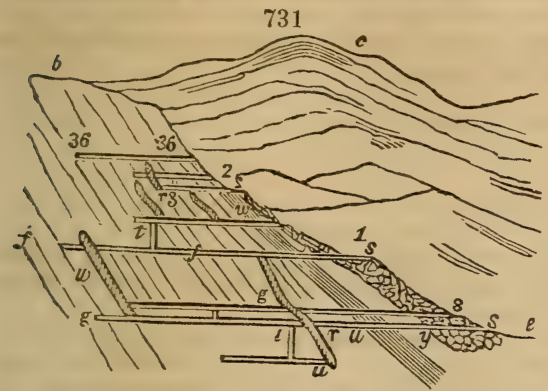
in the space $w, w$, to the west of the line $1, s$, it would appear that a great portion of this system of mountains has suffered a slip between $1, s$, and a parallel one towards the east; whereby, probably, that vertical position of the strata has been produced, which exists through a considerable extent. The metalliferous limestone is covered to a certain thickness with a marly schist, and other more recent rocks. It is in this schist that the fine marble known under the name of the lumachello of Bleyberg is quarried.

The galena occurs in the bosom of this rock in flattened masses, or blocks of a considerable volume, which are not separated from the rest of the calcareous beds by any seam. It is accompanied by zinc ore (calamine), especially in the upper parts of the mountain.

Several of the workable masses are indicated by $r, r 3$; each presents itself as a solid analogous to a very elongated ellipse, whose axis dips, not according to the inclination of the surrounding rock, but to an oblique or intermediate line between this inclination, and the direction of the beds of limestone; as shown by $r w, r^{\prime} u$. Every thing indicates the contemporaneous formation of the limestone, and the lying beds of the lead ore.

The accidents or faults called kluft (rent) at Bleyberg are visible on the surface of the ground. Experienced miners have remarked that the rich masses occur more frequently in the direction of these accidents than elsewhere.

It is in general by galleries eut horizontally in the body of the mountain, and at different levels, $s, g, s f$, that the miner advances towards the masses of ore $r, r 3$. Many of these galleries are 500 fathoms long before they reach a workable mass. The several galleries are placed in communication by a few shafts, such as $t$; but few of these are sunk deeper than the level of the valley $e$.

The total length of the mines of Bleyberg is about 10,000 yards, parallel to the valley $e$ : in which space there are 500 concessions granted by the government to various individuals or joint stock societies, either by themselves or associated with the government.

The metalliferous valley contains 5000 inhabitants, all deriving subsistence from the mines; 300 of whom are occupied in the government works.

Each concession has a number and a name; as Antoni, Christoph, Matthæus, Oswaldi, $2,8,36$, s.e.

Fig. 732 is a section in the quicksilver mine of Idria. 1. is the gray limestone; 2. is a blackish slate; 5 . is a grayish slate. Immediately above these transition rocks lies the bed containing the ores called corallenerz, which consist of an intimate mixture of sulphuret of mercury and argillaceous limestone; in which four men can cut out, in a month, $2 \frac{1}{2}$ toises cube of rock.
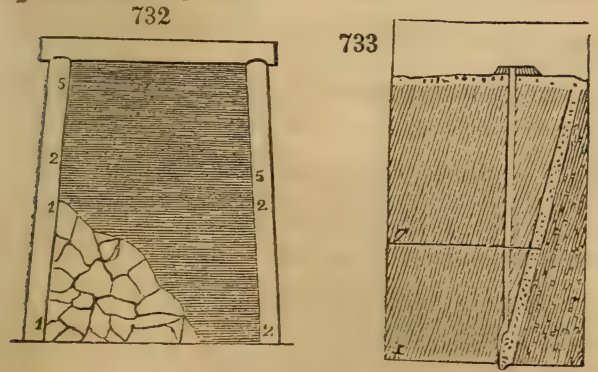

Fig. 733 represents a section of part of the copper mine of Mansfeldt ; containing the cellular limestone, called rauchwacke, always with the compact marl-limestone called zechstein ; the cupreous schist, or kupferschiefer; the wall of grayish-white sandstone, called the weisse liegende; and the wall of red sandstone, or the rothelie gende. The thin dotted stratum at top is vegetable mould; the large dotted portion to the right of the figure is oolite; the vein at its side is sand; next is rauchwacke; and lastly, the main body of fetid limestone, or stinkstein. 
Fig. 734 represents one of the Mansfeldt copper schist mines in the district ealled Burgoerner, or Preusshoheit.

1. Vegetable mould, with silicious gravel.

2. Ferruginous clay or la if.

3. Sand, with fragments of quartz.

4. Red clay, a bed of variable thickness as well as the lower strata, according as the cupreous schist is nearer or farther from the surface.

5. Ooolite (roogenstein).

6. Newer variegated sandstone (bunter sandstein).

7. Newer gypsum; below which, there is

8. A bluish marly clay.

9. Stinkstone, or lucullite.

10. Friable grayish marl.

11. Older gypsum, a rock totally wanting in the other districts of the mines of Rothenberg; but abounding in Saxon Mansfeldt, where it includes vast caverns known among the miners by the name of schlotten, as indicated in the figure.

12. The calcareous rock called zechstein. The lower part of this stratum shows symptoms of the cupriferous schist that lies underneath. It presents three thin bands, differently modified, which the miner distinguishes as he descends by the names of the sterile or rotten ( faüle) rock; the roof (dachklotz); and the main rock (oberberg.)
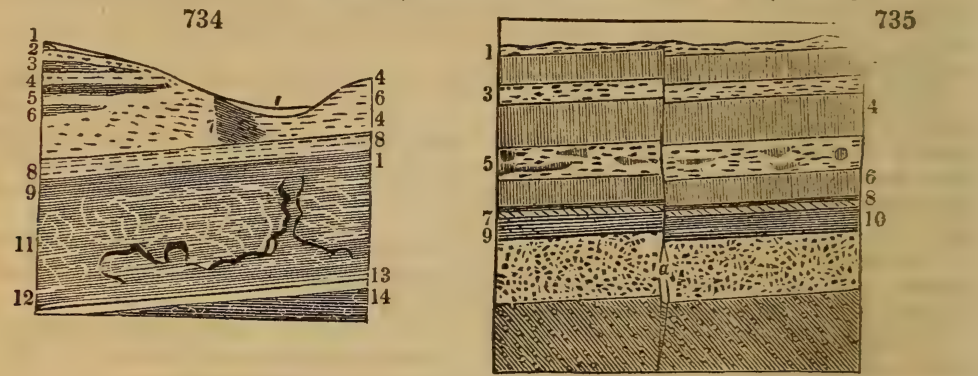

13. Is a bed of cupriferous schist (kupferschiefer), also called the bitumino-marty schist, in which may be noted, in going down, but not marked in the figure :-

$a$, the lochberg, a seam 4 inches thick.

$b$, the kammschale, $\frac{1}{4}$ of an inch thick.

c, the kopfschale, one inch thick.

These seams are not worth smelting; the following, however, are :-

$d$, the schiefer kopf, the main copper schist, 2 inches thick.

$e$, a layer called lochen, one inch thick.

14. The wall of sandstone, resting upon a porphyry.

Fig. 735 is a section of the mines of Kiegelsdorf in Hessia, presenting-

1. Vegetable mould.

2. Limestone distinctly stratified, frequently of a yellowish color, called lagerhafter kalkstein.

3. Clay, sometimes red, sometimes blue, sometimes a mixture of red, blue, and yellow.

4. The cellular limestone (rauhkalk). This rock differs both in nature and position from the rock of the same name at Mansfeldt. nite.

5. Clay, usually red, containing veins of white gypsum, and fine crystals of selo-

6. Massive gypsum of recent formation.

7. Fetid limestone, compact and blackish gray, or cellular and yellowish gray.

8. Pulverulent limestone, with solid fragments interspersed.

9. Compact marl-limestone, or zechstein, which changes from a brownish color above to a blackish schist below, as it comes nearer the cupreous schist, which seems to form a part of it.

10. Cupreous schist (kupferschiefer), of which the bottom portion, from 4 to 6 inches thick, is that selected for metallurgic operations. Beneath it, is found the usual wall or bed of sandstone. A vein of cobalt ore $a$, which is rich only in the grayish-white sandstone (weisse liegende), traverses and deranges all the beds wherever it comes.

of working mines by fire.-The celebrated mine worked since the tenth century in the mountain called Rammelsberg, in the Hartz, to the south of Goslar, presents a stras 
tified mass of ores, among the beds of the rock which constitute that mountain. The mineral deposite is situated in the earth, like an enormous inverted wedge, so that its thickness (power), inconsiderable near the surface of the ground, increases as it descends. At about 100 yards from its outcrop, reckoning in the ${ }^{\prime}$ ction of the slope of the deposite, it is divided into two portions or branches, which are separated from each other, throughout the whole known depth, by a mass of very hard clay slate, which passes into flinty slate. The substances composing the workable mass are copper and iron pyrites with sulphuret of lead (galena), accompanied by quartz, carbonate of lime, compact sulphate of baryta, and sometimes gray copper ore, sulphuret of zinc, and arsenical pyrites. The ores of leac? and copper contain silver and gold, but in small proportion, particularly as to the last.

A mine so ancient as that of Rammelsberg, and which was formerly divided among several adventurous companies, cannot fail to present a great many shafts and excavations; but out of the 15 pits, only two are employed for the present workings ; namely, those marked A B and E F, in fig. 736, by which the whole extraction and drainage ar

736

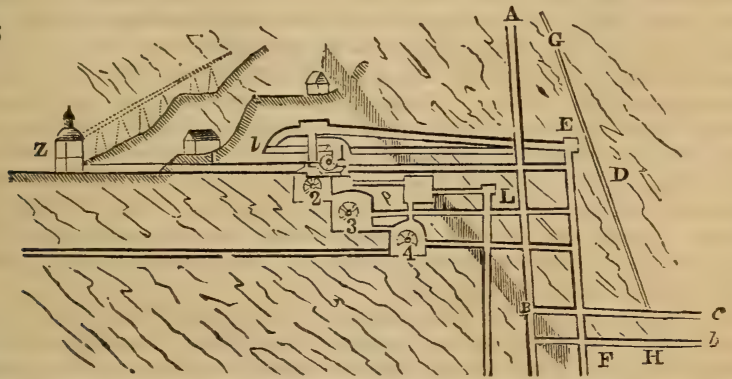

executed. The general system of exploitation by fire, as practised in this mine, consists of the following operations :-

1. An advance is made towards the deposites of ore, successively at different levels, by transverse galleries which proceed from the shaft of extraction, and terminate at the wall of the stratiform mass.

2. There is formed in the level to be worked, large vaults in the heart of the ore, by means of fire, as we shall presently describe.

3. The floor of these vaults is raised up by means of terraces formed from the rubbish, in proportion as the roof is scooped out.

4. The ores detached by the fire from their bed, are picked and gathered; sometimes the larger blocks are blasted with gunpowder.

5. Lastly, the ores thus obtained are wheeled towards the shaft of extraction, and turned out to the day.

Let us now see how the excavation by fire is practised; and in that view, let ns consider the state of the workings in the mines of Rammelsberg in 1809. We may remark in fig. 736 the regularity of the vaults previously scooped out above the level B c, and the other vaults which are in full activity of operation. It is, therefore, towards the lower levels that the new workings must be directed. For this purpose, the transverse gallery being already completed, there is prepared on the first of these fioors a vault of exploitation at $b$, which eventually is to become similar to those of the superior levels. At the same time, there is commenced at the starting point below it, reached by a small well dug in the line of the mineral deposite, a transverse gallery in the rock, by means of blasting with gunpowder. The rock is also attacked at the starting-point by a similar cut, which advances to meet the first perforation. In this way, whenever the vaults of the level $\mathrm{c}$ are exhausted of ore and terraced up with rubbish, those of the level beneath it will be in full activity.

Others will then be prepared at a lower level; and the exploitation may afterwards he driven below this level by pursuing the same plan, by which the actual depth of excavation has been gained.

In workings by fire we must distinguish, 1 . The case where it is neecessary to open a vault immediately from the floor; 2 . The case where the vault having already a certain elevation, it is necessary to heighten its roof. In the former case, the wall or floor of the mineral deposite is first penetrated by blasting with gunpowder. As soon as this penetration is effected over a certain length, parallel to the direction of the future vault, as happens at $b$, there is arranged on the bottom a horizontal laver of hillets of firwood, over which other billets are piled in nearly a vertical position, which rest upon the ore, so that the flame in its expansion comes to play against the mineral mass to be 
detached. When after some similar operations, the flame of the pile san no longet reach the ore of the roof on account of its height, a small terrace of rubbish must be raised on the floor of the deposite; and over this terrace, a new pile of fagots is to be heaped up as above described. The ancient miners committed the fault of constantly placing such terraces close to the roof, and consequently arranging the fagots against this portion of the ore, so that the flame circulated from the roof down to the floor. The result of such procedure was the weakening of the roof, and the loss of much of the ore which could not be extracted from so unstable a fabric; and besides, much more wood was burned than at the present day, because the action of the flame was dissipated in part against the whole mass of the roof, instead of being concentred on the portion of the ore which it was desired to dislodge. Now, the flame is usually made to circulate from the floor to the roof, in commencing a new vault.

When the vault has already a certain height, care is always taken that between the roof of the vault and the rubbish on which the pile is arranged, no more than two yards of space should intervene, in order that the flame may embrace equally the whole concavity of the vault, and produce a uniform effect on all its parts. Here, the pile is formed of horizontal beds, disposed crosswise above one another, and presents four free vertical faces, whence it has been called a chest by the miners.

It is usually on Saturday that the fire is applied to all the piles of fagots distributed through the course of the week. Those in the upper floors of exploitation are first burned, in order that the inferior piles may not obstruct, by their vitiated air, the combustion of the former. Thus, at 4 o'clock in the morning, the fires are kindled in the upper ranges; from pile to pile, the fireman and his assistant descend towards the lower foors, which occupies them till 3 o'clock in the afternoon. Vainly should we endeavor to describe the majestic and terrific spectacle which the fire presents, as it unfolds its wings under its metallic vaults, soon filled with vast volumes of smoke and flame. Let us mark the useful effect which it produces.

When the flame has beat for a few instants on the beds of ore, a strong odor of sulphur, and sometimes of arsenic is perceived; and soon thereafter loud detonations are heard in the vaults. Suddenly the flame is seen to assume a blue color, or even a white; and at this period, after a slight explosion, flakes of the ore, of greater or less magnitude, usually fall down on the fire, but the chief portion of the heated mineral still remains fixed to the vault. The ores pass now into a shattered and divided condition, which allows them afterwards to be detached by long forks of iron. In this manner the fire, volatilizing entirely some principles, such as sulphur, zinc, arsenic, and water, changing the aggregation of the constituent parts of the ore, and causing fissures by their unequal expansibilities, facilitates the excavation of such materials as resist by their tenacity the action of gunpowder.

The combustion goes on without any person entering the mine from Saturday evenIng till Monday morning, on which day, the fireman and his assistants proceed to extinguish the remains of the bonfires. On Monday also some piles are constructed in the parts where the effect of the former ones has been incomplete; and they are kindled after the workmen have quitted the mine. On Tuesday all hands are employed in detaching the ores, in sorting them, taking them out, and preparing new piles against the next Saturday.

The labor of a week consists for every man of five posts during the day, each of 8 hours, and of one post of four hours for Saturday. Moreover, an extra allowance is made to such workmen as employ themselves some posts during the night.

The labor of one compartment or atelier of the mine consists thercfore in arranging the fagots, in detaching the ore which has already experienced the action of the fire, in breaking the blocks obtained, in separating the ore from the debris of the pile, and, whenever it may be practicable or useful, in boring holes for blasting with gunpowder. The heat is so great in this kind of mine, that the men are obliged to work in it without clothing.

We have already remarked, that besides the working by fire, which is chiefly used here, recourse is sometimes had to blasting by gunpowder. This is done in order either to recover the bottom part or ground of the vaults on which the fire can act but imperfectly, to clear away some projections which would interfere with the effect of the pile, or lastly to strip the surrounding rock from the mass of the ore, and thence to obtain schist proper for the construction of the rubbish-terraces.

The blasting process is employed when the foremen of the workshop or minechamber judge that a hole well placed may separate enough of ore to pay the time, the repair of tools, and the gunpowder expended. But this indemnification is rar ely obtained. The following statement will give an idea of the tenacity which the mineral deposite often presents.

In 1808, in a portion of the Rammelsberg mine, the ore, consisting of extremely compact iron and copper pyrites, was attacked by a single man, who bored a mining hole, 
After 11 posts of obstinate labor, occupying altogether 88 hours, the workman, being vigilantly superintended, had been able to advance the hole to a depth of no more than 4 inches; in doing which he had rendered entirely unserviceable 126 punches or borers, besides 26 others which had been re-tipped with steel, and 201 which had been sharpened; $6 \frac{1}{4}$ pounds of oil had been consumed in giving him light; and half a pound of gunpowder was required for blasting the bore. It was found from a calculation made upon these facts by the administration of mines, that every inch deep of this hole cost, at their low price of labor, nearly a florin, value two shillings and sixpence.

It is therefore evident that though the timber, of which the consumption is prodigiously great, were much less abundant and dearer than it still is at Rammelsberg, mining by fire would be preferable to every other mode of exploitation. It is even certain, that on any supposition, the employment of gunpowder would not be practicable for every part of the mine; and if fuel came to fail, it would be requisite to renounce the workings at Rammelsberg, although this mountain still contains a large quantity of metals.

If in all mines the free circulation of air be an object of the highest importance, we must perceive how indispensable it must be in every part of a mine where the mode of exploitation maintains the temperature of the air at $112^{\circ} \mathrm{Fahr}$, when the workmen return into it after the combustion of the piles, and in which besides it is necessary that this combustion be effected with activity in their absence. But in consequence of the extent and mutual ramifications of the workings, the number of the shafts, galleries, and their differences of level, the ventilation of the mine is in a manner spontaneously maintained. The high temperature is peculiarly favorable to it. The aid of art consists merely in placing some doors judiciously, which may be opened or shut at pleasure, to carry on the circulation of the air.

In considering the Rammelsberg from its summit, which rises about 400 yards above the town of Goslar, we observe, first, beds of slaty sandstone, which become the more horizontal the nearer they approach to the surface. At about 160 yards below the top level there occurs, in the bosom of the slaty graywacke, a powerful stratum of shells impasted in a ferruginous sandstone. See $\mathrm{D}$, fig. 730. In descending towards the

737

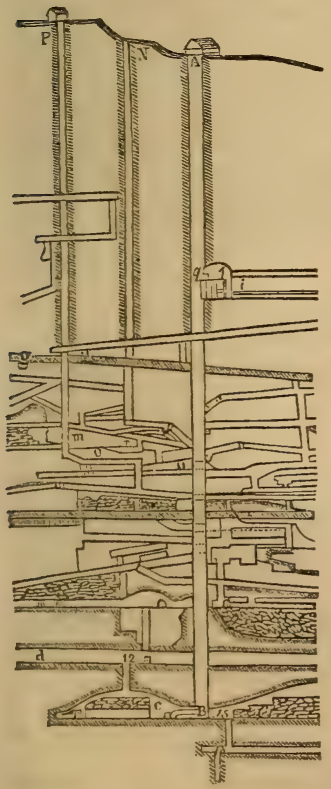

face of the ore, the parallel stratification of the clay-slate which forms its walls and roof grows more and more manifest. Here the slate is black, compact, and thinly foliated. The inclination of the different beds of rock is indicated at $\mathrm{B}$. The substance of the workable mass is copper and iron pyrites, along with sulphuret of lead, accompanied by quartz, carbonate of lime, compact sulphate of baryta, and occasionally gray copper ( $f a h l e r z)$, sulphuret of zinc, and arsenical pyrites.

The ores are argentiferous and auriferous, but very slightly so, especially as to the gold. It is the ores of lead and copper which contain the silver, and in the latter the gold is found, but without its being well ascertained in what mineral it is deposited. Sometimes the copper occurs in the native state, or as copper of cementation. Beautiful crystals of sulphate of lime are found in the old workings.

In figs. 736, 737, A $\mathbf{B}$ is the shaft of extraction, called the Kahnenkuhler; $\mathrm{N}$ is the ventilation shaft, called Breitlingerwetterschacht; P is the extraction shaft, called Innierschacht.

E F, is a new extraction-shaft, called Neuer treibschacht, by which also the water is pumped up; by $\mathrm{A} B$, and $\mathrm{E} F$, the whole extraction and draining are carried on. The ores are raised in these shafts to the level of the wagongallery (gaierie de roulage) $i$, by the whims $1, q$, provided with ropes and buckets. 1, 2, 3, 4, fig. 736, represent the positions of four water-wheels for working the whims; the first two being employed in extracting the or $\epsilon$, the last two in draining. The driving stream is led to the wheel 1 , along the drift $l$; whence it falls in succession upon the wheels $2,3,4$. The general system of working consists of the following operation :-

1. The bed of ore is got at by the transverse galleries, $m, n, o, q, \mathrm{R}, s$, which branch off from the extraction shaft, and terminate at the wall of the main bed;

2. Great vaults are scooped out at the level of the workings, by means of fire ; 
2. Great vaults are scooped out at the level of the workings, by means of fire ;

3. The roofs of these vaults are progressively propped with mounds of rubbish;

4. The ores thus detached, or by blasting with gunpowder, are then collected;

5. Lastly, they are wheeled out to the day; and washed near $z$.

Comparative Table of celebrated Mines in Europe and America. By F. Burr, Esq (Quarterly Mining Review for July, 1835, p. 60.)

\begin{tabular}{|c|c|c|c|c|}
\hline & $\begin{array}{l}\text { Consolidited AND } \\
\text { UNITED MiNes. } \\
\text { (At present the richest } \\
\text { mines in Cornwall.) }\end{array}$ & $\begin{array}{c}\text { Veta Grande Mines. } \\
\text { (At present the richest } \\
\text { mines in Mexico.) }\end{array}$ & $\begin{array}{c}\text { Mrine or Valenctana } \\
\text { 'Richest of itie Mexican } \\
\text { mines at the beginning of } \\
\text { the present century.) }\end{array}$ & $\begin{array}{c}\text { MrNe of } \\
\text { HIMMELs ripsT. } \\
\text { (Richest of the } \\
\text { Saxon mines at the } \\
\text { beginning of the } \\
\text { present century.) }\end{array}$ \\
\hline Situation - - & Two miles east of Redruth. & $\begin{array}{l}\text { Four miles north of } \mathrm{Za}- \\
\text { catecas. }\end{array}$ & $\begin{array}{l}\text { One mile north of Gua- } \\
\text { naxuato. }\end{array}$ & $\begin{array}{l}\text { Two miles south- } \\
\text { east of Freyburg. }\end{array}$ \\
\hline Slevation & $\begin{array}{l}\text { Elevation of the surface } \\
\text { above the level of the } \\
\text { sea, from } 200 \text { to } 300 \text { ft. } \\
\text { depth of the bottom of } \\
\text { the mine below the level } \\
\text { of the eea, about } 1,370 \\
\text { feet. }\end{array}$ & $\begin{array}{l}\text { Elevation of the surface } \\
\text { above the level of the } \\
\text { nea, aupposed to be about } \\
6000 \text { feet. Elevation of } \\
\text { the bottom of the mine } \\
\text { above the level of the sea, } \\
\text { probably nelar } 5,000 \text { feet. }\end{array}$ & $\begin{array}{l}\text { Elevation of the surface } \\
\text { above the level of the sea, } \\
7,617 \text { feet. Elevation of } \\
\text { the bottom of the mine } \\
\text { above the level of the } \\
\text { sea, } 5,730 \text { feet. }\end{array}$ & $\begin{array}{l}\text { Elevation of the sur- } \\
\text { face ate ve the } \\
\text { level of the sea, } \\
1,346 \text { feet } \text { Eleva- } \\
\text { tion of the bot- } \\
\text { tom of the mine } \\
\text { above the level of } \\
\text { the sea, } 263 \text { feet. }\end{array}$ \\
\hline Nature of the rock & $\begin{array}{l}\text { Primary clay slate resting } \\
\text { immediately on granite, } \\
\text { a short distance west- } \\
\text { ward of the mines. The } \\
\text { clay slate is intersected } \\
\text { by numerous channels of } \\
\text { porphyry, which have } \\
\text { nearly the same direction } \\
\text { as the mineral veins, and } \\
\text { are often of considerable } \\
\text { width. The porphyry } \\
\text { sometimes appears also } \\
\text { to form large irregular } \\
\text { masses in the clay slate. } \\
\text { Both rocks are traversed } \\
\text { by veins of quartz and } \\
\text { clay intersecting the me- } \\
\text { talliferous veins. }\end{array}$ & $\begin{array}{l}\text { Transition clay slate, siter- } \\
\text { nating with doiomite, } \\
\text { and occasionally with } \\
\text { greywacke. This clay } \\
\text { slate is sometimes de- } \\
\text { composed; it rests on } \\
\text { syenitic rocks, and is in } \\
\text { some places covered with } \\
\text { porphyry. }\end{array}$ & $\begin{array}{l}\text { The Veta Madre of Gua- } \\
\text { naxuato, upon which } \\
\text { this mine is worked, tra- } \\
\text { verses both clay slate } \\
\text { and porphyry, but it is } \\
\text { most productive in the } \\
\text { former rock. The clay } \\
\text { slate is considered by } \\
\text { Humboldt to belong to } \\
\text { the transition class, but } \\
\text { situate near the limits of } \\
\text { primary formations. This } \\
\text { rock in depth passes into } \\
\text { chlorite slate, and talc } \\
\text { slate. It contains sub- } \\
\text { ordinate beds of syenite, } \\
\text { hornblende slate, and } \\
\text { serpentine. The por- } \\
\text { phyry rest8 upon the clay } \\
\text { slate, and is conforinable } \\
\text { to it, both in direction } \\
\text { and stratification. }\end{array}$ & $\begin{array}{l}\text { The rock prevailing } \\
\text { in the neighbour- } \\
\text { hood of Freyberg, } \\
\text { in which this and } \\
\text { most of the ocher } \\
\text { mines are situate, } \\
\text { is a formation of } \\
\text { primary gneiss. }\end{array}$ \\
\hline $\begin{array}{l}\text { Nature of the me- } \\
\text { talliferous deposite }\end{array}$ & $\begin{array}{l}\text { In the eonsolidated mines, } \\
\text { the eight following lodes } \\
\text { are extensively worked:- } \\
\text { Wheal Fortune lode, } \\
\text { Cusvea lode, Deeble's } \\
\text { lode, Old lode, Taylor's } \\
\text { lode, Tregonning's lode, } \\
\text { Martin's. lode, and Glo- } \\
\text { ver's lode. In the united } \\
\text { mines, the principal } \\
\text { workings are upon the } \\
\text { Old lode, and about five } \\
\text { or six others are more or } \\
\text { less productive. Nume- } \\
\text { rous smaller lodes or } \\
\text { "branches" occur also } \\
\text { in both mines. The } \\
\text { principal lodes are from } \\
2 \text { or } 3 \text {, to } 7 \text { or } 8 \text { feet wide } \\
\text { the "branches" are gen- } \\
\text { erally } 12 \text { or } 18 \text { inches } \\
\text { wide. The direction of } \\
\text { the lodes varies from } \\
\text { nearly east and west to } \\
\text { about } 20 \text { degrees north of } \\
\text { east and south of west. } \\
\text { The underlie of the prin- } \\
\text { cipal lodes is from } 2 \text { to } \\
3 \text { feet per fathom north, } \\
\text { that of the smaller ones } \\
\text { about the same south. }\end{array}$ & $\begin{array}{l}\text { One principal vein (the } \\
\text { Veta Grande) which is } \\
\text { generally separated into } \\
\text { three branches, and } \\
\text { sometimes into four. } \\
\text { When ramified, the } \\
\text { width extends to } 60 \text { or } \\
70 \text { feet; when united, } \\
\text { it varies from } 8 \text { or } 10 \text { to } \\
20 \text { or } 30 \text { feet. The } \\
\text { branches are generally } \\
\text { about } 10 \text { or } 12 \text { feet wide } \\
\text { and the upper one is } \\
\text { most productive. The } \\
\text { direction of the Veta } \\
\text { Grande is from } 30 \text { to } \\
40 \text { degrees south of east, } \\
\text { and north of west, and } \\
\text { its underlie, from two to } \\
\text { three feet per fathom } \\
\text { south. Other veins of } \\
\text { less size occur in the } \\
\text { neighbourhood of the } \\
\text { Veta Grande, which } \\
\text { cross it at an acute angle. } \\
\text { One of these appears } \\
\text { to heave the vein for } \\
\text { about 700 feet, being the } \\
\text { most remarkatle de- } \\
\text { rangement of the kind } \\
\text { on record. }\end{array}$ & $\begin{array}{l}\text { One Veta (the Veta } \\
\text { Madre) which is often } \\
\text { separated into three } \\
\text { branches, extending from } \\
130 \text { to } 160 \text { feet in width. } \\
\text { When not ramified, its } \\
\text { width varies from } 20 \text { or } \\
30 \text { to } 60 \text { or } 70 \text { feet, but } \\
\text { is more commonly from } \\
40 \text { to } 50 \text { feet. The } \\
\text { direction of the vein is } \\
\text { north-west and south- } \\
\text { east, its underlie is } \\
\text { south, and about five or } \\
\text { six feet per fathom. }\end{array}$ & $\begin{array}{l}\text { There are tre veins } \\
\text { worked in this } \\
\text { mine. The prin- } \\
\text { cipal vein ( Teich- } \\
\text { flache) is from one } \\
\text { foot six inches to } \\
\text { three feet in width, } \\
\text { the others are from } \\
\text { six to twelve inches } \\
\text { wide. The direc- } \\
\text { tion of this vein } \\
\text { is nearly north } \\
\text { and south, its un- } \\
\text { derlie is west, and } \\
\text { about three feet } \\
\text { per fathom. Some } \\
\text { of the other veins } \\
\text { intersect it. }\end{array}$ \\
\hline Ores & $\begin{array}{l}\text { Chiefly copper ore, occa- } \\
\text { sionally native copper, } \\
\text { blue and green carbonate } \\
\text { of copper. Tin, or oxide } \\
\text { of tin, also occurs, but } \\
\text { not in very great abun- } \\
\text { dance. }\end{array}$ & $\begin{array}{l}\text { Chiefly red silver, native } \\
\text { silver, sulphuret of silver, } \\
\text { and argentiferous pyrites. } \\
\end{array}$ & $\begin{array}{l}\text { Sulphuret of silver, natire } \\
\text { silver, prismatic black } \\
\text { silver, red silver, native } \\
\text { gold, argentiferous ga- } \\
\text { lena. }\end{array}$ & 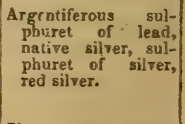 \\
\hline Produce of the ores & $\begin{array}{l}94 \text { per cent of fine copper; } \\
\text { average produce in } 100 \\
\text { parts of ore. }\end{array}$ & 3/ oz. per quinta! & $\begin{array}{l}\text { Four ounces of silver per } \\
\text { quinta! of } 100 \mathrm{lbs} \text {, equi- } \\
\text { valent to } 21 \text { parts of } \\
\text { metal in } 1,000 \text { of ore, or } \\
\$ \text { per cent. }\end{array}$ & 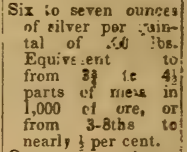 \\
\hline Veinsteae & $\begin{array}{c}\text { Chiefly quartz, of which } \\
\text { many varieties occur. }\end{array}$ & $\begin{array}{l}\text { Chiefly quartz, occasionally } \\
\text { amethyst, carbonate of } \\
\text { lime, and sulphate of } \\
\text { barytes. }\end{array}$ & $\begin{array}{l}\text { Quartz, amethyst, car- } \\
\text { bonate of lime, pearlspar, } \\
\text { and hornstone. }\end{array}$ & $\begin{array}{l}\text { Quartz, pearlspar, } \\
\text { and } \\
\text { spar. }\end{array}$ \\
\hline $\begin{array}{l}\text { Mineral substances } \\
\text { accorapanying the } \\
\text { ores - }\end{array}$ & $\begin{array}{l}\text { The ores are generally ac- } \\
\text { companied by "gossan"* } \\
\text { in the backs of the lodes, } \\
\text { by blende, and by iron, } \\
\text { and arsenical pyrites in } \\
\text { depth. }\end{array}$ & $\begin{array}{l}\text { The ores are generally ac- } \\
\text { companied by blende, } \\
\text { sulphuret of antimony, } \\
\text { and iron pyrites. }\end{array}$ & $\begin{array}{c}\text { The ores are accompanied } \\
\text { by blende, spathose iron, } \\
\text { copper and iron pyrites. }\end{array}$ & $\begin{array}{l}\text { The ores are ac- } \\
\text { companied apathose } \\
\text { blende, } \\
\text { iron, and a little } \\
\text { iron and arsenical } \\
\text { pyrites. }\end{array}$ \\
\hline $\begin{array}{l}\text { Depth of the princi- } \\
\text { pal shafts }\end{array}$ & $\begin{array}{l}\text { Woolf's engine-shaft, 248 } \\
\text { fathoms; Pearce's en- } \\
\text { gine-shaft, } 275 \text { fathoms. } \\
\text { Some of the other en- } \\
\text { gine shafts are scarcely } \\
\text { inferior in depth. }\end{array}$ & $\begin{array}{l}\text { Tiro General, } 182 \text { fathoms; } \\
\text { Gallega shaft, } 138 \text { fa- } \\
\text { thoms. }\end{array}$ & Tiro General, 310 fathoms. & $\begin{array}{l}\text { Frankenschacht, } 180 \\
\text { fathoms. }\end{array}$ \\
\hline
\end{tabular}

* Gossan, or Gozzan; oxide of iron and quartz. 
MINES.

Comparative Table of celebrated Mines in Europe and America.

By F. Burr, Esq.-Continued.

\begin{tabular}{|c|c|c|c|c|}
\hline & $\begin{array}{l}\text { CoNsolid } \\
\text { UniTED Minies. } \\
\text { (At present the richest } \\
\text { mines in Cornwall.) }\end{array}$ & $\begin{array}{c}\text { Veta Grande Mines. } \\
\text { (At present the richest } \\
\text { mines in Mexico.) }\end{array}$ & $\begin{array}{c}\text { MINE of VALEnciana. } \\
\text { (Richest of the Mexisan } \\
\text { mines at the beginning of } \\
\text { the present century.) }\end{array}$ & \begin{tabular}{|} 
MINE of \\
HrMmeLsFïrsT. \\
(Richest of the \\
Saxon mines at the \\
beginning of the \\
present century.)
\end{tabular} \\
\hline $\begin{array}{c}\text { Depth of adit at the } \\
\text { principal shafts }\end{array}$ & $\begin{array}{l}\text { At Woolf's engine-shaft, } \\
13 \text { fathorns. The average } \\
\text { depth of the adit at the } \\
\text { other engine-shafts is } \\
\text { about } 30 \text { or } 40 \text { fathoms. }\end{array}$ & $\begin{array}{l}\text { There is no adit to this } \\
\text { mine. }\end{array}$ & $\begin{array}{l}\text { There is no adit to this } \\
\text { mine. }\end{array}$ & $\begin{array}{l}\text { The adit at the staft } \\
\text { called Franken- } \\
\text { schacht, is } 47 \text { fa- } \\
\text { thoms in dept } \leq \text {. }\end{array}$ \\
\hline Quantity of water & $\begin{array}{l}\text { Varies from 2,000 to } 3,000 \\
\text { gallons per minute. }\end{array}$ & $\begin{array}{l}\text { About } 80 \text { gallons per } \\
\text { minute. }\end{array}$ & $\begin{array}{l}\text { The Valenciana was a dry } \\
\text { mine from its com- } \\
\text { mencement in } 1760 \text { to } \\
1780 \text {, when it first be- } \\
\text { came troubled with } \\
\text { water, in consequence of } \\
\text { some of the workings } \\
\text { being inadvertently com- } \\
\text { municated with the ad- } \\
\text { joining mine of Tepeyac; } \\
\text { which, although upon } \\
\text { the same vein, was ex- } \\
\text { tremely wet. The quan- } \\
\text { tity of waterraised during } \\
\text { the late working appears } \\
\text { to have been about } 110 \\
\text { gallons per minute, but } \\
\text { the regular influx was } \\
\text { much less. }\end{array}$ & $\begin{array}{l}50 \text { gallons } \\
\text { minute. } \\
\end{array}$ \\
\hline $\begin{array}{l}\text { Height te which the } \\
\text { water is raised }\end{array}$ & $\begin{array}{l}\text { About } 230 \text { fathoms at the } \\
\text { consolidated mines, at. } \\
\text { the united mines, about } \\
110 \text { fathoms. }\end{array}$ & $\begin{array}{l}\text { On an average about } 150 \\
\text { fathoms. }\end{array}$ & 310 fathoms. & 133 fathoms. \\
\hline $\begin{array}{l}\text { Power employed in } \\
\text { drainage }\end{array}$ & $\begin{array}{l}9 \text { steam-engines; } 3 \text { of } 90- \\
\text { inch cylinder, } 3 \text { of } 85,1 \\
\text { of } 80 \text {, and } 2 \text { of } 65 \text {. A } \\
\text { water wheel, } 48 \text { feet in } \\
\text { diameter. }\end{array}$ & Usually 10 malacates. & $\begin{array}{l}\text { A steam-engine of } 30 \mathrm{inch}- \\
\text { cylinder, and } 7 \text { mala- } \\
\text { cates. }\end{array}$ & $\begin{array}{l}\text { Two water-wheels, } \\
\text { each } 42 \text { feet in } \\
\text { diameter. }\end{array}$ \\
\hline $\begin{array}{l}\text { Probable equivalent } \\
\text { in actual horse- } \\
\text { power }\end{array}$ & $\begin{array}{l}1,500 \text { constantly at work, } \\
\text { or a total number of } \\
\text { above } 4,500 \text {. }\end{array}$ & $\begin{array}{l}\text { horses constantly } \\
\text { working, or a total } \\
\text { number of about } 100 \\
\text { horses. }\end{array}$ & $\begin{array}{l}65 \text { horses constantly at } \\
\text { work, or a total number } \\
\text { of about } 200 \text {. }\end{array}$ & $\begin{array}{l}16 \text { horses con- } \\
\text { stantly at work, } \\
\text { or a total num- } \\
\text { ber of about }\end{array}$ \\
\hline $\begin{array}{l}\text { Average annual ex- } \\
\text { pense in drainage }\end{array}$ & $\begin{array}{l}12,700 \text {. taking the } \\
\text { average of the last } \\
\text { ten years. }\end{array}$ & 20,000l. per annum. & $\begin{array}{l}\text { About } 40,000 l \text {. per } \\
\text { annum. }\end{array}$ & 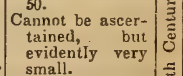 \\
\hline $\begin{array}{l}\text { Quantity of ore an- } \\
\text { nually produced }\end{array}$ & $\begin{array}{l}16,400 \text { tons of copper } \\
\text { ore, a few tons of tin } \\
\text { ore. }\end{array}$ & 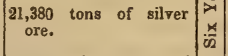 & 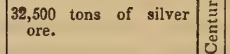 & $\begin{array}{l}630 \text { tons of silver } \\
\text { ore. }\end{array}$ \\
\hline Produce in metal & $\begin{array}{l}1,517 \text { tons of fine cop- } \\
\text { per, a little tin. }\end{array}$ & 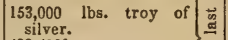 & 222,900 lbs. troy silver. & $\begin{array}{l}\text { 6,160 lbs. troy of } \\
\text { silver. }\end{array}$ \\
\hline $\begin{array}{l}\text { Total returns, or } \\
\text { value of the above }\end{array}$ & 119,8006 & 423,400l. per annum. & About $600,000 l$. & A bout $18,000 l$. \\
\hline $\begin{array}{l}\text { Total costs of the } \\
\text { mine }\end{array}$ & $\begin{array}{c}93,500 l \text { exclusive of } \\
\text { lord's dues; 98,500l. } \\
\text { including lord's dues. }\end{array} \mid \begin{array}{c}\vec{\varpi} \\
\Xi \\
\Xi\end{array}$ & 252,170l. per annum. & 197,900l. per annum. & $\begin{array}{l}9,500 l \text {. per an- } \\
\text { num. }\end{array}$ \\
\hline $\begin{array}{l}\text { Clear profit to the } \\
\text { proprietors }\end{array}$ & $21,000 l_{\text {. per annum. }}$ & 171,240l. per annum & $118,750 l$. per annum. & $\begin{array}{c}3,560 l \text {. per an- } \\
\text { num. }\end{array}$ \\
\hline $\begin{array}{l}\text { Amount of capital } \\
\text { invested }\end{array}$ & 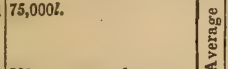 & 130,0002 & $\begin{array}{l}\text { Cannot be ascertained, } \\
\text { but known to have } \\
\text { been very small. }\end{array}\left|\begin{array}{c}0 \\
\Xi \\
\Xi \\
\Xi\end{array}\right|$ & $\begin{array}{l}\text { Cannot be ascer- } \\
\text { tained, } \\
\text { probably but } \\
\text { small. }\end{array}$ \\
\hline $\begin{array}{l}\text { Interest on capital } \\
\text { invested }\end{array}$ & $\begin{array}{l}280 \text { per cent. after pay- } \\
\text { ing back the original } \\
\text { capital. }\end{array}$ & $\begin{array}{c}\text { Nearly } 700 \text { per cent. } \\
\text { after paying back the } \\
\text { original capital. }\end{array}$ & $\begin{array}{l}\text { Not known, but cer- } \\
\text { tainly many hundred } \\
\text { per cent. }\end{array}$ & $\begin{array}{l}\text { Not known, but } \\
\text { probably very } \\
\text { high. }\end{array}$ \\
\hline $\begin{array}{l}\text { Proportion of cos:s } \\
\text { to returns }\end{array}$ & $\begin{array}{l}\text { Costs exclusive of lord's } \\
\text { dues, } 78 \text { per cent. }\end{array}$ & About $59 \frac{1}{8}$ per cent. & $\begin{array}{l}\text { Costs } 60 \text { per ceint. In } \\
\text { the nine years follow- } \\
\text { ing, the proportion } \\
\text { was } 80 \text { per cent., at } \\
\text { the end of that time } \\
\text { the working of the } \\
\text { mine was stopped by } \\
\text { the revolution, in the } \\
\text { year } 1809 \text {. }\end{array}$ & $\begin{array}{ll}\text { Costs } 73 & \text { per } \\
\text { cent. } & \end{array}$ \\
\hline $\begin{array}{l}\text { Number of men em- } \\
\text { ployed }\end{array}$ & $\begin{array}{c}\text { A bout } 8,500 \text { persons, of } \\
\text { whom about } 1,450 \text { are } \\
\text { employed under ground. }\end{array}$ & $\begin{array}{l}\text { About } 900 \text {, of whom nearly } \\
600 \text { are employed under } \\
\text { ground. }\end{array}$ & $\begin{array}{l}3,100 \text { Indians and Mesti- } \\
\text { zoes, of whom } 1800 \text { are } \\
\text { employed under ground. }\end{array}$ & $\begin{array}{l}700 \text { miners, of whom } \\
550 \text { are employed } \\
\text { under ground. }\end{array}$ \\
\hline $\begin{array}{l}\text { Wages of the miners } \\
\text { per day } \\
\text { Quantity and ex- } \\
\text { pense of powder }\end{array}$ & $\begin{array}{c}\text { Probably about } 3 \text { shillings } \\
\text { on an average. }\end{array}$ & $\begin{array}{l}\text { About } 8 \text { or } 9 \text { shillings per } \\
\text { day. }\end{array}$ & $\begin{array}{l}\text { From } 4 \text { to } 5 \text { shillings. } \\
1,480 \mathrm{cwt} \text {.; value } 15,830 l \text {. }\end{array}$ & $\begin{array}{l}\text { A bout is. } 6 d \text {. per } \\
\text { day. } \\
240 \text { cwt.; value } \\
1,070 l \text {. }\end{array}$ \\
\hline $\begin{array}{l}\text { Manner in which } \\
\text { the ores are dis- } \\
\text { posed of }\end{array}$ & $\begin{array}{l}\text { Sold to the smelting com- } \\
\text { panies, and smelted by } \\
\text { them at Swansea, in } \\
\text { South Wales. }\end{array}$ & $\begin{array}{l}\text { Chiefly reduced by the } \\
\text { company at the hacienda } \\
\text { of Sanceda, by smelting } \\
\text { and amalgamation. }\end{array}$ & $\begin{array}{l}\text { Sold to the Resentadores, } \\
\text { and reduced by smelting } \\
\text { and amalgamation at } \\
\text { haciendas, in the neigh- } \\
\text { bourhood of Guanaxuato. }\end{array}$ & $\begin{array}{l}\text { Delivered to the } \\
\text { Government, re- } \\
\text { duction works in } \\
\text { the neighbourhood } \\
\text { of Freyberg, where } \\
\text { they are partly } \\
\text { smelted, and part- } \\
\text { ly amalgamated. }\end{array}$ \\
\hline
\end{tabular}

VENTILATION OF MINES.

When men penetrate by narrow passages into the interior of the earth, their respiration, joined to the combustion of candle and gunpowder, are not long of vitiating the an. The decomposition of wood contributes to the same effect, as also the mineral bed itself, especially in coal mines, by the carburetted hydrogen and carbonic acid evolved, and from the absorption of oxygen by pyrites. In many cases, arsenical and mercurial vapours are disengaged. Hence the necessity of maintaining in sub. 
serranean cavities a contınual circulation of air, which may renew the atmosphere round the miners. The whole of the means employed to produce this effect, constitutes what is called the ventilation of mines.

These means are divided into natural and artificial. The natural means are the currents produced by the difference of density between the air of mines and the external air ; the artificial are air-exhausters or condensers, fires, \&c.

The temperature of the air of the subterranean workings surpasses the mean temperature of the place in which the mine is opened. Hence it is lighter in winter, but in summer often heavier than the air of the atmosphere. For this reason, when the mine presents two openings at different levels, the air naturally flows out by the most elevated in winter, and by the lowest in summer. We may take advantage of this circumstance, to lead the air into the bottom of even a very long gallery, opening into the side of the mountain, by piercing a shaft into its roof at some distance from the entrance, and diriding the gallery by a horizontal floor into two parts, which have no mutual communication, except at the furthest extremity-the upper part communica ting with the shaft, and the under with the mouth of the gallery. If the two compartments have different dimensions, the air in the smaller sooner comes into an equilibrium of temperature with the rock; and the difference of temperature of the two compart. ments is sufficient to produce a current. If a streamlet of water flow $\mathrm{Chrough}$ this gallery, it facilitates the flow of the air along the lower compartment. If a mine has sereral openings situated on the same level, it rarely happens but some peculiar circumstance destroys, during the colds of winter and the heats of summer, the equilibrium of the air. But in spring and autumn, when the external air is nearly of the same temperature with that of the mines, the abore-named causes are almost alwars too feeble to excite an issuing current. This effect is, howerer, frequently obtained br raising orer one of the shafts a chimney 20 or 30 yards high, which alone produces the effect of an opening at a different level. It has been remarked that stormy weather usually deranges every system of ventilation. See Pitcoal and Ventilation.

MINIUM. (Eng. and Fr.; Red lead; Mennige, Germ.) This pigment is a peculiar oxyde of lead, consisting of two atoms of the protoxyde and one of the peroxyde ; but, as found in commerce, it always contains a little extra protoxyde, or yellow massicot. It is prepared by calcining lead upon a reverberatory hearth with a slow fire, and frequent renewal of the surface with a rake, till it becomes an oxrde, taking care not to fuse it. The calcined mass is triturated into a fine powder in a paint mill, where it is elutriated with a stream of water, to carry off the finely levigated particles, and to deposite them afterwards in tanks. The powder thus obtained, being dried, is called massicot. It is converted into minium, by being put in quantities of about 50 pounds into iron trays, 1 foot square, and 4 or 5 inches deep. These are piled up upon the reverberatory hearih, and exposed during the night, for economy of fuel, to the residuary heat of the furnace, whereby the massicot absorbs more oxygen, and becomes partially red lead. This, after being stirred about, and subjected to a similar low calcining heat once and again, will be found to form a marketable red lead.

The best minium, howerer, called orange mine, is made by the slow calcination of good white lead (carbonate) in iron trass. If the lead contains either iron or copper, it affords a minium which cannot be employed with advantage in the manufacture of flint-glass, for pottery glazes, or for house-painting.

Dumas found several samples of red lead which he examined to consist of the chemical sesquioxyde and the protoxyde, in proportions varring from 50 of the former and 50 of the latter, to 95.3 of the former and $4 \cdot 7$ of the latter. The more oxrgen gas it gives out when heated, the better it is, generally speaking. See NAPLes YELLOW.

MINT. (Monnaie, Fr.; Munze, Germ.) The chief use of gold and silver is to serve for the medium of exchange in the sale and purchase of commodities, a function for which they are pre-eminently fitted by their scarcity, by being unalterable by common agents, and condensing a great value in a small volume. It would be rery inconvenient in general to barter objects of consumption against each other, because their carriage would be expensive, and their qualities, in many cases, easily injured by external agents, \&c. Gold is exempt from spontaneous change, and little costly in converance. Mankind at a very early period recognised how much easier it was to exchange a certain weight of gold or silver for objects of commerce, than to barter these objects themselves; and thenceforth all agreed to par for their purchases in bars or ingots of these precious metals. But as their intrinsic value depends upon their purity, it became necessary to stamp on these bars their standard quality and their weight.

The inconvenience of using ingots in general trade, on account of the difficulty of defining fractional ralues, has determined gorernments to coin pieces of money, that is, quantities of metal whose weight and standard were made known and guarantied by the effigies of the prince. It is true, indeed, that kings have become frequently coiners 
of base money, by altering the weight and purity of the pieces apparently guarantied by their impress. By such reductions, modern coins represent less of the precious metal than they did long ago. The ordonnance of 755, for the coining of sous in Frarce, proves that there was then as much fine silver in a single sous as there is now in a piece of 5 francs. During the last two centuries, indeed, silver coins have been diminished two thirds in weight.

But since knowledge has become more generally diffused, it has been shown that these rrauds are equally injurious to the prince and to public faith. A sovereign may, it is true, declare by a decree that a shilling-piece is to be held worth five; but let us consider the consequences of this decree. All the individuals who have rents or capital sums to receive will be ruined, by getting in metallic value only one fifth of what is due to them: for although the nominal value should be the same as what they are entitled to, tine intrinsic value would be but a fifth of the former; so that when they go to purchase the necessaries or comforts of life, the dealer who sells them will at once raise their price five-fold. Each article of merchandise would thus acquire a nominal price 5 times greater; and he who had received payment of a debt in that money could not with it procure more than one fifth of the goods he could have previously commanded. That fraudulent law would, therefore, favor the debtors at the expense of the creditors; and as the state is commonly a great debtor, especially when it has recourse to the depreciation of the currency, it is obvious, that however illicit the gain which it makes, it still does gain; and this is the reason why princes have so often tampered with the mint. But let us examine the other consequences of this decree.

If the sovereign is a debtor, he is also a creditor and consumer, and even the most considerable of any. The taxes which he imposes are paid him in this deteriorated money, returned to him at its nominal value; and the purveyors of his armies, his buildings, and his household, sell him their commodities only at the actual market price. We may infer from this simple development that the coin with which he pays for any object has the same intrinsic value as the object; and that the name given to the coin is of no consequence. The prince may call it a crown, a ducat, or a rix-dollar at his pleasure; and he may assign any value to it that his caprice may suggest, yet this will not affect its value; for this is fixed beyond his control by the general nature of things. The prince may, indeed, at the outset, have profited by defrauding his creditors, and by authorizing each debtor to imitate him, but he will soon lose whatever he may have gained; and he will thus learn to his cost that it was bad policy to sacrifice his character by giving an example of a fraud so truly unprofitable in the issue. Moreover, he will lose still as much in the following years, because his treasury will receive only one fifth part of the taxes, unless he has quintupled the imposts. It may be said, indeed, that he might do the one thing along with the other. But every one knows that this power is neither generally permitted to princes, nor, if it were, could it be safely exercised. Serious political crises would combine to endanger the stability of the government; which besides, as the main consumer in the nation, must lose always as much as it seems to gain.

It is therefore manifest, that the alteration of the standard and weight of the coinage is at once a crime and a ruinous action for the sovereign power to commit; and hence such disastrous measures have been long abandoned in all well-regulated states. A gold sovereign is intrinsically worth 20 shillings, minus the cost of coinage; for were it worth more, all our sovereign pieces would be exported or melted down, to obtain the difference of value, however trifling it might be; and were it worth less, it would be the source of loss similar to what the state occasions when it depreciates the coin.

To comprehend the true value of a coin, we must regard this piece as an article of merchandise, whose value depends, as that of every thing else, on its usefulness, the esteem in which it is held, and the demand for it in the market. Grain increases in value when there are few sellers and many buyers; gold and silver are in the same predicament. The value of these metals is much augmented, indeed, by the universal currency they obtain when struck into money; a value additional to what they possess as objects of the arts. This value of the precious metals changes with time and place, like that of every merchandise; their abundance, since the discovery of America, has greatly lowered their value; that is, with the same weight of metal, we cannot at the present day purchase the same quantity of corn, land, wool, \&c. as formerly. In the countries where silver abounds, this netal has less value, or, in other terms, commodities are dearer. Hence the metal tends to resume its equilibrium in flowing into those places where it is rarer; which means, that the consumer prefers purchasing his commodities there, rather than in another place, if he can easily transport them to where they are dearer.

It was formerly believed that a country is rich when it has a great deal of wold and silver; but this popular illusion has passed away. Spain has never been poorer than since the discovery of America, because its national industry has been ruined, and the 
capitals merely passed through its hands to spread over the rest of Europe, from which it was obliged to import every thing that its want of home manufactures made it necessary to procure from abroad. We may add to these the prodigalities of the court, which, supposing its wealth inexhaustible, tried to corrupt all the ministers of the other powers, in furtherance of the chimera of universal dominion. The richest state is that in which there is most industry, whereby the inhabitants may procure every thing indispensable to the conveniences and comforts of life. Gold as a useful metal, and a medium of exchange, is undoubtedly very precious, and an adequate quantity for these exchanges must be had; but as it is good for very little besides, nay, as an excess is even hurtful, it soon begins to fly of itself towards the places where it is more needed or less common.

With regard to the relative value of gold and silver, several details have already been given in our view of the mineral wealth of the globe. Three centuries ago, an ounce of gold was worth at London or Paris 10 ounces of silver; now it may be exchanged for 15 ounces and a half.

The par of two coins results from the comparison of their weight and standard fineness. Let us take for an example the conversion of English gold sovereigns worth 20 shillings or a pound sterling, in relation to the French louis of 20 francs. The standard of the sovereign gold is 0.917 , fine gold being 1000 ; its weight is $125,256 \mathrm{gr}$. English, or 7.980855 grammes; by multiplying this weight into its standard, we have a product of $7 \cdot 318444035$; this is, in grammes, the quantity of pure gold contained in the sovereign piece. The piece of 20 francs has a legal standard of 0.9 ; and multiplying this number by the weight of the louis, 6.45161 grammes, we find that it contains $5 \cdot 806449$ of pure metal. We then make this proportion:-

As 5.806449: 20 francs : : $7 \cdot 31844: 25 \cdot 2079$ franes; or the value of the English sovereign is nearly $25 \cdot 21$ francs, in French gold coin. A similar calculation may be made for silver coins. The French rule for finding the par of a foreign gold coin, or its intrinsic value in francs, is to multiply its weight by its standard or titre, and that product by $3 \frac{4}{9}$. The par of foreign silver money, or its intrinsic value in francs, is obtained by multiplying its weight in grammes by its standard in thousand parts, and by $\frac{2}{9}$. The French 5 -franc piece has its standard or titre at 0.9 , and weighs 25 grammes.

The assaying of gold for coin and trinkets requires very delicate management. The French take half a gramme at most (about $7 \frac{2}{2}$ grains) of gold, and fuse it with thrice its weight of silver, as already described under AssAy. The parting is the next operation. For this purpose the button of gold and silver alloy is first hammered flat on a piece of steel, and then made feebly red hot in burning charcoal or over a lamp flame. After being thus annealed, the metal is passed through the rolling press, till it be converted into a plate about $\frac{1}{70}$ of an inch thick. After annealing this riband, it is coiled into a spiral form, introduced immediately into a small matrass of a pear shape, an assay matrass, and about 500 grains of nitric acid, sp. grav. $1 \cdot 185$, are poured over it. Heat being now applied to the vessel, the solution of the silver and copper alloys ensues, and after 22 minutes of constant ebullition, the liquid is poured off and replaced by an equal quantity of nitric acid, likewise very pure, but of the density $1 \cdot 28$. This is made to boil for about 10 minutes, and is then poured off, when the matrass is filled up with distilled water to the brim. In conclusion, a small annealing crucible is inverted as a cup over the mouth of the matrass, which is now turned upside down with a steady hand; the slip of metal falls into the crucible through the water; which by sustaining a part of its weight softens its descent and prevents its tearing. The matrass is then dexterously removed, without letting its water overflow the crucible. The water is gently decanted from the crucible, which is next covered, placed in the middle of burning charcoal, and withdrawn whenever it becomes red hot. After cooling, the metal slip is weighed very exactly, whence the weight of fine gold in the alloy is known. Stronger acid than that prescribed above would be apt to tear the metallic riband to pieces, and it would be difficult to gather the fine particles of gold together again. The metallic plate becomes at last merely a golden sieve, with very little cohesion. When copper is to be separated from gold by cupellation, a higher temperature is requisite than in cupelling silver coin.

The coining apparatus of the Royal Mint of London is justly esteemed a masterpiece of mechanical skill and workmanship. It was erected in 1811, under the direction of the inventor, Mr. Boulton; and has since been kept in almost constant employment.

The melting pots ( $f \mathrm{~g}$. 738) are made of cast-iron, and hold conveniently 400 poinds of metal. They are furnished with a spout or lip for pouring out the metal, and with two ears, on which the tongs of the crane lay hold in lifting them out of the furnace. The pot rests on pedestals on the grate of the furnace, and has a ring cast on its edge to prevent the fuel falling into it. Whenever it becomes red hot, the metal properly prepared and mixed, so as to produce an alloy containing 0.915 parts of gold, is put in, and during the melting, which occupies some hours, it is occasionally stirred. The moulds are meanwhile prepared by warming them in a stove, and thereafter by rubbing their 
insiac surfaces with a cloth dipped in oil, by which means the ingots cast in them get a better surface. Fig. 739 represents a side view of the carriage, charged with its

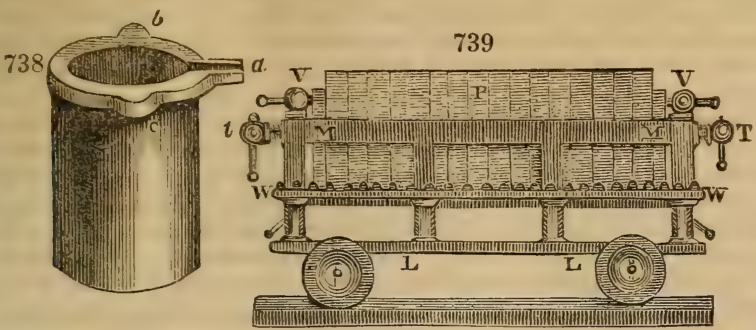

moulds. When the proper number of moulds is introduced, the screws at the end, represented at $t$, are screwed fast, to fix them all tight.

The pot of fused metal is lifted out of the furnace $b$; the crane (fig. 740) inen

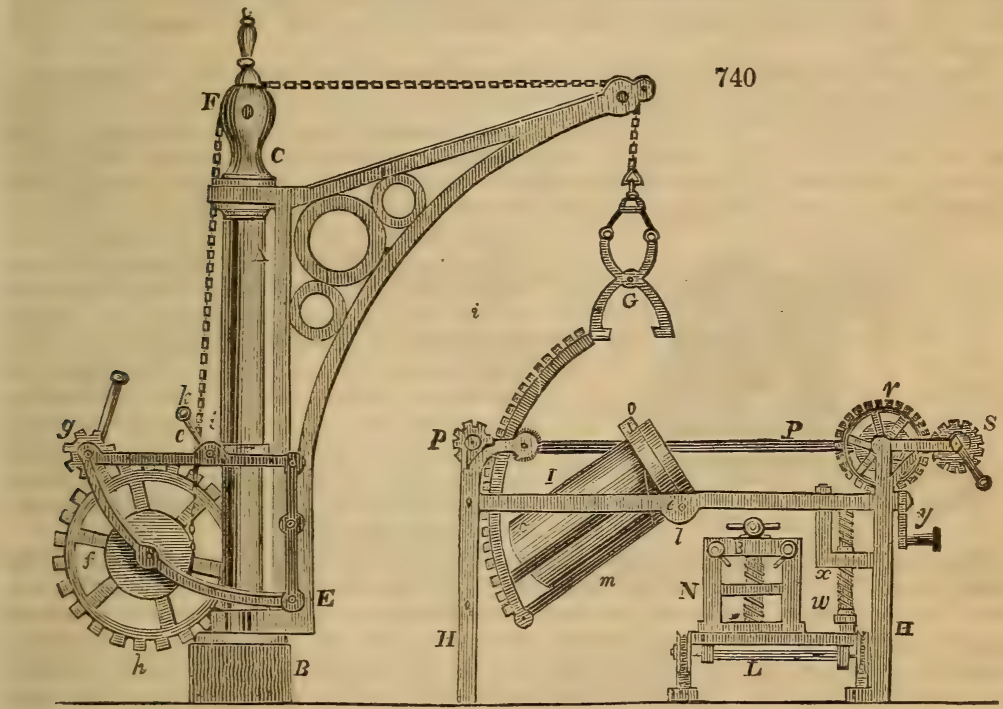

swung round, and lowered down into the cradle $l, m, n, 0$ of the pouring machine, until the ring on the edge of it rests on the iron hoop $n$, which, being screwed tight up, holds it secure, and the crane-tongs are removed. One of the assistants now takes the winch handle $s$ in one hand, and $y$ in the other. By turning $y$ he moves the car. riage forward, so as to bring the first mould beneath the lip of the melting pot; and by turning $s$, he inclines the pot, and pours the metal into the mould. He then fills the other moulds in succession. The first portion of liquid metal is received in a small iron spoon, and is reserved for the assay-master; a second sample is taken from the centre of the pot, and a third from the bottom part. Each of these is examined as to its quality.

The ingots, which are about 10 inches long, 7 broad, and 6 tenths of an inch thick, are sow carried to the rolling mill,

Fig. 741, where A represents a large spur wheel, fixed on the extremity of a long

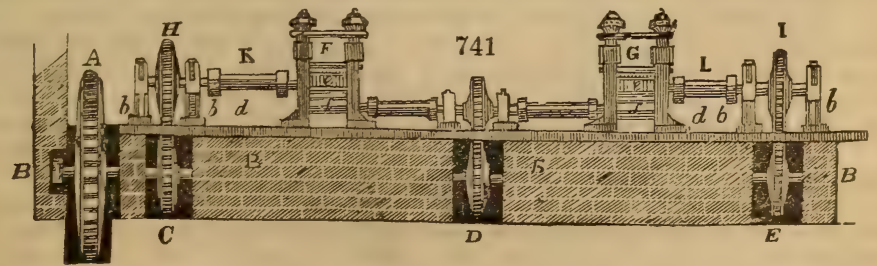


horizontal shaft в в, extending beneath the whole mill. This wheel and shaft are driven by a smaller wheel, fixed on the main or fly-wheel shaft of a steam engine of 36-horse power. The main shaft $\mathrm{B}$ of the rolling mill has wheels $\mathrm{C}, \mathrm{D}, \mathrm{E}$ fixed upon it, to give motion to the respective rollers, which are mounted at $F$ and $G$, in strong iron frames, bolted to the iron sills $a a$, which extend through the whole length of the mill, and rest upon the masonry, in which the wheels are concealed. The two large wheels $\mathrm{c}$ and $\mathrm{r}$ give motion to the wheels $\mathrm{H}, \mathrm{I}$, which are supported on bearings between two standards $b, b$, bolted down to the ground sills. On the ends of the axes of these wheels are heads for the reception of compling boxes $d$, $d$, which unite them to short connecting shafts $\mathbf{K} \mathrm{L}$; and these again, by means of coupling boxes, convey motion to the upper rollers $e, e$, of each pair, at $\mathrm{F}$ and $\mathrm{G}$. The middle wheel $\mathrm{D}$ upon the main shaft $\mathrm{B}$ gives motion to the lower rollers in a similar manner. Thus both the rollers $e, f$ of each frame receive their motion from the main shaft with equal velocity, by means of wheels of large radius, which act with much more certainty than the small pinions usually empluyed in rolling mills to connect the upper and lower rollers, and cause them to move together.

The rolling mill contains four pairs of rollers, each driven by its train of wheel work : the mill, therefore, consists of two such sets of wheels and rollers as are represented in our figure. The two shafts are situated parallel to each other, and receive their motion from the same steam engine. This admirable rolling mill was erected by John Rennie, Esq.

The ingots are heated to redness in a furnace before they are rolled. The two furnaces for this purpose are situated before two pairs of rollers, which, from being used to consolidate the metal by rolling whilst hot, are termed breaking-down rollers. Two men are employed in this operation; one taking the metal from the furnace with a pair of tongs, introduces it between the rollers; and the other, catching it as it comes through, lifts it over the top roller, and returns it to his fellow, who puts it through 742

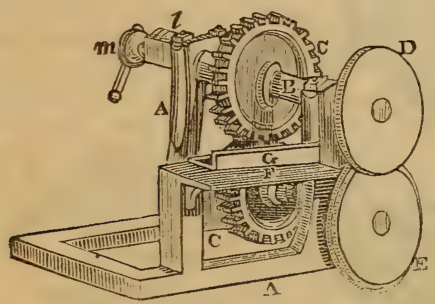
again, having previously approximated the rollers ' a little by their adjusting screws. After having been rolled in this manner four or five times, they are reduced to nearly two tenths of an inch thick, and increased lengthwise to about four times the breadth of the ingot. These plates, while still warm, are rubbed over with a dilute acid or pickle, to remove the color produced by the heat, and are then cut up into narrow slips across the breadth of the plate, by means of the circular shears $f i g .742$.

This machine is worked by a spur-wheel at the extremity of the main shaft B of the rolling mill ( $f g .741$.) It consists of a framing of iron A A, supporting two shafts B B, which are parallel to each other, and move together by means of two equal spur-wheels c c, the lower one of which works with the teeth of the great wheel abore mentioned, upon the main shaft of the rolling mill. At the extremities of the two shafts, wheels or circular cutters are fixed with their edges overlapping each other a little way. F represents a shelf on which the plate is laid, and adranced forward to present it to the cutter ; and G is a ledge or guide, screwed down on it, to conduct the metal and to regulate the breadth of the piece to be cut off. Hence the screws which fasten down the ledge are fitted in oblong holes, which admit of adjustment. The workman holds the plate flat upon the surface $\mathrm{F}$, and pushing it towards the shears, they will lay hold of it, and draw it through until they have cut the whole length. The dirided parts are also prevented from curling up into scrolls, as they do when cut by a common pair of shears; because small shoulders on $\mathrm{E}$ and $\mathrm{D}$, behind the cutting edge, keep them straight. Behind the standard, supporting the back pivots of the shafts в в of the cutter, is a frame l, with a screw $m$ tapped through it. This is used to draw the axis of the upper cutter $\mathbf{D}$ endwise, and keep its edge in close contact with the edge of the other cutter $\mathbf{E}$. The slips $\mathrm{r}$ ribands of plate are now earried to the other two pairs of rollers in the rolling mill, which are made of case-hardened iron, and better polished than the breaking-down rollers. The plates are passed cold between these, to bring them to exactly the same thickness; whence they are called adjusting or planishing rollers. The workman here tries every piece by a common gauge, as it comes through. This is a piece of steel having a notch in it; the inside lines of which are very straight, and inclined to one another at a very acute angle. They are divided by fine lines, so that the edge of the plate being pressed into the notch, will have its thickness truly determined by the depth to which it enters, the divisions showing the thickness in fractions of an inch.

In rolling the plate the second time, all the plates are successively passed through the rollers; then the rollers being adjusted, they are passed through another time. This is repeated thrice or even four times; after which they are all tried by the gauge, and 
thus sorted into as many parcels as there are different thicknesses. It is a curious circumstance, that though the rollers are no less than 14 inches in diameter, and their frame proportionally strong, they will yield in some degree, so as to reduce a thick plate in a less degree than a thin one; thus the plates which have all passed through the same rollers, may be of 3 or 4 different degrees of thickness, which being sorted by the gauge into as many parcels, are next reduced to the exact dimension, by adapting the rollers to each parcel. The first of the parcel which now comes through is tried, by cutting out a circular piece with a small hand machine, and weighing it. If it proves either too light or too heary, the rollers are adjusted accordingly, till by a few such trials they are found to be correct, when all the parcel is rolled through. The trial plates which turn out to be too thin, are returned as waste to the melting-house. By these numerous precautions, the blanks or circular discs, when cut out by the next machine, will be very nearly of the same weight; which they would scarcely be, even if the gauge determined all the plates to the same thickness, because some being more condensed than others, they would weigh differently under the same volume.

A great improvement has been made on that mode of lamination, by the late $\mathrm{Mr}$. Barton's machine for equalizing the thickness of slips of metal for making coin, which has been for several years introduced into the British mint. A side elevation is shown in fig. 743 , and a plan in fig. 744. It operates in the same way as wire-drawing mechanisms; namely, pulls the slips of metal forcibly through an oblong opening, left between two surfaces of hardened steel. The box or ease which contains the steel dies, composed of two hardened cylinders, is represented at $\mathrm{c}$ in fig. 743. The pincers employed to hold the metal, and draw it through, are shown at $s$.

743

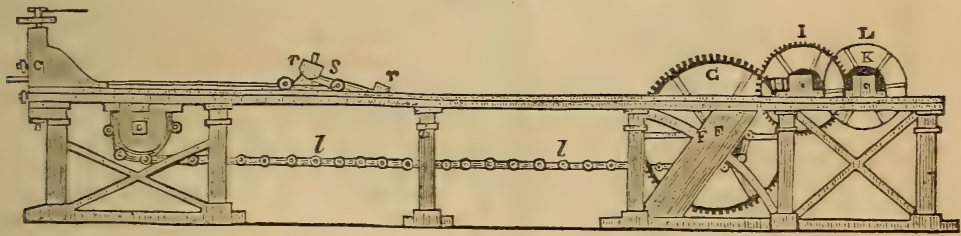

744

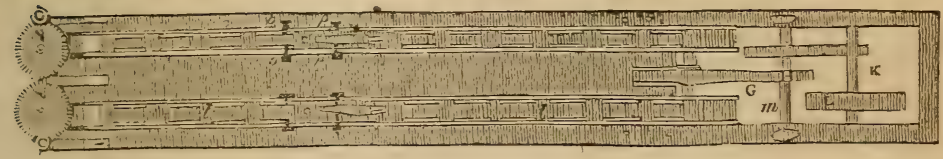

The slips of metal to be operated on by the drawing machine, are first rendered thinner at one end, that they may be introduced between the dies, and also between the jaws of the pincers. This thinning of the ends is effected by another machine, consisting of a small pair of rollers, mounted in an iron frame, similar to a rolling-mill. The upper roller is cylindrical, but the lower is formed with 3 flat sides, leaving merely portions of the cylinder entire, between these flat sides. The distance between the centres of the rollers is regulated by screws, furnished with wheels on their upper ends, similar to what is seen in the drawing dies at $\mathbf{c}$. The two rollers have pinions on their axes, which make them revolve together; they are set in motion by an endless strap passing round a drum, upon whose axis is a pinion working into the teeth of a wheel fixed upon the axis of the lower roller.

The end of a slip of metal is presented between the rollers while they are in motion, not on that side of the roller which would operate to draw in the slip between them, as in the rolling-press above described, but on the contrary side, so that when one of the flat sides of the under roller fronts horizontally the circumference of the upper roller, an opening is formed, through which the slip of metal is to be inserted until it bears against a fixed stop at the back of the rollers. As the rollers continue to turn round, the cr?indrical portions come opposite to each other, and press the metal between them, forcing it outwards, and rendering the part which has been introduced between the rollers as thin as the space between their cylindrical surfaces. Thus the end of the slip of metal becomes attenuated enough to pass between the dies of the drawing machine, and to be seized by the pincers.

In using the drawing machine, a boy takes hold of the handle $s$ of the pincers, their hook of connexion with the endless chain $l, l$, not shown in the present figure, being disengaged, and he moves them upon their wheels towards the die-box c. In this movement the jaws of the pincers get opened, and they are pushed up so close to the 
die-bux that their jaws enter a hollow, which brungs them near the dies, enabling them to seize the end of the slip of metal introduced between them $b_{j}$ the acion of the pre. paratory roilers. The boy now holds the handle $s$ on the top of the pincers fast, and with his other hand draws the handle $x$ backwards. Thus the jaws are closed, and the meta? firmly griped. He now presses down the handle $x$ till a hook on the under side of the pincers seizes the endless chain as it mores along, when it carries the pincers, and their slip of metal, onwards with it. Whenever the whole length of the metallie riband has passed through between the dies, the strain on the pincers is suddenly relieved, which causes the weight $r$ to raise their hook out of the chain, and stop their motion. The machine in the mint has two sets of dies, and two endless chains, as represented in the plan, fig. 74 . $\mathrm{N} \mathrm{x}$, are toothed wheels in the upper end of the die-box, furnished with pinions and levers, for turning them round, and adjusting the distance between the dies. A large spur-wheel $G$, is fixed unon the axis $F$, to gire motion to the endless chains; see both figures. This spur-wheel is turned by a pinion $\mathrm{H}$, fixed upon an axis $m$, extending across the top of the frame, and working in bearings at each end. A spur-wheel $\mathrm{r}$, is fixed upon the axis $m$, and worlis into the teeth of a pinion $\mathbf{K}$, upon a second axis across the frame, which carries likewise a drum wheel $\mathbf{L}$, through which motion is communicated to the whole mechanism by an endless strap.

The cutting-out machine is exhibited in fig. 745 . A A is a basement of stone to support 745

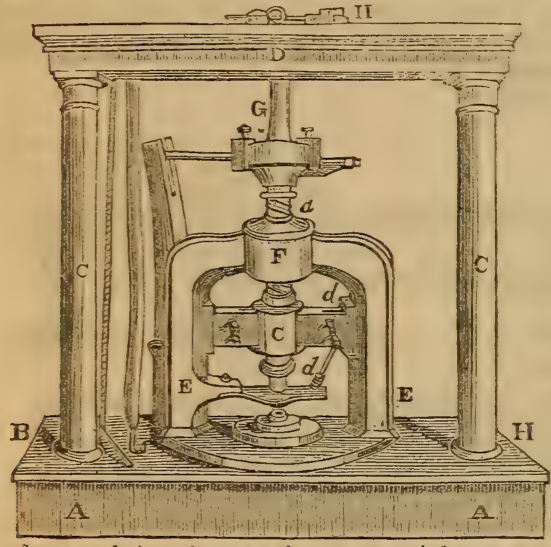
an iron plate $B$, on which stand the columns $\mathrm{c}$, that bear the upper part $D$ of the frame. The iron frame of the machine $E, F, E$, is fixed down upon the iron plate $\mathrm{B}, \mathrm{B}$. The punch $d$ is fixed in the lower part of the inner frame, and is mored up and down by the screw $a$, which is worked by wipers turned by a steam engine, impelling the lever $\mathbf{H}$, and turning backwards and forwards the axis $\mathrm{G}$, through a sufficient space for cutting the thichness of the metallic lamina. A boy manages this machinc. There are twelve of them mounted on the same basement frame in a circular range contained in an elegant room, lighted from the roof. The whole are mored by a steam engine of 16 horse power.

The blanks or planchels thus eut out, were formerly adjusted by filing the edges, to bring them to the exact weight; a step which Mr. Barton's ingenious mechanism has rendered in a great measure unnecessary. The edge is then milled, by a process which Mr. Boulton desires to keep secret, and which is therefore not shown in our mint.

But the French mint emplots a very elegant machine for the purpose of lettering or milling the edges, called the cordon des monnaies, invented by M. Gengembre, which has entirely superseded the older milling machine of $M$. Castaing, described in the

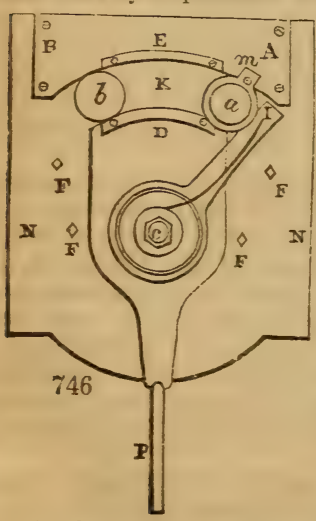
Encyclopedias. The Napoleon coins of France bear on the edge, in sunk letters, the legend, Dieu protege la France; and those of the king, Domine salvum fac regem. This is marked before striking the blank or flan. One machine imprints this legend, and its service is so prompt and easy, that a single man marks in a day 20,000 pieces of 5 francs, or 100,000 franes.

Each of the two arc dies E, D, ( fig. 746,) carries one half of the legend, engraved in relief on the eurved face; these ares are pieces of steel tempered very hard, and fixed with two screws, one immoveably at $\mathrm{E}$, on the sill which bears the apparatus; the other at $D$, at the extremity of the lever $\mathbf{P}, \mathbf{D}$, which turns round the axis c. The letters of these demilegends are exactly parallel, and inseribed in an inverse order on the dies. An alternating circular motion is communicated to the handle $P$. The curvatures of the two dies are ares of circles described from the centre $c$; and the interval which separates them, or the difference of the radii, is precisely the diameter of the piece to be milled.

As the centre c sustains the whole strain of the milling, and produces, of consequence, a hard friction, this axis must possess a considerable size. It is composed of a squat 
truncated cone of tempered steel, which enters into an eye of the moveable piece $\mathrm{P}, \mathrm{D}$. This cone is liept on the plate of the metal $\mathrm{N} N$, which bears the whole machine, by a nut, whose screw, by being tightened or slackened, gives as much freedom as is requisite for the murement of rotation, or remores the shake which hard service gives to the cone in its eve. The middle thickness of the hole of the moveable piece $P, D$, and the axis of the lever $P$, which terminates it, are exactly on a level with the engraved letters of the die, so that no strain can derange the moreable piece, or disturb the centre by its oscillations.

At $a$ is a rertical tube, containing a pile of blanks for milling. It is kept constantly full; the tube being open at both ends, a little elevated abore the circular space $a$, $E, b$, which separates the dies, and fixed by a tail $m$ with a screw to the motionless piece $A, B$. The branch $I, c$, moreable with the piece $P, D$, passes under the tube, and pushes bcfore it the blank at the bottom of the column, which is received into a small exearation in the form of a circular step, and carried forwards. Matters are thus so arranged as to regulate the issue of the blanks, one by one, on the small step, called the posoir (bed.)

As soon as the blank is pushed forwards into contact with the lower edge of the engrared groores, it is seized by them, and carried on by the strain of milling, without exposing the upper or under surfaces of the blank to any action which may obstruct the vrinting on its edge.

The blank is obserred to rerolre between the two dies according as the lerer $\mathrm{P}$ completes its course, and this blank passing from $a$ to $\mathrm{k}$, then to $b$, meets a circular aperture $b$, through which it falls into a drawer placed under the sill.

The range of the moreable lerer $P$ is regulated by four pieces, $F, F, F, F$, solidly sunk in the plate $\mathrm{x}, \mathrm{N}$, which bears the whole apparatus. A stud placed on this lever towards $\mathrm{D}$, makes the arm of the posoir I $c$ retire no farther than is necessary for the little blank to issue from the column; and a spring fixed to the centre $c$, and supported on a peg, brings back the posoir; so that when a screw I comes to strike against the column, the posoir stops, and the moreable die $\mathrm{D}$, which continues its progress, finds the blank in a fit position for pressing, seizing, and carrying it on, by reaction of the fixed die E. Thus the edge of the blank is lettered in half a second. A hundred may easily be marked in about three minutes.

The coining press is the most beautiful part of the whole mechanism in the British mint; but the limits of this volume will not allow of its being figured upon an adequate seale. An engraving of it may be seen in the Enevelopedia Britannica.

The only attention which this noble machine requires is that of a little boy, who stands in a sunk place before the press, and always keeps the tube full of blanks. He has two strings, one of which, when pulled, will put the press in motion br the concealed mechanisin in the apartment above; and the other string, when snatched, stops the press. This coining operation goes on at the rate of 60 or 70 strokes per minute; and with sers few interruptions during the whole day. The press-room at the Royal Mint contains eioht machines, all supported on the same stone base; and the iron beams between the columns serve equally for the presses on each side. The whole has therefore a magnificent appearance. The eight presses will strike more than 19,000 coins in an hour, with only a child to supply each. The grand improvement in these presses consists; 1. in the precision with which ther operate to strike every coin with equal force, which could not be ensured by the oid press impelled by manual labor; 2. The rising coliar or steel ring in which they are struck, keeps them all of one size, and makes a fair edge, which was not the ease with the old coins, as they were often rounded and defaced by the expansion of the metal under the blow; 3 . The twisting motion of the upper die is thought to produce a better surface on the flat parts of the coin; but this is somewhat doubiful; 4 . The feeding mechanism is very complete, and enables the machine to work much quicker than the old press did, where the workman, being in constant danger of having his fingers eaught, was obliged to proceed cautiously, as well as to place the coin true on the die, which was seldom perfectly done. The feeding mechanism of the abore press is a French invention; but Mr. Boulton is supposed to have improved rpon it.

MIRrORS. See Copper and Glass.

MISPICKEL is arsenical pyrites.

MOHAIR is the hair of a goat which inhabits the mountains in the vicinity of Angora, in Asia Minor.

MOIRE'E METALLIQLE, called in this country crystallized tin-plate, is a rariegated primrose appearance, produced upon the surface of tin-plate, br applying to it in a heated state some dilute nitro-muriatic acid for a few seconds, then washing it with water, drying, and coating it with lacker. The figures are more or less beau. tiful and dirersified, according to the degree of heat, and relative dilution of the acid. This mode of ornamenting tin-plate is much less in vogue now than it was a few years ago, 
MOLASSE is a sandstone belonging to the tertiary strata, employed under that name by the Swiss for building.

MOLASSES is the brown viscid uncrystallizable liquor, which drains from cane sugar in the colonies. See Sugar.

MOLYBDENUM (Molybdène, Fr.; Molybdan, Germ.) is a rare metal which occurs in nature sometimes as a sulphuret, sometimes as molybdic acrd, and at others as molybdate of lead. Its reduction from the acid state by charcoal requires a rery high heat, and affords not very satisfactory results. When reduced br passing hydrogen over the ignited acid, it appears as an ash-gray powder, susceptible of acquiring metallic Justre by being rubbed with a steel burnisher; when reduced and fused with charcoal, it rossesses a silver white color, is rery brilliant, hard, brittle, of specific gravity 8.6 ; it melts in a powerful air-furnace, oxydizes with heat and air, burns at an intense heat into molybdic acid, dissolres in neither dilute sulphuric, muriatic, nor fluoric acids, but in the concentrated sulphuric and nitric.

The protoxyde consists of 85.69 of metal, and 14.31 of oxygen: the deutoxyde con. sists of 75 of metal, and 25 of oxygen; and the peroxyde, or molybdic acid, of $66 \cdot 6$ of metal, and 33.4 of oxygen. These substances are too rare at present to be used in any manufacture.

MORDANT, in dyeing and calico-printing, denotes a body which, having a twofold attraction for organic fibres and coloring particles, serves as a bond of union between them, and thus gives fixity to dyes; or it signifies a substance which, by combining with coloring particles in the pores of textile filaments, renders them insoluble in hot soapy and weak alkaline solutions. In order properly to appreciate the utility and the true functions of mordants, we must bear in mind that coloring matters are peculiar compounds possessed of certain affinities, their distinctive characters being not to be either acid or alkaline, and yet to be capable of combining with many bodies, and especially with salifiable bases, and of receiring from each of them modifications in their color, solubility, and alterability. Organic coloring substances, when pure, have a rery energetic attraction for certain bodies, feeble for others, and none at all for some. Among these immediate products of animal or vegetable life, some are soluble in jure water, and others become so only through peculiar agents. We may thus readily conceive, that whenever a dye-stuff possesses a certain affinity for the organic fibre, it will be able to become fixed on it, or to dye it without the intervention of mordants, if it be insoluble by itself in water, which, in fact, is the case with the coloring matters of safflower, annotto, and indigo. The first two are soluble in alkalis; hence, in order to use them, they need only be dissolved in a weak alkaline Jer, be thus applied to the stuffs, and then hare their tinctorial substance precipitated within their pores, by abstracting their solvent alkali with an acid. The coloring matter, at the instant of ceasing to be liquid, is in an extremely divided state, and is in contact with the organic fibres for which it has a certain affinity. It therefore unites with them, and, being naturally insoluble in water, that is, haring no affinitr for this rehicle, the subsequent washings have no effect upon the dye. The same thing may be said of indigo, although its solubility in the dye-bath does not depend upon a similar cause, but is due to a modification of its constituent elements, in consequence of which it becomes soluble in allialis. Stuffs plunged into this indigo bath get impregnated with the solution, so that when again exposed to the air, the dyeing substance resumes at once its primitive color and insolubility, and washing can carry of only the portions in excess above the intimate combination, or which are merely deposited upon the surface of the stuff.

Such is the result with insoluble coloring matters; but for those which are soluble it should be quite the reverse, since ther do not possess an affinity for the organic fibres, which can counterbalance their affinity for water. In such circumstances, the drer must have recourse to intermediate bodies, which add their affinity for the coloring matter to that possessed by the particles of the stuff, and increase by this twofold action the intimacy and the stability of the combination. These intermediate bodies are the true mordants.

Mordants are in general found among the metallic bases or oxrdes; whence they might be supposed to be rery numerous, like the metals; but as they must unite the twofold condition of possessing a strong affinity for both the coloring matter and the organic fibre, and as the insoluble bases are almost the only ones fit to form insoluble combinations, we may thus perceire that their number may be rerr limited. It is well linown, that although lime and magnesia, for example, hare a considerahle affinitr for coloring particles, and form insoluble compounds with them, ret they cannot be employed as mordants, because they possess no affinity for the textile fibres.

Experience has proved, that of all the bases, those which succeed best as mordants are alumina, tin, and oxyde of iron; the first two of which, being naturally white, are the only ones which can be employed for preserving to the color its original tint, at least 
without much variation. But whenever the mordant is itself colored, it will cause the dye to talie a compound color quite different from its own. If, as is usually said, the mordant enic's into a real chemical union with the stuff to be dyed, the application of the morlatisi should obriously be made in such circumstances as are linown to be most favorable to the combination taking place; and this is the principle of every day's practice in the dire-house.

In order that a combination may result between two bodies, they must not only be in contact, but they must be reduced to their ultimate molecules. The mordants that are to be united with stuft's are, as we have seen, insoluble of themselves, for which reason their particles must be divided by solution in an appropriate vehicle. Now this solvent or menstruum will exert in its own favor an affinity for the mordant, which will prove to that extent an obstacle to its attraction for the stuff. Hence we must select such solvents as have a wealier affinity for the mordants than the mordants have for the stuffs. Of all the acids which can be employed to dissolve alumina, for example, vinegar is the one which will retain it with least energy, for which reason the acetate of alumina is now generally substituted for alum, because the acetic acid gives up the alumina with such readiness, that mere elevation of temperature is sufficient to effect the separation of these two substances. Before this substitution of the acetate, alum alone was employed; but without knowing the true reason, all the French dyers preferred the alum of Rome, simply regarding it to be the purest; it is only within these few years that they have understood the real grounds of this preference. This alum has not, in fact, the same composition as the alums of France, England, and Germany, but it consists chiefly of cubic alum having a larger proportion of base. Now this extra portion of base is held by the sulphuric acid more feebly than the rest, and hence is more readily detached in the form of a mordant. Nay, when a solution of cubic alum is heated, this redundant alumina falls down in the state of a subsulphate, long before it reaches the boiling point. This difference had not, however, been recognised, because Roman alum, being usually soiled with ochre on the surface, gives a turbid solution, whereby the precipitate of subsulphate of alumina escaped observation. When the liquid was filtered, and crystallized afresh, common octahedral alum alone was obtained; whence it vas most erroneously concluded, that the preference given to Roman alum was unjustifiable, and that its only superiority was in being freer from iron.

Here a remarkable anecdote illustrates the necessity of extreme caution, before we venture to condemn from theory a practice found to be useful in the arts, or set about changing it. When the French were masters in Rome, one of their ablest chemists swas sent thither to inspect the different manufactures, and to place them upon a level with the state of chemical knowledge. One of the fabrics, which seemed to him furthest behindhand, was precisely that of alum, and he was particularly hostile to the construction of the furnaces, in which vast boilers received heat merely at their bottoms, and could not be made to boil. He strenuously advised them to be new modelled upon a plan of his own; but, notwithstanding his advice, which was no doubt very scientific, the old routine kept its ground, supported by utility and reputation, and very fortunately $\$ 00$, for the manufacture; for had the higher heat been given to the boilers, no more genuine eubical alum would have been made, since it is decomposed at a temperature of about $i 20^{\circ} \mathrm{F}$, and common octahedral alum would alone have been produced. The addition of a little alkali to common alum brings it into the same basic state as the alum of Rome.

The two principal conditions, namely, extreme tenuity of particles, and liberty of action, keing found in a mordant, its operation is certain. But as the combination to be effeeted is merely the result of a play of affinity between the solvent and the stuff to be dised, a sort of partition must take place, proportioned to the mass of the solvent, as well as to its attractive force. Hence the stuff will retain more of the mordant when its solution is more concentrated, that is, when the base uslused through it is not so muck protected by a large mass of menstruum; a fact applied to very valuable uses by the practical man. On impregnating in calico printing, for example, different spots of the same web with the same mordant in different degrees of concentration, there is obtained in the dye-bath a depth of color upon these spots intense in proportion to the strength of their various mordants. Thus, with solution of acetate of alumina in different grades of density, and with madder, every shade can be produced, from the fullest zed to the lightest pink; and, with acetate of iron and madder, every shade from black to pale violet.

We hereby perceive that recourse must indispensably be had to mordants at different stages of concentration; a circumstance readily realized by varying the proportions of the watery vehicle. See CALICO-PRINTING and MADDER. When these mordants are to be topically applied, to produce partial dyes upon cloth, they must be thickened with stareh or gum, to prevent their spreading, and to permit a sufficient body of them to become attached to the stuff. Starch answers best for the more neutral mordants, and 
gum for the acidulous; but so much of them should never be used, as to impede the attraction of the mordant for the cloth. Nor should the thickened mordants be of toc desiccative a nature, lest they become hard, and imprison the chemical agent before it has had an opportunity of combining with the cloth, during the slow evaporation of its water and acid. Hence the mordanted goods, in such a case, should be hung up to dry in a gradual manner, and when oxygen is necessary to the fixation of the hase, they should be largely exposed to the atmosphere. 'The foreman of the factory ought, therefore, to be thoroughly conversant with all the minutiæ of chemical reaction. In cold and damp weather he must raise the temperature of his drying-house, in order to command a more decided evaporation; and when the atmosphere is unusually dry and warm, he should add deliquescent correctives to his thickening, as I have particularized in treating of some styles of calico-printing. But, supposing the application of the mordant and its desiccation to have been properly managed, the operation is by no means complete; nay, what remains to be done is not the least important to success, nor the least delicate of execution. Let us bear in mind that the mordant is intended to combine not only with the organic fibre, but afterwards also with the coloring matter, and that, consequently, it must be laid entirely bare, or scraped clean, so to speak, that is, completely disengaged from all foreign substances which might invest it, and obstruct its intimate contact with the coloring matters. This is the principle and the object of two operations, to which the names of dunging and clearing have been given.

If the mordant applied to the surface of the cloth were completely decomposid, and the whole of its base brought into chemical union with it, a mere rinsing or scouring in water would suffice for removing the viscid substances added to it, but this nerer happens, whatsoever precautions may be taken; one portion of the mordant remains untouched, and besides, one part of the base of the portion decomposed does not enter into combination with the stuff, but continues loose and superfluous. All these particles, therefore, must be removed without causing any injury to the dyes. If in this predicament the stuff were merely immersed in water, the free portion of the mordant would dissolve, and would combine indiscriminately with all the parts of the cloth not mordanted, and which should be carefully protected from such combination, as well as the action of the dye. We must therefore add to the scouring water some substance that is capable of seizing the mordant as soon as it is separated from the cloth, and of forming with it an insoluble compound; by which means we shall withdraw it from the sphere of action, and prevent its affecting the rest of the stuff, or interfering with the other dyes. This result is obtained by the addition of cow-dung to the scouring bath; a substance which contains a sufficiently great proportion of soluble animal matters, and of coloring particles, for absorbing the aluminous and ferruginous salts. The heat given to the dung-bath accelerates this combination, and determines an insoluble and perfectly inert coagulum.

Thus the dung-bath produces at once the solution of the thickening paste; a more intimate union between the alumina or iron and the stuff, in proportion to its elevation of temperature, which promotes that union; an effectual subtraction of the undecomposed and superfluous part of the mordant, and perhaps a commencement of mechanical separation of the particles of alumina, which are merely dispersed among the fibres; a separation, however, which can be completed only by the proper scouring, which is done by the dash-wheel with such agitation and pressure (see BLEAcHING and DUNGIKG) as vastly facilitate the expulsion of foreign particles. See also Bran.

Before concluding this article, we may say a word or two about astringents, ant? especially gall-nuts, which have been ranked by some writers among mordants. It is rather difficult to account for the part which they play. Of course we do not allude to their operation in the black dye, where they give the well known purple-black color with salts of iron; but w . a circumstance of their employment for madder dyes, and especially the Adrianople red. All that seems to be clearly established is, that the astringent principle or tannin, whose peculiar nature in this respect is unknown, combines like mordants with the stuff's and the coloring substance, so as to fix it; but as this tannin has itself a brown tint, it will not suit for white grounds, though it answers quite well for pink grounds. When white spots are desired upon a cloth prepared with oil and galls, they are produced by an oxygenous discharge, effected either through chlorine os chromic acid.

MORDANT is also the name sometimes given to the adhesive matter by which gold-leaf is made to adhere to surfaces of wood and metal in gilding. Paper, vellum, taffety, \&c., are easily gilt by the aid of different mordants, such as the following : 1 . beer in which some honey and gum arabic have been dissolved; 2 . gum arabic, sugar, and water; 3 . the viscid juice of onion or hyacinth, strengthened with a little gum arabic. When too much gum is employed, the silver or gold leaf is apt to crack in the drying of the mordant. A little carmine should be mixed with the above colorless 
liquids, to mark the places where they are applied. The foil is applied by means of a dossil of cotton wool, and when the mordant has become hard, the foil is polished with the same.

The best medium for stiching gold and silver leaf to wood is the following, called mixfion by the French artists:-1 pound of amber is to be fused, with 4 ounces of mastic in tears, and 1 ounce of Jewish pitch, and the whole dissolved in 1 pound of linseed oil rendered drying by litharge.

Painters in distemper sometimes increase the effect of their work, by patches of gold leaf, which they place in favorable positions; they employ the abore mordant. The manufacturers of paper hangings of the finer kinds attach gold and silver leaf to them by the same varnish.

MOROCCO. See LEATHER.

MORPHIA (Morphine, Fr.; Morphin, Germ.) is a vegeto-alkali which exists assoeiated with opian, codeine, narcotine, meconine, meconic acid, resin, gum, bassorine, lignine, fat oil, caoutchouc, extractive, \&c. in opium. Morphia is prepared as follows: Opium in powder is to be repeatedly digested with dilute muriatic acid, slightly heated, and sea-salt is to be added, to precipitate the opian. The filtered liquid is to be supersaturated with ammonia, which throws down the morphia, along with the meconine, resin, and extractive. The precipitate is to be washed with water, heated, and dissolved in dilute muriatic acid; the solution is to be filtered, whereby the foreign matters are separated from the salt of morphia, which concretes upon cooling, while the meconine remains in the acid liquid. The muriate of morphia having been squeezed between folds of blotting paper, is to be sprinkled with water, again squeezed, next dissolved in water, and decomposed by water of ammonia. The precipitate, when washed, dried, dissolred in alcohol, and crystallized, is morphia.

These crystals, which contain $6 \cdot 32$ per cent. of combined water, are transparent, olorless, four-sided prisms, without smell, and nearly void of taste, fusible at a moderate heat, and they concrete into a radiated translucent mass, but at a higher temperature they grow purple-red. Morphia consists of 72.34 of carbon; 6.366 of hydrogen; 5 of azote; and $16 \cdot 3$ of oxygen. It burns with a red and very smoky flame, is stained red by nitric acid, is soluble in 30 parts of boiling anhydrous alcohol, in 500 parts of boiling water, but hardly if at all in cold water, and is insoluble in ether and oils. The solutions have a strong bitter taste, and an alkaline reaction upon litmus paper. The saline compounds hare a bitter taste, are mostly crystallizable, are soluble in water and alcohol (but not in ether), and give a blue color to the peroxyde salts of iron. It is a rery poisonous substance. Acetate of morphia is sometimes prescribed, instead of opium, in medicine.

MORTAR, HYDRAULIC, called also Roman Cement, is the kind of mortar used for building piers, or walls under or exposed to water, such as those of harbors, dochs, \&c. The poorer sorts of limestone are best adapted for this purpose, such as contain from 8 to 25 per cent. of foreign matter, in silica, alumina, magnesia, \&c. These, though calcined, do not slake when moistened; but if pulverized they absorb water without swelling up or heating, like fat lime, and afford a paste which hardens in a few days under water, but in the air they never acquire much solidity. Smeaton first discovered these remarkable facts, and described them in 1759.

The following analyses of different hydraulic limestones, by Berthier, merit confidence :-

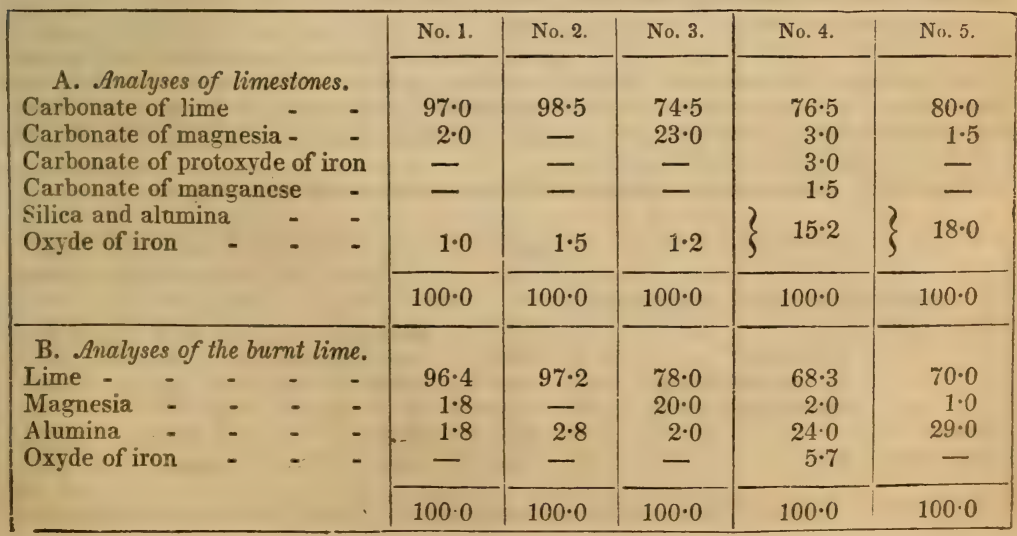


No. 1 is from the fresh-water lime formation of Chateau-Landon, near Nemours. No. 2, the large-grained limestone of Paris; both of these afford a fat lime when burnt. Duomite affords a pretty fat lime, though it contains 42 per cent. of carbonate of magnesia; No. 3 is a limestone from the neighborhood of Paris, which yields a poor lime, possessing no hydraulic property; No. 4 is the secondary limestone of Metz; No. 5 is the lime marl of Senonches, near Dreux; both the latter have the property of hardening under water, particularly the last, which is much used at Paris on this account.

All good hydraulic mortars must contain alumina and silica; the oxydes of iron and manganese, at one time considered essential, are rather prejudicial ingredients. By adding silica and alumina, or merely the former, in certain circumstances, to fat lime, a water-cement may be artificially formed; as also by adding to lime any of the following native productions, which contain silicates; puzzolana, trass or tarras, pumice-stone, basalt-tuff, slate-clay. Puzzolana is a volcanic product, which forms hills of considerable extent to the south-west of the Appenines, in the district of Rome, the Pontine marshes, Viterbo, Bolsena, and in the Neapolitan region of Puzzuolo, whence the name. A similar voleanic tufa is found in many other parts of the world. According to Berthier, the Italian puzzolana consists of 44.5 silica; 15.0 alumina; 8.8 lime ; 4.7 magnesia ; 1.4 potash; 4.1 soda ; 12 oxydes of iron and titanium; 9.2 water; in 100 parts.

The tufa stone, which when ground forms trass, is composed of $57 \cdot 0$ silica, $16 \cdot 0$ clay, 2.6 lime, 1.0 magnesia, $7 \cdot 0$ potash, 1.0 soda, 5 oxydes of iron and titanium, 9.6 water. This tuff is found abundantly filling up valleys in beds of 10 or 20 feet deep, in the north of Ireland, among the schistose formations upon the banks of the Rhine, and at Monheim in Bavaria.

The fatter the lime, the less of it must be added to the ground puzzolana or trass, to form a hydraulic mortar; the mixture should be made extemporaneously, and must at any rate be kept dry till about to be applied. Sometimes a proportion of common sand mortar instead of lime is mixed with the trass. When the hydraulic cement hardens too soon, as in 12 hours, it is apt to crack; it is better when it takes 8 days to concrete. Through the agency of the water, silicates of lime, alumina, (magnesia,) and oxyde of iron are formed, which assume a stony hardness.

Besides the above two volcanic products, other native earthy compounds are used in making water cements. To this head belong all limestones which contain from 20 to 30 per cent. of clay and silica. By gentle calcination, a portion of the carbonic acid is expelled, and a little lime is combined with the clay, while a silicate of clay and lime zesults, associated with lime in a subcarbonated state. A lime-marl containing less clay will hear a stronger calcining heat without prejudice to its qualities as a hydraulic cement; but much also depends upon the proportion of silica present, and the physical structure of all the constituents.

The mineral substance most used in England for making such mortar is vulgarly called cement-stone. It is a reniform limestone, which occurs distributed in single nodules or rather lenticular cakes, in beds of clay. They are mostly found in those argillaceous strata which alternate with the limestone beds of the oolite formation, as also in the clay strata above the chalk, and sometimes in the London clay. On the coasts of Kent, in the isles of Sheppey and Thanet, on the coasts of Yorkshire, Somersetshire, and the Isle of Wight, \&c., these nodular concretions are found in considerable quantities, having been laid bare by the action of the sea and weather. They were called by the older mineralogists Septaria and Ludus Helmontii (Van Helmont's coits.) When sawn across, ther show reins of calc-spar traversing the silicious clay, and are then sometimes placed in the eabinets of virtuosi. They are found also in several places on the Continent, as at Neustadt-Eberswalde, near Antwerp, near Altdorf in Bavaria; as also at Boulognesur-mer, where they are called Boulogne-pebbles (galets). These nodules vary in size from that of a fist to a man's head; they are of a yellow-gray or brown cnlor, interspersed with veins of calc-spar, and sometimes contain cavities bestudded with crystals.

Their specific gravity is $2 \cdot 59$.

Analyses of several cement-stones, and of the cement made with them :-

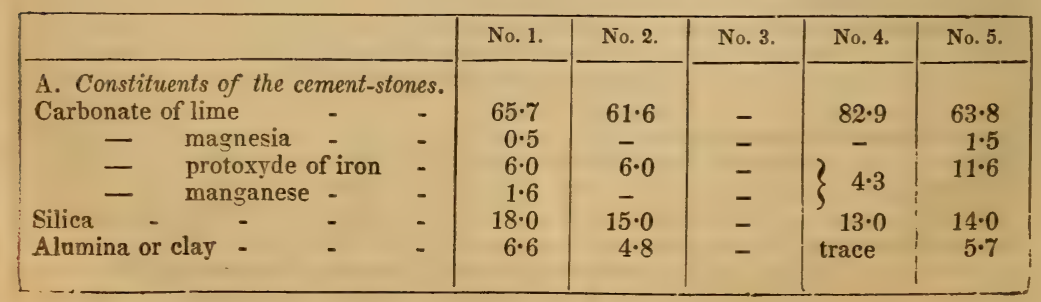


Analyses of several cement-stones, \&c.-Continued.

\begin{tabular}{|c|c|c|c|c|c|c|c|c|}
\hline & & & & No. 1. & No. 2. & No. 3. & No. 4. & No. 5. \\
\hline Oxyde of iron & - & - & - & - & $3 \cdot 0$ & & & \\
\hline Water - & - & - & - & $1 \cdot 2$ & $6 \cdot 6$ & - & - & $3 \cdot 4$ \\
\hline \multicolumn{9}{|c|}{ B. Constituents of the Cement. } \\
\hline Lime & - & - & - & $55 \cdot 4$ & $54 \cdot 0$ & $55 \cdot 0$ & - & $56 \cdot 6$ \\
\hline Magnesia & - & - & - & - & - & - & - & $1 \cdot 1$ \\
\hline Alumina or clay & - & - & - & $36 \cdot 0$ & $31 \cdot 0$ & $38 \cdot 0$ & - & $21 \cdot 0$ \\
\hline Oxyde of iron & - & - & - & $8 \cdot 6$ & $15 \cdot 0$ & $13 \cdot 0$ & - & $13 \cdot 7$ \\
\hline
\end{tabular}

No. 1. English cement-stone, analyzed by Berthier; No. 2. Boulogne stone, by Drapiez; No. 3. English ditto, by Davy; No. 4. reniform limestone nodules from Arkona, by Hühnefeld; No. 5. cement-stone of Avallon, by Dumas.

In England the stones are calcined in shaft-kilns, or sometimes in mound-kilns, then ground, sifted, and packied in casks. The color of the powder is dark-brown-red. When made into a thick paste with water, it absorbs little of it, evolves hardly any heat, and soon indurates. It is mixed with sharp sand in various proportions, immediately before using it; and is employed in all marine and river embankments, for securing the seams of stone or brick floors or arches from the percolation of moisture, and also for facing walls to protect them from damp.

The cement of Pouilly is prepared from a Jurassic (secondary) limestone, which contains 39 per cent. of silica, with alumina, magnesia, and iron oxyde. Vicat forms a factitious Roman cement by making bricks with a pasty mixture of 4 parts of chalk, and 1 part of dry clay, drying, burning, and grinding them. River sand must be added to this powder; and eren with this addition, its efficacy is somewhat doubtful; though it has, for want of a better substitute, been much employed at Paris.

The cement of Dihl consists of porcelain or salt-glaze potsherds ground fine, and mixed with boiled linseed oil.

Hamelin's mastic or lithic paint to cover the façades of brick buildings, \&c., is composed of 50 measures of silicious sand, 50 of lime-marl, and 9 of litharge or red-lead ground up with linseed oil.

MOSAIC GOLD. For the composition of this peculiar alloy of copper and zine, called also Or-molu, Messrs. Parker and Hamilton obtained a patent in Norember, 1825. Equal quantities of copper and zinc are to be "melted at the lowest temperature that copper will fuse," which being stirred together so as to produce a perfect admixture of the metals, a further quantity of zinc is added in small portions, until the alloy in the melting pot becomes of the color required. If the temperature of the copper be too high, a portion of the zinc will fly off in vapor, and the result will be merely spelter or hard solder; but if the operation be carried on at as low a heat as possible, the alloy will assume first a brassy yellow color; then, by the introduction of small portions of zinc, it will take a purple or violet hue, and will ultimately become perfectly white; which is the appearance of the proper compound in its fused state. This allor may be poured into ingots; but as it is difficult to preserve its character when re-melted, it should be east directly into the figured moulds. The patentees claim the exclusive right of compounding a metal consisting of from 52 to 55 parts of zinc out of 100 .

Mosaic gold, the aurum musivum of the old chemists, is a sulphuret of tin.

MOSAIC. (Mosaique, Fr.; Mosaisch, Germ.) There are several linds of mosalc, but all of them consist in imbedding fragments of different colored substances, usually glass or stones, in a cement, so as to produce the effect of a picture. The beautiful chapel of Saint Lawrence in Florence, which contains the tombs of the Medici, has been greatly arlmired by artists, on account of the vast multitude of precious marbles, jaspers, agates, aranturines, malachites, \&c., applied in mosaic upon its walls. The detailed discussion of this subject belongs to a treatise upon the fine arts.

MOTHER OF PEARL (Nacre de Perles, Fr.; Perlen mutter, Germ.) is the hard, silvery, brilliant internal layer of several kinds of shells, particularly oysters, which is often variegated with changing purple and azure colors. The large oysters of the Indian seas alone secrete this coat of sufficient thickness to render their shells available to the purposes of manufactures. The genus of shell fish called pentadince furnishes the finest pearls, as well as mother of pearl; it is found in greatest perfection round the coasts of Cerlon, near Ormus in the Persian Gulf, at Cape Con.orin, and among some of the Australian seas. The brilliant hues of mother of pearl do not depend upon the nature of the substance, but upon its structure. The microscopic wrinkles or furrows which run across the surface of every slice, act upon the reflected light in such a way as to produce the chromatic effect; for Sir David Brewster has shown, that if we take, witb 
very fine black wax, or with the fusible alloy of D'Arcet, an impression of mother of pearl, it will possess the iridescent appearance. Mother of pearl is very delicate to work, but it may be fashioned by saws, files, and drills, with the aid sometimes of a corrosive acid, such as the dilute sulphuric or muriatic; and it is polished by colcothar of vitrios.

MOTHER-WATER is the name of the liquid which remains after all the salts that will regularly crystallize have been extracted, by evaporation and cooling, from any saline solution.

MOUNTAIN SOAP (Savon de montagne, Fr.; Bergseife, Germ.) is a tender mineral, soft to the touch, which assumes a greasy lustre when rubbed, and falls to pieces in water. It consists of silica 44 , alumina 26.5 , water 20.5 , oxyde of iron 8 , lime 0.5 . It occurs in beds, alternating with different sorts of clay, in the Isle of Skye, at Billin in Bohemia, \&c. It has been often, but improperly, confounded with steatite.

MUCIC ACID (Acide mucique, Fr.; Schleimsaïre, Germ.) is the same as the saclactic acid of Scheele, and may be obtained by digesting one part of gum arabic, sugar of milk, or pectic acid, with twice or thrice their weight of nitric acid. It forms white granular crystals, and has not been applied to any use in the arts.

MUCILAGE is a solution in water of gummy matter of any kind.

MUFFLE is the earthenware case or box, in the assay furnaces, for receiving the cupels, and protecting them from being disturbed by the fuel. See AssaY and FURNace.

MUNDIC is the name of copper pyrites among English miners.

MUNJEET is a kind of madder grown in several parts of India.

MURIATIC or HYDROCHLORIC ACID ; anciently marine acid, and spirit of salt. (Acide hydrochlorique, and Chlorhydrique, Fr. ; Salzsaïre, Germ.) This acid is now extracted from sea-salt, by the action of sulphuric acid and a moderate heat; but it was originally obtained from the salt by exposing a mixture of it and of common clay to ignition in an earthen retort. The acid gas which exhales, is rapidly condensed by water. 100 cubic inches of water are eapable of absorbing no less than 48,000 cubic inches of the acid gas, whereby the liquid acquires a specific gravity of $1 \cdot 2109$; and a volume of 142 cubic inches. This vast condensation is accompanied with a great production of heat, whence it becomes necessary to apply artificial refrigeration, especially if so strong an acid as the above is to be prepared. In general, the muriatic acid of commerce has a specific gravity varying from $1 \cdot 15$ to 1.20 ; and contains, for the most part, considerably less than 40 parts by weight of acid gas in the hundred. The above stronger acid contains 42.68 per cent. by weight; for since a cubic inch of water, which weighs 252.5 grains, has absorbed 480 cubic inches $=188$ grains of gas; and $252.5+188=440.5$; then $440 \cdot 5: 188:: 100: 42 \cdot 68$. In general a very good approximation may be found to the per centage of real muriatic acid, in any liquid sample, by multiplying the decimal figures of the specific gravity by 200 . Thus for example, at 1.162 we shall have by this rule $0.162 \times 200=32.4$, for the quantity of gas in 100 parts of the liquid. Muriatic acid gas consists of chlorine and hydrogen combined, without condensation, in equal volumes. Its specific gravity is $1 \cdot 247$, air $=1 \cdot 000$.

By sealing up muriate of ammonia and sulphuric acid, apart, in a strong glass tube recurved, and then causing them to act on each other, Sir H. Davy procured liquid muriatic acid. He justly observes, that the generation of elastic substances in close vessels, either with or without heat, offers much more powerful means of approximating their molecules than those dependant on the application of cold, whether natural or artificial; for as gases diminish only $\frac{1}{480}$ in volume for every degree of Fahrenheit's scale, beginning at ordinary temperatures, a very slight condensation only can be produced by the most powerful freezing mixtures, not half as much as would result from the application of a strong flame to one part of a glass tube, the other part being of ordinary temperature; and when attempts are made to condense gases into liquids by sudden mechanical compression, the heat instantly generated presents a formidable obstacle to the success of the experiment; whereas in the compression resulting from their slow generation in close vessels, if the process be conducted with common precautions, there is no source of difficulty or danger; and it may be easily assisted by artificial cold, in cases where gases approach near to that point of compression and temperature at which they become vapors. - Phil. Trans. 1823.

The muriatic acid of commerce has usually a yellowish tinge, but when chemically pure it is colorless. It fumes strongly in the air, emitting a corrosive vapor of a peculiar smell. The characteristic test of muriatic acid in the most dilute state, is nitrate of silver, which causes a curdy precipitate of chloride of silver.

The preparation of this acid upon the great scale is frequently effected in this country by acting upon sea-salt in hemispherical iron pots, or in cast-iron cylinders, with concentrated sulphuric acid; taking 6 parts of the salt to 5 of the acid. The mouth of the pot may be covered with a slab of silicious freestone, perforated with two holes of abous two inches diameter each, into the one of which the acid is poured by a funnel in successive 
portions, and into the other, a bent glass, or stone-ware tuke, is fixed, for conducting the disengaged muriatic gas into a series of large globes of bottle glass, one third filled with water, and laid on a sloping sand-bed. A week is commonly employed for working off each pot; no heat being applied to it till the second day.

ihe decomposition of sea-salt by sulphuric acid, was at one time carried on by some French manufacturers in large leaden pans, 10 feet long, 5 feet broad, and a foot deep, zovered with sheets of lead, and luted. The disengaged acid gas was made to circulate in a conduit of glazed bricks, nearly 650 yards long, where it was condensed by a sheet of water exceedingly thin, which flowed slowly in the opposite direction of the gas down a slope of 1 in 200. At the end of this eanal nearest the apparatus, the muriatic acid was as strong as possible, and pretty pure; but towards the other end, the water was hardly acidulous. The condensing part of this apparatus was therefore tolerably complete; but as the decomposition of the salt could not be finished in the leaden pans, the acid mixture had to be drawn out of them, in order to be completely decomposed in a reverberatory furnace; in this way nearly 50 per cent. of the muriatic acid was lost. And besides, the great quantity of gas given off during the emptying of the lead-chambers was apt to suffocate the workmen, or seriously injured their lungs, causing severe hemoptysis. The employment of muriatic acid is so inconsiderable, and the loss of it incurred in the preceding process is of so little consequence, that subsequently, both in France and in England, sulphate of soda, for the soda manufacture, has been procured with the dissipation of the muriatic acid in the air. In the method more lately resorted to, the gaseous products are discharged into extensive vaults, where currents of water condense them and carry them off into the river. The surrounding vegetation is thereby sared in some measure from being burned up, an accident which was previously sure to happen when fogs precipitated the floating gases upon the ground. At Newcastle, Lirerpool, and Marseilles, where the consumption of muriatic acid bears no proportion to the manufacture of soda, this process is now practised upon a vast scale.

The apparatus for condensing muriatic acid gas has been modified and changed, of late years, in many different ways.

The Bastringue apparatus. At the end of a reverberatory furnace, (see CoPPER, SMELTiNg of, and SodA, MANUFACTURE of,) a rectangular lead trough or pan, about 1 foot deep, of a width equal to that of the interior of the furnace, that is, about 5 feet wide, and $6 \frac{1}{2}$ feet long, is incased in masonry, having its upper edges covered with cast-iron plates or fire tiles, and placed upon a level with the passage of the flame, as it escapes from the reverberatory. The arch which covers that pan forms a continuation of the roof of the reverberatory, and is of the same height. The flame which proceeds from the furnace containing the mixture of salt and sulphuric acid is made to escape between the vault and the surface of the iron plates or fire tiles, through a passage only 4 inches in height. When the burned air and vapors reach the extremity of the pan, they are reflected downwards, and made to return beneath the bottom of the pan, in a flue, which is afterwards divided so as to lead the smoke into two lateral flues, which terminate in the chimner. The pan is thus surrounded as it were with the heat and flame discharged from the reverberatory furnace. See Evaporation. A door is opened near the end of the pan, for introducing the charge of sea-salt, amounting to 12 bags of 2 cwts. each, or $24 \mathrm{cwts}$. This door is then luted on as tightly as possible, and for every 100 parts of salt, 110 of sulphuric acid are poured in, of specific gravity 1.594 , containing 57 per cent. of dry acid. This acid is introduced through a funnel inserted in the roof of the furnace. Decomposition ensues, muriatic acid gas mingled with steam is disengaged, and is conducted through 4 stone-ware tubes into the refigerators, where it is finally condensed. These refrigerators consist of large stone-ware carboys, called dame-jeans in France, to the number of 7 or 8 for each pipe, and arranged so that the neck of the one communicates with the body of the other; thus the gas must traverse the whole series, and gets in a good measure condensed by the water in them, before reaching the last.

When the operation is finished, the door opposite the pan is opened, and the residuum in it is discharged, in the form of a fluid magma, upon a square bed of bricks, exterior to the furnace. This paste speedily concretes on cooling, and is then sroken into fragments and carried to the soda manufactory. The immense quantity of gas exhaled in discharging the pan, renders this part of the operation very painful to the workmen; and wasteful in reference to the production of muriatic acid. The difficulty of luting securely the cast-iron plates or fire tiles which cover the pan, the impossibility of completing the decomposition of the salt, since the residuum must be run off in a liquid state, finally, the damage sustained by the melting and corrosion of the lead, \&c., are among the causes why no more than 80 or 90 parts of muriatic acid at $1 \cdot 170$ are collected, equivalent to 25 per cent. of real acid for every 100 of salt employed, instead of much more than double that quantity, which it may be made to yield by a well-conducted chemical process.

The cylinder apparatus is now much esteemed by many manufacturers. Fig. 74? 
represents, in transverse section, a bench of iron cylinder retorts, as built up in a proper furnace for producing muriatic acid; and fig. 748 a longitudinal section of one retort

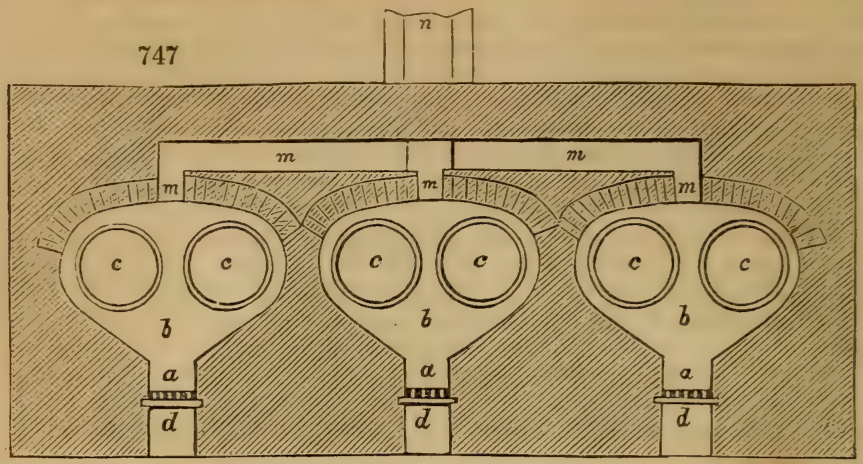

with one of its carboys of condensation. $a$ is the grate; $b$, a fireplace, in which two iron cylinders, $c c$, are set alongside of each other.

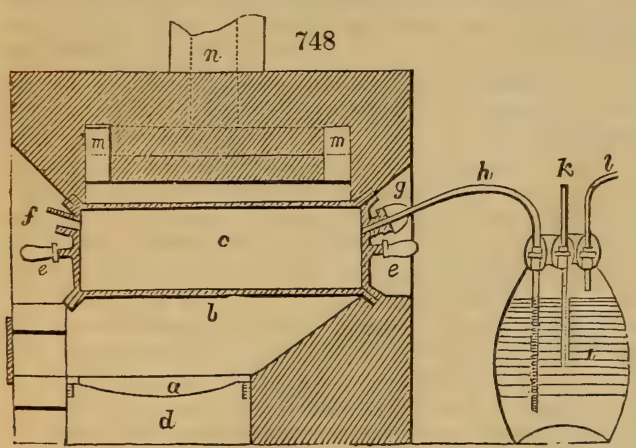

They are $5 \frac{1}{2}$ feet long, 20 inches in diameter, about $\frac{1}{4}$ of an inch thick, and take 1.6 ewts. of salt for a charge : $d$ is the ashpit ; $e, e$, are cast-iron lids, for closing both ends of the cylinders; $f$ is a tube in the posterior lid, for pouring in the sulphuric acid; $g$ is another tube, in the anterior lid, for the insertion of the bent pipe of hard glazed stone-ware $h$; $i$ is a three-necked stone-ware carboy; $k$ is a tube of safety ; $l$, a tube of communication with the second carboy ; $m m, m m$, are the flues leading to the chimney $n$.

After the salt has been introduced, and the fire kindled, $83 \frac{1}{4}$ per cent. of its weight of sulphuric acid, of spec. grav. $1 \cdot 80$, should be slowly poured into the cylinder through a lead funnel, with a syphon-formed pipe. The three-necked carboys may be either placed in a series for each retort, like a range of Woulfe's bottles, or all the carboys of the front range may be placed in communication with one another, while the last carboy at one end is joined to the first of the second range; and thus in succession. They must be half filled with cold water; and when convenient, those of the front row at least, should be plunged in an oblong trough of running water. The acid which condenses in the carboys of that row is apt to be somewhat contaminated with sulphuric acid, muriate of iron, or even sulphate of soda; but that in the second and third will be found to be pure. In this way 100 parts of sea-salt will yield 130 parts of muriatic acid, of spec. grav. 1.19; while the sulphate of soda in the retort will afford from 208 to 210 of that salt in crystals.

It is proper to heat all the parts of the cylinders equably, to ensure the simultaneous decomposition of the salt, and to protect it from the acid; for the hotter the iron, and the stronger the acid, the less ersion ensues.

Some manufacturers, with the view of saving fuel by the construction of their furnaces, oppose to the flame as many obstacles as they can, and make it perform numerous circulations round the cylinders; but this system is bad, and does not even effect the desired economy, because the passages, being narrow, impair the draught, and become speedily choked up with the soot, which would be burned profitably in a freer space; the decomposition also, being unequally performed, is less perfect, and the cylinders are more injured. It is better to make the flame envelope at once the body of the cylinder; after which it may circulate beneath the vault, in order to give out a portion of its caloric before it escapes at the chimney.

The fire should be briskly kindled, but lowered as soon as the distillation commences; and then continued moderate till the evolution of gas diminishes, when it must be heated somewhat strongly to finish the decomposition. The iron door is now removed, 
to extract the sulphate of soda, and to recommence another operation. This sulphate ought to be white and uniform, exhibiting in its fracture no undecomposed sea-salt.

Liquid muriatic acid has a very sour corrosive taste, a pungent suffocating smell, and acts very powerfully upon a vast number of mineral, vegetable, and animal substances. It is much employed for making many metallic solutions; and in combination with nitric acid, it forms the aqua regia of the alchemists, so called from its property of dissolving gold.

Table of Muriatic Acid, by Dr. Ure.

\begin{tabular}{|c|c|c|c|c|c|c|c|c|c|c|c|}
\hline $\begin{array}{l}\text { Acid } \\
\text { of } 120 \\
\text { in } 100\end{array}$ & $\begin{array}{l}\text { Specific } \\
\text { gravity. }\end{array}$ & $\begin{array}{l}\text { Chlo- } \\
\text { rine. }\end{array}$ & $\begin{array}{c}\text { Muriatic } \\
\text { Gas. }\end{array}$ & $\begin{array}{l}\text { Acid } \\
\text { of } 120 \\
\text { in } 100\end{array}$ & $\begin{array}{l}\text { Specific } \\
\text { gravity. }\end{array}$ & $\begin{array}{l}\text { Chlo- } \\
\text { rine. }\end{array}$ & $\begin{array}{c}\text { Muriatic } \\
\text { Gas. }\end{array}$ & $\begin{array}{l}\text { A cid } \\
\text { of } 120 \\
\text { in } 100\end{array}$ & $\begin{array}{l}\text { Specific } \\
\text { gravity. }\end{array}$ & $\begin{array}{l}\text { Chlo- } \\
\text { rine. }\end{array}$ & $\begin{array}{c}\text { Muriatic } \\
\text { Gas. }\end{array}$ \\
\hline 100 & $1 \cdot 2000$ & $39 \cdot 675$ & $40 \cdot 777$ & 66 & $1 \cdot 1328$ & $26 \cdot 186$ & $26 \cdot 913$ & 32 & 1.0637 & $12 \cdot 697$ & $13 \cdot 049$ \\
\hline 99 & $1 \cdot 1982$ & $39 \cdot 278$ & $40 \cdot 369$ & 65 & $1 \cdot 1308$ & $25 \cdot 789$ & $26 \cdot 505$ & 31 & 1.0617 & $12 \cdot 300$ & $12 \cdot 641$ \\
\hline 98 & $1 \cdot 1964$ & $38 \cdot 882$ & $39 \cdot 961$ & 64 & $1 \cdot 1287$ & $25 \cdot 392$ & $26 \cdot 098$ & 30 & $1 \cdot 0597$ & 11.903 & $12 \cdot 233$ \\
\hline 97 & $1 \cdot 1946$ & $38 \cdot 485$ & $39 \cdot 554$ & 63 & $1 \cdot 1267$ & $24 \cdot 996$ & $25 \cdot 690$ & 29 & 1.0577 & $11 \cdot 506$ & $11 \cdot 825$ \\
\hline 96 & $1 \cdot 1928$ & $38 \cdot 089$ & $39 \cdot 146$ & 62 & $1 \cdot 1247$ & $24 \cdot 5599$ & $25 \cdot 282$ & 28 & 1.0557 & $11 \cdot 109$ & $11 \cdot 418$ \\
\hline 95 & $1 \cdot 1910$ & $37 \cdot 692$ & $38 \cdot 738$ & 61 & $1 \cdot 1226$ & $24 \cdot 202$ & $24 \cdot 874$ & 27 & $1 \cdot 0537$ & $10 \cdot 712$ & $11 \cdot 010$ \\
\hline 94 & $1 \cdot 1893$ & $37 \cdot 296$ & $38 \cdot 330$ & 60 & $1 \cdot 1206$ & $23 \cdot 805$ & $24 \cdot 466$ & 26 & 1.0517 & $10 \cdot 316$ & $10 \cdot 602$ \\
\hline 93 & $1 \cdot 1875$ & $36 \cdot 900$ & $3 \% \cdot 923$ & 59 & $1 \cdot 1185$ & $23 \cdot 408$ & $24 \cdot 058$ & 25 & 1.0497 & $9 \cdot 919$ & $10 \cdot 194$ \\
\hline 92 & $1 \cdot 1857$ & 36.503 & $37 \cdot 516$ & 58 & $1 \cdot 1164$ & $23 \cdot 012$ & $23 \cdot 050$ & 24 & 1.0477 & $9 \cdot 522$ & $9 \cdot 786$ \\
\hline 91 & $1 \cdot 1846$ & $36 \cdot 107$ & $37 \cdot 108$ & 57 & $1 \cdot 1143$ & $22 \cdot 615$ & $23 \cdot 242$ & 23 & $1 \cdot 0457$ & $9 \cdot 126$ & $9 \cdot 379$ \\
\hline 90 & $1 \cdot 1822$ & $35 \cdot 707$ & $36 \cdot 700$ & 56 & $1 \cdot 1123$ & $22 \cdot 218$ & $22 \cdot 834$ & 22 & $1 \cdot 0437$ & $8 \cdot 729$ & 8.971 \\
\hline 89 & $1 \cdot 1802$ & $35 \cdot 310$ & $36 \cdot 292$ & 55 & $1 \cdot 1102$ & $21 \cdot 822$ & $22 \cdot 426$ & 21 & $1 \cdot 0417$ & $8 \cdot 332$ & 8.553 \\
\hline 88 & $1 \cdot 1782$ & $34 \cdot 913$ & $35 \cdot 884$ & 54 & $1 \cdot 1082$ & $21 \cdot 425$ & $22 \cdot 019$ & 20 & 1.0397 & 7.935 & $8 \cdot 155$ \\
\hline 87 & $1 \cdot 1762$ & $34 \cdot 517$ & 35.476 & 53 & $1 \cdot 1061$ & 21.028 & $21 \cdot 611$ & 19 & 1.0377 & $7 \cdot 538$ & $7 \cdot 747$ \\
\hline 86 & $1 \cdot 1741$ & $34 \cdot 121$ & $35 \cdot 068$ & 52 & $1 \cdot 1041$ & $20 \cdot 632$ & $21 \cdot 203$ & 18 & $1 \cdot 0357$ & $7 \cdot 141$ & $7 \cdot 340$ \\
\hline 85 & $1 \cdot 1721$ & $33 \cdot 724$ & $34 \cdot 660$ & 51 & $1 \cdot 1020$ & $20 \cdot 235$ & $20 \cdot 796$ & 17 & 1.0337 & $6 \cdot 745$ & $6 \cdot 932$ \\
\hline 84 & 1.1701 & $33 \cdot 328$ & $34 \cdot 252$ & 50 & $1 \cdot 1000$ & $19 \cdot 837$ & $20 \cdot 388$ & 16 & 1.0318 & $6 \cdot 348$ & $6 \cdot 524$ \\
\hline 83 & $1 \cdot 1681$ & 32.931 & $33 \cdot 845$ & 49 & 1.0980 & $19 \cdot 440$ & $19 \cdot 980$ & 15 & $1 \cdot 0298$ & 5.951 & $6 \cdot 116$ \\
\hline 82 & $1 \cdot 1661$ & $32 \cdot 535$ & $33 \cdot 437$ & 48 & 1.0960 & $19 \cdot 044$ & $19 \cdot 572$ & 14 & $1 \cdot 0279$ & $5 \cdot 554$ & $5 \cdot 709$ \\
\hline 81 & $1 \cdot 1641$ & $32 \cdot 136$ & 33.029 & 47 & 1.0939 & $18 \cdot 647$ & $19 \cdot 165$ & 13 & $1 \cdot 0259$ & $5 \cdot 158$ & $5 \cdot 301$ \\
\hline 80 & $1 \cdot 1620$ & $31 \cdot 746$ & $32 \cdot 621$ & 46 & 1.0919 & $18 \cdot 250$ & $18 \cdot 757$ & 12 & 1.0239 & $4 \cdot 762$ & $4 \cdot 893$ \\
\hline 79 & 1.1599 & $31 \cdot 343$ & $32 \cdot 213$ & 45 & 1.0899 & $17 \cdot 854$ & $18 \cdot 349$ & 11 & $1.022 C$ & $4 \cdot 365$ & $4 \cdot 486$ \\
\hline 78 & $1 \cdot 1578$ & $30 \cdot 946$ & $31 \cdot 805$ & 44 & 1.0879 & $17 \cdot 457$ & $17 \cdot 941$ & 10 & 1.0200 & 3.968 & 4.078 \\
\hline 77 & $1 \cdot 1557$ & $30 \cdot 550$ & $31 \cdot 398$ & 43 & 1.0859 & $17 \cdot 060$ & $17 \cdot 534$ & 9 & $1 \cdot 0180$ & 3.571 & $3 \cdot 670$ \\
\hline 76 & $1 \cdot 1536$ & $30 \cdot 153$ & $30 \cdot 990$ & 42 & 1.0838 & $16 \cdot 664$ & $17 \cdot 126$ & 8 & $1 \cdot 0160$ & $3 \cdot 174$ & $3 \cdot 262$ \\
\hline 75 & $1 \cdot 1515$ & $29 \cdot 757$ & 30.582 & 41 & $1 \cdot 0818$ & $16 \cdot 26 \pi$ & $16 \cdot 718$ & 7 & 1.0140 & $2 \cdot 778$ & $2 \cdot 854$ \\
\hline 74 & $1 \cdot 1494$ & $29 \cdot 361$ & $30 \cdot 174$ & 40 & $1 \cdot 0798$ & $15 \cdot 870$ & $16 \cdot 310$ & 6 & $1 \cdot 0120$ & $2 \cdot 381$ & $2 \cdot 447$ \\
\hline 73 & $1 \cdot 1473$ & 28.964 & $29 \cdot 767$ & 39 & 1.0778 & $15 \cdot 474$ & 15.902 & 5 & $1 \cdot 0100$ & 1.984 & $2 \cdot 039$ \\
\hline 72 & $1 \cdot 1452$ & 28.567 & $29 \cdot 359$ & 38 & 1.0758 & $15 \cdot 077$ & $15 \cdot 494$ & 4 & $1 \cdot 0080$ & 1.588 & $1 \cdot 631$ \\
\hline 71 & $1 \cdot 1431$ & $28 \cdot 171$ & 28.951 & 37 & 1.0738 & $14 \cdot 680$ & $15 \cdot 087$ & 3 & 1.0060 & $1 \cdot 191$ & $1 \cdot 224$ \\
\hline 70 & $1 \cdot 1410$ & $27 \cdot 772$ & $28 \cdot 544$ & 36 & 1.0718 & $14 \cdot 284$ & $14 \cdot 679$ & 2 & 1.0040 & 0.795 & 0.816 \\
\hline 69 & $1 \cdot 1389$ & $27 \cdot 376$ & $28 \cdot 136$ & 35 & $1 \cdot 0697$ & $13 \cdot 887$ & $14 \cdot 271$ & 1 & $1 \cdot 0020$ & 0.397 & 0.408 \\
\hline 68 & $1 \cdot 1369$ & 26.979 & $27 \cdot 728$ & 34 & 1.0677 & $13 \cdot 490$ & $13 \cdot 863$ & & & & \\
\hline 67 & $1 \cdot 1349$ & 26.583 & $27 \cdot 321$ & 33 & $1 \cdot 0657$ & $13 \cdot 094$ & $13 \cdot 456$ & & & & \\
\hline
\end{tabular}

MURIATES were, till the great chemical era of Sir H. Davy's researches upon chlorine, considered to be compounds of an undecompounded acid, the muriatic, with the different hases; but he proved them to be in reality compounds of chlorine with the metals. They are all, however, still known in commerce by their former appellation. The oniy muriates much used in the manufactures are, Muriate of ammonia, or SAI AmMoniac; muriated peroxyde of mercury, MERCURy, bichloride of; muriate of soda, or chlorde of sodium, see SALT; muriate of tin, see CALICO-PRINTING and TIN.

MUSK (Musc, Fr.; Moschus, Germ.) is a peculiar aromatic substance, found in a sac between the navel and the parts of generation of a small male quadruped of the deer kınd, called by Linnæus, Moschus moschiferus, which inhabits Tonquin and Thibet. The color of musk is blackish-brown; it is lumpy or granular, somewhat like dried blood, with which substance, indeed, it is often adulterated. The intensity of its smell is almost the only criterion of its genuineness. When thoroughly dried it becomes nearly scentless; but it recovers its odor when slightly moistened with water of ammonia. 'The 'Tonquin musk is most esteemed. It comes to us in small hags covered with a reddish-brown hair; the bag of the Thibet musk is covered with a silver-gray hair. All the analyses of musk hitherto made teach little or nothing concerning its active or essential constituent. It is used in medicines, and is an ingredient in a great many perfumes. 
MUSLIN is a fine cotton fabric, used for ladies' robes; which is worn either white, dyed, or printed.

MUST is the sweet juice of the grape.

MUSTARD (Moutarde, Fr.; Senf, Germ.) is a plant which yields the well-known seed used as a condiment to food. M. Lenormand gives the following preseription for preparing mustard for the table.

With 2 pounds of very fine flour of mustard, mix half an ounce of each of the following fiesh plants; parslev, chervil, celery, and tarragon, along with a clove of garlic, and twelve salt anchovies, all well minced. The whole is to be triturated with the flour of mustard till the mixture becomes uniform. A little grape-must or sugar is to be addled, to give the requisite sweetness; then one ounce of salt, with sufficient water to form a thinnish paste by rubbing in a mortar. With this paste the mustard pots being nearly filled, a redhot poker is to be thrust down into the contents of each, which removes (it is said) some of the acrimony of the mustard, and evaporates a little water, so as to make room for pouring a little vinegar upon the surface of the paste. Such table mustard not only keeps perfectly well, but improves with age.

The mode of preparing table mustard patented by M. Soyés, consisted in steeping mustard seed in twice its bulk of weak wood vinegar for eight days, then grinding the whole into paste in a mill, putting it into pots, and thrusting a redhot poker into each of them.

MUTAGE is a process used in the south of France to arrest the progress of fermentation in the must of the grape. It consists either in diffusing sulphurous acid, from burning sulphur matches in the cask containing the must, or in adding a little sulphite (not sulphate) of lime to it. The last is the best process. See FErmentation.

MYRICINE is a vegetable principle which constitutes from 20 to 30 per cent. of the weight of bees-wax, being the residuum from the solvent action of alcohol upon that substance. It is a grayish-white solid, which may be vaporized almost without alteration.

MYRRH is a gum-resin, which occurs in tears of different sizes; they are reddishbrown, semi-transparent, brittle, of a shining fracture, appear as if greasy under the pestle, they have a very acrid and bitter taste, and a strong, not disagreeable, smell. Myrrh flows from the incisions of a tree not well known, which grows in Arabia and Abyssinia, supposed to be a species of amyris or mimosa. It consists of resin and gum in proportions stated by Pelletier at 31 of the former and 66 of the latter; but by Braconot at 23 and 77. It is used only in medicine.

N.

NACARAT is a term derived from the Spanish word nacar, which signifies mother of pearl; and is applied to a pale red color, with an orange cast. See CaLrco-PRINTING. The nacarat of Portugal or Bezetta is a crape or fine linen fabric, dyed fugitively of the above tint, which ladies rub upon their countenances to give them a roseate hue. The Turks of Censtantinople manufacture the brightest red crapes of this kind. See Rouge.

NAILS, MANUFACTURE OF. (Clou, Fr. ; Nagel, Germ.)

The forging of nails was till of late years a handicraft operation, and therefore belonged to a book of trades, rather than to a dictionary of arts. But several combinations of machinery have been recently employed, under the protection of patents, for making these useful implements, with little or no aid of the human hand; and these deserve to be noticed, on account both of their ingenuity and importance.

As nails are objects of prodigious consumption in building their block-houses, the citizens of the United States very early turned their mechanical genius to good account in the construction of various machines for making them. So long since as the year 1810 , it appears, from the report of the secretary of their treasury, that they possessed a machine which performed the cutting and heading at one operation, with such rapidity that it could turn out upwards of 100 nails per minute. "Twenty years ago," says the secretary of the state of Massachusetts, in that report, "some men, then unknown, and then in obscurity, began by cutting slices out of old hoops, and, by a common vice griping these pieces, headed them with several strokes of the hammer. By progressive improvements, slitting-mills were built, and the shears and the heading tools were perfected; yet much labor and expense were requisite to make nails. In a little time Jacob Perkins, Jonathan Ellis, and a few others, put into execution the thought of cutting and of heading nails by water power ; but, being more intent upon their machinery than upon their pecuniary affairs, they were unable to prosecute the business. At different times other men have spent fortunes in improvements and it may be said with truth that more than one mil- 
lion of dollars has been expended ; but at length these joint efforts are crowned with com. plete success, and we are now able to manufacture, at about one third of the expense that wrought nails can be manufactured for, nails which are superior to them for at least three fourths of the purposes to which nails are applied, and for most of those purposes they are full as good. The machines made use of by Odiorne, those invented by Jonathan Ellis, and a few others, present very fine specimens of American genius.

"To northern carpenters, it is well known that in almost all instances it is unnecessary to bore a hole before driving a cut nail; all that is requisite is, to place the cutting edge of the nail across the grain of the wood; it is also true, that cut nails will hold better in the wood. These qualities are, in some rough building works, worth twenty per cent. of the value of the article, which is equal to the whole expense of manufacturing. For sheathing and drawing, cut nails are full as good as wrought nails; only in one respect are the best wrought nails a little superior to cut nails, and that is where it is necessary they should be clinched. The manufacture of cut nails was born in our country, and has advanced, within its bosom, through all the various stages of infancy to manhood; and no doubt we shall soon be able, by receiving proper encouragement, to render them superior to wrought nails in every particular.

" The principal business of rolling and slitting-mills, is rolling nail plates; they also serve to make nail rods, hoops, tires, sheet iron, and sheet copper. In this State we have not less than twelve.

"These mills could roll and slit 7000 tons of iron a year; they now, it is presumed, roil and slit each year about 3500 tons, 2400 tons of which, probably, are cut up into nails and brads, of such a quality that they are good substitutes for hammered nails, and, in fact, have the preference with most people, for the following reasons; viz., on account of the sharp corner and true taper with which cut nails are formed; they may be driven into harder wood without bending or breaking, or hazard of splitting the wood, by which the labor of boring is saved, the nail one way being of the same breadth or thickness from head to point."

Since the year 1820, the following patents have been obtained in England for making nails ; many of them of American origin :-

Alexander Law, September, 1821, for nails and bolts for ships' fastenings, made in a twisted form, by hand labor.

Glascott and Mitchell, December, 1823, for ship nails with rounded heads, by hand labor.

Wilks and Ecroyd, November, 1825, for an engine for cutting wedge-form pieces from plates.

Ledsom and Jones, December 11, 1827, for machinery for cutting brads and sprigs from plates; it does not form heads.

The first nail apparatus to which I shall particularly advert, is due to Dr. Church ; it was patented in his absence by his correspondent, Mr. Thomas Tyndall, of Birmingham, in December, 182\%. It consists of two parts; the first is a mode of forming nails, and the shafts of screws, by pinching or pressing ignited rods of iron between indented rollers; the second produces the threads on the shafts of the screws previously pressed. The metallic rods, by being passed between a pair of rollers, are rudely shaped, and then cut asunder between a pair of shears ; after which they are pointed and headed, or otherwise brought to their finished forms, by the agency of dies placed in a revolving cylinder. The several parts of the mechanism are worked by toothed wheels, cams, and levers. The second part of Dr. Church's invention consists of a mechanism for cutting the threads of screws to any degree of obliquity or form.*

Mr. L. W. Wright's (American) apparatus should have been mentioned before the preeeding, as the patent for it was sealed in March of the same year; though an amended patent was obtained in September, 1828. Its object was to form metal screws for wood. I have seen the machinery, but consider it much too complex to be described in the present work.

Mr. Edward Hancorne, of Skinner street, London, nail manufacturer, obtained a patent in October, 1828 , for a nail making-machine, of which a brief description may give my readers a conception of this kind of manufacture. Its principles are similar to those of Dr. Church's more eleborate apparatus.

The rods or hars having been prepared in the usual way, either by rolling or hammering, or by cutting from sheets or plates of iron, called slitting, are then to be made redhot, and in that state passed through the following machine, whereby they are at once cut into suitable lengths, pressed into wedge forms for pointing at the one end, and stamped at the other end to produce the head. A longitudinal view of the machine is shown in fig. 749. A strong iron frame-work, of which one side is shown at $a a_{\text {s }}$ supports the whole of the mechanism. $b$ is a table capable of sliding to and fro horizontally. Upon 
this table are the clamps, which lay hold of the sides of the rod as it advances : as arso the shears which cut the rod into nail lengths.

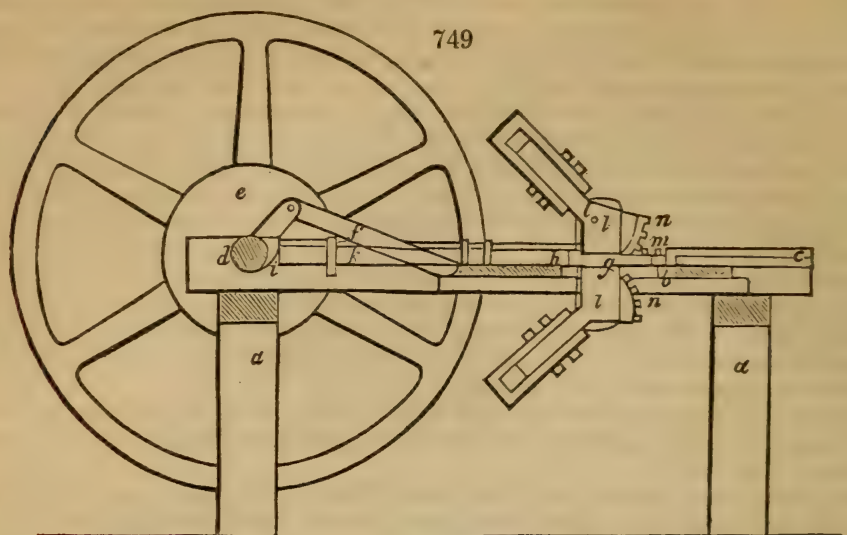

These clamps or holders consist of a fixed piece and a moveable piece; the latter being brought into action by a lever. The rod or bar of iron shown at $c$, having been made redhot, is introduced into the machine by sliding it forward upon the table $b$, when the table is in its most advanced position; rotatory motion is then given to the crank shaft $d$, by means of a band passing round the rigger pulley $e$, which causes the table $b$ to be drawn back by the crank rod $f$ : and as the table recedes, the horizontal lever is acted upon, which closes the clamps. By these means the clamps take fast hold of the sides of the heated rod, and draw it forward, when the moveable chap of the shears, also acted upon by a lever, slides laterally, and cuts off the end of the rod held by the clamps: the piece thus separated is destined to form one nail.

Suppose that the nail placed at $g$, having been thus brought into the machine and cut off, is held between clamps, which press it sideways (these clamps are not visible in this view); in this state it is ready to be headed and pointed.

The header is a steel die $h$, which is to be pressed up against the end of the nail by a cam $i$, upon the crank-shaft; which cam, at this period of the operation, acts against the end of a rod $k$, forming a continuation of the die $h$, and forces up the die, thus compressing the metal into the shape of a nail-head.

The pointing is performed by two rolling snail pieces or spirals $l, l$. These pieces are somewhat broader than the breadth of the nail; they turn upon axles in the side frames. As the table $b$ advances, the racks $m$, on the edge of this table, take into the toothed segments $n, n$, upon the axles of the spirals, and cause them to turn round.

These spirals pinch the nail at first close under its head with very little force; but as they turn round, the longer radius of the spiral comes into operation upon the nail, so as to press its substance very strongly, and squeeze it into a wedge form. Thus the nail is completed, and is immediately discharged from the clamps or holders. The carriage is then again put in motion by the rotation of the crank-shaft, which brings another portion of the rod $c$ forward, cuts it off, and then forms it into a nail.

Richard Prosser, July, 1831, for making tacks for ornamental furniture, by soldering or wedging the spike into the head. This also is the invention of Dr. Church.

Dr. William Church, February, 1832, for improvements in machinery for making nails. These consist, first, in apparatus for forming rods, bars, or plates of iron, or other metals; secondly, in apparatus for converting the rods, \&c., into nails; thirdly, in improvements upon Prosser's patent. The machinery consists in laminating rollers, and compressing dies.

The method of forming the rods from which the nails are to be made, is very advantageous. It consists in passing the bar or plate iron through pressing rollers, which have indentations upon the peripheries of one or both of them, so as to form the bar or plate into the required shape for the rods, which may be afterwards separated into rods of any desired breadth, by common slitting rollers.

The principal object of rolling the rods into these wedge forms, is to measure out a quantity of metal duly proportioned to the required thickness or strength of the nail in its several parts; which quantity corresponds to the indentations of the rollers.

Thomas John Fuller, February 27, 1834, for an improved apparatus for making squarepointed, and also flat-pointed nails. He claims as his invention, the application of vertical and horizontal hammers (mounted in his machine) combined for the purpose of 
tapering and forming the points of the nails; which, being made to act alternately, resemble hand work, and are therefore not so apt to injure the fibrous texture of the iron, he imagines, as the rolling machinery is. He finishes the points by rollers.

Hiles Berry, February 19, 1834, for machinery for forming metal into bolts, rivets, nails, and other articles ; being a communication from a foreigner residing abroad. He empıuys in his machine holding chaps, heading dies, toggle joints, cams, \&c., mechanisms apparently skilfully contrived, but too complex for admission under the article nail in this volume.

William Southwood Stocker, July, 1836. This is a machine apparently of American parentage, as it has the same set of features as the old American mechanisms of Perkins and Dyer, at the Britannia Nail-works, Birmingham, and all the other American machines since described, for pressing metal into the forms of nails, pins, screw-shafts, rivets, \&c. ; for example, it possesses pressers or hammers for squeezing the rods of metal, and forming the shanks, which are all worked by a rotatory action; cutters for separating the appropriate lengths, and dies for forming the heads by compression, also actuated by revolving cams or cranks.

Mr. Stocker intends, in fact, to effect the same sorts of operations by automatic mechanisms as are usually performed by the hands of a nail-maker with his hammer and anvil; viz., the shaping of a nail from a heated rod of iron, cutting it off at the proper length, and then compressing the end of the metal into the form of the head. His machine may be said to consist of two parts, connected in the same frame; the one for shaping the shank of the nail, the other for cutting it off and heading it. The frame consists of a strong table to bear the machinery. Two pairs of hammers, formed as levers, the one pair made to approach each other by horizontal movements, the other pair by vertical movements, are the implements by which a portion at the end of a redhot rod of iron is beaten or pressed into the wedge-like shape of the shaft of a nail. This having been done, and the rod being still hot, is withdrawn from the beaters, and placed in the other part of the machine, consisting of a pair of jaws like those of a vice, which pinch the shank of the nail and hold it fast. A cutter upon the side of a wheel now comes round, and, by acting as the moving chap of a pair of shears, cuts the nail off from the rod. The nail shank being still firmly held in the jaws of the vice, with a portion of its end projecting outwardly, the heading die is slidden laterally until it comes opposite to the end of the nail; the die is then projected forward with great force, for the purpose of what is termed upsetting the metal at the projecting end of the nail, and thereby blocking out the head.

A main shaft, driven by a band and rigger as usual, brings, as it revolves, a cam into operation upon a lever which carries a double inclined plane or wedge in its front or acting part. This wedge being by the rotatory cam projected forwards between the tails of one of the pairs of hammers, causes the faces of these hammers to approach each other, and to beat or press the redhot iron introduced between them, so as to flatten it upon two opposite sides. The rotatory cam passing round, the wedge lever is relieved, when springs instantly throw back the hammers; another cam and wedge-lever now brings the second pair of hammers to act upon the other two sides of the nail in a similar way. This is repeated several times, until the end of the redhot iron rod, gradually advanced by the hands of the workman, has assumed the desired form, that is, has received the bevel and point of the intended nail.

The rod is then withdrawn from between the hammers, and in its heated state is introduced between the jaws of the holders, for cutting off and finishing the nail. A bevel pinion upon the end of the main shaft, takes into and drives a wheel upon a transverse shaft, which carries a cam that works the lever of the holding jaws. The end of the rod being so held in the jaws or vice, a cutter at the side of a wheel upon the transverse shaft separates, as it revolves, the nail from the end of the rod, leaving the nail firmly held by the jaws. By means of a cam, the heading die is now slidden laterally opposite to the end of the nail in the holding jaws, and by another cam, upon the main shaft, the die is forced forward, which compresses the end of the nail, and spreads out the nail into the form of a head. As the main shaft continues to revolve, the cams pass away, and allow the spring to throw the jaws of the vice open, when the nails fall out; but to guard against the chance of a nail sticking in the jaws, a picker is provided, which pushes the nail out as soon as it is finished.

In order to produce round shafts, as for screw blanks, bolts, or rivets, the faces of the hammers, and the dies for heading, must be made with suitable concavities.

In $1835,5,180$, and in $1836,5,580$ tons of iron nails were exported from the Unted Kingdom.

NANKIN is a peculiarly colored cotton cloth, originally manufactured in the above named ancient capital of China, from a native cotton of a brown yel'ow hue. Nankin cloth has been long imitated in perfection by our own manufacturers; and is now exported in considerable quantities from England to Canton. The following is the process for dyeing calico a nankin color. 
1. Take 300 pounds of cotton yarn in hanks, being the quantity which four workmen can dye in a day. The yarn for the warp may be about No. 27 's, and that for the weft 23 's or 24 's.

2. For aluming that quantity, take 10 pounds of saturated alum, free from iron (see MORDANT); divide this into two portions; dissolve the first by itself in hot water, so as to form a solution of spec. grav. $1^{\circ}$ Baumé. The second portion is to be reserved for the galling bath.

3. Galling is given with about 80 pounds of oak bark finely ground. This bark may serve for two quantities, if it be applied a little longer the second time.

4. Take 30 pounds of fresh slaked quicklime, and form with it a large bath of limewater.

5. Nitro-muriate of tin. For the last bath, 10 or 12 pounds of solution of tin are used, which is prepared as follows:

Take 10 pounds of strong nitric acid, and dilute with pure water till its specific gravity

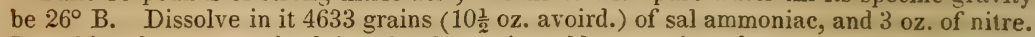
Into this solvent, contained in a bottle set in cold water, introduce successively, in very small portions, 28 ounces of grain-tin granulated. This solution, when made, must be kept in a well stoppered bottle.

Three coppers are required, one round, about five feet in diameter, and 32 inches deep, for scouring the cotton; 2 . two rectangular coppers tinned inside, each 5 feet long and 20 inches deep. Two boxes or cisterns of white wood are to be provided, the one for the lime-water bath, and the other for the solution of tin, each about 7 feet long, 32 inches wide, and 14 inches deep; they are set upon a platform 28 inches high. In the middle between these two chests, a plank is fixed, mounted with twenty-two pegs for wringing the hanks upon, as they are taken out of the bath.

6. Aluming. After the cotton yarn has been scoured with water, in the round copper, by being boiled in successive portions of 100 pounds, it must be winced in one of the square tinned coppers, containing two pounds of alum dissolved in 96 gallons of water, at a temperature of $165^{\circ} \mathrm{F}$. It is to be then drained over the copper, exposed for some time upon the grass, rinsed in clear water, and wrung.

7. The galling. Having filled four-fifths of the second square copper with water, 40 pounds of ground oak bark are to be introduced, tied up in a bag of open canvass, and boiled for two hours. The bag being withdrawn, the cotton yarn is to be winced through the boiling $\tan$ bath for a quarter of an hour. While the yarn is set to drain above the bath, 28 ounces of alum are to be dissolved in it, and the yarn being once more winced through it for a quarter of an hour, is then taken out, drained, wrung, and exposed to the air. It has now acquired a deep but rather dull yellowish color, and is ready without washing for the next process. Bablah may be substituted for oak bark with advantage.

8. The liming. Into the cistern filled with fresh made lime-water, the hanks of cotton yarn, suspended upon a series of wooden rods, are to be dipped freely three times in rapid succession; then each hank is to be separately moved by hand through the lime bath, till the desired carmelite shade appear. A weak soda ley may be used instead of lime water.

9. The brightening is given by passing the above hanks, after squeezing, rinsing, and airing them, through a dilute bath of solution of tin. The color thus produced is said to resemble perfectly the nankin of China.

Another kind of nankin color is given by oxyde of iron, precipitated upon the fibre of the cloth, from a solution of the sulphate, by a solution of soda. See CalicoPRINTING.

NAPLES YELLOW (Jaune minéral, Fr.; Neapelgelb, Germ.), is a fine yellow pigment, called giallolino, in Italy, where it has been long prepared by a secret process; for few of the recipes which have been published produce a good color. It is employed not only in oil painting, but also for porcelain and enamel. It has a fresh, brilliant, rich hue, but is apt to be very unequal in different samples.

The following prescription has been confidently recommended. Twelve parts of metallic antimony are to be calcined in a reverberatory furnace, along with eight parts of red lead, and four parts of oxyde of zinc. These mixed oxydes, being well rubbed together, are to be fused; and the fused mass is to be triturated and elutriated into a fine powder. Chromate of lead has in a great measure superseded Naples yellow.

NAPTHA, or ROCK-OIL (Huile pétrole, Fr., Steinöl, Germ.); the Seneca oil of North America is an ethereous or volatile oil, which is generated within the crust of the earth, and issues in many different localities. The colorless kind, called naptha, occurs at Baku, near the Caspian Sea, where the vapors which it exhales are kindled, and the flame is applied to domestic and other economical purposes. Wells are also dug in that neighborhood, in which the naptha is collected. Similar petroleum wells exist in the territory of the Birmans, at Yananghoung, upon the river Erawaddy, 80 hours' journey north-east of Pegu, where no less than 520 such springs issue from a pale blue clay, soaked with oil, which rests upon roofing slate. Under the slate is coal 
contanning much pyrites. Each spring yields annually 173 casks of 950 pounds each. Petroleum is also found at Amiano in the dutchy of Parma, at Saint Zibio in the grand dutchy of Modena, at Neufchatel in Switzerland, at Clermont in France, upon some points of the banlis of the Iser, at Gabian, a village near Bezières, at Tegernsee in Bavaria, at Val di Noto in Sicily, in Zante, Gallicia, Wallachia, Trinidad, Barbadoes, the Lnited States, Rangoon, near Ava, Sc. What is found in the market comes mostly from Trinidad. The eity of Parma is lighted with naptha.

The Persian roch-oil is colorless, limpid, very fluid, of a penetrating odor, a hot taste, and a specific gravity of 0.753 ; it is said to boil at $160^{\circ} \mathrm{F}$. The common petroleum has a reduish-yellow color, which appears blue by reflected light, is transparent, has a spec. grai. of 0.836 , and contains, according to Unverdorben, several oils of different degrees of rolatility, a little oleine and stearine, resin, with a brown indifferent substance held in solution. By repeated rectifications its derisity may be reduced to 0.758 at $60^{\circ} \mathrm{F}$. Native naptha, of specific gravity 0.749 , is said by some to boil at $201^{\circ} \mathbf{F}$. The condensed vapor consists of 85.05 carbon, and $14 \cdot 30$ hydrogen.

The naptha procured by distilling the coal oil of the gas-works, is of specific gravity $0.85 \%$, boils at $316^{\circ} \mathrm{F}$, and consists of, carbon 83.04 , hydrogen 12.31 , and oxygen $4 \cdot 65$, by my experiments.

Rock-oil is very inflammable; its vapor forms with oxygen gas a mixture which violently detonates, and produces water and carbonic acid gas. It does not unite with water, but it imparts a peculiar smell and taste to it; it combines in all proportions with strong alcohol, with ether and oils, both essential and unctuous; it dissolves sulphur, phospliorus, iodine, camphor, most of the resins, wax, fats, and softens caoutchouc into a glairy varnish. When adulterated with oil of turpentine, it becomes thick and reddish brown, on being agitated in contact with strong sulphuric acid. A very fine black pigment may be prepared from the soot of petroleum lamps.

IAPHALINE is a peculiar white crystallizable substance, which may be extracted by distillation from coal tar. It has a pungent aromatic smell and taste, and a specific gravity of 1.048 . It is a solid bicarburet of hydrogen, consisting, by my experiments, of 92.9 of earbon, and $7 \cdot 1$ of hydrogen. It has not been applied to any use.

NATRON is the name of the native sesquicarbonate of soda, which occurs in Egypt, in the west of the Delta; also in the neighborhood of Fessan, in the province of Sukena in Northern Africa, where it exists under the name of Trona, crystallized along with sulphate of soda; near Symrna, in Tartary, Siberia, Hungary, Hindostan, and Mexico. In the last eountry, there are several natron lakes, a little to the north of Zucatecas, as well as in many other provinces. In Columbia, 48 miles from Merida, native mineral natron is dug up from the bottom of lakes in large quantities, under the name of Urao.

Aceording to Laugier, the Egyptian natron consists of carbonate of soda $22 \cdot 44$, sulphate of soda $18 \cdot 35$, muriate of soda $38 \cdot 64$, water $14 \cdot 0$, insoluble matter $6 \cdot 0$. Trona is composed of carbonate of soda $65 \cdot 75$, sulphate of soda $7 \cdot 65$, muriate of soda $2 \cdot 63$, water 24 , insoluble matter 1. The sesquicarbonate may be artificially prepared by boiling for a short time a solution of the bicarbonate.

NEALING. See ANNEALING.

$\mathrm{NEB}-\mathrm{NEB}$ is the East Indian name of Bablah.

TEEDLE MANUFACTURE. When we consider the simplicity, smallness, and moderate price of a needle, we would be naturally led to suppose that this little instrument requires neither much labor nor complicated manipulations in its construction; but when we learn that every sewing needle, however inconsiderable its size, the hands of 120 different operatives, before it is ready for sale, we cannot fail to be surprised.

The best steel, reduced by a wire-drawing machine to the suitable diameter, is the material of which needles are formed. It is brought in bundles to the needle factory, and carefully examined. For this purpose, the ends of a few wires in each bundle are cut off, ignited, and hardened by plunging them into cold water. They are now snapped between the fingers, in order to judge of their quality; the bundles belonging to the most brittle wires are set aside, to be employed in making a peculiar kind of needles.

After the quality of the steel wire has been properly ascertained, it is calibred by means of a gauge, to see if it be equally thick and round throughout, for which purpose merely some of the coils of the bundle of wires are tried. Those that are too thick are returned to the wire-drawer, or set apart for another size of needles.

The first operation, properly speaking, of the needle factory, is unwinding the bundles of wires. With this view the operative places the coil upon a somewhat conical reel, fig. 750 , whereon he may fix it at a height proportioned to its diameter. The wire is wound off upon a wheel $\mathbf{B}$, formed of eight equal arms, placed at equal distances round a nave, which is supported by a polished round axle of iron, made fast to a strong apright $\mathrm{c}$, fixed to the floor of the workshop. Each of the arms is 54 inches long; and 
one of them, D, consists of two parts; of an upper part, which bears the cross bar $\mathrm{E}$, to which the wire is applied; and of an under part, connected with the nave. The part $F_{1}$ lides in a slot in the fixed part $\mathbf{F}$, and is made fast to it by a peg at a proper height for
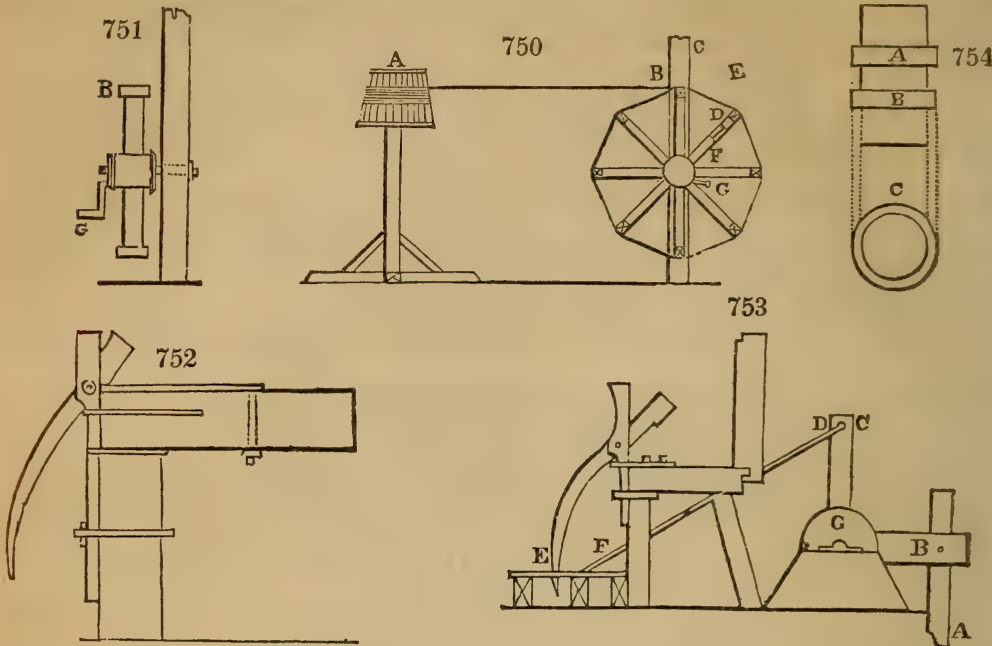

753

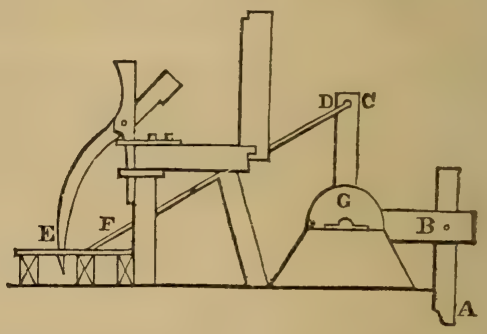

placing the ends of all the spokes in the circumference of a circle. This arrangement is necessary, to permit the wire to be readily taken off the reel, after being wound tight round its eight branches. The peg is then removed, the branch pushed down, and the coil of wire released. Fig. 751 shows the wheel in profile. It is driven by the winchhandle $\mathrm{G}$.

The new made coil is cut in two points diametrically opposite, either by hand shears, of which one of the branches is fixed in a block by a bolt and a nut, as shown in fig. 752 , or by means of the mechanical shears, represented in fig. 753. The crank $\mathrm{A}$ is moved by a hydraulic wheel, or steam power, and rises and falls alternately. The extremity of this crank enters into a mortise cut in the $\operatorname{arm}$ B of a bent lever $\mathbf{B}$ G $\mathbf{c}$, and is made fast to it by a bolt. An iron rod D F, hinged at one of its extremities to the end of the arm c, and at the other to the tail of the shears or chisel $E$, forces it to open and shut alternately. The operative placed upon the floor under $F$ presents the coil to the action of the shears, which cut it into two bundles, composed each of 90 or 100 wires, upwards of 8 feet long. The chisel strikes 21 blows in the minute.

These bundles are afterwards cut with the same shears into the desired needle lengths, these being regulated by the diameter. For this purpose the wires are put into a semicylinder of the proper length, with their ends at the bottom of it, and are all cut across by this gauge. The wires, thus cut, are deposited into a box placed alongside of the workman.

Two successive incisions are required to cut 100 wires, the third is lost; hence the shears, striking 21 blows in a minute, cut in 10 hours fully 400,000 ends of steel wire, which produce more than 800,000 needles. The wires thus cut are more or less bent, and require to be straightened. This operation is executed with great promptitude, by means of an appropriate instrument. In two strong iron rings A B, fig. 754, of which one is shown in front view at c, 5000 or 6000 wires, closely packed together, are put ; and the bundle is placed upon a flat smooth bench $\mathrm{L} M$, fig. $75 \%$, covered with a cast-iron plate D E, in which there are two grooves of sufficient depth for receiving the two ring bundles of wire, or two openings like the rule $F$, fig. 757 , upon which is placed the open iron rule $F$, shown in front in fig. 756 upon a greater scale. The two rings must be carefully set in the intervals of the rule. By making this rule come and go five or six times with such pressure upon the bundles of wires as causes it to turn upon its axis, all the wires are straightened almost instantaneously.

The construction of the machine, represented in fig. 757, may require explanation. It consists of a frame in the form of a table, of which $L M$ is the top; the castiron plate DE is inserted solidly into it. Above the table, seen in fig. 755 in plan, there are two uprights $\mathrm{C} \mathrm{H}$, to support the cross bar A A, which is held in forks cut out in the top of each of the two uprights. This cross bar A A, enters tightly into a 
mortse cut in the swing piece $\mathrm{N}$, at the point $\mathrm{N}$, where it is 6xed by a strong pin, 60 that 757

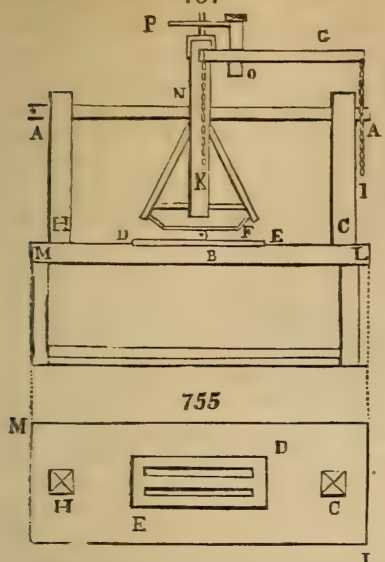
the horizontal traverse communicated to the cross bar $\mathbf{A} \mathrm{A}$ affects at the same time the swing piece $\mathrm{N}$. At the bottom of this piece is fixed, as shown in the figure, the open rule $F$, seen upon a greater scale in fig. 756 .

When the workman wishes to introduce the bundle $\mathbf{B}$, he raises, by means of two chains $\mathrm{I} K$, fig. $75 \%$, and the lever $\mathrm{G} 0$, the swing piece and the cross bar. For this purpose he draws down the chain I; and when he has placed the bundle properly, so that the two rings enter into the groove E D, fig. 755, he allows the swing piece to fall back, so that the same rings enter the open clefts of the rule $F$; he then seizes one of the projecting arms of the cross bar A, alternately pulling and pushing it in the horizontal direction, whereby he effects, as already stated, the straightening of the wires.

The wires are now taken to the pointing-tools, which usually consist of about 30 grindstones arranged in two rows, driven by a water-wheel. Each stone is about 18 inches in diameter, and 4 L inches thick. As they revolve with great velocity, and are liable to fly in pieces, they are partially incased by iron plates, having a proper slit in them to admit of the application of the wires. The worliman seated in front

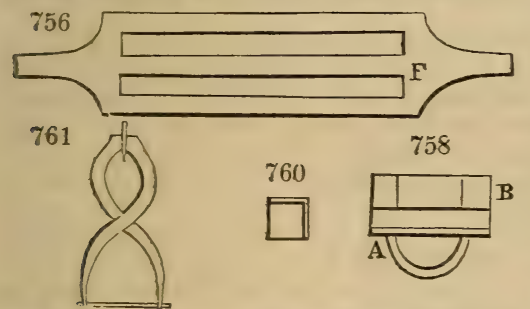
of the grindstone, seizes 50 or 60 wires between the thumb and forefinger of his right hand, and directs one end of the bundle to the stone. By means of a bit of stout leather called a thumb-piece, of which A, fig. 758, represents the profile, and $\mathrm{s}$ the plan, the workman presses the wires, and lurns them about with his forefinger, giving them such a rotatory motion as to make their points conical. This operation, which is called roughing down, is dry grinding; because, if water were made use of, the points of the needles would be rapidly rusted. It has been observed long ago, that the silicious and steel dust thrown off by the stones, was injurious to the eres and lungs of the grinders; and many methods have been proposed for preventing its bad effects. The machine invented for this purpose by Mr. Prior, for which the Society of Arts voted a premium, deserves to be generally known.

A A, fig. 759 , is the fly-wheel of an ordinary lathe, round which the endless cord B B passes, and embraces the pulley c, mounted upon the axle of the grindstone $\mathrm{D}$. The flywheel is supported by a strong frame E E, and may be turned by a winch-handle, as usual, or by mechanical power. In the needle factories, the pointing-shops are in general very large, and contain several grindstones running on the same long horizontal shaft, placed near the floor of the apartment, and driven by water or steam power. One of the extremities of the shaft of the wheel $A$ has a lineed or bent winch $F$, which by means of an intermediate crank $\mathrm{G} \mathrm{G}$, sets in action a double bellows $\mathrm{H}$, with a continuous blast, consisting of the air feeder $\mathrm{H}$ below, and the air regulator $\mathrm{I}$ above. The first is com posed of two flaps, one of them, $a, a$, being fast and attached to the floor, and the other, $e e$, moring with a hinge-joint; both being joined by strong leather nailed to their edges. This flap has a tail $g$, of which the end is forked to receive the end of the crank $\mathrm{G}$. Both flaps are perforated with openings furnished with valves for the admission of the air, which is thence driven into a horizontal pipe $\mathrm{k}$, placed beneath the floor of the workshop, and may be afterwards directed in an uninterrupted blast upon the grindstone, by means of the tin tubes $\mathbf{N} 0 \mathrm{o}$, which embrace it, and have longitudinal slits in them. $\dot{\mathbf{A}}$ brass sochet is supposed to be fixed upon the ground; it communicates with the pipe $\mathbf{k}$, by means of a small copper tube, into which one of the extremities of the pipe $\mathrm{N}$ is fitted; the other is supported by the point of a screw $\mathrm{Q}$, and moves round it as a pivot, so as to allow the two upright branches 00 , to be placed at the same distance from the grindstone. These branches are soldered to the borizontal pipe $\mathrm{N}$, and connected at their top by the tube $\mathbf{P}$.

The wind which escapes through the slits of these pipes, blows upon the grindstone, and carries off its dust into a conduit $\mathrm{R}$, fig. 759, which may be extended to $\mathrm{s}$, beyond 
the wall of the building, or bent at right angles, as at $\mathrm{x}$, to receire the conduits of the other grindstones of the factory.

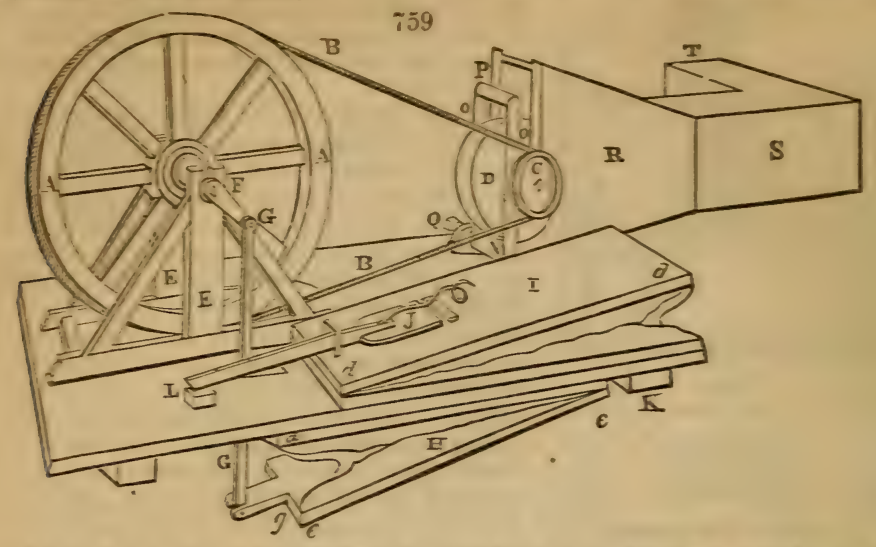

A safetr ralre $\mathrm{J}$, placed in an orifice formed in the regulator flap $\mathrm{r}$, is kept shut br a spiral spring of strong iron wire. It opens to allow the superfluous air to escape, when, by the rising of the bellows, the tail I presses upon a small piece of mood, and thereby prevents their being injured.

The wires thus pointed at both ends are transferred to the first morkshop, and cat in two, to form two needles, so that all of one qualitr mar be of equal length. For each sort a small instrument, fig. 750 , is emplored, being a copper plate nearly square, haring a turnel $u_{p}$ edze only upon two of its sides; the one of which is intended to receive all the points, and the other to resist the pressure of the shears. In this small tocl a certain number of wires are put with their points in contact with the border, and they are eut together fush with the plate by means of the shears, fig. 752, which are mered by the linee of the workman. The remainder of the wires are then laid upon the same copper or brass tocl, and are cut also eren; there being a trilling waste in this overation. The pieces of wire out of which two needles are formed, are alwars left a little too long, as the pointer ean never hit exact uniformity in his work.

These pointed wires are laid parallel to each other in little wooden boxes, and transferred to the head-flattener. This worliman, seated at a table with a block of steel before him, about 3 inches cube, seizes in his left hand 20 or 25 needles, between his finger and thumb, spreading them out like a fan, with the points under the thumb, and the heads projecting; he lars these heads upon the steel block, and with a small flat-faced hammer strikes successire blows upon all the heads, so as to Hatten each in an instant. He then arranges them in a bor with the points tarned the same war.

The Eatted heads hare become hardened br the blow of the hammer: when annealed by heating and slow cooling, they are handed to the prercer. This is commonly a child, who laring the head upon a blcck of steel, and applring the point of a small puneh to it, pierces the ere with a smart tap of a hammer, applied first upon the one side, and then exactly opposite apon the other.

Another child trims the eves, which he does br laying the needle upon a lnmp of lead, and driving a proper punch throngh its ere; then laying it sidewise upon a flat piece of steel, with the punch sticking in it, he gives it a tap on each side with his hammer, and causes the ere to take the shape of the panch. The operation of piercing and trimming the eyes, is performed by clever children with astonishing rapility; who become so derterous as to pierce with their punch a human hair, and thread it with another, for the amusement of risiters.

The nert operatire makes the groore at the ere, and rounds the head. He fires the needle in pincers, $f \mathrm{~g} g .761$, so that the ere corresponds to their lat side; he then rests the head of the needle in an angular groore, cut in a piece of hard wood fixed in a rice, with the eve in an upright position. He now forms the groore with a single stroke of a small file, derterously appliet, first to the one side of the needle, and then to the other. He next rounds and smooths the head with a small flat file. Haring finished, he opens the pincers, throws the needle upon the bench, and puts another in its place. A still more expeditions method of making the groores and finishing the heads has been long nsed in most English factories. A small ram is so mounted as to be made to rise and fall br a pedal lever, so that the chill works the tool with his font: in the same way as the heads of pins are fired. A small die of tempered steel bears the form 
of the one channel or groore, another similar die, that of the other, both being in relief; these being worked by the lever pedal, finish the grooring of the eye at a single blow, by striking against each other, with the head of the needle between them.

The whule of the needles this prepared are thrown pell-mell into a sort of drawer or box, in which they are, by a few dexterous jerks of the workman's hand, made to arrange themselves parallel to each other.

The needles are now ready for the tempering; for which purpose ther are weighed out in quantities of about 30 pounds, which contain from 250,000 to 500,000 needles, and are earried in boxes to the temperer. He arranges these upon sheet-iron plates, about 10 inches long, and 5 inches broad, having borders only upon the two longer sides. These plates are heated in a proper furnace to bright redness for the larger needles, and to a less intense degree for the smaller; ther are taken out, and inverted smartly orer a cistern of water, so that all the needles may be immersed at the same moment, yet distinct from one another. The water being run off from the cistern, the needles are remored, and arranged by agitation in a box, as above described. Instead of heating the needles in a furnace, some manufacturers heat them by means of a bath of melted lead in a state of ignition.

After being suddenly plunged in the cold water, they are verr hard and excessirely brittle. The following mode of tempering them is practised at Neustadt. The needles are thrown into a sort of frying-pan along with a quantity of grease. The pan being placed on the fire, the fatt! matter soon inflames, and is allowed to burn out; the needles are now found to be sufficiently well tempered. They must, however, be re-adjusted upon the steel anvil, because mant of them get twisted in the hardening and tempering.

Polishing is the longest and not the least expensire process in the needle manufacture. This is done upon bundles containing 500,000 needles; and the same machine, under the guidance of one man, polishes from 20 to 30 bundles at a time ; either by water or steam power. The needles are rolled up in canvass along with some quartzose sand interstratified between their larers, and the mixture is besmeared with rape-seed oil. Fig. 762 represents one of the rolls or packets of needles 12 inches long, strongly

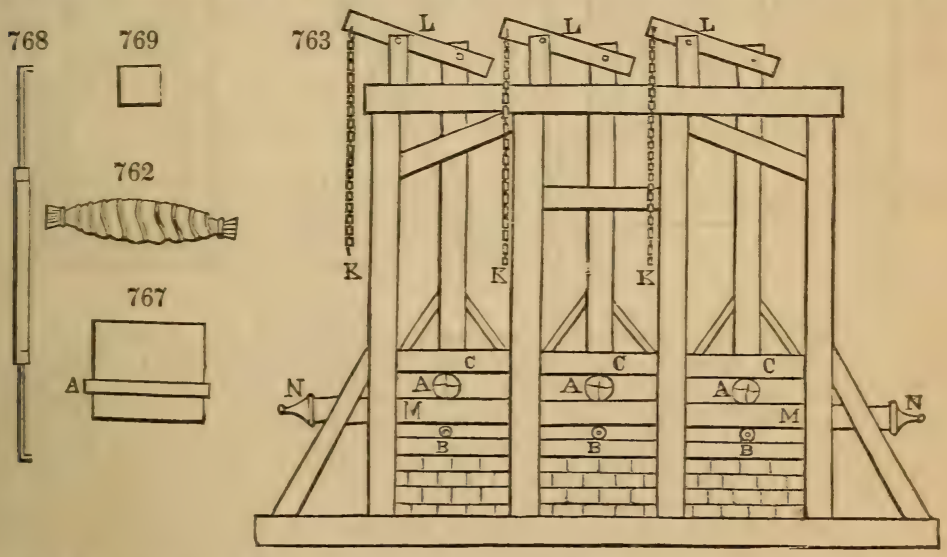

bound with cords. These packets are exposed to the to-and-fro pressure of wooden tables, by which they are rolled about, with the effect of causing every needle in the bundle to rub against its fellow, and against the silicious matter, or emery, enclosed in the bag. Fig. 763 represents an improved table for polishing the needles by attrition-bags. The lower table м $\mathrm{m}$ is moveable, whereas in the old constructions it was fixed; the table c has merely a vertical motion, of pressure upon the bundles, whereas formerly it had both a rertical and horizontal motion. Sereral bundles may obrionsly be polished at once in the present machine. The table $\mathrm{m} \mathrm{m}$ mar be of any length that is required, and from 24 to 27 inches broad; resting upon the wooden rollers B, B, B, placed at suitable distances, it receires a horizontal motion, either br hand or other convenient power; the packets of needles $A, A, A$, are laid upon it, and orer then the tables $\mathrm{c}, \mathrm{c}, \mathrm{c}$, which are lifted by means of the chains $\mathrm{K}, \mathrm{K}$, $\mathrm{K}$, and the levers $\mathrm{L}, \mathrm{L}, \mathrm{L}$, in order to allow the needles to be introduced or remored. The see-saw motion forces the rouleaux to turn upon their own axes, and thereby creates such attrition among heir contents as to polish them. The workman has merely to distribute these rolls 
upon the table $\mathrm{m}$, in a direction perpendicular to that in which the table moves; and whenever one of them gets displaced, he sets it right, lifting by the help of the chain the loaded table. The table makes about 20 horizontal double vibrations in the minute; whereby each bundle, running over 24 inches each time, passes through 40 feet per minute, or 800 yards in the hour.

Scouring by the cask. After being worked during 18 or 20 hours under the tables, the needles are taken out of the packets, and put into wooden bowls, where they are mixed with sawdust to absorb the black grease upon their surfaces. They are next introduced into a cask, fig. 764, and a workman seizing the winch $\mathbf{P}$, turns it round a little; he now puts in some more sawdust at the door, A, B, which is then shut by the clasps $\mathrm{G}_{\text {. }}$. and continues the rotation till the needles be quite clean and clear in their eyes; which he ascertains by taking out a sample of them from time to time.

Winnowing is the next process, by means of a mechanical ventilator similar to that by which corn is winnowed. The sawdust is blown away, and the grinding powder is separated from the needles, which remain apart clean and bright.

The needles are in the next place arranged in order, by being shaken, as above described, in a small somewhat concave iron tray. After being thus laid parallel to each other, they are shaken up against the end of the tray, and accumulated in a nearly upright position, so that they can be seized in a heap and removed in a body upon a pallet knife, with the help of the forefinger.

The preceding five operations, of making up the rouleaux, rolling them under the tables, scouring the needles in the cask, winnowing, and arranging them, are repeated ten times in succession, in manufacturing the best articles; the only variation being in the first process. Originally the bundles of needles are formed with alternate layers of silicious schistus and needles; but after the seventh time, bran freed from flour by sifting is substituted for the schistus. The subsequent four processes are, however, repeat. ed as described. It has been found in England, that emery powder mixed with quartz and
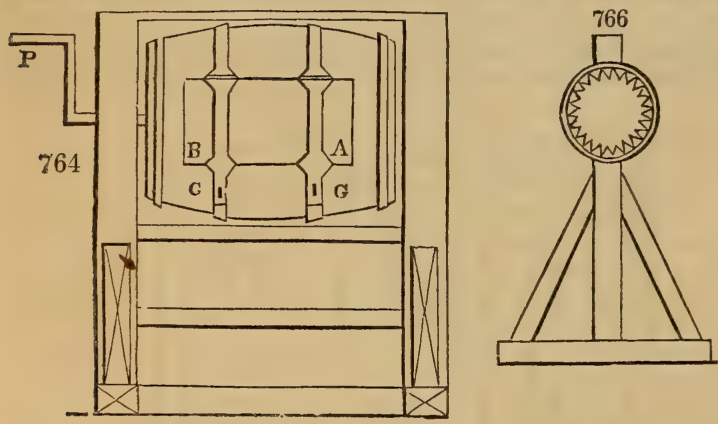

765

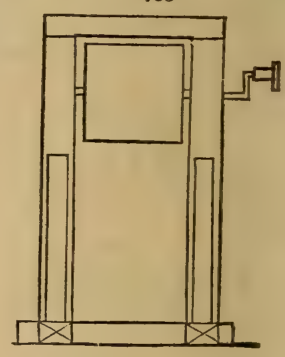

mica or pounded granite, is preferable to everything else for polishing needles at first by attrition in the bags; at the second and following operations, emery mixed with olive oil is used, up to the eighth and ninth, for which putty or oxyde of tin with oil is substituted for the emery; at the tenth the putty is used with very little oil ; and lastly bran is employed to give a finish. In this mode of operating, the needles are scoured in the copper cask shown in elevation in fig. 765, and in section in fig. 766. The inner surface of this cask is studded with points to increase the friction among the needles; and a quantity of hot soap suds is repeatedly introduced to wash them clean. The cask must be slowly turned upon its axis, for fear of injuring the mass of needles which it contains. They are finally dried in the wooden cask by attrition with sawdust; then wiped individually with a linen rag or soft leather; when the damaged ones are thrown aside.

Sorting of the needles. This operation is performed in a dry upper chamber, kept free from damp by proper stoves. Here all the points are first laid the same way; and the needles are then picked out from each other in the order of their polish. The sorting is effected with surprising facility. The workman places 2000 or 3000 needles in an jron ring, fig. 767, two inches in diameter, and sets all their heads in one plane; then on looking carefully at their points, he easily recognises the broken ones; and by means of a small hook fixed in a wooden handle, fig. 768, he lays hold of the broken needle, and turns it out. These defective needles pass into the hands of another workman, who points them anew upon a grindstone, and they form articles of inferior value. The needles which have got bent in the polishing must now be straightened. The whole are finally arranged exactly according to their lengths by the tact of the finger and thumb of the sorter.

The needles are divided into quantities for packing in blue papers, by putting into a 
small balance the equivalent weight of 100 needles, and so measuring them out without the trouble of counting them individually.

The bluer receives these packets, and taking 25 of their needles at a time between the forefinger and thumb, he presses their points against a rery small hone-stone of compact micaceous schist, mounted in a little lathe, as shown in fig. 769, he turns tiem briskly round, giving the points a bluish cast, while he polishes and improves them. This partial polish is in the direction of the axis; that of the rest of the needle is transverse, which distinguishes the boundaries of the two. The little hone-stone is not cylindrical, but quadrangular, so that it strikes successive blows with its corners upon the needles as it revolves, producing the effect of filing lengthwise. Whenever these angles seem to be blunted, they are set again by the bluer.

It is easy to distinguish good English needles from spurious imitations; because the former have their axis coincident with their points, which is readily observed by turning them round between the finger and thumb.

The construction of a needle requires, as already stated, about 120 operations; but they are rapidly and uninterruptedly successive. A child can trim the eyes of 4000 needles per hour.

When we survey a manufacture of this kind, we cannot fail to observe, that the diversity of operations which the needles undergo bears the impress of great mechanical refinement. In the arts, to divide labor, is to abridge it; to multiply operations, is to simplify them; and to attach an operative exclusively to one process, is to render him much more economical and productive.

NEROLI is the name given by perfumers to the essential oil of orange flowers. It is procured by distillation with water, in the same way as the other volatile oils. Since in distilling water from neroli, an aroma is obtained different from that of the orange-flower, it has been concluded that the distilled water of orange-flowers owes its scent to some principle different from an essential oil.

NET (Filet, reseau, Fr.; Netz, Germ.) is a textile fabric of knotted meshes, for catching fish, and other purposes. Each mesh should be so secured as to be incapable of enlargement or diminution. The French government offered in 1802 a prize of 10,000 francs to the person who should invent a machine for making nets upon automatic principles, and adjudged it to M. Buron, who presented his mechanical invention to the Conservatoire des Arts et Métiers. It does not appear, however, that this machine has accomplished the object in view ; for no establishment was ever mounted to carry it into execution. Nets are usually made by the fishermen and their families during periods of leisure. The formation of a mesh is too simple a matter to require description in this Dictionary.

NEUTRALIZATION is the state produced when acid and alkaline matters are combined in such proportions that neither predominates, as evinced by the color of tincture of litmus and cabbage remaining unaffected by the combination.

NICARAGUA WOOD is the wood of the Casalpinia echinata, a tree which grows in Nicaraca. It is used with solution of tin as a mordant to dye a bright but fugitive red. It is an inferior sort of Brazil wood.

NICKEL is a metal rather sparingly found, and in few localities; being usually associated with cobalt. Native nickel occurs at Westerwald in the Erzegebirge, in Bohemia, combined with arsenic, under the significant name of Kupfernickel; with cobalt, iron, and copper, as Arsenic-nickel, in the Harz; at Riechelsdorf in Hessia; as an oxyde, in Nickelschwärtze; as a sulphuret of nickel in Haarkies; as a sulphuret and arseniate of nickel in Nickelglanz; and with sulphur and antimony in Nickelspiess glanzerz at Siegen. Nickel is always present in meteoric stones. Kupfernickel occurs in numerous external shapes; as reniform, globular, botroidal, arborescent, massive, and disseminated; fracture, coarse or fine grained, with metallic lustre; color, copper red, occasionally brown and gray; in silver and cobalt veins, in gneiss, sienite, mica-slate, kupfer-schiefer, accompanied by speisse cobalt, native silver, quartz, \&c. It is found in Westphalia near Olpe, in Hessia at Riechelsdorf, and Biber, in Baden; in the Saxon Erzegebirge near Schneeberg, and Freiberg; in Bohemia, at Joachimsthal; in Thuringia, at Saalfeld; in Steyermark near Schladming; in Hungary, France, and England.

Since the manufacture of German silver, or Argentane, became an object of commercial importance, the extraction of nickel has been undertaken upon a considerable scale. The cobalt ores are its most fruitful sources, and they are now treated by the method of Wishler, to effect the separation of the two metals. The arsenic is expelled by roasting the powdered speise, first by itself, next with the addition of charcoal powder, till the garlic smell be no longer perceived. The residuum is to be mixed with three parts of sulphur and one of potash, melted in a crucible with a gentle heat, and the product being edulcorated with water, leaves a powder of metallic lustre, which is a sulphuret of nickel free from arsenic; while the arsenic associated with the sulphur, and combined with the resulting sulphuret of potassium, remains dissolved. Should any arsenic still be found 
in the sulphuret, as may happen if the first roasting heat was too great, the above pro. cess must be repeated. The sulphuret must be finally washed, dissolved in concentrated sulpnuric acid, with the addition of a little nitric, the metal must be precipitated by a carbonated alkali, and the carbonate reduced with charcoal.

In operating upon kupfernickel, or speise, in which nickel predominates, after the arsenic, iron, and copper have been separated, ammonia is to be digested upon the mixed oxydes of cobalt and nickel, which will dissolve them into a blue liquor. This being diluted with distilled water deprived of its air by boiling, is to be decomposed by caustic potash, till the blue color disappears, when the whole is to be put into a bottle tightly stoppered, and set aside to settle. The green precipitate of oxyde of nickel, which slowly forms, being freed by decantation from the supernatant red solution of oxyde of cobalt, is to be edulcorated and reduced to the metallic state in a crucible containing crown glass. Pure nickel in the form of a metallic powder is readily obtained by exposing its oxalate to modern ignition.

The reduction of the oxyde of nickel with charcoal requires the heat of a powerful air furnace or smith's forge.

Nickel possesses a fine silver white color and lustre; it is hard, but malleable, both hot and cold; may be drawn into wire $\frac{1}{50}$ of an inch, and rolled into plates $\frac{1}{50}$ of an inch thick. A small quantity of arsenic destroys its ductility. When fused it has a specinic gravity of 8.279 , and when hammered, of 8.66 or 8.82 ; it is susceptible of magnetism, in a somewhat inferior degree to iron, but superior to cobalt. Mariners' compasses may be made of it. Its melting point is nearly as high as that of manganese. It is not oxydized by contact of air, but may be burned in oxygen gas.

There is one oxyde and two suroxydes of nickel. The oxyde is of an ash-gray color, and is obtained by precipitation with an alkali from the solution of the muriate or nitrate. The niccolous suroxyde of Berzelius is black, and may be procured by exposing the nitrate to a heat under redness. The niccolic suroxyde has a dirty pale green color; but its identity is doubtful.

NICOTIANINE is the name of an oil recently extracted from the leaves of tobacco, which possesses the smell of tobacco smoke.

NICOTINE is a peculiar principle, obtainable from the leaves and seeds of tobacco (nicotiuna tabacum), by infusing them in acidulous water, evaporating the infusion to a certain point, adding lime to it, distilling and treating the product which comes over with ether. It is colorless, has an acrimonious taste, a pungent smell, remains liquid at $20^{\circ} \mathrm{F}$., mixes in all proportions with water, but is in a great measure separable from it by ether, which dissolves it abundantly. It combines with acids, and forms salts acrid and pungent like itself; the phosphate, oxalate, and tartrate being crystallizable. Nicotine causes the pupils to contract. A single drop of it is sufficient to kill a dog.

NITRATE OF AMMONIA is prepared by neutralizing nitric acid with carbonate of ammonia, and erystallizing the solution.

NITRATE OF LEAD (Nitrate de plomb, Fr.; Salpetersaures bleioxyd, Germ.) is made by saturating somewhat dilute nitric acid with oxyde of lead (litharge), evaporating the neutral solution till a pellicle appears, and then exposing it in a hot chamber till it be converted into crystals, which are sometimes transparent, but generally opaque white octahedrons. Their spec. grav. is 4.068 ; they have a cooling, streetish, pungent taste. They dissolve in 7 parts of cold, and in much less boiling water; they fuse at a moderate elevation of temperature, emit oxygen gas, and pass into oxyde of jead. Their constitu ents are $67 \cdot 3$ oxyde, and $32 \cdot \%$ acid. Nitrate of lead is much employed in the chrom. yellow style of CaLico-Printing; which see.

There are three other compounds of nitric acid and lead oxyde; viz., the bi-basic the tri-basic, and the se-basic; which contain respectively 2,3 , and 6 atoms of base tu 1 of acid.

NITRATE OF POTASH, Nitre, Saltpetre. (Nitrate de potasse, Fr.; Salpoter saures kali, Germ.) This salt occurs native as an efflorescence upon limestones, sand stones, marls, chalk, and calctuff; it forms a saline srust in caverns, as also upon thi surface of the ground in certain places, especially where animal matters have been decomposed. Such caverns exist in Germany near Homburg (Burkardush); in Apulia upon the Adriatic sea (Pulo di Mofetta); in France; in the East Indies; in Ceylon, where 22 nitriferous caverns are mentioned; in North America, at Crooked river, Tennessee, Kentucky, and upon the Missouri; in Brazil, Teneriffe, and Africa. Nitre occurs as an efflorescence upon the ground in Arragon, Hungary, Podolia, Sicily, Egypt, Persia, Bengal, China, Arabia, North America, and South America. Sereral plants contain saltpetre; particularly borage, dill, tobacco, sunflowers, stalks of maize, beet-root, bugloss, parietaria, \&c. It has not hitherto been found in animal substances.

The question has been frequently put; how is nitre annually reproduced upon the surface of limestones, and the ground, after it has been removed by washing ? har 
been said, in reply, that as secondary limestones contain remains of animal matters, the oxyen of the atmosphere, absorbed in virtue of the porous structure, will com. birir with their azote to form nitric acid; whence nitrate of lime will result. Where po'ash is present in the ground, a nitrate of that base will be next formed. The generation oi nitre is in all cases limited to a very small distance from the surface of porous stones; no further, indeed, than where atmospherical air and moisture can penetrate; and none is ever produced upon the surface of compact stones, such as marble and quartz, or of argillaceous minerals. Dr. John Davy and M. Longchamp have advanced an opinion, that the presence of azotized matter is not necessary for the generation of nitric acid or nitrous salts, but that the oxygen and azote of the atmosphere, when condensed by capillarity, will combine in such proportions as to form nitric acid, through the agency of moisture and of neutralizing bases, such as lime, magnesia, potash, or soda. They conceive that as spongy platina serves to combine oxygen and hydrogen into water, or the vapor of alcohol and oxygen into acetic acid, and as the peroxyde as well as the hydrate of iron, and argillaceous minerals, serve to generate ammonia from the oxygen of the air and the hydrogen of water; in like manner, porous limestones, through the agency of water, operate upon the constituents of the atmosphere to produce nitric acid, without the presence of animal matter. This opinion may certainly be maintained; for in India, Spain, and several other countries, at a distance from all habitations, immense quantities of saltpetre are reproduced in soils which have been washed the year before. But, on the other hand, it is known that the production of this salt may be greatly facilitated and increased by the admixture of animal offals with calcareous earths.

The spontaneous generation of nitre in Spain, Egypt, and especially in India, is sufficient to supply the wants of the whole world. There this salt is observed to form upon the surface of the ground in silky tufts, or even in slender prismatic crystals, particularly during the continuance of the hot weather that succeeds copious rains. 'These saline efflorescences, after being collected by rude besoms of broom, are lixiviated, allowed to settle, evaporated, and crystallized. In France, Germany, Sweden, Hungary, \&c., vast quantities of nitrous salts are obtained by artificial arrangements called nitriaries, or nitre-beds. Very little nitrate of potash, indeed, is obtained in the first place; but the nitrates of lime and magnesia, which being deliquescent, remain in the nitrous earths in a semi-liquid state. The operation of converting these salts into good nitre is often sufficiently complex, in consequence of the presence of several muriates, which are difficult to eliminate.

The following instructions have been given by the consulting committee of poudres et salpêtres in France, for the construction of their nitrières artificielles. The permeability of the materials to the atmospherical air, being found to be as indispensable as is the presence of a base to fix the nitric acid at the instant of its formation, the first measure is to select a light friable earth, containing as much carbonate of lime or old mortarrubbish as possible; and to interstratify it with beds of dung, five or six inches thick, till a considerable heap be raised in the shape of a truncated pyramid, which should be placed under an open shed, and kept moist by watering it from time to time. When the whole appears to be decomposed into a kind of mould, it is to be spread under sheds in layers of from two to three feet thick; which are to be watered occasionally with urine and the drainings of dunghills, taking care not to soak them too much, lest they should be rendered impermeable to the air, though they should be always damp enough to favor the absorption and mutual action of the atmospherical gases. Moist garden mould affords an example of the physical condition most favorable to nitre-beds. The compost should be turned over, and well mixed with the spade once at least in every fortnight, and the sides of the shed shuuld be partially closed; for although air be essential, wind is injurious, by carrying off the acid vapors, instead of allowing them to rest incumbent upon, and combine with, the bases. The chemical reaction is slow and successive, and can be made effective only by keeping the agents and materials in a state of quiescence. The whole process lasts two years; but since organic matters would yield in the lixiviation several soluble substances detrimental to the extraction of saltpetre, they must not be added during the operations of the latter six months; nor must any thing except clear water be used for watering during this period; at the end of which the whole organic ingredients of the beds will be totally decomposed. Where dung is not sufficiently abundant for the above stratifications, a nitre-bed should be formed in a stable with friable earth, covered with a layer of litter; after four months the litter is to be lifted off, the earth is to be turned over, then another layer of fresh earth, 8 or 9 inches thick, is to be placed over it, and a layer of the old and fresh litter over all. At the end of other four months, this operation is to be repeated; and in the course of a year the whole is ready to be transferred into the regular nitre-beds under a shed, as above described. Such are the laborious and disagreeable processes practised by the peasants of Sweden, each of whom is bound by law to have a nitre-bed, and to furnish a certain quantity of nitre to the state every year. "His nitriary commonly con- 
sists of a small hut built of boards, with a bottom of rammed clay, covered by a wuoder floor, upon which is spread a mixture of ordinary earth with calcareous sand or marl. and lixiviated wood-ashes. This mixture is watered with stable urine, and its surfice is turned over once a week in summer, and once a fortnight in winter. In some countries, walls 2 or 3 feet thick, and 6 or 7 high, are raised with the nitrifying compost, interspersed with weeds and branches of trees, in order at once to bind them together, and to favor the circulation of air. These walls are thatched with straw; they are placed with one of their faces in the direction of the rains; and must be moistened with water not rich in animal matter. One side of the walls is upright and smooth; while the other is sloped or terraced, to favor the admission of humidity into their interior. The nitre eventually forms a copious efflorescence upon the smooth side, whence it may be easily scraped off.

M. Longchamp, convinced that organic matters are a useless expense, and not in the least essential to nitrification, proposes to establish nitre-beds where fuel and labor are cheapest, as amidst forests, choosing as dry and low a piece of ground as possible, laying them out upon a square space of about 1000 feet in each side, in the middle of which the graduation-house may be built, and alongside of it sheds for the evaporation furnaces and pans. Upon each of the four sides the nitrifying sheds are to be erected, 130 feet long by 30 feet wide, where the lixiviation would be carried on, and whence the water would be conducted in gutters to the graduation-house. The sheds are to be closed at the sides by walls of pisé, and covered with thatch. No substance is so favorable to nitrification as the natural stony concretion known under the name of lime-tuf. In Touraine, where it is used as a building stone, the saltpetre makers re-establish the foundations of old houses at their own expense, provided they are allowed to carry off the old tuf, which owes its nitrifying properties not only to its chemical nature, but to its texture, which being of a homogeneous porosity, permits elastic fluids and vapors to pass through it fieely in all directions. With the rough blocks of such tuf, walls about 20 inches thick, and moderately high, are to be raised, upon the principles above prescribed; in the absence of tuf, porous walls may be raised with a mixture of arable soil, sand, and mortar-rubbish, chalk or rich marl. The walls ought to be kept moist.

In France, the greater part of the indigenous saltpetre is obtained by lixiviating the mortar-rubbish of old buildings, especially of those upon the ground-floor, and in sunk cellars; which are by law reserved for this purpose. The first object of the manufacturer is then to ascertain the richness of his materials in nitrous salts, to see if they be worth the trouble of working; and this point he commonly determines merely by their saline, bitter, and pungent taste, though he might readily have recourse to the far surer criteria of lixiviation and evaporation. He next pounds them coarsely, and puts them into large casks open at top, and covered with straw at bottom; which are placed in three successive levels. Water is poured into the casks till they are full, and after 12 hours' digestion it is run off, loaded with the salts, by a spigot near the bottom. A fresh quantity of water is then added, and drawn off after an interval of four hours; even a third and fourth lixiviation are had recourse to; but these weak liquors are reserved for lixiviating fresh rubbish. The contents of the casks upon the second and third lower levels are lixiviated with the liquors of the upper cask, till the leys indicate from 12 to 14 degrees of Baumé's hydrometer. They are now fit for evaporating to a greater density, and of then receiving the dose of wood-ashes requisite to convert the materials of lime and magnesia into nitrate of potash, with the precipitation of the carbonates of magnesia and lime. The solution of nitre is evaporated in a copper pan, and as it boils, the scum which rises to the surface must be diligently skimmed oft into a cistern alongside. Muriate of soda being hardly more soluble in boiling than in cold water, separates during the concentration of the nitre, and is progressively removed with cullender-shaped ladles. The fire is withdrawn whenever the liquor has acquired the density of $80^{\circ} \mathrm{B}$.; it is allowed to settle for a little while, and is then drawn off, by a lead syphon adjusted some way above the bottom, into iron vessels, to cool and crystallize. The crystals thus obtained are set to drain, then re-dissolved and re-crystallized. The further purification of nitre,.is fully described under the article GunPowder.

The annual production of saltpetre in France, by the above-described processes, duing the wars of the Revolution, amounted to 2000 tons ( 2 millions of kilogrammes) of an article fit for the manufacture of gunpowder; of which seven twentieths were furnished by the saltpetre works of Paris alone. Considerably upwards of six times that quantity of common and cubic nitre were imported into the United Kingdom, for home consumption, luring the year ending January 5, 1838.

Nitrate of potash crystallizes in six-sided prisms, with four narrow and two broad faces : the last being terminated by a dihedral summit, or two-sided acumination; they are striated lengthwise, and have fissures in their long axis, which are apt to contain mother water. The spec. gravity of nitre, varies from 1.93 to 2.00 . It possesses 
a cooling, bitterish-pungent taste, is void of smell, permanent in the air when pure, fuses $\mathrm{a}$ : $\%$ heat of about 662 , into an oily-looking liquid, and concretes upon cooling into a solid mass, with a coarsely radiating fracture. This has got the unmeaning names of sal-prunelle and mineral crystal. At a red heat, nitre gives out at first a great deal of pretty pure oxygen gas; but afterwards nitrous acid fumes, while potash remains in the retort. It is soluble in 7 parts of water at $32^{\circ}$; in about $3 \frac{1}{2}$ at $60^{\circ} \mathrm{F}$, in less than half a part at $194^{\circ}$, and in four tenths at $212^{\circ}$. It is very slightly soluble in spirit of wine, and not at all in absolute alcohol. It causes a powerful deflagration when thrown upon burning coals; and when a mixture of it with sulphur is thrown into a red-hot crucible, a very vivid light is emitted. Its constituents are, 46.55 potash, and 53.45 nitric acid.

Nitre is applied to many purposes:-1. to the manufacture of gunpowder; 2 . to that of sulphuric acid; 3 . to that of nitric acid, though nitrate of soda or cubic nitre has lately superseded this use of it to a considerable extent; 4 . to that of flint-glass; 5 . it is used in medicine; 6. for many chemical and pharmaceutical preparations; 7. for procuring by deflagration with charcoal or cream of tartar, pure carbonate of potash, as also black and white fluxes; 8 . for mixing with salt in curing butcher meat; 9 . in some countries for sprinliling in solution upon grain, to preserve it from insects; 10 . for making fireworks. See Fire-works.

An account of the quantities of Saltpetre and Cubic Nitre imported into, exported from and retained for consumption in the United Kingdom. Duty 6d. per cwt:-

\begin{tabular}{ccccc} 
Imported in & \multicolumn{3}{c}{ Exported in } & \multicolumn{2}{c}{ Retained for consumption in } \\
1835. 1836. & 1837. & $1835 . \quad 1836$. & 1837. & 1835.1836 .1837$.
\end{tabular} cwts. 264,338 ; 279,902; 349,993. 73,379; 38,414;93,024. 204,580; 242,131; 256,969 .

Duty received in $1837, £ 6,424$.

NITRATE OF SILVER (Nitrate d'argent, Fr.; Silbersalpeter, Germ.), is prepared by saturating pure nitric acid of specific grav. $1 \cdot 25$ with pure silver, evaporating the solution, and crystallizing the nitrate. When the drained crystals are fused in a platina capsule, and cast into slender cylinders in silver moulds, they constitute the lunar caustic of the surgeon. This should be white, and unchangeable by light. It is deliquescent in moist air. The crystals are colorless, transparent 4 and 6 sided tables; they possess a bitter, acrid, and most disagreeable metallic taste; they dissolve in their own weight of cold, and in much less of hot water; are soluble in four parts of boiling alcohol, but not in nitric acid; they deflagrate on redhot coals, like all the nitrates; and detonate with phosphorus when the two are struck together upon an anvil. They consist of 68.2 of oxyde, and 31.8 of acid. Nitrate of silver, when swallowed, is a very energetic poison; but it may be readily counteracted, by the administration of a dose of sea-salt, which converts the corrosive nitrate into the inert chloride of silver. Animal matter, immersed in a weak solution of neutral nitrate of silver, will keep unchanged for any length of time; and so will polished iron or steel. Nitrate of silver is such a delicate reagent of hydrochloric or muriatic acid, as to show by a sensible cloud, the presence of one 113 millionth part of it, or one 7 millionth part of sea-salt in distilled water. It is much used under the name of indelible ink, for writing upon linen with a pen ; for which purpose one drachm of the fused salt should be dissolved in three quarters of an ounce of water, adding to the solution as much water of ammonia as will re-dissolve the precipitated oxyde, with sap-green to color it, and gum-water to make the volume amount to one ounce. Traces written with this liquid should be first heated before a fire to expel the excess of ammonia, and then exposed to the sun-beam to blacken. Another mode of using nitrate of silver as an indelible ink, is to imbue the linen first with solution of carbonate of soda, to dry the spot, and write upon it with a solution of nitrate of silver, thickened with gum, and tinted with sapgreen.

NITRATE OF SODA, Cubical Nitre (Nitrate de soude, Fr.; Würfelsalpeter, Germ.), occurs under the nitre upon the lands in Spain, India, Chile, and remarkably in Peru, in the districts of Atacama and Taracapa, where it forms a bed several feet thick. It appears in several places upon the surface, and extends over a space of more than 40 leagues, approaching near to the frontiers of Chile. It is sometimes efflorescent, sometimes crystallized, but oftener confusedly mixed with clay and sand. This immensely valuable deposite is only three days' journey from the port of Con. ception in Chile, and from Iquiqui, another harbor situated in the southern part of Peru.

Nitrate of soda may be artificially prepared by neutralizing nitric acid with soda, and crystallizing the solution. It crystallizes in rhomboids, has a cooling, pungent, bitterish taste, less disagreeable than nitre; it becomes moist in the air; dissolves in 3 parts of water at $60^{\circ} \mathrm{F}$., in less than 1 part of boiling water; deflagrates more slowly than nitre, and with an orange yellow flame. It consists, in its dry state, of 36.6 soda and 63.4 nitrie 
acid; but its crystals contain one prime equivalent of water; hence they are composed of, acid $56 \cdot 84$, base $33 \cdot 68$, water $9 \cdot 47$.

It is susceptible of the same applications as nitre, with the exception of making gunpowder; for which it is not adapted, on account of its deliquescent property.

NITRATE OF STRONTIA. (Niirate de Strontiane, Fr.; Salyclersaïrer strontian, Germ.) This salt is usually prepared from the sulphuret of strontium, obtained by de. composing sulphate of strontia with charcoal, by strong ignition of the mixed powders in a crucible. This sulphuret being treated with water, and the solution being filtered, is to be neutralized with nitric acid, as indicated by the test of turmeric paper; care being taken to aroid breathing the noxious sulphureted hydrogen gas, which is copiously disengaged. The neutral nitrate being properly evaporated and set aside, affords colorless, transparent, slender octahedral crystals. It has a cooling, yet somewhat acrid taste; is soluble in 5 parts of cold, and in one half part of boiling water, as also in alcohol; is permanent in the air, deflagrates upon burning coals, gives off oxtgen when calcined, and leaves caustic strontia. The salt consists of 48.9 strontia and $51 \cdot 1$ nitric acid. That salt is anhydrous; but there is another variety of it, which contains nearly 40 per cent. of water of crystallization, which occurs in large octahedrons. This is preferred for fireworks, because by efflorescence it is easily obtained in a fine powder, which mixes more intimately with the chlorate of potash and charcoal, for the composition of the brilliant red fires, now so much admired in theatrical conflagrations.

NITRIC ACID, Aquafortis (Acide nitrique, Fr.; Salpetersaüre, Germ.), exists, in com bination with the bases, potash, soda, lime, magnesia, in both the mineral and regetable kingdoms. This acid is never found insulated. It was distilled from saltpetre so long ago as the 13th century, by igniting that salt, mixed with copperas or clay, in a retort. Nitric acid is generated when a mixture of oxygen and nitrogen gases, confined over water or an alkaline solution, has a series of electrical explosions passed through it. In this way the salubrious atmosphere may be converted into corrosive aquafortis. When a little hydrogen is introduced into the mixed gases, standing over water, the chemical agency of the electricity becomes more intense, and the acid is more rapidly formed from its elements, with the production of some nitrate of ammonia.

Nitric acid is usually made on the small scale by distilling, with the heat of a sandbath, a mixture of 3 parts of pure nitre, and 2 parts of strong sulphuric acid, in a large glass retort, connected by a long glass tube with a globular receiver surrounded by cold water. By a well-regulated distillation, a pure acid, of specific gravity $1 \cdot 500$, may be thus obtained, amounting in weight to about two thirds of the nitre employed. To obtain easily the whole nitric acid, equal weights of nitre and concentrated sulphuric acid may be taken; in which case but a moderate heat need be applied to the retort. The residuum will be bisulphate of potash. When only the single equivalent proportion of sulphuric acid is used, namely, 48 parts for 100 of nitre, a much higher heat is required to complete the distillation, whereby more or less of the nitric acid is decomposed, while a compact neutral sulphate of potash is left in the retort, very difficult to remove by solution in water, and therefore apt to destroy the vessel.

Aquafortis is manufactured upon the great scale in iron pots or cylinders of the same construction as I have described under muriatic acid. The more concentrated the sulphuric acid is, the less corrosively will it act upon the metal; and it is commonly used in the proportion of one part by weight to two of nitre. The salt being introduced into the cool retort, and the lid being luted tight, the acid is to be slowly poured in through the aperture $f, f g .748$; while the aperture $g$ is connected by a long glass tube with a range of balloons inserted into each other, and laid upon a sloping berl of sand. The bottle $i$, with 3 tubulures partly filled with water, which is required for condensing muriatic acid gas, must, for the present purpose, be replaced by a series of empty receivers, either of glass or salt-glazed stoneware. The cylinders should be onl and be worked off by a gradually raised heat.

Commercial aquafortis is very generally contaminated with sulphuric and muriatic acids, as also with alkaline sulphates and muriates. The qtantity of these salts may be readily ascertained by evaporating in a glass capsule a given weight of the aquafortis ; while that of the muriatic acid may be determined by nitrate of silver; and of sulphuric acid, by nitrate of baryta. Aquafortis may be purified in a great measure, by re-distillation at a gentle heat; rejecting the first liquid which comes over, as it contains the chlorine impregnation; receiving the middle portion as genuine nitric acid; and leaving a residuum in the retort, as being contaminated with sulphuric acid.

Since nitrate of soda has been so abundantly imported into Europe from Peru, it has been employed by many manufacturers in preference to nitre for the extraction of nitric acid, because it is cheaper, and because the residuum of the distillation, being sulphate of soda, is more readily removed by solution from glass retorts, when a range of these set in a gallery furnace is the apparatus employed. Nitric acid of specific gravity $1 \cdot 47$ may be obtained colorless; but by further concentration a portion of it is decomposed 
whereby some nitrous acid is produced, which gives it a straw-yellow tinge. At this streneth it exhales white or orange fumes, which have a peculiar, though not very disagreeable smell; and even when largely diluted with water, it tastes extremely sour. The greatest density at which it can be obtained is 1.51 or perhaps 1.52 , at $60^{\circ} \mathrm{F}$, in which state, or even when much weaker, it powerfully corrodes all animal, vegetable, and most metallic bodies. When slightly diluted it is applied, with many precautions, to silk and woollen stuffs, to stain them of a bright yellow hue. See Calico-printing, page 246.

In the dry state, as it exists in nitre, this acid consists of $26 \cdot 15$ parts by weight of azote, and 73.85 of oxygen; or of 2 volumes of the first gas, and 5 volumes of the second.

When of specific gravity 1.5 , it boils at about $210^{\circ}$ Fahr.; of $1 \cdot 45$, it boils at about $240^{\circ}$; of 1.42 , it boils at $253^{\circ}$; and of $1 \cdot 40$, at $246^{\circ} \mathrm{F}$. If on acid stronger than $1 \cdot 420$ be distilled in a retort, it gradually becomes weaker; and if weaker than 1.42 , it gradually becomes stronger, till it assumes that standard density. Acid of specific gravity 1.485 has no more action upon tin than water has, though when either stronger or weaker it oxydizes it rapidly, and evolves fumes of nitrous gas with explosive violence. In my two papers upon nitric acid published in the fourth and sixth volumes of the Journal of Science (1818 and 1819), I investigated the chemical relations of these phenomena. Acid of 1.420 consists of 1 atom of dry acid, and 4 of water; acid of $1 \cdot 485$, of 1 atom of dry acid, and 2 of water; the latter compound possesses a stable equilibrium as to chemical agency; the former as to calorific. Acid of specific gravity $1 \cdot 334$, consisting of 7 atoms of water, and 1 of dry acid, resists the decomposing agency of light. Nitric acid acts with great energy upon most combustible substances, simple or compound, giving up oxygen to them, and resolving itself into nitrous gas, or even azote. Such is the result of its action upon hydrogen, phosphorus, sulphur, charcoal, sugar. gum, starch, silver, mercury, copper, iron, tin, and most other metals.

A Table of Nitric Acid, by Dr. Ure.

\begin{tabular}{|c|c|c|c|c|c|c|c|c|c|c|c|}
\hline $\begin{array}{l}\text { Specific } \\
\text { gravity. }\end{array}$ & $\begin{array}{l}\text { Liq. } \\
\text { Acid } \\
\text { in } 100\end{array}$ & $\begin{array}{c}\text { Dry acid } \\
\text { in } 100 .\end{array}$ & $\begin{array}{l}\text { Specific } \\
\text { gravity. }\end{array}$ & $\begin{array}{c}\text { Liq. } \\
\text { Acid } \\
\text { in } 100\end{array}$ & $\begin{array}{l}\text { Dry acid } \\
\text { in } 100 .\end{array}$ & $\begin{array}{l}\text { Specific } \\
\text { gravity. }\end{array}$ & $\begin{array}{c}\text { Liq. } \\
\text { Acid } \\
\text { in } 100\end{array}$ & $\begin{array}{l}\text { Dry acid } \\
\text { in } 100 .\end{array}$ & $\begin{array}{l}\text { Specific } \\
\text { gravity. }\end{array}$ & \begin{tabular}{|} 
Liq. \\
Acid \\
in 100
\end{tabular} & $\begin{array}{l}\text { Dry acid } \\
\text { in } 100 .\end{array}$ \\
\hline 1.5000 & 100 & $79 \cdot 700$ & $1 \cdot 4189$ & 75 & $59 \cdot 775$ & $1 \cdot 2947$ & 50 & $39 \cdot 850$ & $1 \cdot 1403$ & 25 & $19 \cdot 925$ \\
\hline $1 \cdot 4980$ & 99 & $78 \cdot 903$ & $1 \cdot 4147$ & 74 & 58.978 & $1 \cdot 2887$ & 49 & $39 \cdot 053$ & $1 \cdot 1345$ & 24 & $19 \cdot 128$ \\
\hline 1.4960 & 98 & $78 \cdot 106$ & 1.4107 & 73 & $58 \cdot 181$ & $1 \cdot 2826$ & 48 & $38 \cdot 256$ & $1 \cdot 1286$ & 23 & $18 \cdot 331$ \\
\hline $1 \cdot 4940$ & 97 & $77 \cdot 309$ & $1 \cdot 4065$ & 72 & $57 \cdot 384$ & $1 \cdot 2765$ & 47 & $37 \cdot 459$ & $1 \cdot 1227$ & 22 & $17 \cdot 534$ \\
\hline $1 \cdot 4910$ & 96 & $76 \cdot 512$ & $1 \cdot 4023$ & 71 & 56.587 & $1 \cdot 2705$ & 46 & 36.662 & $1 \cdot 1168$ & 21 & $16 \cdot 737$ \\
\hline $1 \cdot 4880$ & 95 & $75 \cdot 715$ & $1 \cdot 3978$ & 70 & $55 \cdot 790$ & $1 \cdot 2644$ & 45 & 35.865 & 1.1109 & 20 & $15 \cdot 940$ \\
\hline $1 \cdot 4850$ & 94 & 74.918 & $1 \cdot 3945$ & 69 & $54 \cdot 993$ & $1 \cdot 2583$ & 44 & $35 \cdot 068$ & $1 \cdot 1051$ & 19 & $15 \cdot 143$ \\
\hline $1 \cdot 4820$ & 93 & $74 \cdot 121$ & $1 \cdot 3882$ & 68 & $54 \cdot 196$ & $1 \cdot 2523$ & 43 & $34 \cdot 271$ & $1 \cdot 0993$ & 18 & $14 \cdot 346$ \\
\hline $1 \cdot 4790$ & 92 & $73 \cdot 324$ & $1 \cdot 3833$ & 67 & $53 \cdot 399$ & $1 \cdot 2462$ & 42 & $33 \cdot 474$ & $1 \cdot 0$ & 17 & $-13 \cdot 549$ \\
\hline $1 \cdot 4760$ & 91 & 72.527 & $1 \cdot 3783$ & 66 & $52 \cdot 602$ & $1 \cdot 2402$ & 41 & $32 \cdot 677$ & $1 \cdot 0$ & 16 & $12 \cdot 752$ \\
\hline $1 \cdot 4730$ & 90 & $71 \cdot 730$ & $1 \cdot 3732$ & 65 & $51 \cdot 805$ & $1 \cdot 2341$ & 40 & $31 \cdot 880$ & $1 \cdot 0821$ & 15 & 11.955 \\
\hline $1 \cdot 4700$ & 89 & $70 \cdot 933$ & $1 \cdot 3681$ & 64 & $51 \cdot 068$ & $1 \cdot 2277^{\circ}$ & 39 & $31 \cdot 083$ & $1 \cdot 0764$ & 14 & $11 \cdot 158$ \\
\hline 1.4670 & 88 & $70 \cdot 136$ & $1 \cdot 3630$ & 63 & $50 \cdot 211$ & $1 \cdot 2212$ & 38 & $30 \cdot 286$ & $1 \cdot 0708$ & 13 & $10 \cdot 361$ \\
\hline $1 \cdot 4640$ & 87 & $69 \cdot 339$ & $1 \cdot 3579$ & 62 & $49 \cdot 414$ & $1 \cdot 2148$ & 37 & $29 \cdot 489$ & $1 \cdot 0651$ & 12 & $9 \cdot 564$ \\
\hline 1.4600 & 86 & $68 \cdot 542$ & $1 \cdot 3529$ & 61 & $48 \cdot 617$ & $1 \cdot 2084$ & 36 & $28 \cdot 692$ & $1 \cdot 0595$ & 11 & $8 \cdot 767$ \\
\hline $1 \cdot 4570$ & 85 & $67 \cdot 745$ & $1 \cdot 3477$ & 60 & $47 \cdot 820$ & $1 \cdot 2019$ & 35 & $27 \cdot 895$ & $1 \cdot 0540$ & 10 & $7 \cdot 970$ \\
\hline 1.4530 & 84 & $66 \cdot 948$ & $1 \cdot 3427$ & 59 & $47 \cdot 023$ & $1 \cdot 1958$ & 34 & $27 \cdot 098$ & $1 \cdot 0485$ & 9 & $7 \cdot 173$ \\
\hline $1 \cdot 4500$ & 83 & $66 \cdot 155$ & $1 \cdot 3376$ & 58 & $46 \cdot 226$ & $1 \cdot 1895$ & 33 & $26 \cdot 301$ & $1 \cdot 0430$ & 8 & $6 \cdot 376$ \\
\hline 1.4460 & 82 & $65 \cdot 354$ & $1 \cdot 3323$ & 57 & $45 \cdot 429$ & $1 \cdot 1833$ & 32 & $25 \cdot 504$ & $1 \cdot 0375$ & 7 & 5.579 \\
\hline 1.4424 & 81 & $64 \cdot 557$ & $1 \cdot 3270$ & 56 & $44 \cdot 632$ & $1 \cdot 1770$ & 31 & $24 \cdot 707$ & $1 \cdot 0320$ & 6 & $4 \cdot 782$ \\
\hline 1.4385 & 80 & $63 \cdot 760$ & $1 \cdot 3216$ & 55 & $43 \cdot 835$ & $1 \cdot 1709$ & 30 & $23 \cdot 900$ & $1 \cdot 0267$ & 5 & $3 \cdot 985$ \\
\hline $1 \cdot 4346$ & 79 & $62 \cdot 963$ & $1 \cdot 3163$ & 54 & $43 \cdot 038$ & $1 \cdot 1648$ & 29 & $23 \cdot 113$ & $1 \cdot 0212$ & 4 & $3 \cdot 188$ \\
\hline $1 \cdot 4306$ & 78 & $62 \cdot 166$ & $1 \cdot 3110$ & 53 & $42 \cdot 241$ & $1 \cdot 1587$ & 28 & $22 \cdot 316$ & $1 \cdot 0159$ & 3 & $2 \cdot 391$ \\
\hline $1 \cdot 4269$ & 77 & $61 \cdot 369$ & $1 \cdot 3056$ & 52 & $41 \cdot 444$ & $1 \cdot 1526$ & 27 & $21 \cdot 519$ & 1.0106 & 2 & 1.594 \\
\hline $1 \cdot 4228$ & 76 & 60.572 & $1 \cdot 3001$ & 51 & $40 \cdot 647$ & $1 \cdot 1465$ & 26 & 20.722 & 1.0053 & 1 & 0.797 \\
\hline
\end{tabular}

NITROGEN, DEUTOXYDE OF ; Nitrous gas, Nitric oxyde (Deutoxyde d'azote, Fr. : Stichstoffoxyd, Germ.) is a gaseous body which may be obtained by pouring upon copper or mercury, in a retort, nitric acid of moderate strength. The nitrous gas comes over in abundance without the aid of heat, and may be received over water freed from air, or over mercury, in the pneumatic trough. It is elastic and colorless; what taste and smell. it possesses are unknown, because the moment it is exposed to the mouth or nostrils, it absorbs atmospherical oxygen, and becomes nitrous or nitric acid. Its specific gravity is 1.0393 , or 1.04 ; whence 100 cubic inches weigh $36.66 \mathrm{gr}$. Water condenses not more than $\frac{1}{20}$ of its volume of this gas. It extinguishes animal life, and the flame of many combustibles; but of phosphorus well kindled, it brightens the flame in a most remarkable degree. It consists of 47 parts of nitrogen gas, and 53 of oxygen gas, by weight; 
and of equal parts in bulk, without any condensation; so that the specific gravity of deutoxyde of nitrogen is the arithmetical mean of the two constituents. The constitu. tion of this gas, and tue play of affinities which it exercises in the formation of sulphuric acid, are deeply interesting to the chemical manufacturer.

The Hyponitrons acid (Salpetrigesaüre, Germ.), like the preceding compound, deserves notice here, on account of the part it plays in the conversion of sulphur into sulphuric acid, by the agency of nitre. It is formed by mingling four volumes of deutoxyde of nitrogen with one volume of oxygen; and appears as a dark orange vapor which is condensable into a liquid at a temperature of $4^{\circ}-$ zero, Fahr. When distilled, this liquid leaves a dark yellow fluid. The pure hyponitrous acid consists of $37 \cdot 12$ nitrogen, and 62.88 oxygen; or of two volumes of the first, and three of the second. Water converts it into nitric acid and deutoxyde of nitrogen; the latter of which escapes with effervescence. This acid oxydizes most combustible bodies with peculiar energy; and though its vapor does not operate upon dry sulphurous acid, yet, through the agency of steam, it converts it into sulphuric acid, itself being simultaneously transformed into deutoxyde of nitrogen; ready to become hyponitrous acid again, and to perform a circulating series of important metamorphoses. See Sulphuric Acid.

NITROGEN GAS, or AZOTE (Eng. and Fr.; Stickstoffgas, Germ.), constitutes about 79 hundredths of the bulk of the atmospheric air; it is copiously disengaged fom several mineral springs, as from the natural basins of hot water which supply the baths of Leuk, near the Gemmi in Switzerland, and from other springs, in the Pyrenees, in Ceylon, South and North America, \&c. It exists also in flesh and most animal substances, as well as in some vegetable products, being one of their essential constituents. When phosphorus is burnt within a jar filled with air, standing over water in the pneumatic trough, it consumes or absorbs the oxygen, and leaves nitrogen, which may be rendered pure by agitation with water. By exposing nitrite of ammonia to heat in a retori, nitrogen comes over alone in great abundance; for the hydrogen of the ammonia is sufficient to saturate the oxygen of the acid, and to convert it into water; while the nitrogen of both constituents is set at liberty. By transmitting chlorine through water of ammonia, or digesting lean flesh in warm nitric acid, nitrogen may also be obtained. This permanently elastic gas is destitute of color, taste, and smell; it has a specific gravity of 0.976 , air being $1 \cdot 000$. Hence 100 cubic inches of it weigh $29 \cdot 7$ gr. It extinguishes all burning bodies, and when respired without oxygen is fatal to animal life.

NITROGEN, PROTOXYDE OF, Nitrous oxyde (Protoxyde d'azote, Fr.; Stickstoffoxydul, Germ.), is a gas which displays remarkable powers when breathed, causing in many persons unrestrainable feelings of exhilaration, whence it has been called the laughing or intoxicating gas. It is prepared by exposing crystallized nitrate of ammonia to a heat of about $350^{\circ} \mathrm{Fahr}$. in a glass retort. It is much denser than the air of the atmosphere, having a spec. grav. of 1.527 ; whence 100 cubic inches weigh 46.6 grains. It consists of 63.64 parts of nitrogen, and 36.36 of oxygen, by weight; or of two volumes of nitrogen and one volume of oxygen, condensed by reciprocal attraction into two volumes. It is colorless, and possesses all the mechanical properties of the atmosphere. Water previously freed from air absorbs its own volume of this gas; and thus affords a ready criterion for estimating its freedom from incondensable gases, as oxygen, nitrogen, and its deutoxyde. Several combustibles burn in this gas with an enlarged blue and very vivid flame; and it relumes a taper which has been blown out, provided its tip be redhot. By powerful pressure it may be liquefied. See Gas.

NITRO-MURIATIC ACID, Aqua regia (Acide nitro-muriatique, Fr.; Salpeter sulzsaüre, Königswasser, Germ.) is the compound menstruum invented by the alchemists for dissolving gold. If strong nitric acid, orange-colored by saturation with nitrous gas (deutoxyde of azote), be mixed with the strongest liquid muriatic acid, no other effect is produced than might be expected from the action of nitrous acid of the same strength upon an equal quantity of water; nor has the mixed acid so formed any power of acting upon gold or platina. But if colorless aquafortis and ordinary muriatic acid be mixed together, the mixture immediately becomes yellow, and acquires the power of dissolving these two noble metals. When gently heated, pure chlorine gas rises from it, and its color becomes deeper; when further heated, chlorine still rises, but now mixed with nitrous acid gas. If the process has been very long continued, till the color becomes very dark, no more chlorine can be procured, and the liquor has lost the power of dissolving gold. It then consists of nitrous and muriatic acids. It appears, therefore, that aqua regia owes its peculiar properties to the mutual decomposition of the nitric and muriatic acids; and that water, chlorine, and nitrous acid gas are the results of that reaction. Aqua regia does not, strictly speaking, oxydize gold and platinum; it causes merely their combination with chlorine. It may be composed of very different proportions of the two acids; the nitric being commonly of specific gravity 1.34 ; the muriatic, of specific gravity $1 \cdot 18$ or $1 \cdot 19$. Sometimes 3 parts, and at others 6 parts of the muriatic acid are mixed with 1 of nitric; and occasionally mu 
riate of ammonia, instead of muriatic acid, is added to nitric acid for particular purposes, as for making a solution of tin for the dyers. An aqua regia may also be prepared by dissolving nitre in muriatic acid.

NITROL'S ACID (Acide nitreux, Fr.; Salpetrige salpetersaüre, Germ.) may be procured by distilling, in a coated glass retort, perfectly dry nitrate of lead, into a glass receiver surrounded with a freezing mixture. The acid passes over in vapor, and condenses into a liquid; oxygen gas escapes through the safety tube; while oxyde of lead remains in the bottom of the retort. Nitrous acid may also be obtained by distilling strong fuming nitric acid, at the lowest possible temperature, and rectifying what comes over. At $4^{\circ}-$ zero, Fahr., this acid is colorless; at $32^{\circ}$ it is wax yellow; at $60^{\circ}$ it has an orange hue. It possesses a strong smell, has a very pungent, acrid, sour taste, and a specific gravity of 1.42 . It powerfully decomposes organic bodies, staining them yellow. It boils at $82^{\circ}$ Fahr. with the disengagement of red or orange fumes. Its constituents are, 41.34 of hy"ponitrous acid, and 58.66 of anhydrous nitric acid; or ultimately, 30.68 nitrogen $=1$ volume, and $69 \cdot 32$ oxygen $=2$ volumes. In its other habitudes, it is quite analogous to hyponitrous acid.

A mixture of this double or compound acid with nitric acid, constitutes the orangebrown fuming nitrous acid of the British apothecaries.

The hyponitrous and nitrous are two acids remarkable for containing no water in their composition ; being therefore dry liquids.

NOPAL is the Mexican name of the plant cactus opuntia, upon which the cochineal insect breeds.

NUTMEG (Muscade, Fr.; Muskatennuss, Germ.) is the fruit of the myristica moschata, a beautiful tree of the family of the laurinea of Jussieu, which grows in the Molucea islands. All the parts of this tree are very aromatic; but only those portions of the fruit called mace and nutmeg are sent into the market. The entire fruit is a species of drupa, of an ovoid form, of the size of a peach, and furrowed longitudinally. The nutmeg is the innermost kernel, or seed, contained in a thin shell, which is surrounded by the mace; and this again is enclosed in a tough fleshy skin, which opening at the tip, separates into two valves. The nutmeg tree yields three crops annually; one in April, which is the best; one in August; and one in December.

Good nutmegs should be dense, and feel heavy in the hand. When they have been perforated by worms, they feel light, and though the holes have been fraudulently stopped, the unsound ones may easily be detected by this criterion.

Nutmegs afford two oily products. 1. Butter of nutmeg, vulgarly called oil of mace, is obtained in the Moluccas, by expression, from the fresh nutmegs, to the amount of 50 per cent. of their weight. It is a reddish yellow butter-like substance, interspersed with light and dark streaks, and possesses the agreeable smell and taste of the nutmeg, from the presence of a volatile oil. It consists of two fats; one reddish and soft, soluble in cold alcohol; another white and solid, soluble in hot alcohol. 2. The volatile oil is solid, or a stereoptene, and has been styled Myristicine.

NUT OIL. See Oils, Unctuous.

NUY, VOMICA, a poisonous nut, remarkable for containing the regeto-alkals StruchNia.

O.

OAK BARK. See TAN.

OATS. (Avoine, Fr.; Hafer, Germ.) The composition of oats is less known than that of the other Cerealia. Vogel found that 100 parts of oats afforded 66 parts of flour or meal, and 34 parts of bran; but this proportion would depend upon the quality of the grain. The flour contains 2 parts of a greenish-yellow fat oil ; 8.25 of bitterish sweet extractive; 2.5 of gum; 4.30 of a gray substance, more like coagulated albumen than gluten; 59 of starch ; 24 of moisture (inclusive of the loss). Schrader found in the ashes of oats, silica, carbonate of lime, carbonate of magnesia, alumina, with oxydes of manganese and iron.

OBSIDIAN is a glassy looking mineral, with a large conchoidal fracture, and of a blackish color, which froths much at the blow-pipe before it melts into a white enamel.

OCHRE, yellow and brown (Ocre, Fr.; Ocker, Germ.), is a native earthy mixture of silica and alumina, colored by oxyde of iron, with occasionally a little calcareous matter and magnesia. Ochre occurs in beds some feet thick, which lie generally above the oolite, are covered by sandstone and quartzose sands more or less ferruginous, and are accompanied by gray plastic clays, of a yellowish or reddish color; all of them substances which contribute more or less to its formation. The ochry earths are prepared for use by grinding under edge millstones, and elutriation. The yellow ochres 
may be easily renderel red or reddish brown by calcination in a reverberatory oven, which oxydizes their iron to a higher degree.

Native red ochre is called red chalk and reddle in England. It is an intimate mixture of clay and red iron ochre; is massive; of an earthy fracture; is brownish-red, bloodred, stains and writes red. The oxyde of iron is sometimes so considerable, that the ochre may be reckoned an ore of that metal.

The ochre beds of England are in the iron sand, the lowest of the formations which intervene between the chalk and oolites. Beds of fuller's earth alternate with the iron sand. The following is a section of the ochre pits at Shotover Hill, near Oxford :-

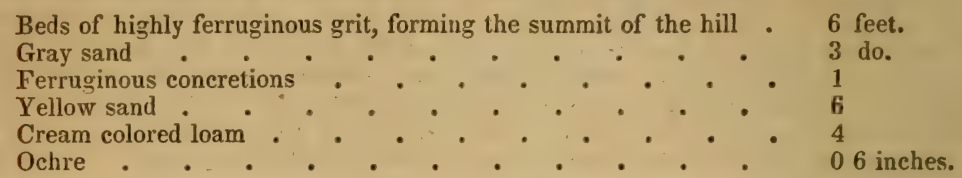

Beneath this, there is a second bed of ochre, separated by a thin bed of clay.

Bole, or Armenian bole; called also Lemnian earth, and terra sigillata, because when refined it was stamped with a seal ; is massive, with a conchoidal fracture, a feeble lustre, reddish-yellow or brown, a greasy feel; adheres to the tongue, spec. grav. 1.4 to 2.0 . It occurs in the island Stalimene (the ancient Lesbos), and in several other places, especially at Sienna; whence the brown pigment called terra di Siena.

OILS (Huiles, Fr.; Oele, Germ.), are divisible into two great classes: the fat or fixed oils, huiles grasses, Fr.; Fette oele, Germ. ; and the essential or volatile oils, Huiles volatiles, Fr.; Flüchtige, aetherische oele, Germ. The former are usually bland and mild to the taste; the latter hot and pungent. The term distilled, applied also to the last class, is not so correct, since some of them are obtained by expression, as the whole of the first class may be, and commonly are.

All the known fatty substances found in organic bodies, without reference to their vegetable or animal origin, are, according to their consistence, arranged under the chemical heads of oils, butters, and tallows. They all possess the same ultimate constituents, carbon, hydrogen, and generally oxygen, and in nearly the same proportions.

The fat oils are widely distributed through the organs of vegetable and animal nature. They are found in the seeds of many plants, associated with mucilage, especially in those of the bicotyledinous class, occasionally in the fleshy pulp surrounding some seeds, as the olive; also in the kernels of many fruits, as of the nut and almond tree, and finally in the roots, barks, and other parts of plants. In animal bodies, the oily matter occurs enclosed in thin membraneous cells, between the skin and the flesh, between the muscular fibres, within the abdominal cavity in the omentum, upon the intestines, and round the kidneys, and in a bony reccptacle of the scull of the spermaceti whale; sometimes in special organs, as of the beaver; in the gall-bladder, \&c., or mixed in a liquid state with other animal matters, as in the milk.

Braconnot, but particularly Raspail, have shown that animal fats consist of small microscopic, partly polygonal, and partly reniform particles, associated by means of their containing sacs. These may be separated from each other by tearing the recent fat asunder, rinsing it with water, and passing it through a sieve. The membranes being thus retained, the granular particles are observed to float in the water, and afterwards to separate, like the globules of starch, in a white pulverulent semi-erystalline form. The particles consist of a strong membraneous skin, enclosing stearine and elaine, or solid and liquid fat, which may be extracted by trituration and pressure. These are lighter than water, but sink readily in spirit of wine. When boiled in strong alcohol, the oily principle dissolves, but the fatty membrane remains. These granules have different sizes and shapes in dif ferent animals; in the calf, the ox, the sheep, they are polygonal, and from $\frac{1}{70}$ to $\frac{1}{45} \overline{0}$ of an inch in diameter; in the hog they are kidney-shaped, and from $\frac{1}{70}$ to $\frac{1}{7} \frac{1}{4}$ of an inch; in man, they are polygonal, and from $\frac{1}{70}$ to $\frac{1}{900}$ of an inch; in inseets they are usually spherical, and not more than $\frac{1}{600}$ of an inch.

The following is a list of the Plants which yield the ordinary Unctuous Oils of commerce :

\begin{tabular}{|c|c|c|c|c|c|c|c|c|}
\hline No. & \multicolumn{3}{|c|}{ Plants. } & \multicolumn{4}{|c|}{ Oils. } & Spec. gravity.| \\
\hline 1. & Linum usitatissum e & perenne & - D. & Linseed oil & - & - & - & 0.9347 \\
\hline 2. & $\left.\begin{array}{l}\text { Corylcus avellana } \\
\text { Juglans regia }\end{array}\right\}$ & $=$ & $=D$. & Nut oil & - & - & - & 0.9260 \\
\hline 4. & Papaver somniferum & - & - D. & Poppy oil & - & - & - & 0.9243 \\
\hline 5. & Cannabis sativa & - & - D. & Hemp oil & - & - & - & 0.9276 \\
\hline
\end{tabular}


List of the Plants which yield the ordinary Unctuous Oils of commerce-continued.

\begin{tabular}{|c|c|c|c|c|c|c|c|}
\hline No. & Flants. & & & Oils. & & & Spec. gravity. \\
\hline 6. & Sesamum orientale & - & - G. & Oil of sesamum & - & - & \\
\hline 7. & Olea Europea - & - & & Olive oil -- & - & - & 0.9176 \\
\hline 8. & Amygdalus communis & - & - G. & Almond oil & - & - & 0.9180 \\
\hline 9. & Guilandina mohringa & & & Oil of behen or be & & & \\
\hline 10. & Cucurbita pepo, and $n$ & melapepo & o D. & Cucumber oil & - & - & $0 \cdot 9231$ \\
\hline 11. & Fagus silvatica & - & - G. & Beech oil - & - & - & 0.9225 \\
\hline 12. & Sinapis nigra et arvens & & - G. & Oil of mustard & - & - & 0.9160 \\
\hline 13. & Helianthus annuus et & perenni & is $\mathrm{D}$. & Oil of sunflower & - & - & 0.9262 \\
\hline 14. & Brassica napus et cam & pestris & - G. & Rape-seed oil & - & - & 0.9136 \\
\hline 15. & Ricinus communis & - & - D. & Castor oil - & - & - & $0 \cdot 9611$ \\
\hline 16. & Nicotiana tabacum et $\mathrm{r}$ & rustica & - D. & Tobacco-seed oil & - & - & 0.9232 \\
\hline 17. & Prunus domestica & - & - G. & Plum-kernel oil & - & & 0.9127 \\
\hline 18. & Vitis vinifera - & - & - D. & Grape-seed oil & - & & 0.9202 \\
\hline 19. & Theobroma cacao & - & - G. & Butter of cacao & - & & 0.892 \\
\hline 20. & Cocos nucifera & & - G. & Cocoa-nut oil & - & & \\
\hline 21. & Cocus butyracea vel av & voira elai & is $\mathrm{G}$. & Palm oil - & - & - & 0.968 \\
\hline 22. & Laurus nobilis - & - & - G. & Laurel oil - & - & - & \\
\hline 23. & Arachis hypogæa & - & $-\mathrm{G}$. & Ground-nut oil & - & - & \\
\hline 24. & Vateria indica - & - & - G. & Piney tallow & - & - & 0.926 \\
\hline 25. & Hesperis matronalis & - & $-D$. & Oil of Julienne & - & - & 0.9281 \\
\hline 26. & Myagrum sativa & - & $-\mathrm{D}$. & Oil of camelina & - & - & 0.9252 \\
\hline 27. & Reseda luteola & - & - D. & Oil of weld-seed & - & - & 0.9358 \\
\hline 28. & Lepidium sativum & - & - D. & Oil of garden cres & sses & - & $0 \cdot 9240$ \\
\hline 29. & Atropa belladonna & - & $-\mathrm{D}$. & Oil of deadly nigh & htshade - & - & 0.9250 \\
\hline 30. & Gossypium Barbadense & & - D. & Cotton-seed oil & - & - & \\
\hline 31. & Brassica campestris ol & leifera & - G. & Colza oil - & - & - & $0 \cdot 9136$ \\
\hline 32. & Brassiea præcox & - & - G. & Summer rape-seed & oil & & 39 \\
\hline 33. & Raphanus sativus oleif & & - G. & Oil of radish-seed & $1-$ & - & 0.9187 \\
\hline 34. & Prunus cerasus & - & - G. & Cherry-stone oil & - & - & 0.9239 \\
\hline 35. & Pyrus malus - & - & - G. & Apple-seed oil & - & - & \\
\hline 36. & Euonymus Europæus & - & - G. & Spindle-tree oil & - & - & $0 \cdot 9380$ \\
\hline 37. & Cornus sanguinea & - & G. & Cornil-berry tree & & & \\
\hline 38. & Cyperus esculenta & - & - G. & Oil of the roots of & cyper gra & & $0 \cdot 9180$ \\
\hline 39. & Hyociamus niger & - & - $\mathrm{G}$. & Henbane-seed oil & & & $0 \cdot 9130$ \\
\hline 40. & Esculus hippocastanu & & - $\mathrm{G}$. & Horse-chestnut oil & 1 - & - & 0.927 \\
\hline 41. & Pinus abies - & - & $-D$. & Pinetop oil & & & $0 \cdot 285$ \\
\hline
\end{tabular}

The fat oils are contained in that part of the seed which gives birth to the cotyledons; they are not found in the plumula and radicle. Of all the families of plants, the cruciform is the richest in oleiferous seeds; and next to that are the drupaceæ, amentaceæ, and solaneæ. The seeds of the gramineæ and leguminosæ contain rarely more than a trace of fat oil. One root alone, that of the cyperus esculenta, contains a fat oil. The quantity of oil furnished by seeds varies not only with the species, but in the same seed, with culture and climate. Nuts contain about half their weight of oil ; the seeds of the brassica oleracea and campestris, one third; the variety called colza in France, two fifths; hempseed, one fourth; and linseed from one fourth to one fifth. Unverdorben states that a last or ten quarters of linseed yields $40 \mathrm{ahms}=120$ gallons English of oil; which is about $1 \mathrm{cwt}$. of oil per quarter.

The fat oils, when first expressed without much heat, taste merely unctuous on the tongue, and exhale the odor of their respective plants. They appear quite neutral by litmus paper. Their fluidity is very various, some being solid at ordinary temperatures, and others remaining fluid at the freezing point of water. Linseed oil indeed does not congeal till cooled from $4^{\circ}$ to $18^{\circ}$ below $0^{\circ} \mathrm{F}$. The same kind of seed usually affords oils of different degrees of fusibility; so that in the progress of refrigeration one portion concretes before another. Chevreul, who was the first to observe this fact, considers all the oils to be composed of two species, one of which resembles suet, and was thence styled by him stearine; and another which is liquid at ordinary temperatures, and was called elaine, or oleine. By refrigeration and pressure between the folds of blotting paper, or in linen bags, the fluid•part is separated, and the solid remains. By heating the paper in water, the liquid oil may be obtained separate. When alcohol is boiled with the natural oil, the greater part of the stearine remains undissolved. 
Oleine may also be procured by digesting the oil with a quantity of caustic soda equal to one half of what is requisite to saponify the whole; the stearine is first transformed into soap, then a portion of the oleine undergoes the same change, but a great part of it remains in a pure state. This process succeeds nnly with recently expressed or very fresh oils. The properties of these two principles of the fat oils vary with the nature of the respective oils, so that the sole difference does not consist, as many suppose, in the different proportions of these two bodies, but also in peculiarities of the several stearines and oleines, which, as extracted from different seeds, solidify at very different temperatures.

In close vessels, oils may be preserved fresh for a very long time, but with contact of air they undergo progressive changes. Certain oils thicken and eventually dry into a transparent, yellowish, flexible substance; which forms a skin upon the surface of the oil, and retards its further alteration. Such oils are said to be drying or siccutive, and are used on this account in the preparation of varnishes and painters' colors. Other vils do not grow dry, though they turn thick, become less combustible, and assume an offensive smell. They are then called rancid. In this state, they exhibit an acid reaction, and irritate the fauces when swallowed, in consequence of the presence of a peculiar acid, which may be removed in a great measure by boiling the oil along with water and a little common magnesia for a quarter of an hour, or till it has lost the property of reddening litmus. While oils undergo the above changes, they absorb a quantity of oxygen equal to several times their volume. Saussure found that a laver of nut oil, one quarter of an inch thick, enclosed along with oxygen gas over the surface of quicksilver in the shade, absorbed only three times its bulk of that gas in the course of eight months; but when exposed to the sun in August, it absorbed 60 volumes additional in the course of ten days. This absorption of oxygen diminished progressively, and stopped altogether at the end of three months, when it had amounted to 145 times the bulk of the oil. No water was generated, but 21.9 volumes of carbonic acid were disengaged, while the oil was transformed in an anomalous manner into a gelatinous mass, which did not stain paper. To a like absorption we may ascribe the elevation of temperature which happens when wool or hemp, besmeared with olive or rapeseed oil, is left in a heap; circumstances under which it has frequently taken fire, and caused the destruction of both cloth-mills and dock-yards.

In illustration of these accidents, if paper, linen, tow, wool, cotton, mats, straw, wood shavings, moss, or soot, be imbued slightly with linseed or hempseed oil, and placed in contact with the sun and air, especially when wrapped or piled in a heap, they very soon become spontaneously hot, emit smoke, and finally burst into flames. If linseed oil and ground manganese be triturated together, the soft lump so formed will speedily become firm, and ere long take fire.

The fat oils are completely insoluble in water. When agitated with it, the mixture becomes turbid, but if it be allowed to settle the oil collects by itself upon the surface. This method of washing is often employed to purify oils. Oils are little soluble in alcohol, except at high temperatures. Castor oil is the only one which dissolves in cold alcohol. Ether, however, is an excellent solvent of oils, and is therefore employed to extract them from other bodies in analysis; after which it is withdrawn by distillation.

Fat oils may be exposed to a considerably high temperature, without undergoing much alteration; but when they are raised to nearly their boiling point, they begin to be decomposed. The vapors that then rise are not the oil itself, but certain products generated in it by the heat. These changes begin somewhere under $600^{\circ}$ of Fahr., and require for their continuance temperatures alwars increasing. The products consist at first in aqueous vapor, then a very inflammable volatile oil, which causes boiling oil to take fire spontaneously; and next carbureted hydrogen gas, with carbonic acid gas. In a lamp, a small portion of oil is raised in the wick by capillarity, which being heated, boils and burns. See Rosin-Gas.

Several fat oils, mixed with one or two per cent. of sulphuric acid, assume instantly a dark green or brown hue, and, when allowed to stand quietly, deposite a coloring matter after some time. It consists in a chemical combination of the sulphuric acid, with a body thus separated from the oil, which becomes in consequence more limpid, and burns with a brighter flame, especially after it is washed with steam, and clarified by repose or filtration. Any remaining moisture may be expelled by the heat of a water bath.

The oils combine with the salifiable bases, and give birth to the substance called glycerine (the sweet principle), and to the margaric, oleic, and stearic acids. The general product of their combination with potash or soda, is SoAP, which see. Caustic ammonia changes the oils very difficultly and slowly into a soap; but if readily unites with then into a milky emulsion called volatile liniment, used as a rubefacient in 
medicine. Ipon mixing water with this liquor, the oil separates in an unchanged state. By l-nger contact, ammonia acts upon oils like the other allialis. Sea salt dissolves in small quantity in the oils, and so does verdigris. The latter solution is green. Oils dissolve also several of the vegetable alkalis, as morphia, cinchonia, quinia, strychia, and delphia.

Olive oil consists of 77.2 carbon, 13.4 hydrogen, and $9 \cdot 4$ oxygen, in 100 parts. Spermaceti oil, by my analysis, of 78.9 carbon, 10.97 hydrogen, and 10.13 oxygen.

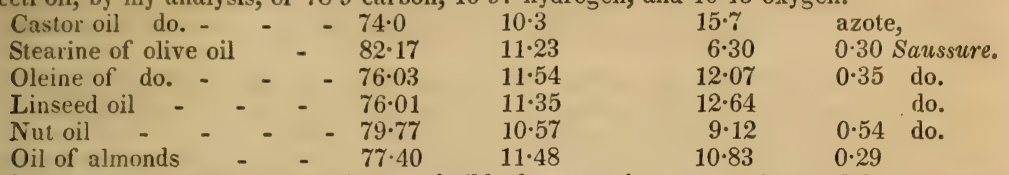

De Saussure concludes that the less fusible fats contain more carbon and less oxygen, and that oils are more soluble in alcohol, the more oxygen they contain.

I shall now take a short view of the peculiarities of the principal expressed oils.

Oil of almonds, according to Gusseron, contains no stearine; at least he could obtain none by cooling it and squeczing it successively till it all congealed. Braconnot had, on the contrary, said, that it contains 24 per cent. of stearine. I believe that Gusseron is right, and that Braconnot had made fallacious experiments on an impure oil.

Oil of colza is obtained from the seeds of brassica campestris, to the amount of 39 per cent. of their weight. It forms an excellent lamp oil, and is much employed in France.

The corylus avellana furnishes in oil 60 per cent. of the weight of the nuts.

Hempeed oil resembles the preceding, but has a disagreeable smell, and a mawkish taste. It is used extensively for making both soft soap and varnishes.

Linseed oil is obtained in greatest purity by cold pressure; but by a steam heat of ahout $200^{\circ} \mathrm{F}$. a very good oil may be procured in larger quantity. The proportion of oil usually stated by authors is 22 per cent. of the weight of the seed; but $\mathrm{Mr}$. Blundell informs me, that, by his plan of hydraulic pressure, he obtains from 26 to 27 . In the Encyclopædia Metropolitana, under Oil Press, a quarter of seed (whose average weight is 400 lbs.) is said to yield 20 gallons of oil. Now as the gallon of linseed oil weighs $9 \cdot 3 \mathrm{lbs}$., the total product will be $186 \mathrm{lbs}$., which amounts to more than 45 per cent.-an extravagant statement, about double the ordinary product in oil mills. Even supposing the gallons not to be imperial, but old English, we should have upwards of 38 per cent. of oil by weight, which is still an impossible quantity. Such are the errors introduced into respectable books, by adonting without practical knowledge, the puffing statements of a patentee. It dissolves in j̃ parts of bolling alcohol, in 40 parts of cold alcohol, and in 1.6 parts of ether. When kept long cool in a cask partly open, it deposites masses of white stearine along with a brownish powder. That stearine is very difficult of saponification.

Mustard-seed oil. The white or yellow seed affords 36 per cent. of oil, and the black seed 18 per cent. The oil concretes when cooled a little below $32^{\circ} \mathrm{F}$.

Nut oil is at first greenish colored, but becomes pale yellow by time. It congeals at the same low temperature as linseed oil, into a white mass, and has a more drying quality than it.

Oil of olives is sometimes of a greenish and at others of a pale yellow color. A few degrees above $32^{\circ} \mathrm{F}$. it begins to deposite some white granules of stearine, especially if the oil have been originally expressed with heat. At $22^{\circ}$ it deposites 28 per cent. of its weight in stearine, which is fusible again at $68^{\circ}$, and affords 72 per cent. of oleine. According to Kerwych, oleine of singular beauty may be obtained by mixing 2 parts of olive oil with 1 part of caustic soda ley, and macerating the mixture for 24 hours with frequent agitation. Weak alcohol must then be poured into it, to dissolve the stearine soap, whereby the oleine, which remains meanwhile unsaponified, is separated, and floats on the surface of the liquid. This being drawn off, a fresh quantity of spirits is to be poured in, lill the separation of all the oleine be completed. It has a slightly yellowish tint, which may be removed by means of a little animal charcoal mixed with it in a warm place for 24 hours. By subsequent filtration, the oleine is obtained limpid and colorless, of such quality that it does not thicken with the greatest cold, nor does it affect either iron or copper instruments immersed in it.

There are three kinds of olive oil in the market. The best, called virgin salad oil, is obtained by a gentle pressure in the cold; the more common sort is procured by stronger pressure, aided with the heat of boiling water; and thirdly, an inferior kind, by boiling the olive residuum or marc, with water, whereby a good deal of mucilaginous oil rises and floats on the surface. The latter serves chiefly for making soaps. A still worse oil is got by allowing the mass of bruised olives to ferment before subjecting it to rressure. 
Oil of olives is refined for the watchmakers by the following simple process. Into 8 bottle or vial containing it, a slip of sheet lead is immersed, and the bottle is placed at a window, where it may receive the rays of the sun. The oil by degrees gets covered with a curdy mass, which after some time settles to the bottom, while itself becomes limpid and colorless. As soon as the lead ceases to separate any more of that white substance, the oil is decanted off into another vial for use.

Palm oil melts at $117 \cdot 5^{\circ} \mathrm{F}$, and is said to consist of 31 parts of stearine and 69 of oleine in 100 . It becomes readily rancid by exposure to air, and is whitened at the same time.

The oil extracted from the plucked tops of the pinus abies, in the Black Forest in Germany, is limpid, of a golden yellow color, and resembles in smell and taste the oil of turpentine. It answers well for the preparation of varnishes.

The oil of plum-stones is made chiefly in Wurtemberg, and is found to answer very well for lamps.

Poppy-seed oil has none of the narcotic properties of the poppy juice. It is soluble in ether in every proportion.

Rape-seed oil has a yellow color, and a peculiar smell. At $25^{\circ} \mathrm{F}$. it becomes a yellow mass, consisting of 46 parts of stearine, which fuses at $50^{\circ}$, and 54 of oleine, in which the smell resides.

The oils of belladonna seeds, and tobacco seeds, are perfectly bland. The former is much used for lamps in Swabia and Wurtemburg. The oil-cakes of both are poisonous.

Oil of wine-stones is extracted to the amount of 10 or 11 per cent. from the seeds of the grape. Its color is at first pale yellow, but it darkens with age. It is used as an article of diet.

\section{FAT OIL MANUFACTURE.}

It is the practice of almost all the proprietors in the neighborhood of Aix, in Provence, to preserve the olives for 15 days in barns or cellars, till they have undergone a species of fermentation, in order to facilitate the extraction of their oil. If this practice were really prejudicial to the product, as some theorists have said, would not the high reputation and price of the oil of Aix have long ago suffered, and have induced them to change their system of working? In fact, all depends upon the degree of fermentation excited. They must not be allowed to mould in damp places, to lie in heaps, to soften so as to stick to each other, and discharge a reddish liquor, or to become so hot as to raise a thermometer plunged into the mass up to $96^{\circ} \mathrm{F}$. In such a case they would afford an acrid nauseous oil, fit only for the woollen or soap manufactories. A slight fermentative action, however, is useful, towards separating the oil from the mucilage. The olives are then crushed under the stones of an edge-mill, and next put into a screw-press, being enclosed in bulrush-mat bags (cabas), laid over each other to the number of eighteen. The oil is run off from the channels of the ground-sill, into casks, or into stone cisterns called pizes, two thirds filled with water. The pressure applied to the cabas should be slowly graduated.

What comes over first, without heat, is the virgin oil already mentioned. The cabas being now removed from the press, their contents are shovelled out, mixed with some boiling water, again put in the bags, and pressed anew. The hot water helps to carry off the oil, which is received in other casks or pizes. The oil ere long accumlates at the surface, and is skimmed off with large flat ladles; a process which is called lever l'huile. When used fresh, this is a very good article, and quite fit for table use, but is apt to get rancid when kept. The subjacent water retains a good deal of oil, by the interven tion of the mucilage; but by long repose in a large general cistern, called l'enfer, it parts with it, and is then drawn off from the bottom by a plug-hole. The oil which remains after the water is run off, is of an inferior quality, and can be used only for factory purposes.

The marc being crushed in a mill, boiled with water, and expressed, yields a still coarser article.

All the oil must be fined by keeping in clean tuns, in an apartment heated to the 60 th degree Fahr. at least, for twenty days; after which it is run off into strong casks, which are cooled in a cellar, and then sent into the market.

Oil of almonds is manufactured by agitating the kernels in bags, so as to separate their brown skins, grinding them in a mill, then enclosing them in bags, and squeezing them strongly between a series of cast iron plates, in a hydraulic press; without heat at first, and then between heated plates. The first oil is the purest, and least apt to become rancid. It should be refined by filtering through porous paper. Next to olive vil, this species is the most easy to saponify. Bitter almonds, being cheaper than the sweet, are used in preference for obtaining this oil, and they afford an article equally bland, wholesome, and inodorous. But a strongly scented oil may be procured, accord. ing to M. Planché, by macerating the almonds in hot water, so as to blanch them. 
then drying them in a stove, and afterwards subjecting them to pressure. The volatile oil of almonds is obtained by distilling the marc or bitter almond cake, along with water. See Press Hydradlic, and Stearine.

Linseed, rapeseed, poppyseed, and other oleiferous seeds were formerly treated for the extraction of their oil, by pounding in hard wooden mortars with pestles shod with iron, set in motion by cams driven by a shaft turned with horse or water power, then the triturated seed was put into woollen bags which were wrapped up in hair-cloths, and squeezed between upright wedges in press-boxes by the impulsion of vertical rams driven also by a cam mechanism. In the best mills upon the old construction, the cakes obtained by this first wedge pressure were thrown upon the bed of an edge-mill, ground anew and subjected to a second pressure, aided by heat now, as in the first case. These mortars and press-boxes constitute what are called Dutch mills. They are still in very general use both in this country and on the Continent; and are by many persons supposed to be preferable to the hydraulic presses.

The roller-mill, for merely bruising the linseed, \&c., previous to grinding it under edgestones, and to heating and crushing it in a Dutch or a hydraulic oil-mill, is represented

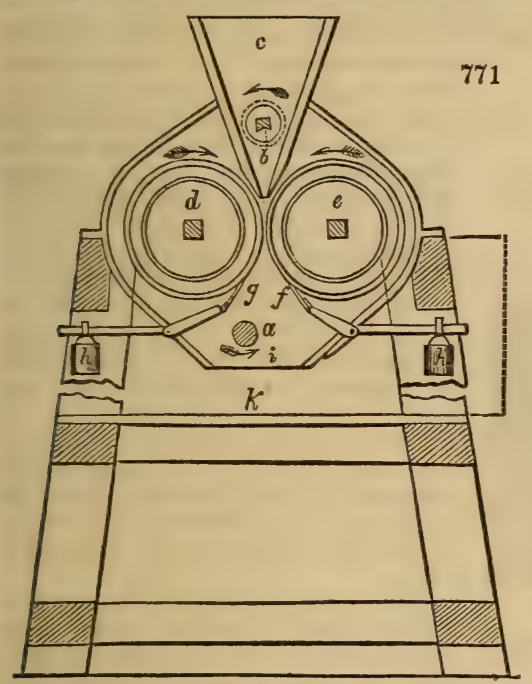

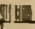

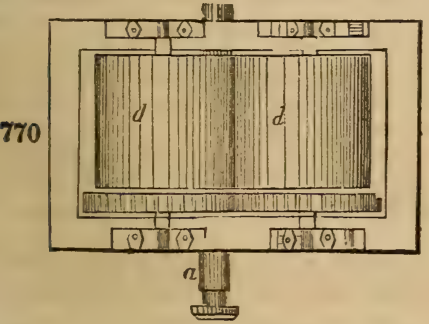
in figs. 770 , and 771 . The iron shaft $a$, has a winch at each end, with a heavy fly-wheel upon the one of them, when the machine is to be worked ry hand. Upon the opposite end is a pu ley, with an endless cord which passts round a pulley on the end of the fluted roller $b$, and thereby drives it. This fluted roller $b$, lies across the hopper $c$, and by its agitation causes the seeds to descend equably through the hopper, between the crushing rollers $d, e$. Upon the shaft $a$, there is also a pinjon which works into two toothed wheels on the shafts of the crushing cylinders $d$ and $e$, thus communicating to these cylinders motion in opposite directions. $f, g$ are two seraperblades, which by means of the two weights $h, h$, hanging upon levers, are pressed against the surfaces of the cylinders, and remove any seed-cake from them. The bruised seeds fall through the slit $i$ of the case, and are received into a chest which stands upon the board $k$.

Machines of this kind are now usually driven by power. Hydraulic presses have been of late years introduced into many seed-oil mills in this country; but it is still a matter of dispute whether they, or the old Dutch oil-mill, with bags of seed compressed between wedges, driven by cam-stamps, be the preferable; that is, afford the largest product of oil with the same expenditure of capital and power. For figures of hydraulic presses, see Press, and Stearine.

This bruising of the seed is merely a preparation for its proper grinding under a pair of heavy edge-stones, of granite, from 5 to 7 feet in diameter; because unbruised seed is apt to slide away before the vertical rolling wheel, and thus escape trituration. The edge-mill, for grinding seeds, is quite analogous to the gunpowder-mill represented in fig. 531, page 636. Some hoop the stones with an iron rim, but others prefer, and I think justly, the rough surface of granite, and dress it from time to time with hammers, as it becomes irregular. These stones make from 30 to 36 revolutions upon their horizontal bed of masonry or iron in a minute. The centre of the bed, where it is perforated for the passage of the strong vertical shaft which turns the stones, is enclosed by a circular box of cast iron, firmly bolted to the bed-stone, and furnished with a cover. This box serves to prevent any seeds or powder getting into the step or socket, and obstructing the movement. The circumference of the mill-bed is formed of an upright rim of oak-plank, bound with iron. There is a rectangular notch left in the edge of the bed, and corresponding part of the rim, which is usually closed with a slide-plate, and $s$ opened only at the end of the operation, to let the pasty seed-cake be turned out by 
the oblique arm of the bottom scraper. The two parallel stones, which are set near each other, and travel round their circular path upon the bed, grind the seeds not merely by their weight, of three tons each, but also by a rubbing motion, or attrition; because theip periphery being not conical, but cylindrical, by its rolling upon a plane surface, must at every instant turn round with friction upon their resting points. Strong cast-iron boxes are bolted upon the centre of the stones, which by means of screw clamps scize firmly the horizontal iron shafts that traverse and drive them, by passing into a slit-groove the verticai curning shaft. This groove is lined with strong plates of steel, which wear rapidly by the friction, and need to be frequently renewed.

The seeds which have been burst between the rolls, or in the mortars of the Dutch mills, are to be spread as equably as possible by a shovel upon the circular path of the edge-stones, and in about half an hour the charge will be sufficiently ground into a paste. This should be put directly into the press, when fine cold-drawn oil is wanted. But in general the paste is heated before being subjected to the pressure. The pressed cake is again thrown under the edge-stones, and, after being ground the second time, should be exposed to a heat of $212^{\circ}$ Fahr., in a proper pan, called a steam-kettle, before being subjected to the second and final pressure in the woollen bags and hair-cloths.

Fig. 772 , is a vertical section of the steam-kettle of Hallette, and fig. 773 is a view of

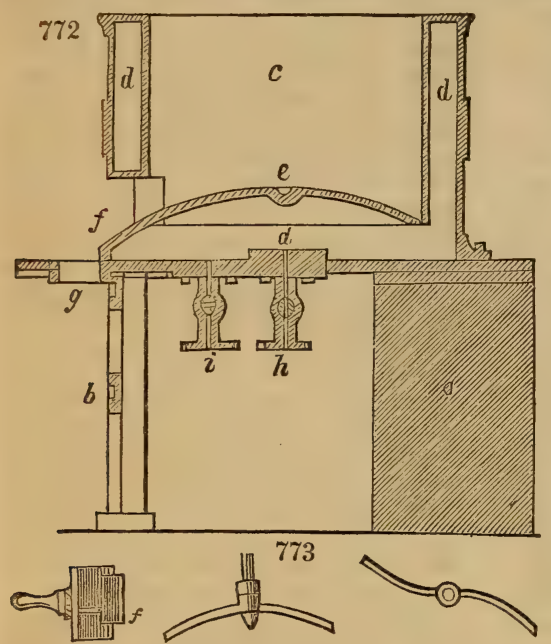
the seed-stirrer. $a$, is the wall of masonry, upon which, and the iron pillars $b$, the pan is supported. It is enclosed in a jacket, for admitting steam into the intermediate space $d, d, d$, at its sides and bottom. $c$, is the middle of the pan in which the shaft of the stirrer is planted upright, resting by its lower end in the step $e ; f$, is an opening, by which the contents of the pan may be emptied; $g$, is an orifice into which the mouth of the hair or worsted bag is inserted, in order to receive the heated seed, when it is turned out by the rotation of the stirrer and the withdrawal of the plug $f$ from the discharge aperture; $h$, is the steam induction pipe; and $t$, the eduction pipe, which serves also to run off the condensed water.

The hydraulic oil-press is generally double; that is, it has two vertical rams placed parallel to each other, so that while one side is under pressure, the other side is being discharged. The bags of heated seed-paste or meal are put into cast-iron cases, which are piled over each other to the number of 6 or 8 , upon the press sill, and subjected to a force of 300 or 400 tons, by pumps worked with a steam engine. The first pump has usually 2 or $2 \frac{1}{2}$ inches diameter for a ram of 10 inches, and the second pump one inch. Each side of the press, in a well-going establishment, should work 38 pounds of seed-flour every 5 minutes. Such a press will do 70 quarters of linseed in the days' work of one week, with the labor of one man at $20 \mathrm{~s}$. and three boys at $5 \mathrm{~s}$. each; and will require a 12-horse power to work it well, along with the rolls and the edge-stones.

I am indebted to my excellent friend Mr. E. Woosey, for the following most valuable notes, taken by him at sundry mills for pressing oil; and remarks upon the subject of seed-crushing in general.

"The chief point of difference depends upon the quality of seed employed. Heavy seed will yield most oil, and seed ripened under a hot sun, and where the flax is not gathered too green, is the best. The weight of linseed varies from 48 to $52 \mathrm{lbs}$. per imperial bushel; probably a very fair average is $49 \mathrm{lbs}$., or $392 \mathrm{lbs}$. per imperia] quarter. I inspected one of the seed-crusher's books, and the average of 15 trials of a quarter each of different seeds in the season averaged $14 \frac{1}{2}$ galls. of $7 \frac{1}{2}$ lbs. each; say, 109 lbs. of oil per quarter. This crusher, who uses only the hydraulic press, and one pressing, informed me that-

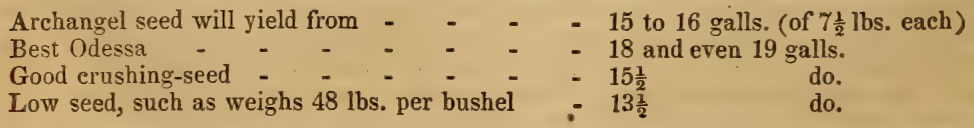


"6 The average of the seed he has worked, which he represents to be of an inferior quality, for the sake of its cheapness, yields $14 \frac{1}{2}$ galls. per quarter. I had some American seed which weighed $52 \frac{1}{4}$ lbs. per imperial bushel, ground and pressed under my own observation, and it gave me $111 \mathrm{lbs}$. oil; that is to say, $418 \mathrm{lbs}$. of seed gave 111 lbs. oil $=26 \frac{56}{100}$ per cent. A friend of mine, who is a London crusher, told me the oil varied according to the seed from 14 to 17 galls.; and when you consider the relative value of seeds, and remember that oil and cake from any kind of seed is of the same value, it will be apparent that the yield is very different; for example,

25th July, 1836, \{ E. India linseed worth $52 s . \quad$ per quarter. prices of seed. Petersburg linseed 48 to 52 do.

The difference of $4 s$. must be paid for in the quantity of oil, which at $38 \mathrm{~s}$. $6 d$. per cwt. (the then price) requires about $11 \frac{1}{2}$ lbs. more oil expressed to pay for the differenco in the market value of the seed. Another London crusher informed me that East India linseed will produce 17 gallons, and he seemed to think that that was the extreme quantity that could be expressed from any seed. The average of last year's Russian seed would be about 14 galls. ; Sicilian seed 16 galls.

\begin{tabular}{|c|c|c|c|c|c|c|c|c|}
\hline Place. & Engine Power. & $\begin{array}{c}\text { Hydraulic } \\
\text { Presses. }\end{array}$ & Stampers. & Rollers. & $\begin{array}{l}\text { Edge- } \\
\text { stones. }\end{array}$ & Kettles & $\begin{array}{l}\text { Work done, } \\
\text { reduced to } \\
\text { an hour. }\end{array}$ & $\begin{array}{l}\text { Num- } \\
\text { ber of } \\
\text { press- } \\
\text { ings. }\end{array}$ \\
\hline France & 10 horse power & $\begin{array}{ll}1 & \text { hydrau- } \\
& \text { lic, } 200 \\
& \text { tons. }\end{array}$ & $\begin{array}{l}5 \text { light } \\
\text { stamp- } \\
\text { ers. }\end{array}$ & $\begin{array}{l}1 \text { pair } \\
\text { rolls. }\end{array}$ & $\begin{array}{l}1 \text { pr. edge- } \\
\text { stones. }\end{array}$ & $\begin{array}{l}5 \text { table kettles } \\
\text { small size } \\
\text { heated by }\end{array}$ & $\begin{array}{l}1 \text { English } \\
\text { quarter per } \\
\text { working }\end{array}$ & $\begin{array}{l}2 \text { pres- } \\
\text { sings. }\end{array}$ \\
\hline London & 20 horse power & $\begin{cases}1 & \text { hydrau- } \\
& \text { lic, } 800 \\
& \text { tons. }\end{cases}$ & $\begin{array}{l}13 \text { light } \\
\text { stamp- } \\
\text { ers. }\end{array}$ & $\begin{array}{l}1 \text { pair } \\
\text { rolls. }\end{array}$ & $\begin{array}{l}2 \text { pr. edge- } \\
\text { stones. }\end{array}$ & $\begin{array}{l}8 \text { table kettles } \\
\text { small size } \\
\text { heated by }\end{array}$ & $\begin{array}{l}2 \text { English } \\
\text { quarters per } \\
\text { working }\end{array}$ & 2 ditto \\
\hline London & $\begin{array}{c}12 \text { horse power, } \\
\text { but the engine } \\
\text { is used also for } \\
\text { other work. }\end{array}$ & none & $\begin{array}{l}9 \text { light } \\
\text { stamp- } \\
\text { ers. }\end{array}$ & $\begin{array}{l}2 \text { pair } \\
\text { rolls, } \\
\text { used } \\
\text { also for } \\
\text { other } \\
\text { purposes }\end{array}$ & $\begin{array}{l}2 \text { pr. edge- } \\
\text { stones, } \\
\text { used also } \\
\text { for other } \\
\text { purposes. }\end{array}$ & $\begin{array}{l}4 \text { table kettles } \\
\text { small size } \\
\text { heated by } \\
\text { fire. } \\
\end{array}$ & $\begin{array}{l}\frac{7}{8} \text { English } \\
\text { quarter per } \\
\text { working } \\
\text { hour. }\end{array}$ & 2 ditto \\
\hline Hull & $\begin{array}{l}18 \text { horse engine, } \\
\text { old construc- } \\
\text { tion. }\end{array}$ & none & 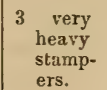 & $\begin{array}{l}1 \text { pair } \\
\text { rolls. }\end{array}$ & $\left\{\begin{array}{l}1 \text { pr. edge- } \\
\text { stones. }\end{array}\right.$ & \begin{tabular}{|l}
3 double case \\
large size \\
steam \\
kettles.
\end{tabular} & $\begin{array}{l}1 \frac{1}{4} \text { English } \\
\text { quarter per } \\
\text { working } \\
\text { hour. }\end{array}$ & 1 ditto \\
\hline Ditto & 22 horse engine & none & 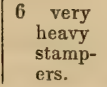 & $\begin{array}{l}2 \text { pair } \\
\text { rolls. }\end{array}$ & $\begin{array}{l}2 \text { pr. edge- } \\
\text { stones. }\end{array}$ & $\left|\begin{array}{l}6 \text { double case } \\
\text { large size } \\
\text { steam } \\
\text { kettles. }\end{array}\right|$ & Not known. & 1 ditto \\
\hline
\end{tabular}

"Rape-seed.-I have not turned my attention to the quantity of oil extracted from this seed; but a French crusher (M. Geremboret), on whom I think one may place considerable dependance, told me that

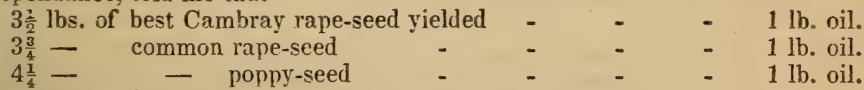

" Rape-seed weighs from 52 to 56 lbs. per imperial bushel."

The following are the heads of a reference of machinery for a seed oil-mill :-

1. Two pairs of cast-iron rollers, 19 inches long, and 10 inches in diameter, fixed in a cast-iron frame, with brasses, wheels, shafts, bolts, scrapers, hoppers, shoes, \&c.

2. Two pairs of edge-stones, 7 feet diameter each, with two bottom stones, 6 feet diameter each, cast-iron upright shafts, sweepers, wheels, shafts, ehairs, brasses, bolts, and scrapers, with driving spur-wheels, \&c.

3. Five steam kettles, with wheels, shafts, and brasses, bolts, breeches, and steam pipes, an upright cast-iron shaft, with chairs and brasses at each end; and a large bevel wheel upon the bottom end of upright shaft, and another, smaller, upon fly-wheel shaft, for the first motions.

4. Five stamper presses, with press plates of cast-iron, cast-iron stamper shaft with 10 arms and 10 rollers, with bosses, brasses, bolts, driving bevel-wheels.

A well made oil-mill, consisting of the above specified parts, will manufacture 200 quarters of seed per week.

I have been assured by practical engineers, conversant in oil-mills, that a donble hydraulic press, with 2 ten-inch rams, will do the work of no more than two of the stamper presses; that is to say, it will work 22 quarters in 24 hours; while three stamper presses will work 33 quarters in the same time, and produce one half more oil 


\section{Castor oil, quantity of,}

\begin{tabular}{|c|c|c|c|}
\hline & Imported. & Retained for consumption. & Exported \\
\hline Year. & Cwts. & Cwts. & Cwts. \\
1835. & $1,109,307$ & 670,205 & 61,296 \\
1836. & 981,585 & 809,559 & 68,515 \\
\hline
\end{tabular}

Duty, from British possessions, 2s. $6 d$. per cwt. ; from foreign, 1s. per lb.

Cocoa-nut oil, quantity of,

\begin{tabular}{|c|c|c|c|}
\hline & Imported. & Retained for consumption. & Exported. \\
\hline Year. & Cwts. & Cwts. & Cwts. \\
1835. & 19,838 & 14,015 & 2,238 \\
1836. & 26,058 & 26,062 & 3,158 \\
1837. & 41,218 & 28,836 & \\
\hline
\end{tabular}

Olive oil, quantity of,

\begin{tabular}{|c|c|c|c|}
\hline & Imported. & Retained for consumption. & Exported. \\
\hline Year. & Galls. & Galls. & Galls. \\
1835. & 606,166 & 554,196 & 283,734 \\
1836. & $2,682,016$ & $1,844,622$ & 150,561 \\
1837. & $1,720,397$ & $1,499,122$ & \\
\hline
\end{tabular}

Duties on olive oil, not of Naples and Sicily, $4 d$. ; of Naples and Sicily, $8 d$. ; and if in ships of these countries, $10 d$. per gallon.

Train oil, spermaceti, and blubber, quantity of,

\begin{tabular}{|c|c|c|c|}
\hline & Imported. & Retained for consumptic & Exported. \\
\hline Year. & Tuns. & Tuns. & Tuns. \\
1835. & 24,197 & 16,114 & 8,035 \\
1836. & 19,489 & 18,722 & 1,365 \\
1837. & 21,823 & 21,286 & \\
\hline
\end{tabular}

Duties on oil taken by British ships, $1 s$.; by foreign fishers, $£ 2618 s$. per tun.

OILS, VOLATILE OR ESSENTIAL; Manufacture of. The volatile oils occur in every part of odoriferous plants, whose aroma they diffuse by their exhalation; but in different organs of different species. Certain plants, such as thyme and the scented labiatce, in general contain volatile oil in all their parts; but others contain it only in the blossoms, the seeds, the leaves, the root, or the bark. It sometimes happens that different parts of the same plant contain different oils; the orange, for example, furnishes three different oils, one of which resides in the flowers, another in the leaves, and a third in the skin or epidermis of the fruit. The quantity of oil varies not only with the species, but also in the same plant, with the soil, and especially the climate; thus in hot countries it is generated most profusely. In several plants, the volatile oil is contained in peculiar orders of vessels, which confine it so closely that it does not escape in the drying, nor is dissipated by keeping the plants for many years. In other species, and particularly in flowers, it is formed continually upon their surface, and flies off at the moment of its formation.

Volatile oils are usually obtained by distillation. For this purpose the plant is introduced into a still, water is poured upon it, and heat being applied, the oil is volatilized by the aid of the watery vapor, at the temperature of $212^{\circ}$, though when alone it would probably not distil over unless the heat were $100^{\circ}$ more. This curious fact was first explained in my New Researches upon Heat, published in the Philosophical Transactions for 1818. Most of the essential oils employed in medicine and perfumery are extracted by distillation from dried plants; only a few, such as those of the rose and orange flower, are obtained from fresh or succulent salted plants. When the mingled vapors of the oil and water are condensed into the liquid state, by the refrigerator of the still, the oil separates, and either floats on the surface or sinks to the bottom of the water. Some oils of a less volatile nature require a higher heat than $212^{\circ}$ to raise them in vapor, and must be dislodsed by adding common salt to the water, whereby the heat being augmented by $15^{\circ}$, they readily come over. If in such distillations too much 
water be added, no oil will be obtained, because it is partially soluble in water; and thus merely an aromatic water is produced. If on the other hand too little water be used, the slant may happen to adhere to the bottom of the still, get partially charred, and thus imjart an emprreumatic odor to the product. But as the quality of water distilled depends less upon the quantity employed, than upon that of the surface exposed to the heat, it is c bvious that by giving a suitable form to the still, we may get rid of every inconvenience. Hence the narrower and taller the alembic is, within certain limits, the greater will be the proportion of oil relative to that of the aromatic water, from like proportions of aqueous and vegetable matter employed. Some place the plants in baskets, and suspend these ummediately over the bottom of the still under the water, or above its surface in the steam. But the best mode in my opinion is to stuff an upright cylinder full of the plants, and to drive down through them, steam of any desired force; its tension and temperature being further regulated by the size of the outlet orifice leading to the condenser. The cylinder should be made of strong copper tinned inside, and incased in the worst conducting species of wood, such as soft deal or sycamore.

The distillation is to be continued as long as the water comes over of a millky appearance. Certain plants yield so little oil by the ordinary processes, notwithstanding every care, that nothing but a distilled water is obtained. In this case, the same water must be poured upon a fresh quantity of the plants in the still; which being drawn over, is again to be poured upon fresh plants; and thus repeatedly, till a certain dose of oil be separated. This being +-2ken off, the saturated water is reserved for a like distillation.

The refrigeratory vessel is usually a worm or serpentine plunged in a tub of water, whose temperature should be generally cold; but for distilling the oils of anise-seed, fennel, \&c., which become concrete at low temperatures, the water should not be cooler than $45^{\circ} \mathrm{F}$.

The liquid product is commonly made to run at the worm end, into a vessel called an Italian or Florentine receiver, which is a conical matrass, standing on its base, with a pipe rising out of the side close to the bottom, and recurved a little above the middle of the flask like the spout of a coffee-pot. The water and the oil collected in this vessel soon separate from each other, according to their respective specific gravities; the one floating above the other. If the water be the denser, it occupies the under portion of the vessel, and continually overflows by the spout in communication with the bottom, while the lighter oil is left. When the oil is the heavier of the two, the receiver should be a large inverted cone, with a stopcock at its apex to run off the oil from the water when the separation has been completed by repose. A funnel, having a glass stopcock attached to its narrow stem, is the most convenient apparatus for freeing the oil finally from any adhering particles of water. A cotton wick dipped in the oil may also serve the same purpose by its capillary action. 'The less the oil is transvased the better, as a portion of it is lost at every transfer. It may occasionally be useful to cool the distilled water by surrounding it with ice, because it thus parts with more of the oil with which it is impregnated.

There are a few essential oils which may be obtained by expression, from the substances which contain them; such as the oils of lemons and bergamot, found in the pellicle of the ripe fruits of the citrus aurantium and medica; or the orange and the citron. The oil comes out in this case with the juice of the peel, and collects upon its surface.

For collecting the oils of odoriferous flowers which have no peculiar organs for imprisoning them, and therefore specdily let them exhale, such as violets, jasmine, tuberose, and hyacinth, another process must be resorted to. Alternate layers are formed of the fresh flowers, and thin cotton fleece or woollen cloth-wadding, previously soaked in a pure and inodorous fat oil. Whenever the flowers have given out all their volatile oil to the fixed oil upon the fibrous matter, they are replaced by fresh flowers in succession, till the fat oil has become saturated with the odorous particles. The cotton or wool wadding being next submitted to distillation along with water, gives up the volatile oil. Perfumers alone use these oils; they employ them either mixed as above, or dissolve them out by means of alcohol. In order to extract the oils of certain flowers, as for instance of white lilies, infusion in a fat oil is sufficient.

Essential oils differ much from each other in their physical properties. Most of them are yellow, others are colorless, red, or brown; some again are green, and a few are blue. They have a powerful smell, more or less agreeable, which immediately after their distillation is occasionally a little rank, but becomes less so by keeping. The odor is seldom as pleasant as that of the recent plant. Their taste is acrid, irritating, and heating, or merely aromatic when they are largely diluted with water or other substances. They are not greasy to the touch, like the fat oils, but on the contrary make the skin fcel rough. They are almost all lighter than water, only a very few falling to the bottom of this liquid; their specific gravity lies between 0.847 and 1.096 ; the first number denoting the density of oil of citron, and the second that of oil of sassafras. Although 
styled volatile oils, the tension of their vapor, as well as its specific heat, is much less than that of water. The boiling point differs in different kinds, but it is usually about $316^{\circ}$ or $320^{\circ}$ Fahr. Their vapors sometimes render reddened litmus paper blue, ajthough they contain no ammonia. When distilled by themselves, the volatile oils are partially decomposed; and the gaseous products of the portion decomposed always carry off a little of the oil. When they are mixed with clay or sand, and exposed to a distilling heat, they are in a great measure decomposed; or when they are passed in vapor through a redhot tube, combustible gases are obtained, and a brilliant porous charcoal is deposited in the tube. On the other hand, they distil readily with water, because the aqueous vapor formed at the surface of the boiling fluid carries along with it the vapor of the oil produced in virtue of the tension which it possesses at the 212th degree Fahr. In the open air, the rolatile oils burn with a shining flame, which deposites a great deal of soct. The congealing point of the essential oils varies greatly; some do not solidify till cooled below $32^{\circ}$, others at this point, and some are concrete at the ordinary temperature of the atmosphere. They comport themselves in this respect like the fat oils; and they probably consist, like them, of two different oils, a solid and a fluid; to which the names stearoptine and eleoptine, or stearessence and oleiessence, may be given. These may be separated from each other by compressing the cooled concrete oil between the folds of porous paper; the stearessence remains as a solid upon the paper; the oleiessence penetrates the paper, and may be recovered by distilling it along with water.

When exposed to the air, the volatile oils change their color, become darker, and gradually absorb oxygen. This absorption commences whenever they are extracted from the plant containing them; it is at first considerable, and diminishes in rapidity as it goes on. Light contributes powerfully to this action, during which the oil disengages a little carbonic acid, but much less than the oxygen absorbed; no water is formed. The oil turns gradually thicker, loses its smell, and is transformed into a resin, which becomes eventually hard. De Saussure found that oil of lavender, recently distilled, had absorbed in four winter months, and at a temperature below $54^{\circ} \mathrm{F} ., 52$ times its volume of oxygen, and had disengaged twice its volume of carbonic acid gases; nor was it yet completely saturated with oxygen. The stearessence of anise-seed oil absorbed at its liquefying temperature, in the space of two years, 156 times its rolume of oxyen gas, and disengaged 26 times its volume of carbonic acid gas. An oil which has begun to experience such an oxydizement is composed of a resin dissolved in the unaltered oil ; and the oil may be separated by distilling the solution along with water. To preserve oils in an unchanged state, they must be put in vials, filled to the top, closed with ground glass stopples, and placed in the dark.

Volatile oils are little soluble in water, yet enough so as to impart to it by agitation their characteristic smell and taste. The water which distils with any oil is in general a saturated solution of it, and as such is used in medicine under the name of distilled water. It often contains other volatile substances contained in the plants, and hence is apt to putrefy and acquire a nauseous smell when kept in perfectly corked bottles; but in vessels partially open, these parts exhale, and the water remains sweet. The waters, however, which are made by agitating volatile oil with simple distilled water, are not apt to spoil by keeping in well-corked bottles.

The volatile oils are soluble in alcohol, and the more so the stronger the spirit is. Some volatile oils, devoid of oxygen, such as the oils of turpentine and citron, are very sparingly soluble in dilute alcohol; while the oils of lavender, pepper, \&c. are considerably so. De Saussure has inferred from his experiments that the rolatile oils are the more soluble in alcohol, the more oxygen they contain. Such combinations form the odoriferous spirits which the perfumers incorrectly call waters, as lavender water, eau de Cologne, eau de jasmin, \&c. They become turbid by admixture of water, which seizes the alcohol, and separates the volatile oils. Ether also dissolves all the essential oils.

These oils combine with several regetable acids, such as the acetic, the oxalic, the succinic, the fat acids (stearic, margaric, oleic), the camphoric, and suberic.

With the exception of the oil of cloves, the volatile oils do not combine with the salifiable bases. They have been partially combined with caustic alkali, as in the case of Starkey's soap. This is prepared by triturating recently fused caustic soda in a mortar, with a little oil of turpentine, added drop by drop, till the mixture has acquired the consistence of soap. The compound is to be dissolved in spirits of wine, filtered, and distilled. What remains after the spirit is drawn off, consists of soda combined with a resin formed in the oil during the act of trituration.

The volatile oils in general absorb six or eight times their bulk of ammoniacal gas; but that of lavender absorbs 47 times.

The essential oils dissolve all the fat oils, the resins, and the animal fats.

In commerce these oils are often adulterated with fat oils, resins, or balsam of capivs dissolved in rolatile oil. This fraud may be detected by putting a drop of the oil on paper, and exposing it to heat. A pure essential oil evaporates without leaving any residuum, 
whilst an oil mixed with any of the above substances leaves a translucent stain upon the paper. If fat oil be present, it will remain undissolved, on mixing the adulterated essential oil with thrice its volume of spirit of wine of specific gravity 0.840 . Resinous matter mixed with rolatile oil is easily detected, being left in the alembic after distillation. ()il diluted with spirit of wine, forms a milky emulsion on the addition of water; the alcoholic part is absorbed by the water, and the oil afterwards found on the surface, in a graduated glass tube, will show by its quantity the amount of the adulteration.

But it is more difficult to detect the presence of a cheap essential oil in a dear one, which it resembles. Here the taste and smell are our principal guides. A few drops of the suspected oil are to be poured upon a bit of cloth, which is to be shaken in the air, and smelled to from time to time. In this way we may succeed in distinguishing the odor of the oil which exhales at the beginning, and that which exhales at the end; a method which serves perfectly to detect oil of turpentine in the finer essential oils. Moreover, when the debased oil is mixed with spirits of wine at sp. gr. $0 \cdot 840$, the oil of turpentine remains in a great measure undissolved. If an oil heavier than water, and an oil lighter than water, be mixed, they may be separated by agitation for some time with that liquid, and then leaving the mixture at rest. Essential oils may also be distinguished by ? careful examination of their respective densities.

Oi! of bitter almonds is prepared by exposing the bitter almond cake, from whicis the bland oil has been expressed, in a sieve to the vapor of water rising within the still. The steam, as it passes up through the bruised almond parenchyma, carries off its volatile oil, and con! unses along with it in the worm. The oil which first comes over, and which falls to the bottom of the water, has so pungent and penetrating a smell, that it is more like cyanogen gas than hydrocyanıc or prussic acid. This oil has a golden yellow color, it is heavier than water; when much diluted, it has an agreeable smell, and a bitter burning taste. When exposed to the air, it absorbs oxygen, and lets fall a heap of crystals of benzoic acid. This oil consists of a mixture of two oils; one of which is volatile, contains hydrocyanic acid, and is poisonous; the other is less volatile, is not poisonous, absorbs oxygen, and becomes benzoic acid. If we dissolve 100 parts of the oil of bitter almonds in spirit of wine, mix with the solution an alcoholic solution of potash, and then precipitate the oil with water, we shall obtain a quantity of cyanide of potash, capable of producing $22 \frac{1}{2}$ parts of Prussian blue. Oil of bitter almonds combines with the alkalis. Perfumers employ a great quantity of this oil in scenting their soaps. One manufacturer in Paris is said to prepare annually $3 \mathrm{cwts}$. of this oil. A similar poisonous oil is obtained by distilling the following substances with water:-the leaves of the peach (amygdalus persica), the leaves of the bay-laurel (prunus lauro-cerasus), the bark of the plum-tree (prunus pudus), and the bruised kerneis of cherry and plumstones. All these oils contain hydrocyanic acid, which renders them poisonous, and they also generate benzoic acid, by absorbing oxygen on exposure to air.

Oil of anise-seed is extracted by distillation from the seeds of the pimpinella anisum. It is either colorless, or has merely a faint yellow color, with the smell and taste of the seed. It concretes in lamellar crystals at the temperature of $50^{\circ}$, and does not melt again till heated to $64^{\circ}$ nearly. Its specific gravity at $61^{\circ}$ is 0.9958 , and at $77^{\circ}, 0.9857$. It is sol uble in all proportions in alcohol of 0.806 ; but only to the extent of 42 per cent. in alco. hol of 0.84 . When it becomes resinous by long exposure to the air, it loses its congealing property. It consists of two oils; a solid stearessence, and a liquid oleiessence, which may be separated by compression of the cold concrete oil.

Oil of bergamot is extracted by pressure from the rind of the ripe fruit of the citrus bergamium and aurantium. It is a limpid, vellowish fluid, having a smell resembling that of oranges. Its specific gravity varies from 0.888 to 0.885 . It bzcomes concrete when cooled a little below $32^{\circ}$.

Oil of cajeput is prepared in the Moluccas, by distilling the dry leaves of the melaleuca leucadendron. Cajeput is a native word, signifying merely a white tree. This oil is green; it has a burning taste, a strong smell of camphor, turpentine, and savine. It is very fluid, and at $48^{\circ}$ has a specific gravity of 0.948 . The color seems to be derived from the copper vessels in which it is imported, so that it is removed by distillation with water, which also separates the oil into two sorts; the first which comes over having a density of 0.897 , the last of 0.920 . This has a green color.

The oil of caraway is extracted from the seeds of the carum carui. It has a pale yellow color, and the smell and taste of the plant. Its specific gravity is 0.960 . The seeds of the cuminum cyminum (cumin) afford an oil similar to the preceding, but not so agreeable. Its specific gravity is 0.975 .

The oil of cassia, from the laurus cassia, is yellow passing into brown, has a specific gravity of 1.071, and affords a crystalline stearessence by keeping in a somewhat open vessel.

The oil of chamomile is extracted by distillation from the flowers of the matricaria chamomilla. It has a deep blue color, is almost opaque, and thick; and possesses the 
peculiar smell of the plant. In the atmosphere it becomes brown and unctuous. If an ounce of oil of lemons be added to 3 pounds of this oil, they make it separate more readily from the adhering water.

Other blue oils, having much analogy with oil of chamomile, are obtained by distilling the following plants : Roman chamomile (anthemis nobilis), the flowers of arnica montana, and those of milfoil (achillaa millefolia). The last has a spec. grav. of 0.852 .

Oil of cinnamon is extracted by distillation from the bark of the laurus cirnamomum. It is produced chiefly in Ceylon, from the pieces of bark unfit for exportation. It is distilled over with difficulty, and the process is promoted by the addition of salt water, and the use of a low still. It has at first a pale yellow color, but it becomes brown with age. It possesses in a high degree both the sweet burning taste, and the agreeable smell of cinnamon. It is heavier than water; its specific gravity being 1.035. It concretes below $32^{\circ} \mathrm{F}$, and does not fuse again till heated to $41^{\circ}$. It is very sparingly soluble in water, and when agitated with it readily separates by repose. It dissolves abundantly in alcohol, and combines with ammonia into a viscid mass, not decomposed on exposure to air.

When oil of cinnamon is kept for a long time, it deposites a stearessence in large regular colorless or yellow crystals, which may be pulverized, and which melt at a very gentle heat into a colorless liquid, which crystallizes on cooling. It has an odor intermediate between that of cinnamon and vanilla; and a taste at first greasy, but afterwards burning and aromatic. It crackles between the teeth. It requires a high temperature for distillation, and becomes then brown and empyreumatic. It is very soluble in alcohol.

The oil of cloves is extracted from the dried flower buds of the caryophyllus aromaticus. It is colorless, or yellowish, has a strong smell of the cloves, and a burning taste. Its specific gravity is 1.061 . It is one of the least volatile oils, and the most difficult to distil. At the end of a certain time it deposites a crystalline concrete $\cdot \mathbf{A}$ similar stearessence is obtained by boiling the bruised cloves in alcohol, and letting the solution cool. The crystals thus formed are brilliant, white, grouped in globules, with out taste and smell. Oil of cloves has remarkable chemical properties. It dissolres in alcohol, ether, and acetic acid. It does not solidify at a temperature of $4^{\circ}$ under $0^{\circ} \mathrm{F}$., even when exposed to that cold for several hours. It absorbs chlorine gas, becomes green, then hrown, and turns resinous. Nitric acid makes it red, and if heated upon it, converts it into oxalic acid. If mixed by slow degrees with one third of its weight of sulphuric acid, an acid liquor is formed, at whose bottom a resin of a fine purple color is found. After being washed, this resin becomes hard and brittle. Alcohol dissolves it, and takes a red color; and water precipitates it of a blood red hue. It dissolres also in ether. When we agitate a mixture of strong caustic soda ley and oil of cloves in equal parts, the mass thickens very soon, and forms delicate lamellar crystals. If we then pour water upon it, and distil, there passes along with the water, a smali quantity of an oil which differs from oil of cloves both in taste and chemical properties. During the cooling, the liquor left in the retort lets fall a quantity of crystalline needles, which being separated by expression from the alkaline liquid, are almost inodorous, but possess an alkaline taste, joined to the burning taste of the oil. These crrstals require for solution from 10 to 12 parts of cold water. Potash ley produces similar effects. Ammoniacal gas transmitted through the oil is absorbed and makes it thick. The concrete combination thus formed remains solid as long as the vial containing it is corked, but when opened, the compound becomes liquid; and these phenomena may be reproduced as many times as we please. Such combinations are decomposed by acids, and the oil set at liberty has the same taste and smell as at first, but it has a deep red color. The alkalis enable us to detect the presence of other oils, as that of turpentine or sassafras, in that of cloves, because they fix the latter, while the former may be volatilized with water by distilling the mixture. The oil of cloves found in commerce is not pure, but contains a mixture of the tincture of pinks or clove-gillyflowers, whose acrid.resin is thereby introduced. It is sometimes sophisticated with other oils.

The oil of elder is extracted by distillation from the flowers of the sambucus nigra. It has the consistence of butter. The watery solution is used in medicine.

Oil of fennel is extracted by distillation from the seeds of the anethum faniculum. It is either colorless or of a yellow tint, has the smell of the plant, and a specific gravity of 0.997. When treated with nitric acid, it affords benzoin. It congeals at the temperature of $14^{\circ} \mathrm{F}$, and then yields by pressure a solid and a liquid oil; the former appearing in erystalline plates. It is used in this country for scenting soap.

Oils of fermented liquors. The substances usually fermented contain a small quantity of essential oils, which become volatile along with the alcoholic vapors in distillation, and progressively increase as the spirits become weaker towards the end of the process. The vapors then condense into a milky liquor. These oils adhere strongly to the alcohol, and give it a peculiar acrid taste. Thev differ according to the vinous wash 
from which they are obtained, and combine with greater or less facility with caustic alkalis.

1. Oil of grain spirits. At the ordinary temperature it is partially a white solid; when cooled lower it assumes the aspect of suet, and therefore consists chiefly of stearessence. Its taste and smell are most offensive; it swims upon the surface of water, and even of spirit containing 30 per cent. of alcohol. It sometimes derives a green color from the copper worm of the still. When heated it fuses and turns yellow. When it has become resinous by the agency of the atmosphere, it gives a greasy stain to paper. It dissolves in 6 parts of anhydrous alcohol, and in two of ether; and is said to crystallize when the spirit solution has been saturated with it hot, and is allowed to cool. By exposure to a freezing mixture, the whiskey which contains it lets it fall. Caustic potash dissolves it very slowly, and forms a soap soluble in 60 parts of water It is absorbed by wood charcoal, and still better by bone black; whereby it may be completely abstracted from bad whiskey. According to Buchner, another oil may also be obtained from the residuum of the second distillation of whiskey, if saturated with sea salt, and again distilled. Thus we obtain a pale yellow fluid oil, which does not concrete with cold, possessed of a disagreeable smell and acrid taste. Its specific gravity is 0.835 . It is soluble inoalcohol and ether.

2. The oil from potato spirits has properties quite different from the preceding. It is obtained in considerable quantity by continuing the distillation after most of the alcohol has come over, and it appears in the form of a yellowish oil, mixed with water and spirits. After being agitated first with water, then with a strong solution of muriate of lime, and distilled afresh, it possesses the following properties ; it is colorless, limpid, has a peculiar smell, and a bitter hot taste of considerable permanence. It leaves no greasy stain upon paper, remains liquid at $0^{\circ} \mathrm{F}$., but cooled below that point it crystallizes like oil of anise-seed. When pure it boils at $257^{\circ} \mathrm{F}$; but at a lower degree, if it contains alcohol. Its specific gravity is 0.821 , or 0.823 when it contains a little water. It burns with a clear flame without smoke, but it easily goes out, if not burned with a wick. It dissolves in small quantity in water, to which it imparts its taste and the properties of forming a lather by agitation. It dissolves in all proportions in alcohol. Chlorine renders it green. Concentrated sulphuric acid converts it into a crimson solution, from which it is precipitated yellow by water. It dissolves in all proportions in acetic acid. Concentrated caustic leys dissolve it, but give it up to water. It does not appear to be poisonous, like the oil of corn spirits; because, when given by spoonfuls to dogs, it produced no other effect but vomiting.

3. The oil of brandy or grape spirits is obtained during the distillation of the fermented residuum of expressed grapes; being produced immediately after the spirituous liquor has passed over. It is very fluid, limpid, of a penetrating odor, and an acrid disagreeable taste. It grows soon yellow in the air. When this oil is distilled, the first portions of it pass unchanged, but afterwards it is decomposed and becomes empyreumatic. It dissolves in 1000 parts of water, and communicates to it its peculiar taste and smell. One drop of it is capable of giving a disagreeable flavor to ten old English gallons of spirits. It combines with the caustic alkalis, and dissolves sulphur.

Oil of juniper is obtained by distilling juniper berries along with water. These should be bruised, because their oil is contained in small sacs or reservoirs, which must be laid open before the oil can escape. It is limpid and colorless, or sometimes of a faint greenish yellow color. Its specific gravity is 0.911 . It has the smell and taste of the juniper. Water, or even alcohol, dissolves very little of it. Gin contains a very minute quantity of this oil. Like oil of turpentine, it imparts to the urine of persons who swallow it, the smell of violets. Oil of juniper is frequently sophisticated with oil of turpentine introduced into the still with the berries; a fraud easily detected by the diminished density of the mixture.

The oil of lavender is extracted from the flowering spike of the lavandula spica. It is yellow, very fluid, has a strong odor of the lavender, and a burning taste. The specific gravity of the oil found in commerce is 0.898 at the temperature of $72^{\circ} \mathrm{F}$., and of 0.877 when it has been rectified. It is soluble in all proportions in alcohol of 0.830 , but alcohol of 0.887 dissolves only 42 per cent. of its weight. The fresh oil detonates slightly when mixed with iodine, with the production of a yellow cloud. There occurs in commerce a kind of oil of lavender known under the name of oil of aspic or oil of spike, extracted by distillation from a wild variety of the lavandula spica, which has large leaves, and is therefore called latifolia. This oil is manufactured in the south of Europe. Its odor is less characteristic than that of the lavender, resembling somewhat that of oil of turpentine, with which it is indeed often adulterated. It is also so cheap as to be sometimes used instead of the latter oil. Oil of lavender deposites, when partially exposed to the air, a concrete oil, which resembles camphor, to the amount of one fourth of its weight.

Oil of lemons is extracted by pressure from the yellow peel of the fruit of the lemon, or 
cutrus medica. In this state it is a yellowish fluid, having a specific gravity of 0.8517 ; but when distilled along with water till thrce fifths of the oil have come over, it is obtained in a colorless state, and of a specific gravity of 0.847 at $72^{\circ} \mathrm{F}$. This oil does $z$ ot become concrete till cooled to $4^{\circ}$ below $0^{\circ} \mathrm{F}$.

The oil of lemons has a very agreeable smell of the fruit, which is injured by distillation. It is soluble in all proportions in anhydrous alcohol, but only 14 parts dissolve in 100 of spirits of wine of specific gravity 0.837 . 'This oil, especially when distilled, forms with muriatic acid similar camphorated compounds with oil of turpentine, absorbing no less than 280 volumes of the acid gas.

Oil of lemons kept long, in ill-corked bottles, generates a quantity of stearessence, which when dissolved in alcohol, precipitated by water, and evaporated, affords brilliant, colorless, transparent needles. Some acetic acid is also generated in the old oil. Accord ing to Brandes, the specific gravity of oil of lemons is 0.8786 .

The oil of mace lets fall, after a certain time, a concrete oil under the form of a crystalline crust, called by John myristicine.

The oil of nutmegs is extracted chiefly from mace, which is the inner epidermis of these nuts. It is colorless, or yellowish, a little viscid with a strong aromatic odor of nutmegs, an acrid taste, and a specific gravity of 0.948. It consists of two oils, which may be easily separated from each other by agitation with water; for one of them, which is more volatile and aromatic, comes to the surface, while the other, which is denser, white, and of a buttery consistence, falls to the bottom. The latter liquefies by the heat of the hand.

The oil of orange flowers, called neroli, is extracted from the fresh flowers of the citrus aurantium. When recently prepared it is yellow; but when exposed for two hours to the rays of the sun, or for a longer time to diffuse daylight, it becomes of a yellowishred. It is very fluid, lighter than water, and has a most agreeable smell. The aqueous solution known under the name of orange-flower water, is used as a perfume. It is obtained either by dissolving the oil in water, or by distilling with water the leaves either fresh or salted; the first being the stronger, but the last being the more fragrant preparation. Orange-flower water obtained by distillation, contains besides the oil, a principle which comes over with it, of a nature hitherto unknown; it possesses the property of imparting to water the faculty of becoming red with a few drops of sulphuric acid. The water formed from the oil alone, is destitute of this property. The intensity of the rosecolor is a test in some measure of the richness of the water in oil.

The oil of parsley is extracted from the apium petroselinum. It is of a pale yellow color, having the smell of the plant, and consists of two oils separable by agitation in water. Its liquid part floats upon the surface in a very fluid form; its stearessence, which falls to the bottom, is butyraceous and crystallizes at a low temperature. This concrete oil melts at $86^{\circ} \mathrm{F}$.

The oil of pepper is extracted from the piper nigrum. In the recent state it is limpid and colorless, but by keeping it becomes yellow. It swims upon the surface of water. In odor it resembles pepper, but is devoid of its hot taste.

The oil. of peppermint is extracted from the mentha piperita. It is yellowish, and endued with a very acrid burning taste. Its specific gravity is 0.920 . At $6^{\circ}$ or $7^{\circ}$ below $0^{\circ} \mathrm{F}$., it deposites small capillary crystals. After long keeping it affords a stearessence resembling camphor, provided the oil had been obtained from the dry plant gathered in flower, but not from distillation of the fresh plant. When artificially cooled, it yields 6 per cent. of stearessence, which crystallizes in prisms with three sides, has an acrid, somewhat rank taste, is soluble in ether and alcoliol, and is thrown down from the latter solution by water in the form of a white powder. Peppermint water is characterized by the sensation of coolness which it diffuses in the mouth.

The oil of pimento is extracted from the envelopes of the fruits of the myrtus pimenta, which aftord 8 per cent. of it. It is yellowish, almost colorless, of a smell analogous to that of cloves, an acrid burning taste, and a specific gravity greater than water. Nitric acid makes it first red, and after the effervescence, of a rusty brown hue. It combines with the salifiable bases, like oil of cloves.

The oil of rhodium is extracted from the wood of the convolvolus scoparius. It is very fluid, and has a yellow color, which in time becomes red. It has somewhat of the rose odor, and is used to adulterate the genuine otto. Its taste is bitter and aromatic, which it imparts to the otto as well as its fluidity.

The oil of roses, called also the attar or otto, is extracted by distillation from the petals of the rosa centifolia and sempervirens. Our native roses furnish such small quantities of the oil, that they are not worth distilling for the purpose. The best way of operating is to return the distilled water repeatedly upon fresh petals, and eventually to cool the saturated water with ice; whereby a little butyraceous oil is deposited. But the oil thus obtained has not a very agreeable odor, being injured by the action of the air in the repeated distillations. In the East Indies, the attar is obtained by stratifying rose 
leares in earthen pans in alternate layers, with the oleiferous seeds of a species of digitalis, called gengeli, for several days, in a cool situation. The fat oil of the seed absorbs the essential oil of the rose. By repeating this process with fresh leaves and the same seed. this becomes eventually swollen, and being then expressed fumishes the oil. The turbid liquid thus obtained is left at rest, in well-closed vessels, where it gets clarified. The layer of oil that floats on the top is then drawn off by a capillary cotton wicli, and subjected to distillation along with water, whereby the volatile otto is separated from the fat seed-oil.

The oil of roses is colorless, and possesses the smell of roses, which is not however agreeable, unless when diffused, for in its concentrated state it is far from pleasant to the nostrils, and is apt to occasion headaches. Its taste is bland and sweetish. It is lighter than water, and at the temperature of $92^{\circ}$, its specific gravity compared to that of water at $60^{\circ}$ is 0.832 . At lower temperatures it becomes concrete and butyraceous; and afterwards fuses at $90^{\circ}$. It is but slightly soluble in alcohol; 1000 parts of this liquid at 0.806 dissolving only $7 \frac{1}{2}$ parts at $58^{\circ} \mathrm{F}$. This oil consists of two parts, the stearessence and oleiessence; the latter being the more volatile odoriferous portion.

The oil of rosemary is extracted from the rosmarinus officialis. It is as limi nd as water, has the smell of the plant, and in other respects resembles oil of turpentine. The oil found in commerce has a specific gravity of 0.911 , which becomes 0.8886 by rectification. It boils at $320^{\circ} \mathrm{F}$. (occasionally at $329^{\circ}$ ). It is soluble in all portions in alcohol of $0 \cdot 830$. When liept in imperfectly closed vessels, it deposites a stearessence to the amount of one tenth of its weight, resembling camphor. It is sometimes adulterated with oil of turpentine, a fraud easily detected by adding anhydrous alcohol, which dissolves only the oil of rosemary.

The oil of saffion is extracted from the stigmata of the crocus sativus. It is yellow, very fluid, falls to the bottom of water, diffuses the penetrating odor of the plant, and has an acrid and bitter taste. It is narcotic.

The oil of sassafras is extracted from the woody root of the laurus sassafras. It is colorless, but at the end of a certain time it becomes yellow or red. It has a peculiar, sweetish, pretty agreeable, but somewhat burning taste. Its specific gravity is $1 \cdot 094$. According to Bonastre, this oil separates by agitation with water into an oil lighter and an oil heavier than this fluid. When long kept, it deposites a stearessence in transparent and colorless crystals, which have the smell and taste of the liquid oil.

The oil of savine is extracted from the leaves of the juniperus sabina. It is limpid, and has the odor and taste of the plant, which is one more productive of volatile oil than any other.

The oil of tansy has a specific gravity of 0.946 , the penetrating odor of the tanacetum vulgare, with an acrid and bitter taste.

Oil of turpentine, commonly called essence of turpentine. It is extracted from several species of turpentine, a semi-liquid resinous substance which exudes from certain trees of the pine tribe, and is obtained by distilling the resin along with water. This oil is the cheapest of all the volatile species, and, as commonly sold, contains a little resin, from which it may be freed by re-distillation with water. It is colorless, limpid, very fluid, and has a very peculiar smell. Its specific gravity at $60^{\circ}$ is 0.872 ; that of the spirit on sale in the shops is $0 \cdot 876$. This oil always reddens litmus paper, because it contains a little succinic acid.

100 parts of spirits of wine, of specific gravity 0.84 , dissolve only $13 \frac{1}{2}$ of oil of turpentine at $72^{\circ} \mathrm{F}$. When agitated with alcohol at 0.830 the oil retains afterwards one fifth of its bulk of the spirit; hence this proposed method for purifying oil of turpentine is defective. The oil, if left during four months in contact with air, is capable of absorbing 20 times its bulk of oxygen gas. One volume of rectified oil of turpentine absorbs at the temperature of $72^{\circ}$, and under the common atmospheric pressure, 163 times its volume of muriatic acid gas, provided the vessel be kept cool with ice. This mixture being allowed to repose for 24 hours, produces out of the oil from 26 to 47 per cent. of a white crystalline substance, which subsides to the bottom of a brown, smoking, translucent liquor. Others say that 100 parts of oil of turpentine yield 110 of this crystalline matter, which was called by Kind, its discoverer, artificial camphor, from its resemblance in smell and appearance to this substance. Both the solid and the liquid are combinations of muriatic acid and oil of turpentine; indicating the existence of a stearine and an oleine in the latter substance. The liquid compound is lighter than water, and is not decomposed by it, nor does it furnish any more solid matter when more muriatic gas is passed through it. The solid compound, after being washed first with water containing a little carbonate of soda, then with pure water, and finally purified by sublimation with some chalk, lime, ashes, or charcoal, appears as a white, translucent, crystalline body, in the form of flexible, tenacious needles. It swims upon the surface of water, diffuses a faint smell of camphor, commonly mixed with that of oil of turpentine, and has rather an aromatic than a camphorated taste. It does not redden litmus paper. Water 
dissolves a very minute quantitr; but cold alcohol of $0 . \$ 06$ dissolves fully one thi.d of its weight, and lot much more, depositine, as it cools, this excess in the furm of crystals. The solution is not precipitated by nitrate of silver, which shows that the nature of the muriatic acid is perfectly mastied by the combination. It is composed, in 100 ? arts, of i6. 4 carton, 9.6 hydrogen, and 14 muriatic acid. The muriatic acid, or cllorine, das be separated by distilling an alcoholic solution of the artificial camphor 12 or 14 times in succession with slaked lime.

Oil of turpentine is best preserved in casks enclosed within others, with water between the two. Its principal use is for making ramishes, and as a remedy for the tape-worm.

The oil of thyme is extracted from the thymus serpylium. It is reduish rellow, has an agreeable smell, and, after being long kiept, it lets fall a crystalline stearessence. It is used merely as a perfume.

The oil of wormucod is extracted from the artemisia absinthium. It is rellow, or sometimes green, and possesses the odor of the plant. Its taste resembles that of wormwood, but without its bitterness. Its specific gravity is 0.9703, according to Brisson, and 0.9725 , according to Brandes. It detonates with iodine when it is fresh. Treated with nitric acid of $1 \cdot 25$ specific grarity, it becomes first blue, and after some time brown.

OIL OF VITRIOL is the old name of concentrated StLPHCrIC AcrD.

OLEATES are saline compounds of oleic acid with the bases.

OLEFIANT GAS is the name originally giren to bi-carbureted hydrogen.

OLEIC ACID is the acid produced by saponifying olire oil, and then separating the base by dilute sulphuric or muriatic acid. See FATs, and STEArnNe.

OLEINE is the thin oily part of fats, naturally associated in them with glrcerine, margarine, and stearine.

OLIBANCM is a gum-resin, used only as incense in Roman Catholic churches.

OLTE OIL. See OrLs, unctuous.

ONYX, an ornamental stone of little value; a subspecies of quartz.

OOLITE is a species of limestone composed of glcbules clustered together, commonly without any risible cement or base. These vary in size from that of small pin-heads to peas; ther sometimes occur in concentric layers, at others they are compact, or radiated from the centre to the circumference; in which case, the oolite is called roogenstein by the German mineralogists. In geology the oolitic'series includes all the strata between the iron sand abore and the red mari below. It is the great repository of the best architectural materials which the midland and eastern parts of England produce; it is dirided into three systems :-

1. The upper oolite, including the argillo-calcareous Purbeck strata, which separate the iron and oolitic series: the oolitic strata of Portland, Tisburt, and Arlesbury; the caleareous sand and concretions, as of Shotorer and Thame; and the argillo-calcareous formation of Kimmeridge, the oak tree of Smith.

2. The middle colite; the oolitic strata associated with the coral rag; calcareous sand and grit; great Oxford elar, between the oolites of this and the following ststem.

3. The louer oolite; which contains numerous oolitic strata, occasionalit subdirided by thin argillaceous beds; including the cornbrash, forest marble, schistose oolite, and sand of Stonesfield and Hinton, great nolite and inferior oolite; calcareo-silicious sand rassing into the inferior oolite: great argillo-calcareous formation of lias, and lias marl, constituting the base of the whole series.

These formations oceupy a zone 30 miles broad in England.

OOST, or OAST ; tie trivial or prorincial name of the store in which the picked hops are dried.

OPAL; an ornamertal stone of moderate value. See LAPIDARY.

OPERAMETER is the name giren to an apparatus patented in Februarr, 1829, by Samuel Walker, cloth manufacturer, in the parish of Leeds. It consists of a train of toothed wheels and pinions enclosed in a box, haring indexes attached to the central arbor, like the hands of a clock, and a dial plate; wherebr the number of rotations of a shaft projecting from the posterior part of the box is shown. If this shaft be connected by any convenient means to the working parts of a gig mill, shearing frame, or any other machinerr of that kind for dressing cloths, the number of rotations made br the operating machine will be exhibited br the indexes upon the dial plate of this apparatus. In dressing cloths, it is often found that too little or too much work has been expended upon them, in consequence of the unskilfulness or inattention of the workmen. Br the use of the operameter, that eril will be aroided, as the master mar regulate and prescribe beforehand by the dial the number of turns which the wheels should perform.

A similar cloch-work mechanism, called a counter, has been for a great many rears employed in the cotton factories to indicate the number of rerolutions of the main shaft of the mill, and of course the quantity of yarn that might or should be spun, or of cloth that might be woren in the power looms. A common pendulum or spring clock is 
commonly set up alongside of the counter; and sometimes the indexes of both are regulated to go together, when the mill performs its average work.

OPIU M, is the juice which exudes from incisions made in the heads of ripe poppies, (paputer somniferum, ) rendered concrete by exposure to the air and the sun. The best opium which is found in the European markets comes from Asia Minor and Egypt; what is imported from India is reckoned inferior in quality. This is the most valuable of all the vegetable products of the gum-resin family : and very remarkable for the complexity of its chemical composition. Though examined by many able analysts, it still requires further elucidation.

Opium occurs in brown lumps of a rounded form about the size of the fist, and often larger; haring their surface covered with the seeds and leaves of a species of romex, for the purpose of preventing the mutual adhesion of the pieces in their semiindurated state. These seeds are sometimes introduced into the interior of the masses to increase their weight; a fraud easily detected by cutting them across. Good opium Is hard in the cold, but becomes flexible and doughy when it is worked between the hot hands. It has a characteristic smell, which by heat becomes stronger, and very offensive to the nostrils of many persons. It has a very bitter taste. Water first softens, and then reduces it to a pasty magma. Proof spirit digested upon opium forms laudanum, being a better solution of its active parts than can be obtained by either water or strong alcohol alone. Water distilled from it acquires its peculiar smell, but carries over no volatile oil.

Opium was analyzed by Bucholz and Braconnot, but at a period anterior to the knowledge of the alkaline properties of morphia and opian (narcotine). Bucholz found in 100 parts of it, 9.0 of resin; 30.4 of gum; 35.6 of extractive matter; 4.8 of caoutchouc; 11.4 of gluten; 2.0 of ligneous matter, as seeds, leaves, \&c. ; 6.8 of water and loss. John, who made his analysis more recently, obtained $2 \cdot 0$ parts of a rancid nauseous fat; 12.0 of a brown hard resin; 10.0 of a soft resin ; 2 of an elastic substance; 12.0 of morphia and opian; 1.0 of a balsamic extract; 25.0 of extractive matter; 2.5 of the meconates of lime and magnesia; 18.5 of the epidermis of the heads of the poppy; 15 of water, salts, and odorous matter.

In the Numbers of the Quarterly Journal of Science for January and June, 1830, I published two papers upon opium and its tests, containing the results of researches made upon some porter which had been fatally dosed with that drug; for which crime, a man and his wife had been capitally punished, about a year before, in Scotland.* From the first of these papers the following extract is made :-

"Did the anodyne and soporific virtue of opium reside in one definite principle, chemical analysis might furnish a certain criterion of its powers. It has been pretty generally supposed that this desideratum is supplied by Sertürner's discovery of morphia. Of this narcotic alkali not more than 7 parts can be extracted by the most rigid analysis from 100 of the best Turkey opium; a quantity, indeed, somewhat above the arerage result of many skilful chemists. Were morphia the real medicinal essence of the poppy, it should display, when administered in its active saline state of acetate, an operation on the living system commensurate in energy with the fourteen-fold concentration which the opium has undergone. But so far as may be judged from the most authentic recent trials, morphia in the acetate seems to be little, if any, stronger as a narcotic than the heterogeneous drug from which it has been eliminated. Mr. John Murrar's experiments would, in fact, prove it to be greatly weaker; for he gave 2 drachms of superacetate of morphia to a cat, without causing any poisonous disorder. This is perhaps an extreme case, and may seem to indicate either some defect in the preparation, or an uncommon tenacity of life in the animal. To the same effect Lassaigne found that a doo lived 12 hours after 36 grains of acetate of morphia in watery solution had been injected into its jugular vein. The morphia meanwhile was entirely decomposed by the vital forces, for none of it could be detected in the blood drawn from the animal at the end of that period. Now, from the effects produced by 5 grains of watery extract of opium, injected by Orfila into the veins of a dog, we may conclude that a quantity of it, equivalent to the above dose of the acetate of morphia, would have proved speedily fatal.

" Neither can we ascribe the energy of opium to the white crystalline substance called narcotine, or opian, extracted from it by the solvent agency of sulphuric ether; for Orfila assures us that these crystals may be swallowed in various forms by man, even to the amount of 2 drachms in the course of 12 hours, with impunity; and that a drachm of it dissolved in muriatic or nitric acid may be administered in the food of a dog without producing any inconvenience to the animal. It appears, however, on the same authority,

* A country merchant travelling in a steam-boat upon the river Clyde, who had incautiously displaved a good deal of money, was poisoned with porter charged with laudanum. The contents of the dead man's tomach were sent to me for analysis. 
that 30 grains of it dissolved in acetic or sulphuric acid caused dogs that had swallowen the dose to die under convulsions in the space of 24 hours, while the head was thrown backwards on the spine. Oil seems to be the most potent menstruurn of narcotine; for 3 grains dissolved in oil readily kill a dog, whether the dose be introduced into the stom. ach or into the jugular vein.

"Since a bland oil thus seems to develop the peculiar force of narcotine, and since opium affords to ether, and also to ammonia, an unctuous or fatty matter, and a resin (the caoutchouc of Bucholz) to absolute alcohol, we are entitled to infer that the activity of opium is due to its state of composition, to the union of an oleate or margarate of narcotine with morphia. The meconic acid associated with this salifiable base has no narcotic power by itself, but may probably promote the activity of the morphia."

Opian or narcotine, and morphia, may be well prepared by the following process. The watery infusion of opium being evaporated to the consistence of an extract, every 3 parts are to be diluted with one and a half parts in bulk of water, and then mixed in a retort with 20 parts of ether. As soon as 5 parts of the ether have been distilled over, the narcotic salt contained in the extract will be dissolved. The fluid contents of the retort are to be poured hot into a vessel apart, and the residuum being washed with 5 other parts of ether, they are to be added to the former. Crystals of narcotine will be obtained as the solution cools. The remaining extract is to be diluted in the retort with a little water, and the mixture set aside in a cool place. After some time, some narcotine will be found crystallized at the bottom. The supernatant liquid thus freed from narcotine being decanted off, is to be treated with caustic ammonia; and the precipitate thrown upon a filter. This, when well washed and dried, is to be boiled with a quantity of spirit of wine at 0.84 , equal to thrice the weight of the opium employed, containing 6 parts of animal charcoal for every hundred parts of the drug. The alcoholic solution being filtered hot, affords, on cooling, colorless crystals of morphia.

This alkali may be obtained by a more direct process without alcohol or ether. A solution of opium in vinegar, is to be precipitated by ammonia ; the washed precipitate is to be dissolved in dilute muriatic acid, the solution is to be boiled along with powdered bone black, filtered, and then precipitated by ammonia. This, when washed upon a filter and dried, is white morphia, which may be dissolved in hot alcohol, if fine crystals be wanted. See Morphia.

Opium, quantity of,

\begin{tabular}{|c|c|c|c|}
\hline & Imported. & $\begin{array}{l}\text { Retained for con- } \\
\text { sumption. }\end{array}$ & Exported. \\
\hline $\begin{array}{l}\text { Year. } \\
1835 . \\
1836 .\end{array}$ & $\begin{array}{l}\text { Lbs. } \\
85,481 \\
130,794\end{array}$ & $\begin{array}{c}\text { Lbs. } \\
31,181 \\
38,943\end{array}$ & $\begin{array}{l}\text { Lbs. } \\
74,126 \\
70,824\end{array}$ \\
\hline
\end{tabular}

Duty, at present, 1 s. per $1 \mathrm{~b}$.

OPOBALSAM is the balsam of Peru in a dry state.

OPOPONAX is a gum-resin resembling gum ammoniac. It is occasionally used in medicine.

ORANGE DYE is given by a mixture of red and yellow dyes in various proportions. Annotto alone dyes orange; but it is a fugitive color.

ORCINE is the name of the coloring principle of the lichen dealbatus. The lichen dried and pulverized is to be exhausted by boiling alcohol. The solution filtered hot, lets fall in the cooling crystalline flocks, which do not belong to the coloring matter. The supernatant alcohol is to be distilled off, the residuum is to be evaporated to the consistence of an extract, and triturated with water till this liquid will dissolve no more. The aqueous solution reduced to the consistence of sirup; and left to itself in a cool place, lets fall, at the end of a few days, long brown brittle needles, which are to be freed by pressure from the mother water, and dried. That water being treated with animal charcoal, filtered and evaporated, will yield a second crop of crystals. These are orcine. Its taste is sweet and nauseous; it melts readily in a retort into a transparent liquid, and distils without undergoing any changes. It is soluble in water and alcohol. Nitric acid colors it blood-red; which color afterwards disappears. Subacetate of lead precipitates it completely. Its conversion into the archil red is effected by the action of an alkali, in contact with the air. When dissolved, for example, in ammonia, and exposed to the atmosphere, it takes a dirty brown red hue; but when the orcine is exposed to air charged with vapors of ammonia, it assumes by degrees a fine violet color. To obtain this result, the orcine in powder should be placed in a capsule, alongside of a saucer containing water of ammonia; and both should be covered by a large bell glass; whenever the orcine has acquired a dark 
brown cast, it must be withdrawn from under the bell, and the excess of ammonia be allowed to rolatilize. As soun as the smell of ammonia is gone, the orcine is to be dissolvel in water; and then a few drops of ammonia being poured into the brownish liquid, it assumes a magnificent reddish-violet color. Acetic acid precipitates the red lalie of lichen.

ORES (Mines, Fr.; Erze, Germ.), are the mineral bodies which contain so much metal as to be worth the smelting, or being reduced by fire to the metallic state. The substances naturally combined with metals, which mask their metallic characters, are chiefly uxygen, chlorine, sulphur, phosphorus, selenium, arsenic, water, and several acids, of which the carbonic is the most common. Some metals, as gold, silver, platinum, often occur in the metallic state, either alone, or combined with other metals, constituting what are called native alloyss.

I have described in the article MrNe, the general structure of the great metallic repositories within the earth, as well as the most approved methods of bringing them to the surface; and in the article Metaliurgr, the various mechanical and chemical operations requisite to reduce the ores into pure metals. Under each particular metal, moreover, in its alphabetical place, will be found a srstematic account of its most important ores.

Relatively to the theory of the smelting of ores, the following observations may be made. It is probable that the coaly matter employed in that process is not the immediute agent of their reduction; but the charcoal seems first of all to be transformed by the atrospherical oxygen into the oxyde of carbon; which gaseous product then surrounds and penetrates the interior substance of the oxydes, with the effect of decomposing them, and carrying off their oxygen. That this is the true mode of action, is evident from the well-known facts, that bars of iron, stratified with pounded charcoal, in the steel cementation-chest, most readily absorb the carbonaceous principle to their innermost centre, while their surfaces get blistered by the expansion of carbureted gases formed within; and that an intermixture of ores and charcoal is not always necessary to reduction, but merely an interstratification of the two, without intimate contact of the particles. In this case, the carbonic acid which is generated at the lower surfaces of contact of the strata, rising up through the first bed of ignited charcoal, becomes converted into carbonic oxyde; and this gaseous matter, passing up through the next laver of ore, seizes its oxygen, reduces it to metal, and is itself thereby transformed once more into carbonic acid; and so on in continual alternation. It may be laid down, however, as a general rule, that the reluction is the more rapid and complete, the more intimate the mixture of the charcoal and the metallic oxyde has been, because the formation of both the carbonic acid and carbonic oxyde becomes thereby more east and direct. Indeed, the cementation of iron bars into steel will not succeed, unless the charcoal be so porous as to contain, interspersed, enough of air to faror the commencement of its conversion into the gaseous oxyde; thus acting like a ferment in brewing. Hence also finely pulverized charcoal does not answer well; unless a quantity of ground iron cinder or oxyde of manganese be blended with it, to afford enough of oxygen to begin the generation of carbonic oxyde gas; whereby the successive transformations into acid, and oxyde, are put in train.

ORPI.MENT (Eng. and Fr., Yellow sulphuret of arsenic; Operment, Rauschgelb, Germ.), occurs in indistinct crystalline particles, and sometimes in oblique rhomboidal prisms; but for the most part, in kidney and other imitative forms; it has a scaly and granular aspect; texture foliated, or radiated; fracture small granular, passing into conchoidal; splintery, opaque, shining, with a weak diamond lustre; lemon, orange, or honey yellow; sometimes green; specific gravity, $3 \cdot 44$ to $3 \cdot 6$. It is found in floetz rocks, in marl, clay, sand-stone, along with realgar, lead-glance, pyrites, and blende, in many parts of the world. It volatilizes at the blowpipe. It is used as a pigment.

The finest specimens come from Persia, in brilliant yellow masses, of a lamellar texsure, called golden orpiment.

Artificial orpiment is manufactured chiefly in Saxonr, by subliming in cast iron cucurbits, surmounted by conical cast-iron capitals, a mixture in due proportions of sulphur and arsenious acid (white arsenic). As thus obtained, it is in yellow compact opaque masses, of a glassy aspect; affording a powder of a pale yellow color. Genuine orpiment is often adulterated with an ill-made compound; which is sold in this country br the preposterous name of king's yellow. This fictitious substance is frequently nothing else than white arsenic combined with a little sulphur; and is quite soluble in waler. It is therefore a deadly poison, and has been administered with criminal intentions and fatal effects. I had occasion, some rears ago, to examine such a specimen of king's yellow, with which a woman had killed her child. A proper ¿nsoluble sulphuret of arsenic, like the native or the Saxon, may be prepared by transmitting sulphureted hydrogen gas through any arsenical solution. It consists of 38.09 sulphur, and 60.92 of metallic arsenic, and is not remarkably poisonous. The finest 
kinds of native orpiment are reserved for artists; the inferior are used for the indigo vat.

They are all soluble in alkaline leys, and in water of ammonia.

ORYCTNOGNOSY is the name given by Werner to the knowledge of minerals; and is therefore synonymous with the English term Mineralogy.

OSTEOCOLLA is the glue obtained from bones, by removing the earthy phos phates with muriatic acid, and dissolving the cartilaginous residuum in water at a tem perature considerably above the boiling point, by means of a digester. It is a very indifferent article.

OSMIUM is a metal discovered by Mr. Tennant in 1803, among the grains of native platinum. It occurs also associated with the ore of iridium. As it has not been applied to any use in the arts, I shall reserve any chemical observations that the subject may require for the article Platinum.

OXALATES are saline compounds of the bases with

OXALIC ACID (Acide oxalique, Fr.; Sauerkleesaïre, Germ.), which is the object of a considerable chemical manufacture. It is usually prepared upon the small scale by digesting four parts of nitric acid of specific gravity $1 \cdot 4$, upon one part of sugar, in a glass retort; but on the large scale, in a series of salt-glazed stoneware pipkins, two thirds filled, and set in a water bath. The addition of a little sulphuric acid has been found to increase the product. 15 pounds of sugar yield fully 17 pounds $c$. the crystalline acid. This acid exists in the juice of wood sorrel, the oxalis acetosella, in the state of a bioxalate; from which the salt is extracted as an object of commerce in Switzerland, and sold under the name of salt of sorrel, or sometimes, most incorrectly, under that of salt of lemons.

Some prefer to make oxalic acid by acting upon 4 parts of sugar, with 24 parts of nitric acid of specific gravity $1 \cdot 220$, heating the solution in a retort till the acid begins to decompose, and keeping it at this temperature as long as nitrous gas is disengaged. The sugar loses a portion of its carbon, which combining with the oxygen of the nitric acid, becomes carbonic acid, and escapes along with the deutoxyde of nitrogen. The remaining carbon and hydrogen of the sugar being oxydized at the expense of the nitric acid, generate a mixture of two acids, the oxalic and the malic. Whenever gas ceases to issue, the retort must be removed from the source of heat, and set aside to cool; the oxalic acid crystallizes, but the malic remains dissolved. After draining these crystals upon a filter funnel, if the brownish liquid be further evaporated, it will furnish another crop of them. The residuary mother water is generally regarded as malic acid, but it also contains both oxalic and nitric acids; and if heated with 6 parts of the latter acid, it will yield a good deal more oxalic acid at the expense of the malic. The brown crystals now formed being, however, penetrated with nitric, as well as malic acid, must be allowed to dry and effloresce in warm dry air, whereby the nitric acid will be got rid of without injury to the oxalic. A second crystallization and efflorescence will entirely dissipate the remainder of the nitric acid, so as to afford pure oxalic acid at the third crystallization. Sugar affords, with nitric acid, a purer oxalic acid, but in smaller quantity, than saw-dust, glue, silk, hairs, and several other animal and vegetable substances.

Oxalic acid occurs in aggregated prisms when it crystallizes rapidly, but in tables of greater or less thickness when slowly formed. They lose their water of crystallization in the open air, fall into powder, and weigh 0.28 less than before; but still retain 0.14 parts of water, which the acid does not part with except in favor of another oxyde, as when it is combined with oxyde of lead. The effloresced acid contains 20 per cent. of water, according to Berzelius. By my analysis, the crystals consist of three prime equivalents, of water $=27$, combined with one of dry oxalic acid $=36 ;$ or in 100 parts, of 42.86 of water with $57 \cdot 14$ of acid. The acid itself consists of 2 atoms of carbon $=12,+3$ of oxygen $=24$; of which the sum is, as above stated, 36 . This acid has a sharp sour taste, and sets the teeth on edge; half a pint of water, containing only $1 \mathrm{gr}$. of acid, very sensibly reddens litmus paper. Nine parts of water dissolve one part of the crystals at $60^{\circ} \mathrm{F}$. and form a solution, of spec. grav. $1 \cdot 045$, which when swallowed acts as a deadly poison. Alcohol also dissolves this acid. It differs from all the other acid products of the vegetable kingdom, in containing no hydrogen, as I demonstrated (in my paper upon the ultimate analysis of organic bodies, published in the Phil. Trans. for 1822), by its giving out no muriatic acid gas, when heated in a glass tube with calomel or corrosive sublimate.

Oxalic acid is employed chiefly for certain styles of discharge in calico-printing (which see), and for whitening the leather of boot-tops. Oxalate of ammonia is an excellent reagent for detecting lime and its salts in any solution. The acid itself, or the bi-oxalate of potash, is often used for removing ink or iron-mould stains from linen.

A convenient plan of testing the value of peroxyde of manganese for bleachers, \&c., originally proposed by Berthier, has been since simplified by Dr. Thomson, as follows. In a poised Florence flask weigh 600 grains of water, and 75 grains of crystallized oxalic 
acid; add 50 grains of the manganese, and as quickly as possible afterwards from 150 to 200 grains of concentrated sulphuric acid. Cover the mouth of the flask with paper, and leave it at rest for 24 hours. The loss of weight it has now suffered, corresponds cxactly to the weight of peroxyde of manganese present; because the quantity of carbonic acid producible by the reaction of the oxalic acid with the peroxyde, is precisely equal to the weight of the peroxyde, as the doctrine of chemical equivalents shows.

OXYDES are neutral compounds, containing oxygen in equivalent proportion.

OXYSELS are salts, consisting of oxygenated acids and oxydes, to distinguish them from the HaLOSELS, which are salts consisting of one of the archæal elements; such as chlorine, iodine, bromine, \&c. combined with metals. See SALT.

OXYGEN (Oxygène, Fr.; Sauerstoff, Germ.), is a body which can be examined only in the gaseous form; for which purpose it is most conveniently obtained in a pure state by exposing chlorate of potash, or red oxyde of mercury, in a glass retort, or recurved tube, to the heat of a spirit lamp; 100 grains of the salt yield 115 cubic inches of gas. One pound of nitre, ignited in an iron retort, gives out about 1200 cubic inches of oxygen, mixed with a little nitrogen. The peroxyde of manganese also affords it, either by ignition alone in an iron or earthen retort, or by a lamp heat in a glass retort, when mixed with sulphuric acid. Oxygen is void of taste, color, and smell. It possesses all the mechanical properties of the atmosphere. Its specific gravity is $1 \cdot 1026$ compared to air 1.0000; whence 100 cubic inches of it weigh 33.85 grains. Combustibles, even iron and diamonds, once kindled, burn in it most splendidly. It forms 21 parts in $10 t \bar{y}$ volume of air, bcing the constituent essential to the atmospheric functions of supporting animal and vegetable life, as well as flame.

The full development of this subject in its multifarious relations, will be discussed in my forthcoming new system of chemistry.

OXYGENATED-MURIATIC, and OxymuRIATIC, are the names originally given by the French chemists, from false theoretical notions, to chlorine, which Sir H. Davy proved to be an undecompounded substance.

\section{P.}

PACKFONG is the Chinese name of the alloy called white copper, or German silver.

PACO, or PACOS, is the Peruvian name of an earthy-looking ore, which con. sists of brown oxyde of iron, with imperceptible particles of native silver disseminated through it.

PADDING MACHINE (Machine d̀ plaquer, Fr.; Klatsch, or Grundirmaschine, Germ.), in calico-printing, is the apparatus for imbuing a piece of cotton cloth uniformly with any mordant. In fig. 774, A B c D represents in section a cast iron

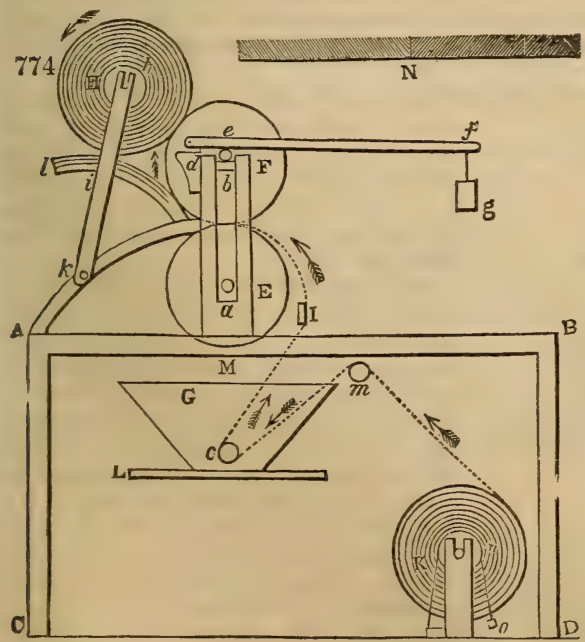
frame, supporting two opposite standards above $\mathrm{M}$, in whose vertical slot the gudgeons $a b$, of two copper or bronze cylinders $\mathbf{E} F$, run; the gudgeons of $\mathbf{E}$ turn upon fixed brasses or plummer blocks; but the superior cylinder $F$ rests upon the surface of the under one, and may be pressed down upon it with greater or less force by means of the weighted lever $d e f g$, whose centre of motion is at $d$, and which bears down upon the axle of $F$. $\mathbf{K}$ is the roller upon which the pieces of cotton cloth intended to be padded are wound; several of them being stitched endwise together. They receive tension from the action of a weighted belt $0, n$, which passes round a pulley $n$, upon the end of the roller $\mathrm{K}$. The trough $\mathrm{G}$, which contains the coloring matter or mordant, rests beneath the cylinder upon the table $\mathbf{L}$, or other convenient support. About two inches above the bottom of the trough, there is a copper dip-roller c, under which the cloth passes, after going round the guide roller $m$. Upon escaping from the trough, it is drawn over the half-round stretcher-bar at $\mathrm{I}$, grooved 
obliquely right and left, as shown at $N$, whereby it acquires a diverging exiension from the middle, and enters with a smooth surface between the two cylinders $\boldsymbol{F} \boldsymbol{F}$. These are lapped round 6 or 7 times with cotton cloth, to soften and equalize their pressure. The piece of goods glides obliquely upwards, in contact with one third of the cylinder $\mathrm{F}$, and is finally wound about the uppermost roller $\mathrm{H}$. The gudgeon of $\mathbf{H}$ revolves in the end of the radius $h, k$, which is jointed at $k$, and moveable by a mortise at $\imath$ along the quadrantal arc towards $l$, as the roller $\mathrm{K}$ becomes enlarged by the convolutions of the web. The under cylinder $\mathrm{E}$ receives motion by a pulley or rigger upon its opposite end, from a band connected with the driving-shaft of the printshop. To ensure perfect equability in the application of the mordant, the goods are in some works passed twice through the trough; the pressure being increased the second time by sliding the weight $g$ to the end of the lever $d f$.

A view of a padding machine in connexion with the driving mechanism is given unde: Hot Flue; see also Starching Machine.

PAINT. See Rougr.

PAINTS, GRINDING OF. There are many pigments, such as common orpiment, or king's yellow, and verdigris, which are strong poisons; others which are very deleterious, and occasion dreadful maladies, such as white lead, red lead, chrome yellow, and vermilion; none of which can be safely ground by hand with the slab and muller,
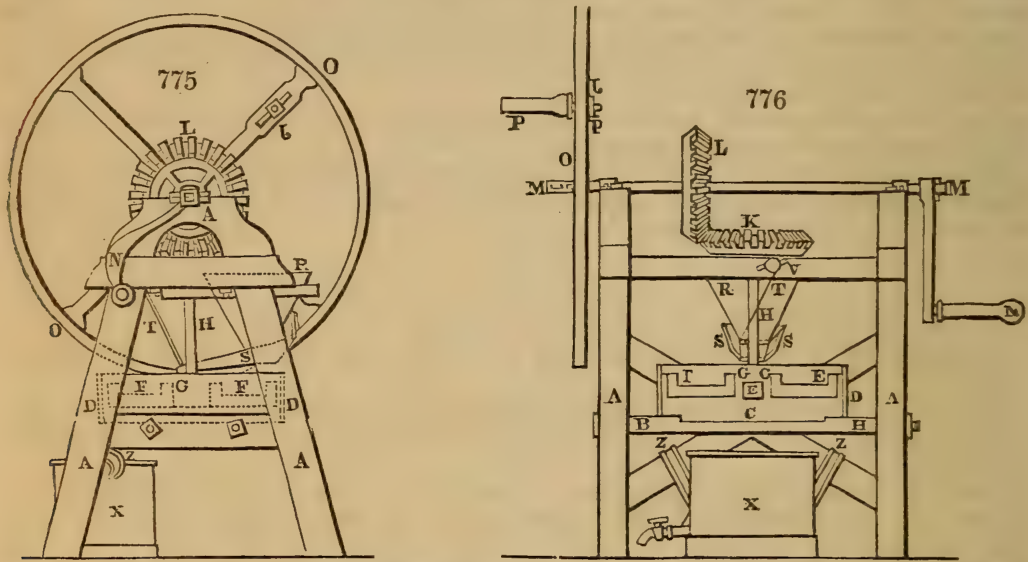

but should always be triturated in a mill. The emanations of white lead cause, first, that dangerous disease the colica pictonum, afterwards paralysis, or premature decrepitude and lingering death.

Figs. 775, 776, 777, 778 exhibit the construction of a good color-mill in three views ;

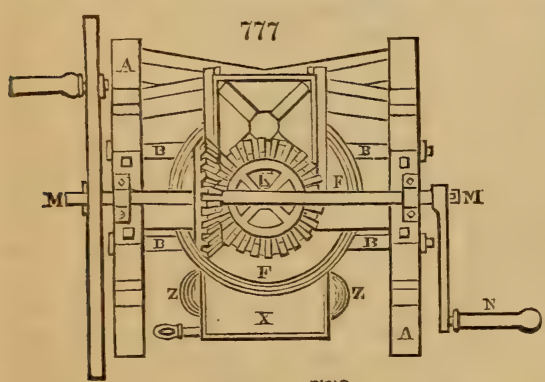

fig. 775 being an elevation shown upon the side of the handle, or where the power is applied to the shaft; fig. 776 a second elevation, taken upon the side of the line $c, d$, of the plan or bird's-eye view, fig. 777 .

The frame-work A A of the mill is made of wood or cast iron, strongly mortised or bolted together; and strengthened by the two cross iron bars B, $\mathbf{P}$ Fig. 778 is a plan of the millstones. The lying or nether millstone c, fig. 776, is of cast iron, and is channelled on its upper face like corn millstones. It is fixed upon the two iron bars $\mathbf{B}, \mathbf{B}$; but may be preferably supported upon the 3 points of arjustable screws, passing up through bearing-bars. The millstone $\mathrm{c}$ is surrounded by a large iron hoop $\mathrm{D}$, for preventing the pastyconsistenced color from running over the edge. It can escape only by the sluice hole $\mathbf{E}$, fig. 776 , formed in the hoop; and is then received in the tub $\mathrm{x}$ placed beneath. 
The upper or moving millstone $F$, is also made of cast iron. The dotted lines indicate its shape. In the centre it has an aperture with ledges G, G; there is als? a ledge upon its outer circumference, sufficiently high to confine the color which may occasionally accurnulate upon its surface. An upright iron shaft $\mathrm{H}$ passes into the turning stone, and gives motion to it. A horizontal iron bevel wheel $\mathrm{K}$, figs. 776,777 , fur nished with 27 wooden teeth, is fixed upon the upper end of the upright shaft $\mathrm{H}$. A similar bevel wheel $\mathrm{x}$, having the same number of teeth, is placed vertically upon the horizontal iron axis $\mathrm{M}$, $\mathrm{M}$, and works into the wheel $\mathrm{K}$. This horizontal axis $\mathrm{M}$, $\mathrm{M}$ bears, at one of its ends, a handle or winch $\mathrm{N}$, by which the workman may turn the millstone $F$ : and on the other end of the same axis, the fly-wheel $o$ is made fast, which serves to regulate the movements of the machine. Upon one of the spokes of the fly-wheel there is fixed, in like manner, a handle $P$, which may serve upon occasion for turning the mill. This handle may be attached at any convenient distance from the centre, by means of the slot and serew-nut $J$.

The color to be ground is put into the hopper $\mathbf{R}$, below which the bucket $\mathbf{s}$ is susnended, for supplying the color uniformly through the orifice in the mil!stone G. A cord or chain $\mathrm{T}$, by means of which the bucket $\mathrm{s}$ is suspended at a proper heioht for pouring out the requisite quantity of color between the stones, pulls the bucket obliquely, and makes its beak rest against the square upright shaft $\mathrm{H}$. By this means the bucket is continually agitated in such a way as to discharge more or less color, according to its degree of inclination. The copper cistern $\mathrm{x}$, receives the color successively as it is grourd; and, when full, it may be carried away by the two handles z, z; it may be emptied by the stopeock $\mathrm{y}$, without removing the tub.

Paints, Vitrifiable. See Porcelain, Pottery, and Stained Glass.

PALLADIUM, a rare metal, possessed of valuable properties, was discovered in 1803, by Dr. Wollaston, in native platinum. It constitutes about 1 per cent. of the Columbian ore, and from $\frac{1}{4}$ to 1 per cent. of the Uralian ore of this metal; occurring nearly pare in loose grains, of a steel-gray color, passing into silver white, and of a specific gravity of from 11.8 to $12 \cdot 14$; also as an alloy with gold in Brazil, and combined with selenium in the Harz near Tilkerode. Into the nitro-muriatic solution of native platinum, if a solution of cranide of mercury be poured, the pale yellow cyanide of palladium will be thrown down, which being ignited affords the metal. This is the ingenious process of Dr. Wollaston. The palladium present in the Brazilian gold ore may be readily separated as follows : melt the ore along with two or three parts of silver, granulate the alloy, and di gest it with heat in nitric acid of specific gravity $1 \cdot 3$. The solution containing the silver and palladium, for the gold does not dissolve, being treated with common salt or muriatie acid, will part with all its silver in the form of a chloride. The supernatant liquor being concentrated and neutralized with ammonia, will yield a rose-colored salt in long silky crystals, the ammonia-muriate of palladium, which being washed in ice-cold water, and ignited, will afford 40 per cent. of metal.

Tlie metal obtained by this process is purer than that by the former; and if it be fised in a crucible along with borax, by the heat of a powerful air-furnace or forge, a button of malleable and ductile palladium will be produced. When a slip of it is heated to redness, it takes a bronze-blue shade of greater or less intensity, as the slip is coolerl more or less slowly; but if it be surdenly chilled, as by plunging it into water, it resumes instantly its white lustre. This curious phenomenon depending upon oxydizement and de-oxydizement, in different circumstances, serves at once to distinguish palladium from platinum.

Pure palladium resembles platinum, but has more of a silver hue; when planished by the hammer into a cup, such as that of M. Breant, in the museum of the Mint at Paris, it is a splendid steel-white metal, not liable, like silver, to tarnish in the air. Another cup made by M. Breant, weighing 2 lbs. (1 kilogramme), was purchased by Charles X., and is now in the garde-meuble of the French crown. The specific gravity of this metal, when laminated, is stated by Dr. Wollaston at $11 \cdot 8$, and by Vauquelin o.t $12 \cdot 1$. It melts at from $150^{\circ}$ to $160^{\circ}$ Wedgewood; and does not oxydize at a white heat. When a drop of tincture of iodine is let fall upon the surface of this metal, and dissipated over a lamp flame, a black spot remains, which does not happen with platinum. A slip of palladium has been used with advantage to inlay the limbs of astronomical instruments, where the fine graduated lines are cut, because it is bright, and not liable to alteration, like silver.

There are a protoxyde and peroxyde of palladium. The proto-chloride consists of 60 of metal and 40 of chlorine; the cyanide, of 67 of metal, and 33 of cyanogen.

PALM OIL (Huile de palme, Fr.; Palmöl, Germ.), is obtained, in Guinea and Guyana, by expressing, as also by boiling, the fruit of the aroira elois. It has an orange color, a smell of violets, a bland taste, is lighter than water, melts at $84^{\circ} \mathrm{Fahr}$, becomes rancid and pale by exposure to air, dissolves in boiling alcohol, and consists of 69 parts of oleine, and 31 of stearine, in 100. It is employed chiefly for making yellow 
soap. It may be bleached by the action of either chlorine or oxygen gas, as also by that of light and heat.

Palm oil, quantity of,

\begin{tabular}{|c|c|c|c|}
\hline & Imported. & Retained for consumption. & Exported. \\
\cline { 2 - 4 } Year. & Cwts. & Cwts. & Cwts. \\
1835. & 260,151 & 242,733 & 30,915 \\
1836. & 277,017 & 234,357 & 34,379 \\
1837. & 223,329 & 214,000 & \\
\hline
\end{tabular}

Duty, 1 s. $3 d$. per ewt.

PAPER CUTTING. Mr. T. B. Crompton, of Farnworth, Lancashire, who obtained a patent in May, 1821, for proposing to conduct the newly formed web of paper in the Fourdrinier machine over heated cylinders, for the purpose of drying it expeditiously, in imitation of the niode so long practised in drying calicoes, obtained, along with Enoch Miller, another, in May, 1828, for cutting the endless web of paper lengthwise, by revolving circular blades, fixed upon a roller, parallel to a cylinder, round which the paper is lapped, and progressively unwound.

A patent had been obtained two months before, for certain improvements in cutting paper, by Mr. Edward Cowper, consisting of a machine, with a reel on which the weh of paper of very considerable length has been previously wound, in the act of being made in a Fourdrinier's machine; this web of paper being of sufficient width to produce two, three, or more sheets, when cut.

The several operative parts of the machine are mounted upon standards, or frame-work of any convenient form or dimensions, and consist of travelling endless tapes to conduct the paper over and under a series of guide rollers; of circular rotatory cutters for the purpose of separating the web of paper into strips equal to the widths of the intended sheets; and of a saw-edged knife, which is made to slide horizontaliy for the purpose of separating the strips into such portions or lengths as shall bring them to the dimensions of a sheet of paper.

The end of the web of paper from the reel $a, f i g .779$, is first conducted up an inclined

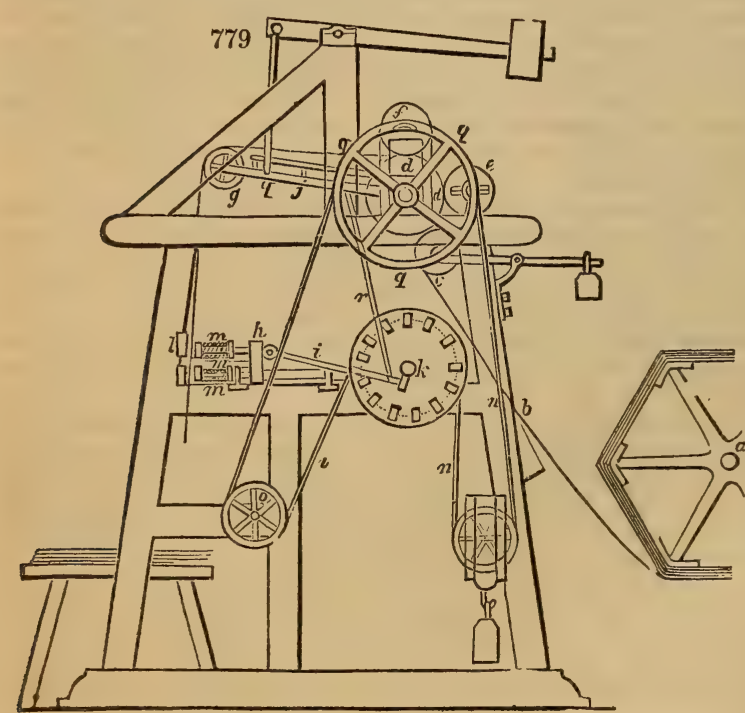
plane $b$ by hand; it is then taken hold of by endless tapes extended upon rollers, as in Mr. Cowper's Printing Machine, which see. These endless tapes carry the web of paper to the roller $c$, which is press. ed against the roller $d$ by weighted levers, acting upon the plummer blocks that its axle is mounted in. The second roller $d$ may be either of wood or metal, having several grooves formed round its periphery for the purpose of receiving the edges of the circular cutters $e$, (see CARD-cuttring)mounted upon an axle turning upon bearings in the standards or frame.

In order to allow the web of paper to proceed smoothly between the two rollers $c, d$, a narrow rib of leather is placed round the edges of one or both of these rollers, for the purpose of leaving a free space between them, through which the paper may pass without wrinkling.

From the first roller $c$, the endless tapes conduct the paper over the second $d$, and then under a pressing roller $f$, in which progress the edges of the circular knives $e$, revolving in the grooves of the second roller $d$, cut the web of paper longitudinally into strips of such 
widths as may be required, according to the number of the circular cutters and distances between them.

The strips of paper proceed onward from between the knife roller $d$ and pressing rolio: $f$, conducted by tapes, until they reach a fourth roller $g$, when they are allowed to descend, and to pass through the apparatus designed to cut them transversely; that is, into sheet 1:ngths.

The apparatus for cutting the strips into sheets is a sliding knife, placed horizontally upon a frame at $h$, which frame, with the knife $e$, is moved to and fro by a jointed rod $i$, connected to a crank on the axle of the pulley $k$. A flat board or plate $l$ is fixed to the standard frame in an upright position, across the entire width of the machine; and this board or plate has a groove or opening cut along it opposite to the edge of the knife. The paper descending from the fourth roller $g$ passes against the face of this board, and as the carriage with the knife advances, two small blocks, mounted upon rods with springs $m \mathrm{~m}$, come against the paper, and hold it tight to the board or plate $l$, while the edge of the knife is prisituded forwards into the groove of that board or plate, and its sharp saw-shaped teeth passing through the paper, cut one row of sheets from the descending strips; which, on the withdrawing of the blocks, falls down, and is collected on the heap below.

The power for actuating this machine is applied to the reverse end of the axle, on which the pulley $k$ is fixed, and a band $n, n, n, n$, passing from this pulley over tension wheels 0 , drives the wheel $q$ fixed to the axle of the knife roller $d$; hence this roller receives the rotatory motion which causes it to conduct forward the web of paper, but the other rollers $c$ and $f$, are impelled solely by the friction of contact.

The rotation of the crank on the axle of $k$, through the intervention of the crank-rod $i$, moves the carriage $h$, with the knife, to and fro at certain periods, and when the spring blocks $m$ come against the gronved plate $l$, they slide their guide rods into them, while the knife advances to sever the sheets of paper. But as sheets of different dimensions are occasionally required, the lengths of the slips delivered between each return of the knife are to be regulated by enlarging or diminishing the diameter of the pulley $k$, which will of course retard or facilitate the rotation of the three conducting rollers, $c, d, f$, and cause a greater or less length of the paper to descend between each movement of the linife carriage.

The groove of this pulley $k$, which is susceptible of enlargement, is constructed of wedge-formed blocks passed through its sides, and meeting each other in opposite directions, so that on drawing out the wedges a short distance, the diameter of the pulley becomes diminished; or by pushing the wedges further in, the diameter is increased; and a tension wheel $p$ being suspended in a weighted frame, keeps the band always tight.

As it is necessary that the paper should not continue descending while it is held by the blocks $m, m$ to be cut, and yet that it should be led on progressively over the knife roller $d$, the fourth roller $g$, which hangs in a lever $j$, is made to rise at that time, so as to take up the length of paper delivered, and to descend again when the paper is withdrawn. 'This is effected by a rod $r$, connected to the crank on the shaft of the aforesaid roller $k$, and also to the under part of the lever $j$, which lever hanging loosely upon the axle of the knife roller $d$, as its fulcrum, vibrates with the under roller $g$, so as to effect the object in the way described.

The patentee states that several individual parts of this machine are not new, and that some of them are to be found included in the specifications of other persons, such as the circular cutters $e$, which are employed by Mr. Dickinson (CARD-CuTTING), and the horizontal cutter $h$, by Mr. Hansard; he therefore claims only the general arrangement of the parts in the form of a machine for the purpose of cutting paper, as the subject of his invention.

The machine for cutting paper contrived by John Dickinson, Esq. of Nash Mill was patented in January, 1829. The paper is wound upon a cylindrical roller $a$, fig. 780 .

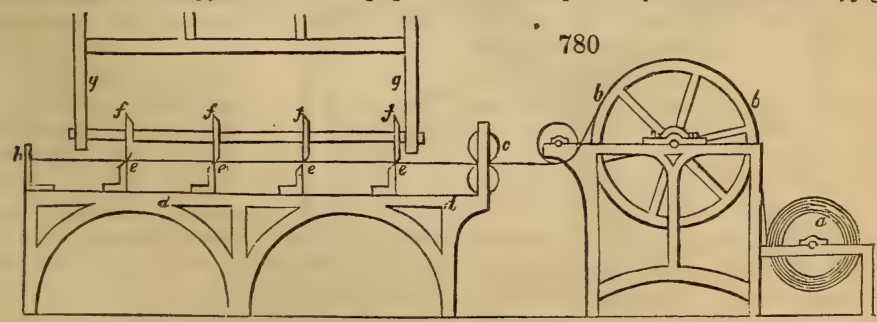

mounted upon an axle, supported in an iron frame or standard. From this roller the paper in its breadth is extended over a conducting drum $b$, also mounted upon an axle turning in the frame or standard, and after passing under a small guide roller, it proceeds through a pair of drawing or feeding rollers $c$, which carry it into the outting machine. 
Upon a table $a, d$, firmly fixed to the floor of the building, there is a series of chisel-edged knives $e_{,} e, e$, placed at such distances apart as the dimensions of the cut sheets of paper are intended to be. These knives are made fast to the table, and against them a scries of circular cutters $f, f, f$, mounted in a swinging frame $g, g$, are intended to act. The length of paper being brought along the table over the edges of the knives, up to a stop $h$, the cutters are then swung forwards, and by passing over the paper against the stationary knives, the length of paper becomes cut into three separate sheets.

The frame $g, g$, which carries the circular cutters $f, f, f$, hangs upon a very elevated axle, in order that its pendulous swing may move the cutters as nearly in a horizontal line as possible; and it is made to vibrate to and fro by an eccentric, or crank, fixed upon a horizontal rotatory shaft extending over the drum $b$, considerably above it, which may be driven by any convenient machinery.

The workmen draw the paper from between the rollers $c$, and bring it up to the stop $h$, in the intervals between the passing to and fro of the swing-cutters.

The following very ingenious apparatus for cutting the paper web transversely into any desired lengths, was made the subject of a patent by Mr. E. N. Fourdrinier, in June, 1831, and has since been performing its duty well in many establishments.

Fig. 781 is an elevation, taken upon one side of the machine; and fig. 782 is a longi

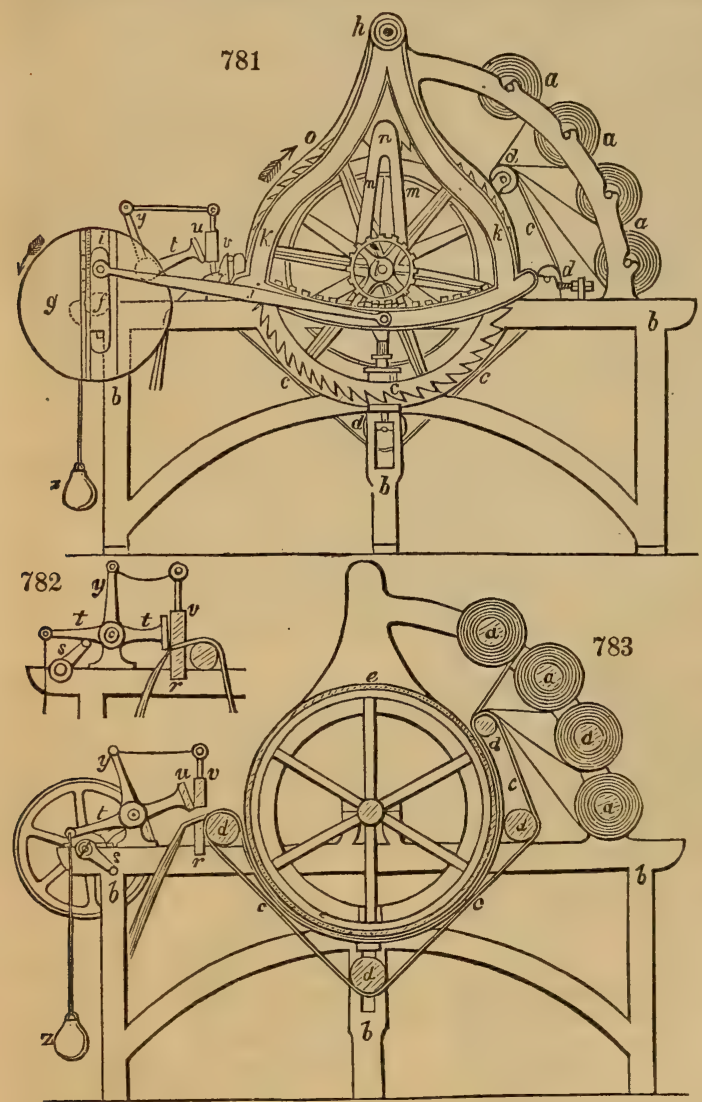
tudinal section. $\quad \alpha, a, a, a$, are four reels, each covered with one continuous sheet of paper; which reels are supported upon bearings in the frame-work $b, b, b . \quad c, c, c$, is an endless web of felt-cloth passed over the rollers $d, d, d, d$, which is kept in close contact with the under side of the drum $e, e$, seen best in fig. 782 .

The several parallel layers of paper to be cut, being passed between the drum $e$, and the endless felt $c$, will be drawn off their respective reels, and fed into the machine, whenever the driving-band is slid from the loose to the fast pulley upon the end of the main shaft $f$. But since the progressive advance of the paper-webs must be arrested during the time of making the cross cut through it, the following apparatus becomes necessary. A dise $g$, which carries the pin or stud of a crank $i$, is made fast to the end of the driving shaft $f$. This pin is set in an adjust. able sliding piece, which may be confined by a screw within the bevelled graduated groove, upon the face of the disc $g$, at variable distances from the axis, whereby the eccentricity of the stud $i$, and of course the throw of the crank, may be considerably varied. The crank stud $i$, is connected by its rod $j$, to the swinging curvilinear rack $k$, which takes into the toothed wheel $l$, that turns freely upon the axle of the feed drum $e, e$. From that wheel the arms $m, m$, rise, and bear one or more palls $n$, which work in the teeth of the great ratchet wheel $o, 0$, mounted upon the shaft of the drum $e$.

The crank-plate $g$ being driven round in the direction of its arrow, will communicate a see-saw movement to the toothed are $k$, next to the toothed wheel $l$ in gearing with it, and an oscillatory motion to the arms $m, m$, as also to their surmounting pall $n$. 
In its swing to the left hand, the catch of the pall will slide over the slope of the teeth of the ratchet wheel 0 ; but in its return to the right hand, it will lay hold of these teeth and pull them, with their attached drum, round a part of a revolution. The layers of paper in close contact with the under half of the drum will be thus drawn forward at intervals, from the reels, by the friction between its surface and the endless felt, and in lengths corresponding to the arc of vibration of the pall. The knife for cutting these lengths transversely is brought into action at the time when the swing are is making its inactive stroke, viz., when it is sliding to the left over the siopes of the ratchet teeth 0 . The extent of this vibration varies according to the distance of the crank stud $i$, from the centre $f$, of the plate $g$, because that distance regulates the extent of the oscillations of the curvilinear rack, and that of the rotation of the drum $e$, by which the paper is fed forwards to the knife apparatus. The proper length of its several layers being by the above described mechanism carried forward over the bed $r$ of the cutting knife or shears $r, r$, whose under blade $r$ is fixed, the wiper $s$, in its revolution with the shaft $f$, lifts the tail of the lever $t$, consequently depresses the transverse moveable blade $v$ (as shown in fig. 783), and slides the slanting blades across each other obliquely, like a pair of scissors, so as to cause a clean cut across the plies of paper. But just before the shears begin to operate, the transverse hoard $u$ descends to press the paper with its edge, and hold it fast upon the bed $r$. During the action of the upper blade $v$, against the under $r$, the fall board $u$, is suspended by a cord passing across pulleys from the arm $y$ of the bell-crank lever $t, t$. Whenever the lifter cam $s$, has passed away from the tail of the bell-crank $t$, the weilit $z$, hung upon it, will cause the blade $v$, and the pinching board $u$, to be moved up out of the way of the next length of paper, which is regularly brought forward by the rotation of the drum $e$, as above described. The upper blade of the shears is not set parallel to the shaft of the drum, but obliquely to it, and is, moreover, somewhat curved, so as to close its edge progressively upon that of the fixed blade. The blade $v$ malso be set between two guide pieces, and have the necessary motion given so it by levers.

PAPER-HANGINGS, called more properly by the French, papiers peints. The art of making paper-hangings, papier de tenture, has been copied fiom the Chinese, among whom it has been practised from time immemorial. The English first imported and began to imitate the Chinese paper-hangings; but being exposed till very lately to a high excise duty upon the manufacture, they have not carried it to that extent and pitch of refinement which the French genius has been enabled to do, unchecked by taxation. The first method of making this paper was stencilling; by laying upon it, in an extended state, a piece of pasteboard having spaces cut out of various figured devices, and applying different water colors with the brush. Another piece of pasteboard with other patterns cut out was next applied, when the former figures were dry, and new designs were thus imparted. By a series of such operations, a tolerable pattern was executed, but with no little labor and expense. The processes of the calico printer were next resorted to, in which engraved blocks of the pear or sycamore were employed to impress the colored designs.

Paper-hangings may be distinguished into two classes; 1 . those which are really painted, and which are designed in France under the title of papiers peints, with brilliant flowers and figures; and 2 . those in which the designs are formed by foreign matters ppplied to the paper, under the name of papier tontisse, or flock paper.

The operations common to paper-hangings of both kinds, may be stated as follows:-

1. The paper should be well sized.

2. The edges should be evenly cut by an apparatus like the bookbinder's press.

3. The ends of each of the 24 sheets which form a piece, should be nicely pasted together; or a Fourdrinier web of paper should be taken.

4. Laying the grounds, is done with earthy colors or colored lakes thickened with size, and applied with brushes.

An expert workman, with one or two children, can lay the grounds of 300 pieces in a dav. The pieces are now suspended upon poles near the ceiling, in order to be dried. They ars then rolled up and carried to the apartment where they are polished, by being laid upon a smooth table, with the painted side undermost, and rubbed with the polisher. Pieces interded to be satined, are grounded with fine Paris plaster, instead of Spanish white; and are not smoothed with a brass polisher, but with a hard brush attached to the lower end of the swing polishing rod. After spreading the piece upon the table with the giounded side undermost, the paper-stainer dusts the upper surface with finely powdered chalk of Briançon, commonly called talc, and rubs it strongly with the brush. In this way the satiny lustre is produced.

\section{THE PRINTING OPERATIONS.}

Blocks about two inches thick, formed of three separate boards glued together, of which two are made of poplar, and one (that which is engraved) of pear-tree or syca 
more, are used for printing paper-hangings, as for calicoes. The grain of the upper layer of wood should be laid across that of the layer below. As many blocks are required as there are colors and shades of color. To make the figure of a rose, for example, three several reds must be applied in succession, the one deeper than the other, a white for the clear spaces, two and sometimes three greens for the leaves, and two wood colors for the stems; altogether from 9 to 12 for a rose. Each block carries small pin points fixed at its corners to guide the workman in the insertion of the figure exactly in its place. An expert hand places these guide pins so that their marks are corered and concealed by the impression of the next block; and the finished piece shows merely those belonging to the first and last blocks.

In printing, the workman employs the same swimming-tub apparatus which has been described under block printing (see CALICo-PRINTING), takes off the color upon his blocks, and impresses them on the paper extended upon a table in the very same way. The tub in which the drum or frame covered with calf-skin is inverted, contains simply water thickened with parings of paper from the bookbinder, instead of the pasty mixture employed by the calico-printers. In impressing the color by the block upon the paper, he employs a lever of the second kind, to increase the power of his arm, making it act upon the block through the intervention of a piece of wood, shaped like the bridge of a violin. This tool is called tasseau by the French. A child is constantly occupied in spreading color with a brush upon the calf-skin head of the drum or siere, and in sliding off the paper upon a wooden trestle or horse, in proportion as it is finished. When the piece has received one set of colored impressions, the workman, assisted by his little aid, called a tireur (drawer), hooks it upon the drying-poles under the ceiling. A sufficient number of pieces should be provided to keep the printer occupied during the whole at least of one day, so that they will be dried and ready to receive another set of colored impressions by the following morning.

All the colors are applied in the same manner, every shade being formed by means of the blocks, which determine all the beauty and regularity of the design. A pattern drawer of taste may produce a very beautiful effect. The history of Psyche and Cupid, by $\mathbf{M}$. Dufour, has been considered a masterpiece in this art, rivalling the productions of the pencil in the gradation, softness, and brilliancy of the tints.

When the piece is completely printed, the workman looks it all over, and if there be any defects, he corrects them by the brush or pencil, applying first the correction of one color, and afterwards of the rest.

A final satining, after the colors are dried, is communicated by the friction of a finely polished brass roller, attached by its end gudgeons to the lower extremity of a long swing-frame; and acting along the cylindrical surface of a smooth table, upon which the paper is spread.

The fondu or rainbow style of paper-hangings, which I have referred to this place in the article CALICO-PRINTING, is produced by means of an assortment of oblong narrow tin pans, fixed in a frame, close side to side, each being about one inch wide, two inches deep, and eight inches long; the colors of the prismatic spectrum, red, orange, yellow, green, \&c., are put, in a liquid state, successively in these pans; so that when the oblong brush $\mathrm{A}, \mathrm{B}$, with guide ledges $a, c, d$, is dipped into them across the whole of the

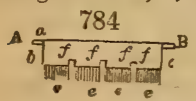
parallel row at once, it comes out impressed with the different colors at successive points $e, e, e, e$, of its length, and is then drawn by the paper-stainer over the face of the woollen drum head, or siere of the swimming tub, upon which it leaves a corresponding series of stripes in colors, graduating into one another like those of the prismatic spectrum. By applying his block to the tear, the workman takes up the color in rainbow hues, and transfers these to the paper. $f, f, f, f$ show the separate brushes in tin sheaths, set in one frame.

At M. Zuber's magnificent establishment in the ancient château of Rixheim, near Mulhouse, where the most beatiful French papiers peints are produced, and where I was informed that no less than 3000 blocks are required for one pattern, I saw a two. color calico machine employed with great advantage, both as to taste and expedition. Steam-charged cylinders were used to dry the paper immediately after it was printed, as the colors, not being so rapidly absorbed as they are by calico, would be very apt to spread.

The operations employed for common paper-hangings, are also used for making flock paper, only a stronger size is necessary for the ground. The flocks are obtained from the woollen cloth manufacturers, being cut off by their shearing machines, called levises by the English workmen, and are preferred in a white state by the French paper-hanging makers, who scour them well, and dye them of the proper colors themselves. When they are thoroughly stove-dried, they are put into a conical fluted mill, like that for making snuff, and are properly ground. The powder thus obtained is afterwards siftea by a bolting-machine, like that of the flour mill, whereby flocks of different degrees of fineness are produced. These are applied to the paper after it has undergone all the 
asual printing operations. Upon the workman's left hand, and in a line with his printIng 1able, a large chest is placed for receiving the flock powders : it is seven or eight feet long, two feet wide at the bottom, three feet and a half at top, and from 15 to 18 inches deep. It has a hinged lid. Its bottom is made of tense calf-skin. This chest is called the drum ; it rests upon four strong feet, so as to stand from 24 to 28 inches above the floor.

The block which serves to apply the adhesive basis of the velvet-powders, bears in relief only the pattern corresponding to that basis, which is formed with linseed oil, rendered drying by being boiled with litharge, and afterwards ground up with white lead. The French workmen call this mordant the encaustic. It is put upon the cloth which covers the inverted swimming tub, in the same way as the common colors are, and is spread with a brush by the tireur (corruptly styled tearer by some English writers). The workman daubs the blocks upon the mordant, spreads the pigment even with a kind of brush, and then applies it by impression to the paper. Whenever a sufficient surface of the paper has been thus covered, the child draws it along into the great chest, sprinkling the flock powder over it with his hands ; and when a length of 7 feet is printed, he covers it up within the drum, and beats upon the calf-skin bottom with a couple of rods to raise a cloud of flock inside, and to make it cover the prepared portion of the paper uniformly. He now lifts the lid of the chest, inverts the paper, and beats its back lightly, in order to detach all the loose particles of the woolly powder.

By the operation just described, the velvet-down being applied everywhere of the same color, would not be agreeable to the eye, if shades could not be introduced to relieve the pattern. To give the effect of drapery, for example, the appearance of folds must be introduced. For this purpose, when the piece is perfectly dry, the workman stretches it upon his table, and by the guidance of the pins in his blocks, he applies to the flock surface a color in distemper, of a deep tint, suited to the intended shades, so that he dyes the wool in its place. Light shades are produced by applying some of his lighter water-colors.

Gold leaf is applied upon the above mordant, when nearly dry; which then forms a proper gold size; and the same method of application is resorted to, as for the ordinary gilding of wood. When the size has become perfectly hard, the superfluous gold leaf is brushed off with a dossil of cotton wool or fine linen.

The colors used by the paper-hangers are the following :-

1. Whites. These are either white-lead, good whitening, or a mixture of the two.

2. Yellous. These are frequently vegetable extracts; as those of weld, or of Avignon or Persian berries, and are made by boiling the substances with water. Chrome yellow is also frequently used, as well as the terra di Sienna and yellow ochre.

3. Reds are almost exclusively decoctions of Brazil wood.

4. Blues are either Prussian blue, or blue verditer.

5. Greens are Scheele's green, a combination of arsenious acid, and oxyde of copper; the green of Schweinfurth, or green verditer; as also a mixture of blues and yellows.

6. Violets are produced by a mixture of blue and red in various proportions, or they may be obtained directly by mixing a decoction of $\log$ wood with alum.

7. Brouns, blacks, and grays. Umber furnishes the brown tints. Blacks are either common ivory or Frankfort black; and grays are formed by mixtures of Prussian blue and Spanish white.

All the colors are rendered adhesive and consistent, by being worked up with gelatinous size or a weak solution of glue, liquefied in a kettle. Many of the colors are previously thickened, however, with starch. Sometimes colored lakes are employed. See LAKES.

PAPER, MANUFACTURE OF. (Papeterie, Fr.; Papiermacherkunst, Germ.) This most useful substance, which has procured for the moderns an incalculable advantage over the ancients, in the means of diffusing and perpetuating knowledge, seems to have been first invented in China, about the commencement of the Christian era, and was thence brought to Mecca, along with the article itself, about the beginning of the 8th century; whence the Arabs carried it, in their rapid career of conquest and colonization, to the coasts of Barbary, and into Spain, about the end of the 9th or beginning of the 10th century.

By other accounts, this art originated in Greece, where it was first made from cotton fibres, in the course of the tenth century, and continued there in common use during the next three hundred years. It was not till the beginning of the 14th century that paper was made from linen in Europe, by the establishment of a paper-mill in 1390, at Nurcmberg in Germany. The fir's English paper-mill was erected at Dartford by a German jeweller in the service of Queen Elizabeth, about the year 1588. But the business was not very successful; in consequence of which, for a long period afterwards, indeed till within the last 70 years, this country derived its supplies of fine writing papers from France and Holland. Nothing places in a more striking light the 
vast improvement which has taken place in all the mechanical arts of England since the era of Arkwright, than the condition of our paper-machine factories now, compared with those on the Continent. Almost every good automatic paper mechanism at present mounted in France, Germany, Belgium, Italy, Russia, Sweden, and the United States, has either been made in Great Britain, and exported to these countries, or has been constructed in them closely upon the English models.

Till within the last 30 years, the linen and hempen rags from which paper was made, were reduced to the pasty state of comminution requisite for this manufacture by mashing them with water, and setting the mixture to ferment for many days in close vessels, whereby they underwent in reality a species of putrefaction. It is easy to see that the organic structure of the fibres would be thus unnecessarily altered, nay, frequently destroyed. The next methor employed, was to beat the rags into a pulp by stamping rods, shod with iron, working in strong oak mortars, and moved by water-wheel machinery. So rude and ineffective was the apparatus, that forty pairs of stamps were required to operate a night and a day, in preparing one hundred weight of rags. The pulp or paste was then diffused through water, and made into paper by methods similar to those still practised in the small hand-mills.

About the middle of the last century, the cylinder or engine mode, as it is called, of comminuting rags into paper pulp, was invented in Holland; which was soon afterwards adopted in France, and at a later period in England.

The first step in the paper manufacture, is the sorting of the rags into four or five qualities. They are imported into this country chiefly from Germany, and the ports of the Mediterranean. At the mill they are sorted again more carefully, and cut into shreds by women. For this purpose a table frame is covered at top with wire cloth, containing about nine meshes to the square inch. To this frame a long steel blade is attached in a slanting position, against whose sharp edge the rags are cut into squares or fillets, after having their dust thoroughly shaken out through the wire cloth. Each piece of rag is thrown into a certain compartment of a box, according to its fineness; seven or eight sorts being distinguished. An active woman can cut and sort nearly one cwt. in a day.

The sorted rags are next dusted in a revolving cylinder surrounded with wire cloth, about six feet long, and four feet in diameter, having spokes about 20 inches long, attached at right angles to its axis. These prevent the rags from being carried round with the case, and beat them during its rotation; so that in half an hour, being pretty clean, they are taken out by the side door of the cylinder, and transferred to the engine, to be first washed, and next reduced into a pulp. For fine paper, they should be previously boiled .or some time in a caustic ley, to cleanse and separate their filaments.

The construction of the stuff-engine is represented in figs.785, 786. Fig. 785 is the lon-

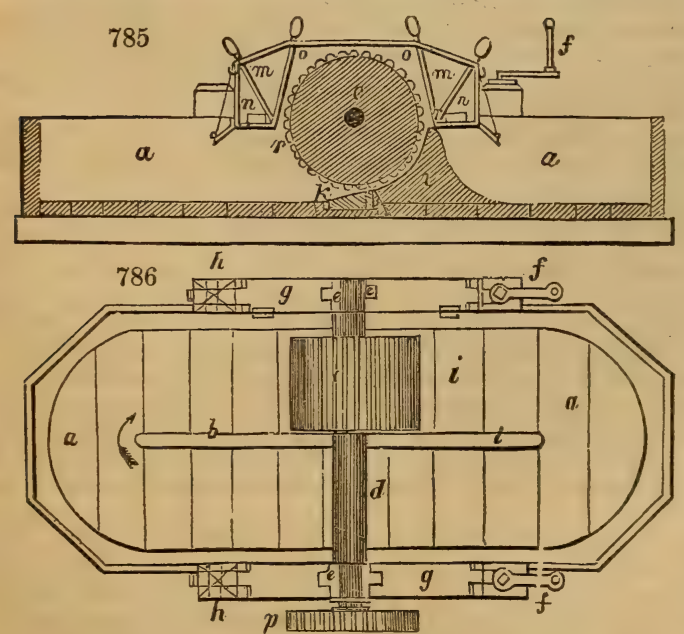
gitudinal section, and fig. 786 the plan of the engine. The large vat is an oblong cistern rounded at the angles. It is divided by the partition $b, b$, and the whole inside is lined with lead. The cylinder $c$, is made fast to the spindle $d$, which extends across the engine, and is put in motion by the pinion $p$, fixed to its extremity. The cylinder is made of wood, and furnished with a number of blades or cutters, secured to its circumference, parallel to the axis, and projecting about an inch above its surface. Immediately beneath the cylinder a block of wood $k$ is placed. This is mounted with cutters like those of the cylinder, which in their revolution pass very near to the teeth of the block, but must not touch it. The distance between these fixed and moving blades is capable of adjustment by elevating or depressing the bearings upon which the necks $e, e$, of the shaft are supported. These bearings rest upon two levers $g$, $g$, which have tenons at their ends, fitted into upright mortises, made in short beams $h, h$, bolted to the sides of the engine. The one end of 
the levers $g, g$, is moveable, while the other end is adapted to rise and fall upon bolts in the beams $h, h$, as centres. The front lever, or that nearest to the cylinder $c$, is capable of being elevated $\mathrm{rr}$ depressed, by turning the handle of a serew (not seen in this view), which acts in a nut fixed to the tenon of $y$, and comes up through the top of the beam $h$, upon which the head of the screw takes its bearing. Two brasses are let into the middle of the levers $\mathrm{g}, \mathrm{g}$, and form the bearings for the shaft of the engine to turn upon. The above-mentioned vertical screw is used to raise or lower the cylinder, and cause it to cut coarser or finer, by enlarging or diminishing the space between the fixed cutters in the block and those in the cylinder.

To the left hand of $i$, fig. 785, is a circular breasting made of boards, and covered with sheet lead; it is curved to fit the cylinder very truly, and leaves but very little space between the teeth and breasting; at its bottom, the block $k$ is fixed. The engine is supplied with water from a pump, by a pipe, which delivers it into a small cistern, near to and corr.municating with the engine. A stopcock cuts off or regulates the supply of water at pleasure, and a grating covered with hair-cloth is fixed across that small cistern, to intercept any filth that may be floating in the water; in other cases a flannel bag is tied round the nose of the stopcock, to act as a filter.

The rags being put into the engine filled with water, are drawn by the rapid rotation of the cylinder between the two sets of cutters, whereby they are torn into the finest filaments, and by the impulsion of the cylinder they are floated over the top of the breasting upon the inclined plane. In a short time more rags and water are raised into that part of the engine vat. The tendency in the liquid to maintain an equilibrium, puts the whole contents of the cistern in slow motion down the inclined plane, to the left hand of $i$, and round the partition $b, b$, (see the arrow), whereby the rags come to the cylinder again in the space of about 20 minutes; so that they are repeatedly drawn out and separated in all directions till they are reduced to the appearance of a pulp.

This circulation is particularly useful, by turning over the rags in the engine, causing them to be presented to the cutter at different angles every time; otherwise, as the blades always act in one direction, the comminution would not be so complete. The cutting is performed as follows: The teeth of the block are set somewhat obliquely to the axes of the cylinder, as shown by fig. 787 ; but the teeth of the cylinder $c$ itself are set 787

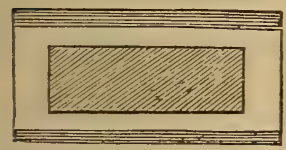
parallel to its axis; therefore the cutting edges meet at a small angle, and come in contact, first at the one end, and then lowards the other, by successive degrees, so that any rags coming between them, are torn as if between the blades of a pair of forceps. Sometimes the blades $k$ in the block are bent to an angle in the middle, instead of being straight and inclined to the cylinder. These are called elbow plates; their two ends being inclined in opposite directions to the axis of the cylinder. In either case, the edges of the plates of the block cannot be straight lines, but must be curved, to adapt themselves to the curve which a line traced on the cylinder will necessarily have. The plates or blades are united by screwing them together, and fitting them into a cavity cut into the wooden block $k$. Their edges are bevelled away upon one side only.

The block is fixed in its place by being made dovetailed, and truly fitted into the bottom of the cistern, so that the water will not leak through its junction. The end of it comes through the woodwork of the chest, and projects to a small distance on its outside, being kept in its place by a wedge. By withdrawing this wedge, the block becomes loose, and can be removed in order to sharpen the cutters, as occasion may be. This is done at a grindstone, after detaching the plates from each other.

The cutters of the cylinder are fixed into grooves, cut in the wood of the cylinder, at equal distances asunder, round its periphery, in a direction parallel to its axis. The number of these grooves is twenty, in the machine here represented. For the washer, each groove has two cutters put into it; then a fillet of wood is driven fast in between them, to hold them firm; and the fillets are secured by spikes driven into the solid wood of the cylinder. The beater is made in the same manner, except that each groove contains three bars and two fillets.

In the operation of the cylinder, it is necessary that it should be enclosed in a case, or it would throw all the water and rags out of the engine, in consequence of its great velocity. This case is a wooden box $m, m, f i g .785$, enclosed on every side except the bottom; one side of it rests upon the edge of the vat, and the other upon the edge of the partition $b, b, f i g .786$. The diagonal lines $m, r$, represent the edges of wooden frames, which are covered with hair or wire-cloth, and immediately behind these the box is furnished with a bottom and a ledge towards the cylinder, so as to form a complete trough. The square figures under $n, n$, in $f i g$. 785 , show the situation of two openings or spouts through the side of the case, which conduct to flat lead-pipes, one of which is seen near the upper $g$ in fig. 786, placed by the side of the vat; the beam being cut away from them. These are waste pipes to discharge the foul water from the engine; because the 
cylinder, as it turns, throws a great quantity of water and rags up against the sieves; the water goes through them, and runs down to the trough under $n$, $n$, and thence into the ends of the flat leaden pipes, through which it is discharged. $0,0, f i g .785$, are grooves for two boards, which, when put down in their places, cover the hair sieves, and stop the water from going through them, should it be required in the engine. This is always the case in the beating engines, and therefore they are seldom provided with these waste pipes, or at most on one side only; the other side of the cover being curved to conform to the cylinder. Except this, the only difference between the washing engine and the beater, is that the teeth of the latter are finer, there being 60 instead of 40 blades in the periphery; and it revolves quicker than the washer, so that it will tear out and comminute those particles which pass through the teeth of the washer. In small mills, when the supply of water is limited, there is frequently but one engine. which may be used both for washing and beating, by adjusting the screw so as to let the cylinder down and make its teeth work finer. But the system in all considerable works, is to have two engines at least, or four if the supply of water be great. The power re. quired for a 5 or 6 vat mill, is about 20 horses in a water-wheel or steam engine.

In the above figures only one engine is shown, namely, the finisher; there is another, quite similar, placed at its end, but on a level with its surface, which is called the washer, in which the rags are first worked coarsely with a stream of water, running through them to wash and open their fibres; after this washing they are called halfstuff, and are then let down into the bleaching engine, and next into the beating engine, above described.

By the arrangements of the mill gearing, the two cylinders of the washer and beater engines make from 120 to 150 revolutions per minute, when the water-wheel moves with due velocity. The beating engine is always made to move, however, much faster than the washing one, and nearly in the ratio of the above numbers.

The vibratory noise of a washing engine is very great; for when it revolves 120 times per minute, and has 40 teeth, each of which passes by 12 or 14 teeth in the block at every revolution, it will make nearly 60,000 cuts in a minute, each of them sufficiently loud to produce a most grating growling sound. As the beater revolves quicker, having perhaps 60 teeth, instead of 40 , and 20 or 24 cutters in the block, it will make 180,000 cuts in a minute. This astonishing rapidity produces a coarse musical humming, which may be heard at a great distance from the mill. From this statement, we may easily understand how a modern engine is able to turn out a vastly greater quantity of paper pulp in a day than an old mortar machine.

The operation of grinding the rags requires nice management. When first put snto the washing engine they should be worked gently, so as not to be cut, but only powerfully scrubbed, in order to enable the water to carry off the impurities. This effect is obtained by raising the cylinder upon its shaft, so that its teeth are separated considerably from those of the block. When the rags are comminuted too much in the washer, they would be apt to be carried off in part with the stream, and be lost; for at this time the water-cock is fully open. After washing in this way for 20 or 30 minutes, the bearings of the cylinder are lowered, so that its weight rests upon the eutters. Now the supply of water is reduced, and the rags begin to be torn, at first with considerable agitation of the mass, and stress upon the machinery. In about three or four hours, the engine comes to work very smoothly, because it has by this time reduced the rags to the state of half-stuff. They are then discharged into a large basket, through which the water drains away.

The bleaching is usually performed upon the half-stuff. At the celebrated manufactory of Messrs. Montgolfier, at Annonay, near Lyons, chlorine gas is employed for this purpose with the best effect upon the paper, since no lime or muriate of lime can be thus left in it; a circumstance which often happens to English paper, bleached in the washing engine by the introduction of chloride of lime among the rags, after they have been well washed for three or four hours by the rotation of the engine. The current of water is stopped whenever the chloride of lime is put in. From 1 to 2 pounds of that chemical compound are sufficient to bleach $1 \mathrm{cwt}$. of fine rags, but more must be employed for the coarser and darker colored. During the bleaching operation the two sliders $0,0, f i g .785$, are put down in the cover of the cylinder, to prevent the water getting away. The engine must be worked an hour longer with the chloride of lime, to. promote its uniform operation upon the rags. The cylinder is usually raised a little during this period, as its only purpose is to agitate the mass, but not to triturate it. The water-cock is then opened, the boards $m, m$ are removed, and the washing is continued for about an hour, to wash the salt away; a precaution which ought to be better attended to than it always is by paper manufacturers.

The half-stuff thus bleached is now transferred to the beating engine, and worked into a fine pulp. This operation takes from 4 to 5 hours, a little water being admitted from time to time, but no current being allowed to pass through, as in the washing engine 
The softest and fairest water should be selected for this purpose; and it should be administered in nicely regulated quantities, so as to produce a proper spissitude of stuff for making paper.

For printing paper, the sizing is given in the beating engine, towards the end of its operation. The size is formed of alum in fine powder, ground up with oil; of which mixture about a pint and a half are thrown into the engine at intervals, during the last half-hour's beatıng. Sometimes a little indigo blue or smalt is also added, when a peculiar bloom color is desired. The pulp is now run off into the stuff chest, where the different kinds are mixed; whence it is taken out as wanted. The chest is usually a rectangular vessel of stone or wood lined with lead, capable of containing 300 cubic feet at least, or 3 engines full of stuff. Many paper-makers prefer round chests, as they admit of rotatory agitators.

When the paper is made in single sheets, by hand labor, as in the older establishments, a small quantity of the stuff is transferred to the working-vat by means of a pipe, and there diluted properly with water. This vat is a vessel of stone or wood, about 5 feet square, and 4 deep, with sides somewhat slanting. Along the top of the vat a board is laid, with copper fillets fastened lengthwise upon it, to make the mould slide more easily along. This board is called the bridge. The maker stands on one side; and has to his left hand a smaller board, one end of which is made fast to the bridge, while the other rests on the side of the vat. In the bridge opposite to this, a nearly upright piece of wood, called the ass, is fastened. In the vat there is a copper, which communicates with a steam pipe to keep it hot; there is also an agitator, to maintain the stuff in a uniform consistence.

The moulds consist of frames of wood, neatly joined at the corners, with wooden bars running across, about an inch and a half apart. Across these, in the length of the moulds, the wires run, from fifteen to twenty per inch. A strong raised wire is laid along each of the cross bars, to which the other wures are fastened; this gives the laid paper its ribbed appearance.

The water-mark is made by sewing a raised piece of wire in the form of letters, or any figured device, upon the wires of the mould, which makes the paper thinner in these places. The frame-work of a wove mould is nearly the same; but instead of sewing on separate wires, the frame is covered with fine wire cloth, containing from 48 to 64 meshes per inch square. Upon both moulds a deckel, or moveable raised edge-frame, is used; which must fit very neatly, otherwise the edges of the paper will be rough.

A pair of moulds being laid upon the bridge, the workman puts on the deckel, brings the mould into a vertical position, dips it about half way down into the stuff before him, then turning it into a horizontal position, covers the mould with the stuff and shakes it gently. This is a very delicate operation; for if the mould be not held perfectly level, one part of the sheet will be thicker than another. The sheet thus formed has, however, no coherence; so that by turning the mould, and dipping the wire cloth surface in the vat, it is again reduced to pulp if necessary. He now pushes the mould along the small board to the left, and removes the deckel. Here another workman called the coucher receives it, and places it at rest upon the ass, to drain off some of the water. Meanwhile the vat-man puts the deckel upon the other mould, and makes another sheet. The coucher stands to the left side of the vat, with his face towards the vat-man or maker, on his right is the press furnished with felt cloths, or porous flannels; a three-inch thick plank lies before him on the ground. On this he lays a cushion of felts, and on this another felt; he then turns the paper wire mould, and presses it upon the felt, where the sheet remains. He now returns the mould by pushing it along the bridge. The maker has by this time another sheet ready for the coucher; which, like the preceding, is laid upon the ass, and then couched or inverted upon another felt, laid down for the purpose.

In this way, felts and paper are alternately stratified, till a heap of six or eight quires is former, which is from 15 to 18 inches high. This mass is drawn into the press, and exposed to a force of 100 tons or upwards. After it is sufficiently compressed, the machine is relaxed, and the elasticity of the flannel makes the rammer descend (if a hydraulic press be used) with considerable rapidity. The felts are then drawn out on the other side by an operative called a layer, who places each felt in succession upon one board, and eash sheet of paper upon another. The coucher takes immediate possession of the felts for his further operations.

Two men at a vat, and a boy as a layer or lifter, can make about 6 or 8 reams in 10 hours. In the evening the whole paper made during the day is put into another press, and subjected to moderate compression, in order to get quit of the mark of the felt, and more of the water. Next day it is all separated, a process called parting, and being again pressed, is carried into the loft. Fine papers are often twice parted and pressed, in order to give them a proper surface.

The next operation is the drying, which is performed in the following way. Posts 
about 10 or 12 feet high are erected at the distance of ten feet from each other, and pierced with holes six inches apart; two spars with ropes stretched between them, at the distance of 5 inches from one another, called a treble or tribble, are placed about 5 feet high between these posts, supported by pins pushed into the holes in the posts. The workman takes up 4 or 8 sheets of paper, and puts them upon a piece of wood in the form of a $T$; passing this $T$ between the ropes, he shifts the sheets upon them, and proceeds thus till all the ropes are full. He then raises the treble, and puts another in its place, which he fills and raises in like manner. Nine or ten trebles are placed in every set of posts. The sides of the drying-room have proper shutters, which can be opened to any angle at pleasure.

When the paper is dry, it is taken down, and laid neatly in heaps to be sized. Size is made of pieces of skin, cut off by the curriers before tanning, or sheep's feet, or any other matter containing much gelatine. These substances are boiled in a copper to a jelly ; to which, when strained, a small quantity of alum is added. The workman then takes about 4 quires of paper, spreads them out in the size properly diluted with water, taking care that they be equally moistened. This is rather a nice operation. The superfluous size is then pressed out, and the paper is parted into sheets. After being once more pressed, it is transferred to the drying-room, but must not be dried too quickly. Three days are required for this purpose. When the paper is thoroughly dry, it is carried to the finishing-house, and is again pressed pretty hard. It is then picked by women with small knives, in order to take out the knots, and separate the perfect from the imperfect sheets. It is again pressed, given to the finisher, to be counted into reams, and done up. These reams are compressed, tied up, and sent to the warehouse for sale. A good finisher can count 200 reams, or 96,000 sheets in a day.

Hot pressing is executed by placing a sheet of paper between two smoothed pasteboards, alternately, and between every 50 pasteboards a heated plate of iron, and subjecting the pile to the press. This communicates a fine smooth surface to writingpaper.

The grain of the paper is often disfigured by the feits, when they are too much used, or when the loose fibres do not cover the twisted thread. The two sides of the felt are differently raised, and that on which the fibres are longest is applied to the sheets which are laid down. As the felts have to resist the reiterated action of the press, their warp should be made stout, of long combed wool, and well twisted. The wocf, however, should be of carded wool, and spun into a soft thread, so as to render the fabrie spongy, and capable of imbibing much water.

This operose and delicate process of moulding the sheets of paper by hand, has for nearly thirty years past been performed, in many manufactories, by a machine which produces it in a continuous sheet of indefinite length which is afterwards cut into suitable sizes, by the Paper-cutting Machine.

In 1799, Louis Robert, then employed in the paper works of Essonne in France, con. trived a machine to make paper of a great size, $\mathrm{k} v$ a continuous motion, and obtained for it a patent for 15 years, with a sum of 8000 francs from the French government, as a reward for his ingenuity. The specification of this patent is published in the second volume of Brevets d' Invention expirés. M. Leger-Didot, then director of the said works, bought Robert's machine and patent for 25,000 francs, to be paid by instalments. Having become proprietor of this machine, which, though imperfect, contained the germ of a valuable improvement in paper-making, M. Didot came over with it to England, where he entered into several contracts for constructing and working it.

Meanwhile M. L. Didot having failed to fulfil his obligations to Robert, the latter instituted a law-suit, and recovered possession of his patent by a decision dated 23d June, 1810. Didot then sent over to Paris the Repertory of Arts, for Sept. 1808, which contained the specification of the English patent, with instructions to a friend to secure the improved machines described in it, by a French patent. The patent was obtained, but became inoperative in consequence of M. L. Didot failing to return to France, as he had promised, so as to mount the patent machine within the two years required by the French patent law. It was not till 1815, that $M$. Calla, machinemaker at Paris, constructed the paper apparatus known in England by the name of Fourdrinier's, and which, on the authority of the Dictionnaire Technologique, was very imperfect in comparison of an English made machine imported about that time into France. La construction de ces machines, qui n'offre pourtant rien de difficile, est restée jusqu' à ce jour exclusivement dans les mains des. Anglais, is the painful acknowledgment made in 1829 , for his countrymen, by the author of the elaborate article Papeterie in that national work. If there be nothing difficult in the construction of these machines, the French mechanicians ought to be ashamed of forcing their countrymen to seek the sole supply of them in England; for the principal paper works in France, as those of MM. Canson, Montgolfier, Thomas Varenne, Firmin Didot, Delcambre, De Maupeon, \&c., are mounted with English-made machines. 
The following, for example, are a few of the paper-mills in France which are mounted with the self-rcting machines of Messrs. Bryan Donkin \& Co.

Messrs. Cianson, at Annonay.

M. de la Place, at Jean d'Heures, Bar-le-duc.

Société anunyme, at Sainte Marie, under M. Delatouche.

Echarcon près Mennecy, (Seine et Oise).

Firmin Didot, Mesnil sur l'Estrée.

M. F. M. Montgolfier, à Annonay.

Muller, Bouchard, Ondin and Co's., at Gueures, near Dieppe.

MM. Richard et Comp. à Plainfoing.

M. Callot-Bellisle; Vieuze et Chantoiseau.

M. Bechétaile, near St. Etienne, at Bourg Argental.

It deserves particularly to be remarked, to the honor of English mechanism, that the proprietors of the first five of the above works received gold medals at the last exposition of their papers at the Louvre, and all the rest received medals either of silver or bronze.*

The following is a true narrative of the rise and progress of the paper automaton.

M. Leger Didot, accompanied by Mr. John Gamble, an Englishman who had resided for several years in Paris, obtained permission from the French government, in 1800, to carry over the small workin $r$ model of Robert's continuous machine, with the view of getting the benefit of English capital and mechanical skill to bring it into an operative state upon the great scale. Fortunately for the vigorous development of this embryo project, which had proved an abortion in France, they addressed themselves, on the one hand, to a mercantile firm equally opulent and public spirited, and on the other, to engineers distinguished for persevering energy and mechanical resource. A first patent was granted to Mr. Gamble on the 20th of April, 1801, and a second, for certain improvements upon the former, on the 7th of June, 1803. In January, 1804, Mr. Gamble, for certain considerations, assigned these two patents to Messrs. Henry and Sealy Fourdrinier, the house above alluded to, who were at that period, and for several years afterwards, the most considerable stationers and paper-makers in Great Britain. By an act of parliament passed on the 4th of August, 1807, Mr. Gamble's privilege of 14 years from April, 1801, was prolonged to 15 years after the date of the act, being an extension of about 7 years upon the original patent.

The proprietors showed good reasons, in the enormous expense of their experiments, and the national importance of the object, why the patent should have been extended 14 years from the latter date, and would have obtained justice from parliament in this respect, but for an unworthy artifice of Lord Lauderdale in the House of Lords. " $\mathrm{He}$, and he only, was the person who took the objection," and, by introducing a regulation in a stand. ing order of the House of Lords, that none but the original inventor should have an extension, though Mr. H. Fourdrinier was the inventor substantially of the operative machine, he defeated the honorable intentions of his brother peers, whose committee said, "We will give seven years, and Mr. Fourdrinier may apply again, if it should turn out that the seven years that we propose to give to Mr. Fourdrinier should not give sufficient time to afford any chance of his receiving any remuneration for the expense that he has meurred in introducing this invention." The bill passed in the House of Commons for 14 years, but it was limited by this ruse of Lord Lauderdale to 7, "6 who put the standing order upon the books (of the upper house) which prevented Messrs. Fourdrinier from having any benefit from the invention. $\dagger$

In February, 1808, Mr. Gamble, after losing both his time and money savings during eight years of irksome diligence, assigned over to Messrs. Fourdrinier the whole right of his share in the patent to which he was entitled under the act of parliament.

Dartford in Kent, which had been long conspicuous as the seat of a good manufactory of paper and paper monlds, was selected by the proprietors of the patent as the fittest place for realizing their plans; and happily for them it possessed, in Mr. Hall's engineering establishment, every tool requisite for constructing the novel automaton, and in his assistant, Mr. Bryan Donkin, a young and zealous mechanist, who, combining precision of workmanship with fertility of invention, could turn his local advantages to the best acconnt. To this gentleman, aided by the generous confidence of Messrs. Fourdrinier, the glory of rearing to a stately manhood the helpless bantling of M. L. Didot is entirely due. In 1803, after nearly three years of intense application, he produced a self-acting machine for making an endless web of paper, which was erected at St. Neot's under the. superintendense of $\mathrm{Mr}$. Gamble, and performed in such a manner as to surprise every beholder.

Since that important era Mr. Donkin has steadily devoted his whole mind and meane:

* Rapport de Jury Central, par M. Le Baron Charles Dupin, vol. ii. p. 278 ; Paris, 1836.

+ See this shabby piece of diplomacy unveiled in the Minutes of Evidence taken before the Select Com mittee of the House of Commons on Fourdrinier's patent; Mav, 1837. 
to the progressire improvement of this admirable apparatus; and has, by the unfailing -egularity, precision, promptitude, and productiveness of its work, earned for himself a place along with Watt, Wedgewood, and Arkwright, in the temple of mechanical fame.

"La France," sars a late official eulogist of her arts, and interpreter of her sentiments, "ne craint plus la rivalite des autres peuples pour la fabrication des divers genres de papiers et de cartons." After this boast, one would not expect to hear him immediately confess that in 1823 his country possessed only one manufactory of the pupier continu, containing one of the Fourdrinier machines made at London by Mr. Donkin, for M. Canson, at Vidalon-les-Annonay; that in $182 \tau$ there were only 4 of these machines in France, and that in 1834 there were not many more than a dozen. He justly observes, that " this mode, being more economical, more rapid, and more powerful, will become henceforth the only one which can be practised without loss. Then will disappear the ancient system of hand-work, which likewise involved the inconreniences, we may say dangers, resulting from combinations among the operatives. The machine-made papers possess many adrantages : they can receire, so to speak, unlimited dimensions; they preserve a perfectly uniform thickness throughout all their length; they may be fabricated in every season of the rear; nor do they require to be sorted, trimmed, and hung up in the drring-house, operations which occasioned great waste, amounting to no less than one defective sheet out of every five. The continuous paper at one time retained the impression of the wire-wore web on its under side; a defect from which it has been freed by a pressure apparatus of Mr. Donkin, recently imported from England by M. Delatouche."

It appears from documents presented to a committee of the House of Lords in 1807 . that the Messrs. Fourdrinier had, by that time, withdrawn from their stationary business the large sum of $60,000 l$., to further the object of their patent; so many difficulties did they encounter in bringing the machinery to its then comparatirely complete state, and so little encouragement or support did they receive from the paper manufacturers throughout the kingdom.

The patentees laid a statement before the public in 1806 , containing the following comparative estimate of the expense attending seren rats, and that attending a machine emplojed upon paper sized in the engine, performing the same quantity of work as seren vats, at the rate of 12 hours daily.

A MACHINE.

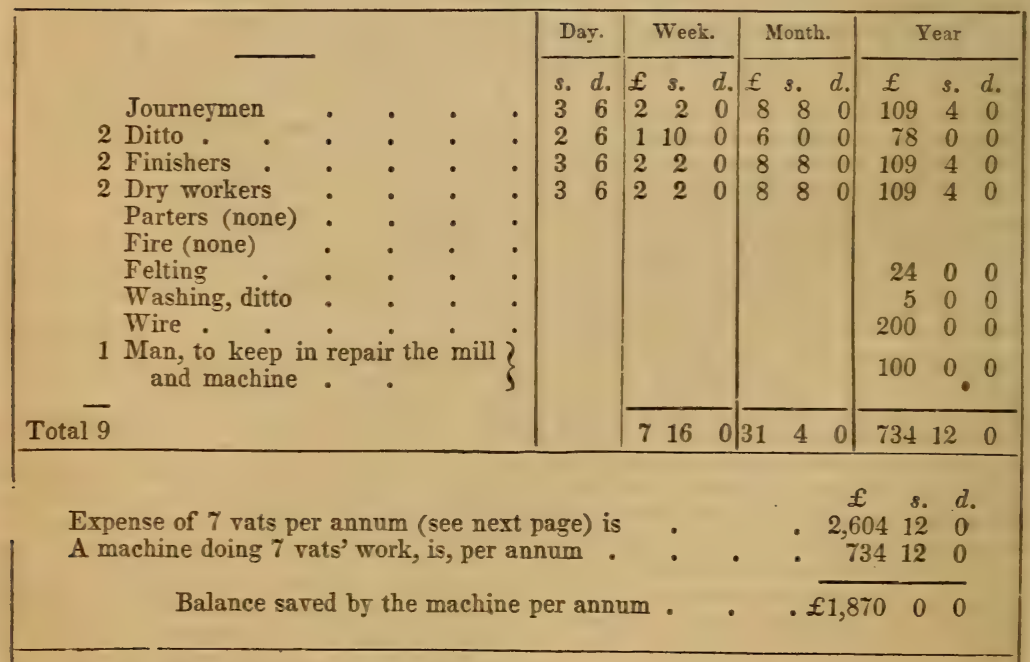

N. B.-There are other adrantages, to the amount of full $400 \mathrm{l}$. per annum, of which manufacturers are well aware, although not taken into this calculation.

* Rapport de Jury Central, sur les Produits de l'Industrie Française exposé en 1834, par Le Baron Charles Dupun, Membre de l'Institut, Rapporteur-général et Vice President du Jury Central; ii. 278. 
SEVEN VATS.

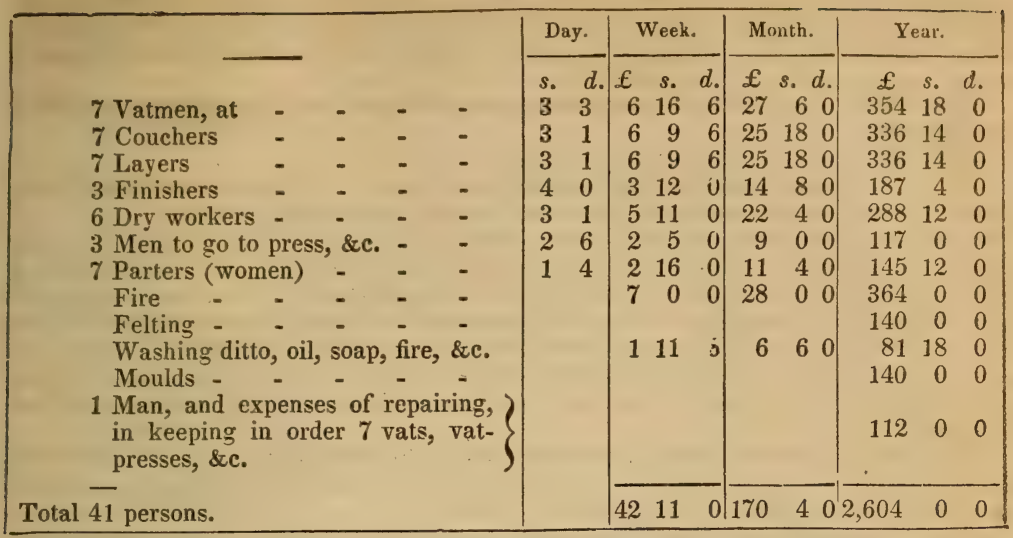

In the same statement, it was shown that the expense of making paper by hand is 16 s. per cwt., whereas by their machine it is only $3 s .9 d$.; so that upon 432,000 evts. the quantity annually made in Great Britain and Ireland (as founded upon the fact that one vat can make $480 \mathrm{cwts}$. of paper, and that there were 900 vats in the kingdom), the annual saving by the machine would be $264,600 l$., or $345,600 l$. $-81,000 l$.

In a second statement laid before the public in 1807 , the patentees observe that their recently improved machine, from its greater simplicity, may be erected at a considerably reduced expense. "Mr. Donkin, the engineer, will engage to furnish machines of the dimensions specified below, with all the present improvements, at the prices specified below.

\begin{tabular}{|c|c|c|c|c|c|c|c|}
\hline & & & & & Inches. & If driven by straps. & $£$ \\
\hline 3 or 4 vats & - & - & - & - & 30 & between the deckles & 715 \\
\hline 6 ditto & - & - & - & - & 40 & ditto & 845 \\
\hline 8 ditto & - & - & - & - & 44 & ditto & 940 \\
\hline 12 ditto & - & - & - & - & 54 & ditto & 995 \\
\hline & & & & & & If driven by wheels. & \\
\hline 3 or 4 vats & - & - & - & - & 30 & between the deckles & 750 \\
\hline 6 ditto & - & - & - & - & 40 & ditto & 880 \\
\hline 8 ditto & - & - & - & - & 44 & ditto & 980 \\
\hline 12 ditto & - & - & - & - & 54 & ditto & 1,040 \\
\hline
\end{tabular}

"Instead of 5 men, formerly employed upon 1 machine, 3 are now (in 1813) fully sufficient, without requiring that degree of attention and skill which was formerly indispensable.

"In 1806 the machine was capable of doing the work of 6 vats in twelve hours; it is, however, now capable of doing double that quantity, at one fourth of the expense. For by the various improvements enumerated above, the consumption of wire is reduced nearly one half, and lasts above double the time; the quantity of paper produced is doubled; and, taking into consideration the work which is now performed by the men over and above thair immediate attendance upon the machine, it may be fairly stated, that the number of men is reduced to one half; consequently tìe expense of wire and labor is reduced to one fourth of what it was.

"6 The other advantages incidental to the nature of the process of making paper by this machine, may be classed in the following order:-

" 1 st. That the paper is much superior in strength, firmess, and appearance, to any which can be made by hand of the same material.

" $2 \mathrm{~d}$. It requires less drying, less pressing and parting, and consequently comes sonner to market; for it receives a much harder pressure from the machine than can possibly be given by any vat press, and is therefore not only drier, but, on account of the closeness and firmness of texture, even the moisture which remains is far sooner evaporated, on exposure to the air, than it would be from the more spongy or bibulous paper made by hand. 
"'The superior pressure, and the circumstance of one side of the paper passing under the polished surface of one of the pressing rollers, contribute to that smoothness which in hand-made papers can only be obtained by repeated parting and pressing; consequently a great part of the time necessarily spent in these operations is saved, and the paper sooner finished and ready for market.

" $3 \mathrm{dly}$. The quantity of broken paper and retree is almost nothing compared with what is made at the vats.

" 4 th. The machine makes paper with cold water.

" 5 th. It is durable, and little subject to be out of repair. The machine at Two Waters, in Hertfordshire, for the last three years, has not cost $10 l$. a year in repairs.

"6 6 th. As paper mills are almost universally wrought by streams, which vary considerably in their power from time to time, there will result from this circumstance a very important advantage in the adoption of the machine. The common paper mill being limited by its number of vats, no advantage can be taken of the frequent accessions of power which generally happen in the course of the year; but, on the contrary, as scarcely any mills are capable of preparing stuff for twelve vats, every accession of power to the mill, where a machine is employed, will increase its produce without any additional expense.

" 7 th. The manufacturer can suspend or resume his work at pleasure; and he is besides effectually relieved from the perplexing difficulties and loss consequent upon the perpetual combinations for the increase of wages."

It is a lamentable fact, that the attention required to mature this valuable invention, and the large capital which it absorbed, led ultimately to the bankruptcy of this rpulent and public-spirited company; after which disaster no patent dues were collected, though twelve suits in Chancery were instituted; these being mostly unsuccessful, on account of some paltry technical objections made to their well-specified patent, by that unscientific judge Lord Tenterden. The piratical tricks practised by many considerable paper-makers against the patentees are humiliating to human nature in a civilized and soi-disant Christian community. Many of them have owned, since the bankruptey of the house removed the fear of prosecution, that they owed them from $2000 l$. to 3000 . apiece.

Nothing can place the advantage of the Fourdrinier machine in a stronger point of view, than the fact of there being 280 of them now at work in the United Kingdom, making collectively 1600 miles of paper, of from 4 to 5 feet broad, every day; that they have lowered the price of paper 50 per cent., and that they have increased the revenue, directly and indirectly, by a sum of probably $400,000 l$. per annum. The tissue paper made by the machine is particularly useful for communicating engraved impressions to pottery ware; before the introduction of which there was but a miserable substitute. Messrs. R. and J. Clewes, of Cobridge potteries, in a letter to Messrs. Fourdrinier, state, " that had not an improvement taken place in the manufacture of paper, the new style of engraving would have been of no use, as the paper previously used was of too coarse a nature to draw from the fair engravings any thing like a clear or perfect impression; and the Staffordshire potteries, in our opinion, as well as the public at large, are deeply indebted to you for the astonishing improvement that has recently taken place, both as regards china and earthenware, more particularly the latter." The following rates of prices justify the above statement:-

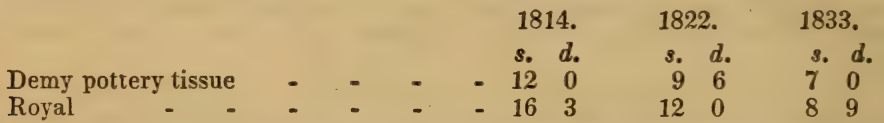

${ }^{65}$ We have adopted a new mode of printing on china and earthenware, which, but for your improved system of making tissue paper, must have utterly failed; our patent machine requiring the paper in such lengths as were impossible to make on the old plan. On referring to our present stock, we find we have one sheet of your paper more than 1200 yards long. Signed, Machin and Potts ; Burslem, February 25th, 1834."

I have had the pleasure of visiting more than once the mechanical workshops of Messrs. Bryan Donkin and Co. in Bermondsey, and have never witnessed a more admirable assortment of exquisite and expensive tools, each adapted to perform its part with despatch and mathematical exactness, though I have seen probably the best machine factories of this country and the Continent. The man of science will appreciate this statement, and may perhaps be surprised to learn that the grand mural circle of 7 feet diameter, made by Troughton, for the Royal Observatory of Greenwich, was turned with final truth upon a noble lathe in the said establishment. It has supplied no fewer than 133 complete automatic paper machines, each of a value of from $1200 l$. to 2000l., to different manufactories, not only in the United Kingdom, but in all parts of the civilized world; as mentioned in the second paragraph of the present article. Each 


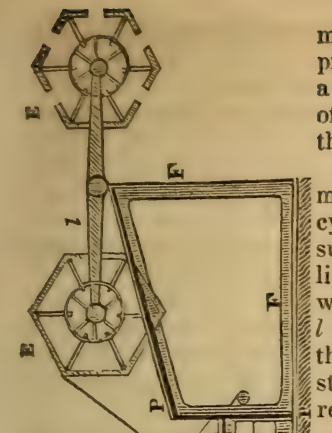

machine is capable of making, under the impulsion of any prime mover, all unwatched by a human eye, and unguided by a human hand, from 20 to 50 feet in length, by 5 feet broad, of most equable paper in one minute. Of paper of average thickness, it turns off 30 feet.

Fig. 788 is an upright longitudinal section, representing the machine in its most complete state, including the drying steam eylinders, and the compound channelled rollers of Mr. Wilks, subsequently to be described in detail. The figure in the upper line shows it all in train, when the paper is to be wound up wet upon the reels $\mathrm{E}, \mathrm{E}$, which being moveable round the centre $l$ of a swing-bar, are presented empty, time about, to receive the tender web. The figure in the under line contains the steam or drying cylinders; the points 00 , of whose frame, replace, at the points $P$, $P$, the wet reel frame, F F, P.

$A$ is the vat, or receiver of pulp from the stuff-chest.

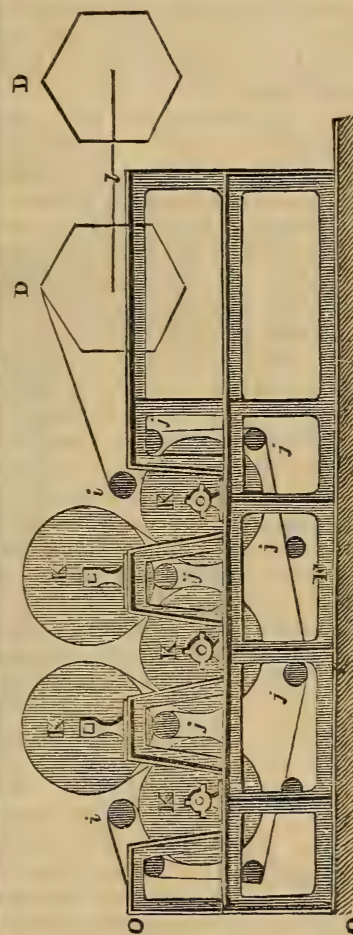

$B$ is the knot strainer of Ibotson (p. 967), to clear the pulp before passing on to the wire.

$\mathrm{G}$ is the hog, or agitator in the vat. The arrows show the course of the currents of the pulp in the vat.

$\mathrm{I}$ is the apron, or receiver of the water and pulp which escape through the endless wire, and which are returned by a scoop-wheel into the rat.

$b$ is the copper lip of the vat, over which the pulp flows to the endless wire, on a leathern apron extending from this lip to about nine inches over the wire, to support the pulp and prevent its eseaping.

$c, c$ are the bars which bear up the small tube rollers that support the wire.

$d, d$ are ruler bars to suppos: the copper rollers over whica the wire revolves.

$\mathbf{K}$ is the breast roller, round which the endless wire turns.

$\mathrm{N}$ is the point where the shaking motion is given to the machine.

$\boldsymbol{M}$ is the guide roller, having its pivots moveable laterally to adjust the wire and keep it o parallel.

I is the pulp roller, or, "dandy," to press out water, and to set the paper. $r$, is the place of the second, when it is used.

$\mathbf{H}$ is the first or wet press, or couching rollers; the wire leaves the paper here, which latter is couched upon the endless felt $p$; and the endless wire o returns, passing round the lower couch roller. By Mr. Donkin's happy invention of placing these rollers obliquely, the water runs freely away, which it did not do when their axes were in a vertical line.

$e, e$ are the deckles, which form the edges of the sheet of paper, and prevent the pulp passing away laterally. They regulate the width of the endless sheet.

$f, f$ are the revolving deckle straps.

$\mathbf{R}$ is the deckle guide, or driving-pulley.

$\mathrm{g}, \mathrm{g}$ are tube rollers, over which the wire passes, which do not partake of the shaking motion; and, 
$h, h$ are moveable rollers for stretching the wire, or orass carriages for keeping the rollers $g, g$ in a proper position.

c is the second press, or dry press, to expel the water in a cold state.

$\mathbf{K}, \mathbf{k}$, sc., in the view of the lower line, are the steam cylinders for drying the endless sheet.

$i, i$ are rollers to convey the paper.

$j, j$ are rollers to conduct the felt; which serves to support the paper, and prevent it wrinkling or becoming cockled.

D, D are the hexagonal expanding reels for the steam-dried paper web, one only being used at a time, and made to suit different sizes of sheets. $l$ is their swing fulcrum.

$F, F, F, F$, is the frame of the machine.

The deckle straps are worthy of particular notice in this beautiful machine. They are composed of many layers of cotton tape, each one inch broad, and together one half inch thick, cemented with caoutchouc, so as to be at once perfectly flexible and water-tight.

The upper end of each of the two carriages of the roller $\mathrm{L}$ is of a forked shape, and the pivots of the roller are made to turn in the cleft of the forked carriages in such a manner, that the roller may be prevented from having any lateral motion, while it possesses a free vibratory motion upwards and downwards; the whole weight of the roller $\mathrm{L}$ being borne by the endless web of woven wire.

The greatest difficulty formerly experienced in the paper manufacture upon the continuous system of Fourdrinier, was to remove the moisture from the pulp, and condense it with sufficient rapidity, so as to prevent its becoming what is called water-galled, and to permit the web to proceed directly to the drying cylinders. Hitherto no in rention has answered so well in practice to remove this difficulty as the channelled and perforated pulp rollers or dandies of Mr. John Wilks, the ingenious partner of Mr. Donlin; for which a patent was obtained in 1830 . Suppose one of these rollers (see L, in fig. 788, and $\mathrm{M}, \mathrm{M}$, in fig. 793) is required for a machine which is to make paper 54 inches wice, it must be about 60 inches long, so that its extremities (see figs. 789 and 790) may extend over or beyond each edge of the sheet of paper upon which it is laid. Its diameter may be 7 inches. About 8 grooves, each 1-16th of an inch wide, are made in every inch of the tube; and they are cut to half the thickness of the copper, with a rectangularly shaped tool. A succession of ribs and grooves are thus formed throughout the whole length of the tube. A similar succession is then made across the former, but of 24 in the inch, and on the opposite surface of the metal, which by a peculiar mode of management had been prepared for that purpose. As the latter grooves are cut as deep as the former, those on the inside meet those on the outside, crossing each other at right angles, and thereby producing so many square holes; leaving a series of straight copper ribs on the interior surface of the said tube, traversed by another series of ribs coiled round them on the outside, forming a cylindrical sieve made of one piece of metal. The rough edges of all the ribs must be rounded off with a smooth file into a semi-circular
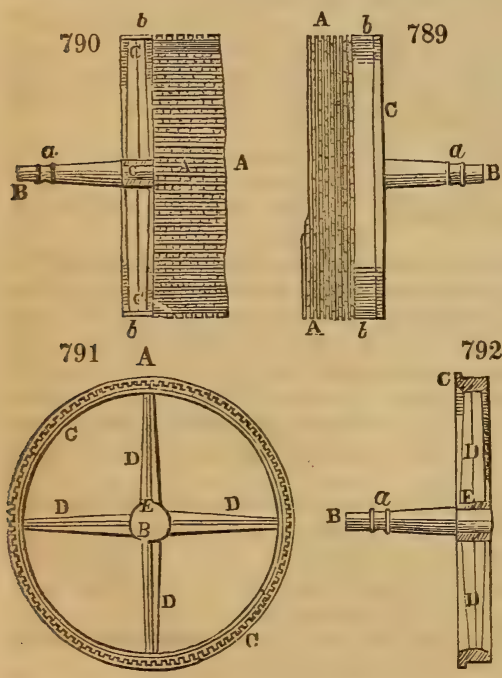

792

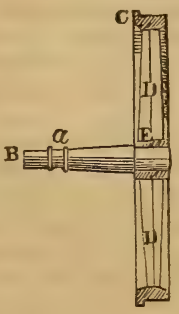
form. Figs. 789 and 790, A A, are portions of the ribbed copper tube. Fig. 789 shows the exterior, and fig. 790 the interior surface; $b, b$ and $b, b$ show the plain part at each of the ends, where it is made fast to the brass rings by rivets or screws; $c, c$ are the rings with arms, and a centre piece in each, for fixing the iron pivot or shaft $\mathbf{B}$; one such pivot is fixed by riveting it in each of the centre pieces of the rings, as shown at $c$, fig. 790 ; so that both the said pieces shall be concentric with the rings, and have one common axis with each other, and with the roller. At $a, a, a$ groove is turned in each of the pivots, for the purpose of suspending a weight by a hook, in order to increase the pressure upon the paper, whenever it may be found necessary.

Fig. 791 is an end view, showing the copper tube and its internal ribs $A, A$; the brass rings $\mathrm{C}, \mathrm{C}$; $\operatorname{arm} \mathrm{D}, \mathrm{D}, \mathrm{D}$; centre piece $\mathrm{E}$, and pivot B. Fig. 792 is a section of the said ring, with the arms, \&c.

The roller is shown at $\mathbf{L}, \mathrm{fig} .788$, as lying upon the surface of the wire-web. 
The relative position of that perforated roller, and the little roller $b$, over which it lies, is such that the axis of $\mathrm{L}$ is a little to one side of the axis of $b$, and not in the same vertical plane, the latter being about an inch nearer the rat end. Hence, whencver the wire-web is set in progressive motion, it will cause the roller $L$ to revolve upon its surface; and as the paper is progressively made, it will pass onwards with the web inder the surface of the roller. Thus the pulpy layer of paper is condensed by compression under the ribbed roller; while it transmits its moisture through the rerforations, it becomes sufficiently compact to endure the action of the wet press rollers $\mathrm{H}, \mathrm{H}$, and also acquires the appearance of parallel lines, as if made by hand in a laid mould.

Mr. Wilks occasionally employs a second perforated roller in the same paper machine, which is then placed at the dotted lines $i, i, i$.

The patentee has described in the same specification a most ingenious modification of the said roller, by which he can exhaust the air from a hollowed portion of its periphery, and cause the paper in its passage over the roller to undergo the sucking operation of the partial void, so as to be remarkably condensed; but he has not been called upon to apply this second invention, in consequence of the perfect success which he has experienced in the working of the first.

The following is a more detailed illustration of Mr. Wilks' improved rolk.r.

Fig. 793 represents two parts of his double-cased exhausting cylinder.

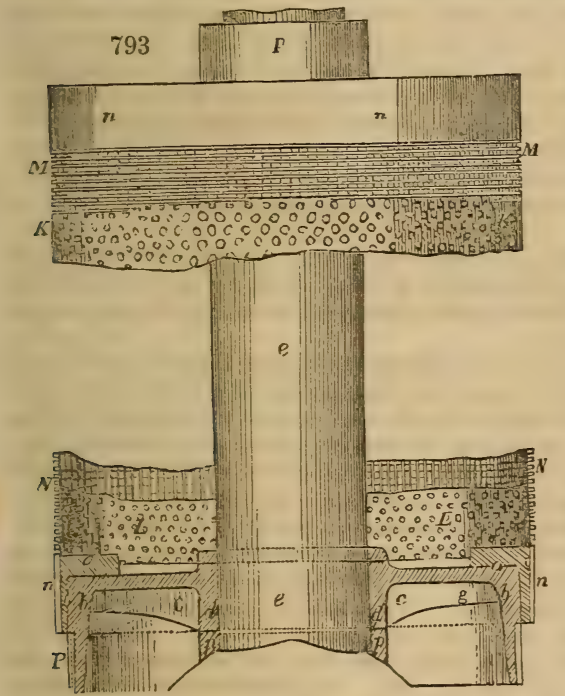

This consists of two copper tubes, one nicely lining the other; the inner being punched full of round holes, as at $\mathbf{K}, \mathbf{K}$, where that tube is shown uncovered; a portion of the inner surface of the same tube is shown at $\mathbf{L}, \mathbf{L}$. In this figure also, two portions of the outer tube are shown at $\mathbf{M}, \mathbf{M}$, and $\mathrm{N}, \mathrm{N}$; the former being an external, and the latter an internal view. Here we see that the external tube is the ribbed perforated one already described; the holes in the inner tube being made in rows to correspond with the grooves in the outer. The holes are so distributed that every hole in one row shall be opposite to the middle of the space left between two holes in the next row, as will appear from inspection of the figure. The diameter of each of the prinched holes somewhat exceeds the width of each rib in the inside of the outer cylinder, and every inside groove of this tube coincides with a row of holes in the former, which construction permits the free transudation or perco lation of the water out of the pulp. At each end of this double-case cylinder, a part is left at $\mathrm{N}, \mathrm{N}$, plain without, and grooved merely in the inside of the outer tube. The smooth surface allows the brass ends to be securely fixed; the outer edge of the brass ring fits tight into the inside of the end of the cylinders.

On the inside of each of these rings there are four pieces which project towards the centre or axis of the cylinder; two of which pieces are shown at $a, a, f i g .793$, in section. $b, b$, is a brass ring with four arms $c, c, c, c$, and a boss or centre piece $d, d$. The outer edge of the last-mentioned ring is also turned cylindrical, and of such a diameter as to fit the interior of the former ring 0,0 . The two rings are securely held together by four screws. $e, e$ is the hollow iron axle or shaft upon which the cylinder revolves. Its outside is made truly cylindrical, so as to fit the circular holes in the bosses $d, d$, of the rings and arms at each end of the cylinder. Hence, if the hollow shaft be so fixed that it will not turn, the perforated cylinder is capable of having a rotatory motion given to it round that shaft. This motion is had recourse to, when the vacuum apparatus is employed. But otherwise the cylinder is made fast to the hollow axle by means of two screw clamps. To one end of the cylinder, as at $p$, a toothed wheel is attached, for communicating a rotatory motion to it, so that its surface motion shall be the same as that of the paper web; otherwise a rubbing motion might ensue, which woxld wear and injure both.

The paper stuff or pulp is allowed to flow from the vat A, fig. 788, on to the surface of the endless wire-web, as this is moring along. The lines $0,0, f i g .788$, show the course of 
the motion of the web, which operates as a sieve, separating to a certain degree the water from the pulp, yet leaving the latter in a wet state till it arrives at the first pair of prese ing rollers $\mathrm{H}, \mathrm{H}$, hetween which the web with its sheet of paper is squeezed. Thick paper, in passing through these rollers, was formerly often injured by becoming watergalled, from the greater retention of water in certain places than in others. But Messrs. Donkin's cylinder, as above described, has facilitated vastly the discharge of the water, and enabled the manufacturer to turn off a perfectly uniform smooth paper.

In fig. 788 , immediately below the perforated cylinder, there is a wooden watertrough. Along one side of the trough a copper pipe is laid, of the same length as the cylinder, and parallel to it; the distance between them being about one fourth of an inch. The side of the pipe facing the cylinder is perforated with a line of small holes, which transmit a great many jets of water against the surface of the cylinder, in order to wash it and keep it clean during the whole continuance of the process.

The principle adopted by John Dickinson, Esq., of Nash Mill, for making paper, is different from that of Fourdrinier It consists in causing a polished hollow brass cylinder, perforated with holes or slits, and covered with wire cloth, to revolve over and just in contact with the prepared pulp: so that by connecting the cylinder with a vessel exhausted of its air, the film of pulp, which adheres to the cylinder during its rotation, becomes gently pressed, whereby the paper is supposed to be rendered drier, and of more uniform thickness, than upon the horizontal hand munids, or travelling wire cloth of Fourdrinier. When subjected merely to agitation, the water is sucked inwards through the cylindric cage, leaving the textile filaments so completely interwoven as, if felted among each other, that they will not separate without breaking, and, when dry, they will form a sheet of paper of a strength and quality relative to the nature and preparation of the pulp. The roll of paper thus formed upon the hollow cylinder is turned off continuously upon a second solid one covered with felt, upon which it is condensed by the pressure of a third revolving cylinder, and is thence delivered to the drying rollers.

Such is the general plan of Mr. Dickinson's paper machines, into which he has introduced numerous improvements since its invention in 1809, many of them secured by patent right; whereby he has been enabled to make papers of first-rate quality, more particularly for the printing-press. See infra.

In July, 1830, Mr. Ibotson of Poyle, paper manufacturer, obtained a patent, see B, fig. 788 , which has proved very successful, for a peculiar construction of a sieve or strainer. Instead of wire meshes, he uses a series of bars of gun-metal, laid in the bottom of a box, very closely together, so that the upper surfaces or the flat sides may be in the same plane, the edge of each bar being parallel with its neighbor, leaving parallel slits between them of from about 1-70th to 1-100th of an inch in width, according to the fineness or coarseness of the paper-stuft to be strained. As this stuff is known to consist of an assemblage of very fine flexible fibres of hemp, flax, cotton, \&c., mixed with water, and as, even in the pulp of which the best paper is made, the length of the said fibres considerably exceeds the diameter of the meshes of which common strainers are formed, consequently the longest and most useful fibres were formerly lost to the paper manufacturer. Mr. Ibotson's improved sieve is employed to strain the paper-stuff previously to its being used in the machine above described, (see its place at в in the vat.) When the strainer is at work, a quick vertical and lateral jogging motion is given to it. by machinery similar to the jogging screens of corn mills.

Since the lateral shaking motion of the wrre-web in the Fourdrinier machine, as originally made, was injurious to the fabric of the paper, by bringing its fibies more closely together breadthwise than lengthwise, thus tending to produce long ribs, or thick streaks in its substance, Mr. George Dickinson, of Buckland Mill, near Dover, proposed, in the specification of a patent obtained in February, 1828, to give a rapid up-and-down movement to the travelling web of pulp. He does not, however, define with much precision any proper mechanism for effecting this purpose, but claims every plan which may answer this end. He proposes generally to mount the rollers, which conduct the horizontal endless web, upon a vibrating frame. The forepart of this frame is attached to the standards of the machine, by hinge joints, and the hinder part, or that upon which the pulp is first poured out, is supported by vertical rods, connected with a crank on a shaft below. Rapid rotatory motion being given to this crank-shaft, the hinder part of the frame necessarily receives a quick up-and-down vibratory movement, which causes the water to be shaken out from the web of pulp, and thus sets the fibres of the paper with much greater equality than in the machines formerly constructed. A plan similar to this was long ago introduced into Mr. Donkin's machines, in which the vibrations were actuated in a much more mechanical way.

John Dickinson, Esq., of Nash Mill, obtained a patent in October, 1830, for a method of uniting face to face two sheets of pulp by means of machinery, in order to produce paper 
of extraordinary thickness. Two vats are to be supplied with paper stuff as usual; in which two hollow barrels or drums are made to revolve upon axles driven by any first mover; an endless felt is conducted by guide rollers, and brought into contact with the drums; the first drum gives off the sheet of paper pulp from its periphery to the felt, which massing over a pressing roller, is conducted by the felt to that part of a second drum which is in contact with another pressing roller. A similar sheet of paper pulp is now given off from the second drum, and it is brought into contact with the former by the pressure of its own roller. The two sheets of paper pulp thus united are carried forward by the felt over a guide roller, and onward to a pair of pressing rollers, where by contact the moist surfaces of the pulp are made to adhere, and to constitute one double thick sheet of paper, which, after passing over the surfaces of hollow drums, heated by steam, becomes dry and compact. The rotatory movements of the two pulp-lifting drums must obriously be simultaneous, but that of the pressing rollers should be a little faster, because the sheets extend by the pressure, and they should be drawn forward as fast as they are deliverei, otherwise creases would be formed. Upon this invention is founded Mr. Dickinson's ingenious method of making safety-paper for Post-office stamps, by introducing silk fibres, \&c., between the two laminæ.

The following contrivance of the same inventive manufacturer is a peculiarly elegant mechanical arrangement, and is likely to conduce to the perfection of machine-made paper. I have already described Mr. Ibotson's excellent plan of parallel slits, or gridiron strainers, which has been found to form paper of superior quality, because it permits all the elon "ated tenacious fibres to pass, which give strength to the paper, while it intercepts the coarser knots and lumps of the paste, that were apt to spoil its surface. Mr. Turner's circular wire sieves, presently to be noticed, may do good work, but they cannot compete with Mr. Dickinson's present invention, which consists in causing the diluted paper pulp to pass between longitudinal apertures, about the hundred-and-fifteenth part of an inch wide, upon the surface of a revolving cylinder.

The pulp being diluted to a consistency suitable for the paper machine, is delivered into a vat, of which the level is regulated by a waste pipe, so as to keep it nearly full. From this vat there is no other outlet for the pulp, except through the wire-work periphery of the revolving cylinder, and thence out of each of its ends into troughs placed alongside, from which it is conducted to the machine destined to convert it into a paper web.

The revolving cylinder is constructed somewhat like a squirrel cage, of circular rods, or an endless spiral wire, strengthened by transverse metallic bars, and so formed that the spaces between the rings are sufficient to allow the slender fibres of the pulp to pass through, but are narrow enough to intercept the knots and other coarse impurities, which must of course remain, and accumulate in the vat. The spaces between the wires of the squirrel cage may vary from the interval above stated, which is intended for the finest paper, to double the distance for the coarser kinds.

It has been stated that the pulp enters the revolving cylinders solely through the intervals of the wires in the circumference of the cylinder; these wires or rods are about three eighths of an inch broad without, and two eighths within, so that the circular slits diverge internally. The rods are one quarter of an inch thick, and are riveted to the transverse bars in each quadrant of their revolution, as well as at their ends to the necks of the cylinder.

During the rotation of the cylinder, its interstices would soon get clogged with the pulp, were not a contrivance introduced for creating a continual vertical agitation in the inside of the cylinder. This is effected by the up-and-down motion of an interior agitator or plunger, nearly long enough to reach from the one end of the cylinder to the other, made of stout copper, and hollow, but water-tight. A metal bar passes through it, to whose projecting arm at each end a strong link is fixed; by these two links it is hung to two levers, in such a way that when the levers move up and down, they raise and depress the agitator, but they can never make it strike the sides of the cylinder. Being heavier than its own bulk of water, the agitator, after being lifted by the levers, sinks suddenly afterwards by its weight alone.

The agitator's range of up-and-down movement should be about one inch and a quarter, and the number of its vibrations about 80 or 100 per minute; the flow of the pulp through the apertures is suddenly checked in its descent and promoted in its ascent, with the effect of counteracting obstructions between the ribs of the cylinder.

The sieve cylinder has a toothed wheel fixed upon the tubular part of one of its ends, which works between two metal flanches made fast to the wooden side of the vat, for the purpose of keeping the pulp away from the wheel; and it is made to revolve by a pinion fixed on a spindle, which going across the vat, is secured by two plummer blocks on the outside of the troughs, and has a rotatory motion given to it by an outside rigger or pulley, by means of a strap from the driving shaft, at the rate of 40 or 50 revolutions 
per minute. This spindle has also two double eccentrics fixed upon it, immediately under the levers, so that in every revolution it lifts those levers twice, and at the same time lifts the agitator.

The diameter of the sieve cylinder is not very material, but 14 inches have been found a convenient size; its length must be regulated according to the magnitude of the machine which it is destined to supply with pulp. One, four feet long in the cage part, is suflicient to supply a machine of the largest size in ordinary use, viz., one capable of making paper 4 feet 6 inches wide. When the cylinder is of this length, it should have a wheel and pinion at each end.

Metal flanches are firmly fixed to the sides of the vat, with a water-tight joint, and form the bearings in which the cylinder works.

Mr. Turner of Bermondsey, paper-maker, obtained a patent in March, 1831, for a peculiar strainer, designed to arrest the lumps mixed with the finer paper pulp, whereby he can dispense with the usual vat and hog in which the pulp is agitated immediately before it is floated upon the endless wire-web of the Fourdrinier apparatus. His strainer may also be applied advantageously to hand paper machines. He constructs his sieves of a circular form, by combining any desirable number of concentric rings of metal, with small openings between them, from the 50 th to the 100 th part of an inch wide. In order to facilitate the passage of the fine pulp and water, the sieves receive a vibratory motion up and down, which supersedes the hog employed in other paper-making machines.

A mechanism to serve the same purpose as the preceding, in which Mr. Ibotson's plan of a parallel rod-strainer is modified, was made the sabject of a patent by Mr. Henry Brewer, of Surrey Place, Southwark, in March, 1832. He constructs square boxes with gridiron bottoms, and gives a powerful up-and-down vibration in the pulp tub, by levers, rotatory shafts, and cranks.

As the contrivance is not deficient in ingenuity, and may be useful, I shall describe this mode of adapting his improved strainers to a vat in which paper is to be made by hand moulds. A hog (or churning rotator) is employed for the purpose of agitating the pulp at the bottom of the rat, in which the sieve is suspended from a crank-shaft, or in any other way, so as to receive the up-and-down vibratory motion for the purpose of straining the pulp. The pulp may be supplied from a chest, and passed through a cuck into a trough, by which it is conveyed to the strainers.

A pipe from the bottom of the vat leads into a lifter-box, which is designed to convey thin pulp into the sieve, in order to dilute that which is delivered from the chest. This pipe also allows the small lumps, called rolls, to be re-sifted. The pressure of the pulp and water in the vat forces the pulp up the pipe into the lifter-box, whence it is taken by rotatory lifters, and discharged into a trough, where it runs down and mixes with the thick pulp from the chest, as before mentioned. By these means the contents of the vat are completely strained or sifted over again in the course of almost every hour.

A patent was obtained for a paper-pulp strainer by Mr. Joseph Amies, of Loose, in the county of Kent, paper manufacturer, who makes the bottoms of his improred strainers with plates of brass or other suitable metal, and forms the apertures for the fine fibres of pulp to pass through, by cutting short slits through such plates, taking care that as much metal is left between the ends of each short slit and the next following as will properly brace or stiffen the ribs of the strainer; and he prefers that the end of one slit shall be nearly opposite to the middle of the two slits next adjoining it, which is commonly called blocking the joints. This is for giving rigidity to the bottom of the strainer, and constitutes the main feature of his improvement. The hottoms of sieves previously constructed with long metallic rods, he considers to be liable to lateral vibration in use, and thus to have permitted knots and lumps to pass through their expanded intervals. This objection is not applicable to Mr. Dickinson's squirrel-cage strainer, of which the ribs may be made rigid by a sufficient number of transverse bars; nor in fact is it applicable to Mr. Ibotson's original strainer, as it is admirably constructed by Messrs. Donkin and Co. Each bar which they make being inflexible by a feathered rib, is rendered perfectly straight in its edge by grinding with emery upon a flat disc-wheel of block tin, and of invariable length, by a most ingenious method of turning each set of bars in a lathe. The bars are afterwards adjusted in the metallic sieve-frame, or chest, at any desired distance apart, from the 120 th to the 60 th of an inch, in such a manner as secures them from all risk of derangement by the vibratory or jogging motion in shaking the pulpy fibres through the lineal intervals between them.

Mr. James Brown, paper manufacturer, of Esk mills, near Edinburgh, obtained a patent in May, 1836, for a particular mode of applying suction to the pasty web in the Fourdrinier's machine. He places a rectangular box transversely beneath the horizontal wire cloth, without the interposition of any perforated covering, such as had been 
tried in the previously constructed vacuum machines, and which he considers to have impeded their efficacy in condensing the pulp and extracting the water.

Upon this and all similar contrivances for making a partial vacuum under the pulpy paper web, it may be justly remarked, that they are more apt to injure than improve the texture of the article; since when the suction is unequally operative, it draws down not only the moisture, but many of the vegetable fibres, causing roughnesses, and even numerous small perforations in the paper.

A modification of Mr. Dickinson's cylinder-mould continuous paper machine was made the subject of a patent in Nov. 1830, by Mr. John Hall, jun., of Dartford, as communicated to him by a foreigner residing abroad. The leading feature of the invention is a mode of supplying the vat in which the wire cylinder is immersed with a copious flow of water, for the purpose of creating a considerable pressure upon the external surface of the cylinder, and thereby causing the fibres of the paper pulp to adhere to the mould.

There is a seml-cylindrical trough, in which the mould is immersed, and made to revolve by any convenient means. The pulp is transferred from the vat into that vessel at its bottom part. On the side of the drum-mould opposite to the vat, these is a cistern into which a copious flow of water is delivered, which passes thence into the semicrlindrical trough. In the interior of the cylindrical mould, a bent or syphon tube is introduced, on the horizontal part of which tube, inside, the mould revolves. This tube is connected at the outside to a pump, by which the water is drawn from the interior of the cylindrical mould. Thus the water in the semi-cylindrical trough, on the outside of the drum, is kept at a considerably higher level than it is within; and consequently the pressure of the water, as it passes through the wire gauze, will, it is supposer, cause the fibres of the paper pulp to adhere to the circumference of the mould. The water which is withdrawn from the interior of the drum by the recurved tube, is conducted round into the cistern, where its discharge is impeded by several vertical partitions, which make the water flow in a gentle stream into the semi-cylindrical mould vat. In order to keep the pulp properly agitated in the mould vat, a segment frame, having rails extended across the vat, is moved to and fro; as the drum mould goes round, the fibres of the pulp are forced against its circumference, and as the water passes through, the fibres adhere, forming the sheet of paper, which, on arriving at a couching roller above, is taken up as usual by an endless felt, conducted away to the drying apparatus, and thence to the reel to be wound up.

The patentee claims merely the application of a pump to draw the water from the interior of the mould drum, and to throw it upon its external surface.

A rag-cutting and lacerating machine was patented by Mr. Henry Davy, of Camberwell, in September, 1833, being a communication from a foreigner residing abroad. The machine consists of an endless feeding-cloth, by which the rough rags supplied by the attendants are progressively conducted forwards to a pair of fecd-rollers (see CotToN, spinning), and on passing through these rollers, the rags are subjected to the operation of rotatory cutters, acting against a fixed or ledger blade, which cut and tear them to pieces. Thence the rags pass down an inclined sieve, upon which they are agitated to separate the dust. The cleaned fragments are delivered on to a horizontal screen or sorting table, to suffer examination. When picked here, they are ready for the pulpengine. A distinct representation of this machine is given in Newton's Journal, conjoined series, vol. iv. pl. ix. fig. 1.

Mr. Jean Jacques Jequier obtained a patent in August, 1831, for a mode of making paper on the continuous machine with wire-marks. The proposed improvement consists merely in the introduction of a felted pressing roller, to act upon the paper after it has been discharged from the mould, and need not therefore be particularly described.

In August, 1830, Mr. Thomas Barratt, paper-maker, of St. Mary Cray, in the county of Kent, obtained a patent for an apparatus by which paper may be manufactured in a continuous sheet, with the water-mark and maker's name, so as to resemble in every respect paper made by hand, in moulds the size of each separate sheet. On the wire web, at equal distances apart, repetitions of the maker's name or other device is placed, according to the size of the paper when cut up into single sheets. In manufacturing such paper, the ordinary method of winding upon a reel cannot be einployed; and therefore the patentee has contrived a compensating reel, whose diameter diminishes at each rcvolution, equal to the thickness of a sheet of paper. See Newton's Journal, C. S. vol. vii. p. 285.

For Mr. Lemuel Wellman Wright's series of improvements in the manufacture of paper, specified in his patent of November, 1834, I must refer to the above Journal, C. S., vol, viii. p. 86 .

A committee of the Sociéte d'Encouragement, of Paris, made researches upon the best composition for sizing paper in the vat, and gave the following recipe :- 


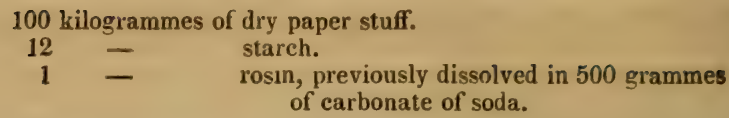

18 pails of water.

M. Braconnot proposed the following formula in the 23d volume of the Annales de Chimie et de Physique :-To 100 parts of dry stuff, properly diffused through water, add a boiling uniform solution of 8 parts of flour, with as much caustic potash as will render the liquor clear. Add to it one part of white soap previously dissolved in hot water. At the same time heat half a part of rosin with the requisite "uantity of weak potash ley for dissolving the rosin; mix both solutions together, and pour into them one part of alum dissolved in a little water.

Those who color prints, size them previously with the following composition : -4 ounces of glue, and 4 ounces of white soap dissolved in 3 English pints of hot water. When the solution is complete, two ounces of pounded alum must be added, and as soon as the composition is made homogeneous by stirring, it is ready for use. It is applied cold with a sponge, or rather with a flat camel's hair brush. Ackermann's liquor, as analyzed by Vauquelin, may be made for sizing paper as follows :-

$$
\begin{array}{ccl}
100 \text { kilogrammes of dry stuff. } \\
4 & - & \text { glue. } \\
8 & - & \text { resinous soap. } \\
8 & - & \text { alum. }
\end{array}
$$

The soap is made from 4.8 kilos. of pounded rosin, and 2.22 crystals of carbonate of soda, dissolved in 100 litres of water. It is then boiled till the mixture becomes quite uniform; the glue, previously softened by 12 hours' maceration in cold water, is to be next added; and when this is totally dissolved, the solution of alum in hot water is poured in. Three quarts of this size were introduced into the vat with the stuff, and well mixed with it. The paper manufactured with this paste seemed to be of excellent quality, and well sized.

The Chinese, in manufacturing paper, sometimes employ linen rags, as we do; at other times, the fibres of the young bamboo; of the mulberry; the envelope of the silk-worm cocoon; also a tree, unknown to our botanists, which the natives call chu or ko-chu; cotton down, and especially the cotton tree. The processes pursued in China to make paper with the inner bark of their paper-tree (Broussonetia-papyrifera), or Chinese mulberry, have been described at great length in the bulletin of the Société d' Encouragement, for 1826 , p. 226; but they will hardly prove serviceable to a European manufacturer. That tree has been acclimated in France.

Chinese paper is not so well made as the good paper of Europe; it is not so white, it is thinner, and more brittle, but extremely soft and silky. The longitudinal tenacity of its filaments, however, renders it fitter for the engraver than our best paper. The Chinese, after triturating, grinding, and boiling the bamboo, set the paste to ferment in a heap covered with mats. Chinese paper is readily recognised, because it is smooth on one side, and bears on the other the marks of the brush with which it is finished, upon smooth tables, in order to dry it flat. The kind employed for engravings is in sheets four feet long, and two broad. It is made of the bamboo; their myrtle-tree paper would be too strong for this purpose.

\section{Tracing paper.}

The best paper of this kind, sometimes superfluously called vegetable paper, is made of the refuse of the flax mills, and prepared by the engine without fermentation. It thus forms a semi-transparent paste, and affords a transparent paper. Bank-note paper is made of the same materials, but they always undergo a bleaching with chloride of lime. Great nicety is required in drying this kind of paper. For this purpose, each sheet must be put between two sheets of gray paper in the press; and this gray paper must be renewed several times, to prevent the bank-note paper from creasing.

\section{Paper of Safety or Surety; Papier de Sureté.}

This subject has occupied the attention of the French Academy for many years, in consequence of the number of fiauds committed upon the stamp revenue in France. One of the best methods of making a paper which would evince whether any part of a writing traced upon it had been tampered with or discharged, is to mix in the vat two kinds of pulp, the one perfectly white, the other dyed of any color easily affected by chlorine, acids, and alkalis. The latter stuff being mingled with the former in any desired proportion, will furnish a material for making a paper which will contain colored points distributed throughout all its substance, ready to show, by the changes they suffer, whether any chemical reaction has been employed. 
Quantity of Paper charged with Duties of Excise, in the United Kingdom, in

\begin{tabular}{|c|c|c|c|}
\hline & 1834. & 1835. & 1836. \\
\hline $\begin{array}{l}\text { First class - } \\
\text { Second class }- \\
\text { Pasteboard, millboard, \&c. - } \\
\text { Stained - - - - }\end{array}$ & $\begin{array}{c}\text { lbs. } \\
54,053,721 \\
16,552,168 \\
49,392 \\
\text { yards. } \\
8,749,144\end{array}$ & $\begin{array}{c}\text { lbs. } \\
56,179,555 \\
17,863,095 \\
49,772 \\
\text { yards. } \\
8,247,931\end{array}$ & $\begin{array}{c}\text { lbs. } \\
66,202,689 \\
15,906,258 \\
36,340 \\
\text { yards. } \\
8,032,577\end{array}$ \\
\hline $\begin{array}{cl}\text { Amount of duty, first class - } \\
- & \text { second class } \\
- & \text { pasteboard, \&c. } \\
- & \text { stained - } \\
\end{array}$ & $\begin{array}{rrr}£ & s . & d . \\
675,671 & 10 & 0 \\
103,451 & 0 & 0 \\
54,689 & 0 & 0 \\
63,795 & 16 & 0 \\
\end{array}$ & $\begin{array}{rrr}£ & s . & d . \\
702,244 & 9 & 0 \\
111,644 & 0 & 0 \\
54,548 & 15 & 0 \\
60,141 & 0 & 0\end{array}$ & $\begin{array}{rrr}£ & s . & d . \\
651,699 & 0 & 0 \\
99,414 & 0 & 0 \\
39,557 & 0 & 0 \\
22,112 & 0 & 0 \\
\end{array}$ \\
\hline
\end{tabular}

The late reduction of the duty, from $3 d$. to $1 \frac{1}{2} d$. per lb., upon paper of the first class, viz., on all descriptions of it, except that made out of tarred ropes only, has been already attended with considerable benefit to the manufacture, and would have acted with much greater effect, but for the American crisis. The gross amount of the paper duty in the year ending 5th January, 1836, was 831,057l., and in the year ending 5th January, 1838, it was 554,497l.; instead of being little more than one half, as might have been the case from the reduction of the duty, which only came into full operation in the year 1837. At the same time that the tax on common paper was reduced, that upon stained paper was repealed altogether. The effect of the diminution consequently made in the price of paper-hangings, has been so great as nearly to double the consumption of the country, while the manufacture appears to be still rapidly on the increase.

Declared Value of Stationary and Printed Books exported in

\begin{tabular}{|c|r|r|r|}
\hline Years. & Stationary. & Printed Books. & \multicolumn{1}{c|}{ Total. } \\
\cline { 3 - 4 } 1827 & $£ 195,110$ & $£ 107,199$ & $£ 302,309$ \\
1828 & 208,532 & 102,874 & 311,406 \\
1829 & 190,652 & 109,878 & 300,530 \\
1830 & 171,848 & 95,874 & 267,722 \\
1831 & 179,216 & 101,110 & 280,326 \\
1832 & 177,718 & 93,038 & 270,756 \\
1833 & 211,518 & 124,535 & 336,053 \\
1834 & 211,459 & 122,595 & 334,054 \\
1835 & 259,105 & 148,318 & 407,423 \\
1836 & 301,121 & 178,945 & 480,066 \\
\hline
\end{tabular}

Till the paper trade shall escape entirely from the clutches of its ancient dry-nurse, the excise, neither it nor the book trade can acquire the same ascendency in exportation which all wther articles of British manufactures have over the French.

The value of Stationary exported in France, from 1833, was-

Cartons lustrés (polished pasteboards for the cloth manufacture)

Cartons en feuilles (pasteboard in sheets) - - - -

Cartons moulés (papier-maché)

Cartons coupés et assemblés

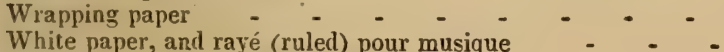

Colored paper in reams

Stained paper (paper nangings) in rouleaux, - - - -

Silk paper

$$
\text { Total }(=£ 208,000)
$$$$
\begin{array}{r}
18,992 \text { francs. } \\
6,352= \\
215,376= \\
54,184= \\
178,544= \\
2,903,075= \\
58,541= \\
1,885,387= \\
3,240=
\end{array}
$$

PARAFFINE. Distil beech-tar to dryness, rectify the heavy oil which collects at the and urge the heat moderately as long as any thing more distils. Pyrélaine passes over, containing crystalline scales of paraffine. This mixture being digested with its own volume of alcohol of 0.833 , forms a limpid solution, which is to be gradually diluted with more alcohol, till its bulk becomes 6 or 8 times greater. The alcohol, which at first dissolves the whole, lets the paraffine gradually fall. The precipitate being washed 
with cold alcohol till it becomes nearly coloriess, and then dissolved in boiling alcohol, is deposited on cooling in minute spangles and needles of pure paraffine.

Or the above mixture may be mixed with from $\frac{1}{4}$ to $\frac{1}{2}$ its weight of concentrated sulphuric acid, and subjected for 12 hours to digestion, at a heat of $150^{\circ} \mathrm{F}$., till, on cooling, crys. tals of paraffine appear upon the surface. These are to be washed with water, dissolved in hot alcohol, and crystallized. Paraffine is a white substance, void of taste and smell, feels soft between the fingers, has a specific gravity of 0.87 , melts at $112^{\circ}$ Fahr., boils at a higher temperalure with the exhalation of white fumes, is not decomposed by dry distillation, burns with a clear white flame without smoke or residuum, does not stain paper, and consists of 85.22 carbon, and 14.78 hydrogen; having the same composition as olefiant gas. It is decomposed neither by chlorine, strong acids, alkalis, nor potassium; and unites by fusion with sulphur, phosphorus, wax, and rosin. It dissolves readily in warm fat oils, in cold essential oils, in ether, but sparingly in boiling absolute alcohol. Paraffine is a singular solid bicarburet of hydrogen; it has not hitherto been applied to any use, but it would form admirable candles.

PARCHMENT. (Parchemin, Fr.; Pergament, Germ.) This writing material has been known since the earliest times, but is now made in a very superior manner to what it was anciently, as we may judge by inspection of the old vellum and parchment manuscripts. The art of making parchment consists in certain manipulations necessary to prepare the skins of animals of such thinness, flexibility, and firmness, as may be required for the different uses to which this substance is applied. Though the skins of all animals might be converted into writing materials, only those of the sheep or the shegoat are used for parchment; those of calves, kids, and dead-born lambs for vellum; those of the he-goat, she-goat, and wolves for drum-heads ; and those of the ass for battledoors. All these skins are prepared in the same way, with slight variations, which need no particular detail.

They are first of all prepared by the leather-dresser. After they are taken out of the lime-pit, shaved, and well washed, they must be set to dry in such a way as to prevent their puckering, and to render them easily worked. The small manufacturers make use of hoops for this purpose, but the greater employ a herse, or stout wooden frame. This is formed of two uprights and two cross-bars solidly joined together by tenons and mortises, so as to form a strong piece of carpentry, which is to be fixed up against a wall. These four bars are perforated all over with a series of holes, of such dimensions as to receive slightly tapered box-wood pins, truly turned, or even iron bolts. Each of these pins is transpierced with a hole like the pin of a violin, by means of which the strings employed in stretching the skin may be tightened. Above the herse, a sheli is placed, for receiving the tools which the workman needs to have always at hand. In order to stretch the skin upon the frame, larger or smaller skewers are employed, according as a greater or smaller piece of it is to be laid hold of. Six holes are made in a straight line to receive the larger, and four to receive the smaller skewers or pins. These small slits are made with a tool like a earpenter's chisel, and of the exact size to admit the skewer. The string round the skewer is affixed to one of the bolts in the frame, which are turned round by means of a key, like that by which pianos and harps are tuned. The skewer is threaded through the skin in a state of tension.

Every thing being thus prepared, and the skin being well softened, the workman stretches it powerfully by means of the skewers; he attaches the cords to the skewers, and fixes their ends to the iron pegs or pins. He then stretches the skin, first with his hand applied to the pins, and afterwards with the key. Great care must be taken that no wrinkies are formed. The skin is usually stretched more in length than in breadth, from the custom of the trade; though extension in breadth would be preferable, in order to reduce the thickness of the part opposite the backbone.

The workman now takes the fleshing tool represented under CuRrying. It is a semicircular double-edged knife, made fast into a double wooden handle. Other forms of the fleshing-knife edge are also used. They are sharpened by a steel. The workman seizes the tool in his two hands, so as to place the edge perpendicularly to the skin, and pressing it carefully from above downwards, removes the fleshy excrescences, and lays them aside for making glue. He now turns round the herse upon the wall, in order to get access to the outside of the skin, and to scrape it with the tool inverted, so as to run no risk of cutting the epidermis. He thus removes any adhering filth, and squeezes out some water. The skin must next be ground. For this purpose it is sprinkled upon the fleshy side with sifted chalk or slaked lime, and then rubbed in all directions with a piece of pumice-stone, 4 or 5 inches in area, previously flattened upon a sandstone. The lime gets soon moist from the water contained in the skin. The pumice-stone is then rubbed over the other side of the skin, but without chalk or iime. This operation is necessary only for the best parchment or vellum. The skin is now allowed to dry upon the frame; being carefully protected from sunshine, and from frost. In the arid 
weather of summer a moist cloth needs to be applied to it from time to time, to prevent its drying too suddenly; immediately after which the skewers require to be tightened.

When it is perfectly dry, the white color is to be removed by rubbing it with the woolly side of a lambskin. But great care must be taken not to fray the surface; a circumstance of which some manufacturers are so much afraid, as not to use either chalk or lime in the polishing. Should any grease be detected upon it, it must be removed by steeping it in a lime pit for 10 days, then stretching it anew upon the herse, after which it is transferred to the scraper

This workman employs here an edge tool of the same shape as the fleshing-knife, but larger and sharper. He mounts the skin upon a frame like the herse above described; but he extends it merely with cords, without skewers or pins, and supports it generally upon a piece of raw calfskin, strongly stretched. The tail of the skin being placed towards the bottom of the frame, the workman first pares off, with a sharp linife, any considerable roughnesses, and then scrapes the outside surface obliquely downwards with the proper tools, till it becomes perfectly smooth : the fleshy side needs no such operation, and indeed were both sides scraped, the skin would be apt to become too thin, the only object of the scraper being to equalize its thickness. Whatever irregularities remain, may be removed with a piece of the finest pumice-stone, well flattened beforehand upon a fine sandstone. This process is performed by laying the rough parchment upon an oblong plank of wood, in the form of a stool; the plank being covered with a piece of soft parchment stuffed with wool, to form an elastic cushion for the grinding operation. It is merely the outside surface that requires to be pumiced. The celebrated Strasburgh vellum is prepared with remarkably fine pumice-stones.

If any small holes happen to be made in the parchment, they must be neatly patched, by cutting their edges thin, and pasting on small pieces with gum water.

The skins for drum-heads, sieves, and battledoors are prepared in the same way. For drums, the skins of asses, calves, or wolves are employed; the last being preferred. Ass skins are used for battledoors. For sieves, the skins of calves, she-goats, and, best of all, he-goats are employed. Church books are covered with the dressed skins of pigs.

Parchment is colored only green. The following is the process. In 500 parts of rain water, boil 8 of cream of tartar, and 30 of crystallized verdigris; when this solution is cold, pour into it 4 parts of nitric acid. Moisten the parchment with a brush, and then apply the above liquid evenly over its surface. Lastly, the necessary lustre may be given with white of eggs, or mucilage of gum arabic.

PARTING (Départ, Fr.; Scheidung, Germ.), is the process by which gold is separated from silver. See Assay, Gold, Refining, and Silver.

PASTEL, is the French name of colored crayons.

PASTEL, is a dye stuff, allied to Indigo, which see.

PASTES, or FACTITIOUS GEMS. (Pierres précieuses artificielles, Fr.; Glaspasten, Germ.) The general vitreous body called Strass, (from the name of its German invenfor,) preferred by Fontanier in his treatise on this subject, and which he styles the Mayence base, is prepared in the following manner :-8 ounces of pure rock-crystal or flint in powder, mixed with 24 ounces of salt of tartar, are to be baked and left to cool. The mixture is to be afterwards poured into a basin of hot water, and treated with dilute nitric acid till it ceases to effervesce; and then the frit is to be washed till the water comes off tasteless. This is to be dried, and mixed with 12 ounces of fine white-lead, and the mixture is to be levigated and elutriated with a little distilled water. An ounce of calcined borax being added to about 12 ounces of the preceding mixture in a dry state, the whole is to be rubbed together in a porcelain mortar, melted in a clean crucible, and poured out into cold water. This vitreous matter must be dried, and melted a second and a third time, alwars in a new crucible, and after each melting poured into cold water, as at first, taking care to separate the lead that may be revived. To the third frit, ground to powder, 5 drachms of nitre are to be added; and the mixture being melted for the last time, a mass of crystal will be found in the crucible, of a beautiful lustre. The diamond may be well imitated by this Mayence base. Another very fine white crystal may be obtained, according to M. Fontanier, from 8 ounces of white-lead, 2 ounces of powdered borax, $\frac{2}{2}$ grain of manganese, and 3 ounces of rock-crystal, treated as ahove.

The colors of artificial gems are obtained from metallic oxydes. The oriental topaz is prepared by adding oxyde of antimony to the base; the amethyst, by manganese with a little of the purple of Cassius; the beryl, by antimony and a very little cobalt; yellow artificial diamond and opal, by horn-silver (chloride of silver); blue-stone or sapphire, by cobalt. The following proportions have been given :-

For the yellow diamond. To 1 ounce of strass add 24 grains of chloride of silver, or 10 grains of glass of antimony.

For the sapphire. To 24 ounces of strass, add 2 drachms and 26 grains of the oxyde of cobalt.

For the oriental ruby. To 16 ounces of strass, add a mixture of 2 drachms and 48 
grains of the precipitate of Cassius, the same quantity of peroxyde of iron prepared by nitric acid, the same quantity of golden sulphuret of antimony and of manganese calcined with nitre, and 2 ounces of rock crystal. Manganese alone, combined with the base in proper quantity, is said to give a ruby color.

For the emerald. To 15 ounces of strass, add 1 drachm of mountain blue (carbonate of copper), and 6 grains of glass of antimony ; or, to 1 ounce of base, add 20 grains of glass of antimony, and 3 grains of oxyde of cobalt.

For the common opal. To 1 ounce of strass, add 10 grains of horn-silver, 2 grains of calcined magnetic ore, and 26 grains of an absorbent earth (probably chalk-marl) Fontanier.

M. Douault-Wiéland, in an experimental memoir on the preparation of artificial colored stones, has offered the following instructions, as being more cxact than what were pub. lished before.

The base of all artificial stones is a colorless glass, which he calls fondant, or flux; and he unites it to metallic oxydes, in order to produce the imitations. If it be wrorked alone on the lapidary's wheel, it counterfeits brilliants and rose diamonds remarkably well.

This base or strass is composed of silex, potash, borax, oxyde of lead, and sometimes arsenic. The silicious matter should be perfectly pure; and if obtained from sand, it ought to be calcined and washed, first with dilute muriatic acid, and then with water. The crystal or flint should be made redhot, quenched in water, and ground, as in the notteries. The potash should be purified from the best pearlash; and the borax should be refined by one or two crystallizations. The oxyde of lead should be absolutely free from tin, for the least portion of this latter metal causes milkiness. Good red-lead is preferable to litharge. The arsenic should also be pure. Hessian crucibles are preferable to those of porcelain, for they are not so apt to crack and run out. Either a pottery or porcelain kiln will answer, and the fusion should be continued 24 hours; for the more tranquil and continuous it is, the denser is the paste, and the greater its beauty. The following four recipes have afforded good strass :-

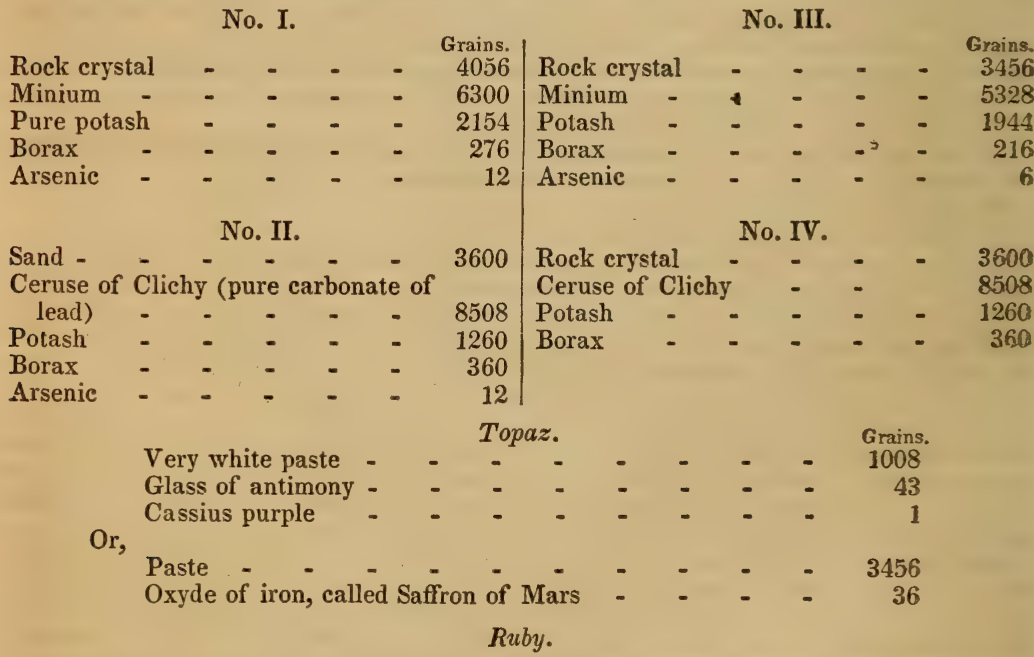

M. Wiéland succeeded in obtaining excellent imitations of rubies, by making use of the topaz materials. It often happened that the mixture for topazes gave only an opaque mass, translucent at the edges, and in thin plates of a red color. 1 part of this substanee being mixed with 8 parts of strass, and fused for 30 hours, gave a fine yellowish crystal. like paste, and fragments of this fused before the blowpipe, afforded the finest imitation of rubies. The result was always the same.

The following are other proportions for rubies :Paste -

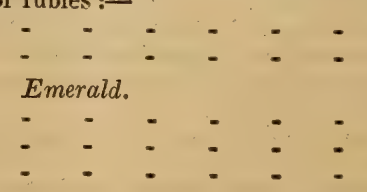

Paste

Green oxyde of pire copper

Oxyde of chrome 


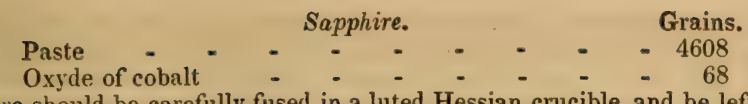

This mixture should be carefully fused in a luted Hessian crucible, and be lef 50 hours in the fire.

$$
\text { Amethyst. }
$$

Syrian Garnet, or Ancient Carbuncle.

$\begin{aligned} & \text { Paste } \\ & \text { Oxyde of manganese }\end{aligned}-$
$\begin{aligned} & \text { Oxyde of cobalt } \\ & \text { Purple of Cassius }\end{aligned}$

In all these mixtures, the substances should be mixed by sifting, fused very carefully, and cooled very slowly, after having been left in the fire from 24 to 30 hours.

M. Lançon has also made many experiments on the same subject. The following are a few of his proportions :-

$$
\begin{aligned}
& \text { Litharge Paste. } \\
& \text { White sand } \\
& \text { White tartar, or potash } \\
& \text { Paste } \\
& \text { Acetate of copper } \\
& \text { Peroxyde of iron, or saffron of Mars }
\end{aligned}
$$

PASTILLE is the English name of small cones made of gum benzoin, with powder of cinnamon, and other aromatics, which are burned as incense, to diffuse a grateful odor, and conceal unpleasant smells in apartments. See Perfumery.

PASTILLE is the French name of certain aromatic sugared confections; called also tablettes.

PEARLASH, a commercial form of Potash, which see.

PEARLS (Perles Fr. ; Perlen, Germ.), are the productions of certain shell-fish. These molluscæ are subject to a kind of disease caused by the introduction of foreign bodies within their shells. In this case, their pearly secretion, instead of being spread in layers upon the inside of their habitation, is accumulated round these particles in concentric layers. Pearl consists of carbonate of lime, interstratified with animal membrane.

The oysters whose shells are lichest in mother of pearl, are most productive of these highly prized spherical concretions. The most valuable pearl fisheries are on the coast of Ceylon, and at Olmutz in the Persian Gulf, and their finest specimens are more highly prized in the East than diamonds, but in Europe they are liable to be rated very differently, according to the caprice of fashion. When the pearls are large, truly spherical, reflecting and decomposing the light with much vivacity, they are much admired. But one of the causes which renders their value fluctuating, is the occasional loss of their peculiar lustre, without our being able to assign a satisfactory reason for it. Besides, they can be now so well imitated, that the artificial pearls have nearly as rich an appearance as the real.

PEARLS, ARTIFICIAL. These are small globules or pear-shaped spheroids of thin glass, perforated with two opposite holes, through which they are strung, and mounted into necklaces, \&c., like real pearl ornaments. They must not only be white. and brilliant, but exhibit the iridescent reflections of mother of pearl. The liquor employed to imitate the pearly lustre, is called the essence of the East (essence d' orient), which is prepared by throwing into water of ammonia the brilliant scales, or rather the lamella, separated by washing and friction, of the scales of a small river fish, the blay, called in French ablette. These scales digested in ammonia, having acquired a degree of softness and flexibility which allow of their application to the inner surfaces of the glass globules, they are introduced by suction of the liquor containing them in suspension. The ammonia is volatilized in the act of drying the globules.

It is said that some manufacturers employ ammonia merely to prevent the alteration of the scales; that when they wish to make use of them, they suspend them in a well clarified solution of isinglass, then pour a drop of the mixture into each bead, and spread it round the inner surface. It is doubtful whether, by this method, the same lustre and play of colors can be obtained as by the former. It seems moreover to be of importance for the success of the imitation, that the globules be formed of a bluish, opalescent, very thin glass, containing but little potash and oxyde of lead. In everv manu- 
factory of artificial pearls, there must be some workmen possessed of great experience and dexterity. The French are supposed to excel in this ingenious branch of industry.

PEARL WHITE is a submuriate of bismuth, obtained by pouring a solution of the nitrate of that metal into a dilute solution of sea-salt, whereby a light and very white powder is obtained, which is to be well washed and dried. See Bismutr.

PECTIC ACID (Acide pectique, Fr.; Gallertsaüre, Germ.), so named on account of its jellying property, from $\pi \eta \wedge \tau \iota$, coagulum, exists in a vast number of vegelables. The easiest way of preparing it, is to grate the roots of carrots into a pulp, to express their juice, to wash the marc with rain or distilled water, and to squeeze it well; 50 parts of the marc are next to be diffused through 300 of rain-water, adding by slow degrees a solution of one part of pure potash, or two of bicarbonate. This mixture is to be heated, so as to be made to boil for about a quarter of an hour, and is then to be thrown boiling-hot upon a filter cloth. It is known to have been well enough boiled, when a sample of the filtered liquor becomes gelatinous by neutralizing it with an acid. This liquor contains pectate of potassa, in addition to other matters extricated from the root. The pectate may be decomposed by a stronger acid, but it is better to decornpose it by muriate of lime; whereby a pectate of lime, in a gelatinous form, quite insoluble in water, is obtained. This having been washed with cold water upon a cloth, is to be boiled in water containing as much muriatic acid as will saturate the lime. The pectic acid thus liberated, remains under the form of a colorless jelly, which reddens litmus paper, and tastes sour, even after it is entirely deprived of the muriatic acid. Cold water dissolves very little of it; it is more soluble in boiling water. The solution is colorless, does not coagulate on cooling, and hardly reddens litmus paper; but it gelatinizes when alcohol, acids, alkalis, or salts are added to it. Even sugar transforms it, after some time, into a gclatinous state, a circumstance which serves to explain the preparation of apple, cherry, raspberry, gooseberry, and other jellies.

PECTINE, or vegetable jelly, is obtained by mixing alcohol with the juice of ripe currants, or any similar fruit, till a gelatinous precipitate takes place; which is to be gently squeezed in a cloth, washed with a little weak alcohol, and dried. Thus prepared, pectine is insipid, without action upon litmus, in small pieces, semi-transparent, and of a membranous aspect, like isinglass. Its mucilagınous solution in cold water is not tinged blue with iodine. A very small addition of potash, or its carbonate, converts pectine into pectic acid; both of which substances are transformed into mucic and oxalic acids by the nitric.

PELTRY (Pelleterie, Fr.; Pelzwerk, Germ.), is nearly synonymous with fur, and comprehends the skins of different kinds of wild animals that are found in high northern latitudes, particularly in the American continent; such as the beaver, bear, moosedeer, marten, mink, sable, wolverine, wolf, \&c. When these skins have received no preparation but from the hunters, they are most properly called peltry; but when they have had the inner side tawed or tanned (see LEATHER) by an aluminous process, they may then be denominated furs.

The scouring or cleaning of peltry is performed in a large cask, or truncated cone laid on its side, and traversed by a revolving shaft, which is furnished with a few rectangular rounded pegs. These are intended to stir round the skins, while they are dusted over with Paris plaster, whitening, or sometimes sand, made as hot as the hand can bear. The bottom of the cask should be grated, to allow the impurities to fall out. The lustrage, which the cleansed skins next undergo, is merely a species of dyeing, either topical, to modify certain disagreeable shades, or general, to impart a more beautiful color to the fur. Under the articles DyerNG, and the several colors, as also HaIR and Morocco, sufficient instructions will be found for dyeing fur. The mordants should be applied pretty hot by a brush, on the hair of the skin, stretched upon a solid table; and after two or three applications, with drying between, the tinctorial infusions may be rubbed on in the same way. The hair must be freed beforehand from all greasiness, by lime water, or a weak solution of carbonate of soda; then well washed. Much nicety, and many successive applications of the dye-stuff, are sometimes requisite to bring out the desired shade.

Under Hat Mandfacture, I referred to this article for a description of the process of sécretage, whereby the hairs of rabbit and hare skins are rendered fit for felting. Dissolve 32 parts of quicksilver in 500 of common aquafortis; and dilute the solution with one half or two thirds of its bulk of water, according to the strength of the acid. The skin being laid upon a table with the hair side uppermost, a brush, made with the bristles of the wild boar, is to be slightly moistened with the mercurial solution, and passed over the smooth surface of the hairs with strong pressure. This application must be repeated several times in succession, till every part of the fur be equally touched, and till about two thirds of the length of the hairs be moistened, or a little more, should they be rigid. In order to complete this impregnation, the skins are laid together in pairs with the hairy sides in contact, put in this state into the stove-room, 
and exposed to a heat higher in proportion to the weakness of the mercarial solution. The drying should be rapidly effected, otherwise the concentration of the nitrate will not take due effect in causing the retraction and curling of the hairs.

No other acid, or metallic solution, but the above, has been found to answer the desiret purpose of the hatmaker. After the hairs are properly secreted, they are plucked off by hand, or shorn off by a machine.

PENCIL MANUFACTURE. (Crayons, fabrique de, Fr.; Bleistifte, verfertigung, Germ.) The word pencil is used in two senses. It signifies either a small hair brush employed by painters in oil and water colors, or a slender cylinder, of black lead or plumbago, either nalied or enclosed in a wooden case, for drawing black lines upon paper. The last sort, which is the one to be considered here, corresponds nearly to the French term crajon, though this includes also pencils made of differently colored earthy compositions. See Crayon.

The best black-lead pencils of this. country are formed of slender parallelopipeds, cut out by a saw from sound pieces of plumbago, which have been previously calcined in close vessels at a bright red heat. These parallelopipeds are generally enclosed in cases made of cedar wood, though of late years they are also used alone, in peculiar pencil-cases, under the name of ever-pointed pencils, provided with an iron wire and serew, to protrude a minute portion of the plumbago beyond the tubular metallic ease, in proportion as it is wanted.

In the year 1795, M. Conté, a French gentleman, well acquainted with the mechanical arts, invented an ingenious process for making artificial black-lead pencils of superior qualty, by which he and his successor and son-in-law, M. Humblot, have realized large fortunes.

Pure clay, or clay containing the smallest proportion of calcareous or silicious matter, is the substance which he employed to give aggregation and solidity, not only to plumbago dust, but to all sorts of colored powders. That earth has the property of diminishing in bulk, and increasing in hardness, in exact proportion to the degree of heat it is exposed to, and hence may be made to give every degree of solidity to crayons. The clay is prepared by diffusing it in large tubs through clear river water, and letting the thin mixture settle for two minutes. The supernatant milky liquor is drawn off by a syphon from near the surface, so that only the finest particles of clay are transferred into the second tub, upon a lower level. The sediment, which falls very slowly in this tub, is extremely soft and plastic. The clear water being run off, the deposite is placed upon a linen filter, and allowed to dry. It is now ready for use.

The plumbago must be reduced to a fine powder in an iron mortar, then put into a crucible, and calcined at a heat approaching to whiteness. The action of the fire gives it a brilliancy and softness which it would not otherwise possess, and prevents it from being affected by the clay, which it is apt to be in its natural state. The less clay is mixed with the plumbago, and the less the mixture is calcined, the softer are the pencils made of it; the more clay is used the harder are the pencris. Some of the best pencils made by M. Conté, were formed of two parts of plumbago and three parts of clay ; others of equal parts. This composition admits of indefinite variations, both as to the shade and hardness; advantages not possessed by the native mineral. While the traces may be made as black as those of pure plumbago, they have not that glistening aspect.which often impairs the beauty of black-lead drawings. The same lustre may, however, be obtained by increasing the proportion of powdered plumbago relatively to the clay.

The materials having been carefully sifted, a little of the clay is to be mixed with the plumbago, and the mixture is to be triturated with water into a perfectly uniform paste. A portion of this paste may be tested by calcination. If on cutting the indurated mass, particles of plumbago appear, the whole must be further levigated. The remainder of the clay is now to be introduced, and the paste is to be ground with a muller upon a porphyry slab, till it be quite homogeneous, and of the consistence of thin dough. It is now to be made into a ball, put upon a support, and placed under a bell glass inverted in a basin of water, so as to be exposed merely to the moist air.

Small grooves are to be made in a smooth board, similar to the pencil parallelopipeds, but a little longer and wider, to allow for the contraction of volume. The wood must be boiled in grease, to prevent the paste from sticking to it. The above described paste being pressed with a spatula into these grooves, another board, also boiled in grease, is to be laid over them very closely, and secured by means of screw-clamps. As the atmospheric air can get access only to the ends of the grooves, the ends of the pencil-pieces become dry first, and by their contraction in volume get loose in the grooves, allowing the air to insinuate further, and to dry the remainder of the paste in succession. When the whole piece is dried, it becomes loose, and might be turned out of the grooves. But before this is done, the mould must be put into an oven moderately heated, in order to render the pencil pieces still drier. The mould should now be taken out, and emptied upon a table covered with cloth. The greater part of the pieces will be 
entire, and oniy a few will have been broken, if the above precautions have been duly observed. They are all, however, perfectly straight, which is a matter of the first importance.

In order to give solidity to these pencils, they must be set upright in a crucible till it is filled with them, and then surrounded with charcoal powder, fine sand, or sifted wood ashes. The crucible, after having a luted cover applied, is to be put into a furnace, and exposed to a degree of heat regulated by the pyrometer of Wedgewood; which degree is proportional to the intended hardness of the pencils. When they have been thus baked, the crucible is to be removed from the fire, and allowed to cool with the pencils in it.

Should the pencils be intended for drawing architectural plans, or for very fine lines, they must be immersed in melted wax or suet nearly boiling hot, before they are put into the cedar cases. This immersion is best done by heating the pencils first upon a grid-iron, and then plunging them into the melted wax or tallow. They acquire by this means a certain degree of softness, are less apt to be abraded by use, and preserve their points much better.

When these pencils are intended to draw ornamental subjects with much shading, they should not be dipped as above.

Second process for making artificial pencils, somewhat different from the preceding.-All the operations are the same, except that some lamp-black is introduced along with the plumbago powder and the clay. In calcining these pencils in the crucible, the contact of air must be carefully' excluded, to prevent the lamp-black from being burned away on the surface. An indefinite variety of pencils, of every possible black tint, may thus be produced, admirably adapted to draw from nature.

Another ingenious form of mould is the following:

Models of the pencil-pieces must be made in iron, and stuck upright upon an iron tray, haring edges raised as high as the intended length of the pencils. A metallic alloy is made of tin, lead, bismuth, and antimony, which melts at a moderate heat. This is poured into the sheet-iron tray, and after it is cooled and concreted, it is inverted, and shaken off from the model bars, so as to form a mass of metal perforated throughout with tubular carities, corresponding to the intended pencil-pieces. The paste is introduced by pressure into these carities, and set aside to dry slowly. When nearly dry, the pieces get so much shrunk that they may be readily turned out of the moulds upon a cloth table. Ther are then to be completely desiccated in the shade, afterwards in a stove-room, next in the oven, and lastly ignited in the crucible, with the precautions above prescribed.

M. Conté recommends the hardest pencils of the architect to be made of lead melted with some antimony and a little quicksilver.

In their further researches upon this subject, M. Conté and M. Humblot found that the different degrees of hardness of crayons could not be obtained in a uniform manner by the mere mixture of plumbago and clay in determinate doses. But they discovered a remedy for this defect in the use of saline solutions, more or less concentrated, into which they plunged the pencils, in order to modify their hardness, and increase the uniformity of their texture. The non-deliquescent sulphates were preferred for this purpose; such as sulphate of soda, \&c. Even sirup was found useful in this way.

PENS, STEEL. The best metal, made from Dannemora or hoop (L) iron, is selected, and laminated into slips about 3 feet long, and 4 inches broad, of a thickness corresponding to the desired stiffness and flexibility of the pens. These slips are subjected to the action of a stamping-press, somewhat similar to that for making buttons. (See ButToN, and PlateD WARE.) The point destined for the nib is next introduced into an appropriate gauged hole of a little machine, and pressed into the semi-crlindrical shape; where it is also pierced with the middle slit, and the lateral ones, prorided the latter are to be giren. The pens are now cleaned, by being tossed about among each other, in a tin cylinder, about 3 feet long, and 9 inches in diameter; which is suspended at each end upon joints, to two cranks, formed one on each of two shafts. The cylinder, by the rotation of a fly-wheel, acting upon the crank-shafts, is made to describe such revolutions as agitate the pens in all directions, and polish them by mutual attrition. In the course of 4 hours several thousand pens may be finished upon this machine.

PEPPER. (Poivre, Fr.; Pfeffer, Germ.) Black pepper is composed, according to $\mathbf{M}$. Pelletier, of the vegetable principle, piperine, of a very acrid concrete oil, a volatile balsamic oil, a colored gummy matter, an extractive principle analogous to legumine, malic and tartaric acids, starch, bassorine, ligneous matter, with earthy and alkaline salts in small quantity. Cubebs pepper has nearly the same composition.

Pepper imported for home consumption, in 1835, 1836, 1837.-Duty 6d. per lb. Lbs. $2,359,575 ; 2,800,980 ; 2,626,298$. 
PERFUMERY, ART OF (Parfumerie, Fr.; Wohlriechende-kunst, Germ.), consists in the preparation of different products, such as fats or pommades, essential oils, distilled spirits, pastes, pastilles, and essence.

Fats ought to be pounded in a marble mortar, without addition of water, till all the membranes be completely torn; then subjected to the heat of a water-bath in a proper resel. The fat soon melts, and the albumen of the blood coagulating, carries with it all the foreign substances; the liquid matter should be skimmed, and passed through a canvass filter.

Of pommades by infusion.-Rose, orange-flower, and cassia. Take 334 pounds of hog's lard, and 166 of beef suet. These 500 pounds are put into a pan called bugadier; and when melted, 150 pounds of rose-leares nicely plucked are added, taking care to stir the mixture every hour. The infusion thus prepared is to remain at rest for 24 hours; at the end of this time, the pommade is again melted, and well stirsed to present its adherence to the bottom of the melting-pan. The mass is now to be poured out into canrass, and made into rectangular bricks or loaves, which are subjected to a press, in order to separate the solid matter from the soft pommade. These brick-shaped pieces being put into an iron-bound barrel perforated all over its staves, the pommade is to be allowed to exude on all sides, and flow down into a copper vessel placed under the trough of the press. This manipulation should be repeated with the same fat ten or twelte times; or in other woids, 3000 pounds of fresh rose-leaves should be employed to make a good pommade.

The pommade of orange-flowers is made in the same manner, as also the pommade of cassia.

Of pommades without infusion.-Jasmine, tuberose, jonquil, narcissus, and riolet.

A square frame, called tiame, is made of four pieces of wood, well joined together, 2 or 3 inches deep, into which a pane of glass is laid, resting upon inside ledges near the bottom. Upon the surface of the pane the simple pommade of hog's lard and suet is spread with a pallet knife; and into this pommade the sweet-scented flowers are stach fresh in different points each successive day, during two or three months, till the pommade has acquired the desired richness of perfume. The above-described frames are piled closely over each other. Some establishments at Grasse possess from 3000 to 4000 of them.

Of oils.-Rose, orange-flower, and cassia oils, are made by infusion, like the pom. mades of the same perfumes; taking care to select oils perfectly fresh. As to those of jasmine, tuberose, jonquil, violet, and generally all delicate flowers, they are made in the following manner. Upon an iron frame, a piece of cotton cloth is stretched, imbued with olive oil of the first quality, and covered completely with a thin bed of flowers. Another frame is similarly treated, and in this way a pile is made. The flowers must be renewed till the oil is saturated with their odor. The pieces of eotton cloth are then carefully pressed to extrude the oil. This last operation requires commonly $\tau$ or 8 dass.

of distillation.-The essential oils or essences, of which the great manufacture is in the south of Frauce, are of rose, neroli, lavender, lemon thyme, common thyme, and rosemary. For the mode of distilling the essential oils, see OiLs, essentraL.

The essence of roses being obtained in a peculiar manner, I shall deseribe it here, Put into the body of a still 40 pounds of roses, and 60 quarts of water; distil off one half of the water. When a considerable quantity of such water of the first distillation is obtained, it must be used as water upon fresh rose-leares; a process of repetition to be carried to the fifth time. In the distillation of orange-flower, to obtain the essence of neroli, the same process is to be followed; but if orange-flower water merely be wanted, then it is obtained at one distillation, by reserving the first fifth part of water that comes orer. What is called the essence of petit-grain, is obtained by distilling the leaves of the orange shrub. The essences of lavender, thyme, \&c., present nothing peculiar in theis mode of extraction.

\section{OF SCENTED SPIRITS,}

From oil of rose, orange, jasmine, tuberose, cassia, violet, and other flowers.

Into each of three digesters, immersed in water-haths, put $25 \mathrm{lbs}$. of any one of these oils, and pour into the first digester 25 quarts of spirit of wine; agitate every quarter of an hour during three days, and at the end of this period, draw off the perfumed spirit, and pour it into the second digester; then transfer it after 3 days into the third digester, treating the mixture in the same way; and the spirit thus obtained will be perfect. The digesters must be carefully covered during the progress of these operations. On pursuing the same process with the same oil and fresh alcohol, essences of inferior qualities may be obtained, called Nos. 2, 3, and 4 .

Some perfumers state that it is better to use highly scented pommades than oils; but there is probably little difference in this respect. 
Esprit de Suave.

7 Eng. qrts. of spirit of jasmine,3d operation.

3

2

$1 \frac{1}{2}$ ounce essence of cloves.

$\frac{2}{2}$ ounce fine neroli.

$1 \frac{1}{2}$ ounce essence of bergamot.

8 ounces essence of musk, $2 d$ infusion.

3 quarts rose water.
Spirit of Cytherea.

1 quart spirit of violets.

Spirit of flowers of Italy.

2 quarts spirit of jasmine, $2 d$ operation.

2 2 quarts spirit of cassia, $2 d$ operation. $1 \frac{1}{2} \quad-\quad$ orange flower water.

The above spirits mark usually $\overline{28}$ alcometric degrees of Gay Lussac. See AlcoHor.

\section{POMMADES.}

No less than 20 scented pommades are distinguished by the perfumers of Paris. The essences commonly employed in the manufacture of pommades, are those of bergamot, lemons, cédrat, limette (sweet lemon), Portugal, rosemary, thyme, lemon thyme, lavender, marjoram, and cinnamon.

The following may serve as an example:-

Pommade à la vanille, commonly called Roman.

12 pounds of pommade à la rose.

$$
\begin{array}{ll}
3 \text { - } & \text { oil à la rose. } \\
6 \text { ounces } & \text { vanilla, first quality, pulverized. } \\
\text { bergamot. }
\end{array}
$$

The pommade being melted at the heat of a water-bath, the vanilla is to be introduced with continual stirring for an hour. The mixture is left to settle during two hours. The pommade is then to be drawn off, and will be found to have a fine yellow color, instead of the brown shade which it commonly has.

In making odoriferous extracts and waters, the spirits of the flowers prepared by macerating the flowers in alcohol should be preferred to their distillation, as forming the foundation of good perfumery. The specific gravity of these spirits should be always under $0 \cdot 88$.

Extract of Nosegay (bouquet). 2 quarts spirit of jasmine, 1st operation.

2 - extract of violets.

1 - spirit of cassia, 1st

1 - roses, - 1 st -

1 - $\quad$ Exange, - 1 st -

4 drms. of flowers of benzoin (benzoic acid).

8 ounces of essence of amber, 1st infusion.

\section{Extract of peuch blossoms.}

6 quarts of spirits of wine.

6 pounds of bitter almonds.

2 quarts of spirits of orange flower, $2 d$ operation.

4 drachms of essence of bitter almonds.

4 drachms of balsam of Peru.

4 ounces of essence of lemons.

\section{Eau de Cologne.}

Two processes have been adopted for the preparation of this perfume, distillation and infusion; the first of which, though generally abandoned, is, however, the preferable one. The only essences which should be employed, and which have giren such celebrity to this water, are the following; bergamot, lemon, rosemary, Portugal, neroli. The whole of them ought to be of the best quality, but their proportions may be varied according to the taste of the consumers.

Thirty different odors are enumerated by perfumers; the three following recipes will form a sufficient specimen of their combinations.

Honey-water.

6 quarts of spirit of roses, $3 d$ operation.

$\begin{array}{lll}3 & \text { do } \\ 3 & \text { do. } & \text { jasmine. } \\ 3 & \text { spirits of wine. }\end{array}$

3 ounces essence of Portugal.

4 drachms flowers of benzoin.

12 ounces of essence of vanilla, $3 \mathrm{~d}$ infusion.

12 do. musk, do.

3 quarts good orange-flower water.
Eau de mille feurs.

18 quarts of spirits of wine. 4 ounces balsam of Peru.

8 do. essence of bergamot.

4 do. cloves.

1 do. ordinary neroli.

1 do. thyme.

8 do. musk, $3 \mathrm{~d}$ infusion.

4 quarts orange flower water. 
Eau de mousseline.

2 quarts spirit of roses, $3 \mathrm{~d}$ infusion.

2 do.

1 do.

2 do. jasmine, th do.

clove gillyflower.

orange flower, 4 th do
2 ounces essence of ranilla, $3 \mathrm{~d}$ infusion.

2 do. musk, do.

4 drachms of sanders wood.

1 quart of orange-flower water.

Almond pastes.

These are, gray, sweet white, and bitter white.

The first is made either with the kernels of apricots, or with bitter almonds. They are winnowed, ground, and formed into loares of 5 or 6 pounds weight, which are put into the press in order to extract their oil; 300 pounds of almonds affording about 130 of oil. The pressure is increased upon them erery two hours during three dars; at the end of which time the loaves or calies are takien out of the press to be dried, ground, and sifted.

The second paste is obtained by boiling the almonds in water till their skins are completely loosened; they are next put into a basket, washed and blanched; then dried, and pressed as above.

The third paste is prepared like the second, only using bitter almonds.

Liquid almond pastes, such as those of the rose, orange, vanilla, and nosegay. The honey paste is most admired. It is prepared as follows;-

6 pounds of honey. $\quad 12$ pounds oil of bitter almonds.

6 do. white bitter paste. 26 yolks of eggs.

The honey should be heated apart and strained; 6 pounds of almond paste must then be kneaded with it, adding towards the conclusion, alternately, the quan:ity of jolks of eggs and almond oil indicated.

\section{Pastilles a la rose, orange flower, and vanilla.}

\section{Pastilles à la rose.}

12 ounces of gum.

12 do. olibanum, in tears.

12 do. storax, do.

8 do. nitre.

16 do. powder of pale roses.

3 pounds 14 do. charcoal powder.

1 do. essence of roses.
Pastilles of orange flower.

12 ounces of gum galbanum.

12 do. olibanum, in tears.

12 do. storax, do.

8 do. nitre.

1 pound of pure orange powder.

3 do. 14 ounces charcoal powder.

1 ounce superfine neroli.

\section{Pastilles à la vanille.}

12 ounces of gum galbanum. 16 ounces powder of vanilla.

\begin{tabular}{rll|l}
12 & do. & olibanum, in tears. & 3 pounds 14 ounces charcoal powder. \\
12 & do. & storax do. & 4 drms. essence of cloves. \\
8 & do. & nitre. & 8 ounces do. vanilla, 1 st infusion.
\end{tabular}

The above mixture in each case is to be thickened with 2 ounces of gum tragacanth dissolved in 2 pints of rose water. It is needless to say that the ingredients of the mixture should be impalpable powders.

Scented cassolettes.

8 pounds of black amber (ambergris).

4 do. Tose powder.

2 ounces of benzoin.

\section{ESSENCES BY INFUSION.}

Essence of musk.

5 ounces of musk from the bladder, cut small.

1 do. civet.

4 quarts of spirit of ambrette (purple sweet sultan).

The whole are put into a matrass, and exposed to the sun for two months during the hottest season of the jear. In winter, the heat of a water bath must be reo sorted to.

\section{Essence of vanilla.}

3 pounds of vanilla in branches, 1st quality, cut small.

4 quarts spirit of ambrette.

2 drachms of cloves.

$\frac{3}{2}$ do. musk from the bladder.

The same process must be followed as for the essenee of musk. 


\section{Essence of Ambergris. \\ 4 ounces of ambergris. \\ 2 ounces of bladder musk. \\ 8 quarts of spirit of ambrette.}

Spirit of ambrette (purple sweet sultan).

25 pounds of ambrette are to be distilled with 25 quarts of spirits of wine, adding 12 quarts of water, so as to be able to draw off the 25 quarts.

PEJ RY is the fermented juice of pears, prepared in exactly the same way as CIDER.

PEKSIAN BERRIES. See BERRIES, PERSIAN.

PETROLEUM. See NAPTHA.

PE-TUNT-SE is the Chinese name of the fusible earthy matter of their porcelain. It is analogous to our Cornish stone.

PEWTER, PEWTERER. (Potier d'étain, Fr.) Pewter is, generally speaking, an alloy of tin and lead, sometimes with a little antimony or copper, combined in several different proportions, according to the purposes which the metal is to serve. The English tradesmen distinguish three sorts, which they call plate, trifle, and ley pewter; the first and hardest being used for plates and dishes; the second for beer-pots; and the third for larger wine measures. The plate pewter has a bright silvery lustre when polished; the best is composed of 100 parts of tin, 8 parts of antimony, 2 parts of bismuth, and 2 of copper. The trifle is said by some to consist of 83 of tin, and 17 of antimony ; but it generally contains a good deal of lead. The ley pewter is composed of 4 of tin, and 1 of lead. As the tendency of the covetous pewterer is always to put in as much of the cheap metal as is compatible with the appearance of his metal in the market, and as an excess of lead mar cause it to act poisonously upon all rinegars and many wines, the French government long ago appointed Foureror, Vauquelin, and other chemists, to ascertain by experiment the proper proportions of a safe pewter alloy. These commissioners found that 18 parts of lead might, without danger of affecting wines, \&c., be allored with 82 parts of tin; and the French government in consequence passed a law requiring pewterers to use $83 \frac{1}{2}$ of tin in 100 parts, with a tolerance of error amounting to $1 \frac{1}{2}$ per cent. This ordonnance, allowing not more than 18 per cent. of lead at a maximum, has been extended to all ressels destined to contain alimentary substances. A table of specific gravities was also published, on purpose to test the quality of the alloy; the density of which, at the legal standard, is $7 \cdot 764$. Any excess of lead is immediately indicated by an increase in the specific gravity above that number.

The pewterer fashions almost all his articles by casting them in moulds of brass or bronze, which are made both inside and outside in various pieces, nicely fitted together, and locked in their positions by ears and catches or pins of various kinds. The moulds must be moderately heated before the pewter is poured into them, and their surfaces should be brushed evenly over with pounce powder (sandarach) beaten up with white of egg. Sometimes a film of oil is preferred. The pieces, after being cast, are turned and polished; and if any part needs soldering, it must be done with a fusible alloy of tin, bismuth, and lead.

Britannia metal, the kind of pewter of which English tea-pots are made, is said to be an alloy of equal parts of brass, tin, antimonr, and bismuth; but the proportions differ in different workshops, and much more tin is commonly introduced. Queen's metal is said to consist of 9 parts of tin, 1 of antimony, 1 of bismuth, and 1 of lead; it serves also for teapots and other domestic utensils.

$A$ much safer and better alloy for these purposes may be compounded by adding to 100 parts of the French pewter, 5 parts of antimony, and 5 of brass to harden it. The English ley pewter contains often much more than 20 per cent. of lead. Under TiN, will be found the description of an easy method of analyzing its lead alloys.

PHOSPHORIC ACID is the acid formed by the virid combustion of

PHOSPHORUS. This interesting simple combustible, being an object of extensive consumption, and therefore of a considerable chemical manufacture, I shall describe the requisite manipulations for preparing it at some detail. Put $1 \mathrm{cwt}$. of finely ground boneash, such as is used by the assayers, into a stout tub, and let one person work it into a thin pap with twice its weight of water, and let him continue to stir it constantly with a wooden bar, while another person pours into it, in a uniform but very slender stream, 78 pounds of concentrated sulphuric acid.

The heat thus excited in the dilution of the acid, and in its reaction upon the calcareons base, is favorable to the decomposition of the bone phosphate. Should the resulting sulphate of lime become lumpy, it must be reduced into a uniform paste, by the addition of a little water from time to time. This mixture must be made out of doors, as under an open shed, on account of the carbonic acid and other offensive gases which are - extricated. At the end of 24 hours, the pap may be thinned with water, and, if con- 
venient, heated, with careful stirring, to complete the chemical change, in a square pan made of sheet lead, simply folded up at the sides. Whenever the paste has lost its granular character, it is ready for transfer into a series of tall casks, to be further diluted and settled, whereby the clear superphosphate of lime may be run of by a syphon from the deposite of gypsum. More water must then be mixed with the precipitate, after subsidence of which, the supernatant liquor is again to be drawn off. The slitful operator employs the weak acid from one cask to wash the deposite in another, and thereby saves fuel in evaporation.

The collected liquors being put into a leaden, or preferably a copper pan, of proper dimensions, are to be concentrated by steady ebullition, till the calcareous deposite becomes considerable; after the whole has been allowed to cool, the clear liquor is to be run off, the sediment removed, and thrown on a filter. The evaporation of the clear liquor is to be urged till it acquires the consistence of honey. Being now weighed, it should amount to 37 pounds. One fourth of its weight of charcoal in fine powder, that is, about 9 pounds, are then to be incorporated with it, and the mixture is to be eraporated to dryness in a cast-iron pot. A good deal of sulphurous acid is disengaged along with the steam at first, from the reaction of the sulphuric acid upon the charcoal, and silerwards some sulphureted hydrogen. When the mixture has become perfectly dry, as shown by the redness of the bottom of the pot, it is to be allowed to cool, and packed tight into stoneware jars fitted with close covers, till it is to be subjected to distillation. For this purpose, earthen retorts of the best quality, and free from air-boles, must be taken, snd evenly luted over their surface with a compost of fire-clay and horse-dung. When the coating is dry and sound, the retort is to be two thirds filled with the powder, and placed upon proper supports in the laboratory of an air-furnace, haring its fire placed not immediately beneath the retort, but to one side, after the plan of a rererberatory; whereby the flame may play uniformly round the retort, and the fuel may be supplied as it is wanted, without admitting cold air to endanger its cracking. The gallery furnace of the palatinate (under MERcery) will show how several retorts may be operated upon together, with one fire.

To the beak of the retort properly inclined, the one end of a bent copper tube is to be tightly luted, while the other end is plunged not more than one quarter of an inch beneath the surface of water contained in a small copper or tin trough placed beneath, close to the side of the furnace, or in a wide-mouthed bottle. It is of adrantage to let the water be somewhat warm, in order to prevent the concretion of the phosphorus in the copper tube, and the consequent obstruction of the passage. Should the beak of the retort appear to get filled with solid phosphorus, a bent rod of iron may be heated, and passed up the copper tube, without remoring its end from the water. The heat of the furnace should be most slowly raised at first, but afterwards equably maintained in a state of bright ignition. After 3 or 4 hours of steady firing, carbonic acid and sulphurous acid gases are evolved in considerable abundance, provided the materials had not been well dried in the iron pot; then sulphureted hydrogen makes its appearance, and next phosphureted hydrogen, which last should continue during the whole of the distillation.

The firing should be regulated by the escape of this remarkable gas, which ought to be at the rate of about 2 bubbles per second. If the discharge comes to be interrupted, it is to be ascribed either to the temperature being too low, or to the retort getting cracked; and if upon raising the heat sufficicntly no bubbles appear, it is a proof that the apparatus has become defective, and that it is needless to continue the operation. In fact, the great nicety in distilling phosphorus lies in the management of the fire, which must be incessantly watched, and fed by the successive introduction of fuel, consisting of coke with a mixture of dry wood and coal.

We may infer that the process approaches its conclusion by the increasing slowness with which gas is disengaged under a powerful heat; and when it ceases to come over, we may cease firing, taking care to prevent reflux of water into the retort, from condensation of its gaseous contents, by admitting air into it through a recurved glass tube, or through the lute of the copper adopter.

The usual period of the operation upon the great scale is from 24 to 30 hours. Its theory is very obvious. The charcoal at an elevated temperature disoxygenates the phosphoric acid with the production of carbonic acid gas at first, and afterwards carbonic oxyde gas, along with sulphureted, carbureted, and phosphureted hydrogen, from the reaction of the water present in the charcoal upon the other ingredients.

The phosphorus falls down in drops, like melted wax, and concretes at the bottom of the water in the receiver. It requires to be purified by squeezing in a shamoy leather bag, while immersed under the surface of warm water, contained in an earthen pan. Each bag must be firmly tied into a ball form, of the size of the fist, and compressed, under the water heated to $130^{\circ}$, bv a pair of flat wooden pincers, like those with which oranges are squeezed. 
The purified phosphorus is moulded for sale into little cylinders, by melting it at the bottom of a deep jar filled with water, then plunging the wider end of a slightly tapering but straight glass tube into the water, sucking this up to the top of the glass, so as to warm it, next immersing the end in the liquid phosphorus, and sucking it up to any desired height.

The tube being now shut at bottom by the application of the point of the left index, may be taken from the mouth and transferred into a pan of cold water to congeal the phosphorus; which then will commonly fall out of itself, if the tube be nicely tapered, or may at any rate be pushed out with a stiff wire. Were the glass tube not duly warmed before sucking up the phosphorus, this would be apt to congeal at the sides, befure the middle be filled, and thus form hollow cylinders, very troublesome and even dangerous to the makers of phosphoric match-bottles. The moulded sticks of phosphorus are finally to be cut with scissors under water to the requisite lengths, and put up in vials of a proper size ; which should be filled up with water, closed with ground stoppers, and kept in a dark place. For carriage to a distance, each vial should be wrapped in paper, and fitted into a tin-plate case.

Phosphorus has a pale yellow color, is nearly transparent, brittle when cold, soft and pliable, like wax, at the temperature of $70^{\circ} \mathrm{F}$., crystallizing in rhombo-dodecahedrons out of its combination with sulphur, and of specific gravity 1.77. It exhales white fumes in the air, which have a garlic smell, appear luminous in the dark, and spontaneously condense into liquid phosphorous acid. Phosphorus melts in close vessels, at $95^{\circ} \mathrm{F}$., into an oily-looking colorless fluid, begins to evaporate at $217.5^{\circ}$, boils at $554^{\circ}$, and if poured in the liquid state into ice-cold water, it becomes black, but resumes its former color when again melted and slowly cooled. It has an acrid disagreeable taste, and acts deleteriously in the stomach, though it has been administered as a medicine by some of the poison-doctors of the present day. It takes fire in the open air at the temperature of $165^{\circ}$, but at a lower degree if partially oxydized, and burns with great vehemence and splendor.

Inflammable match-boxes (briquets phosphoriques) are usually prepared by putting into a small vial of glass or lead a bit of phosphorus, and oxydizing it slightly by stirring it round with a redhot iron wire. The vial should be unstoppered only at the instant of plunging into it the tip of the sulphur match which we wish to kindle. Bendix has given the following recipe for charging such match-vials. Take one part of fine dry cork raspings, one part of yellow wax, eight parts of petroleum, and four of phosphorus, incorporate them by fusion, and when the mixture has concreted by cooling, it is capable of kindling a sulphur match dipped into it. Phosphorus dissolves in fat oils, forming a solution luminous in the dark at ordinary temperatures. A vial half filled with this oil, being shaken and suddenly uncorked, will give light enough to see the dial of a watch by night.

There are five combinations of phosphorus and oxygen :-1. the white oxyde; 2. the red oxyde; 3 . hypophosphorous acid; 4. phosphorous acid; 5. phosphoric acid. The last is the only one of interest in the arts. It may be obtained from the sirupy superphosphate of lime above described, by diluting it with water, saturating with carbonate of ammonia ; evaporating, crystallizing, and gently igniting the salt in a retort. The ammonia is volatilized, and may be condensed into water by a Woulfe's apparatus, while the phosphoric acid remains in the bottom of the retort. Phosphoric acid may be more readily produced by burning successive bits of phosphorus in a silver saucer, under a great bell jar inverted upon a glass plate, so as to admit a little air to carry on the combustion. The acid is obtained in a fine white snowy deposite; consisting, in this its dry state, of 44 of phosphorus and 56 of oxygen. That obtained from the sirupy solution is a hydrate, and contains 9.44 per cent. of water. If the atom of phosphorus be called 32 upon the hydrogen radix, then 5 atoms of oxygen $=40$ will be associated with it in the dry acid, =72; and an additional atom of water $=9$, in the hydrate, will make its prime equivalent 81 . Phosphorous acid seems to contain no more than 3 atoms of oxygen.

The only salts of this acid much in demand, are the phosphate of soda, and the ammonia phosphate of soda. The former is prepared by slightly supersaturating superphosphate of lime with crystals of carbonate of soda ; warming the solution, filtering, evaporating, and crystallizing. It is an excellent purgative, and not unpalatable. The triple phosphate is used in docimastic operations; and is described under Metallurgy.

PICAMARE, is a thick oil, one of the six new principles detected by M. Reichenbach, in wood-tar. See Creosote and Parafrine. Picamare constitutes 1-6th of beech-tar.

PICROMEL, is the name given by M. Thenard to a black bitter principle which he supposed to be peculiar to the bile. MM. Gmelin and Tiedemann have since called its identity in question.

PICROTOXINE, is an intensely bitter poisonous vegetable principle, extracted from the seeds of the Menispermum cocculus, (Cocculus Indicus). It erystallizes in small white 
needles, or columns; dissolves in water and alcohol. It does not combine with acids, but with some bases, and is not, therefore, of an alkaline nature, as had been at first supposed.

PIGMENTs, VITRIFIABLE, belong to five different styles of work: 1 . to enamei painting; 2. to painting on metals; 3 . to painting on stoneware; 4 . to painting on porcelain; 5 . to stained glass.

PIMENTO (Myrtus pimenta, or Jamaica pepper) consists, according to Bonastre's complicated andlysis, of-

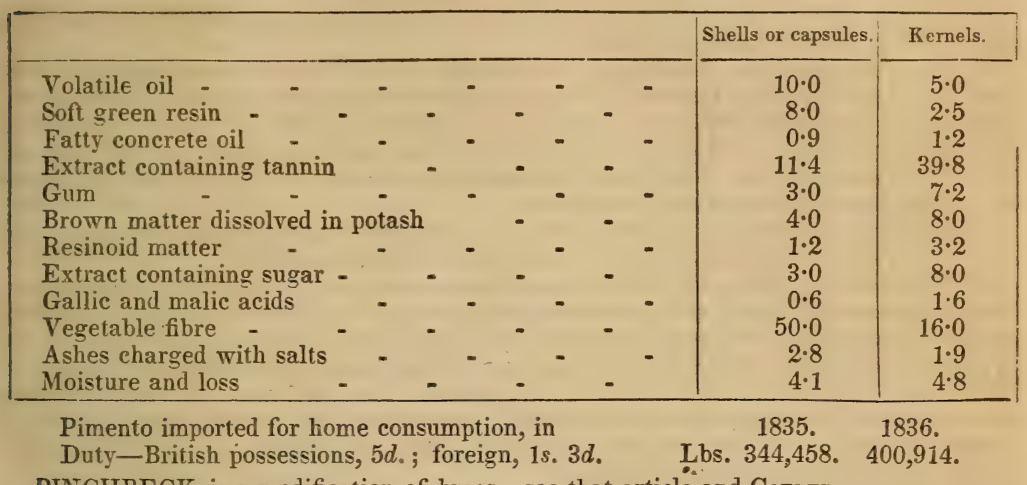

PINCHBECK is a modification of brass; see that article and CoPPER.

PINE-APPLE YARN and CLOTH. In Mr. Zincke's process, patented in December, 1836, for preparing the filaments of this plant, the Bromelia ananas, the leaves being plucked, and deprived of the prickles round their edges by a cutting instrument, are then beaten upon a wooden block with a wooden mallet, till a silky-looking mass of fibres be obtained, which are to be freed by washing from the green fecula. The fibrous part must next be laid straight, and passed between wooden rollers. The leaves should be gathered between the time of their full maturity and the ripening of the fruit. If earlier or later, the fibres will not be so flexible, and will need to be cleared by a boil in soapy water for some hours; after being laid straight under the pressure of a wooden grating, to prerent their becoming entangled. When well washed and dried, with occasional shaking out, they will now appear of a silky fineness. They may be then spun into porous rovings, in which state they are most conveniently bleached by the ordinary methods.

Specimens of cambric, both bleached and unbleached, woven with these fibres, have been recently exhibited, which excited hopes of their rivalling the finest flax fabrics, but in my opinion without good reason, on account of their want of strength.

PINEY TALLOW is a concrete fat obtained by boiling with water the fruit of the Vateria indica, a tree common upon the Malabar coast. It seems to be a substance intermediate between tallow and wax; partaking of the nature of stearine. It melts at $97 \frac{1}{2} \mathrm{~F}$., is white or yellowish, has a spec. grav. of 0.926 ; is saponified by alkalis, and forms excellent candles. Dr. Benjamin Babington, to whom we are indebted for all our knowledge of piney tallow, found its ultimate constituents to be, 77 of carbon, 12.3 of hydrogen, and $10 \cdot 7$ of oxygen.

PIN MANUFACTURE. (Fabrique d'épingles, Fr.; Nadelfabrik, Germ.) A pin is a small bit of wire, commonly brass, with a point at one end, and a spherical head at the other. In making this little article, there are no less than fourteen distinct operations.

1. Straightening the wire. The wire, as obtained from the drawing-frame, is wound about a bobbin or barrel, about 6 inches diameter, which gives it a curvature that must be removed. The straightening engine is formed by fixing 6 or 7 nails upright in \& waving line on a board, so that the void space measured in a straight line between the first three nails may have exactly the thickness of the wire to be trimmed; and that the other nails may make the wire take a certain curve line, which must vary with its thicknes.3. The workman pulls the wire with pincers through among these nails, to the length of about 30 feet, at a running draught; and after he cuts that off, he returns for as much more; he can thus finish 600 fathoms in the hour. He next cuts these long pieces into engths of 3 or 4 pins. A day's work of one man amounts to 18 or 20 thousand dozen. of pin-lengths.

2. Pointing is executed on two iron or steel grmilstones, by two workmen, one of whom roughens down, and the other finishes. 'Thirty or forty of the pin wires are applied to the grindstone at once, arranged in one plane, between the two forefingers and thumbs of both hands, which dexterously give them a rotatory movement. 
3. Cutting these wires into pin-lengths. This is done by an adjusted chisel. The intermediate portions are handed over to the pointer.

4. Twisting of the wire for the pin-heads. These are made of a much finer wire, coiled into a compact spiral, round a wire of the size of the pins, by means of a small lathe constructed for the purpose.

5. Culting the heuds. Two turns are dexterously cut off for each head, by a regulated chisel. A skilful workman may turn off 12,000 in the hour.

6. Annealing the heads. They are put into an iron ladle, made redhot over an open fire, and then thrown into cold water.

7. Stamping or shaping the heads. This is done by the blow of a small ram, raised by means of a pedal lever and a cord. The pin-heads are also fixed on by the same operative, who makes about 1500 pins in the hour, or from 12,000 to 15,000 per diem; exclusive of one thirteenth, which is always deducted for waste in this department, as well as in the rest of the manufacture. Cast heads, of an alloy of tin and antimony, were introduced by patent, but never came into general use.

8. Yellowing or cleaning the pins is effected by boiling them for half an hour in sour beer, wine lees, or solution of tartar; after which they are washed.

9. Whitening or tinning. A stratum of about 6 pounds of pins is laid in a copper pan, then a stratum of about 7 or 8 pounds of grain tin; and so alternately till the vessel be filled; a pipe being left inserted at one side, to permit the introduction of water slowly at the bottom, without deranging the contents. When the pipe is withdrawn, its space is filled up with grain tin. The vessel being now set on the fire, and the water becoming hot, its surface is sprinkled with 4 ounces of cream of tartar; after which it is allowed to boil for an hour. The pins and tin grains are, lastly, separated by a kind of cullender.

10. Washing the pins in pure water.

11. Drying and polishing them, in a leather sack filled with coarse bran, which is agitated to and fro by two men.

12. Winnowing, by fanners.

13. Pricking the papers for receiving the pins.

14. Papering, or fixing them in the paper. This is done by children, who acquire the habit of putting up 36,000 per day.

The pin manufacture is one of the greatest prodigies of the division of labor; it furnishes 12,000 articles for the sum of three shillings, which have required the united diligence of fourteen skilful operatives.

The above is an outline of the mode of manufacturing pins by hand labor, but several beautiful inventions have been employed to make them entirely or in a great measure by machinery; the consumption for home sale and export amounting to 15 millions daily, for this country alone. One of the most elaborate and apparently complete is that for which Mr. L. W. Wright obtained a patent in May, 1824. A detailed description of it will be found in the 9th volume of Newton's London Journal. The following outline will give my readers an idea of the structure of this ingenious machine:-

The rotation of a principal shaft, mounted with several cams, gives motion to various sliders, levers, and wheels, which work the different parts. A slider pushes pincers forwards, which draw wire from a reel, at every rotation of the shaft, and advance such a length of wire as will produce one pin. A die cuts off the said length of wire by the descent of its upper chap; the chap then opens a carrier, which takes the pin to the pointing apparatus. Here it is received by a holder, which turns round, while a beveledged file-wheel rapidly revolves, and tapers the end of the wire to a point. The pin is now conducted by a se-ond carrier to a finer file-wheel, in order to finish the point by a second grinding. A third carrier then transfers the pin to the first heading die, and by the advance of a steel punch, the end of the pin wire is forced into a recess, whereby the head is partially swelled out. A fourth carrier removes the pin to a second die, where the heading is perfected. When the heading-bar retires, a forked lever draws the finished pin from the die, and drops it into a receptacle below.

I believe the chief objection to the raising of the heads by strong mechanical compression upon the pins, is the necessity of softening the wire previously; whereby the pins thus made, however beautiful to the eye, are deficient in that stiffness which is so essential to their employment in many operations of the toilet.

PIPERINE is a crystalline principle extracted from black pepper by means of alcohol. It is colorless, has hardly any taste, fuses at $212^{\circ} \mathrm{F}$; is insoluble in water, but soluble in acetic acid, ether, and most readily in alcohol.

PITCH, MINERAL, is the same as Bitumen and Asphalt.

PITCH of wood-tar (Poix, Fr.; Pech, Germ.) is obtained by boiling tar in an open iron pot, or in a still, till the volatile matters be driven off. Pitch contains pyroligneous resin, along with colophany (common rosin), but its principal ingredient is the former, called by Berzelius pyretine. It is brittle in the cold, but softens and becomes ductile 
with heat. It melts in boiling water, and dissc'ves in alcohol and oil of turpentine, as well as in carbonated or caustic alkaline leys. For Pyretrne, see the mode of preparing it from birch wood, for the purpose of preparing Russia LEATHER.

PITCOAL. (Houille, Fr.; Steinkohle, Germ.) This is by far the most valuable of mineral treasures, and the one which, at least in Great Britain, makes all the others available to the use and comfort of man. Hence it has been searched after with unremitting diligence, and worked with all the lights of science, and the resources of art.

The Brora coal-field in Sutherlandshire is the most remarkable example in this, or in perhaps any country hitherto investigated, of a pseudo coal-basin among the deeper secondary strata, but above the new sandstone or red marl formation. The Rev. Dr. Bucliland and Mr. C. Lyell, after visiting it in 1824, had expressed an opinion that the strata there were wholly unconnected with the proper coal formation below the new red sandstone, and were in fact the equivalent of the oolitic series; an opinion fully confirmed by the subsequent researches of Mr. Murchison. (Geol. Trans. for 1827, p. 293.) The Brora coal-field forms a part of those secondary deposites which range along the southeast coast of Sutherlandshire, nccupying a narrow tract of about twenty miles in length, and three in its greatest breädth.

One stratum of the Brora coal-pit is a coal-shale, composed of a reed-like striated plant of the natural order Equisetum, which seems to have contributed largely towards the formation of that variety of coal. From this coal-shale, the next transition upwards is into a purer bituminous substance approaching to jet, which constitutes the great bed of coal. This is from 3 feet 3 inches to 3 feet 8 inches thick, and is divided nearly in the middle by a thin layer of impure indurated shale charged with pyrites, which, if not carefully excluded from the mass, sometimes occasions spontaneous combustion upon exposure to the atmosphere; and so much, indeed, is that mineral disseminated throughout the district, that the shales might be generally termed "pyritiferous." Inattention on the part of the workmen, in 1817 , in leaving a large quantity of this pyritous matter to accumulate in the pit, occasioned a spontaneous combustion, which was extinguished only by excluding the air ; indeed, the coal-pit was closed in and remained unworked for four years. The fires broke out again in the pit in 1827.

The purer part of the Brora coal resembles common pitcoal; but its powder has the red ferruginous tinge of pulverized lignites. It may be considered one of the last links between lignite and true coal, approaching very nearly in character to jet, though less tenacious than that mineral; and, when burnt, exhaling but slightly the vegetable odor so peculiar to all imperfectly bituminized substances. The fossil remains of shells and plants prove the Brora coal to be analogous to that of the eastern moorlands of Yorkshire, although the extraordinary thickness of the former, compared with any similar deposite of the latter (which never exceeds from 12 to 17 inches), might have formerly led to the belief that it was a detached and anomalous deposite of true coal, rather than a lignite of any of the formations above the new red sandstone : such misconception might more easily arise in the infancy of 'geology, when the strata were not identified by their fossil organic remains.

On the coast of Yorkshire the strata of this pseudo coal formation appear in the follow ing descending order, from Filey Bay to Whitby. 1. Coral-rag. 2. Calcareous grit. 3. Shale, with fossils of the Oxford clay. 4. Kelloway rock (swelling out into an important a.maceous formation). 5. Cornbrash. 6. Coaly grit of Smith. 7. Piersione (according to Mr. Smith, the equivalent of the great oolite). 8. Sandstone and shale, with peculiar plants and various seams of coal. 9. A bed with fossils of the inferior oolite. 10. Marl-stone? 11. Alum-shale or lias. All the above strata are identified by abundant organic remains.

In the oolitic series, therefore, where the several strata are developed in conformity with the more ordinary type of these formations, we may venture to predict with certainty, that no carboniferous deposites of any great value will ever be discovered, at all events in Great Britain. A want of such knowledge has induced many persons to make trials for coal in beds subordinate to the English oolites, and even superior to thern, in places where the type of formation did not offer the least warrant for such attempts.

The third great class of terrestrial strata, is the proper coal-measures, called the carboniferous rocks, our leading object here, and to which we shall presently return.

The transition rocks which lie beneath the coal-measures, and above the primitive rocks, or are anterior to the carboniferous order, and posterior to the primitive, contain a peculiar kind of coal, called anthracite or stone-coal, approaching closely in its nature to carbon. It is chiefly in the transition clay-slate that the anthracite occurs in considerable masses. There is one in the transition slate of the little Saint Bernard, near the village of la Thuile (in the Alps). It is 100 feet long, and 2 or 3 yards thick. 
The coal burns with difficulty, and is used only for burning lime. There are several of the same kind in that country, which extend down the reverse slope of the mountains looking to Savoy. The slate enclosing them presents vegetable impressions of reeds or analogous plants. To the transition clay-slate we must likewise refer the beds of anthracite that $M$. Hericart de Thury observed at very great heights in the Alps of Dauphiny, in a formation of schist and gray-wacke with vegetable impressions, which reposes directly on the primitive rocks.

The great carboniferous formation may be subdivided into four orders of rocks: 1 . the coal-measures, including their manifold alternations of coal-beds, sandstones, and shales; 2. the millstone grit and shale towards the bottom of the coal measures; 3 . the carboniferous limestone, which projecting to considerable heights above the outcrop of the coal and grit, acquires the title of mountain limestone; 4 . the old red sandstone, or connecting link with the transition and primary rock basin in which the coal system lies.

The coal-fields of England, from geographical position, naturally fall under the following arrangement:- 1 . The great northern district; including all the coal-fields north of Trent. 2. The central district; including Leicester, Warwick, Stafford, and Shropshire. 3. The western district; subdivided into north-western, including North Wales, and the south-western, including South Wales, Gloucester, and Somersetshire.

There are three principal coal-basins in Scotland: 1. that of Ayrshire; 2. that of Clydesdale; and 3. that of the valley of the Forth, which runs into the second in the line of the Union Canal. If two lines be drawn, one from Saint Andrews on the northeast coast, to Kilpatrick on the Clyde, and another from Aberlady, in Haddingtonshire, to a point a few miles south of Kirkoswald in Ayrshire, they will include between them the whole space where pitcoal has been discovered and worked in Scotland.

The great coal-series consists of a regular alternation of mineral strata deposited in a great concavity or basin, the sides and bottom of which are composed of transition rocks. This arrangement will be clearly understood by inspecting fig. 794, which represents a section of the coal-field south of Malmsbury.

$\begin{array}{lll}\text { Mendip hills. } \quad \text { Dundry hill. Wick rocks. } & \text { Fog hill, N. of Lansdowne }\end{array}$

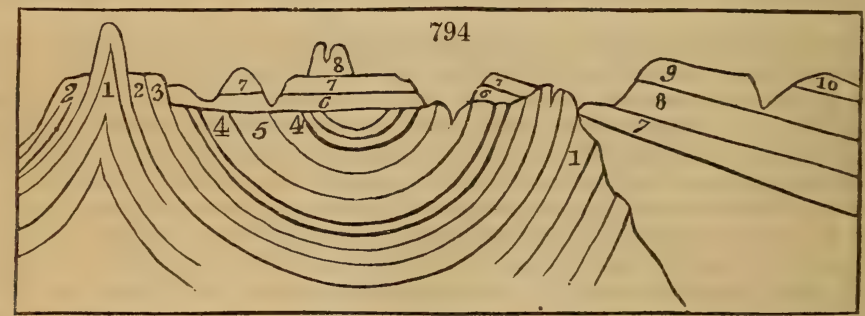

1,1 , old red sandstone; 2 , mountain limestone; 3 , millstone grit; 4,4 , coal seams ; 5 , Pennant, or coarse sandstone; 6 , new red sandstone, or red marl; 7,7 , lias; 8 , 8 , inferior oolite; 9, great oolite; 10, cornbrash and Forest marbie.

No. 1, or the old red sandstone, may therefore be regarded as the characteristic lining of the coal basins; but this sandstone rests on transition limestone, and this limestone on gray-wacke. This methodical distribution of the carboniferous series is well exemplified in the coal-basin of the Forest of Dean in the south-west of England, and has been accurately described by $\mathrm{Mr}$. Mushet.

The gray-wacke consists of highly inclined beds of slaty micaceous sandstone, which on the one hand alternates with and passes into a coarse breccia, having grains as large as peas; on the other, into a soft argillaceous slate. The gray-wacke stands bare on the north-eastern border of the Forest, near the southern extremity of the chain of transition limestone, which extends from Stoke Edith, near Hereford, to Flaxley on the Severn. It is traversed by a defile, through which the road from Gloucester to Ross winds. The abruptness of this pass gives it a wild and mountainous charaeter, and affords the best op portunity of examining the varieties of the rock.

The Transition limestone consists in its lower beds of fine-grained, tender, extremely argillaceous slate, known in the district by the name of water-stone, in consequence of the wet soil that is found wherever it appears at the surface. Calcareous matter is interspersed in it but sparingly. Its upper beds consist of shale alternating with extensive beds of stratified limestone. The lowest of the calcareous strata are thin, and alternate with shale. On these repose thicker strata of more compact limestone, often of a dull blue color. The beds are often dolomitic, which is indicated by straw yellow color, or dark pink color, and by the sandy or glimmering aspect of the rock.

The old red sandstone, whose limits are so restricted in other parts of England, here 
occupres an extensive area. The space which it covers, its great thickness, its high inclination, the abrupt character of the surface over which it prevails, and the consequent display of its strata in many natural sections, present in this district advantages for studying the formation, which are not to be met with elsewhere in South Britain. In the neighborhood of Mitchel Dean, the total thickness of this formation, interposed conformably between the transition and mountain limestone, is from 600 to 800 fathoms. The old red sandstone is characterized in its upper portion by the presence of silicious conglomerate, containing silicious pebbles, which is applied extensively to the fabrication of millstones near Moninouth, and on the banks of the Wye. This sandstone encircles the Forest with a ring of very elevated ground, whose long and lofty ridges on the eastern frontier overhang the valley of the Severn.

The mountain limestone, or carboniferous, is distinguished from transition limestone, rather by its position than by any very wide difference in its general character or organic remains. According to the measurements of $\mathrm{Mr}$. Mushet, the total thickness of the mountain limestone is about 120 fathoms. The zone of limestone belonging to this coal-basin, is from a furlong to a mile in breadth on the surface of the ground, according as the dip of the strata is more or less rapid. The angle of dip on the northern and western border is often no more than $10^{\circ}$, but on the eastern it frequently amounts to $80^{\circ}$. The calcareous zone that defines the outer circle of the basin, suffers only one short interruption, scarcely three miles in length, where in consequence of a fault the limestone disappears, and the coal-measures are seen in contact with the old red sandstone.

Coal measures.-Their aggregate thickness amounts, according to Mr. Mushet, to about 500 fathoms. 1. The lowest beds, which repose on the mountain limestone, are about 40 fathoms thick, and consist here, as in the Bristol coal-basin, of a red silicious grit, alternating with conglomerate, used for millstones; and with clay, occasionally used for ochre. 2. These beds are succeeded by a series about 120 fathoms thick, in which a gray gritstone predominates, alternating in the lower part with shale, and containing 6 seams of coal. The grits are of a fissile character, and are quarried extensively for flag-stone, ashlers, and fire-stone. 3. A bed of grit, 25 fathoms thick, quarried for hearth-stone, separates the preceding series from the following, or the 4 th, which is about 115 fathoms thick, and consists of from 12 to 14 seams of coal alternating with shale. 5. To this suceeds a straw-colored sandstone, nearly 100 fathoms thick, forming a high ridge in the interior of the basin. It contains several thin seams of coal, from 6 to 16 inches in thickness. 6. On this reposes a series of about 12 fathoms thick, consisting of 3 seams of coal alternating with shale. 7. This is covered with alternate beds of grit and shale, whose aggregate thickness is about 100 fathoms, occupying a tract in the centre of the basin about 4 miles long, and 2 miles broad. The sandstone No. 5 is probably the equivalent of the Pennant in the preceding figure.

The floor, or pavement, immediately under the coal beds is, almost without exception, a grarish slate-clay, which, when made into bricks, strongly resists the fire. This fireclay varies in thickness from a fraction of an inch to several fathoms. Clay-ironstone is often disseminated through the shale.

The most complete and simplest form of a coal-field is the entire basin-shape, which we find in some instances without a dislocation. A beautiful example of this is to be seen at Blairengone, in the county of Perth, immediately adjoining the western boundary of Clackmannanshire, as represented in fig. 795, where the outer elliptical line, marked
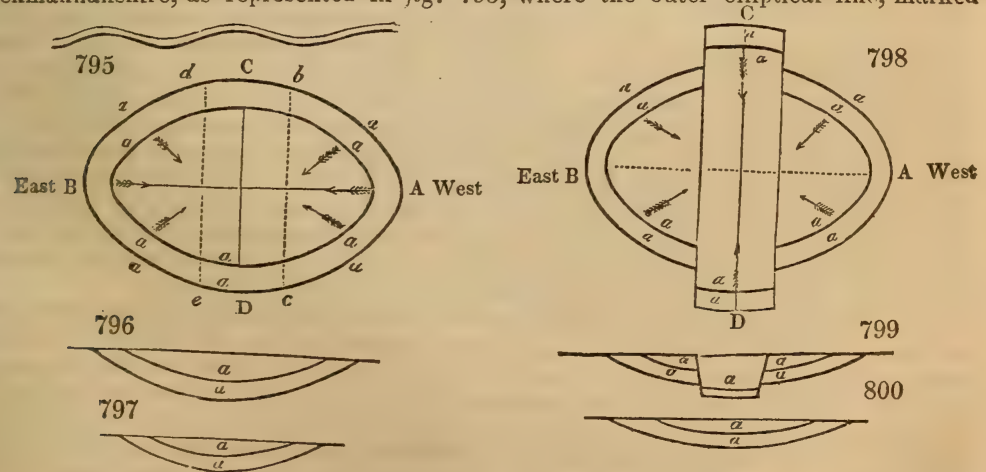

A, B, C, D, represents the crop, outburst, or basset edge of the lower coal, and the inner elliptical line represents the crop or basset edge of the superior coal. Fig. 796 is the 
.ongitudinal section of the line $\mathbf{A} \mathbf{B}$; and $f g .797$, the transverse section of the line $\mathbf{C} \mathbf{D}_{\text {. }}$ All the accompanying coal strata partake of the samc form and parallelism. These basins are generally elliptical, sometimes nearly circular, but are often very eccentric, being much greater in length than in breadth; and frequently one side of the basin on the short diameter has a much greater dip than the other, which circumstance throws the trough or lower part of the basin concavity much nearer to the one side than to the other. From this view of one entire basin, it is evident that the dip of the coal strata belonging to it runs in opposite directions, on the opposite sides, and that all the strata regularly crop out, and meet the alluvial cover in every point of the circumferential space, like the edges of a nest of common basins. The waving line marks the river Devon.

It is from this basin shape that all the other coal-fields are formed, which are segments of a basin produced by slips, dikes, or dislocations of the strata. If the coals (fig. 795) were dislocated by two slips $b c$ and $d e$, the slip $b c$ throwing the strata down to the east, and the slip $d e$ throwing them as much $u p$ in the same direction, the outcrops of the coals would be found in the form represented in fig. 798, of which fig. 799 is the section in the line $\mathbf{A ~ B}$, and fig. 800 the section in the line $\mathbf{c}$.

The chief difficulty in exploring a country in search of coal, or one where coal-fields are known to exist, arises from the great thickness of alluvial and other cover, which completely hides the outcrop or basset edge of the strata, called by miners the rock-head; as also the fissures, dikes, and dislocations of the strata, which so entirely change the structure and bearings of coal-fields, and cause often great loss to the mining adventurer. The alluvial cover on the other hand is beneficial, by protecting the seams of the strata from the superficial waters and rains, which would be apt to drown them, if they were naked. In all these figures of coal-basins, the letter $a$ indicates coal.

The absolute shape of the coal-fields in Great Britain has been ascertained with surprising precision. To whatever depth a coal-mine is drained of its water, from that depth it is worked, up to the rise of the water-level line, and each miner continues to advance his room or working-place, till his seam of coal meets the alluvial cover of the outcrop, or is cut off by a dislocation of the strata. In this way the miner travels in succession over every point of his field, and can portray its basin-shape most minutely.

Fig. 801 represents a horizontal plan of the Clackmannanshire coal-field, as if the 801

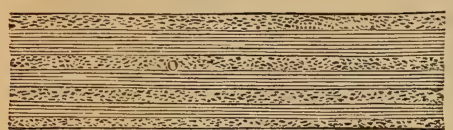
strata at the outcrop all around were denuded of the alluvial cover. Only two of the concentric beds, or of their edges $a, \alpha$, are represented, to avoid perplexity. It is to be remembered, however, that all the series of attendant strata lie parallel to the above lines.

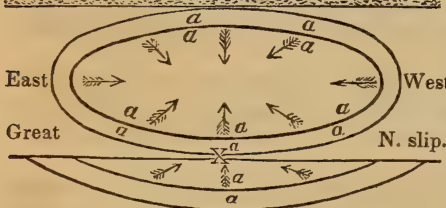
This plan shows the Ochill mountains, with the north coal-fields, of an oblong elliptical shape, est the side of the basin next the mountains being precipitous, as if upheaved by the eruptive p.trap-rocks; while the south, the east, and the west edges of the basin shelve out at a great distance from the lower part of the concavity or trough, as miners call it. Thus the alternate beds of coal, shale, and sandstone, all nearly concentric in the north coal-field, dip inwards from all sides towards the central area of the trough. The middle coal-field of this district, however, which is formed by the great north

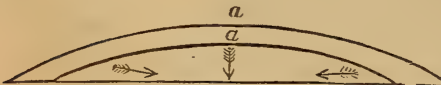
slip, is merely the segment of an elliptical basin, where the strata dip in every direction to the middle of the axis marked with the letter $\mathrm{x}$; being the deepest part of the segment. The south coal-field, formed by the great south slip, is likewise the segment of another elliptical basin, similar in all respects to the middle coal-field. Beyond the outcrop of the coals and subordinate strata of the south coalfields, the counter dip of the strata takes place, producing the mantle-shaped form; whence the coal strata in the Dunmore field, in Stirlingshire, lie in a direction contrary to those of the south coal-field of Clackmannanshire. 0 , are the Ochill mountains.

Fig. 802 is intended to represent an extensive district of country, containing a great coal-basin, divided into numerous subordinate coal-fields by these dislocations. The lines marked $b$ are slips, or faults; the broad lines marked $c$ denote dikes; the former dislocate the strata, and change their level, while dikes disjoin the strata with a wall, but do not in general affect their elevation. The two parallel lines marked $\alpha$, represent two seams of coal, variously heaved up and down by the faults; whereas the dikes are seen to pass through the strata without altering their relative position. In this manner, partial coalGelds are distributed over a wide area of country, in every direction.

The only exception to this general form of the coal-fields in Great Britain, is the in- 
verted basin shape; but this is rare. A few examples occur in some districts of Eng land, and in the county of life; but eren in extensive coal-fields, this convex form is but a partial occurrence, or a deviation by local violence from the ordinary basin. Fig. 803 is an instance of a convex coal-field exhibited in Staffordshire, at the Castle-
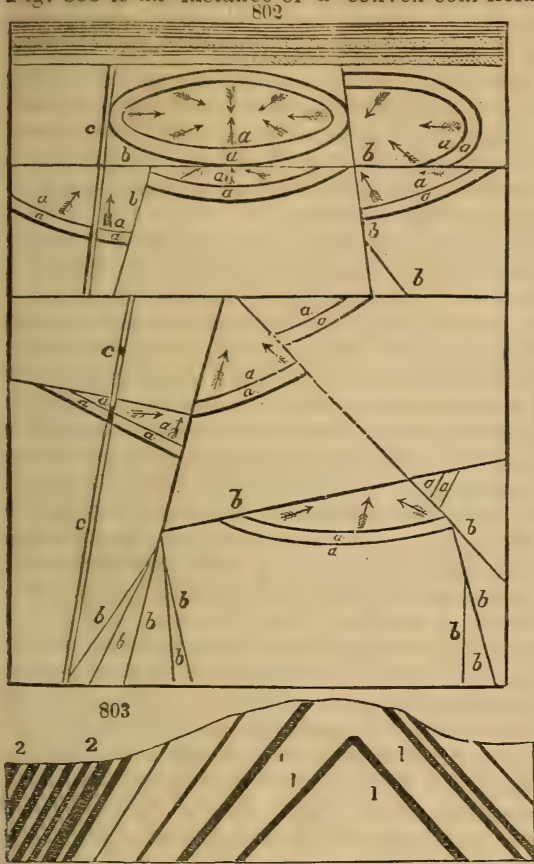
hill, close to the town of Dudley. 1,1 , are limestone strata; 2,2 , are coal. Through this hill, canals have been cut, for working the immense beds of carboniferous limestone. These occur in the lower series of the strata of the coal-field, and therefore at a distance of many miles from the Castlehill, beyond the outcrop of all the workable coals in the proper basinshaped part of the field; but by this apparently inverted basin-form, these limestone beds are elevated far above the level of the general surface of the country, and consequently above the level of all the coals. We must regard this seeming inversion as resulting from the approximation of two coal-basins, separated by the basset edges of their mountain limestone repository.

Fig. 804 is a vertical section of the Dudley coal-basin, the upper coal-bed of which has the astonishing thickness of 30 feet; and this mass extends 7 miles in length, and 4 in breadth. Coal-seams 5 or 6 feet thick, are called thin in that district.

Fig. 805 is a very interesting section of the main coal-basin of Clackmannanshire, as given by Mr. Bald in the Wernerian Society's Memoirs, vol. iii. Here we see it broken into three subordinate coal-fields, formed by two great faults or dislocations of the strata; but independently of these fractures across the whole series, the strata continie quite

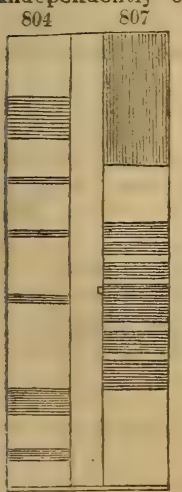
regular in their respective alternations, and preserve nearly unchanged their angle of inclination to the horizon. The section shows the south coal-field dipping northerly, till it is cut across by the great south slip $x$, which dislocates the coal and the parallel strata to the enormous extent of 1230 feet, by which all the coals have been thrown up, not sirnply to the day, but are not found again till we advance nearly a mile northward, on the line of the dip, where the identical seams of coal, shale, \&e. are observed once more with their regular inclination. These coals of the middle area, dip regularly northward till interrupted by the great north slip $y$, which dislocates the strata, and throws them up 700 feet; that is to say, a line prolonged in the direction of any one well-known seam, will run 700 feet above the line of the same seam as it emerges after the middle slip. Immediately adjoining the norih slip, the coals and coal-field resume their course, and dip regularly northward, running through a longer range than either of the other two members of the basin, till they arrive at the valley of the Devon, at the foot of the Ochill mountains, where they form a concave curvature, or trough, $a$, and thence rise rapidly in an almost vertical direction at $b$. Here the coals, with all their associate strata, assume conformity and parallelism with the face of the sienitic-greenstone strata of the Ochill mountains $c$; being raised to the high angle of 73 degrees with the horizon. The coal-seams thus upheaved, are called edge-metals by the miners.

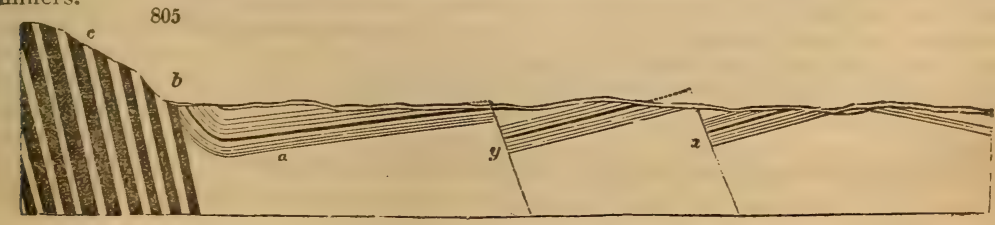


In this remarkable coal-field, which has been accurately explored by pitting and boring to the depth of 703 feet, there are no fewer than 142 beds, or distinct strata of coal, shale, and sandstone, \&c., variously alternating, an idea of which may be had

806 by inspecting fig. 806 . Among these are 24 beds of coal, which would constitute an agregate thickness of 59 feet 4 inches; the thinnest seam of coal being 2 inches, and the thickest 9 feet. The strata of this section contain numerous varieties of sandstone, slate-slay, bituminous shale, indurated clay, or fire-clay, and clay ironstone. Neither trap-rock nor limestone is found in connexion with the workable coals; but an immense bed of greenstone, named Abbey Craig, occurs in the western boundary of Clackmannanshire, under which lie regular strata of slate-clay, sandstone, thin beds of limestone, and large spheroidal masses of clay ironstone, with a mixture of lime.

"With regard to slips in coal-fields," says Mr. Bald, "we find that there is a general law connected with them as to the position of the dislocated strata, which is this:-When a slip is met with in the course of working the minesif when looking to it, the vertical line of the slip or fissure, it forms an acute angle with the line of the pavement upon which the observer stands, we are certain that the strata are dislocated downwards upon the other side of the fissure. On the contrary, if the angle formed by the two lines above mentioned is obtuse, we are certain that the strata are dislocated or thrown upwards upon the other side of the fissure. When the angle is $90^{\circ}$, or a right angle, it is altogether uncertain whether the dislocation throws up or down on the opposite side of the slip. When dikes intercept the strata, they generally only separate the strata the width of the dike, without any dislocation, either up or down; so that if a coal is intercepted by a dike, it is found again by running a mine directly forward, corresponding to the angle or inclination of the coal with the horizon." Wernerian Society's Memoirs, vol. iii. p. 133.*

The Johnstone coal-field, in Renfrewshire, is both singular and interesting. The upper stratum of rock is a mass of compact greenstone or trap, above 100 feet in thickness, not at all in a conformable position with the coal strata, but overlying; next there are a few fathoms of soft sandstone and slate-clay, alternating, and uncommonly soft Beneath these beds, there are no fewer than ten seams of coal, lying on each other, with a few divisions of dark indurated clay. These coal-seams have an aggregate thickness of no less than 100 feet; a mass of combustible matter, in the form of coal, unparalleled for its accumulation in so narrow a space. The greater part of this field contains only 5 beds of eoal; but - at the place where the section shown in fig. 807 is taken, these five coals seem to have been overlapped or made to slide over each other by violence. This strueture is represented in fig. 808, which is a section of the Quarrelton coal in the Johnstone field, showing the orerlapped coal and the double coal, with the thick bed of greenstone, overlying the coal-field.

808

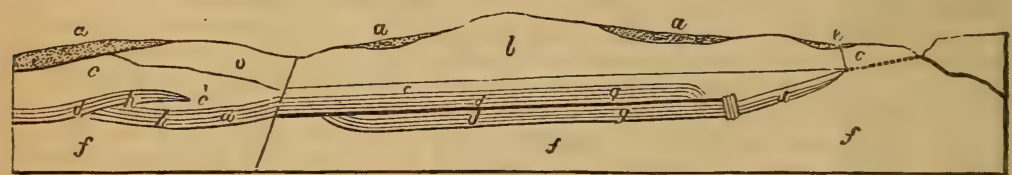

a. Alluvial cover.

b. Bed of trap or greenstone.

c. Alternating coal strata.

d. Coal-seams. e. Position of greenstone, not ascertained.

$f$. Strata in which no coals have been found.

g. The overlapped coal.

$h$. The double coal.

Before proceeding to examine the modes of working coal, I shall introduce here a description of the two principal species of this mineral.

1. Cubical coal.-It is black, shining, compact, moderately hard, but easily frangible. When extracted in the mine, it comes out in rectangular masses, of which the smaller fragments are cubical. The lamellæ (reed of the coal) are alwars parallel to the bed or plane on which the coal rests; a fact which holds generally with this substance. There are two varieties of cubical coal; the open-burning and the caking. The latter, however small its fragments may be, is quite available for fuel, in consequence of its agglutinating into a mass at a moderate heat, by the abundance of its bitumen. This lind is the true smithy or forge-coal, because it readily forms itself into a vault round the blast of the bellows, which serves for a cupola in concentrating the heat on objects thrust into the savity.

The open-burning cubical coals are known by several local names; the rough coal or

* This paper does honor to its author, the eminent coal-viewer of Scotland. 
clod coal, from the large masses in which they may be had and the cherry coal, from the cheerful blaze with which they spontaneously burn; whereas the caking coals, such as most of the Newcastle qualities, require to be frequently poked in the grate. Its specific gravity varies from $1 \cdot 25$ to $1 \cdot 4$.

2. Slate or splint coal.-This is dull-black, very compact, much harder, and more difficultly frangible than the preceding. It is readily fissile, like slate, but powerfully resists the cross fracture, which is conchoidal. Specific gravity from 1:26 to $1 \cdot 40$. In workıng, at separates in large quadrangular sharp-edged masses. It burns without caking, produces much flame and.smoke, unless judiciously supplied with air, and leaves frequently a considerable bulk of white ashes. It is the best fuel for distilleries and all large grates, as it makes an open fire, and does not elog up the bars with glassy scoriæ. I found good splint coal of the Glasgow field to have a specific gravity of $1 \cdot 266$, and to consist of-carbon, 70.9 ; hydrogen, $4 \cdot 3$; oxygen, $24 \cdot 8$.

3. Cannel coul.-Color between velvet and grayish-black; lustre resinous; fracture even; fragments trapezoidal; hard as splint coal; spec. grav. 1.23 to 1.28. In working, it is detached in four-sided columnar masses, often breaks conchoidal, like pitch, kindles very readily, and burns with a bright white projective flame, like the wick of a candle, whence its name. It occurs most abundantly in the coal-field of Wigan, in Lancashire, in a bed 4 feet thick; and there is a good deal of it in the Clydesdale coal-field, of which it forms the lowest seam that is worked. It produces very little dust in the mine, and hardly soils the fingers with carbonaceous matter. Cannel coal from Woodhall, near Glasgow, spec. grav. $1 \cdot 228$, consists by my analysis of-carbon, $72 \cdot 22$; hydrogen, 3.93 ; oxygen, 21.05 ; with a little azote (about 2.8 in 100 parts.) This coal has been found to afford, in the Scotch gas-works, a very rich-burning gas. The azote is there converted into ammonia, of which a considerable quantity is distilled over into the tar-pit.

4. Glance coal.-This species has an iron-black color, with an occasional iridiscence, like that of tempered steel; lustre in general splendent, shining, and imperfect metallic; loes not soil; easily frangible ; fracture flat conchoidal; fragments sharp-edged. It burns without flame or smell, except when it is sulphureous; and it leaves a white-colored ash. It produces no soot, and seems, indeed, to be merely carbon, or coal deprived of its volatile matter or bitumen, and converted into coke by subterranean calcination, frequently from contact with whin-dikes. Glance coal abounds in Ireland, under the name of Kilkenny coal; in Scotland it is called blind coal, from its burning without flame or smoke; and in Wales, it is the malting or stone coal. It contains from 90 to 97 per cent. of carbon. Specific gravity from 1.3 to 1.5 ; increasing with the proportion of earthy impurities.

The dislocations and obstructions found in coal-fields, which render the search for coal so difficult, and their mining so laborious and uncertain, are the following :-

1. Dikes. 2. Slips or Faults. 3. Hitches. 4. Troubles.

The first three infer dislocation of the strata; the fourth changes in the bed of coal itself.

1. A dike is a wall of extraneous matter, which divides all the beds in a coal-field.

Dikes extend not only in one line of bearing through coal-fields for many miles, but run sometimes in different directions, and have often irregular bendings, but no sharp angular turns. When from a few feet to a few fathoms in thickness, they occur sometimes in numbers within a small area of a coal basin, running in various directions, and even crossing each other. Fig. 809 represents a ground plan of a coal-field, intersected

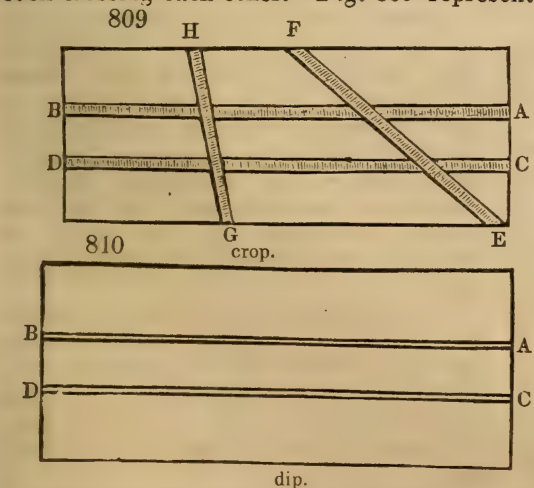
with greenstone dikes. A B and c are two dikes standing parallel to each other; E F and $\mathrm{G} \mathbf{H}$ are cross or oblique dikes, which divide both the coal strata and the primary dikes $A \mathrm{~B}$ and $\mathrm{C} D$.

2. Slips or faults run in straight lines through coal-measures, and at every angle of incidence to each other. Fig. 810 represents a ground plan of a coal-field, with two slips A B and C D in the line of bearing of the planes of the strata, which throw them down to A the outcrop. This is the simplest form of a slip. Fig. 811 exhibits part of a coal-field intersected with slips, like a cracked sheet of ice. Here $\mathbf{A}$ B is a dike; while the narrow lines show faults of every kind, producing disloeations varying in amount of slip from a few feet to a great many fathoms. The faults nt the points $a, a, a$ vanish; and the lines at c denote four small partial slips called hitches. 
The effects of slips and dikes on the coal strata appear more prominently when viewed in a vertical section, than in a ground plan, where they seem to be merely walls, veins, and lines of demarcation. Fig. 812 is a vertical section of a coal-field, from dip

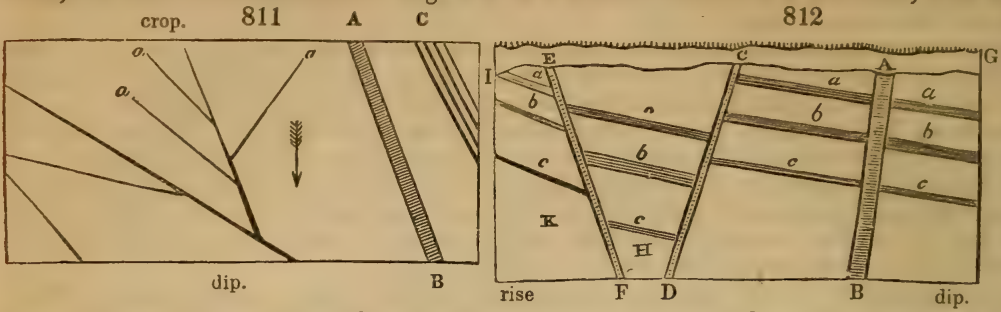

to rise, showing three strata of coal $a, b, c$. A B represents a dike at right angles to the plane of the coal-beds. This rectangular wall merely separates the coal-measures, affecting their line of rise; but further to the rise, the oblique dike $\mathrm{c} \mathrm{D}$ interrupts the coals $a, b, c$, and not only disjoins them, but throves them and their concomitant strata greatly lower down; but still, with this depression, the strata retain their parallelism and general slope. Nearer to the outcrop, another dike $\mathrm{E}, \mathrm{F}$, interrupts the coals $a, b, c$, not merely breaking the continuity of the planes, but throwing them moderately up, so as to produce a steeper inclination, as shown in the figure. It sometimes happens that the coals in the compartment $\mathrm{H}$, betwixt the dikes $\mathrm{c}$ and $\mathrm{x}$, may lie nearly horizontal, and the effect of the dike $\mathbf{E}, \mathrm{F}$, is then to throw out the coals altogether, leaving nc vestige of them in the compartment $\mathrm{K}$. "Such," says Mr. Bald, from whom these illustrations are borrowed, "are the most prominent changes in the strata, as to their line of direction, produced by dikes; but of these changes there are various modifica tions."

The effect of slips on the strata is also represented in the vertical section, $f \mathrm{~g} g .813$, wher $a, b, c$ are coals with their associated strata. A, B, is an intersecting slip, which throws al 813

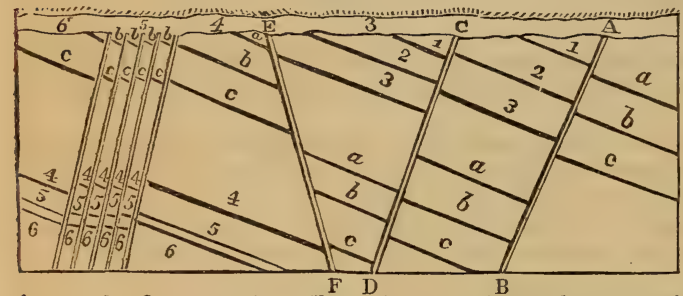

the coals of the first com partment much lower, as $i_{2}$ observable in the second No. 2 ; and from the amoun of the slip, it brings in othe coal-seams, marked 1, 2, 3 not in the compartmen: No. 1 . c, D, is a slip producing a similar result, but not of the same magnitude. E, F represents a slip across the strata, reverse in direc.

tion to the former; the effect of which is to throw up the coals, as shown in the area No. 4. Such a slip occasionally brings into play seams seated under those marked $a, b, c$, 814
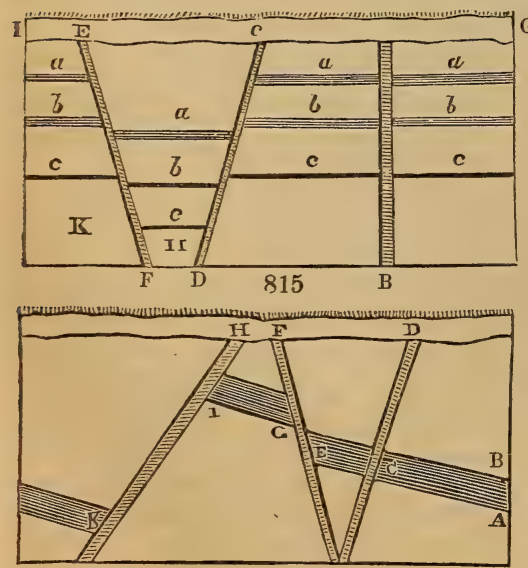
as seen at $4,5,6$; and it may happen $G$ that the coal marked 4 lies in the prolongation of a well-known seam, as c, in the compartment No. 3, when the case becomes puzzling to the miner. In addition to the above varieties, a number of slips or hitches are often seen near one another, as in the area marked No. 5, where the individual displacements are inconsiderable, but the aggregate dislocation may be great, in reference to the seams of the 6th compartment.

The results of dikes and slips on a horizontal portion of a field are exemplified in fig. 814. Where the coal-measures are horizontal, and the faults run at a greater angle than $45^{\circ}$ to the line of bearing, they are termed dip and rise faults, as A B, C D, E $F$.

Coal-viewers or engineers regard the dislocations now described as being subject in one respect to a general law, which may be thus explained :-Let fig. 815 
be a purtion of a coal-measure; $A$, being the pavement and $\mathrm{B}$ the roof of the coal-seam. If, in pursuing the stratum at $c$, a dike $D$ occurs, standing at right angles with the pavement, they conclude that the dike is merely a partition-wall between the beds by its own thicliness, leaving the coal-seam underanged on either side; but if a dike $F$ forms, as at E, an obtuse angle with the pavement, they conclude that the dike is not a simple partition between the strata, but has thrown up the several seams into the predicament shown at $\mathrm{G}$. Finally, should a dike $\mathbf{H}$ make at $\mathbf{I}$ an acute angle with the parement, they conclude that the dike has thrown down the coal-measures into the position of $\mathbf{k}$.

The same important law holds with slips, as I formerly stated; only when they form right angles with the pavement, the case is ambiguous; that is, the strata mas be dislocated either upwards or downwards.

Dikes and faults are denominated upthrow or downthrow, according to the position they are met with in working the mine. Thus, in fig. 812, if the miner in advancing to the rise, the dike $\mathrm{A}, \mathrm{B}$ obriously does not change the direction; but $\mathrm{C}, \mathrm{D}$ is a downthrow dike of a certain number of fathoms towards the rise of the basin, and $F, F$ is an upthrow dike likewise towards the rise. On the other hand, when the dikes are met with by the miner in working from the rise to the dip, the names of the above dikes would be reversed; for what is an upthrow in the first case, becomes a downthrow in the second, relative to the mining operations.

3. We have seen that hitches are small and partial slips, where the dislocation does not cxceed the thickness of the coal-seam; and they are correctly enough called steps by the miner. Fig. 816 represents the operation of the hitches A, B, C, D, E, F, G, H, on the coal816

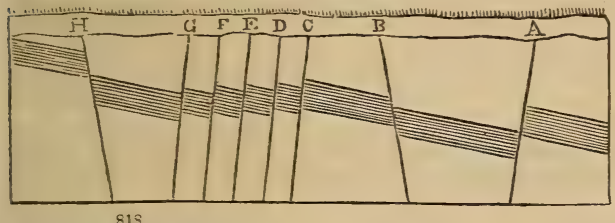

815

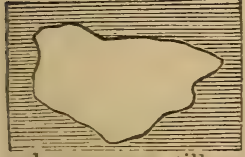

817

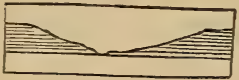
measures. Though observed ir. one or two seams of a field, they may not appear in the rest, as is the case with dikes and faults.

4. Troubles in coal-fields are of various kinds.

1. Irregular layers of sandstone, appearing in the middle of the coal-seam, and gradually increasing in thickness till they separate the coal into two distinct seams, too thin to continue workable.

2. Nips, occasioned by the gradual approximation of the roof and parement, till not a vestige of coal is left between them; the softer shale disappearing also at the same time. Figs. 817 and 818 represent this accident, which is fortunately rare; the first being a vertical, and the second a horizontal view.

3. Shaken coal. It resembles the rubbish of an old waste, being a confused heap of coal-dust, mixed with small pieces of cubical coal, so soft that it can frequently be dug with the spade. This shattering is analogous to that observed occasionally in the flint nodules of the chalk formation; and seems like the effect of some electric tremor of the strata.

In searching for coal in any country, its concomitant rocks ought to be looked for, especially the carboniferous or mountain limestone, known by its organic issils; (see Ure's Geology, p. 175, and corresponding plate of fossils;) likewise the oucurop of the millstone grit, and the newer red sandstone, among some rifts or facades of which, seams of coal may be discerned. But no assurance of coal can be had without boring or pitting.

Skill in boring judiciously for coal, distinguishes the genuine miner from the empirical adventure!; who, ignorant of the general structure of coal-basins, expends labor, time, and money at random, and usually to no purpose; missing the proper coal-field, and leading his employer to sink a shaft where no productive seams can be had. A shilful viewer, therefore, should always direct the boring operations, especially in an unexplored country.

The boring rods should be made of the best and most tenacious Swedish iron; in area, about an inch and a quarter square. Each rod is usually 3 feet long, terminating in a male screw at one end, and a female screw at the other. The boring ehisels are commonly 18 inches long, and from 2 inches and a half to 3 inches and a quarter at their cutting edge, which must be tipped with good steel. The chisel is screwed to an intermediate 18-inch rod, called the double box-rod, forming together a rod 3 feet long. There are, moreover, three short rods, a foot, 18 inches, and 2 feet long each, which may be screwed, as occasion requires, to the brace-head, to make the height above the 
mouth of the bore convenient for the hands of the men in working the rods. Hence the series of rods becomes a scale of measurement for noting the depth of the hore, and keeping a journal of the strata that are perforated. The brace-head rod, also 18 inches long, has two large eyes or rings at its top, set at right angles to each other, through which arms of wood are fixed for the men to lift and turn the rods by, in the boring process.

When the bore is intended to penetrate but a few fathoms, the whole work may be performed directly by the hands; but when the bore is to be of considerable depth, a lofty triangle of wood is set above the bore hole, with a pulley depending at its summit angle, for conducting the rope to the barrel of a windlass or wheel and axle, secured to the ground with heavy stones. The loose end of the rope is connected to the rods by an oval iron ring, called a runner; and by this mechanism they may be raised and let fall in the boring; or the same effect may be more simply produced by substituting for the wheel and axle, a number of ropes attached to the rod rope, each of which may be pulled by a man, as in raising the ram of the pile engine.

In the Newcastle coal district there are professional master-borers, who undertake to search for coal, and furnish an accurate register of the strata perforated. The average price of boring in England or Scotland, where no uncommon difficulties occur, is six shillings for each of the first five fathoms, twice 6 shillings for each of the second five fathoms, thrice 6 shillings for each of the third five fathoms, and so on; hence the series will be-

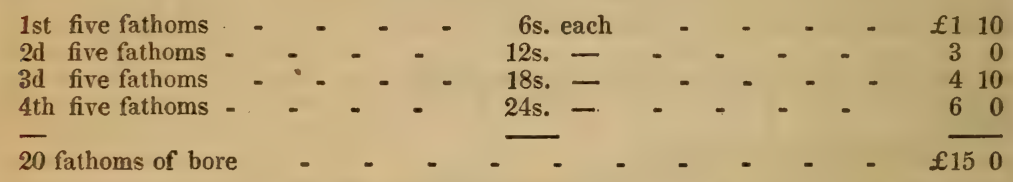

Thus the price increases equably with the depth and labor of the bore, and the undertaker usually upholds his rods. There are peculiar cases, however, in which the expense greatly exceeds the above rate.

The boring tools are represented in the following figures :-

819

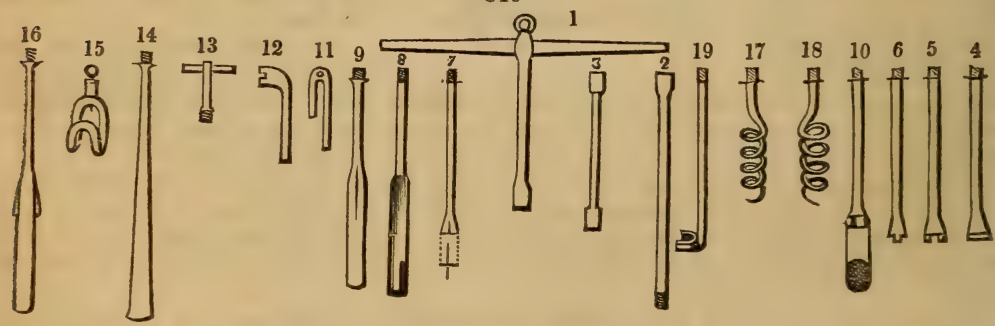

Fig. 819.

1. The brace-head.

2. The common rod.

3. The double-box rod; intermediate piece.

4. The common chisel.

5. The indented chisel.

6. Another of the same.

7. The cross-mouthed chisel.

8. The wimble.

9. The sludger, for bringing up the mud.

10. The rounder.
11. The key for supporting the train of rods at the bore-mouth.

12. The key for screwing together and asunder the rods.

13. The topit, or top-piece.

14. The beché, for catching the rod when it breaks in the bore.

15. The runner, for taking hold of the topit.

16. The tongued chisel.

17. The right-handed worm screw.

18. The left-handed do.

19. The finger grip or eatch.

We shall now explain the manner of conducting a series of bores in searching ground for coal.

Fig. 820 represents a district of country in which a regular survey has proved the existence and general distribution of coal strata, with a dip to the south, as here shown. In this case, a convenient spot should be pitched upon in the north part 
of the district, so that the successive bores put down may advance in the line of the

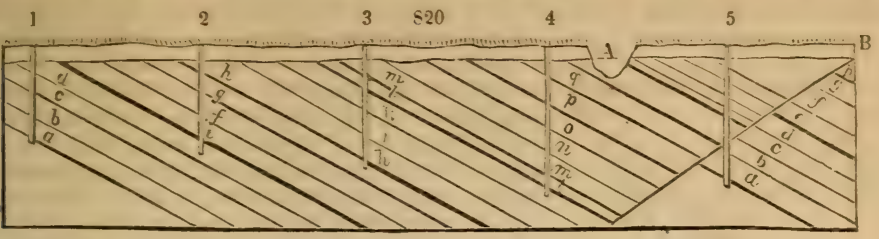

dip. The first bore mar therefore be made at No. 1 , to the depth of sixty yards. In the progress of this perforation, manr diversities and alternations of strata will be probably passed through, as we see in the sections of the strata; each of which, as to quality and thickness, is noted in the journal, and specimens are preserved. This bore is seen to penetrate the strata $d, c, b, a$, without encountering any coal. Now, suppose that the dip of the strata be one yard in ten, the question is, at what distance from bore No. 1 , in a south direction, will a second bore of 60 vards strike the first stratum, $d$, of the preceding? The rule obriously is, to multiply the depth of the bore by the dip, that is, $60 \mathrm{by} 10$, and the product, 600 , gives the distance required; for, by the rule of three, if 1 yard of depression corresponds to 10 in horizontal length, 60 rards of depression will correspond to 600 in length. Hence the bores marked 1, 2, 3, 4, and 5, are successirely distributed as in the figure, the spot where the first is let down being regarded as the point of lerel to which the summits of all the succeeding bores are referred. Should the top of No. 2 bore be 10 rards higher or lower than the top of No. 1, allowance must be made for this difference in the operation; and hence a surface lerel surtey is requisite. Sometimes rarines cut down the strata, and advantage should be taken of them, when they are considerable.

In Yo. 2, a coal is seen to occur near the surface, and another at the bottom of the bore; the latter seam resting on the first stratum $d$, that occurred in bore No. 1 ; and No. 2 perforation must be continued a little farther, till it has certainly descended to the stratum $d$. Thus these two bores hare, together, prored the beds to the depth of 120 Jards.

No. 3 bore being placed according to the preceding rule, will pass through two coalseams near the surface, and after reaching to nearly its depth of 60 jards, it will touch the stratum $h$, which is the upper stratum of bore No. 2 ; but since a seam of coal was detected in No. 2, under the stratum $h$, the proof is confirmed by running the borer down through that coal. The field has now been probed to the depth of 180 rards. The fourth bore is next proceeded with, till the two coal-seams met in No. 3 hare been penetrated; when a depth of 240 yards has been explored. Hence No. 4 bore could not reach the lower stratum $\alpha$, unless it were sunk 240 yards.

The fifth bore (No. 5) being sunk in like manner, a new coal-seam occurs within a few yards of the surface; but after sinking to the depth at which the coal at the top of the fourth bore was found, an entirely different order of strata will occur. In this dilemma, the bore should be pushed 10 or 20 yards deeper than the 60 yards, to ascertain the alternations of the new range of superposition. It may happen that no coals of any value shall be found, as the figure indicates, in consequence of a slip or dislocation of the strata at $\mathrm{B}$, whict has thrown up all the coals registered in the former borings, to such an extent that the strata $b, a$, of the first bore present themselves immediately on perforating the slip, instead of lying at the depth of 300 yards $(5 \times 60)$, as they would hare done, had no dislocation interrened. Some coal-fields, indeed, are so intersected with slips as to bewilder the most experienced miner, which will particularly happen when a lower coal is thrown upon one side of a slip, directly opposite to an upper coal situated on the other side of it; so that if the two seams be of the same thickness, erroneous conclusions are almost inevitable.

When a line of bores is to be conducted from the dip of the strata towards their outcrop, they should be placed a few yards nearer each other than the rule prescribes, lest the strata last passed through be overstepped, so that ther may disappear from the register, and a raluable coal-seam may therebr escape notice. In fact, each successive bore should be so set down, that the first of the strata perforated should be the last passed through in the preceding bore; as is exemplified by viewing the bores in the retrograde direction, Nos. 4, 3, and 2. But if the bore No. 2 had gone no deeper than $f$, and the bore No. 1 had been as represented, then, the stratum $e$, with its immediately subjacent coal, would have been overstepped, since none of the bores would have touched it; and they would have remained unnoticed in the journal, and unknown.

When the line of dip, and consequently the line of bearing which is at right angles to it, are unknown, they are sought for by making three bores in the following position. -Let fig. 821 be a horizontal diagram, in which the place of a bore, No. 1, is 
shown, which reaches a coal-seam at the depth of 50 yards; bore No. 2 may be made at $\mathrm{B}, 300$ yards from the former; and bore No. 3 at c, equidistant from Nos. 1 and 2,

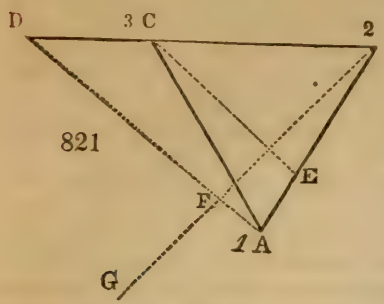
so that the bores are sunk at the three angles of an 2 B equilateral triangle. If the coal occur in No. 2 at the depth of 30 yards, and in No. 3 of 44 yards, it is manifest that none of the lines $A B, B C$, or $C A$, is in the line of level, which for short distances may be taken for the line of bearing, with coal-seams of moderate dip. But since No. 1 is the deepest of the three bores, and No. 3 next in depth, the line A $\mathrm{C}$ joining them must be nearer the line of level than either of the lines A B or B c. The question is, therefore, at what distance on the prolonged line $\mathrm{B} \mathrm{c}$ is the point for sinking a bore which would reach the coal at the same depth as No. 1, namely, 50 yards. This problem is solved by the following rule of proportion: as 14 yards (the difference of depth between bores 2 and 3 ) is to 300 yards (the distance between them), so is 20 (the difference of depth betwixt 1 and 2 ) to a fourth proportion, or $x=428$ yards, 1 foot, and 8 inches. Now, this distance, measured from No. 2, reaches to the point $\mathrm{D}$ on the prolonged line B $\mathrm{C}$, under which point $\mathrm{D}$ the coal will be found at a depth of 50 yards, the same as under $\Lambda$. Hence the line A D is the true level line of the coal-field; and a line $\mathbf{B}$ F G, drawn at right angles to it, is the true dip-line of the plane which leads to the outcrop. In the present example the dip is 1 yard in $14 \frac{1}{2}$; or 1 in $14 \frac{1}{2}$, to adopt the judicious language of the miner; or the sine is 1 to a radius of $14 \frac{1}{2}$, measured along the line from $\mathrm{s}$ to $\mathrm{F}$. By this theorem for finding the lines of dip and level, the most eligible spot in a coal-field for sinking a shaft may be ascertained.

Suppose the distance from B to $\mathrm{G}$ in the line of dip to be 455 yards; then, since every $14 \frac{1}{2}$ gives a yard of depression, 455 will give 30 yards, which added to 30 yards, the depth of the bore at $\mathrm{B}$, will make 60 yards for the depth of the same coal-seam at $\mathrm{G}$. Since any line drawn at right angles to the line of level A D is the line of dip, so any line drawn parallel to $\mathrm{A} D$ is a level line. Hence, if from $\mathrm{C}$ the line $\mathrm{C} \mathrm{E}$ be drawn parallel to $\mathrm{D} A$, the coal-seam at the points $\mathrm{E}$ and $\mathrm{c}$ will be found in the same horizontal plane, or 44 rards beneath the surface level, over these two points. The point $\mathrm{E}$ level with $\mathrm{c}$ may also be found by this proportion: as 20 yards (the difference in depth of the bores under $\mathrm{B}$ and $\mathrm{A}$ ) is to 300 yards (the distance between them), so is 14 yards (the difference of depth under B and c) to 210 yards, or the distance from $\mathrm{B}$ to $\mathrm{E}$.

As boring for coal is necessarily carried on in a line perpendicular to the horizon, and as coal-seams lie at every angle of inclination to it, the thickness of the seam as given obliquely by the borer, is always greater than the direct thickness of the coal; and hence the length of that line must be multiplied by the cosine of the angle of dip, in order to find the true power of the seam.

Of fitting or winning a coal-field.-In sinking a shaft for working coal, the great obstacle to be encountered is water, particularly in the first opening of a field, which proceeds from the surface of the adjacent country; for every coal-stratum, howerer deep it may lie in one part of the basin, always rises till it meets the allurial corer, or crops out, unless it be met by a slip or dike. When the basset-edge of the strata is corered with gravel or sand, any body or stream of water will readily percolate downwards through it, and fill up the porous interstices between the coal-measures, till arrested by the face of a slip, which acts as a valve or flood-gate, and confines the water to one compartment of the basin, which may, howerer, be of considerable area, and require a great power of drainage.

In reference to water, coal-fields are divided into two kinds: 1 . level free coal; 2. coal not level free. In the practice of mining, if a coal-field, or portion of it, is so situated above the surface of the ocean that a level can be carried from that plane till it intersects the coal, all the coal above the plane of intersection is said to be level free; but if a coal-field, though placed above the surface of the ocean, cannot, on account of the expense, be drained by a level or gallery, but by mechanical power, such a coal-field is said to be not level free.

Besides these general levels of drainage, there are subsidiary levels, called off-takes or drifts, which discharge the water of a mine, not at the mouth of the pit, but at some depth beneath the surface, where, from the form of the country, it may be run off level free. From 20 to 30 fathoms off-take is an object of considerable economy in pumping; but even less is often had recourse to; and when judiciously contrived, may serve to intercept much of the crop water, and prevent it from getting down to the dip part of the coal, where it would become a heavy load on a hydraulic engine.

Day levels were an object of eprimary importance with the early miners, who had not the gigantic pumping power of the steam-engine at their command. Levels ought to be no less than 4 feet wide, and from 5 feet and a half to 6 feet high; which is large 
enongh for carrying off water, and admitting workmen to make repairs and clear out depositions. When a day-level, however, is to serve the double purpose of drainage and an outlet for coals, it should be neariy 5 feet wide, and have its bottom gutler covered over. In other instances a level not only carries off the water from the colliery, but is converted into a canal for bearing boats loaded with coals for the market. Some subterranean canals are nine feet wide, and twelve feet high, with 5 feet depth of water.

If in the progress of driving a level, workable coals are intersected before reaching the seam which is the main object of the mining adventure, an air-pit may be sunk, of such dimension as to serve for raising the coals. These air-pits do not in general exceed 7 feet in diameter; and they ought to be always cylindrical. Fig. 822 represents a coal-field where the winning is made by a day-level; $a$ is the mouth of the galiery on a level with the sea; $b, c, d$, $e$ are intersected coal-seams, to be drained by the gallery. But the coals beneath this level must obviously be drained by pumping. A represents a coal-pit sunk on the coal $e$; and if the gallery be pushed
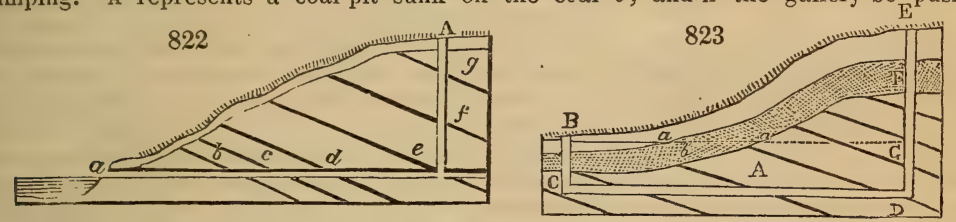

forward, the coal-seams $f, g$, and any others which lie in that direction, will also be drained, and then worked by the pit A. The chief obstacle to the execution of daylevels, is presented by quicksands in the alluvial cover, near the entrance of the gallery. The best expedient to be adopted amid this difficulty is the following:-Fig. 823 represents the strata of a coal-field $A$, with the alluvial earth $a$, $b$, containing the bed of quicksand $b$. The lower part, from which the gallery is required to be carried, is shown by the line B $d$. . But the quicksand makes it impossible to push forward this day-level directly. The pit в c must therefore be sunk through the quicksand by means of tubbing (to be presently described), and when the pit has descended a few yards into the rock, the gallery or drift may then be pushed forward to the point $\mathrm{D}$, when the shaft E D is put down, after it has been ascertained by boring that the rockhead or bottom of the quicksand at $\mathrm{F}$ is a few yards higher than the mouth of the small pit B. During this operation, all the water and mine-stuff are drawn off by the pit $B$; but whenever the shaft $\mathrm{ED}$ is brought into communication with the gallery, the water is allowed to fill it from $\mathrm{C}$ to $\mathrm{D}$, and rise up both shafts till it overflows at the orifice $\mathrm{B}$. From the surface of the water in the deep shaft at G, a gallery is begun of the common dimensions, and pushed onwards till the coal sought after is intersected. In this way no drainage level is lost. This kind of drainage gallery, in the form of an inverted syphon, is called a drowned or a blind level.

When a coal-basin is so situated that it cannot be rendered level free, the winning must be made by the aid of machinery. The engines at present employed in the drainage of coal-mines are :-

1. The water-wheel, and water-pressure engine.

2. The atmospheric steam-engine of Newcomen.

3. The steam-engine, both atmospheric and double stroke, of Watt.

4. The expansion steam-engine of Woolf.

5. The high-pressure steam-engine, without a condenser.

The depth at which the coal is to be won, or to be drained of moisture, regulates the power of the engine to be applied, taking into account the probable quantity of water which may be found, a circumstance which governs the diameter of the working barrels of the pumps. Experience has proved, that in opening collieries, even in new fields, the water may generally be drawn off by pumps of from 10 to 15 inches diameter: excepting where the strata are connected with rivers, sand-beds filled with water, or marsh-lands. As feeders of water from rivers or sand-beds may be hindered from descending coal-pits, the growth proceeding from these sources need not be taken into account; and it is observed, in sinking shafts, that though the influx which cannot be cut off from the mine, may be at first very great, even beyond the power of the engine for a little while, yet as this excessive flow of water is frequently derived from the drainage of fissures, it eventually becomes manageable. An engine working the pumps for 8 or 10 hours out of the 24 , is reckoned adequate to the winning of a new colliery, which reaps no advantage from neighboring hydraulic powers. In the course of years, however, many water-logged fissures come to be cut by the workings, and the coal-seams get excavated towards the outcrop, so that a constant increase of water ensues, and thus a colliery which has been long in operation, frequently becomes heavily 
loaded with water, and requires the action of its hydraulic machinery both night and day.

Of Engine Pits.-In every winning of coal, the shape of the engine-pit deserves much consideration. For shafts of moderate depth, many forms are in use; as circular, oval, square, octagonal, oblong rectangular, and oblong elliptical. In pits of inconsiderable depth, and where the earthy cover is firm and dry, any shape deemed most convenient may be preferred; but in all deep shafts, no shape but the circular should be admitted. Indeed, when a water-run requires to be stopped by tubbing or cribbing, the circular is the only shape which presents a uniform resistance in every point to the equable circumambient pressure. The elliptical form is the next best, when it deriates little from the circle; but even it has almost always given way to a considerable pressure of water. The circular shape has the advantage, moreover, of strengthening the shaft walls, and is less likely to suffer injury than other figures, should any failure of the pillars left in working out the coal cause the shaft to be shaken by subsidence of the strata. The smallest engine-pit should be ten feet in diameter, to admit of the $826 \quad 825 \quad 824$ pumps being placed in the lesser segment, and the

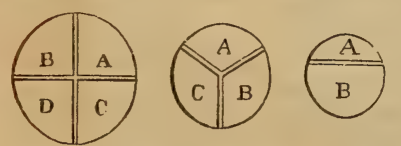
coals to be raised in the larger one, as shown in fig: 824 , which is called a double pit. If much work is contemplated in drawing coals, particularly if their masses be large, it would be advantageous to make the pit more than 10 feet wide. When the area of a shaft is to be divided into three compartments, one for the engine pumps, and two for raising coals, as in fig. 825 , which is denominated a triple pit, it should be 12 feet in diameter. If it is to be divided into four compartments, and made a quadrant shaft, as in fig. 826 , with one space for the pumps, and three for ventilation and coal drawing, the total circle should be 15 feet in diameter. These dimensions are, however, governed by local circumstances, and by the proposed daily discharge of coals.

The shaft, as it passes through the earthy cover, should be securely faced with masonry of jointed ashler, having its joints accurately bevelled to the centre of the circle. Specific directions for building the successive masses of masonry, on a series of rings or cribs of oak or elm, are given by Mr. Bald, article MINE, Breuster's Encyclopadia, p. 336.

When the alluvial cover is a soft mud, recourse must be had to the operation of tubbing. A circular tub, of the requisite diameter, is made of planks from 2 to 3 inches thick, with the joints bevelled by the radius of the shaft, inside of which are cribs of hard wood, placed from 2 to 4 feet asunder, as circumstances may require. These cribs are constructed of the best heart of oak, sawn out of the natural curvature of the wood, adapted to the radius, in segments from 4 to 6 feet long, from 8 to 10 inches in the bed, and 5 or 6 inches thick. The length of the tub is from 9 to 12 feet, if the layer of mud have that thickness; but a succession of such tubs must be set on each other, provided the body of mud be thicker. The first tub must have its lower edge thinned all round, and shod with sharp iron. If the pit be previously secured to a certain depth, the tnb is made to pass within the cradling, and is lowered down with tackles till it rests fair among the soft alluvium. It is then loaded with iron weights at top, to cause it to sink down progressively as the mud is removed from its interior. Should a single tub not reach the solid rock (sandstone or basalt), then another of like 827

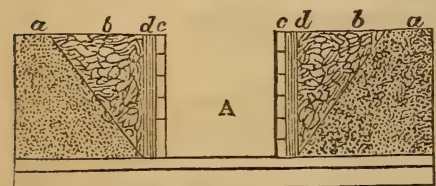
construction is set on, and the gravitating force is transferred to the top. Fig. 827 represents a bed of quicksand resting on a bed of impervious clay, that immediately covers the rock. $A$ is the finished shaft; $\dot{a} a$, the quicksand; $b \quad b$, the excavation necessarily sloping much outwards; $c c$, the lining of masonry ; $d d$, the moating or puddle of clay, hard rammed in behind the stone-work, to render the latter water-tight. In this case, the quicksand, being thin in body, has been kept under for a short period, by the hands of many men scooping it rapidly away as it filled in. But the most effectual method of passing through beds of quicksand, is by means of cast-iron cylinders; called, therefore, cast-iron tubbing. When the pit has a small diameter, these tubs are made about 4 feet high, with strong flanges, and bolt holes inside of the cylinder, and a counterfort ring at the neck of the flange, with brackets; the first tub, however, has no flange at its lower edge, but is rounded to facilitate its descent through the mud. Should the pit be of large diameter, then the cylinders must be cast in segments of 3,4 , or more pieces, joined together with inside vertical flanges, well jointed with oakum and white lead. When the sand-bed is thick, eighty feet, for instance, it is customary to divide that length into three sets of sylinders, each thirty feet long, and so sized as to slide within each other, like the eye tubes of a telescope. These cylinders are pressed down by heavy weights, taking care to 
keep the lower part always further down than the top of the quicksand, where the men are at work with their shovels, and where the bottom of the pumps hangs for withdrawing the surface water. This is an improvement adopted of late years in the Newcastle district, with remarkable success.

The engine pit being secured, the process of sinking through the rock is ready to be commenced, as soon as the divisions of the pit formed of carpentry, called brattices, are mate. In common practice, and where great tightness of jointing is not required, for ventilating inflammable air, bars of wood, called buntons, about 6 inches thick, and 9 deep, are fixed in a horizontal position across the pit, at distances from each other of 10 , 20 , or 30 feet, according to circumstances. Being all ranged in the same vertical plane, deals an inch and a half thick are nailed to them, with their joints perfectly close; one half of the breadth of a bunton being covered by the ends of the deals. In deep pits, where the ventilation is to be conducted through the brattice, the side of the buntons next the pumps is covered with deals in the same way, and the joints are rendered secure by being callied with oakum. Fillets of wood are also fixed all the way down on each side of the brattice, constituting what is called a double pit.

When a shaft is to have 3 compartments, it requires more care to form the brattice, as none of the buntons stretch across the whole space, but merely meet near the middle, and join at certain angles with each other. As the buntons must therefore sustain each other, on the principle of the arch, they are not laid in a horizontal plane, but have a rise from the sides towards the place of junction of 8 or 9 inches, and are bound together by a three-tongued iron strap. Fillets of wood are carried down the whole depth, not merely at the joinings of the brattice with the sides of the pit, but also at their central place of union; while wooden pillars connect the centre of each set of buntons with those above and below. Thus the carpentry work acquires sufficient strength and stiffness.

In quadrant shafts the buntons cross each other towards the middle of the pit, and are generally let into each other about an inch, instead of being half-checked. Fig. 824 is a double shaft: A, the pump pit; B, the pit for raising coal. Fig. 825 is a triple shaft; in which $\mathrm{A}$ is the pump compartment; $\mathrm{B}$ and $\mathrm{C}$ are coal-pits. Fig. 826 is a quadrant shaft: $A$, the pump pit; $B$, pit of ventilation or upcast for the smoke; $C$ and $D$, pits for raising coals.

A depth of 75 fathoms is fully the average of engine pits in Great Britain. In practice, it embraces three sets of pumps. Whenever the shaft is sunk so low that the engine is neerled to remove the water, the first set of pumps may be let down by the method represented in fig. 828 ; where $\mathrm{A}$ is the pump; $a, a$, strong ears through which

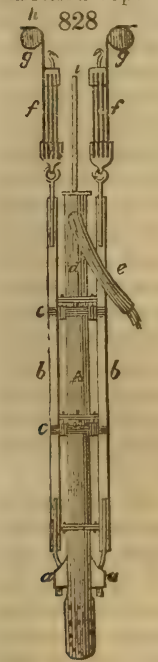
pass the iron rods connected with the spears $b b ; c c$ are the lashings; $d$, the hoggar pump; $e$, the hoggar; $f f$, the tackles; $g$, the single pulleys; $h h$, the tackle fold leading to the capstans; and $i$, the pumpspears. By this mechanical arrangement the pumps are sunk in the most gradual manner, and of their own accord, so to speak, as the pit descends. To the arms of the capstans, sledges are fastened with ropes or chains; these sledges are loaded with weights, as counterpoises to the weight of the column of pumps, and when additional pumps are joined in, more weight is laid on the sledges. As the sinking set of pumps is constantly descending, and the point for the delivery of the water above always varying, a pipe of equal diameter with the pumps, and about 11 feet long, but much lighter in the metal, is attached to $e$, and is terminated by a hose of leather, of sufficient length to reach the cistern where the water is delivered. This is called the hoggar-pipe. In sinking, a vast quantity of air enters with the water, at every stroke of the engine; and therefore the lifting stroke should be very slow, and a momentary stop should take place before the returning stroke, to suffer all the air to escape. As the working barrels are generally 9 or 10 feet long, and the full stroke of the engine from 7 to 8 feet, when at regular work, it is customary to diminish the length of stroke, in sinking, to about 6 feet; because, while the pumps are constantly getting lower, the bucket in the working barrel has its working range progressively higher.

The usual length for a set of pumps, is from 25 to 30 fathoms. Whenever this depth is arrived at by the first set, preparations are made for fixing firmly the upper pit-cistern, into which the upper set of pumps is to be placed, and the water of the second set is to be thrown. If a strong bed of sandstone occurs, a scarcement of it is left projecting about 3 feet into the shaft, which is formed in the course of sinking into a strong chin or bracket, to sustain that part of the cistern in which the superior set of pumps stands. A few feet beneath this scarcement the shaft resumes its usual shape. 
But although from 20 to 30 fathoms be the common length of a pump-lift, it sometimes becomes necessary to malie it much longer, when no place can be found in the 829 shaft for lodging a cistern, on account of the tubbing. Hence a jun r-lift has $T$ been occasionally extended to $t 0$ fathoms; which requires extracirdinary sirength of materials. The best plan for collaring the pumps in the rit, anil keejing them steady in a perpendicular line, is to fix a strong bunton of timber under the joints of each pipe; and to attach the pipes firmly to these buntons by an iron collar, with screws and nuts, as represented in fig. 829.

The water obtained in sinking through the successive strata is, in ordinary cases, eonducted down the walls of the shaft; and if the strata are compact, a spiral groore is cut down the sides of the shaft, and when it can hold no more, the water is drawn off in a spout to the nearest pump-cistern; or a perpendicular groore. is cut in the side of the shaft, and a square box-pipe either sunk in it, flush with the sides of the pit, or it is covered with deal boards well fitted orer the cavity. Similar spiral rings are formed in succession downwards, which collect the trickling strearns, and conduct them into the nearest cistern; or rings, made of wood or cast iron, are inserted flush with the sides of the pipe; and the water is led from one ring to another, through perpendicular pipes, until the undermost ring is full, when it delivers its water into the nearest pump-cistern. Keeping the shaft dry is very important to the comfort of the miners, and the durability of the work.

When an engine shaft happens to pass through a great many beds of coal, a gallery a few yards long is driven into each coal-seam, and a bore then put down from one coal to another, so that the water of each may pass down through these bores to the pumpcisterns.

While a deep pit is sinking, a register is kept of erery part of the excarations, and each feeder of water is measured daily, to ascertain its rate of discharge, and whether it increases or abates. The mode of measurement, is by noting the time, with a seconds watch, in which a cistern of 40 or 50 gallons gets filled. There are three modes of keeping back or stopping up these feeders, by plank tubbing; iron tubbing; and by oak cribs. Let fig. 830 represent the sinking of a shaft through a rarietr of strata,

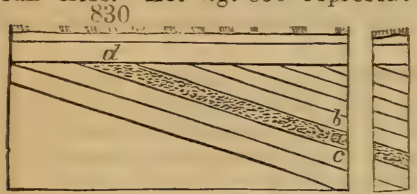
having a top cover of sand, with much water resting on the rock summit. Each plane of the coal-measure rises in a certain direction till it meets the alluvial cover. Hence, the pressure of the water at the bottom of the tubbing that rests on the summit of the rock, is as the depth of water in the superficial allurium; and if a stratum $a$ affords a great bodr of water, while the superjacent stratum $b$, and the subjacent $c$, are imperrious to water; if the porous bed $a$ be 12 feet thick, while no water occurs in the strata passed through from the rock head, until that depth (supposed to be 50 fathoms from the surface of the water in the cover); in this case, the tubbing or cribbing mus sustain the sum of the two water pressures, or 62 fathoms; since the stratum $a$ meets the alluvial corer at $d$, the fountain head of all the water that occurs in sinking. Thus we perceire, that though no water-feeder of any magnitude should present itself till the shaft had been sunk 100 fathoms; if this water required to be stopped up or tubbed of through the breadth of a stratum only 3 feet thick, the tubbing floodgate would need to have a strength to resist 100 fathoms of water-pressure. For though the water at first oozes merely in discontinuous particles through the open pores of the sands and sandstones, yet it soon fills them up, like a mrriad of tubes, which transfer to the botiom the tota] weight of the hydrostatic column of 100 fathoms; and experience shows, as we have already stated, that whaterer water occurs in coal-pits or in mines, generally speaking, proceeds from the surface of the ground. Hence, if the corer be an imperrious bed of clar, very little water will be met with among the strata, in comparison of what would be found under sand.

When sereral fathoms of the strata must be tubbed, in order to stop up the waterflow, the shaft must be widened regularly to admit the kind of tubbing that is to be inserted; the greatest width being needed for plank-tubbing, and the least for irontubbing. Fig. 831 represents a shaft excarated for plank-tubbing, where $a, a, a$ are the 831 impervious strata, $b, b$ the porous beds water-logged, and $c, c$ the bottom

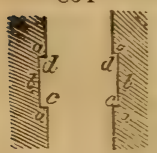
of the excavation, made level and perfectly smooth with mason-chisels. The same precautions are taken in working off the upper part of the excavation $d, d$. In this operation, three kinds of cribs are emplored; called wedging, spiking, and main cribs. Besides the stout plank for making the tub, a quantity of well-seasoned and clean reeded deal is required for forming the joints; called sheeting deal by the workmen. This sheeting deal is alwars applied in pieces laid endwise, with the end of the fibres towards the area of the pit. Since much of the security from water depends on the 
tightness of the tub at its jointing with the rock, several plans have been contrived to ellect this object; the most approved being represented in fig. 832 . To malie room 832 for the lower wedging crib, the recess is excavated a few inches wider, as at $c$; $7 i_{3} h$ and from $b$ to $c$, sheeting deals are laid all around the circle, or a thin stratum of oakum is introduced. On this the wedging crib $d$ is applied, and neatly joint$\mathbb{N}$. ed in the radius-line of the pit, each segment being drawn exactly to the circle; 7. and at each of its segments sheeting deal is inserted. This wedging crib must be 10 inches in the bed, and 6 inches deep. The vacuity $e$, at the back of the

$h$ crib, about 2 and a half inches wide, is filled with pieces of dry clean reeded deal, inserted endwise; which is regularly wedged with one set of wedges all

9 round, and then with a second and a third set of wedges, in the same regular style, to keep the crib in a truly circular posture. By this process, well executed, no water can pass downwards by the back of the crib. The next operation is to fix spiking cribs $f$, to the rock, about 10 or 12 feet from the lower crib, according to the length of the planks to be used for the tubs. They must be set fair to the sweep of the shaft, as on them its true circular figure depends. The tubbing deals $k$, must now be fixed. They are 3 inches thick, 6 broad, and planed on all sides, with the joints accurately worked to the proper bevel for the circle of the pit. The main cribs $g, g$, are then to be placed as counterforts, for the support and strength of the tubbing. The upper ends of the first set of tub-planks being cut square and level all round, the second spiking crib $l$, is fixed, and another set of tubbing deals put round like the former, haring sheeting deal inserted betwixt the ends of the two sets at $f$. When this is wedged, the cribs $h, h$, are placed.

Oak cribbing is made with pieces of the best oak, from 3 to 4 feet long, 10 inches in the bed, and 7 or 8 inches deep.

The third mode of tubbing, by means of iron cylinders cast in segments, is likely henceforth to supersede the wooden tubbing, from the great reduction in the price of iron, and its superior strength and durability. Each segment is adjusted piece to piece in the circular recess of the pit cut out for their reception. The flange for the wedging ioint is best turned inwards. In late improvements of this plan, executed by $\mathrm{Mr}$. Buddle, where the pressure amounted to several hundred feet, the segments were 6 feet long, 2 feet broad, and an inch thick, counterforted with ribs or raised work on the back; the lip of the flange was strong, and supported by brackets. These segments of the iron cylinder are set true to the radius of the pit; and every horizontal and perpendicular joint is made tight with a layer of sheeting deal. A wedging crib is fixed at the bottom, and the segments are built up regularly with joints like ashlerwork. This kind of tubbing can be carried to any height, till the water finds an outlet at the surface, or till strata containing water can be tubbed off, as by the modes of tubbing already described. A shaft finished in this manner presents a smooth lining-wall of iron, the flanges being turned towards the outside of the cylinders. In this iron tubbing, no screw bolts are needed for joining the segments together; as they are packed hard within the pit, like the staves of a cask. There is a shaft in the Newcastle district, where 70 fathoms have been executed in this way, under the direction of $\mathrm{Mr}$. Buddle.

When a porous thin bed or parting betwixt two impervious strata gives out much water, or when the fissures of the strata, called cutters, are very leaky, the water can be 833 completely stopped up by the improved process of wedging. The fissure is min' cut open wi..: chisels, to a width of two, and a depth of seven inches, as represented in fig. 833. The lips being rounded off about an inch and a half, pieces of clean deal are then driven in, whose face projects no further than the contour of the lips; when the whole is firmly wedged, till the water is entirely stopped. By sloping back the edges of the fissures, and wedging back from the face of the stone, it is not liable to burst or crack off in the operation, as took place in the old way, of driving in the wedge directly.

Ventilation of Engine pits.-In ordinary cases, while the sinking of the shaft is going on, the brattice walls produce a circulation, in consequence of the air being slightly

lighter in one compartment than in another. If this does not occur, the

834 circulation of air must be produced by artificial means. The most aprroved contrivance is, to cover the engine compartment of the shaft with deals, leaving apertures for the pump-spears and tackling to pass through, J with hatch-doors for the men, and to carry a brick flue at leat 3 feet square, in a horizontal direction, from the mouth of that compartment to an adjoining high chimney connected with a furnace, as represented in $f i g .834 . a, a$, are double doors, for the fireman to supply fuel by ; $b$, the mouth of the horizontal flue; $c$, the furnace; $d$, the ash-pit; $e$, the furnace; $f$, the upright chimney for draught, from 50 to 100 feet high, from 8 to 10 feet square at bottom, and tapering upwards to 3 or 4 feet 
square inside. Such a furnace and chimney are also needed for ventilating the coal. mine through all its underoround workings. When a great quantity of gas issues from one place in a pit, it is proper to carry it up in a square wooden pipe, which terminating at some distance above the surface in a helmet-shaped funnel, fitted to turn like a vane, may cause considerable ventilation of itself; or the top of such a pipe may be connected with a small fireplace, which will cause a rapid current up through it, from the pit. The stones and rubbish produced in sinking are drawn up with horse-gins, when the pit is not deep; but in all shafts of considerable depth, a steam encine is used, and the workmen have now more confidence in them, as to personal safety, than in machines impelled by horses.

The great collieries of Newcastle are frequently worked by means of one shaft diridod into compartments, which serves as an engine-pit, and coal-pits, and by these the whole ventilation is carried on to an extent and through ramifications altogether astonishing. This sistem has been adopted on account of the vast expense of a large shaft, often amounting to $60,000 \mathrm{l}$. or $80,000 \mathrm{l}$, including the machinery. The British collieries, however, are in general worked by means of an engine-pit, and a series of other pits, sunk at proper distances for the wants of the colliery.

\section{WORKING OF COAL.}

A stratum, bed, or seam of coal, is not a solid mass, of uniform texture, nor always of homogeneous quality in burning. It is often dirided and intersected, with its concomitant strata, by what are named partings, backs, cutters, reeds, or ends. Besides the chief partings at the roof and pavement of the coal seam, there are suburdinate lines of parting in the coal mass, parallel to these, of variable dimensions. These divisions are delineated in fig. 835, where $\mathrm{A}, \mathrm{B}, \mathrm{C}, \mathrm{D}, \mathrm{E}$ F G D, represent a portion of a bed of coal,

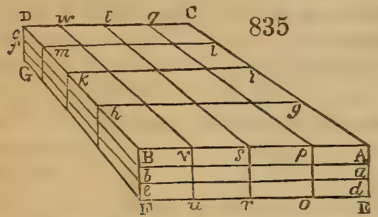
the parallelogram A B D c the parting at the roof, and $\mathbf{E} \mathrm{F}$ G the parting at the parement; $a b, b c, d e$, and $e f$, are the subordinate or intermediate partings; $g h, i k, l m$, the backs; $o p, p q, r s, s t, u v$, and $v w$, the cutters. It is thus manifest that a bed of coal, according to the number of these natural divisions, is subdivided into solid figures of various dimensions, and of a cubical or rhomboidal shape.

When the engine-pit is sunk, and the lodgment formed, a mine is then run in the coal to the rise of the field, or a cropping from the engine-pit to the second pit. This mine may be 6 or 8 feet wide, and carried either in a line directly to the pit bottom, or at right angles to the backs or web of the coal, until it is on a line with the pit, where a mine is set off, upon one side, to the pit bottom. This mine or gallery is carried as nearly parallel to the backs as possible, till the pit is gained. Fig. 836 represents this 836 mining operation. $A$ is the engine-pit. $B$, the second or by-pit. A c, the gallery driven at right angles to the backs. B The next step is to drive the drip-head or main-levels from the engine-pit bottom, or from the dip-hand of the backset immediately contiguous to the engine-pit bottom. In this $\wedge$ business, the best colliers are always employed, as the object is to drive the gallery in a truly level direction, independently of all sinkings or risings of the parement. For coal seams of ordinary thickness, this gallery is usually not more than 6 feet wide; observing to have on the dip side of the gallery a small quantity of water, like that of a gutter, so that it will always be about 4 or 6 inches deep at the forehead upon the dip-wall. When the level is driven correctly, with the proper depth of water, it is said to have dead water at the forehead. In this operation, therefore, the minor pays no regard to the backs or cutters of the coal; but is guided in his line of direction entirely by the water-level, which he must attend to solely, without regard to slips or dislocations of the strata throwing the coal up or down. In the last figure, the coal-field is a portion of a basin; so that if the shape be uniform and unbroken, and if any point be assumed a dipping from the crop, as $\mathrm{D}$, the level lines from that point will be parallel to the line of crop, as D E, D F, and the levels from any point whatever a-dipping, will be also parallel to these; and hence, were the coal-field an entire elliptical basin, the dip-head levels carried from any point would be elliptical, and parallel to the crop. If, as is more commonly the case, the coal-field be merely a portion of a basin,

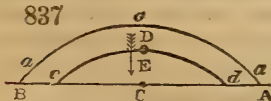
formed by a slip of the strata, as represented in fig. 837, where $a, a, a$, is the crop, and A B, a slip of great magnitude, forming another coal-field on the side $c$, then the crop not A only meets the alluvial cover, but is cut off by the slip at $A$ and at $\mathrm{B}$. Should any point, therefore, be assigned for an engine-pit, the levels from it will proceed in a line parallel to the crop, as $\mathrm{D} d, \mathrm{D} c$, and the level on both sides of 
the engine-pit will be also cut off by the slip A B. In this figure, the part included between the two curve lines, is the breadth or breast of coal-field won by the engine-pit D; what is not included, is termed the under-dip coal, and can be worked only by one or inore new winnings towards the dip, according to circumstances.

In British practice, there are four different systems of working coal-mines :-

1. Working with pillars and rooms, styled post and stall, where the pillars left bear such proportion to the coal excarated, as is just adequate to the support of the incumbent strata.

2. Working with post and stall, where the pillars are left of an extra size, and stronger than may be requisite for bearing the superior strata, with the intention of removing a cunsiderable portion of each massive pillar, whenever the regular working of post and stall has been finished in the colliery.

3. Working with post and stall, or with comparatively narrow rooms or boards, whereby an uncommonly large proportion of coal is left, with the view of working back towards the pits, whenerer the colliery is worked in this manner to the extent of the coal-field, and then taling away erery pillar completely, if possible, and allowing the whole superincumbent strata to crush down, and follow the miners in their retreat.

4. Working the long way, being the Shropshire method; which leaves no pillars, but takes out all the coal progressively as the workings advance. On this plan, the incumbent strata crush down, creeping very close to the heads of the miners.

The post and stall system is practised with coals of every thickness. The Shropshire method is adopted generally with thin coals; for when the thickness exceeds 6 or 7 feet, this mode has been found impracticable.

The following considerations must be had in view in establishing a coal-mine :-

1. The lowest coal of the winning should be worked in such a manner as not to injure the working or the ralue of the upper coals of the field; but if this cannot be done, the upper coals should be worked in the first place.

2. The coals must be examined as to texture, hardness, softness, the number and openness of the backs and cutters.

3. The nature of the pavement of the coal seam, particularly as to hardness and softness; and if soft, to what depth it may be so.

4. The nature of the roof of the coal-seam, whether compact, firm, and strong; or weak and liable to fall; as also the nature of the superincumbent strata.

5. The nature of the alluvial cover of the ground, as to water, quicksands, \&c.

6. The situation of rirers, lakes, or marshes, particularly if any be near the outcrop of the coal strata.

\%. The situation of towns, villages, and mansion-houses, upon a coal-field, as to the chance of their being injured by any particular mode of mining the coal.

Mr. Bald gires the following general rules for determining the best mode of working coal :-

"1. If the coal, pavement, and roof are of ordinary hardness, the pillars and rooms may be proportioned to each other, corresponding to the depth of the superincumbent strata, providing all the coal proposed to be wrought is taken away by the first working, as in the first systew; but if the pillars are te be winged afterwards, they must be left of an extra strength, as in the second system.

" 2 . If the parement is soft, and the coal and roof strong, pillars of an extra size must be left, t; nrevent the pillars sinking into the pavement, and producing a creep.

"3. If the coal is very soft, or has numerous open backs and cutters, the pillars must be left of an extra size, otherwise the pressure of the superincumbent strata will make the pillars fly or break off at the backs and cutters, the result of which would be a total destruction of the pillars, termed a crush or sit, in which the roof sinks to the pavement, and closes up the work.

" 4 . If the roof is very bad, and of a soft texture, pillars of an extra size are required, and the rooms or boards comparatively very narrow.

"In short, lieeping in view all the circumstances, it may be stated generally, that when the coal, pavement, and roof are good, any of the systems before mentioned may be pursued in the working; but if they are soft, the plan is to work with rooms of a moderate width, and with pillars of great extra strength, by which the greater part of the coal may be got out at the last of the work, when the miners retreat to the pit bottom, and there finish the workings of a pit."

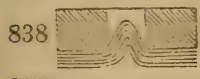

839

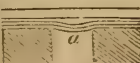

Fig. 838 represents the effects of pillars sinking into the pavement, and producing a creep; and fig. 839 exhibits large pillars and a room, with the roof stratum bending down before it falls at $a$. Thus the roads will be shut up, the air-courses destroyed, and the whole economy of the mining operations deranged.

The proportion of coal worked out, to that left in the pillars, when all the coal intended to be removed is taken out at the first working, varies from 
four fifths to two thirds; but as the loss of even one third of the whole area of coal is far too much, the better mode of working suggested in the third system ought to be adopted.

The proportion of a winning to be worked may be thus calculated. Let fig. 840 be a 8t0 small portion of the pillars, rooms, and thirlings formed in a coal-field; $\leqslant a c$ c $a, a$, are two rooms; $b$, the pillars; $c$, the thirlings (or area worked out),

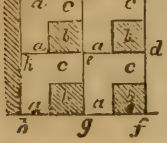
Suppose the rooms to be 12 feet wide, the thirlings to be the same, and the pillars 12 feet on each side; adding the face of the pillar to the width of the room, the sum is 24 ; and also the end of the pillar to the width of the thirling, the sum is likewise 24 : then $24 \times 24=576$; and the area of the pillar is $12 \times 12=144$; and as 576 divided by 144 gives 4 for a quotient, the result is, that one fourth of the coal is left in pillars, and three fourths extracted. Let $d, e, f, g$, be one winning, and $g, e, k, h$, another. By inspeeting the figure, we perceive the workings of a coal-field are resolved into quadrangular areas, having a pillar situated in one of the angles.

In forming the pillars and carrying forwards the boards with regularity, especially where the backs and cutters are very distinct and numerous, it is of importance to work the rooms at right angles to the backs, and the thirlings in the direction of the cutters, however oblique these may be to the backs, as the rooms are by this means condueted with the greatest regularity with regard to each other, kept equidistant, and the pillars 841 are strongest under a given area. At the same time, however, it seldom

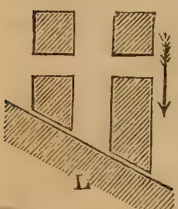
happens that a back or cutter occurs exactly at the place where a pillar is formed; but this is of no consequence, as the shearing or cutting made by the miner ought to be in a line parallel to the backs and cutters. It frequently happens that the dip-head level intersects the eutters in its progress at a very oblique angle. In this ease, when rooms and pillars are set off, the face of the pillar and width of the room must be measured off an extra breadth in proportion to the obliquity, as in fig. 841. By neglect of this rule, much confusion and irregular work are often produced. It is, moreover, proper to make the first set of pillars next the dip-head level much stronger, even where treere is no obniquity, in order to protect that level from being injured by any accidental crush of the strata.

We shall now explain the different systems of working : one of the simplest of which is shown in fig. 842; where A represents the engine-pit, B the by-pit, c D the dip-head 842

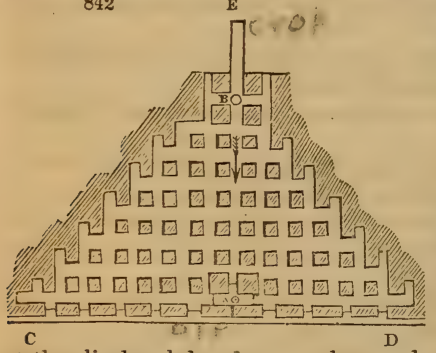
levels, always carried in advance of the rooms, and $\mathrm{e}$, the rise or crop gallery, also carried in advance. These galleries not only open out the work for the miners in the coal-bed, but, being in advance, afford sufficient time for any reguisite operation, should the mines be obstructed by dikes or hitches. In the example before us, the rooms or boards are worked from the dip to the crop; the leading rooms, or those most in advance, are on each side of the crop allery $E$; all the other rooms follow in succession $n_{2}$ as shown in the figure; consequently, as the rooms advance to the crop, additional rooms are begun at the dip-head level, towards c. and D. Should the coal work better in a level-course direction, then the level rooms are next the dip-head level, and the other rooms follow in succession. Hence the rooms are carried a cropping in the one case, till the coal is cropped out, or is no longer workable; and in the other, they are extended as far as the extremity of the dip-head level, which is finally cut off, either by a dike or slip, or by the boundary of the coal-field.

When the winnings are so very deep as from 100 to 200 fathoms, the first workings are carried forward with rooms, pillars, and thirlings, but under a different arrangement, on account of the great depth of the superincumbent strata, the enormous expense incident to sinking a pit, and the order and severity of discipline indispensable to the due ventilation of the mines, the preservation of the workmen, and the prosperity of the whole establishment. To the celebrated Mr. Buddle the British nation is under the greatest obligations for devising a new system of working coal-mines, whereby nearly one third of the coals has been rescued from waste and permanent destruction. This system is named panel work; because, instead of carrying on the coal-field winning in one extended area of rooms and pillars, it is divided into quadrangular panels, each panel containing an area of from 8 to 12 acres; and round each panel is left at first a solid wall of coal from 40 to 50 yards thick. Through the panel walls roads and air-courses are driven, in order to work the coal contained within these walls. Thus all the panels are connected together with the shaft, as to roads and ventilation. Fach district or 
panel has a prorticular name; so that any circumstance relative to the details of the colliery, castial:ius as to falls and crushes, ventilation, and the safety of the workmen, can be referred 10 : $s$ specific place.

Fig. 81:3 represents a part of a colliery laid out in four panels, according to the umproved method. To renler it as distinct as possible, the line of the boards is at right

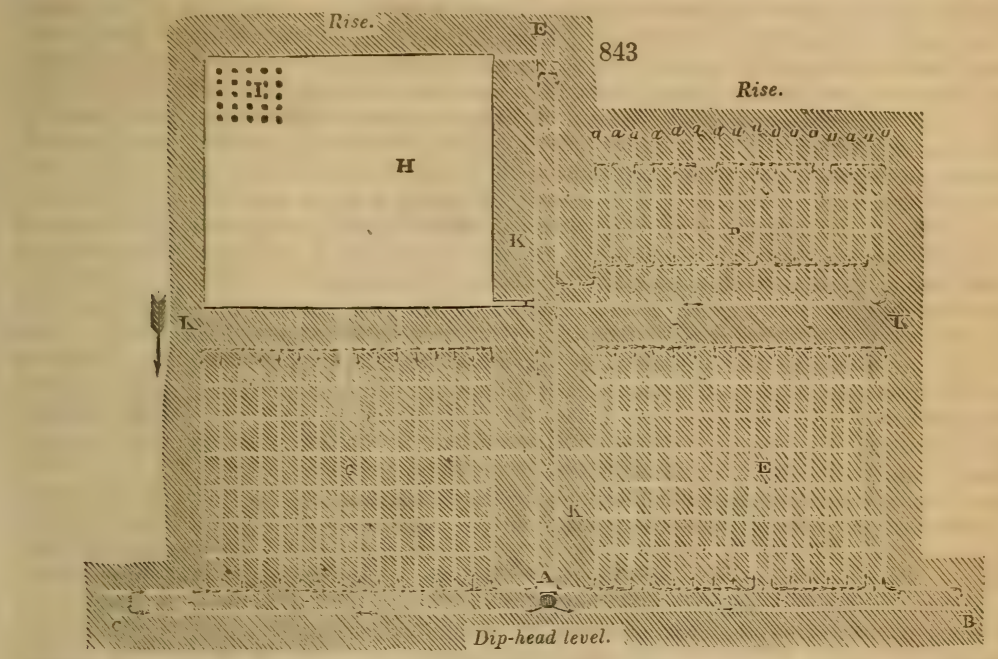

angles with the dip-head level, or level course of the coal. A is the engine-shaft, divided into three compartments, an engine-pit and two coal-pits, like fig. 825. One of the coal-pits is the down-cast, by which the atmospheric air is drawn down to ventilate the worlis : the other coal-pit is the up-cast shaft, at whose bottom the furnace for rarefying the air is placed. B $\mathbf{c}$, is the dip-head level ; A $\mathbf{E}$, the rise or crop gallery; $\mathbf{K}$, $\mathbf{K}$, the panel walls; $F$, G, are two panels completed as to the first work; $D$, is a panel, with the rooms $a, a, a$, in regular progress to the rise; $\mathrm{H}$, is a panel fully worked out, whence nearly all the coal has been extracted; the loss amounting in general to no more than a tenth, instead of a third, or even a half, by the old method. By this plan of Mr. Buddle's, also, the pillars of a panel may be worked out at any time most sulatie for the ecorrom of the mining operation; whereas formerly, though the size of the pillars and gemerai arrangement of the mine were made, with the riew of taking out ultimately a great proportion of the pillars, yet it frequently happened that, before the worlings were pushea to the proposed extent, some part of the mine gave way, and produced a crush; but the most common misfortune was the pillars sinking into the parement, and deranging the whole economy of the field. Indeed, the crush or creep often orerran the whole of the pillars, and was resisted only by the entire body of coal at the wall faces; so that the rentilation was entirely destrosed, the roads leading from the wall faces to the pit-buttom shut up and rendered useless, and the recovery of the colliery by means of new air-courses, new roarls, and by opening up the wall faces or rooms, was attended with jurodigious expense and danger. Even when the pillars stood well, the old method was attended with other rery great inconveniences. If water broke out in any particular spot of the colliery, it was quite impossible to arrest its progress to the engine-pit; and if the rentilation was thereby obstructed, no idea could be formed where the cause might be found, there being instances of no less than 30 miles of air-courses in one colliery. And if from obstructed ventilation an explosion of the fire-damp occurred while many workmen were occupied a) ong the extended wall faces, it was not possible to determine where the disaster had tacen place; nor could the viewers and managers know where to bring relief to the forlorn and mutilated survivers.

In Mr. Buddle's system all these evils are guarded against, as far as human science and foresight can go. He makes the pillars very large, and the rooms or hoards narrow ; the pillars being in general 12 yards broad, and 24 yards long; the boards 4 yards wide, anit the walls or thirlings cut through the pillars from one board to another, only 5 feet wirle, for the purpose of ventilation. In the figure, the rooms are represented as proceeding from the dip to the crop, and the panel walls act as barriers thrown round the area of the panel, to prevent the weight of the superincumbent strata from overrunning the adjoining panels. Again, when the pillars of a panel are to be writied, one range of pillars, as at $\mathrm{I}$ (in $\mathrm{H}$ ), is first attacked; and as the workmen cut away the furthest 
pillars, columns of prop-wood are erected betwixt the pavement and the roof, within a few feet of each other (as shown by the dots), till an area of above 100 square yards is cleared of pillars, presenting a body of strata perhaps 130 fathoms thick, suspended clear and without support, except at the line of the surrounding pillars. This operation is termed working the goaff. The only use of the prop-wood is to prevent the seam, which forms the ceiling over the workmen's heads, from falling down and lilling them by its splintery fragments. Experience has proved, that before proceeding to take away another set of pillars, it is necessary to allow the last-made goaff to fall. The workmen then becin to draw out the props, which is a most hazardous employment. They begin at the more remote props, and knock them down one after another, retreating quickly under the protection of the remaining props. Meanwhile the roof-stratum berins to break by the sides of the pillars, and falls down in immense pieces; while the workmen still perserere, boldly drawing and retreating till every prop is remored. Nay, should any props be so firmly fixed by the top pressure, that they will not give way to the blows of heary mauls, they are cut through with axes; the workmen making a point of honor to leave not a single prop in the goaff. The miners next proceed to cut away the pillars nearest to the sides of the goaff, setting prop-wood, then drawing it, and retiring as before, until every panel is remored, excepting small portions of pillars which require to be left under dangerous stones to protect the retreat of the workmen. While this operation is going forward, and the goaff extending, the superincumbent strata being exposed without support over a large area, break progressively higher up; and when strong beds of sandstone are thus giving way, the noise of the rending rocks is very peculiar and terrific; at one time loud and sharp, at another hollow and deep.

As the piliars of the panels are taken away, the panel walls are also worked progressively backwards to the pit bottom; so that only a very small proportion of coal is eren. tually lost. This method is undoubtedly the best for working such coals as those of Newcastle, considering their great depth beneath the surface, their comparative softness, and the profusion of inflammable air. It is evident that the larger the pillars and panel walls are, in the first working, the greater will be the security of the miners, and the greater the certainty of taking out, in the second stage, the largest proportion of coal. This system may be applied to many of the British collieries; and it will produce a vast quantity of coals beyond the post and stall methods, so generally persisted.in.

In thus tearing to pieces the massive rocks over his head, the miner displays a determined and cool intrepidity; but his ingenuity is no less to be admired in contriving modes of carrying currents of pure atmospheric air through every turning of his gloomy labyrinth, so as to sweep away the explosive spirit of the mine.

The fourth system of working coal, is called the long way, the long-wall, and the Shropshire method. The plan must at first have been extremely hazardous; though now it is so improved as to be reckoned as safe, if not safer, to the workmen, than the other methods, with rooms and pillars.

The object of the Shropshire system, is to begin at the pit-bottom pillars, and to cut away at once every inch of coal progressively forward, and to allow the whole superincumbent strata to crush down behind and over the heads of the workmen. This plan is pursued chiefly with coals that are thin, and is very seldom adopted when the seam is 7 feet thick; from 4 to 5 feet being reckoned the most favorable thickness for proceeding with comfort, amidst ordinary circumstances, as to roof, pavement, \&c. When a pit is opened on a coal to be treated by this method, the position of the coals above the lowest seam sunk to, must first be considered; if the coal beds be contiguous, it will be proper to work the upper one first, and the rest in succession downwards; but if they are 8 fathoms or more apart, with strata of strong texture betwixt them, the

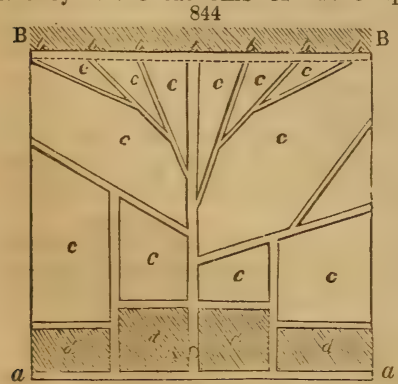
working of the lower coals in the first place will do no injury to that of the upper coals, except breaking them, perhaps, a little. In many instances, indeed, by this operation on a lower coal, upper coals are rendered more easily worked.

When the operation is commenced by working on the Shropshire plan, the dip-head levels are driven in the usual manner, and very large bottom pillars are formed, as represented in fig. 844. Along the rise side of the dip-head level, chains of wall, or long pillars, are also made, from 8 to 10 yards in breadth, and only mined through occasionally, for the sake of ventilation, or of forming new roads. In other cases no $a=a$ pillars are left upon the rise side of the level; but, instead of them, buildings of stone are reared, 4 feet broad at the base, and 9 or 10 feet from the dip side of the level. Though the roads are made 9 feet wide at first, they are reduced to half that width after the full pressure of the strata is upon them. When 
ever these points are secured, the operation of cutting away the whole boly of the coal becrins. The place where the coal is removed, is named the gobb waste; and gobjill, or gobb-stull, is stones or rubbish taken away from the coal, pavement, or roof, to fill up that excavation as much as possible, in order to prevent the crush of superincumbent strata from causing heavy falls, or following the workmen too fast in their desceut. Coals mined in this manner work most easily according to the way in which the widest backs and cutters are; and therefore, in the Shropshire mode, the walls stand sumetimes in one direction, and sometimes in another; the mine always turning out the best coals when the open backs and cutters face the workmen. As roads must be maintained through the crushed strata, the miners in the first place cut away about 15 feet of coal round the pit-bottom pillars, and along the upper sides of the dip-head chain walls; and then, at the distance of 9 or 10 feet, carry regular buildings of stone 3 feet broad, with props set flush with the faces of these, if necessary. As the miners advance, they erect small pillars of roof or pavement stone in regular lines with the wall face, and sometimes with props intermediate.

There are two principal modifications of the Shropshire plan. The first, or the original system, was to open out the wall round the pit-bottom; and, as the wall face extended, to set off main roads and branches, very like the branches of a tree. These roads were so distributed, that between the ends of any two branches there should be a distance of 30 or 40 yards, as might be most convenient. (See fig. 844.) Each space of coal betwixt the roads is called a wall; and one half of the coals produced from each wall is carried to the one road, and the other half to the other road. This is a great convenience when the roof is bad; and hence a distance of only 20 yards betwixt the roads is in many instances preferred. In fig. $844 \mathrm{~A}$ represents the shaft; B B, the wall-face; $a$, the dip-head level; $\vec{b}$, the roads, from 20 to 40 yards asunder; $c$, the gobb or waste, with buildings along the sides of the roads; and $d$, the pillars.

The other Shropshire system is represented in fig. 845, where A shows the pit, with the bottom pillars; $b$, the dip-head levels; $c$, the off-break from the level, where no

\section{5}

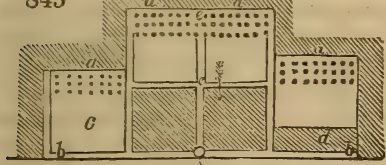
pillars are left; $d$, the off-break, where pillars remain to secure the level. All roads are protected in the sides by stone buildings, if they can be had, laid off 9 feet wide. After the crush settles, the roads generally remain permanently good, and can, in many cases, be travelled through as easily 50 years after they have been made, as at the first. Should stones not be forthcoming, coals must be substituted, which are built about 20 inches in the base. In this method, the roads are likewise from 20 to 40 yards apart; but instead of ramifying, they are arranged parallel to each other. The miners secure the waste by gobbing; and three rows of props are carried forwards next the wall faces $a$, with pillaus of stone or of coal reared betwixt them. This mode has a more regular appearance than the other; though it is not so generally practised.

In the post and stall system, each man has his own room, and performs all the labor of it; but in that of Shropshire, there is a division of labor among the workmen, who are generally divided into three companies. The first set curves or pools the coal along the whole line of walls, laying in or pooling at least $3 \mathrm{feet}$, and frequently 45 inches, or 5 quarters, as it is called. These men are named holers. As the crush is constantly following them, and impending over their heads, causing frequent falls of coal, they plant props of wood for their protection at regular distances $m$ an oblique direction between the pavement and wall face. Indeed, as a further precaution, staples of coal, about 10 inches square, are left at every 6 or 8 yards, till the line of holing or curving is completed. The walls are then marked off into spaces of from 6 to 8 yards in length ; and at each space a shearing or vertical cut is made, as deep as the holing; and when this is done, the holer's work is finished. The set who succeed the holers, are called getters. These commence their operations at the centre of the wall divisions, and drive out the gibbs and staples. They next set wedyes along the roof, and bring down progressively each division of coal; or, if the roof be hard-bound, the coal is blown down with gunpowder. When the roof has a good parting, the coals frequently fall down the moment the gibbs are struck; which makes the work very easy. The getters are relieved in their turn by the third set, named butty-men, who break down the coals into pieces of a proper size for sending up the shaft, and take charge of turning out the coal from the wall face to the ends of the roads. This being done, they build up the stone pillars, fill up the gobb, set the trees, clear the wall faces of all obstructions, set the gibbs, and make every thing clear and open for the holers to resume their work. If the roads are to be heightened by taking down the roof, or removing the pavement, these butty-men do this work also, building forwards the sides of the roads, and securing them with the requisite props. When a coal has a following or roof stone, which regularly separates with the coal, this facilitates the labor, and saves much of the coal; 
and should a soft bed of fire-clay occur a foot or two beneath the coal-seam, the holing is made in it, instead of into the coal, and the stone betwixt the holing and the coal benched down, which serves for pillars and gobbing. In this way all the vendible coa. becomes available.

Another form of the Shropshire system is, for each miner to have from 6 to 12 feet of coal before him, with a leading-hand man; as: for the several workmen to follow in succession, like the steps of a stair. When the coal has open backs and cutters, this work goes on very regularly, as represented in fig. 846 , where the leading miner is at $a$

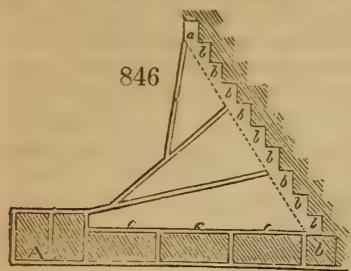
next to the outcrop, and $b \boldsymbol{b}$, \&c. are the wall faces of each workman; A being the shaft, and $\mathrm{B}$ the dip-head level. In this case the roads are carried either progressively through the gobb, or the gobb is entirely shut up; and the whole of the coals are brought down the wall-faces, either to the dip-head level or the road $c, c$. This method may be varied by making the walls broad enough to hold two, three, or four men when each set of miners performs the whole work of holing, getting, breaking down, and carrying off the coals.

It is estimated that from one eighth to one twelfth part only of the coals remains under ground by the Shropshire plan; nay, in favorable circumstances, almost every inch of coal may be taken out, as its principle is to leare no solid pillars nor anf coal below, except what may be indispensable for securing the gobb. Indeed, this system might be applied to coal-seams of almost any ordinary thicliness, providing stuff to fill up the gobb could be conveniently procured.

In Great Britain, seams of coal are mined when they are only 18 inches thick; but if thinner, the working of fire-clay or ironstone immediately adjoining must be included. A few instances may be adduced, indeed, where caking coals of a fine quality for blacksmiths have been worked, though only in 12 -inch seams.

Eighteen-inch seams are best workel by young lads and boys. The coal itself may be mined without lifting the pavemeni, or taking down the roof in the rooms; but roads must be cut either in the pavement or the roof, for removing the coals to the pitbottom. All coals less than 2 feet 3 inches thick, are worked with the view of taling out all the coal, either on the Shropshire system, or with pillar-walls and rooms; with this peculiarity, that, on account of the thinness of the seam, the rooms are worked as wide as the roof will bear up; or if a following of the roof-stone, or fall of it, can be brought on, it proves advantageous, by not only giving head-room, but by filling up the waste, and rendering the roads easily kept for the working of the pillars. Where no folIowing takes place, small temporary pillars, about 8 feet square, are left along the chainwall side. The walls may vary in thickness from 4 to 16 yards, according to circumstances, and they are holed through only for ventilation.

Coals from 5 to 8 feet thick are the best suited in every point of view for the effective work of the miner, and for the general economy of underground operations. When they exceed that thichness, they require very excellent roofs and pavements, to render the worling either safe or comfortable; or to enable those who superintend the field to get out a fair proportion of coal from a given area. In such powerful beds the Shropshire method is impracticable, from want of gobbin; and long props, unless of prodigious girth, would present an inadequate resistance to the pressure of the massive ceiling.

When coals do not exceed 20 feet in thickness, and have good roofs, they are sometimes worked as one bed of coal; but if the coal be tender or free, it is worked as two beds. One half of such thick coal, however, is in general lost in pillars; and it is very seldor. that less than one third can be left. When the coal is free and ready to crumble by the incumbent pressure, as well as by the action of the air, the upper portion of the coal is first worked, then a scaffolding of coal is left, 2 or 3 feet thick, according to the compactness of the coal; and the lower part of the coal is now worked, as shown in $84 \tau$

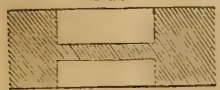
fig. 817. As soon as the workings are completed to the proposed extent, the coal scaffoldings are worked away, and as much of the pillars as can be removed with safety. As propwood is of no use in coal-seams of such a height, and as falls from the roof would prove fresuently fatal to the miners, it is customsry with tender roofs to leave a ceiling of coal from 2 to 3 feet thick. This malies ar excellent roof; and should it break, gives warning beforehand, by a peculiar crackling noise, very different from that of roof-stones crushing down.

One of the thickest coals in Great Britain, worked as one bed from roof to pavement, is the very remarkable seam near the town of Dudley, known by the name of the tenyard coal, about 7 miles long, and 4 broad. No similar coal has been found in the island; and the mode of working it is quite peculiar, being a species of panel work 
totally diflerent from the modern Newcastle system. A compartment, or panel, formed in working the coal, is called a side of work and as the whole operation is exhibited in one of these compartments, it will be proper to describe the mode of taking the coal from one of them, before describing the whole extent of the workings of a mine.

Let fig. 848 represent a sile of work; $\lambda$, the ribs or walls of coal left standing round, constituting the side of work; $a$, the pillars, 8 yards square; $c$, the stalls, 11 yards wide;

848

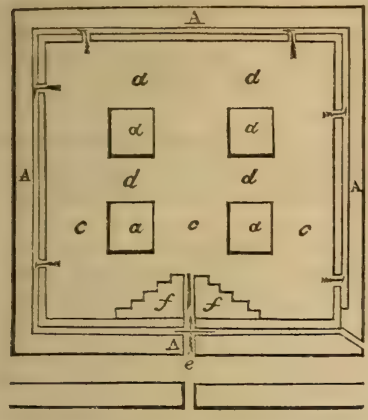
$d$, the cross-openings, or through puts, also 11 yards wide; $e$, the bolt-hole, cut through the rib from the main road, by which bolt-hole the side of work is opened up, and all the coals removed. Two, three, or even four bolt-holes open into a side of work, according to its extent; they are about 8 feet wide, and 9 feet high. The working is in a great measure regulated by the natural fissures and joints of the coalseam; and though it is 30 feet thick, the lower band, of 2 feet 3 inches, is worked first ; the miners choosing to confine themselves within this narrow opening, in order to gain the greater advantage afterwards, in working the superjacent coal. Whenever the bolt hole is cut through, the work is opened up by driving a gallery forward, 4 feet wide, as shown by the dotted lines. At the sides of this gallery next the bolt-hole, each miner breaks off in succession a breast of coal, two yards broad, as at $f, f$, by means of which the sides of the rib-walls a are formed, and the area of the pillars. In this way each collier follows another, as in one of the systems of the Shropshire plan. When the side of work is laid open along the rib-walls, and the faces and sides of the pillars have been formed, the upper coals are then begun to be worked, next the rib-wall. This is done by shearing up to a bed next the bolt-hole, and on each side, whereby the head coals are brought regularly down in large cubical masses, of such thickness as suits with the free partings or subordinate divisions of the coals and bands. Props of wood, or even stone pillars, are placed at convenient distances for the security of the miners.

In working the ten-yard coal, a very large proportion of it is left under ground, not merely in pillars and rib-walls, but in the state of small coal produced in breaking out the coal. Hence, from four tenths to a half of the total amount is lost for ever.

Another method of working coal of uncommon thickness is by scafioldings or stages of coals, as practised in the great coal bed at Johnstone, near Paisley, of which a section has already been giren. In one part of the field the coal is from 50 to 60 feet thick, and in another it amounts to 90 feet. The seams of stone interspersed through the

849 coal are generally inconsiderable, and amount in only two cases to 27 inches

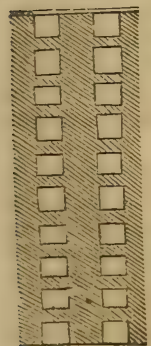
in thickness. The roof of the coal is so unsound, and the height so prodigious, that it could not possibly be worked in one seam, like that of Staffordshire. About 3 feet of the upper coal is therefore left as a roof, under which a band of coal, from 6 to 7 feet thick, is worked on the post and stall plan, with square pillars of extra strength, which are thereafter penetrated. A platform about 3 feet high is left at the sole; under which the rooms and pillars are set off and worked in another portion of the coal, from 5 to 7 feet thick, great care being had to place pillar under pillar, and partition under partition, to prevent a crush. Where the coal is thickest, no less than 10 bands of it are worked in this way, as is shown in fig. 849 . When any band of the coal is foul from sulphur or other causes, it is left for the next platform, so that a large proportion of it is lost, as in the Staffordshire mines. Much attention must here be paid to the vertical distribution of the pillars and apartments; the miner's compass must be continually consultrd, and bore-holes must be put down through the coal scaffoldings, to regulate correctly the position of the pillars under one another.

Edge coals, which are nearly perpendicular, are worked in a peculiar manner; for the collies stands upon the coal, having the roof on the one hand, and the floor on the other, 850

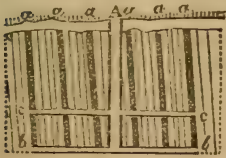

like two vertical walls. The engine-pit is sunk in the most powerful stratum. In some instances the same stratum is so vertica] as to be sunk through for the whole depth of the shaft.

Whenever the shaft has descended to the required depth, galleries are driven across the strata from its bottom, till the coals are intersected, as is shown in fig. 850 , where we see the edge-coals at $a, a ; A$, the engine-pit; $b, b$, the transverse galleries from the bottom of the suitit and $c, c$, upper transverse galleries, for the greater conveniency of working the coal. The principal edge coal works in Great Britain lie in the neigh 
borhood of Edinburgh, and the coals are carried on the backs of women from the wall. face to the bottom of the engine-pit.

The modes of carrying coals from the point where they are excavated to the pit bottom are nearly as diversified as the systems of working.

One method employs hutches, or baskets, having slips or cradle feet shod with iron, containing from 2 to 3 hundred weight of coals. These baskets are dragged along the floor by ropes or leather harness attached to the shoulders of the workmen, who are either the colliers or persons hired on purpose. This method is used in several small collieries; but it is extremely injudicious, exercising the muscular action of a man in the most unprofitable manner. Instead of men, horses are sometimes yoked to these basket-hurdles, which are then made to contain from 4 to 6 hundred weight of coals; but from the magnitude of the friction, this plan cannot be commended.

An improvement on this system, where men draw the coals, is to place the basket or corve on a small four-wheeled carriage, called a tram, or to attach wheels to the corve itself. Thus much more work is performed, provided the floor be hard; but not on a soft pavement, unless some kind of wooden railway be laid.

The transport of coals from the wall-face to the bottom of the shaft was greatly facilitated by the introduction of cast-iron railways, in place of wooden roads, first brought into practice by Mr. John Curr of Sheffield. The rails are called tram-rails, or plate-rails, consisting of a plate from 3 to 4 inches broad, with an edge at right angles to it about two inches and a half high. Each rail is from 3 to 4 feet long, and is fixed either to cross bearers of iron, called sleepers, or more usually to wooden bearers. In some collieries, the miners, after working out the coals, drag them along these railways to the pit bottom; but in others, two persons called trammers are employed to transport the coals; the one of whom, in front of the corve, draws with harness; and the other, called the patter, pushes behind. The instant each corve arrives, from the wall-face, at a central spot in the system of the railways, it is lifted from the tram by a crane placed there, and placed on a carriage called a rolley, which generally holds two corves. Whenever three or four rolleys are loaded, they are hooked together, and the rolley driver, 851 with his horse, takes them to the bottom of the engine-shaft. The rolley horses have a peculiar kind of shafts, commonly made of iron, named limbers, the purpose of which is to prevent the carriage from overrunning them. One of these shafts is represented in $f \mathrm{~g} .851$. The hole shown at $\alpha$ passes over an iron peg or stud in front of the rolley, so that the horse may be quickly attached or disengaged. By these arrangements the work is carried on with surprising regularity and despatch.

The power of the engine for drawing the coals up the shaft is made proportional to the depth of the pit and the quantity to be raised, the corves ascending at an average velocity of about 12 feet per second. So admirable is the modern arrangement of this operation, that the corves are transported from the wall-faces to the pit bottom, and moved up the shaft, as fast as the onsetters at the bottom, and the banksmen at the top, can hook the loaded and empty corves on and off the engine ropes. Thus 100 corves of coals have been raised every hour up a shaft 100 fathoms deep, constituting a lift of 27 tons per hour, or 324 tons in a day, or shif of 12 hours. Coals mined in large cubical masses cannot, however, be so rapidly raised as the smaller coal of the Newcastle district.

When coals have so great a rise from the pit bottom to the crop that horses cannot be used on the rolley ways, the corves descend along the tram-roads, by means of inclinedplane machines, which are moved either by vertical rope-barrels, or horizontal ropesheaves. These inclined planes are frequently divided into successive stages, 200 or 300 yards long, at the end of each of which is an inclined-plane machine, whereby the coals are lowered from one level to another.

The wheels of the trams and rolleys vary in diameter from 8 to 16 inches, according to the thickness of the coal. In some, the axles not only revolve on their journals, but the wheels also revolve on their axles.

Various forms of machines have been employed for raising the coals out of the pits. The stearn engine with fly-wheel and rope-barrels is, however, now preferred in all considerable establishments. When of small power, they are usually constructed with a fly wheel, and short fly-wheel shaft, on which there is a small pinion working into the teeth of a large wheel, fixed upon the rope-barrel. Thus the engine may move with great rapidity, while it imparts an equable slow motion to the corves ascending in the shaft. When the engines are of great power, however, they are directly connected with the rope-barrel; some of these being of such dimensions, that each revolution of the ropebarrel produces an elevation of 12 yards in the corve. A powerful brake is usually con. nected with the circumference of the fly-wheel or rope-barrel, whereby the brakeman, by applying his foot to the governing lever of the brake, and by shutting at the same time the steam valves with his hands, can arrest the corve, or pitch its arrival withın a 
few inches of the required height of every delivery. An endless chain, suspended from the buttom to the top of the shaft, has, in a few pits of moderate depth, been worlieil by a steam encine, for raising corres in constant succession; but the practice has not been found hitherto applicable on the greater scale.

There is a hind of water engines for raising coals, strictly admissible only in level free jits, where the ascent of the loaded corve is produced by the descent of a cassoon filled with water. When the ascent and descent are through equal spaces, the rope barrels for the eassuon and the corres are of equal diameter; but when the point from which the coals have to be lifted is deeper than the point of discharge for the water into the dry level, the cassoon must be larger, and the rope barrel smaller; so that by the time the cassoun reaches to the half-depth, for example, the corve may have mounted through double the space. The cassoon is filled with water at the pit mouth, and is emptied by a self-acting valve whenever it gets to the bottom. The loaded corre is replaced by an empty one at the pit mouth, and its weight, with that of the descending rope, pull up the empty cassoon; the motions of the whole mechanism being regulated by a powerful brake.

Various plans have been devised to prevent collision between the ascending and descending corres, which sometimes pass each other with a joint relocity of 20 or 30 feet per second. One method is by diriding the pit from top to bottom, so that each corve moves in a separate compartment. Another mode was invented by $\mathrm{Mr}$. Curr of Shetfield, in which wooden guides were attached from top to bottom of the pit; being spars of deal about 4 inches square, attached perpendicularly to the sides of the shaft, and to buntons in the middle of the pit. Betwixt these guides, friction-roller sliders are placed, attached to the gin-ropes, to which sliders the corves are suspended. In this way, the corres can be raised with great rapidity; but there is a considerable loss of time in banking the corve at the pit mouth, where shutters or sliding boards must be used. This plan is highly beneficial where the coals are in large lumps.

Both ropes and chains are used for lifling coals. The round ropes are shroud-laid; but the preferable rope is the flat band, made of four ropes placed horizontally together, the ropes being laid alternately right and left. In this way, the ropes counteract one another in the twist, hanging like a riband down the shaft; and are stitched strongly ogether by a small cord. Such rope bands are not only very pliable for their strength, which protects the heart of the rope from breaking, but as they lap upon themselves, a simple sheare serves as a rope-barrel. They possess the additional adrantge, that by so sapping, they enlarge the diameter of the axle in which they coil, and thus make a compensation mechanically against the increasing length of rope descending with its corve. Thus the counterpoise chains, used in deep pits to regulate the descent, have been superseded. See Rope-SPINNING.

When chains are preferred to ropes, as in very deep pits, the short pudding-link chains are mostly used. See CABLE.

The corres, after being landed or banked at the pit mouth, are drawn to the bin or coal-hill, either upon slips by horses, or by trammers on a tram-road. But with small coals, like the Newcastle, the pit head is raised 8 or 9 feet above the common levol of the ground, and the coal-heap slopes downwards from that height. As the bins increase, tram-roads are laid outwards upon them.

I shall nor lescribe the ventilation of coal mines. Into their furthest recesses, an adequate supply or fresh air must be carried forwards, for the purposes of respiration, and the combustion of candles; as also for clearing off the carbonic acid and carbureted hydrogen gases, so destructive to the miners, who call these noxious airs, from their most obvious qualities, choke-damp and fire-damp.

Before the steam engine was applied to the drainage of the mines, and the extraction of the coal, the excavations were of such limited extent, that when inflammable air accumulated in the foreheads, it was usual in many collieries to fire it erery morning. This was done by fixing a lighted candle to the end of a long pole, which being extended towards the roof by a person lying flat on the floor, the gas was fired, and the blast passed safely over him. If the gas was abundant, the explosive miner put on a wet jacket, to prevent the fire from scorching him. In other situations, where the fire-damp was still more copious, the candle was drawn forwards into it, by a cord passing over a catch at the end of the gallery, while the operator stood at a distance. This very rude and dangerous mode of exploding the inflammable gas is still practised, in a few mines, under the name of the firing line.

The carbonic acid or choke-damp, having a greater specific gravity than atmospheric air, in the proportion of about 3 to 2 , occupies the lower part of the worlings, and gives comparatirely little annoyance. Its jresence may moreover be always safely ascertained by the lighted candle. This cannot, however, be said of the fire-damp, which being lighter and more moveable, diffuses readily through the atmospheric air, so as to form a most dangerous explosive mixture, even at a considerable distance from 
the blowers or sources of its extrication from the coal strata. Pure subcarbureted hy. drogen has a specific gravity $=0.555$, air being 1 ; and consists of a volume of vapor of carbon, and two volumes of hydrogen, condensed by mutual affinity into one volume. The choke-damp is a mixture of the abuve, with a little carbonic acid gas, and variable proportions of atmospheric air. As the pure subcarbureted hydrogen requires twice its bulk of oxygen to consume it completely, it will take for the same effect about 10 times its bulk of atmospheric air, since this volume of air contains about two volumes of oxygen. Ten volumes of air, therefore, mixed with one volume of subcarbureted hydrogen, form the most powerfully explosive mixture. If either less or more air be intermixed, the explosive force will be impaired; till 3 volumes of air below or above that ratio, constitute non-explosive mixtures; that is, 1 of the pure fire-damp mixed with either 7 or 13 of air, or any quantity below the first, or above the second number, will afford an unexplosive mixture. With the first proportion, a candle will not burn; with the second, it burns with a very elongated blue flame. The fire-damp should therefore be still further diluted with common air, considerably beyond the above proportion of 1 to 13 , to render the working of the mine perfectly safe.

These noxious gases are disengaged from the cutters, fissures, and minute pores of the coal ; and if the quantity be considerable, relative to the orifice, a hissing noise is heard.

Though the choke-damp, or carbonic acid gas, be invisible, yet its line of division from the common air is distinctly observable on aprreaching a lighted candle to the

852

1952
lower level, where it accumulates, which becomes extinguished the instant it comes within its sphere, as if it were plunged in water. The stratum of carbonic acid sometimes lies 1 or 2 feet thick on the floor, while the superincumbent air is perfectly good. When the coal has a considerable dip and rise, the choke-damp will be found occupying the lower parts of the mine, in a wedge form, as represented in fig. 852, where $a$ shows the place of the carbonic acid gas, and $b$ that of the common air.

When a gallery is driven in advance of the other workings, and a discharge of this gas takes place, it soon fills the whole mine, if its direction be in the line of lerel, and the mine is rendered unworkable until a supply of fresh air is introduced to dislodge it. As the flame of a candle indicates correctly the existence of the choke-damp, the miners may have sufficient warning of its presence, so as to avoid the place which it occupies, till adequate means be taken to drive it away.

The fire-damp is not an inmate of every mine, and is seldom found, indeed, where the carbonic acid prevails. It occurs in the greatest quantities in the coal mines of the counties of Northumberland, Durham, Cumberland, Staffordshire, and Shropshire. It is more abundant in coals of the caking kind, with a bright steel-grained fracture, than in cubic coals of an open-burning quality. Splint coals are still less liable to disengage this gas. In some extensive coal-fields it exists copiously on one range of the line of bearing, while on the other range none of it is observed, but abundance of carbonic acid gas.

In the numerous collieries in the Lothians, south from the city of Edinburgh, the fire damp is unknown; while in the coal-fields round the city of Glasgow, and along the coasi of Ayrshire, it frequently appears.

The violent discharge of the gas from a crevice or cutter of the coal, is called a blower; and if this be ignited, it burns like an immense blowpipe, inflaming the coal at the opposite side of the gallery. The gas evidently exists in a highly compressed and elastic state; whence it seems to loosen the texture of the coals replete with it, and renders them more easily worked. The gas is often peculiarly abundant near a great dislocation or slip of the strata; so that the fissure $d^{2}$ the dislocation will sometimes emit a copious stream of gas for many years. It has also happened, that from certain coals, newly worked, and let fall from a height into the hold of a vessel, so much inflammable gas has been extricated that, after the hatches were secured, and the ship ready to proceed to sea, the gas has ignited with the flame of a candle, so as to scorch the seamen, to blow up the decks, and otherwise damage the vessel. In like manner, when the pillars in a mine are crushed by sudden pressure, a great discharge of gas ensues. This gas, being lighter than common air, always ascends to the roof or to the rise of the galleries; and, where the dip is considerable, occupies the forehead of the mine, in a wedge form, as shown in 853 fig. 853, where $a$ represents the fire-damp, and $b$ the common air. In this case, a candle will burn without danger near the point $\mathbf{c}$, close to the floor; but if it be advanced a few feet further towards the roof, an explosion will immediately ensue; since at the line where the two elastic fluids are in contact, they mix, and form an explosive body.

When this gas is largely diluted with air, the workmen do not seem to feel any inconvenience from breathing the mixture for a period of many years; but on inhaling pure carbureted hydrogen, the miner instantly drops down insensible, and, if not speedily removed into fresh air, he dies. 
The production of these noxious gases renders ventilation a primary object in the srstem of mining. The most easily managed is the carbonic acid. If an air-pipe has been carried down the engine pit for the purpose of ventilation in the sinking, other pipes are comnected with it, and laid along the parement, or are attached to an angle of the mine next the roof. These pipes are prolonged with the galleries, by which means the air at the forehead is drawn up the pipes and replaced by atmospheric air, which descends by the shaft in an equable current, regulated by the draught of the furnace at the pit mouth. This circulation is continued till the miners cut through upon the second shaft, when the air-pipes become superfuous; for it is well known that the instant such communication is made, as is represented in fig. 854, the air spontaneously descends in the engine pit $A$, and, passing along the gallery $a$, ascends in a steady current in the second

854

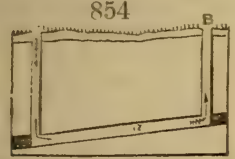

pit B. The air, in sinking through $A$, has at first the atmospheric temperature, which in winter may be at or under the freezing point of water; but its temperature increases in passing down through the relatively warmer earth, and ascends in the shaft $\mathrm{B}$, warmer than the atmosphere. When shafts are of unequal depths, as represented in the figure, the current of air flows pretty uniformly in onte direction. If the second shaft has the same depth with the first, and the bottom and inouth of both be in the same horizontal plane, the air would sometimes remain at rest, as water would do in an inverted syphon, and at other times would circulate down one pit and $\mathrm{up}$ another, not always in the same direction, but sometimes up the one, and sometir: s up the other, according to the variations of temperature at the surface, and the barometrical pressures, as modified by winds. There is in mines a proper heat, proportional to their depth, increasing about one degree of Fahrenheit's scale for every 60 feet of descent.

There is a simple mode of conducting air from the pit bottom to the forehead of the mine, by cutting a ragglin, or trumpeting, as it is termed, in the side of the gallery as rep-

855 resented in fig. 855, where A exhibits the gallery in the coal, and B the 855 A ragglin, which is from 15 to 18 inches square. The coal itself forms three sides of the air-pipe, and the fourth is composed of thin deals applied air-tight, and nailed to small props of wood fixed between the top and bottom of the lips of the ragglin. This mode is very generally adopted in running galleries of communication, and dip-head level galleries, where carbonic acid abounds, or when from the stagnation of the air the miners' lights burn dimly.

When the ragglin or air-pipes are not made spontaneously active, the air is sometimes impelled through them by means of ventilating fanners, having their tube placed at the pit bottom, while the vanes are driven with great velocity by a wheel and pinion worked with the hand. In other cases, large bellows like those of the blacksmith, furnished with a wide nozzle, are made to act in a similar way with the fanners. But these are merely temporary expedients for small mines. A very slight circulation of air can be effected by propulsion, in comparison of what may be done by exhaustion; and hence it is better to attach the air-pipe to the valve of the bellows, than to their nozzle.

Ventilation of collieries has been likewise effected on a small scale, by attaching a horizontal funnel to the top of air-pipes elevated a considerable height above the pit mouth. The funnel revolves on a pivot, and by its tail-piece places its mouth so as to receive the wind. At other times, a circulation of air is produced by placing coal-fires in iron grates, either at the bottom of an upcast pit, or suspended by a chain a few fathoms down.

Such are some of the more common methods practised in collieries of moderate depth, where carbonic acid abounds, or where there is a total stagnation of air. But in all great coal mines the aerial circulation is regulated and directed by double doors, called main or bearing doors. These are true air-valves, which intercept a current of air moving in one direction from mixing with another moving in a diferent direction. Such valves are placed on the main roads and passages of the

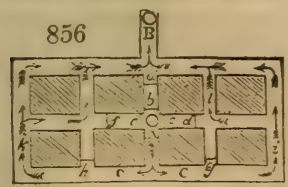
galleries, and are essential to a just ventilation. Their functions are represented in the annexed fig. 856, where $\mathrm{A}$ shows the downcast shaft, in which the aerial current is made to descend; $\mathbf{B}$ is the upcast shaft, sunk towards the rise of the coal; and c, the dip-head level. Were the mine here figured to be worked without any attention to the circulation, the air would flow down the pit $\mathbf{A}$, and proceed in a direct line up the rise mine to the shaft $B$, in which it would ascend. The consequence would therefore be, that all the galleries and boards to the dip of the pit $A$, and those lying on each side of the pits, would have no circulation of air; or, in the language of the collier, would be laid dead. To obviate this result, double doors are placed in three of the galleries arljoining the pit; viz., at $a$ and $b, c$ and $d, e$ and $f$; all of which open invarts to the shaft $A$. By this plan, as the air is not suffered to pass directly from the shaft $A$ to the shaft $B$, through 
the doors $a$ and $b$, it would have taken the next shortest direction by $e d$ and $e f$; but the doors in these galleries prevent this course, and compel it to proceed downwards to the dip-head level $\mathrm{c}$, where it will spread or divide, one portion pursuing a route tc the right, another to the left. On arriving at the boards $g$ and $h$, it would have naturally ascended by them; but this it cannot do, by reason of the building or stopping placed at $g$ and $h$. By means of such stoppings placed in the boards next the dip-head level, the air can be transported to the right hand or to the left for many miles, if necessary, providing there be a train or circle of aerial communication frum the pit $\mathrm{A}$ to the pit $\mathbf{B}$. If the boards $i$ and $k$ are open, the air will ascend in them, as traced out by the arrows; and after being diffused through the workings, will again meet in a body at $a$, and mount the gallery to the pit $\mathbf{B}$, sweeping away with it the deleterious air which it meets in its path. Without double doors on each main passage, the regular circulation of the air would be constantly liable to interruptions and derangements; thus, suppose the door $c$ to be removed, and only $d$ to remain in the left hand gallery, all the other doors being as represented, it is obvious, that whenever the door $d$ is opened, the air, finding a more direct passage in that direction, would mount by the nearest channel $l$, to the shaft $B$, and lay dead all the other parts of the work, stopping all circulation. As the passages on which the doors are placed constitute the main roads by which the miners go to and from their work, and as the corves are also constantly wheeling along all the time, were a single door, such as $d$, so often opened, the ventilation would be rendered precarious or languid. But the double doors obviate this inconvenience; for both men and horses, with the corves, in going to or from the pit bottom $A$, no sooner enter the door $d$, than it shuts behind them, and encloses them in the still air contained between the doors $d$ and $c$; $c$ having prevented the air from changing its proper course while $d$ was open. When $d$ is again shut, the door $c$ may be opened without inconvenience, to allow the men and horses to pass on to the pit bottom at $\mathrm{A}$; the door $d$ preventing any change in the aerial circulation while the door $c$ is open. In returning from the pit, the same rule is observed, of shutting one of the double doors, before the other is opened.

If this mode of disjoining and insulating air-courses from each other be once fairly conceived, the continuance of the separation through a working of any extent, may be easily understood.

When carbonic acid gas abounds, or when the fire-damp is in very small quantity, the air may be conducted from the shaft to the dip-head level, and by placing stoppings of each room next the level, it may be carried to any distance along the diphead levels; and the furthest room on each side being left open, the air is suffered to diffuse itself through the wastes, along the wall faces, and mount in the upcast pit, as is represented in fig. 842. But should the air become stagnant along the wall faces, stoppings are set up throughout the galleries, in such a way as to direct the main body of fresh air along the wall faces for the workmen, while a partial stream of air is allowed to pass through the stoppings, to prevent any accumulation of foul air in the wastes.

In very deep and extensive collieries more elaborate arrangements for ventilation are introduced. Here the circulation is made active by rarefying the air at the upcast shaft, by means of a very large furnace placed either at the bottom or top of the shaft. The former position is generally preferred. Fig. 834 exhibits a furnace placed at the top of the pit. When it surmounts a single pit, or a single division. of the pit, the compartment intended for the upcast is made air-tight at top, by placing strong buntons or beams across it, at any suitable distance from the mouth. On these buntons a close scaffolding of plank is laid, which is well plastered or moated over with adhesive plastic clay. A little way below the scaffold, a passage is previously cut, either in a sloping direction, to connect the current of air with the furnace, or it is laid horizontally, and then communicates with the furnace by a vertical opening. If any obstacle prevent the scaffold from being erected within the pit, this can be made air-tight at top, and a brick flue carried thence along the surface to the furnace.

The furnace has a size proportional to the magnitude of the ventilation, and the chimneys are either round or square, being from 50 to 100 feet high, with an inside diameter of from 5 to 9 feet at bottom, tapering upwards to a diameter of from $2 \frac{1}{2}$ feet to 5 feet. Such stalks are made 9 inches thick in the body of the building, and a little thicker at bottom, where they are lined with fire-bricks.

The plan of placing the furnace at the bottom of the pit is, however, more advantageous, because the shaft through which the air ascends to the furnace at the pit mouth, is always at the ordinary temperature; so that whenever the top furnace is neglected, the circulation of air throughout the mine becomes languid, and dangerous to the workmen; whereas, when the furnace is situated at the bottom of the shaft, its sides get heated, like those of a chimney, through its total length, so that though the heat of the furnace be accidentally allowed to decline or become extinct for a little, the circu- 
lation will still go on, the air of the upcast pit being rarefied by the heat remaining in the sides of the shaft.

To prevent the annoyance to the onsetters at the bottom, from the hot smoke, the fol. lowing plan has been adopted, as shown in the wood-cut, fig. 857, where a represents

857

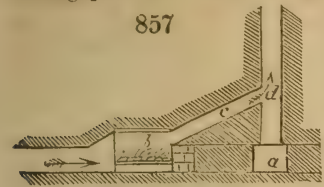
the lower part of the upcast shaft; $b$, the furnace, built of brick, arched at top, with its sides insulated from the solid mass of coal which surrounds it. Between the furnace wall and the coal beds, a current of air constantly passes towards the shaft, in order to prevent the coal catching fire. From the end of the furnace a gallery is cut in a rising direction at $c$, which communicates with the shaft at $d$, about 7 or 8 fathoms trom the bottom of the pit. Thus the furnace and furnacekeeper are completely disjoined from the shaft; and the pit bottom is not only free from all encumbrances, but remains comfortably cool. 'To ob27 viate the inconveniences from the smoke to the banksmen in 858 landing the coals at the pit mouth, the following plan has been contrived for the Newcastle collieries. Fig. 858 represents the mouth of the pit; $a$ is the upcast shaft, provided with a furnace at bottom; $b$, the downcast shaft, by which the supply of atmospheric air descends ; and $d$, the brattice carried above the pit mouth. A little way below the settle-boards, a gallery $c$ is pushed, in communication with the surface from the downcast shaft, over which a brick tube or chimney is built from 60 to 80 feet high, 7 or 8 feet diameter at bottom, and 4 or 5 feet diameter at top. On the top of this chimney a deal funnel is suspended horizontally on a pivot, like a turn-cap. The vane $f$, made also of deal, keeps the mouth of the funnel always in the same direction with the wind. The same mechanism is mounted at the upcast shaft $u$, only here the funnel is made to present its mouth in the wind's eye. It is obvious from the figure, that a high wind will rather aid than check the ventilation by this plan.

The principle of ventilation being thus established, the next object in opening up a colliery, and in driving all galleries whatever, is the double mine or double headways course; on the simple but very ingenious distribution of which, the circulation of air depends at the commencement of the excavations.

The double headways course is represented in fig. 859, where $a$ is the one heading or gallery, and $b$ the other; the former being immediately connected with

\section{9}

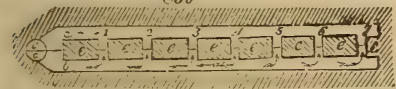
the upcast side of the pit $c$, and the latter with the downcast side of the pit $d$. The pit itself is made completely air-tight by its division of deals from top to bottom, called the brattice wall; so that no air can pass through the brattice from $d$ to $c$, and the intercourse betwixt the two currents of air is completely intercepted by a stopping betwixt the pit bottom and the end of the first pillar of coal; the pillars or walls of coal, marked $e$, are called stenting walls; and the openings betwixt them, walls or thirlings. The arrows show the direction of the air. The headings $a$ and $b$ are generally made about 9 feet wide, the stenting walls 6 or 8 yards thick, and are holed or thirled at such a distance as may be most suitable for the state of the air. The thirlings are 5 feet wide.

When the headings are set off from the pit bottom, an aperture is left in the brattice at the end of the pillar next the pit, through which the circulation betwixt the upeast and downcast pits is carried on; but whenever the workmen cut through the first thirling No. 1, the aperture in the brattice at the pit bottom is shut; in consequence of which the air is immediately drawn by the power of the upcast shaft through that thirling as represented by the dotted arrow. Thus a direct stream of fresh air is obviously brought close to the forehead where the mines are at work. The two headings $a$ and $b$ are then advanced, and as soon as the thirling No. 2 is cut through, a wall of brick and mortar, $4 \frac{1}{2}$ inches thick, is built across the thirling No. 1 . This wall is termed a stopping ; and being air-tight, it forces the whole circulation through the thirling No. 2. In this manner the air is always led forward, and caused to circulate always by the lastmade thirling next the forehead; care being had, that whenever a new thirling is made, the last thirling through which the air was circulated, be secured with an air-tight stopping. In the woodcut, the stoppings are placed in the thirlings numbered $1,2,3$, $4,5,6$, and of consequence the whole circulation passes through the thirling No. 7 , which lies nearest the foreheads of the healings $a, b$. By inspecting the figure, we observe, that on this very simple plan, a stream of air may be circulated to any required distance, and in any direction, however tortuous. Thus, for example, if while the double headways course $a, b$, is pushed forward, other double headways courses are rejuired to be carried on at the same time on both sides of the first headway, the same general principles have only to be attended to as shown in fig. 860, where 


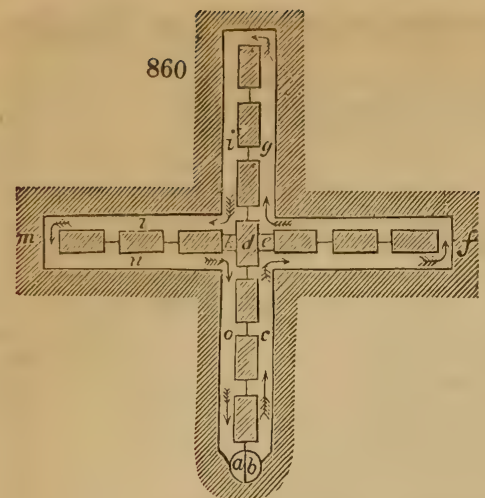

$a$ is the upcast, and $b$ the downeast shaft. The air advances along the heading $c$, but cannot proceed further in that direction than the pillar $d$, being obstructed by the double doors at $e$. It therefore advances in the direction of the arrows to the foreheads at $f$, and passing through the last thirling made there, returns to the opposite side of the double doors, ascends now the heading $g$, to the foreheads at $h$, passes through the last-made thirling at that point, and descends, in the heading $i$, till it is interrupted by the double doors at $k$. The aerial current now moves along the heading $l$, to the foreheads at $m$, returns by the last-made thirling there, along the heading $n$, and finally goes down the heading 0 , and mounts by the upcast shaft $a$, carrying with it all the noxious gases which it encountered during its circui-

tous journey. This wood-cut is a faithful representation of the system by which collieries of the greatest extent are worked and ventilated. In some of these, the air courses are from 30 to 40 miles long. Thus the air conducted by the medium of a shaft divided by a brattice wall only a few inches thick, after descending in the downcast in

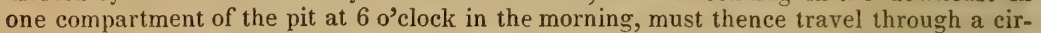
cuit of nearly 30 miles, and cannot arrive at its reascending compartment on the other side of the brattice, or pit partition, till $6 o^{9}$ clock in the evening, supposing it to move all the time at the rate of $2 \frac{1}{2}$ miles per hour. Hence we see that the primum mobile of this mighty circulation, the furnace, must be carefully looked after, since its irregularities may affect the comfort, or even the existence of hundreds of miners spread over these vast subterraneous labyrinths. On the principles just laid down, it appears that if any number of boards be set off from any side of these galleries, either in a level, dip, or rise direction, the circulation of air may be advanced to each forehead, by an ingoing and returning current.

Yet while the circulation of fresh air is thus advanced to the last-made thirling next the foreheads $f, h$, and $m, f \mathrm{~g} .860$, and moves through the thirling which is nearest to the face of every board and room, the emission of fire-damp is frequently so abundant from the coaly strata, that the miners dare not proceed forwards more than a few feet from that aerial circulation, without hazard of being burned by the combustion of the gas at their candles. To guard against this accident, temporary shifting brattices are employed. These are formed of deal, about $\frac{3}{4}$ of an inch thick, 3 or 4 feet broad, and 10 feet long; and are furnished with cross-bars for binding the deals together, and a few finger loops cut through them, for lifting them more expeditiously, in order to place them in a proper position. Where inflammable air abounds, a store of such brattice deals should be kept ready for emergencies.

The mode of applying these temporary brattices, or deal partitions, is shown in the accompanying figure ( $f$ g. 861 ), which shows how the air circulates freely through the

R!il

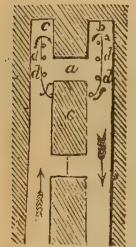
thirling $d, d$, before the brattices are placed. At $b$ and $c$, we see two heading boards or rooms, which are so full of inflammable air as to be unworkable. Props are now erected near the upper end of the pillar $e$, betwixt the roof and pavement, about two feet clear of the sides of the next pillar, leaving room for the miner to pass along between the pillar side and the brattice. The brattices are then fastened with nails to the props, the lower edge of the under brattice resting on the pavement, while the upper edge of the upper is in contact with the roof. By this means any variation of the height in the bed of coal is compensated by the overlap of the brattice boards; and as these are advanced, shifting brattices are laid close to, and alongside of, the first set. The miner next sets up additional props in the same parallel line with the former, and slides the brattices forwards, to make the air circulate close to the forehead where he is working; and he regulates the distance betwixt the brattice and the forehead by the disengagement of fire-damp and the velocity of the aerial circulation. The props are shown at $d, d$, and the brattices at $f, f$. By this arrangement the air is prevented from passing directly through the thirling $a$, and is forced along the right-hand side of the brattice, and, sweeping over the wall face or forehead, returns by the back of the brattice, and passes through the thiling $a$. It is prevented, however, froin returning in its former direction by the brattice planted in the foreneas $c$, whereisy it mounts up and accomplishes its return close to that forehead. Thus headways and boards are ventilated till another thirling is made at the upper part of the pillar. The thirling $a$ is then closed by a brick stopping, and the brattice boards removed forwards for a similar operation. 
When blowers occur in the roof, and force the strata down, so as to nroduce a large vaulted excavation, the accumulated gas must be swept away; because, after filling that space, it would descend in an unmixed state under the common roof of the coal. The manner of removing it is represented in $f \mathrm{~g} .862$, where $a$ is the bed of coal,
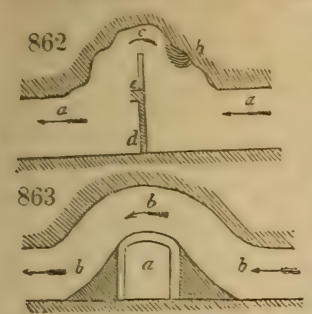
$b$ the blower, $c$ the excavation Jeft by the downfall of the roof, $d$ is a passing door, and $e$ a brattice. By this arrangement the aerial current is carried close to the roof, and constantly sweeps off or dilutes the inflammable gas of the blow$\mathrm{er}$, as fast as it issues. The arrows show the direction of the current ; but for which, the accumulating gas would be mixed in explosive proportions with the atmospheric air, and destroy the miners.

There is another modification of the ventilating system, where the air-courses are traversed across; that is, when one air-course is advanced at right angles to another, and must pass it in order to ventilate the workings on the further side. This is accomplished on the plan shown in fig. 863 , where $a$ is a main road with an aircourse, over which the other air-course $b$, has to pass. The sides of this air channel are built of brichs archerl over so as to be air-tight, and a gallery is driven in the roof strata as shown in the figure. If an air-course, as $a$, be laid orer with planlis made air-tight, crossing and recrossing mar be effected with facilits. The general velocity of the air in these rentilating channels is from 3 to 4 feet per second, or about $2 \frac{1}{2}$ miles per hour, and their internal dimensions vary from 5 to 6 feet square, affording an area of from 25 to 36 square feet.

Mr. Taylor's hydraulic air-pump, formerly described, p. 845, deserves to be noticed

$86 t$ among the rarious ingenious contrirances for rentilating mines, particularly d. when they are of moderate extent. $a$ is a large wooden tub, nearly filled with water, through whose bottom the rentilating pipe $b$ passes down into the recesses of the mine. Upon the top of $b$, there is a valve $e$, opening upwards. Over $b$, the gasometer vessel is inverted in $a$, having a ralve also opening outwards at $d$. When this resse] is depressed by any moring force, the air contained within it is expelled through $d$; and when it is raised, it diminishes the atmospherical pressure in the pipe $b$, and thus draws air out of the mine into the gasometer; which cannot return on account of the valve at $e$, but is thrown out into the atmosphere through $d$ at the next descent.

The general plan of distributing the air, in all cases, is to send the first of the current that descends in the downcast shaft among the horses in the stables, next among the workmen in the foreheads, after which the air, loaded with whatever mixtures it may have received, is made to traverse the old wastes. It then passes throngh the furnace with all the inflammable gas it has collected, ascends the upcast shaft, and is dispersed into the atmosphere. This system, styled coursing the air, was inrented by Mr. Spedding of Cumberland. According to the quantity of the fire-damp, the coursing is conducted either up one room, and returned by the next alternately, through the whole extent of the rorks, or it passes along 2 or 3 connected rooms, and returns by the same number.

This admirable system has receired the greatest improrements from the mining engineers of the Newcastle district, and especially from Mr. Buddle of Wallsend. His plan being a most complete scale of rentilation, where the aerial current is made to sweep away every corner of the workings, is shown in fig. 865; in which $a$

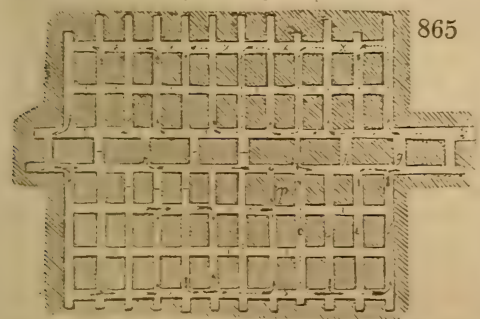
represents the downcast, and $b$ the upcast shaft. By pursuing the track of the arrows, we may observe that the air passes first along the two rooms $c, d$, having free access to each through the walls, but is hindered from entering into the adjoining rooms by the stoppings which form the air-courses. It sweeps along the wall faces of the rooms $c, d$, and makes a return down the rooms $e, f$, but is not allowed to proceed further in that direction by the stoppings $g, h$. It then proceeds to the foreheads $i, k$, and single courses all the rooms to the foreheads $l, m$; from this point it would go directly to the upcast pit $b$, were it not prerented by the stopping $n$, which throws it again into double coursing the rooms, till it arrives at 0 , whence it goes directly to t? ascends the shaft $b$. The lines across each other represent the passing doors; snd these may ne sunsituted in any place for a passage where there is a stoping. The stopping $p$, near the bottum of the downcast shaft, is termed a main stopping; because if it were remored, the whole circulation would instantly cease, and the air, instead of 
traversing in the direction of the arrows, would go directly from the downcast pit $a$, to the upcast pit $b$, along the gallery $q$. Hence every gallery and room of the workings would be laid dead, as it is termed, and be immediately filled with fire-damp, which might talie fire either at the workmen's candles, or at the furnace next the ujcast shaft $b$. Thus also a partial stagnation in one district of the colliery, would be produced by any of the common stoppings being accidentally removed or destroyed, since the air would thereby always pursue the nearest route to the upeast pit. Miain stoppings are matie particularly securc, by strong additional stone buildings, and they are set up at different places, to maintain the main air courses entire in the event of an explosion; by which precautions great security is given to human life. This system of ventilation may be extended to almost any distance from the pit-bottom, provided the volume of fresh air introduced be adequate to dilute sufticiently the fire-damp, so that the mixture shall not reach the explosive point. The air, by this management, rentilates first one panel of work, and then other panels in succession, passing onwards through the barriers or panel walls, by means of galleries, as in fig. 843 , by the principle either of single, double, or triple coursing, according to the quantity of gas in the mine.

In ventilating the very thick coal of Staffordshre, though there is much inflammable air, less care is needed than in the north of England collieries, as the workings are very roomy, and the air courses of comparatively small extent. The air is conducted down one shaft, carried along the main roads, and distributed into the sides of work, as shown in fig. 818. A narrow gallery, termed the air-head, is carried in the upper part of the coal, in the rib walls, along one or more of the sides. In the example here figured, it is carried all round, and the air enters at the bolt-hole $e$. Lateral openings, named sponts, are led from the air-head gallery into the side of work; and the circulating stream mixed with the gas in the workings, enters by these spouts, as represented by the arrows, and returns by the air-head at $g$, to the upcast pit.

When the fire-damp comes off suddenly in any case, rendering the air foul and explosive at the foreheads, if no other remedy be found effectual, the working of the coal must be suspended, and a current of air sent directly from the fresh in-going stream, in order to dilute the explosive mixture, before it reaches the furnace. This is termed skuiling the air ; for otherwise the gas would kindle at the furnace, and flame backwards, like a train of gunpowder, through all the windings of the work, carrying devastation and death in its track. By skiailing the air, however, time is given for running forward with water, and drowning the furnace. A cascade of water from the steam engine pumps is then allowed to fall down the pit, the power of which, through a fall of 500 or 600 feet, is so great in carrying down a body of air, that it impels a sufficient current through every part of the workings. The ventilation is afterwards put into its usual train at leisure.

In collieries which have oeen worked for a considerable tıme, and particularly in such as have goaves, creeps, or crushed wastes, the disengagement of the fire-damp from these recesses is much influenced by the state of atmospheric pressure. Should this be suddenly diminished, as shown by the fall of the barometer, the fire-damp suddenly expands and comes forth from its retirement, polluting the galleries of the mine with its noxious presence. But an increase of barometric pressure condenses the gases of the mine, and restrains them within their sequestered limits. It is therefore requisite that the coalviewer should consult the barometer before inspecting the subterraneous workings of an old mine, on the Monday mornings, in order to know what precautions must be observed in his personal survey.

The catastrophe of an explosion in an extensive coal-mine is horrible in the extreme. Let us imagine a mine upwards of 100 fathoms deep, with the workings extended to a great distance under the surrounding country, with machinery complete in all its parts, the mining operations under regular discipline, and railways conducted through all its ramifications; the stoppings, passing doors, brattices, and the entire economy of the mine, so arranged that every thing mores like a well-regulated machine. A mine of this magnitude at full work is a scene of cheering animation, and happy industry; the sound of the hammer resounds in every quarter, and the numerous carriages, loaded or empty, passing swiftly to and fro from the wall faces to the pit bottom, enliren the gloomiest recesses. At each door a little boy, called a trapper, is stationed, to open and shut it. Every person is at his post, displaring an alacrity and happiness pleasingly contrasted with the surrounding gloom. While things are in this merry train, it has but too frequently happened that from some unforeseen cause, the ventifation has partially stagnated, allowing a quantity of the fire-damp to accumulate in one space to the explo sive pitch; or a blower has suddenly sprung forth, and the unsuspecting miner, entering this fatal region with his candle, sets the whole in a blaze of burning air, which immediately suffocates and scorches to death erery living creature within its sphere, while multitudes beyond the reach of the flame are dashed to pieces by the force of the explosion, rolling like thunder along the winding galleries. Sometimes the explosive flame 
seoms to linger in one district for a few moments; then gathering strength for a giant eflort, it rushes forth from its cell with the violence of a hurricane, and the spced of lightning, destroying every obstacle in its way to the upcast shaft. Its power seems to be irresistible. The stoppings are burst through, the doors are shivered into a thousand pieces; whiie the unfortunate miners, men, women, and boys, are swept along with an inconceirable relocity, in one body, with the horses, carriages, corves, and coals. Should a massive pillar obstruct the direct course of the aerial torrent, all these objects are dashed against it, and there prostrated or heaped up in a mass of common ruin, mutilation, and death. Others are carried directly to the shaft, and are either buried there amid the wreck, or are blown up and ejected from the pit mouth. Even at this distance from the explosive den, the blast is often so powerful, that it frequently tears the brattice walls of the shaft to pieces, and blows the corves suspended in the shaft as high up into the open air as the ropes will permit. Not unfrequently, indeed, the ponderous pulleywhecls are blown from the pit-head frame, and carried to a considerable distance in the bosom of a thicli cloud of coals and coal dust brought up from the mine by the fire-damp, whose explosion shakes absolutely the superincumbent solid earth itself, with a mimic earthquake. The dust of the ruins is sometimes thrown to such a height above the pit as to obscure the light of the sun. The silence which succeeds to this awful turmoil is no less formidable; for the atmospheric back-draught, rushing down the shaft, denotes the consumption of vital air in the mine, and the production of the deleterious choke-damp and azote.

Though many of the miners may have escaped by their distance in the workings from the destructive blast and the fire, yet their fate may perhaps be more deplorable. They hear the explosion, and are well aware of its certain consequences. Every one, anxious to secure his personal safety, strains every faculty to reach the pit-bottom. As the lights are usually extinguished by the explosion, they have to grope their way in utter darkness. Some have made most marvellous escapes, after clambering over the rubbish of fallen roofs, under which their companions are entombed; but others, wandering into uncertain alleys, tremble lest they should encounter the pestilential airs. At last they feel their power, and aware that their fate is sealed, they cease to struggle with their inevitable doom; they deliberately assume the posture of repose, and fall asleep in death. Such has been too often the fate of the hardy and intelligent miners who immure themselves deep beneath the ground, and venture their lives for the comfort of their fellowmen; and such frequently is the ruinous issue of the best ordered and most prosperous mining concerns.

In such circumstances the mining engineers or coal viewers have a dangerous and difficult duty to perform. The pit into which they must descend as soon as possible, is rendered unsafe by many causes; by the wrecks of loose timber torn away by the eruption, or by the unrespirable gases; by the ignition perhaps of a portion of the coal itself, or by the flame of a blower of fire-damp ; either of which would produce violent and repeated explosions whenever the gas may again accumulate to the proper degree. Such a predicament is not uncommon, and it is one against which no human skill can suard. Yet even here, the sense of duty, and the hope of saving some workmen from a lingering death by wounds or suffocation, lead this intrepid class of men to descend amid the very demons of the mine.

As soon as the ventilation is restored by temporary brattices, the stoppings and doors are rebuilt in a substantial manner, and the workings are resumed with the wonted activity. From an inspection of fig. 864 , p. 1035 , it is obvious that the stability of the main stopping $p$, is an important point; for which reason it is counterforted by strong walls of stone, to resist the explosive force of fire-damp.

When it is known that fire exists in the wastes, either by the burning of the small coal-dust along the roads, or from the ignition of the solid coal by a blower of gas, the inspection of the mine is incomparably more hazardous, as safety cannot be ensured for an instant; for if the extrication of gas be great, it rapidly accumulates, and whenever it reaches the place where the fire exists, a new explosion takes place. There have been examples of the most furious detonations occurring regularly after the interval of about an hour, and being thus repeated 36 times in less than two days, each eruption appearing at the pit mouth like the blast of a volcano. It would be madness for any one to attempt a descent in such circumstances. The only resource is to moat up the pit, and check the combustion by exclusion of atmospheric air, or to drown the workings by letting the water accumulate below ground.

When fire exists in the wastes, with less apparent risk of life, water is driven upon it by portable fire-extinguishing engines, or small cannon are discharged near the burning coal, and the concussion thus produced in the air sometimes helps to extinguish the flame.

Since the primary cause of these tremendous catastrophes is the accension of the explosive gases by the candle of the miner, it has been long a desideratum to procuse light of such a nature as may not possess the power of kindling the fire-damp. The train of light producible from the friction of flint and steel, by a mechanism called 
a sleel mill, has been long $\mathrm{knc} w \mathrm{n}$, and afforded a tolerable gleam, with which the miners were cbliged to content themselres in hazardous atmospheres.

It consists of a small frame of iron, mountel with a wheel and pinion, which gire rapid -otation to a disk of hard steel place.j upright, to whose edge a piece of thint is aprlied. The use of this machine entailed on the miner the expense of an attendant, calied the miller, who gave him light. Nor was the light altogether safe, fur occa-ionally the ignited shower of steel particles attained to a sufficient heat to set fire to the fire-damp.

At length the attention of the scientific world was powerfully attracted to the means of lighting the miner with safety, by an awful catastrophe which happened at Feling Colliery, near Jewcastle, on the 25 th May, 1312. This mine was working with great vigor, under a well-regulatel system of rentilation, set in action by a furnace and air-tube, placed orer a rise pit in elerated ground. The depth of winning was above 100 fathoms; 25 acres of coal had been excavated, and one pit was rielling at the rate of 1700 toms per week. At $110^{\circ}$ cloch in the forenoon the night shift of miners was relieved by the day shift: 121 persons were in the mine, at their sereral stations, when, at half-past 11 , the gas fired, with a most awful explosion, which alarmed all the neighboring rillaces. The subterraneous fire brolke forth with two heart discharges from the dip-pit, and these were instantly followed br one from the rise-pit. A slight trembling, as from an earthriake, was felt for about half a mile round the collierr, and the noise of the explosion, though dull, was heard at from 3 to 4 miles' distance. Immense quantities of dust and small coal accompanied these blasts, and rose high into the air, in the form of an inverted cone. The heaviest part of the ejected matter, such as corres, wood, and small coal, fell near the pits; but the dust, borne away by a strong west wind, fell in a continnous shower a mile and a half from the pit. In the adjoining village of Heworth it caused a darkness like that of early twilight, corering the roads where it fell so thichly that the footsteps of passengers were imprinted in it. The heads of both shaft-frames were blown off, their sides set on fire, and their pulleys shattered to pieces. The coal-dust ejected from the rise-pit into the horizontal part of the rentilating tube, was about 3 inches thich, and speedily burnt to a cinder; pieces of burning coal, driren off the solid stratum of the mine, were also blown out of this shaft. Of the 121 persons in the mine at the time of the explosion, only 32 were drawn up the pit alive, 3 of whom died a fer hours after the accident. Thus no less than 92 raluable lires were instantaneously destroyed by this pestilential fire damp. The scene of distress among the relatires at the pit mouth was indescribably sorrowful.

Dr. W. Reid Clanny, of Sunderland, was the first to contrire a lamp which might burn among explosire air without communicating flame to the gas in which it was plunged. This he effected, in 1813, br means of an air-tight lamp, with a glass front, the flame of which was supnorted by blowing fresh air from a small pair of bellows throngh a stratum of water in the bottom of the lamp, while the heated air passed out through water by a recurred tube at ton. By this means the air within the lamp was completely insulated from the surrounding atmosphere. This lamp was the first erer taken into a body of inflammable air in a coal-mine, at the exploding point, without setting fire to the gas around it. Dr. Clanny made another lamp upon an improred plan, by introducing into it the steam of water generated in a small ressel at the top of the lamp, heated by the flame. The chief objection to these lamps is their inconvenience in use.

Various other schemes of safe-lamps were offered to the miner br ingenious mechanicians, but ther hare been all superseded br the admirable inrention of Sir H. Darr, founded on his fine researches upon flame. The lamp of Dart was instantly tried and approred of br. Mr. Buddle and the principal mining engineers of the Neweastle district. A nerfect security of accident is therefore afforded to the miner in the use of a lamp which transmits its light, and is fed with air, through a crlinder of wire gauze; and this inrention has the advantage of requiring no machinerr; no philosophical knowledge to direct its use, and is made at a very cheap rate.

In the course of a long and laborions inrestigation on the properties of the fire-damp, and the niture and communication of flame, Sir H. Darr ascertained that the explosions of inflammable oases were incapable of being passed through long narrow metallic tulies; and that this principle of security was still obtained by diminishing their length and dianeter at the same time, and likerlse diminishing their length, and increasing their number, so that a great number of small apertures would not pass an explosion, $\pi$ her their denth was equal to their diameter. This fact led him to trials unon sieres made of wire-gauze, or metallic plates perforated with numerous small holes; and he frund it was impossible to pass explosions through them.

The anertures in the gauze should nerer be more than 1-20th of an inch square. Ir the working models sent by Sir H. to the mines, there were 748 apertures in the square inch, and the wire was about the $40 \mathrm{th}$ of an inch diameter. The cage or crlinder of wrre-gauze should be made br double joinings, the gauze being folded orer in such a manner as to leare no apertures. It should not be more than two inches in diameter; for in large cylinders the combustion of the fire-damp renders the top inconveniently 
hot; and a louble top is alvays a proper precaution, fixed at a distance of about half an inch above tie first top. The gauze cylinder should be fastened to the lamp by a screw of 4 or 5 1:: is. All joinings in the lamp should be made with hard solder; and the security dep ads upon the condition, that no aperture exists in the apparatus larger than in the wire auze.

The form of the lamp and eage, and the mode of burning the wick, may be greatly dirersifie.l: but the principle which ensures their safety must be strictly attended to. See Lavip of Diry, SAFetr Limp, and Vextilatiox.

The state of the air in coal mines, from very early periods till the discovery of the safe-lamp, was judged of by the appearances exhibited by the flame of a candle; and this test mist in many circumstances be still had recourse to. When there is merely a defect of atm sppheric oxygen, the air being also partially vitiated by a little carbonic acid, either from chote-damp or the lungs and candles of the miners, the lights burn with a rery dull fin:ne, the tallow ceases to melt in the cup formed round the wick, till the flame flichers and expires. In this case the candle may be kept burning by slanting it more or less towarits a horizontal position, which causes the tallow to melt with the edge of the flame. The cantle is thus :apidly wasted, however; and therefore an oil lamp is preferable, as it continues to bum. where a candle would be extinguished. The candles of the collier are generally small, with a rery small wick; such being found to produce a more distinct flame than candles of a large size with a thick wick.

In trying the quality of the air by the flame of a candle, the wick must be trimmed br taking off the snuff, so as to produee a clear, distinct, and steady burning flame. When a candle thus trimmed is looked at in common air, a distinct and well-defined cone of flame is seen, of a fine sliy-blue at the bottom next the wick, and thence of a bright rellow to the anex of the cone. Besides this appearance, there is another, surrounding the cone, which the brightness of the flame prerents the eye from discerning. This may be seen by placing one of the hands expanded as a sereen betwixt the eves and the candle, and at the distance of about an inch, so that the least point of the apex of the rellow flame may be seen, and no more. By this method, a top, as the miners term it, will be distinctly observed close to the apex of the vellow flame, from an eighth to a quarter of an inch in length. This top is of a yellowish-brown color, and like a misty haze. This haze is seen not only on the top, but it extends downwards and surrounds the flame fully half war, about a twentieth of an inch in thickness; here it assumes a riolet color, which passes into a beautiful blue at the bottom next the wick. The test of the state of the air in mines, or "trying the candle," as practised by miners, depends entirels on the appearance which this haze assumes in shape and color at the top of the flame. In fact, this top has distinct appearances when burning in atmospheric air, carbonated air, azotized air, or fire-damp air ; displaying many modifications, according to the proportions of the various admixtures.

When azote or carbonic acid abounds, the top is frequently an inch or two in length, of a decided brown color, and the flame is short and dim. When they are still more copious, the flame goes out, and the miners immediately retire.

When inflammable air is imagined to exist in considerable quantity, the miner trims his candle, and adrances with cautious step, holding the candle with the left hand, and screening the flame with the right; and as the fire-damp floats in the upper part of the gallery next the roof, he holds the candle as low as he can, and keeping his ere fixed on the tip, he mores forwards. If the gas be small in quantity, he may reach the forehead without observing any material change in his light. But if in his adrance he perceives the tip to elongate, and take a bluish-gray color, he is put on his guard, and steps on with much caution; and if the tip begins to spire, he drops down on one knee, and hold-

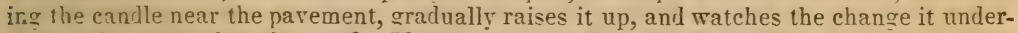
goes as it approaches the roof. If the gas be copious, the flame elongates into a sharp spire, as well as the top. It is in general reckoned dangerous when the tip changes from the bluish-grar to a fine bluc color, accompanied with minute luminous points, which pass rapilly upwards through the flame and top. When the symptoms are manifestly dangerous, a sudden morement of the hands or body is liable to produce ignition by agitation of the fire-damp. The experienced miner therefore slowly and cautiously lowers his cantle to the parement, and then turning round, effects his retreat slowly, or slips up his right hand and extinguishes the flame with his finger and thumb. Should he renture too far, and approach the body of gas in an explosive condition, the tip of the candle rapilly elongates, and the whole rises in a sharp spire sereral inches in length; and then the whole surrounding atmosphere is in a blaze, an explosion ensucs, and destructive ravag: is the consequence, to an extent proportioned to the quantity of fire-damp. See SAFETY LAMP, and Vextulation.

This trying the candle is a delicate operation, requiring much practical sagacity, where the lives of so many men, and the welfare of the whole establishment, are at stake. Almost every colliery, after having been worked for some time, gives a peculiar top to 
the candle; so that while in one mine liable to fre-damp an explosion will take place with a top less than an inch long, in another mine the top may be two inches high, and yet the air be considerably under the point of accension. These differences depend on several particulars. If the gas has not passed through a long course of rentilation, and is little inixed with air, it will ignite with a very short top; while, on the other hand, a gas which has run through a ventilation of 20 or 30 miles may cause the production of a long top without hazard. It is hence obvious, that skilful experience, and thorutigh practical knowledge, are the only sure guides in these cases.

We shall now describe briefly the modern modes of working coals a-dipping of, and deeper than, the engine-pit bottom. One of these consists in laying a working pump barrel with a long wind-bore at the bottom of the downset mine, furnished with a smooth rod working through a collar at the top of the working barrel. At one side of this, near the to', a kneed pipe is attached, and from it pipes are carried to the point of delivery, either at the engine pit bottom or day level, as represented in fig. 866. The spears are worked sometimes by rods connected with the machinery at the $866 \quad 867 \quad$ surface; in which case the spears, if very long, are either sus.

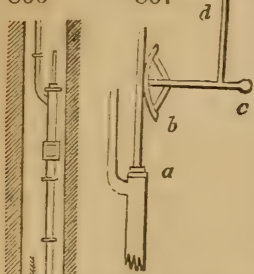
pended from swing or pendulum rods, or move on friction rollers. But since the action of the spears, running with great velocity the total length of the engine stroke, very soon tears every thing to pieces, the motion of the spears under ground has been reduced from 6 or 8 feet, the length of the engine stroke, to about 15 inches; and the due speed in the pump is effected by the centring of a beam, and the attachment of the spears to it, as represented in fig. 867, where $a$ is the working barrel, $b$ the beam centred at $c$, having an arc-head and martingale sinking-chain. The spears $d$ are fastened by a strong bolt, which passes through the beam; and there are several holes, by means of which the stroke in the pumps can be lengthened or shortened at convenience. The movement of the spears is regulated by a strong iron quadrant or wheel at the bottom.

In level-free coals, these pumps may be worked by a water-wheel, stationed near the bottom of the pit, impelled by water falling down the shaft, to be discharged by the level to the day (day-level).

But the preferable plan of working under-dip coal, is that recently adopted by the Newcastle engineers; and consists in running a mine a-dipping of the engine-pit, in such direction of the dip as is most convenient; and both coals and water are brought up the rise of the coal by means of high-pressure engines, working with a power of from 30 to 50 pounds on the square inch. These machines are quite under command, and, producing much power in little space, they are the most applicable for underground work. An excavation is made for them in the strata above the coal, and the air used for the furnace under the boiler, is the returned air of the mine ventilation. In the dip-mine a double tram-road is laid; so that while a number of loaded corves are ascending, an equal number of empty ones are going down. Although this improved method has been introduced only a few rears back, under-dip workings have been already executed more than an English mile under-dip of the engine-pit bottom, by means of three of these high-pressure engines, placed at equal distances in the under-dip mine. It may hence be inferred, that this mode of working is susceptible of most extensive application; and in place of sinking pits of excessive depth upon the dip of the coal, at an almost ruinous expense, much of the under-dip coal will in future be worked by means of the actual engine-pits. In the Newcastle district, coals are now working in an engine pit 115 fathoms deep under-dip of the engine-pit bottom, above 1600 yards, and fully 80 fathoms of perpendicular depth more than the bottom of the pit.

If an engin?-pit be sunk to a given coal at a certain depth, all the other coals of the 868

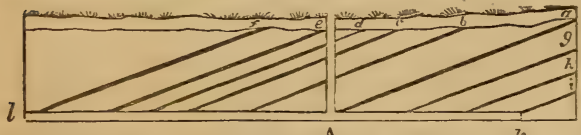
coal-field, both above and below the coal sunk to, ${ }^{\circ}$ can be drained and worked to the same depth, by driving a level cross-cut mine, both to the dip and rise, till all the coals are intersected, as represented in fig. 868 , where $\mathrm{A}$ is the engine-pit bottom reaching to the coal $a$; and $b, c, d, e, f$, coals lying abore the coal $a$; the coals which lie below it, $g, h, i ; k$ is the forehead of the cross-cut mine, intersecting all the lower coals; and $l$, the other forehead of the mine, intersncting all the upper coals.

In the "Report from the select committee of the House of Lords, appointed to take into consideration the state of the coal trade in the Uniter Kingdom," printed in June, 1829, under the head of Mr. Buddle's evidence we have an excellent description of the 
nature and progress of creeps, which we have adverted to in the preceding account. The annexed fig. 869 exhibits the creep in all its progressive stages, from its commsncement until it has completely closed all the workings, and crushed the pillars of coal. The section of the figures supposes us standing on the level of the diffitent galleries which are opened in the seam. The black is the coal pillars between each gallery; when these are weakened too much, or, in other words, when their bases become too narrow for the pavement below, by the pressure of the incumbent stratification, they sink down into the parement, and the first appearance is a little curvature in the bottom of each gallery: that is the first symptom obvious to sight; but it may generally be heard before it is seen. The next stage is when the pavement begins to open with a crack longitudinally. The next stage is when that crack is completed, and it assumes the shape of a metal ridge. The next is when the metal ridge reaches the roof. The next stage is when the peak of the metal ridge becomes flattened by pressure, and forced into a horizontal direction, and becomes quite close ; just at this moment the coal pillars begin to sustain part of the pressure. The next is when the coal pillars take part of the pressure. The lasi stage is when it is dead and settled; that is, when the metal or factitious ridge, formed by the sinking of the pillar into the pavement, bears, in common with the pillars of coal on each side, the full pressure, and the coal becomes crushed or cracked, and can be no longer worked, except by a very expensive and dangerous process. Fig. 869.

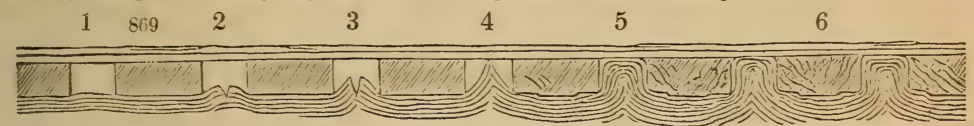

1. First stage of active creep.

2. Second do.

3. Third do.

4. Fourth do.
5. The metal ridge closed, and the creep beginning to settle.

6 . The creep settled, the metal ridges being closely compressed, and supporting the roof.

The quantity of coals, cinders, and culm shipped coastwise, and exported from the sereral ports of the United Kingdom in the year 1837, was 8,204,301 tons; in 1836, the quantity was $7,389,272$ tons, being an increase of 815,029 tons, or 11.03 per cent. in favor of 1837 .

The following $\mathrm{T}_{\mathrm{ABLE}}$ shows the separate proportions of this quantity supplied by England and Wales, Scotland and Ireland :-

\begin{tabular}{|c|c|c|c|}
\hline & 1836. & 1837. & Increase. \\
\hline $\begin{array}{l}\text { England and Wales } \\
\text { Scotland - } \\
\text { Ireland }\end{array}$ & $\begin{array}{r}\text { Tons. } \\
6,757,937 \\
624,308 \\
7,027\end{array}$ & $\begin{array}{r}\text { Tons. } \\
7,570,254 \\
626,532 \\
7,515\end{array}$ & $\begin{array}{l}\text { Tons. } \\
812,317 \text { or } 12 \cdot 02 \text { per cent. } \\
2,204-0 \cdot 36 \\
488-6.94\end{array}$ \\
\hline Total & $7,389,272$ & $8,204,301$ & 815,029 or 11.03 per cent. \\
\hline
\end{tabular}

PITCOAL, COKING OF. See also Charcoal.

Fig. 870 represents a shachtofen, or pit-kiln, for coking coals in Germany. $a$ is the 870

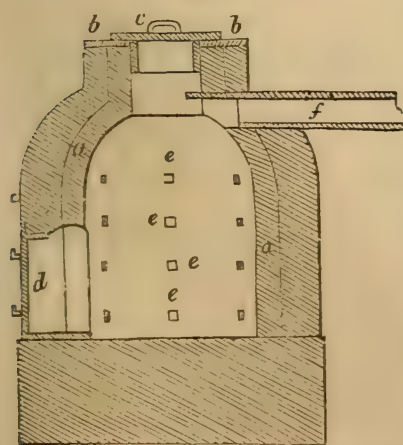

lining (chemise), made of fire-bricks; the enclosing walls are built of the same material; $b, b$, is a castiron ring covered with a cast-iron plate $c$. The floor of the kiln is massive. The coals are introduced, and the coke taken out, through a hole in the side $d$; during the process it is bricked up, and closed with an iron door. In the surrounding walls are 4 horizontal rows of flues $e, e, e, e$, which are usually iron pipes; the lowest row is upon a level with the floor of the kiln; and the others are each respectively one foot and a half higher than the preceding. Near the top of the shaft there is an iron pipe $f$, of from 8 to 10 inches in diameter, which allows the incoercible vapors generated in the coking to escape into the condenser, which consists either of wood or brick chambers. For kindling the coal, a layer of wood is first placed on the bottom of the kiln.

The coking of small coal is performed upon vaulted hearths, somewhat like baliers' ovens, but with still flatter roofs. Of such kilns, several are placed alongside one another, 


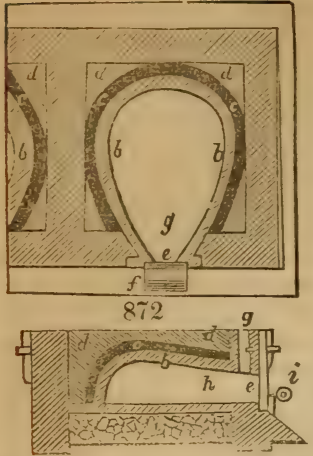

\section{PITCOAL, COKING OF.}

each being an ellipse deviating little from a circle, so that the mouth may project but a small space. The dimensions are such, that froin 10 to 12 cubic feet of coal-culm may be spread in a layer 6 inches deep upon the sole of the furnace. The top of the flat arch of fire brick should be covered with a stratum of loam and sand.

Figs. 871 and 872 represent such a kiln as is mounted at Zabrze, in Upper Silesia, for coking small coal. F 2 g. 871 is the ground plan; fig. 872 the vertical section in the line of the long axis of fig. 871. $a$, is the sand-bed of the hearth, under the brick sole; $b$, is the roof of large firebricks; $c$, the covering of loam; $d$, the top surface of sand; $e$, the orifice in the front wall, for admission of the culm, and removal of the coke, over the sloping stone $f$. The flame and vapors pass off above this orifice, through the chimney marked $g$, or through the aperture $h$, into a lateral chimney. $i$, is a bar of iron laid across the front of the door, as a fulcrum to work the iron rake upon. A layer of coals is first kindled upon the hearth, and when this is in brisk ignition, it is covered with the culm in successive sprinklings. When the coal is sufficiently coked, it is raked out, and quenched with water.

Fig. 873 represents a simple coking meiler or mound, constructed in a circular form

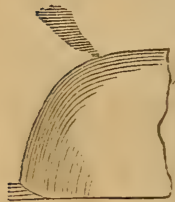

874

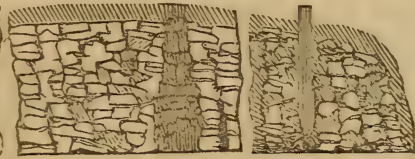

873

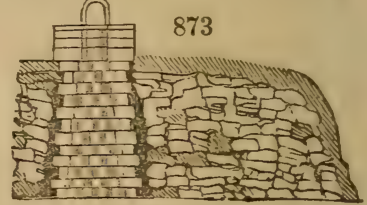

round a central chimney of loose bricks, towards which small horizontal flues are laid among the lumps of coals. The sides and top are covered with culm or slack, and the heap is kindled from certain openings towards the circumference. Fig. 874 represents an oblong meiler, sometimes made 100 or 150 feet in length, and from 10 to 12 in breadth. The section in the middle of the figure shows how the lumps are piled up; the wooden stakes are lifted out when the heap is finished, in order to introduce kindlings at various points; and the rest of the meiler is then covered with slack and clay, to protect it from the rains. A jet of smoke and flame is seen issuing from its left end.

An excellent range of furnaces for making a superior article of cole, for the service of the locomotive engines of the London and Birmingham Railway Company, has been recently erected at the Camden Town station; consisting of 18 ovens in two lines, the whole discharging their products of combustion into a horizontal flue, which terminates

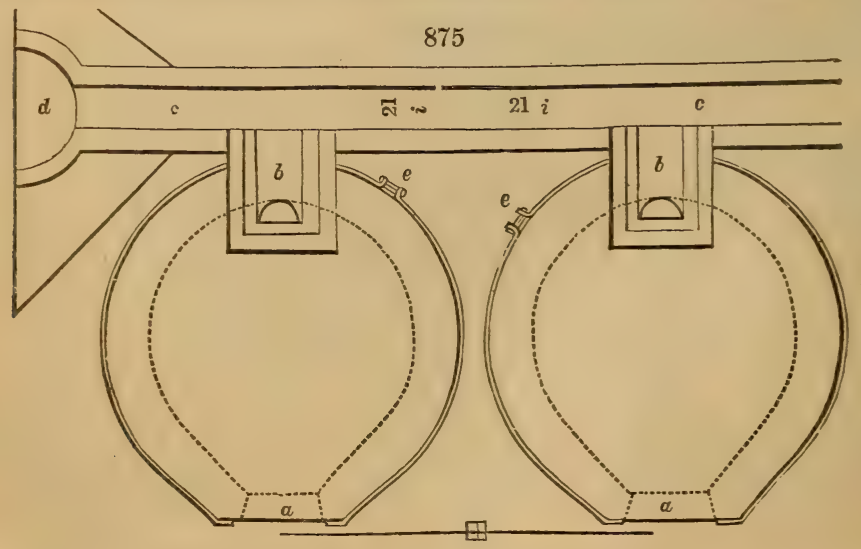

in a chımner-stalk, 115 feet high. Fig. 875 is a ground plan of the elliptical ovens, each being 12 feet by 11 internally, and having 3 feet thickness of walls. $a, a$, is the mouth, $3 \frac{1}{2}$ 
feet wide outside, and about $2 \frac{3}{4}$ feet within. $b, b$, are the entrances into the flue; they may be shut more or less completely by horizontal slabs of fire-brick, resting on iron fiames, pushed in from behind, to modify the draught of air. The grooves of these damperslabs admit a small stream of air to complete the combustion of the volatilized particles of soot. By this means the smoke is well consumed. The flue $c, c$, is $2 \frac{1}{2}$ feet high,

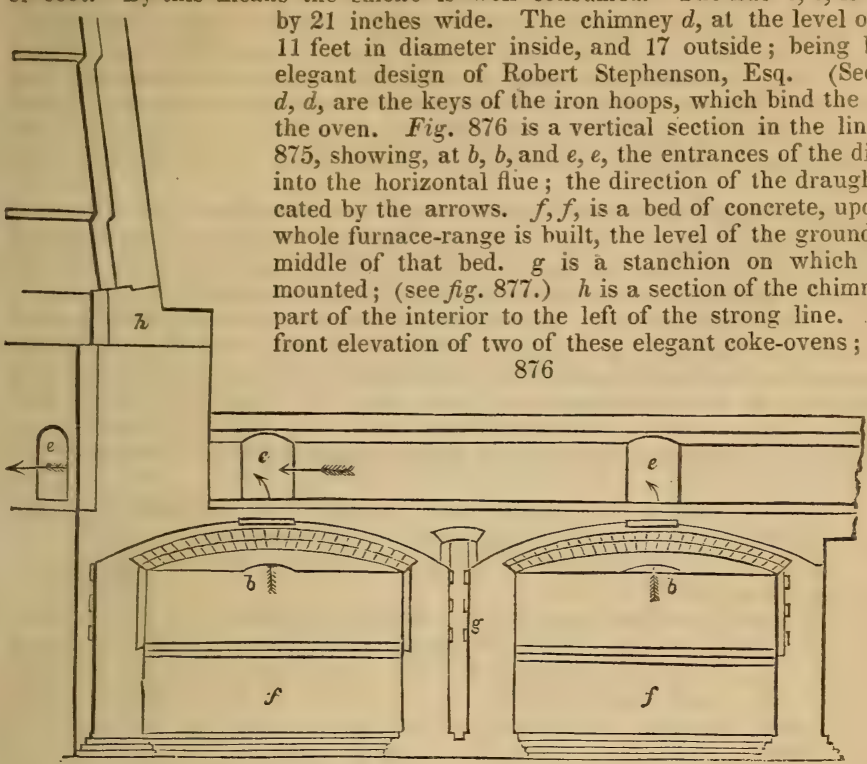

bracing hoops $i, i, i$, are shown; $k, k$, are the cast iron doors, strengthened outside with diagonal ridges; each door being $5 \frac{1}{2}$ feet high, by 4 feet wide, and lined internally with fire-bricks. They are raised and lowered by means of chains and counterweights, moved by the crane $l$.

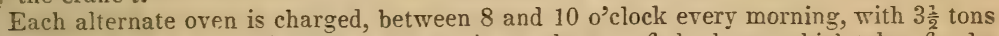
of good coals. A wisp of straw is thrown in on the top of the heap, which takes fire by the radiation from the dome (which is in a state of dull ignition from the preceding operation), and inflames the smoke then rising from the surface, by the re-action of the hot

877

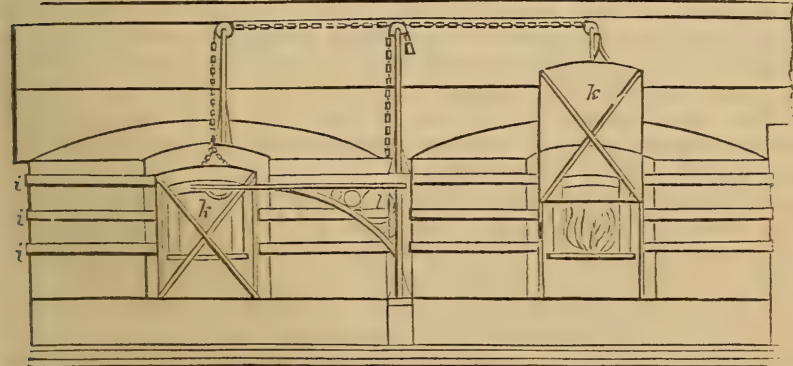
sides and bottum upon the body of the fuel. In this way the smoke is consumed at the very commencement of the process, when it would otherwise be most abundant. A neighbor of the above coking ovens, having lately indicted

them as a nuisance, procured, secundum artem, a parcel of affidavits from sundry chemical and medical men. Two of the former, who had not entered the premises, but had espied the outside of the furnaces' range at some distance, declared that "the coking process, as performed at the orens, is a species of distillation of coal !" How rashly do unpractical theorists affirm what is utterly unfounded, and mislead an unscientific judge! That the said coking process is in no respect a species of distillation, but a complete combustion of the volatile principles of the coal, will be manifest from the following rescription of its actual progress. The mass of coals is first lindled at the surface, as above stated, where it is supplied with abundance of atmospheric oxy yen; because the doors of the ovens in front, and the throat-vents behind, are then left open 
The consequence is, that no more smoke is discharged from the top of the chimney, at this the most sooty period of the process, than is produced by an ordinary litchen fire. In these circumstances, the coal gas, or other gas, supposed to be generated in the slightly heated mass beneath, cannot escape destruction in passing up through the bright open flame of the oren. As the coking of the coal adrances most slowly and regularly from the top of the heap to the bottom, only one layer is affected at a time, and in succession downwards, while the surface is always covered with a stratum of redhot cinders, ready to consume every particle of carbureted or sulphureted hydrogen gases which may escape from below. The greatest mass, when calcined in this downward order, cannot emit into the atmosphere any more of the above-mentioned gases than the smallest heap; and therefore the argument raised on account of the magnitude of the operations, is altogether fallacious.

The colie being perfectly freed from all fuliginous and volatile matters by a calcination of upwards of 40 hours, is cooled down to moderate ignition by sliding in the dampers, and sliding up the doors, which had been partially closed during the latter part of the process. It is now observed to form prismatic concretions, somewhat like a columnar mass of basalt. These are loosened by iron bars, lifted out upon shorels furnished with long iron shanks, which are poised upon swing chains with hooked ends, and the lumps are thrown upon the pavement, to be extinguished by sprinkling water apon them from the rose of a watering-can; or, they might be transferred nto a large ohest of sheet-iron set on wheels, and then covered up. Good coals thus treated, yield 80 per cent. of an excellent compact glistening coke; weighing about $14 \mathrm{cwt}$. per chaldron.

The loss of weight in coking in the ordinary ovens is usually reckoned at 25 per cent.; and coal, which thus loses one fourth in weight, gains one fourth in bulk.

Laborers who have been long employed at rightly-constructed coke ovens, seem to enjoy remarkably good health.

PITTACALL is one of the 6 curious principles detected in wood-tar by Reichenbach. It is a dark-blue solid substance, somewhat like indigo, assumes a metallic fiery lustre on friction, and raries in tint from copper to golden. It is roid of taste and smell, not volatile; carbonizes at a high heat without emitting an ammoniacal smell; is soluble or rather very diffusible in water; gives a green solution with a cast of crimson, in sulphuric acid, with a cast of red blue, in muriatic acid, and with a cast of aurora red, in acetic acid. It is insoluble in alkalis. It dyes a fast blue upon linen and cotton goods, with tin and aluminous mordants.

\section{PLASTER; See MORTAR.}

PLASTER OF PARIS; see GrPSUM.

PLATED MÁNUFACTURE. (Fabrique de plaqué, Fr.; Silber plattirung, Germ.) The silver in this case is not applied to ingots of pure copper, but to an alloy consisting of copper and brass, which possesses the requisite stiffness for the various articles.

The furnace used for melting that alloy, in black-lead crucibles, is a common air-furnace, like that for making brass.

The ingot-moulds are made of cast-iron, in two pieces, fastened together; the carity being of a rectangular shape, 3 inches broad, $1 \frac{1}{2}$ thick, and 18 or 20 long. There is an elevated mouth-piece or gate, to give pressure to the liquid metal, and secure solidity to the ingot. The mould is heated, till the grease with which its carity is besmeared merely begins to smoke, but does not burn. The proper heat of the melted metal for casting, is when it assumes a bluish color, and is quite liquid. Whenever the metal has solidified in the mould, the wedges that tighten its rings are driven out, lest the shrinkage of the ingot should cause the mould to crack. See Brass.

The ingot is now dressed carefully with the file on one or two faces, according as it is to be single or double plated. The thickness of the silver plate is such as to constitute one fortieth of the thickness of the ingot; or when this is an inch and a quarter thick, the silver plate applied is one thirty-second of an inch; being by weight a pound troy of the former, to form 8 to 10 pennyweights of the latter. The silver, which is slightly less in size than the copper, is tied to it truly with iron wire, and a little of a saturated solution of borax is then insinuated at the edges. This salt melts at a low heat, and excludes the atmosphere, which might oxydize the copper, and obstruct the union of the metals. The ingot thus prepared is brought to the plating furnace.

The furnace has an iron door with a small hole to look through; it is fed with cokes, laid upon a grate at a level with the bottom of the door. The ingot is placed immediately upon the colies, the door is shut, and the plater watches at the peep-hole the instant when the proper soldering temperature is attained. During the union of the silver and copper, the surface of the former is seen to be drawn into intimate contact with the latter, and this species of riveting is the signal for removing the compound bar instantly from the furnace. Were it to remain a very little longer, the silver would become alloyed with the copper, and the plating be thus completely spoiled. The adhesion is, in 
fact, accomplished here by the formation of a film of true silver-solder at the surfaces of contaet.

The ingot is next cleaned, and rolled to the proper thinness between cylinders as described under Mint; being in its progress of lamination frequently annealed on a small reverberatory hearth. After the last annealing, the sheets are immersed in hot dilute sulphuric acid, and scoured with fine Calais sand; they are then ready to be fashioned into various articles.

In plating copper wire, the silver is first formed into a tubular shape, with one edge proiecting slightly over the other; through which a redhot copper cylinder being somewhat loosely run, the silver edges are closely pressed together with a steel burnisher, whereby they get firmly united. The tube, thus completed, is cleaned inside, and put on the proper copper rod, which it exactly fits. The copper is left a little longer than its coating tube, and is grooved at the extremities of the latter, so that the silver edges, being worked into the copper groove, may exclude the air from the surface of the rod. The compound cylinder is now heated redhot, and rubbed briskly over with the steel burnisher in a longitudinal direction, whereby the two metals get firmly united, and form a solid rod, ready to be drawn into wire of any requisite fineness and form; as flat, half-round, fluted, or with mouldings, according to the figure of the hole in the draw-plate. Such wire is musn used for making bread-baskets, toast-racks, snuffers, and articles combining elegance with lightness and economy. The wire must be annealed from time to time during the drawing, and finally cleaned, like the plates, with dilute acid.

Formerly the different shaped vessels of plated metal were all fashioned by the hammer; but every one of simple form is now made in dies struck with a drop-hammer or stamp. Some manufacturers employ 8 or 10 drop machines.

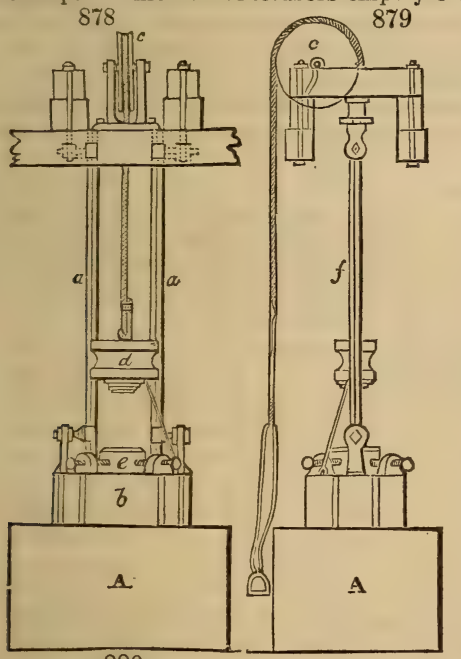

Figs. 878 and 879 are two views of the stamp. $A$ is a large stone, the more massy the better; $b$, the anvil on which the die $e$ is secured by four screws, as shown in the ground plan, fig. 880 . In $f$ ig. 878, $a$ a are two upright square prisms, set diagonally with the angles opposed to each other; between which the hammer or drop $d$ slides truly, by means of nicely fitted angular grooves or recesses in its sides. The hammer is raised by pulling the rope $f$, which passes over the pulley $c$, and is let fall from different heights, according to the impulse required. Vessels which are less in diameter at the top and bottom than in the middle, must either be raised by the stamp in two pieces, or raised with a hand hammer. The die is usually made of cast steel. When it is placed upon the anvil, and the plated metal is cut into pieces of proper size, the top of the die is then surrounded with a lute made of oil and clay, for an inch or two above its surface; and the cavity is filled with melted lead. The under face of the stamp-hammer has a plate of iron called the licker-up fitted into it, about the area of the die. When

880

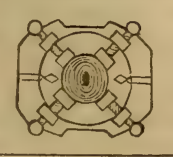
ever the lead has become solid, the hammer is raised to a certair: height, and dropped down upon it; and as the under face of the licker-up is made rough like a rasp, it firmly adheres to the lead, so as to lift it afterward with the hammer. The plated metal is now placed over the die, and the hammer mounted with its lead is let fall repeatedly upon it, till the impression on the metal is complete. If the vessel to be struck be of any considerable depth, two or three dies may be used, of progressive sizes in succession. But it occasionally happens that when the vessel has a long conical neck, recourse must be had to an auxiliary operation, called punching. See the embossing punches, fig. 881 . These

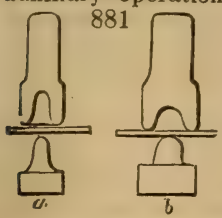
are made of cast steel, with their hollows turned out in the lathe. The pieces $a, b$ are of lead. The punching is performed by a series of these tools, of different sizes, beginning with the largest, and ending with the least. By this means a hollow cone, 3 or 4 inches deep, and an inch diameter, may be raised out of a flat plate. These punches are struck with a hand hammer also, for small articles, of too great delicacy for the drop. Indeed, it frequently happens that one part of an article is executed by the stamp, and another by the hand. 
Cylindrical and conical vessels are mostly formed by bending and soldering. The bending is performed on blocks of wood, with wooden mallets; but the machine so much used by the tin-smiths, to form their tubes and crlindric vessels (see the end section,

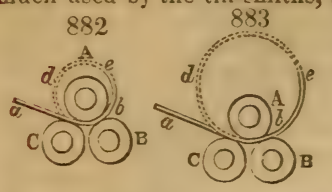
figs. 882 and 883 ), might be employed with advantage. This consists of 3 iron rollers fixed in an iron frame. A, B, c, are the three cylinders, and $a, b, c, d$, the riband or sheet of metal passed through them to receive the cylindrical or conical curvature. The upper roller $\mathrm{A}$ can be raised or lowered at pleasure, in order to modify the diameter of the tube; and when one end of the roller is higher than the other, the conical curvature is given. The edges of the plated crlinders or cones are soldered with an alloy composed of silver and brass. An alloy of silver and copper is somewhat more fusible; but that of brass and silver answers best for plated metal, the brass being in rery small proportion, lest the color of the plate be affected. Calcined borax mixed with sandiver (the salt skimmed from the pots of crown glass) is used along with the alloy, in the act of soldering. The seam of the -lated metal being smeared with that saline mixture made into a pap with water, and the bi:s of laminated solder, cut small with scissors, laid on, the seam is exposed to the flame of an oil blowpipe, or to that of charcoal urged by bellows in a little forge-hearth, till the solder melts and flows evenly along the junction. The use of the sandiver seems to be, to prevent the iron wire that binds the plated metal tube from being soldered to it.

Mouldings are sometimes formed upon the edges of vessels, which are not merely ornamental, but gire strength and stiffness. These are fasnioned by an instrument called a swage, represented in figs. 884 and 885 . The part $\mathrm{A}$ lifts up br a joint, and the metal to be suraged is placed between the dies, as shown in the figures; the tail $b$ being held in the jaws of a rice, while the shear-shaped hammer rests upon it. By striking on the head $\mathrm{A}$, while the metai plate is shifted successirely forwards, the beading is formed. In $f$ g. 884 the tooth $a$ is a guide to regulate the distance between the bead and the edge. A similar effect is produced of late years in a neater and more expe-
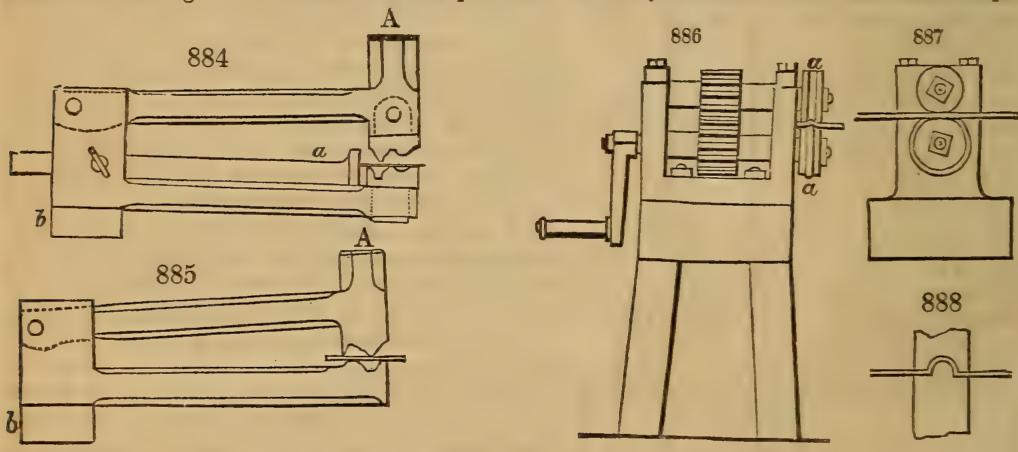

ditious manner by the rollers, figs. 885,886 . Fig. 888 is a section to show the form of the bead. The two wheels $a, a, f i g .886$, are placed upon axes, two of which are furnished with toothed pinions in their middle; the lower one, being turned by the handle, gives motion to the upper. The groove in the upper wheel corresponds with the bead in the lower, so that the slip of metal passed through between them assumes the same figure.

The greatest improvement made in this branch of manufacture, is the introduction of silver edges, beads, and mouldings, instead of the plated ones, which from their prominence had their silver surface speedily worn off, and thus assumed a brassy look. The silver destined to form the ornamental edgings is laminated exceedingly thin; a square inch sometimes weighing no more than 10 or 12 grains. This is too fragile to bear the action of the opposite steel dies of the swage above described. It is necessary, therefore, that the sunk part of the die should be steel, and the opposite side lead, as was observed in the stamping; and this is the method now generally employed to form these silver ornaments. The inside shell of this silver moulding is filled with soft solder, and then bent into the requisite form.

The base of candlesticks is generally made in a die by the stamp, as well as the neck, the dish part of the nczzle or socket, and the tubular stem or pillar. Tive different parts are united, some with soft and others with hard solder. The branches of candlesticks are formed in two semi-cylindrical halves, like the feet of tea-urns. When an article is to be engraved on, an extra plate of silver is applied at the proper part, 
while the plate is still flat, and fixed by burnishing with great pressure over a hot anvil. This is a species of welding.

The last finish of plated goods is given by burnishing-tools of bloodstone, fixed in sheetiron cases, or hardened steel, finely polished.

The ingots for lamination might probably be plated with advantage by the delicate pressure process employed for silvering copper wire.

The total value of the plate, plated ware, jewellery, and watches, exported in the year 1836 , was $338,8891 . ;$ but the value of the plated gonds is not given in the tables of revenue. M. Parquin, the greatest manufacturer of plated goods in Paris (or France, for this business is monopolized by the capital), who makes to the value of $\tau 00,000$ francs per annum, out of the $1,500,000$ which, he says, is the whole internal consumption of the kingdom, states that the internal consumption of the United Kingdom amounts to 30,000,000, or 20 times that of France! He adds, that our common laminated copper costs 26 sous the pound, while theirs costs 34 . Their plated goods are fashioned, not in general with stamps, but by the pressure of tools upon wood moulds in the turning lathe, which is a sreat economy of capital to the manufacturer. There are factories at Birming. ham which possess a heavy stock of 300,000 different die-moulds. See STAmping or Metals.

PLATINA-MOHR. The following easy method of preparing igniferous black plalinum, proposed thirty years ago by Descotil, has been recently recommended by $\mathbf{M}$. Dobereiner :-

Melt : latina ore with double its weight of zinc, reduce the alloy to powder, and treat it first with dilute sulphuric acid, and next with dilute nitric acid, to oxydize and dissolve out all the zinc, which, contrary to one's expectations, is somewhat difficult to do, even at a boiling heat. The insoluble black-gray powder contains some osmiuret of iridium, united with the crude platinum. This compound acts like simple platina-black, after it has been purified by digestion in potash ley, and washing with water. Its oxydizing power is so great, as to transform not only the formic acid into the carbonic, and alcohol into vinegar, but even some osmic acid, from the metallic osmium. The above powder explodes by heat like gunpowder.

When the platina-mohr prepared by means of zinc is moistened with alcohol, it becomes incandescent, and emits osmic acid; but if it be mixed with alcohol into a paste, and spread upon a watch-glass, nothing but acetic acid will be disengaged; affording an elegant means of diffusing the odor of vinegar in an apartment.

PLATINUM is a metal of a grayish-white color, resembling in a good measure polished steel. It is harder than silver, and of about double its density, being of specific gravity 21. It is so infusible, that no considerable portion of it can be melted by the strongest heats of our furnaces. It is unchangeable in the air and water; nor does a white heat impair its polish. The only acid which dissolves it, is the nitro-muriatic; the muriate or chloride thus formed, affords, with pure ammonia or sal ammoniac, a triple salt in a yellow powder, convertible into the pure metal by a red heat. This character distinguishes platinum from every other metal.

Native Platinum.-In the natural state it is never pure, being alloved with several other metals. It occurs only under the form of grains, which are usually flattened, and resemble in shape the gold pepitas. Their size is in general less than linseed, although in some cases they equal hempseed, and, occasionally, peas. One piece brought from Choco, in Peru, and presented to the Cabinet of Berlin, by M. Humboldt, weighs 55 grammes $=850$ grains, or nearly $2 \mathrm{oz}$. avoirdupois. The greatest lump of native platinum known, till of late years, was one in the Royal Museum of Madrid, which was found in 1814 in the gold mine of Condoto, province of Novita, at Choco. Its size is greater than a turkey's egg, (about 2 inches one diameter, and 4 inches the other,) and its weight 760 grammes, $=24 \mathrm{oz}$., or fully $2 \mathrm{lbs}$. troy. See infra.

The color of the grains of native platinum is generally a grayish-white, like tarnished steel. The cavities of the rough grains are often filled with earthy and ferruginous matters, or sometimes with small grains of black oxyde of iron, adhering to the surface of the platinum grains. Their specific gravity is also much lower than that of forged pure platinum; varying from 15 in the small particles, to 18.94 in M. Humboldt's large specimen. This relative lightness is owing to the presence of iron, copper, lead, and chrome; besides its other more lately discovered metallic constituents, palladium, osmium, rhodium, and iridium.

Its main localities in the New Continent, are in the three following districts :-

1. At Choco, in the neighborhood of Barbacoas, and generally on the coasts of the South Sea, or on the western slopes of the Cordillera of the Andes, between the ?d and the 6 th degrees of north latitude. The goid-washings that furnish most platinum, are those of Condoto, in the province of Novita; those of Santa Rita, or Viroviro, of Santa Lucia, of the ravine of Iro, and Apoto, between Novita and Taddo. The deposite of gold and platinum grains is found in alluvial ground, at a depth of about 20 
foet. The gold is separated from the platinum by picking with the hand, and also by amalgamation; formerly, when it was imagined that platinum might be used to dehaso gold, the grains of the former metal were thrown into the rivers, through which mistakev opinion an immense quantity of it was lost.

2. Platinum grains are found in Brazil, but always in the alluvial lands that contain gold, particularly in those of Matto-grosso. The ore of this country is somewhat different from that of Choco. It is in grains, which seem to be fragments of a spongy substance. The whole of the particles are nearly globular, exhibiting a surface formed of small spheroidal protuberances strongly cohering together, whose interstices are clean, and $\epsilon$ ven brilliant.

This platinum includes many small particles of gold, but none of the magnetic ironsand or of the small zircons which accompany the Peruvian ore. It is mixed with small grains of native palladium, which may be recognised by their fibrous or radiated structure, and particularly by their chemical characters.

3. Platinum grains are found in Hayti, or Saint Domingo, in the sand of the river Jacky, near the mountains of Sibao. Like those of Choco, they are in small brilliant grains, as if polished by friction. The sand containing them is quartzose and ferruginous. This native platinum contains, like that of Choco, chromium, copper, osmium, iridium, rhudium, palladium, and probably titanium. Vauquelin could find no gold among the grains.

Platinum has been discovered lately in the Russian territories, in the auriferous sands of Kuschwa, 250 wersts from Ekaterinebourg, and consequently in a geological position which seems to be analogous with that of South America.

These auriferous sands are, indeed, almost all superficial; they cover an argillaceous soil ; and include, along with gold and platinum, débris of dolerite (a kind of green-stone), protoxyde of iron, grains of corundum, \&c. The platinum grains are not so flat as those from Choco, but they are thicker; they have less brilliancy, and more of a leaden hue. This platinum, by M. Laugier's analysis, is similar in purity to that of Choco; hut the leaden-gray grains, which were taken for a mixture of osmium and iridium, are merely an alloy of platinum, containing 25 per cent. of tiese metals.

The mines of Brazil, Columbia, and Saint Domingo furnish altogether only about 400 kilos. of platinum ore per annum; but those of Russia produce above 1800 kilos. The latter were discovered in 1822, and were first worked in 1824. They are all situated in the Ural mountains. The ore is disseminated in an argillaceous sand, of a greenish-gray color, resulting from the disintegration of the surrounding rocks, and constitutes from 1 to 3 parts in 4000 of the sand. Occasionally it has been found in lumps weighing 8 kilogrammes (16 lbs.!), but it generally occurs in blackish angular grains, which contain 70 per cent. of platinum, and 3 to 5 of iridium. The ore of Goro-Blagodatz is in small flattened grains, which contain 88 per cent. of this precious metal. The osmiure of iridium is found upon a great many points of the Urals, throughout a space of 140 leagues, being a product accessory to the gold washings. 32 kilogrammes of osmiure are collected there annually, which contain upon an average 2 per cent. of platinum.

M. Vauquelin found nearly ten per cent. of platinum in an ore of argentiferous copper, which was transmitted to him as coming from Guadalcanal in Spain. This would be the only example of platinum existing in a rock, and in a vein. As the same thing has not again been met with, even in other specimens from Guadalcanal, we must delay drawing geological inferences, till a new example has confirmed the authenticity of the first.

Platinum has been known in Europe only since 1748, though it was noticed by Ulloa in 1741. It was compared at first to gold; and was, in fact, brought into the market under the name of white gold. The term platinum, however, is derived from the Spanish word plata, silver, on account of its resemblance in color to that metal.

The whole of the platinum ore from the Urals is sent to St. Petersburg, where it is treated by the following simple process :-

One part of the ore is put in open platina vessels, capable of containing from 6 to $8 \mathrm{lbs}$, along with 3 parts of muriatic acid at $25^{\circ} \mathrm{B}$. and 1 part of nitric acid at $40^{\circ}$. Thirty of these vessels are placed upon a sand-bath covered with a glazed dome with moveable panes, which is surmounted by a ventilating chimney to carry the vapors out of the laboratory. Heat is applied for 8 or 10 hours, till no more red vapors appear; a proof that the whole nitric acid is decomposed, though some of the muriatic remains. After settling, the supernatant liquid is decanted off into large cylindrical glass vessels, the residuum is washed, and the washing is also decanted off. A fresh quantity of nitro-muriatic acid is now poured upon the residuum. This treatment is repeated till the whole solid matter has eventually disappeared. The ore requires for solution from 10 to 15 times its weight of nitro-muriatic acid, according to the size of its grains.

The solutions thus made are all acid; a circumstance essential to prevent the iridium 
from precipitating with the platinum, by the water of ammonia, which is next added. The deposite being allowed to form, the mother waters are poured off, the precipitate is washed with cold water, dried, and calcined in crucibles of platinum.

The mother-waters and the washings are afterwards treated separately. The first being concentrated to one twelfth of their bulk in glass retorts, on cooling they let fall the iridium in the state of an ammoniacal chloride, constituting a dark-purple powder, oceasionally crystallized in regular octahedrons. The washings are evaporated to dryness in porcelain ressels; the residuum is calcined and treated like fresh ore ; but the platinum it aftords needs a second purification.

For agglomerating the platinum, the spongy mass is pounded in bronze mortars; the powder is passed through a fine sieve, and put into a cylinder of the intended size of the ingot. The cylinder is fitted with a rammer, which is forced in by a coining press, till the powder be much condensed. It is then turned out of the mould, and baked 36 hours in a porcelain liiln, after which it may be readily forged, if it be pure, and may receive any desired form from the hammer. It contracts in volume from 1-6th to 1-5th during the calcination. The cost of the manufacture of platinum is fixed by the administration at 32 francs the Russian pound; but so great a sum is never expended upon it.

For Dr. Wollaston's process, see Phil. Trans. 1829, Part I.

Platinum furnishes most valuable vessels to both analytical and manufacturing chemists. It may be beat out into leaves of such thinness as to be blown about with the breath.

This metal is applied to porcelain by two different processes; sometimes in a rather coarse powder, applied by the brush, like gold, to form ornamental figures; sometimes in a state of extreme division, obtained by decomposing its muriatic solution, by means of an essential oil such as rosemary or lavender. In this case, it must be evenly spread over the whole ground. Both modes of application give rise to a steely lustre.

The properties possessed in common by gold and platinum, have several times given occasion to fraudulent admixtures, which have deceived the assayers. M. Vauquelin having executed a series of experiments to elucidate this subject, drew the following conclusions :-

If the platinum do not exced 30 or 40 parts in the thousand of the alloy, the gold does not retain any of it when the parting is made with nitric acid in the usual way; and when the proportion of platinum is greater, the fraud becomes manifest; 1 st by the higher temperature required to pass it through the cupel, and to form a round button; 2 . by the absence of the lightning, fulguration, or coruscation; 3 . by the dull white color of the button and its crystallized surface; 4. by the straw-yellow color which platinum communicates to the aquafortis in the parting; 5 . by the straw-yellow color, bordering on white, of the cornet, after it is annealed. If the platinum amounts to one fourth of the gold, we must add to the alloy at least 3 times its weight of fine silver, laminate it very thin, anneal somewhat strongly, boil it half an hour in the first aquafortis, and at least a quarter of an hour in the second, in order that the acid may dissolve the whole of the platinum.

Were it required to determine exactly the proportions of platinum contained in an alloy of copper, silver, gold, and platinum, the amount of the copper may be found in the first place by cupellation, then the respective quantities of the three other metals may be learned by a process founded, 1 . upon the property possessed by sulphuric acid of dissclving silver without affecting gold or platinum; and, 2 . upon the property of platinum being soluble in the nitric acid, when it is alloyed with a certain quantity of gold and silver.

According to Boussingault, the annual product of platinum in America does not exceed $8 \frac{1}{3} \mathrm{cwts}$. At Niszhne-Tagilsk, in 1824, a lump of native platinum weighing fully $10 \mathrm{lbs}$. was found; and in 1830, another lump, of nearly double size, which weighed $35 \frac{3}{4}$ Prus. sian mares; fully $18 \mathrm{lbs}$. avoirdupois.

PRODUCTION OF PLATINUM IN THE URAL.

From 1822 to 1827 inclusively, 52 puds* and $22 \frac{1}{2}$ pounds.

$\begin{array}{lrc}1828 & 94 & \\ 1829 & 78 & 31 \frac{1}{2} \\ 1830 & 105 & 1 \\ 1831 \text { to } 1833 & 348 & 15\end{array}$

- One pud $=40$ Russian pounds $,=69,956$ Prussian marcs (See SILver) ; 1 pound $=96$ zolotniks 
Analyses of the Pritinum Ores of the Urals, and of that from Barbacoas on the Pacific, between the $2 \mathrm{~d}$ and 6 th degrees of northern latitude.

\begin{tabular}{|c|c|c|c|c|c|c|}
\hline & \multicolumn{2}{|c|}{$\begin{array}{c}\text { From Nischne-Tagilsk. } \\
\text { Berzelius. }\end{array}$} & \multirow{2}{*}{\multicolumn{2}{|c|}{$\begin{array}{l}\text { Goroblagodat. } \\
\text { Osann. }\end{array}$}} & \multirow[t]{2}{*}{$\begin{array}{l}\text { Barbacoas. } \\
\text { Berzelius. }\end{array}$} \\
\hline & & Magnetic. & Not Magnetic. & & & \\
\hline Platinum & - & $73 \cdot 58$ & 78.94 & $83 \cdot 07$ & $86 \cdot 50$ & $84 \cdot 30$ \\
\hline Iridiun - & - & $2 \cdot 35$ & 4.97 & 1.91 & - & $1 \cdot 46$ \\
\hline Rhodium & - & $1 \cdot 15$ & $0 \cdot 86$ & 0.59 & $1 \cdot 15$ & $3 \cdot 46$ \\
\hline Palladium & - & $0 \cdot 30$ & $0 \cdot 28$ & $0 \cdot 26$ & $1 \cdot 10$ & $1 \cdot 06$ \\
\hline Iron - & - & $12 \cdot 98$ & $11 \cdot 04$ & $10 \cdot 79$ & $8 \cdot 32$ & $5 \cdot 31$ \\
\hline Copper - & - & $5 \cdot 20$ & 0.70 & $1 \cdot 30$ & 0.45 & 0.74 \\
\hline \multirow{2}{*}{\multicolumn{2}{|c|}{$\left.\begin{array}{l}\text { Undissolved } \\
\text { Osmium and } \\
\text { Iridium }\end{array}\right\}$}} & & & & - & \\
\hline & & $2 \cdot 30$ & $1 \cdot 96$ & $1 \cdot 80$ & $1 \cdot 40$ & - \\
\hline Osmium & - & - & - & - & - & 1.03 \\
\hline Quartz - & - & - & - & - & - & $0 \cdot 60$ \\
\hline \multirow[t]{2}{*}{ Lime - } & - & - & - & - & - & $0 \cdot 12$ \\
\hline & & $97 \cdot 86$ & $98 \cdot 75$ & $99 \cdot 72$ & $98 \cdot 92$ & $98 \cdot 08$ \\
\hline
\end{tabular}

PLUMBAGO. See Graphite, for its mineralogical and chemical characters. The mountain at Borrowdale, in which the black-lead is mined, is 2000 feet high, and the entrance to the mine is 1000 feet below its summit. This valuable mineral became so common a subject of robbery about a century ago, as to have enriched, it was said, a great many persons living in the neighborhood. Even the guard stationed over it by the proprietors was of little avail against men infuriated with the love of plunder; since in those days a body of miners broke into the mine by main force, and held possession of it for a considerable time.

The treasure is now protected by a strong building, consisting of four rooms upon the ground floor; and immediately under one of them is the opening, secured by a trapdoor, through which alone workmen can enter the interior of the mountain. In this apartment, called the dressing-room, the miners change their ordinary clothes for their working dress, as they come in, and after their six hours' post or journey, they again change their dress, under the superintendence of the steward, before they are suffered to go out. In the innermost of the four rooms, two men are seated at a large table, sorting and dressing the plumbago, who are locked in while at work, and watched by the steward from an adjoining room, who is armed with two loaded blunderbusses. Such formidable apparatus of security is deemed requisite to check the pilfering spirit of the Cumberland mountaineers.

The cleansed black-lead is packed up into strong casks, which hold $1 \mathrm{cwt}$. each. These are all despatched to the warehouse of the proprietors in London, where the black-lead is sold monthly by auction, at a price of from $35 s$. to $45 \mathrm{~s}$. a pound.

In some years, the net produce of the six weeks' annual working of the mine has, it is said, amounted to $30,000 l$. or $40,000 l$.

PLUsH (Panne, Peluche, Fr.; Wollsammet, Plüsch, Germ.) is a textile fabric, having - a sort of velvet nap or shag upon one side. It is composed regularly of a woof of a single woollen thread, and a two-fold warp, the one, wool of two threads twisted, the other, 'goat's or camel's hair. There are also several sorts of plush made entirely of worsted. It is manufactured, like velvet, in a loom with three treadles; two of which separate and depress the woollen warp, and the third raises the hair-warp, whereupon the weaver, throwing the shuttlc, passes the woof between the woollen and hair warp; afterwards, laying a brass broach or needle under that of the hair, he cuts it with a knife (see FustiAin) dnitined for that use, running its fine slender point along in the hollow of the guide-bruach, to the end of a piece extended upon a table. Thus the surface of the plush receives its velvety appearance. This stuff is also made of cotton and silk.

POINT NET is a style of lace formerly much in vogue, but now superseded by the bobbin-net manufacture.

PORCELAIN is the finest kind of pottery-ware. It is considered under that title.

PORPHYRY is a compound mineral or rock, composed essentially of a base of hornstone, interspersed with crystals of feldspar. It frequently contains also quartz, mica, and hornblende. That most esteemed is the ancient porphyry of Egypt, with a ground of a fine red color passing into purple, having snow-white crystals of feldspar imbedded in it. Most beautiful sp ecimens of it ale iv ie seen in the antıque coiossai statues in the British Museum. 
Porphyry occurs in Arran, and in Perthshire between Dalnacardoch and Tummel bridse. It is much used for making slabs, mullers, and mortars.

PORTER is a malt liquor, so called from being the favorite beverage of the porters and workipeople of the metropolis and other large towns of the British empire; it is characterized by its dark-brown color, its transparency, its moderately bitter taste, and peculiar aromatic flavor, which, along with its tonic and intoxicating qualities, make it be keenly relished by thirsty palates accustomed to its use. At first the essential distinction of porter arose from its wort being made with highly-kilned brown malt, while other kinds of beer and ale were brewed from a paler article; but of late years, the taste of the public having run in favor of sweeter and lighter beverages, the actual porter is brewed with a less proportion of brown malt, is less strongly hopped, and not allowed to get hard by long leeping in huge ripening tuns. Some brewers color the porter with burnt sugar; but in general the most respectable concentrate a quantity of their first and best wort to an extract, in an iron pan, and burn this into a coloring stuff, whereby they can lay claim to the merit of using nothing in their manufacture but malt and hops. The singular flavor of good London porter seems to proceed, in a great degree, from that of the old casks and fermenting tuns in which it is prepared. Though not much addicted to vinous potations of any hind, I feel warranted by long experience to opine, that the porter brewed by the eminent London houses, when drunk in moderation, is a far wholesomer beverage for the people than the thin acidulous wines of France and Germany. See BEER.

PORTLAND STONE is a fine compact oolite, so named from the island where it is quarried. It is a convenient but not a durable building-stone.

POTATO (Pomme de terre, Fr.; Kartoffel, Germ.) is the well known root of the Solanum tuberosum.

The following TABLE exhibits several good analyses of the potato:-

\begin{tabular}{|c|c|c|c|c|c|c|c|}
\hline Sort. & Fibrine. & Starch. & $\begin{array}{l}\text { Veg. } \\
\text { album. }\end{array}$ & Gum. & $\begin{array}{l}\text { Acids and } \\
\text { Salts. }\end{array}$ & Water. & Analyst. \\
\hline Red potatoes - & $7 \cdot 0$ & $15 \cdot 0$ & $1 \cdot 4$ & $4 \cdot 1$ & $5 \cdot 1$ & $75 \cdot 0$ & Einhof. \\
\hline Id. germinated - & $6 \cdot 8$ & $15 \cdot 2$ & $1 \cdot 3$ & $3 \cdot 7$ & - & $73 \cdot 0$ & - \\
\hline Potato sprouts - & $2 \cdot 8$ & $0 \cdot 4$ & $0 \cdot 4$ & $3 \cdot 3$ & - & $93 \cdot 0$ & - \\
\hline Kidney potatoes & $8 \cdot 8$ & $9 \cdot 1$ & $0 \cdot 8$ & - & - & $81 \cdot 3$ & - \\
\hline Large red do. - - & $6 \cdot 0$ & 12.9 & 0.7 & - & - & $78 \cdot 0$ & - \\
\hline Sweet do. - - & $8 \cdot 2$ & $15 \cdot 1$ & $0 \cdot 8$ & - & - & $74 \cdot 3$ & - \\
\hline Potato of Peru & $5 \cdot 2$ & $15 \cdot 0$ & 1.9 & & $1 \cdot 9$ & $76 \cdot 0$ & Lampad. \\
\hline - England & $6 \cdot 8$ & $12 \cdot 9$ & $1 \cdot 1$ & & $1 \cdot 7$ & $77 \cdot 5$ & - \\
\hline Onion potato - & $8 \cdot 4$ & $18 \cdot 7$ & 0.9 & & $1 \cdot 7$ & $70 \cdot 3$ & - \\
\hline - Voigtland & $7 \cdot 1$ & $15 \cdot 4$ & $1 \cdot 2$ & & $2 \cdot 0$ & $74 \cdot 3$ & - \\
\hline $\begin{array}{l}\text { - cultivated in the } \\
\text { - environs of Paris }\end{array}$ & $6 \cdot 79$ & $13 \cdot 3$ & 0.92 & $3 \cdot 3$ & $1 \cdot 4$ & $73 \cdot 12$ & Henry. \\
\hline
\end{tabular}

POTASH, or POTASSA. (Potasse, Fr.; Kali, Germ.) This substance was so named from being prepared for commercial purposes by evaporating in iron pots the lixivium of the ashes of wood fuel. In the crude state called potashes, it consists, therefore, of such constituents of burned regetables as are very soluble in water, and fixed in the fire. The potash salts of plants which originally contained vegetable acids, will be converted into carbonates, the sulphates will become sulphites, sulphurets, or even carbonates, according to the manner of incineration; the nitrates will be changed into pure carbonates, while the muriates or chlorides will remain unaltered. Should quicklime be added to the solution of the ashes, a corresponding portion of caustic potassa will be introduced into the product, with more or less lime, according to the care taken in decanting off the clear ley for evaporation.

In America, where timber is in many places an incumbrance upon the soil, it is ielled, piled up in pyramids, and burned, solely with a view to the manufacture of potashes. The ashes are put into wooden cisterns, having a plug at the bottom of one of the sides under a false bottom; a moderate quantity of water is then poured on the mass, and some quicklime is stirred in. After standing for a few hours, so as to take up the soluble matter, the clear liquor is drawn off, evaporated to dryness in iron pots, and finally fused at a red heat into compact masses, which are gray on the outside, and pink-colored within.

Pearlash is prepared by calcining potashes upon a reverberatory hearth, till the whole carbinnaceous motter. and the oreater rart ct the sulnhur, be dissipaied; then lixiviating the mass, in a cistern naving a faise jotrom covered witn straw, evaporating the clear ley 
to dryness in flat iron pans, and stirring it towards the end into white lumpy granula. tions.

I find the best pink Canadian potashes, as imported in casks containing about $5 \mathrm{cwts}$, to contain pretty uniformly 60 per cent. of absolute potassa; and the best pearl-ashes to contain 50 per cent. ; the alkali in the former being nearly in a caustic state; in the latter, carbonated.

All kinds of vegetables do not yield the same proportion of potassa. The more succulent the plant, the more does it afford; for it is only in the juices that the vegutable salts reside, which are converted by incineration into alkaline matter. Herbaceous weeds are more productive of potash than the graminiferous species, or shrubs, and these than trees; and for a like reason, twigs and leaves are more productive than timber. But plants in all cases are richest in alkaline salts when they have arrived at maturity. The soil in which they grow also influences the quantity of saline matter.

The following TABLE exhibits the average product in potassa of several plants, according to the researches of Vauquelin, Pertuis, Kirwan, and De Saussure :-

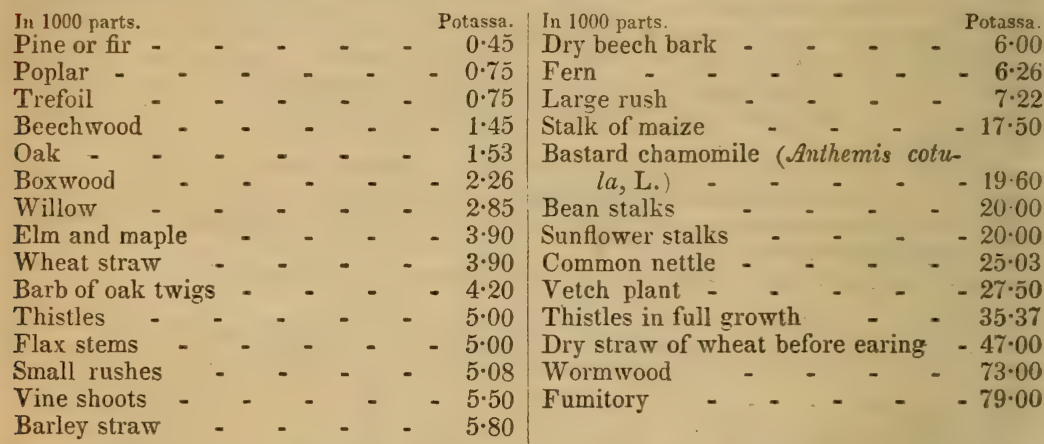

Stalks of tobacco, potatoes, chestnuts, chestnut husks, broom, heath, furze, tansy, sorrel, vine leaves, beet leaves, orach, and many other plants, abound in potash salts. In Burgundy, the well-known cendres gravelées are made by incinerating the lees of wine pressed into cakes, and dried in the sun; the ashes contain fully 16 per cent. of potassa.

The purification of pearlash is founded upon the fact of its being more soluble in water than the neutral salts which debase it. Unon any given quantity of that substance, in an iron pot, let one and a half times its weight of water be poured, and let a gentle heat be applied for a short time. When the whole has again cooled, the bottom will be incrusted with the salts, while a solution of nearly pure carbonate of potash will be found floating above, which may be drawn off clear by a syphon. The salts may be afterwards thrown upon a filter of gravel. If this ley be diluted with 6 times its bulk of water mixed with as much slaked lime as there was pearlash employed, and the mixture be boiled for an hour, the potash will become caustic, by giving up its carbonic acid to the lime. If the clear settled lixivium be now syphoned off, and concentrated by boiling in a covered iron pan, till it assumes the appearance of oil, it will constitute the common caustic of the surgeon, the potassa fusa of the shops. But to obtain potassa chemically pure, recourse must be had to the bicarbonate, nitrate, or tartrate of potassa, salts which, when carefully crystallized, are exempt from any thing to render the potassa derived from them impure. The bicarbonate having been gently ignited in a silver basin, is to be dissolved in 6 times its weight of water, and the solution is to be boiled for an hour, along with one pound of slaked lime for every pound of the bicarbonate used. The whole must be left to settle without contact of air. The supernatant ley is to be drawn off by a syphon, and evaporated in an iron or silver vessel provided with a small orifice in its close cover for the escape of the steam, till it assumes, as above, the appearance oi oil, or till it be nearly redhot. Let the fused potassa be now poured out upon a hright plate of iron, cut into pieces as soon as it concretes, and put up immediately in a bottle furnished with a well-ground stopper. It is hydrate of potassa, being composed of 1 atom of potassa $48,+1$ atom of water $9,=57$.

A pure carbonate of potassa may be also prepared by fusing pure nitre in an earthen crucible, and projecting charcoal into it by small bits at a time, till it ceases to cause deflagration. Or a mixture of 10 parts of nitre and 1 of charcoal may be deflagrated in small successive portions in a redhot deep crucible. When a mixture of 2 parts of tartrate of potassa, or crystals of tartar, and 1 of nitre, is deflagrated, pure carbonate of 
potassa remains mixed with charcoal, which by lixiviation, and the agency of quicklime, will afford a pure hydrate. Crystals of tartar calcined alone yield also a pure carbonate.

Caustic potassa, as I have said, after being fused in a silver crucible at a red heat, retains 1 prime equivalent of water. Hence its composition in 100 parts is, potassium 70, oxygen 14, water 16 . Anhydrous potassa, or the oxyde free from water, can be obtained unly by the combustion of potassium in the open air. It is composed of $83 \frac{1}{3}$ of netal, and $16 \frac{2}{3}$ of oxygen. Berzelius's numbers are, 83.05 and 16.95 .

Caustic potassa may be crystallized; but in general it occurs as a white brittle substance of spec. grav. 1.708, which melts at a red heat, evaporates at a white heat, deliquesces into a liquid in the air, and attracts carbonic acid; is soluble in water and alcohol, forms soft soaps with fat oils, and soapy-looking compounds with resins and wax; dissolves sulphur, some metallic sulphurets, as those of antimony, arsenic, \&c., as also silica, alumina, and certain other bases; and decomposes animal textures, as hair, wool, silk, horn, skin, \&c. It should never be touched with the tongue or the fingers.

The following TaBLE exhibits the quantity of Fused Potassa in 100 parts of caustic ley, at the respective densities :-

\begin{tabular}{|c|c|c|c|c|c|c|c|c|c|}
\hline $\mathrm{s}_{\mathrm{P}} \cdot \mathrm{gr}$. & Pot. in 100. & Sp. gr. & Pot. in 100 & Sp. gr. & Pot. in 100 & Sp. gr. & Pot, in 100. & Sp. gr. & Pot.in10 \\
\hline 1.58 & 66 & 16 & & 1 & & & & $1 \cdot 10$ & $11 \cdot 28$ \\
\hline 1.5 & & & & & & & & & $9 \cdot 20$ \\
\hline 1.54 & 9 & $1 \cdot 42$ & 37. & & & & & 6 & $7 \cdot 02$ \\
\hline $1 \cdot 52$ & & $1 \cdot 40$ & 35.99 & $1 \cdot 28$ & & $1 \cdot 16$ & & $1 \cdot 04$ & $4 \cdot 77$ \\
\hline 1.50 & $46 \cdot 45$ & $1 \cdot 38$ & $34 \cdot 74$ & $1 \cdot 26$ & $26 \cdot 34$ & $1 \cdot 14$ & 15 & $1 \cdot 02$ & $2 \cdot 44$ \\
\hline $1 \cdot 48$ & $44 \cdot 40$ & $1 \cdot 36$ & $33 \cdot 46$ & $1 \cdot 24$ & $24 \cdot 77$ & $1 \cdot 12$ & $13 \cdot 30$ & $1 \cdot 00$ & 0.00 \\
\hline
\end{tabular}

The only certain way of determining the quantity of free potassa in any solid or liquid, is from the quantity of a dilute acid of known strength which it can saturate.

The hydrate of potassa, or its ley, often contains a notable quantity of carbonate, the presence of which may be detected by lime water, and its amount be ascertained by the loss of weight which it suffers, when a weighed portion of the ley is poured into a weighed portion of dilute sulphuric acid poised in the scale of a balance.

There are two other oxydes of potassium; the suboxyde, which consists, according to Berzelius, of $90 \cdot 74$ of metal, and $9 \cdot 26$ oxygen; and the hyperoxyde, an orange-yellow substance, which gives off oxygen in the act of dissolving in water, and becomes potassa. It consists of 62 of metal, and 38 of oxygen.

Carbonate of potassa is composed of 48 parts of base, and 22 of acid, according to most British authorities; or, in 100 parts, of 68.57 and 31.43 ; but according to Berzelius, of $68 \cdot 09$ and $31 \cdot 91$.

Carbonate of potassa, as it exists associated with carbon in calcined tartar, passes very rearlily into the Bicarbonate, on being moistened with water, and having a current of carbonic acid sas passed through it. The absorption takes place so rapidly, that the mass becomes hot, and therefore ought to be surrounded with cold water. The salt should then be dissolved in the smallest quantity of water at $120^{\circ} \mathrm{F}$., filtered, and crystallized.

POTASSIUM (Eng. and Fr.; Kalium, Germ.) is a metal deeply interesting, not only from its own marvellous properties, but from its having been the first link in the chain of discovery which conducted Sir H. Davy through many of the formerly mysterious and untrodden labyrinths of chemistry.

The easiest and best mode of obtaining this elementary substance, is that contrived by Brunner, which I have often practised upon a considerable scale. Into the orifice of one of the iron bottles, as $\mathrm{A}, \mathrm{fig} .889$, in which mercury is imported, adapt, by screwing, a piece of gun-barrel tube, 9 inches long; having brazed into its side, about 3 inches from its outer end, a similar piece of iron tube. Fill this retort two thirds with a mixture of 10 parts of cream of tartar, previously calcined in a covered crucible, and 1 of charcoal, both in powder; and lay it horizontally in an air-furnace, so that while the screw orifice is at the inside wah, ihe extremity of the straight or nozzle tube may project a few inches besond the brickwork, and the tube brazed into it at right angles may descend pretty close to the outside wall, so as to din its lower end a quarter of an inch beneath the surface of some rectified naptha contained in a copper bottle surrounded by ice-cold water. By bringing the condenser-vessel so near the furnace, the tubes along which the potassium vapor requires to pass, run less risk of getting obstructed. The horizontal straight end of the nozzle tube should be shut by screwing a stopcock air-tight into it. By opening the cock momentarily, and thrusting in a hot wire, this tube may be readily kept free, without permitting any considerable waste of potassium. The heat should be slowly applied at first, but eventually urged to whiteness, and continued as long 
as potassureted hydrogen continues to be disengaged. The retort, and the part of the nozzle tube exposed to the fire, should be covered with a good refractory lute, as described under the article Phosphorus. The joints must be perfectly air-tight; and the vessel freed from every trace of mercury, by ignition, before it is charged with the tartar-ash.

'Tartar' skilfully treated in this way will afford 3 per cent. of potassium; and when it is observed to send forth green fumes, it has commenced the production of the metal. Instead of the construction above described, the following form of apparatus may be employed.

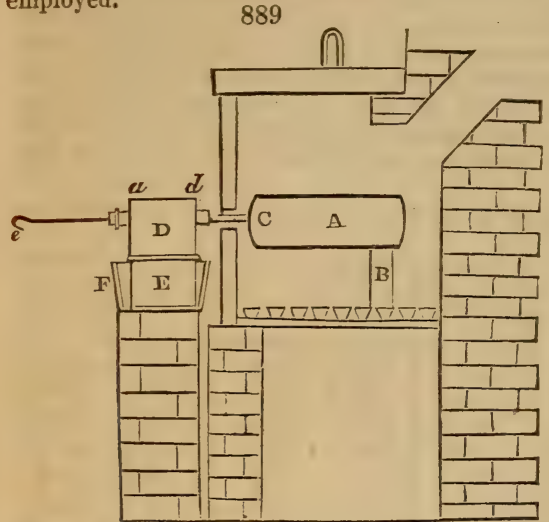

A, fig. 889, represents the iron bottle, charged with the incinerated tartar; and $B$ is a fire-brick support. A piece of fire. tile should also be placed between the bottom of the bottle and the back wal. of the furnace, to keep the apparatus steady during the operation. Whenever the moisture is expelled, and the mass faintly ignited, the tube $\mathrm{c}$ should be screwed into the mouth of the bottle, through a small hole left for this purpose in the side of the furnace. That tube should be no longer, and the front wall of the furnace no thicker, than what is absolutely necessary. As soon as the reduction is indicated by the emission of green vapors, the receiver must be adapted, $d, a, \mathrm{D}, \mathbf{E}$, shown in a large scale in fig. 890 .

This is a condenser, in 1 wo pieces, made of thin sheet copper; $D$, the upper part, is a rectangular box, open at bottom, about 10 inches high, by 5 or 6 long, and 2 wide; near to the side $a$, it is divided inside into two equal compartments, up to two thirds 890
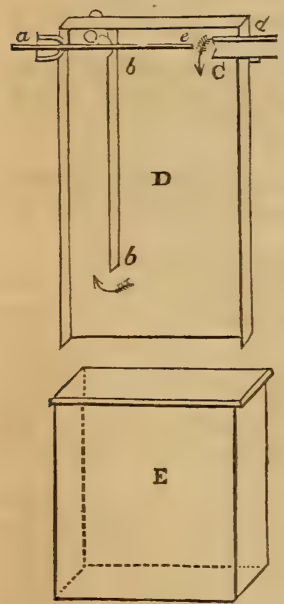
of its height, by a partition $b, b$, in order to make the vapors that issue from $\mathrm{c}$ pursue a downward and circuitous path. In each of its narrow sides, near the top, a short tube is soldered, at $d$ and $a$; the former being fitted air-tight into the end of the nozzle of the retort, while the latter is elosed with a cork traversed by a stiff iron probe $e$, which passes through a small hole in the partition $b, b$, under $c$, and is employed to keep the tube c clear, by its drill-shaped steel point. In one of the broad sides of the box $D$, near the top, a bit of pipe is soldered on at $c$, for receiving the end of a bent glass tube of safety, which dips its other and lower end into a glass containing naptha. $\mathrm{E}$, the bottom copper box, with naptha, which receives pretty closely the upper case $D$, is to be inmersed in a cistern of cold water containing some lumps of ice.

The chemical action by which potassa is reduced in this process seems to be somewhat complicated, and has not been thoroughly explained. A very small proportion of pure potassium is obtained; a great deal of it is converted into a black infusible mass, which passes over with the metal, and is very apt to block up the tube. Should this resist clearing out with the probe, the fire must be immediately withdrawn from the furnace, otherwise the apparatus will probably burst or blow up. Care must be taken to prevent any moisture getting into the nozzle, for it would probably produce a violent detonation.

When the operation has proceeded regularly, accompanied to the end with a constant evolution of gas, the retort becomes nearly emptr, or contains merely a littlc charcoal, or carbonate of potassa, and the potassium collects in the naptha at the bottom of the receirer $\mathrm{E}$, in the form of globules or rounded lumps, of greater or less size, and of a learlen hue. But the greater part of the metal escapes with the gas, in a state of combination not well understood. This gaseous compound burns with a white or reddish-white flame, and deposites potassa. Sereral ounces of potassium may be produced in this way at one operation; but, as thus obtained, it always contains some combined charcoal, which must be separated by distilling it in an iron retort, haring its beak plunged in naptha.

Pure potassium, as procured in Sir H. Darr's original method, by acting upon fused potassa under a film of naptha, with the negative wire of a powerful voltaic battery, is very like quicksilver. It is semi-fiuid at $60^{\circ} \mathrm{Fahr}$, nearly liquid at $92^{\circ}$, and entireli 
so at $120^{\circ}$. It $50^{\circ}$ it is malleable, and has the lustre of polished silver; at $32^{\circ}$ it is brittle, with a crystalline fracture; and at a heat approaching to redness, it begins to boil, is volatilized, aid converted into a green colored gas, which condenses into globules upon the surface of a cold body. Its specific gravity in the purest state is 0.865 at $60^{\circ}$. When heated in the air, it takes fire, and burns very vividly. It has a stronger affinity for oxygen than any other linown substance; and is hence very difficult to preserve in the metallic state. At a high temperature it reduces almost every oxygenated body. When thrown upon water, it lindles, and moves about violently upon the surface, burning with a red flame till it be consumed; that is to say, converted into potassa. When thrown upon a cake of ice, it likewise kindles, and burns a hole in it. If a globule of it be laid upon wet turmeric paper, it takes fire, and runs about, marking its desultory path with red lines. The flame observed in these cases is owing chiefly to hydrogen, for it is at the expense of the water that the potassium burns.

Potassa, even in a pretty dilute solution, produces a precipitate with muriate of platinum, a phenomenon which distinguishes it from soda. It forms, moreover, with sulphuric and acetic acids, salts which crystallize very differently from the sulphates and acetates of soda.

POTTERY, PORCELAIN. (Eng. and Fr.; Steingut, Porzellun, Germ.) The French, who are fond of giving far-fetched names to the most ordinary things, have

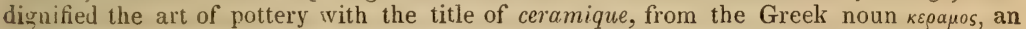
earthen pot, compounded of two words which signify, in that language, burned clay. In reference to chemical constitution, there are only two genera of baked stoneware. The first consists of a fusible earthy mixture, along with an infusible, which when combined are susceptible of becoming semi-vitrified and translucent in the kiln. This constitutes porcelain or china-ware; which is either hard and genuine, or tender and spurious, according to the quality and quantity of the fusible ingredient. The second kind consists of an infusible mixture of earths, which is refractory in the kiln, and continues opaque. This is pottery, properly so called; but it comprehends several subspecies, which graduate into each other by imperceptible shades of difference. To this head belong earthenware, stoneware, flintware, fayence, delftware, iron-stone china, \&c.

The earliest attempts to make a compact stoneware, with a painted glaze, seem to have originated with the Arabians in Spain, about the 9 th century, and to have passed thence into Majorea, in which island they were carried on with no little success. In the 14th century, these articles, and the art of imitating them, were highly prized by the Italians, under the name of Majolica, and porcelana, from the Portuguese word for a cup. The first fabric of stoneware possessed by them was erected at Fayenza, in the ecclesiastical state, whence the French term fayence is derived. The body of the ware was usually a red clay, and the glaze was opaque, being, formed of the oxydes of lead and tin, along with potash and sand. Bernhard de Pallissy, about the middle of the 16th century, manufactured the first white fayence, at Saintes, in France; and not long afterwards the Dutch produced a similar article, of substantial make, under the name of delftware, and delft porceluin, but destitute of those graceful forms and paintings for which the ware of Fajenza was distinguished. Common fayence may be, therefore, regarded as a strong, well-burned, but rather coarse-grained kind of stoneware.

It was in the 17 th century that a small work for making earthenware of a coarse description, coated with a common lead glaze, was formed at Burslem, in Staffordshire, which may be considered as the germ of the vast potteries now established in that county. The manufacture was improved about the year 1690 , by two Dutchmen, the brothers Elers, who introduced the mode of glazing ware by the vapor of salt, which they threw by nandfuls at a certain period among the ignited goods in the kiln. But these were rude, unscientific, and desultory efforts. It is to the late Josiah Wedgewood, Esq. that this country and the world at large are mainly indebted for the great modern advancement of the ceramic art. It was he who first erected magnificent factories, where every resource of mechanical and cr $n$ mical science was made to co-operate with the arts of painting, sculpture, and statuar, in perfecting this valuable department of the industry of nations. So sound were his principles, so judicious his plans of procedure, and $s 0$ ably have they been prosecuted by his successors in Staffordshire, that a population of 60,000 operatives now derives a comfortable subsistence within a district formerly bleak and barren, of 8 miles long by 6 broad, which contains 150 kilns, and is significantly called the Potteries.

\section{OF THE MATERIALS OF POTTERY OR PORCELAIN, AND THEIR PREPARATION.}

1. Clay.-The best clay from which the Staffordshire ware is made, comes from Dorsetshire; and a second quality from Devonshire; but both are well adapted for working, being refractory in the fire, and becoming very white when burnt. The clay is cleaned as much as possible by hand, and freed from loosely adhering stones at th 
pits where it is lug. In the factory mounted by Mr. Wedgewood, which may be re garded as a type of excellence, the clay is cut to pieces, and then kneaded into a pulp with water, by engines; instead of being bioken down with pickaxes, and worked with water by hand-paddles, in a square pit or water-tank, an old process, called blunging. The clay is now thrown into a cast-iron cylinder, 20 inches wide, and 4 feet high, or into a cone 2 feet wide at top, and 6 feet deep, in whose axis an upright shaft revolves, bearing knives as radii to the shaft. The knives are so arranged, that their flat sides lie in the plane of a spiral line; so that by the revolution of the shaft, they not only cut through everything in their way, but constantly press the soft contents of the erlinder or cone obliquely downwards, on the principle of a screw. Another set of knives stands out motionless at right angles from the inner surface of the cylinder, and projects nearly to the central shaft, having their edges looking opposite to the line of motion of the revolving blades. Thus the two sets of slicing implements, the one active, and the other passive, operate like shears in cutting the clay into small pieces, while the active blades, by their spiral form, force the clay in its comminuted state out at an aperture at the bottom of the cylinder or cone, whence it is conveyed into a cylindrical vat, to be worked into a pap with water. This cylinder is tub-shaped, being about 4 times wider than it is deep. A perpendicular shaft turns also in the axis of this vat, bearing eross spokes one below another, of which the vertical set on each side is connected by apright staves, giving the moveable arms the appearance of two or four opposite square paddleboards revolving with the shaft. This wooden framework, or large blunger, as it is called, turns round amidst the water and clay lumps, so as to beat them into a fine pap, from which the stony and coarse sandy particles separate, and subside to the bottom. Whenever the pap has acquired a cream-consistenced uniformity, it is run off through a series of wire, lawn, and silk sieves, of different degrees of fineness, which are kept in continual agitation backwards and forward by a crank mechanism; and thus all the grosser parts are completely separated, and hindered from entering into the composition of the ware. This clay liquor is set aside in proper cisterns, and diluted with water to a standard density.

2. But clay alone cannot form a proper material for stoneware, on account of its great contractility by heat, and the consequent cracking and splitting in the kiln of the vessels made of it; for which reason, a silicious substance incapable of contraction must enter into the body of pottery. For this purpose, ground flints, called fintpowder by the potters, is universally preferred. The nodules of flint extracted fiom the chalk formation are washed, heated redhot in a kiln, like that for burning lime, and thrown in this state into water, by which treatment they lose their translucency, and become exceeding brittle. They are then reduced to a coarse powder in a stampingmill, similar to that for stamping ores; see MEtallurgy. The pieces of flint are laid on a strong grating, and pass through its meshes whenever they are reduced by the stamps to a certain state of comminution. This granular matter is now transferred to the proper flint-mill, which consists of a strong cylindrical wooden tub, bottomed with flat pieces of massive chert, or hornstone, over which are laid large flat blocks of similar chert, that are moved round over the others by strong iron or wooden arms projecting from an upright shaft made to revolve in the axis of the mill-tub. Sometimes the active blocks are fixed to these cross arms, and thus carried round over the passive blocks at the bottom. See infrù, under PorcexaIn, figures of the flint and feldspar mill. Into this cr?indrical vessel a small stream of water constantly trickles, which facilitates the grinding motion and action of the stones, and works the flint powder and water into a species of pap. Near the surface of the water there is a plug-hole in the side of the tub, by which the creamy-looking flint liquor is run off from time to time, to be passed through lawn or silk sieves, similur to to se used for the clay liquor; while the particles that remain on the sieves are returned into the mill. This pap is also reduced to a standard density by dilution with water; whence the weight of dry silicious earth present, may be deduced from the measure of the liquor.

The standard clay and flint liquors are now mixed together, in such proportion by measure, that the flint powder may bear to the dry clay the ratio of one to five, or occasionally one to six, according to the richness or plasticity of the clay; and the liquors are intimately incorporated in a revolving churn, similar to that employed for making the claypap. This mixture is next freed from its excess of water, by evaporation in oblong stone troughs, called slip-kilns, bottomed with fire-tiles, under which a furnace flue runs. The breadth of this evaporating trough varies from 2 to 6 feet; its length from 20 to 50 ; and its depth from 8 to 12 inches, or more.

By the dissipation of the water, and careful agitation of the pap, a uniform doughy mass is obtained; which, being taken out of the trough, is cut into cubical lumps. These are piled in heaps, and left in a damp cellar for a considerable time; that is, several months, in large manufactories. Here the dough suffers disintegration, pror doted by a kind of fermentative action, due probabiy to some vegetable matter in the water 
and the clay; for it becomes black, and exhales a fetid odor. The argillaceous and silicious particles get disintegrated also by the action of the water, in such a way that the ware made with old paste is found to be more homogeneous, finer grained, and not so apt to erack or to get disfigured in the baking, as the ware made with newer paste.

But this chemical comminution must be aided by mechanical operations; the first of which is called the potter's sloping or wedging. It consists in seizing a mass of clay in the hands, and, with a twist of both at once, tearing it into two pieces, or cutting it with a wire. These are again slapped together with force, but in a different direction from that in which they adhered before, and then dashed down on a board. The mass is once more torn or cut asunder at right angles, again slapped together, and so worked repeatedly for 20 or 30 times, which ensures so complete an incorporation of the different parts, that if the mass had been at first half black and half white clay, it would now be of a uniform gray color. A similar effect is produced in some large establishments by a slicing machine, like that used for eutting down the clay lumps as they come from the pit.

In the axis of a cast iron cylinder or cone, an upright shaft is made to revolve, from which the spiral-shaped blades extend, with their edges placed in the direction of rotation. The pieces of clay subjected to the action of these knives (with the reaction of fixed ones) are minced to small morcels, which are forced pell-mell by the screw-like pressure into an opening of the bottom of the cylinder or cone, from which a horizontal pipe about 6 inches square proceeds. The dough is made to issue through this outlet, and is then cut into lengths of about 12 inches. These clay pillars or prisms are thrown back into the cylinder, and subjected to the same operation again and again, till the lumps have their particles perfectly blended together. This process mar advantageously precede their being set aside to ripen in a damp cellar. In France the stoneware dough is not worked in such a machine; but after being beat with wooden mallets, a practice common also in England, it is laid down on a clean floor, and a workman is set to tread upon it with naked feet for a considerable time, walking in a spiral direction from the centre to the circumference, and from the circumference to the centre. In Sweden, and also in China (to judge from the Chinese paintings which represent their manner of making porcelain), the clay is trodden to a uniform mass by oxen. It is afterwards, in all cases, kneaded like baker's dough, by folding back the cake upon itself, and kneading it out, alternately.

The process of slapping consists in cutting through a large mass with a wire, lifting up either half in both hands, and casting it down with great violence on the other; and this violent treatment of the clay is repeated till every appearance of air-bubbles is removed, for the smallest remaining vesicle expanding in the kiln, would be apt to cause blisters or warts upon the ware.

Having thus detailed the preparation of the stoneware paste, we have next to describe the methods of forming it into articles of various forms.

Throwing is performed upon a tool called the potter's lathe. (See fig., infrà.) This consists of an upright iron shaft, about the height of a common table, on the top of which is fixed, by its centre, a horizontal disc or circular piece of wood, of an area sufficiently great for the largest stoneware vessel to stand upon. The lower end of the shaft is pointed, and runs in a conical step, and its collar, a little below the top-board, being truly turned, is embraced in a socket attached to the wooden frame of the lathe. The shaft has a pulley fixed upon it, with grooves for 3 speeds, over which an endless band passes from a fly-wheel, by whose revolution any desired rapidity of rotation may be given to the shaft and its top-board. This wheel, when small, may be placed alongside, as in the turner's lathe, and then it is driven by a treadle and crank; or when of larger dimensions, it is turned by the arms of a laborer. Sometimes, indeed, the wooden plate is replaced by a large thick disc of Paris plaster, which is whirled round by the hand of the potter, without the intervention of a pulley and fly-wheel, and affords sufficient centrifugal power for fashioning small vessels. The mass of dough to be thrown, is weighed out or gauged by an experienced hand. The thrower dashes down the lump on the centre of the revolving board, and dipping his hands frequently in an adjoining tub of water, he works up the clay into a tall irregular cylinder, and then down into a cake, alternately, till he has secured the final extrication of air-bubbles, and then gives the proper form to the vessel under a less speed of rotation, regulating its dimensions by wooden pegs and gauges. He now cuts it off at the base with a piece of fine brass wire, fastened to a handle at either end. The vessel thus rudely fashioned is placed in a situation where it may dry gradually to a proper point. At a certain stage of the drying, called the green state, it possesses a greater tenacity than at any other, till it is baked. It is then taken to another lathe, called the turning lathe, where it is attached by a little moisture to the vertical face of a wooden chuck, and turned nicely into its proper shape with a very sharp tool, which also smooths it. After this it is slightly burnished with a smooth steel surface. 
DESCRIPTION OF THE POTTER'S LATHE.

A, fig. 891, is the profile of the English potter's lathe, for blocking out round ware; $\mathrm{c}$ is the table or tray ; $a$ is the head of the lathe, with its horizontal disc ; $a, b$, is the upright shaft of the head; $d$, palleys with several grooves of different diameters, fixed upon the shaft, for receiving the driving-cord or band; $k$ is a bench upon which the workman sits astride; $e$, the treadle foot-board; $l$ is a ledge-board,

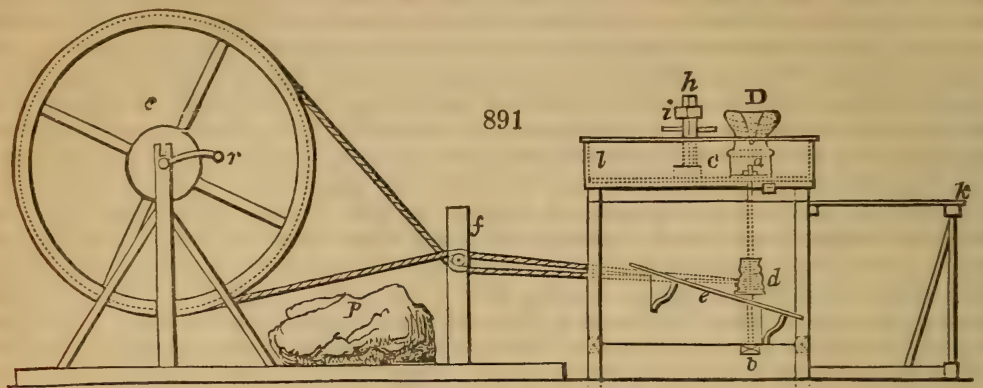

for catching the sharings of clay which fly off from the lathe; $h$ is an instrument, with a slide-nut $i$, for measuring the objects in the blocking out; $c$ is the fly-wheel with its winch-handle $r$, turned by an assistant; the sole-frame is secured in its place by the heavy stone $p ; f$ is the oblong guide-pulley, having also sereral grooves for converting the vertical movement of the fly-wheel into the horizontal movement of the head of the lathe.

$D$ is one of the intermediate forms given by the potter to the ball of clay, as it revolves upon the head of the lathe.

In large potteries, the whole of the lathes, both for throwing and turning, are put in motion by a steam-engine. The vertical spindle of the lathe has a bevel wheel on it, which works in another bevel toothed wheel fixed to a horizontal shaft. This shaft is prorided with a long conical wooden drum, from which a strap ascends to a similar conical drum on the main lying shaft. The apex of the one cone corresponds to the base of the other, which allows the strap to retain the same degree of tension (see the conical drum apparatus of the Stearine-press), while it is made to traverse horizontally, in order to vary the speed of the lathe at pleasure. When the belt is at the base of the drivingcone, it works near the vertex of the driven one, so as to give a maximum velocity to the lathe, and vice versâ.

During the throwing of any article, a separate mechanism is conducted by a boy, which makes the strap move parallel to itself along these conical drums, and nicely regulates the speed of the lathe. When the strap runs at the middle of the cones, the velocity of each shaft is equal. By this elegant contrivance of parallel cones reversed, the velocity rises gradually to its maximum, and returns to its minimum or slower motion when the workman is about finishing the article thrown. The strap is then transferred to a pair of loose pulleys, and the lathe stops. The vessel is now cut off at the base with small wire; is dried, turned on a power lathe, and polished as above described.

The same degree of dryness which admits of the clay being turned on the larhe, also suits for fixing on the handles and other appendages to the vessels. The parts to be attached, being previously prepared, are joined to the circular work by means of a thin paste which the workmen call slip, and the seams are then smoothed off with a wet sponge. They are now taken to a stove-room heated to $80^{\circ}$ or $90^{\circ} \mathrm{F}$., and fitted up with a great many shelves. When they are fully dried, they are smoothed over with a small bundle of hemp, if the articles be fine, and are then ready for the kiln, which is to convert the tender clay into the hard biscuit.

A great variety of pottery wares, however, cannot be fashioned on the lathe, as they are not of a circular form. These are made by two different methods, the one called press-work, and the other casting. The press-work is done in moulds made of Paris plaster, the one half of the pattern being formed in the one side of the mould, and the other half in the other side: these moulding-pieces fit accurately together. All vessels of an oval form, and such as have flat sides, are made in this way. Handles of teapots, and fluted solid rods of various shapes, are formed by pressure also; viz., by squeezing the dongh contained in a pump-barrel through different shaped orifices at its hottom, by working a screw applied to the piston-rod. The worm-shaped dongh, as it issues, is cut to proper lengths, and bent into the desired form. Tubes may be also made on the same pressure principle, only a tubular opening must be provided in the bottom plate of the clay-forcing pump. 
The other method of fashioning earthenware articles is called casting, and is, perhaps the most elegant for such as have an irregular shape. This operation consists in pouring the clay, in the state of pap or slip, into plaster moulds, which are kept in a desiccated state. These moulds, as well as the pressure ones, are made in halves, which nicely correspond together. The slip is poured in till the cavity is quite full, and is left in the mould for a certain time, more or less, according to the intended thickness of the vessel. The absorbent power of the plaster soon abstracts the water, and makes the coat of clay in contact with it quite doughy and stiff, so that the part still liquid being poured out, a hollow shape remains, which when removed from the mould constitutes the half of the vessel, bearing externally the exact impress of the mould. The thickness of the clay varies with the time that the paste has stood upon the plaster. These cust articles are dried to the green state, like the preceding, and then joined accurately with slip. Imitations of flowers and foliage are elegantly executed in this way. This operation, which is called furnishing, requires very delicate and dexterous manipulation.

The saggers for the unglazed colored stoneware should be covered inside with a glaze composed of 12 parts of common salt and 30 of potash, or 6 parts of potash and 14 of salt; which may be mixed with a little of the common enamel for the glazed pottery saggers. The bottom of each sagger has some bits of flints sprinkled upon it, which become so adherent after the first firing as to form a multitude of little prominences for setting the ware upon, when this does not consist of plates. It is the duty of the workmen belonging to the glaze kiln to make the saggers during the intervals of their work; or, if there be a relay of hands, the man who is not firing makes the saggers.

The English kilns differ from those of France and Germany, in their construction, in the nature of their fuel, and in the high temperature required to produce a surface sufficiently hard for a perfectly fine glaze.

When the ware is sufficiently dry, and in sufficient quantity to fill a kiln, the next process is placing the various articles in the baked fire-clay vessels, which may be either of a cylindrical or oval shape; called gazettes, Fr. ; kapseln, Germ. These are from 6 to 8 inches deep, and from 12 to 18 inches in diameter. When packed full of the dry ware, they are piled over each other in the kiln. The bottom of the upper sagger forms the lid of its fellow below ; and the junction of the two is luted with a ring of soft clay applied between them. These dishes protect the ware from being suddenly and unequally heated, and from being soiled by the smoke and vapors of the fuel. Each pile of saggers is called a bung.

POTTERY KILN OF STAFFORDSHIRE.

Figs. 892, 893, 894, 895, 896, represent the kiln for baking the biscuit, and also for rumning the glaze, in the English potteries.

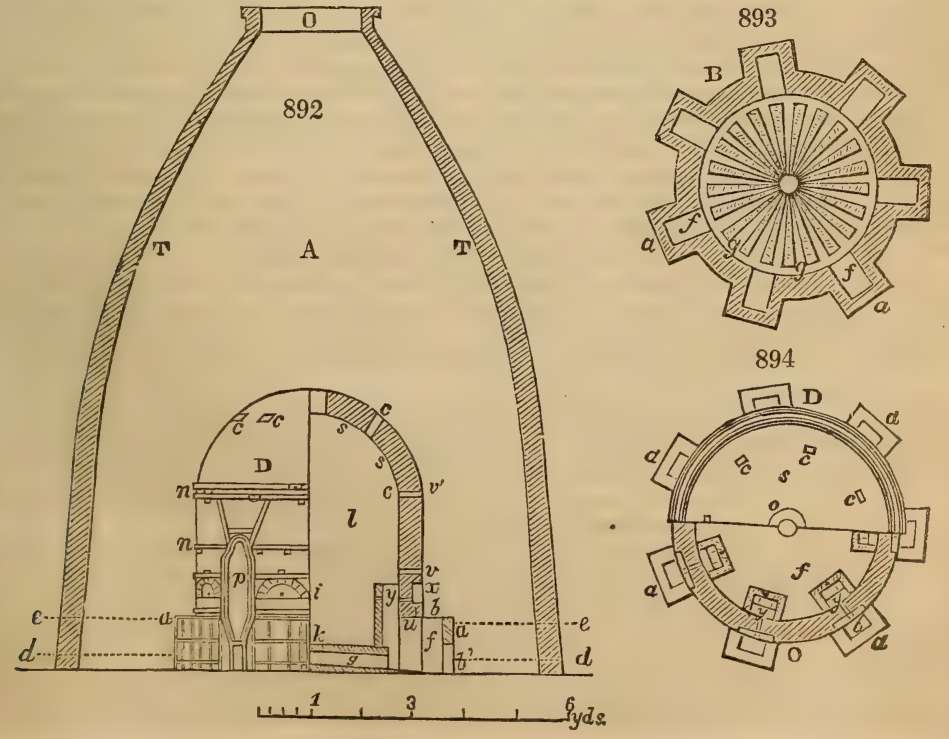


$a, a$, figs. 892, 893, and 894, are the furnaces which heat the kiln; of which $b$, in fig 892 , are the upper mouths, and $b^{\prime}$ the lower; the former being closed more or less by the fire-tile $z$, shown in fig. 896 .

$f$ is one fireplace; for the manner of distributing the fuel in it, see fig. 896 .

g, y, figs. 892 and 896, are the horizontal and vertical flues and chimneys for conducting the flame and smoke. $l$ is the laboratory, or body of the kiln; having its floor $k$ sloping slightly downwards from the centre to the circumference. $x, y$, is the slit of the horizontal register, leading to the chimney flue $y$ of the furnace, being the first regu lator; $x, u$, is the vertical register conduit, leading to the furnace or mouth $f$, being the second regulator; $v$ is the register slit above the furnace, and its vertical flue leading into the borly of the kiln; $v^{\prime}$, $c$, slit for regulating flue at the shoulder of the kiln; $i$ is an arch which supports the walls of the kiln, when the furnace is under repair; $c, c$, are small flues in the vault $s$ of the laboratory. $h, f i g .893$, is the central flue, called iunette, of the laboratory.

$\mathbf{T}, \mathbf{T}$, is the conical tower or howell, strengthened with a series of iron hoops. $0^{\prime}$ is the great chimney or lunette of the tower; $p$ is the door of the laboratory, bound inside with an iron frame.

895
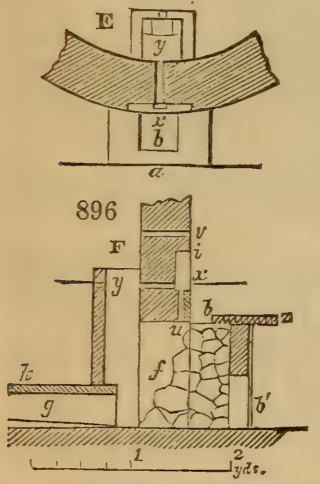

$A$, is the complete kiln and howell, with all its appurte. nances.

B, fig. 893, is the plan at the level $d, d$, of the floor, to show the arrangement and distribution of all the horizonta? flues, both circular and radiating.

c, fig. 894, is a plan at the level $e$, $e$, of the upper mouths $b$, of the furnaces, to show the disposition of the fireplaces of the vertical flues, and of the horizontal registers, or peep-holes. $\mathrm{D}$, fig. 894 , is a bird's-eye view of the top of the vault or dome $s$, to show the disposition of the vent-holes $c, c$.

$\mathbf{E}$, fig. 895, is a detailed plan at the level $c, c$, of one furnace and its dependencies.

F, fig. 896, is a transverse section, in detail, of one furnace and its dependencies.

The same letters in all the figures indicate the same objects.

Charging of the kiln.-The saggers are piled up first in the space between each of the upright furnaces, till they rise to the top of the flues. These contain the smaller articles. Above this level, large fire tiles are laid, for supporting other saggers, filled with teacups, sugar-basins, \&c. In the bottom part of the pile, within the preceding, the same sorts of articles are put; but in the upper part all such articles are placed as require a high heat. Four piles of small saggers, with a middle one 10 inches in height, complete the charge. As there are 6 piles between each furnace, and as the biscuit kiln has 8 furnaces, a charge consequently amounts to 48 or 50 bungs, each composed of from 18 to 19 saggers. The inclination of the bungs ought always to follow the form of the kiln, and should therefore tend towards the centre, lest the strong draught of the furnaces should make the saggers fall against the walls of the kiln, an accident apt to happen were these piles perpendicular. The last sagger of each bung is covered with an unbaked one, three inches deep, in place of a round lid The watches are small cups, of the same biscuit as the charge, placed in saggers, four in number, above the level of the flue-tops. They are taken hastily ovi of the sagyers, lest they should get smoked, and are thrown into cold water.

When the charging is completed, the firing is commenced, with coal of the best quality. The management of the furnace is a matter of great consequence to the success of the process. No greater heat should be employed for some time than may be necessary to agglutinate the particles which enter into the composition of the paste, by evaporating all the humidity ; and the heat should never be raised so high as to endanger the fusion of the ware, which would make it very brittle.

Whenever the mouth or door of the kiln is built up, a child prepares several fires in the neighborhood of the howell, while a laborer transports in a wheelbarrow a supply of coals, and introduces into each furnace a number of lumps. These lumps divide the furnace into two parts; those for the upper flues being placed above, and those for the ground flues below, whick must be kept -unobstructed.

The fire-mouths being charged, they are kindled to begin the baking, the regulator tile $z$, fig. 896, being now opened; an hour afterwards the bricks at the bottom of the

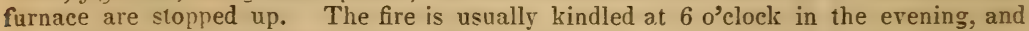
progressively increased till 10, when it begins to gain force, and the flame rises half-way up the chimner. The second charge is put in at $80^{\prime} \mathrm{clock}$, and the mouths of the furnaces are then covered with tiles; by which time the flame issues through the vent of the tower. An hour afterwards a fresh charge is made; the tiles $z$, which cover the furnaces, are slipped 
back; the cinders are drawn to the front, and replaced with small coal. About half past 11 o'clock the kiln-man examines his furuaces, to see that their dranght is pro. perly regulated. An hour afterwards a new change of coal is applied; a practice repeated hourly till 6 o'clock in the morning. At this moment he takes out his first ratch, to see how the baking goes on. It should be at a very pale-red heat; but the watch of 7 o'cluck should be a deeper red. He removes the tiles from those furnaces which appear to have been burning too strongly, or whose flame issues by the orifices made in the shoulder of the kiln; and puts tiles upon those which are not hot enough. The flames glide along brislily in a regular manner. At this period he draws out the watches every quarter of an hour, and compares them with those reserved from a previous standard liln: and if he observes a similarity of appearance, he allows the furnaces to burn a little longer; then opens the mouths carefully and by slow degrees; so as to lower the heat, and finish the round.

The baling usually lasts from 40 to 42 hours; in which time the biscuit kiln may consume $1 \pm$ tons of coals; of which four are put in the first day, seven the next day and following night, and the four last give the strong finishing heat.

Emptying the kiln.-The kiln is allowed to cool very slowly. On taking the ware out of the saggers, the biscuit is not subjected to friction, as in the foreign potteries, because it is smooth enough; but is immediately transported to the place where it is to be dipped in the glaze or enamel tub. A child makes the pieces ring, by striking with the handle of the brush, as he dusts them, and then immerses them into the glaze cream: from which tub they are taken out by the enameller, and shaken in the air. The tub usually contains no more than 4 or 5 inches depth of the glaze, to enable the workman to pich out the articles more readily, and to lay them upon a board, whence they are taken by a child to the glaze kiln.

Glazing.-A good enamel is an essential element of fine stoneware; it should exper1ence the same dilatation and contraction by heat and cold as the biscuit which it covers. The English enamels contain nothing prejudicial to health, as many of the foreign glazes do; no more lead being added to the former than is absolutely necessary to convert the silicious and aluminous matters with which it is mixed into a perfectly neutral glass.

Three kinds of glazes are used in Staffordshire; one for the common pipe-clay or cream-colored ware; another for the finer pipe-clay ware to receive impressions, called printing body; a third for the ware which is to be ornamented by painting with the pencil.

The glaze of the first or common ware is composed of 53 parts of white lead, 16 of Cornish stone, 36 of ground flints, and 4 of flint glass; or of 40 of white lead, 36 of Cornish stone, 12 of flints, and 4 of flint or crystal glass. These compositions are not fritted; but are employed after being simply triturated with water into a thin paste.

The following is the composition of the glaze intended to cover all kinds of figures printed in metaliic colors; 26 parts of white feldspar are fritted with 6 parts of soda, 2 of nitre, and 1 of burax; to 20 pounds of this frit, 26 parts of feldspar, 20 of white lead, 6 of ground flints, 4 of chalk, 1 of oxyde of tin, and a small quantity of oxyde of cobalt, to take off the brown cast, and give a faint azure tint, are added.

The following recipe may also be used. Frit together 20 parts of flint glass, 6 of flints, 2 of nitre, and 1 of brrax; add to 12 parts of that frit, 40 parts of white lead, 36 of feld spar, 8 of flints, and 6 of flint glass; then grind the whole together into a uniform creamconsistenced paste.

As to the stoneware which is to be painted, it is covered with a glaze composed of 13 parts of the printing-color frit, to which are added 50 parts of red lead, 40 of white lead, and 12 of flint; the whole having been ground together.

The above compositions produce a very hard glaze, which cannot be scratched by the knife, is not acted upon by regetable acids, and does no injury to potable or edible articles kept in the vessels covered with it. It preserves for an indefinite time the glassy lustre, and is not subject to crack and exfoliate, like most of the Continental stoneware, made from common pipe-clay.

In order that the saggers in which the articles are baked, after receiving the glaze, may not absorb some of the vitrifying matter, they are themselves coated, as above mentioned, with a glaze composed of 13 parts of common salt, and 30 parts of potash, simply dissolved in water, and brushed over them.

Glaze kilu. - This is usually smaller than the biscuit kiln, and contains no more than 40 or 45 bungs or columns, each composed of 16 or 17 saggers. Those of the first bung rest upon round tiles, and are well luted together with a finely ground fire-clay of only moderate cohesion; those of the second bung are supported by an additional tile. The iower saggers contain the cream-colored articles, in which the glaze is softer than that which covers the blue printed ware; this being always placed in the intervals between the furnaces, and in the uppermost saggers of the columns. The bottom of the kiln, where the glazed ware is not baked, is occupied by printed biscuit ware. 
Pyrometric balls of red clay, coated with a very fusible lead enamel, are employed in the binglish potteries to ascertain the temperature of the glaze kilns. This enamel is so rich, and the clay upon which it is spread is so fine-grained and compact, that even when exposed for three hours to the briskest flame, it does not lose its lustre. The color of the clay alone changes, whereby the workman is enabled to judge of the degree of heat within the kiln. At first the balls have a pale red appearance; but they become browner with the increase of the temperature. The balls, when of a slightly dark-red color, indicate the degree of baking for the hard glaze of pipe-clay ware; but if they become dark brown, the glaze will be much too hard, being that suited for ironstone ware; lastly, when they acquire an almost black hue, they show a degree of heat suited to the formation of a glaze upon porcelain.

The glazier provides himself at each round with a stock of these ball watches, reserved from the preceding baking, to serve as objects of comparison; and he never slackiens the firing till he has obtained the same depth of shade, or even somewhat more; for it may be remarked, that the more rounds a glaze kiln has made, the browner the balls are apt to become. A new kiln bakes a round of enamel-ware sooner than an old one; as also with less fuel, and at a lower temperature. The watch-balls of these first rounds have generally not so deep a color as if they were tried in a furnace three or four months old. After this period, cracks begin to appear in the furnaces; the horizontal flues get partially obstructed, the joinings of the brickwork become loose; in consequence of which there is a loss of heat and waste of fuel; the baking of the glaze takes a longer time, and the pyrometric balls assume a different shade from what they had on being takien out of the new kiln, so that the first watches are of no comparable use after two months. The baking of enamel is commenced at a low temperature, and the heat is progressively increased; when it reaches the melting point of the glaze, it must be maintained steadily, and the furnace mouths be carefully looked after, lest the heat should be suffered to fall. The firing is continued 14 hours, and then gradually lowered by slight additions of fuel; after which the kiln is allowed from 5 to 6 hours to cool.

Muffles.-The paintings and the printed figures applied to the glaze of stone-

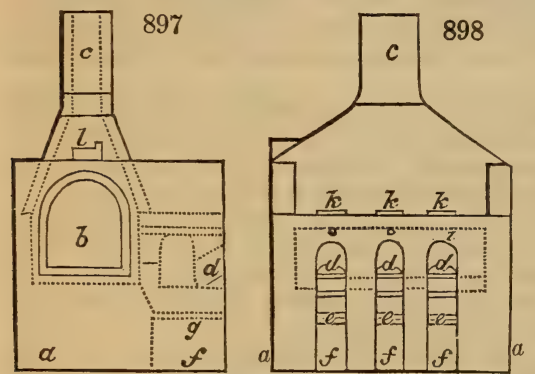
ware and norcelain are baked in muffles of a peculiar form. Fig. 897 is a lateral elevation of one of these muffles; fig. 898 is a front view. The same letters denote the same parts in the two figures.

$a$ is the furnace; $b$, the oblong muffle, made of fire-clay, surmounted with a dome pierced with three apertures $k, k, k$, for the escape of the vaporous matters of the colors and volatile oils with which they are ground up; $c$ is the chimney; $d, d$, feedholes, by which the fuel is introduced; $e$, the fire-grate; $f$, the ash-pit ; channels are left in the bottom of the furnace to facilitate the passage of the flame beneath the mutlle; $g$ is a lateral hole, which makes a communication across the furnace in the muffle, enabling the kiln man to ascertain what is passing within; $k, k$, are the lateral chinks for observing the progress of the firing or flame; $l$, is an opening $s:$ oped out in the front of the chimney to modify its draught.

The articles which are printed or painted upon the glaze are placed in the muffe without saggers, upon tripods, or moveable supports furnished with feet. The muffe being charged, its mouth is closed with a fire-tile well luted round its edges. The fuel is then kindled in the fire-places $d, d$, and the door of the furnace is closed with bricks, in which a small opening is left for taking out samples, and for examining the interior of the muffle. These sample or trial pieces, attached to a strong iron wire, show the progress of the baking operation. The front of the fireplaces is covered with a sheet-iron plate, which slides to one side, and may be shut whenever the kiln is charged. Soon after the fire is lighted, the flame, which communicates laterally from one furnace to another, en velopes the muffle on all sides, and thence rises up the chimney.

Printing of stoneware.-The printing under the stoneware glaze is generally performed by means of cobalt, and has different shades of blue according to the quantity of coloring matter employed. After having subjected this oxyde to the processes requisite for its purification, it is mixed with a certain quantity of ground flints and sulphate of baryta, proportioned to the dilution of the shade. These materials are fritted ana ground; bui helore they are "aeu, they must he inixsd with a fnx cussisisg of aqual paris by weight of flint glass and ground tints, which serves to fix the color upon the biscuit, so that the immersion in the glaze liquor may not displace the lines printed on, as also to aid in fluxing the cobalt. 
The following are the processes usually practised in Staffordshire for printing under the glaze.

The cobalt, or whatever color is employed, should be ground upon a porphyry slab, with a rarnish prepared as follows:-A pint of linseed oil is to be boiled to the consistence of thick honey, along with 4 ounces of rosin, half a pound of tar, and half a pint of oil of amber. This is very tenacious, and can be used only when liquefied by heat; which the printer effects by spreading it upon a hot cast-iron plate.

The printing plates are made of copper, engraved with pretty deep lines in the common war. The printer, with a leather muller, spreads upon the engraved plate, previously healed, his color, mixed up with the above oil varnish, and removes what is superfluous with a pallet knife ; then cleans the plate with a dossil filled with bran, tapping and wiping as if he were removing dust from it. This operation being finished, he takes the paper intended to receive the impression, soaks it with soap-water, and lays it moist upon the copper-plate. The soap makes the paper part more readily from the copper, and the thick ink part more readily from the biscuit. The copper-plate is now passed through the engraver's cylinder press, the proof leaf is lifted off and handed to the women, who cut it into detached pieces, which they apply to the surface of the biscuit. The paper best fitted for this purpose is made entirely of linen rags; it is very thin, of a yellow color, and unsized, like tissue blotting-paper.

The stoneware biscuit never receives any preparation before being imprinted, the oil of the color being of such a nature as to fix the figures firmly. The printed paper is pressed and rubbed on with a roll of flannel, about an inch and a half in diameter, and 12 or 15 inches long, bound round with twine, like a roll of tobacco. This is used as a burnisher, one end of it being rested against the shoulder, and the other end being rubbed upon the paper; by which means it transfers all the engraved traces to the biscuit. The piece of biscuit is laid aside for a little, in order that the color may take fast hold; it is then plunged into water, and the paper is washed away with a sponge.

When the paper is detached, the piece of ware is dipped into a caustic alkaline ley to saponify the oil, after which it is immersed in the glaze liquor, with which the printed figures readily adhere. This process, which is easy to execute, and very economical, is much preferable to the old plan of passing the biscuit into the muffle after it had been printed, for the purpose of fixing and volatilizing the oils. When the paper impression is applied to pieces of porcelain, they are heated before being dipped in the water, because, being already semi-vitrified, the paper sticks more closely to them than to the biscuit, and can be removed only by a hard brush.

The impression above the glaze is done by quite a different process, which dispenses with the use of the press. A quantity of fine clean glue is melted and poured hot upon a large flat dish, so as to form a layer about a quarter of an inch thick, and of the consistence of jelly. When cold it is divided into cakes of the size of the copper-plates it is intended to cover.

The operative (a woman) rubs the engraved copper-plate gently over with linseed oil boiled thick, immediately after which she applies the cake of glue, which she presses down with a silk dossil filled with bran. The cake licks up all the oil out of the engraved lines; it is then cautiously lifted off, and transferred to the surface of the glazed ware which it is intended to print. The glue cake being removed, the enamel surface must be rubbed with a little cotton, whereby the metallic colors are attached only on the lines charged with oil : the piece is then heated under the muffle. The same cake of glue may serve for several impressions.

Ornaments and coloring.-Common stoneware is colored by means of two kinds of apparatus; the one called the blowing-pot, the other the worming-pot. The ornaments made in relief in France, are made hollow (intaglio) in England, by means of a mould engraved in relief, which is passed over the article. The impression which it produces is filled with a thick clay paste, which the workman throws on with the blowing-pot. This is a vessel like a tea-pot, having a spout, but it is hermetically sealed at top with a clay plug, after being filled with the pasty liquor. The workman, by blowing in at the spout, causes the liquor to fly out through a quill pipe which goes down through the clay plug into the liquor. The jet is made to play upon the piece while it is being turned upon the lathe; so that the hollows previously made in it by the mould or stamp are filled with a paste of a color different from that of the body. When the piece has acquired sufficient firmness to bear working, the excess of the paste is removed by an instrument called a cournasin, till the ornamental figure produced by the stamp be laid bare; in which casc merely the color appears at the bottom of the impression. By passing in this manner several layers of clay liquor of different colors over each other with the blowing-pot, net-work, auù decurativis of different cuiurs and siacies, are very rapiuiy pro'inc ${ }^{-1}$

The serpentine or snake pots, established on the same principle, are made of tin plate in three compartments, each containing a different color. These open at the top of 
the vessel in a common orifice, terminated by small quill tubes. On inclining the vessel, the three colors flow out at once in the same proportion at the one orifice, and are let fall upon the piece while it is heing slowly turned upon the lathe; whereby curious serpent-like ornarnents may be readily obtained. The clay liquor ought to be in lieeping with the stoneware paste. The blues succeed best when the ornaments are made with the finer pottery mixtures given above.

Metullic lustres applied to stoneware.-The metallic lustre being applied only to the outer surface of ressels, can have no bad effect on health, whatever substances be employed for the purpose; and as the glaze intended to receive it is sufficiently fusible, from the quantity of lead it contains, there is no need of adding a flux to the metallic coating. The glaze is in this case composed of 60 parts of litharge, 36 of feldspar, and 15 of flints.

The silver and platina lustres are usually laid upon a white ground, while those of gold and copper, on account of their transparency, succeed only upon a colored ground. The darli-colored stoneware is, however, preferable, as it shows off the colors to most advantage; and thus the shades may be varied by varying the colors of the ornamental figures applied by the blowing-pot.

The gold and platina lustre is almost always applied to a paste body made on purpose, and coated with the above-described lead glaze. This paste is brown, and consisis of 4 parts of clay, 4 parts of flints, an equal quantity of kaolin (china clay), and 6 parts of feldspar. To make brown figures in relief upon a body of white paste, a liquor is mixed up with this paste, which ought to weigh 26 ounces per pint, in order to unite well with the other paste, and not to exfoliate after it is baked.

Preparation of gold lustre.-Dissolve first in the cold, and then with heat, 48 grains of fine gold in 288 grains of an aqua regia, composed of 1 ounce of nitric acid and 3 ounces of muriatic acid; add to that solution $4 \frac{1}{2}$ grains of grain tin, bit by bit; and then pour some of that compound solution into 20 grains of balsam of sulphur diluted with 10 grains of oil of turpentine. The balsam of sulphur is prepared by heating a pint of linseed oil, and 2 ounces of flowers of sulphur, stirring them continually till the mixture begins to boil; it is then cooled, by setting the vessel in cold water; after which it is stirred afresh, and strained through linen. The above ingredients, after being well mixed, are to be allowed to settle for a few minutes; then the remainder of the solution of gold is to be poured in, and the whole is to be triturated till the mass has assumed such a consistence that the pestle will stand upright in it; lastly, there must be added to the mixture 30 grains of oil of turpentine, which being ground in, the gold lustre is ready to be applied. If the lustre is too light or pale, more gold must be added, and if it have not a sufficiently violet or purple tint, more tin must be used.

Platina lustre.-Of this there are two kinds; one similar to polished steel, another lighter and of a silver-white hue. To give stoneware the steel color with platina, this metal must be dissolved in an aqua regia composed of 2 parts of muriatic acid, and 1 part of nitric. The solution being cooled, and poured into a capsule, there must be added to it, drop by drop, with continual stirring with a glass rod, a spirit of tar, composed of equal parts of tar and sulphur boiled in linseed oil and filtered. If the platina solution be too strong, more spirit of tar must be added to it; but if too weak, it must be concentrated by boiling. Thus being brought to the proper pitch, the mixture may be spread over the piece, which being put into the muffle, will take the aspect of steel.

The oxyde of platina, by means of which the silver lustre is given to stoneware, is prepared as follows:-After having dissolved to saturation the metal in an aqua regia composed of equal parts of nitric and muriatic acid, the solution is to be poured into a quantity of boiling water. At the same time a capsule, containing solution of sal-ammoniac, is placed upon a sand-bath, and the platina solution being poured into it, the inetal will fall down in the form of the well-known yellow precipitate, which is to be washed with cold water till it is perfectly edulcorated, then dried, and put up for use.

This metallic lustre is applied very smoothly by means of a flat camel's hair brush. It is then to be passed through the muffle kiln; but it requires a second application of the platinum to have a sufficient body of lustre. The articles sometimes come black out of the kiln, but they get their proper appearance by being rubbed with cotton.

Platina and gold lustre; by other recipes.

Platina lustre.-Dissolve 1 ounce of platinum in aqua regia formed of 2 parts of muriatic acid and 1 part of nitric acid, with heat upon a sand-bath, till the liquid is reduced to two thirds of its volume; let it cool; decant into a clean vessel, and pour into it, drop by drop, with constant stirring, some distilled tar, until such a mixture is produced as will give a good result in a trial upon the ware in the kiln. If the lustre be too intense, more tar must be added; if it be too weak, the mixture must be concentrated by further evaporation.

Gold lustre.-Dissolve four shillings' worth of gold in aqua regia with a gentle heat. 
To the solution, when conl, add 2 grains of grain tin, which will immediately dissolve. Prepare a mixture of half an ounce of balsam of sulphur with a little essence of turpentine, beating them together till they assume the appearance of milk. Pour this mixture into the solution of gold and tin, drop by drop, with continual stirring; and place the whole in a warm situation for some time.

It is absolutely necessary to apply this lustre only upon an enamel or glaze which has aiready passed through the fire, otherwise the sulphur would tarnish the composition.

These lustres are applied with most advantage upon chocolate and other dark grounds. Much skill is required in their firing, and a perfect acquaintance with the quality of the glaze on which they are applied.

An tron lustre is obtained by dissolving a bit of steel or iron in muriatic acid, mixing this solution with the spirit of tar, and applying it to the surface of the ware.

Aventurine glaze.-Mix a certain quantity of silver leaf with the above-described soft glaze, grind the mixture along with some honey and boiling water, till the metal assume the aprearance of fine particles of sand. The glaze, being naturally of a yellowish hue, gires a golden tint to the small fragments of silver disseminated through it. Molybdena may also be applied to produce the aventurine aspect.

The granite-like gold lustre is produced by throwing lightly with a brush a few drops of oil of turpentine upon the goods already covered with the preparation for gold lustre. These cause it to separate and appear in particles resembling the surface of granite. When marbling is to be given to stoneware, the lustres of gold, platina, and iron are used at once, which blending in the fusion, form veins like those of marble.

Pottery and stoneware of the Wedgewood color.-This is a kind of semi-vitrified ware, called dry bodies, which is not susceptible of receiving a superficial glaze. This pottery is composed in two ways : the first is with harytic earths, which act as fluxes upon the clays, and form enamels : thus the Wedgewood jasper ware is made.

The white vitrifying pastes, fit for receiving all sorts of metallic colors, are composed of 47 parts of sulphate of barytes, 15 of feldspar, 26 of Devonshire clay, 6 of sulphate of lime, 15 of flints, and 10 of sulphate of strontites. This composition is capable of receiving the tints of the metallic oxydes and of the ochrous metallic earths. Manganese produces the dark purple color; gold precipitated by tin, a rose color; antimony, orange; cobalt, different shades of blue; copper is employed for the browns and the dead-leaf greens; nickel gives, with potash, greenish colors.

One per cent. of oxyde of cobalt is added; but one half, or even one quarter, of a per cent. would be sufficient to produce the fine Wedgewood blue, when the nickel and manganese constitute 3 per cent., as well as the carbonate of iron. For the blacks of this kind, some English manufacturers mix black oxyde of manganese with the black oxyde of iron, or with ochre. Nickel and umber afford a fine brown. Carbonate of iron, mixed with bole or terra di Sienna, gives a beautiful tint to the paste; as also manganese with cobalt, or cobalt with nickel. Antinnony produces a very fine color when combined with the carbonate of iron in the proportion of 2 per cent., along with the ingredients necessary to form the above-described vitrifying paste.

The following is another vitrifying paste, of a much softer nature than the preceding. Feldspar, 30 parts; sulphate of lime, 23 ; silex, 17; potter's clay, 15; kaolin of Cornwall (china clay), 15 ; sulphate of baryta, 10 .

These vitrifying pastes are very plastic, and may be worked with as much facility as English pipe-clay. The round ware is usually turned upon the lathe. It may, however, be moulded, as the oval pieces always are. The more delicate ornaments are cast in hollow moulds of baked clay, by women and children, and applied with remarkable dexterity upon the turned and moulded articles. The colored pastes have such an affinity for each other, that the detached ornaments may be applied not only with a little gum water upon the convex and concave forms, but they may be made to adhere without experiencing the least cracking or chinks. The colored pastes receive only one fire, unless the inner surface is to be glazed; but a gloss is given to the outer surface. The enamel for the interior of the black odgewood ware is composed of 6 parts of red lead, 1 of silex, and 2 ounces of manganese, when the mixture is made in poundss weight.

The operation called smearing, consists in giving an external lustre to the unglazed semi-virifed ware. The articles do not in this way receive any immersion, nor even the aid of the brush or pencil of the artist; but they require a second fire. The saggers are coated with the salt glaze already described. These cases, or saggers, communicate by reverberation the lustre so remarkable on the surface of the English stoneware; which one might suppose to be the result of the glaze tub, or of the brush. Occasionally also a very fusible composition is thrown upon the inner surface of the muffle, and 5 or 6 pieces called refraclories are set in the middle of it, coated with the same composition. The intensity of the heat converts the flux into vapor; a part of 
this is condensed upon the surfaces of the contiguous articles; so as to give them the de. sired brilliancy.

Mortur body is a paste composed of 6 parts of clay, 3 of feldspar, 2 of silex, and 1 of china clay.

White and yellow figures upon dark-colored grounds are a good deal employed. To produce yellow impressions upon brown stoneware, ochre is ground up with a small quantity of antimony. The flux consists of flint glass and flints in equal weights. The composition for white designs is made by grinding silex up with that flux, and printIng it on, as for blue colors, upon brown or other colored stoneware, which shows off the light hues.

English porcelain or china.-Most of this belongs to the class called tender or soft porcelain by the French and German manufacturers. It is not, therefore, composed simply of kaolin and petuntse. The English china is generally baked at a much lower heat than that of Sèvres, Dresden, and Berlin; and it is covered with a mere glass. Being manufactured upon a prodigious scale, with great economy and certainty, and little expenditure of fuel, it is sold at a very moderate price compared with the foreign porcelain, and in external appearance is now not much inferior.

Some of the English porcelain has been called ironstone china. This is composed usually of 60 parts of Cornish stone, 40 of china clay, and 2 of flint glass; or of 42 of the feldspar, the same quantity of clay, 10 parts of flints ground, and 8 of flint glass.

The glaze for the first composition is made with 20 parts of feldspar, 15 of flints, 6 of red lead, and 5 of soda, which are fritted together; with 44 parts of the frit, 22 parts of flint glass, and 15 parts of white lead, are ground.

The glaze for the second composition is formed of 8 parts of flint glass, 36 of feldspar, 40 of white lead, and 20 of silex (ground flints.)

The English manufacturers employ three sorts of compositions for the porcelain biscuit; namely, two compositions not fritted; one of them for the ordinary table service; another for the dessert service and tea dishes; the third, which is fritted, corresponds to the paste used in France for sculpture; and with it all delicate kinds of ornaments are made.

\begin{tabular}{|c|c|c|c|c|c|c|c|}
\hline \multirow{3}{*}{$\begin{array}{l}\text { Ground flints } \\
\text { Calcined bones }\end{array}$} & \multirow{3}{*}{ - } & \multirow{3}{*}{ - } & \multirow{3}{*}{ 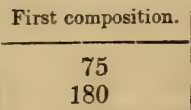 } & \multicolumn{2}{|c|}{ Second composition. } & \multicolumn{2}{|c|}{ Third composition. } \\
\hline & & & & - & 66 & Lynn sand & 150 \\
\hline & & & & - & 100 & $-\quad \quad-$ & 300 \\
\hline China clay & - & - & 40 & - & 96 & - & 100 \\
\hline Clay & - & - & 70 & Granite & 80 & Potash - & 10 \\
\hline
\end{tabular}

The glaze for the first two of the preceding compositions consists of, feldspar 45 , flints 9 , borax 21, flint glass 20, nickel 4. After fritting that mixture, add 12 parts of red lead. For the third composition, which is the most fusible, the glaze must receive 12 parts of ground flints, instead of 9 ; and there should be only 15 parts of borax, instead of 21 .

\section{PLAN OF AN ENGLISH POTTERY.}

A stoneware manufactory should be placed by the side of a canal or navigable river, because the articles manufactured do not well bear land carriage.

A Staffordshire pottery is usually built as a quadrangle, each side being about 100 feet long, the walls 10 feet high, and the ridge of the roof 5 feet more. The base of the edifice consists of a bed of bricks, 18 inches high, and 16 inches thick; upon which a mud wall in a wooden frame, called pisé, is raised. Cellars are formed in front of the build. ings, as depots for the pastes prepared in the establishment. The wall of the yard or court is 9 feet high, an 18 inches thick.

Fig. 899 , A, is the entrance door; B, the porter's lodge; c, a particular warehouse, $\mathrm{D}$, workshop of the plaster-moulder. $\mathrm{F}$, ine clay depot; F, F, large gates, 6 feet 8 inches high; G, the winter evaporation stove; $\mathrm{H}$, the shop for sifting the paste liquors; $\mathrm{I}$, sheds for the paste liquor tubs; J, paste liquor pits; $\mathbf{K}$, workshop for the moulder of hollow ware; $L$, ditto of the dish or plate moulder; $M$, the plate drying-stove; $N$, workshop of the biscuit-printers; 0 , ditto of the biscuit, with $o^{\prime}$, a long window; $P$, passage leading to the paste liquor pits; $\mathbf{Q}$, biscuit warehouse; $\mathbf{R}$, place where the biscuit is cleaned as it comes out of the biscuit-kilns, s, S; T, T, enamel or glaze-kilns; $\mathrm{U}$, long passage ; v, space left for supplementary workshops; $\mathbf{x}$. space appointed as a depot for the sagger fire-clay, as also for making the saggers; $z$, the workshop for applying the glaze liquor to the biscuits; $a$, apartment for cleaning the glazed ware; $b, b$, pumps; $c$, basin; $d$, muffles ; $e$, warehouse for the finished stoneware; $f$, that of the glazed goods; $g, g$, another warehouse, $\boldsymbol{h}$, a large space for the smith's forge, carpenter's shop, packing room, depot of clays, kaggers, \&c. The packing and loading of the goods are performed in front of the 
warehouse, which has two outlets, in order to facilitate the work; $\imath$, a passage to the court or yard; $l$, a space for the wooden sheds for keeping hay, clay, and other miscel899

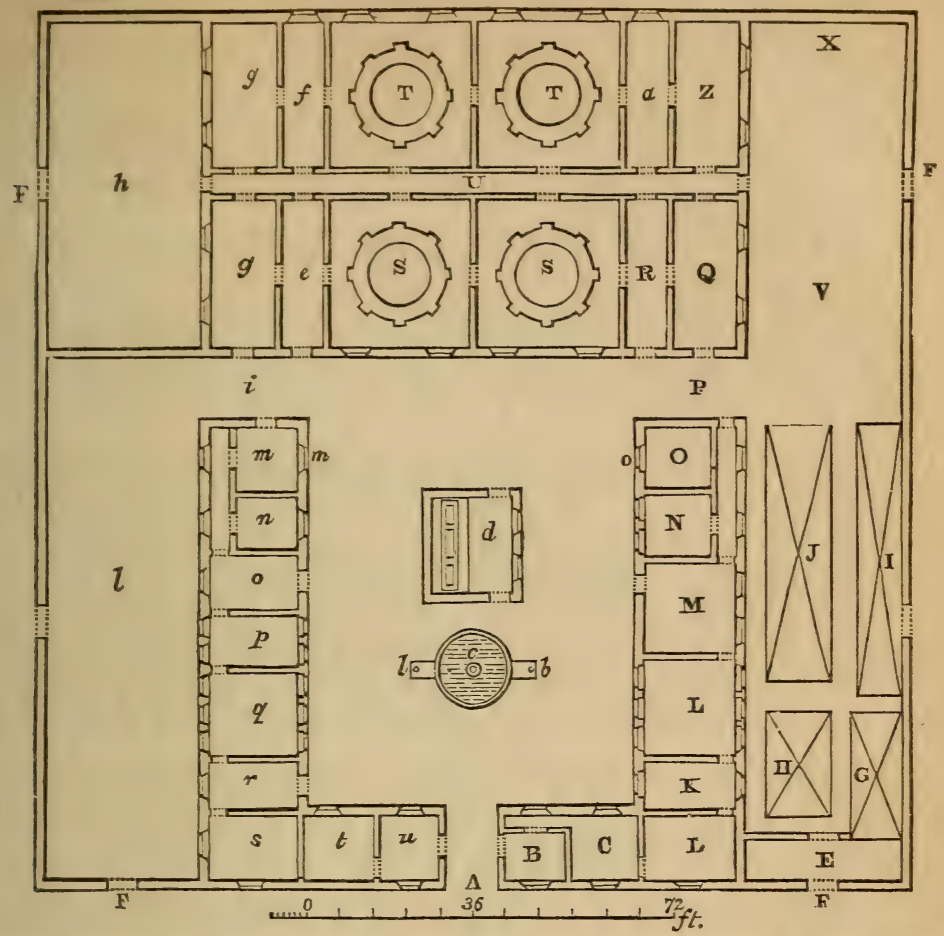

laneous articles; $m$, room for putting the biscuit into the saggers; $m^{\prime}$, a long window; $n$, workshop with lathes and fy-wheels; 0 , drying-room; $p$, room for mounting or furnishing the pieces; $q$, repairing-room; $r$, drying-room of the goods roughly turned; $s$, rough turning or blocking-out room; $t$, room for beating the paste or dough; $u$, counting-house.

The declared value of the earthenware exported in 1836 , was $837,774 l$.; in 1837 , 558,6821 .

There are from 33,000 to 35,000 tons of clay exported annually from Poole, in Dorsetshire, to the English and Scotch potteries. A good deal of clay is also sent from Devonshire and Cornwail.

The Spanish alcarazzas, or cooling vessels, are made porous, to favor the exudation of water through them, and maintain a constantly moist evaporating surface, Lasteyrie sars, that granular sea salt is an ingredient of the paste of the Spanish alcarazzas; which being expelled partly by the heat of the baking, and partly by the subsequent watery percalation, leaves the body very open. The biscuit should be charged with a considerable proportion of sand, and very moderately fired.

\section{OF PORCELAIN.}

Porcelain is a kind of pottery ware whose paste is fine grained, compact, very hard, and faintly translucid; and whose biscuit softens slightly in the kiln. Its ordinary whiter.sss cannot form a definite character, since there are porcelain pastes variously colored. There are two species of porcelain, very different in their nature, the essential properties of which it is of consequence to establish; the one is called hard, and the other tender; important distinctions, the neglect of which has introduced great confusion into many treatises on this elegant manufacture.

Hard porcelain is essentially composed, first, of a natural clay containing some silica, infusible, and preserving its whiteness in a strong heat; this is almost always a true kaolin; secondly, of a flux, consisting of silica and lime, composing a quartzose feldspar rock, called pe-tun-ise. The glaze of this porcelain, likewise earthy, admits of no metallic substance or alkali. 
Tender porcelain, styled also vitreous porcelain, has no relation with the preveding in its composition; it always consists of a vitreous frit, rendered oparque and less fusible by the addition of a calcareous or marly clay. Its glaze is an artificial glass or crystal, into which silica, alkalis, and lead enter.

This porcelain has a more vitreous biscuit, more transparent, a little less hard, and 'ess fragile, but much more fusible than that of the hard porcelain. Its glaze is more glossy, more transparent, a little less white, much tenderer, and more fusible.

The biscuit of the hard porcelain made at the French national manufactory of Sèvres is generally composed of a kaolin clay, and of a decomposed feldspar rock; analogous to the china clay of Cornwall, and Cornish stone. Both of the above French materials come from Saint Yriex-la-perche, near Limoges.

After many experiments, the following composition has been adopted for the service paste of the royal manufactory of Sèvres ; that is, for all the ware which is to be glazed; silica, 59 ; alumina, $35 \cdot 2$; potash, $2 \cdot 2$; lime, $3 \cdot 3$. The conditions of such a compound are pretty nearly fulfilled by taking from 63 to 70 of the washed kaolin or china clay, 22 to 15 of the feldspar, nearly 10 of flint powder, and about 5 of chalk. The glaze is composed solely of solid feldspar, calcined, crushed, and then ground fine at the mill. This rock pretty uniformly consists of silica 73 , alumina $16 \cdot 2$, potash $8 \cdot 4$, and water $0 \cdot 6$.

The kaolin is washed at the pit, and sent in this state to Sèvres, under the name of decanted eurth. At the manufactory it is washed and elutriated with care; and its slip is passed through fine sieves. This forms the plastic, infusible, and opaque ingredient to which the substance must be added which gives it a certain degree of fusibility and semi-transparency. The feldspar rock used for this purpose, should contain neither dark mica nor iron, either as an oxyde or sulphuret. It is calcined to make it crushable, under stamp-pestles driven by machinery, then ground fine in hornstone mills, as represented in figs. 897, 898, 899, and 900 . This pulverulent matter, being diffused through water, is mixed in certain proportions, regulated by its quality, with the argillaceous slip. The mixture is deprived of the chief part of its water in shallow plaster pans without heat; and the resulting paste is set aside to ripen, in damp cellars, for many months.

When wanted for use, it is placed in hemispherical pans of plaster, which absorb the redundant moisture; after which it is divided into small lumps, and completely dried. It is next pulverized, moistened a little, and laid on a floor, and trodden upon by a workman marching over it with bare feet in every direction; the parings and fragments of soft moulded articles being intermixed, which improve the plasticity of the whole. When sufficiently tramped, it is made up into masses of the size of a man's head, and kept damp till required.

The dough is now in a state fit for the potter's lathe; but it is much less plastic than stoneware paste, and is more difficult to fashion into the various articles; and hence one cause of the higher price of porcelain.

The round plates and dishes are shaped on plaster moulds; but sometimes the paste is laid on as a crust, and at others it is turned into shape on the lathe. When a crust is to be made, a moistened sheep-skin is spread on a marble table; and over this the dough is extended with a rolling-pin supported on two guide-rules. The crust is then transferred over the plaster mould, by lifting it upon the skin; for it wants tenacity to bear raising by itself. When the piece is to be fashioned on the lathe, a lump of the dough is thrown on the centre of the horizontal wooden disc, and turned into form as directed in treating of stoneware, only it must be left much thicker than in its finished state. After it dries to a certain degree on the plaster mould, the workman replaces it on the lathe, by moistening it on its base with a wet sponge, and finishes its form with an iron tool. A good workman at Sèvres makes no more than from 15 to 20 porcelain plates in a day; whereas an English potter, with two boys, makes from 1000 to 1200 plates of stoneware in the same time. The pieces which are not round, are shaped in plaster moulds, and finished by hand. When the articles are very large, as wash-hand basins, salads, \&c., a flat cake is spread above a skin on the marble slab, which is then applied to the mould with the sponge, as for plates; and they are finished by hand.

The projecting pieces, such as handles, beaks, spouts, and ornaments, are moulded and adjusted separately; and are cemented to the bodies of china-ware with slip, or porcelain dough thinned with water. In fact, the mechanical processes with porcelain and the finer stoneware are substantially the same; only they require more time and greater nicety. The least defect in the fabrication, the smallest bit added, an unequal pressure, the cracks of the moulds, although well repaired, and seemingly effaced in the clay shape, re-appear after it is baked. The articles should be allowed to dry very slow. ly; if hurried but a little, they are liable to be spoiled. When quite dry, they are taken to the kiln.

The kiln for hard porcelain at Sèvres, is a kind of tower in two flats, constructed of 
fire-bricks; and resembles, in other respects, the stoneware kiln already figured and deseribed. The fucl is young aspin wood, very dry, and cleft very small; it is put into the apertures of the four outside furnaces or fire-mouths, which discharge their flame into the inside of the kiln; each floor being closed in above, by a dome pierced with holes. The whole is covered in by a roof with an open passage, placed at a proper distance fiom the uppermost dome. There is, therefore, no chimney proper so called. See STONF, ARTIFICIAL.

The raw pieces are put into the upper floor of the kiln; where they receive a heat of about the 60th degree of Wedgewood's pyrometer, and a commencement of baking which, without altering their shape, or causing a perceptible shrinking of their bulk, makes them completely dry, and gives them sufficient solidity to bear handling. By this preliminary baking, the clay loses its property of forming a paste with water; and the pieces become fit for receiving the glazing ooat, as they may be dipped in water without risk of breakage.

The glaze of hard porcelain is a feldspar rock ; this being ground to a very fine powder, is worked into a paste with water mingled with a little vinegar. All the articles are dipped into this milky liquid for an instant; and as they are very porous, they absorb the water greedily, whereby a layer of the feldspar glaze is deposited on their surface, in a nearly dry state, as soon as they are lifted out. Glaze-pap is afterwards applied with a hair brush to the projecting edges, or any points where it had not taken; and the powder is then removed from the part on which the article is to stand, lest it should get fixed to its support in the fire. After these operations it is replaced in the kiln, to be completely baked.

The articles are put into saggers, like those of fine stoneware; and this operation is one of the most delicate and expensive in the manufacture of porcelain. The saggers are made of the plastic or potter's clay of Abondant, to which about a third part of cement of broken saggers has been added.

As the poreelain pieces soften somewhat in the fire, they cannot be set above each other, even were they free from glaze; for the same reason, they cannot be baked on tripods, several of them being in one case, as is done with stoneware. Every piece of porcelain requires a sagger for itself. They must, moreover, be placed on a perfectly flat surface, because in softening they would be apt to conform to the irregularities of a rough one. When therefore any piece, a soup plate for example, is to be saggered, there is laid on the bottom of the case a perfectly true disc or round cake of stoneware, made of the sagger material, and it is secured in its place on three small props of a clay-lute, consisting of potter's clay mixed with a great deal of sand. When the cake is carefully levelled, it is moistened, and dusted over with sand, or coated with a film of fire-clay slip, and the porcelain is carefully set on it. The sand or fire-clay hinders it from sticking to the cahe. Several small articles may be set on the same cake, provided they do not touch one another.

The saggers containing the pieces thus arranged, are piled up in the kiln over each other, in the columnar form, till the whole space be occupied; leaving very moderate intervals between the columns to favor the draught of the fires. The whole being arranged with these precautions, and several others, too minute to be specified here, the door of the kiln is built up with 3 rows of bricks, leaving merely an opening 8 inches square, through which there is access to a sagger with the nearest side cut off. In this sagger are put fragment: of porcelain intended to be withdrawn from time to time, in order to judge of the progress of the baking. These are called lime-pieces or watches (montres). This opening into the watches is closed by a stopper of stoneware.

The firing begins by throwing into the furnace-mouths some pretty large pieces of white wood, and the heat is maintained for about 15 hours, gradually raising it by the addition of a larger quantity of the wood, till at the end of that period the kiln has a cherry-red color within. The heat is now greatly increased by the operation termed covering the fire. Instead of throwing billets vertically into the four furnaces, there is placed horizontally on the openings of these furnaces, aspin wood of a sound texture, cleft small, laid in a sloping position. The brisk and long flame which it vields dins into the funnels, penetrates the kiln, and circulates round the sagger-piles. The heat augments rapidly, and, at the end of 13 or 15 hours of this firing, the interior of the kiln is so white, that the watches can hardly be distinguished. The draught, indeed, is su rapid at this time, that one may place his hand on the slope of the wood without feeling incommoded by the heat. Everything is consumed, no small charcoal remains, smoke is no longer produced, and even the wood-ash is dissipated. It is obrious that the liln and the saggers must be composed of a very refractory clay, in order to resist such a fire. The heat in the Sères kilns mounts so high as the 134th degree of Wedgewond.

At the end of 15 or 20 hours of the great fire, that is, after from 30 to 36 hours? firing, the porcelain is baked; as is ascertained by taking out and examining the 
watches. The kiln is suffered to cool during 3 or 4 day 3 , and is then opened and discharged. The sand strewed on the cakes, to prevent the adhesion of the articles to them, gets attached to their sole, and is removed by friction with a hard sandstone; an operation which one woman can perform for a whole kiln in less than 10 days; and is the last applied to hard porcelain, unless it needs to be returned into the hot kiln to have some defects repaired.

The materials of fine porcelain are very rare; and there would be no advantage ir making a gray-white porcelain with coarser and somewhat cheaper materials, for the other sources of expense above detailed, and which are of most consequence, wiuld still exist; while the porcelain, losing much of its brightness, would lose the main part of its value.

Its pap or dough, which requires tedious grinding and manipulation, is also more difficult to work into shapes, in the ratio of 80 to 1 , compared to fine stoneware. Each porcelain plate requires a separate sagger; so that 12 occupy in the kiln a space sufficient for at least 38 stoneware plates. The temperature of a hard porcelain kiln being very high, involves a proportionate consumption of fuel and waste of saggers. With 40 steres (cubic metres) of wood, 12,000 stoneware plates may be completely fired, both in the biscuit and glaze kilns; while the same quantity of wood would bake at most only 1000 plates of porcelain.

To these causes of high price, which are constant and essential, we ought to add the numerous accidents to which porcelain is exposed at every step of its preparation, and particularly in the kiln; these accidents damage upwards of one third of the pieces, and frequently more, when articles of singular form and large dimensions are adventured.

The best English porcelain is made from a mixture of the Cornish kaolin (called china clay), ground flints, ground Cornish stone, and calcined bones in powder, or bone-ash, besides some other materials, according to the fancy of the manufacturers. A liquid pap is made with these materials, compounded in certain proportions, and diluted with water. The fluid part is then withdrawn by the absorbent action of dry stucco basins or pans. The dough, brought to a proper stiffness, and perfectly worked and kneaded on the principles detailed above, is fashioned on the lathe, by the hands of modellers, or by pressure in moulds. The pieces are then baked to the state of biscuit in a kiln, being enclosed, of course, in saggers.

This biscuit has the aspect of white sugar, and being very porous, must receive a vitreous coating. The glaze consists of ground feldspar or Cornish stone. Into this, diffused in water, along with a little flunt-powder and potash, the biscuit ware is dipped, as already described, under stoneware. The pieces are then fired in the glaze-kiln, care being taken, before putting them into their saggers, to remove the glaze powder from their bottom parts, to prevent their adhesion to the fire-clay vessel.

\section{TENDER PORCELAIN.}

Tender porcelain, or soft china-ware, is made with a vitreous frit, rendered less fusible and opaque by an addition of white marl or bone-ash. The frit is, therefore, first prepared. This, at Sèvres, is a composition, made with some nitre, a little sea salt, Alieant barilla, alum, gypsum, and much silicious sand or ground flints. That mixture is subjected to an incipient pasty fusion in a furnace, where it is stirred about to blend the materials well; and thus a very white spongy frit is obtained. It is pulverized, and to every three parts of it, one of the white marl of Argenteuil is added; and when the whole are well ground, and intimately mixed, the paste of tender porcelain is formed.

As this paste has no tenacity, it cannot bear working till a mucilage of gum or black soap be added, which gives it a kind of plasticity, though even then it will not bear the lathe. Hence it must be fashioned in the press, between two moulds of plaster. The pieces are left thicke? than they should be; and when dried, are finished on the lathe with iron tools.

In this state they are bared, without any glaze being applied; but as this porcelain softens far more during the baking than the hard porcelain, it needs to be supported on every side. This is done by baking on earthen moulds all such pieces as can be treated in this way, namely, plates, saucers, \&c. The pieces are reversed on these moulds, and undergo their shrinkage without losing their form. Beneath other articles, supports of a like paste are laid, which suffer in baking the same contraction as the articles, and of course can serve only once. In this operation saggers are used, in which the pieces and their supports are fired.

The kiln for the tender porcelain at Sèvres is absolutely similar to that for the common stoneware; but it has two floors; and while the biscuit is baked in the lower story, the glaze is fused in the upper one; which causes considerable economy of fuel. The glaze of soft porcelain is a species of glass or crystal prepared on purpose. It is composed of flint, silicious sand, a little potash or soda, and about two fifth parts 
of lead oxyle. This mixture is melted in crucibles or pots beneath the kiln. The resulting glass is ground fine, and diffused through water mixed with a little vinegar to the consistence of cream. All the pieces of biscuit are covered with this glazy matter, by pouring this slip over them, since their substance is not absorbent enough to take it on by immersion.

The pieces are encased once more each in a separate sagger, but without any supports; for the heat of the upper floor of the kiln, though adequate to melt the glaze, is not strong enough to suften the biscuit. But as this first vitreous coat is not very equal, a second one is appliel, and the pieces are returned to the kiln for the third time. See STONE, AR. TIFICral, for a view of this kiln.

The manufacture of soft porcelain is longer and more difficult than that of hard; its biscuit is deacer, although the raw materials may be found everywhere; and it furnishes also more refiuse. Many of the pieces split asunder, receive fissures, or become deformed in the biscuit-kiln, in spite of the supports; and this vitreous porcelain, moreover, is always yellower, more transparent, and incapable of bearing rapid transitions of temperature, so that even the heat of boiling water frequently cracks it. It possesses some advantages as to painting, and may be made so gaudy and brilliant in its decorations, as to captivate the vulgar eye.

\section{DHSCRIPTION OF THE PORCELAIN MILL.}

1. The following figures of a feldspar and flint mill are taken from plans of apparatus lately constructed by Mr. Hall of Dartford, and erected by him in the royal manufactory of Serres. There are two similar sets of apparatus, fig. 900, which may be employed together or in succession; composed each of an elevated tub A, and of three successive vats

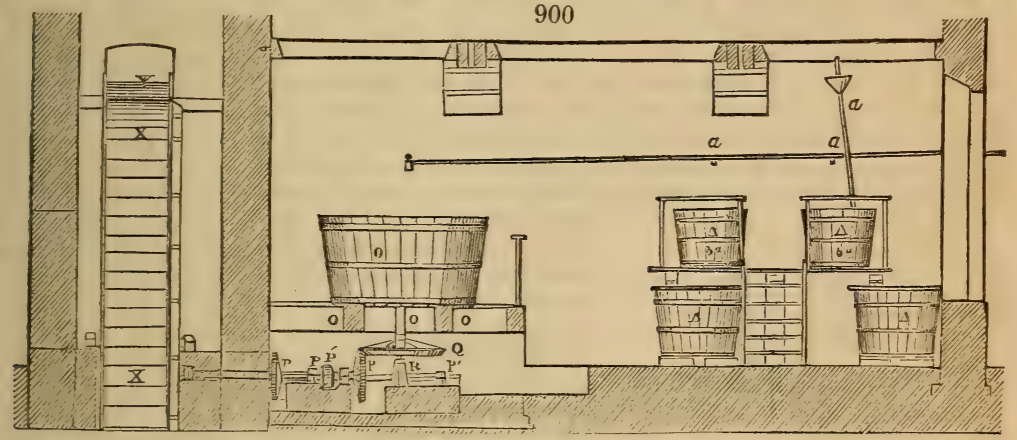

of reception $\mathbf{A}^{\prime}$, and two behind it, whose top edges are upon a lower level than the bottom of the casks A, A, to allow of the liquid running out of them with a sufficient slope. A proper charge of kaolin is first put into the cask $A$, then water is gradually run into it by the gutter adapted to the stopeock $a$, after which the mixture is agitated powerfully in every direction by hand with the stirring-bar, which is hung within a hole in the ceiling, and has at its upper end a small tin-plate funnel to prevent dirt or rust from dropping down into the clay. The stirrer may be raised or lowered so as to touch any part of the eask. The semi-fluid mass is left to settle for a few minutes, and then the finer argillaceous pap is run off by the stopcock $a^{\prime}$, placed a little above the gritty deposite, into the zinc pipe which conveys it into one of the tubs $A^{\prime}$; but as this semiliquid matter mav still contain some granular substances, it must be passed through a sieve before it is admitted into the tub. There is, therefore, at the spot upon the tub where the zinc pipe terminates, a wire-cloth sieve, of an extremely close texture, to receive the liquid paste. This sieve is shaken upon its support, in order to make it discharge the washed argillaceous kaolin. After the clay has subsided, the water is drawn off from its surface by a zinc syphon. The vats $\mathbf{A}^{\prime}$ have covers, to protect their contents from dust. In the pottery factories of England, the agitation is produced by machinery, instead of the hand. A vertical shaft, with horizontal or oblique paddles, is made to revolve in the vats for this purpose.

The small triturating mill is represented in fig. 901. There are three similar grinding. tubs on the same line. The details of the construction are shown in figs. 902, 903, where it is seen to consist principally of a revolving millstone B ( $f$ g. 902), of a fast or sleeper millstone $\mathbf{B}^{\prime}$, and of a vat $\mathrm{c}$, hooped with iron, with its top raised above the upper millstone. The lower block of hornstone rests upon a very firm basis, $b^{\prime}$; it is surrounded immediately by the strong wooden circle $c$, which slopes out funnel-wise above, in order to throw back the earthy matters as they are pushed up by the attrition 
of the stones. That piece is hollowed out, partially to admit the key c, opposite tc which is the faucet and spigot $c^{\prime}$, for emptying the tub. When one operation is com-

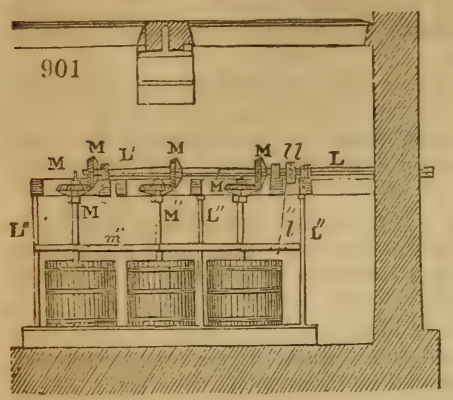
pleted, the key $\mathbf{c}$ is lifted out by means of a peg put into the holes at its top; the spigot is then drawn, and the thin paste is run out into vats. The upper grindstone, в $d$, like the lower one, is about two feet in diameter, and must be cut in a peculiar manner. At first there is scooped out a hollowing in the form of a sector, denoted by $d e f, f i g .903$; the are $d f$ is about one sixth of the circumference, so that the vacuity of the turning grindstone is one sixth of its surface; moreover, the stone must be channelled, in order to grind or erush the hard gritty substances. For this purpose, a wedge-shaped groove $d e \mathrm{~g}$, about an inch and a quarter deep, is made on its under face, whereby the stone, as it turns in the direction indicated by the arrow, acts with this inclined plane upon all the particles in its course, crushing them and forcing them in between the stones, till they be triturated to an impalpable powder. When the grindstone wears unequally on its lower surface, it is useful to trace upon it little furrows, proceeding from the centre to the circumference, like those shown by the dotted lines $e^{\prime} e^{\prime \prime}$. It must, moreover, be indented with rough points by the hammer.

The turning horn-stone block is set in motion by the vertical shaft $\mathrm{H}$, which is fixed by the clamp-iron cross I to the top of the stone. When the stone is new, its thiekness is about 14 inches, and it is made to answer for grinding till it be reduced to about 8 inches, by lowering the clamp $\mathrm{I}$ upon the shaft, so that it may continue to keep its hold of the stone. The manner in which the grindstones are turned, is obrious from inspection of fig. 901, where the horizontal axis $\mathrm{x}$, which receives its impulsion from the great water-wheel, turns the prolonged shaft $L^{\prime}$, or leaves it at rest, according as the clutch $l, l^{\prime}$, is locked or opened. This second shaft bears the three bevel wheels $\mathrm{M}, \mathrm{M}, \mathrm{M}$. These work in three corresponding bevel wheels $\mathrm{N}^{\prime} \mathrm{M}^{\prime} \mathrm{M}^{\prime}$, made fast respectively to the

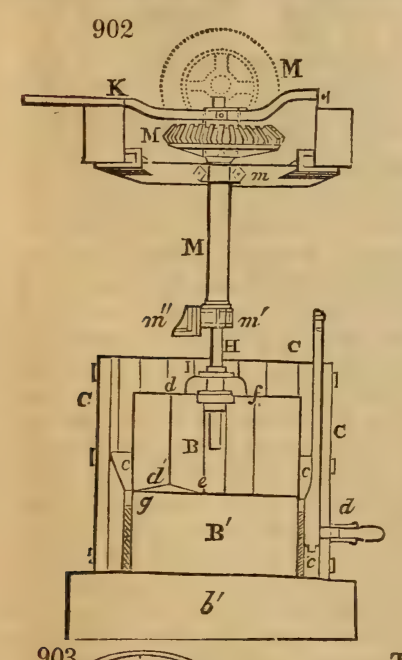
three vertical shafts of the millstones, which pass through the cast iron guide tubes $\mathbf{m}^{\prime \prime} \mathbf{m}^{\prime \prime}$. These are fixed in a truly vertical position by the collarbar $m^{\prime \prime}, m^{\prime}, f i g .902$. In this figure we see at $m$ how the strong cross-bar of cast iron is made fast to the wooden beams which support all the upper mechanism of the mill-work. The bearing $m^{\prime}$ is disposed in an analogous manner; but it is supported against two cast iron columns, shown at $\mathrm{L}^{\prime \prime} \mathrm{L}^{\prime \prime}$, in fig. 901. The guide tubes $\mathrm{m}^{\prime \prime}$ are bored smonth for a small distance from each of their extremities, and their interjacent calibre is wider, so that the vertical shafts touch only at two places. It is obvious, that whenever the shaft $\mathbf{L}^{\prime}$ is set a-going, it necessarily turns the wheels $M$ and $M^{\prime}$, and their guide tubes $M^{\prime \prime}$; but the vertical shaft may remain either at rest, or revolve, according to the position of the lever click or catch $\mathbf{K}$, at the top, which is made to slide upon the shaft, and can let fall a finger into a vertical groove cut in the surface of that shaft. The clamp-fork of the click is thus made to catch upon the horizontal bevelwh eel $\mathbf{M}^{\prime}$, or to release it, according as the lever $\mathrm{K}$ is lowered or lifted up. Thus each millstone may be thrown out of or into gear at pleasure.

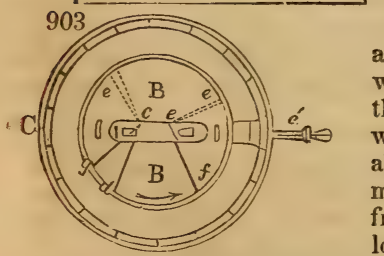

These stones make upon an average 11 or 12 turns in a minute, corresponding to thrce revolutions of the waterwheel, which moves through a space of 3 feet 4 inches in the second, its outer circumference being 66 feet. The weight of the upper stone, with its iron mountings, is about 6 cwts., when new. The charge of each mill in dry material is $2 \mathrm{cwts}$; and the water may be estimated at from one half to the whole of this weight; whence the total load may be reckoned to be at least 3 cwts.; the stone, by displacement of the magma, loses fully 400 pounds of its weight, and weighs therefore in reality only 2 cwts. It is charged in successive portions, but it is discharged all at once. When the grinding of the silicious or feldspar matters is nearly complete, a remarkable 
phenomenon occurs; the substance precipitates to the bottom, and assumes ${ }_{2} \mathrm{n}$ a few seconds so strong a degree of cohesion, that it is hardly possible to restore it again to the pasty or mamga state; hence if a millstone turns too slowly, or if it be accidentally stoppel for a few minutes, the upper stone gets so firmly cemented to the under one, that it is difficult to separate them. It has been discovered, but without knowing why, that a little vinegar added to the water of the magma almost infallibly prevents that sudilen stiffening of the deposite and stoppage of the stones. If the mills come to be set fast in this way, the shafts or gearing would be certainly broken, werf not some safety provision to be made in the machinery against such accidents. Mr. Hall's contrivance to obriate the above danger is highly ingenious. The clutch $l, l^{\prime}, f$ ig. 901 , is not a locking crab, fixed in the common way, upon the shaft $L$; but it is composed, as 905
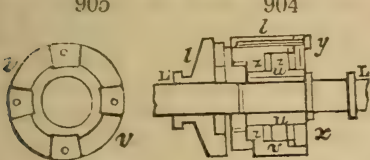

906
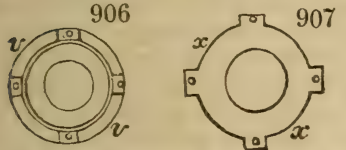
shown in figs. 904, 905, 906, 907, of a hoop $u$, fixed upon the shaft by means of a key, of a collar $v$, and of a flat ring or washer $x$, with four projections, which are fitted to the collar $v$, by four bolts $y$. Fig. 905 represents the collar $v$ seen in front; that is, by the face which carries the clutch teeth; and fig. 906 represents its other face, which receives the flat ring $x$, fig. 907, in four notches corresponding to the four projections of the washer-ring. Since the ring $u$ is fixed upon the shaft $L$, and necessarily turns with it, it has the two other pieces at its disposal, namely, the collar $v$, and the washer $x$, because they are always connected with it by the four bolts $y$, so as to turn with the ring $u$, when the resistance they encounter upon the shaft $L^{\prime}$ is not too great, and to remain at rest, letting the ring $u$ turn br itself, when that resistance increases to a certain pitch. To give this degree of friction, we need only interpose the leather washers $z, z^{\prime}$, fig. 904; and now as the collar couplingbox, $v$, slides pretty freely upon the ring $u$, it is obvious that by tightening more or less the screw bolts $y$, these washers will become as it were a lateral brake, to tighten more or less the bearing of the ring $u$, to which they are applied; by regulating this pressure, everything may be easily adjusted. When the resistance becomes too great, the leather washers, pressed upon one side by the collar $v$, of the washer $x$, and rubbed upon the other side by the prominence of the ring $u$, get heated to such a degree, that they are apt to become carbonized, and require replacement.

This safety clutch may be recommended to the notice of mechanicians, as susceptible of beneficial application in a variety of circumstances.

\section{GREAT PORCELAIN MILL.}

The large feldspar and kaolin mill, made by Mr. Hall, for Sèvres, has a flat bed of hornstone, in one block, laid at the bottom of a great tub, hooped strongly with iron. In most of the English potteries, however, that bed consists of several flat pieces of cher: or hornstone, laid level with each other. There are, as usual, a spigot and faucet at the side, for drawing off the liquid paste. The whole system of the mechanism is very sul. stantial, and is supported by wooden beams.

The following is the manner of turning the upper blocks. In fig. 900 the mai. horizontal shaft $\mathrm{P}$ bears at one of its extremities a toothed wheel, usually mounted upon 908

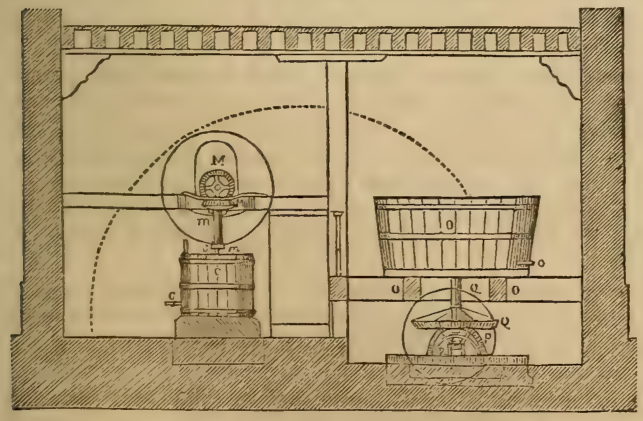
the periphery of the great water-wheel ( fig. 908 shows this toothed wheel by a dotted line) at its other end; $\mathbf{P}$ carries the fixed portion $p$ of a coupling-box, similar to the one just described as belonging to the little mill. On the prolongation of $\mathrm{P}$, there is a second shaft $P^{\prime}$, which bears the moveable portion of that box, and an upright bevel wheel $P^{\prime}$ Lastly, in figs. 900 and 908, there is shown the vertical shaft $Q$, which carries at its upper end a large horizontal cast-iron wheel $Q^{\prime}$, not seen in

this view, because it is sunk within the upper surface of the turning hornstone, like the clamp $d, f$, in $f i g .902$. At the lower end of the shaft $Q$, there is the bevel wheel $Q^{\prime \prime}$, which receives motion from the wheel $\mathbf{P}^{\prime \prime}$, fig. 900 .

The shaft P always revolves with the water-wheel; but transmits its motion to the 
shaft $\mathbf{P}^{\prime}$ only when the latter is thrown into gear with the coupling-box $p^{\prime}$, by means of its forked lever. Then the bevel wheel $\mathbf{P}^{\prime}$ turns round with the shaft $\mathbf{P}^{\prime}$, and communicates its rotation to the bevel wheel $Q^{\prime \prime}$, which transmits it to the shaft $Q$, and to the large castiron wheel, which is sunk into the upper surface of the revolving hornstone.

The shaft $Q$ is supported and centred by a simple and solid adjustment; at its lower part, it rests in a step $\mathbf{R}^{\prime}$, which is supported upon a cast-iron arch $Q^{\prime}$, seen in profile in fig. 900 ; its base is solidly fixed by four strong bolts. Four set screws above R, fig. 900, serve to set the shaft $\mathrm{Q}$ truly perpendicular; thus supported, and held securely at its lower end, in the step at $\mathbf{R}$, figs. 900 and 908 , it is embraced near the upper end by a brass bush or collar, composed of two pieces, which may be drawn closer together by means of a screw. This collar is set into the summit of a great truncated cone of cast-iron, which rises within the tub through two thirds of the thickness of the hornstone bed; having its base firmly fixed by bolts to the bottom of the tub, and having a brass collet to secure its top. The iron cone is cased in wood. When all these pieces are well adjusted and properly screwed up, the shaft $Q$ revolves without the least vacillation, and carries round with it the large iron wheel $Q^{\prime}$, cast in one piece, and which consists of an outer rim, three arms or radii, and a strong central nave, made fast by a key to the top of the shaft $Q$, and resting upon a shoulder nicely turned to receive it. Upon each of the three arms, there are adjusted, with bolts, three upright substantial bars of oak, which descend vertically through the body of the revolving mill to within a small distance of the bed-stone; and upon each of the three ares of that wheel-ring, comprised between its three strong arms, there are adjusted, in like manner, five similar uprights, which fit into hollows cut in the periphery of the moving stone. They ought to be cut to a level at their lower part, to suit the slope of the bottom of the tub o, figs. 900 and 908, so as to glide past it pretty closely, without touching.

The speed of this large mill is eight revolutions in the minute. The turning hornstone describes a mean circumference of $141 \frac{1}{3}$ inches (its diameter being 45 inches), and of course moves through about 100 feet per second. The $t u b 0$, is 52 inches wide at bottom, 56 at the surface of the sleeper block (which is 16 inches thick), and 64 at top, inside measure. It sometimes happens that the millstone throws the pasty mixture out of the vessel, though its top is 6 inches under the lip of the tub 0 ; an inconvenience which can be obviated only by making the pap a little thicker; that is, by allowing only from 25 to 30 per cent. of water; then its density becomes nearly equal to $2 \cdot 00$, while that of the millstones themselves is only 2.7 ; whence, supposing them to weigh only 2 cwts., there would remain an effective weight of less than $\frac{1}{2}$ cwt. for pressing upon the bottom and grinding the granular particles. This weight appears to be somewhat too small to do much work in a short time; and therefore it would be better to increase the quantity of water, and put covers of some convenient form over the tubs. It is estimated that this mill will grind nearly 5 cwts. of hard kaolin or feldspar gravel, in 24 hours, into a proper pap.

To the preceding methodical account of the porcelain manufacture, I shall now subjoin some practical details relative to certain styles of work, with comparisons between the methods pursued in this country and upon the Continent, but chiefly by our jealous rivals the French.

The blue printed ware of England has been hitherto a hopeless object of emulation in France. M. Alexandre Brongniart, membre de l'Institut, and director of the Manufacture Royal de Sères, characterizes the French imitations of the Fayence fine, ou Anglaise, in the following terms: "Les défauts de cette poterie, qui tiennent à sa nature, sont de ne pouvoir aller sur le feu pour les usages domestiques, et d'aroir un vernis tendre, qui se laisse aisément entamer par les instruments d'acier et de fer. Mais lorsque cette poterie est mal fabriquée, ou fabriquée avec une économie mal entendue, ses défauts deviennent bien plus graves; son vernis jaunâtre et tendre tressaille souvent; il se laisse entamer ou user avec la plus grande facilité par les instruments de fer, ou par l'usage ordinaire. Les fissures que ce tressaillement ou ces rayres ouvrent dans le vernis permettent aux matières grasses de pénétrer dans le biscuit, que dans les poteries affectées de ce défaut, a presque toujours une texture láche; les pièces se salissent, s'empuantissent, et se brisent même avec la plus grande facilité."

What a slaze, to be scratched or grooved with soft iron; to fly off in scales, so as to let grease soak into the biscuit or body of the ware; to become foul, stink, and break with the ntmost ease! The refuse crockery of the coarsest pottery works in the United King. dom would hardly deserve such censure.

In the minutes of evidence of the Enquête Ministériclle, published in 1835, MM. de Saint Cricq and Lebeuf, large manufacturers of pottery-ware at Creil and Montereau, give a very oratifying account of the English stoneware manufacture. They declare that the English possess magnificent mines of potter's clay, many leagues in extent; while those of the 
Frenct. are mere patches or pots. Besides, England, they say, having upwards of 200 potteries, can constantly employ a great many public flint-mills, and thereby obtain tha! indispensable material of the best quality, and at the lowest rate. "The mill erected by M. Brongniart, at Sèvres, does its work at twice the price or the English mills. The ruel costs in England one fourth of what it does in France. The expense of a kiln-round, in the latter country, is 200 francs; while in the former it is not more than 60." After a two-months tour among the English potteries, these gentlemen made the following additional observations to their first official statement :-

"The clay, which goes by water carriage from the counties of Devon and Dorset, into Staffordshire, to supply more than 200 potteries, clustered together, is delivered to them at a cost of 4 francs ( 3 s. $2 d$.) the 100 kilogrammes ( $2 \mathrm{cwt}$.); at Creil, it costs $4 f$. $50 \mathrm{c}$., and at Mintereau, only $2 f$. $40 c$. There appears, therefore, to be no essential difference in the price of the clay; but the quality of the English is much superior, being incontestably whiter, purer, more homogeneous, and not turning red at a high heat, like the French." The grinding of the flints costs the English potter $4 \frac{1}{2} d$. per 100 kilos., and the French $6 d$.; but as that of the latter is in general ground dry, it is a coarser artiele. The laolin, or china clay, is imported from Cornwall for the use of many French potteries; but the transport of inerchandise is so ill managed in France, that while $2 \mathrm{cwts}$. cost in Staffordshire only $8 f$. $75 c$. (about $7 s .1 d$.), they cost $12 f$. at Creil, and $13 f$. $50 c$. at Montereau. The white lead and massicot, so much employed for glazes, are 62 per cent. dearer to the French potters than the English. As no French mill has succeeded in making unsized paper fit for printing upon stoneware, our potters are under the necessity of fetching it from England; and, under favor of our own custom-house, are allowed to import it at a duty of $165 f$. per 100 kilogrammes, or about $8 d$. per pound English. No large stock of materials need be kept by the English, because every article may be had when wanted from its appropriate wholesale dealers; but the case is quite different with the French, whose stocks, even in small works, can never safely be less in value than $150,000 f$. or $200,000 f$.; constituting a loss to them, in interest upon their capital, of from 7,500f. to $10,000 \mathrm{f}$. per annum. The capital sunk in buildings is far less in England than in France, in consequence of the different styles of erecting stoneware factories in the two countries. M. de Saint Cricq informs us, that Mr. Clewes, of Shelton, rents his works for 10,000f. (380l.) per annum; while the similar ones of Creil and Montereau, in France, have cost each a capital outlay of from $500,000 f$. to $600,000 f$., and in which the products are not more than one half of $\mathrm{Mr}$. Clewes'. "This forms a balance against us," says M. St. C., " of about 20,000f. per annum; or nearly 8007 . sterling. Finally, we have the most formidable rival to our potteries in the extreme dexterity of the English artisans. An enormous fabrication permits the manufacturers to employ the same workmen during the whole year upon the same piece: thus I have seen at Shelton a furnisher, for sixpence, turn off 100 pieces, which cost at Creil and Montereau 30 sous ( 1 s. $2 \frac{1}{2} d$.); yet the English workman earns $18 f$. $75 c$. a week, while the French never earns more than $15 f$. I have likewise seen an English moulder expert enough to make 25 waterpots a day, which, at the rate of $2 d$. a piece, bring him $4 s$. $2 d$. of daily wages; while the French moulder, at daily wages also of $4 s .2 d$, turns out of his hands only 7, or at most 8 pots. In regard to hollow wares, the English may be fairly allowed to have an advantage over us, in the cost of labor, of 100 per cent.; which they derive from the circumstance, that there are in Staffordshire 60,000 operatives, men, women, and children, entirely dedicated to the stoneware manufacture; concentrating all their energies within a space of 10 square leagues. Hence a most auspicious choice of good practical potters, which cannot be found in France."

M. Saint Amans, a French gentleman, who spent some years in Staffordshire, and has lately erected a large pottery in France, says the English surpass all other nations in manufacturing a peculiar stoneware, remarkable for its lightness, strength, and elegance; as also in printing blue figures upon it of every tint, equal to that of the Chinese, by processes of singular facility and promptitude. After the biscuit is taken out of the kiln, the fresh impression of the engraving is transferred to it from thin unsized paper, previously immersed in strong soap water; the ink for this purpose being a compound of arseniate of cobalt with a flux, ground up with properly boiled linseed oil. The copper-plates are formed by the graving tool with deeper or shallower lines, according to the variable depth of shades in the design. The cobalt pigment, on melting, spreads so as to give the soft effect of water-color drawing. The paper, being still moist, is readily applied to the slightly rough and adhesive surface of the biscuit, and may be rubbed on more closely by a dossil of flannel. The picce is then dipped in a tub of water, whereby the paper gets soft, and may be easily removed, leaving upon the pottery the piyment of the engraved impression. After being gently dried, the piece is dipped into the glaze mixture, and put into the enamel oven. 


\section{Composition of the Earthy Mixtures.}

The basis of the English stoneware is, as formerly stated, a bluish clay, brought from Dorsetshire and Devonshire, which lies at the depth of from 25 to 30 feet beneath the surfice. It is composed of about 24 parts of alumina, and 76 of silica, with some other ingredients in very small proportions. This clay is very refractory in high heats, a property which, joined to its whiteness when burned, renders it peculiarly valuable for pottery. It is also the basis of all the yellow biscuit-ware called cream color, and in general of what is called the printing body; as also for the semi-vitrified porcelain of Wedgewood's invention, and of the tender porcelain.

The constituents of the stoneware are, that clay, the powder of calcined flints, and of the decomposed feldspar called Cornish stone. The proportions are varied by the different manufacturers. The following are those generally adopted in one of the principal establishments of Staffordshire :-

For cream color, Silex or ground flints -
Clay - - - - - - - - -
Cornish stone

\section{Composition of the Paste for receiving the Printing Body under the Glaze.}

For this purpose the proportions of the flint and the feldspar must be increased. The substances are mixed separately with water into the consistence of a thick cream, which weighs per pint, for the flints 32 ounces, and for the Cornish stone 28. The china clay of Cornwall is added to the same mixture of flint and feldspar, when a finer pottery or porcelain is required. That clay cream weighs 24 ounces per pint. These 24 ounces in weight are reduced to one third of their bulk by evaporation. The pint of dry Cornish clay weighs 17 ounces, and in its first pasty state 24 , as just stated. The dry flint powder weighs $14 \frac{1}{2}$ ounces per pint; which when made into a cream weighs 32 ounces. To 40 measures of Devonshire clay-cream there are added,

$$
\begin{aligned}
& 13 \text { measures of flint liquor. } \\
& 12 \quad-\quad \text { Cornish clay ditto. } \\
& 1 \quad-\quad \text { Cornish stone ditto. }
\end{aligned}
$$

The whole are well mixed by proper agitation, half dried in the troughs of the slip-kiln, and then subjected to the machine for cutting up the clay into junks. The above paste, when baked, is very white, hard, sonorous, and susceptible of receiving all sorts of impressions from the paper engravings. When the silica is mixed with the alumina in the above proportions, it forms a compact ware, and the impression remains fixed between the bisenit and the glaze, without communicating to either any portion of the tint of the metallic color employed in the engraver's press. The feldspar gives strength to the biscuit, and renders it sonorous after being baked; while the china clay has the double advantage of imparting an agreeable whiteness and great closeness of grain.

PRECIPITATE, is any matter separated in minute particles from the bosom of a fluid, which subsides to the bottom of the vessel in a pulverulent form.

PRECIPITATION, is the actual subsidence of a precipitate.

PRESS, HYDRAULIC. Though the explanation of the principles of this powerful machine belongs to a work upon mechanical engineering, rather than to one zoon 909

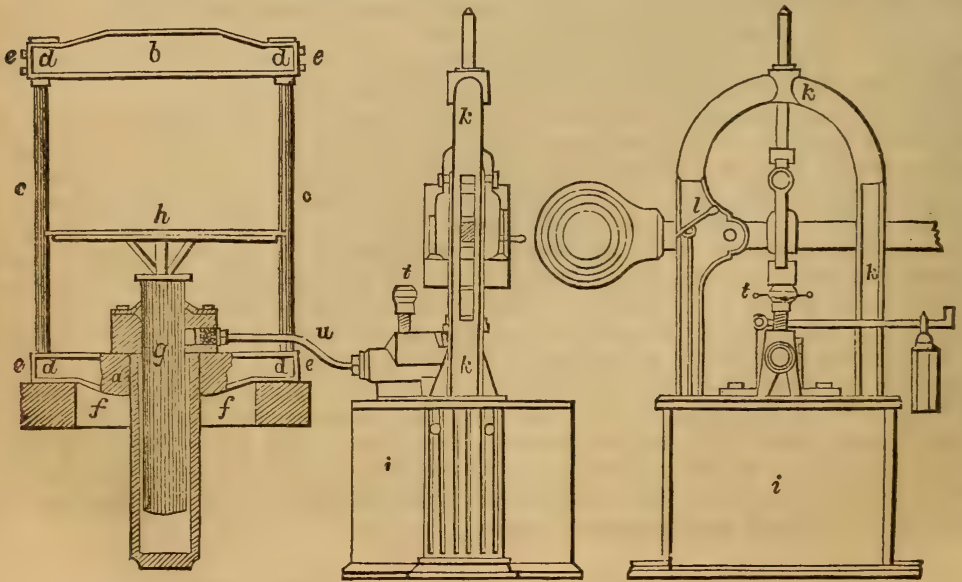


manufactures, yet as it is often referred to in this volume, a brief description of it cannot be unaccepiable to many of my readers.

The framing consists of two stout cast iron plates $a, b$, which are strengthened by pro. jecting ribs, not seen in the section, fig. 909. The top or crown plate $b$, and the baseplate $a, a$, are bound most firmly together by 4 cylinders of the best wrought iron, $c, c$, which pass up through holes near the ends of the said plates, and are fast wedged in them. The flat pieces $e, e$, are screwed to the ends of the crown and base plates, so as to bind the columns laterally. $f$, is the hollow cylinder of the press, which, as well as the ram g, is made of cast iron. The upper part of the cavity of the cylinder is cast narrow, but is truly and smoothly rounded at the boring-mill, so as to fit pretty closely ronnd a well-turned ram or piston; the under part of it is left somewhat wider in the casting. A stout cup of leather, perforated in the middle, is put upon the ram, and serves as a valve to render the neck of the cylinder perfectly water-tight, by filling up the sprace between it and the ram; and since the mouth of the cup is turned downwards, the greater the pressure of water upwards, the more forcibly are the edges of the leather valve pressed against the inside of the cylinder, and the tighter does the joint become. This was Bramah's beautiful invention.

Unon the top of the ram, the press-plate or table, strengthened with projecting ridges, rests, which is commonly called the follower, because it follows the ram closely in its

911

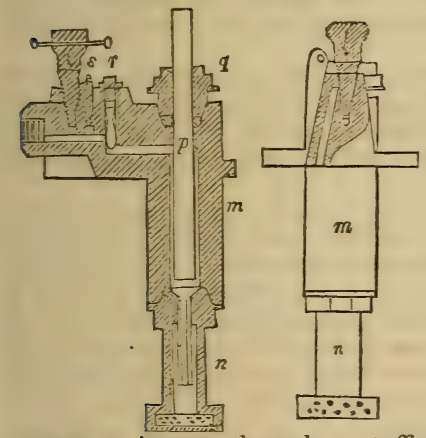

descent. This plate has a half-round hole at each of its four corners, corresponding to the shape of the four iron columns along which it glides in its up-and-down motions of compression and relaxation.

$k, k$, figs. 909 and 910 , is the framing of a force pump with a narrow barrel; $i$ is the well for containing water to supply the pump. To spare room in the engraving, the pump is set close to the press, but it may be removed to any convenient distance by lengthening the water-pipe $u$, which connects the discharge of the force pump with the inside of the cylinder of the press. Fig. 911 is a section of the pump and its valves. The pump $m$, is of bronze; the suction-pipe $n$, has a conical valve with a long tail; the solid piston or plunger $p$, is smaller than the barrel in which it plays, and passes at its top through a stuffing-box $q ; r$ is the pressure-valve, $s$ is the safetyvalre, which, in fig. 910 , is seen to be loaded with a weighted lever; $t$ is the discharge-valve, for letting the water escape, from the cylinder beneath the ram, back into the weli. See the winding passages in fig. 912. $u$ is the tube which conveys the water from the pump into the press-cylinder. In fig. 910 two centres of motion for the pump-lever are shown. By shifting the bolt into the centre nearest the pump-rod, the mechanical advantage of the workman may be doubled. Two pumps are generally mounted in one frame for one hydraulic press; the larger to give a rapid motion to the ram at the beginning, when the resistance is small; the smaller to give a slower but more powerful impulsion, when the resistance is much increased. A pressure of 500 tons may be obtained from a well-made hydraulic press with a ten-inch ram, and a two and a one inch set of pumps. See Stearine Press.

PRINCE'S METAL, or Prince Rupert's metal, is a modification of brass.

PRINTING INK. (Encre d'imprimerie, Fr.; Buchdruckerfarbe, Germ.) After reriewing the different prescriptions given by Moxon, Breton, Papillon, Lewis, those in Nicholson's and the Messrs. Aikins' Dictionaries, in Rees' Cyclopæedia, and in the French Printer's Manual, Mr. Savage* says, that the Encyclopædia Britannica is the only work, to his knowledge, which has given a recipe by which a printing ink might be made, that could be used, though it would be of inferior quality, as acknowledged by the editor; for it specifies neither the qualities of the materials, nor their due proportions. The fine black ink made by Mr. Savage, has, he informs us, been pronounced by some of our first printers to be unrivalled; and has procured for him the large medal from the Society for the Encouragement of Arts.

1. Linseed oil.-Mr. Sarage says, that the linseed oil, however long boiled, unless set fire to, cannot be brought into a proper state for forming printing ink; and that the flame may be most readily extinguished by the application of a pretty tight tin cover to the top of the boiler, which should never be more than half full. The French prefer nut oil to linseed; but if the latter be old, it is fully as good, and much cheaper, in this country at least.

2. Black rosin is an important article in the composition of good ink; as by melting

* In his work or the Preparation of Printing Ink ; Sro., London. 1832. 
it in the oil, when that ingredient is sufficiently boiled and burnt, the two combine, and form a compound approximating to a natural nalsam, like that of Canada, which is itself one of the best varnishes that can be used for printing ink.

3. Soap.-This is a most important ingredient in printers' ink, which is not even mentioned in any of the recipes prior to that in the Encyclopædia Britannica. For want of soap, ink accumulates upon the face of the types, so as completely to clog them up after comparatively few impressions have been taken; it will not wash off without alkaline leys, and it skins over very soon in the pot. Yellow rosin soap is the best for black inks; for those of light and delicate shades, white curd soap is preferable. Too much soap is apt to render the impression irregular, and to prevent the ink from drying quickly. The proper proportion has been hit, when the ink works clean, without clogging the surface of the types.

4. Lamp black.-The vegetable lamp black, sold in firkins, takes by far the most varnish, and answers for making the best ink. See BLAck.

5. Ivory black is too heavy to be used alone as a pigment for printing ink; but it may be added with advantage by grinding a little of it upon a muller with the lamp black, for certain purposes; for instance, if an engraving on wood is required to be printed so as to produce the best possible effect.

6. Indigo alone, or with an equal weight of Prussian blue, added in small proportion, takes off the brown tone of certain lamp black inks. Mr. Savage recommends a little Indian red to be ground in with the indigo and Prussian blue, to give a rich tone to the black ink.

7. Balsam of capivi, as sold by Mr. Allen, Plough-court, Lombard-street, mixed, by a stone and a muller, with a due proportion of soap and pigment, forms an extem. poraneous ink, which the printer may employ very advantageously when he wishes to execute a job in a peculiarly neat manner. Canada balsam does not answer quite so well.

After the smoke begins to rise from the boiling oil, a bit of burning paper stuck in the cleft end of a long stick should be applied to the surface, to set it on fire, as soon as the vapor will burn; and the flame should be allowed to continue (the pot being meanwhile removed from over the fire, or the fire taken from under the pot), till a sample of the varnish, cooled upon a pallet-knife, draws out into strings of about half an inch long between the fingers. To six quarts of linseed oil thus treated, six pounds of rosin should be gradually added, as soon as the froth of the ebullition has subsided. Whenever the rosin is dissolved, one pound and three quarters of dry brown soap, of the best quality, cut into slices, is to be introduced cautiously, for its water of combination causes a violent intumescence. Both the rosin and soap should be well stirred with the spatula. The pot is to be now set upon the fire, in order to complete the combination of all the constituents.

Put next of well ground indigo and Prussian blue, each $2 \frac{1}{2}$ ounces, into an earthen pan, sufficiently large to hold all the ink, along with 4 pounds of the best mineral lamp black, and $3 \frac{1}{2}$ pounds of good vegetable lamp black; then add the warm varnish by slow degrees, carefully stirring, to produce a perfect incorporation of all the ingredients. This mixture is next to be subjected to a mill, or slab and muller, till it be levigated into a smooth uniform paste.

One pound of a superfine printing ink may he made by the following recipe of $\mathrm{Mr}$. Savage :-Balsam of capivi, 9 oz.; lamp black, $3 \mathrm{oz}$; indigo and Prussian blue, together, p. æq. $1 \frac{1}{4}$ oz.; Indian red, $\frac{3}{4}$ oz.; turpentine (yellow) soap, dry, $3 \mathrm{oz}$. This mixture is to he ground upon a slab, with a muller, to an impalpable smoothness. The pigments use. for colored printing inks are, carmine, lakes, vermilion, red lead, Indian red, Venetian red, chrome yellow, chrome red or orange, burnt terra di Sienna, gall-stone, Roman ochre, yellow ochre, verdigris, blues and yellows mixed for greens, indigo, Prussian blue, Antwerp blue, lustre, umber, sepia, browns mixed with Venetian red, \&c.

PRINTING MACHINE. (Typographie mécanique, Fr.; Druckmaschine, Germ.) In reviewing those great eras of national industry, when the productive arts, after a long period of irksome vassalage, have suddenly achieved some new conquest over the inertia of rnatter, the contemplative mind cannot fail to be struck with the insignificant part which the academical philosopher has generally played in such memorable events.

Engrossed with barren syllogisms, or equational theorems, often little better than truisms in disguise, he nevertheless believes in the perfection of his attainments, and disdains to soil his hands with those handicraft operations at which all improvements in the arts must necessarily begin. He does not deem a manufacture worthy of his regard, till it has worked out its own grandeur and independence with patient labor and consummate skill. In this spirit the men of speculative science neglected for 60 years the steam engine of Newcomen, till the artisan Watt transformed it into an automatic prodigy; they have never deigned to illustrate by dynamical investigations the factory mechanisms 
of Arkwright, yet nothing in the whole compass of art deserves it so well; and though perfectly aware that revolvency is the leading law in the system of the universe, they have never thought of showing the workman that this was also the true principle of every automatic machine.

These remarks seem to be peculiarly applicable to book-printing, an art invented for the honor of learning and the glory of the learned, though they have done nothing for its advancement; yet by the overruling bounty of Providence it has eventually served as the great teacher and guardian of the whole family of man.

It has been justly observed by $\mathrm{Mr}$. Cowper, in his ingenious lecture, ${ }^{*}$ that no improvement had been introduced in this important art, from its invention till the year 1798, a period of nearly 350 years. In Dr. Dibdin's interesting account of printing, in the Bibliographical Decameron, may be seen representations of the early printing-presses, which exactly resemble the wooden presses in use at the present day. A new era has, however, now arrived, when the demands for prompt circulation of political intelligence require powers of printing newspapers beyond the reach of the most expeditious hand presswork.

For the first essential modification of the old press, the world is indebted to the late Earl Stanhope. $\dagger$ His press is formed of iron, without any wood; the table upon which the form of types is laid, as well as the platen or surface which immediately gives the impression, is of cast iron, made perfectly level; the platen being large enough to print a whole sheet at one pull. The compression is applied by a beautiful combination of levers, which give motion to the screw, cause the platen to descend with progressively increasing force till it reaches the type, when the power approaches the maximum; upon the infinite lever principle, the power being applied to straighten an obtuse-angled jointed lever. This press, however, like all its flat-faced predecessors, does not act by a continuous, but a reciprocating motion, and can hardly be made automatic; nor does it much exceed the old presses in productiveness, since it can turn off only 250 impressions per hour.

The first person who publicly projected a self-acting printing-press, was $\mathbf{M r}$. William Nicholson, the able editor of the Philosophical Journal, who obtained a patent in 1790-1, for imposing types upon a cylindrical surface; this disposition of types, plates, and blocks, being a new invention (see $f \mathrm{~g} .913$ ) ; 2, for applying the ink upon the surface of the types, \&c., by causing the surface of a cylinder smeared with the coloring-matter to roll over them; or else causing the types to apply themselves to the said cylinder. For the purpose of spreading the ink evenly over this cylinder, he

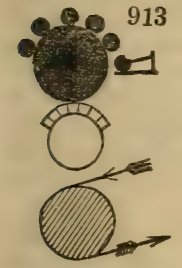

Nicholson's for arched type.

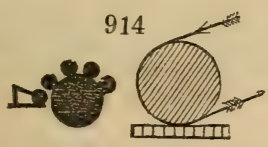

Nicholson's for proposed to apply three or more distributing rollers longitudinally against the inking cylinder, so that they might be turned by the motion of the latter. 3. "I perform," he says, "all my impressions by the action of a cylinder, or cylindrical surface; that is, I cause the paper to pass between two cylinders, one of which has the form of types attached to it, and forming part of its surface; and the other is faced with cloth, and serves to press the paper so as to take off an impression of the color previously applied; or otherwise I cause the form of types, previously colored, to pass in close and successive contact with the paper wrapped round a cylinder with woollen." (See figs. 913 and 914.) f

In this description Mr. Nicholson indicates pretty plainly the principal parts of modern printing machines; and had he paid the same attention to any one part of his invention which he fruitlessly bestowed upon attempts to attach types to a cylinder, or had he bethought himself of curving stereotype plates, which were then beginning to be talked of, he would in all probability have realized a working apparatus, instead of scheming merely ideal plans.

The first operative printing machine was undoubtedly contrived by, and constructed under the direction of, M. König, a clockmaker from Saxony, who, so early as the year 1804, was occupied in improving printing-presses. Having failed to interest the continental printers in his views, he came to London soon after that period, and submitted his plans to Mr. T. Bensley, our celebrated printer, and to Mr. R. Taylor, now one of the editors of the Philosophical Magazine.

* On the recent improvements in printing, first delivered at the Royal Institution, Folruary 22,1828

+ Lord Stanhope is the only man of learning whose name firures in the annals of typography.

\$ The black parts in these little diagrams, 913-922, indir:ate the inking apparatus; the diaronal lines. the oylinders upon which the paper to be printed is applied; the perpendicular lines, the plates or types . and the arrows show the track pursued by the sheet of paper. 
These gentlemen afforded Mr. König and his assistant Bauer, a German mechanic, liberal pecuniary support. In 1811 , he obtained a patent for a method of working a common hand-press by power; but after much expense and labor he was glad to renounce the scheme. He then turned his mind to the use of a cylinder for communicating the pressure, instead of a flat plate; and he finally succeeded, some time before the 28th November, 181.t, in completing his printing automaton; for on that day the editors of the Times informed their readers that they were perusing for the first time a newspaper printed by steam-impelled machinery; it is a day, therefore, which will be ever memorable in the annals of typography.

In that machine the form of type was made to traverse horizontally under the pressure cylinder, with which the sheet of paper was held in close embrace by means of a series

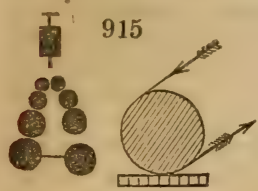

Konı's single, for one side of the sheet. of endless tapes. The ink was placed in a cylindrical box, from which it was extruded by means of a powerful screw, depressing a well-fitted piston; it then fell between two iron rollers, and was by their rotation transferred to several other subjacent rollers, which had not only a motion round their axes, but an alternating traverse motion (endwise). This system of equalizing rollers terminated in two which applied the ink to the types. (See fig. 915.) This plan of inking evidently involved a rather complex mechanism, was hence difficult to manage, and sometimes required two hours to get into good working trim. It has been superseded by a happy invention of Mr. Cowper, to be presently described.

In order to obtain a great many impressions rapidly from the same form, a paper-conducting cylinder (one embraced by the paper) was mounted upon each side of the inking apparatus, the form being made to traverse under both of them. This double-action machine threw off 1100 impressions per hour when first finished; and by a subsequent improvement, no less than 1800 .

Mr. König's next feat was the construction of a machine for printing both sides of 916

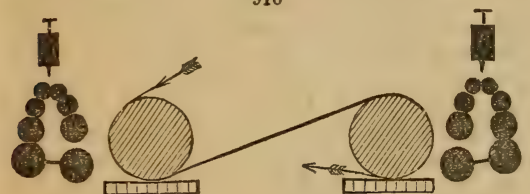
the newspaper at each complete traverse of the forms. This resembled two single machines, placed with their cylinders towards each other, at a distance of two or three feet; the sheet was conveyed from one paper cylinder to another, as before, by means of tapes; the track of the sheet exactly resembled Konig's double, for both sides of the sheet. the letter S laid horizontally, thus, on; and the sheet was turned over or reversed in the course of its passage. At the first paper cylinder it received the impression from the first form, and at the second it received it from the second form; whereby the machine could print 750 sheets of book letter-press on both sides in an hour. This new register apparatus was erected for $\mathrm{Mr}$. T. Bensley, in the year 1815 , being the only machine made by Mr. König for printing upon both sides. See fig. 916 .

Messrs. Donkin and Bacon had for some years previous to this date been busily engaged with printing machines, and had indeed, in 1813, obtained a patent for an

917

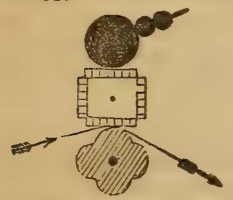

Donkin and Bacon's for type.

apparatus, in which the types were placed upon the sides of a revolving prism; the ink was applied by a roller, which rose and fell with the eccentricities of the prismatic surface, and the sheet was wrapped upon another prism fashioned so as to coincide with the eccentricities of the type prism. One such machine was erected for the University of Cambridge. (See fig. 917.) It was a beautiful specimen of ingenious contrivance and good workman. ship. Though it was found to be too complicated for common operatives, and defective in the mechanism of the inking process; yet it exhibited for the first time the elastic inking rollers, composed of glue combined with treacle, which alone constitute one of the finest inventions of modern typography. In König's machine the rollers were of metal covered with leather, and never answered their purpose very well.

Before proceeding further, I may state that the above elastic composition, which re. sembles caoutchouc not a little, but is not so firm, is made by dissolving with heat in two pounds of ordinary treacle, one pound of good glue, previously soaked during a night in cold water.

In the year 1815, Mr. Cowper turned his scientific and inventive mind to the subject of printing machines, and has since, in co-operation with his partner, Mr. Applegath, carried them to an unlooked-for degree of perfection. In $1815 \mathrm{Mr}$. Cowper obtained a patent for curving stereotype plates, for the purpose of fixing them on a crlinder 
Several machines so mounted, capable of printing 1000 sheets per hour upon both sides, are at work at the present day; twelve machines on this principle having been

918

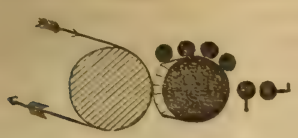

Cowper's single, for curved stereotype.

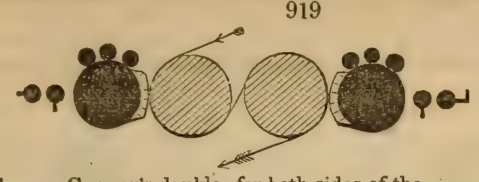

Cowper's dopble, for both sides of the made for the Directors of the Bank of England a short time previous to their re-issuing gold. See figs. 918, and 919.

It deserves to be remarked here, that the same object seems to have occupied the attention of Nicholson, Donkin, Bacon, and Cowper; viz., the revolution of the form. of types. Nicholson sought to effect this by giving to the shank of a type a shape like the stone of an arch; Donkin and Bacon by attaching types to the sides of a revolving prism; and Cowper, more successfully, by curving a stereotype plate. (See fig. 918.) In these machines Mr. Cowper places two paper cylinders side by side, and against each of them a cylinder for holding the plates; each of these four cylinders is about two feet in diameter. Upon the surface of the stereotype-plate cylinder, four or five inking rollers of about three inches in diameter are placer; they are kept in their position by a frame at each end of the said cylinder, and the axles of the rollers rest in vertical slots of the frame, whereby, having perfect freedom of motion, they act by their gravity alone, and require no adjustment.

The frame which supports the inking rollers, called the waving-frame, is attached by hinges to the general framework of the machine; the edge of the stereotype-plate cylinder is indented, and rubs against the waving-frame, causing it to vibrate to and fro, and consequently to carry the inking rollers with it, so as to give them an unceasing traverse movement. These rollers distribute the ink over three fourths of the surface of the cylinder, the other quarter being occupied by the curved stereotype plates. The ink is contained in a trough, which stands parallel to the said cylinder, and is formed by a metal roller revolving against the edge of a plate of iron; in its revolution it gets covered with a thin film of ink, which is conveyed to the plate cylinder by a distributing roller vibrating between both. The ink is diffused upon the plate cylinder as before described; the plates in passing under the inking rollers become charged with the colored varnish; and as the cylinder continues to revolve, the plates come into contact with a sheet of paper on the first paper cylinder, which is then carried by means of tapes to the second paper cylinder, where it receives an impression upon its opposite side from the plates upon the second cylinder.

Thus the printing of the sheet is completed. Though the above machine be applicable oniy to stereotype plates, it has been of general importance, because it formed the foundation of the future success of Messrs. Cowper and Applegath's printing machinery, by showing them the best method of serving out, distributing, and applying the colored varnish to the types.

In order to adapt this method of inking to a flat type-form machine, it was merely requisite to do the same thing upon an extended flat surface or table, which had been performed upon an extended cylindrical surface. Accordingly, Messrs. Cowper and Applegath constructed a machine for printing both sides of the sheets from type, including the inking apparatus, and the mode of conveying the sheet from the one paper cylinder to the other, by means of drums and tapes. It is highly creditable to the scientif: judgment of these patentees, that in new modelling the printing machine they dispensed with forty wheels, which existed in Mr. König's apparatus, when Mr. Bensley requested them to apply their improvements to it

The distinctive advantages of these machines, and which have not hitherto been equalled, are the uniform distribution of the ink, the equality as well as delicacy with which it is laid upon the types, the diminution in its expenditure, amounting to

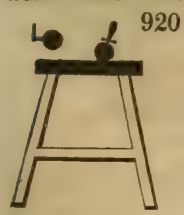
one half upon a given quantity of letter-press, and the facility with which the whole mechanism is managed. The band inking-roller and distributing-table, now so common in every printing-office in Europe and America, is the invention of $\mathrm{Mr}$. Cowper, and was specified in his patent. The vast superiority of the inking apparatus in his machines, over the balls used of old, induced him to apply it forthwith to the common press, and most successfully for the public; but with little or no profit to the inventor, as the plan was unceremoniously infringed throughout the kingdom, by such a multitude of printers, whether rich or poor, as to render all attempts at reclaiming his rights by prose cution hopeless. See fig. 920.

To construct a printing machine which shall throw off two sides at a

Cowper's inkine sable and roller. ame with exact register, that is, with the second side placed precisely upon the back of the 
first, is a very difficult problem, which was first practically solved by Messrs. Applegath and Cowper. It is comparatively easy to make a machine which shall print the one side of a sheet of paper first, and then the other side, by the removal of one form, and the introduction of another; and thus far did Mr. König advance. A correct register requires the sheet, after it has received its first impression from one cylinder, to travel

921

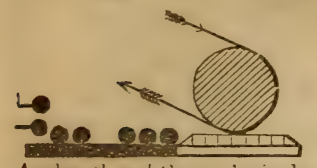

Applerath and c'owper's single.
922

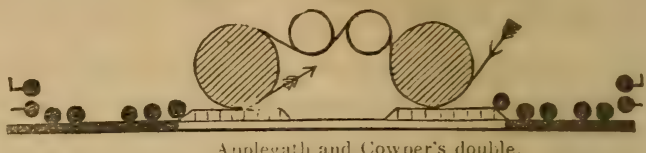

Applegath and Cowper's double.

round the peripheries of the cylinders and drums, at such a rate as to meet the types of the second side at the exact point which will ensure this side falling with geometrical nicety upon the back of the first. For this purpose, the cylinders and drums must revolve at the very same speed as the carriage underneath; hence the least incorrectness in the workmanship will produce such defective typography as will not be endured in book-printing at the present day, though it may be tolerated in newspapers. An equable distribution of the ink is of no less importance to beautiful letter-press. See figs. 921,922 .

The machines represented in figs. 923, 924, 925, are different forms of those which have been patented by Messrs. Applegath and Cowper. That shown in figs. $9 \dot{2} 3$ and 925 , prints both sides of the sheet during its passage, and is capable of throwing off nearly 1000 finished sheets per hour. The moistened quires of blank paper being piled upon a table A, the boy, who stands on the adjoining platform, takes up one sheet after another, and lays them upon the feeder $B$, which has several linen girths passing across its surface, and round a pulley at each end of the feeder; so that whenever the pulleys begin to revolve, the motion of the girths carries forward the sheet, and delivers it over the entering roller $\mathrm{E}$, where it is embraced between two series of endless tapes, that pass round a series of tension rollers. These tapes are so placed as to fall partly between, and partly exterior to, the pages of the printing; whereby they remain in close contact with the sheet of paper on both of its sides during its progress through the machine. The paper is thus conducted from the first printing cylinder $F$, to the second cylinder $G$, without having the truth of its register impaired, so that the coincidence of the two pages is perfect. These two great cylinders, or drums, are made of cast iron, turned perfectly true upon a self-acting lathe ;* they are clothed in these parts, corresponding to the typographic impression, with fine woollen cloth, called blankets by the pressmen, and revolve upon powerful shafts which rest in brass bearings of the strong framing of the

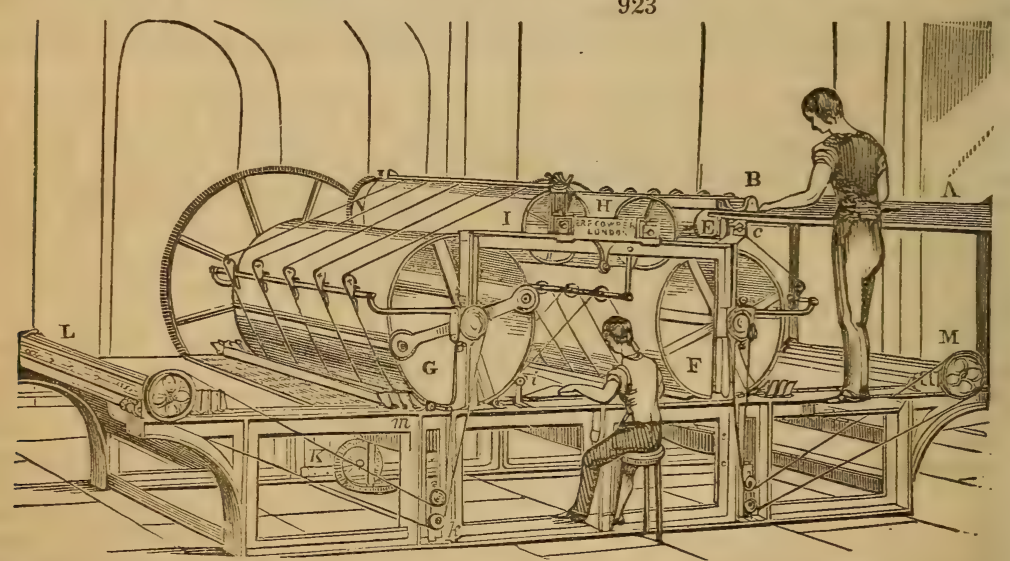

machine. These bearings, or plummer blocks, are susceptible of any degree of adjustment, by set screws. The drums $\mathrm{H}$ and $\mathrm{I}$ are made of wood; they serve to conduct the sheet evenly from the one printing cylinder to the other.

One series of tapes commences at the upper part of the entering drum E, proceeds in contact with the right-hand side and under surface of the printing cylinder $F$, passes * I have witnessed with much pleasure the turning of these great cylinders in Messrs. Cowper's factory at
Manchester. 
next over the carrier-drum $H$, and under the carrier-drum $I$; then encompassing the sefthand side and under portion of the printing drum G, it passes in contact with the

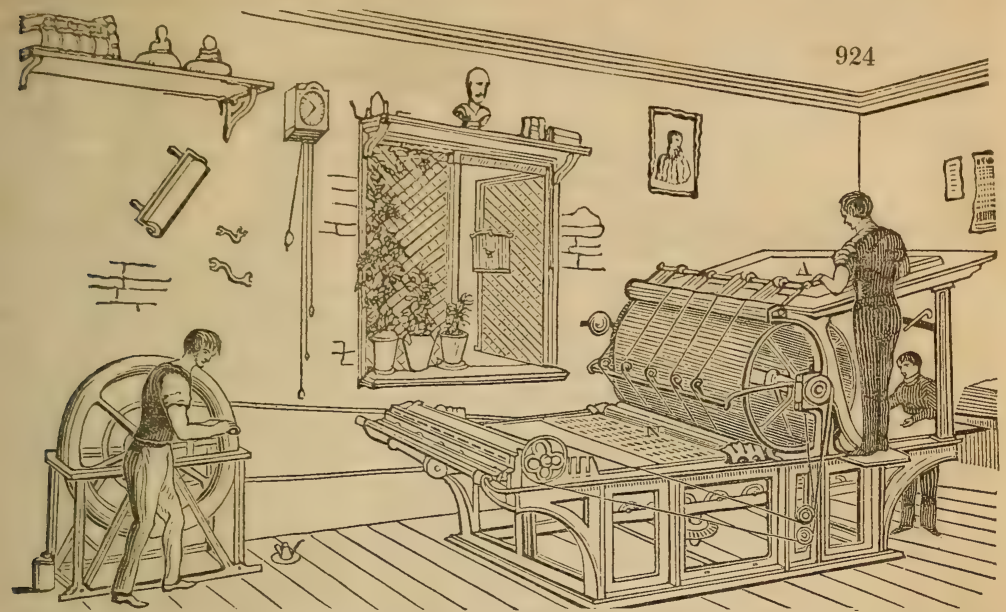

small tension rollers $a, b, c, d, f i g$. 925, and finally arrives at the roller $\mathrm{E}$, which may be called the commencement of the one series of endless tapes. The other series may be supposed to commence at the roller $h$; it has an equal number of tapes, and corresponds with the former in being placed upon the cylinders so that the sheets of paper may be held securely between them. This second series descends from the roller $h$, fig. 925, to the entering drum E, where it meets and coincides with the first series in such a way that both sets of tapes proceed together under the printing cylinder $\mathbf{F}$, over $\mathbf{H}$, under $\mathrm{I}$, and round $\mathrm{G}$, until they arrive at the roller $i$, fig. 923 , where they separate, after having continued in contact, except at the places where the sheets of paper are held between them. The tapes descend from the roller $i$, to a roller at $k$, and, after passing in contact with rollers at $l, m, n$, they finally arrive at the roller $h$, where they were supposed to commence. Hence two series of tapes act invariably in contact, without the least mutual interference, as may be seen by inspection of the fig's. 923, 924, 925 .

The various cylinders and drums revolve very truly by means of a system of toothed wheels and pinions mounted at their ends. Two horizontal forms of types are laid at a certain distance apart upon the long carriage $\mathrm{M}$, adjoining to each of which there is a nat metallic plate, or inking table, in the same plane. The common carriage, bearing its two forms of type and two inking tables, is moved backwards and forwards, from one end of the printing machine to the other, upon rollers attached to the frame-work, and in its traverse brings the types into contact with the sheet of paper clasped by the tapes round the surfaces of the printing cylinders. This alternate movement of the carriage is produced by a pinion working alternately into the opposite sides of a rack under the table. The pinion is driven by the bevel wheels $\mathrm{K}$.

The mechanism for supplying the ink, and distributing it over the forms, is one of the most ingenious and valuable inventions belonging to this incomparable machine, and is so nicely adjusted, that a single grain of the pigment may suffice for printing one side of a sheet. Two similar sets of inking apparatus are provided; one at each end of the machine, adapted to ink its own form of type. The metal roller $\mathrm{L}$, called the ductor roller, as it draws out the supply of ink, has a slow rotatory motion communicated to it by a catgut cord, which passes round a small pulley upon the end of the shaft of the printing cylinder G. A horizontal plate of metal, with a straight-ground edge, is adjusted by set screws, so as to stand nearly in contact with the ductor roller. This plate lias an upright ledge behind, converting it into a sort of trough or magazine, ready to impart a coating of ink to the roller, as it revolves over the table. Another roller, corered with elastic composition (see suprà), called the vibrating roller, is made to travel between the ductor roller and the inking table; the vibrating roller, as it rises, touches the ductor roller for an instant, abstracts a film of ink from it, and then descends to transfer it to the table. There are 3 or 4 small rollers of distribution, placed somewhat diagorally across the table at $\mathrm{M}$, (inclined only 2 inches from a parallel to the end of the frame, furnished with long slender axles, resting in vertical slots, whereby they are left at liberty to revolve and to traverse at the same time; by which compound movement they are enabled to efface all inequality in the surface of the varnish, or to effect a per- 
fect distribution of the ink along the table. The table thus evenly sineared, being made 11 pass under the 3 or 4 proper inking rollers $\mathrm{N}$, fig. 924, imparts to them a uniferm
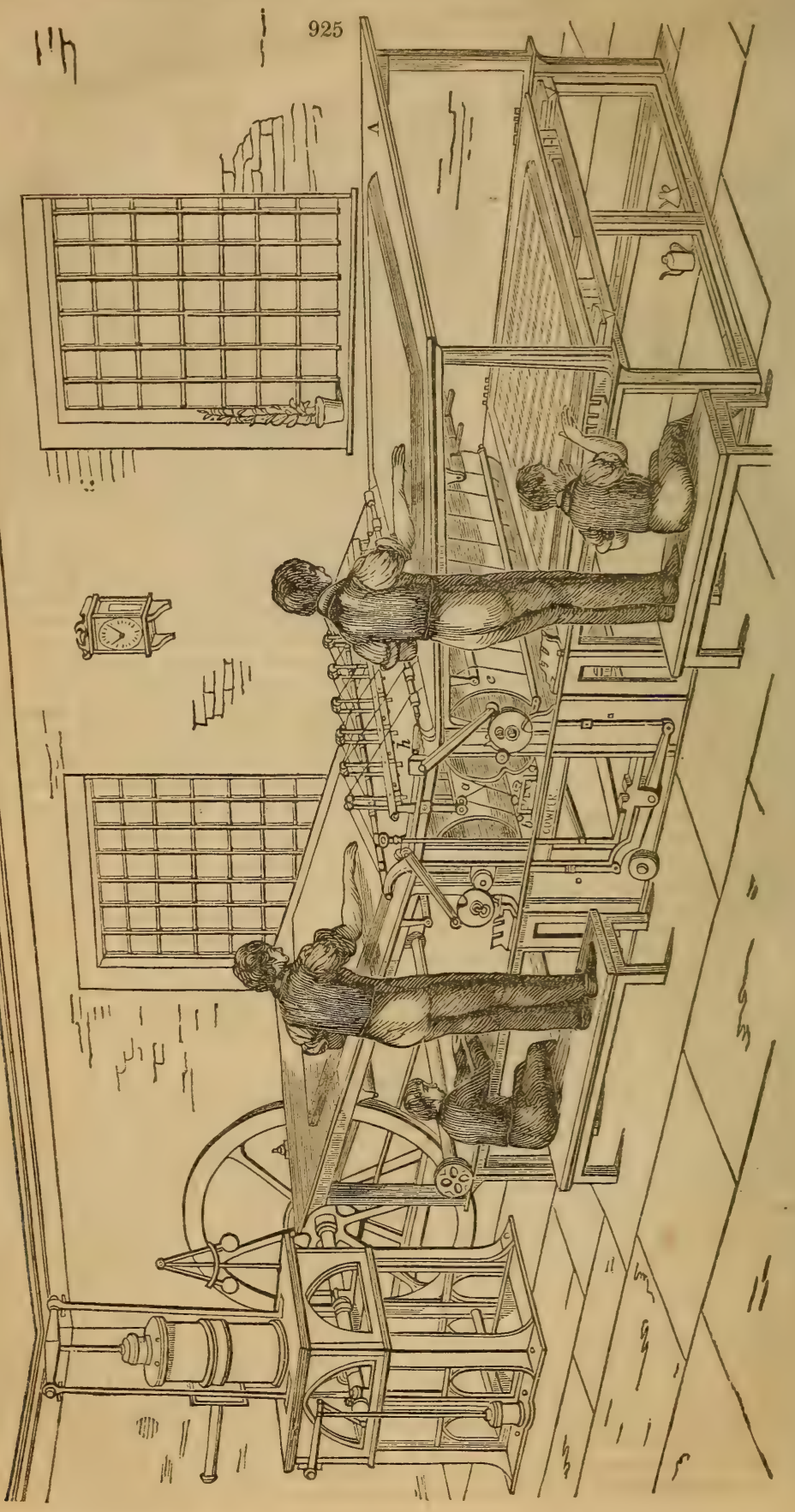
film of ink, to be immediately transferred by them to the types. Hence each time that the forms make a complete traverse to and fro, which is requisite for the printing of every sheet, they are touched no less than eight times by the inking rollers. Both the distributing and inking rollers turn in slots, which permit them to rise and fall so as to bear with their whole weight upon the inking table and the form, whereby they never stand in need of any adjustment by screws, but are always ready for work when dropped into their respective places.

Wotion is given to the whole system of apparatus by a strap from a steam engine going round a pulley placed at the end of the axle at the back of the frame; one steam-horse power being adequate to drive two double printing machines; while a single machine may be driven by the power of two men acting upon a fly-wheel. In Messrs. Clowes' establishment, in Stamford-street, two five-horse engines actuate nineteen of the above described machines.

The operation of printing is performed as follows :-See fig. 926.

The sheets being carefully laid, one by one, upon the linen girths, at the feeder B, the rollers $\mathrm{C}$ and $\mathrm{D}$ are made to more, by means of a segment wheel, through a portion of a rerolution. This morement carries on the sheet of paper sufficiently to introduce it between the two series of endless tapes at the point where they meet each other upon the entering drum E. As soon as the sheet is fairly embraced between the tapes, the rollers $c$ and $D$ are drawn back, by the operation of a weight, to their original position, so as to be ready to introduce another sheet into the machine. The sheet, advancing between the endless tapes, applies itself to the blanket upon the printing cylinder $F$, and as it revolves meets the first form of types, and receives their impression; after being thus printed on one side, it is carried, orer $\mathrm{H}$ and under $\mathrm{I}$, to the blanket upon the printing $\mathrm{cy}$ linder G, where it is placed in an inrerted position; the printed side being now in contact with the blanket, and the white side heing outwards, meets the second form of types at the proper instant, so as to receive the second impression, and get completely printed. The perfect sheet, on arriring at the point $i$, where the two series of tapes separate, is tossed out by centrifugal force into the hands of a boy.

The diagram, fig. 926, shows the arrangement of the tapes, agreeably to the preced-

926

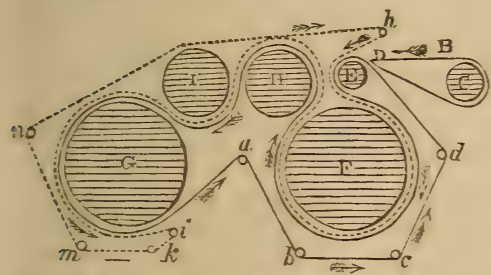

ing description; the feeder $\mathbf{B}$, with the rollers $\mathrm{c}$ and $\mathrm{D}$, is seento have an independent endless girth.

The diagram, fig. 927, explains the structure of the great machine contrived by Messrs. Applegath and Cowper for printing the Times newspaper. Here there are four places to lay on the sheets, and four to take them off; consequently, the assistance of eight lads is required. $P, P, P, P$, are the four piles of paper; $F, F, F, F$, are the four feeding-boards; $E, E, E, E$, are the four entering drums, upon which the sheets are introduced between the tapes $t, t, t, t$, whence they are conducted to the

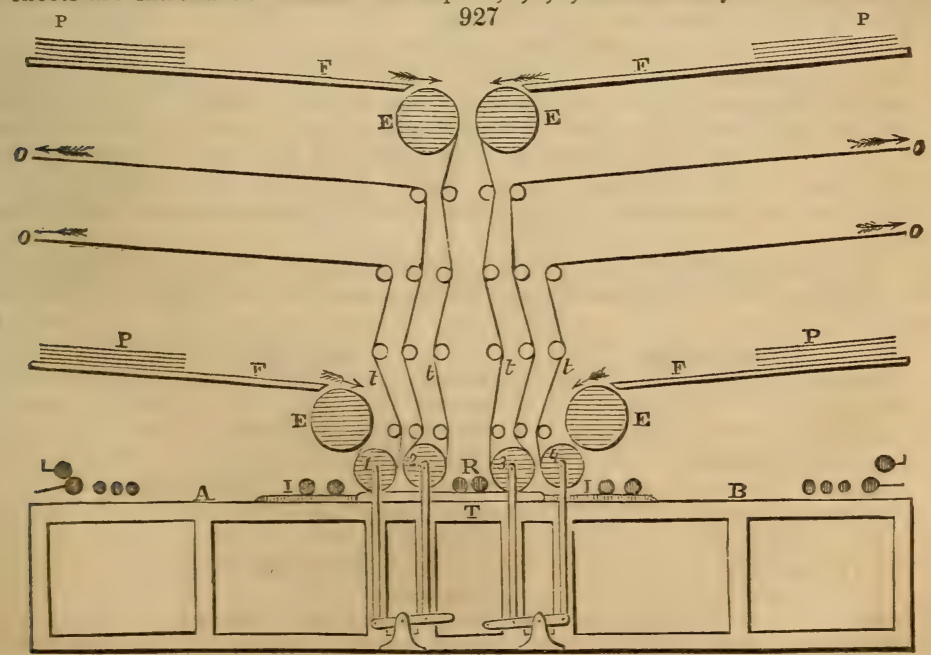


four printing cylinders $1,2,3,4 ; \mathrm{T}$ is the form of type; $\mathrm{I}, \mathrm{I}$, are two inking tables, of which one is placed at each end of the form. The inking apparatus is similar to that above described, with the addition of two central inking rollers $\mathrm{R}$, which likewise receive their ink from the inking tables. The printing cylinders $1,2,3,4$, are made to rise and fall about half an inch; the first and third simultaneously, as also the second and fourth. The form of type, in passing from $\mathrm{A}$ to $\mathrm{B}$, prints sheets at 1 and 3 ; in returning from $\mathrm{B}$ to $A$, it prints sheets at 4 and 2 ; while the cylinder alternately falls to give the impression, and rises to permit the form to pass untouched.

Each of the lines marked $t$, consists of two endless tapes, which run in contact at the parts shown, but separate at the entering drums $\mathbf{E}$, and at the taking off parts $0,0,0,0$. The return of the tapes to the entering drum is omitted in the diagram, to avoid confusion of the lines.

The sheets of paper being laid upon their respective feeding-boards, with the fore edges just in contact with the entering drum, a small roller, called the drop-down roller, falls, at proper intervals, down upon the edges of the sheets; the drum and the roller being then removed, instantly carry on the sheet, between the tapes $t$, downwards to the printing cylinder, and thence upwards to $0,0,0,0$, where the tapes are parted, and the sheet falls into the hands of the attendant boy. This noble mechanism is so perfectly equipped, that it is generally in full work within four minutes after the form is brought into the machine-room. The speed of König's machine, by which the Times was formerly printed, was such as to turn out 1800 papers per hour; that of Applegath and Cowper throws off 4200 per hour, and it has been daily in use during eight years.

PRUSSIAN BLUE, and PRUSSIATE OF POTASH, are two important articles of chemical manufacture, which must be considered together. The first is called by English chemists, Ferrocyanodide of iron, the Cyanure ferroso-ferrique of Berzelius; Eisenblausaures eisenoxyd, or eisencyanür +eisencyunid, Germ.; the second is called Ferrocycnodide of potassium, the Cyanure ferroso-potassique of Berzelius; Eisencyanur-kalium, cyaneisen + cyankalium or Blausaures eisenoxydul-kali, Germ.

Prussian blue (Berliner-blau, Germ.), is a chemical compound of iron and cyanogen. When organic matters, abounding in nitrogen, as dried blood, horns, hair, skins, or hoofs of animals, are triturated along with potash in a strongly ignited iron pot, a dark gray mass is obtained, that affords to water the liquor originally called lixivium sanguinis, or blood-ley, which, by evaporation, yields lemon-colored crystals in large rectangular tables, bevelled at the edges. This salt is called in commerce, prussiate of potash, and has for its ultimate constituents, potassium, iron, oxygen, and hydrogen (the latter two in such proportions as to form water), and the peculiar compound Cranogen, the blaustoff of the Germans.

These crystals consist, in 100 parts, of potassium $37 \cdot 02$, iron $12 \cdot 82$, cyanogen $37 \cdot 40$, water $12 \cdot 76$; or, cyanide of potassium $61 \cdot 96$, cyanide of iron $25 \cdot 28$, and water $12 \cdot 76$. They may be represented also by the following composition : 44.58 of potassa, 38.82 of hydrocyanic or prussic acid, and 16.60 of oxyde of iron, in 100 parts; but the first appears to be their true chemical constitution. Dry ferrocyanodide of potassium is a compound of one atom of cyanide of iron, $54=(28+26)$, and 2 atoms of cyanide of potassium, $132,=$ $\overline{(26 \times 2}+40 \times 2)$; the sum being 186 ; hydrogen being 1.0 in the scale of equivalents. The crystals of prussiate of potash are nearly transparent, soft, of a sweetish saline and somewhat bitterish taste, soluble in 4 parts of water at $52^{\circ} \mathrm{F}$., and in 1 part of boiling water, but insoluble in alcohol. They are permanent in the air at ordinary temperatures, but in a moderately warm stove-room they part with $12 \frac{3}{4}$ per cent. of water, without losing their form or coherence, and becomes thereby a white friable anhydrous ferrocyanodide of potassium, consisting of 42.44 potassium, 42.87 cyanogen, and 14.69 iron, in 100 parts.

This salt is an excellent reagent for distinguishing metals from each other, as the following TABLE of the precipitates which it throws down from their saline solutions will slow :-

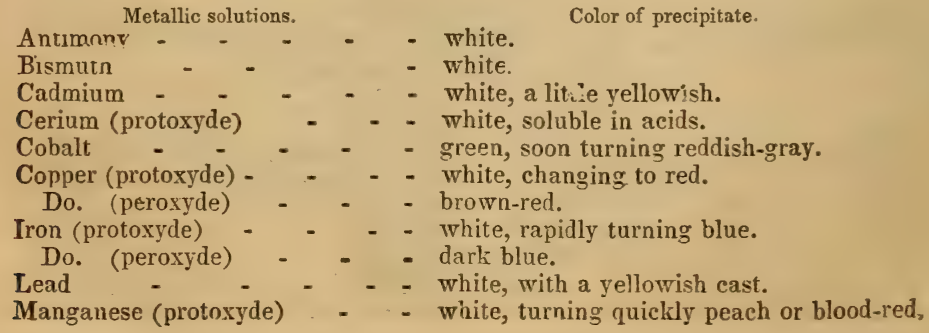




$\begin{aligned} & \text { Metallic solutions. } \\ & \text { Manganese (deutoxyde) }\end{aligned}$
Mercury (protoxyde)
Do. (peroxyde)

No precipitations ensue with solutions of the alkaline or earthy salts, except that of yttria, which is white; nor with those of gold, platinum, rhodium, iridium, osmium, (in concentrated solutions) tellurium, chromium, tungstenium. All the precipitates oy the ferrocyanodide of iron, are double compounds of cyanide of iron with cyanide of the metal thrown down, which is produced by the reciprocal decomposition of the yanile of potassium and the peculiar metallic oxyde present in the solution. The precipitate from the sulphate of copper has a fine brown color, and has been used as a pigment; but it is somewhat transparent, and therefore does not cover well. The precipitate from the peroxyde salts of iron is a very intense Prussian blue, called on the continent, Paris blue. It may be regarded as a compound of prussiate of protoxyde and prussiate of peroxyde of iron; or as a double cyanide of the protoxyde and peroxyde of iron, as the denomination cyanure ferroso-ferrique denotes. In numbers, its composition may be therefore stated thus : prussic or hydrocyanic acid, $48 \cdot 48$; protoxyde of iron, 20.73; peroxyde of iron, $30 \cdot 79$; or eyanogen, $46 \cdot 71$; iron, $37 \cdot 36$; water, 15.93 ; which represent its constitution when it is formed by precipitation with the prussiate of potash or a salt of iron that contains no protoxyde. If the iron be but partially peroxydized in the salt, it will afford a precipitate, at first pale blue, which turns dark blue in the air, consisting of a mixture of prussiate of protoxyde and prussiate of peroxyde. In fact, the white cranide of iron (the prussiate of the pure protoxyde), when exposed to the air in a moist condition, becomes, as above stated, dark blue; yet the new combination formed in this case through absorption of oxygen, is essentially different from that resulting from the precipitation by the peroxyde of iron, since it contains an excess of the peroxyde in addition to the usual two cyanides of iron. It has been therefore called basic Prussian blue, and, from its dissolving in pure water, soluble Prussian blue.

Both kinds of Prussian blue agree in being void of taste and smell, in attracting humidity from the air when they are artificially dried, and being decomposed at a heat abore $348^{\circ} \mathrm{F}$. The neutral or insoluble Prussian blue is not affected by alcohol; the basic, when dissolved in water, is not precipitated by that liquid. Neither is acted upon by dilute acids; but they form with concentrated sulphuric acid a white pasty mass, from which they are again reproduced by the action of cold water. They are decomposed by strong sulphuric acid at a boiling heat, and by strong nitric acid at common temperatures; but they are hardly affected by the muriatic. They become green with eniorine, but resume their blue color when treated with disoxydizing reagents. When Prussian blue is digested in warm water along with potash, soda, or lime, peroxyde of iron is separated, and a ferroprussiate of potash, soda, or lime remains in solution. If the Prussian blue has been previously purified by boiling in dilute muriatic acid, and washing with water, it will afford by this treatment a solution of ferrocyanodide of potassium, from which by evaporation this salt may be obtained in its purest crystalline state. When the powdered Prussian blue is diffused in boiling water, and digested with red oxyde of mercury, it parts with all its oxyde of iron, and forms a solution of bi-cyanodide, improperly called prussiate of mercury ; consisting of $79 \cdot 33$ mercury, and 20.67 cyanozen; or, upon the hydrogen equivalent scale, of 200 mercury, and $52=(26 \times 2)$ cyanozen. When this salt is gently ignited, it affords gaseous cyanogen. Hydrocyanic or prussic acid, which consists of 1 atom of cyanngen $=26,+1$ of hydrogen $=1$, is prepared hy distilling the mercurial bi-cyanide in a glass retort with the saturating quantity of dilute muriatic acid. Prussic acid may also be obtained by precipitating the mercury by sulphureted hydrogen gas from the solution of its cyanide; as also by distilling the ferrocyanide of potassium along with dilute sulphuric acid. Prussic acid is a very volatile light fluid, eminently poisonous, and is spontaneously decomposed by keeping, especially when somewhat concentrated.

Having expounded the chemical constitution of Prussian blue and prussiate of potash, I shall now treat of their manufacture upon the commercial scale.

1. Of blood-ley, the phlogisticated alkali of Scheele. Among the animal substances used for the preparation of this lixivium, blood deserves the preference, where it can be had ahes- snnigh It must he evanorated to nerfect dryness, reduced to nowder, and sifted 
Hoofs, parings of horns, hides, old woollen rags, and other animal offals, are, however, generally had recourse to, as condensing most azotized matter in the smallest bulk. Dried funguses have been also prescribed. These animal inatters may either be first carbonized in cast iron cylinders, as for the manufacture of sal ammoniac (which see), and the residual charcoal may be then taken for making the ferroprussiate; or the dry animal matters may be directly employed. The latter process is apt to be exceedingly offensive to the workmen and neighborhood, from the nauseous vapors that are exhaled in it. Eight pounds of horn (hoofs), or ten pounds of dry blood, afford upon an average one pound of charcoal. This must be mixed well with good pearlash, (freed previously from most of the sulphate of potassa, with which it is always contaminated), either in the dry way, or by soaking the bruised charcoal with a strong solution of the alkali; the proportion being one prart of carbonate of potassa to from $1 \frac{1}{2}$ to 2 parts of charcoal, or to about eight parts of hard animal matter. Gautier has proposed to calcine three parts of dry blood with one of ni tre; with what advantage to the manufacturer, I cannot discover.

The pot for calcining the mixture of animal and alkaline matter is egg-shajed as represented at $a, f i g .928$, and is considerably narrowed at the neck $e$, to facilitate the closing of the mouth with a lid $i$. It is made of cast iron, about two inches thich in the

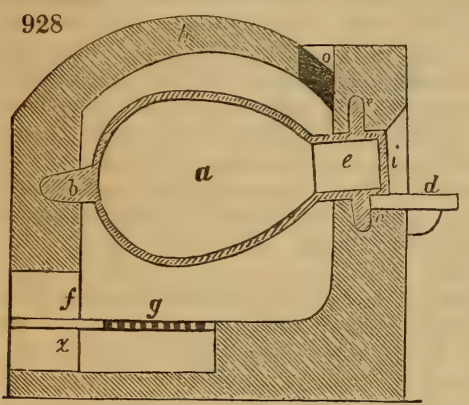
belly and bottom; this strength being requisite because the chemical action of the materials wears the metal fast away. It should be built into the furnace in a direction sloping downwards, (more than is shown in the figure), and have a strong knob $b$, projecting from its bottom to support it upon the back wall, while its shoulder is embraced at the $\operatorname{arms} c, c$, by the brickwork in front. The interior of the furnace is so formed as to leave but a space of a few inches round the pot, in order to make the flame play closely over its whole surface. The fire-door $f$, and the draughthole $z$, of the ash-pit, are placed in the posterior part of the furnace, in order that the workmen may not be incommoded by the heat. The smoke vent 0 , issues through the arched top $h$ of the furnace, towards the front, and is thence led backwards by a flue to the main chimney of the factory. $d$ is an iron or stone shelf, inserted before the mouth of the pot, to prevent loss in shovelling out the semi-liquid paste. The pot may be half filled with the materials.

The calcining process is different, according as the animal substances are fresh or carbonized. In the first case, the pot must remain open, to allow of diligent stirring of its contents, with a slightly bent flat iron bar or scoop, and of introducing more of the mixture as the intumescence subsides, during a period of five or six hours, till the nauseous vapors cease to rise, till the flame becomes smaller and brighter, and till a smell of ammonia be perceived. At this time, the heat should be increased, the mouth of the pot should be shut, and opened only once every half hour, for the purpose of working the mass with the iron paddle. When on opening the mouth of the pot, and stirring the pasty mixture, no more flame rises, the process is finished.

If the animal ingredients are employed in a carbonized state, the pot must be shut as soon as its contents are brought to ignition by a briskly urged fire, and opened for a few seconds only every quarter of an hour, during the action of stirring. At first, a body of flame bursts forth every time that the lid is removed; but by degrees this ceases, and the mixture soon agglomerates, and then softens into a paste. Though the fire be steadily kept up, the flame becomes less and less each time that the pot is opened; and when it ceases, the process is at an end. The operation, with a mass of 50 pounds of charcoal and 50 pounds of purified pearlash, lasts about 12 hours, the first time that the furnace is kindled; but when the pot has been previously brought to a state of ignition, it takes only 7 or 8 hours. In a well-appointed factory, the fire should be invariably maintained at the proper pitch, and the pots should be worked with relays of operatives.

The molten mass is now to be scooped out with an appropriate iron shovel, having a long shank, and caused to cool in small portions, as quickly as possible; but not by throwing it into water, as has sometimes been prescribed; for in this way a good deal of the cyanogen is converted into ammonia. If it be heaped up and kept hot in contact with air, some of the ferrocyanide is also decomposed, with diminution of the product. The crude mass is to be then put into a pan with cold water, dissolved by the application of a moderate heat, and filtered through cloths. The charcoal which remains upon the filter possesses the properties of decoloring sirups, vinegars, \&c., and of destroying smells in a pre-eminent degree. It may also serve, when mixed with fresh animal coal, for another calcining operation. 
As the iron requisite for the formation of the ferrocyanide is in general derived from the sides of the pot, this is apt to wear out into holes, especially at its under side, where the heat is greatest. In this event, it may be taken out of the furnace, patched up with iron-rust cement, and re-inserted with the sound side undermost. The erosion of the pot inay be obviated in some measure by mixing iron borings or cinder (hammerschlag) with the other materials, to the amount of one or two hundredths of the potash.

The above lixivium is not a solution of pure ferroprussiate; it contains not a little cyanide of potassium, which in the course of the process had not absorberl the proper dose of iron to form a ferrocyanide; it contains also more or less carbonate of potash, with phosphate, sulphate, hydrogenated sulphuret, muriate, and sulpho-cyanide of the same base, as well as phosphate of lime; substances derived partly fiom the impure potash, and partly from the incinerated animal matters. Formerly that very complex impure solution was employed directly for the precipitation of Prussian blue; but now, in all well regulated works, it is conrerted by evaporation and cooling into crystallized ferroprussiate of potash. The mother-water is again evaporated and crystallized, whereby a somewhat inferior ferroprussiate is obtained. Before evaporating the ley, however, it is advisable to add as much solution of green sulphate of iron to it, as will re-dissolve the white precipitate of cyanide of iron which first falls, and thereby convert the cyanide of potassium, which is present in the liquor, into ferrocyanide of potassium. The commercial prussiate of potash may be rendered chemically pure by making its crystals effloresce in a stove, fusing them with a gentle heat in a glass retort, dissolving the mass in water, neutralizing any carbonate and cyanide of potash that may be present with acetic acid, then precipitating the ferroprussiate of potash by the addition of a sufficient quantity of alcohol, and finally erystallizing the precipitated salt twice over in water. The sulphate of potassa may be decomposed by acetate of baryta, and the resulting acetate of potassa removed by alcohol.

2. The precipitation of Prussian blue.-Green sulphate of iron is always employed by the manufacturer, on account of its cheapness, for mixing with solution of the ferroprussiate, in forming Prussian blue, though the red sulphate, nitrate, or muriate of iron would afford a much richer blue pigment. Whatever salt of iron be preferred, should be carefully freed from any cupreous impregnation, as this would give the pure blue a dirty brownish cast. The green sulphate of iron is the most advantageous precipitant, on account of its affording protoxyde, to convert into ferrocyanide any cyanide of potassium that may happen to be present in the uncrystallized lixivium. The carbonate of potash in that lixivium might be saturated with sulphuric acid before adding the solution of sulphate of iron; but it is more commonly done by adding a certain portion of alum; in which case, alumina falls along with the Prussian blue; and though it renders it somewhat paler, yet it proportionally increases its weight; whilst the acid of the alum saturates the carbonate of potash, and prevents its throwing down iron-oxyde, to degrade by its brown-red tint the tone of the blue. For every pound of pearlash used in the calcination, from two to three pounds of alum are employed in the precipitation. When a rich blue is wished for, the free alkali in the Prussian ley may be partly saturated with sulphuric acid, before adding the mingled solutions of copperas and alum. One part of the sulphate of iron is generally allowed for 15 or 20 parts of dried blood, and 2 or 3 of horn-shavings or hoofs. But the proportion will depend very: much upon the manipulations, which, if skilfully conducted, will produce more of the cyanides of iron, and require more copperas to neutralize them. The mixed solutions of alum and copperas should be progressively added to the ley as long as they produce any precipitate. This is not at first a fine blue, but a greenish gray, in consequence of the admixture of some white cyanide of iron; it becomes gradually blue by the absorption of oxygen from the air, which is favored by agitation of the liquor. Whenever the color seems to be as beautiful as it is likely to become, the liquor is to be run off by a spigot or cock from the bottom of the precipitation vats, into flat cisterns, to settle. The clear supernatant fluid, which is chiefly a solution of sulphate of potash, is then drawn off by a syphon; more water is run on with agitation to wash it, which after settling is again drawn off; and whenever the washings become tasteless, the sediment is thrown . xpon filter sieves, and exposed to dry, first in the air of a stove, but finally upon slabs of chalk or Paris plaster. But for several purposes, Prussian blue may be best employed in the fresh pasty state, as it then spreads more evenly over paper and other surfaces.

A good article is known by the following tests : it feels light in the hand, adheres to the tongue, has a dark lively blue color, and gives a smooth deep trace; it should not efferresce with acids, as when adulterated with chalk; nor become pasty with boiling water, as when adulterated with starch. The Paris blue, prepared without alum, with a peroxyile salt of iron, displays, when rubbed, a copper-red lustre, like indigo. Prussian blie, degraded in its color by an admixture of free oxyde of iron, may be im. 
proved by digestion in dilute sulphuric or muriatic acid, washing, and drying. Its relative richness in the real ferroprussiate of iron may be estimated by the quantity of potash or soda which a given quantity of it requires to destroy its blue color.

Sulphureted hydrogen passed through Prussian blue diffused in water, whitens it: while prussic acid is eliminated, sulphur is thrown down, and the sesquicyanide of iron is conrerted into the single cranide. Iron and tin operate in the same way. When Prussian blue is made with two atoms of ferrocyanide of potassium, instead of one, it becomes soluble in water.

For the mode of applying this pigment in dyeing, see CALIco-printing.

Sesquiferrocyanate of potash is prepared by passing chlorine gas through a solution of ferrocyanide of potassium, till it becomes red, and ceases to precipitate the peroxyde salts of iron. : The liquor yields, by evaporation, prismatic crystals, of a ruby-red transparency. They are soluble in 38 parts of water, and consist of 40.42 parts of sesquicyanide of iron, and 59.58 of eyanide of potassium. The solution of this salt precipitates the following metals, as stated in the table:-

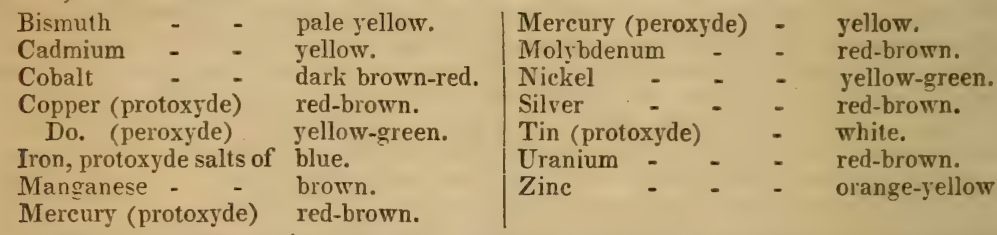

PUMICE-STONE (Pierre-ponce, Fr.; Bimstein, Germ.), is a spongy, vitreous-looking mineral, consisting of fibres of a silky lustre, interlaced with each other in all directions. It floats upon water, is harsh to the touch, having in mass a mean sp. grav. of 0.914 ; though brittle, it is hard enough to scratch glass and most metals. Its color is usually grayish white; but it is sometimes bluish, greenish, reddish, or brownish. It fuses without addition at the blowpipe into a white enamel. According to Klaproth, it is composed of, silica, $77 \cdot 5$; alumina, $17 \cdot 5$; oxyde of iron, 2 ; potassa and soda, 3 ; in 100 parts. The acids have hardly any action upon pumice-stone. It is used for polishing ivory, wood, marble, metals, glass, \&c.; as also skins and parchment. Pumice-stone is usually reckoned to be a volcanic product, resulting, probably, from the action of fire upon obsidians. The chief localities of this mineral are the islands of Lipari, Ponza, Ischia, and Vulcano. It is also found in the neighborhood of Andernach, upon the banks of the Rhine, in Teneriffe, Iceland, Auvergne, \&c. It is sometimes so spongy as to be of specific gravity 0.37 .

PCOZZOLANA is a volcanic gravelly product, used in making hydraulic mortar. See Cements and Mortars.

PURPLE OF CASSIUS. Gold purple (Pourpre de Cassius, Fr.; Gold-purpur, Germ.), is a ritrifiable pigment, which stains glass and porcelain of a beautiful red or purple hue. Its preparation has been deemed a process of such nicety, as to be liable to fail in the most experienced hands. The following observations will, I hope, place the subject upon a surer footing.

The proper pigment can be obtained only by adding to a neutral muriate of gold a mixture of the protochloride and perchloride of tin. Everything depends upon this intermediate state of the tin; for the protochloride does not afford, even with a concentrated solution of gold, either a chestnut-brown, a blue, a green, a metallic precipitate, or one of a purple tone; the perchloride occasions no precipitate whatever, whether the solution of gold be strong or dilute; but a properly neutral mixture, of 1 part of crystallized protochloride of tin, with 2 parts of crystallized perchloride, produces, with 1 part of crystallized chloride of gold (all being in solution), a beautiful purple-colored precipitate. An excess of the protosalt of tin gives a yellow, blue, or green cast; an excess of the persalt gives a red and violet cast; an excess in the gold salt occasions, with heat (but not otherwise), a change from the violet and chestnutbrown precipitate into red. According to Fuchs, a solution of the sesquioxyde of tin in muriatic acid, or of the sesquichloride in water, serves the same purpose, when dropped into a very dilute solution of gold.

Buisson prepares gold-purple in the following way. He dissolves, first, 1 gramme of the best tin in a sufficient quantity of muriatic acid, taking care that the solu tion is neutral; next, 2 grammes of tin in aqua regia, composed of 3 parts of nitric acid, and 1 part of muriatic, so that the solution can contain no protoxyde; lastly, 7 grammes of fine gold in a mixture of 1 part of nitric acid, and 6 of muriatic, observing to make the solution neutral. This solution of gold being diluted with $3 \frac{1}{2}$ litres of water (about three quarts), the solution of the perchloride of tin is to be added at once, and afterwards that of the protochloride, drop by drop, till the precipitate thereby formed 
acquires the wished-for tone; after which it should be edulcorated by washing, as quickly .s possible.

Frick gives the following prescription:-Let tin be set to dissolve in very dilute aqua regia without heat, till the fluid becomes faintly opalescent, when the metal must be taken out, and weighed. The liquor is to be diluted largely with water, and a definite weight of a dilute solution of gold, and dilute sulphuric acid, is to be simultaneously stirred into the nitro-muriate of tin. The quantity of solution of gold to be poured irto the tin liquor must be such, that the gold in the one is to the tin in the other in the ratio of 36 to 10.

Gold-purple becomes brighter when it is dry, but appears still as a dirty-brown powder. Muriatic acid takes the tin out of the fresh-made precipitate, and leaves the gold either in the state of metal or of a blue powder. At a temperature between $212^{\circ}$ and $300^{\circ} \mathrm{Fahr}$., mercury dissolves out all the gold from the ordinary purple of Cassius.

Relative to the constitution of gold-purple, two views are entertained: according to the first, the gold is associated in the metallic state along with the oxyde of tin; according to the second, the gold exists as a purple oxyde along with the sesquioxyde or peroxyde of tin. Its composition is differently reported by different chemists. The constituents. according to-

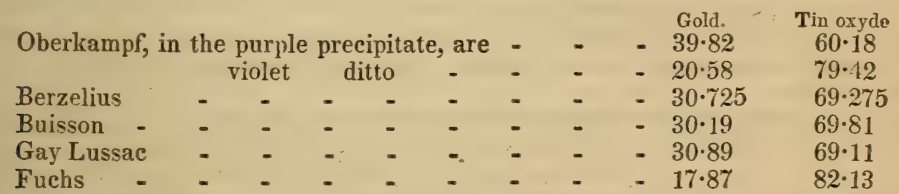

If to a mixture of protochloride of tin, and perchloride of iron, a properly diluted solution of gold be added, a very beautiful purple precipitate of Cassius will immediately fall, while the iron will be left in the liquid in the state of a protochloride. The purple thus prepared keeps in the air for a long time without alteration. Mercury does not take from it the smallest trace of gold.-F Fuchs' Journal für Chemie, t. xv.

PURPLE OF MOLLUSCA is a viscid liquor, secreted by certain shell-fish, the Buccinum lapillus, and others, which dyes wool, scc. of a purple color, and is supposed to be the substance of the Trrian dye, so highly prized in ancient Rome for producing the imperial purple. See Dyeing.

PURPURIC ACID is an acid obtained by treating uric or lithic acid with dilute nitric acid. It has a fine purple color; but has hitherto been applied to no use in the arts.

PURPURINE is the name of a coloring principle, supposed by Robiquet and Colin to exist in madder. Its identity is questionable.

PUTREFACTION, and its Prevention. The decomposition of animal bodies, or of such ylants as contain azote in their composition, which takes place spontaneously when they are exposed to the air, under the influence of moisture and warmth, is called putrefaction. During this process, there is a complete transposition of the proximate principles, the elementary substances combining in new and principally gaseous comrounds. Oxygen is absorbed from the atmosphere, and converted into carbonic acid; one portion of the hydrogen forms water with the oxygen; another portion forms, with the azote, the carbon, the phosphorus, and the sulphur respectively, ammonia, carbureted, phosphureted, and sulphureted hydrogen gases, which occasion the nauseous smell evolved by putrefying bodies. There remains a friable earthy-looking residuum, consisting of rotten mould and charcoal. Vegetables which contain no azote, like the ligneous part of plants, suffer their corresponding decomposition much more slowly, and with different modifications, but they are finally converted into regetable mould. In this process, the juices with which the plants are filled first enter into the acetous fermentation under the action of heat and moisture; the acid thereby generated destroys the cohesion of the fibrous matter, and thus reduces the solids to a pulpy state. In the progress of the decomposition, a substance is lastly produced which resembles oxydized extractive, is soluble in alkalis, and is sometimes called mould. This decomposition of the plants which contain no azote, goes on without any offensive smell, as none of the abovenamed nauseous gases are disengaged. When vegetable matters are mixed with animal, as in the dung of cattle, this decomposition proceeds more rapidly, because the animalizecu portion serres as a ferment to the regetable. Vegetable acids, resins, fats, and volatilized oils, are not of themselves subject to putrefaction.

The object of the present article is to detail the principles and processes, according to which, for various purposes in the arts, the destruction of bodies by putrefaction may be prevented, and their preservation in a sound state secured for a longer or a shorter time. 


\section{CONDITIONS OF THE PREVENTION OF PUTREFACTION.}

The circumstances by which putrefaction is counteracted, are, 1. the chemical change of the azotized juices; 2 . the abstraction of the water; 3 . the lowering of the temperature; and 4. the exclusion of oxygen.

1. The chernical change of the azotized juices.-The substance which in dead animal matter is first attacked with putridity, and which serves to communicate it to the solid fibrous parts, is albumen, as it exists combined with more or less water in all the animal fluids and soft parts. In those vegetables also which putrefy, it is the albumen which first suffers decomposition; and hence those plants which contain most of that proximate principle, are most apt to become putrid, and most resemble, in this respect, animal substances; of which fact, mushrooms, cabbages, coleworts, \&c., afford illustrations. The albumen, when dissolved in water, very readily putrefies in a moderately warm air; but when coagulated, it seems as little liable to putridity as fibrin itself. By this change, it throws off the superfluous water, becomes solid, and may then be easily dried. Hence, those means which by coagulation make the albumen insoluble, or form with it a new compound, which does not dissolve in water, but which resists putrefaction, are powerful antiseptics. Whenever the albumen is coagulated, the uncombined water may be easily eraporated away, and the residuary solid matter may be readily dried in the air, so as to be rendered unsusceptible of decomposition.

In this way acids operate, which combine with the albumen, and fix it in a coagulated state, without separating it from its solution : such is the effect of vinegar, citric acid, tartaric acid, \&c.

Tannin combines with the albuminous and gelatinous parts of animals, and forms insoluble compounds, which resist putrefaction; on which fact the art of tanning is founded.

Alcohol, oil of turpentine, and some other volatile oils, likewise coagulate albumen, and thereby protect it from putrescence. The most remarkable operation of this hind is exhibiled by wood vinegar, in consequence of the creosote contained in it, according to the discovery of Reichenbach. This peculiar volatile oil has so decided a power of coagulating albumen, that even the minute portion of it present in pyroligneons vinegar is sufficient to preserve animal parts from putrefaction, when they are simply soaked in it. Thus, also, flesh is cured by wood smoke. Wood tar likewise protects animal matter from change, by the creosote it contains. The ordinary pyroligneous acid sometimes contains 5 per cent. of creosote.

In circumstances where a stronger impregnation with this antiseptic oil may be necessary, common wood vinegar may be heated to $16 \tau^{\circ} \mathrm{F}$., and saturated with effloresced Glauber's salts, by which expedient the oil is separated and made to float upon the surface of the warm liquid; whence it should be immediately skimmed off; because, by cooling and crystallizing, the solution would so diminish in density as to allow the oil to sink to the bottom; for its specific gravity is considerably greater than that of water. This oil, which contains, besides creosote, some other volatile constituents, may be liept dissolved ready for use in strong vinegar or alcohol. Water takes up of pure creosote only $1 \frac{3}{4}$ per cent.; but alcohol dissolves it in every proportion.

The earthy and metallic salts afford likewise powerful means for separating albumen from its watery solution, their bases having the property of forming insoluble compounds with it. The more completely they produce this separation, the more effectually do they counteract putrefaction. The alkaline salts also, as common salt, sal ammoniac, salıpetre, and tartar, operate against putrescence, though in a smaller degree, because they do not precipitate the albumen; but, by abstracting a part of its water, they render it less liable to become putrid. Among the earthy salts, alum is the most energetic, as it forms a subsalt which combines with albumen; it is three times more antiseptic than common salt, and from seven to eight times more so than saltpetre. Muriate of soda, however, may be employed along with alum, as is done in the tawing of sheepskins.

The metallic salts operate still more effectually as antiseptics, because they form with albumen still more intimate combinations. Under this head we class the green and red sulphates of iron, the chloride of zinc, the acetate of lead, and corrosive sublimate; the latier, however, from its poisonous qualities, can be employed only on special uccasions. Nitrate of silver, though equally noxious to life, is so antiseptic, that a solution containing only $\frac{1}{500}$ of the salt is capable of preserving animal matters from corruption.

2. Abstraction of water.-Even in those cases where no separation of the albumen takes place in a coagulated form, or as a solid precipitate, by the operation of a substance foreign to the animal juices, putrefaction cannot go on, any more than other kinds of fermentation, in bodies wholly or in a great measure deprived of their water. For the albumen itself runs so much more slowly into putrefaction, the less water it is dissolved in ; and in the desiccated state, it is as little susceptible of alteration as any other dry vegetable or animal matter. Hence, the proper drying of an animal substance becomes a universal preventive of putrescence. In this way fruits, herbs, cabbages, fish, flesh, 
may be preserved from corruption. If the air be not cold and dry enough to cause the evaporation of the fluids before putrescence may come on, the organic substance must be dried by artificial means, as by being exposed in thin slices in properly constructed airstores. At temperatures under $140^{\circ} \mathrm{F}$, the albumen dries up without coagulation, and may then be re-dissolved in cold water, with its valuable properties unaltered. By such artificial desiccation, if flesh is to be preserved for cooking or boiling, it must not be exposed, however, to so high a degree of heat, which would harden it permanently, like the balied mummies of Egypt. Mere desiccation, indeed, can hardly ever be employrd upon flesh. Culinary salt is generally had recourse to, either alone or with the addition of saltpetre or sugar.

These alkaline salts abstract water in their solution, and, consequently, concentrate the aqueous solution of the albumen; whence, by converting the simple watery fluid into salt water, which is in general less favorable to the fermentation of animal matter than pure water, and by expelling the air, they counteract putridity. On this account, salted meat may be dried in the air much more speedily and safely than fresh meat. The drying is promoted by heating the meat merely to such a degree as to consolidate the albumen, and eliminate the superfluous water.

Alcohol operates similarly, in abstracting the water essential to the putrefaction of animal substances, taling it not only from the liquid albumen, but counteracting its decomposition, when mixed among the animal solids. Sugar acts in the same way, fixing in an unchangeable sirup the water which would otherwise be accessory to the fermentation of the organic bodies. The preserves of fruits and regetable juices are made upon this principle. When animal substances are rubbed with charcoal powder or sand, perfectly dry, and are afterwards freely exposed to the air, they become deprived of their moisture, and will keep for any length of time.

3. Defect of warmth.-As a certain degree of heat is requisite for the vinous fermentation, so is it for the putrefactive. In a damp atmosphere, or in one saturated with moisture, if the temperature stand at from $70^{\circ}$ to $80^{\circ} \mathrm{F}$, the putrefaction goes on most rapidly; but it proceeds languidly at a few degrees above freezing, and is supended altogether at that point. The elephants preserved in the polar ices are proofs of the antiseptic influence of low temperature. In temperate climates, ice-houses serve the purpose of keeping meat fresh and sweet for any length of time.

4. Abstraction of oxygen gas.-As the putrefactive decomposition of a bouly first commences with the absorption of oxygen from the atmosphere, so it may be retarded by the exclusion of this gas. It is not, however, enough to remove the aerial oxygen from the surface of the hody, but we must expel all the oxygen that may be diffused among the vessels and other solids, as this portion suffices in general to excite putrefaction, if other circumstances be favorable. The expulsion is most readily accomplished by a moderate degree of heat, which, by expanding the air, evolves it in a great measure, and at the same time favors the fixation of the oxygen in the extractive matter, so as to make it no longer available towards the putrefaction of the other substances. Milk, soup, solution of gelatine, \&c., may be kept long in a fresh state, if they be subjected in an air-tight vessel every other day to a boiling heat. Oxygenation may be prevented in several ways: by burning sulphur or phosphorus in the air of the meat receiver; by filling this with compressed carbonic acid; or with oils, fats, sirups, \&c., and then sealing it hermetically. Charcoal powder recently calcined is efficacious in preserving meat, as it not only excludes air from the bodies surrounded hy it, but intercepts the oxygen by condensing it. When butcher-meat is enclosed in a vessel filleu with sulphurous acid, it absorbs the gas, and remains for a considerable time proof against corruption. The same result is obtained if the vessel be filled with ammo. niacal gas. At the end of 76 days such meat has still a fresh look, and may be safely dried in the atmosphere.

\section{PECULIAR ANTISEPTIC PROCESSES.}

Upon the preceding principles and experiments depend the several processes erployed for protecting substances from putrescence and corruption. Here we must distinguish between those bodies which may be preserved by any media suitable to the purpose, as anatomical preparations or objects of natural history, and those bodies which, being intended for food, can be cured only by wholesome and agreeable means.

A common method for preserving animal substances unchanged in property and texture, is to immerse them in a spirituous liquor containing about 65 or 70 per cent. of real alcohol. Camphor may also be dissolved in it, and as much common salt as its water will take up. A double fold of ox-bladder should be bound over the mouth of the vessel, in order to impede the evaporation of the watery portion of the liquid, and its upper surface should be coated with a turpentine varnish. Undoubtedly a little creosote would be of use to counteract the decomposing influence of the alcohol upon the 
animal substances. With such an addition, a weaker spirit, containing no more than 30 per cent. of alcohol, would answer the purpose.

Instead of alcohol, a much cheaper vehicle is water saturated with sulphurous acid; and if a few drops of creosote be added, the mixture will become very efficacious. A solution of red sulphate of iron is powerfully antiseptic; but after some time it gives a deposite of the oxyde, which disguises the preparation in a great degree.

According to Tauffier, animal substances may be preserved more permanently by a solution of one part of chloride of tin in 20 parts of water, sharpened with a little muriatic acid, than even by alcohol.

For preserving animal bodies in an embalmed form, mummy-like, a solution of chloride of mercury and wood vinegar is most efficacious. As there is danger in manipulating with that mercurial salt, and as in the present state of our knowledge of creosote we have it in our power to make a suitably strong solution of this substance in vinegar or spirit of wine, I am led to suppose that it will become the basis of most antiseptic preparations for the future. From the statements of Pliny, it is plain that wood vinegar was the essential means employed by the ancient Egyptians in preparing their mummies, and that the odoriferous resins were of inferior consequence.

CURING OF PROVISIONS.

Flesh.-The ordinary means employed for preserving butcher meat are, drying, smoking, salting, and pickling or souring.

Drying of animal fibre. - The best mode of operating is as follows:-The flesh must be cut into slices from 2 to 6 ounces in weight, immersed in boiling water for 5 or 6 minutes, and then laid on open trellis-work in a drying-stove, at a temperature kept steadily about $122^{\circ} \mathrm{F}$., with a constant stream of warm dry air. That the boiling water may not dissipate the soluble animal matters, very little of it should be used, just enough for the meat to be immersed by portions in succession, whereby it will speedily become a rich soup, fresh water being added only as evaporation takes place. It is adrantageous to add a little salt, and some spices, especially coriander seeds, to the water. After the parboiling of the flesh has been completed, the soup should be evaporated to a gelatinous consistence, in order to fit it for forming a varnish to the meat after it is dried, which may be completely effected within two days in the oven. By this process two thirds of the weight is lost. The perfectly dry flesh must be plunged piece by piece in the fatty gelatinous matter liquefied by a gentle heat; then placed once more in the stove, to dry the layer of varnish. This operation may be repeated two or three times, in order to render the coat sufficiently uniform and thick. Butcher's meat dried in this way keeps for a year, affords, when cooked, a dish similar to that of fresh meat, and is therefore much preferable to salted provisions. The drying may be facilitated, so that larger lumps of flesh may be used, if they be imbued with some common salt immediately after the parboiling process, by stratifying them with salt, and leaving them in a proper pickling-tub for 12 hours before they are transferred to the stove. The first method, however, affords the more agreeable article.

Smolking.-This process consists in exposing meat previously salted, or merely rubbed over with salt, to wood smoke, in an apartment so distant from the fire as not to be unduly heated by it, and into which the smoke is admitted by flues at the bottom of the side walls. Here the meat combines with the empyreumatic acid of the smoke, and gets dried at the same ${ }_{c}$ ime. The quality of the wood has an influence upon the smell and taste of the smoke-aried meat; smoke from beech wood and oak being preferable to that from fir and larch. Smoke from the twigs and berries of juniper, from rosemary, peppermint, \&c., imparts somewhat of the aromatic flavor of these plants. A slow smoking with a slender fire is preferable to a rapid and powerful one, as it allows the empyreumatic principles time to penetrate into the interior substance, without drying the outside too much. To prevent soot from attaching itself to the provisions, they may be wrapped in cloth, or rubbed over with bran, which may be easily removed at the end of the operation.

The process of smoking depends upon the action of the wood acid, or the creosote volatilized with it, which operates upon the flesh. The same change may be produced in a much shorter time by immersing the meat for a few hours in pyroligneous acid, then hanging it up in a dry air, which, though moderately warm, makes it fit for keeping, without any taint of putrescence. After a few days exposure, it loses the empyreumatic smell, and then resembles thoroughly smoked provisions. The meat dried in this way is in general somewhat harder than by the application of smoke, and therefore softens less when cooked, a difference to be ascribed to the more sudden and concentrated operation of the wood vinegar, which effects in a few hours what would require smoking for scveral weeks. By the judicious employment of pyroligneous acid diluted to successive degrees, we might probably succeed in imitating perfectly the effect of smoke in curıng provisions. 
Salting.-The meat should be rubbed well with coinmon salt, containing about one sixteenth of saltpetre, and one thirty-secondth of sugar, till every crevice has been impremated with it; then sprinkled over with salt, laid down for 24 or 48 hours, and, lastly, subjected to pressure. It must next be sprinkled anew with sali, packed into propier ressels, and covered with the brine obtained in the act of pressing, rendered stronger by boiling down. For household purposes it is sufficient to rub the meat well with good salt, to put it into vessels, and load it with heavy weights, in order to squeeze ont as much pickle as will cover its surface. If this cannot be had, a pickle must be poured on it, composed of 4 pounds of salt, 1 pound of sugar, and 2 oź. of saltpetre, dissolved in 2 gallons of water.

Pickiing with vinegar.-Vinegar dissolves or coagulates the albumen of flesh, and thereby counteracts its putrescence. The meat should be washed, dricd, and then laid in strong vinegar. Or it may be boiled in the vinegar, allowed to cool in it, and then set aside with it in a cold cellar, where it will keep sound for several months.

Fresh meat may be kept for some months in water deprived of its air. If we strew on the bottom of a ressel a mixture of iron filings and flowers of sulphur, and pour over them some water which has been boiled, so as to expel its air, meat immersed in it will keep a long time, if the water be covered with a layer of oil, from half an inch to an inch thick. Meat will also keep fresh for a considerable period when surrounded with oil, or fat of any hind, so purified as not to turn rancid of itself, especially if the meat be previously boiled. This process is called potting, and is applied successfully to fish, fowls, s.c.

Prechtl says that living fish may be preserved 14 days without water, by stopping their mouths with crumbs of bread steeped in brandy, pouring a little brandy into them, and paching them in this torpid state in straw. When put into fresh water, they come alive again after a few hours! Prechtl, Encyclop. Technologisches, art. Faülniss Abhalıung.

Eggs.-These ought to be taken new laid. The essential point towards their preservation is the exclusion of the atmospheric oxygen, as their shells are porous, and permit the external air to pass inwards, and to excite putrefaction in the albumen. There is also some oxygen always in the air-cell of the eggs, which ought to be expelled or rendered inoperative, which may be done by plunging them for 5 minutes in water heated to $140^{\circ} \mathrm{F}$. The egos must be then taken out, wiped dry, besmeared with some oil (not apt to turn rancid) or other unctuous matter, packed into a vessel with their narrow ends uppermost, and covered with sawdust, fine sand, or powdered charcoal. Eggs coated with gum arabic, and packed in charcoal, will keep fresh for a year. Lime water, or rather rnilk of lime, is an excellent vehicle for keeping eggs in, as I have verified by long experience. Some persons coagulate the albumen partially, and also expel the air by boiling the eggs for 2 minutes, and find the method successful. When eggs are intended for hatching, they should be lrept in a cool cellar; for example, in a chamber adjoining an ice-house. Eggs exposed, in the holes of perforated shelves, to a constant current of air, lose about $\frac{3}{4}$ of a grain of their weight daily, and become concentrated in their albuminous part. so as to be little liable to putrefy. For long sea voyages, the surest means of preserving eggs, is to dry up the albumen and yolk, by first triturating them into a homogeneous paste, then evaporating this in an air-stove or a water-bath heated to $125^{\circ}$, and putting up the dried mass in vessels which may be made air-tight. When used, it should be dissolved in three parts of cold or tepid water.

Grain of all kinds, as wheat, barley, rye, \&c., and their flour, may be preserved for an indefinite length of time, if they be kiln-dried, put up in vessels or chambers free from damp, and excluded from the air. Well dried grain is not liable to the depredations of insects.

To preserve fruits in a fresh state, various plans are adopted. Pears, apples, plums, \&c. should be gathered in a sound state, altogether exempt from bruises, and plucked, in dry weather, before they are fully ripe. One mode of preservation is, to expose them in an airy place to dry a little for eight or ten days, and then to lay them in dry sawdust, or chopped straw, spread upon shelves in a cool apartment, so as not to touch each other. Another method consists in surrounding them with fine dry sand in a vessel which should be unade air-tight, and kept in a cool place. Some persons coat the fruit, including their stalks, with melted wax; others lay the apples, \&c., upon wicker-work shelves in a vaulted chamber, and smoke them daily during 4 or 5 days with vine branches or juniper wood. Apples thus treated, and afterwards stratified in dry sawdust, without touching each other, will keep fresh for a whole year.

The drying of garden fruits in the air, or by a kiln, is a well-known method of preservation. Apples and pears of large size should be cut into thin slices. From 5 to 6 meas ures of fresh apples, and from 6 to 7 of pears, afford in general one measure of dry fruit, (biffins). Dried plums, grapes, and currants are a common article of commerce.

Herbs, cabbages, \&c., may he kept a long time in a cool cellar, provided they are covered with dry sand. Such vegetables are in general preserved for the purposes of 
food, by means of drying, salting, pickling with vinegar, or beating up with sugar. Cab. bages should be scalded in hot water previously to drying; and all such plants, when dried, should be compactly pressed together, and liept in air-tight vessels. Tuberous and other roots are better kept in an airy place, where they may dry a little without being exposed to the winter's frost.

A partial drying is given to various vegetable juices by evaporating them to the consistence of a sirup, called a rob, in which so much of the water is dissipated as to prevent them from running into fermentation. The fruits must be crushed, squeezed in bags to expel the juices, which must then be inspissated either over the naked fire, or on a water or steam bath, in the air or in vacuo. Sometimes a small proportion of spices is added, which tends to prevent mouldiness. Such extracts may be conveniently mixed with sugar into what are called conserves.

Salting is employed for certain fruits, as small cucumbers or gherkins, capers, olives, \&c. Even for peas such a method is had recourse to, for preserving them a certain time. They must be scalded in hot water, put up in bottles, and covered with saturated brine, having a film of oil on its surface, to exclude the agency of the atmospheric air. Before being used, they must be soaked for a short time in warm water, to extract the salt. The most important article of diet of this class, is the sour kraut of the northern nations of Europe (made from white cabbage), which is prepared simp]y by salting; a little vinegar being formed spontaneously by fermentation. The cabbage must be cut into small pieces, stratified in a cask along with salt, to which juniper berries and carui seeds are added, and packed as hard as possible by means of a wooden rammer. The cabbage is then covered with a lid, on which a heavy weight is laid. A fermentation commences, which causes the cabbage to become more compact, while a quantity of juice exudes and floats on the surface, and a sour smell is perceived towards the end of the fermentation. In this condition the cask is transported into a cool cellar, where it is allowed to stand for a year; and indeed, where, if well made and packed, it may be kept for several years.

The excellent process for preserving all kinds of butcher meat, fish, and poultry, first contrived by M. Appert in France, and afterwards successfully practised upon the great commercial scale by Messrs. Donkin and Gamble, for keeping beef, salmon, soups, \&c. perfectly fresh and sweet for exportation from this country, as also turtle for importation thither from the West Indies, deserves a brief description.

Let the substance to be preserved be first parboiled, or rather somewhat more, the bones of the meat being previously removed. Put the meat into a tin cylinder, fill up the vessel with seasoned rich soup, and then solder on the lid, pierced with a small hole. When this has been done, let the tin vessel thus prepared be placed in brine and heated to the boiling point, to complete the remainder of the cooking of the meat. The hole of the lid is now to be closed perfectly by soldering, while the air is rarefied. The vessel is then allowed to cool, and from the diminution of the volume, in consequence of the reduction of temperature, both ends of the cylinder are pressed inwards, and become concave. The tin cases, thus hermetically sealed, are exposed in a test-chamber, for at least a month, to a temperature above what they are ever likely to encounter; from $90^{\circ}$ to $110^{\circ}$ of Fahrenheit. If the process has failed, putrefaction takes place, and gas is erolved. which, in process of time, will cause both ends of the case to bulge, so as to render them convex, instead of concave. But the contents of those cases which stand the test will infallibly keep perfectly sweet and good in any climate, and for any number of years. If there be any taint about the meat when put up, it inevitably ferments, and is detected in the proving process. Mr. Gamble's turtle is delicious.

This preservative process is founded upon the fact, that the small quantity of oxygen contained within the vessel gets into a state of combination, in consequence of the high temperature 10 which the animal substances are exposed, and upon the chemical principle. that free oxygen is necessary as a ferment to commence or give birth to the process of putrefaction.

I shall conclude this article with some observations upon the means of preserving water fresh on sea voyages. When long kept in wooden casks, it undergoes a kind of putrefaction, contracts a disagreeable sulphurous smell, and becomes undrinkable. The influence of the external air is by no means necessary to this change, for it happens in close vessels even more readily than when freely exposed to the atmospherical oxygen. The origin of this impurity lies in the animal and vegetable juices which the water originally contained in the source from which it was drawn, or from the cask, or insects, \&c. These matters easily occasion, with a sufficient warmth, fermentation in the stagnant water, and thereby cause the evolution of offensive gases. It would appear that the gypsum of hard waters is decomposed, and gives up its sulphur, which aggravates the disagreeable odor; for selenitic waters are more apt to take this putrid taint, than those which contain merely carbonate of lime.

As the corrupted water has become unfit for use merely in consequence of the admix 
ture of these foreign matters, for water in itself is not liable to corruption, so it may be purified aqain by their separation. This purification may be accomplished most easily by passing the water through charcoal powder, or through the powder of rightly calcined bone-black. The carbon talies away not only the finely diffused coriupt particles, but also the gaseous impurities. By adding to the water a very little sulphuric acid, about 30 drops to 4 pounds, Lowitz says that tw: 0 thirds of the charcoal may be saved. Undoubtedly the sulphuric acid acts here, as in other similar cases, by the coagulation and separation of the albuminous matters, combining with them, and rendering them more apt to be seized by the charcoal. A more effectual agent for the purification of foul water is to be found in alum. A drachm of pounded alum should be dissolved with agitation in a gallon of the water, and then left to operate quietly for $2 t$ hours. A sediment falls to the bottom, while the water becomes clear above, and may be poured off. The alum combines here with the substances dissolved in the water, as it does with the stuffs in the dyeing copper. In order to decompose any alum which may remain in solution, the equiralent quantity of crystals of carbonate of soda may be added to it.

The red sulphate of iron acts in the same way as alum. A few drops of its solution are sufficient to purge a pound of foul water. The foreign matters dissolved in the water, which occasion putrefaction, become insoluble, in consequence of oxydizement, like regetable extractive, and are precipitated. On this account, also, foul water may be purified, by driving atmospheric air through it with bellows, or by agitating it in contact with fresh air, so that all its particles are exposed to oxvgen. Thus we can explain the influence of streams and winds, in counteracting the corruption of water exposed to them. Chlorine acts still more energetically than the air in purifying water. A little aqueous chlorine added to foul water, or the transmission of a little gaseous chlorine through it, cleanses it immediately.

Water-casks ought to be charred inside, whereby no fermentable stuff will be extracted from the wood. British ships, however, are now commonly provided with iron tanks for holding their water in long voyages.

PYRITES, is the native bisulphuret of iron. Copper pyrites, called vulgarly mundick, is a bisulphuret of copper.

PYRO-ACETIC SPIRIT. (Esprit pyro-acétique, Acétone, Fr.; Brennzlicher Essiggeist, Mesit, Germ.) This liquid was discorered and described by Chenerix long before pyroligneous spirit was known. It may he obtained by subjecting to dry distillation the acetates of copper, lead, alkalis, and earths; and as it is formed especially during the second half of the process, the liquor which comes over then should be set apart, separated br decantation from the emprreumatic oil, and distilled a second time by the heat of a water-bath. The fine light fluid which now comes over first, is to be rectified along with carbonate of potassa, or chloride of calcinm. As pyro-acetic spirit usually retains, even after repeatel distillations, a disagreeable emprreumatic smell, like garlic, a little good bone-black should be employed in its final rectification. According to Reichenbach, prro-acetic spirit mar be extracted in considerable quantity from beech tar. (See the next article.) The spirit thus prepared is a colorless limpid liquid, of an acrid and burning taste at first, but afterwards cooling; of a penetrating aromatic smell, different from that of alcohol; of the spec. gravity 0.7921 at $60^{\circ} \mathrm{F}$., boiling at $132^{\circ} \mathrm{F}$., and remaining fluid at $5^{\circ}$. It consists ultimately of-carbon, $62 \cdot 148$; hydrogen, $10 \cdot 453$; oxtgen, $2 \tau \cdot 329$; or, of 1 proportion of carbonic acid +2 prop. of olefiant gas $\div 1$ prop. of water; or, 1 prop. of acetic acid -1 prop. of carbonic acid. Accor:ing to another view, it is composed of, 51.52 parts of concentrated acetic acid, and 48.488 of oil of wine, being doute of the quantity in acetic ether. It is very combustible, anu barns with a brilliant flame, without smoke. When treated by chlorine, it loses an atorn of its hydrogen, and absorbs 2 atoms of chlorine. It is soluble in water, alcohol, ether, and is not convertible into ether by strong sulphuric acid. It is used for dissolving the resins commonly called gums, with which the bodies of hats are stuftened.

PYROLIGNEOUS ACID. In addition to what has been said under AcETIC AcID I shall here describe the process as conducted upon a great scale at an establishment near Manchester. The retorts are of cast iron, 6 feet long, and 3 feet 8 inches in diameter Two of these cylinders are heated by one fire, the flame of which plays round their sidas and upper surface; but the bottom is shielded by fire-tiles from the direct action of the fire. $2 \mathrm{cwts}$. of coals are sufficient to complete the distillation of one charge of wood: 36 imperial gallons of crude vinegar, of specific gravity $1 \cdot 025$, being obtained from each retort. The process occupies 24 hours. The retort-mouth is then remored, and the ignited charcoal is raked out for extinction into an iron chest, having a groove round its odges, into which a lid is fitted.

When this pyroligneous acid is saturated with quicklime, and distilled, it yields one per 
sent. of pyroxilic spirit (sometimes called naptha); which is rectified by two or tnree successive distillations with quicklime.

The tarry deposite of the crude pyroligneous acid, being subjected to distillation by itself, aflords a crude pyro-acetic ether, which may also be purified by re-distillation with quicklime, and subsequent agitation with water.

The pyrolignite of lime is made by boiling the pyroligneous acid in a large copper, which has a sloping spout at its lip, by which the tarry scum freely flows over, as it froths up with the heat. The fluid compound thus purified is syphoned off in to another copper, and mixed with a quantity of alum equivalent to its strength, in order to form the red liquor, or acetate of alumina, of the calico-printer. The acetate of lime, and sulphate of alumina and potash, mutually decompose each other; with the formation of sulphate of lime, which falls immediately to the bottom.

M. Kestner, of Thann, in Alsace, obtains, in his manufictory of pyroligneous acid, 5 hectolitres (112 gallons imperial, nearly) from a cord containing 93 cubic feet of wood. The acid is very brown, much loaded with tar, and marks $5^{\circ}$ Baumé; 220 kilogrammes of charcoal are left in the cylinders; 500 litres of that brown acid produce, after several distillations, 375 of the pyroligneous acid of commerce, containing 7 per cent. of acid, with a residuum of 40 kilogrammes of pitch. For the purpose of making a crude acetate of lead (pyrolignite) he dries pyrolignite of lime upon iron plates, mixes it with the equivalent decomposing quantity of sulphuric acid, previously diluted with its own weight of water, and cooled; and transfers the mixture as quickly as possible into a cast-iron cylindric still, built horizontally in a furnace; the under half of the mouth of the cylinder being always cast with a semicircle of iron. The acetic acid is received into large saltglazed stone bottles. From 100 parts of acetate of lime, he obtains 133 of acetic acid, at $38^{\circ}$ Baumé. It contains always a little sulphurous acid from the reaction of the tar and the sulphuric acid.

The apparatus represented in figs, 929 and 930 is a convenient modification of that exhibited under acetic acid, for producing pyroligneous acid. Fig. 929 shows the fur929

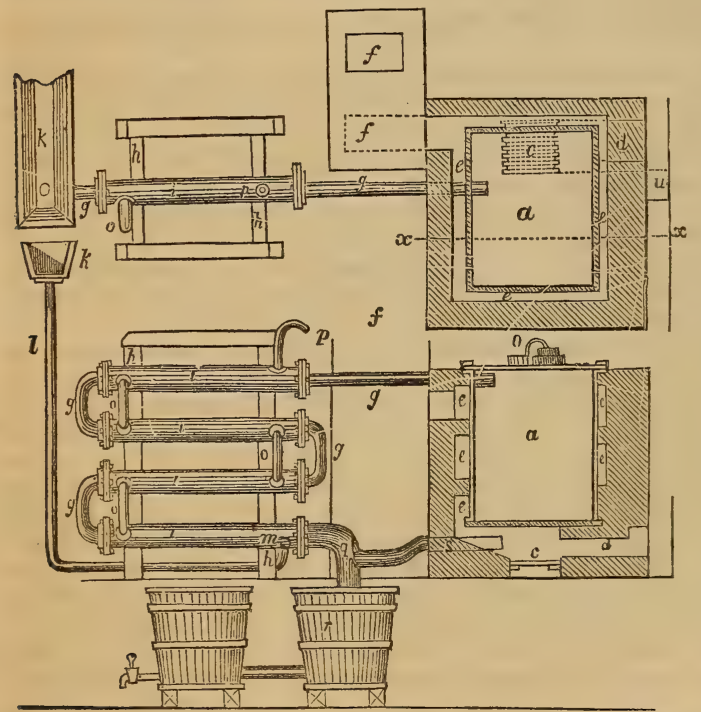
nace in a horizontal section drawn through the middle of the flue which leads to the ehimney. Fig. 930 is a vertical section taken in the dotted line $\mathrm{x}, \mathrm{x}$, of fig. 929 . The chest $a$ is constructed with cast-iron plates bolted together, and has a capacity of 100 cubic feet. The wood is introduced into it through the opening $b$, in the cover, for which purpose it is cleft into billets of moderate length. The chest is heated from the subjacent grate $c$, upon which the fuel is laid, through the fire-door $d$. The flame ascends spirally through the flues $e, e$, round the chest, which terminate in the chimney $f$. An iron pipe $g$ conveys the vapors and gaseous products from the iron chest to the con-

donser. This consists of a series of pipes laid zigzag over each other, which rest upon a framework of wood. The condensing tubes are enclosed in larger pipes $i, i$; a stream of cold water being caused to circulate in the interstitial spaces between them. The water passes down from a trough $k$, through a conducting tube $l$, enters the lowest cylindrical case at $m$, flows thence along the series of jackets $i, i, i$, being transmitted from the one row to the next above it, by the junction tubes $0,0,0$, till at $p$ it runs off in a boiling-hot state. The vapors proceeding downwards in an opposite direction to the cooling stream of water, get condensed into the liquid state, and pass off at $q$, through a discharge pipe, into the first close receiver $r$, while the combustible gases flow off through the tube $s$, which is provided with a stopcock to regulate the magnitude of their flame under the chest. As soon as the distillation is fully set agoing, the stopeock upon the gas-pipe is opened; and after it is finished, it must be shut. The fire should be 
supplied with fuel at first, but after some time the gas generated lieeps up the distilling heat. The charcoal is allowed to coul during 5 or 6 hours, and is then taken out through an aperture in the back of the chest, which corresponds to the opening $u, j i g .929$, in the bricn work of the furnace. About 60 per cent. of charcoal may be obtained from 100 feet of fir-wood, with a consumption of as much brush-wood for fuel.

Stoltze has ascertained, by numerous experiments, that one pound of wood yields from 6 to $\tau \frac{1}{2}$ ounces of liquid products; but in acetic acid it affords a quantity varying from 2 to 5 , according to the nature of the wood. Hard timber, which has grown slowly upon a dry soil, gives the strongest vinegar. White birch and red beech afford per pound $7 \frac{1}{3}$ ounces of wood rinegar, $1 \frac{1}{3}$ ounce of combustible oil, and 4 ounces of charcoal. One ounce of that vinegar saturates 110 grains of carbonate of potassa. Red pine yields per pound $6 \frac{1}{2}$ ounces of rinegar, $2 \frac{1}{4}$ ounces of oil, $3 \frac{3}{4}$ ounces of charcoal ; but one ounce of the vinegar saturates only 44 grains of carbonate of potassa, and has therefore only twc fifths of the strength of the vinegar from the birch. An ounce of the vinegar from the white beech, holly oak (Ilex), common ash, and horse chestnut, saturates from 90 to 100 grains of the carbonate. In the same circumstances, an ounce of the vinegar of the alder and white pine saturates from 58 to 60 grains.

PYROLIGNEOUS or PYROXILIC SPIRIT, improperly called naptha. This is employed, as well as pyro-acetic ether, to dissolve the sandarach, mastic, and other resinous substances, which, under the name of gums, are used for stiffening the bodies of hats. I have already described, in the article Pyroligneors AcID, how this spirit is obtained. Berzelius has found that the crude spirit may be best purified by agitating it with a fat oil, in order to abstract the empyreumatic oil ; then to decant the spirit, distil it, first with fresh calcined charcoal, and next with chloride of calcium. The pyroligneous spirit, thus purified, is colorless, and limpid like alcohol; has an ethereous smell, somewhat resembling that of ants. Its taste is hot, and analogous to that of oil of peppermint. Its specific gravity, by my experiments, is 0.824 . It readily takes fire, and burns with a blue flame, without smoke. It combines with water in any proportion; a property which distinguishes it from pyro-acetic ether and spirit.

It is not easy to say what is the real chemical nature of pyroxilic spirit. There is no ultimate analysis of it that can be depended upon. The properties of the spirit examined by MM. Marcet and Macaire, differ from those of our spirit, in refusing to combine with water, like alcohol. The article on sale in this country readily unites with water, and in all proportions with alcohol.

PYROMETER is the name of an instrument for measuring high degrees of heat above the range of the mercurial thermometer. Wedgewood's is the one commonly referred to by writers upon porcelain and metallurgy; but a better one might be easily contrived.

PYROPHORUS is the generic name of any chemical preparation, generally a powder, which inflames spontaneously when exposed to the air.

PYROTECHNY. See FIRE-Works.

PYROXILINE is a name which I have ventured to give to a substance detected in pyroxylic spirit, by Mr. Scanlan, while residing in Dublin, and therefore called by him Eblanin. I am indebted to that ingenious chemist for the following facts.

If potash water be added to raw wood-spirit (pyroligneous), as long as it throws down anything, a precipitate is produced, which is pyroxiline, mixed with tarry matter. This precipitate is to be collected on a filter cloth, and submitted to strong pressure between folds of blotting-paper; it is next to be washed with cold alcohol, spec. grav. $0 \cdot 840$, in order to iree it from any adhering tarry matter; when the pyroxiline is left nearly pure. If it be dissolved in boiling alcohol, or hot oil of turpentine, it crystallizes regularly on cooling, in right square prisms, of a fine yellow color, that look opaque to the naked eye, but when examined under the microscope, have the transparency and color of ferroprussiate of potash. Its turpentine solution affords crystals of a splendid orangered color, having the appearance of minute plates, whose form is not discernible by the naked eye, but when examined by the microscope, they are seen to be thin right rectangular prisms. The orange-red color is only the effect of aggregation; for when ground to powder, these crystals become yellow; and under the microscope, the difference in color between the two is very slight. Its melting point is $318^{\circ} \mathrm{F}$. It sublimes at $300^{\circ}$ in free air; heated in a close tube in a bath of mercury, it emits rapor at $400^{\circ}$; it then begirs to decompose, and is totally decomposed at $500^{\circ}$. Sulphuric acid decomposes it, producing a beautiful blue color, which passes into crimson, as the acid attracts water from the atmosphere, and it totally disappears on plentiful dilution with water, leaving carbon of a dirty-brown color. Its alcoholic or turpentine solution imparts a permanent yellow dye to vegetable or animal matter.

Pyroxiline consists, according to the analysis of Drs. Apjohn and Gregory, of-carbon, $75 \cdot 275$; hydrogen, $5 \cdot 609$; oxygen, $19 \cdot 116$, in 100 parts. 
Q.

QUARTATION is the alloying of one pan of gold that is to be refined, along wits three parts of silver, so that the gold shall constitute one quarter of the whole, and there. by have its particles too far separated to be able to protect the other metals originally as. sociated with it, such as silver, copper, lead, tin, palladium, \&c., from the action of the nitric or sulphuric acid employed in the subsequent parting process. See REFINING.

QUARTZ has been described in the article LAPIDARY.

QUASSIA is the wood of the root of the Quassia excelsa, a tree which grows in Surinam, the East Indies, \&c. It affords to water an intensely bitter decoction, which is occasionally used in medicine, and was formerly substituted by some brewers for hops, but is now prohibited under severe penalties. It affords a safe and efficacious fly-water, or poison for flies.

QUEEN'S WARE. See PotTery.

QUEEN'S YELLOW is an ancient name of Turbith Mineral, or yellow subsulphate of mercury.

QUERCITRON is the bark of the Quercus nigra, or yellow oak, a tree which grows in North America. The coloring principle of this yellow dye-stuff has been called Quercitrin, by its discoverer Chevreul. It forms small pale Jellow spangles, like those of Aurum musivum, has a faint acid reaction, is pretty soluble in alcohol, hardly in ether, and little in water. Solution of alum developes from it, by degrees, a beautiful yellow dye. See Calico-rinting and Yellow Dye.

QUICKLIME; see LIME.

QUICKSILVER; see MERcURY.

QUILL; see FEATHERS.

QUININA. This medicine is now prepared in such quantities as to constitute a chemical manufacture. Quinina and cinchonina are two vegetable alkalis, which exist in Peruvian bark or cinchona; the pale or gray bark contains most cinchonina, and the yellow bark most quinina. The methods of extracting these bases are very various. In general, water does not take them out completely, because it transforms the neutral salts in the barks into more soluble acidulous salts, and into less soluble sub-salts. To exhaust the bark completely, one or other of the following solvents is employed:

1. Alcohol.-An extract by this menstruum, is to be treated with very dilute warm muriatic acid, in order to dissolve everything thus soluble; the acid liquor is to be saturated with magnesia, by boiling it with an excess of this earth ; the precipitate is to be dried, filtered, and then exhausted by boiling-hot alcohol.

2. Dilute acids.-Boil the bark, coarsely pounded, with eight times its weight of water, containing 5 per cent. of the weight of the bark of sulphuric acid. This treatment is to be repeated with a fresh quantity of dilute acid. The whole liquors must be filtered, the residuum strained, and the solution mixed with quicklime, equal to one fourth of the bark employed. This mixture, after having been well stirred, is to be strained, whenever it acquires an alkaline reaction, that is, tinges reddened litmus paper blue, or turmeric brown. The calcareous mass is to be now washed with a little water, and dried, and then boiled thrice with spirit of wine of spec. grav. 0.836. This solution being filtered, is to be mixed with a little water, and distilled. The bases, cinchonina and quinina, remain under the form of a brown viscid mass, and must be purified by subsequent crystallization, after being converted into sulphates.

3. An alkali, and then an acid.-The object of this process is, to retain the vegetable alkalis in the bark, while with the alkaline water we dissolve out the acids, the coloring matters, the extractive, the gum, \&c. Boil for an hour one pound of the bark with six pounds of water, adding by degrees a little solution of potash, so that the liquor may have still an alkaline taste when the boiling is over. Allow it to cool, filter, wash the residuum with a little water, and squeeze it. Diffuse it next in tepid water, to which add by degrees a little muriatic acid, till after a prolonged digestion the mixture shall perceptibly redden litmus paper. Filter the liquor, and boil it with magnesia. The precipitate being washed and dried, is to be treated with hot alcohol, which dissolves the quinina and cinchonina.

Obtained by any of the above methods, the quinina and cinchonina are more or less colored, and may be blanched by dissolving them in dilute muriatic acid, and treating the solution with animal ohareoa].

There are several methods of separating these two vegetable alkalis.

1. When their solution in spirit of wine is evaporated by heat to a certain point, the greater part of the cinchonina cryst a lizes on cooling, while the quinina remains dissolved. 
2. Digestion in ether dissolves the quinina, and leaves the cinchonina.

3. We may supersaturate slightly the two bases with sulphuric acid. Now as the supersulphate of quinina is sparingly soluble, the liquor need only to be evaporated to a proper point to crystallize out that salt, while the supersulphate of cinchonina continues in solution with very little of the other salt. Even this may be separated by precipitating the bases, and treating them, as above preseribed, with alcohol or ether.

One pound of bark rarely yields more than 2 drachms of the bases. One pound of red bark afforded, to Pelletier and Caventou, 74 grains of cinchonina, and 107 grains of quinina.

Quinina is composed of 75.76 carbon, $7 \cdot 52$ hydrogen, 8.11 azote, and 8.61 oxygen.

The salts of quinina are distinguished by their strong taste of Peruvian bark, and if crystallized, by their pearly lustre. Most of them are soluble in water, and some also in ether and alcohol. The soluble salts are precipitated by the oxalic, gallic, and tartaric acids, and by the salts of these acids. Infusion of nutgalls also precipitates them.

The sulphate of quinina is the only object of manufacturing operations. Upon the brownish viscid mass obtained in any of the above processes for obtaining quinina, pour very dilute sulphuric acid, in sufficient quantity to produce saturation. The solution must be then treated with animal charcoal, filtered, evaporated, allowed to cool, when it deposites crystals. 1000 parts of bark afford, upon an average, 12 parts of sulphate. The sulphate of cinchonina, which is formed at the same time, remains dissolved in the mother-waters.

The neutral sulphate of quinina occurs in small transparent right prismatic needles. By spontaneous evaporation of their solution, larger crystals may be procured. They contain $24 \frac{2}{3}$ per cent. of water; and, therefore, melt when exposed to heat. They dissolve in 11 parts of water at ordinary temperatures; are much more soluble in hot spirit of wine, somewhat dilute, than in cold; and are nearly insoluhle in anhydrous alcohol. If they be well dried, they possess the property of becoming luminous when heated a little abore the boiling point of water, especially when they are rubbed. The sulphate is, in this ease, charged with vitreous electricity.

There is a sub-sulphate, but it is applied to no use. The effloresced sulphate, called by some bisulphate, is preferred for medical practice. The extensive sale and high price of sulphate of quinina, have given rise to many modes of adulteration. It has been mixed with boracic acid, margaric acid, sugar, sugar of manna, gypsum, \&c. By incinerating a little of the salt upon a slip of platina, the boracic acid and gypsum remain, while the quinine is dissipated; sugar and margaric acid exhale their peculiar smoke and smell; or they may be dissolved out by a few drops of water. Cinchonina may be detected by adding ammonia to the solution, and treating the precipitate with ether, which leaves that vegeto-alkali.

QUINTESSENCE. The alchemists understood by this term, now no longer in scientific use, the solution in alcohol of the principles which this menstruum can extract from aromatic plants or flowers, by digestion, during some days, in the sun, a stove, or upon a sand-bath slightly warmed. A quintessence, therefore, corresponds to the alcoholic tincture or essence (not essential oil) of the present day. See Perfumrry.

R.

RAISINS, are grapes allowed to ripen and dry upon the vine. The best come from the south of Europe, as from Roquevaire in Provence, Calabria, Spain, and Portugal. Fine raisins are also imported from Smyrna, Damascus, and Egypt. Sweet fleshy grapes are selected for maturing into raisins, and such as grow upon the sunny slopes of hills sheltered from the north winds. The bunches are pruned, and the vine is stripped of its leares, when the fruit has become ripe; the sun then beaming full upon the grapes, completes their saccharification, and expels the superfluous water. The raisins are piucked, cleansed, and dipped for a few seconds in a boiling ley of wood ashes and quicklime, at 12 or 13 degrees of Baumés areometer. The wrinkled fruit is lastly drained, lrieu, an : exposed in the sun upon hurdles of basket-work during 14 or 15 days.

The fint st raisins are those of the sun, so called; being the plumpest bunches, which are left to ripen fully upon the vine, after their stalks have been half cut through.

The amount of raisins imported for home consumption was, in the year $1836,156,495$ cwts. ; in 1837, 152,635 ewts.

RAPF-SEED, imported for home consumption in $1836,561,457$ bushels; in 1837 , 937,526 bushels. See OiLs, unctuous.

RASP, MECHANICAL, is the name given by the French to an important machine much used for mashing beet-roots. See SugAr. 
RATAFIA, is the generic name, in France, of liqucurs compounded with alcohol sugar, and the odoriferous or flavoring principles of vegetables. Bruised cherries with their stones are infused in spirit of wine to make the ratafia of Granoble de Teyssère. The liquor being boiled and filtered, is flavored, when cold, with spirit of noyun, made by distilling water off the bruised bitter kernels of apricots, and mixing it with alcuhol. Sirup of bay laurel and galango are also added.

REALGAR, Red Orpiment. (Arsenic rouge sulphuré, Fr.; Rothes schwefelarsenik, Germ.) This ore occurs in primitive mountains, associated sometimes with native arsenic, under the form of veins, efflorescences, very rarely crystalline; as also in volcanic districts; for example, at Solfaterra near Naples; or sublimed in the shape of stalactites, in the rents and craters of Etna, Vesuvius, and other volcanoes. Its spec. grav. varies from $3 \cdot 3$ to $3 \cdot 6$. It has a fine scarlet color in mass, but orange-red in powder, whereby it is distinguishable from cinnabar. It is soft, sectile, readily scratched by the nail; its fracture is vitreous and conchoidal. It volatilizes easily before the blowpipe, emitting the garlic smell of arsenic, along with that of burning sulphur. It consists of, arsenic 70 , sulphur 30 , in 100 parts. It is employed sometimes as a pigment. Factitious orpiment is made by distilling, in an earthen retort, a mixture of sulphur and arsenic, of orpiment and sulphur, or of arsenious acid, sulphur, and charcoal. It has not the rich color of the native pigment, and is much more poisonous; since, like factitious orpiment, it always contains more or less arsenious acid.

RECTIFICATION, is a second distillation of alcoholic liquors, to free them from whatever impurities may have passed over in the first.

RED LIQUOR, is a crude acetate of alumina, employed in calico-printing, and prepared from pyroligneous acid; which see.

REED, is the well-known implement of the weaver, made of parallel slips of metal or reeds, called dents. A thorough knowledge of the adaption of yarn of a proper degree of fineness to any given measure of reed, constitutes one of the principal objects of the manufacturer of cloths; as upon this depends entirely the appearance, and in a great degree the durability, of the cloth when finished. The art of performing this properly, is known by the names of examining, setting, or sleying, which are used indiscriminately, and mean exactly the same thing. The reed consists of two parallel pieces of wood, set a few inches apart, and they are of any given length, as a yard, a yard and a quarter, \&c. The division of the yard being into halves, quarters, eighths, and sixteenths; the breadth of a web is generally expressed by a vulgar fraction, as $\frac{1}{4}, \frac{4}{4}, \frac{5}{4}, \frac{6}{4}$; and the subdivisions by the eighths or sixteenths, or nails, as they are usually called, as $\frac{7}{8}, \frac{9}{8}, \frac{11}{8}$ \&c., or $\frac{13}{16}, \frac{15}{1} \frac{1}{6}, \frac{19}{16}$, \&c. In Scotland, the splits of cane which pass between the longitudinal pieces or ribs of the reed, are expressed by hundreds, porters, and splits. The porter is 20 splits, or $\frac{1}{5}$ th of a hundred.

In Lancashire and Cheshire a different mode is adopted, both as to the measure and divisions of the reed. The Manchester and Bolton reeds are counted by the number of splits, or, as they are there called, dents, contained in $24 \frac{1}{4}$ inches of the reed. These dents, instead of being arranged in hundreds, porters, and splits, as in Scotland, are calculated by what is there termed hares or bears, each containing 20 dents, or the same number as the porter in the Scotch reeds: The Cheshire or Stockport reeds, again, receive their designation from the number of ends or threads contained in one inch, two ends being allowed for every dent, that being the almost universal number in every species and description of plain cloth, according to the modern practice of weaving, and also for a great proportion of fanciful articles.

The number of threads in the warp of a web is generally ascertained with considerable precision by means of a small manifying glass, fitted into a socket of brass, under which is drilled a small round hole in the bottom plate of the standard. The number of threads visible in this perforation, ascertains the number of threads in the standard measure of the reed. Those used in Scotland have sometimes four perforations, over any one of which the glass may he shifted. The first perforation is $\frac{1}{4}$ of an inch in diameter, and is therefore well adapted to the Stockport mode of counting; that is to say, for ascertaining the number of ends or threads per inch; the second is adapted for the Holland reed, being $\frac{1}{200}$ th part of 40 inches; the third is $\frac{1}{7} \frac{1}{0}$ th of 37 inches, and is adapted for the now almost universal construction of Scotch reeds ; and the fourth, being $\frac{1}{2} \frac{1}{0}{ }^{0}$ th of 34 inches, is intended for the French cambrics. Every thread appearing in these respeclive mea sures, of course represents 200 threads, or 100 splits, in the standard breadth; and thus the quality of the fabric may be ascertained with considerable presision, even after the cloth has undergone repeated wettings, either at the bleachingground or dye-work. By counting the other way, the proportion which the woof bears to the warp is also known, and this forms the chief use of the glass to the manufacturer and operative weaver, both of whom are previously acquainted with the exact measurs of the reed. 
Comparative TABLE of 37 -inch reeds, being the standard used throughout Europe, for linens, with the Lancashire and Cheshire reeds, and the foreign reeds used for holland and cambric.

\begin{tabular}{|c|c|c|c|c|}
\hline Scotch. & Lancashire. & Cheshire. & Dutch holland. & French cambric. \\
\hline 600 & 20 & 34 & 550 & 653 \\
\hline 700 & 24 & 38 & 650 & 761 \\
\hline 800 & 26 & 44 & 740 & 870 \\
\hline 900 & 30 & 50 & 832 & 979 \\
\hline 1000 & 34 & 54 & 925 & 1089 \\
\hline 1100 & 36 & 60 & 1014 & 1197 \\
\hline 1200 & 40 & 64 & 1110 & 1300 \\
\hline 1300 & 42 & 70 & 1202 & 1414 \\
\hline 1400 & 46 & 76 & 1295 & 1464 \\
\hline 1500 & 50 & 80 & 1387 & 1602 \\
\hline 1600 & 52 & 86 & 1480 & 1752 \\
\hline 1700 & 56 & 92 & 1571 & 1820 \\
\hline 1800 & 58 & 96 & 1665 & 1958 \\
\hline 1900 & 62 & 104 & 1757 & 2067 \\
\hline 2000 & 66 & 110 & 1850 & 2176 \\
\hline
\end{tabular}

In the above table, the 37 -inch is placed first. It is called Scotch, not because it either originated or is exclusively used in that country. It is the general linen reed of all Europe; but in Scotland it has also been adopted as the regulator of her cotton mantfactures.

REFINING OF GOLD AND SILVER; called also Parting. (Affinage d'argent, Départ, Fr.; Scheidung in die quart, Germ.) For several uses in the arts, these precious metals are required in an absolutely pure state, in which alone they possess their malleability and peculiar properties in the most eminent degree. Thus, for example, neither gold nor silver leaf can be made of the requisite fineness, if the metals contain the smallest portion of copper alloy. Till within these ten or twelve years, the parting of silver from gold was effected everywhere by nitric acid; it is still done so in all the establishments of this country, except the Royal Mint; and in the small refining-houses abroad. The following apparatus may be advantageously employed in this operation. It will serve the double purpose of manufacturing nitric acid of the utmost purity, and of separating silver from gold by its means.

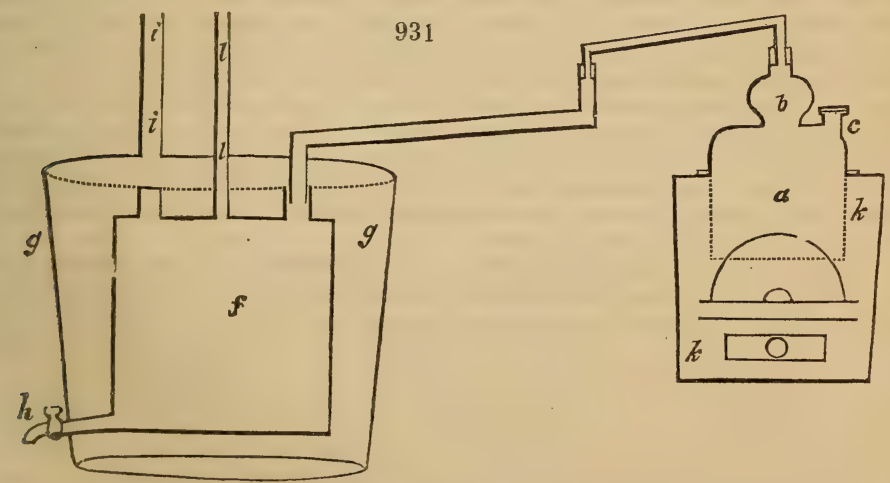

1. On procuring nitric acid for parting. $-a$ is a platinum retort or alembic; $b$ is its capital, terminating above in a tubulure, to which a kneed tube of platinum, about 2 feet long, is adapted; $c$ is the tubulure of the retort, for supplying acid during the process, and for inspecting its progress. It is furnished with a lid ground air-light, which may be secured in its place by a weight. $e$ is a stoneware pipe, about two inches diameter, and several feet long, according to the locality in which the operation is to be carried on. It is made in lengths fitted to one another, and secured at the joints with loam-lute. The one bend of this earthenware hard salt-glazed pipe is adapted to receive the platinum tube, and the other bend is inserted into a tubulure in the top of the stoneware $\operatorname{drum} f$. The opening $l, l$, in the middle of the top of $f$, is 
for inspecting the progress of the condensation of acid; and the third tubulure termi nates in a prolonged pipe $i, i$, consisting of several pieces, each of which enters from above conically into the one below. The joinings of the upper pieces need not be tighty luted, as it is desirable that some atmospherical oxygen should enter, to convert the relatively light nitrous gas into nitrous or nitric acid vapor, which when supplied with moisture will condense and fall down in a liquid state. To supply this moisture in the most diflusive form, the upright stoneware pipes $i, i, l, l$, (at least 3 inches diameter, and 12 feet high), should be obstructed partially with flint nodules, or with silicious pebbles; and water should be allowed to trickle upon the top pebble from a cistern placed above. Care must be taken to let the water drop so slowly as merely to preserve the pebbles in a state of humidity. $h$ is a stopcock, of glass or stoneware, for drawing off the acid from the cistern $f . \quad k$ is a section of a small air-furnace, covered in at top with an iron ring, on which the flat iron ring of the platinum frame rests.

$g, g$, is a tub in which the stoneware cistern stands, surrounded with water, kept constantly as cold as possible by passing a stream through it; the spring water entering by a pipe that dips near to the bottom, and the hot water escaping at the upper edge.

With the above apparatus, the manufacture of pure nitric acid is comparatively easy and economical. Into the alembic $a, 100$ pounds (or thereby) of pure nitre, coarsely bruised if the crystals be large, are to be put; the capital is then to be adapted, and the platinum tube (the only moveable one) luted into its place. Twenty pounds of strong sulphuric acid are now to be introduced by the tubulure $c$, and then its lid must be put on. No heat must yet be applied to the alembic. In about an hour, another ten pounds of acid may be poured in, and so every hour, till 60 pounds of acid have been added. A few hours after the affusion of the last portion of acid, a slight fire may be kindled in the furnace $k$.

By judicious regulation of the heat, the whole acid may be drawn off in 24 hours ; its final expulsion being aided by the dexterous introduction of a quart or two of boiling water, in small successive portions, by the tubulure $c$, whose lid must be instantly shut after every inspersion. The most convenient strength of acid for the parting process, is when its specific gravity is about $1 \cdot 320$, or when a vessel that contains 16 ounces of pure water, will contain $21 \frac{1}{8}$ of the aquafortis. To this strength it should be brought very exactly by the aid of a hydrometer.

Its purity is easily ascertained by letting fall into it a few drops of solution of silver; and if no perceptible milkiness ensues, it may be accounted good. Should a white cloud appear, a few particles of silver may be introduced, to separate whatever muriatic acid may be present, in the form of chloride of silver. Though a minute quantity of sulphuric acid should exist in the nitric, it will be of no consequence in the operation of parting.

2. On parting by the nitric acid, called by the Mexicans, " $I l$ apartado." - The principle on which this process is founded, is the fact of silver being soluble in nitric acid, while gold is insoluble in that menstruum. If the proportion of gold to that of silver be greater than one to two, then the particles of the former metal so protect or envelop those of the latter, that the nitric acid, even at a boiling heat, remains quite inactive on the alloy. It is indispensable, therefore, that the weight of the silver be at least double that of the gold. 100 pounds of silver take 38 pounds of nitric acid, of specific gravity $1 \cdot 320$, for oxydizement, and 111 for solution of the oxyde; being together 149; but the refiner often consumes, in acid of the above strength, more than double the weight of silver, which shows great waste, owing to the imperfect means of condensation employed for recovering the vapors of the boiling and very volatile acid.

By the apparatus above delineated, the 38 pounds of acid expended in oxydizing the silver, become nitrous gas in the first place, and are afterwards reconverted in a great measure into nitric acid by absorption of atmospherical oxygen; so that not one fifth need be lost, under good management. As the acid must be boiled on the granulated garble, or alloy, to effect the solution of the silver, by proper arrangements the vapors may be entirely condensed, and nearly the whole acid be recovered, except the 111 parts indispensable to constitute nitrate of silver. Hence, with economical management, 120 pounds of such acid may be assigned as adequate to dissolve 100 of silver associated with 50 of gold.

It must here be particularly observed, that 100 pounds of copper require 130 pounds of the above acid for oxydizement; and 390 for solution of the oxyde; being 520 pounds in whole, of which less than $\frac{1}{4}$ part could be recovered by the above apparatus. It is therefore manifest that it is desirable to employ silver pretty well freed from copper by a previous process; and always, if practicable, a silver containing some gold.

These data being assumed as the bases of the parting operation, 60 pounds of gold and silver alloy or garble finely granulated, containing not less than 40 pounds of silver, are to be introduced into the ten-gallon alembic of platinum, fig. 931, and 80 pounds of nitric acid, of $1 \cdot 320$, is to be poured over the alloy; a quantity which will measure 6 gallons imperial. As for the bulk of the alloy, it is considerably less than half a gallon. Abun 
danoe of space therefore remains in the alembic for effervescence and ebullition, provided the fire be rightly tempered.

$\mathrm{Bv}$ the extent of stoneware conducting pipe $e$, which should not be less than 40 feet, by the dimensions and coldness of the cistern $f$, and by the regenerating influence of the vertical aerial pipe filled with moist pebbles $i$, $i$, it is clear, that out of the 80 pounds of nitric acid, specific gravity 1.320 , introduced at first, from 20 to 30 will be recovered.

Whenever the effervescence and disengagement of nitrous red fumes no longer appear on opening the orifice $c$, the fire must be removed, and the vessel may be cooled by the application of moist cloths. The alembic may be then disengaged from the platinum tube, and lifted out of its seat. Its liquid contents must be cautiously decanted off, through the orifice $c$, into a tub nearly filled with soft water. On the heavy pulverulent gold which remains in the vessel, some more acid should be boiled, to carry off any residuary silver. This metallic powder, after being well washed with water, is to be dried, fused along with a little nitre or borax, and cast into ingots.

Plates of copper being immersed in the nitric solution contained in wooden or stoneware cisterns, will throw metallic silver down, while a solution of nitrate of copper, called blue water, will float above. The pasty silver precipitate is to be freed from the nitrate of copper, first, by washing with soft water, and next, by strong hydraulic pressure in cast iron cylinders. The condensed mass, when now melted in a crucible along with a little nitre and borax, is fine silver.

The above apparatus has the further advantage of enabling the operator to recover a great portion of his nitric acid, by evaporating the blue water to a state approaching to dryness, with the orifices at $c$, and at the top of the capital, open. In the progress of this evaporation, nothing but aqueous vapor escapes. Whenever the whole liquid is dissipatel, the pipe $d$ is to be re-adjusted, and the lid applied closely to $c$. The heat being now continued, and gradually increased, the whole nitric acid will be expelled from the copper oxyde, which will remain in a black mass at the bottom of the alembic. The contrivance for letting water trickle upon the pebbles, must be carefully kept in play, otherwise much of the evolved acid would be dissipated in nitrous fumes. With due attention to the regenerative plan, a great part of the acid may be recovered, at no expense but that of a little fuel.

The black oxyde of copper thus obtained, is an economical form of employing that metal for the production of the sulphate; 100 pounds of it, with $122 \frac{1}{2}$ of sulphuric acid diluted with water, produce $312 \frac{1}{2}$ pounds of crystallized sulphate of copper. A leaden boiler is best adapted for that operation. 100 pounds of silver are precipitable from its solution in nitric acid, by 29 of copper. If more be needed, it is a proof that a wasteful excess of acid has existed in the solution.

In parting by nitric acid, the gold generally retains a little silver; as is proved by the cloud of chloride of silver which it affords, at the end of some hours, when dissolved in aqua regia. And on the other hand, the silver retains a little gold. These facts indr:ed M. Dizé, when he was inspector of the French mint, to adopt some other process, which would give more accurate analytical results; and after numerous experiments, he ascertained that sulphuric acid presented great advantages in this point of view, since with it he succeeded in detecting, in silver, quantities of gold which had elured the other plan of parting. The suggestion of M. Dizé has been since universally adopted in France. M. Costell, about nine or ten years ago, erected in Pomeroystreet, Old Kent-road, a laboratory upon the French plan, for parting by sulphuric acid; but he was not successful in his enterprise; and since he relinquished the business, Mr. Matheson introduced the same system into our Royal Mint, under the management of M. Costell's French operatives. In the Parisian refineries, gold, to the amount of one thousandth part of the weight, has been extracted from all the silver which had been previously parted by the nitric acid process; being 3500 francs in value upon every thousand kilogrammes of silver.

I shall give first a general outline of the method of parting by sulphuric acid, and then describe its details as I have lately seen them executed upon a magnificent scale in an establishment near Paris.

The most suitable alloy for refining gold, by the sulphuric acid process, is the compound of oold, silver, and copper, having a standard quality, by the cupel, of from 900 to 950 millièmes, and containing one fifth of its weight of gold. The best proportions of the three metals are the following:-silver, 725 ; gold, 200 ; copper, $75 ;=1000$. It has been found that alloys which contain more copper, afford solutions that hold some anhydrous sulphate of that metal in solution, which prevents the gold from being readily separated; and that alloys containing more gold, are not acted on easily by the sulphuric acid. The refiner ought, therefore, when at all convenient, to reduce the alloys that he has to treat to the above-stated proportions. He may effect this purpose either by fusing the coarser alloys with nitre in a crucible, or by adding finer alloy, or even fine silver, or finally, by subjecting the coarser alloys to a previous cupellation with lead on 
the great scale. As to gold or silver bullion, which contains lead and other easily oxytizable metals besides copper, the refiner ungnt always to avoid treatıng tnem oy sul. phuric acid; and should separate, first of all, these foreign metals by the agency of nitre; if they exist in minute quantity; but if in larger, he should have recourse to the cupel. Great advantage will therefore be derived from the judicious preparation of the alloy tc be refined.

For an alloy of the above description, the principal Parisian refiners are in the habit of employing thrice its weight of sulphuric acid, in order to obtain a clear solution of sulphate of silver, which does not too suddenly concrete on cooling, so as to obstruct its discharge from the alembic by decantation. A small increase in the quantity of copper, calls for a considerable increase in the quantity of acid.

Generally speaking, one half of the sulphuric acid strictly required for converting the silver and copper into sulphates, is decomposed into sulphurous acid, which is lost to the manufacturer, unless he has recourse to the agency of nitrous acid.

The process for silver containing but little gold, consists of five different operations.

1. Upon several furnaces, one foot in diameter, egg-shaped alembics of platinum are mounted, into each of which are put 3 kilogrammes ( 8 lbs. troy) of the granulated silver, containing a few grains of gold per pound, and 6 kilogrammes of concentrated sulphuric acid. The alembics are covered with conical capitals, ending in bent tubes, which conduct the acid vapors into lead pipes of condensation; and the furnaces are erected under a proper hood. As the cold acid is inoperative, it must be set a boiling, at which temperature it gives up one atom of its oxygen to the metal, and is transformed into sulphurous acid, which escapes in a gaseous state. Some of the undecomposed sulphuric acid immediately combines with the oxyde into a sulphate, which subsides, in the state of a crystalline powder; to the bottom of the vessel. The solution goes on vigorously, with a copious disengagement of sulphurous acid gas, only during the two or three first hours; after which it proceeds slowly, and is not completed till after a digestion of nearly twelve hours more. During the ebullition a considerable quantity of sulphuric acid vapor escapes along with the sulphurous acid gas; the former of which is readily condensed in a large leaden receiver immersed in a cistern of cold water, if need be. It has been proposed to condense the sulphurous acid, by leading it over extensive surfaces of lime-pap, as in the coal-gas purifiers.

2. When the whole silver has been converted into sulphate, this is to be emptied out of the alembic into water contained in a round-bottomed receiver lined with lead, and diluted till the density of the solution marks from $15^{\circ}$ to $20^{\circ}$ Baume. The small portion of gold, in the form of a brown powder, which remains undissolved, having been allowed to settle to the bottom, the supernatant solution of silver is to be decanted carefully off into a leaden cistern, and the powder being repeatedly edulcorated with water, the washings are to be added to it. The silver is now to be precipitated by plunging plates of copper in the solution, and the magma which falls is to be well washed, and freed from the residuary particles of sulphate of copper by powerful compression.

3. The silver, precipitated and dried as above described, is melted in a crucible, and east into an ingot.

4. The gold powder is also dried and cast into an ingot, a little nitre being added in the fusion, to oxydize and separate any minute particles of copper that may perchance have been protected from the solvent action of the acid.

5. As the sulphate of copper is of considerable ralue, its solution is to be neutralized, evaporated in leaden pans to a proper strength, and set aside to crystallize in leaden cisterns. The farmers throughout France consume an immense quantity of this salt. They sprinkle a weak solution of it (at $2^{\circ}$ or $3^{\circ}$ Baumé) orer their grain before sowing it, in order to protect it against the ravages of birds and insects.

The pure gold, at the instant of its separation from the allor by the action of sulphuric acid, being in a very fine powder, and lying in close contact with the platinum, under the influence of a boiling menstruum, which brightens the surfaces of the two metals, and raises their temperature to fully the 600th degree oi Fahrenheit's scale, tends to become partially soldered to the platinum, and may thus progressirely thicken the bottom of the still. The importance of preserving this vessel entire, and of economizing the fuel requisite to heat its contents, induces the refiner to detach the crust of gold from time to time, by passing over the bottom of the still, in small quantities, a dilute nitromuriatic acid, which acts readily on gold, but not on platinum. But as this nperation is a very delicate one, it must be conducted with great circumspection. The danger of such adhering deposites is much increased by using too high a heat, and too small a body of acid, relatively to the metals dissolved. Hence it is advantageous to employ alembics of large size. Should any lead or tin get into the platinum still, while the hot acid is in it, the precions vessel would be speedily destroyed; an accident which has not unfrequently happened. Each operation may be conveniently finished in twelve hours; 
so that each alembic may refine with ease 160 mares daily. Some persons work more rapilly, but such haste is hazardous.

The Parisian refiners restore to the owners the whole of the gold and silver contained in the ingots, reserving to themselves the copper which formed the alloy, and charging only the sum of $5 \frac{1}{2}$ francs per kilogramme $(2.68 \mathrm{lbs}$. troy) for the expense of the parting of the metals.

If they are employed to refine an ingot of silver containing less than one tenth of gold, they retain for themselves a two thousandth part of the gold, and all the copper, existing in the alloy; return all the rest of the gold, with the whole of the silver, in the ingot; and give, besides, to the owners a premium or bonus, which amounted lately to $\frac{3}{4}$ of a franc on the kilogramme of metal. Should the owner desire to have the whole of the gold and silver contained in his ingot, the refiner then demands from him 2 francs and 68 centimes per kilogramme, retaining the copper of the alloy. As to silver ingots of low standard, the perfection of the refining processes is such, that the mere copper contained in them pars all the costs; for in this case, the refiner restores to the proprietor of the ingot as much fine silver as the assay indicated to exist in the ingot, contenting himself with the copper of the alloy. See infra.

The chemical worlis of M. Poizat, called affinage d'argent, on the bank of the canal de l'Ourcq, in the vicinity of Paris, are undoubtedly the most spacious and best arranged for refining the precious metals, which exist in the world. On being introduced to this gentleman, by my friend and companion M. Clement-Desormes, he immediately expressed his readiness to conduct me through his fabrique, politely alluding to the French translation of my Dictionary of Chemistry, which lay upon the desk of his bureau. The principal room is 240 feet long, 40 feet wide, and about 30 feet high. A lofty chimney rises up through the middle of the apartment, and another at each of its ends. The one space, 120 feet long, to the rioht of the central chimney, is allotied to the processes of dissolving the silver, and parting the gold; the other, to the left, to the evaporation and crystallization of the sulphate of copper, and the concentration of the recovered sulphuric acid.

M. Poizat melts his great masses of silver in pots made of malleable iron, capable of holding sereral cwts. each; and granulates it by pouring it into water contained in large iron pans. The granulated silver is dried with heat, and carried into a well liohted office enclosed by glazed casements, to be weighed, registered, and divided into determinate portions. Each of these is put into a cast-iron pot, of a flattened hemispherical shape, about 2 feet in diameter, covered with an iron lid, made in halves, and hinged together in the middle line. From the top of the fixed lid a bent pipe issues, and proceeds downwards into an oblong leaden chest sunk bepeath the floor. Four of the above cast-iron pots stand in a line across the room, divided into two ranges, with an intervening space for passing between them. The bottoms of the pots are directly heated by the flame, one fire serving for two pots. Two parts of concentrated sulphuric acid by weight are poured upon ever part of granulated silver, and liept gently boiling till the whole silv ${ }^{-}$ be converted intc a pasty sulphate.

From the underground leaden chests, a leaden, pipe 4 inches in diameter, rises vertically, and enters the side of a leaden chamber, which is supported upon strong cross-beams or rafters, a little way beneath the roof of the apartment. This chamber, which is 30 feet long, 10 feet wide, and 6 feet high, is intended to condense the sulphuric acid varors, along with some of the sulphurous acid; that of the latter being promoted by the admission of nitrous gas and air, which convert it into sulphuric acid. From the further end of this chamber, a large square leaden pipe returns with a slight slope towards the middle of the room, and terminates at the right-hand side of the central chimney, in a small leaden chest, for receiving the drops of acid which are condensed in the pipe. From that chest a pipe issues, to discharge into the high central chimney the incondensable gases, and also to maintain a constant draught through the whole series of leaden chambers back to the cast-iron hemispherical pots.

Besides the above cast-iron pots, destined to dissolve only the coarse cupreous silver, containing a few grains of gold per pound, there are, in the centre of the apartment, at the right-hand side of the chimney, 6 alembics of platinum, in which the rich alloys of gold and silver are treated in the process of refining gold.

The pasty sulphate of silver ohtained in the iron pots, is transferred by cast-iron ladles with long handles into large leaden cisterns, adjoining the pots, and there diluted with a little water to the density of $36^{\circ}$ Baumé. Into this liquor, steam is admitted through a series of upright leaden pipes arranged along the side of the cistern, which speedily canses ebullition, and dilutes the solution eventually to the $22 d$ deoree of Baumé. In this state, the liquid supersulphate is run off by leaden syphons into large oblong leaden cisterns, rouncled at the bottom; and is there exposed to the action of ribands of copper, like thin wood shavings. The metallic silver precipitates in a pasty form; and the 
supernatant sulphate of copper is then run off into a cistern, upon a somewhat lower level, where it is left to settle and become clear.

The precipitate of silver, called by the English, water-silver, and by the French, chaux d'argent, is drained, then strongly squeezed in a square box of cast-iron, by the action of a hydraulic press; in which 60 pounds of silver are operated upon at once.

The silver lumps are dried, melted in black lead crucibles, in a furnace built near the silver end of the room, where the superintendent sits in his bureau-a closet enclosed by glazed casements, like a green-house. The whole course of the operations is so planned, that they are made to commence near the centre with the mixed metals, and progressively approach towards the office end of the apartment as the parting processes advance. Here the raw material, after being granulated and weighed, was given out, and here the pure gold and silver are finally eliminated in a separate state.

In the other half of the hall, the solutions of sulphate of copper are evaporated in large shallow leaden pans, placed over a range of furnaces; from which, at the proper degree of concentration, they are run off by syphons into crystallizing pans of the same metal. From the mother-waters, duly evaporated, a second crop of crystals is obtained; and also a third, the last being anhydrous, from the great affinity for water possessed by the strong sulphuric acid with which they are now surrounded. The acid in this way parts with almost the whole of the cupreous oxyde, and is then transferred into a large alembic of platinum (value $1000 l$.), to be rendered fit, by re-concentration, for acting upon fresh portions of granulated silver. The capital of that alembic is connected with a leaden worm, which traverses an oblong vessel, through which a stream of cold water flows.

The crystallized sulphate of copper fetched, two years ago, 30l. a ton. It is almost all sold to the grocers in the towns of the agricultural districts of France. In the above establishment of M. Poizat, silver to the value of $10,000 l$. can be operated upon daily.

There is a steam engine of 6 -horse power placed in a small glazed chamber at one side of the parting hall, which serves to work all his leaden pumps for lifting the dilute sulphuric acid and acidulous solutions of copper into their appropriate cisterns of concentration, as also to grind his old crucibles, and drive his amalgamation mill, consisting of a pair of vertical round-edged wheels, working upon one shaft, in a groove formed round a central hemisphere-of cast-iron. After the mercury has dissolved out of the ground crucibles all the particles of silver which it can find, the residuary earthy matter is sold to the sweep-washers. The floor of the hall around the alembics, pots, and cisterns, is covered with an iron grating, made of bars having one of their angles uppermost, to act as scrapers upon the shoes of the operatives. The dust collects in a vacant space left beneath the grating, whence it is taken to the amalgamation mill. The processes are so well arranged and conducted by M. Poizat, that he can execute as much business in his establishment with 10 workmen as is elsewhere done with from 40 to 50 ; and with less than 3 grains of gold, in one Paris pound or 7561 grains of silver, he can defray the whole expenses of the parting or refining.

Since 26 parts of copper afford 100 of the crystallized sulphate, the tenth of copper present in the dollars, and most foreign coins, will yield nearly four times its weight of blue vitriol; a subsidiary product of considerable value to the refiner.

The works of $M$. Poizat are so judiciously fitted up as to be quite salubrious, and have not those "very mischievous effects upon the trachea," which Mr. Matheson states as being common in his refinery works in the Roya ${ }_{\perp}$ Mint.* But, in fact, as refining by sulphuric acid is always a nuisance to a neighborhood, it is not suffered in the Monnaie Royale of Paris; but is best and most economically performed by private enterprise and fair competition, which is impossible in London, on account of the anomalous privilege, worth at least 2000l. a year, possessed by Mr. Matheson, who works most extensively for private profit on a public plant, fitted up with a lofty chimney, platinum vessels to the value of $3000 l$., and other apparatus, at the cost of the government. His charge to the crown for refining gold per lb. troy, is $6 s .6 d$; ; that of the refiners in London, who are obliged, for fear of prosecution, to employ the more expensive, but more condensable, nitric acid, is only 4s. That of the Parisian refiners is regulated as follows. For the dealers in the precious metals :-

For gold bullion containing silver, and more than $\frac{1}{1} \frac{0}{0} 00$ of gold, 6 fr. 12 c. per kilogramme, $=2$ fr. 29 c. per lb. troy.

For silver bullion, containing from $\frac{1}{1000}$ to $\frac{1}{1000}$ of gold (called dorés), $3 \mathrm{fr} .27 \mathrm{c}$. per kilogramme, $=1 \mathrm{fr}$. $22 \mathrm{c}$. per $1 \mathrm{~b}$. troy.

For the Monnaie, the charges are-

For gold refined by sulphuric acid, when alloyed with copper only, from $\frac{898}{1000}$ to $\frac{1}{100 \overline{0}}$.

fr. per kilogramme, $=1 \mathrm{fr} .86 \mathrm{c}$. per lb. troy.

For gold alloyed with copper and silver, whatever be the quantity of silver, $5 \mathrm{fr} .75 \mathrm{c}$. per kilogramme, $=2$ fr. 12 c. per lb. troy.

* Roport of Committee of House of Commons on the Mint, in 1837, p. 91. 
There are about ten bullion refiners by sulphuric acid in the environs of Paris; two of whom, M. Puizat St. Andre, and M. Chauvière, are by far the most considerable; the former working about 300 hilogrammes ( $=804 \mathrm{lbs}$. troy) daily, and the latter about two thirds of that quantity. In former times, when competition was open in London, Messrs. Bruwne and Brinde were wont to treat 6 cwts. of silver, or $9 \mathrm{cwts}$. of gold alloy, daily, for several months in succession.

The result of free trude in refining bullion at Paris is, that the silver bars imported into London from South America, \&c., are mostly sent off to Paris to be stripped of the few grains of gold which they may contain, and are then brought back to be sold here. Three grains of gold in one Paris lb. of silver, pay the refiners there for taking them out. What a disgrace is thus brought upon our manufacturing industry and slill, by the monopoly charges in refining and assaying granted to two individuals in our Roval Mint.

Mr. Bingley's charges for assaying at the Royal Mint in London, are-

For an assay of gold, $4 s$. ; for a parting assay of gold and silver, $6 s$. ; for a silver assay, 2s. 6d.-charges which absorb the profits of many a transaction.

The charges at the Royal Mint of Paris, for assays made under the following distinguished chemical savants-Darcet, Directeur; Bréant, Verificateur; Chevillot and Pelouze, Essuyeurs ; are-

For an assay of gold, or doré (a parting assay), 3 francs.

silver - $-0.80 \mathrm{c} .=8 \mathrm{~d}$. English.

M. Gay Lussac is the assayer of the Bureau de Garantie at the Monnaie Royale, an office which corresponds to the Goldsmiths' Hall at London. The silver assays in all the official establishments of Europe, except the two in London, are made by the humid method, and are free from those errors and blunders which daily annoy and despoil the British bullion merchant, who is compelled by the Mint and Bank of England to buy and sell by the cupellation assay of Mr. Bingley. See Assay and Silver.

REFRIGERATION OF WORTS, \&c. In August, 1826, Mr. Yandall obtained a patent for an appararus designed for cooling worts and other hot fluids, without exposing them to evaporation. Utensils employed for this purpose, are generally called refrigerators, and are so constructed, that a quantity of cold water shall be brought in contact with the vessel which contains the heated fluid. But in every construction of refrigerator heretofore used, the quantity of cold water necessarily employed in the operation, greatly exceeded the quantity of the fluid cooled, which, in some situations, where water cannot be readily obtained, was a serious impediment and objection to the use of such apparatus.

The inventor has contrived a mode of constructing a refrigerator, so that any quantity of wort or other hot fluid may be cooled by an equal quantity of cool water; the process being performed with great expedition, simply by passing the two fluids through very narrow passages, in opposite directions, the result of which is, that the cold liquor imbibes the heat from the wort, or other fluid, and the temperature of the hot fluid is reduced in the same ratio.

Figs. 932, 933, and 934 represent different forms in which the apparatus is proposed to be made. The two first have zigzag passages; the third, channels running in convolute curves. These channels or passages are of very small capacity in thickness, but of great length, and of any breadth that may be required, according to the quantity of fluid intended to be cooled or heated.

Fig. 935 is the section of a portion of the apparatus shown at figs. 932 and 933 upon an enlarged scale; it is made by connecting three sheets of copper or any other thin metallic plates together, leavi $\mathrm{g}$ parallel spaces between each plate for the passage of the fluids, represented by the biack lines.

These spaces are formed by occasionally introducing between the plates thin straps, ribs, or portions of metal, by which means very thin channels are produced, and through these channels the fluids are intended to be passed, the cold liquor running in one direction, and the hot in the reverse direction.

Supposing that the passages for the fluids are each one eighth of an inch thick, then the entire length for the run of the fluid should be about 80 feet, the breadth of the apparatus being made according to the quantity of fluid intended to be passed through it in a given time. If the channels are made a quarter of an inch thick, then their length should be extended to 160 feet; and any other dimensions in similar proportions; but a larger channel than one quarter of an inch, the patentee considers would be objectionable. It is, however, to be observed, that the length here recommended, is under the consideration, that the fluids are driven through the apparatus by some degree of hydrostatic pressure from a head in the delivery-vats above; but if the fluids flow without pressure, then the lengths of the passages need not be quite so great.

In the apparatus constructed as shown in perspective at fig, 932, and further 
developed by the section, fig. 935, cold water is to be introduced at the funnel $a$,

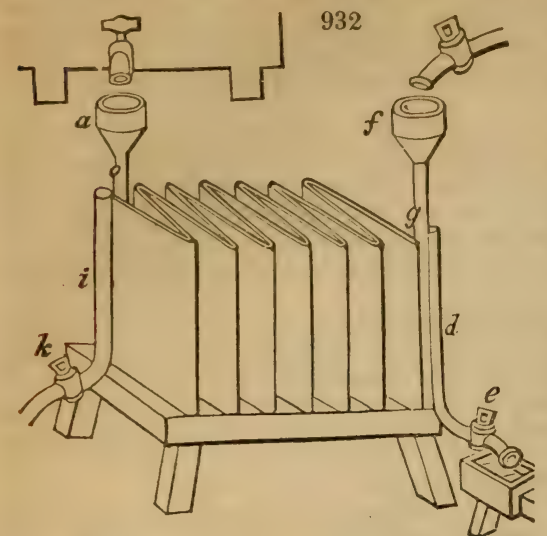
whence it passes down the pipe $b$, and through a long slit or opening in the side of the pipe, into the passage $c, c$ (see fig. 935 ), between the plates, where it flows in a horizontal direction through the channel towards the discharge-pipe $d$. When such a quantity of cold water has passed through the funnel $a$, as shall have filled the channel $c, c$, up to the level of the top of the apparatus, the cock $e$ being shut, then the hot wort or liquor intended to be cooled, may be introduced at the funnel $f$, and which, descending in the pipe $g$, passes in a similar manner to the former, through a long slit or opening in the side of the pipe $g$, into the extended passage $h, h$ (see fig. 935), and from thence proceeds horizontally into the dischargepipe $i$.

or The two cocks $e$ and $k$, being now cock $k$, and the water through $e$. If the apertures of the two cocks $e$ and $k$ are equal, and the channels equal also, it follows that the same quantity of wort, \&c., will flow through the channel $h, h, h$, in a given time, as of water through the channel $c$, $c$; and by the hot fluid passing through the apertures in contact with the side of the channel which contains the cold fluid, the heat becomes abstracted from the former, and communicated to the latter; and as the hot fluid enters the apparatus at that part which is in immediate contact with the part where the cooling fluid is discharged, and the cold fluid enters the apparatus at that part where the wort is discharged, the consequence is, that the wort or other hot liquor becomes cooled down towards its exit-pipe nearly to the temperature of cold water; and the temperature of the water, at the reverse end of the apparatus, becomes raised nearly to that of the boiling wort.

It only remains to observe, that by partially closing either of the exit-cocks, the quantity of heat abstracted from one fluid, and communicated to the other, may be regulated; for instance, if the cock $e$ of the water-passage be partially closed, so as to diminish the quantity of cold water passed through the apparatus, the wort or other hot fluid conducted through the other passages will be discharged at a higher temperature, which in some eases will be desirable, when the refrigerated liquor is to be fermented.

Fig. 933 exhibits an apparatus precisely similar to the foregoing, but different in its position; for instance, the zigzag channels are made in obliquely descending planes.

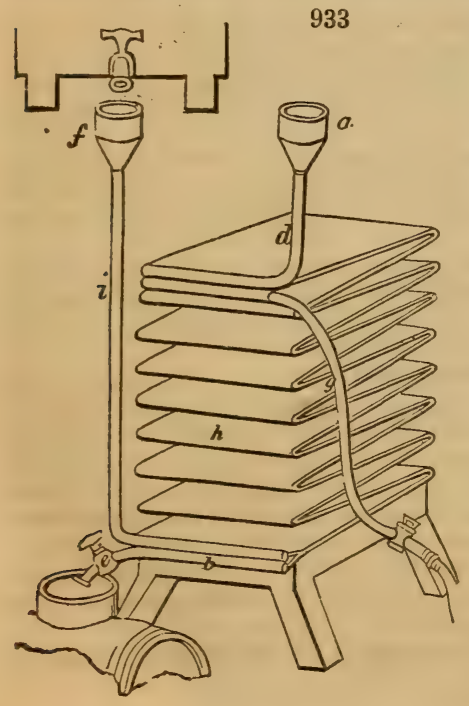
$a$ is the funnel for the hot liquor, whence it descends through the pipe $d$ into the channel $c, c$ (see fig. 935), and ultimately is discharged through the pipe $b$, at the cock $e$. The cold water being introduced into the funnel $f$, and passing down the pipe $i$, enters the zigzag channel $h, h$, and, rising through the apparatus, runs off by the pipe $g$, and is discharged at the cock below.

The passages of this apparatus for heating and cooling fluids, may be bent into various contorted figures; one form found particularly convenient under some applications, is that represented at fig. 934, which is contained in a cylindrical case. The passages here run in convolute curves, the one winding in a spiral to the centre, the other receding from the centre.

The wort or other hot liquor intended to be cooled, is to be introduced at the funnel $a$, and passing down the pipe $b$, is delivered into the open passage $c$, which winds round to the central chamber $d$, and is thence discharged through the pipe $e$, at the cock $f$. The cold water enters the apparatus at the funnel $g$, and proceeding down the pipe $h$, enters the 
elosed channel $i$, and after traversing round through the apparatus, is in like manner discharged through the pipe $k$, at the cock $l$. Or the hot liquor may be passed througin

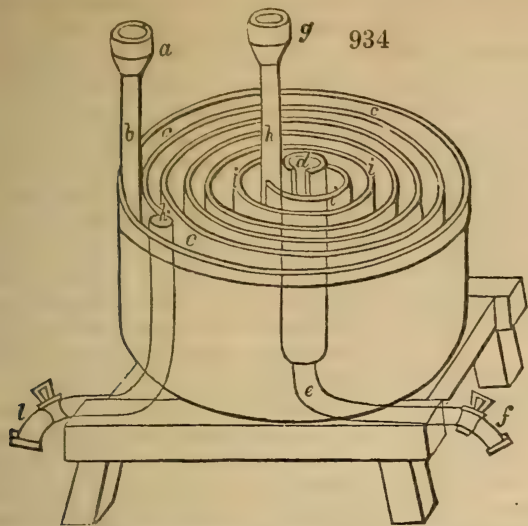

the closed channel, and the cold through the open one; or these chambe s may be both of them open at top, and he apparatus covered by a lid when at work, the principal design of which is to afford the convenience of cleaning them more readily than could be done if they were closed; or they may be both closed.

A similar ingenious apparatus for cooling brewer's worts, or wash for distillers, and also for condensing spirits, in place of the ordinary worm tub, is callo' by the inventor, Mr. Wheeler, an Archimedes condenser, or refrigerator, the peculiar novelty of which consists in forming the chambers for the passage of the fluids in spiral channels, winding round a central tube, through which spiral channels the hot and cold fluids are to be passed in opposite directions.

Fig. 936 represents the external appearance of the refrigerator, enclosed in a cylindrical case; fig. 937 , the same, one half of the case being removed to show the form 935 of the apparatus within; and fig. 938, a $d$ section cut through the middle of the appa-

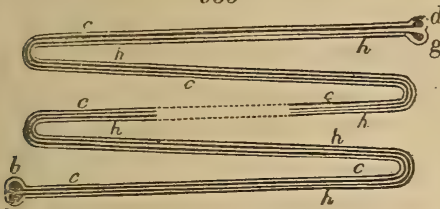
ratus perpendicularly, for the-purpose of displaying the internal figure of the spiral channels.

The apparatus is proposed to be made of sheet copper, tinned on its surface, and is formed by cutting circular pieces of thin copper, or segments of circles, and connecting $\stackrel{b}{b}$ together by rivets, solder, or by any other convenient means, as coppersmiths usually do; these circular pieces of copper being united to one another, in the way of a spiral or screw, form the chambers through which the fluids are to pass within, in an ascending or descending inclined plane.
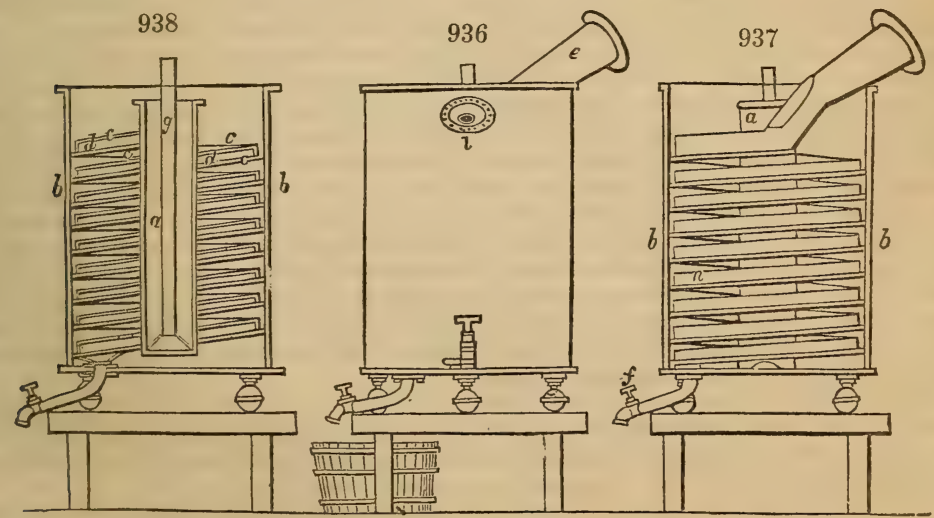

In figs. 937 and $938, a, a$, is the central tube or standard (of any diameter that may be found convenient), round which the spiral chambers are to be formed; $b, b$, are the sides of the outer case, to which the edges of the spiral fit closely, but need not be attached; $c, c$, are two of the circular plates of copper, connected together by rivets at the edges, in the manner shown, or by any other suitable means; $d$, is the chamber, formed by the two sheets of copper, and which is carried round from top to bottom in a spiral or circular inclined plane, by a succession of circular plates connected to each other. 
The hot fluid is admitted into the spiral chamber $d$, through a trumpet or wide.
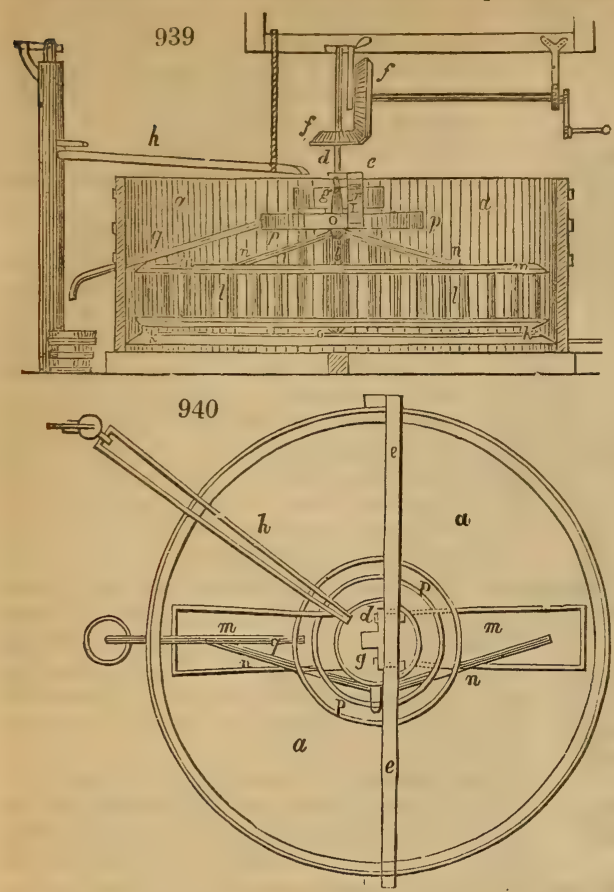

mouthed tube $e$, at top, and is discharged at bottom by an aperture and cock $f$. The cold water which is to be employed as the cooling material, is to be introduced through the pipe $g$, in the centre, from whence discharging itself by a hole at bottom, the cold water occupies the interior of the cylindrical case $b$, and rises in the spiral passage $h$, between the coils of the chamber, until it ascends to the top of the vessel, and then it flows away by a spout $i$, seen in fig. 936.

It will be perceived that the hot fluid enters the apparatus at top, and the cold fluid at bottom, passing each other, by means of which an interchange of temperatures takes place through the plates of copper, the cooling fluid passing off at top in a heated state, by means of the caloric which it has abstracted from the hot fluid; and the hot fluid passing off through the pipe and cock at bottom, in a very reduced state of temperature, by reason of the caloric which it held having been given out to the cooling fluid.

Fig. 939 is a side view and section of Wagenmann's apparatus for cooling worts; fig. 940 , a view from above. The preceding contrivances seem to be far preferable.

$a, a$, is the tub for receiving the apparatus, whose central upright shaft $b$, rests upon a step $c$, in the bottom, and revolves at top in a bush at $d$, made fast to a bar $e$, fixed flat across the mouth of the tub. The shaft may be driven by the two bevel wheels $f, f$, at right angles to each other, and the horizontal rod turned by hand; or the whole may be impelled by any power. $g$, is an iron basin for receiving the cold water from the spout $h$, supplied by a well; it flows out of the basin through two tubes $i, i$, down into the lower part of the cooler $k k$. The cooler consists of two flat vessels, both of which are formed of a flat interior plate, and an arched exterior one, so that their transverse section is planoconvex. The water which flows along the tubes $i i$, spreads itself upon the bottom of the cooler, and then rises through the scabbard-shaped tubes $l l$, sc., into the upper annular vessel $m \mathrm{~m}$; whence it is urged by hydrostatic pressure, in a now heated state, through the slanting tubes $n n$, which terminate in the common pipe 0 , of the annular basin $p p$, and is thence discharged by the pipe $q$. The basin $p p$, is supported br the two bearers $r$, made fast to the cross-beam $e$. There is in the lowest part of the hollow ring at bottom, a screw plug, which may be opened when it is desired to discharge the whole contents, and to wash it with a-stream of water.

REGULUS is a term introduced by the alchemists, now nearly obsolete. It means literally a little king, and refers to the metallic state as one of royalty, compared with the native earthy condition. Antimony is the only metal now known by the name of regulus.

RESINS (Résines, Fr.; Harze, Germ.), are proximate principles found in most vege tables, and in almost every part of them; but the only resins which merit a particular de scription, are those which occur naturally in such quantities as to be easily collected or extracted. They are obtained chiefly in two ways, either by spontaneous exudation from the plants, or by extraction by heat and alcohol. In the first case, the discharge of resin in the liquid state is sometimes promoted by artificial incisions made in summer through the bark into the wood of the tree.

Resins possess the following general properties:-They are soluble in alcohol, insoluble in water, and melt by the application of heat, but do not volatilize without partial decomposition. They have rarely a crystalline structure, but, like gums, they 
seldom affect any peculiar form. They are almost all translucid, not often coloriess, sut generally brown, occasionally red or ureen. Any remarkable taste or smell which hey sonetimes possess, may be aseribed to some foreign matter, commonly an essential il. Their specific gravity varies from 0.92 to 1.2 . Their consistence is also very variable. The greater part are hard, with a vitreous fracture, and so brittle as to be calily pulverized in the coal. Some of them are soft, a circumstance probably do. renlant upon the presence of a heterogeneous substance. The hard resins do not zonutuct electricity, and they become negatively electrical by friction. When heated, they melt more or less easily into a thick viscid liquid, and concrete, on cooling, into a mooth shining mass, of a vitreous fracture, which occasionally flies off into pieces, like Prince Rupert's drops; especially after being quickly cooled, and scratched with a sharp point. They talie fire by contact of an ignited body, and burn with a bright flame, and the diffusion of much sooty smoke. When distilled by themselves in close vessels, they afford carbonic acid and carbureted gases, empyreumatic oil of a less lisagreeable smell than that emitted by other such oils, a little acidulous water, and a very little shining charcoal. See Rosin Gas.

Resins are insoluble in water, but dissolve in considerable quantities in alcoliol, bcth hot and cold. This solution reddens tincture of litmus, but not sirup of violets; it is decomposed by water, and a milkiness ensues, out of which the particles of the resin gradually agglomerate. In this state it contains water, so as to be soft, and easily knearled between the fingers; but it becomes hard and brittle again when fieed by fusion from the water. The resins dissolve in ether and the volatile oils, and, with the aid of heat, combine with the unctuous oils. They may be combined by fusion with sulphur, and with a little phosphorus. Chlorine water bleaches several colored resins, if they be diffused in a milky state through water. The carburet of sulphur dissolves them.

Resins are little acted upon by acids, except by the nitric, which converts them into artificial tan. They combine readily with the alkalis and alkaline earths, and form what were formerly reckoned soaps : but the resins are not truly saponified; they rather represent the acid constitution themselves, and, as such, saturate the salifiable bases.

Every resin is a natural mixture of several other resins, as is the case also with oils; one principle being soluble in cold alcohol, another in hot, a third in ether, a fourth in oil of turpentine, a fifth in naptha, \&c. The soft resins, which retain a certain portion of volatile oil, constitute what are called balsams. Certain other balsams contain benzoic acid. The solid resins are, amber, anime, benzoin, colophony (common rosin), copal, dummara, dragon's blood, elemi, guaiac, lac, resin of jalap, ladanum, mastic, sandarach, storax, tekamahac.

RESIN, KATRI or COWDEE, is a new and very peculiar substance, recently im. ported in considerable quantities from New Zealand, which promises to be useful in the arts. It oozes from the trunk of a noble tree called Dammara australis, or Pinus kauri, which rises sometimes to the height of 90 feet without a branch, with a diameter of 12 feet. and furnishes a log of heart timber of 11 feet. The resin, which is called Cowdee gum by the importers, is brought to us in pieces varying in size from that of a nutmeg to a block of 2 or 3 cwts. The color varies from milk-white to amber, or even deep brown; some pieces are transparent and colorless. In hardness it is intermediate between copal and resin. The white milky pieces are somewhat fragrant, like elemi. Specific gravity, 1.04 to 1.06 . It is very inflammable, burns all away with a clear bright flame, but does not drop. When cautiously fused, it concretes into a transparent hard tough mass, like shellac. It affords a fine varnish with alcohol, being harder and less colored than mastic, while it is as soluble, and may be had probably at one tenth of the price. A solution in alcohol, mixed with one fourth of its bulk of a solution in oil of turpentine, forms an excellent varnish, which dries quickly, is quite colorless, clear and hard. It is insoluble in pyro-acetic (pyroxilic?) spirit. Combined with shellac and turpentine, it forms a good sealing-wax.

\section{REVERBERATORY FURNACE; see COPPER, IRON, and SODA.}

RETORT. For producing coal gas, there are many modifications, varying in dimension and shape with the caprice of the constructor, and in many cases without any definite idea of the principle to be aimed at.

They may be divided into three general classes :

1st. The circular retort, from twelve to twenty inches in diameter, and from six to nine feet in length. This retort is used in Manchester and some other places, in general for the distillation of cannel, or Scotch parrot coal. It answers for the distillation of a coal which retains its form in lumps, and is advantageous only from the facility with which its position is changed, when partially destroyed by the action of fire on the under side.

2d. The small or London $\mathrm{D}$ retort, so called in consequence of its having first Jeen used by the chartered company in Loncion, being still in use at their works, and re- 
cornmendel by their engineer. This retort is 12 inches broad on the base, 11 inches high, and 7 feet long, carbonizing one and a half to two bushels at a charge.

3d. The York D retort, (so called in consequence of its having been introduced by Mr. Outhit, of York,) and the modifications of it, ainnng which I should include the elliptic retort, as having the same general purpose in view. The difference between the London and York D retorts, consists only in an extension of surface upon which the coal is spread. See Gas-LIGHT.

RHODIUM, is a metal discovered by Dr, Wollaston in 1803, in the ore of platinum. It is contained to the amount of three per cent. in the platinum ore of Antioguia in Colombia, near Barbacoas; it occurs in the Ural ore, and, alloyed with gold, in Mexico. The palladium having been precipitated from the muriatic solution of the platinum ore previously saturated with soda, by the cyanide of mercury, muriatic acid is to be poured into the residuary liquid, and the mixture is to be evaporated to dryness, to expel the hydrocyanic acid, and convert the metallic salts into chlorides. The dry mass is to be reduced to a very fine powder, and washed with alcohol of specific gravity 0.837 . This solvent takes possession of the double chlorides which the sodium forms with the platinum, iridium, copper, and mercury, and does not dissolve the double chloride of rhodium and sodium, but leaves it in the form of a powder, of a fine dark-red color. This salt being washed with alcohol, and then exposed to a very strong heat, affords the rhodium. But a better mode of reducing the metal upon the small scale, consists in heating the double chloride gently in a glass tube, while a stream of hydrogen passes over it, and then to wash away the chloride of sodium with water.

Rhodium resembles platinum in appearance. Any heat which can be produced in a chemical furnace is incapable of fusing it; and the only way of giving it cohesive solidity, is to calcine the sulphuret or arseniuret of rhodium in an open vessel at a white heat, till all the sulphur or arsenic be expelled. A button may thus be obtained, somewhat spongy, having the color and lustre of silver. According to Wollaston, the specific gravity of rhodium is 11 . It is insoluble by itself in any acid; but when an alloy of it with certain metals, as platinum, copper, bismuth, or lead, is treated with aqua regia, the rhodium dissolves along with the other metals; but when alloyed with gold or silver it will not dissolve along with them. It may, however, be rendered very soluble by mixing it in the state of a fine powder with chloride of potassium or sodium, and heating the mixture to a dull-red heat, in a stream of chlorine gas. It thus forms a triple salt, very soluble in water. The solutions of rhodium are of a beautiful rose color, whence its name. In the dry way, it dissolves by heat in bisulphate of potassa; and disengages sulphurous acid gas in the act of solution. There are two oxydes of rhodium. Rhodium combines with almost all the metals; and, in small quantity, melted with steel, it has been supposed to improve the hardness, closeness, and toughness of this metal. Its chief use at present is for making the inalterable nibs of the so-named rhodium pens.

RIBAND MANUFACTURE, is a modification of WEAVING, which see.

RICE, of Carolina, analyzed by Braconnot, was found to be composed of starch 35.07 , of gluten 3.60 , of gum 0.71 , of uncrystallizable sugar 0.29 , of a colorless rancid at like suet $0 \cdot 13$, of vegetable fibre 4.8 , of salts with potash and lime bases 0.4 , and 5.0 cf water.

The quantity of rice entered for home consumption in the year 1836, was-

$$
\text { Cwts. } 81,610 \text {. In } 1837,126,739 .
$$

Ditto in the husk, Bushels 292,444. 282,377 .

Rice Paper, as it is called, on which the Chinese and Hindoos paint flowers so prettily, is a membrane of the bread-fruit tree, the Artocarpus incisifolia of naturalists.

RICE CLEANING. Various machines have been contrived for effecting this purpose, of which the following, secured by patent to $\mathrm{Mr}$. Melvil Wilson, in 1826, may be regarded as a good specimen. It consists of an oblong hollow cylinder, laid in an inclined position, having a great many teeth stuck in its internal surface, and a central shaft also furnished with teeth. By the rapid revolution of the shaft, its teeth are carried across the intervals of those of the cylinder with the effect of parting the grains of rice, and detaching whatever husks or impurities may adhere to them. A hopper is set above to receive the rice, and conduct it down into the cleansing cylinder.

About 80 teeth are supposed to be set in the cylinder, projecting so as to reach very nearly the central shaft; in which there is a corresponding number of teeth, that pass freely between the former.

The cylinder is shown inclined in the figure which accompanies the specification; but it may be placed also upright or horizontal, and may be mounted in any convenient frame-work. The central sia.t should be put in rapid rotation, while the cylinder receives a slow motion in the opposite direction. The rice, as cleaned by that action, is discharged at the lower end of the cylinder, where it falls into a shute (shoot), and is ccnducted to the ground. The machine may be driven by hand, or by any other convenient power. 
Rice consists chiefy of starch, and therefore cannot by itself make a proper bread. It is used in the cotton factories to form weavers' dressings for warps. The Chinese reduce its flour into a puly with hot water, and mould it into figures and plates, which they afterwards harden, and ornament with engravings, resembling those of mother-of-pearl. When a decoction of rice is fermented and distilled, it affords the scri of ardent spirit called arrack in the East Indies.

RIFLE; see FIRE-ARMS.

RINSING MACHINE is one of those ingenious automatic contrivances for economizing labor, and securing uniformity of action, now so common in the factories

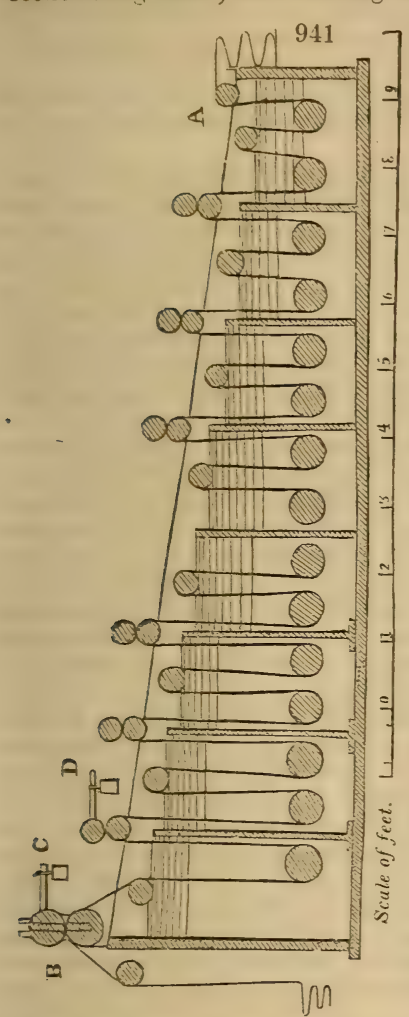
of Lancashire. Fig. 941 is a longitudinal middle section of an approved mechanism for rinsing pieces of calico dyed with spirit or fancy colors, and which require more delicate treatment than is compatible with hand-washing. A, E, F, B, is a wooden cistern, about 12 feet long, 4 feet high at one end, 2 feet at the other, and of the ordinary width of calico cloth. It is divided transversely into a series of equal compartments by partitions, decreasing in height from the upper to the lower end, the top of each of them, however, being an inch at least under the top of the enclosing side at its line of junction. Above the highest end of the trough, a pair of squeezing rollers is mounted at $\mathbf{B}$; the lower one having a pulley upon the end of its shaft, for turning it, by means of a band from one of the drivingshafts of the factory; and the upper one is pressed down upon it by weighted levers acting on the ends of its axis. The roller above the second highest partition has also a pair of squeezing rollers, with a weighted lever D. The pieces of cloth, stitched endwise, being laid upon a platform to the right hand of the cistern, are introduced over the roller A, passed down under the roller beneath it, and so up and down in a serpent-like path, from the lowest compartment of the cistern to the uppermost, being drawn through the series by the traction of the rotatory roller at $B$. While the long web is thus proceeding upwards from $A$ to $B$, a stream of pure water is made to flow along in the opposite direction from $B$ to $A$, running over the top of each partition in a thin sheet. By this contrivance, the goods which enter at $A$, having much loose color upon their surface, impregnate the water strongly, but as they advance they continually get cleaner by the immersion, and pressure of the successive rollers, being exposed to purer water, till at last they reach the limpid stream, and are discharged at $\mathbf{B}$ perfectly bright. The rinsing operation may be modified by varying the quantity of water admitted, the speed with which the pieces are drawn through the cells, or the pressure apon the series of top rollers.

ROCKETS. M. de Montgers, captain of a frigate in the French service, has written a Truité sur les Fusćes de Guerre, in which he discusses the merits of the Congreve rockets, and describes methods of imitating them. As the subject of military projectiles is foreign to :his Dictionary, I refer my readers to the above work, which is commended by the editor of the Dictionnaire Technologique.

ROLLING-MiLL. See Iron, Mint, and Plated Manufacture.

ROPE-MAKING. The fibres of hemp which compose a rope, seldom exceed in length three feet and a half, at an average. They must, therefore, be twined together so as to unite them into one; and this union is effected by the mutual circumtorsion of the two fibres. If the compression thereby produced be too great, the strength of the fibres at the points where they join will be diminished; so that it becomes a matter of great consequence to give them only such a degree of twist as is essential to their union.

The first part of the process of rope-maling by hand, is that of spinning the yarns or threads, which is done in a manner analogous to that of ordinary spinning. The spinner carries a bundle of dressed hemp round his waist; the two ends of the bundlc being assembled in front. Having drawn out a proper number of fibres with his hand, he twists them with his fingers, and fixing this twisted part to the hook of a whirl, which 
is driven by a wheel put in motion by an assistant, he walks backwards down the ropewalk, the twisted part always serving to draw out more fibres fiom the bundle round his waist, as in the flax-spinning wheel. The spinner takes care that these fibres are equably supplied, and that they always enter the twisted parts by their ends, and never by their middle. As soon as he has reached the termination of the walk, a second spinner takes the yarn off the whirl, and gives it to another person to put upon a reel, while he himself attaches his own hemp to the whill hook, and proceeds down the walk. When the person at the reel begins to turn, the first spinner, who has completed his yarn, holds it firmly at the end, and adrances slowly up the walk, while the reel is turning, lieeping it equally tight all the way, till he reaches the reel, where he waits till the second syinner takes his yarn ofl the whirl hook, and joins it to the end of that of the first spinner, in order that it may follow it on the reel.

The next part of the process previous to tarring, is that of warping the yarns, or stretching them all to one length, which is about 200 fathoms in full-length rope-grounds, and also in putting a slight turn or twist into them.

The third process in rope-making, is the tarring of the yarn. Sometimes the yarns are made to wind off one reel, and, having passed through a vessel of hot tar, are wound upon another, the superfluous tar being removed by causing the yarn to pass through a hole surrounded with spongy oakum; but the ordinary method is to tar it in skeins or hanks, which are drawn by a capstan with a uniform motion through the tar-keltle. In this process, great care must be taken that the tar is boiling neither too fast nor tou slow. Yarn for cables requires more tar than for hawser-laid ropes; and for standing and running rigging, it requires to be merely well covered. Tarred cordage has been found to be weaker than what is untarred, when it is new; but the tarred rope is not so easily injured by immersion in water.

The last part of the process of rope-making, is to lay the cordage. For this purpose two or more yarns are attached at one end to a hook. The hook is then turned the contrary way from the twist of the individual yarn, and thus forms what is called a strand. Three strands, sometimes four, besides a central one, are then stretched at length, and attached at one end to three contiguous but separate hooks, but at the other end to a single hook; and the process of combining them together, which is effected by turning the single hook in a direction contrary to that of the other three, consists in so regulating the progress of the twists of the strands round their common axis, that the three strands receive separately at their opposite ends just as much twist as is taken out of them by their twisting the contrary way, in the process of combination.

Large ropes are distinguished into two main classes, the cable-laid and havser-laid. The former are composed of nine strands, namely, three great strands, each of these consisting of three smaller secondary strands, which are individually formed with an equal number of primitive yarns. A cable-laid rope eight inches in circumference, is made up of 333 yarns or threads, equally divided among the nine secondary strands. A hauser-laid rope consists of only three strands, each composed of a number of primitive yarns, proportioned to the size of the rope; for example, if it be eight inches in circumference, it may have 414 yarns, equally divided among three strands. Thirty fathoms of yarn are reckoned equivalent in length to eighteen fathoms of rope cable-laid, and to twenty fathoms hawser-laid. Ropes of fiom one inch to two inches and a half in circumference are usually hawser-laid; of from three to ten inches, are either hawser or cable-laid; but when more than ten inches, they are always cable-laid.

Every hand-spinner in the dock-yard is required to spin, out of the best hemp, six threads, each 160 fathoms long, for a quarter of a day's work. A hawl of yarn, in the warping process, contains 336 threads.

The following are Captain Huddart's improved principles of the rope manufacture :-

1. To keep the yarns separate from each other, and to draw them from bobbins revolving upon sliewers, so as to maintain the twist while the strand or primary cord is forming.

2. To pass them through a register, which dirides them by circular shells of holes; the number in each concave shell being conformable to the distance from the centre of the strand, and the angle which the yarns make with a line parallel to it, and which gives them a proper position to enter.

3. To employ a tube for compressing the strand, and preserving the cylindrical figure of its surface.

4. To use a gauge for determining the angle which the yarns in the outside shell make with a line parallel to the centre of the strand, when registering; because according to the angle made by the yarns in this shell, the relative lengths of all the yarns in the strand will be determined.

5. To harden up the strand, and thereby increase the angle in the outside shell; which compensates for the stretching of the yarns, and the compression of the strands.

A great many patents have been obtained, and worked with various degrees of success, for rnaking ropes. Messrs. Cartwright, Fothergill, Curr, Chapman, Balfour, and Hud. 
dart, have been the most conspicuous inventors in this country; but the limits of this work preclude us doing justice to their respective merits.

All the improvements in the manufacture of cordage at present in use, either in her Majesty's yards or in private rope-grounds, owe their superiority over the old meihod of maling cordage to Captain Huddart's invention of the register plate and tube.

Mr. Balfour took out a patent for the manufacture of cordage about a month before Captain Huddart; hut the formation of his strand was to be accomplished by what he called a top minor (in the form of a common top, with pins to divide the yarns), which upon trial could not make cordage so good as by the common mode. On seeing Captain Huddart's specification, Mr. Balfour, five years after, procured another patent, in which he included a plate and tube, but which was not sufficiently correct, and experience in the navy prored the insufficiency of the cordage. Captain Huddart's plate and tube were then adopted in the king's yards, and he gave his assistance for the parpose.

Captain Huddart then inrented and took a patent for a machine, which by registering the strand at a short length from the tube, and winding it up as made, preserved a uniformily of twist, or angle of formation, from end to end of the rope, which cannot be accomplished by the method of forming the strands down the ground, where the twist is communicated from one end to the other of an elastic body upwards of 300 yards in length. This registering-machine was constructed with such correctness, that when some were afterwards required, no alteration could be made with advantage by the most skilful and scientific mechanic of that day, Mr. Rennie. Thus the cold register was carried to the greatest perfection.

A number of yarns cannot be put together in a cold state, without considerable vacancies, into which water may gain admission; Captain Huddart, therefore, formed the yarns into a strand immediately as they came from the tar-kettle, which he was enabled to do br his registering-machine, and the result was most satisfactory. This combination of yarns was found by experiment to be 14 per cent. stronger than the cold register; it constituted a body of hemp and tar impervious to water, and had great advantage over any other cordage, particularly for shrouds, as after they were settled on the mast-head, and properly set up, they had scarcely any tendency to stretch, effectually secured the mast, and enabled the ship to carry the greatest press of sail.

In order more effectually to obtain correctness in the formation of cables and large cordage, Cantain Huddart constructed a laying-machine, which has carried his inventions in rope-making to the greatest perfection, and which, founded on true mathematical principles, and the most laborious calculations, is one of the noblest monuments of mechanical ability since the improvement of the steam-engine by $\mathrm{Mr}$. Watt. By this machine, the strands receive that degree of twist only which is necessary, and are laid at any angle with the greatest regularity; the pressure is regulated to give the required elasticity, and all parts of the rope are made to bear equally. In no one instance has a rope or cable thus formed been found defective in the lay, or stiff, or difficult to coil.

Such a revolution in the manufacture of cordage could not be accomplished without great expense, as the works at Limehouse fully testify; and considerable opposition necessarily arose. Captain Huddart's first invention was, however, generally adopted, as soon as the patent expired; and experience has established the great importance of his subsequent improvements.

His cordage has been supplied in large quantities to her Majesty's navy, and has received the most satisfactory reports.

The following description of one of the best modern machines for making ropes on Captain Huddart's plan, will gratify the intelligent reader.

$F i g .942$ exhibits a side elevation of the tackle-board and bobbin-frame at the head

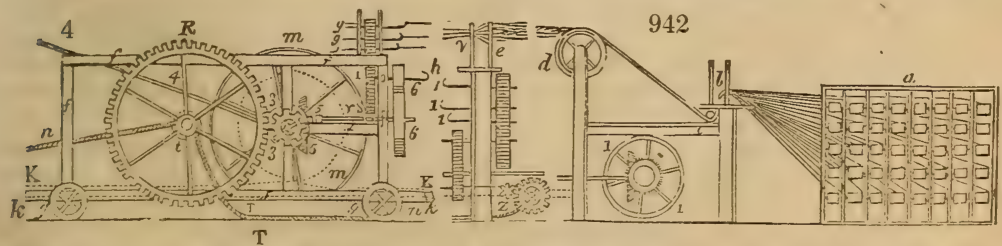

of the ropes, and also of the carriage or rope-machine in the act of hauling out and twisting the strands.

F'ig. 943 is a front elevation of the carriage.

Fig. 944 is a yarn-guide, or board, or plate, with perforated holes for the yarns to pass through before entering the nipper.

Figs. 945 and 946 are side and front views of the nipper for pressing the ropeyarns. 
$a$ is he frame for containing the yarn bohbins. The yarns are brought from the frame, and pass through a yarn-guide at $b . \quad c$ is a small roller, under which the ropeyarns pass; they are then brought over the reel $d$, and through another yarn-guide $e$, after which they enter the nippers at $v$, and are drawn out and formed into strands by the carriage. The roller and reel may be made to traverse up and down, so as to regulate the motion of the yarns.

The carriage runs on a railway. $f, f$, is the frame of the carriage; $g, g$, are the small wheels on which it is supported; $k, k$, is an endless rope, reaching from the head to
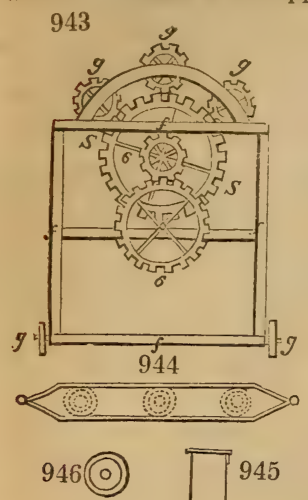
the bottom of the railway, and is driven by a steam-engine; $m, m$, is a wheel with gubs at the back of it, over which the endless rope passes, and gives motion to the machinery of the carriage. $n$, is the ground rope for taking out the carriage, as will be afterwards described. On the shaft of $m, m$, are two bevel wheels 3,3 , with a shifting catch between them; these bevel wheels are loose upon the shaft, but when the catch is put into either of them, this last then keeps motion with the shaft, while the other runs loose. One of these wheels serves to communicate the twist to the strand in drawing out; the other gives the opposite or after turn to the rope in closing. 4,4 , is a lever for shifting the eatch accordingly. 5, is a third bevel wheel, which receives its motion from either of the other two, and communicates the same to the $t$ wo spur wheels 6,6 , by means of the shaft $x$. These can be shifted at pleasure; so that by applying wheels of a greater or less number of teeth above and beneath, the twist given to the strands can be increased or diminished accordingly. The upper of these two communicates motion, by means of the shatt 0 , to another spur wheel 8 , which working in the three pinions above, 9,9 , gives the twist to the strand hooks.

The carriage is drawn out in the following manner. On the end of the shaft of $m, m$, is the pinion 3, which, working in the large wheel $\mathrm{R}$, gives motion to the ground-rope shaft upon its axis. In the centre of this shaft is a curved pulley or drum $t$, round which the ground-rope takes one turn. This rope is fixed at the head and foot of the ropery ; so that when the machinery of the carriage is set a-going by the endless rope $k$, $k$, and gives motion to the ground-rope shaft, as above described, the carriage will necessarily move along the railway; and the speed may be regulated either by the diameter of the circle formed by the gubs on the wheel $m, m$, or by the number of teeth in the pinion 3. At $T$, is a small roller, merely for preventing the ground-rope from coming up among the machinery. At the head of the railway, and under the taclile-board, is a wheel and pinion $z$, with a crank for tightening the ground-rope. The fixed machinery at the head, for hardening or tempering the strands, is similar to that on the carriage, with the exception of the ground-rope year, which is unnecessary. The motion is communicated by another endless rope (or short band, as it is called, to distinguish it from the other), which passes over gubs at the back of the wheel 1,1 .

When the strands are drawn out by the carriage to the requisite length, the spur wheels $3, \mathrm{R}$, are put out of gear. The strands are cut at the tackle-board, and fixed to the hooks $1,1,1$; after which they are hardened or tempered, being twisted at hoth ends. When this operation is finished, three strands are united on the large hook $h$, the top put in, and the rope finisherl in the usual way.

In preparing the hemp for spinning and ordinary thread or rope yarn, it is only heckled over a large keg or clearer, until the fibres are siraightened and separated, so as to run freely in the spinning. In this case, the hemp is not stripped of the tow, or cropped, unless it is designed to spin beneath the usual grist, which is about 20 yarns for the strand of a three-inch strap-laid rope. The spinning is still performed by harid, being found not only to be more economical, but also to make a smoother thread, than has yet been effecied by machinery. Various ways have been tried for preparing the yarns for tarring. That which seems now to be most generally in use, is, to warp the yarns upon the stretch as they are spun. This is accomplished by having a wheel at the foot, as well as the head of the walk, so that the men are able to spin both up and down, and also to splice their threads at both ends. By this means, they are formed into a haul, resemb ing the warp of a common web, and a little turn is hove into the haul, to preserve it from getting foul in the tarring. The advantages of warping from the spinners, as above, instead of winding on winches, as formerly, are, 1st, the saring of this last operation altogether; 2dly, the complete check which the foreman has of the quantity of yarn spun in the day; 3dly, that the quality of the work can be subjected to the minutest inspection at any time. In tarring the yarn, it is found favorahle to the fairness of the strip, to allow it to pass around or under a reel or roller in the 
bottom of the kettle while boiling, instead of coiling the yarn in by hand. The tar is then pressed from the yarn, by means of a sliding nipper, with a lever over the upper part, and to the end which the necessary weight is suspended. The usual proportion of tar in orlinary ropes, is something less than a fifth. In large strap-laid ropes, which are necessirily subjected to a greater press in the laying of them, the quantity of tar can scarcely exceed a sixth, without injuring the appearance of the rope when laid.

For a long period, the manner of laying the yarns into ropes, was by stretching the haul on the rope-ground, parting the number of yarns required for each strand, and wisting the strands at both ends, by means of hand-hooks, or cranks. It will be ubrious that this method, especially in ropes of any considerable size, is attended with serious disadvantages. The strand must always be very uneven; but the principal disidvantage, and that which gave rise to the many attempts at improvement, was, that he yarns being all of the same length before being twisted, it frlowed, when the rope was finished, that while those which occupied the circumference of the strand were perfectly tight, the centre yarns, on the other hand, as they were now greatly slackened by the $o_{\text {; }}$ eration of hardening or twisting the strands, would actually bear little or no part of the strain when the rope was stretched, until the former gare way. The method lisplayed in the preceding figures and description, is among the latest and most -mproved. Every yarn is given out from the bobbin frame as it is required in twisting the rope; and the twist communicated in the out-going of the carriage, can be increased or diminished at pleasure. In order to obtain a smooth and well-filled strand, it is necessary also, in passing the yarns through the upper board, to proportion the number of centre to that of outside yarns. In ordinary sized ropes, the strand seems to hare the fairest appearance, when the outside yarns form from $\frac{2}{3} \mathrm{ds}$ to $\frac{3}{4}$ ths of the whole quantity, in the portion of twist given by the carriage in drawing out and forming the strands.

In laying cables, torsion must be given both behind and before the laying top. Figs. $947,948,949$ represent the powerful patent apparatus employed for this purpose. A, is

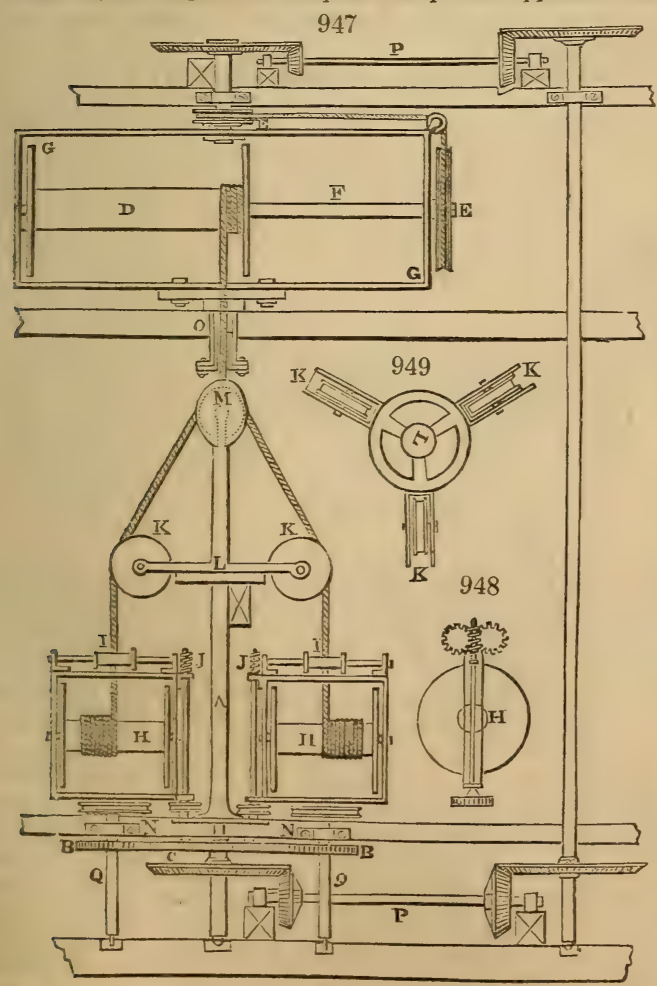
a strong upright iron pillar, supported upon the great horizontal beam $\mathrm{N}, \mathrm{N}$, and bearing at its upper end the three-grooved laying top $\mathbf{m}$. $\mathrm{H}, \mathrm{H}$, are two of the three great bobbins or reels round which the three secondary strands or small haw sers are wound. These are drawn up by the rotation of the three feeding rollers I, I, I, thence proceed over the three guide pulleys $\mathbf{K}, \mathbf{k}, \mathbf{K}$, towards the laying top $M$, and finally pass through the tube 0 , to be wound upon the cablereel $\mathrm{D}$. The frames of the three bobbins $\mathrm{H}, \mathrm{H}, \mathrm{H}$, do not revolve about the fast pillar $A$, as a common axis; but each bobbin revolves round its own shaft $Q$, which is steadied by a bracing collet at $\mathrm{N}$, and a conical step at its bottom. The three bobbins are placed at an angle of 120 degrees apart, and each receives a rotatory motion upon its axis from the toothed spar wheel $B$, which is driven by the common central spur wheel c. Thus each of the three secondary cords has a proper degree of twist put into it in one direction, while the cable is laid, by getting a suitable degree of twist in an opposite direction, from the revolution of the frame or cage $G, G$, round two pivots, the one under the puller $E$, and the other over o. 
The reel $\mathrm{D}$ has thus, like the bobbins $\mathrm{H}, \mathrm{H}$, two movements; that in common with ite frame, and that upon its axis, produced by the action of the endless band round the pulley $\mathbf{E}$, upon one of its ends, and the pulley $\mathrm{E}^{\prime}$ above its centre of rotation. The pulley $\mathrm{E}$ is driven by the bevel mill-gearing $P, P, P$, as also the uniler spur-wheel c. $\quad L$, in fig. 949 , is the place of the ring $\mathrm{L}$, fig. 947 , which bears the three guide pulleys $\mathrm{K}, \mathrm{K}, \mathrm{K}$. Fig. 948 is an end view of the bobbin $\mathrm{H}$, to show the worm or endless screw $\mathrm{J}$, of fig. 949 , working into the two snail-toothed wheels, upon the ends of the two feed-rullers $I$, $I$, which serve to turn them. The upright shafts of $\mathrm{J}, \mathrm{J}$, receive their motion from pulleys and cords near their bottom. Instead of these pulleys, and the others $\mathbf{E}, \mathbf{E}$, bevel-wheel gearing has been substituted with advantage, not being liable to slip, like the pulley-band mechanism. The axis of the great reel is made twice the length of the brobin $\mathrm{D}$, in order to allow of the latter moving from right to left, and hack again alternately, in winding on the cable with uniformity as it is laid. The traverse mechanism of this part is, for the sake of perspicuity, suppressed in the figure.

Mr. William Norvell, of Newcastle, obtained a patent in May, 1833, for an improvement adapted to the ordinary machines employed for twisting hempen yarns into strands, affording, it is said, a simpler and more eligible mode of accomplishing that object, and also of laying the strands together, than has been hitherto effected by machinery. The yarns spun from the fibres of heinp are wound upon bobbins, and these bubbins are mounted upon axles, and hung in the frame of the machine, as shown in the elevation, fig. 950, from which bobbins the sereral ends of yarn are passed upwards through slanting tubes; by the rotation of which tubes, and of the carriages in which the bobbins are suspended, the yarns become twisted into strands, and also the strands are laid so as to form ropes.

His improvements consist, first, in the application of three or more tubes, two of which are shown in fig. 950, placed in inclined positions, so as to receire the strands immediately above the press-block $a, a$, and nearly in a line with $\mathrm{A}$, the point of closing or laying the rope. $\mathbf{B}^{\prime}$, and $\mathbf{B}_{3}$, are opposite side views; $\mathbf{B} 2$, an edge view; and $\mathbf{B}$, a side section of the same. He does not claim any exclusive right of patent for the tubes themselves, but only for their form and angular position.

Secondly, in attaching two common flat sheaves, or pulleys, c, c, fig. 950, to each

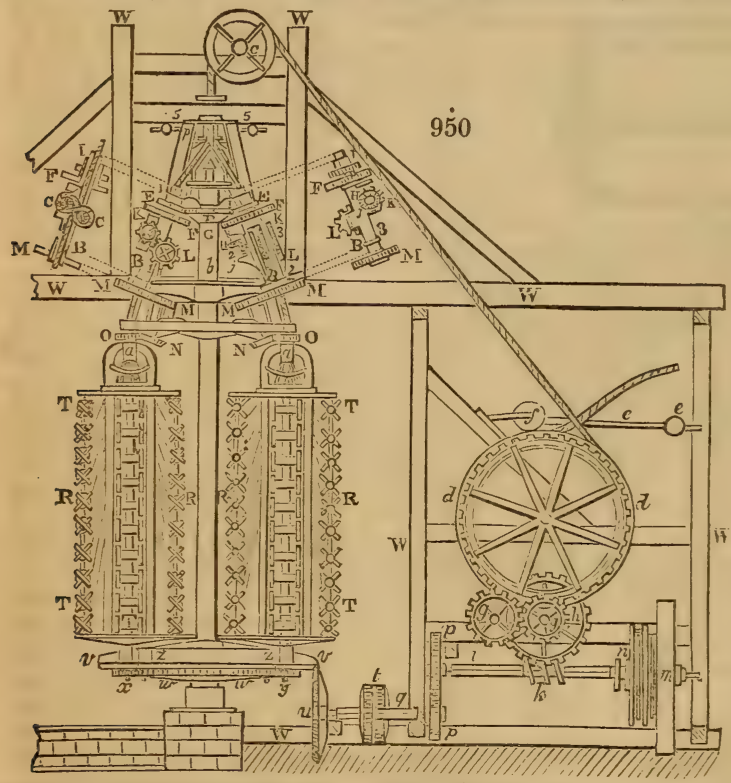
of the said tubes, nearly round which each strand is lapped or coiled, to prevent it from slipping, as shown in the section $\mathbf{B}^{\prime}$. The said sheaves or pulleys are connected by $\mathbf{a}$ crown or centre wheel $D$, loose upon $b, b$, the main or upright axle; $\mathbf{E}, \mathbf{E}$, is a smaller wheel upon each tuhe, working into the said crown or centre wheel, and fixed upon the loose box $I$, on each of the tubes.

$F, F$, is a toothed or spur wheel, fixed also upon each of the loose boxes I, and working into a smaller wheel $\mathrm{G}$, upon the axis 2 , of each tube; $\mathrm{H}$, is a bevel wheel fixed upon the same axis with G, and working into another bevel wheel $j$, fixed upon the cross axle 3 , of each tube; $\mathbf{k}$, is a spur wheel attached to the same axis with $j$, at the opposite end, and working into $\mathrm{L}$, another spur wheel of the same size upon each of the tubes. By wheels thus arranged and connected with the sheaves or pulleys, as above described, a perfectly equal strain or tension is put upon each strand as drawn forward over the pulley $\mathrm{c}$.

Thirdly, the invention consists in the introduction of change wheels $\mathbf{M}, \mathbf{M}, \mathbf{M}, \mathbf{M}$, fig. 50, for putting the forehard or proper twist into each strand before the rope is 
laid; this is effected by small spindles on axles 4,4 , placed parallel with the line of carh tube $\mathrm{B}$.

Upon the lower end of each spindle the bevel wheels $\mathrm{N}, \mathrm{N}$, are atlached, and driven by other bevel wheels 0 , 0 , fixed immediately above each press-block $a, \|$. On the top end of each spindle or axle 4,4 , is attached one of the change wheels, working into the other change wheel fixed upon the bottom end of each of the tubes, whereby the furchard or proper twist in the strands for all sizes of ropes, is at once attaineil, by simply changing the sizes of those two last described wheels, which can he very reqalily effected, from the manner in which they are attached to the tubes $B, B$, and 4, 4.

From the angular position of the tubes towards the centre, the strands are nearly in contact at their upper ends, where the rope is laid, immediately below which the forehard or proper twist is given to the strands.

Fourthly, in the application of a press-block $\mathbf{P}$, of metal, in two parts, placed directly above and close down to where the rope is laid at $A$, the inside of which is polished, and the under end is bell-mouthed; to prevent the rope from being chafed in entering it, a sufficient grip or pressure is put upon the rope by one or two levers and weights 5, 5, acting upon the press-block, so as to adjust any ifling irregularity in the strand or in the laying; the inside of which being polished, gives smoothness, and by the said levers and weights, a proper tension to the rope, as it is drawn forward through: the press-block. By the application of this block, ropes may be made at once properly stretched, rendering them decidedly preferable and extremely advantageous, particularly for shipping, inclined planes, mines, \&c.

The preceding description includes the whole of Mr. Norvell's improvements; the remaining parts of the machine, being similar to those now in use, may be briefly described as follows:-A wheel or pulley $c$, is fixed independently of the machine, orer which the rope passes to the drawing motion represented at the side; $d, d$, is a groored wheel, round which the rope is passed, and pressed into the groove by means of the lever and weight $e$, $e$, acting upon the binding sheaf $f$, to prevent the rope from slipping. After the rope leaves the said sheave, it is coiled away at pleasure. $g, g$, are two change wheels, for varring the speed of the grooved wheel $d, d$, to answer the various sizes of ropes; $h$, is a spiral wheel, driven by the screw $l$, fixed upon the axle $l$; $m$, is a bandwheel, which is driven by a belt from the shaft of the engine, or any other communicating power ; $n, n$, is a friction strap and striking clutch. The axle $q$, is driven by two change wheels $p, p$; by changing the sizes of those wheels, the different speeds of the drum $\mathrm{R}, \mathrm{R}$, for any sizes of ropes, are at once cffected.

The additional axle $s$, and wheels $t, t$, shown in fig. 951, are applied occasionally for

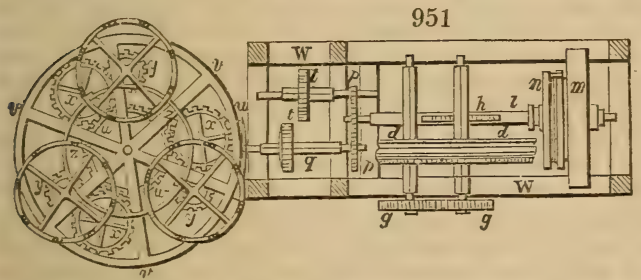
reversing the motion of the said drums, and making what is usually termed left-hand ropes; $u$, figs. 950, and 951, show a bevelled pinion, driving the main crown wheel $v, v$, which wheel carries and gives. motion to the drums $\mathbf{R}, \mathbf{R}$; $w, w$, is a fixed or sun wheel, which gives a reverse motion to the drums, as they revolve round the same, by means of the intervening wheels $x, x, x$, whereby the reverse or retrograding motion is produced, and which gives to the strands the right twist. The various retrograding motions, or right twists for all sizes and descriptions of ropes, may be obtained by changing the diameters of the pinions $y, y, y$, on the under ends of the dium spindles; the carriages of the intervening wheels $x, x, x$, being made to slide round the ring $z, z ; w, w$, is the framework of the machine and drawing motion; $T, T, T$, are the bobbins containing the yarns; their number is varied to correspond with the different sizes of the machines.

The machine here described, in elevation and plan, is calculated to make ropes from three to seven and one half inches in circumference, and to an indefinite lenoth.

Messrs. Chapman of Newcastle, to whom the art of rope-making is deeply indebted, naving observed that rope-yarn is considerably weakened by passing through the tar-kettle, that tarred cordage loses its strength progressively in cold climates, and so rapilly in hot climates as to be scarcely fit for use in three years, discovered that the deterioration was due to the reaction of the mucilage and acid of the tar. Ther accordingly proposed the following means of amelioration. 1. Boiling it with water, in order to reinove these iwo soluble constifuents. 2. Concentrating the washed tar by heat, till it becomes pitchy, and then restoring the plasticity which it thereby loses, by the addition of tallow, or animal or expressed oils.

In 1807 , the same able engineers obtained a patent for a method of making a 
belt or flat band, of two, three, or more strands of shroud or hawser-laid rope, placed side by side, so as to form a band of any desired breadth, which may be used for hoisting the kibbles and corves in mine-shafts, without any risk of its losing twist by rotation. The ropes should be laid with the twist of the one strand directed to the right hand, that of the other to the left, and that of the yarns the opposite way to the strands, whereby perfect flatness is secured to the band. This parallel assemblage of strands has been found also to be stronger than when they are all twisted into one cylinder. The patentees at the same time contrived a mechanism for piercing the strands transversely, in order to brace them firmly together with twine. Flat ropes are usually formed of hawsers with three strands, softly laid, each containing 33 yarns, which with four ropes, cnmpose a cordage four and a half inches broad, and an inch and a quarter thick, being the ordinary dimensions of the grooves in the whim-pulleys round which they pass.

Relative Strength of Cordage, shroud laid.

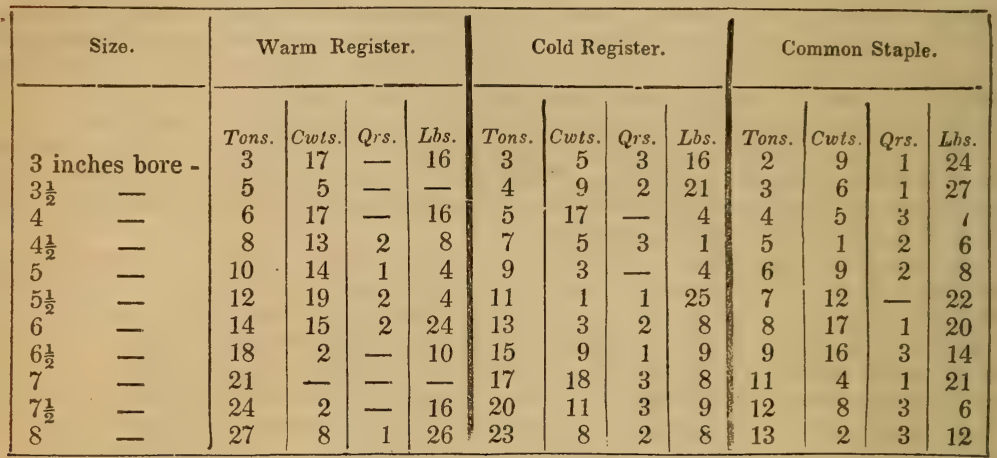

The above statement is the result of several hundred experiments.

ROSIN, or COLOPHANY (Galipot, Fr.; Fichtenharz, Germ.), is the rosin left after distilling off the volatile oil from the different species of turpentine. Yellow rosin contains some water, which black rosin does not. See Turpentine.

ROSIN GAS. Fig. 952 exhibits the retort and its appendages, as erected by Messrs. Taylor and Martineau, under the direction of the patentee, Professor Daniel, F. R. S.

I have introduced this manufacturing project, not as a pattern to imitate, but as an example to deter; as affording a very instructive lesson of the danger of rushing headlong into most extensive enterprises, without fully verifying, upon a moderate scale, the probability of their ultimate success. The capital, labor, and time annually wasted upon visionary schemes of this sort, got up by chamber chemists, are incalculably great. No more essential service could be rendered to the cause of productive industry, than to unmask the thousand and one chimerical inventions which disgrace our lists of patents during the last thirty years. These remarks have been suggested by the circumstance, that $50,000 l$. were squandered upon the rosin-gas concern; a fact communicated to me by an eminent capitalist, who was induced by fallacious statements to embark largely in the speculation. Had $100 l$. been employed beforehand, by a disnassionate practical man, in making judicious trials, and in calculating the chances of eventual profit and loss, it would have been demonstrated, as clearly as noonday, that rosin could never compete with pitcoal in the production of gas-light. Whatever ingenuity was expended in getting up the following apparatus, may be regarded as an additional ignis fu/uus to mislead the public, and divert their thoughts from the abyss that lay before them. The main preliminary to be settled, in all new undertakings, is the soundness of the principle. By neglecting this point, projectors perpetually realize the expiatory fable of the Danaïds.

The retort $e, e$, fig. 952, is seen charged with coke, which is in the first instance raised to a bright red heat, by means of the furnace beneath. The common brown rosin of commerce, which is deposited in the tank $a$, is to be mixed with the essential oil (condensed from the rosin vapors in a preceding ope: ation) in the proportion of one hundred pounds oi the former to ten gallons of the latter. The influence of the flams and heated air beneath serves to preserve this in a fluid state, and by a damper passing across the aperture in the chimney the temperature of the fluid may be exactly regulated. A wire-gauze screen at $f$, reaches to the bottom of the iann, and prevents the solid rosin, or any impurity with which it may be mixed, from choking the stopeock.

The melted rosin having passed by the stopcock $b$, funnel $c$, and syphon $d$, in 11 the retort, fails on the coke, and in its passage through the ignited mass, becomes deconposed. On arriving at the other end of the retort, a large portion of the oil of turpentine, in 
the form of condensable vapor, is separated by the refrigerator $g$; this is supplied with water from a cistern above, and the non-condensable vapor or gas passes up the tube 952

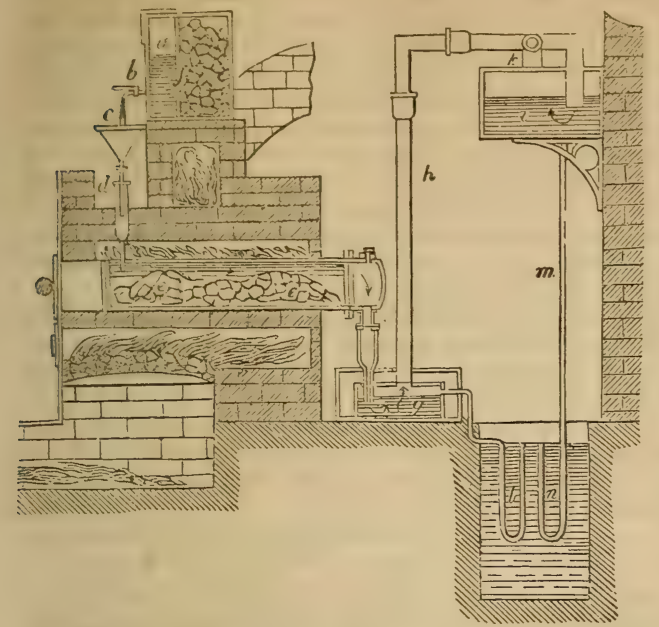
$h$, and dips beneath the sur. face of the fluid in the vessel $i$. This completes the condensation; and the gas proceeds in a perfectly pure state, by the pipe $k$, to the gasometer, or rather to the floating reservoir, for use.

The essential oil, when it leaves the refrigerator, is conveyed, by the syphon $l$, to a cistern beneath. The necessity for employing a syphon will be apparent, when it is borne in mind that the tube prevents the escape of the gas, which would otherwise pass away from the box with the essential oil. Another pipe and syphon $m, n$, serve to convey the condensed essential oil from the top cistern.

ROTTEN-STONE. See Tripoli.

ROUGE. (Fard, Fr.) The only cosmetic which can be applied without injury to brighten a lady's complexion, is that prepared, by the following process, from safflower, (Carthamus tinctorius.) The flowers, after being washed with pure water till it comes off colorless, are dried, pulverized, and digested with a weak solution of crystals of soda, which assumes thereby a yellow color. Into this liquor a quantity of finely carded white cotton wool is plunged, and then so much lemon juice or pure vinegar is added as to supersaturate the soda. The coloring matter is disengaged, and falls down in an impalpable powder upon the cotton filaments. The cotton, after being washed in cold water, to remove some yellow coloring particles, is to be treated with a fresh solution of carbonate of soda, which takes up the red coloring matter in a state of purity. Before precipitating this pigment a second time by the acid of lemons, some soft powdered talc should be laid in the bottom of the vessel, for the purpose of absorbing the fine rouge, in proportion as it is separated from the carbonate of soda, which now holds it dissolved. The colored mixture must be finally triturated with a few drops of olive oil, in order to make it smooth and marrowy. Upon the fineness of the talc, and the proportion of the safflower precipitate which it contains, depend the beauty and value of the cosmetic. The rouge of the above second precipitation is received sometimes upon bits of fine-twisted woollen stuff, called crepons, which ladies rub upon their cheeks.

RUBY. See LAPIDARY.

RUM, is a variety of ardent spirits, distilled in the West Indies, from the fermented skimmings of the sugar teaches, mixed with molasses, and diluted with water to the proper degree. A sugar plantation in Jamaica or Antigua, which makes 200 hogsheads of sugar of about $16 \mathrm{cwts}$. each, requires, for the manufacture of its rum, two ropper stills; one of 1000 gallons for the wash, and one of 600 gallons for the low wines, with corresponding worm refrigeratories. It also requires two cisterns, one of 3000 gallons for the lees or spent wash of former distillations, called dunder (Quasi redundur, Span.), another for the skimmings of the clarifiers and teaches of the sugarhouse; along with twelve, or more, fermenting cisterns or tuns.

Lees that have been used more than three or four times, are not considered to be equally fit for exciting fermentation, when mixerl with the sweets, as fresher lees. The wort is malle, in Jamaica, by arlding to 1000 gallons of dunder, 120 gallons of molasses, 720 gallons of skimmings ( $=120$ of molasses in sweetness), and 160 gallons of water; so that there may be in the liquid nearly 12 per cent. of solid saccharum. Another proportion, often used, is 100 gallons of molasses, 200 gallons of lees, 300 gallons of skimmings, and 400 of water; the mixture containing, therefore, 15 per cent. or sweets. These two formulæ prescribe so much spent wash, according to my opinion, as would be apt to communicate an unpleasant flavor to the spirits. Both the fermenting and flavoring principles reside chiefy in the fresh cane juice, and in the skimmines of the clarifier; because, after the sirup has been boiled, ther are in a great measure dissipatcd. I have made many experiments upon fermentation and distillation from West India molasses, and always found the spirits to be perfectly exempt from any rum flavor. 
The fermentation goes on most uniformly and kindly in very large masses, and requires from 9 to 15 days to complete; the difference of time depending upon the strength of the wort, the condition of its fermentable stuff, and the state of the weather. The progress of the attenuation of the wash should be examined from day to day with a hydrometer, as I have described in the article Distrllation. When it has reached nearly to its maximuin, the wash should be as soon as possible transferred by pumps into the still, and worked off by a properly regulated heat; for if allowed to stand over, it will deteriorate y acetification. Dr. Higgins's plan, of suspending a basket full of limestone in the wash tuns, to counteract the acidity, has not, I believe, been found to be of much use. It would be better to cover up the wash from the contact of atmospheric air, and to add perhaps a very little sulphite of lime to it, both of which means would tend to arrest the acetous fermentation. But one of the best precautions against the wash becoming sour, is to preserve the utmost cleanliness among all the vessels in the distillery. They should be scalded at the end of every round with boiling water and quicklime.

About 115 gallons of proof rum are usually obtained from 1200 gallons of wash. The proportion which the product of rum bears to that of sugar, in very rich moist plantations, is rated, by Edwards, at 82 gallons of the former to $16 \mathrm{cwt}$. of the latter ; but the more usual ratio is 200 gallons of rum to 3 hogsheads of sugar. But this proportion will necessarily vary with the value of rum and molasses in the market, since whicherer fetches the most remunerating price, will be brought forward in the greatest quantity. In one considerable estate in the island of Grenada, 92 gallons of rum were made for everv hogshead (16 cwts.) of sugar. See STiLL.

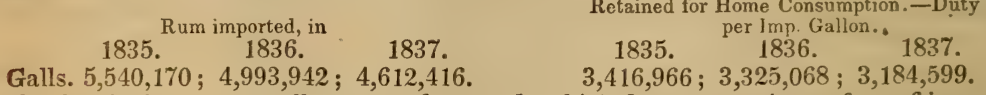

RUST, is the orange-yellow coat of peroxyde which forms upon the surface of iron exposed to moist air. Oil-paint, varnish, plumbago, or a film of caoutchouc, may be em ployed, according to circumstances, to prevent the rusting of iron utensils.

RYE, consists, according to the analysis of Einhof, of 21.2 of husk, 65.6 of flour, and 10.2 of water, in 100 parts. This chemist found in 100 parts of the flour, 61.07 of starch, $9 \cdot 48$ of gluten, 3.28 of vegetable albumen, 3.28 of uncrystallizable sugar, 11.09 of gum, 6.38 of vegetable fibre, and the loss was 5.62 , including a vegetable acid not yet investigated. Some phosphate of lime and magnesia are also present. See GrN.

SAFETY LAMP. I have reserved for this place an account of the patented improvement made upon Davy's lamp, by Messis. Upton and Roberts; the latter of whom, having worked in coal mines from a boy, and having observed, that in peculiar circumstances the Davy was insecure, was led to contrive certain modifications of it, for which he received, some years ago, a reward from the Society of Arts. It appears from undoubted experiments, that if a jet of carbureted hydrogen (coal gas for example) be impelled with very moderate force against the side of the Davy, it will first fill the wire cylinder of the burning lamp with flame, and then take fire itself exteriorly. This passage of the flame of explosive gases through the meshes of wire gauze of the fineness prescribed for safety lamps by Sir H. Davy was demonstrated in several trials before the select committee of the House of Commons on accidents in mines, by Mr. Pereira, at the London University.* While the gas is at rest, relatively to Davy's lamp, the explosion has never been known to pass; but "if," says Mr. Pereira, "a lamp be held before a jet of gas until it becomes hot (a red heat is not essential), and then gently moved, the flame will pass, and the experiment may be repeated successively a number or times in the minute." Two layers of wire gauze, though they greatly impede the transmission of light, will still permit that of flame, in the above circumstances. In Upton and Roberts' lamp, there is but one coat of wire gauze, but it is enclosed in a glass cylinder, in such a manner as to admit the air which feeds the flame only under its bottom, first through an annular range of holes, and next through one disc, or sereral, of wire gauze, fixed a little way below the wick. The explosive air, after passing up through these wire-oauze discs, enters a little hrass cupola, and is reflected inwards from the orifice at its top unon the flame, whereby it is completely burned before it reaches the cavity of the surmounting cylinder. By this reverberatory action of the air unon the wick, the intensity of the light is at the same time greatly augmented. Since the feed orifices of the lamp are small in comparison with the capacity of the surmounting cage, the latter does not get filled with flame on being plunged in an explosive gaseous mixture, as happens tc the naked cage of Davy. The wire-gauze can never, therefore, become very hut, far lesi ignited, in the new lamp. There are, in fact, three impediments to the passage of the flame 
sut of the lamp; first, the stratum of carbunic acid round the light; secondly, the wire. gauze cylinder; and thirdly, the grlass cylinder. The entrance at the bottom may be made secure in any desired degree, by multiplying the layers of wire cloth. The top is protected, moreuver, by a brass hood, throush which the currents of carbonic acid and nitrogen gases, continually ascending from the burning wick, oppose certain obstacles to the transmission of flame downwards. Even should the glass be accidentally broken, the lamp is still a complete Davy.

In the experiments made before the honorable committee at the London University, Mr. Pereira showed, first, that when a jet of coal-gas alone, or an explosive mixture of coal-gas and air, impinged upon the wire-gauze cylinder of one of Dary's lamps with a certain force, the flaine generally passed through the meshes, of which there were from 950 to 1024 in the square inch. When a mixture of four parts of hydrogen and one of coal-gas was directed in a jet upon the lighted lamps of Davy, Stevenson, Dillon, Wood of Killingworth (called the refrigerating lamp), Robson, and Clanny the flame readily passed; but when thrown upon the lamp of Upton and Roberts, it did not once pass, causing merely slight detonations within the lamp. When the force of the jet was augmented, it extinguished the light. This lamp was finally subjected to the still severer test of a mixture of four parts of atmospherical air, and one of hydrogen; yet it did not explode it. When exposed to a mixture of two thirds of air, and one of hydrogen, the lamp was immediately extinguished.

The following, out of many certificates, appears to me decisive in favor of this improve ment of Davy's lamp. It comes from an experienced pitman, in a very deep and exten sive coal mine, which I know to be replete with explosive gas, as I have myself visited it in company with its accomplished engineer, John Buddle, Esq.

"I hereby certify that I have this day tried Messrs. Upton and Roberts' new patent safety lamp, in the Jarrow colliery; and I state, as an experienced pitman, having been thirty-two years master wasteman in that colliery, that I greatly prefer this new lamp to the common Davy lamp. I had it between five and six hours on trial in the pit. I consider that it gives about three times the light of the Dary lamp, as I could see at least ten yards before me in a straight line; and of its great safety I can have no doubt, as it does not fill with flame, as the Davy does. And although I had this extra light, there was much less oil consumed. I consider it a good working lamp."

"Jarrow Colliery, near Neweastle on Tyne, March 31, 1836." (Signed) " ROBERT FAIRLY."

Fig. 953 is a vertical section through the middle of the lamp. $a, a$, is the oil-cistern,

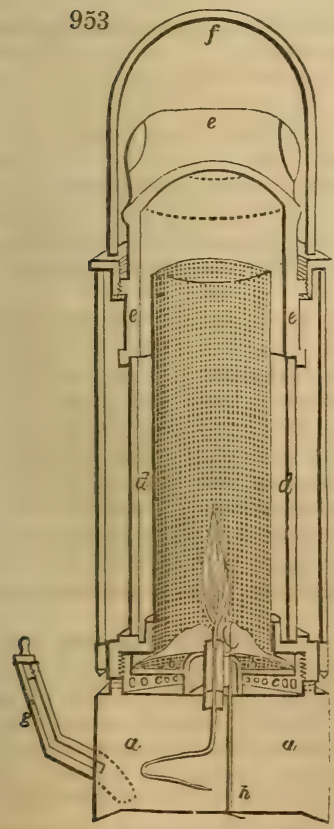
showing the fold of the wick; it is covered at top with $b, b$, several layers of wire gauze; $c, c$, is the perforated brass ring, under these layers, for admitting air, which is reverberated upon the burning wick by the cupola $\mathrm{c} ; d, d$, is the cylinder of glass, surrounding the wire-cloth one; $e, e$, is the safety brass hood, which screws down in the frame, so as to cover in the top of the glass chimney; $f$, is the arched wire for suspending the lamp to the girdle of the miner; $g$, is the bent tube for supplying oil to the cistern; and $h$ is the safety-trimmer, shown more distinctly in the figure illustrative of the LAMP of DAvY.

Between the glass and the cage there should be a space of about one tenth of an inch, forming an annular chimney for the free ventilation of the flame; and between the under edge of the hood $e$, and the upper rim of the glass, there should likewise be an interval, as also ventholes in the top of the hood, for the free escape of the smoke. The orifice of the little tube $g$, should be rather lower than the ring of holes $c$, otherwise the oil, when incautiously pourea into it, might overflow them, and prevent the lamp from burning. The figure is drawn somewhat in perspective.

As the naked cage of Davy often gets redhot with flame; as it is sometimes used for hours by miners in this most hazardous state; as this lamp gives so little light as to tempt rash men to remove its safety-cage;" as " it is upon record, that taking the average of ten years previous to the introduction of Sir H. Davy's safety lamp, and allowing one clear year for its introduction, and of ten years after it was properly introduced, there had been double the number of accidents, and at least double the number of deaths, of

- At Rowol Harrator June 3n. 1817, thurtv-ejght lives were lost bv the wilfulness of one man 
what took place in the ten years previous to its introduction ; as his lamp in explosive air-courses needs to be carried close upon the bosom, or under the coat of the miner; as it was declared by its illustrious inventor to be dangerous when exposed to such currents of explosive gas; and as the above described modification of it is free from all these defocts and dangers, I humbly apprehend that no conscientious proprietor or viewer of coal. mines will delay to substitute the lamp of $\mathrm{U}_{\mathrm{p}}$ ton and Roberts for the naked Davr, for otherwise he will certainly stand in a very painful predicament before a coroner's inquest, at the next mortal casualty from explosion.

The patentees have, I am told, been put to so much trouble and expense in trying t: introduce this life-protector into our coal-mines, that they have in a great measure abandoned the business. Messrs. Smith of Birmingham have meanwhile undertaken to make the lamps.

SAFFLOWER. This dye-stuff has been fully described under Carthamus and Rocge.

SAFFRON (Saffran, Fr. and Germ.) is a filamentous cake, composed of the stigmata of the flowers of the Crocus sativus. It contains a yellow matter called polychrö̈te, because a small quantity of it is capahle of coloring a great body of water. This is obtained by eraporating the watery infusion of saftion to the consistence of an extract, digesting the extract with alcohol, and concentrating the alcoholic solution. The polychroite remains in the form of a brilliant mass, of a reddish-yellow color, transparent, and of the consistence of honey. It has the agreeable smell, with the bitter pungent taste, of saftiron. It is very soluble in water; and if it be stove-dried, it deliquesces speedily in the air. According to M. Henry père, polychroïte consists of eighty parts of coloring matter, combined with 20 parts of a volatile oil, which cannot be separated by distillation till the coloring matter has been combined with an alkali. By mixing one part of shred saffron with eight parts of saturatei brine, and one half part of caustic ley, and distilling the mixture, the oil comes over into the receiver, and leaves the coloring matter in the retort, which may be precipitated from the alkaline solution by an acid. The pure coloring matter, when dried, is of a scarlet hue, and then readily dissolves in alcohol, as also in the fat and volatile oils, but sparinoly in water. Light blanches the reddish-ytllow of saffron, even when it is contained in a full vial well corked. Polychroïte, when combined with fat oil, and subjected to dry distillation, affords ammonia, which shows that azote is one of its constituents. Sulphuric acid colors the solution of polychroite indigo blue, with a lilach cast ; nitric acid turns it green, of various shades, according to the state of dilution. Protochloride (muriate) of tin prodnces a reddish precipitate.

Saffron is employed as a seasoning in French cookery. It is also used to tinge confectionary articles, liqueurs, and varnishes; but rarely as a pigment.

SAGO (Sagou, Fr. and Germ.) is a species of starch, extracted from the pith of the sago palm, a tree which grows to the height of 30 feet in the Moluccas and the Philippines. The tree is cut down, cleft lengthwise, and deprived of its pith, which being washed with water upon a sieve, the starchy matter comes out, and soon forms a deposite. This is dried to the consistence of dough, pressed through a metal sieve to corn it (which is called pearling), and then dried over a fire with agitation in a shallow copper fan. Sago is sometimes imported in the pulverulent state, in which it can be distinguished from arrowroot only by microscopic examination of its particies. These are uniform and spherical, not unequal and ovoid, like those of arrow-root.

SAL AMMONIAC. The manufacture of this salt may be traced to the remotest era. Its name is derived from Ammonia, or the temple of Jupiter Ammon, in Egypt, near to which the salt was originally made. Sal ammoniac exists ready formed in sereral animal products. The dung and urine of camels contain a sufficient quantity to have rendered its extraction from them a profitable Egyptian art in former times, in order to supply Europe with the article. In that part of Africa, fuel being very scarce, recourse is had to the dung of these animals, which is dried for that purpose, by plastering it upon the walls. When this is afterwards burned in a peculiar kind of furnace, it exhales a thick smoke, replete with sal ammoniac in vapor; the soot of course contains a portion of that salt, condensed along with other products of combustion. In every part of Egypt, but especially in the Delta, peasants are seen driving asses loaded with bags of that soot, on their way to the sal ammoniac works.

Here it is extracted in the following manner. Glass globes coated with loam are filled with the soot pressed down by wooden rammers, a space of only two or three inches heing left vacant, near their mouths. These globes are set in round orifices formed in the ridge of a long vault, or large horizontal furnace flue. Heat is gradually applied by a fire of dry camels' dung, and it is eventually increased till the globes become obscurely

unscrewing it, though he was well forewarned of the danger. He said, "he could not see with that thing," meaning the Davy.-Buddle, in Report of House of Commons, p. 215.

* Dr. Reid Clanny, in Report on Accidents in Mines, p. 32. I obseıve that in Sykes' Local Records of the counties of Durham and Northumberland, corrected by .I. Buddle, Es 7. , there are 540 deaths by explosions, between June, 1817, and June, 1835. What a mass of misery to the families of the sufferers 
red. As the muriate of ammonia is volatile at a temperature much below ignition, is rises out of the soot in vapor, and gets condensed into a calie upon the inner surface of the top of the globe. A consilerable portion, however, escapes into the air; and another portiun concretes in the mouth, which must be cleared from time to time by an iron rod. Tuwards the end, the obstruction becomes very troublesome, and must be most carefully attended to and obriated, otherwise the globes would explode by the uncondensed vapors. In all cases, when the subliming process approaches to a conclusion, the glubes crack or split; and when they come to be removed, after the heat has subsided, they usually fall to pieces. The upper portion of the mass is separated, because to it the white salt adheres; and on detaching the pieces of glass with a hatchet, it is ready for the market. At the bottom of each balloon a nucleus of salt remains, surrounded with fixed pulverulent matter. This is reserved, and after being bruised, is put in along with the charge of soot in a fresh operation.

The sal ammoniac obtained by this process is dull, spongy, and of a grayish hue; but nothing better was for a long period known in commerce. Forty years ago, it fetched $2 s .6 d$. a pound; now, perfectly pure sal ammoniac may be had at one fifth part of that price.

Vailous animal offals develop during their spontaneous putrefactive fermentation, or their decomposition by heat, a large quantity of free or carbonated ammonia, among their volatile products. Upon this principle many sal ammoniac works have been established. In the destructive distillation of pitcoal, there is a considerable quantity of ammoniacal products, which are also worked up into sal ammoniac.

The tirst attempts made in France to obtain sal ammoniac profitably in this manner, failed. A very extensive factory of the kind, which experienced the same fate, was under the superintendence of the celebrated Baumé, and affords one out of a thousand instances where theoretical chemists have shown their total incapacity for conducting operations on the scale of manufacturing economy. It was established at Gravelle near Charenton, and caused a loss to the shareholders in the speculation of upwards of 400,000 francs. This result closed the concern in 1787 , after a foolish manipulation of 27 years. For ten years after that event, all the sal ammoniac consumed in France was imported into it from foreign countries. Since then the two works of MM. Payen and Pluvinet were mounted, and seem to have been tolerably successful. Coal soot was, prior to the introduction of the gas-works, a good deal used in Great Britain for obtaining sal ammoniac. In France, bones and other animal matters are distilled in large iron retorts, for the manufacture of both animal charcoal and sal ammoniac.

These retorts are iron cylinders, 2 or 3 feet in diameter, and 6 feet long. Figs. 954, and 955 , show the form of the furnace, and the manner in which the cylinders are 954 955
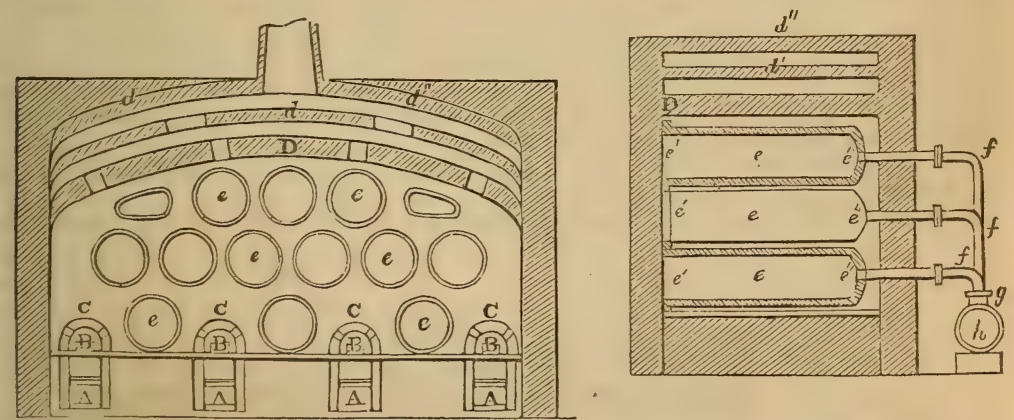

arranged; the first being a longitudinal, the second a transverse section of it. A, the ashpits under the grates; $B$, the fireplaces, arched over at top; $c$, the vault or bench of firebricks, perforated inside with eight flues for distributing the flame; $D$, a great arch, with a triple voussojr $\mathrm{D} d^{\prime}, d^{\prime \prime}$, under which the retorts are set. The first arch $\mathrm{D}$, is perforated with twenty vent-holes; the second, with four vent-holes; through which the flame passes to the thurd arch, and thence to the common chimney-stalk. The retorts $e$, are shut by the door $e^{\prime}$ ( $f$ g. 955), luted, and made fast with screw-bolts. Their other ends $e^{\prime \prime}$ terminate in tubes $f, f, f$, which all enter the main pipe $h$. The condensing pipe proceeds slantingly downwards from the further end of $h$, and dips into a large sloping iron cylinder immersed in cold water. See GAS-LIGHT and STOvE, for a better plan of furnace.

The filters used in the large sal ammoniac works in France are represented in fig. 956. The apparatus consists-1. of a wooden chest $a$, lined with lead, and which is turned over at the edges; a socliet of learl $b$, soldered into the lowest part of the bottom, serves to discharge the liquid; 2 . of a wooden crib or grating formed of rounded rods. 
as shown in the section $c, c$, and the plan $d$; this grating is supported one inch at least above the bottom, and set truly horizontal, by a series of wedses; 3 . of an open fabric of canvass or strong calico, laid on the grating, and secured over the edges, so as to keep it tense. A large wooden reservoir $f$, lined with lead, furnished with a

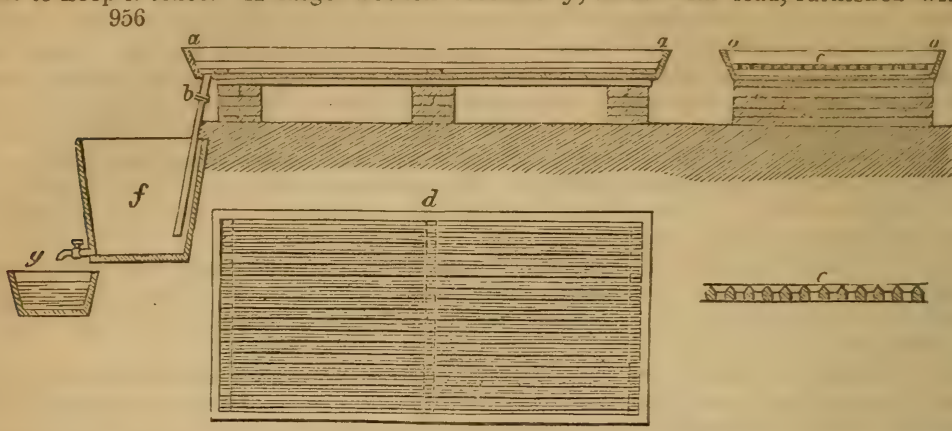

cover, is placed under each of the filters; a pump throws back once or twice upon tne filters what has already passed through. A common reservoir g, below the others, may be made to communicate at pleasure with one of them, by means of intermediate stopcocks.

The two boilers for evaporating and decomposing are made of lead, about one quarter of an inch thick, set upon a fire-brick vault, to protect them from the direct action of the flame. Through the whole extent of their bottoms above the rault, horizontal cast-iron plates, supported by ledges and brick compartments, compel the flame and burned air, as they issue from the arch, to percur many sinuosities before they pass up the chimney. This floor of cast iron is intended to support the bottom of the boiler, and to diffuse the heat more equably. The leaden boilers are surrounded with brickwork, and supported at their edges with a wooden frame. Ther may be emptied at pleasure into lower receivers, called crystallizers, by means of leaden syphons and longnecked funnels.

The crystallizers are wooden chests lined with lead, 15 inches deep, 3 or 4 feet. broad, and from 6 to 8 feet long; and may be inclined to one side at pleasure. A round cistern receives the drainings of the mother-waters. The pump is made of lead, hardened with antimony and tin.

The subliming furnace is shown in figs. 957 and 958 , by a transverse and longitudinal section. $a$ is the ash-pit; $b$, the grate and fire-place; $c$, the arch above them. This arch, destined to protect the bottles from the direct action of the fire, is perforated with vent-holes, to give a passage to the products of combustion between the subliming ressels. $d, d$, are bars of iron, upon which the bottoms of the bottles rest; $e$, stoneware bottles, protected by a coating of loam from the flame.

Fig. 959 shows the cast-iron plates, $a, b, c$, which, placed above the vaults, receive each two bottles in a double circular opening.

At the extremity of the above furnace, a second one, called the drier, fig.960, receives the 957 960
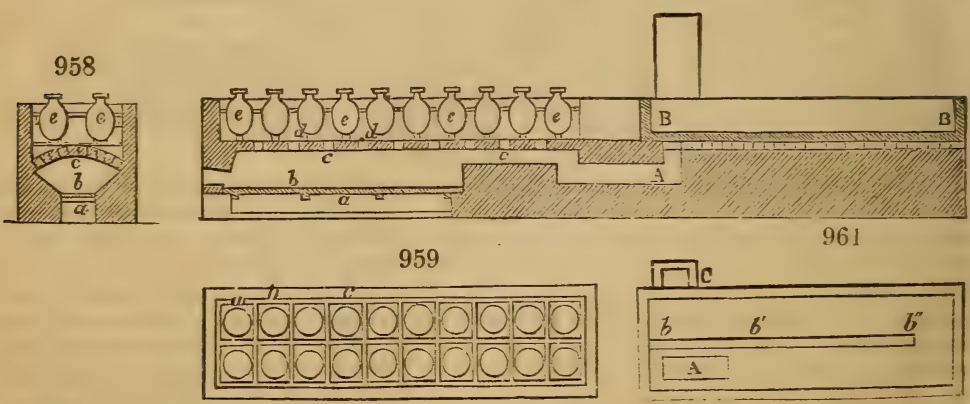

prodncts of the combustion of the first, at $A$, under horizontal cast-iron plates, and upon which the bottom of a rather shallow boiler b, rests. After passing twice under these plates, round a longitudinal brick partition $b, b^{\prime}, b^{\prime \prime}$. the products of combustion enter the smoke chimney c. See plan, fig. 961 .

The boiler set over this furnace should have no soldered joints. It may be $3 \frac{1}{2}$ feet 
broad, 9 or 10 feet long, and 1 foot deep. The concrete sal ammoniac may be crushed uncler a pair of edge mill-stones, when it is to be sold in powder.

Bones, blood, flesh, hoins, hoofs, woollen rags, silk, hair, scrapings of t.ides and leather, sc., may be distilled for procuring ammonia. When hones are used, the resiluum in the retort is bone black. The charcoal from the other substances will serve for the manufacture of Prussian blue. The bones should undergo a degree of culcination beyond what the ammoniacal process requires, in order to convert them into the vest bone black; but the other animal matters should not be calcined up to that point, otherwise they are of little use in the Prussian blue works. If the bones be calcined, however, so highly as to become glazed, their decoloring power on sirups is nearly destroyed. The other substances should not be charred beyond a red-brown heat.

The condensed rapors from the cylinder retorts afford a compound liquor hoiding car bonate of ammonia in solution, mixed with a large quantity of empyreumatic oil, which floats at top. Lest incrustations of salt should at any time tend to obstruct the tubes, a pipe should be inserted within them, and connected with a steam boiler, so as to blow steam through them occasionally.

The whole liquors mixed have usually a density of $8^{\circ}$ or $9^{\circ}$ Baumé $(1.060)$. The simplest process for converting their carbonate of ammonia into muriate, is to saturate them with muriatic acid, to evaporate the solution in a leaden boiler till a pellicle appears, to run it off into crystallizers, and to drain the crystals. Another process is, to decompose the carbonate of ammonia, by passing its crude liquor through a layer of sulphate of lime, 3 or 4 inches thick, spread upon the filters, fig. 956. The liquor may be laid on with a pump; it should never stand higher than 1 or 2 inches above the surface of the briised gypsum, and it should be closely covered with boards, to prevent the dissipation of the volatile alkali in the air. When the liquor has passed througb the first filter, it must be pumped upon the second; or the filters being placed in a terrace form, the liquor from the first may flow down upon the second, and thus in succession. The last filter should be formed of nearly fresh gypsum, so as to ensure the thorough conrersion of the carbonate into sulphate. The resulting layers of carbonate of lime should he washed with a little water, to extract the sulphate of ammonia interposed among its particles. The ammoniacal liquor thus obtained must be completely saturated, by adding the requisite quantity of sulphuric acid; even a slight excess of acid can do no harm. It is then to be eraporated, and the oil must be skimmed off in the course of the concentration. When the liquid sulphate has acquired the density of about $1 \cdot 160$, sea salt should be added, with constant stirring, till the whole quantity equivalent to the double decomposition be introduced into the lead boiler.

The fluid part must now be drawn off by a syphon into a somewhat deep reservoir, where the impurities are allowed to subside; it is then evaporated by boiling, till the sulphate of soda falls down in granular crystals, as the result of the mutual reaction of the sulphate of ammonia and muriate of soda; while the more soluble muriate of ammonia remains in the liquor. During this precipitation, the whole must be occasionaıy agitated with wooden paddles; the precipitate being in the intervals removed to the cooler portion of the pan, in order to be taken out by copper rakes and shovels, and thrown into draininghoppers, placed near the edges of the pan. The drained sulphate of soda must be afterwards washed with cold water, to extract all the adhering sal ammoniac.

The liquor thus freed from the greater part of the sulphate, when sufficiently concentrated, is to be drawn off by a lead syphon, into the crystallizers, where, at the end of 20 or 30 hours, it affords an abundant crop of crystals of sal ammoniac. The motherwater may then be run off, the crystallizers set aslope to drain the salt, and the salt itself must be washed, first by a weak solution of sal ammoniac, and lastly with water. It must be next desiccated, by the apparatus fig. 960, into a perfectly dry powder, then put into the subliming stoneware balloons, by means of a funnel, and well rammed down. The mouth of the bottle is to be closed with a plate.or inverted pot of any kind. The fire must be nicely regulated, so as to effect the sublimation of the pure salt from the under part of the bottle, with due regularity, into a white cake in the upper part. The neck of the bottle should be cleared from time to time with a long steel skewer, to prevent the risk of choking, and consequent bursting; but in spite of every precaution, several of the bottles crack almost in every operation. In Scotland, sai ammoniac is sablimed in cast-iron pots lined with thin fire-tiles, made in segments accommodated to the internal surface of the pots; the vapor being received and condensed into cakes, within balloons of green glass set over their mouths. The salt, when taken out, and freed by scraping from any adhering ochreous or other impurities, is ready for the market, being sold in hollow spherical masses. The residuum in the pots or bottles may pe partially worked up in another operation. The greatest evil is produced by the mixture or even contact of iron, because its peroxyde readily rises in vapor with the sal ammoniac, and tinges it of a red or yellow color.

The most ordinary process for converting the ammoniacal liquor of the gas-works into 
sal ammoniac, is to saturate it with sulphuric acid, and to decompose the sulphate, thus iormed, by the processes above described. But muriatic acid will be preferred, where it is as cheap as sulphuric of equivalent saturating power; because a tolerably pure sal ammoniac is thereby directly obtained. As the coal-gas liquor contains a good deal of sulphureted hydrogen, the saturation of it with acid should be so conducted as to burn the disengraged noxious gases in a chimney. Formerly human urine was very extensively employed, both in this country and in France, in the manufacture of sal ammoniac; but since the general establishment of gas-works, it has been, I believe, abandoned. The process was exceedingly offensive.

The best white sal ammoniac is in spheroidal cakes of about one foot diameter, three or four inches thick in the middle, sumewhat thinner at the edges, and is semi-transparent or translucent. Each lump weighs about one quarter of a cwt. As it is easily volatilized by heat, it may be readily examined as to its sophistication with other salts. Sal ammoniac has a certain tenacity, and is flexible under the hammer or pestle. It is principally used In tinning of cast-iron, wrought iron, copper, brass, and for making the various ammoniacal preparations of pharmacy.

In a chemical factory near Glasgow, 7200 gallons of ammoniacal liquor, obtained weekly from the gas-works, are treated as follows:-The liquor is first rectified by distillation from a wagon-shaped wrought-iron boiler, into a square cistern of iron lined with lead. $4500 \mathrm{lbs}$. of sulphuric acid, of specific gravity 1.625 , are then slowly added to the somewhat concentrated distilled water of ammonia. The produce is 2400 gallons of sulphate of ammonia, slightly acidulous, of specific gravity $1 \cdot 150$, being of such strength as to deposite a few crystals upon the sides of the lead-lined iron tank in which the saline combination is made. It is decomposed by common salt.

From the 7200 gallons of the first crude liquor, 900 gallons of tar are got by subsidence, and 200 gallons of petroleum are slimmed off the surface. The tar is converted, by a moderate boiling in iron pans, into good pitch.

SALAMSTONE. See LAPIDARY.

SALEP, or SALOUP, is the name of the dried tuberous roots of the Orchis, imported from Persia and Asia Minor, which are the product of a great many species of the plant, but especially of the Orchis mascula. Salep occurs in commerce in small oval grains, of a whitish-yellow color, at times semi-transparent, of a horny aspect, very hard, with a faint peculiar smell, and a taste like that of gum tragacanth, but slightly saline. These are composed almost entirely of starchy matter, well adapted for making a thick pap with water or milk, and are hence in great repute in the Levant, as restorers of the animal forces. Their aphrodisiacal properties are apocryphal. If the largest roots of the Orchis mascula of our own country were cleaned, scraped, steeped for a short time in hot, and then for a few minutes in boiling water, to extract their rank flavor, afterwards suspended upon strings to dry in the air, they would afford as nourishing and palatable an article as the Turkey saloup, and at a vastly lower price.

SALICINE, is a febrifuge substance, which may be obtained in white pearly crystals from the bark of the white willow (Salix alba), of the aspen tree (Salix helis), as also of some other willows, and some poplars. It has a very bitter taste.

SAL PRUNELLA, is fused nitre cast into cakes or balls.

SAL VOLATILE, is sesquicarbonate of ammonia.

SALT, EPSOM, is sulphate of magnesia.

SALT, MICROCOSMIC, is the triple phosphate of soda and ammonia.

SALT OF AMBER, is succinic acid.

SALT OF LEMONS, is citric acid.

SALT OF SATURN, is acetate of lead.

SALT OF SODA, is carbonate of soda.

SALT OF SORREL, is bi-oxalate of potassa.

SALT OF TARTAR, is carbonate of potassa.

SALT OF VITRIOL, is sulphate of zinc.

SALT PERLATE, is phosphate of soda.

SALTPETRE, is nitre, or nitrate of potassa.

SALT, SEDATIVE, is boracic acid.

SALTS, are an important class of chemical compounds, anciently studied under the Greek title of Halurgy. At one period every inorganic substance readily soluble in mater, was regarded as a salt; and afterwards, every substance soluble in five hundred imes its weight of water. Thus both acid and alkaline indies cars? to be enrolled among salts; but latterly, the combinat ins of the acils witi alkalis, eazths, and metaliiz calces (now styled oxydes), were aloce thought to be entitled to the denomination of salts, in conseque :ce of therr resemblance in appearance, and supposed analogy in composision, to culinary salt. Since Sir H. Davy demonstrated that this substance contained neither acid nor alkaline matter, but that it consisted of chlorine and the metal sodium, the generality of chemists found it impossible to include salts under one category of consti- 
- rion; while a few have rashly offered to cut the knot, by excluding from the saline

1. nily, chloride of sodium, the patriarch of the whole.

Saits may be justly divided into three orders:

1. The binary, consisting of two single members; such as the bromides, chlorides, cyanides, fluorides, iodides, carburets, phosphurets, sulphurets, \&c.

2. The bi-binary, consisting of two double members; such as the borates, bromates. carbonates, chlorates, sulphates, sulphites, hyposulphites, sulphohydrates, \&c.

3. The ternary, consisting of two single members of one genus, and one member of another; such as the boro-fluorides, silico-flnorides, sulpho-cyanides, chloriodides, \&c.

The species of each order may exist in three states, constituting neutral salts, supersalts, and subsalts; as for example, the chloride of sodium, the bisulphate of potassa, the subnitrate of lead, \&c.

In the above arrangement, cyanogen is allowed to represent a simple substance, from its forming analogous compounds with chlorine and iodine. The neutral state of salts is commonly indicated by their solutions not changing the colors of litmus, violets, or red cabbage; the sub-state of salts, by their turning the violet and cabbage green; and the super-state of salts, by their changing the purple of litmus, violets, and cabbage, red; but to the generality of this criterion there are some exceptions. The atomic theory may be adrantageously resorted to, in this predicament. 1. When one prime equivalent of the one member (whether single or double) of a salt, combines with one prime of the other member, a neutral salt is the result, as in chloride of sodium or nitrate of potassa. 2. When two primes of the electro-negative member combine with one prime of the electro-positive, a supersalt is formed, as bichloride of tin, or bisulphate of potassa. 3. When one prime of the electro-negative member combines with two or more primes of the electro-positive, a subsalt is produced, as the subacetate and subchromate of lead, \&c.

SALT, SEA, or CULINARY; chloride of sodium; muriate of soda. (Hydrochlorate de soude, Fr.; Chlornatrium, Germ.) Sea salt, or rock salt, in a state of purity, consists of 60 of chlorine +40 of sodium, in 100 parts.

This important species of the saline class possesses, even in mass, a crystallıne structure, derived from the cube, which is its primitive form. It has generally a foliated texture, and a distinct cleavage; but it has also sometimes a fibrous structure. The massive salt has a vitreous lustre. It is not so brittle as nitre; it is nearly as hard as alum, a little harder than gypsum, and softer than calcareous spar. Its specific gravity varies from 2.0 to 2.25 . When pure, it is colorless, translucent, or transparent. On exposure to heat, it commonly decrepitates; but some kinds of rock salt enter quietly into fusion at an elevated temperature, a circumstance which has been ascribed to their having been originally subjected to the action of fire.

According to M. Gay Lussac, 100 parts of water dissolve-

$35 \cdot 81$ parts of the salt, at temperature $57 \cdot 0^{\circ} \mathrm{Fahr}$.

$\begin{array}{rrr}35.88 & - & 62.5^{\circ} \\ 37 \cdot 14 & - & 140 \cdot 0^{\circ} \\ 40.38 & - & 229.5^{\circ}\end{array}$

Natire chloride of sodium, whether obtained from the waters of the ocean, from saline lakes, from salt springs, or mineral masses, is never perfectly pure. The foreign matters present in it vary with its different origins and qualities. These are, the sulphates of lime, magnesia, soda, muriates of magnesia and potash, bitumen, oxyde of iron, clay in a state of diffusion, \&c.

Muriate of potash has been detected, in the waters of the ocean, in the sal-gem of Berchtesgaden in Bavaria, of Hallein in the territory of Salzbourg, and in the salt springs of Rosenheim.

The more heterogeneous the salt, the more soluble is it, by the reciprocal affinity of its different saline constituents; and thus a delicate hydrometer, plunged in saturated brine, may serve to show approximately the quality of the salt. I find that the specific gravity of a saturated solution of large-grained cubical salt, is 1.1962 at $60^{\circ} \mathrm{F}$. 100 parts of this brine contain $25 \frac{1}{2}$ of salt, $(100 \mathrm{w} .+34.2 \mathrm{~s}$.) From mutual penetration, 100 zolumes of the aqueous and saline constituents form rather less than 96 of the solution.

Among the varieties in the form of this salt, the octahedral, the cubo-octahedral, and the dolecahedral, have been mentioned; but there is another, called the funnel or hoppershaped, which is very common. It is a hollow rectangular pyramid, which forms at the surface of the saline solution in the course of its evaporation, commencing with a small floating cube, upon which lines of nther little cubes attach themselves to the edges of the upper face; whereby they form and enlarge the sides of a hollow pyramid, whose apex, the single cubic crystal, is downward. This sinks by degrees as the aggregation goes on above, till a pyramidal boat of considerable size is constructed. 
A Table of the results of the Analyses of several varieties of Culinary SALt.

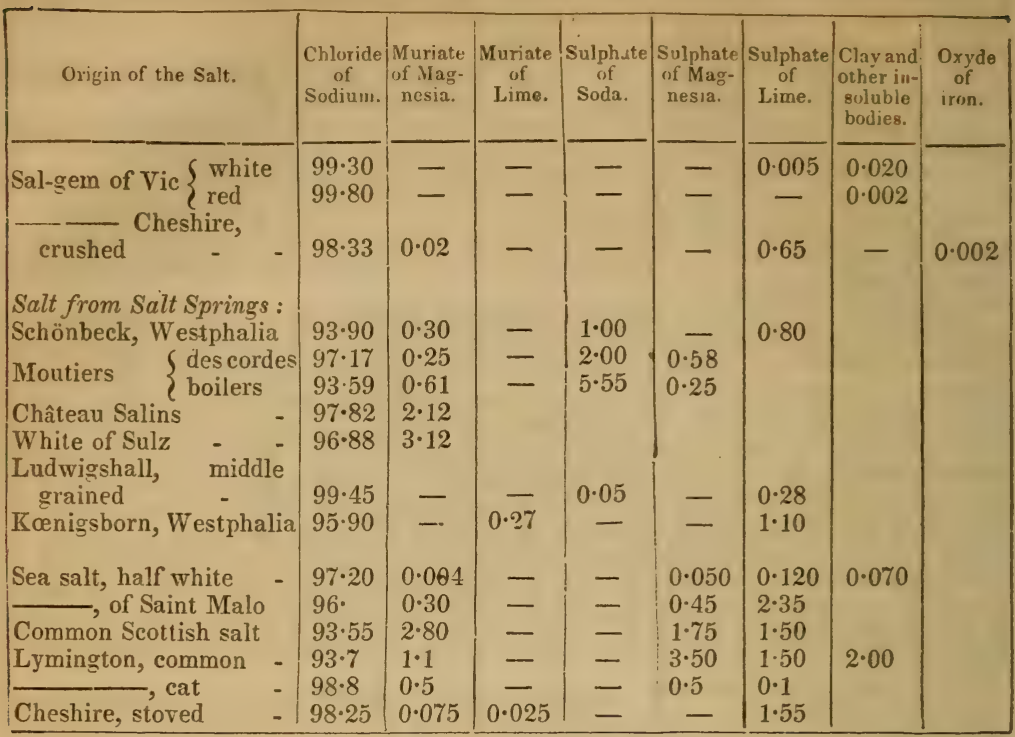

The geological position of rock salt is between the coal formation and the lias. The great rock-salt formation of England occurs within the red marl, or new red sandstone, the bunter-sandstein of the Germans, so called, because its colors vary from red to salmon and chocolate. This mineral stratum frequently presents streaks of light blue, verdigris, buff, or cream color; and is chiefly remarkable for containing considerable masses or beds of gypsum. At Northwich, in the vale of the Weaver, the rock salt consists of two beds, together not less than 60 feet thick, which are supposed to constitute large insulated masses, about a mile and a half long, and nearly 1300 yards broad. There are other deposites of rock salt in the same valley, but of inferior importance. The uppermost bed occurs at 75 feet beneath the surface, and is covered with many layers of indurated red, blue, and brown clay, interstratified more or less with sulphate of lime, and interspersed with argillaceous marl. The second bed of rock salt lies $31 \frac{1}{2}$ feet below the first, being separated from it by layers of indurated clay, with veins of rock salt running through them. The lowest bed of salt was excarated to a depth of 110 feet, several years ago.

The beds or masses of rock salt are occasionally so thick, that they have not been yet bored through, though mined for many centuries. This is the case with the inmense mass of Wieliczka, and the lower bed at Northwich. But in ordinary cases, this thickness varies from an inch or two to 12 or 15 yards. When the strata are thin. they are usually numerous; but the beds, layers, or masses never exhibit throughout a great extent any more than an illusory appearance of parallelism; for when they are explored at several points, enlargements are obserred, and such diminutions as cause the salt to disappear sometimes altogether. This mineral is not deposited, therefore, in a geological stratum, but rather in lenticular masses, of very variable extent and thickness, placed alongside of each other at unequal distances, and interposed between the courses of the other formations.

Sometimes the rock salt is disseminated in small masses or little veins among the calcareous and argillaceous marls which accompany or orerlie the greater deposites. Bitumen, in small particles, hardly visible, but distinguishable by the smell, occurs in all the minerals of the saliferous system.

It has been remarked, that the plants which grow generally on the sea shores, such as the Triglochinum maritimum, the Salicomia, the Salsola kali, the Aster trifolium, or farewell to summer, the Glaux maritima, \&c., occur also in the neighborhood of salt mines and salt springs, even of those which are most deeply buried beneath the surface.

The interior of rock-salt mines, after digging through the strata of clay marl, \&cc. is extremely dry; so that the dust produced in the workings becomes an annoyance to the miners, though in other respects the excavations are not at all insalubrious.

Salt springs occur nearly in the same circumstances, and in the same geological form. 
ation as the salt rock. It has been noticed that salt spings 1ssue, in general, from the upper portion of the saliferous strata, principally from the saline clay marls. Cases however occur, where the salt springs are not accompanied by rock salt, and where the whole saline matter is derived from the marls themselves, which thus constitute the only saliferous beds.

It has been imagined that there are two other periods of geological formation of this substance; one much more ancient, belonging to the transition series of rocks; the other relalively modern, among secondary strata. To the former has been referred the salt formation of Bex, that of Cardonne, \&c. But M. Brongniart assigns valid rensons for rejecting this supposition. M. Beudant, indeed, refers to the secondary strata above the chalk, the rock-salt formation of Wieliczlia, and of the base of the Carpathians; placing these among the plastic clay and lignites.

The mines of rock salt do not appear to possess any determinate elevation upon the surface of the earth. Inmense masses of it are met with at very great depths below the level of the sea, (the mine of Wieliczlia is excavated 860 feet beneath the soil,) and others exist at a considerable altitude, as that of Hallein near Salzbourg, which is 3300 Seet ahove the level of the sea, and the saline rock of Arbonne in Savoy, which is nearly 4000 feet higher, situated at the great elevation of 7200 feet above the level of the sea, and cunsequently in the reoion of perpetual snow. The rock is a mass of saccharoid and anhydrous gypsum, imbued with commun salt, which is extracted by lixiviation; after which the gypsum remains porous and light.

The inland seas, salt lalies, and salt marshes, have their several localities obviously independent of peculiar geological formations. The ocean is, howerer, the most masnificent mine of salt, since this chloride constitutes about one thirtieth part of its weight; being pretty evenly diffused throughout its waters, when no local cause disturbs the equilibrium. The largest proportion of salt held in solution in the open sea, is 38 parts in 1000 , and the smallest 32 . In a specimen taken by $\mathrm{Mr}$. Wilkinson, out of the Red Sea, at Berenice, I found 43 parts of salt in 1000 . The specific gravity of the water was 1.035.

Were it requisite to extract the chloride of sodium from sea-water by fuel alone, many countries, even maritime, would find the process too costly. The salt is therefore obtained from it in two different manners; 1 . by natural evaloration alone; 2 . by natural and artificial eraporation combined. The first method is empluyed in warm reoions, under the form of saline tanks, or brine reservoirs, called also brine-pits. These are large shallow basins, the bollom of which is very smooth, and formed of clay. They are excavated along the sea-shore, and consist of -

1st. A large reservoir, deeper than the proper brine-pits, which is dug between them and the sea. This reservoir communicates with the sea by ineans of a channel provided with a slnice. On the sea-shore, these reservoirs may be filled at high water, though the tides are rather inconvenient than advantageous to brine-pits.

2 dly. The brine-pits, properly so called, which are divided into a number of compartments hy means of litile banks. All these compartments have a communication with each other, but so that the water frequently has a long circuit to make, from one set to another. Sometimes it must flow 400 or 500 yards, before it reaches the extremity of this sort of labyrinth. The various divisions have a number of singular names, by which they are technically distinguished. They should be exposed to the north, north-east, or northwest winds.

The water of the sea is let into these reservoirs in the month of March, where it is exposed on a vast surface to evaporation. The first reservoir is intended to detain the water till its impurities have subsided, and from it the other reservoirs are supplied, as their water evaporates. The salt is considered to be on the point of crystallizing when the water begins to grow re Soon after this, a pellicle forms on the surface, which breaks, and falls to the bottom. Sometimes the salt is allowed to subside in the first compartment; at others, the strong brine is made to pass on to the others, where a larger surface is exposed to the air. In either case the salt is drawn out, and left upon the borders to drain and dry.

The salt thus obtained partakes of the color of the bottom on which it is formed; and is hence white, red, or gray.

Sea water contains, in 1000 parts, 25 of chloride of sodium, $5 \cdot 3$ sulphate of magnesia, 3.5 chloride of magnesium, 0.2 carbonate of lime and magnesia, 0.1 sulphate of lime, besides $\overline{2} \overline{00}$ of sulphate and muriate of potash. It also contains iodide of sodium, and bromide of magnesium. Its average spec. grav. is from $1 \cdot 029$ to $1 \cdot 030$.

Sea-water and weak brines may be cancentrated either by the addition of rock salt, by spontaneous evaporation in brine-pits (see suprà), or by graduation. Houses for the last purpose are extensively employed in France and Germany. The weak brine is pumped into an inmense cistern on the top of a tower, and is thence allowed to flow down the surface of bundles of thorns built up in regular walls, between parallel wooden frames. At Salza, near Schönebeck, the graduation-house is 5817 feet long, the thorn 
walls are from 33 to 52 feet high, in different parts, and present a total surface of 25,000 square feet. Under the thorns, a great brine cistern, made of strong wooden planks, is placed, to receive the perpetual shower of water. Upon the ridge of the graduation-house there is a long spout, perforated on each side with numerous holes, and furnished with spigots or stopcoclis for distributing the brine, either over the surface of the thorns, or down through their mass; the latter method affording larger evaporation. The graduation-house should be built lengthivise in the direction of the prevailing wind, with its ends open. An experience of many years at Salza and Dürrenberg has shown, that in the former place graduation can go on 258, and in the latter 207 dars, on an average, in the year; the best season being from May till August. At Dürrenberg, $3,596,561$ cubic feet of water are evaporated annually. According to the weakness of the brine, it must be the more frequently pumped up, and made to flow down over the thorns in different compartments of the building, called the $1 \mathrm{st}, 2 \mathrm{~d}$, and $3 \mathrm{~d}$ graduation. A deposite of gypsum incrusts the twigs, which requires them to be renewed at the end of a certain time. Figs. 962 and 963 represent the graduation-house of the salt-works at Dürrenberg. $a, a, a$, are low stone pillars for supporting the brine cistern $b$, called

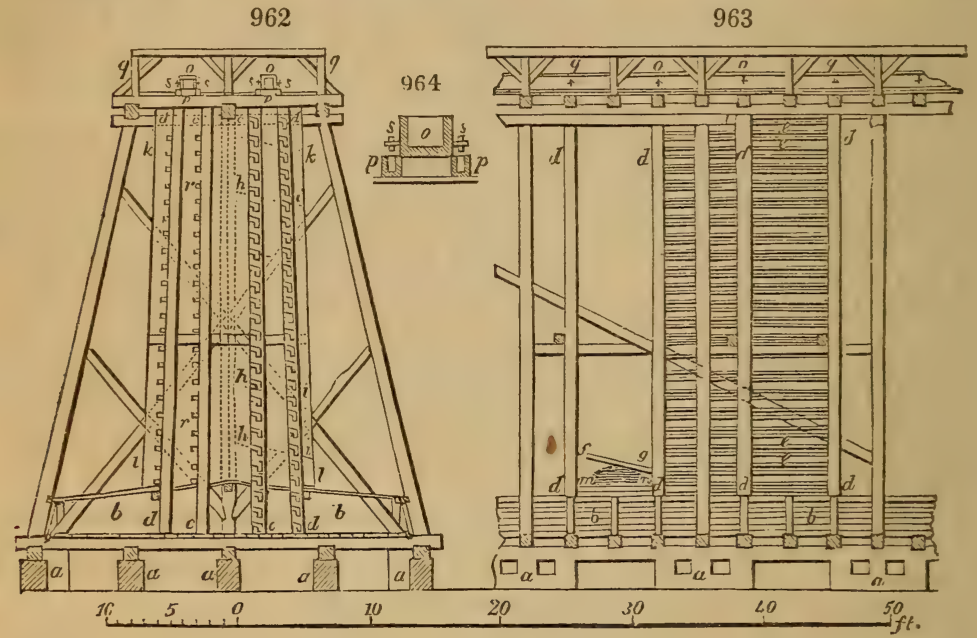

the soole-schiff. $\quad c, c$ are the inner, $d, d$ the outer, walls of thorns; the first have perpendicular sides, the last sloping. The spars $e, e$, which support the thorns, are longer than the interval between two thorn walls from $f$ to $g$, fig. 963 , whereby they are readily fastened by their tenons and mortises. The spars are laid at a slope of 2 inches in the foot, as shown by the line $h, i$. The bundles of thorns are each $1 \frac{1}{2}$ foot thick, from 5 to 7 feet long, and are piled up in the following way : - Guide-bars are first placed in the line $k, l$, to define the outer surface of the thorn wall; the undermost spars $m, n$, are fastened upon them; and the thorns are evenly spread, after the willow-withs of the bundles have been cut. Over the top of the thorn walls are laid, through the whole length of the graduation-house, the brine spouts 0,0 , which are secured to the upper beams; and at both sides of these spouts are the drop-spouts $p, p$, for discharging the brine by the spigots $s, s$, as shown upon a larger scale in $f g .964$. The drop-spouts are 6 feet long, have on each side small notches, 5 inches apart, and are each supplied by a spigot. The space above the ridge of the graduation-house is covered with boards, supported at their ends by binding-beams $q . \quad r, r$, show the tenons of the thorn-spars. Over the sooleschiff $b$, inclined planes of boards are laid for conducting downwards the innumerable showers. The brine, which contains at first $7 \cdot 692$ per cent. of salt, indicates, after the first shower, 11.473; after the second, 16.108; and after the third, 22. The brine, thus concentrated to such a degree as to be fit for boiling, is kent in great reservoirs, of which the eight at Saiza, near Schöneheck, have a capacity of $2,421,720$ cubic feet, and are furnished with pipes leading to the sheet-iron salt-pans. The capacity of these is very different at different works. At Schönebeck there are 22, the smallest having a square surface of 400 feet, the largest of 1250, and are enclosed within walls, to prevent their being affected by the cold external air. They are covered with a funnel-formed or pyramidal trunk of deals, ending in a square chimney, to carry oft the steam.

Figs. 965, 966, 967 represent the construction of a salt-pan, its furnace, and the salt store-room of the works at Dürrenberg; fig. 967 being the ground plan, fig. 966 
the longitudinal section, and fig. 965 the transverse section. $a$ is the fire-grate, which slopes upwirils to the back part, and is $31 \frac{1}{2}$ inches distant from the bottom of the pan. The ratio uf the surface of the grate to that of the bottom of the pan, is as 1 to $59 \cdot 5$; that of the air-hole into the ash-pit, as 1 to 306 . The bed under the pan is laid with brichs, smoorthy plastered over, from $b$ to $c$, in fig. 966 . Upon this bed the pillars $d, r$, \&c., are built in a radiated direction, heing 6 inches broad at the bottom, and tapering to $1 \frac{1}{2}$ inch at top. The pan is so laid that its bottom has a fall towards the midule of 965

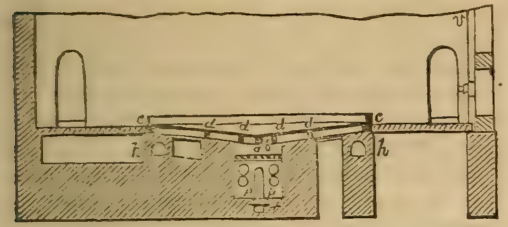
$2 \frac{1}{2}$ inches; see e, $f$, fig. 966 . The fire diffuses itself in all directions under the pan, proceeds thence through several holes $g, g, g$, into flues $h, h, h$, which run round three sides of the pan; the burnt air then passes through $i$, fig. 967, under other pans, from which it is collected in the chimneys $k, k$, to be conducted into the drying-room. At $l, l$, there is a transverse flue, through which, by means of dampers, the fire-draught may be conducted into an extra chimney $m$. From the flues $k, k$, four square iron pipes $n, n$, issue and conduct the burnt air into the main chimneys in the opposite wall.

The bottoms of the several flues have a gradual ascent above the level of the fire-grate. A special chimney 0 , rises above the ash-pit, to carry off the smoke, which may chance to regurgitate in certain states of the wind. $p, p$, are iron pipes laid upon each side of the ash-pit (see figs. 966 and 96\%), into which cold air is admitted by the flue $q, r$
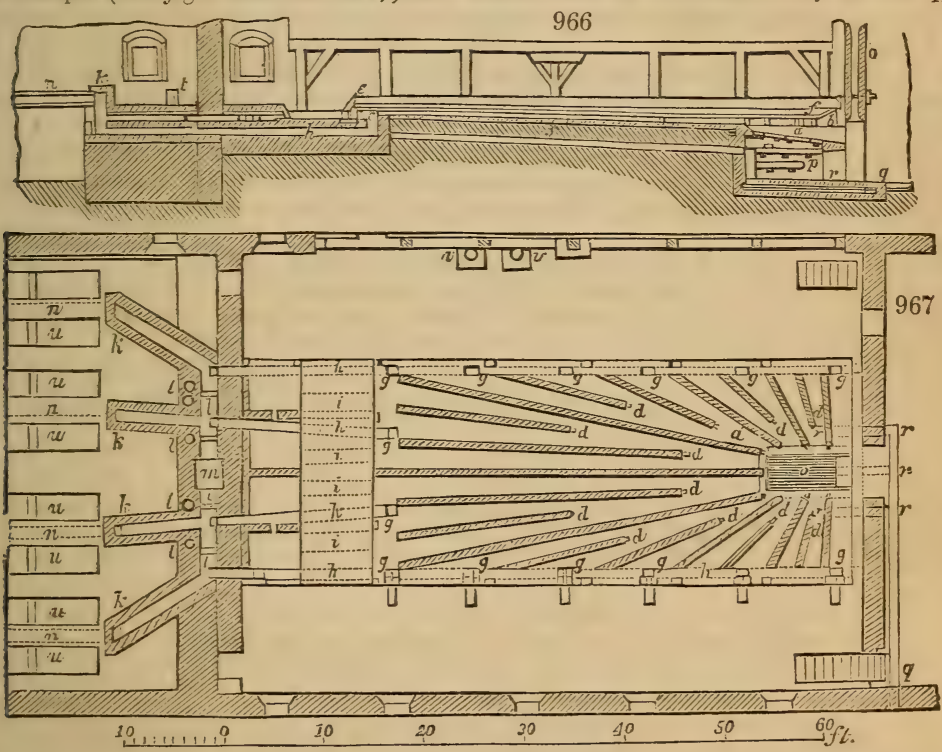

where, becoming heated, it is conducted through iron pipes $s$, and thence escapes at $t_{\text {, }}$ into the stove-room. Upon both sides of the hot flues in the stove-room, hurdle-frames $s u, u$, are laid, each of which contains 11 baskets, and every basket, except the under most, holds 60 pounds of salt, spread in a layer 2 inches thick. $v$, $v$, show the pipes by which the pan is supplied with graduated brine.

Description of the Steam-trunk, in fig. 968.

In front of the pan $a, a$, there are two upright posts, upon which, and in holes of the back wall, two horizontal beams $b, b$, are supported. The pillars $c, c$, are sustained upon the bearers $d, d$. At $e, e$, a deep quarrangular groove is made in the beams, for fixing down the four boards which form the bottom of the steam-way. In this groove any condensed water from the steam collects, and is carried off by a pipe $f$, to prevent it falling back into the pan. Upon the three sides of the pan not in contact with the wall, there are three rows of boards hinged upon planks $b, h$. Behind the apper one, a board is hung on at $g$, upon which the boiled salt is laid to drain. The 
two other rows of boards are hooked on so as to cover the pan, as shown at $h$ Whenever the salt is sufficiently drained, the upper shelves are placed in a horizonta!

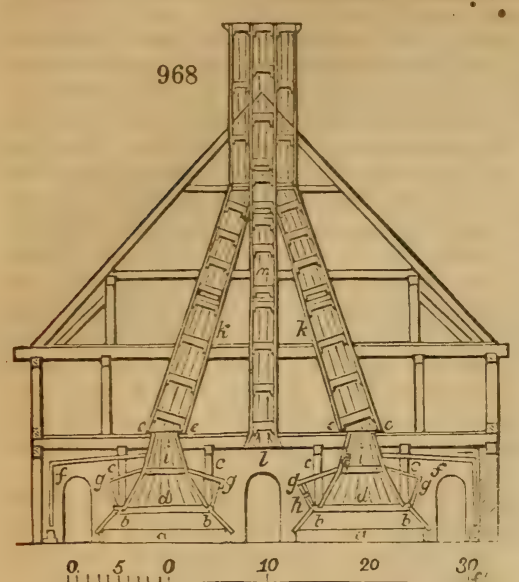
position; the salt is put into small baskets, and carried into the stove-room. $i, k$, is the steam-trunk; $l, m$, is a tunnel for carrying off the steam from the middle of the pan, when this is uncovered by lifting the boards.

In proportion as the brine becomes concentrated by evaporation, more is added - from the settling reservoir of the graduation-house, till finally small crystals appear on the surface. No more weak brine is now added, but the charge is worked off care being taken to remove the scum as it appears. In some places the first pan is called a schlot-plan, in which the concentration is carried only so far as to cause the deposition of the sludge, from which the saline solution is run into another pan, and gently evaporated, to produce the precipitation of the fine salt. This salt should be continually raked towards the cooler and more elevated sides of the pan, and then lifted out with cullender-shovels into large conical baskets, arranged in wooden frames round the border of the pan, so that the drainage may flow back into the boiling liquor. The drained salt is transferred to the hurdles or bastiets in the stoveroom, which ought to be kept at a temperature of from $120^{\circ}$ to $130^{\circ} \mathrm{Fahr}$. The salt is then stowed away in the warehouse.

The graduation range should be divided lengthwise into several sections; the first to receive the water of the spring, the lake, or the sea; the second, the water from the first shower-receiver; the third, the water from the second receiver; and so on. The pumps are usually placed in the middle of the building, and lift the brine from the several receivers below into the alternate elevated cisterns. The square wooden sjouts of distri. bution may be conveniently furnished with a slide-board, attached to each of their sides, to serve as a general valve for opening or shutting many trickling orifices at once. The rate of evaporation at Moutiers is exhibited by the following table :-

\begin{tabular}{|c|c|c|c|c|c|}
\hline \multicolumn{2}{|l|}{ Number of Showers. } & \multicolumn{2}{|c|}{ Total Surface of the Fagots. } & $\begin{array}{l}\text { Specific Gravity } \\
\text { of the Brine. }\end{array}$ & $\begin{array}{l}\text { Water } \\
\text { evaporated. }\end{array}$ \\
\hline $\begin{array}{l}1 \text { and } 2 \\
3,4,5,6,7,8 \text {, and } 9 \\
10\end{array}$ & $\begin{array}{l}- \\
-\end{array}$ & $\begin{array}{l}5158 \text { square feet } \\
2 \tau 20 \\
550\end{array}$ & $\begin{array}{ll}- & - \\
- & - \\
- & -\end{array}$ & $\begin{array}{l}1 \cdot 010 \\
1 \cdot 023 \\
1 \cdot 072 \\
1 \cdot 140\end{array}$ & $\begin{array}{l}0.000 \\
0.540 \\
0.333 \\
0.062\end{array}$ \\
\hline \multicolumn{5}{|c|}{$\begin{array}{l}\text { Total evaporation } \\
\text { Water remaining in the brine at the density of } 1 \cdot \overline{140}\end{array}$} & $\begin{array}{l}0.935 \\
1.065\end{array}$ \\
\hline \multicolumn{5}{|c|}{ Water assigned at the density of $1 \cdot 010$} & 1.000 \\
\hline
\end{tabular}

From the above table it appears that no less than 10 falls of the brine have been quired to bring the water from the specific gravity 1.010 to $1 \cdot 140$, or $18^{\circ}$ Baumé. The aporation is found to proceed at nearly the same rate with the wealier water, and with e stronger, within the above limits. When it arrires at a density of from $1 \cdot 140$ to $1 \cdot 16$, is run off into the settling cisterns. M. Berthier calculates, that upon an average, in .dinary weather, at Moutiers, 60 kilogrammes of water (13 gallons, imp.) are eraporated from the fagots, in the course of 24 nours, for every square foot of their surface. Without the aid of currents of air artificially warmed, such an amount of eraporation could not be reckoned upon in this countrs. In the schiotting, or throwing down of the sediment, a little bullock's blood, previously beaten up with some cold brine, promotes the clarification. When the brine acquires, by brisk ebullition, the density of $1 \cdot 200$, it should be run off from the preparation, to the finishing or salting pans.

The mother-water contains a great deal of chloride of magnesium, along with chloride of sodium, and sulphate of magnesia. Since the last two salts mutually decompuse each other at a low temperature, and are transformed into sulphate of soda, which crvstallizes, and muriate of magnesia, which remains dissolved, the mother-water with 
this new may be exposed in tanks to the frost during winter, when it affords three suc. cessive erystalline deposites, the last being sulphate of soda, nearly pure.

The chloride of mannesium, or bittern, not only deteriorates the salt very much, but oczasions a considerable loss of weight. It may, however, be most adrantageously cot rid of, and converted into chloride of sodium, by the following simple expedient : - Let quichlime be introduced in cquivalent quantity to the magnesia present, and it will precipitate this earth, and form chloride of calcium, which will immediately react upon the sulphate of soda in the mother-water, with the production of sulphate of lime and chloride of sodium. The former being sparingly soluble, is easily separated. Lime, moreover, decomposes directly the chloride of magnesium, but with the eflect of merely substituting chloride of calcium in its stead. But in general there is abundance of sulphate of soda in brine springs to decompose the chloride of calcium. A still better way of proceeding with sea-water, would be to add to it, in the settling tank, the quantity of lime equiralent to the magnesia, whereby an available deposite of this earth would be obtained, at the same time that the brine would be sweetened. Water thus purified may be safely crystallized by rapid evaporation.

In summer, the saturated boiling brine is crystallized by passing it over vertical ropes; for which purpose 100,000 metres (110,000 yards) are mounted in an apartment 70 metres ( 77 yards) long. When the salt has formed a crust upon the ropes abont $2 \frac{1}{2}$ inches thick, it is broken off, allowed to fall upon the clean floor of the apartment, and then gathered up. The salting of a charge, which would take 5 or 6 days in the pan, is completed in this way in 17 hours; but the mother-waters are more abundant. 'The salt is, however, remarkably pure.

The boilers constructed at Rosenheim, in Bavaria, evaporate $3 \frac{1}{2}$ pounds of water for every pound of wood burned; which is reckoned a favorable result; but some of those described under Evaporation, would throw off much more.

"T The rock salt mines and principal brine springs are in Cheshire; and the chief part of the Cheshire salt, both fossil and manufactured, is sent by the river Wearer to Lirerpool, a very small proportion of it being conveyed elsewhere, by canal or land carriage. There are brine springs in Staffordshire, from which Hull is furnished with white salt; and in Worcestershire, from which Gloucester is supplied. If to the quantity shipped by the Weaver, 100,000 tons of white salt are adrled annually for internal consumption and exports, exclusive of Liverpool, the total manufacture will be approached rery nearIy; but as there is now no check from the excise, it is impossible to ascertain it exactly. Fossil salt is used in small quantities at some of the Cheshire manufactories, to strenothen the brine, but is principally exported; some to Ireland, but chiefly to Belgium and Holland.9* The average quantity of rock salt sent annually down the river Weaver from the mines in Cheshire, between the years 1803 and 1834 inclusive, was 86,000 tons, of $2,600 \mathrm{lbs}$. each; the greatest being 125,658 , in the year 1823 , and the least 47,230 , in the year 1813. The arerage quantity of white salt sent annually down the Weaver from the manufactories in Cheshire during the same period, was 221,351; the greatest being 383,669 , in the year 1832 , and the least being 120,486 , in the year 1811 .

M. Clement-Desormes, engineer and chief actionnaire of the great salt-works of Dieuze. in France, informs me that the internal consumption of that kingdom is rather mere than 200,000 tons per annum, being at the rate of $6 \frac{1}{2}$ kilogrammes for each individuąl of a population estimated at $32,000,000$. As the retail price of salt in France is 10 sous per kilogramme (of $2 \frac{1}{2} \mathrm{lbs}$. a voird.), while in this country it is not more than 2 sous ( 1 penny), its consumption per head will be much greater with us; and, taking into account the immense quantity of salted provisions that are used, it may be reckoned at 22 lbs.; whence our internal consumption will be 240,000 tons, instead of 100,000 , as quoted above, from the tables published by the Board of Trade.

In $1836,9,622,427$ bushels, of $56 \mathrm{lbs} .=240,560$ tons of salt, value 173,923l., were exported from the United Kingdom, of which 1,350,849 bushels went to Russia; $1,235,086$ to Bclgium; 314,132 to the Western coast of Africa; $1,293,560$ to the British North American colonies; 2,8\%0,808 to the United States of America; 53,299 to New South Wales, Van Diemen's Land, and other Australian settlements; 58,735 to the British West Indies ; and 90,655 to Guernsey, Jersey, Alderney, and Man.

SAND (Eng. and Germ.; Sable, Fr.), is the name given to any mineral substance in a hard granular or pulverulent form, whether strewed upon the surface of the ground, found in strata at a certain depth, forming the beds of rivers, or the shores of the sea. The silicious sands seem to be either original crystalline formations, like the sand of Neuilly, in 6-sided prisms, terminated by two 6-sided pyramids, or the dibris of granitic, schistose, quartzose, or other primitive crystalline rocks, and are abundantly distributed over the globe; as in the immense plains known under the names of downs, deserts, steppes, iandes, \&c., which, in Africa, Asia, Europe, and America, are entirely covered with

* Tables of the Revenue, Population, Commerce, \&c., for 1835, p. I22. 
loose sterile sand. Valuable metallic ores, those of gold, platinum, tin, copper, iron, titanium, often occur in the form of sand, or mixed with that earthy substance. Pure silicious sands are very valuable for the manufacture of glass, for making mortars, filters; ameliorating dense clay soils, and many other purposes. For moulder's sand, See FoundING. Lynn and Ryegate furnish our purest silicious sand.

SANDAL or RED SAUNDERS WOOD (Santal, Fr.; Sandelhole, Germ.), is the wood of the Plerocurpus suntadinus, a tree which grows in Ceylon, and on the coast of Coromandel. The old wood is preferred by dyers. Its coloring matter is of a resinous nature; and is, therefore, quite soluble in alcohol, essential oils, and alkaline leys; but sparingly in boiling water, and hardly if at all in cold water. The coloring matter which is obtained by evaporating the alcoholic infusion to dryness, has been called santaline; it is a red resin, which is fusible at $212^{\circ} \mathrm{F}$. It may also be obtained by digesting the rasped sandal wood in water of ammonia, and afterwards saturating the ammonia with an acid. The santaline falls, and the supernatant liquor, which is yellow by transmitted, appears blue by reflected light. Its spirituous solution affords a fine purple precipitate with the protochloride of tin, and a violet one with the salts of lead. santaline is very soluble in acetic acid, and the solution forms permanent stains upon the shin.

Sandal wood is used in India, along with one tenth of sapan wood (the Casalpinia sapan of Japan, Jara, Siam, Celebes, and the Philippine isles), principally for dyeing silk and cotton. Trommsdorf dyed wool, cotton, and linen a carmine hue by dipping them alternately in allialine solution of the sandal wood, and in an acidulous bath. Bancroft obrained a fast and brilliant reddish-yellow, by preparing wool with an alum and tartar bath, and then passing it through a boiling bath of sandal wood and sumac. Pelletier did not succeed in repeating this experiment. According to Togler, wool, silk, cotton, and linen, mordanted with salt of tin, and dipped in a cold alcoholic tincture of the wood, or the same tincture mixed with 8 parts of boiling water, become of a superb ponçeau-red color. With alum, they took a scarlet-red; with sulphate of iron, a deep violet, or brown-red. Unluckily, these dyes do not stand exposure to light well.

SANDARACH, is a peculiar resinous substance, the product of the Thuya articulata, a small tree of the coniferous family, which grows in the northern parts of Africa, especially round Mount Atlas.

The resin comes to us in pale yellow, transparent, brittle, small tears, of a spherica] or cylindrical shape. It has a faint aromatic smell, does not sofien, but breaks between the teeth, fuses readily with heat, and has a specific gravity of from 1.05 to 1.09. It contains three different resins; one soluble in spirit of wine, somewhat resembling pinic acid (see TuRPENTINi.); one not soluble in that menstruum; and a third, soluble only in alcohol of 90 per cent. It is used as pounce-powder for strewing over paper erasures, as incense, and in varnishes.

SAPAN WOOD, is a species of the Casalpinia genus, to which Brazil wood belongs. It is so called by the French, because it comes to them from Japan, which they corruptly pronounce Sapan. As all the species of this tree are natives of either the East Indies or the New World, one would imagine that they could not have been used as dye-stufis in Europe before the beginning of the 16 th century. Yet the author of the article "Brazil," in Rees' Cyclopædia, and Mr. Southey, in his History of Brazil, say that Brazil wood is mentioned nearly one hundred years before the discoveries of Columbus and Vasco de Gama, by Chaucer, who died in 1400 ; that it was known many ages before his time; and that it gave the name to the country, instead of the country giving the name to the wood, as I have stated, with Berthollet and other writers on dyeing. The Casalyinia sappan, being a native of the Coromandel coast, may possibly have been transported along with other Malabar merchandise to the Mediterranean marts in the middle ages; but the importation of so lumbering an article in any considerable quantity by that channel, is so improbable, that I am disposed to belive that Brazil wood was not commonly used by the dyers of Europe before the discovery of the New World.

SARD ; see LAPIDARY. ${ }^{\circ}$

SATIN (Eng., Fr., and Germ.), is the name of a silk stuff, first imported from China, which is distinguished by its very smooth, polished, and glossy surface. It is woven upon a loom with at least five-leaved healds or heddles, and as many corresponding treddles. These are so mounted as to rise and fall four at a time, raising and depressing alternately four yarns of the warp, across the whole of which the weft is thrown by the shuttle, so as to produce a uniform smooth texture, instead of the checkered work resulting from intermediate decussations, as in common webs. See Textrle Fabrics. Satins are woven with the glossy or right side undermost, because the four-fifths of the warp, which are always left there during the action of the healds, serve to support the shuttle in its race. Were they woven in the reverse way, the scanty fifth part of the warp threads could either not support, or would be too much worn by the shuttle. 
SATURATION is the term at which any body has taken its full dose or chemical pro portion of any other with which it can combine; as water with a salt, or an acid with an alkali in the neutro-saline state.

SCALIOLA is merely ornamental plaster-work, produced by applying a pap made of finely-ground calcined gypsum, mixed with a weak solution of Flanders' glue, upion any figure formed of laths nailed together, or occasionally upon brickwork, and bestudding its surface, while soft, with splinters (scagliole) of spar, marble, granite, bits of concrete colored gyjsum, or veins of clay, in a semi-fluid state. The substances employed to color the spots and patches, are the several ochres, boles, terra di Sienna, chrome yellow, \&c. The surface of the columr is turned smooth upon a lathe, polished with stones of diflerent fineness, and finished with some plaster-jap, to give it lustre. Pillars and other flat surfaces are smoothed by a carpenter's plane, with the chisel finely serrated, and afterwards polished with plaster by friction. The glue is the cause of the gloss, but makes the surface apt to be injured by moisture, or even damp air.

SCARLET DYE. (Teinture en écarlate, Fr.; Scharlachfärberei, Germ.) Scarlet is usually given at two successive operations. The boiler (see figs. 364, 365, article DyeING) is made of block tin, but its bottom is formed occasionally of copper.

1. The bouillon, or the coloring-bath.-For 100 pounds of cloth, put into the water, when it is little more than lukewarm, 6 pounds of argal, and stir it well. When the water becomes too hot for the hand, throw into it, with agitation, one pound of cochineal in fine powder. An instant afterwards, pour in 5 pounds of the clear mordant G (see TIN MorDANTS), stir the whole thoroughly as soon as the bath begins to boil, introduce the eloth, and wince it briskly for two or three rotations, and then more slowly. At the end of a two-hours' boil, the cloth is to be taken out, allowed to become perfectly cool, and well washed at the river, or winced in a current of pure water. (See an automatic plan of washing described under the article Rinsing Machine.)

2. The rougie, or finishing dye.-The bouillon bath is emptied, and replaced with water for the rougie. When it is on the point of boiling, $5 \frac{1}{2}$ pounds of cochineal in fine powder are to be thrown in, and mixed with care; when the crust, which forms upon the surface, opens of itself in several places, 14 pounds of solution of tin (as above) are to be added. Should the liquor be likely to boil over the edges of the kettle, it must be refreshed with a little cold water. When the bath has become uniform, the cloth is to be put in, taking care to wince it briskly for two or three turns; then to boil it bodily for an hour, thrusting it under the liquor with a rod whenever it rises to the surface. It is lastly taken out, aired, washed at the river, and dried.

As no person has done more for the improvement of the scarlet dyes than Poërner, I shall here give his processes in detail.

Bouillon, or coloring.-For every pound of cloth or wool, take 14 drachms of cream of tartar. When the bath is boiling, and the tartar all dissolved, pour in surcessively 14 drachms of solution of tin (Mordant $F$, TIN), and let the whole boil together during a few minutes. Now introduce the cloth, and boil it for 2 hours; then take it out, and let it drain and cool.

Rou gie, or dye.-For every pound of woollen stuff, take 2 drachms of eream of tartar. When the bath begins to boil, add 1 ounce of cochineal reduced to fine powder, stir the mixture well with a rod of willow or any white wood, and let it boil for a few minutes. Then pour in, by successive portions, 1 ounce of solution of tin (Mordant F), stirring continually with the rod. Lastly, dye as quickly as possible. The color will be a beautiful scarlet.

Second scarlet process of Poërner, the bouillon being the same as above given, and always estimated for 1 pound of cloth or wool. Rougie.-Take 1 ounce of cochineal in fine powder, and 2 ounces of solution of tin without tartar.

Third scarlet process of Poërner; the bouillon being as above. Rougie for a pound of cloth. - Take two drachms of cream of tartar, one ounce of cochineal, one ounce of solu. tion of tin, and 2 ounces of sea salt; dye as in process 1 . The salt helps the dye to pene. trate into the cloth.

Tables of the Composition of the Bouizlon and Rougie, by different Authors, for 100 pounds of Cloth or Wool.

Composition of the Bouillon.

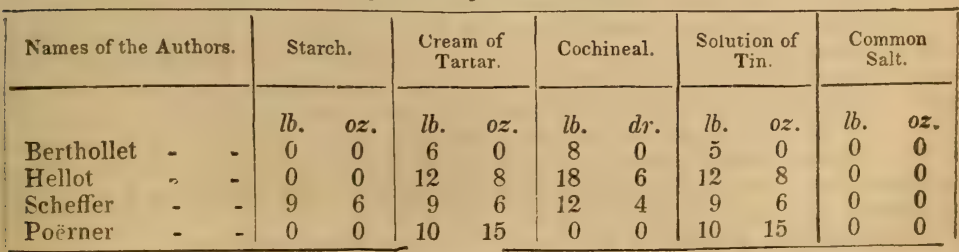


Composition of the Rongre.

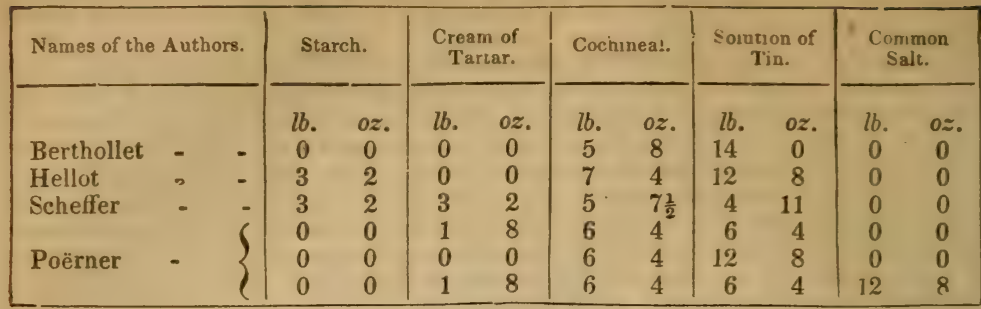

M. Lenormand states that he has made experiments of verification upon all the formu læ of the preceding tables, and declares his conviction that the finest tint may be obtained by taking the bouillon of Scheffer, and the rougie No. 4 of Poërner. The solution which produced the most brilliant red, is that made according to the process of mordant $\mathrm{B}$ (Ti\%.) M. Robiquet has given the following prescription for making a printing scurlet, for wellwhitened woollen cloth.

Boil a pound of pulverized cochineal in four pints of water down to 2 pints, and pass the decoction through a sieve. Repeat the boiling three times upon the resirluum, mix the eight pints of decoction, thicken them properly with two pounds of starch, and boil into a paste. Let it cool down to $104^{\circ} \mathrm{F}$., then add four ounces of the subjoined solution of tin, and two ounces of ordinary salt of tin (muriate.) When a ponçeau red is wanted, two ounces of pounded curcuma (turmeric) should be added.

The solution of tin above prescribed, is made by taking-one ounce of nitric acid, of specific gravity $36^{\circ} \mathrm{B},=1.33$; one ounce of sal ammoniac; four ounces of grain tin. The tin is to be divided into eight portions, and one of them is to be put into the acid mixture every quarter of an hour.

A solution of chlorate of potassa (chloride?) is said to beautify scarlet cloth in a re markable manner.

Bancroft proposed to supplant the nitro-muriatic acid, by a mixture of sulphuric and murialic acids, for dissolving tin; but I do not find that he succeeded in persuading scarletdyers to adopt his plans. In fact, the proper base is, in my opinion, a mixture of the protoxyde and peroxyde of tin; and this cannot be obtained by acting upon the metal with the murio-sulphuric acid. He also prescribed the extensive use of the quercitron yellow to change the natural crimson of the cochincal into scarlet, thereby economizing the quautity of this expensive dye-stuff. See Lac Dye.

SCHEELE'S GREEN is a pulverulent arsenite of copper, which may be prepared as follows :-Form, first, an arsenite of potassa, by adding gradually 11 ounces of arsenious acid to 2 pounds of carbonate of potassa, dissolved in 10 pounds of boiling water; next, dissolve 2 pounds of crystallized sulphate of copper in 30 pounds of water; filter each solution, then pour the first progressively into the second, as long as it produces a rich grass-green precipitate. This being thrown upon a filter-cloth, and edulcorated with warm water, will afford 1 pound 6 ounces of this heautiful pigment. It consists of, oxyde or copper 23.51 , an of arsenious acid $71 \cdot 46$. This green is applied by an analogous double decomposition to cloth. See Calico-PRINTING.

SCHWEINFURTH GREEN is a more beautiful and velvety pigment than the preceling, which was discovered in 1814, by MM. Rusz and Saltler, at Schweinfurth, and remained for many years a profitable secret in their hands. M. Liebig having made its composition known, in 1822 , it has been since prepared in a great many color-works. Braconnot published, about the same time, another process for manufacturing the same pigment. Its preparation is very simple; but its formation is accompanied with some interesting circumstances. On mixing equal parts of acetate of copper and arsenious acid, each in a boiling concentrated solution, a bulky olive-green precipitate is immediately produced; while much acetic acid is set free. The powder thus obtained, appears to be a compound of arsenious acid and oxyde of copper, in a peculiar state; since when decomposed by sulphuric acid, no acetic odor is exhaled. Its color is ne $t$ changed by drying, by exposure to air, or by being heated in water. But, if it be boiled in the acidulous liquor from which it was precipitated, it soon changes its color, as well as its state of aggregation, and forms a new deposite in the form of a dense granular beautiful green powder. As fine a color is produced by ebullition during five or six minutes, as is obtained at the end of several hours by mixing the two boiling solutions, and allowing the whole to ccol together. In the latter case, the precipitate, which is slight and flocky at first, becomes denser by deorees; it next betrays green spots, which progressively increase, till the mass grows altogether of a crystalline constitution, and of a still more beautiful tint than if formed by ebullition.

When cold water is added to the mixed solutions, immediately after the precipitato 
takes place, the development of the color is retarded, with the effect of maling it much finer. The best mode of procedure, is to add to the blended soluticns, their uwn bulk of cold water, and to fill a globe up to the neck with the mixture, in order to prevent the formation of any such pellicle on the surface as might, by falling to the hottom, excite premature crystallization. Thus the reaction continues during two or three days with the happiest effect. The difference of tint produced by these variations, arises merely from the different sizes of the crystalline particles; for when the several powders are levigated upon a porphyry slab to the same degree, they have the same shade. Schweinfurth green, according to M. Ehrmann's researches, in the 31st Bulletin de la Société Indiustrielle de Mulhausen, consisis of, oxyde of copper $31 \cdot 666$, arsenious acid 58.699, acetic acid $10 \cdot 294$. Kastner has given the following prescription for making this pigment: - For 8 parts of arsenious acid, take from 9 to 10 of verdigris; difluse the latter through water at $120^{\circ} \mathrm{F}$., and pass the papthrough a sieve; then mix it with the arsenical solution, and set the mixture aside, till the reaction of the ingredients shall produce the wished-for shade of color. If a yellowish tint be desired, more arsenic must be used. By digesting Scheele's green in acetic acid, a variety of Schweinfurth green may be obtained.

Both of the above colors are rank poisons. The first was detected a few years ago, as the coloring-matter of some Parisian bombons, by the conseil de salubrité; since which the confectioners were prohibited from using it, by the French government.

SCOLRING, or renovating articles of dress. This art has been much more studied by Frenchmen, who wear the same coats for two or three years, than by Englishmen, who generally cast them off after so many months. The workmen who remore greasy stains from dress, are called, in France, teinturiers-degraisseurs, because they are often obliged to combine dyeing with scouring operations. The art of cleansing clothes being founded upon the linowledge of solvents, the practitioner of it should, as we shall presently illustrate by examples, be acquainted with the laws of chemical affinity.

Among the spots which alter the colors fixed upon stuffs, some are caused by a substance which may be described as simple, in common language; and others by a substance which results from the combination of two or more bodies, that may act separately or together upon the stuff, and which may therefore be called compound.

Simple stains.- Oils and fats are the substances which form the greater part of simple stains. They give a deep shade to the ground of the cloth; they continue to spread for several dars; they attract the dust, and retain it so strongly, that it is not remoreable by the brush; and they eventually render the stain lighter colored upon a dark ground, and of a disagreeable gray tint upon a pale or light ground.

The general principle of cleansing all spots, consists in applying to them a sutstance which shall have a stronger affinity for the matter composing them, than this has for the cloth, and which shall render them soluble in some liquid menstruum, such as water, spirits, naptha, oil of turpentine, \&c. See BLEAcHING.

Allialis would seem to be proper in this point of view, as they are the most powerful solvents of grease; but they act too strongly upon silk and wool, as well as change too powerfully the colors of dyed stuffs, to be safely applicable in removi..z stains. The best substances for this purpose are-1. Soap. 2. Chalk, fuller's earth, soap-stone or steatite (called in this country French chalk). These should be merely diffused through a little water into a thin paste, spread upon the stain, and allowed to dry. The spot requires now to be merely brushed. 3. Ox-gall and yoll of egg have the property of dissolving fatty bodies without affecting perceptibly the texture or colors of cloth, and may therefore be employed with advantage. The ox-gall should be purified, to prevent its greenish tint from degrading the brilliancy of dyed stuffs, or the purity of whites. Thus prepared (see GALL), it is the most precious of all substances known for removing these kinds of stains. 4. The volatile oil of turpentine will take out only recent stains; for which purpose it ought to be previously purified by distillation over quichlime. Wax, rosin, turpentine, pitch, and all resinous bodies in general, form stains of greater or less adhesion, which may be dissolved out by pure alcohol. The juices of fruits, and the colored juices of all vegetables in general, deposite upon clothes marks in their peculiar hues. Stains of wine, mulberries, black currants, morellos, liquors, and weld, yield only to soaping with the hand, followed by fumigation with sulphurous acid; but the latter process is inadmissible with certain colored stuffs. Iron mould or rust stains may be taken out almost instantaneously with a strong solution of oxalic acid. If the stain is recent, cream of tartar will remove it.

Compound spots. - That mixture of rust of iron and grease called cambouis by the French, is an example of this kind, and requires Iwo distinct operations; first, the removal of the grease, and then of the rust, by the means above indicated.

Mud, especially that of cities, is a compound of vegetable remains, and of ferruginous matter in a state of black oxyde. Washing with pure water, followed if necessary with soaping, will take away the vegetable juices; and then the iron may be removed with 
creain of tartar, which itself must, however, be well washed out. Ink stains, when recent, may be taken out by washing, first with pure water, next with soapy water, and lastly with lemon juice; but if old, they must be treated with oxalic acid. Stains occasioned by smoke, or by sauces browned in a frying-pan, may be supposed to cronsist of a mixture of pitch, black oxyde of iron, empyreumatic oil, and some saline matters dissolved in pyroligneous acid. In this case several reagents must be employed to remove the stains. Water and soap dissolve perfectly well the vegetable matters, the salts, the pyroligneous acid, and even the empyreumatic oils in a great measure; the essence of turpentine will remove the rest of the oils and all the pitchy matter; then oxalic acid may be used to discharge the iron. Coffee stains require a washing with water, with a careful soaping, at the temperature of $120^{\circ} \mathrm{F}$., followed by sulphuration. The two latter processes may be repeated twice or tbrice. Chocolate stains may be removed by the same means, and more easily.

As to those stains which change the color of the stuff, they must be corrected by appropriate chemical reagents or dyes. When black or brown cloth is reddened by an acid, the stain is best counteracted by the application of water of ammonia. If delicate silk colors are injured by soapy or alkaline matters, the stains must be treated with colorless vinegar of moderate force. An earthy compound for removing grease spots is made as follows : - Take fuller's earth, free it from all gritty matter by elutriation with water; mix with half a pound of the earth so prepared, half a pound of soda, as much soap, and eight yolks of eggs well beat up with half a pound of purified ox-gall. The whole must be carefully triturated upon a porphyry slab; the soda with the soap in the same manner as colors are ground, mixing in gradually the eggs and the ox-gall previously beat together. Incorporate next the soft earth by slow degrees, till a uniform thick paste be formed, which should be made into balls or cakes of a convenient size, and laid out to dry. A little of this detergent being scraped off with a knife, made into a paste with water, and applied to the stain, will remove it. Purified ox-gall is to be diffused through its own bulk of water, applied to the spots, rubbed well into them with the hands till they disappear, after which the stuff is to be washed with soft water. It is the best substance for removing stains on woollen clothes.

The redistilled oil of turpentine may also be rubbed upon the dry clothes with a sponge or a tuft of cotton till the spot disappear; but it must be immediately afterwards covered with some plastic clay reduced to powder. Without this precaution, a cloud would be formed round the stain, as large as the part moistened with the turpentine.

Oxalic acid may be applied in powder upon the spot previously moistened with water, well rubbed on, and then washed off with pure water.

Sulphurous acid is best generated at the moment of using it. If the clothes be much stained, they should be suspended in an ordinary fumigating chamber. For trifling stains, the sulphur may be burned under the wide end of a small card or paper funnel, whose upper orifice is applied near the cloth.

Manipulations of the scourer. - These consist, first, in washing the clothes in clear soft water, or in soap-water. The cloth must be next stretched on a sloping board, and rubbed with the appropriate reagent as above described, either by a sponge or a small

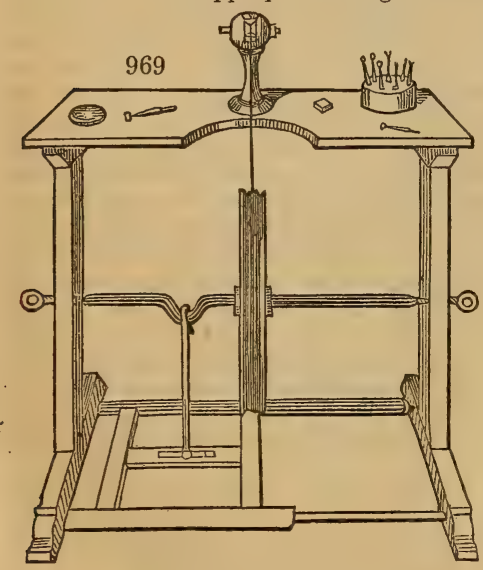

972 hard brush. The application of a redhot iron a little way above a moistened spot often volatilizes the greasy matter out of it. Stains of pitch, varnish, or oil paint, which have become dry, must first be softened with a little fresh butter or lard, and then treated with the powder of the scouring ball. When the gloss has been taken from silk, it may be restored by applying the filtered mucilage of gum tragacanth; stretching it upon a frame to dry. Ribands are glossed with isinglass. Lemon juice is used to brighten scarlet spots, after they have been cleaned.

SEAL ENGRAVING. The art of en graving gems is one of extreme nicety. The stone having received its desired form from the lapidary, the engraver fixes it by cement to the end of a wooden handle, and then draws the outline of his subject, with a brass reedle or a diamond, upon its smooth surface.

Fig. 969 represents the whole of the sea engraver's lathe. It consists of a table on which is fixed the miil, a small horizontal 
eylinder of steel, into one of whose extremities the tool is inserted, and which is made to revolve by the usual fly-wheel, driven by a treddle. The tools that may be fitted to the mill-cylinder, are the following; fig. 970 a hollow cylinder, for describing circles, and for borir.g; fig. 971 a knobbed tool, or rod terminated by a small ball; fig. 972 a stem terminated with a cutting disc, whose edge may be either rounded, square, oi sharp; being in the last case called a saw.

Having fixed the tool best adapted to his style of work in the mill, the artist applies to its cutting point, or edge, some diamond-powder, mixed up with olive oil; and turning the wheel, he holds the stone against the tool, so as to produce the wished-for delineation and erosion. A similar apparatus is used for engraving on glass.

In order to give the highest degree of polish to the engraving, tools of boxwood, pewter, or copper, bedaubed with moistened tripoli or rotten-stone, and lastly, a brush, are fastened to the mill. These are worked like the above steel instruments. Modern engravings on precious stones have not in general the same fine polish as the ancient. The article Geus, in Rees' Cyclopæedia, contains a variety of valuable information on this subject, equally interesting to the artist and the scholar.

SEALING-WAX. (Cire à cacheter, Fr.; Siegellack, Germ.) The Hindoos from time immemorial hare possessed the resin lac, and were long accustomed to use it for sealing manuscripts before it was linown in Europe. It was first imported from the East into Tenice, and then into Spain; in which country sealing-wax became the object of a con. siderable commerce, under the name of Spanish wax.

If shrilac be compounded into sealing-wax, immediately after it has been separated by fusion fiom the palest qualities of stick or seed lac, it then forms a better and less brittle article, than when the shellac is fused a second time. Hence sealing-wax, rightly prepared in the East Indies, deserves a preference over what can be made in other countries, where the lac is not indigenous. Shellac can be restored in some degree, howerer, to a plastic and tenacious state by melting it with a very small portion of turpentine. The palest shellac is to be selected for bright-colored sealing-wax, the dark kind being reserved for black.

The following prescription may be followed for making red sealing-wax:-Take 4 ounces of shellac, 1 ounce of Venice turpentine (some say $1 \frac{1}{2}$ ounces), and 3 ounces of vermilion. Melt the lac in a copper pan suspended over a clear charcoal fire, then pour the turpentine slowly into it, and soon afterwards add the vermilion, stirring briskly all the time of the mixture with a rod in either hand. In forming the round sticks of sealing-wax, a certain portion of the mass should be weighed while it is ductile, dirided into the desired number of pieces, and then rolled out upon a warm marble slab, by means of a smooth wooden block, like that used by apothecaries for rolling a mass of pills. The oral sticks of sealing-wax are cast in moulds, with the above compound in a state of fusion. The marks of the lines of junction of the mould-box may be afterwards removed by holding the sticks over a clear fire, or passing them over a blue gas. flame. Marbled sealing-wax is made by mixing $t w 0$, three, or more colored linds of it, while they are in a semi-fluid state. From the viscidity of the several masses, their incorporation is left incomplete, so as to produce the appearance of marbling. Gold sealing-wax is made simply by stirring gold-colored mica spanoles into the melted resins. Wax may be scented by introducing a little essential oil, essence of musk, or other perfume. If 1 part of balsam of Peru be melted along with 99 parts of the sealing-wax composition, an agreeable fragrance will be exhaled in the act of sealing with it. Either lamp black or ivory black serves for the coloring-matter of black wax. Sealing-rvax is often adulterated with rosin; in which case it runs into thin drops at the flame of a candle.

SEA WATER, is composed as follows, according to the author of the article Salines, in the Dictionnaire Technologique:-Chloride of sodium, 2.50; chloride of magnesium, 0.35 ; sulphate of magn.sia, 0.58 ; carbonates of lime and magnesia, 0.02 ; sulphate of lime, 0.01 ; water, 96.54 , in 100 parts. See SALT, SEA.

SEGGAR, or SAGGER, is the cylindric case, of fire-clay, in which fine stoneware is enclosed while being baked in the kiln.

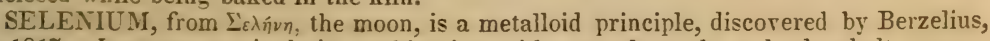
in 1817. It occurs sparingly in combination with several metals, as lead, cobalt, copper, and quichsilver, in the Harz, at Tilkerode; with copper and silver (Eulcairite) in Sweden, with tellurium and bismuth in Norway, with tellurium and gold in Siebenbürgen, in several copper and iron pyrites, and with sulphur in the rolcanic products of the Lipari islands. Selenium has been found likewise in a red sediment which foims upon the bottoms of the lead chambers in which ril of vitriol has been made frotin peculiar pyrites, or pyritous sulphur. The extraction of selenium from that deposite is a very complex process.

Selenium, after being fused and slowly cooled, appears of a bluish-gray color, with a glistening surface; but it is reddish brown, and of metallic lustre when quiclily cooled. 
It is brittle, not very nard, and has little tendency to assume the crystalline state. Selenium is dark-red in powder, and transparent, with a ruby cast, in thin scales. Its specific vravity is $4 \cdot 30$. It softens at the temperature of $176^{\circ} \mathrm{F}$., is of a pasty consistence at $212^{\circ}$, becomes liquid at a somewhat higher heat, forming in close vessels dark yellow vapors, which condense into black drops; but in the air, the fumes have a cinnabar-red color.

This singular substance, apparently intermediate in its constitution between sulphur and metals, has not hitherto been applied to any use in the arts.

SELTZER WATER. See Soda-Water, and Waters, Mineral.

SEPI.A, is a pigment prepared from a black juice secreted by certain glands of the cuttle-fish, which the animal ejects to darken the water when it is pursued. One part of it is capable of making 1000 parts of water nearly opaque. All the varieties of this mollusca secrete the same juice; but the Sepia officinalis, the Sepia ioligo, and the Sepia tunicata, are chiefly sought after for making the pigment. The first, which occurs abundantly in the Mediterranean, affords most color; the sac containing it being extracted, the juice is to be dried as quickly as possible, because it runs rapidly into putrefaction. Though insoluble in water, it is extremely diffusible through it, and is very slowly deposited. Caustic alkalis dissolve the sepia, and turn it brown; but in proportion as the allali becomes carbonated by exposure to air, the sepia falls to the bottom of the vessel. Chlorine blanches it slowly. It consists of carbon in an extremely divided state, along with albumine, gelatine, and phosphate of lime.

The dried native sepia is prepared for the painter, by first triturating it with a little caustic ley, then adding more ley, boiling the liquid for half an hour, filtering, next saturating the alkali with an acid, separating the precipitate, washing it with water, and finally drying it with a gentle heat. The pigment is of a brown color, and a fine grain.

SEPTARIA, called anciently ludus Helmontii, (the quoits of Van Helmont, froin their form,) are lenticular concretions of clay ironstone, intersected by veins of calc-spar, which, when calcined, and ground to powder, form an excellent hydraulic cement. See MorTAR, HYDRAULIC.

SERPENTINE, is a mineral of the magnesian family, of a green color ; it is scratched by calcareous spar, is sectile, tough, and therefore easily cut into ornamental forms. It oecurs in Unst and Fetlar, in Shetland; at Portsoy, in Banffshire; in Cornwall; and the Isle of Holyhead. The floors of bakers' ovens are advantageously laid with slabs of serpentine.

SHAFT, in mining, signifies a perpendicular or slightly inclined pit.

SHAGREEN. (Chagrin, Fr. and Germ.) The true oriental shagreen is essentially different from all modifications of leather and parchment. It approaches the latter somewhat, indeed, in its nature, since it consists of a dried skin, not combined with any tanning or foreign matter whatever. Its distinguishing characteristic is having the grain or hair side covered over with small rough round specks or granulations.

It is prepared from the skins of horses, wild asses, and camels; of strips cut along the chine, from the neck towards the tail, apparently because this stronger and thicker portion of the skin is best adapted to the operations about to be described. These fillets are to be steeped in water till the epidermis becomes loose, and the hairs easily come away by the roots; after which they are to be stretched upon a board, and dressed with the currier's fleshing-knife. They must be kept continually moist, and extended by cords attached to their edges, with the flesh side uppermost upon the board. Each strip now resembles a wet bladder, and is to be stretched in an open square wooden frame by means of strings tied to its edges, till it be as smooth and tense as a drum-head. For this purpose it must be moistened and extended from time to time in the frame.

The grain or hair side of the moist strip of skin must next be sprinkled over with a kind of seeds called Allabuta, which are to be forced into its surface either by tramping with the feet, or with a simple press, a piece of felt or other thick stuff being laid upon the seeds. These seeds belong probably to the Chenapodium album. They are lenticular, hard, of a shining black color, farinaceous within, about the size of poppy seed, and are sometimes used to represent the eyes in wax figures.

The skin is exposed to dry in the shade, with the seeds indented into its surface; after which it is freed from them by shaking it, and beating upon its other side with a stick. The outside will be then horny, and pitted with small hollows corresponding to the shape and number of the seeds.

In order to make the next process intelligible, we must advert to another analogous and well-known operation. When we make impressions in fine-grained dry wood with steel punches or letters of any kind, then plane away the wood till we come to the level of the bottom of these impressions, afterwards steep the wood in water, the condensed or punched points will swell above the surface, and place the letters in relief. Snuff-boxes have sometimes been marked with prominent figures in this way. Now shagreen is treatel in a similar manner. 
The strip of skin is stretched in an inclined plane, witn its upper edge attached to hooks, and its under one loaded with weights, in which position it is thinned off with a proper seini-lunar knife, but not so much as to touch the bottom of the seed-pits or depressions. By Inaceration in water, the skin is then made to swell, and the pits become prominent over the surface which had been shaved. The swelling is completed by steeping the strips in a warm solution of soda. after which they are cleansed by the action of salt brine, and then dyed.

In the East the following processes are pursued. Entirely white shagreen is obtained by imbuing the skin with a solution of alum, covering it with the dough made with Turkey wheat, and after a time washing this away with a solution of alum. The strips are now rubbed with grease or suet, to diminish their rigidity, then worked carefully in hot water, curried with a blunt knife, and afterwards dried. They are died red with decoc. tion of cochineal or kermes, and green with fine copper filings and sal ammoniac, the solution of this salt being first applied, then the filings being strewed upon the skin, which must be rolled up and loaded with weights for some time; blue is given with indigo, quicklime, soda, and honey; and black, with galls and copperas.

SHALE, or SLATE CLAY, is an important stratiform member of the coal-measures. See Pitcoal.

SHAMOY LEATHER. See LEATHER.

SHEATHING OF SHIPS. For this purpose many different metals and metallic alloys have been lately proposed. From a train of researches which I made for an eminent copper company, a few years ago, upon various specimens of sheathing which had been exposed upon ships during many voyages, it appeared that copper containing a minute but definite proportion of tin, was by far the most durable.

SHELlaC. See Lac, and Sealing-wax.

SIENITE is a granular aggregated compound rock, consisting of feldspar and hornblende, sometimes mixed with a little quartz and mica. The hornblende is the characteristic ingredient, and serves to distinguish sienite from granite, with which it has been sometimes confounded; though the feldspar, which is generally red, is the more abundant constituent. The Egyptian sienite, containing but little hornblende, with a good deal of quartz and mica, approaches most nearly to granite. It is equally metalliferous with porphyry ; in the island of Cyprus, it is rich in copper; and in Hungary, it contains many valuable gold and silver mines.

Sienite forms a considerable part of the Criffle, a hill in Galloway. It takes its name from the city of Syene, in the Thebaid, near the cataracts of the Nile, where this rock abounds. It is an excellent building-stone, and was imported in large quantities from Egypt by the Romans, for the architectural and statuary decorations of their capital.

SII.ICA and SILICON. (Silice, silicium, Fr.; Kieselerde, kiesel, Germ.) Silica was till lately ranked among the earths proper; but since the researches of Davy and Berzelius, it has been transferred to the chemical class of acids. It constitutes the principal portion of most of the hard stones and minerals which compose the crust of the globe; occurring nearly pure in rock crystal, quartz, agate, calcedony, flint, \&c. Silica or silicic acid may be obtained perfectly pure, and also in the finest state of comminution, by taking the precipitate formed by passing silicated fluoric gas through water, filtering, washing, and igniting it, to expel the last traces of the fluoride of silicon. The powder thus obtained is so light as to be blown away with the least breath of air. Silica may be more conveniently procured, however, by fusing ground flint with four times its weight of a mixture, in equal parts, of dry carbonate of potassa and carbonate of soda, in a platinum or silver crucible. The alkaline carbonates should be first fused, and the flint powder sprinkled into the liquid, as long as it dissolves with effervescence. The mass is to be then allowed to cool, dissolved in dilute muriatic acid; the solution is to be filtered, and evaporated to dryness; the dry crust is to be pulverized, digested for two hours with a little muriatic acid, to remove any iron and alumina that may be present, next washed with hot water, drained, dried, and ignited.

The above silicate of potassa and soda is the compound called soluble glass, which applied in solution to the surface of wood, calico, paper, \&c., renders them unsusceptible of taking fire on the contact of an ignited body.

Silica, as thus prepared, is a white powder, rough to the touch, gritty between the teeth, absolutely insoluble in water, acids, and most liquids. Its specific gravity is 2.66. It cannot be fused by the most intense heat of our furnaces, but at the flame of the oxy-hydrogen blowpipe it melts into a limpid colorless glass. By peculiar chemieal rnethods, an aqueous solution of it may be made artificially, similar to what nature presents us with in many thermal springs, as in those of Reikum and of Geyser in Iceland, and of most mineral waters, in minute quantity. There is no acid except the fluoric which can directly dissolve dry or calcined silica. Silica is composed of 48.04 silicon, and 51.96 oxygen. 
SILICATES are compounds of silicic acid (silica), with the buses alumina, lime, magnesia, potassa, soda, \&c. They constitute the greater number by far of the hard miner. als which incrust the terrestriai globe. Thus cyanite is a subsilicate of alumina; feldspar and leucite, are silicates of alumina and potassa ; albite and analcime, are silicates of alumina and soda; stilhite, prehnite, mesolite, labradorite, tourmaline, mica, \&c., are silicates of alumina and lime; chrysolite, steatite, serpentine, and meerschaum, are silicates of magnesia; augite and hornblende, are silicates of lime and magnesia, \&c.

SILICON, called also silicium, may be obtained by burning potassium in silicated fluoric gas. The product of the combustion is a brown cinder, which, on being thrown into water, disengages hydrogen with violence, and lets fall a dark liver-brown powder, upon which water exercises no action. This matter is silicon mixed with a salt of difficult solution, which is composed of fluorine, potassium, and silicon. This salt may, however, be removed by a great deal of washing. The further details of this curious subiect will be given in my forthcoming system of chemistry.

SILK MANUFACTURE. (Fabrique de soie, Fr.; Seidenfabrik, Germ.) This may be divided into two branches; 1 . the production of raw silk; 2 . its filature and preparation in the mill, for the purposes of the weaver and nther textile artisans. The threads, as spun by the silkworm, and wound up in its cocoon, are all twins, in consequence of the twin orifice in the nose of the insect through which they are projected. These two threads are laid paralle] to each other, and are glued inore or less evenly together by a kind of glossy varnish, which also envelopes them, constituting nearly 25 per cent. of their weight. Each ultimate filament measures about $\frac{1}{2000}$ of an inch in average fine silk, and the pair measures of course fully $\frac{1}{3} \frac{1}{0} 0$ of an inch. In the raw silk, as imported from Italy, France, China, \&c., several of these twin filaments are slightly twisted and agglutinated to form one thread, called a single.

The specific gravity of silk is $1 \cdot 300$, water being $1 \cdot 000$. It is by far the most tenacious or the strongest of all textile fibres, a thread of it of a certain diameter being nearly three times stronger than a thread of flax, and twice stronger than hemp. Some varieties of silk are perfectly white, but the general color in the native state is a golden yellow.

The production of silk was unknown in Europe till the sixth century, when two monks, who brought some eggs of the silkworm from China or India to Constantinople, were encouraged to breed the insect, and cultivate its cocoons, by the Emperor Justinian. Several silk manufactures were in consequence established in Athens, Thebes, and Corinth, not only for rearing the worm upon mulberry-leaves, but for unwinding its cocoons, for twisting their filaments into stronger threads, and weaving these into robes. The Venetians having then and long afterwards intimate commercial relations with the Greek empire, supplied the whole of western Europe with silk goods, and derived great riches from the trade.

About 1130, Roger II., king of Sicily, set up a silk manufacture at Palermo, and another in Calabria, conducted by artisans whom he had seized and carried off as prisoners of war in his expedition tô the Holy Land. From these countries, the silk industry soon spread throughout Italy. It seems to have been introduced into Spain at a very early period, by the Moors, particularly in Murcia, Cordova, and Granada. The last town, indeed, possessed a flourishing silk trade when it was taken by Ferdinand in the 15th century. The French having been supplied with workmen from Milan, commenced, in 1521, the silk manufacture; but it was not till 1564 that they began successfully to produce the silk itself, when Traucat, a working gardener at Nismes, formed the first nursery of white mulberry-trees, and with such success, that in a few years he was enabled to propagate them over many of the southern provinces of France. Prior to this time, some French noblemen, on their return from the conquest of Naples, had introduced a few silkworms with the mulberry into Dauphiny; but the business had not prospered in their hands. The mulberry plantations were greatly encouraged by Henry IV.; and since then they have been the source of most beneficial employment to the French people. James I. was most solicitous to introduce the breeding of silkworms into England, and in a speech from the throne he earnestly recommended his subjects to plant mulberry-trees; but he totally failed in the project. This country does not seem to be well adapted for this species of husbandry, on account of the great prevalence of blighting east winds during the months of April and May, when the worms require a plentiful supply of mulberry-leaves. The manufacture of silk goods, however, made great progress during that king's peaceful and pompous reign. In 1629 it had become so considerable in London, that the silk-throwsters of the city and suburbs were formed into a public corporation. So early as 1661 , they employed 40,000 persons. The rerocation of the edict of Nantes, in 1685, contributed in a remarkable manner to the increase of the English silk trade, by the influx of a large colony of skilful French weavers, who settled in Spitalfields. The great silk-throwing mill mounted at Derby, in 1719 , also served to promote the extension of this branch of manufacture; for soon 
afterwards, in the year 1730, the English silk goods bore a higher price in Italy than those made by the Italians, according to the testimony of Keysler.

Till the year 1826, however, oux silk manufactures in general labored under very grievuus fiscal burdens. Foreign organzine, or twisted raw silk, paid an import duty of 14s. $\tau \frac{1}{2} d$. per pound; Raw Bengal silk, $4 s$.; and that from other places, $5 s .7 \frac{1}{2} d$. $\mathrm{Mr}$. Huslisison introduced a bill at that time, reducing the duty on organzine to $5 \mathrm{~s}$., and the duty on other raw silk to 3 . per pound. The total prohibition of the import of French manufactured silks, which gave rise to so much contraband trade, was also converted into a duty of 30 per cent. ad valorem. During the reign of the prohibitory system, when our silk weavers had no variety of patterns to imitate, and no adequate stimulus to excel, on account of the monopoly which they possessed in the home market, the inferiority of their productions was a subject of constant pride and congratulation among the Lyonnais; and accordingly the English could not stand their competition any where. At that time, the disadvantage on English silk goods, compared to French, was estimated in foreign markets at 40 per cent. ; of late years it certainly does not exceed 20, notwithstanding the many peculiar facilities which France enjoys for this her favorite staple.

The silkworm, called by entomologists Phalana bombyx mori, is, like its kindred species, subject to four metamorphoses. The egg, fostered by the genial warmth of spring, sends forth a caterpillar, which, in its progressive enlargement, casis its skin either three or four times, according to the variety of the insect. Having acquired its full size in the course of 25 or 30 days, and ceasing to eat during the remainder of its life, it begins to discharge a viscid secretion, in the form of pulpy twin flaments, from its nose, which harden in the air. These threads are instinctively coiled into an ovoid nest round itself, called a cocoon, which serves as a defence against liring enemies and changes of temperature. Here it soon changes into the chrvsalis or nymph state, in which it lies swaddled, as it were, for about 15 or 20 days. Then it bursts its cerements, and comes forth furnished with appropriate wings, antennæ, and feet, for living in its new element, the atmosphere. The male and the female moths couple tozether at this time, and terminate their union by a speedy death, their whole existence being limited to two months. The cocoons are completely formed in the course of three or four days; the finest being reserved as seed worms. From these cocoons, after an interval of 18 or 20 days, the moth makes its appearance, perforating its tomb by knocking with its head against one end of the cocoon, after softening it with saliva, and thus rendering the filaments more easily torn asunder by its claws. Such moths or aurelias are collected and placed upon a piece of soft cloth, where they couple and lay their eggs.

The eggs, or grains, as they are usually termed, are enveloped in a liquid which causes them to adhere to the piece of cloth or paper on which the female lays them. From this glue they are readily fieed, by dipping them in cold water, and wiping them dry. They are best preserved in the ovum state at a temperature of about $55^{\circ} \mathrm{F}$. If the heat of spring advances rapidly in April, it must not be suffered to act on the eggs; otherwise it might hatch the caterpillars long before the mulberry has sent forth its leaves to nourish them. Another reason for keeping back their incubation is, that they may be hatched together in large broods, and not by small numbers in succession. The eggs are made up into small packets, of an ounce, or somewhat more, which in the south of France are generally attached to the girdles of the women during the day, and placed under their pillows at night. They are, of course, carefully examined from time to time. In large establishments, they are placed in an appropriate stove-room, where they are exposed to a temperature gradually increased till it reaches the 86th degree of Fahrenheit's scale, which term it must not exceed. Aided by this heat, nature completes her mysterious work of incubation in eight or ten days. The teeming eggs are now covered with a sheet of paper pierced with numerous holes, about one twelfth of an inch in diameter. Through these apertures the new-hatched worms creep upwards instinctively, to get at the tender mulberry leaves strewed over the paper.

The nursery where the worms are reared is called by the French a magnanière; it ought to be a well-aired chamber, fiee from damp, excess of cold or heat, rats, and other vermin. It should be ventilated occasionally, to purify the atmosphere from the noisome emanations produced by the excrements of the caterpillars and the decayed zaves. The scaffolding of the wicker-work shelves should be substantial; and they snould be from 15 to 18 inches apart. A separate small apartment should be allotted to the sickly worms. Immediately before each moulting, the appetite of the worms begins to flag; it ceases altogether at that period of cutaneous metamorphosis, but revives speedily after the skin is fairly cast, because the internal parts of the animal are thereby allowed freely to develop themselves. At the end of the second age, the worms are half an inch long; and then should be transferred from the small room in which they were first hatched, into the proper apartment where they are to 
be brought to maturity and set to spin their balls. On occasion of changing ther abode, they must be well cleansed from the litter, laid upon beds of fresh leaves, and supplied with an abundance of food every six hours in succession. In shifting their berl, a piece of network being laid over the wicker plates, and covered with leares, the worms will creep up over them; when they may be transferred in a body upon the net. The litter, as well as the sickly worms, may thus be readily removed, without handling a single healthy one. After the third age, they may be fed with entire leaves; because they are now exceedingly voracious, and must not be subsequently stinted in their diet. The exposure of chloride of lime, spread thin upon plates, to the air of the magnaniere, has been found useful in counteracting the tendency which sometimes appears of an epidemic disease among the silkworms, from the fetid exhalations of the dead and dying.

When they have ceased to eat, either in the fourth or fifth age, agreeably to the variety of the bombyx, and when they display the spinning instinct by crawling up among the twigs of heath, \&c., they are not long of beginning to construct their cocoons, by throwing the thread in different directions, so as to form the floss, filoselle, or outer open network, which constitutes the bourre or silk for carding and spinning.

The cocoons destined for filature, must not be allowed to remain for many days with the worms alive within them; for should the chrysalis have leisure to grow mature or come out, the filaments at one end would be cut through, and thus lose almost all their value. It is therefore necessary to extinguish the life of the animal by heat, which is done either by exposing the cocoons for a few days to sunshine, by placing them in a hot oven, or in the steam of boiling water. A heat of $202^{\circ} \mathrm{F}$. is sufficient for effecting this purpose, and it may be best administered by plunging tin cases filled with the cocoons into water heated to that pitch.

80 pounds French ( $88 \mathrm{Eng}$.) of cocoons, are the average produce from one ounce of eggs, or 100 from one ounce and a quarter; but M. Folzer of Alsace obtained no less than 165 pounds. The silk obtained from a cocoon is from 750 to 1150 feet long. The varnish by which the coils are glued slightly together, is soluble in warm water.

The silk husbandry, as it may be called, is completed in France within six weeks from the end of April, and thus affords the most rapid of agricultural returns, requiring merely the advance of a little capital for the purchase of the leaf. In buying up cocoons, and in the filature, indeed, capital may be often laid out to great advantage. The most hazardous period in the process of breeding the worms, is at the third and fourth moulting; for upon the sixth day of the third age, and the seventh day of the fourth, they in general eat nothing at all. On the first day of the fourth age, the worms proceeding from one ounce of eggs will, according to Bonafons, consume upon an average twenty-three pounds and a quarter of mulberry leaves; on the first of the fifth age, they will consume fortytwo pounds; and on the sixth day of the same age, they acquire their maximum voracity, devouring no less than 223 pounds. From this date their appetite continually decreases, till on the tenth day of this age they consume only fifty-six pounds. The space which they occupy upon the wicker, tables, being at their birth only nine feet square, becomes eventually 239 feet. In general, the more food they consume, the more silk will they produce.

A mulberry-tree is valued, in Provence, at from $6 d$. to $10 d$; it is planted out of the nursery at four years of age; it is begun to be stripped in the fifth year, and affords an increasing crop of leaves till the twentieth. It yields from $1 \mathrm{cwt}$. to $30 \mathrm{cwts}$. of leaves, according to its maguitude and mode of cultivation. One ounce of silkworm eggs is worth in France about $2 \frac{1}{2}$ franes; it requires for its due development into cocoons about 15 cwts. of mulberry leaves, which cost upon an average 3 francs per cwt. in a favorable season. One ounce of eggs is calculated, as I have said, to produce from 80 to 100 pounds of cocoons, of the value of $1 \mathrm{fr} .52$ centimes per pound, or 125 francs in whole. About 8 pounds of reeled raw silk, worth 18 franes a pound, are obtained from these 100 pounds of cocoons.

There are three denominations of raw silk; viz., organzine, trame (shute or tram), and floss. Organzine serves for the warp of the best silk stuff, and is considerably twisted; tram is made usually from inferior silk, and is very slightly twisted, in order that it may spread more, and cover better in the weft; floss, or bourre, consists of the shorter broken silk, which is carded and spun like cotton. Organzine and trame may contain from 3 to 30 twin filaments of the worm; the former possesses a double twist, the component filaments being first twisted in one direction, and the compound thread in the opposite; the latter receives merely a slender single twist. Each twin filament gradually diminishes in thickness and strength, from the surface of the cocoon, where the animal begins its work in a state of vigor, to the centre, where it finishes it, in a state of debility and exhaustion; because it can receive no food from the moment of its beginning to spin by spouting forth its silky substance. The winder is attentive to this progressive attenuation, and introduces the commancement of some cocoons to compensate for the 
termination of others. The quality of raw silli depends, therefore, very much upon the slill and care bestowed upon its filature. The softest and purest water should be used in the cocion kettle.

The yuality of the raw silk is determined by first winding off 400 ells of it, equal to 475 metres, round a drum one ell in circumference, and then weighing that length. The weight is expressed in grains, 24 of which constitute one denier; 24 deniers constitute one ounce; and 16 ounces make one pound, poids de marc. This is the Lyons rule for valuing silk. The weight of a thread of raw silk 400 ells long, is two grains and a half, when five twin filaments have been reeled and associated together.

Raw silk is so absorbent of moisture, that it may be increased ten per cent. in weight by this means. This property has led to falsifications; which are detected by enclosing weighed portions of the suspected silk in a wire-cloth cage, and exposing it to a stove-heat of about $78^{\circ} \mathrm{F}$. for 24 hours, with a current of air. The loss of weight which it thereby undergoes, demonstrates the amount of the fraud. There is an office in Lyons called the Condirioil, where this assay is made, and by the report of which the silk is bought and sold. The law in France requires, that all the silk tried by the Condition must be worked up into fabrics in that country.

In the Journal of the Asiatic Society of Bengal, for January, 1837, there are two very valuable papers upon silkworms; the first, upon those of Assam, by Mr. Thomas Hugun, stationed at Nowgong; the second by Dr. Helfer, upon those which are indigenous to India. Besides the Bombyx mori, the Doctor enumerates the following seven species, formerly unknown: -1 . The wil silkworm of the central provinces, a motl not larger than the Bombyx mori. 2. The Joree silkworm of Assam, Bombyx religiosa, which spins a cocoon of a fine filament, with much lustre. It lives upon the pipul tree (Ficus religiosa), which abounds in India, and ought therefore to be turned to account in breeding this valuable moth. 3. Saturnia silhetica, which inhabits the cassia mountains in Silhet and Dacea, where its large cocoons are spun into silk. 4. A still larger Saturnia, one of the greatest moths in existence, measuring ten inches from the one end of the wing to the other; observed by Mr. Grant, in Chirra punjee. 5. Suturnia paphia, or the Tusseh silkworm, is the most common of the native species, and furnishes the cloth usually worn by Europeans in India. It has not hitherto been domesticated, but millions of its cocoons are annually cullected in the jungles, and bruught to the silk factories near Calcutta and Bhagelpur. It feeds most commonly on the liair-tree (Zizyphus jujubu), but it prefers the Terminalia alata, or Assam tree, and the Bombux heptaphyllum. It is called Koutkuri mooga, in Assam. 6. Another Saturitia, from the neighborhood of Comercolly. 7. Saturnia assamensis, with a cocoon of a yellow-brown color, different from all others, called mooga, in Assam; which, although it can be reared in houses, thrives best in the open air upon trees, of which seven different kinds afford it food. The Mazankoory mooga, which feeds on the Adakoory tree, produces a fine silk, which is nearly white, and fetches 50 per cent. more than the fawn-colored. The trees of the first year's growth produce by far the most valuable cocoons. The mooga which inhabits the soom-tree, is found principally in the forests of the plains, and in the villages. The tree grows to a large size, and yields three crops of leaves in the year. The silk is of a light fawn color, and ranks next in value to the Mazankoory. There are generally five breeds of mooga worms in the year; 1. in January and February; 2. in May and June ; 3. in June and July ; 4. in August and September; 5 . in October and November; the first and last being the most valuable.

The Assamese select for breeding, such cocoons only as have been begun to be formed in the largest number on the same day, usually the second or third after the commencement; those which contain males being distinguishable by a more pointed end. They are put in a closed basket suspended fiom the roof; the moths, as they come forth, having room to move about, after a day, the females (known only by their large body) are taken out, and tied to small wisps of thatching-straw, selected always from over the hearth, its darkened color being thought more acceptable to the insect. If out of a batch, there should be but few males, the wisps with the females tied to them are exposed outside at night; and the males thrown away in the neighborhood find their way to them. These wisps are hung upon a string tied across the roof, to keep them fiom vermin. The eggs laid after the first three days are said to produce weak worms. The wisps are taken out morning and erening, and exposed to the sunshine, and in ten days after being laid, a few of them are hatched. The wisps being then hung up to the tree, the young worms find their way to the leaves. The ants, whose bite is fatal to the worm in its early stages, are destroyed by rubbing the trunk of the tree with molasses, and tying dead fish and toads to it, to attract these rapacious insects in large numbers, when they are destroyed with fire; a process which needs to be repeated sereral times. The ground under the trees is also well cleared, to render it easy so pick up and replace the worms which fall down. They are prevented from coming to 
the ground by tying fresh plantain-leaves round the trunk, over whose slippery surface they cannot crawl; and they are transferred from exhausted trees to fresh ones, on bam. boo platters tied to long poles. The worms require to be constantly watched and pro. tected from the depredations of both day and night birds, as well as rats and other vermin. During their moultings, they remain on the branches; but when about beginning to spin, they come down the trunk, and being stopped by the plantain-leaves, are there collected in baskets, which are afterwards put under bunches of dry leaves, suspended from the roof, into which the worms crawl, and form their cocoons-several being elustered together : this accident, due to the practice of crowding the worms together, which is most injudicious, rendering it impossible to wind off their silk in continuous threads, as in the filatures of Italy, France, and even Bengal. The silk is, therefore, spun like flax, instead of being unwound in single filaments. After four days the proper cocoons are selected for the next breed, and the rest are uncoiled. The total duration of a breed varies from 60 to 70 days; divided into the following periods :-

Four moultings, with one day's illness attending each

From fourth moulting to beginning of cocoon

In the cocoon 20, as a moth 6 , hatching of eggs 10

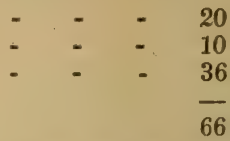

On being tapped with the finger, the body renders a hollow sound; the quality of which shows whether they have come down for want of leaves on the tree, or from their having ceased feeding.

As the chrysalis is not soon killed by exposure to the sun, the cocoons are put on stages, covered up with leaves, and exposed to the hot air from grass burned under them; they are next boiled for about an hour in a solution of the potash, made from incinerated rice-stalks; then taken out, and laid on cloth folded over them to keep them warm. The floss being removed by hand, they are then thrown into a basin of hot water to be unwound; which is done in a very rude and wasteful way.

The plantations for the mooga silkworm in Lower Assam, amount to 5000 acres, besides what the forests contain; and yield 1500 maunds of 84 lbs. each per annum. Upper Assam is more productive.

The cocoon of the Koutkuri mooga is of the size of a fowl's egg. It is a wild species, and affords filaments much valued for fishing-lines. See Sinкworm Gut.

8. The Arrindy, or Eria worm, and moth, is reared over a great part of Hindostan, but entirely within doors. It is fed principally on the Hera, or Palma christi leaves, and gives sometimes 12 broods of spun silk in the course of a year. It affords a fibre which looks rough at first; but when woven, becomes soft and silky, after repeated washings. The poorest people are clothed with stuff made of it, which is so durable as to descend from mother to daughter. The cocoons are put in a closed basket, and hung up in the house, out of reach of rats and insects. When the moths come forth, they are allowed to move about in the basket for twenty-four hours; after which the females are tied to long reeds or canes, twenty or twenty-five to each, and these are hung up in the house. The eggs that are laid the first three days, amounting to about 200, alone are kept; they are tiéd up in a cloth, and suspended to the roof till a few begin to hatch. These eggs are white, and of the size of turnip-seed. When a few of the worms are hatched, the cloths are put on small bamboo platters hung up in the house, in which they are fed with tender leaves. After the second moulting, they are removed to bunches of leaves suspended above the ground, beneath which a mat is laid to receive them when they fall. When they cease to feed, they are thrown into baskets full of dry leaves, among which they form their cocoons, two or three being often found joined together. Upon this injudicious practice I have already animadverted.

9. The Saturnia trifenestrata has a yellow cocoon of a remarkably silky lustre. It lives on the soom-tree in Assam, but seems not to be much used.

The mechanism of the silk filature, as lately improved in France, is very ingeniors. Figs. 973 and 974 exhibit it in plan and longitudinal view. $a$ is an oblong copper basin containing water heated by a stove or by steam. It is usually divided by i-ansverse partitions into several compartments, containing 20 cocoons, of which there ars 5 in one group, as shown in the figure. $b, b$, are wires with hooks or eyelets at their ends, through which the filaments run, apart, and are kept from ravelling. $c, c$, the points where the filaments cross and rub each other, on purpose to clean their surfaces. $d_{\text {, }}$ is a spiral groove, workiıg upon a pin point, to give the traverse motion alternately to right and left, whereby the thread is spread evenly over the surface of the reel $e$. $f, f$, are the pulleys, which by means of cords transmit the rotatory movement of the cylinder $d$, to the reel $e . \mathrm{g}$, is a friction lever or tumbler, for lightening or slackening the endless 
cord, in the net of starting or stopping the winding operation. Every apartment of a large filature contains usually a series of such reels as the above, all driven by one prime mover; each of which, however, may by means of the tumbling lever be stopped at 973
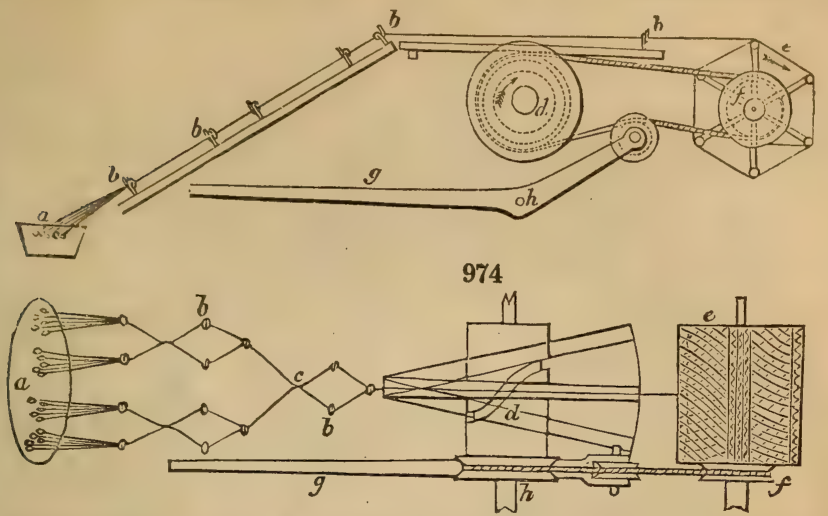

pieasure. The reeler is careful to remove any slight adhesions, by the application of a brush in the progress of her work.

The expense of reeling the excellent Cevennes silk is only 3 francs and 50 centimes per Alais pound; from 4 to 5 cocoons going to one thread. That pound is 92 hundredths of our avoirdupois pound. In Italy, the cost of reeling silk is much higher, being 7 Italian livres per pound, when 3 to 4 cocoons go to the formation of one thread; and 6 livres when there are from 4 to 5 cocoons. The first of these raw silks will have a titre of 20 to 24 deniers; the last, of 24 to 28 . If 5 to 6 cocoons go to one thread, the titre will be from 26 to 32 deniers, according to the quality of the cocoons. The Italian livre is worth $7 \frac{1}{2} d$. English. The woman employed at the kettle receives one livre and five sous per day; and the girl who turns the reel, gets thirteen sous a day; both receiving board and lodging in addition. In June, July, and August, they work 16 hours a day, and then they wind a rubo or ten pounds weight of cocoons, which yield from 1-5th to 1-6th of silk, when the quality is good. The whole expenses amount to from 6 to 7 livres upon every ten pounds of cocoons: which is about $2 s$. $8 d$. per English pound of raw silk.

The raw silk, as imported into this country in hanks from the filatures, requires to be regularly wound upon bobbins, doubled, twisted, and reeled in our silk-mills. These processes are called throwing silk, and their proprietors are called silk throwsters; terms probably derived from the appearance of swinging or tossing which the silk threads exhibit during their rapid movements among the machinery of the mills.

A representation of a French mill for throwing silk, is given in the Dictionnaire Technologique, under the article Moulinage de Soie. But it is a most awkward, operose, and defective piece of machinery, quite unworthy of being presented to my readers. It was in Manchester that throwing-mills received the grand improvement upon the ancient Italian plan, which had been originally introduced into this country by Sir Thomas Lombe, and erected at Derby. That improvement is chiefly due to the eminent factory engineers, Messrs. Fairbairn and Lillie, who transferred to silk the elegant mechanism of the throstle, so well known in the cotton trade. Still, throughout the silk districts of France, the throwing mills are generally small, not many of them turning off more than 1000 pounds of organzine per annum, and not involving $5000 \mathrm{l}$. of capital. The average price of throwing organzine in that country, where the throwster is not answerable for loss, is 7 franes; of throwing trame, from $4 \mathrm{fr}$. to $5 \mathrm{fr}$. (per kilogramme?) Where the throwster is accountable for loss, the price is from $10 \mathrm{fr}$. to $11 \mathrm{fr}$. for organzine, and from 6 to 7 for trame. In Italy, throwing adds $3 s .9 d$. to the price of raw silk, upon an average. I should imagine, from the perfection and speed of the silk-throwing machinery in this country, as about to be described, that the cost of converting a pound of raw silk either into organzine or trame must be considerably under any of the above sums.

SILK-THROWING MILL.

The first process to which the silk is subjected, is winding the skeins, as imported, off upon bobbins. The mechanism which effects this winding off and on, is technically' colled the engine, or swift. The bobbins to which the silk is transferred, are wooden: 
cylinders, of such thickness as may not injure the silk by sudden flexure, and which may also receive a great length of thread without having their diameter materially increased, or their surface velocity changed. Fig. 975 is an end view of the silk throwing machine, or engine, in which the two large hexagonal reels, called swifts, are 975

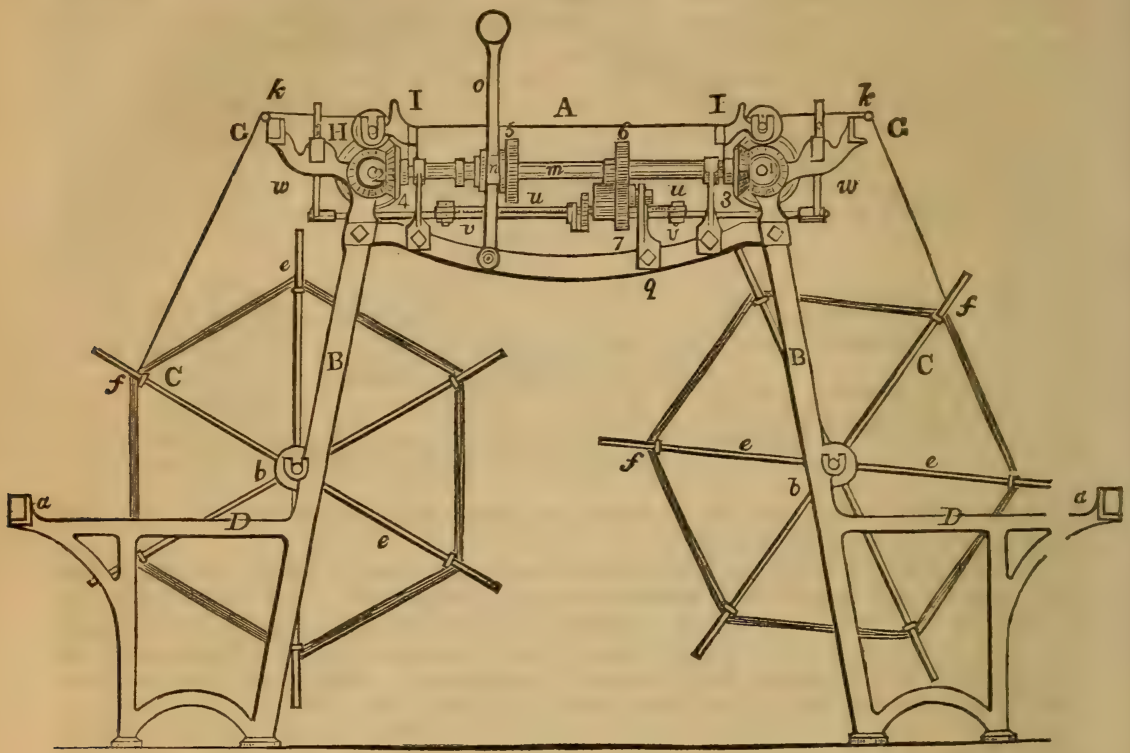

seen in section, as well as the table between them, to which the bobbins and impelling mechanism are attached. The skeins are put upon these reels, from which the silk is gradually unwound by the traction of the revolving bobbins. One principal object of attention, is to distribute the thread over the length of the bobbin-cylinder in a spiral or oblique direction, so that the end of the slender semi-transparent thread may be readily found when it breaks. As the bobbins revolve with uniform velocity, they would soon wind on too fast, were their diameters so small at first as to become greatly thicker when they are filled. They are therefore made large, are not covered thick, but are frequently changed. The motion is communicated to that end of the engine shown in the figure.

The wooden table A, shown here in cross section, is sometimes of great length, extending 20 feet, or more, according to the size of the apartment. Upon this the skeins are laid out. It is supported by the two strong slanting legs $\mathrm{B}, \mathrm{B}$, to which the bearings of the light reel $\mathrm{c}$ are made fast. These reels are called swifts, apparently by the same etymological casuistry as lucus à non lucendo; for they turn with reluctant and irregular slowness; yet they do their work much quicker than any of the old apparatus, and in this respect may deserve their name. At every eighth or tenth leg there is a projecting horizontal piece $\mathrm{D}$, which carries at its end another horizontal bar $a$, called the knee rail, at right angles to the former. This protects the slender reels or swifts from the knees of the operatives.

These swifts have a strong wooden shaft $b$, with an iron axis passing longitudinally through it, round which they revolve, in brass bearings fixed near to the middle of the legs в. Upon the middle of the shaft $b$, a loose ring is hung, shown under $c$, in fig. 976, to which a light weight $d$, is suspended, for imparting friction to the reel, and thus preventing it from turning round, unless it be drawn with a gentle force, such as the traction of the thread in the act of winding upon the bobbin.

Fig. 796 is a front view of the engine. B, B, are the legs, placed at their appropriate distances (scale $1 \frac{1}{2}$ inch to the foot); c, c, are the swifts. By comparing figs. 975 and 976, the structure of the swifts will be fully understood. From the wooden shaft $b$, six slender wooden (or iron) spokes $e, e$, proceed, at equal angles to each other; which are bound together by a cord $f$, near their free ends, upon the transverse line $f$ of which cord, the silk thread is wound, in a hexagonal form; due tension being given to the circumferential cords, by sliding them out from the centre. Slender wooden rods 
are set between each pair of spokes, to stay them, and to keep the cord tight. $\Sigma$ is one of the two horizontal shafts, placed upon each side of the engine, to which are affixed

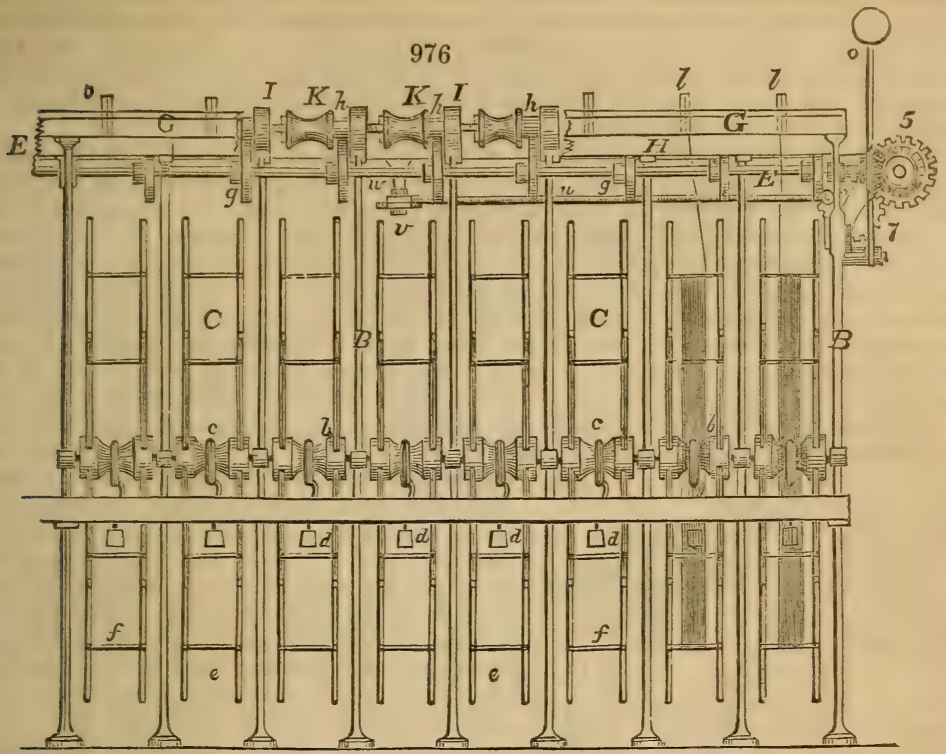

a number of light iron pulleys $g, g$ (shown on a double scale in fig. 977. (These serve, by friction, to drive the bobbins which rest upon their peripheries.

To the table A, fig. 975, are screwed the light cast-iron slot-bearings I, I, wherein the horizontal spindles or skewers rest, upon which the bobbins revolve. The spindles (see F, fig. 981) carry upon one end a little wooden pulley $h$, whereby they press and revolve upon the larger driving pulleys $g$, of the shaft $\Sigma$. These pulleys are called stars by our workmen. The other ends of the spindles, or skewers, are cut into serews, for attaching the swivel nuts $i$ ( $f \mathrm{~g} .981$ ), by which the bobbins $\mathrm{K}, \mathrm{K}$, are made fast to

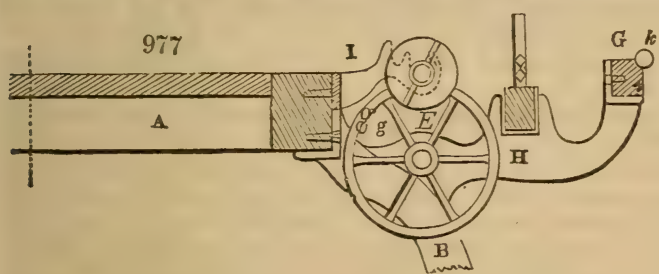

their respective spindles. Besides the slots, above described, in which the spindles rest when their friction pulleys $h$, are in contact with the moving stars $g$, there is another set of slots in the bearings, into which the ends of the spindles may be occasionally laid, so as to be above the line of conact of the rubbing periphery of the star $g$, in case the thread of any bohbin brealis. Whenever the girl has mended the thread, she replaces the bobbin-spindle in its deeper slot-bear-

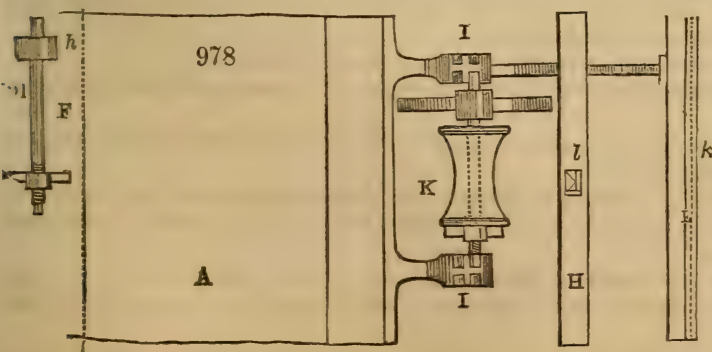
ings, thereby bringing its pulley once more into contact with the star, and causing it to revolve.

$\mathrm{G}$ is a long ruler or b bar of wood, which is supported upon every eighth or twelfth leg $\mathbf{B}$, B. (The figure being, for convenience of the page, contracted in length, shows it at every sixth leg.) To the edge of that bar the smooth glass rods $k$, are made fast, over which the threads glide from the swifts, in 
their way to the bobbins. $\mathbf{H}$ is the guide bar, which has a slow traverse or seesaw mo. tion, sliding in slots at the top of the legs B, where they support the bars G. Upon the guide bar $\mathrm{H}$, the guide pieces $l, l$, are made fast. These consist of two narrow, thin, upright plates of iron, placed endwise together, their contiguous edges being smooth, parallel, and capable of approximation to any degree by a screw, so as to increase or diminish at pleasure the ordinary width of the vertical slit that separates them. Through this slit the silk thread must pass, and, if rough or knotty, will be either cleaned or broken; in the latter case, it is neatly mended by the attendant girl.

The motions of the various parts of the engine are given as follows. Upon the end of the machine, represented in fig. 975, there are attached to the shafts $\mathrm{E}$ ( fig. 976$)$, the bevel wheels 1 and 2, which are set in motion by the bevel wheels 3 and 4 , respectively. These latter wheels are fixed upon the shaft $m$, fig. 975. $m$ is moved by the main steam shaft which runs parallel to it, and at the same height, through the length of the engine apartment, so as to drive the whole range of the machines. 5 is a loose wheel or pulley upon the shaft $m$, working in gear with a wheel upon the steam shaft, and which may be connected by the clutch $n$, through the hand lever or gearing rod 0 ( $f$ igs. 975 and 976), when the engine is to be set at work. 6 is a spur wheel upon the shaft $m$, by which the stud wheel 7 is driven, in order to give the traverse motion to the guide bar H. This wheel is represented, with its appendages, in double size, figs. 979 and 980 , with its boss upon a stud $p$, secured to the bracket $q$. In an eccentric hole

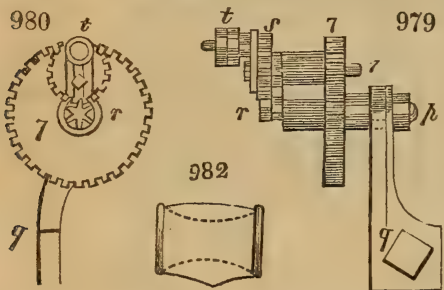
of the same boss, another stud $r$, revolves, upon which the little wheel $s$, is fixed. This wheel $s$, is in gear with a pinion cut upon the end of the fixed stud $p$; and upon it is screwed the little crank $t$, whose collar is connected by two rods $u$ (figs. 975 and 976 ), to a cross-piece $v$ which unites the two arms $w$, that are fixed upon the guide bar $\mathbf{H}$, on both sides of the machine. By the revolution of wheel 7 , the wheel $s$ will cause the pinion of the fixed stud $p$ to turn round. If that wheel bear to the pinion the proportion of 4 to 1 , then the wheel $s$ will make, at each revolution of the wheel 7 , one fourth of a revolution; whereby the crank $t$ will also rotate through one fourth of a turn, so as to be brought nearer to the centre of the stud, and to draw the guide bar so much less to one side of its mean position. At the next revolution of wheel 7 , the crank $t$ will move through another quadrant, and come still nearer to the central position, drawing the guide bars still less aside, and therefore causing the bobbins to wind on more thread in their middle than towards their ends. The contrary effect would ensue, were the guide bars moved by a single or simple crank. After four revolutions of the wheel 7 , the crank $t$ will stand once more as shown in $f \mathrm{gg} .980$, having moved the bar $\mathrm{H}$ through the whole extent of its traverse. The bobbins, when filled, have the appearance repre sented in fig. 982 ; the thread having been laid on them all the time in diagonal lines, so as never to coincide with each other.

Doubling is the next operation of the silk throwster. In this process, the threads of two or three of the bobbins, filled as above, are wound together in contact upon a single bobbin. An ingenious device is here employed to stop the winding-on the moment that one of these parallel threads happens to break. Instead of the swifts or reels, a creel is here mounted for receiving the bobbins from the former machine, two or three being placed in one line over each other, according as the threads are to be doubled or trebled. Though this machine is in many respects like the engine, it has some additional parts, whereby the bobbins are set at rest, as above mentioned, when one of the doubling threads gets broken.

Fig. 983 is an end view, from which it will be perceived that the machine is, like the preceding, a double one, with two working sides.

Fig. 984 is a front view of a considerable portion of the machine.

Fig. 985 shows part of a cross section, to explain minutely the mode of winding upon $\mathrm{a}$ E.ingle bobbin.

Fig. 986 is the plan of the parts shown in fig. 985 ; these two figures being drawn to double the scale of figs. 983 and 984.

A, A, figs. 983 and 984, are the end frames, connected at their tops by a wooden stretcher, or bar-beam, $a$, which extends through the whole length of the machine; this bar is shown also in figs. 985 and 986.

B, B, are the creels upon each side of the machine, or bobbin bearers, resting upon wooden beams or boards, made fast to the arms or brackets $c$, about the middle of the frames $A$.

$D, D$, are two horizontal iron shafts, which pervade the whole machıne, and carry a series of light moveable pulleys, called stars, $c, c$, ( $f$ gss. 985, 986, ) which serve to rrive the 
Dobbins E, E, whose fixed pulleys rest upon their peripheries, and are therefore turned simply by friction. These bobbins are screwed by swivel nuts $e, e$, upon spindles, as in

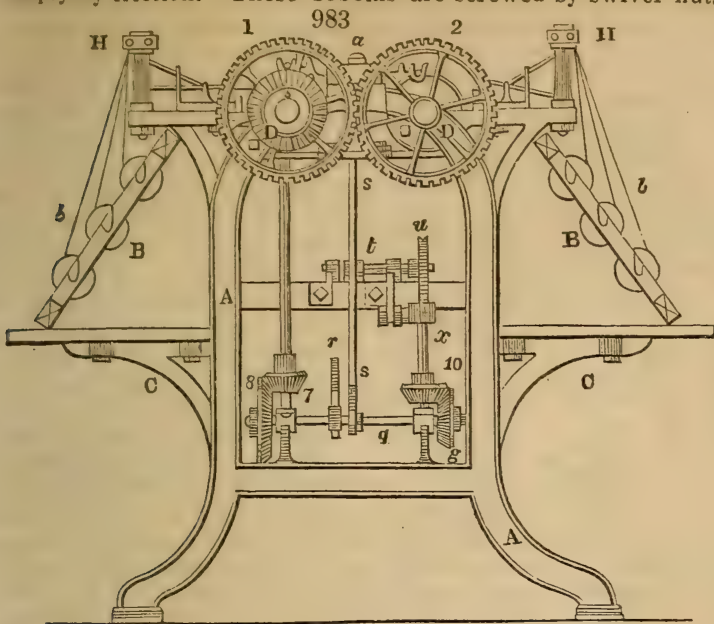

the silk engine. Besides the small friction pulley or boss, $d$, seen best in fig. 986 , by which they rest upon the star pulleys $c, c$, a little ratchet wheel $f$, is attached to the other end of each bobbin. This is also shown by itself at $f$, in fig. 987 .

The spindles with their bobbins revolve in two slot-bearings F, F, fig. 986, screwed to the bar-beam $a$, which is supported by two or three intermediate upright frames, such as $A^{\prime}$. The slot bearings $\mathrm{F}$, have alse a second slot, in which the spindle with the bobbin is laid at rest, out of contact of the star wheel, while its broken thread is being mended. $G$ is the guide bar (to which the cleaner slit pieces $g, g$, are attached), for

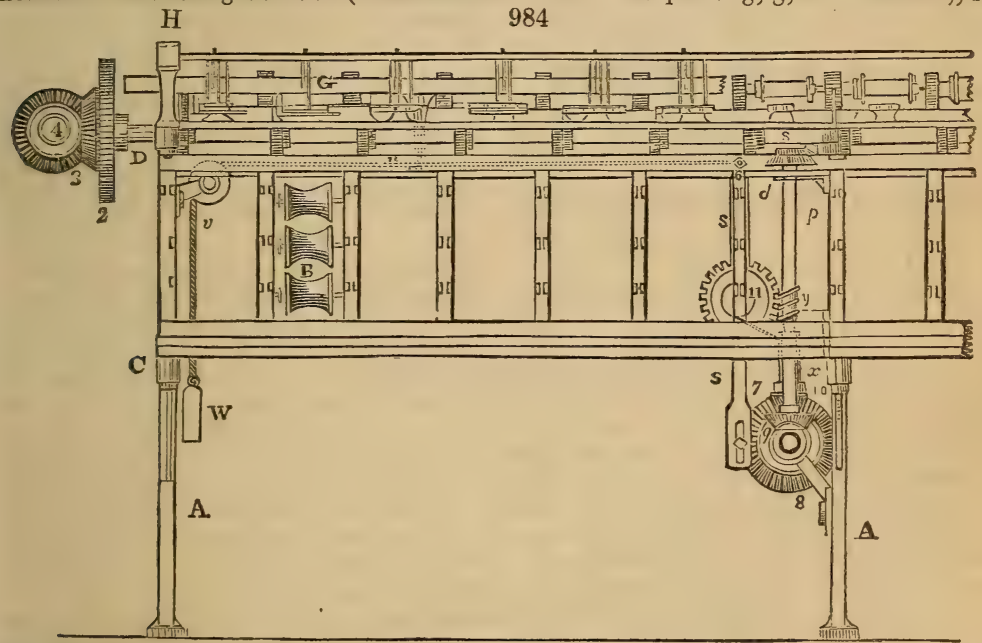

making the thread traverse to the right and the left, for its proper distribution over the surface of the bobbin. The guide bar of the doubling machine is moved with a slower traverse than in the engine; otherwise, in consequence of the different obliquities of the paths, the single threads would be readily broken. $h, h$, is a pair of smonth rods of iron or brass, placed parallel to each of the two sides of the machine, and made fast to the standards $\mathrm{H}, \mathrm{H}$, which are screwed to brackets projecting from the frames $\mathrm{A}, \mathrm{A}^{\prime}$. Over these rods the silk threads glide, in their passage to the guide wires $g, g$, and the bobbins $E, E_{\text {. }}$

$I$, $I$, is the lever bourd upon each side of the machine, upon which the slight brass bearings or fulcrums $i$, $i$, one for each bobbin in the creel, are made fast. This board bears the balnnce-lever $k, l$, with the fallers $n, n, n$, which act as dexterous fingers, and stop the bobbin from winding-on the instant a thread may chance to break. The levers $k$, $l$, swing upon a fine wire axis, which passes through their props $i, i$, their arms being shaped rectangularly, as shown at $k, k^{\prime}, f i g .986$. The $\operatorname{arm} l$, being heavier than the arm $k$, naturally rests upon the ridge bar $m$, of the lever board $I_{\text {. }} n, n, n$, are three wies, resting at une of their ends upon the axis of the fulcrum $i$, $i$, and having each of their other hooked ends suspended by one of the silk threads, as it passes over the front steel rod $h$. and under 
$h^{\prime}$. These faller wires, or stop fingers, are guided truly in their up-and-down motions with the thread, by a cleaner-plate 0 , having a vertical slit in its middle. Hence, whenever any thread happens to break, in its way to a winding-on bobbin $\Sigma$, the wire $n$,

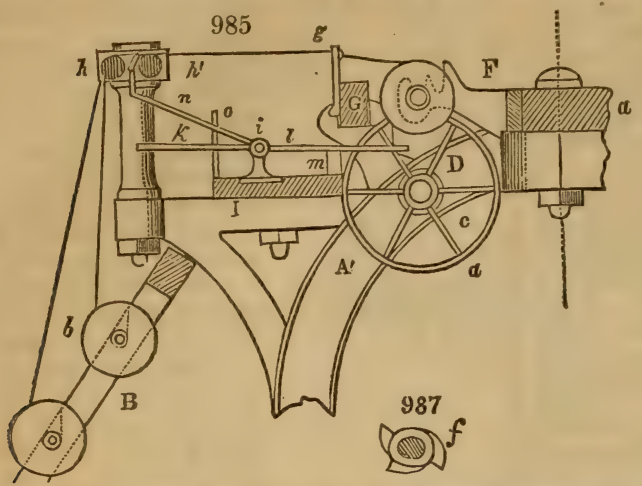
which hung by its eyelet end to that thread, as it passed through between the steel rods in the line of $h, h^{\prime}$, falls upon the lighter arm of the balanee lever $k$, $l$, weighs down that $\operatorname{arm} k$, consequently jerks up the $\operatorname{arm} l$, which pitches its tip or end into one of the three notches of the ratchet or eatch wheel $f$ ( $f$ igs. 986 and 987), fixed to the end of the bobbin. Thus its motion is instantaneously arrested, till the girl has had leisure to mend the thread, when she again hangs up the faller wire $n$, and restores the lever $k, l$, to its horizontal position. If meanwhile, she took occasion to remove the wiriding bobbin out of the sunk slot-bearing, where pulley $d$ touches the star wheel $c$, into the right-hand upper slot of repose, she must now shift it into its slot of rotation.

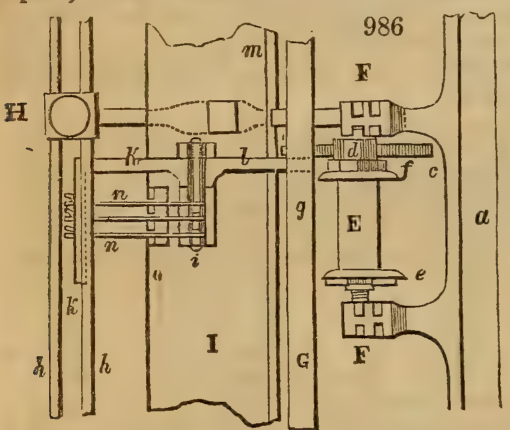

The motions are given to the doubling machine in a very simple way. Upon the end of the framing, represented in fig. 983, the shafts D, D, bear two spur wheels 1 and 2, which work into each other. To the wheel 1 , is attached the bevel wheel 3 , driven by another bevel wheel 4 ( $\mathrm{fig}$. 984), fixed to a shaft that extends the whole length of the apartment, and serves, therefore, to drive a whole range of machines. The wheel 4 may be put in gear with the shaft, by a clutch and gearhandle, as in the silk engine, and thereby it drives two shafts, by the one transmitting its movement to the other.

The traverse motion of the guide bar $G$, is effected as follows: - Upon one of the shafts $\mathrm{D}$, there is a bevel wheel 5 , driving the bevel wheel 6, upon the top of the upright shaft $p$ ( $f \mathrm{gg} .981$, to the right of the middle); whence the motion is transmitted to the horizontal shaft $\varphi$, below, by means of the bevel wheels 7 and 8 . Upon this shaft $q$, there is a heart-wheel $r$, working against a roller which is fixed to the end of the lever $s$, whose fulcrum is at $t, f i g .983$. The other end of the lever $s$, is connected by two rods (shown by dotted lines in fig. 984) to a brass piece which joins the arms $u$ ( $f \mathrm{~g}$. 984), of the guide bars G. To the same cross piece a cord is attached, which goes over a roller $v$, wind suspends a weight $w$, by means of which the level $s$, is pressed into contact with the heart-wheel $r$. The fulcrum $t$, of the lever $s$, is a shaft which is turned somewhat eccentric, and has a very slow rotatory motion. Thus the guide bar, after each traverse, neccessarily winds the silk in variable lines, to the side of the preceding threads.

The motion is given to this shaft in the following way. Upon the horizontal shaft $q$, there is a bevel wheel $g$ ( $f$ igs. 983 and 984), which drives the wheel 10 upon the shaft $x$; on whose upper end, the worm $y$ works in the wheel 11, made fast to the said eccentric shaft $t$; round which the lever $s$ swings or oscillates, causing the guide bars to traverse.

The spinning silk-mill.--The machine which twists the silk threads, either in their single or doubled state, is called the spinning mill. When the raw singles are first twis'ed in one direction, next doubled, and then twisted together in the opposite direction, an exceedingly wiry, compact thread, is produced, called organzine. In the spinning mill, either the singles or the doubled silk, while being unwound from one set of bobbins, and wound upon another set, is subjected to a regular twisting operation; in which process the thread is conducted as usual through guides, and coiled diagonally upon the bobbins by a proper mechanism.

Fig. 988 exhibits an end view of the spinning mill; in which four working lines are shown; two tiers upon each side, one above the other. Some spinning mills have 
three working tiers upon each side; but as the highest tier must be reached by a ladder or platform, this construction is considered by many to be injudicious.

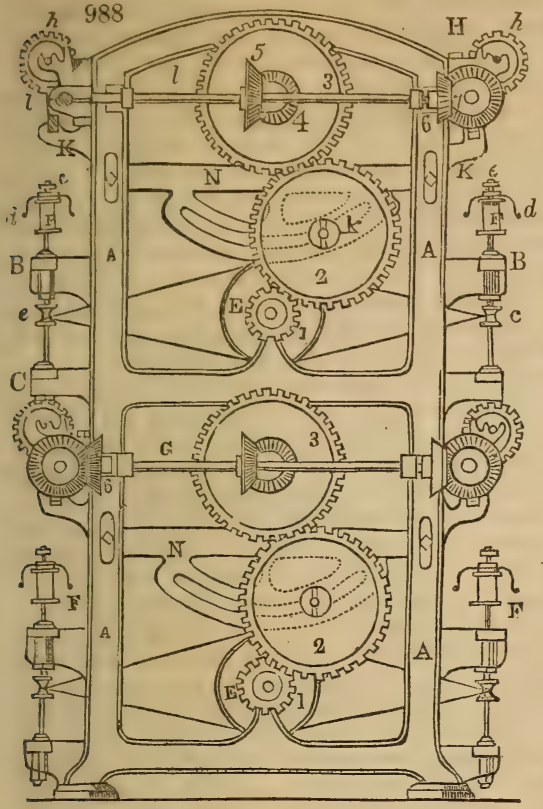

Fig. 989, is a front view, where, as in the former figure, the two working lines are shown.

Fig. 990, is a cross section of a part of the machine, to illustrate the construction and play of the working parts; figs. 996, 997, are other views of fig. 990.

Fig. 991, shows a single part of the machine, by which the bobbins are made to revolve.

Figs. 992, and 993, show a different mode of giving the traverse to the guide bars, than that represented in fig. 990 .

Figs. 994, and 995, show the shape of the full bobbins, produced by the action of these two different traverse motions.

The upper part of the machine being exactly the same as the under part, it will be sufficient to explain the construction and operation of one of them.

$A, A$, are the end upright frames or standards, between which are two or three intermediate standards, according to the length of the machine. They are all connected at their sides by beams $\mathrm{B}$ and $\mathrm{c}$, which extend the whole length of the machines. $D, D$, are the spindles, whose top bearings $a, a$, are made fast to the beams $\mathrm{B}$, and their bottoms turn in hard brass steps, fixed to the bar c. These two bars together are

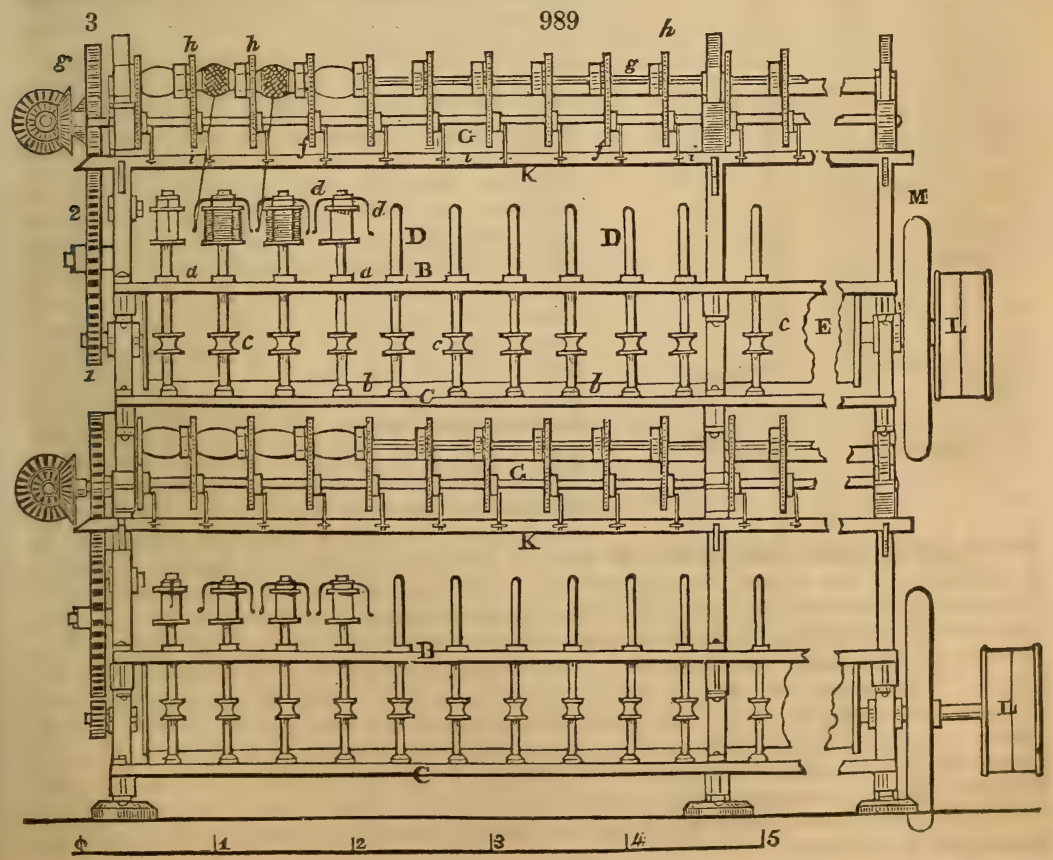


called, by the workmen, the spindle box. The standards $A, A$, are bound with cross bars $\mathrm{N}, \mathrm{N}$.

$c, c$, are the wharves or whorls, turned by a band from the horizontal tin cylinder in the lines of $\mathrm{E}, \mathrm{E}$, fig. 988, lying in the middle line between the two parallel rows of spindles $\mathrm{D}, \mathrm{D}$. F, F, are the bobbins containing the untwisted doubled silk, which are simply pressed down upon the taper end of the spindles. $d, d$, are little fliers, or forked wings of wire, attached to washers of wood, which revolve loose upon the tops of the said bobbins $F$, and round the spindles. One of the wings is sometimes bent upwards, to serve as a guide to the silk, as shown by dotted lines in fig. 990 . e, e, are pieces of wood pressed upon the tops of the spindles, to prevent the fiers from starting off by the centrifugal force. G, are horizontal shafts bearing a number of little spur wheels $f, f . \quad \mathrm{H}$, are slot-bearings, similar to those of the doubling-machine, which are fixed to the end and middle frames. In these slots, the light square cast-iron shafts or spindles $g$, fig. 989, are laid, on whose end the spur wheel $h$ is cast ; $z r_{c} d$ when the shaft $g$ lies in the front slot of its bearing, it is in gear with the wheel $f$, upon the snait G; but when it is laid in the back slot, it is out of gear, and at rest. See F, F, fig. 986.

Upon these little cast-iron shafts or spindles $g$, fig. 991, the bobbins or blocks $\mathrm{I}$, are

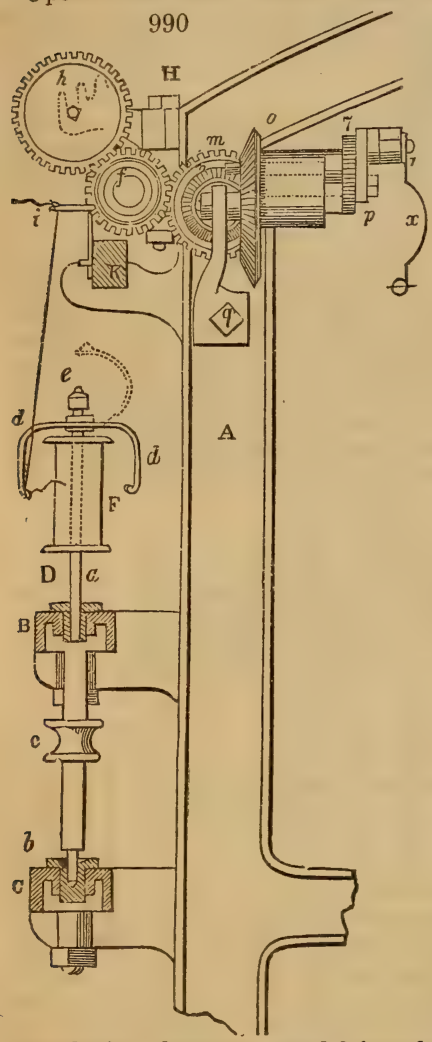
thrust, for receiving, by winding-on, the twisted or spun silk. These blocks are made of a large diameter, in order that the silk fibres may not be too much bent; and they are but slightly filled, at each successive charge, lest, by increasing their diameter too much, they should produce too rapid an increase in the rate of winding, with proportional diminution in the twist, and risk of stretehing or tearing the silk. They are therefore the more frequently changed. $k, k$, are the guide bars, with the guides $i, i$, through which the silk passes, being drawn by the revolving bobbins $\mathrm{I}$, and delivered or laid on by the fliers $d, d$, from the rotatory twisting bobbins $F$. The operation of the machine is therefore simple, and the motions are given to the parts in a manner equally so.

Upon the shaft of the tin cylinder or drum, exterior to the frame, the usual fast and loose pulleys, or riggers, $\mathrm{L}, \mathrm{L}^{\prime}$, are mounted, for driving the whole machine. These riggers are often called steam-pulleys by the workmen, from their being connected by bands with the steam-driven shaft of the factory. In order to allow the riggers upon the shafts of the upper and the under drums to be driven from the same pulley upon the main shaft, the axis of the under drum is prolonged at $\mathbf{L}, \mathbf{L}^{\prime}$, and supported at its end, directly from the floor, by an upright bearing. Upon the shafts of the tin cylinders there is also a fly-wheel $\mathrm{M}$, to equalize the motion. Upon the other ends of these shafts, namely, at the end of the spinningmill, represented in fig. 988, the pinions 1 are fixed, which drive the wheels 3 , by means of the intermediate or carrier wheel 2 ; called also the plate wheel, from its being hollowed somewhat like a trencher. 1 , is called the change-pinion, because it is changed for another, of a different size and different number of teeth, when a change in the velocity of wheels 2 and 3 is to be made. To allow a greater or smaller pinion to be applied at 1 , the wheel 2 is mounted upon a stud $k$, which is moveable in a slot concentric with the axis of the wheel 3. This slot is a branch from the cross bar N. The smaller the change-pinion is, the nearer will the stud $k$ approach to the vertical line joining the centres of wheels 1 and 3 ; and the more slowly will the plate wheel 2 be driven. To the spur wheel 3 , a bevel wheel 4 , is fixed, with which the other also revolves loose upon the stud. The bevel wheel 5 , upon the shaft $l$, is driven by the bevel wheel 4 ; and it communicates motion, by the bevel wheels 6 and 7, to each of the horizontal shafts $\mathrm{G}, \mathrm{G}$, extending along the upper and under tiers of the machine. At the left-hand side of the top part of fig. 988, the two wheels 6 and 7 are omitted, on purpose to show the bearings of the shaft $\mathrm{G}$, as also the slot-bearings for carrying the shafts or skewers of the bobbins. 
If it be desired to communicate twist in the opposite direction to that which would be given by the actual arrangement of the wheels, it is necessary merely to manspose the carrier wheel 2, from its present position on the right hand of pinion 1 , to the left of it, and to drive the tin cylinder by a crossed or close strap, instead of a straight or open one.

The traverse motion of the guide is given here in a similar way to that of the engine, ( $f i g .975$.$) Near one of the middle or cross-frames of the machine (see fig. 990) the$ wheel $f$, in gear with a spur wheel $h$, upon one of the block-shafts, drires also a spur wheel $m$, that revolves upon a stud, to which wheel is fixed a berel wheel $n$, in gear with the bevel wheel 0 . To wheel 0 , the same mechanism is attached as was described under figs. 979 and 980, and which is here marked with the same letters.

To the crank-knob $r$, fig. 990, a rod $x$, is attached, which moves or traverses the guide

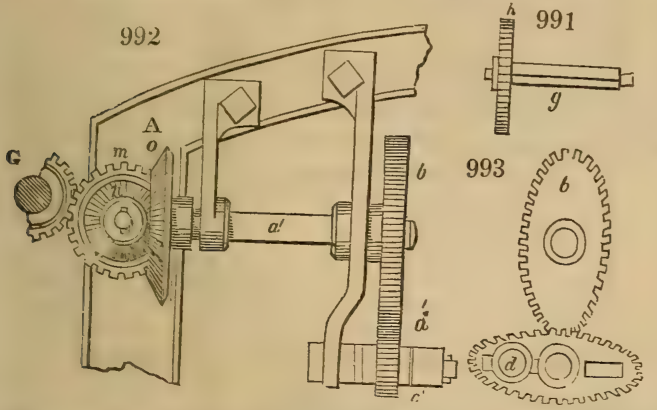
bar belonging to that part of the machine; to each machine one such apparatus is fitted. In figs. 992 and 993 another mode of traversing the guide bar is shown, which is generally used for the coarser qualities of silk. Near to one of the middle frames, one of the wheels $f$, in gear with the spur wheel $m$, and the bevel wheel $n$, both revolving on one stud, gives motion also to the wheel $o$, fixed upon a shaft $a^{\prime}$, at whose other end the elliptical wheel $b^{\prime}$ is fixed, which drives a second ${ }^{c^{\prime}}$ elliptical wheel $c^{\prime}$, in such a way that the larger diameter of the one plays in gear with the smaller liameter of the other; the teeth being so cut as to take into each other in all positions. The crank-piece $d^{\prime}$ is screwed

$994 \quad 995$

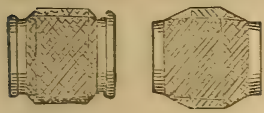
upon the face of the wheel $c^{\prime}$, at such a distance from its centre as may be necessary to give the desired length of traverse motion to the guide bar for laying the silk spirally upon the blocks. The purpose of the elliptical wheel is to modify the simple crank motion, which would wind on more silk at the ends of the bobbins than in their middle, and to effect an equality of windingon over the whole surface of the blocks. In fig. 993 the elliptical wheels are shown in front, to illustrate their mode of operating upon each other. Fig. 994 is a block filled

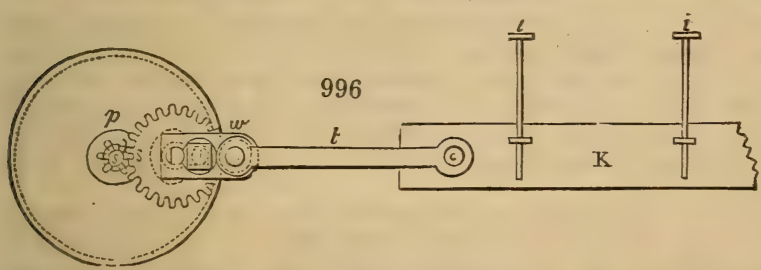

by the motion of the eccentric, fig. 990 ; and fig. 995 is a block filled by the elliptical mechanism. As the length of the motions of the bar in the latter construction re-

mains the same during the whole operation, the silk, as it is wound on the blocks, will slide over the edges, and thereby produce the flat ends of the barrel in fig. 995. The

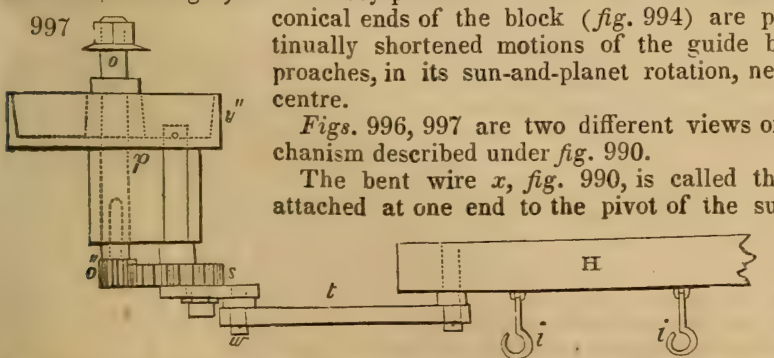
ntre.

anism deseribed under fig. 990. rotion, nearer to the general

The bent wire $x$, fig. 990 , is called the guider iron. It is -and-planet wheel.

work $t, s, o$, and at the other to the suide bar $f, f$, fig. 989. The silk threads pass

through the guides, as already explained. By the motion communicated to the guide bar (guider), the diamond pattern is produced, as shown in fig. 994. 
THE SILK AUTOMATIC REEL.

In this machine, the silk is unwound from the blocks of the throwing-mill, and formed into hanks for the market. The blocks being of a large size, would be productive of much friction, if made to revolve upon skewers thrust through them, and would cause frequent breakage of the silk. They are, therefore, set with their axes upright upon a board, and the silk is drawn from their surface, just as the weft is from a cop in the shuttle. On this account the previous winding-on must be executed in a very regular manner; and preferably as represented in fig. 994.

Fig. 998 is a front view of the reel; little more than one half of it being shown. Fig. 999 is an end view. Here the steam pulleys are omitted, for fear of obstructing the

\section{D} 998

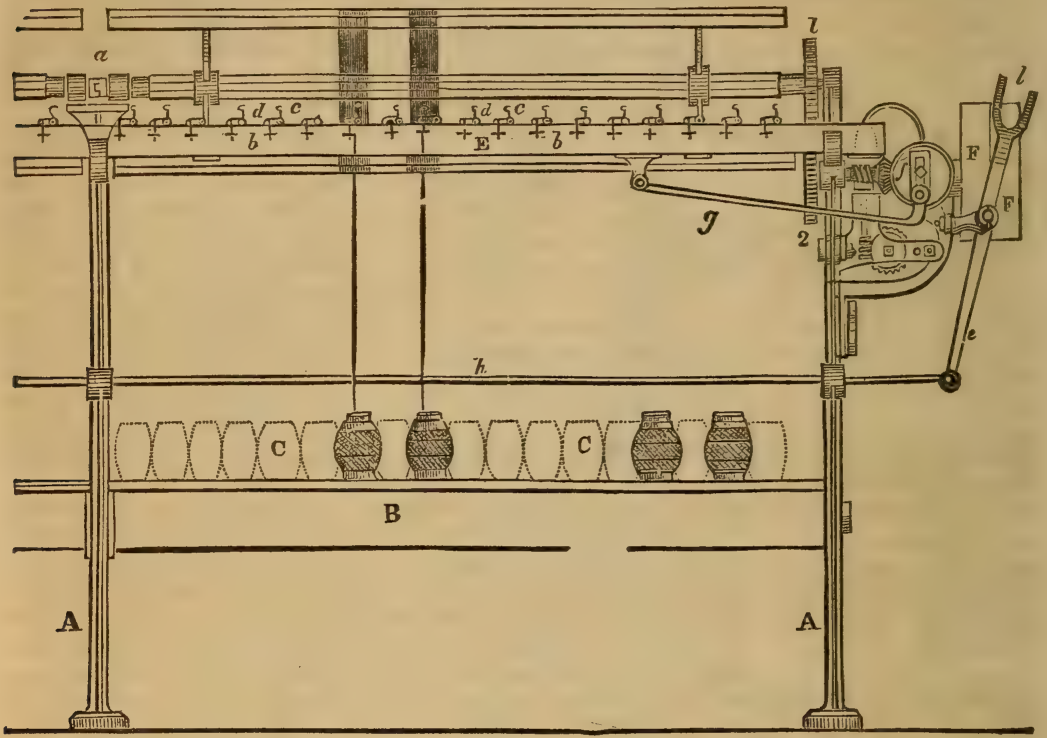

view of the more essential parts. A, A, are the two end framings, connected by mahogany stretchers, which form the table $\mathrm{B}$, for receiving the bobbins $\mathrm{C}$, $\mathrm{c}$, which are sometimes weighted at top with a lump of lead, to prevent their tumbling. D is the reel, consisting of four long laths of wood, which are fixed upon iron frames, attached to an octagonal wooden shaft. The arm which sustains one of these laths is capable of being bent inwards, by loosening a tightening hook, so as to permit the hanks, when finished, to be. taken off, as in every common reel.

The machine consists of two equal parts, coupled together at $a$, to facilitate the removal of the silk from either half of the reel; the attendant first lifting the one part, and then the other. $\mathrm{E}$ is the guide bar, which by a traverse motion causes the silk to be wound on in a cross direction. $b$ and $c$ are the wire guides, and $d$ are little levers lying upon the cloth covered guide bar E. The silk, in its way from the block to the reel, passes under these levers, by which it is cleaned from loose fibres.

On the other end of the shaft of the reel, the spur wheel 1 is fixed, which derives mo tion from wheel 2, attached to the shaft of the steam-pulley $F$. Upon the same shaft there is a bevel wheel 3 , which impels the wheel 4 upon the shaft $e$; to whose end a plate is attached, to which the crank $f$ is screwed, in such a way as to give the prorier length of traverse motion to the guide bar $\mathrm{E}$, connected to that crank or eccentric stud by the jointed rod $g$. Upon the shaft of the steam-pulleys $F$, there is a worm or endless screw, to the left of $f$, fig. 999, which works in a wheel 5, attached to the short upright shaft $h$ ( $f g .998$ ). At the end of $h$, there is another worm, which works in a wheel 6 ; at whose circumference there is a stud $i$, which strikes once at every revolution against an arm attached to a bell, seen to the left of G; thus announcing to the reel tenter that a measured length of silk has been wound upon her reel. $e$ is a rod or handle, by which the fork $l$, with the strap, may be moved upon the fast or loose pulley, so as to set on or arrest the motion at pleasure.

Throwsters submit their silk to scouring and steaming processes. They soak the 
hanks, as imported, in lukewarm soap-water in a tub; but the bobbins of the twisted single silk from the spinning mill are enclosed within a wooden chest, and exposed to

$$
999
$$

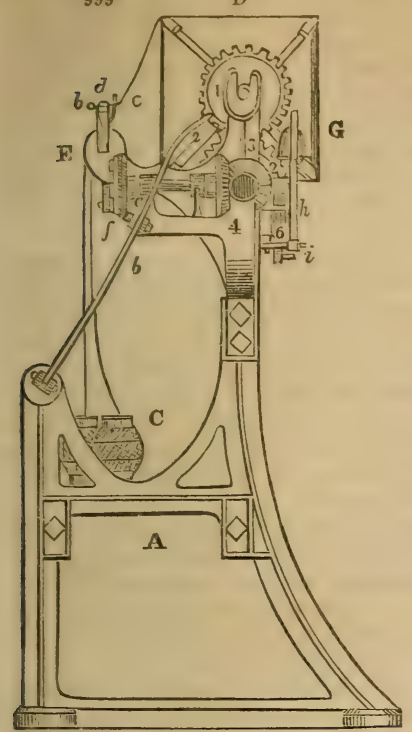
the opening action of steam for about ten minutes. They are then immersed in a cistern of warm water, from which they are transferred to the doubling frame.

The wages of the workpeople in the silk-throwing mills of Italy are about one half of their wages in Manchester; but this difference is much more than counterbalanced by the protecting duty of $2 s$. $10 d$. a pound upon thrown silk, and the superior machinery of our mills. In 1832, there was a power equal to 342 horses engaged in the silkthrowing mills of Manchester, and of about 100 in the mills of Derby. The power employed in the other silk mills of England and Scotland has not been recorded.

There is a peculiar kind of silk called marabout, containing generally three threads, made from the white Novi raw silk. From its whiteness, it takes the most lively and delicate colors without the discharge of its gum. After being made into tram by the single twist upon the spinning mill, it is reeled into hanks, and sent to the dyer without further preparation. After being dyed, the throwster re-winds and re-twists it upon the spinning mill, in order to give it the whipcord hardness which constitutes the peculiar feature of marabout. The cost of the raw Novi silk is $19 \mathrm{~s} .6 \mathrm{~d}$. a pound; of throw-. ing it into tram, 2s. $6 \mathrm{~d}$.; of dyeing, $2 s$. ; of re-winding and re-twisting, after it has been dyed, about 5 s.; of waste, $2 s_{\text {., }}$ or 10 per cent. : the total of which sum is $31 \mathrm{~s} . ;$ being the price of one pound of marabout in 1832 .

An Estimate of the Annual Quantities of Silk produced or exported from the several Countries in the World, exhibiting also the Countries to which exported.

\begin{tabular}{|c|c|c|c|}
\hline Countries whence exported. & Quantities. & $\begin{array}{c}\text { Countries to } \\
\text { which exported. }\end{array}$ & Quantities \\
\hline Italy exports & 34,000 bales of 225 small lbs. & & Bales. \\
\hline France produces - & $10,500 \ldots 573 \frac{1}{8}$ kils., or & 2 England - & 28,000 \\
\hline India and Bengal export - & $9,500 \ldots\left\{128 \frac{1}{2}\right.$ Vienna lbs. & France - & 22,000 \\
\hline Persia - $\quad-\quad-$ & . $\quad 162$ lbs. English & Prussia - & 7,600 \\
\hline China - & 4,000 & Russia - & 6,400 \\
\hline Asia Minor & 3,500 & Austria and & \\
\hline Levant, Turkey, and Ar- & & Germany - & 5,000 \\
\hline chipelago export & 3,500 & Switzerland & 5,000 \\
\hline Spain exports & $1,500 \ldots$ & Total & \\
\hline Total & 74,000 bales. & $10 t a 1$ & \\
\hline
\end{tabular}

Note.-These estimates exclude the silk manufactured in Italy.

The declared value of the silk manufactures exported from the United Kingdom in 1836, was $917,822 l$.; and in 1837 , only 494,569. The deficit in the last year was owing o the commercial crisis in the United States; which country took, the preceding year, our silk goods to the value of 524,3011 .

SILKWORM GUT, for angling, is made as follows:-Select a number of the best and largest silkworms, just when they are beginning to spin; which is known by their refusing to eat, and having a fine silk thread hanging from their mouths. Immerse them in strong vinegar, and cover them closely for twelve hours, if the weather be warm, but two or three hours longer, if it be cool. When taken out, and pulled asunder, two transparent guts will be observed, of a yellow green color, as thick as a small straw, bent double. The rest of the entrails resembles boiled spinage, and therefore can occasion no mistake as to the silk-gut. If this be sof, or break upon stretching it, it is a proof that the worm has not been long enough $1 n^{\prime}$ ? is fit to draw out, the one end of it is to be dipped into the vinegar, and the other end 
is to be stretched gently to the proper length. When thus drawn out, it must be kept extended on a thin piece of board, by putting its extremities into slits in the end of the

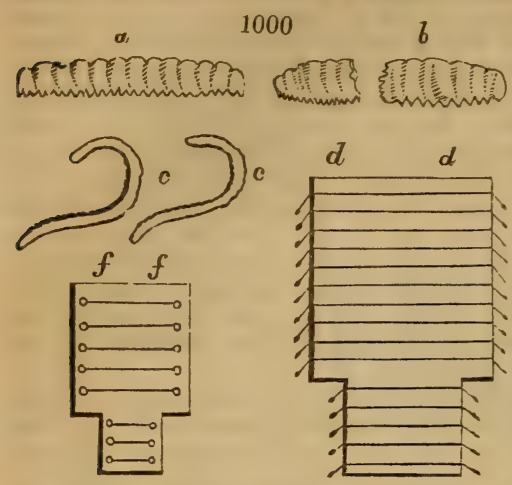
wood, or fastening them to pins, and then exposed in the sun to dry. Thus genuine silk-gut is made in Spain. From the manner in which it is dried, the ends are always more or less compressed or attenuated.* Fig. 1000, $a$, is the silkworm; $b$, the worm torn asunder; $c, c$, the guts; $d, d$, a board slit at the ends, with the gut to dry; $f, f$, a board with wooden pegs, for the same purpose.

SILVER (Argent, Fr.; Silber, Germ.) was formerly called a perfect metal, because heat alone revived its oxyde, and secause it could pass unchanged through fiery trials, which apparently destroved most other metals. The distinctions, perfect, imperfect, and noble, are now justly rejected. The bodies of this class are all equal in metallic nature, each being endowed merely with different relations to other forms of matter, which serve to characterize it, and to give it a peculiar value.

When pure and planished, silver is the brightest of the metals. Its specific gravity in the ingot is 10.47 ; but, when condensed under the hammer or in the coining press, it becomes $10^{\circ}$. It melts at a bright red heat, a temperature estimated by some as equal to $1280^{\circ}$ Fahr., and by others to $22^{\circ}$ Wedgewood. It is exceedingly malleable and ductile; affording leaves not more than $\frac{1}{1000} \overline{0}$ of an inch thick, and wire far finer than a human hair.

By Sickingen's experiments, its tenacity is, to that of gold and platinum, as the numbers 19,15 , and $26 \frac{1}{4}$; so that it has an intermediate strength between these two metals. Pure atmospheric air does not affect silver, but that of houses impregnated with sulphureted hydrogen, soon tarnishes it with a film of brown sulphuret. It is distinguished chemically from gold and platinum by its ready solubility in nitric acid, and from almost all other metals, by its saline solutions affording a curdy precipitate with a most minute quantity of sea salt, or any soluble chloride.

Silver occurs under many forms in nature :-

1. Native silver possesses the greater part of the above properties; yet, on account of its being more or less alloyed with other metals, it differs a little in malleability, lustre, density, \&c. It sometimes occurs crystallized in wedge-form octahedrons, in cubes, and cubo-octahedrons. At other times it is found in dendritic shapes, or arborescences, resulting from minute crystals implanted upon each other. But more usually it presents itself in small grains without determinable form, or in amorphous masses of various magnitude.

The gangues (mineral matrices) of native silver are so numerous, that it may be said to occur in all kinds of rocks. At one time it appears as if filtered into their fissures, at another as having vegetated on their surface, and at a third, as if impasted in their substance. Such varieties are met with principally in the mines of Peru.

The native metal is found in almost all the silver mines now worked; but especially in that of Kongsberg in Norway, in carbonate and fluate of lime, \&c.; at Schlangenberg in Siberia, in a sulphate of barytes; at Allémont, in a ferruginous clay, \&c. In the article Mrnes, I have mentioned several large masses of native silver that have been discovered in various localities.

The metals most usually associated with silver in the native alloy are gold, copper, arsenic, and iron. At Andreasberg and Guadalcanal it is alloyed with about 5 per cent. of arsenic. The auriferous native silver is the rarest; it has a brass-yellow color.

2. Antimonial silver. - This rare ore is yellowish-blue; destitute of malleability; even very brittle; spec. grav. 9.5. It melts before the blowpipe, and affords white fumes of oxyde of antimony ; being readily distinguished from arsenical iron, and arsenical cobalt, by its lamellar fracture. It consists of from 76 to 84 of silver, and from 24 to 16 of antimony.

3. Mixed antimonial silver.-At the blowpipe it emits a strong garlic smell. Its constituents are, silver 16, iron 44, arsenic 35, antimony 4. It occurs at Andreasberg.

4. Sulphuret of silver.-This is an opague substance, of a dark-gray or leaden hue; slightly nalleable, and easily cut with a knife. wh... it betrays a meialin lustre. The silver is easily separated by the blowpipe. Il consists of, 13 of sulphur to 89 of silver, 
by experiment; 13 to 87 are the theoretic proportions. Its spec. grav. is 6.9 . It occurs crystallized in most silver mines, but especially in those of Freyberg, Joachimsthal in Bohemia, Schemnitzin, Hungary, and Mexico.

5. Ret sulphuret of silver; silver glance.-Its spec. grav. is $5 \cdot 7$. It contains from 84 to 86 of silver.

6. Sulphureted silver, with bismuth.-Its constituents are, lead 35, bismuth 27, silver 15 , sulphur 16, with a little iron and copper. It is rare.

7. Intimoniated sulphuret of silver, the red silver of many mineralogists, is an ore remarkable for its lustre, color, and the variety of its forms. It is friable, easily scraped by the knife, and affords a powder of a lively crimson red. Its color in mass is brilliant red, dark red, or even metallic reddish-black. It crystallizes in a variety of forms. Its constituents are,-silver from 56 to 62 ; antimony from 16 to 20 ; sulphur from 11 to 14 ; and oxygen from 8 to 10 . The antimony being in the state of a purple oxyde in this ore, is reckoned to be its coloring principle. It is found in almost all silver mines; but principally in those of Freyberg, Sainte-Marie-aux-Mines, and Guadalcanal.

8. Black sulphuret of silver, is blackish, brittle, cellular, affording globules of silver at the blowpipe. It is found only in certain mines, at Allémont, Freyberg; more abundantly in the silver mines of Peru and Mexico. The Spaniards call it negrillo.

9. Chloride of silver, or horn silver.-In consequence of its semi-transparent aspect, its yellowish or greenish color, and such softness that it may be cut with the nail, this ore has been compared to horn, and may be easily recognised. It melts at the flame of a caindle, and may be reduced when heated along with iron or black flux, which are distinctive characters. It is seldom crystallized; but occurs chiefly in irregular forms, sometimes covering the native silver as with a thick crust, as in Peru and Mexico. . Its density is only $4 \cdot 74$.

Chloride of silver sometimes contains 60 or 70 per cent. of clay; and is then called butter-milk ore, by the German miners. The blowpipe causes globules of silver to sweat out of it. This ore is rather rare. It occurs in the mines of Potosi, of Annaberg, Freyberg, Allémont, Schlangenberg, in Siberia, \&c.

10. Carbonate of silver, a species little known, has been found hitherto only in the mine of S. Wenceslas, near Wolfache.

TABLE of the Quantities of SILver brought into the Market every year, on an average, from 1790 to 1802 .

\begin{tabular}{|c|c|c|c|c|c|c|}
\hline \multicolumn{3}{|c|}{ Old Continent. } & Lbs. Avoird. & \multicolumn{2}{|c|}{ New Comtinent. } & Lbs. Avoird. \\
\hline Siberia & \multicolumn{2}{|l|}{ ASIA. } & 38,500 & Central America & - & $1,320,000$ \\
\hline Hungary & $y^{\text {EUROPE. }}$ & - & 44,000 & South America & - & 605,000 \\
\hline Austrian & n States - & - & 11,000 & & & \\
\hline Hartz an & nd Hessia & - & 11,000 & & & \\
\hline Saxony & - & - & 22,000 & & & \\
\hline Norway & - & - & 22,000 & & & \\
\hline Sweden & - & -2 & & & & \\
\hline France & - & $-\}$ & 11,000 & & & \\
\hline \multicolumn{3}{|c|}{ Total of the Old Continent } & 159,500 & \multicolumn{2}{|c|}{ Total of the New Continent } & $1,925,000$ \\
\hline
\end{tabular}

Thus the New Continent furnished twelve times more silver than the old. For more detailed statistics of silver, see the end of the article.

The following is Mr. Ward's description of the treatment of silver ores in Mexico:-

"After returning from San Augustin," says he, "I passed the whole of the after noon at the hacienda (metallurgic works) of Salgado, in which the ores of the Valenciana mine are reduced. The hacienda, of which a representation is given below, fig. 1001, contains forty-two crushing-mills, called arrastres, and thirty-six stampers. The ore, on being extracted from the mine, is placed in the hands of the pepenadores, men and women, who break all the larger pieces with hammers, and after rejecting those in which no metallic particles are contained, divide the rest into three classes" (inferior, middling, and rich). "These are submitted to the action of the morteros (stamps), one of which, of eight stampers, is capable of reducing to powder ten cargas of ore (each of 350 lhs.) in twenty-four hours. This powder not being thought sufficiently fine for the quicksilver to act upon with proper effect, it is transferred from the morteros to the arrastres (crushing-mills, see wood-cut), in which water is used. Each of these redices to a fine impa!nable metalliferous mud, six quintals (600 lbs.) of powder in 
24 hours At Guanajuato, where water-power cannot be obtained, the arrastres are worlied by mules (see fig. 1001), which are kept constantly in motion at a slow pace, and are changed every 6 hours. The grinding-stones, as well as the sides and bottom of the mill itself, are composed of granite; four blocks of which revolve in each crushing-mill, attached to cross-bars of wood. This part of the operation is thought of great importance, for it is upon the perfection of the grinding that the saving of the quicksilver is supposed in a great measure to depend, in the subsequent amalramation. The grinding is performed usually in a covered shed or gallery, which in a largehurienda. like Salgado, from the number of arrastres at work at the same time, is neecssarily of considerable extent."

1001 The Gallera of the Hacienda of Salgado.

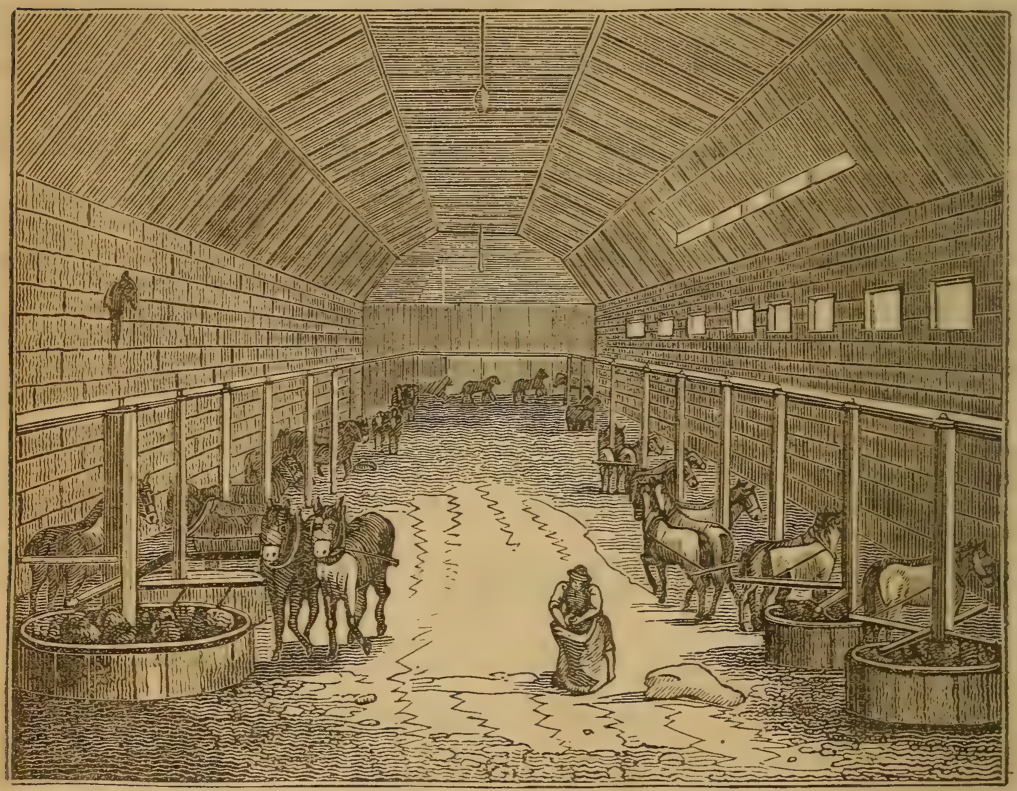

Fig. 1002, represents the rude grinding apparatus used at the lavaderos, or gold washings, in Chile. The streamlet of water conveyed to the hut of the gold washer, is received upon

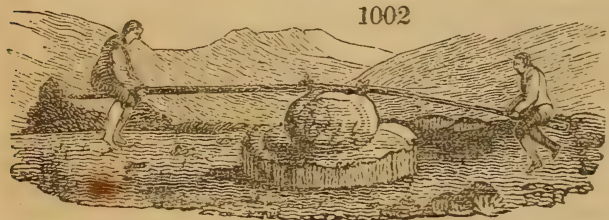

a large rude stone, whose flat surface has been hollowed out into a shallow basin, and in the same manner into 3 or 4 others in succession; the auriferous particles are thus allowed to deposite themselves in these receptacles, while the lighter earthy atoms, still suspended, are carried off by the running water. The gold thus collected is mixed with a quantity of ferruginous black sand and stony matter, which requires the process of trituration, eflected by the very rude and simple trapiche shown in the figure; consisting of two stones, the under one being about three feet in diameter, and slightly concave. The upper stone is a large spherical boulder of syenitic granite, about two feet in diameter, having on its upper part two iron plugs fixed oppositely, to which is secured, by lashings of hide, a transverse horizontal pole of canelu (cinnamon) wood, about 10 feet long; two men seated on the extremities of this lever, work it up and down alternatelr, so as to give to the stone a rolling motion, which is sufficient to crush and grind the materials placed beneath it. The washings thus ground, are subjected to the action of running water, upon inelined planes formed of skins, by which process the silicious particles are carried off, while a portion of the ferruginous matter, mixed with the heavier grains of gold, is extracted by a loadstone; it is again washed, till nothing but pure gold-dust remain. The whole process is managed with much dexterity; and if there were much gold to be separated, it 
would afford very profitable employment; but generally the small quantity collected is sufficient only to afford subsistence to a few miserable families.

The trapiche, ingenio, or mill, for grinding the ores of silver, is a very simple piece of mechanism. A place is chosen where a small current of water, whose section will present a surface of six inches diameter, can be brought to a spot where it can fall perpendicularly ten or twelve feet; at this place a well is built of this depth, about 6 feet in diameter; in its centre is fixed an upright shaft, upon a central brass pin ; it is confined above by a wooden collar. A little above its foot, the shaft has a small wheel affixed to it, round which are fixed a number of radiating spokes, shaped at the end somewhat Jilie cups, and forming altogether a horizontal wheel, four feet in diameter. Upon the slanting edges of the cups, the water is made to strike with the force it has acquired in falling down a nearly perpendicular trough, scooped out of the solid trunk of a tree. This impression makes the wheel turn with a quick rotatory motion. The upright axis rises about 6 feet above the top of the well, at about half which height is inserted a small horizontal arm, four feet long, which serves as an axle to a ponderous mis.-stone of granite, of from four to six feet diameter, which is made to roll on its edge in a circular trough, sometimes made of the same material, and sometimes of hard wood.

The weight of this quickly rolling stone effects the pulverization of the ore. In some cases, it is taken out in the dry state, and sifted; but more generally the separation of the fine? ground particles is accomplished by the action of running water. For this purpose a small stream is made to trickle into the circular trough, by which the pounded ore is worked up into a muddy consistence, and the finer particles flow off with the excess of water, through a notch cut in the margin of the trough. This fine matter is received in little pools, where the pounded ore is left to settle; and the clear water 1003

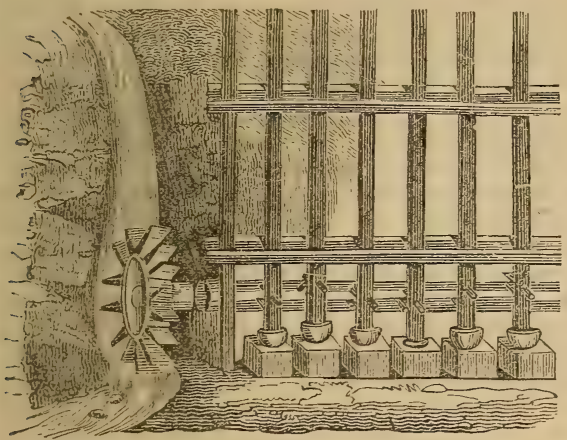
being run off, the powder is re moved from the bottom, and car ried to the place of amalgamation.

The ingenios, or stamping-mills, are driven by a small breast waterwheel, of five feet diameter, and one foot broad. Fig. 1003 will give a sufficient idea of their construction. The long horizontal shaft, fixed on the axis of the wheel, is furnished with 5 or 6 cams placed at different situations round the shaft, so as to act in succession on the projecting teeth of the upright rods or pestles. Each of these weighs 200 pounds, and works in a corresponding oblong mortar of stone or wood.

The patio, or amalgamation floor, fig. 1004, is a large flat space, open to the sky, 312 feet in length, by 236 in brearth, and securely surrounded by strong walls. It is 1004

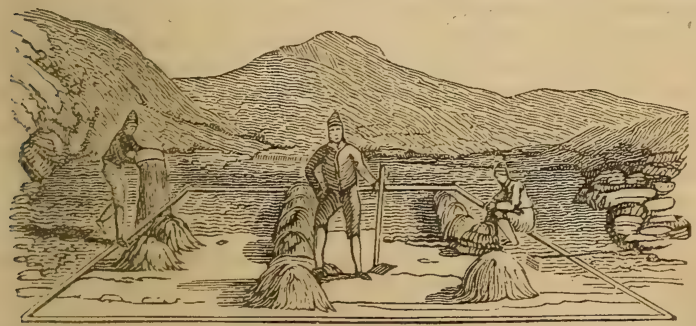
paved with large unhewn blocks of porphyry, and is capable of containing 24 tortas, or flat circular collections of lama, of about 50 feet diameter, and 7 inches deep, when the patio is not filled, (but of somewhat smaller dimensions when nearly so,) ranged in 4 rows, and numbered from the left-hand corner. At one end a small space is generally set apart for the assays, which are made each on one monton.

The following description of Mexican amalgamation is given by Captain Lyon.

A torta of Zacatecas contains 60 montons of 20 quintals each, and is thus formed:In the first instance, a square space, of the requisite size for a torta, is marked out, and enclosed by a number of rough planks, which are propped in their places on the patio floor by large stones, and dried horse-dung and dust are piled round their edges to prevent the escape of the lama. A heap of saltierra (salt mixed with earthy impurities) is then piled in the centre, in the proportion of 2 fanegas (each $=1.6$ English bushels) and a half to the monton, $=150$ for the torta. After this, the lama, or ore ground into a 
fine paste, is poured in. When the last or $60 \mathrm{th}$ monton is delivered, the saltierra is shovelled down and well mixed with the lama, by treading it with horses, and turning it with shovels; after which the preparation is left at rest for the remainder of the day. On the fullowing day comes the el incorporo. After about one hour's treading by horses, the magistral or roasted and pulverized copper ore is mixed with the lama, (the repuso or trearling-mill still continuing,) in summer in the proportion of 15 cargas of 12 arrubas ( $25 \mathrm{lbs}$. each) to the torta, if the ore be of 6 mares to the monton, and in winter in only half the quantity. For it is a singular fact, that in summer the mixture cools, and requires more warmth; while in winter it acquires of itself additional heat. With poorer ores, as for instance those of 4 marcs to the monton, 12 cargas are applied in summer, and 6 in winter. From November to February, lime is also occasionally used to cool the lama, in the proportion of about a peck per monton.

The repaso, or treading out, is continued by six horses, which are guided by one man, who stands in the lama, and directs them all by holding all their long halters. This operation is much more effectual in a morning than an evening, and occupies about five or six hours. When the magistral is well mixed, the quicksilver is applied by being sprinkled through pieces of coarse cloth doubled up like a bag, so that it spurts out in very minute particles. The second treading of the horses then follows; after which the whole mixture is turned over by six men with wooden shovels, who perform the operation in an hour. The torta is then smoothed and left at rest for one entire day, to allow the incorporation to take place. It undergoes the turning by shovels and treading by horses every other day, until the amalgamator ascertains that the first admixture of quicksilver is found to be all taken up by the silver; and this he does by ranning or washing a small quantity of the torta in a little bowl. A new supply is then added, and when this has done its duty, another is applied to catch any stray particles of silver. On the same day, after a good repaso, the torta is removed on hand-barrows by the laborers, to the lavaderos, in order that it may receive its final cleansing. The general method of proportioning the quicksilver to the tortas, is by allowing that every marco of silver which is promised by trial of the ores as the probable produce of a monton, will require in the whole process $4 \mathrm{lbs}$.

In metals of five to six mares and a half per monton (of the average richness of Zacatecas), $16 \mathrm{lbs}$. of quicksilver were incorporated for every monton, $=900 \mathrm{lbs}$. for the torta. On the day of the second addition, the proportion is $5 \mathrm{lbs}$. the monton; and when the torta is ready to receive the last dose of quicksilver, it is applied at the rate of 7 lbs. the monton, $=420 \mathrm{lbs}$. ; making a total of $1620 \mathrm{lbs}$. of quicksilver. With poorer ores, less quicksilver and less magistral are required.

The usual time for the completion of the process of amalgamation, is from 12 to 15 days in the summer, and 20 to 25 in the winter. This is less than a third of the time taken at some other mines in Mexico. This rapidity is owing to the tortas being spread very flat, and receiving thereby the stronger influence of the sun. In the Mexican mines, only one monton is commonly mixed at a time; and the lama is then piled in a small conical heap or monton.

Lavadero, or washing vat.-Here the prepared tortas are washed, in order to carry off the earthy matters, and favor the deposition of the amalgam at the bottom. Each vat is about 8 feet deep, and 9 in diameter; and solidly built in masonry.

A large horizontal wheel, worked by mules, drives a vertical one, which turns a horizontal wheel fitted round a perpendicular wooden shaft, revolving upon an iron pivot at the bottom of the vat. To the lower end of this shaft, four cross-beams are fitted, from which long wooden teeth rise to the height of 5 feet. Their motion throngh the water being rapid, keeps all the lighter particles afloat, while the heavier sink to the bottom. The large wheel is worked by four mules, two at each extremity of the cross-heam. Water iscupplied from an elevated tank. It requires 12 hours' work of one tub to wash a torta. Eight porters are employed in carrying the prepared lama of the torta in handbarrows to the vats. The earthy matter receives a second washing.

The amalgam is carried in bowls into the azogueria, where it is subjected to straining through the strong canvass bottom of a leather bag. The hard mass left in the bag is

1005

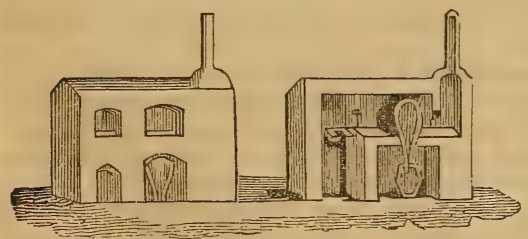

moulded into wedge-shaped masses of 30 lbs., which are arranged in the burninghouse, (fig. 1005), to the number of 11 , upon a solid copper stand, ealled baso, having a round hole in its centre. Over this row of wedges several others are built ; and the whole pile is called pina. Each circular range is firmly bound round with a rope. The base is placed over a pipe which leads to a small tank of water for con-

densing the quicksilver; a cylindrical space being left in the middle of the pina, to give free egress to the mercurial vapors. 
A large b. ll-shaped cover, called capellina, is now hoisted up, and carefully lowered over the pir.t, by means of pulleys. A surong lute of ashes, saltierra, and lama is applied to its lower lge, and made to fit very closely to the plate on which the base stands. A wall of lire-bricks is then built loosely round the capellina, and this space is filled with hurning charcoal, which is thrice replenished, to keep it burning all night. After the heat his been applied 20 hours, the bricks and ashes are removed, the luting broken, and the capellina hoisted up. The burned silver is then found in a hard mass, which is broken up, weighed, and carried to the casting-house, to be formed into bars of about 1080 ounces each. The luss of silver in burning is about 5 ounces to each bar (barra), and the loss of quicksilver, from $2 \frac{1}{2}$ upon the good metals, to 9 upon the coarse.

Molina told Mr. Miers, that the produce of the galena ores of Uspaltata did not average more than 2 mares per caxon of $5000 \mathrm{lbs}$., which is an excessively poor ore. The argentiferous salena ores of Cumberland afford 11 mares per caxon; while the average produce of the Potosi silver ores is ouly 5 or 6 mares in the same quantity. These comparisons afford the clearest eridence that the English mode of smelting can never be brought into com petition with the process of amalgamation as practised in America.

Humboldt, Gay Lussac, Boussingault, Karsten, and several other chemists of note, have offered solutions of the amalgamation enigma of Mexico and Peru. The following seems to be the most probable rationale of the successive steps of the process:-

The addition of the magistral (powder of the roasted copper pyrites), is not for the purpose of disengaging muriatic acid from the sea salt (saltierra), as has been supposed, since nothing of the kind actually takes place; but, by recipiocal or compound affinity, is serves to form chloride of copper, and chloride of iron, upon the one hand, and sulphate of soda, upon the other. Were sulphuric acid to be used instead of the magistral, as certain norices have prescribed, it would certainly prove injurious, by causing muriatic acid to exhale. Since the ores contain only at times oxyde of silver, but always a great abundance of oxyde of iron, the acid would carry off both partly, but leave the chloride of silver in a freer state. A magistral, such as sulphate of iron, which is not in a condition to generate the chlorides, will not suit the present purpose; only such metallic sulphates are useful as are ready to be transformed into chlorides by the saltierra. This is peculiarly the case with sulphate of copper. Its deuto-chloride gives up chlorine to the silver, becomes in consequence a protochloride, while the chloride of silver, thus formed, is revived, and amalgamated with the quicksilver present, by electro-chemical agency which is excited by the saline menstruum; just as the voltaic pile of copper and silver is rendered active by a solution of sea salt. A portion of chloride of mercury will be simultaneously formed, to be decomposed in its turn by the sulphate of silver resulting from the mutual action of the acidified pyrites, and the silver or its oxyde in the ore. An addition of quicklime counteracts the injurious effect of too much mavistral, by decomposing the resulting sulphate of copper. Quicksilver being an excellent conductor of heat, when introduced in too great quantities, is apt to cool the mass too much, and thereby enfeebles the operation of the deuto-chloride of copper upon the silver.

There is a method of extracting silver from its ores by what is called imbibition. This is exceedingly simple, cousisting in depriving, as far as possible, the silver of its gangue, then melting it with about its own weight of lead. The alloy thus procured, contains from 30 to 35 per cent. of silver, which is separated by cupellation on the great scale, as described under ores of lead. In his way the silver is obtained at Kongsberg in Norway.

The amalgamation works at Halsbrücke, near Freyberg, for the treatment of silver ores by mercury, have been justly admired as a model of arrangement, convenience, and regularity; and I shall conclude this subject with a sketch of their general distribution.

Fig. 1006 presents a vertical section of this great usine or hüttenwerk, subdivided into 1006

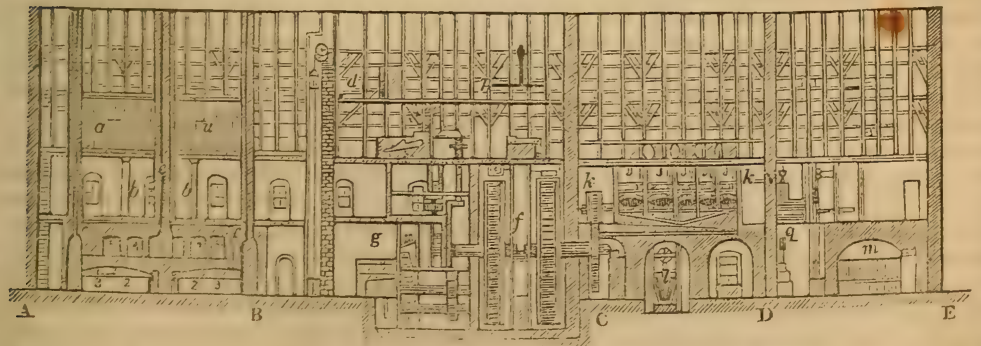

four main departments. The first, A, B, is devoted to the preparation and roasting of the matters intended for amalgamation. The second, $B, c$, is occupied with two successive 
siftings and the milling. The third, $\mathrm{c}, \mathrm{D}$, includes the amalgamation apartment above, and the wash-house of the residuum below. And in the fourth, $D, E$, the distilling apparatus is placed, where the amalgam is finally delivered.

Thus, from one extremity of this building to the other, the workshops follow in the order of the processes; and the whole, over a length of 180 feet, seems to be a natural labura tory, through which the materials pass, as it were of themselves, from their crude to their refined condition; so skilfully economized and methodical are the labors of the workmen; such are the regularity, precision, concert, and facility, which pervade this long series of combinations, carriages, movements, and metamorphoses of matter.

Here we distinguish the following objects :-

1. In division $A, B ; a, a$, is the magazine of salt ; $b, b$, is the hall of preparation of the ores; on the floor of which they are sorted, interstratified, and mixed up with salt ; $c, c$, are the roasting furnaces; in each of which we see, 1 , the fireplace; 2,3 , the reverberatory hearth, divided into two portions, one a little higher than the other, and more distant from the fireplace, called the drier. The materials to be calcined fall into it, through a chimney 6 . The other part 2 , of the hearth, is the calcining area. Above the furnace are chambers of sublimation 4,5 , for condensing some volatile matters which escape by the opening 7. $e$ is the main chimney.

2. In the division $\mathrm{B}, \mathrm{c}$, we have $d$, the floor for the coarse sifting; beneath, that for the fine sieves; from which the matters fall into the hopper, whence they pass down $t o \mathrm{~g}$, the mill-house, in which they are ground to flour, exactly as in a corn-mill, and are after wards bolted through sieves. $\quad p, f$, is the wheel machinery of the mill.

3 . The compartment $\mathrm{C}, \mathrm{D}$, is the amalgamation work, properly speaking, where the casks are seen in their places. The washing of the residuums is effected in the shop $l$, below. $k, k$, is the compartment of revolving casks.

4. In the division $D, E$, the distillation process is carried on. There are four similar furnaces, represented in different states, for the sake of illustration. The wooden drawer is seen below, supporting the cast-iron basin, in which the tripod with its candelabra for bearing the amalgam saucers is placed. $q$ is a store chamber.

At $\mathrm{B}$, are placed the pulleys and windlass for raising the roasted ore, to be sifted and ground; as also for raising the milled flour, to be transported to the amalgamation casks. At $\mathrm{D}$, the crane stands for raising the iron bells that cover the amalgamation candelabra.

Details of the Amalgamation Process, as practised at Halsbrücke. - All ores which contain more than $7 \mathrm{lbs}$. of lead, or $1 \mathrm{lb}$. of copper, per cent., are excluded from this reviving operation (anquickverfahren); because the lead would render the amalgam very impure, and the copper would be wasted. They are sorted for the amalgamation, in such a way that the mixture of the poorer and richer ores may contain $7 \frac{1}{2}$, or, at most, 8 loths (of $\frac{1}{2} \mathrm{oz}$. each) of silver per $100 \mathrm{lbs}$. The most usual constituents of the ores are, sulphur, silver, antimonial silver (speissglanzsilber), bismuth, sulphurets of arsenic, of copper, iron, lead (nickel, cobalt), zinc, with several earthy minerals. It is essential that the ores to be amalgamated shall contain a certain proportion of sulphur, in order that they may decompose enough of sea salt in the roasting to disengage as much chlorine as to convert all the silver present into a chloride. With this view, ores poor in sulphur are mixed with those that are richer, to make up a determinate average. The ore-post is laid upon the bed-floor, in a reetangular heap, about 17 ells long, and $4 \frac{1}{2}$ ells broad ( 13 yards and $\left.3 \frac{1}{2}\right)$; and upon that layer the requisite quantity of salt is let down from the floor above, through a wooden tunnel; $40 \mathrm{cwts}$. of salt being allotted to $400 \mathrm{cwts}$. of ore. The heap being made up with alternate strata to the desired magnitude, must be then well mixed, and formed into small bings, called roast-posts, weighing each from $3 \frac{1}{2}$ to $4 \frac{1}{2}$ cwts. The annual consumption of salt at Halshrücke is 6000 ewts.; it is supplied by the Prussian salt-works.

Roasing of the Amalgamation Ores.-- The furnaces appropriated to the roasting of the ore-posts are of the reverberatory class, provided with soot chambers. They are built up alongside of the bed-floor, and connected with it by a brick tunnel. The prepared ground ore (erzmehl) is spread out upon the hearth, and dried with incessant turning over; then the fire is raised so as to kindle the sulphur, and keep the ore redhot for one or two hours; during which time, dense white-gray vapors of arsenic, antimony, and water, are exhaled. The desulphuration next begins, with the appearance of a blue flame. This continues for three hours, during which the ignition is kept up; and the mass is diligently turned over, in order to present new surfaces, and to prevent any caking. Whenever sulphurous acid ceases to be formed, the finishing calcination is to be commenced with increased firing; the object being now to decompose the sea salt by means of the metallic sulphates that hare been generated, to convert them into chlorides, with the simultaneous production of sulphate of soda. The stirring is to be continued till the proofs taken from the hearth no longer betray the smell of sulphurous, but only of muriatic acid gas. This roasting stage lasts commonly three quarters of an hour, 13 or 14 furnaces are worked at the same time at Halsbrücke; and each turns out in a 
week 5 tons upon an average. Out of the nicht chambers or soot vaults of the furnaces. from .96 to $100 \mathrm{cwts}$. of ore-dust are obtained, containing 32 mares ( 16 lbs.) of silvert. This dust is to be treated like unroasted ore. 'The fuel of the first fire is pitcoal; of tite finishing one, fir-wood. Of the former $115 \frac{1}{2}$ cubic feet, and of the latter, $294 \frac{1}{4}$, are, upon an average, consumed for every $100 \mathrm{cwts}$. of ore.

During the last roasting, the ore increases in bulk by one fourth, becomes in conse quence a lighter powder, and of a brown color. When this process is completed, the ore is raked out upon the stone pavement, allowed to cool, then screened in close sieve-boxes. in orter to separate the finer powder from the lumps. These are to be bruised, mixed with sea salt, and subjected to another calcination. The finer powder alone is taken 10 the millstones, of which there are 14 pairs in the establishment. The stones are of granite, and make from 100 to 120 revolutions per minute. The roasted ore, after it has passed through the bolter of the mill, must be $s:$ impalpable as the finest flour.

The Amalgamation.-This (the verquicken) is performed in 20 horizontal casks. arranged in 4 rows, each turning upon a shaft which passes through its axis; and al driven by the water-wheel shown in the middle of fig. 1006. The cash are 2 feet 10 inches long, 2 feet 8 inches wide, inside measure, and are provided with iron ends. The staves are $3 \frac{1}{2}$ inches thick, and are bound together with iron hoops. They have a double bung-hole, one formed within the other, secured by an iron plug fastened with serews. They are filled by means of a wooden spout terminated hy a canvass hose; through which $10 \mathrm{cwts}$. of the bolted ore-flour (erzmehl) are introduced after $3 \mathrm{cwts}$. of watez have been poured in. To this mixture, from $\frac{3}{4}$ to $\frac{7}{8}$ of a cwt. of pieces of iron, $1 \frac{1}{2}$ inch square, and $\frac{3}{8}$ thick, are added. When these pieces get dissolved, they are replaced by others from time to time. The casks being two thirds full, are set to revolve for $1 \frac{2}{2}$ or 2 hours, till the ore-powder and water become a uniform pap; when 5 ewts. of quicksilver are poured into each of them. The casks being again made tight, are put in gear with the driving machinery, and kept constantly revolving for 14 or 16 hours, at the rate of 20 or 22 turns in the minute. During this time they are twice stopped and opened, in order to see whether the pap be of the proper consistence; for if too thick, the globules of quichsilver do not readily combine with the particles of ore; and if too thin, they fall and rest at the bottom. In the first case, some water must be added; in the second, some ore. During the rotation, the temperature rises, so that even in winter it sometimes stands so high as $104^{\circ} \mathrm{F}$.

The chemical changes which occur in the casks are the following:-The metallic chlorides present in the roasted ore are decomposed by the iron, whence results murjate of iron, whilst the deutochloride of copper is reduced partly to protochloride, and partly to metallic copper, which throw down metallic silver. The mercury dissolves the silver, copper, lead, antimony, into a complex amalgam. If the iron is not present in sufficient quantity, or if it has not been worked with the ore long enough to convert the copper deutochloride into a protochloride, previously to the addition of the mercury, more or less of the last metal will be wasted by its conversion into protochloride (calomel.) The water holds in solution sulphate of soda, undecomposed sea salt, with chlorides of iron, manganese, \&c.

As soon as the revivification is complete, the casks must be filled with water, set to revolve slowly (about 6 or 8 times in the minute), whereby in the course of an hour, or an hour and a half at most, a great part of the amalgam will have collected at the bottom; and in consequence of the dilution, the portion of horn silver held in solution by the sea salt will fall down and be decomposed. Into the smail plug in the centrc of the bung, a small tube with a stopcock is now to be inserted, to discharge the amalgam into its appropriate chamber. The cock must be stopped whenever the brown muddy residuum begins to flow. The main bung being then opened, the remaining contents of the casks are emptied into the wash-tun, while the pieces of iron are kept back. The residuary ore is found to be stripped of its silver within $\frac{5}{3} \overline{2}$ or $\frac{7}{40}$ of an ounce per cwt. The emptying of all the casks, and charging them again, takes 2 hours; and the whole process is finished within 18 or 20 hours; namely, 1 hour for charging, 14 to 16 nours for amalgamating, $1 \frac{1}{2}$ hour for diluting, 1 hour for emptying. In 14 days, 3200 cwts. of ore are amalgamated. For working $100 \mathrm{cwts}$. of ore, $14 \frac{1}{2} \mathrm{lbs}$. of iron, and 2 lbs. $12 \frac{1}{2}$ ounces of mercury are required; whence, for every pound of silver obtained, 0.95 of an ounce of mercury are consumed.

Trials have been made to conduct the amalgamation process in iron casks, heated to $150^{\circ}$ or $160^{\circ}$ Fahrenheit, over a fire; but, though the de-silvering was more complete, the loss by mercury was so much greater as to more than counterbalance that advantage.

Treatment of the Amalgam.- It is first received in a moist canvass bag, through which the thin uncombined quicksilver spontaneously passes. The bao is then tied up and subjected to pressure. Out of 20 casks, from 3 to $3 \frac{1}{2} \mathrm{cwts}$. of solid amaleam are thus procured, which usually consist of 1 part of an alloy, containing silver of 12 or 13 loths (in 16), and 6 parts of quicksilver. The foreign metals in that alloy are, copper, lead, 
gold, antimony, cobalt, nickel, bismuth, zinc, arsenic, and iron. The filtered quicksilver contains moreover 2 to 3 loths of silver in the cwt.

Fig. 1007 represents the apparatus for distilling the amalgam in the Halsbrücke works; marked $m$ in fig. 1006. $a$ is the wooden drawer, sliding in grooves upon the

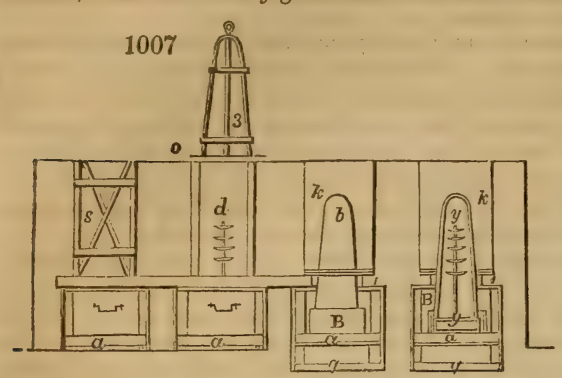
basis $q$; $\mathrm{B}$ is an open basin or box of cast iron, laid in the wooden drawer; $y$ is a kind of iron candelabra, supported upon four feet, and set in the basin B; under $d$ are five dishes, or plates of wrought iron, with a hole in the centre of each, whereby they are fitted upon the stem of the candelabra, 3 inches apart, each plate being success̀ively smaller than the one below it. 3 indicates a cast-iron bell, furnished with a wrought-iron frame and hook, for raising it by means of a pullev and cord. $s$ is a sheet-iron door for closing the

stove, whenever the bell has been set in its place.

The box $a$, and the basin B above it, are filled with water, which must be continually renewed, through a pipe in the side of the wooden box, so that the iron basin may be kept always submersed and cool. The drawer $a$, being properly placed, and the plates under $d$ being charged with balls of amalgam (weighing altogether 3 cwts.), the bell 3 is to be let down into the water, as at $y$, and rested upon the lower part of the candelabra. Upon the ledge 1, which defines the bottom of the fire-place, a circular plate of iron is laid, having a hole in its middle for the bell to pass through. Upon this plate chips of fir-wood are kindled, then the door $s$, which is lined with clay, is closed and luted tight. The fuel is now placed in the vacant space $k$, round the upper part of the bell. The fire must be fed in most gradually, first with turf, then with charcoal; whenerer the bell gets red, the mercury volatilizes, and condenses in globules into the bottom of the basin $\mathbf{B}$. At the end of 8 hours, should no more drops of mercury be heard to fall into the water, the fire is stopped. When the bell has become cool, it is lifted off; the plates are removed from the candelabra $d$; and this being taken out, the drawer $a$ is slid away from the furnace. The mercury is drained, dried, and sent again into the amalgamation works. The silver is fused and refined by cupellation.

The solid amalgam which is distilled in the above apparatus, would be distilled more profitably out of iron trays set in the mercurial retorts described and figured in pages $815,816$.

From 3 ewts. of amalgam, distilled under the bell, from 95 to 100 mares ( $\frac{1}{2}$ lbs.) of teller silver (dish silver) are procured, containing from 10 to $13 \frac{1}{2}$ parts of fine silver out of 16 ; one fifth part of the metal being copper. The leller silver is refined in quantities of 160 or 170 marcs, in black-lead crucibles filled within two inches of their brims, and submitted to brisk ignition. The molten mass exhales some vapors, and throws up a liquid slag, which being skimmed off, the surface is to be strewed over with charcoal powder, and corered with a lid. The heat having been briskly urged for a short time, the charcoal is then removed along with any fresh slag that may have risen, in order to observe whether the vapors have ceased. If not, fresh charcoal must be again applied, the crucible must be covered, and the heat increased, till fumes are no longer produced, and the surface of the silver becomes tranquil. Finally, the alloy, which contains a little gold and much copper, being now from 11 to 13 lothig (that is, holding from 11 to 13 parts of fine silver in 16 parts), is cast into iron moulds, in ingots of 60 marcs. The loss of weight by eraporation and skimming of the slag amounts to 2 per cent.; the loss in silver is quite inconsiderable.

The dust from the furnace (tiegelöfen) is collected in a large condensation chamber of the chimney, and affords from 40 to 50 marcs of silver per cwt. The slags and old cru cibles are ground and sent to the small amalgamation mill.

The earthy residuum of the amalgamation casks being submitted to a second amalga mation, affords out of $100 \mathrm{cwts}$. 2bout $2 \mathrm{lbs}$. of coarse silver. This is first fused along with three or four per cent. of a mixture of potashes and calcined quicksalz (impure sulphate of soda), and then refined. The supernatant liquor that is drawn out of the tanks in which the contents of the casks are allowed to settle, consists chiefly of sulphate of soda, along with some common salt, sulphates of iron and manganese, and a little phosphate, arseniate, and fluate of soda. The earthy deposite contains from $\frac{1}{4}$ to $\frac{9}{32}$ of a loth of silver per cwt., but no economical method of extracting this small quantity has yet been contrived.

The argentiferous or rich lead is treated in Germany by the cupellation furnace represented in figs. 1008, 1009, 1010, and 1011. These figures exhibit the cupellation 
lurnace of the principa] smelting works in the Hartz, where the following parts must be distinguished; (fig. 1010); 1. masonry of the foundation; 2. flues for the escape of moisture; 3. stone covers of the flues; 4 . bed of hard rammed scoriæ; 5 . brichis set on edige, to form the permanent area of the furnace; 6 . the sole, formed of wood ashes, washed, dried, and beaten down; $k$, dome of iron plate, moveable by a crane, and susceptible of being lined two inches thick with loam; $n$, n, tuyeres for two belluws $s$; having valves suspended before their orifices to break and spread the blast; $q$, door for introducing into the furnace the charge of lead, equal to 84 quintals at a time; $s, f i g$.
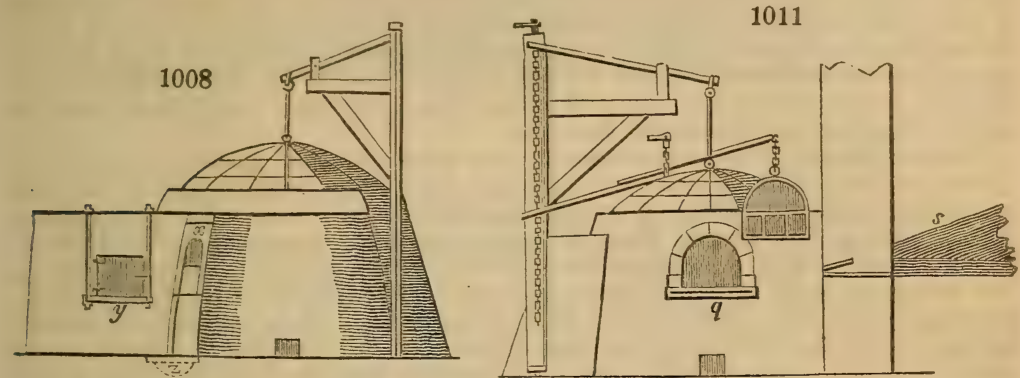

1011, two bellows, like those of a smith's forge; $y$, door of the fireplace, through which billets of wood are thrown on the grate; $x$, small aperture or door, for giving issue to the

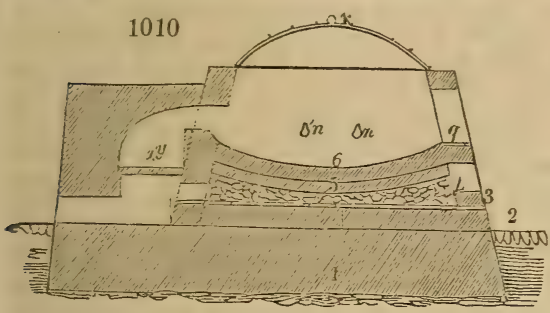
frothy scum of the eupellation, and the litharge; $z$, basin of safety, usually covered with a stone slab, ovey which the litharge falls; in case of accident the basin is laid open to admit the rich lead.

The following is the mode of con. ducting the cupellation. Before putting the lead into the furnace, a floor is made in it of ashes beat earefully down (see 6, fig. 1010); and there is left in the centre of this floor a circular space, somewhat lower than the rest of the hearth, where the silver ought to gather at the end of the operation. The cupel is fully six feet in diameter.

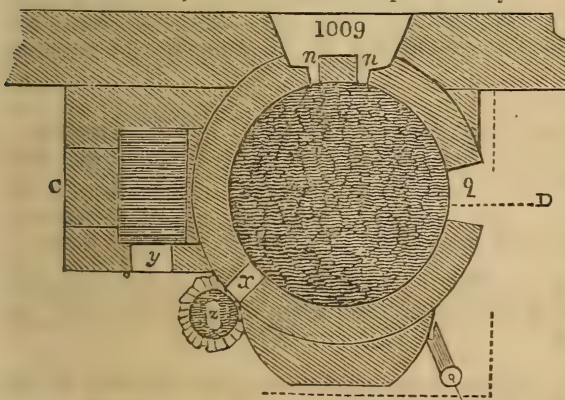

In forming the floor of a cupel, 35 cubic feet of washed wood ashes, usually got from the soap works, are employed. The preparation of the floor requires two and a half hours' work; and when it is completed, and the moveable dome of iron plate has been lined with loam, 84 quintals (cwts.) of lead are laid on the fioor, 42 quintals being placed in the part of the furnace farthest from the bellows, and 42 near to the fire-bridge; to these, scoriæ containing lead and silver are added, in order to lose nothing. The moveable lid is now luted on the furnace, ana heat is slowly applied in the fireplace, by burning fagots of fir-wood. which is graduaily raised. Section 1010 is in the line $c, D$, of 1009 .

At the end of three hours, the whole lead being melted, the instant is watched for when $n$ more ebullition can be perceived on the surface of the bath or melied metal; then, but not sooner, the bellows are set a playing on the surface at the rate of 4 or 5 strokes per minute, to favor the oxydizement.

In five hours, reckoned from the commencement of the process, the fire is smartly raised; when a grayish froth (abs/rich) is made to issue from the small aperture $x$ of the furnace. This is found to be a brittle mixture of oxydized metals and impurities. The workman now glides the rake over the surface of the bath, so as to draw the froth out of the furnace; and, as it issues, powdered charcoal is strewed unon it, at the aperture $x$, to cause its coagulation. The froth skimming lasts for about an hour and a half. 
After this time, the litharge brgins to form, and it is also let off by the small opening $x$; its issue being aided by a hook. In proportion as the floor of the furnace gets im. pregnated with litharge, the workman digs in it a gutter for the escaje of the liquid litharge; it falls in front of the small aperture, and concretes in stalactitic forms.

By means of the two moveable valves suspended before the tuyeres $n, n,(f i g .1010)$, the workman can direct the blast as he will over the surface of the metal. The wind should be made to cause a slight curl on the liquid, so as to produce circular undulations, and gradually propel a portion of the litharge generated towards the edges of the cupel, and allow this to retain its shape till the end of the operation. The stream of air should drive the greater part of the litharge towards the small opening $x$, where the workman deepens the outlet for it, in proportion as the level of the metal bath descends, and the bottom of the floor rises by the apposition of the litharge formed. $\mathrm{Li}$. tharge is thus obtained during about 12 hours; after which period the cake of silver be. gins to take shape in the centre of the cupel.

Towards the end of the operation, when no more than four additional quintals of litharge can be looked for, and when it forms solely in the neighborhood of the silver cake in the middle of the floor, great care must be taken to set apart the latter portions, because they contain silver. About this neriod, the fire is increased, and the workman places before the little opening $x$ a brick, to serve as a mound to the efflux of litharge. The use of this brick is, -1 . to hinder the escape of the silver in case of any accident; for example, should an explosion take place in the furnace; 2 . to reserve a magazine of litharge, should that still circulating round the silver cake be suddenly absorbed by the eupel, for in this dilemma the litharge must be raked back on the silver; 3 . to prevent the escape of the water that must be thrown on the silver at the end of the procrss.

When the argentiferous litharge, collected in the above small magazine, is to be removed, it is let out in the form of a jet, by the dexterous use of the iron hook.

Lastly, after 20 hours, the silver cake is seen to be well formed, and nearly circular. The moment for stopping the fire and the bellows is indicated by the sudden disappearance of the colored particles of oxyde of lead, which, in the latter moments of oxydation, undulate with extreme rapidity over the slightly convex surface of the silver bath, moving from the centre to the circumference. The phenomenon of their total disappearance is called the lightning, or fulguration. Whenever this occurs, the plate of silver being perfectly clean, there is introduced into the furnace, by the door $q$, a wooden spout, along which water, previously heated, is carefully poured on the silver.

The cupellation of 84 quintals of argentiferous lead takes in general 18 or 20 hours' working. The promptitude of the operation depends on the degree of purity of the leads employed, and on the address of the operator, with whom also lies the economy of fuel. A good workman completes the cupellation of 84 quintals with 300 billets, each equivalent to a cubic foot and eight tenths of wood (Hartz measure); others consume 400 billets, or more. In general, the cupellation of 100 quintals of lead, executed at the rate of 84 quintal charges, occasions a consumption of 790 cubic feet of resinous wood billets.

The products of the charge are as follows :-

1. Silver, holding in 100 mares, 7 mares and 3 loths of alloy -

2. Pure litharge, containing from 88 to 90 per cent. of lead -

3. Impure litharge, holding a little silver

4. Skimmings of the cupellation -

5. Floor of the furnace impregnated with litharge

24 to 30 mares.

$50-60$ quintals.

Note.-The marc is 7 oz. 2 duts. $4 \mathrm{gr}$. English troy; and the loth is half an ounce. 16 loths make a marc. 100 pounds Cologne are equal to 103 pounds aroirdupors; and the above quintal contains 116 Cologne pounds.

The loss of lead inevitable by this operation, is estimated at 4 parts in 100 . It has been diminished as much as possible in the Frankenscharn works of the Hartz, by lcading the smoke into long flues, where the lead fumes are condensed into a metallic soot. The silver cake receives a final purification at the Mint, in a cupel on a smaller scale.

From numerous experiments in the great way, it has been found that not more than 100 quintals of lead can be profitably cupelled at one operation, however large the furnace, and however powerful and multiplied the bellows and tuyères may be; for the loss on either the lead or the silver, or on both, would be increased. In one attempt, no less than 500 quintals were acted on, in a furnace with two fireplaces, and four escapes for the litharge; but the silver remained disseminated through the lead, and the lightning could not be brought on. The chief object in view was economy of fuel.

Reduction of the Litharge. - This is executed in a slag-hearth, with the aid of wood charcoal.

Such is the train of operations by which the cupriferous galena schlich, or ground ore 
is reduced, in the district of Clausthal, into lead, copper, and silver. The works ot Frankenscharn have a front fully 400 feet long.

Fiv. 1011, exhibits the plan and elevation of these smelting-works, near Clausthal, in the Hartz, for lead ores containing copper and silver, where about $84,000 \mathrm{cwts}$. of schlich

Silver-smelting Works of Frankenscharn, near Clausthal.

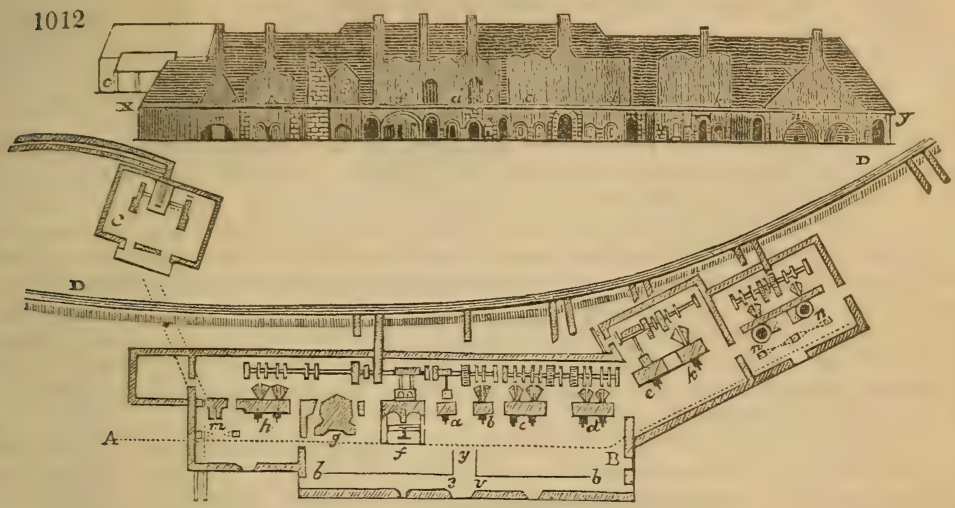

(each of 123 Cologne pounds) are treated every year. This quantity is the prodire of thirty distinct mines, as also of nearly as many stamp and preparation works. All these different schlichs, which belong to so many different joint-stock companies, are confounded and worked up together in the same series of metallurgic operations; the resulting mixture being considered as one and the same ore belonging to a single undertaking; but in rirtue of the order which prevails in this royal establishment, the rights of each of the companies, and consequently of each shareholder, are equitably regulated. A vigorous control is exercised between the mines and the stamps, as also between the stamps and the smelting-houses; while the cost of the metallurgic operations is placed under the officers of the crown, and distributed, upon just principles, among the several mines, according to the quantities of metal furnished by each.

From these arrangements, the following important advantages flow:-

1. The poor ores may be smelted with profit, without putting the companies to any risk or expense in the erection of new works; 2 , by the mixture of many different ores, the smelting and metallic product become more easy and abundant; 3 , the train of the operations is conducted with all the lights and resources of science; and 4 , the amount of metal brought into the market is not subject to such fluctuations as might prove injurious to their sale.

The following is the series of operations;-

1. The fusion of the schlich (sludge); 2 , the roasting of the mattes under a shed, and their treatment by four successive re-meltings; 3 , the treatment of the resulting black copper; 4 , the liquation; 5 , the re-liquation (ressuage); 6 , the refining of the copper ; 7 , the cupellation of the silver; 8 , the reduction of the litharge into lead. The 5th and 6 th processes are carried on at the smelting works of Altenau.

The buildings are shown at $A, B, C$, and the impelling stream of water at $D$; the upper figure being the elevation; the lower, the plan of the works.

$a$, is the melting furnace, with a cylinder bellows behind it; $b, c, d$, furnaces similar to the preceding, with wooden bellows, such as fig. $1013 ; e$, is a furnace for the same

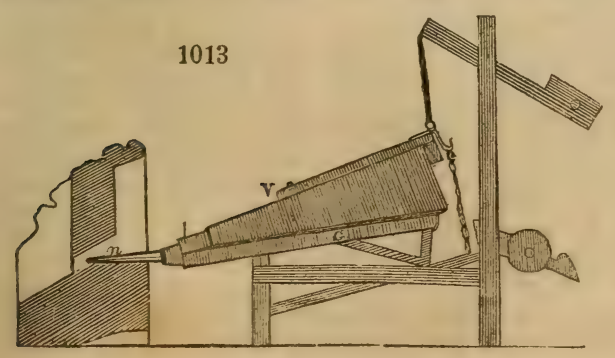
purpose, with three tuyères, and a cylinder bellows; $f$, the large furnace of fusion, also with three tuyères; $g$, a furnace with seven tuyères, now seldom used; $h$, low furnaces, like the English slaghearths, (krummofen, ) employed for working the last mattes; $k$, slag-hearths for reducing the litharge; $m$, the area of the liquation; $n, p$, cupellation furnaces.

$x, y$, a floor which separates the principal smelting-house inte 
two stories; the materials aestined for charging the furnaces being deposited in beds upon the upper floor, to which they are carried by means of two inclined planes, terrazed in front of the range of buildings.

Here 89,600 quintals of schlich are annually smelted, which furnish-

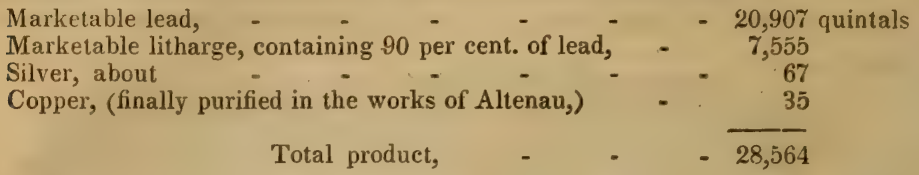

This weight amounts to one twenty-fifth of the weight of ore raised for the service of the establishment. Eight parts of ore furnish, on an average, about one of schlich. The bellows are constructed wholly of wood, without any leather; an improvement made by a bishop of Bamberg, about the year 1620. After receiving different modifications, they were adopted, towards 1730 , in almost all the smelting-works of the cor,inent, except in a few places, as Carniola, where local circumstances permitted a water blowing-machine to be erected. These pyramidal shaped bellows, composer of moveabie wooden boxes, have, however, many imperfections; their size must often be inconveniently large, in order to furnish an adequate stream of air; they do not drive into the furnace all the air which they contain; they require frequent repairs; and, working with great friction, they waste much mechanical power.

Fig. 1014, represents such wooden bellows, consisting of two chests or boxes fitted into each other; the upper or moving one being called the $f y$, the lower or fixed one,

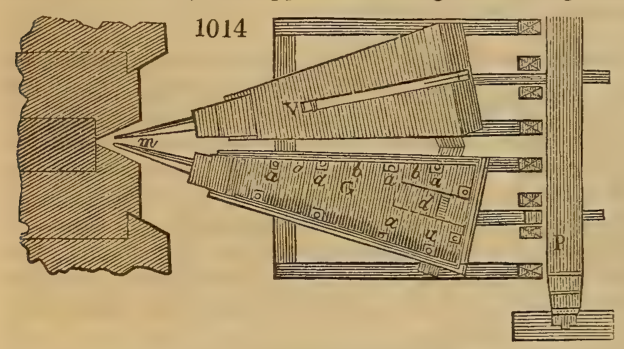
the seat, (gite.) In the bottom of the gile, there is an orifice furnished with a clack-valve $d$, opening in wards when the $f l y$ is raised, and shutting when it falls. In order that the air included in the capacity of the two chests may have no other outlet than the nose-pipe $m$, the upper portion of the gite is provided at its four sides with small square slips of wood, $c, c, c$, which are pressed against the sides of the fly by strong springs of iron wire, $b, b, b$, while they are retained upon the gite by means of small square pieces of wood, $a$, $a, a, a$. The latter $a, a$, are perforated in the centre, and adjusted upon rectangular stems, called buchettes; they are attached, at their lower ends, to the upright sides of the gite $\mathrm{G}$. $\mathrm{P}$, is the driving-shaft of a water-wheel, which, by means of cams or tappets, depresses the fly, while the counterweight $\mathrm{Q}$, fig. 1013, raises it again.

Figs. 1015, 1016, 1017, 1018, represent the moderately high (demihauts, or half-blast) furnaces employed in the works of the lower Hartz, near Goslar, for smelting the silvery lead ores extracted from the mine of Rammelsberg. See its section in fig. 737.

Fig. 1015, is the front elevation of the twin furnaces, built in one body of masonry; fig. 1016, is a plan taken at the level of the tuyères, in the line v, l, 6, of fig. 1017;

1015

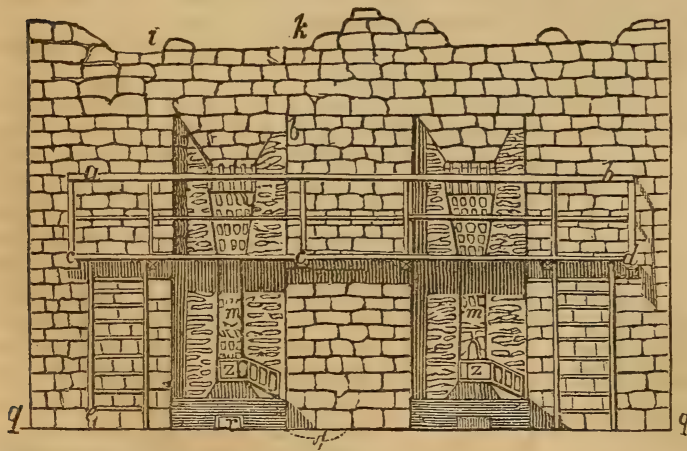
figs. 1017 and 1018, exhibit two vertical sections; the former in the line $A, B$, the latter in the line $\mathbf{c}, \mathbf{D}$, of $f \mathrm{~g} .1016$. In these four figures the following ob jects may be distinguished. $a, b, c, d$, a balcony or platform which leads to the place of charging, $n$; $e, f$,wooden stairs, by which the charging workmen mount from the ground $p, q$, of the works, to the platform; $g, h$, brick-work of the furnaces; $i, k$, wall of the smelting-works, against which they are

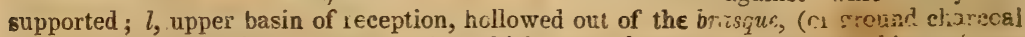
bed,) $6 ; m$, arch of the tuyère $v$, by which each furnace recerves the blast ci two 
bellows; $n$, place of charging, which takes place through the upper orifice $n, o$, of the basin $n, 0, v, t$, of the furnace; $t$, a slab of clay, placed in such a way that, during the

A 1016

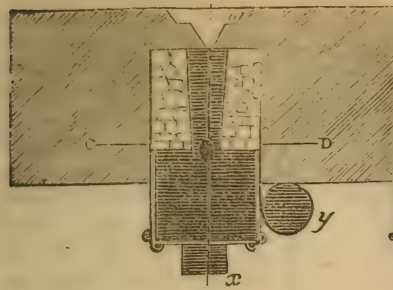

$\mathrm{B}$

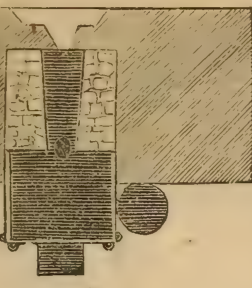
treatment of the lead, a little metallic zine may run together in a sloping yutter, seen in fig. $100 \mathrm{l}$, formed of slates cemented together with clay.

In $f$ igs. 1015 and $1017,1,2$ is the brick-work of the fcandations; $m$, conduits (called evaporatory) for the exhalation of the moisture; 4 , a layer of slags, rammed above; 5 , a bed of clay, rammed above the slags; 6 , a brasque, composed of one part of clar, and two parts of ground charcoal, which forms the sole of the furnace. The excellent refinery furnace, or treibheerd, of Frellerıckshütte, near Tarnowitz, in

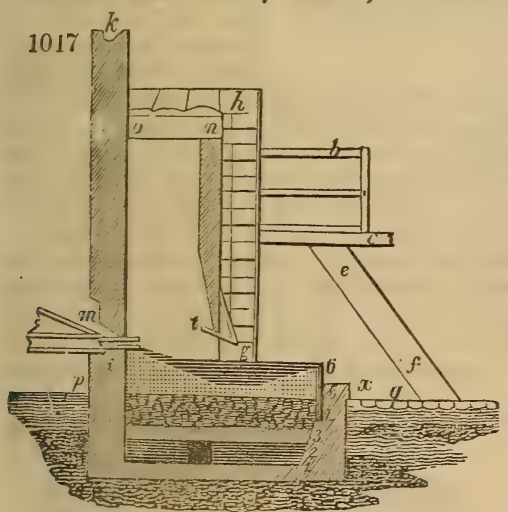
Upper Silesia, is represented in fig? 1019 and 1020. $a$, is the hottom, made of slag or cinders; $b$, the foundation of fire-bricks; $c$, the body of the hearth proper, composed of a mixture of 7 parts of dolomite, and 1 of fire-clay, in bulk; $d$, the grate of the air furnace; $e$, the fire-bridge; $f$, the dome or cap, made of iron plate strengthened with bars, and lined with clay-lute, to protect the metal from burning; $g$, the door of the fireplace; $h$, the ash-pit; $i$, the tap-hole; $k, k$, the flue, which is divided by partitions into several channels; $l$, the chimney; $m$, a damper-plate for regulating the draught; $n$, a back valve, for admitting air to cool the furnace, and brushes to sweep the flues; 0 , tuyère of copper, which by means of an iron wedge may be sloped more or less towards the hearth; $p$, the schnepper, a round

1018

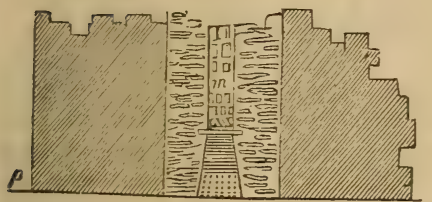
piece of sheet iron, hung before the eye of the tuyère, to break and spread the blast; $q$, the outlet for the glassy litharge.

Lime-marl has been found to answer well for making the body of the hearth-sole, as it absorbs the vitrified litharge freely, without combining with it. A basin-shaped hollow is formed in the centre, for receiving the silver at the end of the process ; and a gutter is made across the hearth for running off the glätte or fluid litharge.

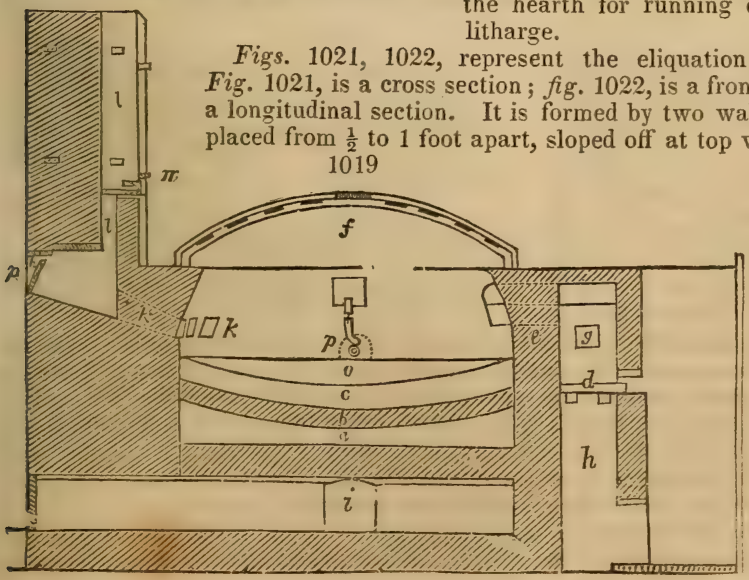

the eliquation hearth of Neustadt. Fig. 1021, is a cross section; fig. 1022, is a front view; and fig. 1023 , a longitudinal section. It is formed by two walls $\alpha, a, 3 \frac{1}{2}$ feet high, $\frac{1}{2}$ to 1 foot apart, sloped off at top with iron plates, three inches thick, and 18 inches broad, called saigers-charten, or refining plates, $b, b$, inclined three inches to. wards each other in the middle, so as to leave at the lowest point a slit two and a half inches wide between them, throngh wrlich the seain, as it sweais out by the heat, is allowed to fall into 
the space between the two walls c, called the saigergasse, (sweating gutter.) The sole of this channel slopes down towards the front, so that the liquefied metal may run off into

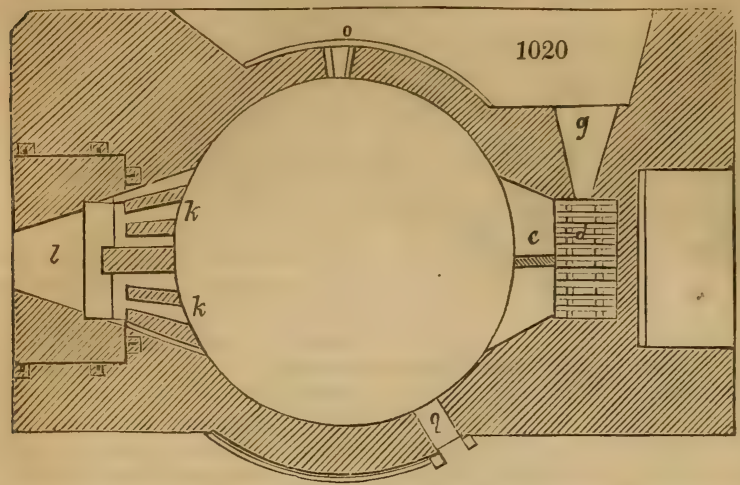
a crucible or pot. Up. on one of the long sides, and each of the shorter ones, of the hearth, the walls $d, d$, are raised two feet high, and upon these the liquation lumps rest; upon the other long side, where there is no wall, there is an opening for admitting these jumps into the hearth. The openings are then shut with a sheet or cast iron plate $e$, which, by means of a chain, pulley, and counterweight, may be easily raised and lowered. $f$, is a passage for increasing the draught of air.

Figs. 1023 and 1026, represent the refining furnaces of Frede-

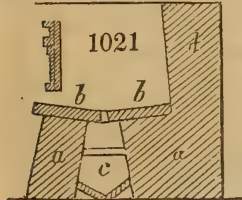
rickshütie by Tarnowitz; $a$, is the fire door ; $b$, the grate; $c$, the door for introducing the silver; $d$, the moveable test; resting upon a couple of iron rods $e, e$, which are let at their ends into the brick-work. They lie lower than would seem to be necessary; but this is done in order to be able to place the surface of the test at any desired level, by placing tiles $f, f$, under it ; $g$, the flue, leading to a chimney 18 feet high. For the refining of 100 marks of blicksilber, of the fineness of $15 \frac{1}{2}$ loths (half ounces) per cwt., 3 eubic feet of pit-coal are required. The test or cupel must be heated before the impure silver and soft lead are put into it.

At these smelting-houses, from 150 to $160 \mathrm{cwts}$. of very pure workable lead (lead con-

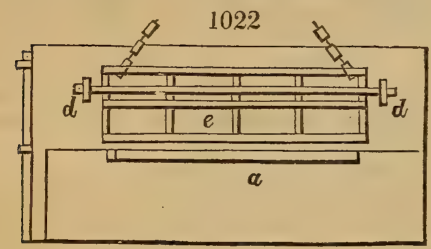
1023

Iainıng merely a little silver) are put into the furnace at once, and from 10 to $14 \mathrm{cwts}$. run off in vitrified oxyde; the remainder is then refined with some pure lead, when an alloy containing from $14 \frac{1}{2}$ to $15 \frac{1}{2}$ loths of blicksilber per cwt. is obtained.
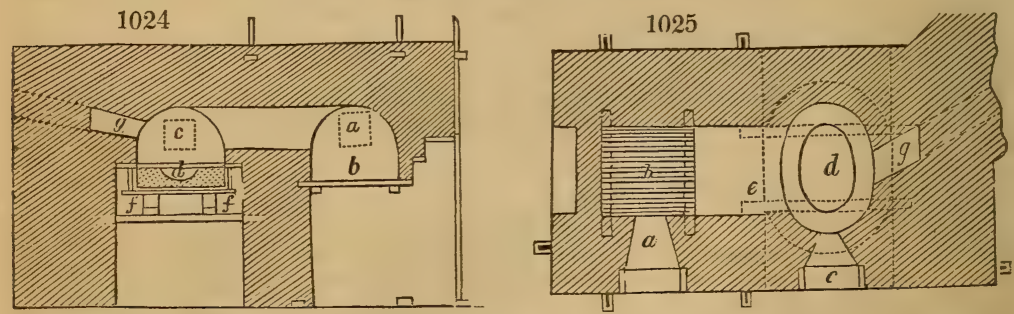

English refining furnaces.-The refining of lead is well performed in some works in the neighborhood of Alston-moor, in reverberatory furnaces, figs. 1026 and 1027, whose fireplace is 22 inches square, and is separated from the sole by a fire-bridge, 14 inches in breadth. The flame, after having passed over the surface of the lead in the cupel, enters two flues $e, e$, on the opposite side of the furnace, which terminate in a chimney $i, i$, $i, i, 40$ feet high. At the bottom of the chimney are openings $f, f$, for taking out the metallic dust deposited within. These openings are shut during the process.

The cupel or test, which constitutes, in fact, the sole of the hearth in which the operation takes place, is moveable. It consists of a vertical elliptical ring of iron, 
A, B, C, D, figs. 1028 and $1029,3 \frac{3}{4}$ inches high, the greatest diameter of the elipse neing 4 feet, and the smallest 2 . Four iron bars ( $A, D, m, m^{\prime}, \mathrm{B}, \mathrm{C}, n, n^{\prime}$ ) are fixed across its
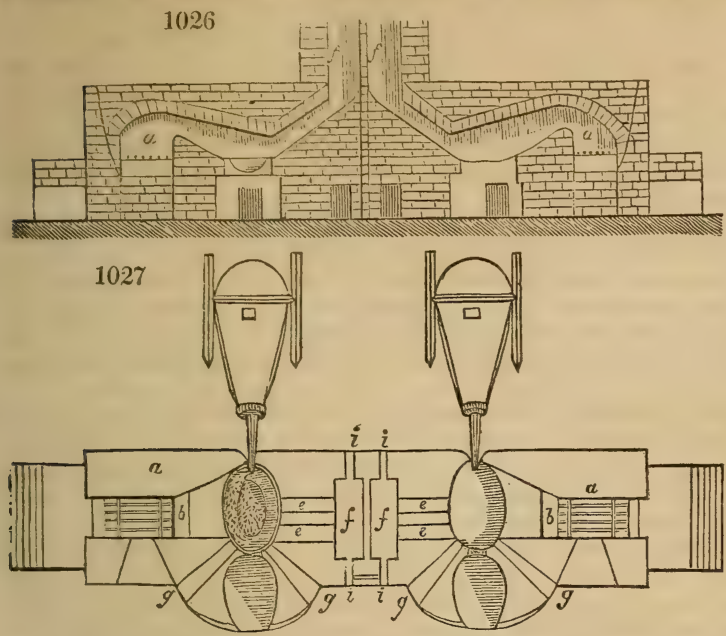
bottom, which we also $3 \frac{3}{4}$ inches broad, and an inch thick. The first of these bars is placed 9 inches from the end of the elliptic ring neares: the fireplace, and the three others are equally distributed between this bar and the back end.

In forming the cupel, several layers of a mixture of moistened bone ashes, and fern ashes, in very fine powder, are put into the test-frame. The bone ash constitutes from $\frac{1}{8}$ to $\frac{1}{16}$ of the bulk of the mixture, according to the purity of the fern ashes employed, estimated by the proportion of potash they contain, which has the property of semi-vitrifying the powder of burnt bones, of thus removing its friability, and

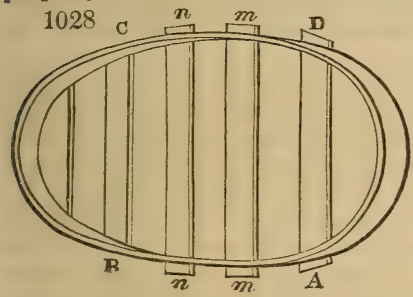
1029

rendering it more durable. The layers of ashes are strongly beat down, till the frame is: entirely filled. The mass thus formed is then hollowed out by means of a little spade, made on purpose, till it is only three quarters of an inch thick above the iron bars near the centre of the bottom. A flange, 2 inches broad, is made at the upper part, and $2 \frac{2}{2}$ inches at the lower part, except on the front or breast, which is 5 inches thick. In this anterior part, there is hollowed out an opening of an inch and a quarter broad, and 6 inches long, with which the outlet or gateway of the litharge communicates.

The cupel thus prepared is placed in the refining furnace. It rests in an iron ring built into the brickwork. The arched roof of the furnace is 12 inches above the cupel near the fire-bridge, and 9 inches near the flue at the other end.

The tuyère is placed in the back of the furnace, opposite to the side at which the litharge is allowed to overflow.

Openings $g, g$, are left at the sides of each cupel, either for running off or for introducing melted lead.

Refining of lead to extract its silver.-This operation, which the lead of Derbyshire cannot be submitted to with advantage, is performed in a certain number of the smeltinghouses at Alston-moor, and always upon leads reduced in the Scotch furnace.

The cupel furnace above described must be slowly heated, in order to dry the cupel Wathout causing it to crack, which would infallibly be produced by sudden evaporation of the moisture in it. When it has been thus slowly brought to the verge of a red heat, it is almost completely filled with lead previously melted in an iron pot. The cupel may be charged with about $5 \mathrm{cwts}$. At the temperature at whicn the lead is introduced, it is immediately covered with a gray pellicle of oxyde; but when the heat of the furnace has been progressively raised to the proper pitch, t becomes whitish-red, and has its surface covered over with litharge. Now is the time to set in action the blowing-machine, the blast of which, impellec in the direction of une great axis of the cupel, drives the litharge towards the breast of the cupel, and makes it flow out by the 
way prepared for it, through which it falls upon a cast-iron plate, on a level with the floor of the aplartment, and is dispersed into tears. It is carried in this state to the furnace of reduction, and revived. As by the effect of the continual oxydization which it undergoes, the surface of the metal necessarily falls below the level of the gateway of the litharge, melted lead must be added anew by ladling it into the furnace from the iron boiler, as occasion may require. The operation is carried on in this manner till $84 \mathrm{cw}$ ts. or 4 Newcastle fodders of lead have been introduced, which takes from 16 to 18 hours, if the tuyère has been properly set. The whole quantity of silver which this mass of lead contains, is left in combination with about $1 \mathrm{cwt}$. of lead, which, under the name of rich lead, is taken out of the cupel.

When a sufficient number of these pieces of rich lead have been procured, so that by their respective quality, as determined by assaying, they contain in whole from 1000 to 2000 ounces of silver, they are re-melted to extract their silver, in the same furnace, but in a cupel which differs from the former in having at its bottom a depression capable of receiving at the end of the process the cake of silver. In this case a portion of the bottom remains uncovered, on which the scoriæ may be pushed aside with a little rake, from the edges of the silver.

The experiments of MM. Lucas and Gay Lussac have proved that fine silver, exposed to the air in a state of fusion, absorbs oxygen gas, and gives it out again in the act of consolidation. The quantity of oxygen thus absorbed may amount to twenty-two times the volume of the silver. The following phenomena are observed when the mass of metal is considerable; for example, from 40 to 50 pounds.

The solidification commences at the edges, and advances towards the centre. The liquid silver, at the moment of its passage to the solid state, experiences a slight agitation, and then becomes motionless. The surface, after remaining thus tranquil for a little, gets all at once irregularly perturbed, fissures appear in one or several lines, from which flow, in different directions, streams of very fluid silver, which increase the original agitation. The first stage does not yet clearly manifest the presence of gas, and seems to arise from some intestine motion of the particles in their tendency to group, on entering upon the process of crystallization, and thus causing the rupture of the envelop or external crust, and the ejection of some liquid portions.

After remaining some time tranquil, the metal presents a fresh appearance, precisely analogous to volcanic phenomena. As the crystallization continues, the oxygen gas is given out with violence at one or more points, carrying with it melted silver from the interior of the surface, producing a series of cones, generally surmounted by a small crater, vomiting out streams of the metal, which may be seen boiling violently within them.

These cones gradually increase in height by the accumulation of metal thrown up, and that which becomes consolidated on their sloping sides. The thin crust of metal on which they rest, consequently experiences violent impulses, being alternately raised and depressed by such violent agitation, that were it not for the tenacity and elasticity of the metal, there would evidently arise dislocation, fissures, and other analogous accidents. At length several of the craters permanently close, while others continue to allow the gas a passage. The more difficult this is, the more the craters become elevated, and the more their funnels contract by the adhesion or coagulation of a portion of the metal. The projection of globules of silver now becomes more violent; the latter being carried to great distances, even beyond the furnace, and accompanied by a series of explosions, repeated at short intervals. It is generally the last of these little volcanoes that attains the greatest altitude, and exhibits the foregoing phenomena with the greatest eneroy. It is, moreover, observable, that these cones do not all arise at the same time, some having spent their force, when others commence forming at other points. Some reach the height of an inch, forming bases of two or three inches in diameter. The time occupied by this exhibition is at least from half to three quarters of an hour.

During the formation of these cones, by the evolution of gas, portions of silver are shot forth, which assume, on induration, a form somewhat cylindrical, and often very fantastic, notwithstanding the incompatibility which appears to exist between the fluidity of the silver and these elongated figures. Their appearance is momentary, and without any symptoms of gas, although it is impossible to decide whether they may not arise from its influence; they seem, in fact, to resemble the phenomena of the first volcanic period.

Till very recently, the only operations employed for separating silver from lead in the English smelting-works, were the following :-

1. Cupellation, in which the lead was converted into a vitreous oxyde, which was floated off from the surface of the silver.

2. Reduction of that oxyde, commonly called litharge.

3. Smelting the bottoms of the cupels, to extract the lead which had soaked into them, in a glassy state. 
Cupellation and its two complementary operations were, in many respects, objectionable rrucesses, from the injurions effects of the lead vapors upon the heallh of the workmen; from the very considerable loss of metallic lead, amounting to 7 per cent. at least; and, lastly, from the immense consumption of fuel, as well as from the vast amount of manual labor incurred in such complicated operations. Hence, unless the lead were tolerably rich in silver, it would not bear the expense of cupellation.

The patent process lately introduced by $\mathrm{Mr}$. Pattinson, of Newcastle, is not at all prejudicial to the health of workmen; it does not occasion more than 2 per cent. of loss of lead, and in other respects it is so econcmical, that it is now profitably applied in Northumberland to alloys too poor in silver to be treated by cupellation. This process is founded upon the following phenomena.

After melting completely an alloy of lead and silver, if we allow it to cool very slowly, continually stirring it meanwhile with a rake, we shall observe at a certain period a continually increasing number of imperfect little crystals, which may be taken out with a drainer, exactly as we may remove the crystals of sea salt deposited during the concentration of brine, or those of sulphate of soda, as its agitated solution cools. On submitting to analysis the metallic crystals thus separated, and also the liquid metal deprived of them, we find the former to be lead almost alone, but the latter to be rich in silver, when compared with the original alloy. The more of the crystalline particles are drained from the metallic bath, the richer does the mother liquid become in silver. In practice, the poor lead is raised by this means to the standard of the ordinary lead of the litharge works; and the better lead is made ten times richer. 'This very valuable alloy is then submitted to cupellation; but as it contains only a tenth part of the quantity of lead subjected to crystallization, the loss in the cupel will be obviously reduced to one tenth of what it was by the former process; that is, seven tenths of a per cent., instead of seven.

These nine tenths of the lead separated by the drainer, are immediately sent into the market, without other loss than the trifling one, of about one half per cent., involved in reviving a little dross skimmed off the surface of the melted metal at the beginning of the operation. Hence the total waste of lead in this method does not exceed two per cent. And as only a small quantity of lead requires to be cupelled, this may be done with the utmost slowness and circumspection; whereby loss of the precious metal, and injury to the health of the work-people, are equally avoided.

The crystallization refinery of Mr. Pattinson is an extremely simple smelting-house. It contains 3 hemispherical cast-iron pans, 41 inches in diameter, and $\frac{1}{4}$ of an inch thick. The 3 pans are built in one straight line, the broad flange at their edge being supported upon brick-work. Each pan has a discharge pipe, proceeding laterally from one side of its bottom, by which the melted metal may be run out when a plug is withdrawn, and each is heated by a small separate fire.

Three tons of the argentiferous lead constitute one charge of each pan; and as soon as it is melted, the fire is withdrawn; the flue, grate-door, and ash-pit, are immediately closed, and made air-tight with bricks and clay-lute. The agitation is now commenced, with a round bar of iron, terminated with a chisel-point, the workman being instructed merely to keep moring that simple rake constantly in the pan, but more especially towards the edges, where the solidification is apt to begin. He must be careful to take out the crystals, progressively as they appear, with an iron drainer, heated a little higher than the temperature of the metal bath. The liquid metal lifted in the drainer; flows readily back through its perforations, and may be at any rate effectually detached by giving the ladle two or three jogs. The solid portion remains in the form of a spongy, semicrystalline, semi-pasty mass.

The proportion of crystals separated at each melting, depends upon the original quality of the alloy. If it be poor, it is usually divided in the proportion of two thirds of poor crystals, and one third of rich liquid metal; but this proportion is reversed if the alloy contain a good deal of silver.

Let us exemplify, by the common case of a lead containing 10 ounces of silver per ton. Operating upon 3 tons of this alloy, or $60 \mathrm{cwts.}$, containing 30 oz. of silver, there will be obtained in the first operation-

(a) 40 ewts. at $4 \frac{x}{4}$ ounces of silver per ton; in whole $9 \mathrm{oz}$. ?

(b) $20 \mathrm{cwts}$. at 21 21 oz. $30 \mathrm{oz}$.

Each of these alloys, $(a)$ and $(b)$, will be joined to alloys of like quality obtained in the treatment of one or several other portions of three tons of the primitive alloy. Again, three tons of each of these rich alloys are subjected to the crystallization process, and thus in succession. Thus poorer and poorer lead is got on the one hand, and richer and richer alloys on the other. Sometimes the mother metal is parted from a great body of poor crystals, by opening the discharge-pipe, and running off the liquid, while the workman keeps stirring, to facilitate the separation of the two.

25 fodders, 15 civts., 49 lbs. $=540 \mathrm{cwts}$., $49 \mathrm{lbs}$. of alloy, holding $5 \mathrm{oz}$. of silver per fodder, in the whole $130 \mathrm{oz}$., afforded, after three successive crystallizations, - 
440 ewts. of poor lead, holding $\frac{1}{2}$ oz. of silver per fodder; in all ${ }^{10 z}$

$15 \mathrm{cwts.} 49$ - holding the original quantity, nearly -

$84 \mathrm{cwts}$. of lead for the cupel, holding $29 \mathrm{oz}$.

Total - - - - - - - $-\overline{130}$

$1 \mathrm{cwt}$ of loss, principally in the reduction of dross.

The expenses of the new method altogether, including $3 \mathrm{~s}$. per fodder of patent dues, are about one third of the old; being $17 \mathrm{l}$. 13s. and 54l. 16s. respectively, upon $84 \mathrm{cwts}$. of lead, at $29 \mathrm{oz}$. per fodder.

In the conditions above stated, the treatment of argentiferous lead occasions the following expenses:-

\section{By the new process} FOR ONE FODDER.

By the old process

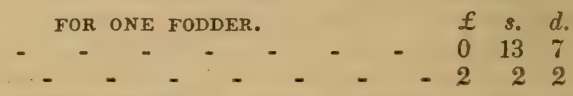

Admitting that the treatment of silver holding lead is economically possible only when the profit is equal to one tenth of the gross expenses of the process, we may easily calculate, with the preceding data, that it is sufficient for the lead to have the following con ients in silver:-

With the new process, 3 ounces per fodder; or, - - 0.000078

With the old process, $8 \frac{4}{70}$ ounces per fodder; or, - - 0.000218

To conclude, the refining by crystallization reduces the cost of the parting of lead and silver, in the proportion of three to one; and allows of extracting silver from a lead which contains only about three oz. per ton. In England, the new method produces at present very advantageous results, especially in reference to the great masses to which it may be applied. In 1828, the quantity of lead annually extracted from the mines in the United Kingdom had been progressively raised to 47,000 tons. Reduced almost to one half of this amount in 1832, by the competition of the mines of la Sierra de Gador, the English production began again to increase in 1833 . In $1835,35,000$ tons of lead were obtained, one half of which only having a mean content of eight and a half ounces of silver per ton, was subjected to cupellation, and produced $14,000 \mathrm{oz}$. of that precious metal. The details of this production are-

Silver extracted from 17,500 tons of lead, holding upon the average eight ? and a half ounces per ton, - _ _ _ _ _ _ _ $\} 140,000 \mathrm{oz}$.

Silver extracted from silver ores, properly so called, in Cornwall, _ _ 36,000

In $183 \%$, the production of lead amounted probably to 40,000 tons; upon which the introduction of the new method would have the effect not only of reducing considerably the cost of parting the 20,000 tons of lead containing $8 \mathrm{oz}$. of silver per ton, but of permitting the extraction of 4 or $5 \mathrm{oz}$. of silver, which may be supposed to exist upon an average in the greater portion of the remaining 20,000 tons. Otherwise, this mass of the precious metal would have had no value, or have been unproductive.

There are two oxydes of silver; called argentic oxyde, and suroxyde, by Berzelius. 1. The first is obtained by adding solution of eaustic potassa, or lime-water, to a solution of nitrate of silver. The precipitate has a brownish-gray color, which darkens when dried, and contains no combined water. Its specific gravity is $7 \cdot 143$. On exposure to the sun, it gives out a certain quantity of oxygen, and becomes a black powder. This oxyde is an energetic base; being slightly soluble in pure water, reacting like the alkalis upon reddened litmus paper, and displacing, from their combinations with the alkalis, a portion of the acids, with which it forms insoluble compounds. It is insoluble in the caustic leys of potassa or soda. By combination with caustic ammonia, it forms fulminating silver. This formidable substance may be prepared by precipitating the nitrate of silver with lime-water, washing the oxyde upon a filter, and spreading it upon gray paper, to make it nearly dry. Upon the oxyde, still moist, water of ammonia is to be poured, and allowed to remain for several hours. The powder which becomes black, is to be freed from the supernatant liquor by decantation, divided into small portions while moist, and set aside to dry upon bits of porous paper. Fulminating silver may be made more expeditiously by dissolving the nitrate in water of pure ammonia, and precipitating by the addition of caustic potassa ley in slight excess. If fulminating silver be pressed with a hard body in its moist state, it detonates with unparalleled violence; nay, when touched even with a feather, in its dry state, it frequently explodes. As many persons have been seriously wounded, and some have been killed, by these explosions, the utmost precautions should be taken, especially by joung chemists, in its preparation. This violent phenomenon is caused by the sudden production of water and nitrogen, at the instant when the metallic oxyde is reduced. The quiescent and 
livellent affinities seem to be so nicely balanced in this curious compound, that the slightest disturbance is suflicient to incite the hydrogen of the ammonia to snatch the oxygen from the silver. The oxyde of silver dissolves in glassy fluxes, and renders them yellow. It consists, according to Berzelius, of $93 \cdot 11$ parts of silver, and 6.89 of oxygen. 2. The suroxyde of silver is obtained by passing a voltaic current through a weak solution of the nitrate; it being deposited, of course, at the positrve or oxy genating pole. It is said to crystallize in needles of a metallic lustre, interlacing one another, which are one third of an inch long. When thrown into muriatic acid, it causes the disengage ment of chlorine, and the formation of chloride of silver; into water of ammonia, $i$ occasions such a rapid production of nitrogen gas, with a hissing sound, as to conver the whole liquid into froth. If a little of it, mixed with phosphorus, be struck with a hammer, a loud detonation ensues. With heat it decrepitates, and becomes metallic silver.

Sulphuret of silver, which exists native, may be readily prepared by fusing the constituents logether; and it forms spontaneously upon the surface of silver exposed to the air of inhabited places, or plunged into eggs, especially rotten ones. The tarnish may be easily removed, by rubbing the metal with a solution of cameleon mineral, prepared by calcining peroxyde of manganese with nitre. Sulphuret of silver is a powerful sulphobase; since though it he heated to redness in close vessels, it retains the volatile sulphides, whose combinations with the alkalis are decomposed at that temperature. It consists of 87.04 of silver, and 12.96 of oxygen.

A small quantity of tin, alloyed with silver, destroys its ductility. The best method of separating these two metals, is to lami.ıate the alloy into thin plates, and distil them along with corrosive sublimate. The bichloride of tin comes over in vapors, and condenses in the receiver. Silver and lead, when combined, are separated by heat alone in the process of cupellation, as described in the article Assay, and in the reduction of silver ores. See suprà.

An alloy, containing from one twelfth to one tenth of copper, constitutes the silver coin of most nations; being a harder and more durable metal under friction than pure silver. When this alloy is boiled with a solution of cream of tartar and sea-salt, or scrubbed with water of ammonia, the superficial particles of copper are removed, and a surface of fine silver is left.

Chloride of silver is obtained by adding muriatic acid, or any soluble muriate, to a solution of nitrate of silver. A curdy precipitate falls, quite insoluble in water, which being dried and heated to dull redness, fuses into a semi-transparent gray mass, called, from its appearance, horn-silver. Chloride of silver dissolves readily in water of ammonia, and crystallizes in proportion as the ammonia evaporates. It is not decomposed by a red heat, even when mixed with calcined charcoal; but when hydrogen or steam is passed over the fused chloride, muriatic acid exhales, and silver remains. When fused along with potassa, (or its carbonate, the silver is also revived; while oxygen (or also carbonic acid) gas is liberated, and chloride of potassium is formed. Alkaline soiutions do not decompose chloride of silver. When this compound is exposed to light, it suffers a partial decomposition, muriatic acid being disengaged. See AssAy by the humid method.

The best way of reducing the chloride of silver, says Mohr, is to mix it with one third of its weight of colophony, (black rosin,) and to heat the mixture moderately in a crucible till the flame ceases to have a greenish-blue color; then suddenly to increase the fire, so as to melt the metal into an ingot.

The subchloride may be directly formed, by pouring a solution of deuto-chloride of copper or iron up n silver leaf. The metal is speedily changed into black spangles, which, being immediately washed and dried, constitute subchloride of silver. If the contact of the solutions be prolonged, chloride would be formed.

The bromide, cyanide, fluoride, and iodide of silver, have not been applied to any use in the arts. Sulphate of silver may be prepared by boiling sulphuric acid upon the metal. See Refining of Gold and Silver. It dissolves in 88 parts of boiling water, but the greater part of the salt crystallizes in small needles, as the solution cools. It consists of 118 parts of oxyde, combined with 40 parts of dry acid. Solutions of the hyposnlphite of potassa, soda, and lime, which are bitter salts, dissolve chloride of silver, a tasteless sithstance, into liquids possessed of the most palling sweetness, but not at all of any metallic taste.

The iudide of silver is remarkable, like some other metallic compounds, for changing its color alternately with heat and cold. If a sheet of white paper be washed over with a solution of nitrate of silver, and afterwards with a somewhat dilute solution of hydrio date of potasn, it will immediately assume the pale yellow tint of the cold silver iodide. On placing the paper before the fire, it will change color from a pale primrose to a gaudy brilliant yellow, like the sun-flower; and on being cooled, it will aqain resume the primrose hue. These alternations may be reneated indefinitely, like those with the 
salts of cobalt, provided too great a heat be not applied. The pressure of a finger upos the hot yellow paper makes a white spot, by cooling it quickly.

Fulminate of silver is prepared in the sarne way as Fulminate of Mercury, which see.

On the 10th of February, 1798, the Lords of the Privy Council appointed the Hon. Charles Cavendish, F. R. S., and Charles Hatchett, Esq., F. R. S., to make investigations upon the wear of gold coin by friction. Their admirable experiments were begun in the latter end of 1798, and completed in April, 1801, having been instituted and conducted with every mechanical aid, as devised by these rnost eminent chemical philosophers, and provided at no small expense, by the government. The following are the important conclusions of their official report :- *

"Gold made standard by a mixture of equal parts of silver and copper, is not so soft as gold alloyed only with silver; neither is it so pale; for it appears to be less removed from the color of fine gold, than either the former or the following metal.

"Gold, when alloyed with silver and copper, when annealed, does not become black, but brown; and this color is more easily removed by the blanching liquor, or solution of alum, than when the whole of the alloy consists of copper. It may also be rolled and stamped with great facility; and, under many circumstances, it appears to suffer less by friction than gold alloyed by silver or copper alone.

"If copper alone forms the alloy, it must be dissolved and separated from the surface of each piece of coin, in the process of annealing and blanching.

"Upon a comparison of the different qualities of the three kinds of standarl gold, it appears (strictly speaking) that gold made standard by silver and copper is rather to be preferred for coin."

It will, undoubtedly, seem not a little strange to the uninitiated, that this report, and its important deductions, should have been of late years entirely set at naught, without any scientific reason or research, apparently for the purpose of giving a certain official in our Mint a good job, in sweating out all the silver from our sovereigns, and replacing it, in the new coinage, with copper, taking on an average $3 d$. worth of silver out of each ounce of our excellent gold coin, and charging the country $6 \frac{1}{2} d$. for its extraction, besides the very considerable expense in providing fine copper to replace the silver. The pretence set up for this extraordinary deoradation of the gold, was, that our coin might peradventure be exported, in order to be de-silvered abroad, a danger which could have been most readily averted, by leaving out as much gold in every sovereign as was equivalent to the silver introduced, and thus preserving its intrinsic value in precions metal. When the film of fine gold which covers each of our present pieces has been rubbed off from the prominent parts, these must appear of a very different and deeper color than the flat part or ground of the coin. "The reason, therefore, is sufficiently apparent, says Mr. Hatchett, why gold which is alloyed with silver only, cannot be liable to this blemish ;" and with one half of silver alloy, it must be much less liable to it, than with copper alone. Why did the political economists in the recent Committee of the House of Commons on the Mint, blink this question of public economy and expediency?

Gold, as imported from America, Asia, and Africa, contains on an average nearly the right proportion of silver for maling the best coin; and were it alloyed to our national standard, of 22 parts of gold, 1 of silver, and 1 of copper, as defined by Messrs. Cavendish and Hatchett, then by simply adding the deficient quantities of one or two of these metals, by the rule of alligation, the very considerable expense would be saved to the nation, and sulphureous nuisance to the Tower Hamlets, now foolishly incurred in de-silvering and cuprifying sovereigns at the Royal Mint.

It was long imagined in Europe, that the average metallic contents of the silver ores of Mexico and Peru, were considerably greater than those of Saxony and Hungary. Much poorer ores, however, are worked among the Cordilleras than in any part of Europe. The mean product of the whole silver ores that are annually reduced in Mexico, amounts only to from 0.18 to 0.25 of a per cent.; that is, from 3 to 4 ounces in 100 lbs.; the true average being, perhaps, not more than $2 \frac{1}{2}$. It is by their greater profusion of ores, not their superior richness, that the mines of South America surpass those of Enrope.

GoLd and Silver produced in Forty Years, from 1790 to 1830.

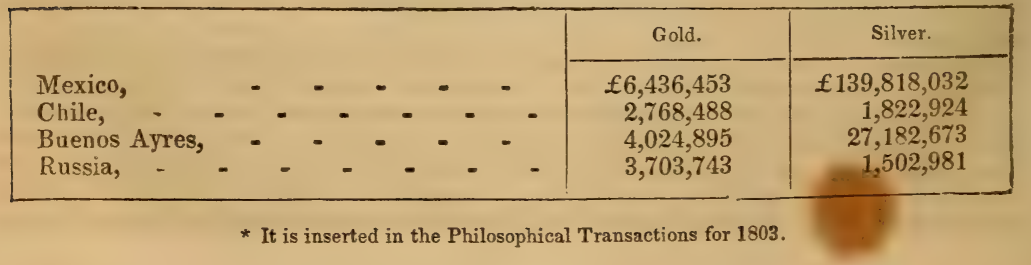


Raturns of the Dorrans coined at the different Mints in Mexrco.

\begin{tabular}{|lcc|r|r|r|r|}
\hline & & \multicolumn{1}{|c|}{1829.} & \multicolumn{1}{|c|}{1830.} & \multicolumn{1}{c|}{1831.} & \multicolumn{1}{c|}{1834.} \\
\cline { 3 - 4 } Mexico & - & - & $1,280,000$ & $1,090,000$ & $1,386,000$ & 952,000 \\
Guanajuato & - & - & $2,406,000$ & $2,560,000$ & $2,603,000$ & $2,703,000$ \\
Zacateciss & - & - & $4,505,000$ & $5,190,000$ & $4,965,000$ & $5,527,000$ \\
Guadalaxara & - & - & 596,000 & 592,000 & 590,000 & 715,000 \\
Durango & - & - & 659,000 & 453,000 & 358,000 & $1,215,000$ \\
San Luis & - & - & $1,613,000$ & $1,320,000$ & $1,497,000$ & 928,000 \\
Ilalpan & - & - & 728,000 & 90,000 & 323,000 & - \\
\hline \multicolumn{1}{|c|}{ Total } & - & - & $11,787,000$ & $11,295,000$ & $11,722,000$ & $12,040,000$ \\
\hline
\end{tabular}

The returns for 1832 and 1833 are wanting.

Perd.-Returns of Gold and Silver coined at the Mints of Lima and Casco.

\begin{tabular}{|c|c|c|c|}
\hline & Gold. & Silver. & Total, in Dollars. \\
\cline { 2 - 4 } 1830 & 180,000 & $2,015,000$ & $2,195,000$ \\
1831 & 92,000 & $2,384,000$ & $2,476,000$ \\
1832 & 94,000 & $3,210,000$ & $3,284,000$ \\
1833 & 150,000 & $2,990,000$ & $3,140,000$ \\
1834 & 110,000 & $3,150,000$ & $3,260,000$ \\
\hline
\end{tabular}

Returns of Silver in Bars produced at the different Smelting-works in Peru.

\begin{tabular}{|r|r|r|r|r|r|r|r|}
\hline & Lima. & Truxillo. & Pasco. & Ayacucho. & Puno. & Arequipa. & Tital, in Dollars. \\
\cline { 2 - 6 } 1830 & 270,000 & 190,000 & 780,000 & 120,000 & 250,000 & 150,000 & $1,760,000$ \\
1831 & 270,000 & 60,000 & $1,110,000$ & 70,000 & 310,000 & 110,000 & $1,930,000$ \\
1832 & 290,000 & 100,000 & $1,800,000$ & 70,000 & 345,000 & 25,000 & $2,640,000$ \\
1833 & 222,000 & 70,000 & $2,130,000$ & 50,000 & 25,000 & 65,000 & $2,562,000$ \\
\hline
\end{tabular}

Returns of Silver in Dollars exported from the Provinces of Chilt.

\begin{tabular}{|c|c|c|c|}
\hline & Coquimbo. & Ifuasco. & Copiano. \\
\cline { 2 - 3 } 1831 & 785,000 & 115,000 & 670,000 \\
1832 & 316,000 & - & 36,000 \\
1833 & 490,000 & 100,000 & 585,000 \\
\hline & $1,591,000$ & 215,000 & $1,291,000$ \\
\hline
\end{tabular}

Santiago-Mint Coinage.

Gold. $\quad$ Silver.

Total.

$1832,174,000 ; 1833,392,500|1832,42,000 ; 1833,92,000| 700,500$

The production of SiLver in the kingdom of SAXoNy amounted to-

\begin{tabular}{|c|c|c|c|c|}
\hline 55,023 & - & & - & - \\
\hline 60,034 & - & & - & - \\
\hline 61,361 & - & & - & - \\
\hline 65,176 & - & and 10 & loths & - \\
\hline 65,886 & - & & - & - \\
\hline
\end{tabular}

The mine of Himmelfürst alone produces annually 10,000 mares.

The quantity of SrLver produced in the Prussian States was-

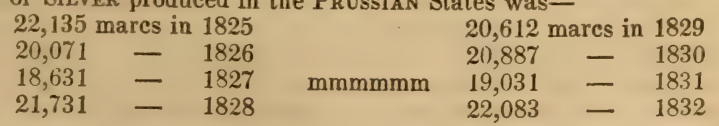

The whole annual production of Europe, and Asiatic Russia, has been rated by Hum. boldt at 292,000 mares, by other authorities at 310,000 mares; while at the beginning of the present century, that of the Spanish colonies in America was 3,349,160 mares, of 
nearly twelve times as much. The sum total is $3,704,160$ marcs, of 3609 grains troy each; which is nearly $1,900,000$ lbs. aroirdupois; that is, little less than 9000 tons.

The Enslish Mint silver contains 222 pennyweights of fine silver, and 18 of copper, in the troy pound of 240 pennyweights: or 92.5 in 100 parts. 1 pound troy $=5760$ grains, contains 65.8 shillings, each weighing 87.55 grains. The French silver coin contains one tenth of copper, and a franc weighs 5 grammes $=77.222$ grains troy. The Prussian dollar (thaler) is the standard coin; $10 \frac{1}{2}$ thaler weigh 1 marc; hence, 1 thaler weighs 343.7 grains troy, and contains 257.9 grains of fine silver; being 75 per cent. of silver, and 25 of alloy. The Austrian coin contains $\frac{13}{288}$ of alloy, according to Wasserberg; which is only $4 \frac{1}{2}$ per cent.

SILVER LEAF is made in precisely the same way as gold leaf, to which article I must therefore refer the reader.

SILVERING is the art of covering the surfaces of bodies with a thin film of silver. When silver leaf is to be applied, the methods prescribed for gold leaf are suitable. Among the metals, copper or brass are those on which the silverer most commonly operates. Iron is seldom silvered; but the processes for both metals are essentially the same.

The principal steps of this operation are the following :-

1. The smoothing doun the sharp edges, and polishing the surface of the copper; called émorfiler by the French artists.

2. The annealing; or making the piece to be silvered red-hot, and then plunging it in very dilute nitric acid, till it be bright and clean.

3. Pumicing; or clearing up the surface with pumice-stone and water.

4. The warming, to such a degree merely as, when it touches water, it may make a slight hissing sound; in which state it is dipped in the very weak aquafortis, whereby it acquires minute insensible asperities, sufficient to retain the silver leaves that are to be applied.

5. The hatching. When these small asperities are inadequate for giving due solidity to the silvering, the plane surfaces must be hatched all over with a graving tool; but the chased surfaces need not be touched.

6. The blueing consists in heating the piece till its copper or brass color changes to b]ue. In heating, they are placed in hot tools made of iron, called mandrins in France.

7. The charging, the workman's term for silvering. This operation consists in placing the silver leaves on the heated piece, and fixing them to its surface by burnishers of steel, of various forms. The workman begins by applying the leaves double. Should any part darken in the heating, it must be cleared up by the scratch-brush.

The silverer always works two pieces at once; so that he may heat the one while burnishing the other. After applying two silver leaves, he must heat up the piece to the same degree as at first, and he then fixes on with the burnisher four additional leaves of silver; and he goes on charging in the same way, 4 or 6 leaves at a time, till he has applied, one over another, $30,40,50$, or 60 leaves, according to the desired solidity of the silvering. He then burnishes down with great pressure and address, till he has given the surface a uniform silvery aspect.

Silvering by the precipitated chloride of silver.-The white curd obtained by adding a solution of common salt to one of nitrate of silver, is to be well washed and dried. One part of this powder is to be mixed with 3 parts of good pearlash, one of washed whiting, and one and a half of sea salt. After clearing the surface of the brass, it is to be rubbed with a bit of soft leather, or cork moistened with water, and dipped in the above powder. After the silvering, it should be thoroughly washed with water, dried, and immediately varnished. Some use a mixture of 1 part of the silver precipitate with 10 of creamtof tartar, and this mixture also answers very well.

Others give a coating of silver by applying with friction, in the moistened state, a mixture of 1 part of silver-powder precipitated by copper, 2 parts of cream of tartar, and as much common salt. The piece must be immediately washed in tepid water very faintly alkalized, then in slightly warm pure water, and finally wiped dry before the fire. See Plated Manufacture.

The inferior kinds of plated buttons get their silver coating in the following way :-

2 ounces of chloride of silver are mixed up with 1 ounce of corrosive sublimate, 3 pounds of common salt, and 3 pounds of sulphate of zinc, with water, into a paste. The buttons being cleaned, are smeared over with that mixture, and exposed to a moderate degree of heat, which is eventually raised nearly to redness, so as to expel the mercury from the amalgam, formed by the reaction of the horn silver and the corrosive sublimate. The copper button thus acquires a silvery surface, which is brightened by clearing and burnishing.

Leather is silvered by applying a coat of parchment size, or spirit varnis, to the sur. ace, and then the silver leaf, with pressure.

SIMILOR is a golden-colored variety of brass. 
SINGEING OF WFBS. The old furnace for singeing cotton gonds is represented in longitudinal section, fig. 1030, and in a transverse une in fig. 1031. $a$ is the 1030

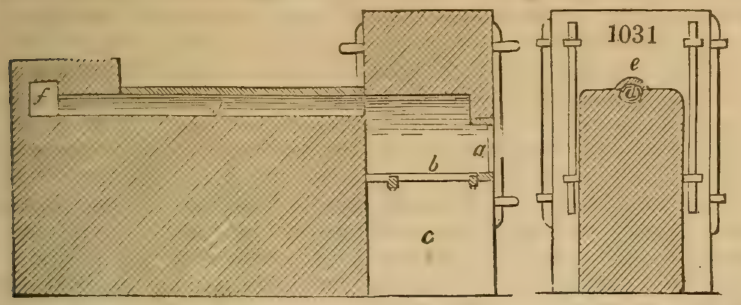

fire-door; $b$ the grate; $c$, the ashpit; $d$, a flue 6 inches broad, and $2 \frac{1}{2}$ high, over which a hollow semi-cylindrical mass of cast iron $e$, is laid, one inch thick at the sides, and $2 \frac{1}{2}$ thick at the top curvature. The flame passes along the fire-flue $d$, into a side opening $f$, in the chimney. The goods are swept swiftly orer this ignited piece of iron, with considerable fruction, by means of a wooden roller. and a swing frame for raising them at any moment out of contact.

In some shops, semi-cylinders of copper, three quarters of an inch thick, have been substituted for those of iron, in singeing goods prior to bleaching them. The former last three months, and do 1500 pieces with one ton of coal; while the latter, which are an inch and a half thick, wear out in a week, and do no more than from 500 to 600 pjeces with the same weight of fuel.

In the early part of the year $1818, \mathrm{Mr}$. Samuel Hall enrolled the specification of a patent for removing the downy fibres of the cotton thread from the interstices of bobbinet lace, or muslins, which he effected by singeing the lace with the flame of a gasburner. The second patent granted to Mr. Hall, in April, 1823, is for an improvement in the abore process; viz., causing a strong current of air to draw the flame of the gas through the interstices of the lace, as it passes over the burner, by means of an aperture in a tube placed immediately above the row of gas-jets, which tube communicates with an air-pump or exhanster.

Fig. 1032 shows the construction of the apparatus complete, and manner in which it operates; $a, a$, is a gas-pipe, supplied by an ordinary gasometer; from this pipe, 1032

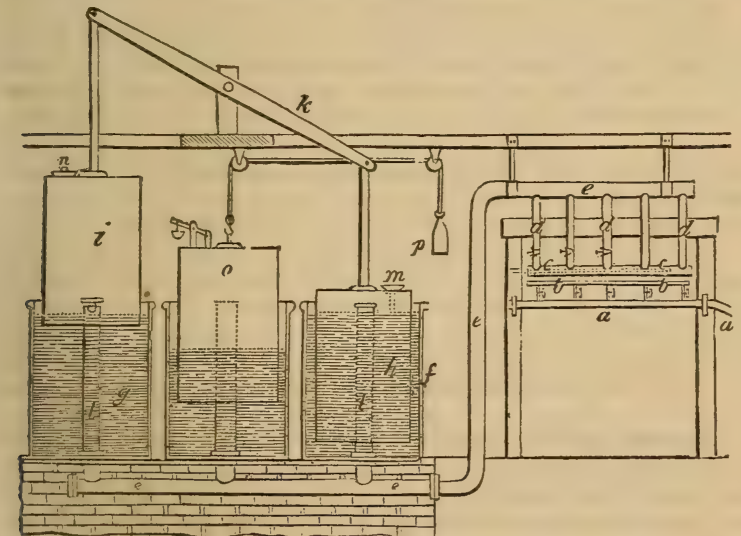

several small ones extend upwards to the long burner $b, b$. This burner is a horizontal tube, perforated with many small holes on the upper side, through which, as jets, the gas passes; and when it is ignited, the bobbinet lace, or other material intended to be singed, is extended and drawn rapidly over the flame, by means of rollers, which are not shown in the figure.

The simple burning of the gas, even with a draught chimney, as in the former specification, is found not to be at all times efficacious; the patentee, therefore, now introduces a hollow tube $c$, $c$, with a slit or opening, immediately over the row of burners; and this tube, by means of the pipes $d, d, d$, communicates with the pipe $e, e, e$, which leads to the exhausting apparatus.

This exhausting apparatus consists of two tanks, $f$ and $g$, nearly filled with water, and two inverted boxes or vessels, $h$ and $i$, which are suspended by rods to the vibrating beam $k$; each of the boxes is furnished with a valve opening upwards; $l$, $l$, are pipes extending from the horizontal part of the pipe $e$, up into the hoxes or vessels $h$ and $i$, which pipes have valves at their tops, also opening upward. When the ressel $h$ descends, the water in the tank forces out the air contained within the vessel at the valve $m$; but whemat vessel rises again, the valve $m$ being closed, the air is drawn from the pipe $e$, th pugh the pipe $l$. The same takes place in the ressel $i$, from which the air in its descent is expelled through the valve $n$, and, in its ascent, draws the air 
through the pipe $l$, from the pipe $e$. By these means, a partial exhaustion is effected in the pipe $e, e$, and the tube $c, c$; to supply which, the air rushes with considerable force through the long opening of the tube $c, c$, and earries with it the flame of the gas-burners. The bobbinet lace, or other goods, being now drawn over the flame between the burner $b, b$, and the exhausted tube $c, c$, by means of rollers, as above said, the flame of the gas is forced through the interstices of the fabric, and all the fine filaments and loose fibres of the thread are burnt off, without damaging the substance of the goods.

To adjust the draught from the gas-burners, there are stopcocks introduced into several of the pipes $d$; and to regulate the action of the exhansting apparatus, an air vessel $o$, is suspended by a cord or chain passing over pulleys, and balanced by a weight $p$. There is also a scraper introduced into the tube $c$, which is made, by any convenient contrivance, to revolve and slide backwards and forwards, for the purpose of removing any light matter that may arise from the goods singed, and which would otherwise obstruct the air passage. Two of these draught tubes $c$, may be adapted and united to the exhausting apparatus, when a double row of burners is employed, and the inclination of the flame may be directed upwards, downwards, or sideways, according to the position of the slit in the draft tube, by which means any description of goods may, if required, be singed on both sides at one operation.

The greater part of the bobbinet lace made in England, is sent to Mr. Hall's works, at Basford, near Nottingham, to be singed; and at a reduction of price truly wonderful. He receives now only one farthing for what he originally was paid one shilling.

SKIN (Peau, Fr.; Haut, Germ.), the external membrane of animal bodies, consists of three layers: 1. the epidermis, scarf-skin, (Oberhaut, Germ.); 2. the vascular organ, or papillary body, which performs the secretions; and 3. the true skin, (Lfderhuut, Germ.), of which leather is made. The skin proper, or dermoid substance, is a tissue of innumerable very delicate fibres, crossing each other in every possible direction, with small orifices between them, which are larger on its internal than on its external surface. The conical channels thus produced are not straight, but oblique, and filled with cellular membrane; they receive vessels and nerves which pass out through the skin (cutis vera), and are distributed upon the secretory organ. The fibrous texture of the skin is composed of the same animal matter as the serous membranes, the cartilages, and the cellular tissue ; the whole possessing the property of dissolving in boiling water, and being, thereby, converted into glue. See GLUE, LkAther, and TAN.

SLAG (Laitier, Fr.; Schlacke, Germ.), is the vitreous mass which covers the fused metals in the smelting-hearths. In the iron-works it is commonly cal'ed cinder. Slags consist, in general, of bi-silicates of lime and magnesia, along with the oxydes of iron and other metals; being analogous in composition, and having the same crystalline form as the mineral, pyroxene. See Copper and Iron.

SLATES (Ardoises, Fr.; Schiefern, Germ.) The substances belonging to this class may be distributed into the following species :-

1. Mica-slate, occasionally used for co- 5. Drawing-slate, or black chalk. vering houses.

2. Clay-slate, the proper roofing-slate.

3. Whet-slate.

4. Polishing-slate.

6. Adhesive slate.

7. Bituminous shale.

8. Slate-clay.

1. Mica-slate. - This is a mountain rock of vast continuity and extent, of a schistore texture, composed of the minerals mica and quartz, the mica being generally predominant.

2. Clay-slate. - This substance is closely connected with mica; so that uninterrupted transitions may be found between these two rocks in many mountain chains. It is a simple schistose mass, of a bluish-gray or grayish-black color, of various shades, and a shining, somewhat pearly internal lustre on the faces, but of a dead color in the cross fracture.

Clay-slate is extensively distributed in Great Britain. It skirts the Highlands of Scotland, from Lochlomond by Callender, Comrie, and Dunkeld; resting on, and gradually passing into mica-slate throughout the whole of that territory. Roofingslate occurs, on the western side of England, in the counties of Cornwall and Devon; in various parts of North Wales and Anglesea; in the north-east parts of Yorkshire, near Ingleton, and in Swaledale; as also in the counties of Cumberland and Westmoreland. It is likewise met with in the county of Wicklow and other mountainous districts of Ireland.

All the best beds of roofing-slate improve in quality as they lie deeper under the sur face; near to which, indeed, they have little value.

A good roofing-slate should split readily into thin even laminæ; it should not be absorbent of water either on its face or endwise, a property evincerl by its not increasing perceptibly in weight after immersion in water; and it should be sound, compact, and 
not apt to disintegrate in the air. The slate raised at Eisdale, on the west coast of Aroyllshire, is very durable.

Cleaving and dressing of the slates.-The splitter begins by dividing the block, cut lengthwise, to a proper size, which he rests on end, and steadies between his linees. $\mathrm{He}$ uses a mallet and a chisel, which he introduces into the stone in a direction parallel to the folia. By this means he reduces it into several manageable pieces, and he gives to each the reyuisite length, by cutting cross arooves on the flat face, and then striking the slab with the chisel. It is afterwards split into thinner sections, by finer chisels dexterously applied to the edges. The slate is then dressed to the proper shape, by being laid on a block of wood, and having its projecting parts at the ends and sides cut off with a species of hatchet or chopping-knife. It deserves to be noticed, that blocks of slate may lose their property of divisibility into thin laminæ. This happens from long exposure to the air, after they have been quarried. The workmen say, then, that they have lost their waters. For this reason, the number of splitters ought to be always proportioned to the number of block-hewers. Frost renders the blocks more fissile; but a supervening thaw renders them quite refractory. A new frost restores the faculty of splitting, though not to the same degree; and the workmen therefore avail themselves of it without lelay. A succession of frosts and thaws renders the quarried blocks quite intractable.

3. Whet-slate, or Turkey hone, is a slaty rock, containing a great proportion of quartz, in which the component particles, the same as in clay-slate and mica-slate, but in different proportions, are so very small as to be indiscernible.

4. Polishing slate. Color, cream-yellow, in alternate stripes; massive; composition umpalpable; principal fracture, slaty, thin, and straight ; cross fracture, fine earthy; feels fine, but meager; adheres little, if at all, to the tongue; is very soft, passing into friable; specific grarity in the dry state, 0.6 ; when imbued with moisture, 1.9 . It is supposed to have been formed from the ashes of burnt coal. It is found at Planitz, aear Zwickau, and at Kutschlin near Bilin in Bohemia.

5. Drawing-slule, or black chalk; has a grayish-black color; is very soft, sectile, easily broken, and adheres slightly to the tongue; spec. grav. $2 \cdot 11$. The streak. is glistening. It occurs in beds in primitive and transition clay-slate; also in secondary formations, as in the coal-measures of most countries. It is used in crayon drawing. Its trace upon paper is regular and black. The best kinds are found in Spain, Italy, and France. Some good black chalk occurs also in Caernarvonshire and in the island of Islay.

6. Adhesive slate, has a light greenish-gray color, is easily broken or exfoliated, has a shining streak, adheres strongly to the tongue, and absorbs water rapidly, with the emission of air-bubbles and a erackling sound.

7. Bituminous shale, is a species of soft, sectile slate-clay, much impregnated with bitumen, which occurs in the coal-measures.

8. Slute-clay, has a gray or grayish-yellow color; is massive, with a dull glimmering lustre from spangles of mica interspersed. Its slaty fracture approaches at times to earthy; fragments, tabular; soft, sectile, and very frangible; specific gravity, $2 \cdot 6$. It adheres to the tongue, and crumbles down when immersed for some time in water. It is found as an alternating bed in the coal-measures. (See the sections of the strata under Pitcoal.) When breathed upon, it emits a strong argillaceous odor. When free from lime and iron, it forms an excellent material for making refractory fire-brichs, being an infusible compound of alumina and silica; one of the best examples of which is the schist known by the name of Stourbridge clay.

SMALL WARES, is the name given in this country to textile articles of the tape kind, narrow bindings of cotton, linen, silk, or woollen fabric; plaited sash cord, braid. \&c. Tapes are woven upon a loom like that for weaving ribands, which is now generally driven by mechanical power. Messrs. Worthington and Mulliner obtained a patent, in June, 1825, for improvements in such a loom, which have answered the purposes of their large factory in Manchester very well; and in May, 1831, Mr. Whitehead, of the same town, patented certain improvements in the manufacture of small wares. The objects of the latter patent are, the regular taking up of the tape or cloth, as it is woven, a greater facility of varying the vibration of the lay, together with the saving of room re. quired for a range of looms to stand in.* See Braiding Machine.

SMALT, see Azure and Cobalt.

Imported for home consumption in $1834,162,232 \mathrm{lbs}$; in $1835,96,649$; in 1836 , 79,531; duty, 4d. per lb.

SMELTING, is the operation by which the ores of iron, copper, lead, \&c., are reduced to the metallic state. See Metaludrgy, OREs, and the respective metals.

SOAP (Saron, Fr.; Seife, Germ.), is a chemical compound, of saponified fats or oils with potash or soda, prepared for the purposes of washing linen, \&c. Fatty 
matters, when subjecled to the action of alkaline leys, undergo a remarkable change, being converted into three different acids, called stearic, margaric, and oleic; and it is these acids, in fact, which combine with the bases, in a efinite proportions, to form cumpounds analogous to the neutro-saline. Some chemical writers describe under the tille soap, every compound which may result from the union of fats with the various earths and metallic oxydes $\rightarrow$ a latitude of nomenclature which common language cannot recognise, and which would perplex the manufacturer.

Soaps are distinguished into two great classes, according to their consistence; the hard and the soft; the former being produced by the action of soda upon fats, the latter by that of potash. The nature of the fats contributes also somewhat to the consistence of soaps; thus tallow, which contains much stearine and margarine, forms with potash a more consistent soap than liquid oils will do, which consist chiefly of oleine. The drying oils, such as those of linseed and poppy, produce the softest soaps.

1. Of the manufacture of hard soap.-The fat of this soap, in the northern countries of Europe, is usually tallow, and in the southern, coarse olive oil. Different species of grease are saponified by soda, with different degrees of facility; among oils, the olive, sweet almond, rapeseed, and castor oil ; and among solid fats, tallow, bone grease, and butter, are most easily saponified. According to the practice of the United Kingdom, six or seven days are required to complete the formation of a pan of hard soap, and a day or two more for settling the impurities, if it contains rosin. From 12 to $13 \mathrm{cwts}$. of tallow are estimated to produce one ton of good soap. Some years ago, in many manufactories the tallow used to be saponified with potash leys, and the resulting soft soap was converted, in the course of the process, into hard soap, by the introduction of muriate of soda, or weak kelp leys, in sufficient quantity to furnish the proper quantity of soda by the reaction of the potash upon the neutral salts. But the high price of potash, and the diminished price as well as improved quality of the crude sodas, have led to their general adoption in soap-works. The soda-ash used by the soap-boiler, contains in general about 36 per cent. of real soda, in the state of dry carbonate, mixed with muriate of soda, and more or less undecomposed sulphate. I have met lately with sodaash, made from sulphate of soda, in which the materjals had been so ill workel, and so imperfectly decomposed, as to contain 16 per cent. of sulphate, a circumstance equally disgraceful, as it was ruinous to the soda manufacturer. The barillas from Spain and Teneriffe contain from 18 to 24 per cent. of real soda. The alkali in both states is employed in England; barilla being supposed by many to yield a finer white or curd soap, on account of its frecdom from sulphur.

The crude soda of either kind being ground, is to be stratified with lime in eylindrical cast-iron vats, from 6 to 7 feet wide, and from 4 to 5 feet deep; the lowest layer consisting, of course, of unslaked or shell quicklime. The vats have a false bottom, perforated with holes, and a lateral tubulure under it, closed commonly with a wooden plug, similar to the épine of the French soap pans, by which the leys trickle off clear and caustic, after infiltration through the beds of lime. The quantity of lime must be proportional to the carbonic acid in the soda.

Upon 1 ton of tallow put into the soap pan, about 200 gallons of soda ley, of specific gravity $1 \cdot 040$, being poured, heat is applied, and after a very gentle ebullition of about 4 hours, the fat will be found to be completely saponified, by the test of the spatula, trowel, or pallet knife; for the fluid ley will be seen to separate at once upon the steel blade, from the soapy paste. Such leys, if composed of pure caustic soda, would contain 4 per cent. of alkali; but from the presence of neutro-saline matter, they seldom contain so much as 2 per cent.; in fact, a gallon may be estimated to contain not more than 2 ounces; so that 200 gallons contain 25 pounds of real soda. The fire being withdrawn from the soap pan, the mass is allowed to cool during one hour, or a little more, after which the spent leys, which are not at all alkaline, are run off by a spigot below, or pumped off above, by a pump set into the pan. A second similar charge of ley is now introduced into the pan, and a similar boiling process is renewed. Three such boils may be given in the course of one day's work, by an active soap-maker. Next day the same routine is resumed with somewhat stronger leys, and so progressively, till, towards the sixth day, the ley may have the density of $1 \cdot 160$, and will be found to contain 6 per cent. of real soda.* Were the ley a solution of pure caustic soda, it would contain at this density no less than $14 \frac{3}{4}$ per cent. of alkali. The neutro-saline matter present in the spent ley is essential to the proper granulation and separation of the saponaceous compound; for otherwise the watery menstruum would dilute and even liquefy the soap. Supposing $12 \frac{1}{2} \mathrm{cwts}$. of tallow to yield upon an average $20 \mathrm{cwts}$. of hard soap, then $20 \mathrm{cwts}$. of tallow will produce $32 \mathrm{cwts.;}$ and as its average contents in soda are 6 per cent., these $32 \mathrm{cwts}$. should require $1.52 \mathrm{cwts}$. of real soda for their production. If barilla at 20 per cent. be the alkali employed, then $7 \cdot 6 \mathrm{cwts}$. of barilla must be consumed in the said process.

* According to my own experiments upon the soda ley used in the London soap-works. 
If the alkali be soda-ash of 40 per cent., half the weight will of course suffice. I have reason to believe that there is great waste of alkali incurred in many soap-workis, as $6 \mathrm{cwts}$. of sola-ash, of at least 30 per cent., are often expended in making 1 ton of suap, being 50 per cent. more than really enters into the composition of the soap.

The barillas always contain a small proportion of potash, to which their peculiar value, in making a less brittle or more plastic hard soap than the factitious sodas, may with great probability be ascribed. Chemistry affords many analogies, especially in mineral raters, where salts, apparently incompatible, co-exist in dilute solutions. We may thus conceive how a small quantity of stearate or oleate of potash may resist the decomposing action of the soda salts. The same modification of the consistence of hard soap may, however, be always more conveniently produced by a proper admixture of oleine with stearine.

Soda which contains sulphurets is preferred for making the mottled or marbled soap, whereas the desulphureted soda makes the best white curd soap. Mottling is usually given in the London soap-works, by introducing into the nearly finished soap in the pan a certain quantity of the strong ley of crude soda, through the rose spout of a watering-can. The dense sulphureted liquor, in descending through the pasty mass, causes the marbled appearance. In France a small quantity of solution of sulphate of iron is added during the boiling of the soap, or rather with the first service of the leys. The alkali seizes the acid of the sulphate, and sets the protoxyde of iron free, to mingle with the paste, to absorb more or less oxygen, and to produce thereby a variety of tints. A portion of oxyde combines also with the stearine to form a metallic soap. When the oxyde passes into the red state, it gives the tint called manteau 1sabelle. As soon as the moltler has broken the paste, and made it pervious in all directions, he ceases to push his rake from right to left, but only plunges it perpendicularly, till he reaches the ley; then he raises it suddenly in a vertical line, making it act like the stroke of a piston in a pump, whereby he lifts some of the ley, and spreads it over the surface of the paste. In its subsequent descent through the numerous fissures and channels, on its way to the bottom of the pan, the colored ley impregnates the soapy particles in various forms and degrees, whence a varied marbling results.

Three pounds of olive oil afford five pounds of marbled Marseilles soap of good quality, and only four pounds four ounces of white soap; showing that more water is retained by the former than the latter. Oils of grains, as linseed and rapeseed, do not afford so solid a soda soap as oil of olives; but tallow affords a still harder soap with soda. Some of the best Windsor soap made in London contains one part of olive oil (gallipoli) for every nine parts of tallow. Much of the English hard soap is made .with kitchen and bone fat, of a very coarse quality; the washing of the numerous successive leys, however, purifies the foul fats, and deprives them of their offensive smell in a great degree. It is common now at Marseilles to mix ten per cent. of the oil of grains with olive oil; for which purpose a large proportion of the oils extracted from seeds in the mills of the Department du Nord is sent to Marseilles ; but five per cent. of poppyseed oil, mixed with tallow, renders the soap made with the mixture stringy and unfit, for washing; because the two species of fat refuse to amalgamate.

The affinity between the stearine of tallow and the alkali, is so great that a soap may be speedily made from them in the cold. If we melt tallow at the lowest possible temperature, and let it cool to the fixing point, then add to it half 'its weight of caustic ley, at $36^{\circ}$ B., agitating meanwhile incessantly with a pallet knife, we shall perceive, at the end of some hours of contact, the mixture suddenly acquire a very solid consistence, and at the same moment assume a marked elevation of temperature, proving the phenomenon to be due to chemical attraction. In some trials of this kind, the thermometer has risen from $54^{\circ}$ to $140^{\circ} \mathrm{F}$.

According to recent experiments made in Marseilles, 100 pounds of olive oil take, for their conversion into soap, 54 pounds of crude soda, of 36 per cent. alkaline strength. One part of lime is employed for rendering three parts of the soda caustic. The richer the oil is in stearine, the more dilute should be the ley used in the saponification; and vice versâ when it abounds in oleine. For oil of the former kind, the first leys added have a density of from $8^{\circ}$ to $9^{\circ} \mathrm{B}$.; but for the latter kind, the density is from $10^{\circ}$ to $11^{\circ}$. When four parts of olive oil are mixed with one part of poppy, rape, or linseed oil, as is now the general practice at Marseilles. then for such a mixture the first leys have usually a specific gravity of from $20^{\circ}$ to $25^{\circ}$, the second from $10^{\circ}$ to $15^{\circ}$, and the third from $4^{\circ}$ to $5^{\circ}$, constituting a great difference from the practice in Great Britain, where the weaker leys are generally employed at the commencement. The chief reason for this practice is, however, to be found in the more complete causticity of the weak than of the strong leys, according to the slovenly way in which most of our soap-boilers prepare them. Indeed, one very extensive manufacturer of soap in London assured me that the leys should not be caustic; an extraordinary assertion, upon which no comment need be zade. In common cases, I would recommend the first combination of the ingredients 
to be made with somewhat weak, but perfectly caustic ley, and when the saponification is fairly established, to introduce the stronger ley.

In a Marseilles soap-house, there are four ley-vats in each set: No. 1 is the fresh vat, into which the fresh alkali and lime are introduced; No. 2 is called the urançaire, being one step in advance; No. 3 is the small avançaire, being two steps in advance, and therefore containing weaker liquor; No. 4 is called the water vat, because it receives the water directly.

Into No. 3 the moderately exhausted or somewhat spent leys are thrown. From No. 3 the ley is run or pumped into No. 2, to be strengthened; and in like manner from No. 3 into No. 1. Upon the lime paste in No. 4, which has been taken from No. 3, water is poured; the ley thus obtained is poured upon the paste of No. 3 , which has been taken frorn No. 2. No. 3 is twice lixiviated; and No. 2, once. Thereceiver under No. 1 has four compartments; into No. 1 of which the first and strongest ley is run; into No. 2 the second ley; into No. 3 the third ley; and into No. 4 the fourth ley, which is so weak as to be used for lixiviation, instead of water ; (pour d'avances).

The lime of rat No. 4, when exhausted, is emptied out of the window near to which it stands; in which case the water is poured upon the contents of No. 3 ; and upon No. 2 the somewhat spent leys.

No. 1 is now the avançaire of No. 4 ; because this has become, in its turn, the fresh vat, into which the fresh soda and quicklime are put. The ley discharged from No. 3 comes, in this case, upon No. 2 ; and after being run through it, is thrown upon No. 1.

144 pounds of oil yield at Marseilles, upon an average, not more than from 240 to 244 pounds of soap ; or 100 pounds yield about 168 ; so that in making 100 pounds of soap, at this rate nearly 60 pounds of oil are consumed.

\section{OF YELLOW OR ROSIN SOAP.}

Rosin, although very soluble in alkaline menstrua, is not however susceptible, like fats, of being transformed into an acid, and will not of course saponify, or form a proper soap by itself. The more caustic the alkali, the less consistence has the resinous compound which is made with it. Hence fat of some kind, in considerable proportion, must be used along with the rosin, the minimum heing equal parts; and then the soap is far from being good. As alkaline matter cannot be neutralized by rosin, it preserves its peculiar acrimony in a soap poor in fat, and is ready to act too powerfully upon woollen and all other animal fibres to which it is applied. It is said that rancid tallow serves to mask the strong odor of rosin in soap, more than any oil or other species of fat. From what we have just said, it is obviously needless to make the rosin used for yellow soaps pass through all the stages of the saponifying process; nor would this indeed be proper, as a portion of the rosin would be carried away, and wasted with the spent leys. The best mode of proceeding, therefore, is first of all to make the hard soap in the usual manner, and at the last service or charge of ley, namely, when this ceases to be absorbed, and preserves in the boiling-pan its entire causticity, to add the proportion of rosin intended for the soap. In order to facilitate the solution of the rosin in the soap, it should be reduced to coarse powder, and well incorporated by stirring with the rake. The proportion of rosin is - usually from one third to one fourth the weight of the tallow. The boil must be kept up for some time with an excess of caustic ley; and when the paste is found, on cooling a sample of it, to aequire a solid consistence, and when diffused in a little water, not to leave a resinous varnish on the skin, we may consider the soap to be finished. We next proceed to draw off the superfluous leys, and to purify the paste. For this purpose, a quantity of leys at $80^{\circ} \mathrm{B}$. being poured in, the mass is heated, worked well with a rake, then allowed to settle, and drained of its leys. A second service of leys, at $4^{\circ} \mathrm{B}$., is now introduced, and finally one at $2^{\circ}$; after each of which, there is the usual agitation and period of repose. The pan being now skimmed, and the scurn removed for another operation, the soap is laded off by hand-pails into its frame-moulds. A little palm oil is usually employed in the manufacture of yellow soap, in order to correct the flavor of the rosin, and brighten the color. This soap, when well made, ought to te of a fine wax-yellow hue, be transparent upon the edges of the bars, dissolve readily in water, and afford, even with hard pump-water, an excellent lather.

The frame-moulds for hard soap are composed of strong wooden bars, made into the form of a parallelogram, which are piled over each other, and bound together by screwed iron rods, that pass down through them. A square well is thus formed, which in large soap factories is sometimes 10 feet deep, and capable of containing a cr.uple of tons of soap.

Mr. Sheridan some time since obtained a patent for combining silicate of soda with hard soap, by triturating them together in the hot and pasty state with a crutch in an iron pan. In this way from 10 to 30 per cent. of the silicate may be introduced. Such soap possesses very powerful detergent qualities, but it is apt to feel hard and be somewhat gritty in use. The silicated soda is prepared by boiling ground flints in a strong caustic ley, till the specific gravity of the compound rises to nearly double the 
density of water. It then contains about 35 grains of silica, and 46 of soda-hydrate in 100 grains."

Hard soap, after remaining two days in the frames, is at first divided horizontally into parallel tablets, 3 or 4 inches thick, by a brass wire; and these tablets are again cut vertically into oblong nearly square bars, called wedges in Scotland.

The soap-pans used in the United Kingdom are made of cast iron, and in three separate pieces joined together by iron-rust cement. The following is their general form :The two upper frusta of cones are called curbs; the third, or undermost, is the prn, to which alone the heat is applied, and which, if it gets eracked in the course of boiling, may easily be lifted up within the conical pieces, by attaching chains or cords for raising it, without disturbing the masonry, in which the curbs are firmly set. The surface of the hemispherical pan at the bottom, is in general about one tenth part of the surface of the conical sides.

The white ordinary tallow soap of the London manufacturers, called curd soap, consists, by my experiments, of - fat, 52 ; soda, 6 ; water, 42 ; $=100$. Nine tenths of the fat, at least, is tallow.

I have examined several other soaps, and have found their composition somewhat different.

The foreign Castile soap of the apothecary has a specific gravity of 1.0705 , and consists of-

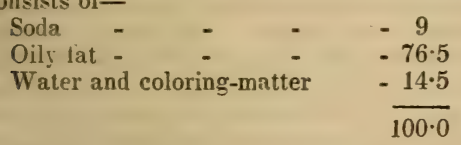

English imitation of Castile soap, spec. grav. 0.9669 , consists of-

$\begin{array}{ll}\text { Soda - } & - \\ \text { Pasty consistenced fat - } & - \\ \text { Water, with a little coloring - } & - \\ \text { matter - } & -\frac{14 \cdot 3}{100 \cdot 0}\end{array}$

A perfumer's white soap was found to consist of-

\begin{tabular}{llll} 
Soda - & - & - & -9 \\
Fatty matter & - & - & -75 \\
Water - & - & - & -16 \\
\hline & & &
\end{tabular}

$\begin{array}{llll}\text { Glasgow white soap- } & & \\ \text { Soda - } & - & - & -6.4 \\ \text { Tallow - } & - & - & -60 \cdot 0 \\ \text { Water - } & - & - & -33 \cdot 6\end{array}$

Glasgow brown rosin soapSoda -
Fat and rosin -
Water
A London cocoa-nut oil soap was found to consist of-

$\begin{array}{llll}\text { Soda - } & - & - & -4.5 \\ \text { Cocoa-nut lard - } & - & -22.0 \\ \text { Water - } & - & -73.5 \\ & & & 100.0\end{array}$

This remarkable soap was sufficiently solid; but it dissolved in hot water with extreme facility. It is calied marine soap, because it washes linen with sea water.

A poppy-nut-oil hard soap consisted of-

\begin{tabular}{lllll} 
Soda & - & - & - & -7 \\
Oil & - & - & - & -76 \\
Water & - & - & - & -17 \\
\hline $100 \dagger$
\end{tabular}

The soap known in France by the name of soap in tables, consists, according to M. Thenard's analysis, of-

$\begin{array}{llll}\text { Soda } & - & - & 4.6 \\ \text { Fatty matter } & - & - & 50 \cdot 2 \\ \text { Water } & - & - & 45 \cdot 2 \\ & & & \\ & & \end{array}$

M. D'Arcet states the analysis of Marseilles soap at-

\begin{tabular}{l|lllll}
-6.5 & Soda & - & - & - & 6 \\
-70.0 & Oil & - & - & - & 60 \\
-23.5 & Water & - & - & - & 34 \\
\hline 100.0 & & & & &
\end{tabular}

SOFT SOAP.

The principal difference between soaps with base of soda, and soaps with base of potash, depends upon their mode of combination with water. The former absorb a large quantity of it, and become solid; they are chemical hydrates. The others experience a much feebler cohesive attraction; but they retain much more water in a state of mere mixture.

Three parts of fat afford, in general, fully five parts of soda soap, well dried in the open air ; but three parts of fat or oil will afford from six to seven parts of potash soap of moderate consistence. This feebler cohesive force renders it apt to deliquesce, especially if there be a small excess of the alkali. It is, therefore, impossible to separate it from the leys; and the washing or relargage, practised on the hard-soap process, is inadmissible in the soft. Perhaps, however, this concentration or abstraction of water might be effected by using dense leys of muriate of potash. Those of muriate or sulphate of soda change the potash into a soda soap, by double decomposition. From its superior

* By my own experiments unor. the linuid sılicate made at Mr. Gibbs' excellent soap factory.

+ My own experiments. See Fats, Oils, and Stearine. 
solubility, more alkaline reaction, and lower price, potash soap is preferred for many purposes, and especially for scouring woollen yarns and stuffs.

Soft soaps are usually made in this country with whale, seal, olive, and linseed oils, and a certain quantity of tallow; on the continent, with the oils of hempseed, sesame, rapeseed, linseed, poppy-seed, and colza ; or with mixtures of several of these oils. When tallow is added, as in Great Britain, the object is to produce white and somewhat solid grains of stearic soap in the transparent mass, called figging, because the suap then resembles the granular texture of a fig.

The potash leys should be made perfectly caustic, and of at least two different strengths; the weakest being of specific gravity 1.05 ; and the strongest, $1 \cdot 20$, or even 1.25. Being made from the potashes of commerce, which contain seldom more than 60 per cent., and often less, of real alkali, the leys correspond in specific gravity to double their alkaline strength; that is to say, a solution of pure potash, of the same density, would be fully twice as strong. The following is the process followed by re. spectable manufacturers of soft soap (savon vert, being naturally or artificially green) upon the continent.

A portion of the oil being poured into the pan, and heated to nearly the boiling puint of water, a certain quantity of the weaker ley is introduced; the fire being kept up so as to bring the mixture to a boiling state. Then some more oil and les are added alternately, till the whole quantity of oil destined for the pan is introduced. The ebullition is liept up in the gentlest manner possible, and some stronger ley is occasionally added, till the workman judges the saponification to be perfect. The boiling becomes progressively less tumultuous, the frothy mass subsides, the paste grows transparent, and it gradually thickens. The operation is considered to be finished when the paste ceases to affect the tongue with an acrid pungency, when all milkiness and opacity disappear, and when a little of the soap placed to cool upon a glass plate, assumes the proper consistency.

A peculiar phenomenon may be remarked in the cooling, which affords a good criterion of the quality of the soap. When there is formed around the little patch, an opaque zone, a fraction of an inch broad, this is supposed to indicate complete saponification, and is called the strength; when it is absent, the soap is said to want its strength. When this zone soon vanishes after being distinctly seen, the soap is said to have false strength. When it occurs in the best form, the soap is perfect, and may be secured in that state by removing the fire, and then adding some good soap of a previous round, to cool it down, and prevent further change by evaporation.

200 pounds of oil require for their saponification-72 pounds of American potash of moderate quality, in leys at $15^{\circ} \mathrm{B}$.; and the product is 460 pounds of well-boiled soap.

If hempseed oil have not been employed, the soap will have a yellow color, instead of the green, so much in request on the continent. This tint is then given by the addition of a little indigo. This dye-stuff is reduced to fine powder, and boiled for some hours in a considerable quantity of water, till the stick with which the water is stirred presents, on withdrawing it, a gilded pellicle over its whole surface. The indigo paste diffused through the liquid, is now ready to be incorporated with the soap in the pan, before it stiffens by cooling.

M. Thenard states the composition of soft soap at-potash $9 \cdot 5,+$ oil $44 \cdot 0$, + water $46 \cdot 5,=100$.

Good soft soap of London manufacture, yielded to me-potash $8 \cdot 5,+$ oil and tallow $45,+$ water $46 \cdot 5$.

Belgian soft or green soap afforded me-potash $7,+$ oil $36,+$ water $57,=100$.

Scotch soft soap, being analyzed, gave me-potash $8,+$ oil and tallow $47,+$ water 45 . Another well-made soap-potash $9,+$ oil and fat $34,+$ water 57.

A rapeseed-oil soft soap, from Scotland, consisted of -potash 10, + oil $51 \cdot 66,+$ water $38 \cdot 33$.

An olive-oil (gallipoli) soft soap, from ditto, contained-potash with a good deal of carbonic acid 10 , oil 48 , water $42,=100$.

A semi-hard soap, from Verviers, for fulling woollen cloth, called savon économique, consisted of, potash $11 \cdot 5,+$ fat (solid) $62,+$ water $26 \cdot 5,=100$.

The following is a common process, in Scotland, by which good soft soap is made :-

273 gallons of whale or cod oil, and $4 \mathrm{cwts}$. of tallow, are put into the soap-pan, with 250 gallons of ley from American potash, of such alkaline strength that 1 gallon contains 6600 grains of real potash. Heat heing applied to the bottom pan, the mixture froths up very much as it approaches the boiling temperature, but is prevented from boiling over by being beat down on the surface, within the iron curb or crib which surmounts the caldron. Should it soon subside into a doughy-looking paste, we may infer that the ley has been too strong. Its proper appearance is that of a thin glue. We should now introduce about 42 gallons of a stronger ley, equivalent to $8700 \mathrm{gr}$. of potash per gallon; and after a short interval, an additional 42 gallons; and thus sue. 
cessively till nearly 600 such gallons have been added in the whole. After suitable boil. ing to saponify the fats, the proper quality of soap will be obtained, amounting in quantity to 100 firkins of 64 pounds each, from the above quantity of materials.

It is generally supposed, and I believe it to be true, from my own numerous experiments upon the subject, that it is a more difficult and delicate operation to make a fine soft soap of glassy transparency, interspersed with the figged granulations of stearate of potash, than to make hard soap of any kind.

Soft soap is made in Belgium as follows:-For a boil of 18 or 20 tons, of 100 kilogrammes each, there is employed for the leys- 1500 pounds of American potashes, and 500 to 600 pounds of quicklime.

The ley is prepared cold in cisterns of hewn stone, of which there are usually five in a range. The first contains the materials nearly exhausted of their alkali; and the last the potash in its entire state. The ley run off from the first, is transferred into the second; that of the second into the third; and so on to the fifth.

In conducting the empatage of the soap, they put into the pan, on the eve of the boiling-day, 6 aimes ( $1 \mathrm{ohm},=30$ gallons imperial) of oil of colza, in summer, but a mixture of that oil with linseed oil in winter, along with 2 aimes of potash ley at $13^{\circ} \mathrm{B}$, and leave the mixture without heat during eight hours. After applying the fire, they continue to boil gently till the materials cease to swell up with the heat; after which, ley of $16^{\circ}$ or $17^{\circ}$ must be introduced successively, in quantities of $\frac{1}{4}$ of an aime after another, till fiom 2 to 4 aimes be used. The boil is finished by pouring some ley of $20^{\circ} \mathrm{B}$., so that the whole quantity may amount to $9 \frac{1}{2}$ aimes.

It is considered that the operation will be successful, if from the time of kindling the fire till the finish of the boil, only five hours elapse. In order to prevent the soap from boiling over, a wheel is kept revolving in the pan. The operative considers the soap to be finished, when it can no longer be drawn out into threads between the finger and thumb. He determines if it contains an excess of alkali, by taking a sample out during the boil, which he puts into a tin dish; where if it gets covered with a skin, he pours fresh oil into the pan, and continues the boil till the soap be perfect. No wonder the Belgian soap is bad, amid such groping in the dark, without one ray of science!

\section{SOFT TOILET SOAPS.}

The soft fancy toilet soaps are divisible into two classes : 1. good potash soap, colored and scented in various ways, forms the basis of the Naples and other ordinary soft soaps of the perfumer; 2. pearl soap, (savon nacré,) which differs from the other both in physical aspect and in mode of preparation.

Ordinary soft Toilet Soup.-Its manufacture being conducted on the principles already laid down, presents no difficulty to a man of ordinary skill and experience; the only point to be strictly attended to, is the degree of evaporation, so as to obtain soap always of uniform consistence. The fat generally preferred is good hog's lard; of which thirty pounds are to be mixed with forty-five pounds of a caustic ley marking $17^{\circ}$ on Baumés scale; the temperature is to be gradually raised to ebullition, but the boil must not be kept up too long or too briskly, till after the empatage or saponification is completed, and the whole of the ley intimately combined with the fatty particles; after this, the evaporation of the water may be pushed pretty quickly, by a steady boil, till copious vapors cease to rise. This eriterion is observed when the paste has become too stiff to be stirred freely. The soap should have a dazzling snowy whiteness, provided the lard has been well refined, by being previously triturated in a mortar, melted by a steam heat, and then strained. The lard soap so prepared, is semi-solid, and preserves always the same appearance. If the paste is not sufficiently boiled, however, it will show the circumstance very soon; for in a few days the soap will become gluey and stringy, like a tenacious mass of birdlime. This defect may not only be easily avoided, but easily remedied, by subjecting the paste to an adequate evaporation. Such soaps are in great request for shaving, and are most convenient in use, especially for travellers. Hence their sale has become very considerable.

Pearl soft Scap.-It is only a few years since the process for making this elegant soap became known in France. It differs little from the preceding, and owes its beautiful aspect merely to minute manipulations, about to be described. Weigh out 20 pounds of purified hog's lard on the one hand, and 10 pounds of potash ley at $36^{\circ} \mathrm{B}$. on the other. Put the lard into a porcelain capsule, gently heated upon a sand-bath, stirring

constantly with a wooden spatula; and when it is half melted, and has a milky appearance, pour into it only one half of the ley, still stirring, and keeping up the same temperature, with as little variation as possible. While the saponification advances gradually, we shall perceive, after an hour, some fat floating on the surface, like a film of oil, and at the same time the soapy granulations falling to the bottom. We must then add the second portion of the ley; whereon the granulations immediately disappeas 
and the paste is formed. After conducting this operation during four hours, the paste becomes so stiff and compact, that it cannot be stirred; and must then be lighty beaten. At this time the capsule must be transferred from the sand-bath into a basin of warm water, and allowed to cool very slowly.

The suap, though completely made, has yet no pearly appearance. This phrisicaj property is developed only by pounding it strongly in a marble mortar; whereby all its particles, which seemed previously separated, combine to form a homogeneous paste. The perfume given to it, is always essence of bitter almonds; on which account the soap is called almond cream, crème d'amandes.

\section{HARD SOAPS FOR THE TOILET.}

The soaps prepared for the perfumer, are distinguished into different species, according to the fat which forms their basis. Thus there is soap of tallow, of hog's lard, of oil of olives, of almonds, and palm oil.

It is fiom the combination of these different sorts, mingled in various proportions, and perfumed agreeably to the taste of the consumer, that we owe the vast number of toilet soaps sold under so many fantastic names. One sort is rarely scented by itself, as a mixture of several is generally preferred; in which respect every perfumer has his peculiar secret. Some toilet soaps, however, require the employment of one kind more than of another.

Formerly the Windsor soap was made in France, wholly with mutton suet; and it was accordingly of inferior value. Now, by mixing some olive oil or lard with the suet, a very good Windsor soap is produced. I have already stated, that the fat of the London Windsor is, nine parts of good ox tallow, and one of olive oil. A soap made entirely with oil and soda, does not afford so good a lather as when it contains a considerable proportion of tallow.

The soaps made with palm oil are much used; when well made, they are of excellent quality, and ought to enter largely into all the colored sorts. They naturally possess the odor of violets.

The soaps made with oil of almonds are very beautiful, and preserve the agreeable smell of their perfume; but being expensive, are introduced sparingly into the mixtures by most manufacturers.

Some perfumers are in the habit of making what may be called extempore soaps, employing leys at $36^{\circ}$ Baumé in their formation. This method, however, ought never to be adopted by any person who prefers quality to beauty of appearance. Such soap is, indeed, admirably white, glistening, contains no more water than is necessary to its constitution, and may therefore be sold the day after it is made. But it has counter-balancing disadvantages. It becomes soon very hard, is difficultly soluble in water, and, if not made with tallow, does not lather well. Hog's lard is very commonly used for making that soap. Twenty kilogrammes of the fat are taken, to ten kilogrammes of soda ley, at $36^{\circ} \mathrm{B}$. (specific gravity $1 \cdot 324$ ); as soon as the former is nearly fluid, five kilogrammes of the ley are introduced, and the mixture is continually agitated during an hour with a wooden spatula. The temperature should never be raised ahore $150^{\circ} \mathrm{Fahr}$. at the commencement of the operation; at the end of one hour, five other kilogrammes of ley are to be added, with careful regulation of the heat. The paste thus formed by the union of the fat and alkali, ought to be perfectly homogeneous, and should increase in consistence every hour, fill it becomes firm enough to be poured into the frame; during which transfer, the essential oils destined to scent it, should he introduced. Next day the suap is hard enough; nor does it differ in appearance from ordinary soap, only it requilrs prompt manipulation to be cut into bars and cakes; for when neglected a day or two, it may become too brittle for that purpose, and too hard to take the impression of the stamps in relief. Such an article gets the name of little-pan soap, on account of the small quantity in which it is usually manufactured. Hard soap, made in the common way, is, on the contrary, called large-pan soap. This extemporaneous compound is now seldom or never made by respectable manufacturers. In making Windsor soap, the admixture of olive oil is advantageous ; because, being richer in oleine than suet, it saponifies less readily than it, and thus favors the formation of a more perfect neutral combination. When the soap cuts, or parts from the ley, when the paste becomes clotty, or, in the language of the operative, when the grain makes its appearance, the fire should be immediately withdrawn, that the impurities may be allowed to subside. This part of the operation lasts twelve hours at least; after which, the soap, still hot, becomes altogether fluid and perfectly neutral.

For every 1000 pounds of the paste, there must be introduced nine pounds of essences, mingled in the following proportions:-six pounds of essence of carui; one and a half ditto lavender, (finest); one and a half ditto rosemary.

The mixture must be well stirred, in order to get completely saturated with the perfumes; and this may be readily done without at all touching or stirring up the 
subjacent leys; in the course of two hours, the soap may be transferred into the ordinary frames. In twenty-four hours, the mass is usually solidified enough for cutting into bars and cakes, ready to be stamped for sale.

The above method of scenting Windsor soap is practised only in the largest establishments; in the smaller, the soap is pailed out of the soap-pans, into a pan provided with a steam case or jacket, and there mixed with the essential oils, by means of appropriate heat and agitation.

The most fashionable toilet soaps are, the rose, the bouquet, the cinnamon, the orangeflower, the musk, and the bitter almond or peach blossom.

Soup c̀ la rose.- This is made of the following ingredients : 30 pounds of olive-oil soap; 20 of good tallow soap.

Toilet soaps must be reduced to thin shavings, by means of a plane, with its under face turned up, so that the bars may be slid along it. These shavings must be put into an untinned copper pan, which is surrounded by a water-bath, or steam. If the soap be old and hard, 5 pounds of water must be added to them; but it is preferable to take fresh-made soaps, which may melt without addition, as soap some time kept does not readily form a homogeneous paste. The fusion is commonly completed in an hour, or thereby, the heat being applied at $212^{\circ} \mathrm{F}$., to accelerate the progress, and prevent the dissolution of the constituent water of the soap. For this purpose the interior pan may be covered. Whenever the mass is sufficiently liquefied, $1 \frac{1}{2}$ ounces of finely ground vermilion are to be introduced, and thoroughly mixed, after which the heat may be taken off the pan; when the following perfumes may be added with due trituration :- 3 ounces of essence of rose; 1 ditto cloves; 1 ditto cinnamon; $2 \frac{1}{2}$ ditto bergamot; $=7 \frac{1}{2}$.

The scented soap being put into the frames, speedily consolidates. Some recommend to pass the finished fused soap through a tammy cloth, in order to free it from all clots and impurities; a very proper precaution in the act of transferring it to the frame. If the preceding instructions be observed, we obtain a soap perfect in every point of view; possessing a delicious fragrance, equally rich and agreeable, a beautiful roseate hue, and the softest detergent qualities, which lieeping cannot impair. Such a soap has, in fact, been known to retain every property in perfection during four or five years. When the essential oils are particularly volatile, they should not be added to the soap till its temperature has fallen to about $140^{\circ} \mathrm{Fahr}$; but in this case a more careful trituration is required. The economy is, however, ill bestowed; for the cakes made of such cooler soap are never so homogeneous and glossy.

Soap au bouquet. -30 pounds of good tallow soap; 4 ounces of essence of bergamot; oil of cloves, sassafras, and thyme, 1 ounce each; neroli, $\frac{1}{2}$ ounce. The color is given with 7 ounces of brown ochre.

Cinnamon Soap.-30 pounds of good tallow soap; 20 ditto of palm-oil soap. Perfumes:-7 ounces of essence of cinnamon; $1 \frac{1}{4}$ ditto sassafras; $1 \frac{1}{4}$ ditto bergamot. Color :-1 pound of yellow ochre.

Orange-flower Soap.-30 pounds of good tallow soap; 20 ditto palm-oil soap. Perfumes :- $7 \frac{1}{2}$ ounces essence of Portugal; $7 \frac{1}{2}$ ditto amber. Color :- $9 \frac{1}{2}$ ounces, consisting of $8 \frac{1}{4}$ of a yellow-green pigment, and $1 \frac{1}{4}$ of red lead.

Musk Soap.-30 pounds of good tallow soap; 20 ditto palm-oil soap. Perfumes :Powder of clores, of pale roses, gilliflower, each $4 \frac{1}{2}$ ounces; essence of bergamot, and essence of musk, each $3 \frac{1}{2}$ ounces. Color : -4 ounces of brown ochre, or Spanish brown.

Bitter. Almond Soap-Is r sade by compounding, with 50 pounds of the best white soap, 10 ounces of the essence of bitter almonds.

\section{IIGHT SOAPS.}

The apparatus employed for making these soaps is a copper pan, heated by a waterbath; in the bottom of the pan there is a step, to receive the lower end of a vertical shaft, to which arms or paddles are attached, for producing constant agitation, by causing them to revolre arnong the liquefied mass. Into a pan so mounted, 50 pounds of good oil soap of any hind are put (for a tallow soap does not become frothy enough), and melted by proper heat, with the addition of 3 or 4 pounds of water. By the rapid rotation of the machine, an abundant thick lather is produced, beginning first at the bottom, and creeping gradually upwards to the top of the pan, when the operation should be stopped; the soap haring by this time doubled its rolume. It must now be pailed off into the frame, allowed to cool, and then cut into cakes. Such soap is exceedingly pleasant at the washstand, feeling very soft upon the skin, affording a copious thick lather, and dissolving with the greatest ease.

TRANSPARENT SOAPS.

These soaps were for a long time manufactured only in England, where the process was kept a profound secret. They are now made every where. 
Equal parts of tallow soap, made perfectly dry, and spirit of wine, are to be put into a copper still, which is plunged in a water-bath, and furnished with its capital and refrigeratory. The heat applied to effect the solution should be as slight as possible, to avoid evaporating too much of the alcohol. The solution being effected, must be suffered to settle; and after a few hours' repose, the clear supernatant liquid is drawn off into tin frames, of the form desired for the cakes of soap. These bars do not acquire their proper degree of transparency till after a few weeks' exposure to dry air. They are now planed, and subjected to the proper mechanical treatment for making calies of any form. The soap is colored with strong alcoholic solution of archil for the rose tint, and of turmeric for the deep yellow. Transparent soaps, however pleasing to the eye, are always of indifferent quality; they are never so detergent as ordinary soaps, and they eventually acquire a disagreeable smell.

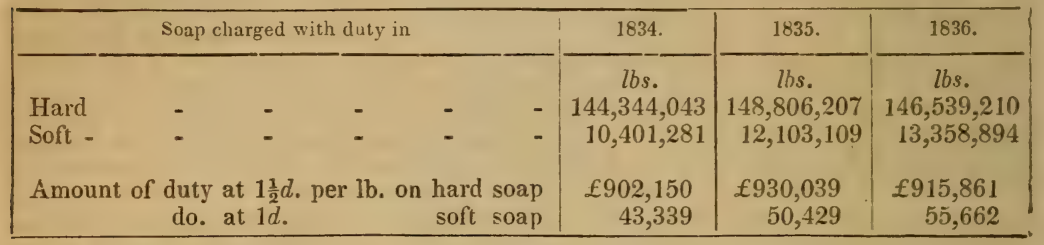

SOAPSTONE; see STEATrTE.

SODA, Causiic soda (Hydrate de soude, Fr.; Aetznatron, Germ.), is an alkaline sub stance, used in chemical researches, in bleaching, and in the manufacture of soap. It is prepared by boiling a solution of crystallized carbonate of soda in 4 or 5 parts of water, with half its weight of recently slaked and sifted lime. At the end of half an hour, the vessel of iron, porcelain, or preferably silver, may be removed from the fire, and covered carefully, till the calcarecus matter has settled into a solid magma at the bottom. The clear supernatant ley may be then decanted into bottles for use in the liquid state, or evaporated, out of contact of air, till it assumes an oily appearance, then poured upon an iron or marble slab, broken into pieces, and put up in vials secured with greased stoppers or corks.

Caustic soda is a white brittle mass, of a fibrous texture, a specific gravity of $1 \cdot 536$, melting at a heat under redness, having a most corrosive taste and action upon animal matters, dissolving readily in both water and alcohol, attracting carbonic acid when exposed to the atmosphere, but hardly any water, and falling thereby into an efflorescent carbonate; it forms soaps with tallow, oils, wax, rosin; dissolves wool, hair, silk, horn, alumina, silica, sulphur, and some metallic sulphurets. It consists of 77.66 soda, and 22.34 water. A solution of caustic soda affords no precipitate with solution of chloride of platinum, or tartaric acid, as a solution of caustic potash never fails to do.

The following TABLE of the quantity of CAustic SoDA contained in LEYs of different densities, has been given by Richter :-

\begin{tabular}{|c|c|c|c|c|c|c|c|}
\hline $\begin{array}{l}\text { Spec. } \\
\text { grav. }\end{array}$ & $\begin{array}{c}\text { Sorla } \\
\text { per cent. }\end{array}$ & $\begin{array}{l}\text { Spec. } \\
\text { grav. }\end{array}$ & $\begin{array}{c}\text { Soda } \\
\text { per cent. }\end{array}$ & $\begin{array}{l}\text { Spec. } \\
\text { grar: }\end{array}$ & $\begin{array}{c}\text { Soda } \\
\text { per cent. }\end{array}$ & $\begin{array}{l}\text { Spec. } \\
\text { grav. }\end{array}$ & $\begin{array}{c}\text { Soda } \\
\text { per cent. }\end{array}$ \\
\hline $1 \cdot 00$ & 0.00 & $1 \cdot 12$ & $11 \cdot 10$ & $1 \cdot 22$ & $20 \cdot 66$ & $1 \cdot 32$ & $29 \cdot 96$ \\
\hline $1 \cdot 02$ & $2 \cdot 07$ & $1 \cdot 14$ & $12 \cdot 81$ & $1 \cdot 24$ & $22 \cdot 58$ & $1 \cdot 34$ & $31 \cdot 67$ \\
\hline $1 \cdot 04$ & $4 \cdot 02$ & $1 \cdot 16$ & $14 \cdot 73$ & $1 \cdot 26$ & $24 \cdot 47$ & $1 \cdot 35$ & $32 \cdot 40$ \\
\hline $1 \cdot 06$ & $5 \cdot 89$ & $1 \cdot 18$ & $16 \cdot 73$ & $1 \cdot 28$ & $26 \cdot 33$ & $1 \cdot 36$ & $33 \cdot 08$ \\
\hline $1 \cdot 08$ & $7 \cdot 69$ & $1 \cdot 20$ & $18 \cdot 71$ & $1 \cdot 30$ & $28 \cdot 16$ & $1 \cdot 38$ & $34 \cdot 41$ \\
\hline $1 \cdot 10$ & $9 \cdot 43$ & & & & & & \\
\hline
\end{tabular}

Soda free from water can be obtained only by the combustion of sodium, which see.

SODA, CARBONATE OF (Kohlensaures natron, Germ.), is the soda of commerce in various states, either crystallized, in lumps, or in a crude powder called soda-ash. It exists in small quantities in certain mineral waters; as, for example, in those of Seltzer, Seydschutz, Carlsbad, and the volcanic springs of Iceland, especially the Geyser; it frequently occurs as an efflorescence in slender needles upon damp walls, being produced by the action of the lime upon the sea salt present in the mortar. The mineral soda is the sesquicarbonate, to be afterwards described.

Of manufactured soda, the variety most anciently known is barilla, the incinerated ash of the Salsola soda. This plant is cultivated with great care by the Spaniards, especially in the vicinity of Alicant. The seed is sown in light low soils, which are embanked towards the sea shore, and furnished with sluices, for admitting an occasional overflow of salt water. When the plants are ripe, the crop is cut down and dried; the 
seeds are rubbed out and preserved; the rest of the plant is burned in rude furnaces, at a temperature just sulficient to cause the ashes to enter into a state of semi-fusion, so as to concrete on cooling into cellular masses moderately compact. The most valuable variety of this article is called sucet burilla. It has a grayish-blue color, and gets covered with a saline eftlorescence when exposed for some time to the air. It is hard and difficult to break; when applied to the tongue, it excites a pungent allialine taste.

I have analyzed many varieties of barilla. Their average quantity of free or alkalimetrical soda is about 17 per cent. ; though several contain only 14 parts in the hundred, and a few upwards of 20 . This soda is chiefly a carbonate, with a little sulphuret and sulphite; and is mixed with sulphate and muriate of soda, carbonate of lime, vegetable carbon, \&c.

Another mode of manufacturing crude soda, is by burning sea-weed into kelp. Formerly very large revenues were derived by the proprietors of the shores of the Scottish islands and Highlands, from the incineration of sea-wred by their tenants, who usually paid their rents in kelp); but since the tax has been taken off salt, and the manufacture of a crude soda from it has been generally. established, the price of kelp has fallen extremely low.

The crystals of soda-carbonate, as well as the soda-ash of British commerce, are now made altogether by the decomposition of sea salt. SODA MANUFACTURE.

The manufacture divides itself into three branches:-1. The conversion of sea salt, or chloride of sodium, into sulphate of soda. 2. The decomposition of this sulphate into crude soda, called black balls by the workmen. 3. The purification of these balls, either into a dry white soda-ash or into erystals.

1. The preparation of the sulphate of soda.-Figs. 1033, 1034, 1035, represent the furnace for converting the muriate of soda into the sulphale. The furnace must be built interiorly of the most refiactory fire-bricks, such as are used for glasshouses, but of the ordinary brick size; except the bridges c, G, N, which should be formed of one mass, such as what is called a Welsh lump. A is the ash-pit; $B$, the grate; $c$, the first bridge, between the fire and the first calcining hearth $D, D ; F, F$, is its roof; G, the second bridoe, between the calcining hearth and the decomposing hearth I, I, I; the roof of which is $\mathrm{K}, \mathrm{k}$. This hearth $\mathrm{r}, \mathrm{r}$, is lined with a lead square pan, 5 or 6 inches deep, sloped at the back opening, in fig. 1035, marked $\mathrm{r}^{\prime}$; which deficient part of the upright side is filled up with two briclis placed one over the other, as shown at $m, m$, fig. 10:34, and luted with elay, to confine the semi-liquid mass in the pan, $\mathrm{I}$, $\mathrm{I}$. Some manufacturers make this pan 8 inches deep, and line its bottom and sides with bricks

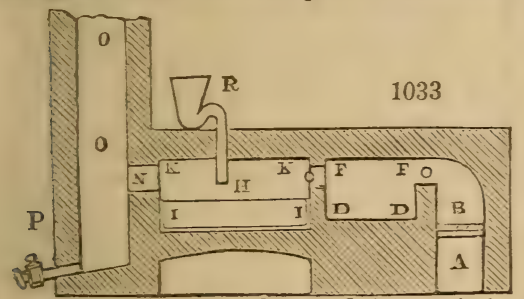
or silicious sandstone, to protect the lead from the corrosive action of the acid. There are others who consider this, precaution troublesome, as the points of the pan which become leaky are thereby concealed. In the roof of the decomposing hearth, one or two syphon funnels $\mathbf{R}$, of lead, are inserted when the charge of acid (sulphuric) is to be poured down upon the salt in $r, I$, to save the risk of any annuyance from the fumes of the muriatic acid. 0,0 , is a chimney filled with round flint nodules, which are kept continually moist by the trickling of a streamlet of water

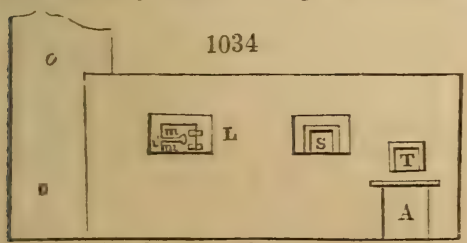
upon the topmost layer. The muriatic gas, meeting this descending film of water upon so extensive a surface, becomes absorbed, and runs out below in a liquid form. When the acis is required in a somewhat concentrated state, this chimney should be made both high and capacious. Such a plan, moreover, is very valuable for abating the nuisance caused by the disengagement of the muriatic acid gas; which is otherwise apt to sterilize the surrounding vegetation.

A fire being lindled in the grate $\mathrm{B}, f \mathrm{gs} .1033$ and $1034,3 \mathrm{cwts}$. of salt in powder are to be thrown by a shovel into the pan $\mathrm{I}$, through the door $\mathrm{M}, \mathrm{fig}$. 1035, or $\mathrm{m}, \mathrm{m}$, fig. 1034. Two hunlred weights and a half of oil of vitriol, of specific gravity $1 \cdot 844$, having been diluted with from 25 to 30 per cent. of water, and well mixed, or 3 cwts. at $56^{\circ}$ Baumé, are to be slowly poured in by the funnel, and ciffused among the muriate of soda, by an occasional stir with an iron rake cased with sheet lead. Fumes of muriatic acid will now plentifully escape, and, passing up the condensing-shaft o, will flow down 
in the form of liquid spirit of salt, and escape by the stoneware stopcock $P$, into the pipe of a sunk cistern. The fire having been steadily kept up at a moderate degree, the chemical reaction will be tolerably complete in the course of two hours; but as this is relative to the nature of the fuel, and the draught of the furnace, no very precise rule in point of time can be laid down; but it is sufficient for this stage of the process, when the fumes cease to be very dense and copious, as may be ascertained by opening the door $\mathbf{M}$, and looking in, or by the appearance at the top of the shaft o. Over the door $\mathbf{M}^{\prime}$, in the opposite side of the decomposing hearth, fig. 1035, there must be an arch or hood terminating in a small chimney, 15 or 20 feet high, for the ascent of the muriatic vapors, when the charge is drawn or run out of the hearth, and allowed to fall into a square shallow iron tray, placed on the ground at the back of the furnace. For this discharge, the two bricks which serve as stoppers to that orifice, must be unluted and removed.

As soon as that charge is taken out, (the fire being meanwhile checked by opening the door $\mathrm{T}, \mathrm{fig} .1034$, and shutting partially the ash-pit opening at $\mathrm{A}_{9}$ ) a fresh charge must be introduced as above described. The nearly decomposed saline matter, during the second charging of the hearth $\mathrm{I}$, will have grown cool and concrete. It must be shovelled into the calcining hearth $\mathrm{D}, \mathrm{D}, \mathrm{fig}$. 1033, by the back door $\mathrm{Q}$, fig. 1035 , where it will receive a higher degree of heat; and, by the expulsion of the remaining part of 1035 ar muriatic acid, it will become a

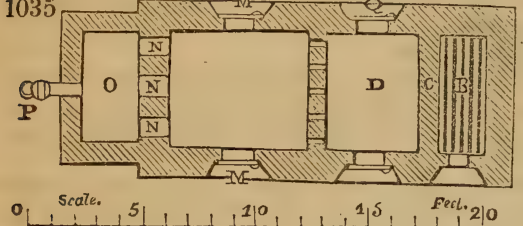
perfect sulphate of soda. It should be finally brought into a state of semifusion. When a sample of it, taken out on the end of the rake or trowelshaped scraper, emits no fumes, the conversion is accomplished.

From 3 ewts. of common salt, or muriate of soda, rather more than $3 \frac{1}{2} \mathrm{cwts}$. of perfect sulphate should be obtained, quite free from metallic impurity.

The next step is the conversion of the sulphate into a crude soda.

One of the most improved soda furnaces is that employed in a few factories, represented in figs. 1036, 1037, and 1038. In the section fig. 1037, there are two hearths in one furnace, the one elevated above the level of the other by the thickness of a brick, or about 3 inches. A is the preparatory shelf, where the mixture to be decomposed is first laid in order to be thoroughly heated, so that when transferred to the lower or decomposing hearth $\mathrm{B}$, it may not essentially chill it, and throw back the operation.

1036
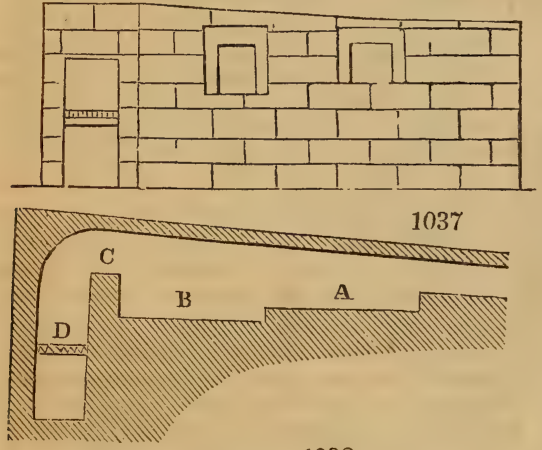

1038

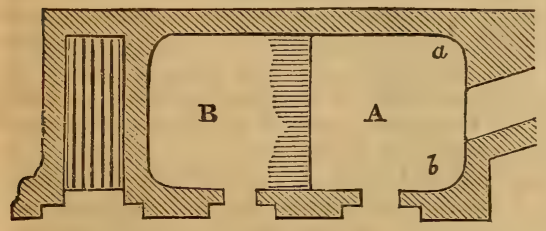

T, 1, $1,5_{1}, 1,1,1$, Feet,

The materials with which the sulphate is decomposed into a rough carbonate of soda, are chalk or ground limestone, and ground coal or charcoal. The proportions in which

these three substances are mixed, influence in a remarkable degree the success of the $\mathrm{C}$ is the fire-bridge, and $\mathrm{D}$ is the grate. In the horizontal section, or ground plan, fig. 1038, we see an opening in the front corresponding to each hearth. This is a door, as shown in the side view or elevation of the furnace, fig. 1036 ; and each door is shut by an iron square frame filled with a fire-tile or bricks, and suspended by a chain over a pulley fixed in any convenient place. See Prtcoar, COKING of, p. 1047. The workman, on pushing up the door lightly, makes it rise, because there is a counterweight at the other end of each chain, which balanees the weight of the frame and bricks. In the ground plan, only one smoke-flie is shown; and this construction is preferred by many manufacturers; but others choose to have two flues, one from each shoulder, as at $a, b$; which two flues afterwards unite in one vertical chimney, from 25 to 40 feet high; because the draught of a soda-furnace must be very sharp. Having sufficiently explained the construction of this improved furnace, I shall now proceed to describe the mode of making soda with it. 
decomposing :rocess. I hare known a false proportion introduced, and persevered in, at a factory, with the most prejudicial effect to the product; the soda-ash produced being in a small yustity relatively to the sulphate employed, and being much chatred with sulphur. Alier very numerous trials which I have made on the great scale, anu many in ranted to offer the following proportions as the most profitable :-

Sulphate of soda, 100 parls; carbonate of lime (chalk or limestone), from 110 to 120 parts; if pure, 110 ; if a little impure or lamp, 120 ; pitcoal, 50 parts.

These miterials must be separately giound by an edge-stone mill, and sifted into a tolerably fine powder. They must be then very carefully mixed. Attention to these particulars is of no little importance to the success of the soda process.

One hundred parts or pounds of sulphate of soda are equivalent to 75 parts of carbonate, and when skilfully decomposed, will generally yield fully 70 pounds. A charge for the decomposing furnace with the preparatory shelf should not exceed $200 \mathrm{lbs}$., or perhaps 180 ; therefore if 75 pounds of ground sulphate of soda, with 80 pounds of chalk or limestone (ground), and 37 pounds of ground coal, be well mixed, they will constitute one charge. This charge must be shovelled in upon the hearth $\mathrm{A}$, or shelf of preparation, ( $f$. . . 103\%); and whenever it has become hot (the furnace having been previously brought to bright ignition), it is to be transferred to the decomposing hearth or laboratory $\mathrm{B}$, by an iron tool, shaped exactly iike an oar, called the spreader. This tool has the flattened part from 2 to 3 feet long, and the round part, for laying hold of and working by, from 6 to 7 feet long. Two other tools are used; one, a rake, bent down like a garden hoe at the end; and another, a small shovel, consisting of a long iron rod terminated with a piece of iron plate, about 6 inches lon $q, 4$ broad, sharpened and tipped with steel, for cleaning the bottom of the hearth from adhering cakes or crusts. Whenever the charge is shoved by the sliding motion of the oar down upon the working hearth, a fresh charge should be thrown into the preparation shelf, and evenly spread over its surface.

The hot and partially carbonized charge being also evenly spread upon the hearth $\mathbf{B}$, is to be left untouched for about ten minutes, during which time it becomes ignited, and begins to fuse upon the surface. A view may be taken of it through a peep-hole in the door, which should be shut immediately, in order to prevent the reduction of the temperature. When the mass is seen to be in a state of incipient fusion, the workman takes the oar and turns it over breadth by breadth in regular layers, till he has reversed the position of the whole mass, placing on the surface the particles which were formerly in contact with the hearth. Having done this, he immediately shuts the door, and lets the whole get another decomposing heat. After five or six minutes, jets of flame begin to issue from various parts of the pasty-consistenced mass. Now is the time to incorporate the materials together, turning and spreading by the oar, gathering them together by the rake, and then distributing them on the reverse part of the hearth; that is, the oar should transfer to the part next the fire-bridge the portion of the mass lying next the shelf, and vice versa. The dexlerous management of this transposition characterizes a good soda-furnacer. A little practice an' instruction will render this operation easy to a robust clever workman. After this transposition, incorporation, and spreading, the door mar be shut again for a fer minutes, to raise the heat for the finishing off. Lastly, the rake must be dexterously employed to mix, shift, spread, and incorporate. The jets, called candles, are very numerous, and bright at first; and whenever they begin to fade, the mass must be raked out into cast-iron moulds, placed under the door of the laboratory to receive the ignited paste.

One batch being thus worked off, the other, which has lain undisturbed on the shelf, is to be shoved down from A to B, and spread equally upon it, in order to be treated as above described. A third batch is then to be placed on the shelf.

The article thus oocained should contain at least 22 per cent. of real soda, equivalent to 37 per cent. of dry carbonate, or to 100 of crystals. A skilful workman can turn out a balch in from three quarters of an hour to an hour, producing a perfect carbonate, which yields on solution an almost colorless liquid, nearly destitute of sulphur, and containing hardly any decomposed sulphate.

In some soda-works, where the decomposing furnace is very large, and is charged with a ton of materials at a time, it takes two men to work it, and from five to six hours to complete a batch. Having superintended the operation of the above-described small furnace, and examined its products, I feel warranted to recommend its adoption.

The following materials and products show the average state of this soda process :Materiuls. - 100 parts of sulphate of soda, ground, equivalent to 75 of carbonate;

110 of chalk or ground limestone ; 55 of ground coal ; in the whole, 265.

Products. -168 parts of crude soda, at 33 per cent. $=55.5$ of dry carbonate.

Or, $\{130$ - crystals of carbonate of soda $=48$ of dry carbonate; and $\{100$ - insoluble matter.

But these products necessarily vary with the skill of the workman. 
In another manufactory the following proportions are used:-Six stones, of $14 \mathrm{lbs}$. each, of ily ground sulphate of soda, are mixed with 3 of chalk and 3 of coal. This mixture, weiohing $1^{\frac{1}{2}} \mathrm{cwt}$, forms a batch, which is spread upon the preparation shelf of the furnace (figs. 1037 and 1038), as above described, and gradually heated to incipier.t ignition. It is then swept forwards to the lower area $\mathrm{B}$, by the iron oar, and spread evenly by the rake. Whenever it begins to soften under the rising heat of the laboratory (the side doors being meanwhile shut), the mess must be laboriously turned over and incorporated; the small shovel, or paddle, being employed to Iransfer, by the interchange of small portions at a time, in rapid but orderly succession, the whole materials from the colder to the hotter, and from the hotter to the colder parts of the hearth. The process of working one batch takes about an hour, during the first half of which period it remains upon the preparation shelf. The average weight of the finished ball is $1 \mathrm{cwt}$., and its contents in alkalimetrical soda are 33 pounds.

Where the acidulous sulphate of iron from pyrites may be had at a cheap rate, it has been long ago employed, as at Hurlett in Scotland, instead of sulphuric acid, for decomposing the chloride of sodium. Mr. Turner's process of preparing soda, by decomposing sea salt with litharge and quicklime, has bcen long abandoned, the resulting parent yellow, or sub-chlnride of lead, having a very limited sale.

2. The extraction of pure soda from the crude article.-The black balls must be broken into fragments, and thrown into large square iron cisterns, furnished with false bottoms of wooden spars; when the cisterns are nearly full of these lumps, water is pumped in upon them, till they are all covered. After a few days, the lixiviation is effected, and the ley is drawn off either by a syphon or by a plug-hole near the bottom of the eistern, and run into evaporating vessels. These may be of two kinds. The surface-evaporating furnace, shown in fig. 1039, is a very

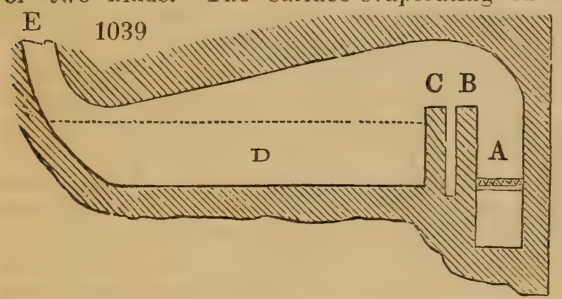

admirable invention for economizing vessels, lime, and fuel. The grate $\mathbf{A}$, and fireplace, are separated from the evaporating laboratory $\mathrm{D}$, by a double fire-bridge $B, c$, having an interstitial space in the middle, to arrest the comınunication ố a melting or ig. niting heat towards the lead-lined cistern $\mathrm{D}$. This cistern may be 8 , 10 , or 20 feet long, according to the magnitude of the soda-work, and 4 feet or more wide. Its depth should be about 4 feet. It consists of sheet lead, of about 6 pounds weight to the square foot, and it is lined with one layer of bricks, set in Roman or hydraulic cement, both along the bottom and up the sides and ends. The lead comes up to the top of $\mathrm{c}$, and the liquor, or ley, may be filled in to nearly that height. Things being thus arranged, a fire is kindled upon the grate $\mathrm{A}$; the flame and hot air sweep along the surface of the liquor, raise its temperature there rapidly to the boiling point, and carry off the watery parts in vapor up the chimney $\mathrm{E}$, which should be 15 or 20 feet high, to command a good draught. But, indeed, it will be most economical to build one high capacious chimney stalk, as is now done at Glasgow, Manchester, and Newcastle, and to lead the flues of the several furnaces above described into it. In this eraporating furnace the heavier and stronger ley goes to the bottom, as well as the impurities, where they remain undisturbed. Whenerer the liquor has attained 10 the density of $1 \cdot 3$, or thereb\%, it is pumped up into evaporating cast-iron pans, of a flattened somewhat hemispherical shape, and evaporated to dryness while being diligently stirred with an iron rake and iron scraper.

This alkali gets partially carbonated by the above surface-evaporating furnace, and is an excellent article.

When pure carbonate is wanted, that dry mass must be mixed with its own bulk of ground coal, sawdust, or charcoal, and thrown into a reverberatory furnace, like fig. 1038, but with the sole all upon one level. Here it must be exposed to a heat not exceeding $650^{\circ}$ or $700^{\circ} \mathrm{F}$; that is, a little above the melting heat of lead; the only object being to volatilize the sulphur present in the mass, and carbonate the alkali. Now, it has been found, that if the heat be raised to distinct redness, the sulphur will not go off, but will continue in intimate union with the soda. This process is called calking, and the furnace is called a calker furnace. It may be six or eight feet long, and four or five feet broad in the hearth, and requires only one door in its side, with a hanging iron frame filled with a fire-tile or bricks, as above described.

This carbonating process may be performed upon several cwts. of the impure soda, mixed with sawdust, at a time. It takes three or four hours to finish the desulphuration; and it must he carefully turned over by the oar and the rake, in order to burn the coal into carbonic acid, and to present the carbonic acid to the particles of caustic soda diffu. sed through the mass, so that it may combine with them. 
When the blue flames cease, and the saline matters become white, in the millst of the soaly matter, the batch may be considered as completed. It is ralied out, :und when couled, lixiriated in great iron cisterns with false bottoms, covered with mats. 'The watery solution being drawn off clear by a plug-hole, is evaporated either to drymess, in hemispherical cast-iton pans, as above deseribed, or only to such a strength that it shows a pellicle upon its surface, when it may be run off into crystallizing cisterns of cast iron, or lead-lined wooden cisterns. The above dry carbonate is the best article for the glass manufacture.

Crystallized carbonate of soda contains $62 \frac{3}{4}$ per cent. of water. The crystals are colorless transparent rhomboids, which readily effloresce in the air, and melt in their own water of crystallization. On decanting the liquid from the fused mass, it is found that one part of the salt has given up its water of crystallization to another. By evaporation of that fluid, crystals containing one fifth less water than the common carbonate are obtained. These do not effloresce in the air.

Mineral soda, the sesquicarbonate (Anderthalb kohlensaures natron, Germ.), is found in the province of Suliena, in Africa, between Tripoli and Fezzan. It forms a stratum no more than an inch thick, just below the surface of the soil. Its texture is striated crystalline, like fibrous gypsum. Several hundred tons of it are collected annually, which are chiefly consumed in Africa. This species of soda does not effloresce like the Egyptian, or the manufactured soda crystals, owing to its peculiar state of composition and density. It was analyzed by Klaproth, under its native name of trona, and was found to consist, in 100 parts, of-soda, 37 ; carbonic acid, 38 ; sulphate of soda, 2.5 ; water, $22 \cdot 5$, in 100 .

This soda is, therefore, composed of -3 atoms of carbonic acid, associated with 2 atoms of soda, and 4 of water; while our commercial soda crystals are composed of -1 atom of carbonic acid, 1 atom of soda, and 10 atoms of water.

There are six natron lakes in Egypt. They are situated in a barren valley, called Bahrbela-ma, about thirty miles to the west of the Delta.

There are natron lakes also in Hungary, which afford in summer a white saline efflorescent crust of carbonate of soda, mixed with a little sulphate.

There are several soda lakes in Mexico, especially to the north of Zacatecas, as also in many other provinces. In Columbia, 48 English miles from Merida, mineral soda is extracted from the earth in great abundance, under the name of urao.

Bicarbonate of soda (Doppelt kohlensaures natron, Germ.), is prepared, like bicarbonate of potassa, by transmitting carbonic acid gas through a cold saturated solution of pure carbonate of soda, till crystalline crusts be formed. The bicarbonate may also be ohtained in four-sided tables grouped together. It has an alkaline taste and reaction upon litmus paper, dissolves in 13 parts of cold water, and is converted by boiling water into the sesquicarbonate, with the disengagement of one fourth of its carbonic acid. It consists of -37 of soda, 52.35 carbonic acid, and $10 \cdot 65$ water.

SOD A-WATER, is the name given to water containing a minute quantity of soda, and highly charged with carbonic acid gas, whereby it acquires a sparliling appearance, an agreeable pungent taste, an exhilarating quality, and certain medicinal powers. It constitutes a considerable object of manufacture in this kingdom. The following figure represents, I understand, the best system of apparatus for preparing it. A very dilute solution of soda is put into the globular vessel $\mathrm{H}$, and the carbonic acid gas is forced into it from the gasometer $\mathrm{E}$, by means of the powerful pump-work, as will be understood from the subjoined explanation.

The same apparatus may serve for making any species of aerated water, in imitation of any natural spring. All that is necessary for this purpose, is to put into the cistern $\mathbf{Q}$, the neutro-saline matter, earths, metallic oxydes, pure water, \&c., each in due proportion, according to the most accredited analysis of the mineral water to be imitated, to agitate that mixture, to suck it into the condenser $\mathrm{H}$, through the pipe $\mathrm{R}$, and then to impregnate it to the due degree, by pumping in the appropriate gas, previously contained in the gasometer $\mathbf{F}$.

Thus, to make Seltzer water, for each 12 pounds troy, $=69,120$ grains, or 1 gallon imperial very nearly, take 55 grains of dry carbonate of soda, 17 of carbonate of lime, 18 of carbonate of magnesia, $3 \frac{1}{2}$ of subphosphate of alumina, 3 of chloride of potassium, 155 of chloride of sodium, and 3 of finely precipitated silica. Put these materials into the cistern $Q$, and charge the gasometer $\mathbf{F}$ with 353 cubic inches of carbonic acid gas. Then work the machine by the handle of the wheel $x$, as explained below, and regulate the introduction of the liquid and the gas in aliquot portions; for example, if the condenser $\mathrm{H}$ admits half a gallon of water at a time, that quantity of liquid should be charged with 176 cubic inches of the gas, being one half of the whole quantity. The sulphureted mineral waters may be imitated in like manner, by taking the proportions of their constituents, as given in Table II. of WATERs, Mineral. 
IMPROVED SO1)A-WATER APPARATUS, AS MADE BY MR. HAYWARD TYLEA, OF MILTON STREET.

Fig. 1040, front view of the soda water machine. Fig. 1041, end view of the same
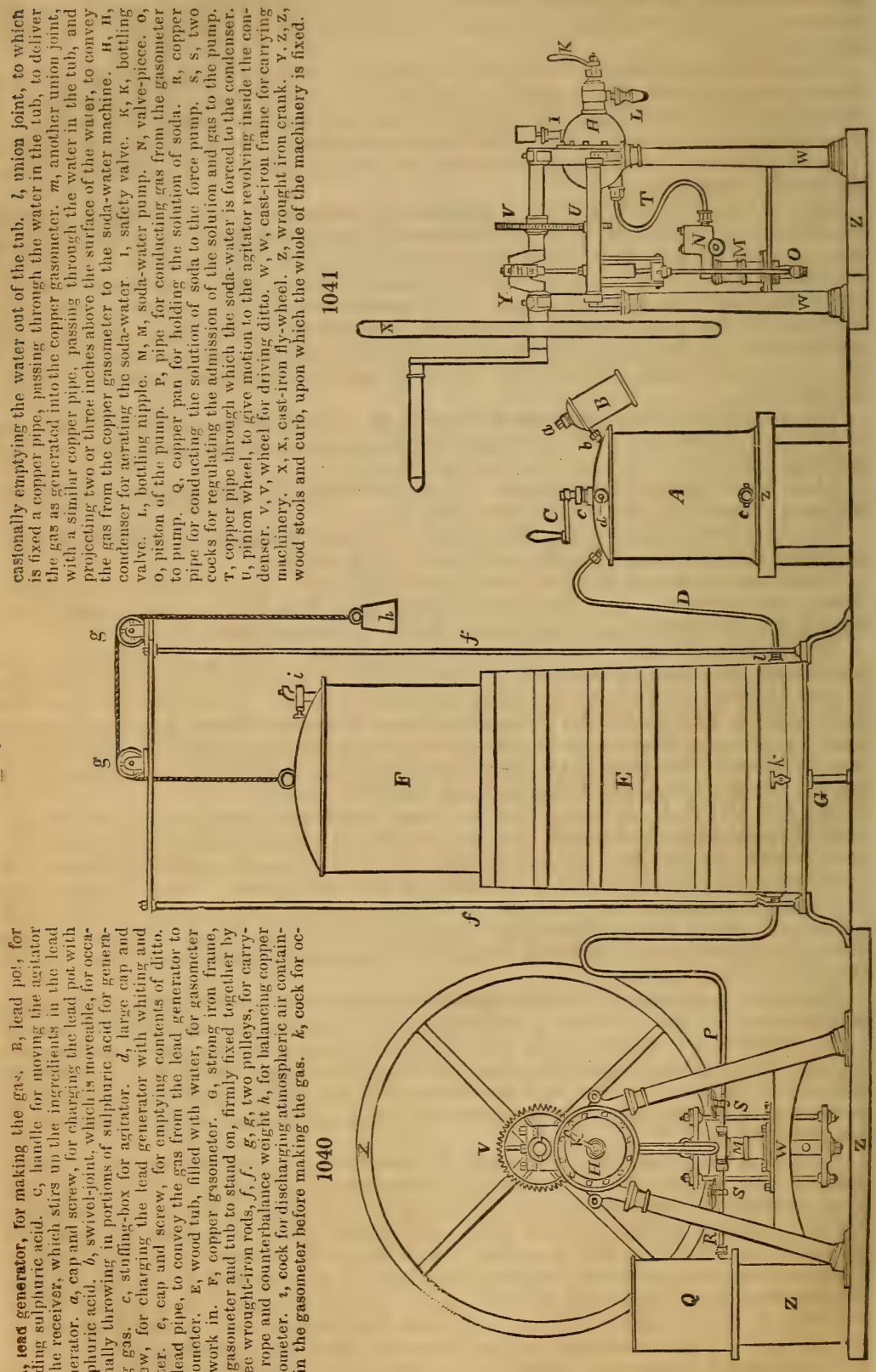

ちั

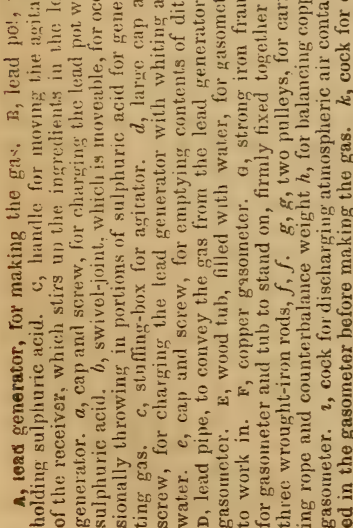


SODIUM, the metallic basis of soda, is obtained by processes similar to those by which potassium is procured. By fusing hydrate of soda with a little hydrate of potassa, a mixture is obtained, which yields more readily than suda by itself to the decomposing action of iron-turnings at a high heat, in a bent gun-barrel. The portion of potassium produced may be got rid of, by digesting the alloy for a few days in some naptha or oil of turpentine contained in an open vessel. The sodium remains at the bottom of the liquid. Pure sodium may, however, be prepared at once, by subjecting incinerated tartrate of soda to heat in the apparatus of Brunner, described under PotAssitm. It is white, like silver; softer and more malleable than any other metal, and may be readily reduced into rery thin leaves. It preserves its malleability till it approaches the melting point. Its specific gravity is 0.970 . It softens at the temperature of $122^{\circ} \mathrm{F}$, and at 20()$^{\circ}$ it is perfectly fluid; but it will not rise in vapor until heated to nearly the melting point of glass. In the air it oxydizes slowly, and gets covered with a crust of soda; but it does not take fire till it is made nearly red-hot; and then it emits brilliant scintillations. When thrown upon water, it is rapidly oxydized, but without lindling, like potassium. If a drop of water be thrown upon it, it becomes so hot by the chemical action as to take fire. There are three oxydes of sodium; 1 . the suboxyde; 2 . the oxyde, or the basis of common soda; and, 3. the suroxyde; the last being formed when sodium is heated to redness upon a plate of silver.

SOLDERING (Souder, Fr.; Lölhen, Germ.;, is the process of uniting the surfaces of metals, by the intervention of a more fusible metal, which being melted upon each surface, serves, partly by chemical attraction, and partly by cohesive force, to bind them together. The metals thus united may be either the same or dissimilar; but the uniting metal must always have an affinity for both. Solders must be, therefore, selected in reference to their appropriate metals. Thus tin-plates are soldered with an alloy consisting of from 1 to 2 parts of tin, with 1 of lead; pewter is soldered with a more fusible alloy, containing a certain proportion of bismuth added to the lead and tin; iron, copper, and brass are soldered with spelter, an alloy of zine and copper, in nearly equal parts; silver, sometimes with pure tin, but generally with silver-solder, an alloy consisting of 5 parts of silver, 6 of brass, and 2 of zine; zine and lead, with an alloy of from 1 to 2 parts of lead with 1 of tin; platinum, with fine gold; gold, with an alloy of silver and gold, or of copper and gold; \&c.

In all soldering processes, the following conditions must be observed; 1 . the surfaces to be united must be entirely free from oxyde, bright, smooth, and level; 2. the contact of air must be excluded during the soldering, because it is apt to oxydize one or other of the surfaces, and thus to prevent the formation of an alloy at the points of union. This exclusion of air is effected in rarious ways. The locksmith encases in loam the objects of iron, or brass, that he wishes to subject to a soldering heat; the silversmitb and brasier mix their respective solders with moistened borax powder; the coppersmith and tinman apply sal ammoniac, rosin, or both, to the cleaned metallic surfaces, before using the soldering-iron to fuse them together with the tin alloy. The strong solder of the coppersmith consists of 8 parts of brass and 1 of ziric; the latter being added to the former, previously brought into a state of fusion. The crucible must be immediately covered up for two minutes till the combination be completed. The melted alloy is to be then poured out upon a bundle of twios held over a tub of water, into which it falls in granulations. An alloy of 3 parts of copper and 1 of zine forms a still stronger solder for the coppersmiths. When several parts are to be soldered successivin's upon the same piece, the more fusible alloys, containing more zinc, should be used first. A soiter solder for coppersmiths is made with 6 parts of brass, 1 of tin, and 1 of zinc; the tin being first added to the melted brass, then the zinc; and the whole well incorporated by stirring.

The edges of sheet lead for sulphuric acid chambers, and its concentration pams, are joined together by melted lead itself, because any solder containing tin would soon be corroded. With this view, the two edges being placed in contact, are flattened down into a long wooden groove, and secured in their situation by a few brass pins driven into the wood. The surfaces are next brightened with a triangular scraper, rubbed over with candle grease, and then covered with a stream of hot melted lead. The riband of lead thus applied is finally equalized by being brought into partial fusion with the plumber's conical iron heated to redness; the contact of air being prevented by sprinkling rosin over the surface. The sheets of lead are thus burned together, in the language of the workmen.

SOOT (Noir de fumée, Suie, Fr.; Rus, Flatterrus Germ.), is the pulverulent charcoal condensed from the smoke of wood or coal fuel. A watery infusion of the former is said to be antiseptic, probably from its containing some creosote.

The soot of pitcoal has not been analyzed with any minuteness. It contains some sulphate and carbonate of ammonia, along with bituminous matter. 
SORBIC ACID, is the same with malic acid; which see.

SOY, is a liquid condiment, or sauce, imported chiefly from China. It is preparea with a species of white haricots, wheat flour, common salt, and water; in the proportions respectively of $50,60,50$, and 250 pounds. The haricots are washed, and boiled in water till they become so soft as to yield to the fingers. They are then laid in a flat dish to cool, and kneaded along with the flour, a little of the hot water of the decoction being added from time to time. This dough is next spread an inch or an inch and a half thick upon the flat vessel (made of thin staves of bamboo), and when it becomes hot and mouldy, in two or three days, the cover is raised upon bits of stick, to give free access of air. If a rancid odor is exhaled, and the mass grows green, the process goes on well; but if it grows black, it must be more freely exposed to the air. As soon as all the surface is covered with green mouldiness, which usually happens in eight or ten days, the cover is removed, and the matter is placed in the sunshine for several days. When it has become as hard as a stone, it is cut into small fragments, thrown into an earthen vessel, and covered with the 250 pounds of water having the salt dissolved in it. The whole is stirred together, and the height at which the water stands is noted. The vessel being placed in the sun, its contents are stirred up every morning and evening; and a cover is applied at night, to keep it warm and exclude rain. The more powerful the sun, the sooner the soy will be completed; but it generally requires two or three of the hottest summer months. As the mass diminishes by evaporation, well water is added; and the digestion is continued till the salt water has dissolved the whole of the flour and the haricots; after which the vessel is left in the sun for a few days, as the good quality of the soy depends on the completeness of the solution, which is promoted by regular stirring. When it has at length assumed an oily appearance, it is poured into bags, and strained. The clear black liquid is the soy, ready for use. It is not boiled, but is put up into bottles, which must be carefully corked. Genuine soy was made in this way at Canton, by Michael de Grubbens. See Memoirs of Academy of Sciences of Stockholm for 1803.

SPECIFIC GRAVITY, designates the relative weights of different bodies under the same bulk; thus a cubic foot of water weighs 1000 ounces avoirdupois; a cubic foot of coal, 1350 ; a cubic foot of cast iron, 7280 ; a cubic foot of silver, 10,400; and a cubic foot of pure gold, 19,200 ; numbers which represent the specific gravities of the respective substances, compared to water $=1 \cdot 000$. See Alloy.

SPECULUM METAL, is an alloy of copper and tin; described under CopPER.

SPERMACETI; the Cetine of Chevreul. In certain species of the cachalot whale, as the Physeter macrocephalus, tursio, microps, and orthodon, as also the Delphinus edentulus, the fat of some parts of their bodies contains a peculiar kind of stearine, called spermaceti. The oil obtained from cavities in the bones of the cranium of the above cetaceæ is the richest in this kind of stearine. This being thrown into great filter-bags, the spermaceti oil prasses through, and is subsequently purified by the addition of a small quantity of potash ley, which precipitates certain matters by neutralizing the acid that held them in solution. The solid which remains on the filter is next squeezed in bags, by means of a horizontal hydraulic press incased in steam, then digested with a weak potash ley, in order to dissolve out any oil which may continue to adhere to it, washed with water, finally dissolved in a tub by the agency of steam, laded into tin pans, and allowed slowly to concrete into a white, semi-transparent, brittle, lamellar crystalline mass, which forms elegant candles.

At $60^{\circ}$ its specifie gravity is 0.943 . It melts at $112.5^{\circ} ; 100$ parts of alcohol at 0.821 dissolve $3 \frac{1}{2}$ of it, of which 0.9 are deposited on cooling. Warm ether dissolves it in very large quantities. It is soluble also in the fat of volatile oils; and if the solutions have been saturated while hot, the greater part of the spermaceti crystallizes on cooling. When this substance has been purified by digesting alcohol upon it repeatedly, what remains is the cetine of Chevreul, or pure spermaceti. Its melting point has now become $116^{\circ} \mathrm{F}$., and its boiling point $616^{\circ} \mathrm{F}$, at which it distils without alteration. Caustic alkaline leys saponify it with difficulty.

SPIRIT OF AMMONIA is, properly speaking, alcohol combined with ammonia gas; but the term is often applied to water of ammonia.

SPIRITS, VINOUS. This subject has been fuily discussed in the articles AlcoHor, Distillation, and Fermentation. I have shown that the progressive increase of alcohol in the wash tends progressively to prevent the conversion of the wort into spirit, or checks the fermenting process, though a great deal of fermentable matter remains unchanged. Mr. Sheridan has sought to remove this obstacle to the thorough transmutation of saccharine matter into alcohol, by drawing off the spirit as it is formed. For this purpose he ferments his wash in close tuns, connected with a powerful airpump worked by machinery, thus continually removing the carbonic acid as it is formed, and maintaining a diminished pressure under which the alcohol readily distils at a temperature of $120^{\circ}$ or $130^{\circ} \mathrm{F}$. He finds that this degree of heat is not injurious to the 
fermentation, provided that it be communicated by the air of a stove-room, and not by water or steam pipes traversing the liquid, which would inevitably scald or sceth the particles in succession, and thereby extinguish the fermenting principle.

By the above ingenious plan, Mr. Sheridan tells me he has obtained 28 gallons of pront spirit from a quarter of grain, instead of the average product 21 , being an increase of 25 per cent. The experiment was tried upon a considerable scale at Messis. Currie's great distillery near London; but could not be established as a mode of manufacture, on acount of the excise laws, which prohibit the distillers from carrying on the two processes of fermentation and distillation at the same time.

SPIRIT OF WINE; Alcohol.

SPONGE. (Eponge, Fr.; Schwamm, Germ.), is a cellular fibrous tissue produced by small animals, almost imperceptible, called polypi by naturalists, which live in the sea. This tissue is said to be covered in its recent state with a kind of semi-fluid thin coat of animal jelly, susceptible of a slight contraction or trembling on being touched; which is the only symptom of vitality displayed by the sponge. After death, this jelly disappears, and leaves merely the sponge; formed by the combination of a multitude of small capillary tubes, capable of receiving water in their interior, and of becoming thereby distended. Sponges occur attached to stones at the bottom of the sea; and abound particularly upon the shores of the islands in the Grecian Archipelago. Although analogous in their origin to coral, sponges are quite different in their nature; the former being composed almost entirely of carbonate of lime; while the latter are formed of the same elements as animal matters, and afford, on distillation, a considerable quantity of ammonia.

Dilute sulphuric acid has been recommended for bleaching sponges, after the calcareous impurities have been removed by muriatic acid. Chlorine water answers better.

SPOON MANUFACTURE. See Stamprng of Metals.

STAINED GLASS. When certain metallic oxydes or chlorides, ground up with proper fluxes, are painted upon glass, their colors fuse into its surface at a moderate heat, and make durable pictures, which are frequently employed in ornamenting the windows of churches as well as of other public and private buildings. The colors of stained glass are all transparent, and are therefore to be viewed only by transmitted light. Many metallic pigments, which afford a fine effect when applied cold on canvass or paper, are so changed by vitreous fusion as to be quite inapplicable to painting in stained glass.

The glass proper for receiving these vitrifying pigments, should be colorless, uniform, and difficult of fusion; for which reason crown glass, made with little alkali, or with kelp, is preferred. When the design is too large to be contained on a single pane, several are fitted together, and fixed in a bed of soft cement while painting, and then taken asunder to be separately subjected to the fire. In arranging the glass pieces, care must be taken to distribute the joinings so that the lead frame-work may interfere as little as possible with the effect.

A design must be drawn upon paper, and placed beneath the plate of glass; though the artist cannot regulate his tints directly by his palette, but by specimens of the colors producible from his palette pigments after they are fired. The upper side of the glass being sponged over with gum-water, affords, when dry, a surface proper for receiving the colors, without the risk of their running irregularly, as they would be apt to do, on the slippery glass. The artist first draws on the plate, with a fine pencil, all the traces which mark the great outlines and shades of the figures. This is usually done in black, or, at least, some strong color, such as brown, blue, green, or red. In laying on these, the painter is guided by the same principles as the engraver, when he produces the effect of light and shade by dots, lines, or hatches; and he employs that color to produce the shades, which will harmonize best with the color which is to be afterwards applied; but for the deeper shades, black is in general used. When this is finished, the whole picture will be represented in lines or hatches similar to an engraving finished up to the highest effect possible ; and afterwards, when it is dry, the vitrifying colors are laid on by means of larger hair pencils; their selection being regulated by the burnt specimen tints. When he finds it necessary to lay two colors adjoining, which are apt to run together in the kiln, he must apply one of them to the back of the glass. But the few principal colors to be presently mentioned, are all fast colors, which do not run, except the yellow, which must therefore be laid on the opposite side. After coloring, the artist proceeds to bring out the lighter effects by taking off the color in the proper place, with a goose quill cut like a pen without a slit. By working this upon the glass, he removes the color from the parts where the lights should be the strongest; such as the hair, eyes, the reflection of bright surfaces and light parts of draperies. The blank pen may be employed either to make the lights by lines, or hatches and dots, as is most suitable to the subject. 
By the metallic preparations now laid upon it, the glass is made ready for being fired, in order to fix and bring out the proper colors. The furnace or kiln best adapted for this purpose, is similar to that used by enamellers. See Enames, and the Glaze-kiln, under PotTury. It consists of a muffle or arch of fire-clay, or pottery, so set over a fireplace, and so surrounded by flues, as to receive a very considerable heat within, in the most equable and regular manner; otherwise some parts of the glass will be melted; while, on others, the superficial film of colors will remain unvitrified. The mouth of the muffe, and the entry for introducing fuel to the fire, should be on opposite sides, to prevent as much as possible the admission of dust into the muffle, whose mouth should be closed with double folding-doors of iron, furnished with small peep-holes, to allow the artist to watch the progress of the staining, and to withdraw small trial slips of glass, painted with the principal tints used in the picture.

The muffle must be made of very refractory fire-clay, flat at its bottom, and only 5 or 6 inches high, with such an arched top as may make the roof strong, and so close on all sides as to exclude entirely the smoke and flame. On the bottom of the muffe a smooth bed of sifted lime, freed from water, about half an inch thick, must be prepared for receiving the pane of glass. Sometimes several plates of glass are laid over each other with a layer of diy pulverulent lime between each. The fire is now lighted, and most gradually raised, lest the glass should be broken; and after it has attained to its full heat, it must be kept up for 3 or 4 hours, more or less, according to the indications of the trial slips; the yellow color being principally watched, as it is found to be the best criterion of the state of the others. When the colors are properly burnt in, the fire is suffered to die away, so as to anneal the glass.

\section{STAINED-GLASS PIGMENTS.}

Flesh color.-Take an ounce of red lead, 2 ounces of red enamel, (Venetian glass enamel, from alum and copperas calcined together, ) grind them to fine powder, and work this up with spirits (alcohol) upon a hard stone. When slightly baked, this produces a fine tlesh color.

Black color.--Take $14 \frac{1}{2}$ ounces of smithy scales of iron, mix them with two ounces of white glass, (crystal,) an ounce of antimony, and half an ounce of manganese; pound and grind these ingredients together with strong vinegar. A brilliant black may also be obtained by a mixture of cobalt blue with the oxydes of manganese and iron. Another black is made from three parts of crystal glass, two parts of oxyde of copper, and one of (glass of) antimony worked up together, as above.

Brown color.-An ounce of white glass or enamel, half an ounce of good manganese; ground together.

Red, rose, and brown colors, are made from peroxyde of iron, prepared by nitric acid. The flux consists of borax, sand, and minium in small quantity.

Red color, may be likewise obtained from one ounce of red chalk pounded, mixed with two ounces of white hard enamel, and a little peroxyde of copper.

$A$ red, may also be composed of rust of iron, glass of antimony, yellow glass of lead, such as is used by potters, (or litharge,) each in equal quantity; to which a little sulphuret of silver is added. This composition, well ground, produces a very fine red color on glass. When protoxyde of copper is used to stain glass, it assumes a bright red or green color, according as the glass is more or less heated in the furnace, the former corresponding to the orange protoxyde, the latter having the copper in the state of peroxyde.

Bis' "es and brown reds, may be obtained by mixtures of manganese, orange oxyde of copper, and the oxyde of iron called umber, in different proportions. They must be previously fused with vitreous solvents.

Green color.- Two ounces of brass calcined into an oxyde, two ounces of minium, and eight ounces of white sand; reduce them to a fine powder, which is to be enclosed in a well luted crucible, and heated strongly in an air-furnace for an hour. When the mixture is cold, grind it in a brass mortar. Green may, however, be advantageously produced by a yellow on one side, and a blue on the other. Oxyde of chrome has been also employed to stain glass green.

A fine yellow color.-Take fine silver laminated thin, dissolve in nitric acid, dilute with abundance of water, and precipitate with solution of sea salt. Mix this chloride of silver, in a dry powder, with three times its weight of pipe-clay well burnt and pounded. The back of the glass pane is to be painted with this powder; for when painted on the face, it is apt to run into the other colors.

Another yellow can be made by mixing sulphuret of silver with glass of antimony, and yellow ochre previously calcined to a red-brown tint. Work all these powders together, and paint on the back of the glass. Or silver lamince melted with sulpliur, and glass of antimony, thrown into cold water, and afterwards ground to powder, afford a yellow. 
A paie yellow may be made with the powder resulting from brass, sulphur, and rlass of antimony, calcined together in a crucible, till they cease to smoke; and then mixed with a little burnt yellow ochre.

The fine yellow of M. Merand is prepared from chloride of silver, oxyde of zinc, whiteclay, and rust of iron. This mixture, simply ground, is applied on the glass.

Orange color.-Take 1 part of silver powder, as precipitated fiom the nitrate of that metal by plates of copper, and washed; mix it with 1 part of red ochre and 1 of yellow, by careful trituration; grind into a thin pap with oil of turpentine or lavender, and apply this with a brush, dry, and burn in.

In the Philosophical Magazine, of December, 1836, the anonymous author of an in. genious essay, "On the Art of Glass-painting," says, that if a large proportion of ochre has been employed with the silver, the stain is yellow; if a small proportion, it is orangecolored; and by repeated exposure to the fire, without any additional coloring-matter, the orange may be converted into red; but this conversion requires a nice management of the heat. Artists often make use of panes colored throughout their substance in the glass-house pots, because the perfect transparency of such glass gives a brilliancy of eflect, which enamel painting, always more or less opaque, cannot rival. It was to a glass of this kind that the old glass-painters owed their splendid red. This is, in fact, the only point in which the modern and ancient processes differ; and this is the only part of the art which was ever really lost. Instead of blowing plates of solid red, the old olass-makers (like those of Bohemia, for some time back) used to flash a thin layer of brilliant red over a substratum of colorless glass; by gathering a lump of the latter upon the end of their iron rod in one pot, covering it with a layer of the former in another pot, then blowing out the two together into a globe or cylinder, to be opened into cucular tables, or into rectangular plates. The elecant art of tinging glass red by protoxyde of copper, and flashing it on common crown glass, has become general within these few years.

That gold melted with flint glass stains it purple, was originally discovered and practised, as a profitable secret, by Kunckel. Gold has been recently used at Birmingham for giving a beautiful rose-color to scent bottles. The proportion of gold should be very small, and the heat very great, to produce a good effect. The glass must contain either the oxyde of lead, bismuth, zinc, or antimony; for crown glass will take no color from gold. Glass combined with this metal, when removed from the crucible, is generally of a pale rose-color; nay, sometimes is as colorless as water, and does not assume its ruby color till it has been exposed to a low red heat, either under a muffle or at the lamp. This operation must be nicely regulated; because a slight excess of fire destroys the color, learing the glass of a dingy brown, but with a blue (green ?) transparency, like that of gold leaf. It is metallic gold which gives the color; and, indeed, the oxyde is too easily reduced, not to be converted into the metal by the intense heat which is necessarily required.

Upon the kindred art of painting in enamel, Mr. A. Essex has published an interesting paper in the same journal, for June, 1837, in which he says that the ancient muby glass, on being exposed to the heat of a glass-kiln, preserves its color unimpairel, while the modern suffers considerable injury, and in some cases becomes almost black. Hence the latter cannot be painted upon, as the heat required to fix the fresh color would destroy the beauty of the original basis. To obviate this difficulty, the artist paints upon a piece of plain glass the tints and shadows necessary for blending the rich ruby g'ow with the other parts of his picture, leaving those parts untouched where he wishes the ruby to appear in undiminished brilliancy, and fixes the ruby glass in the picture behind the painted piece, so that in such parts the window is double glazed. Mr. Essex em. ploys, as did the late Mr. Muss, chrome oxyde alone for greens; and he rejects the use of iron and manganese in his enamel colors.

Colored transparent glass is applied as enamel in silver and gold bijouterie, previously bright-cul in the metal with the graver or the rose-engine. The cuts, reflecting the rays of light from their numerous surfaces, exhibit through the glass, richly stained with gold, silver, copper, cobalt, \&c., a gorgeous play of prismatic colors, varied with every change of aspect. When the enamel is to be painted on, it should be made opalescent by oxyde of arsenic, in order to produce the most agreeable effect.

The artist in enamel has obtained from modern chemistry, preparations of the meials platinum, uranium, and chromium, which furnish four of the richest and most useful colors of his palette. Oxyde of platinum produces a substantive rich brown, formerly unknown in enamel painting; a beautiful transparent tint, which no intensity or repetition of fire can injure. Colors proper for enamel painting, he says, are not to be purchased; those sold for the purpose, are adapted only for painting upon china. The constituents of the green enamel used by his brother, Mr. W. Essex, are, silica, borax, oxyde of lead, and oxyde of chrome. 
Mr. Essex's enamelling furnace is a cubic space of about 12 inches, and contains a fire-clay muffle, without either bottom or back, which is surrounderl with colie, except in front. The entire draught of air which supplies the furnace, rasses through the muffle; the plates and paintings being placed on a thin slab, made of tenjered fireclay, technically termed planche, which rests on the bed of coke-fuel. As the greatest heat is at the back of the muffle, the picture must be turned round while in the fire, by means of a pair of spring tongs. The above furnace serves for ohjects up to five inches in diameter; but for larger works a different furnace is required, for the description of which I must refer to the original paper.

Relatively to the receipts for enamel colors, and for staining and gilding on glass, for which twenty guineas were voted by the Society for the Encouragement of Aits, in the session of 1817, to Mr. R. Wynn, Mr. A. Essex says, in p. 446 of his essay" the unfortunate artist who shall attempt to make colors for the purpose of jainting in enamel from these receipts, will assuredly find, to his disajointment, that they are utterly useless." In page 449 he institutes a comparison between Mr. Wynn's complex farrago for green, as published in the Transactions of the Society, with the simple receipt of his brother, as given above. It is a remarkable circumstance, that not one of our enamel artists, during a period of twenty years. should have denounced the fallacy of these receipts, and the folly of sanctioning imposture by a public reward. Shuld Mr. Essex's animadversions be just, the well-intentioned Society in the Adelphi may, from the negligence of its committee, come to merit the sobriquet, "For the Discouragement of Arts."

STAMPING OF METALS. The following ingenious machine for manufacturing metal spoons, forks, and other articles, was made the subject of a patent by Jonathan Hayne, of Clerkenwell, in May, 1833. He employs a stamping-machine with dies, in which the hammer is raised to a height between guides, and is let fall.by a trigoer. He prefers fixing the protuberant or relief portion of the die to the stationary block or bed of the stamping-machine, and the counterpart or intaglio to the falling hammer or ram.

The peculiar feature of improvement in this manufacture consists in producing the spoon, ladle, or fork perfect at one blow in the stamping-machine, and requiring no further manipulation of shaping, but simply trimming off the barb or fin, and polishing the surface, to render the article perfect and finished.

Heretofore, in employing a stamping-machine, or fly-press, for manufacturing spoons, ladles, and forks, it has been the practice to give the impressions to the handles, and to the bowls or prongs, by distinct operations of different dies, and after having so partially produced the pattern upon the article, the handles had to be bent and formed by the operations of filing and hammering.

By his improved form of dies, which, having curved surfaces and bevelled edges, allow of no parts of the faces of the die and counter-die to come into contact, he is enabled to produce considerable elevations of pattern and form, and to bring up the article perfect at one blow, with only a slight barb or fin upon its edge.

In the accompanying drawings, fig. 1042 is the lower or bed die for producing a spoon, seen edgewise; fig. 1043 is the face of the upper or counter-die, corresponding; 1044
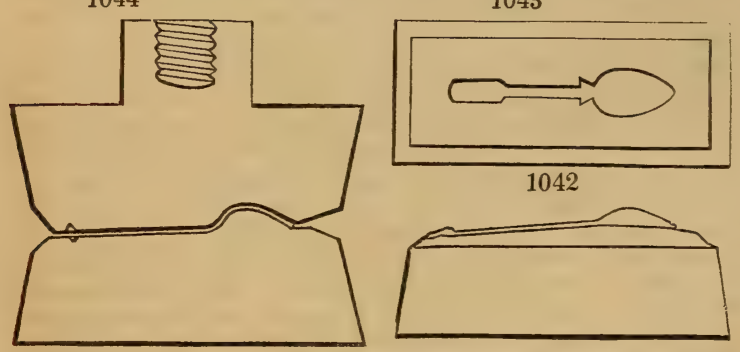

fig. 1044 is a section, taken through the middle of the pair of dies, showing the space in which the metal is pressed to form the spoon.

To manufacture spoons, ladles, or forks according to his improved process, he first forges out the ingot into flat pieces, of the shape and di-

mensions of the die of the intended article; and if a spoon or ladle is to be made, gives a slight degree of concavity to the bowl part; but, if necessary, bends the back, in order that it may lie more steadily, and bend more accurately, upon the lower die; if a fork, he cuts or otherwise removes portions of the metal at those parts which will intervene between the prongs; and, having thus produced the rude embryo of the intended article, scrapes its entire surface clean and free from oxydation-scale or fire-strain, when it is ready to be introduced into the stamping-machine.

He now fixes the lower die in the bed of the stamping-machine, shown at $a$, $a$, in the elevations figs. 1045 and 1046, and fixes, in the hammer $b$, the upper or counter-die 
c, accurately adjusting thim both, so that they may correspond exactly when brought iogether. He then places the rudely-formed article above described upon the lower
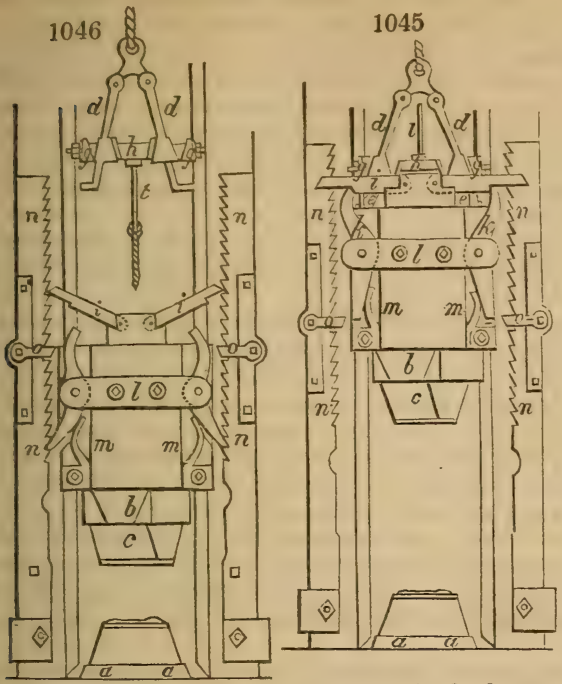
die, and having drawn up the hammer to a sufficient elevation by a windlass and rope, or other ordinary means, lets go the trigger, and allows the hammer with the counter-die to fall upon the under die, on which the article is placed; when, by the blow thus given to the metal, the true and perfect figure and pattern of the spoon, ladle, or fork is produced, and which, as before said, will only require the removal of the slight edging of barb or fin, with polishing, to finish it.

On striking the blow, in the operation of stamping the article, the hammer will recoil and fly up some distance, and if allowed to fall again with reiterated blows, would injure both the article and the dies; therefore, to avoid this inconvenience, he causes the hammer on recoiling to be caught by a pair of palls locking into racks on the face of the standards, seen in figs. 1045 and 1046. In fig. 1045 the hammer $b$, of the stamping-machine, is seen raised and suspended by a rope attached to a pair of jointed hooks or holders $d, d$, the lower ends of which pass into eyes $e, e$, extending from the top of the hammer. When the lever or trigger.t is drawn forward, as in fig. 1046, the two inclined planes $g$, g, on the axle $h$, press the two legs of the holders $d$, $d$, inward, and cause their hooks or lower ends to be withdrawn from the eyes $e, e$, when the hammer instantly falls, and brings the dies together: such is the ordinary construction of the stamping-machine.

On the hammer falling from a considerable elevation, the violence of the blow causes it to recoil and bound upwards, as before mentioned; it therefore becomes necessary to catch the hammer when it has rebounded, in order to prevent the dies coming again together; this is done by the following mechanism:-

Two latch levers $i, i$, are connected by joints to the upper part of the hammer, and two pall levers $k, k$, turning upon pins, are mounted in the bridge $l$, affixed to the hammer. Two springs $m, m$, act against the lower arms of these levers, and press them outwards, for the purpose of throwing the palls at the lower ends of the levers into the teeth of the ratchet racks $n, n$, fixed on the sides of the upright standards.

Previously to raising the hammer, the upper ends of the pall levers $k$, are drawn back, and the latches $i$, being brought down upon them, as in fig. 1045, the levers $k$ are confined, and their palls prevented from striking into the side racks; but as the hammer falls, the ends of the latches $i$ strike upon the fingers 0,0 , fixed to the side standards, and liberate the palls, the lower ends of which, when the hammer rebounds, after stamping, catch into the teeth of the racks, as in fig. 1046, and thereby prevent the hammer from again descending.

STARCH (Amidon, Fecule, Fr; Stärke, Germ.), is a white pulverulent substance, composed of microscopic spheroids, which are bags containing the amylaceous matter. It exists in a great many different plants, and varies merely in the form and size of its microscopic particles; as found in some plants, it consists of spherical particles $\frac{1}{10} 00$ of an inch in diameter; and in others, of ovoid particles, of $\frac{1}{300}$ or $\frac{1}{400}$ of an inch. It occurs, 1 . in the seeds of all the acotyledinous plants, among which are the several species of corns, and those of other graminea; 2. in the round perennial tap roots, which shoot up an annual stem; in the tuberose roots, such as potatoes, the Convolvulus batatas and edulis, the Helianthus tuberosus, the Jatropha manihot, \&c., which contain a great quantity of it; 3. in the stems of several monocotyledinous plants, especially of the palm tribe, whence saoo comes; but it is very rarely found in the stems and branches of the dicotyledinous plants; 4. it occurs in many species of lichen. Three kinds of starch have been distinguished by chemists; that of wheat, that called inuline, and lichen starch. These three agree in being insoluble in cold water, alcohol, ether, and oils, and in being converted into sugar by either dilute sulphuric acid or diastase. The main difference between them consists in their habitudes with water and iodine. The first 
forms with hot water a mucilaginous solution, which constitutes, when cold, the paste of the laundress, and is tinged blue by iodine; the second forms a granular precipitate, when its solution in boiling-hot water is suflered to cool, which is tinged yellow by iedine; the third affords, by cooling the concentrated solution, a gelatinous mass, with a clear liquor floating orer it, that contains little starch. Its jelly becomes brown-gray with iodine.

1. Ordinury slarch. - This may be extracted from the fullowing grains:-wheat, rye, barley, oats, buckwheat, rice, maize, millet, spelt ; from the siliquose seeds, as peas beans, lentiles, \&c. ; from tuberous and tap roots, as those of the potato, the orchis, manioc, arrowroot, batata, \&c. Different kinds of corn yield very variable quantities of starch. Wheat diflers in this respect, according to the varieties of the plant, as well as the soil manure, season, and climate. See BrEAD.

Wheat partly damaged by long keeping in granaries, may be employed for the inanufacture of starch, as this constituent suffers less injury than the gluten; and it may be used either in the ground or unground state.

1. With unground uheat.-The wheat being sifted clean, is to be put into cisterns, covered with soft water, and left to steep till it becomes swollen and so soft as to be easily crushed between the fingers. It is now to be taken out, and immersed in clear water of a temperature equal to that of malting-barley, whence it is to be transferred into bags, which are placed in a wooden chest containing some water, and exposed to strong pressure. The water rendered milky bI the starch being drawn off by a tap, fresh water is poured in, and the pressure is repeated. Instead of putting the swollen grain into bags, some prefer to grind it under vertical edge-stones, or between a pair of horizontal roilers, and ther to lay it in a cistern, and separate the starchy liquor by elutriation with successire quantities of water well stired up with it. The residuary mitter in the saclis or cisterns contains much vegetable albumen and gluten, along with the husks; when exposed to fermentation, it affords a small quantity of starch of rather inferior quality.

The above milky liquor, obtained by expression or elutriation, is run into large cisterns, where it deposites its starch in layers successively less and less dense; the uppermost containing a considerable proportion of gluten. The supernatant liquor being drawn off, and fresh water poured on it, the whole must be well stirred up, allowed again to settle, and the surface-liquor again withdrawn. This washing should be repeated as long as the water takes any perceptible color. As the first turbid liquor contains a mixture of gluten, sugar, gum, albumen, \&c., it ferments readily, and produces a certain portion of vinegar, which helps to dissolve out the rest of the mingled gluten, and thus to bleach the starch. It is, in fact, by the action of this fermented or soured water, and repeated washing, that it is purified. After the last deposition and decantation, there appears on the surface of the starch a thin layer of a slimy mixture of gluten and albumen, which, being scraped off, serves for feeding pigs or oxen; underneath will be found a starch of good quality. The layers of different sorts are then talien up with a wooden shovel, transferred into separate cisterns, where they are agitated with water, and passed through fine sieves. After this pap is once more well settled, the clear water is drawn off, the starchy mass is taken out, and laid on linen cloths in wicker baskets, to drain and become partially dry. When sufficiently firm, it is cut into pieces, which are spread upon other cloths, and thoroughly desiccated in a proper drying-room, which in winter is heated by stores. The upper surface of the starch is generally scraped, to remove any dusty matter, and the resulting powder is sold in that state. Wheat yields, upon an average, only from 35 to 40 per cent. of good starch. It should afford more by skilful management.

2. In this country, wheat crushed between iron rollers is laid to steep in as much water as will wet it thoroughly ; in four or five days the mixture ferments, soon afterwards settles, and is ready to be washed out with a quantity of water into the proper fermenting vats. The common time allowed for the steep, is from 14 to 20 dars. The next process consists in removing the stuff from the rats into a stout round basket set across a back below a pump. One or two men keep gcing round the basket, stirring up the stuff with strong wooden shovels, while another lieeps pumping water, till all the farinx is completely washed from the bran. Whenever the subjacent back is filled, the liquor is taken out and strained through hair sieves into square frames or cisterns, where it is allowed to settle for 24 hours; after which the water is run off from the deposited starch by plug taps at different levels in the side. The thin stuff called slimes, upon the surface of the starch, is remored by a tray of a peculiar form. Fresh water is now introduced, and the whole being well mixed by proper agitation, is then poured upon fine silk sieres. What passes through is allowed to settle for 24 hours; the liquor being withdrawn, and then the slimes, as before, more water is again poured in, with agitation, when the mixture is again thrown upon the silk sieve. The milky liquor is now suffered to rest for several days, 4 or 5 , till the starch becomes set1led pretty firmly at the bottom of the square cistern. If the starch is to have the blue tint, 
called Poland, fine smalt must be mixed in the liquor of the last sieve, in the proportion of two or three pounds to the cwt. A considerable portion of these slimes may, by good management, be worked up into starch by elutriation and straining.

The starch is now fit for boxing, by shorelling the cleaned deposite into wooden chests, about 4 feet long, 12 inches broad, and 6 inches deep, perforated throughout, and lined with thin canvass. When it is drained and dried into a compact mass, it is turned out by inverting the chests upon a clean table, where it is broken into pieces four or five inches square, by laying a ruler underneath the calie, and giving its surface a cut with a knife, after which the slightest pressure with the hand will make the fracture. These pieces are set upon half-burned briclis, which by their porous capillarity imbibe the moisture of the starch, so that its under surface may not become hard and horny. When sufficiently dried upon the briclis, it is put into a stove, (which resembles that of a sugar refinery; ) and left there till tolerably dry. It is now removed to a table, when all the sides are carefully scraped with a linife; it is next packed up in the papers in which it is sold; these packages are returned into the stove, and subjected to a gentle heat during some days; a point which requires to be skilfully regulated.

Mr. Samuel Hall obtained a patent for bleaching starch by chloride of lime in 1821 . Chlorine water would probably be preferable, and might prove useful in operating upon damaged wheat.

The sour water of the starch manufacture contains, according to Vauquelin, acetic acid, acelate of ammonia, alcohol, phosphate of lime, and gluten.

During the drying, starch splits into small prismatic columns, of considerable regularity. When kept diy, it remains unaltered for a very long period. When it is heated to a certain degree in water, the envelopes of its spheroidal particles burst, and the farina forms a mucilaginous emulsion, magma, or paste. When this apparent solution is evaporated to dryness, a brittle horny-looking substance is obtained, quite different in aspect from starch, but similar in chemical habitudes. When the moist paste is exposed for two or three months to the air in summer, the starch is converted into sugar to the amount of one third or one half of its weight, into gum, and gelatinous starch called amidine by De Saussure, with occasionally a resinous matter. This curious change goes on even in close vessels.

Starch from potatoes.-From the following table of analyses, it appears that potatoes contain from 24 to 30 per cent. of dry substance :-

\begin{tabular}{|c|c|c|c|c|c|c|c|c|}
\hline & & & & Starch. & \begin{tabular}{|c|} 
Fibrous $\mathrm{Pa}-$ \\
renchyma.
\end{tabular} & $\begin{array}{l}\text { Vegetable } \\
\text { Albumen. }\end{array}$ & $\mid \begin{array}{c}\text { Gum, Sugar, } \\
\text { and Salts. }\end{array}$ & Water. \\
\hline Red potatoes, - & - & & - & $15 \cdot 0$ & $7 \cdot 0$ & $1 \cdot 4$ & $9 \cdot 2$ & $75 \cdot 0$ \\
\hline Germinating potato & bes, & & - & $15 \cdot 2$ & $6 \cdot 8$ & $1 \cdot 3$ & $3 \cdot 7$ & $73 \cdot 0$ \\
\hline Kidney potatoes, & - & & - & $9 \cdot 1$ & $8 \cdot 8$ & $0 \cdot 8$ & - & $81 \cdot 3$ \\
\hline Large red potatoes, & & - & - & $12 \cdot 9$ & $6 \cdot 0$ & $0 \cdot 7$ & - & $78 \cdot 0$ \\
\hline Sweet potatoes, & - & & - & $15 \cdot 1$ & $8 \cdot 2$ & 0.8 & - & $74 \cdot 3$ \\
\hline Peruvian potatoes, & & & - & $15 \cdot 0$ & $5 \cdot 2$ & $1 \cdot 9$ & 1.9 & $76 \cdot 0$ \\
\hline English potatoes, & - & & - & $12 \cdot 9$ & $6 \cdot 8$ & $1 \cdot 1$ & $1 \cdot 7$ & $77 \cdot 5$ \\
\hline Parisian potatoes, & & & - & $13 \cdot 3$ & $6 \cdot 8$ & 0.9 & $4 \cdot 8$ & $73 \cdot 1$ \\
\hline
\end{tabular}

Manufacture of potato starch.-The potatoes are first washed in a cylindrical cage formed of wooden spars, made to revolve upon a horizontal axis, in a trough filled with water to the level of the axis. They are then reduced to a pulp by a rasping machine, similar to that represented in figs. 1047, 1048, where $a$ is a wooden drum covered with sheet-iron, roughened outside with numerous prominences, made by punching out holes from the opposite side. It is turned by a winch fixed upon each end of the shaft. The drum is enclosed in a square wooden box, to prevent the potato-mash from being scattered ahont. The hopper $b$ is attached to the upper frame, has its bottom concentric with the rasp-arum, and nearly in contact with it. The pulp chest $c$ is made to slide out, so as $\pi$ lien full to be readily replaced by another. The two slanting boards $d$, $d$, conduct the pulp into it. A moderate stream of water should be made to play into the hopper upon the potatoes, to prevent the surface of the rasp from getting foul with fibrous matter. Two men, with one for a relay, will rasp, with such a machine, from $2 \frac{1}{2}$ to 3 tons of poatoes in 12 hours.

The potato pulp must be now elutriated upon a fine wire or hair seive, which is set upon a frame in the mouth of a large vat, while water is made to flow upon it from a spout with many jets. The pulp meanwhile must be stirred and kneaded by the hand, or by a mechanical brush-agitator, till almost nothing but fibrous particles are left upon the sieve. These, however, generally retain about five per cent. of starch, which cannot be separated in this way. This parenchyma should therefore be subjected to a separate. rasping upon another cylinder. The water turbid with starch is allowed to settle for sone 
time in a back; the supernatant liquor is then run by a cock into a second back, and after 1047

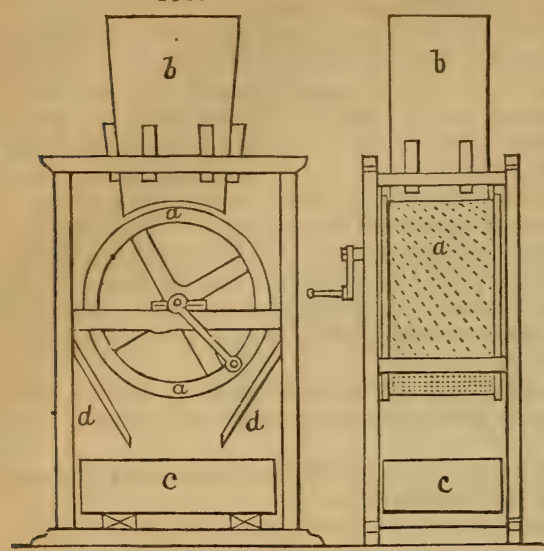
some time into a third, whereby the whole starch will be precipitated. The finest powder collects in the last vessel. The starch thus obtained, containing 33 per cent. of water, may be used either in the moist state, under the name of green fecu$i a$, for various purposes, as for the preparation of dextrine, and starch sirup; or it may be preserved under a thin layer of water, which must be renewed from time to time, to prevent fermentation; or lastly, it may be taken out and dried.

In trials made with St. Etienne's rasp and starch machinery, in Paris, which was driven by two horses, nearly $18 \mathrm{ewts}$. of potatoes were put through all the requisite operations in one hour, including the pumping of the water. The produet in starch amounted to from 17 to 18 per cent. of the potatoes. The quicker the process of "potato-starch making, the better is its quality.

Starch from certain foreign plants.-1. From the pith of the sago palm. See SAgo.

2. From the roots of the Maranta arundinacea, of Jamaica, the Bahamas, and other West India islands, the powder called arrow-root is obtained, by a process analogous to that for making potato starch.

3. From the root of the Manioc, which also grows in the West Indies, as well as in Africa, the cassava is procured by a similar process. The juice of this plant is poisonous, from which the wholesome starch is deposited. When dried with stirring upon hot iron plates, it agglomerates into small lumps, called tapioca; being a gummy fecula.

The characters of the different varieties of starch can be learned only from microscopic observation; by which means also their sophistication or admixture may be readily ascertained.

Starch, from whatever source obtained, is a white soft powder, which feels crispy, like flowers of sulphur, when pressed between the fingers; it is destitute of taste and smell, unchangeable in the atmosphere, and has a specific gravity of 1.53 . I have already described the particles as spheroids enclosed in a membrane. The potato contains some of the largest, and the millet the smallest. Potato starch consists of truncated ovoids, varying in size from $\frac{1}{300}$ to $\frac{1}{3000}$ of an inch; arrow-root, of ovoids varying in size from $\frac{1}{800}$ to $\frac{1}{2000}$ of an inch; flower starch, of insulated globules about $\frac{1}{1000}$ of an inch; cassava, of simular globules assembled in groups. These measurements I have made with a good achromatic microscope, and a divided glass-slip micrometer of Tully.

For the saccharine changes which starch undergoes by the action of diustase, see FERMENTATION.

Lichenine, a species of starch obtained from Iceland moss, (Cetraria islandica, as well as inuline, from elecampane, (Inula Helenium,) are rather objects of chemical curiosity, than of manufactures.

There is a kind of starch made in order to be converted into gum for the calico-printer. This conversion having been first made upon the great scale in this country, has occasioned the product to be called British gum. The following is the process pursued in a large and well conducted establishment near Manchester. A range of four wooden cis.erns, each about 7 or 8 feet square, and 4 feet deep, is provided. Into each of them 2000 gallons of water being introduced, $12 \frac{1}{2}$ loads of flour are stirred in. This mixture is set to ferment upon old leaven left at the bottom of the backs, during 2 or 3 days. The sontents are then stirred up, and pumped off into 3 stone cisterns, 7 feet square and 4 feet deep; as much water being added, with agitation, as will fill the cisterns to the brim. In the course of 24 hours the starch forms a firm deposite at the bottom; and the water is then syphoned off. The gluten is next seraped fiom the surface, and the starch is transferred into wooden boxes pierced with holes, which may be lined with coarse eloth, or not, at the pleasure of the operator.

The starch, cut into cubical masses, is put into iron trays, and set to dry in a large apartment, two stories high, heated by a horizontal cylinder of cast iron traversed by the flame of a furnace. The drying occupies two days. It is now ready for conversion into gum, for which purpose it is put into oblong trays of sheet iron, and heated to the temperature of $300^{\circ} \mathrm{F}$. in a cast-iron oven, which holds four of these trays. Here it concretes into irregular semi-transparent yellow-brown lumps, which are ground into 


\section{STARCHING APPARATUS.}

fine flour between mill-stones, and in this state brought to the market. In this roasted starch, the vesicles being burst, their contents become soluble in cold water. British gum is not convertible into sugar, as starch is, by the action of dilute sulphuric acid; nor into mucic acid, by nitric acid; but into the oxalic; and it is tinged purple-red by iodine. It is composed, in 100 parts, of 35.7 carbon, 6.2 hydrogen, and 58.1 oxygen; while starch is composed of, 43.5 carbon, $6 \cdot 8$ hydrogen, and $49 \cdot 7$ oxyogen.

To prove whether starch be quite free from gluten, or whether it be mixed with any

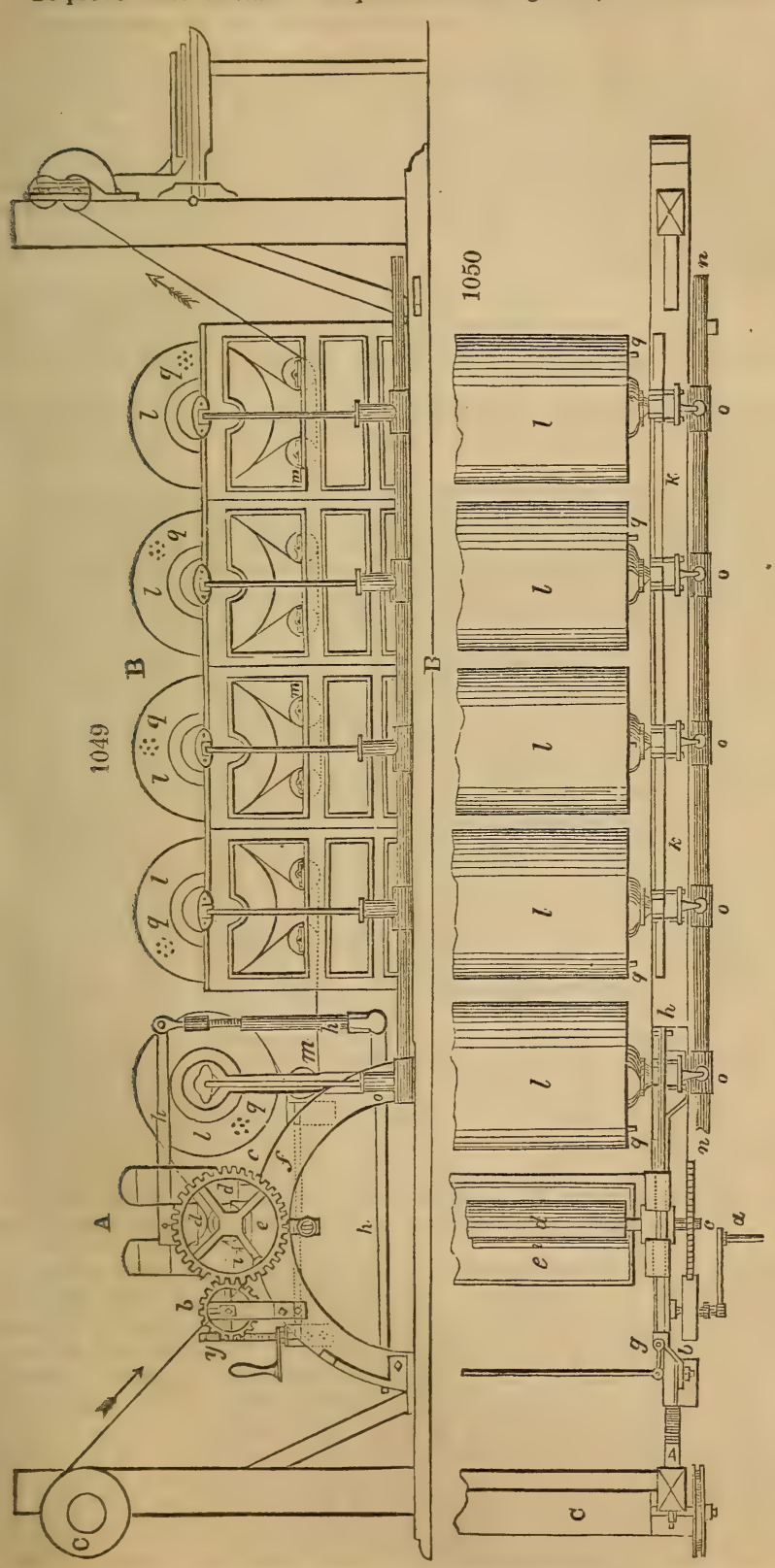
wheat flour, diffuse 12 grains of it through six ounces of water, heat the mixture to boiling, stirring it meanwhile with a glass slip. If the starch be pure, no froth will be seen upon the surface of the pasty fluid; or if any be produced during the stirring, it will immediately subside after it; but if the smallest portion of gluten be present, much froth will be permanently formed, which may be raised by stirring into the appearance of soap-suds.

STARCHING AND STF:AM-DRYING APPARATUS. The system of hollow eylinders, for drying goods in the processes of bleaching or calico-printing, is represented in fig. 1049, in a longitudinal section, and in fig. 1050 , in a top view; but the cylinders are supposed to be broken off in the middle, as it was needless to repeat the parts at the other end, which are sufficiently shown in the section.

$A$ is the box containing the paste, when the goods are to be starched or stiffened; $a$, a winch, when it is desired to turn the ma. 
chine by hand, though it is always moved by power in considerable factories; $b$, ts the driving pinion; $d, d^{\prime}$, two brass rollers with iron shafts, the undermost of which is moved by the wheel $c$, in gear with the pinion $b$. The uppermost roller $d^{\prime}$, is turned by the friction with the former, $d$, being pressed upon it by the weighted lever $h$; $e$ is the trough filled with the paste, which rests upon the bars $f$, and may be placed higher or lower by means of the adjusting screws $g$, according as the roller $d$ is to be plunged more or less deeply. A brass roller $i$ serves to force down the cloth into the paste.

$\mathrm{B}$, is the drying part of the machine; $k, k$, its iron framing; $l, l$, \&c., five drums, or hollow copper cylinders, heated with steam; $m, m, m$, \&c., small copper drums, in pairs, turning freely on shafts under the former, for stretching the goods, and airing them, during their passage through the machine; $n, n$, is the main steam-pipe, from which branch off small copper tubes, $0,0, \& c$., which conduct the steam through stuffing-boxes into the cavity of the drying-drums. There are similar tubes upon the other ends of the drums, for discharging the condensed water through similar stuffing-boxes; $q, q$, are Valves, opening internally, for admitting the air whenever the steam is taken off, or becomes feeble, to prevent the druins from being crushed by the unbalanced pressure of the atmosphere upon their external surfaces.

c, is the cloth-beam, from which the starching roller draws forward the goods; $D$, are two rollers, of which the lower is provided with a band-pulley or rigger, driven by a similar pulley fixed upon the shaft of the starching roller $d$. These two rollers pull the goods through the drying machine, and then let them fall either upon a table or the floor.

STEAM, is the vapor of hot water; the discussion of which belongs to chemistry, physics, and engineering. Certain practical applications of the subject will be found in the article Evaporation.

STEARIC ACID, improperly called StEARINe (Talgsaüre, Germ.), is the solid constituent of fatty substances, as of tallow and olive oil, converted into a crystalline mass by saponification with alkalinc matter, and abstraction of the alkali by an acid. By this process, fats are convertible into three acids, called Stearic, Margaric, and Oleic; the first two being solid, and the last liquid. The stearine, of which factitious wax candles are made, consists of the stearic and margaric acids combined. These can be separated from each other only by the agency of alcohol, which holds the margaric acid in solution after it has deposited the stearic in crystals. Pure stearic acid is prepared, according to its discoverer, Chevreul, in the following way:-Make a soap, by boiling a solution of potash and mutton-suet in the proper equivalent proportions (see SoAp); dissolve one part of that soap in 6 parts of hot water, then ad to the solntion 40 or 50 parts of cold water, and set the whole into a place whose temperature is about $52^{\circ}$ Fahrenheit. A substance falls to the bottom, possessed of pearly lustre, consisting of the bi-stearate and bi-margarate of potash; which is to be drained and washed upon a filter. The filtered liquor is to be evaporated, and mixed with the small quantity of acid necessary to saturate the alkali left free by the precipitation of the above bi-salts. On adding water to it afterwards, the liquor affords a fresh quantity of bi-stearate and bi-margarate. By repeating this operation with precaution, we finally arrive at a poins when the solution contains no more of these solid acids, but only the oleic. The precipitated bi-salts are to be washed and dissolved in hot alcohol, of specific gravity 0.820 , of which they require about 24 times their weight. During the cooling of the solntion, the bi-stearate falls down, while the greater part of the bi-margarate, and the rerrainder of the oleate, remain dissolved. By once more dissolving in alcohol, and crystallizing, the bi-stearate will be obtained alone; as may be proved by decomposing a little of it in water at a boiling heat, with muriatic acid, letting it cool, washing the stearic acid obtained, and exposing it to heat, when, if pure, it will not fuse in water nnder the 158 th degree of Fahrenheit's scale. If it melts at a lower heat, it contains more or less margaric acid. The purified bi-stearate being decomposed by boiling in water along with any acid, as the muriatic, the disengaged stearic acid is to be washed by melting in water, then cooled and dried.

Stearic acid, prepared by the above process, contains combined water, from which it cannot be freed. It is insipid and inodorous. After being melted by heat, it solidifies at the temperature of $158^{\circ}$ Fahrenheit, and affects the form of white brilliant needles grouped together. It is insoluble in water, but dissolves in all proportions in boiling anhydrous alcohol, and on cooling to $122^{\circ}$, crystallizes therefrom, in pearly plates; but if the concentrated solution be quickly cooled to $112^{\circ}$, it forms a crystalline mass. A dilute solution affords the acid crystallized in large white brilliant seales. It dissolves in its own weight of boiling ether of 0.727 , and crystallizes on cooling in beautiful scales, of changing colors. It distils over in vacuo without alteration; but if the retort contains a little atmospheric air, a small portion of the acid is decomposed during the distillation; while the greater part passes over unchanged, but slightly tinged prown, and mixed with traces of empyreumatic oil. When heated in the open air, and 
kindled, stearic acid burns like wax. It contains 3.4 per cent. of water, from which it may be fired by combining it with oxyde of lead. When this anhydrous acid is subjected to uhimate analysis, it is found to consist of -80 of carbon, 12.5 hydrogen, and $7 \cdot 5$ oxy zen, in 100 parts. Stearic acid displaces, at a boiling heat in water, carbonic acil from its combinations with the bases; but in operating upon an alkaline carbonate, a portion of the stearic acid is dissolved in the liquor before the carbonic acid is expelled. This decomposition is founded upon the principle, that the stearic acid transforms the salt into a bicarbonate, which is decomposed by the ebullition.

Stearic acid put into a strong watery infusion of litmus, has no action upon it in the cold; but when hot, the acid combines with the alkali of the litmus, and changes its blue color to red; so that it has sufficient energy to abstract from the concentrated tincture all the alkali required for its neutralization. If we dissolve bi-stearate of potash in weak alcuhol, and pour litmus water, drop by drop, into the solution, this will become red, because the litmus will give up its alkali to a portion of the bi-stearate, and will convert it into neutral stearate. If we now add cold water, the reddened mixture will resume its blue tint, and will deposite bi-stearate of potash in small spangles. In order that the alcoholic solution of the bi-stearate may redden the litmus, the alcohol should not be very strong.

From the composition of stearate of potash, the atomic weight of the acid appears to be $106^{\circ} \cdot{ }^{3}$; hydrogen being 1 ; for $18: 48 \times 2:: 100: 533 \cdot 3=5$ atoms of acid.

From the stearate of soda, it appears to be 104; and from that of lime, 102. The stearate of lead, by Chevreul, gives 109 for the atomic weight of the acid.

The margaric and oleic acids seem to have the same neutralizing power, and the same atomic weight.

The preceding numbers will serve to regulate the manufacture of stearic acid for the purpose of making candles. Potash and soda were first prescribed for saponifying fat, as may be seen in M. Gay Lussac's patent, under the article CANDLE; and were it not for the cost of these articles, they are undoubtedly preferable to all others in a chemical point of view. Of late years lime has been had recourse to, with perfect success, and has become subservient to a great improvernent in candle-making. The stearine block now made by many London houses, though containing not more than 2 or 3 per cent. of wax, is hardly to be distinguished from the purified produce of the bee. The first process is to boil the fat with quicklime and water in a large tub, by means of perforated steam pipes distributed over its bottom. From the above statements we see that about 11 parts of dry lime are fully equivalent to 100 of stearine and oleine mixed : but as the lime is in the state of hydrate, 14 parts of it will be required when it is perfectly pure; in the ordinary state, however, as made from average good limestone, 16 parts may be allowed. After a vigorous ebullition of 3 or 4 hours, the combination is pretty complete. The stearate being allowed to cool to such a degree as to allow of its being handled, becomes a concrete mass, which must be dug out with a spade, and transferred into a contiguous tub, in order to be decomposed with the equivalent quantity of sulphuric acid diluted with water, and also heated with steam. Four parts of concentrated acid will be sufficient to neutralize three parts of slaked lime. The saponified fat now liberated from the lime, which is thrown down to the bottom of the tub in the state of sulphate, is skimmed off the surface of the watery menstruum into a third contiouous tub, where it is washed with water and steam.

The washed mixture of stearic, margaric, and oleic acids, is next cooled in tin pans ; then shaved by large knives, fixed on the face of a fly-wheel, called a tallow cutter, preparatory to its being subjected in canvass or caya bags to the action of a powerful hydraulic press. Here a large portion of the oleic acid is expelled, carrying with it a little of the margarie. The pressed cakes are row subjected to the action of water and steam once more, after which the supernatant stearic acid is run off; and cooled in moulds. The cakes are then ground by a rotatory rasping-machine to a sort of mealy powder, which is put into canvass bags, and subjected to the joint action of steam and pressure in a horizontal hydraulic press of a peculiar construction, somewhat similar to that which has been long used in London for pressing spermaceti. The cakes of stearic acid thus freed completely from the margaric and oleic acids, are subjected to a final cleansing in a tub with sterm, and then melted into hemispherical masses called blocks. When these blocks are broken, they display a highly crystalline texture, which would render them unfit formaking eandles. This texture is therefore broken down or comminuted by fusing the? stearine in a plated copper pan, along with one thousandth part of pulverized arsenious. acid, after which it is ready to be cast into candles in appropriate moulds. See CANDLE.

STEARINE COLD PRESS. The cold hydraulic press, as mounted by Messrs. Maudslay and Field, for squeezing out the oleic acid from saponified fat, or the oleine from cocoa-nut lard, is represented in plan in fig. 1051 ; in side view of pump in fig. 1052 ; and in elevation, fig. 1053; where the same letters refer to like objects. 

draulic presses; 8 the

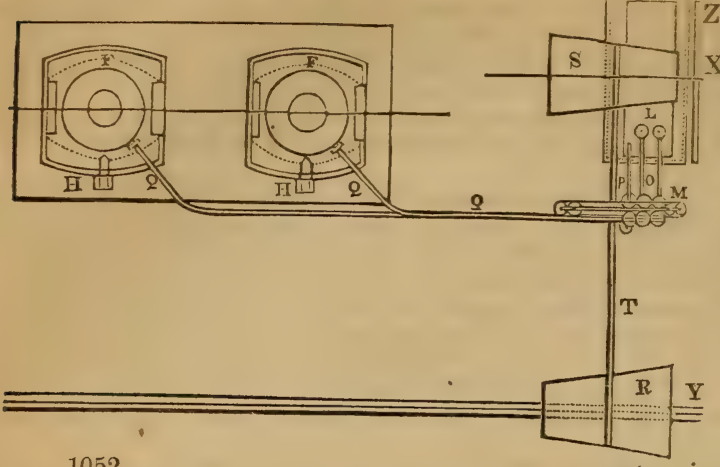

$Z$ frame; $c$, the eylinder; $D$, the piston or

ram; $E$, the follower; $\boldsymbol{F}$, the recess in the bottom to receive the oil; G, twilled woollen bags with the material to be pressed, having a thin plate of wrought iron between each; $\mathbf{H}$, apertures for the discharge of the oil; I, cistern in which the pumps are fixed; $\mathbf{k}$, framing for machinery to work in; L, two pumps, large and small, to inject the

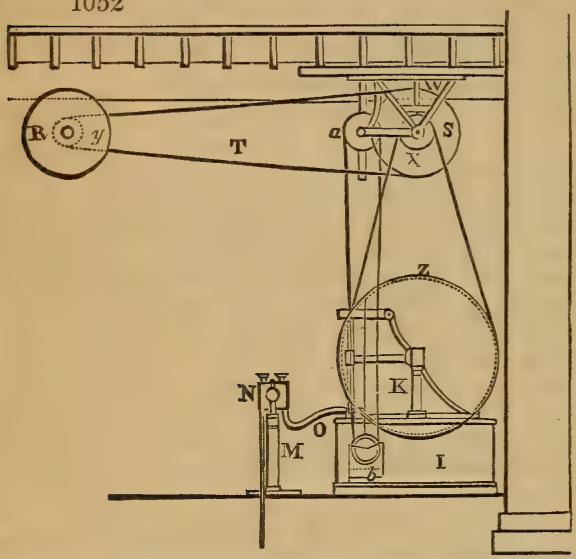
water into the eylinders; $M, a$ frame containing three double branches; $N$, three branches, each having two stops or plugs, by which the action of one of the pumps may be intercepted from, or communicated to, one or both of the presses; the large pump is worked at the beginning of the operation, and the small one towards the end; by these branches, one or both presses may be discharged when the operation is finished; 0 , two pipes from the pumps to the branches; $P$, pipe to return the water from the cylinders to the cisterns; $\mathbf{Q}$, pipes leading from the pumps through the branches to the cylinders; $\mathbf{R}$, conical drum, fixed upon the main shaft $\mathbf{T}$, driven by the steam-engine of the factory;

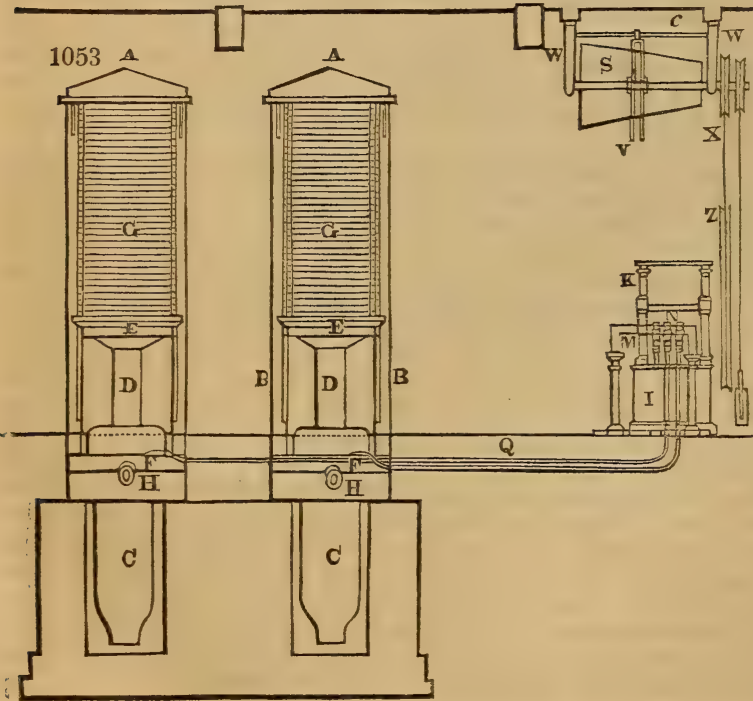
$s$, a like conical drum to work the pumps; $T$, a narrow leather strap to communicate the motion from $\mathrm{R}$ to $\mathrm{s}$; $\mathrm{U}$, a long screw hearing a nut, which works along the whole length of the drum; $v$, the fork or guide for moving the strap $T ; W, w$, two hanging bearings to carry the drum 8 ; $\mathrm{x}$, a pulley on the spindle of the drum $\mathbf{s ;} \mathbf{x}$, the main shaft; $z$, fly-wheel with groove on the edge, driven by the pulley $x$; on the axis of $s$, is a double crank, which works the two pumps L. $a$, is a 
pulley on the end of the long screw 0 ; an endless cord passes twice round this pulley, and under a pulley fixed in the weight $b$; by laying hold of both sides of this cord, and raising or lowering it, the forked guide $\mathrm{v}$, and the leather strap $\mathrm{r}$, are moved backwards or forwards, by means of the nut fixed in the guide, so as to accelerate or retard at pleasure the speed of the worling of the pumps; $c$, is a piece of iron, with a long slit, in which a pin, attached to the fork $\mathrm{v}$, travels, to keep it in the vertical position.

STEATITE (Suupstone; Craie de Briançon, Fr.; Speckstein, Germ.), is a mineral of the magnesian family. It has a grayish-white or greenish-white color, often marked with dendritic delineations, and occurs massive, as also in various supposititious crystalline forms; it has a dull or fatty lustre; a coarse splintery fracture, with translucent edges; a shining streak; it writes feebly; is soft, and easily cut with a knife; but somewhat tough; does not adhere to the tongue; feels very greasy ; infusible before the blowpipe; specific gravity from 2.6 to 2.8 . It consists of-silica, 44 ; magnesia, 44 ; alumina, 2 ; iron, $\tau \cdot 3$; manganese, $1 \cdot \overline{5}$; chrome, 2 ; with a trace of lime. It is found frequently in small contemporaneous veins that traverse serpentine in all directions, as at Portsoy, in Shetland, in the limestone of Icolmkiln, in the serpentine of Cornwall, in Anglesey, in Saxony, Bararia, (at Bayruth,) Hungary, \&c. It is used in the manufacture of porcelain. It makes the buiscuit semi-transparent, but rather brittle, and apt to crack with slight changes of heat. It is employed for polishing serpentine, marble, gypseous alabaster, and mirror glass; as the basis of cosmetic powders; as an ingredient in arti-attrition pastes; it is dusted in powder upon the inside of boots, to make the feet glide easily into them; when rubbed upon grease-spots in silk and woollen clothes, it removes the stains by absorption; it enters into the composition of certain crayons, and is used itself for making traces upon glass, silk, \&c. The spotted steatite, cut into cameos and calcined, assumes an onyx aspect. Soft steatite forms excellent stoppers for the chemical apparatus used in distilling or subliming corrosive vapors. Lamellar steatite is TAI.c.

STEEL (Acier, Fr.; Stahl, Germ.), as a carburet of iron, has already been considered under that metal. I shall treat in this article more particularly of its manufacture and technical relations.

1. Steel of cementation, bar or blistered steel.-With the exception of the Ulverstone charcoal iron, no bars are manufactured in Great Britain capable of conversion into steel at all approaching in quality to that made from the Madras, Swedish, and Russian irons, so largely imported for that purpose. The first rank is assigned to the Swedish iron stamped with a circle enclosing the letter $\mathrm{L}$ (hence called hoop L); which fetches the high price of $36 \mathrm{l}$. $10 \mathrm{~s}$. per ton, while excellent English coke-iron may be had for one fifth of the price. The other Swedish irons are sold at a much lower rate, though said to be manufactured in the same way; and therefore the superiority of the Dannemora iron must be owing to some peculiarity in the ore from which it is smelted. The steel recently made in the Indian steel-works at Chelsea, from Mr. Heath's Madras iron, rivals that from the hoop $L$.

The Sheffield furnace for making bar or blistered steel, called the furnace of cementation, is represented in fig. 1054, in a cross section, and in fig. 105.

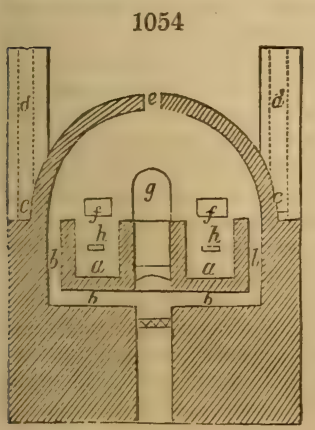

1055

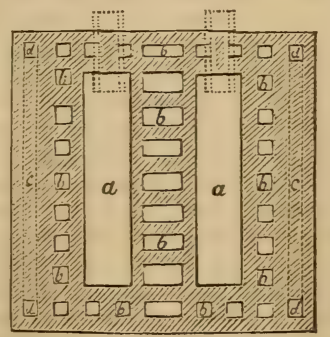

flat arch of the furnace. In one of its shorter sides (ends), there are orifices $f, f$, through which the long bars of iron may be put in and taken out; $g$, is the door by which the steel-maker enters, in filling or emptying the trough; $h$, is a proof hole, at which small samples of the steel, in the act of its conversion, may be drawn out. The firnace is built under a conical hood or chimney, from thirty to fifty feet high, for aiding the draught, and carrying off the smoke.

The two chests are built of fire-stone grit. They are 8,10 , or even 15 feet long, and from 26 to 36 inches in width and depth; the lower and smaller they are, the mors uniform will the quality of the steel be. A great breadth and height of trough are $\mathrm{m}$ - 
compatible with equability of the cementing temperature. The sides are a few inches thick. The sprace between them is at least a foot wide. They should never rest di-ectly upon the sole of the furnace, but must have their bottom freely played upon sy the flame, as well as the sides and top. The degree of heat is regulated by openings in the arch, or upon the long sides of the furnace, which lead to the chimney; as also by the greater or: less quantity of air admitted below the grate, as in glass-house furnaces.

The cement consists of ground charcoal (sometimes of soot), mixed with one tenth of ashes, and some common salt; the charcoal of hard wood being preferred. Ground coke is inadınissible, on account of the sulphur, silica, and clay, which it generally contains. Possibly the salt serres to vitrify the particles of silica in the charcoal, and thus to prevent their entering into combination with the steel. As for the ashes, it is difficult to discover their use. The best steel may be made without their presence. The bottom of the trough being corered with two inches of the powder of cementation, the bars are laid along in it, upon their narrow edge, the side bar being one inch from the trough, and the rest being from one half to three fourths of an inch apart. Above this first layer of iron bars, fully half an inch depth of the powder is spread, then a new series of bars is stratified, and so on till the trough is filled within six inches of the top. This space is partially filled with old cement powder, and is covered with refractory damp sand. Sometimes the trough is filled to the surface with the old cement, and then closely covered with firetiles. The bars should never be allowed to touch each other, or the trough. The fire must be carefully urgei from two to four days, till it acquires the temperature of $100^{\circ}$ Wedgewood; which must be steadily maintained during the four, six, eight, or ten dars requisite for the cementation; a period dependant on the size of the furnace, and which is determined by the examination of the proof pieces, taken out from time to time.

In the front or remote end of the furriace, fig. 1054, a door is left in the outer building, corresponding to a similar one in the end of the interior vault, through which the workman enters for charging the furnace with charcoal and iron bars, as also for taking out the steel after the conversion. Small openings are likewise made in the ends of the chests, through which the extremities of a few bars are left projectins, so that they may be pulled out and examined, through small doors opposite to them in the exterior wails. These tap holes, as they are called, should he placed near the centre of the end stones of the chests, that the bars may indicate the average state of the process. The joinings of the fire-stones are secured with a finely ground Stourbridge clay.

The interval between the two chests (in furnaces crntaining two, for many have oniy one) being covered with an iron platform, the workman stands on it, and sifis a layer of charcoal on the hottom of the chests evenly, about half an inch thick; he then lays a row of bars, cut to the proper length, orer the charcoal, about an inch from each other; he next sifts on a second stratum of charcoal-dust, which, as it must serve for the bars above, as well as below, is made an inch thick; thus, he continues to stratify, till the chest be filled within tro inches of the top; and he covers the whole with the earthy detritus found at the bottom of grindstone troughs, or any convenient fire-loam. It is obrious that the second series of bars should correspond vertically with the interstices between the first series, and so in succession. The trial-rods are left longer than the others, and their projecting ends are incrusted with fire-clay, or imbedded in sand. The iron platform being remored, and all the openings into the rault closed, the fire is lighted, and very gradually increased, to aroid erery risk of cracking the grit-stone br 100 sudden a change of temperature; and the ignition being finally raised to about $100^{\circ}$ Wedgewood, but not higher, for fear of melting the metal, must be maintained at a uniform pitch, till the iron have absorbed the desired quantity of carbon, and hare been converted as highly as the manufacturer intends for his peculiar object. From six to eight days may be reckioned a sufficient period for the production of steel of moderate hardness, and fit for tilting into shear steel. A softer steel, for saws and springs, takes a shorter period; and a harder steel, for fabricating chisels used in cutting iron, will need longer exposure to the ignited charcoal. But, for a few purposes, such as the bits for boring cast iron, the bars are exposed to two or three successive processes of cementation, and are hence said to be twice or thice converted into steels. The higher the heat of the furnace, the quicker is the process of conversion.

The furnace being suffered to cool, the workman enters it again, and hands out the steel bars, which being corered with blisters, from the formation and bursting of resicles on the surface filled with gaseous carbon, is called blistered steel. This steel is tery irregular in is interior texture, has a white color, like frosted silver, and displays cristalline angles and facettes, which are larger the further the cementation has been urged, or the greater the dose of carbon. The central particles are always smaller than those near the surface of the bar.

In such a furnace as the above, twelve tons of bar iron may be converted at a charge 
But other furnaces are constructed with one chest, which receives six or eight tons at a time; the small furnaces, however, consume more fuel in proportion than the larger.

The absorption and action of the carbonaceous matter, to the amount of about a half per cent., occasions fissures and cavities in the substance of the blistered bars, which render the steel uiffit for any useful purpose in tool-making, till it be condensed and r'sidered uniform by the operation of tilting, under a powerful hammer driven by machinery. See IRON.*

The heads of the tilt-hammers for steel weigh from one and a half to two hundred pounds. Those in the neighborhood of Sheffield are much simpler than the one referred to in the note. Ther are worked by a small water-wheel, on whose axis is another wheel, bearing a great number of cams or wipers on its circumference, which strike the tail of the hammer in rapid succession, raise its head, and then let it fall smartly on the hot metal rod, dexterously presented on its several parts to the anvil beneath it, by the workman. The machinery is adapted to produce from 300 to 400 blows per minute; which on this plan requires an undue and wasteful velocity of the float-boards. Were an intermediate toothed wheel substituted between the water-wheel and the wiperwheel, so that while the former made one turn, the latter might make three, a much smaller force of water would do the work. The anvils of the tilt-hammer are placed nearly on a level with the fioor of the mill-house; and the workman sits in a fosse, dug on purpose, in a direction perpendicular to the line of the helve, on a board suspended from the roof of the building by a couple of iron rods. On this swinging seat, he can adrance or retire with the least impulse of his feet, pushing forward the steel bar, or drawing it back with equal rapidity and convenience.

At a small distance from each tilt, stands the forge-hearth, for heating the steel. The bellows for blowing the fire are placed above-head, and are worked by a small crank fixed on the end of the axis of the wheel, the air being conveyed by a copper pipe down to the nozzle. Each workman at the tilt has two boys in attendance, to serve him with hot rods, and to take them away after they are hammered. In small rods, the bright ignition originally given at the forge soon declines to darkness; but the rapid impulsions of the tilt revive the redness again in all the points near the hammer; so that the rod, skilfully handled by the workman, progressively ignites where it advances to the strokes. Personal inspection alone can communicate an adequate idea of the precision and celerity with which a rude steel rod is stretched and fashioned into an even, smooth, and sharp-edged prism, under the operation of the tilt-hammer. The heat may be clearly referred to the prodigious friction among the particles of so cohesive a metal, when they are made to slide so rapidly over each other in every direction during the elongation and squaring of the rol.

2. Shear steel derives its name from the accidental circumstance of the shears for dressing woollen cloth being usually forged from it. It is made by binding into a bundle, with a slender steel rod, four parallel bars of blistered steel, previously brolien into lengths of about 18 inches, including a fifth of double length, whose projecting end may serve as a handle. This fagot, as it is called, is then heated in the forge-hearth to a good welding-heat, being sprinkled over with sand to form a protecting film of iron siag, carried forthwith to the tilt, and notched down on both sides to unite all the bars together, and close up every internal flaw or fissure. The mass being again heated, and the binding rings knocked off it, is drawn out into a uniform rod of the size required. Manufacturers of cutlery are in the habit of purchasing the blistered bars at the conversion furnaces, and sending them to tilt-mills to have them drawn out to the proper size, which is done at regular prices to the trade; from 5 to 8 per cent. discount being allowerl on the rude bars for waste in the tilting. The metal is rendered so compact by the welding and hammering, as to become susceptible of a much finer polish than blistered steel can take; while the uniformity of its body, tenacity, and malleability are at the same time much increased; by which properties it becomes well adapted for making table knires and powerful springs, such as those of gun-locks. The steel is also softened down by this process, probably from the expulsion of a portion of its carbon during the welding and subsequent heats; and if these be frequently or awkwardly applied, it may pass back into common iron.

3. Cast steel is made by melting, in the best fire-clay crucibles, blistered steel, broken down into small pieces of convenient size for packing; and as some carbon is always dissipated in the fusion, a somewhat highly converted steel is used for this purpose. The furnace is a square prismatic cavity, lined with fire-briclis, 12 inches in each side, and 24 deep, with a flue immediately under the cover, $3 \frac{1}{2}$ inches by 6 , for conducting the smoke into all adjoining chimney of considerable beight. In some establishments a dozen such furnaces are constructed in one or two ranges, their tops being on a level with the floor of the laboratory, as in brass-foundries, for enabling the workmen more

* For minute details of the parts, see the excellent article TILTING-HAMmER, in Rees's Cyclopadia. 
conveniently to inspect, and lift out, the crucibles with tongs. The ash-pits terminate in a subterraneous passage, which supjilies the grate with a current of cool air, and serves for emptying out the ashes. The crucible stands, of course, on a sole-piece of baked fire-clay; and its mouth is closed with a well-fitted lid. Sometimes a little bottle-glass, or blast-furnace slag, is put into the crucible, above the steel pieces, to form a vitreous coating, that may thoroughly exclude the air from oxydizing the metal. The fuel employed in the cast-steel furnace is a dense coke, brilliant and sonorous, broken into pieces about the size of an egg, one good charge of which is sufficient. The tongs are furnished at the fire end with a pair of concave jaws, for embracing the curvature of the crucible, and lifting it out whenever the fusion is complete. The lid is then removed, the slag or scoriæ cleared away, and the liquid metal poured into cast-iron octagonal or rectangular moulds, during which it throws out brilliant scintillations.

Cast-steel works much harder under the hammer than shear steel, and will not, in its usual state, bear much more than a cherry-red heat without becoming brittle; nor can it bear the fatigue incident to the welding operation. It may, however, be firmly welded to iron, through the intervention of a thin film of vitreous boracic acid, at a moderate degree of ignition. Cast steel, indeed, made from a less carbureted bar steel, would be susceptible of welding and hammering at a higher temperature; but it would require a very high heat for its preparation in the crucible.

Iron may be very elegantly plated with cast steel, by pouring the liguid metal from the erucible into a mould containing a bar of iron polished on one face. In this circumstance the adhesion is so perfect as to admit of the two metals being rolled out together; and in this way the chisels of planes and other tools may be made, at a moderate rate and of excellent quality, the cutting-edge being formed in the steel side. Such instruments combine the toughness of iron with the hardness of steel.

For correcting the too high carbonization of steel, or equalizing the too highly converted exterior of a bar with the softer steel of the interior, the metal requires merely to be imbedded, at a cementing heat, in oxyde of iron or manganese; the oxygen of which soon abstracts the injurious excess of carbon, so that the outer layers may be even converted into soft iı on, while the axis continues steely ; because the decarbonizing advances far more rapidly than the earbonizing.

Fig. 1056 represents the mould for making crucibles for the cast-steel works. M M, is a solid block of wood, to support the two-handled ontside mould $\mathrm{N}, \mathrm{N}$. This being 1056

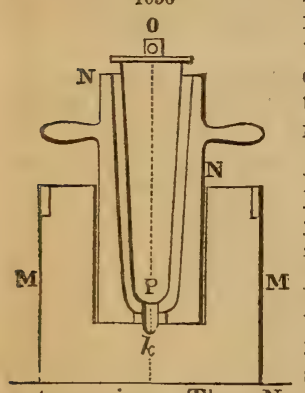
rammed full of the proper clay dough or compost (see CRUCIBLE), the inner mould is to be then pressed vertically into it, till it reaches the bottom $P$, being directed and facilitated in its descent by the point $k$. A cord passes through 0 , by which the inner mould is suspended over a pulley, and guided in its motions.

When a plate of polished steel is exposed to a progressive heat, it takes the following colors in succession: 1 , a faint yellow; 2 , a pale straw-color; 3 , a full yellow; 4 , a brown yellow; 5 , a brown with purple spots; 6 , a purple; 7 , a bright blue; 8 , a full blue; 9 , a dark blue, verging on black; after which the approach to ignition supersedes all these colors. If the steel plate has been previously hardened by being dipped in cold water or mercury when red-hot, then those successive shades indicate or correspond to successive degrees of softening or tempering. Thus, No. 1 suits the hard temper of a lancet, which requires the finest edge, but little strength of metal; No. 2 a little softer, for razors and surgeons' amputating instruments; No. 3, somewhat more toughness, for penknives; No. 4, for cold chisels and shears for cutting iron; No. 5, for axes and plane-irons; No. 6, for table knives and cloth shears; No. 7, for swords and watch-springs; No. 8 , for small fine saws and daggers; No. 9, for large saws, whose teeth need to be set with pliers, and sharpened with a file. After ignition, if the steel be very slowly cooled, it becomes exceedingly soft, and fit for the engraver's purposes. Hardened steel may be tempered to the desired pitch, by plunging it in metallic baths heated to the proper thermometric degree, as follows : for No. 1, $430^{\circ}$ Fahr.; No. 2, $450^{\circ}$; No. $3,470^{\circ}$; No. 4, $490^{\circ}$; No. 5, $510^{\circ}$; No. $6,530^{\circ}$; No. $7,550^{\circ}$; No. $8,560^{\circ}$; No. $9,600^{\circ}$.

Small steel tools are most frequently tempered, after hardening, by covering their surface with a thin coat of tallow, and heating them in the flame of a candle till the tallow diffuses a faint smoke, and then thrusting them into the cold tallow. Rinman long ago defined steel to be any kind of iron which, when heated to redness, and then plunged in cold water, becomes harder. But several kinds of cast iron are susceptible of such hardening. Every malleable and flexible iron, however, which may be hardened in that way is a steel. Moreover, steel may be distinguished from pure iron by its giving a 
dark-oray spot when a drop of dilute nitric acid is let fall on its surface, while iron aftords a green one. Exposed to the air, steel rusts less rapidly than iron; and the more highly carbureted, the more slowly does it rust, and the blacker is the spot left by an acid.

After hardening, steel seems to be quite a different body; even its granular texture becomes coarser or finer, according to the degree of heat to which it was raised; it grows so hard as to scratch slass, and resist the keenest file, while it turns exceedingly brittle. When a slowly cooled steel rod is forged and filed, it becomes capable of affording agreeahle and harmonious sounds by its vibrations; but hard-tempered steel affords only dull deafened tones, like those emitted by a cracked instrument.

The good quality of steel is shown by its being homogeneous; being easily worked at the fore ; by its hardening and tempering well; by its resisting or overcoming forces; and by its elasticity. To ascertain the first point, the surface should be ground and polished on the wheel; -when its lustre and texture will appear. The second test requires a skilful workman to give it a heat suitable to its nature and state of conversion. The size and color of the grain are best shown by taking a bar forged into a razor form; hardening and tempering it; and then breaking off the thin edge in successive bits with a hammer and anvil. If it had been fully ignited only at the end, theis, after the hardening, it will display, on fracture, a succession in the aspect of its grains from that extremity to the other; as they are whiter and larger at the former than the latter. The other qualities become manifest on filing the steel; using it as a chisel for cutting iron; or bending it under a heavy weight.

Much interest was excited a few years back by the experiments of Messrs. Stodart and Faraday on the alloys of steel with silver, platinum, rhodium, and iridium. Steel refuses to take up in fusion more than one five-hundredth part of silver; but with this minute quantity of alloy, it is said to bear a harder temper, without losing its tenacity. When pure iron is substituted for steel, the alloys so formed are much less subject to oxydation in damp air than before. With three per cent. of iridium and osmium, an alloy was obtained which had the property of tempering like steel, and of remaining clean and bright, in circumstances when simple iron became covered with rust. "Upon the whole," says the editor of the Quarterly Journal of Science, giving a report of these experiments in his 14 th volume, p. 378 , "6 though we consider these researches upon the alloys of steel as very interesting, we are not sanguine as to their important influence upon the improvement of the manufacture of cutlery, and suspect that a bar of the best ordinary steel, se. lected with precaution, and most carefully forged, wrought, and tempered, under the immediate inspection of the master, would afford cutting instruments as perfect and excellent as those composed of wootz, or of the alloys."

Case-hardening of iron, is a process for converting a thin film of the outer surface into steel, while the interior remains as before. Fine keys are generally finished in this way. See Case-Hardening.

So great is the affinity of iron for carbon, that, in certain circumstances, it will absorb it from carbureted hydrogen, or coal-gas, and thus become converted into steel. On this principle, Mr. Mackintosh of Glasgow obtained a patent for making steel. His furnace consists of one cylinder of bricks built concentrically within another. The bars of iron are suspended in the innermost, from the top; a stream of purified coal-gas circulates freely round them, entering below and escaping slowly above, while the bars are maintained in a state of bright ignition by a fire burning in the annular space between the cylinders. The steel so produced is of excellent quality; but the process does not seem to be so economical as the ordinary cementation with charcoal powder.

Damasking of steel, is the art of giving to sabre blades a variety of figures in the style of watering. See Damascus Brades.

Several explanations have been offered of the change in the constitution of steel, which accompanies the tempering operation; but none of them seems quite satisfactory. It seems to be probable that the ultimate molecules are thrown by the sudden cooling into a constrained state, so that their poles are not allowed to take the position of strongest attraction and greatest proximity; and hence the mass becomes hard, brittle, and somewhat less dense. An analogous condition may be justly imputed to hastily cooled glass, which, like hardened steel, requires to be annealed by a subsequent nicely graduated heat, under the influence of which the particles assume the position of repose, and constitute a denser, softer, and more tenacious body. The more sudden the cooling of ignited steel, the more unnatural and constrained will be the distribution of its particles, and also the more refractory, an effect produced by plunging it into cold mercury. This excess of hardness is removed in any required degree by judicious annealing or temper ing. The state of the carbon present in the steel may also be modified by the rate ot refrigeration, as $\mathbf{M r}$. Karsten and $\mathbf{M}$. Bréant conceive happens with cast iron and the damask metal. If the uniform distribution and combination of the carbon through the r.ass, determine the peculiarity of white cast iron, which is a hard and brittle substance 
and if its transition to the dark-gray and softer cast metal be effected by a partial forms tion of plumbago during slow cooling, why may not something similar be supposed to orcur with stcel, an analogous compound?

Mr. Oldham, printing encrincer of the Bank of England, who has had great experience in the treatment of steel for dies and mills, says that, for hardening it, the fire should never be hea:ed above the redness of "sealing-wax, and kept at that pitch for a sufficient time. On taking it out, he hardens it by plunging it, not in water, but in olive oil, 0. rather naptha, previously heated to $200^{\circ} \mathrm{F}$. It is kept immersed only till the ebullition ceases, then instantly transfered into cold spring water, and kept there till quite culd. By this treatment the tools come out perfectly clean, and as hard as it is possible to make cast-steel, while they are perfectly free from cracks, flaws, or twist. Large tools are readily brought down in temper by being suspended in the red-hot muffle till they show a straw color; but for small tools, he prefers plunging them in the oil heated to 400 degrees; and leaves them in till they become cold.

Mr. Oldham softens his steel dies by exposing them to ignition for vire requisite time, imbedded in a ixture of chalk and charcoal.

"The common mode of softening steel," says Mr. Baynes, "is to put it into an iron case, surrounded with a paste made of lime, cow's gall, and a little nitre and water; then to expose the case to a slow fire, which is gradually increased to a considerable heat, and afterwards allowed to go out, when the steel is found to be soft and ready for the engraver."

Indian steel or wootz.-The wootz ore consists of the magnetic oxyde of iron, united with quartz, in proportions which do not seem to differ much, being generally about 42 of quartz, and 58 of magnetic oxyde. Its grains are of various size, down to a sandy texture. The natives prepare it for smelting by pounding the ore, and winnowing away the stony matrix, a task at which the Hindoo females are very dexterous. The manner in which iron ore is smelted and converted into wootz or Indian steel, by the natives at the present day, is probably the very same that was practised by them at the time of the invasion of Alexander; and it is a uniform process, from the Himalaya mountains to Cape Comorin. The furnace or bloomery in which the ore is smelted, is from four to five feet high; it is somewhat pear-shaped, being about two feet wide at bottom, and one foot at top; it is built entirely of clay, so that a couple of men can finish its erection in a few hours, and have it ready for use the next day. There is an opening in front about a foot or more in height, which is built up with clay at the commencement, and broken down at the end, of each smelting operation. The bellows are usually made of a goat's skin, which has been stripped from the animal without ripping open the part covering the belly. The apertures at the legs are tied up, and a nozzle of bamboo is fastened in the opening formed by the neck. The orifice of the tail is enlarged and distended by two slips of bamboo. These are grasped in the hand, and kept close together in making the stroke for the blast; in the returning stroke they are separated to admit the air. By working a bellows of this kind with each hand, making alternate strokes, a pretty uniform blast is produced. The bamboo nozzles of the bellows are inserted into tubes of clay, which pass into the furnace at the bottom corners of the temporary wall in front. The furnace is filled with charcoal, and a lighted coal heing introduced before the nozzles, the mass in the interior is soon kindled. As soon as this is accomplished, a small portion of the ore, previously moistened with water, to prevent it from running through the charcoal, but without any flux whatever, is laid on the top of the coals, and covered with charcoal to fill up the furnace.

In this manner ore and fuel are supplied; and the bellows are urged for 3 or 4 hours, when the process is stepped; and the temporary wall in front being broken down, the bloom is removed by a pair of tongs from the bottom of the furnace. It is then beaten with a wooden mallet, to separate as much of the scoriæ as possible from it, and, while still red-hot, it is cut through the middle, but not separated, in order merely to show the quality of the interior of the mass. In this state it is sold to the blacksmiths, who make it into bar iron. The proportion of such iron made by the natives from 100 parts of ore, is about 15 parts. In converting the iron into steel, the natives cut it into pieces, to enable it to pack better in the crucible, which is formed of refractory clay, mixed with a large quantity of charred husk of rice. It is seldom charged with more than a pound of iron, which is put in with a proper weight of dried wood chopped small, and both are covered with one or two green leaves; the proportions being in general 10 parts of iron to 1 of wood and leaves. The mouth of the crucible is then stopped with a handful of tempered clay, rammed in very closely, to exclude the air. The wood preferred is the Cassia auriculata, and the leaf that of the Asclepias gigantea, or

* History of the Cotton Manufacture, p. 275. If that strange farrago be employed by Mr. Locket of Manchester, for softening his dies and mills, it deserves consideration. Should the nitre be used in too great quantity to be all carbonated by the gall, its oxygen may serve to consume some of the carbon of the steel and thus bring it nearer to iron. The recipe may be old, but it is a novelty to me 
the Convolvulus laurifolius. As soon as the clay plugs of the crucibles are dry, from 20 to : $t$ of them are built up in the form of an arch, in a small blast furnace; they are nept covered with charcoal, and subjected to heat urged by a blast for abunt two hours and a half, when the process is considered to be complete. The crucibles being now taken out of the furnace and allowed to cool, are broken, and the steel is found in the firm of a cake, rounded by the bottom of the crucible. When the fusion has been perfect, the top of the calie is covered wirh striæ, radiating from the centre, and is free frum holes and rough projections; but if the fusion has been imperfect, the surface of the cake has a honercomb appearance, with projecting lumps of malleable iron. On in averave, four out of five cakes are more or less defective. These imperfections have been tried 10 be corrected in London by re-melting the cakes, and running them into ingots; but it is obrious, that when the cakes consist partially of malleable iron and of unreduced oxyde, simple fusion cannot convert them into good steel. When care is taken, however, to select only such cakes as are perfect, to re-melt them thoroughly, and tilt them carefully into rods, an article has been produced which possesses all the requisites of fine steel in an eminent degree. In the Supplement to the Encyclopædia Britannica, article Cutlery, the late Mr. Stodart, of the Strand, a very competent judge, has declared "that for the purposes of fine cutlery, it is infinitely superior to the best English cast steel."

The natives prepare the cakes for being drawn into bars by annealing them for several hours in a small charcoal furnace, actuated by bellows; the current of air being made to play inon the calies while turned over before it; whereby a portion of the combined carboin is probably dissipated, and the steel is softened; without which operation the cakes would break in the attempt to draw them. They are drawn by a hammer of a few pounds weight.

The natires weld two pieces of cast steel, by giving to each a sloping face jagged all over with a small chisel; then applying them with some calcined borax between, and tying them together with a wire, they are brought to a full red heat, and united by a few smart blows of a hammer.

The ordinary bar iron of Sweden and England, when converted by cementation into steel, exhibits upon its surface numerous small warty points, but few or no distinct vesicular eruptions; whereas the Dannemora and the Ulverston steels present, all over the surface of the bars, well raised blisters, upwards of three eighths of an inch in diameter horizontally, but somewhat flattened at top. Iron of an inferior description, when highly converted in the cementing-chest, becomes gray on the outer edges of the fracture; while that of Dannemora acquires a silvery color and lustre on the edges, with crystalline facets within. The highly converted steel is used for tools that require to be made very hard; the slightly converted, for softer and more elastic articles, such as springs and sword blades.

STEREOTYPE PRINTING signifies printing by fixed types, or by a cast typographic plate. This plate is made as follows:-The form, composed in ordinary types, and containing one, two, three, or more pages, inversely as the size of the book, being laid flat upon a slab, with the letters looking upwards, the faces of the types are brushed over with oil, or preferably, with plumbago (black lead.) A heavy brass rectangular fraine of three sides, with bevelled borders, adapted exactly to the size of the pages, is then laid down upon the chase, ${ }^{*}$ to circumscribe three siles of its typography; but the fourth side, which is one end of the rectangle, is formed by placing near the types, and over the hollows of the chase, a single brass bar, having the same inwards sloping bevel as the other three sides. The complete frame resembles that of a picture, and serves to define the area and thickness of the cast, which is made by pouring the pap of Paris plaster into its interior space, up to a given line on its edges. The plaster mould, which soon sets, or b-comes concrete, is lifted gently off the types, and immediately placed upright on its edge in one of the cells of a sheet iron rack, mounted within the cast-iron oven. An able workman will mould ten sheets octavo in a day, or 160 pages. The moulds are here exposed to air heated to fully $400^{\circ} \mathrm{F}$., and become perfectly dry in the course of two hours. As they are now friable and porous, they require to be delicately handled. Each mould, containing generally two pages octavo, is laid, with the impression downwards, upon a flat cast-iron plate, called the floating-plate; this plate being itself laid on the bottom of the dipping-pan, which is a cast-iron square tray, with its upright edges sloping outwards. A cast-iron lid is applied to the dipping-pan, and secured in its place by a screw. The pan having been heated to $400^{\circ}$ in a cell of the oven, under the mould-rack, previous to receiving the hot mould, is ready to be plunged into the bath of melted alloy contained in an iron pot placed over a furnace, and it is dipped with a slight deviation from the horizontal plane, in order to facilitate the escape of the air. As there is a minute space between the back or top surface of

* Chase (chassis, frame, Fr.), quoin.-(coin, wedge, Fr.), are terms which show that the art of prmtine came directly from France to England. 
the mould and the lid of the dipping-pan, the liquid metal, on entering into the pan through the orifices in its corners, floats uj) the plaster along with the iron plate on which it had been laid, thence called the floating-plate, whereby it flows freely into every line of the mould, through notches cut in its edge, and forms a layer or lamina upon its face, of a thickness corresponding to the depth of the border. Only a thin metal film is left upon the back of the mould. The dipping-pan is suspended, plunged, and removed, by means of a powerful crane, susceptible of vertical and horizuntal motions in al] directions. When lifted out of the bath, it is set in a water-cistern, upon bearers so placed as to allow its bottom only to touch the surface. Thus the metal first concretes below, while, by remaining fuid above, it continues to impart hydrostatic pressure during the shrinkage attendant upon refrigeration. As it thus progressively contracts in volume, more melted metal is fed into the corners of the pan by a ladle, in order to keep up the hydrostatic pressure upon the mould, and to secure a perfect impression, as well as a solid cast. Were the pan more slowly and oquably cooled, by being left in the air, the thin film of metal upon the back of the inverted plaster cake would be apt to solidify fist, and intercept the hydrostatic action indispensable to the purpose of filling all the lines in its face. A skilful workman makes five dips, containing two pages octavo each, in the course of an hour, or about nine and a half octavo sheets per day. The pan being taken asunder, the compound cake of mould and metal is removed, and beat upon its edoes with a wooden mallet, to detach the superfluous metal. The stereotype plate is then handed over to the picker, who planes its edges truly square, turns its back flat unon a lathe to a determinate thickness, and carefully removes the little imperfections occasioned by dirt or air left among the letters when the mould was cast. Should any of them be damaged in the course of the operation, they must be cut out, and replaced by soldering in separate types of the same size and form.

STILL (Alambic, Fr.; Blase, Germ.), is a chemical apparatus, for vaporizing liquids by heat in one part, called the cucurbit, and condensing the vapors into liquids in another part, called the refrigeratory; the general purpose of both combined being to separate the more volatile fluid particles from the less volatile. In its simplest form, it consists of a retort and a receiver, or of a pear-shaped matrass and a capital, furnished with a slanting tube for conducting away the condensed vapors in drops; whence the term still, from the Latin verb stillare, to drop. Its chief employment in this country being to eliminate alcohol, of greater or less strength, from fermented wash, I shall devote this article to a description of the stills best adapted to the manufacture of British spirits, referring to chemical authors* for those fitted for peculiar objects.

In respect of rapidity and extent of work, stills had attained to an extraordinary pitch of perfection in Scotland about thirty years ago, when legislative wisdom thought fit to levy the spirits duty, per annum, from each distiller, according to the capacity of his still. It having been shown, in a report presented to the House of Commons in 1799, that an 80-gallon still could be worked off in eight minutes, this fact was made the basis of a new fiscal law, on the supposition that the maximum of velocity had been reached. But, instigated by the hopes of enormous gains at the expense of the revence, the distillers soon contrived to do the same thing in three minutes, by means of broad-buttomed shallow stills, with stirring-chains, and lofty capitals. In the year 1815, that preposterous law, which encouraged fraud and deteriorated the manufacture, was repealed. The whiskey duties having been since levied, independently of the capacity of the still, upon the quantity produced, such rapid operations have been abandoned, and processes of econ. omy in fuel, and purity in product, have been sought after.

One of the greatest improvements in modern distilleries, is completing the analysis of crude spirit at one operation. Chemists had been long familiar with the contrivance of Woulfe, for impregnating with gaseous matter, water contained in a range of boltles; but they had not thought of applying that plan to distillation, when Edouard Adam, an illiterate workman of Montpellier, after hearing accidentally a chemical lecture upon that apparatus, bethought himself of converting it into a still. He caused the boiling-hot vapors to chase the spirits successively out of one bottle into another, so as to obtain in the successive vessels alcohol of any desired strength and purity, "at one and the same heat." He obtained a patent for this invention in 1801, and was soon afterwards enabled, by his success on the small scale, to set up in his native city a magnificent distillery, which excited the admiration of all the practical chemists of that day. In November, 1805, he obtained a certificate of certain improvements for extracting from wine, at one process, the whole of its alcohol. Adam was so overjoyed, after making his first experiments, that he ran about the streets of Montpellier, telling everybody of the surprising results of his invention. Several competitors soon entered the lists with him, especially Solimani, professor of chemistry in that city, and Isaac

* The treatises of Le Normand and Dubrunfaut may also be consulted. The French stills are in general 80 much complicated with a great many small pipes and passages, as to be unfit for distilling the glutinous wash of grains. 
Berard, distiller in the department of Gard; who, having contrived other forms of continuous stills, divided the profits with the first inventor.

The principles of spirituous distillation may be stated as follows :-The boiling point of alcohol varies with its density or strength, in conformity with the numbers in the fol. lowing table:-

\begin{tabular}{|c|c|c|c|}
\hline Specific gravity. & $\begin{array}{l}\text { Boiling point, by Fahrenheit's } \\
\text { scule. }\end{array}$ & Specific gravity. & $\begin{array}{l}\text { Boiling point, by Fahrenheit's } \\
\text { scale. }\end{array}$ \\
\hline 0.7939 & $168 \cdot 5^{\circ}$ & 0.8875 & $181 \cdot 0^{\circ}$ \\
\hline $0 \cdot 8034$ & $168 \cdot 0$ & 0.8631 & $183 \cdot 0$ \\
\hline 0.8118 & $168 \cdot 5$ & 0.8765 & $187 \cdot 0$ \\
\hline 0.8194 & $169 \cdot 0$ & 0.8892 & $190^{\circ} 0$ \\
\hline 0.8265 & 172.5 & 0.9013 & $194 \cdot 0$ \\
\hline 0.8332 & $173 \cdot 5$ & 0.9126 & $197 \cdot 0$ \\
\hline 0.8397 & 175.0 & 0.9234 & $199 \cdot 0$ \\
\hline 0.8458 & $177 \cdot 0$ & 0.9335 & $201 \cdot 0$ \\
\hline 0.8518 & $179 \cdot 0$ & & \\
\hline
\end{tabular}

See also the table under AlcoHor, page 22.

Hence, the lower the temperature of the spirituous vapor which enters the refrigeratory apparatus, the stronger and purer will the condensed spirit be; because the offensive oils, which are present in the wash or wine, are less volatile than alcohol, and are brought over chiefly with the aqueous vapor. A perfect still should, therefore, consist of three distinct memhers; first, the cucurbit, or kettle; second, the rectifier, for intercepting more or less of the watery and oily particles; and third, the refrigerator, or condenser of the alcoholic vapors.

These principles are illustrated in the construction of the still represented in figs. 1057 , $1058,1059,1060,1061$; in which the resources of the most refined French stills are combined with a simplicity and solidity suited to the grain distilleries of the United Kingdom. Three principal objects are obtained by the arrangement here shown; first, the extraction from fermented wort or wine, at one operation, of a spirit of any desired cleanness and strength; second, great economy of time, labor, and fuel; third, freedom from all danger of blowing up or boiling over, by mismanaged firing. When a combination of water, alcohol, and essential oil, in the state of vapor, is passed upwards through a series of winding passages, maintained at a determinate degree of heat, between $170^{\circ}$ and $180^{\circ}$, the alcohol alone, in any notable proportion, will retain the elastic form, and will proceed onwards into the refrigeratory tube, in which the said passages terminate; while the water and the oil will be in a great measure condensed, arrested, and thrown back into the body of the still, to be discharged with the effete residuum.

The system of passages or channels, represented in fig. 1058, is so contrived as to bring the mingled vapors which rise from the alembic $\alpha$, into ample and intimate contact with metallic surfaces, maintained, in a water-bath, at a temperature self-regulated by a heatgovernor. See Thermostat.

The neck of the alembic tapers upwards, as shown at $b, f i g .1057$; and at $c, f i g .1058$, it enters the bottom, or ingress vestibule, of the rectifier $c, f$. $f$ is its top or egress vestibule, which communicates with the bottom one by parallel cases or rectangular channels $d, d, d$, of which the width is small, compared with the length and height. These cases are open at top and bottom, where they are soldered or riveted into a general frame within the cavity, enclosed by the two covers $f, c$, which are secured round their edges $e, e, e, e$, with bolts and packing. Each case is occupied with a numerous series of shelves or trays, placed at small distances over each other, in a horizontal or slightiy inclined position, of which a side view is given in fig. 1059, and cross sections at $d, d, d$, fig. 1058. Each shelf is turned up a little at the two edges, and at one end, but sloped down at the other end, that the liquor admitted at the top may be made to flow slowly backwards and forwards in its descent through the system of shelves or trays, as $\mathrm{m}$ dicated by the darts and spouts in fig. 1059. The shelves of each case are framed together by two or more vertical metallic rods, which pass down through them, and are fixed to each shelf by solder, or by serew-nuts. By this means, if the cover $f$, be removed, the sets of shelves may be readily lifted out of the cases and cleaned; for which reason they are called moveable.

The intervals $i, i$, $i$, fig. 1058, between the cases, are left for the free circulation of the water contained in the bath-vessel $g, g$; these intervals being considerably narrower than the cases.

Fig. 1060 represents in plan the surface of the rectifying cistern, shown in two different sections in figs, 1058 and 1059. h, $k$, figs. 1058 and 1060, is the heat-governor, 
shaped somewhat like a pair of tongs. Each leg is a compound bar, consisting of a flat bar or ruler of steel, and one of brass alloy, riveted facewise together, having their edges up and down. The links, at $k$, are joined to the free ends of these compound bars, which, receling by increase and approaching by decrease of temperature, act by a lever or

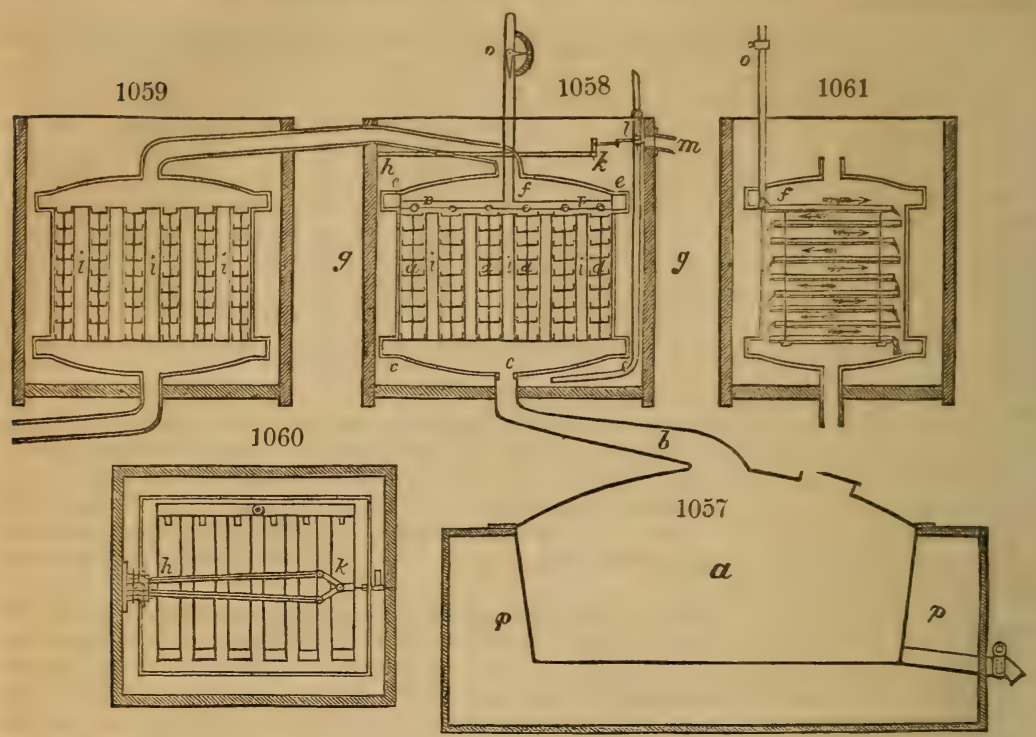

the stopcock 1 , fixed to the pipe of a cold-water back, and are so adjusted by a screw-nut, that whenever the water in the bath vessel $g, g$, rises above the desired temperature, cold water will be admitted, through the stopcock $l$, and pipe $n$, into the bottom of the cistern, and will displace the over-heated water by the overflow-pipe $m$. Thus a perfect equilibrium of caloric may be maintained, and alcoholic vapor of correspondent uniformity transmitted to the refrigeratory.

Fig. 1061 is the cold condenser, of similar construction to the rectifier, fig. 1058; only the water cells should be here larger in proportion to the vapor channels $d, d$. This refrigeratory system will be found very powerful, and it presents the great adrantage of permitting its interior to be readily inspected and cleansed. It is best made of laminated tin, hardened with a little copper alloy.

The mode of working the preceding apparatus will be understood by the following instructions. Into the alembic, $a$, let as much fermented liquor be admitted as will protect its bottom from being injured by the fire, reserving the main body in the chargingback. Whenever the ebullition in the alembic has raised the temperature of the waterbath $g, g$, to the desired pitch, whether that be $170^{\circ}, 175^{\circ}$, or $180^{\circ}$, the thermostatic instrument is to be adjusted by its screw-nut, and then the communication with the charging-back is to be opened by moving the index of the stopcock 0 , over a proper portion of its quadrantal arch. The wash will now descend in a slender equable stream, through the pipe $0, f$, thence spread into the horizontal tube $p, p$, and issue from the orifices of distribution, as seen in the figure, into the respective flat trays or spouts. The manner of its progress is seen for one set of trars, in fig. 1059. The direction of the stream in each shelf is evidently the reverse of that in the shelf above and below it; the turned-up end of one shelf corresponding to the discharge slope of its neighbor.

By diffusing the cool wash or wine in a thin film over such an ample range of surfaces, the constant tendency of the bath to exceed the proper limit of temperature is counteracted to the utmost, without waste of time or fuel; for the wash itself, in transitu, becomes boiling-hot, and experiences a powerful steam distillation. By this arrangement a very moderate influx of cold water, through the thermostatic stopcock, suffices to temper the bath; such an extensive vaporization of the wash producing a far more powerful refrigerant influence than its simple heating to ebullition. It deserves to be remarked, that the maximum distillatory effect, or the bringing orer the greatest quantity of pure spirits in the least time, and with the least labor and fuel, is here accomplished without the least steam pressure in the alembic; for the passages are 
Ill pervious to the vapor; whereas, in almost every wash-still heretofore contrived for similar purposes, the spirituous vapors must force their way through successive layers of liquid, the total pressure produced by which causes undue elevation of temperature, and ubstruction to the process. Whatever supplementary refrigeration of the vapors in their passage through the bath may be deemed proper, will be administered by the thermostatic regulator.

Towards the end of the process, after all the wash has entered the alembic, it may be sometimes desirable, for the sake of despatch, to modify the thermostat, by its adjustingscrew, so that the bath may take a higher temperature, and allow the residuary feints to run rapidly over, into a separate cistern. This weair fluid may be pumped back into the alembic, as the preliminary charge of a fresh operation.

The above plan of a water-bath regulated by the thermostat, may be used simply as a rectifying cistern, without transmitting the spirit or wash down through it. The series of shelves will cause the vapors from the still to impinge against a most extensire system of metallic surfaces, maintained at a steady temperature, whereby their watery and crude constituents will be condensed and thrown back, while their fine alcoholic particles will proceed forwards to the refrigeratory. Any ordinary still may be readily converted into this self-rectifying form, by merely interposing the cistern, fig. 1058, between the alembic and the worm-tub. The leading novelty of the present invention is the moveable system of shelves or trays, enclosed in metallic cases, separated by water, combined with the thermostatic regulator. By this combination, any quality of spirits may be procured at one step from wash or wine, by an apparatus, simple, strong, and easily kept in order.

The empyreumatic taint which spirits are apt to contract from the action of the naked fire on the bottom of the still, may be entirely prevented by the use of a bath of potash lej, $p, p$, fig. 1057 ; for thus a safe and effectual range of temperature, of $300^{\circ} \mathrm{F}$., may be conveniently obtained. The still may also be used without the bath vessel.

Mr. D. T. Shears, of Southwark, obtained a patent in March, 1830, for certain improvements and additions to stills, which are ingenious. They are founded upon a previous patent, granted to Joseph Corty, in 1818; a section of whose contrivance is shown in fig. 1062, consisting of a first still $a$, a second still $b$, a connecting tube $c$, from 1062 the one end to the other, and the tube $d$, which leads
from the second still-head down through the bent tube $k$ I $f$ f $e, e$, to the lower part of the condensing apparatus.

The original improvements described under Corty's patent, consisted further, in placing boxes $f, f, f$, of the

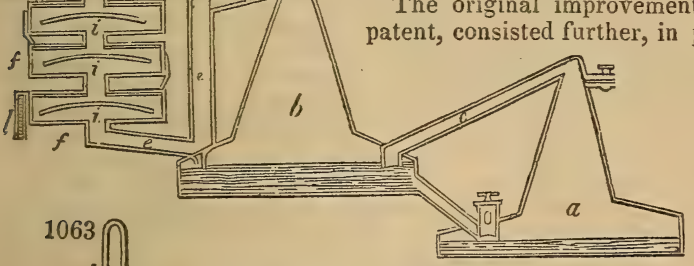

condensing apparatus in horizontal positions, and at a distance from each other, in order that the vapor might ascend through them, for the purpose of discharging the spirit by the top tube $g$, and pipe $h$, into the worm, in a highly rectified or concentrated state. In each of the boxes $f$, there is a convex plate or inverted dish $i$, $i, i$, and the vapor in rising from the tube $e$, strikes against the concave or under part of the first dish, and then escapes round its edges, and over its convex surface, to the under part of the second dish, and so on to the top, the condensed part of the vapor flowing down again into the still, and the spirit passing off by the pipe $h$, at top; and as the process of condensation will be assisted by cooling the vapor as it rises, cold water is made to flow over the tops of the boxes $f$, from a cock $k$, and through small channels or tubes on the sides of the boxes, and is ultimately discharged by the pipe $l$, at bottom.

Fig. 1063 represents a peculiarly shaped tube $a$, through which the spirit is described as passing after leaving the end of the worm at $b$, which tube is open to the atmospheric 
air at $z ; c$, is the passage through which the carbonic acid gas is described as escaping into the vessel of water $d$.

Now the improvements claimed under the present patent, are exhibited in figs. 1064, 1065, and 1066. Fig. 1064 represents the external appearance of a still, the head of which is made very capacious, to guard against over-boiling by any mismanagement of the fire; fig. 1065 is the same, partly in section. On the top of the still-head is formed the first-described rectifying apparatus, or series of condensing boxes. The vapor from the body of the still filling the head, meets with the first check from the dish or lower vessel $i$, and after passing under its edges, ascends and strikes against the lower part of the second dish or vessel $i$, and so on, till it ultimately leaves the still-head by the pipe at top.

This part of the apparatus is slightly altered from the former, by the substitution of hollow convex vessels, instead of the inverted dishes before described, which vessels have rims descending from their under surfaces, for the purpose of retaining the vapor. The cold water, which, as above described, flowed over the tops of the boxes $f$, for the purpose of cooling them, now flows also through the hollow convex vessels $i$, within the boxes, and by that means greatly assists the refrigerating process, by which the aqueous parts of the vapor are more readily condensed, and made to fall down and flow back again into the body of the still, while the spirituous parts pass off at top to the worm, in a very high state of rectification.

After the water employed for the refrigeration has passed over all the boxes, and through all the vessels, it is carried off by the pipe $m$, through the vessel $n$, called the wash-heater; that is, the vessel in which the wash is placed previous to introducing it into the still. The pipe $m$, is coiled round in the lower part of the vessel $n$, in order that the heated water may communicate its caloric to the wash, instead of losing the heat by allowing the water to flow away. After the heated water has made several turns round the wash heater, it passes out at the curved pipe 0 , which is bent up, in order to keep the coils of the pipe within always full of water.

Instead of the coiled pipe $n$, last described, the patentee proposes sometimes to pass the hot water into a chamber in a tub or wooden vessel, as at $n$, in fig. 1061, in which the wash to be heated occupies the upper part of the vessel, and is separated from the lower part by a thin metallic partition.

The swan-neck $h$, figs. 1064 and 1065, which leads from the head of the still, conduets the spirit from the still through the wash-heater, where it becomes partially cooled, and gives out its heat to the wash; and from thence the spirit passes to the worm tub, and being finally condensed, is passed through a safety tube, as ( fig. 1058) before described, and by the funnel is conducted into the cask below.

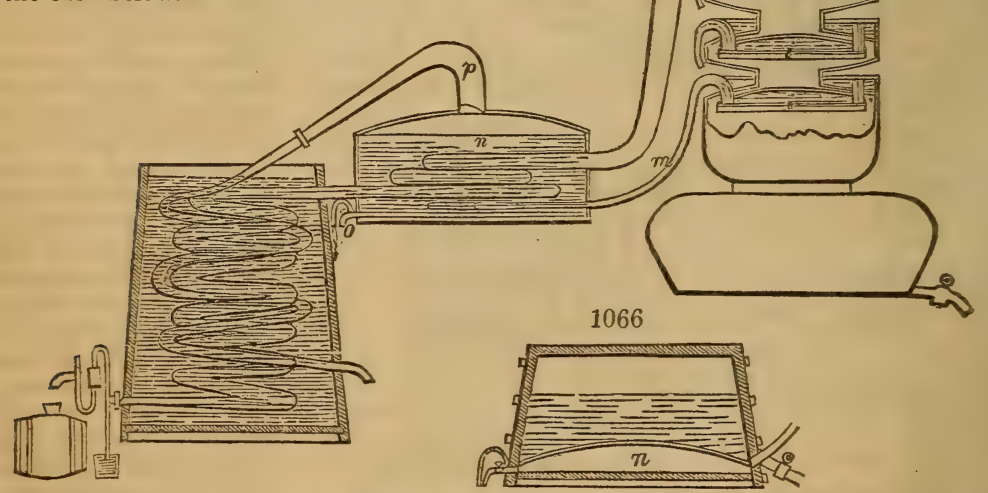

Should any spirit rise in the wash-heater during the above operation, it will be carried down to the worm by the neck $p$, and coiled pipe, and discharged at its lower end; or it may be passed into the still-head, as shown in fig. 1062.

A patent was obtained by Mr. Eneas Coffey, in Augast, 1830, for a still, which has been since mounted in several distilleries. It is economical in fuel, labor, and time, but is said not to produce a clean spirit, without peculiar attention.

The apparatus is represented in fig. 1067. $a, b, c, d$, is a sectional view of that part of the still wherein the wash is deprived of its alcohol, and the vapors analyzed. It is described as consisting of a chamber or vessel $a$, with the vertical chamber $b, c$, placed above it; the lower half of this chamber is divided into compartments by horizon 
tal plates $e, e, e$, of thin copper or other metal; each of these plates is turned down at one side, until it nearly touches the plate next underneath it, as shown in the figure; thus

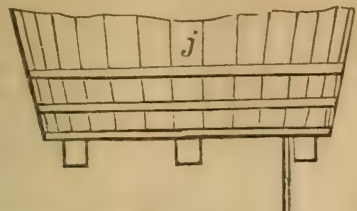

leaving a passage throughout the whole of them, by which any liquid falling on the top plate may descend into the next under it, and from that to the third, and $60 \mathrm{on}$, from plate to plate, at the alternate ends, until it arrives at the last plate, wherein it falls into thi vessel $\alpha$, by the pipe $f$; each of these plates is furnished with several light valves, opening upwards, through which any steam or vapor may ascend; it may also be perforated with holes, but they must not be so numerous or so large as to allow of all the steam passing through them without raising the valves; $c$ is a pipe by which the alcoholic vapor, after it has been analyzed, and has acquired the proper strength, is conducted into the vessel $d$, which is made perfectly close; the vapor will here be condensed on the surface of the pipe $g, g, g$; from this chamber it will descend in a liquid state into the pipe $h$, shence it may be conducted to a worm or refrigerator, to be cooled in the ordinary way; $i$ is a ressel through which the spent wash flows, after being operated upon in the distilling apparatus, and is discharged in a state of ebullition; $j$ is a vessel or chamber containing the wash to be distilled. A force pump may be substituted, to force the wash through the pipes $k$, and distilling apparatus, with the velocity required.

The patentee states that it is requisite the wash should be passed through the pipe $k$ with sufficient velocity and force, so as to prevent the deposition of sediment in the pipe; the wash, in its passage through the pipe $k$, will gradually become increased in temperature as it passes through the spent wash in the chamber, and the close vessel $d$, until it is discharged nearly at the boiling point on the upper plate in the chamber, where it comes in contaet with the vapors arising from the vessel $a$.

It is to be observed that the wort does not reach the boiling point while in the pipe $k, k$; to ascertain which, a thermometer is placed on the pipe, and by increasing or diminishing the quantity of wash, its temperature may be regulated. The wash, after being discharged from the pipe $k$, descends from plate to plate as before mentioned, at which time a supply of steam from a boiler, or generator, is admitted into the apparatus, through the pipe.

The lower part of this pipe in the vessel $a$ is pierced with a number of small holes, so as to spread the steam over the vessel; it then rises upwards, passing through the plate by the small holes and valves, and through the stratum or sheet of wash flowing over them; the wash, as it descends, gives out a portion of its alcohol to the steam, as it passes over every plate, until it is entirely deprived of its spirit, which it will generally do by the time it arrives at the 7 th or 8 th plate; but it is better to employ a greater number, to guard against accidents or neglect.

A small steam pipe rises from the chamber $a$, with its upper end opening into the box or chamber; into this chamber the end of a worm projects from the cistern of cold water; the steam rising up the pipe is nearly all condensed in the worm, and flows back Into the chamber $a$, by the pipe. The small portion of the steam uncondensed is allowed to escape at the upper end of the worm, and the flame of a small lamp or taper is to be constantly kept orer the orifice; when, should the least quantity of alcohol descend with the wash into the chamber $a$, it will rise with the steam through the pipe and worm, and immediately take fire from the flame of the lamp or taper, thereby warn. ing the attendant to increase the supply of steam or diminish the quantity of wash, as may seem necessary. 
I shall conclude this article with a description of the cheap still which is commonly enployed by the chemists in Berlin for rectifying alcohol. $a$ is the ash-pit; $b$, the

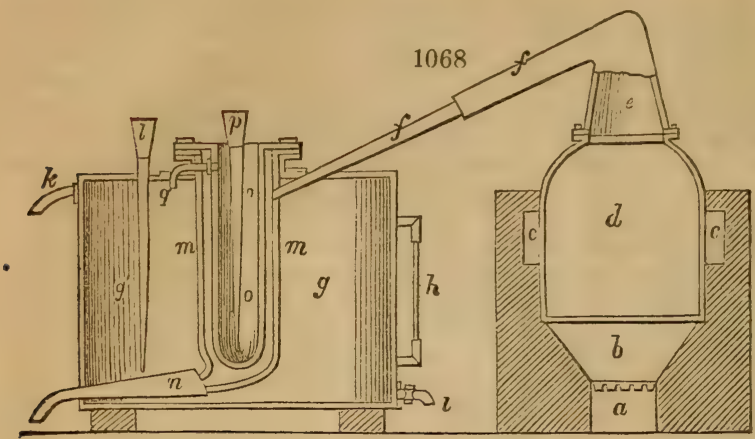

fire-place; $c, c$, the flues, which go spirally round the sides of the cucurbit $d ; e_{3}$ the capital, made of block tin, and furnished with a brass edge, which fits tight to a corresponding edge on the mouth of $d ; f, f$, the slanting pipes of the capital $g$, the oval refrigeratory, made of copper ; $h$, the water-gauge glass tube; $i$, a stopcock for emptying the vessel; $k$, ditto for drawing off the hot water from the surface; $l$, tube for the supply of cold water. A double cylinder of tin is placed in the refrigeratory, of which the outer one, $m, m$, stands upon three feet, and is furnished with a discharge pipe $n$. The inner one, 0 , 0 , which is open above, receives cold water through the pipe $p$, and lets the warm water flow off through the short tube $q$ into the refrigeratory. In the narrow space between the two cylinders, the vapors proceeding from the capital are condensed, and pass off in the liquid state through $n$. The refrigeratory is made oval, in order to receive two condensers alongside of each other in the line of the longer axis; though only one, and that in the middle, is represented in the figure.

STOCKING MANUFACTURE. See HosIERY.

STONE is earthy matter, condensed into so hard a state as to yield only to the blows of a hammer, and therefore well adapted to the purposes of building. Such was the care of the ancients to provide strong and durable materials for their public edifices, tha but for the desolating hands of modern barbarians, in peace and in war, most of the temples and other public monuments of Greece and of Rome would have remained perfect at the present day, uninjured by the elements during 2000 years. The contrast, in this respect, of the works of modern architects, especially in Great Britain, is very humiliating to those who boast so loudly of social advancement; for there is scarcely a public building of recent date, which will be in existence one thousand years hence. Many of the most splendid works of modern architecture are hastening to decar, in what may be justly called the very infancy of their existence, if compared with the date of those erected in ancient Italy, Greece, and Egypt. This is remarkably the case with the three bridges of London, Westminster, and Blackfriars; the foundations of which began to perish most visibly in the very lifetime of their constructors. Every stone intended for a durable edifice, ought to be tested as to its durability, by immersion in a saturated. solution of sulphate of soda, and exposure during some days to the air. The crystallization which ensues in its interior, will cause the same disintegration of its substance which frost would occasion in a series of years.

STONE, ARTIFICIAL, for statuary and other decorations of architecture, has been made for several years with singular success at Berlin, by Mr. Feilner. His materials are nearly the same with those of English pottery; and the plastic mass is fashioned either in moulds or by hand. His kilns, which are peculiar in form, and economical in fuel, deserve to be generally known. Figs. 1069 and $10 \% 0$ represent his round riln; fig. 1069 being an oblique section in the line A, B, c, of fig. 10 0 , which is a ground plan in the line $\mathrm{D}, a, b, \mathrm{E}$, of fig. 1069. The inner circular space $c$, covered with the elliptical arch, is filled with the figures to be baked, set upon brick supports. The hearth is a few feet above the ground; and there are steps before the door $d$, for the workmen to mount by, in charging the liln. The fire is applied on the four sides under the bearth. The flame of each passe along the straight flues $f i, f i$, and $f k$. In the second annular flue $g, g$, as also in the third $l, l$, the flame of each fire is kept apart, being separated from the adjoining by the stones $h$ and $m$. In the fourth flue $n$, the flames again come together, as also in 0 , and ascend by the middle opening. Besides this large orifice, there are several small holes, $p, p$, in the hearth over the above flues, to lead the flames from the other points into contact with the various articles. There are also channels; $q, q$, in the sides, enclosed by thin walls, $r$, to promote the equable distribution of the heat; and these are placed right ofer the first fire-flues $e$. The 
partitions $r$, are perforated with many holes, through which, as well as from their tops, the flame may be directed inwards and downwards; s are the vents for carrying off the

1069

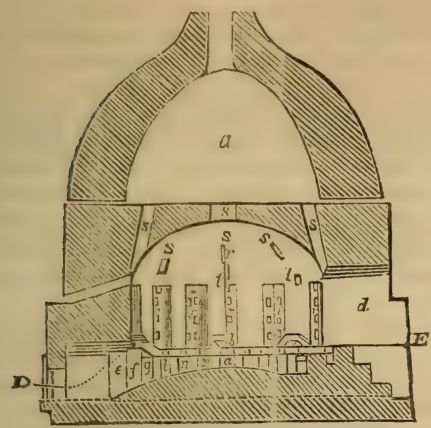

1070

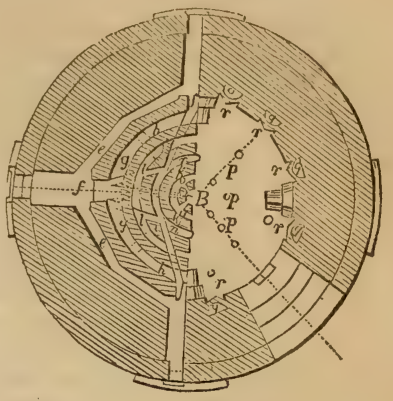

flames into the upper space i , which is usually left empty. These vents can be closed כy iron damper-plates, pushed in through the side-slits of the dome. $t, t$, are peep-holes, or observing the state of ignition in the furnace; but they are most commonly bricked ap. Fig. 1071 is a vertical section, and fig. 1072, a plan of an excellent kiln for baking elay to a stony consistence, for the above purpose, or for burning of bricks. A, is the

1071

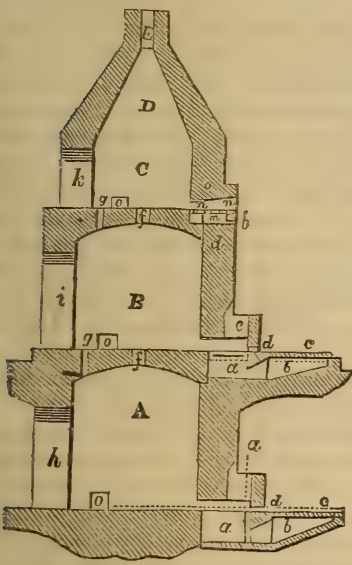
lower; $\mathrm{B}$, the middle ; $\mathrm{c}$, the upper kiln ; and $\mathrm{E}$, the hood, terminating in the chimney; $a, a$, is the ash-pit; $b, b$, the vault for raking out the ashes; it is covered with an iron door $c . \quad d$, is the peep-hole, filled with a clay stopper; $e$, is the fire-place; $f, f$, a vent in the middle of each arch; $g, g$, flues at the sides of the arches, situated between the two fire-places; $h, i, k$, are apertures for introducing the articles to be baked; $l$, a grate for the fire in the uppermost kiln; $m$, the ash-pit; $n$, the fire-door; $o$, open-

1072

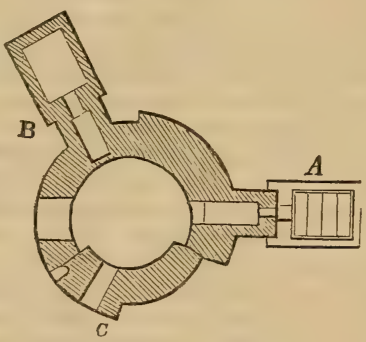
ings through which the flames of a second fire are thrown in. At first, only the ground kiln $A$, is fired, with cleft billets of pine-wood, introduced at the opening $e$; when this is finished, the se cond is fired; and then the third, in like man ner. This kiln is verv like the porcelain kiln or Sèvres, and is employed in many places for ba. king stoneware.

Mr. Keene obtained a patent, about a year ago, for making a factitious stone-paste in the following way:- $\mathrm{He}$ dissolves one pound of alum in a gallon of water, and in this solution he soaks eighty-four pounds of gypsum calcined in small lumps. He exposes these lumps in the open air for about eight days, till they become apparently dry, and then calcines them in an oven at a dull-red heat. The waste heat of a coke oven is well adapted for this purpose. (See Pitconl, coking of.) These lumps being ground and sifted, afford a fine powder, which, when made up into a paste with the proper quantity of water, forms the petrifying ground. The mass soon concretes, and after being brushed over with a thin layer of the petrifying paste, may be polished with pumice, \&c., in the usual way. It then affords a body of great compactness and durability. If half a pound of copperas be added to the solution of the alum, the gypsum paste, treated as above, has a fine cream or yellow color. This stone stands the weather well.

STONEWARE. (Fayence, Fr.; Steingut, Germ.) See PotTery.

STORAX, STYRAX, flows from the twigs and the trunk of the Liquidamber styraciflua, a tree which grows in Louisiana, Virginia, and Mexico. Liquidamber, as this resin is alsn called, is a brown or ash-gray substance, of the consistence of turpentine, which dries up rapidly, has an agreeable smell, like benzoin, and a bitterish, sharp, burning taste. It dissolves in four parts of alcohol, and affords 1.4 per cent. of benzoic acid. 
STOVE (Fuële, Calorifère, Fr.; Ofen, Germ.), is a fire-place, more or less close, for warming apartments. When it allows the burning coals to be seen, it is called a stovegrate. Hitherto stoves have rarely been had recourse to in this country for heating our sitting-rooms; the cheerful blaze and ventilation of an open fire being generally preferred. But last winter, by its inclemency, gave birth to a vast multitude of projects for increasing warmth and economizing fuel, many of them eminently insalubrious, by preventing due renewal of the air, and by the introduction of noxious fumes into it. When coke is burned very slowly in an iron box, the carbonic acid gas which is generated, being half as heavy again as the atmospherical air, cannot ascend in the chimney at the temperature of $300^{\circ} \mathrm{F}$.; but regurgitates into the apartment through every pore of the stove, and poisons the atmosphere. The large stoneware stoves of France and Germany are free from this vice; because, being fed with fuel from the outside, they cannot produce a reflux of carbonic acid into the apartment, when their draught becomes feeble, as inevitably results from the obscurely burning stoves which have the doors of the fire-place and ash-pit immediately above the hearth-stone.

I have recently performed some careful experiments upon this subject, and find that when the fuel is burning so slowly in the stove as not to heat the iron surface above the 250th or 300th degree of Fahr., there is a constant deflux of carbonic acid gas from the ash-pit into the room. This noxious emanation is most easily evinced by applying the beak of a matrass, containing a little Goulard's extract (solution of subacetate of lead), to a round hole in the door of the ash-pit of a stove in this languid state of combustion. In a few seconds the liquid will become milky, by the reception of carbonic acid gas. I shall be happy to afford ocular demonstration of this fact to any incredulous votary of the pseudo-economical, anti-ventilation stoves, now so much in vogue. There is no mode in which the health and life of a person can be placed in more insidious jeopardy, than by sitting in a room with its chimney closed up with such a cholke-damp-vomiting stove.

That fuel may be consumed by an obscure species of combustion, with the emission of very little heat, was clearly shown in Sir H. Davy's Researches on Flame. "The facts detailed on insensible combustion," says he, "explain why so much more heat is obtained from fuel when it is burned quickly, than slowly; and they show that, in all cases, the temperature of the acting bodies should be kept as high as possible; not only because the general increment of heat is greater, but likewise because those combinations are prevented, which, at lower temperatures, take place without any considerable production of heat. These facts likewise indicate the source of the great error into which experimenters have fallen, in estimating the heat given out in the combustion of charcoal; and they indicate methods by which the temperature may be increased, and the limits to certain methods." These conclusions are placed in a strong practical light by the following simple experiments :-I set upon the top orifice of a small cylindrical stove, a hemispherical copper pan, containing six pounds of water, at $60^{\circ} \mathrm{F}$., and burned briskly under it three and a half pounds of coke in an hour; at the end of which time, four and a half pounds of water were boiled off. On burning the same weight of coke slowly in the same furnace, mounted by the same pan, in the course of twelve hours, little more than one half the quantity of water was exhaled. Yet, in the first case, the aerial products of combustion swept so rapidly over the bottom of the 1073 pan, as to communicate to it not more than one fourth of

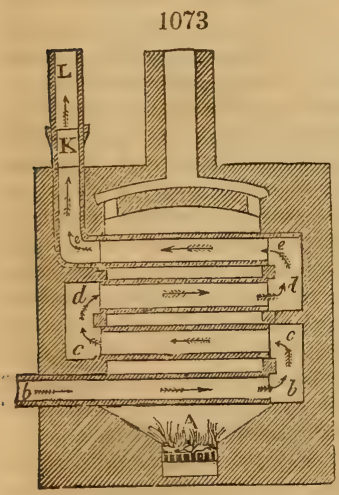
the effective heat which might have been obtained by one of the plans described in the article Evaporation; while in the second case, these products moved at least twelve times more slowly across the bottom of the pan, and ought therefore to have been so much the more effective in evaporation, had they possessed the same power or quantity of heat.

Stoves, when properly constructed, may be employed both safely and advantageously to heat entrance-halls upon the ground story of a house; but care should be taken not to vitiate the air by passing it over ignited surfaces, as is the case with most of the patent stoves now foisted upon the public. Fig. 1073 exhibits a vertical section of a stove which has been recommended for power and econo$\mathrm{my}$; but it is highly objectionable, as being apt to scorch the air. The flame of the fire $A$, circulates round the horizontal pipes of cast-iron, $b, b, c, c, d, d, e, e$, which receive the external air at the orifice $b$, and conduct it up through the series, till it issues highly heated at $\mathrm{K}, \mathrm{L}$, and may be thence conducted wherever it is wanted. The smoke escapes through the chimney B. This stove has evidently two prominent faults; first, it heats the air-pipes very unequally, 
and the undermust far too much; secondly, the air, by the time it has ascended throngh. the zigzag rance to the pipe $e e$, will be nearly of the same temperature with it, and will therefore abstract none of its heat. Thus the upper pipes, if there be several in the range, will be quite inoperative, wasting their warmth upon the sooty air.

Fig. 1074 exhibits a transverse vertical section of a far more economical and powerfu] stove, in which the above evils are avoided. The products of combustion of the fire $A$,

1071

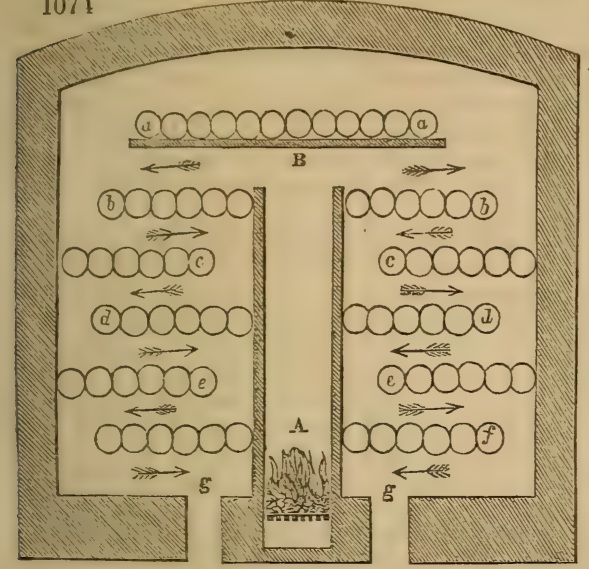
rise up between two brick walls, so as to play upon the bed of tiles $B$, where, after communicating a moderate heat to the series of slanting pipes whose areas are represented by the small circles, $a, \alpha$, they turn to the right and left, and circulate round the successive rows of pipes $b b, c c, d d, e e$, and finally es. cape at the bottom by the flues $g, g$, pursuing a somewhat similar path to that of the burned air among a bench of gas-light retorts. It is known, that two thirds of the fue? have been saved in the gas-works by this distribution of the furnace. For the purpose of heating apartments, the great object is to supply a vas? body of genial air; and, therefore, merely such a moderate fire should be kept up in $A$, as will suffice to warm all the pipes pretty equably to the temperature of $220^{\circ} \mathrm{Fahr}$; and, indeed, as they are laid with a slight slope, are open to the air at their under ends, and terminate at the upper in a common main pipe or tunnel, they can hardly be rendered very hot by any intemperance of firing. I can safely recommend this stove to my readers. If the tubes be made of stoneware, its construction will cost very little; and they may be made of any size, and multiplied so as to carry off the whole effective heat of the fuel, leaving merely so much of it in the burned air, as to waft it fairly up the chimney.

I shall conclude this article by a short extract of a paper which was read before the Royal Society, on the 16th of June, 1836, upon warming and ventilating apartments; $\mathbf{a}$ subject to which my mind had been particularly turned at that time, by the Directors of the Customs Fund of Life Assurance, on account of the very general state of indisposition and disease prevailing among those of their officers (nearly 100 in number) engaged on duty in the Long Room of the Custom House, London.

"The symptoms of disorder experienced by the several gentlemen (about twenty in number) whom I examined, out of a great many who were indisposed, were of a very uniform character. The following is the result of my researches :-

"A sense of tension or fulness of the head, with occasional flushings of the conntenance, throbbing of the temples, and vertigo, followed, not unfrequently, with a confusion of ideas, very disagreeable to officers occupied with important and sometimes intricate calculations. A few are affected with unpleasant perspiration on their sides. The whole of them complain of a remarkable coldness and languor in their extremities, more especially the legs and feet, which has become habitual, denoting languid circulation in these parts, which requires to be counteracted by the application of warm flannels on going to bed. The pulse is, in many instances, more feeble, frequent, sharp, and irritable, than it ought to be, according to the natural constitution of the individuals. The sensations in the head occasionally rise to such a height, notwithstanding the most temperate regimen of life, as to require cupping, and at other times depletory remedies. Costiveness, though not a uniform, is yet a prevailing symptom.

"The sameness of the above ailments, in upwards of one hundred gentlemen, at very various periods of life, and of various temperaments, indicates clearly sameness in the cause.

"The temperature of the air in the Long Room ranged, in the three days of my experimental inquiry, from $62^{\circ}$ to $64^{\circ}$ of Fahrenheit's scale; and in the Examiner's Room it was about $60^{\circ}$, being kept somewhat lower by the occasional shutting of the hot-air valve, which is here placed under the control of the gentlemen; whereas that of the Long Room is designed to be regulated in the sunk story, by the fireman of the stove, who seems sufficiently careful to maintain an equable temperature amidst all the vicissitudes of our winter weather. Upon the 7 th of January, ths temperature of the open 
air was $50^{\circ}$; and on the 11 th it was only $35^{\circ}$; yet upon both days the thermometer in the Long Room indicated the same heat, of from $62^{\circ}$ to $64^{\circ}$.

"The hot air discharged from the two cylindrical stove-tunnels into the Long Room was at $90^{\circ}$ upon the 7 th, and at $110^{\circ}$ upon the 11 th. This air is diluted, however, and disguised, by admixture with a column of cold air, before it is allowed to escape. The air, on the contrary, which heats the Examiner's Room, undergoes no such mollification, and comes forth at once in an ardent blast of fully $170^{\circ}$; not unlike the simoorn of the desert, as described by travellers. Had a similar nuisance, on the greater scale, existed in the Long Room, it could not have been endured by the merchants and other visiters on business: but the disguise of an evil is a very different thing from its removal. The direct air of the stove, as it enters the Examiner's Room, possesses, in an eminent degree, the disagreeable smell and flavor imparted to air by the action of red-hot iron; and, in spite of every attention on the part of the fireman to sweep the stove apparatus from time to time, it carries along with it abundance of burned dusty particles.

"The leading characteristic of the air in these two rooms, is its dryness and disagreeable smell. In the Long Room, upon the 11 th, the air indicated, by Daniell's hygrometer, 70 per cent. of dryness, while the external atmosphere was n: arly saturated with moisture. The thermometer connected with the dark bulb of that instrument stood at $30^{\circ}$ when dew began to be deposited upon it; while the thermometer in the air stood at $64^{\circ}$. In the court behind the Custom-house, the external air being at $35^{\circ}$, dew was deposited on the dark bulb of the hygrometer by a depression of only $3^{\circ}$; whereas in the Long Room, on the same day, a depression of $34^{\circ}$ was required to produce that deposition. Air, in such a dry state, would evaporate 0.44 in depth of water from a cistern in the course of twenty-four hours; and its influence on the cutaneous exhalants must be proportionably great.

"As cast iron always contains, besides the metal itself, more or less carbon, sulphur, phosphorus, or even arsenic, it is possible that the smell of air passed over it in an incandescent state, may be owing to some of these impregnations; for a quantity of noxious effluvia, inappreciably small, is capable of affecting not only the olfactory nerves, but the pulmonary organs. I endeavored to test the air as it issued from the valre in the Examiner's Room, by presenting to it pieces of white paper moistened with a solution of nitrate of silver, and perceived a slight darkening to take place, as if by sulphurous fumes. White paper, moistened with sulphureted hydrogen water, was not in the least discolored. The faint impression on the first test paper, may be, probably, aseribed to sulphurous fumes, proceeding from the ignition of the myriads of animal and vegetable matters which constantly float in the atmosphere, as may be seen in the sunbeam admitted into a dark chamber: to this cause, likewise, the offensive smell of air, transmitted over red-hot iron, may in some measure be attributed, as well as to the hydrogen resulting from the decomposition of aqueous vapor, always present in our atmosphere in abundance; especially close to the banks of the Thames, below London Bridge.

"When a column of air sweeps furiously across the burning deserts of Africa and Arabia, constituting the phenomenon called simoom by the natives, the air becomes not only very hot and dry, but highly electrical, as is evinced by lightning and thunder. Dry sands, devoid of vegetation, cannot be conceived to communicate any noxious gas or vapor to the atmosphere, like the malaria of marshes, called miasmata ; it is, hence, highly probable that the blast of the simoom owes its deadly malignity, in reference to animal as well as vegetable life, simply to extreme heat, dryness, and electrical disturbance. Simi'ar conditions, though on a smaller scale, exist in what is called the bell, or cockle, apparatus for heating the Long Room and the Examiner's apartment in the Custom-house. It consists of a series of inverted, hollow, flattened pyramids of cast iron, with an oblong base, rather small in their dimensions, to do their work sufficiently in cold weather, when moderately heated. The inside of the pyramids is exposed to the flames of coke furnaces, which heat them frequently to incandescence, while currents of cold air are directed to their exterior surfaces by numerous sheet-iron channels. The incandescence of these pyramids, or bells, as they are vulgarly called, was provec: by pieces of paper taking fire when I laid them on the summits. Again, since air be comes electrical when it is rapidly blown upon the surfaces of certain bodies, it oc curred to me that the air which escapes into the Examiner's Room might be in this predicament. It certainly excites the sensation of a cobweb playing round the head, which is well known to all who are familiar with electrical machines. To determine this point, I presented a condensing gold-leaf electrometer to the said current of hot air, and obtained faint divergence with negative electricity. The electricity must be impaired in its tension, however, in consequence of the air escaping through an iron grating, and striking against the flat iron valves, both of which tend to restore the eiectric equilibrium. The air blast, moreover, by being diffused round the glass of the 
eondensel apparatus, would somewhat mask the appearances. Were it worth while, an apparatus might be readily constructed for determining this point, without any such sources of fallacy. The influence of an atmosphere charged with electricity in exciting headache and confusion of thought in many persons, is universally known.

"The fetid burned odor of the stove air, and its excessive avidity for moisture, are of themselves, however, sufficient causes of the general indisposition produced among the gentlemen who are permanently exposed to it in the discharge of their public duties.

"From there being nearly" a vacuum, as to aqueous vapor, in the said air, while there is nearly a plenum in the external atmosphere round about the Custom-house, the vicissitudes of feeling in those who have occasion to go out and in frequently, must be highly detrimental to health. The permanent action of an artificial desiccated air on the ani mal economy may be stated as follows :-

"The living body is continually emitting a transpirable matter, the quantity of which, in a grown up man, will depend partly on the activity of the cutaneous exhalants, and partly on the relative dryness or moisture of the circumambient medium. Its average amount, in common circumstances, has been estimated at 20 ounces in twenty-four hours.

"When plunged in a very dry air, the insensible perspiration will be increased; and, as it is a true evaporation or gasefaction, it will generate cold proportionably to its amount. Those parts of the body which are most insulated in the air, and furthest from the heart, such as the extremities, will feel this refrigerating influence most powerfully. Hence the coldness of the hands and feet, so generally felt by the inmates of the apartment, though its temperature be at or above $60^{\circ}$. The brain, being screened by the scull from this evaporating influence, will remain relatively hot, and will get surcharged, besides, with the fluids which are repelled from the extremities by the condensation, or contraction, of the blood-vessels, caused by cold. Hence the affections of the head, such as tension, and its dangerous consequences. If sensible perspiration hapnen, from debility, to break forth from a system previously relaxed, and plunged into dry air, so attractive of vapor, it will be of the kind called a cold clammy sweat on the sides and back, as experienced by many inmates of the Long Room.

"Such, in my humble apprehension, is a rationale of the phenomena observed at the Custom-house. Similar effects have resulted from hot-air stoves of a similar kind in many other situations.

"After the most mature physical and medical investigation, I am of opinion that the circumstances above specified cannot act permanently upon human beings, without impairing their constitutions, and reducing the value of their lives. The Directors of the Customs Fund are therefore justified in their apprehensions, 'that the mode of heating the Long Room is injurious to the health of persons employed therein, and that it must unduly shorten the duration of life.'

"It may be admitted, as a general principle, that the comfort of sedentary individuals, occupying large apartments during the winter months, cannot be adequately secured by the mere influx of hot air from separate stove rooms: it requires the genial influence of radiating surfaces in the apartments themselves, such as of open fires, of pipes, or other vessels filled with hot water or steam. The clothing of our bodies, exposed to such radiation in a pure, fresh, somewhat cool and bracing air, absorbs a much more agreeable warmth than it could acquire by being merely immersed in an atmosphere heated even to $62^{\circ} \mathrm{Fahr}$, like that of the Long Room. In the former predicament, the lungs are supplied with a relatively dense air, say at $52^{\circ} \mathrm{Fahr}$.; while the external surface of the body or the clothing is maintained at, perhaps, $70^{\circ}$ or $75^{\circ}$. This distinctive circumstance has not, I believe, been hitherto duly considered by the stove doctors, each intent on puffing his own pecuniary interest; but it is obviously one of great importance, and which the English people would do well to keep in view; because it is owing to our domestic apartments being heated by open fires, and our factories by steam pipes, that the health of our population, and the expectation of life among all orders in this country, are so much better than in France and Germany, where hot-air stores, neither agreeable nor inoffensive, and in endless variety of form, are generally employed.

"In conclusion, I take leave to state to you my firm conviction that the only method of warming your Long Room and subsidiary apartments, combining salubrity, safety, and economy, with convenience in erection and durable comfort in use, is by a series of steam pipes laid along the floor, at the line of the desk partitions, in suitahle lengths, with small arched junction-pipes rising over the several doorways, to keep the nassages clear, and at the same time to allow a free expansion and contraction in the pipes, thereby providing for the permanent soundness of the joints."

It would not be difficult to construct a stove or stove-grate which should combine econoun and comfort of warming an apartment, with hriskness of combustion and dura. bility of the fire, without any noxious deflux of carbonic acid. See Chimney. 
STRASS; see PAstes.

STRAW-HAT MANUFACTURE. The mode of preparing the Tuscany or Italian straw, is by pulling the bearded wheat while the ear is in a soft milky state, the corn having been sown very close, and of consequence produced in a thin, short, and dwindled condition. The straw, with its ears and roots, is spread out thinly upon the ground in fine hot weather, for three or four days or more, in order to dry the sap: it is then tied up in bundles and stacked, for the purpose of enabling the heat of the mow to drive off any remaining moisture. It is important to keep the ends of the straw airtight, in order to retain the pith, and prevent its gummy particles from passing off by evaporation.

After the straw has been about a month in the mow, it is removed to a meadow and spread out, that the dew may act upon it, together with the sun and air, and promote the bleaching, it being necessary frequently to turn the straw while this process is going on. The first process of bleaching being complete, the lower joint and root is pulled from the straw, leaving the upper part fit for use, which is then sorted according to qualities; and after being submitted to the action of steam, for the purpose of extracting its color, and then to a fumigation of sulphur, to complete the bleaching, the straws are in a condition to be platted or woven into hats and bonnets, and are in that state imported into England in bundles, the dried ears of the wheat being still on the straw.

Straw may be easily bleached by a solution of chloride of lime, and also by sulphuring. For the latter purpose, a cask open at both ends, with its seams papered, is to be set upright a few inches from the ground, having a hoop nailed to its inside, ahout six inches beneath the top, to support another hoop with a net stretched across it, upon which the straw is to be laid in successive handfuls loosely crossing each other. 'The cask having been covered with a tight overlapping lid, stuffed with lists of cloth, a brasier of burning chareoal is to be inserted within the bottom, and an iron dish containing pieces of brimstone is to be put upon the brasier. The brimstone soon takes fire, and fills the cask with sulphurous acid gas, whereby the straw gets bleached in the course of three or four hours. Care should be taken to prerent such a violent combustion of the sulphur as might cause black burned spots, for these cannot be afterwards removed. The straw, after being aired and softened by spreading it upon the grass for a night, is ready to be split, preparatory to dyeing. Blue is given by a boiling-hot solution of indigo in sulphuric acid, called Saxon blue, diluted to the desired shade; yellow, by decoction of turmeric ; red, by boiling hanks of coarse scarlet wool in a bath of weak alum water, containing the straw; or directly, by cochineal, salt of tin, and tartar. Brazil wood and archil are also employed for dyeing straw. For the other colors, see their respective titles in this Dictionary.

STRETCHING MACHINE. Cotton goods and other textile fabrics, either white or printed, are prepared for the market by being stretched in a proper machine, which lays all their warp and woof yarns in truly parallel positions. A very ingenious and effective mechanism of this kind was made the subject of a patent by Mr. Samuel Morand, of Manchester, in April, 1834, which serves to extend the width of calico pieces, or of other cloths woven of cotton, wool, silk, or flax, after they have become shrunk in the processes of bleaching, dyeing, \&c. I regret that the limits of this volume will not admit of its description. The specification of the patent is published in Newton's Journal, for December, 1835 .

STRONTIA, one of the alkaline earths, of which strontium is the metallic basis, occurs in a crystalline state, as a carbonate, in the lead mines of Strontian in Argyleshire, whence its name. The sulphate is found crystallized near Bristol, and in several other parts of the world; but strontitic minerals are rather rare. The pure earth is prepared exactly like baryta, from either the carbonate or the sulphate. It is a grayishwhite powder, infusible in the furnace, of a specific gravity approaching that of baryta, having an acrid, burning taste, but not so corrosive as baryta, though sharper than lime. It becomes hot when moistened, and slakes into a pulverulent hydrate, dissolves in 150 parts of water at $60^{\circ}$, and in much less at the boiling point, forming an alkaline solution ealled strontia water, which deposites crystals in four-sided tables as it cools. These contain 68 per cent. of water, are soluble in 52 parts of water at $60^{\circ}$, and in about 2 parts of boiling water; when heated they part with 53 parts of water, but retain the other 15 parts, even at a red heat. The dry earth consists of 84.55 of base, and 15.45 of oxygen. It is readily distinguished from baryta, by its inferior solubility, and by its soluble salts giving a red tinge to flame, while those of baryta give a yellow tinge. Fluosilicic acid and iodate of soda precipitate the salts of the latter earth, but not those of the former. The compounds of strontia are not poisonous, like those of baryta. The only preparation of strontia used in the arts is the Nitrate, which see.

STRYCHNIA is an alkaline base, extracted from the Strychnos nux vomica, Strychnos ignatia, and the Upas tiente; which has been employed in medicine by some of the poison doctors, but is of no use in any of the arts. When introduced into the stomach, 
otrychnia acts with fearful energy, causing lock-jaw immediately, and the denth of the animal in a very short time. Half a grain, blown into the throat of a rabbit, proves fatal in fire minutes.

STLCCO. See Grpsum.

SUBERIC ACID, is prepared by digesting grated cork with nitric acid. It forms crystals, which sublime in white vapors when heated.

SUBLIMATE, is any solid matter resulting from condensed vapors, and,

SUBLIMATION, is the process by which the rolatile particles are raised by heat, and condensed into a crystalline mass. See Calomel and Sal-ammoniac, for examples.

SUBSALT, is a salt in which the base is not saturated with acid; as subacetate of lead.

SUCCINIC ACID, Acid of amber (Acide succinique, Fr.; Bernsteinsaüre, Germ.), is obtained by distilling coarsely pounded amber in a retort by itself, with a heat gradually raised; or mixed with one twelfth of its weight of sulphuric acid, diluted with half its weight of water. The acid which sublimes is to be dissolved in hot water, to be saturated with potassa or soda, boiled with bone black, to remove the foul empyreumatic oily matter, filtered, and precipitated by nitrate of lead, to convert it into an insoluble succinate; which being washed, is to be decomposed by the equivalent quantity of sulphuric acid. Pure succinic acid forms transparent prisms. The succinate of ammonia is an excellent reagent for detecting and separating iron.

SUGAR (Sucre, Fr.; Zucher, Germ.), is the sweet constituent of vegetable and animal products. It may be distinguished into two principal species. The first, which occurs in the sugar-cane, the beet-root, and the maple, crystallizes in oblique four-sided prisms, terminated by two-sided summits; it has a sweetening power which may be represented by 100 ; and in circumpolarization it bends the luminous rays to the right. The second occurs ready formed in ripe grapes and other fruits; it is also produced by treating starch with diastase or sulphuric acid. This species forms cauliflower concretions, but not true crystals; it has a sweetening power which may be represented by 60 , and in circumpolarization it bends the rays to the left. Besides these two principal kinds of sugar, some others are distinguished by chemists; as the sugar of milk, of manna, of certain mushrooms, of liquorice-root, and that obtained from saw-dust and glue by the action of sulphuric acid; but they have no importance in a manufacturing point of view.

Sugar, extracted either from the cane, the beet, or the maple, is identical in its properties and composition, when refined to the same pitch of purity; only that of the beet seems to surpass the other two in col esive force, since larger and firmer crystals of it are obtained from a clarified solution of equal density. It contains 5.3 per cent. of combined water, which can be separated only by uniting it with oxyde of lead, into what has been called a saccharate; made by mixing sirup with finely ground litharge, and evaporating the mixture to dryness upon a steam-bath. When sugar is exposed to a heat of $400^{\circ}$ F., it melts into a brown pasty mass, but still retains its water of composition. Sugar thus fused is no longer capable of crystallization, and is called caramel by the French. It is used for coloring liqueurs. Indeed, sugar is so susceptible of change by heat, that if a colorless solution of it be exposed for some time to the temperature of boiling water, it becomes brown and partially uncrystallizable. Acids exercise such an injurious influence upon sugar, that after remaining in contact with it for a little while, though they be rendered thoroughly neutral, a great part of the sugar will refuse to crystallize. Thus, if three parts of oxalic or tartaric acid be added to sugar in solution, no crystals of sugar can be obtained by evaporation, even though the acids be neutralized by chalk or carbonate of lime. By boiling cane sugar with dilute sulphuric acid, it is changed into starch sugar. Manufacturers of sugar should be, therefore, particularly watchful against every acidulous taint or impregnation. Nitric acid converts sugar into oxalic and malic acids. Alkaline matter is likewise most detrimental to the grain of sugar; as is always evinced by the large quantity of molasses formed, when an excess of temper lime has been used in clarifying the juice of the cane or the beet. When one piece of lump sugar is rubbed against another in the dark, a phosphorescent light is emitted.

Sugar is soluble in all proportions in water; but it takes four parts of spirits of wine, of spec. grav. 0.830 , and eighty of absolute alcohol, to dissolve it, both being at a boiling temperature. As the alcohol cools, it deposites the sugar in small crystals. Caramelized and uncrystallizable sugar dissolves readily in alcohol. Pure sugar is unchangeable in the air, even when dissolved in a good deal of water, if the solution be kept covered and in the dark; but with a very small addition of gluten, the solution soon begins to ferment, whereby the sugar is decomposed into alcohol and carbonic acid, and ultimately into acetic acid.

Sugar forms chemical compounds with the salifiable bases. It dissolves readily in 
caustic potash ley, whereby it loses its sweet taste, and affords on evaporation a mass which is insoluble in alcohol. When the ley is neutralized by sulphuric acid, the sugar recovers its sweet taste, and may be separated from the sulphate of potash by alcohol, but it will no longer crystallize.

That sirup possesses the property of dissolving the alkaline earths, lime, magnesia, strontites, barytes, was demonstrated long ago by Mr. Ramsay of Glasgow, by experiments published in Nicholson's Journal, volume xviii. page 9, for September, 1807 . He found that sirup is capable of dissolving half as much lime as it contains of sugar ; and as much strontites as sugar. Magnesia dissolved in much smaller quantity, and barytes, seemed to decompose the sugar entirely. These results have been since confirmed by Professor Daniell. Mr. Ramsay characterized sugar treated with lime as weak, from its sweetening power being impaired; from its solution he obtained, after some time, a deposite of calcareous carbonate. M. Pelouze has lately shown, that the carbonic acid in this case is derived from the atmosphere, and is not formed at the expense of the elements of the sugar, as Mr. Daniell had asserted.

Sugar forn.s with oxyde of lead two combinations; the one soluble, the other insoluble. Oxyde of lead digested in sirup dissolves to a certain amount, forms a yellowish liquor, which possesses an alkaline reaction, and leaves after evaporation an uncrystallizable, viscid, deliquescent mass. If sirup be boiled with oxyde of lead in excess, if the solution be filtered boiling hot, and if the vial be corked in which it is received, white bulky flocks will fall to its bottom in the course of $2 t$ hours. This compound is best dried in vacuo. It is in both cases light, tasteless, and insoluble in cold and boiling water; it takes fire like German tinder, (AMADOU,) when touched at one point with an ignited body, and burns away, leaving small globules of lead. It dissolves in acids, and also in neutral acetate of lead, which forms with the oxyde a subsalt, and sets the sugar free. Carbonic acid gas passed through water, in which the above saccharate is diffused, decomposes it with precipitation of carbonate of lead. It consists of 58.26 parts of oxyde of lead, and 41.74 sugar, in 100 parts. From the powerful action exercised upon sugar by acids and oxyde of lead, we may see the fallacy and danger of using these chemical reagents in sugar-refining. Sugar possesses the remarkable property of dissolving the oxyde, as well as the subacetate of copper, (verdigris, ) and of counteracting their poisonous operation. Orfila found that a dose of verdigris, which would kill a dog in an hour or two, might be swallowed with impunity, provided it was mixed with a considerable quantity of sugar. When a solution of sugar is boiled with the acetate of copper, it causes an abundant precipitate of protoxyde of copper; when boiled with the nitrates of mercury and silver, or the chloride of gold, it reduces the respective bases to the metallic state.

The following TABLE shows the quantities of Sugar contained in Sirups of the annexed specific gravities.* It was the result of experiments carefully made.

\begin{tabular}{|c|c|c|c|}
\hline $\begin{array}{c}\text { Experimental specific gra- } \\
\text { vity of solution at } 60^{\circ} \mathrm{F} \text {. }\end{array}$ & $\begin{array}{l}\text { Sugar in } 100, \text { by } \\
\text { weight. }\end{array}$ & $\begin{array}{l}\text { Experimental specific gra- } \\
\text { vity of solution at } 60^{\circ} \mathrm{F}\end{array}$ & $\begin{array}{l}\text { Sugar in } 100, \text { by } \\
\text { weight. }\end{array}$ \\
\hline $\begin{array}{l}1 \cdot 3260 \\
1 \cdot 2310 \\
1 \cdot 1777 \\
1 \cdot 4400 \\
1 \cdot 1340 \\
1 \cdot 1250 \\
1 \cdot 1110\end{array}$ & $\begin{array}{l}66 \cdot 666 \\
50 \cdot 000 \\
40 \cdot 000 \\
33 \cdot 333 \\
31 \cdot 250 \\
29 \cdot 412 \\
26 \cdot 316\end{array}$ & $\begin{array}{l}1 \cdot 1045 \\
1 \cdot 0905 \\
1 \cdot 0820 \\
1 \cdot 0685 \\
1 \cdot 0500 \\
1 \cdot 0395\end{array}$ & $\begin{array}{l}25 \cdot 000 \\
21 \cdot 740 \\
20 \cdot 000 \\
16 \cdot 666 \\
12.500 \\
10.000\end{array}$ \\
\hline
\end{tabular}

If the decimal part of the number denoting the specific gravity of sirup be multiplied by 26 , the product will denote very nearly the quantity of sugar per gallon in pounds weight, at the given specific gravity. $\dagger$

Sugar has been analyzed by several chemists; the following. TABLE exhibits some of their results :-

\begin{tabular}{|c|c|c|c|c|c|}
\hline & $\begin{array}{c}\text { Gay Lussac } \\
\text { and Thenard. }\end{array}$ & Berzelius. & Prout. & Ure. & \\
\hline $\begin{array}{l}\text { Oxygen, - - } \\
\text { Carbon, - - - } \\
\text { Hydrogen, - - }\end{array}$ & $\begin{array}{r}56 \cdot 63 \\
42 \cdot 47 \\
6 \cdot 90\end{array}$ & $\begin{array}{r}49 \cdot 856 \\
43 \cdot 265 \\
6 \cdot 875\end{array}$ & $\begin{array}{r}53 \cdot 35 \\
39 \cdot 99 \\
6 \cdot 66\end{array}$ & $\begin{array}{r}50 \cdot 33 \\
43 \cdot 38 \\
6 \cdot 29\end{array}$ & in 100 \\
\hline
\end{tabular}

* The author, in minutes of evidence of Molasses Committee of the House of Commons, 1831, p. 142.

+ This rule was annexed to an extensive table, representing the quantities of sugar per gallon corresponding to the specific gravities of the sirup, constructed by the author for the Excise, in subserviency to the Beet-root Bill. 
Of the sugar cane, and the extraction of sugur from it.-Humboldt, after the most elaborate historical and botanical researches in the New World, has arrired at the conclusion that before America was discovered by the Spaniards, the inhabitants of that continent and the adjacent islands were entirely unacquainted with the sugar canes, with any of our corn plants, and with rice. The progressive diffusion of the cane has been thus traced out by the partisans of its oriental origin. From the interior of Asia it was \{ransplanted first into Cyprus, and thence into Sicily, or possibly by the Saracens directly into the latter island, in which a large quantity of sugar was manufactured in he year 1148. Lafitau relates the donation made by William the Second, ling of Sicily, to the convent of St. Benoit, of a mill for crushing sugar canes, along with all its privileges, workmen, and dependencies: which remarkable gift bears the date of 1166 . According to this author, the sugar cane must have been imported into Europe at the period of the Crusades. The monk Albertus Aquensis, in the description which he has given of the processes employed at Acre and at Tripoli to extract sugar, says, that in the Holy Land, the Christian soldiers being short of provisions, had recourse to sugar canes, which they chewed for subsistence. Towards the year 1420, Dom Henry, regent of Portural, caused the sugar cane to be imported into Madeira from Sicily. This plant succeeded perfectly in Madeira and the Canaries; and until the discovery of America these islands supplied Europe with the greater portion of the sugar which it consumed.

The cane is said by some to have passed from the Canaries into the Brazils; but by others, f:om the coast of Angola in Africa, where the Portuguese had a sugar colony. It was transported in 1506, from the Brazils and the Canaries, into Hispaniola or Hayti, where several crushing-mills were constructed in a short time. It would appear, moreover, from the statement of Peter Martyr, in the third book of his first Decade, written during the second expedition of Christopher Columbus, which happened between 1493 and 1495 , that even at this date the cultivation of the sugar cane was widely spread in St. Domingo. It may therefore be supposed to have been introduced here by Columbus himself, at his first voyage, along with other productions of Spain and the Canaries, and that its cultivation had come into considerable activity at the period of his second expedition. Towards the middle of the 17 th century, the sugar cane was imported into Barbadoes from Brazil, then into the other English West Indian possessions, into the Spanish Islands on the coast of America, into Mexico, Peru, Chile, and, last of all, into the French, Dutch, and Danish colonies.

The sugar cane, Arundo saccharifera, is a plant of the graminiferous family, which varies in height from 8 to 10 , or even to 20 feet. Its diameter is about an inch and a half; its stem is dense, brittle, and of a green hue, which verges to yellow at the approach of maturity. It is divided by prominent annular joints of a whitish-yellow color, the plane of which is perpendicular to the axis of the stem. These joints are placed about 3 inches apart; and send forth leaves, which fall off with the ripening of the plant. The leaves are 3 or 4 feet long, flat, straight, pointed, from 1 to 2 inches in breadth, of a seagreen tint, striated in their length, alternate, embracing the stem by their base. They are marked along their edges with almost imperceptible teeth. In the 11 th or 12 th month of their growth, the canes push forth at their top a sprout 7 or 8 feet in height, nearly half an inch in diameter, smooth, and without joints, to which the name arrow is given. This is terminated by an ample panicle, about 2 feet long, divided into several knotty ramifications, composed of very numerous flowers, of a white color, apetalous, and furnished with 3 stamens, the anthers of which are a little oblong. The roots of the sugar cane are jointed and nearly cylindrical; in diameter they are about one twelfth of an inch; in their utmost length 1 foot, presenting over their surface a few short radicles.

The stem of the cane in its ripe state is heavy, very smooth, brittle, of a yellowishviolet, or whitish color, according to the variety. It is filled with a fibrous, spongy, dirty-white pith, which contains very abundant sweet juice. This juice is elaborated separately in each internodary portion, the functions of which are in this respect independent of the portions above and below. The cane may be propagated by seeds or buds with equal facility; but it is usually done by cuttings or joints of proper lengths, from 15 to 20 inches, in proportion to the nearness of the joints, which are generally taken from the tops of the canes, just below the leaves.

There are several varieties of the singar-cane plant. The first, and longest known, is the creole, or common sugar cane, which was originally introduced at Madeira. It grows freely in every region within the tropics, on a moist soil, even at an eleration of 3000 feet above the level of the sea. In Mexico, among the mountains of CaudinaMasca, it is cultivated to a height of more than 5000 feet. The quantity and quality of sugar which it yiclds, is proportional to the heat of the place where it grows, provided it be not too moist and marshy. 
The second variety of this plant is the Otaheitan cane. It was introduced into the West Indies about the end of the 18 th century. This variety, stronger, taller, with longer spaces between the joints, quicker in its growth, and much more productive in sugar, succeeds perfectly well in lands which seem too much imporerished to grow the ordinary cane. It sends forth shoots at temperatures which chill the growth and development of the creole plant. Its maturation does not take more than a year, and is accomplished sometimes in nine months. From the strength of its stem, and the woodiness of its fibres, it better resists the storms. It displays a better inflorescence, weighs a third more, affcrds a sixth more juice, and a fourth more sugar, than the common variety. Its main advantage, however, is to yield four crops in the same time that the cresle cane yields only three. Its juice contains less feculency and mucilage, whence its sugar is more easily crystallized, and of a fairer color.

Besides these two varieties, another kind is described by Humboldt and Bonpland, under the name of the violet sugar-cane, for its haum and leaves are of this color. It was transported from Batavia in 1782. It flowers a month sooner than the rest, that is, in August, but it yields less solid sugar, and more liquid, both of which have a violet tint.

In saying that the cane may be propagated by seeds as well as huds, we must remark that in all the colonies of the New World, the plant flowers, indeed, but it then sends forth a shoot (arrow), that is, its stem elongates, and the seed-ressel prores abortive. For this reason, the bud-joints must there be used for its propagation. It grows to seed. however, in India. This circumstance occurs with some other plants, which, when propagated by their roots, cease to yield fertile seeds; such as the banana, the bread-fruit, the lily, and the tulip.

In the proper season for planting, the ground is marked out by a line into rows three or four feet asunder, in which rows the canes are planted about two feet apart. The series of rows is divided into pieces of land 60 or 70 feet broad, leaving spaces of about 20 feet, for the convenience of passage, and for the admission of sun and air between the stems. Canes are usually planted in trenches, about 6 or 8 inches deep, made with the hand-hoe, the raised soil being heaped to one side, for covering-in the young cane; into the holes a negro drops the number of cuttings intended to be inserted, the digging being performed by other negroes. The earth is then drawn about the hillocks with the hoe. This labor has been, however, in many places better and more cheaply performed by the plough; a deep furrow being made, into which the cuttings are regularly planted, and the mould then properly turned in. If the ground is to be afterwards kept clear by the horse-hoe, the rows of canes should be 5 feet asunder, and the hillocks $2 \frac{1}{2}$ feet distant, with only one cane left in one hillock. After some shoots appear, the sooner the horse-hoe is used, the more will the plants thrive, by keeping the weeds under, and stirring up the soil. Plant-canes of the first growth have been known to yield, on the brick-mould of Jamaica, in very fine seasons, $2 \frac{1}{2}$ tons of sugar per acre. The proper season for plinting the cane slips, containing the buds, namely, the top part of the cane, stripped of its leaves, and the two or three upper joints, is in the interval between August and the beginning of November. Favored by the autumnal weather, the young plants become luxuriant enough to shade the ground before the dry season sets in; thereby lieeping the roots cool and moderately moist. By this arrangement the creole canes are ripe for the mill in the beginning of the second year, so as to enable the manager to finish his crop early in June. There is no greater error in the colonist than planting canes at an improper season of the year, whereby his whole system of operations becomes disturbed, and, in a certain degree, abortive.

The withering and fall of a leaf afford a good criterion of the maturity of the canejoint to which it belonged; so that the eight last leafless joints of two canes, which are cut the same day, have exactly the same age and the same ripeness, though one of the canes be 15 and the other only 10 months old. Those, however, cut towards the end of the dry season, before the rains begin to fall, produce better sugar than those cut in the rainy season, as they are then somewhat diluted with watery juice, and require more evaporation to form sugar. It may be reckoned a fair average product, when one pound of sugar is obtained from one gallon (English) of juice.

Rattoons (a word corrupted from rejettons) are the sprouts or suckers that spring from the roots or stoles of the canes that have been previously cut for sugar. They are commonly ripe in 12 months; but canes of the first growth are called plant-canes, heing the direct produce of the original cuttings or germs placed in the ground, and require a longer period to bring them to maturity. The first yearly return from the roots that are cut over, are called first rattoons; the second year's growth, second rattoons; and so on, according to their age. Instead of stocking up his rattoons, holing, and planting the land anew, the planter suffers the stoles to continue in the ground, and contente 
himself, as the cane-fields become thin and impoverished, with supplying the vacant places with fresh plants. By these means, and with the aid of manure, the produce of sugar per acre, if not apparently equal to that from plant-canes, gives perhaps in the long run as great returns to the owner, considering the relative proportion of the labor and expense attending the different systems. The common yielding on proper land, such as the red soil of Trelawney, in Jamaica, is 7 hogsheads, of $16 \mathrm{cwts}$. each, to 10 acres of rattoons cut annually; and such a plantation lasts from 6 to 10 years.

When the planted canes are ripe, they are cut close above the ground, by an oblique section, into lengths of 3 or 4 feet, and transported in bundles to the mill-house. If the roots be then cut off, a few inches below the surface of the soil, and covered up with fine mould, they will push forth more prolific offsets or rattoons, than when left projecting in the common way.

OF SUGAR MILLS.

The first machines employed to squeeze the canes, were mills similar to those which serve to crush apples in some cider districts, or somewhat like tan-mills. In, he centre of a circular area, of about 7 or 8 feet in diameter, a vertical heavy wheel was made to revolve on its edge, by attaching a horse to a cross beam projecting horizontally from it, and making it move in a circular path. The cane pieces were strewed on the somewhat concave bed in the path of the wheel, and the juice expressed flowed away through a channel or gutter in the lowest part. This machine was tedious and unproductive. It was replaced by the vertical cyli ider-mill of Gonzales de Velosa; which has continued till modern times, with little variation of external form, but is now generally superseded by the sugar-mill with horizontal cylinders.

SUGAR-CANE MILL.

\section{Specification of, and Observations on, the Construction and Use of the best} Horizontal Sugar-mill.

Fig. 1075. Front elevation of the entire mill. Fig. 1076. Horizontal plan. Fig. 1077. End elevation. Fig. 1078. Diagram, showing the dispositions of the feeding and delivering rollers, feeding board, returner, and delivering board.

Fig. 1075. A, A, solid foundation of masonry; B, B, bed plate; c, c, headstocks or standards; $\mathrm{D}$, main shaft (seen only in fig. 1076); $\mathrm{E}$, intermediate shaft; F, F, plummerblocks of main shaft $\mathrm{D}$ (seen only in fig. 1076); $\mathrm{H}$, driving pinion on the fly-wheel shaft of cngine; I, first motion mortise wheel driven by the pinion; $\mathbf{k}$, second motion pinion, on the same shaft; $L$, second motion mortise-wheel, on the main shaft; $M$, brays of wood, holding the plummer-blocks for shaft $\mathrm{D} ; \mathrm{N}$, wrought-iron straps connecting the brays to the standards $\mathrm{c}, \mathrm{C}$; $\mathrm{O}, \mathrm{o}$, regulating screws for the brays; $\mathrm{P}$, top roller and gudgeons; $Q$ and $R$, the lower or feeding and delivering rollers; $s$, clutch for the connexion of the side of lower rollers $\mathrm{Q}$ and $\mathrm{R}$, to the main shaft (seen only in fig. 1076); $\mathbf{T}$, $\mathbf{T}$, the drain gutters of the mill-bed (seen only in fig. 1076).

The same letters of reference are placed respectively on the same parts of the mill in each of figs. 1075,1076 , and 1077 .

The relative disposition of the rollers is shown in the diagram, fig. 1078, in which A is the top roller; $\mathrm{B}$, the feeding roller; $\mathrm{C}$, the delivering roller; $\mathrm{D}$, the returner ; $\mathbf{E}$, the feed bnard; $F$, the delivering board.

The rollers are made two inches and a quarter to two inches and a half thick, and ribbed in the centre. The feeding and delivering rollers have small flanges at their ends (as shown in fig. 1075), between which the top roller is placed; these flanges prevent the pressed canes or begass from working into the mill-bed. The feeding and top rollers are generally fluted, and sometimes diagonally, enabling them the better to seize the canes from the feed-board. It is, however, on the whole, considered better to flute the feeding roller only, leaving the top and delivering rollers plane; when the top roller is fluted, it should be very slightly, for, after the work of a few weeks, its surface becomes sufficiently rough to bite the canes effectively. The practical disadvantage of fluting the delivering rollers, is in the grooves carrying round a portion of liquor, which is speedily absorbed by the spongy begass, as well as in breaking the begass itself, and thus causing great waste.

The feed board is now generally made of cast iron, and is placed at a considerable inclination, to allow the canes to slip the more easily down to the rollers. The returner is also of cast iron, serrated on the edge, to admit the free flowing of the liquor to the mill-bed. The concave returner, formerly used, was pierced with holes to drain off the. liquor, but it had the serious disadvantage of the holes choking up with the splinters of the cane, and has therefore been discarded. The delivering board is of cast iron, fitted close to the roller, to detach any begass that may adhere to it, and otherwise mix with: the liquor. 
In Demerara, Surinam, Cayenne, and the alluvial district of Trinidad, it is usual to attach to the mill a liquor-pump, with two barrels and three adjustments of stroke. Thir
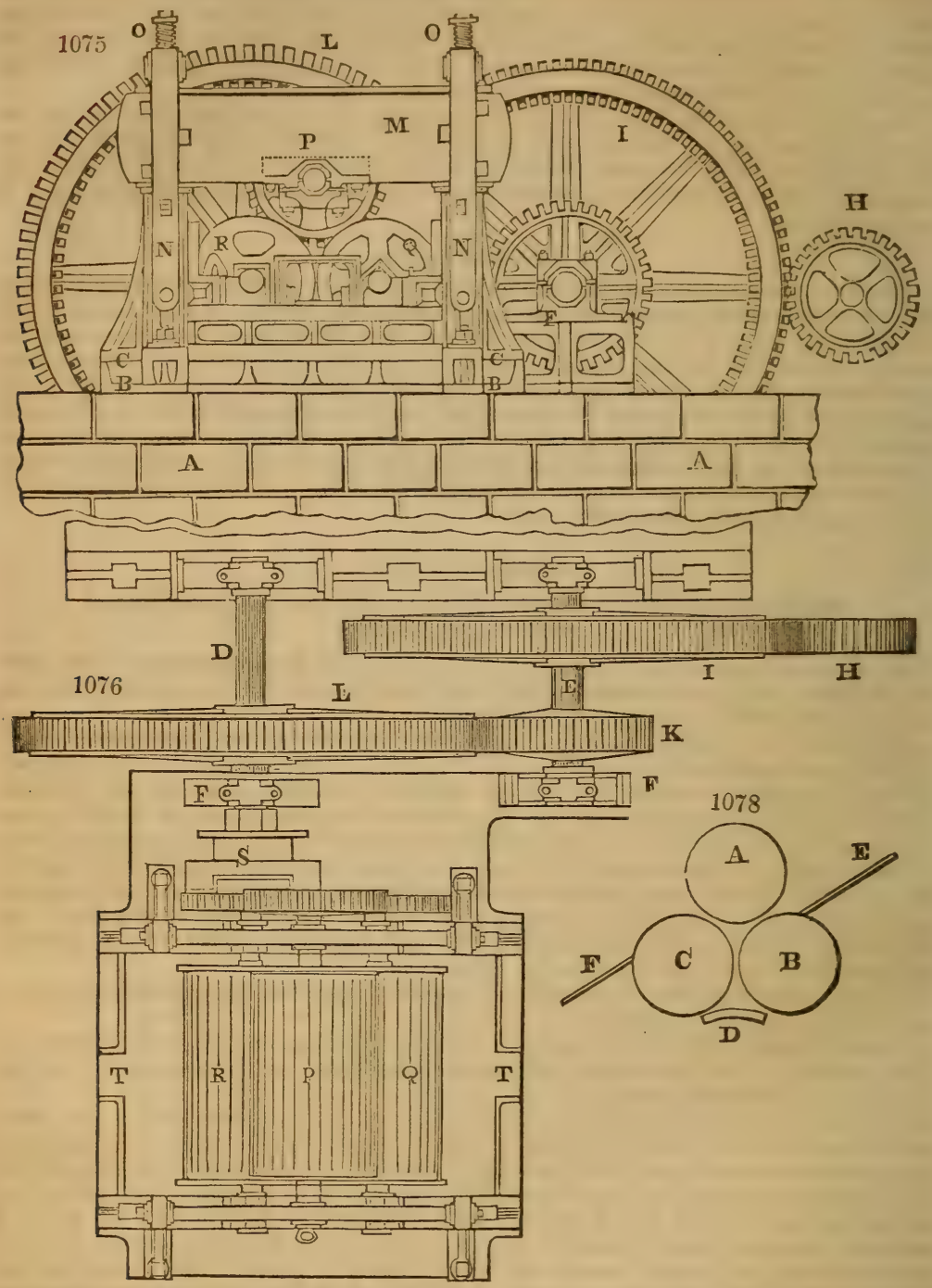

Is worked fiom the gudgeon of the top roller. In action, the liquor from the gutter of the mili-bed runs into the cistern of the pump, and is raised by the pump to the gutter which leads to the clarifier or coppers. Such pumps have brass barrels and copper discharging pipes, are worked with a very slow motion, and require to be carefully adjusted to the quantity of liquor to be raised, which, without such precaution, is either not drawn off sufficiently quick, or is agitated with air in the barrels, and delivered to the gutter in a state of fermentation.

In working this mill, the feeding roller is kept about half an inch distant from the upper roller, but the delivering roller is placed so close to it, as to allow the begass to pass through unbroken.

The practice with this mill is to cut the sugar canes into short lengths of abont three feet, and bring them to the mill tied up in small bundles; there the feeder unites them, throws them on the feed board, and spreads them so that they may cross each 
o:her as little as possible. They are taken in by the feed rollers, which split and slightly press them; the liquor flows down, and, the returner guiding the canes between the top

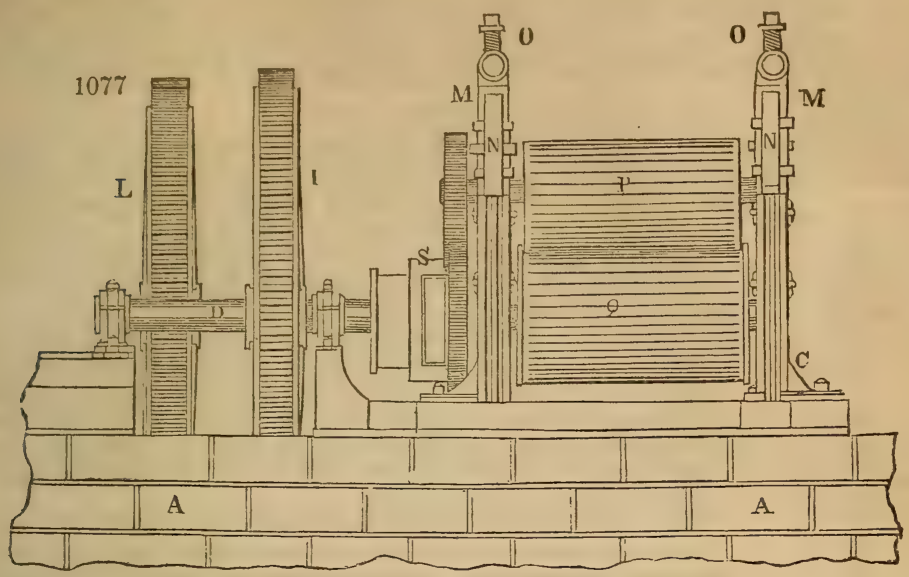

and delivering rollers, they receive the final pressure, and are turned out on the mill-floor, while the liquor runs back and falls into the mill-bed. The begass, then in the state of pith, adhering to the skin of the cane, is tied up in bundles, and after being exposed a short time to the sun, is finally stored in the begass-house for fuel. By an important improvement in this stage of the process, recently introduced, the begass is carried to the begass-house by a carrier chain, worked by the engine.

The relative merits of horizontal and vertical sugar-mills on this construction may be thus stated:-The horizontal mill is cheaper in construction, and is more easily fixed; the process of feeding is performed at about one half of the labor, and in a much superior manner; the returner guides the canes to receive the last pressure more perfectly; and the begass is not so much broken as in the vertical mill, but left tolerably entire, so as to be tied, dried, and stored, with less trouble and waste.

The vertical mill has a considerable advantage, in being more easily washed; and it can be readily and cheaply mounted in wooden framing; but the great labor of feeding the vertical mill renders it nearly inapplicable to any higher power than that of about ten horses. In situations where the moving power is a windmill, or a cattle-gin, the vertical mill may be preferred.

The scale of produce of such mills varies according to the climate and soil. In Demerara, a well-constructed engine and mill will produce about 100 gallons of liquor per hour for each horse power.

The dimensions of the most approved horizontal mills are these :-

\begin{tabular}{|c|cc|c|}
\hline Horse-power of Engine. & \multicolumn{2}{|c|}{ Length of Rollers. } & Diameter of Rollers. \\
\cline { 1 - 3 } & yt. & in. & inches. \\
8 & 4 & 0 & 25 \\
10 & 4 & 6 & 27 \\
12 & 4 & 8 & 28 \\
\hline
\end{tabular}

The surface speed of the rollers is 3.4 or 3.6 feet per minute; and to provide for the varying resistance arising from irregular feeding, or the accidental crossing of the canes, by which the engine is often brought up so suddenly as to break the fly-wheel shaft, it is necessary to make both the shaft and the fly-wheel of unusual strength and weight.

Sugar is manufactured in the East Indies by two distinct classes of persons; the ryots, who raise the sugar cane, extract its juice, and inspissate it to a sirupy consistence; and the goldars, who complete the conversion into sugar.

The ryots are the farmers, or actual cultivators of the soil; but, properly speaking, they are merely peasants, toiling under oppressive landlords, and miserably poor. After they cut the canes, they extract the juice by one $c i$ other of the rude mills or mortars presently to be described, and boil it down to an entire mass, which is generally called goor, without making any attempt to clarify it, or separate the granular sugar from the uncrystallizable molasses. This goor is of various qualities; one of 
which, in most common use for making sugar, is known amongst the English settler under' the name of jaggery. There is a caste in Ceylon, called jaggeraros, whe make sugar from the produce of the Caryota urens, or Kitul tree; and the sugar is styled jaggery. Sugar is not usually made in Ceylon from the sugar cane; but either fiom the juice of the Kitul, from the Cocos nucifera, or the Borassus flabelliformis (the Palmyra tree.)

Several sorts of cane are cultivated in India.

The Cadjoolee ( fig. 1079) is a purple-colored cane; yields a sweeter and richer juice than the yellow or light-colored, but in less quantities, and is harder to press. It grows

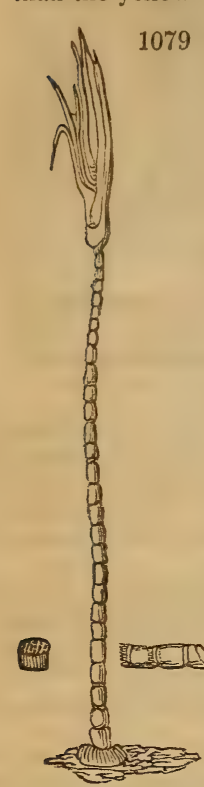
in dry lands. When eaten raw, it is somewhat dry and pithy in the mouth, but is esteemed very good for making sugar. It is not known to the West India planter. The leaves rise from a point 6 feet above the ground. An oblique and transverse section of the cane is represented by the parts near the bottom of the figure.

The Pooree is a light-colored cane, yellow, inclining to white, deeper yellow when ripe and on rich ground. West India planters consider it the same sort as one of theirs. It is softer and more juicy than the preceding, but the juice is less rich, and produces a weaker sugtr. It requires seven parts of pooree juice to make as much goor as is produced from six of the cadjoolee. Much of this cane is brought to the Calcutta market, and eaten raw.

The Cullorah thrives in swampy lands, is light-colored, and grows to a great height. Its juice is more watery, and yields a weaker sugar also than the cadjoolee. However, since much of Bengal consists of low grounds, and since the upland canes are apt to suffer from drought, it deserves encouragement in certain localities.

It is only large farms that cut an acre of cane in a year; one mill, therefore, and one set of the implements used in inspissating the juice, although very rude and simple, serve for several farms, and generally belong to some wealthy man, who lets them out for hire to his poorer neighbors, the whole of whom unite to clear each other's fields by turns; so that though many people anil cattle are employec at one of these miserable sets of works, very few indeed are hired, and the greater part of the labor is performed by the common stock of the farms.

The inspissated juice, or extract of cane, called by the natives goor is of two kinds; one of which may be termed cake extract, and the other pot extract; both being often denominated jaggery, as above stated, by the English residents.

One third of an acre of good land in the southern districts, is reckoned by the

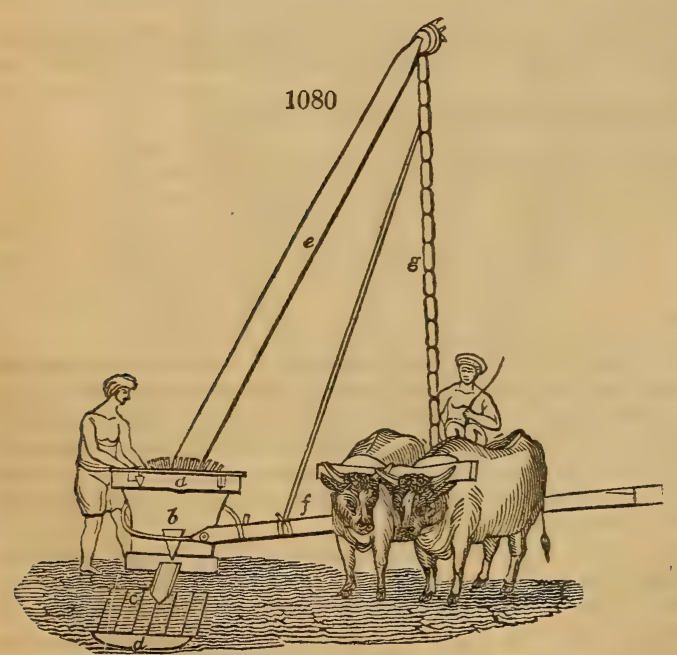
farmers to produce 18,891 pounds of cane, and 1,159 pounds of pot extract. Its produce in cake extract is about 952 pounds.

I shall now describe the primitive rude mill and boiler used in preparing the extract of sugar cane, and which are usually let to the ryots by the day. The mill in Dinajpur, fig. 1080, is on the principle of a pestle and mortar. The pestle, however, does not beat the canes, but is rubbed against them, as is done in many chemical triturations; and the moving force is two oxen. The mortar is generally a tamarind tree, one end of which is sunk deep in the ground, to give it firmness. The part projecting, $a, a, a, a$, may De about two feet high, and a foot and a half in diameter; and in the upper end a hollow is cut, like the small segment of a sphere. In the centre of this, a 
channel descends a little way perpendicularly, and then obliquely to one side of the mortar, so that the juice, as squeezed from the cane, runs off, by means of a spout $b$, into a strainer $c$, through which it falls into an earthen pot, that stands in a hole $d$, under the spout. The pestle $e$, is a tree about 18 feet in length, and 1 foot in diameter, rounded at its bottom, which rubs against the mortar, and which is secured in its place by a button or knob, that goes into the channel of the mortar. The moving force is applied to a horizontal beam $f$, about 16 feet in length, which turns round about the mortar, and is fastened to it by a bent bamboo $b$. It is suspended from the upper end of the pestle by a bamboo $g$, which has been cur with part of the root, in which is formed a pivot that hangs on the upper point of the pestle. The cattle are yoled to the horizontal beam, at about ten feet from the mortar, move round it in a circle, and are driven by a man, who sits on the beam, to increase the weight of the triturating power. Scarcely any machine more miserable can be conceived; and it would be totally ineffectual, were not the cane cut into thin slices. This is a troublesome part of the operation. The grinder sits on the ground, having before him a bamboo stake, which is driven into the earth, with a deep notch formed in its upper end. He passes the canes gradually through this notch, and at the same time cuts off the slices with a kind of rude chopper.

The boiling apparatus is somewhat better contrived, and is placed under a shed, though the mill is without shelter. The fireplace is a considerable cavity dug in the ground, and covered with an iron boiler $p$, fig. 1081. At one side of this, is an opening q, for throwing in fuel; and opposite to this, is another opening, which communicates

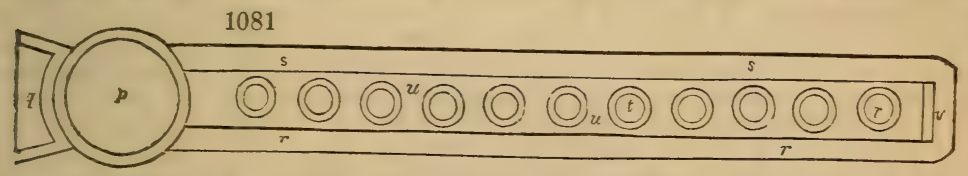

with the horizontal flue. This is formed by two parallel mud walls $r, r, s, s$, about 20 feet long, 2 feet high, and 18 inches distant from each other. A row of eleven earthen boiless $t$, is placed on these walls, and the interstices $u$, are filled with clay, which completes the furnace-flue, an opening $v$, being left at the end, for giving vent to the smoke.

The juice, as it comes from the mill, is first put into the earthen boiler that is most distant from the fire, and is gradually removed from one boiler to another, until it reaches the iron one, where the process is completed. The fireplace is manifestly on the same model as the boiler range in the WVest Indies, and mar possibly have suggested it, since the Hindostan furnace is, no doubt, of immemorial usage. The execution of its parts is rery rude and imperfect. The inspissated juice that can be prepared in 24 hours by such a mill, with 16 men and 20 oxen, amounts to no more than $476 \mathrm{lbs}$; and it is only in the southern parts of the district, where the people work night and day, that the sugar-works are so productive. In the northern districts, the people work only during the day, and inspissate ahout one half the quantity of juice. The average daily make of a West India sugar-house, is from 2 to 3 hogsheads, of $16 \mathrm{cwts}$. each.

The Indian manufacturers of sugar purchase the abore inspissated juice or goor from the farmers, and generally prefer that of a granular honey consistence, which is offered for sale in pots. As this, however, cannot conveniently be brought from a distance, some of the calie kind is also employed. The boilers are of two sizes; one adapted for making at each operation about ten cwts.; the other, about eight and a half. 'The latter is the segment of a sphere, nine feet diameter at the mouth; the former is larger. The boiler is sunk into a cylindrical cavity in the oround, which serves as a fireplace, so that its edge is just above the floor of the boiling-house. The fuel is thrown in by an aperture close to one side of the boiler, and the smoke escapes by a horizontal chimney that passes out on the opposite side of the hut, and has a small round aperture, about ten feet distant from the wall, in order to lessen the danger from fire. Some manufacturers hare only one boiler; others as many as four ; but each boiler has a separate hut, in one end of which is some spare fuel; and in the other, some bamboo stages, which support cloth strainers, that are used in the operation. This hut is about twenty-four cubits long, and ten broad; has mud walls, six cubits high; and is raised about one cubit above the ground.

For each boiler, two other houses are required: one in which the cane extract is separated by straining from the molasses, is about twenty cubits long by ten wide; another, about thirty cubits long, by eight wide, is that in which, after the extract has beew strained, boiled, and clarified, the treacle is separated from the sugar by an operation an. alogous to claying. 
Each sugar manufacturer has a warehouse besides, of a size proportional to the num. ber of his boilers.

About 960 pounds of pot extract being divided into four parts, each is put into a bag of coarse sackcloth, hung over an erual number of wide-mouthed earthen vessels, and is besprinkled with a little water. These drain from the bags about 240 pounds of a substance analogous to West Indian molasses. The remainder in the bags is a kind of coarse muscorado sugar; but is far from being so well drained and freed from molasses as that of the Antilles. The 720 pounds of this substance are then put into a boiler with 270 pounds of water, and the mixture is boiled brislily for 144 minutes, when 180 additional pounds of water are added, and the boiling is continued for 48 minutes more. An alkaline solution is prepared from the ashes of the plantain tree, strewed over straw placed in the bottom of an earthen pot perforated with holes. Ninety pounds of water are passed through; and 6 pounds of the clear lixivium are added to the boiling sirup; whereby a thick scum is raised, which is remored. After 24 minutes, four and a haif pounds of alkaline solution, and about two fifths of a pound of raw milk, are added; after which the boiling and skimming are continued 24 minutes. This must be repeated from five to seven times, until no more scum appears. 240 pounds of water being now added, the liquor is to be poured into a number of strainers. These are bags of coarse cotton cloth, in the form of inverted quadrangular pyramids, each of which is suspended from a frame of wood, about two feet square. The operation of straining oceupies about 96 minutes. The strained liquor is divided into three parts : one of these is put into a boiler, with from half a pound to a pound and a half of alkaline solution, one twelfth of a pound of milk, and 12 pounds of water. After having boiled for between 48 and $\tau 2$ minutes, three quarters of a pound of milk are added, and the liquor is poured, in equal portions, into four refining pots. These are wide at the mouth, and pointed at the bottom; but are not conical, for the sides are curved. The bottom is perforated, and the stem of a plantain leaf forms a plug for closing the aperture. The two remaining portions of the strained liquor are managed in exactly the same manner; so that each refining pot has its share of each portion. When they have cooled a little, the refining pot is removed to the curing-house, and placed on the ground for 24 hours; next day they are placed on a frame, which supports them at some distance from the ground. A wide-mouthed vessel is placed under each, to receive the viscid liquor that drains from them. In order to draw off this more completely, moist leaves of the Valisneria spiralis are placed over the mouth of the pot, to the thickness of two inches; after 10 or 12 days, these are removed; when a crust of sugar, about half an inch in thichness, is found on the surface of the boiled liquor. The crust being broken and removed, fresh leaves are repeatedly added, until the whole sugar has formed; which requires from 75 to 90 days. When cake extract is used, it does not require to be strained before it be put into the boiler.

On the above-described operose and preposterous process, it is needless to make any remarks. While it is adhered to with the tenacity of Hincloo habit, the West Indies has no reason to fear the competition of the East, in the manufacture of sugar, provided the former avail themselves of the aids which chemical and mechanical science are ready to supply.

In every part of the Behar and Putna districts, several of the confectioners prepare the coarse article called shukkur, which is entirely similar in appearance to the inferior Jamaica sugars. They prepare it by putting some of the thin extract of sugar cane into coarse sackcloth bags, and by laying weights on them, they squeeze out the molasses;

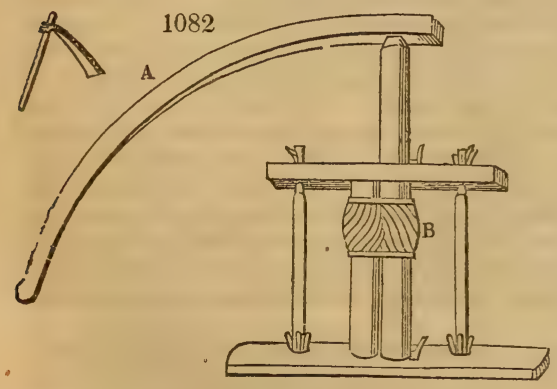
a process perfectly analogous to that contemplated in several English patents.

The sugar-mill at Chica Ballapura is worked by a single pair of buffaloes or oxen, fig. 1082, going round with the lever $A$, which is fixed on the top of the right-hand roller. The two rollers have endless screw heads $\mathrm{B}$, which are formed of 4 spiral grooves and 4 spiral ridges, cut in opposite directions, which turn into one another, when the mill is working. These rollers and their heads are of one piece, made of the toughest and hardest wood that can be got, and suck ns will not impart any bad taste to the juice. They are supported in a thick strong wooden frame, and their distance from each other is regulated by means of wedges, which pass through mortises in the frame planks, and a groove made in a bit of some 
sort of hard wood, and press upon the axis of one of the rollers. The axis of the other presses arainst the left-hand side of the hole in the frame-boards. The cane juice runs down the ruliers, and through a hole in the lower frame-board, into a wooden conductor, which carries it into an earthen pot. Two long-pointed stakes or piles are driven into the earth, to keep the mill steady, which is all the fixing it requires. The under part of the lowermost plank of the frame rests upon the surface of the ground, which is chosen level and very firm, that the piles may hold the faster. A hole is dug in the earth, immediately below the snout of the conductor, to receive the pot.

The mill used in Burdwan and near Calcutta, is simply two small wooden cylinders, grooved, placed horizontally, close to each other, and turned by two men, one at each end. This simple engine is said completely, but slowly, to express the juice. It is very cheap, the prime cost not being two rupees; and being easily moved from firld to field, it saves much labor in the carriage of the cane. Notwithstanding this advantage, so rude a machine must leave a large proportion of the richest juice in the cane-trash.

It is curious to find in the ancient arts of Hindostan exact prototypes of the sugar-rollers, horizontal and upright, of relatively modern invention in the New World.

The sugal-mill of Chinaratam, fig. 1083, consists of a mortar, lever, pestle, and regulator. The mortar is a tree about 10 feet in length, and 14 inches in diameter : $a$ is a

1083
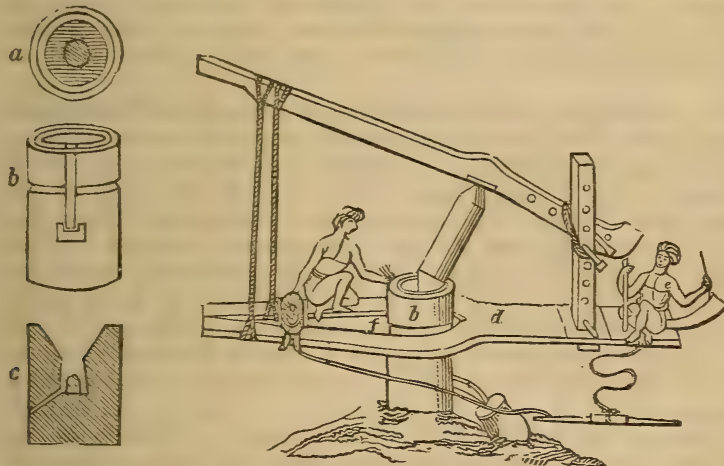
plan of its upper end; $b$ is an outside view; and $c$ is a vertical section. It is sunk perpendicularly into the earth, leaving one end 2 feet above the surface. The hollow is conical, truncated downwards, and then becomes cylindrical, with a hemispherical projection in its bottom, to allow the juice to run freely to the small opening that conveys it to a spout, from which it falls into an earthen pot. Round the upper mouth of the cone is a circular cavity, which collects any of the juice that may run over from the upper ends of the pie. ces of cane; and thence a canal conveys this juice, down the outside of the mortar, tc the spout. The beam $d$, is about 16 feet in length, and 6 inches in thickness, being cut out from a large tree that is divided by a fork into two arms. In the fork an excavation is made for the mortar $b$, round which the beam turns horizontally. The surface of this excaration is secured by a semi-circle of strong wood. The end towards the fork is quite open, for changing the beam without trouble. On the undivided end of the beam sits the bullock-driver $\varepsilon$, whose cattle are yoked by a rope which comes from the end of the beam; and they are prevented from dragging out of the circle by another rope, which passes from the yoke to the forked end of the beam. On the arms $f$, a basket is placed, to hold the cuttings of cane; and between this and the mortar sits the man who feeds the mill. Just as the pestle comes round, he places the pieces of cane sloping down into the cavity of the mortar; and after the pestle has passed, he removes those that have been squeezed.

\section{OF THE MANUFACTURE OF SUGAR IN THE WEST INDIES,}

Cane-juice varies exceedingly in richness, with the nature of the soil, the culture, the season, and variety of the plant. It is an opaque fluid, of a dull gray, olive, or olivegreen color; in taste, balmy and saccharine; exhaling the balsamic odor of the cane; slightly viscid; and of a specific gravity varying from 1.033 to 1.106 , according to circumstances. When fresh, it consists of two parts; the one liquid, the other solid; the latter of which being merely suspended in the former, and, therefore, separable in a great measure by filtration or repose. The solid matter consists of fragments of the cellular parenchyma of the cane, its fibres, and bark, mechanically protruded throngh the mill; mixed with a very abundant greenish substance, like that called chlorophyle by chemists.

When left to itself in the colonial climates, the juice runs rapidly into the acetous fermentation; twenty minutes being, in many cases, sufficient to bring on this destructive change. Hence arises the necessity of subjecting it immediately to clarifying pro- 
cesses, speedy in their action. When deprived of its green fecula and glutınous extractive, it is still subject to fermentation; but this is now of the vinous kind. The juice flows from the mill through a wooden gutter lined with lead, and being conducted into the sugar-house, is received in a set of large pans or caldrons, called clarifiers. On estates which make on an average, during crop time, from 15 to 20 hogsheads of sugar a week, three clarifiers, of from 300 to 400 gallons' capacity each, are sufficient. With pans of this dimension, the liquor may be drawn off at once by a stop-cock or syphon, without disturbing the feculencies after they subside. Each clarifier is hung over a separate fire, the flue being furnished with a damper for checking the combustion, or extinguishing it altogether. The clarifiers are sometimes placed at one end, and sometimes in the middle of the house, particularly if it possesses a double set of evaporating pans.

Whenever the stream from the mill cistern has filled the clarifier with fresh juice, the fire is lighted, and the temper, or dose of slaked lime, diffused uniformly through a little juice, is added. If an albuminous emulsion be used to promote the clarifying, very little lime will be required; for recent cane-liquor contains no appreciable portion of acid to be saturated. In fact, the lime and alkalis in general, when used in small quantity, seem to coagulate the glutinous extractive matter of the juice, and thus tend to brighten it up. But if an excess of temper be used, the gluten is taken up again by the strong affinity which is known to exist between sugar and lime. Excess of lime may always be corrected by a little alum-water. Where canes grow on a calcareous marly soil, in a favorable season, the saccharine matter gets so thoroughly elaborated, and the glutinous mucilage so completely condensed, that a clear juice and a fine sugar may be obtained without the use of lime.

As the liquor grows hot in the clarifier, a scum is thrown up, consisting of the coagulated feculencies of the cane-juice. The fire is now gradually urged till the temperature approaches the boiling point; to which, however, it must not be suffered to rise. It is known to be sufficiently heated, when the scum rises in blisters, which break into white foth; an appearance observable in about forty minutes after kindling the fire. The damper being shut down, the fire dies out; and after an hour's repose, the clarified liquor is ready to be drawn off into the last and largest in the series of evaporating pans. In the British colonies, these are merely numbered $1,2,3,4,5$, beginning at the smallest, which hangs right over the fire, and is called the teache; because in it the trial of the sirup, by touch, is made. The flame and smoke proceed in a straight line along a flue to the chimney-stalk at the other end of the furnace. The area of this flue proceeds, with a slight ascent from the fire to the aperture at the bottom of the chimney; so that beiween the surface of the grate and the bottom of the teache, there is a distance of 28 inches; while between the bottom of the flue and that of the grand, No. 5, at the other end of the range, there are barely 18 inches.

In some sugar-houses there is planted, in the angular space between each boiler, a basin, one foot wide and a few inches deep, for the purpose of receiving the scum which thence flows off into the grand copper, along a gutter scooped out on the margin of the brick-work. The skimmings of the grand are thrown into a separate pan, placed at its side. A large cylindrical cooler, about six feet wide and two feet deep, has been plaeed in certain sugar-works near the teache, for receiving successive charges of its inspissated sirup. Each finished charge is called a skipping, because it is skipped or laded out. The term striking is also applied to the act of emptying the teache. When upon one skipping of sirup in a state of incipient granulation in the cooler, a second skipping is poured, this second congeries of saccharine particles agglomerates round the first as nuclei of crystallization, and produces a larger grain ; a result improved by each successive skipping. This principle has been long known to the chemist, but does not seem to have been always properly considered or appreciated by the sugar-planter.

From the above described cooler, the sirup is transferred into wooden chests or boxes, open at top, and of a rectangular shape; also called coolers, bnt which are more properly crystallizers or granulators. These are commonly six in number; each being about one foot deep, seven feet long, and five or six feet wide. When filled, such a mass is collected, as to favor slow cooling, and consequent large-grained crystallization. If these boxes be too shallow, the grain is exceedingly injured, as may be easily shown by pouring some of the same sirup on a small tray ; when, on cooling, the sugar will appear like a muddy soft sand.

The criterion by which the negro boilers judge of the due concentration of the sirur in the teache, is difficult to describe, and depends almost entirely on the sagacity and experience of the individual. Some of them judge by the appearance of the incipient grain on the back of the cooling ladle; but most decide by "the torch," that is, the fee and appearance of a drop of the sirup pressed and then drawn into a thread between the thumb and fore-finger. The thread eventually breaks at a certain limit of exten sion, shrinking from the thumb to the suspended finger, in lengths somewhat propor 
tional to the inspissation of the sirup. But the appearance of granulation in the thread must also be considered; for a viscid and damaged sirup may give a long enough thread, and yet yield almost no crystalline grains when cooled. Tenacity and granular aspect must, therefore, be both taken into the account, and will continue to constitute the practical guides to the negro boiler, till a less barbarous mode of concentrating cane-juice be substituted for the present naked teache, or sugar frying-pan.

That weak sugars are such as contain an inferior proportion of carbon in their composition, was first deduced by me from my experiments on the ultimate analysis of vegetable and animal bodies; an account of which was published in the Philosophical Trans. actions of the Royal Society for 1822. Since then, Dr. Prout has arrived at results confirmatory of my views. See Philosophical Transactions for 1827. Thus, he found pure sugar-candy, and the best refined sugar, to contain 42.85 parts of carbon per cent.; East India sugar-candy, 41.9 parts ; East India raw sugar in a thoroughly dry state, but of a low quality, 40.88 ; manna sugar, well refined, $28 \cdot 7$; sugar from Narbonne honey, $36 \cdot 36$; sugar from starch, $36 \cdot 2$. Hence, by caramelizing the sirup in the teache, not only is the crystaliizable sugar blackened, but its faculty of crystallizing impaired, and the granular portion rendered weaker.

A viscous sirup containing much gluten and sugar, alicred by lime, requires a higher temperature to enable it to granulate than a pure saccharine sirup; and therefore the thermometer, though a useful adjuvant, can by no means be regarded as a sure guide, in determining the proper instant for striking the teache.

The colonial curing-house is a capacious building, of which the earthen floor is excavated to form the molasses reservoir. This is lined with sheet lead, boards, tarras, or other retentive cement; its bottom slopes a little, and it is partially covered by an open massive frame of joist-work, on which the potting casks are set upright. These are merely empty sugar hogsheads, without headings, having 8 or 10 holes bored in their bottoms, through each of which the stalk of a plantain leaf is stuck, so as to protrude downwards 6 or 8 inches below the level of the joists, and to rise above the top of the cask. The act of transferring the crude concrete sugar from the crystallizers into these hogsheads is called potting. The bottom holes, and the spongy stalks stuck in them, allow the molasses to drain slowly downwards into the sunk cistern. In the common mode of procedure, sugar of average quality is kept from 3 to 4 weeks in the curinghouse; that which is soft-grained and glutinous must remain 5 or 6 weeks. The curinghouse should be close and warm, to favor the liquefaction and drainage of the viscid caramel.

Out of 120 millions of pounds of raw sugar, which used to be annually shipped by the St. Domingo planters, only 96 millions were landed in France, according to the authority of Dutrone, constituting a loss by drainage in the ships of 20 per cent. The average transport waste at present in the sugars of the British colonies cannot be estimated at less than 12 per cent., or altogether upwards of 27,000 tons! What a tremendous sacrifice of property !

Within these few years a very considerable quantity of sugar has been imported into Great Britain in the state of concentrated cane-juice, containing nearly half its weight of granular sugar, along with more or less molasses, according to the care taken in the boiling operations. I was at first apprehensive that the sirup might undergo some change on the voyage; but among more than a hundred samples which I have analyzed for the custom-house, I have not perceived any traces of fermentation. Since sugar softens in its grain at each successive solution, whatever portion of the crop may be destined for the refiner, should upon no account be granulated in the colonies; but should be transported in the state of a rich cane-sirup to Europe, transferred at once into the blowing-up cistern, subjected there to the reaction of bone black, and passed through bag-filters, or through layers of the coarsely ground black, previously to its final concentration in the vacuum pan. Were this means generally adopted, I am convinced that 30 per cent. would be added to the amount of home-made sugar loaves corresponding to a given quantity of average cane-juice; while 30 per cent. would be taken from the amount of molasses. The saccharine matter now lost by drainage from the hogsheads in the ships, amounting to from 10 to 15 per cent., would also be saved. The produce of the cane would, on this plan, require less labor in the colonies, and might be exported 5 or 6 weeks earlier than at present, because the period of drainage in the curing-house would be spared.

It does not appear that our sugar colonists have availed themselves of the proper chemical method of counteracting that incipient fermentation of the cane-juice, which sometimes supervenes, and proves so injurious to their products. It is known that grape. must, feebly impregnated with sulphurous acid, by running it slowly into a cask in which a few sulphur matches have been burned, will keep without alteration for a year; and if must, so muted, is boiled into a sirup within a week or ten days, it retains no sulphureous odor. A very slight muting would suffice for the most fermentable cane. 
juice; and it could be easily given, by burning a sulphur match within the cistern imme. diately before charging it from the mill. The cane-juice should, in this case, be heated in the clarifier, so as to expel the sulphurous acid, before adding the temper lime; for otherwise a little calcareous sulphite inight be introduced into the sugar. Thus the acescence so prejudicial to the saccharine granulation would be certainly prevented.

An Accounv of Sugar Imported into the United Kingdom during the years ending 5 th January, 1837, and 5th January, 1838.

\begin{tabular}{|c|c|c|c|c|c|c|}
\hline \multirow{6}{*}{$\begin{array}{l}\text { Sugar, unrefined; } \\
\quad \text { viz.-of the } \\
\text { British posses- } \\
\text { sions in America, } \\
\text { Of Mauritius, } \\
\text { East India Brit- } \\
\text { ish possessions, } \\
\text { East India For- } \\
\text { eign possessions, } \\
\text { Other sorts }\end{array}$} & \multicolumn{2}{|c|}{ Quantities imported. } & \multicolumn{2}{|c|}{$\begin{array}{l}\text { Quantities entered for Home } \\
\text { Consumption. }\end{array}$} & \multicolumn{2}{|c|}{$\begin{array}{c}\text { Gross amount of Duty } \\
\text { received. }\end{array}$} \\
\hline & $183 \%$. & 1838. & 1837. & 1838. & 1837. & 1838. \\
\hline & & & & & $£$. & f. \\
\hline & $\left|\begin{array}{rrr}3,600,516 & 3 & 2 \\
497,303 & 0 & 8\end{array}\right|$ & $\begin{array}{rlr}3,304,092 & 2 & 2 \\
537,054 & 1 & 21\end{array}$ & $\begin{array}{rrr}3.296,641 & 1 & 19 \\
518,228 & 0 & 5\end{array}$ & $\left|\begin{array}{rrrr}3,562,703 & 1 & 24 \\
522,348 & 3 & 11\end{array}\right|$ & $\begin{array}{r}3,956,879 \\
621,596\end{array}$ & $\begin{array}{r}4,275,207 \\
626,131\end{array}$ \\
\hline & 152,229113 & 296,677212 & 110,23620 & $270,146 \quad 1 \quad 2$ & 176,376 & 368,672 \\
\hline & $\begin{array}{rrrr}71,464 & 2 & 0 \\
327,647 & 1 & 12\end{array}$ & 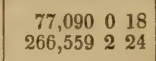 & $\begin{array}{rrr}20 & 3 & 18 \\
31 & 1 & 6\end{array} \mid$ & \begin{tabular}{rrr|}
3 & 3 & 11 \\
37 & 3 & 10
\end{tabular} & $\begin{array}{l}66 \\
41\end{array}$ & $\begin{array}{l}12 \\
95\end{array}$ \\
\hline Total, & $4,649,161 \quad 0 \quad \tau$ & $4,481,474 \quad 1 \quad 21$ & $3.925,140 \quad 0 \quad 20$ & $4,355,240 \quad 1 \quad 2$ & $4,754,958$ & $5,270.117$ \\
\hline
\end{tabular}

An Account of Sugar Exported in the year ending 5th January, 1838, compared with the Exports of the preceding Year.

\begin{tabular}{|c|c|c|}
\hline & 1837. & 1838. \\
\hline $\begin{array}{c}\text { Sugar, of the British possessions in America } \\
\text { Mauritius - - } \\
\text { East India, of British possessions } \\
\text { Foreign do. }\end{array}$ & $\begin{array}{rrr}\text { Cwts. } & \text { qrs. } & \text { lbs. } \\
8,774 & 1 & 15 \\
2,687 & 3 & 14 \\
22,290 & 3 & 16 \\
52,384 & 0 & 4\end{array}$ & $\begin{array}{rrr}\text { Cwts. qrs. lbs. } \\
9,267 & 0 & 21 \\
3,065 & 0 & 19 \\
13,283 & 0 & 22 \\
68,252 & 2 & 18\end{array}$ \\
\hline Other sorts & $191,961 \quad 0 \quad 20$ & 354,513123 \\
\hline
\end{tabular}

Sirup intended for forming clayed sugar must be somewhat more concentrated in the teache, and run off into a copper cooler, capable of receiving three or four successive skippings. Here it is stirred to ensure uniformity of product, and is then transferred by ladles into conical moulds, or formes, made of coarse pottery, having a small orifice at the apex, which is stopped with a plug of wood wrapped in a leaf of maize. These pots are arranged with the base upwards. As their capacity, when largest, is greatly less than that of the smallest potting-casks, and as the process lasts several weeks, the claring-house requires to have very considerable dimensions. Whenever the sirup is properly granulated, which happens usually in about 18 or 20 hours, the pluos are removed from the apices of the cones, and each is set on an earthen pot to receive the drainings. At the end of 24 hours, the cones are transferred over empty pots, and the molasses contained in the former ones is either sent to the fermenting-house or sold. The claying now begins, which consists in applying to the smoothed surface of the sugar at the base of the cone, a plaster of argillaceous earth, or tolerably tenacious loam in a pasty state. The water diffused among the clay escapes from it by slow infiltration, and descending with like slowness through the body of the sugar, carries along with it the residuary viscid sirup which is more readily soluble than the granulated particles. Whenerer the first magma of clay has become dry, it is replaced by a second; and this occasionally in its turn by a third, whereby the sugar cone gets tolerably white and clean. It is then dried in a stove, cut transversely into frusta, crushed into a coarse powder, on wooden trays, and shipped off for Europe. Clayed sugars are sorted into different shades of color, according to the part of the cone from which they were cut; under the denomination in French commerce of premier, second, troisième, petit, comrnun, and tete; the last or the tip being an indifferent article. The clayed sugar of Cuba is called Havana sugar, from the name of the shipping port.

Clayed sugar can be made only from the ripest cane-juice, for that which contains much gluten would be apt to get too much burned by the ordinary process of boiling, to bear the claying operation. The sirups that run off from the second, third, and fourth applications of the clay-paste, are concentrated afresh in a small building apart, called the refinery, and yield tolerable sugars. Their drainings go to the molasses cistern. The cones remain for 20 days in the claying-house, before the sugar is taken out of them.

Claying is seldom had recourse to in the British plantalions, on account of the increase of labor, and diminution of weight in the produce, for which the improvement 
in quality yields no adequate compensation. Such, however, was the esteem in which the French consumers held clayed sugar, that it was prepared in 400 plantations of St. Domingo alone.

\section{SUGAR REFINING.}

Raw, or muscovado sugar, as imported. from the colonies, is contaminated more or less with gluten, lime, but particularly caramel, which give its grains a yellow brown tint, an emprreumatic odor, and a soft clammy feel in the hand. If such sugar be dissolved in water, and the sirup be evaporated by a gentle heat, it will afford a sugar of still inferior quality and appearance. This rapid deterioration is in some measure owing to the injurious operation of a prolonged heat upon the crystalline structure, but chiefly to the chemical reaction of the glutinous ferment and lime upon the sugar. The first care of the refiner should therefore be the immediate abstraction of these noxious alteratives, which he effects by the process called meltings; that is, mixing up the sugar in a pan with hot water or steam into a pap, and transferring this pap intc large sugar-moulds. Whenever these become cool, their points are unplugged, and they are set $t$. Irain for a few days in a warm apartment. Sugar thus cleansed is well prepared for the next refining process; which consists in putting it into a large square copper cistern along with some lime-water, (a little bullock's blood,) and from 5 to 20 per cent. of bone black, and blowing it up with steam; or, in other words, injecting steam through the mixture from numerous orifices in copper pipes laid along the bottom and sides of the vessel. Under the influence of the heat and agitation thus occasioned, the saccharine matter is perfectly dissolved and incorporated with the albumen of the blood and the bone black. Instead of the blood, many refiners employ a mixture of gelatinous alumina and gypsum, called finings, prepared by adding a solution of alum to a bodr of lime-water, collecting, washing, and draining the precipitate upon a filter. 1081

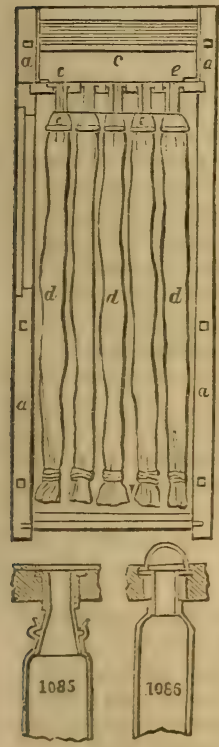
Other refiners use both the blood and finings, with advantage. Bone black is now very frequently employed by the sugar-refiner, not in a fine meal, but in a granular state, like corned gunpowder, for the purpose of decoloring his sirups; in which ease, he places it in a box, in a stratum 8 or 10 inches thick, and makes the sirup percolate downwards through it, into a cistern placed beneath. By this means it is deprived of color, and forms the clairce of the French refiner. When the blowing up eistern is charged with sugar, finely ground bone black, and blood, the mixture must be passed through a proper system of filters. That now most in use is the creased bag filter, represented in figs. 1084 $1085,1086$.

The apparatus consists of an upright square wooden ease $a, a$, about 6 or 8 feet high, furnished with a door of admission to arrange the interior objects; beneath is a cistern with an educting-pipe for receiving and carrying off the filtered liquor; and above the case is another cistern $e$, which, like the rest, is lined with tinned sheet copper. Into the upper cistern, the sirup mixed with animal charcoal is introduced, and passes thence into the mouths $e, e$, of the several filters $d, d$. These consist each of a bag of thick tweeled cotton cloth, about 12 or 15 inches in diameter, and 6 or 8 feet long, which is inserted into a narrow bottomless bag of canvass, about 5 inches in diameter, for the purpose of folding the filter-bag up into a small space, and thus enabling a great extent of filtering surfaces to be compressed into one box. The orifice of each compound bag is tied round a conical brass mouth-piece or nozzle $e$, which screws tight into a corresponding opening in the copper bottom of the upper cistern. From 40 to 60 bags are mounted in each filter case. The liquor which first passes is generally tinged a little with the bone black, and must he pumped back into the upper cistern, for refiltration. In cold weather the interior of the case may be kept warm by a proper distribution of steam-pipes. Fig. 1085 shows one mode of forming the funnel-shaped nozzles of the bags, in which they are fixed by a bayonet catch. Fig. 1086 shows the same made fast by means of a screwed cap, which is more secure.

The next process in sugar-refining is the evaporation of the clarified sirup to the granulating or crystallizing pitch. The more rapidly this is effected, and with the less scorching injury from fire, the better and greater is the product in sugar-loaves. No apparatus answers the refiner's double purpose of safety and expedition so well as the acuum-pan of Howard.

Fig. 1087 shows the structure of a single vacuum-pan. The horizontal diameter of the copper spheroid $\mathrm{A}$, is not less than 5 feet; the depth of the under hemisphere is a 
least 18 inches from the level of the plane; and the height of the dome-cover is 2 feet. The two hemispheres (of which the inferior one is double, or has a steam-jacket) are put together by bolts and screws, with packing between the flanges to preserve the joints tight against atmospheric pressure. The jacket of the lower hemisphere forms the case of the steam, which communicates heat to the sirup enclosed in the inner hemisphere. In general, the pans contain, when filled to the flange, 100 gallons of sirup, and yield about 11 cwts. of granulated sugar, at every charge.

$A$, represents the vacuum spheroid; $\mathbf{B}$, the neck with the lid. From the side of $\mathbf{B}, \mathbf{a}$ pipe passes into the lower extremity of the bent pipe $C, D$, which terminates in the 1087

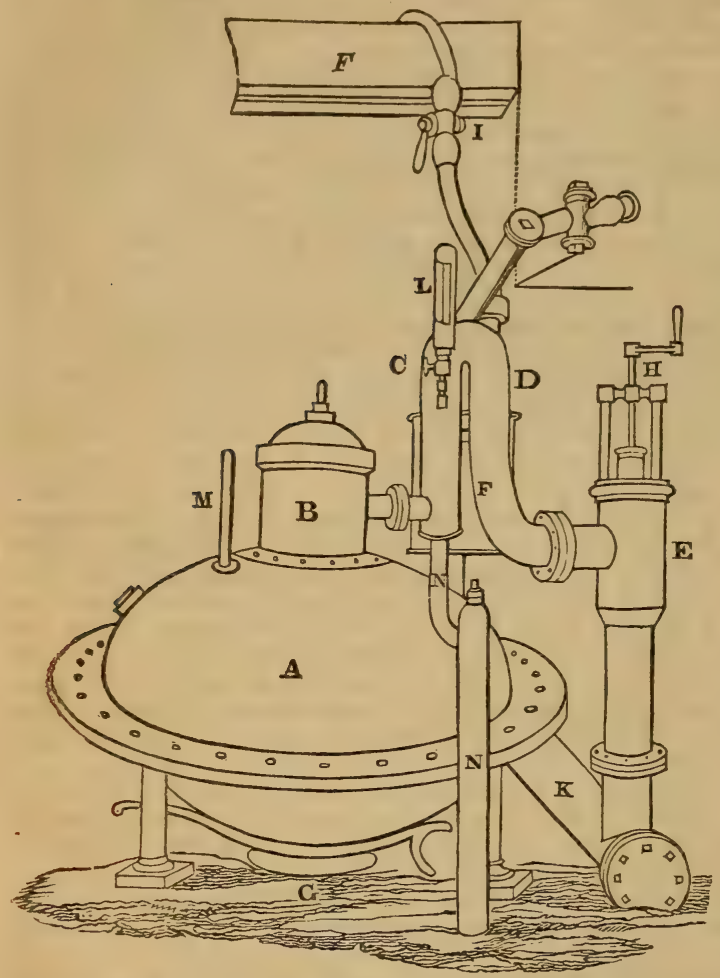
vertical pipe $\mathbf{E}$, connected with the vacuum main-pipe $\mathbf{K}$, proceeding horizontally from the air-pump (not shown in the figure). At the top of $x$, a valve, moveable by a screw $\mathrm{H}$, is placed for establishing or cutting off the connexion with the air-pump at pleasure. Behind $F$, is the mersure cistern, from which the successive charges are admitted into the pan. This measure is filled with the clear sirup, by opening the stopcock $I$, on the pipe under the ceiling, which communicates with the filter-cistern placed above. $G$ is the valve or plug-hole, at the bottom of the pan, for discharging the granulating sirup. This plug is opened by means of a powerful lever attached to it; the connexion with the air-pump being previously intercepted. $\mathbf{L}$, is the barometer, or ma nometer, for showing the state of the vacuum corresponding to the temperature. $\mathbf{N}, \mathbf{N}$, is a cistern-pipe for receiving any little sirup which may accidentally boil over the neck $\mathrm{B}$. Its contents are let off by a stopcock at its bottom from time to time. M shows the place of the proof-stick, an ingenious brass rod for taking out a sample of sirup without admitting air. See infrà.

The charging-cistern contains about 20 gallons. This quantity of sirup being first admitted, and brought to a certain pitch of concentration, a second measure is introduced, the inspissation of which is supposed by some refiners to cause an agglomeration of saccharine matter round the first crystalline particles. The repetition of this process for two or three times is imagined to produce the large brilliant grain of vacuum-pan sugar. This hypothesis is more specious than sound, because the granulating sirup discharged from the pan is subjected to a heat of $180^{\circ}$ or $190^{\circ}$ in the subjacent steamcased receiver, whereby the granulations are again reduced to a very small size. Into this receiver, two or three skippings or discharges of the pan are admitted in succession, and the whole are diligently mixed and agitated by a stirring oar. It is by this process that the granulating tendency is promoted and determined. From this receiver (absurdly enough called a cooler) the moulds are filled in the usual way, by means of copper basins or large ladles.

The case of the under hemisphere of the vacuum-pan is filled with steam, generated under a pressure of four or five pounds on the square inch ; the heat of which causes the interior sirup to boil rapidly while the air-pump is kept in action. A small escape-pipe for waste steam must be placed at the opposite side of the case or jacket, to ensure its equal 
distribution; as also a stopeock below, to let off the water of condensation. The pans are mounted on iron feet, or short pillars, which insulate them fiom the floor, and allow their whole surface to be inspected, and any flaw to be repaired. The air-pump usually stands in a cold-water cistern, to favor the condensation of the aqueous vapor, which it draws out of the pans; and it is kept in constant action by the steam-engine, being attached to the working-beam of its piston.

Fig. 1088 exhibits the general arrangement of the vacuum-pans, and their subsidiary w.pparatus. Here are shown, on the ground floor, the heaters $e, e$, (miscalled coolers), into

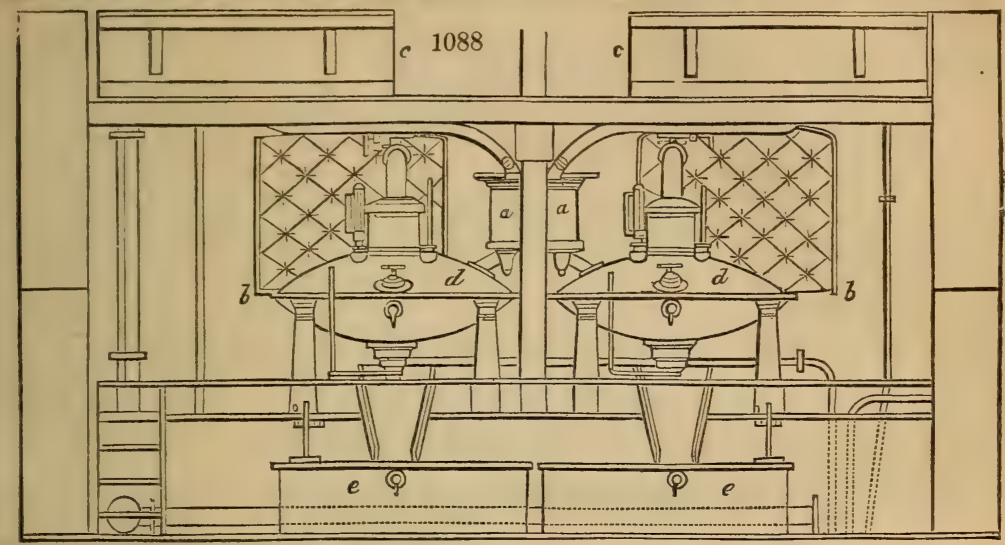

which the concentrated sirup is let down. These heaters are made of copper, in one piece, surrounded with a cast-iron jacket, bolted at the flange or brim to it. Each pan contains, when full, about 350 gallons, equivalent to nearly $35 \mathrm{cwts}$. of crystallized sugar. They are furnished with steam-cocks and waste steam-pipes. Under the level of the spheroids $d, d$, the horizontal main-pipe is seen, for supplying the cases with steam. In the face of each pan, above the line $b, b$, the handle of the proof-stick appears, like that of a stopcock. The distribution of the measure cisterns, and some other parts of the pans, is slightly varied in this representation from the former. From the bottom of the liquor cisterns $\mathrm{c}$, c, pipes descend to the charging measures $a, a$, below. The cisterns $\mathrm{c}, \mathrm{c}$, are made of copper, and contain each about 400 gallons. Six tons of refined sugar can be turned out daily in a three-pan house.

Fig. 1089 represents in section another form of the vacuum-pan. $\quad a$ is the spheroidal copper vessel, supported by four iron columns $b, b$. It may be discharged by means of

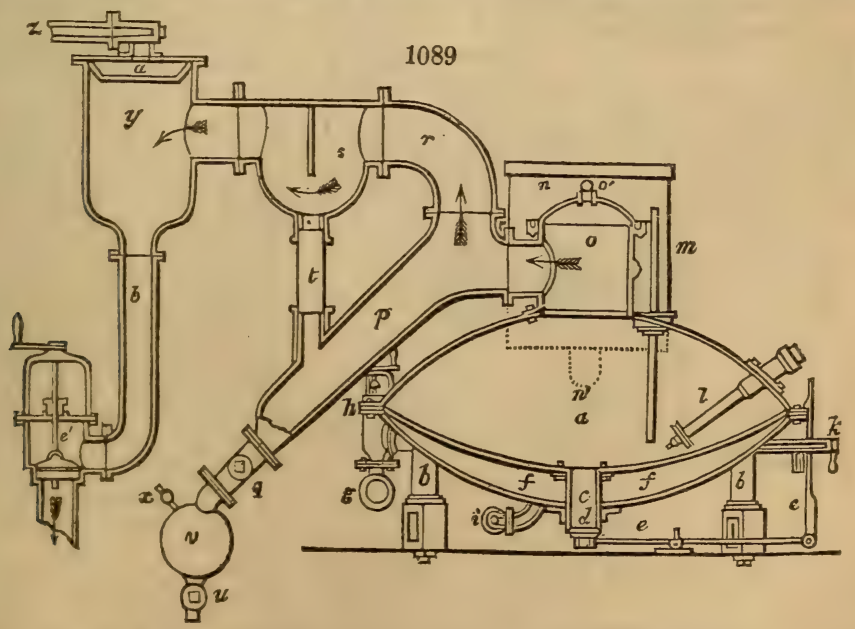

the pipe $c$, which is secured with a conical valve $d$. This may be opened or shut, by acting on the lever $e$. The lower of the two hemispheres of which the pan is composed 
is double, and the interstitial space $f, f$, is filled with steam by the pipe $g$, as the heeting and evaporating agent. $h$, is the steam valve; $i$, the pipe for the efflux of the condensed water. $k$, a tube for the escape of the air at the commencement of the operation. $l$, is an apparatus inserted air-tight into the cover of the vacuum-pan, and wlich dips down into the sirmp; serving to take out a sample of it, without allowing air to enter, and hence called the proof-stick. The construction of this instrument is exhibited in figs. 1091, 1092, 1093, 1094, 1095, which will be presently explained. $m$, is the thermometer, which is also plunged into the sugar; behind it, is the barometer. $n$, is the charger or gauge-vessel, filled with the filtered sirup, which it discharges by the pipe $n^{\prime} . \quad 0$, is the cover or capilal of the vacuum-pan. $o^{\prime}$, is a safety-valve, through which the air may be adritted, after the completion of the process. $p$, is a bent pipe, slanting downwards with a stopcock $q$, at its end, to receive the superfluous sirup. The vapor, which is disengaged from the sirup during its concentration, is extracted from the top of the pan into the pipe $r$, passes from this into the vessel $s$, which is divided by a plate of copper into two compartments. The sirup forced over a cidentally in the ebullition, goes into the ressel $s$, and passes by the glass tube $t$, into the pipe $p$. The glass tube serves to show the quantity of the sirup that has boiled over, so that it may be drawn off when necessary. For this purpose, the stopcock $u$, of the vessel $v$, must be closed, and $q$ must be opened, in order to fill $v$, while the air contained in it escapes into the pan. The stopcock $q$, being then shut, and $u$, with the little air-cock $x$, opened, the sirup will flow into the large receiver placed beneath it, commonly but erroneously called a cooler; because it is a double copper basin, with steam in the interstitial space. The hot steam rushes from $s$, into the cast-iron vessel $y$, where it is condensed. $z$, is a pipe for introducing the water of condensation through the copper rose $a^{\prime}$. The condensed water flows through the pipe $b^{\prime}$, and the valve $e^{\prime}$, to the air-pump, which receives motion from the shaft of the steam-engine.

The vacuum-pan was originally heated solely by the admission of steam between the 1090

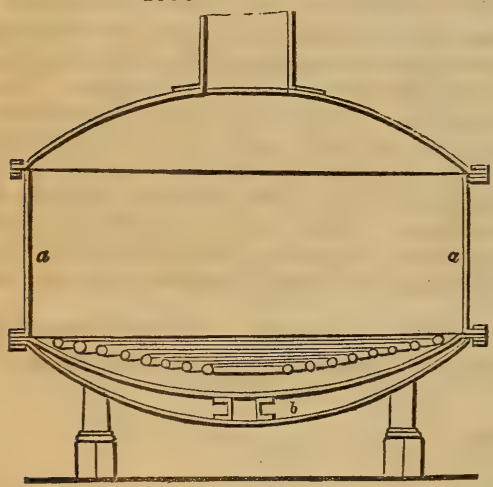
double bottom; but of late years the heat has been also applied to the sirup through several coils of pipe placed within the pan, filled with steam at a temperature many degrees above $212^{\circ} \mathrm{F}$., sometimes so high as $250^{\circ}$. By this double application of heat, the evaporating power of a pan has been vastly increased. The latest made pans have a considerably flat bottom, fig. 1090 ; a spiral pipe, laid close upon it; and between the under hemisphere and the upper one, there is a space $a, a, 2 \frac{1}{2}$ feet high, to give the sirup room for frothing up without boiling over. The space $b$, of the bottom receives steam of eommon pressure, and the spiral tubes, of high pressure. A pan like this is now making for a house in London, which is to work off 16 tons of sugar-loaves daily.

The proof-stick, fig. 1095, consists of a cylindrical rod, capable of being screwed airtight into the pan in an oblique direction downwards. The upper or exterior end is open; the under, which dips into the sirup, is closed, and has on one side a slit $a$ ( $f i g s .1091,1092)$ or notch, about $\frac{1}{2}$ inch wide. In this external tube, there is another shorter tube $b$, capable
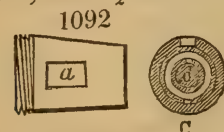

$\square$
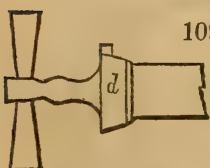

1094
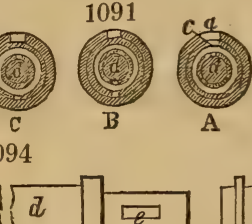

A 1093
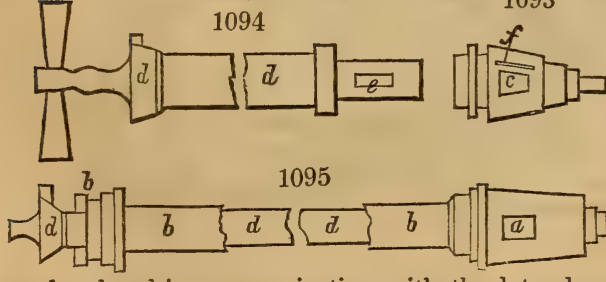

1095

of moving round in it, through an are of $180^{\circ}$. An opening upon the under end $e$, corresponds with the slit in the outer tube, so that both may be made to coincide, fig. 1091, A. A wooden plug $d$, is put in the interior tube, but so as not to shut it entirely. Upon the upper end there is a projection or pin, which eatches in a slit of the inner tube, by which this may be turned round at pleasure. In the lower end of the plug there is a hole $e$, which can be placed in communication with the lateral openings in both tubes. Hence it is possible, when the plug and the inner tube are brought into the proper position, A, fig. 1091, to fill the cavity of the wooden rod with the sirup, and to take it out without 
allowing any air to enter. In order to facilitate the turning of the inner tube within the euter, there is a grocive in the under part, into which a little grease may be introduced.

Whenever a proof lias been taken, the wooden plug must be placed in reference to the inner tube, as shown in fig. $1091, \mathrm{c}$, and then be turned into the position $\mathrm{A}$; when the cavity of the plug will again be filled with sirup. $\quad \mathrm{c}$ must be now turned back to the former position, whereby all intercourse with the vacuum-pan is cut off; the plug being drawn out a little, and placed out of communication with the inner tube. The plug is then turned into the position B, drawn out, and the proof examined by the fingers.

TABLE showing the boiling point of sirup, at the corresponding atmospheric pressure within the vacuum-pan:-

Height of the mercury (inches) in one leg of the syphon, above that in the other-

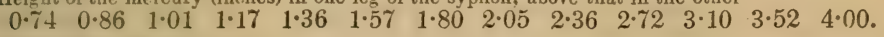

Boiling point, Fahr.-

$115^{\circ} 120^{\circ} 125^{\circ} \quad 130^{\circ} \quad 135^{\circ} 140^{\circ} 145^{\circ} 150^{\circ} \quad 155^{\circ} \quad 160^{\circ} \quad 165^{\circ} \quad 170^{\circ} \quad 175^{\circ}$.

The large double steam-basin, which receives several successive skippings of the concentrated granulating sirup, serves to heat it from the temperature of $160^{\circ}$ or $170^{\circ}$, at which it leares the vacuum-pan, up to $200^{\circ}$ or thereby, before it is filled out into the moulds; for were it introduced in the cooler state, it would not concrete into sufficiently compact loaves.

The following apparatus is used in many French sugar-houses, for concentrating sirups, called the swing pan, or chaudière a bascule. It is represented in fig. 1096, in 1096

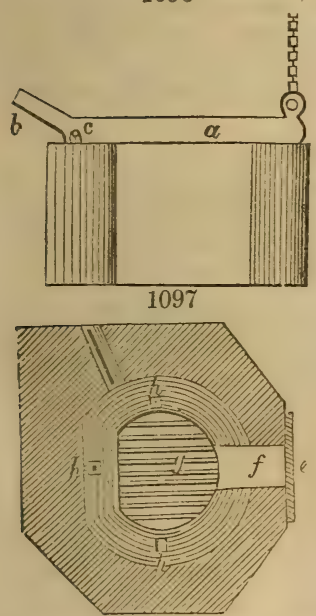
elevation, and in fig. 1097, in ground plan. $a$, is the pan; $b$, its spout; $c$, the axis or pivot round which it swings, so as to empty itself, when raised behind by the chain $d$; $e$, is the furnace door; $f$, the passage to the fireplace and grate $g ; h, h, h$, side fiues for conducting the smoke into the chimney.

The duly clarified, concentrated, granulated, and reheated sirup, is transferred, by means of copper basins, from the coolers into conical moulds, made either of brown and somewhat porous earthenware, or of sheet iron, strongly painted. The sizes of the moulds vary, from a capacity of 10 pound loaves, to that of 56 pound bastards-a kind of soft brown sugar obtained by the concentration of the inferior sirups. These moulds have the orifices at their tips closed with bits of twisted paper, and are set up in rows close to each other, in an airy apartment adjoining the coolers. Here they are left several hours, commonly the whole night, after being filled, till their contents become solid, and they are lifted next morning into an upper floor, kept at a temperature of about $80^{\circ}$ by means of steam pipes, and placed each over a pot to receive the sirup drainings-the paper plug being first removed, and a steel wire, called a piercer, being thrust up to clear away any concretion from the tip. Instead of setting the lower portion of the inverted cones in pots, some refiners arrango them in wooden racks, with their apices suspended over longitudinal gutters of lead or zinc, laid with a slight slope upon the floor, and terminating in a sunk cistern. The sirup whick flows off spontaneously is called green sirup. It is kept separate. In the course of two or three days, when the drainage is nearly complete, some finely clarified sirup, made from loaf sugar, called liquor by the refiners, is poured to the depth of about an inch upon the base of each cone, the surface having been previously rendered level and solid by an iron tool, called a bottoming trowel. The liquor, in percolating downwards, being already a saturated sirup, can dissolve none of the crystalline sugar, but only the colored molassy matter; whereby, at each successive liquoring, the loaf becomes whiter, from the base to the apex. A few moulds, taken promiscuously, are emptied from time to time, to inspect the progress of the blanching operation; and when the loaves appear to have acquired as much color, according to the language of refiners, as is wanted for the particular market, they are removed from the moulds, turned on a lathe at the tips, if necessary, set for a short time upon their bases, to diffuse their moisture equally through them, and then transferred into a stove heated to $130^{\circ}$ or $140^{\circ}$ br steam pipes, where they are allowed to remain for two or three days, till they be baked thoroughly dry. They are then taken out of the stove, and put up in blue paper for sale.

In the above description of sugar-refining, I have said nothing of the process of claying the loaves, because $\mathrm{jt}$ is now nearly obsolete, and abandoned in all well-aplointea 
sugar-houses. Those of my readers who desire to become acquainted with sugar-refining upon the old plan, may consult my Report made upon the subject to the Honorable HousE of Commons in July, 1833 ; where they will find every step detailed, and the numerical results stated with minute accuracy. The experiments subservient to that official report were instituted purposely to determine the average yield or product, in double and single refined loaves, lumps, bastards and treacle, which different kinds of sugar would afford per cwt., when refined by decoloring with not more than 5 per cent. of bone black, boiling in an open pan, and clearing the loaves with clay-pap.

BEET-ROOT SUGAR.

The physical characters which serve to show that a beet-root is of good quality, are its being firm, brittle, emitting a creaking noise when cut, and being perfectly sound within; the degree of sweetness is also a good indication. The 45 th degree of latitude appears to be the southern limit of the successful growth of beet in reference to the extraction of sugar.

Extruction of $S u g a r$ from the Beet. - The first manipulations to which the beets are exposed, are intended to clear them from the adhering earth and stones, as well as the fibrous ronts and portions of the neck. It is desirable to expose the roots, after this operation, to the action of a cylinder washing-machine.

The parenchyrna of the beet is a spongy mass, whose cells are filled with juice. The cellular tissue itself, which forms isually only a twentieth or twenty-fifth of the whole weight, consists of ligneous fibre. Compression alone, however powerful, is inadequate to force out all the liquor which this tissue contains. To effect this object, the roots must be subjected to the actior of an instrument which will tear and open up the greatest possible number of these cells. Experiments have, indeed, proved, that by the most considerable pressure, not more than 40 or 50 per cent. in juice from the beet can be obtained; whilst the pulp procured by the action of a grater produces from 75 to 80 per cent.

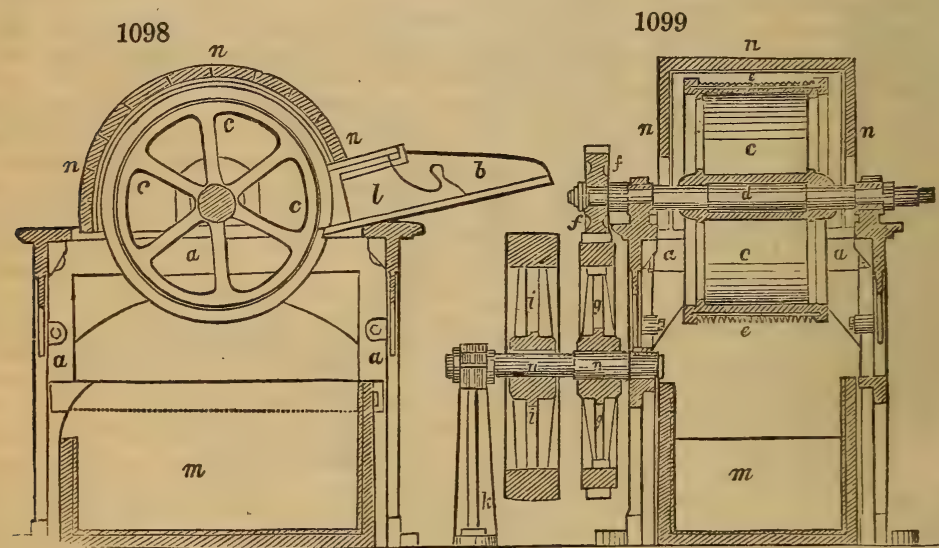

The beet-root rasp of Moulfarine is represented in fiss. 1098, 1099. a, a, is the framework of the machine; $b$, the feed-plate, made of cast iron, divided by a ridge into two parts; $c$, the hollow drum; $d$, its shaft, upon either side of whose periphery nuts are screwed for securing the saw blades $e, e$, which are packed tight against each other by means of laths of wood; $f$, is a pinion upon the shaft of the drum, into which the wheel $g$ works, and which is keyed upon the shaft $h ; i$, is the driving rigger; $k$, pillar of support; $l$, blocks of wood, with which the workman pushes the beet-roots against the revolvingrasp; $m$, the chest for receiving the beet-pap; $n$, the wooden cover of the drum, lined with sheet iron. The drum should make 500 or 600 turns in the minute.

A few years ago, M. Dombasle introduced a process of extracting the juice from the beet without either rasping or hydraulic pressure. The beets were cut into thin slices, by a proper rotatory blade-machine; these slices were put into a macerating cistern, with about their own bulk of water, at a temperature of $212^{\circ} \mathrm{F}$. After half an hour's maceration, the liquor was said to have a density of $2^{\circ} \mathrm{B}$., when it was run off into a second similar cistern, upon other beet-roots; from the second it was let into a third, and so on to a fifth; by which time, its density having risen to $5 \frac{1}{2}^{\circ}$, it was ready for the process of defecation. Juice procured in this way is transparent, and requires little lime for its purification; but it is apt to ferment, or to have its granulating power im. 
parred by the watery dilution. The process has been accordingly abandoned in most establishments.

I have seen the following operations successfully executed in a beet-root factory near Lille, and have since verified their propriety in my own laboratory upon white beets, grown near Mitcham in Surrey. My product was nearly 5 per cent.; it was very fair, and large grained, like the vacuum-pan sugar of Demerara, but without its clamminess.

The roots were washed by a rotatory movement upon a grating made like an Archimedes' screw, formed round the axis of a squirrel-cage cylinder, which was laid horizontally beneath the surface of water in an oblong trough. It was turned by hand rapidly, with the intervention of a toothed wheel and pinion. The roots, after being sufficiently agitated in the water, were tossed out by the rotation at the end of the cylinder furthest from the winch. They were next hoisted in a basket up through a trap-hole into the floor above, by means of a cord and pulley moved by mechanical power; a six-horse steam engine, upon Woolfe's expansive principle, being employed to do all the heavy work. They were here subjected to the mechanical grater (rape mécanique), see figs. 1098, 1099, which had, upon its sloping feed-table, two square holes for receiving at least two beets at a time, which were pushed forwards by a square block of wood held in the workman's hand by means of a strap. The rasp was a drum, having rows of straight saws placed half an inch apart round its periphery, parallel to the axis, with teeth projecting about $\frac{1}{8}$ of an inch. The space between each pair of saws was filled with a wedge of wood. The steel slips, or saw plates, were half an inch broad, twelve inches long, and serrated on both their longitudinal edges, so that when the one line of teeth was blunted, the other could be turned out. The drum made 750 turns per minute.

The pulp from the rasp fell into a flat trough placed beneath, whence it was shovelled into small bags. Each bag had its mouth folded over, was laid upon a wircker plate, and spread flat with a rolling-pin. The bags and hurdles were then piled in the hydraulic press. There were three presses, of which the two allotted to the first pressure were charged alternately, and the third was reserved for a final and more durable pressure of the marc. See Press, Hydraulic, and Stearine Press.

The juice flowed over the edges of the wicker plates, and fell into the sill-plate of the press, which was furnished with upright borders, like a tray, through whose front side a pipe issued, that terminated in a leathern hose, for conducting the juice into an elevatel cistern in the boiling-house. Here one pound of slaked lime was mixed with every four hectolitres (about 88 gallons imp.) of juice. The mixture was made to boil for a little while in a round pan alongside, whence it was decanted into oblong flat filters, of blanket stuff. The filtered liquor, which had in general a spec. gravity of $15^{\circ}$ Baumé (about double that of the fresh juice), was now briskly concentrated by boiling, in an oblong pan, till it acquired the density of $28^{\circ} \mathrm{B}$. The fire being damped with raw coal, the sirup was run off rapidly by a stopcock into a large basin with a swing handle, and immediately replaced by fresh defecated liquor. The basin was carried by two men to the opposite side of the boiling-house, and emptied into a cistern sec on a high platform, whose horizontal discharge-pipe was provided with a series (five) of stopcocks, placed respectively over five copper chests (inverted truncated pyramids), containing a thick bed of granular bone black, covered with a perforated copper plate. The hot sirup thus filtered had a pale straw-color, and was subsequently evaporated in swing pans, $f$ gs. 1096, 1097, over a brisk fire, in quantities equivalent to half a cwt. of sugar, or four. hectolitres of average juice.

\section{MAPLE SUGAR.}

The manufacture of sugar from the juice of a species of maple tree, which grows spontaneously in many of the uncultivated parts of North America, appears to have been first attempted about 1752, by some of the farmers of New England, as a branch of rural economy.

The sugar maple, the Acer saccharinum of Linnæus, thrives especially in the States of New York and Pennsylvania, and yields a larger proportion of sugar than that which grows upon the Ohio. It is found sometimes in thickets which cover five or six acres of land; but it is more usually interspersed among other trees. They are supposed to arrive at perfection in forty years.

The extraction of maple sugar is a great resource to the inhabitants of districts far removed from the sea; and the process is very simple. After selecting a spot among surrounding maple trees, a shed is erected, called the sugar-camp, to protect the boilers and the operators from the vicissitudes of the weather. One or more augers, three fourths of an inch in diameter; small troughs for receiving the sap; tubes of elder or sumach, 8 or 10 inches long, laid open through two thirds of their length, and corres. ponding in size to the auger-bits; pails for emptying the troughs, and carrying the sap to the camp; boilers capable of holding 15 or 16 gallons; moulds for receiving the sirup inspissated to the proper consistence for concreting into a loaf of sugar; and, 
lastly, hatchets to cut and cleave the fuel, are the principal utensils requisite for this manufacture. The whole of February and beginning of March are the sugar season.

The trees are bored obliquely from below upwards, at 18 or 20 inches above the ground, with two holes 4 or 5 inches asunder. Care must be taken that the auger penetrates no more than half an inch into the alburnum, or white barls; as experience has proved that a greater discharge of sap takes place at this depth than at any other. It is also advisa ble to perforate in the south face of the trunk.

The trough, which contains from two to three gallons, and is made commonly of white pine, is set on the ground at the foot of each tree, to receive the sap which flows through the two tubes inserted into the holes made with the auger; it is collected together daily, and carried to the camp, where it is poured into casks, out of which the boilers are supplied. In every case, it ought to be boiled within the course of two or three days from flowing out of the tree, as it is liable to run quickly into fermentation, if the weather become mild. The evaporation is urged by an active fire, with careful skimming during the boiling; and the pot is continually replenished with more sap, till a large body has at length assumed a sirupy consistence. It is then allowed to cool, and passed through a woollen cloth, to free it from impurities.

The sirup is transferred into a boiler to three fourths of its capacity, and it is urged with a brisk fire, till it acquires the requisite consistence for being poured into the moulds or troughs prepared to receive it. This point is ascertained, as usual, by its exhibiting a granular aspect, when a few drops are drawn out into a thread between the finger and the thumb. If in the course of the last boiling, the liquor froth up considerably, a small bit of butter or fat is thrown into it. After the molasses have been drained from the concreted loaves, the sugar is not at all deliquescent, like equally brown sugar from the cane. Maple sugar is in taste equally agreeable with cane sugar, and it sweetens as well. When refined, it is equally fair with the loaf sugar of Europe.

The period during which the trees discharge their juices is limited to about six weeks. Towards the end of the flow, it is less abundant, less saccharine, and more difficult to be crystallized.

QUANTITY of Sugar brought into the Markets of the World, in the year 1838 .

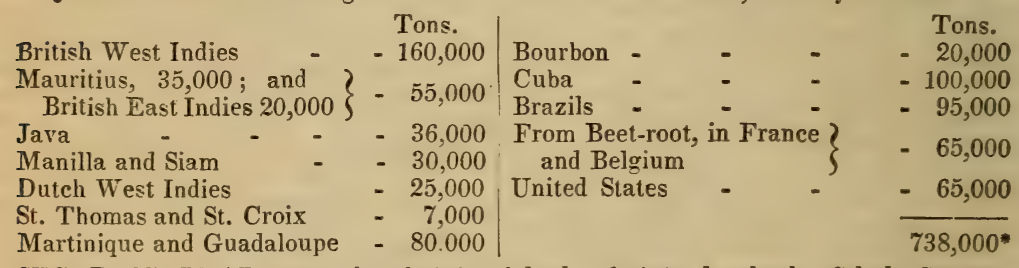

SUGAR OF LEAD, properly Acetate of lead (Acetate de plomb; Sel de Saturne, Fr.; Essigsaures Bleioxyd, Bleizucker, Germ.), is prepared by dissolving pure litharge, with heat, in strong vinegar, made of malt, wood, or wine, till the acid be saturated. A copper boiler, rendered negatively electrical by soldering a strap of lead within it, is the best adapted to this process on the great scale. 325 parts of finely ground and sifted oxyde of lead, require 575 parts of strong acetic acid, of spec. grav. $7^{\circ}$ Baumé, for neutralization, and afford 960 parts of crystallized sugar of lead. The oxyde should be gradually sprinkled into the moderately hot vinegar, with constant stirring, to prevent adhesion to the bottom; and when the proper quantity is dissolved, the solution may be weakened with some of the washings of a preceding process, to dilute the acetate, after which the whole should be heated to the boiling point, and allowed to cool slowly, in order to settle. The limpid solution is to be drawn off by a syphon, concentrated by boiling to the density of $32^{\circ} \mathrm{B}$., taking care that there be always a faint excess of acid, to prevent the possibility of any basic salt being formed, which would interfere with the formation of regular crystals. Should the concentrated liquor be colored, it may be whitened by filtration through granular bone black.

Stoneware vessels, with salt glaze, answer best for crystallizers. Their edges should be smeared with candle-grease, to prevent the salt creeping over them by efflorescent vegetation. The crystals are to be drained, and dried in a stove-room very slightly heated. It deserves remark, that linen, mats, wood, and paper, imbued with sugar of lead, and strongly dried, readily take fire, and burn away like tinder. When the mother waters cease to afford good crystals, they should be decomposed by carbonate of soda, or by lime skilfully applied, when a carbonate or an oxyde will be obtained, fit for treating with fresh vinegar. The supernatant acetate of soda may be empioyed for the extraction of pure acetic acid. 
A main point in the preparation of sugar of lead, is to use a strong acid; otherwise much time and acid are wasted in concentrating the solution. This salt crystallizes in colorless, transparent, four and six sided prisms, from a moderately concentrated solution; but fiom a stronger solution, in small needles, which have a yellow cast if the acid has been slightly impure. It has no smell, a sweetish astringent metallic taste, a specific. gravity of $2 \cdot 345$; it is permanent in the air at ordinary temperatures, but effloresces when heated to $95^{\circ}$, with the loss of its water of crystallization and some acid, falling into a powder, which passes, in the air, slowly into carbonate of lead. The crystals dissolve in $1 \frac{1}{2}$ limes their weight of water at $60^{\circ}$, but in much less of boiling water, and in 8 parts of alcohol. The solution feebly reddens litmus paper, but has an alkaline reaction upon the colors of violets and turmeric. The constituents of the salt are, 58.71 oxyde of lead, 27.08 acetic acid, and $14 \cdot 21$ water, in 100 .

Acetate of lead is much used in calico-printing. It is poisonous, and ought to be prepared and handled with attention to this circumstance.

There are two subacetates of lead; the first of which, the ter-subacetate, has three atoms of base to one of acid, and is the substance long known by the name of Goulard's extract. It may be obtained by digesting with heat a solution of the neutral acetate, upon pure litharge or massicot. The solution affords white crystalline scales, which do not taste so sweet as sugar of lead, dissolve in not less than 30 parts of water, are insoluble in alcohol, and have a decided alkaline reaction upon test paper. Carbonic acid, transmitted through the solution, precipitates the excess of the oxyde of lead, in the state of a carbonate, a process long ago prescribed by Thenard for making white-lead. This subacetate consists of 88.66 of oxyde, and 13.34 acid, in 100 parts. It is employed for making the orange sub-chromate of lead, as also sometimes in surgery.

A sex-subacetate, containing 6 atoms of base, may be obtained by adding ammonia in excess to a solution of the preceding salt, and washing the precipitate with dilute water of ammonia. A white powder is thus formed, that dissolves sparingly in cold water, but gives a solution in boiling water, from which white silky needles are deposited. It consists of $92 \cdot 86$ oxyde, and $7 \cdot 14$ acid.

SULPHATES, are saline compounds of sulphuric acid with oxydized bases. The minutest quantity of them present in any solution, may be detected by the precipitate, insoluble in nitric or muriatic acid, which they afford with nitrate or muriate of baryta. They are mostly insoluble in alcohol.

SULPHATE OF ALUMINA AND POTASSA, is alum.

SULPHATE OF AMMONIA, is a salt sometimes formed by saturating the ammonia liquor of the gas-works with sulphuric acid; and it is employed for making carbonate of ammonia. See AMmonia and SAL AMmoniac.

SULPHATE OF BARYTA, is the mineral called heavy-spar, which frequently forms the gangue or vein-stone of lead and other metallic oars.

SULPHATE OF COPPER, Roman or Blue Vitriol (Vitriol de Chypre, Fr. ; Kup. fervitriol, Germ.), is a salt composed of sulphuric acid and oxyde of copper, and may be formed by boiling the concentrated acid upon the metal, in an iron pot. It is, however, a natural product of many copper mines, from which it flows out in the form of a blue water, being the result of the infiltration of water over copper pyrites, which has become oxygenated by long exposure to the air in subterranean excavations. The liquid is concentrated by heat in copper vessels, then set aside to crystallize. The salt forms in oblique four-sided tables, of a fine blue color; has a spec. gravity of $2 \cdot 104$; an acerb, disagreeable, metallic taste; and, when swallowed, it causes violent vomiting. It becomes of a pale dirty blue, and efforesces slightly, on long exposure to the air; when morlerately heated, it loses 36 per cent. of water, and falls into a white powder. It dissolves in 4 parts of water, at $60^{\circ}$, and in 2 of boiling water, but not in alcohol; the solution has an acid reaction upon litmus paper. When strongly ignited, the acid flies off, and the black oxyde of copper remains. The constituents of crystallized sulphate of copper are-oxyde, 31.80 ; acid, 32.14 ; and water, 36.06 . Its chief employment in this country is in dyeing, and for preparing certain green pigments. See ScHEELE's and Schweinfurth Green. In France, the farmers sprinkle a weak solution of it upon their grains and seeds before sowing them, to prevent their being attacked by birds and insects.

SULPHATE OF IRON, Green vitriol, Copperas (Couperose verte, Fr.; Eisen-vitriol, Schwefelsures Eisenoxydul, Germ.), is a crystalline compound of sulphuric acid and protoxyde of iron; hence called, by chemists, the protosulphate; consisting of, $26 \cdot 10$ of base, 29.90 of acid, and 44.00 of water, in 100 parts; or of 1 prime equivalent of protoxyde, $36,+1$ of acid, $40,+7$ of water, $63,=139$. It may be prepared by dissolving iron to saturation in dilute sulphuric acid, evaporating the solution till a pellicle forms upon its eurface, and setting it aside to crystallize. The copperas of commerce is made in a much cheaper way, by stratifying the pyrites found in the coal 
measures (Vitriolkies and S/rahlkies of the Germans), upon a sloping puddled platform of stone, leaving the sulphuret exposed to the weather, till, by the absorption of oxygen, il effloresces, lixivitling with water the supersulphate of iron thus formed, saturating the excess of acid with plates of old iron, then evaporating and crystallizing. The other pyrites, which occurs often crystallized, called by the Germans Schwefelkies or Eisenkies, must be deprived of a part of its sulphur by calcination, before it acquires the property of absorbing oxygen from the atmosphere, and thereby passing from a bisulphuret into a bisulphate. Alum schist very commonly contains vitriolkies, and affords, after heing roasted and weather-worn, a considerable quantity of copperas, which must be carefully separated by crystallization from the alum.

This liquor used formerly to be concentrated directly in leaden vessels; but the first stage of the operation is now carried on in stone canals of considerable length, vaulted over with bricks, into which the liquor is admitted, and subjected at the surface to the action of flame and heated air, from a furnace of the reverberatory kind, constructed at one end, and discharging its smoke by a high chimney raised at the other. See SoDA Manufacture. Into this oblong trough, resting on dense clay, and rendered tight in the joints by water-cement, old iron is mixed with the liquor, to neutralize the excess of acid generated from the pyrites, as also to correct the tendency to superoxydizement in copperas, which would injure the fine green color of the crystals. After due concentration and saturation in this surface evaporator, the solution is run off into leaden boilers, where it is brought to the proper density for affording regular erystals, which it does by slow cooling, in stone cisterns.

Copperas forms sea-green, transparent, rhomboidal prisms, which are without smell, but have an astringent, acerb, inky taste; they speedily become yellowish-brown in the air, by peroxydizement of the iron, and effloresce in a warm atmosphere: they dissolve in 1.43 parts of water at $60^{\circ}$, in 0.27 at $190^{\circ}$, and in their own water of erystallization at a higher heat. This salt is extensively used in dyeing black, especially hats, in making ink and Prussian blue, for reducing indigo in the blue vat, in the China blue dye, for making the German oil of vitriol, and in many chemical and medicinal preparations.

There is a persulphate and subpersulphate of iron, but they belong to the domain of chemistry. The first may be formed, either by dissolving with heat one part of red oxyde of iron (colcothar) in one and a half of concentrated sulphuric acid, or by adding some nitric acid to a boiling-hot solution of copperas. It forms with galls and logwood a very black ink, which is apt to become brown-black. When evaporated to dryness, it appears as a dirty white pulverulent substance, which is soluble in alcohol. It consists, in 100 parts, of 39.42 of red oxyde of iron, and 60.58 sulphuric acid.

Hydrated peroxyde of iron, prepared by precipitation with alkali from solution of the persulphate, is an excellent antidote against poisoning by arsenic. A French perruquier, who had swallowed two drachms of arsenious acid, was, after an interval of twenty minutes, treated with the oxyde precipitated from 6 ounces of that salt by caustic potash. It was diffused in 20 quarts of weak sirup, and administered in successive doses. After repeated vomiting and purging, the patient felt no more pain, and was pronounced by the physician to be quite convalescent.

In the copperas and alum works, a very large quantity of ochrey sediment is obtained; which is a peroxyde of iron, containing a little sulphuric acid and alumina. This deposite, calcined in reverberatory hearths, becomes of a bright-red color ; and when ground and elutriated, in the same way as is described under white lead, forms a cheap pigment, in very considerable demand, called English red, in the French market.

Colcothar of Vitriol, and Crocus of Mars, are old names for red oxyde of iron. This brown-red powder is obtained in its purest state, by calcining dried sulphate of iron in a furnace till all its acid be expelled, and its base become peroxydized. It must be levigated, elutriated, and dried. This powder is employed extensively in the steel manufacture, for giving the finishing lustre to fine articles; it is used by silversmiths under the name of plate powder and rouge; and by the opticians for polishing the specula of zeflecting telescopes. Much of the crocus in the market, is made, however, from the copperas and alum sediments, and is greatly inferior to the article prepared by the last process. The finest rouge is made by precipitating the oxyde with soda, then washing and calcining the powder.

An excellent powder for applying to razor-strops, is made by igniting together in a crucible equal parts of well-dried copperas and sea salt. The heat must be slowly raised and well regulated, otherwise the materials will boil over in a pasty state, and the product will be in a great measure lost. When well made, out of contact of air, it has the brilliant aspect of plumbago. It has a satiny feel, and is a true fer olegiste, similar in composition to the Elba iron ore. It requires to be ground and elutriated; after which it affords, on drying, an impalpable powder, that may be either rubbed on a strop of smooth buff leather, or mixed up with hog's-lard or tallow into a stiff cerate. 


\section{SULPHATE OF LIME. See GyPsum.}

SULPHATE OF MAGNESIA, Epsom Salt (Sel amer, Fr.; Bittersalz, Germ.), exists in sea-water, as also in the waters of Saidschütz, Sedlitz, and Püllna; and in many saline springs, besides Epsom in Surrey, whence it has derived its trivial name, and from which it was first extracted, in the year 1695 , and continued to be so, till modern chemistry pointed out cheaper and more abundant sources of this useful purgative salt. The sulphate of magnesia, occasionally found effloresced on the surface of minerals in crystalline filaments, was called haarsalz (hair salt) by the older writers. The bittern of the Scotch sea-salt works is muriate of magnesia, mixed with a little sulphate of magnesia and chloride of sodium. If the proper decomposing quantity (found by trial) of sulphate of soda be added to it, and the mixed solution be evaporated at the temperature of $122^{\circ} \mathrm{F}$., chloride of sodium will form by double affinity, and fall down in cubical erystals; while the solution of sulphate of magnesia which remains, being evaporated to the proper point, will afford regular crystals in four-sided prisms with four-sided acuminations. Or, if bittern be treated in a retort with the equivalent quantity of sulphuric acid, the muriatic acid may be distilled off into a series of Woulfe's bottles, and the sulphate of magnesia, soda, and lime, will remain in the retort, from which mixture the sulphate of magnesia may be separated by filtration and crystallization.

Magnesian limestone being digested with as much muriatic acid as will dissolve out its lime only, will, after washing, afford, with the equivalent quantity of sulphuric acid, a pure sulphate of magnesia; and this is certainly the simplest and most profitable process for manufacturing this salt upon the great scale. Many prepare it directly, by digesting upon magnesian limestone the equivalent saturating quantity of dilute sulphuric acid. The sulphate of lime being separated by subsidence, the supernatant solution of sulphate of magnesia is evaporated and crystaliized.

This salt is composed of, magnesia $16 \cdot 72$, sulphuric acid $32 \cdot 39$, and water 50.89 . When free from muriate, it tends to effloresce in the air. It dissolves in four parts of water at $32^{\circ}$, in 3 parts at $60^{\circ}$, in 1.4 at $200^{\circ}$, and in its own water of crystallization at a higher heat.

SULPHATE OF MANGANESE is prepared on the great scale for the calico. printers, by exposing the peroxyde of the metal and pitcoal ground together, and made into a paste with sulphuric acid, to a heat of $400^{\circ} \mathrm{F}$. On lixiviating the calcined mass, a solution of the salt is obtained, which is to be evaporated and crystallized. It forms pale arnethyst-colored prisms, which have an astringent bitter taste, dissolve in $2 \frac{1}{2}$ parts of water, and consist of, protoxyde of manganese $31 \cdot 93$, sulphuric acid $35 \cdot 87$, and water $32 \cdot 20$, in 100 parts.

SULPHATE OF MERCURY is a white salt which is used in making corrosive sublimate. See Mercury. The subsulphate, called Turbith Mineral, is a pale yellow pigment, and may be prepared by washing the white sulphated peroxyde with hot water, which resolves it into the soluble supersulphate, and the insoluble subsulphate, or Turbith. It is poisonous.

SULPHATE OF POTASSA is obtained by first igniting and then crystallizing the residuum of the distillation of nitric acid from nitre.

SLLPHATE OF SODA is commonly called Glauber's salt, from the name of the chemist who first prepared it. It is obtained by igniting and then crystallizing the residuum of the distillation of muriatic acid from common salt. It crystallizes in channelled 6-sided prisms. See Soda Manufacture.

SULPHATE OF ZINC, called also White Vitriol, is commonly prepared in the Harz, by washing the calcined and effloresced sulphuret of zinc or blende, on the same principle as green and blue vitriol are obtained from the sulphurets of iron and copper. Pure sulphate of zinc may be made most readily by dissolving the metal in dilute sulphuric acid, evaporating and crystallizing the solution. It forms prismatic crystals, which have an astringent, disagreeable, metallic taste; they effloresce in a dry air, dissolve in $2 \cdot 3$ parts of water at $60^{\circ}$, and consist of-oxyde of zinc, 2829 ; acid, $28 \cdot 18$; water, $43 \cdot \check{3}$. Sulphate of zine is used for preparing drying oils for varnishes, and in the reserve or resist pastes of the calico-printer.

SULPHITES are a class of salts, consisting of sulphurous acid, combined in equivalent proportions with the oxydized bases.

SULPHOSELS is the name given by Berzelius to a class of salts which may be prepared as follows :- 1 . Dissolve a salt consisting of an oxyde and an acid (an oxysalt) in a very small quantity of water, and pass through the solution a stream of sulphureted hydrogen, till the salt be entirely decomposed. In this operation, the oxysalt is transformed into a sulphosalt, by the sulphur of the compound gas; while its hydrogen forms water with the oxygen of the saline base. This process is applicable only to the metallic salts; and among these, not to the nitrates, carbonates, or phosphates. 2 . Another method of preparing sulphosalts is, to add to a watery solution of sulphuret of 
potassium, an electro-negative metallic sulphuret, which will dissolve in the liquid till the sulphuret of potassium be saturated. This saline compound is to be employed to effect double decumpositions with the oxysalts; that is, to convert the radical of another bese, combined with an oxacid, into a sulphosalt. 3. If the electro-negative sulphuret be put in powder into a solution of the hydısulyhuret of potassa, it will dissolve and expel the sulphureted hydrogen with effervescence: just as carbonic acid is displaced by a stronger acid. For his other three methods of preparing sulphosalts, see his Elements, vol. iii. p. 336, Fr. translation.

SULPHUR, Brimstone (Sonfre, Fr.; Schwefel, Germ.), is a simple combustible, solid, non-metallic, of a peculiar yellow color, very brittle, melting at the temperature of $226^{\circ}$ Fahr., and possessing, after it has been fused, a specific gravity of $1 \cdot 99$. W hen held in a warm hand, a roll of sulphur emits a crackling sound, by the fracture of its interior parts; and when it is rubbed, it emits a peculiar well-hnown smell, and acquires it the same time negative electricity. When heated to the temperature of $560^{\circ} \mathrm{F}$. it takes fire, burns away with a dull blue flame of a suffocating odor, and leaves no residuum. When more strongly heated, sulphur burns with a vivid white flame. It is not aflected by air rr water.

Sulphur is an abundant product of nature; existing sometimes pure or merely mixed, and at others in intinate chemical combination with oxygen, and various metals, forming sulphates and sulphurets. See ores of COPPER, IRON, LEAD, \&c., under these metals.

Fig. 1100 represents one of the cast-iron retorts used at Marseilles for refining sulphur, wherein it is melted and converted into vapors, which are led into a large chamber for condensation. The body $a$, of the retort is an iron pot, 3 feet in diameter outside, 22 inches deep, half an inch thick, which weighs $14 \mathrm{cwts}$., and receives a charge of $8 \mathrm{cwts}$. of crude sulphur. The grate is 8 inches under its bottom, whence the flame rises and plays round its sides. A cast-iron capital $b$, being luted to the pot, and covered with sand, the opening in front is shut with an iron plate. The chamber $d$, is 23 feet long, 11 feet wide, and 13 feet high, with walls 32 inches thick. In the roof,

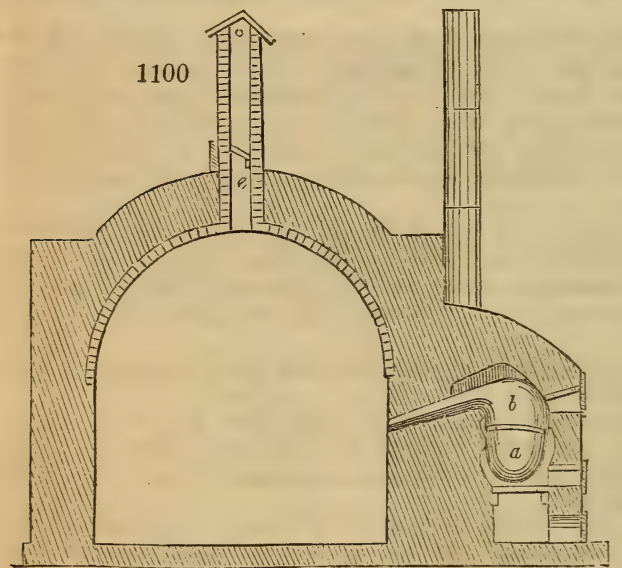
at each gable, valves or flapdoors, $e, 10$ inches square, are placed at the bottom of the chimney $c$. The cords for opening the valves are led down to the side of the furnace. The entrance to the chamber is shut with an iron door. In the wall opposite to the retorts, there are two apertures near the floor, for taking out the sulphur. Each of the two retorts belonging to a chamber is charged with $7 \frac{1}{2}$ or 8 cwts. of sulphur; but one is fired first, and with a gentle heat, lest the brimstone froth should overflow; but when the fumes begin to rise copiously, with a stronger flame. The distillation commences within an hour of kindling the fire, and is completed in six hours. Three hours after putting fire to the first retort, the second is in like manner set in operation.

When the process of distillation is resumed, after having been some time suspended, explosions may be apprehended, from the presence of atmospherical air ; to obviate the danger of which, the flap-doors must be opened every ten minutes; but they should remain closed during the setting of the retorts, and the reflux of sulphurous fumes or acid should be carried off by a draught-hood over the retorts. The distillation is carried on without interruption during the week, the charges being repeated four times in the day. By the third day, the chamber acquires such a degree of heat as to preserve the sujphur in a liquid state; on the sixth, its temperature becoming nearly $300^{\circ} \mathrm{F}$., gives the sulphur a dark hue, on which account the furnace is allowed to cool on the Sunday. The fittest distilling temperature is about $248^{\circ}$. The sulphur is drawn off throngh two Iron pipes cast in the iron doors of the orifices on the side of the chamher opposite to the furnace. The iron stoppers being taken out of the mouths of the pipes, the sulphur is allowed to run along an iron spout placed over red-hot charcoal, into the appropriate wooden moulds.

Native sulphur in its pure state is solid, brittle, transparent, yellow, or yellow border 
ing on green, and of a glassy lustre when newly broken. It occurs frequently in crystalline masses, and sometimes in complete and regular crystals, which are all derivable from the rhomboidal octakedron. The fracture is usually conchoidal and shining. Its specific gravity is 2.072 , exceeding somewhat the density of melted sulphur. It possesses a very consilerable refractive power; and doubles the images of objects even across two parallel faces. Sulphur, crystallized by artificial means, presents a very remarkable phenomenon; for by varying the processes, crystals are obtained whose forms belong to two different srstems of crystallization. The red tint, so common in the crystals of Sicily, and of volcanic districts, has been ascribed by some inineralogists to the presence of realgar, and by other's to iron; but Stromeyer has found the sublimed orange-red sulphur of Vulcano, one of the Lipari islands, to result from a natural combination of sulphur and selenium.

It is extracted from the minerals containing it, at Solfatara, by the following pro cess :-

Ten earthen pots, of about a yard in height, and four and a half gallons imperial in oa pacity, bulqing in the middle, are ranged in a furnace called a gallery; five being set on th: one side, and five on the other. These are so distributed in the body of the walls of th: gallery, that their belly projects partly without, and partly within, while their top rises out of the vault of the roof. The pots are filled with lumps of the sulphur ore of the size of the fist; their tops are closed with earthenware lids, and from their shoulder proceeds a pipe of about two inches diameter, which bends down, and enters into another covered pot, with a hole in its bottom, standing over a tub filled with water. On applying heat to the gallery, the sulphur melts, volatilizes, and runs down in a liquid state into the tubs, where it congeals. When one operation is finished, the pots are re-charged, and the process is repeated.

In Saxony and Bohemia, the sulphurets of iron and copper are introduced into large earthenware pipes, which traverse a furnace-gallery; and the sulphur exhaled flows into pipes filled with cold water, on the outside of the furnace. 900 parts of sulphuret afford from 100 to 150 of sulphur, and a residuum of metallic protosulphuret. See METALLUR GY and Copper.

Volcanic sulphur is purer than that extracted from pyrites; and as the latter is commonly mixed with arsenic, and some other metallic impregnations, sulphuric acid made of it would not answer for many purposes of the arts; though a tolerably good sulphuric acid may be made directly from the combustion of pyrites, instead of sulphur, in the lead chambers. The present high price of the Sicilian sulphur is a great encouragement to its extraction from pyrites. It is said that the common English brimstone, such as was extracted from the copper pyrites of the Parys mine of Anglesey, contained fully a fifteenth of residuum, insoluble in boiling oil of turpentine, which was chiefly orpiment ; while the fine Sicilian sulphur, now imported in vast quantities by the manufacturers of oil of vitriol, contains not more than three per cent. of foreign matter, chiefly earthy, but not at all arsenical.

Sulphur has been known from the most remote antiquity. From its kindling at a moderate temperature, it is employed for readily procuring fire, and lighting by its flame other bodies not so combustible. At Paris, the preparation of sulphur matches constitutes a considerable branch of industry. The sulphurous acid formed by the combustion of sulphur in the atmospheric air, is employed to bleach woollen and silken goods, as also cotton strckings; to disinfect vitiated air, though it is inferior in power to nitric acid vapor and chlorine; to kill mites, moths, and other destructive insects in collections of zoology; and to counteract too rapid fermentation in wine-vats, \&c. As the same acid gas has the property of suddenly extinguishing flame, sulphur has been thrown into a chimney on fire, with the best effect; a handful ; $\therefore$ being sometimes sufficient. Sulphur is also employed for cementing iron bars in stone, for laking impressions from seals and cameos, for which purpose it is kept previously melted for some time, to give the casts an appearance of bronze. Its principal uses, however, are for the manufactures of vermilion, or cinnabar, gunpowder, and sulphuric acid.

See Metalicugr, page 829, for the description of Gahn's furnace for extracting sulphur from pyrites.

Pyrites as a bi-sulphuret, consisting of 45.5 parts of iron, and 54.5 of sulphur, may, by proper chemical means, be made to give off one half of its sulphur, or about 27 per cent. but great care must be taken not to generate sulphurous acid, as is done very wastefully by the Fahlun and the Goslar processes. By the latter, indeed, not more than one or two purts of sulphur are obtained, by roasting 100 parts of the pyritous ores of the Rammelsberg mines. In these cases, the sulphur is burned, instead of being sublimed. The residuum of the operation, when it is well conducted, is black sulphuret of iron, which may be profitably employed for making copperas. The apparatus for extracting sulphur from pyrites should admit no more air than is barely necessary to promote the sublimation. Sicily produced last year 70,000 tons of sulphur, and Tuscany 1200; of which Great Brit. 
ain consumed 46,000 ; France, 18,000 ; other places, 6,000. In 1820, Great vistain con. sumed only 5,000 tons.

SULPHÚRATION, is the process by which woollen, silk, and cotton goods are ex. posed to the vapors of burning sulphur, or to sulphurous acid gas. In the article STRAw. HAT MANufacture, I have described a simple and cheap apparatus, well adapted to this operation.

Sulphuring-rooms are sometimes constructed upon a great scale, in which blankets, shawls, and woollen clothes may be suspended freely upon poles or cords. The floor should be flagged with a sloping pavement, to favor the drainage of the water that drops down from the moistened cloth. The iron or stoneware vessels, in which the sulphur is burned, are set in the corners of the apartment. They should be increased in number according to the dimensions of the place, and distributed uniformly over it. The windows and the entrance door must be made to shut hermetically close. In the lower part of the door there should be a small opening, with a sliding shutter, which may be raised or lowered by the mechanism of a cord passing over a pulley.

The aperture by which the sulphurous acid and azotic gases are let off, in order to earry on the combustion, should be somewhat larger than the opening at the bottom. A lofty chimney carries the noxious gases above the building, and diffuses them over a wide space, their ascension being promoted by means of a draught-pipe of iron, connected with an ordinary stove, provided with a valve to close its orifice when not kindled.

When the chamber is to be used, the goods are hung up, and a small fire is made in the draught-stove. The proper quantity of sulphur being next put into the shallow pans, it is kindled, the entrance door is closed, as well as its shutter, while a vent-hole near the ground is opened by drawing its cord, which passes over a pulley. After a few minutes, when the sulphur is fully kindled, that vent-hole must be almost entirely shut, by relaxing the cord; when the whole apparatus is to be let alone for a sufficient time.

The object of the preceding precautions is to prevent the sulphurous acid gas escaping from the chamber by the seams of the principal doorway. This is secured by closing it imperfectly, so that it may admit of the passage of somewhat more air than can enter by the upper seams, and the smallest quantity of fresh air that can support the combustion. The velocity of the current of air may be increased at pleasure, by enlarging the nnder vent-hole a little, and quickening the fire of the draught-stove.

Before opening the entrance door of the apartment, for the discharge of the goods, a small fire must be lighted in the draught furnace, the vent-hole must be thrown entirely oper, and the sliding shutter of the door must be slid up, gradually more and more every quarter of an hour, and finally left wide open for a proper time. By this means the air of the chamber will become soon respirable.

SULPHURETED HYDROGEN, is a gas, composed of one part of hydrogen and sixteen parts of sulphur, by weight. Its specinic gravity is $1 \cdot 1912$, compared to $\mathrm{air}=1 \cdot 0000$. It is the active constituent of the sulphurous mineral waters. When breathed, it is very deleterious to animal life; and being nearly twice as dense as air, it may he poured from its generating bottle into cavities; a scheme successfully employed by $\mathbf{M}$. Thenard to destroy rats in their holes.

SULPHURIC ACID, Vitriolic Acid, or Oil of Vitriol, (Acide sulfurique, Fr.; Schwefelsaüre, Germ.) This important product, the agent of many chemical operations, was formerly procured by the distillation of dried sulphate of iron, called green vitriol, whence the corrosive liquid which came over, having an oily consistence, was denominated oil of vitriol. This methan has been superseded in Great Britain, France, and most other countries, by the coin $u$ tion of sulphur along with nitre, in large leaden chambers; but as the former process, whicn is still practised at Bleyl in Bohemia, and Nordhausen in Saxony, gives birth to some interesting results, I shall describe it briefly.

Into a long horizontal furnace, or gallery of brickwork, a series of earthenware retorts, of a pcar shape, is arranged, with curved necks fitted into stoneware bottles or condensers. Each retort is charged with sulphate of iron, which has been previously heated to molerate redness. The first product of the distillation, a slightly acidulous phlegm, is llowed to escape; then the retort and receiver are securely luted together. The fire is now raised, and urged briskly for thirty-six hours, whereby the strong sulphuric acid is expelled, in the form of heavy white vapors, which condense in the cold receiver into an - oily-looking liquid. The latter portions, when received in a separate refrigerator, frequently concrete into a crystalline mass, formerly called glacial oil of vitriol. About six. ty four pounds of strong acid may be obtained from six huridred pounds of copperas. It is brown-colored; and varies in specific gravity from 1.842 to $1 \cdot 896$. Its boiling point is so low as $120^{\circ} \mathrm{Fahr}$. When re-distilled in a glass retort, into a receiver surrounded with ice, a very moderate heat sends over white fumes, which condense into a soft solid, in silky filaments, like asbestos, tough, and difficnlt to cut. When this is exposed to 
the air, it emits copious fumes of sulphuric (not sulphurous) acid. It burns holes in paper as rapidly as a red-hot iron. Dropped in small quantities into water, it excites a hissing noise, like ignited metal; and in larger quantities, it occasions an explosion. By dropping a fragment of it into a poised vial containing water, and stoppering instantly, to prevent the ejection of liquid, by the ebullition which always ensues, I got a dilute acid, containing a known portion of the solid acid, from the specific gravity of which, as well as from its saturating power, I ascertained that the above solid sulphuric acid was truly anhydrous (void of water), consisting of 1 equivalent proportion of sulphur, and 3 of oxygen; or, by weight, of 16 of the former, and 24 of the latter. This acid makes a red solution of indigo.

The production of sulphuric acid from sulphur and nitre may be elegantly illustrated by means of a glass globe with a stoppered hole at its side, and four hent glass tubes inserced into a leaden cap in its upper orifice. The first tuhe is to be connected with a heated matrass, disengaging sulphurous acid from copper filings and sulphuric acid; the second with a retort, disengaging more slowly deutoxyde of azote (nitric oxyde) from copper filings and nitric acid; the third with a vessel for furnishing steam in a moderate current towarls the end of the process, when no water has been previously admitted into the balloon; the fourth tube may be upright, and terminate in a small funnel. Through the opening in the side of the globe, atinospherical air is to be admitted from time to time, by removing the stopper; after which, the residuary lighter azote may be allowed to escape by the funnel orifice.

The nitric oxyde first absorbs oxygen from the air, becomes, in consequence, nitrous acid vapor, which giving up one third of its oxygen to the sulphurous acid, converts this, with the ail of water, into sulphuric acid, while itself returning to the state of nitric oxyde, is again qualified to take oxygen from the air, and to transfer it to the sulphwrous acid gas; and thus in perpetual rotation. These oxygenating and disoxygenating processes continue until nearly the whole oxrgen of the atmospheric air contained in the globe is consumed. Were there little aqueous vapor present, those gases would soon cease to operate upon each other; fur though the nitric oxyde became nitrous acid, this would oxgenate litlle of the sulphurous acid, because the three substances would condense into white crystals upon the sides of the balloon, like hoar frost upon a windowpane in winter. These indicate a deficiency of aqueous vapor, and an excess of nitrous acid. On the admission of steam, the crystals disappear, the sulphuric acid is liquefied, the nitrous acid is converted into nitric acid and nitric oxrde; the former of which combines with the water, while the latter is converted by the atmospheric oxron into nitrous acid vapor. A certain quantity of water is therefore requisite to prevent the formation of that crystalline compound, which condenses the nitrous acid, and renders it inoperative in transfurming fiesh portions of sulphurous acid into sulphuric. On these principles alone is it possible to oxygenate the sulphurous acid, by the nitrous acid resuming and surrendering a dose of oxygen, in perpetual alternation.

It was MM. Clement and Desormes who first had the sagacity to trace these complicated changes. They showed that nitrous acid gas and sulphurous acid gas mixed, react on each other throngh the intervention of moisture; that there resulted thence a combination of sulphuric acid, dentoxyde of azote (nitrous gas), and water; that this crystalline compound was instantly destroyed by more water, with the separation of the sulphuric acid in a liquid state, and the disengagement of nitrous gas; that this gas re-constituted nitrous acid at the expense of the atmospheric oxygen of the leaden chamber, and thus brought matters to their primary condition. From this point, starting again, the particles of sulphur in the sulphurous acid, through the agency of water, became fully oxyenated by the nitrous acil, and fell down in heavy drops of sulphuric acid, while the nitrous gas derived from the nitrous acid, had again recourse to the air for its lost dose of oxygen. This beautiful interchange of the oxygenous principle was found to go on, in their experiments, till either the sulphurous acid, or oxygen in the air, was exhausted.

They verified this proposition, with regard to what occurs in sulphuric acid chambers, by mixing in a crystal globe the three substances, deutoxyde of azote, sulphurous acid, and atmospheric air. The immediate production of red rapors indicated the transformation of the deutoxyde into nitrous acid gas; and now the introduction of a very little water cause the proper reaction, for opaque vapors rose, which deposited white starform erystalion the surface of the glass. The gases were once more transparent and colorless; but another addition of water melted these crystals with effervescence, when ruddy vapors appared. In this manner the phenomena were made to alternate, till the oxygen of the includel air was expended, or all the sulphurous acid was converted into sulphuric. The resiluary gases were found to be nitrous acid gas and azote, without sulphurous acil gas; while unctuous sulphuric acid bedewed the inner surface of the zlobe. Hence, they justly concluded their new theory of the manufacture of oil of vitriol to be demonstrated.

In consequence of their discovery, the manufacture of this acid has received such 
improvements, that a nearly double product of it may now be obtained from the same weight of materials. Indeed, the economy may be reckoned to be much grcater; for one half of the more costly ingredient, the nitre, formerly employed with a given weight of sulphur, suffices at present.

In the manufacture of sulphuric acid upon the great scale, two different systems of working were long prevalent; the intermittent or periodical, and the continuous or uniform. Both were carried on in large leaden chambers. In the former, the chambers were closed during the period of combustion and gaseous combination, but were opened from time to time to introduce fresh atmospheric air. This method is, I believe, generally abandoned now, on account of the difficulties and delays attending it, though it afforded large products in skilful hands. In the latter, a continuous current of air is allowed to enter at the oven in front of the chamber for the combustion of the sulphur, and there is a constant escape of nitrogen gas, with a little sulphurous acid gas, at the remote end of the roof.

Fig. 1101 represents a sulphuric acid chamber. $a, a$, are the brick or stone pillars upon which it rests; $b, b$, are the sustaining wooden beams or joists; $c$, is the chimney for the discharge of the nitrogen; $d$, is the roof, and $e$, the sole of the hearth for the combustion of the sulphur; $f$, is the cylindrical tunnel, or pipe of lead or cast iron, for conducting the gasiform materials into the chamber; $g$, is the steam boiler; and $h$, the steam-pipe. That plan is rariously modified, by different oil of vitriol makers in this country and in France. Very frequently, the oven $e, d$, is not situated under the chamber, but is built at the end of it, as at $i$, and arched over with brick, the crown being 9 inches thick. The pipe $f, 18$ inches in diameter, is then placed outside of the chamber, being inserted into a brick chimney, and, turning rectangularly, enters it opposite $k$. The sole of the hearth $e$, is a thick plate of cast iron (not hollowed as shown in the figure), 5 or 6 feet long, and 3 or 4 broad, with a small fireplace constructed beneath it, whose smoke-flue runs outwards, under the floor, to the side wall of the building. The oven is in this case about 2 feet in height, from the sole to the roof; and it has an iron door, abcut 12 inches by 15, which slides up and down in a tightly-fitted iron

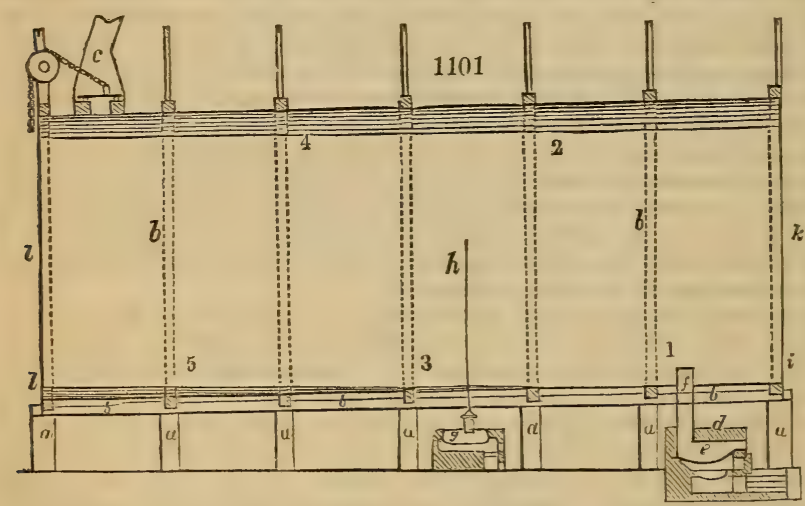

frame. This door is frequently placed in the side of the oven, parallel to the long side of the leaden chamber. A stout collar $k$ of lead is bolted to the chamber, where the pipe enters it. A the middle of the side of the chamber, about 2 feet above the ground, a leaden trough is fixed, which serves as

a syphon-funnel and water-trap for introducing water to the acid gases.

Several manufacturers divide the chamber into a series of rectangular compartments, by parallel leaden screens, 10 or 12 feet asunder, and allow these compartments to communicate by a narrow opening, or a hole 1 foot square, in the top and bottom of each screen alternately. Thus the fumes, which enter from the chimney-pipe over $k$, will be forced, by the screen at $b$, to descend to 1 , and pass through the opening there, to get into the second compartment, whence they will escape near the top at 2 , thus circulating up and down, so as to occasion a complete agitation and intermixture of their heterogeneous particles. Into the side of the chamner, opposite to the centre of each compartment, a lead pipe enters, and proceeds towards the middle of the area, terminating in a narrow orifice, for discharging a jet of high-pressure steam from a boiler loaded with 40 nounds upon the square inch. This boiler should be placed under a shed exterior to the building. It deserves to be noted, that the incessant tremors produced in this pipe by the escape of the steam, cause the orifice to contract, and eventually to close almost entirely, just as the point of a glass tube does when exposed directly to the flame of a blowpipe. Provision should therefore be made against this event, by the chemical engineer.

Equidistant between the middle point and each end of the chamber, two round holes are cut out in its side, about 16 inches in diameter, and 2 feet from the floor; the sheet 
lead being folded back over the face of the strong deals which strengthen the chamber in that place. The edges of the holes are bevelled outwards, so as to fit a large conical plug of wood faced with lead, called a man-hole door. One or other of these doors is opened from time to time, to allow the superintendent to inspect the process, or workmen to enter, after the chamber is well ventilated, for the purpose of making repairs. The joists or tie-beams, that bind the rafters of the roof of both the leaden chamber and the house, must be at least 7 inches deep, by 3 broad, and of such length as to have their ends supported upon the outer wall, or the columnar supports of the roof, in case a number of chambers are enclosed together in parallel ranges under a vast shed. These beams, which lie two feet apart, suspend the leaden roof, by means of leaden straps soldered to its upper surface and edges. The sides of the chamber are sustained by means of similar leaden straps affixed to the wooden posts (uprights), 4 inches broad by 3 thick, placed two or three leet apart along the sides of the chamber; resting on the ground below, and mortised into the tie-beams above. Some chambers rest upon a sand-floor; but they are preferably placed upon wooden joists, supported by pillars stretching over an open area, as shown in the figure, into which the workmen may descend readily, to examine the bottom.

The outlet $c$, on the top of the chamber, is sometimes joined to a long pipe of lead laid nearly horizontally, with a slight inclination upwards, along the roof, for favoring the condensation and return of acid matter.

At the extremity $l$, of the chamber, which, having a downward slope of 1 inch in every 20 feet, should stand from 3 to 6 inches (according to its length) lower than $i$, one leg of an inverted syphon pipe is fixed by fusion, into which the liquid of the chamber passing, will show by its altitude the depth on the bottom within. From the cup-shaped orifice of that bent-up pipe, the acid of the chamber is drawn off by an ordinary leaden syphon into the concentration pans.

The sheet lead of which the sides and top are made should weigh from 5 to 6 pounds per square foot; that of the bottom should be nearly of double thickness.

Having now detailed, with sufficient minuteness, the construction of the chamber, $\mathbf{I}$ shall next describe the mode of operating with it. There are at least two plans at present in use for burning the sulphur coutinuously in the oven. In the one, the suiphur is laid on the hearth $e$, (or rather on the flat hearth in the separate oven, above deseribed,) and is kindled by a slight fire placed under it; which fire, however, is allowed to go out after the first day, because the oven becomes by that time sufficiently heated by the sulphur flames to carry on the subsequent combustion. Upon the hearth, an iron tripod is set, supporting, a few inches above it, a hemispherical cast-iron bowl (basin) charged with nitre and its decomposing proportion of strong sulphuric acid. In the other plan, 12 parts of bruised sulphur, and 1 of nitre, are mixed in a leaden trough on the floor with 1 of strong sulphuric acid, and the mixture is shovelled through the sliding iron door upon the hot hearth. The successive charges of sulphur are proportioned, of course, to the size of the chamber. In one of the largest, which is 120 feet long, 20 broad, and 16 high, $12 \mathrm{cwts}$. are burned in the course of 24 hours, divided into 6 charges, every fourth hour, of 2 cwts. each. In chambers of one sixth greater capacity, containing 1400 metres cube, 1 ton of sulphur is burned in 24 hours. This immense production was first introduced at Chaunay and Dieuze, under the management of $\mathbf{M}$. Clement-Desormes. The bottom of the chamber should be covered at first with a thin stratum of sulphurie acid, of spec. grav. 1.07, which decomposes nitrous acid into oxygen and nitrous gas; but not with more water, which would absorb the nitrous acid vapors, and withdraw them from their aerial sphere of action. The vapor of nitric acid, disengaged from the nitre on the hearth of the oven, when brought into intimate contact with the sulphurous acid, either gives up oxygen to it, becomes itself nitrous gas, and converts it into sulphuric acid; or combines with the sulphurous acid into the crystalline compound above described, which, the moment it meets with moisture, is decomposed into sulphuric acid and nitrous gas. The atmospherical oxygen of the chamber immediately reconverts this gas into nitrous or nitric acid fumes, which are again ready, with the co-operation of sulphurous acid gas and aqueous vapor, to produce fresh quantities of hydrous sulphuric acid (oil of vitriol) and nitrous gas. At low temperatures, this curious play of chemical affinities has a great tendency to form the crystalline compound, and to deposite it in a crust of considerable thichness (from one half to one inch) on the sides of the chamber, so as to render the process inoperative. A circumstance of this kind occurred, in a very striking manner, during winter, in a manufacture of oil of vitriol in Russia; and it has sometimes occurred, to a moderate extent, in Scotland. It is called, at Marseilles, the maladie des chambres. It may be certainly prevented, by maintaining the interior of the chamber, by a jet of steam, at a temperature of $100^{\circ} \mathrm{F}$. When these crystals fall into the dilute acid at the bottom, they are decomposed with a violent efferrescence, and a hissing gurgling noise, somewhat like that of a tun of beer in brisk fermentation. 
M. Clement-Desormes demonstrated the proposition relative to the influence of temperature ly a decisive experiment. He took a glitss globe, furnished with three tubulures, and put a bit of ice into it. Through the first opening he then introduced sulphurous acid gas; through the second, oxygen; and through the third, nitrous gas (deutoxyde of azote). While the globe was kept cool, by being plunged in iced water, no sulphuric acid was formed, though all the ingredients essential to its production were present. But on exposing the globe to a temperature of $100^{\circ}$ Fahr., the four bodies began immediately to react on each other, and oil of vittiol was condensed in visible stria.

The introduction of steam is a modern invention, which has vastly facilitated and increasel the production of oil of vitriul. It serves, by powerful agitation, not only to mix the different gaseous molecules intimately together, but to impel them against each other, and thus bring them within the sphere of their mutual chemical attraction. This is its mechanical effect. Its chemical agency is still more important. By supplying moisture at every point of the immense included space, it determines the formation of lydrous sulphuric acid, from the compound of nitric, nitrous, sulphurous, and dry sulphuric aciils. No sooner is this reaction accomplished, than the nitrous gas resumes its oxygen, from the continuous atmospherical current, and becomes again fit to operate a like round of transmutations with sulphurous acid, steam, and oxygen. The nitrogen (azote), which onght to be the only residuun in a perfectly regulated vitriol chamber, escapes, by its relative lightness, at the opening $c$, in the rouf, or, inore properly speaking, is displaced by the influx of the heavier gases at the entrance-pipe.

On the intermittent plan, after the consumption of each charge, and condensation of the product, the chamber was opened, and freely ventilated, so as to expel the residuary azote, and replenish it with fresh atmospheric air. In this system there were four distinct stages or periods:-1. Combustion for two hours; 2. Admission of steam, and settling, for an hour and a half; 3 . Conversion, for three hours, during which interval the drops of strong acid were heard falling like heavy hailstones on the bottom; 4. Purging of the chamber, for three quarters of an hour.

By the continuous method, sulphuric acid may be currently obtained in the chambers, of the specific gravity 1.350 , or 1.450 at most: for, when stronger, it absorbs and retains permanently much nitrous acid gas; hut by the intermittent, so dense as 1.550 , or even $1 \cdot 620$; whence in a district where fuel is high priced, as near Paris, this method recommended itself by economy in the concentration of the acid. In Great Britain, and even in most parts of France, however, where time, workmen's wages, and interest of capital, are the paramount considerations, manufacturers do not find it for their interest in general to raise the density of the acid in the chambers above 1.400 , or at most 1.500 ; as the further Increase goes on at a retarded rate, and its concentration from 1.400 to 1.600 , in leaden pans, costs very little.

At about the specific gravity of 1.35 , in Great Britain, the liquid of the chambers is run off; by the syphon above described, into a leaden gutter or spout, which discharges it into a series of rectangular vessels made of large sheels of lead, of 12 or $14 \mathrm{lbs}$. to the square foot, simply lolded up at the anoles into pans 8 or 10 inches deep, resting upon a grate made of a pretty close row of wrought-iron bars of considerable strengih, under which the flame of a furnace plays. Where coals are very cheap, each pan may have a separate fire; but where they are somewhat dear, the flame, after passing under the lowest pan of the range, which contains the strongest acid (at about 1.600 ), proceeds upwards with a slight slope to heat the pans of weaker acid, which, as it concentrates, is gradually run down by syphons to replenish the lower pans, in proportion as their aqueous matter is dissipated. The 3 or 4 pans constituting the range are thus placed in a straight line, but each at a different level, terrace like; en gradins, as the French say.

When the acid has thereby acquired the density of 1.650 , or 1.700 at most, it must be remove I from the learlen evaporators, because, when of greater strength, it would begin to corrote them; and it is transferred into lealen coolers, or run through a long refigeratory worm-nipe surrounded by cold water. In this state it is introduced into glass or platinuin retorts, to untergo a final concentration. up to the specific gravity of $1 \cdot 842$, or even occasionally 1.845 , in consequence of slight saline impurities. When glass retorts are usel, they are set in a long sand-bath over a gallery furnace, resting on fire tiles, under which a nowerful flame plays; and as the flue gradually ascend; fiom the fireplace, near to which it is most distant from the tiles, to the remoter end, the heat acts with tolerable equality on the first and last retor in the range. When platinum stills are employed, they are fitted into the insile of cast-iron pots, which protect the thin bottom and siles of the precious metal. The fire being applied directly to the iron, causes a safe, rapil, and econonical concentration of the acid. The iron pots, with their platinnm interior, filled with concentrated boiling-hot oil of vitriol, are lifted out of the fire-seat by rackle, and let down into a cistern of cold water, to effect the speedy refrigeration of the acid, and facilitate its transvasion into carboys packed in asier baskets lined with straw. Sornetimes, however, the acid is cooled by running it 
slowly off through a long platinum syphon, surrounded by another pipe filled with cold water. Fig. 1102 shows my contrivance for this purpose.

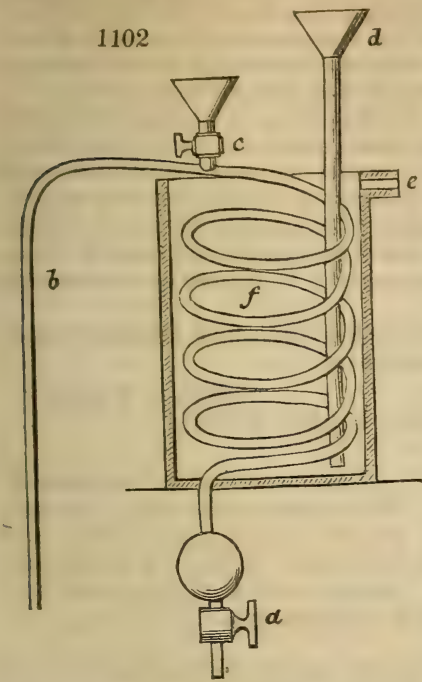

The under stopeck $a$, being shut, and the leg $b$, being plunged to nearly the bottom of the still, the worm is to be filled with concentrated cold acid through the funnel $c$. If that stopcock is now shut, and $a$ opened, the acid wil] flow out in such quantity as to rarefy the small portion of air in the upper part of the pipe $b$, sufficiently to make the hot acid rise up over the bend, and set the syphon in action. The flow of the fluid is to be so regulated by the stopcock $\alpha$, that it may be greatly cooled in its passage by the surrounding cold water in the vessel $f$, which may be replenished by means of the tube and funnel $d$, and overflow at $e$.

A manufacturer of acid in Scotland, who burns in each chamber 210 pounds of sulphur in 24 hours, being at the rate of 420 pounds for 20,000 cubic feet ( $=$ nearly 2000 metres cube), has a product of nearly 3 pounds of concentrated oil of vitriol for every pound of sulphur and twelfth of a pound of nitre. The advantage of his process results, I conceive, from the lower concentration of the acid in the chambers, which favors its more rapid production.

The platinum retort admits of from 4 to 6 operations in a day, when it is well mounted and managed. It has a capital of platinum, furnished with a short neck, which conducts the disengaged vapors into a lead worm of condensation; and the liquid thus obtained is returned into the lead pans. Great care must be taken to prevent any particles of lead from getting into the platinum vessel, since at the temperature of boiling sulphuric acid, the lead unites with the precious metal, and thus causes holes in the retort. These must be repaired by soldering-on a plate of platinum with gold.

Before the separate oven or hearth for burning the sulphur in contact with the nitre was adopted, this combustible mixture was introduced into the chamber itself, spread on iron trays or earthen pans, supported above the water on iron stands. But this plan was very laborious and unproductive. It is no longer followed.

One of the characters of the good quality of sulphuric acid, is its dissolving indigo without altering its fine blue color.

Sulphuric acid, when well prepared, is a colorless and inodorous liquid, of an oily aspect, possessing a specific gravity, in its most concentrated state, of $1 \cdot 842$, when redistilled, but as found in commerce, of 1.845 . It is eminently acid and corrosive, so that a single drop will communicate the power of reddening litmus to a gallon of water, and will produce an ulcer of the skin when allowed to remain upon it. If swallowed in its strongest state, in even a small quantity, it acts so furiously on the throat and stomach as to cause intolerable agony and speedy death. Watery diluents, mixed with chalk or magnesia, are the readiest antidotes. At a temperature of about $600^{\circ} \mathrm{F}$., or a few degrees below the melting point of lead, it boils and distils over like water. This is the best method of procuring sulphuric acid free from the saline and metallic matters with which it is sometimes contaminated.

The affinity of sulphuric acid for water is so strong, that when exposed in an open saucer, it imbibes one-third of its weight from the atmosphere in 24 hours, and fully six times its weight in a few months. Hence it should be kept excluded from the air. If four parts, by weight, of the strongest acid be suddenly mixed with one part of water, both being at $50^{\circ} \mathbf{F}$, the temperature of the mixture will rise to $300^{\circ}$; while, on the other hand, if four parts of ice be mixed with one of sulphuric acid, they immediately liquefy and sink the thermometer to $4^{\circ}$ below zero. From the great attraction existing between this acid and water, a saucer of it is employed to effect the rapid condensation of aqueous vapor as it exhales from a cup of water placed over it; both standing under the exhausted receiver of an air-pump. By the cold produced by this unchecked evaporation in vacuo, the water is speedily frozen.

To determine the purity of sulphuric acid, let it be slowly heated to the boiling point of water, and if any volatile acid matter be present, it will evaporate, with its characteristic smell. The presence of saline impurity, which is the common one, is discovered by evaporating a given weight of it in a small capsule of platinum placed on red-hot cinders. If more than two grains remain out of 500 , the acid may be reckoned to be 
impure. The best test for sulphuric acid, and the soluble salts into which it enters, is the nitrate of baryta, of which 182 parts are equivalent to 49 of the strongest Jiquid acid, or to 40 of the dry, as it exists in crystallized sulphate of potassa. One twenty thousandth pait of a grain of the acid may be detected by the grayish-white cloud which baryta forms with it. 100 parts of the concentrated acid are neutralized by 143 parts of dry carbonate of potassa, and by 110 of dry carbonate of soda, both perfectly pure.

of all the acids, the sulphuric is most extensively used in the arts, and is, in fact, the primary agent for obtaining almost all the others, by disengaging them from their saline combinations. In this way, nitric, muriatic, tartaric, acetic, and many other acids, are procured. It is employed in the direct formation of alum, of the sulphates of copper, zine, potassa, soda; in that of sulphuric ether, of sugar by the saccharification of starch, and in the preparation of phosphorus, \&c. It serves also for opening the pores of skins in tanning, for clearing the surfaces of metals, for determining the nature of several salts by the acid characters that are disengaged, \&c.

According to the analysis of Dr. Thomson, the crystalline compound deposited occasionally in the leaden chambers above described consists of-

Sulphurous acid, 0.6387 , or 3 atoms. | Water _ _ 0.0733 , or 1 atom.

Sulphuric acid, $0.5290 \quad 2 \quad$ Sulphate of lead, 0.0140 .

Nitric acid $0.3450 \quad 1$ atom.

He admits that the proportion of water is a little uncertain; and that the presence of sulphurous acid was not proved by direct analysis. When heated with water, the crystalline matter disengages nitrous gas in abundance; lets fall some sulphate of lead; and the liquid is found to be sulphuric acid. When heated without water, it is decomposed with emission of nitrous gas ard fuming nitric acid; leaving a liquid which, mixed with water, produces a brisk effervescence, consisting chiefly of nitrous gas.

The following TABLE shows the quantity of concentrated and dry sulphuric acid in 100 parts of dilute, at different densities, by my experiments, published in the Quarterly Journal of Science, for October, 1817 :-

\begin{tabular}{|c|c|c|c|c|c|c|c|c|}
\hline Liquid. & Spec. gravity. & Dry. & Liquid. & Spec. gravity. & Dry. & Liquid. & Spec. gravity. & Dry. \\
\hline 100 & $1 \cdot 8460$ & $81 \cdot 54$ & 66 & $1 \cdot 5503$ & $53 \cdot 82$ & 32 & $1 \cdot 2334$ & $26 \cdot 09$ \\
\hline 99 & $1 \cdot 8438$ & $80 \cdot 72$ & 65 & 1.5390 & $53 \cdot 00$ & 31 & $1 \cdot 2260$ & $25 \cdot 28$ \\
\hline 98 & $1 \cdot 8415$ & $79 \cdot 90$ & 64 & $1 \cdot 5280$ & $52 \cdot 18$ & 30 & $1 \cdot 2184$ & $24 \cdot 46$ \\
\hline 97 & $1 \cdot 8391$ & $79 \cdot 09$ & 63 & 1.5170 & $51 \cdot 37$ & 29 & $1 \cdot 2108$ & $23 \cdot 65$ \\
\hline 96 & $1 \cdot 8366$ & $78 \cdot 28$ & 62 & $1 \cdot 5066$ & $50 \cdot 55$ & 28 & $1 \cdot 2032$ & $22 \cdot 83$ \\
\hline 95 & $1 \cdot 8340$ & $77 \cdot 46$ & 61 & $1 \cdot 4960$ & $49 \cdot 74$ & 27 & $1 \cdot 1956$ & $22 \cdot 01$ \\
\hline 94 & $1 \cdot 8288$ & $76 \cdot 65$ & 60 & $1 \cdot 4860$ & $48 \cdot 92$ & 26 & $1 \cdot 1876$ & $21 \cdot 20$ \\
\hline 93 & $1 \cdot 8235$ & $75 \cdot 83$ & 59 & $1 \cdot 4760$ & $48 \cdot 11$ & 25 & $1 \cdot 1792$ & $20 \cdot 38$ \\
\hline 92 & $1 \cdot 8181$ & $75 \cdot 02$ & 58 & $1 \cdot 4660$ & $47 \cdot 29$ & 24 & $1 \cdot 1706$ & $19 \cdot 57$ \\
\hline 91 & $1 \cdot 8026$ & $74 \cdot 20$ & 57 & $1 \cdot 4560$ & $46 \cdot 48$ & 23 & $1 \cdot 1626$ & $18 \cdot 75$ \\
\hline 90 & 1.8070 & $73 \cdot 39$ & 56 & $1 \cdot 4460$ & $45 \cdot 66$ & 22 & $1 \cdot 1549$ & $17 \cdot 94$ \\
\hline 89 & $1 \cdot 7986$ & 72.57 & 55 & $1 \cdot 4360$ & $44 \cdot 85$ & 21 & $1 \cdot 1480$ & $17 \cdot 12$ \\
\hline 88 & $1 \cdot 7901$ & $71 \cdot 75$ & 54 & $1 \cdot 4265$ & $44 \cdot 03$ & 20 & $1 \cdot 1410$ & $16 \cdot 31$ \\
\hline 87 & $1 \cdot 7815$ & $70 \cdot 94$ & 53 & $1 \cdot 4170$ & $43 \cdot 22$ & 19 & $1 \cdot 1330$ & $15 \cdot 49$ \\
\hline 86 & 1.7728 & $70 \cdot 12$ & 52 & $1 \cdot 4073$ & $42 \cdot 40$ & 18 & $1 \cdot 1246$ & $14 \cdot 68$ \\
\hline 85 & $1 \cdot 7640$ & $69 \cdot 31$ & 51 & $1 \cdot 3977$ & $41 \cdot 58$ & 17 & $1 \cdot 1165$ & $13 \cdot 86$ \\
\hline 84 & 1.75ั40 & $68 \cdot 49$ & 50 & $1 \cdot 3884$ & $40 \cdot 77$ & 16 & $1 \cdot 1090$ & $13 \cdot 05$ \\
\hline 83 & $1 \cdot 7425$ & $67 \cdot 68$ & 49 & $1 \cdot 3788$ & $39 \cdot 95$ & 15 & $1 \cdot 1019$ & $12 \cdot 23$ \\
\hline 82 & $1 \cdot 7315$ & $66 \cdot 86$ & 48 & $1 \cdot 3697$ & $39 \cdot 14$ & 14 & 1.0953 & $11 \cdot 41$ \\
\hline 81 & 1.7200 & $66 \cdot 05$ & 47 & $1 \cdot 3612$ & $38 \cdot 32$ & 13 & $1 \cdot 0887$ & $10 \cdot 60$ \\
\hline 80 & $1 \cdot 7080$ & $65 \cdot 23$ & 46 & $1 \cdot 3530$ & $37 \cdot 51$ & 12 & 1.0809 & $9 \cdot 78$ \\
\hline 79 & $1 \cdot 6972$ & $64 \cdot 42$ & 45 & $1 \cdot 3440$ & $36 \cdot 69$ & 11 & 1.0743 & 8.97 \\
\hline 78 & $1 \cdot 6860$ & $63 \cdot 60$ & 44 & $1 \cdot 3345$ & $35 \cdot 88$ & 10 & 1.0682 & $8 \cdot 15$ \\
\hline 77 & $1 \cdot 6744$ & 62.78 & 43 & $1 \cdot 3255$ & 35.06 & 9 & $1 \cdot 0614$ & $7 \cdot 34$ \\
\hline 76 & $1 \cdot 6624$ & $61 \cdot 97$ & 42 & $1 \cdot 3165$ & $34 \cdot 25$ & 8 & 1.0544 & $6 \cdot 52$ \\
\hline 75 & $1 \cdot 6500$ & $61 \cdot 15$ & 41 & $1 \cdot 3080$ & $33 \cdot 43$ & 7 & 1.0477 & $5 \cdot 71$ \\
\hline 74 & $1 \cdot 6415$ & $60 \cdot 34$ & 40 & $1 \cdot 2999$ & $32 \cdot 61$ & 6 & $1 \cdot 0405$ & $4 \cdot 89$ \\
\hline 73 & $1 \cdot 6321$ & $59 \cdot 52$ & 39 & $1 \cdot 2913$ & $31 \cdot 80$ & 5 & $1 \cdot 0336$ & $4 \cdot 08$ \\
\hline 72 & $1 \cdot 6204$ & $58 \cdot 71$ & 38 & $1 \cdot 2826$ & 30.98 & 4 & $1 \cdot \cap 268$ & $3 \cdot 26$ \\
\hline 71 & $1 \cdot 6090$ & $57 \cdot 89$ & 37 & $1 \cdot 2740$ & $30 \cdot 17$ & 3 & $1 \cdot 0206$ & $2 \cdot 446$ \\
\hline 70 & 1.5975 & $57 \cdot 08$ & 36 & $1 \cdot 2654$ & $29 \cdot 35$ & 2 & $1 \cdot 0140$ & $1 \cdot 63$ \\
\hline 69 & 1.5868 & $56 \cdot 26$ & 35 & $1 \cdot 2572$ & 28.54 & 1 & 1.0074 & 0.8154 \\
\hline 68 & 1.5760 & 55.45 & 34 & $1 \cdot 2490$ & $27 \cdot 72$ & & & \\
\hline 67 & $1 \cdot 5648$ & $54 \cdot 63$ & 33 & $1 \cdot 2409$ & $26 \cdot 91$ & & & \\
\hline
\end{tabular}


SUMACH (Eng. and Fr.; Schmack, Germ.) is the powder of the leaves, peduncles, and young branches of the Rhus coriaria, and Rhus cotinus, shrubs which grow in Hungary, the Bannat, and the Illyrian provinces. Both kinds contain tannin, with a little yellow coluring matter, and are a good deal employed for tanning light-colored leathers; but the first is the best. With mordants, it dyes nearly the same colors as galls. In calicoprinting, sumach affords, with a mordant of tin, a yellow color; with acetate of iron, weak or strong, a gray or black; and with sulphate of zinc, a brownish-yellow. A decoction of sumach reddens litmus paper strongly; gives white flocks with the protomuriate of tin; pale yellow flocks with alum; blue flocks with red sulphate of iron, with an abundant precipitate. In the south of France, the twigs and leaves of the Coriaria myrthifolia are used for dyeing, under the name of rédoul, or rodou.

SWEEP-WASHER is the person who extracts from the sweepings, potsherds, \&c., of refineries of silver and gold, the small residuum of precious metal.

SYNTHESIS is a Greek word, which signifies combination, and is applied to the chemical action which unites dissimilar bodies into a uniform compound; as sulphuric acid and lime, into gypsum; or chlorine and sodium, into culinary salt.

SIRUP is a solution of sugar in water. Cane-juice, concentrated to a density of $1 \cdot 300$, forms a sirup which does not ferment in the transport home from the West Indies, and may be boiled and refined at one step into superior sugar-loaves, with eminent advantage to the planter, the refiner, and the revenue.

\section{$\mathrm{T}$.}

TABBYING, or WATERING, is the process of giving stuffs a wavy appearance with the calender.

TACAMAHAC is a resin obtained from the Fagura octandra, a tree which grows in Mexico and the West Indies. It occurs in yellowish pieces, of a strong smell, and a bitterish aromatic taste. That from the island of Madagascar has a greenish tint.

TAFFETA is a light silk fabric, with a considerable lustre or gloss.

TAFIA is a variety of rum.

TALC is a mineral genus, which is divided into two species, the common and the indurated. The first occurs massive, disseminated in plates, imitative, or crystallized in small six-sided tables. It is splendent, pearly, or semi-metallic, translucent, flexible, but not elastic. It yields to the nail; spec. grav. $2 \cdot 77$. Before the blowpipe, it first whitens and then fuses into an enamel globule. It consists of - silica, 62 ; magnesia, 27; alumina, 1.5 ; oxyde of iron, 3.5 ; water, 6 . Klaproth found $2 \frac{1}{2}$ per cent. of potash in it. It is found in beds of clay-slate and mica slate, in Aberdeenshire, Banffshire, Perthshire, Salzburg, the Tyrol, and St. Gothard. It is an ingredient in rouge for the toilette, communicating softness to the skin. It gives the flesh polish to soft alabaster figures, and is also used in porcelain paste.

The second species, or talc-slate, has a greenish-gray color; is massive, with tabular fragments, translucent on the edges, soft, with a white streak; easily cut or broken, but is not flexible; and has a greasy feel. It occurs in the same localities as the preceding. It is employed in the porcelain and crayon manufactures; as also as a crayon itself, by carpenters, tailors, and glaziers.

TALLOW (Suif, Fr.; Talg, Germ.) is the concrete fat of quadrupeds and man. That of the ox consists of 76 parts of stearine, and 24 of oleine; that of the shecp contains somewhat more stearine. See Fat and SteArine.

Tallow imported into the United Kingdom, in 1836, 1,186,364 cwts. 1 qr. 4 lbs.; in $1837,1,308,734$ cwts. 1 qr. 4 lbs. Retained for home consumption, in $1836,1,318,678$ cwts. 1 qr. 25 ibs.; in 1837, 1,294,009 cwts. 2 qrs. 21 lbs. Duty received, in 1836, $£ 208,284$; in $1837, £ 204,377$.

TALlOW, PINEY. See Piney Tallow.

TAMPING is a term used by miners to express the filling up of the hole which they have bored in a rock, for the purpose of blasting it with gunpowder. See MrNFs.

TAN, or TANNIC ACID. (Tannin, Fr.; Gerbstoff, Germ.) See its preparation and properties described under GaLLs.

The barks replete with this principle should be stripped with hatchets and bills, from the trunk and branches of trees, not less than 30 years of age, in spring, when their sap flows most freely. Trees are also sometimes barked in autumn, and left stanüing, whereby they cease to vegetate, and perish ere long; but afford, it is thought, a more compact timber. This operation is, however, too troublesome to be generally practised, and therefore the bark is commonly obtained from felled trees; and it is richer in tannin the older they are. The bark mill is described in Gregory's Mechanics, and other similas works. 
The following TABLE shows the quantity of extractive matter and $\tan$ in 100 parts of the several substances:-

\begin{tabular}{|c|c|c|c|c|c|c|c|}
\hline Substances. & 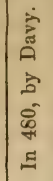 & 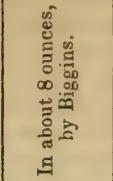 & 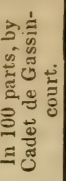 & Substances. & 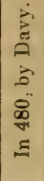 & 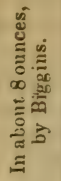 & 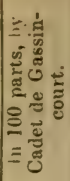 \\
\hline White inner bark of old oak & 72 & - & 21 & Bark of Cherry-tree & - & 59 & 24 \\
\hline Do. young oak - - & 77 & & & Do. Sallow - - - & - & 59 & \\
\hline Do. Spanish chestnut & 63 & 30 & & Do. Poplar - & - & 76 & \\
\hline Do. Leicester willow & 79 & & & Do. Hazel & - & 79 & \\
\hline Colored or middle bark of & 19 & & & Do. Ash - - - - & -- & 82 & \\
\hline oak - - - & 19 & & & Do. trunk of Span. chestnut & - & 98 & \\
\hline $\begin{array}{l}\text { Do. Spanish chestnut } \\
\text { Do. Leicester willow }\end{array}$ & $\begin{array}{l}14 \\
16\end{array}$ & & & $\begin{array}{l}\text { Do. Smooth oak - } \\
\text { Do. Oak, cul in spring }\end{array}$ & - & $\begin{array}{l}104 \\
108\end{array}$ & \\
\hline Entire bark of oak - & 29 & & & Root of Tormentil - & - & - & 46 \\
\hline Do. Spanish chestnut & 21 & & & Cornus sanguienea of Canada & - & - & 44 \\
\hline Do. Leictster willow & 33 & 109 & & Bark of Alder & - & - & 36 \\
\hline Do. Elm - - - & 13 & 28 & & Do. A pricot & - & - & 32 \\
\hline Do. Common willow - & 11 & boughs, 31 & & Do. Pomegranate & - & - & 32 \\
\hline Sicilian sumach - & 78 & 158 & & Do. Cornish cherry-tree & - & - & 19 \\
\hline Malaga sumach - & 79 & & & Do. Weeping willow - & - & - & 16 \\
\hline Souchong tea. - & 48 & & & Do. Bohemian olive - & - & - & 14 \\
\hline Green tea - - & 41 & & & Do. Tan shrub with myrtle & & & 13 \\
\hline Bombay catechu - & 261 & & & leaves $-\quad\}$ & - & & 13 \\
\hline Bengal catechu - & 231 & & & Do. Virginian sumach & - & - & 10 \\
\hline Nut-galls - & 127 & - & 46 & Do. Green oak - & - & - & 10 \\
\hline Bark of oak, cut in winter - & - & 30 & & Do. Service-tree - & - & - & 8 \\
\hline Do. beech - & - & 31 & & Do. Rose chestnut of Amer. & - & - & 8 \\
\hline Do. Elder - & - & 41 & & Do. Rose chestnut & - & - & 6 \\
\hline Do. Plum-tree - & - & 58 & & Do. Rose chestnut of Caro- ? & & & \\
\hline Bark of the trunk of willow - & - & 52 & & lina - - - & - & - & 6 \\
\hline Do. Sycamore - & - & 53 & 16 & Do. Sumach of Carolina - & - & - & 5 \\
\hline Bark of Birch - & -1 & 54 & & & & & \\
\hline
\end{tabular}

TANNING (Tanner, Fr.; Gärberei, Germ.) is the art of converting skin into LEATHER, which see. It has been ascertained, beyond a doubt, that "the saturated infusions of astringent barks contain much less extractive matter, in proportion to their tannin, than the weak infusions; and when skin is quickly tanned (in the former), common experience shows that it produces leather less durable than leather slowly formed."** The older tanners, who prided themselves on producing a substantial article, were so much impressed with the advantages of slowly impregnating skin with astringent matter, that they employed no concentrated infusion (ooze) in their pits, but stratified the skins with abundance of ground bark, and covered them with soft water, knowing that its active principles are very soluble, and that, by being gradually extracted, they would penetrate uniformly the whole of the animal fibres, instead of acting chiefly upon the surface, and making brittle leather, as the strong infusions never fail to do. In fact, 100 pounds of skin, quickly tanned in a strong infusion of bark, produce 137 of leather; while 100 pounds, slowly tanned in a weak infusion, produce only $117 \frac{1}{2}$. The additional $19 \frac{1}{2}$ pounds weight in the former case serve merely to swell the tanner's bill, while they deteriorate his leather, and cause it to contain much less of the textile animal solid. Leather thus highly charged with tannin is, moreover, so spongy as to allow moisture to pass readily through its pores, to the great discomfort and danger of persons who wear shoes made of it. That the saving of time, and the increase of product, are temptations strong enough to induce many modern tanners to steep their skins in a succession of strong infusions of bark, is sufficiently intelligible; but that any shoemaker should be so ignorant or so foolish as to proclaim that his leather is made by a process so injurious to its quality, is unaccountably stupid.

TAN'TALUM is the rare metal, also called CoLUmriom.

TAPESTRY is an ornamental figured textile fabric of worsted or silk, for lining the walls of apartments; of which the most famous is that of the Gobelins Royal Manufactory, near Paris.

TAPIOCA is a modification of starch, partially converted into gum, by heating and stirring cassava upon iron plates. See CASSava and STarch.

TAR (Goudron, Fr.; Ther, Germ.) is the viscid, brown-black, resino-oleaginous compound, obtained by distilling wood in close vessels, or in ovens of a peculiar construction. See Charcoal, Pitcoal, coking of, and Pyroligneous Acid. According to Reichenbach, tar contains the peculiar proximate principles, paraffine, eupion, creosote, picamar, pittacal, besides pyrogenous resin, or pyretine, pyrogenous oil, or pyroleine, and vinegar. The resin, oil, and vinegar are called empyreumatic, in common language.

Tar imported into the United Kingdom, in 1836, 9,797 lsts. 8 barrels; in 1837 * Sir H. Davy, on the Oneration of Astringent Vegetables in Tanning.-Phil. Trans. 1803. 
11,480 lsts. 1 brl. Retained for home consumption, in $1836,9,6391$ sts. 8 brls. ; in 1837, 11,6 . 6 ists. 2 brls. Duty received, in $1836, £ \tau, 231$; in $1837, £ 8,775$.

TARRAS; see Cenent, and Mortar, hydraulic.

TARTAR (Tarlre, Fr.; Weinstein, Germ.), called also argal or argol, is the crude bitartrate of potassa, which exists in the juice of the grape, and is deposited from wines in their fermenting casks, being precipitated in proportion as the alcohol is formed, in consequence of its insolubility in that liquid. There are two sorts of argal known in commerce, the white, and the red; the former, which is of a pale-pinkish color, is the crust let fall by white wines; the latter is a dark-red, from red wines.

The crude tartar is purified, or converted into cream of tartar, at Montpellier, by the following process:-

The argal having been ground under vertical mill-stones, and sifted, one part of it is boiled with 15 of water, in conical copper kettles, tinned on the inside. As soon as it is dissolved, $3 \frac{1}{2}$ parts of ground pipe-clay are introduced. The solution being well stirred, and then settled, is drawn off into crystallizing vessels, to cool; the crystals found concreted on the sides and bottom are picked out, washed with water, and dried. The mother water is employed upon a fresh portion of argal. The crystals of the first crop are re-dissolved, re-crystallized, and exposed upon stretched canvass to the sun and air, to be bleached. The clay serves to abstract the coloring matter. The crystals formed upon the surface are the whitest, whence the name cream of tartar is derived.

Purified tartar, the bitartrate of potassa, is thus obtained in hard clusters of small colorless crrstals, which, examined by a lens, are seen tỏ be transparent 4 -sided prisms. It has no sinell, but a feebly acid taste; is unchangeable in the air, has a specific gravity of 1.953 , dissolves in 16 parts of boiling water, and in 200 parts at $60^{\circ} \mathrm{F}$. It is insoluble in alcohol. It consists of 24.956 potassa, 70.276 tartaric acid, and 4.768 water. It affords, by dry distillation, pyrotartaric acid, and an empyreumatic oil ; while carbonate of potassa remains associated with much charcoal in the retort, constituting black flux. Tartar is used in dyeing, medicine, and for extracting-

TARTARIC ACID. (Acide tartarique, Fr.; Weinsteinsäure, Germ.) This is prepared by adding gradually to a boiling-hot solution of 100 parts of tartar, in a large copper boiler, 26 of chalk, made into a smooth pap with water. A brisk effervescence ensues, by the disengagement of the carbonic acid of the chalk, while its base combines with the acid excess in the tartar, and forms an insoluble precipitate of tartrate of lime. The supernatant liquor, which is a solution of neutral tartrate of potassa, must be drawn off by a syphon, and decomposed by a solution of chloride of calcium (muriate of lime.) $28 \frac{1}{2}$ parts of the dry chloride are sufficient for 100 of tartar. The tartrate of lime, from both processes, is to be washed with water, drained, and then subjected, in a leaden cistern, to the action of 49 parts of sulphuric acid, previously diluted with 8 times its weight of water; 100 of dry tartrate take 75 of oil of vitriol. This mixture, after digestion for a few days, is converted into sulphate of lime and tartaric acid. The latter is to be separated from the former by decantation, filtration through canvass, and edulcoration of the sulphate of lime upon the filter.

The clear acid is to be concentrated in leaden pans, by a moderate heat, till it acquires the density of $40^{\circ} \mathrm{B}$. (spec grav. $1 \cdot 38$ ), and then it is run off, clear from any sediment, into leaden or stoneware vessels, which are set in a dry stove-room for it to crystallize. The crystals, being re-dissolved and re-crystallized, become colorless 6-sided prisms. In decomposing the tartrate of lime, a very slight excess of sulphuric acid must be employed; because pure tartaric acid would dissolve any tartrate of lime that may escape decomposition. Bone black, previously freed from its carbonate and phosphate of lime, by muriatic acid, is sometimes employed to blanch the colored solutions of the first crystals. Tartaric acid contains nearly 9 per cent. of combined water. It is soluble in two parts of water at $60^{\circ}$, and in its own weight of boiling water. In its dry state, as it exists in the tartrate of lime or lead, it consists of 36.8 of carbon, 3 of hydrogen, and 60.2 of oxygen. It is much employed in calico-printing, and for making sodaic powders.

TARTRATES are salts composed of tartaric acid, and oxydized bases, in equivalent proportions.

TAWING is the process of preparing the white skins of the sheep, doe, \&c. See LEATHER.

TEA, green, contains 34.6 parts of tannin, 5.9 of gum, 5.7 of vegetable albumine, 51.3 of ligneous fibre, with 2.5 of loss; and black tea contains 40.6 of tannin, 6.3 of gum, 6.4 of vegetable albumine, 44.8 of ligneous fibre, with 2 of loss. The ashes contain silica, carbonate of lime, magnesia, and chloride of potassium.-Frank. Davy obtained 32.5 of extract from Souchong tea; of which 10 were precipitated by gelatine. He found 8.5 only of tannin in green tea. The latter chemist is most to be depended upon. Chemical analysis has not yet discovered that principle in tea, to which its exciting property is due. 
The Chinese method of making Black Teu in Upper Assam.*-In the first place, the ;oungest and most tender leaves are cathered; but when there are many hands and $a$ great quantity of leaves to be collected, the people employed nip off with the forefinger and thumb the fine end of the branch with about four leaves on, and sometimes even more, if they look tender. These are all brought to the place where they are to be converted into tea; they are then put into a large, circular, open-worked bamboo basket, having a rim all round, two fingers broad. The leaves are thinly scattered in these baskets, and then placed in a frame-work of bamboo, in all appearance like the side of an Indian hut without grass, resting on posts, 2 feet from the ground, with an angle of about $25^{\circ}$. The baskets with leaves are put in this frame to dry in the sun, and are pushed up and brought down by a long bamboo with a circular piece of wood at the end. The leaves are permitted to dry about two hours, being occasionally turned; but the time required for this process depends on the heat of the sun. When they begin to have a slightly withered appearance, they are taken down and brought into the house, where they are placed on a frame to cool for half an hour. They are then put into smaller baskets of the same kind as the former, and placed on a stand. People are now employed to soften the leaves still more, by gently clapping them between their hands, with their fingers and thumb extended, and tossing them up and letting them fall, for about five or ten minutes. They are then again put on the frame during half an hour, and brought down and clapped with the hands as before. This is done three successive times, until the leaves become to the touch like soft leather; the beating and putting away being said to give the tea the black color and bitter flavor. After this the tea is put into hot cast-iron pans, which are fixed in a circular mud fireplace, so that the flame cannot ascend round the pan to incommode the operator. This pan is well heated by a straw or bamboo fire to a certain degree. About two pounds of the leaves are then put into each hot pan, and spread in such a manner that all the leaves may get the same degree of heat. They are every now and then briskly turned with the naked hand, to prevent a leaf from being burnt. When the leaves become inconveniently hot to the hand, they are quickly taken out and delivered to another man with a close-worked bamboo basket ready to receive them. A few leaves that may have been left behind are smartly brushed out with a bamboo broom; all this time a brisk fire is kept up under the pan. After the pan has been used in this manner three or four times, a bucket of cold water is thrown in, and a soft briclibat and bamboo broom used, to give it a good scouring out; the water is thrown out of the pan by the brush on one side, the pan itself being never taken off. The leaves, all hot on the bamboo basket, are laid on a table that has a narrow rim on its back, to prevent these baskets from slipping off when pushed against it. The two pounds of hot leaves are now divided into two or three parcels, and distributed to as many men, who stand up to the table with the leaves right before them, and each placing his legs close together; the leaves are next collected into a ball, which he gently grasps in his left hand, with the thumb extended, the fingers close together, and the hand resting on the little finger. The right hand must be extended in the same manner as the left, but with the palm turned downwards, resting on the top of the ball of tea leaves. Both hands are now employed to roll and propel the ball along; the left hand pushing it on, and allowing it to revolve as it moves; the right hand also pushes it forward, resting on it with some force, and keeping it down to express the juice which the leaves contain. The art lies here in giving the ball a circular motion, and permitting it to turn under and in the hand two or three whole rerolutions, before the arms are extended to their full length, and drawing the ball of leaves quickly back without leaving a leaf behind, being rolled for about five minutes in this way. The ball of tea leaves is from time to time gently and delicately opened with the fingers, lifted as high as the face, and then allowed to fall again. This is done two or three times, to separate the leaves; and afterwards the basket with the leaves is lifted up as often, and receives a circular shake to bring these towards the centre. The leaves are now taken back to the hot pans, and spread out in them as before, being again turned with the naked hand, and when hot taken out and rolled; after which they are put into the drying basket, and spread on a sieve which is in the centre of the basket, and the whole placed over a charcoal fire. The fire is very nicely regulated; there must not be the least smoke, and the charcoal should be well picked.

When the fire is lighted, it is fanned until it gets a fine red glare, and the smoke is all gone off; being every now and then stirred and the coals brought into the centre, so as to leave the outer edge low. When the leaves are put into the drying basket, they are gently separated by lifting them up with the fingers of both hands extended far apart, and allowing them to fall down again; they are placed 3 or 4 inches deep on the sieve, leaving a passage in the centre for the hot air to pass. Before it is put over the fire, the drying basket receives a smart slap with both hands in the act of lifing it un, which is done to shake down any leaves that might otherwise drop through the sieve, or to pre* By C. A. Bruce, superintendent of tea cuiture. 
vent them from falling into the fire and occasioning a smoke, which would affect and spoil the tea. This slap on the basket is invariably applied throughout the stages of the tea manufacture. There is always a large basket underneath to receive the small leaves that fall, which are afterwards collected, dried, and added to the other tea; in no case are the baskets or sieves permitted to touch or remain on the ground, but always laid on a receiver with three legs. After the leaves have been half dried in the drying basket, and while they are still soft, they are taken off the fire and put into large open-worked baskets, and then put on the shelf, in order that the tea may improve in color.

Next day the leares are all sorted into large, middling, and small; sometimes there are four sorts. All these, the Chinese informed me, become so many different kinds of teas; the smallest leaves they called Pha-ho, the second, Pow-chong, the third Su-chong, and the fourth, or the largest leaves, Toy-chong. After this assortment they are again put on the sieve in the drying basket (taking great care not to mix the sorts), and on the fire, as on the preceding day; but now very little more than will cover the bottom of the sieve is put in at one time, the same care of the fire is taken as before, and the same precaution of tapping the drying basket every now and then. The tea is taken off the fire with the nicest care, for fear of any particle of the tea falling into it. Whenever the drying basket is taken off, it is put on the receiver, the sieve in the drying basket taken out, the tea turned over, the sieve replaced, the tap given, and the basket placed again over the fire. As the tea becomes crisp, it is taken out and thrown into a large receiving basket, until all the quantity on hand has become alike dried and crisp; from which basket it is again removed into the drying basket, but now in much larger quantities. It is then piled up eight and ten inches high on the sieve in the drying basket; in the centre a small passage is left for the hot air to ascend; the fire that was before bright and clear, has now ashes thrown on it to deaden its effect, and the shakings that have been collected are put on the top of all; the tap is given, and the basket with the greatest care is put over the fire. Another basket is placed over the whole, to throw back any heat that may ascend. Now and then it is taken off, and put on the receiver; the hands, with the fingers wide apart, are run down the sides of the basket to the sieve, and the tea gently turned over, the passage in the centre again made, \&c., and the basket again placed on the fire. It is from time to time examined, and when the leaves have become so crisp that they break by the slightest pressure of the fingers, it is taken off, when the tea is ready. All the different kinds of leaves underwent the same operation. The tea is now little by little put into boxes, and first pressed down with the hands and then with the feet (clean stockings having bcen previously put on).

There is a small room inside of the tea-house, 7 cubits square and 5 high, having bamboos laid across on the top to support a net-work of bamboo, and the sides of the room smeared with mud to exclude the air. When there is wet weather, and the leaves cannot be dried in the sun, they are laid out on the top of this room, on the network, on an iron pan, the same as is used to heat the leaves; some fire is put into it, either of grass or bamboo, so that the flame may ascend high; the pan is put on a square wooden frame, that has wooden rollers on its legs, and pushed round and round this little room by one man, while another feeds the fire, the leaves on the top being occasionally turned; when they are a little withered, the fire is taken away, and the leaves brought down and manufactured into tea, in the same manner as if it had been dried. in the sun. But this is not a good plan, and never had recourse to, if it can possibly be avoided.

Tea imported into the United Kingdom, in 1836, 49,307,701 lbs. ; in 1837, 36,765,735 lbs. Retained for home comsumption, in 1836, $49,841,507$ lbs. ; in 1837, 31,872lbs. Duty received, in $1836, £ 4,728,600$; in $1837, £ 3,319,665$.

TEASEL, the head of the thistle (Dipsacus), is employed to raise the nap of cloth. See WOOLLEN MANUfacture.

TEETH. See Bones.

TELLURIUM, is a metal, too rare and high-priced to be used in the arts.

TERRA-COTTA, literally baked clay, is the name given to statues, architectural decorations, figures, vases, \&c., modelled or cast in a paste made of pipe or potter's clay and a fine-grained colorless sand, from Ryegate, with pulverized potsherds, slowly dried in the air, and afterwards fired to a steny hardness in a proper kiln. See STONE, ARTIFICIAL.

TERRA DI SIENA, is a brown ferruginous ochre, employed in painting.

TESTS, are chemical reagents of any kind, which indicate, by special characters, the nature of any substance, simple or compound. See AssAr, the several metals, acids, \&c.

TEX TILE FABRICS. The first business of the weaver is to adapt those paris of his loom which move the warp, to the formation of the various kinds of ornamental figures which the cloth is intended to exhibit. This subject is called the draught, drawing or reading in, and the cording of looms. In every species of weaving, whether direct or cross, the whole difference of pattern or effect is produced, either by the succession in which the 
threads of warp are introduced into the heddles, or by the succession in which those heddlos are moved in the working. The heddles being stretched between two shafts of wood, all the heddles connected by the same shafts are called a leaf; and as the operation of introducing the warp into any number of leaves is called drawing a warp, the plan of succession is called the draught. When this operation has been performed correctly, the next part of the weaver's business is to connect the different leaves with the levers or treddles by which they are to be moved, so that one or more may be raised or sunk by every treddle successively, as may be required to produce the peculiar pattern. These connexions being made by coupling the diflerent parts of the apparatus by cords, this operation is called the cording. In order to direct the operator in this part of his business, especially if previously unacquainted with the particular pattern upon which he is employed, plans are drawn upon paper, specimens of which will be found in figs. 1103, 1104, \&c.

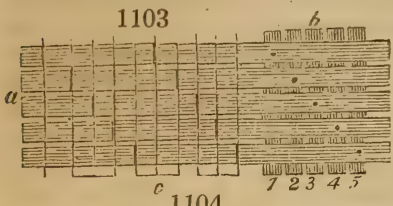

1104

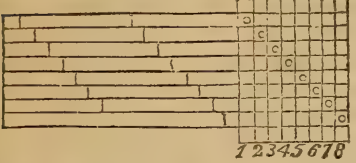
These plans are horizontal sections of a loom, the heddles being represented across the paper at $a$, and the treddles under them, and crossing them at right angles, at $b$. In figs. 1103 and 1104 , they are represented as if they were distinct pieces of wood, those across being the under shaft of each leaf of heddles, and those at the left hand the treddles. See WEAvING. In actual weaving, the treddles are placed at right angles to the heddles, the sinking cords descending perpendicularly as nearly as possible to the centre of the latter. Placing them at the left hand, therefore, is only for ready inspection, and for practical convenience. At $c$ a few threads of warp are shown as they pass through the heddles, and the thick lines denote the leaf with which each thread is connected. Thus, in fig. 1103, the right-hand thread, next to $a$, passes throunh the eye of a heddle upon the back leaf, and is disconnected with all the other leaves; the next thread passes through a heddle on the second leaf; the third, through the third leaf; the fourth, through the fourth leaf; and the fifth, through the fifth or front leaf. One set of the draught being now completed, the weaver recommences with the back leaf, and proceeds in the same succession again to the front. Two sets of the draught are represented in this figure, and the same succession, it is understood by weavers (who seldom draw more than one set), must be repeated until all the warp is included. When they proceed to apply the cords, the right-hand part of the plan at $b$ serves as a guide. In all the plans shown by these figures, excepting one which shall be noticed, a connexion must be formed, by cording, between every leaf of heddles and every treddle; for all the leaves must either rise or sink. The raising motion is effected by coupling the leaf to one end of its correspondent top lever; the other end of this lever is tied to the long march below, and this to the treddle. The sinking connexion is carried directly from under the leaf to the treddle. To direct a weaver which of these connexions is to be formed with each treddle, a black spot is placed when a leaf is to be rajsed, where the leaf and treddle intersect each other upon the plan, and the sinking connexions are left blank. For example, to cord the treddle 1 , to the back leaf, put a raising cord, and to each of the other four, sinking cords; for the treddle 2 , raise the second leaf, and sink the remaining four, and so of the rest; the spot always denoting the leaf or leaves to be raised. The figs. 1103, and 1104, are drawn for the purpose of rendering the general principle of this kind of plans familiar to those who have not been previously acquainted with them; but those who have been accustomed to manufacture and weave ornamented cloths, never consume time by representing either heddles or treddles as solid or distinct bodies. They content themselves with ruling a number of lines across a piece of paper, sufficient to make the intervals between these lines represent the number of leaves required. Upon these intervals, they merely mark the succession of the draught, without producing every line to resemble a thread of warp. At the left hand, they draw as many lines across the former as will afford an interval for each treddle; and in the squares produced by the intersections of these lines, they place the dots, spots, or ciphers which denote the raising cords. It is also common to continue the cross lines which denote the treddle a considerable length beyond the intersections, and to mark by dots, placed diagonally in the intervals, the order or succession in which the treddles are to be pressed down in weaving. The former of these modes has been adopted in the remaining figs. to 1112; but to save room, the latter has been avoided, and the succession marked by the order of the figures under the intervals which denote the treddles.

Some explanation of the various kinds of fanciful cloths represented by these plans, may serve further to illustrate this subject, which is, perhaps, the most important of any connected with the manufacture of cloth, and will also enable a person who thoroughly studies them, readily to acquire a competent knowledge of the other varieties in weaving, 
which are boundless. Fins. 1103 and 1104 represent the draught and cording of the two varieties of tweeled cloth wrought with five leaves of heddles. The first is the regular or run tweel, which, as every leaf rises in regular succession, while the rest are sunk, interweaves the warp and woof only at every fifth interval, and as the succession is unifirm, the cloth, when woven, presents the appearance of parallel diagonal lines, at an anrle of about $45^{\circ}$ over the whole surface. A tweel may have the regularity of its diaconal lines broken by applying the cording as in fig. 1104. It will be observed, that in both figures the draught of the warp is precisely the same, and that the whole difference of the two plans consists in the order of placing the spots denoting the raising cords, the first being regular and successive, and the second alternate.

Figs. 1105 and 1106 are the regular and broken tweels which may be produced with eight leaves. This properly is the tweel denominated satin in the silk manufacture, 1105

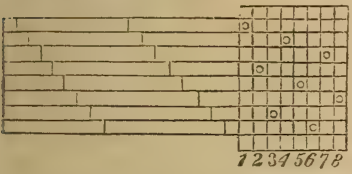

\section{6}

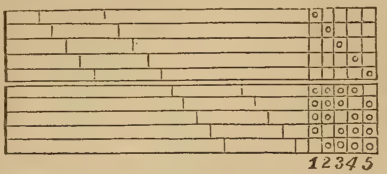

although many webs of silk wrought with only five leaves receive that appellation. Some of the finest Florentine silks are tweeled with sixteen leaves. When the brolien tweel of eight leaves is used, the effect is much superior to what could be produced by a smaller number; for in this, two leaves are passed in every interval, which gives a much nearer resemblance to plain cloth than the others. For this reason it is preferred in weaving the finest damasks. The draught of the eight-leaf tweel differs in nothing from the others, excepting in the number of leaves. The difference of the cording in the broken tweel, will appear by inspecting the ciphers which mark the raising cords, and comparing them with those of the broken tweel of five leares. Fig. 1107 represents the draught and cording of striped dimity of a tweel of five leaves. This is the most simple species of fanciful tweeling. It consists of ten 1107

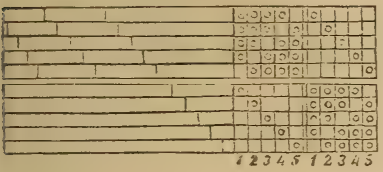
leaves, or double the number of the common tweel. These ten leaves are moved by only five treddles, in the same manner as a common tweel. The stripe is formed by one set of the leaves flushing the warp, and the other set, the woof. The figure represents a stripe formed by ten threads, alternately drawn through each of the two sets of leaves. In this case, the stripe and the intervals will be equally broad, and what is the stripe upon one side of the cloth, will be the interval upon the other, and vice versâ. But great variety of patterns may be introduced by drawing the warp in greater or smaller portions through either set. The tweel is of the regular kind, but may be broken by placing the cording as in fig. 1104. It will be observed that the cording-marks of the lower or front leaves are exactly the converse of the other set; for where a raising mark is placed upon one, it is marked for sinking in the other; that is to say, the mark is omitted; and all leaves which sink in the one, are marked for raising in the other: thus, one thread rises in succession in the back set, and 1108

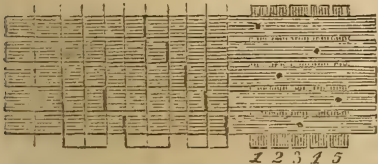

four sink; but in the front set, four rise, and only one sinks. The woof, of course, passing over the four sunk threads, and under the raised one, in the first instance, is flushed above; but where the reverse takes place, as in the second, it is flushed below ; and thus the appearance of a stripe is formed. The analogy subsisting between striped dimity and dornock is so great, that before noticing the plan for fancy dimity, it may be proper to allude to the dornock, the plan of which is represented by fig. 1108 .

The draught of dornock is precisely the same in every respect with that of striped dimity. It also consists of two sets of tweeling-heddles, whether three, four, or five leaves are used for each set. The right hand set of treddles is also corded exactly in the same way, as will appear by comparing them. But as the dimity is a continued stripe from the beginning to the end of the web, only five treddles are required to move ten leares. The dornock being checker-wuri, the weaver must possess the power of reversing this at pleasure. He therefore adds five more treddles, the cording of which is exactly the reverse of the former; that is to say, the back leaves in the former case, having one leaf raised, and four sunk, have, by working with these additional treddles, one leaf sunk and four leaves raised. The front leaves are in the same manner reversed, and the mounting is complete. So long as the weaver continues to work with either set, a stripe will be formed, as in the dimity; but when he changes his feet from one set 
to the other, the whole effect is reversed, and the checkers formed. The dornock pat tern upon the design-paper, fig. 1105 , may be thus explained: let every square of the design represent five threats uinon tither set of the hedlles, which are said by weavers to be once over the draught, supposing the tweel to be one of five leares; draw thiee parallel lines, as under, to form two intervals, each representing one of the spts; the diaught will then be as follows:-

\begin{tabular}{|l|lllllllll}
\hline 4 & 1 & 4 & 1 & 1 & 4 & 1 \\
\hline 4 & 4 & 1 & 1 & 1 & 4 & 4 \\
\hline
\end{tabular}

The above is exactly so much of the pattern as is there laid down, to show its appearance; but one whole range of the pattern is completed by the figure 1 . nearest to the right hand upon the lower interval betwe n the lines, and the remaining figures, nearer to the right, form the beginning of a second range or set. These are to be repeated in the same was across the whole warp. The lower interval represents the fire iront leares; the uprer interval, the five back ones. The first figure 4 , denctcs that fire threads are to be sticcessively diawn upon the back leaves, and this operation repeated four times. The first figure $t$, in the lower interral, expresses that the same is to be done unon the front leares: and each figure, br its liagonal position, shows how often, and in what succession, five threads are to be drawn upon the leares which the interval in which it is placed represents.

Dornochs of more extensive paiterns are sometimes woren with $3,4,5$, and eren 6 sets of leaves: but after the Jeaves exceed 15 in number, the both occuny an inconrenient space, and are rery unwieldy to work. For these reasons the diaper harness is in almost every instance preferred.

Fig. 1109 represents the draught and cording of a fanciful species of dimity, in which it will be cbserved that the rarp is not drawn directly from the back 1109

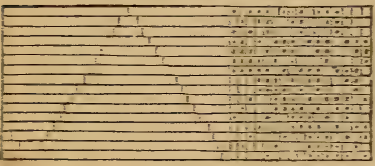
to the front leaf, as in the former examples; but when it has arrived at either external leaf, the draught is reversed, and returns gradually to the other. The same draught is frequently used in tweeling, when it is rished that the diagonal lines should aprear unon the cluth in a zigzag direction. This plan exhibits the draught and cording which will produce the pattern upon the design-paper in fig. 1103, a. Were all the squares produced by the intersection of the lines denoting the leares and tredules where the raised dots are placed, filled the same as on the design, they would produce the effect of exactiy one fourth of that pattern. This is caused br the reversing of the draught, which gives the other side reversed as on the design; and when all the treddies, from 1 tc 16, hare been successirely used in the worling, one half of the pattern $\pi$ ill become com;lete. The wearer then goes again orer his tredlles, in the reversed order of the numbers, from 17 to 30 , when the other half of the pattern will be eompleted. From this similarits of the cording to the design, it is easr, when a desiun is given, to make out the draught and cording proper to work it; and when the cording is giren, to see its effect upon the design.

Fig. 1110 represents the draught of the dianer mounting. and the cording of the front 1110

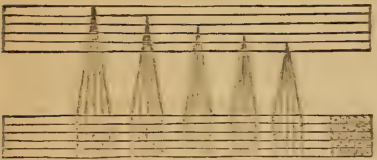
leaves, which are moved br treddles. From the plan, it will appear that 5 threads are included in every mail of the harress, and that these are dramn in single threads through the front leaves. The eording forms an exception to the general rules. that when une or more leares are raised, all the rest must be sunk; for in this instance, one leaf rises, one sinks, and three remain stationary. An additional mark, therefore, is used in this plan. The dots, as formerly, denote raising cords; the blanks, sinking conds; and where the cord is to be totally unitted, the cross marks $X$ are placed.

Fig. 1111 is the draught and corling of a snot whose tro sides are similar, hut reversed. That upon the plan forms a diamond, similar to the one drawn upon the design paper in the diagram, but smaller in size. The dransht here is reversed, as in the dimitr plan, and the treading is also to be reversed, after arriving at 6 , to complete the diamond. Like it, too, the raising marlis form one fourth of the pattern. In wearing spots, ther are commonly placed at interrals, with a portion of plain cloth betreen them, and in alternate rows, the spots of one row being between those of the other. But as intervals of plain cloth must take place, both bT the warp and woof, two leares are added for that purpose. The front, or ground leaf, inclndes erers second thrcad of the whole warp; the second, or plain leaf, that part which forms the inter. 
vals by the warp. The remaining leaves form the spots; the first six being allotted to one row of snots, and the sceond six tu the next row; where each spot is in the centre be-

1111

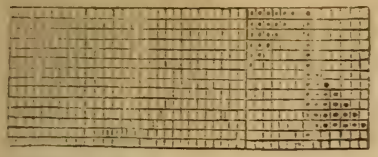

tween the former. The reversed draught of the first is shown entire, and is succeeded by 12 threads of plain. One half of the draught of the next row is then given, which is to be completed exactly like the first, and succeeded by 12 threads more of plain; when, one set of the pattern being finished, the same succession is to be repeated over the whole warp. As spots are formed by inserting woof of coarser dimensions than that which forms the fabric, every second thread only is allotted for the spotting. Those included in the front, or ground leaf, are represented by lines, and the spot threads between them, by marks in the intervals, as in the other plans.

The tredilles necessary (1) work this spot are, in number, 14. Of these, the two in the artre $\imath, b$, when pressed alternately, will produce plain cloth; for $b$ raises the front leaf, which includes half of the warp, and sinks all the rest; while $a$ exactly reverses the opera-

1112

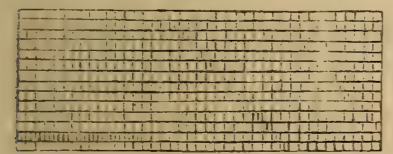

tion. The spot-treddles on the right hand work the row contained in the first six-spot leaves; and those upon the left hand, the row contained in the second six. In working spots, one thread, or shot of spottingwoof, and two of plain, are successively inserted, by means of two separate shuttles.

Dissimilar spots, are those whose sides are quite different from each other. The draught only of these is represented by fig. 1112. The eording depends entirely upon the figure.

Fig. 1113 represents any solid body composed of parts lashed together. If the darkened squares be supposed to be beams of wood, connected by cordage, they will give a precise idea of textile fabric. The beams cannot come into actual contact, because, if the lashing cords were as fine even as human hairs, they must still require

\section{3}

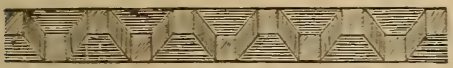

space. The thickness is that of one beam and one cord; but if the cords touch each other, it may then be one beam and two cords; but it is not possible in praetical weaving to bring every thread of weft into actual contact. It mar therefore be assumed, that the thichness is equal to the diameter of one thread of the warp, adrled to that of one yarn of the weft; and when these are equal, the thick.

1114

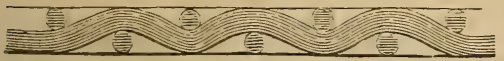

ness of the cloth is double of that diameter. Denser cloth would not be sufficiently pliant or flexible.

Fig. 1114 is a representation of a sec-

tion uf cluth of an open fabric, where the round dots which represent the warp are placed at a considerable distance from each other.

Fig. 1115 may be supposed a plain fabric of that description which approaches the most nearly to any idea we can form of the most dense or close contact of which rarn ean be made susceptible. Here the warp is supposed to be so tightly stretched in

\section{5}

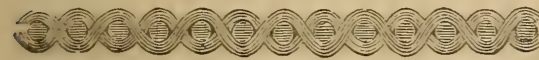
the loom as to retain entirely the parallel state, without any currature, and the whole flexure is therefore given to the woof. This mode of wearing can never really exist; but if the warp be sufficiently strong to bear any tight stretehing, and the woof be spun very soft and flexible, something very near it may be produced. This way of making cloth is well fitted for those goods which require to give considerable warmth; but they are sometimes the means of very gross fraud and imposition; for if the warp is made of very slender threads, and the woof of slackly twisted cotton or woollen yarn, where the fibrils of the stuff, being but slightly brought into contact, are rough and oozy, a great appearance of thickness and strength may he given to the eye, when the cloth is absolutely so flimsy, that it mar be torn asunder as easily as a sheet of writine-paper. Many frauds of this kind are practised.

In $r$ g. 1116 is given a representation of the position of a fabric of cloth in section, as it is in the loom before the warp has been closed upon the woof, which still appears as a

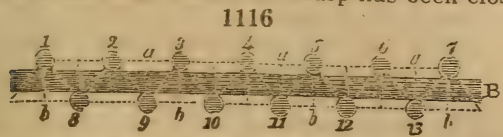
straight line. This figure may usefully illustrate the direction and ratio of contraction which must unavoidably take place in every kind of cloth, according to the density of the texture, the dinensions of the threarls, and the descrintion of the cloth. Let $A, B$, represent one thread of woof completely stretched by the relocity of the shuttle in passing between 
the threads of warp which are represented by the round dots 1, 2, \&c., and those distinguished by 8,9 , \&c. When these threads are closed by the operation of the heddles to form the inner texture, the first tendency will be to move in the direction $1, b, 2, b, \& c$., for those above, and in that of $8 a, 9 u$, \&c., for those below ; but the contraction for $A, B$, by its deviation from a straight to a curved line, in consequence of the compression of the warp threads $1 b, 2 b$, \&c., and $1 a, 2 a$, \&c., in closing, will produce, by the action of the two powers at right angles to each other, the oblique or diagonal direction denoted by the lines $1,8-2,9$, to the left, for the threads abore, and that expresserl by the lines $2,8-3,9, \& c$., to the right, for the threads below. Now, as the whole deviation is produced by the flexure of the thread $A, B$, if $A$ is supposed to be placed at the middle of the cloth, equidistant from the two extremities, or selvages, as they are called by weavers, the thread at 1 may be supposed to move really in the direction $1 \mathrm{~b}$, and all the others to approach to it in the directions represented, whilst those to the right would approach in the same ratio, but the line of approxima1117

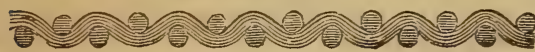
tion would be inverted. Fig. 1117 represents that common fabric used for lawns, muslins, and the middle kin ' of gouds, the excellence of which weither consists in the greatest strength, nor in the greaiset transparency. It is entirely a medium between fig. 1114 and fig. 1115 .

In the efforts to give great strength and thicliness to cloth, it will be obvious that the common mode of wearing, by constant intersection of warp and woof, although it may be perhaps the best which can be derised for the former, presents invincible obstructions to the latter, beyond a certain limit. To remedy this, two modes of weaving are in common use, which, while they add to the power of compressing a great quantity of materials in a small compass, possess the additional advantage of affording much facility for adding ornament to the superficies of the fabric. The first of these is double cloth, or two webs woven together, and joined by the operation. This is chiefly used for 1118 carpets; and its geometrical principles are entirely the same as those of plain eloth, supposing the webs

to be sewed together. A section of the cloth will be found in fig. 1118. See CARPET.

Of the simplest kind of tweeled fabrics, a section is given in fig. 1119 .

The great and prominent adrantage of the tweeled fabric, in point of texture, arises from the facility with which a very great quantity of materials may be put elosely to-

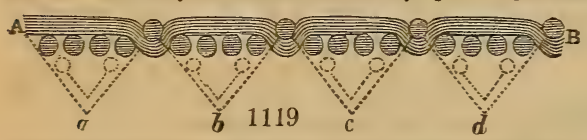
gether. In the figure, the warp is represented by the dots in the same straight line as in the plain fabries; but if we consider the direction and ratio of contraction, upon principles similar to those laid down in the explanation given of fig. 1116, we shall readily discover the very different way in which the tweeled fabric is affected.

When the dotted lines are drawn at $a, b, c, d$, their direction of contraction, instead of being upon every second or alternate thread, is only upon every fifth thread, and the natural tendency would consequently be, to bring the whole into the form represented by the lines and dotted circles at $a, b, c, d$. In point, then, of thickness, from the upper to the under superficies, it is evident that the whole fabric has increased in the ratio of nearly three to one. On the other hand, it will appear, that four threads or cylinders being thus put together in one solid mass, might be supposed only one thread, or like the strands of a rope before it is twisted; but, to remedy this, the thread being shifted every time, the whole forms a hody in which much aggregate matter is compressed; but where, being less firmly united, the accession of strength acquired by the accumulation of materials is partially counteracted by the want of equal firmness of junction.

The second quality of the tweeled fabric, susceptibility of receiving ornament, arises 1120

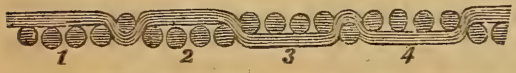
from its capability of being inverted at pleasure, as in $f \mathrm{~g} .1120$. In inis figure we have, as before, four threads, and one atternately intersected; but here the four threads marked 1 and 2 are under the woof, while those marked 3 and 4 are above.

Fig. 1121 represents that kind of tweeled work which produces an ornamental effect, and adds even to the strength of a fabric, in so far as accumulation of matter can be 1121

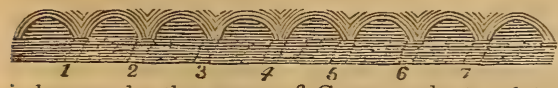
considered in that light. The figure represents a piece of velvet cut in section, and of that kind which, being woven upon a tweeled ground,

is known by the name of Genoa velvet. 1st. Because, by combining a great quantity 
of material in a small compass, they aflord great warmth. 2d. From the great resistance which they oppose to external friction, they are very durable. And 3d. Because, from the very nature of the texture, they afford the finest means of rich ornarnental decoration.

The use of velvet cloths in cold weather is a sufficient proof of the truth of the first. The manufacture of plush, corduroy, and other stuffs for the dress of those exposed to the rccidents of laborious employment, evinces the second; and the ornamented velvets and Wilton carpeting are demonstrative of the third of these positions.

In the figure, the diagonal form which both the warp and woof of cloth assume, is very apparent from the smallness of the scale. Besides what this adds to the strength of the cloth, the flushed part, which appears interwoven at the darkly shaded interva]s, 1, 2, \&c., forms, when finished, the whole covering or upper surface. The principle, in so far as regards texture, is entirely the same as any other tweeled fabric.

Fig. 1122, which represents corduroy, or king's cord, is merely striped velvet. The principle is the same, and the figure shows that the one is a copy of the other. The re-

1122

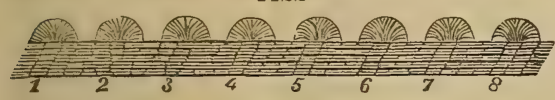
maining figures represent those kinds of vork which are of the most flimsy and open description of texture; those in which neither strength, warmth, nor durability is much required, and of which

openness and transparency are the chief recommendations.

Fig. 1123 represents common gauze, or linau, a substance very much used for various purposes. The essential difference between this description of cloth and all others, con-

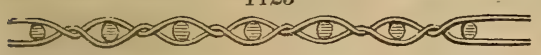
sists in the warp being turned or twisted like a rope during the operation of weaving, and hence it bears a considerable analogy to lace. The twining of gauze is not continued in the same direction, but is alternately from right to left, and vice versâ, between every intersection of the woof. The fabric of gauze is always open, flimsy, and transparent; but, from the turning of the warp, it possesses an uncommon degree of strength and tenacity in proportion to the quantity of material which it contains. This quality, together with the transparency of the fabric, renders it peculiarly adapted for ornamental purposes of various kinds, particularly for flowering or figuring, either in the loom, or by the needle. In the warp of gauze, there arises a much greater degree of contraction during the weaving, than in any other species of eloth; and this is produced by the turning. The twisting between every intersection of weft amounts precisely to one complete revolution of both threads; hence this difference exists between this and every other species of weaving, namely, that the one thread of warp is always above the woof, and

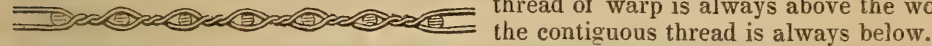

Fig. 1124 represents a section of another species of twisted cloth, which is known by the name of catgut, and which differs from the gauze only by being subjected to a greater degree of twine in weaving; for in place of one revolution between each intersection, a revolution and a half is always given; and thus the warp is alternately above and below, as in other kinds of weaving.

$F i g .1125$ is a superficial representation of the most simple kind of ornamental net-work produced in the loom. It is called a whip-net by weavers, who use the term whip for

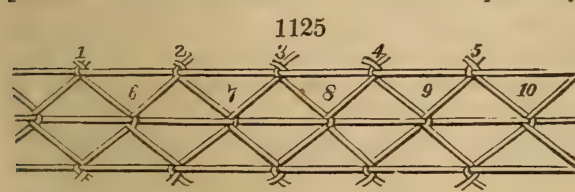
any substance interwoven in cloth for ornamental purposes, when it is distinct from the ground of the fabric. In this, the difference is merely in the crossing of the warp; for it is very evident that the crossings at 1 , $2,3,4$, and 5 , are of different threads from those at $6,7,8$, and 9 .

Fig. 1126 represents, superficially, what is called the mail-net, and is merely a combination of common gauze and the whip-net in the same fabric. The gauze here being in

\section{6}

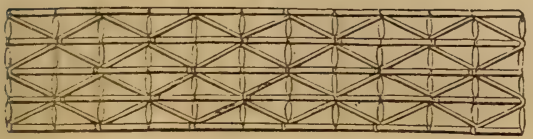
the same direction as the dotted line in the former figure, the whole fabric is evidently a continued succession of rightangled triangles, of which the woof forms the basis, the gauze part the perpendiculars, and the whip part the hypotenuses. The contraction here being very different, it is necessary that the gauze

and whip parts should be stretched upon separate beams.

In order to design ornamental figures upon cloths. the lines which are drawn from the 
top to the bottom of the paper may be supposed to represent the warp; and those drawn across, the woof of the web; any number of threads being supposed to be included between every two lines. The paper thus forms a double scale, by which, in the first instance, the size and form of the pattern may be determined with great precision; and the whole subsequent operations of the weaver regulated, both in mounting and working his loom. To enable the projector of a new pattern to judge properly of its effects, when transferred from the paper to the cloth, it will be essentially necessary that he should bear constantly in his view the comparative scale of magnitude which the design will bear in each, regulating his ideas always by square or superficial measurement. Thus, in the large design, fig. 1127, representing a bird perched upon the branch of a tree, it will

1127

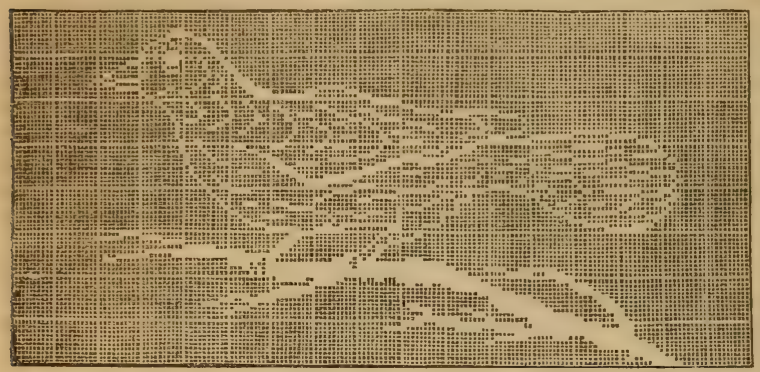
be proper, in the first place, to count the number of spaces from the point of the bill to the extremity of the tail; and to render this the more easy, it is to be observed that eve. ry tenth line is drawn considerably bolder than the others. This number in the design is 135 spaces. Counting again, from the stem of the branch to the $11+1 \cdots+1$ inl of the bid's head, he will find 76 spaces. Between these spaces, there fore, the whole superficial measure of the pattern is contained. By the measure of the paper, this may be easily tried with a pair of compasses, and will be found to be nearly $6 \frac{5}{10}$ inches in length, by $3 \frac{3}{16}$ inches in breadth. Now, if this is to be woven in a reed containing 800 intervals in 37 inches, and if every interval contains five threads, supposed to be contained between every two parallel lines, the length will be 6.24 inches, and the breadth 3.52 inches nearly; so that the figure upon the cloth would be very nearly of the same dimensions as that upon the paper; but if a 1200 reed were used, instead of an 800 , the dimensions would be proportionally contracted.

A correct idea being formed of the design, the weaver may proceed to mount his loom according to the pattern; and this is done by two persons, one of whom takes from the desion the instructions necessary for the other to follow in tying his cords.

Fig. 1128 is a representation of the most simple species of table-linen, which is merely 1128

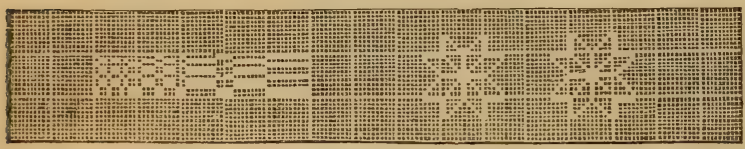
an imitation of checker-. work of various sizes; and is known in Scotland, where the manufacture is chiefly prac. tised, by the name of Dornock. When a pattern is formed upon tweeled cloth, by reversing the flushing, the two sides of the fabric being dissimilar, one may be supposed to be represented by the black marks, and the other by the part of the figure which is left uncolored. For such a pattern as this, two sets of common tweel-heddles, moved in the ordinary way, by a double succession of heddles, are sufficient. The other part of $f \mathrm{~g} .1128$, is a design of that intermediate lind of ornamental work which is called diaper, and which parlakes partly of the nature of the dornock, and partly of that of the damask and tapestry. The principle upon which all these descriptions of goods are woven is entirely the same, and the only difference is in

1129

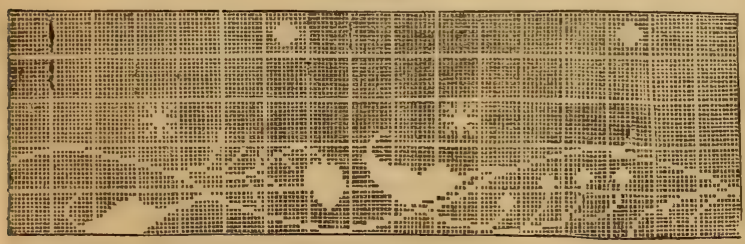

the extent of the design, and the means by which it is executed. Fig. 1129 is a design for a border of a handkerchief or napkin, which may be execnted either in the manner of damask, or as the spotting is practised in the lighter fabrics.

THENARD'S BLUE, or COBALT BLUE, is prepared by digesting the oxyde of cobalt use:l in the potteries, with nitric acid, evaporating the nitrate almost to dryness, diluting it with water, and filtering, to separate some arseniate of iron, which usually 
precipitates. The clear liquor is to be poured into a solution of phosphate of soda, whence an insulnble phosphate of cobalt falls. This being well washed, is to be intimately mixed in its soft state with eight times its weight of well-washed gelatinous alumina, which has been obtained by pouring a solution of alum into water of ammonia in excess. The uniformly colored paste is to be spread upon plates, dried in a stove, then bruised dry in a mortar, enclosed in a crucible, and subjected to a cherry-red heat for half an hour. On taking out the crucible, and letting it cool, the fine blue pigment is to be removed into a bottle, which is to be stopped till used.

The arseniate of cobalt may be substituted, in the above process, for the phosphate, but it must be mixed with sixteen times its weight of the washed gelatinous alumina. The arseniate is procured by pouring the dilute nitrate of cobalt into a solution of arseniate of potassa. If nitrate of cobalt be mixed with the alumina, and the mixture be treated as above described, a blue pigment will also be obtained, but paler than the preceding, showing that the color consists essentially of alumina stained with oxyde of cobalt.

THERMOMETER, signifies the measure of heat. Its description belongs to a treatise on chemical physics.

THERMOSTAT, is the name of an apparatus for regulating temperature, in vaporization, distillation, heating baths or hot-houses, and ventilating apartments, \&c.; for which I obtained a patent in the year 1831. It operates upon the physical principle, that when two thin metallic bars of different expansibilities are riveted or soldered facewise together, any change of temperature in them will cause a sensible movement of flexure in the compound bar, to one side or other; which movement may be made to operate, by the intervention of levers, \&c., in any desired degree, upon valves, stop-cocks, stove-registers, air-ventilators, \&c.; so as to regulate the temperature of the media in which the sail compound bars are placed. Two long rulers, one of steel, and one of hard hammered brass, rireterl together, answer very well; the object being not simply to indicate, but to control or modify temperature. The following diagrams will illustrate a few out of the numerous applications of this instrument :-

Fig. 1130, $a, b$, is a single thermostatic bar, consisting of two or more bars or rulers of differently expansible solids (of which, in certain cases, wood may be one) : these bars

\section{0}

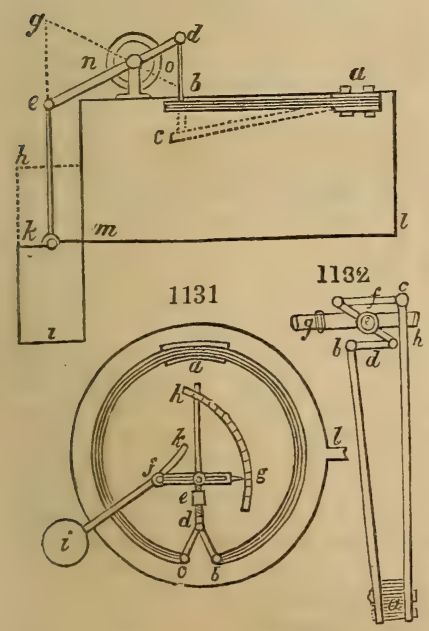
or rulers are firmly riveted or soldered together, face to face. One end of the compound bar is fixed by bolts at $a$, to the interior of the containing cistern. boiler, or apartment, $a, l, m, b$, whereof the temperature has to be regulated, and the other end of the compound bar at $b$, is Jeft free to move down towards $c$, by the flexure which will take place when its temperature is raised.

The end $b$, is connected by a link, $b, d$, with a lever $d, e$, which is moved by the flexure into the dotted position $b, g$, causing the turning-valve, air-ventilator, or register, $0, n$, to revolve with a corresponding angular motion, whereby the lever will raise the equipoised slide-damper $k, i$, which is suspended by a link from the end $e$, of the lever $e, d$, into the position $k, h$. Thus a hot-house or a water-bath may have its temperature regulated by the contemporaneous admission of warm, and discharge of cold air, or water.

Fig. $1131, a, b, c$, is a thermostatic hoop, immersed horizontally beneath the surface of the water-bath of a still. The hoop is fixed at $a$, and the two ends $b, c$, are connected by two links $b, d$, $c, d$, with a straight sliding rod $d, h$, to which the hoop will give an endwise motion, when its temperature is altered; $e$, is an adjusting screw-nut on the $\operatorname{rod} d, h$, for setting the lever $f, g$, which is fixed on the axis of the turning-valve or cock $f$, at any desired position, so that the valve may be opened or shut at any desired temperature, corresponding to the widening of the points $b, c$, and the consentaneous retraction of the point $d$, towards the circumference $a, b, c$, of the hoop. $g, h$, is an arc graduated by a thermometer, after the screw-piece $e$ has been adjusted. Through a hole a: $h$, the guide-rod passes. $i$, is the cold-water cistern; $i, f, k$, the pipe to admit cold water; $l$, the overflow pipe, at which the excess of hot water runs off.

Fig. 1132 shows a pair of thermostatic bars, bolted fast together at the ends $a$. The free ends $b, c$, are of unequal length, so as to act by the cross links $d, f$, on the stop-cock $e_{\text {. }}$ The links are jointed to the handle of the turning plug of the cock, on opposite sides of 
its centre; whereby that plug will be turned round in proportion to the widening of the points $b, c . h, g$, is the pipe communicating with the stop-cock.

Suppose that for certain purposes in pharmacy, dyeing, or any other chemical art, a water-bath is required to be maintained steadily at a temperature of $150^{\circ} \mathrm{F}$; ; let the combined thermostatic bars, hinged together at $e, f, f i g .1133$, be placed in the bath, be

1133

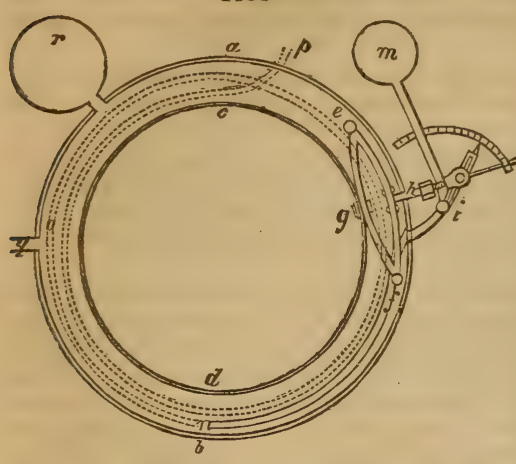
tween the outer and inner vessels $a, b, c, d$, being bolted fast to the inner vessel at $g$; and have their sliding rod $k$, connected by a link with a lever fixed upon the turning plug of the stop-cock $i$, which introduces cold water from a cistern $m$, through a pipe $m$, $i, n$, into the bottom part of the bath. The length of the link must be so adjusted that the flexure of the bars, when they are at a temperature of $150^{\circ}$, will open the said stopcock, and admit cold water to pass into the bottom of the bath through the pipe $i, n$, whereby hot water will be displaced at the top of the bath through an open overflowpipe at $q$. An oil bath may be regulated on the same plan; the hot oil overflowing from $q$, into a refrigeratory worm, from which it may be restored to the cistern $m$. When a water bath is heated by the distribution of a tortuous steam pipe through it, as $i, n, o, p$, it will be necessary to connect the link of the thermostatic bars with the lever of the turning plug of the steam-cock, or of the throttle valve $i$, in order that the bars, by their flexure, may shut or open the steam passage more or less, according as the temperature of the water in the bath shall tend inore or less to deviate from the pitch to which the apparatus has been adjusted. The water of the condensed steam will pass off from the sloping winding-pipe $i, n, 0, p$, through the sloping orifice $p$. A saline, acid, or alkaline bath has a boiling temperature proportional to its degree of concentration, and may therefore have its heat regulated by immersing a thermostat in it, and connecting the working part of the instrument with a stop-cock $i$, which will admit water to dilute the bath whenever by evaporation it has become concentrated, and has acquired a higher boiling point. The space for the bath, between the outer and inner pans, should communicate by one pipe with the water-cistern $m$; and by another pipe, with a safety cistern $r$, into which the bath may be allowed to overflow during any sudden excess of ebullition.

Fig. 1136 is a thermostatic apparatus, composed of three pairs of bars, $d, d, d$, which are represented in a state of flexure by heat; but they become nearly straight and parallel when cold. $a, b, c$, is a guide rod, fixed at one end by an adjusting screw $e$, in the strong

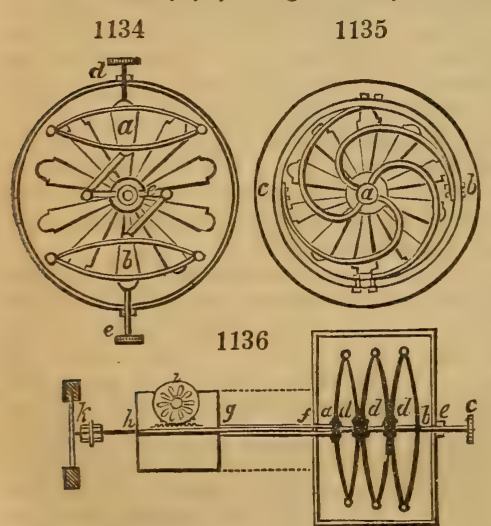
frame $f, e$, having deep guide grooves at the sides. $f, g$, is the working-rod, which moves endways when the bars $d, d, d$, operate by heat or cold. A square register-plate $h, \mathrm{~g}$, may be affixed to the $\operatorname{rod} f, g$, so as to be moved backwards and forwards thereby, according to the variations of temperature; or the $\operatorname{rod} f, g$, may cause the circular turning air-register $i$, to revolve by rack and wheelwork, or by a chain and pulley. The register-plate $h, g$, or turning register $i$, is situated at the ceiling or upper part of the chamber, and serves to let out hot air. $k$, is a pulley, over which a cord runs to raise or lower a hot-air register $l$, which may be situated near the floor of the apartment or hot-house, to admit hot air into the room. $c$, is a milled head for adjusting the thermostat, by means of the screw at $e$, in order that it may regu. late the temperature to any degree.

Fig. 1137 represents a chimney, furnished with a pyrostat, $\alpha, b, c$, acting by the links $b, d, e, c$, on a damper $f, h, g$. The more expansible metal is in the present example supposed to be on the outside. The plane of the damper-plate will, in this case, be turned more directly into the passage of the draught through the chimney by increase of tempe. rature.

Fig. 1135 represents a circular turning register, such as is used for a stove, or stove 
Erate, or for ventilating apartments; it is furnished with a series of spiral thermostatic hars, each bar being fixed fast at the circumference of the circle $b, c$, of the fixed plate 1137 of the air-register; and all the bars act in concert at the centre $a$, of the

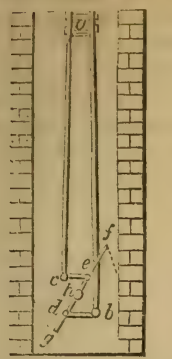
twining part of the register, by their ends being inserted between the teeth of a small pinion, or by being jointed to the central part of the turning plate by small pins.

Fig. 1134 represents another arrangement of my thermostatic apparatus applied to a circular turning register, like the preceding, for ventilating apartments. Two pairs of compound bars are applied so as to act in concert, by means of the links $a c, b c$, on the opposite ends of a short lever, which is fixed on the central part of the turning plate of the air-register. The two pairs of compound bars $a \cdot b$, are fastened to the circumference of the fixed plate of the turning register, by two sliding rods $a d, b e$, which are furnished with adjusting screws. Their motion or flexure is transmitted by the links $a c$, and $b c$, to the turning plate, about its centre, for the purpose of shutting or opening the ventilating sectorial apertures, more or less, according to the temperature of the air which surrounds the thermostatic turning register. By adjusting the screws $a d$, and $b c$, the turning register is made to close all its apertures at any desired degree of temperature; but whenever the air is above that temperature, the flexure of the compound bars will open the apertures.

THIMBLE (Dé à coudre, Fr. ; Fingerhut (fingerhat), Germ.), is a small truncated metallic cone, deviating little from a cylinder, smooth within, and symmetrically pitted on the outside with numerous rows of indentations, which is put upon the tip of the middle finger of the right hand, to enable it to push the needle readily and safely thiough cloth or leather, in the act of sewing. This little instrument is fashioned in two ways; either with a pitted round end, or without one; the latter, called the open thimble, being employed by tailors, upholsterers, and, generally speaking, by needle-men. The following ingenious process for making this essential implement, the contrivance of MM. Rouy and Berthier, of Paris, has been much celebrated, and very successful. Sheet-iron, one twenty-fourth of an inch thick, is cut into strips, of dimensions suited to the intended size of the thimbles. These strips are passed under a punch-press, whereby they are cut into discs of about 2 inches diameter, tagged together by a tail. Each strip contains one dozen of these blanks. A child is employed to make them redhot, and to lay them on a mandril nicely fitted to their size. The workman now strikes the middle of each with a round-faced punch, about the thickness of his finger, and thus sinks it into the concavity of the first mandril. He then transfers it successively to another mandril, which has five hollows of successively increasing depth; and, by striking it into them, brings it to the proper shape.

A second workman takes this rude thimble, sticks it in the chuck of his lathe, in order to polish it within, then turns it outside, marks the circles for the gold ornament, and indents the pits most cleverly with a kind of milling tool. The thimbles are next annealed, brightened, and gilt inside, with a very thin cone of gold leaf, which is firmly united to the surface of the iron, simply by the strong pressure of a smooth steel mandril. A gold fillet is applied to the outside, in an annular space turned to receive it, being fixed, by pressure at the edges, into a minute groove formed on the lathe.

Thimbles are made in this country by means of moulds in the stamping-machine. See StAMPING of Metals.

THORINA is a primitive earth, with a metallic basis, discovered in 1828 , by Berzelius. It was extracted from the mineral thorite, of which it constitutes 58 per cent., and where it is associated with the oxydes of iron, lead, manganese, tin, and uranium, besides earths and alkalis, in all 12 substances. Pure thorina is a white powder, without. taste, smell, or alkaline reaction on litmus. When dried and calcined, it is not affected $\mathrm{ky}$ either the nitric or muriatic acid. It may be fused with borax into a transparent glass, but not with potash or soda. Fresh precipitated thorina is a hydrate, which dissolves readily in the above acids, as well as in solutions of the carbonates of potash, soda, and ammonia, but not in these alkalis in a pure state. This earth consists of 74.5 parts of the metal thorinum, combined with 100 of oxygen. Its hydrate contains one equivalent prime of water. It is hitherto mereiy a chemical curiosity, remarkable chiefly for a density of 9.402 , far greater than that of all the earths, and even of copper.

THREAD MANUFACTURE. The doubling and twisting of cotton or linen yarn into a compact thread, for weaving bobbinet, or for sewing garments, is performed by a machine resembling the throstle of the cotton-spinner. Fig. 1138 shows the thread-frame in a transverse section, perpendicular to its length. $a$, is the strong framing of cast-iron; $b$, is the creel, or shelf, in which the bobbins of yarn $l, l$, are set loosely upon their respective skewers, along the whole iine of the machine, their lower ends turning in oiled steps, and their upper in wire eyes; $c$, is a glass rod, across which the yarn runs as it is unwound; $d, d$, are oblong narrow troughs, lined with lead, and 
filled with water, for moistening the thread during its torsion; the threads being made to pass through eyes at the bottom of the fork $e$, which has an upright stem for lifting it out, without wetting the fingers, when any thing goes amiss; $f, f$, are the pressing rollers, the under one $g$, being of smouth iron, and the upper one $h$, of box-wood; the former extends from end to end of the frame, in lengths comprehending 18 threads, which are joined by square pieces, as in the drawing-rollers of the mule-jenny. The necks of the under rollers are supported, at the ends and the middle, by the standards $i$, secured to square bases $j$, both made of cast iron. The upper cylinder has an iron axis, and is formed of as many rollers as there are threads; each roller being kept in its place unon the lower one by the guides $k$, whose vertical slots receive the ends of the axes

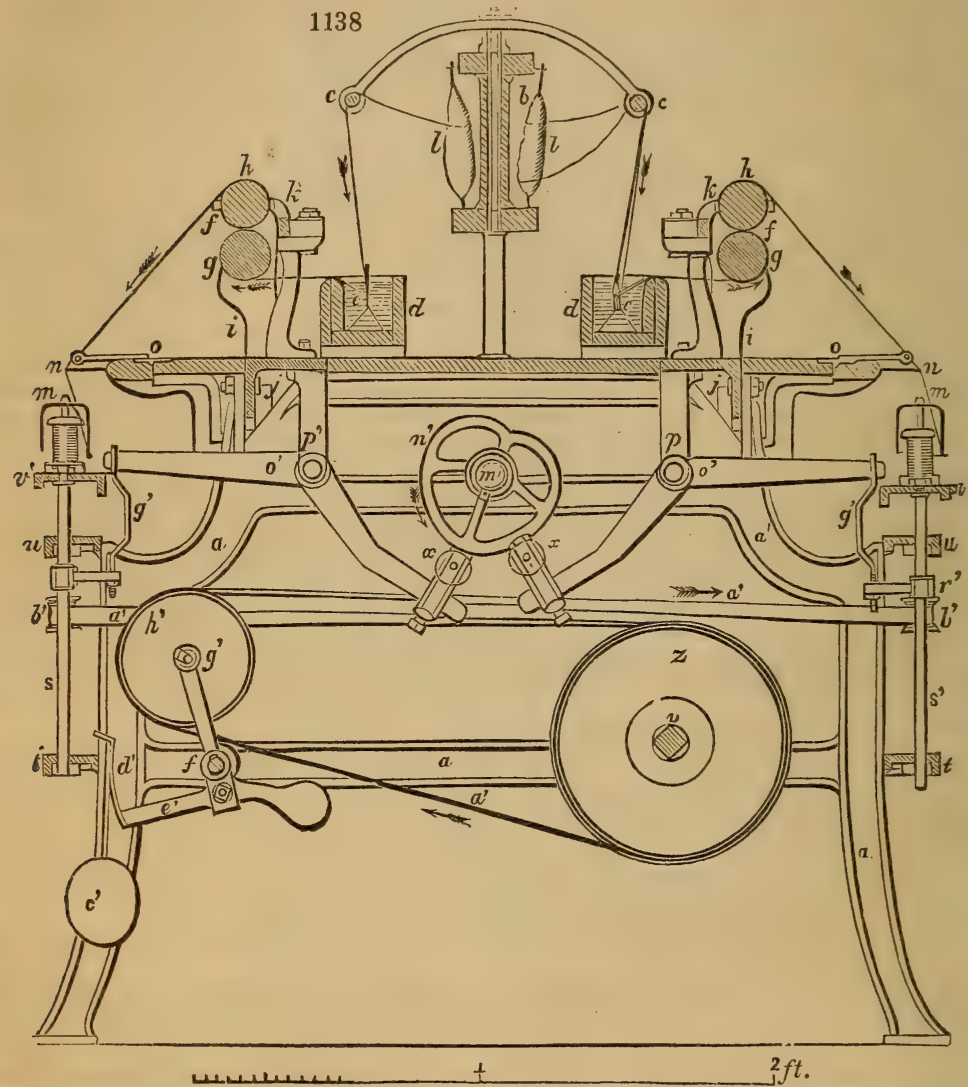

The yarn delivered by the bobbin $l$, glides over the rod $c$, and descends into the trough $d, e$, where it gets wetted; on emerging, it goes along the bottom of the roller $g$, turns up, so as to pass between. it and $h$, then turns round the top of $h$, and finally procecds obliquely downwards, to be wound upon the bobbin $m$, after traversing the guide-eye $n$. These guides are fixed to the end of a plate, which may be turned up by a hinge-joint at 0 , to make room for the bobbins to be changed.

There are three distinct simultaneous movements to be considered in this mashine : 1. that of the rollers, or rather of the under roller, for the upper one revolves merely by friction; 2 . that of the spindles $m, s^{\prime} ; 3$. the up-and-down motion of the bobbins upon the spindles.

The first of these motions is produced by means of toothed wheels, upon the right hand of the under set of rollers. The second motion, that of the spindles, is effected by the drum $z$, which extends the whole length of the frame, turning upon the shaft $v$, and communicating its rotatory movement (derived from the steam pulley) to the whorl $b^{\prime}$, of the spindles, by means of the endless band or cord $a .^{\prime}$ Each of these cords turns four spindles, two upon each side of the frame. They are kept in a proper state of tension 
by the weights $c^{\prime}$, which act tangentially upon the circular are $d^{\prime}$, fixed to the extremity of the bell-crank lever $e^{\prime} f^{\prime} g^{\prime}$, and draw in a horizontal direction the tension pulleys $h$, embraced by the cords. The third movement, or the vertical traverse of the bobbins, along the spindles $m$, takes place as follows :-

The end of one of the under rollers carries a pinion, which takes into a carrier wheel, that communicates motion to a pinion upon the extremity of the shaft $m^{\prime}$, of the heartshaped pulley $u^{\prime}$. As this eccentric revolves, it gives a reciprocating motion to the lever's $0^{\prime}, o^{\prime}$, which oscillate in a vertical plane round the points $p^{\prime} p^{\prime}$. The extremities of these levers, on either side, act by means of the links $q^{\prime}$, upon the arms of the sliding socliets $r^{\prime}$, and cause the vertical rod $s^{\prime}$, to slide up and down in guide-holes at $t^{\prime}, u^{\prime}$, along with the cast-iron step $v^{\prime}$, which bears the bottom washer of the bobbins. The periphery of the heart-wheel $n^{\prime}$, is seen to bear upon friction wheels $x, x^{\prime}$, set in frames adjusted by screvs upon the lower end of the bent levers, at such a distance from the point $p^{\prime}$, as that the traverse of the bobbins may be equal to the length of their barrel.

$\mathrm{By}$ adapting change pinions and their corresponding wheel to the rollers, the delivery of the yarn may be increased or diminished in any degree, so as to vary the degree of twist put into it by the uniform rotation of the drum and spindles. The heart motion, being derived from that of the rollers, will necessarily vary with it.

Silk thread is commonly twisted in lengths of from 50 to 100 feet, with hand reels, somewhat similar to those employed for making ropes by hand.

TILES. See BRICKs.

TII'TiNG OF STEEL. See Steel. Rees's Cyclopædia contains an excellent article on this subject.

TIN (Etain, Fr.; Zinn, Germ.), in its pure state, has nearly the color and lustre of silver. In hardness it is intermediate between gold and lead; it is very malleable, and may be laminated into foil less than the thousandth of an inch in thickness; it has an unpleasant taste, and exhales on friction a peculiar odor; it is flexible in rods or straps of considerable strength, and emits in the act of bending a crackling somnd, as if sandy particles were intermixed, called the creaking of tin. A small quantity of lead, or other metal, deprives it of this characteristic quality. Tin melts at $442^{\circ} \mathrm{Fahr}$, and is very fixed in the fire at higher heats. Its specific gravity is $\mathbf{7 \cdot 2 9}$. When heated to redness with free access of air, it absorbs oxygen with rapidity, and changes first into a pulverulent gray protoxyde, and by longer ignition, into a yellow-white powder, called putty of tin. This is the peroxyde, consisting of 100 of metal $+27 \cdot 2$ of oxygen.

Tin has been known from the most remote antiquity; being mentioned in the books of Moses. The Phœnicians carried on a lucrative trade in it with Spain and Cornwall.

There are only two ores of tin; the peroxyde, or tin-stone, and tin pyrites; the former of which alone has been found in sufficient abundance for metallurgic purposes. The external aspect of tin-stone has nothing very remarkable. It occurs sometimes in twin crystals; its lustre is adamantine; its colors are very various, as white, gray, yellow, red, brown, black; specific gravity 6.9 at least; which is, perhaps, its most striking feature. It does not melt by itself before the blowpipe; but is reducible in the sinoky flame or on charcoal. It is insoluble in acids. It has somewhat of a greasy aspect, and strikes fire with steel.

Tin-stone occurs disseminated in the ancient rocks, particularly granite; also in beds and veins, in large irregular masses, called stockwertes; and in pebbles, an assemblage of which is called stream-works, where it occasionally takes a ligneous aspect, and is termed wood-tin.

This ore has been found in few countries in a workable quantity. Its principal localities are, Cornwall, Bohemia, Saxony, in Europe; and Malacea and Banca, in Asia. The tin-mines of the Malay peninsula lie between the 10 th and 61 h degree of south lacitude; and are most productive in the island of Junck-Ceylon, where they yield sometimes 800 tons per annum, which are sold at the rate of $48 l$. each. The ores are found in large cares near the surface: and though actively mined for many centuries, still there is easy access to the unexhausted parts. The mines in the island of Banca, to the east of Si matra, discovered in 1710 , are said to have furnished, in some years, nearly 3500 tons of tin. Small quantities occur in Gallicia in Spain, in the department of Haute Vienne ili France, and in the mountain chains of the Fichtel and Riesengebürge in Germany. The columnar pieces of pyramidal tin-ore from Mexico and Chile, are products of streamworks. Small groups of black twin crystals have been lately discovered in the albite rock of Chesterfield in Massachusetts.

The Cornish ores occur-1. in small strata or veins, or in masses ; $z$. in stockwerks, or congeries of small veins; 3 . in laige veins; 4 . disseminated in alluvial denosites.

The stanniferous small veins, or thin flat masses, though of small extent, are sometimes very numerous. interposed between certain rocks, parallel to their beds, and are commonly called tin.Hoors. The same name is occasionaliy given to stockwerks. In 
the mine of Botlalack, a tin-floor has been found in the killas (primitive schistose rock), thirty-six fathous below the level of the sea; it is about a foot and a half thick, and oceupies the space between a principal vein and its ramification; but there seems to be no connexion between the floor and the great vein.

2. Stocliwerks occur in granite and in the feldspar porphyry, called in Cornwall, elvan. The most remarkable of these in the granite, is at the tin-mine of Carclase, near St. Austle. 'The works are carried on in the open air, in a friable granite, containing feldspar disintegrated into kuolin, or china clay, which is traversed by a great rnany small veins, composed of tourmaline, quartz, and a little tin-stone, that form black delineations on the face of the light-gray granite. The thickness of these little veins rarely exceeds 6 inches, including the adhering solidified granite, and is occasionally much less. Some of them run nearly east and west, with an almost vertical dip; others, with the same direction, incline to the south at an angle with the horizon of 70 degrees.

Stanniferous stockwerks are much more frequent in the elvan (porphyry), of which the mine of Trewidden-ball is a remarkable example. It is worked among flattened rnasses of elvan, separated by strata of killas, which dip to the east-north-east at a considerable angle. The tin ore occurs in small veins, varying in thickness from half an inch to 8 or 9 inches, which are irregular, and so much interrupted, that it is difficult to determine either their direction or their inclination.

3. The large and proper metalliferous veins are not equally distributed over the surface of Cornwall and the adjoining part of Devonshire; but are grouped into three districts ; namely, 1. In the south-west of Cornwall, beyond Truro; 2. In the neighborhood of St. Austle; and 3. In the neiohborhood of Tavistock in Devonshire.

The first group is by far the richest, and the best explored. The formation most abundant in tin mines is principally granitic; whilst that of the copper mines is most frequently schistose or killas; though with numerous exceptions. The great tin veins are the most ancient metalliferous veins in Cornwall ; yet they are not all of one formation, but belong to two different systems. Their direction is, however, nearly the same, but some of them dip towards the north, and others towards the south. The first are older than the second; for in all the mines where these two sets of veins are associated, the one which dips to the north, cuts across and throws out the one which dips to the south. See Mrnes, p. 841.

At Trevannance mines, the two systems of tin veins are both intersected by the oldest of the copper veins; indicating the prior existence of the tin veins. In fig. 1139, 1139

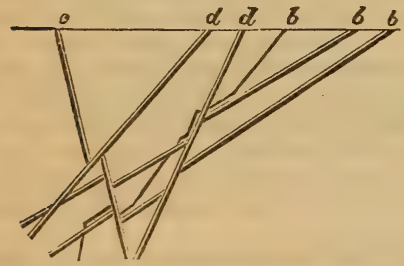
$b$, marks the first system of tin veins; $c$, the second; and $d$, the east and west copper veins. Some of these tin veins, as at Poldice, have been traced over an extent of two miles; and they vary in thickness from a small fraction of an inch to several feet, the average width being from 2 to 4 feet; though this does not continue uniform for any length, as these veins are subject to continual narrowings and expansions. The gangue is quartz, chlorite, tourmaline, and sometimes decomposed granite and fluor spar.

4. Alluvial tin ore, stream tin.-Peroxyde of tin occurs disseminated both in the alluvium which covers the gentle slopes of the hills adjoining the rich tin-mines, and also in the alluvium which fills the valleys that wind round their base; but in these numerous deposites the tin-stone is rarely distributed in sufficient quantities to make it worth the working. The most important explorations of alluvial tin ore are grouped in the environs of St. Just and St. Austle; where they are called stream-works; because water is the principal agent employed to separate the metallic oxyde from the sand and gravel.

The tin mine of Altenberg, in Saxony ( $f \mathrm{~g} .1140$, which is a vertical projection in a plane passing from west to east), is remarkable for a stockwerk, or interlaced mass of ramifying veins, which has been worked ever since the year 1458. The including rock is a primitive porphyry, superposed upon gneiss; becoming very quartzose as it approaches the lode. This is usually disseminated in minute particles, and accom. panied with wolfram, copper, and arsenical pyrites, fer oligiste, sulphuret of molybdenum, and bismuth, having gangues of lithomarge, fluor spar, mica, and feldspar. The spare which the ore occupies in the heart of the quartz, is a kind of dædalus, the former being often so dispersed among the latter as to seem to merge into it; whence it is called by the workmen zuitter, or ambiguous. In 1620, the mine was worked by 21 independent companies, in a most irregular manner, whereby it was damaged to a depth of 170 fathoms by a dreadful downfall of the roofs. This happened on a Sunday, providentially, when the pious miners were all at church. The depth of this abyss, marked by the curved line 
$b, b, b$, is 66 fathoms; but the devastation is manifest to a depth of 95 fathoms below that curve, and 35 fathoms below the actual workings, represented at the bottom of the shaft under $\mathbf{B}$. The parts excavated are shaded black in the figure. There are two masses of ore, one under the shaft $B$, and another under the shaft $\mathrm{C}$; which at the levels 5 and 10 are in communication, but not at 6,7 . There is a direct descent from 8 to 9 . The deposites are by no means in one vertical plane, but at a considerable horizontal distance from each other. $A$ is the descending shaft; $\mathrm{B}$ is the extraction shaft, near the mouth of which there is a water-wheel; $\mathbf{c}$ is another extraction shaft, worked also by means of a water-wheel. $A$ and $c$ are furnished with ladders, but for $B$ the ladder's are placed in an accessory shaft $b^{\prime}$; under $D$ a shaft is sunk for pumping out the water, by means of an hydraulic wheel at D; $\mathrm{E}$ is the gallery or drift for admitting the water which drives the wheels. This falls 300 feet, and ought to be applied to a water-pressure engine, instead of the paddles of a wheel. At $D$ is the gallery of discharge for the waters, which serves also to ventilate the mine, being cut to the day, through 936 toises of syenitic porphyry and gneiss. $\mathrm{J}$ is a great vaulted excavation. The mine has 13 stages of galleries, of which 11 serve for extracting the ore; 1 is the mill-course; the rest are marked with the numbers 2, 3, 4, \&c.; each having besides a characteristic German name. The rare mineral called topaz pycnite is found in this mine, above 10 , between the shafts $\mathrm{C}$ and $\mathrm{D}$.

The only rule observed in taking ore from this mine has been to work as much out of each of these levels as is possible, without endangering the superincumbent or collateral galleries; on which account many pillars are constructed to support the roofs. The mine yields annually 1600 quintals (Leipzick) of tin, being four fifths of the whole furnished by the district of Altenberg; to produce which, 400,000 quintals of ore are raised. 1000 parts of the ruck rield 8 of concentrated schlich, equivalent to only 4 of metal; being only 1 in 250 parts.

But the most extensive and productive stream-works are those of Pentowan, near St. Austle.

Fig. 1141 represents a vertical section of the Pentowan mine, taken from the stream-uo,k, Happy Union. A vast excavation, $\mathrm{R}, \mathrm{T}, \mathrm{U}, \mathrm{s}$, has been hollowed out in

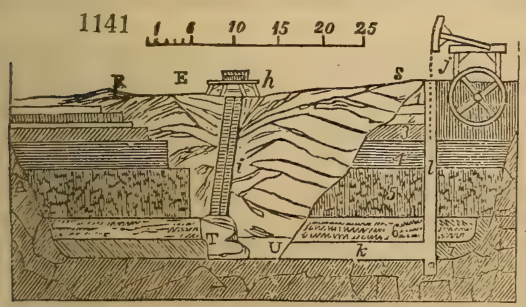
the open air, in quest of the alluvial tin ore, $\mathbf{T}$, which occurs here at an unusual depth, below the level of the strata $\mathrm{R}, s_{\text {: }}$ Before getting at this deposite, several successive layers had to be sunk through; namely, 1, 2, 3 ; the gravel, containing in its middle a band of ochreous earth 2 , or ferruginous clay; 4, a black peat, perfectly combustible, of a coarse texture, composed of reeds and woody fibres, cemented into a mass by a fine loam; 5 , coarse sea-sand, mingled with marine shells;

6, a blackish marine mud, filled with shells. Below these the deposite of tin-stone occurs, including fragments of various size, of clay slate, flinty slate, quartz, iron ore, jasper: in a word, of all the rocks and gangues to be met with in the surrounding territory, with the exception of granite. Among these fragments there occur, in rounded particles, a coarse quartzose sand, and the tin-stone, commonly in small grains and crystals. Beneath the bed $\mathrm{T}$, the clay slate occurs, called killas, $(A, \mathbf{x}, \mathbf{x}$,$) which supports all the deposites$ of more recent formation.

The system of mining is very simple. The successive beds, whose thickness is shown in the figure, are visibly cut out into steps or platforms. By a level or gallery of efflux, $\boldsymbol{k}$, the waters flow into the bottom of the well $l, m$, which contains the drainage pumps; and these are put in action by a machine, $j$, moved by a water-wheel. The extraction of the ore is effected by an inclined plane, $i$, cut out of one of the sides of the excavation, at an angle of about 45 degrees. At the lower end of this sloping pathway there is a 
place of loading; and at its upper end $h$, a horse-gır, for alternately raising and lowenng the two baskets of extraction on the pathway $i$.

Mine tin requires peculiar care in its mechanical preparation or dressing, on account of the presence of foreign metals, from which, as we have stated, the stream tin is fiee.

1. As the mine tin is for the most part extremely dispersed through the gangue, it must be all stamped and reduced to a very fine powder, to allow the metallic particles to be separated from the stony matters.

2. As the density of tin-stone is much greater than that of most other metallic ores, it is less apt to run off in the washing; and may, therefore, be dressed so as to be completely stripped of every matter not chemically combined.

3. As the peroxyde of tin is not affected by a moderate heat, it may bs exposed to calcination; whereby the specific gravity of the associated sulphurets and arseniurets is so diminished as to facilitate their separation.

We may therefore conclude, that tin ore should be first of all pounded very fine in the stamp-mill, then subjected to reiterated washings, and afterwards calcined. The order of proceeding in Cornwall is as follows:-

1. Clewning the ore.--This is usually done at the mouth of the gallery of efflux, by agitating the ore in the stream of water as it runs out. Sometimes the ore is laid on a grating, under a fall of water.

2. Sorting.- The ore thus cleaned, is sorted on the grate, into four heaps: 1 . stones rich in tin; 2. stones containing both tin and copper ore; 3 . copper ore; 4 . sterile pieces, composed in a great measure of stony gangue, with iron and arsenical pyrites. In those veins where there is no copper ore, the second and third heaps are obviously absent. When present, the compound ore is broken into smaller pieces with a mallet, and the fragments are sorted anew.

3. Stumping.-The stanniferous fragments (No. 1) are stamped into a sand, of greater or less fineness, according to the dissemination of the tin-stone in the gangue. The determination of the size of the sand is an object of great importance. It is regulated by a copper plate pierced with small holes, through which every thing from the stampingmill must run off with the rapid stream introduced for this purpose. This plate forms the front of the stamp cistern.

Several years ago, all the stamp-mills were driven by water-wheels, which limited the quantity of ore that could be worked to the hydraulic power of the stream or waterfall; but since the steam engine has been applied to this purpose, the annual product of tin has been greatly increased. On the mine of Huel Vor, there are three steam engines appropriated to the stamping-mills. Their force is 25 horses at least. One of these machines, called sorth stamps, drives 48 pestles; a second, called old stamps, drives 36 ; and a third, 24. The weight of these pestles varies from $3 \% 0$ to 387 pounds; and they generally rise through a space of $10 \frac{1}{2}$ inches. The machine called south stamps, the strongest of the three, gives $17 \frac{1}{2}$ blows in the minute, each pestle being lifted twice for every stroke of the piston. The steam engine of this mill has a power of 25 horses, and it consumes 1062 bushels of coals in the month. Three pestles constitute a battery, or stamp-box.

Washing and stamping of tin ores at Polgooth, near St. Austle.-The stamps or pestles are of wood, 6 inches by $5 \frac{1}{2}$ in the square: they carry lifting bars $b$, secured

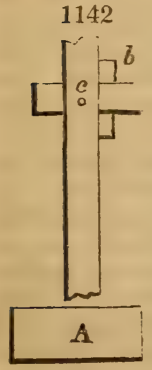
with a wooden wedge and a bolt of iron, and they terminate below in a lump of cast iron A, called the head, which is fastened to them by a tail, and weighs about $2 \frac{1}{2} \mathrm{cwts}$. The shank of the pestle is strengthened with iron hoops. A turning-shaft communicates motion to the stamps by cams stuck round its circumference, so arranged that the second falls while the first and third of each set are uplifted. There are 4 cams on one periphery, and the shaft makes 7 turns in the minute. Each stamp, therefore, gives 28 strokes per minute, and falls through a space of $7 \frac{1}{2}$ inches. The stamp chest is open behind, so that the ore slips away under the pestles, by its weight, along the inclined plane with the stream of water. The bottom of the troughs consists of stamped ores. With 6 batteries of 6 pestles each, at Poldice, near Redruth, 120 bags of ore are stamped in 12 hours; each bag containing 18 gallons of 282 cubic inches; measuring altogether 352 cubic feet, and 864 cubic inches.

The openings in the front sides of the troughs are nearly eight inches by seven and a half; they are fitted with an iron frame, which is closed with sheet iron, pier ced with aivout 160 holes in the square inch, bored conically, being narrower within. The ore, ca issuing, deposites its ronyh in the first basin, and its slimes in the following basins. The rough is washed in buddles (see LEAD, page 757), and in tossing-tubs; the slimes in trunks, and up on a kind of twin tables, called racks. Into the tossing-tub; or dolly, fig. 1143, the stamp ed ore is thrown, along with a certain quantity of water, and a workman stirs it about 
with an iron shovel for three or four minutes. He then removes a little of the water with a handled pitcher, and strikes the sides of the tub for 8 or 10 minutes with a hammer,

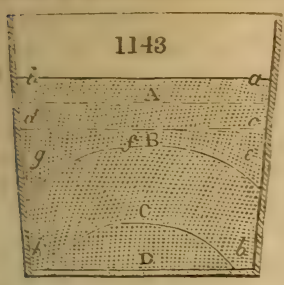
which hastens the subsidence of the denser parts. The water is next poured oft by inclining the tub to one side. In one operation of this kind, four distinct strata of the ores may be procured, as indicated by the lines $a b, c d, e f g, h i k$, in the figure. The portion $B$ is to be washed again in the trunking-box, figs. 1144,$1145 ; \mathrm{B}$ is to be washed upon the German chests or racks, fig. 1146 ; c, the most considerable, is put aside, as schlich fit for the market; $\mathrm{D}$, forming a nucleus in the centre of the tub, is to be passed through sieves of copper wire, having 18 meshes in the square inch. This product thus affords a portion. $D^{\prime}$, which passes through the sieve, and D" which remains upon it; the latter is sometimes thrown away, and at others is subjected to the operation called the tie, viz., a washing upon the sloping bottom of a long trough.

The slimes are freed from the lighter mud in the trunking-box, figs. 1144, 1145 ; which is from 7 to 8 feet long. Being accumulated at $M$, the workman pushes them
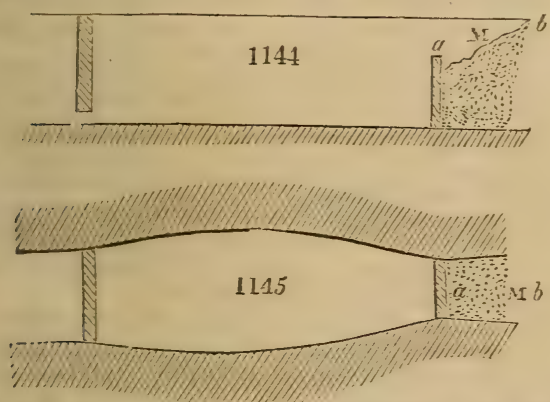
back with a shovel from $a$ towards $b$. The metallic portion is carried off, and deposited by the stream of water upon the table; but the earthy matters are floated along into a basin beyond it. The product collected in the chest is divided into two portions; the one of which is washed once, and the other twice, upon the rack, fig. 1146. This is composed of a frame c, which carries a sloping board or table, susceptible of turning round to the right or left upon two pivots, $к$, $к$. The head of the table is the inclined plane T. A small board $\mathbf{P}$, which is attached by a band of leather L, forms the communication with the lower table c, whose slope is generally 5 inches in its whole length of 9 feet; but this may vary with the nature of the ore, being somewhat less when it is finely pulverized. The ore is thrown upon $T$, in small

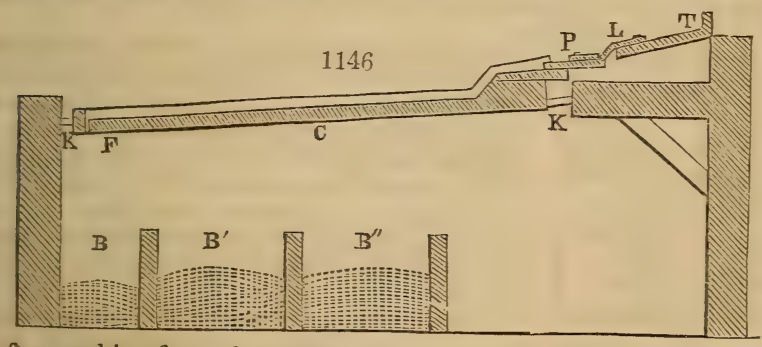
portions of 20 or 25 lbs. A woman spreads it with a rake, while a stream of water sweeps a part of it upon the table, where it gets washed. The fine mud falls through a cross slit near the lower end, into a basin $\mathbf{B}$.

After working for a few minutes, should the schlich seem tolerably rich, the operative turns the table round its axis $\mathrm{K}, \mathrm{K}$, so as to tumble it into the boxes below. The mud is in $\mathbf{B}$; an impure schlich in $\mathbf{B}^{\prime}$, which must be washed again upon the rack; and a schlich fit for roasting in $\mathbf{B}^{\prime \prime}$. feet.

The slope of the rack-table for washing the roasted tin ore, is $7 \frac{3}{4}$ inches in the nine

Crashing rolls at the Pembroke mines.-Wagons, moved on a railway by an endless rope, bring the ore to be crushed immediately over the rolls, as shown in fig. 1147. A trap being opened in the side of the wagon, the ore falls into the hopper $T$, whence it passes directly between the twin cylinders $c, c$, and next upon the sieve $D$, which receives a seesaw motion horizontally, by means of the rod $L$, and the crank of the upright turning-shaft. The finer portion of ore, which passes through that sieve, forms ine neap s. The coarser portion is tossed over the edge of the sieve, and falls between the cylinders $c^{\prime} c^{\prime}$, upon a lower level, and forms the second heap $s^{\prime}$ of sifted,
and $s^{\prime \prime}$ of unsifted, ore.

The holes of the sieves $D, D^{\prime}$, being of the same size, the products $s, s^{\prime}$, are of the same fineness. $s^{\prime \prime}$ is ground again, being mixed, in the uppermost hopper $T$, along with the limps from the wagons. 
The diameter and length of the under rolls (see fig. 1148) are each 16 inches. $a b$, is the square end of the gudgeon $t$, which prevents the shaft shifting laterally out

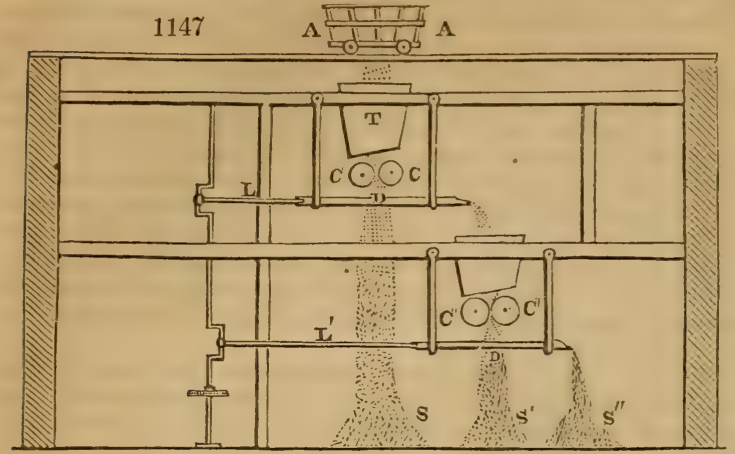
of its place. The diameter of the upper rolls is 18 inches, but their length is the same. Both are made of white cast iron, chilled or case-hardened by being cast in iron moulds instead of sand; and they last a month, at least, when of good quality. They make from 10 to 15 turns in a minute, according to the hardness of the ores of $\mathrm{in}$ or copper; and can grind about 50 tons of rich copper ore in 12 hours; but less of the poorer sort.

The next process is the calcination in the burning-house; which includes severai

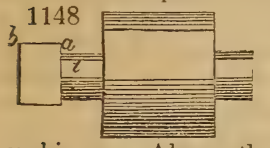
reverberatory furnaces. At the mine of Poldice, they are 4 or 5 yards long, by from $2 \frac{1}{2}$ to 3 yards wide. Their hearth is horizontal; the elevation, about 26 inches high near the fireplace, sinks slightly towards the chimney. There is but one opening, which is in the front; it is closed by a plate-iron door, turning on hinges. Above the door there is a chimney, to let the sulphurous and arsenical vapors fly off, which escape out of the hearth, without annoying the workmen. This chimney leads to horizontal flues, in which the arsenious acid is condensed.

Six hundred weights of ore are introduced; the calcination of which takes from 12 to 18 hours, according to the quantity of pyrites contained in the ore. At the beginning of the operation, a moderate heat is applied; after which it is pushed to a dull red, and kept so during several hours. The door is shut; the materials are stirred from time to time with an iron rake, to expose new surfaces, and prevent them from agglutinating or kerning, as the workmen say. The more pyrites is present, the more turning is neces. sary. Should the ore contain black oxyde of iron, it becomes peroxydized, and is then easily removed by a subsequent washing.

Figs. 1149, 11 o 0 represent the furnace employed at Altenberg, in Saxony, for roasting tin ores. $a$ is the grate ; $b$, the sole of the roasting hearth; $c$, an opening in the arched

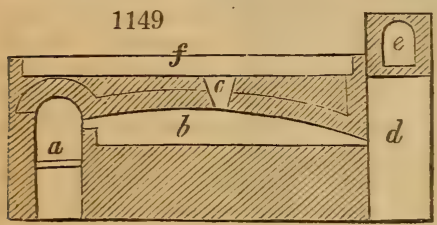

1150

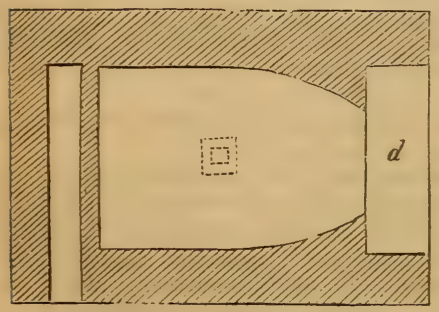
roof for introducing the dried sehlich (the ground and elutriated ore); $d$, is the smoke-mantle or chimney-hood, at the end of the furnace, under which the workmen turn over the spread schlich, with long iron rods bent at their ends; $e$, is the poison vent, which conducts the arsenical vapors to the poison chamber (gifthaus) of condensation.

When the ore is sufficiently calcined, as is shown by its ceasing to exhale vapors, it is taken out, and exposed for some days to the action of the air, which decomposes the sulphurets, or changes them into sulphates. The ore is next put into a tub filled with water, stirred up with a wooden rake, and left to settle; by which means the sulphate of copper that may have been formed, is dissolved out. After some time, this water is drawn off into a large tank, and its copper recovered by precipitation with pieces of old iron. In this way, almost all the copper contained in the tin ore is extracted.

The calcined ore is sifted, and treated again on the racks, as above described. The pure schlich, called black tin, is sold under this name to the smelters; and that which collects on the middle part of the inclined wash-tables, being much mixed with wolfram, is called mock lead. This is passed once more through the stamps, and washed; when it also is sold as black tin.

Stream tin is dressed by similar methods; 1 . by washing in a trunking-box, of such dimensions that the workman stands upon it in thick bosts, and makes a skilful use 
of the rake; 2, by separating the larger conglomerate pebbles fiom the smaller pure ones; picking, stamping, and washing, on a kind of sleeping-tables. See METALLuRGr. figs. $67 \%, 678$.

The tin ores of Cornwall and Devonshire are all reduced within the counties where they are mined, as the laws prohibit their exportation out of them. Private interests suffer no injury from this prohibition; because the vessels which bring the fuel from Wales, for smelting these ore, return to Swansea and Neath loaded with copper ores.

The smelting-works belong in general to individuals who possess no tin mines, but who purchase at the cheapest rate the ores from the mining proprietors. The ores are appraised according to their contents in metal, and its fineness; conditions which they determine by the following mode of assay :-When a certain number of bags of ore, of nearly the same quality, are brought to the works, a small sample is taken from each bag, and the whole are well blended. Two ounces of this average ore are mixed with about four per cent. of ground coal, put into an open earthen crucible, and heated in an air furnace (in area about ten inches square) till reduction takes place. As the furnace is very hot when the crucible is introduced, the assay is finished in about a quarter of an hour. The metal thus revived is poured into a mould, and what remains in the crucible is pounded in a mortar, that the grains of tin may be added to the ingot.

This method, though imperfect in a chemical point of view, serves the smelter's purpose, as it affords him a similar result to what he would get on the great scale. A more exact assay would be obtained by fusing, in a crucible lined with hard-rammed charcoal, the ore mixed with five per cent. of ground glass of borax. To the crucible a gentle heat should be applied during the first hour, then a strong heat during the second hour, and, lastly, an intense heat for a quarter of an hour. This process brings out from four to five per cent. more tin than the other; but it has the inconvenience of reducing the iron, should any be present; which by subsequent solution in nitric acid will be readily shown. This assay w-uld be too tedious for the smelter, who may have occasion to try a great many samples in one day.

The smelting of tin ores is effected by two different methods :-

In the first, a mixture of the ore with charcoal is exposed to heat on the hearth of a reverberatory furnace fired with coal.

In the second, the tin ore is fused in a blast furnace, called a blowing-house, supplied with wood charcoal. This method is practised in only a few works, in order to obtain a very pure quality of tin, called grain tin in England, and étain en larmes in France; a metal required for certain arts, as dyeing, Scc. This method is applied merely to stream sin.

In the smelting-liouses, where the tin is worked in reverberatories, two kinds of furnaces are employed; the reduction and the refining furnaces.

Figs. 1151, 1152, represent the furnaces for smelting tin at St. Austle, in Cornwall;

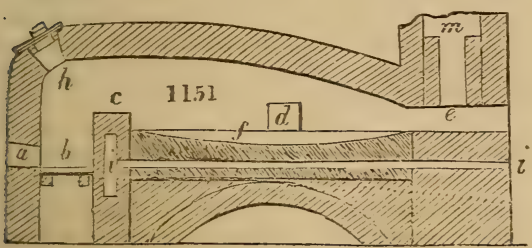

1152

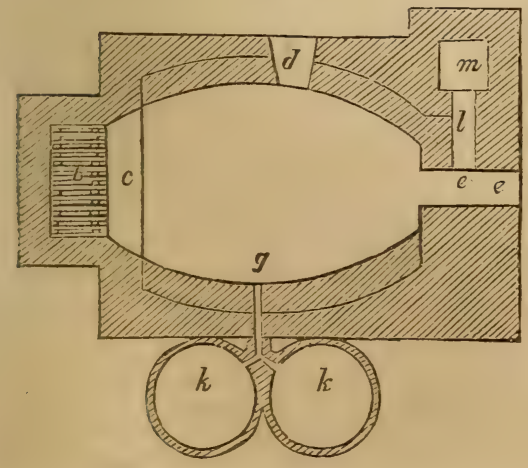
the former being a longitudinal section, the latter a ground plan. $a$, is the firedoor, through which pitcoal is laid upon the grate $b ; c$, is the fire-bridge; $d$, the door for introducing the ore; $e$, the door through which the ore is worked upon the hearth $f$; $g$, the stoke-hole; $h$, an anerture in the vault or roof, which is opened at the discharge of the waste schlich, to secure the free escape of the fumes up the chimney; $i, i$, air channels for admitting cold air under the firebridge and the sole of the hearth, with the view of protecting them from injury by the intensity of the heat above. $k, k$, are basins into which the melted tin is drawn off; $l$, the flue; $m$, the chimney, from 35 to 50 feet high. The roasted and washed schlich is mixed with small coal or culm, along with a little slaked lime, or fluor spar, as a flux; each charge of ore amounts to from 15 to 24 ewts., and contains from 60 to 70 per cent. of metal.

Fig. 1153 represents in a vertical section through the tuyère, and fig. 1154, in a horizontal section, in the dotted line $x$, $x$, of fig. 1153, the furnace employed 
for smelting tin at the Erzegebirge mines, in Saxony. $a$, are the furnace piliars, of gneiss; $b, b$, are shrouding or casing walls; $c$, the tuyère wall; $d$, front wall, both of

1153

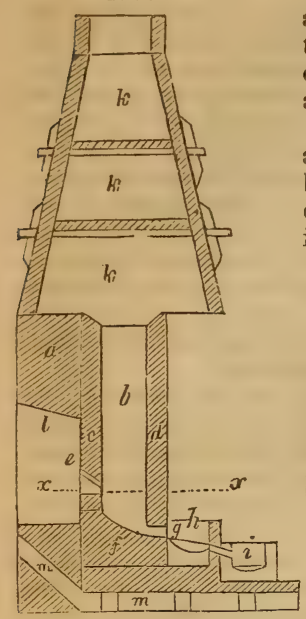
granite; as also the tuyère $e$. $f$, the sole-stone, of granite, hewn out basin-shaped; g, the eye, through which the tin and slag are drawn off into the fore-hearth $h ; i$, the stoke-hearth; $k, k$, the light ash chambers; $l$, the arch of the tuyère; $m, m$, the common flue, which is placed under the furnace and the hearths, and has its outlet under the vault of the tuyère.

In the smelting furnaces at Geyer, the following dimensions are preferred:-Length of the tuyère wall, 11 inches; of the breast wall, 11 inches; depth of the furnace, 17 inches. High chimney-stalks are advantageous where a great quantity of ores is to be reduced, but not otherwise.

The refining furnaces are similar to those which serve for re-

1154

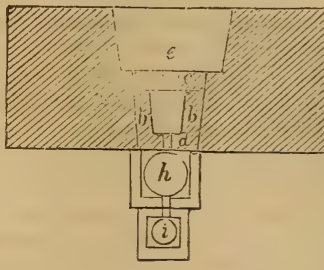

ducing the ore; only, instead of a basin of reception, they have a refining basin placed alongside, into which the tin is run. This basin is about four feet in diameter, and thirty-two inches deep; it consists of an iron pan, placed over a grate, in which a fire may be kindled. Above this pan there is a turning gib, by means of which a billet of wood may be thrust down into the bath of metal, and kept there by wheeling the gibbet

over it, lowering a rod, and fixing it in that position.

The works in which the blast furnaces are employed, are called blowing-honses. The smelting furnaces are six feet high, from the bottom of the crucible (concar hearth) to the throat, which is placed at the origin of a long and narrow chimney, interrupted by a chamber, where the metallic dust, carried off by the blast, is deposited. This chamber is not placed vertically over the furnace; but the lower portion of the chimney has an oblique direction from it. The furnace is lined with an upright cylinder of cast iron, coated internally with loam, with an opening in it for the blast. This opening, which corresponds to the lateral face opposite to the charoing side, receires a tuyerre, in which the nozzles of two cylinder single bellows, driven by a water-wheel, are planted. The tuyere opens at a small height above the sole of the furnace. On a level with the sole, the iron cylinder presents a slope, below which is the hemispherical basin of reception, set partly beneath the interior space of the furnace, and partly without. Near the corner of the building there is a second basin of reception, larger than the first, which car discharge itself into the former by a sloping gutter. Near this basin there is another, for the refining operation. These are all made either of brick or cast iron.

The quality of the average ground-tin ore prepared for smelting is such, that 20 parts of it yield from $12 \frac{1}{2}$ to 13 of metallic tin, ( $62 \frac{1}{2}$ to 65 per cent.) The treatment consists of two operations, smelting and refining.

First operation; deoxydization of the ore and fusion of the tin.-Before throwing the ore into the smelting furnace, it is mixed with from one fifth to one eighth of its weight of blind coal, in powder, called culm; and a little slaked lime is sometimes added, to render the ore more fusible. These matters are carefully blended, and damped with water, to render the charging easier, and to prevent the biast from sweeping any of it away at the commencement. From 12 to $16 \mathrm{cwts}$. are introduced at a charge; and the doors are immediately closed and luted, while the heat is progressively raised. Were the fire too strong at first, the tin oxyde would unite with the quartz of the gangue, and form an enamel. The heat is applied for 6 or 8 hours, during which the doors are not opened; of course the materials are not stirred. By this time the reduction is, in general, finished; the door of the furnace is removed, and the melted mass is worked up to complete the separation of the tin from the scoriæ, and to ascertain if the operation be in sufficient forwardness. When the reduction seems to be finished, the scoriæ are taken out at the same door, with an iron rake, and divided into three sorts; those of the first class $A$, which constitute at least three fourths of the whole, are as poor as possible, and may be thrown away; the scoriæ of the second class $\mathrm{B}$, which contrin some small grains of tin, are sent to the stamps; those of the third class $c$, which are last removed from the surface of the bath of tin, are set apart, and re-smelted, as containing a considerable quantity of metal in the form of grain tin. These scoriæ are in small quantity. The stamp slag contains fully five per cent. of metallic tin.

As soon as the scoriæ are cleared away, the channel is opened which leads to the 
basin of rece:'tion, into which the tin consequently flows out. Here it is left for some time, that the scoria which may be still mixed with the metal, may separate, in virtue of the dilleresce of their specific gravities. When the tin has sufficiently settled, it is litted utut witu ladles, and poured into cast-iron moulds, in each of which a bit of wood is rixed, to form a hole in the ingot, for the purpose of drawing it out when it becomes cold.

Relining of tin.-The object of this operation is to separate from the tin, as completely as possible, the metals reduced and alloyed along with it. These are, principally, iron, culper, arsenic, and tungsten; to which are joined, in small quantities, some sulphurets and arseniurets that have escaped decomposition, a little unreduced oxyde of tin, and also some earthy matters which have not passed off with the scoriæ.

Liquution.- The refining of tin consists of two opesations; the first being a liquation, which, in the interior, is effected in a reverberatory furnace, similar to that employed in smelting the ore, (figs. 1151, 1152.) The blocks being arranged on the hearth of the furnace, near the bridge, are moderately heated; the tin melts, and flows away into the retining-basin; but, after a certain time, the blocks cease to aflord tin, and leave on the hearth a residuum, consisting of a very ferruginous alloy.

Fresh tin blucks are now arranged on the remains of the first; and thus the liquation is continued till the refinin:-basin be sutriciently full, when it contains about five tons. The residuums are set aside, to be treated as shall be presently pointed out.

Refining proper.-Now begins the second part of the process. Into the tin-bath, billets of green wood are plunged, by aid of the gibbet above described. The disensagement of gas from the green wood produces a constant ebullition in the tin; bringing up to its surface a species of froth, and causing the impurest and densest parts to fall to the bottum. That froth, composed almost wholly of the oxydes of tin and foreign metals, is successively skimmed off, and thrown back into the furnace. When it is judged that the tin has boiled long enough, the green wood is lifted out, and the bath is allowed to seltle. It separates into diflerent zones, the upper being the purest; those of the middle are charged with a little of the foreign metals; and the lower are much contaminated with them. When the tin begins to cool, and when a more complete separation of its different qualities cannot be looked for, it is lifted out in ladles, and poured into cast-iron moulds. It is obvious, that the order in which the successive blocks are obtained, is that of their purity; those formed from the bottom of the basin being usually so impure, that they inust be subjected anew to the refining process, as if they had been directly smelted from the ore.

The refining operation takes 5 or 6 hours; namely, an hour to fill the basin, three hours to boil the tin with the green wood, and from one to two hours for the subsidence.

Sometimes a simpler operation, called tossing, is substituted for the above artificial ebullition. To effect it, a workman lifts some tin in a ladle, and lets it fall back into the boiler, from a considerable height, so as to agitate the whole mass. He continues this manipulation for a certain time; after which, he skims with care the surface of the bath. The tin is afterwards poured into moulds, unless it be still impure. In this case, the separation of the metals is completed by keeping the tin in a fused state in the boiler fur a certain period, without agitation; whereby the upper portion of the bath (at least one half) is pure enough for the market.

The moulds into which the tin blocks are cast, are usually made of granite. Their capacity is such, that each block shall weigh a little more than three hundred weights. This metal is called block tin. The law requires them to be stamped or coined by public officers, before being exposed to sale. The purest block tin is called refined tin.

The treatment just detailed gives rise to two stanniferous residuums, which have to be smelted again. These are-

1. The scoriæ $\mathbf{B}$ and $\mathbf{c}$, which contain some granulated particles of tin.

2. The dross found on the bottom of the reverberatory furnace, after re-melting the tin to refine it.

The scoriæ c, are smelted without any preparation; but those marked $\mathrm{B}$, are stamped in the mill, and washed, to concentrate the tin grains; and from this rich mixture, called prillion, smelted by itself, a tin is procured of very inferior quality. This may be readily imagined, since the metal which forms these granulations is what, being less fusible than the pure tin, solidified quickly, and could not flow off into the metallic bath.

Whenever all the tin blocks have thoroughly undergone the process of liquation, the fire is increased, to melt the less fusible residuary alloy of tin with iron and some other metals, and this is run out into a small basin, totally distinct from the refining basin. After this alloy has reposed for some time, the upper portion is lifted out into block moulds, as impure tin, which needs to be refined anew. On the bottom and sides of the basin there is deposited a white, brittle alloy, with a crystalline fracture, which contains so great a proportion of foreign metals, that no use can be made of it. About three and a half tons of coal are consumed in producing 2 of tin.

Smelting of tin by the blast furnace. - This mode of reduction employs only wood. 
charcoal, and its object is to obtain tin of the maximum purity to which it can be brought by manufacturing processes. The better ores of the stream-works, and the finer tin sands, are selected for this operation. The washings being always well performed, the oxyde of tin is exempt from every arsenical or sulphurous impurity, and is associated with nothing but a little hematite. It is therefore never calcined.

The smelting is effected without addition; only, in a few cases, some of the residuary matters of a former operation are added to the ore. About a ton and six tenths of wood charcoal are burned for one ton of fine smelted tin. The only rule is, to keep the furnace always full of charcoal and ore. The revived tin is received immediately in the first basin; then run off into the second, where it is allowed to settle for some tinae. The scoriæ that run off' into the first basin, are removed as soon as they fix. These scoriæ are divided into two classes; namely; such as still retain tin oxyde, and such as hold none of the metal in that state, but only in granulations. The metallic bath is divided, by repose, into horizontal zones, of different degrees of purity; the more compound and denser matters falling naturally to the bottom of the basin. The tin which forms the superior zones, being judged to be pure enough, is transrased by ladles into the refining basin, previously heated, and under which, if it is of cast-iron, a moderate fire is applied. The tin near the bottom of the receiving hasin is always laded out apart, to be again smelted; sometimes, indeed, when the furnace is turning out very impure tin, none of it is transvased into the second basin; but the whole is cast into moulds, to be again treated in the blast furnace.

In general they receive no other preparation, but the green wood ebullition, before passing into the market. Sometimes, however, the block of metal is heated till it becomes brittle, when it is lifted to a considerable height, and let fall, by which it is broken to pieces, and presents an agglomeration of elongated grains or tears; whence it is called grain tin.

On making a comparative estimate of the expense by the blowing-hcuse process, and by the reverberatory furnace, it has been found that the former yields about 66 per cent. of tin, in smelting the stream or alluvial ore, whose absolute contents are from 75 to 78 parts of metal in the hundred. One ton of tin consumes a ton and six tenths of wood charcoal, and sufiers a loss of 15 per cent. In working with the reverberatory furnace, it is calculated that ore whose mean contents by an exact analysis are 70 per cent., yields 65 per cent. on the great scale. The arerage value of tin ore, as sold to the smelter, is 50 pounds sterling per ton; but it fluctuates, of course, with the market prices. In 1824, the ore of inferior quality cost $30 l$., while the purest sold for $60 l$. One ton of tin, obtained from the reverberatory furnace, cost-

\begin{tabular}{lllllrrr}
$1 \frac{1}{2}$ tons of ore, worth $-\quad-$ & - & - & - & $£ 75$ & 0 & 0 \\
$1 \frac{3}{4}$ tons of coals, at $10 \mathrm{~s}$. per ton & - & - & - & - & 0 & 17 & 6 \\
Wages of lahor, interest on capital, \&c. & - & - & - & 3 & 0 \\
\hline 78 & & & &
\end{tabular}

On comparing these results with the former, we perceive that in a blowing-house the loss of tin is 15 per cent., whereas it is only 5 in the reverberatory furnace. The expense in fuel is likewise much less relatirely in the latter process; for only $1 \frac{3}{4}$ tons of coals are consumed for one ton of tin; while a ton and six tenths of wood charcoal are burned to obtain the same quantity of tin in the blowing-house; and it is admitted that one ton of wood charcoal is equiralent to two tons of coal, in calorific effect. Hence every thing conspires to turn the balance in faror of the reverberatory plan. The operation is also, in this way, much simpler. and may be carried on by itself. The scoriæ, besides, from the reverberatory hearth, contain less tin than those derived from the same ores treated with charcoal by the blast, as is done at Altenberg. It must be remembered, however, that the grain tin procured by the charcoal process is reckoned to be finer, and fetches a higher price; a superiority partly due to the purity of the ore reduced, and partly to the purity of the fuel.

To test the quality of tin, dissolve a certain weight of it with heat in muriatic acid; should it contain arsenic, brown-black flocks will be separated during the solution, and arseniureted hydrogen gas will be disengaged, which, on being burned at a jet, will deposite the usual gray film of metallic arsenic upon a white saucer held a little way above the flame. Other metals present in the tin are to be sought for, by treating the abore solution with nitric acid of spec. grav. $1 \cdot 16$, first in the cold, and at last with heat and a small excess of acid. When the action is over, the supernatant liquid is to be decanted off the peroxydized tin, which is to be washed with very dilute nitric acid, and both liquors are to be evaporated to dissipate the acid excess. If, on the addition of water to the concentrated liquor, a white powder falls, it is a proof that the tin contains bismuth; if on adding sulphate of ammonia, a white precipitate appears, the tin contains lead; water of ammonia added to supersaturation, will occasion reddish-brown 
flocks, if iron is present; and on evaporating the supernatant liquid to dryness, the copper will be obtained.

The uses of tin are very numerous. Combined with copper, in different proportions, it forms bronze, and a series of other useful alloys; for an account of which see COPPER. With iron, it forms tin-plate; with lead, it constitutes pewter, and solder of various kinds. (See LEAD.) Tin-foil coated with quicksilver makes the reflecting surface of glass mirrurs. (See GLass.) Nitrate of tin affords the basis of the scarlet dye on wool, and of many bright colors to the calico-printer and the cotton-dyer. (See ScARLET and TrN Mordants.) A compound of tin with gold gives the fine crimson and purple colors to stained glass and artificial gems. (See PURPLE of Cassius.) Enamel is made by fusing oxyde of tin with the materials of flint glass. This oxyde is also an ingredient in the white and yellow glazes of pottery-ware.

An Account of Trn coined in Cornwall and Devon, from 1817 to 1829 , inclusive :-

\begin{tabular}{|c|c|c|c|c|c|}
\hline Years. & Blocks. & Tons. & Years. & Blocks. & Tons. \\
\cline { 2 - 4 } 1817 & 25,379 & 4,120 & 1824 & 28,602 & 4,819 \\
1818 & 23,048 & $3,745 \frac{1}{3}$ & 1825 & 24,902 & 4,170 \\
1819 & 18,881 & 3,065 & 1826 & 26,299 & 4,406 \\
1820 & 17,084 & $2,773 \frac{1}{2}$ & 1827 & 31,744 & 5,316 \\
1821 & 19,273 & 3,128 & 1828 & 28,179 & 4,696 \\
1822 & 18,732 & 3,137 & 1829 & 26,344 & 4,396 \\
1823 & 24,077 & 4,031 & & & \\
\hline
\end{tabular}

\begin{tabular}{|c|c|c|c|c|c|}
\hline \multicolumn{2}{|c|}{$\begin{array}{c}\text { Tin imported. } \\
\text { Duty, } 50 s \text {. per cwt. }\end{array}$} & \multirow{2}{*}{$\begin{array}{c}\text { Tin exported. } \\
\text { Cwts. } \\
2,938\end{array}$} & \multicolumn{2}{|c|}{$\begin{array}{c}\text { Tin imported. } \\
\text { Duty, 50s. per cwt }\end{array}$} & \multirow{2}{*}{$\begin{array}{c}\text { Tin exported. } \\
\text { Cwts. } \\
21,720\end{array}$} \\
\hline 1827 & $\begin{array}{l}\text { Cwts. } \\
2,217\end{array}$ & & 1832 & $\begin{array}{c}\text { Cwts. } \\
29,203\end{array}$ & \\
\hline 1828 & 3,386 & 3,258 & 1833 & 35,124 & 39,850 \\
\hline 1829 & 2,674 & 2,581 & 1834 & 46,769 & 46,685 \\
\hline 1830 & 15,539 & 10,426 & 1835 & 17,705 & 23,796 \\
\hline 1831 & 8,099 & 12,226 & 1836 & 23,236 & 17,231 \\
\hline
\end{tabular}

The principal importations are from the East India Company's territories and Ceylon: they amounted in 1832 to 24,585 cwts. ; in 1833 to 27,928 ; in 1834 to 33,611 ; in 1835 to 10,104 ; and in 1836 to 17,729 . From Sumatra and Java 1961 ewts. were imported in 1832 , and 1145 in 1834 , but in the other years greatly less.

\begin{tabular}{|c|c|c|c|c|c|}
\hline $\begin{array}{l}\text { Declared value of tin } \\
\text { and pewter wares }\end{array}$ & $\begin{array}{c}1827 . \\
302,255 l .\end{array}$ & $\begin{array}{c}1829 . \\
235,178 l .\end{array}$ & $\begin{array}{c}1831 . \\
239,143 l .\end{array}$ & $\begin{array}{c}1833 . \\
282,176 l .\end{array}$ & $\begin{array}{c}1835 . \\
381,076 l .\end{array}$ \\
\hline $\begin{array}{l}\text { and tin-plates ex } \\
\text { ported in- }\end{array}$ & $\begin{array}{c}1828 . \\
266,6511 .\end{array}$ & $\begin{array}{c}1830 \\
249,657 l .\end{array}$ & $\begin{array}{c}1832 . \\
243,259 l .\end{array}$ & $\begin{array}{c}1834 . \\
337,056 l .\end{array}$ & $\begin{array}{c}1836 . \\
387,951 l .\end{array}$ \\
\hline
\end{tabular}

Of these goods, from two fifths to three fifths go to the United States of America.

Abstract of Tin coined in Cornwall and Devon, in the year ending June 30, 1835; from the Mining Review, vol. iii.

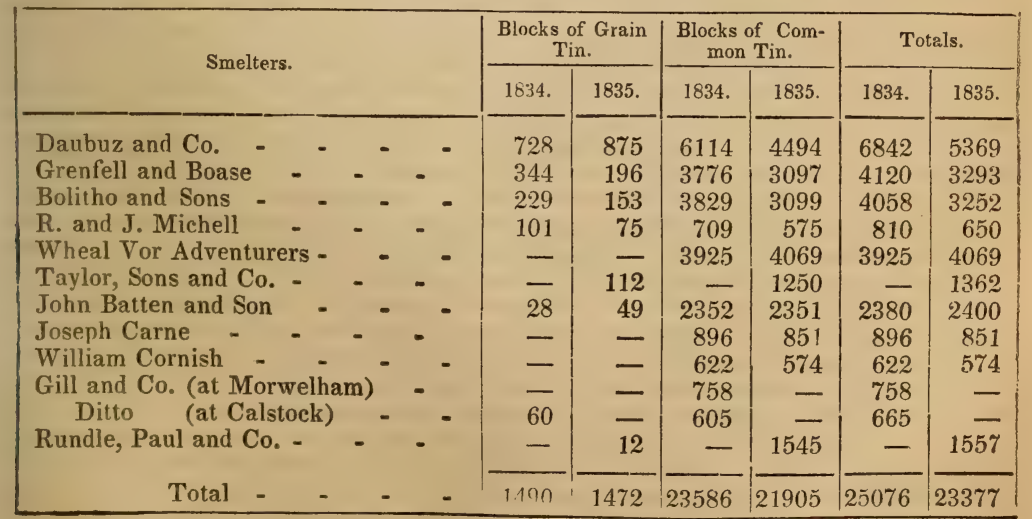

Total, in 1834,4180 tons; in 1835,3899 tons. $(6$ blocks $=1$ ton. $)$ 
TINCAL, crude borax.

TINCTORIAL MATTER. One of the most curious and valuable facts ascertained apon this subject, is, that madder kept in casks, in a warm place, undergoes a species of fermentation, which, by ripening, or rather deoxydizing the coloring-matter, increases its dyeing power by no less than from 20 to 50 per cent. See M. H. Schlumberger's memoir read to the Société Industrielle de Mulhausen, 24 November, 1837.

IINCTURE is a title used by apothecaries to designate alcohol, in a somewhat dilute state, impregnated with the active principles of either vegetable or animal substances.

TIN-GLASS is a name of bismuth.

TIN MORDANTS, for dyeing scarlet:-

Mordant A, as commonly made by the dyers, is composed of 8 parts of aquafortis, 1 part of common salt or sal ammoniac, and 1 of granulated tin. This preparation is very uncertain.

Mordunt B.-Pour into a glass globe, with a long neck, 3 parts of pure nitric acid at $30^{\circ} \mathrm{B}_{\circ}$; and 1 part of muriatic acid at $17^{\circ}$; shake the globe gently; avoiding the corrosive rapors, and put a loose stopper in its mouth. Throw into this nitro-muriatic acid one eighth of its weight of pure tin, in small bits at a time. When the solution is com. plese, and settled, decant it into bottles, and close them with ground stoppers. It should be diluted only when about to be used.

Mordant c, by Dambourney.-In two drachms Fr. (144 grs.) of pure muriatic acid, dissolve 18 grains of Malacca tin. This is reckoned a good mordant for brightening or fixing the color of peachwood.

Mordant $\mathrm{D}$, br Hellot.-Take 8 ounces of nitric acid, diluted with as much water; dissolve in it half an ounce of sal ammoniac, and 2 drachms of nitre. In this acid solution dissolve one ounce of granulated tin of Cornwall, observing not to put in a fresh piece till the preceding be dissolved.

Mordant E, by Scheffer.-Dissolve one part of tin in four of a nitro-muriatic acid, prepared with nitric acid diluted with its own weight of water, and one thirty-secondth of sal ammoniac.

Mordant $\mathrm{F}$, by Poerner.-Mix one pound of nitric acid with one pound of water, and dissolve in it an ounce and a half of sal ammoniac. Stir it well, and add, by very slow degrees, two ounces of tin turned into thin ribands upon the lathe.

Mordant G, by Berthollet.-Dissolve in nitric acid of $30^{\circ} \mathrm{B}$. one eighth of its weight of sal ammoniac, then add by degrees one eighth of its weight of tin, and dilute the solution with one fourth of its weight of water.

Mordant $\mathrm{K}$, by Dambourney.-In one drachm (72 grs.) of muriatic acid at $17^{\circ}$, one of nitric acid at $30^{\circ}$, and 18 grains of water, dissolve, slowly and with some heat, 18 grains of fine Malacea tin.

Mordant $\mathrm{L}$ is the birch bark prescribed by Dambourney.-This bark, dried and ground, Is said to be a very valuable substance for fixing the otherwise fugitive colors produced by woods, roots, archil, \&c.

TIN-PLATE. The only alloy of iron interesting to the arts is that with tin, in the formation of tin-plate or white-iron.

The sheet iron intended for this manufacture is refined with charcoal instead of coke, subsequently rolled to various degrees of thinness, and cut into rectangles of different sizes, by means of a shearing-machine driven by a water-wheel, which will turn out 100 boxes a day, or four times the number cut by hand labor. The first step towards tinning is to fiee the metallic surface from every particle of oxyde or impurity, for any such would inevitably prevent the iron from alloying with the tin. The plates are next bent separately by hand into a saddle or $\Lambda$ shape, and ranged in a reverheratory oven, so that the flame may play freely among them, and heat them to redness. They are then plunged into a bath, composed of four pounds of muriatic acid diluted with three gallons of water, for a few minutes, taken out and drained on the floor, and once more exposed to ignition in a furnace, whereby they are scaled, that is to say, cast their scales. The above bath will suffice for scaling $\$ 00$ plates. When taken out, they are beat level and smooth on a cast-iron block, after which they appear mottled blue and white, if the scaling has been thoroughly done. They are next passed through chilled rolls or castiron cyl nders, rendered very hard by being cast in thick iron moulds, as has been long practised br the Scotch founders in casting bushes for cart-wheels. After this process of cold rolling, the plates are immersed, for ten or twelve hours, in an acidulous ley, made by fermenting bran-water, taking care to set them separately on edge, and to turn them at least once, so that each may receive a due share of the operation. From this ley-steep they are transferred-into a leaden trough, divided by partitions, and charged with dilute sulphuric acid. Each compartment is called a hole by the workmen, and is calcu lated to receive about 225 plates, the number afterwards packed up together in a box. In this liquid they are agitated about an hour, till they become perfectly bright, and fres 
from such black spots as might stain their surface at the time of immersion. This process, called pickling, is both delicate and disagreeable, requiring a good workman, at high wages. The temperature of the last two steeps should be at least $90^{\circ}$ or $100^{\circ} \mathrm{F}$., which is kept up by stoves in the apartments. The plates are finally scoured with hemp and sand in a body of water, and then put aside for use in a vessel of pure water, under which they remain bright and free from rust for many months, a very remarkable circumstance.

The timning follows these preparatory steps. A range of rectangular cast-iron pots is set over a fire-flue in an apartment called the stow, the workmen stationing themselves opposite to the narrow ends. The first rectangle in the range is the tin-pot; the second is the wash-pot, with a partition in it; the third is the grease-pot; the fourth is the pan, grated at bottom; the fifth is the list-pot, and is greatly narrower than any of the rest: they are all of the same length.

The prepared plates, dried by rubbing bran upon them, are first immersed one by one in a pot filled with melted tallow alone, and are left there for nearly an hour. They are thence removed, with the adhering grease, into pot No. 1, filled with a melted mixture of block and grain tin, covered with about four inches of tallow, slightly carbonized. This pot is heated by a fire, playing under its bottom and "ound its sides, till the metal becomes so hot as nearly to inflame the grease. Here about 340 plates are exposed, upright, to the action of the tin for an hour and a half, or more, according to their thickness. They are next lifted out, and placed upon an iron grating, to let the superfluous metal drain off; but this is more completely removed in the next process, called washing.

Into the wash-pot No. 2, filled with melted grain tin, the workman puts the above plates, where the heat detaches the ribs, and drops. There is a longitudinal partition in it, for keeping the drop of tin that rises in washing from entering the vessel where the last dip is given. Indeed, the metal in the wash-pot, after having acted on 60 or 70 boxes, becomes so foul, that the weight of a block ( 300 cwts.) of it is transferred into the tin-pot, No. 1, and replaced by a fresh block of grain tin. The plates being lifted out of the wash-pot, with tongs held in the left hand of the workman, are scrubbed on each side with a peculiar hempen brush, held in his right hand, then dipped for a moment in the hot tin, and forthwith immersed in the adjoining grease-pot, No. 3. This requires manual dexterity; and though only three-pence be paid for brushing and tinwashing 225 plates, yet a good workman can earn six shillings and three-pence in twelve hours, by putting 5625 plates through his hands. The final tin-dip is useful to remove the marks of the brush, and to make the surface uniformly bright. To regulate the temperature of the tallow-pot, and time during which the plates are left in it, requires great skill and circumspection on the part of the workman. If kept in it too long, they would be deprived, to a certain extent, of their silvery lustre; and if too short, streaks of tin would disfigure their surface. As a thick plate retains more heat after being lifted out of the washing-pot, it requires a proportionally cooler grease-pot. This pot has pins fixed within it, to keep the plates asunder; and whenever the workman has transferred five plates to it, a boy lifts the first out into the cold adjoining pan, No. 4 ; as soon as the workman transfers a sixth plate, the boy removes the second; and so on. The manufacture is completed by removing the wire of tin left on the under edge of the plates, in consequence of their vertical position in the preceding operations. This is the business of the list-boy, who seizes the plates when they are cool enough to handle, and puts the lower edge of each, one by one, into the list-pot, No. 5, which contains a very little melted tin, not exceeding a quarter of an inch in depth. When he observes the wire-edye to be melted, he takes out the plate, and, striking it smartly with a thin stick, detaches the superfluous metal, which leaves merely a faint stripe where it lay. This mark may be perceived on every tin-plate in the market.

The plates are finally prepared for packing up in their boxes, by being well cleansed from the tallow, by friction with bran.

Mr. Thomas Morgan obtained a patent, in September, 1829, for clearing the sheet-iron plates with dilute sulphuric acid in a hole, instead of scaling them in the usual way, previous to their heing cold rolled, annealed, and tinned; whereby, he says, a better article is produced at a cheaper rate.

Crystallized tin-plate, see Morree Metallique. It would seem that the acid merely lays bare the crystalline structure really present on every sheet, but masked by a film of redundant tin. Though this showy article has become of late years vulgarized by its cheapness, it is still interesting in the eyes of the practical chemist. The English tinplates marlied $\mathrm{F}$ answer well for producing the Moirée, by the following process. Place the tin-plate, slightly heated, over a tub of water, and rub its surface with a sponge dipped in a liquor composed of four parts of aquafortis, and two of distilled water, holding one part of common salt or sal ammoniac in solution. Whenever the crystalline spangles seem to be thoroughly brought out, the plate must be immersed in water, 
washed either with a feather or a little cotton (taking care not to rub off the film of tin that forms the feathering), forthwith dried with a low heat, and coated with a lacker varnish, otherwise it loses its lustre in the air. If the whole surface is not plunged at once in cold water, but if it be partially cooled by sprinkling water on it, the crystallization will be finely variegated with large and small figures. Similar results will be obtained by blowing cold air through a pipe on the tinned surface, while it is just passing from the fused to the solid state; or a variety of delineations may be traced, by playing over the surface of the plate with the pointed flame of a blowpipe.

The following TABLE shows the several sizes of tin-plates, the marks by which they are distinguished, and their current wholesale prices in London:-

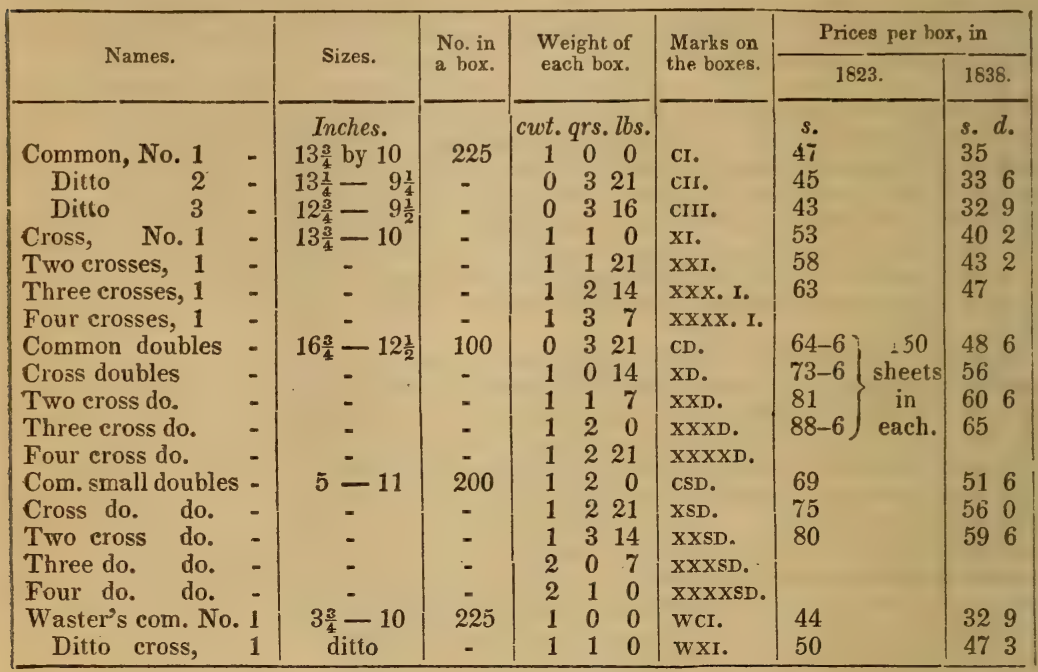

These are the cash prices of one wholesale warehouse in Thames street; an immediately adjoining warehouse charges fully $1 s$. more upon the standard $\mathrm{Cr}$, and proportionally upon the others.

TITANIUM is a rare metal, discovered by Klaproth, in menachanite, in 1794 . It has been detected since in the form of small cubes of a copper-red color, in some of the blast furnaces in Yorkshire. According to Hassenfratz, its presence in small quantity does not impair the malleability of iron. It is very brittle, so hard as to scratch steel, and very light, having a specific gravity of only $5 \cdot 3$. It will not melt in the heat of any furnace, nor dissolve, when crystallized, even in nitro-muriatic acid; but only when in fine powder. By calcination with nitre, it becomes oxygenated, and forms titanate of potassa. Traces of this metal may be detected in many irons, both wrought and cast. The principal ores of titanium are sphene, common and foliated, rutile, iserine, menachanite, and octahedrite or pyramidal titanium ore. None of them has been hitherto applied to any use.

TOBACCO. It is said that the name tobacco was given by the Spaniards to the plant, because it was first observed by them at Tabasco, or Tabaco, a province of Yucatan in Mexico. In 1560, Nicot, the French ambassador to Portugal, having received some iobacco from a Flemish merchant, showed it, on his arrival in Lisbon, to the grand prior, and, on his return into France, to Catherine of Medicis, whence it has been called Nicotiana by the botanists. Admiral Sir Francis Drake, having, on his way home from the Spanish Main in 1586, touched at Virginia, and brought away some forlorn colonists, is reported to have first imported tobacco into England. But, according to Lobel, this plant was cultirated in Britain before the year 1570 ; and was consumed by smoking in pipes by Sir Walter Raleigh and companions, so early as the year 1584 .

The plants are hung up to dry during four or five weeks; taken down out of the sheds in damp weather, for in dry they would be apt to crumble into pieces; stratified in heaps, covered up, and left to sweat for a week or two, according to their quality and the state of the season; during which time they must be examined frequently, opened up, and turned over, lest they become too hot, take fire, or run into putrefactive fermentation. This process needs to be conducted by skilful and attentive operatives. An experienced negro can form a sufficiently accurate judgment of the temperature, by thrusting his har down into the heap. 
The tobacco thus prepared, or often withont fermentation, is sent into the market; but, before being sold, it must undergo the inspection of officers, appointed by the state with very liberal salaries, who determine its quality, and brand an appropriate stamp upon its casks, if it be sound; but if it be bad, it is burned.

Our respectable tobacconists are very careful to separate all the damaged leaves, before they proceed to their preparation, which they do by spreading them in a heap upon a stone parement, watering each layer in succession with a solution of sea salt, of spec. grav. 1·107, called suuce, till a ton or more be laid; and leaving their principles to react on each other for tiree or four days, according to the temperature, and the nature of the tobacco. It is highly probable that ammonia is the volatilizing agent of many odors, and especially of those ji tobacco and musk. If a fresh green leaf of tobacco be crushed between the fingers, it emits merely the herbaceous smell common to many plants; 'but if it be triturated in a mortar, along with a little quicklime or caustic potash, it will immediately exhale the peculiar odor of sruff. Now analysis shows the presence of muriate of ammonia in this plant, and fermentation serves further to generate free ammonia in it; whence, by means of this process, and lime, the odoriferous vehicle is abundantly developed. If, on the other hand, the excess of alkaline matter in the tobacco of the shops be saturated by a mild dry acid, as the tartaric, its peculiar aroma will entirely disappear.

Tubacco contains a great quantity of an azotized principle, which by fermentaiton roduces abundance of ammonia ; the first portions of which saturate the acid juices of the plant, and the rest serve to volatilize its odorous principles. The salt water is useful chielly in moderating the fermentation, and preventing it from passing into the putrefactive stage; just as salt is sometimes added to saccharine worts in tropical countries, to temper the fermentative action. The sea salt, or concentrated sea water, which contains some muriate of lime, tends to keep the tobacco moist, and is therefore preferable to pure chloride of sodium for this purpose. Some tobacconists mix molasses with the salt sauce, and ascribe to this addition the violet color of the macouba snuff of Martinique; and others add a solution of extract of liquorice. The following prescription is that used by a skilful manufacturer :- In a solution of the liquorice juice, a few figs are to be boiled for a couple of hours; 10 the decoction, while hot, a few bruised anise-seeds are to be added, and when cold, common salt to saturation. A little silent spirit of wine being poured in, the mixture is to be equably, but sparingly, sprinkled with the rose of a watering-pot, over the leaves of the tobacco, as they are successively stratified upon the preparation floor.

The fermented leaves, being next stripped of their middle ribs by the hands of children, are sorted anew, and the large ones are set apart for making cigars. Most of the tobaccos on sale in our shops are mixtures of different growths: one kind of smoking tobacco, for example, consists of 70 parts of Maryland, and 30 of meager Virginia ; and one kind of snuff consists of 80 parts of Virginia, and 30 parts of either Humesfort or Warwick. The Maryland is a very light tobacco, in thin yellow leaves; that of Virginia is in large brown leaves, unctuous or somewhat gluey on the surface, having a smell somewhat like the figs of Malaga; that of Havana is in brownish, light leaves, of an agreeable and rather spicy smell; it forms the best cigars. The Carolina tobacco is less unctuous than the Virginian; but in the United States it ranks next to the Maryland.

The shag tobacco is dried to the proper point upon sheets of copper.

Tobacco is cut into what is called shag tobacco by knife-edged chopping stamps, a machine somewhat similar to that represented under Metallurgy, fig. 670. For grinding the tobacco leaves into snuff, conical mortars are employed, somewhat like that used by the Hindoos for grinding sugar-canes, fig. 1080; but the sides of the snuff-mill have sharp ridues from the top to near the bottom.

Mr. L. W. Wright obtained a patent in August, 1827, for a tobacco-cutting machine, which bears a close resemblance to the well-known machines with revolving knives, for culting straw into chaff. The tobacco, after being squeezed into cakes, is placed upon a smooth bed within a horizontal trough, and pressed by a follower and screws to keep it compact. These cakes are progressively advanced upon the bed, or fed in, to meet the revolving blades. The speed of the feeding-screw determines the degree of fineness of the sections or particles into which the tobacco is cut.

I was employed some years ago by the Excise to analyze a quantity of snuff, seized on suspicion of having been adulterated by the manufacturer. I found it to be largely drugged with pearl-ashes, and to be thereby rendered very pungent, and absorbent of moisture; an economical method of rendering an effete article at the same time active and aqueous.

According to the recent analysis of Possett and Reimann, 10,000 parts of tobaccoleaves contain -6 of the peculiar chemical principle nicotine; 1 . of nicotiunine; 287 of slightly bitter extractive; 174 of gum, mixed with a little malic acid; 26.7 of a green resin; 26 of vegetable albumen; 104.8 of a substance analogous to gluten; 51 of 
malic acid; 12 of malate of ammonia ; 4.8 of sulphate of potassa; 6.3 of chloride of potassium; 9.5 of potassa, which had been combined with malic and nitric acids; 16.6 of phosphate of lime; 24.2 of lime, which had been combined with malic acid; 8.8 of silica ; 496.9 of fibrous or ligneous matter; traces of starch ; and 88.28 of water.

Nicotine is a transparent colorless liquid, of an alkaline nature. It may be distilled in a retort plunged into a bath heated to $290^{\circ}$ Fahrenheit. It has a pricking, burning taste, which is very durable; and a pungent, disagreeable smell. It burns by means of a wick, with the diffusion of a vivid light, and much smoke. It may be mixed with water in all proportions. It is soluble also in acetic acid, oil of almonds, alcohol, and ether, but not in oil of turpentine. It acts upon the animal economy with extreme violence; and in the dose of one drop it kills a dog. It forms salts with the acids. About one part of it may be obtained by very skilful treatment from one thousand of good tobacco.

Tobacco imported into the United Kingdom, viz. - unmanufactured, in 1836, $52,232,9(1) 7 \mathrm{lbs} . ;$ in $1837,27,070,448 \mathrm{lb}$..; - manufactured, and snuff, in 1836, 182,218 lbs.; in 1837, 642,287 lbs. Retained for home consumption, unmanufactured, in 18:36, 22,309,021 lbs.; in 1837, 22,504,343 lbs.;-manufactured, and snuff, in 1836, 159,226 lbs.; in $1837,145,045 \mathrm{lbs}$. Duty received,-on unmanufactured tobacco, in $1836, £ 3,344,703$; in $1837, £ 3,375,125$; on manufactured tobacco, and snuff, in 1836 , $£ 71,560$; in $1837, £ 65,220$.

TOBACCO-PIPES. The practice of smoking tobacco has become so general in many nations as to render the manufacture of tobacco-pipes a considerable branch of industry. Some seek in the inhalation of tobacco-smoke a pleasurable narcotism; others imagine it to be beneficial to their health; but, in general, smoking is merely a dreamy resource against ennui, which ere long becomes an indispensable stimulus. The filthiness of this habit, the offensive odor which persons under its influence emit from their mouths and clothes, the stupor it too often occasions, as well as the sallow complexion, black or carious teeth, and impaired digestion, all prove the great consumption of tobacco to be akin in evil influence upon mankind to the use of ardent spirits.

Tobacco-pipes are made of a fine-grained plastic white clay, to which they have given the name. It is worked with water into a thin paste, which is allowed to settle in pits, or it may be passed through a sieve, to separate the silicious or other stony impurities; the water is afterwards evaporated till the clay becomes of a doughy consistence, when it must be well kneaded to make it uniform. Pipe-clay is found chiefly in the isle of Purbeck and Dorsetshire. It is distinguished by its perfectly white color, and its great adhesion to the tongue after it is baked; owing to the large proportion of alumina which it contains.

A child fashions a ball of clay from the heap, rolls it out into a slender cylinder upon a plank, with the palms of his hands, in order to form the stem of the pipe. He sticks a small lump to the end of the cylinder for forming the bowl; which having done, he lays the pieces aside for a day or two, to get more consistence. In proportion as he makes these rough figures, he arranges them by dozens on a board, and hands them to the pipemaker.

The pipe is finished by means of a folding brass or iron mould, channelled inside of the shape of the stem and the bowl, and capable of being opened at the two ends. It is formed of two pieces, each hollowed out like a half-pipe, cut as it were lengthwise; and these two jaws, when brought together, constitute the exact space for making one pipe. There are small pins in one side of the mould, corresponding to holes in the other, which serve as guides for applying the two together with precision.

The workman takes a long iron wire, with its end oiled, and pushes it through the soft clay in the direction of the stem, to form the bore, and he directs the wire by feeling with his left hand the progress of its point. He lays the pipe in the groove of one of the jaws of the mould, with the wire sticking in it; applies the other jaw, brings them smartly together, and unites them by a clamp or vice, which produces the external form. A lever is now brought down, which presses an oiled stopper into the bowl of the pipe, while it is in the mould, forcing it sufficiently down to form the cavity; the wire being meanwhile thrust backwards and forwards so as to pierce the tabe completely through. The wire must become visible at the bottom of the bowl, otherwise the pipe will be imperfect. The wire is now withdrawn, the jaws of the mould opened, the pipe taken out, and the redundant clay removed with a knife. After drying for a day or two, the pipes are scraped, polished with a piece of hard wood, and the stems being bont into the ciesired form, they are carried to the baking kiln, which is crpahle of firing fity grcss in from 8 to 12 hours. A workman and a child can easily make five gross of pipes in a day.

No tobacco-pipes are so highly prized as those made in Natolia, in Turkey, out of meerschaum, a somewhat plastic magnesian stone, of a soft greasy feel, which is formed into pipes after having been softened with water. It becomes white and hard in the kiln 
A wobacco-pipe kiln should diffuse an equal heat te every part of its interior, while It excludes the smoke of the fire. The crucible, or large sagger, A, A, figs. 1155 and

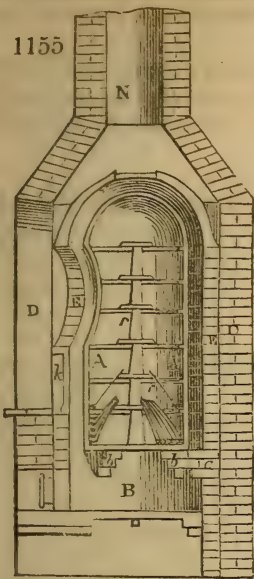

1156, is a cylinder, covered in with a dome. It is placed over the fireplace $\mathbf{b}$, and enclosed within a furmace of ordinary brickwork D, D, lined with fire-bricks E, E. Between this lining and the cylinder, a space of about 4 inches all round is left for the circulation of the flame. There are 12 supports or ribs between the cylinder and the furnace lining, which form so many flues, indicated by the dotted lines $x$, in fig. 1156 (the dotted circle representing the cylinder). These ribs are perforated with occasional apertures, as shown in fig. 1155 , for the purpose of connecting the adjoining flues; but the main 1156 bearing of the hollow cylinder is given by five piers, $b, b, c$, formed of bricks

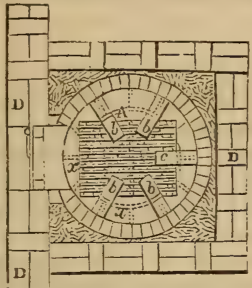
projecting over and beyond each other. One of these piers $c$, is placed at the back of the fireplace, and the other four at the sides $b, b$. These project nearly into the centre, in order to support and strengthen the bottom; while the flues pass up between them, unite at the top of the cýlinder in the dome $\mathbf{L}$, and discharge the smoke by the chimney $N$.

The lining $\mathbf{F}, \mathbf{E}, \mathbf{E}$, of the chimney is open on one side to form the door, by which the cylinder is charged and discharged. The opening is permanently closed as high as $k$, fig. 1155 , by an iron plate plastered over with fire clay; above this it is left open, and shut merely with temporary brick-work while the furnace is yoing. When this is removed, the furnace can be filled or emptied through the opening, the cylindric crucible having a correspondent aperture in its side, which is closed in the following ingenious way, while the furnace is in action. The workman first spreads a layer of clay round the edge of the opening, he then sticks the stems of broken pipes across from one side to the other, and plasters up the interstices with clay, exactly like the lath-and-plaster work of a ceiling. The whole of the cylinder, indeed, is constructed in this manner, the bottom being composed of a great many fragments of pipe stems, radiating to the centre; these are coated at the circumference with a layer of clay. A number of bowls of broken pipes are inserted in the clay; in these other fragments are placed upright, to form the sides of the cylinder. The ribs round the outside, which form the flues, are made in the same way, as well as the dome I ; by which means the cylindric case may be made very strong, and yet so thin as to require little clay in the building, a moderate fire to heat it, while it is not apt to split asunder. The pipes are arranged within, as shown in the figure, with their bowls resting against the circumference, and their ends supported on circular pieces of clay $r$, which are set up in the centre for that purpose. Six small ribs are made to project inwards all round the crucible, at the proper heights, to support the different ranges of pipes, without having so many resting on each other as to endanger their being crushed by the weight. $\mathrm{By}$ this mode of distribution, the furnace may contain 50 gross, or 7200 pipes, all baked within 8 or 9 hours; the fire being gradually raised, or damped if occasion be, by a plate partially slid over the chimney top.

TODDY, Sura, Mee-ra, sweet juice.-The proprietors of cocoa-nut plantations in the peninsula of India, and in the Island of Ceylon, instead of collecting a crop of nuts, frequently reap the produce of the trees by extracting sweet juice from the flowerstalk. When the flowering branch is half shot, the toddy-drawers bind the stock round with a young cocoa-nut leaf in several places, and heat the spadix with a short baton of ebony. This beating is repeated daily for ten or twelve days, and about the end of that period a portion of the flower-stalk is cut off. The stump then begins to bleed, and an earthen vessel (chatty) or a calabash is suspended under it, to receive the juice, which is by the Europeans called toddy.

A thin slice is taken from the stump daily, and the toddy is removed tivice a day. A cocoa-nut frequently pushes out a new spadix once a month; and after each spadix begins to bleed, it continues to produce freely for a month, by which time another is ready to supply its place. The old spadix continues to give a little juice for another month, after which it withers; so that th. are are sometimes two pots attached to a tree at one time, but never mors. Each of these spadices, if allowed to grow, would produce a bunch of nuts from two to twenty. Trees in a good soil produce twelve bunches in the year; but when less favorably situated, they often do not give more than six bunches. The quantity of six English pints of toddy is sometimes yielled by a tree daily. 
Toddy is much in demand as a beverage in the neighborhood of villages, especially where European troops are stationed. When it is drunk before sunrise, it is a cool, delicious, and particularly wholesome beverage; but by eight or nine o'clock fermentation has made some progress, and it is then highly intoxicating.*

TOLU, is a brownish-red balsam, extracted from the stem of the Myroxilon toluiferum, a tree which grows in South America. It is composed of resin, oil, and benzoic acid. Having an agreeable odor, it is sometimes used in perfumery. It has a place in the Materia Medica, but for what good reason I know not.

TOMBAC, is a white alloy of copper.

TONKA BEAN, the fruit of the Dipterix odorata, affords a concrete crystalline volatile oil (stearoptene), called coumarine by the French. It is extracted by digestion with alcohol, which dissolves the stearoptène, and leaves a fat oil. It has an agreeable smell, and a warm taste. It is fusible at $122^{\circ}$ Fahrenheit, and volatile at higher heats.

TOPAZ. See LAPIDARY.

TORTOISE-SHELL, or rather scales, a horny substance, that covers the hard strong covering of a bony contexture, which encloses the Testudo imbricata, Linn. The lamellæ or plates of this tortoise are 13 in number, and may be readily separated from the bony part by placing fire beneath the shell, whereby they start asunder. They vary in thickness from one eighth to one quarter of an inch, according to the age and size of the animal, and weigh from. 5 to 25 pounds. The larger the animal, the better is the shell. This substance may be softened by the heat of boiling water; and if compressed in this state by screws in iron or brass moulds, it may be bent into any shape. The moulds being then plunged in cold water, the shell becomes fixed in the form imparted by the mould. If the turnings or filings of tortoise-shell be subjected skilfully to gradually increased compression between moulds immersed in boiling water, compact objects of any desired ornamental figure or device may be produced. The soldering of two pieces of scale is easily effected, by placing their edges together, after they are nicely filed to one bevel, and then squeezing them strongly between the long flat jaws of hot iron pincers, made somewhat like a hairdresser's curling-tongs. The pincers should be strong, thick, and just hot enough to brown paper slightly, without burning it. They may be soldered also by the heat of boiling water, applied along with skilful pressure. But in whatever way this process is attempted, the surfaces to be united should be made very smooth, level, and clean; the least foulness, even the touch of the finger, or breathing upon them, would prevent their coalescence. See HorN.

TOUCH-NEEDLES, and TOUCH-STONE, are means of aseertaining the quality of gold trinkets. See Assay.

TOW. See FLAX.

TRAGACANTH, GUM. (Gomme adracante, Fr.; Traganth Germ.) See GuM.

TRAVERTINO. See TUFA.

TREACLE, is the viscid brown uncrystallizable sirup which drains from the sugar-refinng moulds. Its specific gravity is generally $1 \cdot 4$, and it contains upon an average 75 pe : cent. of solid matter, by my experiments.

TRIPOLI (Terre pourrie, Fr.; Tripel, Germ.), rotten-stone, is a mineral of an earthy fracture, a yellowish-gray or white color, composition impalpably fine, meager to the touch, does not adhere to the tongue, and burns white. Its analogue, the Polierschiefer, occurs in thin flat foliated pieces, of the above colors, occasionally striped; soft, absorbent of water; spec. grav. $1 \cdot 9$ to $2 \cdot 2$.

M. Ehrenberg has shown that both of these friable homogeneous rocks, which consist almost entirely of silica, are actually composed of the exuviæ or rather the skeletons of infusoria (animaliula) of the family of Barcillaria, and the genera Coeconema, Gonphonema, \&c. They are recognised with such distinctness in the microscope, that their analogies with living species may be readily traced; and in many cases there are no appreciable differences between the living and the petrified. The species are distinguished by the number of partitions or transverse lines upon their bodies. The length is about $\frac{1}{2} \frac{1}{88}$ of a line. M. Ehrenberg made his observations upon the tripolis of Billen in Bohemia, of Santafiora in Tuscany, of the Isle of France, and of Francisbad, near Eger.

The meadow iron ore (Fer limoneux des marais) is composed almost wholly of the Gaellonella ferruginea. Most of these infusoria are lacustrine; but others are marine, particularly the tripolis of the Isle of France.

According to the chemical analysis of Bucholz, tripoli consists of-silica, 81 ; alumina, 1.5 ; oxyde of iron, 8 ; sulphuric acid, 3.45 ; water, 4.55 . This specimen was probably found in a coal-field. The tripoli of Corfu is reckoned the best for scouring or brightenng brass and other metals. Mr. Phillips found in the Derbyshire rotten-stnne (near

* Contributions to the History of the Cocoa-nut Tree. By Henry Marshall, Esq., Deputy Inspector 0 Hospitals. 
Bakewell), 86 of alumina, 4 of silica, and 10 of carbon-being a remarkable difference in composition from the Bohemian.

TUFA, or TUF, is a gray deposite of calcareous carbonate, from springs and streams. TULA METAL, is an alloy of silver, copper, and lead.

TUNGSTEN (Eng. and Fr.; Wolfram, Germ.), is a peculiar metal, which occurs in the state of an acid (the tungstic), combined with various bases, as with lime, the oxydes of iron, manganese, and lead. The metal is obtained by reduction of the ore, or the dooxydizement of the acid, in the form of a dark steel-gray powder, which assumes under the burnisher a feeble metallic lustre. Its specific gravity is $17 \cdot 22$.

TURBITH MINERAI, is the yellow subsulphate of mercury.

TURF (Peat, Scotch; Tourbe, Fr.; Torf, Germ.), consists of vegetable matter, chiefly of the moss family, in a state of partial decomposition by the action of water. Cut, during summer, into brick-shaped pieces, and dried, it is extensively used as fuel by the peasantry in every region where it abounds. The dense black turf, which forms the lower stratum of a peat-moss, is much contaminated with iron, sulphur, sand, \&c., while the lighter turf of the upper strata, though nearly pure vegetable matter, is too bulky for transportation, and too porous for factory fuel. These defects have been happily removed by Mr. Williams, managing director of the Dublin Steam Navigation Company, who has recently obtained a patent for a method of converting the lightest and purest beds of peat-moss, or bog, into the four following products: 1. A brown combustible solid, denser than oak; 2. A charcoal, twice as compact as that of hard wood; 3. A factitious coal; and 4. A factitious coke; each of which possesses very valuable properties.

Mr. D'Ernst, artificer of fire-works to Vauxhall, has proved, by the severe test of co lored fires, that the turf charcoal of $\mathrm{Mr}$. Williams is 20 per cent. more combustible than that of oak. Mr. Oldham, engineer of the Bank of England, has applied it in softening his steel plates and dies, with remarkable success. But one of the most important results of Mr. Williams's invention is, that with $10 \mathrm{cwts}$. of pitcoal, and $2 \frac{1}{2} \mathrm{cwts}$. of his factitious coal, the same steam power is now obtained, in navigating the Company's ships, as with $17 \frac{1}{2} \mathrm{cwts}$. of pitcoal alone; thereby saving 30 per cent. in the stowage of fuel. What a prospect is thus opened up of turning to admirable account the unprofitable bogs of Ireland; and of producing, from their inexhaustible stores, a superior fuel for every purpose of arts and engineering!

The turf is treated as follows:-Immediately after being dug, it is triturated under revolving edge-wheels, faced with iron plates perforated all over their surface, and is forced by the pressure through these apertures, till it becomes a species of pap, which is freed from the greater part of its moisture by squeezing in a hydraulic press between layers of eara cloth, then dried, and coked in suitable ovens.- (See Charcoal, and PrTCOAL, COKING OF.) Mr. Williams makes his factitious coal by incorporating with pitch or rosin, melted in a caldron, as much of the above charcoal, ground to powder, as will form a doughy mass, which is moulded into bricks in its hot and plastic state. From the experiments of M. Le Sage, detailed in the 5th volume of "The Repertory of Arts," charred ordinary turf seems to be capable of producing a far more intense heat than common charcoal. It has been found preferable to all other fuel for case-hardening iron, tempering steel, forging horse-shoes, and welding gun-barrels. Since turf is partially carbonized in its native state, when it is condensed by the hydraulic press, and fully charred, it must evidently afford a charcoal very superior in calorific power to the porous substance generated from wood hy fire.

TURKEY RED, is a brilliant dye produced on cotton goods by MADDER.

TURMERIC, Curcuma, T'erra merita, (Souchet, or Safran des Indes, Fr.; Gelbuurzel, Germ.), is the root of the Curcuma longa and rotunda, a plant which grows in the East Indies, where it is much employed in dyeing yellow, as also as a condiment in curry sauce or powder. The root is knotty, tubercular, oblong, and wrinkled; pale-yellow without, and brown-yellow within; of a peculiar smell, a taste bitterish and somewhat spicy. It contains a peculiar yellow principle, called curcumine, a brown coloring-matter, a volatile oil, starch, \&c. The yellow tint of turmeric is changed to brown-red by alkalis, alkaline earths, subacetate of lead, and several metallic oxydes; for which reason, paper stained with it is employed as a chemical test.

Turmeric is employed by the wool-dyers for compound colors which require an admixture of yellow, as for cheap browns and olives. As a yellow dye, it is employed only upon silk. It is a very fugitive color. A yellow lake may be made by boiling turmeric powder with a solution of alum, and pouring the filtered decoction upon pounded chalk.

TURNSOLE. See Archil and Litmus.

TURQUOIS. See LAPIDARY.

TURPENTINE (Térébinthine, Fr.; Terpenthin, Germ.), is a substance whieh flowi 
out of incisions made in the stems of several species of pines. It has the consistence and gray-yellow color of honey. It has a smell which is not disagreeable to many persons, a warm, sharp, bitterish taste; dries into a solid in the air, with the evaporation of its volatile oil. It becomes quite fluid at a molerate elevation of temperature, and burns at a higher heat, with a bright but a very fuliginous flame. There are several varieties of turpentine.

1. Common turpentine, is extracted from incisions in the Pinus abies and Pinus silvestris. It has little smell; but a bitter burning taste. It consists of the volatile oil of turpentine to the amount of from 5 to 25 per cent.; and of rosin or colophony.

2. Venice turpentine, is extracted fiom the Pinus larix (larch), and the French turpentine from the Pinus maritima. The first comes from Styria, Hungary, the Tyrol, and Switzerland, and contains from 18 to 25 per cent. of oil; the second, from the south of France, and contains no mure than 12 per cent. of oil. The oil of all the turpentines is extracted by distilling them along with water. They dissolve in all proportions in alcohol, without leaving any residuum. They also combine with alkaline leys, and in general with the salifiable bases. Venice turpentine contains also succinic acid.

3. Turpentine of Strasbourg is extracted from the Pinus picea and Abies excelsa. It affords 33.5 per cent. of volatile oil, and some volatile or crystallizable resin, with extractive matter and succinic acid.

4. Turpentine of the Carpathian mountains, and of Hungary; the first of which comes from the Pinus cembra, and the second from the Pinus mugos. They resemble that of Strasbourg.

5. Turpentine of Canada, called Canada balsam, is extracted from the Pinus canadensis and balsamea. Its smell is much more agreeable than that of the preceding species.

6. Turpentine of Cyprus or Chio, is extracted from the Pistacea terebinthus. It has a yellow, greenish, or blue-green color. Its smell is more agreeable, and taste less acrid, than those of the preceding sorts.

Common turpentine imported into the United Kingdom, in 1836, 370,981 cwts. 1 qr. $26 \mathrm{lbs}$; in 1837, 415,023 cwts. 1 qr. $10 \mathrm{lbs}$. Retained for home consumption, in 1836, 341,693 cwts. $18 \mathrm{lbs}$; in 1837, 405,772 cwts. 2 qrs. 14 lbs. Duty received, in 1836, $£ 74,052$; in $1837, £ 87,918$.

TURPENTINE, OIL OF, sometimes called essence of turpentine. As found in commerce, it contains more or less rosin, from which it may be freed by re-distillation along with water. It is colorless, limpid, very fluid, and possessed of a very peculiar smell. Its specific gravity, when pure, is 0.870 ; that of the oil commonly sold in London, is 0.875 . It always reddens litmus paper, from containing a little succinic acid. According to Oppermann, the oil which has been repeatedly rectified over chloride of calcium, consists of 84.60 carbon, 11.735 hydrogen, and 3.67 oxygen. When oil of turpentine contains a little alcohol, it burns with a clnn flame; but otherwise it affords a very smoky flame. Chlorine inflames this oil; and muriatic acid converts it into a crystalline substance, like camphor. It is employed extensively in varnishes, paints, \&c., as also in medicine.

TUTENAG, is an alloy of copper and zinc.

TYPE, (Caractère, Fr.; Druckbuchstabe, Germ.) The first care of the letter-cutter is to prepare well-tempered steel punches, upon which he draws or marks the exact shape

1157

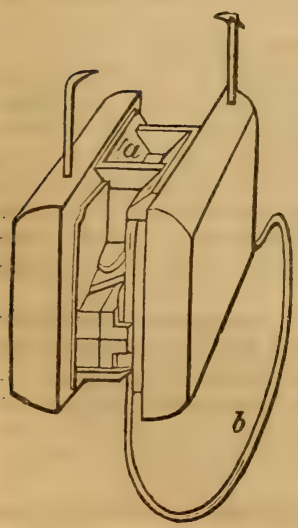
of the letter, with pen and ink if it be large, but with a smooth blunted point of a needle if it be small; and then, with a proper sized and shaped graver and sculptor, he digs or scoops out the metal between the strokes upon the face of the punch, leaving the marks untouched and prominent. He next works the outside with files till it be fit for the matrix. Punches are also made by hammering down the hollows, filing up the edges, and then hardening the soft steel. Before he proceeds to sink and justify the matrix, he provides a mould to justify them by, of which a good figure is shown in plate xv., Miscellany, figs. 2, 3, of Rees's Cyclopadia.

A matrix is a piece of brass or copper, about an inch and a half long, and thick in proportion to the size of the letter which it is to contain. In this metal the face of the letter intended to be cast is sunk, by striking it with the punch to a depth of about one eighth of an inch. The mould, fig. 1157 , in which the types are cast, is composed of two parts. The outer part is made of wood, the inner of steel. At the top it has a hopper-mouth $a$, into which the fused type-metal is poured. The interior cavity is as uniform as if it had been hollowed out of a single piece of steel; because each 
half, wh.ch forms two of the four sides of the letter, is exactly fitted to the other. The matrix is placed at the bottom of the mould, directly under the centre of the orifice, and is held in its position by a spring $b$. Every letier that is cast can be loosened from the matrix only by remoring the pressure on the spring.

A cood type-foundry is always provided with several furnaces, each surmounted with an iron pot containing the melted alloy, of 3 parts of lead and 1 of antimony. Into this pot the founder dips the very small iron ladle, to lift merely as much metal as will cast a single letter at a time. Having poured in the metal with his right hand, and returned the ladle to the melting-pot, the founder throws up his left hand, which holds the mould, abore his head, with a sudden jerk, supporting it with his right hand. It is this movement which forces the metal into all the interstices of the matrix; for without it, the metal, especially in the smaller moulds, would not be able to expel the air and reach the bottom. The pouring in the metal, the throwing up the mould, the unclosing it, removing the pressure of the spring, picking ont the cast letter, closing the mould again, and reapplying the spring to be ready for a new operation, are all performed with such astonishing rapidity and precision, that a skilful workman will turn out five hundred good letters in an hour, being at the rate of one every eighth part of a minute. A considerable piece of metal remains attached to the end of the type as it quits the mould. There are nicks upon the lower edge of the types, to enable the compositor to place them upright, without looking at them.

From the table of the caster, the heap of types turned out of his mould, is transferred from time to time to another table, by a boy, whose business it is to break off the superfluous metal, and that he does so rapidly as to clear from 2000 to 5000 types in an hour; a very remarkable despatch, since he must seize them by their edges, and not by their feeble flat sides. From the breaking-off boy, the types are taken to the rubber, a man who sits in the centre of the workshop with a grit-stone slab on a table before him, and having on the fore and middle finger of his right hand a piece of tarred leather, passes each broad side of the type smartly over the stone, turning it in the movement, and that so dexterously, as to be able to rub 2000 types in an hour.

From the rubber, the types are conveyed to a boy, who, with equal rapidity, sets them up in lines, in a long shallow frame, with their faces uppermost and nicks outwards. This frame, containing a full line, is put into the dresser's hands, who polishes them on each side, and turning them with their faces downwards, cuts a groove or channel in their bottom, to make them stand steadily on end. It is essential that each letter be perfectly symmetrical and square; the least inequality of their length would prevent them from making a fair impression; and were there the least obliquity in their sides, it would be quite impossible, when 200,000 single letters are combined, as in one side of the Times newspaper, that they could hold together as they require to do, when wedged up in the chases, as securely as if that side of type formed a solid plate of metal. Each letter is finally tied up in lines of convenient length, the proportionate numbers of each variety, small letters, points, large capitals, small capitals, and figures, being selected, when the fount of type is ready for delivery to the printer.

The sizes of types cast in this country vary from the smallest, called diamond, of which 205 lines are contained in a foot length, to those letters employed in placards, of which a single letter may be three or four inches high. The names of the different letters and their dimensions, or the number of lines which each occupies in a foot, are stated in the following table:-

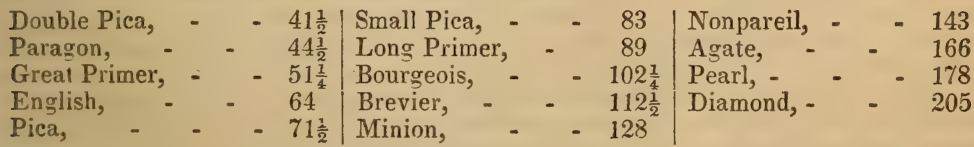

T. Aspinwall, Esq., the American Consul, obtained, in May, 1828, a patent for an improved method of casting printing types by means of a mechanical process, being a communication from a foreigner residing abroad. The machine is described, with six explanatory figures, in the second series of Newton's Journal, vol. v. page 212. The patentee does not claim, as his invention, any of the parts separately, but the general process and arrangement of machinery; more particularly the manner of suspending a swing table (upon which the working parts are mounted) out of the horizontal and perpendicular position; the mode of moving the table with the parts of the mould towards the melting pot; the manner of bringing the parts of the mould together, and keeping then closed during the operation of casting the types. Several other mechanical schemes have been proposed for founding types, but I have been informed by very competent judges, Messrs. Clowes, that none of them can compete in prastical utility with that dexterity and precision of handiwork, which I have often seen practiscd in their great printing establishment in Stamford street. 
U.

ULTRAMARINE (Outremer, Fr.; Ultramarins, Germ.), is a beautiful blue pigmen obtained from the variegated blun mineral, called lazulite (lapis lazuli), by the follow. ing process:-Grind the stone to fragments, rejecting all the colorless bits, calcine at a red heat, quench in water, and then grind to an impalpable powder along with water, in a paint-mill (see PAINTs, GRINDING of), or with a porphyry slab and muller. The paste, being dried, is to be rubbed to powder, and passed through a silk sieve. 100 parts of it are to be mixed with 40 of rosin, 20 of white wax, 25 of linseed oil, and 15 of Burgundy pitch, previously melted together. This resinous compound is to be poured hot into cold water; kneaded well first with two spatulas, then with the hands, and then formed into one or inore small rolls. Some persons prescribe leaving these pieces in the water during fifteen days, and then kneading them in it, whereby they give out the blue pigment, apparently because the ultramarine matter adheres less strongly than the gangue, or merely silicious matter of the mineral, to the resinous paste. MM. Clement and Desormes, who were the first to divine the true nature of this pigment, think that the soda contained in the lazulite, uniting with the oil and the rosin, forms a species of soap, which serves to wash out the coloring-matter. If it should not separate readily, water heated to about $150^{\circ} \mathrm{F}$. should be had recourse to. When the water is sufficiently charged with blue color, it is poured off and replaced by fresh water; and the kneading and change of water are repeated till the whole of the color is extracted. Others knead the mixed resinous mass under a slender stream of water, which runs off with the color into a large earthen pan. The first waters afford, by rest, a deposite of the finest ultramarine; the second, a somewhat inferior article, and so on. Each must be washed afterwards with several more waters, before they acquire the highest quality of tone; then dried separately, and freed from any adhering particles of the pitchy compound by digestion in alcohol. The remainder of the mass being melted with oil, and kneaded in water containing a little soda or potash, yields an inferior pigment, called ultramarine ashes. The best ultramarine is a splendid blue pigment, which works well with oil, and is not liable to change by time. Its price in Italy was five guineas the ounce, a few years ago, but it is now greatly reduced.

The blue color of lazulite had been always ascribed to iron, till MM. Clement and Desormes, by a most careful analysis, showed it to consist of-silica, 34; alumina, 33; sulphur, 3 ; soda, 22 ; and that the iron, carbonate of lime, \&c., were accidental ingredients, essential neither to the mineral, nor to the pigment made from it. By another analyst, the constituents are said to be-silica, 44 ; alumina, 35 ; and soda, 21 ; and by a third, potassa was found instead of soda, showing shades of difference in the composition of the stone.

Till a few years ago, every attempt failed to make ultramarine artificially. At length, in 1828, M. Guimet resolved the problem, guided by the analysis of MM. Clement and Desormes, and by an observation of M. Tassaert, that a blue substance like ultramarine was occasionally produced on the sandstone hearths of his reverberatory soda furnaces. Of M. Guimet's finest pioment I received a bottle several years ago, from my friend $M$. Merimée, Secretary of the Ecole de Beaux Arts, which has been found by artists little, if any, inferior to the lazulite ultramarine. M. Guimet sells it at sixty francs per pound French, - which is little more than two guineas the English pound. $\mathrm{He}$ has kept his process secret. But M. Gmelin, of Tübingen, has published a prescription for making it ; which consists in enclosing carefully in a Hessian crucible a mixture of two parts of sulphur, and one of dry carbonate of soda, heating them gradually to redness till the mass fuses, and then sprinkling into it by degrees another mixture, of silicate of soda, and aluminate of soda; the first containing seventy-two parts of silica, and the second seventy parts of alumina. The crucible must be exposed after this for an hour to the fire. The ultramarine will be formed by this time; only it contains a little sulphur, which ean be separated by means of water. M. Persoz, professor of chemistry at Strasbourg, has likewise succeeded in making an ultramarine, of perhaps still better quality than that of M. Guimet. Lastly, M. Robiquet has announced, that it is easy to form ultramarine, by heating to redness a proper mixture of kaolin (China clay), sulphur, and carbonate of soda. It would therefore appear, from the preceding details, that ultramarine may be regarded as a compound of silicate of alumina, silicate of soda, with sulphuret of sodium; and that to the reaction of the last constituent upon the former two, it owes its color.

UMBER, is a massive mineral ; fracture large and flat; conchoidal in the great, very fine earthy in the small; dull; color, liver, chestnut,-dark yellowish brown; opaque; does not soil, but writes; adheres strongly to the tongue, feels a little rough and meager, and is very soft; specific gravity $2 \cdot 2$. It occurs in beds with brown jasper in the Island of Cyprus, and is used by painters as a brown color, and to make varnish dry quickly 
URANIUM, is a rare metal, first discovered by Klaproth, in the black mineral called sechblende, found in a mine near Johann-Georgen-Stadt, in Saxony, and which is a sulphuret of uranium. A double phosphate of uranium and copper, called green uranite, and urun micu, occurs in Cornwall. It has been reduced to the metallic state by various devices, but it has hardly the appearance of metal to the naked eye, and from the rarity of its ores is not likely to be of any importance in the arts.

UR $\mathrm{AO}$, is the native name of a sesquicarbonate of soda found at the bottom of certain lakes in Mexico, especially to the north of Zacatecas, and in several other provinces; also in South America at Columbia, 48 English miles from Merida.

V.

VALONIA, is a kind of acorn, imported from the Levant and the Morea for the use of tanners, as the husk or cup contains abundance of tannin. The quantity imported for home consumption in 1836 , was $80,511 \mathrm{cwts.;} \mathrm{of} \mathrm{which} \mathrm{Turkey} \mathrm{furnished} \mathrm{58,724,}$ Italy and the Italian islands, 7209.

VANADIUM, is a metal discovered by Sefström, in 1830, in a Swedish iron, remarkable for its ductility, extracted from the iron mine of Jaberg, not far from Jönköping. Its name is derived from Vanadis, a Scandinavian idol. This metal has been found in the state of vanadic acid, in a lead ore from Zimapan, in Mexico. The finery cinders of the Jaberg iron contain more vanadium than the metal itself. It exists in it as vanactic acil. For the reduction of this acid to vanadium, see Berzelius's Traite de Chimie, vol. iv. p. 644. Vanadium is white, and when its surface is polished, it resembles silver or molsblenum more than any other metal. It combines with oxygen into two oxydes and an acid.

The vanadate of ammonia, mixed with infusion of nutgalls, forms a black liquid, which is the best writing-ink hitherto known. The quantity of the salt requisite is so small as to be of no importance when the vanadium comes to be more extensively extracted. The writing is perfectly black. The acids color it blue, but do not remove $i t$, as they do tannate of iron: the alkalis, diluted so far as not to injure the paper, do not dissolve it; and chlorine, which destroys the black color, does not, however, make the traces illegible, even when they are subsequently washed with a stream of water. It is perfectly fluent, and, being a chemical solution, stands in want of no viscid gum to suspend the color, like common ink. The influence of time upon it remains to be tried.

VANILI.A, is the oblong narrow pod of the Epidendron vanilla, Linn., of the natural family Orchidea, which grows in Mexico, Colombia, Peru, and on the banks of the Oronoco.

The best comes from the forests round the village of Zentila, in the intendancy of Oaxaca.

The vanilla plant is cultivated in Brazil, in the West Indies, and some other tropical countries, but does not produce fruit of such a delicious aroma as in Mexico. It clings like a parasite to the trunks of old trees, and sucks the moisture which their bark derives from the lichens, and other cryptogamia, but without drawing nourishment from the tree itself, like the ivy and mistletoe. The fruit is subcylindric, about 8 inches long, one-celled, siliquose, and pulpy within. It should be gathered before it is fully ripe.

When about 12000 of these pods are collected, they are strung like a garland by their lower end, as near as possible to the foot-stalk; the whole are plunged for an instant in boiling water to blanch them; they are then hung up in the open air, and exposed to the sun for a few hours. Next day they are lightly smeared with oil, by means of a feather, or the fingers; and are surrounded with oiled cotton, to prevent the valves from opening. As they become dry, on inverting their upper end, they discharge a viscid liquid from it, and they are pressed at several times with oiled fingers to promote its flow. The dried pods lose their appearance, grow brown, wrinkled, soft, and shrink into one fourth of their original size. In this state they are touched a second time with oil, but very sparingly; because, with too much oil, they would lose much of their delicious perfume. They are then packed for the market, in small bundles of 50 or 100 in each, enclosed in lead foil, or tight metallic cases. As it comes to us, vanilla is a capsular fruit, of the thickness of a swan's quill, straight, cylindrical, but somewhat flattened, truncated at the top, thinned off at the ends, glistening, wrinkled, furrowed lengthwise, flexible, from 5 to 10 inches long, and of a reddish-brown color. It contains a pulpy parenchyma, soft, unctuous, very brown, in which are imbedded black, brilliant, very small seeds. Its smell is ambrosiacal and aromatic; its taste hot, and 
rather sweetish. These properties seem to depend upon an essential oil, and also upow benzoic acid, which forms efflorescences upon the surface of the fruit. The pulpy part possesses alone the aromatic quality; the pericarpium has hardly any smell.

The kind most esteemed in France, is called leq vanilla; it is about 6 inches long, from $\frac{1}{4}$ to $\frac{1}{3}$ of an inch broad, narrowed at the two ends, and curved at the base; somewhat soft and viscid, of a dark-reddish color, and of a most delicious flavor, like that of Balsam of Peru. It is called vanilla givrées, when it is covered with efforescences of benzoic acid, after having been kept in a dry place, and in vessels not hermeticaliy closed.

The second sort, called vanilla simarona, or bastard, is a little smaller than the preceding, of a less deep brown hue, drier, less aromatic, destitute of efflorescence. It is said to be the produce of the wild plant, and is brought from St. Domingo.

A third sort, which comes from Brazil, is the vanillon, or large vanilla of the French market; the vanilla pamprona or bova of the Spaniards. Its length is from 5 to 6 inches; its breadth from one half to three quarters of an inch. It is brown, soft, viscid, almost always open, of a strong smell, but less agreeable than the leq. It is sometimes a little spoiled by an incipient fermentation. It is cured with sugar, and enclosed in tin-plate boxes, which contain from 20 to 60 pods.

Vanilla, as an aromatic, is much sought after by makers of chocolate, ices, and creams; by confectioners, perfumers, and li juorists, or distillers. It is difficultly reduced to fine particles; but it may be sufficiently attenuated by cutting it into small bits, and grinding these along with sugar. The odorous principle can, for some purposes, be extracted by alcohol. Their analysis by Bucholz is unsatisfactory, and refers obviously to the coarsest sort. Berzelius says that the efflorescences are not acid.

VAPOR (Vapeur, Fr.; Dampf, Germ.), is the state of elastıc or aëriform fluidity into which any substance, naturally solid or liquid at ordinary temperatures, may be converted by the agency of heat. See Evaporation.

VARNISH (Vernis, Fr.; Firniss, Germ.), is a solution of resinous matter, which is spread over the surface of any body, in order to give it a shining, transparent, and hard coat, capable of resisting, in a greater or less degree, the influences of air and moisture. Such a coat consists of the resinous parts of the solution, which remain in a thin layer upon the surface, after the liquid solvent, has either evaporated away, or has dried up. When large quantities of spirit varnish are to be made, a common still, mounted with its capital and worm, is the vessel employed for containing the materials, and it is placed in a steam or water bath. The capital should be provided with a stuffing-box, through which a stirring-rod may pass down to the bottom of the still, with a cross-piece at its lower end, and a handle or winch at its top. After heating the bath till the alcohol boils and begins to distil, the heat ought to be lowered, that the solution may continue to proceed in an equable manner, with as little evaporation of spirit as possible. The operation may be supposed to be complete when the rod can be easily turned round. The varnish must be passed through a silk sieve of proper fineness; then filtered through porous paper, or allowed to clear leisurely in stone jars. The alcohol which has come over should be added to the varnish, if the just proportions of the resins have been introduced at first. The following are reckoned good French recipes for varnishes :-

White spirit varnish.--Sandarach, 250 parts; mastic in tears, 64 ; elemi resin, 32 ; turpentine (Venice), 64 ; alcohol, of 85 per cent., 1000 parts by measure.

The turpentine is to be added after the resins are dissolved. This is a brilliant varnish, but not so hard as to bear polishing.

Varnish for the wood toys of Spa. Tender copal, 75 parts; mastic, 12.5 ; Venice turpentine, 6.5 ; alcohol, of 95 per cent., 100 parts by measure ; water ounces, for example, if the other parts be taken in ounces.

The alcohol must be first made to act upon the copal, with the aid of a little oil of lavender or camphor, if thought fit; and the solution being passed through a linen cloth, the mastic must be introduced. After it is dissolved, the Venice turpentine, previously melted in a water-bath, should be added; the lower the temperature at which these operations are carried on, the more beautiful whl the varnish be. This varnish ought to be very white, very drying, and capable of being smoothed with pumice-stone and polished.

Varnish for certain parts of carriages.-Sandarach, 190 parts; pale shellac, 95 ; rosin, 125 ; turpentine, 190 ; alcohol, at 85 per cent., 1000 parts by measure.

Varnish for cabinet-makers.-Pale shellac, 750 parts; mastic, 64 ; alcohol, of 90 per cent., 1000 parts by measure. The solution is made in the cold, with the aid of fiequent stirring. It is always muddy, and is employed without being filtered.

With the same resins and proof spirit a varnish is made for the bookbinders to do over their morocco leather.

The varnish of Watin, for gilded articles.-Gum lac, in grain, 125 parts ; gamboge, 125; dragon's blood, 125 ; annotto, 125 ; saffron, 32 . Each resin must be dissolved in 
1000 parts by measure, of alcohol of 90 per cent.; two separate tinctures must be male with lie dragon's blood and annotto, in 1000 parts of such alcohol; and a proper proportion wi each should be added to the varnish, according to the shade of golden color wanted.

For fixing engravings or lithographs upon wood, a varnish called mordant is used in France, which differs from others chiefly in containing more Venice turpentine, to make it sticky; it consists of-sandarach, 250 parts; mastic in tears, 64 ; rosin, 125 ; Venice turpentine, 250 ; alcohol, 1000 parts by measure.

Copal varnish.-Hard copal, 300 parts; drying linseed or nut oil, from 125 to 250 parts; oil of turpentine, 500 ; these three substances are to be put into three separate vessels; the copal is to be fused by a somewhat sudden application of heat ; the drying oil is to be heated to a temperature a little under ebullition, and is to be added by small portions at a time to the melted copal. When this combination is made, and the heat a little abated, the essence of turpentine, likewise previously heated, is to be introduced by degrees; some of the volatile oil will be dissipated at first; but more being added, the union will take place. Great care must be taken to prevent the turpentine vapor from catching fire, which might occasion serious accidents to the operator. When the varnish is made, and has cooled down to about the 130th degree of Fahr., it may be strained through a filter, to separate the impurities and undissolved copal.

Almost all varnish-maker's think it indispensable to combine the dryiny oil with the copal, before adding the oil of turpentine; but in this they are mistaken. Boiling oil of turpentine combines very readily with fused copal; and, in some cases, it would probablv be preferable to commence the operation with it, adding it in successive small quantities. Indeed, the whitest copal varnish can be made only in this way ; for if the drying oil have been heated to nearly its boiling point, it becomes colored, and darkens the varnish.

This varnish improves in clearness by keeping. Its consistence may be varied by varying the proportions of the ingredients, within moderate limits. Good varnish, applied in summer, should become so dry in 24 hours that the dust will not stick to it, nor receive an impression from the fingers. To render it sufficiently dry and hard for polishing, it must be subjected for several days to the heat of a stove.

Milk of wax is a valuable varnish, which may be prepared as follows:-Melt in a porcelain capsule a certain quantity of white wax, and add to it, while in fusion, an equal quantity of spirit of wine, of sp. grav. 0.830 ; stir the mixture, and pour it upon a large porphyry slab. The granular mass is to be converted into a paste by the muller, with the addition, from time to time, of a little alcohol; and as soon as it appears to be smooth and homogeneous, water is to be introduced in small quantities successively, to the amount of four times the weight of the wax. This emulsion is to be then passed through canvass, in order to separate such particles as may be imperfectly incorporated.

The milk of wax, thus prepared, may be spread with a smooth brush upon the surface of a painting, allowed to dry, and then fused by passing a hot iron (salamander) over its surface. When cold, it is to be rubbed with a linen cloth to bring out the lustre. It is to the unchangeable quality of an encaustic of this nature, that the ancient paintings upon the walls of Herculaneum and Pompeii owe their freshness at the present day.

The most recent practical account of the manufacture of varnishes, is that communicated by Mr. J. Wilson Neil to the Society of Arts, and published in the 49th volume of their "Transactions."

The building or shed wherein varnish is made, ought to be quite detached from any buildings whatever, to avoid accidents by fire. For general purposes, a building about 18 feet by 16 is sufficiently large for manufacturing 4000 gallons and upwards annually, provided there are other convenient buildings for the purpose of holding the utensils, and warehousing the necessary stock.

Procure a copper pan, made like a common washing-copper, which will contain from fifty to eighty gallons, as occasion may require; when wanted, set it upon the boiling furnace, and fill it up with linseed oil within five inches of the brim. Kindle a fire in the furnace underneath, and manage the fire so that the oil shall gradually, but slowly, increase in heat for the first two hours; then increase the heat to a gentle simmer; and if there is any scum on the surface, skim it off with a copper ladle, and put the skimming away. Let the oil boil gently for three hours longer; then introduce, by a little at a time, one quarter of an ounce of the best calcined magnesia for every sallon of oil, occasionally stirring the oil from the bottom. When the magnesia is all in, let the oil boil rather smartly for one hour; it will then be sufficient. Lay a cover over the oil, to keep out the dust while the fire is withdrawn and extinguished by water; next uncover the oil, and leave it till next morning; and then, while it is yet hot, ladle it into the carrying-jack, or let it out through the pipe and cock; carry it away, and deposite it in either a tir. or leaden cistern, for wooden vessels will not hold it; let it remain to settle for at least three months. The magnesia will absorb all the acid and mucilage from the oil, and 
fall to the bottom of the cistern, leaving the oil clear and transparent, and fit for use. Recollect, when the oil is taken out, not to disturb the bottoms, which are only fit for black paint.

\section{GENERAL OBSERVATIONS AND PRECAUTIONS TO BE OBSERVED IN MAKING VARNISHES.}

Set on the boiling-pot with 8 gallons of oil ; kindle the fire; then lay the fire in the gum-furnace; have as many $8 \mathrm{lb}$. hags of gum-copal all ready weighed up, as will be wanted; put one $8 \mathrm{lb}$. into the pot, put fire to the furnace, set on the gum-pot; in three minutes (if the fire is brisk) the gum will begin to fuse and give out its gas, steam, and acid; stir and divide the gum, and attend to the rising of it, as before directed. Eight pounds of copal take in general from sixteen to twenty minutes in fusing, from the beginning till it gets clear like oil, but the time depends very much on the heat of the fire, and the attention of the operator. During the first twelve minutes, while the gum is fusing, the assistant must look to the oil, and bring it to a smart simmer; for it ought to be weither too hot, nor yet too cold, but in appearance beginning to boil, which he is strictly to observe, and, when ready, to call out, "Bear a hand!" Then immediately both lay hold of a handle of the boiling-pot, lift it right up, so as to clear the plate, carry it out and place it on the ash-bed, the maker instantly returning to the gum-pot, while the assistant puts three copper ladlefuls of oil in to the copper pouring-jack, bringins it in and placing it on the iron plate at the back of the gum-pot to keep hot until wanted. When the maker finds the gum is nearly all completely fused, and that it will in a few minutes be ready for the oil, let him call out, "Ready oil !" The assistant is then to lift up the oil-jack with both hands; one under the bottom and the other on the handle, laying the spout over the edge of the pot, and wait until the maker calls out, "Oil !" The assistant is then to pour in the oil as before directed, and the boiling to be continued until the oil and gum become concentrated, and the mixture looks clear on the glass; the gum pot is now to be set upon the brick-stand until the assistant puts three more ladlefuls of hot oil into the pouring-jack, and three more into a spare tin for the third run of gum. There will remain in the boiling-pot still $3 \frac{1}{2}$ gallons of oil. Let the maker put his right hand down the handle of the gum-pot near to the side, with his left hand near the end of the handle, and with a firm grip lift the gum-pot, and deliberately lay the edge of the gum-pot over the edge of the boiling-pot until all its contents run into the boiling-pot. Let the gum-pot be held, with its bottom turned upwards, for a minute right over the boiling-pot. Observe, that whenever the maker is beginning to pour, the assistant stands ready with a thick piece of old carpet, without holes, and sufficiently large to cover the mouth of the boiling-pot should it catch fire during the pouring, which will sometimes happen if the gum-pot is very hot; should the gum-pot fire, it has only to be kept bottom upwards, and it will go out of itself; but if the boiling-pot should catch fire, during the pouring, let the assistant throw the piece of carpet quickly over the blazing pot, holding it down al! round the edges; in a few minutes it will be smothered. The moment the maker has emptied the gum-pot, he throws into it half a gallon of turpentine, and with the swish immediately washes it from top to bottom, and instantly empties it into the flat tin jack : he wipes the pot dry, and puts in 8 pounds more gum, and sets it upon the furnace; proceeding with this run exactly as with the last, and afterwards with the third run. There will then be 8 gallons of oil and 21 pounds of gum in the boiling-pot, under which keep up a brisk strong fire until a scum or froth rises and covers all the surface of the contents, when it will begin to rise rapidly. Observe, when it rises near the rivets of the handles, carry it from the fire, and set it on the ash-bed, stir it down-again, and scatter in the driers by a little at a time; leep stirring, and if the frothy head goes down, put it upon the furnace, and introduce gruduclly the remainder of the driers, alway's carrying out the pot when the froth rises near the rivets. In general, if the fre be good, all the time a pot requires to boil, from the time of the last gum being poised in, is about three and a half or four hours; but time is no criterion for a beginner to judge by, as it may vary according to the weather, the quality of the oil, the quality of the gum, the driers, or the heat of the fire, \&c.; therefore, about the third bour of boiling, try it on a bit of glass, and keep it boiling until it feels strong and stringy between the fingers; it is then boiled sufficiently to carry it on the ash-bed, and to be stirred down until it is cold enough to mix, which will depend much on the weather, varying from half an hour, in dry frosty weather, to one hour in warm summer weather. Previous to beginning to mix, have a sufficient quantity of turpentine ready, fill the pot, and pour in, stirring all the time at the top or surface, as before directed, unti] there are fifteen gallons, or five tins of oil of turpentine introduced, which will leave it quite thick enough if the gum is good, and has been well run; but if the gum was of a weak quality, and has not been well fused, there ought to be no more than twelve gallons of turpentine mixed, and even that may be too much. Therefore, when twelve gallons of turpentine have been introdused, have a flat saucer at hand, nd pour into it 
a portion of the varnish, and in two or three minutes it will show whether it is toc thicis; if not sufficiently thin, add a little more turpentine, and strain it off quichly. $A=$ soun as the whole is stored away, pour in the turpentine washings, with which the gum-pots have been washed, into the boiling-pot, and with the swish quickly wash down all the varnish from the pot sides; afterwards, with a large piece of woollen rag lipplin pumice-powder, wash and polish every part of the inside of the boiling-pot, perrioming the same operation on the ladle and stirters; rinse them with the turpentine washings, and at last rinse them altogether in clean turpentine, which also put to the washings; wipe dry with a clean soft rag the pot, ladle, stirrer, and funnels, and lay the sieve so as to be completely covered with turpentine, which will alwars beep it from gumming up. The foregoing directions concerning running the gum, and pouring in the oil, and also boiling off and mixing, are, with rery little difference, to be observed in tire maliing of all sorts of copal varnishes, except the differences of the quantities of oil, sun, sc., which will be found under the various descriptions by name, which will be hereafter described.

The choice of linseed oil is of peculiar consequence to the varnish-maker. Oil from fine full-grown ripe seed, when riewed in a vial, will appear limpid, pale, and brilliant; it is mellow and sweet to the taste, has very little smell, is specincally lighter than impure oil, and, when clarified, dries quickly and firmly, and does not materially change the color of the varnish when made, but appears limpid and brilliant.

Copul vurnishes for fine paintings, \&c.-Fuse 8 pounds of the very cleanest pale African gum copal, and, when completely run fluid, pour in two gallons of hot oil, old ineasure let it boil until it will string very strong; and in about fifteen minutes, or while it is Jet rery hot, pour in three gallons of turpentine, old measure, and got from the top of a cistern. Perhaps, during the mixing, a considerable quantity of the turpentine will escape; but the varnish will be so much the brighter, transparent, and fluid; and will work freer, dry more quickly, and be very solid and durable when dry. After the rarnish has been strained, if it is found too thick, before it is quite cold, heat as much turpentine, and mix with it, as will bring it to a proper consistence.

Cubinet varnish.-Fuse 7 pounds of very fine African gum copal, and pour in half a gallon of pale clarified oil; in three or four minutes after, if it feel stringy, take it out of doors, or into another building where there is no fire, and mix with it three gallons of turpentine; afterwards strain it, and put it aside for use. This, if properly boiled, will dry in ten minutes; but if too strongly boiled, will not mix at all with the turpentine; and sometimes, when boiled with the turpentine, will mix, and yet refuse to incorporate with any other varnish less boiled than itself: therefore it requires a nicety which is only to be learned from practice. This varnish is chiefly intended for the use of japanners, cabinet-painters, coach-painters, \&c.

Best borly copal varnish for coach-makers, \&c.-This is intended for the bodJ parts of coaches and other similar vehicles, intended for polishing.

Fuse $8 \mathrm{lbs}$. of fine African gum copal; add two gallons of clarified oil (old measure); boil it very slowly for four or five hours, until quite stringy; mix with three gallons and a half of iurpentine; strain off, and pour it into a cistern. As they are too slow in drying, coach-makers, painters, and varnish-makers, have introduced to two pots of the bre. ceding varnish, one made as follows :-

8 lbs. of fine pale gum animé;

2 gallons of clarified oil;

$3 \frac{1}{2}$ gallons of turpentine.

To be boiled four hours.

Quick dryıng body copal varnish, for coaches, \&c.

(1.) 8 lbs. of the best African copal;

2 gallons of clarified oil ;

$\frac{1}{2}$ lb. of dried sugar of lead;

$3 \frac{1}{2}$ gallons of turpentine.

Boiled till stringy, and mixed and strained.
(2.) 8 lbs. of fine gum animé;

2 gallons of clarified oil ;

$\frac{1}{4}$ lb. of white copperas;

$3 \frac{1}{2}$ gallons of turpentine.

Boiled as before.

To be mixed and strained while hot into the other pot. These two pots mixed togetnex. will dry in six hours in winter, and in four in summer; it is very useful for varnishing old work on dark colors, \&c.

\section{Best pale carriage varnish.}

(1.) 8 lbs. 2d sorted African copal;

$2 \frac{1}{2}$ gallons of clarified oil. Boiled till very stringy.

1. lb. of dried copperas;

$\frac{1}{1} \mathrm{lb}$. of lit'arge;

$5 \frac{1}{2}$ gallons of turpentine.

Strained \&.c.
(2.) $8 \mathrm{lbs}$. of $2 \mathrm{~d}$ sorted gum animé; $2 \frac{1}{2}$ gallons of clarified oil ;

$\frac{x}{4}$ lb. of dried sugar of lead;

$\frac{1}{4} 1 \mathrm{~b}$. of litharge ;

$5 \frac{1}{2}$ gallons of turpentine.

Mix this to the first while hot. 
This varnish will dry hard, if well boiled, in four hours in summer, and in six in win. ter. As the name denotes, it is intended for the varnishing of the wheels, springs, and carriage parts of coaches, chaises, \&c.; also, it is that description of varnish which is generally sold to and used by house-painters, decorators, \&c.; as from its drying quality and strong gloss, it suits their general purposes well.

\section{Second carriage varnish.}

$8 \mathrm{lbs}$. of $2 \mathrm{~d}$ sorted gum animé; $2 \frac{3}{4}$ gallons of fine clarified oil ; $5 \frac{1}{4}$ gallons of turpentine;

$\frac{1}{4} \mathrm{lb}$. of litharge;

8 lbs. of $2 \mathrm{~d}$ sorted gum animé ;

3 gallons of clarified oil ;

$\frac{1}{4} \mathrm{lb}$. of litharge ;

$\frac{1}{4} \mathrm{lb}$. of dried sugar of lead; $\frac{1}{4} 1 \mathrm{~b}$. of dried sugar of lead;

$\frac{1}{4}$ lb. of dried copperas.

Boiled and mixed as before.

Wainscot varnish.

$5 \frac{1}{2}$ gallons of turpentine.

To be well boiled until it strings very strong, and then mixed and strained.

Mahogany varnish is made either with the same proportions, with a little darker gum; otherwise it is wainscot varnish, with a small portion of gold size.

Black japun is made by putting into the set-pot 48 pounds of Naples, or any other of the foreign asphaltums, (except the Egyptian.) As soon as it is melted, pour in 10 gallons of raw linseed oil; keep a moderate fire, and fuse 8 pounds of dark gum animé in the gum-pot; mix it with 2 gallons of hot oil, and pour it into the set-pot. Afterwards fuse 10 pounds of dark or sea amber in the 10 gallon iron pot; keep stirring it while fusing; and whenever it appears to be overheated, and risirg too high in the pot, lift it from the fire for a few minutes. When it appears completely fused, mix in 2 gallons of hot oil, and pour the mixture into the set-pot; continue the boiling for 3 hours longer, and during that time introduce the same quantity of driers as before directed; dratw out the fire, and let it remain until morning; then boil it until it rolls hard, as before directed; leave it to cool, and afterwards mix with turpentine.

Pale amber varnish.-Fuse 6 pounds of fine picked very pale transparent amber in the gum-pot, and pour in 2 gallons of hot clarified oil. Boil it until it strings very strong. Mix with 4 gallons of turpentine. This will be as fine as body copal, will work very free, and flow well upon any work it is applied to ; it becomes very hard, and is the most durable of all varnishes; it is very excellent to mix in copal varnishes, to give them a hard and durable quality. Observe; amber varnish will always require a long time before it is ready for polishing.

Best Brunswick black.-In an iron pot, over a slow fire, boil 45 pounds of foreign asphaltum for at least 6 hours; and during the same time boil in another iron pot 6 gallons of oil which has been previously boiled. During the boiling of the 6 gallons, introduce 6 pounds of litharge gradually, and boil until it feels stringy between the fingers; then ladle or pour it into the pot containing the boiling asphalıum. Let the mixture boil until, upon trial, it will roll into hard pills; then let it cool, and mix it with 25 gallons of turpentine, or until it is of a proper consistence.

Iron-work black.-Put 48 pounds of foreign asphaltum into an iron pot, and boil for 4 hours. During the first 2 hours, introduce 7 pounds of red lead, 7 pounds of litharge, 3 pounds of dried copperas, and 10 gallons of boiled oil ; add 1 eight-pound run of dark gum, with 2 gallons of hot oil. After pouring the oil and gum, continue the boiling two hours, or until it will roll into hard pills like japan. When cool, thin it off with thirty gallons of turpentine, or until it is of a proper consistence. This varnish is intended for blacking the iron-work of coaches and other carriages, \&c.

A cheap Brunswick black.-Put 28 pounds of common black pitch, and 28 pounds of common asphaltum made from gas tar, into an iron pot; boil both for 8 or 10 hours, which will evaporate the gas and moisture; let it stand all night, and early next morning, as soon as it boils, put in 8 gallons of boiled oil; then introduce, gradually, 10 pounds of red lead, and 10 pounds of litharge, and boil for 3 hours, or until it will roll very hard. When ready for mixing, introduce 20 gallons of turpentine, or more, until of a proper consistence. This is intended for engineers, founders, ironmongers, \&c. ; it will dry in half an hour, or less, if properly boiled.

Axioms observed in the making of copul varnishes.-The more minutely the gum is run, or fused, the greater the quantity, and the stronger the produce. The more regular and longer the boiling of the oil and gum together is continued, the more fluid or free the varnish will extend on whatever it is applied to. When the mixture of oil and gum is too suddenly brought to string by too strong a heat, the varnish requires more than its just proportion of turpentine to thin it, whereby its oily and guniny quality is reduced, which renders it less durable; neither will it flow so well in laying on. The greater proportion of oil there is used in varnishes, the less they are liable to crack, because the tougher and sofier they are. By increasing the proportion of gum in varnishes, 
the th.cker will be the stratum, the firmer they will set solid, and the quicker they will dry. When varnishes are quite new made, and must be sent out for use before they are of suthicient age, they must always be left thicker than if they were to be kept the pioper time. Varnish rnade from African copal alone possesses the most elasticity and transparency. Tuo much driers in varnish render it opaque and unfit for delicate colors. Copperas does not combine with rarnish, but only hardens it. Sugar of lead does combine with rarnish. Turpentine improves by age; and varnish by being kept in a warm place. All copal or oil varnishes require age before they are used.

Concluding observations. - All body varnishes are intended and ought to have $1 \frac{1}{2} \mathrm{lbs}$. of gum to each gallon of varnish, when the varnish is strained off, and cold; but as the thinring $u p$, or yuantity of turpentine required to bring it to its proper consistence, depena's very much upon the degree of boiling the varnish has undergone, therefore, when the gum and oil have not been strongly boiled, it requires less turpentine for that purpose ; whereas, when the gum and oil are very strongly boiled together, a pot of 20 gallons will require perhaps 3 gallons above the regular proportionate quantity; and if mixing the turpentine is commenced too soon, and the pot not sufficiently cool, there will be frequently above $\&$ gallon and a half of turpentine lost by evaporation.

All carriage, wainscot, and mahogany varnish ought to have fully one pound of gum for each gallon, when strained and cold; and should one pot require more than its proportion of mirpentine, the following pot can easily be left not quite so strongly boiled; then it will require less turpentine to thin it up.

Gold sizes, whether pale or dark, ought to have fully half a pound of good gum copal to each gallon, when it is finished; and the best black japan, to have half a pound of good gum, or upwards, besides the quantity of asphaltum.

Fine mastic, or picture varnish.-Put 5 pounds of fine picked gum mastic into a new four-gallon tin bottle; get ready 2 pounds of glass, bruised as small as barley; wash it sereral times; afterwards dry it perfectly, and put it into the bottle with 2 gallons of turpentine that has settled some time; put a piece of soft leather under the bung; lay the tin on a sack upon the counter, table, or any thing that stands solid; begin to agitate the in, smartly rolling it bacliward and forward, causing the gum, glass, and turpentine, to work as if in a barrel churn for at least 4 hours, when the varnish may be emptied out into any thing sufficiently clean, and large enough to hold it. If the gum is not all dissolved, return the whole into the bottle, and agitate as before, until all the gum is dissolved; then strain it through fine thin muslin into a clean tin bottle: leave it uncorked, so that the air can get in, but no dust; let it stand for 9 months, at least, before it is used; for the lunger it is liept, the tougher it will be, and less liable to chill or bloom. To prevent mastic varnish from chilling, boil one quart of river sand with two ounces of pearl-ashes; afterwards wash the sand three or four times with hot water, straining it each time; put the sand on a soup-plate to dry, in an oven; and when it is of a good heat, pour half a pint of hot sand into each gallon of varnish, and shake it well for five minutes; it will soon settle, and carry down the moisture of the gum and turpentine, which is the general cause of mastic varnish chilling on paintings.

Common mastic varnish.- Put as much gum mastic, unpicked, into the gum-pot as may be required, and to every $2 \frac{3}{4}$ pounds of gum pour in 1 gallon of cold turpentine; set the pot over a rery moderate fire, and stir it with the stirrer; be careful, when the steam of the turpentine rises near the mouth of the pot, to cover it with the carpet, and carry it out of doors, as the vapor is very apt to catch fire. A few minutes' low heat will perfectly dissolve 8 pounds of gum, which will, with 4 gallons of turpentine, produce, when strained, $4 \frac{1}{2}$ gallons of varnish; to which add, while yet hot, 5 pints of pale turpentine varnish, which improves the body and hardness of the mastic varnish.

Crystal varmish, may he made either in the varnish-house, drawing-room, or parlor. Procure a bottle of Canada balsam, which can be had at any druggist's; draw out the cork, and set the bottle of balsam at a little distance from the fire, turning it round several tines, urtil the heat has thinned it; then have something that will hold as much as donble the quantity of balsam; carry the balsam from the fire, and, while fluid, mix it with the same quantity of good turpentine, and shake them together until they are well incorporated; in a few days the varnish is fit for use, particularly if it is poured into a half-gallon glass or stone bottle, and kept in a gentle warmth. This varnish is used for maps, prints, charts, drawings, paper ornaments, \&c.; and when made upon a larger scale, requires only warming the balsam to mix with the turpentine.

White hard spirit-of-uine varnish.-Put 5 pounds of gum sandarach into a four-gallon tin bottle, with 2 gallons of spirits of wine, 60 over proof, and agitate it until dissolved, exactly as directed for the best mastic varnish, recollecting, if washed glass is used, that it is convenient to dip the bottle containing the gum and spirits into a copperful of hot water every 10 minutes-the bottle to be immersed only 2 minutes at a time-which will greatly assist the dissolving of the gum; but, above all, be careful to keep a firm hold over the cork of the bottle, otherwise the rarefaction will drive the cork out with the 
force of a shot, and perhaps set fire to the place. The bottle, every time it is heated, ought to be carried away from the fire; the cork should be eased a little, to allow the rarefied air to escape; then driven tight, and the agitation continued in this manner until all the gum is properly dissolved; which is easily known by having an ernpty tin can to pour the varnish into, until near the last, which is to be poured into a gallon measure. If the gum is not all dissolved, return the whole into the four-gallon tin, and continue the agitation until it is ready to be strained, when every thing ought to be quite ready, and perfectly clean and dry, as oily tins, funnels, strainers, or any thing damp, or even cold weather, will chill and spoil the varnish. After it is strained off, put into the varnish one quart of very pale turpentine varnish, and shake and mix the two well together. Spirit varnishes should be kept well corked; they are fit to use the day after being made.

Brown hard spirit varnish, is made by putting into a bottle 3 pounds of gum sandarach, with 2 pounds of shellac, and ' 2 gallons of spirits of wine, 60 over proof; proceeding exactly as before directed for the white hard varnish, and agitating it when cold, which requires about 4 hours' time, without any danger of fire; whereas, making any spirit varnish by heat is always attended with danger. No spirit varnish ought to be made either near a fire or by candle light. When this brown hard is strained, add one quart of turpentine varnish, and shake and mix it well: next day it is fit for use.

The Chinese varnish, comes from a tree which grows in Cochin-China, China, and Siam. It forms the best of all varnishes.

Gold lacker.-Put into a clean four-gallon tin, 1 pound of ground turmeric, $1 \frac{1}{2}$ ounces of powdered gamboge, $3 \frac{1}{2}$ pounds of powdered gum sandarach, $\frac{3}{4}$ of a pound of shellac, and 2 gallons of spirits of wine. After being agitated, dissolved, and strained, add 1 pint of turpentine varnish, well mixed.

\section{Red spirit lacker.}

2 gallons of sperits of wine;

1 pound of dragon's blood;

3 pounds of Spejaish annotto;

$3 \frac{1}{4}$ pounds of gum sandarach;

2 pints of turpentine.

Made exactly as the yellow gold lacker.
Pale brass lacker.

2 gallons of spirits of wine;

3 ounces of Cape aloes, cut small;

1 pound of fine pale shellac;

1 ounce gamboge, cut small.

No turpentine varnish. Made exactly as before.

But observe, that those who make lackers, frequently want some paler, and some darker, and sometimes inclining more to the particular tint of certain of the component ingredients. Therefore, if a four-ounce vial of a strong solution of each ingredient be prepared, a lacker of any tint can be produced at any time.

Preparation of linseed oil for making varnishes.-Put 25 gallons of linseed oil into an iron or copper pot that will hold at least 30 gallons; put a fire under, and gradually increase the heat, so that the oil may only simmer, for 2 hours; during that time the greatest part of its moisture evaporates; if any scum arises on the surface, skim it off, and put that aside for inferior purposes. Then increase the fire gradually, and sprinkle in, by a little at a time, $3 \mathrm{lbs}$. of seale litharge, $3 \mathrm{lbs}$. of good red lead, and $2 \mathrm{lbs}$. of Turkey umber, all well dried and free from moisture. If any moist driers are added, they will cause the oil to tumefy; and, at the same time, darken it, causing it to look opaque and thick, ropy and clammy, and hindering it from drying and hardening in proper time; besides, it will lie on the working painting like a piece of bladder skin, and be very apt to rise in blisters. As soon as all the driers are added to the oil, keep quietly stirring the driers from the bottom of the pot; otherwise they will burn, which will cause the oil to blacken and thicken before it is boiled enough. Let the fire be so regulated that the oil shall only boil slowly for three hours from the time all the driers were added; if it then ceases to throw up any scum, and emits little or no smoke, it is necessary to test its temperature by a few quill tops or feathers. Dip a quill top in the oil every two minutes, for when the oil is boiled enough, the quill top will crackle or curl up quite burnt; if so, draw out the fire immediatcly, and let the oil remain in the pot at least from 10 to 24 hours, or longer if convenient, for the driers settle much sooner when the oil is $l \in f$ to cool in the pot, than when it is immediately taken out.

Poppy oil.-Into four pints of pure soft water, put two ounces of foreign white vitriol; warm the water in a clean copper pan, or glazed earthen jar, until the vitriol is dissolved; pour the mixture into a clean glass or stone bottle, large enough to contain three gallons; then add to the solution of vitriol one gallon and a half of poppy oil, cork and agitate the bottle regularly and smartly for at least two hours; then pour out the contents into a wide earthenware dish : leave it at rest for eight days, when the oil will be clear and brilliant on the surface, and may be taken off with a spoon or flat skimmer, and pus up in a glass bottle and exposed to the light, which in a few weeks renders the oil exceed. ingly limpid and colorless. 
Vut-oil, o: onl of walnuis, is extracted by expression; and that which is extracted without heat, is certainly the most pale, puite, and nutritive seasoning, and retains an exquisite taste of the fruit. That designed for the arts is of inferior quality, and is plentifully imported to us from France; the heat it undergoes in its torrefaction, previous to its expression, disposes it to dry more quickly than that expressed by the cold process; but, at the same time, the heat, though it frees it from its unctuous quality, gives it more color. When it has been extracted by the cold process, it may be prepared in the same way as directed for the poppy oil.

In the above article I have retained the workmen's names-gum, white vitriol, \&c.; instead of resin, sulphate of zinc, \&c.

VEINS (Filons, Fr.; Gïnge, Germ.), are the fissures or rents in rocks, which are filled with peculiar mineral substances, most commonly metallic ores.

VEIN STONES, or GANGITES, are the mineral substances which accompany, and fre. quently enclose, the metallic ores.

VELLUM, is a fine sort of PARCHMENT, which see.

VELVET (Velours, Fr.; Sammet, Germ.), a peculiar stuff, the nature of which is ex plained under Fustran and Textile Fabrics.

VENETIAN CHALK, is STEATITE.

VENUS, is the mythological name of copper.

VENTILATION, or the renewal of fresh air in stagnant places, is nowhere exhibited to such advantage as in the coal mines of Northumberland and Durham, where Mr. Buddle has carried well nigh to systematic perfection the plan of coursing the air through the winding galleries, originally contrived about the year 1760, by $\mathrm{Mr}$. James Spedding, of Workington, the ablest pitman of his day.* $\mathrm{He}$ converted the whole of the passages into air-pipes, so to speak, drew the current of air from the downcast pit, then traversed it up and down, and round about, through the several sheaths of the workings, so that no particular gallery was left without a current of air. $\mathrm{He}$ thereby succeeded in actually expelling the noxious gases from the mines; those demons, which in Germany, at no remote era, were wont to be combated by the priests with impotent exorcisms or pious frauds. Before $\mathrm{Mr}$. Buddle introduced his improvements, he has known the air to be led through a series of workings, thirty miles long, before it made its exit. There is in every coal mine an experienced corps, called wastemen, because they travel over the waste, or the exhausted regions, who can tell at once, by the whistling sound which the air makes at the crevices in certain partitions and doors, whether the ventilation be in good condition or not. They hear these stoppings begin to $\operatorname{sing}$ or call, as they say, whenever an interruption takes place in any point of the labyrinthian line. Another indication of something being wrong, is when the doors get so heavy, that the boys in attendance upon them find them difficult to shut or open. The instant such a defect is discovered by any one, he cries aloud, "Holloa, there is something wrong - the doors are calling !"

In Mr. Spedding's system, the whole of the return air came in one current to his rarefying furnace (see letter c, fig. 1158), whether it was at the explosive point or not. This distribution was often fraught with such danger, that a torrent of water had to be kept in readiness, under the name of the waterfall, to be let down to extinguish the fire in a moment. Many explosions at that time occurred, from the furnaces below, and also down through tubes from the furnaces above-ground.

About the year 180\%, Mr. Buddle had his attention intensely occupied with this most important object, and then devised his plan of a divided current, carrying that portion through the active furnace $\mathrm{c}, \mathrm{fig}$. 1158, and the portion of the air from the foul workings of the air which, descending in the downcast pit A, coursed through the clean workings, up the dumb furnace $D$, till it reached a certain elevation in $B$, the upcast pit, above the fireplace. The pitmen had a great aversion, however, at first, to adopt this plan, as they thought that the current of air, by being split, would lose its ventilating power; but they were, ere long, convinced by Mr. Buddle to the contrary. He divides the main current into two separate streams, at the bottom of the pit A, as shown by darts in the figure; the feathered ones, representing that part of the pit in which the course of the current of air is free from explosive mixture, or does not contain above one thirtieth of carbureted hydrogen, as indicated by its effect upon the flame of a candle. The naked darts denote the portions of the mine where the air, being charged to the firing point, is led off towards $\mathrm{D}$, the dumb furnace, which communicates with the hot upcast shaft, out of reach of the flame, and thence derives its power of draught. By suitable alterations in the stoppings (see the various transverse lines, and the crosses), any portion of the workings may, by the agency of the furnace, be laid out of, or brought within, the course of the vitiated current, at the pleasure of the skilful mine viewer; so that, if he fo and it necessary, he could confine, by proper arrangements if his furnace, all the vitiated current to a mere gas-pipe or drift, and direct it wholly :hrough the dumb fur-

* Mining engineers use the term good pitman, as admirals do good seaman, denote a proficient in kis celling. 
nace. During a practice of twenty years, Mr. Buddle has not met with any accident in: consequence of a defect in the stoppings preventing the complete division of the air. The engineer has it thus within his power to detach or insulate those portions of the mine in which there is a great exudation of gas, from the rest; and, indeed, he is continually making changes, borrowing and lending currents, so to speak; sometimes laying one division or panel upon the one air-course, and sometimes upon the other, just to suit the immediate emergency. As soon as any district has ceased to be dangerous, by ibe exhaustion of the gas-blowers, it is transferred from the foul to the pure air course, where gunpowder may be safely used, as also candles, instead of Davy's lamps, which give less light.

The quantity of air put down into the Wallsend colliery, at the time of the last dreadfu] iccident, 18 th June, 1835, was not less than 5000 cubic feet per minute, whence it has been justly inferred that the explosion was caused by the rashness of a wasteman carrying a light through a door into a foul drift.

Till the cutting out of the pillars commences (see the right end of the diagram), the ventilation of the several passages, boards, \&c., may be kept perfect, supposing the working extended no further than $a$, or $b$; because, as long as there are pillars standing,

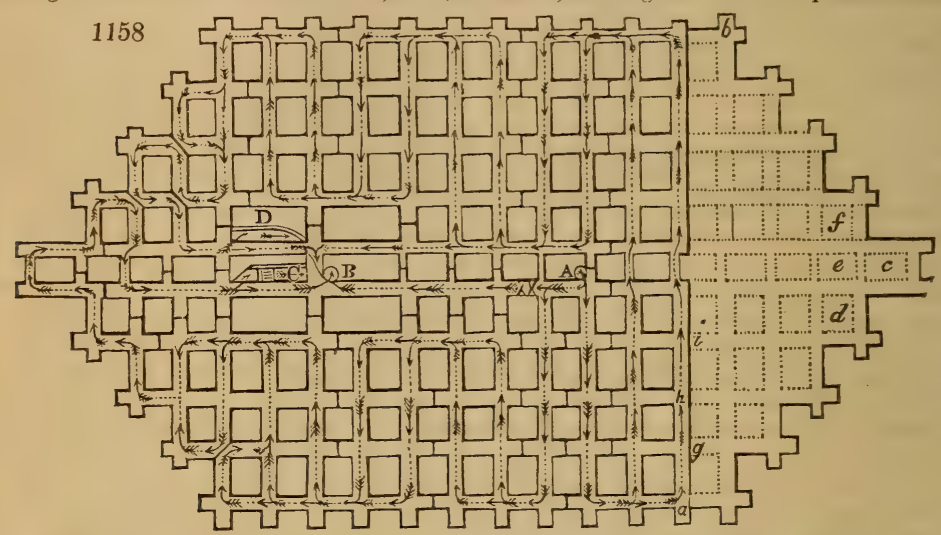

every passage may be converted into an air-conduit, for leading a current of air in any direction, either to $\mathrm{c}$, the burning, or $\mathrm{D}$, the dumb furnace. But the first pillar that is removed deranges the ventilation at that spot, and takes away the means of carrying the air into the further recess towards $c$. In taking out the pillars, the miners always work to windward, that is to say, against the stream of air; so that whatever gas may be evolved shall be immediately carried off from the people at work. When a range of pillars has been removed, as at $d, e, f$, no power remains of dislodging the gas from the section of the mine beyond $a, b$; and as the pillars are successively cut away to the left hand of the line $a, b$, the size of the goaf, or void, is increased. This vacuity is a true gas-holder, or reservoir, continually discharging itself at the points $g, h, i$, into the circulating current, to be carried off by the gas-pipe drift at the dumb furnace, but not to be suffered ever to come in contact with flame of any description. The next range of working, is the line of pillars to the left of $a, b$; the coal having been entirely cleared out of the space to the right, where the place of the pillars is marked by dotted lines. The roof in the waste soon falls down, and gets fractured up to the next sean of coal, called the yard-coal seam, which, abounding in gas, sends it down in large quantities, and keeps the immense gasometer, or goaf below, continually replenished. See STove.

VERATRINE, is a vegetable alkali, of a poisonous nature, extracted from the seeds of the Veratrum sabadilla, the roots of the Veratrum album, or white hellebore, and of Colchicum autumnale, or meadow saffron, in which plants it exists combined chiefly with gallic acid. It is obtained in the form of a white powder. It has an acrid, burning taste, but without any bitterness; it has no smell; but when snuffed into the nostrils, it excites violent and dangerous sneezing. It melts at a heat of $122^{\circ} \mathrm{F}$., and concretes, on cooling, into a transparent yellowish mass. It restores the blue color of reddened litmus paper. It is hardly soluble in water or ether, but abundantly in alcohol. It consists of-carbon $66 \cdot 75$, hydrogen 8.54 , nitrogen $5 \cdot 04$, and oxygen $19 \cdot 60$. Its saline compounds have an acrid and burning taste. Veratrine resembles strychnine and brucine, in its effects upon living bodies, producing tetanus and death in a moderate dose; notwithstanding which, it has been prescribed by some of our poison doctors, especially mixed with hog's lard, in the form of frictions on the forehead, for nervous maladies ; but seldom, I belitve, with any good effects. 
VERDIGRIS. (Vert-de-gris, Fr.; Grünspan, Germ.) The copper used in this manulicture, is formed into round sheets, from 20 to 25 inches diameter, by one twentyfowtin of an inch in thicliness. Each sheet is then divided into oblong squares, firom 4 to 6 inches in length, by 3 broad; and weighing about 4 ounces. They are separately beaten upon an anvil, to smooth their surfaces, to consolidate the metal, and to free it from scales. The refuse of the grapes, after the extraction of their juice, formerly thrown on to the lunghill, is now preserved for the purpose of making verdigris. It is put loosely into earthen vessels, which are usually 16 inches high, 14 in diameter at the widest part, and about 12 at the mouth. The vessels are then covered with lids, which are surrounded by straw mats. In this situation the materials soon become heated, and exhale an acid odor; the fermentation beginning at the bottom of the cask, and rradually rising till it actuate the whole mass. At the end of two or three days, the manufacturer removes the fermenting materials into other vessels, in order to check the prucess, lest putrefaction should ensue. The copper plates, if new, are now prepared, by rubbing them over with a linen cloth dipped in a solution of verdigris; and they are laid up alongside of one another to dry. If the plates are not subjected to this kind of preparation, they will become black, instead of green, by the first operation. When the plates are ready, and the materials in a fermenting state, one of them is put into the earthen vessel for 24 hours, in order to ascertain whether it be a proper period to pruceed to the remaining part of the process. If, at the end of this period, the plate be covered with a uniform green layer, concealing the whole copper, everything is right; b it if, on the contrary, liquid drops hang on the surface of the metal, the worknen say the plates are sweating, and conclude that the heat of the fermented mass has been inadequate; on which account another day is allowed to pass before making a similar trial. When the materials are finally found to be ready, the strata are formed in the following manner. The plates are laid on a horizontal wooden grating, fixed in the midlle of a vat, on whose bottom a pan full of burning charcoal is placed, which heats them to such a degree, that the women who manage this work are obliged to lay hold of them frequently with a cloth when they lift them out. They are in this state put into earthen vessels, in alternate strata with the fermented materials, the uppermost and undermost layers being composed of the expressed grapes. The vessels are covered with their straw mats, and left at rest. From 30 to 40 pounds of copper are put intc one vessel.

At the end of $10,12,15$, or 20 days the vessels are opened, to ascertain, by the materials having become white, if the operation be completed.

Detached glossy crystals will be perceived on the surface of the plates; in which case the grapes are thrown away, and the plates are placed upright in a corner of the verdigris cellar, one against the other, upon pieces of wood laid on the ground. At the end of two or three days they are moistened by dipping in a vessel of water, after which they are replaced in their former situation, where they remain seven or eight days, and are then subjected to momentary immersion, as before. This alternate moistening and exposure to air is performed six or eight times, at regular intervals of about a week. As these plates are sometimes dipped into damaged wine, the workmen term these immersions, one wine, two wines, \&c.

By this treatment, the plates swell, become green, and covered with a stratum of verdigris, which is readily scraped off with a knife. At each operation every vessel yields from ñve to six pounds of verdigris, in a fresh or humid state; which is sold to wholesale dealers, who dry it for exportation. For this purpose, they knead the paste in wooden troughs, and then transfer it to leathern bags, a foot and a half long, and ten inches in diameter. These bags are exposed to the sun and air till the verdigris has attained a sufficient degree of hardness. It loses about half its weight in this operation; and it is said to be knife-proof, when this instrument, plunged through the leathern bag, cannot penetrate the loaf of verdigris.

The manufacture of verdigris at Montpellier is altogether domestic. In most wine farm-houses there is a verdigris cellar; and its principal operations are conducted by the fcmales of the family. They consider the forming the strata, and scraping off the verdigris, the most troublesome part. Chaptal says that this mode of making verdigris would admit of some improvements; for example, the acetification requires a warmer temperature than what usually arises in the earthen vessels; and the plates, when set aside to generate the coat of verdigris, require a different degree of heat and moisture from that requisite for the other operations.

Verdigris is a mixture of the crystallized acetate of copper and the sub-acetate, in varying proportions. According to Vauquelin's researches, there are three compounds of oxyde of copper and acetic acid; 1. a subacetate, insoluble in water, but decomposing in that Huid, at common temperatures changing into peroxyde and acetate; 2 . a neutral acetate, the solution of which is not altered at common temperatures, but is decomposed by ebullition, becoming peroxyde and superacetale; and, 3 superacetate, which ip 
solution is not decomposed, either at common temperatures or at the boiling point, and which cannot be obtained in crystals, except by slow spontaneous evaporation, in air or in vacuo. The first salt, in the dry state, contains 66.51 of oxyde; the second, $44 \cdot 44$; and the third, $33 \cdot 34$.

Mr. Phillips has given the following analyses of French and English verdigris; Annuls of Philosophy, No. 21.-

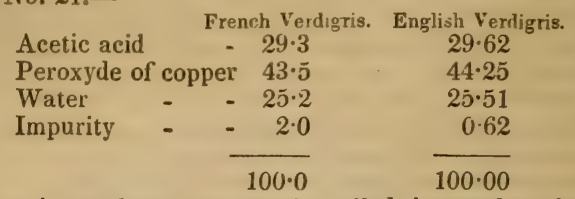

Distilled verdigris, as it was long erroneously called, is merely a binacetate or superacetate of copper, made by dissolving, in a copper kettle, one part of verdigris in two of distilled vinegar; aiding the mutual action by slight heat and agitation with a wooden spatula. When the liquor has taken its utmost depth of color, it is allowed to settle, and the ciear portion is decanted off into well-glazed earthen vessels. Fresh vinegar is poured on the residuum, and if its color does not become deep enough, more verdigris is added. The clear and saturated solution is then slowly evaporated, in a vessel kept uniformly filled, till it acquires the consistence of sirup, and shows a pellicle on its surface; when it is transferred into glazed earthen pans, called oulas in the country. In each of these dishes, two or three sticks are placed, about a foot long, cleft till within two inches of their upper end, and having the base of the cleft kept asunder by a bit of wood. This kind of pyramid is suspended by its summit in the liquid. All these vessels are transported into crystallizing rooms, moderately heated with a stove, and left in the same state for 15 days, taking care to maintain a uniform temperature. Thus are obtained very fine groups of crystals of acetate of copper, clustered round the wooden rods; on which they are dried, taken off, and sent into the market. They are distinctly rhomboidal in form, and of a lively deep blue color. Each cluster of crystals weighs from five to six pounds; and, in general, their total weight is equal to about one third of the verdigris employed.

The crystallized binacetate of commerce consists, by my analysis, of-acetic acid, 52 ; oxyde of copper, 39.6 ; water, 8.4 , in 100 . I have prepared crystals which contain no water. There is a triple acetate of copper and lime, which resembles distilled rerdigris in color. It was manufactured pretty extensively in Scotland some years ago, and fetched a high price, till I published an analysis of it in the Edinburgh Philosophical Journal. It is much inferior, for all uses in the arts, to the proper binacetate.

VERDITER, or BLUE VERDITER. This is a precipitate of oxyde of copper with lime, made by adding that earth, in its purest state, to the solution of nitrate of copper, obtained in quantities by the refiners, in parting gold and silver from copper by nitric acid. The cupreous precipitate must be triturated with lime, after it is nearly dry, to bring out the fine velvety blue color. The process is delicate, and readily misgives in unskilful hands.

The cendres bleues en pâte of the French, though analogous, are in some respects a different preparation. To make it, dissolve sulphate of copper in hot water, in such proportions that the liquid may have a density of $1 \cdot 3$. Take 240 pound measures of this solution, and divide it equally into 4 open-headed casks; add to each of these 45 pound measures of a boiling-hot solution of muriate of lime, of specific gravity, $1 \cdot 31 \%$, whereby a double decomposition will ensue; with the formation of muriate of copper and sulphate of lime, which precipitates. It is of consequence to work the materials well together at the moment of mixture, to prevent the precipitate agglomerating in unequal masses. After learing it to settle for 12 hours, a small quantity of the clear liquor may be examined, to see whether the just proportions of the two salts have been employed, which is done by adding either sulphate of copper or muriate of lime. Should either cause much precipitation, some of the other must be poured in till the equivalent decomposition be accomplished; though less harm results from an excess of sulphate of copper than of muriate of lime.

The muriate of copper is to be decanted from the subsided gypsum, which must be drained and washed in a filter; and these blue liquors are to be added to the stronger: and the whole distributed, as before, into 4 casks; composing in all 670 pound measures of a green liquor, of $1 \cdot 151$ specific gravity.

Meanwhile, a magma of lime is to be prepared as follows:-100 pounds of quicklime are to be mixed up with 300 pounds of water, and the mixture is to be passed through a wire-gauze sieve, to separate the sandy and stony particles, and then to be ground in a proper mill to an impalpable paste. About 70 or 80 pounds of this mixture (the beauty of the color is inversely as the quantity of lime) are to be distributed 
in equal portions between the four casks, strongly stirring all the time with a wooden spatula. It is then left to settle, and the limpid liquor is tested by ammonia, which ought to occasion only a faint blue tinge; but if the color be deep blue, more of the lime paste must be added. The precipitate is now to be washed by decantation, employing for this purpose the weak washings of a former operation; and it is lastly to be Irained and washed on a cloth filter. The proportions of material prescribed above, furnish from 500 to 540 pounds of green paste.

Before making further use of this paste, the quantity of water present in it must be determined by drying 100 or 200 grains. If it contain 27 per cent. of dry matter, 12 pounds of it may be put into a wooden bucket (and more or less in the ratio of 12 to 27 per cent.) capable of containing $17 \frac{1}{2}$ pints; a pound (measure) of the lime paste is then to be rapidly mixed into it; immediately afterwards, a pint and a quarter of a watery solution of the pearlash of commerce, of spec. grav. 1.114, previously prepared; and the whole mixture is to be well stirred, and immediately transferred to a color-mill. The quicker this is done, the more beautiful is the shade.

On the other hand, two solutions must have been previously made ready, one of salammoniac ( $4 \mathrm{oz}$. troy dissolved in $3 \frac{1}{2}$ pints of water), and another of sulphate of copper ( $8 \mathrm{oz}$. troy dissolved in $3 \frac{1}{2}$ pints of water).

When the paste has come entirely through the mill, it is to be quickly put into a jar, and the two preceding solutions are to be simultaneously poured into it; when a cork is to be inserted, and the jar is to be powerfully agitated. The cork must now be secured with a fat lute. At the end of four days this jar and three of its fellows are to be emptied into a large hogshead nearly full of clear water, and stirred well with a paddle. After repose, the supernatant liquid is run off; when it is filled up again with water, and elutriated several times in succession, till the liquid no longer tinges turmeric paper brown. The deposite may be then drained on a cloth filter. The pigment is sold in the state of a paste; and is used for painting, or printing paper-hangings for the

walls of apartments.

The above prescribed proportions furnish the superfine blue paste: for the second quality, one half more quicklime paste is used; and for the third, double of the lime and tal ammoniac; but the mode of preparation is in every case the same.

This paste may be dried into a blue powder, or into crayons for painters, by exposing it on white deals to a very gentle heat in a shady place. This is called cendres bleues in pierre.

VERDITER, or BREMEN GREEN. This pigment is a light powder, like magnesia, having a blue or bluish green color. The first is most esteemed. When worked up with oil or glue, it resists the air very well; but when touched with lime, it is easily affected, provided it has not been long and carefully dried. A strong heat deprives it of its lustre, and gives it a brown or blackish-green tint.

The following is, according to M. J. G. Gentele, the process of fabrication in Bremen, Cassel, Eisenach, Minden, \&c. :-

a. $225 \mathrm{lbs}$. of sea salt, and $222 \mathrm{lbs}$. of blue vitriol, both free from iron, are mixed in the dry state, then reduced between mill-stones with water to a thick homogeneous paste.

b. $225 \mathrm{lbs}$. of plates of old copper are cut by scissors into bits of an inch square, then thrown and agitated in a wooden tub containing two lbs. of sulphuric acid, diluted with a sufficient quantity of water, for the purpose of separating the impurities; they are afterwards washed with pure water in casks made to revolve upon their axes.

$c$. The bits of copper being placed in oxydation-chests, along with the magma of common salt and blue vitriol previously prepared in strata of half an inch thick, they are left for some time to their mutual reaction. The above chests are made of oaken planks joined without iron nails, and set aside in a cellar, or other place of moderate temperature.

The saline mixture, which is partially converted into sulphate of soda and chloride of copper, absorbs oxygen from the air, whereby the metallic copper passes into a hydrated oxyde, with a rapidity proportioned to the extent of the surfaces exposed to the atmosphere. In order to increase this exposure, during the three months that the process requires, the whole mass must be turned over once every week, with a copper shovel, transferring it into an empty chest alongside, and then back into the former one.

At the end of three months, the corroded copper scales must be picked out, and the saline particles separated from the slimy oxyde with the help of as little water as possible.

d. This oxydized schlam, or mud, is filtered, then thrown, by means of a bucket containing 30 pounds, into a tub, where it is carefully divided or comminuted.

e. For every six pailfuls of schlam thus thrown into the large tub, 12 pounds of muriatic acid, at $15^{\circ}$ Baumé, are to be added; the mixture is to be stirred, and then left at rest for 24 or 36 hours. 
$f$. Into another tub, called the blue back, there is to be introduced, in like manner for every six pailfuls of the acidified schlam, 15 similar pailfuls of a solution of colorless clear caustic alkali, at $19^{\circ}$ Baumé.

$\mathrm{g}$. When the back (e) has remained long enough at rest, there is to be poured into it a pail of pure water for every pail of schlam.

$h$. When all is thus prepared, the set of workmen who are to empty the back $(e)$, and those who are to stir $(f)$, must be placed alongside of each. The first set transfer the schlum rapidly into the latter back; where the second set mix and agitate it all the time requisite to convert the mass into a consistent state, and then leave it at rest from 36 to 48 hours.

The whole mass is to be now washed; with which view it is to be stirred about with the affusion of water, allowed to settle, and the supernatant liquor is drawn off. This process is to be repeated till no more traces of potash remain among the blue. The deposite must be then thrown upon a filter, where it is to be kept moist, and exposed freely to the air. The pigment is now squeezed in the filter-bags, cut into bits, and dried in the atmosphere, or at a temperature not exceeding $78^{\circ} \mathrm{Fahr}$. It is only after the most complete desiccation that the color acquires its greatest lustre.

VERMICELLI, is a paste of wheat flour, drawn out and dried in slender cylinders, more or less tortuous, like worms, whence the Italian name. The gruau of the French is wheat coarsely ground, so as to free it from the husk; the hardest and whitest part, being separated by sifting, is preferred for making the finest bread. When this gruau is a little more ground, and the dust separated from it by the bolting-machine, the granular substance called semoule is obtained, which is the basis of the best pastes. The softest and purest water is said to be necessary for making the most plastic vermicelli dough; 12 pounds of it being usually added to 50 pounds of semoule. It is better to add more semoule to the water, than water to the semoule, in the act of kneading. The water should be hot, and the dough briskly worked while still warm. The Italians pile one piece of this dough upon another, and then tread it well with their feet for two or three minutes. They afterwards work it for two hours with a powerful rolling-pin, a bar of wood from 10 to 12 feet long, larger at the one end than the other, having a sharp cutting edge at the extremity, attached to the large kneadingtrough.

When the dough is properly prepared, it is reduced to thin ribands, cylinders, or tubes, to form vermicelli and macaroni of different kinds. This operation is performed by means of a powerful press. This is vertical, and the iron plate or follower carried by the end of the screw fits exactly into a cast-iron cylinder, called the bell, like a sausagemachine, of which the bottom is perforated with small holes, of the shape and size intended for the vermicelli. The bell being filled, and warmed with a charcoal fire to thin the dough into a paste, this is forced slowly through the holes, and is immediately cooled and dried by a fanner as it protrudes. When the threads or fillets have acquired the length of a foot, they are grasped by the hand, broken off, and twisted, while still flexible, into any desired shape upon a piece of paper.

The macaroni requires to be made of a less compact dough than the vermicelli. The former is forced through the perforated bottom, usually in fillets, which are afterwards formed into tubes by joining their edges together before they have had time to become dry. The lazagnes are macaroni left in the fillet or riband shape.

VERMILION, or Cinnabar, is a compound of mercury and sulphur in the nroportion of 100 pais of the former to 16 of the latter, which occurs in nature as a common ore of quicksilver, and is prepared by the chemist as a pigment, under the name of Vermilion. It is, properly speaking, a bisulphuret of mercury. This artificial compound being extensively employed, on account of the beauty of its color, in painting, for making red sealing-wax, and other purposes, is the object of an important manufacture. When vermilion is prepared by means of sublimation, it concretes in masses of considerable thickness, concave on one side, convex on the other, of a needle-form texture; brownish-red in the lump, but when reduced to powder, of a lively red color. On exposure to a moderate heat, it evaporates without leaving a residuum, if it be not contaminated with red lead; and at a higher heat, it takes fire, and burns entirely away, with a blue flame.

Holland long kept a monopoly of the manufacture of vermilion, from being alone in possession of the art of giving it a fine flame color. Meanwhile the French chemists examined this product with great care, under an idea that the failure of other nations to rival the Dutch arose from ignorance of its true composition; some, with Berthollet, imagined that it contained a little hydrogen; and others, with Fourcroy, believed that the mercury contained in it was oxydized; but, eventually, Seguin proved that both of these opinions were erroneous; having ascertained, on the one hand, that no hydrogenous matter was given out in the decomposition of cinnabar, and on the other that 
sulphur and mercury, by combining, were transformed into the red sulphuret in close vessels, without the access of any oxygen whatever. It was likewise supposed that the solution of the problem might be found in the difference of composition between the red and black sulphurets of mercury; and many conjectures were made with this view, the whole of which were refuted by Seguin. He demonstrated, in fact, that a mere change of temperature was sufficient to convert the one sulphuret into the other, without occasioning any variation in the proportion of the two elements. Cinnabar, moderately heated in a glass tube, is convertible into ethiops, which in its turn is changed intu cinnabar by exposing the tube to a higher temperature; and thence he was led to con clude that the difference between these two sulphurets was owing principally to the state of the combination of the constituents. It would seem to result, from all these researches, that cinnabar is only an intimate compound of pure sulphur and mercury, in the proportions pointed out by analysis; and it is therefore reasonable to conclude, that in order to make fine vermilion, it should be sufficient to effect the union of its elements at a high enough temperature, and to exclude the influence of all foreign matters; but, notwithstanding these discoveries, the art of making good vermilion is nearly as much a mystery as ever. M. Seguin, indeed, announced in his Memoirs, that he had succeeded in obtaining, in his laboratory, as good a cinnabar as that of Holland, and at a remunerative price; but whatever truth may be in this assertion, or however much the author may have been excited by the love of honor and profit, no manufacture on the great scale sprung up under his auspices. France is still as tributary as ever to foreign nations for this chemical product. At an exposition some years ago, indeed, a sample of good French vermilion was brought forward to prove that the problem was nearly solved; but that it is not so completely, may be inferred from the silence on this subject in M. Dupin's report of the last exposition, in 1834, where we see so many chemical trifies honored with eulogiums and medals by the judges of the show. The English vermilion is now most highly prized by the French manufacturers of sealing-wax.

M. Tuckert, apothecary of the Dutch court, published, long ago, in the Annales de Chimie, vol. iv., the best account we yet have of the manufacture of vermilion in Holland; one which has been since verified by M. Payssé, who saw the process practised on the great scale with success.

"The establishment in which I saw, several times, the fabrication of sublimed sulphuret of mercury," says M. Tuckert, "was that of Mr. Brand, at Amsterdam, beyond the gate of Utrecht; it is one of the most considerable in Holland, producing annually, from three furnaces, by means of four workmen, 48,000 pounds of cinnabar, besides other mercurial preparations. The following process is pursued here :-

"The ethiops is first prepared by mixing together 150 pounds of sulphur, with 1080 pounds of pure mercury, and exposing this mixture to a moderate heat in a flat polished iron pot, one foot deep, and two feet and a half in diameter. It never takes fire, provided the workman understands his business. The black sulphuret, thus prepared, is ground, to facilitate the filling with it of small earthen bottles capable of holding about 24 ounces of water; from 30 to 40 of which bottles are filled beforehand, to be ready when wanted.

"Three great subliming pots or vessels, made of very pure clay and sand, have been previously coated over with a proper lute, and allowed to dry slowly. These pots are set upon three furnaces bound with iron hoops, and they are covered with a kind of iron dome. The furnaces are constructed so that the flame may freely circulate and play upon the pots, over two thirds of their height.

"The subliming vessels having been set in their places, a moderate fire is kindled in the evening, which is gradually augmented till the pots become red. A bottle of the black sulphuret is then poured into the first in the series, next into the second and third, in succession; but eventually, two, three, or even more, bottles may be emptied in at once; this circumstance depends on the stronger or weaker combustion of the sulphuret of mercury thus projected. After its introduction, the flame rises 4 and sometimes 6 feet high; when it has diminished a little, the vessels are covered with a plate of iron, a foot square, and an inch and a half thick, made to fit perfectly close. In this manner, the whole materials which have been prepared are introdnced, in the course of 34 hours, into the three pots; being for each pot 360 pounds of mercury, and 50 of sulphur ; in all, 410 pounds."

The degree of firing is judged of, from time to time, by lifting off the cover; for if the flame rise several feet above the mouth of the pot, the heat is too great; if it be hardly visible, the heat is too low. The proper criterion being a vigorous flame playing a few inches above the vessel. In the last of the 36 hours' process, the mass should be dexterously stirred up every 15 or 20 minutes, to quicken the sublimation. The subliming pots are then allowed to cool, and broken to pieces in order to collect all the vermilion incrusted within them; and which usually amounts to 400 lbs., being a loss of 
only 60 on each vessel. The lumps are to be ground along with water between horizonta, stones, elutriated, passed through sieves, and dried. It is said that the rich tone of the Chinese vermilion may be imitated by adding to the materials for subliming one per cent. of sulphuret of antimony, and by digesting the ground article first in a solution of sulphuret of potassa, and, finally, in diluted muriatic acid.

The humid process of Kirchoff has of late years been so much improved, as to furnish a vermilion quite equal in brilliancy to the Chinese. The following process has been recommended. Mercury is triturated for several hours with sulphur, in the cold, till a perfect ethiops is formed; potash ley is then added, and the trituration is continued for some time. The mixture is now heated in iron vessels, with constant stirring at first, but afterwards only from time to time. The temperature must be kept up as steadily as possible at $130^{\circ}$ Fahr., adding fresh supplies of water as it evaporates. When the mixture which was black, becomes, at the end of some hours, brown-red, the greatest caution is requisite, to prevent the temperature from being raised above $114^{\circ}$, and to preserve the mixture quite liquid, while the compound of sulpnur and mercury should always be pulverulent. The color becomes red, and brightens in its hue, often with surprising rapidity. When the tint is nearly fine, the process should be continued at a gentler heat, during some hours. Finally, the vermilion is to be elutriated, in order to separate any particles of running mercury. The three ingredients should be very pure. The proportion of product varies with that of the constituents, as we see from the following results of experiments, in which 300 parts of mercury were always em ployed, and from 400 to 450 of water :-

$\begin{array}{ccc}\text { Sulphur. } & \text { Potash. } & \text { Vermilion obtained. } \\ 114 & 75 & 330 \\ 115 & 75 & 331 \\ 120 & 120 & 321 \\ 150 & 152 & 382 \\ 120 & 180 & 245 \\ 100 & 180 & 244 \\ 60 & 180 & 142\end{array}$

The first proportions are therefore the most advantageous; the last, which are those of M. Kirchoff himseif, are not so good.

Brunner found that 300 parts of quicksilver, 114 of sulphur, 75 of caustic potassa, and from 400 to 450 of water, form very suitable proportions for the moist process; that the best temperature was $113^{\circ} \mathrm{F}$.; and that $122^{\circ}$ was the highest limit of heat compatible with the production of a fine color.

The theory of this process is by no means clear. We may suppose that a sulphuret of potassium and mercury is first formed, which is eventually destroyed, in proportion as the oxygen of the air acts upon the sulphuret of potassium itself. There may also be produced some hyposulphite of mercury, which, under the same influence, would be trans. formed into sulphuret of mercury and sulphate of potash.

Sulphuret of potassium and mercury furnish also vermilion, but it is not beautiful. Red oxyde of mereury, calomel, turbith mineral, and the soluble mercury of Hahnemann, treated with the sulphuret of potassium, or the hydrosulphuret of ammonia, are all capable of giving birth to vermilion by the humid way.

The vermilion of commerce is often adulterated with red lead, brickdust, dragon's blood, and realgar. The first two, not being volatile, remain when the vermilion is heated to its subliming point; the third gives a red tincture to alcohol; the fourth exhales its peculiar garlic smell with heat; and when calcined in a crucible with carbonate of soda, and nitre in excess, affords arsenic acid, which may be detected by the usual chemical tests.

VINEGAR MANUFACTORY, BY MALT. Annual produce, 100,000 gallons.

$$
\text { Expenses for one month. } \quad \& \text { s. } d \text {. }
$$

Cost of material and fuel for 8,333 gallons, at $8 \frac{3}{4} d$. _ _ $\quad-303162$

Wages to 8 workmen, at 25s. per week - - - $\quad 4000$

Salaries to clerks, manager, and traveller - _ _ - $\quad-\quad 8368$

Travelling expenses - - - - - - - $\quad-3000$

Three horses' keep - - - - - - - - $\quad$ - 7100

Rent and taxes - - - - - - - -2500

$£ 4891210$

Expenses for 5 months, at 489 l. 12s 10d. - - - - $-\overline{244842}$

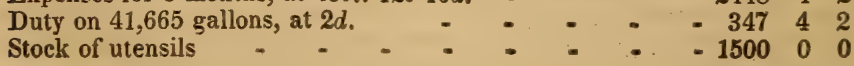

$\lcm{£ 229584}$ 


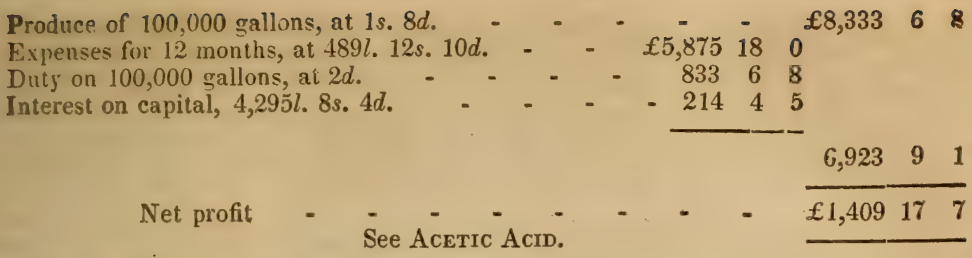

VIOLET DYE, is produced by a mixture of red and blue coloring-matters, which are applied in succession. Silk is dyed a fugitive violet with either archil or Brazil wood; but a fine fast violet, first by a crimson with cochineal, without tartar or tin mordant, and after washing, it is dipped in the indigo vat. A finish is sometimes given with archil. A violet is also given to silk, by passing it through a solution of verdigris, then through a bath of logwood, and, lastly, through alum water. A more beautiful violet may be communicated by passing the alumed silk through a bath of Brazil wood, and after washing it in the river, through a bath of archil.

To produce violets on printed calicoes, a dilute acetate of iron is the mordant, and the dye is madder. The mordanted goods should be well dunged.

A good process for dyeing cottons violet, is-first, to gall, with 18 or 20 pounds of nutgalls for every 100 pounds of cotton; second, to pass the stuff, still hot, through a mordant composed of-alum, 10 pounds; iron-liquor; at $1 \frac{1}{2}^{\circ} \mathrm{B}$, and sulpnate of copper, each 5 or 5 pounds; water, from 24 to 28 gallons; working it well, with alternate steeping, squeezing, airing, dipping, squeezing, and washing; third, to madder, with its own weight of the root; and fourth, to brighten with soap. If soda be used at the end, instead of soap, the color called prune de monsiewr will be produced; and by varying the doses of the in gredients, a variety of violet tints may be given.

The best riolets are produced by dyeing yarn or cloth which has been prepared with oil as for the Turkey-red process. See MADDER.

For the violet pruneau, a little nitrate of iron is mixed with the alum mordant, which malies a black; but this is changed into violet pruneau, by a madder. bath, followed by a brightening with soap.

VItrifiable COLORS; see Enamels, Pastes, Pottery, and Stained Glass.

VITRIOL, from vitrum, glass, is the old chemical, and still the vulgar appellation of sulphuric acid, and of many of its compounds, which in certain states have a glassy appearance : thas -

Vitriolic acid, or oil of vitriol, is sulphuric acid; blue vitriol, is sulphate of copper; green vitriol, is green sulphate of iron; vitriol of Mars, is red sulphate of iron; and white vitriol, is sulphate of zinc.

W.

WACKE, is a massive mineral, intermediate between claystone and basalt. It is of a greenish-gray color; vesicular in structure; dull, opaque; streak shining; soft, easily frangible; spec. grav. 2.55 to 2.9 ; it fuses like basalt.

WADD, is the provincial name of plumbago in Cumberland; and also of an ore of manganese in Derbyshire, which consists of the peroxyde of that metal, associated with nearly its own weight of oxyde of iron.

WAIDING (Ouate, Fr.; Watte, Germ.), is the spongy web which serves to line 'adies' pelisses, \&c. Ouate, or Wat, was the name originally given to the glossy downy tufts found in the pods of the plant commonly called Apocyn, and by botanists Asclepias Syriact, which was imported from Egypt and Asia Minor for the purpose of stuffing cushions, \&c. Wadding is now made with a lap or fleece of cotton prepared by the carding. engine (see Carding, Cotton Manufacture), which is applied to tissue paper by a coat of size, marle by boiling the cuttings of hare-skins, and adding a little alum to the gelatinous soluiion. When two laps are glued with their faces together, they form the most downy kind of wadding.

WAFERS. There are two manners of manufacturing wafers: 1, with wheat flour and water, for the ordinary kind; and 2, with gelatine. 1. A certain quantity of fine flour is to be diffused through pure water, and so mixed as to leave no clotty particles. This thin pap is then colored with one or other of the matters to be particularly described under the second head; and which are, vermilion, sulphate of indigo, and gamboge. The pap is not allowed to ferment. but must be employed immediately after it is mixed. For this purpose a tool is employed, consisting of two plates of iron, which 
come together like pincers or a pair of tongs, leaving a certain small definite space betwixt them. These plates are first slightly heated, greased with butter, filled with the pap, closed, and then exposed for a short time to the heat of a charcoal fire. The iron plates being allowed to cool, on opening them, the thin cake appears dry, solid, brittle, and about as thick as a playing-card. By means of annular punches of different sizes, with sharp edges, the cake is cut into wafers. 2. The transparent wafers are made as follows :-

Dissolve fine glue, or ising-glass, in such a quantity of water, that the solution, when cold, may be consistent. Let it be poured hot unon a plate of mirror glass, (previously warmed with steam, and slightly greased,) which is fitted in a metallic frame, with edges just as high as the wafers should be thich. A second plate of glass, heated and greased, is laid on the surface, so as to touch every point of the gelatine, resting on the edoes of the frame. By this pressure, the thin calie of gelatine is made perfectly uniform. When the two plates of glass get cold, the gelatine becomes solid, and may easily be removed. It is then cut with proper punches into dises of different sizes.

The coloring-matters ought not to be of an insalubrious kind.

For red wafers, carmine is well adapted, when they are not to be transparent; but this color is dear, and can be used only for the finer kinds. Instead of it, a decoction of Brazil wood, brightened with a little alum, may be employed.

For yellow, an infusion of saffron or turmeric has been prescribed; but a decoction of weld, fustic, or Persian berries, might be used.

Sulphate of indigo, partially saturated with potash, is used for the blne wafers; and this mixed with yellow, for the greens. Some recommend the sulphate to be nearly neutralized with chalk, and to treat the liquor with alcohol, in order to obtain the best blue dye for wafers.

Common wafers are, nowever, colored with the substances mentioned at the beginning of the article; and for the cheaper kinds, red lead is used instead of vermilion, and turmeric instead of gamboge.

WALNUT HUSKS, or PEELS (Brout des noix, Fr.), are much employed by the French dyers for rooting or giving dun colors.

WARP (Chaine, Fr.; Kette, Anschweif, Zettel, Germ.), is the name of the longitudinal threads or yarns, whether of cotton, linen, silk, or wool, which being deeussated at right angles by the woof or weft threads, form a piece of cloth. The warp yarns are parallel, and continuous from end to end of the web. See Weaving, for a description of the warping-mill.

WASH, is the fermented wort of the distiller.

WASHING. See Bleaching, and Scouring.

WATERING OF STUFFS (Moirage, Fr.), is a process to which silk and other textile fabrics are subjected, for causing them to exhibit a variety of undulated reflections, and plays of light. It is produced by sprinkling water upon the goods, and then passing them through a calender, either with cold or hot rollexs, plain or variously indented.

WATER-PROOF CLOTH. See Caovtchouc, and Grlatine.

A patent was obtained, in August, 1830, by Mr. Thomas Hancoek, for rendering textile fabrics impervious to water and air, by spreading the liquid juice of the eaoutehoue tree upon the surfaces of the goods, and then exposing them to the air to dry. It does not appear that this project has been realized in our manufactures.

Mr. William Simpson Potter proposes, in his patent of April, 1835, to render fabries water-proof by imbuing them with a solution of ising-glass, alum, and soap, by means of a brush applied to the wrong side of the cloth, distended upon a table. After it is dry, it must be brushed on the wrong side, against the grain. Then the brush is to be dipped in clean water, and passed. lightly over the cloth. The gloss caused by the above application can be taken off by brushing the goods when they are dry. Cloth so prepared is said to be impervious to water, but not to air.

I have examined woollen cloth now on sale in a shop in the Strand, which may be breathed through with the greatest facility, but which retains water upon its surface, as is evinced by a body of water standing upon a concave piece of it tied over a show-glass in the window.

Mr. Sievier's plan of rendering cloth water-proof, for which he obtained a patent in December, 1835, consists in spreading over it, with a brush, a solution of India rmbber in spirits of turpentine, at one or more applications, ana then applying a similar solution mixed with acetate of lead, litharge, sulphate of zinc, gum mastic, or other drying material. He next takes wool, or other textile material, cut into proper lengths, and spreads if upon the surface of the fabric varnished in this manner, for the purpose of forming the nap or pile. He then presses the cloth by means of rollers, or brushes, so as to fix the nap firmly to its surface. 
WATERS, MINERAL.

\begin{tabular}{|c|c|c|c|c|c|}
\hline 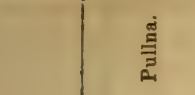 & 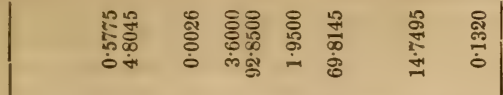 & 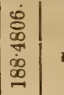 & $\sim$ & $\stackrel{\circ}{\circ}$ & 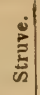 \\
\hline 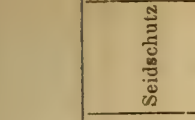 & 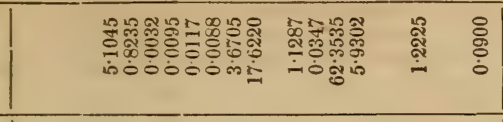 & 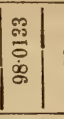 & สి & 品 & 离 \\
\hline 岕 & 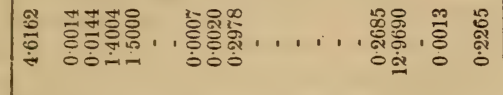 & 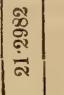 & $\stackrel{\mathscr{2}}{\stackrel{2}{2}}$ & 品 & 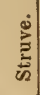 \\
\hline 气ึँ & 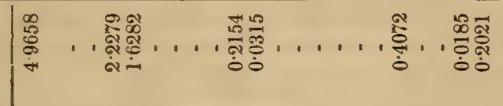 & 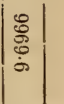 & ஜ & $\frac{0}{3}$ & 离 \\
\hline 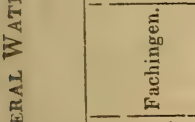 & 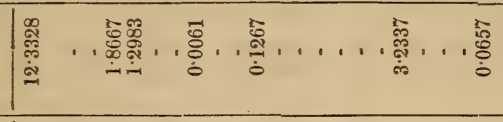 & 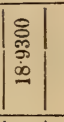 & 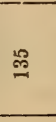 & 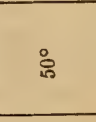 & 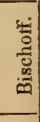 \\
\hline के & 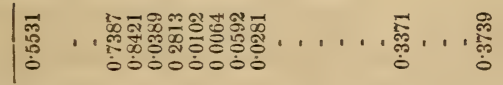 & 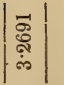 & 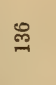 & 우 & 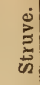 \\
\hline हैं & 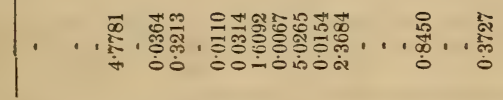 & 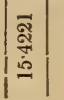 & $\stackrel{8}{\stackrel{-}{0}}$ & ¿̊ & $\stackrel{\Delta}{\mathscr{D}}$ \\
\hline 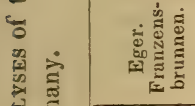 & 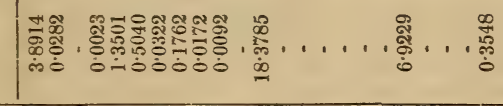 & $\mid \begin{array}{c}8 \\
\vdots \\
\dot{m} \\
\dot{m}\end{array}$ & 节 & ్ㅐㅁำ & 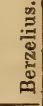 \\
\hline 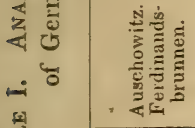 & 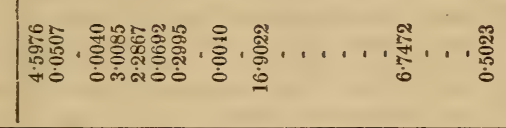 & 站 & $\stackrel{0}{ \pm}$ & $\stackrel{8}{\circ}$ & 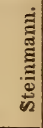 \\
\hline . & 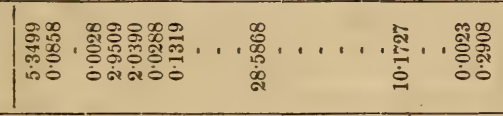 & 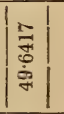 & $\stackrel{\circ}{\circ}$ & ถి & 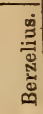 \\
\hline 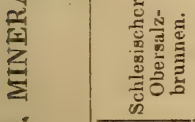 & 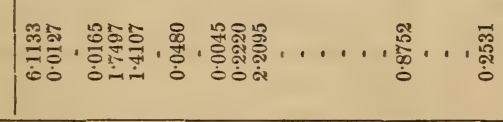 & 政 & $\stackrel{\circ}{\circ}$ & $\stackrel{\circ}{\circ}$ & 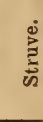 \\
\hline 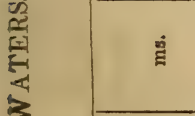 & 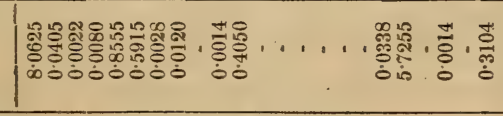 & 趈 & $\overrightarrow{5}$ & 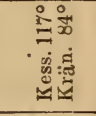 & 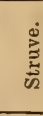 \\
\hline कृ & 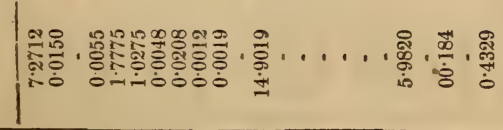 & |⿱宀⿻心㇒日 & 象 & 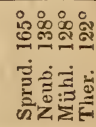 & 宽 \\
\hline 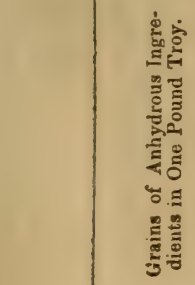 & 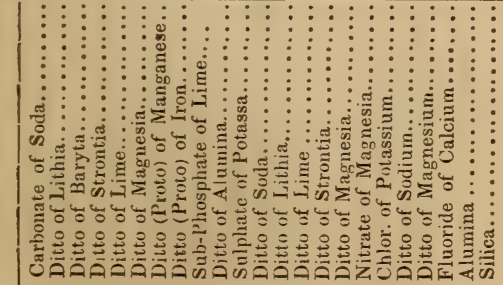 & 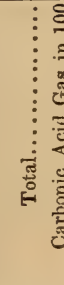 & 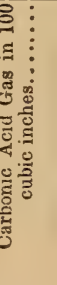 & 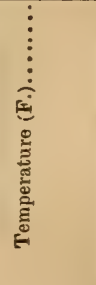 & है \\
\hline
\end{tabular}


WATERS, MINERAL.

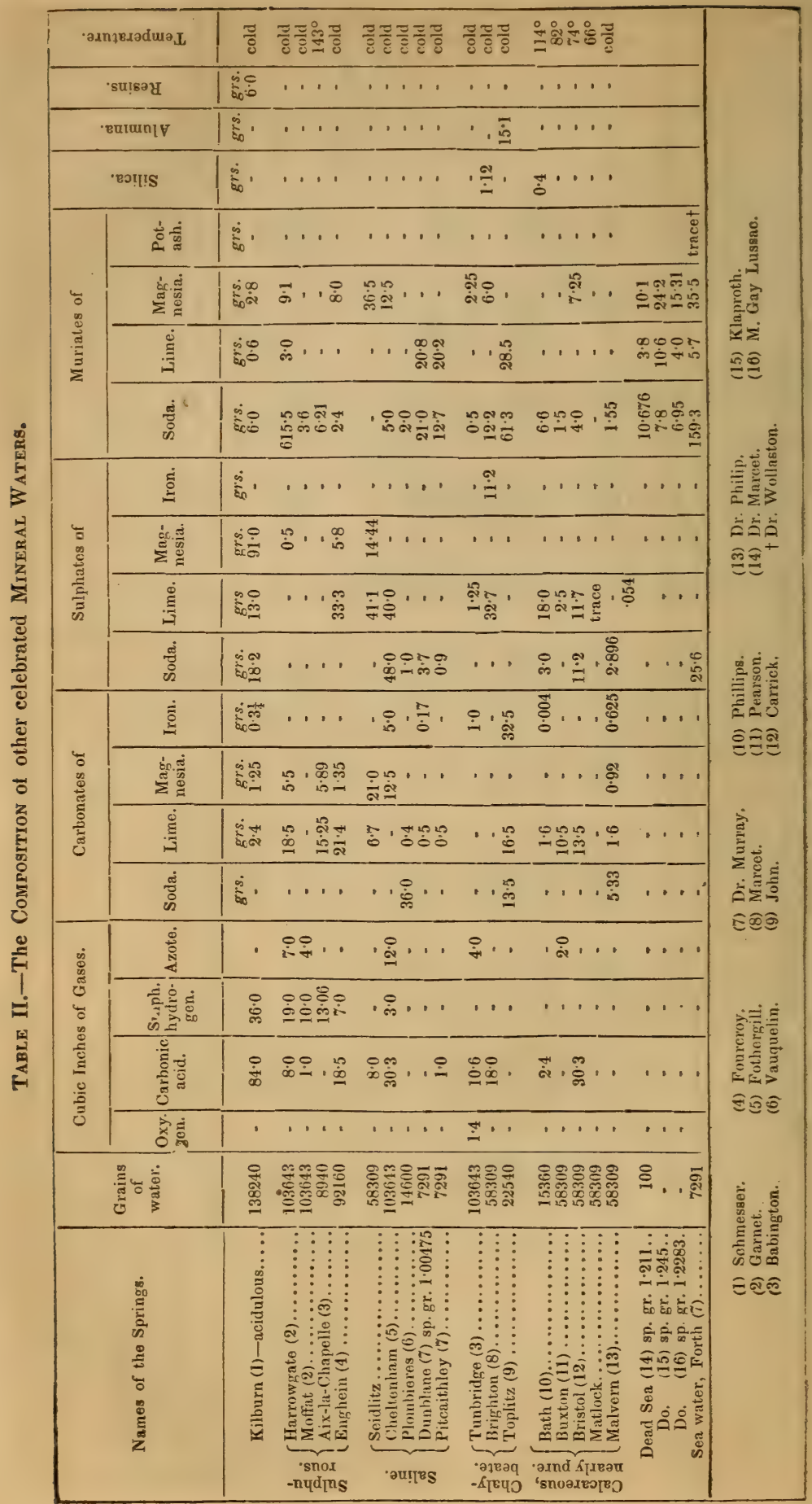


Mineral waters may, in most cases, be artificially prepared, by the skilful application of the knowledge derived from analysis, with such precision as to imitate very closelv the native springs. When the various earthy or metallic constituents are held in solution by carbonic acid, or sulphureted, they should be placed, along with their due proportious of water, in the receiver of the aerating machine (see SODA WATER), and then the proper quantity of gas should be injected into the water. Sufficient agitation will be given by the action of the forcing-pump to promote their solution.

IVAX (Cire, Fr.; Wachs, Germ.), is the substance which forms the cells of bees. It was long supposed to be derived from the pollen of plants, swallowed by these insects, and merely voided under this new form; but it has been proved by the experiments, first of Mr. Hunter, and more especially of M. Huber, to be the peculiar secretion of a certain organ, which forms a part of the small sacs, situated on the sides of the median line of the abdomen of the bee. On raising the lower segments of the abdomen, these sacs may be observed, as also scales or spangles of wax, arranged in pairs upon each segment. There are none, howęver, under the rings of the males and the queen. Each individual has only eight wax sacs, or pouches; for the first and the last ring are not provided with them. M. Huber satisfied himself by precise experiments that bees, though fed with honey, or sugar alone, produced revertheless a very considerable quantity of wax; thus proving that they were not mere collectors of this substance from the vegetable kingdom. The pollen of plants serves for the nourishment of the larvæ.

But wax exists also as a vegetable product, and may, in this point of view, be regarded as a conerete fixed oil. It forms a part of the green fecula of many plants, particularly of the cabbage; it may be extracted from the pollen of most flowers; as also from the skins of plums, and many stone fruits. It constitutes a varnish upon the upper surface of the leaves of many trees, and it has been observed in the juice of the cow-tree. The berries of the Myrica angustifolia, latifolia, as well as the cerifera, afford abundance of wax.

Bees' wax, as obtained by washing and melting the comb, is yellow. It has a peeuliar smell, resembling honey, and derived from it, for the cells in which no honey has been deposited, yield a scentless white wax. Wax is freed from its impurities, and bleached, by melting it with hot water or steam, in a tinned copper or wooden vessel, letting it settle, running off the clear supernatant oily-looking liquid into an oblong trough with a line of holes in its bottom, so as to distribute it upon horizontal wooden cylinders, made to revolve half immersed in cold water, and then exposing the thin ribands of films thus obtained to the blanching action of air, light, and moisture. For this purpose, the ribands are laid upon long webs of canvass stretched horizontally between standards, two feet above the surface of a sheltered field, having a free exposure to the sunbeams. Here they are frequently turned over, then covered by nets to prevent their being blown away by winds, and watered from time to time, like linen upon the grass field in the o's method of bleaching. Whenever the color of the wax seems stationary, it is collected, remelted, and thrown again into ribands upon the wet cylinder, in order to expose new surfaces to the blanching operation. By several repetitions of these processes, if the weather proves favorable, the wax eventually loses its yellow tint entirely, and becomes fit for forming white candles. If it be finished under rain, it will become gray on keeping, and also lose in weight.

In France, where the purification of wax is a considerable object of manufacture, about four ounces of cream of tartar, or alum, are added to the water in the first meltingcopper, and the solution is incorporated with the wax by diligent manipulation. The whole is left at rest for some time, and then the supernatant wax is run off into a settling cistern, whence it is discharged by a stopcock or tap, over the wooden cylinder revolving at the surface of a large water-cistern, kept cool by passing a stream continually through it.

The bleached wax is finally melted, strained through silk sieves, and then run into circular cavities in a moistened table, to be cast or moulded into thin disc pieces, weighing from two to three ounces each, and three or four inches in diameter.

Neither chlorine, nor even the chlorides of lime and alkalis, can be employed with any advantage to bleach wax, because they render it brittle, and impair its burning quality.

Wax purified, as above, is white and translucent in thin seoments; it has neither taste nor smell; it has a specific gravity of from 0.960 to 0.966 ; it does not liquefy till it be heated to $154 \frac{1}{2}^{\circ} \mathrm{F}$.; but it softens at $86^{\circ}$, becoming so plastic, that it may be moulded by the hand into any form. At $32^{\circ}$ it is hard and brittle.

It is not a simple substance, but consists of two species of wax, which may be easily separated by boiling alcohol. The resulting solution deposites, on cooling, the waxy body called cerine. The undissolved wax, being once and again treated with boiling alcohol, finally affords from 70 to 90 per cent. of its weight of cerine. The insoluble residuum is the myricine of Dr. John, so called because it exists in a much larger pro- 
portion in the wax of the Myrica cerifera. It is greatly denser than wax, being of the same specific gravity as water; nnd may be distilled without decomposition, which cerine undergoes. See these two articles.

Wax is adulterated sometimes with starch; a fraud easily detected by oil of turpentine, which dissolves the former, and leaves the latter substance; and more frequently with mutton suet. This fraud may be discovered by dry distillation; for wax does not thereby afford, like tallow, sebacic acid (benzoic), which is known by its occasioning a precipitate in a solution of acetate of lead. It is said that two per cent. of a tallow sophistication may be discovered in this way.

Bees' wax imported for home consumption:-in 1835, unbleached, 4,449 cwts.; bleached, 243 cwts.; -in 1836, unbleached, 4,673 cwts.; bleached, 121 cwts. Duty, when from British possessions, 10s. ; from foreign, 30s.

WAX, MINERAL, or Ozocerite, is a solid, of a brown color, of various shades, translucent, and fusible like bees' wax; slightly bituminous to the smell, of a foliated texture, a conchoidal fracture, but wanting tenacity, so that it can be pulverized in a mortar. Its specific gravity varies from 0.900 to 0.953 . Candles have been made of it in Moldavia, which give a tolerable light. It occurs at the foot of the Carpathians near Slanik, beneath a bed of bituminous slate-clay, in masses of from 80 to 100 pounds weight. Layers of brown amber are found in the neighborhood. It is associated with variegated sandstone, rock salt, and beds of coal (lignite?). It is analogous to hatchetine. Something similar has been discovered in a trouble at Urpeth colliery, near Newcastle, 60 fathoms beneath the surface. Ozocerite consists of different hydro-carbureted compounds associated together; the whole being composed, ultimately, of-hydrogen 14 , carbon 86 , very nearly.

WEAVING (Tissage, Fr.; Weberei, Germ.), is performed by the implement called loom in English, métier à tisser in French, and weberstuhl in German. The process of warping must always precede weaving. Its object is to arrange all the longitudinal threads, which are to form the chain of the web, alongside of each other in one parallel plane. Such a number of bobbins, filled with yarn, must therefore be taken as will furnish the quantity required for the kength of the intended piece of cloth. One sixth of that number of bobbins is usually mounted at once in the warp mill, being set loosely in a horizontal direction upon wire skewers, or spindles, in a square frame, so that they may revolve, and give off the yarn freely. The warper sits at A, fig. 1159, and causes the reel в to revolve, by turning round with his hand the wheel $\mathrm{c}$, with the

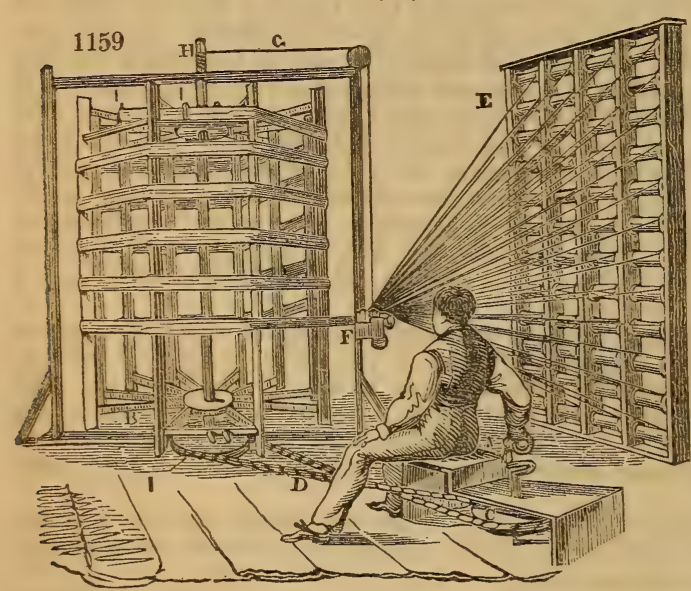
endless rope or band $D$. The bobbins filled with yarn are placed in the frame $\mathbf{E}$. There is a sliding piece at $\mathbf{F}$; called the heck box, which rises and falls by the coiling and uncoiling of the cord $G$, round the central shaft of the reef H. By this simple contrivance, the band of warpyarns is wound spirally, from top to bottom, upon the reel. I, I, I, are wooden pins which separate the different bands. Most warping mills are of a prismatic form; having twelve, eighteen, or more sides. The reel is commonly about six feet in diameter, and seven feet in height, so as to serve for measuring exactly upon its periphery the total length of the warp. All the threads from the frame $E$, pass through the heck $F$, which consists of a series of finely-polished hard-tempered steel pins, with a small hole at the upper part of each, to receive and guide one thread. The heck is divided into two parts, either of which may be lifted by a small handle below, while their eyes are placed alternately. Hence, when one of them is raised a little, a vacuity is formed between the two bands of the warp; but when the other is raised, the vacuity is reversed. In this way, the lease is produced at each end of the warp, and it is preserved by appropriate wooden pegs. The lease being carefully tied up, affords a guide to the weaver for inserting his lease-rods. The warping mill is turned alternately from right to left, and from left to right, till a sufficient number of yarns are coiled round it to form the 
breadth that is wanted; the warper's principal care being to tie immediately every thread as it brealis, otherwise deficiencies would be occasioned in the chain, injurious to the appearance of the web, or productive of much annoyance to the weaver.

The simplest and probably the most ancient of looms, now to be seen in action, is that of the Hindoo tanty, shown in fig. 1160. It consists of two bamboo rollers; one for

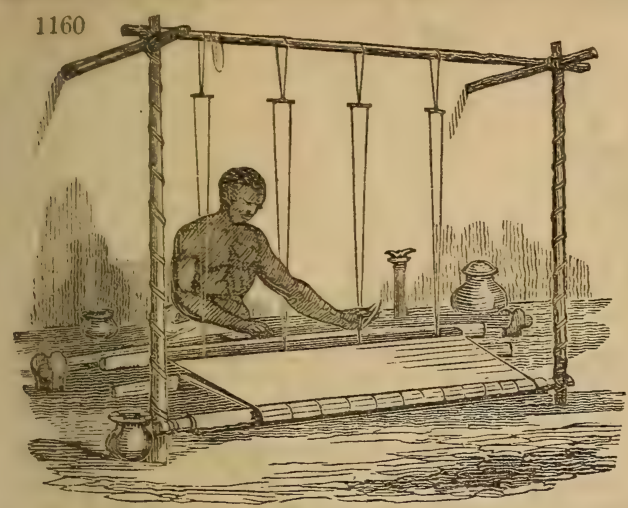
the warp, and another for the woven cloth; with a pair of heddles, for parting the warp, to permit the weft to be drawn across between its upper and under threads. The shuttle is a slender rod, like a large netting needle, rather longer than the web is broad, and is made use of as a batten or lay, to strike home or fondense each successive thread of weft, against the closed fabric. The Hindoo carries this simple implement, with his water pitcher, rice pot, and hooka, to the foot of any tree which can afford him a comfortable shade; he there digs a large hole, to receive his legs, along with the treddles or lower part of the harness; he next extends his warp, by fastening his two bamboo rollers, at a proper distance from each other, with pins, into the sward; he attaches the heddles to a convenient branch of the tree overhead; inserts his great toes into two loops under the gear, to serve him for treddles; lastly, he sineds the warp, draws through the weft, and beats it close up to the web with his rodshuttle or batten.

The European loom is represented in its plainest state, as it has existed for several centuries, in fig. 1161. A is the warp-beam, round which the chain has been wound; B represents the flat rods, usually three in number, which pass across between its threads, to preserve the lease, or the plane of decussation for the weft; $\mathrm{c}$ shows the heddles or healds, consisting of twines looped in the middle, through which loops the warp yarns are drawn one half through the front heddle, and the other through the back one; by

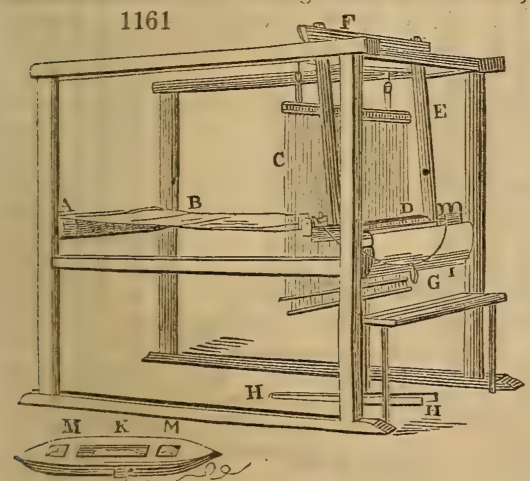
moving which, the decussation is readily effected. The yarns then pass through the dents of the REED under $D$, which is set in a moveable swing-frame $\mathbf{E}$, called the lathe, lay, and also batten, because it beats home the weft to the web. The lay is freely suspended to a cross-bar $F$, attached by rulers, called the swords, to the top of the lateral standards of the loom, so as to oscillate upon it. The weaver, sitting on the bench $\mathrm{G}$, presses down one of the treddles at $\mathbf{H}$, with one of his feet, whereby he raises the corresponding heddle, but sinks the alternate one; thus sheds the warp, by lifting and depressing each alternate thread, through a little space, and opens a pathway or race-course for the shuttle to traverse the middle of the warp, upon its two friction rollers $\mathrm{M}$, M. For this purpose, he lays hold of the picking-peg in his right hand, and, with a smart jerk of his wrist, drives the fly-shuttle swiftly from one side of the loom to the other, between the shed warp yarns. The shoot of weft being thereby left behind from the shettle pirn or cop, the weaver brings home, by pulling, the lay with its reed towards him by his left hand, with such force as the closeness of the texture requires. The web, as thus woven, is wound up by turning round the cloth beam I, furnished with a ratchet-wheel, which takes into a holding tooth. The plan of throwing the shuttle by the picking-peg and enrd, is a great improvement upon the old way of throwing it by hand. It was contrived exactly a century ago, by John Kay, of Bury in Lancashire, but then resident in Colchester, and was called the fly-shuttle, from its speed, as it enabled the weaver to make double the quantity of narrow cloth, and much more broadcloth, in the same time.

The cloth is kept distended, during the operation of weaving, by means of two pieces 
of hard wood, called a templet, furnished with sharp iron points in their ends, which take hold of the opposite selvages or lists of the web. The warp and web are kept longitudinally stretched by a weighted cord, which passes round the warp-beam, and which tends continually to draw back the cloth from its beam, where it is held fast by the ratchet tooth. See Fustian, Jacquard Loom, Reed, and Textile Fabrics.

The greater part of plain weaving, and much even of the figured, is now performed bv

1164

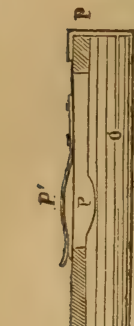

1162

the power loom, called métier mécanique à tisser, in French. Fig. 1162, represents the cast-iron power loom of Sharp and Roberts. A, $\mathbf{A}^{\prime}$, are the two side uprights, or standards, on the front of the loom. $\mathrm{D}$, is the great arch of cast iron, which binds the two sides together. $\mathrm{E}$, is the front cross-beam, terminating in the forks $e, e$; whose 
ends are bolted to the opposite standards $A, A^{\prime}$, so as to bind the framework most firmly together. $G^{\prime}$, is the breast beam, of wood, nearly square; its upper surface is sloped a litile towards the front, and its edge rounded off, for the web to slide smoothly over it, in its progress to the cloth beam. The beam is supported at its end upon brackets, and is secured by the bolts $g^{\prime}, g^{\prime}$. $H$, is the cloth beam, a wooden cylinder, mounted with iron gudgeons at its ends, that on the right hand being prolonged to carry the toothed winding wheel $\mathbf{H}^{\prime} . \quad k^{\prime}$, is a pinion in gear with $\mathbf{H}^{\prime} . \mathbf{H}^{\prime \prime}$, is a ratchet wheel, mounted unon the same shaft $h^{\prime \prime \prime}$, as the pinion $h^{\prime} . h^{\prime}$, is the click of the ratchet wheel $\mathbf{H}^{\prime \prime}$. $h^{\prime \prime \prime}$, is a long bolt fixed to the frame, serving as a shaft to the ratchet wheel $\mathbf{H}^{\prime \prime}$, and the pinion $h^{\prime}$. I, is the fiont heddle-leaf, and $\mathrm{r}^{\prime}$, the back one. $\mathrm{J}, \mathrm{J}, \mathrm{J}^{\prime}, \mathrm{J}^{\prime}$, jacks or pulleys and straps, for raising and depressing the leaves of the heddles. $\mathrm{J}^{\prime \prime}$, is the iron shaft which carries the jacks or system of pulleys $\mathrm{J}, \mathrm{J}, \mathrm{J}^{\prime}, \mathrm{J}^{\prime} . \mathbf{K}$, a strong wooden ruler, connecting the front heddle with its treddle. $\mathbf{L}, \mathrm{L}^{\prime}$, the front and rear marches or treddlejieces, for depressing the heddle leaves alternately, by the intervention of the rods $k$, (and $\hat{k}$, hid behind $k$ ). M, M, are the two swords (swing bars) of the lay or batten. $\mathbf{N}$, is the upper cross-bar of the lay, made of wood, and supported upon the squares of the levers $n, n^{\prime}$, to which it is firmly bolted. $N^{\prime}$, is the lay-cap, which is placed higher or lower, according to the breadth of the reed; it is the part of the lay which the handloom wearer seizes with his hand, in order to swing it towards him. $n^{\prime}$ is the reed contained between the bar $\mathrm{N}$, and the lay-cap $\mathrm{N}^{\prime}$. 0,0 , are two rods of iron, perfectly round and straight, mounted near the ends of the batten-bar $N$, which serve as guides to the drivers or peckers 0,0 , which impel the shuttle. These are made of buffalo hide, and should slide freely on their guide-rods. $o^{\prime}, o^{\prime}$, are the fronts of the shuttle-boxes; they have a slight inclination backwards. $\quad$, is the back of them. See figs. 1163 and 1164 . $0^{\prime \prime}, 0^{\prime \prime}$, are iron plates, forming the bottoms of the shuttle-boxes. $p$, small pegs or pins, planted in the posterior faces $\mathbf{P}$ ( fig. 1164) of the boxes, round which the levers $\mathbf{P}^{\prime}$ turn. These levers are sunk in the substance of the faces $\mathbf{P}$, turn round pegs $p$, being pressed from without inwards, by the springs $p^{\prime}$. $\mathbf{p}^{\prime \prime}$, fig. 1162 , (to the right of $\mathbf{K}$,) is the whip or lever, (and $\mathrm{Q}^{\prime \prime}$, its centre of motion, corresponding to the right arm and elbow of the weaver,) which serves to throw the shuttle, by means of the pecking-cord $p^{\prime \prime}$, attached at its other end to the drivers 0,0 .

On the axis of $Q^{\prime \prime}$, a kind of eccentric or heart wheel is mounted, to whose concave part, the middle of the double band or strap $r$, being attached, receives impulsion; its two ends are attached to the heads of the bolts $r^{\prime}$, which carry the stirrups $r^{\prime \prime}$, that may be adjusted at any suitable height, by set screws.

$\mathrm{s}$ (see the left-hand side of fig. 1162) is the moving shaft, of wrought iron, resting on the two ends of the frame. 's' (see the right-hand side) is a toothed wheel, mounted exteriorly to the frame, upon the end of the shaft $s$. $s^{\prime \prime}$ (near $s^{\prime}$ ) are two equal elbows, in the same direction, and in the same plane, as the shaft s, opposite to the swords M, M, of the lay.

$z$, is the loose, and $z^{\prime}$, the fast pulley, or riggers, which receive motion from the steamshaft of the factory. $z^{\prime \prime}$, a small fiy-wheel, to regulate the movements of the main shaft of the loom.

$T$, is the shaft of the eccentric tappets, cams, or wipers, which press the treddle levers alternately up and down; on its right end is mounted $\mathrm{T}^{\prime}$, a toothed wheel in gear with the wheel $\mathrm{s}^{\prime}$, of one half its diameter. $\mathrm{T}^{\prime \prime}$, is a cleft clamping collar, which serves to support the shaft $T$.

$\mathrm{U}$, is a lever, which turns round the bolt $u$, as well as the click $h^{\prime \prime}$. $\mathrm{U}^{\prime}$, is the click of traction, for turning round the cloth beam, jointed to the upper extremity of the lever $\mathrm{U}$; its tooth $u^{\prime}$, catches in the teeth of the ratchet wheel $\mathrm{H}^{\prime \prime} . u^{\prime \prime}$, is a long slender rod, fixed to one of the swords of the lay $\mathrm{m}$, serving to push the lower end of the lever $u$, when the lay retires towards the heddle leaves.

$\mathrm{x}$, is a wrought-iron shaft, extending from the one shuttle-box to the other, supported at its ends by the bearings $x, x$.

$\mathrm{Y}$, is a bearing, affixed exteriorly to the frame, against which the spring bar $\mathbf{z}$, rests; near its top, but is fixed to the frame at its bottom. The spring falls into a notch in the bar $\mathbf{Y}$, and is thereby held at a distance from the upright $\mathbf{A}$, as long as the band is upon the loose pulley $z^{\prime}$; but when the spring bar is disengaged, it falls towards $A$, and carries the band upon the fast pulley $z$, so as to put the loom in gear with the steam-shaft of the factory.

Weaving, by this powerful machine, consists of four operations: 1 . to shed the warp by means of the heddle leaves, actuated by the tappet wheels upon the axis $Q^{\prime}$, the rods $k$, $k^{\prime}$, the cross-bar $\mathbf{E}$, and the eyes of the heddle leaves $\mathrm{I}, \mathbf{I}^{\prime} ; 2$. to throw the shuttle (see fig. 1161), by means of the whip lever $\mathbf{P}^{\prime \prime}$, the driver cord $p$, and the pecker $0 ; 3$. to drive home the weft by the batten $N, N^{\prime} ; 4$. to unwind the chain from the warp beam, and io draw it progressively forwards, and wind the finished web upon the cloth beam $\mathbf{H}$, by the click and toothed wheel mechanism at the right-hand side of the frame. For more minute details, the reader may consult The Cotton Manufacture of Great Britain, vol. ii p. 291. 
WEFT (Trame, Fr.; Eintrag, Germ.), is the name of the yarns or threads which run froun selvage to selvage in a web.

WELD (Voü̈de, Fr.; Wau, Gelbkraut, Germ.), is an annual herbaceous plant, which grows all orer Eurnpe, called by botanists Reseda luteola. The stems and the leaves dye vellow; and among the dyes of organic nature, they rank next to the Persian berry for the beauty and fastness of color. The whole plant is cropped when in seed, at which period its dyeing power is greatest; and after being simply dried, is brought into the market.

Cherreul has discorered a yellow coloring principle in weld, which he has called luteoline. It may be sublimed, and thus obtained in long needle-form, transparent, tellow crystals. Luteoline is but sparingly soluble in water; but it nevertheless dyes alumed silk and wool of a fine jonquil color. It is soluble in alcohol and ether; it combines with acids, and especially with bases.

When weld is to be emplored in the dye-bath, it should be boiled for three quarters of an hour; after which the exhausted plant is taken out, because it occupies too much room. The decoction is rapidly decomposed in the air, and ought therefore to be made only when it is wanted. It produces, with

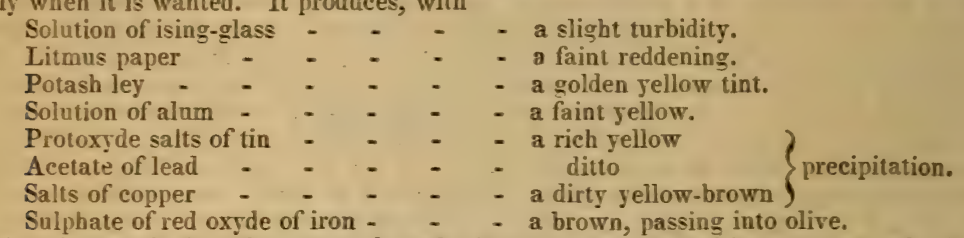

A lack is made from decoction of weld with alum, precipitated by carbonate of soda or potassa. See Yellow Dre.

WELDING (Souder, Fr.; Schweissen, Germ.), is the property which piees of wrought iron possess, when heated to whiteness, of uniting intimatelr and permanently under the hammer, into one bndy, without ant appearance of junction. The welding temperature is usually estimated at from $60^{\circ}$ to $90^{\circ}$ of Wedgewood. When a slilful blacksmith is about to perform the welding operation, he watches minutely the effect of the heat in his forge-fire upon the two iron bars; and if he perceives them beginning to burn, he pulls them out, rolls them in sand, which forms a glassy silicate of iron upon the surface, so as to prerent further oxydizement; and then laying the one properly upon the other, he incorporates them by his right-hand hammer, being assisted by another workman, who strikes the metal at the same time with a heavy forgehammer.

Platinum is not susceptible of being welded, as many chemical authors have erroneously asserted.

Mr. T. H. Russell, of Handsworth, near Birmingham, obtained a patent, in Mar, 1836, for manufacturing welded iron tubes, by drawing or passing the skelp, or fillet of sheet iron, five feet long, between dies or holes, formed by a pair of grooved rollers, placed with their sides contiguous; for which process, he does not previousl? turn up the skelp from end to end, but he does this so as to bring the edges together at the time when the welding is performed. He draws the skelp through two or more pairs of the above pincers or dies, each of less dimension than the preceding. In making tubes of an inch of internai diameter, a skelp four inches and a half broad is employed. The twin rollers revolre on rertical axes, which mar be made to approach each other to give pressure; and ther are kept cool br a stream of water, while the skelp, ignited to the welding heat, is passed between them. They are affixed at about a foot in front of the mouth of the furnace, on a draw-bench; there being a suitable stop within a few inches of the rollers, against which the workman may place a pair of pincers, having a bellmouthed hole or die, for welding and shaping the tube. In the first passage between the rollers, a circular revolving plate of iron is let down vertically between them, to prevent the edges of the skelp from overlapping, or even meeting. The welding is performed at the last passage.

WELLS, ARTESIAN. See also Artesian Wells. The following account of a successful operation of this kind, lately performed at Mortlake, in Surrey, deserres to be recorded. The spot at which this undertaking was begun, is within 100 feet of the Thames. In the first instance, an auger, seren inches in diameter, was used in penetrating 20 feet of superficial detritus, and 200 feet of London clar. An iron tube, 8 inches in diameter, was then driren into the opening, to dam out the land-springs and the percolation from the river. A 4-inch auger was next introduced through the iron tube, and the boring was continued until, the London clay haring been perforated to the depth of 240 feet, the sands of the plastic clay were reached, and water of the softest and purest nature was obtained; but the supply was not sufficient, and it did not reach 
the surface. The work was proceeded with accordingly; and after 55 feet of alternating beds of sand and clay had been penetrated, the chalk was touched upon. A second tube, $t \frac{1}{2}$ inches in diameter, was then driven into the chalk, to stop out the water of the plastic sands; and through this tube an auger, $3 \frac{1}{2}$ inches in diameter, was introduced, and worked down through 35 feet of hard chalk, abounding with flints. To this succeeded a bed of soft chalk, into which the instrument suddenly penetrated to the depth of lis feet. On the auger being withdrawn, water gradually rose to the surface, and overflowed. The expense of the work did not exceed 300l. The general summary of the strata penetratud is as follows:-Gravel, 20 feet; London clay, 250; plastic sands and clays, 55 ; hard chalk with flints, 35 ; soft chalk, $15 ;=375$ feet.

WHALEBONE (Baleine, Fr.; Fischbeine, Germ.), is the name of the horny laminæ, consisting of fibres laid lengthwise, found in the mouth of the whale, which, by the fringes upon their edges, enable the animal to allow the water to flow out, as through rows of teetk (which it wants), from between its capacious jaws, but to catch and detain the minute creatures upon which it feeds. 'The fibres of whalebone have little lateral cohesion, as they are not transversely decussated, and may, therefore, be readily detached in the form of long filaments or bristles. The blades, or scythe-shaped plates, are externally compact, smooth, and susceptible of a good polish. They are connected, in a parallel series, by what is called the gum of the animal, and are arranged along each side of its inouth, to the number of about 300 . The length of the longest blade, which is usually found near the middle of the series, is the gauge adopted by the fishermen to design? i the size of the fish. The greatest length hitherto known has been 15 feet, but it rarely exceeds 12 or 13 . The breadth, at the root end, is from 10 to 12 inches; and the average thickness, from four to five tenths of an inch. The series, riewed altogether in the mouth of the whale, resemble, in general form, the roof of a house. They are cleansed and softened before cutting, by boiling for two hours in a long copper.

Whalebone, as brought from Greenland, is commonly divided into portable junks or pieces, comprising ten or twelve blades in each; but it is occasionally subdivided into separate blades, the gum and the hairy fringes having been removed by the sailors during the voyage. The price of whalebone fluctuates from 50l. to 150l. per ton. The blade is cut into parallel prismatic slips, as follows:-It is clamped horizontally, with its edge up and down, in the large wooden vice of a carpenter's bench, and is then planed by the following tool: fig. 1165, A, B, are its two handles; c, D, is an iron plate, with a guide-notch $\mathbf{E} ; \mathrm{F}$, is a semicircular knife, screwed firmly at each end to 1165

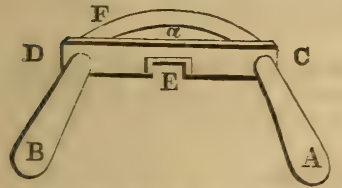
the ends of the iron plate $c, D$, having its cutting edge adjusted in a plane, so much lower than the bottom of the notch $E$, as the thickness of the whalebone slip is intended to be; for different thicknesses, the knife may be set by the screws at different levels, but always in a plane parallel to the lower guide surface of the plate $c, D$. The workman, taking hold of the handles $A, B$, applies the noteh of the tool at the end of the whalebone blade furthest from him, and with his two hands pulls it steadily along, so as to shave off a slice in the direction of the fibres; being careful to cut none of them across. These prismatic slips are then dried, and planed level upon their other $\mathrm{tw}$ o surfaces. The fibrous matter detached in this operation, is used, instead of hair, for stuffing mattresses.

From its flexibili-v, strength, elasticity, and lightness, whalebone is employed for many purposes : for ribs to umbrellas or parasols; for stiffening stays; for the frame-work of hats, \&c. When heated by steam, or a sand-bath, it softens, and may be bent or moulded, like born, into various shapes, which it retains, if cooled under compression. In this way, snuff-boxes, and knobs of walking-sticks, may be made from the thicker parts of the blade. The surface is polished at first with ground pumice-stone, felt, and water; and finished with dry quicklime, spontaneously slaked, and sifted.

WHEAT. (Triticum vulgare, Linn.; Froment, Fr.; Waizen, Germ.) See Bresd, Gloten, and Starch.

WHEEL CARRIAGES. Though this manufacture belongs most properly to a treatise upon mechanical engineering, I shall endeavor to describe the parts of a carriage, so as to enable gentlemen to judge of its make and relative merits. The external form may vary with every freak of fashion; but the general structure of a vehicle, as to lightness, elegance, and strength, may be judged of from the following figure and description.

Fig. 1i66, shows the body of a chariot, hung upon an iron carriage, with iron wheels, axletrees, and boxes; the latter, by a simple contrivance, is close at the out-head, by which means the oil cannot escape; and the fastening of the wheel being at the in-head, as will be explained afterwards, gives great security, and prevents the possibility of the wheel being taker off by any other carrage running against it. 
Fig. 1167, shows the arm of an axletree, turned perfectly true, with two collars in the solid, as seen at $\mathrm{G}$ and $\mathrm{H}$. The parts from $\mathrm{G}$ to $\mathrm{B}$ are made cylindrical. At $\mathrm{K}$ is a screw nail, the purpose of which will be explained in fig. 1171 .

\section{$\mathbf{1 1 6 6}$}

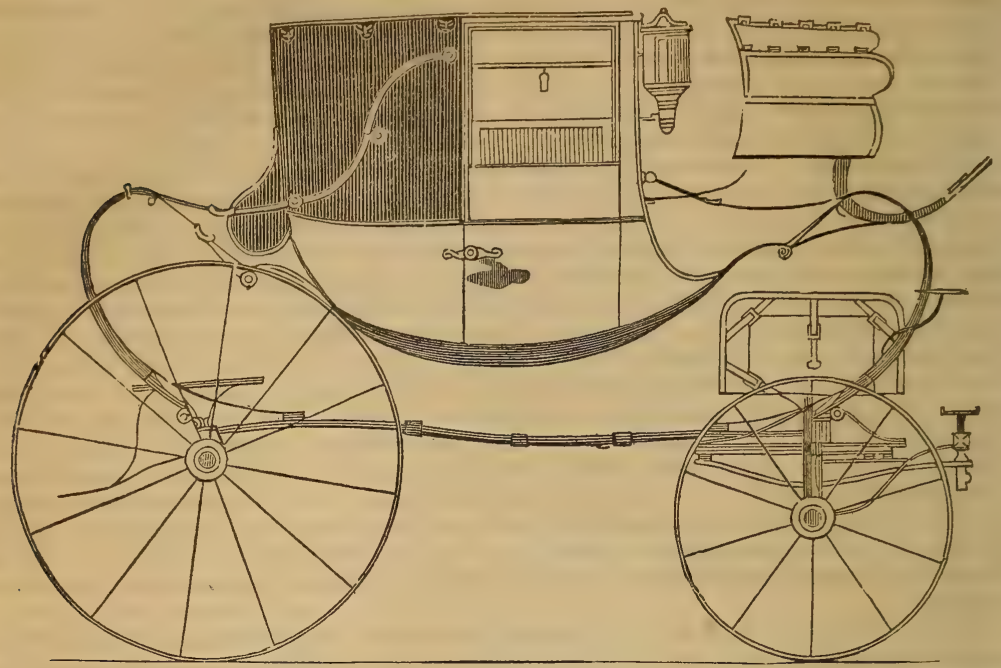

Fig. 1168, is the longitudinal section of a metal nave, which also forms the bush, for the better fitting of which to the axletree, it is bored out of the solid, and made quite airtight upon the pin; and for retaining the oil, it is left close at the out-head $\mathrm{D}$.

Fig. 1169, represents a collet, made of metal, turned perfectly true, the least diameter of which is made the same with that part of the axletree $\mathrm{M}$, fig. 1167, and its greatest diameter the same with that of the solid collar G, fig. 1167. This collet is made with a joint at $\mathrm{s}$, and opens at $p$. Two grooves are represented at $q q, q q$, which are seen at the same letters in $f i g .1170$, as also the dovetail $r$, in both figures.

Fig. 1170, is an edge view of the collet, fig. 1169 .

Fig. $117 \mathrm{l}$, is a longitudinal section of an axletree arm, nave or bush, and fastening. $A, B$, is the arm of the axletree, bored up the centre from B to E. C, C, D, the nave, which 1169 1170

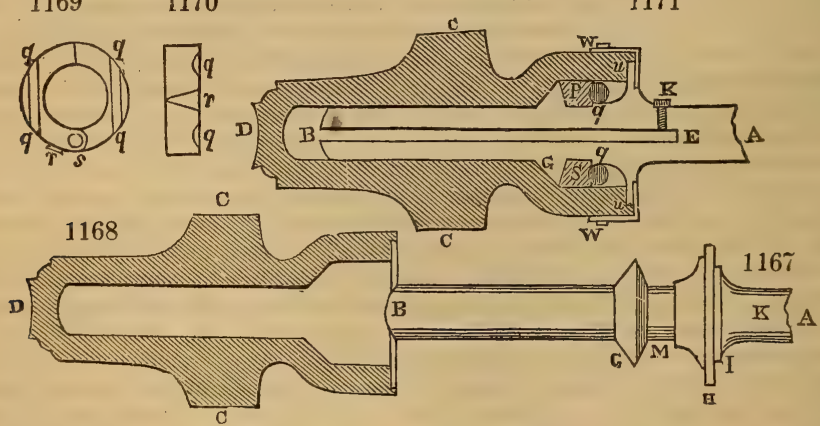

answers also for the bush. P, s, the collet (see figs. 1169 and 1170), put into its place. $q, q$, two steel pins, passing through the in-head of the bush; and filling up the grooves in the collet. W, w, a caped hoop, sufficiently broad to cover the ends of said pins, and made fast to the bush by screws. This hoop, when so fastened to the bush, prevents the possibility of the pins $q$, from getting out of their places. $u, u$, is a leather washer, interposed betwixt the in-head of the bush and the larger solid collar of the axletree, to prevent the escape of orl at the in-head. $\mathrm{r}$, is a screw, the head of which is near the letter $\mathrm{K}$, in fig. 1167. This screw being undone, and oil poured into the hole, it flows down the bore in the centre of the axletree arm, and fills the space $B$, left by the arm, being about one inch shorter than the bore of the bush, and the screw, being afterwards replaced, keeps all tight. In putting on the wheel, a little oil ought to be 
put into the space betwixt the collet $\mathrm{P}, \mathrm{s}$, and the larger collar. The collar $\mathbf{P}, \mathrm{s}$, being moveable round the axletree arm, and being made fast to the bush by means of the two pins 1, 1\%, revolves along with the bush, acting against the solid collar G, of the arm, and keeps the wheel fast to the axletree, until by removing the caped hoop $\mathrm{w}^{\prime}$, $w$, and driving nut the pins $q, q$, the collet becomes disengaged from the bush.

The dovetail, seen upon the collet at $r, f i g .11 \%$, has a corresponding groove cut in the bush, to receive it, in consequence of which the wheel must of necessity be put on so that the collet and pins fit exactly. These wheels very rarely require to be taken off, and they will run a thousand miles without requiring fresh oiling.

The spokes of the wheel, made of malleable iron, are screwed into the bush or nave at c, c, igs. 1168, 1171, all round. The felloes, composed merely of two bars of iron, bent into a circle edgeways, are put on, the one on the front, the other on the back, of the spokes, which have shouliers on both sides to support the felloes, and all three are attached ogether by rivets through them. The space between the two iron rings forming the felloes, should be filled up with light wood, the tire then put on, and fastened to the felloes by bolts and glands clasping both felloes.

This is a carriage without a mortise or tenon, or wooden joint of any kind. It is, at an average, one seventh lighter than any of those built on the ordinary construction.

The design of $\mathrm{Mr}$. W. Mason's patent invention, of 1827 , is to give any required pressure to the ends of what are called mail axletrees, in order to prevent their shaking in the boxes of the wheels. This object is effected by the introduction of leather collars in certain parts of the box, and by a contrivance, in which the outer cap is screwed up, so as to bear against the end of the axletree with any degree of tightness, and is held in that situation, without the possibility of turning round, or allowing the axletree to become loose.

Fig. 1172, shows the section of the box of a wheel, with the end of the axletree secured in it. The general form of the box, and of the axle, is the same as other mail

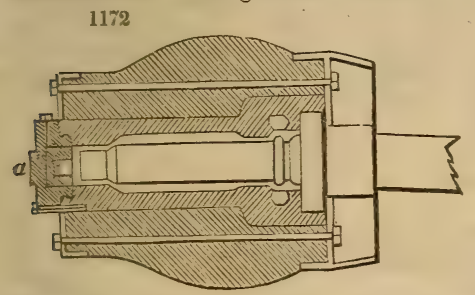

1173

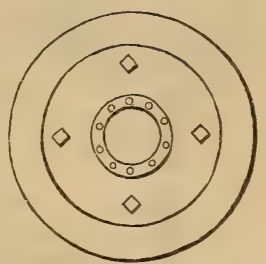
axles, there being recesses in the box for the reception of oil. At the end of the axle, a cap $a$, is inserted, with a leather collar enclosed in it, bearing against the end of the axle; which cap, when screwed up suffieiently tight, is held in that situation by a pin or screw passed through the cap $a$, into the end of the iron box; a representation of this end of the iron box being shown at fig. 1173 .

In the cap $a$, there is also a groove for conducting the oil to the interior of the box, with a screw at the opening, to prevent it running out as the wheel goes round.

The particular claims of improvement are, the leather collar against the end of the axle; the pin going through one of the holes in the end of the box, to fix it; and the channel for conducting the oil.

Mr. Mason's patent, of August, 1830, applies also to the boxes and axles of that construction of carriage wheels which are fitted with the so called mail-boxes; but part of the invention applies to other axles.

Fig. 1174, represents the nave of a wheel, with the box for the axle within it, both shown in section longitudinally; fig. 1175, is a section of the axle, taken in the same direction; and fig. 1176, represents the screw cap and oil-box, which attaches to the outer extremity of the axle-box. Supposing the parts were put together, that is, the axle inserted into the box, then the intention of the different parts will be perceived.

The cylindrical recess $a$, in the box of the nave, is designed to fit the cylindrical part of the axle $b$; and the conical part $c$, of the axle, to shoulder up against a corresponding conical cavity in the box, with a washer of leather to prevent its shaking. A collar $d$, formed by a metallic ring, fits loosely upon a cylindrical part of the axle, and is kept there by a flange or rim, fixed behind the cone $c$. Several strong pins $f, f$, are cast into the back part of the box; which pins, when the wheel is attached, pass through corresponding holes in the collar $d$; and nuts being screwed on to the ends of the pins $f$, behind the collar, keep the wheel securely attached to the axle. 'The screw-cap g, is then inserted into the recess $h$, at the outer part of the box, its conical end and small tube $i$, passing into the recess $\boldsymbol{k}$, in the end of the axle.

The parts being thus connected, the oil contained within the cap g, will flow through the small tube $i$, in its end, into the recess or eylindrical channel $l$, within the axle, and will thence pass throngh a small hole in the side of the axle, into the cylindrical recess $a$, of the box; and then lodging in the groove and other cavities within the box, will lu. 
bricate the axle as the wheel goes round. There is also a small groove cut on the outside of the axle, for conducting the oil, in order that it may be more equally dis.

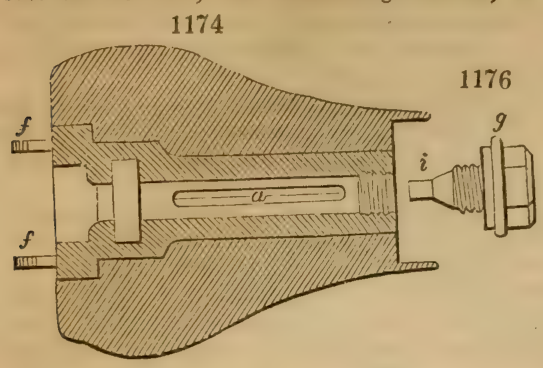
tributed over the surface and the bearings. This construction of the box and axle, as far as the lubrication goes, may be applied to the axles of wheels in general; but that part of the invention which is designed to give greater security in the attachment of the wheel to the carriage, applies particularly to mail axles.

Mr. William Mason's patent invention for wheel earriages, of August, 1831 , will be understood by reference to the annexed figures. Fig. 1177, is a plan showing the fore-axletree bed $a, a$, of a four-wheeled carriage, to which the axletrees $b, b$, are jointed at each end; fig. 1178 is an enlarged plan; and fig. 1179 an elevation, or side view of one end of the said fore-axletree bed, having a Collinge's axletree jointed to the axletree bed, by means of the cylindrical pin or bolt $c$, which passes through and turns in n cylindrical hole $d$, formed at the end of the axletree bed, shown also in the plan view, fig. 1180 , and section, fig. 1181 .
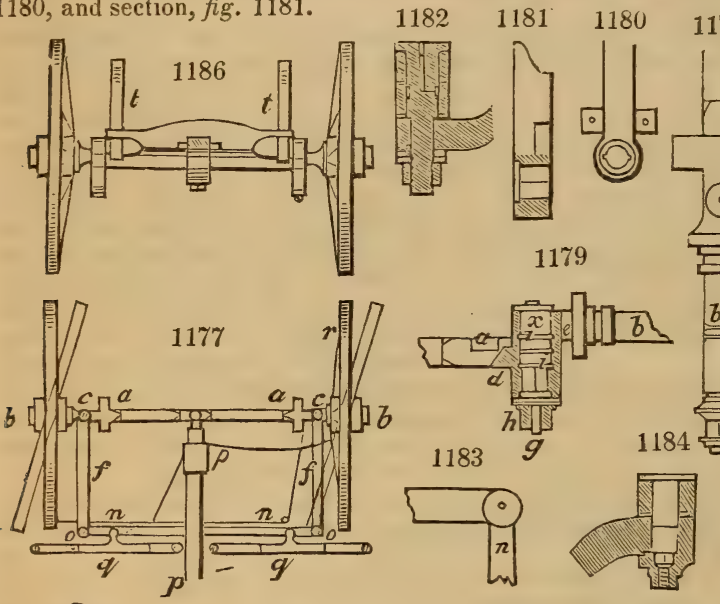

1178
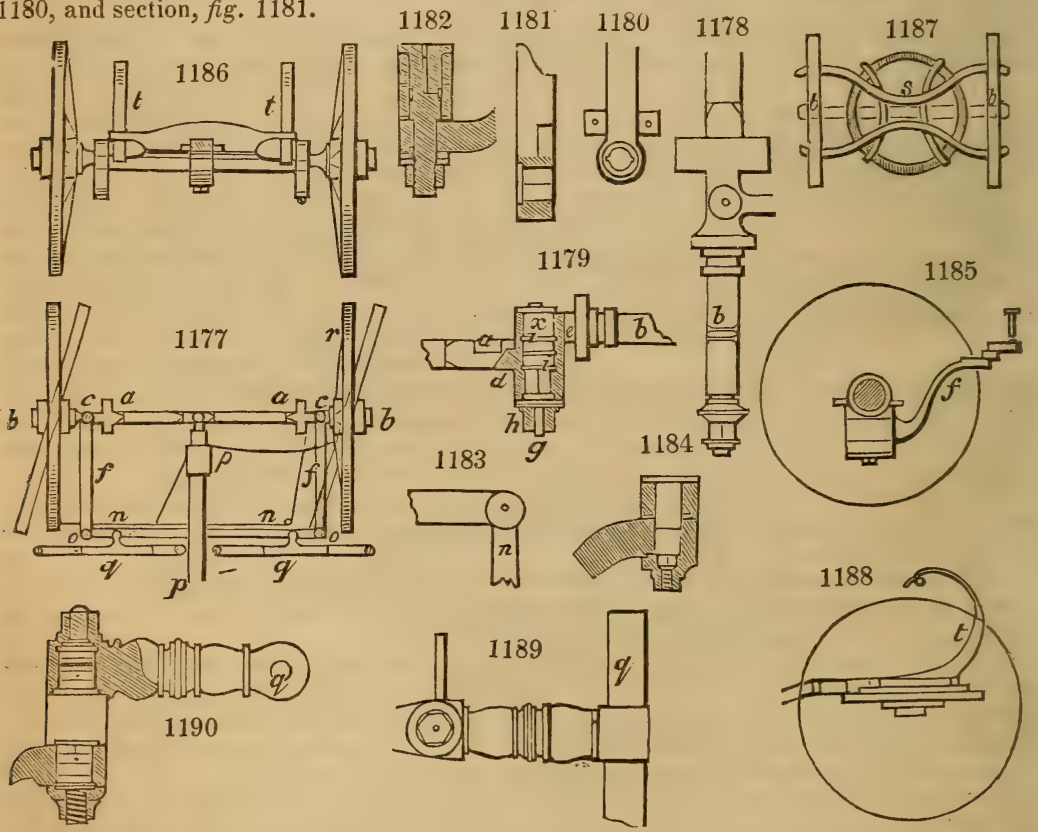

The axletree $b$, is firmly united with the upper end $e$, of the pin or bolt $c$; and to the lower end of it, which is squared, the guide piece $f$, is also fitted, and secured by the screw $g$, and cap or nut $h$, seen in fig. 1179, and in section in $f i g .1182$. There are leather washers $i, i$, let into recesses made to receive them in the parts $a, b$, and $f$, the intent of which is to prevent the oil from escaping that is introduced through the central perpendicular hole seen in fig. 1182, which hole is closed by means of a screw inserted into it. The oil is diffused, or spread over the surface of the cylinder $c$, by means of a side branch leading from the bottom of the hole into a groove formed around the cylinder, and also by means of two longitudinal gaps or cavities made within the hole, as shown in figs. 1180, and 1181. The guide piece $f$, is affixed at right angles with the axletree $b$. as shown in fig. 1178, and turns freely and steadily in the cylindrical hole $d$, made to receive one end of the iron fore-axletree bed $a$. In like manner, the opposite fore axletree $b, f i g .1177$, is jointed to the other end of the iron fore-axletree bed. 
The outer ends of the guide pieces $f, f$, are jointed to the splinter-bar $n, f i g .1181$, as follows:-Fig. 1183, is a plan, and itg. 118t, a section of the joint 0 , in fig. 1177, shown on an enlarued scale; a cylindrieal pin or bolt $c$, is firmly secured in the splinter-bar, and round the luwer part of the said pin or bolt the guide piece $f$, turns, and is made fast in its place by the screw $g$, and screwed nut $h$.

$\mathrm{O}_{1} \mathrm{l}$ is conveyed to the lower part of the cylindrical pin $c$, in a similar manner to that already described, and two leather washers are likewise furnished, to prevent its escape The connecting joint at the opposite end of the splinter bar $n$, is constructed in a sinilar manner. The futchel or socket $p, p$, for the pole of the carriage, must also be jointed to the midille of the fore-axletree bed and splinter-bar, in a similar manner. The swingletrees $q, q, f i g .11 \% 7$, are likewise jointed in the same way to the splinter-bar. Fig. 1185 , is a side view of these parts. The fore wheels of the carriage, fig. 1177, are furnished with cast-iron boxes, as usual. The dotted lines show the action of the pole $p, p$, upon the splinter-bar $n$, and as communicated through the latter to the guide pieces $f, f$, connected with the axletrees $b, b$, so as to lock the wheels $r, r$, as shown in that figure.

The axletree may be incased in the woodwork of the fore-bed of the carriage, as usual, and as shown by dotted lines in the back end view thereof, fig. 1186; and the framing $s$, fig. 1187, may be affixed firmly upon the said woodwork, in any fit and proper manner, as well as the fore-springs $t, t$, shown in figs. 1186 and 1187, and like. wise in the side riew, fig. 1188. In certain cases it may be desirable to fix the cylindrical pin or bult $c$, firmly in the splinter-bar $n$, in the manner shown in figs. 1189 and 1190 ; the swingletrees $q, q$, and guide pieces $f, f$, turning fieely above and below upon the said pin or bolt, and secured in their places thereon by screws and screwed nuts, oil being also sitiplied through holes formed in both ends of the said pin or bolt, and leather washers provided, as in the above-described instances.

Mr. Gibbs, engineer, and Mr. Chaplin, coach-maker, obtained a patent, in 1832, for the construction of a four-wheeled carriage which shall be enabled to turn within a small compass, by throwing the axles of all the four wheels simultaneously into different positions. They efféct this object by mounting each wheel upon a separate jointed

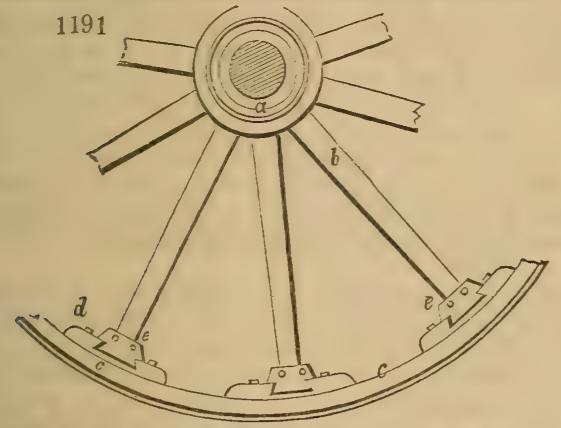
axle, and by connecting the free ends of the four axles by jointed rods or chains, with the pole and splinterbar in front of the carriage.

To fix the ends of the spokes of wheels to the felloe or rim, with greater security than had been effected by previous methods, is the object of a contrivance for which William Howard obtained a patent, in February, 1830. Fig. 1191 shows a portion of a wheel constructed on this new method; $a$, is the nave, of wood; $b, b, b$, wooden spokes, inserted into the nave in the usual way; $c, c$, is the rim or felloe, intended to be formed by one entire circle of wrought iron; $d$, and $e, e$, are the shoes or blocks, of cast iron, for receiving the ends of the spokes, which are secured by bolts to the rim on the inner circumference. The cap of the block $d$, is removed, for the purpose of showing the internal form of the block; $e, e$, have their caps fixed on, as they would appear when the spoles are fitted in. One of the caps or shoes is shown detached, upon a larger scale, at fig. 1192, by which it will be perceived that the end

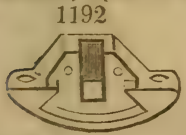
of the spoke is introduced into the shoe on the side. It is proposed that the end of the spoke shall not reach quite to the end of the recess formed in the block, and that it shall be made tight by a wedge driven in. The wedge piece is to be of wood, as fig. 1193, with a small slip of iron within it; and a hole is perforated in the back of the block or shoe, for the wedge to be driven through. When this is done, the ends of the spokes become confined and tight; and the projecting extremities of the

1193 wedges being cut off, the caps are then attached on the face of the block, as at $e, e$, by pins riveted at their ends, which secures the spolies, and renders it impossible for them to be loosened by the vibrations as the wheel passes srer the ground. One important use of the wedges, is to correct the eccentric figure of the wheel, which may be readily forced out in any part that may be out of the true form, by driving the wedge up further; and this, it is considered, will be a very important advantage, as the nearer a wheel can be brought to a true circle, the easier it will run upon the road. The periphery of the wheel is to be protected by a tire, 
which may be put on in pieces, and bolted through the felloe; or it may be made in one ring, and attached, while hot, in the usual way.

Mr. Reedhead's patent improvements in the construction of carriages, are represented in the following figures. They were specified in July, 1833.

Fig. 1194, is a plan or horizontal view of the fore part of a carriage, intended to be drawn by horses, showing the fore wheels in their position when running in a straight course; fig. 1195, is a similar view, showing the wheels as locked, wher, in the act of

1194

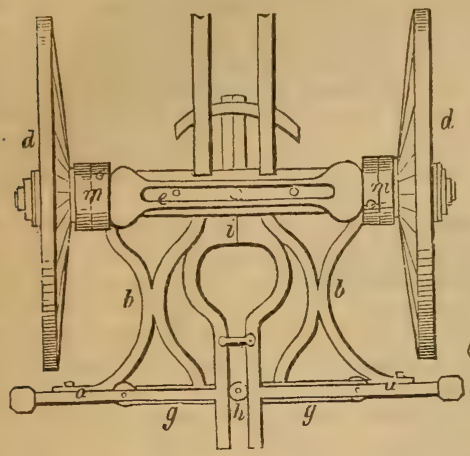

1195

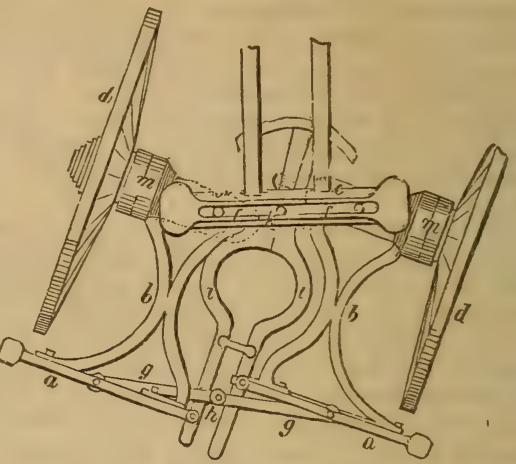

turning; fig. 1196, is a front end elevation of the same; fig. 1197, is a section taken through the centre of the fore axletree; and fig. 1198, is a side elevation of the general appearance of a stage-coach, with the improvements appended: $a, a$, are two splinterbars, with their roller-bolts, for connecting the traces of the harness; these splinter-bars are attached, by the bent irons $b, b$, to two short axletrees or axle-boxes $c$, $c$, which carry the axles of the fore wheels $d, d$, and turn upon vertical pins or bolts $e, e$, passed through the fore axletree $f$, the splinter-bars and axle-boxes being mounted so as to move parallel to each other, the latter partaking of any motion given to the splinter-bars by the horses in drawing the carriage forward, and thereby producing the locking of the wheels, as shown in fig. $1195 ;$ and in order that the two wheels, and their axles and axle-boxes, together with the splinter-bars $a, \alpha$, may move simultaneously, the latter are connected by pivots to the end of the links or levers $g, g$, which are attached to the arms $i, i$, which receive the pole of the coach by a hinge-joint or pin $h$; the arms $i, i$, turning on a vertical fulcrum-pin $k$, passed through the main axletree $f$, as the pole is moved from one side to the other.

The axles 0,0 , are firmly fixed into the naves of the wheels, as represented in the side view of a wheel detached, at fig. 1200, the axles being mounted so as to revolve within their boxes in the following manner:- The axle-boxes, which answer the purpose of short axletrees, are formed of iron, and consist of one main or bottom plate $l$, seen best in figs. 1200 and 1199 ; upon this bottom plate is formed the chamber $m, m$, carrying the two anti-friction rollers $n, n$, which turn on short axles passed through the sides and partition at the upper part of the chambers. These anti-friction rollers bear upon the cylindrical parts of the axle $o_{\mathrm{s}}$ of each wheel, and support the weight of the coach; $p$, is a bearing firmly secured in the axle-box to the plate $l$, for the end of the axle 0 to run 1196

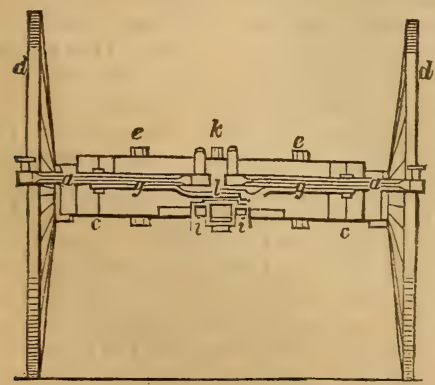

1197

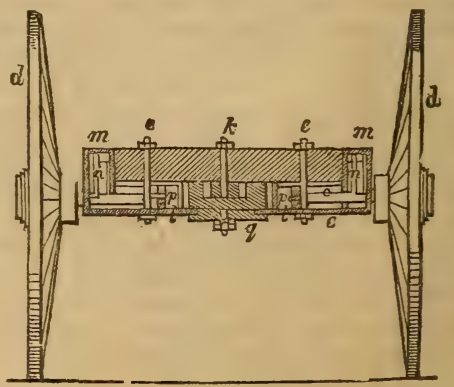

in, the axle being confinel in its proper situation by a collar and screw-nut on its end ; e, is the vertical pin or bolt before mentioned, upon which the axle-bar turns when the 
wheels are locking, which bolt is enlarged within the hox, and has an eye for the axle to pass through, being firmly secured to the plate $l$, and also to the sides of the box. Fig.

1200

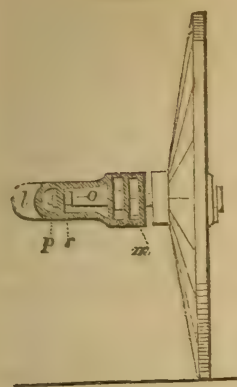
1200 , is a plan or horizontal view of an axle and its box, belonging to one of the fore wheels; a piece $q$, is fixed to the under side of the main axletree, which supports the ends of the plates $l$, and thereby relieves the pins $e, e$, of the strain they would otherwise have to withstand. The axles of the hind wheels are mounted upon similar plates $l, l$, with bearings and chambers with anti-friction rollers; but as these are not required to lock, the plates $l, l$, are fixed on to the under side of the hind axletree by screw-nuts; there are small openings or doors, which can be removed for the purpose of unscrewing the nuts and collars of the bearings $p$, when the wheel is required to be taken off the carriage, when the axle can be withdrawn from the boxes. If it should be thought necessary, other chambers with friction rollers may be placed on the under side of the plate $l$, to bear up the end of the axles, and relieve the bearing p. In order to stop or impede the progress of a carriage in passing down hills, there is a grooved friction or brake wheel $t$, fixed, by clamps or otherwise, on to the spokes of one of the hind wheels; $\boldsymbol{~}$, is a brake-band or spring, of metal, encircling the friction wheel, one end of which band is fired into the standard $v$, upon the hind axletree, and the other end connected by a joint to the shorter end of the lever $w$, which has its fulcrum in the standard $v$; this leser extends up to the hind seat of the coach, as shown in fig. 1198, and is inrenied to be under the command of the guard or passengers of the coach, and when deseending a hill, or on occasion of the horses running away, the longer end of the lever is to be depressed, which will raise the shorter end, and, consequently, bring the band or spring $u$, in contact with the surface of the friction wheel, and thereby retard its revolution, and prevent the coach travelling too fast; or, instead of attaching the friction brake to the hind wheel, as represented in fig. 1198, it may be adapted to the fore
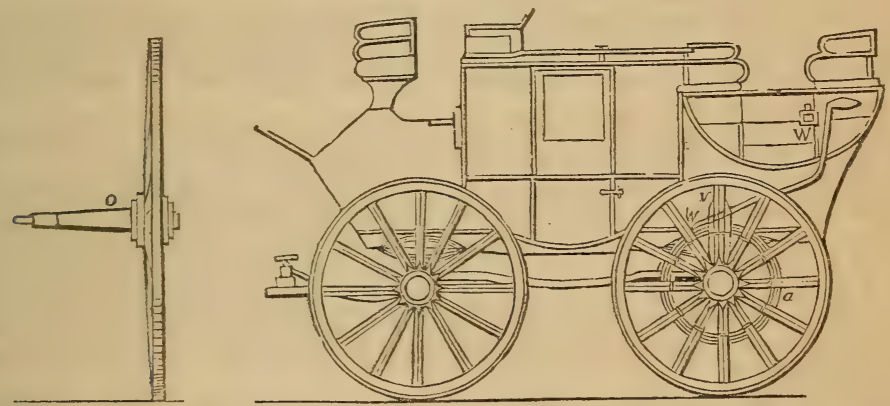

wheels, and the end of the lever brought up to the side of the foot-board, or under it, and within command of the coachman, the standard which carries the fulcrum being made to mose upon a pirot, to accommodate the locking of the wheels. It will be observed, that by these improved constructions of the carriage, and mode of locking, the patentee is enabled to use much larger fore wheels than in common, and that the splinter-bars will always be in the position of right angles with the track or way of the horses in drawing the carriage, by which they are much relieved, and always pull in a direct and equal manner.

A manifest defect in all four-wheeled carriages, involving vast superfluous friction, is the small size of the front wheels; a defect which has existed ever since Walter Rippon made "she first hollow turning coach with pillars and arches for her majesty Queen Nary, being then her servant," until the railroad era, when our engineers remedied the defect by equalizing the wheels, at the expense of another defect-sacrificing the power of turning, and thus producing great lateral friction; whence a train of evil consequences result :-necessarily increased strength, and consequently increased weight of the carriages; increased power and weight of the .engine to draw them, and overcome the friction; and, of course, inereased strength of rails, and greater solidity of railway.

These defects are at last remedied by an invention patented by Mr. William Adams, author of a work entitled "English Pleasure Carriages." Instead of placing the perchbolt, or turning centre, as is commonly done, over the front axle, he places it at a convenient distance between the front and hind axles; so that when turning the carriage the 
front wheels, instead of turning beneath the body, as is common, turn outside of it, ano the driver's seat turns with them; thus giving him a perfect command over his horses in all positions, instead of the usual dangerous plan, which renders a driver liable to be pulled off his box by a restiff horse, when in the act of turning. A carriage constructed on Mr. Adains's plan may also be driven round a corner at full speed, without any risk of overturning, as the weight is erjually poised on the axles in all positions. It is well known that the oversetting of stage coaches usually takes place when turning a corner, the momentum urging the vehicle in a right line, while the horses are pulling at an angle. By the new arrancement the front wheels may be made equal to the hind ones, or of any desirable height, and at the same time the body may be kept as low as inay be thought convenient, even almost close to the ground, if desired. Thus two important objects, hitherto deemed incompatible, are combined-high wheels and a low centre of grarity. These carriages are therefore essentially safety carriages, while the friction is reduced to a minimum. The principle, in its varions nodifications, is applicable to every variety of carriage, both those of the simply useful kind, and those where beauty of form and color are prime requisites.

Another most important part of Mr. Adams's invention, is his new mode of spring suspension; applying the principle of the bow and string, for the first time, to obriate the effects of concussion in wheel carriages. All the springs hitherto in use for wheel carriages, have been friction springs, composed of long sliding surfaces, uncertain in their action, and liable to quick destruction by rust. But Mr. Adams's springs are essentially elastic, being formed of single plates alutting endways, so that all friction is remored, and they can be hermeticaliy sealed within paint to prevent their corrosion. He has various modes of applying the bow, either single or double, above or below the axle; but one most important feature is, that the axle being attached to the flexible cords or braces, the concussion which affects the wheels, either laterally, vertically, or in the line of progress, is perfectiy intercepted, without the unpleasant oscillation experienced in carriages where the same purpose is accomplished by the use of the curved or $\mathrm{C}$ spring. Mr. Adams's brace being, at the same time, a non-conductor of sound, the rattling of the wheels does not annoy the rider as in ordinary carriages. His springs are equally applicable to vehicles with two and four wheels.

The advantages of these carriages may be thus summed up :-A great diminution of the total weight; a diminution of resistance in draught equal to about one third; inerease of safety to the riders; increased durability of the vehicle; absence of noise and vibration; absence of oscillation.

To these qualities, so desirable to all, and especially those of delicate nervous temperament, may be added-greater economy, both in the first cost and maintenance.

The whirling public so blindly follows fashionable caprice in the choice of a carriage, as to have hitherto paid too little attention to this fundamental improrement; but many intelligent individuals have fully verified its practical reality. Having inspected various forms of two-wheeled and four-wheeled carriages, in the patentee's premises in Drury Lane, I feel justified in recommending them as being constructed on the soundest mechanical principles; and have no doubt, that if reason be allowed to decide upon their merits, they will ere long be universally preferred by all who seek for easy-moving, safe, anc? comfortable vehicles.

WHETSLATE, is a massive mineral of a greenish-gray color; feebly glimmering; fracture, slaty or splintery; fragments tabular; translucent on the edges; feels rather greasy; and has a spec. grav. of $2 \cdot 722$. It occurs in beds, in primitive and transition slates. Very fine varieties of whetslate are brought from Turkey, called hone-stones, which are in much esteem for sharpening steel instruments.

WHEY (Petit lait, Fr.; Molken, Germ.), is the greenish-gray liquor which exudes from the curd of milk. Scheele states, that when a pound of milk is mixed with a spoonful of proof spirit, and allowed to become sour, the whes filtered off, at the end of a month or a little more, is a good vinegar, devoid of lactic acid.

WHISKEY, is dilute alcohol, distilled from the fermented worts of malt or grains.

WHITE LE.AD, Carbonate of lead, or Ceruse. (Blunc de plomb, Fr.; Bleiweiss, Germ.) This preparation is the only one in general use for painting wood and the plaster walls of apartments white. It mixes well with oil, without having its bright color impaired, spreads easily under the brush, and gives a uniform coat to wond, stone, metal, \&c. It is employed either alone, or with olher nigments, to serve as their basis, and to give them. body. This article has been long manufactured with much success at Klagenfurth in Carinthia, and its mode of preparation has been lately described with precision by Marcel de Serres. The great white-lead establishments at Krems, whence, though incorrectly, the terms white of Kremnitz became current on the continent, have been abandoned.

1. The lead comes from Bleyberg; it is very pure, and particularly free from contamination with iron, a point essential to the beauty of its factitious carbonate. It is melted 
In ordinary pits of cast iron, and cast into sheets of varying thickness, according to the pleasuce of the manufacturer. These sheets are made by pouring the melted lead upon an irun plinte placed over the boiler; and whenever the surface of the metal begins to consolidite, he plate is slightly sloped to one side, so as to run off the still liquid metal, an.I leitre a lead sheet of the desired thinness. It is then lifted off like a sheet of paper; and as the iron plate is cooled in water, several hundred weights of lead can he readily cas! in a day. In certain white-lead works these sheets are one twenty-fourth of an inch thick; in others, half that quantity; in some, one of these sheets takes up the whole widih of the conversion-box; in others, four sheets are employed. It is of consequence not 10 smooth down the faces of the leaden sheets; because a rongh surface presents more points of contact, and is more readily attacked by acid vapors, than a polished one.

2. These plates are now placed so as to expose an extensive surface to the acid fumes, by folding each other over a square slip of wood. Being suspended by their middle, like a sheet of praper, they are arranged in wooden boxes, from $4 \frac{1}{2}$ to 5 feet long, 12 to 14 inches broal, and from 9 to 11 inches deep. The boxes are very substantialiy constructed; their joints being morlised; and whatever nails are used being carefully covered. Their bottom is made tight with a coat of pitch about an inch thick. The mouths of the boxes are luted over with paper, in the works where fermenting horse-dung is employed as the means of procuring heat, to prevent the sulphureted and phosphureted hydrogen from injuring the purity of the white lead. In Carinthia it was formerly the practice, as also in Holland, to form the lead sheets into spiral rolls, and to place them so coiled up in the chests; but this plan is not to be recommended, because these rolls resent obvionsly less surface to the action of the vapors, are apt to fall down into the liquid at the bottom, and thus to impair the whiteness of the lead. The lower edges of the sheets are suspended about two inches and a half from the bottom of the box; and they must not touch either one another or its sides, for fear of obstructing the vapors in the first case, or of injuring the color in the second. Before introducing the lead, a peculiar acid liquor is put into the box, which differs in different works. In some, the proportions are four quarts of vinegar, with four quarts of wine-lees; and in others, a mixture is made of twenty pounds of wine-lees, with eight and a half pounds of vinegar, and a pound of carbonate of potash. It is evident that in the manufactories where no carbonate of potash is employed in the mixture, and no dung for heating the boxes, it is not necessary to lute them.

3. The mixture being poured into the boxes, and the sheets of lead suspended within them, they are carried into a stove-room, to receive the requisite heat for raising round the lead the corrosive vapors, and thus converting it into carbonate. This apartment is heated generally by stoves, is about 9 feet high, 30 feet long, and 24 feet wide, or of such a size as to receive about 90 boxes. It has only one door.

The heat should never be raised above $86^{\circ} \mathrm{Fahr}$; and it is usually kept up for fifteen days, in which time the operation is, for the most part, completed. If the heat be too high, and the vapors too copious, the carbonic acid escapes in a great measure, and the metallic lead, less acted upon, affords a much smaller product.

When the process is well managed, as much carbonate of lead is obtained, as there was employed of metal; or, for 300 pounds of lead, 300 of ceruse are procured, besides a certain quantity of metal after the crusts are removed, which is returned to the meltingpot. The mixture introduced into the boxes serves only once; and if carbonate of potash has been used, the residuary matter is sold to the hatters.

4. When the preceding operation is supposed to be complete, the sheets, being removed from the boxes, are found to have grown a quarter of an inch thick, though previously not absre a twelfth of that thickness. A few pretty large crystals of acetate of lead are sometimes observed on their edges. The plates are now shaken smartly, to cause the crust of carbonate of lead formed on their surfaces to fall off. This carbonate is put into large cisterns, and washed very clean. The cistern is of wood, most commonly of a square shaje, and dirided into from seven to nine compartments. These are of equal capacity, but inequal height, so that the liquid may be made to overflow from one to the other. Thereby, if the first chest is too full, it decants its excess into the second, and so on in succession. See Rinsing Machine.

The water poured into the first chest passes successively into the others, a slight agitation being meanwhile kept up, and there deposites the white lead diffused in it proportionally, so that the deposite of the last compartment is the finest and lightest. After this washing, the white lead receives another, in large vats, where it is always kept under water. It is lastly lifted out in the state of a liquid paste, with wooden spoons, and laid on drying-tables to prepare it for the market.

The white lead of the last compartment is of the first quality, and is called on the con. tinent silver white. It is employed in fine painting.

When white lead is mixed in equal quantities with ground sulphate of barytes, it is 
known in France and Germany by the name of Venice white. Another quality, adulter. ated with double its weight of sulphate of barytes, is styled Hamburgh white; and a fourth, having three parts of sulphate to one of white lead, gets the name of Dutch white. When the sulphate of barytes is very white, like that of the Tyrol, these mixtures are reckoned preferable for certain kinds of painting, as the barytes communicates opacity to the color, and protects the lead from being speedily darkened by sulphureous smoke or vapors.

The high reputation of the white lead of Krems was by no means due to the barytes, for the first and whitest quality was mere carbonate of lead. The freedom from silver of the lead of Villach, a very rare circumstance, is one cause of the superiority of its carbonate; as well as the sliilful and laborious manner in which it is washed, and separated from any adhering particle of metal or sulphuret.

In England, lead is converted into carbonate in the following way :-The metal is cast into the form of a net-work grating, in moulds about fifteen inches long, and four or five broad. Several rows of these are placed over cylindrical glazed earthen pots, about four or five inches in diameter, containing some treacle-vinegar, which are then covered with straw; above these pots another range is piled, and so in succession, to a convenient height. The whole are imbedded in spent bark from the tan-pit, brought into a fermenting state by being mixed with some bark used in a previous process. The pots are left undisturbed under the influence of a fermenting temperature for eight or nine weeks. In the course of this time the lead gratings become, generally speaking, converted throughout into a solid carbonate, which when removed is levigated in a proper mill, and elutriated with abundance of pure water. The plan of inserting coils of sheet lead into earthenware pipkins containing vinegar, and imbedding the pile of pipkins in fermenting horsedung and litter, is now little used; because the coil is not uniformly acted on by the acid vapors, and the sulphureted hydrogen evolved from the dung is apt to darken the white lead.

In the above processes, the conversion of lead into carbonate seems to be effected by keeping the metal immersed in a warm, humid atmosphere, loaded with carbonic and acetic acids; and hence a pure vinegar does not answer well; but one which is susceptible, by its spontaneous decomposition in these circumstances, of yielding carbonic acid. Such are tartar, wine-lees, molasses, \&c.

Another process has lately been practised to a considerable extent in France, though it does not afford a white lead equal in body and opacity to the products of the preceding operations. M. Thenard first established the principle, and MM. Brechoz and Leseur contrived the arrangements of this new method, which was subsequently executed on a great scale by MM. Roard and Brechoz.

A subacetate of lead is formed by digesting a cold solution of uncrystallized acetate, over litharge, with frequent agitation. It is said that 65 pounds of purified pyroligneous acid, of specific gravity $1 \cdot 056$, require, for making a neutral acetate, 58 pounds of litharge ; and hence, to form the subacetate, three times that quantity of base, or 174 pounds, must be used. 'The compound is diluted with water as soon as it is formed, and being decanted off quite limpid, is exposed to a current of carbonic acid gas, which, uniting with the two extra proportions of oxyde of lead in the subacetate, precipitates them in the form of a white carbonate, while the liquid becomes a faintly acidalous acetate. The carbonic acid may be extricated from chalk, or other compounds, or generated by combustion of chalcoal, as at Clichy; but in the latter case, it must be transmitted throngh a solution of acetate of lead before being admitted into the subacetate, to deprive it of any particles of sulphureted hydrogen. When the precipitation of the earbonate of lead is completed, and well settled down, the supernatant acetate is decanted off, and made to act on another dose of litharge. The denosite being first rinsed with a little water, this washing is added to the acetate; after which the white lead is thoroughly elutriated. This repetition of the process may be indefinitely made; but there is alwar's a small loss of acetate, which must be repaired, either directly or by adding some vinegar.

In order to obtain the finest white lead by the process with earthen pots containing vinegar buried in fermenting tan, and covered by a grating of lead, the metal should be so thin as to be entirely convertible into carbonate; for whenever any of it remains, it is ant to give a gray tint to the product; if the temnerature of the fermenting mass is less than $90^{\circ}$ Fahr., some particles of the metal will resist the action of the vinegar, and degrade the color; and if it exceeds $122^{\circ}$, the white verges into yellow, in con sequence of some carbonaceous compound being develoned from the principles of the acetic acid. The dung and tan have been generally supposed to act in this process by supplying carbonic acid, the result of their fermentation; but it is now said that this explanation is inexact, because the best white lead can be obtained by the entire exclusion of air from the pots in which the carbonization of the metal is carried on. We are thence led to conclude that the lead is oxydized at the expense of the oxygen 0 , the vinegar, and carbonated by the agency of its oxygen and carbon; the hydrogen 0 . 
the acil being left to associate itself with the remaining oxygen and carbon, so as to constilute an ethereous compound: thus, supposing the three atoms of oxrgen to form, with one of leal and one of carbon, an atom of carbonate, then the remaining three atoms of carbon and three of hydrogen would compose olefiant gas.

It is eustomary on the continent to mould the white lead into conjanl loaves, before senling them intu the market. This is done by stuffing well-drained white lead into unilazed earthen pots, of the requisite size and shape, and drying it to a solid mass, by expusing these pots in stove rooms. The moulds beirig now inverted on tables, discharge their contents, which then receive a final desiccation; and are afterwards put up in pale. blue parer, to set off the white color by contrast. Nothing in all the white-lead process is so injurious as this pot operation; a useless step, fortunately unknown in Great Britain. Neither greasing the skin, nor wearing thick gloves, can protect the op rators from the diseases induced by the poisonous action of the white lead; and hence they must be soon sent off to sume other department of the work.

It has been sapposel that the differences observed between the ceruse of Clichy and the connun kinds, depend on the greater compactness of the particles of the latter, produce 1 by their slower aggregation; as also, according to $\mathbf{M}$. Robiquet, on the former containing considerably less carbonic acid. See infra.

Mr. Ham proposed, in a patent dated June, 1826 , to produce white lead with the ard of the folluwing apparatus. $a, a(f i g .1201)$ are the side-walls of a stove-room, constructed of 1201

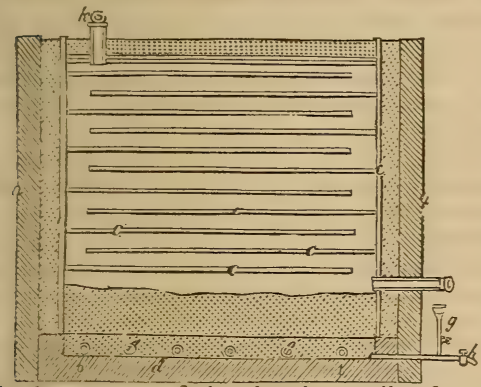
bricks; $b$, is the floor of bricks laid in Roman cement; $c, c$, are the side-plates, between which and the walls, a quantity of refuse tanner's bark, or other suitable vegetable matter, is to be introduced. The same material is to be put also into the lower part at $d$ (upon a false bottom of graling ?) The tan should rise to a considerable height, and have a series of strips of sheet lead $e, e, e$, placed upon it, which are kept apart by blocks or some other convenient means, with a space open at one end of the plates, for the passage of the vapors; but above the upper plates, boards are placed, and covered with tan, to confine them there. In the lower pait of the chamber, coils of steam-pipe $f, f$, are laid in different directions to distribute heat; $g$, is a funnel-pipe, to conduct vinegar into the lower part of the vessel; and $h$, is a cock to draw it off, when the operation is suspended. The acid vapors raised by the heat, pass up through the spent bark, and on coming into contact with the sheets of lead, corrode them. The quantity of acid liquor should not be in exeess; a point to be ascertained by means of the small tube $i$, at top, which is intended for testing it by the tongue. $k$, is a tube for inserting a thermometer, to watch the temperature, which should not exceed $170^{\circ}$ Fahr. I am not aware of what success has attended this patented arrangement. The heat prescribed is far too great.

A magnificent factory has been recently erected at West Bromwich, near Birmingham, to work a patent lately granted to Messrs. Gossage and Benson, for making white lead by mixing a small quantity of acetate of lead in solution with slightly damped litharge, contained in a long stone trough, and passing over the surface of the trough currents of hot carbonic acid, while its contents are powerfully stirred up by a travelling-wheel mechanism. The product is afterwards ground and elutriated, as usual. The carbonic acid gas is produced from the combustion of coke. I am told that 40 tons of excellent white lead are made weekly by these chemico-mechanical operations.

Messrs. Button and Dyer obtained a patent about a year and a half ago, for making white lead by transmitting a current of purified carbonic acid gas, from the combustion of coke, through a mixture of litharge and nitrate of lead, diffused and dissolved in water, which is kept in constant agitation and ebullition by steam introduced through a perforated coil of pipes at the bottom of the tub. The carbonate of lead is formed here upon the principle of Thenard's old process with the subacetate; for the nitrate of lead forms with the litharge a subnitrate, which is forthwith transformed into carbonate and neutral nitrate, by the agency of the carbonic acid gas. I have discovered that all sorts of white lead produced by precipitation from a liquid, are in a semi-crystalline condition; they appear, therefore, semi-transparent, when vieved in the microscope; and do not cover so ivell as white lead made by the process of vinegar and tan, in which the lead has remained always solid during its transition from the blue to the white state; and hence consists of opaque particles.

A patent was obtained, in December, 1833, by John Baptiste Constantine Torassa, 
and others, for making white lear by agitating the granulated metal, or shot, in trays or barrels, aleng with water, and exposing the mixture of lead-dust and water to the air, to be oxydized and carbonated. It is said that upwards of $100,000 \%$. have been cxpended at Chelsea, by a joint stock company, in a factory constructed for executing the preceding most operose and defective process; which has been, many years ago, tried without success in Germany. I am convinced that the whole of these recent pro. jects for preparing white lead, are inferior in economy, and quality of produce, to the old Dutch process, which may be so arranged as to convert sheets of blue lead thoroughly into the best white lead, within the space of 12 days, at less expense of labor than by any other plan.

White lead, as obtained by precipitation from the acetate, subacetate, and subnitrate, is a true carbonate of the metal, consisting of one prime equivalent of lead 104, one of oxygen 8 , and one of carbonic acid 22 ; whose sum is 134 , the atomic weight of the compound; or, of lead, 77.6 ; oxygen, 6 ; carbonic acid, 16.4 ; in 100 parts. It nas been supposed, by some authors, that the denser and better-covering white lead of Krems and Holland is a kind of subcarbonate, containing only 9 per cent. of carbonic acid; but this view of the subject does not accord with my researches.

Wick (Mèche, Fr.; Docht, Germ.), is the spongy cord, usually made of soft spun cotton threads, which by capillary action draws up the oil in lamps, or the melted tallow or wax in candles, in sinall successive portions, to be burned. In common wax and tallow candles, the wick is formed of parallel threads; in the stearine candles, the wick is plaited upon the brailing machine, mostened with a very dilute sulphuric acid, and dried, whereby, as it burns, it falls to one side and consumes without requiring to be snuffed; in the patent candles of $\mathrm{Mr}$. Palmer, one tenth of the wick is first imbued with subnitrate of bismuth ground up with oil, the whole is then bound round in the manner called gimping; and of this wick, twice the length of the intended candle is twisted double round a rod, like the caduceus of Mercury. This rod with its coil being inserted in the axis of the candle mould, is to be enclosed by pouring in the melted tallow; and when the tallow is set, the rod is to be drawn out at top, leaving the wick in the candle. As this candle is burned, the ends of the double wick stand out sideways beyond the flame; and the bismuth attached to the cotton being acted upon by the oxygen of the atmosphere, causes the wick to be completely consumed, and, therefore, saves the trouble of snuffing it.

WINCING-MACHINE, is the English name of the dyer's reel, which he suspends horizontally, by the ends of its iron axis in bearings, over the edge of his vat, so that the line of the axis, being placed uver the middle partition in the copper, will permit the piece of eloth which is wound upon the reel to descend alternately into either compartment of the bath, according as it is turned by hand to the right or the left. For an excellent selfacting or mechanical wince, see Dyerng.

WINE, is the fermented juice of the grape. In the more southern states of Europe, the grapes, being more saccharine, afford a more abundant production of alcohol, and stronger wines, as exemplified in the best port, sherry, and madeira. The influence of solar heat upon the vines may, however, be mitigated by growing them to moderate heights on level ground, and by training them in festoons under the shelter of trees. In the more temperate climates, such as the district of Burgundy, the finer flavored wines are produced; and there the vines are usually grown upon hilly slopes fronting the south, with more or less of an easterly or westerly direction, as on the Côte $\mathrm{d}^{\prime} \mathrm{Or}$, at a distance from marshes, forests, and rivers, whose vapors might deteriorate the air. The plains of this district, even when possessing a similar or analogous soil, do not produce wines of so agreeable a flavor. The influence of temperature becomes very manifest in countries further north, where, in consequence of a few degrees of thermometric depression, the production of generous agreeable wine becomes impossible.

The land most farorable to the vine is light, easily permeable to water, but somewhat retentive by its composition; with a sandy subsoil, to allow the excess of moisture to drain readily off. Calcareous soils produce the highly esteemed wines of the Cote d'Or; a granite debris forms the foundations of the lands where the Hermitage wines are grown; silicious soil interspersed with flints furnishes the celebrated wines of Château-Neıtf, Ferté, and La Gaude; schistose districts afford also good wine, as that called la Malgue. Thus we see that lands differing in chemical composition, but possessel of the proper physical qualities, may produce most agreeable wines; and so alsu may lands of like chemical and physical constitution produce various linds of w $\mathbf{i n e}$, according to their varied exposure. As a striking example of these effects, we may adduce the slopes of the hills which grow the wines of Montrachet. The insulated part towards the top furnishes the wine called Chevalier Montrachet, which is less esteerned, and sells at a much lower price, than the delicious wine grown on the middle height, called true Montrachet. Beneath this district, and in the surrounding plains, 
the vines afford a far inferior article, called bastard Montrachet. The opposite side of the hills produces very indifferent wine. Similar differences, in a greater or less degree, are ubservable relatively to the districts which grow the Pomard, Volnay, Beaune, Nusts, Vutyeot, Chambertin, Romanée, \&c. Everywhere it is found, that the reverse side of the hill, the summit, and the plain, although generally consisting of like soil, afford inferior wine to the middle southern slopes.

Ameliorution of the soil. - When the vine lands are too light or too dense, they may be modified, within certain limits, by introducing into them either argillaceous or silicious matter. Marl is excellent for almost all grounds which are not previously too calcareous, being alike useful to open dense soils, and to render porous ones more retentive.

Manure.-Fcr the vine, as well as all cultivated plants, a manure supplying azotized or animal nutriment may be used with great advantage, provided care be taken to ripen it by previous fermentation, so that it may not, by absorption in too crude a state, impart any disagreeable odor to the grape; as sometimes happens to the vines grown in the vicinity of great towns, like Paris, and near Argenteuil. There is a comjost used in France, called animalized black, of which from one fifth to one half of a litre (old English quart) serves sufficiently to fertilize the root of one vine, when applied every year, or two years. An excess of manure, in rainy seasons especially, has the effect of rendering the grapes large and insipid.

The ground is tilled at the same time as the manure is applied, towards the month of March; the plants are then dressed, and the props are inserted. The weakness of the plants renders this practice useful; but in some southern districts, the stem of the vine, when supported at a proper height, acquires after a while sufficient size and strength to stand alone. The ends of the props or poles are either dipped in tar, or charred, to prevent their rotting. The bottom of the stem must be covered over with soil, after the spring rains hare washed it down. The principal husbandry of the vineyard consists in digging or ploughing to destroy the weeds, and to expose the soil to the influence of the air, during the months of May, June, and occasionally in August.

The vintage, in the temperate provinces, generally takes place about the end of September; and it is alwars deteriorated whenever the fruit is not ripe enough before the 15 th or 20 th of October; for, in this case, not only is the must more acid, and less saccharine, but the atmospherical temperature is apt to fall so low during the nights, as to obstruct more or less its fermentation into wine. The grapes should be plucked in dry weather, at the interval of a few days after they are ripe; being usually gathered in baskets, and transported to the vats in dorsels, sufficiently tight to prevent the juice from runring out. Whenever a layer about 14 or 15 inches thick has been spread on the bottom of the vat, the treading operation begins, which is usually repeated after macerating the grapes for some time, when an incipient fermentation has softened the texture of the skin and the interior cells. When the whole bruised grapes are collected in the vat, the juice, by means of a slight fermentation, reacts, through the acidity thus generated, upon the coloring matter of the husks, and also upon the tannin contained in the stones and the fruit-stalks. The process of fermentation is suffered to proceed without any other precaution, except forcing down from time to time the pellicles and pedicles floated up by the carbonic acid to the top; but it would be less apt to become acetous, were the mouths of the vats covered. With this view, M. Sebille Auger introduced with success his elastic bung in the manufacture of wine in the department of the Maine-et-Loire.

With whatever kiud of apparatus the fermentation may have been regulated, as soon as it ceases to be tumultuous, and the wine is not sensibly saccharine or muddy, it must be racked off from the lees, by means of a spigot, and run into the ripening tuns. The mare being then gently squeezed in a press, affords a tolerably clear wine, which is distributed among the tuns in equal proportions; but the liquor obtained by stronger pressure is reserved for the caslis of inferior wine.

In the soith of France the fermentation sometimes proceerls too slowly, on account of the must being too saccharine; an accident which is best counteracted by maintaining a temperature of about $65^{\circ}$ or $68^{\circ} \mathrm{F}$., in the tun-room. When the must, on the other hani, is too thin, and deficient in sugar, it must be partially concentrated by rapid boiling, before the whole can be made to ferment into a good wine. By boiling up a part of the must for this purpose, the excess of ferment is at the same time destroyed. Should this cuncentration be inconvenient, a certain propo:tion of sugar must be introduced, immediately after racking it off.

The specific gravity of must varies with the richness and ripeness of the grapes which afford it; b-ing in some cases so low as $1 \cdot 0627$, and in others so high as $1 \cdot 1283$. This happens particularly in the south of France. In the district of the Necker in Germany, the specific gravity varies from 1.050 to 1.090 ; in Heidelberg, frcm 1.039 , to 1.091 ; but it varies much in different years. 
After the fermentation is complete, the vinous part consists of water, alcohol, a coloring-matter, a peculiar aromatic principle, a little undecomposed sugar, bitartrate and malate of potash, tartiate of lime, muriate of soda, and tannin; the latter substances be. ing in small proportions.

It is known that a few green grapes are capable of spoiling a whole cask of wine, and therefore they are always allowed to become completely ripe, and even sometimes to undergo a species of slight fermentation, before being plucked, which completes the development of the saccharine principle. At other times the grapes are gathered whenever they are ripe, but are left for a few days on wicker-floors, to sweeten, hefore being pressed.

In general the whole vintage of the day is pressed in the evening, and the resulting must is received in separate vats. At the end usually of 6 or 8 hours, if the temperature be above $50^{\circ} \mathrm{F}$, and if the grapes have not been too cold when plucked, a froth or scum is formed at the surface, which rapidly increases in thickness. After it xequires such a consistence as tc crack in several places, it is taken off with a skimmer and drained; and the thin liquor is returned to the vat. A few hours afterwards another coat of froth is formed, which is removed in like manner, and someimes a third may be produced. The regular vinous fermentation now begins, characterized by air-bubbles rising up the sides of the staves, with a peculiar whizzing as they break ai the surface. At this period all the remaining froth should be quickly skimmed off, and the clear subjacent must be transferred into barrels, where it is left to ripen by a regular fermentation.

The while wines, which might be disposed to become stringy, from a deficient supply of tannin, may be preserved from this malady by a due addition of the footstalks of ripe grapes. The tannin, while it tends to preserve the wines, renders them also more easy to clarify, by the addition of white of egg, or ising-glass.

The white wines should be racked oft as soon as the first frosts have made them clear, and at the latest by the end of the February moon. By thus separating the wine from the lees, we avoid, or render of little consequence, the fermentation which takes place on the return of spring, and which, if too brisk, would destroy all its sweetness, by decomposing the remaining portion of sugar.

The characteristic odor possessed by all wines, in a greater or less degree, is produced by a peculiar substance, which possesses the characters of an essential oil. As it is not volatile, it cannot be confounded with the aroma of wine. When large quantities of wine are distilled, an oily substance is obtained towards the end of the operation. This may also be procured from the wine lees which are deposited in the casks after the fermentation has commenced. It forms one forty thousandih part of the wine; and consists of a peculiar new acid, and ether, each of which has been called the ananthic. The acid is analogous to the fatty acids, and the ether is liquid, but insoluble in water. The acid is perfectly white when pure, of the consistence of butter at $60^{\circ}$, melts with a moderate heat, reddens litmus, and dissolves in caustic and carbonated alkalis, as well as in alcohol and ether. Enanthic ether is colorless, has an extremely strong smell of wine, which is almost intoxicating when inhaled, and a powerful disagreeable taste. Liebig and Pelouze.

Sparkling wines.-In the manufacture of these, black grapes of the first quality are usually employed, especially those gathered upon the vine called by the French noirien, cultivated on the best exposures. As it is important, however, to prevent the coloringmatter of the skin from entering into the wine, the juice should be squeezed as gently and rapidly as possible. The liquor obtained by a second and third pressing is reserved for inferior wines, on account of the reddish tint which it acquires. The marc is then mixed with the grapes of the red-wine vats.

The above nearly colorless must is immediately poured into tuns or casks, till about three fourths of their capacity are filled, when fermentation soon begins. This is allowed to continue under the control of the elastic bung, above mentioned, for about 15 days, and then three fourths of the casks are filled up with wine from the rest. The casks are now closed by a bung secured with a piece of hoop iron nailed to two contiguous staves. The casks should be made of new wood, but not of oak-though old white wine casks are occasionally used.

In the month of January the clear wine is racked off, and is fined by a small quantity of ising-glass dissolved in old wine of the same kind. Forty days afterwards a second fining is required. Sometimes a third may be useful, if the lees be considerable. In the month of May the clear wine is drawn off into bottles, taking care to add to each of them a small measure of what is called liquor, which is merely about 3 per cent. of a sirup made by dissolving sugar-candy in white wine. The bottles being filled, and their corks secured by packthread and wire, they are laid on their sides, in this month, with their mouths sloping downwards at an angle of about twenty degrees, in 
order that any sediment may fall into the neck. At the end of 8 or 10 days, the inclina. tion of the bottles is increased, when they are slightly tapped, and placed in a vertical position; so that after the lees are all collected in the neck, the cork is partially removed for an instant, to allow the sediment to be expelled by the pressure of the gas. If the wine be still muddy in the bottles, along with a new dose of liquor, a small quantity of fining should be adiled to each, and the bottles should be placed again in the inverted position. At the end of 2 or 3 months, the sediment collected over the cork is dexterously discharged; and if the wine be still deficient in transparency, the same process of fining must be repeated.

Strarkling wine (vin mousseux), prepared as above described, is fit for drinking usually at the end of from 18 to 30 months, according to the state of the seasons. It is in Champagne that the lightest, most transparent, and most highly flavored wines have been hilherto made. The breakage of the bottles in these sparkling wines amounts frequently to thirty per cent., a circumstance which adds greatly to their cost of production.

Weak wines of bad growths ought to be consumed within 12 or 15 months after being manufactured; and should be kept meanwhile in cool cellars. White wines of middling strength ought to be kept in casks constantly full, and carefully excluded from contact of air, and the racking off should be done as quickly as possible. As the most of them are injured by too much fermentation, this process should be so regulated as always to leave a little sugar undecomposed. It is useful to counteract the absorption of oxygen, and the consequent tendency to acidity, by burning a sulphur match in the casks into which they are about to be run. This is done by hooking the match to a bent wire, kindling and suspending it within the cask through the bung-hole. Immediately on withdrawing the match, the cask should be corked, if the wine be not ready for transfer. If the burning sulphur be extinguished on plunging it into the cask, it is a proof of the cask being unsound, and unfit for receiving the wine; in which case it should be well cleansed, first with lime-water, then with very dilute sulphuric acid, and lastly with boiling water.

Wine-cellars ought to be dry at bottom, floored with flags, have windows opening to the north, be so much sunk below the level of the adjoining ground as to possess a nearly uniform temperature in summer and winter; and be at such a distance from a frequented highway or street as not to suffer vibration from the motion of carriages.

Wines should be racked off in cool weather; the end of February being the fittest time for light wines. Strong wines are not racked off till they have stood a year or eighteen months upon the lees, to promote their slow or insensible fermentation. A syphon well managed serves better than a faucet to draw off wine clear from the sediment. White wines, before being bottled, should be fined with ising-glass; red wines are usually fined with whites of egos beat up into a froth, and mixed with two or three times their bulk of water. But some strong wines, which are a little harsh from excess of tannin, are fined with a little sheep or bullock's blood. Occasionally a small quantity of sweet glue is used for this purpose.

The following maladies of wines, are certain accidental deteriorations, to which remedies should be speedily applied.

La-pousse (pushing out of the cask), is the name given to a violent fermentative movement, which occasionally supervenes after the wine has been run off inte the casks. If these have been tightly closed, the interior pressure may increase to such a degree as to burst the hoops, oreause the seams of the staves or ends to open. The elastic bungs already described will prevent the bursting of the casks; but something must be done to repress the fermentation, lest it should destroy the whole of the sugar, and make the wine unpalatably harsh. One remedy is, to transfer the wine into a cask previously fumigated with burning sulphur; another is, to add to it about one thousandth part of sulphite of lime; and a third, and perhaps the safest, is to introduce half a pound of mustard-seed into each barrel. At any rate, the wines should be fined whenever the movements are allayed, to remove the floating ferment which has been the cause of the mischief.

Turning sour.-The production of too much acid in a wine, is a proof of its containing originaliy too little alcohol, of its being exposed too largely to the air, or to vibrations, or to too high a temperature in the cellar. The best thing to be done in this case is, to mix it with its bulk of a stronger wine in a less advanced state, to fine the mixture, to bottle it, and to consume it as soon as possible, for it will never prove a good keeping wine. This distemper in wines formerly gave rise to the very dangerous practice of add. ing litharge as a sweetener; whereby a quantity of acetate or sugar of lead was formed in the liquor, productive of the most deleterious consequences to those who drank of it. In France, the regulations of police, and the enlightened surveillance of the council of salubrity, have completely put down this gross abuse. The saturation of the 
acid bỵ lime and other alkaline bases has generally a prejudicial effect, and injures more or less the vinous flaror and taste.

Ropiness or viscidity of wines. - The cause of this phenomenon, which renders wine unfit for drinking, was altogether unknown, till M. François, an apothecary of Nantes, demonstrated that it was owing to an azotized matter, analogous to gliadine (gluten); and in fact it is the white wines, especially those which contain the least tannin, which are sutject to this malady. He also pointed out the proper remedy, in the addition of tannis under a rather agreeable form, narely, the bruised berries of the mour sain-ash (sortier), in a somewhat unripe slate; of which one pound, well stirred in, is sufficient for a barrel. After agitation, the wine is to be left in repose for a day or two, and then racked off. The tannin by this time will have seprarated the azotized matter from the liquor, and removed the ropiness. The wine is to be fined and bottled off.

The tusle of the cask, which sometimes happens to wine put into casks which had remained long empty, is best remedied by agitating the wine for some time with a spoonful of olive oil. An essential oil, the chief cause of the bad taste, combines with the fixed oil, and rises with it to the surface.

According to a statement in the Dictionnaire Technologique, the annual produce of a hectare of vineyard, upon the average of 113 years, in the district of Volnay, is 1779 litres, which fetch 0.877 francs each, or 200 francs the piece of 228 litres, amounting in all to $16 \% 2$ francs. Deducting for expenses and taxes (contributions) 572 francs, there remain 1.100 francs of net proceeds; and as the ralue of the capital mar be estimated at 23,000 francs, the profit turns out to be no more than 5 per cent. The net proceeds in the growths of Beaune, Nuits, \&c., does not exceed 600 francs per hectare (2.4 acres), and therefore is equivalent to only $2 \frac{1}{2}$ per cent. upon the capital.

The quantity of alcohol contained in different wines, has been made the subject of elaborate experiments by Brande and Fontenelle; but as it must evidently vary with different seasons, the results can be receired merely as approximative. The only apparatus required for this research, is a small still and refrigeratorr, so well fitted up as to permit none of the spirituous rapors to be dissipated. The distilled liquor should be receired in a glass tube, graduated into one hundred measures, of such capacity as to contain the whole of the alcohol which the given measure of wine employed is capable of yielding. In the successive experiments, the quantity of wine used, and of spirit distilled orer, being the same in volume, the relative densities of the latter will show at once the relative strengths of the wines. A very neat small apparatus has been contrived for the purpose of analyzing wines in this manner, by 11 . Gay Lussac. It is constructerl, and sold at a moderate price, by M. Collardeau, No. 56, Rue Faubourg St. Martin, Paris. The proportion given by Brande (Table I.), has been reduced to the standard of absolute alcohol by Fesser; and that by Fontenelle (Table II.), to the same standard by Schubarth; as in the following tables:-

\section{TABLE I.}

W

\begin{tabular}{|c|c|c|c|c|c|c|c|}
\hline \multirow{2}{*}{ Name of the wine. } & \multirow{2}{*}{ Sp. grav. } & \multicolumn{2}{|c|}{$\begin{array}{c}100 \text { measures } \\
\text { contain at } 60^{\circ} \mathrm{F} \text {. }\end{array}$} & \multirow{2}{*}{ Name of the wine. } & \multirow{2}{*}{ Sp. grav. } & \multicolumn{2}{|c|}{$\begin{array}{l}100 \text { measures } \\
\text { contain at } 60^{\circ} \mathrm{F} \text {. }\end{array}$} \\
\hline & & $\begin{array}{l}\text { Alcohol } \\
\text { of } 0.825 \text {. }\end{array}$ & $\begin{array}{l}\text { Absolute } \\
\text { alcohol. }\end{array}$ & & & $\begin{array}{l}\text { Alcohol } \\
\text { of } 0.825 \text {. }\end{array}$ & $\begin{array}{l}\text { Absolute } \\
\text { alcohol. }\end{array}$ \\
\hline Port Wine,. & 0.97616 & $21 \cdot 40$ & $19 \cdot 82$ & Frontignac, . & 0.98452 & $17 \cdot 79$ & 11.84 \\
\hline Port Wine,. & 0.97200 & $25 \cdot 83$ & 23.92 & 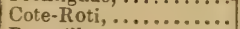 & 0.95495 & $12 \cdot 2 \tau$ & $11 \cdot 36$ \\
\hline Mean.... & 0.97460 & $23 \cdot 49$ & $21 \cdot 75$ & Roussillon, .... & 0.95005 & $17 \cdot 24$ & $15 \cdot 96$ \\
\hline Madeira,........ & 0.97810 & $19 \cdot 34$ & 17.91 & Cape Madeira, .............. & 0.97924 & $18 \cdot 11$ & $16 \cdot 77$ \\
\hline Madeıra,......... & 0.97333 & $21 \cdot 42$ & $22 \cdot 61$ & Muscat, $\ldots \ldots \ldots \ldots \ldots$ & 0.97913 & $18 \cdot 25$ & $1 \% \cdot 00$ \\
\hline Sherry,..... & 0.97913 & $18 \cdot 25$ & $17 \cdot 00$ & Constantia, ............. & $0.97 \approx 0$ & $19 \cdot 75$ & $18 \cdot 29$ \\
\hline Sherry.... & 0.97700 & $19 \cdot 83$ & $18 \cdot 37$ & Tinto,......... & 0.98399 & $13 \cdot 30$ & $12 \cdot 32$ \\
\hline Bordeaux, Claret, . & 0.97410 & $12 \cdot 91$ & 11.95 & Schiraz,...... & 0.98176 & $15 \cdot 52$ & $14 \cdot 35$ \\
\hline Bordeaux, Claret, .. & 0.97092 & $16 \cdot 32$ & $15 \cdot 11$ & Syracuse,.... & 0.98200 & $15 \cdot 28$ & $14 \cdot 15$ \\
\hline Calcavella,........ & 0.97920 & $18 \cdot 10$ & $16 \cdot \% 6$ & Nice,....... & 0.98263 & $14 \cdot 63$ & $13 \cdot 64$ \\
\hline Lisbon, ..... & 0.97846 & 18.94 & $17 \cdot 45$ & Tokay, ........ & $0.98 \div 60$ & $9 \cdot 88$ & $9 \cdot 15$ \\
\hline Malaযa, ... & 0.98000 & $17 \cdot 26$ & 15.98 & Raisin Wine,... & 0.97205 & $25 \cdot 77$ & $23 \cdot 86$ \\
\hline Bucellas, . . & $0.9 \div 890$ & $18 \cdot 49$ & $17 \cdot 22$ & Drained grape Wine,... & 0.97925 & $18 \cdot 11$ & $16 \cdot 77$ \\
\hline Renl Madeira,.. & 0.97899 & $18 \cdot 40$ & $17 \cdot 04$ & Lachrymæ Christi, .... & - & $19 \div 0$ & $18 \cdot 24$ \\
\hline Malmsey, ...... & 0.98090 & 16.40 & $15 \cdot 91$ & Currant Wine,.... & 0.97696 & $20 \cdot 55$ & $19 \cdot 03$ \\
\hline Marsala,.... & $\begin{array}{l}0.98190 \\
0.98000\end{array}$ & $\begin{array}{l}15 \cdot 26 \\
17 \cdot 96\end{array}$ & $\begin{array}{l}14 \cdot 31 \\
15 \cdot 08\end{array}$ & Gooseberry Wine, & 0.98550 & $11 \cdot 84$ & $10 \cdot 96$ \\
\hline $\begin{array}{l}\text { Marsala,........... } \\
\text { Champagne, [rose], }\end{array}$ & $\begin{array}{l}0.98000 \\
0.98608\end{array}$ & $\begin{array}{l}17 \cdot 26 \\
11 \cdot 30\end{array}$ & $\begin{array}{l}15 \cdot 98 \\
10 \cdot 46\end{array}$ & $\begin{array}{l}\text { Elder Wine,... } \\
\text { Cider........ }\end{array}$ & 0 & $9 \cdot 8$ & 0.14 \\
\hline Champagne, [white],... & 0.98150 & $12 \cdot 80$ & $11 \cdot 84$ & Perry,............. & 100 & 98 & \\
\hline Burgundy,............. & 0.98300 & $14 \cdot 53$ & $13 \cdot 34$ & Brown Stout,... & 0.99116 & $6 \cdot 80$ & $6 \cdot 30$ \\
\hline Burgundy,.. & 0.98540 & $11 \cdot 95$ & 11.06 & Ale, ........... & $0.988 \% 3$ & 888 & $8 \cdot 00$ \\
\hline White Herm & 0.97990 & $1 \tau \cdot 43$ & $16 \cdot 14$ & Purter, ... & - & $4 \cdot 20$ & $3 \cdot 8 y$ \\
\hline Red Hermitage,... & $2.9 \$ 495$ & $12 \cdot 32$ & $11 \cdot 40$ & Rum,... & 0.93494 & $53 \cdot 68$ & $49 \cdot 71$ \\
\hline Hock, ......... & 0.98290 & $14 \cdot 37$ & $13 \cdot 31$ & Hollands, : & 0.93855 & $51 \cdot 60$ & $47 \cdot 77$ \\
\hline Hock, ..... & $0.988 \% 3$ & 8.88 & $8 \cdot 00$ & Scotch Whiskey, & - & $54 \cdot 32$ & $50 \cdot 20$ \\
\hline Vin de Grave,.... & 0.98450 & $12 \cdot 80$ & $11 \cdot 84$ & Irish Whiskey,........ & - & $53 \cdot 90$ & $49 \cdot 91$ \\
\hline
\end{tabular}


TABLE II.

\begin{tabular}{|c|c|c|c|c|c|}
\hline Name of the Wine. & $\begin{array}{l}\text { Absolute } \\
\text { alcohol. }\end{array}$ & Name of the Wine. & $\begin{array}{l}\text { Absolute } \\
\text { alcohol. }\end{array}$ & Name of the Wine. & $\begin{array}{l}\text { Absolute } \\
\text { alcohol. }\end{array}$ \\
\hline $\begin{array}{l}\text { Roussillon (Eastern } \\
\quad \text { Pyrenees.) } \\
\text { Rive-saltes } 18 \text { yrs. old } \\
\text { Banyulls } 18 \\
\text { Collyouvre } 15 \\
\text { Salces "10 " } \\
\text { " } \\
\text { Department of the } \\
\text { Aude. } \\
\text { Fitou and Leu - } \\
\text { caté } 10 \text { yxs, old } \\
\text { Lapalme } 10 \text { " }\end{array}$ & $\begin{array}{l}9 \cdot 156 \\
9 \cdot 223 \\
9 \cdot 080 \\
8 \cdot 580\end{array}$ & 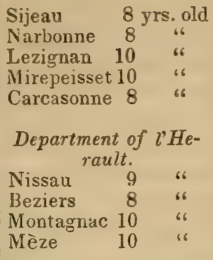 & $\begin{array}{l}8 \cdot 635 \\
8 \cdot 379 \\
8 \cdot 173 \\
8 \cdot 589 \\
7 \cdot 190\end{array}$ & $\begin{array}{lll}\text { Montpellier } & 5 & \text { yrs. old } \\
\text { Lunel } & 8 & \text { "6 } \\
\text { Frontignan } & 5 & \text { "6 } \\
\text { Red Hermitage } & 4 & \text { " } \\
\text { White do. } & - \\
\text { burgundy } & 4 & \text { "، } \\
\text { Grave } & 3 & \text { " } \\
\text { Charnpagne (sparkling) } \\
\text { Do. white } & \text { do. } \\
\text { Do. rose } & - \\
\text { Bordeaux } & - \\
\text { Toulouse } & -\end{array}$ & $\begin{array}{l}7 \cdot 413 \\
7 \cdot 564 \\
7 \cdot 098 \\
5 \cdot 838 \\
7 \cdot 056 \\
6 \cdot 195 \\
5 \cdot 838 \\
5 \cdot 880 \\
5 \cdot 145 \\
4 \cdot 956 \\
6 \cdot 186 \\
5 \cdot 027\end{array}$ \\
\hline
\end{tabular}

WINE, FAMILY, may be made by the following recipe:-Take black, red, white currants, ripe cherries (black hearts are the best), and raspherries, of each an equal quantity. To 4 pounds of the mixed fruit, well bruised, put 1 gallon of clear soft water; steep three days and nights, in open vessels, frequently stirring up the magma; then strain through a hair sieve; press the residuary pulp to dryness, and add its juice to the former. In each gallon of the mixed liquors dissolve 3 pounds of good yellow muscovado sui ar; let the solution stand other three days and nights, frequently skimming and stirring it up; then tun it into casks, which should remain full, and purging at the bunghole, about two weeks. Lastly, to every 9 gallons put 1 quart of good Cognac brandy (but not the drugged imitations made in London with grain whiskey), and bung down. If it does not soon become fine, a steeping of ising-glass may be stirred into the liquid, in the proportion of about half an ounce to 9 gallons. I have found that the addition of an ounce of cream of tartar to each gallon of the fermentable liquor, improves the quality of the wine, and makes it resemble more nearly the produce of the grape.

WINE-STONE is the deposite of crude tartar, called argal, which seitles on the sides and bottoms of wine casks.

WIRE-DRAWING. (Tréfilerie, Fr.; Draht-ziehen, Drahtzug, Germ.) When an oblong lump of metal is forced through a series of progressively diminishing apertures in a steel plate, so as to assume in its cross section the form and dimensions of the last hole, and to be augmented in length at the expense of its thickness, it is said to be wiredrawn. The piece of steel called the draw-plate is pierced with a regular gradation of holes, from the largest to the smallest ; and the machine for overcoming the lateral adhesion of the metallic particles to one another, is called the draw-bench. The pincers which lay hold of the extremity of the wire, to pull it through the successive holes, are adapted to bite it firmly, by having the inside of the jaws cut like a file. For drawing thick rods of gilt silver down into stout wire, the hydraulic press has been had recourse to with advantage.

Fig. 1202 represents a convenient form of the draw-bench, where the power is applied by a toothed wheel, pinion, and rack-work, moved by the hands of one or two men work-

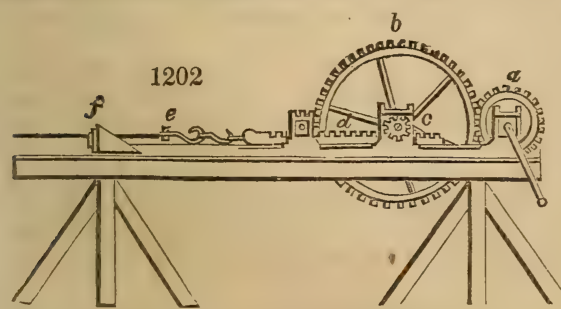
ing at a winch; the motion being so regulated by a fly-wheel, that it does not proceed in fits and starts, and cause inequalities in the wire. The metal requires to be annealed, now and then, between successive drawings, otherwise it would become too hard and brittle for further extension. The reel upon which it is wound is sometimes mounted in a cistern of sour small beer, for the purpose of clearing off, or loosening at least, any crust of oxyde formed in the annealing, before the wire enters the draw-plate.

When, for very accurate purposes of science or the arts, a considerable length of uniform wire is to be drawn, a plate with one or more jewelled holes, that is, filled with one or more perforated rubies, sapphires, or chrysolites, can alone be trusted to, because the holes even in the best steel become rapidly wider by the abrasion. Through a hole in $\approx$ ruby, 0.0033 of an inch in diameter, a silver wire $1 \tilde{7} 0$ miles long has been drawn, which possessed at the end the very same section as at the heginning; a result determined by weighing portions of equal length, as also by measuring it with a micrometer. The hole in an ordinary draw-plate of soft steel becomes so wide by drawing 14,000 fathoms of brass wire, that it requires to be narrowed before the orioinal sized wire ean be again obtained.

Wire, by being diminished one half; one third, "ne fourth, \&c., in diameter, is ausmented in length respectivelv. fonr. nine. sixtepn times. \&c. The speed with which it 
may be prudently drawn out, depends upon the ductility and tenacity of the metal; but may be always increased the more the wire becomes attenuated, because its particles progressively assume more and more of the filamentous form, and accommodate themselve's more readily to the extending force. Iron and brass wires, of 0.3 inch in diameter, bear drawing at the rate of from 12 to 15 inches per second; but when of $0.025\left(\frac{1}{40}\right)$ of an inch, at the rate of from 40 to 45 inches in the same time. Finer silver and copper wire may be extended from 60 to 70 inches per second.

By enclosing a wire of platinum within one of silver ten times thicker, and drawing down the compound wire till it be $\frac{1}{30}$ of an inch, a wire of platinum of $\frac{1}{300}$ of an inch will exist in its centre, which may be obtained apart, by dissolving the silver away in nitric acid. This pretty experiment was first made by Dr. Wollaston.

The French draw-plates are so much esteemed, that one of the best of them used to be sold in this country, during the late war, for its weight in silver. The holes are formed with a steel punch; being made large on that side where the wire enters, and diminishing with a regular taper to the other side. In the act of drawing, they must be well supplied with grease for the larger kinds of wire, and with wax for the smaller.

WOAD (Vouëde, Pastel, Fr.; Wuid, Germ.; Isatis tinctoria, Linn.), the glastum of the ancient Gauls and Germans, is an herbaceous plant which was formerly much culti valed, as affording a permanent blue dye, but it has been in modern times well nigh superseded by indigo. Pliny says, "A certain plant which resembles plantago, called glastum, is employed by the women and girls in Great Britain for dyeing their bodies all over, when they assist at certain religious ceremonies; they have then the color of Ethiopians."-Hist. Nat. cap. xxii. § 2.

When the arts, which had perished with the Roman empire, were revived, in the middle ages, woad began to be generally used for dyeing blue, and became an object of most extensive cultivation in many countries of Europe. The environs of Toulouse and Mirepoix, in Upper Languedoc, produced annually $40,000,000$ pounds of the prepared woad, or pastel, of which 200,000 bales were consumed at Bordeaux. Beruni, a rich manufacturer of this drug, became surety for the payment of the ransom of his king, Francis I., then the prisoner of Charles V. in Spain.

The leaves of woad are fermented in heaps, to destroy certain vegetable principles injurious to the beauty of the dye, as also to elaborate the indigoferors matter present, before they are brought into the market; but they should be carefully watched during this process. Whenever the leaves have arrived at maturity, a point judged of very differently in different countries, they are stripped off the plant, a cropping which is repeated as often as they shoot, being three or four times in Germany, and eight or ten times in Italy. The leaves are dried as quickly as possible, but not so much as to become black; and they are ground before they get quite dry. The resulting paste is laid upon a sloping pavement, with gutters for conducting the juice, which exudes into a tank; the heap being tramped from time to time, to promote the discharge of the juice. The woad ferments, swells, and cracks in many places, which fissures must be closed; the whole being occasionally watered. The fermentation is continued for twenty or thirty days, in cold weather; and if the leaves have been gathered dry, as in Italy, for four months. When the fermented heap has become moderately dry, it is ground again, and put up in calies of from one to three pounds; which are then fully dried, and packed up in bundles for the market. Many dyers subject the pastel to a second fermentation.

1,600 square toises (fathoms) of land afford in two cuttings at least 19,000 pounds of leaves, of which weight four fifths are lost in the fermentation, leaving 3,880 pounds of pastel, in loaves or cakes. When good, it has rather a yellow, or greenish-yellow, than a blue color; it is light, and slightly humid; it gives to paper a pale-green trace; and improves by age, in consequence of an obscure fermentation; for if kept four years, it dyes twice as much as after two years. According to Hellot, 4 pounds of Guatimala indigo produce the same effect as 210 pounds of the pastel of Albi. At Quins, in Piedmont, the dyers estimate that 6 pounds of indigo are equivalent to 300 of pastel; but Chaptal thinks the indigo underrated.

Pastel will dye blue of itself, but it is commonly employed as a fermentative addition to the proper blue vat, as described under INDrgo.

Fresh woad, analyzed by Chevreul, afforded, in 100 parts, 65.4 of juice. After being steeped in water, the remaining mass yielded, on expression, 29.65 of liquid; being in whole, 95.05 parts, leaving 4.95 of ligneous fibre. The juice, by filtration, gave 1.95 of grecn fecula. 100 parts of fresh woad, when dried, are reduced to $13 \cdot 76$ parts. Alcohol, boiled upon dry woad, deposites, after cooling, indigo in microscopic needles; but these cannot be separated from the vegetable albumine, which retains a greeni.h. gra color.

WOLFRAM is the native tungstate of iron and manganese, a mineral which occurs in primitive formations, along with the ores of tin, antimony, and lead, in the Bohenian Erzegebirge, in Cornwall, Switzerland, North America \&c. It is used by chemists for obtaining tungstic acid and tungsten 
WI)OD (Bois, Fr.; Holz, Germ.), is the hard but porous tissue between the pith and the bark of trees and shrubs, through which the chief part of the juices is conducted from the root towards the branches and leaves, during the life of the vegetable. The ligneous fibre is the substance which remains, after the plant has been subjected to the solvent action of ether, alcohol, water, dilute acids, and caustic alkaline leys. It is considered by chemists that dry timber consists, on an average, of 96 parts of fibrous, and $t$ of soluble matter, in 100 ; but that these proportions vary somewhat with the seasons, the soil, and the plant. All kinds of wood sink in water, when placed in a basin of it under the exhausted receiver of an air-pump; showing their specific gravity to be greater than 1.000 . That of fir and maple is stated, by chemical authors, to be $1 \cdot 46$; and that of oak and beech, at 1.53 ; but I believe them to have all the same spec. grav. as the fibre of flax; namely, $1 \cdot 50$, as determined by me some years ago.*

Wood becomes snow-white, when exposed to the action of chlorine; digested with sulphuric acid, it is transformed first into gum, and, by ebullition with water, afterwards into grape-sugar; with concentrated nitric acid, it grows yellow, loses its coherence, falls into a pulverulent mass, but eventually dissolves, and is converted into oxalic acid; with strong caustic alkaline leys, in a hot state, it swells up excessively, dissolves into a homogeneous liquid, and changes into a blackish-brown mass, containing oxalic and acetic acids.

The composition of wood has been examined by Gay Lussac and Thenard, and Dr. Prout. The first two chemists found it to consist, in 100 parts, of-

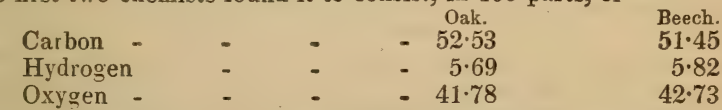

According to Dr. Prout, the oxygen and hydrogen are in the exact proportions to form water. Willow contains 50 , and box $49 \cdot 8$ per cent. of carbon; each containing, thereore, very nearly 44.444 of oxygen, and 5.555 of hydrogen. In the analyses of Gay Lussac and Thenard, there is a great excess of hydrogen above what the oxygen requires :o form water. Authenrieth stated, some years ago, that he found that fine sawdust, nixed with a sufficient quantity of wheat flour, made a coherent dough with water, which ormed an excellent food for pigs; apparently showing that the digestive organs of this nnimal could operate the same sort of change upon wood as sulphuric acid does.

Table of the Distillation of One Pound of Wood, dried, at $86^{\circ}$ Fahr.

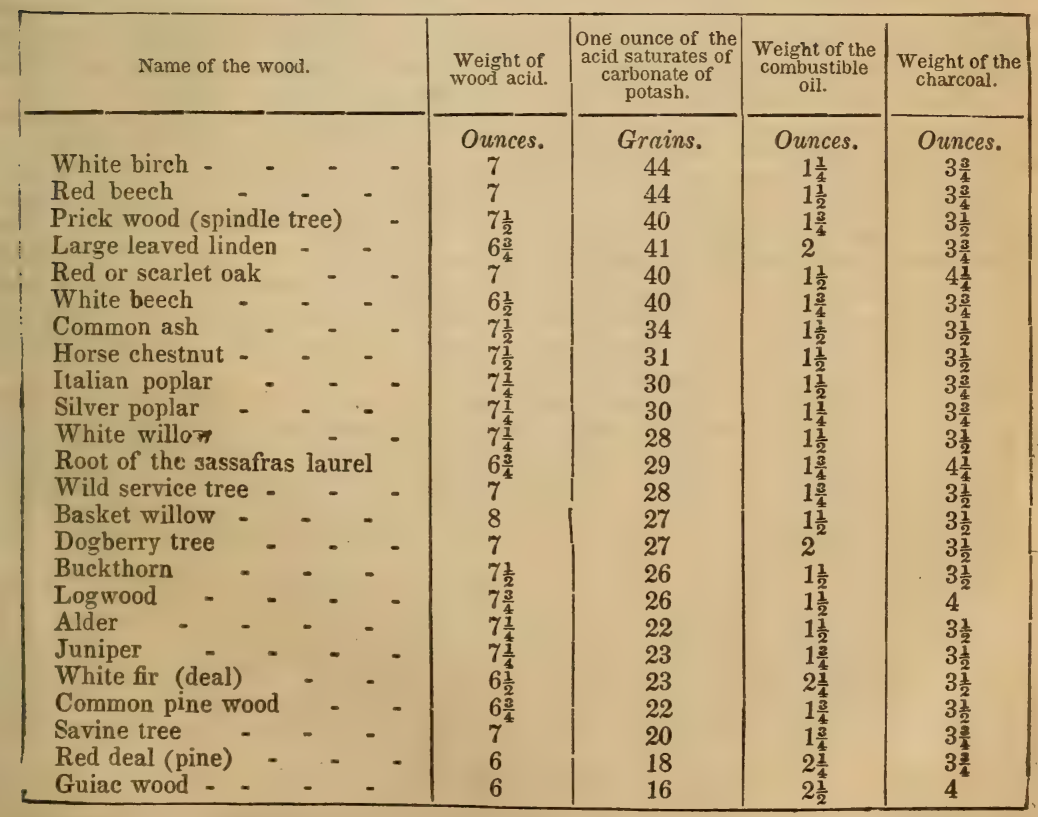

+ "From the small difference found by experiment between the specific gravity of flax $(1 \cdot 50)$ and of cot ton $(1 \cdot 47)$, I ain inclined to think that the density of both may be considered to be equal," or $1.50 .-P h \delta$ losophy of Manufactures. 2d edition, pp. $97,98,99$. 
WO()F is the same as WFrr.

WOOLIEN MANUFACTURE. In reference to textile fabries, sheep's wool is of iwo different sorts, the short and the long stapled; each of which requires different modes of manufacture in the preparation and spinning processes, as also in the treatment of the cloth after it is woven, to fit it for the market. Each of these is, moreover, distinguished in commerce by the names of fleece wools and dead wools, according as they have been shorn at the usual annual period from the living animal, or are cut from its skin after death. The latter are comparatively harsh, weak, and incapable of imbibing the dyeing principles, more especially if the sheep has died of some malignant distemper. The annular pores, leading into the tubular cavities of the filaments, seem, in this case, to have shrunk and becorne obstructed. The time of year for sheep-shearing most favorable to the quality of the wool, and the comfort of the animal, is towards the end of June and beginning of July; - the period when Lord Leicester holds his celebrated rural fête for that interesting purpose.

The wool of the sheep has been surprisingly improved by its domestic culture. The mouflon (Ovis aries), the parent stock from which our sheep is undoubtedly derived, and which is still found in a wild state upon the mountains of Sardinia, Corsica, Barbary, Greece, and Asia Minor, has a very short and coarse Heece, more like hair than wool. When this animal is brought under the fostering care of man, the rank fibres gradually disappear; while the soft wool round their roots, little conspicuous in the wild animal, becomes singularly developed. The male most speedily undergoes this change, and continues ever afterwards to possess far more power in modifying the fleece of the offspring, than the female parent. The produce of a breed from a coarse-woolled ewe and a finewoolled ram is not of a inean quality between the two, but half-way nearer that of the sire. By coupling the female thus generated with such a male as the former, another improvement of one half will be obtained, affording a staple three fourths finer than that of the grandam. By proceeding inversely, the wool would be as rapidly deteriorated. It is, therefore, a matter of the first consequence in wool husbandry, to exclude from the flock all coarse-fleeced rams.

Long wool is the produce of a peculiar variety of sheep, and varies in the length of its fibres from 3 to 8 inches. Such wool is not carded like cotton, but combed like flax, either by hand or appropriate machinery. Short wool is seldom longer than 3 or 4 inches; it is susceptible of carding and felting, by which processes the filaments become first convoluted, and then densely matted together. The shorter sorts of the combing wool are used principally for hosiery, though of late years the finer kinds have been extensively worked up into Merino and mousseline-de-laine fabrics. The longer wools of the Leicestershire breed are manufactured into hard yarns, for worsted pieces, such as waistcoats, earpets, bombazines, poplins, crapes, \&\&.

The wool of which good broadcloth is made should be not only shorter, but, generally sperking, finer and softer than the worsted wools, in order to fit them for the fulling process. Some wool-sorters and wool-staplers acquire hy practice great nicety of discernment in judging of wools by the touch and traction of the fingers. Two years ago, I made a series of observations upon different wools, and published the results. The filaments of the finer qualities varied in thickness from $\frac{1}{100}$ to $\frac{1}{150} \overline{0}$ of an inch; their structure is very curious, exhibiting, in a good achromatic microscope, at intervals of about $\frac{1}{300}$ of an inch, a series of serrated rings, imbricated towards each other, like the joints of Equisetum, or rather like the scaly zones of a serpent's skin. See Philosophy of Manufactures, gs. 11, 12, page 91, second edition.

There are four distinct qualities of wool upon every sheep; the finest being upon the spine, from the neck to within 6 inches of the tail, including one third of the breadth of the back; the second covers the flanks between the thighs and the shoulders; the third clothes the neck and the rump; and the fourth extends upon the lower part of the neck and breast down to the feet, as also upon a part of the shoulders and the thighs, to the bottom of the hind quarter. These should be torn asunder, and sorted, immediately after the shearing.

The harshness of wools is dependant not solely upon the breed of the animal, or the climate, but is owing to certain peculiarities in the pasture, derived from the soil. It is known, ihat in sheep fed upon chalky districts, wool is apt to get coarse; but in those upon a rich loamy soil, it becomes soft and silky. The ardent sun of Spain renders the fleece of the Merino breed harsher than it is in the milder climate of Saxony. Smearing sheep with a mixture of tar and butter is deemed favorable to the softness of their wool.

All wool, in its natural state, contains a quantity of a peculiar potash-scap, secretsd by the animal, called in this country the yoik; which may be washed out by water aione, with which it forms a sort of lather. It constitutes from 25 to 50 per cent. of the wool, being most abundant in the Merino breed of sheep; and however favorable to the growth of the wool on the living animal, should be taken out soon after it is shorn, lest 
it injure the fibres by fermentation, and cause them to become hard and brittle. After being washed in water, somewhat more than lukewarm, the wool should be well pressed, and carefully dried. England grows annually about 1,000,000 packs of wool, and imports 100,000 bags.

Wool imported into the United Kingdom, in 1836, 64,239,977 lbs.; in 1837, 48,356,121. lbs. Retained for home consumption, in 1836, 60,724,795 lbs.; in 1837, 43,148,297 lbs. Duty received, in $1836, £ 190,075$; in $1837, £ 118,519$.

Having premised these general observations on wool, I shall now proceed to treat of its manufacture, beginning with that of wool-combing, or

\section{THE WORSTED MANUFACTURE.}

In this branch of business, a long stapled and firm fibre is required to form a smooth sevel yarn, little liable to shrink, curl, or felt in weaving and finishing the cloth. It must not be entangled by carding, but stretched in lines as parallel as possible, by a suitable system of combing, manual or mechanical.

When the long wool is brought into the worsted factory, it is first of all washed by men with soap and water, who are paid for their labor by the piece, and are each assisted by a boy, who receives the wool as it issues from between the drying squeezers, (see BLEACHING.) The boy carries off the wool in baskets, and spreads it evenly upon the floor of the drying-room, usually an apartment over the boilers of the steam-engine, which is thus economically heated to the proper temperature. The health of the boys employed in this business is found to be not at all injured.

The wool, when properly•dried, is transferred to a machine called the piucker, which is always superintended by a be'y of 12 or 14 years of age, being very light work. $\mathrm{He}$ lays the tresses of wool pretty evenly upon the feed-apron, or table covered with an endless moving web of canvass, which, as it advances, delivers the ends of the long tufts to a pair of fluted rollers, whence it is introduced into a fanning apparatus, somewhat similar to the willow empioyed in the cotton manufacture, which see. The filaments are turned out, at the opposite end of this winnowing machine, straightened, cleaned, and ready for the combing operation. According to the old practice of the trade, and still

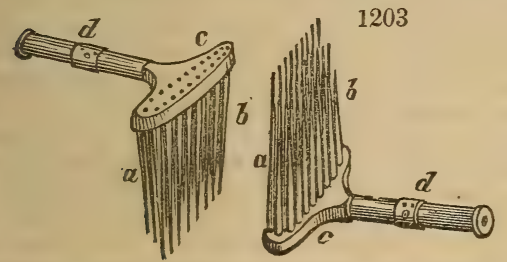
for the finer descriptions of the long staple, according to the present practice, the wool is carded by hand. This is far more severe labor than any subservient to machinery, and is carried on in rooms rendered close and hot by the number of stoves requisite to heat the combs, and so enable them to render the fibres soft, flexible, and elastic. This is a task at which only robust men are engaged. They use three implements; 1. a pair of combs for each person; 2. a post, to which one of the combs can be fixed; 3. a comb-pot, or small stove for heating the teeth of the combs. Each comb is composed

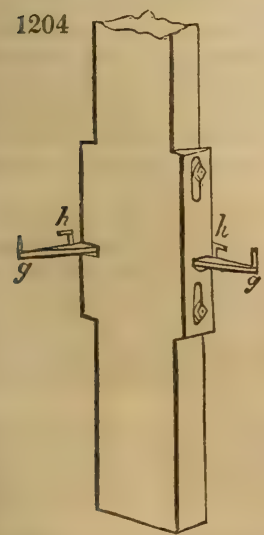
either of two or three rows of pointed tapering steel teeth $b$, fig. 1203, disposed in two or three parallel planes, each row being a little longer than the preceding. They are made fast at the roots to a wooden stock or head $\iota$, which is covered with horn, and has a handle $d$, fixed into it at right angles to the lines of the teeth. The spaces between these two or three planes of teeth, is about one third of an inch at their bottoms, but somewhat more at their tips. The first combing, when the fibres are most entangled, is performed with the two-row toothed combs; the second, or finishing combing, with the three-row toothed.

In the workshop a post is planted ( $f \mathrm{~g}$. 1204), upright, for resting the combs occasionally upon, during the operation. An iron stem $g$, projects from it horizontally, having its end turned up, so as to pass through a hole in the handle of the comb. Near its point of insertion into the post, there is another staple point $h$, which enters into the hollow end of the handle; which, between these two catches, is firmly secured to the post. The stove is a very simple affair, cousisting merely of a flat iron plate, heated by fire or steam, and surmounted with a similar plate, at an interval sufficient to allow the teeth to be inserted between them at one side, which is left open, while the space between their edges, on the other sides, is closed to confine the heat.

In combing the wool, the workman takes it up in tresses of about four ounces each, sprinkles it with oil, and rolls it about in his hands, to render all the filaments equally 
unctuous. Some harsh dry wools require one sixteenth their weight of oil, otliers no more than a fortieth. He next attaches a heated comb to the post, with its teeth pointed upwards, seizes one half of the tress of wool in his hands, throws it over the teeth, then draws it through them, and lhus repeatedly, leaving a few straight filaments each time upon the comb. When the comb has in this way collected all the wool, it is placed with its points inserted into the cell of the stove, with the wool hanging down outside, exposed to the influence of the heat. The other comb, just removed in a heated state from the stove, is planted upon the post, and furnished in its turn with the remaining two-ounce tress of wool; after which it supplants the preceding at the stove. Having both combs now hot, he holds one of them with his left hand orer his knee, being seated upon a low stool, and seizing the other with his right hand, he combs the wool upon the first, by introducing the teeth of one comb into the wool stuck in the other, and drawing them through it. This manipulation is skilfully repeated, till the fibres are laid truly parallel, like a flat tress of hair. It is proper to begin by combing the tips of the tress, and to advance progressively, from the one end towards the other, till at length the combs are worked with their teeth as closely together as is possible, without bringing them into collision. If the workman proceeded otherwise, he would be apt to rupture the filaments, or tear their ends entirely out of one of the combs. The flocks left at the end of the process, because they are tno short for the comber to grasp them in his hand, are called noyls. They are unfit for the worsted spinner, ard are reserved for the coarse cloth manufacture.

The wool finally drawn off from the comb, though it may form a uniform tress of straight filaments, must yet be combed again at a somewhat lower temperature, to prepare it perfectly for the spinning operation. From ten to twelve slivers are then arranged in one parcel.

To relieve the workman from this laborious and not very salubrious task, has been the object of many mechanical inventions. One of these, considerably employed in this country and in France, is the invention of the late Mr. John Collier, of Paris, for which a patent was obtained in England, under the name of John Platt, of Salford, in November, 1827. It consists of two comb-wheels, about ten feet in diameter, having hollow iron spokes filled with steam, in order to keep the whole apparatus at a proper combing heat. The comb forms a circle, made fast to the periphery of the wheel, the teeth being at right angles to the plane of the wheel. The shafts of the two wheels are mounted in a strong frame of cast iron; not, howerer, in horizontal positions, but inclined at acute angles to the horizon, and in planes crossing each other, so that the teeth of one circular comb sweep with a steady obliquity over the teeth of the other, in a most ingenious manner, with the effect of combing the tresses of wool hung upon them. The proper quantity of long wool, in its ordinary state, is stuck in handfuls upon the wheel, revoiving slowly, by a boy, seated upon the ground at one side of the machine. Whenever the wheel is dressed, the machine is made to revolve more rapidly, by shifting its driving-band on another pulley; and it is heautiful to observe the delicacy and precision with which it smooths the tangled tress. When the wools are set in rapid rotation, the loose ends of the fleece, by the centrifugal force, are thrown out, in the direction of radii, upon the teeth of the other revolving comb-wheel, so as to be drawn out and made truly straight. The operation commences upon the tips of the tresses, where the wheels, by the oblique posture of their shafts, are at the greatest distance apart; but as the planes slowly approach to parallelism, the teeth enter more deeply into the wool, till they progressively comb the whole length of its fibres. The machines being then thrown out of gear, the teeth are stripped of the tresses by the hand of the attendant; the noyls, or short refuse wool, being also removed, and kept by itself.

This operation being one of simple superintendence, not of handicraft effort and skill, like the old combing of long wool, is now performed by boys or girls of 13 and 14 years of age ; and places in a striking point of view the influence of automatic mechanism, in so embodying dexterity and intelligence in a machine, as to render the cheap and tractable labor of children a substitute for the high-priced and often refractory exertions of workmen too prone to capricious combinations. The chief precaution to be taken with this machine, is to keep the steam-joints tight, so as not to wet the apartments, and to provide due ventilation for the operatives.

The following machine, patented by James Noble, of Halifax, worsted-spinner, in February, 1834, deserves particular notice, as its mode of operation adapts it well also for heckling flax. In fig. 1205 the internal structure is exhibited. The frame-work $a, a$, suppurts the axle of a wheel, $b, b$, in suitable bearings on each side. To the face of this whee, is affixed the eccentric or heart-wheel cam $c, c$. On the upper part of the periphery of this cam or heart-wheel, a lever $d, d$, bears merely by its gravity; one end of which lever is connected by a joint to the crank $e$. By the rotation of the crank $e$, it will be perceived that the lever $d$, will be slidden to and fro on the upper part of the periphery of the eccentric or heart-wheel cam $c$, the outer end of the lever $d$, carrying 
the upper or working comb or needle-points $f$, as it moves, performing an elliptical 1205

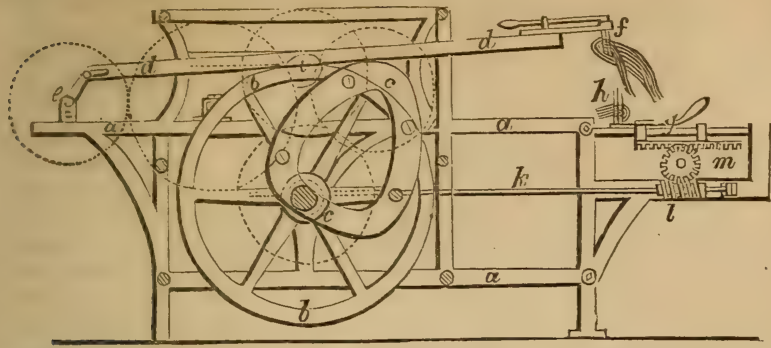
curve, which curve will be dependant upon the position of the heart-wheel cam $c$, that guides it. A moveable frame $g$, carries a series of points $h$, which are to constitute the lower comb or frame of needles. Into these lower needles the rough uncombed wool is to be fell by hand, and to be drawn out and combed straight by the movements of the upper or working comb.

As it is important, in order to prevent waste, that the ends of the wool should be first combed out, and that the needle-points should be made to penetrate the wool progressively, the moreable frame $g$, is in the first instance placed as far back as possible; and the action of the lever $d$, during the whole operation, is so directed by the varying positions of the cam-wheel, as to allow the upper comb to enter at first a very little way only into the wool; but as the operation of combing goes on, the frame with the Lower combs is made to advance gradually, and the relative positions of the revolving heart cam-whcel $c$, being also gradually changed, the upper or working needles are at length alluwed to be drawn completely throngh the wool, for the purpose of combing out straight the whole length of its fibre.

In order to give to the machine the necessary movements, a train of toothed wheels and pinions is mounted, mostly on studs attached to the side of the frame; which train of wheels and pinions is shown by dots in the figure, to avoid confusion. The driving power, a horse or steam-engine, is communicated by a band to a rigger on the short axle $i$; which axle carries a pinion, taking into one of the wheels of the train. From this wheel the crank $e$, that works the lever $d$, is driven; and also by gear from the same pinion, the axle of the wheel $b$, carrying the eccentric or heart-wheel eam, is also actuated, but slower than the crank-axle.

At the end of the axle of the wheel $b$, and cam $c$, a bevel pinion is affixed, which gears into a correspunding hevel pinion on the end of the lateral shaft $k$. The reverse end of this shaft has a worm or endless screw $l$, taking into a toothed wheel $m$; and this last-mentioned toothed wheel gears into a rack at the under part of the frame $g$.

It will hence be perceived, that by the movements of the train of wheels, a slow

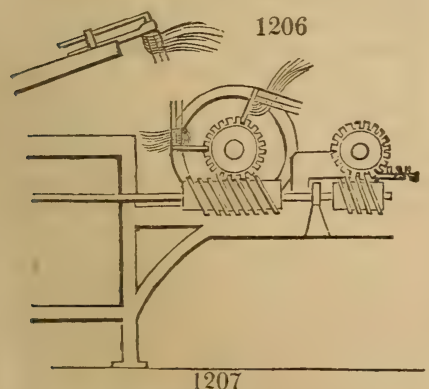
motion is given to the frame $g$, by which the lower needles carrying the wool are progressively advanced as the operation goes on; and also, that by the other wheels of the train, the heart-wheel cam is made to rotate, for the purpose of giving such varying directions to the stroke of the lever which slides upon its periphery, and to the working comb, as shall cause the comb to operate gradually upon the wool as it is brought forward. The construction of the frames which hold the needles, and the manner of fixing them in the machine, present no features of importance; it is therefore unnecessary to describe them farther, than to say, that the heckles are to be heated when used for combing wool. Instead of introducing the wool to be combed into the lower needles by hand, it is sometimes fed in, by means of an endless feedinycloth, as shown in fig. 1206. This endless cloth is distended over two rollers, which are made to revolve, for the purpose of carrying the cloth with the wool forward, by means of the endless screw and pinions.

A slight variation in the machine is shown at fig. 1207, for the purpose of combing wool of long fibre, which differs from the former only in placing the combs or needle points upon a revolving cylinder or shaft. At the end of the axle of this shaft there is a toothed wheel, which is actuated by an 
endless screw upon a lateral shaft. The axle of the cylinder on which the needles are fixed, is mounted in a moveable frame or carriage, in order that the points of the needles may, in the first instance, be brought to act upon the ends of the wool only, and ultimately be so advanced as to enable the whole length of the fibres to be drawn through. The progressive advancement of this carriage, with the needle cylinder, is effected by the agency of the endless screw on the lateral shaft before mentioned.

Some combing-machines reduce the wool into a continuous sliver, which is ready for the drawing-frame; but the short slivers produced br the hand combing, must be first joined together, by what is called planking. The slivers are rolled up by the combers ten or twelve together, in balls called tops, each of which weighs half a pound. At the spinning-mill these are unrolled, and the slivers are laid on a long plank or trough, with the ends lapping over, in order to splice the long end of one sliver into the short end of another. The long end is that which was drawn off first from the comb, and contains the longer fibres; the short is that which comes last from the comb, and contains the shorter. The wool-comber lays all the slivers of each ball the same way, and marks the long end of each by twisting up the end of the sliver. It is a curious circumstance, that when a top or ball of slivers is unrolled and stretched out straight, they will not separate from each other without tearing and breaking, if the separation is begun at the short ends; but if they are first parted at the long ends, they will readily separate.

The machine for combing long wool, for which Messrs. Donisthorpe and Rawson obtained a patent in April, 1835, has been found to work well, and therefore merits

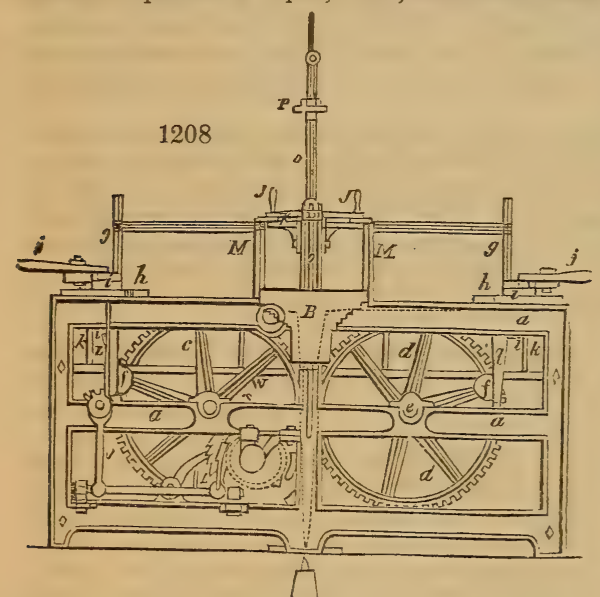
a detailed description :-

Fig. 1208 , is an elevation; fig. 1209 an end view; and fig. 1210 a plan; in which $a, a$, is the framing; $b$, the main shaf, bearing a pinion which drives the wheel and shaft $c$, in gear with the wheel $d$, on the shaft $e$. Upon each of the wheels $c$ and $d$, there are two projections or studs $f$, which cause the action of the combs $g$, $g$, of which $h, h$, are the tables or carriages. These are eapable of sliding along the upper guide rails of the framing $a$. Through these carriages or tables $h, h$, there are openings or slits, shown by dotted lines, which act as guides to the holders $i, i$, of the combs $g$, $g$, rendering the holders susceptible of motion at right angles to the course pursued by the

tables $h$. The combs are retained in the holders $i, i$, by means of the lever handles $j, j$, which move upon inclined surfaces, and are made to press on the surface of the heads of the combs $g, g$, so as to be retained in their places; and they are also held by studs affixed to the holders, which pass into the comb-heads. From the under side of the tables, forked projections $i, i$, stand out, which pass through the openings or slits formed in the tables $h h$; these projections are worked from side to side by the frame $k, k$, which turning on the axis or shaft $l, l$, is caused to vibrate, or rock to and fro, by the arms $m$, moved by the eccentric groove $n$, made fast to the shaft $e$. The tables $\bar{h}$, are drawn inwards, by weights suspended on cords or straps 0,0 , which pass over frietion pulleys $p, p$; whereby the weights have a constant tendency to draw the combs into the centre of the machine, as soon as it is released by the studs $f$, passing beyond the projecting arms $g$, on the tables. On the shaft $c$, a driving-tooth or eatch $r$, is fixed, which takes into the ratchet wheel $s$, and propels one of its teeth at every revolution of the shaft $c$. This ratchet wheel turns on an axis at $t$; to the ratchet the pulley $v$ is made fast, to which the cord or band $w$ is secured, as also to the pulley $x$, on the shaft $y$. On the shaft $y$, there are two other pulleys $z$, $z$, having the cords or bands $A, A$, made fast to them, and also to the end of the gauge-plates $B$, furnished with graauated steps, against which the tables $h, h$, are drawing at each operation of the machine. In proportion as these gauge-plates are raised, the nearer the carriages or tables $h$, will be able to advance to the centre of the machine, and thus permit the combs $g, g$, to lay hold of, and comb, additional lengths of the woolly fibres. The gauge-plates $\mathbf{B}$, are guided up by the bars $\mathrm{c}$, which pass through openings, slots, or guides, made in the framing $\alpha$, as shown by D. 
To the ratchet wheel $s$, an inclined projection $\mathrm{E}$, is made fast, which in the course of the rotation of the ratchet wheel, comes under the lever $\mathrm{F}$, fixed to the shaft $\mathrm{G}$, that turns

in bearings $\mathrm{H}$. To this shaft the levers $\mathrm{I}$ and $\mathrm{J}$, are also fixed; $\mathrm{I}$ serv1209 ing to throw out the click or catch $\mathrm{K}$, from the ratchet wheel, by which the parts of the machine will be released, and restored to positions ready for starting again. The lever $\mathrm{J}$, serves to slide the drum upon the driving shaft $b$, out of gear, by means of the forked handle $\mathrm{L}$, wher. the machine is to be stopped, whenever it has finished combing a certain quantity of wool. The combs which hold the wool have a motion upwards, in order to take the wool out of the way of the combs $g, g$, as these are drawn into the centre of the machine; while the holding combs descend to lay the wool among the points of the combs $g, g$. For obtaining this upward and downward motion, the combs $M, M$, are placed upon the frame $N$, and retained there just as the combs $g, g$, are upon the holders $i, i$. The framing $\mathrm{N}$ is made fast to the $\mathrm{ba}_{\mathrm{s}}$ or spindle 0 , which moves vertically through openings in the cross-head $\mathrm{P}$, and the cross-framing of the machine $Q$; from the top of which, there is a strap passes over pulleys with a weight suspended to it; the cross-head being supported by the two guide-rods $\mathrm{R}$, fixed to the cross-framing $\mathrm{Q}$. It is by the guide-rods $\mathrm{R}$, and the spindle 0 , that the frame $\mathrm{N}$ is made to move up and down; while the spindle is made to rise by the studs $f$, as the wheels $c$ and $d$ come successively under the studs $s$, on the spindle 0 .

A quantity of wool is to be placed on each of the combs $\mathrm{g}, \mathrm{g}$, and $\mathrm{M}, \mathrm{M}$, the machine being in the position shown in fig. 1210. When the main shaft $b$, is set in motion, it will drive by its pinion the toothed wheel $c$, and therefrom the remaining parts of the machine. The first effect of the movement will be to raise the combs $M, M$, sufficiently high to

1210

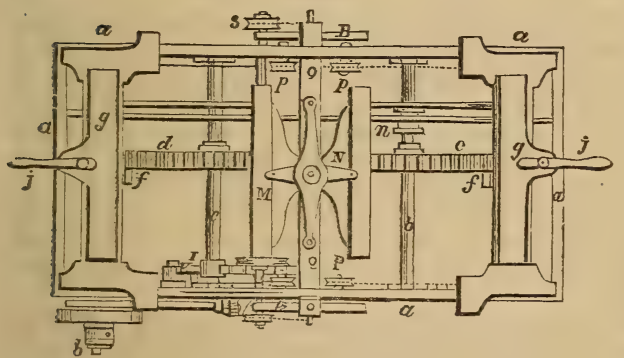
remove the wood out of the way of the combs $g, g$, which will be drawn towards the centre of the machine, as soon as they are released by the studs $f$, passing the projecting arms $q$, on the tables $h$; but the distance between the combs $g, g$, and the combs $\mathrm{H}, \mathrm{H}$, will depend on the height to which the gauge-plates $\mathrm{B}$, have been raised. These plates are raised one step at each revolution of the shaft $c$; the combs $g$, $g$, will therefore be continually approaching more nearly to the combs $\mathrm{M}$, $\mathrm{M}$, till the plates $\mathrm{B}$, are so much raised as to permit the tables $h$, to approach the plates $\mathbf{B}$, below the lowest step or graduation, when the machine will continue to work. Notwithstanding the plates $\mathbf{B}$, continuing to rise, there being only perallel surfaces against which the tables come, the combs $g$, $g$, will successively come to the same position, till the inclined projection $\mathrm{E}$, on the ratchet wheel $s$, comes under the lever $F$, which will stop the machine. The wool which has been combed is then to be removed, and a fresh quantity introduced. It should be remarked, that the combs $g$, $g$, are continually moving from side to side of the machine, at the sarne time that they are combing out the wool. The chief object of the invention is obviously to give the above peculiar motions to the combs $\mathrm{g}, \mathrm{g}$, and $\mathrm{M}, \mathrm{M}$; which may be applied also to combing goathair.

For the purposes of the worsted manufacture, wool should be rendered inelastic to a considerable degree, so that its fibres may form long lines, capable of being twisted into siraight level yarn. Mr. Bayliffe, of Kendal, has sought to accomplish this object, first, by introducing into the drawing machine a rapidly revolving wheel, in contact with the front drawing roller, by whose friction the filaments are heated, and at the same time denrived of their curling elasticity; secondly, by employing a moveable regulating roller, by which the extent of surfice on the periphery of the wheel that the lengths of 
wool is to act upon, may be increased or diminished at pleasure, and, consequently, the effect regulated or tempered as the quality of the wool may require; thirdly, the em-

1211

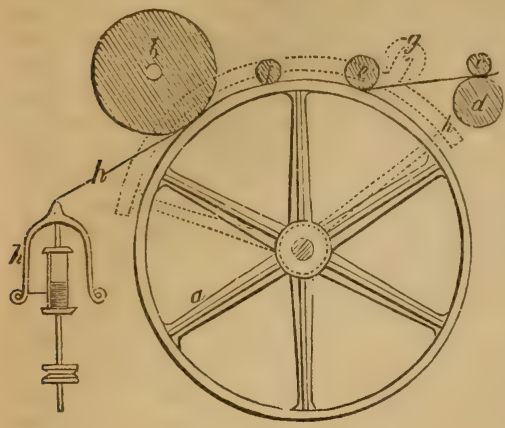
ployment of steam in a rotatory drum, or hollowed wheel, in place of the wheel first described, for the purpose of heating the wool, in the process of drawing, in order to facilitate the operation of straightening the fibres.

These objects may be effected in several ways; that is, the machinery may be variously constructed, and still embrace the principles proposed. Fig. 1211, shows one mode:- $a$, is the friction wheel; $b$, the front drawing roller, placed in the drawing frame in the same way as usual; the larger wheel $a$, constituting the lower roller of the pair of front drawing rollers; $c$, and $d$, are the pair of back drawing rollers, which are actuated by gear connected to the front rollers, as in the ordinary construction of drawing machines, the front rollers moving very considerably faster than the back rollers, and, consequently, drawing or extending the fibres of the sliver of wool, as it passes through between them; $e$, is a guide roller, bearing upon the periphery of the large wheel; $f$, is a tension roller, which presses the fibres of the wool down upon the wheel $a$.

Now, supposing the back rollers $c$ and $d$ to be turned with a given velocity, and the front roller $b$ to be driven much faster, the effect would be, that the fibres of wool constituting the sliver, passing through the machine, would be considerably extended between $b$ and $d$, which is precisely the effect accomplished in the ordinary drawing frame; but the wheel $a$, introduced into the machine in place of the lower front drawing roller, being made to revolve much faster than $b$, the sliver of wool extended over the upper part of its periphery from $b$, to the tension roller $f$, will be subjected to very considerable friction from the contact; and, consequently, the natural curl of the wool will be taken out, and its elasticity destroyed, which will enable the wool to proceed in a connected roving down to the spindle or flier $h$, where it becomes twisted or spun into a worsted thread.

In order to increase or diminish the extent to which the fibres of wool are spread over the periphery of the wheel $a$, a regulating roller is adapted to the machine, as shown at $\mathrm{g}$, in place of the tension roller $f$. This regulating roller $g$, is mounted by its pivots in bearings on the circular arms $h$, shown by dots. These circular arms turn loosely upon the axle of the wheel $a$, and are raised or depressed by a rack and a winch, not shown in the figure; the rack taking into teeth on the periphery of the cireular arms. It will hence be perceived, that by raising the circular arms, the roller $\mathrm{g}$, will be carried backward, and the fibres of wool pressed upon the periphery of the wheel to a greater extent. On the contrary, the depression of the circular arms will draw the roller $g$, forward, and cause the wool to be acted upon by a smaller portion of the periphery of the wheel $a$, and consequently subject it to less friction.

When it is desired to employ steam for the purpose of heating the wool, the wheel $a$, is formed as a hollow drum, and steam from a boiler, in any convenient situation, is conveyed through the hollow axle to the interior of the drum, which, becoming heated by that means, communicates heat also to the wool, and thereby destroys its curl and elasticity.

Breaking-frame.-Here the slivers are planked, or spliced together, the long end of one to the short end of another; after which they are drawn out and extended by the rollers of the breaking-frame. A sketch of this machine is given in fig. 1212. It consists of 4 pairs of rollers, A, B, c, D. The first pair A, receives the wool from the inclined trough $\mathrm{E}$, which is the planking-table. The slivers are unrolled, parted, and hung loosely over a pin, in reach of the attendant, who takes a sliver, and lays it flat in the trough, and the . end is presentel to the rollers $A$, which being in motion, will draw the wool in; the sliver is then conductad through the other rollers, as shown in the figure: when the sliver has passed half through, the end of another sliver is placed upon the middle of the first, and they pass through together; when this second is passed half through, the end of a third is applied upon the middle of it, and in this way the short slivers produced by the combing are joined into one regular and even sliver.

The lower roller $\mathrm{c}$ receives its motion from the mill, by means of a pulley upon the , end of its axis, and an endless strap. The roller which is immediately over it, is borne down by a heavy weight, suspended from hooks, which are over the pivots of the uprer rolier. The fourth pair of rollers $D$, moves with the same velocity as $\mathrm{c}$, being turned 
by n.eans of a small wheel upon the end of the axis of the roller $\mathrm{c}$, which turns a wheel of the same size upon the axis of the roller D, by means of an intermediate wheel $d$, which makes both rollers turn the same way round. The first and second pairs of

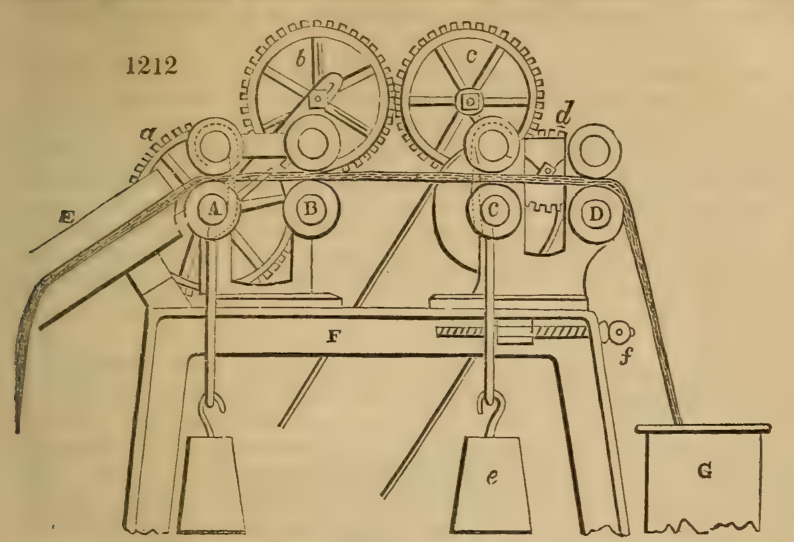
rollers, $\mathrm{A}$ and $\boldsymbol{B}$, move only one third as quick as $\mathrm{C}$ and $\mathrm{D}$, in order to draw out the sliver between $\mathbf{B}$ and $\mathbf{c}$ to three times the length it was when put on the plankingtable. The slow motion of the rollers A, is given by a large wheel $a$, fixed upon the axis of the roller $A$, and turned by the intermediate cog-wheels $b, c$, and $d$; the latter comrnunicates between the rollers $\mathrm{C}$ and $\mathrm{D}$. The pinions on the rollers $\mathrm{c}$ and $\mathrm{D}$ being only one third the size of the wheel $a, \mathrm{C}$ and $\mathrm{D}$ turn three times as fast as $\mathrm{A}$, for $b, c$, and $d$, are only intermediate wheels. The rollers $B$ turn at the same rate as A. The upper roller $e$ is loaded with a heavy weight, similar to the rollers A; but the other rollers, B and D, are no further loaded than the weight of the rollers.

The $t$ wo pairs of rollers A, B, and $c$, D, are mounted in separate frames; and that frame which contains the third and fourth pairs $\mathrm{c}, \mathrm{D}$, slides upon the cast-iron frame $\mathrm{F}$, which supports the machine, in order to increase or diminish the distance between the rollers $\mathrm{B}$ and $\mathrm{c}$. There is a screw $f$, by which the frame of the rollers is moved, so as to adjust the machine according to the length of the fibre of the wool. The space between $B$ and $c$ should be rather more than the length of the fibres of the wool. The intermediate wheels $b$ and $c$, are supported upon pieces of iron, which are moveable on centres; the centre for the piece which supports the wheel $b$ is concentric with the axis of the roller $A$; and the supporting piece for the wheel $c$ is fitted on the centre of the wheel $d$. By moving these pieces the intermediate wheels $b$ and $c$ can be always kept in contact, although the distance between the rollers is varied at times. By means of this breakingframe, the perpetual sliver, which is made up by planking the sliver together, is equalized, and drawn out three times in length, and delivered into the can G.

Drawing-frame.-Three of these cans are removed to the drawing-frame, which is similar to the breaking-trame, except that there is no planking-table $\mathbf{E}$. There are five sets of rollers, all fixed upon one common frame $F$, the breaking-frame, which we have dnscribed, being the first. As fast as the sliver comes through one set of rollers, it is received into a can, and then three of these cans are put together, and passed again through another set of rollers. In the whole, the wool must pass through the breaker and four drawing-frames before the roving is begun. The draught being usually four times at each operation of drawing, and three times in the breaking, the whole will be $3 \times 4 \times 4 \times 4 \times 4=768$; but to suit different sorts of wool, the three last drawingframes are capable of making a greater draught, even to five times, by changing the pinions; accordingly the draught will be $3 \times 4 \times 5 \times 5 \times 5=1500$ times.

The size of the sliver is diminished by these repeated drawings, because only three slivers aie put together, and they are drawn out four times; so that, in the whole, the sliver is reduced to a fourth or a ninth of its original bulk.

The breaking-frame and drawing-frame which are used when the slivers are prepared by the combing-machines, are differently constructed; they have no plankingtable, but receive three of the perpetual slivers of the combing-machine from as many tin cans, and draw them out from ten to twelve times. In this case, all the four rollers contribute to the operation of drawing: thus the second rollers $\mathrm{B}$, more $2 \frac{1}{2}$ times as fast as the rollers $A$; the third rollers $c$, move 8 times as fast as $A$; and the fourth rollers $\mathrm{E}$, move $10_{2}^{\frac{1}{2}}$ times as fast as $\mathrm{A}$. In this case, the motion is given to the different rollers by means of bevelled wheels, and a horizontal axis, which extends across the ends of all the four rollers, to communicate motion from one pair of rollers to another.

There are three of these systems of rollers, which are all mounted on tne same frame; and the first one through which the wool passes, is called the breaking-fiame; 
but it does not differ from the others, which are called drawing-frames. The slivers which have passed through one system of rollers, are collected four or five together, and put through the drawing-rollers. In all, the slivers pass through three drawings, and the whole extension is seldom less than 1000 times, and for some kinds of wool much greater.

After the drawing of the slivers is finished, a pound weight is taken, and is measured by means of a cylinder, in order to ascertain if the drawing has been properly conducted; if the sliver does not prove of the length proposed, according to the size of worsted which is intended to be spun, the pinions of some of the drawing-frames are changed, to make the draught more or less, until it is found by experiment that one pound of the sliver measures the required length.

Roving-frame.-This is provided with rollers, the same as the drawing-frames; it takes in one or two slivers together, and diaws them out four times. By this extension, the sliver becomes so small, that it would break with the slightest force, and it is therefore necessary to give some twist; this is done by a spindle and flier. See Roving, unde: Cotton Manufacture.

Spinning-frame.-This is so much like the roving-frame, that a short description will be sufficient. The spindles are more delicate, and there are three pairs of rollers, instead of two; the bobbins, which are taken off from the spindles of the rovingframe, when they are quite full, are stuck upon skewers, and the roving which proceeds from them is conducted between the rollers. The back pair turns round slowly; the middle pair turns about twice for once of the back rollers; and the front pair makes from twelve to seventeen turns for one turn of the back roller, according to the degree of extension which is required.

The spindles must revolve very quickly in the spinning-frame, in order to give the requisite degree of twist to the worsted. The hardest twisted worsted is called tammy warp; and when the size of this worsted is such as to be 20 or 24 hanks to the pound weight, the twist is about 10 turns in each inch of length. The least twist is given to the worsted for fine hosiery, which is from 18 to 24 hanks to the pound. The tivist is from 5 to 6 turns per inch. The degree of twist is regulated by the size of the whirls or pilleys upon the spindle, and by the wheel-work which communicates the motion to the front rollers from the band-wheel, which turns the spindles.

It is needless to enter more minutely into the description of the spinning machinery, because the fluted roller construction, invented by Sir Richard Arkwright, fully described under Cotton Manufacture, is equally applicable to worsted. The difference between the two is chiefly in the distance between the rollers, which, in the worsted-frame, is capable of being increased or diminished at pleasure, according to the length of the fibres of the wool; and the draught or extension of the roving is far greater than in the cotton.

Reeling.- The bobbins of the spinning-frame are placed in a row upon wires before a long horizontal reel, and the threads from 20 bobbins are wound off together. The reel is exactly a yard in circumference, and when it has wound off 80 turns, it rings a bell; the motion of the reel is then stopped, and a thread is passed round the 80 turns or folds which each thread has made. The reeling is then continued till another 80 yards is wound off, which is also separated by interweaving the same thread; each of chese separate parcels is called a ley, and when 7 such leys are reeled, it is called a hank, which contains 560 yards. When this quantity is reeled off, the ends of the binding thread are tied together, to bind each hank fast, and one of the rails of the reel is struck to loosen the hanks, and they are drawn off at the end of the reel. These hanks are next hung upon a hook, and twisted up hard by a stick; then doubled, and the two parts twisted together to make a firm bundle. In this state, the hanks are weighed by a small index machine, which denotes what number of the hanks will weigh a pound, and they are sorted accordingly into different parcels. It is by this means that the number of the worsted is ascertained as the denomination for its fineness : thus No. 24 means, that 24 hanks, each containing 560 yards, will weigh a pound, and so on.

This denomination is different from that used for cotton, because the hank of cotton contains 840 yards, instead of 560 ; but in some places the worsted hank is made of the same length as the cotton.

To pack up the worsted for market, the proper number of hanks is collected to make a pound, according to the number which has been ascertained; these are weighed, as a proof of the correctness of the sorting, then tied up in bundles of one pound each, and four of these bundles are again tied together. Then 60 such bundles are packed up in a sheet, making a bale of 240 pounds, ready for market.

Of the treatment of short wool for the cloth manufacture.-Short wool resembles cotton not a little in the structure of its filaments, and is cleaned by the willy, as cotton is by the willow, which nrens up the matted fleece of the wool-stapler, and cleans it from accidental impurities. Sheep's wool for working into coarse goods, must be passed re- 
peatedly through this machine, both before and after it is dyed; the second last time for the purpose of blending the different sorts together, and the last for imbuing the fibres intimately with oil. The oiled wool is next subjected to a first carding operation called scribbling, whereby it is converted into a broad thin fleece or lap, as cotton is by the breaker-cards of a cotton mill. The woollen lap is then worked by the cards proper, which deliver it in a narrow band or sliver. By this process the wool expands greatly in all its dimensions; while the broken or short filaments get entangled by crossing in every possible direction, which prepares them for the fulling operation. See Carding, under Cotton Manufacture.

The slubbing machine, or billy, reduces the separate rolls of cardings into a continuous s]ightly twisted spongy cord, which is sometimes ealled a roving. Fig. 1213 is a per-

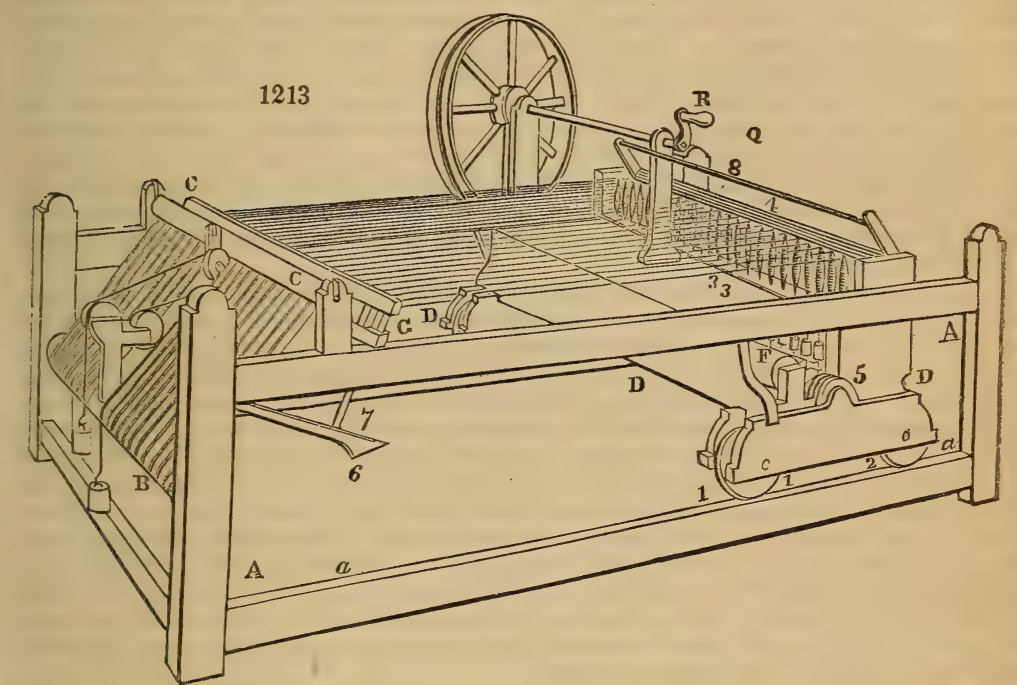

spective representation of the slubbing machine in most common use. A, $A$, is the wooden frame; within which is the moveable carriage $\mathrm{D}, \mathrm{D}$, which runs upon the lower side rails at $a, a$, on friction wheels at 1,2 , to make it move easily, backwards and forwards from one end of the frame to the other. The carriage contains a series of steel spindles, marked 3,3 , which receive rapil rotation from a long tin drum $F$, by means of a series of cords passing round the pulley or whorl of each spindle. This drum, 6 inches in diameter, is covered with paper, and extends across the whole breath of the carriage. The spindles are set nearly upright in a frame, and about 4 inches apart; their under ends being pointed conically, turn in brass sockets called steps, and are retained in their position hy a small brass collet, which embraces each spindle at about the middle of its length. The upper half of each spindle projects above the top of the frarne. The drum revolves horizontally before the spindles, having its axis a little below the line of the whorls; and receives motion, by a pulley at one of its ends, from an endless banc which passes round a wheel $\mathrm{E}$, like the large domestic wheel formerly used in spinning wool by hand, and of similar dimensions. This wheel is placed upon the outside of the main frame of the machine, and has its shafts supported by upright standards upon the carriage $\mathrm{D}$. It is turned by the spinner placed at $\mathrm{Q}$, with his right hand applied to a winch $\mathrm{R}$, which gives motion to the drum, and thereby causes the spindles to revolve with great velocity.

Each spindle receives a soft cylinder or carding of wool, which comes through beneath a wooden roller c, c, at the one end of the frame. This is the billy roller, so much talked of in the controversies between the operatives and masters in the cotton factories, as an instrument of cruel punishment to children, though no such machine has been used in cotton mills for half a century at least. These woollen rolls proceed to the series of spindles, standing in the carriage, in nearly a horizontal plane. By the alternate ajvance and retreat of the carriage upon its railway, the spindles are made to approach to, and recede from, the roller $c$, with the effect of drawing out a given length of the soft cord, with any desired degree of twist, in the following manner:-

The carding rolls are laid down straight, side by side, upon the endless cloth, strained in an inclined direciion between two rollers, one of which is seen at $\mathrm{B}$, and the 
other lies behind c. One carding is allotted to a spindle; the total number of each in one machine being from 50 to 100. The roller c, of light wood, presses gently with its weight upon the cardings, while they move on wards over the endless cloth, with the running out of the spindle carriage. Immediately in front of the said roller, there is a horizontal wooden rail or bar $G$, with another beneath it, placed across the frame. The carding is conducted through between these two bars, the moveable upper one being raised to let any aliquot portion of the roll pass fieely. When this bar is again let down, it pinches the spongy carding fast; whence this mechanism is called the clasp. It is in fact the clove, originally used by Hargreaves in his cotton-jenny. The moveable upper rail G, is guided between sliders, and a wire 7 , descends from it to a lever c. When the spindle carriage $\mathrm{D}, \mathrm{D}$, is wheeled close home to the billy roller, a wheel 5 , lifts the end 6 of the lever, which, by the wire 7, raises the upper bar or rail $\mathrm{G}$, so as to open the clasp, and release all the card rolls. Should the carriage be now drawn a little way from the clasp bars, it would tend to pull a corresponding length of the cardings forward from the inclined plane $\mathrm{B}, \mathrm{c}$. There is a small catch, which lays hold of the upper bar of the clasp G, and hinders it from falling till the carriage has receded to a certain distance, and has thereby allowed from 7 to 8 inches of the cardings to be taken out. A stop upon the carriage then comes against the catch, and withdraws it ; thus allowing the upper rail to fall and pinch the carding, while the carriage, continuing to recede, draws out or stretches that portion of the roll which is between the clasp and the spindle points. But during this time the wheel has been turned to keep the spindles revolving, communicating the proper degree of twist to the cardings in proportion to their extension, so as to prevent them from breaking.

It might be imagined that the slubbing cords would be apt to coil round the spindles; but as they proceed in a somewhat inclined direction to the clasp, they receive merely a twisting motion, continually slipping over the points of the spindles, without getting wound upon them. Whenever the operative or slubber has given a due degree of twist to the rovings, he sets about winding them upon the spindles into a conical shape, for which purpose he presses down the faller-wire 8 , with his left hand, so as to bear it down from the points of the spindles, and place it opposite to their middle part. He next makes the spindles revolve, while he pushes in the carriage slowly, so as to coil the slubbing upon the spindle into a conical cop. The wire 8 , regulates the winding-on of the whole series of slubbings at once, and receives its proper angle of depression for this purpose from the horizontal rail 4, which turns upon pivots in its ends, in brasses fixed on the standards, which rise from the carriage $\mathrm{D}$. By turning this rail on its pivots, the wire 8 may be raised or lowered in any degree. The slubber seizes the rail 4 in his left hand, to draw the carriage out; but in returning it, he depresses the faller-wire, at the same time that he pushes the carriage before him.

The cardings are so exceedingly tender, that they would readily draw out, or even break, if they were dragged with friction upon the endless cloth of the inclined plane. To save this injurious traction, a contrivance is introduced for moving the apron. A cord is applied round the groove in the middle part of the upper roller, and after passing over pulleys, as shown in the figure, it has a heavy weight hung at the one end, and a light weight at the other, to keep it constantly extended, while the heavy weight tends to turn the rollers with their endless cloth round in such a direction as to bring forward the rovings, without putting any strain upon them. Every time that the carriage is pushed home, the larger weight gets wound up; and when the carriage is drawn out, the greater weight turns the roller, and advances the endless apron, so as to deliver the carding at the same rate as the carriage runs out; but when the proper quantity is delivered, a knot in the rope arrives at a fixed stop, which does not permit it to move any further; while at the same instant the roller 5 quits the lever 6 , and allows the upper rail $G$, of the clasp to fall, and pinch the carding fast; the wheel $\mathrm{E}$, being then set in motion, makes the spindles revolve; and the carriage being simultaneously drawn out, extends the slubbings while under the influence of twisting. In winding up the slubbings, the operative must take care to push in the carriage, and to turn the wheel round at such rates that the spindles will not take up faster than the carriage moves on its railway, or he wculd injure the slubbings. The machine requires the attendance of a child, to bring the cardings from the card-engine, to place them upon the sloping feed-cloth, ard to join the ends of the fresh ones carefully to the ends of the others newly drawn under the roller. Slubbings intended for warp-yarn must be more twisted than those for weft; but each must receive a degree of torsion relative to the quality of wool and of the cloth intended to be made. In general, however, no more twist should be given to the slubbings than is indispensable for enabling them to be drawn out to the requisite slenderness without breaking. This twist forms no part of the twist of the finished yarn, for the slubbing will be twisted in the contrary direction, when spun afterwards in the jenny or mule.

I may here remark, that various machines have been constructed of late years $f_{1}$ 
making continuous card-ends, and slubbings, in imitation of the carding and roving of the Cotros MANUfacturf; to which alticle I therefore refer my readers. The wool slubhings are now spun into yarn, in many factories, by means of the mule. Indeed, I have seen in France the finest yarn, for the mousseline-de-laine fabrics, beautifully spun upon the self-actor mule of Sharp and Roberts.*

Tentering.-- When the clolh is returned from the fulling-mill (which see), it is stretched upon the tenter-frame, and left in the open air till dry.

In the woollen manufacture, as the cloth suffers, by the operation of the fulling-mill, a shrinliage of its breadth to well nigh one half, it must at first be woven of nearly double its intended width when finished. Superfine six-quarter broad cloths must therefore be turned out of the loom twelve quarters wide.

Burling is the name of a process, in which the dried cloth is examined minutely in every part, freel from linots or uneven threads, and repaired by sewing any little rents, or inserting sound yarns in the place of defective ones.

Teasling.- The object of this operation is to raise up the loose filaments of the woollen yarn into a nap upon one of the surfaces of the cloth, by scratching it either with thistle-heads, called teasels, or with teasling-cards or brushes, made of wire. The natural teasels are the balls which contain the seeds of the plant called Dipsacus fullorum; the scales which form the balls project on all sides, and end in sharp elastic points, that turn downwards like hooks. In teasling by hand, a number of these balls are put into a small wooden frame, having crossed handles, eight or ten inches long; and when thus filled, form an implement not unlike a curry-comb, which is used by two men, who seize the teasel-frame by the handies, and scrub the face of the cloth, hung in a vertical position from two horizontal rails, made fast to the ceiling of the workshop. First, they wet the cloth, and work three times over, by strokes in the direction of the warp, and next of that of the weft, so as to raise all the loose fibres from the felt, and to prepare it for shearing. In large manufactories, this dressing operation is performed by a machine called a gig-mill, which originally consisted, and in most places still consists, of a cylinder bristled all over with the thistleheads, and made to revolve rapidly while the cloth is drawn over it in a variety of directions. If the thistle be drawn in the line of the warp, the points act more efficaciously upon the weft, being perpendicular to its softer spun yarns. Inventors who have tried to give the points a circular or oblique action between the warp and the weft, proceed apparently upon a false principle, as if the cloth were like a plate of metal, whose substance could be pushed in any direction. Teasling really consists in drawing out one end of the filaments, and Jeaving the body of them entangled in the cloth; and it should seize and pull them perpendicularly to their length, because in this way it acts upon the ends, which being least implicated, may be most readily disengaged.

When the hooks of the thistles become clogged with flocks of wool, they must be taken out of the frame or cylinder, and cleaned by children with a small comb. Moisture, moreover, softens their points, and impairs their teasling powers; an effect which needs to be counterbalanced, by taking them out, and drying them from time to time. Many contrivances have, therefore, been proposed in which metallic teasels of an unchangeable nature, mounted in rotatory machines, driven by power, have been substituted for the vegetable, which being required in prodigious quantities, becomes sometimes excessively scarce and dear in the clothing districts. In 1818, several schemes of that kind were patented in France, of which those of M. Arnold-Merick, and of MM. Taurin frères, of Elbœuf, are described in the 16th volume of Brevets d'Invention expirés. Mr. Daniell, cloth manufaciurer in Wilts, renewed this invention under another form, by making his rotatory cards with two kinds of metallic wires, of unequal length; the one set, long, thin, and delicate, representing the points of the thistle; the other, shorter, stiffer, and blunter, bein in- $^{-}$ tended to stay the cloth, and to hinder the former from entering too far into it. But none of these processes have succeeded in discarding the natural teasel from the most eminent manufactories.

The French government purchased, in 1807, the patent of Douglas, an English mechanist, who had, in 1802, imported into France the best system of gig-mills then used in the west of England. A working set of his machines having been placed in the Conservaioire des Arts, for public inspection, they were soon introduced into most of the French establishments, so as generally to supersede teasling (lainage) by hand. A description of them was published in the third volume of the Brevets d'Invention. The following is an outline of some subsequent improvements :-

1. As it was imagined that the seesaw action of the hand operative was in some respects more effectual than the uniform rotation of a gig-mill, this was attempted to be iinitated by an alternating movement.

* See this admirable machine fully dewcribed and delineated in my Cotton Manufacture of Great Britarm rol. ii. 
2. Others conceived that the seesaw motion was not essential, but that it was advan. tageous to make the teasels or cards act in a rectilinear direction, as in working by hand: this action was attempted by placing the two ends of the teasel-frame in grooves formed like the letter $\mathrm{D}$, so that the teasel should act on the cloth only when it came into the rectillinear part. Mr. Wells, machine-maker, of Manchester, obtained a patent, in 1832, for this construction.

3. It was supposed that the teasels should not act perpendicularly to the weft, but obliquely or circularly upon the face of the cloth. Mr. Ferrabee, of Gloucester, patented, in 1830, a scheme of this kind, in which the teasels are mounted upon two endless chains, which traverse from the middle of the web to the selvage or list, one to the right, and another to the left hand, while the cloth itself passes under them with such a relocity, that the effect, or resultant, is a diagonal action, dividing into two equal parts the rectangle formed by the weft and warp yarns. Three patent machines of Mr. George Oldland-the first in 1830, the second and third in 1832-all proceed upon this principle In the first, the teasels are mounted upon dises made to turn flat upon the surface of the cloth; in the second, the rotating dises are pressed by corkscrew spiral springs against the cloth, which is supported by an elastic cushion, also pressed against the discs by springs; and in the third machine, the revolving dises have a larger diameter, and they turn, not in a horizontal, but a vertical plane.

4. Others fancied that it would be-beneficial to support the reverse side of the cloth by flat hard surfaces, while acting upon its face with cards, or teasels. Mr. Joseph Cliseld Daniell, having stretched the cloth upon smooth level stones, teasels them by hand. 5. Messrs. Charlesworth and Mellor obtained a patent, in 1829, for supporting the back of the cloth with elastic surfaces, while the part was exposed to the teasling action. 6. Elasticity has also been imparted to the teasels, in the three patent inventions of Mr. Sevill, Mr. J. C. Daniell, and Mr. R. Atkinson. 7. It has been thought useful to separate the teasel-frames upon the drum of the gig-mill, by simple rollers, or by rollers heated with steam, in order to obtain the combined effect of calendering and teasling. Mr. J. C. Daniell, Mr. G. Haden, and Mr. J. Rayner, have obtained patents for contrivances of this kind. 8. Several French schemes have been mounted for making the gig-drum act upon the two sides of the cloth, or eren to mount two drums on the same machine.

Mr. Jones, of Leeds, contrived a very excellent method of stretching the cloth, so as to prevent the formation of folds or wrinkles. (See Newton's Journal, vol. viii., 2d series, page 126.) Mr. Collier, of Paris, obtained a patent, in 1830, for a greatly improved gig-mill, upon Douglas's plan, which is now much esteemed by the French clothiers. The following figures and description exhibit one of the latest and best teasling machines. It is the invention of M. Dubois and Co., of Louviers, and is now doing excellent work in that celebrated seat of the cloth manufacture.

In the fulling-mill, the woollen web acquires body and thickness, at the expense of its other dimensions; for being thereby reduced about one third in length, and one half in breadth, its surface is diminished to one third of its size as it comes out of the loom; and it has, of course, increased threefold in thickness. As the filaments drawn forth by teasling are of very unequal lengths, they inust be shorn to make them level, and with different degrees of closeness, according to the quality of the stuff, and the appearance it is desired to have. But, in general, a single operation of each kind is insufficient; whence, after having passed the cloth once through the gig-mill and once through the shearing-machine (tondeuse), it is ready to receive a second teasling, deepel than the first, and then to suffer a second shearing. Thus, by the alternate repetition of these processes, as often as is deemed proper, the cloth finally acquires its wished-for appearance. Both of these operations are very delicate, especially the first; and if they be ill conducted, the cloth is weakened, so as to tear or wear most readily. On the other hand, if they be skilfully executed, the fabric becomes not only more sightly, but it $\mathrm{xc}$ quires strength and durability, because its face is changed into a species of fur, which protects it from friction and humidity.

Figs. 1214, 1215, represent the gig-mill in section, and in front elevation. A, B, C, D, $\mathbf{A}^{\prime}, \mathbf{B}^{\prime}, \mathbf{C}^{\prime}, \mathrm{D}^{\prime}$, being the strong frame of iron, cast in one piece, having its feet enlarged a little more to the inside than to the outside, and bolted to large blocks in the stone pavement. The two uprights are bound together below by two eross-beams A", being fastened with screw-bolts at the ears $a^{\prime \prime}, a^{\prime \prime}$; and at top, by the wrought-iron stretcher-rod $\mathrm{D}$, whose ends are secured by screw-nuts at $\mathrm{D}, \mathrm{D}^{\prime}$. The drum is mounted upon a wrought-iron shaft F, which bears at its right end ( $f i g .1215$ ), exterior to the frame, the usual riggers, or fast and loose pulley, $f f^{\prime \prime}, f^{\prime}$ which give motion to the machine by a band from the main shaft of the mill. On its right end, within the frame, the shaft $F$, has a bevel wheel $F^{\prime}$, for transmitting movement to the cloth, $x$ s shall be afterwards explained. Three crown wheels $G$, of which one is shown in the section, fig 1214, are, as usual, keyed by a wedge to the shaft $F$. Their contour is a sinvous 
WOOLLEN MANUFACTURE.
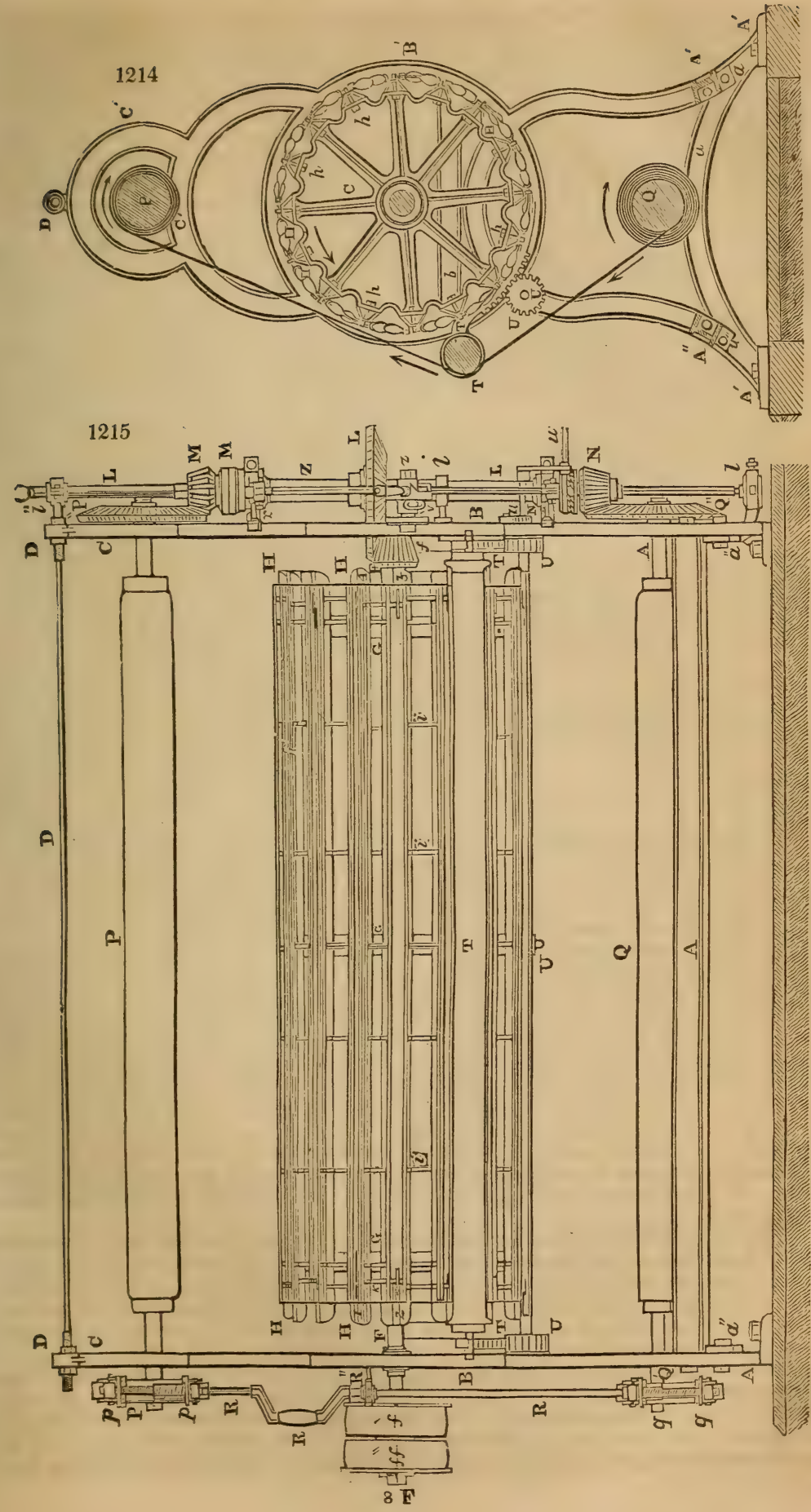
band, with six semi-cylindrical hollows, separated alternately by as many portions of the periphery. One of these three wheels is placed in the middle of the shaft $F$, and the other iwo, towards its extremities. Their size may be judged of, from inspection of fig. 1214. Alter having set them so that all their spokes or radii correspond exactly, the 16 sides $\mathrm{H}$, are made fast to the 16 portions of the periphery, which correspond in the three wheels. These sides are made of sheet iron, curved inio a gutter form, fig. 1214, but round. ed ofl at the end, fig. 1215, and each of them is fixed to the three felloes of the wheels b. three bolts $h$. The elastic part of the plate iron allows of their being sufficiently well ad justed, so that their flat portions furthest from the centre may lie pretty truly on a cylindrica] surface, whose axis would coincide with that of the shaft $F$.

Between the 16 sides there are 16 intervals, which correspond to the 16 hollowings of each of the wheels. Into these intervals are adjusted, with proper precautions, 16 frames bearing the teasels which are to act upon the cloth. These are fitted in as follows:-Each has the shape of a rectangie, of a length equal to that of the drum, but their breadth only large enough to contain two thistle-heads set end to end, thus making two rows of parallel teasels throughout the entire length, (see the contour in fig. 1214.) A portion of the frame is represented in fig. 1216. The large side $\mathbf{r}$, a ainst which the tops of the teasels rest, is hollowed out into a semi-cylinder, and its

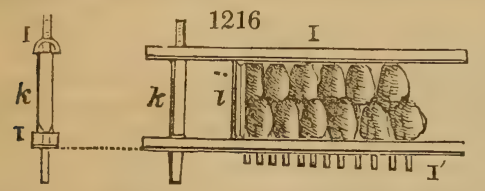
opposite side is cleft throughout its whole length, to. receive the tails of the teasels, which are seated and compressed in it. There are, moreover, cross-bars $i$, which serve to maintain the sides of the frame $r$, at an invariable distance, and to form short compartments for keeping the thistles compact. The ends are fortified by stronger bars $k, k$, with projecting bolts to fasten the frames between the ribs. The distance of the sides of the frame $I, I^{\prime}$, ought to be such, that if a frame be laid upon the drum, in the interval of two ribs, the side I will rest upon the inclined plane of one of the ribs, and the side $x^{\prime}$ upon the inclined plane of the other (see fig. 1214); while at the same time the bars $k$, of the two ends of the frame,

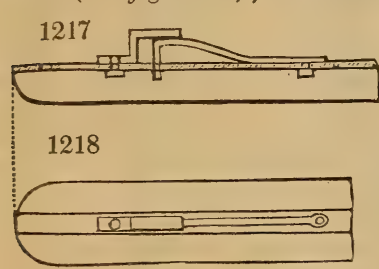
rest upon the flat parts of the ribs themselves. This point being secured, it is obvious, that if the ends of the bars $k$ be stopped, the frame will be made fast. But they need not be fixed in a permanent manner, because they must be frequently removed and replaced. They are fastened by the clamp (figs. 1217, 1218), which is shut at the one end, and furnished at the other with a spring, which can be opened or shut at pleasure. 2 and 4 , in fig. 1215 (near the right end of the shaft $F$ ), shows the place of the clamp, figs. 1217, 1218. The bar of the right hand is first set in the clamp, by holding up its other end; the frame is then let down into the left-hand clamp.

The cloth is wound upon the lower beam Q, fig. 1214; thence it passes in contact with a wooden cylinder $\mathbf{T}$, turning upon an axis, and proceeds to the upper beam $\mathbf{P}$, on to which it is wound : by a contrary movement, the cloth returns from the beam $\mathbf{P}$ to $\mathbf{Q}$, over the cylinder $\mathrm{T}$; and may thus go from the one to the other as many times as shall be requisite. In these successive circuits it is presented to the action of the teasels, under certain conditions. In order to be properly teasled, it must have an equal tension throughout its whole breadth during its traverse; it must be brought into more or less close contact with the drum, according to the nature of the cloth, and the stage of the operations; sometimes being a tangent to the surface, and sometimes embracing a greater or smaller portion of its contour, it must travel with a determinate speed, dependant upon the velocity of the drum, and calculated so as to produce the best result: the machine itself must make the stuff pass alternately from one winding beam to the other.

In fig. 1215, before the front end of the machine, there is a vertical shaft $\mathrm{L}$, as high as the framework, which revolves with great facility, in the bottom step $l$, the middle collet $l^{\prime}$, and top collet $l^{\prime \prime}$, in the prolongation of the stretcher D. Upon this upright shaft are mounted-1. a bevel wheel $\mathbf{L}^{\prime} ; 2$. an upper bevel pinion $\mathrm{M}$, with its boss $\mathrm{M}^{\prime}$; 3 . a lower bevel pinion $N$, with its boss $N^{\prime}$. The bevel wheel $L^{\prime}$ is keyed upon the shaft $\mathrm{L}$, and communicates to it the movement of rotation which it receives from the pi-:$f$, with which it is in gear; but the pinion $f$, which is mounted upon the shaft $\mathbf{F}$ of the drum, participates in the rotation which this shaft receives from the prime mover, by means of the fast rigger-pulley $f^{\prime}$. The upper pinion $\mathrm{m}$ is independent upon the shaft $\mathrm{L}$; that is to say, it may be slidden along it, up and down, withont being driven by it; but it may be turned in an indirect manner by means of six curved teeth, projecting from 
its bottom, and which may be rendered active or not, at pleasure; these curved teeth, and their intervals, correspond to similar teeth and intervals upon the top of the boss $\mathbf{M}^{\prime}$, which is dependent, by feathered indentations, upon the rotation of $\mathbf{L}$, though it can slide fieely up and down upon it. When it is raised, therefore, it comes into gear with M. The pinion $N$, and its boss, have a similar mode of being thrown into and out of gear with each other. The bosses $\mathrm{M}^{\prime}$ and $\mathrm{N}^{\prime}$, ought always to be moved simultaneously, in -- - ter to throw one of them into gear, and the other out of gear. The shaft $\mathrm{L}$ serves to put the cloth in motion, by means of the beve! wheels $P^{\prime \prime}$ and $Q^{\prime \prime}$, upon the ends of the beams $P, Q$, which take into the pinions $M$ and $N$.

The mechanism destined to stretch the cloth is placed at the other end of the machine, where the shafts of the beams $P, Q$, are prolonged beyond the frame, and bear at their extremities $\mathrm{P}^{\prime}$ and $\mathrm{Q}^{\prime}$, armed each with a brake. The beam $\mathbf{P}$ ( $f$ ig. 1214), turns in an opposite direction to the drum; consequently the cloth is wound upon $\mathbf{P}$, and unwound from $Q$. If, at the same time as this is going on, the handle $\mathrm{R}^{\prime}$, of the brakeshaft, be turned so as to clasp the brake of the pulley $Q^{\prime}$, and release that of the pulley $\mathbf{P}^{\prime}$, it is obvious that a greater or smaller resistance will be occasioned in the beam $Q$, and the cloth which pulls it in unwinding, will be able to make it turn only when it has acquired the requisite tension; hence it will be necessary, in order to increase or diminish the tension, to turn the handle $\mathrm{R}^{\prime}$ a little more or a little less in the direction which clasps the brake of the pulley $Q^{\prime}$; and as the brake acts in a very equable manner, a very equable tension will take place all the tirne that the cloth takes to pass. Besides, should the diminution of the diameter of the beam $Q$, render the tension less efficacious in any considerable degree, the brake would need to be unclamped a very little, to restore the primitive tension.

When the cloth is to be returned from the beam $\mathbf{P}$, to the beam $\mathbf{Q}, \mathbf{z}$ must be lowered, to put the shaft $L$ out of gear above, and in gear below; then the cloth-beam $Q$, being driven by that vertical shaft, it will turn in the same direction as the drum, and. will wind the cloth round its surface. In order that it may do so, with a suitable tension, the pulley $Q^{\prime}$ must be left free, by clasping the brake of the pulley $P^{\prime}$, so as to oppose an adequate resistance.

The cloth is brought into more or less close contact with the drum as follows :-There is for this purpose a wooden roller $T$, against which it presses in passing from the one winding beam to the other, and which may have its position changed relatively to the drum. It is obvious, for example, that in departing from the position represented in fig. 1214, where the cloth is nearly a tangent to the drum, if the roller ' $T^{\prime}$ be raised, the cloth will cease to touch it; and if it be lowered, the cloth will, on the contrary, embrace the drum over a greater or less portion of its periphery. For it to produce these effects, the roller is borne at each end, by iron gudgeons, upon the heads of an arched rack $\mathrm{T}^{\prime \prime}$ ( $f \mathrm{ig} .1214$ ), where it is held merely by pins. These racks have the same curvature as the circle of the frame, against which they are adjusted by two bolts; and by means of slits, which these bolts traverse, they may be slidden upwards or downwards, and consequently raise or depress the roller $\mathrm{T}$. But to graduate the movements, and to render them equal in the two racks, there is a shaft $\mathrm{U}$, supported by the uprights of the frame, and which carries, at each end, pinions $\mathrm{U}^{\prime}, \mathrm{U}^{\prime \prime}$, which work into the two racks $T^{\prime}, T^{\prime \prime}$; this shaft is extended in front of the frame, upon the side of the head of the machine ( $f$ ig. 1215), and there it carries a ratchet wheel $u$, and a handle $u^{\prime}$. The workman, therefore, requires merely to lay hold of the handle, and turn it in the direction of the ratchet wheel, to raise the racks, and the roller $\mathbf{r}$, which they carry; or to lift the click or catch, and turn the handle in the opposite direction, when he wishes to lower the roller, so as to apply the cloth to a larger portion of the drum.

\section{CLOTH CROPPING.}

Of machines for cropping or shearing woollen cloths, those of Lewis and Davis have been very generally used.

Fig. 1219 is an end view, and fig. 1220 is a side view, of Lewis's machine for shearing cloth from list to list. Fig. 1221 is an end view of the carriage, with the rotatory cutter detached from the frame of the machine, and upon a larger scale; $a$, is a cylinder of metal, on which is fixed a triangular steel wire; this wire is previously bent round the cylinder in the form of a screw, as represented at $a, a$, in $f \mathrm{~g} .1219$, and, being hardened, is intended vo constitute one edge of the shear or cutter.

The axis of the cylindrical cutter $a$, turns in the frame $b$, which, having proper adjustments, is mounted upon pivots $c$, in the standard of the travelling carriage $d, d$; and $e$, is the fixed or ledger blade, attached to a bar $f$, which constitutes the other edge of the cutter; that is, the stationary blade, against which the edges of the rotatory cutter act; $f$ and $g$, are flat springs, intended to keep the eloth (shown by dots) up against the cutting edges. The form of these flat springs $f, g$, is shown at figs. 1222 and 1223, 
as consisting of plates of thin metal cut into narrow slips ( $\mathrm{ig}$. 1222), or perforated with long hules, (fig. 1223.) 'Iheir object is to support the cloth, which is intended to pass between them, and operate as a spring bed, bearing the surface of the cloth against

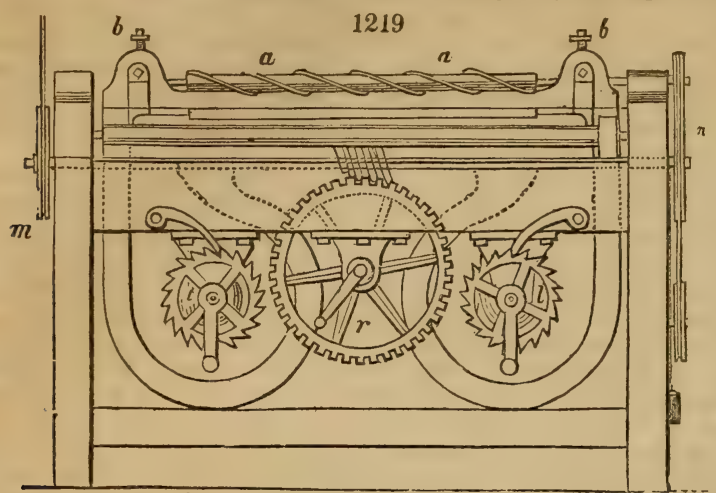

the cutters, so that its pile or nap may je cropped off or shorn as the carriage $d$ is drawn along the top rails of the standard or frame of the machine $h, h$, by means of cords.

The piece of cloth to be shorn, is wound upon the beam $k$, and its end is then conducted through the machine, between the flat springs $f$ and $g$ 'as shown in fig. 1221), to the other beam $l$, and is then made fast; the sides or lists of the cloth being held and stretched by small hooks, called habiting hooks. The cloth being thus placed in the machine, and drawn tight, is held dis-

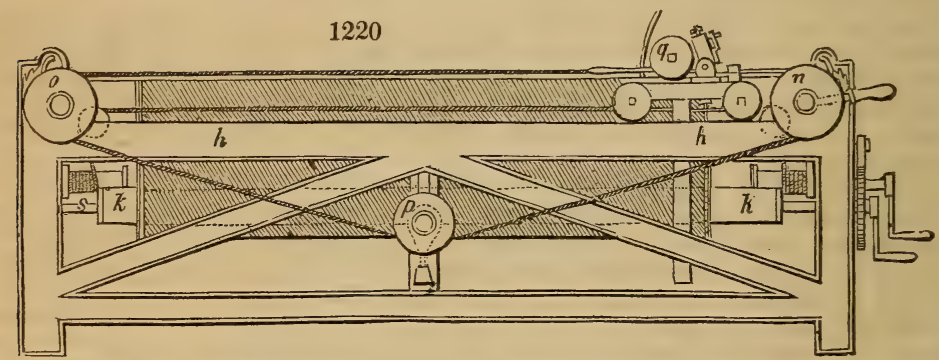

tended by means of ratchets on the ends of the beams $k$ and $l$, and palls. In commencing the operation of shearing, the carriage $d$, must be brought back, as in fig. 1221, so

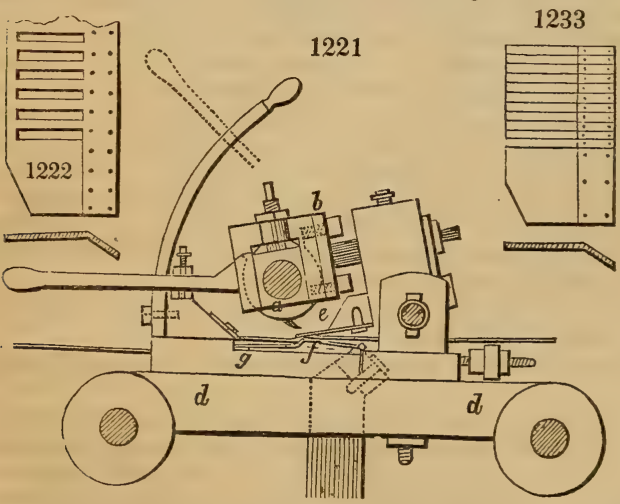
that the cutters shall be close to the list; the frame of the cutters is raised up on its pivots as it recedes, in order to keep the cloth from injury, but is lowered again previously to being put in action. A band or winch is applied to the rigger or pulley $m$, which, by means of an endless cord passed round the pulley $n$, at the reverse end of the axle of $m$, and round the other pulleys 0 and $p$, and the small pulley $q$, on the axle of the cylindrical cutter, gives the cylindrical cutter a very rapid rotatory motion; at the same time a worm, or endless screw, on the axle of $m$ and $n$, taking into the teeth of the large wheel $r$, causes that wheel to revolve, and a small drum $s$, upon its axle, to coil up the cord, by which the carriage $d$, with the cutters $a$ and $e$, and the spring bed $f$ and $g$, are slowly, but progressively, made to advance, and to carry the cutters over the face of the cloth, from list to list; the rapid rotation of the cutting cylinder $a$, producing the operation of cropping or shearing the pile.

Upon the cutting cylinder, betwcen the spiral blades, it is proposed to place stripes of plush, to answer the purpose of brushes, to raise the vap or pile as the cylinder goet 1. round, and thereby assist in bringing the points of the wool up to the cutters.

The same contrivance is adapted to a machire for shearing the cloth lengthwise. 
Fig. 1224, is a geometrical elevation of one side of Mr. Davis's machine. Fig. 1225, a plan or horizontal representation of the same, as seen in the top; and fig. 1226, a section taken vertically across the machine near the middle, for the purpose of displaying the working parts more perfectly than in the two preceding figures. These three figures represent a complete machine in working condition, the cutters being worked by a rotatory motion, and the cloth so placed in the carriage as to be cut from list to list. $a, a, a$, is a frame or standard, of wood or iron, firmly bolted together by - cross braces at the ends and in the middle. In the upper side-rails of the standard,

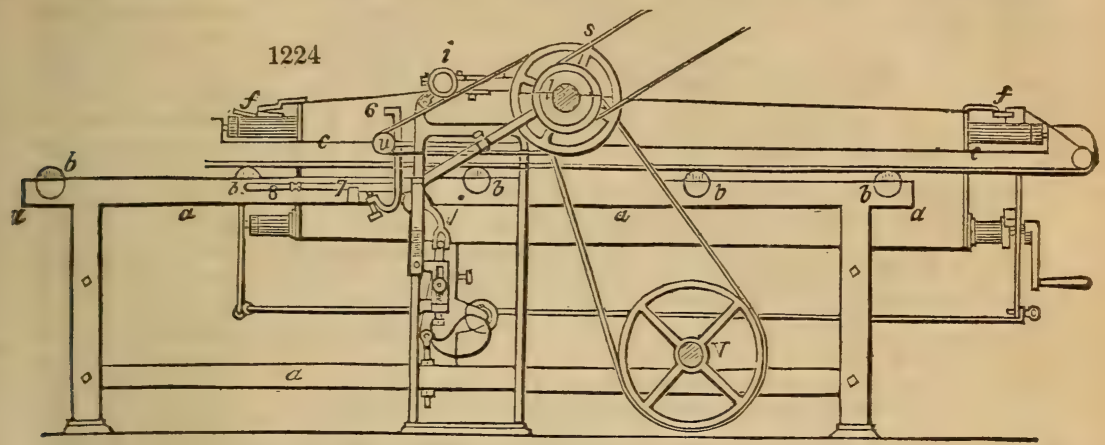

there is a series of axles carrying anti-friction wheels $b, b, b$, upon which the side-rails $c, c$, of the carriage or frame that bears the cloth runs, when it is passing under the cutters in the operation of shearing. The side-rails $c, c$, are straight bars of iron, formed with edges $v$, on their under sides, which run smoothly in the grooves of the rollers $b, b, b$. These side-rails are firmly held together by the end stretchers $d, d$.

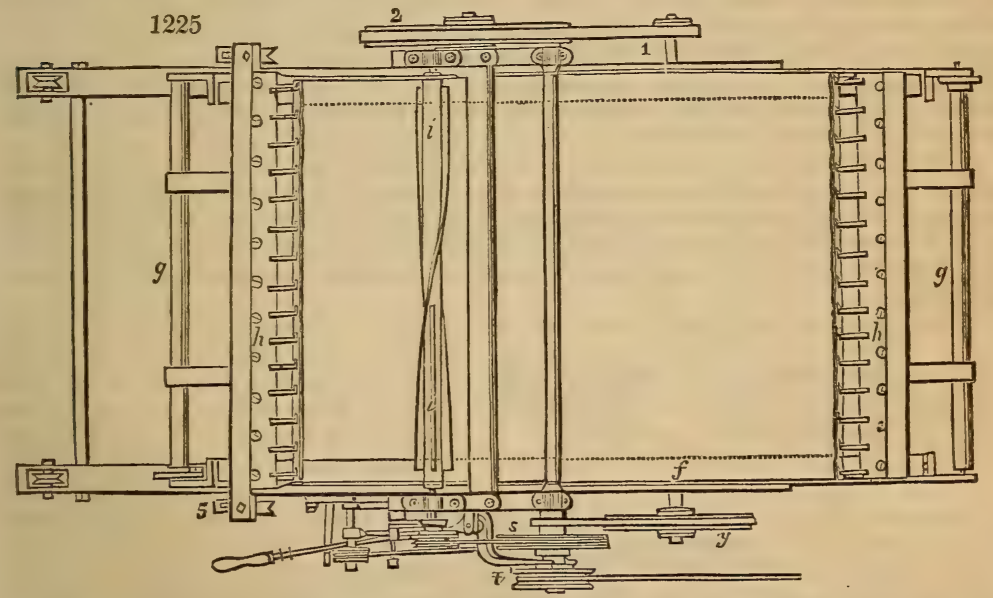

The sliding frame has attached to it the two lower rollers $e, e$, upon which the eloth intended to be shorn is wound; the two upper lateral rollers $f$, $f$, over which the cloth is conducted and held up; and the two end rollers $g$, $g$, by which the habiting rails $h, h$, are drawn tight.

In preparing to shear a piece of cloth, the whole length of the piece is, in the first place, tightly rolled upon one of the lower rollers $e$, which must be something longer than the breadth of the cloth from list to list. The end of the piece is then raised, and passed over the top of the lateral rollers $f, f$, whence it is carried down to the other roller $e$, and its end or farral is made fast to that roller. The hooks of the habiting rails $h, h$, are then put into the lists, and the two lower rollers $e, e$, with the two end rollers $g, g$, are then turned, for the purpose of drawing up the cloth, and straining it tight, which tension is preserved hy ratchet wheels attached to the cnds of the respective rollers, with palls dropping into their teeth. The frame carrying the cloth is now slidden along upon the top standard rails by hand, so that the list shall be brought 
nearly up to the cutter $i$, $i$, ready to commence the shearing operation; the bed is then raised, which brings the cloth up against the edges of the shears.

The construction of the bed will be seen by reference to the cross section, fig. 1226 .

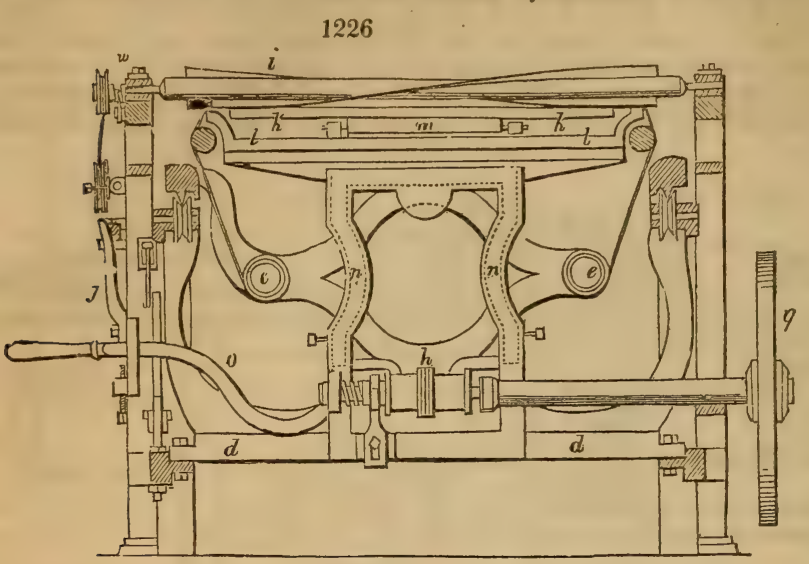

It consists of an iron or other metal roller $k, k$, turned to a truly cylindricalfigure, and covered with cloth or leather, to afford a smali? degree of elas. ticity. This roller is mounted upon pivots in a frame $l, l$, and is suppor. ted by a smaller roller $m$, similarly mounted, which roller $m$, is intended merely to prevent any bending or depression of the central part of

the upper roller or bed $k, k$, so that the cloth may be kept in close contact with the whole length of the cutting blades.

In order to allow the bed $k$ to rise and fall, for the purpose of bringing the cloth up to the cutters to be shorn, or lowering it away from them after the operation, the frame $l, l_{3}$ is made to slide up and down in the grooved standard $n, n$, the moveable part enclosed within the standard being shown by dots. This standard $n$, is situated about the middle of the machine, crossing it immediately under the cutters, and is made fast to the frame $a_{3}$ by bolts and screws. There is a lever 0 , attached to the lower cross-rail of the standard, which turns upon a fulcrum-pin, the extremity of the shorter arm of which lever acts under the centre of the sliding-fiame, so that by the lever 0 , the sliding-frame, with the bed, may be raised or lowered, and when so raised, be held up by a spring catch $j$.

It being now explained by what means the bed which supports the cloth is constructed, and brought up, so as to keep the cloth in close contact with the cutters, while the operation of shearing is going on; it is necessary in the next place to describe the construction of the cutters, and their mode of working; for which purpose, in addition to what is shown in the first three figures, the cutters are also represented detached, and upon a larger scale, in fig. 1227.

In this figure is exhibited a portion of the cutters in the same situation as in fig.

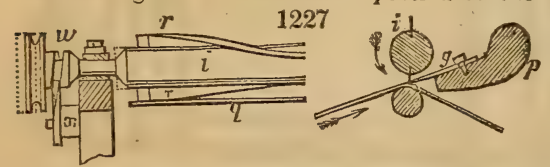
1221 ; and alongside of it is a section of the same, taken through it at right angles to the former; $p$, is a metallic bar or rib, somewhat of a wedge form, which is fastened to the top part of the standard $a, a$, seen best in fig. 1220 .

To this bar a straight blade of steel $g$, is attached by screws, the edge of which stands forward even with the centre or axis of the cylindrical cutter $i$, and forms the ledger blade, or lower fixed edge of the shears. This blade remains stationary, and is in close contact with the pile or nap of the cloth, when the bed $k$ is raised, in the manner above deseribed.

The cutter or upper blade of the shears is formed by inserting two or more strips of plate steel $r, r$, in twisted directions, into grooves in the metallic cylinder $i$, $i$, the edges of which blades $\gamma$, as the cylinder $i$ revolves, traverse along the edge of the fixed or ledger blade $g$, and by their obliquity produce a cutting action like shears; the edges of the two blades taking hold of the pile or raised nap, as the cloth passes under it, shaves off the superfluous ends of the wool, and leaves the face smooth.

Rotatory motion is given to the cutting cylinder $i$, by means of a band leading from the wheel $s$, which passes round the pulley fixed on the end of the cylinder $i$, the wheel $s$ being driven by a band leading from the rotatory part of a steam-engine, or any other first mover, and passed round the rigger $t$, fixed on the axle $s$. Tension is given to this band by a tightening pulley $u$, mounted on an adjustable sliding-piece $v$, which is secured to the standard by a screw; and this rigger is thrown in and out of gear by a clutch-box and lever, which sets the machine going, or stops it.

In order to give a drawing stroke to the cutter, which will cause the piece of cloth to be shorn off with better effect, the upper cutter has a slight lateral action, produced 
by the axle of the cutting cylinder being made sufficiently long to allow of its sliding laterally about an inch in its bearings; which sliding is effected by a cam $w$, fixed at one end. This cam is formed by an oblique groove, cut round the axle (see $w$, fig. 1227), and a looth $x$, fixed to the frame or standard which works in it, as the cylinder revolves. By means of this tooth, the cylinder is made to slide laterally, a distance equal to the obliquity of the groove $w$, which produces the drawing stroke of the upper shear. In orler that the rotation of the shearing cylinder may not be obstructed by friction, the tooth $x$, is made of two pieces, set a little apart, so as to afford a small degree of elasticity.

The manner of passing the cloth progressively under the cutters is as follows:-On the axle of the wheel $s$, and immediately behind that wheel, there is a small rigger, from which a band passes to a wheel $y$, mounted in an axle turning in bearings on the lower vide-rail of the standard $a$. At the reverse extremity of this axle, there is another small rigger 1 , from which a band passes to a wheel 2 , fixed on the axle 3 , which crosses near the middle of the machine, seen in fig. 1226. Upon this axle there is a sliding pulley 4, round which a cord is passed several times, whose extremities are made fast to the ends of the sliding carriage $d$; when, therefore, this pulley is locked to the axle, which is done by a clutch box, the previously described movements of the machine cause the pulley 4 to revolve, and by means of the rope passed round it, 5 draw the frame, with the cloth, slowly and progressively along under the cutters.

It remains only to point out the contrivance whereby the machinery throws itself out of gear, and stops its operations, when the edge of the cloth or list arrives at the cutters.

At the end of one of the habiting rails $h$, there is a stop affixed by a nut and screw 5 , which, by the advance of the carriage, is brought up and made to press against a lever 6 ; when an arm from this lever 6, acting under the catch 7 , raises the catch up, and allows the hand-lever 8 , which is pressed upon by a strong spring, to throw the clutchbox 10 , out of gear with the wheel 8 ; whereby the evolution of the machine instantly ceases. The lower part of the lever 6 , being connected by a joint to the top of the lever $j$, the receding of the lever 6 , draws back the lower catch $j$, and allows the sliding frame $i, l$, within the bed $k$, to descend. By now turning the lower rollers $e, e$, another portion of the cloth is brought up to be shorn; and when it is properly habited and strained, by the means above described, the carriage is slidden back, and, the parts being all throw? into gear, the operation goes on as before.

Mr. Hirst's improvements in manufacturing woollen cloths, for which a patent was obfained in February, 1830, apply to that part of the process where a permanent lustre is given usually by what is ealled roll-boiling; that is, stewing the cloth, when tightly wound upon a roller, in a vessel of hot water or steam. As there are many disadvantages attendant upon the operation of roll-boiling, such as injuring the cloths, by overheating them, which weakens the fibre of the wool, and also changes some colors, he substituted, in place of it, a particular mode of acting upon the cloths, by occasional or intermitted immersion in hot water, and also in cold water, which operations may be performed either with or without pressure upon the cloth, as circumstances may require.

The apparatus which he proposes to employ for carrying on his improved process, is shown in the accompanying drawing. Fig. 1228, is a front view of the apparatus, com-

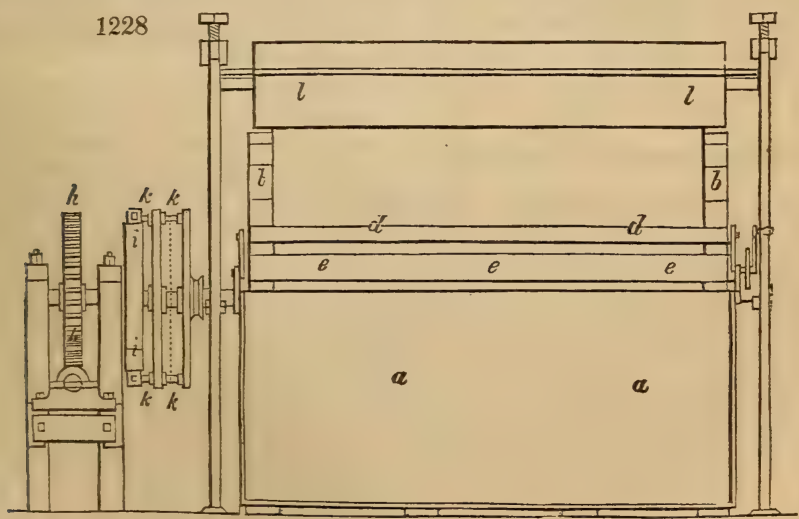

plete, and in working order; $f i g .1229$, is a section, taken transversely through the middle of the machine, in the direction of fig. 1230 ; and fig. 1230, is an end view of the same; $a, a, a$, is a vessel or tank, made of iron or wood, or any other suitable material : sloping at the back and front, and perpendicular at the end. This tank must be suffi- 
ciently large to admit of half the diameter of the cylinder or drum $b, b, b$, being immersed into it, which drum is about four feet diameter, and about six feet long, or something more than the width of the piece of cloth intended to be operated upon. This cylinder

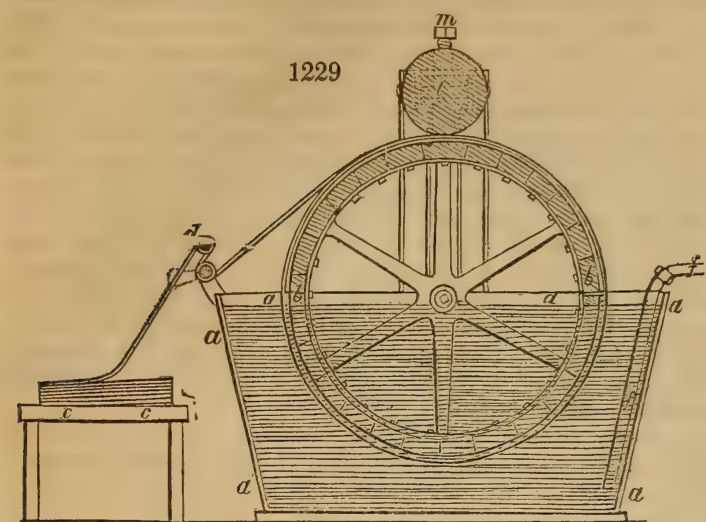
or drum $b, b$, is constructed by combining segments of wood cut radially on their edges, secured by screw-bolts to the rims of the iron wheels, having arms, with an axle passing through the middle.

The eylinder or drum being thus formed, rendered smooth on its periphery, and mounted upon its axle in the tank, the piece of cloth is wound upon it as tightly as possible, which is done by placing it in a heap upon a stool, as at $c$, fig. 1229, passing its end over and between the tensionrollers $d, e$, and then securing it to the drum, the cloth is progressively drawn from the heap, between the tension-rollers, which are confined by a pall and ratehet, on to the periphery of the drum, by causing the drum to revolve upon its axis, until the whole prece of cloth is tightly wound upon the drum; it is then bound round with canvass or other wrappers, to keep it secure.

If the tank has not been previously charged with clean and pure water, it is now filled to the brim, as shown at fig. 1229, and opening the stop-cock of the pipe $f$, which leads

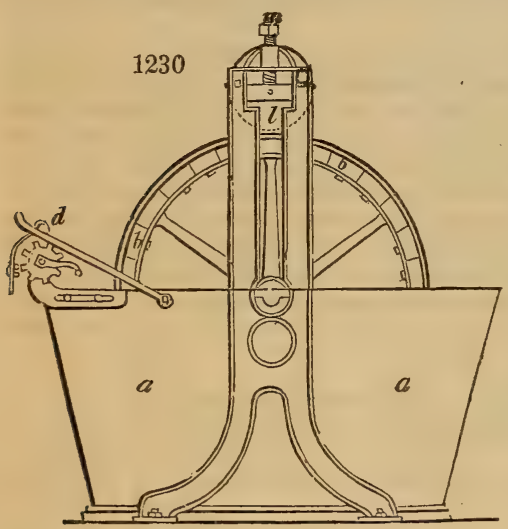
from a boiler, the steam is allowed to blow through the pipe, and discharge itself at the lower end, by which means the tem. perature of the water is raised in the tank to about $170^{\circ}$ Fahr. Before the temperature of the water has got up, the drum is set in slow rotatory motion, in order that the cloth may be uniformly heated throughout; the drum making about one rotation per minute. The cloth, by immersion in the hot water, and passing through the cold air, in succession, for the space of about eight hours, gets a smooth soft face, the texture not being rendered harsh, or otherwise injured, as is frequently the case by roll-boiling.

Uniform rotatory motion to the drum is shown in fig. 1228, in which $g$ is an endless screw or worm, placed horizontally, and driven by a steam-engine or any other first mover employed in the factory. This endless screw takes into the teeth of, and drives, the vertical wheel $h$, upon the axle of which the coupling-box $i$, $i$, is fixed, and, consequently, continually revolves with it. At the end of the shaft of the drum, a pair of sliding clutches $k, k$, are mounted, which, when projected forward, as shown by dots in fig. 1228, produce the coupling or locking of the drum-shaft to the driving wheel, by which the drum is put in motion; but on withdrawing the clutches $k, k$, from the coupling-box $i, i$, as in the figure, the drum immediately stands still.

After operating upon the cloth in the way described, by passing it through hot water for the space of time required, the hot water is to be withdrawn by a cock at the bottom, or otherwise, and cold water introduced into the tank in its stead; in which cold water the cloth is to be continued turning, in the manner above described, for the space of twenty-four hours, which will perfectly fix the lustre that the face of the cloth has acquired by its immersion in the hot water, and leave the pile or nap, to the touch, in a soft silky state.

In the cold-water operation he sometimes employs a heavy pressing roller $l$, which, being mounted in slots in the frame or standard, revolves with the large drum, rolling over the back of the cloth as it goes round. This roller may be made to act upon the 
eloth with any required pressure, by depressing the screws $m, m$, or by the employment of weighted levers, if that should be thought necessary.

Pressing is the last finish of cloth to give it a smooth level surface. The piece is folded backivards and forwards in yard lengths, so as to form a thick package on the board of a screw or hydraulic press. Between every fold sheets of glazed paper are placed to prevent the contiguous surfaces of cloth from coming into contact; and at the end of every twenty yards, three hot iron plates are inserted between the folds, the plates being laid side by side, so as to occupy the whole surface of the folds. Thin sheets of iron not heated are also inserted above and below the hot plates to moderate the heat. When the packs of eloth are properly folded, and piled in sufficient number in the press, they are subjected to a severe compression, and left under its influence till the plates get cold. The eloth is now taken out and folded again, so that the creases of the former folds may come opposite to the flat faces of the paper, and be removed by a second pressure. In finishing superfine cloths, however, a very slight pressure is given with iron plates but moderately warmed. The satiny lustre and smoothness given by strong compression with mich heat is objectionable, as it renders the surface apt to be come spotted and disfigared by rain.

WOO IZ, is the Indian name of steel.

WORT, is the fermentable infusion of malt or grains. See BEER.

WOULFE'S APPARA'TUS, is a series of vessels, connected by tubes, for the purpose of condensing gaseous products in water. See Acetic Acid, fig. 1 ; also Muriatrc Acru.

$\mathrm{X}$.

XANTHINE, is the name given by Kuhlmann to the yellow dyeing-matter centained in madder.

Y.

YEAST, is the froth of fermenting worts. See BEER and Fermentation.

YELLOW DYE. (Teinture jaune, Fr.; Gelbfärben, Germ.) Annotto, dyer's-broom, (Genista tinctoria,) fustic, fustet, Persian or French berries, quercitron bark, saw-wort, (Sorratula tinctoria,) turmeric, weld, and willow leaves, are the principal yellow dyes of the vegetable kingdom; chromate of lead, iron-oxyde, nitric acid, (for silk,) sulphuret of antimony, and sulphuret of arsenic, are those of the mineral kingdom. Under these articles, as also under Calico-printing, Dyeing, and Mordants, ample instructions will be found for communicating this color to textile and other fibrous substances. Alumina and oxyde of tin are the most approved bases of the above vegetable dyes. A mankin dye may be given with bablah, especially to cotton oiled preparatory to the Turkey red process. See MADDER.

YELLOW, KING'S, is a poisonous yellow pigment. See Arsenic and Orprment.

YTTRIA, is a rare earth, extracted from the minerals gadolinite and yttrotantalite, being an oxyde of the metal yttrium.

\section{Z.}

ZAFFRE. See CoBALT.

ZFDOARY, is the root of a plant which grows in Malabar, Ceylon, \&c. It occurs in wrinkled pieces, externally ash-colored, internally brownish-red; possessed of a fragrant odor, somewhat resembling camphor; and of a pungent, aromatic, bitterish taste. It contains, according to Bucholz, 1.42 of volatile oil, of a burning camphorated taste; 3.60 of a soft, bitter, aromatic resin ; 11.75 of a bitter aromatic extract, mixed with a little resin and potash-salts; 4.5 of gum; 9 of vegetable mucilage; 3.60 of starch; 8.0 of a starchy extract from the woody fibre, by means of caustic potassa, along with 31.2 of another matter, 12.89 of wooly fibre, and 15 of water. According to Morin, this root contains, besides, an azotized substance, analcgous to the extract of beef.

ZIMOME, is a principle supposed by Taddei to exist in the gluten of wheat-flour. Its identity is not recognised by later chemists.

ZIRCON. See Hyacinth and LAPIDARY.

ZIRCONIA, is a rare earth, extracted from the minerals zircon and hyacinth; it is an oxyde of zirconium, a substance possessing externally none of the metallic characters, but resembling rather charcoal powder, which burns briskly, and almost with explosive violence. 
ZINC, is a metal of a bluish-white color, of considerable lustre when broken across, but easily tarnished by the air; its fracture is hackly, and foliated with small facets, irregularly set. It has little cohesion, and breaks in thin plates before the hammer, unless it has been previously subjected to a regulated process of lamination, at the temperature of from $220^{\circ}$ to $300^{\circ} \mathrm{F}$., whereby it becomes malloable, and retains its malleability and ductility afterwards. On this singular property, a patent was taken out by Messis. Hobson and Sylvester, of Sheffield, many years ago, for manufacturing sheet zinc, for covering the roofs of houses, and sheathing ships; but the low price of copper at that time, and its superior tenacity, rendered their patent ineffective. The specific gravity of zinc varies from 6.9 to $7 \cdot 2$, according to the condensation it has received. It melts under a red heat, at about the 680 th or 700 th degree of Fahrenheit's scale. When exposed to this heat with contact of air, the metal takes fire, and burns with a brilliant bluish-white light, while a few flocculi, of a woolly-looking white matter, rise out of the crucible, and float in the air. The result of the combustion is a white powder, formerly called flowers, but now oxyde of zinc; consisting of 34 of metal, and 8 of oxygen, being their respective prime equivalents; or, in 100 parts, of 81 and 19 .

The principal ores of zinc are, the sulphuret called blende, the silicate called calamine, and the sparry calamine, or the carbonate.

1. Blende crystallizes in the garnet-dodecahedron; its fracture is highly conchoidal: lustre, adamantine; colors, black, brown, red, yellow, and green; transparent or trans. lucent; specific gravity, 4 . It is a simple sulphuret of the metal; and, therefore, consists, in its pure state, of 34 of zinc, and 16 of sulphur. It dissolves in nitric acid, with disengagement of sulphureted hydrogen gas. It occurs in beds and veins, accompanied chiefly by galena, iron pyrites, copper pyrites, and heavy spar. There is a radiated variety found at Przibram, remarkable for containing a large proportion of cadmium. Blende is found in great quantities in Derbyshire and Cumberland, as also in Cornwall.

2. Calamine, or silicate of zinc, is divided into two species; the prismatic or electric calamine, and the rhomboidal; though they both agree in metallurgic treatment. The first has a vitreous lustre, inclining to pearly; color, white, but occasionally blue, green, yellow, or brown; spec. grav. 3.38. It often occurs massive, and in botroidal shapes. This species is a compound of oxyde of zine with silica and water; and its constituents are-zinc oxyde, $66 \cdot 37$; silica, $26 \cdot 23$; water, $7 \cdot 4$; in 100 parts. Reduced to powder, it is soluble in dilute sulphuric or nitric acid, and the solution gelatinizes on cooling. It emits a green phosphorescent light before the blowpipe. The second species, or rhombohedral calamine, is a carbonate of zinc. Its specific gravity is $4 \cdot 442$, much denser than the preceding. It occurs in kidney-shaped, botroidal, stalactitic, and other imitative shapes; surface generally rough, composition columnar. Massive, with a granular texture, sometimes impalpable; strongly coherent. According to Smithson's analysis, Derbyshire calamine consists of - oxyde of zinc, $65 \cdot 2$; carbonic acid, $34 \cdot 8$; which coincides almost exactly with a prime equivalent of the oxyde and acid, or $42+$ $22=64$.

The mineral genus called zinc-ore, or red oxyde of zinc, is denser than either of the above, its spec. grav. being 5.432 . It is a compound of oxyde of zinc 88 , and oxyde of iron and manganese 12. It is found massive, of a granular texture, in large quantities, in several localities, in New Jersey. It is set free in several metallurgic processes, and occurs crystallized in six-sided prisms of a yellow color, in the smelting-works of Kœnigshutte in Silesia, according to Mitscherlich.

The zinc ores of England, like those of France, Flanders, and Silesia, occur in two geological localities.

The first is in veins in the carboniferous or mountain limestone. The blende and the calamine most usually accompany the numerous veins of galena which traverse that limestone; though there are many lead mines that yield no calamine; and, on the other hand, there are veins of calamine alone, as at Matlock, whence a very considerable quantity of this ore is obtained.

In almost every point of England where that metalliferous limestone appears, there are explorations for lead and zinc ores. The neighborhood of Alston-moor in Cumberland, of Castleton and Matlock in Derbyshire, and the small metalliferous belt of Flintshire, are peculiarly marked for their mineral riches. On the north side of the last county, calamine is mined in a rich veir of galena at Holywell, where it presents the singular appearance of occurring only in the ramifications that the lead vein makes from east to west, ard never in those from north to south; while the blende, abundantly present in this mine, is found indifferently in all directions.

The second locality of calamine is in the magnesian limestone formation of the English geologists, the alpine limestone of the French, and the zechstein of the Germans. The calamine is disseminated through it in small contemporaneous veins, which, running in all directions, form the appearance of network. These veins have commonly a thickness of only a few inches; but in certain cases they extend to four feet, 
in consequence of the union of several small ones into a mass. The explorations of calimine in the maonesian limestone, are situated chiefly on the flanks of the Mendip Hill-, a chain which extends in a northwest and southeast direction, from the canal of Bristul to Frome. The calamine is worked mostly in the parishes of Phipham and Roborough, as also near Rickford and Broadfield-Doron, by means of a great multitude of small shafts. The miners pay, for the privilege of working, a tax of $1 l$. sterling per annum to the Lords of the Treasury; and they sell the ores, mixed with a considerable quantity of carbonate of lime, for 11 . per ton, at Phipham, after washing it slightly in a sieve. They are despatched to Bristol, where they receive a new washing, in order to separate the galena.

OF THE SMELTING OF THE ORES OF ZINC.

The greater part of the zinc works are situated in the neighborhood of Birmingham and Bristol. The manufacture of brass, which has been long one of the staple articles of these towns, was probally the cause of the introduction of this branch of industry, at the period when brass began to be made by the direct union of copper with metallic zinc, instead of calamine. A few zinc furnaces exist also in the neighborhood of Shelfield, amid the coal-pits surrounding that town. Bristol and Birmingham derive their chief supply of ores from the Mendip Hills and Flintshire; and Sheffield, from Alston-moor.

The calamine, freed from the galena by sorting with the hand, is calcined before its introduction into the smelting-furnaces, by being exposed, coarsely bruised, in reverberatory ovens, 10 feet long, and 8 broad, in a layer 6 inches thick. In some establishments the calcination is omitted, and the calamine, broken into pieces about the size of a pigeon's egg, is mixed with its bulk of small coal.

Zinc is smelted in England, likewise from blende, (sulphuret of zinc.) This ore, after being washed, and broken into pieces of the size of a filbert, was sold a few years ago at the mine of Holywell for $3 l$. a ton, or half the price of calamine. It is roasted, without any other preparation, in reverberatory furnaces; which are about 8 feet wide, and 10 long; the distance between the roof and the sole being 30 inches, and the height of the fire-bridge, 18. The layer of blende, which is placed on the hearth, is about 4 or 5 inches thick; and it is cuntinually stirred up with rakes. One ton of it requires, for roasting, four tons of coals; and it suffers a loss of 20 per cent. The operation takes from 10 to 12 hours. The mixture of reducing consists of one fourth part of the desulphureted oxyde, one fourth of calcined calamine, and one half part of charcoal; which affords commonly 30 per cent. of zinc.

The English furnaces for smelting zinc ores are sometimes quadrangular, sometimes round; the latter form being preferable. They are mounted with from 6 to 8 crucibles or pots (see fig. 1231), arched over with a cupola $a$, placed under a conical chimney $b$, which

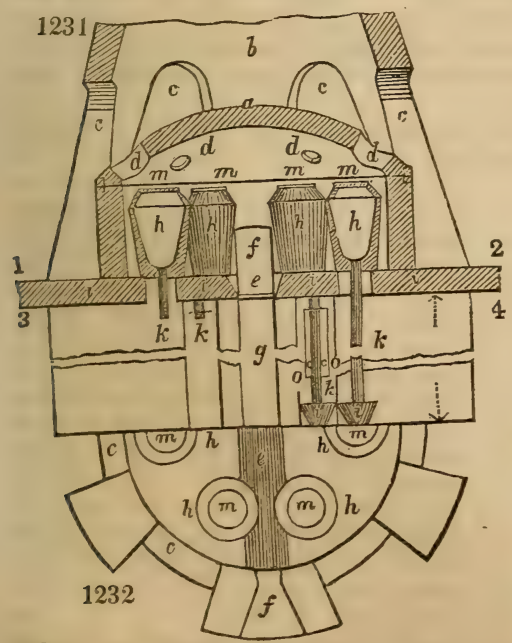
serves to give a strong draught, and to carry off the smoke. In this cone there are as many doors $c, c, c$, as there are pots in the furnace; and an equal number of vents $d, d, d$, in the cupola, through which the smoke may escape, and the pots may be set. In the surrounding walls there are holes for taking out the pots, when they become unserviceable; after the pots are set, these holes are bricked up. The pots are heated to ignition in a reverberatory furnace before being set, and are put in by means of iron tong machinery supported upon two wheels, as is the case with glasshouse pots. $e$, is the grate; $f$, the door for the fuel; g, the ash-pit. The pots $h, h, h$, have a hole in the centre of their bottom, which is closed with a wooden plug, when they are set charged with calamine, mixed with one seventh of coal ; which coal prevents the mixture from falling through the orifice, when the heat rises and consumes the plug. The sole of the hearth $i, i$, upon which the crucibles stand, is perforated under each of them, so that they can be reached from below; to the bottom orifice of the pot, when the distillation begins, a long sheet-iron pipe $k$, is joined, which dips at its end into a water vessel $l$, for receiving in drops the condensed vapors of the zinc. The pot 
is charged from above, through an orifice in the lid of the pot, which is left open after the firing, till the bluish color of the flame shows the volatilization of the metal; immediately whereupon the hole is covered with a fire-tile $m$. The iron tubes are apt to get obstructed during the distillation, and must therefore be occasionally cleared out with a redhot rod. When the distillation is finished, the iron pipes must be removed; the coaly and other contents of the pot cleared away. A pot lasts about four months upon an average. Five distillations may be made in the course of 14 days, in which from 6 to 10 tons of calamine may be worked up, and from 22 to 24 tons of coals consumed, with a product of two tons of zinc. The metal amounts to from 25 to 40 per cent. of the ore.

1,2 , is the level of the upper floor; 3,4 , level of the lower ceiling of the lower floor.

Fig. 1232, ground plan on the level of 1,2 ; only one half is here shown.

The zinc collected in this operation is in the form of drops, and a very fine powder, mingled with some oxyde. It must be melted in an iron pot or boiler, set in a proper furnace; and the oxyde is skimmea off the surface, to be returned into the crucibles. The inetal is, lastly, cast into square bars or ingots.

The crucibles are discharged at the end of each operation, by withdrawing the condenser, breaking with a rake the piece of charcoal which shuts their bottom, and then emptying them completely, by shaking thei: upper part. In replacing the condenser-pipe $k$ (see second pot from the right hand, fig. 1230), the flange at its top is covered with a ring of loam-lute, pressed against the conical bottom of the crucible, and secured in its place by means of two parallel rods 0,0 , which can be clamped by screws projecting horizontally from the vertical tunnel. See their flaces, indicated by two open dots near 0,0 .

A smelter and two laborers are employed in conducting a furnace; who make, with a mixture of equal parts of fire-clay, and cement of old pounds finely ground, the pots or crucibles, which last about four months. Five charges are made in 15 days; these work up from 6 to 10 tons of calamine, consume from 22 to 24 tons of coals, and produce 2 tons of zinc, upon an average. The following estimate of prices was malle a few years ago:3 tons of calamine, at $£ 6$

24 ditto coal, at $5 \mathrm{~s}$.

A smelter, at 2 guineas a week.

Two laborers, each at $4 s$. per day

Incidental expenses

$$
\begin{array}{lllll}
- & - & - & - & - \\
- & - & - & - & - \\
- & - & - & - & - \\
- & - & - & - & - \\
- & - & - & - & -
\end{array}
$$

$\begin{array}{rrr}£ 18 & 0 & 0 \\ 6 & 0 & 0 \\ 2 & 2 & 0 \\ 2 & 16 & 0 \\ 1 & 0 & 0\end{array}$

$£ 29 \quad 18 \quad 0$

The calamine of Alston-moor, used at Sheffield, is not so rich; it produces at most only 25 per cent. of zinc. The coals are laid down at a cost of 5 s. $8 d$. per ton; and the calamine laid down there $5 l$. ; whence the zinc will amount to $32 l$. $14 s$. per ton. The considerable importations of zinc from Belgium and Germany, for some years back, have caused a considerable fall in its price.

At Lüttich, where the calamine of Altenberg, near Aix-la-Chapelle, is smelted, a reduction furnace, containing long horizontal earthen tubes, is employed. The roasted calamine is finely ground, and mixed with from one third to two thirds its volume of coke or charcoal, broken to pieces the size of nuts.

Fig. 1233 represents this zinc furnace in elevation; and fig. 1234 in a vertical section through the middle. From the hearth to the bottom of the chimney it is

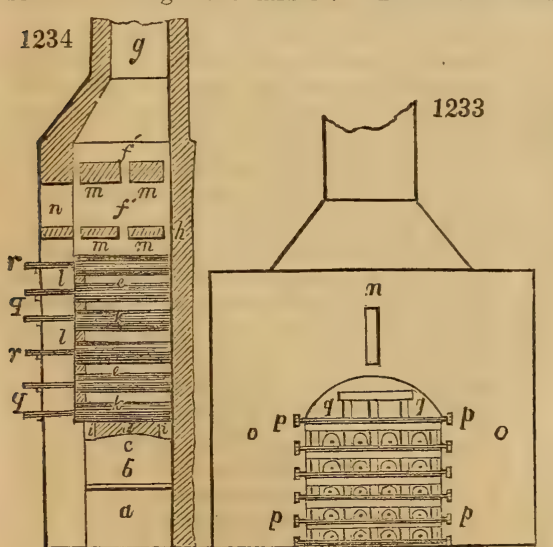

there are 22 tubes altogether in one furnace. 9 feet high, and the chimney itself is 18 or 20 feet high. $a$, is the ash-pit; $b$, the grate; $c$, the fireplace; $d$, the hearth; $e, e$, the laboratory; $f$, the upper arch, which closes in the laboratory; $f^{\prime}$, the second arch, which forms the hood-cap of the furnace; $g$, the chimney; $h$, the fire-wall, which rests against a supporting wall of the smelting-house. Through the vaulted hearth the flame of the fire draws through ten flues $i, i$, two placed in one line; betwixt these five pairs of draught-openings, upon the sole of the hearth, the undermost earthen tubes $k$, immediately rest. The second and third rows of tubes $k, k$, lie in a parallel direction over each other, at about one inch apart; in the sixth row there are only two tubes; so that At their two ends these tubes rest 
upon fire-tiles, which form, with the side-walls, a kind of checker-work $l, l$. The tubes are 4 feet long, 4 to 5 inches in diameter within, five fourths of an inch thick. The fire, which arrives at the laboratory through the flues $i, i$, plays round the tubes, and passes off through the apertures $m, m$, in both arches of the furnace, into the chimney. $n$, is an opening in the front wall between the two arches, which serves to modify the draught, by admitting more or less of the external air.

The two slender side walls 0,0 , of the furnace, are a foot distant from the checkerwork, so that on the horizontal iron bars $q, q$, supported by the hooks $p$, $p$, the iron receivers $r, r$, may have room to rest at their fore part. These receivers are conical pipes of cast iron, $1 \frac{1}{2}$ foot long, posteriorly $1 \frac{1}{2}$ inch, and anteriorly 1 inch wide at the utmost. After the earthen tubes have been filled with the ore to be smelted, these conical pipes are luted to them in a slightly slanting position. These cones last no more than three weeks; and are generally lengthened with narrow-mouthed wroughtiron tubes, to prevent the combustion of the zinc, by contact of air. When the furnace is in activity, a blue flame is to be seen at the mouths of all these pipes. Every two

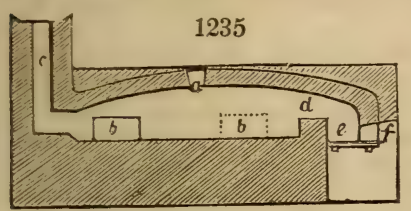

1236

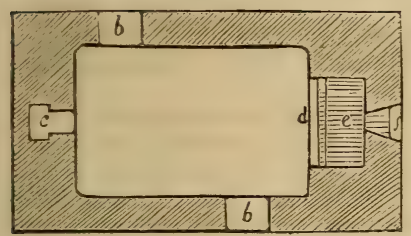

cwts. at a time; and for roasting e

English bushels, are employed. 1237

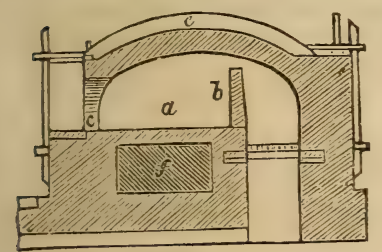

1238

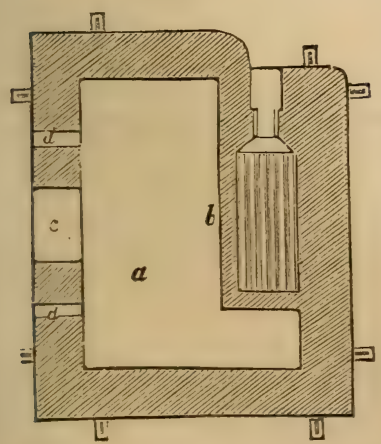

hours the liquefied metal is raked out into a shovel placed beneath; and in 12 hours the charge is distilled; after which the tubes are cleared out, and re-charged. 100 pounds of metallic zine are the product of one operation. It is remelted at a loss of ten per cent., and cast into moulds for sale.

Fig. 1235 is a longitudinal section of the furnace for calcining calamine in Upper Silesia; fig. 1236 is a ground plan of the furnace. $a$, is the orifice in the vault or dome, for the introduction of the ore; $b, b$, apertures in the side-walls, shut with doors, through which the matter may be turned over; $c$, the chimney; $d$, the fire-bridge ; $e$, the grate; $f$, the feed opening of the fire, the fuel being pitcoal. The calamine is stirred about every hour; and after being well calcined during 5 or 6 hours, it is withdrawn; and a new charge is put in. These Silesian furnaces admit of 30 every 100 cwts. 15 Prussian bushels of fuel, equal to 23 These calcining furnaces are sometimes built alongside of the zinc smelting-furnaces, and are heated by the waste flame of the latter. The roastiny is performed in the Netherlands in shafts, like small blast iron-furnaces, called schachtofen.

The hearth $a$, in figs. 1237, 1238, is constructed for working with 5 muffles, one of which is long, and four short. The muffles are made upon moulds, of fireclay mixed with ground potsherds. The receivers are stoneware bottles. The grate is ten inches beneath the level of the hearth. $b$, the firebridge, is proportionally high to diminish the force of the flame upon the hearth, that it may not strike the muffles. $c$, is the opening through which the muffles are put in and taken out; during the firing it is partly filled with bricks, so that the smoke and flame may escape between them; $d, d$, are openings for adjusting the positions of the muffles; $e$, cross hoops of iron, to strengthen the brick arch; $f$, is a bed of sand under the sole of the hearth. During the first two days, the fire is applied under the grating; the heat must be very slowly raised to redness, at which pitch it must be maintained during two days. From 8 to 10 days are required for the firing of the muffles.

The furnace shown in figs. $1239,1240,1241$, is for the melting of the metallic zinc. Fig. 1240 is a front view ; fig. 1239 a transverse section; fig. 1241 a view from above; $a$, is the fire-door; $b$, the grate; $c$, the fire-bridge; $d$, the flue; $e$, the chimney; $f, f, f$, cast-iron melting-pots, which contain each about $10 \mathrm{cwts}$. of the metal. The heat is moderated by the successive addition of pieces of cold zinc. The inside of the pots should be coated with loam, to prevent the iron being attacked by the zinc. When the 
zinc is intended to be aminated, it should be melted with the lowest possible heat, and poured into hot moulds.

When the zinc ores contain cadmium, this metal distils over in the form of brown

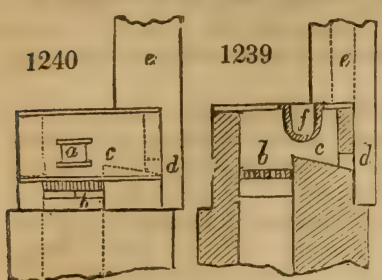
oxyde, with the first portions, being more volatile than zinc.

Under Brass and Copper, the most useful alloys of zinc are described. The sulphate, vulgarly called white vitriol, is made from the sulphuret, by roasting it gently, and then exposing it upon sloping terraces to the action of air and moisture, as has been fully detailed under Sulphate of Iron. The purest sulphate of zine is made by dissolving the metal in dilute sulphuric acid, digesting the solution over some of the metal, filtering, evaporating, and crystallizing.

Sulphate of zinc is added as a drier to japan varnishes.

1241

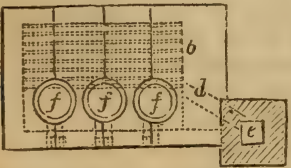

The ordinary zine found in the market is never pure; but contains lead, cadmium, arsenic, copper, iron, and carbon; from some of which, it may be freed in a great degree by distillation; but even after this process it retains a little lead, with all the arsenic and cadmium. The separation of the latter is described under CADMıUm. Zinc, free from other metals, may be obtained by distilling a mixture of charcoal and its subcarbonate, precipitated from the crystallized sulphate by carbonate of soda. By holding a porcelain saucer over the flame of hydrogen produced from the action of dilute sulphuric acid upon any sample of the zinc of commerce, the presence of arsenic in it may be made manifest by the deposite of a gray film of the latier metal. Antimony, however, produces a somewhat similar effect to arsenic.

Zinc is extensively employed for making water-cisterns, baths, spouts, pipes, plates for the zineographer, for voltaic batteries, filings for fire-works, covering roofs, and a great many architectural purposes, especially in Berlin; because this metal, after it gets covered with a thin film of oxyde or carbonate, suffers no further change by long exposure to the weather. One capital objection to zinc as a roofing material, is its combustibility.

Chloride of zinc has been recently used with great advantage as an escharotic for removing cancerous tumors, and healing various ill-constitutioned ulcers. It, as also the nitrate, forms an ingredient in the resist pastes for the pale blues of the indigo vat.

Spelter (zinc) imported for home consumption-in 1835, 52,604 cwts. ; in 1836, 47,436 ewts. Duty-in cakes, $2 s$, ; not in cakes, 10 s. per cwt. 


\title{
RECENT IMPROVEMENTS
}

IN

\section{ARTS, MANUFACTURES,}

\author{
AND \\ M I N E S:
}

BEING

\section{A SUPPLEMENT TO HIS DICTIONARY}

B Y

A N D R E W URE, M. D.,

r.R.g. M.G.S. M.A.S. LOND.; M. ACAD. N.S. PHILAD.; S. PH. SOC. N. GERM.

HANOV.; MULL. ETC., ETC.

ILLUSTRATED WITH ONE HUNDRED AND NINETY ENGRAVINGS,

N E W Y R K:

D. A PPLETON \& COMPANY, 200 BROADWA $\mathbf{X}$ PH I L A D E L P H I A :

GEORAE S. APPLETON. 164 CHESNUT ST. 



\section{PREF ACE.}

Is laying this Supplement to my Dictionary of Arts, Manufactures, and Mines, before the world, while I gratefully acknowledge the indulgence with which that work has been received, may I be permitted to advert very briefly to some of my present endeavors to render it less undeserving of public favor, though, after all my efforts it will by no means realize either my own wishes and intentions, or the expectations of all my readers?

To investigate thoroughly any single branch of art, we should examine it in its origin, objects, connexion with kindred arts, its progressive advancement, latest improved state, and theoretical perfection. The general principles on which it is founded, whether belonging to the mechanical, the physical, the chemical sciences, or to natural history, should be fully expounded, and tested by an application to its practical working on the great scale. The maximum effect of the machinery which it employs, and the maximum product of the chemical mixtures and operations which it involves, should in every case be calculated and compared with the actual results.

Such have been my motives in the numerous consultations I have had with manufacturers relatively to the establishment or amelioration of their factories, and when they are kept steadily in view, they seldom fail to disclose whatever is erroneous or defective, and thereby to lead to improvement. It will not be denied by any one conversant with the productive arts, that very few of them bave been either cultivated or described in this spirit. It is to be hoped, however, that the period is not remote, under the intellectual excitement and emulation now so prevalent in a peaceful world, when manufactories will be erected, and conducted upon the most rational and economical principles, for the common benefit of mankind. Meanwhile it is the duty of every professor of practical science to contribute his mite toward this desirable consummation.

It is under a sense of this responsibility that I have written the leading articles of this Supplement, having enjoyed some peculiar advantages in my profession for making the requisite researches and comparisons. I trust that not many of them deserve to be regarded as trite compilations or as frivolous novelties, with the exception of a few of the notices of recent patents, which I have intentionally exhibited as beacons to deter from treacherous quicksands, not as lights to friendly havens. I have sought sincerely to make them all conducive, more or less, to utility ; being either new contributions to the old stock of knowledge, or additions and corrections to the miscellaneous volume of which the present is the sequel.

ArRow Root is here for the first time treated as a well-organized manufacture, in conformity with my quondam definition: "Manufacture is a word which, in the vicissitude of language, has come to signify the reverse of its intrinsic meaning, for it now denotes every extensive product of art which is made by machinery, with little or no aid of the human hand; so that the most perfect manufacture is that which dispenses entirely with manual labor."*

Arrow root being the purest and most agreeable variety of fecula, and therefore one of the most powerful nutriments, deserves more attention from the colonial planter than it seems hitherto to have received. As it has been now so judiciously prepared in the island of St. Vincent, by the proprietor of the Hor estate,

" " Philosophy of Manufactures," page 1. 
it will, I hope, amply repay his enterprising and liberal spirit, seeing that he sup plies us with an article equal if not superior to the best from Bermudas, at two thirds of the price.

To Artesian Wells an interesting notice has been added of the successful labors of MM. Arago and Malot at Grenelle, near Paris.

I doubt not that should cockneys happen to read what is here said of BAVARIAN BEER, they will feel no little surprise, mixed with scorn and incredulity, when told that the mystery of brewing is more philosophically studied and incomparably better understood in Munich than in London, and indeed throughout all Bavaria, than in Old England; but such is certainly the fact, as every delicate stomach will experience which is cheered with the beverage of the former capital, and loaded with the heavy-v'et of the latter. The brief outline here offered to my readers has been carefully drawn from the best sources of information, obtained during several excursions into Germany. It will, I hope, induce the brewers of this country to set more value on chemical science than they have heretofore done, and thereby eventually lead to a radical reform of our colossal establishments for extracting from malt a beverage more akin to that of fermented grape-juice, in its freedom from vinegar and gluten, so abundant now in the greater part of the British porters and beers.

Under Biscuiss will be found a complete description, with figures, of the grand automatic bakeries of Deptford and Portsmouth, which provide our hardy tars with the staff of life in the soundest state.

The perusal of the article BREAD will prompt the wish that our land-holding legislators would consent to let the people under their domination get, at a moderate cost, some of the wheat of southern Europe, much richer than that of our average home growth in the azotized glutinous principle, so essential to the formation of our blood and muscles; a wheat adapted to make a superior bread, such as that called pain du gruau in Paris, and also a superior macaroni, like the Neapolitan. In this department of industry, so important for the welfare of the population, the French have set us the example of applying to it the economical resources of the factory system, having organized a self-acting bakery, in which bread of the finest quality is made on the great scale, in smokeless ovens of a nicely regulated temperature. Meanwhile, the mass of her majesty's subjects are dependant for their bread upon a multitude of tradesmen of slender means, who earn a scanty livelihood by hard labor, and who work up a weak inferior flour into a bad bread, which they are too often tempted to whiten with alum and other unwholesome drugs. The penalty liable to be inflicted upon bakers for having alum on their premises, is commonly evaded by letting it be added to the flour in the mill. Why do not our wise legislators enact a law for the summary conviction and punishment of a baker selling bread with alum in it; a saline sompound most easily detected by chemical analysis?

I was lately called upon professionally to examine the very white bread of a ashionable baker of high pretensions, and found it to contain a notable quantity of alum; so much so, as to have been directly offensive to the stomachs, and hurtful to the health of several individuals in the family using it. This is no solitary case, but is, I believe, that of a large proportion of the bakers in London, and suburbs, who operate upon a partially damaged flour, as one may easily surmise from the disagreeable odor exhaled from the hot loaves in too many of their shops. Yet what individual will be Quixotic enough to attack the numorous and ever-changing arms of this Briareus? Who would choose to incur the trouble, responsibility, and expense of prosecuting a frequent misdemeanor of this kind, relatively to which the want of fine wheat in the market is a principal motive and apology?

From these evils our grandees are exempt, as they bake their bread at home of the best materials. Though they are apparently regardless of the injury suffered by the public from this source, they are, however, quite alert in the execution of the game and excise laws, the stringent penalties of which are inexorably inflicted against petty transgressors, exposed to temptations often too strong for the infirmity of human nature to resist.

In every well-governed state of continental Europe there exists a Board of Health, or Conseil de Salubritê, composed of eminent physicians, chemists, and 
engineers, appointed to watch over whatever may affect injuriously the public health and comfort. In France, this commission consists, for the capital, of seven m-mbers, who have the surveillance, in this respect, of markets, factories, places of public anusement, bakeries, shambles, secret medicines, \&c. This tribunal has discharged its functions to the entire satisfaction of their fellow-citizens, as appears from the following authentic report: "Non seulement une foule de causes d'insulubrité disparurent, mais beaucoup de moyens, de procédés nouveaux furent proposés your assuinir les Arts et les Métiers. qui jusque là avaient paru inséparables de res causes d'insalubrité; la plûpart de ces moyens eurent un plein succès. Il n'y a pas d'exemple que les membres du Conscil appelês à donner leur avis sur des plaintes formées contre des fabrnques, aient jamais répondu qu'il fallait les suprimer sans avoir cherché eux-mémes à aplanir les difficultés, que prèsentait aux fabricants, l'assainissement de leur art, et presque toujours ils sont parvenu à resoudre le problème. Le Conseil de Salubrité, que l'on ne saurait trop signaler à la reconnaissance de publique, est une institution que les nations étrangères admirent, et s'efforceront d'imiter sans doute."*

From this confident hope of emulation by other nations, the author of these excellent observations would have excepted the United Kingdom, had he known how little paternal care is felt by the government for the general interests of the people. In Germany, indeed, where the fatherland feeling is strong in the breasts even of those rulers whom we are apt to consider despots, similar boards of health are universally established, whereas our legislative oligarchy frames laws chiefly for the benefit of its own class and dependants; as happened in the old time, when there was no king in Israel to regard alike the interests of the poor and the rich.

The Prussian municipal law (Allgemeine Landrecht) contains the following euactments with regard to the sale of spoiled or adulterated victuals. Th. II. Tit. 20 ; Abschnitt 11 ; $\$ \$ 722$ to 725 . "No person shall knowingly sell or communicate to other persons for their use, articles of food or drink which possess properties prejudicial to health, under a penalty of fine or bodily punishment. Whosoever adulterates any such victuals in any manner prejudicial to health or mixes them with unwholesome materials, especially by adding any preparation of lead to liquors, shall, according to the circumstances of the case, and the degree of danger to health, be liable to imprisonment in a correction-house, or in a fortress, during a period varying from one to three years. Besides this punishment, those who are found guilty of knowingly selling victuals which are damaged or spoiled (verdorbener), or mixed with deleterious additions, shall be rendered incapable for ever of carrying on the same branch of business. The articles in question shall be destroyed, if incorrigibly bad, but if otherwise, they are to be improved as far as possible at the cost of the culprit, and then confiscated for the benefit of the poor. Further, whosoever mixes victuals or other goods with foreign materials, for the purpose of increasing their weight or bulk, or their seeming good qualities, in a deceitful manner, shall be punished as a swindler."

It is singular how, amid the law-making mania which has actuated our senators for many sessions, that not even one bill has been framed for the protection of the people from spoiled and adulterated foods and drinks.

For the article on BRick-MAKING, my readers are indebted to a valuable communication to the Institution of Civil Engineers, and the judicious remarks on it by several of its members. At its conclusion, a notice is inserted of one of those abuses which too often recur in our courts of judicature, in consequence of scientific witnesses merging the dispassionate philosopher in the mercenary partisan, and striving to mislead the judge and jury, by giving a one-sided view of the matter submitted to their candid examination. Such procedure is injurious, not merely to the individual casuist, but to the cause of science. What a close affinity is there between these quibblers and the venal philosophists so graphically portrayed by Lucian!

In addition to the sectional view of the four-colored calico-printing machine, given in the Dictionary, an outside view of this admirable mechanism is now presented to my readers ; the two together constituting the only good representation of it hitherto made public.

" "Dictionnaire Technologique," tom. ii., p. 293. 
The production, properties, and manufacture of caoutchouc, are treated here in considerable detail, peculiar facilities having occurred to me for the thorough investigation of this novel branch of industry. If, along with the account now given, the articles Bookbinding, Braining Machinf, and Elastic Bands of the Dictionary be consulted, the student will possess a pretty complete knowledge of caoutchouc.

CHocolate is also a new contribution to my work, which I have been enabled to make in consequence of extensive experimental researches. It is to be hoped that our intrepid sailors will be allowed to reap the full benefit of the investigations which I made in their behalf, by desire of the Lords Commissioners of the Admiralty, and that their daily breakfast beverage will be for the future more solubie, emulsive, and nutritious, than I found it to be on commencing my researches, at which time about three fourths of the cocoa was so coarsely ground, for the service of the navy, as to be left in a state altogether unfit for digestion.

The various new modes of producing pictorial impressions by the agency of light on chemically prepared surfaces are described under the titles, CALOTYPE, Daguerrotype, Photography, \&c. The somewhat kindred copying art by electricity, is treated under the article Electro-Metallurgy.

Fermentation will be found a useful companion to the account of Bavarian beer: both being calculated to invite brewers and distillers to look more narrowly than they seem to have done into the interesting world of organic chemistry, so successfully explored in Germany and France, but so little studied in this country.

Gas Light has been contributed by a most intelligent friend, and deserves tc be regarded as a standard treatise on this important branch of engineering, condensed into the shortest possible space consistent with perspicuity.

Guano, destined ere long to become the chief pabulum of British agriculture and thereby to emancipate our landholders and farmers from their exositophobia their dread of the importation of foreign corn, has been discussed at considerable length from peculiar sources of information.

Under Iron and Smelting are given descriptions, with figures, of the best plans of the apparatus for the hot-air blast, and for feeding the blast furnace with mine, limestone, and fuel; both being original contributions from an eminent engineer.

The SeEn-Crushing oil manufacture is, I believe, now for the first time in this country represented by a complete set of figures, exhibiting the various parts of the wedge stamping-mill; the oldest and probably still the best plan of extracting oil from seeds.

Pepper presents an instructive example of the fallacy of chemical evidence, -sometimes too inconsiderately given in a court of justice. Were the solemn sanctity of an oath rightly impressed on the minds of scientific men, they would not testify to anything but what they did most surely know, and would escape the remorse and obloquy consequent, in feeling minds, on having borne false witness against their neighbor.

The Saccharoneter table printed at first for the Dictionary, but omitted along with some other articles of less importance, for want of room, is now given with certain improvements.

Smoke-prevention is a matter of such moment to the comfort and salubrity of all our large towns, and even of many semi-rural districts, that in promoting the publicity of Mr. Charles Wye Williams's unexceptionably simple and successful plan for effecting a consummation so earnestly to be wished for, I am conscious of merely discharging a professional duty.

SpINing exhibits a short but systematic view of the admirable self-acting system invented by Mr. Bodmer, whereby all the operations in a cotton-factory are linked together in regular succession, and co-operate, with little or no manual aid, toward turning out a perfect product. This invention constitutes a true automatic era in textile factories. I trust the author of these inventions will be duly recompensed for the ingenuity and labors of very many years.

In the spinning of fine flax yarn by machinery, the greatest mechanical conquest which our factories have made in our own days over the industry of rival nations, a capital improvement has been recently made by Mr. Westly of Leeds His former invention, the screw gill or spiral comb described in the Dictionary. 
under $F_{L A X}$, is now universally employed. His new cortrivance is called the Sulver Roving, of which a description will be found in the Appendix, having come too lately to hand for insertion in its proper place. It promises to be a still more valuable improvement in this difficult branch of manufacture than even the spiral comb.

'The table entitled SpIrits exhibits the correspondence between the technical nomenclature of our excise as reckoved in over-proof and under-proof strengths, and the simple scale of specific gravity as understood and agreed upon all over the world. Since alcohol alone, and not water, is the subject of taxation, why not have an alcohol-meter, like that of Gay Lussac, which shows at once the proportion of taxable stuff in any spirit? (See Alcoног in the Dictionary.) As, however, our excise laws, like those of the Medes and Persians, are not likely to be changed in conformity with any scientific remarks, the above table is a desideratum to practical chemists.

Sugar of Potatoes, being a recent manufacture in this country, is fully investigated from my own professional resources.

TuвAсco is discussed at considerable length, valuable materials for this inquiry having been afforded in the Report of the recent Committee of the House of Commons. While this plant is a rank weed, conducive neither to the sustenance nor health of man, and therefore a most fit object for deriving from its consumers a fiscal revenue, it affords instructive lessons on the influence of our fiscal administration on arts and manufactures.

When the duty on an article is more than ten times its intrinsic value, it must become the subject of perpetual and enormous frauds, and engender innumerable misdemeanors and crimes. Toward the prevention and punishment of these transgressions of the fiscal laws, a cumbrous, complex, costly, somewhat arbitrary and despotic system of espionage and prosecution must be organized. The working of this vast machinery is well shown in the committee's report, and must excite uncomfortable feelings in every honorable mind. We here see, on a somewhat magnified scale, the system of interference with, and prying iuto, processes of art and manufacture which accompanies and characterizes all the operations of the excise. This device for collecting revenue for the necessities of the state is the Pandora's box of the dethroned Stuarts, and should have been expatriated with that ill-starred family. We may say of it, Quicquid tangit, deornat. No branch of industry can acquire its due development under its wiry training and fastening. Had the incubus of the excise overlaid our textile manufactures of wool, cotton, flax, and silk, how dwarfish would their stature have remained, and how meanly would they have quailed under the unrestrained labor of rival nations; whereas now they afford employment, with food, raiment, and lodging, to millions of our people. For the manufacture of glass in all its useful and ornamental branches, this country possesses indigenous resources superior to those of every other one, in its stores of fuel and vitrifiable materials of every kind, and yet it is surpassed by France, Switzerland, and Bavaria, in glass for optical purposes, and by Bohemia in the quality and execution of decorative glass. Our sciertific chemists have been obliged to get all their best glass apparatus from Germany, via Hamburgh.

Surely our glass-makers are the same race of people as our manufacturers of iron, fine cotton yarn, muslin, bobbin-net, broad silks, \&c., which defy the competition of the world, and if unshackled by the excise they would ere long turn the scale against their foreign rivals, now their superiors. The incessant and vexatious espionage of the excise is a bar to all invention in every art under its control. Who would expend thought, science, labor, and money, in maturing any discovery or improvement, by experiments necessarily conducted under the cyes of needy excisemen, who would tell all they have seen for a trifling bribe? Perhaps the gigantic scale of our spirit distilleries may be appealed to in proof of the fostering care of the excise, under which they have been reared. But this overgrowth, when well looked into, is no evidence of a sound constitution, but merely of the depravity of a grovelling uneducated people. In fact, our distilleries produced until very lately a very impure and offensive spirit, strongly imbued with noxious fusel-oil, or oil of grains (see AlcoHor in this Supplement), and but for the recent introduction of Mr. Coffey's still into some distilleries, they would 
all have been yet sending forth a similar crude spirit. But though Mr. Coffey was for many years an officer of excise, and therefore did adapt his patent invention to all the just requirements of the revenue laws, he has met with very vexatious obstructions in the erection of his stills and on the most frivolous pretences.

As a general corollary from my long experience in the conduct of arts and manufactures, I feel warranted to declare, that the excise system is totally incompatible with their healthy growth, and is in itself the fruitful parent of fraud, perjury, theft, and occasionally murder. The sooner this portion of the revenue, so oppressively, so expensively, and so offensively collected, is replaced by an equitable tax on property, the better for the welfare of this great country. I have no quarrel with the gentlemen who administer the excise laws; several of them with whom I have been professionally conversant I esteem very highly as intelligent and upright men, who do what they deem their duty in a conscientious manner. But in concluding a very extensive survey of the great branches of our national industry, this vile obstacle to their progressive growth became so manifest, that it would have been pusillanimous to shrink from the task of pointing out the magnitude of the evil.

Ventilation describes the plan now organized in the Reform Club House, which I inculcated in the Philosophy of Manufactures, published in 1835, as also in a paper read before the Royal Society in 1836, and which was copied into several of the scientific journals of that period. About the same time, Dr. Reid was erecting a huge factory furnace and chimney for the ventilation of the House of Commons, which would have been accomplished more effectually at one half of the expense, and without any architectural disfigurement, by my method of ventilating fans, which was, in fact, that long practised in our great textile factories. The doctor has, I understand, renounced his chimney draughts in ventilating the Court of Exchequer at Westminster, and adopted a similar system to that of the Reform Club House. I hope he will pursue the same plan in the new Houses of Parliament, as it may be mounted at a very small cost, and without occasioning the least unsightly appearance or any annoyance.

I have subjoined in an Appendix a brief treatise, entitled Chemistry SimpliFIED, which, being duly studied, will prove a useful guide to practitioners in testing alkalis, acids, and bleaching substances, in several departments of the chemical arts.

A few of the articles marked in the Dictionary for reference to the Supplement, were, on reconsideration, not found susceptible of useful annotation. Most of these references were, indeed, statistical details, which are given from the latest and best sources in Mr. M'Culloch's excellent Dictionary of Commerce.

LoNDON, 13 Chariotte Street, Bedford Equare, 28 th October, 1844. 


\section{S U P P L E M E N T,}

\&c., \&c.

ACETIC ACID. Rapid acetification, or the quick formation of vinegar, was practised upon malt worts in this country long before the rapid conversion of alcohol into vinegar was introduced into Germany. In the year 1842, Mr. Ham obtained his patent for an improved method of making vinegar, which is described in the dictionary. His son, Mr. F. Ham, of Norwich, civil engineer, states that for some years, four of the largest country manufactories in the kingdom have been at work upon his father's plan, and that they are now in successful competition with the great London establishments. The apparatus consists of a huge vat, in the centre of which is a revolving pump, having two or inore shoots pierced with holes, whereby a constant shower of the fermented wort, called wash, is kept falling from the top. The under part of the vat contains the wash; the upper part, birch twigs properly prepared, which are so placed as not to interfere with the revolving shoots. Between the surface of the wash and the rafters which support the twigs, a space of a few inches is left vacant, into which one or more holes in the side of the vat admit the air spontaneously or have it forced in. The wash is maintained, by steam pipes immersed in it, at a temperature of from $90^{\circ}$ to $100^{\circ} \mathrm{F}$., so that, in consequence of the extensive application of the atmospheric oxygen during the trickling through the twigs, it may be made sour in the course of 48 hours; but, in general practice, it is completely acetified in from 15 to 20 days. By this apparatus, a wort brewed from raw grain, with only one seventh of malt, will produce a vinegar equal to that from malt alone; and the acetifying process may be arrested whenever it is completed, thus preventing the risk of the vinegar running into the putrefactive stage, as happens occasionally in the slow plan of fermentation. The admission of air is so moderated as not to dissipate the alcohol of the wash by evaporation. A wort of 24 lbs. gravity per Richardson's instrument, equal to $1.066 \mathrm{sp}$. gravity, will in this way yield an acid of revenue proof.

This old-going process is essentially the same with that for which John W. Neale and James Edouard Duyck obtained a patent in September, 1841, with this difference, that they employ the expressed juice of beet-roots instead of corn-wort.

The total number of vinegar factories in the United Kingdom was a few years ago only 48 , of which five of the principal are in London, four being on the Surrey side of the Thames. In these, malt vinegar-making is associated with the manufacture of British wines, called "sweets" by the excise. The fermented wort or wash is acetified either by "stoving or fielding," By the first plan, casks containing the wash are arranged in close rooms, heated by steam-pipes or stoves. By the second plan, the casks, each holding somewhat more than 100 gallons, are laid on their sides, with the bungholes up, and distributed in long parallel rows, two or more deep, with narrow lanes between. A flexible pipe or hose, in connexion with the great wash tun in the brewhouse, is laid alongside of the casks, for the purpose of filling them, and keeping them suppliea a case of leakage, or evaporation. The wash requires usually several months for its complete acetification, during which time the bung-holes are left open in fine weather, but covered with a tile in the time of rain. When the acetous fermentation is completed, the contents of the casks are transferred by a syphon into a shoot laid on the ground, whence it is drawn by a pump into a store vat within doors. It is next clarified in very large vats, called "rapes," because in them it is filtered slowly and repeatedly throush a compacted heap of the stalks and skins of raisins, called rape, which is the refuse of the British wine manufacture.

In 1838, 2,628,978 gallons of vinegar paid duty in England; in 1839, 2,939,665; 
and in $1840,3,021,130$; upon which the gros; anoun of duty was respectively, 21,908l. 3s.; 24,488l. 17s. 6d. ; and 25,978l. 12s. 9 d.

In Scotland, in the same years, 15,626 gallons; 14,532 ; and 12,967 ; on which the duty charged was, respectively, 130l. $4 \mathrm{~s} .4 d . ; 121 \mathrm{l} .2 \mathrm{~s} . ;$ and $111 \mathrm{l}$. $19 \mathrm{~s} .7 \mathrm{~d}$.

In Ireland, in the same years, 48,158 gallons; 50,508 ; and 56,812 ; on which the duty charged.was, 401l. 6s. 4d.; 420l. 18s.; and 489l. 13s.

In the German process of Schützenbach for the rapid formation of vinegar, 180 measures (of 2 litres, or 2 quarts each) of water are added to 20 of alcohol of from 44 to 45 per cent. by Trailes, and $6 \frac{1}{2}$ of vinegar, containing $3 \frac{1}{2}$ per cent. of acetic acid. These $205 \frac{1}{3}$ measures produce on the average 203 to 204 of rinegar of the above strength. The process of acetification in the graduation tubes (gruilirfuisser) is finished in about 48 hours, and furnishes a vinegar of only 2.75 per cent. of acid strength. The liquid still contains some unchanged alcohol, and it is therefore transferred into tuns, where it completes its oxygenation. The heat of the chamber being about $90^{\circ} \mathrm{F}$, occasions the stream of air that is passed through the abore materials to carry off unproductiveiy one tenth of the alcohol at least. Of the air tha passes through the apparatus, only 3 per cent. of its oxygen is conrerted into carbonic acid.* An increase in the proportion of alcohol in the mixture is not found farorable to increased production of vinegar.

The theory of the acetification of alcohol was first fully cleared up by the researches of Liebig on aldehyde. For the production of 100 pounds of hydrated acetic acid, 53 pounds of oxygen are required, which are contained in 227 pounds of air, and oxygenate 77 pounds of absolute aleohol.

The conversion of the alcohol of fermented liquors into vinegar may be chemically represented as follows: Alcohol is a compound of four atoms of carbon, six of hydrogen, and two of oxygen, or in symbols $\mathrm{C}_{4} \mathrm{H}_{6} \mathrm{O}_{2}^{2}$. In certain circumstances (as the first stage of acetification) it loses two atoms of hydrogen, and becomes aldehyde, or dehydrogenated alcohol, $\mathrm{C} 4 \mathrm{H} 4 \mathrm{O}_{2}$. This body readily absorbs two atoms of oxygen on exposure to air, and thus forms one atom acetic acid; in symbols, $\mathrm{C}_{4} \mathrm{H}_{3} \mathrm{O}_{3}+1$ atom water $(\mathrm{H} \mathrm{O})$. These results are obtained in the exposure of rapor of alcohol to platinum sponge, or platinum mohr. In all cases it is presumed that aldehyde is first produced, then vinegar. The quick vinegar process has been in this country advantageously aprlied to the acetification of a solution of starch sugar made by the agency of either malt or sulphuric acid. But as our excise laws are adverse to the spirituous fermentation of such sugar, the starch liquor, after being boiled with one per cent. of sulphuric acid, is directly fermented into a crude wash, which is then acetified by the following method :-

A very large slightly conical tub or tun, 14 feet wide at bottom, 15 at top, and 13 high, turns out in a given time as much vinegar as is in Germany obtained from six tubs 8 feet high and 4 feet wide. Our larger mass of materials generates and maintains s much heat in the oxidation of the spirit, as to require no store-heating in a properly constructed chamber. Two and a half feet above the bottom of the above tun, a false bottom is laid. 'The space above this bottom is filled with coopers' wood shavings and chips, and the space beneath is destined to receire the liquor as it trichles down on the true bottom, in order to be pumped up in continual circulation. At a moderate height above the tun, the reservoir of the wash is placed, which discharges itself through a regulating stop-cock, or valve, into a pipe in its bottom, which passes down through a pretty large hole in the middle of the lid of the tun, and terminates a few inches under it, in a cross pipe shut at the ends, which is made to revolve slowly by mechanical power, in a horizontal direction round the end of the rertical pipe. This cross pipe is long enough to reach nearly to the sides of the tun, and, being pierced with small holes in its under side, delivers the fermented liquor, in minute streams, equally all over the surface of the chips of wood. It thence falls into the lower compartment of the tun, through holes round the circumference of the false bottom, whence it is zumperi up again, under certain modifications, to be presently described.

Tne air for oxygenating the alcohol into vinegar is supplied from two floating gasometers, which are made to rise and fall aiternately br steam power. The ascending one draws its air from a pipe which passes into the centre of the tun, immediately under the false bottom, and as it redescends it discharges that air through a pipe into a cistern of water, which condenses, and retains the alcoholic vapor drawn off with the air. This water is used in making the next acetifying mixture. The fresh air is admitted in the top of the tun by the sides of the vertical liquor pipe, which is somewhat smaller than the hole through which it passes. Proper valves are placed upon the pipes connected with the gasometer pump, whereby the air drawn from the bottom compart. ment is prevented from returning thither. A small forcing-pump is employed to rais: 
the liquor continually from the bottom of the tun to the cistern overhead. By this arrangement rood vinegar may be made in a few days, without any perceptible loss of materials. The progress of the acetification in this apparatus is ascertained by testing the air for oxygen as it is slowly drawn into the gasometers, or expelled from them. Fo: this puriose a bundle of twine, which has been impregnated with solution of sugar of l:aul, and liried, is set fire to, and plunged into a bottle filled with the air. In reneral, it is so well disoxygenated and carbonated, that the ignition is immediately extinguished. By regulating the warmth of the apartment, the motion of the gasometer, and the admission of air, the due progress of the acetification may be secured. The rine:rar has an arerage strength of $5 \frac{1}{2}$ per cent. of acetic acid hydrate, and is immediately ready for the market.

Hitherto it has been generally imagined that the formation of vinegar is accomplished br a peculiar fermentation, which has been called the acetous, in contradistincfion from the vinous, the panary, the putrefactive, \&c. But this doctrine is doubtful. The experiments which serie as its base, and which should reveal the nature of its peculiar fermen as also the chemical reactions which take place in its progress, all seem to place this phenomenon somewhat out of the sphere of fermentation properly so called. Every fermentation operates by resolving a body into compounds less complex than itself. But the so-called acetic fermentation serves to combine, $\mathrm{c}:$ the contrur:; two bodies, viz., alcchol and aldehyde, with the oxygen of the air; and this is the only case where fermentation produces such an action, which is a true combustion.

Fet it must be confessed that the acetic seems to possess all the characters of the other fermentations; namely, the union of an organized body or ferment with a fermentable organic matter. The former is found in that mucous substance called mother of vinegar, and which is seen floating on the surface of vinegar in the act of its generation. It begins to appear with the acid fermentation, and it continues to be formed during its whole progress. It is at first a pellicle composed of globules much more minute than those which constitute yeast; and they are often irregularly grouped. The pellicle becomes afterward thicker in body and consistence, exhibits more distinct granular forms, and acquires a tendency to be distributed in stripes or narrow bands. The mode of the reproduction of these globules is quite unknown; but they seem somewhat akin to the slimy deposite of sulphureous mineral waters called baregine.

If the study of the acetic ferment be mysterious, it is, however, clear that the conversion of alcohol into vinegar never takes place in the common process without the presence of an albuminous substance, and of the condition favorable to all fermentations, besides the necessary access of air, not only at the commencement (as suffices for the vinous) but during all its course. Hence every weak spirituous liquor which contains an albuminous matter or any ferment may, with contact of air, and a temperature of from $60^{\circ}$ to $90^{\circ} \mathrm{F}$, give birth to vinegar. If the mixture be too rich in alcohol, or if the azotised matter be absent, or if the temperature be much above or below these two points, the phenomenon of acetification stops. There are, therefore, several indications of the existence of a peculiar vinegar fermentation; though it should be observed that the production of lactic acid (as from fermenting cabbage, starch, \&c.) has sometimes misled chemists into the belief of an acetic fermentation. I shall, on this account, point out here briefly the distinction between the two processes.

The acetic fermentation requires the presence of ready formed alcohol and of the air; the lactic, on the contrary, proceeds with starchy or saccharine mixtures, without the intervention of alcohol or of atmospheric oxygen; and when once begun, it will go on of itself. The acetifying process presents, moreover, a striking analogy with the phenomenon of nitrification, in the necessity of an elevated temperature and the influence of porous bodies to divide the particles of the liquids and the air. Thus gaseous ammonia mixed with oxygen, when passed through a tube containing spongy platinum slightly heated, becomes nitric acid; when sulphurous acid gas and oxygen are passed through hot pumice-stone they become sulphuric acid; and when lime or potssh, dilfused through porous matter, is placed in contact with ammoniacal emanatior: 3 , in the artificial nitre beds, or nitrifiable soils, nitrate of lime or potash is formed. In like manner, under the influence of spongy platinum, alcohol $\left(\mathrm{C}_{4} \mathrm{H}_{4} \mathrm{O}+\mathrm{H}_{2} \mathrm{O}\right)$ and air may, by a true oxidisement of the ethereous part of the alcohol $(\mathrm{C} 4 \mathrm{H} 4 \mathrm{O})$ produce aldehyde $\left(\mathrm{C}_{4} \mathrm{H}_{4} \mathrm{O}_{2}\right)$, which passes afterward into acetic acid ( $\mathrm{C}_{4} \mathrm{H}_{3} \mathrm{O}_{3}+$ water, $\mathrm{HO}$ ). On these principles we may conceive that vinegar must be readily formed when alcoholic wash, at a proper temperature, is extensively exposed to atmospheric air, by being spread over the surfaces, of whoden twigs, or chips in the German gradualors. In some districts cider is rapidly acetified by being made to trickle cautiously along strings suspended vertically between two vats. See Acetrc Acid and Graduator in the dictionary. 
AGRICULTURAL CROPS, cumposition of, by M. Boussingault.-Ann. de Chem. es Phys. III. S. p. 234.

\begin{tabular}{|c|c|c|c|c|c|c|c|c|c|c|c|c|}
\hline \multirow{2}{*}{\multicolumn{3}{|c|}{ Substances. }} & & \multicolumn{5}{|c|}{ Ashes inclusive. } & \multicolumn{4}{|c|}{ Exclusive of Ashes. } \\
\hline & & & & 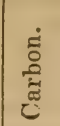 & 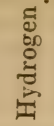 & 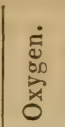 & 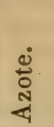 & $\begin{array}{l}\dot{p} \\
\frac{0}{5} \\
\frac{G}{4}\end{array}$ & 莒. & 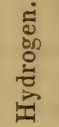 & 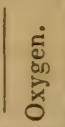 & 造 \\
\hline Wheat & - & - & & $46 \cdot 1$ & $5 \cdot 8$ & $43 \cdot 4$ & $2 \cdot 3$ & $2 \cdot 4$ & $47 \cdot 2$ & $6 \cdot 0$ & $44 \cdot 4$ & $2 \cdot 4$ \\
\hline Rye & - & - & & $46 \cdot 2$ & $5 \cdot 6$ & $44 \cdot 2$ & $1 \cdot 7$ & $2 \cdot 3$ & $47 \cdot 3$ & $5 \cdot 7$ & $45 \cdot 3$ & $1 \cdot 7$ \\
\hline Oats & - & - & 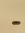 & $50 \cdot 7$ & $6 \cdot 4$ & $36 \cdot 7$ & $2 \cdot 2$ & $4 \cdot 0$ & $52 \cdot 9$ & $6 \cdot 6$ & $38 \cdot 2$ & $2 \cdot 3$ \\
\hline Wheat $\mathrm{s}$ & traw & - & & $48 \cdot 4$ & $5 \cdot 3$ & $38 \cdot 9$ & $0 \cdot \tilde{4}$ & $7 \cdot 0$ & $52 \cdot 1$ & $5 \cdot 7$ & 41.8 & 01 \\
\hline Rye stra? & & - & - & $49 \cdot 9$ & $5 \cdot 6$ & $40 \cdot 6$ & $0 \cdot 3$ & $3 \cdot 6$ & $51 \cdot 8$ & $5 \cdot 8$ & $42 \cdot 1$ & $0 \cdot 3$ \\
\hline Oat strav & & - & & $50 \cdot 1$ & $5 \cdot 4$ & $39 \cdot 0$ & $0 \cdot 4$ & $5 \cdot 1$ & $52 \cdot 8$ & $5 \cdot 7$ & $41 \cdot 1$ & $0 \cdot 4$ \\
\hline Potatoes & 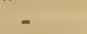 & . & & $44 \cdot 0$ & $5 \cdot 8$ & $44 \cdot 7$ & $1 \cdot 5$ & $4 \cdot 0$ & $45 \cdot 9$ & $6 \cdot 1$ & $46 \cdot 4$ & $1 \cdot 6$ \\
\hline Beetroot & (field) & - & & 42.8 & $5 \cdot 8$ & $43 \cdot 4$ & $1 \cdot 7$ & $6 \cdot 3$ & $45 \cdot 7$ & $6 \cdot 2$ & $46 \cdot 3$ & $\mathrm{I} \cdot 8$ \\
\hline Turnips & - & - & - & 42.9 & $5 \cdot 5$ & $42 \cdot 3$ & $1 \cdot 7$ & $7 \cdot 6$ & $46 \cdot 3$ & $6 \cdot 0$ & $45 \cdot 9$ & $1 \cdot 8$ \\
\hline Jerusaler & $m$ artic & rokes & & $43 \cdot 3$ & $5 \cdot 8$ & $43 \cdot 3$ & $1 \cdot 6$ & $6 \cdot 0$ & $46 \cdot 0$ & $6 \cdot 2$ & 46.1 & 1.7 \\
\hline Yellow p & peas & - & - & $46 \cdot 5$ & $6 \cdot 2$ & $40 \cdot 0$ & $4 \cdot 2$ & $3 \cdot 1$ & $48 \cdot 0$ & $6 \cdot 4$ & $41 \cdot 3$ & $4 \cdot 3$ \\
\hline Pease str & raw & - & & $45 \cdot 8$ & $5 \cdot 0$ & $35 \cdot 6$ & $2 \cdot 3$ & $11 \cdot 3$ & 51.5 & $5 \cdot 6$ & $40 \cdot 3$ & $2 \cdot 6$ \\
\hline Red trefo & oil hay & - & - & $47 \cdot 4$ & $5 \cdot 0$ & $37 \cdot 8$ & $2 \cdot 1$ & $7 \cdot 7$ & $51 \cdot 3$ & $5 \cdot 4$ & $41 \cdot 1$ & $2 \cdot 2$ \\
\hline Jerusaler & $m$ artic & oke & & $45 \cdot 7$ & $5 \cdot 4$ & $45 \cdot 7$ & $0 \cdot 4$ & $2 \cdot 8$ & $47 \cdot 0$ & $5 \cdot 6$ & $47 \cdot 0$ & $0 \cdot 4$ \\
\hline
\end{tabular}

ALCOHOL, as obtained by the distillation of wine, of fermented corn-wort, of potato syrup, possesses different flavors, which arise from what the Germans call Fusel oil, and which oil differs in those several spirituous liquors. That of wine has been called Onanthic ether. It is resolvable into ether (oxide of ethal), and a peculiar fatty acid, the Onanthic.

The œnanthic ether of corn spirit contains an additional oil, called corn oil, which has a most offensive smell. The potato fusel oil differs from that obtained from grain spirit. It has, at the first impression, in its pure state, a strong, not disagreeable smell, which afterward becomes extremely nauseous, and excites an acrid burning taste. The inhalation of its vapor causes a feeling of oppression, and romiting. Al] these fusel oils are readily soluble in alcohol, but not in water, whence, when poured into the latter, they make a milky mixture. The potato fusel oil contains no solanine from the potatoes, as has been alleged, though this noxious principle exists in all potatoes, especially after germination. It is not a volatile product.

Corn damaged by rain in harvest-time affords, after mashing and fermentation, a most offensive fusel oil, which irritates the eyes and nostrils, and smells like a solution of cyanogen in alcohol. Spirits so contaminated intoxicate more powerfully than purer spirits, and are apt to bring on temporary madness and subsequent indisposition. This noxious substance does not combine with gases; and, being more rolatile than alcohol, it may be drawn off by distillation in a concentrated state. It is separated to a considerable degree in Germany by diluting the foul spirits with water, mixing in a portion of olive or other bland oil, letting the oil gather on the top, drawing of the spirituous liquor from below, and subjecting it to rectification. When the spirit so rectified is kept for 2 or 3 months in a cask, corked, but not too closely, the noxious œnanthic compound disappears, in some measure by its spontaneous decomposition. For statistics and excise-proof table, see Sprrits.

The high price of alcohol in this country, in consequence of the heavy fiscal duties, and its low price in most other countries, where it is nearly duty free, has led to its contraband importation under various disguises. Sometimes it is introduced under the mask of oil of turpentine, from which it can be sufficiently freed by rectification for the purpose of the gin manufacturers. Sometimes it is disguised with wood naphtha, or wood vinegar; from the latter of which it may be separated by distillation in a water bath; but from the former it is more difficult to extricate it, as alcohol and wood spirit are nearly equally volatile. It has also been disguised with coal naphtha; but from this it may be easily separated by distillation, on account of the great difference between the boiling points of the two liquids; besides, coal naphtha will not combine with water, as alcohol does.

When the object is to discover whether wood spirit eontains alcohol, we may proceed as follows: Add to the suspec ed liquid a little nitric acid, of specific gravity 1.45. If alcohol be present, in even small proportions, an effervescence will ensue, from the evolution of etherised nitrous gas, with its characteristic ethereous smell. On treating the mixture with a nitrous solution of mercury, as in the process for fulminate of mercury, an effervescence will take place, the dense vapor of etherised mer. 
curial gas will appear, and a certain proportion of fulminate will be formed, corresponding pretty closely to the proportion of alcohol in the wood naphtha mixture.

As the boiling joint of wood spirit is only about $145^{\circ}$, while that of alcohol, of like specific matvity $(0.825)$, is $173^{\circ} \mathrm{F}$., a good criterion of the proportion of the two liquids present in the mixture may be found in its boiling temperature.

Pure wood spirit, when mixed with the above nitric acid, becomes of a ruby tint, but remains tranquil. Alcohol continues colorless, but enters into violent ebullition, and is nearly all dissipated in fumes.

Alcohol diluted with water has a less resultant density than wood spirit of like strength similarly diluted. While alcohol thus becomes of 0.920 , wood spirit becomes 0.926 or 0.927 .

If' wrod spirit be contained in alcohol, it may be detected to the greatest minuteness by the tes: of caustic potash, a little of which, in powder, causing wood spirit to become speedily vellow and brown, while it gives no tint to alcohol. Thus 1 per cent., of woud spirit may be discovered in any sample of spirits of wine. For further details apon this analytical inquiry, see my pamphlet, entitled The Revenue in Jeopardy.

ALGAROVILLA. This substance is called by the Spaniards Algaroba, from the resemblance it bears to the fruit of the Carob (Ceratonia siliqua), which is a native of Europe, in the southern countries of Spain and Portugal. The substance lately analysed by me is the fruit of a tree which grows in Chile, of which the botanical name is Prosopis pallida, according to Captain Bagnald, R. N., who first brought a sample of it to this country in the year 1832. It consists of pods bruised and agglutinated more or less with the extractive exudation of the seeds and husks. According to a more recent determination, algarovilla is said to be the product of the tree Juga Marthæ of Santa Martha, a province of New Carthagena.

It is an astringent substance replete with tannin, capable, by its infusion in water, of tanning leather, for which purpose it possesses more than four times the power of good oak hark. Its active matter is very soluble in water at a boiling temperature. The seeds are merely nutritive and demulcent, but contain no astringent property. This resides in the husks. The seeds in the entire pod constitute about 1-5th of the weight, and they are three or four in number in each oblong pod. Alcohol of 60 per tent. over proofs dissolves 64 parts in 100 of this substance. The solution consists chiefly of tannin, with a very little resinous matter. Water dissolves somewhat more of it, and affords a very styptic-tasted solution, which precipitates solution of isinglass very copiously, like infusion of galls and catechu. Its solution forms with sulphate of iron a black precipitate, which is kept Hoating by means of the gum present, and thereby constitutes good ink. My report to the merchant was written with a combination thus made, in proportions taken at random; and there is no doubt that by using a stronger decoction of the algarovilla, along with a proper proportion of copperas, an exeellent black ink might be prepared without any other addition.

I find that a decoction of the algarovilla affords with cotton cloth, mordanted with tin solution, as also with acetate of alumina liquor, a brilliant yellow die; the former being the brighter and fuller of the two.

A tincture of algarovilla might be used as an astringent in medicine; or probably a decoction of the whole substance would be preferable, on account of the demulcent quality of the seeds when bruised. As an article of commerce it can not be rated at a high price, nor should it pay much duty till its value as an article of manufactures or medicine be fully ascertained.

ALMONDS. Imported in 1839, 28,261 cwt.; in 1840, 27,566. Retained for consumption, 9,785 and 7,935, respectively.

ALUM. In the alum works on the Yorkshire coast, 8 different liquors are met with.

1st. "Raw Liquor." The calcined alum shale is steeped in water till the liquor has acquired a specific gravity of 9 or 10 pennyweights, according to the language of the alum-maker.

2d. "Clarified Liquor." The raw liquor is brought to the boiling point in lead pans, and suffered to stand in a cistern till it has cleared : it is then called clarified liquor. Its gravity is raised to 10 or 11 pennyweights.

3d. "Concentrated Liquor." Clarified liquor is boiled down to about 20 pennyweights. This is kept merely as a test of the comparative value of the potash sasis used by the alum-maker.

4th. "Alum Mother Liquor." The alum pans are fed with clarified liquor, which is boiled down to about 25 or 30 pennyweights, when a proper quantity of potash salt in solution is mixed with it, and the whole run into coolers to erystallize. The liquor pumped from these rough crystals is called "alum mothers."

5th. "Salts Mothers." The alum mothers are boiled down to a crystallizing point, and afford a crop of "Rough Epsom," which is a sulphate of magnesia and protoxide of iron. 
6th and 7th. "Alum Washings." The rough crystals of alum (No. 4), are washed twice in water, the first washing being about 4 pennyweights, the second about $2 \frac{1}{2}$, the diflerence in gravity being due to mother liquor clinging to the crystals.

8th. "Tun Liquor." The washed crystals are now dissolved in boiling water, and run into the "roaching tuns" (wood vessels lined with lead) to crystallize. The mother liquor of the "roach alum" is called "tun liquor;" it is, of course, not quite so pure as a solution of roach alum in water.

The alum-maker's sp. gr. bottle holds 80 pennyweights of water, and by 10 pennyweights he means 10 more than water, or 90 .

The numbers on Twaddle's hydrometer, divided by $2 \cdot 5$, gave alum-maker's pennyweights.

The alum-maker tests his samples of potash salts comparatively by dissolving equal weights of the different samples in equal measures of alum liquor at 20 pennyweights, heated up to the boiling point, and weighing the quantity of alum crystals produced on cooling.

For the above information I am indebted to my friend Mr. Maurice Scanlan, who superintended for some time the Mulgrave alum works.

He informs me that $61 \frac{1}{2}$ tons of the alum rock at the Mulgrave works to the north of Whitby, yield, after calcination, \&c., one ton of alum.

It has been computed that with sulphur at $6 l$. per ton, sulphuric acid of spec. grav. $1 \cdot \% 50$ can be produced at $3 l$. per ton, including the mere cost of making: this acid contains 2 atoms of water : 174 tons of this acid, and $87 \frac{1}{4}$ tons of sulphate of potash, with the pipe-clay, will form 474 tons of alum; so that the neat cost would be $522 l$., for the acid $+1047 l$. for the sulphate of potash $=1569 l$.; which sum, divided by 474 , gives a quotient of $3 l .6 s$. for the neat cost of 1 ton of alum by the direct process.

At the pit 1 ton of alum, rock or mine, coast $3 l$. $4 \mathrm{s.}$; to which, adding the cost of the potash salt for 1 ton of alum, $3 l .15 \mathrm{~s}$., they constitute together an amount of $6 l .19 \mathrm{~s}$. From the latter sum 1l. 10s. must, however, be deducted for the value of rough Epsom salt produced, leaving a balance of $5 l .9 s$. for the cost of a ton of mine-alum, prior to evaporation and crystallization.

A patent was obtained in November, 1839, by Mr. William Wiesmann, of Duesburg, for improvements in the manufacture of alum. He subjects potter's clay to a moderate red heat, grinds it, and subjects the powder, in leaden pans, to the action of concentrated sulphuric acid ( $66^{\circ}$ Beaumé), taking care to use excess of clay and a moderate heat. This mixture is to be stirred till it is dry, then treated with boiling water, in order to dissolve the sulphate of alumina formed. So far the process is old and well-known. The novelty consists in freeing the saline solution from iron by ferrocyanure of potassium (prussiate of potash). When the iron has been all thrown down in the form of prussian blue, the liquor is allowed to settle, the supernatant pure sulphate is drawn off, and evaporated till it forms on cooling a concrete mass, which may be moulded into the shape of bricks, \&c., for the convenience of packing. He proposes to crystallize his alum; but he will find this process rather difficult. The prussian blue obtained may be reconverted by any alkaline solution into a ferrocranide, and again employed on a fresh quantity of the raw sulphate. How he is to precipitate the iron by sulphate of lime, as he states, I can not comprehend.

Dr. Turner's process for making alum from felspar is thus described by him in the specification of his patent, sealed October 8, 1842. If it be desired to make a potash alum, the best substance to operate upon is a potash felspar. This felspar is ground in a common edge-stone mill to the consistency of fine sand (a process which is much assisted by first heating it to redness, and then plunging it in cold water); it is then mixed with its own weight of sulphate of potash, and placed in the upper part of the inclined bed of a reverberatory furnace (being such a furnace as is known in the potteries as a frit furnace), and which furnace has previously been brought to a full white heat. When by the action of the heat a glass has been produced, and is obtained to flow down the inclined bed of the furnace, to such glass is to be added gradually, at the lower end of the furnace, as much carbonate of potash as was berore used of sulphate of potash. And this process of placing the mixture of felspar and sulphate of potash at the upper part of the bed of the furnace is to be repeated, adding at the lower part of the bed, gradually and proportionally, as the glass flows down from the upper part, the carbonate of potash, as before mentioned. This is continued until the sack of the furnace is filled with the glass; this glass is then fit for the next process. The preparation of the glass may also be affected in a reverberatory furnace with a flat bed; and the f́acility of removing the glass from such a furnace is an advantage. In this case no carbonate must be added to the mixture until the sulphate of potash is observed to be completely decomposed. On boiling in water the glass thus obtained, the same quantity of potash as was added to the felspar 
and two thirds of the silica contained in the felspar are dissolved, while the remaining one third of the silica and the alumina, and an equal quantity of potash as the felspar originally contained, are left in the form of a light porous substance, similar in chemical composition to the mineral commonly called elæolite; this porous substance is carefully separated from the safd solution, and washed with water until freed from the silicate of potash, then placed in an open leaden cistern or boiler, and boiled with dilute sulphuric acid of the specific gravity $1 \cdot 2$ (one and two tenths). This acid will contain about the quantity of water required for the solution and crystallization of the alum produced by the decomposition of the elæolite; the quantity of the dilute sulphuric must be such as will contain about $160 \mathrm{lbs}$. of dry sulphuric acid for every $285 \mathrm{lbs}$. of felspar rock (if that rock be. used), and in like proportion to the silica and alumina contained in the substance, if any other substance be used, as it is important that the alum solution thus obtained should not contain an excess of acid. I recommend that only four fifths of the proposed quantity of dilute sulphuric acid should be used in the first operation, which will leave a portion of the elæolite undecomposed; but by acting upon this undecomposed portion, after the solution has been drawn off, with the full quantity of dilute acid to be used in the next operation, it will be completely decomposed, and the alum thus formed becomes part of the next batch. In this way a neutral solution of alum is obtained at each process. The boiling solution, after the sediment subsides, is drawn into coolers, cich as are commonly used for the crystallization of alum; here about four fifths of the alum held in solution will form into crystals. The mother-liquor from the coolers is boiled in any convenient boiler to dryness, in order to render the silica it contains insoluble; the residuum is boiled either in water or in the mother-liquor from the roaching tubs, so as to dissolve the alum it contains, and the process of crystallization repeated. Had the above process been performed with the salts of soda instead of potash, a soda alum would have been formed. For this purpose the soda felspar or albite should be selected. The potash or soda (as the case may be) contained in the liquor, drawn as aforesaid from the elæolite (or nepheline, which is formed when soda is used), may be recovered by either of the following processes: The strong solutions which are obtained, about the specific gravity of $1 \cdot 2$ are placed in any convenient vessel in which a stream of carbonic acid gas, obtained in any convenient method, may be driven through them, the carbonic acid becomes absorbed, and the solution assumes the form of a gelatinous mass : this mass consists of carbonate of potash or soda and hydrate of silica. On drying this mass in a furnace, which must never be allowed to rise to red heat even in the dark, the silica loses its water and becomes insoluble; the potash or soda may then be separated from it in the form of a sesquicarbonate of potash or soda, by solution and evaporation to dryness. The other process, which under most circumstances will be found more economical and convenient, is to allow the boiling solution of silicate of potash or soda to filter through a bed of caustic lime, when it will be found that the lime has combined with the silicate, and a caustic potash or soda ley is obtained. This process may be conveniently conducted in an apparatus similar to that used by soap-makers for the preparation of their caustic leys. The potash or soda may then be readily obtained as caustic potash or soda, or as carbonate, by the known processes used in making soda. The weak solutions of silicate of potash or soda are used to decompose another portion of the glassy substance.

Now I do not claim as new, or as my invention, any particular form of vessel or apparatus in which or with which my operations may be conducted; nor do I claim any particular proportions in which the alkaline salts may be used. But I claim as new, and as my invention, the improvements aforesaid, and the production of substances similar to elæolite and nepheline artificially; by the decomposition by water of the glassy substances produced by the fusing of felspar as aforesaid, or other mineral substances containing silica and alumina, with salts of potash and soda, as aforesaid, and the use and application of such artificial elæolite and nepheline in the production and manufacture of alum, as aforesaid. I also claim the process, as above described, for separating the alkalies from silica by means of caustic lime.

ANCHOVIES, ESSENCE OF. I insert this article to show on what slender pretence of inrention a patent may be obtained. Mr. John Masters, of Leicester, makes his improved transparent preparation, by placing in a kettle any given quantity of anchovies in the state in which they are imported, along with their own weight of water, exposing the kettle to a simmering heat for two or three hours, removing the kettle, and straining its contents when cold, first by suitable pressure through a strong canvas bag, and next filtered through a flannel or paper till a clear liquor is obtained. If it be desired to render the essence thicher, the material used for this purpose should be transparent. He says that flour is used for thickening the common essence. I presume he would prefer gelatine, gum, or arrow root for his transparent thickening, though he does not specify anything. 
ANNOTTO or ANNATTO. Imported in 1839, 303,489 lbs.; in $\mathbf{1 8 1 0 , 1 0 8 , 4 6 9 .}$ Retained for consumption, 224,794 and 330,490, respectively.

ARCHIL has been lately the object of numerous chemical researches, but hitherto, it must be owned, without producing any results useful to the dyer, so as to promote the solidity of this beautiful and now cheap dye. The new experiments of Schunk, perforined in Liebig's laboratory, tend to show that the whole matter is still involved in much mystery. By the action of either on the Variolaria lactea (one of the archil lichens), in an apparatus of displacement, he obtained crystals, which he calls lecanorine. It is convertible into the orcine of Robiquet and Heeren by hot barytes water. When moistened with water of ammonia, and exposed to the air, it gradually assumes the archil tint. Liebig is of opinion, that this product, and some other analogous ones, vary accurding to the nature of the solvents and the temperature of the digestions, so that they probably are mere metamorphoses of the self-same one or two substances, pre-existing in the lichens. Since lecanorine is decomposed by boiling water into carbonic acid, and orcine, it may also undergo this change from the boiling alcohol employed in Schunk's researches.

When ammonia acts upon orcine, it gives it a dark blood-red color, and converts it into orceine, a new compound containing azote, but in a different state of combination from what it is in ammonia. This orceine is the true coloring matter of the archil or orseille; and, according to Robiquet, it is here in the state of an orceate of ammonia, requiring for its production the co-operation of air and water. In these circumstances the orceine absorbs oxygen, and is transformed into orceate of ammonia, without any other product, even carbonic acid, being formed. Variolarine, erythrine, and psuedoerythrine, three products obtained by Robiquet and Heeren; the first from Variolaria dealbata, the second and third from Parmelia roccella, and Lecanora tartarea, are interesting merely in a scientific point of view. The last two are transformed into red coloring matters by ammonia and air. "Latterly," says Liebeg, "Kane has made these two substances objects of a particular investigation; but the researches of this chemist are far, as appears to me, from clearing up their history." I need not therefore, give any account of these researches, which occupy a large portion of a recent volume of the Philosophical Transactions.

Tournesole or litmus, consists, according to Peretti, of a red coloring matter, rendered blue by combination with ammonia.

ARROW ROOT. This plant has been lately cultivated with great success, and its root manufactured in a superior manner, upon the Hopewell estate, in the island of St. Vincent. It grows there to the height of about 3 feet, and it sends down its tap roots from 12 to 18 inches into the ground. Its maturity is known by the flagging and falling down of the leaves, an event which takes place when the plant is from 10 to 12 months old. The roots being dug up with the hoe are transported to the washinghouse, where they are thoroughly freed from all adhering earth, and next taken individually into the hand, and deprived by a knife of every portion of their skins, while every unsound part is cut away. This process must be performed with great nicety, for the cuticle contains a resinous matter, which imparts color and a disagreeable flavor to the ferula, which no subsequent treatment can remove. The shinned roots are thrown into a large cistern, with a perforated bottom, and there exposed to the action of a copious cascade of pure water, till this runs off quite unaltered. The cleansed roots are next put into the hopper of the mill, and are subjected to the powerful pressure of two pairs of polished rollers of hard brass; the lower pairs of rollers being set much closer together than the upper. (See the accompanying figure.) The starchy matter is thus ground into a pulp, which falls into the receiver placed beneath, and is thence transferred to large fixed copper cylinders, tinned inside, and perforated at the bottom with numerous minute orifices, like a kitchen drainer. Within thesc cylinders, wooden paddles are made to revolve with great velocity, by the power of a water-wheel, at the same time that a stream of pure water is admitted from above. The paddle-arms beat out the fecula from the fibres and parenchyma of the pulp, and discharge it in the form of a milk through the perforated bottom of the cylinder. This starchy water runs along pipes, and then through strainers of fine muslin into large reservoirs, where, after the fecula has subsided, the supernatant water is drawn off, and fresh water being let on, the whole is agitated and left again to repose. This process of ablution is repeated till the water no longer acquires anything from the fecula. Finally, all the depusites of fecula of the day's work are collected into one cistern, and, being covered and agitated with a fresh charge of water, are allowed to settle till next morning. The water being now let off, the deposite is skimmed with palette knives of German silver, to remove any of the superficial parts, in the slightest degree colored; and only the lower, purer, and denser portion is prepared by drying for the 
market. The drying-house on the Hopewell estate is constructed like the hot-louse of an English garden. But instead of plants, it contains about 4 dozen of drying pans made of copper, $7 \frac{1}{2}$ feet by $4 \frac{1}{2}$, and tinned inside. Each pan is supported on a carriage, having iron axles, with lignum vitæ wheels, like those of a railway carriage, and they run on rails. Immediately after sunrise, these carriages with their pans corered with white gause, to exclude dust and insects, are run out into the open air, but if rain be apprehended, they are run back under the glazed roof. In about 4 days the fecula is thoroughly dry and ready to be packed, with German silver shovels, into tins or American flour barrels, lined with paper attached with arrow root paste. The packases are never sent to this country in the hold of the ship, as their contents are easily tainted by noisome effuvia, of sugar, \&c. By such a skilful series of operations, and by such precautions, the arrow root thus manufactured may vie with any similar preparation in the Bermudas or any other part of the world. I have found it, on analysis and trial, to be pure, powerful, and agreeable, and a most wholesome article of food.

Fig. 1. Plan of arrow root grinding-mill, and of 2 sets of copper cylinder washing.

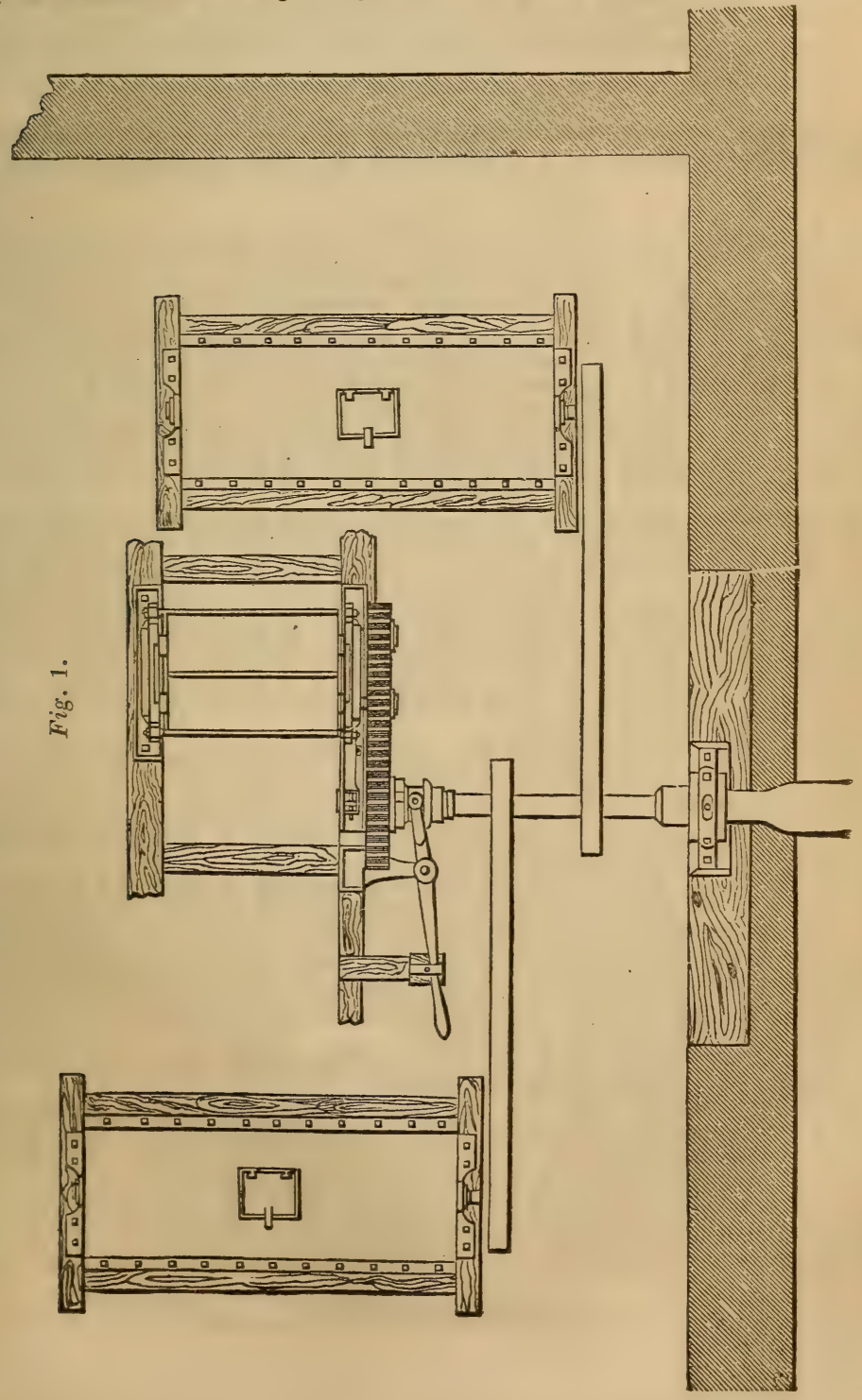


machines, with the connecting machinery for driving them; the washing agitator besny duiven from the connecting shaft with leathern belts. Fig. 2. End elevation of arrow

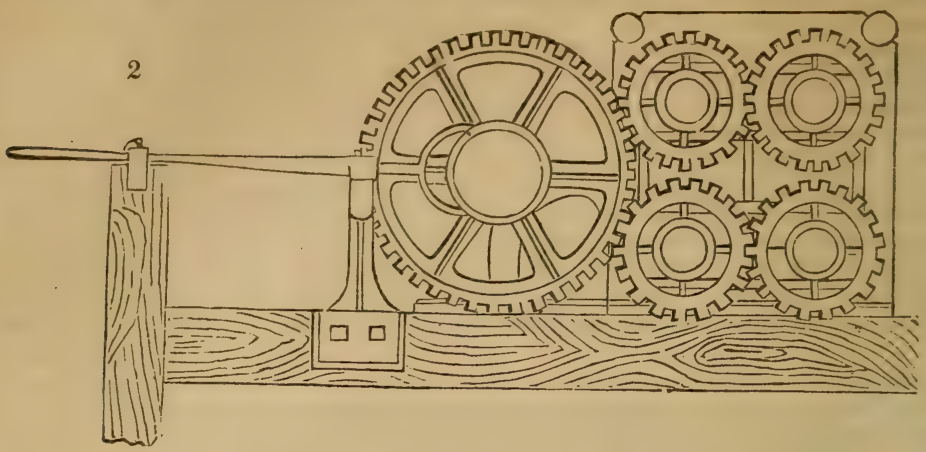

root mill, with wheels and pinions, disengaging lever, \&c. Fig. 3. End elevation of copper washing-cylinders, with press-framing, \&c. The washing-cylinders are $6 \frac{1}{2}$

\section{3}

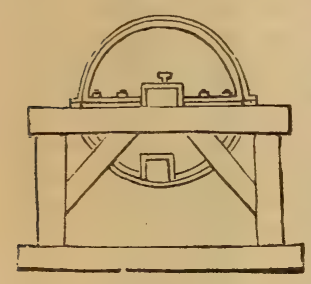

feet long and $3 \frac{1}{2}$ in diameter. The mill-rollers are 3 feet long and 1 foot in diameter.

The uses of arrow root are too well known and acknowledged to require recounting here. It is the most elegant and the richest of all the feculas, and being now manufactured, with the advantage of excellent machinery, and abundance of pure water, in the fertile island of St. Vincent, it may be brought into our market at a much more moderate price than it has heretofore been supplied from less favored localities. The Bermuda arrow root is treated necessarily with rain water collected in tanks, and therefore is occasionally soiled with insects, from which the St. Vincent article is entirely free.

The presence of potato starch in arrow root may be discovered by the microscope. Arrow root consists of regular ovoid particles of nearly equal size, whereas potato starch consists of particles of an irregular ovoid or truncated form, exceedingly irregular in their dimensions, some being so large as $\frac{1}{300}$ of an inch, and others only $\frac{1}{200}$. But the most convenient test is dilute nitric acid of $1 \cdot 10$ (about the strength of single aquafortis), which, when triturated in a mortar with the starch, forms immediately a transparent very viscid paste or jelly. Flour starch exhibits a like appearance. Arrow root, however, forms an opaque paste, and takes a much longer time to become riscid. (Dr. Scharling in Liebig's Annalen.)

Arrow root may be distinguished from potato starch, not only by the different size of its particles, but by the difference of structure. Their surfaces in the arrow root are smooth, and free from the streaks and furrows seen in the potato particles by a good microscope. The arrow root, moreover, is destitute of that fetid unwholesome oil, extractable by alcohol from potato starch.

Liebig places the powers of arrow root, as a nutriment to man, in a very remarkable point of view, when he states that 15 pounds of flesh contain no more carbon for supplying animal heat by its combustion into carbonic acid in the system, than 4 pounds of starch; and that if a savage, with one animal and an equal weight of starch, could maintain life and health for a certain number of days, he would be compelled, if confined to flesh alone, in order to procure the carbon necessary for respiration, during the same time, to consume five such animals.

Arrow root imported in 1839, 303,489; in 1840, 408,469 lbs.; retained for con sumption, 224,794 and 330,490 lbs. respectively.

ARTESIAN WELLS. Fig. $4,{ }^{*}$ represents the manner in which the condensed water of the heavens distributes itself under the surface of our globe. Here we have a geological section, showing the succession of the several formations, and the sheets or laminæ of water that exist at their boundaries, as well as in their sandy beds. The figure shows also very plainly that the height to which the water reascends in the bore of a well, depends upon the height of the reservoir which supplies the sheet of water to which the well is perforated. Thus the two wells, A, $a$, having gone down to

- At the end of the volume. 
the two aquenus expanses below, whose respective waters of supply are derived from the percolations $\mathbf{m} \mathrm{A}^{\prime}$ and $\mathrm{M} \mathrm{B}^{\prime}$, will afford rising waters, which will come to the surface. While in the well $\mathrm{B}$, supplied by the sheet $\mathrm{P}, \mathrm{C}^{\prime} \mathrm{D}^{\prime}$, the waters will spout above the surface, and in the well $c$ they will remain short of it. 'The same figure shows that thise wells often traverse sheets of water that rise to different heights. Thus in the well c, there are five columns of ascending waters, which rise to heights proportional to the points whence they take their origin. Several of these will be spouting or overflowing, but some will remain beneath the surface.

Nogent-

Paris. $\quad$ Provins. Seine. Sroyes. Lusigny. Seine.

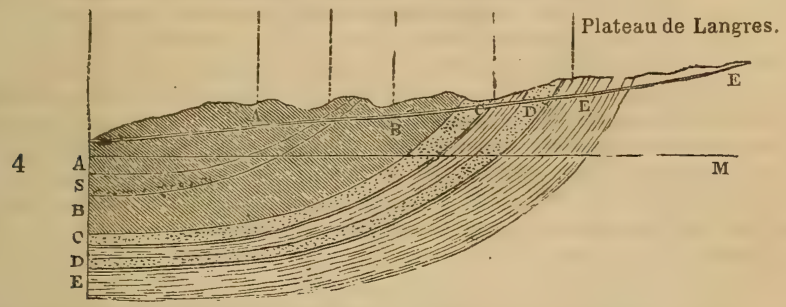

The older geological formations are seldom propitious to the construction of Artesian wells, on account of the compact massiveness of their rocks, of the few fissures or porous places in them, and of the rarity of filtering strata overlying retentive ones. It is therefore vain to attempt the formation of an overflowing spring, upon the above principles, in territories of granite, gneiss, mountain limestone, and basalt. Among transition and secondary formations, such wells will rarely furnish a supply of good water. The latter strata of alternating clay and variegated sandstone contain so much gypsum and rock salt as to impregnate therewith the waters derived from them to an unpalatable degree. It is in the sandy, calcareous, and argillaceous strata of the Jura limestone, indeed, that borings may most probably be made for brine springs. The hot springs which burst out of the ground in primitive rocky districts come undoubtedly from a great depth under the surface, and derive their temperature, and also probably their waters, from the rapors of deep-seated volcanoes in connexion with the sea. A miniature representation of such springs is exhibited in the intermitting fountains of fresh water on the shoulder of Vesuvius. Springs of this kind, which rary with the seasons, may derive a portion of their water from the surface of the earth, from which it may sink through clefts in the primitive rocks, till meeting in its descent with stony obstructions and ascending steam, it is forced to remount in a heated state to the day, like the Geisers in Iceland. The most remarkable example of an Artesian well is that recently formed at Grenelle, a suburb at the southwest of Paris, where there was a great want of water. It cost eight years of difficult labor to perforate. The geological strata round the French capital are all of the tertiary class, and constitute a basin, like that shown in fig. 4 . The bottom of this basin is chalk; A A are tertiary strata above the chalk; $\mathrm{B}$ B, chalk or cretaceous earbonate of lime ; C C, D. D, green sand and clay; E E, oolite and Jura limestone (muschelkalk); $\mathrm{E} \mathrm{A}$, general slope of the surface of the country from Langres to Paris; $\mathrm{MA}$ A, the level of the sea. Over a circular space, of which Paris is the centre, and which is bounded by the towns of Laon, Mantes, Blois, Sancerre, Nogent-surSeine, and Epernay, these strata are found upon the surface, concealing the chalk; but on the other side of these towns, the edge of the basin being passed, the chalk is generally the superficial bed. By looking at the order of these tertiary strata, it is easy to perceive the obstacles that M. Malot, the engineer of the well, had to overcome, and the difficulty and hazard of his undertaking. The surface at Grenelle consists of gravel, pebbles, and fragments of rock, which have been deposited by the waters at some period anterior to any historical record. Below this layer of detritus, it was known to the engineer, by geological induction, as well as previous experience, that at Grenell $\approx$, marl and clay would be found, instead of the limestone which generally forms the immediately subjacent stratum. He was aware that he had to bore about 440 yards! deep before he should arrive at the sheet of water ( $\mathrm{s}$, figure) which flows in the gravel below the limestone, and supplies the wells of St. Ouen, St. Denis, and Seains. Underneath the marl and the clay, the boring rods had to perforate pure gravel, plastic clay, and finally chalk, which forms the bottom of the general tertiary basin, as we have seen. No calculation from geological data could determine the thickness of this stratum of chalk, which, from its powers of resistance, might present an almost insuperable obstacle. The experience acquired in boring the wells of 
Flbeuf, Rouen, and Tours, was in this respect but a very imperfect guide. Bnt supposing this obstacle to be overcome, was he sure of finding a supply of water below this mass of chalk? In the first place, the strata $\mathrm{C}$ D below the chalk possessed, as we shall see, all the necessary conditions for producing Artesian springs, namely, succes. sive layers of clay and gravel, or of pervious and impervious beds. M. Mialot confidently relied on his former experience of the borings of the wells at Rouen, Elbeuf, and Tours, where abundant supplies of water had been found below the chalk, between similar strata of clay and gravel.

But one other condition is requisite to ensure the rising of the water in an Artesian well, namely, that the feeding level of infiltration should be higher than the orifice in the bore above which the water is to ascend. This, however, turned out to be the case with Grenelle. M. Arago had shown that the water of the spring here would necessarily rise to the surface, because in the well at Elbeuf, which is nearly 9 rards above the level of the sea, the water rises from 27 to 29 yards above the surface of the earth, and, consequently, from 36 to 38 yards above the ocean level. Now, as the orifice of the bore at Grenelle is only 34 jards abore the same level, it follows, that if the identical spring be met with, the water must rise above the earth's surface at Grenelle.

The necessary works were commenced with boring rods about 9 yards long, attached to each other, and which could be raised or lowered by mechanical power, while an ingenious method was adopted for giving them a rotary motion. The diameter of the bore was about 6 inches. The instrument affixed to the end of the lowest boring rod was changed according to the different strata which were successiveir attacked; the form suited for passing through the softer materials near the surface being unsuitable for boring through the chalk and flint, as a hollow tube was used for the former, while a chisel-shaped tool was employed to penetrate the latter. The size of the rods was lessened as the depth increased; and, since the subterranean water was not reached so soon as was expected; it became requisite to enlarge five several times the diameter of the bore, in order to permit the work to be successfully prosecuted. Accidents occurred which tried the patience of the projectors. In May, 183\%, when the boring had extended down to a depth of 418 rards, the hollow tube, with nearly 90 rards of the long rods attached to it, broke, and fell to the bottom of the hole, whence it became necessary to extract the broken parts before any further progress could be made. The difficulty of accomplishing this task may be conceived; for the different fragments were not all extracted until after the constant labor of 15 months. Again, in April, 1840 , in passing through the chalk, the chisel attached to the boring rod got detached, and before it could be recovered, sereral months were spent in digging around about it. A similar occurrence created an obstacle which impeded the work for 3 months, but, instead of withdrawing the detached part, it was forcibly driren down among the stratum of gravel. At length, in February, 1841, after 8 years' labor, the rods suddenly descended several yards, having pierced into the vault of the subterranean waters so long sought after by the indefatigable engineer. A few hours afterward, he was rewarded for all his anxious toils; for lo! the water rose to the surface, and dis eharged itself at the rate of 600,000 gallons per hour!

The depth reached down was 602 jards, or about three times the height of St. Paul's. The pipe by which the water reaches the surface has been recently carried to a height nearly level with the source of supply. The portion of the pipe above the ground is surrounded with a monumental pagoda of ornamental carpentry, and it discharges a circular cascade of clear water continually into a circular iron reserroir, to be thence conveyed by a lateral pipe to the ground. The water is well adapted for all domestic uses, and it will be unfailing, being supplied from the infiltration of a surface of country nearly 200 miles in diameter. The Artesian wells of Elbeuf, Rouen, and Tours, which were formed many years ago, overlow in never-varying streams; and the ancient Artesian well at Lillers, in the Pas de Calais, has for about seven centuries furnished a constant and equable supply.

The opportunity of ascertaining the temperature of the earth at different depths, was not neglected during the progress of the works at Grenelle. Thermometers placed at a depth of thirty yards in the wells of the Paris Observatory invariably stand at $53^{\circ}$ Fahrenheit. In the well at Grenelle the thermometer indicated $74^{\circ} \mathrm{F}$, at a depth of 442 yards, and at 550 yards it stood at $79^{\circ}$. At the depth finally arrived at of 602 yards, the temperature of the water which rose to the surface was $81^{\circ}$, corroborating previous calculations on the subject. For a descent of 572 yards there is an increase of temperature equal to $28^{\circ} \mathrm{F}$., which is 20.4 yards, or 61.2 feet for each degree of that scale. Now that the skilful labor of so many years is terminated, the Parisians regret that the subterranean sheet of water had not lain 1,000 yards beneath the surface, that they might have had an overflowing stream of water at $104^{\circ}$, to furnish a cheap supply to their numerous hot-bath establishments.

ASPHALTIC PAVEMENT; see BITUMEN. 


\section{B.}

BALSAM OF CAPIVI, or Copaiva Balsam. This substance, which is extensively used in medicine, is often adulterated. Formerly some unctuous oil was mixed with it, but as this is easily discovered by its insolubility in alcohol, castor oil has since been used. The presence of this cheaper oil may be detected, 1, by agitating the balsam with a solution of caustic soda, and setting the mixture aside to repose; when the balsam will come to float clear on the top, and leave a soapy thick magma of the oil below; 2, when the balsam is boiled with water, in a thin film, for some hours, it will become a brittle resin on cooling, but it will remain viscid if mixed with castor cil ; 3 , if a drop of the oil on white paper be held over a lamp, at a proper distance, its volatile oil will evaporate and leave the brittle resin, without causing any stain aronud, which the presence of oilwill produce; 4 , when three drops of the balsam are poured into a watch-glass, alongside of one drop of sulphuric acid, it becomes yellow at the point of contact, and altogether of a safiron hue when stirred about with a glass rod, but if sophisticated with castor oil, the mixture soon becomes nearly colorless like white honey, though after some time the acid blackens the whole in either case; 5 , if 3 parts in bulk of the balsam be mixed with 1 of good water of ammonia (of $0.970 \mathrm{sp}$. grav.) in a glass tube, it will form a transparent solution, if it be pure, but will form a white liniment if it contain castor oil ; 6 , if the balsam be triturated with a little of the common magnesia alba, it will form a clear solution, from which acids dissolve out the magnesia, and leave the oil transparent if it be pure, but opaque if it be adulterated. When turpentine is employed to falsify the balsam, the fraud is detected by the smell on heating the compound.

BARILLA. Imported in $1841,2,131$ tons; in $1842,2,141$. Retained for consumption, 2,369 and 2,139 , respectively.

BEER. The Germans from time immemorial have been habitually beer-drinkers, and have exercised much of their technical and scientific skill in the production of beer of many different kinds, some of which are little known to our nation, while one at least, called Bararian, possesses excellent qualities, entitling it to the attention of all brewers and consumers of this beverage. The peculiarities in the manufacture of $\mathrm{Ba}$ varian beer have recently attracted the attention of the riost eminent chemists in Germany, especially of Professor Liebig, and much new light has thereby been thrown upon this curious portion of vegetable chemistry, which I shall endeavor to reflect upon the present article.

The following is a list of the principal beers at present brewed in Germany :-

1. Brown beer of Merseburg; of pure barley malt.

2. - - barley malt and beer-root sugar.

3. - barley malt, potatoês, and beet-root syrup.

4. - $\quad$ refined beet-root syrup alone.

5. Covent or thin beer.

6. Berlin white beer, or the Champagne of the north.

7. Broyhan, a famous Hanoverian beer.

8. Double beer of Grünthal.

9. Bavarian beer; 1 . Summer beer; 2. Winter beer.

10. - Bock-beer.

11. Wheat Lager-beer (slowly fermented).

12. White bitter beer of Erlangen.

Considerable interest among men of science, in favor of the Bavarian beer process, has been excited ever since the appearance of Liebig's Organic Chemistry, first published about three years ago. In the introduction to this admirable work, he says :

"The beers of England and France, and the most part of those of Germany, become gradually sour by contact of air. This defect does not belong to the beers of Bavaria, which may be preserved at pleasure in half-full casks, as well as full ones, without alteration in the air. This precious quality must be ascribed to a peculiar process employed for fermenting the wort, called in Germany untergährung, or fermentation from below; which has solved one of the finest theoretical problems.

"Wort is proportionally richer in soluble gluten than in sugar.* When it is set to ferment by the ordinary process, it evolves a large quantity of yeast, in the state of a thick froth, with bubbles of carbonic acid gas attached to it, whereby it is floated to the surface of the liquid. This phenomenon is easily explained. In the body of the wort alongside of particles of sugar decomposing, there are particles of gluten being oxidized

* It does not surely contain more gluten than it does sugar; at least no experiments, known to me, arove this proposition. 
at the same time, and enveloping as it were the former particles, whence the carbonic acid of the sugar and the insoluble ferment from the gluten being simultaneously produced, should mutually adhere. When the metamorphosis of the sugar is completed, there remains still a large quantity of gluten dissolved in the fermented liquor, which gluten, in virtue of its tendency to appropriate oxygen, and to get decomposed, induces also the transformation of the alcohol into acetic acid (vinegar). But were all the matters susceptible of oxidizement as well as this vinegar ferment removed, the beer would thereby lose its faculty of becoming sour. These conditions are duly fulfilled in the process followed in Bavaria.

- "In that country the malt-wort is set to ferment in open backs, with an extensive surface, and placed in cool cellars, having an atmospheric temperature not exceedins $8^{\circ}$ or $10^{\circ}$ centigrade $\left(46 \frac{1}{2}^{\circ}\right.$ or $50^{\circ} \mathrm{F}$.). The operation lasts from 3 to 4 weeks; the carbonic acid is disengaged, not in large bubbles that burst on the surface of the liquid, but in very small vesicles, like those of a mineral water, or of a liquor saturated with carbonic acid, when the pressure is removed. The surface of the fermenting wort is always in contact with the oxygen of the atmosphere, as it is hardly covered with froth, and as all the yeast is deposited at the bottom of the back under the form of a very viscid sediment, called in German unterhefe.

"In order to form an exact idea of the difference between the two processes of fermentation, it must be borne in mind that the metamorphosis of gluten and of azotized bodies in general is accomplished successively in two principal periods, and that it is in the first that the gluten is transformed in the interior of the liquid into an insoluble ferment, and that it separates alongside of the carbonic acid proceeding from the sugar. This separation is the consequence of an absorption of oxygen. It is, however, hardly possible to decide if this oxygen comes from the sugar, from the water, or even from an intestine change of the gluten itself, or, in other words, whether the oxygen corrbines directly with the gluten, to give it a higher degree of oxidation, or whether it lays hold of its hydrogen to form water.

"This oxidation of the gluten, from whichever cause, and the transformation of the sugar into carbonic acid and alcohol, are two actions so correlated, that by an exclusion of the one, the other is immediately stopped."

The superficial ferment (oberhefe in German) which covers the surface of the fermenting works is gluten oxidized in a state of putrefaction; and the ferment of deposite is the gluten oxidized in a state of érémacausie.

The surface yeast, or barm, excites in liquids containing sugar and gluten the same alteration which itself is undergoing, whereby the sugar and the gluten suffer a rapid and tumultuous metamorphosis. We may form an exact idea of the different states of these two kinds of yeast by comparing the superficial to vegetable matters putrefying at the bottom of a marsh, and the bottom yeast to the rotting of wood in a state of érémacausie, that is, of slow combustion. The peculiar condition of the elements of the sediment ferment causes them to act upon the elements of the sugar in an extremely slow manner, and excites the change into alcohol and carbonic acid, without that of the dissolved gluten.

Sugar, which at ordinary temperatures has no tendency to combine with oxygen, enters in the above predicament into fermentation; but the action is rendered much slower by the low temperature, while the affinity of the dissolved gluten for the oxygen of the air is aided by the contact of the sediment. The superficial yeast may be removed without stopping the fermentation, but the under yeast can not be removed without arresting all the phenomena of disoxidation of the second period. These would immediately cease; and if the temperature were now raised, they would be succeeded by the phenomena of the first period. 'The deposite does not excite the phenomena of tumnltuous fermentation, for which reason it is totally unfit for panification (breadbaking), while the superficial yeast alone is suitable to this purpose.

If to wort at a temperature of from $46 \frac{1}{2}^{\circ}$ to $50^{\circ} \mathrm{F}$. the top yeast be added, a quiet slow fermentation is produced, but one accompanied with a rising up of the mass, while yeast collects both at the surface and bottom of the backs. If this deposite be removed to make use of it in other operations, it requires by little and little the characters of the unterhefe, and becomes incapable of exciting the phenomena of the first fermenting period, causing only, of $59^{\circ} \mathrm{F}$., those of the second; namely, sedimentary fermentation. It must be carefully observed that the right unterhefe is not the precipitate which falls to the bottom of backs in the ordinary fermentation of beer, but is a matter entirely different. Peculiar pains must be taken to get it genuine, and in a proper condition at the commencement. Hence the brewers of Hessia and Prussia, who wished to make Bavarian beer, found it more to their interest to send for the article to Wurtzburg, or Bamberg, in Bavaria, than to prepare it themselves. When once the due primary fermentation has been established and well regulated in a brewery, abundance of the true unterhefe may be obtained for all fusure operations. 
In a wort made to ferment at a low temperature with deposite only, the presence of the unterhefe is the first condition essential to the metanorphosis of the saccharum, but it is not competent to bring about the oxidation of the gluten dissolved in the wort, and its transformation into an insoluble state. This change must be accomplished at the cost of the atmospherical oxygen.

In the tendency of soluble gluten to absorb oxygen, and in the free access of the air, all the conditions necessary for its érémacausis, or slow combustion, are to be found. It is known that the presence of oxygen and soluble gluten are also the conditions of acetification (vinegar-making), but they are not the only ones; for this process requires a temperature of a certain elevation for the alcohol to experience this slow combustion. Hence, by excluding that temperature, the combustion (oxidation) of alcohol is obstructed, while the gluten alone combines with the oxygen of the air. This property does not belong to alcohol at a low temperature, so that during the oxidation in this case of the gluten, the alcohol exists alongside of it, in the same condition as the gluten alongside of sulphurous acid in the muted wines. In wines not impregnated with the fumes of burning sulphur, the oxygen which would have combined at the same time with the gluten and the alcohol does not seize either of them in wines which hare been subjected to mutism, but it unites itself to the sulphurous acid to convert it into the sulphuric. The action called sedimentary fermentation is therefore merely a simultaneous metamorphosis of putrefaction and slow combustion; the sugar and the unterhefe putrefy, and the soluble gluten gets oxidized, not at the expense of the oxygen of the water and the sugar, but of the oxygen of the air, and the gluten then falls in the insoluble state. The process of Appert for the preservation of provisions is founded upon the same principle as the Bavarian process of fermentation; in which all the putrescible matters are separated by the intervention of the air at a temperature too low for the alcohol to become oxidized. By removing them in this way, the tendency of the beer to grow sour, or to suffer a further change, is prevented. Appert's method consists in placing in presence of vegetables or meat which we wish to preserve the oxygen at a high temperature, so as to produce slow combustion, but without putrefaction or even fermentation. By removing the residuary oxygen after the combustion is finished, all the causes of an ulterior change are removed. In the sedimentary fermentation of beer, we remove the matter which experiences the combustion; whereas, on the contrary, in the method of Appert, we remove that which produces it.

It is uncertain whether the dissolved gluten, in being converted into insoluble yeast by the action of the oxygen, combines directly with the oxygen; that is to say, whether the yeast differs from the soluble gluten merely by having absorbed an additional quantity of oxygen. This question is in fact very difficult to solve by analysis. If the gluten be regarded as a hydrogenated combination, it is obvious that in the fermentation of wine-must, and malt-wort, the hydrogen will be carried off by the oxygen, and the action will then be the same as the transformation of alcohol into aldehyde. When the contact of the atmosphere is excluded, this oxygen can not evidently be derived from the elements of the air, or from those of the water; for it can not be supposed that oxygen will take hydrogen from the water, in order to recompose water with the hydrogen of the gluten. The elements of the saccharum must therefore furnish this oxygen; or in the course of the formation of the yeast, a portion of the sugar will be decomposed; but thts decomposition is not of the same kind as that which results from the immediate metamorphosis of the sugar into carbonic acid and alcohol; hence a certain portion of the sugar will afford neither alcohol nor carbonic acid, but it will yield less oxgenated products from its elements. These products occasion the great difference in the qualities of fermented liquors, and particularly in their alcoholic strength. In the ordinary fermentation of grape-juice and worts, these liquids do not furnish a quantity of alcohol equivalent to the sugar which they contain, because a certain portion of the sugar serves for the oxidation of the gluten, and is not transformed like the rest. But whenever the liquor has arrived at the second period, the product in alcohol ought to be equivalent to the quantity of sugar present, as happens in all fermentations which are not accompanied with a formation, but a disappearance of the yeast. It is well ascertained that worts furnish in the Bavarian breweries 10 or 20 per cent. more alcohol than they do by the ordinary process of fermentation. It is also a well-established fact that in the manufacture of spirits from potatoes, where no yeast is produced, or merely a quantity corresponding to the proportion of barley-malt added to the potato-wort, a quantity of alcohol may be produced, as also of carbonic acid, corresponding exactly to the quantity of carbon in the fecula employed. But, on the contrart, in the fermentation of beet-root juice, it is hardly possible to determine precisely, from the quantity of carbonic acid erolved, the quantity of sugar contained in the beets, for there is always less carbonic acid than the juice of the fresh root would furnish. In equal volumes, the beer made by the unterhefe process contains more alcohol, and is therefore more heady than that formed by the ordinary process. 
The tempersture at which fermentation is carried on has a very marzed influence upon the quantity of alcohol produced. It is known that the juice of beets set to ferment between $86^{\circ}$ and 95 Fahr. does not yield alcohol, and its sugar is replaced by a less oxygenated substance, mannite, and lactic acid, resulting from the mucilage. In proportion as the temperature is lowered the mannite fermentation diminishes. As to azotized juices, however, it is hardly possible to define the conditions under which the transformation of the sugar will take place, without being accompanied with another decomposition which modifies its products. The fermentation of beer by deposite demonstrates that by the simultaneous action of the oxygen of the air and a low temperature, the metamorphosis of sugar is effected in a complete manner; for the vessels in which the operation is carried on are so disposed that the oxygen of the air may act upon a surface great enough to transform all the gluten into insoluble yeast, and thus to present to the sugar a matter constantly undergoing decomposition. The oxidizement of the dissolved gluten goes on, but that of the alcohol requires a higher temperature; whence it can not suffer eremacausis, that is, acetification, or conversion into vinegar.

At the beginning of the fermentation of must and wort, the quantity of matter undergoing change is obviously the largest. All the phenomena which accompany it, the disengagement of gas and the rise of temperature, are most active at this period, and in proportion as the decomposition adrances, the external signs of it become less perceptible, without, however, disappearing completely before the transformation has reached its limit. The slow and continuous decomposition which succeeds to the rapid and violent disengagement of gases is denominated the after or complementary fermentation. For wine and beer it lasts till all the sugar has disappeared, so that the specific gravity of the liquors progressively diminishes during several months. This slow fermentation is in most cases a truly depositary fermentation; for by the progressive decomposition of the less, the sugar still in solution gets completely transformed; but when the air is excluded, that decomposition does not occasion the com. plete separation of the azotized matters in an insoluble shape.

In several states of the German confederation, the favorable influence of a rationa] process of fermentation upon the quality of the beers has been fully recognised. In the Grand Dutchy of Hesse considerable premiums were proposed for the brewing of beer according to the process pursued in Bararia, which were decreed to those brewers who were able to prove that their product (neither strong nor highly hopped) had hept six months in the casks without becoming at all sour. When the first trials were being made several thousand barrels were spoiled, till eventually experience led to the discovery of the true practical conditions which theory had foreseen and prescribed.

Neither the richness in alcohol, nor in hops, nor both combined, can hinder ordinary beer from getting tart. In England, says Liebig, an immense capital is sacrificed to preserve the better sorts of ale and porter from souring, by leaving them for several years in enormous tuns quite full, and very well closed, while their tops are covered with sand. This treatment is identical with that applied to wines to make them deposite the wine-stone. A slight transpiration of air goes on in this case through the pores of the wood; but the quantity of azotized matter contained in the beer is so great, relatively to the proportion of oxygen admitted, that this element can not act upon the alcohol. And yet the beer thus managed will not keep sweet more than two months in smaller casks to which air has access. The grand secret of the Munich brewers is to conduct the fermentation of the wort at $100 \mathrm{low}$ a temperature to permit of the acetification of the alcohol, and to cause all the azotized matters to be completely separated by the intervention of the oxygen of the air, and not by the sacrifice of the sugar, It is only in March and October that the good store beer is begun to be made in Bavaria.

In our ordinary breweries, the copious disengagement of carbonic acid from the frothy top of the fermenting tuns and gyles prevents the contact of oxygen from the worts; so that, as the gluten can not be oxidized by the air, it attracts oxygen from the sugar, and thus gives rise to several adventitious hydrogenated products, just as the fetid oil is generated in the rapid fermentation of spirit-wash by the distillers. In this case no inconsiderable portion of the gluten remains undecomposed in the beer, which, by its extreme proneness to corruption, afterward attracts oxygen greedily from the air, and, at temperature above $52^{\circ}$, imparts this contact action to the alcohol, and, by a species of infection, changes it into vinegar. Indeed, in most of the rapid fermentations a portion of vinegar is formed, which itself serves as an acetous ferment to the rest of the alcohol; whereas the result of the bottom fermentation is a beer free from vinegar, and certainly hardly a trace of gluten; so that it does not possess the conditions requisite to intestine change or deterioration. This perfection is, however, in my opinion, rarely attained. In my several journeys into Germany I have met with much spurious or ill-made Bavarian beer. The best contains, when brought to England, a little acil, 
but no perceptible gluten on the addition of ammonia in excess. Most of our beers, ales, \&cc., deposite more or less gluten when thus treated.

The following table exhibits the results of the chemical examinations of the undermentioned kinds of beer :-

\begin{tabular}{|c|c|c|c|c|c|}
\hline \multirow{2}{*}{ Name of the Beer. } & \multicolumn{4}{|c|}{ Quantity in 100 parts by weight } & \multirow{2}{*}{ Analyst. } \\
\hline & Water. & Malt extr. & Alcohol. & Carb. acid. & \\
\hline Augustine double beer- & $88 \cdot 86$ & $8 \cdot 0$ & $3 \cdot 6$ & $0 \cdot 14$ & Kaiser. \\
\hline $\begin{array}{l}\text { Munich - } \\
\text { Salvator beer - do. }\end{array}$ & $87 \cdot 62$ & $8 \cdot 0$ & $4 \cdot 2$ & $0 \cdot 18$ & Do. \\
\hline $\left.\begin{array}{l}\text { Bock-beer, from the Royal } \\
\text { brewery - do. }\end{array}\right\}$ & $88 \cdot 64$ & $7 \cdot 2$ & $4 \cdot 0$ & $0 \cdot 16$ & Do. \\
\hline $\left.\begin{array}{l}\text { Scheak (pot) beer, from a Ba- } \\
\text { varian country brewery; a } \\
\text { kind of small beer }\end{array}\right\}$ & $92 \cdot 94$ & $4 \cdot 0$ & $2 \cdot 9$ & $0 \cdot 16$ & Do. \\
\hline $\left.\begin{array}{l}\text { Bock-beer of Brunswick, of } \\
\text { the Bararian kind - }\end{array}\right\}$ & $88 \cdot 50$ & 6.50 & $5 \cdot 0$ & & Balhorn. \\
\hline $\begin{array}{l}\text { Lager (store) beer, of Bruns- } \\
\text { wiek, of the Bavarian kind }\end{array}$ & $91 \cdot 0$ & $5 \cdot 4$ & $3 \cdot 50$ & - & Otto. \\
\hline Brunswick sweet small beer & $84 \cdot 70$ & $14 \cdot 0$ & $1 \cdot 30$ & - - & Do. \\
\hline Brunswick mum & $59 \cdot 2$ & $39 \cdot 0$ & 1.80 & $0 \cdot 1$ & Kaiser. \\
\hline
\end{tabular}

Malting in Munich.-The barley is steeped till the acrospire, embryo, or seed-germ, seems to be quickened; a circumstance denoted by a swelling at the end of that ear which was attached to the foot-stalk, as also when, on pressing a pile between two fingers against the thumb-nail, a slight projection of the embryo is perceptible. As long, however, as the seed-germ sticks too firm to the husk, it has not been steeped enough for exposure on the underground malt-floor. Nor can deficient steeping be safely made up for afterward by sprinkling the malt-couch with a watering-can, which is apt to render the malting irregular. The steep-water should be changed repeatedly, according to the degree of foulness and hardness of the barley; first, six hours after immersion, having preriously stirred the whole mass several times; afterward, in winter, every twenty-four hours, but in summer every twelve hours. It loses none of its substance in this way, whatever vulgar prejudice may think to the contrary. After letting off the last water from the stone cistern, the Bavarians leave the barley to drain in it during four or six hours. It is now taken out, and laid on the couch floor, in a square heap, eight or ten inches high, and it is turned over, morning and evening, with dexterity, so as to throw the middle portion upon the top and bottom of the new-made couch. When the acrospire has become as long as the grain itself, the malt is carried to the withering (welkboden) or drying-floor, in the open air, where it is exposed (in dry weather) during from eight to fourteen days, being daily turned over three times with a winnowing shorel. It is next dried on a well-constructed cylinder or flueheated malt-kin, at a gentle clear heat, without being browned in the slightest degree, while it turns friable into a fine white meal. Smoked malt is entirely rejected by the best Bararian brewers. Their malt is dried on a series of wove wire horizontal shelves, placed orer each other; up through whose interstices or perforations streams of air, heated to only $122^{\circ} \mathrm{Fahr}$., rise from the surfaces of rows of hot sheet-iron pipe-flues, arranged a little way below the shelves. Into these pipes the smoke and burned air of a little furnace on the ground are admitted. The whole is enclosed in a raulted chamber, from whose top a large wooden pipe issues, for conveying away the steam from the drying malt. Each charge of malt may be completely dried on this kiln in the space of from eighteen to twenty-four hours, by a gentle uniorm heat, which does not injure the diastase, or discolor the farina.*

The malt for store-beer should be kept three months at least before using it, and be freed by rubbing and sifting from the acrospires before being sent to the mill, where it should be crushed pretty fine. The barley employed is the kest distichon or common kind, styled hordeum vulgare.

The hops are of the best and freshest growth of Bavaria, called the fine spalter, or raatse, Bohemian townhops, and are twice as dear as the best ordinary hops of the rest of Germany. They are in such esteem as to be exported even into France.

The Bavarians are so much attached to the beer beverage, which they have enjoyed from their remotest ancestry, that they regard the use of distilled spirits, even in moderation, as so immoral a practice, as to disqualify dram-drinkers for decent society.

* I have a set of designs of the Bavarian kiln, but I believe the above description will make its construction sufficiently intelligible. 
Their government has taken great pains to improve this national beverage, by encouraging the growth of the best qualities of hops and barley. The vaults in which the beer is fermented, ripened, and kept, are all underground, and mostly in stony excavations, called felsenkeller or rock-cellars. 'The beer is dirided into two sorts, ealled summer and winter. The latter is light, and, being intended for immediate retail in tankards, is termed schankbier. The other, or the lagerbier, rery sensibly increases in vinous strength in proportion as it decreases in sweetness, by the judicious management of the nachgährung, or fermentation in the casks. In several parts of Germany a keeping quality is communicated to beers by burning sulphur in the casks before filling them, or by the introduction of sulphite of lime. But the flavor thus imparted is disliked in Munich, Bayreuth, Regensburg, Nürnberg, Hof, and the other chief towns of Bararia; instead of which a preservative virtue is sought for in an aromatic mineral or Trrol pitch, with which the insides of the casks are carefully coated, and in which the ripe beer is kept and exported. In December and January, after the casks are charged with the summer or store-beer, the double doors of the cellars are closed, and lumps of ice are piled up against them, to prevent all access of warm air. The cellar is not opened till next August, in order to take out the beer for consumption. In these circumstances the beer becomes transparent like champagne wine; and, since but little carbonic acid gas has been disengaged, little or none of the additionally generated alcohol is lost by evaporation.

The winter or schank (pot) beer is brewed in the months of October, Norember, March, and April; but the summer or store-beer in December, January, and February, or the period of the coldest weather. For the former beer, the hopped worts are cooled down only to from $51^{\circ}$ to $55^{\circ}$, but for the latter to from $41^{\circ}$ to $42 \frac{12^{\circ}}{\mathrm{F}} \mathrm{Fhr}$. The winter beer is also a little weaker than the summer beer, being intended to be sooner consumed; since four bushels* (Berlin measure) of fine, dry, sifted malt, of large heary hordeum vulgare distichon, affords seven eimers of winter beer, but not more than from fire and a half to six of summer beer. $\dagger$ At the second infusion of the worts, small beer is obtained to the amount of twenty quarts from the above quantity of malt. For the above quantity of winter beer, six pounds of middling hops are reckoned sufficient; but for the summer beer, from seren to eight pounds of the finest hops. The winter beer may be sent out to the publicans in barrels five days after the fermentation has been completed in the tuns, and, though not quite clear, it will become so in the course of six dars; jet they generally do not serve it out in pots for two or three weeks. But the summer beer must be perfectly bright and still before it is racked off into casks for sale.

Statement of the Products of a Brewing of Bavarian Beer.-The quantity brewed is 41 Munich eimers (64 maass) $=85 \frac{1}{2}$ Berlin quarts ; and 60 Berlin quarts $=1$ eimer; or 24 Munich barrels (of 100 Berlin quarts each); 1 Munich eimer =15 gallons imperial. The beer contains from 50 to 60 parts by weight, of dry saccharum in 1,000 parts.

\section{Expenditure.}

24 Berlin bushels of white kiln-dried barley, rather finely crashed, weighing from 12 to $13 \mathrm{cwts}$.

36 pounds of new fine spalter (parted) hops at 46 thalers the cwt.

$\frac{1}{2}$ pound of Carageen moss, for clarifying

1 quart of yeast.

1 quart of Tyrol pitch - - - rate of 20 silbergroschen $=2 s$., the cwt.

Cost of erushing

Fuel

Wages of labor, in the brewhouse and vault

Do. Do. for cooper in pitching the casks

Sundry small expenses .

Or 11l. 8 s.

1 thaler $=30$ silbergroschen $=3$ shillings

Thaler. Slbg.

$\begin{array}{lrr}- & 24 & 0 \\ - & 16 & 17\end{array}$

$\begin{array}{rr}- & 16 \\ -\quad & 0\end{array}$

110

\begin{tabular}{rrr}
- & 80 \\
- & 1 & 0 \\
- & 4 & 0 \\
- & 6 & 0 \\
- & 3 & 0 \\
$-\quad 2$ & 10 \\
\hline & 76 & 0
\end{tabular}

Deduct for the grains of $12 \mathrm{cwts}$. of malt, at 10 subergrozcnen, or 1 s. per cwt. $=4$ thalers, and for the value in yeast produced $=2$ thalers more -

Total neat expenditure $=10 l .10 \mathrm{~s}$.

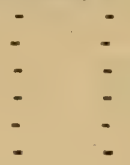

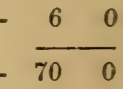

* An English quarter of grain is equal to 5 bushels (scheffel) and nearly one third Prussian measure +1 Eimer Prussian = 15 English imperial gallons ; one Munich scheffel is equal to four Berlin scheffels. 1 Lit Munich $=1 \cdot 235$ Eng. lbs. Avoird.: 1 Lib. Berlin $=1.031$ lbs. Avoird. 
This cost for 42 eimers ( 1 eimer $=14 \frac{3}{4}$ galls. Imp. $)=619 \frac{1}{2}$ gallons $=17 \cdot 2$ London norter barrels, amounts to $4 \frac{1}{2} d$. per gallon, or $12 s$. $2 d$. per barrel. By the above reckioning, a good profit acciues to the brewer, after allowing a liberal sum for the rent of premises, interest of capital, \&c.

He has less profit from the summer beer. For a brewing of 33 eimers $=505$ gallons Imp., containing from 60 to 65 pounds of saccharum in 1,000 pounds of the beer, by Hermstaedt's saccharometer.

\section{Expendilure.}

24 Berlin scheffels of white kiln-dried barley-malt, weighing from 12 to 13 centners* - $\quad$ - $\quad$ - $\quad$ - $\quad$ - $\quad$ - $\quad$ - 240

48 Berlin pounds of fresh Bavarian fine hops, at 46 thaler per centner $\begin{array}{lll}20 & 0\end{array}$

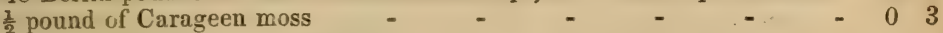
1 quart setting yeast (unterhefe). 1 centner pitch

Malt tax on 12 centners

Crushing ${ }^{\circ}$ the malt Fuel $-$. Wages, 6 thalers; coopers' do., 3 thalers; and sundries, 3 th. 27 sq.

Thaler. Slbg.

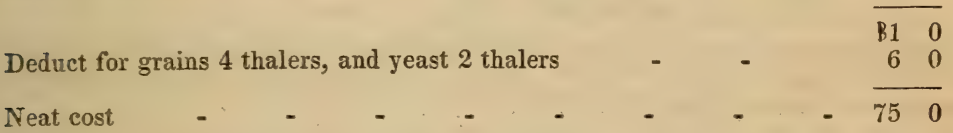

This cost of $1 \mathrm{il}$. $5 \mathrm{~s}$. for 505 gallons amounts to fully $5 \frac{1}{3} d$. per gallon, and $16 \mathrm{~s} .6 d$. the barrel.

The cost at Munich is $2 \frac{1}{2}$ thalers the eimer, and 4 thalers the barrel. The eimer of the summer beer, or lagerbier, is sold for 4 thalers. The publicans there, as in London, are known to add more or less water to their beer before retailing it.

The reast (unterhefe) is carefully frced by a scraper from the portions of light top yeast that may hare fallen to the bottom; the true unterhefe is then carefully sliced off from the slimy sediment on the wood.

In Munich the malt is moistened slightly 12 or 16 hours before crushing it, with from 2 to 3 maast of water for every bushel; the malt being well dried, and several months old. The mash-tun into which the malt is immediately conveyed is, in middle-sized breweries, a round oaken tub, about $4 \frac{1}{2}$ feet deep, 10 feet in diameter at bottom and 9 at top, outside measure, containing about 6,000 Berlin quarts. Into this tun cold water is admitted late in the evening, to the amount of 25 quarts for each scheffel, or 600 quarts for the 24 scheffels of the ground malt, which are then shot in and stirred about and worked well about with the oars and rakes, till a uniform pasty is formed without lumps. It is left thus for three or four houss; 3,000 quarts of water being put into the copper, and made to boil; and 1,800 quarts are gradually run down into the mash-tun, and worked about in it, producing a mean temperature of $142.5^{\circ} \mathrm{Fahr}$. After an hour's interval, during which the copper has been kept full, 1,800 additional quarts of water are run into the tun, with suitable mashing. The copper being now emptied of water, the mash-mixture from the tun is transferred into it, and brought quickly to the boiling point, with careful stirring to prevent its setting on the bottom and getting burned, and it is kept at that temperature for half an hour. When the mash rises by the ebullition, it needs no more stirring. This process is called, in Bavaria, boiling the thick mash, dickmaisch kochen. The mash is next returned to the tun, and well worked about in it. A few barrels of a thin mash-wort are kept ready to be put into the copper the moment it is emptied of the thick mash. After a quarter of an hour's repose the portion of liquid filtered through the sieve-part of the bottom of the tun into the wort-cistern is put into the copper, thrown back boiling hot into the mash in the tun, which is once more worked thoroughly.

The copper is next cleared out, filled up with water, which is made to boil for the after or small-beer brewing. After two hours settling in the open tun, the worts are drawn off clear.

Into the copper, filled up one foot high with the wort, the hops are introduced, and the mixture is made to boil during a quarter of an hour. This is called roasting the hops. The rest of the wort is now put into the copper, and boiled along with the hops during at least an hour or an hour and a half. The mixture is then laded out through the hop-filter into the cooling-cistern, where it stands three or four inches deep, and is exposed upon an extensive surface to natural or artificial currents of cold air, so as to

* 1 Centner $=110$ Prussian pounds $=113.44 \mathrm{lbs}$. Avoird.

+ A Bavarian mas $=1 \frac{1}{4}$ quarts English measure. 
be quickly cooled. For every 20 barrels of lagerbier, there are allowed 10 of small beer; so that 30 barrels of wort are made in all.

For the winter or pot-beer the worts are brought down to about $59^{\circ} \mathrm{Fahr}$. in the cooler, and the beer is to be transferred into the fermenting-tuns at from $54.5^{\circ}$ to $59^{\circ}$ Fahr.; for the summer or lagerbier, the worts must be brought down in the cooler to from $43^{\circ}$ to $45 \frac{1}{2}^{\circ}$, and put into the fermenting-tuns at to from $41^{\circ}$ to $43^{\circ} \mathrm{Fahr}$.

A few hours beforehand, while the wort is still at the temperature of $63 \frac{1}{2}{ }^{\circ}$ Fahr., a quantity of lobb must be made, called vorstellen (fore-selting) in German, by mixing the proportion of unterhefe (yeast) intended for the whole brewing with a barrel or a barrel and a half of the worts, in a small tub called the gähr-tiene, stirring them well together, so that they may immediately run into fermentation. This lobb is in this state to be added to the worts. The lobb is known to be ready when it is covered with a white froth from one quarter to one half an inch thick; during which it must be well covered up. The large fermenting-tun must in like manner be kept covered, even in the vault. The colder the worts, the more yeast must be used. For the above quantity, at

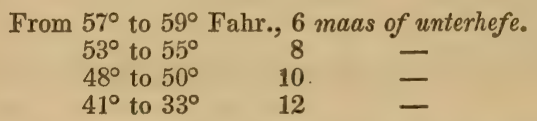

Some recommend that wort for this kind of fermentation (the untergährung) should be set with the yeast at from $48^{\circ}$ to $57^{\circ}$; but the general practice at Munich is to set the summer lager beer at from $41^{\circ}$ to $43^{\circ} \mathrm{F}$.

By following the preceding directions, the wort in the tun should, in the course of from twelve to twenty-four hours, exhibit a white froth round the rim; and even a slight whiteness in the middle. After another twelve or twenty-four hours, the froth should appear in curls; and, in a third like period, these curls should be changed into a stin higher frothy brownish mass. In from twenty-four to forty-eight hours more, the barm should have fallen down in portions through the beer, so as to allow it to be seen in certain points. In this case it may be turned over into the smaller ripening tuns in the course of other five or six days. But when the worts have been set to ferment at from $41^{\circ}$ to $43^{\circ} \mathrm{Fahr}$, they require from eight to nine days. The beer is transferred, after being freed from the top yeast by a skimmer, by means of the stopcock near the bottom of the large tun. It is either first run into an intermediate vessel, in order that the top and bottom portions may be well mixed, or into each of the lager casks, in a numbered series, like quantities of the top and bottom portions are introduced. In the ripening cellars the temperature can not be too low. The best keeping beer can nerer be brewed unless the temperature of the worts at setting, and of course the fermentingvault, be as low as $50^{\circ} \mathrm{F}$. In Bavaria, where this manufacture is carried on under government inspectors, a brewing period is prescribed by law, which is, for the under fermenting lager beer, from Michaelmas (29th September) to St. George (23d April). From the latter to the former period the ordinary top-barm beer alone is to be made. The ripening-casks must not be quite full, and they are to be closed merely with a loose bung, in order to allow of the working over of the ferment. But should the fermentation appear too languid, after six or eight days, a little briskly fermenting lager beer may be introduced. The store lager beer-tuns are not to be quite filled, so as to prevent all the yeasty particles from being discharged in the ripening fermentation: but the pot lager beer-tuns must be made quite full, as this bererage is intended for speedy sale within a few weeks of its being made.

As soon as the summer beer-vaults are charged with their ripening-casks, and with ice-cold air, they are closed air-tight with triple doors, having small intervals between, so that one may be entered and shut again, before the next is opened. These raults are sometimes made in ranges radiating from a centre, and at others in rooms set of at right angles to a main gallery; so that in either case, when the external opening is well secured, with triple air-tight doors, it may be entered at any time, in order to inspect the interior, without the admission of warm air to the beer-barrels. The wooden bungs for loosely stopping them must be coated with the proper pitch, to prevent the possibility of their imparting any acetous ferment. In the Beer Brever* of A. F. Zimmermann, teacher of theoretical and practical brewing, who has deroted thirty-five years to this business, it is stated, that a ripened tun of lager or store-beer must be racked off all at once, for when it is left half full it becomes flat (schaal); and that the tun of pot lager beer must, if possible, be all drunk off in the same day it is tapped; because on the following day the beer gets an unpleasant taste, even when the bung has not been taken out, but only a small hole has been made, which is - opened only at the time of druving the beer, and is immediately closed again with a

- Der Bier-Brauer, als Meister in seinem fache, \&c., illustrates with many plates, Berlin, 1842. 
gpıgot. He ascribes this change to the loss of the carbonic acid gas, with wnich the beer has got strongly impregnated during the latter period of its ripening, while being kept in tightly-bunged casks. The residuums in these casks are, however, bottled up in Bavaria, whereby the beer, after some time, recovers its brisk and pungen taste. But the beer-topers in Bavaria, who are professedly very numerous, indulg: so delicate and fastidious a palate, that when assembled in their favorite pothouse, they wait impatiently for the tapping of a fresh cask, and cease for a while to tipple w: enever it is half empty, puffing the time away with their pipes till another fresh tap be made. In the well-frequented beer-shops of Munich a common-sized cask of lager beer is thus drank off in an hour. A reputation for superior brewing is there the readiest road to fortune.

$B o: k-B e e r$ of Bavaria.-This is a favorite double strong beverage, of the best lager descr ption, which is so named from causing its consumers to prance and tumble about like a buck or a goat; for the German word bock has both these meanings. It is merely a beer having a specific gravity one third greater, and is therefore made with a third greater proportion of malt, but with the same proportion of hops, and flavored with a few coriander-seeds. It has a somewhat darker color than the general lager beer, occasionally brownish, taste less bitter on account of the predominating malt, and somewhat aromatic. It is an eminently intoxicating beverage. It is brewed in December and January, and takes a long time to ferment and ripen; but still it contains too large a quantity of unchanged saccharum and dextrine for its hops, so that it tastes too luscious for habitual topers, and is drunk only from the beginning of May till the end of July, when the fashion and appetite for it are over for the year.

\section{Statement of a Brewing of Bavarian Bock-Beer.}

For 41 Bavarian eimers of 64 maass each (about 15 gallons Imperial) per eimer, or 615 gallons, nearly 17 barrels English in all :-

\section{Expenditure.}

32 Berlin scheffels of the best pale malt freed from its acrospires, weighing $17 \frac{1}{2}$ centners, at 1 thaler per centner

48 lbs. (Berlin) of the best Bavarian hops

$\frac{1}{2}$ lb. Carageen moss for clarifying

1 lb. Coriander-seeds

1 Quart sctting yeast.

1 Centner Tyrolese pitch

Malt-tax

Malt-crushing, fuel, wages, coopering, \&c.

Thalers of $3 s$. each

Deduct for the value of grains and yeast

\section{Thalers of neat cost}

This statement makes the eimer of the Bavarian bock-beer amount to about 2 tha. lers, or 6 shillings; being at the rate of nearly 5 pence per gallon; though without counting rent, interest of capital, or profit. It is, in fact, a malt or barley sweet wine or liqueur; but a very cheap one, as we see by this computation.

The chief difference in the process for making bock-beer lies in the mash-worts, and in the hops being boiled a shorter time, to preserve more of the aroma, and acquire less of the bitterness of the hop. The coriander-seeds are coarsely bruised, and added along with the hops and Carageen moss, to the boiling mash-worts, about twenty or thirty minutes before they are laded or drawn off into the mash-tun. Sometimes the hops are boiled apart in a lictle clear wort, as formerly described. The bock-beer is retailed in Munich at 3 silver groschen, about $3 \frac{1}{2} d$. the seidel, or pot, which is one English pint. The 25 gallon cask (tonne) is sold at 10 thalers, or 30 shillings. The publicans, therefore, have a very remunerating profit per pot, even supposing that they do not reduce the beer with water like our London craftsmen.

Zimmermann assumes the merit of having introduced Carageen moss as a clarifier into the beer manufacture. I do not know whether it may not have been used in this country for the same purpose, or in Ireland, where this fucus (Chondra crispa) grows abundantly. He says that 1 ounce of it is sufficient for 25 gallons of beer; and that it operates, not only in the act of boiling with the hops, but in that of cooling, as also in the squares and backs before the fermentation is begun. Whenever this change, nowever, takes place, the commixture throws up the gluten and moss to the surface of the liquid in a black scum, which is to be skimmed off, so that the proper ycast may 
not be soiled with it. It occasions the separation of much of the vegetable sume, 0 mucilage, called by the German brewers pech (pitch).

On the Clarifying or Clearing of Beers.-Clarifiers act either chemically-by being soluble in the beer, and by forming an insoluble compound with the vegetable gluten, and other viscid vegetable extracts; gelatine and albumen, under one shape or other, have been most used; the former $\mathrm{fc}$ : beer, the latter, as white of egg, for wineor mechanically, by being diffused in fine particles through the turbid lirquor, and, in their precipitation, carrying down with them the floating vegetable matters. To this class belong sand, bone-black (in some measure, but not entirely), and other such articles. The latter means are very imperfect, and can take down only such matters as exist already in an insoluble state; of the former class, milk, blood, glue, calf's-foot jelly, hartshorn-shavings, and isinglass, have been chiefly recommended. Calve's-foot jelly is much used in many parts of Germany, where veal forms so common a kind of butcher-meat; but in summer it is apt to acquire a putrid taint, and to impart the same to the beer. In these islands, isinglass swollen and partly dissolved in vinegar, or sour beer, is almost the sole clarifier, called finings, employed. It is costly, when the best article is used; but an inferior kind of isinglass is imported for the brewers. The solvent or medium through or with which it is administered is eminently injudicious, as it never fails to infect the beer with an acetous ferment. In Germany their tart wine has been used hitherto for dissolving the isinglass; and this has also the same bad property. Mr. Zimmermann professes to have discovered an unexceptionable solrent in tartaric acid, one pound of which dissolved in 24 quarts of water is capable of dissolving two pounds of ordinary isinglass; forming finings which may be afterward diluted with pure water at pleasure. Such isinglass imported from Petersburg into Berlin costs there only $3 s$. per lb. These finings are best added, as already mentioned, to the worts prior to fermentation, as soon as they are let in to the setting. back or tun, immediately after adding the yeast to it. They are best administered by mixing them in a small tub with thrice their rolume of wort, raising the mixture into a froth with a whisk (twig-besom, in German), and then stirring it into the worts. The clarification becomes manifest in the course of a few hours, and when the fermentation is completed, the beer will be as brilliant as can be wished; the test of which with the German topers is when they can read a newspaper while a tall glass beaker of beer is placed between the paper and the candle. One quart of finings of the abore strength will be generally found adequate to the clearing of 100 gallons of well-brewed lager. beer, though it will be surer to use double that proportion of finings. The Carageen moss, as finings, is to be cut in fine shreds, thrown into the boiling thin wort, when the flochs begin to separate, and before adding the hops; after which the boiling is continued for an hour and a half or two hours, as need be. The clarifing with this kind of finings takes place in the cooler, so that a limpid wort may be drawn off into the fermenting back.

Berlin White or Pale Beer (Weiss-bier).-This is the truly patriotic bererage of Prussia Proper, and he is not deemed a friend to his Vaterland who does not swig it. It is brewed from 1 part of barley-malt and 5 parts of wheat-malt, mingled, moistened, and coarsely crushed between rollers. This mixture is worked up first with water at $95^{\circ}$ Fahr., in the proportion of 30 quarts per scheffel of the malt, to which pasty mixture 70 quarts of boiling water are forthwith added, and the whole is mashed in the tun. After it has been left here a little to settle, a portion of the thin liquor is drawn off by the tap, transferred to the copper, and then for each bushel of malt there is added to it a decoction of half a pound of Allmark hops separately prepared. This hopped wort, after half an hour's boiling, is turned back with the hops into the mash-tun, of which the temperature should now be $162 \frac{1}{2}^{\circ}$ Fahr., but not more. In half an hour the wort is to be drawn off from the grains, and pumped into the cooler. The grains are afterward mashed with from 40 to 50 quarts of boiling water per scheffel of malt, and this infusion is drawn off and added to the former worts. The whole mixture is set at $66^{\circ}$ Fahr., with a due proportion of top jeast or ordinary barm, and rery moderately fermented. According to Zimmermann, a very competent judge, this his native beer is very apt to turn sour, and therefore it must be rery speedily consumed. This proneness to acetification is the character of all wheat-malt beers. He recommends, what he himself has made for many jears, a substitution of potato-starch sugar for this sort of malt, and as much tartaric acid as to gire the degree of tartness peculiar to the pale Berlin beer, eren in its best st $z$ t $\triangleq$. This acid moreover prerents the beer from running into the acetous fermentation.

Potato-Beer.- The potatoes being well washed are to be rubbed down to a pulp by such a crating cylinder-machine as is represented in fig. 5, where $a$ is the hopper for receiving the roots (whether potato or beet, as in the French sugar-factories; $b$ is the crushing and grinding-drum; $c$, the handle for turning the spur-wheel $d$, which drives the pinion $e$, and the fly-wheel $f ; g, h$, is the frame. The dotted lines above $c$, are the 


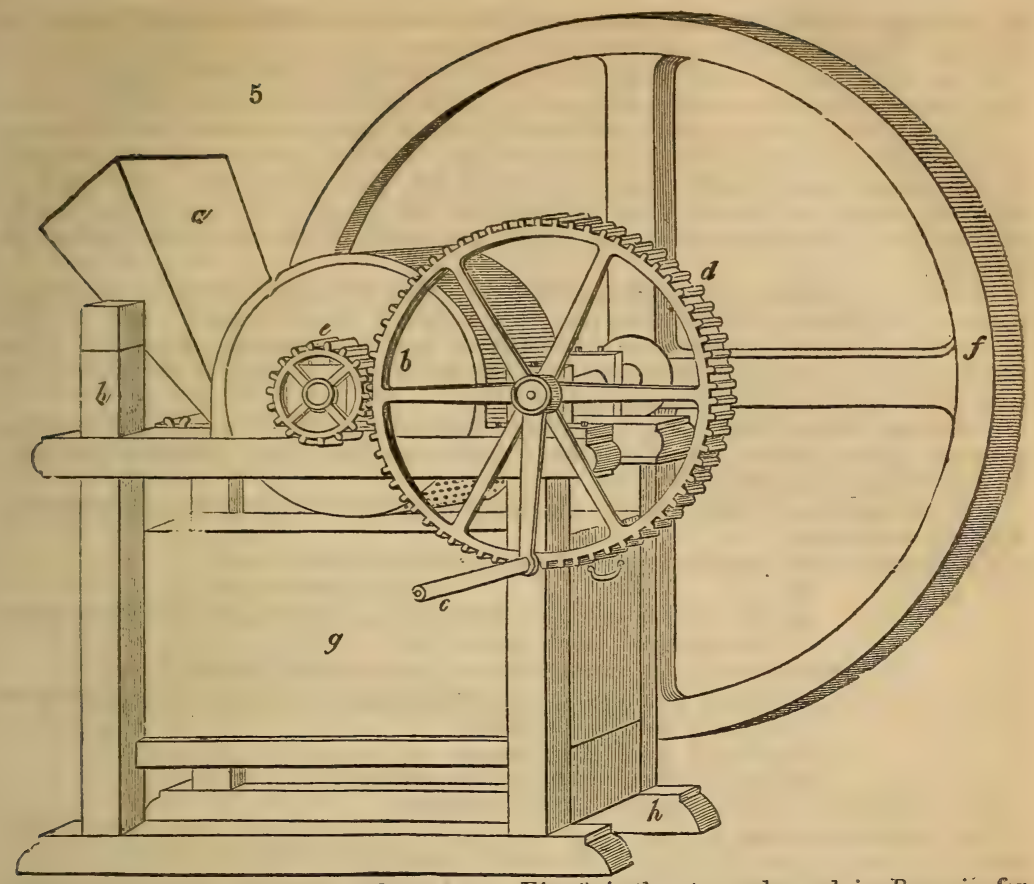

eullender through which the pulp passes. Fig. 6 . is the stopcock used in Bararia for bottling beer. For every scheffel of potatoes 80 quarts of water are to be put with them into the copper, and made to boil.

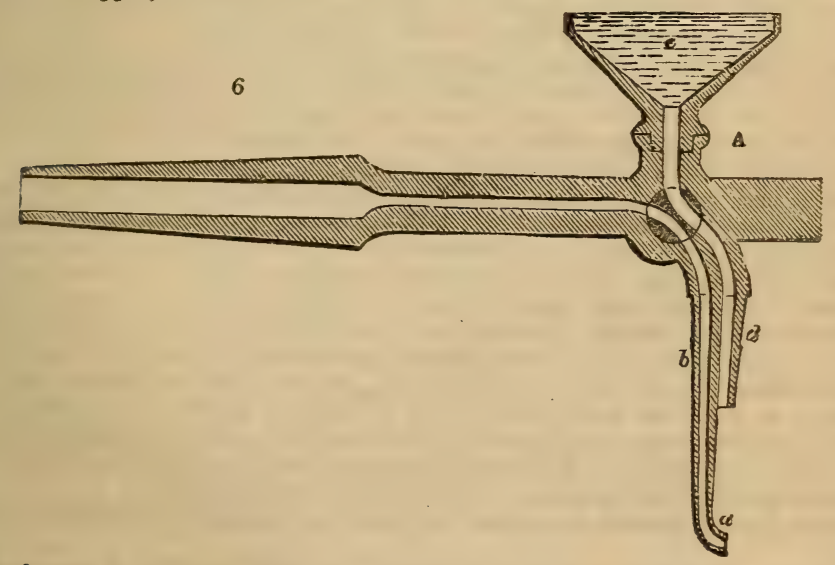

Crushed malt, to the amount of 12 scheffels, is to be well worked about in the unashtun with 360 quarts, or 90 gallons (English) of cold water, to a thick pap, and then 840 additional quarts, or about 6 barrels (English) of cold water are to be successively introduced with constant stirring, and left to stand an hour at rest.

The potatoes having been meanwhile boiled to a fine starch paste, the whole maltmash, thin and thick, is to be speedily laded into the copper, and the mixture in it is to be well stirred for an hour, taking care to keep the temperatnre at from $144^{\circ}$ to $156^{\circ} \mathrm{Fahr}$. all the time, in order that the diastase of the malt may convcrt the starch present in the two substances into sugar and dextrine. This transformation is made manifest by the white pasty liquid becoming transparent and thin. Whenever this 
happens the fire is to be raised, to make the mash boil, and to keep it at this heat for 10 minutes. The fire is then withdrawn, the contents of the copper are to be transferred into the mash, worked well there, and left to settle for half an hour; during which time the copper is to be washed out, and quickly charged once more with boiling water.

The clear wort is to be drawn off from the top of the tun, as usual, and boiled as soon as possible with the due proportion of hops; and the boiling water may be added in any desired quantity to the drained mash, for the second mashing. Wort made in this way is said to have no flavor whatever of the potato, and to clarify more easily than malt-wort, from its containing a smaller proportion of gluten relatively to that of saccharum.

A scheffel of good mealy potatoes affords from 26 to $27 \frac{1}{2}$ pounds of thick well-boiled syrup, of the density of $36^{\circ}$ Baumé (see Areometer in the Dictionary); and 26 lbs. of such syrup are equivalent to a scheffel of malt in saccharine strength. Zimmermann thinks beer so brewed from potatoes quite equal, at least, if not superior, to pure malt beer, both in appearance and quality.

Porter and Brown Stout.-I offer the following statement of the process for brewing genuine London porter, believing it to be more near that really practised than any formula hitherto published.

For 180 barrels of brown stout, containing from 80 to 85 parts of malt extract in 1,000 by weight :-

Components. -530 bushels (English measure) of good barley malt.

$$
10 \text { do. of kiln-browned malt. }
$$

12 cwt. of Essentia-bina, Caramel, or sugar fused over a fire into a dark brown or black syrupy mass.

1500 lbs. of hops, or about three pounds to each bushel of malt.

10 quarts of Calfini, a preparation made with the oil distilled from the outer bark of the birch.

5 quarts of good porter yeast.

finings of isinglass dissolved in sour beer.

For the brewing process see BeER in the Dictionary.

The essentia-bina may be dissolved in hot worts in a separate copper, and mixed with the rest by running it into the cooler, immediately after the boiled wort is strained from the hops in the hop-back. The Calfini (a hocus-pocus term of the brewers) is prepared as follows :-

Put one ounce of birch-bark oil into a bottle with 4 quarts of spirits of wine 60 per cent. over proof; cork the mouth of the bottle, and place it in a slightly warm position till the oil be thoroughly combined with the alcohol, with the aid of occasional shaking. This solution being cooled is to be filtered through paper, and kept for use. The birch oil is an empyreumatic product made in large quantities in Russia and Poland, for the purpose of giving flavor and conservative properties to the Russia leather. It is sold for one shilling the quart. The dose of Calfini in porter is varied according to the taste of the brewers and consumers.

In concluding this supplementary article, I take occasion to refer my readers to the Practical Treatise on Brewing, by Mr. William Black, a gentleman experienced in the business, who has the merit of discovering the evil influence of galvanic combinations in the metallic parts of the fermenting backs and the beer tuns in our breweries. This little work contains much useful information. I have pleasure also in announcing that Messrs. Beamish and Crawford, the eminent porter brewers of Cork, have taken measures to establish the manufacture of genuine Bavarian beer on the best principles: having, with this view, caused their intelligent head brewer, Mr. Topp, to study the practical details of brewing in Munich. They have recently produced excellent brown stout, equal, if not superior, to any in London, by means of the Bavarian fermentation. It is nearly free from gluten, and will therefore prove light and wholesome to weak stomachs. It needs no finings to clarify it.

Professor Leo of Munich has given the following analysis of two kinds of Munich beer :-

\begin{tabular}{|l|r|r|}
\hline & Bock-bier. & Heiliger-Vater \\
\hline Specific gravity - & $1 \cdot 020$ & $1 \cdot 030$ \\
\hline Alcohol - - - - & $4 \cdot 000$ & $5 \cdot 000$ \\
Extract - - - & $8 \cdot 200$ & 13.500 \\
Carbonic Acid - - & 0.085 & 0.077 \\
Water - - - & $87 \cdot 393$ & 81.923 \\
\hline
\end{tabular}


Carl status the alcohol in the Bavarian beer of Bamberg at only 2.840 in 100 . Extract, $6 \cdot 349$.

The following analyses of other German beers are also by Leo :-

\begin{tabular}{|c|c|c|c|c|c|c|c|}
\hline & & & Lichtenhain. & $\begin{array}{c}\text { Upper } \\
\text { Weimar. }\end{array}$ & Ilmenau. & Jena. & Double Jena. \\
\hline Alcohol & - & - & $3 \cdot 168$ & $2 \cdot 567$ & $3 \cdot 096$ & $3 \cdot 018$ & $2 \cdot 080$ \\
\hline Albumen & - & - & 0.048 & 0.020 & 0.079 & 0.045 & 0.028 \\
\hline Extract & - & - & $4 \cdot 485$ & $7 \cdot 316$ & $7 \cdot 072$ & $6 \cdot 14 t$ & $7 \cdot 153$ \\
\hline \multirow[t]{2}{*}{ Water } & 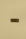 & - & $92 \cdot 299$ & $90 \cdot 097$ & $89 \cdot 753$ & $90 \cdot 793$ & $90 \cdot 739$ \\
\hline & & & $100 \cdot 000$ & $100 \cdot 000$ & $100 \cdot 000$ & $100 \cdot 000$ & $100 \cdot 000$ \\
\hline
\end{tabular}

Under the term extract, in these analyses, is meant a mixture of starch, sugar, dextrine, lactic acid, various salts, certain extractive and aromatic parts of the hop, gluten, and fatty matter.

The following statement is from some of the published analyses of other beers :-

$\begin{array}{lllll} & & & \text { Alcohol. } \\ \text { English ale - } & - & - & - & -8.5 \text { in } 100 \\ \text { Burton - } & - & - & - & -6.2 \\ \text { Scotch - } & - & - & - & -5.8 \\ \text { Common London ale } & - & - & -5.0 \\ \text { Brown stout } & - & - & - & -5.0 \\ \text { London porter } & - & - & - & - \\ \end{array}$

To the above I add the following analyses of certain ales made lately by myself, as follows :-

1. After exposing a portion of the liquor in a wine-glass till the bubbles of carbonic acid were disengaged, I took the specific gravity in a globe with a capillary bored stopper.

2. I then saturated 5,000 grain measures of the ale with a test solution of pure carbonate of soda, to determine the quantity of acid present, after which I added an excess of the alkali to precipitate the gluten; which, however, being but small in amount, I did not separate by a filter, dry, and weigh.

3. I subjected the supersaturated liquid to distillation by the heat of $230^{\circ} \mathrm{F}$. in a chlor-zinc bath till I drew off all its alcohol, of which I noted the quantity in water grain measures, and the specifie gravity.

4. I evaporated to dryness 500 water grain measures slowly in a porcelain capsule, to determine the extract.

\begin{tabular}{|c|c|c|c|c|c|c|}
\hline \multirow{2}{*}{\multicolumn{2}{|c|}{ Specific gravity }} & \multirow[b]{2}{*}{ - } & Bavarian. & Do. Bock. & Allsop's. & Bass's. \\
\hline & & & $1 \cdot 004$ & $1 \cdot 013$ & $1 \cdot 010$ & $1 \cdot 006$ \\
\hline \multirow{5}{*}{$\begin{array}{l}\text { Alcohol } \\
\text { Extract } \\
\text { Acetic acid } \\
\text { Water }\end{array}$} & \multirow{5}{*}{ - } & \multirow{5}{*}{$\begin{array}{l}- \\
- \\
-\end{array}$} & $4 \cdot 00$ & $4 \cdot 50$ & $6 \cdot 00$ & $7 \cdot 00$ \\
\hline & & & $4 \cdot 50$ & $6 \cdot 40$ & $5 \cdot 00$ & $4 \cdot 80$ \\
\hline & & & $0 \cdot 20$ & $0 \cdot 20$ & $0 \cdot 20$ & 0.18 \\
\hline & & & $91 \cdot 30$ & $88 \cdot 90$ & $88 \cdot 80$ & 88.02 \\
\hline & & & $100 \cdot 00$ & $100 \cdot 00$ & $100 \cdot 00$ & $100 \cdot 00$ \\
\hline
\end{tabular}

The Bavarian beers had been recently imported from Germany in casks lined with pitch. The two samples of English ale are those made chiefly for the Indian market, but, being highly hopped, and comparatively clean, as the brewers say, have been recommended as a tonic beverage, by the faculty. Hodgson's bitter beer was the original of this quality.

The above Bavarian beers afford no precipitate of gluten with carbonate of potash; the two English ales become mottled thereby, and yield a small portion of gluten, which had been held in solution by the acid, which is here estimated as the acetic. Common vinegar, excise strength, contains 5 per cent. of such acid as is stated in the above analysis, indicating from 3 to 4 per cent. of table vinegar in the above varieties of beer.

BICARBONATE OF POTASH AND OF SODA. These salts, so much used in medicine, may, according to $\mathbf{M}$. Behrens, be very readily prepared by gradually adding acetic acid to a strong solution of their carbonates; that of soda being hot. The carbonic acid, at the moment of its disengagement, by the stronger affinity of the 
acetic for the alkalis, combines with a portion of them to form bicarbonates, which fall to the bottom of the ressel in which the mixture is made. The supernatant acetate being separated by decantation, the residuary bicarbonate is to be pressed in linen, washed with ice-cold water, and dried. This ingenious process may be practised by the chamber chemist, but will not afford the bicarbonates at so cheap a rate as the ordinary modes of manufacture.

BIRDLIME. All the parts of the misletoe contain a peculiar viscid gluey substance, which they yield by decoction, particularly of the bark and green portions; as also from the expressed juice of the bark or berries, when it is kneaded with the fingers under water. The birdlime is thus obtained in the form of a white opaque mass, sticking to the fingers. It may also be extracted from the berries of the misletoe by means of ether, repeatedly applied, digested with them. It dissolves at first a mixture of green wax and birdlime, but afterward birdlime alone. By distilling off the ether, the birdlime remains colorless and pure. Birdlime may be considered as a hind of viscid resin which does not dry, and resembling in this respect an ointment of oil or lard and rosin melted together-the old basilicon of the surgeon. Alcohol, even boiling hot, dissolves hardly any birdlime; but merely its waxy impurities, which : deposites in flocks on cooling. It is soluble in the oils of rosemary and turpentine, as also in petroleum. Heated with the ley of caustic potash, it forms a compound soluble in alcohol. Nitric acid converts it into oxalic acid, and into a fat which solidifies.

Macaire has examined a substance which exudes from the receptacle and involucre of the atractylis gummifera, and describes it as the pure matter of birdlime, which he styles viscine. It is said to be composed in 100 parts of 75.6 carbon, 9.2 hydrogen, and 15.2 oxygen. Common birdlime may be regarded as a mixture of viscine, vegetable mucilage, and vinegar. The young shoots of the ficus elastica afford a milky juice, which is viscine, while the old branches afford a juice rich in caoutchouc.

BISCUITS. For the following account of the mechanical system of baking biscuits for the royal navy, I am indebted to the ingenious inventor, Thomas Grant, Esq., of Gosport.

Ships' biscuits are now made by machinery; and one of the reasons for this has been that the manual preparation of them was too slow and too costly during the last war. A landsman knows very little of the true value of a biscuit : with a seaman, biscuit is the only bread that he eats for months together. There are many reasons why common loaves of bread could not be used during a long voyage : because, containing a fermenting principle, they would soon become musty and unfit for food, if made previous to the vovage; while the preparation of them on board ship is subject to insuperable objections. Biscuits contain no leaven, and, when well baked through. out, they suffer little change during a long voyage.

The allowance of biscuit to each seaman on board a queen's ship is a pound per day (averaging six biscuits to the pound). The supply of a man-of-war for several months is, consequently, very large; and it often happened during the last war that the difficulty of making biscuits fast enough was so great, that at Portsmouth wagon-loads were unpacked in the streets and conveyed on board ships.

We shall now describe the mode of making biscuits by hand; and afterward speak of the improved method. The bakehouse at Gosport contained nine ovens, and to each was attached a gang of five men-the "turner," the "mate," the "driver," the "breakman," and the "idleman." The requisite proportions of flour and water were put into a large trough, and the "driver," with his naked arms, mixed the whole up together in the form of dough-a very laborious operation. The dough was then taken from the trough and put on a wooden platform called the break: on this platform worked a lever called the break-staff, five or six inches in diameter, and seren feet long; one end of this was loosely attached by a kind of staple to the wall, and the breakman, riding, or sitting on the other end, worked this lever to and fro orer the dough, by an uncouth jumping or shuffling movement. When the dough had become kneaded by this barbarous method into a thin sheet, it was removed to the mouldingboard, and cut into slips by means of an enormous knife; these slips were then broken into pieces, each large enough for one biscuit, and then worked into a circular form by the hand. As each biscuit was shaped it was handed to a second workman, who stamped the king's mark, the number of the oven, \&c., on the biscurt. The biscuit was then docked, that is, pierced with holes by an instrument adapted to the purpose. The finishing part of the process was one in which remarkable dexterity was displayed. A man stood before the open door of the oven, having in his hand the handle of a long shovel called a peel, the other end of which was lying flat in the oven. Another man took the biscuits as fast as they were formed and stamped, and jerked or threw them into the oven with such undeviating accuracy that they should always fall on the peel. The man with the peel then arranged the biscuits side by side over the whole floor of the oven. Nothing could exceed (in manual labor alone) the regu 
larity with which this was all done. Seventy biscuits were thrown into the oven and rexularly arranged in one minute; the attention of each man being vigorously directed to his own department; for a delay of a single second on the part of any one man would have disturbed the whole gang. The biscuits do not require many minutes' baling ; and as the oren is kept open during the time that it is being filled, the biscuits first thrown in would be overbaked were not some precaution taken to prevent it. The moulder therefore made those which were to be first thrown into the oven larger than the subsequent ones, and diminished the size by a nice gradation.

The mode in which, since about the year 1831, ships' biscuits have been made by machinery invented by T. T. Grant, Esq., of the Royal Clarence yard, is this : the meal or flour is conveyed into a hollow cylinder four or five feet long and about three feet in diameter, and the water, the quantity of which is regulated by a gauge admitted to it; a shaft, armed with long knifes, works rapidly round in the cylinder, with such astonishing effect that, in the short space of three minutes, 340 pounds of dough are produced, infinitely better made than that mixed by the naked arms of a man. The dough is removed from the cylinder and placed under the breakingrollers; these latter, which perform the office of kneading, are two in number, and weigh $15 \mathrm{cwt}$. each; they are rolled to and fro over the surface of the dough by means of machinery, and in five minutes the dough is perfectly kneaded. The sheet of dough, which is about two inches thick, is then cut into pieces half a yard square, which pass under a second set of rollers, by which each piece is extended to the size of six feet by three, and reduced to the proper thickness for biscuits. The sheet of dough is now to be cut up into biscuits, and no part of the operation is more beautiful than the mode by which this is accomplished. The dough is brought under a stamping or cutting-out press, similar in effect, but not in detail, to that by which circular pieces for coins are cut out of a sheet of metal. A series of sharp knives are so arranged that, by one movement, they cut out of a piece of dough a yard square about sixty hexagonal biscuits. The reason for a hexagonal (six-sided) shape is, that not a particle of waste is thereby occasioned, as the sides of the hexagonals accurately fit into those of the adjoining biscuits; whereas circular pieces cut out of a large surface always leave vacant spaces between. That a flat sheet can be divided into hexagonal pieces without any waste of material is obvious.

Each biscuit is stamped with the queen's mark, as well as punctured with holes by the same movement which cuts it out of the piece of dough. The hexagonal cutters do not sever the biscuits completely asunder; so that a whole sheet of them can be put into the oven at once on a large peel or shovel adapted for the purpose. About fifteen minutes are sufficient to bake them; they are then withdrawn and broken asunder by the hand.

The corn for the biscuits is purchased at the markets, and cleaned, ground, and dressed; at the government mills; in quality it is a mixture of fine flour and middlings, the bran and pollard being removed. The ovens for baking are formed of fire-brick and ille, with an area of about 160 feet. About 112 lbs. weight of biscuits are put into the ovens at once. This is called a suit, and is reduced to about $110 \mathrm{lbs}$. by the baking. From twelve to sixteen suits can be baked in each oven every day, or after the rate of $224 \mathrm{lbs}$. per hour. The men engaged are dressed in clean check shirts and white linen trowsers, apron, and cap; and every endeavor is made to observe the most scrupulous cleanliness.

We may now make a few remarks on the comparative merits of the hand and the machine processes. If the meal and the water with which the biscuits are made be not thoroughly mixed up, there will be some parts moister than others. Now, it was formerly found that the dough was not well mixed by the arms of the workman; the consequence of which was that the dry parts became burnt up, or else that the moist parts acquired a peculiar kind of hardness which the sailors called "flint :" these defects are now removed by the thorough mixing and kneading which the ingredients receive by the machine.

We have seen that 450 lbs. of dough may be mixed by the machine in four minutes, and kneaded in five or six minutes; we need hardly say how much quicker this is than men's hands could effect it. The biscuits are cut out and stamped sixty at a time, instead of singly: besides the time thus saved, the biscuits become more equally baked, by the oven being more speedily filled. The nine ovens at Gosport used to employ 45 men to produce about 1,500 lbs. of biscuit per hour; 16 men and boys will now produce, by the same number of ovens, 2,241) lbs. of biscuits (one ton) per hour.

The comparative expense is thus stated: Under the old system, wages, and wear and tear of utensils, cost about 1s. $6 \mathrm{~d}$. per cwt. of bis cuit : under the new system, the cost is $5 d$.

The bakehouses at Deptford, Gosport, and Plymouth, could produce 7,000 or 8,000 tons of biscuits annually, at a saving of $12,000 l$. per annum from the cost under the 
old system. The advantages of machine-made over hand-made biscuits, therefore, are many : quality, cleanliness, expedition, cheapness, and independence of w: ernment contractors.

Fig. 7, represents the biscuit machinery, as executed beautifully by Messis. Ronnie,

7

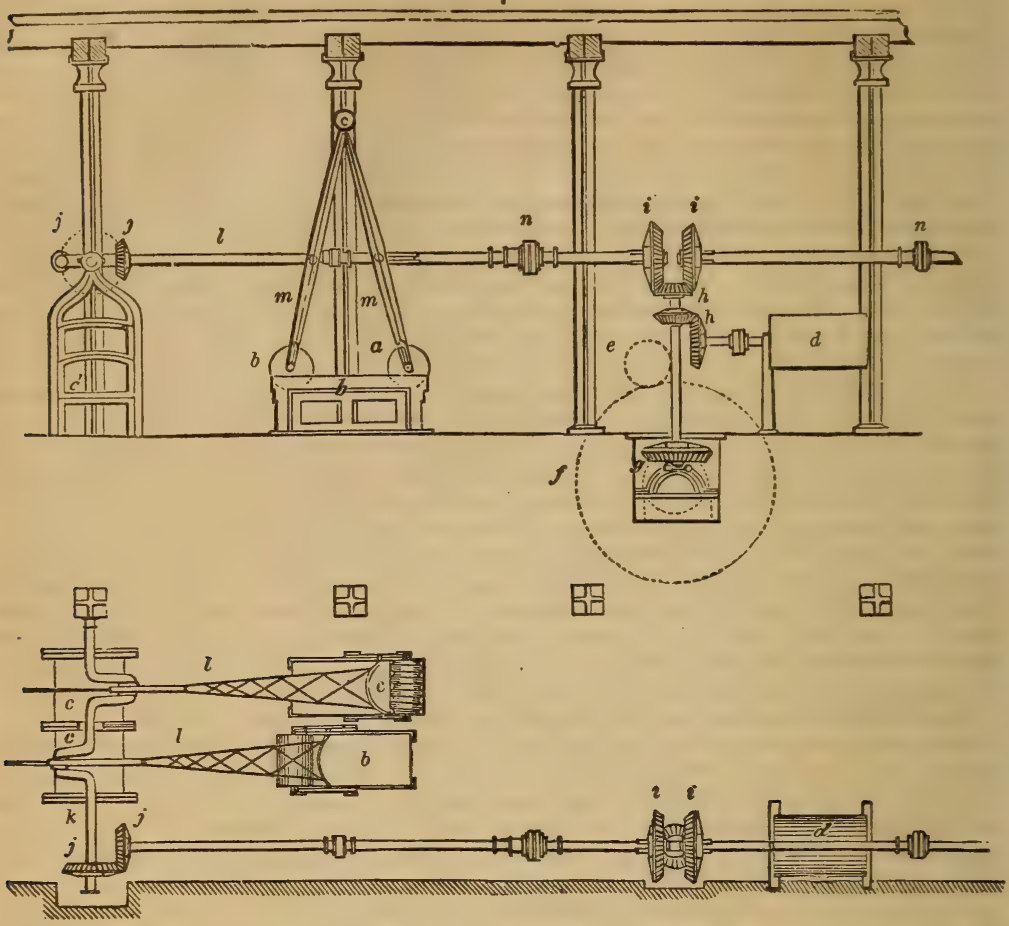

Engineers. $\quad a$, is the breakng roller, table and roller; $b$, the finishing roller, table and roller; $c$, $c$, docking machines for stamping out the biscuits; $d$, mixing machine for making the dough; $e$, spur pinion to engine shaft; $f$, spur-wheel; $g$, g, bevel mitrewheels to give the upright motion; $h, h$, bevel-wheels for working the mixing machine; $i, i, i$, ditto for communicating motion to the rolling machines $j, j ; k$, the crank shaft; $l, l$, connecting rods; $m, m$, pendulums for giving motion to rollers; $n, n$, clutches for connecting either half of the machinery to the other.

BITUMEN. It is a very remarkable fact, in the history of the useful arts, that asphalt, which was so generally employed as a solid and durable cement in the earliest constructions upon record, as in the walls of Babylon, should for so many thousand years have fallen well nigh into disuse among civilized nations. For there is certainly no class of mineral substances so well fitted as the bituminous, by their plasticity, fusibility, tenacity, adhesiveness by surfaces, impenetrability by water, and unchangeableness in the atmosphere, to enter into the composition of terraces, foot-pavements, roofs, and every kind of hydraulic work. Bitumen, combined with calcareous earth, forms a compact, semi-elastic solid, which is not liable to suffer injury by the greatest alternations of frost and thaw, which often disintegrate in a few years the hardest stone, nor can it be ground to dust and worn away by the attrition of the feet of men and animals, as sandstone, flags, and even blocks of granite are. An asphalt pavement, rightly tempered in tenacity, solidity, and elasticity, seems to be incapable of suffering abrasion in the most crowded thoroughfares; a fact exemplified of late in a few places in London, but much more extensively, and for a much longer time, in Paris.

The great Place de la Concorde (formerly Place Louis Quinze) is covered with a beautiful mosaic pavement of asphalt ; many of the promenades on the Boulevards, formerly so filthy in wet weather, are now covered with a thın bed of bituminous mastich, free alike from dust and mud; the foot-paths of the Pont Royal and Pont Carousel, and the areas of the great public slaughter-houses, have been for several 
rears pared in a similar manner with perfect success. It is much to be regretted that the asphalt companies of London made the ill-judged, and nearly abortive attempt, to pave the carriage-way near the east end of Oxford street, and especially at a moist season, most unpropitious to the laying of bituminous mastich. Being formed of blucks not more than three or four inches thick, many of which contained much siliceous sand, such a parement could not possibly resist the crash and vibration of many thousand heavy drays, wagons, and omnibuses, daily rolling over it.* This failure can afford, however, no argument against rightly-constructed foot-pavements and terraces of asphalt. Numerous experiments and observations have led me to conclude that fossil bitumen possesses far more valuable properties, for making a durable mastich, than the solid pitch obtained by boiling wood or coal tar. The latter, when inspissated to a proper degree of hardness, becomes brittle, and may be readily crushed into powder; while the former, in like circumstances, retains sufficient tenacity to resist abrasion. Factitious tar and pitch being generated by the force of fire, seem to have a propensity to decompose by the joint agency of water and air, whereas mineral pitch has been known to remain for ages without alteration.

Bitumen alone is not so well adapted for making a substantial mastich as the native compound of bitumen and calcareous earth, which has been properly called asphaltic rock, of which the richest and most extensive mine is unquestionably that of the Val-de-Travers, in the canton of Neufchâtel. This interesting mineral deposite occurs in the Jurassic limestone formation, the equivalent of the English oolite. The mine is very accessible, and may be readily excavated by blasting with gunpowder. The stone is massive, of irregular fracture, of a liver-brown color, and is interspersed with a few minute spangles of calcareous spar. Though it may be scratched with the nail, it is difficult to break by the hammer. When exposed to a very moderate heat it exhales a fragrant ambrosial smell, a property which at once distinguishes it from all compounds of factitious bitumen. Its specific gravity is $2 \cdot 114$, water being 1,000 , being nearly the density of bricks. It may be most conveniently analyzed by digesting it in successive portions of hot oil of turpentine, whereby it affords 80 parts of a white pulverulent carbonic of lime, and 20 parts of bitumen in 100 . The asphalt rock of Val-de-Travers seems therefore to be far richer than that of Pyrimont, which, according to the statement in the specification of Claridge's patent, of November, 1837, contains " carbonate of lime and bitumen in about the proportion of 90 parts of carbonate of lime to about 10 parts of bitumen."

The calcareous matter is so intimately combined and penetrated with the bitumen, as to resist the action not only of air and water for any length of time, but even of muriatic acid; a circumstance partly due to the total absence of moisture in the mineral, but chiefly to the vast incumbent pressure under which the two materials have been incorporated in the bowels of the earth. It would indeed be a difficult matter to combine, by artificial methods, calcareous earth thus intimately with bitumen, and for this reason the mastichs made in this way are found to be much more perishable. Many of the factitious asphalt cements contain a considerable quantity of siliceous sand, from which they derive the property of cracking and crumbling down when trodden upon. In fact, there seems to be so little attraction between siliceous matter and bitumen, that their parts separate from each other by a very small disruptive force.

Since the asphalt rock of Val-de-Travers is naturally rich enough in concrete bitumen, it may be converted into a plastic workable mastich of excellent quality for foot pavements and hydraulic works at very little expense, merely by the addition of a very small quantity of mineral or coal tar, amounting to not more than 6 or 8 per cent. The union between these materials may be effected in an iron caldron, by the application of a very moderate heat, as the asphalt bitumen readily coalesces with the tar into a tenacious solid.

The mode adopted for making the beautiful asphalt pavement at the Place de la Concorde in Paris was as follows: The ground was made uniformly smooth, either in a horizontal plane or with a gentle slope to carry off the water; the curb-stones were then laid round the margin by the mason, about 4 inches above the level of the ground. This hollow space was filled to a depth of 3 inches with concrete, containing about a sixth part of hydraulic lime, well pressed upon its bed. The surface was next smoothed with a thin coat of mortar. When the whole mass had become perfectly dry, the mosaic pattern was set out on the surface, the moulds being formed of flat iron bars, rings, \&c., about half an inch thick, into which the fluid mastich was poured by ladles from a caldron, and spread evenly over.

The mastich was made in the following way: The asphalt rock was first of all roasted in an oven, about 10 feet long and 3 broad, in order to render it friable. 
The bottom of the oven was sheet iron, heated below by a brisk fire. A volatile matter exhaled, probably of the nature of naphtha, to the amount of one fortieth the weight of aspha't; after roasting, the asphalt became so friable as to be easily reduced to powder, and passed through a sieve, having meshes about one fourth of an inch sipuare.

The bitumen destined to render the asphalt fusible and plastic, was melted in small quantities at a time, in an iron caldron, and then the asphalt in powder was gradually otirred in to the amount of 12 or 13 times the weight of bitumen. When the mixture became fluid, nearly a bucketful of very small, clean gravel, previously heated apart, was stirred into it ; and, as soon as the whole began to simmer with a treacley consistence, it was fit for use. It was transported in buckets, and poured into the moulds.

For the reasons above assigned, I consider this addition of rounded, polished, siliceous stones to be very injudicious. If anything of the kind be wanted to give solidity to the pavement, it should be a granitic or hard calcareous sand, whose angular form will secure the cohesion of the mass. I conceive, also, that tar, in moderate quantity, should be used to give toughness to the asphaltic combination, and prevent its being pulverized and abraded by friction.

In the able report of the Bastenne and Gaujac Bitumen company, drawn up by Messrs. Goldsmid and Russell, these gentlemen have made an interesting comparison between the properties of mineral tar and vegetable tar : the bitumen composed of the latter substance, including various modifications, extracted from coal and gas, hare, so far as ther were able to ascertain, entirely failed. This bitumen, owing to the qualities and defects of regetable tar, becomes soft at $115^{\circ}$ of Fahrenheit's scale, and is brittle at the freezing point; while the bitumen, into which mineral tar enters, will sustain

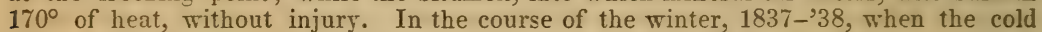
was at $14 \frac{1}{2}$ below zero, C., the bitumen of Bastenne and Gaujac, with which one side of the Pont Neuf at Paris is paved, was not at all impaired, and would, apparently, have resisted any degree of cold; while that in some parts of the Boulerard, which was composed of regetable tar, cracked and opened in white fissures. The French government, instructed by these experiments, has required, when any of the regetable bitrmens are laid, that the pavement should be an inch and a quarter thick; whereas, where the bitumen composed of mineral tar is used, a thickness of three quarters of an inch is deemed sufficient. The pavement of the bonding warehouse at Bordeaux has been laid upward of 15 years by the Bastenne company, and is now in a condition as perfect as when first formed. The reservoirs constructed to contain the waters of the Seine at Batignolles, near Paris, have been mounted 6 years, and, notwithstanding the intense cold of the winter of 1837 , which froze the whole of their contents into one solid mass, and the perpetual water pressure to which they are exposed, they have not betrayed the slightest imperfection in any point. The repairs done to the ancient fortifications at Bayonne, have answered so well, that the government, 2 years ago, entered into a very large contract with the company for additional works, while the whole of the arches of the St. Germain and St. Cloud railwars, and the parements and floorings necessary for these works, are being laid with the Bastenne bitumen.

The mineral tar in the mines of Bastenne and Gaujac is easily separated from the earthy matter with which it is naturally mixed by the process of boiling, and is then transported in barrels to Paris or London, being laid down in the latter place to the company at $1 \% l$. per ton, in virtue of a monopoly of the article purchased by the company at a sum, it is said, of $8,000 l$.

Mr. Harrey, the able superintendent of the Bastenne company, was good enough to supply me with rarious samples of mineral tar, bitumen, and asphaltic rock, for analysis. The tar of Bastenne is an exceedingly viscid mass, without any earthy im purity. It has the consistence of baker's dough at $60^{\circ}$ of Fahrenheit; at $80^{\circ}$ it yields to the slightest pressure of the finger; at 150 degrees it resembles a soft extract; and at 212 degrees it has the fluidity of molasses. It is admirably adapted to give plasticity to the calcareous asphalts.

A specimen of Egyptian asphalt which he brought me, gave, by analysis, the very same composition as the Val-de-Travers, namely, 80 per cent. of pure carbonate of lime, and 20 of bitumen. A specimen of mastich, prepared in France, was found to consist, in 100 parts, of 29 of bitumen, 52 of carbonate of lime, and 19 of siliceous sand. A portion of stone called the natural Bastenne rock, afforded me 80 parts of gritty siliceous matter and 20 of thick tar. The Trinidad bitumen contains a considerable portion of foreign earthy matter: one specimer having yidded me $2 j$ per cent. of siliceous sand; a second, 28 ; a thira, 20 ; and a fourth, 30 : the remaincier was pure pitch. One specimen of Egyptian bitumen, specific grarity $1 \cdot 2$, was found to be perfectly pure, for it dissolved in oil of turpentine without leaving any appreciable residuum. 
Robinson's Parisian Bitumen company use a mastich made with the pitch obtained from boiling coal-tar mixed with chalk. One piece laid down by this company at Knightsbridge and another at Brighton, are said to have gone to pieces. The portion of pavement laid down by them in Oxford street, next Charles street, has been taken up. Claridge's company have laid down their mastich under the archway of the HorseGuards, and in the carriage-entrance at the Ordnance Office; the latter has cracked at the junction with the old pavement of Yorkshire curb-stone. The foot-pavement laid down by Claridge's company at Whitehall has stood well. The Bastenne company has exhibited the best specimen of asphalt pavement in Oxford street; they have lsid down an excellent piece of foot-pavement near Northumberland House; a piece, 40 feet by 7, on Blachfriars' Bridge; they have made a substantial job in paving 830 superficial feet in front of the guard-room at Woolwich, which, though much traversed by foot-passengers, and beat by the guard in grounding arms, remains sound; lastly, the floor of the stalls belonging to the cavalry barracks of the Blues at Knightsbridge, is probably the best example of asphaltic pavement laid down in this country, as it has received no injury from the beating of the horses' feet.

As the specific gravity of properly-made mastich is nearly double that of water, a cubic foot of it will weigh from 125 to $130 \mathrm{lbs}$.; and a square foot, three quarters of an inch thick, will weigh very nearly eight pounds. A ton of it will therefore cover 280 square feet. The prices at which the Bastenne Bitumen company sell their products is as follows :-

Pure Mineral tar, 24l. per ton, or 28s. per cwt.

Mastich $8 l .8 s$. per ton, or 10s. per cwt.

Side Pavement.

From 50 to 100 feet, 1 s. $3 d$. per foot.

100250

$250 \quad 500$

$500 \quad 750$

$750 \quad 1000$

$1000 \quad 2000$

$2000 \quad 5000$
Roofs and Terraces.

- 1 s. $6 d$. per foot.

- $\quad 1 s .4 d$.

- $\quad 1 s .1 d$.

- $\quad 1 s .0 d$.

- $\quad 11 d$.

- $\quad 10 d$.

- $9 d$.

Where the work exceeds 5,000 feet, contracts may be entered into.

For filling up joints of brickwork, \&c., from $1 d$. to $1 \frac{1}{4} d$. per foot, run according tc quantity.

These prices are calculated for half an inch thickness, at which rate a ton will cover 420 square feet.

As the Val-de-Travers company engage to lay down their rich asphaltic rock in London at $5 l$. per ton; and as the mineral tar equal to that of Seissel may probably be had in England at one fourth the price of that foreign article, they may afford to lay their mastich three quarters of an inch thick per the thousand feet, including a substratum of concrete, at a rate of fivepence per square foot, instead of fifteenpence, being the rate charged under that condition by the Bastenne company.

These charges are for London and its immediate vicinity.

\section{Report of the experimental Pavements laid down in Oxford street, from Charles street to Tottenham Court Road, January, 1839.}

1. Robinson's Parisian bitumen, laid in blocks 12 inches square and 5 inches deep; the substance is a compound of bitumen, lime, \&c., and five granite stones are inserted in the top of each block; the work is laid in straight courses, the joints cemented with hot bitumen. The quantity of this is 97 square yards, the length is 20 feet, and the price, if adopted, $9 s$. per square yard.

2. Same as 1 , but the courses laid diagonally. The quantity is 97 square yards, the length is 20 feet.

3. Granite paving, 9 inches deep, jointed with Claridge's asphalt, the work laid in straight courses. The cost to the parish has been $11 s$. $7 d$. per yard superficial for the atone and laying, \&c., no charge being made by Claridge's company for the asphalt. The quantity is 240 yards, the length 54 feet.

4. Granite paving, $4 \frac{1}{2}$ inches deep, jointed with Claridge's asphalt, the work laid in diagonal courses. Cost to the parish $9 s .6 d$. per square yard. No charge made for the asphalt. The quantity is 88 square yards, the length 20 feet.

5. The Bastenne Bitumen company. The blocks are 12 inches long, $6 \frac{1}{4}$ wide, and $3 \frac{3}{4}$ deep, with bevelled joints, close at bottom, and $\frac{1}{2}$ inch open at top; the joints cemented with hot bitumen; the substance is bituminous, with a very large proportion of granite imbedded in each block; the price, if adopted 13s.6d. per square yard; the length, in straight courses, 20 feet. 
6. Same as 5 , but the courses laid diagonally. The length 40 feet; the total quantity in 5 and 6 is 274 square yards.

7. Aberdeen granite paving, 9 inches deep; laid on a concrete bottom, formed of gravel and lime, the joints of the pavement run with hot lime grout, in straight courses. The length is 69 feet; cost, $16 s .5 d$. per square yard.

8. Same as 7, but the courses laid diagonally; length 38 feet.

9. Aberdeen granite paring, 9 inches deep, in straight courses, without a concrete bottom; joints filled with fine gravel; cost, 12s. 5d. per yard; length, 24 feet.

10. The Scotch Asphaltum company. The work is laid in blocks of divers length, 9 inches wide, and $6 \frac{1}{2}$ deep; the side joints are straight, the end joints are bevelled alternately. The work is laid in straight courses, and jointed in Roman cement; the substance is, apparently, a bituminous matter mixed with fine gravel. The length is 50 feet; the number of square yards, 210 ; the price, per yard, if adopted, $13 s .6 d$.

11. The wood-paving. The blocks are sexagon on the plan, and (with the exception of a few courses that are only 8 inches), 12 inches deep. The work is laid endwise of the grain; the blocks are mostly 8 inches diameter-a few courses are 7 inches. The material is Norway fir; there is no prepared bottom-the blocks are laid on the plain ground, a small layer of gravel being spread to bed them in. From the west end, 22 rows of courses of blocks are of wood in its natural state; 31 rows have been Kyanised; 9 rows at the eastern end have been dipped in Claridge's asphalt; 6 rows have been dipped in a solution prepared by the patentee; the remainder are of wood in the natural state. The length of this piece is 60 feet: the number of yards, 230 ; price per yard, if approved, $10 \mathrm{~s} .6 \mathrm{~d}$.

12. Val-de-Travers company. Blocks in straight courses, 12 inches square, 5 inches deep, with square joints. The substance of the blocks is bituminous, with a very large proportion of granite imbedded in each block, the joints cemented with hot bitumen. The length is 25 feet; number of square yards 94 ; the work is performed gra. tuitously.

13. The same company. A layer of clean chippings and hot asphalt poured thereon. The face up, with hot asphalt and broken stone imbedded therein. The length is 25 feet: number of yards, 94 ; the work is gratuitous.

14. Same as 9 . The length 47 feet.

By order of the Committee, H. KENSETT, Chairman.

Statement of the number of carriages passing through Oxford street at the undernamed times and places.

\begin{tabular}{|c|c|c|c|c|c|c|c|c|c|c|c|}
\hline $\begin{array}{l}\text { Date. } \\
1839 .\end{array}$ & Time. & Place. & 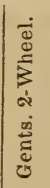 & 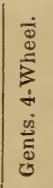 & 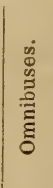 & 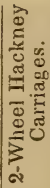 & 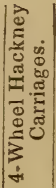 & 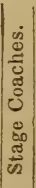 & 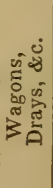 & 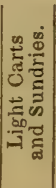 & $\begin{array}{l}\text { त्ड } \\
\text { ०े } \\
\text { E- }\end{array}$ \\
\hline $\begin{array}{r}\text { Jan. } 16 . \\
18 . \\
22 . \\
26 . \\
26 .\end{array}$ & $\begin{array}{l}6 \text { in the morning till } 12 \text { at } \\
\text { do. [night. } \\
\text { do. } \\
\text { do. [morning. } \\
12 \text { at night till } 6 \text { in the }\end{array}$ & $\begin{array}{l}\text { by the Pantheon. } \\
\text { by Stratford place. } \\
\text { by Newman street. } \\
\text { by Stratford place. } \\
\text { do. }\end{array}$ & $\begin{array}{l}347 \\
254 \\
339 \\
371 \\
\end{array}$ & $\begin{array}{r}935 \\
603 \\
1241 \\
766 \\
4\end{array}$ & $\begin{array}{r}890 \\
1213 \\
1015 \\
1337\end{array}$ & $\begin{array}{r}621 \\
401 \\
584 \\
542 \\
82\end{array}$ & $\begin{array}{r}752 \\
728 \\
1288 \\
762 \\
139\end{array}$ & \begin{tabular}{r|}
91 \\
89 \\
85 \\
92 \\
2
\end{tabular} & $\begin{array}{r}372 \\
472 \\
958 \\
881 \\
38\end{array}$ & $\begin{array}{r}1507 \\
993 \\
1382 \\
1292 \\
58\end{array}$ & $\begin{array}{r}5515 \\
4753 \\
6992 \\
5943 \\
324\end{array}$ \\
\hline
\end{tabular}

BLACK DYE. The mordant much employed in some parts of Germany for this dye, with logwood, galls, sumach, \&c., is Iron-Alum, so called on account of its having the crystalline form of alum, though it contains no alumina. It is prepared by dissolving 78 pounds of red oxide of iron in 117 pounds of sulphuric acid, diluting this compound with water, adding to the mixture 87 pounds of sulphate of potash, evaporating the solutinn to the crystallizing point. This potash-sulphate of iron has a fine amethyst color when recently prepared; and though it gets coated in the air with a yellowish crust, it is none the worse on this account. As a mordant, a solution of this salt, in from 6 to 60 parts of water, serves to communicate and fix a great variety of uniform. ground colors, from light gray to brown, blue, or jet black, with quercitron, galls, logwood, sumach, \&c., separate or combined. The above solution may be usefully modified by adding to every 10 pounds of the iron-alum, dissolved in 8 gallons ( 80 pounds) of warm water, 10 pounds of acetate (sugar) of lead, and leaving the mixture, after careful stirring, to settle. Sulphate of lead falls, and the oxide of iron nemains combined with the acetic acid and the potash. After passing through the above mordant, the cotton goods should be quickly dried.

BLACK PIGMENT. A fine lamp-black is obtained by the combustion of a thick torch of coal-gas, supplied with a quantity of air adequate to burn only its hydro- 
gen. In this case, the whole of its carbon is deposited in the form of a very fine black powder of extreme lightness. This black is used in maling the better qualities of printer's ink.

BLACKING FOR SHOES. (Cirage des bottes, Fr.; Schuhschwärze.)

The following prescription for making liquid and paste blacking is given by William Bryaut and Edward James, under the title of a patent, dated December, 1836. Their improvement consists in the introduction of caoutchouc, with the view, possibly, of making the blacking waterproof :-

18 ounces of caoutchouc are to be dissolved in about 9 pounds of hot rape oil. To this solution 60 pounds of fine ivory black, and 45 pounds of molasses, are to be added, along with 1 pound of finely ground gum arabic, previously dissolved in 20 gallons of vinegar, of strength No. 24. These mixed ingredients are to be finely triturated in a paint mill till the mixture becomes perfectly smooth. To this varnish 12 pounds of sulphuric acid are to be now added in small successive quantities, with powerful stirring for half an hour. The blacking thus compounded is allowed to stand for 14 days, it being stirred half an hour daily; at the end of which time, 3 pounds of finely ground gum arabic are added; after which the stirring is repeated half an hour every day for 14 days longer, when the liquid blacking is ready for use.

In making the paste blacking, the patentees prescribe the above quantity of India rubber oil, ivory black, molasses, and gum arabic, the latter being dissolved in only 12 pounds of vinegar. These ingredients are to be well mixed, and then ground together in a mill till they form a perfectly smooth paste. To this paste 12 pounds of sulphuric acid are to be added in small quantities at a time, with powerful stirring, which is to be continued for half an hour after the last portion of the acid has been introduced. This paste will be found fit for use in about seven days.

BLEACHING OF PAPER. The following are the proportions of liquid chloride of lime, at $10^{\circ}$ of Gay Lussac's Chlorometre, employed for the different sorts of rags, consisting of two piles, or 200 pounds French.

Cotton.
No. 1. Fine cotton rags
2. Clean calicoes

It is considered to be much better to bleach the fine rags with liquid chloride of lime, and not with chlorine gas, because they are less injured by the former, and afford a paper of more nerve, less apt to break, and more easily sized. But the coarse or gray rags are much more economically bleached with the gaseous chlorine, without any risk of weakening the fibre too much. Bleaching by the gas is performed always upon the sorted rags, which have been boiled in an alkaline ley, and torn into the fibrous state. They are subjected to the press, in order to form them into damp cakes, which are broken in pieces and placed in large rectangular wooden cisterns. The chlorine gas is introduced by tubes in the lid of the cistern, which falls down by its superior gravity, acting always more strongly upon the rags at the bottom than those above.

When the chlorine, disengaged from 150 kilogrammes (330 lbs.) of manganese and 500 lilos. of muriatic acid, is made to act upon 2,500 kilos. of the stuff (supposed dry), it will hare completed its effect in the course of a few hours. The quantity of gaseous chlorine is equal to what is contained in the quantity of chloride of lime requisite to produce a like bleaching result. The bleached stuff should be forthwith carefully washed, 30 remove all the muriatic acid produced from the chlorine; for if any of this remain in the paper, it destroys lithographic stones, and weakens common ink.

BONE BLACK, or animal charcoal restored. A process for this purpose was made the subject of a patent by Messrs. Bancroft and Mac Innes of Liverpool, which consists in washing the granular charcoal, or digesting it when finely ground, with a weak solution of potash or soda, of specific gravity 1.06 . The bone black which has been used in sugar refining may be thus restored, but it should be first cleared from all the soluble filth by means of water.

Mr. F. Parker's method, patented in June, 1839, for effecting a like purpose, is, by a fresh calcination, as follows :- 
Fig. 8 represents a front section of the furnace and retort; and fig. 9 is a trans verse vertical section of the same. $a$ is a retort, surrounded by the flues of the furnace
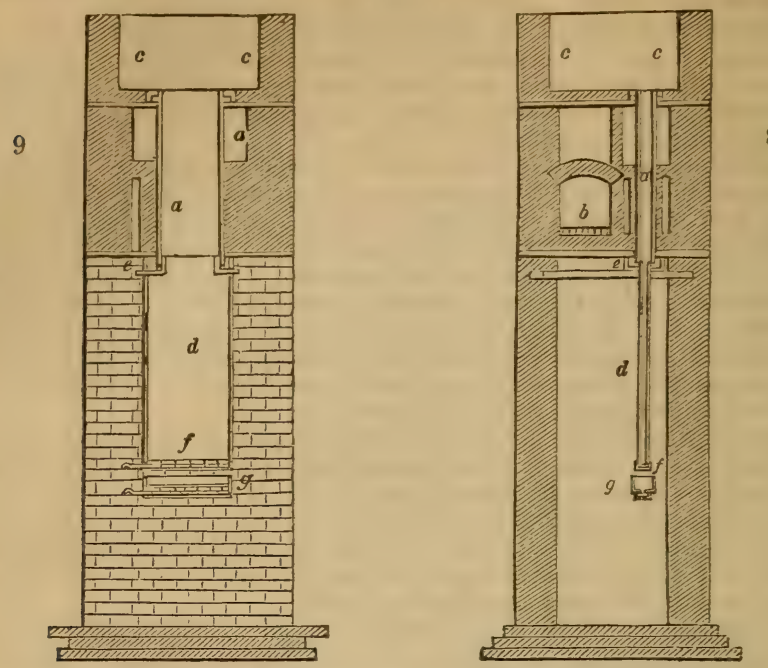

$b$; $c$ is a hopper or chamber, to which a constant fresh supply of the black is furnished, as the preceding portion has been withdrawn, from the lower part of $a . d$ is the cooling vessel, which is connected to the lower part of the retort $a$ by a sand joint $e$. The cooler $d$ is made of thin sheet iron, and is large; its bottom is closed with a slide plate, $f$. The black, after passing slowly through the retort $a$ into the ressel $d$, gets so much cooled by the time it reaches $f$, that a portion of it may be safely withdrawn, so as to allow more to fall progressively down; $g$ is the charcoal-meter, with a slide door.

BOOKBINDING, Mechanical;-An ingenious inrention, for which Mr. Thomas Richards, of Liverpool, bookbinder, obtained a patent in April, 1842. He employs, 1st., a mechanism to sew, weare, or bind a number of sheets together to form a book, instead of stitching them by hand; $2 \mathrm{~d}$, a table which slides to and fro to feed or supply each sheet of paper separately into his machine; also needle-bars, or holders, to present needles with the requisite threads, for stitching such sheets as they are supplied with in succession. He has, moreorer, a series of holding fingers, or pincers, suitably prorided with motions, to enable them to adrance and clasp the needles, draw them through the sheets of paper, and return them into their respective holders, after threading or stitching the sheet; lastly, there are arms or levers for delivering each sheet regularly upon the top of the preceding sheets, in order to form a collection or book of such sheets, ready for boarding and finishing. A minute description of the whole apparatus, with plates, is given in Newton's Journal, C. S., xxiii. $15 \%$.

BORACIC ACID. Imported for consumption in $1839,1,243,868 \mathrm{lbs}$.; in 1840 , 524.205 lbs.

BORAX. Imported for home consumption in 1839, 498,079 Ibs.; in 1840 319,126 lbs.

BRANDY. Imported for consumption in $1839,167,756$ galls.; in $1840,1,108,578$ galls.; duty. 1l. 2s. 10d. per imperial proof gallon.

BRASS, YELLOW. The following table exhibits the composition of several varieties of this species of brass. No. 1 is a cast brass of uncertain origin; 2, the brass of Jemappes; 3, the sheet brass of Stolberg, near Aix-la-Chapelle; 4 and 5, the brass for gilding, according to D'Arcet; 6. the sheet brass of Romilly; 7, English brass wire; 8 , Augsburg brass wire; 9 , brass wire of Neustadt-Eberswald, in the neighborhood of Berlin.

\begin{tabular}{|c|c|c|c|c|c|c|c|c|c|c|}
\hline & & 1. & 2. & 3. & 4. & 5. & 6. & 7. & 8. & 9. \\
\hline Copper & - & $61 \cdot 6$ & $64 \cdot 6$ & 64.8 & $63 \cdot 70$ & $64 \div 5$ & $\tau 0.1$ & 70.29 & $\pi 1.89$ & $\tau 0 \cdot 16$ \\
\hline Zinc & - - & $35 \cdot 3$ & $33 \cdot 7$ & 32.8 & 33.55 & $32 \cdot 44$ & $29-9$ & $29 \cdot 26$ & $2 \pi \cdot 63$ & $2 \pi \cdot 45$ \\
\hline Lead &.- & 2.9 & $1 \cdot 4$ & $2 \cdot 0$ & $0 \cdot 25$ & $2 \cdot 86$ & - & 0.28 & & 020 \\
\hline \multirow[t]{2}{*}{ Tin } & $=$ & 0.2 & 0.2 & 0.4 & 2.50 & $0 \cdot 25$ & $=$ & $0 \cdot 17$ & 0.85 & 0.79 \\
\hline & & $100 \cdot 0$ & 99.9 & $100 \cdot 0$ & $100 \cdot 00$ & $100 \cdot 00$ & - & 100.00 & 100.37 & 9860 \\
\hline
\end{tabular}


The mean proportion of the metals in yellow brass is 30 zinc to 70 copper.

Tombak, or red brass, in the cast state, is an alloy of copper and zinc, containing not more thi: 20 per cent. of the latter constituent. The following varieties are distinguished: 1, 2, 3, tombak for making gilt articles; 4, French tombak for swordhandles, \&c. ; 5, tombak of the Okar, near Goslar, in the Hartz; 6, yellow tombak of Paris for gilt ornaments; 7 , tombak for the same purpose from a factory in Hanover; 8 , chrysochalk ; 9, red tombak from Paris ; 10 , red tombak of Vienna.

\begin{tabular}{|c|c|c|c|c|c|c|c|c|c|c|c|}
\hline \multirow{5}{*}{$\begin{array}{l}\text { Copper } \\
\text { Zinc } \\
\text { Lead } \\
\text { Tin }\end{array}$} & \multirow{4}{*}{$=$} & 1. & 2. & 3. & 4. & 5. & 6. & 7. & 8. & 9. & 10. \\
\hline & & $\begin{array}{l}82 \cdot 0 \\
18 \cdot 0\end{array}$ & $\begin{array}{l}82 \\
18\end{array}$ & $\begin{array}{l}82 \cdot 3 \\
17 \cdot 5\end{array}$ & $\begin{array}{l}80 \\
17\end{array}$ & $\begin{array}{l}85 \\
15\end{array}$ & $\begin{array}{l}85 \cdot 3 \\
14 \cdot 7\end{array}$ & $\begin{array}{l}86 \\
14\end{array}$ & $\begin{array}{r}90.0 \\
7.9\end{array}$ & $\begin{array}{r}92 \\
8\end{array}$ & $\begin{array}{r}97 \cdot 8 \\
2 \cdot 2\end{array}$ \\
\hline & & 1.5 & 3 & - & $-\quad-$ & $-^{10}-$ & -147 & -14. & $1 \cdot 6$ & & \\
\hline & & $3 \cdot 0$ & I & 0.2 & 3 & trace & & & & & \\
\hline & & 104.5 & 104 & $100 \cdot 0$ & 100 & 100 & 100.0 & 100 & 99.5 & 100 & $100 \cdot 0$ \\
\hline
\end{tabular}

Pinchbeck is made of 2 parts copper and 1 yellow brass;

Prince's metal - 3 - 1 zinc.

Mannheim gold (semilor), 28 copper, 12 yellow brass, 3 tin.

Cast white metal buttons are made of an alloy of 32 parts brass (yellow), 4 parts zinc, and 2 tin.

The specific gravity of brass is greater than the mean density of its constituents; varying from 7.82 to 8.73 , according to the proportion of zinc to copper. Sheet brass varies from 8.52 to 8.62 ; brass wire from 8.49 to 8.73 . Brass heated and quickly cooled becomes somewhat less dense. The specific gravity of sheet tombak $(81 \cdot 25$ copper +18.75 zinc) is 8.788 ; of tombak wire $(87.5$ copper +12.5 zinc) has been found so great as $9 \cdot 00$.

BREAD. I believe it may be safely asserted that the art of baking bread, pastry, and confectionary, is carried in Paris to a pitch of refinement which it has never reached in London. I have never seen here any bread which, in flavor, color, and texture, rivalled the French pain de gruau. In fact, our corn monopoly laws prevent us from getting the proper wheat for preparing, at a moderate price, the genuine semoule out of which that bread is baked. Hence, the plebeian bourgeois can daily grace his table with a more beautiful piece of bread than the most affluent English nobleman. The French process of baking has been recently described, with some minuteness, by their distinguished chemist, M. Dumas, ${ }^{*}$ and it merits to be known in this country.

At each operation, the workman (pétrisseur) pours into the kneading-trough the residuary leaven of a former kneading, adding the proportion of water which practice enjoins, and diffuses the leaven through it with his hands. He then introduces into the liquid mass the quantity of flour destined to form the sponge (pâte). This flour is let down from a chamber above, through a linen hose (manche), which may be shut by folding it up at the end.

The workman now introduces the rest of the flour by degrees, diffusing and mingling it, in a direction from the right to the left end of the trough. When he has thus treated the whole mass successively, he repeats the same manipulation from left to right. These operations require no little art for their dexterous performance; hence they have the proper name assigned respectively to each, of frasage and contrefrasage. The workman next subjects the dough to three different kinds of movement, in the kneading process. He malaxates it; that is, works it with his hands and fingers, in order to mix very exactly its component parts, while he adds the requisite quantity of flour. He divides it into six or seven lumps (pâtons), each of which he works successively in the same manner. Then he seizes portions of each, to draw them out, taking only as much as he can readily grasp in his hands. When he has thus kneaded the different lumps, he unites them into one mass, which he extends and folds repeatedly back upon itself. He then lifts up the whole at several times, and dashes it forcibly against the kneading-trough, collecting it finally at its left end. The object of these operations is to effect an intimate mixture of the flour, the water, and the learen. No dry powdery spots, called marrons, should be left in any part of the dough.

The kneader has now completed his work; and after leaving the dough for some time at rest, he turns it upside down. He lays the lumps, of a proper weight, upon a table, rolls them out, and dusts them with a little flour. He next turns over each lump, and puts it in its panneton, where he leaves it to swell. If the flour be of good 
quality, the dough be well made, and the temperature be suitable, the lumps whll swell much and uniformly. If after the surface has risen, it falls to a considerable extent, the flour must be bad, or it must contain other substances, as potato-stareh, bean-meal, \&c.

Whenever the oven is hot enough, and the dough sufficiently fermented, it is subjected to the baking process. Ovens, as at present constructed, are not equably heated throughout, and are particularly liable to be chilled near the door, in consequence of its being occasionally opened and shut. To this cause M. Dumas ascribes many of the defects of ordinary bread; but he adds, that by adopting the patent invention of M. Mouchot these may be obviated. This is called the improved bakery, boulangeric perfectionnée.

Fig. 10 is a ground plan of the aerothermal bakehouse, the granaries being in the

10

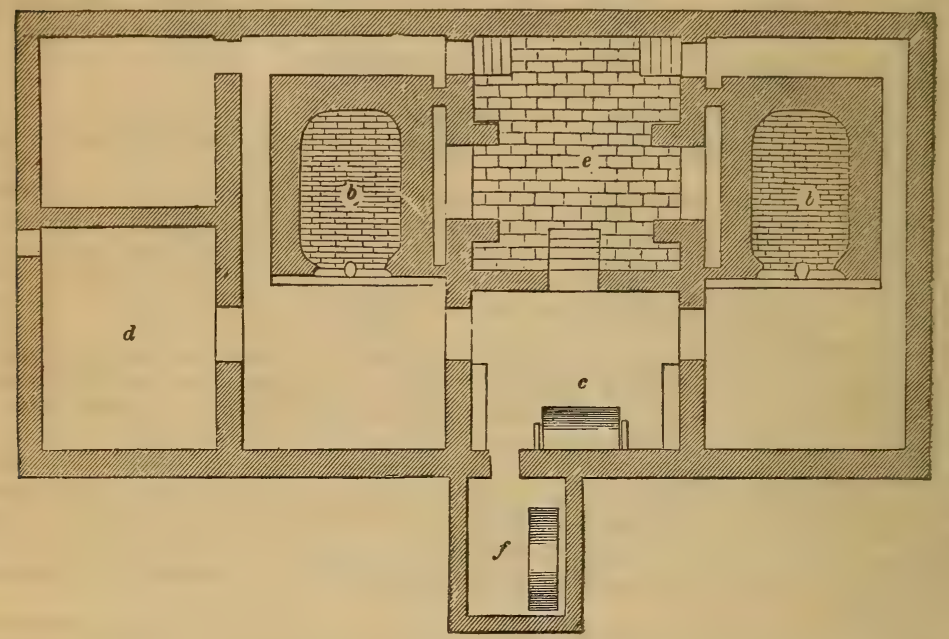

upper stories, and not shown here. $b, b$ are the ovens; $c$, the kneading-machine; $d$, the place where the machinery is mounted for hoisting up the bread into the storeroom above; $e$, a space common to the two ovens, into which the hot air passes; $f$, the place of a wheel driven by dogs, for giving motion to the kneading-machine.

Fig. 11 is a longitudinal section of the oven; A, the grate where coke or even pitcoals may be burned; B, B, void spaces which, becoming heated, serve for warming small pieces of dough in; $c$, c, are flues for conducting the smoke, \&c., from the fireplace; D, seen in fig. 12, is the chimney for carrying off the smoke transmitted by the flues; E, E, void spaces immediately over the flues, and beneath the sole, F, F, of the oven. By this arrangement the air, previously heated, which arrives from the void spaces B through the flues $c$, $c$, gets the benefit of the heat of the flame which circulates in these flues, and, after getting more heated in the spaces $\mathrm{E}, \mathrm{E}$, ascends through channels into the oven F, F, upon the sole of which the loaves to be baked are laid. The hot air is admitted into it through the passages $a$, $a$, being drawn from the reservoirs $\mathrm{B}, \mathrm{B}, \mathrm{B}$, and also by the passage $d, d$, drawn from the reservoirs $\mathrm{E}, \mathrm{E}$. The sole is likewise heated by contact with the hot air contained in the space $\mathrm{E}$, $\mathrm{E}$, placed immediately below it. The hot air, loaded with moisture, issues by the passage $b, b$, and returns directly into the reservoir B, B. G, G, an enclosed space directly over the oven, to obstruct the dissipation of its heat; $g$, vault of the fireplace. Fig. 12, a transverse section through the middle of the oven. Fig. 13, the kneading-machine, a longitudinal section passing through its axis; $\mathrm{P}, \mathrm{P}$, the contour of the machine, made of wood, and divided into three compartments for the reception of the dough. The wooden bars 0,0 , are so placed in the interior of the compartments, as to divide the dough whenever the cylinder is made to revolve. One portion, $\mathrm{D}$, of the cylinder may be opened and laid over upon the other by means of a hinge joint, when the dough and flour are introduced. A, B, C, the three compartments of the inachine, two for making the dough, and one for preparing the sponge, called levain, or leaven, by the French. $a, a$, is the pulley which receives its motion from the engine, and transmits it to the cylindes 
hronsh the pinion $b$, and the spur-wheel $e ; d, d$, the fly-wheel to regulate the motion; $g$, a brake in act upon the fly $d$, by means of a lever $h$; $i$, the pillar of the fly-wheel.

11.

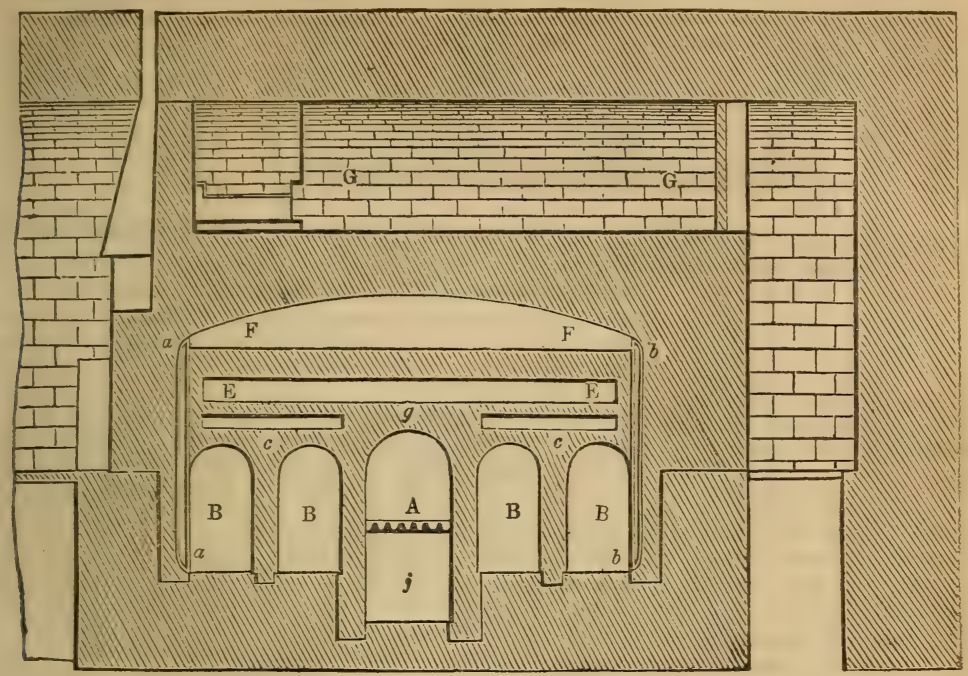

There is a rachet wheel counter for numbering the turns of the kneading machine, but it can not he shown in this view; $n$, cross bars of wood, which are easily removed when the cylinder is opened; they divide the dough.

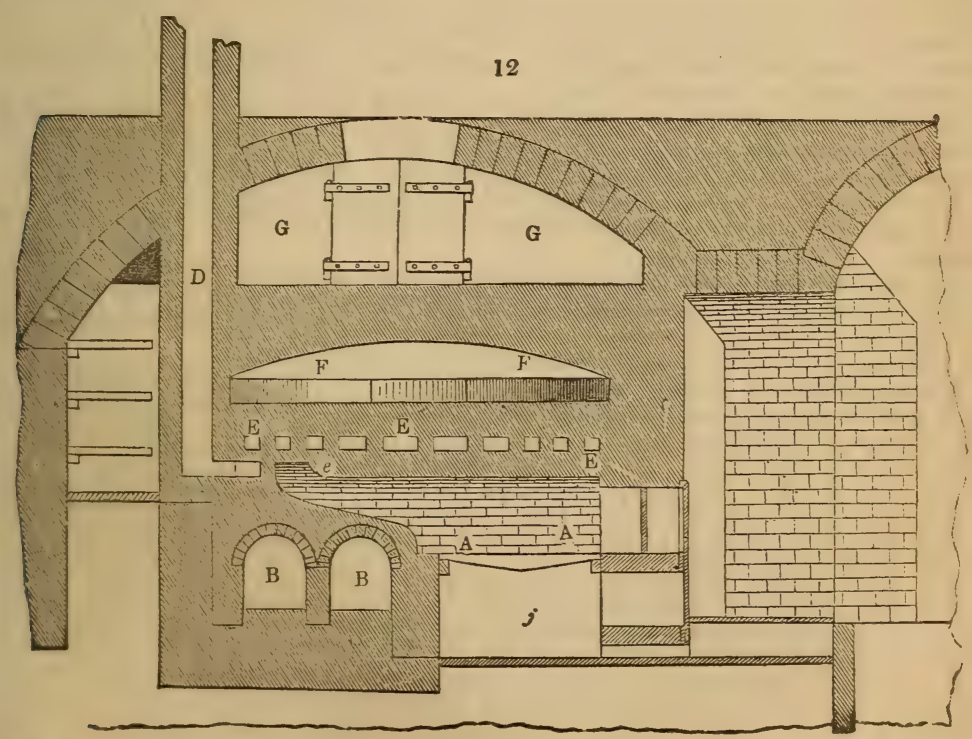

Each of the three compartments of the kneader (fig. 13) is furnished at pleasure with two bars fixed crosswise, but which may be easily removed, whenever the 
cylinder is opened. These bars c nstitute the sole agents for drawing ont the dough.

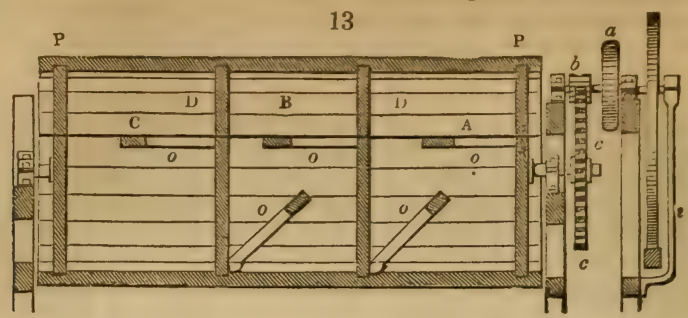

In a continuous operation, the leaven is constantly prepared in the compartment $\mathbf{A}$; with which view there is put into it-

125 kilogrammes of ordinary leaven or yeast.

\section{In all 225 kilogrammes.}

The person in charge of the mechanical kneader shuts down its lid, and sets it a-going. At the end of about seven minutes he hears the bell of the counter sound, announcing that the number of revolutions has been sufficient to call for an inspection of the sponge, in regard to its consistence. The cylinder is therefore opened, and after verifying the right state of the leaven, and adding water to soften, or flour to stiffen it, he closes the lid, and sets the machine once more in motion. In ten minutes more the counter sounds again, and the kneading is completed. The 450 kilogrammes of leaven obtained from the two compartments are adequate to prepare dough enough to supply alternately each of the two ovens. For this purpose 75 kilogrammes of leaven are taken from each of the two compartments $A$ and $A^{\prime}$, and placed in the intermediate compartment $B$. The whole leaven is then $75+75=150$ kilogrammes; to which are added 100 kilogs. of flour and 50 of water $=150$, so that the chest contains 300 kilogrammes. There is now replaced in each of the cavities A and $\mathrm{A}^{\prime}$ the primitive. quantity, by adding 50 kilogrammes of flour and 25 of water $=75$.

The cylinder is again set a-going; and from the nature of the apparatus, it is obvious that the kneading takes place at once on the leavens $A$ and $A^{\prime}$ and on the paste $B$; which last is examined after 7 minutes, and completed in 10 more $=17$, at the second sound of the counter-bell.

The kneader is opened, the paste on the side and on the bars is gathered to the bottom by means of a scraper. The whole paste of the chest $\mathrm{B}$ being removed, 150 kilogs. of the leaven are taken, to which 150 kilogs. of flour and water are added to prepare the 300 kilogs. of paste destined for the supply of the oven No. 2. These 75 kilogs. of leaven from each compartment are replaced as before, and so on in succession.

The water used in this operation is raised to the proper temperature, viz. $25^{\circ}$ or $30^{\circ} \mathrm{C}$. $\left(77^{\circ}\right.$ or $86^{\circ} \mathrm{F}$.) in cold weather, and to about $68^{\circ} \mathrm{F}$. in the hot season, by mixing common cold water with the due proportion of water maintained at the tem. perature of about $160^{\circ} \mathrm{F}$., in the basin $\mathrm{F}$ placed above the ovens.

Through the water poured at each operation upon the flour in the compartment B, there is previously diffused from 200 to 250 grammes of fresh leaven, as obtained from the brewery, after being drained and pressed (German yeast). This quantity is sufficient to raise preperly 300 kilogs. of dough. As soon as this dough is taken out of the kneader, as stated above, and while the machine goes on to work, the quantity res. uisite for each loaf is weighed, turned about on the table $D$, to give it its round or oblong form, and there is impressed upon it with the fore-arm or roller, the cavity which characterizes cleft loaves. All the lots of dough of the size of one kilog., called cleft loaves (pains fendus) are placed upon a cloth a fold of which is raised between two loaves, the cloth being first spread upon a board; which thus charged with 10 or 15 loaves is transferred to the wooden shelves G G in front of the oven.

The whole of them rise easily under the influence of the gentle temperature of this antechamber or fournil. Whenever the dough loaves are sufficiently raised here, they are put into the oven, a process called enfournement in France; which consists in setting each loaf on a wooden shovel dusted with coarse flour, and placing it thereby on the sole of the oven, close 1 . its fellow, without touching it. This operation is made easy, in consequence of the introduction of a long jointed gas-pipe and burner into the interior of the oven, by the light of which all parts of it may be minutely examined. The oven 
is first kept moderately hot, by shutting the dampers; but whenever the thermometer attached to it indicates a temperature of from $300^{\circ}$ to $290^{\circ} \mathrm{C}$. ( $572^{\circ}$ to $554^{\circ} \mathrm{F}$.), the dampers or registers are opened, to restore the heat to its original degree, by allowing of the circulation of the hot air, which rises from the lower carities around the fireplace into the interior of the oven. When the baking is completed the gaslight, which had been withdrawn, is again introduced into the oven, and the bread is taken out; called the process of defournement. If the temperature had been maintained at about $300^{\circ} \mathrm{C}$., the 300 kilogs. of dough divided into loaves of one kilog. ( $2 \frac{1}{4}$ lbs. avoirdupois) will be baked in 27 minutes. The charging having lasted 10 minutes, and the discharging as long, the baking of each batch will take up 47 minutes. But on account of accidental interruptions, an hour may be assigned for each charge of 260 .oaves of 1 kilog. each; being at the rate of 6240 kilogs. (or 6.75 tons) of bread in 24 hours.

Although the outer parts of the loaves be exposed to the radiation of the walls, heated to $280^{\circ}$ or $300^{\circ} \mathrm{C}$., and undergo therefore that kind of caramelization (charring) which produces the color, the taste, and the other special characters of the crust, yet the inner substance of the loaves, or the crumb, never attains to nearly so high a temperature; for a thermometer, whose bulb is inserted into the heart of a loaf, does not indicate more than $100^{\circ} \mathrm{C}$. $\left(212^{\circ} \mathrm{F}\right.$.)

The theory of panification (bread-baking) is easy of comprehension. The flour owes this raluable quality to the gluten, which it contains in greater abundance than any of the other cerealia (kinds of corn). This substance does not constitute, as had been heretofore imagined, the membranes of the tissue of the perisperm of the wheat; but is enclosed in cells of that tissue under the epidermic coats, even to the centre of the grain. In this respect the gluten lies in a situation analogous to that of the starch, and of most of the immediate principles of vegetables. The other immediate principles which play a part in panification are particularly the starch and the sugar; and they all operate as follows:-

The diffusion of the flour through the water, hydrates the starch and dissolves the sugar, the albumen, and some other soluble matters. The kneading of the dough, by completing these reactions through a more intimate union, farors also the fermentation of the sugar, by bringing its particles into close contact with those of the leaven or yeast; and the drawing out and malaxating the dough softens and stratifies it, introducing at the same time oxygen to aid the fermentation. The dough, when distributed and formed into loaves, is kept some time in a gentle warmth, in the folds of the cloth, pans, \&c., a circumstance propitious to the development of their rolume by fermentation. The dimensions of all the lumps of dough now gradually enlarge, from the disengagement of carbonic acid in the decomposition of the sugar; which gas is imprisoned by the glutinous paste. Were these phenomena to continue too long, the dough would become too vesicular; they must, therefore, be stopped at the proper point of sponginess, by placing the loaf lumps in the oven. Though this causes a sudden expansion of the enclosed gaseous globules, it puts an end to the fermentation, and to their growth; as also evaporates a portion of the water.

The fermentation of a small dose of sugar is, therefore, essential to true breadbaking; but the quantity actually fermented is so small as to be almost inapvreciable. It seems probable that in well-made dough the whole carbonic acid that is generated remains in it; amounting to one half the volume of the loaf itself at its baking temperature, or $212^{\circ}$. It thence results that less than one hundredth part of the weight of the flour is all the sugar requisite to produce well-raised bread. What egregious folly was it, therefore, to mount the bakery in Chelsea, twelve years ago, at an expense of 20,000l., for the purpose of catching the volatile spirits in their escape from the loaves in the oven-or, as it was rulgarly termed, "taking the gin out of the bread!" whereas it was nothing but taking the cash out of the pockets of the pseudo-chemical visionaries who swarm in this metropolis.

The richness or nutritive powers of sound flour and also of bread are proportional to the quantity of gluten they contain. It is of great importance to determine this point, for both of these objects are of enormous value and consumption; and it may be accomplished most easily and exactly by digesting in a water-bath, at the temperature of $167^{2}$ F., 1,000 grains of bread (or flour) with 1,000 grains of bruised barler-malt, in 5,000 grains, or in a little more than half a pint, of water. When this mixture ceases to take a hlue color from iodine (that is, when all the starch is converted into soluble dextrine) the gluten left unchanged may be collected on a filter cloth, washed, dried at a heat of 212 , and weighed. The color, texture, and taste of the gluten, ought also to be examined, in forming a judgment of good flour, or bread.

Independently of the skill of the baker, bread varies in quality according to the quantity of water and gluten it contains. A patent of German or French origin was obtained here a few years ago, for manufacturing loaf-bread by using thin boiled flour. 
paste instead of water for setting the sponge, that is, for the preliminary dough fermentation. By this artifice, 104 loaves of $4 \mathrm{lbs}$. each could be nlade out of a saci of flour, instead of 94 , as in ordinary baking; because the boiled paste gave a water-keeping faculty to the bread in that proportion. But this hydrated bread was apt to spoil in warm weather, and became an unprofitable speculation to all concerned.

Bread and flour are often adulterated in France with potato-starch, but almost never, I believe, in this country. This sophistication is easily detected by the microscope, on account of the peculiar ovoid shape and the large size of the particles of the potato fecula. Horse-bean flour gives to wheaten bread a pinkish tint. In spoiled flour (such as is too often used, partially at least, by our inferior bakers) the gluten someimes disappears altcgether, and is replaced by ammoniacal salts." In this case quichlime separates ammonia from the flour without heat; in flour slightly damaged, or ground from damaged wheat, the gluten present is deprived of its elasticity, and is softer than in the natural state. On this account the gluten test of M. Boland is raluable. It consists in putting some gluten into the bottom of a copper tube, and heating that tube in an oven, or in oil, at a temperature of $284^{\circ} \mathrm{F}$. The length to which the cylinder of gluten expands is proportional to and indicates its quality.

It appears that a French sack of flour, which weighs 159 kilogrammes, affords from 102 to 106 loaves of 2 kilogrammes each : and therefore,

$159: 52 \cdot 0:: 280: 91 \cdot 6$ : that is, if 159 kilogs. or lbs. afford 52 loaves of 4 kilogs. or lbs., 280 lbs., a sack English, should afford 91.6 loaves of 4 lbs. each; but our bakers usually make out 94 loaves, which are rated at 4 pounds, though ther seldom weigh so much. The loaves of a baker in my neighborhood; who supplied by family with bread for some time, were found on trial to be from 6 to $8 \mathrm{oz}$. deficient in weight; when challenged for this fraud, he had the effrontery to palliate it br alleging that all his neighbor bakers did the same. It must be borne in mind that a Paris loaf of 2 lbs. or 2 kilogs. contains more dry farina than a London loaf of like weight; for it contains, from its form and texture, more crust. The crumb is to the crust in the Paris long loaves, as 25 to 75 , or 1 to 3 : in our quartern loaves it is as 18 or 20 to 100 .

M. Dumas gives the following table:-

\begin{tabular}{|c|c|c|c|c|}
\hline $\begin{array}{l}\text { Weight of a } \\
\text { sack of flour. }\end{array}$ & $\begin{array}{l}\text { Number of } \\
\text { loaves. }\end{array}$ & $\begin{array}{l}\text { Weight of the } \\
\text { bread. }\end{array}$ & $\begin{array}{c}\text { Increase of } \\
\text { weight of flour. }\end{array}$ & $\begin{array}{l}\text { Ratio of dry flour } \\
=1, \text { to bread. }\end{array}$ \\
\hline $\begin{array}{l}159 \text { Kilogs. } \\
159 \text { do. } \\
159 \text { do. }\end{array}$ & $\begin{array}{l}102 \\
104 \\
106\end{array}$ & $\begin{array}{l}202 \text { Kilogs. } \\
208 \text { do. } \\
212 \text { do. }\end{array}$ & $\begin{array}{l}1 \cdot 283 \\
1 \cdot 300 \\
1 \cdot 333\end{array}$ & $1: 1 \cdot 60$ \\
\hline
\end{tabular}

Thus it would appear that the mean rield would correspond to 130 kilogs. of bread for 100 of the flour employed; and admitting that common flour contains $0 \cdot 17$ of water, the product would be equivalent to 150 of bread for 100 of flour absolutely dry. The whole loaf contains 66 per cent. of dry substance, and the crumb only 44.

BRICKS. Mr. F. W. Simms, C. E., communicated to the Institution of Civil Engineers, in April and May, 1843, an account of the process of brick-making for the Dover railway. The plan adopted is called slop-moulding, because the mould is dipped into water before receiving the clay, instead of being sanded as in making sand-stock bricks. The workman throws the proper lump of clay with some force into the mould, presses it down with his hands to fill the carities, and then strikes off the surplus clay with a stick. An attendant boy, who has previously placed another mould in a water trough by the side of the moulding-table, takes the mould just filled, and carries it to the floor, where he carefully drops the brick from the mould on its flat side, and leaves it to dry; by the time he has returned to the moulding-table, and deposited the empty mould in the water-trough, the brickmaker will have filled the other mould, for the boy to conrey to the floor, where they are allowed to dry, and are then stacked in readiness for being burned in clamps or kilns. The average product is shown in the following table:-

\begin{tabular}{|c|c|c|c|c|}
\hline Force employed. & Area of land. & Duration of season. & Produce per week. & Produce per season. \\
\hline $\begin{array}{l}1 \text { moulder - } \\
1 \text { temperer - } \\
1 \text { wheeler - } \\
1 \text { carrier boy - } \\
1 \text { picker boy - }\end{array}$ & Roods. Perches. & Weeks. & $\begin{array}{l}\text { Bricks. } \\
16,100\end{array}$ & Bricks. \\
\hline
\end{tabular}

*Dumas, Chimı Appliquée, vi. 425. 
It appears that while the produce in sand-stock bricks is to that of slop-bricks in the same time as 30 to 16 , the amount of labor is as 7 to 4 ; while the quantity of land, and the cost of labor per thousand, is nearly the same in both processes. The quantity of coal consumed in the kiln was at the rate of $10 \mathrm{cwt}$. 8lbs. per thousand bricks. The cost of the bricks was $2 l .1 s$. $6 d$. per thousand. The slop-made bricks are fully 1 pound heavier than the sand-stock. Mr. Bennett stated to the meeting, that at his brick-field at Cowley, the average number of sand-stock bricks moulded was 32,000 ; but that frequently so many as 37,000 , or even 50,000 , were formed. The total amount in the shrinkage of his bricks was $\frac{13}{1} \frac{3}{6}$ of an inch upon 10 inches in length; 1 ut this differed with the different clays. Mr. Simms objected to the use of machinery in brick-making, because it caused economy only in the moulding, which constituted no more than about one eighth of the total expense.

The principal rarieties of bricks are called malm, paviors, stocks, grizzles, places, and shuffs. For the first and best kind, the clay was washed and selected with care; stocks were good enough for ordinary building purposes; the rest are inferior. The difference in price between malms, paviors, and stocks, was $15 \mathrm{~s}$. or $20 \mathrm{~s}$. per 1,000; between stocks and places, $10 \mathrm{~s}$. The average weight of a sand-stock brick is fully 5 pounds, that of a slop is 1 pound more.

I believe that the siliceous sand on the surface of the sand-stocks is useful in favoring adhesion of mortar, by the production of a silicate of lime. To smooth aluminous bricks, mortar sometimes forms no stony adhesion.

Mr. Prosser, of Birmingham, makes bricks by pressure. The clay is first ground upon a slip kiln, as if for making pottery, then ground to a fine powder, and in that dry state it is subjected to the heary pressure of about 250 tons, in strong metal moulds, by which means it is reduced to about one third of its original thickness. The clay seems to have retained sufficient moisture to give it cohesion, and the tiles are perfectly sharp at the edges. They being then baked within seggars by the heat of a kiln, seldom crack in the baking. The bricks thus formed are denser than usual, and weigh $6 \frac{3}{4}$ lbss., with a specific gravity of $2 \cdot 5$.

Fig. 14., represents Mr. Hunt's brick-making machine. The principal working

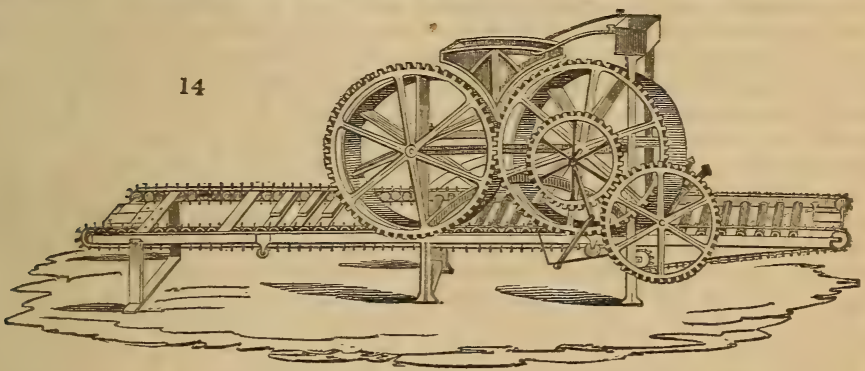

parts consist of 2 cylinders, each covered by an endless web, and so placed as to form the front and back of a hopper, the two sides being iron plates, placed so that when the hopper is filled with tempered clay from the pug-mill, the lower part of the hopper, and consequently the mass of clay within it, has exactly the dimensions of a brick. Beneath the hopper an endless chain traverses simultaneously with the movement of the cylinders. The pallet-boards are laid at given intervals upon the chain, and being thus placed under the hopper, while the clay is brought down with a slight pressure, a frame with a wire stretched across it, is projected through the mass of clay, cutting off exactly the thickness of the brick, which is removed at the same moment by the forward movement of the endless chain. This operation is repeated each time that a pallet-board comes under the hopper.

The chief object of this machine, which is worked by hand, is to produce good square sompact bricks of uniform quality, using only a slight pressure. It has been found to be very difficult to dry bricks made by machinery, where a considerable pressure has been employed, because, before the evaporation from the centre of the clay is completed, the surfaces have become hard and peel off. The present machine is in operation in several parts of England, producing usually about 1,200 bricks per hour, while each machine requires only 2 men and 3 boys to tend it, and to take off the bricks. The clot-moulders are dispensed with, and the workmen are common laborers, so that professed brick-makers at higher wages are not needed.

Fig. 15 shows Mr. Hunt's machine for making tiles, and it is on the same principle. It consists of two iron cylinders, round which webs or bands of cloth revolve, whereby 
the clay is pressed into a slab of uniform thickness, without adhering to the cylinders. It is then earried over a covered wheel, curved on the rim, which gives the tile the semi-cylindrical or other required form; after which the tiles are polished and finished

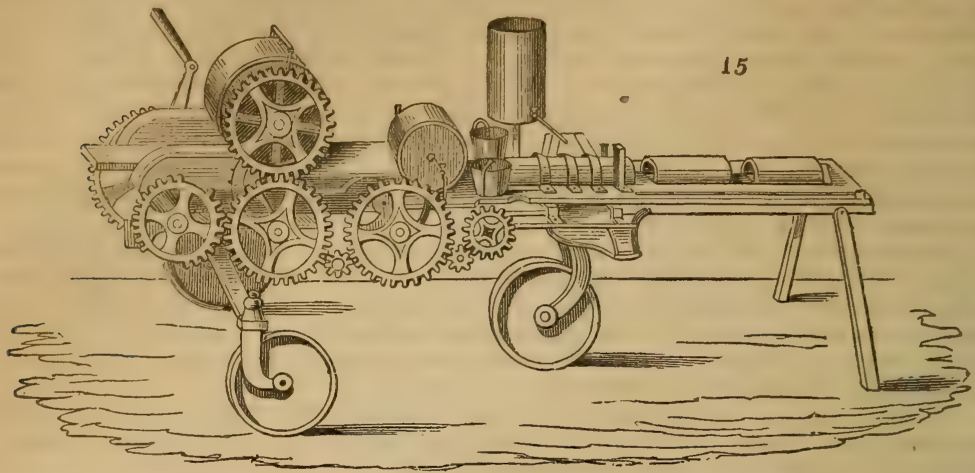

by passing through three iron moulds of a horse-shoe form, as shown in the centre of the cut, while they are at the same time moistened from a water cylinder placed above them. The tiles are next cut off to such lengths as are wanted, and carried away by an endless web, whence they are transferred by boys to the drying shelves.

Flat tiles, for sole pieces to draining tiles, are formed in nearly the same manner, being divided into two portions while passing through the moulds; the quantity of clay used for one draining tile being as much as for two soles.

The method of making bricks in the vicinity of London differed from that of almost all other places, because the material there employed is not pure clay, but a loam of a slightly cohesive nature, which will not admit of its being used in the natural state and buined in close kilns with coal; ; but with an admixture of ashes it becomes sufficiently tenacious to be formed into bricks, by inducing a slight semi-fusion. But the coal-ashes are also of advantage in the process of burning, because they enable the fire to spread gradually from the lower tiers, through the whole mass in the kiln or clamp, and thus obviate the effect of an intense partial heat, where distinct coal fires are trusted to alone, whereby the bricks nearest it get vitrified and glazed.

The brick kilns and clamps round London, and other large cities, which are fired with the breeze-rubbish collected from dust holes, that contain the refuse of kitchens, \&c., emit, in consequence, most unpleasant effluvia; but brick-kilns fired with clean coke or coals, give out no gases of a more noxious nature than common household fires. The consideration of this subject was closely pressed upon my attention on being consulted concerning an injunction issued by the chancellor against a brick clamp in the Isle of Wight, fired with clean coke cinders from the steam-engine furnace at Portsmouth Dock Yard. The bricks being of the description called sand-stock, were of course made in moulds very slightly dusted with sand, to make them fall freely out. The sand was brought from Portsmouth harbor, and on being subjected to a degree of heat, more intense certainly than it would suffer in the clamp, was discovered by two chemical witnesses to give out traces of hydrochloric acid. Not content with this trivial indication, the said chemists, in their evidence before the courts of law, paraded a train of goblin gases, as the probable products of the pre-adjudicated clamp.

As it is well known to the chemist that common salt strongly ignited in contact with moist sand will emit hydrochloric acid, there was nothing remarkable in the above observation, but I ascertained that the sand with which the moulds were strewed would give out no hydrochloric acid, at a heat equal at least to what the bricks were exposed to in a clamp 10 or 12 feet high, and fired at its bottom only with a layer of cinders 3 or 4 inches thick. But I further demonstrated that the entire substance of the brick with its scanty film of sand, on being exposed to ignition in a suitable apparatus, gave out, not hydrochloric or any other corrosive acid, but ammonia gas. Hence, the allegations that the clamp sent forth a host of acid gases to blight the neighboring trees, were shown to be utterly groundless; on the contrary, the ammonia evolved from the heated clay would act beneficially upon vegetation, while it was too small in quantity to annoy any human being. A few yards to leeward of a similar clamp, in full activity, I could perceive no offensive odor. All ferruginous clay, when exposed to the atmosphere, absorbs ammonia from it, and of course emits it again on being gently ignited. It is a reproach to science when, as in the above case, it lends itself to judicial prejudice and oppression. 
BRONZING (of Objects in Imitation of Metallic Bronze). Plaster of Paris, paper, wood, and pisteboard, may be made to resemble pretty closely the appearance of articles of real bronze, modern or antique. The simplest way of giving a brilliant aspect of this kind is with a rarnish made of the waste gold leaf of the beater, ground up on a porphyry slab with honey or gum-water. A coat of drying linseed-oil should be first applied, and then the metallic powder is put on with a linen dossil. Mosaic gold ground up with six parts of bone-ashes has been used in the same way. When it is to be put on paper, it should be ground up alone with white of eggs or spirit varnish, applied with a brush and burnished when dry. When a plate of iron is plunged into a hot solution of sulphate of copper, it throws down fine scales of copper, which being repeatedly washed with water, and ground along with six times its weight of boneashes, forms a tolerable bronzing.

Powdered and sifted tin may be mixed with a clear solution of isinglass, applied with a brush, and burnished or not, according as a bright or dead surface is desired. Gypsum casts are commonly bronzed by rubbing. brilliant black-lead, graphite, upon them with a cloth or brush. Real bronze long exposed the air gets covered with a thin film of carbonate of copper, called by virtuosi antique arugo (patine antique, Fr.) This mas be imitated in a certain degree by several applications skilfully made. The new bronze being turned or filed into a bright surface, and rubbed over with dilute aquafortis by a linen rag or brush, will become at first grayish, and afterward take a greenish blue tint; or we may pass repeatedly over the surface a liquor composed of 1 part of sal ammoniac, three parts of carbonate of potash, and 6 of sea salt, dissolived in 12 parts of boiling water, to which 8 parts of nitrate of copper are to be added; the tint thereby produced is at first unequal and crude, but it becomes more uniform and softer by time. A fine green-blue bronze may be obtained with very strong water of ammonia alone, rubbing it at intervals several times upon the metal.

The base of most of the secret compositions for giving the antique appearance is vinegar with sal ammcniac. Skilful workmen use a solution of 2 ounces of that salt in an English quart of French vinegar. Another compound which gives good results is made with an ounce of sal ammoniac, and a quarter of an ounce of salt of sorrel (binoxalate of potash), dissolved in vinegar. One eminent Parisian sculptor makes use of a mixture of half an ounce of sal ammoniac, half an ounce of common salt, an ounce of spirits of hartshorn, and an English quart of vinegar. A good result will also be obtained by adding half an ounce of sal ammoniac, instead of the spirits of hartshorn. The piece of metal being well cleaned, is to be rubbed with one of these solutions, and then dried by friction with a fresh brush. If the hue be found too pale at the end of two or three days, the operation may be repeated. It is found to be more advantageous to operate in the sunshine than in the shade.

BUDE LIGHT. See GAS LIGHT.

BUTTER is the fatty matter of milk, usually of that of the cow. Milk is composed of butter, caseine, sugar of milk, several salts, and water. The butter exists in the form of very small globules of nearly uniform size, quite transparent, and strongly refractive of light. Milk left in repose throws up the lighter particles of butter to the surface as cream It was imagined that the butter was separated in the process of churning, in consequence of the milk becoming sour; but this is not the case; for milk rendered alkaline by bi-carbonate of potash affords its butter fully more readily than acidulous milk. The best temperature for churning milk or cream is $53^{\circ} \mathrm{F}$.; that of $60^{\circ}$ is too high; and under $50^{\circ}$ it is too low. By the churning action the heat rises from. 3 to 4 degrees $\mathrm{F}$. All the particles of butter are never separated by churning; many remain diffused through the butter-milk, and are easily discoverable by the microscope. These are more numerous in proportion to the bulk of the liquid; and hence it is more economical to churn cream than the whole milk which affords it. It is computed that a cow which gives 1,800 quarts (old English) of milk per annum, eats in that time 8,000 lbs. of hay, and produces 140 lbs. of butter.* Analysis shows that this weight of hay contains 168 pounds of fat. The finest flavored butter is obtained from milk churned not long after it is drawn; but the largest proportion is derived from the cream thrown up by milk after standing 24 hours, in a temperature of about $50^{\circ} \mathrm{F}$. The butter-milk which contains the very fermentable substance, caseine, should be well separated from the butter by washing with cold water, ai $d$ by beating with the hands, or preferably, without water, for the sake of fine flavor, $1 \mathrm{y}$ the action of a press.

The French purify their butter by melting it in pots, plunged into water heated to $200^{\circ}$ or $212^{\circ}$; and sometimes they mix a pure brine with the melting butter, whereby they faror the subsidence of thie zoagulated caseine and other impurities. The supernatant clear butter should be drawn or poured off, and rapidly cooled, to prevent the crystallization of its stearine and separation of its oleine, which injure its flavor an. appearance.

* Two vour:ds 3nd a inatez $\cong$ hay corresnorid to one quart of good milk; and a cow which eats 16,500 ibs. of hay will produce 300 lbs. of butter per arnum. 


\section{C.}

CALCIUM. The atomic weight of this element being an umportant point, both as to pure chemistry and the chemical arts, has been the subject of innumerable researches. Very lately Berzelius, in the Annalen der Chemie und Pharmacie, XLVI. p. 241, has collated the most recent results of the analysis of other philosophers with his own; and while Dumas, Marchand, and Erdmann estimate the weight at 20, that of hydrogen $=1$, or 250 oxygen $=100$, he finds it ought to be, as compared with the latter, $251 \cdot 9$; and to the former, $20 \cdot 152$.

CALICO PRINTING (4-color machine). Of this beautiful and effective mechanism an accurate section is exhibited at p. 220 of the Dictionary. The outside working gear is shown in fig. 16., where $\mathrm{A}, \mathrm{A}$, is a part of the two strong iron frames or

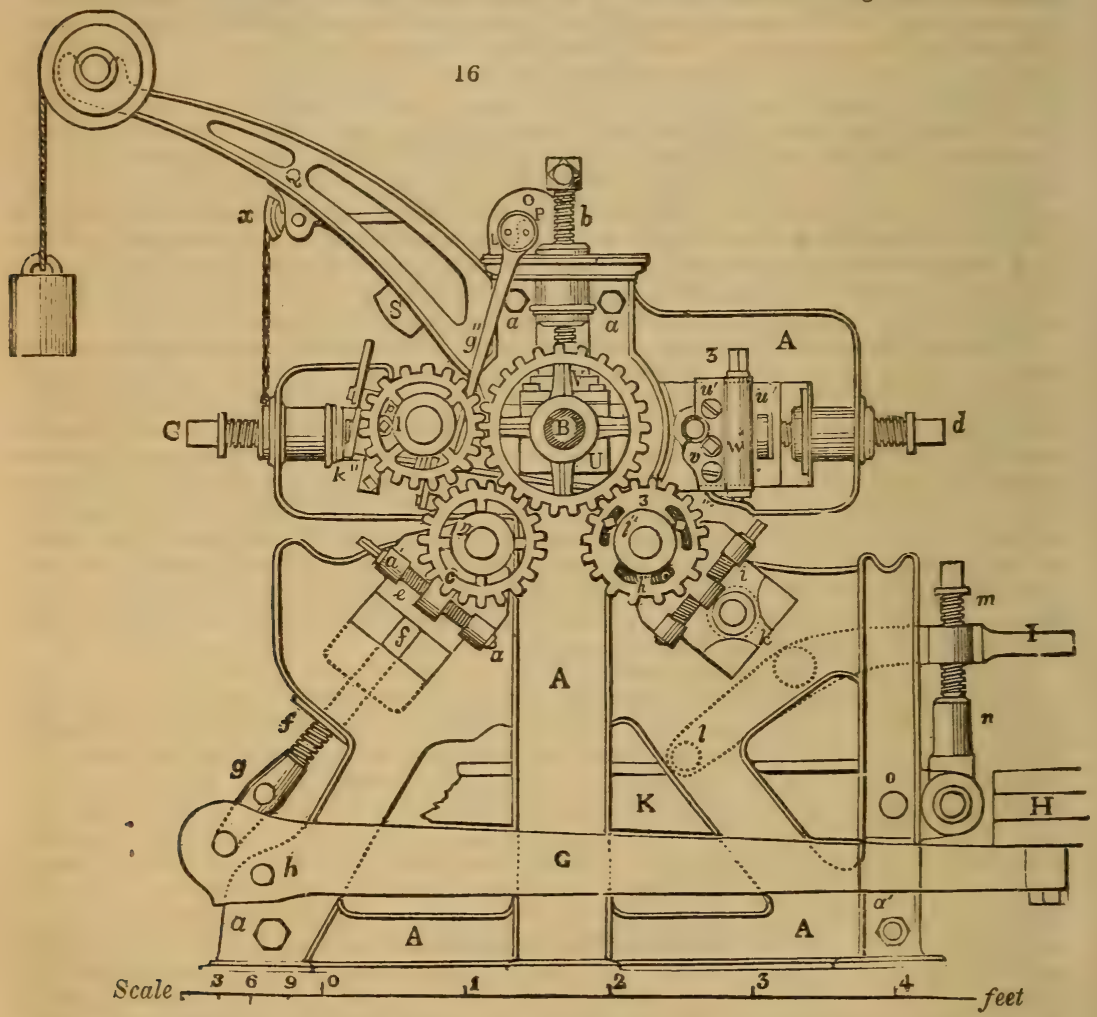

cheeks in which the various rollers are mounted. They are bound together by the rods and bolts $a, a, a . \mathrm{B}$, is the large iron pressure cylinder, which rests with its gudgeons in bearings or bushes, which can be shifted up and down in slots of the side cheeks A, A. These bushes are suspended from powerful screws, $b$, which turn in brass nuts, made fast to the top of the frame A, as is plainly shown in the figure. These screws serve to counteract the strong pressure applied beneath that cylinder by the engraved cylinders D, E.

C, D, E, F, are four printing cylinders, named in the order of their operation. They consist of strong tubes of copper or gun metal, forcibly thrust by a screw press upon the iron mandrels, round which as shafts they revolve. The first and last cylinders, $C$ and F, are mounted in brass bearings, which may be shifted in horizontal slots of the frame A. The pressure roller $B$, against whose surface they bear with a very little obliquity downward, may be nicely adjusted to that pressure by its elevating and depressing screws. "By this means $\mathrm{C}$ and $\mathrm{F}$ can be adjusted to $\mathrm{B}$ with geometrical precision, and made to press it in truly opposite directions. 
The bearings of the cylinders $\mathrm{D}$ and $\mathrm{E}$ are lodged also in slots of the frame $\mathbf{A}$, which point obliquely upward toward the centre of $B$. The pressure of these two print cylinders, $\mathrm{C}$ and $\mathrm{F}$, is produced by two serews, $c$ and $d$, which work in brass nuts made fast to the frame, and very visible in the figure. 'The framework in which these bearings and screws are placed has a curvilinear form, in order to permit the cylinders to be readily removed and replaced, and also to introduce a certain degree of elasticity Hence the pressure applied to the cylinders $\mathrm{C}$ and $\mathrm{F}$ partakes of the nature of a spring, a circumstance essential to their working smoothly, notwithstanding the occasional inequalities in the thickness of the felt web and the calico.

The pressure upon the other two print cylinders, $\mathrm{D}$ and $\mathrm{E}$, is produced by weights acting with levers against the bearings. The bearings of $\mathrm{D}$ are, at each of their ends, acted upon by cylindrical rods, which slide in long tubular bosses of the frame, and press with their nuts $g$, at their under end upon the smaller arms of two strong levers $\mathrm{G}$, which lie on each side of the machine, and whose fulcrum is at $h$ (in the lower corner at the left hand). The longer arms, of these levers, G, are loaded with weights, $\mathrm{H}$, whereby they are made to press up against the bearings of the roller $\mathrm{D}$, with any desired degree of force, by screwing up the nut $g$, and hanging on the requisite weights.

The manner in which the cylinder $\mathrm{E}$ is pressed up against $\mathrm{B}$ is by a similar construction to that just described. With each of its bearings there is connected, by the link $k$, a curved lever, I, whose fulcrum or centre of motion is at $o$. By turning, therefore, the screw $m$, the weight $\mathrm{L}$, laid upon the end of the longer arm of the lever $\mathrm{K}$ (of which there is one on each side of the machine), may be made to act or not at pleasure upon the bearings of the cylinder $\mathrm{E}$. The operation of this exquisite machine is minutely described in the Dictionary, pp. 220, 221.

A patent was obtained in August, 1839, by Mr. J. C. Miller of Manchester, for certain improvements in printing calicoes, consisting of a modified mechanism, by which the same effect can be produced as by block printing.

Figs. 17, 18, 19, are several views of this machine, calculated to print two pieces, or two different patterns (on the same block) of calico, side by side, or four pieces, the carriage printing both ways, the intended device consisting of four colors to be printed from blocks.

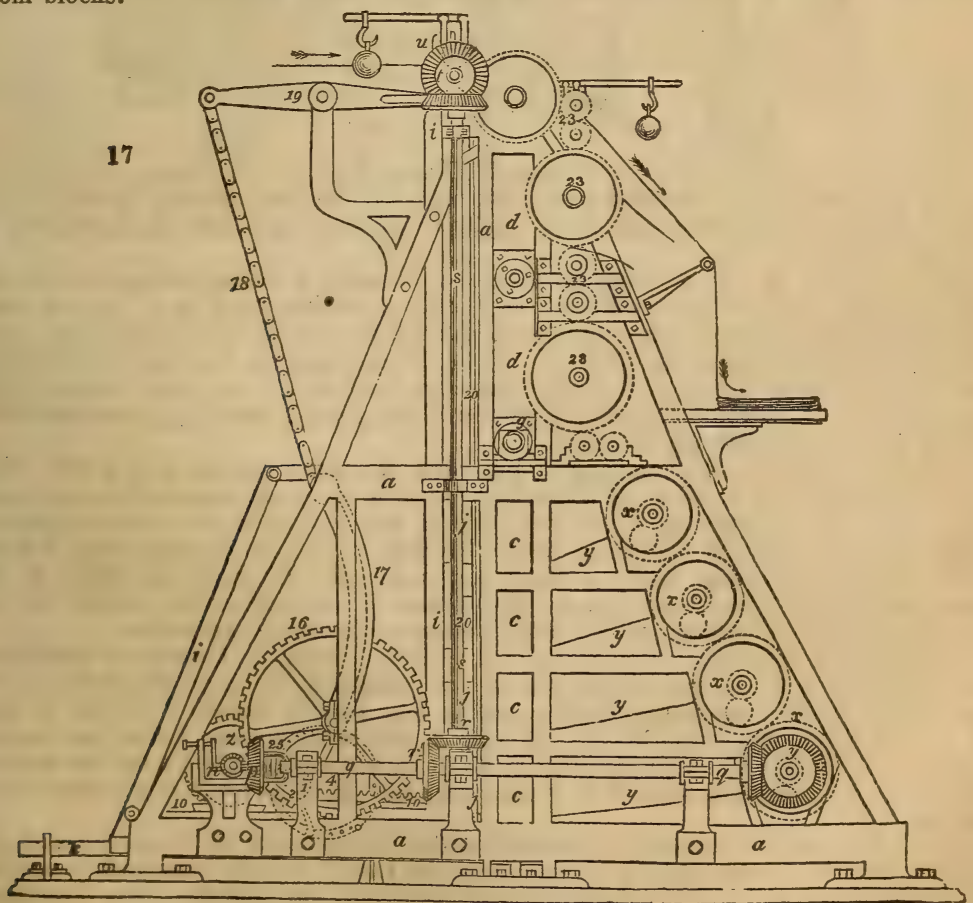

Fig. 17 represents a side elevation, fig. 18 a front view, and fig. 19 a transverse section, taken nearly through the middle of the machine. 
The side or main framing is shown at $a, a$, supporting the color boxes $b, b, b$, with their doclors; the furnishing tables or beds, $c, c, c$ (substitutes for the sieves in ordinary block printing); the printing table, $d, d$; and the feerling drying and coloring rollers, $f, f, g, g, h, h$.

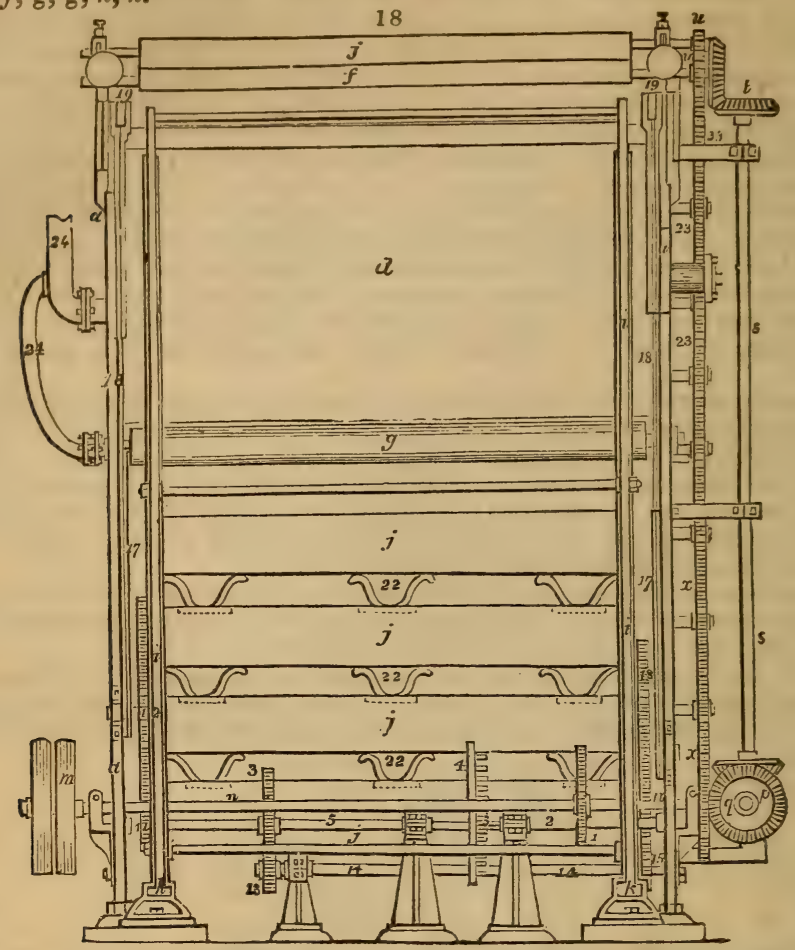

The machine is also provided with a carriage, $i, i$, for the printing blocks, $j, j, j$. This carriage, $i, i$, travels in and out at suitable ihtervals upon rails, $k, k$, attached to the main framing.

The operation of the machine is effected by passing a driving strap, $l$, round the driving pulley $m$, fixed at the extremity of the main driting shaft, $n, n$. At the other end of this shaft, the bevil pinion, 0 , is keyed, gearing at suitable intervals with the bevil wheel $p$,which is mounted upon the end of the cross shaft $q$; at about the middle of this shaft, the mitre wheels $r, r$, driving the upright shaft $s, s$, and mitre wheels $t, t$, above, actuate, by means of the spur pinions $u, u$, the feeding rollers $f, f$, and thus draw the pieces of goods into the machine.

Simultaneously with the progress of the cloth, the mitre wheels $v, v$, at the other end of the cross shaft $q$, drive the furnishing rollers $w, w, w$, by means of the spur gearing $x, x, x$. The furnishing rollers, revolving in their respective color-boxes, spread or apply the colors upon the travelling endless blankets, $y, y, y$, which pass round the top roller and the furnishing tables or beds, $c, c, c$, in order to supply the colors to the surfaces of the printing blocks, $j, j, j$. Either beds or the backs of the printing blocks may be made slightly elastic, to insure the perfect taking up of the colors.

Supposing the carriage, $i, i$, to be run out upon its railways, at the farthest point from: the beds $c, c$, it is drawn inward toward the furnishing beds $c, c$, by means of the spur-wheel $x$, upon the driving-shaft $n$, taking into a small pinion, 1 (shown by dots in fig. 17), upon the shaft, 2. On the end of this shaft is also keyed the mangle pinion, 3, gearing in the mangle wheel, 4, which is keyed upon the end of the shaft, 5 This shaft drives the spur-wheel, 6 , in gear with the pinion, 7 , made fast to the shaft, $s$ (see fig. 19).

Upon either end of the shaft, 5 , is a rack pinion, 9 , taking into the horizontal rack 10 , made fast to the carriage-frame, $i, i$; and thus the blocks, $j, j$, are presented to the furnishing blankets $y, y, y$, and take a supply of color ready for printing. The travellingcarriage and blocks now retire, by the agency of the mangle-wheel and pinion, 3 and 4 , 
the pinion being fired upon the end of the shaft, 2 , and the wheel upon the other shaft in a line with the shaft, 2. At this time another operation of the machine takes place.

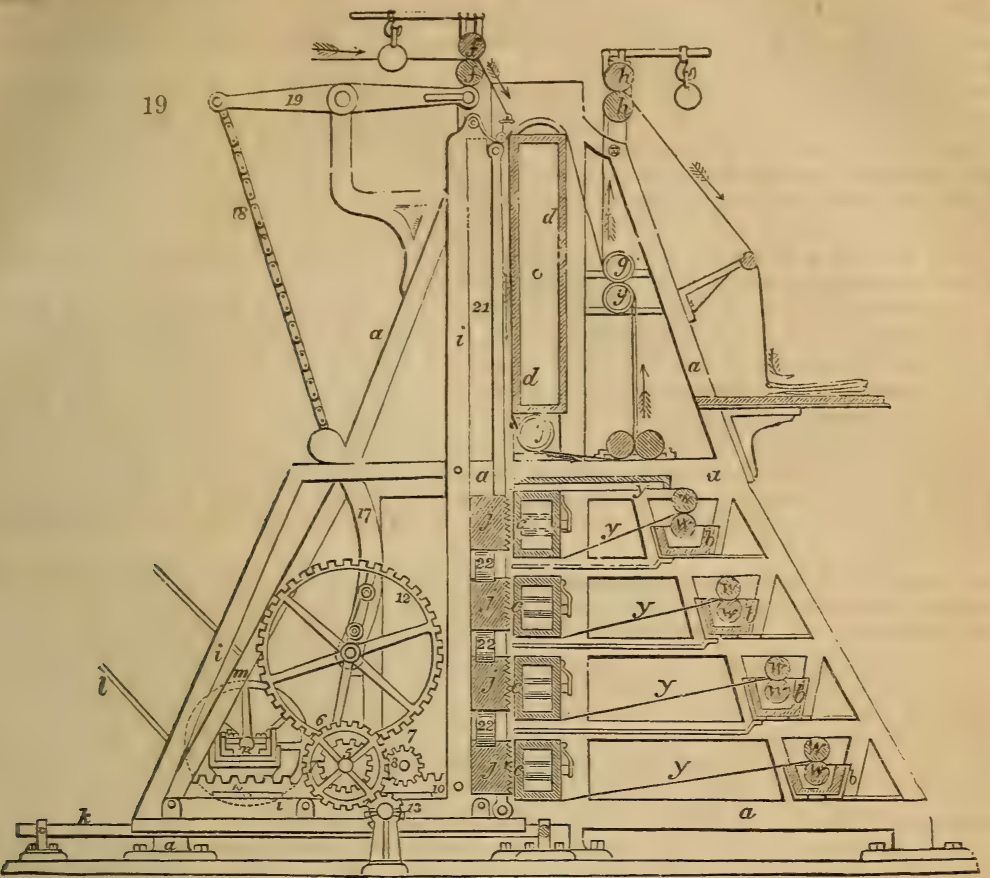

Upon the reverse end of the shaft, 5 , is a pinion, 11 , gearing with the spur-wheel, 12 ; and by means of the spur gearing, 6 and 13, and counter-shaft, 14, the pinion, 15, drives the spur-wheel, 16, which corresponds to the wheel, 12, on the other side of the machine. To one of these spur-wheels are attached by bolts two quadrant levers, 17, 17 ; and as these wheels revolve by means of the gearing just described, the levers, 17, 17, draw down the chains, 18,18 , actuate the levers, 19 and 20 , and thus elevate the whole series of printing blocks in the parallel groores, 21,21 ; at the same time pressing or closing them into one mass or block by expanding the springs 22,22 ; and at the nest of the carriage caused at a proper interval by the agency of the mangle-wheel, the blocks are made to impress the patterns upon the surface of the goods at once, in four or more different colors, and in one, two, or more widths of cloth at one operation.

The cloth is now drawn forward for the space of the exact width of one of the blocks, or slietch of the design, by means of the spur-wheels and pinions, 23, 23, and passed around heated cylinders, $g$, $g$, if necessary, and between the delivering rollers out of the machine. These operations are to be repeated by the continuous rotation of the main driving-shaft, until the printing is completed; the colors making a single advance upon the pattern at every presentation of the blocks, until the whole number of blocks has been presented to the same space or portion of the goods successively.

The steam pipes, 24 , are to be in connexion with the printing table and drying cylinders, in order to supply a degree of heat during the operation, which may be regulated at pleasure.

To give suitable intervals of rest and motion to the various parts of the driving-gear, an ordinary clutched box, 25 (shown in fig. 19), and regulated by suitable stops fixed to the travelling carriage, is used for throwing the wheel, $p$, in and out of gear with the pinion, $\mathrm{O}$; this is to prevent clots of color from being dragged upon the blocks or cloth. -Newton's Journal, xxi. C. S. p. 242.

CALOMEL. A patent was obtained in September, 1841, by Anthony Todd Thom sun, M. D., for an improved method of manufacturing calomel and corrosive sublimate, 23 follows :-

This invention consists in combining chlorine in the state of gas with the vapor of mercury or quicksilver, in order to produce calomel and corrosive sublimate. 
The apparatus employed consists of a glass, earthenware, or other suitable vesse, mounted in brick-work, and communicating at one end with a large air-tight chamber, and at the other end, by means of a bent tube, with an alembic, such as is generally used for generating chlorine gas. The alembic is charged with a mixture of common salt, binoxide of manganese and sulphuric acid, or of binoxide of manganese and muriatic acid, in order to produce chlorine gas.

The mode of operating with this apparatus is as follows : A quantity of mercury or quiclisilver is placed in the glass vessel, and the temperature of the same is raised to between $350^{\circ}$ and $660^{\circ} \mathrm{Fahr}$, by means of an open fire beneath. The chlorine gas, as it is generated, passes from the alembic through the bent tube into the glass ressel, and there combining with the vapor of the mercury, forms either corrosive sublimate or calomel, according to the quantity of chlorine gas employed.

The product is found at the bottom of the air-tight chamber, and may be removed from the same through a door, when the operation is finished.

According to the patent of Mr. Josiah Jewel, the rapor of calomel was to be transmitted into a ressel containing water, in order to condense it at once into an impalpable powder. But this process was beset with many difficulties. The rapor of the calomel was afterward introduced into a large receiver, into which steam was simultaneously admitted; but this plan has also been found to be precarious in the execution. The best way is to sublime the calomel into a very large chamber from an iron pot, in the same way as the flowers of sulphur are formed. The great body of cool air serves to cause the precipitation of the calomel in a finely comminuted state. It is afterward washed with water, till it is no longer colored by sulphuretted hydrogen.

- CALOTYPE is the name giren by Mr. Fox Talbot to the art inrented by him, of making pictures or paper or other such surfaces by the agency of light. It is merely an improved kind of photography. The process is as follows: Dissolve 100 grains of crrstallized nitrate of silver in 6 ounces of distilled water, and brush over the paper (Whatman's sized post answers well) with a soft brush on one side only, with this solution, and mark the side. When nearly dry, dip it into a solution of iodide of potassium (for only a few minutes), containing 500 grains of that salt dissolred in a pint of water. As soon as the paper is completed imbued with this solution, it should be immediately washed in distilled water, drained, and hung up to dry. This paper is to be kept for subsequent use in a portfolio, and carefully secluded from light.

Next dissolve 100 grains of silver-nitrate in 2 ounces of distilled water, and add to the solution one sixth of its volume of strong acetic acid. Keep this solution in the dark. Make a saturated solution of gallic acid in distilled water. When it is required to make a calotype picture, the two liquids last described are to be mixed in equal quantities, but only so much as is needed for the operation. With this gallonitrate of silver, a sheet of the silver iodide paper is to be washed over upon its marked side with a soft brush, an operation to be performed by candle-light. After half a minute, the paper being dipped in water, and dried lightly by pressure between folds of blotting paper, becomes so exceedingly sensitive to light, as to take a pictorial impression in the camera in a space varying from one second to five minutes, according to the brightness of illumination. The camera should be mounted with a meniscus lens, in an adjustible tube, so as to throw the image of the object to be calotyped upon a vertical plate of roughened glass, in the posterior side or wall of the wooden box. Whenever the focus is correctly adjusted, the glass is withdrawn, and replaced b: sliding in a groove a frame with the prepared sheet of paper fixed flat upon it, the pre pared side toward the lens, but screened from light by a card or thin board. The screen being now removed, the light acts upon the paper, and produces a picture. A camera made entirely of metal, in a conical form, and mounted on a stand like a telescope, which has been invented for calotrpe purposes, br Dr. Petzval and M. Voigtlander, of Vienna, is recommended in preference to all others, by Mr. Talbot, especially for taking portraits.

The paper, after exposure for the due time in the camera, is to be again covered from the light, taken out, and subjected to another process; for.as yet it has no pictoral appearance. To bring out this effect, it must be washed with the gallonitrate of silver, and then be gently warmed. In a few seconds the portions of the paper upon which the light has acted will begin to darken, and eventually grown quite black, while the rest of the paper retains its original hue. Even though the pictorial impression be very faint, it mar be brought out br a second application of the same solution. The operator should watch the gradual development of the tints; and wher: it is sufficient, he should fix them by dipping the paper in water, drying it slightly with blotti..g paper, then washing it over with a solution of bromide of potassium. containing 100 grains of that salt, dissolved in 8 or 10 ounces of water. Strong brine will also answer, but not so well. Similar calotype pictures may be made by using 
the bright light emitted from lime ignited by the oxy-hydrogen flame; as is practised in making the Daguerrotype portraits at night.

In all the photographic pictures the lights and shades of the object are reversed; but they may be made conformable to nature by rendering the paper transparent with white wax scraped upon its back, melting this in by rubbing it with a hot smoothingirom, after it is placed between two sheets of common paper, then laying it upon paper imbued with bromide of potassium, and exposing it to sunshine. Portraits are best taken by means of a lens, whose focal length is three or four times only greater than the diameter of the aperture.

CANDLES. Messrs. Hempel and Blundell have given a very minute account of the process for maling palm-oil, stearic and margaric acids, in the specification of their patent for this mode of manufacturing candles:-

1. Their first process is called crystallization, which consists in pouring the melted palm-oil into iron pans, and allowing it to cool slowly, whereby, at about $75^{\circ} \mathrm{F}$., the elaine separates from the crystalline stearine and margarine.

2. The concreted oil is subjected to the action of an hydraulic press, in order to separate the elaine from the solid fats.

3. This process is cilled oxidation. To $104 \mathrm{lbs}$. of the stearine and margarine, melted in an iron pan, about $12 \mathrm{lbs}$. of slaked and sifted quicklime are added, with diligent stirring, during which the temperature is to be slowly raised to $240^{\circ} \mathrm{F}$., and so maintained for about three hours, till a perfect chemical combination takes place. This is shown by the mass becoming thin, transparent, and assuming a glassy appearance when it cools. The fire being now withdrawn, cold water is added very gradually at first, with brisk stirring till the whole mass falls into a state of powdery granulation, when it is passed through a wire sieve to break down any lumps that may remain.

4. Separation of the stearic and margaric acids from the lime. For this purpose, as much muriate of lime (chlorcalcium) is talien as will, with its equivalent quantity of sulphuric acid ( $8 \mathrm{lbs}$. of dry chlorcalcium require $7 \mathrm{lbs}$. of the strongest sulphuric acid), produce as much muriatic acid as will dissolve the lime combined with the fat acids; and therefore that quantity of muriate of lime dissolved in water must be treated with as much sulphuric acid as will saturate its lime and throw it down in the state of sulphate of lime. Add the supernatant solution of muriatic acid in such proportion to the stearate and margarate of lime as will rather more than saturate the lime. Three pounds of muriatic acid diluted with 9 lbs. of water are stated as enough for $1 \mathrm{lb}$. of lime. This mixture is to be let alone for 3 or 4 days, in order to insure the complete separation of the lime from the fat acids; and then the mixture is heated so as to melt and cause them to separate in a stratum on the top of the liquid. The resulting muriate of lime is drawn off into another tub, and decomposed by its dose of sulphuric acid, so as to liberate its muriatic acid for a fresh operation.

5. The fat acids, being well washed by agitation with hot water, are then set to cool and crystallize, in which state they are subjected to the action of the hydraulic press, at a temperature of $75^{\circ} \mathrm{F}$., whereat the margaric acid runs off from the solid stearic acid.

6. Bleaching. The stearic acid is taken from the press, and exposed upon water in large shallow vessels placed in the open air, where it is kept at the melting temperature from 8 to 12 hours, stirring meanwhile, in order to promote the blanching action of the atmosphere. The margaric acid is bleached in a similar manner in separate vessels.

7. Refining process. The fat is warmed again, and poured in a liquid state into an agitating tub; where, for every $1,000 \mathrm{lbs}$. of the stearic acid, about $2 \frac{1}{2} \mathrm{lbs}$. of common black oxide of manganese, and $49 \mathrm{lbs}$. of concentrated sulphuric acid, diluted with $200 \mathrm{lbs}$. of pure water, are to be used. This solution (" mixture"), while warm from the heat erolved in diluting the acid, is placed in a suitable vessel above the agitating tub. The stearic acid being at the melting point, in the vessel below, agitation is to be given with a revolving shaft, while the mixed manganese and acid are run slowly down into it, till the whole be well mixed, which generally requires about two hours. The mass is allowed to lie in this state for 48 hours; after which it may be boiled by Ereal 2 for 2 or 3 hours, when it will be sufficiently refined. The sulphuric acid, which is at the bottom, is now run off, and the stearic acid which remains is well washed with pure water. It is then put into large conical vessels of stoneware, enclosed in a box or jacket, kept warm iy steam-heat, and lined with conical bags of suitable strong filtering paper, through which, being warm, it finds its way; and when the stearic acid has beun thus filtered, it is run into blocks, when it will be found to be a beautiful stearic acid or palm-wax, and is ready to be made into candles in the usual way.

On the above process with manganese and diluted sulphuric acid, it may be observed, that no solution or chemical action takes place between them, and their joint use seems therefore most problematical. The patentees proceed to describe other processes of 
refining, in which sulphate of manganese, with common salt, phosphoric acid (highly concentrated), and oxalic acid, are used, and in my opinion either ignorantly or for the purpose of mystification; for, as prescribed, they can serve no possible purpose of purifying the stearine.

The chief solid constituent of palm-oil is margaric acid. This they direct to be melted with tallow, in the proportion of from 10 to $20 \mathrm{lbs}$. of the former to $100 \mathrm{lbs}$. of the latter. See Newton's Journal, C. S., xi. $20 \%$.

I was told by $M$. Runge, at Berlin, that he was the inventer of the process for making white margaric acid from palm-oil, and that Hempel had got it somehow from him, but most imperfectly, as it would appear. Hempel died here in the midst of the above patent operations; but the specification is, no doubt, a specimen of his manufacture of Runge's margaric acid. He gave me a splendid pearly-looking sample.

CAOUTCHOUC. Hitherto the greater part of the caoutchouc has been imported into Europe from South America, and the best from Para; but of late years a considerable quantity has been brought from Java, Penang, Sincapore, and Assam. About three years ago, Mr. William Griffith published an interesting report upon the Ficus elastica, the caoutchouc-tree of Assam, which he drew up at the request of Captain Jenkins, agent in that country to the governor-general of India. This remarkable species of fig-tree is either solitary, or in twofuld or threefold groups. It is larger and more umbrageous than any of the other trees in the extensive forest where it aboands, and may be distinguished from the other trees, at a distance of several miles, by the picturesque appearance produced by its dense, huge, and lofty crown. The main trunk of one was carefully measured, and was found to have a circumference of no less than 74 feet; while the girth of the main trunk, along with the supports immediately round it, was 120 feet. The area covered by the expanded branches had a circumference of 610 feet. The height of the central tree was 100 feet.

It has been estimated, after an accurate survey, that there are 43,210 snch noble trees within a length of 30 miles, and breadth of 8 miles of forest near Ferozepoor, in the district of Chardwar, in Assam.

Lieutenant Veitch has since discovered that the Ficus elastica is equally abundant in the district of Naudwar. Its geographical range in Assam seems to be between 25 deg. $10 \mathrm{~min}$. and $27 \mathrm{deg} .20 \mathrm{~min}$. of north latitude, and between $90 \mathrm{deg} .40 \mathrm{~min}$. and $95 \mathrm{deg} .30 \mathrm{~min}$. of east longitude. It occurs on the slopes of the hills, up to an elevation of probably 22,500 feet. This tree is of the banyan tribe, famed for "cits pillared shade, where daughters grow about the mother-tree," which has furnished the motto tot rami quot arbores, to the Royal Asiatic Society. Species of this genus afford grateful shade, however, in the tropical regions of America, as well as Asia.

Many species of other trees yield a milky tenacious juice, of which birdlime has been frequently made; as Artocarpas integrifolia, and Lakoocha, Ficus indica, and religiosa, also F. T siela, Roxburghii, glomerata, and oppositifolia. From some of these an inferior kind of caoutchouc has been obtained.

The juice of the Ficus elastica of Chardwar is better when drawn from the old than from the young trees, and richer in the cold season than in the hot. It is extracted by making incisions a foot apart, across the bark down to the wood, all round the trunk, and also the large branches, up to the very top of the tree; the quantity which exndes increasing with the height of the incision. The bleeding may be safely repeated once every fortnight. The fluid, as fresh drawn, is nearly of the consistence of cream, and pure white. Somewhat more than half a maund ( $42 \mathrm{lbs}$.) is reckoned to be the average produce of each bleeding of one tree; or 20,000 trees will yield about 12,000 maunds of juice; which is composed in 10 parts, of from 4 to 6 parts of water, and, of caurse, from 6 to 4 parts of caoutchouc. The bleeding should be confined to the cold months, so as not to interfere with, or obstruct the vigorous vegetation of the tree in the hot months.

Mr. Griffith says that the richest juice is obtained from transverse incisions made into the wood of the larger reflex roots, which are half exposed above ground, and that it proceeds from the bark alone. Beneath the line of incision, the natives of Assam scoop out a hole in the earth, in which they place a leaf of the Phrynium capitatum, Lin., rudely folded up into the shape of a cup. He observes that the various species of Tetranthera, upon which the Moonga silkworm feeds, as also the castor oil plant, which is the chief food of the Eria silkworm, do not afford a milky caoutchouc juice, Hence it would appear that Dr. Royle's notion of caoutchoue forming a necessar: ingredient in the food of silkworms, and being " in some way employed in giving tenacity to their silk," seems to be unfounded. If botany discountenances this idea. chemistry would seem to scout it altogether; for silk contains 11.33 per cent. of azote, and caoutchouc contains none at all; being simply a solid hydro-carburet, and, therefore, widely dissimilar in constitution to silk, which consists of oxygen $34 \cdot 04$, azote $11 \cdot 33$, carbon $50 \cdot 69$, and hydrogen $3 \cdot 94$, in 100 parts. 
This hydro-carburet emulsion is of common occurrence in the ordens Euphorbiacia and 'I ulicer. which may be looked on as the main sources of caoutchouc. The American cautchouc is said to be furnished by the Siphonia elastica, or the Hevea guianensis of Aublet, a tree which grows in Brazil, and also in Surinam.

1)r. Royle sent models of cylinders, of $1 \frac{1}{2}$ to $2 \frac{1}{2}$ inches in diameter, and 4 or 5 inches in length, to both the Asiatic and Agricultural Societies of Bengal, to serve as patterns for the natives to mould their caoutchouc by. Mr. Griffith says that this plan of forming the caoutchonc into tumblers or bottles, as recommended by the committee of the London Joint-stock Caoutchouc company, is, in his opinion, the worst than can possibly be offered; being tedious, laborious, causing the caoutchouc to be blackened in the drying, and not obviating the viscidity of the juice when it is exposed to the sun. He recommends, as a far better mode of treating the juice, to work it up with the hands, to blanch it in water, and then subject it to pressure. I shall presently describe a still better method which has recently occurred to me, in experimenting upon the caoutchouc juice. This fluid, with certain precautions, chiefly exclusion from air and much warmth, may be kept in the state of a creamy emulsion for a very long time.

NEW EXPERIMENTAL RESEARCHES ON CAOUTCHOUC.

The specific gravity of the best compact Para caoutchouc, taken in dilute alcohol, is - _ _ _ _ 0.941567

The specific gravity of the best Assam is - _ - $\quad$ - 0.942972

66 $\quad$ " $\quad$ - $\quad$ Sincapore $\quad 0.936650$

"6 $\quad$ * $\quad$ - 0.919178

Having been favored by Mr. Sievier, formerly managing director of the Joint-stock Caoutchouc company, and by Mr. Beale, engineer, with two different samples of caoutchouc juice, I have subjected each to chemical examination.

That of Mr. Sievier is grayish brown, that of Mr. Beale is of a milky gray color ; the deviation from whiteness in each case being due to the presence of aloetic matter, which accompanies the eaoutchouc in the secretion by the tree. The former juice is of the consistence of thin cream, has a specific gravity of 1.04125 , and yields, by exposure upon a porcelain capsule, in a thin layer, for a few days, or by boiling, for a few minutes, with a little water, 20 per cent. of solid caoutchouc. The latter, though it has the consistence of pretty rich cream, has a specific gravity of only 1.0175 . It yields no less than 37 per cent. of white, solid, and very elastic caoutchouc.

It is interesting to observe how readily and compactly the separate little cloths or threads of caoutchouc coalesce into one spongy mass in the progress of the ebullition, particularly if the emulsive mixture be stirred; but the addition of water is necessary to prerent the coagulated caoutchouc from sticking to the sides or bottom of the vessel and becoming burnt. In order to convert the spongy mass thus formed into good caoutchouc, nothing more is requisite than to expose it to moderate pressure between the folds of a towel. By this process the whole of the aloetic extract, and other vegetable matters, which concrete into the substance of the balls and junks of caoutchouc prepared in Assam and Java, and contaminate it, are entirely separated, and an article nearly white and inodorous is obtained. Some of the cakes of American caoutchouc exhale when cut the fœtor of rotten cheese; a smell which adheres to the threads made of it, after every process of purification.

In the interior of many of the balls which come from both the Brazils and East Indies, spots are frequently found of a viscid tarry-looking matter, which, when exposed to the air, act in some manner as a ferment, and decompose the whole mass into a soft substance, which is good for nothing. Were the plan of boiling the fresh juice along with its own bulk of water, or a little more, adopted, a much purer article would be obtained, and with incomparably less trouble and delay, than has been hitherto brought into the market.

I find that neither of the above two samples of caoutchouc juice affords any appearance of coagulum when mixed in any proportions with alcohol of 0.825 specific gravity; and. therefore, I infer that albumen is not a necessary constituent of the juice, as $\mathrm{Mr}$. Faraday inferred from his experiments published in the 21st vol. of the Journal of the Royal Institution.

The odor of Mr. Sievier's sample is slightly acescent, that of Mr. Beale's, which is by far the richer and purer, has no disagreeable smell whatever. The taste of the latter is at first bland and very slight, but eventually very bitter, from the aloetic impression upon the tongue. The taste of the former is bitter, from the first, in consequence of the great excess of aloes which it contains. When the brown solution which remains in the capsule, after the caoutchouc has been separated in a spongy state by ebullition, from 100 grains of the riches juice is passed through a filter and evaporated. it leaves 4 grains of concrete aloes. 
Buth of these emulsive juices mix readily with water, alcohol, and pyroxilic spirit, though they do not become at all clearer; they will not mix with caoutchoucine (the distilled spirit of caoutchouc), or with petroleum-naphtha, but remain at the bottom of these liquids as distinct as mercury does from water. Soda caustic ley does not dissolve the juice; nitric acid (double aquafortis) converts it into a red curdy magma. The filtered aloetic liquid is not affected by the nitrates of baryta and silver; it affords with oxalate of ammonia minute traces of lime.

\section{CAOUTCHOUC MANUFACTURE:}

This department of operative industry has, within a few years, acquired an importance equal to that of some of the older arts, and promises, ere long, to rival even the ancient textile fabrics in the variety of its designs and applications. The manufacture of caoutchouc has, at present, three principal branches :-1. The condensation of the crude lumps or shreds of caoutchouc, as imported from South America, India, \&c., into compact homogeneous blocks, and the cutting of these blocks into cakes or sheets for the stationer, surgeon, shoemaker, \&c. 2. The filature of either the Indian rubber hottles, or the artificial sheet caoutchouc, into tapes and threads of any requisite length and fineness, which, being clothed with silk, cotton, linen, or woollen yarns, form the basis of elastic tissues of every kind. 3. The conversion of the refuse cuttings and coarser qualities of caoutchouc into a riscid varnish, which, being applied between two surfaces of cloth, constitutes the well-known double fabrics, impervious to water and air.

I. The caoutchouc, as imported in shinny shreds, fibrous balls, twisted concretions, cheese-like cakes, and irregular masses, is, more or less, impure, and sometimes fraudulently interstratified with earthy matter. It is cleansed hr being cut into small pieces and washed in warm water. It is now dried on iron trays, heated with steam, while being carefully stirred about to separate any remaining dirt, and is then passed through, between a pair of iron rolls, under a stream of water, whereby it gets a second rashing, and becomes at the same time equalized by the separate pieces being blended together. The shreds and cuttings thus laminated, if still foul or heterogeneous, are thrown back into a kind of hopper over the rolls, set one sixteenth of an inch apart, and passed sereral times through between them. The abore method of preparation is that practised by Messrs. Keene and Co., of Lambeth, in their excellent manufactory, under a patent granted in October, 1836, to Mr. Christopher Nickels, a partner in the firm.

In the great establishment of the Joint Stock Caoutchoue compant, at Tottenham, originally under the direction of Mr. Sievier, a gentleman distinguished no less by his genius and taste as a sculptor, than by his constructive talents, the preparatory rinsing and lamination are superseded by a process of washing practised in Mr. Nickels's second operation, commonly called the grinding, or, as it should more properly be stried, the kneading. The miil employed for agglutinating or incorporating the separate fragments and shreds of caoutchouc into a homogeneous elastic ball, is a crlindrical box or drum of cast iron, 8 or 9 inches in diameter, set on its side, and traversed in the line of its horizontal axis (also 8 or 9 inches long) by a shaft of wrought iron, furnished with 3 rows of projecting bars, or kneading arms, placed at angles of $120 \mathrm{deg}$. to each other. These act by rotation against 2 chisel-shaped teeth, which stand obliquely up from the front part of the bottom of the drum. The drum itself consists of 2 semi-cylinders; the under of which is made fast to a strong iron framing, and the upper is hinged to the under one behind, but bolted to it before, so as to form a corer or lid, which may be opened or laid back at pleasure, in order to examine the caoutchouc from time to time, and take it out when fully kneaded. In the centre of the lid a funnel is made fast, by which the cuttings and shreds of the Indian rubber are introduced, and a stream of water is made to trickle in, for washing away the foul matter often imbedded in it. The power required to turn the axis of one of these mills, as the drums or boxes are called, may be judged of from the fact, that if it be only 2 inches in diameter, it is readily twisted asunder, and requires to be 3 inches to withstand every strain produced by the fixed teeth holding the caoutchouc against the revolving arms. Five pounds constitute a charge of the material.

One of the most remarkable phenomena of the kneading operation, is the prodigious heat disengaged in the alternate condensation and expansion of the caoutchouc. Though the water be cold as it trickles in, it soon becomes boiling hot, and emits copious rapors. When no water is admitted, the temperature rises much higher, so that the elastic lump, though a bad conductor of heat, can not be safely tonched with the hand. As we shall presently find that caoutchouc suffers no considerable or permanent diminution of its volume by the greatest pressure which can be appiied, we must ascribe the heat evolved in the kneading process to the violent intestine movements excited throughout all the particles of the elastic mass.

During the steaming, much muddy water runs off through apertures in the bottom of the drum. In the course of half an hour's trituration, the various pieces become 
agglutinated into a soft, elastic, ovoid ball, of a reddish brown color. This ball is now trinsterred into another similar iron drum, where it is exposed to the pricking and kneading action of 3 sets of chisel-points, 5 in each set, that project from the revolving shaft at angles of 120 degrees to each other, and which encounter the resistance occasioned by five stationary chisel-teeth, standing obliquely upward from the bottom of the drum. Here the caoutchouc is kneaded dry along with a little quicklime. It soon gets very hot; discharges in steam through the punctures, the air and water which it had. imbibed in the preceding washing operation; becomes, in consequence, more compac; ; and, in about an hour, assumes the dark brown color of stationers' rubber. During all this time irequent explosions take place, from the expansion and sudden extrication of the imprisoned air and steam.

From the second set of drums the ball is transferred into a third set, whose revolving shaft, being furnished both with flat pressing bars, and parallel sharp ehisels, perpendicular to it, exercises the twofold operation of pricking and kneading the mass, so as to condense the caoutchouc into a homogeneous solid. Seven of these finished balls, weighing, as above stated, 5 pounds each, are then introduced into a much larger iron drum of similar construction, but of much greater strength, whose shaft is studded all round with a formidable array of blunt chisels. Here the separate balls become perfectly incorporated into one mass, free from honeycomb cells or pores, and therefore fit for being squeezed into a rectangular or cylindrical form in a suitable cast-iron mould, by the action of a screw-press. When condensed to the utmost in this box, the lid is secured in its place by screw bolts, and the mould is set aside for several days. It is a curious fact, that Mr. Sievier has tried to give this moulding force, by the hydraulic press, without effect, as the cake of caoutchouc, after being so condensed, resiles much more considerably than after the compressing action of the screw. The cake form generally preferred for the recomposed, ground, or milled caoutchoue, is a rectangular mass, about 18 inches long, 9 inches broad, and 5 inches thick.

This is sliced into cakes for the stationer, and into sheets for making tapes and threads of caoutchouc, by an ingenious self-acting machine, in which a straight steel blade, with its edge slanting downward, is made to vibrate most rapidly to and fro in a horizontal plane; while the cake of caoutchouc, clamped or embraced at each side between two strong iron bars, is slowly advanced against the blade by screw-work, like that of the slide rest of a lathe. In cutting caoutchouc by knives of every form, it is essential that either the blade or the incision be constantly moistened with water; for otherwise the tool would immediately stick fast. As the above straight vibrating knife slants obliquely downward, the sheet which it cuts off spontaneously turns up over the blade in proportion as it is detached from the bottom mass of the cake. The thicker slices are afterward cut by hand, with a wetted knife, into small parallelopipeds for the stationer, the sections being guided rectangularly by saw lines in a wooden frame. The wholesale price of these is now reduced to $2 s$. per pound. Slices may be cut off to almost any desired degree of thinness, by means of an adjusting screw-a mechanism that acts against a board which supports the bottom of the cake, and raises it by any aliquot part of an inch, the cutting-blade being caused to vibrate always in the same horizontal plane. These thin slices constitute what is called sheet-caoutchouc, and they serve tolerably for making tubes for pneumatic apparatus, and shcaths of every kind; since, if their two edges be cut obliquely with clean scissors, they may be made 0 coalesce, by gentle pressure, so intimately, that the line of junction can not be discovered either by the eye, or by inflation of a bag or tube thus formed.

The mode of recomposing the cuttings, shreds, and coarse lumps of caoutchouc, into a homogeneous elastic cake, specified by Mr. Nickels, for his patent, sealed October 24, 1836, is not essentially different from that above described. The eylinders of his mill are more capacious, are open at the sides like a cage, and do not require the washingapparatus, as the caoutchouc has been cleansed by previous lamination and rinsing He completes the kneading operation, in this open cylinder, within the space of about two hours, and afterward squeezes the large ball so formed into the cheese form, in $a$ mould subjected to the action of an hydraulic press. As he succeeds perfectly in making compact cakes in this way, his caoutchouc must differ somewhat in its physical constitution from that recomposed by Mr. Sievier's process. He uses a press of the power of 70 tons; such pressure, however, must not be applied suddenly, but progres sively, at intervals of two or three minutes between each stroke; and when the pressing is complete, he suffers the caoutchouc to remain under pressure till it is cold, when he thrusts it out of the mould entirely, or, placing his mould in the slide-rest mechanism, he gradually raises the caoutchouc out of it, while the vibrating knife cuts it into slices in the manner already described. The elegant machine by which these sheets are now so easily and accurately sliced, was, I believe, originally contrived and constructed by $\mathbf{M r}$. Beale, engineer, Church-lane, Whitechapel. 


\section{I1.-FILATURE OF CAOUTCHOUC FOR MAKING ELASTIC FABRICS.}

Messrs. Rattier and Guibal mounted in their factory at St. Denys, so long ago as the year 1826 or 1827 , a machine for cutting a disc of caoutchouc into a continuous fillet spirally, from its circumference toward its centre. This flat dise was made by pressing the bottom part of a bottle of Indian rubber in an iron mould. I have described this machine under the article ExAstic Bands, in the Dictionary. A machine on the same principle was made the subject of a patent by Mr. Joshua Proctor Westhead, of Manchester, in February 16, 1836; and, being constructed with the well-known precision of Manchester workmanship, it has been found to act perfectly well in cutting a disc of canutchouc, from the circumference toward the centre spirally, into one continuous length of tape. For the service of this machine, the bottom of a bottle of Indian rubber of good quality being selected, is cut off and flattened by heat and pressure into a nearly round cake of uniform thickness. This cake is made fast at its centre by a screw nut and washer to the end of a horizontal shaft, which may be made to revolve with any desired velocity by means of appropriate pulleys and bands, at the same time that the edge of the disc of caoutchouc is acted on by a circular knife of cast-steel, made to revolve 3,000 times per minute, in a plane at right angles to that of the disc, and to advance upon its axis progressively, so as to pare off a continuous uniform tape or fillet from the circumference of the cake. During this cutting operation, the knife and caoutchouc are kept constantly moist with a slender stream of water. A succession of threads of any desired fineness is afterward cut out of this fillet, by drawing it in a moist state through a guide slit, against the sharp edge of a revolving steel disc. This operation is dexterously performed by the hands of young girls. MM. Rattier and Guibal employed, at the above-mentioned period, a mechanism consisting of a series of circular steel knives, fixed parallel to each other at minute distances, regulated by interposed washers upon a revolving shaft; which series of knives acted against another similar series, placed upon a parallel adjoining shaft, with the effect of cutting the tape throughout its length into eight or more threads at once. An improved modification of that apparatus is described and figured in the specification of Mr. Nickels's patent of October, 1836. He employs it for cutting into threads the tapes made from the recomposed caoutchouc.

- The body of the bottle of Indian rubber, and in general any hollow cylinder of caoutchouc, is cut into tapes, by being first forced upon a mandril of soft wood of such dimensions as to keep it equally distended. This mandril is then secured to the shaft of a lathe, which has one end formed into a fine-threaded screw, that works in a fixed nut, so as to traverse from right to left by its rotation. A circular disc of steel, kept moist, revolves upon a shaft parallel to the preceding, at such a distance from it as to cut through the caoutchouc, so that, by the traverse movement of the mandril-shaft, the hollow cylinder is cut spirally into a continuous fillet of a breadth equal to the thickness of the side of the cylinder. Mr. Nickels has described two methods of forming hollow cylinders of recomposed caoutchouc, for the purpose of being cut into fillets by such a machine.

It is probable that the threads formed from the best Indian rubber bottles, as imported from Para, are considerably stronger than those made from recomposed caoutchouc, and therefore much better adapted for making Mr. Sievier's patent elastic cordage. When, however, the kneading operation has been skilfully performed, I find that the threads of the ground caoutchouc, as it is incorrectly called by the workmen, answer well for every ordinary purpose of elastic fabrics, and are, of course, greatly more economical, from the much lower price of the material.

Threads of caoutchouc are readily pieced by paring the broken ends obliquely with scissors, and then pressing them together with clean fingers, taking care to admit no grease or moisture within the junction line. These threads must be deprived of their elasticity before they can be made subservient to any torsile or textile manufacture. Each thread is inelasticated individually in the act of reeling, by the tenter boy or girl pressing it between his moist thumb and finger, so as to stretch it to at least eight times its natural length, while it is drawn rapidly through between them by the rotation of the power-driven reel. This extension is accompanied with condensation of the caoutchouc, and with very considerable disengagement of heat, as pointed out in Nicholson's Journal upward of thirty years ago, by Mr. Gough, the blind philosopher of Kendal. I attempted to stretch the thread, in the act of reeling, but found the sensation of heat too painful for my unseasoned fingers. The reels, after being completely filled with the thread, are laid aside for some days, more or fewer, according to the quality of the caoutchouc, the recomposed requiring a longer period than the bottle material. When thus rendered inelastic, it is wound off upon bobbins of various sizes, adapted to various sizes of braiding, or other machines, where it is to be clothed with cotton or other yarn. 
In the process of making the ELASTIC TISSUEs, the threads of caoutchouc being first of all deprived of their elasticity, are prepared for receiving a sheath upon the braiding. machine. For this purpose they are stretched by hand, in the act of winding upon the reel, to 7 or 8 times their natural length, and left two or three weeks in that state of tension upon the reels. Thread thus inelasticated has a specific gravity of no less than 0.4) 48732 ; but when it has its elasticity restored, and its length reduced to its pristine state, by rubbing between the warm palms of the hands, the specific gravity of the same piece of thread is reduced to 0.925939 . This phenomenon is akin to that exhibited in the process of wire-drawing, where the iron or brass gets condensed, hard, and brittle, while it disengages much heat: which the caoutchouc thread also does in a degree intolerable to unpractised fingers, as above mentioned.

The thread of the Joint-Stock Caoutchouc company is numbered from 1 to 8 . No. 1 is the finest, and has about 5,000 yards in a pound weight; No. 4 has 2,000 in the pound weight; and No. 8, 700, being a very powerful thread. The finest is used for the finer elastic tissues, as for ladies' silver and gold elastic bracelets and bands. The ropes made by Mr. Sievier with the strongest of the above threads, clothed with hemp and worked in his gigantic braiding-machine, possess, after they are re-elasticated by heat, an extraordinary strength and elasticity; and, from the nearly rectilinear direction of all the strands, can stand, it is said, double the strain of the best patent cordage of like diameter.

In treating of the manufacture of elastic fabrics, I have great pleasure in adverting to the riband-looms at Holloway, which display to great advantage the mechanical genius of the patentee, Mr. Sievier. Their productive powers may be inferred from the following statement: 5,000 yards of 1 -inch braces are woven weekly in one 18 riband-loom, whereby the female operative, who has nothing to do but watch its automatic morements, earns 10 s. a week; 3,000 yards of 2 -inch braces are woven upon a similar loom in the same time. But one of Mr. Sievier's most curions patent inrentions, is that of producing, by the shrinking of the caoutchouc threads in the foundation or warp of the stuff, the appearance of raised figures, closely resembling coachlace, in the weft. Thus, by a simple physical operation, there is produced, at an expense of one penny, an effect which could not be effected by mechanical means for less than one shilling.

\section{OF THE WATER-PROOF DOUBLE FABRICS,}

The parings, the waste of the kneading operations above described, and the coarsest qualities of imported caoutchouc, such as the inelastic lumps from Para, are worked up into rarnish, wherewith two surfaces of cloth are cemented, so as to form a compound fabric, impervious to air and water. The caoutchouc is dissolved either in petroleum (coal-tar) naphtha, or oil of turpentine, by being triturated with either of the solvents in a close cast-iron ressel, with a stirring apparatus, moved by mechanical power. The heat generated during the attrition of the caoutchouc, is sufficient to favor the solution, without the application of fuel in any way. These triturating cylinders have been called pug mills by the workmen, because they are furnished with obliquely pressing and rerolving arms, but in other respects they differ in construction. They are 4 feet in diameter and depth, receive $13 \mathrm{cwt}$. at a time, have a vertical revolving-shaft of wrought iron 4 inches in diameter, and make one turn in a second. Three days are required to complete the solution of one charge of the varnish materials. The proportion of the solvent oils varies with the object in view, being always much less in weight than the caoutchouc.

When the varnish is to be applied to very nice purposes, as bookbinding, \&c., it must be rubbed into a homogeneous smooth paste, by putting it in a hopper, and letting it fall between a couple of parallel iron rolls, set almost in contact.

The wooden framework of the gallery in which the water-proof cloth is manufactured, should be at least 50 yards long, to give ample room for extending, airing, and drying, the pieces; it should be 2 yards wide, and not less than 5 high. It is formed of upnght standards of wood, bound with three or four horizontal rails at the sides and the ends. At the end of the gallery, where the varnish is applied, the web which is to be smeared must be wound upon a beam, resembling in size and situation the cloth-beam of the weaver's loom. The piece is thence drawn up and stretched in a horizontal direction orer a bar, like the breast-beam of a loom, whence it is extended in a somewhat slanting direction downward, and passed over the edge of a horizontal bar. Above this bar, and parallel to it, a steel-armed edge of wood is adjusted, so closely as to leave but a narrow slit for the passage of the varnish and the cloth. This horizontal slit may be widened or narrowed at pleasure by thumb-screws, which lower or raise the moreable unper board. The caoutchouc paste being plastered thickly with a long spatula of wood upon the down-sloped part of the web, which lies between the breastbeam and the abore-described slit, the cloth is then drawn through the slit by means of 
cords in a horizontal direction along the lowest rails of the gallery, whereby it gets uniformly besmeared. As soon as the whole web, consisting of about 40 yards, is thus coated with the viscid varnish, it is extended horizontally upon rollers, in the upper part of the gallery, and left for a day or two to dry. A second and third coat are then applied in succession. Two such webs, or pieces, are next cemented face to face, by passing them, at the instant of their being brought into contact, through between a pair of wooden rollers, care being taken by the operator to prevent the formation of any creases, or twisting of the twofold web. The under one of the two pieces being intended for the lining, should be a couple of inches broader than the upper one, to insure the uniform covering of the latter, which is destined to form the outside of the garment. The double cloth is finally suspended in a well-ventilated stove-room, till it becomes dry, and nearly free from smell. The parings cut from the broader edges of the under piece, are reserved for cementing the seams of cloaks and other aricles of dress. The tape-like shreds of the double cloth are in great request among gardenere or nailing up the twigs of wall shrubs.

Mr. Walton, of Sowerby-bridge, has recently substituted sheet Indian rubber for leather, in the construction of fillet-cards for the cotton and tow manufactures. The superior elasticity of this article is said to prove advantageous in several respects.

Mr. Charles Keene, proprietor of the extensive and well-organized Indian rubber factory in Lambeth, obtained a patent in March, 1840, for applying a coat of caoutchouc to the outer surface of flexible leather. The varnish of caoutchouc, made with oil of turpentine, has so much lampblack incorporated with it, as to bring it to the consistence of dough. The edge of the doe-skin, buck-skin, or wash-leather, being introduced between a pair of wetted iron rollers, as much of the India rubber compound, softened by a gentle heat, and rolled into a proper length as will cover the leather, is laid in the hollow between the leather and the moist cylinders. By their rotation, the coating is evenly affected. When the surface has become dry, it may be embossed or gilt, and varnished over with a solution of shellac, with a little Venice turpentine, in alcohol. After two or three applications of this kind, the leather is passed through a pair of rollers, either smooth or embossed. When made-up articles, such as shoes or portmanteaus, \&c., are to be covered, the Indian rubber varnish is used in a thinner state.-Newton's Journal, xxiii. 357.

CARMINE. This valuable pigment is often adulterated with starch. Water of ammonia enables us to detect this fraud by dissolving the pure carmine, and learing the starchy matter, as well as most other sophisticating substances. Such debased carmine is apt to spoil with damp.

CASSAVA, or Tapioca, is obtained principally from the Jatropha Manioc. Its extraction is remarkable for the large quantity of hydrocyanic acid which the juice of that plant contains. When distilled it affords, as a first product, a liquor which, in the dose of 30 drops, will cause the death of a man in the course of six minutes; and it is well known that this acid does not pre-exist in the plant, but that it is generated in it, after it is grated down into a pulp. It would be interesting to discover in what state the substance exists, from which it proceeds. After the grating of the root, and washing of the pulp, this is dried upon hot plates, to agglutinate it into the form of concretions, constituting the tapioca of commerce. But the starch of the washed root floated in water, is spontaneously deposited, and, when dried in the sun, forms Cassava, flour, called moussache by the French.

CASTOR OIL. Imported for consumption in 1839, 710,344 lbs.; in 1840, 807,175 lbs.: duty, $1 s$. $3 d$. per cwt.

CEMENTS. See Mortar, Hydraulic.

An excellent cement for resisting moisture is made by incorporating thoroughly eight parts of melted glue, of the consistence used by carpenters, with four parts of linseed oil, boiled into varnish with litharge. This cement hardens in about forty-eight hours, and renders the joints of wooden cisterns and casks air and water tight. A compound of glue with one fourth its weight of Venice turpentine, made as above, serves to cement glass, metal, and wood, to one another. Fresh made cheese-curd, and old slim-milk cheese, boiled in water to a slimy consistence, dissolved in a solution of bicarbonate of potash, are said to form a good cement for glass and porcelain. The gluten of wheat, well prepared, is also a good cement. White of eggs, with flour and water well mixed, and smeared over linen cloth, forms a ready lute for steam joints in small apparatus.

White lead ground upon a slab with linseed oil varnish, and kept out of contact of air, affords a cement capable of repairing fractured bodies of all kinds. It requires a few weeks to harden. When stone and iron are to be cemented together, a compound of equal parts of sulphur with pitch answers very well.

CHALYBEATE is the name given in medicine to preparations of iron. The most agreeable, and one of the most powerful, forms of such medicines, is the im. 
provel chalybeate water, for which Mr. Henry Bewley, apothecary in Dublin, obtained a patent in June, 1842 . 'The following is his valuable recipe:-Eight ounces of crystallized citric acid being dissolved in about four times their weight of water heated to $170^{\circ} \mathrm{F}$., are saturated with pure peroxide of iron, in the washed state, after being precipitated by ammonia from the ferric sulphate. The solution is sweetened, flavored, and charged highly with carbonic acid gas, so as to make a very palatable potion, agreeable also to the stomach.

I find by analysis that 100 parts of Mr. Bewley's brilliant citrate of iron contain $2 Q .5$ of peroxide, 48.5 of citric acid, and 23 of water; and that a six ounce phial of his chalybeate water contains of that citrate a quantity equivalent to nearly 8 grains of peroxide of iron.

Similar compounds are also specified to be made with other organic salts, as the tartrate or lactate of iron.-Newton's Journal, xxii. 470.

CHAMELEON MINERAL. As this compound-so long known in chemistry as a mere curiusity, on account of the surprising changes of color which it spontaneously assumes-has of late been largely employed for whitenirg tallow, palm oil, and decoloring other organic matters, it merits description in this dictionary. It exists in two states; one of which is called by chemists the manganate of potash, and the other the oxymanganate; denoting that the first is a compound of manganic acid with potash, and that the second is a compound of oxymanganic acid with the same base. They are both prepared in nearly the same way; the former by calcining together, at a red heat in a covered crucible, a mixture of one part of the blach peroxide of manganese with three parts of the hydrate of potash (the fused potash of the apothecary). The mass is of a green color when cold. It is to be dissolved in cold water, and the solution allowed to settle, and become clear, but by no means filtered for fear of the decomposition to which it is very prone. When the decanted liquid is exaporated under the exhausted receiver of an air-pump, over a surface of sulphuric acid, it affords crystals of a beautiful green color, which should be laid on a clean porous brick to drain and dry. They may be preserved in dry air, but should be kept in a well-corked bottle. They are decomposed by water, but dissolve in weak water of potash. On diluting this much, decomposition of the salt ensues, with all the chameleon changes of tint; red, blue, and violet. Sometimes a green solution of this salt becomes red on being heated, and preserves this color even when cold, but resumes its green hue the moment it is shaken : it might, therefore, furnish the crafty rotaries of St. Januarius with an admirable means of mystifying the worshippers at his shrine. The original calcined mass, in being dissolved, always deposites a considerable quantity of a brown powder, which is a compound of the acid and peroxide of manganese combined with water. Much of the potash remains unchanged, which may be recovered.

The oxymanganate of potash is made by fusing, with a strong heat, a mixture of equal parts of peroxide of manganese and hydrate of potash, or one part of peroxide and two parts of nitre. The mass is to be dissolved in water, and, if the solution be green, it should be reddened by the cautious addition of a few drops of nitric acid. The clarified liquor is to be evaporated to the point of crystallization. Even the smallest crystals of this salt have such an intense red color, that they appear black with a green metallic reflection. In the air they gradually assume a steel gray hue, without undergoing any essential change of nature. A very little of the salt reddens a large body of water. The least portion of any organic matter added to the solution of this salt reduces the oxymanganic acid to the state of peroxide, which precipitates combined with water; and the liquor becomes green or colorless, according to circumstances.

A more permanent oxymanganic salt may be made as follows:-Melt chlorate of potash over a spirit lamp, and throw into it a few pieces of hydrate of potash, which immediately dissolve, and form a limpid liquid. When peroxide of manganese in fine powder is gradually introduced into that melted mixture, it immediately dissolves, with the production of a rich green color. After adding the manganese in excess, the whole is to be exposed to a gentle red heat, in order to decompose the residuary chlorate of potash. It is now a mixture of manganate of potash, chloride of potassium and peroxide of manganese. It forms with water a deep green-colored solution; which when boiled assumes a fine red color, in consequence of its becoming an oxymanganate, and it ought to be decanted off the sediment while hot. By cooling, and still more after further evaporation, the oxymanganate of potash separates in crystals possessed of great lustre ; but toward the end colorless crystals of chloride of potassium.

Both the above salts are readily decomposed by organic bodies and other combustibles, whereby they have their acid converted into an oxide, with the disengagement of oxygen, and the destruction of many vegetable and animal colors. In this respect they resemble the nitrates and chlorates. 
CHINA INK. (Encre de Chine, Fr.; Chinesischer Tusch, Germ.) The finest kind of this useful pigment is seldoin inet with in our markets. According to a description in a Japanese book, it is made from the condensed smoke or soot of burned camphor; and hence, when of the best quality, it has this odor. Most of the China ink is made from oil-lampblack occasionally disguised, as to smell, with musk, or with a little camphor black. The binding substance is gelatine, commonly made from parchment or ass's skin; but isinglass answers equally well. A good imitation may be made by dissolving isinglass in warm water, with the addition of a very little alkali (soda), to destroy its gelatinizing power; and incorporating with that solution, by levigation on a porphyry slab, as much of the finest lampblack as to produce a mass of the proper consistence. The minute quantity of alkali serves also to saponify the oil which usually adheres to lampblack; and thereby to make a pigment readily miscible with water.

CHLORATE OF POTASH. The following ingenious and easy way of making this valuable compound has been lately suggested by Professor Graham :-Mix equal atomic weights of carbonate of potash and hydrate of lime ( 70 of the former, if pure, and 37 of slaked lime in powder), diffuse them through cold water, and transmit chlnride gas through the mixture. The gas is absorbed with great avidity, and the production of a boiling heat. When the saturation is complete, carbonate of lime remains, and a mixture of muriate and chlorate of potash, which latter salts are to be separated, as usual, by the diflerence of their solubility in water.

It has been remarked on the above process, that it effects no saving of potassa, and therefore is far inferior to the one long practised in several parts of Germany, especially at Giessen, and introduced into this country a good many years ago by Dr. Wagenmann, from Berlin. The chlorine is passed into a mixture of one equivalent of chloride of potassium (76), and 6 equivalents of hydrate of lime (222), previously stirred with water, to the consistence of a thin paste. Thus the calcium of the lime unites with the chlorine to form chloride of calcium, while the chloride of potassium is converted into chlorate of potassa, which salt is easily separated in crystals by its sparing solubility.

Chlorate of petash may also be made by saturating with chlorine a mixture of 74 parts of chloride of potassium (muriate of potash) and 168 parts of quicklime, brought to the consistence of a thin pap by the cautious addition of water. The mass being dissolved in warm water, and evaporated and cooled, yields crystals of chlorate of potash, while a mother water of chloride of calcium (muriate of lime), remains. The following process has likewise been prescribed:-Mix 10 parts of good chloride of lime with water into a pap, and evaporate to dryness, whereby it is converted into a mixture of chloride of calcium and chlorate of lime devoid of bleaching power: dissolve it in water, filter, concentrate the solution by evaporation, then add to it 1 part of chloride of potassium, and cool for crystallization. The salt which may thereby be separated from the chloride of calcium will afford 0.83 of pure chlorate of potash. By this process of Professor Liebig five sixths of the potash are saved, but much oxygen is wasted in the evaporation to dryness of the chloride of lime, and, consequently, much chloric acid is lost toward the production of the salt. Vée mixes the chloride of lime pap, before heating it, with the chloride of potassium, boils the mixture smartly, whereby much oxygen is undoubtedly thrown off, and then sets the liquor aside to crystallize. L. Gmelin suggests that saturation of the liquor with chlorine before boiling might be advantageous. Gay Lussac has suggested to make this valuable salt by precipitating a solution of chloride of lime with carbonate (or sulphate) of potash, saturating the liquor after filtration with chlorine gas, evaporating, and crystallizing.

Professor Juch's process is to pass chlorine gas into a mixture of 1 pound caustic lime and 1 pound carbonate of potash, with 8 pounds of water. The resulting chloride of potash readily separates in the filtered liquid by crystallization, from the rery soluble chloride of calcium. By this method, potash is not wasted in the useless production of chloride of potassium.

CHOCOLATE. About eighteen months ago, samples of chocolate were sent to me for analysis, by order of the lords of the admiralty. It was made at the victuallingyard, Deptford, for the use of the Royal Navy, by the government chocolate-mills, where about 400 tons are annually prepared, to be distributed to the sailors and convicts at the rate of an ounce daily, and to be used at their breakfast. After taking the said chocolate for some time, men in several ships complained of its occasioning sickness, vomiting, purging, and more serious maladies, terminating in a few cases fatally. I examined it with great care, but could find no injurious ingredient in it, and no chemical alteration from the beans of the Guyaquil coco from which it was manufactured. But I observed that it consisted of gritty grains, from very imperfect trituration or milling; that these grains were quite immiscible with water, like so much fine gravel; that they contained many sharp spiculæ of the coco-bean husks, and that bence, when swallowed, they were calculated to form mechanically irritating lodge- 
ments in the villous coats of the stomach and bowels, whereby they could produce the morbid eflects certified by several naval surgeons. It was, moreover obvious that, frum the insoluble condition of the chocolate, it could be of little use as an article of foud, or as a demulcent substitute for milk, and that, in fact, three fourths of it were, on this account, an ineffective article of diet; or were wasted.

Having reported these results and opinions to the Lords of the Admiralty, they were pleased, after a few weeks' consideration, to request me to go down to the victualling yard at Deptford, and superintend the preparation of a quantity of chocolate in the best manner I could with the means there provided. I accordingly repaired thither on the 13th of September, 1842, and experienced the utmost courtesy and cooperation fiom Sir John Hill, the Captain Superintendent, and his subordinate officers. The coco-beans had been heretofore milled, after a slight roasting upon the sole of an oat-kiln, along with their husks. As I was satisfied, from analysis, that the husks were no better food than sawdust, and that they might cause irritation by their minute spiculie left after grinding between rotating millstones, I set about a plan for shelling them, but could find no piece of apparatus destined for the purpose. There was however, a pea-sheling mill, which had been used only for one day some years before, and had stood ever since idle, ${ }^{*}$ which, on being cleaned and having its millstones placed at a proper distance, was found to answer pretty well. The beans for experiment, to the amount of $6 \mathrm{cwt}$, had been previously roasted, under my care, at a well-regulated heat, with much stirring, in the oat-kiln; and, on being cold, were run through the shelling mill, which was put in communication with the fanners of the flour mill. $\mathrm{By}$ this arrangement, the coco-beans were tolerably shelled, and the kernels separated from their scaly husks. The weighings were accurately made.

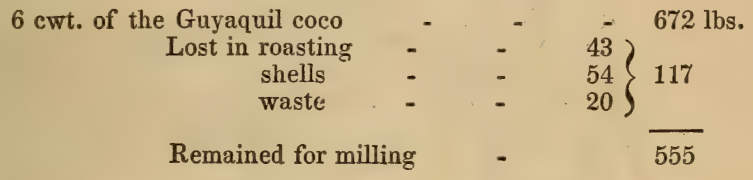

On the 14th September I made a report to the Lords of the Admiralty upon the experiments of the 13 th, of which the following is an outline. After describing the pains taken to regulate the roasting temperature, and to equalize the effect upon the beans by moving them occasionly by a rake, I stated that the oat-kiln was not well adapted to the purpose of roasting the coco, because it was impossible to turn the beans regularly and continuously during the process, so that they could not be equally roasted, and because it was an unwholesome operation for the workmen, who must go into a chamber filled with noxious gases and fumes, to use the rakes. When the door of the kiln was shut, to allow the burned air from the fire below to draw up through it, mischief might be done to the stratum of coco on the sole, and when the door was again opened, to permit a person to go in and stir, time and heat were wasted in replenishing the chamber with fresh air. I understand that a revolving-cylinder-roasting machine had been made by Messrs. Rennie for the chocolate process at Deptford; but, for reasons unknown to me, it had never been employed.

The diminution of weight by roasting and shelling may be estimated at about 17 per cent. A part of this loss is moisture, which should be completely expelled, to prevent its causing the chocolate to become mouldy at sea. But a part of the defalcation was also due to some of the coco remaining in the crevices of the pea-splitting mill and the fanners, which would not be observable if these were in constant employment. I think, therefore, that the roasted kernels may be estimated in general at 85 per cent. of the raw beans.

Fig. 20 represents the chocolate mills at the victualling-yard, Deptford, as mounted by the celebrated engineers, Messrs. Rennie. There are four double millstones, A, B, C, D, each three feet in diameter, of which the nether rests upon a bed of cast iron, like a drum-head, kept at the temperature of about $220^{\circ}$ by the admission of steam to the case below. Over each mill there is a feeding-hopper $1,2,3$, 4 , in communication by the pipes $5,6,7,8$, with the general reservoir $\mathrm{E}$, charged upon the floor above with coco through the funnel placed over it. The vertical shafts which turn these mills are marked F, G, H, L; they are moved by the train of bevil wheels above, which are driven by an arm from the main shaft of the steam engine. Each mill can, of course, be thrown in and out of gear at pleasure. At I, I, I, I, the discharge-spout is shown, which pours out the semi-fiuid hot chocolate into shallow cylindrical tin pans,

* It was found that peas in their skin kept better at sea than the split peas, and they were also pre ferred by the sailors in their natural state 
capable of containing about nine pounds of chocolate each. These four mills are capable of converting upward of a ton of coco into good chocolate in a day, on the system of double trituration which I adopted, and two tons on the former rough plan. I found that the two stones of each mill had been placed so far asunder as would allow entire

20

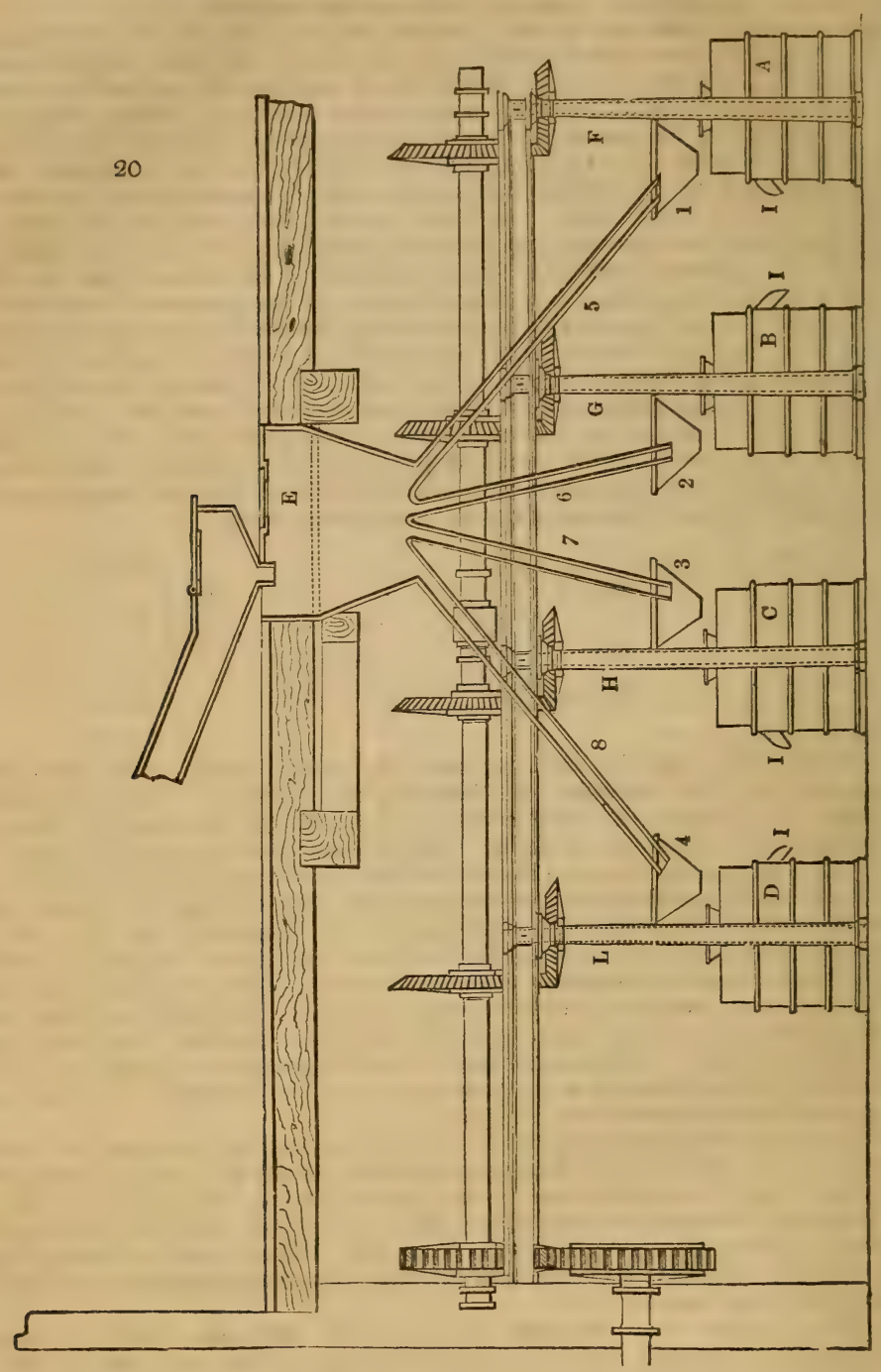

beans to pass through, as spurious chocolate, at one operation; but the chocolate thus discharged was in a very, gritty state, whereas good chocolate in the liquefied state should be smooth and plastic between the fingers, and spread upon the tongue without leaving any granular particles in the mouth. To obtain such a result, I divided the milling into two steps; for the first, two pairs of the stones, $\mathrm{A}$ and c, were set as close together as for a paint mill (which they closely resemble), and the other two pairs, B and D, were left at their ordinary distance. The paste obtained from the first set was transferred, while nearly liquid, into the hoppers of the second pairs, from which it issued at the spouts as thin and smooth as honey from the comb. In subservience to these experiments, I made an analysis of the Guyaquil coco, which $I$ found to be composed as follows :- 
Concrete fat or butter of coco, dissolved out by ether - $\quad$ - $\quad \mathbf{3 7}$

Brown extractive, extractible by hot water, after the operation of ether -

Ligrneous matter, with some albumin

Shells

Water

Loss

$\begin{array}{lllll}- & - & - & - & - \\ - & - & - & - & - \\ - & - & & \end{array}$

-

$\begin{array}{lll}- & - & - \\ - & - & - \\ - & - & -\end{array}$

The solid fat of the coco should be most intimately combined by milling with the extractive, albumine, and ligneous matter, "in order to render it capable of forming an emulsion with water; and, indeed, on account of the large proportion of concrete fat in the beans, some additional substance should be introduced to facilitate this emulsive union of the fat and water. Sugar, gum, and starch or flour, are well adapted for this purpose.

Under this conviction I employed in the first of these trials at Deptford, made with one half of the above roasted kernels $=277 \frac{1}{2}$ lbs. 5 per cent. of sugar, which was first mixed upon a board with shovels, and the mixture was then put progressively into the hoppers of the two mills $B$ and $D$. The paste which ran out of their spouts, was immediately poured into the hoppers of $\mathrm{A}$ and $\mathrm{c}$, from which it flowed smooth and very thin into the concreting pans. The sugar supplied to me was excerdingly moist, whereas it ought to be dry, like the bag sugar of the Mauritius. The other half of the coco kernels was milled alone once by the ordinary mills $\mathbf{B}$ and $\mathbf{D}$. I subjected next day samples of these two varieties of chocolate to the following examination, and compared them with the sample of chocolate as usually made at Deptford, as also with a sample of chocolate soll by a respectable grocer in London. A like quantity of these four samples was treated with eight times its weight of boiling water, the diffusion well stirred, and then left to settle in a conical wineglass. Of the ordinary Deptford coco, four fifths rapidly subsided in coarse grains, incapable of forming anything like an emulsion with water, and therefore of little or no avail in making a breakfast beverage.

1. The single-milled chocolate made under my direction formed a smoother emulsion than the last, on account of the absence of the coco husks; but its particles were gritty, and subsided very soon.

2. The sugared double-milled chocolate, on the contrary, formed a milky-looking emulsion, which remained nearly uniform for some time, and then let fall a soft mucilaginous deposite, free from grittiness.

3. The shop chocolate formed a very indifferent emulsion, though it was well milled, becaus it contained evidently a large admixture of a coarse branny flour, as is too generally the case.

I have given small samples of the above No. 2 chocolate to various persons, and they have considered it superior to what is usually sold by our grocers. The presence of dry sugar in chocolate would also give it a conservative quality at sea, and prevent it from getting musty.

The lords of the admiralty, after seeing the above two samples of chocolate, and my report thereupon, were, about six weeks afterward, pleased to request me to make at their victually-yard further experiments in the preparation of chocolate; and they indicated twe modes, one of milling twice with the husks, and another of milling twice without the usks; permitting me, at the same time, to mill a portion of the kernels with 10 per cent. of sugar, and a second portion of the kernels with 5 per cent. of sugar and 5 per cent. of the excellent flour used in making the biscuits for the royal navy. On the 24th October; 1842, I accordingly performed these experiments upon 12 cwts. of Guyaquil coco as carefully roasted as possible on the kiln.

The loss in drying and slightly roasting the 1,344 lbs. of beans was 5 per cent.

1st experiment, 212 lbs. of roasted coco, milled twice with the husks, produced, of chocolate - _ - _ - _ _ _

$2 \mathrm{~d}$ experiment, $191 \mathrm{lbs}$. ditto, milled twice without husks $\quad$ - $\quad$ - 189

3d experiment, 191 lbs. Kernels, milled once along with 19 lbs. of sugar $=210 \mathrm{lbs}$. 4th experiment, 573 lbs. kernels, milled twice along with 68 lbs. of flour and 34 of sugar $=675 \quad$ - $\quad$ - $\quad-\quad \ldots \quad-669$

Sample cakes of these four varieties of chocolate were subsequently sent to me for examination and report. I found that the chocolate milled twice with the flour and 
sugar formed a complete emulsion with hot water, bland and rich, like the best milk, but the other three were much inferior in this respect. Sugar alone, with proper milling, would serve to give the kernels of well-roasted coco a perfect emulsive property. Instead of merely milling with rotatory stones, I would prefer, for the second or finishing operation, a levigating mill, in which rollers would be rolled either backward and forward, or, when slightly conical, in a circular direction, over a plane metallic, marble, or porphyry slab, as is now indeed, very generally practised by the trade. The coco-beans should be well selected, without musty taint, and possessed of a fine aroma, like the best of that imported from Trinidad. There is a great deal of very coarse coco and chocolate on sale in London and in the provincial towns of the

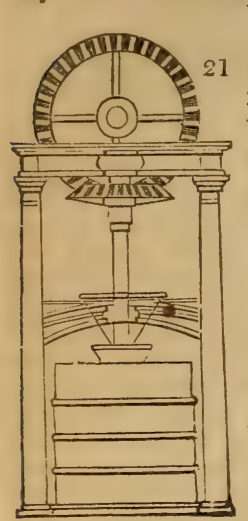

United Kingdom.

Fig. 21 is an end view of one of the chocolate mills with 21 its mitre-gearing. I consider the gritty chocolate hitherto made at Deptford as a very bad substitute for the chocolate which was made from coco by the sailors themselves with a pestle and mortar.

In 1840 the coco cleared for consumption in the United Kingdom was-

$\begin{array}{lccl}\text { British plantation } & - & 2,041,492 \mathrm{lbs} . & 2 d . \text { per lb. } \\ \text { Foreign } & - & 186 & 6 d . \\ \text { Coco-nut husks and shells } & - & 753,580 & 1 d . \\ \text { Chocolate and coco paste } & - & 2,067 & 4 d .\end{array}$

Of the coco-nut shells, 612,122 lbs. were consumed in Ireland! and less than $4,000 \mathrm{lbs}$. of cpco.

Of coco, 726,116 Ibs. were consumed in her majesty's navy.

How scurvily are the people of Ireland treated by their own grocers ! Upward of 600,000 lbs. of worthless coco husks served out to them along with only $4,000 \mathrm{lbs}$. of coco-beans !

CHROMIUM, OXIDE OF. Mix intimately 45 parts of gunpowder with 240 parts of perfectly dry chromate of potash, and 35 parts of hydrochlorate of ammonia (sal ammoniac), reduce to powder, and pass through a fine sieve; fill a conical glass or other mould with this powder, gently pressed, and invert so as to leave the powder on a porcelain slab of any kind. When set on fire at its apex with a lighted match, it will burn down to the bottom with brilliant coruscations. The black residuum, being elutriated with warm water, affords a fine bright green oxide of chromium.

CLOVES. Imported for home consumption in $1840,85,769 \mathrm{cwts}$.

COAL. Under PITCOAL, the composition of several excellent coals, is stated, with their peculiar qualities, as analyzed by me; such as the Llangennoek, Powell's Duffryn steam coal, the Blackley Hurst coal, Lancashire, the Varley Rock vein coal, near Pontypool, \&c.

COCHINEAL. Imported for consumption in 1839, 396,902 lbs. ; in 1840, 325,744; duty, 1 s. per cwt.

COFFEE. If tannin exist in roasted coffee, as maintained long ago by Chenevix, and generally admitted since, it must be very different from the tannin present in tea, catechu, kine oak-bark, willow-bark, and other astringent vegetables; for I find that it is not, like tnem, precipitated by either gelatine, albumen, or sulphate of quinine. With regard to the action, upon the animal economy, of coffee, tea, and coco, which contain one common chemical principle called caffeine or theine, Liebig has lately adranced some ingenious views, and has, in particular, endearored to show that to persons of sedentary habits in the present refined state of society, they afford eminently useful bererages, which contribute to the formation of the characteristic principle of bile. This important secreted fluid, deemed by Liebig to be subservient to the function of respiration, requires for its formation much azotised matter, and that in a state of combination analogous to what exists in caffeine. The quantity of this principle in tea and coffee being only from 2 to 5 per cent., might lead one to suppose that it could have little effect upon the system even of regular drinkers of their infusions; but if the bile contains only one tenth of solid matter, called choleic acid, which contains less than 4 per cent. of azote, then it may be shown that three grains of caffeine would impart to 500 grains of bile the azote which occurs in that crystalline precipitate of bile called taurine, which is thrown down from it by mineral acids.

One atom of caffeine, 9 atoms of oxygen, and 9 of water, being added together, produce the composition of 2 atoms of taurine. Now this is a very simple combination for the living organism to effect; one already paralleled in the generation of hippuric acid in urine, by the introduction of benzoic acid into the stomach; a physio. 
logical discovery male by my son, which is likely to lead to a more successful treatment of some of the most formidable diseases of man, particularly gout and gravel.

If the preceding views be established, they will justify the instinctive love of mankind for tea, coffee, and cocoa, in spite of the denunciations and vetoes of nexropathic, homcopathic, and hydropathic doctors; sorry pathologists-hoc genus omne. See TrA.

Coffee imported for consumption in $1839,26,789,945$ lbs.; in 1840, 28,664,341. Net revenue in $1839,779,115 l$. ; in $1840,921,551 l$.

COPAL and ANIME. Imported for consumption in 1839, 193,066 lbs.; in 1840, 181,388 lbs.: duty, 6s. per cwt., as upon gum arabic and tragacanth.

COPPER. Quantity of copper ore raised in Cornwall in the year 1838-1839, 159,214 tons; value of, $932,090 l$. 15 s. $6 d$.

Quantity raised in the year 1839-1840, 147,049 tons; value of, 792,750l. 14s.

Quantity of metallic copper produced in the former year, 12,469 tons ; standard, $111 l$. : in the latter, 11,056; standard, 108l. $5 \mathrm{~s}$.

Produce per cent., $7 \frac{3}{4}$ and $7 \frac{1}{2}$ respectively. Average price per ton, $5 l$. 17s. in the first ; and $5 l$. $7 s .9 d$. in the second year.

Quantity of unwrought copper imported for home consumption in 1840 , only $2 \frac{1}{2}$ cwts. See Metallic Statistics.

COPPER MEDALS AND MEDALLIONS may be readily made in the following way : Let black oxide of copper, in a fine powder, be reduced to the metallic state, by exposing it to a stream of hydrogen, in a gun-barrel, heated barely to redness. The metallic powder thus obtained is to be sifted through crape, upon the surface of the mould, to the thickness of $\frac{1}{4}$ or $\frac{1}{3}$ of an inch, and is then to be strongly pressed upon it, first by the hand, and lastly by percussion with a hammer. The impression thus formed is beautiful; but it acquires much more solidity by exposure to a red heat, out of contact with air. Such medals are said to have more tenacity than melted copper, and to be sharply defined.

M. Bœttger proposes the following improvement upon the above plan of Mr. Osann : He prepares the powder of copper easier and of better quality, by precipitating a boiling-hot solution of sulphate of copper, with pieces of zinc, boiling the metallic powder thus obtained with dilute sulphuric acid for a little, to remove all traces of the zinc or oxide, washing it next with water, and drying it in a tubulated retort by the heat of a water bath, while a stream of hydrogen is passed over it. This cupreous precipitate possesses so energetic an affinity for oxygen, that it is difficult to prevent its passing into the state of orange oxide. If it be mixed with one half its atomic weight of precipitated sulphur, and the two be ground together, they combine very soon into sulphuret of copper with the evolution of light.

COPPER, Purifying.-Copper may be purified by melting 100 parts of it with 10 parts of copper scales (black oxide), along with 10 parts of ground bottle-glass or other flux. Mr. Lewis Thompson, who received a gold medal from the Society of Arts for this invention, says that after the copper has been kept in fusion for half an hour, it will be found at the bottom of the crucible perfectly pure; while the iron, lead, arsenic, \&c., with which this metal is usually contaminated, will be oxidized by the scales, and will dissolve in the flux, or be volatilized. Thus he has obtained perfectly pure copper from brass, bell-metal, gun-metal, and several other alloys, containing from 4 up to 50 per cent. of Iron, lead, antimony, bismuth, arsenic, \&c. The scales of copper are cheap, being the product of every large manufactory where that metal is worked.

CORK. Unmanufactured, imported in 1840 for home consumption, 59,793 cwts.

CORTEX PERUVIANUS, or CINCHONA. Imported for home consumption in $1840,43,705$ lbs.

COTTON may be distinguished from linen in a cloth fabric by means of a good microscope; the former fibres being flat, riband-like, and more or less contorted or shrivelled, and the latter straight, round, and with cross knots at certain distances. These two fibrous matters may be also distinguished by the action at a boiling heat of a strong caustic ley, made by dissolving fused potash in its own weight of water. By dig..tion in this liquor, linen yarn becomes immediately yellow, while the cotton yarn rem ins white. The best way of operating is to immerse a square inch of the cloth to be tested for two minutes in the above boiling-hot caustic ley, to lift it out on a glass rod, press it dry between folds of blotting-paper, and then to pull out a few of the warp and weft threads-when the linen ones will be found of a deep yellow tint, but the cotton, white or very pale yellow.

COTTON WOOL. Imported for home consumption in $1839,352,000,277 \mathrm{lbs}$.; in $1840,528,142,743$ lbs.

CREOSOTE. Having been employed by a chemical manufacturer to examine his creosote, and compare it with others with a view to the improvement of his process, I found that the article, as made by eminent houses, differed considerably in its properties. 
The specific gravities varied in the several specimens as follows: 1, a specumen give me by Messrs. Zimmer and Sell, at their factory in Sachsenhausen, by Frankforton-the-Maine, had a specific gravity of $1.0524 ; 2$, a sample made in the north of England, sp. gr. 1.057, and its boiling point varied from $370^{\circ}$ to $380^{\circ} \mathrm{Fahr}$. Mr. Morson's creosote, which is much esteemed, has a sp. gr. of 1.070 , and boils first at $280^{\circ}$, but progressively rises in temperature up $10420^{\circ}$, when it remains stationary. The German creosote was distilled from the tar of the pyrolignous acid manufacture. Creosote, I believe, is often made from Stockholm tar. Berzelius gives the sp. gr. of creosote at 1.037 , and its boiling point at $203^{\circ} \mathrm{C}=397.4^{\circ} \mathrm{F}$. I deemed it useless to subject to ultimate analysis products differing so considerably in their physical properties. They were all very soluble in potash ley.

CROSS-FLUCKANS or FLOOKANS. The name given by the Cornish miners to elay veins of more ancient formation.

CYANIDE OF POTASSIUM. This salt, so much used now in the electrotype processes, is prepared, according to Liebig's formula, by mixing 8 parts of pounded prussiate of potash, sharply dried, with 3 parts of pure carbonate of potash, fusing the mixture in an iron crucible, by a moderate red heat, and keeping it so, till the glass or iron rod with which the fluid mass should be occasionally stirred, comes out covered with a white crust. The crucible is then to be removed from the fire; and after the disengaged iron has fallen to the bottom, the supernatant fluid, still obscurely red hot, is to be poured off upon a clean surface of iron or platinum. After concretion and cooling, the white saline mass is to be pounded while hot, and then kept in a wellstopped bottle. It consists of about 5 parts of cyanide of potassium, and 1 of cyanate of potash. For most purposes, and the analysis of ores, the latter ingredient is no ways detrimental.

CYDER. The value of apples to produce this beverage of good quality is proportionate to the specific gravity of their juice. M. Couverchel has given the following table, illustrative of that proposition :-

$\begin{array}{llllll}\text { Juice of the green renette, queen apple (reinette verte) } & - & -1,084 \\ \text { English renette } & - & - & - & - & -1,080 \\ \text { Red renette } & - & - & - & - & -1,072 \\ \text { Musk renette } & - & - & - & & -1,069 \\ \text { Fouillet rayé } & - & - & - & - & -1,064 \\ \text { Orange apple } & - & - & - & - & -1,063 \\ \text { Renette of Caux } & - & - & - & - & -1,060 \\ \text { Water - } & - & - & - & - & - \\ \end{array}$

Cyder-apples may be distributed into three classes, the sweet, the bitter, and the sour. The second are the best; they afford a denser juice, richer in sugar, which clarifies well, and when fermented keeps a long time; the juice of sweet apples is difficult to clarify; but that of the sour ones makes bad cyder. Late apples are in general to be preferred. With regard to the proper soil for raising apple-trees, the reader may consult with advantage an able essay upon "The Cultivation of Orchards, and the making of Cyder and Perry," by Frederick Falkner, Esq., in the fourth volume of the Royal Agricultural Journal. He adverts judiciously to the necessity of the presence of alkaline and earthy bases, in the soils of all deciduous trees, and especially of such as produce acid fruits.

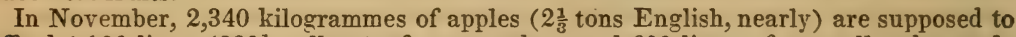
afford 1,000 litres (220 $\frac{1}{2}$ gallons) of pure cyder; and 600 litres of a small cyder made with the mare mixed with water and pressed. But many persons mix all together, and thus manufacture 1,600 litres out of the above weight of fruit. In France, the fermented liquor, as soon as it is clear, is often racked off into casks containing the fumes of burning sulphur, whereby it ceases to ferment, and preserves much of its sugar undecomposed. It is soon afterward bottled. Average cyder should yield 6 per cent. of alcohol on distillation.

\section{D.}

DAGUERROTYPE. This new and most ingenious invention for producing pictures by the action of light, is due to $M$. Daguerre and M. Niepce, two Frenchmen. It was purchased from them by the French government for the benefit of the nation at large; but was made the subject of an exclusive patent in England by M. Daguerre, as that government never purchases any scientific invention.

The fixation of the images, formed in the focus of the camera obscura, is made on very smooth surfaces of pure silver plated on copper. The process is divided into five 
operations. 1. The first consists in polishing and cleaning the silver surface, by friction with cotton fleece imbued with olive oil, upon the plate, previously dusted over with very finely-ground dry pumice-stone out of a muslin bag. The hand of the operator should be moved round in circles, of various dimensions. The plates should be laid upon a sheet of paper solidly supported. The pumice must be ground to an impalpable powder upon a porphyry slab with water, and then dried. The surface is next to be rubbed with a dossil of cotton, slightly moistened with nitric acid, diluted with sixteen parts of water, by applying the tuft to the mouth of the phial of acid, and inverting it for a moment. Two or three such dossils should be used in succession. The plate is lastly to be sprinkled with pumice powder or Venetian tripoli, and rubbed clean with cotton.

The next step is to heat the plate by placing it in a wire frame (fig. 23), with the silver surface uppermost, over a spirit lamp, meanwhile moving it so as to act equally on erery part of the plate. In about five minutes a whitish coating will indicate that this operation is completed. The plate must now be laid upon a flat metal or marble slab to cool it quickly. The white surface is to be brightened by rubbing it with cotton and pumice powder. It must be once more rubbed with the cotton imbued with acid, and afterward dried by friction with cotton and pumice; avoiding to touch the plate with the fingers, or with the part of the cotton held in them, or to breathe upon the plate, since spots would thereby be produced. After cleaning with cotton alone, the plate is ready for the next operation.

2. Here the following implements are required: 1 , the box represented in figs. 24 and $25 ; 2$, the thin board or frame, fig. 26 ; four small metallic bands of the same
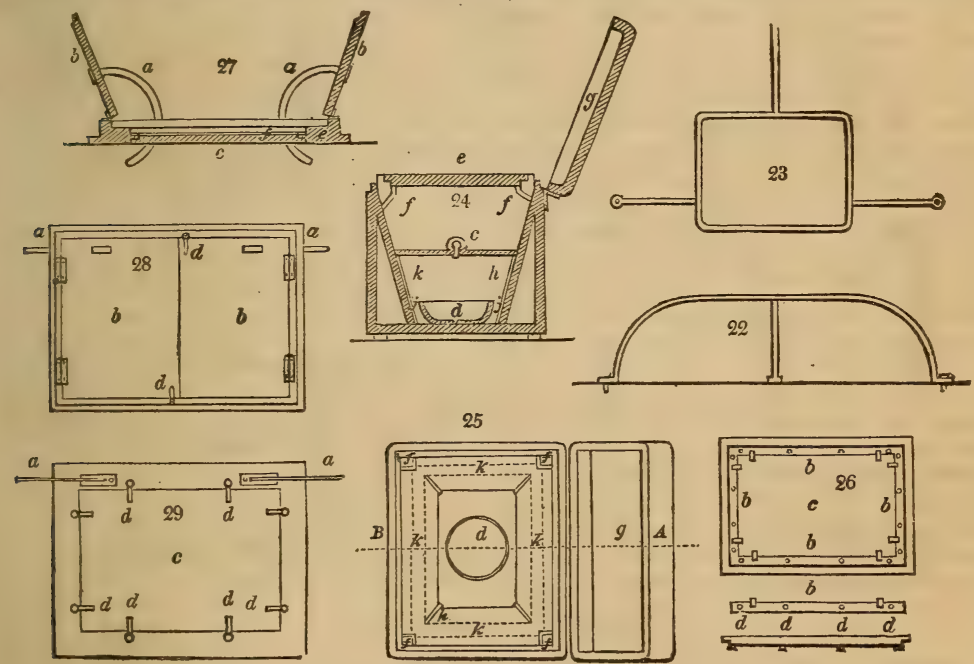

metals as the plates, also shown in $f i g, 26$, a small handle and a box of small nails or tacks, and a phial of iodine.

After fixing, by the metallic bands and the small nails, the plate upon the thin board, with the silver uppermost, several particles of iodine are then to be spread in the dish $d$, at the bottom of the box, figs. 24, and 25. The thin board with the plate, is next placed, with the silver beneath, upon small supports at the four corners of the bo $x$, and its cover is applied. The plate must be left in this position till the surface of the silver acquires a fine golden hue, caused by the vapors of the iodine rising through the gauze cover of the dish, and condensing upon it; but it should not be allowed to assume a violet tint. The room should be darkened, and no heat should be employed. When the box is in constant use it gets impregnated with iodine, and acts more uniformly and rapidly; but in general states of the atmospheric temperature this operation will be effected in about twenty minutes. If the purple color be produced, the plate must be repolished, and the whole process repeated.

The plate with its golden hue is to be introduced with its board into the frame, figs. $27,28,29$, which is adapted to the camera obscura. During this transfer the light must not be suffered to strike upon the surface of the plate; on which account, the camera obscura may be lighted briefly with a small wax taper. 
3. The plate is now submitted to the third operation, that of the camera obscura, figs. 30 and 22; and with the least possible delay. The action of this machine is obviously quicker the brighter the light which acts upon it ; and more correct, according as the focus is previously accurately adjusted to the place of the plate, by moving bacliward and forward a roughened pane of glass, till the focal point be found; and the plate is to be inserted precisely there, see figs. 27, 28, 29. This apparatus exactly replaces the ground glass. While the prepared plate is being fastened, the camera must be closed. The darkening shutters, $b, b$, of the apparatus are opened by means of the two semicircles $a, a$. The plate is now in a proper condition to receive and retain the impression of the image of the objects presented the moment that the camera is opened. Experience alone can teach the proper length of time for submitting the plate to the concentrated rays of light; because that time varies with the climate, the seasons, and the time of day. More time should not be al?'owed to pass than what is necessary for fixing a distinct impression, because the parts meant to be clear would be apt to become clouded.
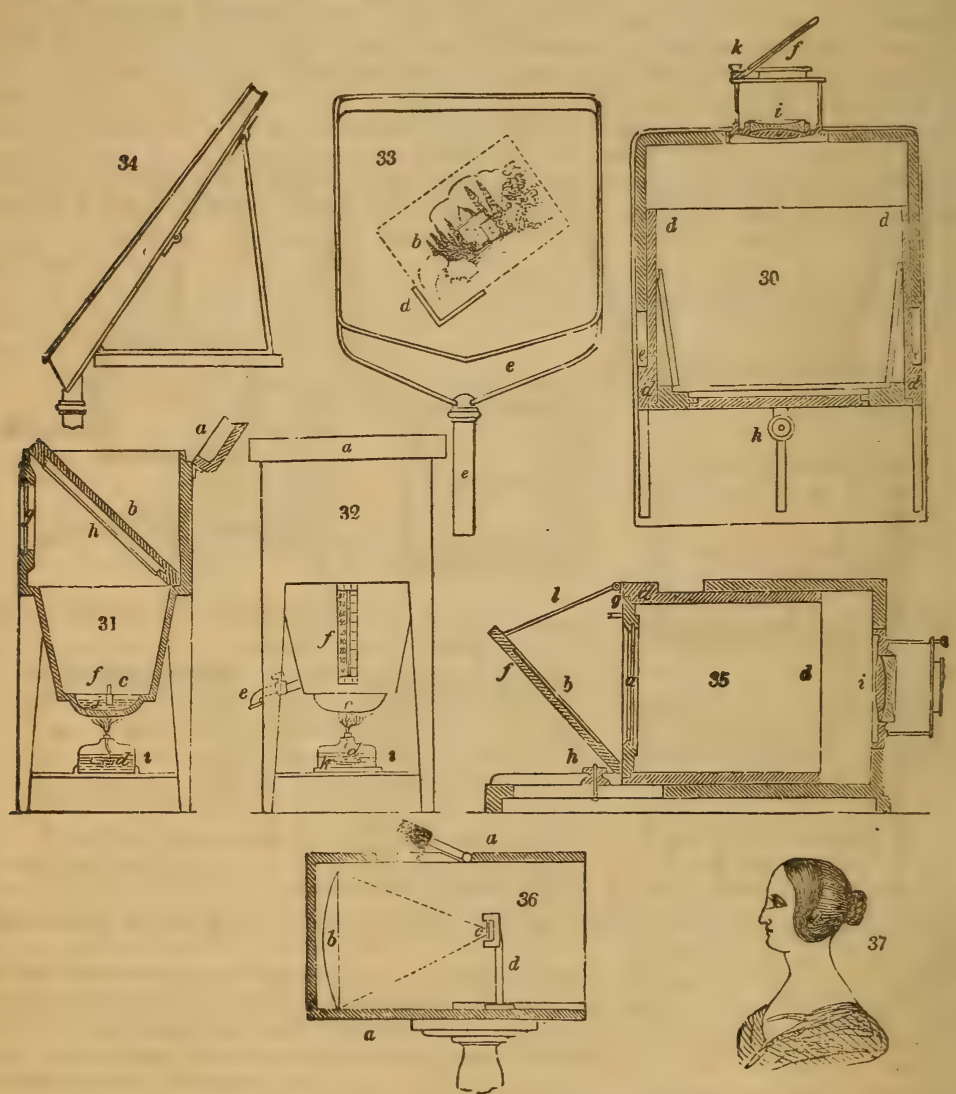

4. The fourth is the operation with quicksilver, which must follow as soon as pos. sible the completion of the third. Here a phial of quicksilver, a spirit lamp (the apparatus represented in figs. 31 and 32 ), and a glass funnel with a long neck are required. The funnel is used for pouring the mercury into the cup $c$, placed in the bottom of the apparatus, so as to cover the bulb of the thermometer $f$. No day-light must now be admitted, but that of a small taper only should be used by the operator in conducting the process. The board with the plate is to be withdrawn from the camera, and inserted into the grooves of the blackened board, $b$ fig. 31 . This black board is laid back into the box at an angle of 45 degrees with the horizon; the prepared metal surface $h$ being placed undermost, so that it may be viewed through the vide glass, $g$; and the cover, $a$, of the box must be put down gently to prevent any par- 
ticles of mercury from being thrown about by the agitation of the air. The whole being thus prepared, the spirit-lamp is lighted, and placed under the cup containing the mercury, and left there until the thermometer indicates a temperature of $110^{\circ}$ Fahr., when the lamp is to be removed. The heat should in no case be permitted to exceed $16 \tau^{\circ} \mathrm{F}$.

The impression of the image of nature is now actually made upon the plate; but it is as yet invisible; and it is only after a lapse of several minutes that faint tracings of the objects begin to be seen through the peep-glass by the momentary gleam of a taper. The plate should be left in the box till the thermometer has cooled to $113^{\circ} \mathrm{F}$., when it is to be taken out.

After each operation, the interior of the apparatus, and the black-board or frame, should be carefully wiped, in order to remove every particle of merc:s; .

The picture may now be inspected in a feeble light, to see how far the process has succeeded. The plate, freed from the metallic bands, is to be placed in a box, provided with a cover and groores, to exclude the light, till it is made to undergo the fifth and last operation, which may be done after any convenient interval of time without detriment, provided the plate be kept in the dark. The following articles are now required: 1, strong brine, or a weak solution of hyposulphite of soda; 2 , the apparatus represented in figs. 33 and $34 ; 3$, two troughs of tin-plate; 4, a jug of distilled water. The object of this process is to fix the photogenic pieture. One of the troughs is to be filled with brine to the depth of an inch, and the other with pure water, both liquids being heated somewhat under the boiling pitch. The solution of hyposulphite of soda is preferable, and does not need to be warm. The plate is to be first immersed in the pure water for a moment, and transferred immediaiely to the saline solution, and moved to and fro in it to equalize the action of the liquor. Whenever the yellow tint of the iodine is removed, the plate is to be lifted out by the edges, and dipped straightway in the water trough. The apparatus of figs. 33 and 34, is then brought into use, with a vessel filled with distilled water, hot, but not boiling. The plate, when lifted out of the water-trough, is to be placed immediately on the inclined plane $e$; and without allowing it time to dry, is to be flooded over with the hot distilled water from the top, so as to carry off all the saline matter. As the quicksilver which traces the images will not bear touching, the silvered plate should be secured by a cover of glass, made tight at the edges by pasting paper round them.

In $f i g .25$, which is a plan-view of the iodine-box apparatus, $c$ is an interior cover; $d$ is the iodine-dish; $e$ is the thin board to which the silvered plate is fixed, as shown at $f i g .24 ; g$ is the cover of the box; $h h$ are small rods, at the four corners of the inclined lining, $k$, of the box, to support the lid $c ; j$ is a gauze of wire-cloth cover, to diffuse the iodine vapor; $k$ is the wooden lining, sloping like a hopper; $d d$, in $f$ ig. 27 , are buttons to fasten the board on the doors; $e$ shows the thichness of the frame; $f$ is the silrered plate. In $f i g .35, a$ is the ground glass of the camera; $b$ is a mirror $x$ :lined about $45^{\circ}$ to the horizon, by means of the rod $l$. The image of the object is easily brought into focus by moving forward or backward the sliding box $d$, in laying hold of it with both hands by the projections $\alpha$, fig. 28. When the focus is adjusted, the thumb-screw, $h$, fixes the whole. The mirror is kept closed by two hooks at $f$, which take into small eyes at $g$. The frame and ground glass plate are withdrawn and replaced by the frame carrying the prepared plate, as represented in fig. 22 , with the shading doors, $b$, open in the camera. These doors and the sliding-box $d$ are lined with black velret. The object-glass is achromatic and periscopic, the concave being outside in the camera; its diameter is about $3 \frac{1}{2}$ inches, and focus about 13 inches. A diaphragm is placed before the object-glass, at $3 \frac{1}{2}$ inches from it, and its aperture may be closed by a plate moving in a pivot. This camera reverses the objects from left to right; but this may be obviated by placing a plane mirror on the outside beyond the aperture of the diaphragm, as at $f$, fig. 30 , where it is fixed by means of a screw, $k$. Loss of light is thereby occasioned.

Fig. 31, is an upright section, and fig. 32, a front elevation of the mercurial apparatus: $a$, the cover; $b$, the black-board, with grooves to receive the board $h ; c$ the cup of qu sisilver; $d$, the spirit-lamp : $e$, a small cock, through which the quicksilver may be $n$ off, if the apparatus be laid to one side; $f$, the thermometer; $g$, a glass window; $h$, the board bearing the metallic plate; $l$, a stand for the spirit-lamp, which is held by the ring $k$, so that its flame may strike the bottom of the cup. The whole of the inside of the apparatus should be blackened and varnished.

Fig. 33 , is a front view of the washing apparatus made of tin plate, varnished. The plates to be washed, are laid on the angular ledge, $d ; e$ is a ledge to conduct the water to the receptacle $c$. Fig. 34 is a side riew of the washing apparatus. The patent was enrolled in February, 1840. (See Newton's Journal, C. S. xvi. 1.)

Mr. Richard Beard haring purchased from M. Daguerre a license to practise his invention above described, received from a foreigner a communication of certain im 
provements for which he obtained a patent in June, 1840. The first of these is the substitution of a concave reflecting mirror for the lens in the camera obscura. Fig. 36 represents in section a slight wooden box, $a$, open at the front, opposite to the person sitting for the portrait. In the back part of the box a concave mirror, $b$, is placed, to reflect the rays coming from the person. A small frame, $c$, is fixed to an adjustable pedestal, $d$, which slides in grooves in the bottom of the box, for the purpose of being set at the focal point of the mirror. In this frame, $c$, a polished surface is first to be placed for trial, to receive the image correctly, as observed by the operator, by looking through the opening, $e$, in the top of the box. The prepared silvered plate is now substituted in the exact place for the trial one. The luminous impression being made, the slide, $d$, is withdrawn, and the plate removed; carefully shut up in a box from the light.

The second object of this patent is making the prepared surface more uniform, by passing two plates, with their silvered faces in contact, several times between hardened rollers, annealing them at a low red heat after each passage.

His third object is to use a compound of bromine and iodine, instead of the latter alone, for coating the silver; which increases its sensibility to light, thereby shortening and improving the operation of taking likenesses. He also recommends to use a combination of iodine with nitric acid. Finally, Mr. Beard finds that by placing a screen of any desired color behind the sitter, the appearance of his Daguerrotype portrait is improved. (Newton's Journal, xxiii. 112.)

M. A. J. F. Claudet, who had also purchased a license from M. Daguerre, obtained a patent in December, 1841 , for certain improvements upon the original process. His first object is to give the front of the camera obscura such an aperture as to admit the largest object-glass intended to be used; and of such he provides a series of different dimensions, each attached to its board, that may be fitted by a slide to the front of the camera.

One of the greatest difficulties in the Daguerrotype process was the impossibility of ascertaining the precise moment at which the light had produced, on the prepared plate, the effect requisite for the vapor of mercury to bring out the image. By applying that rapor to the plate while the silver surface is being acted upon by the light, the operator is enabled to see when his picture is complete. Another advantage of this joint operation is, that the effect of the mercury upon those parts of the plate which have been acted upon by the light, are more perfect when caused to take place immediately under the luminous influence. Hence, instead of using the distinct box with the cup of quicksilver, he places a cup containing that metal in the camera obscura, with its spirit-lamp, and exhales the vapors there. When the mercury has risen to the proper temperature, the aperture of the object-glass is thrown open, and the light, reflected from the object to be delineated, is allowed to operate.

He watches the effect through an opening in the side of the camera, where he views the preparea plate by the light of a lantern passing through a piece of red or orangecolored glass in the (other) side of the camera. Whenever the light and mercury, by their simultaneous action, have produced a good image, the object-glass is corered, and the silver plate, with its picture, removed, in order to be washed and finished. M. Claudet embellishes his Daguerrotype portraits by placing behind the sitter screens of painted scenery, which furnish pleasing back grounds. He specifies also various kinds of artificial illumination, to be used in the absence of solar light. (Newton's Journal, C. S. xx. 430.)

According to M. Barnard, Daguerre's iodized plate should be exposed for half a minute to the action of chlorine, mixed with a large proportion of common air; whereby it becomes so sensitive, that the pictorial impression is produced in the short space of time necessary for removing and replacing the screen of the camera. The mercury is afterward employed; as also the hyposulphite wash. Daguerrotype pictures are colored by dusting over them powders of proper hues, which are immediately washed by passing the plate through water. What remains of the color after this ablntion does not seem in the least to injure the appearance or alter the form of the image. It would seem that those parts of the picture which were at first black, retain, after being washed, a larger proportion of the coloring matter than the lighter parts.

Sereral raluable improvements seem to have been made in Vienna upon the Daguerrotype process; and among others, the mode of using chloriodine.

The best form of box for applying the chloriodic vapor is square, with its bottom of plate glass, supported a little above the table by feet, a thumb-screw being one of them, in order to give a certain inclination to the glass plate for spreading the chloriodine over it uniformly. A sheet of white paper being laid beneath the box, enables the operator to see whether the liquid chloriodine is properly distributed. There is a groove round the top of the box ${ }_{2}$ into which the ledge of the lid fits tight. A thermometer is placed in the box. 
Foigtlar.t's lenses consist of two achromatic object-glasses placed apart ; the first nearest the ubject, having an aperture of 18 lines; the second one of 19 lines; the solar focus of the two is $5 \frac{1}{4}$ inches. A system of lenses of so short a focus with so large apertures aftords from 11 to 12 times more illumination than Daguerre's original apparatus did. The finest portraits can be produced in the course of from 10 to 30 seconds with this arrangement. Such an apparatus, elegantly made in brass, costs only 120 gulden, or about 10 guineas.

Voigtland has recently made a camera with two object-glasses, as above arranged, each having an aperture of 37 lines, and a combined focus of 12 inches. By means of this instrument, portraits $5 \frac{1}{4}$ inches in size can be made. The landscapes produced in them are very beautiful. Its price is 144 gulden, about 12 guineas. Along with the above apparatus, a box with a bottom of amalgamated copper is used for applying the vapor of mercury.

By peculiar methods of polishing the silvered copper plate, peculiar tones and tints may be given to the picture. The olive-oil and pumice-powder are indispensable for remoring the scratches from the plate and to render its surface uniform. If a delicate blue tone be desired, the plate should be a second time polished with sulphuric ether and washed tripoli; and a third time with dilute nitric acid and Paris red, rubbing the plate lastly with a peace of washleather and crocus. But if a brownish black tone be wished for, a like series of operations is to be gone through, only instead of the ether and tripoli, spirit of ammonia and Vienna lime is to be used.

To give the plate the utmost sensibility to light, a film of iodine should be given in the first place. If with dry iodine, this should be strewed, then corered with cotton, and lastly with a sheet of paper, and the plate above the last, but not so as to touch it. This may be done also with a solution of 1 part of iodine in 6 of spirits of wine, put into a saucer, which is laid on the bottom of the box, and covered with gauze. The plate is to be removed whenever it has acquired a faint brazen tint. By this means the plate receives the impressions of light so well as to produce good contrasts between the white and the dark places. 'The application of bromine afterward causes a rapid reception of the image, and occasions the deep black shades of an object. The best form is brome water, made by dissolving the bromine in a little distilled water, and then adding more, when it is wanted, till the solution acquires a straw-yellow color. A delicate thermometer being put into the box, the solution is to be spread uniformly on its glass bottom, the plate being laid on above and covered up, while the time of exposure must be counted by seconds, with a clock or watch. If the temperature be

$$
41^{\circ} \mathrm{F} \text {., the time should be } 258 \text { seconds. }
$$

$\begin{array}{ll}50^{\circ} & 230= \\ 59^{\circ} & 201= \\ 68^{\circ} & 158 \text { - } \\ 77^{\circ} & 113=\end{array}$

By attending to these instruçtions, exact results may be always obtained.

A second mode of experimenting is with bromiodine; prepared by dissolving 1 part of bromine in an alcoholic solution of 5 parts of iodine; and diluting this mixture with water, till it acquires the color of Bavarian beer. The action of this application upon the plate is so rapid as hardly to leave time for consideration. It must be watched every instant till the dark gold yellow tint appear, when it is ready for the camera.

The best time of day for Daguerrotype operations is from an hour after the sun rises till he comes within $45^{\circ}$ of the meridian, and not again till he has passed the meridian by $45^{\circ}$. When the sitting is too long, the parts which should be pure white become of a dirty blue tint, and the dark parts become brown. The picture is burnt, so to speak.

Chloride of gold applied to the picture has the effect of fixing and enlivening the tints. A small grate being fixed by a clamp to the edge of a table, the plate is laid upon it with the image uppermost, and overspread evenly with solution of chloride of gold, by means of a fine broad camel-hair brush, without letting any drop over the edge. A spirit lamp is now brought under the plate, and moved to and fro till a number of small steam bubbles appear upon the image. The spirit lamp must be immediately withdrawn. The remainder of the chloride solution must be poured back into the phial, to be used on another occasion. It is lastly to be washed and examined. This operation has been repeated three or four times with the happiest effect, of giving fixity and force to the picture. It may then be wiped with cotton without injury.

By dusting various pigment powders from small cotton-wool dossils upon the picture, previously coated with an alcoholic solution of copal, and nearly dry, the appearance of a colored miniature has been very successfully imitated. The varnish must be applied delicately with one stroke of a broad brush of badger hair.*

* See Praktische Anweisung znm Daguerrotypiren, Leipzig, \&cc., 1843. 
DEXTRINE. This substance has exactly the same chemical composition as starch, consisting of 24 atoms of carbon, 20 of hydrogen, and 10 of oxygen (Dumas); but it is distinguished from starch by its solubility in cold water, like gum, and nut Deing affected by iodine. British gum, as it is called, or roasted starch, is merely dextrine somewhat discolored; a substance apparently used for the paste on the queen's head postoffice letter-stamps. A process discorered by M. Payen, and patented in France by M. Henzé, for making dextrine, consists in moistening one ton of dry starch with water containing $4 \frac{1}{2} \mathrm{lbs}$. of strong nitric acid. The starch thus uniformly wetted, is made up into small bricks or loaves, and dried in a store. It is then rubbed down into a coarse powder, and exposed in a stove-room to a stream of air heated to about $160^{\circ} \mathrm{F}$. Being now triturated, sifted, and heated in a store to about $228^{\circ} \mathrm{F}$., it forms a perfect dextrine of a fair color; because the acid acts as a substitute for the higher heat, used in making the British gum. Such an article makes a fine dressing for muslin and silk goods, and is much emplored in French surgery, for making a stiff paste-support to the bandages of fractured limbs.

DISTILLATION. Fig. 38 represents one form of the worm-safe, which is a con-

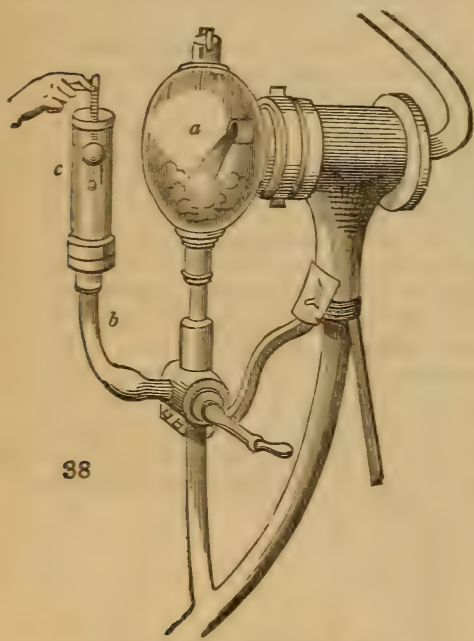
trivance for permitting the distiller to observe and note at any period of the distillation the alcoholic strength or the specific gravity of his spirits, without access to the still or the means of purloining the product before it has paid duty. The nose-pipe of the worm-tub terminates in, and is firmly cemented to the side of the glass globe, $a$, from whose bottom the discharge-pipe descends vertically, but has a stop-cock upon it, and a branch small pipe $b$, turned up parallel to the former. This branch is surmounted with a glass cylinder, $c$, which, when the stop-cock is opened, gets filled with the spirits, and then receives a hydrometer to show the gravity of the fluid. The stop-cock mechanism is so contrired, that only one full of the small glass eylinder can be obtained at a time.

The following is the gross produce of the excise duties on British distilled spirits for the United Kingdom annually from 1830 to 1840 inclusive : $1831,5,196,175 l$; 1832, 5,163,373l.; 1833, 5,258,572l.; 1834, $5,287,032 l . ; 1835,5,073,276 l$. ; 1836, 5, $485,883 l$; 1837, 5,006,697ı ; 1838, 5,451,792l.; 1839, 5,363,220l.; $1840,5,208,040 l$. The net produce is very nearly the same. In $1838,26,486,543$ millions of gallons paid duty; in 1839, 25,190,813; and in 1810, 21,859,33\%. See Rers, SpIRIts, and StILI.

\section{E.}

ELECTRO-METALLURGY. BY this elegant art, perfectly exact copies of any object can be made in copper, silver, gold, and some other metals, through the ageney of roltaic electricity. The earliest application of this hind seems to hare been practised about ten years ago, br Mr. Bessemer, of Camden Town, London, who deposited a coating of copper on lead castings, so as to produce antique heads in relief, about 3 or 4 inches in size. He contented himself with forming a few such ornaments for his mantel-piece; and though he made no secret of his purpose, he published nothing upon the subject. At letter of the $22 \mathrm{~d}$ of Mar, 1839, written br Mr. J. C. Jordan, which appeared in the Mechanics' Mag. for June 8, following, contains the first printed notice of the manipulation requisite for obtaining electro-metallic casts ; and to this gentleman, therefore, the world is indebted for the first discovery of this new and important application of seience to the uses of life. It appears that Mr. Jordan had made his experiments in the preceding summer, and haring become otherwise busily occupied, did not think of publishing till he observed a vague statement in the journals, that Professor Jacobi, of St. Petersburg, had done something of the same kind. Mr. Jordan's apparatus consisted of a glass tube closed at one xtremity with a plug of plaster of Paris, and nearly filled with a solution of sulphate of copper. This tube, and its contents, were immersed in 
a solution of common salt. A plate of copper was plunged in the cupreous solution, and was connected by means of a wire and solder, with a zinc plate dipped in the brine. A slow electric action was thus established through the moist plaster, and copper was deposited on the metal in a thin plate, corresponding to the former in smoothness and polish; so that when he used an engrared metal matrix, he obtamed an impression of it by this electric agency. "On detaching the precipitated metal," says he, "the most delicate and superticial markings, from the fine particles of powder ased in polishing to the deeper touches of a needle or graver, exhibited their correspondent impressions in relief with great fidelity. It is, therefore, evident that this principle will admit of improrement, and that casts and moulds may be obtained from any form of copper. This rendered it probable that impressions might be obtained from those other metals haring an electro-negative relation to the zinc plate of the battery. With this riew a common printing type was substituted for the copper-plate, and treated in the same manner. This, also, was successful; the reduced copper coated that portion of the type immersed in the solution. This, when remored, was found to be a perfect matrix, and might be employed for the purpose of casting, where time is not an object. Casts may probably be obtained from a plaster surface surrounding a plate of copper, \&c."

On the 12th of September following the above publication, Mr. Thomas Spencer read a paper "On Voltaic Electricity applied to the purpose of worling in Metal," before the Polrtechnic Society of Liverpool; which he had intended to present to the British Association at Birmingham in the preceding August, but not being well received there, he exhibited merely some electro-metallic casts which he had prepared. The society published Mr. Spencer's paper, and thereby served to give rapid diffusion to the practice of electro-metallurgy.

One of the most successful cultirators of this art has been Mr. C. V. Walker, secretary to the London Electrical Society. He has published an ingenious little work in two parts, entitled Electrotype Mamipulation, where he presents, in a lucid manner, the theory and practice of working in metals, by precipitating them from their solutions through the agency of roltaic electricity. His first part is deroted to the explanation of principles, to the preparation of moulds, to the description of the voltaic apparatus to be used, to bronzing, to coating busts with copper, to the multiplication of engraved plates, and to the deposition of other metals.

Fig. 39 represents a single cell roltaic apparatus for electro-metallurgy: $\boldsymbol{z}$ is
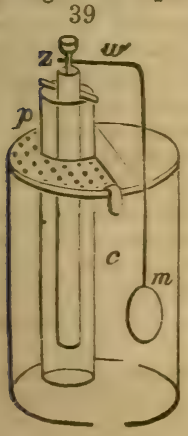
a rod of amalgamated zinc, $m$ is the mould on which the metal is to be deposited; $w$, is the wire joining them; $c$, is a strong solutiou of sulphate of copper in the large vessel; $p$, is a tube or cylinder of porous earthenware, standing in the other, and containing dilute sulphuric acid. The solution of blue vitriol is kept saturated, during the progress of its depositing copper, by piling crystals of the salt upon the shelf, shown by the dots under $p$. The mould to be coated should not be too small in reference to the surface of zinc under voltaic action. The time for the deposition to be effected depends upon the temperature; and is less the higher this is within certain limits; and at a freezing temperature it ceases almost entirely. When a mould of fusible metal is used, it should not be placed in the roltaic apparatus till everything is arranged, otherwise oxide will be dcmosited upon it, and spoit the effect. When the circuit is completed the mould may be immersed, but not before. Wax moulds are rendered electric conductors, and thereby depositors as follows: After breathing on the wax, rub its surface with a soft brush dipped in plumbago; breathing and rubbing alternately till the surface be uniformly covered. Attach a clean wire to the back of the mould, connecting it by plumbago with the blackened wax. Sealing-wax is coated in like manner. Casts of Paris plaster are first well imbued with melted wax or tallow, and then black-leaded. Objects in Paris plaster should be thoroughly penetrated with hot water, but not wet on the surface, before wax casts are made from them. Moulds are best taken from medals in stearine (stearic acid). For plating and gilding by electro-chemical agencr, the following simple plan of apparatus is used. Fig. 40 is a rectangular porcelain vessel, which contains in its centre a porous cell for containing the solution of oxide of silver or gold, by means of cyanide of potassium; and this porous cell is surrounded at a little distance by a similarly formed ressel of zinc. The connexion is formed between the zhc and the suspended object to be coated, either by a pinching screw, or by the pressure of its weight upon the wire. The dilute acid which excites the zinc should, in this case, be rery weak, in reference to the strength of the cyanide solution, which should be recruited occasionally by the addition of oxide. 
It has been found that with cyanide solutions of gold and siorer in the electrochemical apparatus, the nascent cyanogen at the positive pole or plate, in a decomposition cell, will act upon and dissclve gold and silver. Two or three of Daniell's cylindric cells, as shown at $a$ in $f g .41$, of a pint size, for actiss upon solutions of
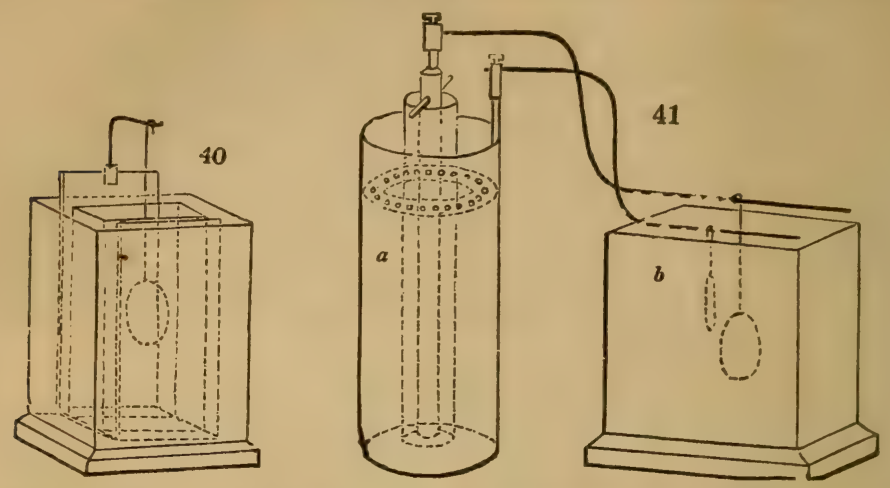

gold or silver, will in general suffice. The decomposition cell $b$ is made of glass or porcelain. The zinc may be amalgamated, and excited with brine; the copper cell contains, as usual, a solution of blue vitriol. To the end of the wire attached to the copper cylinder of the battery, a plate of silver or gold is affixed; and to the end of the wire attached to the zinc cylinder is affixed the mould, or surface, to be plated or gilt. The plates of silver or gold and zinc should be placed face to face as shown in the figure in the decomposition cell; which is filled by the cyanide solution. A certain degree of heat favors the processes of electro-gilding and plating. The surface is dead as first obtained, but it may be easily polished with leather and plate-powder, and burnished in whole or in parts with a steel or agate tool.

In March, 1840, Messrs. Elkington obtained a patent for the use of prussiate of potash, as a solvent for the oxides of gold and silver in the electro-chemical apparatus for plating and gilding metals. They also "sometimes employ a solution of protoxide (purple of Cassius) in the muriates of potash, \&c." The chemical misnomers, in their specification, are very remarkable, and do great discredit to the person employed to draw it up. Prussiate of potash is the ordinary commercial name of a salt very different from the cyanide of potassium - the substance really meant by the patentees-and the purple of Cassius is very different from protoxide of gold.

In plating or gilding great care must be bestowed in making the articles clean, bright, and perfectly free from the least film of grease. For this purpose, ther should be boiled in a solution of caustic alkali, then scoured with sand and water, next dipped into a dilute acid, and finally rinsed with water. A solution of the nitrate or cyanide of mercury may also be used with advantage for cleaning surfaces. The following metals have been deposited by electro-chemistry :-

Gold, platinum, silver, copper, zinc, nickel, antimony, bismuth, cobalt, palladium, cadmium, lead, and tin; of these, the first five are the most important and valuable. The gilding solution may be prepared by placing slips or sheets of gold in a solution of cyanide of potassium, and attaching to the negative pole of a voltaic battery, a small plate of gold, but to the positive pole a much larger one; whereby the latter combines with the cyanogen, under the influence of positive electricity, and forms a solution. Or, oxide of gold, precipitated from the chloride by magnesia, may be dissolved in the solution of the cyanide.

For making copper medals, \&c., a plate of amalgamated zinc is to be put into a vessel of unglazed earthenware, or of any other porous substance, filled with dilute sulphuric acid; which vessel is set into a trough of glass, glazed pottery, or pitched wood, containing blue vitriol in the state of solution, as well as in the state of crystals upon a perforated shelf, near the surface of the liquid.

The moulds to be covered with copper are to be attached by a copper wire to the zinc plate. The surface of zinc excited by the acid should be equal to that of the moulds; with which view a piece of zinc, equivalent in size to the mould, should be suspended in front of it.

For depositing copper upon iron, Messrs. Elkington use a solution of ferrocyanide of copper in cyanide of potassium in the decomposition trough, instead of sulphate of copper, neutralized from time to time with a little caustic alkali, as in the common 
practice of making medals, \&c., of copper. I should imagine that the black oxide of coppes dissolved in solution of cyanide of potassium would answer better; as the iron in the ferrocyanide might be rather injurious. The iron to be coppered being previously well cleaned from rust, \&c., with the aid of a dilute acid, is to be plunged into the cyanide solution heated to $120^{\circ}$ Fahrenheit, and connected by a wire with the negative pole of a voltaic battery, as formerly described. In from five to ten minutes, the iron will be completely coated. It is then to be scoured with sand, and plunged into solution of sulphate of copper; whereby it will show black spots wherever there are any defective places. In this case, it is to be cleaned and replaced under the cyanide solution, in the decomposition cell for a minute or two. Zinc may be deposited from a solution of its sulphate by a like arrangement.

Metallic cloth may be made as follows:-On a plate of copper attach quite smoothly a stout linen, cotton, or woollen cloth, and connect the plate, with the negative pole of a voltaic battery : then immerse it in a solution of copper or other metal, connecting a piece of the same metal as that in the solution with the positive pole; decomposition takes place, and the separated metallic particles in their progress toward the metal plate or negative pole, insinuate themselves into the pores of the tissue, and form a complete sheet of flexible metal. Lace is metallized by coating it with plumbago, and then subjecting it to the electro-metallurgic process.

The gilding solution should be used in the electric process at a temperature of $130^{\circ} \mathrm{F}$. The more intense the electric power, the denser and harder is the metallic coat deposited.

Metallic silver may be combined with cyanogen by subjecting it to the joint action of a solution of cyanide of potassium and positive electricity. Or cyanide of silver may be precipitated from the nitrate by a little cyanide of potassium, and afterward dissolved by means of an excess of cyanide of potassium. The quantity of electric power or surface-size of the battery should in all cases be proportioned to the surface of the articles to be placed or gilt, and the electric intensity or number of sets of jars proportioned to the density of the solution. Plating is accomplished in from 4 to 6 hours. 'The articles should be weighed before and after this operation, to ascertain how much silver they have taken on.

Messrs. Elkington make their moulds with wax, combined with a little phosphorus, which reduces upon their surfaces a thin film of gold or silver, from solutions of these metals, which films are better than the blackleaded surfaces for receiving the copper deposite. They also recommend to add a little alkali to the solution of sulphate of copper, intended to afford a deposite of metal. The single cell, first described above, is best adapted for this purpose.

M. Ruolz employs for gilding, a solution of sulphuret of gold in sulphuret of potassium, which he prepares by precipitating a solution of gold in aqua regia, by sulphuretted hydrogen, and redissolving the precipitate with sulphuret of potassium. By the use of this solution of gold, he obtains a very beautiful and solid gilding, and at less expense than with cyanide of potassium. Every metal which is a negative electrode to gold may be gilded.

Platinizing is effected best by means of a solution of the potash-chloride of platinum in caustic potash. 1 milligramme ( 0.015 grain) covers completely a surface of 50 square centimeters ( 2 inches square); the film of platinum is only one hundr $x^{3}$ th of a milligramme thick.

M. Bœttger has shown that we may easily tin copper and brass in the moist way by dissolving peroxide of tin (putty) in hydrate of potash (caustic potash ley), putting at the bottom of the vessel holding that solution some turnings of tin, setting the piece of copper or brass upon the turnings, and making the liquor boil. An electric current is produced by the contact of the dissimilar metals; and as the tin is withdrawn by the copper or brass from the solution, it is restored to it by the turnings. Zinking may be done in the same way; by putting pieces of zinc into a concentrated solution of chlorine, by setting the piece of metal to be zinked in contact with these pieces, and applying heat to the vessel containing the whole.

For certain new methods of constructing and arranging voltaic batteries for electrometallurgic operations, a patent was obtained by Dr. Leeson in June, 1842.

Fig. 42 , is a longitudinal section of the battery, and fig. 43 , a plan view of the frame to which the metal plates are attached. $a$ is a rectangular wooden trough, containing a wooden frame $b$, formed with vertical grooves in its sides, to receive a series of porous cells $c, c, c$. The plates of the battery are suspended in the fluid or fluids by brass torks $d, d$, fastened to a wooden frame $e$, $e$, which rests upon the trough $a$, and is connected to the other frame $b$, by two pins $f$, when they are required to be raised together out of the trough $a, a$.

The battery may be charged as usual with one or two fluids; one of them in the latte: 
case being contained in the porous cells $c, c, c$; and plates of copper and zinc, or any other suitable metals, may be employed.

The second improvement consists in cleaning copper and zinc plates after they have been used in a battery, by the employment of a voltaic battery; and also in amalgamating or coating with mercury the surfaces of zinc plates, by the same means to render them suitable for being used in the construction of the roltaic apparatus.

The third improvement consists in exciting electricity by a combination of nitric, sulphuric, or muriatic acid, with any of the following substances; riz., impure ammoniacal or lime liquor of the gas works, solutions of alkaline and earthy sulphurets, the alkalies and their carbonates, or lastly the acidulous sulphate of iron generated from iron pyrites.

43
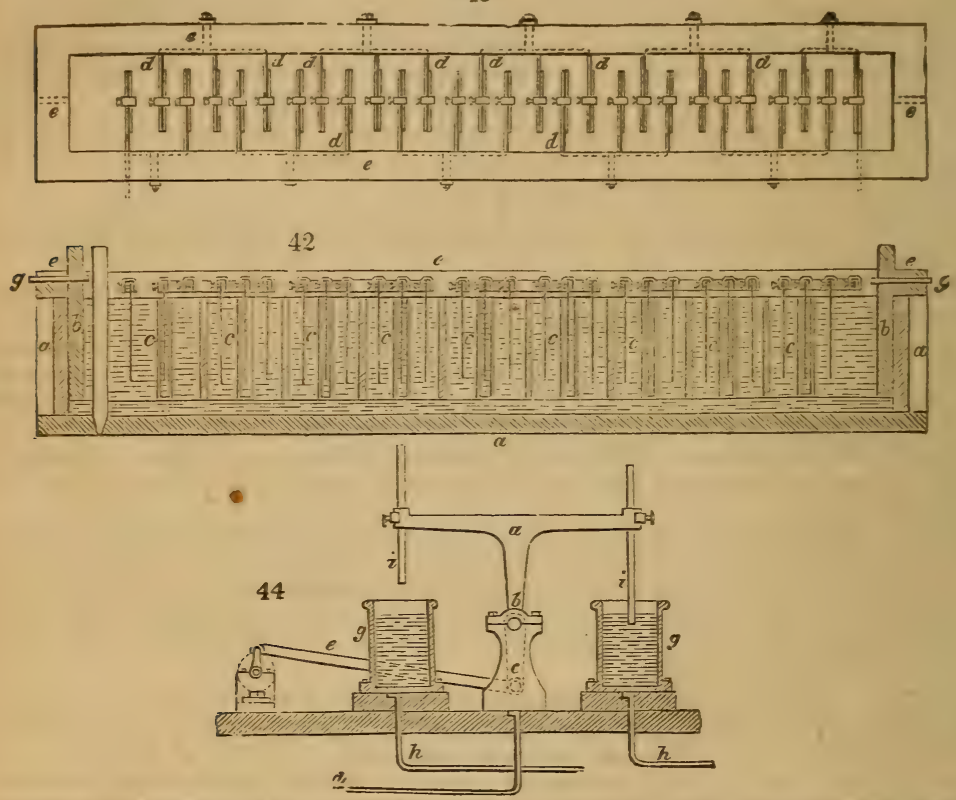

Another of Dr. Leeson's manifold improvements is for depositing metallic alloys, consists in the emplorment of one battery, with the "alternating cathode," represented in fig. 44. It is composed of a beam, $a$, mounted on the shaft, $b$, which turns in bearings carried by standards, $c$; the beam communicates with the anode of the battery by the wire, $d$, and a vibrating motion is given to it by the rod, $e$, from the shaft, $f$, which is driven by an electro-magnetic engine, or any other suitable prime mover. $g, g$, are two vessels containing mercury, connected by wires, $h, h$, with the cathode plates of the two metals comp sing the allor (but if the alloy is to consist of more than two metals, then more ressels, $g$, will be required, for one each cathode plate); these plates are immersed in a solution composed of similar salts of the different metals to be deposited, together with the anode, or surface to be deposited upon, which is connected by a wire with the cathode of the battery. A communication is established between the two cathode plates, or supply metals, and the anode of the battert, by means of the rods, $i, i$, which are caused, by the vibration of the beam, $a$, to dip alternately into either the one or the other of the ressels, $g$; and thus each metal will be deposited on the article to be coated, during the time that the connexion is established between it and the battery, by the immersion of its rod into the ressel of mercury. The relative proportions of the two metals is adjusted by lengthening or shortening the rods, $i, i$, as shown in the figure, so that they may be immersed for a longer or shorter period in the mercury.

Where the electrical current enters the electrolyte, is the anode; where it leaves it, is the cathode.

The patentee describes ten other improvements, which seem to be ingenious. See Newton's Journal, xxii. 292.

ELEMENTS, CHEMICAL. The catalogue given in the Dictionary has been augmented by four new bodies; Lanlhanium, Didymium, from Cerium; Erbium and Terbium, from Yttria. 
EI.TAY. The name given by the Cornish miners to porphryry, as also to the heterogeneuus rocky masses which occur in the granite or in the clay slate, deranging the direction of their metaliic reins, or even the mineral strata : but elvan generally indicates a filspar porphyry.

EMBUSSING OF LEATHER. Beautiful ornaments in basso-reliero for decorating the exteriors or interiors of buildings, medallions, picture-frames, cabinet-mork, sc., have been recently made by the pressure of metallic blocks and dies, for which invention a patent was obtained in June, 1839, by M. Claude Schroth. The dies are made of typ-metal, or of the fusible alloy with bismuth, called d'Arcet's. The leather is beatin soft in water, then wrung, pressed, rolled, and fulled as it were, by working it with the hands till it becomes thicker and quite supple. In this state it is laid on the mould, and forced into all its carities by means of a wooden, bone, or copper tool. In other eases, the embossing is performed by the force of a press. The leather, when it has become dry, is easily taken off the mould, howerer deeply it may be inserted into its erevices, by virtue of its elasticity. A full detail of all the processes is giren in Neuton's Journal, vol. xxii., p. 122.

EYAMELLING of Cast Iron and other Hollow Ware for Saucepans, \&-c. In December, 1799, a patent was obtained for this process by Dr. Samuel Sandy Hickling. His specification is subdivided into two parts :-

1. The coating or lining of iron ressels, \&c., br fusion with a ritrifiable mixture, composed of 6 parts of calcined flints, 2 parts of composition or Cornish stone, 9 parts of litharge, 6 parts of borax, 1 part of argillaceous earth, 1 part of nitre, 6 parts of cals of tin, and 1 part of purified potash. Or, $2 \mathrm{dly}$,

8 parts of calcined flints, 8 red lead, 6 borax, 5 calx of tin, and 1 of nitre. Or, 3 dlr,

12 of potter's composition, 8 borax, 10 white lead, 2 nitre, 1 white marble calcined, 1 argillaceous earth, 2 purified potash, and 5 of calx of tin. Or, 4thly,

4 marts calcined flint, 1 potter's composition, 2 nitre, 8 borar, 1 white marble calcined, $\frac{1}{2}$ argillaceous earth, and 2 calx of tin.

Whicherer of the abore compositions is taken, must be finel! powdered, mixed, fused; the ritreous mass is to be ground when cold, sifted, and levigated with water. It is then made into a pap with water or gum-water. This pap is smeared or brushed orer the interior of the ressel, dried, and fused with a proper heat in a muffe.

Calcined bones are also proposed as an ingredient of the flux.

The fusibility of the vitreous compounds is to vary according to the heat to be applied to the ressel, by using various proportions of the siliceous and fluxing materials. Colors may be given, and also gilding.

The second part or process in his specification describes certain alloys of iron and nickel, which be casts into ressels, and lines or coats them. with copper precipitated from its saline solutions. It also describes a mode of giring the precipitated copper a brassy surface by acting upon it with an amalgum of zinc with the aid of heat.

A factory of such enamelled hollow wares was carried on for some time, but it was given up for want of due encouragement.

A patent was granted to Thomas and Charles Clarke on the 25th of Mar, 1839, for a methor of enamelling or coating the internal surfaces of iron pots and saucepans, in such a way as shall prevent the enamel from cracking or splitting off from the effects of fire. The specification prescribes the ressel to be first cleansed by exposing it to the action of dilute sulphuric acid (sensibly sour to the taste) for three or four hours, then boiling the ressel in pure water for a short time, and next applying the composition. This consists of $100 \mathrm{lbs}$. of calcined ground flints; $50 \mathrm{lbs}$. of boras calcined, and finely ground with the abore. That mixture is to be fused and gradually cooled.

40 lbs. weig of the above product is to be taken with 5 lbs. Weight of potter's clay; to be ground together in water until the mirture forms a pasty-consistenced mass, which will leare or form a coat on the inner surface of the ressel about one sixth of an inch thich. When this coat is set, by placing the ressel in a warm room, the second composition is to be applied. This consists of $125 \mathrm{lbs}$. of white glass (without lead), $25 \mathrm{lbs}$. of borax, $20 \mathrm{lbs}$. of soda (crsstals), all pulrerized together and ritrified by fusion, then ground, cooled in water, and dried. To $45 \mathrm{lbs}$. of that mixture, $1 \mathrm{lb}$. of soda is to be added, the whole mixed together in hot water, and when dry, pounded; then sifted finely and erenly orer the internal surface of the ressel previous] the first coating or composition, while this is still moist. This is the glazing. The ressel thus prepared is to be put into a store, and dried at the temperature of $212^{\circ} \mathrm{F}$. It is then heated in a kiln or muffle, like that used for glazing china. The hiln being brought to its full heat, the ressel is placed first at its mouth to heat it gradually, and then put into the interior of the fusion of the glaze. In practice it has been found adrantageous also to dust the glaze powder orer the fused glaze, and apply a second fluxing heat in the oven. The enamel, by this double application, becomes mucb

ronoother and sounder. 
Messrs. Kenrick of West Bromwich having produced in their factory anu sent into the market some excellent specimens of enamelled saucepans of cast iron, were sued by Messrs. Clarke for an invasion of their patent rights; but after a long litigation in chancery, the patentees were nonsuited in the court of exchequer. The previous pro. cess of cleansing with dilute sulphuric acid appeared by the evidence on the trial to have been given up by the patentees, and it was also shown by their own principal scientific witness that a good enamelled iron saucepan could be made by Hickling's specification. In fact, the formulæ by which a good enamel may be compounded are almost innumerable; so that a patent for such a purpose seems to be untenable, or at least most easily evaded. I have exposed the finely-enamelled saucepans of Messrs. Kenrick to very severe trials, having fused even chloride of calcium in them, and have found them to stand the fire very perfectly without chipping or cracking. I consider such a manufacture to be one of the greatest improvements recently introduced into domestic economy; such vessels being remarkably clean, salubrious, and adapted to the most delicate culinary operations of boiling, stewing, making of jellies, preserves, \&c. They are also admirably fitted for preparing pharmaceutical decoctions, and ordinary extracts.

The enamel of the said saucepans is quite free from lead, in consequence of the glass which enters into its composition being quite free from that metal. In several of the saucepans which were at first sent into the market by Messrs. Clarke, their enamel was found on analysis by several chemists to contain a notable proportion of oxide of lead. In consequence of the quantity of borax and soda in the glaze, this oxide was so readily acted upon by acids, that sugar of lead was formed by digesting vinegar in them with a gentle heat. The presence of this noxious metal formed, in my opinion, a legitimate ground for contesting the patent, being in direct violation of the terms of the specification. Messrs. Kenwick's wares have been always free from this deleterious metal. Messrs. Clarke, I understand, have for some time been careful to reject from their enamel-composition all glass which contains lead; and they now manufacture also wholesome enamelled ware. Thus the public have profited in a most important point by the aforesaid litigation.

Enamelled iron saucepans had been many years ago imported from Germany, and sold in London. I had occasion to analyze their enamel, and found to my surprise that it contains abundance of litharge or oxide of lead. The Prussian government has issued an edict prohibiting the use of lead in the enamelling of saucepans, which are so extensively manufactured in Peiz, Gleiwitz, \&c. Probably the German ware sent to England was fabricated for exportation, with an enamel made to flux easily by a dose of litharge. The composition of the said enamel is nearly the same with that which I found upon some of the earlier saucepans of Messrs. Clarke. Had their patent been sustained, the important legal question would have arisen, whether it gave the patentees the power of preventing dealers from continuing to sell what they had been habitually doing for a great many years.

A suitable oven or muffle for lining or coating metals with enamel may have the following dimensions :-

The outside, 8 feet square, with 14-inch walls; the interior muffle, 4 feet square at bottom, rising 6 inches at the sides, and then arched over; the crown may be 18 inches high from the floor : the muffle should be built of fire-brick, $2 \frac{1}{2}$ inches thick. Another arch is turned over the first one, which second arch is 7 inches wider at the bottom, and 4 inches higher at the top. A 9 -inch wall under the bottom of the muffle at its centre divides the fireplaces into two, of 1 it inches width each, and 3 feet 3 inches long. The flame of the fire plays between the two arches and up through a 3 -inch flue in front, and issues from the top of the arch through three holes, about 4 inches square; these open into a flue, $10+9$ inches, which runs into the chimney.

The materials for the enamel body (ground flint, potter's clay, and borax) are first mixed together and then put into a reverberatory furnace, 6 feet 6 inches long, by 3 feet 4 wide, and 12 inches high. The flame from an 18-inch fireplace passes over the hearth. The materials are spread over the floor of the oven, about 6 inches thick, and ignited or fritted for four or five hours, until they begin to heave and work like yeast, when another coating is put on the top, also 6 inches thick, and fired again, and so on the whole day. If it be fired too mucl, it becomes hard and too refractory to work in the muffles. The glaze is worked in an oven similar to the above. It may be composed of about one half borax and one half of Cornish stone in a yellowish powder procured from the potteries. This is fritted for 10 hours, and then fused into a glass which is ground up for the glaze.

EVAPORATION. For the following scheme of generating, purifying, and conJensing steam, Mr. Charles Clark, merchant, London, obtained a patent in January, 1843. His apparatus for converting sea-water, \&c., economically into good fresh water, is represented in figs. $46,47,48$. A is the supply-cistern, which comm inicate 
with a pipe $a$, with a self-regulating eduction apparatus B. C is a strong wrought iron cylinder, fitted at bottom into a flanged ring-place, $c$, and covered with a conica.

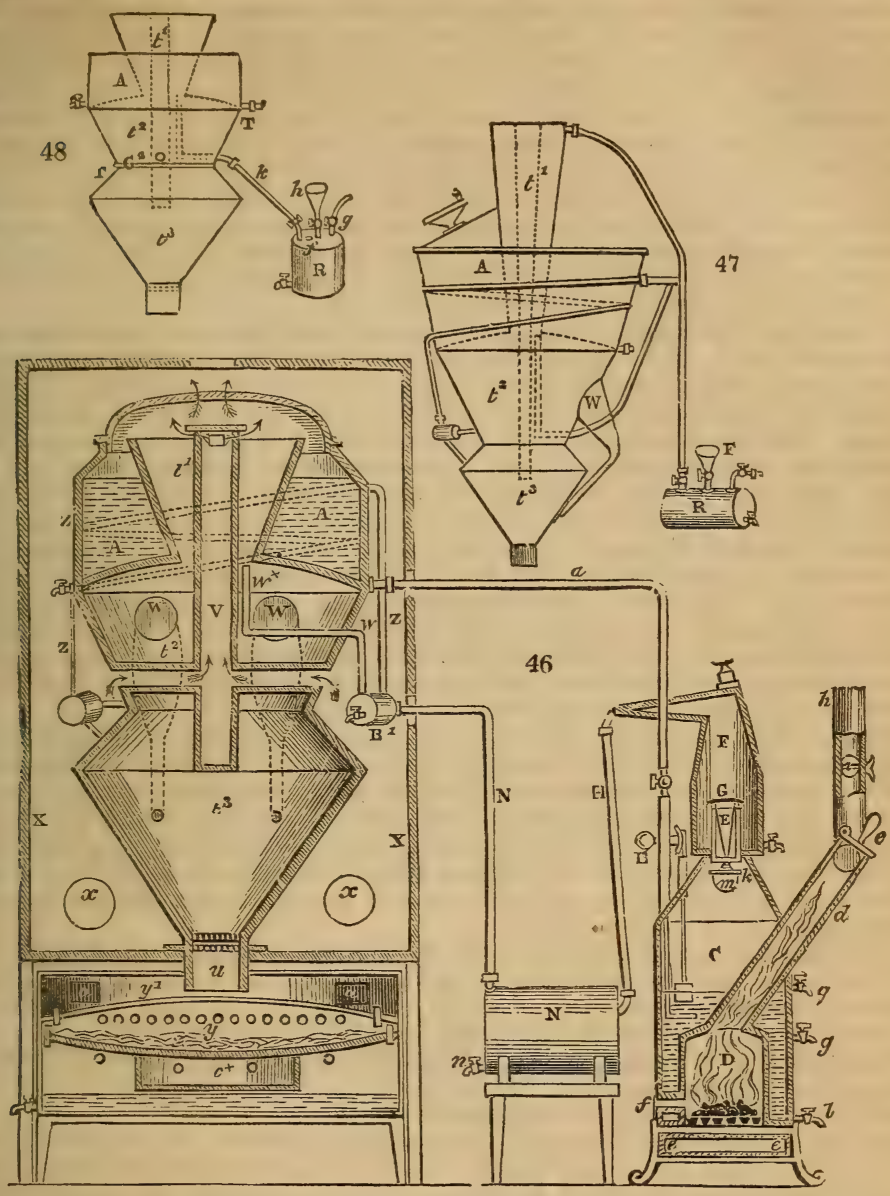

top; it is about two thirds filled with the water, to be operated upon. D is a cylindrical furnace concentric with the water cylinder $\mathrm{C}$; $d$ is an upward air and watertight tube, which serves both as a feed-pipe, through which the fuel is supplied to the furnace, and as a passage for the escape of the smoke, and other gaseous products of combustion; $e$ is a hinged trap door through which the fuel is passed into the tube $d$; $h$ is a chimney ir to which the pipe $d$ terminates; and $i$, a damper, by which the degree of activity given to the furnace can be regulated at pleasure; $f$ is an open air pipe; which leads from the outside, through the boiler into the furnace, a little way above the fire bars, and assists in securing a good draught through the furnace into the chimney. To the water cylinder $\mathrm{C}$ there are attached gauge-cocks, $\mathrm{g} g$, for ascertaining from time to time the height of the water; $l$ ls a cock or tap for drawing off the brine, and other residual matters which collect at the bottom of the boiler; $m$ is a screw-cap and hole, through which access may be had to the interior of the water cylinder $\mathrm{C}$, when it needs to be cleaned; $\mathrm{E}$ is a short pipe fitted into the conical top of the water cylinder $\mathrm{C}$, which conveys the steam generated in it, into the steam-head or receiver $\mathbf{F}$; $\mathbf{G}$ is a concave plate, resting upon the top of the pipe $\mathbf{E}$, a little larger than that pipe, and kept steady by a weight, $k$, of one or more pounds, suspended from it by wires. This plate prevents, in a great measure, the escape water escaping into the steam-head (an accident commonly callcd priming in steam engines; because, till the steam has acquired a pressure exceeding that of the counterweight $k$, it can not 
raise the weight $G$, so as to escape freely into the steam-head $F$, since any particle of water must, during the rising of the cap G, strike against it, and drop back, either into the water cylinder $\mathrm{C}$, through the pipe $\mathrm{E}$, or into the space round that pipe at the bottom of the steam-head $F$; whence it may be withdrawn by the cock shown in the drawing. $\mathrm{H}$ is a pipe which conveys the steam from the steam-head $\mathrm{F}$ to the rectifier R. This consists simply of a cylinder (about one third the size of the cylinder C) laid horizontally, in the lower part of which a body of water speedily collects, and serves to retain any particle of undecomposed matter, which may come over with the steam, as it continues to flow in from the boiler; whereby only its purer portions may pass off from the rectifier $\mathrm{R}$, by the pipe $\mathrm{N}$. $n$ is a cock or tap, at the bottom of the cylinder $\mathrm{R}$ for drawing off its water occasionally; $R 1$ is a second steam-rectifier, like $R$, into which the steam passes from the pipe $N$, and is thereby still further purified; but when the proportion of saline matter is small, $R^{\prime}$ may be dispensed with, and for very foul water, two or three more such rectifiers may be added.

The condenser for liquefying the purified steam, and aerating the resulting water, is shown at $t 1, t 2, t 3$. It is composed of conical upright compartments communicating with each other; the chamber $t^{1}$ is surrounded by the water in the cistern A (slightly heated by the steam in that chamber), while the chambers $t 2$ and $t 3$ are exposed freely to the air. The lowest of these, $t 3$, terminates at bottom in a tube, $u$, containing at the mouth of the cone two or three plates of perforated zinc, for admission of the atmosphere. An upright steam-tight tube of zinc, at about the middle of the lowest chamber, $t^{3}$, and is continued to the top of the uppermost chamber, $t^{\prime}$, having two lateral branches. This tube is closed at its lower end, but open at top, and at the ends of the two branches, to give a draught of cool air into the tube, and a rapid flow of heated air from the top of the tube. W, W, are pipes which pass externally from about the middle of the chamber $t 2$, to near the bottom of the chamber $t 3$. At their tops they are of large dimensions, as represented, but diminish gradually to small pipes at bottom. Of these pipes, there should be as many as can be conveniently applied, in order that the process of condensation may be effectually promoted.

From the second rectifier, $\mathrm{R}^{1}$, the steam is conveyed by a pipe, $w$, of gradually increasing dimensions, to near the top of the middle chamber, $t$, whence it diffuses itself through the three chambers, where it gets condensed. The hottest steam passes into $t 1$, and is there most powerfully condensed. The main body of the water produced therefrom, either drops directly into the bottom of the chamber $t 3$, or runs down the inclined sides of the chambers $t 1, t 2, t 3$, thence through the outer pipes $\mathrm{W}, \mathrm{W}$, and out at the bottom of the tube, getting partially aerated in its progress, by means of the air ascending constantly through the tube $u$.

$Z, Z$, is an auxiliar steam-pipe from the rectifier $\mathrm{R}^{1}$, passing twice or thrice close round the water supplying the cistern, $\mathrm{A}$, and terminating in a cylinder which communicates by pipes with the chambers, $t 2$ and $t 3$; whereby all the water thus condensed may fall through the perforated zinc plates, into the general discharge tube, $u . \quad \mathrm{X}$ is an outer casing of wood or metal, leaving a small space round the condenser, with draughtholes, $x, x$, for the admission of air. The refrigerator is made of protected metal (" tinned copper ?") and divided into three compartments, $y 1, y^{2}, y^{3}$.

In the top of $y^{1}$, the end of the discharged tube $u$ is inserted; and at a little distance from this tube there are air apertures, $a, a$, furnished with shutters in the inside, slanting from the top downward, to prevent as much as possible the escape outward of any vapor which may occasionally be carried down with the water from the condenser. The middle compartment, $y^{2}$, is perforated, convex at top, and coneave at bottom; so that the water that drops from the tube $u$, in the convex top of $y^{2}$, falls off laterally through small pipes into the chamber $y^{2}$, while its concave bottom turns the water into a central filtering-box, $c$, that projects a little into $y^{3}$, set to receive it. For aerating this water, the bottom of $y^{2}$ is covered about an inch deep with small pebbles. $y^{3}$, which is the reservoir of the purified cool water, is perforated with small holes. $c^{1}, c^{1}$, are small pipes for promoting a continual upward flow of cold air. $y^{3}$ is furnished with a tap to draw off its water, as required.

For redistilling or rectifying spirituous liquids, the apparatus, fig. 47, is employed; in which the supply cistern $A$ is much larger, and close at top; the upper condensing chambers, $t^{1}, t^{2}$, are also larger, but the lowest, $t 3$, is narrowed. The second rectifier of fig. 46, is removed. The feints collect in the bottom of the rectifier $\mathrm{R}$, to be drawn off by a cock; while the rectified spirit passes off at top into the condenser. The refrigerator has only two compartments, and no pebbles. F is a funnel into which the spirits may be returned for redistillation.

For extracting the soluble matter of vegetable infusions, the apparatus, shown in fig. 48 , is used. The rectifier is vertical, has a screw-capped hand-hold, $f$, for admitting the vegetables. $g$ is a steam-pipe; and $h$ is a funnel for returning portions of the liquid extract. $\mathbf{R}$ is connected by a pipe, $k$, with the condenser, $\mathbf{T}$, made in two por- 
tions, fitted water-tight together, but separable for the purpose of cleansing. The steam which passes from the boiler into the rectifier $R$ disengages the soluble portion of the regetable substances, and, if they be volatile, carries them off to the condenser; if not, it combines and falls with them to the bottom of the vessel, whence this portion of the extract is drawn off by the cock, and a fresh charge may be introduced. The steam is shut off from the rectifier $R$ by a cock on the pipe $g$. When the steam is afterward admitted to assist the process of maceration, the supply of it is regulated by the stop-cocks in the pipes $g$ and $k$.-Newton's Journal, xxiii. p. 247, C. S.

EXTRACTS. These preparations of vegetables for medicinal use are made either by evaporating the infusions of the dried plant in water, or in alcohol, or the expressed juice of the fresh plant; and this evaporation may be effected by a naked fire, a sand bath, an air bath, a steam heat, or a liquid balneum of any nature, all of which may be carried on either in the open air, or in vacuo. Of late years, since the vacuumpan has been so successfully employed in concentrating syrups in sugar-houses, the same system has been adopted for making pharmaceutical extracts. An elegant apparatus of this kind, invented by Mr. Barry of Plough Court, was made the subject of a patent about 25 years ago. The use of the air-pump for evaporating such chemical substances as are readily injured by heat, has been very common since Professor Leslie's discovery of the efficacy of the combined influence of rarified air, and an absorbing surface of sulphuric acid, in evaporating water at low temperatures. It has been supposed that the virtues of narcotic plants in particular might be better obtained and preserved, by evaporation in vacuo than otherwise, as the decomposing agency of heat and atmospheric oxygen would be thereby excluded. There is no doubt that extracts thus made from the expressed juices of fresh vegetables, possess, for some time at least, the green aspect and odor of the plants in far greater perfection than those usually made in the air, with the aid of artificial heat. Dr. Meurer, in the Archiv. der Pharmacie for April, 1843, has endearored to show that the color and odor are of no value in determining the value of extracts of narcotics, that the albumen left unchanged in the extracts made in vacuo, tends to cause their spontaneous decomposition, and that the extracts made with the aid of alcohol, as is the practice in Germany, are more efficacious at first, and much less apt to be injured by keeping. M. Baldenius has, in the same number of the Archiv., detailed experiments to prove that the juices of recent plants mixed with alcohol, in the homœopathic fashion, are very liable to spontaneous decomposition. To the above expressed juice, the Germans add the alcoholic tincture of the residuary vegetable matter, and evaporating both together, with filtration, prepare very powerful extracts.

\section{F.}

FATS. The following statement is given on the authority of Braconnot :-

\begin{tabular}{|c|c|c|c|c|}
\hline \multirow{2}{*}{\multicolumn{2}{|c|}{ 'resh butter in summer }} & \multirow{3}{*}{ - $\quad$ - } & \multirow{2}{*}{$\frac{\text { Oleine. }}{60}$} & \multirow{2}{*}{$\frac{\text { Stearine. }}{40}$} \\
\hline & & & & \\
\hline & winter & & 37 & 63 \\
\hline og's Lard & $-\quad-$ & - - & 62 & 38 \\
\hline Gr Marrow & - & - & 24 & 76 \\
\hline Goose Fat & - & - & 68 & 32 \\
\hline Duck Fat & - & - & 72 & 28 \\
\hline Ox Tallow & - & - & 25 & 75 \\
\hline Mutton Suet & - & - & 26 & $\cdot 74$ \\
\hline
\end{tabular}

M. Dumas says that butter contains no stearme. The $p$ eation and decoloration $x$ fats has been the object of many patents. Under CAN LE, Hempel's process for refining palm-oil and extracting its margarine is described.

About 30 years ago, palm-oil was deprived of color to a certain degree by mixing with the melted oil, previously freed from its impurities by filtration, some dilute nitric acid, wooden vessels being used, and the oil being in a melted state. This process was both expensive and imperfect. More lately whitening has been prescribed by means of chromic acid, which, in the act of decomposition into chromic oxide, gives out oxygen, and thereby destroys vegetable colors. One pound of bichromate of potash in solution is to be mixed with two pounds of strong sulphuric acid, diluted beforehand with about two gallons of water; and this mixture is to be incorporated by diligent stirring with 2 cwt. of the filtered palm-oil, at a temperature of about $100^{\circ} \mathrm{F}$., contained in a wooden vessel. The palm-oil is afterward to be washed in 
warm lime-water, to which some solution of chloride of lime may be advantageously added. By this process, well managed, a fat may be obtained from palm-oil fit for making white soap. Tallow may be also blanched to a considerable degree by a like operation.

Instead of sulphuric acid, the muriatic may be used to convert the chromic acid into chromic oxide in the above process, and thereby to liberate the blanching oxygen. The resulting solution of green muriate of chrome being freed from some adhering oil, is to be mixed with so much milk of lime as just to neutralize the excess of acid that may be present. The clear green muriate is then to be decomposed in a separatc vessel, by the addition of well-slaked and sifted lime, in some excess. The green mixture of lime and chrome-oxide is now to be dried, and gently ignited, whereby it is converted into yellow chromate of lime, with some unsaturated lime. This compound being decomposed by dilute sulphuric acid, affords chromic acid, to be applied again in the decoloring of palm-oil, on the principles above explained.

Mr. Prynne obtained a patent in March, 1840, for purifying tallow for the candlemaker, by heating it along with a solution of carbonate of potash or soda for 8 hours, letting the whole cool, removing the tallow to another vessel, heating it by means of steam up to $206^{\circ} \mathrm{F}$, along with dry carbonate of potash (pearlash): letting this mixture cool very slowly; and finally removing the tallow to a vessel enclosed in steam, so as to expel any subsidiary moisture.-Newton's Journal, xxi. 258.

A patent for a like purpose was obtained in June, 1842, by Mr. H. H. Watson. He avails himself of the blanching power of oxygen, as evolved from manganate of potash (chameleon mineral), in the act of its decomposition by acids, while in contact with the melted fat. He prescribes a leaden vessel (a well-joined wooden tub will also serve) for operating upon the melted tallow, with one twentieth of its weight of the manganate, dissolved in water, and acidulated to the taste. The whole are to be well mixed, and gradually heated from $150^{\circ}$ up to $212^{\circ} \mathrm{F}$, , and maintained at that temperature for an hour. On account of the tendency of the dissolved manganate to spontaneous decomposition, it should be added to the dilute acid, mixed with the fat previously melted at the lowest temperature consistent with its fluidity.

Palm-oil may be well blanched in the course of 12 hours by heat alone; if it be exposed in a layer of one or two inches to the air and sunshine, upon the surface of water kept up at nearly the boiling point by a coil of steam-pipes laid in the bottom of a square cistern of lead or wood, well jointed.

Tallow imported for home consumption in 1839, 1,148,192 cwt. ; in 1840, 1,131,513. Duty, $3 s .2 d$. per cwt.

FELTED CLOTH. This woollen fabric, made without spinning and weaving, was made the subject of a patent by Mr. T. R. Williams in February, 1840. A copious description of the process is given in Newton's Journal, xxii. 1.

Varnished or Japanned Felt is made by imbuing the stuff of coarse hat-bodies with drying oil, prepared by boiling 50 lbs. of linseed oil with white lead, litharge, and umber, of each one pound. The felt is to be dried in a stove, and then polished by pumice-stone. Five or six coats of oil are required. The surface is at last varnished. When the object is intended to be stiff, like visors, the fabric is to be impregnated first of all with flour-paste, then stove-dried, cut into the desired shape, next imbued with the drying-oil, and pumiced repeatedly; lastly placed, to the number of 20 , in a hot iron mould, and exposed to strong pressure. Japanned hats, made in this way, are sold in France at $1 s .3 d$. a piece; and they will stand several years' wear.

FERMENTATION. This term has been of late extended to several chemical operations, besides those formerly included under it. The phenomena which it exhibits under these different phases, and the changes which it effects among the various subjects of its operation, are no less striking and mysterious in their principle than important in their applications to the arts of life. Fermentations are now arranged into twelve classes-1, the alcoholic; 2 , the glucosic or saccharine; 3 , the viscous or mucous; 4 , the lactic; 5 , the acetic; 6 , the gallic; 7 , the pectic; 8 , the benzoilic; 9 , the sinapic; 10 , the ammoniacal; 11 , the putrid; and 12 , the fatty.

Fermentation, in the most general sense, may be defined to be a spontaneous reaction, a chemical metamorphosis, excited in a mass of organic matter, by the mere presence of another substance, which neither abstracts from nor gives to the matter which it decomposes anything whatever. This process requires the following conditions: 1 , A temperature from $45^{\circ}$ to $90^{\circ} \mathrm{F}$; 2 , Water; 3 , The contact of air; 4 , The presence of a neutral organic azotised matter, in very small quantity, and of a crystallizable non-azotised substance in considerable quantity. The former is the ferment, the latter undergoes fermentation. In ordinary chemical actions we perceive one body unite to another to form a new compound; or one body turn another out of a combination, and take its place, in virtue of a superior affinity. The eflects are foreseen and explained by the intervention of that molecular force which governs all 
chemical operations, that attractive power which unites the partıcles of dissimilar bodies. Thus, also, in the ordinary phenomena of decomposition, we perceive the agency of heat at one time, at another of light, or of electricity; forces of which, though we are not acquainted with the essence, yet we linow the exact effect under determinate circumstances. But fermentation, on the contrary, can be explained neither by the known laws of chemieal affinity nor by the intervention of the powers of light, electricity, or heat. Fermentation reduces complex organic substances to simpler compounds, thereby reducing them nearer to the constitution of mineral nature. It is an operation analogous, in some respects, to that effected by animals upon their vegetable food.

With a good microscope, any person may convince himself that ferment or yeast is an organized matter, formed entirely of globules, or of corpuscles slightly oroid, from the three to the four thousandth part of an inch in diameter. Sometimes their surface seems to have a little tail, which has been regarded as a bud or germ attached to the mother cell. Whenever the fermentation begins, the yeast does not remain an instant idle. These small round bodies become agitated in all directions, and if the substance undergoing fermentation is mixed with an azotized matter, as in beer-worts, the corpuscles become larger, the small tails get developed, and on acquiring a certain size they separate from the parent globule, to live by themselves and give birth to new corpuscles.* In the fermentation of beer from malt, this series of multiplications produces a quantity of jeast seven times greater than what was added at the commencement. Were the above ingenious speculations demonstrated with certainty, we should be led to admit, in all these phenomena, actions truly vital, and a reproduction like that of buds in the regetable kingdom. The existence of a vital force seems to be rendered probable by the fact that in incomplete fermentation, such as that of fine syrup with too little yeast, the ferment loses its properties and powers. If, however, we add to the solution of pure sugar an albuminous substance, a caseous or fleshy matter, the development of yeast becomes manifest, and an additional quantity of it is found at the end of the operation. Thus with nourishment, ferment engenders ferment. It is for this reason that a little fermenting must, added to a body of fresh grape-juice, excites fermentation in the whole mass. These effects are not confined to alcoholic fermentation. The smallest portions of sour milk, of sour dough, or sour juice of beet-root, of putrefied flesh or blood, occasion like alterations in fresh milk, dough, juice of beet-roots, flesh, and blood. But further, and which is a very curious circumstance, if we put into a liquid containing any fermenting substance, another in a sound state, the latter would suffer decomposition under the influence of the former. If we place urea in presence of beer-yeast, it experiences no change ; while if we add to it sugar-water in a fermenting state, the urea is converted into carbonate of ammonia. We thus possess two modes of decomposition, the one direct, the other indirect.

Although yeast has all the appearances of an organized substance, it is merely by analogy that its multiplication by growth is assumed, for this is a phenomenon very difficult of experimental demonstration. When blood, cerebral substance, gall, pus, and such like substances, in a putrid state, are laid upon fresh wounds in animals, vomiting, debility, and death, soon supervene. The scratches from bones in putrid bodies hare been often the causes of disease and death to anatomists. The poison in bad sausages is of the same class of ferments. In Wurtemberg; where sausages are prepared from very miscellaneous matters, as blood, livers, brains, and offal of many other hinds, with bread, meal, salt, and spices, fatal results from eating them are not uncommon. Death in these cases is preceded by the gradual wasting of the muscular fibre, and of all the like constituents of the human body; so that the patient becomes emaciated, dries into a complete mummy, and soon expires. The cadaver is stiff as if frozen, and is not subject to putrefaction. During the progress of the sausage disease, the saliva becomes viscid, and emits an offensive smell. No peculiar poison can be detected by analysis in the sausages; but they are rendered wholesome food for animals by the action of alcr $0^{1}$, or by that of boiling water, which destroy the noxious fomes without acquiring If th. mselves; and thus decompose the putrefactive ferment of the sausages. When t : jowever, passes unchanged through the stomach into the circulating system, it imparts its peculiar action to the constituents of the blood, operating upon it as yeast does up on wort. Poisons of a like kind are produced by the hody itself in some dispases. In plague, small-pox, measles, \&c., substances of a peculiar fermentative nature are generated from the blood, which are capable of inducing in the blood of a healthy person a decomposition like that of which themselves are the subjects. The morbid virus reproduces itself, and multiplies indefinitely, just as the particles of yeast do in the fermentation of beer. The temperature of boiling water, and alcohol applied to matters imbued with such poisonous secretions, render their poison inert. Many 
acids, chlorine, rodine, bromine, empyreumatic oils, smoke, creosote, strong decoction of colfee, have the same salutary effect. All these agents are known to counteract fermeitation, putrefaction, and that dry wasting of organic matter called eremucausis, or slow combustion. It is most deserving of remark that the poisons chemically neutral or alkaline, such as those of small-pox in man, and of typhus ruminantium in cows, lose their baneful power when subjected to the action of the stomach; whereas that of bad sausages, which is acid, resists the modifying power of the digestive organs.

Alcoholic fermentation has been copiously discussed in the Dictionary. I may here add that ammonia, being a product of that change in solution of pure sugar, proves the presence of azote in the yeast; and that sulphuretted hydrogen, being made manifest in the disengaged gaseous products, by their blackening paper imbued with acetate of lead, proves the presence of sulphur. The acid liquor accompanying yeast may be washed away, without impairing materially its fermenting power, while the acid so removed has of itself no such virtue.

Yeast, freed from all soluble matters by water, alcohol, and ether, contains, indepen. dently of ashes-carbon, $50 \cdot 6$; hydrogen, $7 \cdot 3$; azote, 15 ; oxygen, sulphur, and phosphorous, $27 \cdot 1$, in 100 parts. Viewed atomically, yeast bears a close analogy to albumen. Like albuminous matter, yeast takes a violet tint with muriatic acid, and it may be replaced as ferment by gluten. Caseum (the eurd of milk) and flesh operate the same effect. All these fermentative powers have the same globular appearance in the microscope with yeast. When the activity of yeast has been destroyed by heat, \&c., it can be restored by the positive energy of the voltaic battery, which causes its combination with oxygen. The best proportion of sugar and water, for exhibiting the phenomena of fermentation, is 1 of the former to 3 or 4 of the latter, and 5 parts of sugar to 1 of fresh yeast may be added; though in the coarse of fermentation, 100 parts of sugar do not consume 2 parts of yeast, estimated in the dry state. The quickest fermenting temperature is from $68^{\circ}$ to $86^{\circ}$. A very little oil of turpentine or creosote, or of the mineral acids, prevents or stops fermentation completely; oxalic and prussic acids have the same effect, as also corrosive sublimate and verdigris. It has been known from time immemorial in Burgundy, that a little red precipitate of mercury, when added to the must-tun, stopped the fermentation. All alkalies counteract fermentation, but when they are saturated it recommences. The first person who described the microscopic globules of yeast with precision was Désmazières, who arranged them among the mycodermes (fungus-skinned), under the name of mycoderma cerevisia. They have not the flattened form of the globules of blood, but are rather egg-shaped. One small black point may be seen on their surface, which, after some days, is associated with 3,4 , or 5 others. Their average diameter is from $\frac{1}{50 \overline{0}}$ to $\frac{1}{4000}$ of an inch. Sometimes more minute globules cluster round one of ordinary size, and whirl about with it, when the liquor in which the globules float is agitated.

Fresh yeast loses, by drying, 68 parts in the 100, and becomes solid, horny-looking, and semitransparent, breaking readily into gray or reddish fragments. With water, it resumes immediately its pristine appearance. When fresh yeast is triturated with its own weight of white sugar, it forms a liquid possessing the fluidity of oil of almonds, and a yellow color. The globules continue unchanged, except perhaps becoming somewhat smaller. Yeast in the dry state retains its fermentative virtue for a long time.

Saccharine Fermentation is that by which starch and dextrine are converted into sugar, as shown remarkably in the action of diastase upon these bodies. If we mix 2 parts of starch paste with 1 part of dry gluten, and keep the mixture at a temperature of from $122^{\circ}$ to $140^{\circ}$ Fahr., we obtain a good deal of sugar and dextrine. Some lactic acid is also formed. Flour paste, long kept, spontaneously produces sugar by a like reaction. See Fermentation in the Dictionary.

Lactic Fermentation.-Almost all azotized organic matters, after being modified by the contact of air, become capable of giving rise to this fermentation. Oxygen does not come into play, except as the means of transforming the animal substances into a ferment. Diastase and caseum are well adapted to exhibit this change. The body that is to furnish the lactic acid may be any one of the neutral vegetable matters, possessing a like composition with lactic acid, such as cane-sugar, grape or potato sugar, lextrine, and sugar of milk. All the agents which stop the alcoholic, stop also the lactic fermentation; while diastase and caseum are its two best exciters. For producing abundance of lactic acid, we have merely to moisten malt, to expose it to the air for a few days, then to triturate it with a quantity of water, and leave the emulsion for some days more in the air, at a temperature between $67^{\circ}$ and $86^{\circ} \mathrm{F}$. We then saturate the liquor with chalk, after having filtered it, and thereby obtain the lactate of lime; which may be crystallized in alcohol, to deprive it of the dextrine and earthy phosphates; and then decomposed by sulphuric acid.

Lactic Acid, formed from curd (caseum), exhibits more remarkable phenomena. Thus when milk is left alone for some time it becomes sour, and coagulates. The coagulum 
s formed of caseum and butter; while the whey of it contains sugar of milk and some salts. The coagulation of the caseum has been occasioned by the lactic acid, which was generated in consequence of an action which the caseum itself exercised upon the sugar of milk. Thus with the concourse of air, the caseum becomes a ferment, and excites the conversion of the sugar of milli into lactic acid. The lactic acid in its turn coarulates the caseum, which in the consolidation of its particles attracts the butter. The caseum then ceases to act upon the sugar of milk, and consequently produces no more lactic acid.

But now, if the lactic acid already formed be saturated, the caseum will redissolve, and the phenomena will recommence in the same order. This is easily done by adding a due dose of bicarbonate of soda to the soured milk. In the course of 30 hours a fresh portion of lactic acid will be generated, and will have coagulated the milk again. We may also add some sugar of milk to the liquid, and to a certain extent convert it into lactic acid. Milk boiled, and kept from contact of air, will not coagulate, and remains fresh for many months. Animal membranes, modified by exposure to moist air for some time, form a true ferment for the lactic fermentation, and acidify solutions of sugar, dextrine, and gum, but the membranes must not be putrescent. Cane-sugar, starch-sugar, and sugar of milk, by assuming or losing a little water, acquire the constitution of lactic acid.

Viscous or Mucous Fermentation.-Erery one is acquainted with this spontaneous modification of white wine and ale, which gires them a stringy or oily aspect, and is called in French graisse, or fat of wines, and in English the ropiness of beer. The viscous fermentation may be excited by boiling reast with water, and dissolving sugar in the decoction, after it has been filtered. The syrup should have a specific grarity from $1 \cdot 040$ to $1 \cdot 055$, and be kept in a warm place. It soon assumes the consistence and aspect of a thick mucilage, like linseed tea, with the disengagement of a little carbonic acid and hydrogen, in the proportion of 2 or 3 of the former gas to 1 of the latter. A ferment of globular texture like that of yeast is formed, which is capable of producing riscous fermentation in any saccharine solution to which it is added, provided the temperature be suitable. The viscid matter being evaporated to driness forms transparent plates, of a sub-nauseous taste, and soluble in water, but less easily than gum arabic. Its mucilage is, however, thicker than that of gum, and yields with nitric acid, oxalic acid, but no mucic acid. Four parts of sugar, treated as above described, furnish 2.84 of unchanged sugar, and 1.27 of the mucilage; from which it appears that water becomes fixed in the transformation. Muriatic, sulphuric, sulphurous acids, and alum, prevent the production of the riscous fermentation, by precipitating its ferment. It is probably the soluble portion of gluten which is the cause of this species of fermentation. It has been found, accordingly, that tannin, which precipitates the said glutinous ferment, completely stops the viscous fermentation, or graisse, of wines. It is owing to the tannin which the red wines derive from the grape-stallis, with which they are long in contact during fermentation, that they are preserved from this malady of the white wines. The gluten of must is of two kinds, the onc soluble in virtue of the alcohol and tartaric acid, and producing the viscous, the other insoluble, and producing the alcoholic fermentation. The art of the wine-maker consists in precipitating the injurious ferment, without impeding the action of the beneficial one; an art of considerable delicacy with regard to sparkling wines.

Acid Fermentation has been fully discussed under acetic acid. It requires the presence of ready formed alcohol and air. The lactic fermentation, on the contrary, may take place with starchy or saccharine substances, without the intervention of alcahol or constant exposure to the atmosphere; and when once begun, it can go on without air. Acetification has a striking analogy with nitrification, as is shown by the necessity of a high temperature, and the utility of porous bodies for exposing the liquid on a great surface to the air.

Benzoic Fermentation is that which transforms the azotised neutral crystalline matter. existing in bitter almonds, which has no action upon the animal economy, into new and remarkable products, among others the hydrure of benzöile and hydrocjanic (prussic) acid, which together constitute the liquid, called oil, or essence, of bitter almonds, a compound possessed of volatility and poisonous qualities. The attentive study of this fermentation has revealed a great fact in vegetable physiology, the spontaneous production, by means of certain artifices, of certain volatile oils, not pre-existing in the plants, yet capable of being generated in the products of their decomposition. The rolatile oil of bitter almonds constitutes in this respect a starting point, from which have proceeded the oil of mustard, the oil of spiræa, and which will likely lead to other discoveries of the same kind. See Armond and Amygdaline.

Sinapic Fermenlation is that by which the oil of mustard is formed, and which takes place by the contact of water, under certain conditions, of too refined and scientific a nature for this practical work. 
Pectuc Fermentation.-Pectic acid may be obtained from the expressed juice of caz rots, and it seems to be formed in the process of extraction by the reaction of albumine in the carrots upon a substance called pectine; a transformation analogous therefor $-\mathrm{e}$ with that which takes place in the formation of the essence of bitter almonds.

Gallic Fermentation.-Gallic acid does not exist ready formed in nut-galls, but is generated from their tannin when they are ground, made pasty with water, and exposed to the air. This conversion may be counteracted by the red oxide of mercury, alcohol, sulphuric, muriatic, and nitric acids, bromine, essence of turpentine, creosote, oxalic, acetic, and prussic acids. The tannin disappears in the sequel of the above metamorphosis.

Fatty Fermentation.-All fats are transformed by the action of an alkaline or other base into certain acids, the stearic, margaric, the oleic, ethalic, \&c. When these acids are once formed, they can not by any means, hitherto known, be reconverted into the primitive fat. By the fixation of water in the acid and the base (called glycerine), a change is effected which can not be undone, because the glyceric base is incapable by itself to displace the water, once combined in the hydrated fat acid. The circumstances necessary to the fatty fermentation, are like those of other fermentations; namely, the cooperation of an albuminoid matter, along with water, and a temperature of from $60^{\circ}$ to $86^{\circ}$ F.; under these conditions, the matter becomes warm, and assumes speedily the charac. ter of rancidity; acid is generated, and the carbonate of soda can then form salts, while the fatty acid is liberated; a circumstance impossible when the fat was acted upon in the neutral state. This altered fat, treated with water, gives up to it glyceric alcohol.

Digestive Fermentation.-Digestion of food may be considered in its essential features as a peculiar fermentative process. The gastric juice is a genuine ferment. Tiedmann, Gmelin, and Prout, have shown that the gastric juice contains muriatic acid; and Eberli has made interesting experiments on the digestion of food out of the body, with water containing a few drops of the same acid. He observed that when this liquid contained none of the mucous secretion of the stomach, it did not dissolve the aliments put into it; but with a little of that mucus it acquired that property in an eminent degree. Even the mucus of the bladder had a like effect. Schwann and Vogel have produced this digestive principle in a pure state, called by them pepsine, as obtained most abundantly from the stomachs of swine. The glandular part of that viseus being separated from the serous, is cut into small pieces, and washed with cold distilled water. After digestion for 24 hours, that water is poured off, and fresh water is poured on. This operation is repeated for several days, till a putrid odor begins to be felt. The watery infusion thus obtained is precipitated by acetate of lead. This white flaky precipitate contains the pepsine, accompanied with much albumen. It is then washed, mixed with water; and subjected to a stream of sulphuretted hydrogen. The whole being now thrown on a filter, the coagulated albumen remains on the paper, along with the sulphuret of lead, while the pepsine liquor passes, associated with some acetic acid. If to this liquor a very small quantity of muriatic acid be added, it becomes capable of carrying on artificial digestion. Dry pepsine may be obtained by evaporating the above filtered liquor on a water bath, to a syrupy consistence, then adding to it absolute alcohol, which causes a bulky whitish precipitate. This dried in the air constitutes pepsine. It contains a minute quantity of acetic acid, which may be removed completely, by heating it some hours on the water bath. The white powder then obtained is soluble in water, and betrays the presence of no acid whatever. According to Togel, this substance is composed of, carbon, 57.72 ; hydrogen, 5.67 ; azote, 21.09 ; oxygen, $\& c ., 15.52=100$. Vogel has prored the analogy between the action of pepsine and diastase by the following experiment:-

He dissolved two grains of pepsine in very weak muriatic acid, and put into this liquor heated to $81^{\circ} \mathrm{F}$, small bits of boiled beef. In the course of a few hours the pieces became transparent on their edges, and not long after they were completely dissolved. He now added fresh morsels in succession, till those last put in remained unchanged. He found by analysis, that 1.98 grains of the pepsine were left, showing how minute a portion of this ferment was necessary to establish and effect digestion. In fact, we may infer that pepsine, like jeast, serves to accomplish digestion without any waste of its own substance whatever, or probably with its multiplication.

Rennet, with which milk is coagulated in making cheese, is somewhat of the same nature as pepsine. It has been called chymosine. But the simplest digesting liquor is the following :-

If 10,000 parts of water by weight be mixed with 6 parts of ordinary muriatic acid and a little rennet, a liquor is obtained capable of dissolving hard boiled white of egg, beef, gluten, \&c., into a transparent jelly in a few hours.

Ammoniacal Fermentation.- Under this title may be described the conversion of urea into carbonate of ammonia under the influence of water, a ferment, and a favorable temperature. Urea is composed in atoms; reckoned 


In volumes,
which by fixing
give

which is 4 vol. of carbonic acid, and 8 of ammonia; equivalent to ordinary carbonate of ammonia. The fermentation of urea plays an important part in the reciprocal offices of vegetable and animal existence. By its conversion into carbonate of ammonia, urea becomes a food fit for plants; and by the intervention of the mucous ferment which urine contains, that conversion is effected. Thus the urea constitutes a neutral and innocuous substance while it remains in the bladder, but is changed into a volatile, alkaline, and acrid substance, when it is acted upon by the air. Yeast added to pure urea mixed with water, exercises no action on it in the course of several days; but when added to urine, it soon causes decomposition, with the formation of carbonate of ammonia, and disengagement of carbonic acid. The deposite on chamberpots ill-cleaned acts as a very powerful ferment on urine, causing the complete decomposition of fresh urine in one fifth of the time that would otherwise be requisite.

Nitrous Fermentation, as exhibited in the formation of nitric acid from the atmosphere, and consequent production of nitrates in certain soils, has been with much probability traced to the action of ammonia on oxygen, as the intermedium or ferment.

Caseous and putrid Fermentations.-Curd is converted into cheese, when after being coagulated by remet, it is left to itself under certain conditions; and this constitutes the true distinctive character of caseum. In the production of cheese there is evidently the intervention of a peculiar ferment which is gradually formed, and the decomposition of the curd into new products.

For animal and vegetable matters to run into putrefaction, they must be in contact with air and water, at a certain temperature; viz., between the freezing and boiling points of water. The contact of a putrid substance acts as a ferment to fiesh animal and vegetable matters. The reagents which counteract fermentation in general stop also putrefaction. In this process, myriads of microscopic animalcules make their appearance, and contribute to the destruction of the substances.

A dispute having taken place between some distillers in Ireland and officers of Excise, concerning the formation of alcohol in the vats or tuns by spontaneous fermentation, without the presence of yeast, the Commissioners of Excise thought fit to cause a series of experiments to be made upon the subject, and they were placed under my general superintendence. An experiment was made on the 6th of October, with the following mixture of corn :-

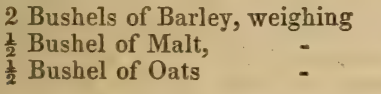

Total, 3 Bushels, weighing -
- 100lbs. $5 \mathrm{oz}$.

- 217

- 20,12

$-1428$

The bruised corn was wetted with 26 gallons of water at the temperature of $160^{\circ} \mathrm{F}$., and, after proper stirring, had 8 gallons more of water added to it at the arerage temperature of $194^{\circ}$. The mash was again well stirred, and at the end of 45 minutes the whole was covered up, having at that time a temperature of $138^{\circ} \mathrm{F}$. Three hours afterward, 16 gallons of wash only were drawn off; being considerably less than should have been obtained, had the apparatus been constructed somewhat differently, as shall be presently pointed out. The gravity of that wash was $1 \cdot 060$, or, in the language of the distiller, $60^{\circ}$. After a delay of two hours more, twenty additisnal gallons of water at the temperature of $200^{\circ}$ were introduced, when the mash was well stirred, and then covered un for two hours, at which period 23 gallons of fine worts of specific gravity 1.042 were drawn off. An hour afterward 12 gallons of water at 200 were added to the residual grains, and in an hour and a half 11 gallons of wort of the density 1.033 were obtained. Nixt morning the several worts were collected in a new mash tun. They consisted of 48 gallons at the temperature $80^{\circ}$, and of a specific gravity 2.0465 when reduced to $60^{\circ}$. Being set at $80^{\circ}$, fermentation soon commenced; in two days the specific gravity had fallen to 1.0317 , in three dars to $1 \cdot 018$, in four days to 1.013 , and in five days to 1.012 , the temperature having at last fallen to $78^{\circ} \mathrm{F}$. The total attenuation was therefore $34 \frac{1}{2} \mathrm{O}$, indicating the production of 3.31 gallons of proof spirit, while the produce by distillation in low wines was 3.22 ; and by rectification in spirits and feints it was 3.05 . The next experiment was commenced on the 12th of October, upon a similar mixture of corn to the preceding. 48 gallons of worts of 1.043 specific gravity were set at $82^{\circ}$ in the tun, which next day was attenuated to 1.0418 , in two days to 1.0202 , in three days to 1.0125 , and in five days to $1 \cdot 0105$, constituting in the whole an attenuation of $32 \frac{1}{2} \circ$, which indicates 
the production of $3 \cdot 12$ gallons of proof spirits; while the produce of the first distllla tion was 2.93 in low wines, and that of the second in feints and spirits was 2.66 . In these experiments the wash, when fermenting most actively, seemed to simmer and boil on the surface, with the emission of a hissing noise, and the copious evolution of carbonic acid gas. They prove beyond all doubt that much alcohol may be generated in grain worts without the addition of yeast, and that, also, at an early period; but the fermentation is never so active as with yeast, nor does it continue so long, or proceéd to nearly the same degree of attenuation. I was never satisfied with the construction of the mash tun used in these experiments, and had accordingly suggested another form, by which the mash mixture could be maintained at the proper temperature during the mashing period. It is known to chemists that the diastase of malt is the true saccharifying ferment which converts the fecula, or starch of barley and other corn, into sugar; but it acts beneficially only between the temperatures of $145^{\circ}$ and $168^{\circ} \mathrm{F}$. When the temperature falls below the former number, saccharification languishes; and when it rises much above the latter, it is entirely checked. The new mash tun was made of sheet zinc, somewhat wider at bottom than top; it was placed in a wooden tun, so much larger as to leave an interstitial space between the two of a couple of inches at the sides and bottom. Through this space a current of water at $160^{\circ}$ was made to circulate slowly during the mashing period. Three bushels of malt, weighing $125 \mathrm{lbs} .3 \mathrm{oz}$., were wetted with 30 gallons of water at $167^{\circ}$, and the mixture being well agitated, the mash was left covered up at a temperature of $140^{\circ}$ during three hours, when 19 gallons of fine worts were drawn off at the specific gravity of 1.0902 or $90 \cdot 2^{\circ}$. Twenty gallons more water at $167^{\circ}$ were then added to the residuum, which afforded after two hours 28 gallons of wort at the gravity $1 \cdot 036 ; 12$ galions of water at $167^{\circ}$ were now poured on, which yielded after other two hours 15 gallons at the gravity $1 \cdot 0185$. Forty gallons of fine worts at $1 \cdot 058$ gravity and $68^{\circ}$ temperature were collected in the evening of the same day, and let into the tun with 5 per cent. of yeast. The attenuation amounted in six days to $54^{\circ}$. The third wort of this brewing, amounting to 15 gallons, being very feeble, was mixed with 7 gallons of the first and second worts, put into a copper, and concentrated by boiling to 11 gallons, which had a gravity 1.058 at $60^{\circ} \mathrm{F}$. They were separately fermented with 5 per cent. of yeast, and suffered an attenuation of $48 \frac{1}{2}{ }^{\circ}$. The produce of spirit from both indicated by the attenuation was $5 \cdot 36$ gallons; the produce in low wines was actually $5 \cdot 52$, and that in spirits and feints was $5 \cdot 33$, being a perfect accord. ance with the Excise tables.

The next experiments were made with a view of determining at what elevation of temperature the activity or efficiency of yeast would be paralysed, and how far the attenuation of worts could be pushed within six hours, which is the time limited by law for worts to be collected into the tun, from the time of beginning to run from the coolers. When worts of the gravity 1.0898 were set at $96^{\circ}$ Fahr., with 5 per cent. of yeast, they attenuated $26.90^{\circ}$ in 6 hours; worts of 1.0535 gravity set at $110^{\circ}$ with 5 per cent. of yeast, attenuated $16^{\circ}$ in about 5 hours; but when worts of 1.0533 were set, as above, at $120^{\circ}$, they neither fermented then, nor when allowed to cool ; showing that the activity of the yeast was destroyed. When fresh yeast was now added to the last portion of worts, the attenuation became 5.8 in 2 hours, and $28.4^{\circ}$ in 3 days; showing that the saccharine matter of the worts still retained its fermentative faculty. Malt worts, being brewed as above specified, were set in the tun, one portion at a temperature of $70^{\circ}$, with a gravity of 1.0939 , and 5 per cent. of yeast, which attenuated $66^{\circ}$ in 3 days; other two portions of the same gravity were set at $120^{\circ}$ with about 10 per cent. of yeast, which underwent no fermentative change or attenuation in 6 hours, all the yeast having fallen to the bottom of the tuns. When these two samples of worts were allowed, however, to cool to from $74^{\circ}$ to $72^{\circ}$, fermentation commenced, and produced in two days an attenuation of about $79^{\circ}$. It would appear, from these last two experiments, that yeast to the amount of 5 per cent. is so powerfully affected by strong worts heated to $120^{\circ}$ as to have its fermentative energy destroyed; but that when yeast is added to the amount of 10 per cent., the 5 parts of excess are not permanently decomposed, but have their activity merely suspended till the saccharine liquid falls to a temperature compatible with fermentation. Yeast, according to my observations, when viewed in a good acromatic microscope, consists altogether of translucent spherical and spheroidal particles, each of about the 6000th part of an inch in diameter. When the beer in which they float is washed away with a little water, they are seen to be colorless; their yellowish tint, when they are examined directly from the fermenting square or round of a porter lrewery, being due to the infusion of the brown malt. The yeast of a square newly set seems to consist of particles smaller than those of older yeast, but the difference of size is not considerable. The researches of Schulze, Cagniard de la Tour, and Schwann, appear to show, that the vinous fermentation, and the putrefaction of animal matters, processes which have been 
bitherto considered as belonging entirely to the domain of chemical affinity-are essentially the results of an organic development of living beings. This pcsition seems to be established by the following experiments: 1. A matrass or flask containing a few bits of flesh, being filled up to one third of its capacity with water, was closed with a cork, into which two slender glass tubes were cemented air-tight. Both of these tubes were passed externally through a metallic bath, kept constantly melted, at a temperature approaching to that of boiling mercury. The end of one of the tubes, on emerging from the bath, was placed in communication with a gasometer. The contents of the matrass were now made to boil briskly, so that the air contained in it and the glass tubes was expelled. The matrass being then allowed to cool, a current of atmospherical air was made constantly to pass through it from the gasometer, while the metallic bath was kept constantly hot enough to decompose the living particles in the air. In these experiments, which were many times repeated, no infusoria or fungi appeared, no putrefaction took place, the flesh underwent no change, and the liquor remained as clear as it was immediately after being boiled. As it was found very troublesome to maintain the metallic bath at the melting pitch, the following modification of the apparatus was adopted in the subsequent researches: A flask of three ounces capacity, being one fourth filled with water and flesh, was closed with a tight cork, secured in its place by wire. Two glass tubes were passed through the cork; the one of them was bent down, and dipped at its end into a small capsule containing quicksilver, covered with a layer of oil; the other was bent on leaving the cork, first into a horizontal direction, and downward for an inch and a half, afterward into a pair of spiral turns, then upward, lastly horizontal, whence it was drawn out to a point. The pores of the cork having been filled with caoutchouc varnish, the contents of the flask were boiled till steam issued copiously through both of the glass tubes, and the quicksilver and oil became as hot as boiling water. In order that no living particles could be generated in the water condensed beneath the oil, a few fragments of corrosive sublimate were laid upon the quicksilver. During the boiling, the flame of a spirit lamp was drawn up over the spiral part of the second glass tube, by means of a glass chimney placed over it, so as to soften the glass, while the further part of the tube was heated by another spirit lamp, to prevent its getting cracked by the condensation of the steam. After the ebullition had been kept up a quarter of an hour, the flask was allowed to cool, and get filled with air through the hot spiral of the second tube. When the contents were quite cold, the end of this tube was hermetically sealed, the part of it between the point and the spiral was heated strongly with the flames, and the lamps were then withdrawn. The matrass contained now nothing but boiled flesh and gently ignited air. The air was renewed occasionally through the second tube, its spiral part being first strongly heated, its point then broken off, and connected with a gasometer, which caused the air to pass onward slowly, and escape at the end of the first tube immersed in the quicksilver. The end of the second tube was again hermetically closed, while the part interjacent between it and the spiral was exposed to the spirit flame. By means of these precautions, decoctions of flesh were preserved, during a period of six weeks, in a temperature of from $14^{\circ}$ to $20^{\circ} \mathrm{R}$. $\left(63 \frac{1}{2}{ }^{\circ}\right.$ to $77^{\circ} \mathrm{F}$.), without any appearance of putrefaction, infusoria, or mouldiness : on opening the vessel, however, the contents fermented in a few days, as if the: had been boiled in the ordinary manner. In conducting such researches, the greatest pains must be taken to render the cork and junctions of the glass tubes perfectly air-tight. The following more convenient modification of the experiment, but one equally successful and demonstrative, was arranged by F. Schulze. The glass tubes connected with the flask were furnished each with a bulb at a little distance from the cork; into one of which globes caustic alkaline ley being put, and into the other strong sulphuric acid, air was slowly sucked through the extremity of the one tube, while it entered at the other, so as to renew the atmosphere over the decoction of flesh in the flask. In another set of experiments, four flasks being filled with a solution of canesugar containing some beer-yeast, were corked and plunged in boiling water till they acquired its temperature. They were then taken out, inverted in a mercurial bath, uncorked, and allowed to cool in that position. From one third to one fourth of their volume of atmospherical air was now introduced into each of the flasks; into two of them through slender glass tubes kept red hot at a certain point, into the other two through glass tubes not heated. By analysis it was found that the air thus heated contained only 19.4 per cent. of oxygen, instead of 20.8 ; but, to compensate for this deficiencr, a little more air was admitted into the two flasks connected with the heated tubes than into the two others. The flasks were now corked and placed in an inverted position, in a temperature of from $10^{\circ}$ to $14^{\circ} \mathrm{R}$. ( $54 \frac{1}{2} \circ$ to $63 \frac{1}{2} \circ \mathrm{F}$.). After a period of from four to six weeks, it was found that fermentation had taken place in both of the flasks which contained the non-ignited air--for, in loosening the corks, some of the contents were projected with force--but, in the other two flasks, there 
was no appcarince of fermentation, either then, or in double the time. As the extract of nux romica is known to be a poison to infusoria (animalcules), but not to vegetating mould, while arsenic is a poison to both, by these tests it was proved that the living particles instrumental to fermentation belenged to the order of plants of the confervoid fumily. Beer yeast, according to Schwann, consists entirely of microscopic fungi, in the shape of small oval grains of a yellowish white color, arranged in rows oblique to each other. Fresh grapes must contain none of them; but after being exposed to the air at $20^{\circ} \mathrm{R}$., for 36 hours, similar grains become visible in the microscope, and may be observed to grow larger in the course of an hour, or even in half that time. A few hours after these plants are first perceived, gas begins to be disengaged. They multiply greatly in the course of fermentation, and at its conclusion subside to the bottom of the beer in the shape of a yellow white powder.

FERRIC ACID. This new compound having been prescribes as a source of supplying oxygen to persons confined in diving-bells and in mines, by M. Payerne, in a patent recently granted to him, merits notice in a practical work. M. Fremy is the discoverer of this new acid, which he obtains in the state of ferrate of potash, by projecting 10 parts of dry nitre in powder upon 5 parts of iron filings, ignited in a crucible; when a reddish mass, containing much ferrate of potash, is formed. The preparation succeeds best when a large crucible, capable of holding about a pint of water, is heated so strongly that the bottom and a couple of inches above it, appear faintly, but distinctly red, in which state the heat is just adequate to effect due deflagration without decomposition. An intimate mixture of about 200 grains of dried nitre with about one half its weight of the finest iron filings, is to be thrown at once upon the side of the crucible. The mixture will soon swell and deflagrate. The crucible being taken from the fire, and the ignited mass being cooled, is to be taken out with an iron spoon, pounded, immediately put into a bottle, and secluded from the air, in which it would speedily attract moisture, and be decomposed. It is resolved by the action of water, especially with heat, into oxygen gas, peroxide, and nitrate of iron.

Mr. J. D. Smith prepares the ferrate of potash by exposing to a full red heat a mixture of finely powdered peroxide of iron with four times its weight of dry nitre. It has an amethyst hue, but so deep as to appear black, except at the edges. Oxygen is rapidly evolved by the action of the sulphuric or nitric acid upon its solution. He considers the atom of iron to exist in this compound, associated with 3 atoms of oxygen, or double the proportion of that in the red oxide. Hence 52 grains of pure ferric acid should give off 12 grains of oxygen, equal to about 35 cubic inches; but how much of the ferrate of potash may be requisite to produce a like quantity of oxgyen, can not be stated, from the uncertainty of the operation by which it is produced.

FERRIC-CYANIDE OF POTASSIUM, or Red Prussiate of Potash. This beautiful and useful salt, discovered by L. Gmelin, is prepared by passing chlorine gas through a weak solution of the prussiate of potash (ferro-cyanide of potassium) till it ceases to affect solution of red sulphate of iron, taking care to agitate the liquid all the while, and not to add an excess of chlorine. On looking through the weak solution to the flame of a candle, one may see the period of change from the greenish to the red hue, which indicates the completion of the process. The liquor being filtered and evaporated in a dish with upright sides, will eventually afford crystalline needles, possessed of an almost metallic lustre, and a yellow color, inclining to red. These being dissolved and recrystallized, will become extremely beautiful. This salt is composed of 33.68 parts of potassium, 16.48 of iron, and 47.84 of cyanogen. It is therefore a dry salt. It dissolves in 38 parts of cold water, and as it forms then the most delicate test of the protoxide of iron, is very useful in Clorometry.-See Appendrx.

The solution of this salt aflords the following colored precipitates with the solutions of the respective metals :-

\begin{tabular}{|c|c|c|c|c|}
\hline Titanium & $=$ & - & & - Brownish yellow. \\
\hline Uranium & - & - & * & - Reddish brown. \\
\hline Manganese & - & - & & - Brownish gray. \\
\hline Cobalt & - & - & & - Deep reddish brow \\
\hline Nickel & - & - & & - Yellowish brown. \\
\hline Copper & $=$ & - & & - Dirty yellowish br \\
\hline Silver & - & - & & - Orange yellow. \\
\hline Mercury & - & - & & $\begin{array}{l}\text { - Yellow, with both } \\
\text { peroxide salts. }\end{array}$ \\
\hline Tin & - & - & & - White. \\
\hline Zine & - & - & & - Orange Yellow. \\
\hline Bismuth & - & $=$ & & - Yellowish brown. \\
\hline Lead . & - & - & & - No precip. \\
\hline Iron protoxi & - & $=$ & & - Blue. \\
\hline & & - & & - No precip. \\
\hline
\end{tabular}


The ferric-cyanide of potassium has been introduced into dyeing and calico-printing.

In ease an excess of chlorine has been used in preparing the above salt, Posselt recommends to add to its solution, when near the crystallizing point, a few drops of potash ley, in order to decompose a green substance that is present, which takes place with the precipitation of a little peroxide of iron.

FIREARMS. Barrel-welding by Machinery.-The barrels of musquets, birdingguns, se., or what are called plain, to distinguish them from those denominated stub or twisted barrels, have of late years been formed by means of rolls, a process in which the welding is first effected on a short slab of thick iron, and then the barrel is brought down to its destined length, and form, by repeatedly passing it between a pair of rolls, that have been previously grooved to the exact shape of the barrel intended to be made.

This method has entirely superseded the skelp-welding by hand described in the Dic. of Man., p. 471, and is conducted as follows :-

The iron being thoroughly refined, and reduced into flat bars by the process described at length at p. 705, is cut by the shears into slabs or lengths of 10 to 12 inches, and 10 to $10 \frac{1}{2}$ lbs. weight, or less, according to the description of gun-barrel that is intended to be made. These slabs are then heated, and bent in their whole length, by means of conveniently grooved bending rolls, until they assume the form of rough tubes,

49 of the kind of section shown by A, fig. 49. They are then placed on the hearth of the reverberatory furnace (Dict. p. 701), and brought to a full

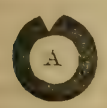
welding heat, and as soon as the edges of a tube come to a semi-fluid state, it is taken out and passed between rolls having grooves somewhat smaller in diameter than the exterior of the tube, by which means the tube is perfectly welded from end to end; and if care be taken in the management of the heat, and the juncture be kept clear of dirt and cinders, the iron will be found perfectly homogeneous in every part, and there will be no appearance whatever of the seam where the edges came together. These tubes are repeatedly heated, and passed between the barrel rolls, which are of sufficient diameter to admit of gradually decreasing grooves, the whole length of the intended barrel being indented on their surfaces.

To preserve the tubular form, and insure regularity in the size of the bore during the welding process, they are taken out of the furnace, bv thrusting into them a tool called a mandril $\mathrm{B}$, which consists of a long rod of iron, having a short steel treblett on its end, of the diameter that the bore of the barrel is meant to bore. This rod is so adjusted by means of a strong iron plate c, near its handle, which is of wood, and long, that when passed with the heated tube on it between two tranverse holding bars, the short steel treblett $\mathrm{D}$, shall be found exactly between the point of impact of the barrelrolls, $\mathbf{E}$.

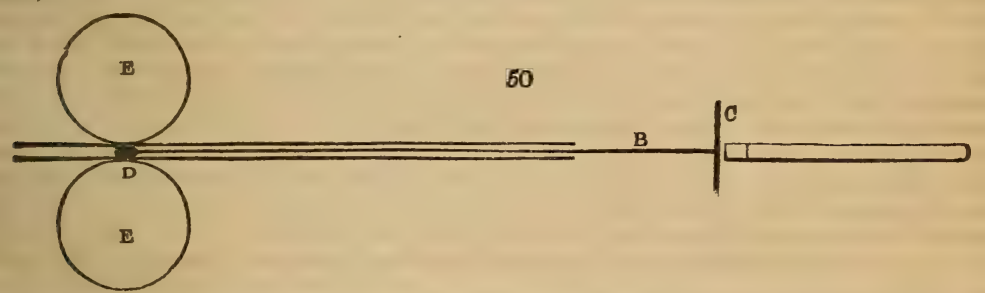

The adhesion of the hot iron to the surface of the rolls is strong enough to draw the tube off the mandril, which thus keeps the bore open from end to end, and by repeating the process through the whole series of grooves in the rolls, the barrel is gradually elongated, and brought down to the exact form required : any superfluous length at the muzzle is then cut off. The breach end is then adjusted by the hammer-a tripple-seat welded on by hand if it be intended for a percussion lock, and then the barrel is ready to go forward to the mill to be bored, turned, and finished.

Gun-barrels formed by this mechanical method are found to stand proof better than those worked by hand, because the heat is more equalized; and any imperfections in the original mass of iron are more dispersed over the whole extent of the tube.

Mr. Wells Ingram, of Bradford street, Birmingham, has lately perfected a very complete lathe for turning the exterior of gun-barrels of all descriptions, a process which is fast superseding the use of the grindstone, for equalizing the barrels of all kinds of firearms.

I am indebted for this article to Mr. Lovell, Director of the Royal Arms Manufactory. See Musedet.

FLAX and TOW, or Codilla of Hemp and Flax, imported for home consumption in $1839,1,216,811$ cwt. ; in $1840,1,256,322 ; 1 d$. per cwt. 
FLOOKAN. The name given by the Cornish miners to a vein of clay-stone, often nearly vertical.

FLOOR-CLOTH MANUFACTURE has become of late years a very large branch of trade. The cloth is a strong somewhat open canvass, woven of flax with a little hemp, and from 6 to 8 yards wide, being manufactured in appropriate lonms chiefly at Dundee. A piece of this canvass from 60 to 100 feet in length, is secured tight in an upright open frame of oaken bars, in which position it receives the foundation coats of painc, 2 or 3 in number, first on the black side, and then on the front; but it previously is brushed over with glue-size, and rubbed smooth with pumice-stones. The foundation paint made with linseed oil and ochre, or any cheap coloring matter, is too thick to be applied by the brush, and is therefore spread evenly by a long narrow trowel, held in the right hand, from a patch of it laid on just before with a brush in the left hand of the workman. Each foundation coat of the front surface is smoothed by pumice whenever it is hard enough to bear the operation. When both sides are dry, the painted cloth is detached from the frame, coiled round a roller, in this state transferred to the proper printing-ronm, where it is spread flat on a table, and variously figured and colored devices are given to it by wooden blocks, exactly as in the block-printing of alicues, and in the wood-printing of books. The blocks of the floor-cloth manufacture are formed of two lajers of white deal and one of pear-tree timber, placed with their grain crossing one another alternately. There is of course a block for each color in the pattern, and in each block those parts are cut away that correspond to the impressions given by the others; a practice now well understood in the printing of two or more colors by the press. The faces of the blocks are so indented with fine lines, that they do not take up the paint in a heavy daub from the flat cushion on which it is spread with a brush, but in minute dots, so as to lay on the paint (somewhat thicker than that of the house-painter) in a congeries of little dots or teeth, with minute interstices between. Applied in this way, the various pigments lie more evenly, are more sightly, and dry much sooner than if the prominent part of the block which takes up the color were a smooth surface. The best kinds of floor-cloth require from two to three months for their production.

FODDER, a weight of $21 \mathrm{cwt}$., by which lead is sold in the north of England.

FUEL. On the measurement of heat, and the qualities of different kinds of coal, I made an elaborate series of experiments, a few years ago, of which the following is an outline :-

The first and most celebrated, though probably not the most accurate apparatus for measuring the quantity of heat transferable from a hotter to a colder body, was the calorimeter of Lavoisier and Laplace. It consisted of three concentric cylinders of tin plate, placed at certain distances asunder; the two outer interstitial spaces being filled with ice, while the innermost cylinder received the hot body, the subject of experiment. The quantity of water discharged from the middle space by the melting of the ice in it, served to measure the quantity of heat given out by the body in the central cylinder. A simpler and better instrument on this principle would be a hollow cylinder of ice of proper thickness, into whose interior the hot body would be introduced, and which would indicate by the quantity of water found meited within it the quantity of heat absorbed by the ice. In this case, the errors occasioned by the retention of water among the fritgments of ice packed into the cylindric cell of the tin calorimeter, would be voided. One pound of water at $172^{\circ} \mathrm{F}$., introduced into the hollow cylinder above descrived, will melt exactly one pound of ice; and one pound of oil heated to $172^{\circ}$ will melt half a pound.

The methor of refrigeration, contrived at first by Meyer, has been in modern times brought to great perfection by Dulong and Petit. It rests on the principle, that two surfaces of like size, and of equal radiating force, lose in like times the same quantity of heat when they are at the same temperature. Suppose for example, that a vessel of polished silver, of small size, and very thin in the metal, is successively filled with different pulverized substances, and that it is allowed to cool from the same elevation of temperature; the quantities of heat lost in the first instant of cooling will be always equal to each other; aud if for one of the substances, the velocity of cooling is double of that for another, we may conclude that its capacity for heat is one half, when its weight is the same; since by losing the same quantity of heat, it sinks in temperature double the number of degrees.

The method of mixtures. - In this method, two bodies are always employed; a hot body which becomes cool, and a cold body, which becomes hot, in such manner that all the caloric which goes out of the former is expended in heating the latter. Suppose for example, that we pour a pound of quicksilver at $212^{\circ} \mathrm{F}$., into a pound of water at $32^{\circ}$; the quicksilver will cool and the water will heat, till the mixture by stirring acquires a common temperature. If this temperature was $122^{\circ}$, the water and mercury would have equal capacities, since the same quantity of heat would produce in an equal 
mass of these two substances equal changes of temperature, viz., an elevation of $90^{\circ}$ in the water and a depression of $90^{\circ}$ in the mercury. But in reality, the mixture is found to have a temperature of only $37 \frac{1}{2}^{\circ}$, showing that while the mercury loses $174 \frac{1}{2}^{\circ}$ the water gains only $5 \frac{1}{2} \circ$; two numbers in the ratio of about 32 to 1 ; whence it is concluded, that the ec, acity of mercury is $\frac{1}{32}$ of that of water. Corrections must be made for the influence of the ressel and for the heat dissipated during the time of the experiment.

The following calorimeter, founded upon the same principle as that of Count Rumford, but with certain improvements, may be considered as an equally correct instrument for measuring heat, with any of the preceding, but one of much more general application, since it can determine the quantity of heat disengaged in combustion, as well as the latent heat of steam and other vapors.

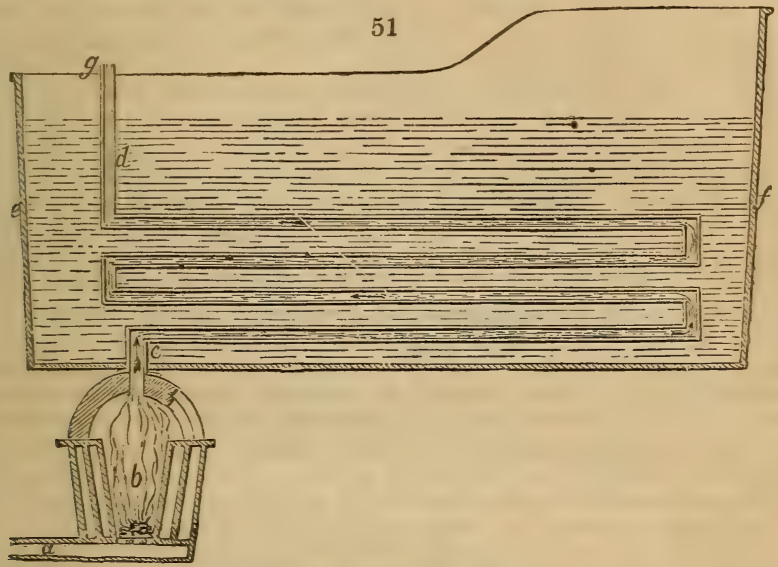

(Scale about $\frac{1}{2}$ inch to the foot.)

It consists of a large copper bath, $e, f$ ( $f i g .51)$, capable of holding 100 gallons of Fater. It is traversed four times, backward and forward, in four different levels, by a zig-zag horizontal flue, or flat pipe $d, c$, nine inches broad and one deep, ending below in a round pipe at $c$, which passes through the bottom of the copper bath $e, f$, and receires there into it the top of a small black lead furnace $b$. The innermost crucible contains the fuel. It is surrounded at the distance of one inch by a second crucible, which is enclosed at the same time by the sides of the outermost furnace; the strata of stagnant air between the crucibles serving to prevent the heat from being dissipated into the atmosphere round the body of the furnace. A pipe $a$, from a pair of cylinder double bellows, enters the ash-pit of the furnace at one side, and supplies a steady but gentle blast, to carry on the combustion, kindled at first by half an ounce of red-hot charcoal. So completely is the heat which is disengaged by the burning fuel absorbed by the waces in the bath, that the air discharged at the top orifice g, has usually the same temperature as the atmosphere.

The vessel is made of copper, weighing two pounds per square foot ; it is $5 \frac{1}{2}$ feet long, $1 \frac{1}{2}$ wide, 2 deep, with a bottom $5 \frac{1}{2}$ feet long, and $1 \frac{3}{4}$ broad, upon an average. Including the zig-zag tin plate flue, and a rim of wrought iron, it weighs altogether 85 pounds. Since the specific heat of copper is to that of water as 94 to 1,000 ; the specific heat of the ressel is equal to that of 8 pounds of water, for which, therefore, the exact correction is made by leaving 8 pounds of water out of the 600 , or 1,000 pounds used in each
experiment.

In the experiments made with former calorimeters of this kind, the combustion was maintained by the current or draft of a chimney, open at bottom, which carried off at the top orifice of the flue a variable quantity of heat, very difficult to estimate.

When the object is to determine the latent heat of steam and other vapors, they mar be introduced through a tube into the orifice $g$, the latent heat being deduced from the elevation of temperature in the water of the bath, and the volume of vapor expended from the quantity of liquid discharged into a measure glass from the bottom outlet $c$. In this case, the furnace is of course removed.

The heating power of the fuel is measured by the number of degrees of temperature which the combustion of one pound of it, raises 600 or 1,000 pounds of water in the bath, the copper substance of the vessel being taken into account. One pound of dry 
wood charcoal by its combustion causes 6,000 pounds of water to become $20^{\circ}$ hotter. For the sake of brevity, we shall call this calorific energy 12,000 unities. In like circumsiances, one pound of Llangennoek coal will y1eld by combustion 11,500 unities of caloric. One pound of charcoal after exposure to the air gives out in burning only 10,500 unities; but when previously deprived of the moisture which it so greedily imbihes from the atmosphere, it affords the above quantity. One pound of Lambton's Wall's-end coals, affords 8,500 unities; and one of anthracite 11,000.

It must be borne in mind that a coal which gives off much unburnt carburetted hydrogen gas, does not afford so much heat, since in the production of the gas a great deal of heat is carried off in the latent state. I have no doubt, that by this distillatory process, from one third to one fourth of the total calorific effect of many coals is dissipated in the air. But by means of such a furnace as the patent Argand invention of Mr. C. W. Williams, the whole heat produceable by the hydrogen as well as the carbon is obtained; and it should be borne in mind that a pound of hydrogen in burning generates as much heat as three pounds of carbon.

Mr. Berthier proposes to determine the proportion of carbon in coals and other kinds of fuel, by igniting in a crucible a mixture of the carbonaceous matter with litharge, both finely comminuted, and observing the quantity of lead which is reduced. For every 34 parts of lead, he estimates 1 part of carbon, apparently on the principle, that when carbon is ignited in contact with abundance of litharge, it is converted into carbonic acid. Each atom of the carbon is therefore supposed to seize two atoms of oxygen, for which it must decompose two atoms of litharge, and revive two atoms of lead. Calling the atom of carbon 6, and that of lead 104, we shall have the following ratio :-6 : $\overline{104 \times 2}:: 1$ : 34.66 , being Berthier's proportion, very nearly.

On subjecting this theory to the touchstone of experiment, I have found it to be en tirely fallacious. Having mixed very intimately 10 grains of recently calcined charcoal with 1,000 grains of litharge, both in fine powder, I placed the mixture in a crucible which was so carefully covered, as to be protected from all fuliginous fumes, and exposed it to distinct ignition. No less than 603 grains of lead were obtained; whereas by Berthier's rule, only 340 or 346.6 were possible. On igniting a mixture of 10 grains of pulverized anthracite from Merthyr Tydfil, with 500 grains of pure litharge (pre. viously fused and pulverized), I obtained 380 grains of metallic lead. In a second similar experiment with the same anthracite and litharge, I obtained 450 grains of lead; and in a third only 350 grains. It is therefore obvious that this method of Berthier is altogether nugatory for ascertaining the quantity of carbon in coals, and is worse than useless for judging of the calorific qualities of different kinds of fuel.

In my researches upon coals, I have also made it one of my principal objects to determine the quantity of sulphur which they may contain; a point which has been hitherto very little investigated in this country at least, but which is of great consequence, not only in reference to their domestic combustion, but to their employment by manufacturers of iron and gas. That good iron can not be produced with a sulphureous coal, however well coked, has been proved in France by a very costly experience. The presence of a notable proportion of sulphur in a gas coal is most injurious to the gaseous products, because so much sulphuretted hydrogen is generated as to require an operose process of washing or purification, which improverishes the gas, and impairs its illuminating powers by the abstraction of its olefiant gas, or bicarburetted hydrogen. In proof of this proposition, I have only to state the fact, that I found in a specimen of coal gas as delivered from the retorts of one of the metropolitan companies, no less than 18 per cent. of olefiant gas, while in the same gas, after being passed through the purifiers, there remained only 11 per cent. of that richly-illuminating gas. By using a gas-coal, nearly free from sulphur, such as No. 4, in the subjoincd list, I think it probable that 10 per cent. of more light may be realized than with the common more sulphureou's coal. This is an important circumstance which the directors of gasworlis have hitherto neglected to investigate with analytical precision, though it is one upon which their success and profits mainly depend.

How little attention indeed has been bestowed upon the sulphureous impregnation of pit-coal may be inferred from the fact that one of our professional chemists of note, in a public report, upon a great commercial enterprise, stated that a certain coal analyzed by him was free from sulphur, which coal I found by infallible chemical evidence to contain no less than 7 per cent. of sulphur, being about the double of what is contained in English coals of average quality. The proportion of sulphur may in general be inferred from the appearance and quantity of the ashes. If these be of a red or ochrey color, and amount to above 10 per cent., we may be sure that the coal is eminently. sulphureous. The coal above referred to afforded from 15 to 16 per cent. of ferruginous ashes. I believe that sulphur exists in coal generally, though not always in the state of pyrites, either in manifest particles, or invisibly disseminated through their substance.

The readiest method of determining rigidly the quantity of sulphur in anv compound, 
s to mix a given weight of it with a proper weight of carbonate of potassa, nitre, and common salt, each chemically pure, and to ignite the mixture in a platinum crucible. A whitish mass, is obtained, in which all the sulphur has been converted into sulphate of potassa. By determining with nitrate of baryta the amount of sulphuric acid produced, that of the sulphur becomes known. By means of this process applied to different samples of coals, I obtained the following results :-

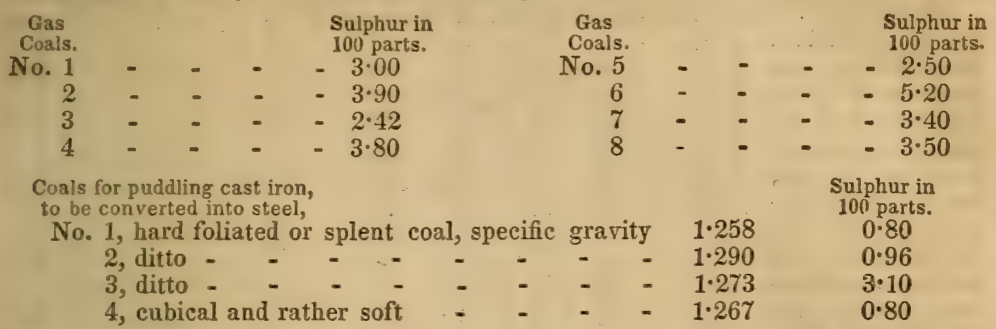

The last coal being rich in bitumen, would prove an excellent one for the production of a pure coal gas. See Prtcoar.

FUEL, ECONOMY OF. In the report of the Transactions of the Institution of Civil Engineers for February, 1838, the results of exact comparisons between the performance of different steam-engines exhibit this economy in a remarkable manner. It is there shown that a condensing engine of the most perfect construction, and in perfect condition, of the common low pressure crank-kind, not working expansively, performs a duty of not more than 20 or 21 millions of lbs. raised one foot high, by 90 or 94 lbs. of coal; or ten lbs. of coal per horse power per head.

The following table exhibits the relative value of different engines in lbs. of coal per horse power per hour :-

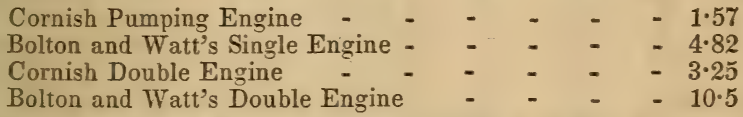

The greatest duty performed by the measured bushel of $84 \mathrm{lbs}$. was $86 \frac{1}{2}$ millions of lbs. There was raised by the Huel Towan engine in Cornwall 1,085 tons (of water) one foot high for one farthing. Hence the weight of a man ( $1 \frac{1}{2}$ cwt.) would be raised ten miles for one penny !

In order to raise steam with economy, the surface of water in the boiler, exposed to the fire, ought not to be less thon 10 square feet per horse power; but the usual allowance in Lancashire is only $7 \frac{1}{2}$; and by Messrs. Boulton and Watt, 5 square feet.

The values of the mean of the Cornish, Warwick, London, Lancashire, and loco-

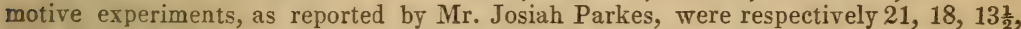
and 10 cubic feet of water evaporated by $112 \mathrm{lbs}$. of coals, from water heated to $212^{\circ} \mathrm{F}$.

FUEL, GRANT'S PATENT. This fuel is composed of coal-dust and coal-tar pitch; these materials are mixed together, under the influence of heat, in the following proportions :-20 lbs. of pitch to 1 crt. of coal-dust, by appropriate machinery ; consisting of crushing-rollers for breaking the coal in the first instance sufficiently small, so that it may pass through a screen the meshes of which do not exceed a quarter of an inch asunder; $2 \mathrm{dly}$, of mixing-pans or cylinders, heated to the temperature of $220^{\circ}$, either by steam or heated air; and, $3 \mathrm{dly}$, of moulding machines, by which the fuel is compressed, under a pressure equal to five tons, into the size of a common brick; the fuel brichs are then whitewashed, which prevents their sticking together, either in the coal bunkers or in hot climates. The advantages of Grant's fuel over even the best coal may be stated to consist, first, in its superior efficacy in generating steam, which may be thus stated-200 tons of this fuel will perform the same work as 300 tons of coal, such as are generally used; secondly, it occupies less space; that is to say, 500 tons of it may be stowed in an area which will contain only 400 tons of coal; thirdly, it is used with much greater ease by the stokers or firemen than coal, and it creates little or no dirt or dust, considerations of some importance when the delicate machinery of a steam-engine is considered; fourthly, it produces a very small proportion of clinkers, and thus it is far less liable to choke and destroy the furnace bars and boilers than coal; fifthly, the ignition is so complete that comparatively little smoke, and only a small quantity of ashes, are produced by it; sixthly, from the mixture of the patent fuel, and the manner of its manufacture, it is not liable to enter into spontaneous ignition. 


\section{G.}

GALVANO-PLASTIC is the German name of ElectroMetallurgy.

GARANCINE, an extract of madder by means of sulphuric acid, prepared in France.

GAS-LIGHT. Since the former edition of this work I have received from Mr. Hedley, an engineer of great eminence and experience, plans and drawings of gas works and of apparatus of the most approved and modern construction, and on the very largest scale as to extent of business or manufacture; also plans and drawings of a gas work on a smaller scale, with its corresponding apparatus. In the first, or large work, purification by wet lime, before described, is used ; in the latter, by dry lime.

The large work referred to is calculated for and is arranged to contain 400 retorts, 12 wet-lime purifiers, \& 2 washers ; 12 large double or telescopic gasholders, capable of storing $1,000,000$ cubic feet of gas; and coal stores capable of holding 10,000 tons of coal. The smaller work is calculated for and will contain 40 retorts, 2 dry lime purifiers, and a wash vessel; 2 gasholders capable of storing 50,000 cubic feet of gas ; and coal stores sufficient for 1000 tons of coal.

Fig. 52 is the side elevation (front view) of a gas work capable of containing 400 retorts, and all their dependencies.
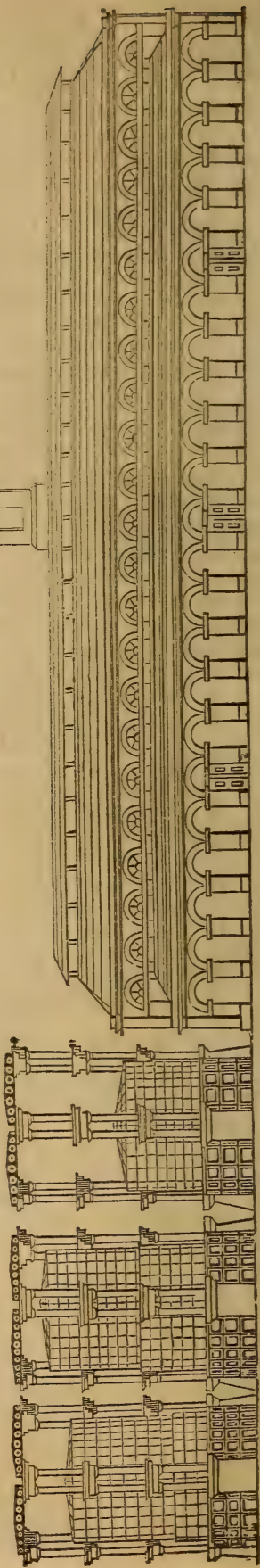
Fig. 53, is the plan of the retort house, coal stores, tanks, gas-holders, \&c., on the largest scale and most approver form, viz., A, the retort house, 300 feet long, 56 feet wide; $\mathrm{B}$, retort beds; $\mathrm{C}$, chimney stack; $\mathrm{D}$, flues; $\mathrm{E}$, hydraulir mains; $\mathrm{F}$, coal otores, each 300 feet long, 30 feet wide; $G$, condensers; $H$, engine houses; $J$, wash

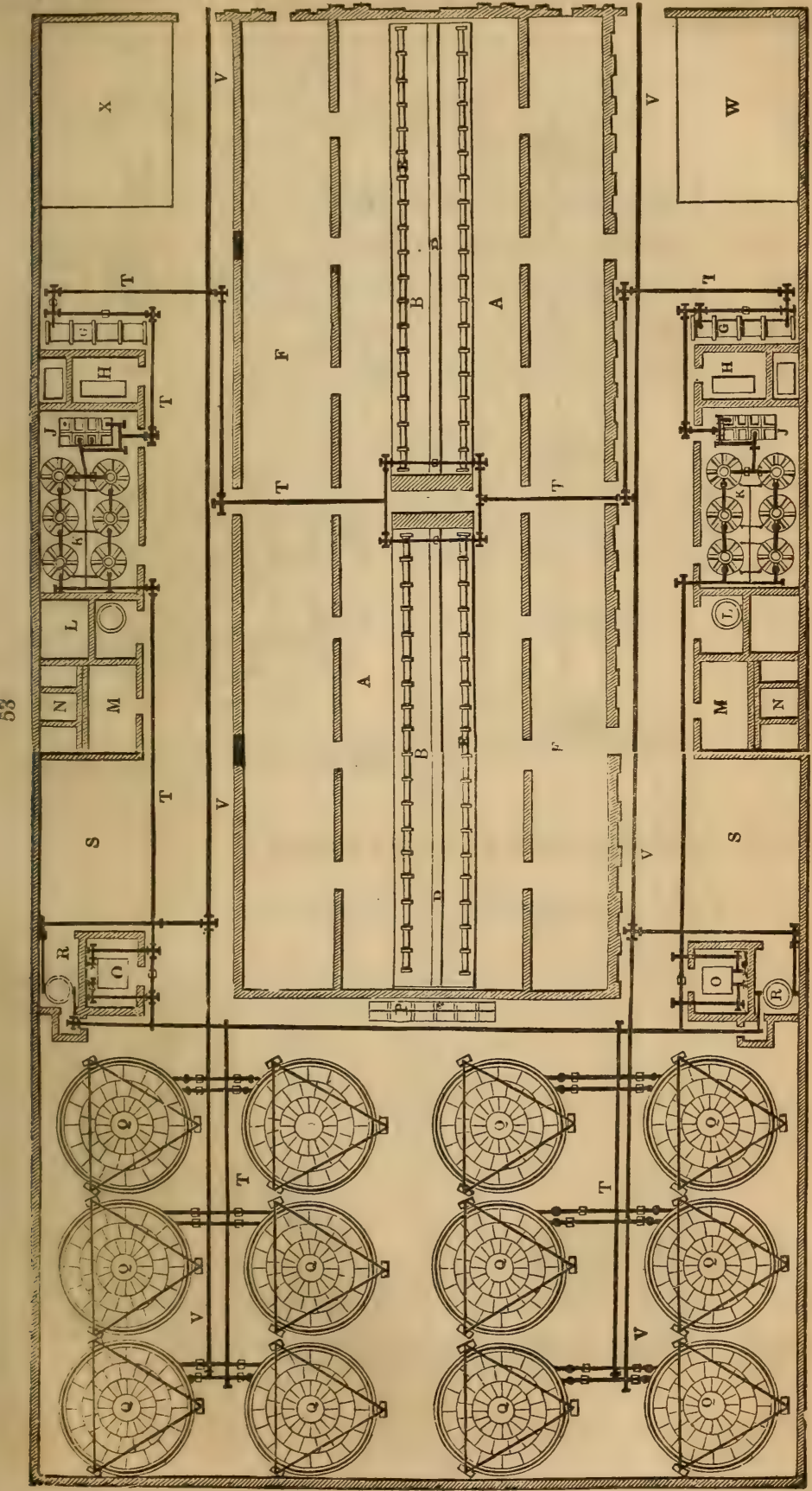


vessels; $\mathbf{K}$, purifiers and connexions; $L$, lime store and muxing tab; $M$, smiths' and fitters' shop; $N$, refuse lime pits; $\mathrm{O}$, meter-house; $\mathrm{P}$, tar tank; $\mathrm{Q}$, tanks, gasholders, bridges, columns, valves, and connexions; R, governors; $S$, coke stores; T, inlet pipes; V, outlêt pipes; W, house and offices; $\mathbf{X}$, stores.

Fig. 54. Transverse section and elevation of a bed of $5 \mathrm{D}$ retorts; $\mathrm{A}$, transverse section; B, elevation.

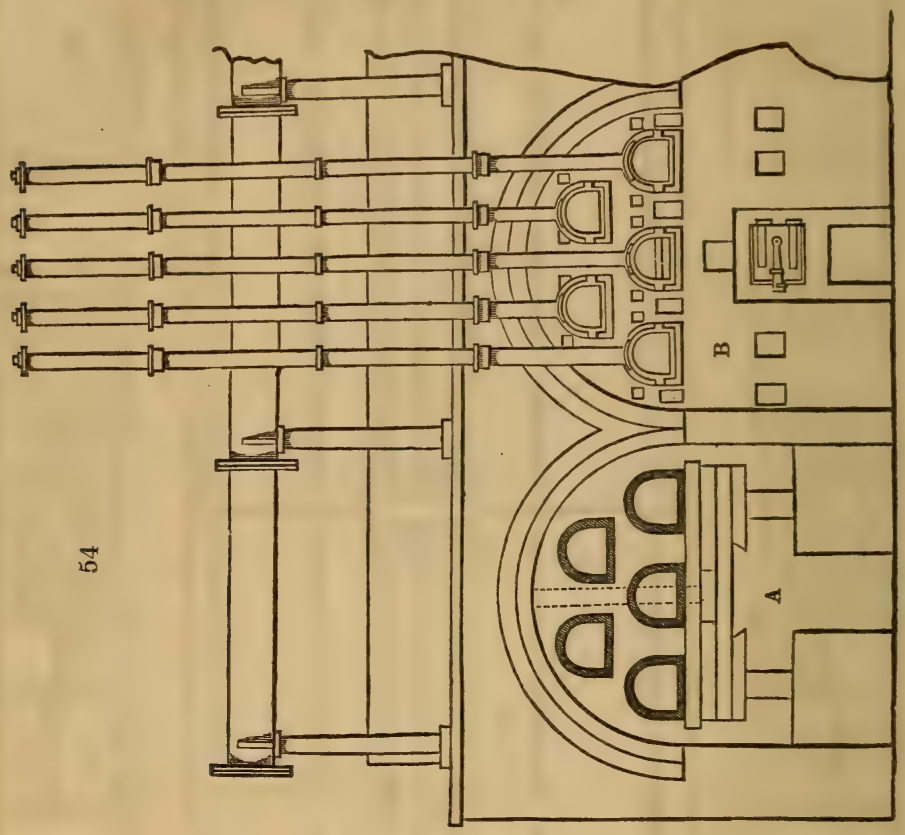

Fig. 55. Longitudinal section of a bed of $5 \mathrm{D}$ retorts.

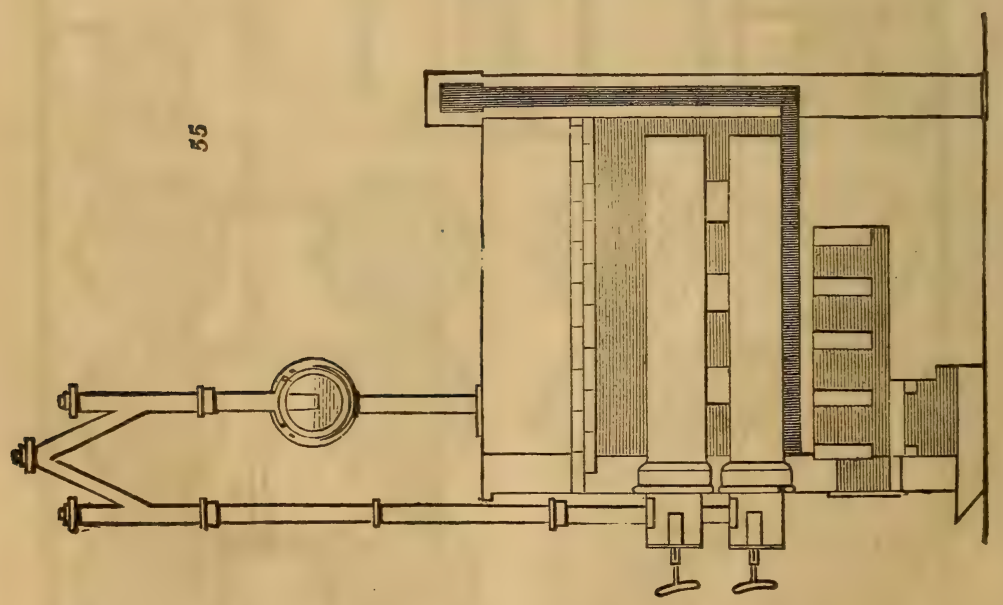


Fig. 56. Elevation of an upright air condenser, consisting of 5 chambers, with a veries of 10 -inch pipes.

56

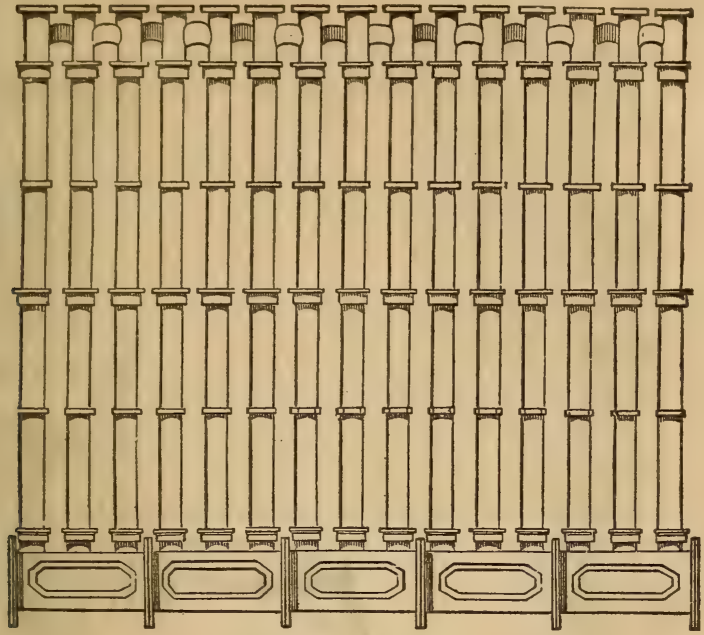

Fig. 57. Elevation of a double or telescopic gas-holder, of a modern and approved form, with part of tank.

57

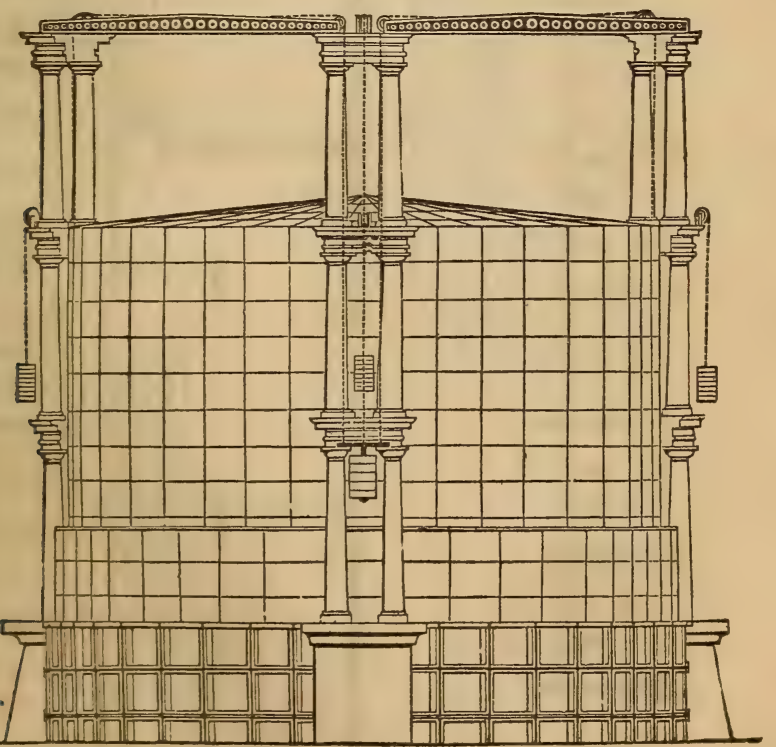


Fig. 58. End elevation and plan of air condenser; A, end elevation; B, plan.

Fig. 59. Set of 3 wet-lime purifiers and wash-vessels in elevation and section, with feed-heads, agitators, valves, and connexions, raised for the lime liquor to run from one purifier to the next below it, and ultimately into the refuse lime-pits, viz $\mathbf{A}$ petion of wash vessel; B, section of purifier; $\mathbf{C}$, elevation of purifier.
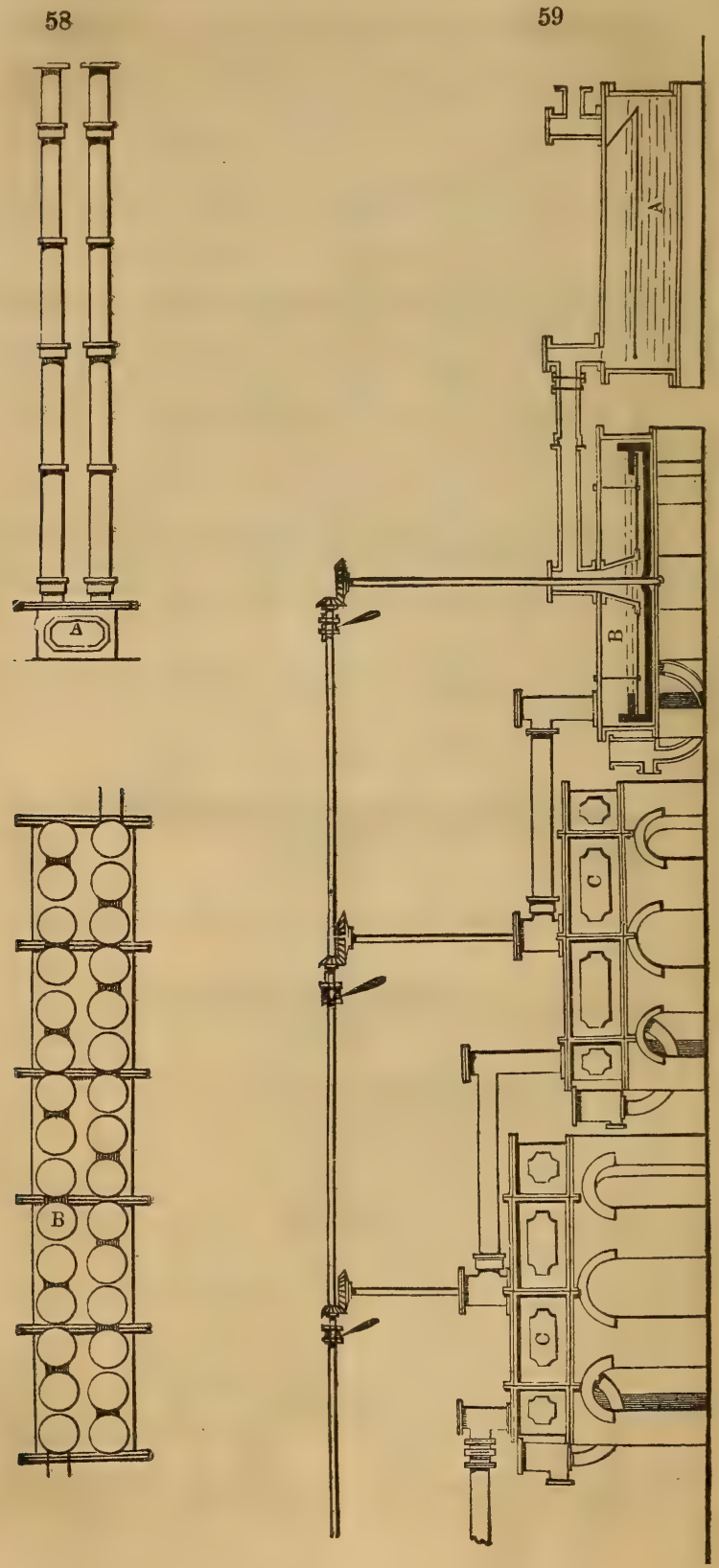
Fig. 60. Front eleration of gas works on a smaller scale, where dry lime is used.

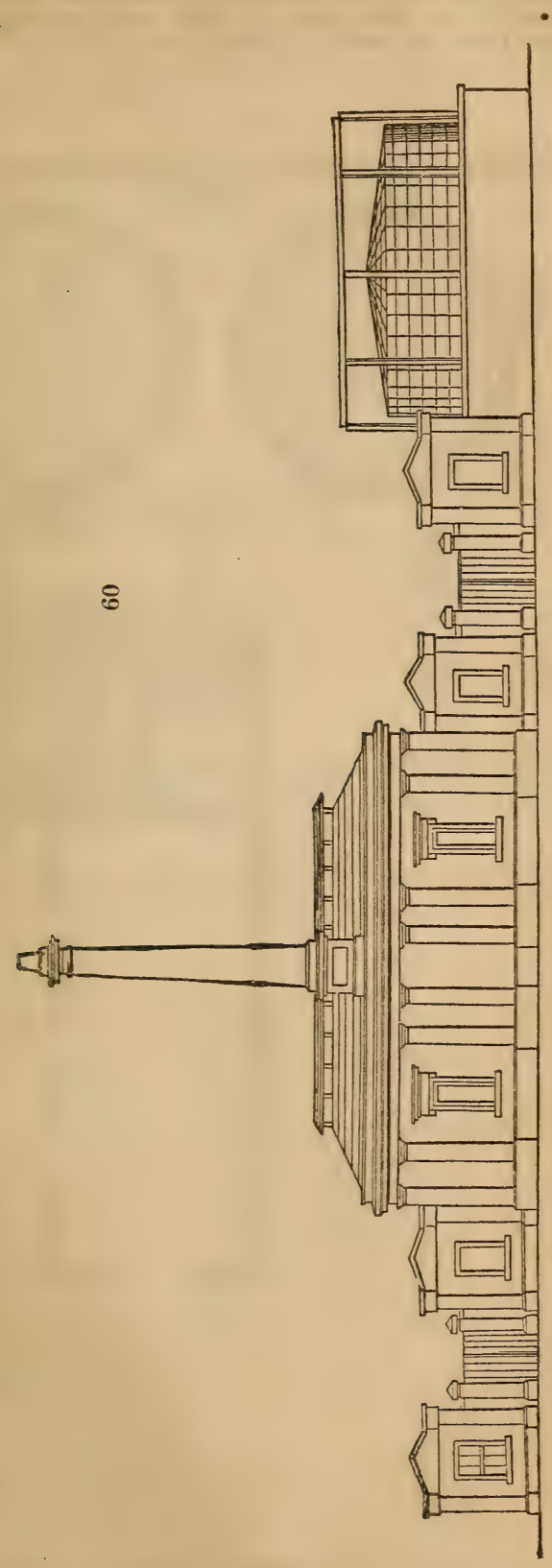


Fig. 61. Plan of gas works, consisting of, viz.: A, retort house; B, retort be ts ; C, chimney stack; D, flue; E, hydraulic main; F, coal store: G, lime store; $\mathbf{H}$, washer and purifiers; $J$, store; $K$, tar-tank; $L$, horizontal condenser laid on the ground; $M$, inlet•pipe; $N$, outlet pipe; $O$, tanks and gas-holders; $P$, meter and governor; $Q$, smith's shop; R, office; S, coke store.

\section{1}

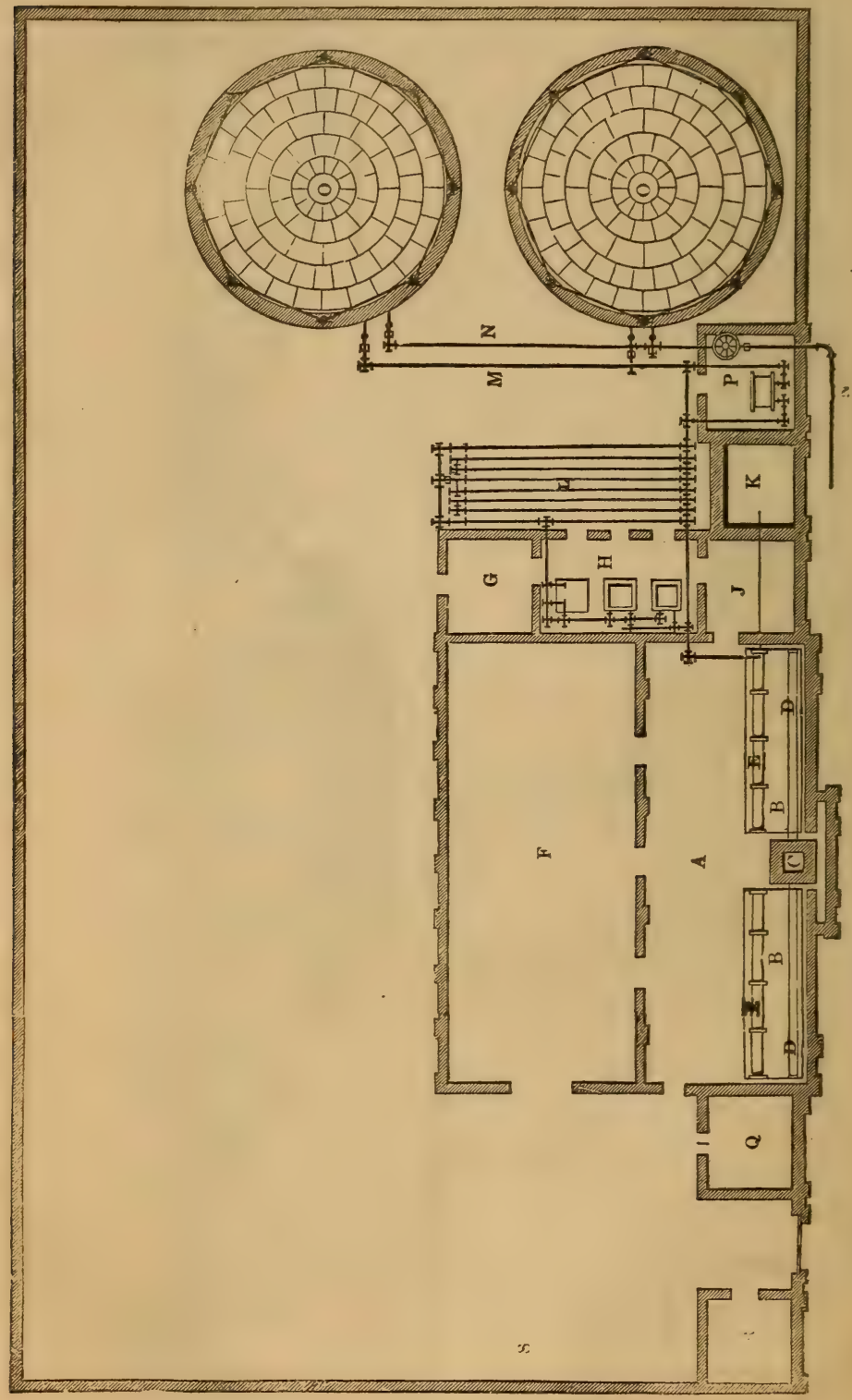


Fig. 62. Elevations and sections of dry-lime purifiers; A, longitudinal elevation B, ditto section; C, transverse elevation; D, ditto section.

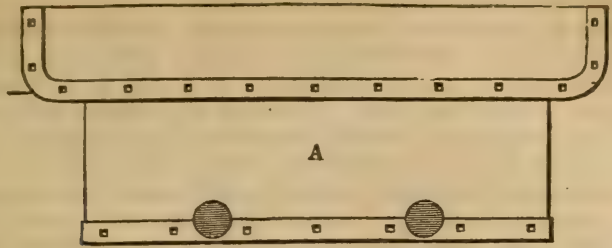

62
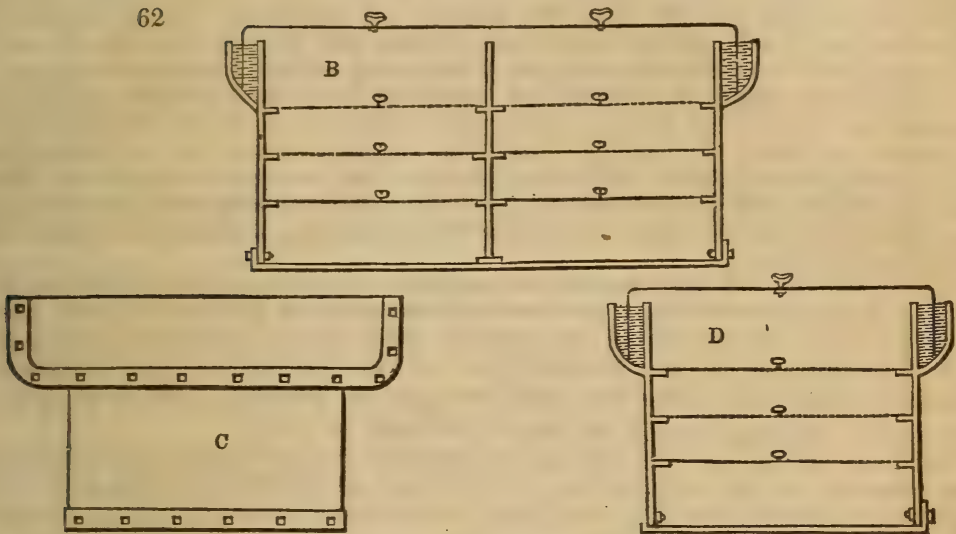

I am well convinced that a distribution and arrangement of gas-works, combining effectiveness, economy, convenience, and elegance, at all equal to the preceding, have never before met the public eye, in this or any other country.

Trials of, and Experiments on, various Kinds of Coal as regards the Production of Gas from each, and its Quality or Illuminating Power; by Joseph Hedley, Esq., Consulting Gas Engineer, London.

Note.-In all the experiments the gas was passed through a governor, on a pressure of 5 10ths of an inch.

\begin{tabular}{|c|c|c|c|c|c|c|c|c|c|c|c|c|c|c|c|c|c|}
\hline & 1. & 2 & 3. & 4. & 5. & 6. & 7. & 8. & 9. & 10. & 11. & 12. & 13. & 14. & 15. & 16. & 17. \\
\hline \multirow[t]{2}{*}{$\begin{array}{l}\text { Name and } \\
\text { Description of } \\
\text { Coal. }\end{array}$} & 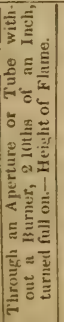 & 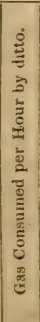 & 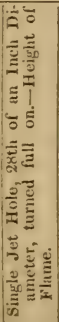 & 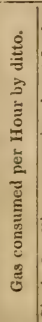 & 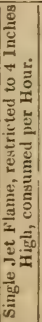 & $\begin{array}{l}\text { Equal in } \\
\text { Illumi- } \\
\text { nating } \\
\text { Power } \\
\text { to }\end{array}$ & 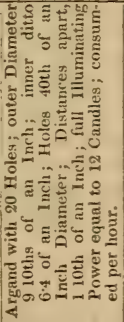 & 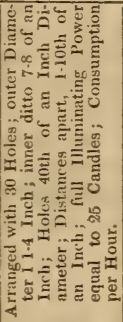 & 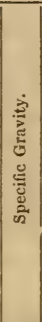 & 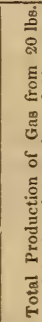 & 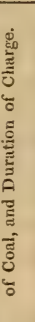 & 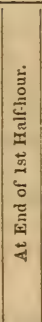 & 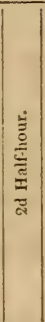 & $\frac{\grave{3}}{3}$ & 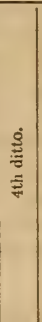 & 总 & 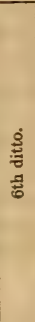 \\
\hline & Inch's & Us & $\frac{\dot{g}}{\stackrel{g}{E}}$ & 号势 & 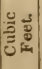 & Candles & Cubic Feet. & Cubic Feet. & 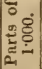 & 鄫 & 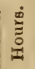 & 造 & 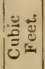 & 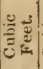 & 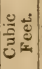 & 造 & 造 \\
\hline $\begin{array}{l}\text { Lismahago, or } \\
\text { Glasgow Can- } \\
\text { nel }\end{array}$ & $21+020$ & & & & & & & & & & & & & & & & \\
\hline Newcastle coal & $\begin{array}{c}21022 \\
15\end{array}$ & $\begin{array}{l}12 \\
16\end{array}$ & $6^{7 \frac{1}{2}}$ & $\begin{array}{l}12-1 \\
16-2\end{array}$ & $\begin{array}{r}7 \cdot 2 \\
11 \cdot 1\end{array} \mid$ & $\begin{array}{l}277 \\
175\end{array}$ & $\begin{array}{l}2 \cdot 3 \\
6\end{array}$ & $\begin{array}{l}3.9 \\
7 \cdot 5\end{array}$ & $\left.\begin{array}{r}737 \\
475\end{array}\right)$ & $\begin{array}{l}101 \\
104\end{array}$ & $\frac{21}{3}$ & $\begin{array}{l}39 \\
30\end{array}$ & $\begin{array}{l}27 \\
20\end{array}$ & $\begin{array}{l}22 \\
18\end{array}$ & $\begin{array}{l}11 \\
15\end{array}$ & $\begin{array}{r}2 \\
15\end{array}$ & 6 \\
\hline & 22 & 11 & D & $12 \cdot 1$ & $7 \cdot 1$ & & 2. & 3. & 737 & 102 & 2 & 50 & 30 & 20 & 2 & & \\
\hline castle Coal. & 18 & 16 & $5 \frac{1}{2}$ & $16 * 1$ & $11 \cdot 1$ & 175 & 5. & $7 \cdot 5$ & -444 & 102 & 3 & 29 & 20 & 17 & 16 & 14 & 6 \\
\hline $\begin{array}{l}\text { Pelton, ditto- } \\
\text { Bickerstaft. I. }\end{array}$ & 28 & 16 & $5 \frac{2}{4}$ & $16-2$ & 112 & 1.73 & 5. & 7.5 & .437 & 102 & 3 & 28 & 20 & 19 & 16 & 14 & 6 \\
\hline verpoul ditto & 19 & 14 & $6 \frac{1}{2}$ & $16-2$ & $11 \cdot 1$ & $2 \cdot 04$ & $4 \cdot 6$ & 6.5 & .475 & 102 & 3 & 30 & 24 & 18 & 16 & 10 & 4 \\
\hline $\begin{array}{l}\text { Wigan Cannel } \\
\text { Blenkinsopp, }\end{array}$ & 22 & 12 & $8 \frac{1}{2}$ & $13 \cdot 1$ & 7.2 & & 2 & 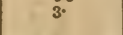 & .606 & 100 & $2 \frac{1}{2}$ & 38 & 30 & 18 & 10 & 4 & \\
\hline Carlisle Coal & 27 & 16 & $6 \frac{1}{2}$ & $15 \cdot 1$ & $11 \cdot 1$ & 1.87 & 4.6 & 7. & .521 & 100 & 3 & 28 & 24 & 18 & 16 & 10 & 8 \\
\hline Neath Coal . & 18 & 16 & $7^{2}$ & $19 \cdot 1$ & $12 \cdot 1$ & 175 & $5-22$ & $7 \cdot 5$ & $\mid 463$ & 100 & 3 & 26 & 21 & 20 & 14 & 12 & 7 \\
\hline
\end{tabular}

NотE.-The candle here used was a composition candle, with plaited wick, requiring no snuffing giving at least one third more light than mould tallow candles. 
Attention to the preceding tabular statement of experiments is important, as exhibit. ing several very important facts, particularly interesting at this moment to the science of gas-lighting, and now for the first time made public.

It will not fail to be observed by these experiments that all the coals produced nearly equal quantities of gas, notwithstanding the variable characters and qualities of the coal. The greatest quantity produced being at the rate of $11 \cdot 648$ cubic feet per ton of $20 \mathrm{cwt}$., the smallest 11.200 cubic feet. All these experiments were performed with the greatest care, and under precisely similar circumstances as to pressure, manufacture, \&c., \&c. The time in which the quantity of gas is produced from the several coals varies considerably, and deserves notice, as it most materially affects the economy of production - that coal being the most valuable, all other things being alike, which yields or gives out its gas in the shorlest time; and particular attention is claimed to this fact. For the more ready reference to the table the columns are numbered. No. 11 exhibits this difference, and it will also be seen by this column that the time varies as the quality of the coal, the best coal yielding its gas in two hours, and the worst in three hours.

Another most important, material, and interesting fact is established by these experi. ments-that the flow of gas is as its density-demonstrated by the variation in the heights of the flames, as shown in column No. 1, being 18 inches in the inferior gases to 22 inches in the superior; while the quantity of gas required to supply these flames is in the inverse ratio of their heights, the longer flame requiring but twelve cubic feet to maintain it, when the shorter flame, from the inferior gas, required sixteen cubic feet. See column No. 2.

Remarkable as this difference in the heights of the flames and the consumption is, it is not so great as the difference caused by the quality or illuminating power of the several gases, shown by columns Nos. 5 and 6 ; where it will be seen that the consumption of the best gas per hour was only $\frac{7}{10}$ of a cubic foot, and its light was equal to 3 candles, while that of the worst gas was $\frac{12}{1} \frac{2}{0}$ of a cubic foot, and its lights equal only to $1 \cdot 75$ candles, or nearly, the best to the worst, as 1 to 3 .

The next column, No. 7, exhibits similar results as to the superior value or illuminating power of one gas over another. In this case an argand burner was used. The best gas required only two feet to be equal to twelve candles, while the inferior required five feet to be equal to the same.

And in column No. 8, in which another and superior argand burner was used, the best gas required only three feet to be equal to twenty-five mould candles, while the inferior required seven and $a$ half feet: from this it results that the $7 \frac{1}{2}$ cubic feet of inferior gas, to be equal to the 3 feet of good gas, should have given light equal to sixty-two and $a$ half candles, whereas they only gave light equal to twenty-five candles; so great is the difference in the QUALITIEs of gas for producing light.

While on the subject of the illuminating power and the value of one gas over another, it will not fail to be observed, by the table, that another great difference also exists, caused by the use of particular burners; as, for example, the best gas in column No. 5, where the single jet was used, required seven tenths of a cubic foot to be equal to three candles, while the same gas in column No. 7, where a 20 -hole argand burner was used, required only two feet to be equal to twelve candles; and in column No. 8, where a 30-hole argand burner was used, only three feet was required to be equal to twenty-five candles; demonstrating the fact that a great and extraordinary improvement in the quantity of illuminating power is effected by the simple increase or enlargement of the burner, affording, where great light in one position is required, a most extraordinary economy in the use of gas, shown in fact practically by the recent introduction of the celebrated "Bude" light, patented by Mr. Goldsworthy Gurney.

Tabular Statement, deduced from the foregoing experiments, showing the cost of candles to produce as much light as 9,000 cubic feet of gas would afford, being the product of one ton of coal. (The candles are moulds, 6 to the pound, 9 inches long, and each candle is calculated to burn $9 \frac{1}{2}$ hours. Cost of candles $7 \frac{1}{2} d$. per pound, or 7s. $6 d$. per dozen pounds.)

\begin{tabular}{|c|c|c|c|c|}
\hline $\begin{array}{l}\text { Candles would cost, to be } \\
\text { equivalent to }\end{array}$ & $\begin{array}{l}\text { Where a single } \\
\text { jet burner is } \\
\text { used. }\end{array}$ & $\begin{array}{l}\text { Where a } 20 \text {-hole } \\
\text { argand burner } \\
\text { is used. }\end{array}$ & $\begin{array}{l}\text { Where a } 30 \text {-hole } \\
\text { argand burner } \\
\text { is used. }\end{array}$ & $\begin{array}{l}\text { Where a Bude } \\
\text { burner is used } \\
\text { according to } \\
\text { statement of } \\
\text { company. }\end{array}$ \\
\hline $\begin{array}{l}\text { Common coal gas } \\
\text { Good do. }\end{array}$ & $\begin{array}{lll}£ & s . & d . \\
10 & 18 & 6 \\
25 & 18 & 4\end{array}$ & $\begin{array}{lrl}£ & s . & d . \\
15 & 15 & 8 \\
39 & 9 & 6\end{array}$ & $\begin{array}{lll}f & s . & d . \\
21 & 18 & 0 \\
54 & 16 & 7\end{array}$ & $\begin{array}{rrr}£ & s & d . \\
59 & 2 & 7 \\
148 & 0 & 9\end{array}$ \\
\hline
\end{tabular}


TABLE, also deduced from the foregoing, showing the Cost of Gas at the several Prices undermentioned, and equivalent to 100 pounds of Mould Candles, costing 3l. $2 s .6 d$.

\begin{tabular}{|c|c|c|c|c|c|c|c|c|c|c|c|c|}
\hline \multirow[t]{2}{*}{$\begin{array}{l}\text { Descrip- } \\
\text { tion of Gas }\end{array}$} & \multirow{2}{*}{$\begin{array}{l}\text { If burnt in } \\
\text { in a singIe } \\
\text { Jet Gas } \\
\text { equal to } \\
100 \mathrm{lbs} \text {. of } \\
\text { Mould } \\
\text { Candles. } \\
\text { Cub. feet }\end{array}$} & \multicolumn{3}{|c|}{$\begin{array}{l}\text { Gas would cost } \\
\text { at per thousand } \\
\text { Cubic Feet. }\end{array}$} & \multirow{2}{*}{$\begin{array}{l}\text { If burnt in } \\
\text { a } 20 \text { hole } \\
\text { Argand } \\
\text { Burner, } \\
\text { Gas equal } \\
\text { to } 100 \text { lbs. } \\
\text { of } \\
\text { Candles. } \\
\text { Cub. feet. }\end{array}$} & \multicolumn{3}{|c|}{$\begin{array}{l}\text { Gas would cost } \\
\text { at per thousand } \\
\text { Cubic Feet. }\end{array}$} & \multirow{2}{*}{$\begin{array}{l}\text { If burnt in } \\
\text { a } 30 \text { hole } \\
\text { Argand } \\
\text { Burner. } \\
\text { Gas equal } \\
\text { to } 100 \text { lbs. } \\
\text { of } \\
\text { Candles. } \\
\text { Cub. feet. }\end{array}$} & \multicolumn{3}{|c|}{$\begin{array}{l}\text { Gas would cost at } \\
\text { per thousand } \\
\text { Cubic Feet. }\end{array}$} \\
\hline & & $5 s$. & $7 s$. & $9 s$. & & $5 s$ & $7 s$ & $9 s$. & & $5 s$. & $7 s$. & $9 s$ \\
\hline $\begin{array}{l}\text { Common } \\
\text { Good - }\end{array}$ & $\begin{array}{l}2,687 \\
1,072\end{array}$ & $\left|\begin{array}{rr}s . & d . \\
13 & 5 \\
5 & 4\end{array}\right|$ & $\begin{array}{rr}\text { s. } & d . \\
18 & 9 \\
7 & 5\end{array}$ & $\mid \begin{array}{rr}s . & d \\
24 & 2 \\
9 & 7\end{array}$ & $\begin{array}{r}1,781 \\
712\end{array}$ & $\begin{array}{lr}s . & d . \\
8 & 10 \\
3 & 6\end{array}$ & $\begin{array}{cc}s . & d . \\
12 & 5 \\
4 & 11\end{array}$ & $\left|\begin{array}{rr}s . & d . \\
16 & 0 \\
6 & 4\end{array}\right|$ & $\begin{array}{r}1,282 \\
513\end{array}$ & $\left|\begin{array}{rl}s . & d \\
6 & 5 \\
2 & 7\end{array}\right|$ & $\begin{array}{cc}s . & d . \\
8 & 11 \\
3 & 7\end{array} \mid$ & $\begin{array}{rl}\text { s. } & d . \\
11 & 6 \\
4 & 7\end{array}$ \\
\hline
\end{tabular}

In the brief description of the meter given in the Dictionary, I omitted to state, that this most ingenious scientific contrivance for measuring aeriform or gaseous fluids as they flow through pipes is the invention of Samuel Clegg, Esq., Civil Engineer, of London, Manchester, Liverpool, Birmingham, Chester, Bristol, \&c., \&c., in all which places he has erected gas-works. To this gentleman's genius and skill the public are mainly indebted for many valuable improvements in the application of gas from coal to purposes of illumination.

Brought up in the great engineering establishment of Messrs. Boulton and Watt, at Soho, near Birmingham, he became connected with Mr. William Murdoch, who most undoubtedly was the author and originator of gas-lighting, as the evidence given before a committee of the house of commons in the year 1809 abundantly verified. He demonstrated that the light produced from gas was superior in economy to all other modes of artificial illumination; and by that evidence, though so long back as 1809 , it will be seen that all the information of the present day was even then known to him, clearly pointed out, and illustrated by his experiments, which strangely contrasted with the statements put forward by the parties then attempting to introduce this mode of lighting into the metropolis. All the ephemeral plans of those parties have, however, long since disappeared, or nearly all. One, unfortunately, remains, and that a most unlucky one-the unprofitable manufacture of coke in gas-making - an article worthless in the scale of value, which should never have been sought for. Messrs. Watt and Murdoch predicted that when the parties became incorporated by parliament, they would resort to their apparatus, notwithstanding their repudiation of it at the time, alleging their own schemes to be so much superior; and they verified this prediction a very few years afterward by engaging the services of $\mathrm{Mr}$. Clegg, to extricate them from their manifold and egregious errors. He began by introducing the very apparatus of Messrs. Murdoch and Watt, so inconsiderately condemned by them.

Mr. Clegg put up the first gas-holder ever erected in London.

To Mr. Clegg is due also the introduction of lime for the purification of the gas, without which gas-lighting would to this day have afforded little comfort and economy. The hydraulic main, for separating the gas making from the gas made, valves, lutes, and many other admirable contrivances, are peculiarly due to $\mathrm{Mr}$. Clegg. But the crowning performance of all his inventions, was that for measuring out the gas to the several parties requiring it exactly according to their demands. The manufacture of gas having by this time been so far mechanically perfected as to be brought to our doors, it became at once apparent that some contrivance should be found by the use of which every person might consume as much or as little gas as he pleased, paying only for what he really used, thus making science subservient to fair dealing.

Mr. Clegg took out a patent for the gas-meter about the year 1814; but great as its merits were, he soon found that serious difficulties remained to be overcome, in inducing parties to support and encourage its use, even where their interests should have prompted them to adopt it. Mr. Clegg had, however, fortunately associated with him, toward the completion of the apparatus, Mr. Samuel Crosley; and by their joint labors it acquired its present precision.

The value of the meter is primarily to the gas companies, next to the public. By its use, the gas companies are enabled to supply gas to all places where light is required, at a rate proportioned to its just value. The public thereby see the economy afforded by gas over candles, oil, or other material; but they gain also in another most important way - by the use of the meter, gas companies, being duly paid, are enabled to reduce the price of gas, and yet realize equal profits, thus bringing it within the reach 
of a much larger class of the community; and it is a well-established fact that in towns where gas is sold by meter, gas companies can and do sell at nearly one half the price they otherwise could do.

Reduction of price increases demand; increased demand increases profits; increased profits again enable prices to be reduced; and again, reduced prices increase the demand, thus benefiting reciprocally companies and consumers.

Notwithstanding, however, all these advantages, there are not wanting persons who have set up an outcry against the use of the meter, by impugning its accuracy, and accusing the gas companies with fraud in charging by it. It would be idle to follow these parties in their baseless allegations. An action for pirating it was brought and tried in the Court of King's Bench, in which not only the novelty of the machine was fully established, but its accuracy and usefulness proved by the ablest mathematicians, mechanicians, and chemists of the dar; and a verdict in its favor obtained. Subsequently very large damages hare been given for the iniringement-in one case as much as $5,000 l$., and in another, in the court of chancery, a decree was made referring it to the master, to take an account of the profits made by the use of the meter; this is not yet finally settled, the master's report finding $6,000 l$. to be due; but this is excepted to by the parties infringing: the chancellor, howerer; allowed the exceptions to be argued, only on parment by the infringers of $4,000 \mathrm{l}$. into court to meet the patentee's law costs. These exceptions have no reference whaterer to the questisn of the accuracy of the meter, but are simply as to whether the advantages of the meter were as great as allowed by the master.

The patent for the meter expired about the year 1828 ; since that period numerous competitors have commenced making the machine.

Mr. Clegg has recently obtained a patent for a dry gas-meter, of which the following are its advantages and construction, as described by the very meritorious inventor :-

1. Working without water.

2. Working without membranes or valves.

3. Working without requiring the least pressure.

4. Working without interference with the perfect steadiness of the lights.

5. Registering more accurately than any other meter.

6. Occupying only one tenth of the space of the common meters.

7. Being subject to little or no wear and tear.

8. And being cheaper.

Prices.-For plain meters-

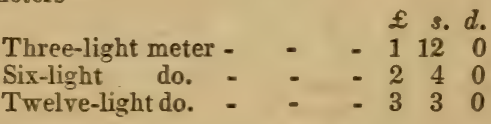

The highest numbers will be still cheaper in proportion.

Ornamental meters, appearing like handsome timepieces, for halls, living-rooms, committee-rooms, offices, counting-houses, \&c., are charged extra, at ten shillings each and upward, according to pattern.

\section{Description of Clegg's patent dry Gas-meter.}

The two figs., 63,64 , are half the full size of the apparatus, and the letters of reference are the same in both.

B, B, fig. 63 , represents a cylindrical vessel, about three inches and three quarters diameter, and four inches deep, being the dimensions of a meter capable of measuring gas for three burners, called a three-light meter. In this ressel are two glass cylinders F, F, connected together by the bent tube $d$. The crlinders being perfectly exhausted of air, and half filled with alcohol, are made to vibrate on centres $e$ and $e$, and are balanced by the weight $f$.

This instrument accurately indicates the excess of heat to which either cylinder may be exposed, upon the principle of Leslie's differential thermometer.

$\mathrm{C}$ is a hollow brass box, called the heater, about four inches long, and half an inch broad, projecting out of the meter about one inch. At $a$ issues a small jet of gas, which, when inflamed, gives motion to the cylinders.

The gas enters the meter by the pipe $A$, and circulates throughout the double case $B$; haring passed round the case $\mathrm{B}$, a portion of it enters the top of the box $\mathrm{C}$, by the pipe $\mathrm{D}$, and passes out again at the bottom by the tube $c$, into the meter; the rest of the gas enters the body of the meter through holes in the curred faces of the hoods E, E, and after blowing on the glass crlinders, passes to the burners by the outlet pipe.

To put the meter in action, let the jet $a$ be lighted about an hour before the burners are wanted. In most cases this jet will be lighted all day as a useful flame. The 
hole $a$ is so situated on the box $\mathrm{C}$, that whatever be the size of the jet, a fixed temperature is giren to the box, that temperature depending on the quantity of flame in

64

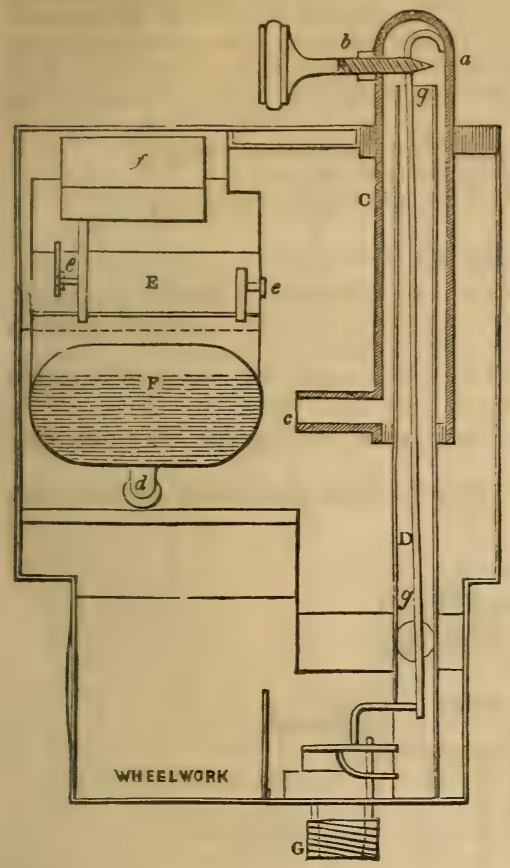

63

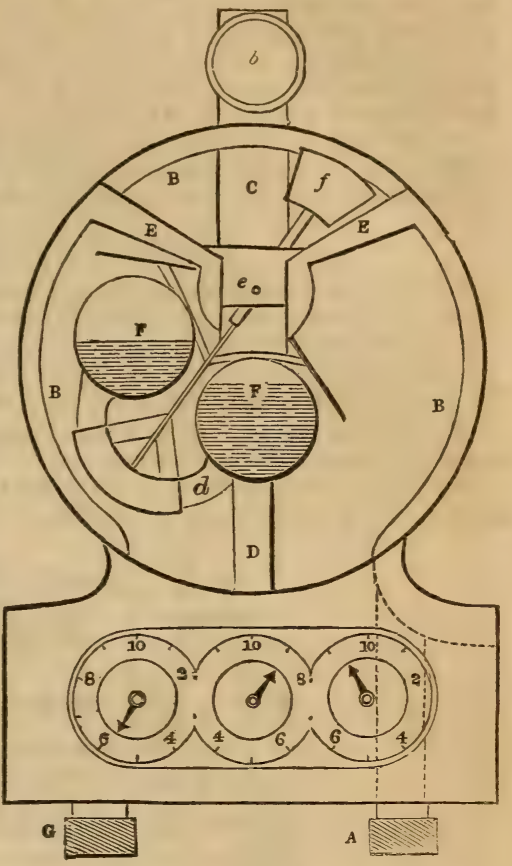

contact with the box, and not at all on the length of the jet. The jet being lighted, and the box $\mathrm{C}$ thereby heated, the gas which passes through it is raised to the same temperature, and, flowing out at the tube $c$, impinges on the glass cylinder which happens for the time to be lowest; the heated gas soon raises a vapor in the lower cylinder, the expansion of which drives the liquid into the upper one, until it becomes heavier than the counterpoise $f$, when the cylinders swing on their centre, the higher one descends, and comes in the line of the current of hot gas, and the lower one ascends; the same motion continues as long as the jet $a$ burns. The same effect on the cylinder is maintained, however the outward temperature may change, by the cold gas, which, issuing from the curved side of the hood EE, impinges on the upper cjlinder, and hastens the condensation of the vapor which it contains.

The cold gas and the heater vary in temperature with the room, and thus counteract each other.

The lighting of the jet $a$ is essential to the action of the meters; in order to insure this, the supply of gas to the burners is made to depend on it in the following manner. The pipe $\mathrm{G}$, by which the gas leaves the meter, is covered by a slide valve, which is opened and shut by the action of the pyrometer $g$; the pyrometer is in communication with and receives heat from the jet, and opens the valve when hot, closing it again when cold.

The speed at which the cylinders vibrate is an index of the quantity of heat communicated to them, and is in exact proportion to the quantity of gas blowing on them through the pipe $c$ and curved side of the hoods EE.

The gas passed through the heater is a fixed proportion of the whole gas passing the meter ; therefore the number of vibrations of the cylinders is in proportion to the gas consumed.

A train of wheel-work, with dials similar to that used in the common meter, registers the vibrations.

Simplicity, accuracy, and compactness, are the most remarkable features of this instrument, and the absence of all corrosive agents will insure its durability. 


\section{Directions for fixing and using Clegg's patent dry Gas-melers.}

Choose a situation for fixing the meter, where the small jet of flame will be of the greatest use, such as an office-desk or counter, taking care to screw the same firm and level on its base. When the jet at the top of the meter is required to be kept constantly burning as a useful flame, press in the brass knob at the front of the meter, and before lighting the burners pull it out; when the small flame is not required, let it be lighted about an hour before you want the burners lighted. Adjust the size of the small flame at pleasure by the screw $b$.

On the back of each meter is marked the number of lights it will supply.

The inlet and outlet pipes are marked at the bottom of the meter.

The quantity of gas consumed is recorded by the index in the usual way.

\section{For testing Clegg's patent dry Gas-meters.}

Pass the gas through two meters at least, and take the mean. Vary the number of lights at pleasure, not exceeding the number marked on the meter, and when one or two hundred cubic feet of gas have been consumed, compare the indices.

These meters are not for measuring small fractional parts; but taking the average for any periodical consumption, are more accurate than any other meter.

Mr. Thomas Edge, of Great Peter Street, Westminster, has contrived the following meter, of which drawings are annexed.

Fig. 65 is a front view of a three-light meter, the front plate being removed, and some of the parts shown in section 65 .

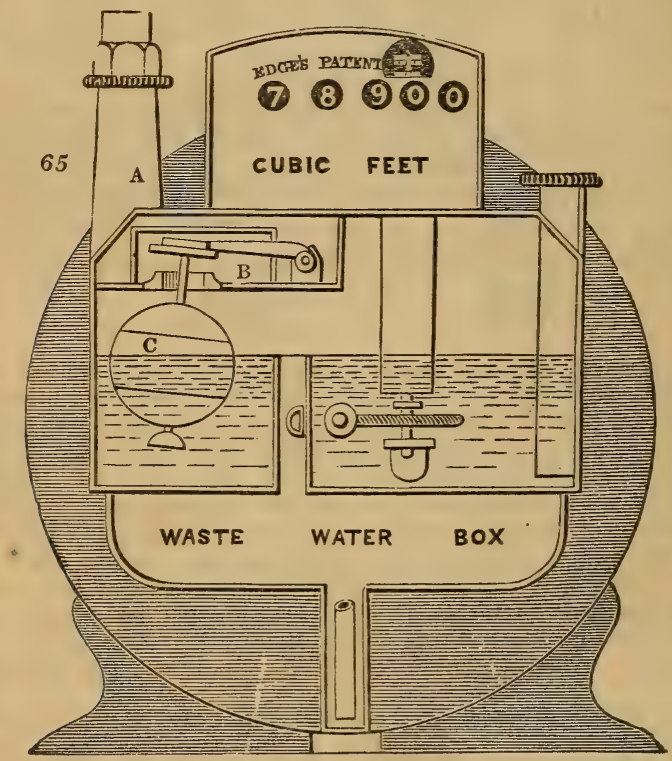

Fig. 66 is a transverse section of the same.

The gas enters at $\mathrm{A}$ into the small chamber $\mathrm{B}$, in the bottom of which is a lever valve (part of Mr. Edge's patent improvements), moving upon its axis and attached by the rod to a metal float $\mathrm{c}$, which in the present drawing is buoyant. The object of this arrangement is to intercept the passage of the gas into the meter, unless a sufficient quantity of water is in it, that being necessary to its proper action; the gas then passes through the inverted syphon or tunnel into the convex cover, whence it passes into the chambers of the drum.

Another of Mr. Edge's improvements consists in the cutting down of this syphon pipe or tunnel to the proper water level, and connecting the bottom of it to a waste waterbox, into which any surplus water must fall. The importance of this precaution will be seen on investigating the drum, as an excessive height of the water will materially interfere with the measurement, the quantity of gas delivered per revolution being considerably less. This, in connexion with the lever valve and float, confines the 
variation of the water levels withn such narrow limits, that the measurement may be considered perfectly just on all occasions.

The last patent by Mr. Edge is for an improved

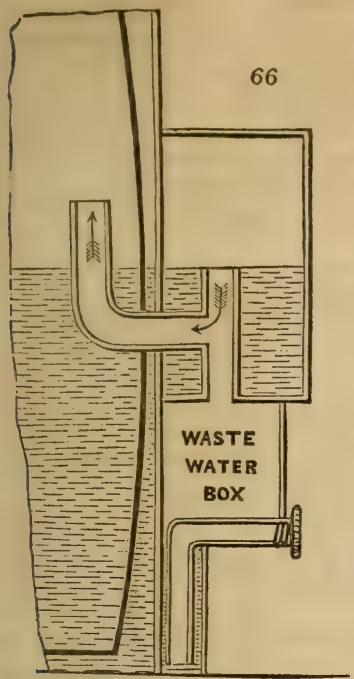
index, which is composed of a series of moving dials, with 10 figures upon each, one figure only appearing of each series at a time.

This contrivance is very ingenious, and will no doubt be applied to other machines, where indexes (indices) of quantity are required.

Recurring to Mr. Clegg, he is also the inventor of an instrument of great value-appropriately called a "governor." Its purpose is to render equal the height of fiame of the several burners in any house or establishment, and to keep them so, notwithstanding any, and whatever alteration may be made in the pressure at the works or elsewhere. This instrument is perfected, and successfully applied, though it is not so jenerally in use as it ought to be. By the use of this instrument a light cnce set at the hcight desired will maintain that height uniformly, and without the least variation the whole evening; and continue to do so till altered.

Without this instrument, it is necessary to pay attention to the burning of gas-lights, as their heights are frequently affected by the most trifling circumstance, such, for example, as their extinction at the hour of closing the shops, which makes a sensible difference in the neighborhood.

All these works have prodigiously increased in the quantity of gas made and supplied. Since the account in the former edition of this work, large additional manufactories have been erected by new companies, and great additions made by the old ones. There are now in the metropolis alone 15 public gas companies, having among them 23 gas establishments. The quantity of gas manufactured by these 23 gas-works, and supplied to the public was during the past year three thousand one hundred millions of cubic feet of gas ; and the coal used to produce this quantity of gas was at the least 400,000 tons!

Baked clay retorts are very generally used in Scotland, and found to be most economical as regards wear and tear; in London, however, they are mostly of cast iron.

The pressure upon the retorts is caused principally by the use of wet lime, used in London, because the process is less expensive and less cumbersome than dry lime. Wet lime can not be used with clay retorts, owing to this excess of pressure.

Merit is due, for enlarging the capacities of double gas-holders, to the late Mr. Joshua Horton, of West Bromwich, near Birmingham; and to Mr. Stephen Hutchinson, engineer of the New London Gas-Works, Vauxhall, where they were first successfully introduced, and manufactured by - $\mathbf{I r}$. Horton. They have now come very generally into use throughout the kingdom, and are manufactured by all gas-holder makers.

Separate gas-holders are advisable and advantageous, but they are nut generally used, except in Glasgow, Manchester, Birmingham, Sheffield, and a few other places.

The annexed drawing represents Mr. Croll's vessels for the purification of gas from ammonia, which is effected by means of dilute sulphuric acid applied between the condensers with the ordinary lime purifiers. The vessels are made of either wood or iron, and lined with lead; have a wash-plate similar to the wet lime purifiers. The radiating bottom formed of wooden bars, as shown in the drawing, is for the purpose of supporting the wash-plate and distributing the gas.

Fig. 67: $a$, is the inlet pipe; $b$, the outlet pipe; $c, c$, the tube with funnel for introducing the sulphuric acid; $d$, the first purifying vat; $e$, the second ditto, both lined with lead, and which are filled up to the dotted line with the dilute acid; $f, f$, the water supply-pipe; $g, g$, the discharging cocks.

Fig. 68 represents a ground-plan of the vats, each 10 feet in diameter; $\mathbf{A}$, the bottom of the middle; $\mathbf{B}$, the inlet of the gas; $\mathbf{C}$, the outlet of ditto.

In commencing the process, these vessels are charged with water and sulphuric acid, in the proportion of seven pounds, or thereabouts, of the latter, to 100 gallons of the former. As the acid is neutralized by the ammonia contained in the gas passing through the vessels, the above proportion, as near as may be, is kept up by a continuous dropping or running of acid, regulated according to the quantity of ammon: a contained 
in the gas, from a reservoir placed on the top of the saturator. 'This mode of supplying the acid is continued until the specific gravity of the solution arrives at 1170 , or close
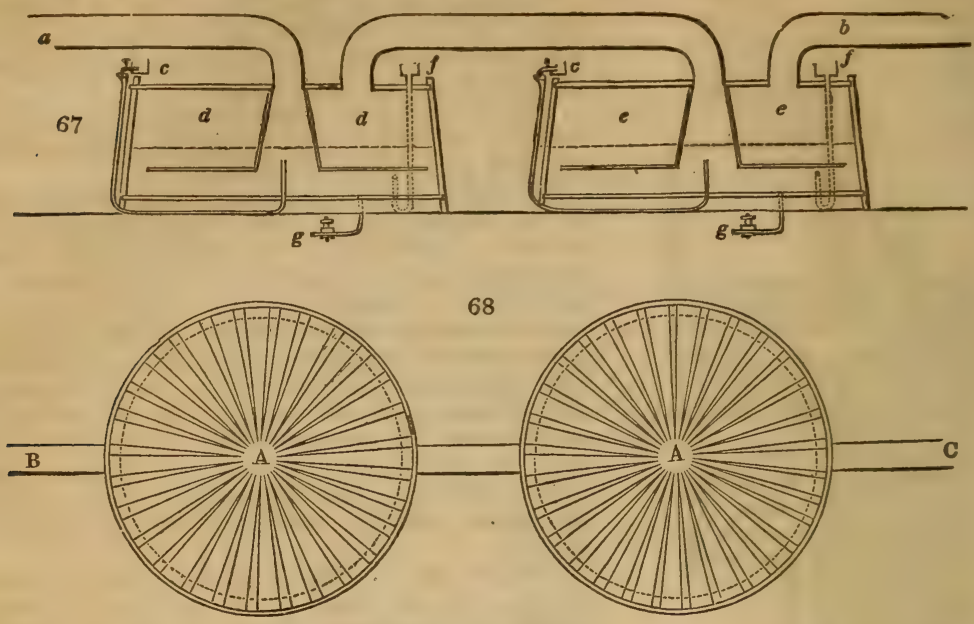

to the point of crystallization, after which the supply of acid is discontinued, and the liquor retained in the vessel until neutral, when it is drawn off and evaporated, and yields a pure sulphate of ammonia.

This process has been introduced at several of the provincial gas-works, the three stations of the Chartered, the Imperial, Phœnix, \&c., \&c. Mr. Croll is also now in treaty with several other companies for its introduction.

The produce-sulphate of ammonia - from the process, by the gas-companies using it, now amounts to several tons per week-and it may be here mentioned, as one of the advantages of science, that the ammonia so produced before the adoption of this process passed along with the gas to the consumer, destroying rapidly the main pipes, fittings, and metres, through which it was transmitted, as well as deteriorating the illuminating power of the gas, and producing a choky effect when consumed in close apartments. It is now employed as a manure, and found to be superior in its effects as a fertilizer, as well as comparatively cheaper than any of the other artificial manures; so that whether Mr. C.'s invention be looked upon as affecting improvements in the manufacture of gas, hitherto unknown, or as producing a valuable manure, the results are alike of the utmost importance.

(When Mr. Croll's process is employed before the lime purifiers, dry lime can be used without creating the nuisance hitherto complained of, and a much less quantity is required for this purification.)

Mr. Croll has recently patented another invention, connected also with the manufacture of gas, which consists in the combination of clay and iron retorts, so that the heat of the furnace first acts on the clay retorts and then passes to those of iron.

The annexed drawing is a transverse section -

$A$ is the fireplace.

B B are piers of fire-bricks, placed at intervals to form nostrils or flues, and the fire tile resting upon them in conjunction with the front and back wall, form the bed or support of the clay retort 1 , and the clay retort 2 is also supported by the front and back brickwork, and a lump, or fire-brick, $E$, placed midway on the crown of the retort 1.

$F$ is a wall which separates the clay retorts 1 and 2 , and the iron retorts $1^{\circ}$ and $2^{\circ}$; a space being left between the top of the said wall $F$, and the under surface of the arch, to allow the fire or heated air to pass freely from the clay to the iron retorts.

G G is the bed, and $\mathrm{H} H$ is the flue under the iron retort $1^{\circ}$. The retort $2^{\circ}$ is supported by the front wall and pieces or lumps.

$J$, placed at the back and crown of the retort $1^{\circ}$, in connexion with the horizontal flue. $\mathrm{H}$ is a vertical flue, forming a passage thence into the shaft or chimney.

The heat passes from the furnace or fireplace $A$, through the spaces or nostrils formed by the piers $\mathrm{B} B$, and around the clay retorts 1 and 2 , over the wall $F$, descends between and around the iron retorts and along the flue $\mathrm{H}$, and escapes by the vertical flue into the chimney. The advantages of this mode of setting retorts are the small quantity of 
brickwork necessary for the erections, the increased durability of the retorts, and the economy in fuel. From adopting this mode of setting a brick lump, it has been found that 12 tons of coke will carbonize 100 tons of coal.

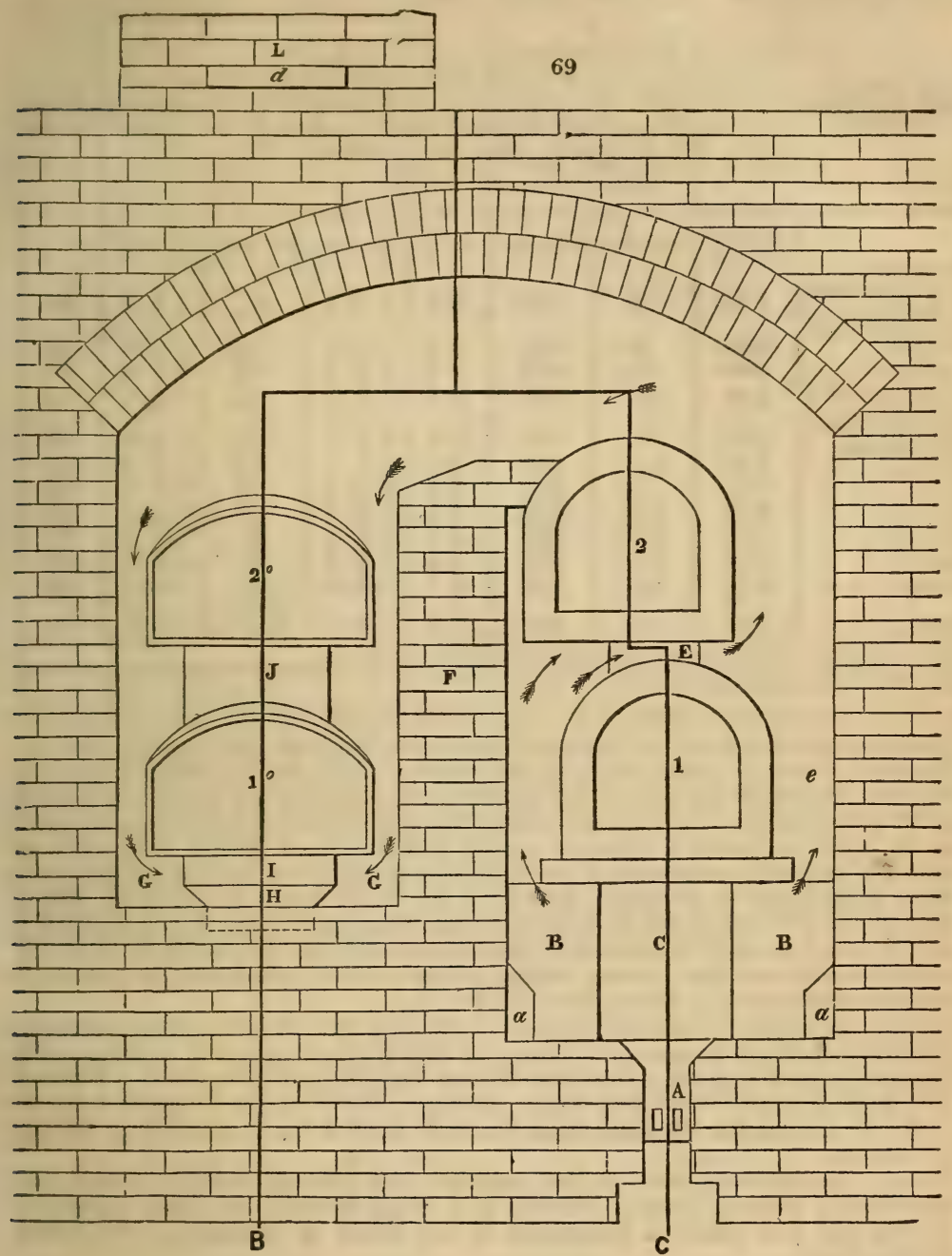

$\mathrm{L}$ is the chimney stalk, and $d$ is a damper or register plate for regulating the chimney draught.

Before dismissing Mr. Croll's patent improvements, it is proper to state, that the sulpharic acid used for condensing the ammonia should be free from iron, otherwise the 8. $^{\prime}$ lphuretted hydrogen of the coal gas is apt to give rise to sulphuret of that metal which will blacken the sulphate of ammonia and reduce its value in the market. An occur. rence of this kind was recently brought professionally before me for investigation. The sulphuric acid had been made from pyrites. 
Copy of a paper laid before a committee of the House of Commons, showing, not only the relative values of the gases produced at the under mentioned places, but showing in like manner the relative economy of gas, as produced at the different places, over candles. By Joseph Hedley, Esq.

\begin{tabular}{|c|c|c|c|c|c|c|c|c|}
\hline $\begin{array}{l}\text { Names of the Places } \\
\text { where. Experiments } \\
\text { were made. }\end{array}$ & $\begin{array}{l}\text { Illuminating } \\
\text { power of a } \\
\text { single Jet of } \\
\text { Gas-flame } \\
\text { four inches } \\
\text { high, taken } \\
\text { by a com. } \\
\text { parison of } \\
\text { Shadow日. }\end{array}$ & $\begin{array}{l}\text { The Jet of } \\
\text { Gas burnt, } \\
\text { four inches } \\
\text { high, con- } \\
\text { sumed per } \\
\text { lour and } \\
\text { was equal to } \\
\text { the Candles } \\
\text { in the last } \\
\text { column. }\end{array}$ & $\begin{array}{l}\text { Gas required } \\
\text { to be equal to } \\
100 \text { lbs. of } \\
\text { mould } \\
\text { Candles } 6 \text { to } \\
\text { the lb., } 9 \\
\text { inches long } \\
\text { each.* }\end{array}$ & $\begin{array}{l}\text { Selling } \\
\text { price of } \\
\text { Gas per } \\
\text { meter per } \\
\text { 1000 cubic } \\
\text { feet. }\end{array}$ & $\begin{array}{l}\text { Cost of Gas } \\
\text { equal in it- } \\
\text { luminating } \\
\text { power to } \\
100 \text { pounds } \\
\text { of candles.t }\end{array}$ & $\begin{array}{l}\text { A verage dis. } \\
\text { count allow- } \\
\text { ed of the } \\
\text { charge for } \\
\text { Gas. }\end{array}$ & $\begin{array}{l}\text { Net cost of } \\
\text { Gas equal to } \\
100 \text { pounds of } \\
\text { Candles. }\end{array}$ & $\begin{array}{l}\text { Dpecific } \\
\text { gravity of } \\
\text { gre Gas. }\end{array}$ \\
\hline & $\begin{array}{l}\text { Equal to } \\
\text { Candles. }\end{array}$ & Cubic Feet. & Cubic Feet. & s. $d$. & L. s. d. & Per Cent. & L. s. d. & \\
\hline $\left.\begin{array}{l}\text { Birmingnam; } \\
\text { Birmingham and } \\
\text { Staffordshire ; } \\
\text { two Companies }\end{array}\right\}$ & $2 \cdot 572$ & $1 \cdot 22$ & 2704 & 100 & 170 & 9 & 147 & .541 \\
\hline $\begin{array}{l}\text { Stockport - - } \\
\text { Manchester - : }\end{array}$ & $\begin{array}{l}3 \cdot 254 \\
3 \cdot 060\end{array}$ & $\begin{array}{l}.85 \\
.825\end{array}$ & $\begin{array}{l}1489 \\
1536\end{array}$ & $\begin{array}{rl}10 & 0 \\
8 & 0\end{array}$ & $\begin{array}{llr}0 & 14 & 11 \\
0 & 12 & 3\end{array}$ & $12 \frac{1}{2}$ & $\begin{array}{rrr}0 & 13 & 0 \\
0 & 10 & 10\end{array}$ & $\cdot 534$ \\
\hline $\begin{array}{l}\text { Liverpool old } \\
\text { Company } \neq\end{array}$ & $2 \cdot 369$ & $1 \cdot 1$ & 2646 & 100 & 165 & $6 \frac{1}{4}$ & 149 & 4 \\
\hline $\begin{array}{l}\text { Liverpool New } \\
\text { Gas Company }\end{array}$ & $4 \cdot 408$ & $\cdot 9$ & 1164 & 100 & 0118 & $6 \frac{1}{4}$ & $\begin{array}{lll}0 & 9 & 10\end{array}$ & 580 \\
\hline Bradford - . - & $2 \cdot 190$ & $1 \cdot 2$ & 3123 & 90 & 181 & $12 \frac{1}{2}$ & 146 & $\cdot 420$ \\
\hline Leeds - - - - & $29 \% 0$ & .855 & 1644 & 80 & 0132 & $6 \frac{t}{4}$ & $\begin{array}{llllll}0 & 12 & 4\end{array}$ & .530 \\
\hline Sheffield - - - & $4 \cdot 434$ & 1.04 & 2440 & 80 & $\begin{array}{lll}0 & 19 & 6\end{array}$ & $6 \frac{1}{4}$ & $\begin{array}{llll}0 & 18 & 3\end{array}$ & $\cdot 466$ \\
\hline Leicester - - & $2 \cdot 435$ & $1 \cdot 1$ & 2575 & 76 & $\begin{array}{lll}0 & 19 & 3\end{array}$ & $15^{2}$ & $\begin{array}{lll}0 & 16 & 5\end{array}$ & .528 \\
\hline Nottingham & 1.645 & $1 \cdot 3$ & 4200 & 90 & $\begin{array}{lll}1 & 17 & 9\end{array}$ & 15 & 1113 & -424 \\
\hline Derby & 1.93 & $1 \cdot 2$ & 3521 & 100 & $\begin{array}{lll}1 & 15 & 4\end{array}$ & 15 & $\begin{array}{lll}110 & 0\end{array}$ & $\cdot 448$ \\
\hline Preston - - - & $2 \cdot 136$ & $1 \cdot 15$ & 3069 & 100 & 1108 & $\begin{array}{c}15 \\
\text { none }\end{array}$ & 162 & -419 \\
\hline London - - - & $2 \cdot 083$ & $1 \cdot 13$ & 3092 & 100 & $\begin{array}{llll}1 & 10 & 11\end{array}$ & allowed. | & $\begin{array}{llll}11 & 10 & 11\end{array}$ & .412 \\
\hline
\end{tabular}

Memorandum.-It will not fail to be observed that in deducing the comparative value between candles and gas by these experiments, the single jet (and in every instance, of course, it was the same), has been the medium. This, however, though decidedly the most correct way of making the comparative estimate of the illuminating power of the several gases, is highly disadvantageous in the economical comparison, inasmuch as gas burnt in a properly regulated argand burner, with its proper sized glass, aur aperture, and sufficient number of holes, gives an advantage in favor of gas consumed in an argand, over a jet burner, of from 30 to 40 per cent. At the same time it must not be overlooked that in many situations where great light is not required, it will be found far more economical to adopt the use of single jets, which by means of swing brackets and light elegant shades, become splendid substitutes for candles, in banking establishments, offices, libraries, \&c., \&c.

Note.-In Glasgow, Edinburgh, Dundee, Perth, and the Scotch towns, generally, the Parrot or Scotch Cannel coal is used; in illuminating power and specific gravity the gas produced is equal to that from the best description of Cannel coal in England. The price per 1,000 cubic feet ranges about $9 \mathrm{~s}$., with from 5 to 30 per cent. off for discounts, leaving the net price abont $9 \mathrm{~s}$., to be equal in the above table to $100 \mathrm{lbs}$. of candles.

Epitome of Experiments made in Gas produced from different qualities of coal, and consumed in different kinds of Burners, tried at the Sheffield Gas Light Company's Works, and laid before a Committee of the House of Commons. By Joseph Hedley, Esq.

\begin{tabular}{|c|c|c|c|c|c|c|c|c|c|c|}
\hline $\begin{array}{l}\text { Ds.te } \\
1835 .\end{array}$ & $\begin{array}{c}\text { Description of } \\
\text { Burner. }\end{array}$ & $\begin{array}{l}\text { Species of } \\
\text { Coal. }\end{array}$ & $\begin{array}{c}\text { Specific } \\
\text { Gravity of } \\
\text { Gas. }\end{array}$ & \begin{tabular}{|l|}
\multicolumn{1}{|c|}{} \\
Dis- \\
tance of \\
Candle \\
from \\
Shadow
\end{tabular} & $\begin{array}{c}\text { Gas } \\
\text { con- } \\
\text { sumed } \\
\text { per } \\
\text { hour. }\end{array}$ & $\begin{array}{l}\text { Height } \\
\text { of Gas. } \\
\text { flame. }\end{array}$ & $\begin{array}{c}\text { Equal to } \\
\text { Mould } \\
\text { Tallow } \\
\text { Candles, } 6 \text { to } \\
\text { the pound, } 9 \\
\text { inches long } \\
\text { each. }\end{array}$ & $\begin{array}{c}\text { Gas equal to } \\
100 \text { pounds } \\
\text { of Mould } \\
\text { Candles. }\end{array}$ & $\begin{array}{l}\text { Cost of Gas } \\
\text { at } 88 . \\
\text { per } 1000 . \\
\text { cubic feet. }\end{array}$ & $\begin{array}{c}\text { Cost of } \\
100 \text { lbs. } \\
\text { Mould } \\
\text { Candles } \\
\text { at } 75.6 d . \\
\text { per dozen } \\
\text { lbs. }\end{array}$ \\
\hline May & & & & Inches & $\begin{array}{l}\text { Cubic } \\
\text { Feet. }\end{array}$ & Inches. & Candles. & Cubic Feet. & L. s. d. & L. s. d. \\
\hline $\begin{array}{l}8 \\
9 \\
9 \\
9\end{array}$ & $\begin{array}{l}\text { Single Jet } \\
\text { Ditto } \\
\text { Ditto }\end{array}$ & $\begin{array}{l}\text { Deep Pit } \\
\text { Mortormley } \\
\text { Cannel }\end{array}$ & $\begin{array}{r}.410 \\
.450 \\
.660\end{array}$ & $\begin{array}{l}75 \\
74 \\
61 \frac{1}{4}\end{array}$ & $\begin{array}{l}1 . \\
.95 \\
\cdot 7\end{array}$ & $\begin{array}{l}4 \\
4 \\
4\end{array}$ & $\begin{array}{l}2 \cdot 36 \\
2 \cdot 434 \\
\cdot 3 \cdot 54\end{array}$ & $\begin{array}{l}2415 \\
2224 \\
1127\end{array}$ & $\left.\begin{array}{rrr}0 & 19 & 3 \frac{1}{2} \\
0 & 17 & 9 \frac{1}{2} \\
0 & 9 & 0\end{array}\right)$ & \\
\hline 8 & Argand $\}$ & Deep Pit. & $\cdot 410$ & 34 & $3 \cdot 3$ & $3 \frac{1}{2}$ & $11 \cdot 53$ & 1631 & $\begin{array}{lll}0 & 13 & 0 \frac{1}{2}\end{array}$ & 326 \\
\hline $\begin{array}{l}9 \\
9\end{array}$ & $\begin{array}{l}\text { Ditto } \\
\text { Ditto }\end{array}$ & $\begin{array}{l}\text { Mortormley } \\
\text { Cannel }\end{array}$ & .450 & $\begin{array}{l}33 \\
29\end{array}$ & $\begin{array}{l}3 \cdot 1 \\
2 \cdot 6\end{array}$ & $\begin{array}{l}3 \frac{1}{2} \\
3 \frac{1}{2}\end{array}$ & $\begin{array}{l}12 \cdot 24 \\
15.85\end{array}$ & $\begin{array}{r}1443 \\
935 \\
\end{array}$ & $\left.\begin{array}{rrrr}0 & 11 & 6 \frac{1}{2} \\
0 & 7 & 5 \frac{3}{4}\end{array}\right]$ & \\
\hline
\end{tabular}


Copy of Experiments made at the Alliance Gas Company's Works in Dublin, during the past year 1837. By Joseph Hedley, Esq.

Results of experiments on the qualities of various coals for the production of gas ; its value in illuminating power; produce of coke, and quality; and other particulars important in gas-making :-

1st Experiment, Saturday, May 27th, 1837.-Deane coal (Cumberland), 2 cwt. of $112 \mathrm{lbs}$. each (or $224 \mathrm{lbs}$.) produced 970 cubic feet of gas ; 4 bushels of coke of middling quality; specific gravity of the gas, 475 . Consumed in a single-jet burner, flame 4 inches high, $1 \frac{4}{10}$ cubic feet per hour; distance from shadow 76 inches or 2.3 mould candles. Average quantity of gas made from the charge (6 hours) 4.33 cubic feet per lb., or 9,700 cubic feet per ton of $20 \mathrm{cwt}$. Increase of coke over coal in measure, not quite 30 per cent. Loss in weight between coal, coke, and breize, 56 lbs., converted into gas, tar, ammonia, \&c.

$2 d$ Experiment, May 28th.-Carlisle coal (Blenkinsopp). 224 lbs. produced 1,010 cubic feet of gas, 4 bushels of coke of good quality though small; increase of coke over coal in measure not quite 30 per cent. Loss in weight, same as foregoing experiment. Average quantity of gas made from the charge ( 6 hours) $4 \cdot 5$ cubic feet per $1 \mathrm{~b}$. or 10,080 per ton.

Illuminating Power of the Gas.

\begin{tabular}{|c|c|c|c|c|}
\hline & $\begin{array}{l}\text { Consumed } \\
\text { per hour, } \\
\text { single jet. }\end{array}$ & $\begin{array}{l}\text { Distance } \\
\text { from candle. }\end{array}$ & $\begin{array}{l}\text { Equal to } \\
\text { candles. }\end{array}$ & $\begin{array}{l}\text { Specific } \\
\text { gravity. }\end{array}$ \\
\hline At the end of the 1 st hour & Feet. & $\begin{array}{l}\text { Inches. } \\
70\end{array}$ & $2 \cdot 72$ & $\cdot 475$ \\
\hline $\begin{array}{l}\text { Ditto ditto with 20-hole } \\
\text { argand burner }\end{array}$ & 5 & 25 & $21 \cdot 33$ & $\cdot 475$ \\
\hline When charge nearly off - & $1 \frac{4}{10}$ & 85 & $1 \cdot 84$ & $\cdot 442$ \\
\hline $\begin{array}{l}\text { When charge quite off, with } 20- \\
\text { hole argand burner }\end{array}$ & 9 & 100 & not 1 & $\cdot 266$ \\
\hline
\end{tabular}

$3 d$ Experiment, May 29th.-Carlisle coal (Blenkinsopp). 112 lbs. produced 556 cubic feet of gas. Other products, loss of weight, \&c., same proportion as foregoing experiment. Average quantity of gas made from the charge (6 hours) 4.96 cubic feet per lb., or 11,120 per ton.

In this experiment the quantity of gas generated every hour was ascertained; the illuminating power, the specific gravity, and the quantity of gas consumed by the single jet with a flame 4 inches high, was tried at the end of each hour, with the respective gases generated at each hour; and the following is a table of results.

RESULTS.

\begin{tabular}{|c|c|c|c|c|c|}
\hline Hour. & Gas produced. & $\begin{array}{c}\text { Consumed } \\
\text { per hour } \\
\text { per single jet, } \\
4 \text { inches high. }\end{array}$ & Specific gravity. & $\begin{array}{l}\text { Distance of } \\
\text { candle from } \\
\text { shadow. }\end{array}$ & $\begin{array}{l}\text { Illuminating } \\
\text { power equal to } \\
\text { mould candles. }\end{array}$ \\
\hline 1st. & $\begin{array}{c}\text { Cubic Feet. } \\
150\end{array}$ & $\begin{array}{l}\text { Cubic Feet. } \\
\left.\begin{array}{c}11 \frac{1}{2} 10 \text { ths. } \\
\text { or } 1 \cdot 15\end{array}\right\}\end{array}$ & $\cdot 534$ & $\begin{array}{c}\text { Inches. } \\
70\end{array}$ & $2 \cdot 72$ \\
\hline $2 \mathrm{~d}$. & 120 & 11 & $\cdot 495$ & 75 & $2 \cdot 36$ \\
\hline $3 \mathrm{~d}$ & 95 & 12 & $\cdot 344$ & 75 & $2 \cdot 36$ \\
\hline 4th. & 95 & 15 & $\cdot 311$ & 80 & $2 \cdot 08$ \\
\hline 5th. & 80 & 17 & $\cdot 270$ & 85 & $1 \cdot 81$ \\
\hline 6 th. & 16 & 29 & .200 & 100 & not one. \\
\hline Total & 556 & & & & \\
\hline
\end{tabular}

\section{Average of the above gas, 6-hour charge.}

$92 \frac{2}{3} \quad 16$ 10ths. nearly $\cdot 359 \quad 81 \quad 2.03$

\footnotetext{
$115 \quad 12 \frac{2}{2}$ 10ths. $\quad .421 \quad 75 \quad 2.36$
}

Production of gas in 6 hours, 556 feet, or at the rate of 11,120 cubic feet per ton. Ditto in 4 hours, 460 feet, or at the rate of 9,200 ditto. 
The relative value of these productions of gas is as follows, viz :-

11,120 at 16 10ths per hour nearly (or 1.5916 accurately), and equal to 22.03 can dies; the 11,120 feet would be equal to and last as long as 1,597 candles, or $266 \frac{1}{6}$ lbs. of candles.

9200 at $12 \frac{1}{8} 10$ ths per hour (or 1.2375 accurately), and equal to 2.36 candles; the 9,200 feet would be equal to 1,949 candles, or $324 \frac{5}{6} \mathrm{lbs}$. candles.

Now $266 \frac{1}{6}$ lbs. of mould candles, at 7s. $6 d$. per ${ }^{6}$ dozen lbs. will cost $8 l .6 s .4 \frac{1}{2} d$; ; while $324 \frac{5}{8} \mathrm{lbs}$ of do. do. at $7 \mathrm{~s} .6 \mathrm{~d}$. per do. do. $10 \mathrm{l}$. $3 \mathrm{~s}$.

Showing the value of 4 -hour charges over 6 -hour charges; and of 9,200 cubic feet over 11,120 cubic feet.

NoTE.-9500 cubic feet of Wigan cannel coal gas are equal in illuminating power to 859 1-6 lbs. of candles, which at $7 s .6 d$. per dozen lbs., will cost $25 l$. 10s. $5 \frac{1}{2} d$. It is also found that any burner with superior gas, will consume only about half the quantity it would do with common gas.

4th Experiment, May 30th.-Cannel and Cardiff coal mixed $\frac{1}{2}$ and $\frac{1}{2}$, together 112 lbs., produced 460 feet of gas, 2 bushels of coke of good quality; inerease of coke over coal in measure about 30 per cent.; loss in weight $41 \mathrm{lbs}$. ; coke weighed $71 \mathrm{lbs.}$, no breize. Average quantity of gas made from the charge ( 4 hours), $4 \cdot 1$ cubic feet per Ib., or $9 \cdot 200$, per ton.

Illnminating Power.-At end of first hour,

$$
\text { Candles. }
$$

Cubic feet.

$\left.\begin{array}{l}\text { Distance of candle from } \\ \text { shadow - }\end{array}\right\} 73$ or $2.49\left\{\begin{array}{c}\text { Consumed per hour, single } \\ \text { jet, } 4 \text { inches high }\end{array}\right\} 1210$ ths. At end of $2 \mathrm{~d}$ hour, do. 70 or 2.72 Do. do. do. $11 \frac{1}{2} 10$ ths. At end of $3 \mathrm{~d}$ hour. This gas very indifferent.

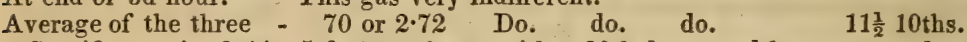

Specific gravity 3.44 ; 5 feet per hour, with a 20 -hole argand burner, equal to $14 \cdot 66$ candles.

5th Experiment, May 31st.-Carlisle coal, $112 \mathrm{lbs}$. produced 410 feet of gas; other products, same as in former experiments with this coal, but heat very low.

Illuminating Power and Produce of Gas.

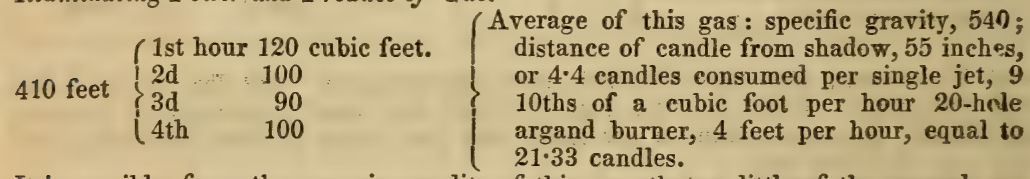

It is possible, from the superior quality of this gas, that a little of the eannel gas made for a particular purpose may have got intermixed with it in the experimental gasholder and apparatus.

Various other experiments were tried on different qualities of coal, and mixtures of ditto, too tedious to insert here, though extremely valuable, and all tending to show the superior value of gas produced at short over long charges; and also showing the importance and value of coal producing gas of the highest illuminating power; among which the cannel coal produced in Lancashire, Yorkshire, and some other counties of England and Wales, and the Parrot or splent coal of Scotland, stand pre-eminent.

NoтE.-In all the foregoing experiments the same single-jet burner was used; its fame in all instances exactly 4 inches high.

The coal when drawn from the retort was slaked with water, and after allowing some short time for drying, was weighed.

A Table of the Number of Hours Gas is burnt in each Month, Quarter, and Year.

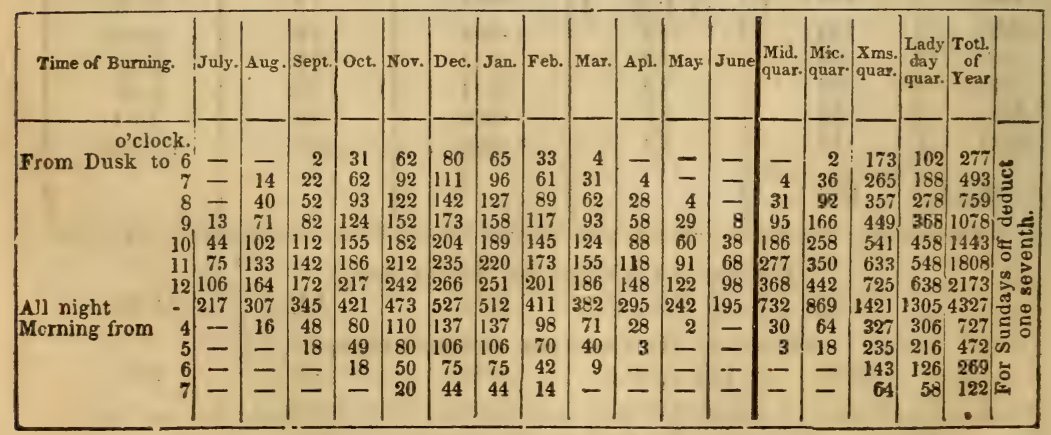


GAS-LIGHT.

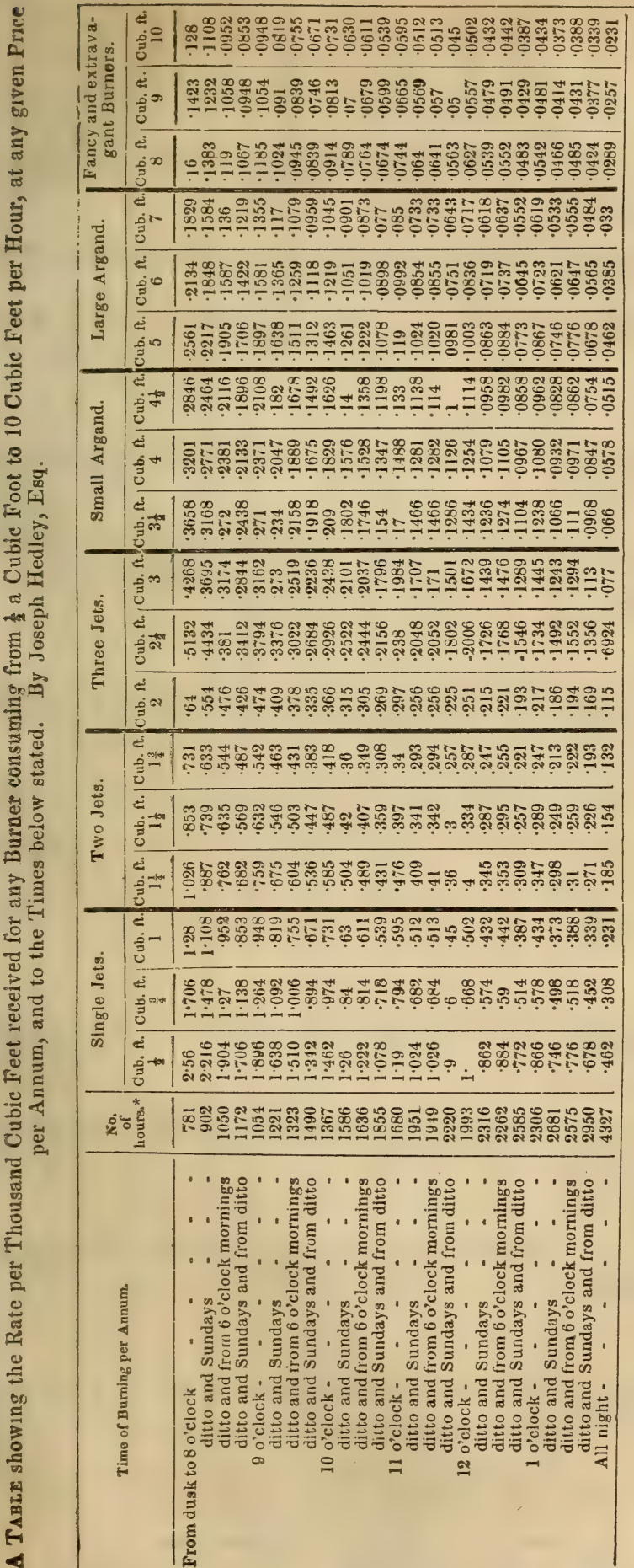

\begin{tabular}{|c|}
\hline 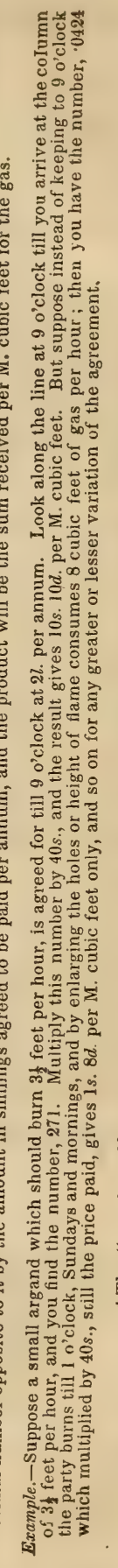 \\
\hline
\end{tabular}


GAS-LIGHT.

Copy of a Paper submitted to a Committee of the House of Commons in the Session of 1837, of England; and procured by actual Survey and

\begin{tabular}{|c|c|c|c|c|c|c|c|c|c|c|c|}
\hline 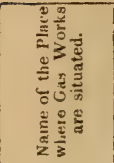 & $\begin{array}{l}\text { Price of Gas per Meter, } \\
\text { and Discounts allowed. }\end{array}$ & 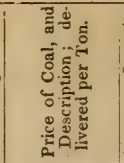 & 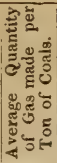 & 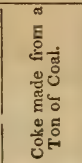 & 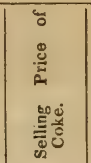 & 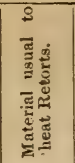 & 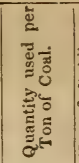 & 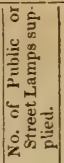 & 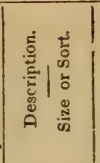 & 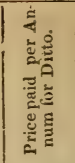 & 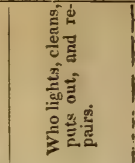 \\
\hline ars & i1ce, pe- 1000 cub, feet & Lump coal & ic. $f:$ & & & Slack & 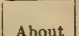 & 400 & Bat & $\begin{array}{ll}\text { L. s. } & d \text {. } \\
1 \text { 10 } & 8\end{array}$ & $8 \mathrm{Cor}$ \\
\hline $\begin{array}{l}\text { Gas Com } \\
\text { pany. }\end{array}$ & $\begin{array}{l}\text { Discounts. } \\
10 l \text {. to } 30 l \text {. \& } 21 / 2 \\
30 l \text {. to } 50 l \text {. \& } 5 \\
50 l \text {. to } 75 i \text {. \& } 71 / 2 \\
75 l \text {. to } 100 l \text {. } 10 \\
100 l \text { \& } 10\end{array}$ & $\begin{array}{l}\text { Brom Wat } \\
\text { pits risen } \\
\text { much of late. } \\
1837,118.10 d .\end{array}$ & & bushels. & $\begin{array}{c}\text { quarter } \\
\text { delivered } \\
\text { or about } \\
3 d . \text { per } \\
\text { busluel. }\end{array}$ & & $\begin{array}{l}5 \text { ewt. } \\
\text { of slack, } \\
\text { at } 6 s \text {. } \\
\text { per ton, } \\
25 \text { per }\end{array}$ & & $\begin{array}{c}460 \\
30\end{array}$ & 203 & $\begin{array}{l}0 \text { provides posta, } \\
\text { services, \&c. }\end{array}$ \\
\hline $\begin{array}{l}\text { Birmingbam } \\
\text { and Staftiord. } \\
\text { shire }\end{array}$ & $\begin{array}{c}108 \text { per } 1000 \text { cub. feet. } \\
\text { Discounts as above. }\end{array}$ & $\begin{array}{c}\text { From West } \\
\text { Bromwich } \\
\text { pits, 1837, } \\
9 s .3 d .\end{array}$ & 6,500 & $\begin{array}{l}24 \text { bush. } \\
\text { but larger } \\
\text { measure } \\
\text { than Bir- } \\
\text { mingham }\end{array}$ & $\left|\begin{array}{c}2 s .10 d . \\
\text { per sack } \\
\text { of } 8 \\
\text { bushels }\end{array}\right|$ & $\begin{array}{l}\text { Slack } \\
\text { and } \\
\text { Tar. }\end{array}$ & $\begin{array}{l}5 \text { cwt. } \\
\text { of slack, } \\
\text { at } 4 a . \\
25 \text { per } \\
\text { cent. }\end{array}$ & 1,500 & Batswings & $\begin{array}{c}\text { average } \\
1180\end{array}$ & Ditto. \\
\hline Macclesfield & 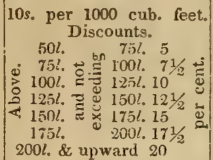 & $\begin{array}{l}\text { Common, } 8 s . \\
\text { average 1834. }\end{array}$ & 6,720 & $12 \mathrm{cwt}$. & $\begin{array}{l}10 s . \\
\text { per ton }\end{array}$ & Coke & $\begin{array}{c}\text { No } \\
\text { account } \\
\text { kept. }\end{array}$ & 220 & Ditto. & 2100 & Company. \\
\hline Stockport & $\begin{array}{l}\text { 10s. per } 1000 \text { cub. feet. } \\
\text { Discounts same as Mac- } \\
\text { clesfield. Macclesfield } \\
\text { discounts taken from } \\
\text { Stockport card. }\end{array}$ & $\begin{array}{c}\text { Coal 10s. } 6 d . \\
\text { cannel } 19 s .6 d . \\
\text { about half and } \\
\text { half used. } \\
\text { Average 15s. } \\
\text { 1834. }\end{array}$ & 7,800 & $7 \mathrm{cwt}$. & $\begin{array}{l}6 s .8 d . \\
\text { per ton. }\end{array}$ & $\begin{array}{c}\text { Coal, } \\
\text { coke, } \\
\text { and tar. }\end{array}$ & Ditto. & 230 & Ditto. & $\begin{array}{l}2100 \\
1834 . \\
200 \\
1837 .\end{array}$ & $\begin{array}{l}0 \text { Comrs-provide } \\
\text { lamps \& posts } \\
0 \text { Company's } \\
\text { service liglit, } \\
\text { repair, clean, } \\
\text { anu extinguish. }\end{array}$ \\
\hline Manchester & 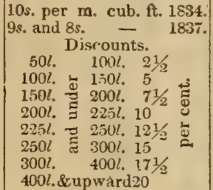 & 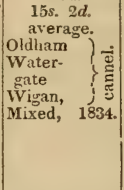 & 9,500 & $14 \mathrm{cwt}$. & Ditto. & Coke. & $\begin{array}{c}4,23 \mathrm{ds} \\
\mathrm{cwt} .\end{array}$ & 2,375 & $\begin{array}{l}\text { Sing'e jets } \\
\text { and fiat } \\
\text { flames. } \\
\text { about lialf } \\
\text { and half. }\end{array}$ & $\begin{array}{lll}1 & 2 & 0 \\
2 & 0 & 0\end{array}$ & $\begin{array}{ll}0 & \text { Commission- } \\
0 & \text { ers of } t 0 l i c e\end{array}$ \\
\hline $\begin{array}{l}\text { Liverpool old } \\
\text { Company, } \\
1834 .\end{array}$ & 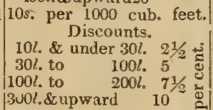 & $\begin{array}{l}7 s, 3 d \text {. per ton } \\
\text { of 112lbs. per } \\
\text { cwt. Ormskirk } \\
\text { or Wigan } \\
\text { slack. }\end{array}$ & 8,200 & $113 / 4 \mathrm{cwt}$. & $\begin{array}{c}8 s .4 d . \\
\text { per ton of } \\
112 \mathrm{lb} \\
\text { per cwt. }\end{array}$ & $\begin{array}{l}\text { Slack. } \\
7 s .3 d . \\
\text { per ton. }\end{array}$ & $61 / 2$ cwt. & $\begin{array}{r}1,700 \\
30\end{array}$ & \begin{tabular}{|c|} 
Batswings \\
1 jet, \\
$2-$ \\
$3-$ \\
$4-$
\end{tabular} & $\begin{array}{rrr}4 & 10 & 0 \\
2 & 5 & 0 \\
2 & 13 & 0 \\
3 & 2 & 9 \\
3 & 13 & 11\end{array}$ & $\begin{array}{l}0 \text { Compat.y } \\
\text { o tiglit, clean,put } \\
\text { o out, and repair. } \\
9 \\
1\end{array}$ \\
\hline
\end{tabular}

Ditto ditto In 1835 this Company resorted to the use of cannel coal similar to the Liverpool New Gas and Coke Company, producing nearly

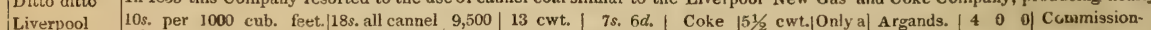
Liverpool $10 s$. per 1000 cub. feet. 18s. all cannel

\begin{tabular}{l|l|l|l|l|l} 
New Gas and Discounts same as Liv- Wigan. & & per ton. & $\begin{array}{l}\text { and } \\
\text { andack. } \\
\text { Coke, 1835. }\end{array}$
\end{tabular}

\begin{tabular}{|l|l|l|l|} 
Bradford, & $9 s$. per 1000 cub. feet to $8 s .6 d$. per ton. 8,000 & 13 cwt. $12 s$.
\end{tabular} and slack.

large consumers. 3 sorts used. 201 Discounts. $20 l$ to $30 l .5$ Slack $5 s .6 d$.

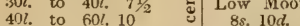
$60 l$. to $80 l .121 / 2$ Catherine $80 l$. to $100 l .15 \%$. lcol.\& npwierd 20 Small consumers.

$10 s$, per 1000 cub. feet,
and 5 per rent. off from

Leeds, $1834\left|\begin{array}{c}\text { and } 10 l \text {, to } 20 l \text {. } \\ \text { 8s. per } 1000 \text { cubic }\end{array}\right|$ os per ton 8s. per 1000 cubic feet.
Discounts.
average. $21 / 2)$ per cent. on $\{15 l$. $23 \mathrm{~d}$ common $71 / 2\}$ half-yearly $\left\{\begin{array}{l}30 \ell . \\ 50 \ell .\end{array} \quad 13 \mathrm{~d}\right.$ cannel, $10 \%$ payments $\left\{\begin{array}{r}50 l .2 \\ 100 l .\end{array}\right.$ 1 3d cannel,

\begin{tabular}{|l|l|l|l|} 
Sheffield, & $8 s$. per 1000 cubic feet. 7s. 9. per ton 8,000 & 10 cwt. $10 s$.
\end{tabular} 1835.

Discounts same as Leeds.
3 sorts used,

Lescester, $75.6 d$. per 1000 cubic feet.

Discounts on half-yearly
re:tal not exceeding
Derbyshire.

lin., 5 per cent.

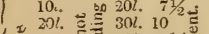

1 \% $30 l$.

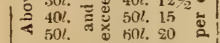

$60 \%$. \& upward 25

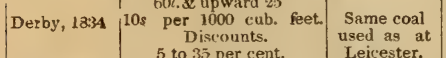

Nottingham, 9s. per 1000 cubic feet

1831. $\quad$ Discounts as above.

Lonuton, 1834. 10s. per 1000 cub. feet. 3 sorts used,
1,2 loth cannel, at $16 \mathrm{~s}$. 8,210 deep pit 7s. $110 \mathrm{th}$ silk stone, 10 s.

$13 s 6 d$.

Leicester.

6,50012 cwt.

7s. $6 d$.

per ton

D
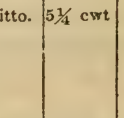

Ditta. $31 / 2$ ewt

per ton.

7,5004 quarters

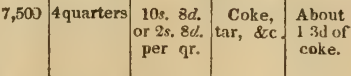

\&ce $13 \mathrm{~d}$ of

17s. average. Newcastle.
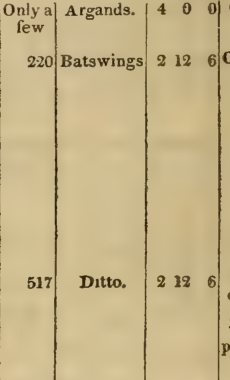

630 Ditto.

210 0 Company pro vite lamps, clear., repair,
put out, \&c. ompany light, guishing, for whir li Comp 3.. $10 x$.

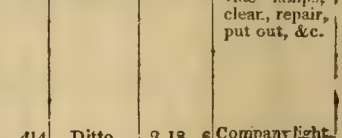

414 Ditto. 218 6ompany light, cleax.

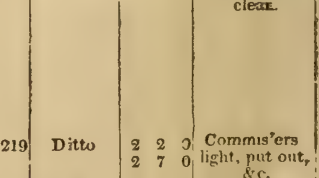

300 Ditto. 33 a Commis'ers light, eleas, Compunt ligh: but not repair, 
GAS-LIGHT.

being a Synupsis of the Proceedings of the under-mentioned principal Gas Light Establishments Experiments between the years 1834 and 1837. By Joseph Hedley, Esq.

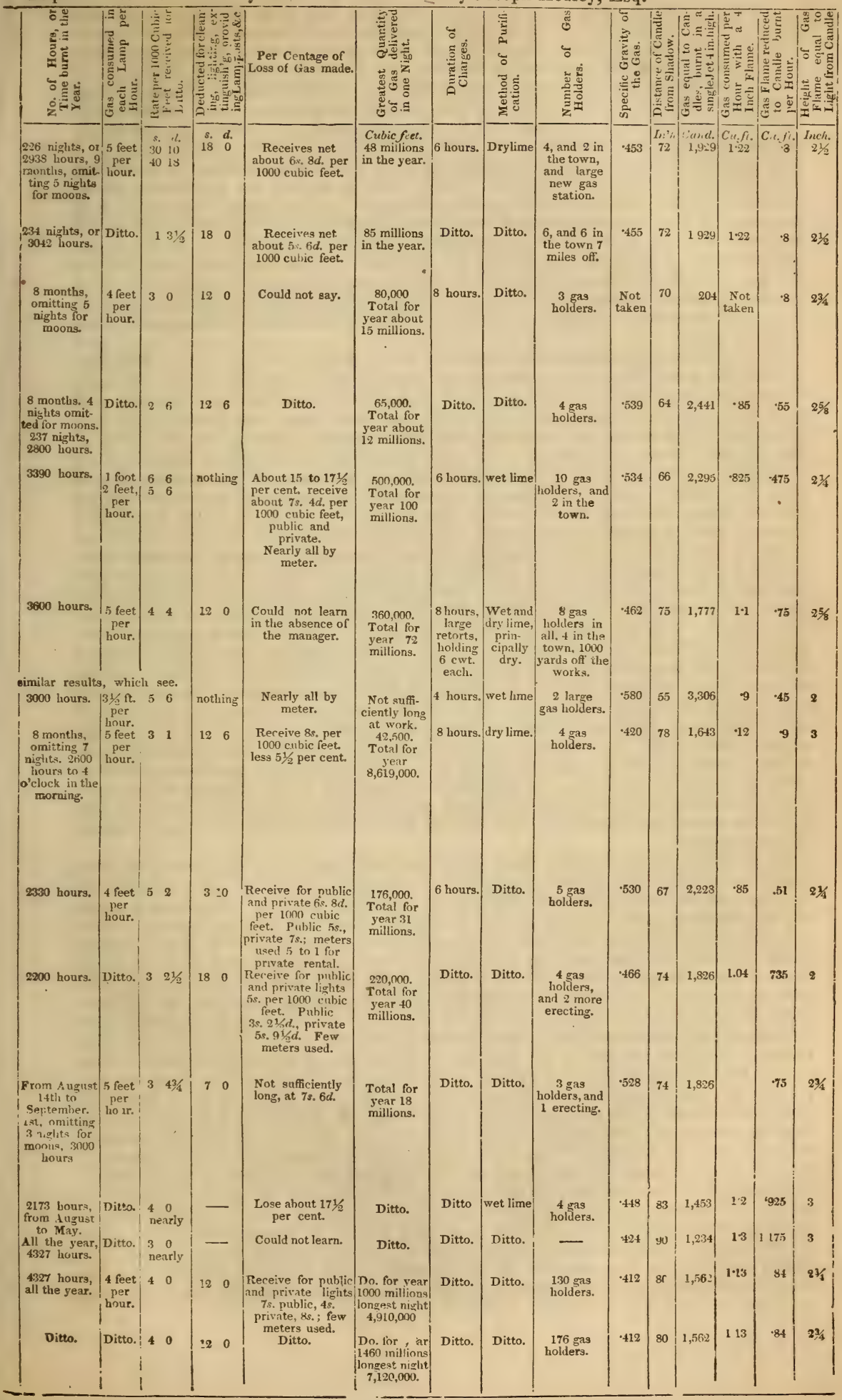


Bule-light.--This brilliant mode of illumination has been so called from the name of the residence in Cornwall of Mr. Goldsworthy Gurney, who obtained a patent for it in the year 1838. In its first form it consisted of a common argand oil flame or lamp of rather narrow circular bore, into the centre of whose wick a jet of oxygen gas was admitted throagh a tube inserted in the middle of the burner. This contrivance was not, however, new in this country, for a similar lamp, similarly supplied with oxygen gas, was employed by the celebrated Dr. Thomas Young in his lectures at the Royal Institution of Great Britain for the purpose of illuminating a solar microscope, or gas microscope, about 40 years ago, and I had done the same thing in Glasgow in the year 1806 or 1807. When used as a light for lighthouses or for other continuous illumination, it has been found to be too expensive and difficult to manage. It was tried upon a good scale a few years ago both by the Trinity House in Tower Hill, and in one of their lighthouses on the coast, as well as by the House of Commons. The Masters of Trinity did not find it to be essentially superior for the use of their lighthouses to their old and ordinary plan of illumination with a number of argand lamps placed in the focus, or near the focus, of reflecting mirrors. It was, after several expensive trials by them and in the House of Commons, aban doned by both.

In the course of numerous experiments in the Trinity House, Tower Hill, Mr. Gurney had occasion to examine the structure and see the performance of Mr. Fresnel's compound argand lamps which are used in the French lighthouses, furnished with refracting lenses of peculiar forms which surround these lamps, and transmit their concentrated light in any desired horizontal direction along the surface of the sea. Two of Mr. Fresnel's lamps are placed in the lamp apartment of the Trinity House. Each consists of a series of 4,5 , or 6 concentric wicks in the same plane, supplied with oil from the fountain below by means of a pumping mechanism, as in the well-known Parisian lamps of Carcel and Gagneau. The effect of 4, 5, or 6 concentric flames thus placed in close proximity to each other, with suitable supply of air through the interior of the innermost tube and the interstices between the exterior ones, is, to increase the heat in a very remarkable degree, and by this augmentation of the heat to increase proportionably the light. For it has been long known that a piece of even incombustible matter, such as a lump of brick, intensely heated, sends forth a most brilliant irradiation of light. This fact was applied first to the purpose of illuminating objects by Professor Hare of Philadelphia, fully 40 years ago. By directing the very feebly luminous flame of the compound jet of hydrogen and oxygen upon a bit of clay, such as one of Wedgwood's pyrometer pieces, a most vivid illumination was sent forth from it as soon as it became intensely heated. More lately, a piece of lime has been used instead of a bit of clay, as it is not so apt to change by the ignition, and affords, therefore, a more durable effect. It is used in our modern gas microscopes. Mr. Gurney suggested the use of lime for the above purpose in a work on chemistry which he published more than twenty years ago. It was afterward adopted by Mr. Drummond, in order to make signal lights in the trigonometrical survey of the Board of Ordnance, and was therefrom called the Drummond light, though he had no share whatever in the merit of the invention.

The structure of the Fresnel lamp would naturally suggest to Mr. Gurney the idea of trying the effect of a similar construction of an argand gas-lamp. But prior to the execution of this scheme, he obtained a second patent in the year 1839, for increasing the illuminating power of coal-gas by feeding its flame in a common argand burner with a stream of oxygen. But here a serious difficulty occurred. The stream of oxygen when admitted into the centre of such a flame, instead of augmenting its quantity of light, destroys it almost entirely. This result might have been predicted by a person well versant in the principles of gas illumination, as long ago expounded in Sir Humphrey Davy's admirable Researches on Flame. This philosopher demonstrated that the white light of gas-lamps, as also of oil-lamps, was due to the vivid ignition of solid particles of carbon evolved by the igneous decomposition of the hydro-carburet, either in the state of gas or vapor; and that if, by any means, these particles were not deposited, but burned more or less completely in the moment or act of their evolution from the hydro-carburet, then the illumination would be more or less impaired. Mr. Gurney, on observing this result, sought to obviate the evil, by charging the coal-gas with the vapor of naphtha. Thus a larger supply of hydro-carburet, and of carbon of course, being obtained, the flame of the naphthalized gas, admitted with advantage the application of oxygen gas, for the increase of its light; on the principle of greater intensity of ignition, and consequently of light being produced by the burning of carbon in oxygen than in common air, as had been long known to the chemical world. But en obstruction to the permanent employment of naphthalized gas was experienced by Mr. Gurney, by the deposition of liquid naphtha in the pipes of distribution. He was therefore induced to renounce this project. He then resorted to the use of coal-gas, 
purified in a peculiar way, and burned in compound Argand lamps, consisting of two or more concentric metallic rings, perforated with rows of holes in their upper surfaces, having intervals between the rings for the admission of a proper quantity of air, the burner being enclosed in a glass chimney at the level of the flame, surmounted by a tall iron chimney. Between these two chimneys, a certain space is left for the admission of air, and to favor draught and ventilation. The intensity and whiteness of the cylinder of light produced by the combustion of coal gas in this lamp are truly admirable, and form such an improvement in illumination for streets, churches, public rooms, and private houses, as to merit the protection of a patent, and the encouragement of the public at large.

General Estimate of Sizes, Number of Concentrics, Consumption of Gas, and Comparative Light.

\begin{tabular}{|c|c|c|c|c|c|c|}
\hline Size. & $\begin{array}{l}\text { Number of } \\
\text { Concentrics. }\end{array}$ & $\begin{array}{l}\text { Bude C } \\
\text { tion pe }\end{array}$ & $\begin{array}{l}\text { nsump- } \\
r \text { hour. }\end{array}$ & $\begin{array}{l}\text { Height of } \\
\text { Flame. }\end{array}$ & Comparative Light. & $\begin{array}{c}\text { Argand } \\
\text { Consumption }\end{array}$ \\
\hline $\begin{array}{l}\text { Inches. } \\
2 \frac{1}{2} \\
3 \\
3 \frac{1}{2} \\
4 \\
4 \frac{1}{2} \\
5 \\
5 \frac{1}{3} \\
6\end{array}$ & $\begin{array}{l}2 \\
2 \\
2 \\
2 \\
2 \\
3 \\
3 \\
3\end{array}$ & $\begin{array}{c}\text { Feet. } \\
10 \\
16 \\
21 \\
26 \\
33 \\
40 \\
43 \\
56\end{array}$ & $\begin{array}{c}\text { Inches. } \\
6 \\
4 \\
6 \\
4 \\
7 \\
0 \\
5 \\
4\end{array}$ & $\begin{array}{c}\text { Inches. } \\
3 \\
3 \\
3 \\
3 \\
3 \\
3 \frac{1}{4} \\
3 \frac{1}{2} \\
4\end{array}$ & $\begin{array}{c}\text { 15-hole argands. } \\
5 \\
8 \\
10 \\
12 \\
15 \\
18 \\
20 \\
24\end{array}$ & $\begin{array}{c}\text { Cubic feet. } \\
30 \\
48 \\
60 \\
72 \\
90 \\
108 \\
120 \\
144\end{array}$ \\
\hline
\end{tabular}

GELATINE. The substance produced by boiling the skin of animals in water, which in its crude but solid state is called glue, and when a tremulous semi-liquid, size. The latter preparation is greatly used by the paper-makers, and was much improved by the following process, for which Mr. William Rattray obtained a patent in May, 1838. The parings and scrows of skins are steeped in water till they begin to putrefy; they are then washed repeatedly in fresh water with the aid of stampers, afterward subjected, in wooden or leaden vessels, to the action of water strongly impregnated with sulphurous acid for from 12 to 24 hours; they are now drained, washed with stampers in cold water, and next washed with water of the temperature of $120^{\circ} \mathrm{F}$., which is poured upon them and run off very soon to complete their purification. The scrows are finally converted into size, by digestion in water of $120^{\circ}$ for 24 hours; and the solution is made perfectly fine by being strained through several thicknesses of woollen cloth. They must be exhausted of their gelatinous substance, by repeated digestions in the warm water. The claim is for the sulphurous acid, which, while it cleanses, acts as an antiseptic.Newton's Journal, xiv. 173.

A fine gelatine for culinary uses, as a substitute for isinglass, is prepared by $\mathrm{Mr}$. Nelson's patent, dated March, 1839. After washing the parings, \&c., of skin, he scores their surfaces, and then digests them in a dilute caustic soda ley during ten days. They are next placed in an air-tight vat, lined with cement, kept at a temperature of $70^{\circ} \mathrm{F}$.; then washed in a revolving cylinder apparatus with plenty of cold water, and afterward exposed to the fumes of burning sulphur (sulphurous acid) in a wooden chamber. They are now squeezed to expel the moisture, and finally converted into soluble gelatine by water in earthen vessels, enclosed in steam cases. The fluid gelatine is purified by straining it at a temperature of $100^{\circ}$ or $120^{\circ} \mathrm{F}$. I have examined this patent gelatine, and found it to be remarkably good, and capable of forming a fine calf's foot jelly.

Very recently a very beautiful sparkling gelatine has been prepared under a patent granted to Messrs. J. \& G. Cox, of Edinburgh. By their process the substance is rendered perfectly pure, while it possesses a gelatinizing force superior even to isinglass. It makes a splendid calves' feet jelly and a milk-white blanc-mange. The patentees also prepare a semi-solid gelatine, resembling jujubes, which readily dissolves in warm water, as also in the mouth, and may be employed to make an extemporaneous jelly.

The gelatine of bones may be extracted best by the combined action of steam and a current of water trickling over their crushed fragments in a properly constructed apparatus. When the gelatine is to be used as an alimentary article, the bones ought to be quite fresh, well preserved in brine, or to be dried strongly by a stove. Bones are best crushed by passing them between grooved iron rolls. The cast-iron cylinders in which they are to be steamed, should be three times greater in length than in diameter. To obtain 1,000 rations of gelatinous soup daily, a charge 
of four cylinders is required; each being $3 \frac{1}{2}$ feet long, by 14 inches wide, capable of holding $70 \mathrm{lbs}$. of bones. These will yield each hour about 20 gallons of a strong jelly, and will require nearly one gallon of water in the form of steam, and 5 gallons of water to be passed through them in the liquid state. The 5 quarts of jelly produced hourly by each cylinder, proceeds from the 1 quart of steam-water and 4 quarts of percolating water.

The boiler should furnish steam of about $223^{\circ} \mathrm{Fahr}$., at a pressure of about 4 lbs. on the square inch.

In fig. $70, \mathrm{~A}, \mathrm{~B}, \mathrm{C}, \mathrm{D}$, represents a vertical section of the eylinder ; $\mathbf{C}, \mathrm{H}, \mathrm{I}, \mathbf{K}$, a

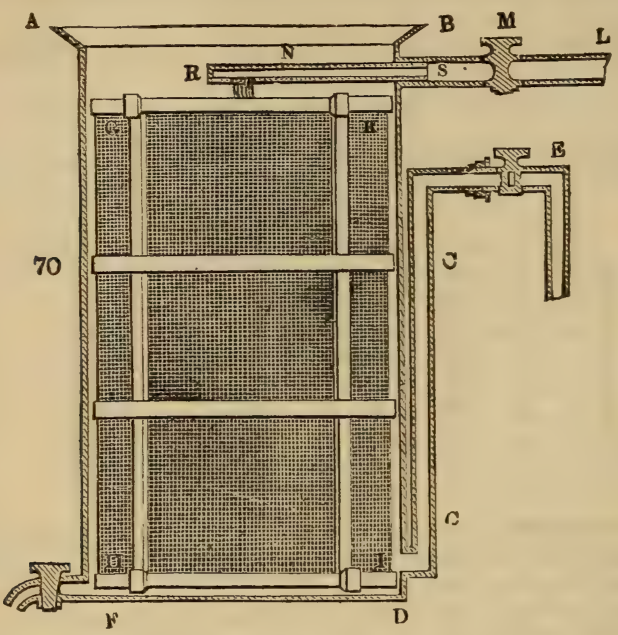

section of the basket or cage, as filled with the bruised bones, enclosed in the cylinder; E, C, C, the pipe which conducts the steam down to the bottom of the cylinder; L, S, 2 pipe for introducing water into the interior; $M$, a stopcock for regulating the quantity of water (according to the force of the steam pressure within the apparatus), which should be $3 \frac{1}{2}$ quarts per hour; $\mathrm{N}$ is a tube of tin plate fitting tightly into the part $S$, of the pipe $L$; it is shut at $R$, and perforated below with a hole; it is inserted in its place, after the cage full of bones has been introduced. Fig. 71 is an

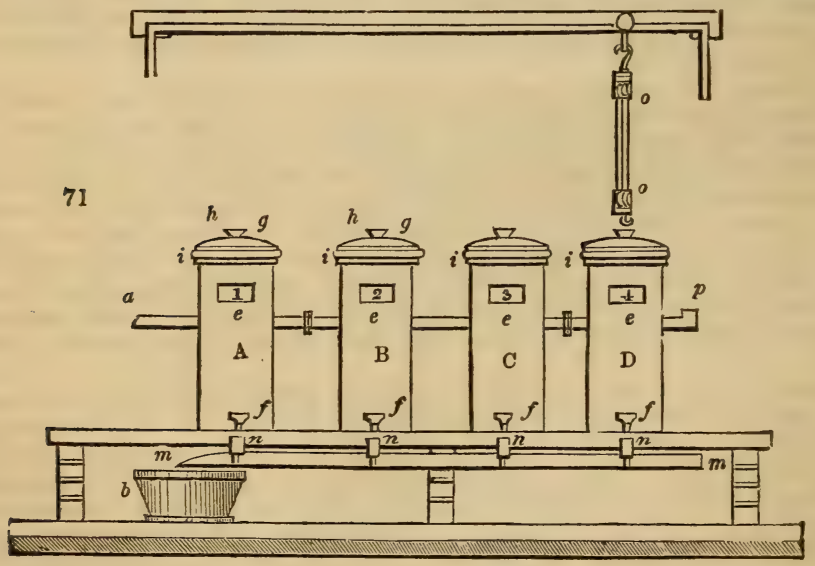

elevation of the apparatus. A, B, C, D, represent the four cylinders raised about 20 inches above the floor, and fixed in their seats by screws; $h, h$, are the lids; $g, g$, tubulures or valves in the lids; $i$, ring junction of the lid; $p$, a thermometer; $f, f$, stop-cocks for drawing off the jelly; $n, n$, small gutters of tin-plate; $m$, the 
general gutter of discharges into the cistern $b ; 0$, a block and tackle for hoisting the cageful of bones in and out. Fig. 72 is an end view of the apparatus; $a$, the main

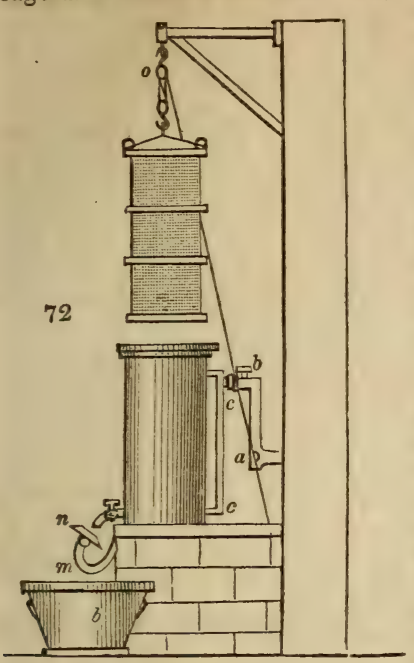
steam-pipe; $a, b, c, c$, branches that conduct the steam to the bottom of the cylinder; 0 , the tackle for raising the cage; $s$, stopeock; $n$, small gutter; $m$, main conduit; $b$, cistern of reception.

When a strong and pure jelly is wished for, the cylinder charged with the bones is to be wrapped in blanket stuff; and whenever the grease ceases to drop, the stopcock which admits the cold water is to be shut, as also that at the bottom of the cylinder, which is to be opened only at the end of every hour, and so little as to let the gelatinous solution run out, without allowing any of the steam to escape with it.

Butcher's meat contains on an average in 100 pounds, 24 of dry flesh, 56 of water, and 20 of bones. These 20 pounds ean furnish 6 pounds of alimentary substance in a dry state; whence it appears that, by the above means, one fourth more nutritious matter can be obtained than is usually got. I am aware that a keen dispute has been carried on for some time in Paris, between the partisans and adversaries of gelatine as an article of food. It is probable that both parties have pushed their arguments too far. Calf's-foot jelly is still deemed a nutritious article by the medical men of this country, at least, though it is not to be trusted to alone, but should have a due admixture or interchange of fibrine, albumine, caseum, \&c.

GILDING. See Electro-Metallurgy.

GLASS. Duty collected on it in the United kingdom: In 1831, 732,455l.; 1832, $751,448 l$.; 1833, 828,558l.; 1834, 916,822l.; 1835, 966,121l.; 1836, 933,281l.; 1837, 903,846l.; 1838, 879,859l.; 1839, 868,193l.; 1840, 965,967l.

Crystal glass is rapidly corroded by the sulphate of ammonia, at a heat of $600^{\circ} \mathrm{F}$.

GLOVE. See LEATHER.

GLUCOSE, the name given to grape and starch sugar by $M$. Dumas.

GROWAN. The name given by the Cornish miners to granite, and to rocks of like structure.

GUANO. This extraordinary excrementitious deposite of certain sea-fowls, which occurs in immense quantities upon some parts of the coasts of Peru, Bolivia, and Africa, has lately become an object of great commercial enterprise, and of intense interest to our agricultural world. Four or five years ago it was exhibited and talked of merely as a natural curiosity. No one could then have imagined that in a short period it would be imported from the coasts of the Pacific in such abundance, and at such a moderate price, as to cheer by its fertilizing powers the languid and depressed spirits of the farmers throughout the United Kingdom. Such, however, is now the result, as attested by the concurring reports of almost all the agricultural societies of Great Britain and Ireland. No less than 28,500 tons of guano have been already imported from Peru and Bolivia, 1,500 from Chili, and 8,000 from Africa, altogether 38,000 tons, while more is on the way. The store of it, laid up from time immemorial in the above localities, seems to be quite inexhaustible; especially since it is receiving constant accessions from myriads of cormorants, cranes, \&c.

Having been much occupied with the chemical analyses of guano during the last two years for Messrs. Gibbs, of London, and Messrs. Myers, of Liverpool, who are the co-agents of the Peruvian and Bolivian governments, I have enjored favorabie opportunities of examining samples of every description, and hope to show that many of the analyses of guano hitherto published have been made upon specimens not in their normal or sound state, like the best imported by the above houses from Chincha and Bolivia, but in a certain state of eremucausis and decay.

Huano, in the language of Peru, signifies dung; a word spelt by the Spaniards guano. The natives have employed it as a manure from the remotest ages, and have by its means given fertility to the otherwise u.ıproductive sandy soils along their coasts. While Peru was governed by its native incas, the birds were protected from violence by severe laws. The punishment of death was decreed to persons who dared to land on the guaniferous islands during the breeding period of the birds, and to all persons who destroyed them at any time. Overseers were appointed by the government to take care of the 
guano districts, and to assign to each claimant his due share of the precious dung. The celebrated Baron Von Humboldt first brought specimens to Europe in 1804, which he sent for examination to Foureroy, Vauquelin, and Klaproth, the best analytical chemists of the day; and he spoke of it in the following terms : "The guano is deposited in layers of 50 or 60 feet thick upon the granite of many of the South sea islands off the coast of Peru. During 300 years the coast birds have deposited guano only a few lines in thickness. This shows how great must have been the number of birds, and how many centuries must have passed over in order to form the present guano-beds." The strata have undergone many changes, according to the length of time they have been deposited. Here and there they are covered with silicious sand, and have thue been protected from the influence of the weather; but in other places, they have lain open to the action of light, air, and water, which have produced important changes upon them. Fresh guano is of a whitish or very pale drab color, but it becomes progressively browner and browner by the joint influence of the above three atmospherical agents. Only one guano examined by Fourcroy and Vauquelin was found to contain a fourth of its weight of uric acid combined with ammonia, whence that appears to have been well selected by Baron Von Humboldt. They also found phosphates of ammonia, of lime, with urate and oxalate of amwunia, and some other constituents of little value in agriculture. Klaproth's analysis reported 16 per cent. of urate of ammonia, no less than 12.75 of oxalate of lime, 10 of phosphate of lime, 32 of clay and sand, with 28.75 of water and indeterminate organic matter. From the great proportion of slay and sand, Klaproth's sample of guano was obviously not genuine. I have met with no specimen of Peruvian guano that contained any apprecrable quantity of clay, and none that contained above 4 or 5 per cent. of silicious sand.

To Mr. Bland, of the firm of Messrs. Myers and Co., I am indebted for the following valuable information :-

The Chincha islands, which afford the best Peruvian guano, are three in number, and lie in one line from north to south, about half a mile apart. Each island is from five to six miles in circumference, and consists of granite covered with guano in some places to a height of 200 feet, in successive horizontal strata, each stratum being from 3 to 10 inches thick, and varying in color from light to dark brown. No earthy matter whatever is mixed with this vast mass of excrement. At Mr. Bland's visit to these islands in 1812, he observed a perpendicular surface of upward of 100 feet of perfectly uniform aspect from top to bottom. In some parts of these islands, however, the deposite does not exceed 3 or 4 feet in thickness. In several plares, where the surface of the guano is 100 feet or more above the level of the sea, it is strewed here and there with masses of granite, like those from the Alpine mountains, which are met with on the slopes of the Jura chain. These seem to indicate an ancient formation for the guano, and terraqueous convulsions since that period. No such granite masses are found imbedded within the guano, but only skeletons of birds.

The good preservation of the Chincha guano is to be ascribed to the absence of rain; which rarely, if ever, falls between the latitude of $14^{\circ}$ south, where these islands lie, about 10 miles from the main land, and the latitude of Paquica, on the island of Bolivia, in $21^{\circ} \mathrm{S}$. L. By far the soundest cargoes of guano which $\mathrm{I}$ have analysed have come from Chincha and Bolivia. Beyond these limits of latitude where rain falls in greater or less abundance, the guano is of less value-and what has been imported from Chili has been found by me far advanced in decay-most of the ammonia and azotised animal substances having been decomposed by moisture, and dissipated in the air (by the eremacausis of Liebig), leaving phosphate of lime largely to predominate along with effete organic matter. The range of the American coast from which the guano is taken must therefore be well considered; and should not extend much beyond the Chıncha islands as the northern limit, and Paquica, in Bolivia, as the southern.

The relative estimation of guano and nitrate of soda among the Peruvians is well shown by the following fact communicated to me by Mr. Bland: "Near the coast of Peru, about 45 miles from Iquique (the shipping port of guano) there is the chief deposite of nitrate of soda. The farmers, who collect and purify this natural product, carry it to the place of shipment, and always require to be paid in return with an equivalent quantity of guano, with which they manure their land, to the exclusion of the far cheaper nitrate of soda. We can not be surprised at this preference, when we learn that in the valley of Chancay, about 40 miles distant from Lima, the soil produces, when farmed with irrigation in the natural way, a return upon maize of only 15 for 1 ; whereas, with the aid of guano, it produces 500 for 1 ! Hence the Peruvian proverb: Huano, though no saint, works many miracles.

In the pamphlet recently published by Messrs. Gibbs and Myers, intitled "Peruvian and Bolivian Guano, its nature, properties, and results," we have a very interesting view of the best established facts with regard to its operation and effects upon every variety of soil, and in every variety of cirı 'umstance, as ascertained by the most 
intelligent agriculturists of the United Kingdom. The general conclusion that may be fairly deduced from the whole evidence is, that good guano will, under judicious application, increase the crops of grain, turnips, potatoes, and grass, by about 33 per cent.; and with its present price of $10 \mathrm{l}$. per ton, at a cost considerably under the average cost of all other manures, whether farm-yard dung and composts, or artificial compounds. Guano is, moreover, peculiarly adapted to horticultural and floricultural improvement, by its relative cleanliness and facility of application.

The following observations upon guano, by Dr. Von Martius, of Munich, addressed to the agricultural society of Bavaria, deserve attention. Among animal manures, says he. it clearly claims the first place. It is uncommonly rich in ammoniacal salts, which act very favorable on vegetation. The ease with which these salts are decomposed, and exhale their ammonia into the air, is by him assigned as the reason why plants manured with guano generally present early in the morning accumulations of dew on the points of their leaves. The guano absorbs the atmospheric vapor, as well as carbonic acid; whereby it becomes so valuable a manure in dry barren regions. If we compare guano with other excrementitious manures, we shall find it far preferable to those afforded by man or other mammalia, which do not generally contain more than 20 per cent. of food that can be appropriated by plants. It is therefore five times better than night-soil, and also very superior to the French poudrette, which, being dried nightsoil, loses, through putrefaction and evaporation, the greater proportion of its ammoniacal elements. In birds, the excretions both of the kidneys and intestines are contained in the cloaca; whereby the volatile elements of the former get combined with the more fixed components of the latter. The guano is also a richer manure, on account of its being produced by sea-fowl, which live entirely on fish, without admixture of vegetable matter. The exposure also of the guano as soon as deposited to the heat of a tropical sun, in a rainless climate, prevents the components from fermenting, and mummifies them, so to speak, immediately into a concrete substance not susceptible of decomposition till it gets moisture; whereas the dung of our dove-cotes suffers a considerable loss by exposure to our humid atmosphere. But in their action on vegetation, and in their chemical composition, these two bird excrements are analogous. Davy found in fresh dove-cote manure 23 parts in 100 soluble in water, which yielded abundance of carbonate of ammonia by distillation, and left carbonaceous matter, saline matter, principally common salt, and carbonate of lime as a residuum. Pigeons' dung readily ferments, but after fermentation afforded only 8 per cent. of soluble matter, which gave proportionably less carbonate of ammonia in distillation than the dung recently voided. Dr. Von Martius proceeds to compare the proportion of soluble salts in guano and pigeons' dung, and thinks that by that comparison alone he can establish the superiority of the former; but he should have considered that the insoluble urate of ammonia, which is so powerful and copious a constituent of good guano, and is present in much smaller proportion in pigeons' dung, is sufficient of itself to turn the balance greatly in favor of the Peruvian manure. His general estimate, however, that the manuring power of genuine guano is four times greater than that of pigeons' dung, is probably not wide of the truth. Besides the above-mentioned constituents, guano derives no small portion of its fertilizing virtue from the great store of phosphoric acid which it contains, in various states of saline combination, with lime, magnesia, and ammonia. Of all the principles furnished to plants by the soil, the phosphates are, according to Liebig, the most important. They afford, so to speak, the bones and sinews of vegetable bodies, while ammonia supplies them with their indispensable element, azote. Their carbon, hydrogen, and oxygen, are derived from the air and water. Those products of vegetation which are most nutritious to man and herbiverous animals, such as bread-corn, beans, peas, and lentils, contain the largest proportion of phosphates. The ashes of these vegetable substances afford no alkaline carbonates. A soil in which phosphates are not present, is totally incapable of producing the above cereals. Agreeably to these views, Liebig believes that the importation of $1 \mathrm{cwt}$. of guano is equivalent to the importation of 8 cwts. of wheat ; so that $1 \mathrm{cwt}$. of that manure assumes, with due culture, the form of $8 \mathrm{cwts}$. of substantial food for man.

Since all these testimonies concur to place this remarkable excrementitious product in such high estimation, it becomes a paramount duty of the chemist to investigate its composition, and to discover certain means of distinguishing what may be termed the sound or normal state of guano, from the decomposed, decayed, and effete condition. The analysis by Fourcroy and Vauquelin of a sample of guano presented to them by Baron Von Humboldt, gave the following composition in 100 parts :-

Urate of ammonia

Oxalate of ammonia

Oxalate of lime

Phosphate of ammonia .

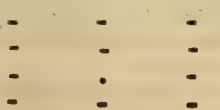

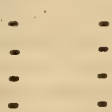

$9 \cdot 0$ 


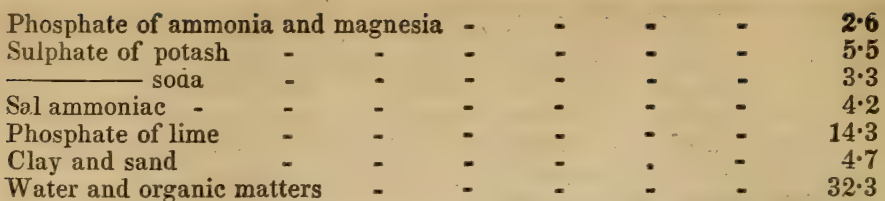

How different are these constituents from those assigned by Klaproth-a no less skılful analyst than the French chemists! and how much this difference shows not only the complexity of the substance, but its very variable nature!

The general results of an analysis by Professor Johnston, published in his paper on guano, in the $3 \mathrm{~d}$ part of the $2 \mathrm{~d}$ vol. of the Journal of the Royal Agricultural Society of England, are as follows :-

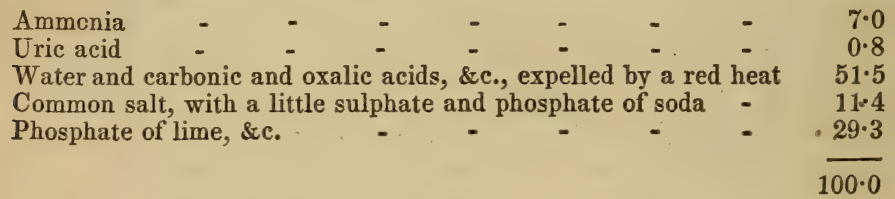

The specimen of guano represented by this analysis must have been far advanced in decomposition, as shown by the very scanty portion of uric acid; and must have been originally impure (spurious?) from the large proportion of common salt, of which I have not found above 4 or 5 per cent. in any of the genuine guanos which I have had os casion to analyze. In another sample, Professor Johnston found 44.4 of phosphate of lime, with a little phosphate of magnesia, and carbonate of lime. These results resemble, to a certain degree, those which I have obtained in analyzing several samples of Chilize and African guanos, especially in the predominance of the earthy phosphates. The propc tion of ammonia which can be extracted by the action of hydrate of soda and quicklime, at an elevated temperature, is the surest criterion of the soundness of the guano; for $\mathrm{h}$ this process we obtain not only the ready-formed ammonia, from its several salise compounds, but also the ammonia producible from its uric acid, and undefined animal matter. These two latter quantities have been hitherto too little regarded by me ut analysts, though they constitute the most durable fund of azote for the nutrition of plants. Uric acid, and urate of ammonia, which contains 1011 ths of uric acid, being both nearly insoluble in water, and fixed at ordinary temperatures, continue to give oat progessively to plants in the soil, the azote, of which they contain fully one third of their weight. Under the influence of oxygen and a certain temperature, uric acid passes through a very remarkable series of transformations; producing allantoin, urea, aud oxalic acid, which eventually becomes carbonic acid. These changes are producible immediately by the action of boiling water and peroxide of lead. From these metamorphose, we can readily understand how so much oxalate of ammonia and of lime is reported in many analyses of guano, though none, I believe, is to be found in the normal state, as it is occasionally imported from the Chincha islands and Bolivia; nor were any oxalates found in the dung of the gannet, as analyzed by Dr. Wollaston, or of the sea-eagle, according to the following analysis of Coindet: ammonia, 9.21 per cent.; uric acid, $84 \cdot 65$; phosphate of lime, $6 \cdot 13=100$. The Peruvian sea-fowl, by feeding exclusively on fish, would seem to swallow a large proportion of earthy phosphates; since, in the purest guano that has come in my way, I have found these salts to amount to from 10 to 15 per cent.

Dr. Von Martius proposes to use the degree of solubility of the guano in water as a good criterion of its quality; but this is a most fallacious test. Sound guano contains from 15 to 25 per cent. of insoluble urate of ammonia ; nearly as much undefined animal matter, along with from 15 to 20 of earthy phosphates, leaving no more than 50 or 55 per cent. of soluble matter, exclusive of moisture; whereas decayed guano yields often 60 or 70 per cent. of its weight to water, in consequence of the uric acid and animal matter being wasted away, and the large portion of moisture in it, the latter amounting very often to from 25 to 35 per cent. The good Peruvian guano does not lose more than from 7 to 9 per cent. by drying, even at a heat of $212^{\circ} \mathrm{F}$.; and this loss necessarily ncludes a little ammonia. Each analysis of guano executed for the information of the farmer should exhibit definitely and accurately to at least 1 per cent. :-

1. The proportion of actual ammonia.

2. The proportion of ammonia producible also from the uric acid and azotized animal matter present; and which may be called the potential ammonia. This is a 
most valuable product, which is, however, to be obtained only from well-preserved dry guano.

3. The proportion of uric acid, to which, if 110 th of the weight be added, the quantity of urate of ammonia is given.

4. The proportion of the phosphates of lime and magnesia.

5. The proportion of fixed alkaline salts, distinguishing the potash from the soda salts ; the former being more valuable, and less readily obtainable, than the latter can be ${ }^{2} y$ the use of common salt. Wheat, peas, rye, and potatoes, require for their successfui cultivation, a soil containing alkaline salts, especially those of potash.

6. The proportion of sandy or other earthy matter, which, in genuine guano, carefully collected, never exceeds 2 per cent. and that is silica.

7 . The proportion of water, separable by the heat of $212^{\circ} \mathrm{F}$.

The farmer should never purchase guano except its composition in the preceding particulars be warranted by the analysis of a competent chemist. He should cork up in a bottle a half-pound sample of each kind of guano that he buys; and if his crop shall disappoint reasonable expectation, he should cause the samples to be analyzed; and should the result not correspond to the analysis exhibited at the sale, he is fairly entitled to damages for the loss of his labor, rent, crop, \&c. The necessity of following this advice will appear on considering the delusive if not utterly false analyses, under which cargoes of guano have been too often sold. In a recent case which came under my cognizance, in consequence of having been employed professionally to analyze the identical cargo, I found the guano to be nearly rotten and effete; containing altogether only $2 \frac{1}{2}$ per cent. of ammonia, $\frac{1}{2}$ per cent. of urate of ammonia, nearly 9 of sea salt, 24 of water, and $45 \frac{1}{2}$ of earthy phosphates. Now, this large cargo, of many hundred tons, fetched a high price at a public sale, under the exhibition of the following analysis by a chemist of some note :-

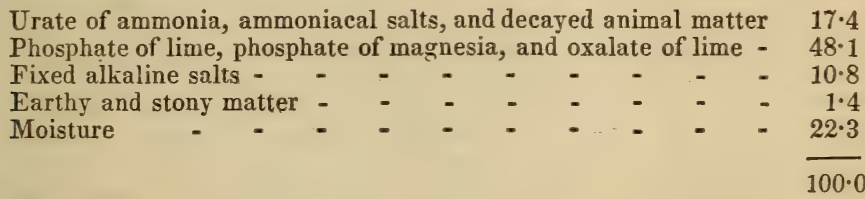

The purchasers, I was told by the broker, bought it readily under a conviction that the guano contained $\mathbf{1 7 \cdot 4}$ of ammonia, though the proportion of ammonia is not stated, but merely mystified, and adroitly confounded with the decayed animal matter.

By the following hypothetical analysis, much guano has been well sold :-

" Bone earth, 35 ; lithic acid, \&c., 15 ; carbonate of ammonia, 14 ; organic matter, 36 $=100 . "$ I am quite certain that no sample of guano can contain 14 per cent. of carbonate of ammonia-a very volatile salt. We shall see presently the state of combination in which the ammonia exists. It may contain at the utmost 4 or 5 per cent. of the carbonate; but such guano must have been acted upon powerfully by humidity, and wiil therefore contain little or no uric acid.

In the very elaborate examination of guano by T. Oellacher, apothecary at Innsbruck, published in a recent number of Buchner's Repertorium of Pharmacy, it is said, that if a glass rod dipped into muriatic acid be held over guano, strong fumes are developed; and the solution of guano has an alkaline reaction with litmus-paper. These phenomena evidently indicate the presence of carbonate of ammonia, and of course a partially decomposed guano; for sound Chincha and Bolivian guanos have an acid reaction, proceeding from the predominance of phosphoric acid. Farmers frequently judge of the goodness of guano by the strength of the ammoniacal odor; but in this judgment they may egregiously err, for the soundest guano has no smell of ammonia whatever; and it begins to give out that smell only when it is more or less decomposed and wasted.

Oellacher could find no evidence of urea in his guano; I have obtained fully 5 per cent. of this substance from good Peruvian guano.

I shall now describe my own system of analysis :-

1. In every case I determine, first of all, the specific gravity of the guano; which I take by means of spirits of turpentine, with a peculiar instrument contrived to render the process easy and precise. If it exceeds 1.75 in density, water being 1.0 , it must contain sandy impurities, or has an excess of earthy phosphates, and a defect of azotized animal matter.

2. I triturate and digest 200 grains of it with distilled water, filter, dry the insoluble matter, and weigh it.

3. The above solution, diffused in 2,000 gr. measures, is examined as to its specific gravity, and then with test paper, to see whether it be acid or alkaline. 
4. One half of this zolution is distilled along with slaked lime in a matrass connected with a small quintuple globe condenser, containing distilled water, and immersed in a basin of the same. As the condensing apparatus terminates in a water-trap, no part of the ammonia can be lost; and it is all afterward estimated by a peculiar meter, whose indications make manifest one hundredth part of a grain.

5. The other half of the solution is mixed with some nitric acid, and divided into 3 equal portions.

$a$, the first portion, is treated with nitrate of barytes, and the resulting sulphate of barytes is collected, ignited, and weighed.

$b$, the second portion, is treated with nitrate of silver, and the resulting chloride of silver ignited and weighed.

$c$, the third portion, has a certain measure of a definite solution of ferric nitrate mixed with it, and then ammonia in excess. From the weight of the precipitated subphosphate of iron after ignition, the known amount of oxide used being deducted, the quantity of phosphoric acid in the soluble portion of the guano becomes known.

$d$, the three above portions are now mixed, freed by a few drops of dilute sulphuric and hydrochloric acids from any barytes and silver left in them, and then tested by nitrate of lime for oxalate of ammonia. The quantity of oxalate of lime obtained, determines that point.

6. The last liquor filtered, being freed from any residuary particles of lime by oxalate of ammonia, is evaporated to dryness and ignited, to obtain the fixed alkaline matter. This being weighed, is then dissolved in a little water, neutralized with acid, and treated with soda-chloride of platinum. From the quantity of potash-chloride of platinum, which precipitates, after being filtered, dried, and weighed, the amount of potash present is deducted-the rest is soda. These bases may be assigned to the sulphuric, hydro-chloric, and phosphoric acids, in proportions corresponding to their respective affinities.

7. The proportion of organic matter in the above solution of guano, is determined directly by evaporating a certain portion of it to dryness, and igniting. The loss of weight, minus the ammonia and oxalic acid, represents the amount of organic matter.

8. A second portion of a solution of the guano is evaporated to dryness by a ger:tle steam heat, weighed, enclosed in a stout well-closed phial along with alcohol of $0 \cdot 825$, and heated to $212^{\circ}$. After cooling, the alcoholic solution is decanted or filtered clear, evaporated to dryness by a gentle $\mathrm{h}$ at, and weighed. This is urea, which may be tested by its conversion into carbonate of a monia, when heated in a test tube or small retort. In this way, I have obtained from Bo .vian guano, 5 per cent. of urea; a certain proof of its entire soundness.

9. Analysis of the insoluble matter. One third of it is digested with heat in abundance of Borax-water, containing $\frac{1}{100}$ of the salt, filtered, and the filter dried by a steam heat. The loss of weight indicates the amount of uric acid, which is verified by supersaturating the filtrate with acetic or hydrochloric acid, thus precipitating the uric acid, throwing it upon a filter, drying, and weighing it. This weight should nearly agree with the above loss of weight, the small difference being due to soluble organic matter, sometimes called geine and ulmic acid. The uric acid is evidenced, 1 , by its specific gravity, which I find to be only $1 \cdot 25$, as also that of the urate of ammonia; 2 , by its affording fine purple murexide when heated in a capsule along with nitric acid, and then exposed to the vapor of ammonia from a feather lield over it; 3 , by its dissipation when heated, without emitting an empyreumatic odor.

10. Another third of the solid matter is distilled along with half its weight of slaked lime, and 10 times its weight of water, in the apparatus already described, and the ammonia obtained from it estimated.

11. The remaining third having been ignited, is digested with a gentle heat in weak hydrochloric acid, and the undissolved silica and alumina washed on a filter, dried, and weighed. To the hydrochloric solution, dilute sulphuric acid is added, and the mixture is heated till all the hydrochloric acid be expelled, with the greater part of the water. Alcohol of 0.850 is now poured upon the pasty residuum, and the whole, after being well stirred, is thrown upon a filter. The phosphoric acid passes through, as also the magnesia in union with sulphuric acid. The sulphate of lime, which is quite insoluble in spirits of wine, being washed with them, is dried, ignited, and weighed. From the weight of sulphate of lime, the quantity of phosphate of that earth, that was present, becomes known.

12. Ammonia in excess is now added to the filtrate, which throws down the granular phosphate of ammonia and magnesia. After washing and drying this powder at a heat of $150^{\circ}$, its weight denotes the quantity of that compound in the guano.

13. To the filtered liquor (of 12), if a little ammonia be added, and then muriate of magnesia be slowly dropped in, phosphate of ammonia and magnesia will precipitate, from the amount of which the quantity of phosphoric acid may be estimated.

14. The proportion of oxalate of lime is determined by igniting the washed residuum 
(of 9) and placing it in an apparatus for estimating the quantity of carbonic acid given off in dissolving carbonate of lime. The apparatus, either fig. 1 or 2 , described in my littie Treatise on Alkalimetry, will serve that purpese well. I have rarely obtained more than $\frac{1}{2} \mathrm{gr}$. of carbonic acid from the insoluble residuuin of $100 \mathrm{gr}$. of good guano, and that corresponds to less than $1 \frac{1}{2}$ per cent. of oxalate of lime in the guano. Sometimes no effervescence at all is to be perceived in treating the washed residuum with acid after ignition.

15. The carbonate of ammonia in guano is readily determined by filtering the solution of it in cold water, and neutralizing the ammonia with a test or alkalimetrical acid. (See the Treatise on Alkalimetry, above referred to.)

16. Besides the above series of operations, the following researches must be made to complete our knowledge of guano. The insoluble residuum (of 10 ) which has been deprived by two successive operations of its uric acid and ammonia, may contain azotized organic matter. It is to be therefore-well dried, mixed with 5 times its weight of the usual mixture of hydrate of soda and quicklime, and subjected to gentle ignition in a glass or iron tube closed at one end, and connected at the other with an ammonia condensing apparatus. The amount of ammonia being estimated by a proper ammonia meter, represents the quantity of azote, allowing 14 of this element for 17 of ammonia, being the potential ammonia corresponding to the undefined animal matter. In a sample of Peruvian guano I obtained 5 per cent. of ammonia from this source.

17. The whole quantity of ammonia producible from guano is to be determined by gently igniting $25 \mathrm{gr}$. of it well dried, and mixed with ten times its weight of the mixture of hydrate of soda and quicklime ( 2 parts of the latter to 1 of the former). The ammonia disengaged is condensed and measured, as described above.

18. The ready-formed ammonia is in all cases determined by distilling a mixture of $100 \mathrm{gr}$. of it with $50 \mathrm{gr}$. of slaked lime, condensing the disengaged ammonia, and estimating it exactly by the meter.

19. The relation of the combustible and volatile to the incombustible and fixed constituents of guano, is determined by igniting $100 \mathrm{gr}$. of it in a poised platinum capsule. The loss of weight denotes the amount of combustible and volatile matter, including the moisture, which is known from a previous experiment.

20. The insoluble matter is digested in hot water, thrown upon a filter, dried, and weighed. The loss of weight is due to the fixed alkaline salts, which, after concentrating their solutions, are investigated by appropriate tests : 1 , nitrate of barytes for the sulphates; 2, nitrate of silver for the chlorides and sulphates; and 3, soda-chloride of platinum, for distinguishing the potash from the soda salts.

21. The insoluble matter (of 20 ) is digested with heat in dilute nitric or hydrochloric acid, and the whole thrown upon a filter. The silica which remains on the filter is washed, ignited, and weighed. The lime, magnesia, and phosphoric acid, may be de'ermined as already pointed out.

22. I have endeavored to ascertain if muriate of ammonia be present in guano, by evaporating its watery solution to dryness, and subliming the residuum, but $I$ have never obtained a satisfactory portion of sal ammoniac; and therefore $I$ am inclined to think there is little of it. The quantity of chlorine to be obtained from guano is too ineonsiderable to lead to a suspicion of its presence, except in combination with sodium and potassium. Phosphate of soda is also a doubtful product-but if present, it may be obtained from the saline matter (of 20), by acidulating it with nitric acid; precipitating first with nitrate of barytes, next with nitrate of silver, taking care to use no excess of these two re-agents, then supersaturating the residuum with ammonia, and adding acetate of magnesia, when the characteristic double phosphate of this earth should fall, in case phosphate of soda be present.

By the preceding train of researche's, all the constituents of this complex product may be exactly disentangled and estimated; but they manifestly require much care, patience, time, and dexterity, and also a delicate balance, particularly in using the appropriate apparatus for generating the potential ammonia, and for measuring the whole of this volatile substance separated in the several steps of the process. It may be easily irnagined how little confidence can be reposed in many of the analyses of guano, framed, I fear, too often with the view of promoting the sale of an indifferent or even spurious article of commerce.

A. I shall now give in detail my analytical results upon three different samples of a good South American guano; and next the general results upon three samples of African and Chilian guanos:-

I. Guano from Bolivia, imported by the Mary and Anne. This sample was taken by myself, as an average out of several bags in the lighter, before the cargo was landed. Pale yellow brown color, dry, partly pulverulent, partly concreted, in small lumps, with a few small fragments of granite interspersed, and which, being obvious, were separated prior to the analysis. Specific gravity of the pulverulent portion without 
the granite, 1.60 ; of the concretions, $1 \cdot 66$; mean $1 \cdot 63$. Water digested on the former portion is neutral to litmus, that on the latter is faintly acid.

2. 100 parts lose 6.5 by the heat of boiling water, and exhale no ammonia. When digested and triturated with cold water, 30.5 parts dissolve, and 69.5 are obtained after drying, at $212^{\circ} \mathrm{F}$. Of those 30.5 parts, 6.5 are therefore water, easily separable, and 24.5 parts are solid matter.

3. 100 parts, mixed with 9 times their weight of water, and 50 of lime, being distilled in an alembic connected with the five-globe condenser, \&c., afforded exactly $4 \cdot 2$ of ammonia. 20 grains in fine powder, along with 200 of a mixture, consisting of 2 parts of dry lime and 1 of hydrate of soda, were gently ignited in a combustion-tube connected with the ammonia-condensing apparatus, and they produced 4.25 grains of ammoniaequivalent to 21.25 from 100 grains of the guano. Thus only 4.2 per cent. of ammonia were ready formed; while 17.05 lurked, so to speak, in their azotized elements.

From its aspect, and its want of ammoniacal odor, this guano, the first cargo received from Bolivia, was imagined by the importers to be of bad quality; and, accordingly, my very favorable report of its analysis surprised them not a little, and rather unsettled the little faith they at that time (January, 1843) had in chemistry. But about a fortnight after the date of my report they received a letter from Peru, apprizing them of the excellence of that cargo of Bolivian guano, and of its being prized by tne Americans, as possessing fertilizing powers in a pre-eminent degree. I consider this guano, therefore, as a type of the substance in its best state.

II. The soluble matter was analyzed, in the manner already detailed, and was found to consist of-

$\begin{array}{lllll}\text { 1. Urea - } & - & - & - & 5 \cdot 00 \\ \text { 2. Sulphate of potash } & - & - & - & 7 \cdot 90 \\ \text { 3. Chloride of sodium } & - & - & 5 \cdot 00 \\ \text { 4. Biphosphate of ammonia } & - & - & 5 \cdot 50 \\ \text { 5. Oxalate of ammonia } & - & - & 0 \cdot 60 \\ & & & \end{array}$

In these ammoniacal salts there are only 1.65 parts of ammonia; but I obtained 2.55 grains in distilling the soluble matter of 100 grains of the guano. The remaining 0.9 parts, therefore, must have proceeded from the partial decomposition of the urea during the long ebullition necessary to extract every particle of ammonia, in distilling the guano along with lime.

III. The insoluble matter $=69 \cdot 5$ parts, was found to consist of-

1. Silica - $\quad-\quad-\quad-\quad-\quad-\quad-\quad-2.25$

2. Subphosphate of lime - - $\quad-\quad-\quad-\quad-9.00$

3. Phosphate of magnesia and ammonia - - $\quad-\quad 1.25$

4. Urate of ammonia - - - - - $\quad 15.27$

5. Undefined azotized organic matter, affording, with the 14 parts of uric acid, by ignition with hydrate of soda, 17.05 parts of ammonia

This result as to the large proportion of organic matter in the dried insoluble residuum was verified by igniting a given quantity of it, when it was found to lose, out of 69.5 parts, 57 ; corresponding to the 15.27 urate of ammonia, 41.73 of undefined organie matter, and 0.08 of ammonia, in the double magnesian phosphate. In the urate and double phosphate are 1.35 of ammonia, which, with the 2.55 , make 3.9 parts ; the other 0.3 parts may be traced to the urea.

As these results differ very considerably in many respects from those of the analyses made by respectable German chemists, I was careful to verify them by manifold variations of the process, as follows :-

1. The soluble matter, with acid reaction, of 100 parts of the lumps of the Bolivian guano, was examined by per-acetate of iron and ammonia, for phosphoric acid, and afforded 4 parts of it, which is more than had been found in the neutral pulverulent guano. After the phosphoric acid was separated by that method, chloride of calcium gave no cloud with the filtered liquor, proving that no oxalic acid was present in these nodules. The washed insoluble matter, when gently ignited, and treated with dilute nitric acid, afforded no effervescence whatever, and therefore showed that no oxalate of lime had been present, for it would have become a carbonate.

It is necessary to determine from time to time the quantity of ferric oxide in the 
acetate or nitrate, as it is liable to be deposited from the solution when this is kept for some time. If this point be not attended to, serious errors would be committed in the estimation of the phosphoric acid.

2. The quantity of uric acid was verified by several repetitions, and found to be 14 per cent.

3. The undefined organic matter, when deprived of the uric acid by prolonged digestion with weak borax, being subjected to ignition along with hydrate of soda, rielded the quantity of ammonia requisite to constitute the whole sum, that producible from the uric acid also being taken into account.

4. The little lumps of the guano afforded, by distillation along with quicklime, $5 \cdot 27$ per cent. of ready-formed ammonia, probably from the uric acid having been partially decomposed by the moisture which had caused them to concrete. It is a curious fact, that the solution of borax, from being of an alkaline, becomes of an acid reaction, after digestion with the Bolivian guano.

5. For distinguishing and separating the soda salts from those of potash, I tried the antimoniate of potash, according to Wackenroder's prescription, but I found reason to prefer very much the crystallized soda-chloride of platinum, for that purpose.

From another specimen of the Bolivian guano, I extracted 3.5 per ct. of the ammoniaphosphate of magnesia.

B. A sample of guano from the Chincha islands, of nearly the same light color as the preceding, and the same dryness, being an early importation of 250 tons in the present year, was subjected by me to a careful analysis.

1. The solution in water of this guano had an alkaline reaction from carbonate of ammonia, which, being neutralized by test acid, indicated 0.34 per cent. of ammonia, equivalent to about 1 of the smelling sesqui-carbonate.

2. Of this guano, 47 per cent. were soluble in water, and 53 per cent. remained, after drying at a heat of $212^{\circ} \mathrm{F}$. Of the above 47 parts, 8.5 were moisture in the guano.

3. The solution being acidulated with nitric acid, was treated with acetate of barytes, in a quantity equivalent to the sulphuric acid present, and it afforded 12 parts of sulphate of barytes. With the filtered liquor, 700 water grain measures of ferric acetate were mixed, and then ammonia in excess ; 18.5 parts of washed and ignited sub-phosphate of iron were obtained, from which deducting 8.8 parts present in the acetate, 9.7 remain as the quantity of phosphoric acid; but 9.7 of acid produce 13.25 of bi-phosphate of ammonia, which contain only 2.3 of ammonia, combined with 0.95 of water, or its elements. From the alkaline excess in the guano, there can be no doubt, however, that it contained the sub-phosphate ( frind in the urine of Carnivora), and not the bi-phosphate of that base. In this case, 9.7 of acid produce 14.32 of dry saline compound, containing 4.62 of ammonia, which, with the 0.34 of ammonia in the carbonate, constitute a sum of $4 \cdot 96$. To the liquor freed from the phosphate of iron, and acidulated with nitric acid, acetate of lime being added, 3.33 parts of oxalate of this base were obtained, which are equivalent to 3.23 oxalate ammonia, containing 0.89 of ammonia.

4. Nitrate of silver now produced from the filtered residual solution 8 parts of chloride, corresponding to nearly 3 of sal ammoniac, which contain nearly 0.95 of ammonia.

5. The 53 parts insoluble in water were digested with weak solution of borax at a boiling heat, thrown on a filter, and the uric acid being precipitated from the filtrate by means of a little hydrochloric acid, washed and dried, was found to weigh 13.5 parts. There were left on the filter 36.5 parts, dried at $212^{\circ} \mathrm{F}$., so that 3 parts of soluble organic matter had passed through the filter. These 36.5 parts lost by ignition only $9 \cdot 7$ parts in organic matter, became white, and afforded a very faint effervescence with hydro-chloric acid, showing that a very little oxalate of lime had been present. 1.25 parts of silica were left after the action of the acid. To the solution of the 26.55 parts, sulphuric acid was added, and the mixture being heated to expel the hydro-chloric acid and the excess of the sulphuric, the residuary matter was digested and washed with dilute alcohol, and thrown on a filter; the solution of magnesia passed through, while the salphate of lime remained. After ignition, this weighed $27 \cdot 5$ parts, equivalent to 22 of sub-phosphate of lime. On supersaturating the filtrate with ammonia, 4.5 parts of the magnesian ammonia phosphate were precipitated, containing 0.32 of ammonia. With the $13 \cdot 5$ parts of uric acid, $1 \cdot 23$ of ammonia had been originally combined, forming $14 \cdot 73$ of urate.

6. 25 grains of the dry guano afforded, by ignition in the combustion-tube along with 200 grains of the mixed lime and hydrate of soda, $4 \cdot 165$ of ammonia, which correspond to 16.66 in 100 parts of the dry, or to 15.244 in the natural state; leaving therefore 5 parts for the quantity of potential ammonia, or of ammonia producible from the de composition of its azotized organic matter. This guano is, therefore, well adapted to promote permanently the fertility of a soil. It yields besides to alcohol a notable quantity of urea, which I did not think it worth while to determine quantitively, and 
from which undoubtedly a portion of the ammonia proceeded, in the distillation with milk of lime.

7. 100 parts afforded by distillation with milk of lime, $10 \cdot 2$ of ammonia.

8. The total constituents of that guano, being tabulated, are-

I. Matter soluble in water

$47 \cdot 00$

consisting of-

1. Sulphate of potash, with a little sulphate of soda

2. Muriate of ammonia

3. Phosphate of ammonia

4. Sesqui-carbonate of ammonia

5. Sulphate of ammonia

6. Oxalate of ammonia

7. Water

8. Soluble organic matter and urea

=-

II. Matter insoluble in water consisting of -

1. Silica

2. Undefined organic matter

3. Urate of ammonia -

4. Oxalate of lime

5. Sub-phosphate of lime

6. Phosphate of magnesia and ammonia

$\begin{array}{ll}- & - \\ - & - \\ - & - \\ - & - \\ - & - \\ - & - \\ - & -\end{array}$

- $6 \cdot 00$

- $\quad 3.00 \quad 0.95$

- 14.32 4.62

- $1.00 \quad 0.34$

- $2.00 \quad 0.50$

- $3.23 \quad 0.89$

- $8 \cdot 50$

- 8.95

$47 \cdot 00$

$53 \cdot 00$

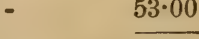

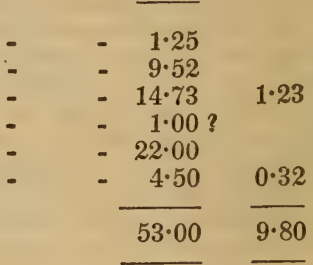

The remaining 1.25 of actual ammonia may be fairly traced to the partial decomposi tion of the urea during the distillation with lime; whereas the 5 per cent. of potential ammonia proceeded from the transforming decomposition of the uric acid.

C. Foliated guano, from Peru, in caked pieces, the layers very thin, parallel, and in. terspersed with white streaks. This guano was somewhat dense for a pure specimen, having a specific gravity of $1 \cdot 7$. The insoluble matter afforded by digestion with borax water, no less than 25.2 per ct. of pale yellow uric acid; 9 of other combustible organic matter, and 15 of eagrthy matter; consisting of silica, $3 \cdot 5$; phosphate of magnesia and ammonia, 6.5 ; and only 5 of sub-phosphate of lime or bone earth. It lost 10 per cent. when dried in a heat of $212^{\circ} \mathrm{F}$. The remaining 30.8 parts soluble in water, had a strong acid reaction, and afforded, by ferric acetate and ammonia, 6 of phosphoric acid, equivalent to 9.7 of crystallized bi-phosphate of ammonia, after acetate of barytes had separated the sulphuric acid. No less than 17 parts of chloride of silver were obtained, by precipitating with nitrate of silver the liquor filtered from the phosphate of iron, and acidulated with nitric acid. As the present is an accidental sample, and not an average of any importation, I did not prosecute the research further.

D. Chincha guano, of a somewhat darker color than the preceding, and alkaline reaction; specific gravity, $1 \cdot 62$. Digested with water and strained, 56.75 parts remained after drying it at $212^{\circ} \mathrm{F}$. The solution, evaporated and dried also at $212^{\circ}$, afforded 31.25 of saline matter. This saline mass being mixed with four fifths of its weight of slaked lime, nine times its weight of water, and distilled, afforded of ammonia 14.28 per cent. Some chemists have prescribed potash instead of lime, for separating the ammonia in distillation; but no person of intelligence who has made the experiment once will choose to repeat it, because the potash forms with the organic matter of the guaro a viscid compound, that froths up like a mass of soap-bubbles, and coming over with the vapors, obstructs and vitiates the result.

2. When dried altogether by a steam heat, 100 parts lost 12 in moisture; whereas by evaporating and drying the soluble matter by itself, the loss amounted to $16 \cdot 3$, no doubt by the dissipation of some of the ammoniacal salts ; for 100 parts of the entire guano afford, by distillation with quicklime, 9 parts of ammonia, and by the transforming decomposition with hydrate of soda and lime, $16 \cdot 25$, indicating $7 \cdot 25$ of potential ammonia, in addition to the 9 of ready formed. The insoluble matter of 100 parts afforded to borax-water a solution containing 16.5 of uric acid, corresponding to 18 of urate of ammonia. There remained on the filter, after drying it at $212^{\circ} \mathrm{F}$., only $33 \cdot 8$ parts; so that about 5 parts of soluble organic matter had passed through the filter in 
the borax water. These 33.8 consisted of subphosphate of lime 17 , magnesian phosphate of ammonia $5 \cdot 5$, silica $0 \cdot 7$, and combustible organic matter $10^{\circ} 6$.

'The ammonia in the soluble portion was in the state chiefly of phosphate; there was merely a faint trace of oxalate of ammonia.

E. African Guano.-Among the many samples of African guano which I have had occasion to analyze for the importers, none has contained any appreciable quantity of uric acid, or by consequence of potential ammonia. The best afforded me 10 per cent. of ready-formed ammonia, existing chiefly in the state of a phosphate, though they all contain carbonate of ammonia, and have of consequence an alkaline reaction. The said sample contained 21.5 of moisture, separable by a heat of $212^{\circ} \mathrm{F}$. Its specific gravity was so low as 1.57 , in consequence of the large proportion of moisture in it. It contained 23 per cent. of subphosphate of lime, 3 of magnesian phosphate of ammonia, 1 of silica, and 1.5 of alkaline sulphate and muriate. The remaining 50 parts consisted of decayed organic matter, with phosphate of ammonia, and a little carbonate, equivalent to half a grain of ammonia, which is the largest quantity in such guanos. Other African guanos have afforded from 24 to 36 of moisture, no uric acid; no potential ammonia; but decayed organic matter; from 5 to 7 of ready-formed ammonia in the state of phosphate, with a little carbonate; from 25 to 35 per cent. of subphosphate of lime; 5 or 6 of the magnesian phosphate of ammonia; more or less oxalates from the decomposition of the uric acid, and 3 to 5 per cent. of fixed alkaline salts.

F. The Chilian Guano gathered on the coast, already adverted to, contained a remarkable proportion of common salt, derived probably from the sea spray.

The following is the general report of the chemical examination of several samples of guano, which I made for Messrs. Gibbs of London, and Messrs. Myers of Liverpool :-

"In these various analyses, performed with the greatest care, and with the aid of the most complete apparatus for both inorganic and organic analysis, my attention has been directed, not only to the constituents of the guano which act as an immediate manure, but to those which are admitted by practical farmers to impart durable fertility to the grounds. The admirable researches of Professor Liebig have demonstrated that AzotE, the indispensable element of the nourishment of plants, and especially of wheat and others abounding in gluten (an azotized product), must be presented to them in the state of ammonia, yet not altogether ammonia in the pure or saline form, for, as such, it is too readily evaporated or washed away; but in the dormant, or as one may say, in the potential condition in contradistinction from the actual. Genuine Peruvian and Bolivian guanos, like those which I have minutely analyzed, surpass very far all other species of manure, whether natural or artificial, in the quantity of potential ammonia, and, therefore, in the permanency of their action upon the roots of plants, while, in consequence of the ample store of actuol ammonia which they contain ready formed, they are qualified to give immediate vigor to vegetation. Urate of ammonia constitutes a considerable portion of the azotized organic matter in well-preserved guano; it is nearly insoluble in water, not at all volatile, and is capable of yielding to the soil, by its slow decomposition, nearly one third of its weight of ammonia. No other manure can rival this animal saline compound. One of the said samples of guano afforded me no less than 17 per cent. of potential ammonia, besides $4 \frac{1}{2}$ per cent. of the actual or ready formed; others from 7 to 8 per cent. of ammonia in each of these states respectively. These guanos which I have examined are the mere excrement of birds, and are quite free from the sand, earth, clay, and common salt, reported in the analyses of some guanos, and one of which (sand) to the amount of 30 per cent. I found myself in a sample of guano from Chile.

"The Peruvian guano, moreover, contains from 10 to 25 per cent. of phosphate of lime, the same substance as bone-earth, but elaborated by the birds into a pulpy consistence, which, while it continues insoluble in water, has been thereby rendered more readily absorbable and digestible (so to speak) by the roots of plants. I have therefore no doubt, that by the judicious application of these genuine guanos, mixed with twice or thrice their weight of a marly or calcareous soil, to convert their phosphate of ammonia into phosphate of lime and carbonate of ammonia, as also to dilute all their armmoniacal compounds-such crops will be produced, even un sterle lands, as the farmer has never raised upon the most improved soil by the best ordinary manure. To the West India planter, guano will prove the greatest boon, since it condenses in a portable and inoffensive shape the means of restoring fertility to his exhausted canefields, a benefit it has long conferred on the poorest districts of Peru.

"I respectfully observe, that no analysis of guano hitherto $\mathrm{n}$ ade public at all exhibits the value of tne cargoes referred to above, while none gives the quantity of ammonia dormant in the azotized animal matter of the birds' dning, which, called into activity with the seeds in the soil, becomes the most valuable of its constituents, as a source of perennial fertility. In the detailed account of $\mathrm{my}$ analyses of this complex 
excretion (now preparing for publication), all the above statements will be brough within the scope of general comprehension. I shall also describe my ' ammonia generator,' based on the process invented in the laboratory of Professor Lipbig, and also my 'ammonia metel,' which, together, can detect and measure one hundredth part of a grain weight of absolute ammonia, whether potential or actual, in any sample of guano.

"Meanwhile the following may be offered as the average result of my analyses of genuine guano in reference to its agricultural value :-

"1. Azotized animal matter, including urate of ammonia, together capable of aftording from 8 to 16 per cent. of ammonia by slow decomposition in the soil - - $\quad-50$

2. Water - - - _ _ _ 8 to 11

3. Phosphate of lime - - - - - - 12 to 25

4. Phosphate of ammonia, sulphate of ammonia, ammoniaphosphate of magnesia, together containing from 5 to 9 parts of ammonia

5. Siliceous sand

$-13$

$-1$

"Very moist guano has in general more actual and less potential ammonia than the dry guano.

"London, 13 Charlotte street, Bedford square,

"ANDREW URE。 "February 14, 1843."

nellacher's analysis of a brownish yellow guano is as follows :-

1. Urate of ammonia

2. Oxalate of ammonia

3. Oxalate of lime

4. Phosphate of ammonia

5. Phosphate of ammonia and magnesia

6. Phosphate of lime -

7. Muriate of ammonia

8. Chloride of sodium (common salt) -

9. Carbonate of ammonia

10. Carbonate of lime

11. Sulphate of potash

12. Sulphate of soda

13. Humate of ammonia

14. Substance resembling wax -

15. Sand

16. Water (hygroscopic)

17. Undefined organic matter - $\begin{array}{r}12 \cdot 20 \\ -17 \cdot 73 \\ -\quad 1 \cdot 30 \\ -\quad 6.90 \\ -11.63 \\ -20 \cdot 16 \\ -\quad 2.25 \\ -\quad 0.40 \\ -\quad 0.80 \\ -\quad 1.65 \\ -\quad 4 \cdot 00 \\ -\quad 4.92 \\ -\quad 1 \cdot 06 \\ -\quad 0.75 \\ -\quad 1 \cdot 68 \\ -\quad 4 \cdot 31 \\ -\quad 8.26 \\ \hline\end{array}$

$100 \cdot 00$
Ammonia.

$1 \cdot 06$

$6 \cdot 50$

$1 \cdot 79$

$1 \cdot 68$

0.72

$0 \cdot 23$

0.09

$12 \cdot 07$

I am satisfied from its large proportion of oxalate of ammonia, that the sample thus analyzed was by no means a fair or normal specimen of guano; and it is in fact widely different from all the fresh samples which have passed through my hands. It is described as "knobby, being mixed with light laminated crystalline portions, in white grains, from the size of a pea to that of a pigeon's egg." Having some lumpy concre tions of a similar aspect in my possession, I submitted them to chemical examination.

G. 1,000 grains being digested in boiling water and strained, afforded a nearly colorless solution. This was concentrated till crystals of oxalate of ammonia appeared. It was then acidulated with hydrochloric acid, to protect the phosphoric acid from precipitation, and next treated carefully with a solution of nitrate of lime equivalent to the oxalic acid present. The oxalate of lime thus obtained being converted into carbonate weighed 80.5 grains, corresponding to 100 of oxalate of ammonia, being 10 per cent. of the weight of the guano.

The liquor filtered from the oxalate was precipitated by nitrate of barytes, and afforded 112 grains of sulphate of barytes $=38$ sulphuric acid; and the last filtrate being mixed with a given measure of ferric acetate, and the mixture supersaturated with ammonia, yielded suhphosphate of iron, equivalent to 5 per cent of phosphoric acid. I digested with heat other 500 grains of the same guano in a weak solution of borax, filtered, acidulated the liquid, but obtained merely a trace of uric acid. It is 
clear therefore that the oxalate of ammonia had been formed in this guano at the expense of the uric acid, and that its concreted state, and the crystalline nodules disseminated through it, were the result of transformation by moisture in a hot climate, which had agglomerated it to a density of 1.75 ; whereas clean fresh guano, friable and dry like the above, is seldom denser than $1 \cdot 65$. The guano contained only $3 \cdot 23$ of arymonia; 65 of insoluble matter, 53 of earthy phosphates, 5 silica, 3 alkaline salts (fixed), and 7 organic matter.

Oxalate of ammonia, being readily washed away, it is a bad substitute for the urate of ammonia, urea, and azotized animal matter, which it has replaced. Oellacher could find no urea in the guano which he analyzed; another proof of its disintegration.

Bartel's analysis of a brown-red guano is as follows :-

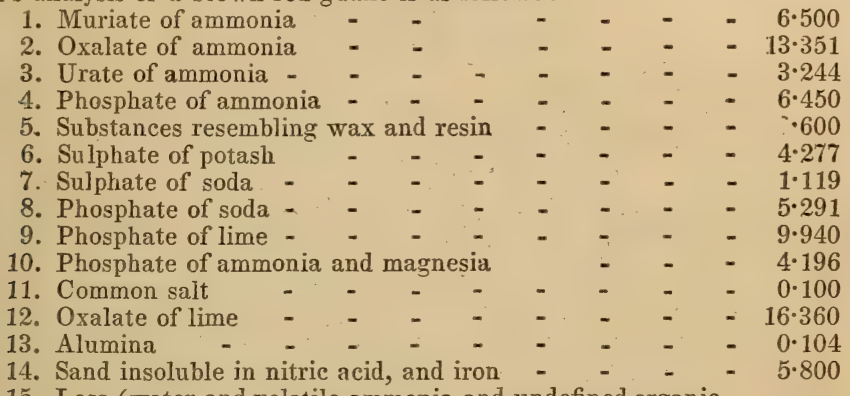

15. Loss (water and volatile ammonia and undefined organic matter)

Voelckel, in his analysis of guano, states 7 per cent. of oxalate of lime-a result quite at variance with all my experience-for I have never found so much as 2 per cent. of carbonate of lime in the washed and gently ignited insoluble matter; whereas, according to Bartels and Voelckel, from 10 to 5 per cent. of carbonate should be obtained, as the equivalents of the proportions of the oxalate assigned by them.

All these analyses are defective moreover in not showing the total quantity of ammonia which the guano is capable of giving out in the soil ; and since it appears that the freshest guano abounds most in what I have called potential ammonia, it must possess, of consequence, the greatest fertilizing virtue.

A sample of decayed dark-brown moist guano from Chile, being examined as above described, for oxalate of ammonia, was found to contain none whatever; and it contained less than 1 per cent. of uric acid.

H. An article offered to the public, by advertisement, as Peruvian guano, was lately sent to me for analysis. I found it to be a spurious composition; it consisted of-
1. Common salts - - - - - - $\quad-32 \cdot 0$

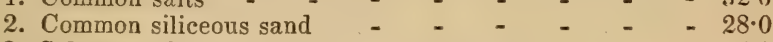
3. Sulphate of iron or copperas - $-\quad-\quad-5.2$
4. Phosphate of lime - _ - _ - _ - $4 \cdot 0$, with
5. Organic matter from bad guano, \&c. (to give it smell) $-23 \cdot 3$
6. Moisture - _ _ _ _ _ _ - - $7 \cdot 5$
$\overline{100 \cdot 0}$

Genuine guano, when burned upon a red hot shovel, leaves a white ash of phosphate of lime and magnesia; whereas this factitious substance left a black fused mass of sea salt, copperas, and sand. The specific gravity of good fresh guano is seldom more than $1 \cdot 66$, water being 100 ; whereas that of the said substance was so high as $2 \cdot 17$; produced by the salt, sand, and copperas.

GUMS. Under the generic name Gum several substances have been classed, which differ essentially, though they possess the following properties in common: viz., forming a thick mucilaginous liquid with water, and being precipitable from that solution by alcohol. Properly speaking, we should style gums only such substances as are transformed into mucic acid by nitric acid; of which bodies there are three: 1. Arabine, which constitutes almost the whole of gum arabic; 2. Bassorine, which forms the chief part of gum tragacanth; and 3. Ceracine, which occurs in cherry-tree gum, and is convertible into gum arabic by hot water.

1. Gum arabic, in its ordinary state, contains 17 per cent. of water, separable from it by a heat of $212^{\circ} \mathrm{F}$. 
2. Cherry-tree gum consists of 52 per cent. of arabine, and 35 of a peculiar gum, which has been called Cerasine. This latter substance is convertible into grape sugar by boiling it with very dilute sulphuric acid.

GUNPOWDER, ANALYSIS OF. M. Bolley dissolves out the sulphur from charcoal in gunpowder (previously freed from its nitre by water), by digesting it, at a boiling heat for 2 hours, with the solution of 20 times its weight of sulphite of soda, which is thereby converted into hyposulphite. To the mixture water must be added, as it is wasted by the boiling. If the residuum be heated on platinum foil, it will exhale sulphur, if this had not been all removed by the sulphurous salt.

\section{H.}

HAIR CLOTH. See WeAving.

HATS. The body of a beaver hat is made of fine wool and coarse fur mixed and felted together, then stiffened and shaped; the covering consists of a coat of beaver fur felted upon the body. Cheap hats have their bodies made of coarse wool, and their coverings of coarse fur or fine wool. The body or foundation of a good bearer hat, is at present made of 8 parts of rabbit's fur, 3 parts of Saxony wool, and 1 part of lama, vicunia, or red wool. About two ounces and a half of the above mixture are sufficient for one hat, and these are placed in the hands of the bower; his tool is a bow or bent ashen staff, from 5 to 7 feet long, having a strong catgut string stretched orer a bridge at each end, and suspended at its middle by a cord to the ceiling, so as to hang nearly level with the work-bench, and a small space above it. The wool and coarser fur are laid in their somewhat matted state upon this bench, when the bower, grasping the bent rod with his left hand, and by means of a small wooden catch plucking the string with his right, makes it ribrate smartly against the fibrous substances, so as to disentangle them, toss them up in the air, and curiously arrange themselves in a pretty uniform layer or fleece. A skilful bower is a valuable workman. The bowed materials of one hat are spread out and divided into two portions, each of which is compressed, first with a light wicker frame, and next under a piece of oil cloth or leather, called a hardening skin, till by pressing the hands backward and forward all orer the skin, the filaments are linked together by their serrations into a somewhat coherent fleece of a triangular shape. The two halves or "bats" are then formed into a cap; one of them is covered in its middle with a 3-cornered piece of paper, smaller than itself, so that its edges may be folded orer the paper, and br overlapping each other a little, form a complete enrelope to the paper; the junctions are then partially felted together by rubbing them hard, care being taken to keep the base of the triangle open by means of the paper; the second bat being made to enclose the first by a similar process of folding and friction. This double cap, with its enclosed sheet of paper, is next rolled up in a damp cloth and kneaded with the hands in erery direction, during which it is unfolded and creased up again in different forms, whereby the two lavers get thoroughly incorporated into one body; thus, on withdrawing the paper, a hollow cone is obtained. The above operations hare been partially described in the body of the Dictionary, and the remaining steps in making a hat are there sufficiently detailed.

In a great hat factory women are employed, at respectable wages, in plucking the beaver slins, cropping off the fur, sorting various qualities of wool, plucking and cutting rabbit's fur, shearing the nap of the blocked hat, picking out unseemly filaments of fur, and in trimming the hats; that is, lining and binding them.

The annual value of the hats manufactured at present in the United Kingdom is estimated at $3,000,000 l$. sterling. The quantity exported in 1840 , was 22,522 dozens, valued at $81,583 l$.

With regard to the stiffening of hats, I have been furnished by a skilful operator with the following valuable information: "All the solutions of gums which I have hitherto seen prepared by hatters, have not been perfect, but, in a certain degree, a mixture, more or less, of the gums, which are merely suspended, owing to the consistency of the composition. When this is thinned by the addition of spirit, and allowed to stand, it lets fall a curdy looking sediment, and to this circumstance may he ascribed the frequent breaking of hats. My method of proceeding is, first to dissolve the gums by agitation in twice the due quantity of spirits, whether of wood or wine, and then, after complete solution, draw off one half the spirits in a still, so as to bring the stiffening to a proper cnnsistency. No sediment subsequently appears on diluting this solution, however much it may be done.

"Both the spirit and alkali stiffenings for hats made by the following two recipes, have been tried by some of the first houses in the trade, and have been much approved of:- 


\section{Spirit Stiffening.}

7 pounds of fine orange shellac.

2 pounds of gum sandarac.

4 ounces of gum mastic.

Half a pound of amber rosin.

1 pint of solution of copal.

1 gallon of spirit of wine or wood naphtha.

"The shellac, sandarac, mastic, and rosin, are dissolved in the spirit, and the solu. tion of copal is added last.

\section{Alkali Stiffening.}

7 pounds of common block shellac.

1 pound of amber rosin.

4 ounces of gum thus.

4 ounces of gum mastic.

6 ounces of borax.

Half a pint of solution of copal.

"The borax is first dissolved in a little warm water (say 1 gallon); this alkalise liquor is now put into a copper pan (heated by steam), together with the shellac, rosin, thus, and mastic, and allowed to boil for some time, more warm water being added occasionally until it is of a proper consistence; this may be known by pouring a little on a cold slab somewhat inclined, and if the liquor runs off at the lower end, it is sufficiently fluid; if, on the contrary, it sets before it reaches the bottom, it requires more water. When the whole of the gums seem dissolved, half a pint of wood naphtha must be introduced, and the solution of copal; then the liquor must be passed through a fine sieve, and it will be perfectly clear and ready for use. This stiffening is used hot. The hat bodies, before they are stiffened, should be stiffened in a weak solution of soda in water, to destroy any acid that may have been left in them (as sulphuric acid is used in the making of the bodies). If this is not attended to, should the hat body contain any acid when it is dipped into the stiffening, the alkali is neutralized, and the gums consequently precipitated. After the body is steeped in the alkaline solution, it must be perfectly dried in the stove before the stiffening is applied; when stiffened and stoved it must be steeped all night in water, to which a small quantity of sulphuric acid has been added; this sets the stiffening in the hat body, and finishes the process. A good workman will stiffen 15 or 16 dozen hats a day. If the proof is required cheaper, more shellac and rosin must be introduced."

HIDES, untanned; buffalo, bull, cow, ox, or horse. Imported in 1840, 352,867; retained for consumption, 302,789. Rates of duty : from west coast of Africa, not exceeding 14 pounds, $2 s .4 d$.; from British possessions, dry, $2 s$. , wet, $1 s .2 d$.; from other places, dry, $4 s .8 d$., wet, $2 s$. $4 d$. Net revenue, 40,139l. in 1840, and 45,328l. in 1839 . In $1839,16,557$ pounds of tanned hides were imported for home consumption, and in 1840 only 5,822 : at the rate from foreign parts of $6 d$. per pound, but if cut and trimmed, $9 d$.; from British possessions $3 d$., if cut and trimmed $4 \frac{1}{2} d$. per pound.

HOPS.

Annual amount of hop duty.

\begin{tabular}{|c|c|c|c|c|c|c|c|c|c|c|c|}
\hline Yrs. & Amount & Yrs. & Amount. & Yrs. & Amount. & Yrs. & Amount & Yrs. & Amount. & Yrs. & Amount. \\
\hline 1711 & $£ 43,437$ & $1 \% 33$ & $£ \neq 0,215$ & 1755 & $£ 82,15 ?$ & 1777 & $£ 43,581$ & 1799 & $£ 73,279$ & 1821 & $£ 154,609$ \\
\hline 1712 & 30,278 & 1734 & 37,716 & 1756 & 48,106 & 1778 & 159,891 & 18110 & 72,928 & 1822 & 203,724 \\
\hline 1713 & 23,018 & 1735 & 42,745 & 1757 & $69, \pi 13$ & $17 \approx 9$ & 55,800 & 1801 & 241,227 & 1823 & 26,058 \\
\hline 1714 & 14,457 & 1736 & 46.482 & 1758 & 72,896 & 1780 & 122,724 & 1802 & 15,463 & 1824 & 148,832 \\
\hline 1715 & 44,975 & 1737 & 56,492 & 1759 & 42,115 & 1781 & 120,215 & 1803 & 199,305 & 1825 & 24,317 \\
\hline 1716 & $20,35+$ & $1 \% 38$ & $86,5 \div 5$ & 1760 & 117,992 & 1782 & 14,895 & $: 804$ & 177,617 & 1826 & 269,331 \\
\hline $1 \% 1 \%$ & 54,669 & 1739 & 70,742 & 1761 & $79,7 \approx 6$ & 1783 & 75,716 & 1805 & 32,904 & 1827 & 140.848 \\
\hline 1718 & 15,005 & 1740 & 37.875 & 1762 & 79,295 & 1784 & 94,359 & 1006 & 153,102 & 1828 & 172,027 \\
\hline 1719 & 90,317 & 1741 & 65,222 & 1763 & 88,315 & 1785 & 112,684 & .307 & $100,0 \tau 1$ & 1829 & 38,398 \\
\hline 1720 & 38,169 & 1742 & 45,550 & 1764 & 17.178 & 1786 & 95,973 & 1808 & 251,089 & 1830 & 88,047 \\
\hline 1721 & 61,362 & 1743 & 61,072 & $1 \% 65$ & 73,778 & 1787 & 42,227 & 1809 & 63,452 & 1831 & 174,864 \\
\hline 1722 & $49,4+3$ & 1744 & 46,708 & 1766 & 116,445 & 1788 & 143,168 & $1 \varepsilon 10$ & $73,5 \mathrm{I} 4$ & 1832 & 139,018 \\
\hline 1723 & 30.279 & 1745 & 34,635 & $1 \pi 6 \tau$ & $25,99 i$ & 1789 & 101,063 & 1811 & 157,025 & 1833 & 156,905 \\
\hline 1724 & 61,271 & 1746 & $91,8 \approx 9$ & 1768 & 114,002 & 1790 & 106,841 & 1812 & 30,633 & 4 & 189.713 \\
\hline 1725 & 6,526 & 1747 & 62,993 & 1769 & 16,201 & 1791 & 90,059 & 1813 & 131,482 & 5 & 235,207 \\
\hline $1 \% 26$ & 80,031 & 1748 & $8 \%, 155$ & $17 \% 0$ & 101,131 & 1792 & 162,112 & 1814 & 140,202 & 1 & 200,332 \\
\hline 1727 & 69,409 & 1749 & 36,805 & 1771 & 33,143 & 1793 & 22,619 & 1815 & 123,878 & 1837 & 178,578 \\
\hline 1728 & 41,494 & 1750 & 72,138 & $1 \% 2$ & 102,650 & 1794 & 203,063 & 1816 & 46,302 & & 171,556 \\
\hline 1729 & 48,441 & 1751 & 73,954 & 1773 & 45,847 & 1795 & 82,342 & 1817 & 66,522 & 1839 & 205,537 \\
\hline 1730 & 44,419 & 1752 & 82,163 & 1774 & 138,887 & 1796 & 75,223 & 1818 & 199,465 & 1840 & 34,091 \\
\hline 1731 & 22.600 & 1753 & 91,214 & 1775 & 41,597 & $1 \% 97$ & $15 \%, 458$ & 1819 & $242,4 \div 6$ & 1841 & 146,159 \\
\hline 1732 & 35,135 & 1754 & 102,012 & $17 \% 6$ & $125,69 \mathrm{I}$ & $1 \% 98$ & 56,032 & 1820 & 138,330 & 1812 & 169,776 \\
\hline
\end{tabular}


Number of acres under the cultivation of hops in England.

\begin{tabular}{|c|c|c|c|c|c|c|c|c|c|c|c|}
\hline $180 \tau$ & 38,218 & 1813 & 39,521 & 1819 & 51,014 & $1 \times 25$ & 46,718 & 1831 & 47,129 & 1837 & 56,323 \\
\hline 1808 & $3\ulcorner, 436$ & 1814 & $40,5 \approx 1$ & 1820 & 50,148 & 1826 & 50,471 & 1832 & 47,101 & 1835 & 55,015 \\
\hline 1809 & 38,357 & 1815 & 42,150 & 1821 & 45,662 & 1827 & 49,485 & 1833 & 49,187 & 1839 & 52,305 \\
\hline 1810 & 38,265 & 1816 & 44,219 & 1822 & 43,766 & 1828 & 48,365 & 1834 & 51,273 & 1840 & 44,805 \\
\hline 1811 & 38,401 & 1817 & 46,493 & 1823 & 41,458 & 1829 & 46,135 & 1835 & 53,816 & 1841 & 45,769 \\
\hline 1812 & 38,700 & 1818 & 48,593 & 1824 & 43,449 & 1830 & 46,726 & 1836 & 55,422 & 1842 & \\
\hline
\end{tabular}

Hop duties of particular districts.

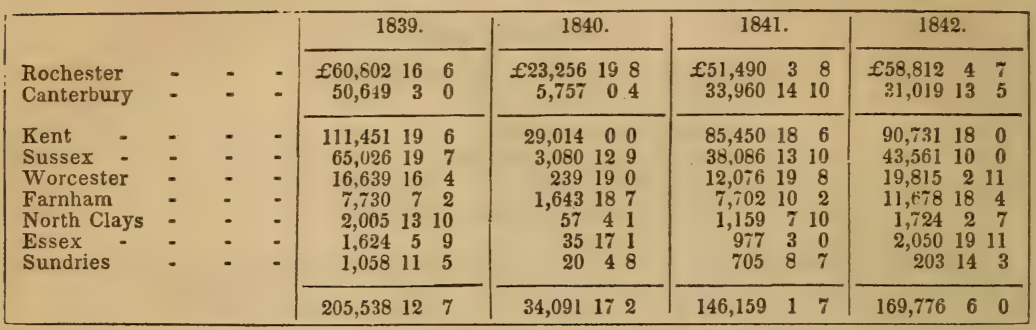

HORN. Mr. J. James has contrived a method of opening up the horns of cattle, by which he avoids the risk of scorching or frizzling, which is apt to happen in heating them over an open fire. He takes a solid block of iron pierced with a conical hole, which is fitted with a conical iron plug, heats them in a stove to the temperature of melting lead, and having previously cut up the horn lengthwise on one side with a saw, he inserts its narrow end into the hole, and drives the plug into it with a mallet. By the heat of the irons, the horn gets so softened in the course of about a minute, as to bear flatting out in the usual.way.

HYPOSULPHITE OF SODA. This salt, so extensively used in the practice of Daguerrotyping, may be easily prepared in quantities by the following process:Mix one pound of finely pulverized ignited carbonate of soda with ten ounces of flowers of sulphur, and heat the mixture slowly in a porcelain dish till the sulphur melts. Stir the fused mass, so as to expose all its parts freely to the atmosphere, whereby it passes from the state of a sulphuret, by the absorption of atmospherical oxygen, into that of a sulphite, with the phenomenon of very slight incandescence. Dissolve in water, filter the solution, and boil it immediately along with flowers of sulphur. The filtered concentrated saline liquid will afford, on cooling, a large quantity of pure and beautiful crystals of hyposulphite of soda.

\section{I.}

ILLUMINATION, COST OF. The production, diffusion, and economy of light, are subjects of the highest interest both to men of science and men of the world; leading the former to contemplate many of the most beautiful phenomena of physics and chemistry, while they provide the latter with the artificial illumination so indispensable to the business and pleasures of modern society. The great cost of light from wax, spermaceti, and even stearic candles, as also the nuisance of the light from tallow ones, have led to the invention of an endless variety of lamps, of which the best hitherto known is undoubtedly the mechanical or Carcel lamp, so generally used by the opulent families in Paris. In this lamp the oil is raised through tubes by clock-work, so as continually to overflow at the bottom of the burning wick; thus keeping it thoroughly soaked, while the excess of the oil drops back into the cistern below. I have possessed for several years an excellent lamp of this description, which performs most satisfactorily ; but it can hardly be trusted in the hands of a servant; and when it gets at all deranged, it must be sent to its constructor in Paris to be repaired. The light of this lamp, when furnished with an appropriate tall glass chimney, is very brilliant, though not perfectly uniform; since it fluctuates a little, but always perceptibly to a nice observer, with the alternating action of the pump-work; becoming dimmer after every successive jet of oil, and brighter just before its return. The flame, moreover, always flickers more or less, owing to the powerful draught, and rectangular reverbatory shoulder of the chimney. The mechenical lamp is, however, remarkable for continuing to burn, not only with unabated but with increasing splendor for seven or eight hours; the vivacity of the combustion increasing evidently with the increased temperature and fluency of 
the oil, which, by its ceaseless circulation through the ignited wick, gets eventually pretty warm. In the comparative experiments made upon different lights by the Parisian philosophers, the mechanical lamp is commonly taken as the standard. I do not think it entitled to this pre-eminence : for it may be made to emit very different quantities of light, according to differences in the nature and supply of the oil, as well as variations in the form and position of the chimney. Besides, such lamps are too rare in this country to be selected as standards of illumination.

After comparing lights of many kinds, I find every reason to conclude that a large wax candle of three to the pound, either long or short, that is, either 12 or 15 inches in length, as manufactured by one of the great wax-chandlers of London, and furnished with a wick containing 27 or 28 threads of the best Turkey cotton, is capable of furnishing a most uniform, or nearly invariable standard of illumination. Its affords one tenth of the light emitted by one of the Argand lamps of the Trinity house, and one eleventh of the light of my mechanical lamp, when each lamp is made to burn with its maximum flame, short of smoking.

The great obstacle to the combustion of lamps, lies in the viscidity, and consequent sluggish supply of oil, to the wicks; an obstacle nearly insuperable with lamps of the common construction during the winter months. The relative viscidity, or relative fluency of different liquids at the same temperature, and of the same liquid at different temperatures, has not, I believe, been hitherto made the subject of accurate researches. I was, therefore, induced to make the following experiments with this view.

Into a hemispherical cup of platinum, resting on the ring of a chemical stand, I introduced 2,000 water-grain measures of the liquid whose viscidity was to be measured, and ran it off through a glass syphon, $\frac{1}{8}$ of an inch in the bore, having the outer leg $3 \frac{1}{4}$ inches, and the inner leg 3 inches long. The time of efflux became the measure of the viscidity; and of two liquids, if the specific gravity, and consequent pressure upon the syphon, were the same, that time would indicate exactly the relative viscidity of the two liquids. Thus, oil of turpentine and sperm oil have each very nearly the same density; the former being, as sold in the shops, $=0.876$, and the latter from 0.876 to 0.880 , when pure and genuine. Now I found that 2,000 grain-measures of oil of turpentine ran off through the small syphon in 95 seconds, while that quantity of sperm oil took 2,700 seconds, being in the ratio of 1 to $28 \frac{1}{2}$; so that the fluency of oil of turpentine is $28 \frac{1}{2}$ times greater than that of sperm oil. Pyroxilic spirit, commonly called naphtha, and alcohol, each of specific gravity 0.825 , were found to run off respectively in 80 and 120 seconds; showing that the former was 50 per cent. more fluent than the latter. Sperm oil, when heated to $265 \mathrm{Fahr}$, runs off in 300 seconds, or one ninth of the time it took when at the temperature of $64^{\circ}$. Southern whale oil, having a greater density than the sperm oil, would flow off faster were it not more viscid.

2,000 grain-measures of water at $60^{\circ}$ run off through the said syphon in 75 seconds, but when heated to $180^{\circ}$, they run off in 61 .

Concentrated sulphuric acid, though possessing the great density of $1 \cdot 840$, yet flows off very slowly at $64^{\circ}$, on account of its viscidity; whence its name of oil of vitriol. 2,000 grain-measures of it took 660 seconds to discharge.

Mr. Samuel Parker, long advantageously known to the public for his sinumbral and pneumatic fountain lamps, as well as other inventions subservient to domestic comfort, having obtained a patent for a new lamp, in which the oil is heated by a very simple contrivance, in the cistern, to any desired degree, before arriving at the wick, I instituted an extensive series of experiments to determine its value in the production of light, and consumption of oil, compared to the value of other lamps, as well as candles, in these respects.

In fig. 73. A, A, B, B, is a section of the cylinder which constitutes the cistern; the oil being contained between the inner and outer cylinders, and receiving heat from the flame of the lamp which passes up through the inner cylinder, and is reverberated more or less against its sides by the top of the metal chimney, being notched and bent back. D is a slide-valve which is opened to allow the oil to descend to the wick, and is shut when the cistern is to be separated from the pipe of supply, at E, for the purpose of recharging it with oil. The flame is modified, not by raising or lowering the wick, as in common lamps, but by raising or lowering the bell-mouthed glass chimney which rests at its bottom on three points, and is moved by means of the rack-work mechanism $F$. The concentric cylindric space $A, A$, and $B, B$, contains a pint imperial, and should be made entirely full before lighting the lamp; so as to leave no air in the cistern, which, by its expansion with the heat, would inevitably cause an overflow of the oil.

The following arrangement was adopted in these experiments for determining the relative illumination of the different lights. Having trimmed, with every precaution, my French mechanical lamp, and charged it with pure sperm oil, I placed it upon an oblong table, at a distance of 10 feet from a wall, on which a white sheet of paper was 
stuck. One of Mr. Parker's hot-nil lamps, charged with a quantity of the same oil, was placed upon the same table; and each being made to burn with its maximum

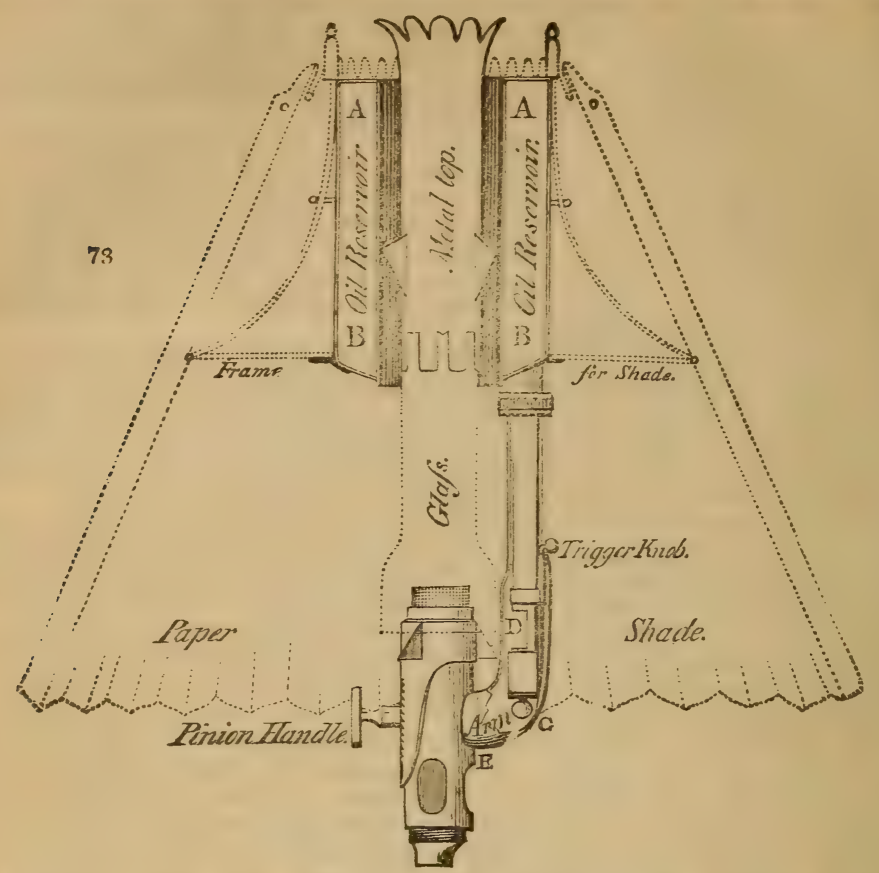

brlliancy, short of smoking, the relative illumination of the two lamps was determined by the well-known method of the comparison of shadows; a wire a few inches long, and of the thickness of a crow-quill, being found suitable for enabling the eje to estimate very nicely the shade of the intercepted light. It was observed in numerous trials, both by my own eyes and those of others, that when one of the lamps was shifted half an inch nearer to or further from the paper screen, it caused a perceptible difference in the tint of the shadow. Professor Wheatstone kindly enabled me to verify the precision of the above method of shadows, by employing, in some of the experiments, a photometer of his own invention, in which the relative brightness of the two lights was determined by the relative brightness of the opposite sides of a revolving silvered ball, illuminated by them.

1. The mechanical lamp was furnished with a glass chimney 1.5 inches in diameter at the base, and 1.2 at top; the wide bottom part was 1.8 inches long, and the narrow upper part 8 inches. When placed at a distance of 10 feet from the wall its light there may be estimated as the square of this number, or 100 . In the first series of experiments, when burning with its maximum flame, with occasioned flickerings of smoke, it emitted a light equal to that of 11 wax candles, and consumed 912 grains of oil per hour. The sperm oil was quite pure, having a specific gravity of 0.874 compared to water at 1,000 . In a subsequent series of experiments, when its light was less flickiering, and equal only to that of 10 wax candles, it consumed only 815 grains, or 0.1164 of a lb. per hour. If we multiply this number into the price of the oil ( $8 s$. per gallon) per lb. $11 d$., the product $1 \cdot 2804 d$. will represent the relative cost of this illumination, estimated at 100 .

2. The hot-oil lamp burns with a much steadier flame than the mechanical, which must be ascribed in no small degree to the rounded slope of the bell-mouthed glass chimney, whereby the air is brought progressively closer and closer into contact with the outer surface of the flame, without being furiously dashed against it, as it is by the rectangular shoulder of the common contracted chimney. When charged with sperm oil, and made to burn with its maximum flame, this lamp required to be placed one foot further from the screen than the mechanical lamp, in order that its shadow should have the same depth of tint. Hence, its relative illumination was, in that case, as the square of 11 to the square of $10 ;$ or as 121 to 100 . Yet its consumption of oil was 
only 696 grains, or somewhat less than $0 \cdot 1$ of a pound per hour. Had its light been reduced to 100 , it would have consumed only 576 grains per hour, or 0.82 of a pound. If we multiply this number by $11 d$., the product $0.902 d$. will represent the relative cost of 100 of this illumination.

3. The hot-oil lamp being charged with the southern whale oil, of specific gravity 0.926 , at $2 s .6 d$. per gallon, or $3 \frac{1}{4} d$. per $1 b$., when burning with its maximum flame, required to be placed 9 feet and 1 inch from the screen to drop the same tint of shadow upon it as the flames of the other two lamps did at 10 and 11 feet with the sperm oil. The square of 9 feet and 1 inch $=82$ is the relative illumination of the hot-oil lamp with the southern whale oil. It consumed 780 grains, or 0.111 of a pound per hour; but had it given 100 of light it would have consumed 911 grains, or $0 \cdot 130$ of a pound, which number being multiplied by its price $3 \frac{3}{4} d$., the product $0.4875 \mathrm{~d}$. will represent the relative cost of 100 of this light.

4. A hot-oil lamp charged with olive oil of specific gravity 0.914 , at $5 s$. $6 d$. per gallon, or $7 \frac{1}{2} d$. per $\mathrm{lb}$. when burning with its maximum flame, required to be placed at 9 feet 6 inches, to obtain the standard tint of shadow upon the screen. It consumed 760 grains per hour. The square of $9 \frac{1}{2}$ feet is $90 \frac{1}{4}$, which is the relative intensity of the light of this lamp. Had it emitted a light $=100$, it would hare consumed 840 grains, or 0.12 of a pound per hour-which number multiplied by the price per pound, gives the product $0.9 d$. as the relative cost of 100 of this light.

5. A hot-oil lamp charged with Price and Co.'s cocoa-nut oil (oleine), of specifie gravity 0.925 , at $4 s .6 d$. per gallon, or $5 \frac{3}{4} d$. per 1 b., had to be placed 9 feet from the screen, and consumed 1,035 grains per hour. Had its light been 100 instead of 81 (92), the consumption would have been $1,27 \tau$ grains, or 0.182 of a pound per hour! which number multiplied by its price per pound, the product $1 \cdot 031 d$. will represent the cost of 100 of this illumination.

6. In comparing the common French annular lamp in general use with the mechanical lamp, it was found to give about one half the light, and to consume two thirds of the oil of the mechanical lamp.

7. Wax candles from some of the most eminent wax-chandlers of the metropolis were next subjected to experiment; and it is very remarkable that, whether they were threes, fours, or sixes in the pound, each afforded very nearly the same quantity of light, for each required to be placed at a distance of three feet from the screen to afford a shadow of the same tint as that dropped from the mechanical lamp, estimated at 100 . The consumption of a genuine wax candle, in still air, is upon an average of many experiments, 125 grains per hour, but as it affords only 111 th of the Jight of the mechanical lamp, 11 times $125=1,375$ grains, or 0.1064 of a pound is the quantity that would need to be consumed to produce a light equal to that of the said lamp. If we multiply that number by the price of the candles per $1 \mathrm{~b} .=30 d$. the product $=5.892 d$. is the cost of 100 of illumination by wax. A wax candle, three in the pound (short), is one inch in diameter, 12 inches in length, and contains 27 or 28 threads, each about 1 10th of an inch in diameter. But the quality of the wicl depends upon the capillarity of the cotton fibrils, which is said to be greatest in the Turkey cotton, and hence the wicks for the best wax candles are always made with cotton yarn imported from the Levant. A wax candle, three in the pound(long), is $\frac{7}{8}$ of an inch in diameter, 15 inches long, and has 26 threads in its wick. A wax candle, six to the pound, is 9 inches long, 4 ths of an inch in diameter, and has 22 threads in its wick. The light of this candle may be reckoned to be, at most, about 111 th less than that of the threes in the pound. A well-made short three burns with surprising regularity in still air, being at the rate of an inch in an hour and a half, so that the whole candle will last 18 hours. A long three will last as long, and a six about $9 \frac{1}{2}$ hours. Specific gravity of wax $=$ 0.960 .

8. A spermaceti candle, three in the pound, is 910 ths of an inch in diameter, 15 inches long, and has a plaited wick, instead of the parallel threads of a wax candle. The same candles four in the pound, are 810 ths of an inch in diameter, and $13 \frac{1}{2}$ inches long. Each gives very nearly the same quantity of light as the corresponding wax candles: viz., 111 th of the light of the above mechanical lamp, and consumes 142 grains per hour. Multiplying the last number by 11 , the product, 1,562 grains $=0.223$ of a pound, would be the consumption of spermaceti requisite to give 100 of illumination. Multiplying the last number by $24 d$., the price of the candles per pound, the product $5 \cdot 352 d$. is the relative cost of 100 of this illumination.

9. Stearic acid candles, commonly called German wax, consume 168.5 grains, or 0.024 of a pound per hour, when emitting the same light as the standard wax candle. Multiplying the latter number by 11 , and by $16 d$. (the price of the candles per pound), the product $4 \cdot 224 d$. will represent the relative cost of 100 of this illumination.

10. Tallow candles: moulds, short threes, 1 inch in diameter, and $12 \frac{1}{2}$ in length; do. long threes, 910 ths of an inch in diameter, and 15 in length; do., long fours, 810 ths of 
an inch in diameter, and $13 \frac{3}{4}$ in length. Each of these candles burns with a most un certain light, which varies from 112 th to 1 16th of the light of the mechanical lampthe average may be taken at $114 \mathrm{th}$. The threes consume each 144 grains, or $0 \cdot 2$ of a pound, per hour; which number, multiplied by 14 , and by $9 d$. (the price per pound), gives the product $2 \cdot 52 d$. for the relative cost of 100 of this illumination.

11. Palmer's spreading wick candles. Distance from the screen 3 feet 4 inches, with a shadow equal to the standard. Consumption of tallow per hour 232.5 grains, or 0.0332 of a pound. The square of 3 feet 4 inches $=11.9$ is the relative illumination of this candle $=11.9:: 0.3332:: 100: 0.28 \times 10 d .=11.9$ is the relative cost of this illumination.

12. Cocoa-nut stearine candles consumed each 168 grains per hour, and emitted a light equal to 116 th of the standard flame. Multiplying 168 by 16 , the product 30.88 grains, oi 0.441 of a pound, is the quantity which would be consumed per hour to afford a light equal to 100 . And 0.441 multiplied by $10 d$., the price per pound, gives the product $4 \cdot 441 d$. as the cost of 100 of this illumination per hour.

A gas argand London lamp, of 12 holes in a circle of $\frac{3}{4}$ of an inch in diameter, with a flame 3 inches long, afforded a light $=78 \frac{1}{2}$ compared to the mechanical lamp: and estimating the light of the said mechanical lamp as before, at 100, that of the hot-oil lamp is 121 , and that of the above gas flame of 78.57 , or in round numbers 80 , and the common French lamp in general use 50.

Collecting the preceding results, we shall have the following tabular view of the cost per hour of an illumination equal to that of the mechanical lamp, reckoned 100, or that of eleven wax candles, three to the pound.

Table of Cost per Hour of One Hundred of Illumination.

1. Parker's hot-oil lamp, with southern whale oil $\quad-0.4875$ or about $\frac{1}{2} d$.

2. Mechanical or Carcel lamp, with sperm oil

3. Parker's hot-oil lamp, with sperm oil - -

4. Ditto ditto

5. Ditto ditto common olive oil

7. Wax candles

8. Spermaceti candles

9. German wax (Stearic acid) ditto

10. Palmer's spreading wick candles

11. Tallow (mould) candles cocoa-nut oleine or oil

12. Cocoa-nut stearine of Price and Co.

- $1 \cdot 2804$

- 0.902

- 0.900

- 1.031

- 1.7072

- 5.892

- 5.352

- 4.224

- 2.800

- 2.520

- 4.41

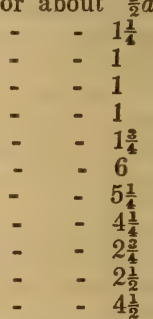

Since the hot-oil lamp affords sufficient light for reading, writing, sewing, \&c., with one fifth of its maximum flame, it will burn at that rate for 10 hours at the cost of about ONE PENNY, and it is hence well entitled to the inventor's designation, "The Economic."

Sir D. Brewster, in his examination lately before the committec of the house of commons on lighting the house, stated, that the French light-house lamp of Fresnel emitted a light equal to that of forty argand flames; whereas, according to other accounts, it gave much less light. With the view of settling this point, before being examined by the said committee, I repaired to the Trinity house, and tried one of the two original Fresnel lamps, which had been deposited there by that eminent French engineer himseif. This lamp consists of four concentric circular wicks, placed in one horizontal plane; the innermost wick being $\frac{7}{8}$ of an inch in diameter, and the outermost $3 \frac{1}{2}$ inches. Being carefully trimmed, supplied with the best sperm oil, surmounted with its great glass chimney, burning with its maximum flame, and placed at a distance of 13 feet 3 inches from the screen, it let fall a shadow of the same tint as that let fall by the flame of my mechanical lamp, placed at a distance of 4 feet 6 inches from the screen. The squares of these two numbers are very nearly as $8 \frac{3}{4}$ to $1(175.5625$ to 20.25) ; showing that the Fresnel lamp gives less than 9 times the light of my mechanical lamp, and about $9 \cdot 6$ times the light of one of the Trinity house argand lamps. The Fresnel lamp is exceedingly troublesome to manage, from the great intensity of its heat, and the frequent fractures of its chimneys-two having been broken in the course of my experiments at the Trinity house.

Mr. Goldsworthy Gurn yy, the ingenious inventor of the new light-house lamp, in which a stream of oxygen gas is sent up through a small tube within the burning circular wick of a small argand lamp, having politely sent two of his lamps to my house, along with a bag of oxygen gas, I made the following experiments, to ascertain their illuminating powess compared to those of the mechanical lamp and wax candles.

His larger lamp has a wick $\frac{5}{8}$ of an inch in diameter, but emits an oxygen flame of only $\frac{3}{8}$ of an inch. The flame is so much whiter than that of the best lamp or candle 
that it becomes difficult to determine, with ultimate precision, the comparative depths of the shadows let fall by them. The mean of several trials showed that the above Bude-light (as Mr. Gurney calls it, from the name of his residence in Cornwall), has an illuminating power of trom 28 to 30 wax candles. His smaller lamp has a flame $\frac{1}{4}$ of an inch in diameter, and a wick $\frac{1}{2}$ of an inch. Its light is equal to that of from 18 to 20 wax candles.

The committee of the house of commons on lighting it, having asked me what was the relative vitiation of air by the breathing of men and the burning of candles, I gave the following answer :-

Wax contains 81.75 parts of carbon in 100 , which generate by combustion 300 parts of carbonic acid gas. Now, since 125 grains of wax constitute the average consumption of a candle per hour, these will generate 375 grains of carbonic acis; equivalent in volume to 800 cubic inches of gas. According to the most exact experiments on respiration, a man of ordinary size discharges from his lungs 1,632 cubis inches of carbonic acid gas per hour, which is very nearly the double of the quantity produced from the wax candle. Hence the combustion of two such candles vitiates the air much the same as the breathing of one man. A tallow candle, three or four in the pound, generates nearly the same quantity of carbonic acid as the wax candle; for though tallnw contains only 79 per cent. of carbon, instead of 81.75 , yet it consumes so much faster, as thereby to compensate fully for this difference.

When a tallow candle of 6 to the lb. is not snuffed, it loses in intensity, in 30 minutes, 80 hundredths; and in 39 minutes 86 hundredths, in which dim state it remains stationary, yet still consuming nearly the same proportion of tallow. A wax candle attains to its greatest intensity of light when its wick has reached the greatest length, and begins to bend out of the flame. The reason of this difference is, that only the lower part of the wick in the tallow candle is charged with the fat, so as to emit luminiferous vapor, while the upper part remains dry; whereas, in the wax candle, the combustible substance being less fusible and volatile, allows a greater length of the wick to be charged by capillary attraction, and of course to emit a longer train of light.

The following table contains, according to Péclet, the illuminating powers of different candles, and their consumption of material in an hour; the light emitted by a Carcel argand lamp, consuming 42 grammes ( $=42 \times 15 \frac{1}{2}$ grains) in an hour, being called 100 :-

\begin{tabular}{|c|c|c|}
\hline & Intensity of Light. & Consumption per Hour. \\
\hline Tallow Candles 6 in $1 \mathrm{~b}$. & $10 \cdot 66$ & $8 \cdot 51$ \\
\hline Stearine, or Pressed Tallow, 8 in lb. - & $8 \cdot 74$ & $7 \cdot 51$ \\
\hline Wax Candles & $\begin{array}{r}7.50 \\
13 \cdot 61\end{array}$ & $\begin{array}{l}7 \cdot 42 \\
8 \cdot 71\end{array}$ \\
\hline Spermaceti ditto, 5 in $\mathrm{lb}$. & $14 \cdot 40$ & $8 \cdot 92$ \\
\hline $\begin{array}{l}\text { Stearic Acid, commonly called Stea- } \\
\text { rine, } 5 \text { in lb. - }\end{array}$ & $14 \cdot 40$ & $9 \cdot 33$ \\
\hline
\end{tabular}

The subjoined table shows the economical ratios of the candles, where the second column gives the quantity of material in grammes which is requisite to produce as much light as the Carcel lamp:-

\begin{tabular}{|c|c|c|c|c|}
\hline \multirow{2}{*}{$\begin{array}{l}\text { Tallow Candle } 6 \text { per } \mathrm{lb} . \\
8 \text { per lb. }\end{array}$} & \multirow{2}{*}{$\ddot{-}$} & $\begin{array}{l}\text { Quantity of } \\
\text { Material. }\end{array}$ & $\begin{array}{l}\text { Price per Kilo- } \\
\text { granme. }\end{array}$ & $\begin{array}{l}\text { Cost of Light per } \\
\text { Hour. }\end{array}$ \\
\hline & & $70 \cdot 35$ & $1 \mathrm{f} . \quad 40 \mathrm{c}$. & $9.8 \mathrm{c}$. \\
\hline Pressed Tallow, 5 per $1 \mathrm{~b}$. & - & $\begin{array}{l}85 \cdot 92 \\
98.93\end{array}$ & $2 \mathrm{f} . \quad 40 \mathrm{c}$. & $23.7 \mathrm{c}$. \\
\hline Wax Candle, 5 per lb. - & - & $64 \cdot 04$ & $7 \mathrm{f} . \quad 60 \mathrm{c}$. & $48 \cdot 6 \mathrm{c}$. \\
\hline Spermaceti ditto, 5 per 1 b. & - & $61 \cdot 94$ & $7 \mathrm{f.} \quad 60 \mathrm{c}$. & $47 \cdot 8 \mathrm{c}$ \\
\hline St & - & $65 \cdot 24$ & $6 \mathrm{f}$. & $37 \cdot 1 \mathrm{c}$. \\
\hline
\end{tabular}

These results may be compared with mine given above. A kilogramme, or 1,000 grammes $=15,440$ grains $=2 \frac{1}{4}$ lbs., avoirdupois.

INDIGO. Imported for home consumption, in 1839, 2,704,396 pounds; in 1840, $2,996,215$; duty $3 d$. on West Indian, $4 d$. on East Indian.

INK. Mr. Stephen's patent blue ink is made by dissolving Prussian blue in a solution of oxalic acid.

IRON. For certain new processes for making malleable iron, Mr. W. N. Clay has obtained two successive patents. Under the first, of December, 1837, he mixed bruised hemestite, with one fifth of its weight of clean carbonaceous matter in coarse powder, 
and subjected the mixture in a $\rho$ shaped retort to a bright red heat for twelve or more hours, till the ore be reduced to the metallic state, as is easily ascertained by applying a file to one of the fragments. When discharged, the metal is to be transferred into a balling or puddling furnace, along with about five per cent. of ground coke or anthracite, and worked therein in the usual way. He also proposes to use a conical kiln, like that for burning lime, instead of the retorts.

In his second patent, dated March, 1840, Mr. Clay prescribes above 28 per cent. (from 30 to 40) of carbonaceous matter to be mixed with the ground-iron ore, containing at least 45 per cent. of metal, which mixture is to be directly treated in a puddling furnace. He also proposes to use a mixture of pig or scrap iron and ore, in equal quantities.

The application of the waste gases (carbonic oxide chiefly) of the blast furnace to the purpose of heating the puddling or balling furnace, was made the subject of a patent in June, 1841 , by a foreigner not named. The process had been previously practised in Germany, and is fully described in the Annales des Mines, about two years ago.

In fig. 74 the manner of conveying the waste carbonic oxide from a blast furnace is shown: $a, a, a$, are openings leading into the vertical channels or passages, $b$,

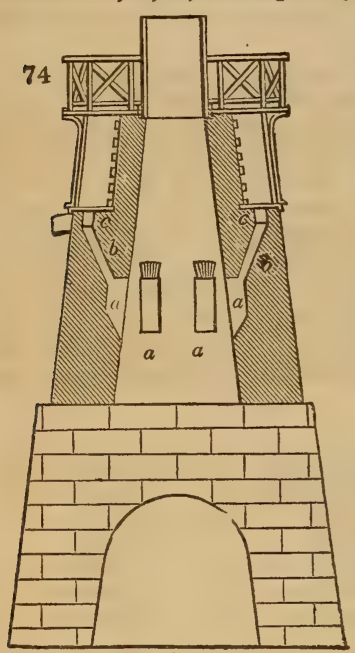
and thence into the chamber $c$. There is a top to this chamber, with openings corresponding to the passages $b$. These openings are closed with castiron plates that can be taken off for the purpose of clearing out the passages $b$, and the chamber $c$. From the chamber $c$, the gas may be conducted in any direction, and to a distance of several hundred feet.

In some localities, and in cases where it is required to take the gas from a blast furnace in operation, a metal cylinder, of a smaller diameter than the top of the furnace, and of a depth equal to its diameter, is suspended vertically within the top of the blast furnace the whole of its length. The space between the cylinder and the furnace at the top or mouth is to be hermetically sealed, and the furnace is to be charged through the cylinder, which must be kept full of minerals and combustibles. Thus the space between the cylinder and the interior of the furnace remains vacant, but the gas may be conducted out of that part laterally, if required. The gases led off from the blast furnace may, if need be, pass through heated pipes, as for the hot blast.

Figs. 75 and 76 represent a refining furnace for iron, with the necessary apparatus for working it with the gases, without the use of other fuel; fig. 75 being a vertical section, and fig. 76 a sectional plan view.

75

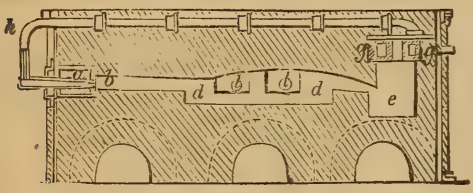

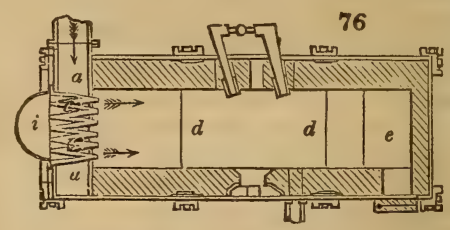

The gas from the blast furnace is brought into the chamber $a a$, and passing through an opening $b b$, it enters the furnace; $c c$ are a series of blow pipes, through which the heated air is forced into the furnace. In the space between the part marked $b$ and the tubes $c$, the gas becomes mixed with the heated atmospherical air.

This combustible gas from the blast furnace, mixed with the heated air, produces an intense heat in the furnace, adequate to the refining of iron. The warm air for burning the gas is usually obtained from the blowing machine and hot blast pipes.

For giving a still greater heat, the air may be carried through the tube $f$, into the iron chambers $g \mathrm{~g}$, or a system of pipes, whence it is led through the tube $h$, into the semi-circtlar chamber $i$, and then through the small pipes $c, c, c$, into the furnace.

The metal to be refined is placed in the space $d d$, in a liquid state, if the arrangement of the furnaces will admit of its being so taken from the blast furnace; if not, it may be nearly melted by the waste heat in the chamber $\epsilon e$. In order to decarbonize 
the metal, a quantity of warm air, from the pipe $h$, is conducted through the pipe $k$, which is divided into two nozzles or tuyères $l l$, and blown upon the fluid metal in the space $d d$. After having been thus exposed for an hour or two, it is run off through the opening $m$, and will be found in a refined state.

Figs. $7 i, 78$, show the application to a puddling furnace. The openings $n n$ admit a stream of cold water to flow through the cast-iron piece 00 , to preserve it from injury by the fire.

77

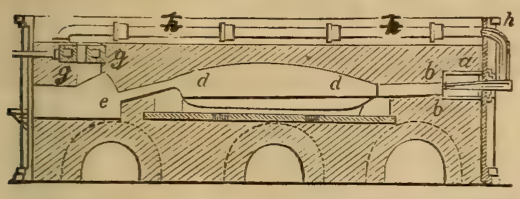

78

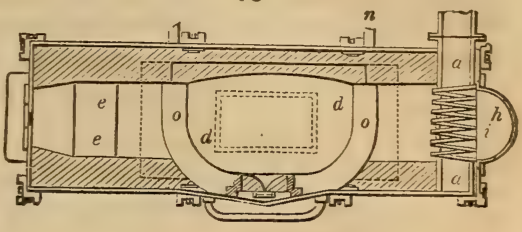

Fig. 79 is a welding furnace; the interior dimensions and the casing of the hearth being different, as well as the fire bridge, from those of the puddling furnace. The pipes for conducting the gases are made of cast-iron, and must have at least a sectional area of one foot for every furnace that is to be heated.

Figs. 80, 81, 82, 83, 84, show the application of this invention to the generation of steam. A chimney is here employed only at the commencement of the operation. The 79

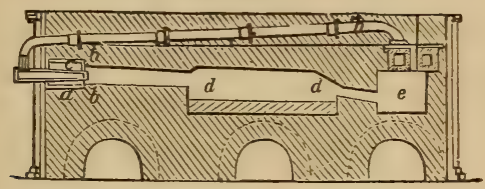

80

84
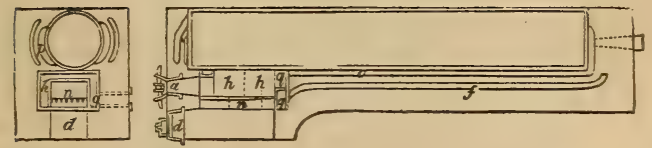

81

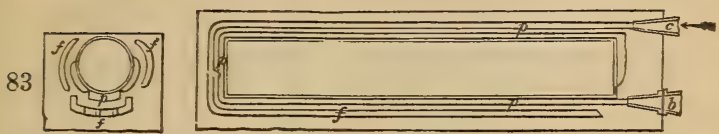

82

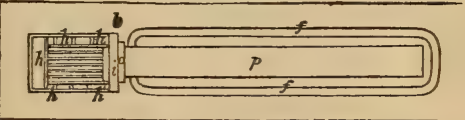

air is forced into the furnace by any sort of blowing machine, or in any other con. venient way. The fuel is introduced into the fireplace, upon the grate $n n$, through the door $a$, which can be closed. The fireplace must contain as much fuel as will last for several hours. When the fire is first lighted, the combustion takes place in the ordinary way, on opening the door $d$, and the slide-valve $b$, and carrying through them a current of air by the chimney draught. This is continued till the steam-engine furnace, or any working (power) engine is in operation, after which a blowing apparatus is employed to force the air through the tube $c$, as shown in $f \mathrm{~g}$. 81. The openings $d$ and $b$ are then closed; the air forced in now passes through the flues $f, f, f$, placed round and beneath the boiler. The air, on arriving at the point $g$, is divided, one portion passes through the opening $h$, regulated by a valve, into the open space beneath the grate $n n$, to assist in the slow combustion of the fuel. The other part of the air passes through $g$, into $h h$, round the fireplace, in order to heat the air to an intense degree. After the second portion of the air has passed into the chamber $h h$, it enters another $i$, thence through a series of blowpipes, or through $o$, into $p p$, beneath the 
boiler. The burnt air goes off through $p p$ into a small chimney, through the opening $b b$, which is regulated by a valve.

IRON, Cast, Strength of.

In the following Table, each bar is reduced to exactly one inch square; and the transverse strength, which may be taken as a criterion of the value of each iron, is obtained from a mean between the experiments upon it, given in the Memoirs;-first on bars $4 \mathrm{ft} .6 \mathrm{in}$. between the supports, and next on those of half the length, or $2 \mathrm{ft} .3 \mathrm{in}$. between the supports. All the other results are deduced from the $4 \mathrm{ft} .6 \mathrm{in}$. bars. In all cases the weights were laid on the middle of the bar.

Table of Results obtained from Experiments on the Strength and other Properties of Cast Iron, from the principal Iron Works in the United Kingdom. By Mr. Wm. Fairbairn.

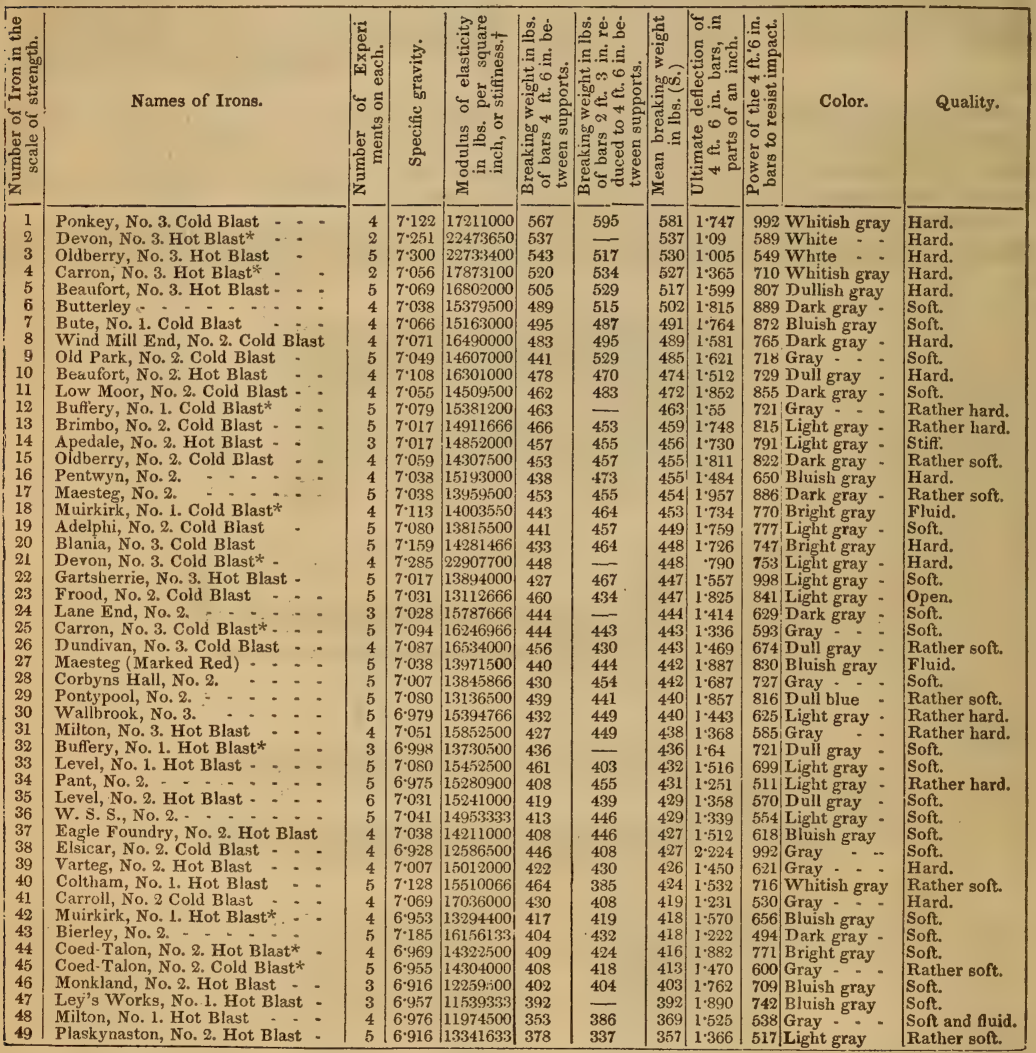

Rule.-To find from the above table the breaking weight in rectangular bars, generally, calling $b$ and $d$ the breadth and depth in inches, and $l$ the distance between the supports in feet, and putting 4.5 for $4 \mathrm{ft} .6 \mathrm{in}$., we have $\frac{4.5 \times b d 2 S}{l}=$ breaking weight in lbs., the value of $\mathrm{S}$ being taken from the table above.

For example:-What weight would be necessary to break a bar of Low Moor iron, 2 inches broad, 3 inches deep, and 6 feet between the supports? According to the rule given above, we have $b=2$ inches, $d=3$ inches, $l=6$ feet, $S=472$ from the table. Then $\frac{4 \cdot 5 \times b d 2 S}{l}=\frac{4.5 \times 2 \times 32 \times 472}{6}=6372 \mathrm{lbs}$, the breaking weight.

* The irons with asterisks are taken from the experiments on hot and cold blast iron, made by Mr. Hodgkinson and myself for the British Association for the Advancement of Science.-See Seventh ort, vol. vi.

+ The modulus of elasticity was usually taken from the deflection caused by $112 \mathrm{lbs}$. on the $4 \mathrm{ft} .6$ in 
IRON. Hot Blast. To the account of this interesting insovation in the smelting of iron ores, given in the dictionary, $I$ have now the pleasure of representing in accurate plans, the complete system mounted at the Codner Park Works belonging to William Jessop, Esq. For the drawings, from which the woodcuts are faithfully copied, I am iadebted to Mr. Joseph Glynn, F.R.S., the distinguished engineer of the Butterly Iron Works.

Figs. 85, 86, 87, exhibit the apparatus of the hot blast in every requisite detail. The smelting furnaces have now generally three tuvères, and three sets of air heating

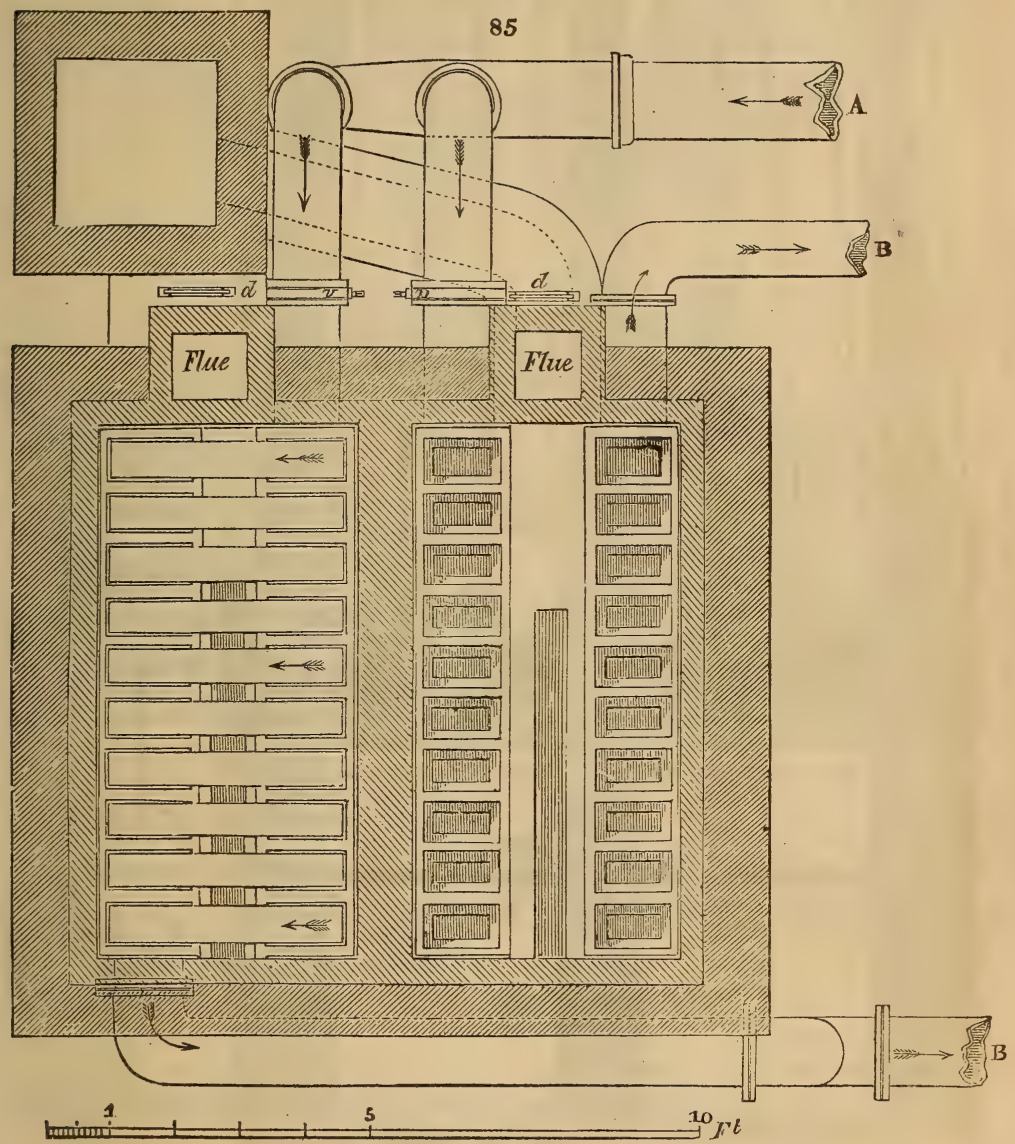

furnaces. The figures show two sets built together; the third set being detached on account of peculiar local circumstances. The air enters the horizontal pipe $A$, in the ground plan, fig. 85, on one side of the arched or syphon pipes, shown in upright section in fig. 86, and passes through these pipes to the horizontal pipe, $B$, on the other side; whence it proceeds to the blast furnace. These syphon pipes are flattened laterally, their section being a parallelogram, to give more heating surface, and also more depth of pipe (in the vertical plane), so as to make it stronger, and less liable to bend by its own weight when softened by the red heat. This system of arched pipe apparatus is set in a kind of oven, from which the flue is taken out at the top of it; but it thence again descends, before it reaches the chimney, entering it nearly at the level of the fire grate (as with coal gas retorts). By this contrivance, the pipes are kept in a bath of ignited air, and not exposed to the corroding influence of a current of flame. The places and directions of these oven flues are plainly marked in the drawing. 
Fig. 87 is a plan of the blast furnace, drawn to a smaller scale than tha preceding figures.

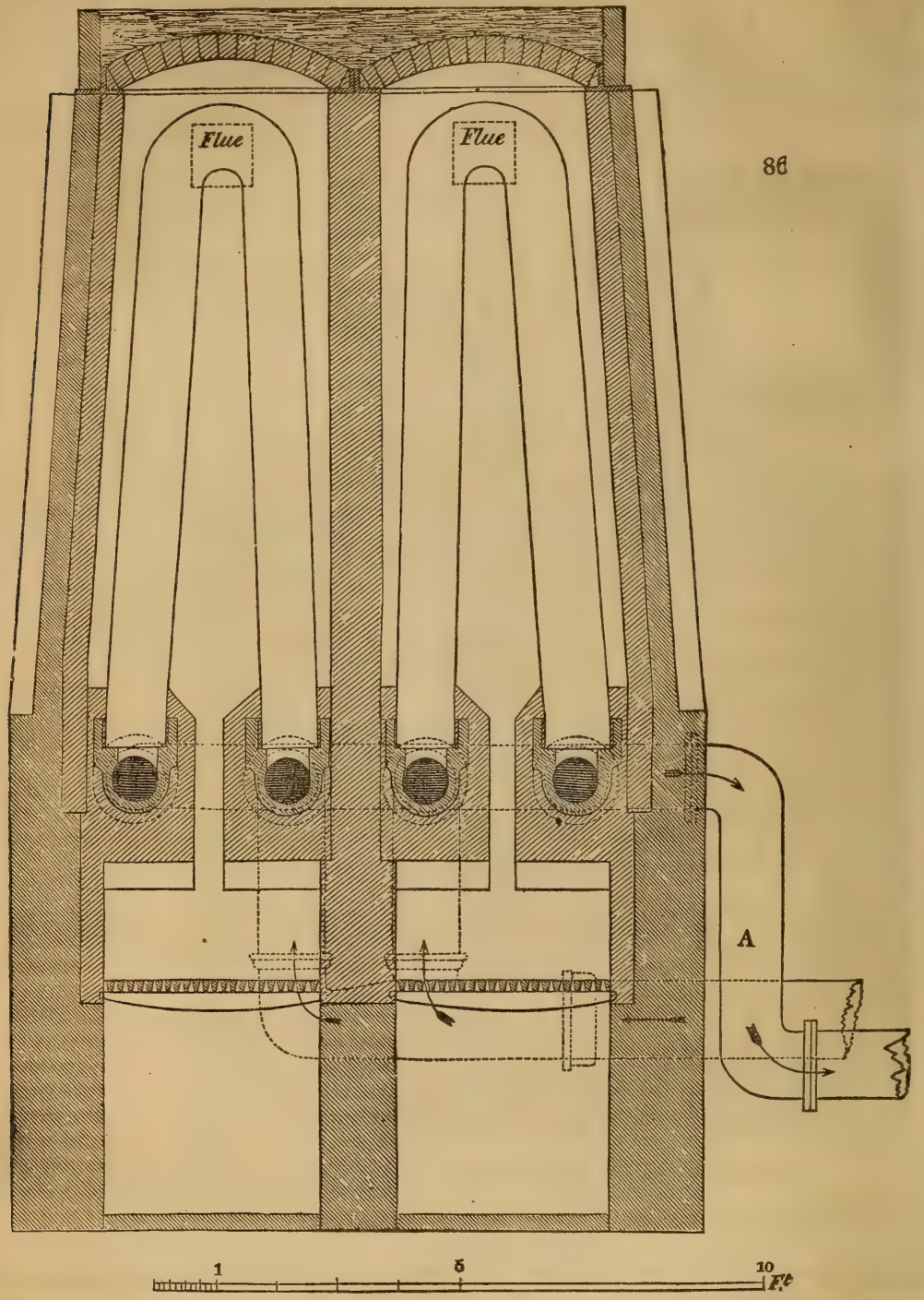

The three sets of hot-blast apparatus, all communicate with one line of conducting pipes, A, which leads to the furnace. Thus in case of repairs being required in one set, the other two may be kept in full activity, capable of supplying abundance of hot air to the blast, though of a somewhat lower temperature. See Smelting for constructions of different blast furnaces; also PudDLING.

During a visit which I have recently made to Mr. Jessop, at Butterley, I found this eminent and very ingenious iron-master had made several improvements upon his hot-blast arrangements, whereby he prevented the alteration of form to which the arched pipes were subject at a high temperature, as also that he was about to employ five tuyères instead of three. For a drawing and explanation of his furnace-feeding apparatus, see Smelting. 


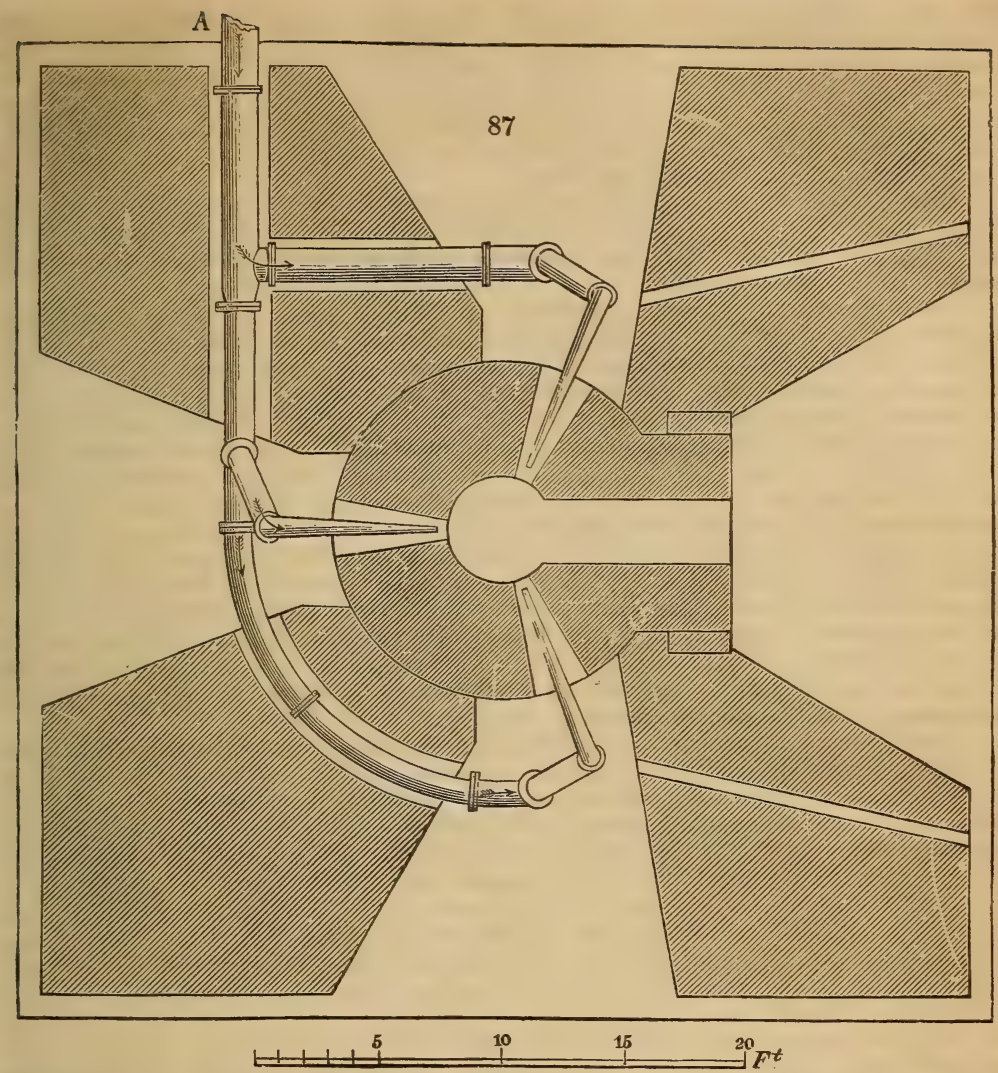

ISINGLASS. Imported for home consumption in 1839, 1,644 cwts.: in 1840, 1,589 cwts. See GeLatine for excellent substitutes for isinglass in culinary operations. Were beer brewed by the Bavarian plan of fermentation, it would require no isinglass for fining it.

IVORY. Imported of elephant's teeth for home consumption in $1839,3,929$ cwts.; in $1840,4,491 \mathrm{cwts}$. Duty $1 \mathrm{~s}$.

\section{K.}

KILLAS. The name given by the Cornish miners to clay slate, commonly of a greenish color, in which the richest deposites of copper and tin occur.

$$
\text { L, }
$$

LAC DYE. Imported for home consumption in $1839,532,881$ pounds; in 1840 , 644,092 pounds; $6 s$. per ewt. duty.

LACTIC ACID. See Fermentation.

LAMPS. The leading novelty under this title, is the construction of lamps for burning spirits of turpentine, in the place of the fat oils which alone have bee. 1 in use from the most remote ages down to the present year. Two patents have recently been obtained for these lamps, under the fantastic title of Camphine; one by Mr. William Young, and another by Messrs. Rayner and Carter, as the invention of a working miner-Roberts. Having been employed by the proprietors of these patents to examine the performances of their respective lamps, I here insert the two reports drawn up by me on these occasions :-

"The Vesta lamp, burning with its utmost brilliancy, without smoke, emits a 
light equal to very nearly twelve wax or sperm candles of three or four to the pound; and in so doing, it consumes exactly one imperial pint of spirits of turpentine (value sixpence retail) in ten hours, hence the cost per hour for a light equal to ten such candles is one halfpenny; whereas that from wax candles would be nearly sixpence, from spermaceti ditto, fivepence; from stearine ditto, fourpence; from Palmer's spreading wick ditto, nearly threepence; from tallow moulds $2 \frac{1}{4} d$. ; from sperm vil in Carcel's Mechanical French Lamp, $1 \frac{1}{2} d$.

"One peculiar advantage of the Vesta lamp is the snowy whiteness of its light, which is such as to display the more delicate colors of natural and artificial objects, flowers, paintings, \&c., in their true tints, instead of the degraded hues visible by the light of candles and ordinary oil lamps.

"The size of the flame from which so much light is emitted in the Vesta lamp, is greatly smaller than that of oil or gas argand flames of equal intensity; a circumstance to be accounted for from the difference in chemical composition, between spirits of turpentine and fat oils. The spirits consist entirely of carbon and hydrogen; in the proportion of $88 \frac{1}{2}$ of the former element, and $11 \frac{1}{2}$ of the latter, in 100 parts ; and they consume 328 parts of oxygen; whereas, sperm and other unctuous oils consist of 78 parts of carbon, $11 \frac{1}{2}$ of hydrogen, and $10 \frac{1}{2}$ of oxygen, in 100 parts; and these consume only 287.2 of oxygen, in being burnt; because the oxygen already present in the oil neutralizes $2 \cdot 6$ parts of the carbon and 0.4 of the hydrogen, thus leaving only $85 \frac{2}{2}$ parts of the combustible elements for the atmosphere to burn. For this reason, $87 \frac{1}{2}$ parts by weight of spirits of turpentine, will consume as much oxygen as 100 parts of sperm oil ; and will afford, moreover, a more vivid light, because they contain no oxide, as fat oils do, which serves to damp the combustion. In the spirits of turpentine, the affinity of its elements for oxygen is entire, whereas in fat oil the affinity is partially neutralized by the oxides it contains; somewhat as the flame of spirits of wine is weakened by their dilution with water.

"Among the many applications of science to the useful arts, for which the present age is so honorably distinguished, few are more meritorious than the Camphine Lamps, by which we can produce a snow-white flame from the cleanly, colorless spirits of turpentine- a pure combustible fluid, in place of the smeary rank oils which contain a seventh part of incombustible matter. Being so rich in hydro-carbon, the spirits require peculiar artifices for complete consumption and the development of their full power of yielding light without smoke or smell. This point of perfection seems to be happily attained by the invention of the two parallel flat rings, in the Paragon Lamp, a larger and smaller, forming a cone round the margin of the wick, which cause a rapid reverberation of the air against the flame: thus consuming every particle of volatilized vapor, and adding energy to the luminous undulations. Hence the Patent Paragon Lamp in full action emits a light equal to that of sixteen wax candles three to the pound, but of better quality, approaching in purity to that of the sun-beam-therefore capable of displaying natural and artificial objects in their true colors.

"One imperial pint of rectified spirits of turpentine, value $6 d$. retail, will burn for twelve hours in this lamp, affording all the time the illumination of eleven wax candles.

"The Paragon Camphine lamp is attended with no danger in use.

"The cost, as compared with other Lamps or Candles, is as follows : viz. :-

PER HOUR.

Paragon Camphine Lamp (equal to 11 wax candles) less than One Halfpenny.

Wax Candles

Spermaceti ditto

Adamantean wax (Stearic Acid)

Palmer's Spread-Wick Candles -

Cocoa Nut Candles

Moulds (Tallow)

Carcel's Lamp, with Sperm Oil

See Illumination, cost of, for a description of an excellent oil lamp.

LEAD. The total produce of the lead mines of Great Britain was estimated in 1822 , at 31,900 tons, which were distributed as follows :-

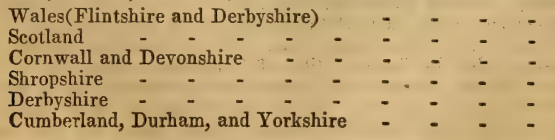

\begin{tabular}{lr}
$-\quad 7,500$ tons. \\
$-\quad 2,800$ \\
$-\quad 800$ \\
$=\quad 800$ \\
$=\quad 1,000$ \\
$-\quad 19,000$ \\
\hline 31,900
\end{tabular}


And in the year 1835 , the total produce was estimated by Mr. John Taylor at 46,112 tons; of which 19,626 were furnished by Northumberland, Durham, and Cumberland; the mines of Mr. Beaumont alone yielding 10,000. See SoLDER.

LEATHER. In the Franklin Institute for February, 1843, Mr. Gideon Lee has published some judicious observations on the process of tanning. He believes that much of the original gelatine of the hides is never combined with the tannin, but is wasted; for he thinks that 100 lbs. of perfectly dry hides, when cleansed from extraneous matter, should, on chemical principles, afford at least $180 \mathrm{lbs}$. of leather. The usual preparation of the hide for tanning he believes to be a wasteful process. In the liming and bating, or the unhairing and the cleansing, the general plan is first to steep the hides in milk of lime for one, two, or three weeks, according to the weather and texture of the skin, until the hair and epidermis be so loosened as to be readily removed by rubbing down, by means of a knife, upon a beam or block. Another mode is to suspend the hides in a close chamber heated slightly by a smouldering fire, till the epidermis gets loosened by incipient putrefaction. A third process, called sweating, used in Germany, consists in laying the hides in a pack or pile, covered with tan, to promote fermentative heat, and to loosen the epidermis and hairs. These plans, especially the two latter, are apt to injure the quality of the hides.

The bate consists in steeping the haired hides in a solution of pigeons' dung, containing, Mr. Lee says, muriate of ammonia, muriate of soda, \&c. ; but most probably phosphates of ammonia and lime, with urate of ammonia, and very fermentable animal matter. The dry hides are often subjected first of all to the operation of the fullingstocks, which opens the pores, but at the same time prepares them for the action of the liming and bate; as also for the introduction of the tanning matter. When the fulling is too violent, the leather is apt to be too limber and thin. Mr. Lee conceires that the liming is injurious, by carrying off more or less of the gelatine and albumen of the skin. High-limed leather is loose, weighs light, and wears out quickly. The subsequent fermentation in the bating aggravates that evil. Another process has therefore been adopted in New York, Maine, New Hampshire, and some parts of Philadelphia, called, but incorrectly, cool sweating, which consists in suspending the hides in a subterranean vault, in a temperature of $50^{\circ} \mathrm{F}$., kept perfectly damp, by the trickling of cold springwater from points in the roof. The hides being first soaked, are suspended in this rault from 6 to 12 days, when the hair is well loosened by the mere softening effects of moisture, without fermentation.

LEATHER, MOROCCO. (Maroquin, Fr.; Saffian, Germ.) Morocco leather of the finer quality is made from goatskins tanned with sumach; inferior morocco leather from sheepskins. The goatskins as imported are covered with hair; to remore which they are soaked in water for a certain time, and they are then subjected to the operation called breaking, which consists in scraping them clean and smooth on the flesh side, and they are next steeped in lime-pits (milk of lime) for several days, during which period they are drawn out, with a hook, from time to time, laid on the side of the pit to drain, and replunged alternately, adding occasionally a little lime, whereby they are eventually deprived of their hair. When this has become sufficiently loose, the skins are taken out one by one, laid on convex beams, the work-benches, which stand in an inclined position, resting on a stool at their upper end, at a height convenient for the workman's breast, who scrapes off the hair with a concave steel blade or knife, having a handle at each end. When unhaired, the skins are once more soaked in milk of lime for a few days, and then scraped on the flesh side to render it very even. For removing the lime which obstructs their pores, and would impede the tanning process, as well as to open these pores, the skins are steeped in a warm semi-putrid alkaline liquor, made with pigeons' and hens' dung diffused in water. Probably some very weak acid, such as fermented bran-water, would answer as well, and not be so offensive to the workmen. (In Germany the skins are first washed in a barrel by a revolving axle and discs.) They are again scraped, and then sewed into bags, the grain outermost, like bladders, leaving a small orifice, into which the neck of a funnel is inserted, and through which is poured a certain quantity of a strong infusion of the sumach; and they are now rendered tight round the orifices, after being filled out with air, like a blown bladder. A parcel of these inflated skins are thrown into a very large tub, containing a weaker infusion of sumach, where they are rolled about in the midst of the liquor, to cause the infusion within to act upon their whole surface, as well as to expose their outsides uniformly to the tanning action of the bath. After a while these bladder-skins are taken out of the bath, and piled over each other upon a wooden rack, whereby they undergo such pressure as to force the enclosed infusion to penetrate through their pores, and to bring the tannin of the sumach into intimate contact, and to form a chemical combination with the skin fibres. The tanning is completed by a repetition of the process, of introducing some infusion or decoction into them, blowing them up, and floating them with agitation in the bath. In this way goatskins may be well tanned in the course of one day. 
The bag 3 are next undone by removing the sewing, the tanned skins are scraped as before on the currier's bench, and hung up in the drying loft or shed; they are sald now to be " in the crust." They are again moistened and smoothed with a rubbingtool before being subjected to the dyeing operations, in which two skins are applied face to face to confine the dye to one of their surfaces only, for the sake of economizing the dyeing materials which may be of several different colors. The dyed skins are grained by being strongly rubbed with a ball of box-wood, finely grooved on its surface.

TAWING of Skins. (Megisserie, Fr.; Weissgerberei, Germ.) The kid, sheep, and lamb slins, are cleansed as has been described under leather in the Dictionary. In some factories they receive the tanning power of the submuriate of alumina (from a solution of alum and common salt) in a large barrel-churn apparatus; in which they are subjected to violent agitation, and thereby take the aluming in the course of a few minutes. In other cases, where the yolks of eggs are added to the above solution, the mixture, with the skins, is put into a large tub, and the whole trampled strongly by the naked feet of the operator, till the emulsion of the egg be forced into the pores of the skin. The tawed skins, when dry, are "staked," that is, stretched, scraped, and smonthed, by friction against the blunt edge of a semicircular knife, fixed to the top of a short beam of wood set upright. The workman holding the extremities of the skin with both hands, pulls it in all directions forcibly, but skilfully, against the smoothing "stake."

In an entertaining article on tanning in the 11th volume of the Penny Magazine, at page 215 , the following description is given of one of the great tawing establishments of London :-

"In the production of 'imitation' kid leather, the skin of lambs is employed; and for this purpose lambskins are imported from the shores of the Mediterranean. They are imported with the wool yet on them; and as this wool is valuable, the leathermanufacturer removes this before the operations on the pelt commence. The wool is of a quality that would be greatly injured by the contact of lime, and therefore a kind of natural fermentation is brought about as a means of loosening the wool from the pelt. At the Neckinger establishment of Messrs. Bevington and Co., Bermondsey, one of the buildings presents, on the ground floor, a flight of stone steps, leading down to a range of subterranean vaults or close rooms, into which the lambskins are introduced in a wet state, after having been steeped in water, 'broken' on the flesh side, and drained. The temperature of these rooms is nearly the same all the year round, a result obtained by having them excluded as much as possible from the variations of the external atmosphere; and the result is that the skins undergo a kind of putrefactive or fermenting process, by which the wool becomes loosened from the pelt. During this chemical change ammonia is evolved in great abundance; the odor is strong and disagreeable; a lighted candle, if introduced, would be instantly extinguished, and injurious effects would be perceived by a person remaining long in one of the rooms. Each room is about ten feet square, and is provided with nails and bars whereon to hang the lambskins. The doors from all the rooms open into one common passage or vault, and are kept close, except when the skins are inspected. It is a point of much nicety to determine when the fermentation has proceeded to such an extent as to loosen the wool from the pelt; for if it be allowed to proceed beyond that stage, the pelt itself would become injured."

When the fermentation is completed, generally in about five days, the skins are removed to a beam, and there 'slimed'-that is, scraped on the flesh side, to remove a slimy substance which exudes from the pores. The wool is then taken off, cleaned, and sold to the hatters, for making the bodies of common hats. The stripped pelts are steeped in limc-water for about a week, to kill the grease ; and are next "fleshed on the beam." After being placed in a "drench." or a solution of sour bran for some days to remove the lime and open the pores, the skins are alumed, and subjected to nearly the same processes as the true kidskins. (See Leather.) These Mediterranean lambskins do not in general measure more than about 20 inches by 12 ; and each one furnishes leather for two pairs of small gloves. These kinds of leather generally leave the leather-dresser in a white state ; but undergo a process of dyeing, softening, "stroking," \&c., before being cut up into gloves.

The tanning of one average-sized skin requires about $1 \frac{1}{4}$ lbs. of good Sicilian sumach; but for leather which is to receive a bright scarlet dye, from one half to three quarters of a pound of gall-nuts are employed in preference. Inferior goatskins are tanned with a willow-bark infusion, in pits, in which they are turned repeatedly, and laid out to drain, as in tanning sole-leather. The finest skins for the brightest scarlet are cured with salt, to prevent, their receiving lamage in the transport, and are dyed before being tanned. This method is practised in Germany and France.

Leather of deer and sheep-skins is prepared with oil, for the purpose of making breeches, \&c., and for wash-leather, used in cleaning plate. After they are completely washed, limed, and beamed, as above described, they have their "grain"-surface re- 
moved, to give them greater softness and pliability. This removal of the grain is called "frizing," and it is done either with the round edge of a blunt knife, or with pumice-stone. After being freed from the lime by steeping in fermented bran-water, they are pressed as dry as may be, and are then impregnated with cod-oil, by beating with stocks in the trough of a kind of fulling-mill. Previously to the application of the oil, they are usually beat for some time alone to open their substance. The oiled skins are stretched, hung up for some time in the air, then fulled with oil as before-a process which is 8 or 9 times repeated. The oil is slowly and evenly poured upon the skins in the trough, during the action of the beaters. One hundred skins usually take up in this way from two to three gallons of oil. The fulled oiled skins are thrown into large tubs, and left for some time to ferment, and thereby to combine more intimately with the oil. They are lastly subjected to a weak potash ley bath, to strip them of the loosely adhering oil. They are then hung up in the air to dry, and dressed for the market.

The quantity of hides and skins converted into leather yearly in England is almost incredibly large. At Messrs. Bevington's establishment alone there are about 250,000 skins annually converted inte leather by the aluming or tawing process; 220,000 by the sumach tanning process; as also a small number by the oil-dressing process. For the importation and exportation of skins, untanned and tanned, see Hides.

In 1839, 5,149 Russian tanned hides were imported for home consumption; and in $1840,4,664$; of $5 s$. of duty on the entire hide; and pieces $2 s .6 d$. per $1 \mathrm{lb}$.

The declared value of leather exported in 1840 was $320,912^{\prime}$; weight, $2,404,667$ lbs Saddlery and harness of $96,167 l$. declared value were exported.

Leather gloves imported for home consumption in 1839, 991,623 pairs; in 1840, $1,503,862$; average duty, 5s. a dozen.

LEATHER SPLITTING. This operation is employed sometimes upon certain sorts of leather for glovers, for bookbinders, sheath-makers, and always to give a uniform thickness to the leather destined for the cotton and wool card-makers.

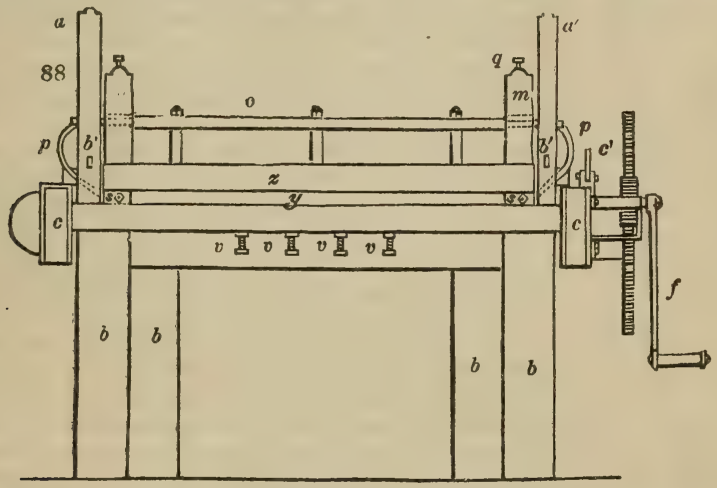

Figs. 88, 89, 90, 91, represent a well contrived machine for that purpose; of which f. 88 , shows the front view, fig. 89 , a view from the left side, fig. 91 , a ground plas
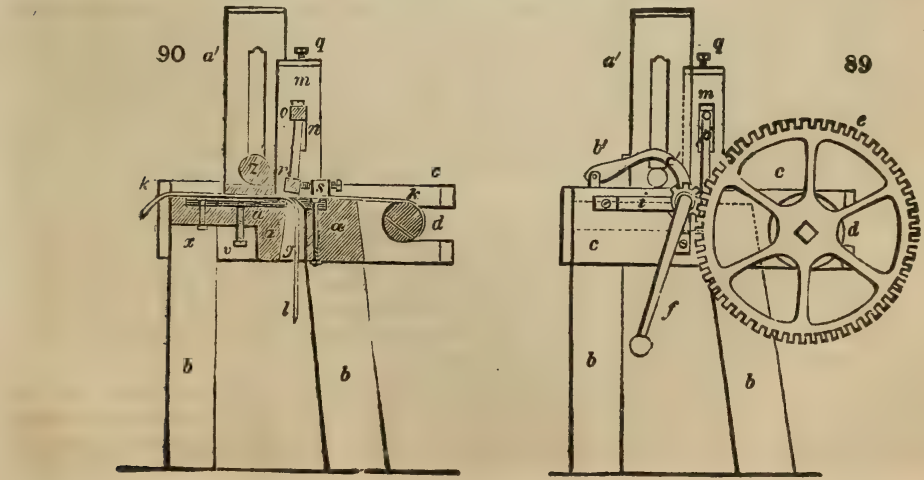
and fig. 90 a vertical section across the machine. $a$ is a strong table, furnished with four legs $b$, which to the right and left hand bears two horizontal pieces $c$. Each of these pieces is cut out in front, so as to form in its substance a half-round fork, that receives a cylinder $d$, carrying on its end a toothed spur-wheel $e$. Motion is communicated to the wheel by means of the handle $f$, upon whose axis the pinion, $i$, is fixed; working into the wheel $d$, made fast to the end of the cylinder round which the leather is rolled. The leather is fixed at one of its ends or edges to the cylinder, either with a wedge pressed into a groove, or by a moveable segment of the cylinder itself.

The table, $a$, is cut out lengthwise with a slot, that is widened below, as shown ir fig. 90 .

The knife $h$ (figs. 90 and 91) is fixed flat upon the table with screw bolts, whose

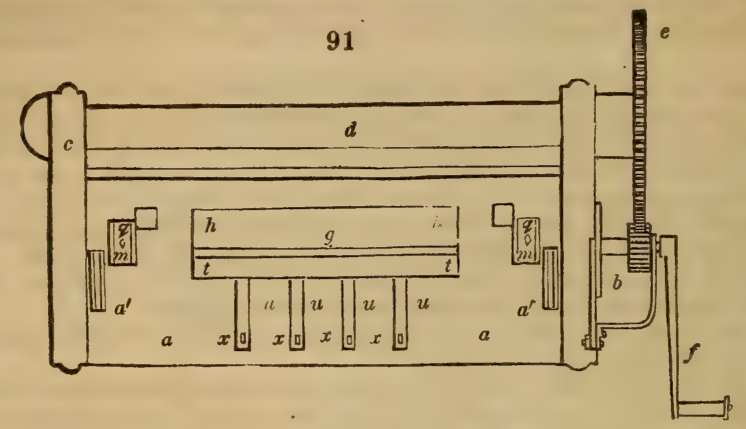

heads are countersunk into the table, and secured with taps beneath ( $f$ g. 90 ), the edge of the knife being placed horizontally over the opening, and parallel with it.

In fig. 90, the Jeather, $k$, is shown advancing against the knife, getting split, and has a portion coiled round the cylinder, which is made to revolve in proportion as the leather is cleft. The upper portion of the leather is rolled upon the cylinder $d$, while the under half, $l$, falls through the oblong opening upon the ground.

In regulating the thickness of the split leather, the two supports, $m$, act; they are made fast to the table $a$ (one on each side of the knife), and are mortised into the table by two tenons secured beneath. These supports are furnished near their tops with keyed slots, by means of which the horizontal iron rod o ( $f$ igs. 88,90 ) is secured, and outside of the uprights they press upon the springs $p, p$, which tend to raise the rod, 0 , in its two end slots; but the adjusting screws $q$, which pass down through the tops of the supports into the mortise $n$ ( fig. 90) and press upon the upper half of the divided tenon, counteract the springs, and, accordingly, keep the rod, $o$, exactly at any desired height or level. The iron rod, $o$, carries another iron bar, $r$, beneath it, parallel and also rectangular, fig. 90. This lower bar, which is rounded at its under face lies upon and presses the leather, by the action of two screws, which pass through two upright pieces $s$ ( figs. 88 and 90), made fast to the table; thus the iron bar, $r$, may be made to press forward the edge of the knife, and it may be adjusted in its degree of pressure, according to the desired thickness of the leaf of split leather, that passes through under it.

Fig. 90 shows that the slant or obliquity of the knife is directed downward, over one of the edges of the oblong opening $g$; the other edge of this opening is provided with an iron plate $t$ ( $f$ igs. 90,91 ), which serves to guide the blade in cutting the leather to the proper depth. For this purpose the plate is made adjustable by means of the four springs $u$ (figs. 90, 91), let into the table, which press it downward. Four screws $v$, pass down through the table, each belonging to its respective springs $u$, and by means of these screws the plate, $t$, may be raised in any desired degree. Each of the screws, $u$, has besides a small rectangular notch, through which a screw bolt, $x$, passes, by which the spring is made fast to the table. Thus also the plate, $t$, may be made to approach to or recede from the knife.

$y$, in $f$ g. .88 and 90 , is a flat board, laid upon the leather a little behind the edge of the plate $t$; this board is pressed by the cylinder $z$, that lies upon it, and whose tenons rest in mortises cut out in the two supports $a^{\prime}$. The cylinder, $z$, is held in its position by a wedge or pin $b$ ( $f$ igs. 88 and 89 ), which passes through the supports. When the leather has been split, these pins are removed, and the cylinder rises then by means of two counter weights, not shown in the figures.

The operation of the machine is as follows-The edge or end of the leather being secured to the cylinder $d$, the leather itself having the direction upon the table, shown in fig. 90, and the bar, $r$, its proper position over the knife, the edge begins to enters in 
this position into the leather, while the cylinder, $d$, is moved by the handle or winch, and the piece gets between the blade and the roller $d$. When the other end of the leather, $k$, advances to the knife, there is, consequently, one half of the leather split; the skin is to be then rolled off the cylinder $d$; it is turned; the already split half, or the end of the leather $k$, is made fast into the wood of the cylinder, and the other half is next split; while the knife now acts from below, in an opposite direction to what it did at first.

That the unrolling of the leather from the cylinder, $d$, may not be obstructed by the pinion $i$, the stop-wedge $e$ ( $f$ igs. 88, 89) is removed from the teeth. In the process of splitting, the grain side of the leather is uppermost, and is therefore cut of a uniform thickness, but the under side varies in thickness with the inequality of the skin.

LINSEED. Imported for home consumption, in 1839, 3,852,359 bushels ; in 1840, $3,256,257 ; 1 \frac{1}{2} d$. duty.

LODES. The name given by the Cornish miners to metallic veins: as, tin lodes, copper lodes, \&c.

LOGWOOD; imported for home consumption in 1839, 17,209 tons; in 1840, 18,683 tons; duty $3 s .$, foreign $4 s .6 d$.

\section{M.}

MACE. Imported for home consumption, in 1839, 21,154 pounds; in 1840, 16,813, duty $2 s .6 d$. per pound.

MADDER, GROUND; imported for honte consumption in $1839,96,702$ cwts.; in $1840,134,179$ cwts. ; duty $2 s$. per cwt.

MADDER ROOT; in $1839,80,259$ cwts.; in 1840, 112,714 cwts.; duty $6 d$. per cwt.

A patent was granted in August, 1843, to Mr. F. Steiner, for the manufacture of Garancine from used madder, formerly thrown away, as being exhausted of its dyeing principle. His process is as follows: "A large filter is constructed outside the building in which the dye-vessels are situated, formed by sinking a hole in the ground, and lining it at the bottom and sides with bricks without any mortar to unite them. A quantity of stones or gravel is placed upon the bricks, and over the stones or gravel common wrappering, such as is used for sacks. Below the bricks is a drain to take off the water which passes through the filter. In the tub adjoining the filter is kept a quantity of dilute sulphuric acid, of about the specific gravity of 105 , water being 100 . Hydrochloric acid will answer the several purposes, but sulphuric acid is preferred as more economical. A channel is made from the dye-vessels to the filter. The madder which has been employed in dyeing is run from the dye-vessels to the filter; and while it is so running, such a portion of the dilute sulphuric acid is run in and mixed with it as changes the color of the solution and the undissolved madder to an orange tint or hue. This acid precipitates the coloring matter which is held in solution, and prevents the undissolved madder from fermenting or otherwise decomposing. When the water has drained from the madder through the filter, the residuum is taken from off the filter and put into bags. The bags are then placed in an hydraulic press, to have as much water as possible expressed from their contents. In order to break the lumps which have been formed by compression, the madder or residuum is passed through a sieve. To $5 \mathrm{cwt}$. of madder in this state, placed in a wood or lead cistern, $1 \mathrm{cwt}$. of sulphuric acid of commerce is sprinkled on the madder through a lead vessel similar in form to the ordinary watering-can used by gardeners. An instrument like a garden spade or rake is next used, to work the madder about so as to mix it intimately with the acid. In this stage the madder is placed upon a perforated lead plate, which is fixed about five or six inches above the bottom of a vessel. Between this plate and the bottom of the vessel is introduced a current of steam by a pipe, so that it passes through the perforated plate and the madder which is upon it. During this process, which occupies from one to two hours, a substance is produced of a dark brown color approaching to black. This substance is garancine and insoluble carbonized matter. When cool, it is placed upon a filter and washed with clear cold water until the water passes from it without an acid taste. It is then put into bags and pressed with an hydraulic press. The substance is dried in a stove and ground to a fine powder under ordinary madder stones, and afterward passed through a sieve. In order to neutralize any acid that may remain, from 4 to $5 \mathrm{lbs}$. of dry carbonate of soda for every hundred weight of this substance is added and intimately mixed. The garancine in this state is ready for use. 
MALT. The Quantity of Malt consumed by the undermentioned Brewers of London and its Vicinity, from 10th October, 1830, to 10th October, 1842.

\begin{tabular}{|c|c|c|c|c|c|c|c|c|c|c|c|c|}
\hline & 1831. & $1+32$. & 1833 & 1834 & 1835. & $1-36$ & $183 \%$. & 1838. & 1839. & 1840. & 1841. & 1842. \\
\hline & & & Qrs. & & Qis. & Qis. & Qrs. & Qrs. & Qrs. & Qrs. & Qrs. & Qrs. \\
\hline $\begin{array}{l}\text { Parclay and Co. - } \\
\text { Hanbury and Co. }\end{array}$ & {$\left[\begin{array}{l}9,198 \\
50,-724\end{array} \mid\right.$} & $\begin{array}{l}96,612 \\
58,512\end{array}$ & $\begin{array}{l}93,175 \\
58,4 y \text {; }\end{array}$ & $\begin{array}{l}99,674 \\
74.982\end{array}$ & $\begin{array}{l}106,098 \\
78,08 i\end{array}$ & $\begin{array}{l}108,715 \\
84,303\end{array}$ & $\begin{array}{r}100,326 \\
81,440\end{array}$ & $\begin{array}{r}107,455 \\
90,140\end{array}$ & $\begin{array}{r}1] 4,827 \\
91,069\end{array}$ & $\begin{array}{r}115,561 \\
98,210\end{array}$ & $\begin{array}{r}106,345 \\
88,132\end{array}$ & $\begin{array}{l}14,090 \\
92,466\end{array}$ \\
\hline Whitbread and Co. & & 53.541 & $50,06:$ & 49,105 & 55,209 & 53,694 & 47,012 & & \begin{tabular}{|l|}
$51,9 \pi 9$ \\
\end{tabular} & 53,622 & 51,457 & 52,098 \\
\hline Reid and Co. & & 44,420 & 40.810 & & 49,430 & & & & 44,010 & 48,130 & & 50,120 \\
\hline Meux and Co. - & & 22,062 & 2 & & 24,376 & 30 , & 30,623 & 35,065 & 38,466 & 87 & 797 & 43,340 \\
\hline Combe and Co. & 3 & 8 & 3 & & 3 & 42, & 40,454 & & 40 & & 36,460 & \\
\hline Calvert and Co. - & 30 & 32,812 & 31,433 & 31,460 & 33,263 & 30,859 & 32,325 & 31,529 & 31,028 & $30,8 \div 2$ & 30,614 & $\begin{array}{l}30,660 \\
30,0\end{array}$ \\
\hline Hoare and Co. & & 2] & $25,40 \%$ & & 31,525 & & & 78 & 31,008 & & & \\
\hline Elliot and Co. & 19,444 & 20,061 & 19,899 & 25,009 & 28,728 & 28,333 & 24,150 & 22,486 & 22,990 & 25,367 & 25,379 & 27,050 \\
\hline Thorne, $T$. and Son & & 2,543 & 5,136 & 8,496 & 10,913 & $12,65 \pi$ & 16,404 & 18,545 & 19,578 & 20,864 & 22,413 & 22,022 \\
\hline Charrington and Co. & 10,531 & 9,648 । & 15,617 & 18,197 & 19,213 & 19,445 & $\mid 18,842$ & 20,290 & 18,688 & 18,328 & 17,840 & 20,423 \\
\hline $\begin{array}{l}\text { Steward and Co. - } \\
\text { Taylor and Co. }\end{array}$ & $\begin{array}{r}8,116 \\
21,845\end{array}$ & $\begin{array}{r}0,072) \\
21,735\end{array}$ & 21,115 & 20,835 & 23 & 24,971 & 23,056 & 27,320 & 25,955 & 27,300 & 21,424 & 19,430 \\
\hline Goding, J. and Co. & & 874 & & 15,256 & & & $\frac{1}{1} \frac{1}{2} 14,023$ & 14,028 & 12,145 & & & \\
\hline $\begin{array}{l}\text { Goding, Thomas - } \\
\text { Remsbottom and C }\end{array}$ & & & 7,630 & & 18 & $\mid \begin{array}{l}4 \\
31,784 \\
\frac{3}{4} 15,364\end{array}$ & $\begin{array}{r}7,095 \\
15,227\end{array}$ & $\begin{array}{r}7,551 \\
13,012\end{array}$ & $\frac{3}{4} 5,758$ & Dit & 16,018 & 17,071 \\
\hline & & & & & & $\mid \frac{0}{4} 15,304$ & & & 10,610 & $y_{2}, t 30$ & 15,791 & 16,688 \\
\hline Gar & 6,666 & 5,904 & 7,471 & 11,429 & 14,699 & 15,369 & 15,256 & 16,921 & 04 & 15,559 & 126 & 14,546 \\
\hline Mar & & & 1,332 & $1,75 \pi$ & 2,780 & 4,840 & & & & 679 & 111 & \\
\hline $\mathrm{Cou}$ & 8,116 & 7,607 & 7,546 & 8.079 & 8,790 & 29 & 86 & 10,723 & 10,456 & 11,532 & 12,328 & 13,016 \\
\hline Wo & 5,469 & 60 & $5,54 \pi$ & 7,602 & $\tau, 320$ & 51 & 34 & 8,506 & 7,607 & 94 & 7,268 & 7,652 \\
\hline Robert & 2,535 & & & 1 & 4,130 & & & 29 & 13 & & 75 & 026 \\
\hline Harris, Thomas - & $4, \pi 78$ & 4 & 4 , & 4,940 & 4,964 & 4, & 42 & 5,888 & 56 & 52 & 91 & 6,022 \\
\hline Hazard and Co. - & & & 6.203 & 7,094 & & 6,597 & & 6.552 & 6,250 & & 58 & 5,556 \\
\hline Tubb, Williarn - & & & & & 200 & & & 65 & 60 & 778 & 44 & 5,503 \\
\hline Richmond a & 3,785 & 3 , & 3.2 & 3,5 & 3,268 & 3 & & & 4,536 & 4,964 & 30 & 5,424 \\
\hline Hodgson and Co. & 4,206 & 3 & 3,870 & 2,0 & 2,414 & 3,400 & 2,400 & 1,790 & 5,358 & 5,704 & 5,862 & \\
\hline $\begin{array}{l}\text { Abbott, E. } \\
\text { Manners and Co. }\end{array}$ & & & & & & & & & & & & $\begin{array}{l}4,983 \\
4,831\end{array}$ \\
\hline Hale, $\mathrm{C}$ & 4,584 & 4,322 & $3,633^{-}$ & 281 & 3,466 & 3,768 & & 39 & 16 & & 4,418 & 4,468 \\
\hline Halfo & 3,215 & 3,187 & 3,330 & 3,545 & & 3,763 & 3,786 & 4,685 & $3,96 \tau$ & 585 & & \\
\hline Ken & & & & & & & & & & 1405 & 155 & 3,878 \\
\hline and Sor & & & & & & & & & & 125 & 3,860 & 3,676 \\
\hline Dugg & & & & & & 2,201 & 2,665 & 2,288 & 3,020 & 3,001 & 2,574 & \\
\hline Gask & & & & $54 \% 0$ & 5 & 600 & & & & & & 3,354 \\
\hline $\begin{array}{l}\mathrm{M}^{6} \\
\text { Plo }\end{array}$ & & & & & 5,360 & 4,689 & 4,960 & 4,700 & 4,300 & $\begin{array}{r}3,410 \\
\approx 88\end{array}$ & 05 & 3,125 \\
\hline d Bryan & 4,048 & 3,020 & 2,941 & 3 & 4 & 3,573 & 3,583 & 3,167 & 3,213 & 2.658 & $\begin{array}{l}1,653 \\
2,579\end{array}$ & $\begin{array}{l}3,001 \\
2,797\end{array}$ \\
\hline Dra & & & & & & & & & $\begin{array}{l}0,20 \\
1,658\end{array}$ & 11 & 87 & 2,777 \\
\hline $\mathrm{Mi}$ & & & & & & & & & 55 & & & 2,685 \\
\hline $\mathrm{Ke}$ & & & & & & & & & 2,326 & 45 & 45 & 2,445 \\
\hline den & & & & & & & 88 & 393 & 55 & & & 32 \\
\hline Co. & & & & & & & & $1,78 \bar{\tau}$ & 5 & & 17 & 56 \\
\hline Clar & 814 & $85 \pi$ & 1,006 & 1,0 & 1,006 & 1,249 & 1,330 & 1,624 & 48 & 34 & & 55 \\
\hline & & & & & & & & & & 1,903 & 2,597 & 2,211 \\
\hline- & 2,235 & 1,832 & 2,163 & 2,266 & b & 3,738 & 3,783 & 3,749 & 72 & 06 & & 2,050 \\
\hline Ver & & & 844 & 1,140 & 1,208 & 1,302 & 1,573 & 1,735 & 1,749 & 1,762 & 25 & 1,840 \\
\hline $\begin{array}{l}\text { Jones, } \mathrm{T} \text {. } \\
\text { Hering ton and We }\end{array}$ & 585 & 4 & 337 & 375 & 248 & 700 & 956 & 1,338 & 1,555 & 1,879 & 1,810 & 1,808 \\
\hline Hil & 2,910 & 1,748 & 1,974 & 1,96 & 2,042 & 1,872 & 1,853 & 1,911 & 1,835 & 77 & 97 & 1,628 \\
\hline Holt and Sons - & & 54 & 717 & . & 734 & 813 & 756 & 46 & & & & \\
\hline John - & 2,302 & $2,2 \pi 9$ & 4,371 & 2,446 & 2,499 & 2,018 & 2,151 & 1,991 & 1,861 & 23 & 1,528 & 1,520 \\
\hline , P. - & 2,146 & 1,530 & 1,063 & 1,6 . & 2,120 & 2,394 & 2,221 & 84 & 53 & 16 & & 1,429 \\
\hline d Co. & & & & 203 & 472 & 731 & 953 & 1,291 & ],241 & 1,201 & 30 & 1,360 \\
\hline Masterman an & 1,704 & 1,803 & 1,830 & 1,810 & 1,877 & 1,789 & 1,914 & 1,847 & 1,789 & & 92 & 1,295 \\
\hline & & & & & & 2,809 & 2,809 & 428 & 2,412 & 2,413 & 2,204 & \\
\hline . & 98 & & & & & & & & & & & \\
\hline - & 001 & 128 & 218 & & 531 & & 2 & & & & & 1,254 \\
\hline & 901 & 719 & 8 & 79 & 838 & $1,03 \pi$ & 1,025 & 1,010 & 1 & & 2 & 5 \\
\hline $\mathrm{H}$ & & & 269 & 47 & 800 & 1,103 & 1,512 & 1,714 & 1,402 & 1,155 & 53 & 1,087 \\
\hline $\mathrm{Je}$ & & 202 & 355 & 529 & 734 & 772 & 833 & 925 & 856 & 929 & 55 & 1,067 \\
\hline C & & & & & & 756 & 742 & 672 & 975 & 949 & & 65 \\
\hline B & 603 & 684 & 59 & 75 & 968 & 1,067 & 943 & 1,006 & 1,143 & 1,034 & 1,113 & 1,045 \\
\hline M & & & & & & 748 & 820 & 978 & 877 & 782 & 797 & 1,025 \\
\hline Satchell and Son & 2,508 & 3,117 & 1,906 & 2,51 & 2,147 & 2,177 & 1,441 & 1,431 & 1,475 & 1,308 & 1,063 & 945 \\
\hline ck, w. & & & & & & & & & & & $8 \times 3$ & 865 \\
\hline John & 674 & 584 & 640 & 677 & 709 & & $\begin{array}{l}169 \\
766\end{array}$ & $\begin{array}{l}361 \\
821\end{array}$ & $\begin{array}{l}532 \\
853\end{array}$ & 775 & 820 & 846 \\
\hline- & & 99 & 259 & 422 & 496 & 620 & 51 & 25 & $\begin{array}{l}85 \\
76\end{array}$ & 776 & $\begin{array}{l}768 \\
765\end{array}$ & $\begin{array}{l}754 \\
737\end{array}$ \\
\hline George - & 1,018 & 985 & 975 & 1,427 & 1.256 & 1,235 & 1,126 & 1,160 & 81 & 791 & 718 & 708 \\
\hline W. L. & 205 & 176 & 254 & 441 & 519 & 527 & 598 & 407 & 36 & 620 & 627 & 705 \\
\hline H. - - & 846 & 57 & & 3 & 406 & 40 & 565 & 7 & 5 & 6 & 70 & 702 \\
\hline and Son. & 1,187 & 84 & 91 & 850 & 757 & 807 & $6 !$ & 650 & 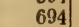 & $\tau 23$ & 641 & 650 \\
\hline i & 756 & 59 & 596 & 65 & 671 & 619 & 768 & 812 & 637 & $\pi 2$ & 638 & 642 \\
\hline and Co. & & & & & & & 397 & 50 & 549 & 592 & & \\
\hline Co & & & 2 & 4 & 671 & 830 & 010 & & & & 637 & 640 \\
\hline rke, W. & & & & & & 839 & 649 & 531 & 504 & 594 & 644 & 624 \\
\hline$\therefore$ & 722 & 841 & & $938^{\circ}$ & 793 & 837 & 74 & 768 & 547 & 450 & $\begin{array}{l}506 \\
302\end{array}$ & $\begin{array}{l}529 \\
520\end{array}$ \\
\hline 3. & & & & & & & 201 & 260 & $\begin{array}{l}340 \\
346\end{array}$ & 433 & 489 & 510 \\
\hline Rudge • • • & & & & & & & & & & & & \\
\hline
\end{tabular}




\begin{tabular}{|c|c|c|c|c|c|c|c|c|c|c|c|c|}
\hline & & 1831. & 1832. & 1833. & 1834 & 1835. & 1836 & $183 \%$ & 1838. & 1839. & 1840. & 1841. \\
\hline enry & - & $\begin{array}{c}\text { Qrs. } \\
5,63 i\end{array}$ & $\begin{array}{l}\text { Qrs. } \\
5,732\end{array}$ & $\begin{array}{c}\text { Qrs. } \\
7,120\end{array}$ & $\begin{array}{l}\text { Qrs. } \\
9,950\end{array}$ & $\begin{array}{l}\text { Qrs. } \\
9, \tau 62\end{array}$ & $\begin{array}{l}\text { Qrs. } \\
9.885\end{array}$ & $\begin{array}{l}\text { Qrs. } \\
9.863\end{array}$ & $\begin{array}{l}\text { Qrs. } \\
8, \times 5 \%\end{array}$ & $\begin{array}{l}\text { Qrs. } \\
\$ .649\end{array}$ & Qrs. & Qrs. \\
\hline and Co. & - & 1,646 & 356 & 883 & $65 \%$ & 403 & 2,085 & 3,600 & 5.251 & & 13,475 & 13,087 \\
\hline and Gooding & $\mathrm{g} \mid-$ & $-\quad-$ & - & - & 1 & - & 1,039 & 1,298 & 1,291 & 1,674 & 1,633 & 1.514 \\
\hline and Co. & - & - & & - & - & - & - & |- & - & 3 & & 1,484 \\
\hline , late Hicks - & - & & - & - & - & - & - & - & 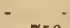 & 1, & & 1.300 \\
\hline Il, Isaac & - & 752 & 713 & 924 & 875 & 834 & 805 & 824 & 756 & 579 & 732 & $7 \% 0$ \\
\hline , E. - & - & 691 & - & 525 & 634 & 654 & $2,3 \div 5$ & 560 & 441 & 312 & $\tau$ & 49 \\
\hline W. & - & $2+4$ & - & 443 & - & 199 & 310 & 315 & $3 \approx 0$ & 4 & 503 & 48 \\
\hline s - & & & - & & - & - & - & - & 81 & 31 & 362 & 47 \\
\hline J. W. - &.- & & - & 179 & 255 & 406 & 295 & 306 & 251 & 2 & 353 & 444 \\
\hline Harris, Robert & $-1-$ & & 1- & 451 & 490 & 557 & 497 & 470 & 456 & 405 & 447 & 441 \\
\hline
\end{tabular}

Barrels of Beer brewed by each of the Twelve principal Brewers in London.

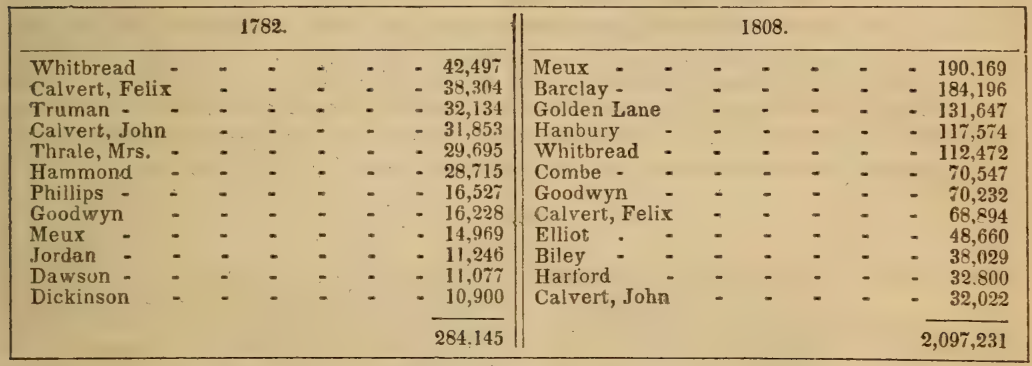

Quarters of Malt consumed in the undermentioned Years, ending 10th October.

\begin{tabular}{|c|c|c|c|c|c|c|c|c|c|c|c|}
\hline \multicolumn{12}{|c|}{ By the Brewers of London and its Vicinity. } \\
\hline $\begin{array}{l}1831 \\
1832\end{array}$ & $\begin{array}{l}622,549 \\
604,47 \pi\end{array}$ & $\begin{array}{l}18.33 \\
18: 34\end{array}$ & \begin{tabular}{|l|}
578,588 \\
662,713 \\
\end{tabular} & \begin{tabular}{|l}
$18 ? 5$ \\
1836 \\
\end{tabular} & \begin{tabular}{|l}
702,533 \\
754,313 \\
\end{tabular} & $\begin{array}{l}1837 \\
1838 \\
\end{array}$ & \begin{tabular}{|l|}
$\pi 14,488$ \\
742,597 \\
\end{tabular} & $\begin{array}{l}1839 \\
1840\end{array}$ & \begin{tabular}{|l|}
750,176 \\
766,219
\end{tabular} & $\begin{array}{l}1841 \\
1842\end{array}$ & $\begin{array}{l}734,295 \\
741,651 \\
\end{array}$ \\
\hline \multicolumn{12}{|c|}{ By the Twelve principal Brewers of London. } \\
\hline $\begin{array}{l}1831 \\
1832\end{array}$ & $\begin{array}{l}432.521 \\
438,046\end{array}$ & $\begin{array}{l}18: 33 \\
18: 34 \\
\end{array}$ & $\begin{array}{l}427,087 \\
470,123\end{array}$ & $\begin{array}{l}1835 \\
1836 \\
\end{array}$ & $\begin{array}{l}503,048 \\
526,092 \\
\end{array}$ & $\begin{array}{l}1837 \\
1838 \\
\end{array}$ & $\begin{array}{l}499.179 \\
517,940 \\
\end{array}$ & $\begin{array}{l}1839 \\
1840\end{array}$ & $\begin{array}{l}528,259 \\
547,908\end{array}$ & $\begin{array}{l}1841 \\
1842\end{array}$ & $\begin{array}{l}517,292 \\
541,710\end{array}$ \\
\hline
\end{tabular}

MANGANESE, OXIDE OF ; for a simple method of ascertaining the value of this substance in the production of chlorine, and the manufacture of the chlorides and chlorates, see Chemistry Simplified, in the Appendix.

MANURE. A patent for an excellent article of this kind was obtained in May, 1842 , by J. B. Lawes, Esq. He decomposes bones, apatite, and other subphosphates of lime, by mixing them in powder with as much sulphuric acid as will liberate enough of the phosphorie to dissolve the phosphate of lime. The free phosphoric acid is thereby ready to combine with the various alkaline earths contained in the soil, while the phosphate of lime is brought to a state of more minute division than is possible by mechanical means. Mr. Lawes also proposes to mix the above soluble superphosphate with such alkalies as are deficient in the soil, and thus to form a manure adapted to fertilize it. His third improvement in manure is the formation and application of a liquor of flints, for such soils as are deficient in soluble silica. The last compound he considers to be valuable for grounds much cropped with wheat and other cereals that require a good deal of silica for their growth.

MARGARIC ACID is obtained most easily by the distillation of stearic acid. The humidity at the beginning of the process must be expelled by a smart heat, otherwise expusive ebullitions are apt to occur. Whenever the ellition becomes uniform, the fire is to be moderated.

MIATCHES, LUCIFER. According to Dr. $\mathrm{R}$ Boettger, in Annalen der Chemie und Fharmacie, vol. xlvii., p. 334, take

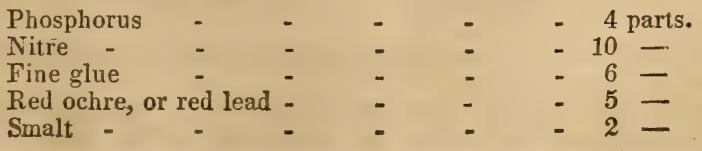


Convert the glue with a little water by a gentle heat into a smooth jelly, put it into a slightly warm porcelain mortar to liquify ; run the phosphorus down through this gelatine at a temperature of about $140^{\circ}$ or $150^{\circ} \mathrm{Fahr}$; add the nitre, then the red powder, and lastly the smalt, till the whole forms a uniform paste. To make writing-paper matches, which burn with a bright flame and diffuse an agreeable odor, moisten each side of the paper with tincture of benzoin, dry it, cut it into slips, and smear one of their ends with a little of the above paste by means of a hair pencil. On rubbing the said end after it is dry, against a rough surface, the paper will take fire without the intervention of sulphur.

To form lucifer wood matches, that act without sulphur, melt in a flat-bottomed tin pan as much white wax as will stand one tenth of an inch deep; take a bundle of wooden matches free from resin, rub their ends against a red hot iron plate till the wood be slightly charred; dip them now in the melted wax for a moment, shake them well on taking them out, and finally dip them separately in the above viscid paste. When dry, they will kindle readily by friction.

For the rapid manufacture of the wooden splints for lucifer matches, a patent was granted to Mr. Reuben Partridge, in March, 1842. He employs a perforated metallic plate, having a steel face, strengthened by a bell metal back; see figs. 92, 93. The size of the perforations must depend on that of the desired splints, but they must be as close together as possible, that there may be a very small blank space between them,
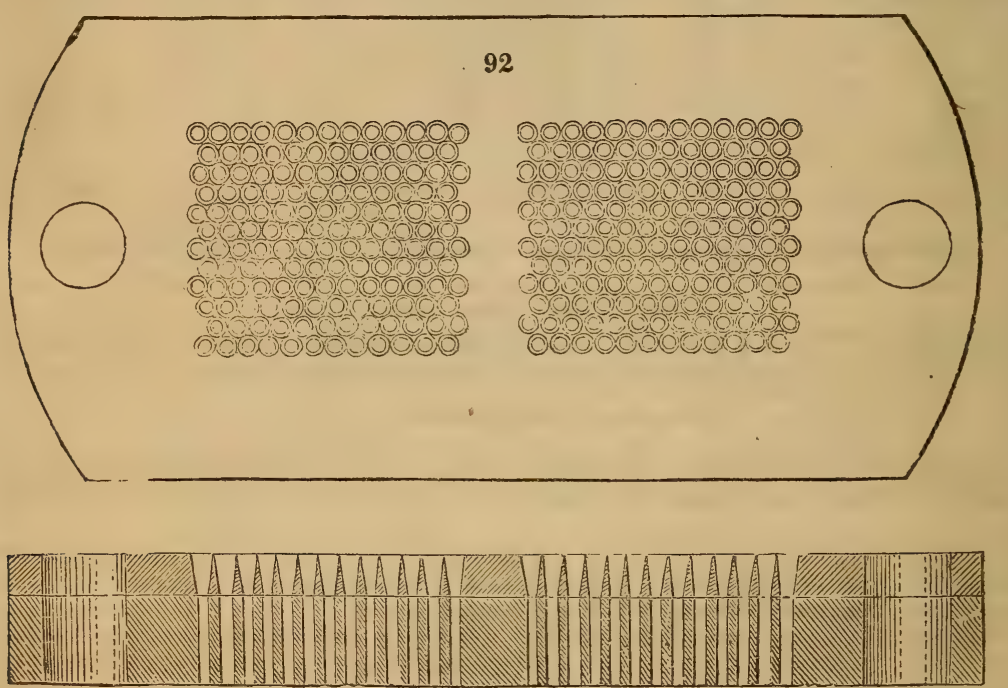

otherwise the plate would afford too great resistance to the passage of the wood. By this construction, the whole area of the block of wood may be compressed laterally into the countersunk openings, and forced through the holes, which are slighty coun. tersunk to favor the entrance and separation of the wooden fibres. Fig. 92 represents the face of one of these plates; and fig. 93 is a rectangular section through the plate. A convenient size of plate is three inches broad, six inches long, and one thick. The mode of pressing is by fixing the back of the plate against a firm resisting block or bearing, having an aperture equal to the area of the perforations in the plate, and then placing the end of the piece or pieces of wood in the direction of the grain against the face of the plate within the area of the perforated portion. A plunger or lever or other suitable mechanical agent being then applied to the back or reverse end of the piece of wood, it may be forced through the perforations in the plate, being first split as it advances by the cutting edges of the holes, and afterward compressed and driven through the perforations in the plate, coming out on the opposite side or back of the plate in the form of a multitude of distinct splints, agreeably to the shapes and dimensions of the perforations.-(Newton's Journal, C. S. vol. xxii. page 268.)

MERCURY; imported for home consumption in 1839, 340,469 pounds; in 1840 , 330,070 pounds; duty $1 d$. per pound.

METALLIC ANALYSIS. Professor Liebig has lately enriched this most useful department of practical chemistry, by the employment of the cyanide of potassium 
prepared in his economical method (see this article). This salt is the best re-agent for detecting nicliel in cobalt. The solution of the two metals being acidulated, the cyanide is $t \prime$ be added until the precipitate that first falls is redissolved. Dilute sulphuric acid is then adlled, and the mixture being warmed and left in repose, a precipitite does not fail to appear sooner or later, which is a compound of nickel. Cyanide of potassium serves well to separate lead, bisinuth, cadmium, and copper, four metals often associated in ores. On adding the cyanide in excess to the solution of these metals in nitric acid, lead and bismuth full as carbonates, and may be parted from each other by sulphuric acid. Sulphuretted hydrogen is passed in excess through the residuary solution, and the mixture being heated, a small quantity of cyanide is added : a yellow precipitate indicates cadmium; and a black precipitate falls on the addition of hydrochloric acid, if copper be present.

If into a crucible (containing the cyanide fused by heat), a little of any metallic ox. ide be thrown at intervals, it will be almost immediately reduced to the reguline state. When the fluid mass is afterward decanted, the metal will be found mixed with the white saline matter, from which it may be separated by water.

Even metallic sulphurets are reduced to the state of pure metals by being projected in a state of fine powder into the fused cyanide. When an iron ore is thus introduced, along with carbonate of potash or soda, and the mixture is heated to fusion, which requires a strong red heat, the alumina and silica of the ore fuse into a slag; from which, on cooling, the metallic iron may be seprrated by the action of water, and then weighed. If manganese exist in the ore, it remains in the state of protoxide; to be determined by a separate process. When oxide of copper is sprinkled on the surface of the fused cyanide, it is immediately reduced, with the disengagement of heat and light. The mixture being poured out of the crucible and concreted, is to be ground and washed, when a pure regulus of copper will be obtained.

The process of reduction is peculiarly interesting with the oxide of antimony and tin ; being accomplished at a low red heat, hardly visible in daylight. Even the sulphurets of these metals are immediately stripped of their sulphur, with the formation of sulphocyanide of potassium.

Cyanide of potassium, mixed with carbonate of soda, is an excellent re-agent in blowpipe operations for distinguishing metals. The reductions take place with the utmost facility, and the fused mixture does not sink into the charcoal, as carbonate of soda alone is apt to do in such cases. Hence the grains or beads of metal are more visible, and can be better examined.

When the cyanide is heated along with the nitrates and chlorates (of potash), it causes a rapid decomposition, accompanied with light and explosions.

Arsenic may be readily detected in the commercial sulphuret of antimony, by fusing it with three fourths of its weight of the cyanide in a porcelain crucible over a spirit lamp, when a regulus of antimony is obtained. The metal may then be easily tested for arsenic, since none of this volatile substance can have been lost, owing to the low temperature employed.

When arsenious acid, or orpiment, or any of the arseniates, are mixed with six times their weight of the mixture of cyanide and carbonate of soda in a tube with a bulb at one end, and heat applied with a spirit lamp to the glass, very beautiful rings of metallic mirror are formed by the reduced arsenic. The arseniates of lead and peroxide of iron, however, do not answer to this test.

When sulphates of lead and barytes, along with silica, are mixed with four or five times their veight of the above mixed cyanide and carbonate, and fused, the sulphate of lead is reduced to the metallic state, the sulphate of barytes becomes a carbonate, and the silica gets combined with the alkali into a soluble glass.

METALLIC STATISTICS. By the returns to five sereral orders made by the house of commons, which were obtained by the exertions and perseverance of Sir J. J. Guest, Sir C. Lemon, and Mr. Evans (M. P. for North Derbyshire), we are enabled to lay before our readers a most correct account of the rarious exports and imports of iron and iron ore, hardware, cutlery, \&c., copper ore, copper, tin, zinc, lead ore, and lead, for the year ending January 5, 1844.

Commencing with iron, it appears there was imported in the year, iron ore, 131 tons; chromate of iron, 1,393 tons; pig-iron, 243 tons; unwrought iron in bars, 12,795 tons; bloom, 563 tons ; rod-iron, 12 tons; old, broken, and cast-iron, 286 tons; cast-iron, only 8 tons; steel, unwrought, 1,697 tons: of these, 97 tons only were entered by weight, the remainder by value--11,035l. $6 \mathrm{~s}$. $9 \mathrm{~d}$. Of the several countries from which these importations came, the principal is Sweden, whence we have received of iron 10,909 tons, and steel 1,558 tons, leaving but a small portion to divide between twenty other places. Our exports of fureign iron have been, unwrought in bars, 3,986 tons; rod, 10 tons; hoops, 2 tons ; cast-iron, $11 \mathrm{cwt}$; steel, unwrought, 1,456 tons. The total quantity of foreign iron retained for home consi mption was 
14,782 tons, apon which the net amount of duty was 14,563l. The exportatinn of that staple produce of our own country, British iron, was as follows : Bar-iron, 176,148 tons; bolt and rod, 22,625 tons; pig-iron, 154,770 tons; cast-iron, 16,449 tons ; iron wire, 1,508 tons; wrought-iron, consisting of anchors, grapnels, \&c., 3,058 tons ; hoops, 14,591 tons; nails, 6,020 tons; and all other sorts, except ordnance, 44,577 tons; old iron for manufacture, 5,924 tons; and unwrought steel, 3,199 tons. Those places which have taken the greatest portions of this produce are-Russia, 10,963 tons of bariron: Denmark, 10,447 tons bar, and 7,010 tons pig; Prussia, 12,009 tons bar, 17,480 suns pig; Germany, 13,298 tons bar, 6,322 tons pig, 1,339 tons cast; Holland, 17,509 tons bar, 75,953 tons pig, 4,317 tons cast; Belgium, 4,279 tons cast; France, 4,237 tons bar, 22,103 tons pig; Italy, 21,930 tons bar, 3,982 tons bolt and rod, 3,005 tons pig; Turkey, and Continental Greece, 6,412 tons bar; East Indies and Ceylon, 20,620 tons bar, 2,967 tons bolt; British North American colonies, 6,837 tons bar, 1,995 tons cast foreign West Indies, 5,043 tons bar, 1,646 tons cast; and to the United States, 21,336 tons bar, and 7,148 tons pig. The largest quantity of unwrought steel has been to the latter place-viz., 1,336 tons.

Of British hardware and cutlery, we exported in the year, 17,183 tons, valued at $1,745,518 l$.; the principal of which has been-to Germany, 1,237 tons, value $159,889 l$.; East Indies, 1,402 tons, value 142,607l. ; British North American colonies, 1,129 tons, value 102,260l.; British West Indies, 997 tons, value 80,040l.; foreign West Indies, 657 tons, value 48,609l.; United States, 4,282 tons, value, 448,341l.; Brazil, 943 tons, value $80,070 l$. ; and divers other places, varying from 100 to 500 tons.

We now come to copper. Of foreign copper ores, we have imported 55,720 tons ; and of metallic copper, unwrought and wrought plates, and coins, 805 tons. Of the ores. the greatest quantities have come from $\mathrm{Cuba}$ and $\mathrm{Chili}$.

We have exported 1,819 tons of British, and 650 cons of foreign tin; of which France has taken 626 tons, Russia 480 tons, Italy 183 tons, Turkey 250 tons, and the remainder distributed among twenty-seven places.

Of foreign zinc, we have imported as follows :-.

Countries whence imported.

$\begin{array}{lll}\text { Denmark } & - & - \\ \text { Prussia - } & - & - \\ \text { Germany } & - & - \\ \text { Holland - } & - & - \\ \text { Belgium } & - & - \\ \text { Syria and Palestine } & -\end{array}$

Total import of foreign zine

$\begin{array}{ll}- & - \\ - & - \\ - & -\end{array}$

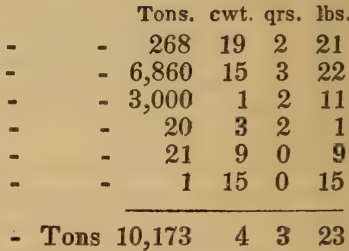

Of this, we retained for home consumption 4,102 tons, on which the net duty was 223l. 2s. 10d.; and we have exported 1,395 tons of British, and 6,445 tons of foreign spelter.

Of foreign lead, we have imported 2,863 tons-of which 2,775 tons were pig and sheet, 68 tons ore, and 19 tons white lead; 157 tons were retained for home consumption, on which the duty was 165l.; and we imported from the Isle of Man, duty free, 2,415 tons of lead ore. Our exportation of foreign lead amounted to 2,439 tons; while of British, we exported 176 tons of ore, 14,610 tons pig and sheet, 378 tons litharge, 707 tons red lead, and 1,224 tons of white lead: making a total of 17,097 tons.-Railuay and Commercial Gazette, May 18, 1844.

METER, GAS. Since the article GAs was printed, I have had occasion to examine very carefully the construction, performance, and comparative merits, of the four gasmetres most generally used in Great Britain, and have been led to conclude that the surmises concerning the correctness of the indications of several of them are but too well founded. The instruments on which my observations were made were all new, and just out of the hands of their respective patentees.

1. The meter of Mr. West is, no doubt, accurate while the water-line is rightly adjusted; but as I find that it will admit an extra pint of water, it may be rendered unjust toward the consumers of gas; and then if it receives a little more water by condensation of vapor, or by accident, its siphon gets filled, which causes the extinction of the lights.

2. The meter of Mr. Bottom has also several defects, and occasions nuisance by letting its overflow-water trickle upon the floor.

3. The meter of Mr. Crossley may be made to err in its measurement fully 26 per cent. by dexterous repletion with water, and that in favor of the gas ccmpanies.

These three meters are furnished with the vertical float-valve, so apt to rust and stick; 
they also allow gas to escape to the discharge plug, to the imminent risk of occasioning fire with ignorant or careless servants; and finally, they have the complex dial-plate indexe, so liable to misapprehension.

4. The meter of Mr. Edge. This instrument is quite exempt from all the above defects, and is equally delicate and just in its indications, being mounted with a lever valve of great mobility, and a new index which any one who knows nuinbers can not miscount. I have subjected this meter to every kind of test and find that it can not be made to give false indications, either by awkwardness or intention. Its inventor is therefore well entitled to the warm patronage both of the public and all gas companies who love their dealing.

MIILK has been adulterated with a solution of potato starch, from which it derives a creamy consistence. This fraud may be detected by pouring a few drops of iodine water into it, which immediately causes it to assume a blue or purple tint. Emulsion of sweet almonds, with which the milk at Paris has been adulterated, may be readily detected by the taste.

MINES. The miner, in sinking into the earth, soon opens up numerous springs, whose waters, percolating into the excavations which he digs, constitutes one of the greatest obstacles that nature opposes to his toils. When his workings are above the level of some valley and at no great distance, it is possible to get rid of the waters by leading them along a treich or a gallery of effux. This forms always the surest means of drainage; and notwithstanding the great outlay which it involves, it is often the most economical. The great adrantages accruing from these galleries, lead to their being always established, and without risk, in mines which promise a long continuance. There are many galleries sereral leagues in length; and sometimes they are so contrired as to discharge the waters of sereral mines, as may be seen in the environs of Freyberg. Merely such a slope should be given them as is barely sufficient to make the water run, at the utmost from $\frac{1}{30} \overline{0}$ to $\frac{1}{400}$, so as to drain the mine at the lowest possible level.

Whenever the workings are driven below the natural means of drainage, or below the level of the plain, recourse must be had to mechanical aids. In the first place, the quantity of percolating water is diminished as much as possible by planking, walling, or calling up with the greatest possible care those pits and excavations which traverse the water levels; and the lower workings are so arranged that all the waters may unite into wells placed at the bottom of the shafts or inclined galleries; whence they may be pumped up to the day, or to the level of the gallery of cfflux. In most mines, simple sucking pumps are empluyed, because they are less subject to give way, and more easy of repair; and as many of these are placed over each other, as the shaft is ten yards deep, below the point where the waters have a natural run.

These draining machines are set in motion by that mechanical power which happens to be the least costly in the place where they are established. In almost the whole of England, and over most of the coal-mines of France and Silesia, the work is done by steam-engines; in the principal metallic mines of France, and in almost the whole of Germany and Hungary, by hydraulic machines; and in other places, by machines moved by horses, oxen, or even by men. If it be requisite to lift the waters merely to the level of a gallery of effux, adrantage may be derived from the waters of the upper parts of the mine, or even from waters turned in from the surface, in establishing in the mine of the gallery-level, water-pressure machines, or overshot water-wheels, for pumping up the lower water. This method is employed with success in several mines of Hungary, Bohemia, Germany, Derbyshire, Cornwall, in those of Poullaouen in Brittany, \&c. It has been remarked, however, that the copious springs are found rather toward the surface of the soil than in the greatest depths.

\section{TRANSPORT OF ORES TO THE SURFACE.}

The ore being extracted from its bed, and having undergone, when requisite, a first sorting, it becomes necessary to bring it to the day, an operation performed in different ways according to circumstances and localities, but too often according to a blind routine. There are mines at the present day, where the interior transport of ores is executed on the backs of men; a practice the most disadvantageous possible, but which is gradually wearing out. The carriage along galleries is usually effected by means of hurdles, barrows, or, still better, by little wagons. These consist of frames resting on four wheels ; two larger, which are placed a little behind the centre of gravity, and two smaller, plazed before it. When this carriage is at rest, it bears on its four wheels, and leans forward. But when the miner, in pushing it before him, rests on its posterior border, he makes it horizontal; in which case it rolls only upon the two larger wheels. Thus, the friction due to four wheels is avoided, and the roller or driver bears no part of the burden, as he would do with ordinary wheelbarrows. To ease the draught still more, two parallel rails of wood or iron are laid along the floor of the gallery, to which 
the wheels of the carriage are adjusted. It is especially in metallic mines, where the ore is heary, and the galleries straight, that these peculiar wagons are employed. In coal mines, carriages formed with a much larger basket, borne on a railroad by four equal wheels, are preferred. Sometimes the above wain, called on the Continent a dog (chien), is merely a simple frame on four wheels, on which a basket is set. In the great mines, such as many of the coal and salt mines of Great Britain, the salt mines of Gallicia, the copper mines of Fahlun, the lead mines of Alston-Moor, horses and asses are introduced Into the workings to drag heavier wagons, or rather a train of wagons attached to one another. These animals often live many years under ground, without ever revisit ing the light of day. In other mines, such as those of Worsley, in Lancashire, subter. ranean canals are cut, upon which the ore is transported in boats.

When the workings of a mine are beginning, when they are still of little depth, and employ few hands, it is sufficient to place over the shaft a simple wheel and axle, by means of which a few men may raise the water-pails, and the baskets or tubs filled with ore; but this method becomes soon inadequate, and should be replaced by more powerful machines.

ACCESSORY DETAILS.

Few mines can be penetrated entirely by means of galleries. More usually there are shafts for mounting and descending. In the pits of many mines, the workmen go down and come up by means of the machines which serve to elevate the ores. In several mines of Mexico, and the north of Europe, pieces of wood, fixed on each side of the pit, form the rude steps of a ladder by which the workmen pass up and down. In other mines, steps are cut in the rock or the ore; as in the quicksilver mines of Idria and the Palatinate, in the salt mines of Wieliczka, and in some of the silver mines of Mexico. In the last they serve for the transport of the ore, which is carried up on men's backs. Lastly, certain mines are entered by means of slopes, some of which have an inclination of more than $30^{\circ}$. The workmen slide down these on a kind of sledge, whose velocity of descent they regulate by a cord firmly fixed at the upper end.

Miners derive light from candles or lamps. They carry the candles in a lump of soft clay, or in a kind of socket terminated by an iron point, which serves to fix it to the rock, or to the timbering. The lamps are made of iron, hermetically closed, and suspended, so that they can not droop, or invert and spill the oil. They are usually hung on the thumb by a hook. Miners also employ small lanterns, suspended to their girdles. Many precautions and much experience are requisite to enable them to carry these lights in a current of air, or in a vitiated atmosphere. It is especially in coal mines liable to the disengagement of carburetted hydrogen, that measures of safety are indispensable against the explosions. The appearance of any halo round the flame should be carefully watched as indicating danger; and the lights should be carried near the bottom of the gallery. The great protector against these deplorable accidents, is the safety lamp. See LAMP of DAVY.

We can not conclude this general outline of the working of mines, without giving some account of the miners. Most men have a horror at the idea of burying themselves, even for a short period, in these gloomy recesses of the earth. Hence mining operations were at first so much dreaded, that, among the ancients, they were assigned to slaves as the punishment of their crimes. This dislike has diminished with the improvements made in mining; and finally, a profitable and respected species of labor has given mining its proper rank among the other departments of industry. The esprit de corps, so conspicuous among seamen, has also arisen among miners, and has given dignity to their body. Like every society of men engaged in perilous enterprises, and cherishing the hopes of great success, miners get attached to their profession, talk of it with pride, and eventually in their old age regard other occupations with contempt. They form, in certain countries, such as Germany and Sweden, a body legally constituted, which enjoys considerable privileges. Miners work usually 6 or 8 hours at a time. This period is called a journey (poste, in French).

Miners wear, in general, a peculiar dress, the purpose of which is to protect them, as much as possible, from the annoyances caused by water, mud, and sharp stones, which occur in the places where they work. One of the most essential parts of the dress of a German miner is an apron of leather fitted on behind, so as to protect them in sitting on moisture or angular rubbish. In England, the miners wear nothing but flannels; though they frequently strip off all their clothes except their trowsers. In many countries the mallet and the pick, or pointerolle (called in German, Schegel and Eisen), disposed in a Saint Andrew's cross, are the badge of miners, and are engraved on their buttons, and on everything belonging to mines.

Several of the enterprises executed in mines, or in subserviency to them, merit a distinguished rank among the histo'y of human labors. Several mines are worked to a depth of more than 600 yards, some even to a thousand yards below the surface of the 
soil. A great many descend beneath the level of the ocean; and a few even extend under its billows, and are separated from them by a thin partition of rock, which allows their noise, and the rolling of the pebbles, to be heard.

In 1792 , there was opened, at Valenciana, in Mexico, an octagonal pit, fully $7 \frac{1}{2}$ yards wide, destined to have a depth of 560 yards, to occupy 23 years in digging, and to cost $240,000 l$.

The great drainage gallery of the mines of Clausthal, in the Hartz, is 11,377 yards, or six and a half miles long, and passes upward of 300 yards below the church of Clausthal. Its excavation lasted from the year 1777 till 1800 , and cost about 66,000l. Several other galleries of efflux might also be adduced, as remarkable for their great length and expense of formation.

The coal and iron mines subservient to the iron works of Mr. Crawshay, at MerthyrTydvil, in Wales, have given birth to the establishment, interiorly and above ground, of iron railways, whose total length, many years ago, was upward of 100 English miles.

The carriage of the coal extracted from the mines in the neighborhood of Newcastle to their points of embarkation, is executed almost entirely, both under ground and on the surface, on iron railways, possessing an extent of upward of 500 miles.

There is no species of labor which calls for so great a development of power as that of mines; and accordingly, it may be doubted if man has ever constructed machines so powerful as those which are now employed for the working of some mineral excavations. The waters of several mines of Cornwall are pumped out by means of steam-engines, whose force is equivalent in some instances to the simultaneous action of many hundred horses.

\section{Mtnes, General Summary of.}

Mines may be divided into three great classes : 1 . Mines in the gealogical formations anterior to the coal strata; 2. Mines in the secondary formations; 3 . Mines in alluvial districts.

The first are opened, for the most part, upon veins, masses, and metalliferous beds.

The second, on strata of combustibles, as coal; and metalliferous or saliferous beds.

The last, on deposites of metallic ores, disseminated in clays, sands, and other alluvial matters, usually superior to the chalk; and even of far more recent formation.

The mines of these three classes, placed, for the most part in very different physical localities, differ no less relatively to the mode of working them, and their mechanical treatment, than in a gealogical point of view.

\section{MINES OF FORMATIONS ANTERIOR TO THE COAL.}

These mines are situated in a few mountainous regions, and their whole amount forms but a small portion of the surface of the earth. The most remarkable of these arethe Cordilleras of South America; the mountains of Hungary; the Altayan mountains; the Ural mountains; the Vosges and the Black Forest; the Hartz, and the east of Germany; the centre of France; the north of Portugal, and the adjacent portions of Spain; Britanny ; the corresponding coasts of Great Britain and Ireland ; the north of Europe; the Allegany chain; the south of Spain; the Pyrenees; the Alps; the schistose districts on the banks of the Rhine and the Ardennes; the calcareous mountains of England and of Daouria.

\section{MINES OF THE CORDILLERAS OF SOUTH AMERICA.}

Few regions are so celebrated for their mineral wealth as the great chain which, under the name of the Cordillera of the Andes, skirts the shores of the Pacific ocean, from the land of the Patagonians to near the northwest point of the American continent. Who has not heard of the mines of Mexico and Potosi? The mineral wealth of Peru has passed into a proverb.

The most important mines of the Cordilleras are those of silver; but several of gold, mercury, copper, and lead, have likewise been opened. These mountains are not equally netalliferous in their whole extent. The workings occur associated in a small number f districts far distant from each other.

In the Andes of Chili, particularly in the province of Coquimbo, some silver mines are explored, which afford chiefly ores of an earthy or ferruginous nature, mingled with imperceptible portions of ores with a silver base, known there under the name of Pacos. The same province also presents copper mines of considerable importance, from which are extracted native copper, orange oxide of copper, carbonate of copper (malachite), and copper pyrites, associated with some muriate of copper. In a few mines, masses of native copper of extraordinary magnitude have been found.

The second metalliferous region of the Andes occurs between the 21st aud 15th Jegrees of south latitude. It includes the celebrated mountain of Potosi, situated in 
nearly the 20th degree of south latitude, on the eastern slope of the chain, and severa. other districts, likewise very rich, which extend principally toward the northwest, as far as the two banks of the lake Titicaca, and even beyond it, through a total length of nearly 150 leagues. All these districts, which formerly depended on Peru, were united in 1778 to the government of Buenos Ayres. The mines of Potosi were discovered in 1545 , and have furnished since that period till our days, a body of silver which M. Hum. boldt values at $230,000,000 l$. sterling. The first years were the most productive. At that time ores were olten found which afforded from 40 to 45 per cent. of silver. Since the beginning of the eighteenth century, the average richness of the ore does not exceed above from 3 to 4 parts in 10,000. These ores are therefore very poor at the present day; they have diminished in richness in proportion as the excavations have become deeper. But the total product of the mines has not diminished in the same proportion : abundance of ore having made up for its poverty. Hence, if the mountain of Potosi is not, as formerly, the richest deposite of ore in the world, it may, however, be still placed immediately after the famous vein of Guanaxuato. The ore lies in veins in a primitive clay state, which composes the principal mass of the mountain, and is covered by a bed of clay porphyry. This rock crowns the summit, giving it the form of a basaltic hill. The veins are very numerous; several, near their outcrop, were almost wholly composed of sulphuret of silver, antimoniated sulphuret of silver, and native silver. Others, which offered near the surface merely sulphuret of tin, became richer as they descended. In 1790, seven copper mines were known in the viceroyalty of Buenos Ayres, seven of lead, and two of tin; the last being merely washings of sands found near the river Oraro.

On the opposite flank of the chain, in a low, desert plain, entirely destitute of water, which adjoins the harbor of Iquiqua, and forms a part of Peru, occur the silver mines of Huantajaya, celebrated for the immense masses of native silver which have been sometimes found in them. In 1758, one was discovered weighing eight ewts.

M. Humboldt quotes 40 cantons of Peru as being at the present day most famous for their subterranean explorations of silver and gold. Those of gold are found in the provinces of Huaailas and Pataz; the silver is chiefly furnished by the districts of Huantajaya, Pasca, and Chota, which far surpass the others in the abundance of their ores.

The silver mines of the district of Pasco are situated about 30 or 40 leagues north of Lima, in $10 \frac{1}{2}$ degrees of south latitude, 4,400 yards above the sea-level, on the eastern. slope of the Cordilleras, and near the sources of the river Amazon. They were discovered in 1630 . These mines, and especially those of Cero of Yauricocha, are actually the richest in all Peru. The ore is an earthy mass of a red color, containing much iron, mingled with particles of native silver, horn silver, \&c., constituting what they call Pacos. At first, nothing but these pacos was collected; and much gray copper and antimoniated. sulphuret of silver were thrown among the rubbish. The mean product of all the ores is $\frac{1}{125}$, ; or an ounce and $\frac{28}{100}$ per cwt. ; although some occur which yield 30 or 40 per cent. These rich deposites do not seem to be extended to a great depth; they have not been pursued further than 130 yards, and in the greater part of the workings only to from 35 to 45 . Forty years ago, these mines, which produced nearly $2,000,000$ of piastres annually, were the worst worked in all South America. The soil seemed as if riddled with an immense number of pits, placed without any order. The drainage of the waters was effected by the manual labor of men, and was extremely expensive. In 1816, some Europeans, among whom were several miners from Cornwall, mounted several high-pressure steam-engines, imported from England, which introduced a considerable improvement in the workings.

The mines of the province of Chota are situated in about seven degrees of south latitude. The principal ones are those of Gualcayoc, near Meeuicampa, discovered in 1771; their outcrop occurs at the height of 4,500 yards above the sea; the city of Mecuicampa itself has 4,000 yards of elevation, that is, higher than the highest summits of the Pyrenees. The climate is hence very cold and uncomfortable. The ore is a mixture of sulphuret of silver and antimoniated sulphuret, with native silver. It constitutes veins, of which the upper portion is formed of pacos, and they sometimes traverse a limestone and sometimes a hornstone, which occurs in subordinate beds. The annual produce of the mines is 67,000 marcs of silver, according to Humboldt.

In the districts of Huaailas and Pataz, which are at a little distance from the former two, gold mines are worked. This metal is extracted chiefly from the veins of quartz, which run across the primitive schistose mountains. The district of Huaailas contains, besides, lead mines. Peru possesses, moreover, some mines of copper.

The quicksilver mines of Huancavelica, the only important mine of this species which has been worked in the New World, occurs on the eastern flank of the Andes of Peru, in 13 degrees of south latitude, at upward of 6,000 yards above the level of the sea. It does not seem referrible to the same class of deposites with the mines hitherto mentioned. 
Indications of mercurial ceposites have been observed in several other points of the Andes of Northern Peru, and of the south of New Granada.

Lastly, mines of sal-gem are known to exist in Peru, especially near the silver mines of Huantajaya.

On receding from the district of Chota, the Cordilleras are very indifferently stored with metallic wealth, to the isthmus of Panama, and even far bejond it. The kingdom of New Granada offers but a very small number of silver mines. There are some auriferous veins in the province of Antioquia, and in the mountains of Guamoco. The province of Caracas, the mountains of which may be considered as a ramification of the Cordilleras, presents at Aroa a copper mine which furnishes annually from 700 to 800 metric quintals $(1,400$ to $1,600 \mathrm{cwt})$ of this metal. Finally, we may state in passing, that there is a very abundant salt mine at Zipaquira, in the province of Sante Fé, and that between this point and the province of Santa-Fé-de-Bogota, a stratum of coal occurs at the extraordinary height of 2,700 yards.

Although Mexico presents a great variety of localities of ores, almost the only ones worked are those of silver. Nearly the whole of these mines are situated on the back or the flanks of the Cordilleras, especially to the west of the chain, nearly at the height of the great table land which traverses this region of the globe, or a little below its level in the chains which divide it. They lie in general between 2,000 and 3,000 yards above the sea; a very considerable elevation, which is favorable to their prosperity, because in this latitude there exists at that height a mean temperature, mild, salubrious, and most propitious to agriculture. There were at the time of Humboldt's visit, from 4,000 to 5,000 deposites of ore exploited. The workings constituted 3,000 distinct mines, which were distributed round 500 head quarters or Reales. These mines are not, however, uniformly spread over the whole extent of the Cordilleras. They may be considered as forming eight groups, which altogether do not include a greater space than 12,000 square leagues; viz., hardly more than the tenth part of the surface of Mexico.

These eight groups are, in proceeding from south to north,

1. The group of Oaxuaca, situated in the province of this name at the southern extremity of Mexico properly so called, toward the 17 th degree of north latitude. Besides silver mines, it contains the only veins of gold explored in Mexico. These veins traverse gneiss and mica-slate.

2. The group of Tasco. The most part of the mines which compose it are situated 20 or 25 leagues to the south west of Mexico, toward the western slope of the great plateau.

3. The group of Biscania, about 20 leagues northeast of Mexico. It is of moderate extent, but it comprehends the rich workings of Pachuca, Real del Monte, and Moram. The district of Real del Monte contains only a single principal vein, named Veta Bezicana of Real del Monte. in which there are several workings; it is, however, reckoned among the richest of Mexico.

4. The group of Zimapan. It is very near the preceding, about 40 leagues northwest of Mexico, toward the eastern slope of the plateau. Besides numerous silver mines, it includes abundant deposites of lead, and some mines of yellow sulphuret of arsenic.

5. The Central group, of which the principal point is Guanaxuato, a city of 70,000 inhabitants, placed at its southern extremity, and 60 leagues N. N. W. of Mexico. It comprises among others the famous mine districts of Gnanaxuato, Catorce, Zacatecas, Sombrerete; the richest in Mexico, and which alone furnish more than half of all the silver which this kingdom brings into circulation.

The district of Guanaxuato presents only one main vein, called the Veta Madre. This vein is enclosed principally in clay-state, to whose beds it runs parallel, but occasionally it issues out of them to intersect more modern rocks. The vein is composed of quartz, carbonate of lime, fragments of clay slate, \&c. ; and includes the sulphurets of iron, of lead, and of zinc in great quantities, some native silver, sulphuret of silver, and red silver ; its power (thickness of the vein) is from 43 to 48 yards. It is recognised and worked throughout a length of upward of 13,000 yards; and contains 19 exploitations, which produced annually well on to 1,200,000l. in siiver. One of the explorations, that of Valenciana, produces $320,000 \mathrm{l}$; being equal to about one fifteenth of the total prodnct of the 3,000 mines of Mexico. Since 1764, the period of its discovery, its neat annual product has never been less than from two to three millions of francs $(80,0007$. to :20,000l.); and its proprietors, at first men of little fortune, became, in ten years, the richest individuals in Mexico, and perhaps in the whole globe.

The workings of this mine are very extensive, and penetrate to a depth of 550 yards. They einploy a great many laborers.

The district of Zacatecas presents in like manner only a single vein in greywacke; which, however, is the seat of several workings.

The deposites mined at Catorce are in limestone; the mine called Purissima de Catorce has been explored to about 650 yards in depth; and yielded, in 1796, nearly 20,000l There are also mines of antimony in the district of Catorce. 
Toward the western part of the group of which we are now speaking, copper mines are worked in the provinces of Valladolid and Guadalaxara ; the ores being chiefly composed of protoxide of copper (orange copper), sulphuret of copper, and native copper. These mines produce about 2,000 metric quintals of copper annually ( $440,000 \mathrm{lbs}$. English). In the same district, ores of tin are collected in the alluvial soils, particularly near Mount Gigante. The concretionary oxide of tin, so rare in Europe, is here the most common variety. This metal occurs also in veins.

The central part of Mexico contains many indications of sulphuret of mercury (cinnabar); but in 1804 it was worked only in two places, and to an inconsiderable extent.

6. The group of new Gallicia is situated in the province of this name, about 100 leagues N. W. from Mexico. It comprises the mines of Balanos, one of the richest districts.

7. The group of Durango and Sonora, in the intendancies of the same name. It is very extensive. The mines are situated in part on the table land, and in part on the western slope. Durango is 140 leagues N. N. W. of Mexico.

8. The group of Chinuahua. It takes its name from the town of Chinuahua, situated 100 leagues $N$. of Durango. It is exceedingly extensive, but of little value; and terminates at $29^{\circ} 10^{\prime}$ of north latitude.

Mexico possesses, besides, several mines which are not included in the eight preceding groups. Thus the new kingdom of Leon, and the province of New Saint-Ander, present abundant mines of lead. New Mexico contains copper mines, and many others.

Lastly, rock salt is mined in several points of New Spain; and coal seems to occur in New Mexico.

The richness of the different districts of the silver mines or reales is extremely unequal. Nineteen twentieths of these reales do not furnish altogether more than one twelfth of the total product. This inequality is owing to the excessive richness of some deposites. The ores of Mexico are principally veins; beds and masses are rare. The veins traverse chiefly, and perhaps only, primitive and transition rocks, among which certain porphyries are remarked as very rich in deposites of gold and silver. The silver ores are mostly sulphuret of silver, black antimoniated sulphuret of silver, muriate of silver (hornsilver), and gray copper. Many explorations are carried on in certain earthy ores, called collorados, similar to the pacos of Peru. Lastly, there are ores of other metals, which are worked principally, and sometimes exclusively, for the silver which they contain; such are the argentiferous sulphuret of lead, argentiferous sulphuret of copper, and argentiferous sulphuret of iron.

Ores of very great richness occur in Mexico; but the average is only from 3 to 4 ounces per cwt., or from 18 to 25 in 10,000 . There are some, indeed, whose estimate does not exceed $2 \frac{1}{5}$ ounces. Almost all the argentiferous veins afford a little gold ; the silver of Guanaxuato, for example, contains $\frac{1}{360}$. The enormous product of the Mexican mines is to be ascribed rather to the great facility of working them, and the abundance of ores, than to their intrinsic richness.

The art of mining was little advanced in this country at the period of Humboldt's journey ; the workings presented a combination of small mines, each of which had only one aperture above, without any lateral communications between the different shafts.

The form of these explorations was too irregular to admit of their being called workings by steps. The shafts and the galleries were much too wide. The interior transport of the ores is generally effected on the back of men; rarely by mules. The machines for raising the ore and drawing off the water are in general ill combined; and the horse gigs for setting them in motion ill constructed. The timbering of the shafts is very imperfectly executed; the walled portions alone are well done. There are some galleries of drainage, but they are too few, and ill directed. Latterly, English capitalists and miners have formed companies for working the silver mines of Mexico; which will probably produce in time a happy revolution.

The silver ores of Spanish America are treated partly by fusion, and partly by amalgamation, but more frequently by the latter mode; hence the importation of mercury forms there an object of the highest importance, especially since the quicksilver mine of Huancavelica fell in, and ceased to be worked. This mine is the only one in Spanish America which belongs to the government. For the modern state of these mines, see Silver.

The following table shows, according to M. de Humboldt, what was the annual prodnet of the silver mines of South America, at the beginning of this century. It is founded in a great measure, upon official documents :-

\begin{tabular}{|c|c|c|c|c|}
\hline Mexico & - & $-\quad 2,196,140$ & 537,512 & $£ 4,778,000$ \\
\hline Peru - & 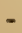 & 573,958 & 140,478 & $1,250,000$ \\
\hline Buenos-Ayres & - & 463,098 & 110,764 & 984,600 \\
\hline Chili - & . & 25,957 & 6,827 & 60,680 \\
\hline Total - & 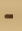 & - $\quad 3,259,153$ & 795,581 & $7,073,280$ \\
\hline
\end{tabular}


To complete our picture of the mineral wealth of Spanish America, it remains to speak of its principal gold mines; hut these belong to a geological locality, alluvial sands and gravel, very different from that of our present objects. The most important of these gold sands are washed on the western slope of the Cordilleras; viz., in New Grenada, from the province of Barbacoas, to the isthmus of Panama, to Chili, and even to the shores of the seas of California. There are likewise some on the eastern slope of the Cordilleras, in the high valley of the river Amazons. The washings of New Granada produce also some platina.

The mines, properly so called, and the washings of South America, furnish, altogether, 42,575 mares, or 10,418 kilogrammes (22,920 libs. Eng.) of gold, worth 1,435,720l.

\section{MINES OF HUNGARY.}

The metallic mines of this kingdom, including those of Transylvania, and the Bannat of Temeschwar, form four principal groups, which we shall denote by the group of the N.W., group of the N.E., group of the E., and group of the S.E.

The group of the N.W. embraces the districts of Schemnitz, Kremnitz, Kœnigsberg, Neuhsohl, and the environs of Schmœlnitz, Bethler, Rosenau, \&c.

Schemnitz, a royal free city of mines, and the principal centre of the mines of Hungary, lies 25 leagues to the north of Buda, 560 yards above the sea, in the midst of a small group of mountains covered with forests. The most part of these mountains, the highest of which reaches an elevation of 1,130 yards above the ocean, are formed of barren trachytes (rough trap rocks); but at their foot below the trachytic formation, a formation is observed, consisting of grten-stone porphyries, connected with syenites, passing into granite and gneiss, and including subordinate beds of mica-slate and limestone. It is in this formation that all the mines occur.

It has been long known that the green-stone porphyries of Schemnitz have intimate relations with the metalliferous porphyries of South America. M. Beudant, on comparing them with those brought by M. de Humboldt from Guanaxuato, Real del Monte, \&.c., has recognised an identity in the minutest details of color, structure, composition, respective situation of the different varieties, and even in the empirical character of effervescence with acids. The metalliferous rocks appear at Schemnitz only in a space of small extent, comprehended partly in a small basin, of which the city occupies the south border. They are traversed by veins which, for the most part, cut across the stratification, but which also are sometimes obviously parallel to it. These veins are in general very powerful; their thickness amounting even to more than 40 yards, but their extent in length seems to be usually inconsiderable. They are numerous and parallel to each other. It appears that they have no side plates of vein-stones (sallebundes), but that the metalliferous mass reposes immediately on the cheeks or sections of the rock, which is usually more or less altered, and includes always much pyrites near the point of contact, and even to a distance of several feet. The substances which constitute the body of these veins, are drusy quartz, carious quartz, ferriferous carbonate of lime, and sulphate of barytes, with which occur sulphuret of silver mixed with native silver containing more or less gold, which is rarely in visible scales; sulphuret of silver, argentiferous galena, blende, copper and iron pyrites, \&c. The sulphuret of silver and the galena are the two most important ores. Sometimes these two substances are insulated, sometimes they are mixed in different manners so as to furnish ores of every degree of richness, from such as yield 60 per cent. of silver down to the poorest galena. The gold seldom occurs alone; it generally accompanies the silver in a very variable proportion, which most usually approaches to that of 1 to 30 .

The ores of Schemnitz are all treated by fusion; the poor galenas at the smelting house of Schemnitz (bleyhutte), and the resulting lead is sent as working lead to the smelting-houses of Kremnitz, Neusohl, and Schernowitz, whither all the silver ores prepared in the different spots of the country are transported in order to be smeited.

The mines of Schemnitz, opened 800 years ago, have been worked to a depth of more than 350 yards. The explorations are in general well conducted. Excellent galleries of efflux have been excavated; the waters for impulsion are collected and applied with skill. It may be remarked, however, that these mines begin to decline from the state of prosperity in which they stood several years ago; a circumstance to be ascribed probably to the same pains being no longer bestowed on the instruction of the officers a pointed to superintend them. Maria Theresa established in 1760, at Schemnitz, a school of mines. This acquired at its origin, throughout Europe, a great celebrity, which it has not been able to maintain.

Kremnitz lies about five leagues N.N.W. of Schemnitz, in a valley flanked on the r.ght by a range of hills formed of rocks quite analogous to the metalliferous rocks of Schemnitz. In the midst of these rocks, veins are worked nearly similar to those of Schemnitz; but the quartz which forms their principal mass is more abundant, and contains more native gold. Here also are found sulphuret and hydrosulphuret of antimony, 
which do not occar at Schemnitz. The metalliferous district is of very moderate extent, and is surrounded by the trachytic district which overlies it, forming to the east and west considerable mountains.

The rity of Kremnitz is one of the most ancient free royal cities of mines in Hungary. It is said that mines were worked there even in the times of the Romans; but it is the Germans who, since the middle ages, have given a great development to these exploitations. There exists at Kremnitz a mint-office, to which all the gold and silver of the mines of Hungary are carried in order to be parted, and where all the chemical processes, such as the fabrication of acids, \&c., are carried on in the large way.

About six leagues N.N.E. from Schemnitz, on the banks of the Gran, lies the little village of Neusohl, founded by a colony of Saxon miners. The mountains surrounding it include mines very different from those of which we have been treating. At Herrengrand, two leagues from Neusohl, greywacke forms pretty lofty mountains; this rock is sovered by transition limestone, and is supported by mica-slate. The lower beds contain bands of copper ores, chiefly copper pyrites. The mica-slate includes likewise masses of ore, apparently constituting veins in it. These ores have been worked since the 13 th century. The copper extracted contains in a hundred weight six ounces of silver.

Eighteen or twenty leagues to the east of Neusohl, we meet with a country very rich in iron and copper mines, situated chiefly in the neighborhood of Bethler, Schmolnitz, Einsiedael, Rosenau, Sc. Talcose and clay slates form the principal body of the mountains here, along with horneblende rocks. The ores occur most usually in strata. Those of iron, or sparry ore, and especially hydrate of iron, compact and in concretions, acsompanied with specular iron ore. They give employment to a great many large smelting-houses. The county of Gœmar alone contains 22 works; and that of Zips also a great number. The copper mines lie chiefly in the neighborhood of Schmœlnitz and Gœelnitz. The copper extracted contains about six or seven ounces of silver in the hundred weight. Near Zalathna there is a quicksilver mine nearly inactive; and near Rosenau one of antimony.

To conclude our enumeration of the mineral wealth of this country, it remains merely to state that there are opal mines in the environs of Czervenitza, placed in the trachytic conglomerate.

GROUP OF THE NORTHEAST, OR OF NAGABANYA.

The mines of this group lie in a somewhat considerable chain of mountains, which, proceeding from the frontiers of Buchowina, where it is united to the Carpathians, finally disappears amid the saliferous sandstones between the Theiss, Lupos, and Nagy Szamos, on the northern frontiers of Transylvania. These mountains are partly composed of rocks analogous to those of Schemnitz, traversed by veins which have much resemblance to the veins of this celebrated spot. Into these veins a great many mines have been opened, the most important of which are those of Nagabanya, Kapnick, Felsobanya, Miszbanya, Laposbanya, Olaposbanya, Ohlalapos. All these mines produce gold. Those of Laposbanya furnish, likewise, argentiferous galena; those of Olaposbanya contain copper and iron; and those of Kapnick copper. Realgar occurs in the mines of Felsobanya; and orpiment in those of Ohlalapos. Several of them produce manganese and sulphuret of antimony. Lastly, toward the north, in the county of Marmarosh, lies the important iron mine of Borscha, and on the frontiers of Buchowina the lead mine of Radna, is which also much zinc ore occurs.

The mines composing the group of the East, or of Abrudbanya, occur almost all in the mountains which rise in the western part of Transylvania, between Lapos and Maros, in the environs of Abrudbanya. M. Beudant notices in this region, limestones, sandstones, trachytes, basalts, and sienite porphyries, apparently quite analogous to the greenstone porphyries of Schemnitz. It seems to be principally in the latter rocks that the mines forming the wealth of this country occur ; but some of them exist also in the micaslate, the greywacke, and even in the limestone. The principal mines are at Nagyag, Korosbanya, Vorospatak, Boitza, Csertesch, Fatzbay, Almas, Porkura, Butschum, and Stonischa. There are, in all, 40 exploitations; the whole of which produce auriferous ores smelted at the foundry of Zalathna. These mines contain also copper, antimony, and manganese. They are celebrated for their tellurium ore, which was peculiar to them prior to the discovery of this metal a few years back in Norway. The auriferous deposites contained in the greenstone porphyry are often very irregular. The mines of Nagyag are the richest and best worked. The numerous reins occur partly in the sienite porphyry, and partly in the greywacke. The auriferous ore is accompanied with galena, realgar, manganese, iron, and zinc. There are iron mines in great beds near Vayda-Huniad and Gyalar. Some Cobalt mines are also noticed.

The group of the $S . E$., or of the Bannat of Temeschwar, occurs in the mountains which block up the valley of the Danube at Orschova, through a narrow gorge of which the river escapes. The principal mines are at Oravitza, Moldawa, Szaska, and Dognaaczka. 
They produce chiefly argentiferous copper, yielding a mare of silver (nearly $\frac{1}{2}$ pound) in the hundred weight, with occasionally a little gold. Ores of lead, zinc, and iron, are also met with. The mines are famous for their beautiful specimens of blue carbonate of copper, and various other minerals. The mine of Moldawa aflords likewise orpiment. These metallic deposites lie in beds and veins; the former occurring particularly between the mica-slate and the limestone, or sometimes between the limestone and the sienite porphyry. Well-defined veins also are known to exist in the sienite und the mica-slate. The Bannat possesses moreover important iron-mines at Domtrana and Ruchersberg; near Dombrawa sulphuret of mercury is found. Cobalt mines occur likewise in these regions.

The mines constituting the four groups now described are not the sole metallic mines possessed by Hungary. A few others, but generally of 'ittle importance, are scattered over different parts of this kingdom. Several have been noticed in the portion of the Carpathians which separates Transylvania from Moldavia and Wallachia. Their principal object is the exploration of some singular deposites of galena.

Besides the mines just noticed, Hungary contains some coal mines, numerous mines of rock salt, and several deposites of golden sands situated chiefly on the banks of the Danube, the Marosch, anil the Nera.

The mines of the kingdom of Hungary produce annually, according to M. Heron de Villefosse, 5,218 marcs, or 2,810 pounds English of gold, worth 175,976l.; and about 85,000 marcs, or 45,767 pounds of silver, worth 186,1321 . The mines of Transrlvania furnish nearly the half of the whole quantity of gold, and one seventeenth of the silver now stated. The other mines of Europe produce together nearly twice as much silver, but merely a few mares of gold. Hungary affords besides from 18,000 to 20,000 metric quintals (about 4,000,000 libs. English) of copper annually, and a great deal of iron.

From these mines proceed likewise from 3,000 to 4,000 metric quintals ( 660,000 to 880,000 libs. Eng.) of lead; a quantity not more than is neetled by the refining-houses for the ores of silver and gold.

\section{MINES OF THE ALTAYAN MOUNTAINS.}

At the western extremity of the chain of the Altayan mountains, which separate Siberia from Chinese Tartary, there exists a number of metalliferous reins, in which several important works have been established since the year 1742. They constitute the locality of the mines of Kolywan; the richest in the precious metals of the three districts of this kind existing in Siberia.

These mines are opened up in the schistose formations which surround to the $N$. and $\mathrm{W}$, and to the S.W. the western declivity of the high granitic chain, from which they are separated by formations consisting of other primitive rocks. These schists alternate in some points with quartzose rocks, called by M. Renovantz hornstone, and with limestone. They are covered by a limestone, replete with ammonites. The metalliferous region forms a semicircle, of which the first lofty mountains occupy the centre.

The most important exploration of this country is the silver mine of Zméof, or Zmeinogarsk, in German Schlangenberg, situated to the N.W. of the high mountains in $51^{\circ} 9^{\prime} 25^{\prime \prime} \mathrm{N}$. L. and $79^{\circ} 49^{\prime} 50^{\prime \prime}$ long. east of Paris. It is opened on a great vein, which contains argentiferous native gold, auriferous native silver, sulphuret of silver, hornsilver, gray copper, sulphuret of copper, green and blue carbonated copper, red oxide of copper, copper pyrites, sulphuret of lead, and great masses of testaceous arsenic slightly argentiferous. There occur likewise sulphuret of zinc, iron pyrites, and sometimes arsenical pyrites. The gangues (vein-stones) of these different ores are sulphate of baryta, carbonate of lime, quartz, but rarely fluate of lime. The principal vein, which is of great power, has been traced through a length of several hundred fathoms, and to a depth of no less than 96 fathoms. In its superior portion, it has an inclination of about 50 degrees; but lower down it becomes nearly vertical. Its roof is always formed of clay-slate. On the floor of the vein, the slate alternates with hornstone. This vein pushes out branches in several directions; it is intersected by barren veins, and presents successive stages of different richness. The first years were the most productire. The German miners employed subsequently by the Russian government have introduced regularity into the workings; and have excavated a gallery of efflux 585 fathoms long.

The most important of the other silver mines of this department are those of Tcherepanofski, 3 leagues S.E. of Zméof; those of Smenofski, 10 leagues S.E.; those of Nicolaiski, 20 leagues to the S.S.W.; and of Philipofski, 90 leagues S.E. of the same place. The last mine lies on the extreme frontier of Chinese Tartary. It is not known whether the southern slope of the Altaic chain within the Chinese territories, contains metalliferous deposites.

The ores extracted from these different mines yield on an average per quintal an 
ounce of silver, which contains 3 per cent. of gold. Their annual product was toward 1786 , according to M. Patrin, 3,000 mares, or 1,615 libs. avoirdupois of gold, worth $101,151 l$. ; and 60,000 marcs, or 31,020 libs. avoird. or silver, worth 130,520l.

The precious metals are not the sole product of this mineral district. There is an important copper-mine 15 leagues N.W. of Zméof, in a chain of hills formed of granitic rocks, schists, porphyries, and shell-limestone, graduating into the plain. The vein presents copper pyrites, sulphuret of copper, and native copper, disseminated in argillaceous substances, more or less ferruginous, and of different degrees of hardness. This mine, which bears the name of Aleiski-Loktefski, furnished annually at the date of $1782,1,500$ quintals (metric), or 330,000 libs. avoird. of copper, which was coined into money in the country itself.

At Tchakirskoy, on the banks of the Tscharisch, toward the northern extremity of the metalliferous semicircle, mentioned above, there is a mine of argentiferous copper and lead, opened in a very large but extremely short vein. Besides the lead and copper ores, including a little silver, this mine affords a great quantity of ealamine (carbonate of zinc), which forms occasionally fine stalactites of a white or green color.

The northern flank of the Altaï mountains presents few mines. Some veins of copper exist 2,000 leagues east of Zméof, near the spot where the river Janissei issues from the Saianean mountains, which are a prolongation of the Altayan chain.

There is no lead-mine, properly so called, in the Altai mountains. Almost all the lead which is required for the treatment of the silver and gold ores is obtained from the department of Nertchinsk, situated 700 leagues off, on the borders of the river Amour.

The first smelting-house erected in this district was in the middle of the metalliferous region at Kolywan, the place from which it takes its name. It has been suppressed on account of the dearth of wood in the neighborhood of the mines. The principal exist ing foundry it that of Bornaoul on the $\mathrm{Ob}, 50$ leagues north of Zméof.

\section{MINES OF THE URAL MOUNTAINS.}

This chain of mountains, which begins on the coasts of the icy sea, and terminates in the 50th degree of latitude amid the steppes of the Kerguis, after having formed, through an extent of more than 40 leagues, the natural limit between Europe and Asia, contains very rich and very remarkable deposites of metallic ores, which have given rise to important mines of iron, copper, and gold. These explorations are situated on the two slopes, but chiefly on the one that looks to Asia, from the environs of Ekaterinbourg to about 120 or 130 leagues north of that city. They constitute the department of the mines of Ekaterinbourg, one of the three belonging to Siberia.

The copper-mines are pretty numerous, and lie almost wholly on the oriental slope of the chain. They are opened into veins of a very peculiar nature, and which although very powerful at the surface, do not extend to any considerable depth. These veins are in general filled with argillaceous matters, penetrated with red oxide of copper, and mingled with green and blue carbonated copper, sulphuret of copper, and native copper. The most important workings are those of Tourinski and Gonmechafski.

The first are situated 120 leagues north of Ekaterinbourg, toward the 60th degree of N. latitude, at the eastern base of the Uralian mountains, near the banks of the Touria. They amount to three, opened in the same vein, which turns round an angle presented by the chain in this place. The ground is composed of a porphyry with a hornstone basis of clay-slate, and of a white or grayish limestone, which form the roof and floor of the vein. The ore yields from 18 to 20 per cent., and these mines produced annually in $1786,10,000$ metric quintals (2,200,000 libs. avoird.) of copper.

The mine of Goumechefski lies 12 or 15 leagues S.W. of Ekaterinbourg, near a lake bordered by primitive mountains, which form in this region the axis of the chain of the Urals. This mine is celebrated for the beautiful malachites that occur in it. It has furnished almost all the fine specimens of this substance employed in jewellery. The vein, of which the sides are calcareous, is vertical, and runs north and south. It does not sink deeper than about 50 yards, and is filled with a species of coarse pudding-stone, composed of masses of primitive rocks. The ore yields from 3 to 4 per cent. of copper, and the mine furnished about the year 1786, 4,400,000 libs. avoird. of this metal per annum.

The beds of iron ore occur generally at a certain distance from the axis of the central chain. Those of the western slope lie sometimes in a gray compact limestone, which contains entrochi and other petrifications, and whose geological age has not been ascertained, but it appears to be much more modern than the rocks of the central chain. Both the one and the other seem to form large veins, which extend little in depth, or rather fill irregular and shallow cavities. The most common ore is the hydrate of iron (bog ore), hematite, or compact iron ore, sometimes mixed or accompanied with hydrate of manganese, and occasionally with ores of zine, copper, and lead. Black oxide of iron, possessing magnetic polarity, likewise frequently occurs, particularly in the 
mines of the eastern slope, on which, in fact, entire mountains of loadstone repose. All these ores, mixed with a greater or less quantity of clay differently colored, are worked by open quarries, and most usually without using gunpowder, or even iron wedges. They yield rarely less than 50 or 60 per cent., and keep in action numerous smelting-houses situated on the two flanks of the chain; the oldest of them have been established since 1628, but the greater number date only from the middle of the 18th century. The most celebrated mines are those of Balgodat and Keskanar, situated on the eastern slope from 30 to 50 leagues north of Ekaterinbourg. In the foundries of the eastern slope, anchors, cannons, bullets, \&c., are fabricated; and in the whole a considerable quantity of bar iron. The products of the works on the western side are directly embarked on the different feeders of the Volga, from which they are at no great distance. Those of the eastern slope are transported during winter on sledges to the same feeder streams, after crossing the least elevated passages of the Urals.

The quantity of materials fabricated by the iron-works of both slopes, amounted annually, toward the year 1790 , to more than $11,000,000$ lbs. avoird. This country is peculiarly favored by nature for this species of industry ; for vast deposites of excellent iron ores occur surrounded by immense forests of firs, pines, and birches; woods, whose charcoal is excellently adapted to the fabrication of iron.

The copper-mines of the Uralian mountains, and the greater part of the iron mines and foundries, form a portion of the properties of some individuals, who may be instanced as among the richest in Europe. The Russian government has neglected no opportunity of promoting these enterprises. It has established at Tourinsky a considerable colony, and at Irbitz a fair which has become celebrated.

There is only one gold mine in the Ural mountains, that of Beresof, situated three leagues N.E. of Elkaterinbourg, at the font of the Urals, on the Asiatic side. It is famous for the chromate of lead, or red lead ore, discovered there in 1776, and worked in the following years, as also for some rare varieties of minerals. The ore of Beresof is a cavernous hydrate of iron (bog ore), presenting here and there some small striated cubes of hepatic iron, and occasionally some pyrites. It contains 5 parts of native gold in 100,000. This deposite appears to have a great analogy with the deposites of iron ore of the same region. It constitutes a large vein, running from $N$. to $S$., encased in a formation of gneiss, hornblende schists, and serpentine, and which does not appear to dip to any considerable depth. It becomes poor in proportion to its distance from the surface. The exploitation, which is in the open air, has dug down 25 yards; having been carried on since the year 1726. The gold is extracted from the ore by stamping and washing. In 1786,500 marcs were collected; but the preceding years had furnished only 200 , because they then worked further from the surface. German miners were called in to direct the operations. On some points of the Ural mountains, and the neighboring countries, deposites of an auriferous clay have been noticed; but they have not hitherto been worked.

Beds of chromate of iron have also been discovered in these mountains.

The beautiful plates of mica, well known in mineral cabinets, and even in commerce, under the name of Muscovy talc, or Russian mica, come from the Urals. There are explorations for them near the lake Tschebarkoul, on the eastern flank of this chain. From the same canton there is exported a very white clay, apparently a kaolin.

25 leagues north of Ekaterinbourg, near the town of Mourzinsk, there occur in a graphic granite, numerous veins, containing amethysts, several varieties of beryl, emeralds, topazes, \&c.

Table of the Production of the Russian Mines during the years 1830, 1831, 1832, 1833, and 1834 ; by $\mathbf{M}$. Teploff, one of their officers.

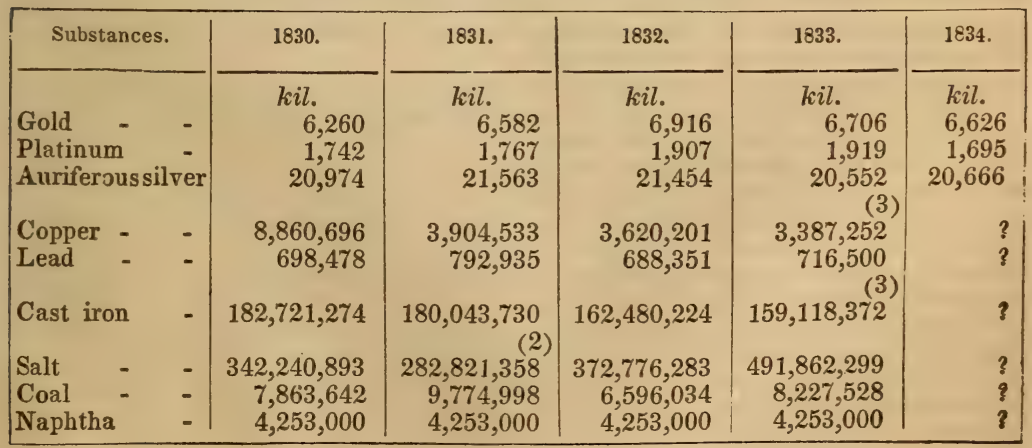


MINES OF THE VOSGES AND THE BLACK FOREST.

These mountains contain several centres of exploration of argentiferous ores of lead and copper, iron ores, and some mines of manganese and anthracite.

At the Crcix-aux-mines, department of the Vosges, a vein of argentiferous lead has been worked, which next to the veins of Spanish America, is one of the greatest known. It is several fathoms thick, and has been traced and mined through an extent of more than a league. It is partly filled with debris, among which occurs some argentiferous galena. It contains also phosphate of lead, antimoniated sulphuret of silver, \&c. It runs from N. to S. nearly parallel to the line of junction of the gneiss, and a porphyroid granite, that passes into sienite and porphyry. In several points it cuts across the gneiss; but it probably occurs also between the two rocks. It has never been worked below the level of the adjoining valley. The mines opened on this vein produced, it is said, at the end of the 16 th century, $26,000 \mathrm{l}$. per annum; they were still very productive in the middle of the last century, and furnished, in 1756, 2,640,000 lbs. avoird. of lead, and 6,000 mares, or 3,230 pounds avoird. of silver.

The veins explored at Sainte Marie of the mines, also traverse the gneiss; but their direction is nearly perpendicular to that of the vein of the Croix, from which they are separated by a barren mountain of sienite. They contain besides galena, several ores of copper, cobalt, and arsenic ; all more or less argentiferous. There is found also at a little distance from Saint Mary of the mines a vein of sulphuret of antimony. The mines of Sainte Marie, opened several centuries ago, are among the most ancient in France; and yet they have been worked only down to the level of the adjoining valleys.

There has been opened up in the environs of Giromagny, on the southern verge of the Vosges, a great number of veins, containing principally argentiferous ores of lead and copper. They run nearly from N. to S., and traverse porphyries and clay-slates ; a system which has some analogy with the metalliferous district of Schemnitz. The workings have been pushed so far as 440 yards below the surface. These mines were in a flourishing state in the 14 th and 16 th centuries; and became so once more at the beginning of the 17th, when they were undertaken by the house of Mazarin. In 1743 they still produced 100 marcs, fully 52 libs. avoird. of silver in the month.

The mines of La Croix, of Sainte-Marie-aux-mines, and of Giromagny, are now abandoned; but it is hoped that those of the first two localities will be resumed ere long.

In the mountains of the Black Forest, separated from the Vosges by the valley of the Rhine, but composed of the same rocks, there occur at Badenweiler and near Hochberg, not far from Freyburg, workings of lead in great activity. These form six distinct mines, and annually afford 88,000 libs. avoird. of lead, and 200 mares of silver. In the Furstenberg near Wolfach, particularly at Wittichen, there are mines of copper, cobalt, and silver. The mines of Wittichen produced, some years ago, 1,600 mares, or near 880 libs. avoird. of silver per annum. They supply a manufactory of smalt, and one of arsenical products. A few other inconsiderable mines of the same kind exist in the grand dutchy of Baden, and in the kingdom of Wurtemberg.

Several important iron mines are explored in the Vosges; the principal are those of Framont, in the department of the Vosges, whose ores are red oxide of iron and brown hematite, which appear to form veins of great thickness, much ramified, and very irregular, in a district composed of greenstone, limestone, and greywacke. The subterranean workings, opened on these deposites, have been hitherto very irregular. There has been discovered lately in these mines, an extremely rich vein of sulphuret of copper. At Rothau, a little to the east of Framont, thin veins of red oxide of iron are worked; sometimes magnetic, owing probably to an admixture of protoxide of iron. These veins run through a granite, that passes into sienite. At Saulnot near Belfort, there are iron mines, analogous to those of Framont.

In the neighborhood of Ihann and Massovaux, near the sources of the Moselle, veins are worked of an iron ore, that traverse formations of greywacke, clay-slate, and porphyry. Lastly, in the north of the Vosges, near Bergzabern, Erelenbach, and Schenau, several mines have been opened on very powerful veins of brown hematite and compact bog ore, accompanied with a little calamine, and a great deal of sand and debris. In some points of these veins, the iron ore is replaced by various ores of lead, the most abundant being the phosphate, which are explored at Erlenbach and Kalzenthal. These veins traverse the sandstone of the Vosges, a formation whose geological position is not altogether well known, but which contains iron mines analogous to the preceding at Langenthal, at the foot of Mount Tonnerre, and in the palatinate. Many analogies seem to approximate to the sandstone of the Vosges, the sandstone of the environs of Saint Avold (Moselle), which include the mine of brown hematite of Creutzwald, and the lead mine of Bleyberg, analogous to the lead mine of Bleyberg, near Aix-laChapelle. 
At Cruttnich and Tholey, to the north of the Sarrebruck, mines of manganese are worlied, famous for the good quality of their products. The deposite exploited at Cruttnich seems to be enclosed in the sandstone of the Vosges, and to constitute a vein in it, analogous to the iron veins mentioned above.

There has been recently opened a manganese mine at Lavelline, near La Croux-auxmines, in a district of gneiss with porphyry.

In the Vosges and the Black Forest there are several deposites of anthracite (stonecoal), of which two are actually worked, the one at Zunswir, near Offenbourg, in the territory of Baden, and the other at Uvoltz, near Cernay, in the department of the Upper Rhine. There are also several deposites of the true coal formation on the flanks of the Vosges.

\section{MINES OF THE HARTZ.}

The name Hartz is given generally to the country of forests, which extends a great many miles round the Brocken, a mountain situated about 55 miles W.S.W. of Mas debourg, and which rises above all the mountains of North Germany, being at its summit 1226 yards above the level of the sea. The Hartz is about 43 miles in length from S.S.E. to N.N.W., 18 miles in breadth, and contains about 450 square miles of surface. It is generally hilly, and covered two thirds over with forests of oaks, beeches, and firs. This rugged and picturesque district corresponds to a portion of the Silva Hercynia of Tacitus. As agriculture furnishes few resources there, the exploration of mines is almost the only means of subsistence to its inhabitants, who amount to about 50,000. The principal cities. Andreasberg, Clausthal, Zellerfeld, Altenau, Lautenthal, Wildemann, Grund, and Goslar, bear the title of mine-cities, and enjoy peculiar privileges; the people deriving their subsistence from working in the mines of lead, silver, and copper, over which their houses are built.

The most common rock in the Hartz is greywacke. It encloses the principal veins, and is covered by a transition limestone. The granite of which the Brocken is formed supports all this system of rocks, forming, as it were, their nucleus. Trap and hornstone rocks appear in certain points.

The veins of lead, silver, and copper, which constitute the principal wealth of the Hartz, do not pervade its whole extent. They occur chiefly near the towns of Andreasberg, Clausthal, Zellerfeld, and Lautenthal; are generally directed from N.W. to S.E., and dip to the S.W., at an angle of $80^{\circ}$ with the horizon.

The richest silver mines are those of the environs of Andreasberg, among which may be distinguished the Samson and Newfang mines, worked to a depth of 560 yards. In the first of them there is the greatest step exploitation to be met with in any mine. It is composed of 80 direct steps, and is more than 650 yards long. These mines were discovered in 1520 , and the city was built in 1521 . They produce argentiferous galena, with silver ores properly so called, such as red silver ore, and ore of cobalt.

The district which yields most argentiferous lead is that of Clausthal ; it comprehends a great many mines, several of which are worked to a depth of 550 yards. Such of the mines as are at the present day most productive, have been explored since the first years of the eighteenth century. The two most remarkable ones are the mines of Dorothy, and the mine of Caroline, which alone furnish a large proportion of the whole net product. The grant of the Dorothy mine extends over a length of 257 yards, in the direction of the vein, and through a breadth of nearly 22 yards perpendicularly to that direction. Out of these bounds, apparently so small, but which however surpass those of the greater part of the concessions in the Hartz, there was extracted from 1709 to 1807 inclusively, 883,722 marcs of silver, 768,845 quintals of lead, and 2,385 quintals of copper. This mine and that of Caroline have brought to their shareholders in the same period of time, more than 1,120,000l.; and have besides powerfully contributed by loans without interest to carry on the exploration of the less productive mines. It was in order to effect the drainage of the mines of the district of Clausthal, and those of the district of Zellerfeld adjoining, that the great gallery of efflux was excavated.

Next to the two districts of Clausthal and Zellerfeld, and Andreasberg, comes that of Goslar, the most important working in which is the copper mine of Rammelsberg, opened since the year 968, on a mass of copper pyrites, disseminated through quartz, and mingled with galena and blende. It is worked by shafts and galleries, with the employment of fire to break down the ore. This mine produces annually from 1,200 to 1,300 metric quintals (about 275,000 libs. avoird.) of copper. The galena extracted from it yields a small quantity of silver, and a very little gold. The latter metal amounts to only the five-millionth part of the mass explored; and yet means are found to separate it with advantage. The mine of Lauterberg is worked solely for the copper, and it furnishes annually near 66,000 libs. avoird. of that metal.

Besides the explorations just noticed, there are a great many mines of iron in different parts of the Hartz, which give activity to important forges, including 21 smelting 
cupolas. The principal o:es are sparry iron, and red and brown hematites, which occur in veins, beds, and masses. Earthy and alluvial ores are also collected. .

The territory of Anhalt-Bernbourg presents, toward the southeast extremity of the Hartz, lead and silver mines, which resemble closely those of the general district. They produce annually 33,000 libs. avoird. of lead.

At the southern foot of the Hartz, at Ilefeld, there is a mine of manganese.

The exploration of the Hartz mines may be traced back for about 900 years. The epoch of their greatest prosperity was the middle of the eighteenth century. Their gross annual amount was in 1808 upward of one million sterling. Lead is their principal product, of which they furnish annually $6,600,000$ libs. avoird., with 36,000 marcs, or 18,700 libs. avoird. of silver, about 360,000 libs. avoird. of copper, and a very great quantity of iron. They are celebrated for the excellence of the mining operations ; and the activity, patience, and skill, of their workmen.

The Hartz is referred to especially for the manner in which the waters are collected and economized for floating down the timber, and impelling the machinery. With this view, dams or lakes, canals, and aqueducts, have been constructed, remarkable for their good execution. The water-courses are formed either in the open air round the mountain-sides, or through their interior as subterranean galleries. The open channels collect the rain-waters, as well as those proceeding from the melting of snows, from the springs and streamlets, or small rivers that fall in their way. The subterıanean conduits are in general the continuation of the preceding, whose circuits they cut short. These water-courses present a development in whole of 125 miles. The banks of some of the reservoirs are of an extraordinary height. In the single district of Clausthal there are 34 tanks, which supply water to 92 wheels of nearly 30 feet diameter; 55 of these serve for the drainage of water, and 37 for the extraction of ores.

MINES OF THE EAST OF GERMANY.

We shall embrace under this head the mines opened in the primitive and transition territories, which constitute the body of a great portion of Bohemia, and the adjacent parts of Saxony, Bavaria, Austria, Moravia, and Silesia.

Among the several chains of small mountains that cross these countries, the richest in deposites of ore is the one known under the name of the Erzgebirge, which separate Saxony from Bohemia on the left bank of the Elbe.

The Erzgebirge contains a great many mines, whose principal products are silver, tin, and cobalt. These mines, whose exploration remounts to the twelfth century, and particularly those situated on the northern slope within the kingdom of Saxony, have been long celebrated. The school of mines established at Freyberg was at one time considered as the first in the world. This is a small city near the most important workings, 8 leagues W.S.W. of Dresden, toward the middle of the northern slope of the Erzgebirge, 440 yards above the level of the sea, in an agricultural and trading district, well cleared of wood. These circumstances have modified the working of the mines, and render it difficult to draw an exact parallel between them and those of the Hartz, which are their rivals in good exploration; they are peculiarly remarkable for the perfection with which the engines are executed both for drainage and extraction of ores, all moved by water or horses ; for the regularity of almost all the subterranean labors; and for the beauty of their walling masonry. In the portion of these mountains belonging to Saxony, the underground workings employ directly from 9,000 to 10,000 men, who labor in more than 400 distinct mines, all associated under the same plan of administration.

The silver mines of the Erzgebirge are opened on veins which traverse gneiss, and though quite different in this respect from the argentiferous veins of Guanaxuato, Schemnitz, and $\mathbf{Z} m e o f$, present but a moderate thickness, never exeeeding a few feet. They form several groups, whose relative importance has varied very much.

For a long time back, those of the environs of Freyberg are much the most productive; and their prosperity has been always on the advance, notwithstanding the increasing depth of the excavations. The deepest of the whole is that of Kuhschacht, which penetrates to 450 yards beneath the surface, that is, nearly down to the sea-level. The most productive and the most celebrated is the mine of Himmelsfürst ; that of Beschertgluck is also very rich.

Among the explorations at Erzgebirge, there are none which were formerly so flourishing as those of Marienberg, a small town situated 7 leagues S.S.W. of Freyberg. In the sixteenth century, ores were frequently found there, even at a short distance from the surface, which yielded 85 per cent. of silver. The disasters of the thirty years' war put a term to their prosperity. Since that period, they have continually languished; and their product now is nearly null.

Our limits do not permit us to describe in detail the silver mines that occur near 
Ehrenfriedersdorf, Johanna-Georgenstadt, Annaberg, Oberwiesenthal, and Schneeberg. Those of the last three localities produce also cobalt.

The mines of Saint-Georges, near Schneeberg, opened in the fifteenth century as iron mines, became celebrated some time after as mines of silver. Toward the end of the fifteenth century, a mass of ore was found there which afforded 400 quintals of silver; on that lump, Duke Albert kept table at the bottom of the mine. Their richness in silver has diminished since then; but they have increased more in importance during the last two hundred years, as mines of cobalt, than they had ever been as silver mines. Saxony is the country where cobalt is mined and extracted in the most extensive manner. It is obtained from the same veins with the silver. Smalt, or cobalt-blue, is the principa] substance manufactured from it. The lead and the copper are in this country only accessory products of the silver mines, from which $120,000 \mathrm{lbs}$. avoird. of the first of these metals are extracted, which are hardly sufficient for the metallurgic operations; and from 50,000 to 60,000 lbs. of copper. A little bismuth is extracted from the mines of Schneeberg and Freyberg. Some manganese is found in the silver mines of the Erzgebirge, and particularly at Johanna-Georgenstadt.

The mines of Saxony produce a little argentiferous galena, and argentiferous gray copper; the minerals with a base of native silver are the principal ores; they are treated in a great measure by amalgamation. All those of Freyberg are carried to the excellent smelting-house of Halsbrück, situated on the Malde, near that city. The arerage richness of the silver ores throughout Saxony is only from 3 to $4 \mathrm{oz}$. per quintal : viz., nearly equal to that of the ores of Mexico, and very superior to the actual richness of the ores of Potosi. The silver extracted from them contains a little gold. The Saxon mines produce annually 52,000 marcs of silver. Of these, the district of Freyberg alone furnishes 46,000; and among the numerous mines of that district, that of Himmelsfurst of itself produces 10,000 mares.

Silver mines exist also on the southern declivity of the Erzgebirge, which belongs to Bohemia, at Joachimsthal and Bleystadt, to the northeast of Eger. Argentiferous galena is chiefly extracted from these. The mines of Joachimsthal have been explored to a depth of 650 yards. They were formerly very flourishing; but in 1805 they were threatened with an impending abandonment. The ancient mines of Kuttenberg, situated in the same region, have been excavated, according to Agricola, to upward of 1,000 yards from the surface soil.

The southern slope of the Erzgebirge possesses cobalt mines like the northern slope ; but they are of much less importance. Some occur, particularly in the neighborhood of Joachimsthal. Lastly, on the same slope, slightly-productive copper mines are mentioned at Gröslitz, near Joachimsthal; at Catharineberg, 8 leagues north of Saatz; and at Kupferberg, lying between the two. At Gröslitz, the ore is a cupreous pyrites, accompanied by blende. The ores of Catharineberg are argentiferous.

Next to the silver mines, the most important explorations of the Erzgebirge are those of tin. This metal occurs in veins, massive, and disseminated in masses of hyalin gray quartz, imbedded in the granite; it is also found in alluvial sands. The most important tin mine of the Erzgebirge is that of Altenberg, in Saxony, which has been under working since the fifteenth century. Some tin is mined also near Gayer, Ehrenfriedersdorf, Johanna-Georgenstadt, Scheibenberg, Annaberg, Seiffen, and Marienberg, in Saxony. At Zinnwald it is also found; where the stanniferous district belongs partly to Saxony and partly to Bohemia; as also important mines occur in the latter territory at Schlackenwald and Abertham, and slightly-productive ones at Platten and Joachimsthal. In several of these mines, particularly at Altenberg and Gayer, fire is employed for attacking the ore, because it is extremely hard. In almost the whole of them, chambers of too great dimensions have been excavated, whence have arisen, at different epochs, vexatious sinkings of the ground. One of these may still be seen at Altenberg, whihh is 130 yards deep, and nearly 50 in breadth. 'The mines of Abertham are explored to a depth of 550 yards, and those of Altenberg to 330. The tin mines of the Erzgebirge produce annually $484,000 \mathrm{lbs}$. avoird. of this metal.

The tin ores are accompanied by arsenical pyrites, which, in the roasting that it undergoes, produces a certain quantity of arsenious acid.

The Erzgebirge presents also a great many iron mines, particularly in Saxony, at Rodenberg, near Cradorf, in the county of Henneberg, where the workings penetrate to a depth of 220 yards, and in Bohemia, at Platten, where may be remarked especially the great explorations opened on the vein of the Irrgang.

There is also in the Erzgebirge a mine of anthracite (stone coal) at Schenfeld, near Frauenstein, in Saxony.

The ancient rock-formations which appear in the remainder of Bohemia, and in the adjacent portions of Bavaria, Austria, Moravia, and Silesia, are much less rich in metals than the Erzgebirge. No explorations of much importance exist there.

The Fichtelgebirge, a group of mountains standing at the western extremity of the 
Erzgebirge, between Hoff and Bayreuth, contains some mines, among which may be noticed, principally, mines of magnetic black oxide of iron.

Argentiferous lead mines have been mentioned at Miess, 25 leagues W.S.W. of Prague, at the N.E. base of the western part of Bomerwaldgebirge, a chain of mountains which separate Bohemia from Bavaria. There are some also at Prszibram, 12 leagues S.W. of Prague, at the extremity of the mountains which separate Behrun from Moldau. In the latter, the argentiferous galena is accompanied by blende, in which the presence of cadmium has been observed. These mines, and those of Joachimsthal and of Bleystadt, furnish annually at present 220,000 lbs. avoird. of lead, and from 2,000 to 3,000 mares of silver. The circle of Behrun, to the S.W. of Prague, contains some inconsiderable mines of mercury. The eastern part of the Bomerwaldgebirge, which separates Bohemia from Austria and Moravia, presents some mines on its southeast slope. Those of the environs of Iglau, in Moravia, and some others situated in Austria, produce annually from 4,000 to 5,000 marcs of silver. The mines of these two countries yield also copper, and in several the copper ores are argentiferous. Moravia comprehends sev. eral iron works, which are in part supplied by magnetic iron ores analogous to those of Sweden.

The northeast slope of the Riesengebirge (giant mountains), which separate Bohemia from Silesia, presents also saveral explorations. The argentiferous copper mines of Rudolstadt, and of Kupferberg, have been stated as producing annually a considerable quantity of copper, and from 600 to 700 mares of silver; as also the cobalt mine of Maria-anna Querback, the whole in the circle of Quaer; and the mines of arsenical pyrites at Reichenstein, in the circle of Glatz. A mine of chrysoprase exists in the mountain of Kosennitz.

\section{MINES OF THE CENTRE OF FRANCE.}

The ancient formations, principally granitic, which constitute the ground of several departments of the centre and south of France, are hardly any richer in explorations than the districts mentioned at the end of the Black Forest. Only some insulated mines are to be observed here, of which a very few possess any importance. These all occur toward the eastern border of the mass of primitive formations, in a zone characterized by a great abundance of schistose rocks.

At Villefort and at Viallaze, in the department of the Lozère, and in some places ad. joining, several veins of argentiferous galena are worked which traverse the gneiss and the granite. These mines, remarkable at present for the regularity of their workings, employ 300 laborers, and produce annually about $220,000 \mathrm{lbs}$. avoird. of lead, and 1,600 mares of silver.

The city of Vienne, in Dauphiny, is built on a hill of gneiss separated by the Rhone from the main body of the primitive formations, and in which veins of galena occur, which are now imperfectly mined. Other lead mines of less importance are observed at St. Julien-Molin-Molette, department of the Loire, and at Joux, department of the Rhone.

At Chessy, a village situated 7 leagues northwest of Lyons, there occur in a talcose schist very extensive veins of cupreous pyrites, by no means rich, but which have, nevertheless, been worked successfully during the latter part of the eighteenth century, and several years of the present; at that period, there was found in a sandstone which covers the talcose schist, and which appears referrible to the red sandstone or the variegated sandstone, a bed containing a great quantity of blue carbonate of copper and protoxide of copper, to the working of which the miners have since directed their principal attention. There exists at Saint-Belle, 2 leagues to the south of Chessy, a deposite of copper pyrites like that of Chessy, which was at one time worked, but is now standing still. At Romanescho, in the department of Saone et Loire, a very abundant deposite of oxide of manganese is observed, apparently forming a mass in the granite, or perhaps above it. The workings are very irregular.

In the mountain of Ecouchettes, near Couches, in the same department, an ore of oxide of chrome has been occasionally worked.

At Malbose, in the department of the Lozère, a feeble vein of sulphuret of antimony is mined.

There are also in the centre of France some explorations of galena, antimony, and manganese, which appear to be of too little importance to be noticed in detail.

Some years ago a tin ore was discovered at Vaubry, 6 leagues N.N.W. of Limoges. At present, researches are making with a view of discovering deposites of such magniude as to pay the expense of working it.

MINES OF THE NORTH OF PORTUGAL AND THE ADJOINING PARTS OF SPAIN.

The Carthaginians appear to have worked tin mines in this part of the peninsula. It is vaid that some formerly existed in Portugal in the environs of Viscu, a province of Beira, at 
a place called Burraco de Stanno. Some veins of the same metal were discovered in 1787 , near Monte-Rey, in the south of Gallicia. They were fully two yards thick, and were incased in granite. This province presents also deposites of sulphuret of antimony. Some analogous ores are found in Castille and Estremadura. Lead ores were worked in the last century not far from Mogadouro, on the banks of the Sabor, in the province of Tras-los-MIontes, and near Longroiva on the banks of the Rio-Prisco. Mines of plumbago occur near Mogadouro. There are also some iron mines in the same country near Felguiera and Torredemnacorvo. They supply the iron-works of Chapa-cunha. Two very ancient establishments of the same kind exist in the Estremadura of Portugal; the one in the district of 'Thomar, and the other in that of Figuiero dos Vinhoss : they are supplied by mines of red oxide of iron, situated on the frontiers of this province and of Beira. One deposite of quichsilver ore occurs at Couna in Portugal. At Rio Tinto in Spain, on the frontiers of Portugal, there is a copper mine which produces abcut 33,000 libs. avoird. of this metal per annum. The ore is a copper $i$ rites. The mountains in the environs of Oporto present everywhere indications of the ores of copper and other metals; and it appears that all this part of the peninsula is in general rich in metallic treasures, but that the want of wood prevents their being mined to advantage.

Besides, many of the deposites which originally existed there must be in a great measure exhausted. It was in these countries chiefly that the gold and silver mines lay, which the Carthaginians and Romans worked with so much advantage, and contested in so keen a manner. Near Loria (the ancient Numantium), Azagala, and Burgos, considerable vestiges of the ancient workings may still be seen.

MINES OF BRITANNY.

Britanny has hardly a better share in mineral wealth than the countries we have just passed in review. There exist in it at this moment only two important exploitations; which are, the lead mines of Poullaouen and Huelgoat, situated near Carhaix. The mine of Huelgoat, celebrated for the plomb-gomme (hydro-aluminate) discovered in it, is opened on a vein of galena, which trarerses transition rocks. The workings have subsisted for about three centuries, and have attained to a depth of 220 yards. The vein of Poullaouen, called the New Mine, was discovered in 1741 . It was powerful and very rich near the surface; but it became subdivided and impoverished with its depth, notwithstanding which the workings have been sunk to upward of 180 yards below the surface. In these mines there are fine hydraulic machines for the drainage of the waters, with wheels from 14 to 15 yards in diameter; and water-pressure machines have been recently constructed. The mines employ more than 900 workmen, and furnish annually more than $1,200,000 \mathrm{lbs}$. aroird. of lead, several thousand pounds of copper, and 2,000 mares, or 1,034 lbs. avoird. of silver. These are the most important metallic mines of France. Several reins of galena exist at Chateläudren, near Saint-Briex, but they are not worked at present. There is also one at Pompean, near Rennes, which has been worked to a depth of 140 yards, but is in like manner now abandoned. It affords, besides the galena, a very large quantity of blende (sulphuret of zinc), of which attempts are making to take advantage. There occurs, also, a lead mine at Pierreville, department of the Channel, in a formation connected with the system of Britanny. It is opened on a vein which trarerses a limestone pretty analogous to that of Derbyshire. The same department presents a deposite of sulphuret of mercury at Ménildot. A few years ago, some tin ore was discorered at Pyriac, near Guérande, in the department of the Loire Inférieur, but the researches since made to find workable deposites have been unsuccessful. A mine of antimony was worked at La Ramée, department of $\mathrm{La}$ Vendée. Several of the coal deposites lately mined in the departments of La Sarthe, La Mayenne, and Mayenneet-Loire, ought probably to be regarded as more ancient than the genuine coal measures.

Table of the production of the French mines, during the year 1832.*

\begin{tabular}{|c|c|c|c|c|c|}
\hline Species of Mine. & $\begin{array}{l}\text { Number of } \\
\text { mines. }\end{array}$ & $\begin{array}{l}\text { Extent of } \\
\text { surface } \\
\text { conceded. }\end{array}$ & $\begin{array}{l}\text { Number of } \\
\text { workmen. }\end{array}$ & $\begin{array}{l}\text { Production is in } \\
\text { l0ths of a ton. }\end{array}$ & $\begin{array}{l}\text { Value of the } \\
\text { rough product } \\
\text { in francs. }\end{array}$ \\
\hline Metallic Substances. & & Kilom. carrés. & & & \\
\hline Antimony & 16 & 93,8954 & 130 & $\begin{array}{c}\text { Melted antim. } \\
1 \cdot 030,98\end{array}$ & $71 \cdot 232,75$ \\
\hline Copper - - & 8 & 274,18 & 258 & $\begin{array}{c}\text { Black copper } \\
1 \cdot 376\end{array}$ & 247.680 \\
\hline Iron & 131 & $1 \cdot 051,391$ & 8917 & $\begin{array}{l}\text { Rough ore } \\
15 \cdot 814,690\end{array}$ & $3,630 \cdot 806,81$ \\
\hline Manganese & 8 & 16,54 & 66 & 6.087 & $66 \cdot 849,88$ \\
\hline Gold - & 1 & 0.49 & & & \\
\hline Lead and silver & 33 & 614,23 & 1259 & $8 \cdot 505$ & $742 \cdot 051$ \\
\hline Zinc & 1 & 6,80 & & & \\
\hline
\end{tabular}

* (Amnales des Mines, tom. v., 1884, p. 676.) 
MINES OF THE CORRESPONDING COASTS OF GREAT BRITAIN AND IRELAND.

The mines comprehended in this section are situated, 1 , in Cornwall and Devonshire; 2 , in the S.E. of Ireland ; 3 , in the island of Anglesey and the adjoining part of Wales 4 , in Cumberland, Westmoreland, and the north of Lancashire, and the Isle of Man, 5 , in the south of Scotland; 6 , in the middle part of the same country.

Cornwall and Devonshire present three principal mining districts; viz., the portion of Cornwall situated in the environs and S.W. of Truro, the environs of St. Austle, and the environs of Tavistock.

The first of these districts is the most important of the three in the number and richness of its mines of copper, tin, and lead. The ores of eopper, which consist almost entirely of copper pyrites and common sulphuret of copper, constitute very regular veins running nearly from east to west, and incased most frequently in a clay-slate of a talcose or hornblende nature, called killas, and sometimes in granite, which forms protuberances in the middle of the schists. The tin occurs principally in veins, which, like the preceding, traverse the killas and the granite. They are also very often directed nearly from east to west, but they have a different inclination, or dip, from that of the copper reins, which cut them across and interrupt them, and are consequently of more recent formation. The tin ore forms also masses, which appear most usually attached to the veins by one of their points. Lastly, it is found in small veins which traverse the granite, principally near the points where this rock touches the killas. Certain veins present the copper and tin ores together; a mixture which occurs chiefly near the points of intersection of the two metallic veins. Certain mines furnish at once both copper and tin; but the most part produce in notable quantity only one of these metals. The most important copper mines are situated near Redruth and Camborn; among which may be noted particularly those called Consolidated Mines, United Mines, Huel-Alfred, Dolcoath, Poldice, \&c. The principal tin mines are situated still farther to the southwest, near Helston, Saint-Yues, \&c. Those called Huel Vor, Great Huas, are particularly noticed. There are several mines in Cornwall of which the crossing veins which at once intersect and throw out the veins of copper and tin, contain argentiferous galena and several ores of silver. There existed formerly mines of argentiferous lead near Helston and Truro. There may be now seen near Saint Michael an ore which, melted and cupelled on the spot, yields from an ounce and a half to two ounces of silver per quintal. Near Calstock a silver mine is worked, called Huel-Saint-Vincent, which has afforded, it is said, in some months, from 900 to $1,000 \mathrm{lbs}$. avoird. of that metal. The ore, consisting of hornsilver and native silver, is treated on the spot.

In the environs of Saint Austle, the copper mines of East Crinnis and West Crinnis deserve to be noticed, as well as the tin mine of Polgooth, opened on a tin vein; and the mine of Carclaise, explored in the open air on a system of small veins of this metal.

Near Tavistock there occur mines of copper, tin, and lead. Among the last may be remarked particularly that called Huel Betsey, of which the ores melted and cupelled on the spot, afford an ounce and a half of silver per cwt.; and that of Beeralston, whose ore is sent to Bristol to be smelted there. It yields from four to five ounces of silver per cwt.

There are mines of antimony at Huel-Boys in Devonshire, and at Saltash in Cornwall.

The tin and copper ores of Cornwall are accompanied with arsenical pyrites, which is turned to some account by the fabrication of white arsenic (arsenious acid).

Cornwall and Devonshire produce annually about $6,160,000$ lbs. avoird. of tin; $18,700,000 \mathrm{lbs}$. avoird. of copper; and 1,760,000 lbs. avoird. of lead. See Copper and TIN.

The tin is treated at the mine localities : but the copper ores are sent in their natural state to Swansea in South Wales, to be smelted.

Wood and labor being very dear in Cornwall and Devonshire, the mineral deposites of these counties can not be worked out so completely, nor can the mechanical preparation of the ore be so far pushed, as in several other parts of the world. But all the operations which appear advantageous are conducted in the most judicious, most economical, and most expeditious manner. Steam-engines are erected there, some of them possessing the power of several hundred horses. Many of the mines are explored to a depth of upward of 400 yards; and several are celebrated for the boldness of their workings. The one called Botallock Mine, situated in the parish of St. Just, near the Cornwall cape, is opened amid rocks which form the seacoast, and stretches several hundred yards under the sea, and upward of 200 yards beneath its level. In some points so small a thickness of rock has been left to support the weight of the waters, that the rolling of pebbles on the bottom is distinctly heard by miners during a storm. The mine of Huel-werry, near Penzance, was worked by means of a single shaft opened on the coast, in a space left dry by the sea only for a few hours at every ebb. A small wooden tower was built over the mouth of the shaft, which, being carefully calked, kept out 
the waters of the ocean when the tide rose, and served to support the machines for raising the ore and drainage. A vessel driven by a storm overturned it during the night, and put a period to this hazardous mode of mining, which has not been resumed.

The most considerable mines of Ireland are those of Cronebane and Tingrony, and of Ballymartagh, situated three leagues S.W. of Wicklow, in the county of the same name. Their object is to work the copper pyrites, accompanied with some other ores of copper, galena, sulphuret of antimony, as well as pyrites of iron, which forms several flattened masses in the clay-slate. Pretty extensive workings have been made here; and the ore was transported in its natural state to Swansea. Veins or masses of copper pyrites and galena are mined in some other points of the southeast of Ireland, but none of them with any notable advantage. The principal is the lead mine situated in the county of Tipperary, near the village called Silver Mines, absurdly enough, because, though silver was sought for in the lead, none was extracted. Many iron mines anciently existed in Ireland, but the destruction of the forests has considerably diminished their number and activity, so that only a few remain in Kilkenny, Wicklow, and Queen's County.

The isle of Anglesey is celebrated for its copper mines, the principal of which are Mona-mine and Parys-mountain. The ore is a copper pyrites, sometimes of considerable volume, lying in masses in a formation containing serpentines and different talcose rocks. For a long time the workings were carried on in the open air, but the exterior exploration has been thereby compromised. The neighboring coasts of Wales present some mines of the same nature. All the ores are treated in a smelting-house established in the isle of Anglesey. The formation of slate-clay and greywacke, which constitutes the greater part of Wales, and some of the adjoining districts of England, includes several lead mines, of which we shall presently speak in noticing those of far greater importance contained in the more recent limestone formations of the same regions.

Pretty important mines of copper pyrites and red hematitic ron are worked in Westmoreland, and in the neighboring parts of Cumberland and Lancashire. The copper ores, and a portion of the iron ones, are embarked for Swansea. The rest of the iron ore is treated on the spot in blast furnaces supplied with wood charcoal. The isle of Man affords indications of lead, copper, and iron, in the mountains of Snafle, which constitute its centre. At Borrowdale in Westmoreland, a mine of graphite (plumbago) has been worked for a long period. It furnishes the black lead of the English pencils, so celebrated over the world. The mineral occurs in mass in a talcose formation.

There are famous lead mines in the south of Scotland, at Leadhills in Lanarkshire; the veins of which are incased in greywacke. Some manganese has also been found. At Cally, in Kirkcudbrightshire, a copper mine has been lately discovered ; and a mine of antimony has been known for some time at West Kirk in Dumfriesshire ; but neither has been turned to good account.

In the middle part of Scotland, the lead mines of Strontian in Argyleshire deserve to be noticed, opposite to the northeast angle of the isle of Mull. They are opened on veins which traverse gneiss. According to Mr. John Taylor, these mines and those of Leadhills produce annually $5,610,000$ lbs. avoird. of lead.

Explorations of manganese were begun at Grantown on the banks of the Don, a river which falls into the German ocean at Aberdeen. A mine of coarse graphite has also been worked at Huntley.

A copper mine was discovered some years back in one of the Shetland isles; and chromate of iron is now extensively worked there in serpentine and talc.

\section{MINES OF THE NORTH OF EUROPE.}

These mines are situated for the most part in the south of Norway, toward the middle of Sweden, and in the south of Finland, a little way from the shortest line drawn from the lake Onega to the southwest angle of Norway. A few mines occur in the northern districts of Norway and Sweden. The main products of these several mines are iron, copper, and silver.

The iron mines of Norway lie on the coasts of the gulf of Christiania, and on the side facing Jutland, principally at Arendal, at Krageroe, and the neighborhood. The ores consist almost solely of black oxide of iron, which forms beds or veins of from 4 to 60 feet thick, incased in gneiss, which is accompanied with pyroxène (augite), epidotes, garnets, \&c. These iron ores are reduced in a great many smelting forges, situated on the same coasts, and particularly in the county of Laurwig. Their annual product is about $16 \frac{2}{2}$ millions of pounds avoird. of iron, in the form of cast iron, bar iron, sheet iron, nails. \&c.; of which one half is exported.

Norway possesses rich copper mines, some of which lie toward the south and the centre of the country, but the most considerable occur in the north, at Quikkne, Lakien, Selboe, and Rœraas, near Drontheim. The mine of Rœraas, 16 miles from Drontheim $t$ 
the S.E. of this city, is opened on a very considerable mass of copper pyrites, and has been worked in the open air since 1664. It has poured into the market from that time, till 1791, 77 millions of pounds avoird. of copper. In 1805, its annual production was 864,600 libs.; while all the other mines of Norway together do not furnish quite one fourth of that amount.

Norway comprehends also some celebrated silver mines. They are situated from 15 to 20 leagues S.W. of Christiania, in a mountainous country near the city of Kongsberg, which owes to them its population. Their discovery goes back to the year 1623, and their objects are veins of carbonate of lime, accompanied with asbestos and other substances in which native silver occurs, usually in small threads or networks, and sometimes in considerable masses, along with sulphuret of silver. These veins are very numerous, and run through a considerable space, divided into four districts (arrondissements), each of which contains more than 15 distinct explorations. When a new mine is opened, an excavation in the open air is first made, which embraces several veins, and they then prosecute by subterranean workings only those that appear to be of consequence. The workings do not exceed 1,000 feet in depth. Fire is employed for attacking the ore. In 1782 , the formation of a new gallery of efflux was commenced, destined to have a length of 10,000 yards, and to cost $60,000 l$. These mines, since their discovery till 1792, have afforded a quantity of silver equivalent to four millions of pounds sterling. The year 1768 was the most productive, having yielded 38,000 marcs of silver. At present they give but a very slender return; in 1804 they were threatened with a complete abandonment. The ore is treated by fusion; the lead necessary for this operation being imported from England. There are, however, lead and silver mines in the county of Jarlsberg, but they are very slenderly worked.

At Edswald, 50 leagues N. of Christiania, a mine is worked of auriferous pyrites, with a very inconsiderable product.

Cobalt mines may be noticed at Modum or Fossum, 8 leagues W. of Christiania ; they are extensive, but of little depth.

Lastly, graphite is explored at Englidal; and chromite of iron deposites have been noticed in some points of Norway.

The irons of Sweden enjoy a merited reputation, and form one of the chief objects of the commerce of that kingdom. Few countries, indeed, combine so many valuable advantages for this species of manufacture. In exhaustible deposites of iron ore are placed amid immense forests of birches and resinous trees, whose charcoal is probably the best for the reduction of iron. The different groups of iron mines and forges form small districts of wealth and animation in the midst of these desolate regions.

The province of Wermeland, including the north bank of the lake Wener, is one of the richest of Sweden in iron mines. The two most important are those of Nordmarck, 3 leagues N. of Philipstadt, and those of Persberg, $2 \frac{1}{2}$ leagues E. from the same city. Philipstadt is about 50 leagues W. $\frac{1}{4}$ N.W. from Stockholm. Both mines are opened on veins or beds of black oxide of iron several yards thick, directed from N. to S. in a ground composed of hornblende, talcose, and granitic rocks. These masses are nearly vertical, and are explored in the opea air to a depth of 130 yards. Formerly this exploitation was effected by iron wedges and pickaxes; but they have been superseded by gunpowder, since 1650 . The province of Wermeland, and that of Dahl which adjoins it, forming the west border of the Wener lake, contained in 1767, 48 smelting cones, each going from 4 to 5 months every year.

The principal iron mines of Rosslagie (part of the province of Upland) are those of Dannemora, situated 11 leagues from Upsal. They stand in the first rank of those of Sweden, and even of Europe. The masses worked upon are flattened and vertical, running from N.E. to S.W., and are incased in a ground formed of primitive rocks, among which gneiss, petrosilex and granite are most conspicuous. They amount to three in number, very distinct, and parallel to each other; an 1 are explored through a length of more than 1,500 yards, and to a depth of above 80 , by the employment of fire, and blasting with gunpowder. The explorations are mere quarries; each presenting an open trench 65 yards wide, by a much more considerable length, and an appalling depth. Magnetic iron ore is extracted thence, which furnishes the best iron of Sweden and Europe; an iron admirably qualified for conversion into steel. In 1767, these minings supplied for a long time, 15 smelting cones situated in Rosslagie, at a distance of 10 leagues.

The island of Utoe, situated near the coast of the province of Upland, presents also rich iron mines. The protoxide of iron there forms a thick bed in the gneiss. It is worked in trenches far below the level of the sea. The ore can not be smelted in the island itself; but is transported in great quantities to the continent.

The province of Smoland includes also very remarkable mines. Near Jonköping, a hill called the Taberg occurs, formed in a great measure of magnetic black oxide of iron, contained in a greenstone reposing on gneiss. 
In several parts of Lapland, the protoxide of iron occurs in great beds, or immense masses. At Gellivara, 200 leagues N. of Stockholm, toward the 67 th degree of latitute, it constitutes a considerable mountain, into which an exploitation has been opened. The iron is despatched on small sledges drawn by reindeer to streams which fall into the Lutea; and thence by water carriage to the port of Lutea, where it is embarked for Stockholm.

There are a great many iron works in Dalecarlia, but a portion of the ores are got from alluvial deposites. Similar deposites exist also in the provinces of Wermeland and Smoland.

The mines and forges of Sweden produce annually about 165 millions of pounds avoird. (74,000 tons nearly) of cast iron or bar iron; of which two thirds are exported chiefly from the harbors of Stockholm, Gottenburg, Geffle, and Norkoping.

The copper mines of Sweden are scarcely less celebrated than its iron mines. The principal is that of Fahlun or Kopparberg, situated in Dalecarlia, near the town of Fahlun, 40 leagues N.W. of Stockholm. It is excavated in an irregular and very powerful mass of pyrites, which in a great many points is almost entirely ferruginous, but in others, particularly near the circumference, it includes a greater or less portion of copper. This mass is enveloped in talcose or hornblende rocks. More to the west there are three other masses almost contiguous to each other, which seem to bend in an arc of a circle around the principal mass. They are explored as well as the last. This was at first worked in the open air; but imprudent operations having caused the walls to crumble and fall in, since 1647 the excavation presents near the surface nothing but frightful precipices. The workings are now prosecuted by shafts and galleries into the lower part of the deposite, and have arrived at a depth of 194 famnars (nearly 430 yards). They display excavations spacious enough to admit the employment of horses, and the establishment of forges for repairing the miners' tools. It is asserted that the exploration of this mine goes back to a period anterior to the Christian era. During its greatest prosperity it is said to have produeed 11 millions of pounds avoird. of copper per annum, or about 5,000 tons. It furnishes now about the seventh part of that quantity; yielding at the same time about $70,000 \mathrm{lbs}$. of lead, with 50 mares of silver, and 3 or 4 of gold. The ores smelted at Fahlun produce from 2 to $2 \frac{1}{2}$ of copper per cent. But the extraction of the metal is not the sole process; the sulphur is also procured; and with it, or the pyrites itself, sulphuric acid and other chemical products are made. Round Fahlun, within the space of a league, 70 furnaces or factories of different kinds may be seen. The black copper obtained at Fahlun is converted into rose copper, in the refining hearths of the small town of Ofwostad.

In the copper mine of Garpenberg, situated 18 leagues from Fahlun, there occur 14 masses of ore quite vertical, and parallel to each other, and to the beds of mica-slate or talc-slate, amid which they stand. This mine has been worked for more than six hundred years.

The mine of Nyakopparberg, in Nericia, 20 leagues W. of Stockholm, presents masses of ores parallel to each other, the form and arrangement of which are very singular. It is worked by open quarrying, and with the aid of fire.

We may notice also the copper mines of Atwidaberg, in Ostrogothia, which furnish annually the sixth part of the whole copper of Sweden.

There are several other copper mines in Sweden. Their whole number is ten; but it was formerly more considerable. They yield at the present day in all, about $2,420,000$ libs. avoird. (1000 tons) of copper.

The number of the silver mines of Sweden has in like manner diminished. In 1767 only 3 were reckoned under exploration, viz., that of Hellefors, in the province of Wermeland; that of Segersfors, in Nericia; and that of Sahla or Sahlberg, in Westmannia, about 23 leagues N.W. of Stockholm. The last is the only one of any importance. It is very ancient, and passes for having been formerly very productive, though at present it yields only from 4,000 to 5,000 mares of silver per annum. Lead very rich in silver is its principal product. It is explored to a depth of more than 200 yards. The soundness of the rock has allowed of vast excavations being made in it, and of even the galleries having great dimensions; so that in the interior of the workings there are winding machines, and carriages drawn by horses for the transport of the ores.

At Sahlberg, there are deposites of sulphuret of antimony.

For the last 30 or 40 years mines of cobalt have been opened in Sweden, principally at Tunaberg and Los, near Nyköping, and at Otward in Ostrogothia. The first are worked upon veins of little power, which become thicker and thinner successively; whence they have been cailed bead-veins. It appears that the products of these mines, though of good quality, are inconsiderable in quantity.

Lastly there is a gold mine in Sweden; it is situated at Adelfors, in the parish of Alsfeda, and province of Smoland. It has been under exploration since 1737, on veins of auriferous iron pyrites, which traverse schistose rocks; presenting but a few inches 
of ore. It formerly yielded from 30 to 40 mares of gold per annum, but for the last few years it has furnished only from 3 to 4 .

The mines and smelting works of Sweden gave annually, in 1809, a gross product worth $1,463,600 l$.

The south of Finland and the bordering parts of Russia contain some mines, but they are far from having any such importance as those of Sweden.

At Orijerwy, near Helsingfors, a mine of copper occurs whose gangue is carbonate of lime, employed as a limestone.

Near Cerdopol, a town situated at the N.W. extremity of the Ladoga lahe, veins of copper pyrites were formerly mined.

Under the reign of Peter the Great, an auriferous vein was discovered in the granitic mountains which border the eastern bank of the lake Ladoga, near Olonetz. It was rich only near the surface; and its working was soon abandoned.

Latterly an attempt has been made to mine copper and iron ores near Eno, above and to the N.W. of Cerdopol, but with little suceess.

Some time ago rich ores of iron, lying in veins, were worked near the lale Shuyna, N.W. from Cerdopol; but this mine has been also relinquished.

On the west bank of the Onega lake, there is an iron work at Petrazavolsk, called a zavode, which is the greatest establishment of this kind existing in the north of Russia.

Nothing is now reduced there except bog iron ore, or swamp ore extracted from small lakes in the neighborhood.

The transition limestone which constitutes the body of Esthonia contains lead ore at Arossaar near Fellin. These ores were worked when these provinces belonged to the Swedes. It was attempted in 1806 to resume the exploitation, but without suecess.

MINES OF THE ALLEGANY MOUNTAINS.

The chain of the Alleganys, which traverses the United States of North America from N.W. to S.E. parallel to the coasts of the Atlantic ocean, includes a considera- ble number of deposites of iron, lead, and copper ores; along with some ores of silver, plumbago, and chromite of iron. Attempts have been made to mine a great many of these deposites; but most of these have been unsuccessful.

A bed of black oxide of iron occurs in gneiss near Franconia in New Hampshire. It has a power of from 5 to 8 feet; and has been mined through a length of 200 feet, and to a depth of 90 feet. The same ore is found in veins in Massachusetts and Vermont, accompanied by copper and iron pyrites. It is met with in immense quantities on the western bank of the lake Champlain, forming beds of from 1 to 20 feet in thickness, almost without mixture, encased in granite. It is also found in the mountains of that territory. These deposites appear to extend without interruption from Canada to the neighborhood of New York, where an exploration on them may be seen at Crown Point. The ore there extracted is in much esteem. Several mines of the same species exist in New Jersey. The primitive mountains which rise in the north of this state near the Delaware, include a bed almost vertical of black oxide of iron, which has been worked to 100 feet in depth. In the county of Sussex the same ore occurs, accompanied with Franklinite. At New Milford, in Connecticut, a pretty abundant mine of sparry iron occurs; the only one of the kind known in the Alleganys. The United States contain a great many iron works, some of which prior to the year 1773, sent over iron to London. They are principally supplied from alluvial iron ore.

The most remarkable lead mines of the Alleganys are those of Southampton, in Massachusetts, and of Perkiomen creek, in Pennsylvania, 8 leagues from Philadelphia. The first furnishes a galena, slightly argentiferous; an ore accompanied with various minerals, with base of lead, copper, and zinc, and with gangues (vein-stones) of quartz, sulphate of baryta, and fluor spar. These substances form a vein which traverses several primitive rocks, and is said to be known over a length of more than 6 leagues. At Perkiomen creek a vein of galena is mined which traverses a sandstone, referred by many geologists to the old red sandstone. Along with galena a great variety of minerals is found with a basis of lead, zinc, copper, and iron. The mines of lead worked in Virginia, on the banks of the Kanahwa, deserve also to be mentioned.

None of the copper mines actually in operation in the United States seem to merit particular attention. The mine of Schuyler, in New Jersey, had excited high hopes, but after the workings had been pushed to a depth of 300 feet, they have been for some years abandoned. The ore, which consisted of sulphuret of copper, with oxide and carbonate of copper, occurred in a red sandstone.

In some points of the Alleganys, deposites have been noticed of chromite of iron and graphite.

Coal-measures occur in several points of the United States, especially on the N.W 
vlope of the Allegany mountains. The coal is mined successfully on the banks of the Ohio, toward the upper part of its course. See Anthracite.

\section{MINES OF THE SOUTH OF SPAIN.}

The mountains which separate Andalusia from Estremadura, Leon and La Mancha; and those of the kingdoms of Murcia and Grenada, include some celebrated mines.

We shall mention first the silver mines of Guadalcanal and Cozalla, situated in the Sierra-Morena, 15 leagues north of Seville. Among the ores, red silver and argentiferous gray copper have been specified. Their product is inconsiderable; but this territory presented formerly much more important mines at Villa-Guttiera, not far from Seville. At the beginning of the serenteenth century they are said to have been worked with such activity, that they furnished daily 170 mares of silver. More to the east, there exists in the mountains of La Mancha a mine of antimony, at Santa-Crux-de-Mudela. On the southern slope of the Sierra-Morena, very important lead mines occur, particularly at Linares, 12 leagues north of Jaen. The veins are rery rich near the surface, which causes them not to be mined much in depth; so that the ground is riddled, as it were, with shafts. More than 5,000 old and new pits may be counted, the greater part of which is ascribed to the Moors. Six of these mines are now explored on account of the crown, and they produce on an annual average, according to M. Laborde, 1,320,000 libs. avoird. (about 600 tons) of lead, which is too poor in silver for this precious metal to be extracted with advantage. Bowles states that there was found at the mines of Linares, a mass of galena, whose dimensions were from 21 to 24 yards in every direction. Abundant mines of zinc occur near Alcaras, 15 leagues northwest of Linares, which supply materials to a brass manufactory established in that town. There are also lead mines in the kingdoms of Murcia and Grenada. Very productive ores have been worked for some time near Almeira, a harbor situated some leagues to the west of the cape of Gates. The ore is in part treated on the spot with coal brought from Newcastle, and in part sent to Newcastle to be reduced there. The kingdoms of Murcia, Grenada, and Cordova, include several iron mines. Near Cazalla and Ronda, in the kingdor of Grenada, mines of plumbago are explored.

On the northern flank of the Sierra-Morena, lie the famous quicksilver mines of Almaden, situated near the town of the same name in La Mancha. They consist of very powerful veins of sulphuret of mercury, which traverse a sandstone, evidently of a geological age as old at least as the coal formation. Hard by, beds of coal are mined.

MINES OF THE PYRENEES.

The Pyrenees, and the mountains of Biscay, of the Asturias, and the north of Galicia, which are their prolongation, are not very rich in deposites of ores : the only important mines that occur there, are of iron; which are widely spread throughout the whole chain, except in its western extremity. We may mention particularly in Biscay, the mine of Sommorostro, opened on a bed of red oxide of iron; and in the province of Guipuscoa, the mines of Mundragon, Oyarzun, and Berha, situated on deposites of sparry iron. There are several analogous mines in Aragon and Catalonia. In the French part of the Pyrenees, veins of sparry iron are worked which traverse the red sandstone of the mountain Ustelleguy, near Baygorry, department of the Basses-Pyrenees. The same department affords in the valley of Asson the mine of Haugaron, which consists of a bed of hydrate of iron, subordinate to transition limestone. The deposite of hydrate of iron, worked for an immemorial time at Rancié, in the valley of Viedessos, department of the Arriège, occurs in a similar position. The ancient workings have been very irregular and very extensive; but the deposite is still far from being exhausted. There are also considerable mines of sparry iron at Lapinouse, at the tower of Batera, at Escaron, and at Fillols, at the foot of the Canigou, in the department of the Oriental Pyrenees. The iron mines of the Pyrenees keep in activity 200 Catalanian forges. Although there exists in these mountains, especially in the part formed of transition rocks, a very great number of veins of lead, copper, cobalt, antimony, \&c., one can hardly mention any workings of these metals; and among the abandoned mines, the only ones which merit notice are-the mine of argentiferous copper of Baygorry, in the department of the Low Pyrenees, the lead and copper mine of Aulus, in the valley of the Erce, department of the Arriège, and the mine of cobalt, of the valley of Gistain, situated in Aragon, on the southorn slope of the Pyrenees. It is asserted, however, that a lead mine is in actual operation near Bilboa, in Biscay. The mines of plumbago opened at Sahun, in Aragon, should not be forgotten. Analogous deposites are known to exist in the department of the Arriège, but they are not mined.

\section{MIVES OF THE ALPS.}

The mines of the Alps by no means correspond in number and richness with the extent and mass of these mountains. On their eastern slope, in the department of the 
high and the low Alps, several lead and copper mines are mentioned, all inconsiderable and abandoned at the present time, with the exception if some workings of galena, which furnish also a little graphite.

During some of the last years of the eighteenth century, there was mined at la Gardette in the Oisans, department of the Isère, a vein of quartz which contained native gold and auriferous pyrites; but the product has never paid the expenses, and the mine has been abandoned. The Oisans presented a more important mine, but it also has been given up; it was the silver mine of Allemont or Chalanches The ore consisted of different mineral species more or less rich in silver, disseminated in a clay which filled the clefts and irregular cavities in the middle of talcose and hornblende rocks. This mine yielded annually toward the conclusion of the eighteenth century, so much as 2,000 marcs of silver, along with some cobalt ore. Among the great number of mineral species, which occurred in too small quantities to be worked to advantage, there was native antimony, sulphuret of mercury, \&c. The Oisans present, moreover, some rather unproductive mines of anthracite. Mines of an analogous nature, but more valuable, are in activity at the western foot of the Alps, at la Mothe, Notre-des-Vaux et Putterille, a few leagues southeast of Grenoble.

From the entrance of the valley of the Oisans to the valley of the Arc in Savoy, there occur on the N.W. slope of the Alps, a great inany mines of sparry iron. The locality of this ore is here very difficult to define. It appears to form sometimes beds or masses, and sometimes veins amid the talcose rocks. Some is also found in small veins in the first course of the calcareous formation which covers these rocks. These mines are very numerous; the most productive occur united in the neighborhood of Allevard, department of the Isère, and of Saint Georges d'Huretières in Savoy. Those of Forneaux and Laprat, in the latter country, are also mentioned. The irregularity of the mining operations surpasses that of the deposites; the mines have been from time immemorial in the hands of the inhabitants of the adjoining villages, who work in them, each on his own account, without any prearrangement, or other rule than following the masses of ore which excite hopes of the most considerable profit in a short space of time. What occurs in almost every mine of sparry iron, is also to be seen here-most imprudent workings. The mine called the Grand Fosse, at Saint Georges d'Huretières, is prolonged without pillars or props, through a height of 130 yards, a length of 220 yards, and a breadth equal to that of the deposite, which amounts in this place to from 8 to 13 yards; thus a void space is exhibited of nearly 300,000 square yards. The sparry iron extracted from these different mines supplies materials to 10 or 12 smelting-furnaces, the cast-iron of which, chiefly adapted for conversion into steel, is manufactured in part in the celebrated steel works of Rives, department of the Isère. There occurs in some parts of the mines of Saint Georges d'Hunctières copper pyrites, which is smelted at Aiguebelle.

Savoy presents celebrated lead mines at Pescy and at Mucot, 7 leagues to the east of Moutiers. Galena, accompanied with quartz, sulphate of baryta, and ferriferous carbonate of lime, occurs in mass in talcose rocks. The mine of Pescy had been restored to activity by the French government, which established there a practical school of mines; and in its hands the mine produced annually as much as 440,000 libs. avoird. of lead, and 2,500 mares of silver. It is now explored on account of the king of Sardinia; but it begins to be exhausted, and yields less products. That of Macot, opened a few years ago, begins to give considerable returns. The mine of copper pyrites of Servoz, in the valley of the Arve, may also be mentioned. The ore occurs both in small veins, and fisseminated in a clay slate; but the exploration is now suspended. Lastly, slightlyproductive workings of anthracite are mentioned in several points of these mountains, and in the conterminous portions of the Alps.

There exist in Piedmont some small mines of argentiferous lead. The copper mines of Allagne, and those of Ollomont, formerly yielded considerable quantities of this metal. Their exploration is now on the decline. The manganese mines of Saint-Marcel have few outlets; whence they have been feebly dereloped. Mines of plumbago, little worked, occur in the neighborhood of Vinay, and in the valley of Pellis, not far from Pignerol. Some mines of auriferous pyrites have also been worked in this district of country; among others, those of Macugnaga, at the eastern foot of Monte-Rosa. The pyrites of this mine afforded by amalgamation only 11 grains of gold per quintal; and this gold, far from being fine, contained one fourth of its weight of silver; they became less rich in proportion as they receded from the surface. The explorations of auriferous pyrites in Piedmont are now abandoned, or nearly so. The only important mines in this country are those of iron. These generally consist of masses of black oxide of iron, of a nature analogous to those of Sweden; the principal ones being those of Cogne and Traverselle, which are worked in open quarries. Some others, less considerable, are explored by shafts and galleries. These ores are reduced in 33 smeltingcupolas, 55 Catalan forges, and 105 refinery-hearths. The whole produce about 10,000 tons of bar-iron. 
There is a mine of black oxide of iron, at present abandoned, at Bovernier, near Martigny, in the Valais. There is also another iron mine at Chamoissons, in a lofty calcareous mountain on the right bank of the Rhone. The ore presents a mixture of oxide of iron and some other substances, of which it has been proposed to make a new mineral species, under the name of Chamoissite.

The district of the Grisons possesses iron mines with very irregular workings, situated a few leagues from Coire.

The mountain of Falkenstein, in the Tyrol, formed of limestone and clay-slate, not far from Schwatz, a little below Inspruck, in the valley of the Inn, contains mines of argentiferous copper. At one of them, that of Kütz-Pühl, the workings reached, in 1759 , according to the report of MM. Jars and Duhamel, nearly 1,100 yards in depth; and were reckoned the deepest in Europe. But it was intended to abandon them. Analogous ores are explored in several other points of the same country. The most part of the products of these mines are carried to the foundry of Brixlegg, 4 leagues from Schwatz. The mines of the Tyrol furnished, on an average of years, toward 1759 , 10,000 mares of silver; at anterior periods, their products had been double; but now it is a little less. This region contains also gold mines whose exploration goes back a century and a half. They occur near the village of Zell, 8 leagues from Schwatz; the auriferous veins traverse clay-slates and quartz rocks. Lastly, a deposite of oxide of chrome, similar to that of the Ecouchets (Saône and Loire) has been discovered in the Tyrol. An unimportant mine of mercury has also been mentioned in that country, near Brenner.

In the territory of Saltzburg there are some copper mines. In the environs of Muerwinkel and of Gastein some veins are worked for the gold they contain; of which the annual return is valued at 118 mares of this metal. There is an inconsiderable mine of quicksilver at Leogang.

In the Tyrol and in Saltzburg there are iron mines in a very active state, principally those of Kleinboden, near Schwatz. But the portion of the Alps most abundant in mines of this metal, is the branch stretching toward Lower Austria. We find here, both in Styria and in Austria, a very great number of explorations of sparry iron. The deposites of the ores of sparry iron of Eisenerz, Erzberg, Admont, and Vordenberg, deserve notice. The latter are situated about 25 leagues southwest of Vienna.

The southern flank of the Alps contains also a great many mines of the same kind, from the Lago Maggiore to Carinthia. Those situated near Bergamo, and those of Huttenberg and Waldenstein, in Carinthia, are especially mentioned.

All these mines of sparry iron are opened in the midst of rocks of different natures, which belong to the old transition district of the Alps. They seem to have close geological relations with those of Allevard.

The branch of the Alps which extends toward Croatia, presents important iron mines, in the mountains of Adelsberg, 10 leagues southwest from Laybach, in Carniola.

The iron mines just now indicated in the part of the Alps that forms a portion of the Austrian states, supply materials to a great many smelting-works. In Styria and in Carinthia, more than 400 furnaces or forges may be enumerated, whose annual product is nearly 25,000 tons of iron. These two provinces are famous for the steel which they produce, and for the steel tools which they fabricate, such as sythes, \&c. Carniola contains also a great many forges, and affords annually about 5,000 tons of iron.

There are mines of argentiferous copper, analogous to those of the Tyrol, at Schladming in Styria, at Kirchdorf in Carinthia, at Agordo in the territory of Venice, and at Zamabor in Croatia. The latter are remarkable for the great irregularity of the deposites, and for the richness of the copper pyrites that is mined; which produces 12 and sometimes 27 per cent. of copper. There are some deposites of antimony, mined to a trifling extent in Carinthia ; and there are a few cobalt mines in Styria, not more actively worked. In the environs of Raibel, in Carinthia, mines of calamine exist, yielding annually about 200 tons of this substance. Of late, some of it has also been explored in Styria.

The limestones that cover the northern slopes of the Alps, present, like those of the departments of the lower and upper Alps, several lead mines of little consequence; they also include several celebrated mines of rock salt.

The analogous limestones which repose on the slopes of the Alps in Carinthia, and in the neighboring provinces, afford likewise lead mines, especially near Willach and Bleyberg. These mines are very numerous, forming more than 500 arrondissements of concessions. They furnish annually about 1,800 tons of a lead too poor in silver to pay the expense of extracting that precious metal. At the mines of Bleyberg, the galena forms 14 beds or strata, inclined at an angle of from 40 to 50 degrees from the horizon, and alternating with a like number of calcareous strata. The latter are extremely full of shells. They of course belong to secondary limestone. 
The limestones surmounting the southern slope of the Alps, contain also some lead mines; but the quicksilver mines of Idria, situated at the foot of the Alps, 10 leagues N.W. of Trieste, is worthy of particular notice ; it lies in a limestone which everything leads us to refer to the zechstein, the most ancient of the secondary limestones.

The Apennines, which may be considered as a dependance of the Alps, present a small number of mines. At Chiavary and Pignone, manganese is mined ; and at the beginning of the eighteenth century a vein of mercury was worked at Levigliani in Tuscany. An antimonial mine is mentioned at Pereta in the marshes of Sienna.

Before quitting these regions, we ought to notice the iron mines of the isle of Elba. They have been famous for 18 centuries; Virgil denotes them as inexhaustible, and supposes them to have been open at the arrival of Eneas in Italy. They are explored by open quarries, working on an enormous mass of specular iron ore, perforated with cavities bespangled with quartz crystals. The island possesses two explorations, called Rio and Terra-Nuova; the last having been brought into play at a recent period. The average amount extracted per annum is 15,000 tons of ore, which are smelted in the foundries of Tuscany, Liguria, the Roman states, the kingdom of Naples, and the island of Corsica.

There has been worked for a few years a mine of chromite of iron, at Carrada, near Gassino, department of the Var.

MINES SITUATED IN THE SCHISTOSE FORMATIONS OF THE BANKS OF THE RHINE, ANR IN THE ARDENNES.

The transition lands, which form, in the northwest of Germany and in Flanders, a pretty extensive range of hills, include several famous mines of iron, zinc, lead, and copper. The latter lie on the right bank of the Rhine, in the territories of Nassau and Berg, at Baden, Augstbach, Rheinbreitenbach, and near Dillenburg. That of Rheinbreitenbach yielded formerly 110,000 libs. avoirdupois of copper per annum, and those of the environs of Dillenburg now furnish annually 176,000 libs. There are also some mines of argentiferous lead in the same regions. The most remarkable are in the territory of Nassau, such as those of Holzapfel, Pfingstiviese, Lœwenburg, and Augstbach on the Wiede, and Ehrenthal on the banks of the Rhine, which all together produce 600 tons of lead, and 3,500 mares of silver. To the above, we must add those of the environs of Siegen and Dillenburg, in the territories of Berg. A little cobalt is explored in the neighborhood of Siegen, and some mines of the same nature are mentioned in the grand dutchy of Hesse-Darmstadt, and in the dutchy of Nassau Usingen.

But iron is the most important product of the mines on the right bank of the Rhine. Veins of hydrate of iron, or brown hematite, are explored in a great many points of Hessia, and the territory of Nassau, Berg, Marck, Tecklenbourg, and Siegen, along with veins or masses of sparry iron, and beds of red oxide of iron. We may note particularly-1. The enormous mass of sparry iron, known under the name of Stahlberg, mined since the beginning of the fourteenth century in the mountain of Martinshardt, near Müssen, where improvident excavations have occasioned, at several times, considerable downfallings of rubbish ; 2 . The abundant and beautiful mines of hydrate of iron and sparry iron on the banks of the Lahn and the Sayn, and among those of the mine of Bendorf; 3. The mine of Hohenkirchen in Hessia, where a powerful bank of manganiferous ore is worked, and where the mines are kept dry by a gallery more than 1,000 yards long, walled over its whole extent. These several mines supply a great many iron works, celebrated for their steel, and for the objects of hardware, sythes, \&c., fabricated there.

The Prussian provinces of the left bank of the Rhine, the dutchy of Luxembourg, and the Low Countries, include also many iron furnaces, of which a great number are supplied, in whole or in part, by ores of hydrate of iron, occasionally zinciferous, ex tracted from the transition rocks, where they form sometimes veins, and sometimes also very irregular deposites. A portion is explored by open quarrying, and a portion by underground workings. Some of these mines penetrate to a depth of 87 yards, and galleries may be observed in them cut in the form of vaults, and timbered with hooped stays. The Hundsrück, the Eiffel, and the territory of Luxembourg, present a great many of them.

The Eiffel formerly possessed important lead mines. Some still exist, which are feebly worked at Berncastle, 8 leagues below Trèves, on the banks of the Moselle. Those of Trarbach, situated 2 leagues lower, are now completely abandoned; the same holds with those of Bleyalf, which were opened on veins incased in the greywacke-slate, 3 leagues W.N.W. of Prüm, not far from the line of separation of the waters of the Mo selle and the Meuse, in a district from which manufactures and comfort have disappeared since the mines were given up which sustained them.

More to the north a great many deposites of calamine occur. The most considerable 
and the one explored with most activity, is situated in the territory of Limburg (kingdom of the Netherlands), and known under the name of the Great mountain. It presents a mass about 45 yards wide, from 400 to 550 long, and of an unknown depth. The first labors, undertaken several centuries age by the Spaniards, were executed by open quarrying, and pushed down 32 yards from the surface. The miners were obliged to renounce this mode of operation, and have since penetrated to the depth of 88 yards by means of subterranean workings. From 50 to 60 men work in this excavation, and exact annually from 700 to 800 tons of calamine, worth from $2,400 l$. to 2, $00 l$. In the adjacent parts of the Prussian territory, not far from Aix-la-Chapelle, calamine is also mined, with ores of lead and iron, with which it is associated, in deposites regarded by $M$. Bouesnel, as analogous to the vein of Vedrin, to be noticed presently. The exploration is effected by means of small round shafts, from 34 to 44 yards deep, which are often wooded only with flexible branches of trees, or a kind of barrel-hoops. These workings may furnish annually from 1,500 to 2,000 tons of calamine, to the brass factories of Stollberg. On the right bank of the Rhine, in the country of la Marck, sereral small zinc mines furnish annually about 130 tons of calamine to the brass manufactures of Iserlohn.

The lead mine of Fedrin, alluded to above, lies at some distance N. of Namur. It is opened on a vein of galena nearly vertical, which crosses from $\mathrm{N}$. to $\mathrm{S}$. a limestone in ntarly vertical strata, probably analogous into the limestone of Derbyshire. The vein is from 4 to 15 feet thick, and is recognised through a length of half a league. The mine, worked for two centuries, presents very extensive excavations; particularly a fine gallery of efflux. It has produced annually 900 tons of lead. At the present day the mine of Vedrin, and some adjoining exploitations, afford per annum only about 200 tons of lead, and 700 mares of silver.

\section{MINES OF THE CALCAREOUS MOUNTAINS OF ENGLAND.}

The limestone formation immediately subjacent to the coal measures, or the mountain limestone, constitutes almost alone several mountainous regions of England and Wales; in which three districts very rich in lead mines deserve to be noted.

The first of these districts comprehends the superior parts of the valleys of the Tyne, the Wear, and the Tees, in the counties of Cumberland, Durham, and York. Its principal mines are situated near the small town of Alston-Moor, in Cumberland. The veins of galena which form the object of the workings, traverse alternate beds of limestone and sandstone; and are very remarkable for their becoming suddenly thin and impoverished on passing from the limestone into the sandstone; and for resuming their richness, and usual size, on returning from the sandstone into the limestone. The exploitations are situated in the flanks of considerably high hills, bare of wood, and almost wholly covered with marshy heaths. The waters are drawn off by galleries of efflux; and the ores are dragged out by horses to the day. The galena extracted from these mines is smelted by means of coal and a little peat, in furnaces of the Scotch construction. The lead is very poor in silver; and there is hardly a single hearth for the purpose of eliminating this metal by cupellation. The mines of this district produce annually 17,200 tons of lead, according to Mr. Taylor's statement, published in the Geology of England and Wales, by Messrs. Conybeare and Phillips. There is more over a copper-mine 2 leagues S.W. of Alston-Moor. The ore is a copper pyrites, accompanied with galena in a very extensive vein, which does not appear to belong tc the same formation as the other veins of this region.

The second metalliferous district lies in the northern part of Derbyshire, and in the conterminous parts of the neighboring counties. The districts called the Peak and King's-Field are the richest in workable deposites. The mines of Derbyshire are getting exhausted; they are very numerous, but in general inconsiderable. The galena extracted from them is treated with coal in reverberatory furnaces; but the silver is not scught for. They yield annually 900 tons of lead; with a certain quantity of calamine, and a little copper ore. A vein of copper pyrites occurs at Ecton, in Staffordshire, on the borders of Derbyshire. The veins of Derbyshire are famous for the beautiful minerals which they have produced; and particularly for the interruption which they almost constantly suffer at the contact of the trap-rock, called toadstone, which is intruded among the limestone.

The third metalliferous distric is situated in Flintshire and Denbighshire, counties forming the N.E. part of Wales. Next to Alston-Moor this is the most productive; furnishing annually 6,900 tons of lead, and a certain quantity of calamine. The galena is smelted in reverberatory furnaces, and affords a lead far from rich in silver, which is therefore seldom subjected to cupellation. The mines occur partly in the metalliferous limestone, and partly in several more ancient rocks.

T'o the S.E. of this district there exist still some lead mines in Shropshire. They 
lie, like the preceding, partly in the metalliferous limestone, and partly in the subjacent rocks. They yield annually from 700 to 800 tons of lead.

Some mines of galena and calamine are mentioned in the Mendip hills, to the south of Bristol ; but they seem to be for the present abandoned.

Besides the metallic mines just enumerated, the formation of the metalliferous limestone presents, in England, especially in the counties of Northumberland and Cumberland, several coal mines, opened on coal strata included by the sandstone, which alternates with the limestone.

MINES OF DAOURIA.

The name Daouria is given to a great region wholly mountainous, which extends from the Baikal Lake to the eastern ocean. There is, perhaps, no other country in the world so rich in deposites of lead ores, as the part of this district which extends from the junction of the rivers Chilca and Argoun, whose united waters form the river Amour, belonging to Russia. The mines opened here constitute the third arrondissement of the Siberian mines, called that of Nertchinsk, from the name of its capital, which lies more than 1,800 leagues east of Saint Petersburg.

The gra and of the metalliferous portion of Daouria is formed of granite, hornschiefer, and schists, on which reposes a gray limestone, sometimes siliceous and argillaceous, which contains a small number of fossils, and in which the veins of lead occur. The plains of these regions, often salt deserts, exhibit remarkable sandstones and pudding-stones; as also vesicular rocks of a volcanic aspect. It appears that the metalliferous limestone is much dislocated, and the lead veins are subject to several irregularities, which render their exploitation difficult and uncertain. The mines lie chiefly near the banks of the Chilca and the Argoun, in several cantons, at a considerable distance from one another; wherefore it was requisite to build a great number of smelting furnaces. The want of wood has placed difficulties in the working of some of them. The ore is galena, sometimes occurring in masses of several yards in diameter; having commonly for vein-stones ores of iron and zinc, of which no use is made. The galena itself, furnished by these mines in enormous quantities, receives a very different treatment from what it would do in a civilized country; for, though the lead which it produces contains only from 6 to 10 gros ( 1 to $1 \frac{1}{2}$ ounce) of silver per quintal, it is for it alone that these mines are worked. The litharge produced by the cupellation is thrown away as useless; so that heaps of it exist near the smelting-furnaces, says M. Patrin, higher than the houses. Only an insignificant quantity of it is reduced to lead for the uses of the country, or for those of the foundries in the arrondissement of Kolywan. The silver extracted from the mines of Daouria, contains a very small proportion of gold. M. Patrin says that their annual product was, toward the year 1784, from 30,000 to 35,000 marcs of silver. The exploitation of some of the mines of Daouria goes back to the end of the 17 th century. It has been commenced in some points by the Chinese, whe were not entirely expelled from this territory till the beginning of the following century. A great part of the mines, however, has been opened up since 1760 .

Besides the lead mines, there are some unimportant mines of copper in Daourra, and in different explorations of this region, arsenical pyrites, from which arsenious acid is sublimed in factories established at Jutlack and at Tchalbutchinsky.

About 45 leagues to the south of Nertchinsk, the mountain of Odon-Tchelon occurs, celebrated for the different gems or precious stones extracted from it. It is formed of a friable granite, including harder nobules or balls which enclose topazes; it is very analogous to the topaz rock of Saxony. In this granite there are several veins filled with a ferruginous clay, which contains a great quantity of wolfram, and many emeralds, aqua-marines, topazes, crystals of smoked quartz, \&c. Multitudes of these minerals have been extracted by means of some very irregular workings. The mountain of Toutt-Kaltoui, situated near the preceding, offers analogous deposites. The presence of wolfram had excited hopes that tin might be found in these mountains; hopes which have not hitherto been realized. There are some unworked deposites of sulphuret of antimony in this country.

ON SOME OTHER LESS KNOWN MINE COUNTRIES.

There seem to exist in Brazil, besides the washings of the sands that produce the diamonds, the precious stones, the platinum, and almost all the gold of this country, some mines of gold, lead, and iron, opened up in very ancient geological formations; but there is no silver mine, which indicates a great difference between the metalliferous deposites of this district and those of Spanish America. The lead mines occur particularly in the captainry of Minas-Geraes, canton of Abaité. Their exploitation has been undertaken within a few years. The captainry of Minas-Geraes contains extremely abundant deposites of black oxide of iron, and specular iron, which constitute beds or enormous masses, forming sometimes entire mountains; along with numerous 
veins of hematite and red oxide of iron. Lately these have been opened up, and smelting-houses have been established at Gaspar-Saarez. There are also iron mines and foundries in the captainry of Saint-Paul. A mine of antimony occurs near Sabara, in the captainry of Minas-Geraes.

In Africa, the inhabitants of the countries adjoining to the cape of Good Hope mine and smelt copper and iron; and the Congo produces considerable quantities of these two metals. It is asserted that a great deal of copper exists in Abyssinia. On the banks of the Senegal the Moors and the Pouls fabricate iron in travelling forges. They employ as the ore the richest portions of a ferruginous sandstone, which seems to be a very modern formation. Lastly, the kingdoms of Morocco and Barbary appear to include several copper and iron mines.

The islands of Cyprus and Negropont, in the Mediterranean, were celebrated, in former times, for their copper mines; and several islands of the Archipelago presented gold mines, now abandoned. The same thing may be said of Macedonia and Thrace. The mountains of Servia and Albania contain iron mines; and lead mines occur in Servia. Natolia possesses iron and copper mines in the neighborhood of Tokat. Some also occur in Arabia and in Persia; and in the territories round Caucasus, the kingdom of Imeretta is distinguished for its iron mines. The celebrity of the Damascus sabres attests the good quality of the products of some of the mines. Persia includes, besides, mines of argentiferous lead at Kervan, a few leagues from Ispahan; and Natolia furnishes orpiment.

Some iron and copper mines have been mentioned in Tartary. Thibet passes for being rich in gold and silver mines. China produces a great quantity of iron and mercury, as well as white brass (tombac), which is much admired. The copper mines of this empire lie principally in the province of $\mathrm{Yu} \mathrm{Nan}$ and the island of Formosa. Japan, likewise, possesses copper mines in the provinces of Kijunack and Sarunga. They seem to be abundant; at a period not far back, they exported their products to Europe. Japan presents, moreover, mines of quicksilver. China and Japan contain also mines of gold, silver, tin, red sulphuret of arsenic, \&c. Large deposites of the latter ore (realgar) are said to occur in the tin mine of Kian-Fu, in China. But in that empire, as in Europe, coal is the most important of the mining products. This combustible is explored, especially in the environs of Pekin, and in the northern parts of the empire.

Iron mines exist in several points of the Burman empire, and of Hindostan. Near Madras there exist excellent ores of sparry iron, and black oxide, analogous to the Swedish ores. The Indian natural steel, named Wootz, has been held in considerable estimation among some eminent London cutlers; but the iron and steel recently manufactured upon a great scale, near Madras, by Messrs. Heath and Co., from the crystallized magnetic ore of that country, will probably ere long rival, and eventually supersede in Europe the product of the Dannemara forges. The islands of Macassar, Borneo, and Timor, include copper mines. As to the tin obtained from the island of Banca, from the peninsula of Malacca, and several other points of southern Asia, it proceeds entirely from the washing of sands. The same is undoubtedly true of the gold furnished by the Philippine isles, Borneo, \&c. It appears, however, that mines of gold and silver are worked in the island of Sumatra.

\section{MINES OF THE SECONDARY ROCK FORMATIONS.}

The most important mines of the secondary rocks, and perhaps of all minerals whatsoever, are those worked in the most ancient of these strata, in the coal-measures.

The British islands, France, and Germany, present several groups of small mountains primitive on the ridge, and transition on the flanks; in the sinuosities between which deposites of coal occur. The principal of these have become great centres of manufactures; for Glasgow, Newcastle, Sheffield, Birmingham, Saint-Etienne, \&c., owe their prosperity and their rapid enlargement to the coal, raised, as it were, at their gates in enormous quantities. Wales, Flanders, Silesia, and the adjacent parts of Gallicia, owe equally to their extensive collieries a great portion of their activity, their wealth, and their population. Other coal districts, less rich, or mined on a less extended scale, have procured for their inhabitants less distinguished, but by no means inconsiderable, advantages; such, for examples in Great Britain, are Derbyshire, Cheshire, Lancashire, Shropshire, Warwickshire, the environs of Bristol \&c.; some parts of Ireland; in France, Litry department of C'alvados, Comanterie, Saint-Georges-Chatelaison, Aubin, Alais, le Creusot; Ronchamps, in the Prussian provinces of the left bank of the Rhine; the environs of Saarebrück; several points of the north of the territory of Berg and Lamarck, of Mansfeld, of Saxony, Hungary, Spain, Portugal, the United States, \&c.

We need not enter here into ampler details on coal mines, reserving these particulars for the article Pitcoal.

Nature has deposited alongside of coal an ore whose intrinsic value alone is very amall, but whose abundance in the neighborhood of fuel becomes extremely precious to 
man; we allude to the clay-ironstone of the coal-measures. It is extracted in enor. mous quantities from the coal-basins of Scotland, Yorkshire, Staffordshire, Shropshire, and South Wales.

Much of it is also raised from the coal strata of Silesia; and the French entertain hopes of finding a supply of this necessary ore in their own country. The iron-works of England, which are supplied almost entirely from this iron-stone reduced with the coke or coal, pour annually into commerce more than one million tons of cast and bar iron, the value of which has been estimated at eight millions sterling; an amount fully equal to the product of all the mines of Spanish America.

The shale or slate-clay of the coal-measures contains sometimes a very large quantity of pyrites, which, decomposing by the action of air, with or without artificial heat, produces sulphate of iron and sulphate of alumina; whence copperas and alum are manufactured in great abundance.

The lead mines of Bleyberg and Gemünd, near Aix-la-Chapelle, are explored in a sandstone referred by many geologists to the red sandstone. The ore consists principally of nodules, of galena disseminated in this rock. They are very abundant, and of very easy exploration. These mines produce annually from 700 to 800 " of lead, which does not contain silver in sufficient proportion to be worth the extracting. 2,000 tons of ore are prepared and sold in the form of black lead dust (alquifoux).

The manganese mines worked in the open air near Exeter, in England, occur in a sandstone analogous to the red.

The calcareous formation which surmounts the coal-sandstone, called by geologists zechstein, magnesian limestone, and older alpine limestone, contains different deposites of metallic ores; the most celebrated being the cupreous schist of Mansfeldt, a stratum of calcareous slate from a few inches to two feet thick, containing copper pyrites in sufficient quantity to afford 2 per cent. of the weight of the ore of an argentiferous copper. This thin layer displays itself in the north of Germany over a length of eighty leagues, from the coasts of the Elbe to the banks of the Rhine. Notwithstanding its thinness and relative poverty, skilful miners have contrived to establish, on dificrent points of this slate, a number of important explorations, the most considerable being in the territory of Mansfeldt, particularly near Rottenburg. They produce annually 2,000 tons of copper, and 20,000 mares of silver. We may also mention those of Hessia, situated near Frankenberg, Bieber, and Riegelsdorf. In the latter, the cupreous schist and its accompanying strata are traversed by veins of cobalt, mined by the same system of underground workings as the schist. These operations are considerable; they extend, in the direction of the strata, through a length of 8,700 yards, and penetrate downward to a very great depth. Three galleries of efflux are to be observed; two of which pour their waters into the Fulde, and the third into the Verra. One of them runs about 20 yards below the most elevated point of the workings. These mines have been in activity since the year 1530. Analogous mines exist near Saalfeld, in Saxony.

To the same geological formation must probably be referred the limestone which contains the sparry iron mine of Schmlacalden, at the western foot of Thuringerwald, where there has been explored from time immemorial a considerable mass of this ore known by the name of Stahlberg. The working is executed in the most irregular manner, and has opened up enormous excavations; whence disastrous ruins have taken place in the mines. It furnishes annually 4,500 tons of ore, which keep in play a great number of furnaces, where a deal of iron and steel is manufactured.

At Tarnowitz, 14 leagues S.E. of Oppeln, in Siberia, the zechstein contains, in some of its strata, considerable quantities of galena and calamine; into which mines have been opened, that yield annually from 600 to 700 tons of lead, 1,000 to 1,100 mares of silver, and much calamine. Mines of argentiferous lead are noticed at Olkutch and Jaworno, in Gallicia, about 6 leagues N.E. of Cracow, and 15 leagues E.N.E. of Tarnowitz. Their position seems to indicate that they belong to the same formation; and possibly those of Willach and Bleyberg in Carinthia have the same locality.

There has been discovered lately near Confolens, in the department of la Charente, in a secondary limestone, calcareous beds, and particularly subordinate beds of quartz, which contain considerable quantities of galena. At Figeac also, in the department of le Lot, deposites of galena, blende, and calamine, occur in a secondary limestone. At la Voulte, on the banks of the Rhone, there is mined, in the lower courses of the limestones that constitute a great portion of the department of the Ardèche, a powerful bed of iron ore.

It is in the zechstein, or in the sandstones, and trap rocks of nearly the same age, that the four great deposites of the sulphuret of mercury, of Idria, the Palatinate, Al maden, and Huancavelica, are mined.

The formation which separates the zechstern from the lias (calcaire a gryphites), called new red sandstone and red marl in England, and bunter-sandstein, muschelkalk, and guadersandstein. in Germany, presents hardly any impsrtant mines except those of rock 
salt; which enrich it, not only in the centre of Europe, as in Cheshire, at Vic, Wieliczka, Buchnia, and Salzbourg, but in many other parts of the world.

'The lias contains often very pyritous lignites, which are mined in many places, and particularly at Whitby and Guisborough in Yorkshire, for the manufacture of alum and copperas.

'The oolitic limestones contain strata of iron ore, which are mined in some districts of France.

The iron sand (Hastings sand) beneath the chalk formation, is often so strongly imbued with iron as to be worth the working.

The lowest beds of the chalk contain iron pyrites, which has become the object of an important exploration at Vissans, on the southern coast of the Pas-de-Calais, where it is converted into sulphate of iron. The waves turn the nodules out of their bed, and roll them on the shore, where they are picked up.

If the chalk be poor in useful minerals, this is not the case with the plastic clay formation above it; for it contains important mines. In it are explored numerous beds of lignite (wood-coal), either as fuel or a vitriolic earth. From these lignite deposites, also, the yellow amber is extracted.

The other tertiary formations present merely a few mines of iron and bitumen.

Several of the secondary or tertiary strata contain deposites of sulphur, which are mined in various countries.

The formations of a decidedly volcanic origin afford few mining materials, if we except sulphur, alum, and opals.

\section{MINES OF THE ALLUVIAL STRATA.}

This formation contains very important mines, since from it are extracted all the diamonds, and alinost all the precious stones, the platinum, and the greatest part of the gold, with a considerable portion of the tin and iron. The diamond mines are confined nearly to Brazil, and to the kingdoms of Golconda and Visapour in the East Indies.

MORTAR, HYDRAULIC. Professor Kuhlmann, of Lisle, obtained a patent in April, 1841, in the name of Mr. Newton, for certain improvements in the manufacture of lime-cement and artificial stone; and of which he gave me a sample, possessed of a hardness and solidity fit for the sculptor.

In operating by the dry method, instead of calcining the limestone with sand and clay alone, as has been hitherto commonly practised, the inventor introduces a sinall quantity of soda, or, preferably, potash, in the state of sulphate, carbonate, or muriate; salts susceptible of forming silicates when the earthy mixture is calcined. The alkaline salt, equal in weight to about one fifth that of the lime, is introduced in solution among the earths.

All sorts of lime are made hydraulic, in the humid way, by mixing slaked lime with solutions of common alum or sulphate of alumina ; but the best method consists in employing a solution of the silicate of potash, called liquor of flints, or soluble glass, to mix in with the lime, or lime and clay. An hydraulic cement may also be made which will serve for the manufacture of architectural ornaments, by making a paste of pulverized chalk, with a solution of the silicate of potash. The said liquor of flints will likewise give chalk and plaster a stony hardness, by merely soaking them in it after they are cut or moulded to a proper shape. On exposure to the air, they get progressively indurated. Superficial hardness may be readily procured by washing over the surface of chalk, \&c., with liquor of flints, by means of a brush. This method affords an easy and elegant method of giving a stony crust to plastered walls and ceilings of apartments; as also to statues and busts, cast in gypsum, mixed with chalk.

The essential constituents of every good hydraulic mortar, are caustic lime and silica; and the hardening of this compound under water consists mainly in a chemical combination of these two constituents through the agency of the water, producing a hydrated silicate of lime. But such mortars may contain other bases besides lime, as for example clay and magnesia, whence double silicates of great solidity are formed; on which account dolomite is a good ingredient of these mortars. But the silica must be in a peculiar state for these purposes; namely, capable of affording a gelatinous paste with acids; and if not so already, it must be brought into this condition, by calcining it along with an alkali or an alkaline earth, at a bright red heat, when it will dissolve, and gelatinize in acids. Quartzose sand, however fine its powder may be, will form no water mortar with lime; but if the powder be ignited with the lime, it then becomes fit for hydraulic work. Ground felspar or clay forms with slaked lime no water cement ; but when they are previously calcined along with the lime, the mixture becomes capable of hardening under water.

The mastic called Hamelin's, and so much employed in London, is composed of ground Portland stone (roe stone), sand, and litharge, in the proportion of 62 of the fist, 35 of the second, and 3 of the third, in 100 parts; but other proportions will also 
answer the purpose. I find that chalk will not make a good mastic; being too com. pact to permit the air to insinuate between the pores, and to produce the concretion of the linseed oil, with which the above mixture is worked up and applied. This mastic soon acquires great hardness, and is totally impervious to water. The surface to which it is to be applied must be dry, and smeared over with linseed oil. Considerable dexterity is required to make good work with it. The fine dust of sandstone alone, mixed with 10 or 12 per cent. of litharge, and 7 per cent. of linseed oil, forms an excellent mastic.

Limestone, which contains so much as 10 per cent. of clay, comports itself after calcination, if all the carbonic acid be expelled, just as pure limestone would do. When it is less strongly burned, it affords, however, a mass which hardens pretty speedily in water. If the argillaceous proportion of a marl amounts to 18 or 20 per cent., it still will slake with water, but it will absorb less of it, and forms a tolerably good hydraulic mortar, especially if a little good Roman cement be added to it. When the proportion of clay is 25 or 30 per eent. after burning, it heats but little with water, nor does it ssake well, and must therefore be ground by stampers or an edge millstone, when it is sa be used as a mortar. This kind of marl yields commonly the best water cement without other addition. Should the quantity of clay be increased further, as up in 40 per cent., the compound will not bear a high or long-continued heat without being zpoiled for making hydraulic mortar after grinding to powder. When more strongly calcined, it forms a vitriform substance, and should, after being pulverized, be mixed up with good lime, to make a water mortar. If the marls, in any locality, differ much in their relative proportions of lime and alumina, as may be readily ascertained by the use of my lime-proof apparatus (see Appendix.), then the several kinds should be mixed in such due proportions as to produce the most speedily-setting and most highly-indurating hydraulic cement.

MUNDICK. The name giren by the Cornish miners to iron or arsenical pyrites.

MUSK. The musk deer, from the male of which animal species the bag containing this valuable drug is obtained, is a native of the mountainous Kirgesian and Langorian steppes of the Altai, on the river Irtish, extending eastward as far as the river Jenesi and Lake Baikal; and generally of the mountains of eastern Asia, between $30^{\circ}$ and $60^{\circ}$ of N. L. Two distinct kinds of musk are known in commerce, the first being the Chinese Tonquin, Thibetian, or Oriental, and the Siberian or Russian. The Chinese is regarded by Dr. Goebel as the result of ingenious adulterations of the genuine article by that crafty people. The Russian musk is genuine, the bags never being opened, are consequently never sewn, nor artificially closed, like those imported into London from China. The former is sometimes so fresh, that moisture may be expressed from the bag by cutting through its fleshy side. The interior mass is frequently of a soft and pappy consistence; but the surface of the bag is perfectly dry. The Chinese bags are found invariably to have been opened and again glued together, more or less neatly; though sometimes the stitches of the sewing are manifest. Mr. Dyrssen, an eminent merchant at St. Petersburgh, states that during the many years he has been in the trade, although he has received at a time from 100 to 200 ounces from London, yet in no case whatever has he met with a bag which had not been opened, and closed with more or less ingenuity. The genuine contents seem to have been first removed, modified, and replaced. M. Guibourt gires the following as the constituents of a Chinese muskbag : 1 , water ; 2 , ammonia ; 3 , solid fat or stearine : 4, liquid fat or elaine ; 5 , cholesterine; 6 , acid oil, combined with ammonia; 7 , volatile oil ; 8-10, hydrochlorates of ammonia, potassa, and lime; 11, an undetermined acid; 12, gelatin; 13, albumen ; 14 , fibrin; 15 , carbonaceous matter soluble in water; 16 , calcareous salt; 17 , carbonate of lime; 18 , hairs and sand.

From June, 1841, to June, 1842 , a duty of $6 d$. per ounce was paid at the port of London alone upon 969 ounces of musk. The prices of grain musk of the best quality (the matter without the bag) varies from $60 \mathrm{~s}$. to $95 \mathrm{~s}$. per ounce.

There is a superior musk imported now from the United States, which is nearly free from the carbonate of lime, so abundant in the bags of the Siberian musk.

MUSQUET. It is now fourteen years since the Hon. Board of Ordnance, with the view of introducing the use of percussion firearms into the British army, employed me to investigate experimentally the be st mode of preparing the priming powder for that purpose. The result of these experiments was presented in a report, the substance of which is given under the article "Fulminate" in the Dictionary. During this long interval, Mr. Lovell, inspector of small-arms for her majesty's service, and director of the Royal Manufactory, at Enfield Chase, has directed his ingenious mind to the construction of a sure, simple, and strong musquet, with which, under his able superintendence, the whole of her majesty's soldiers are now provided. He has also furnished them with a short, but clear set of instructions, for the cleaning and management of these excellent arms, illustrated by a series of wood engravings. From this little work the following notice is copied :- 


\section{MUSQUET.}

Fig. 94. The barrel, reduced to one-seventh size. $a$, the breach; $b$, the nipple-seat or lump; $c$, the back-sight ; $d$, the back loop; $e$, the middle loop; $f$, the swivel-loop; $g$, the front-loop with the bayonet-spring attached; $h$, the front sight; $i$, the muzzle.

Fig. 95. The breech-pin, half size. $a$, the tang; $b$, the neck; $c$, the screw-threads $d$, the face

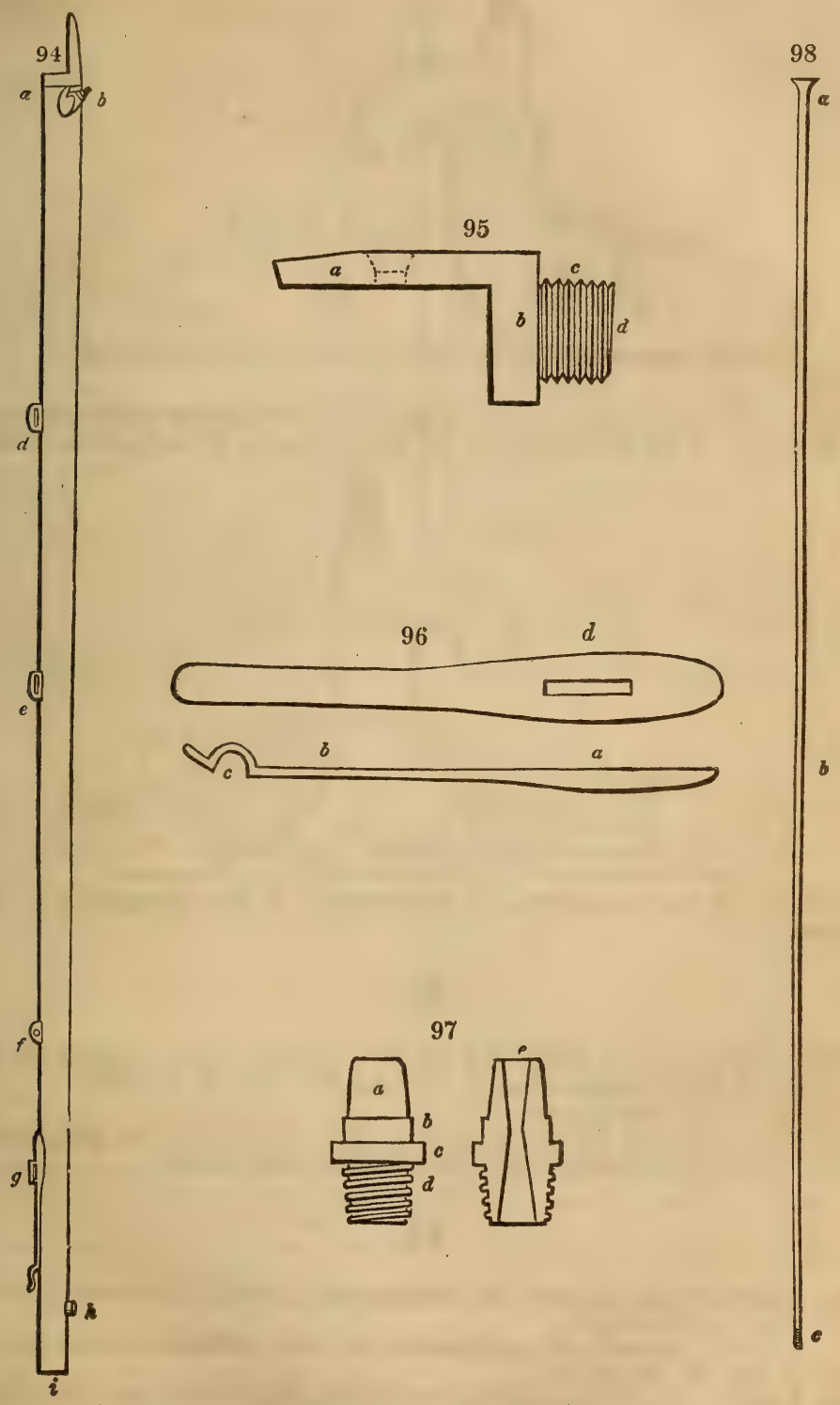
Fig. 96. The bayonet-spring, two ways, half size. $a$, the shank; $b$, the necir; $c$,
the hook; $d$, the mortice.

Fig. 97. The nipple, full size. $a$, the cone; $b$, the squares; $c$, the shoulder; $a_{0}$ the screw-threads; $e$, the touch-hole.

Fig. 98. The rammer, reduced to one-seventh size. $a$, the head; $b$, the shaft; the screw-threads. 
Fig. 99. The lock outside, half size. $a$, the plate; $b$, the cock; $c$, the tumbler-pin $d$, the hollow for the nipple seat.

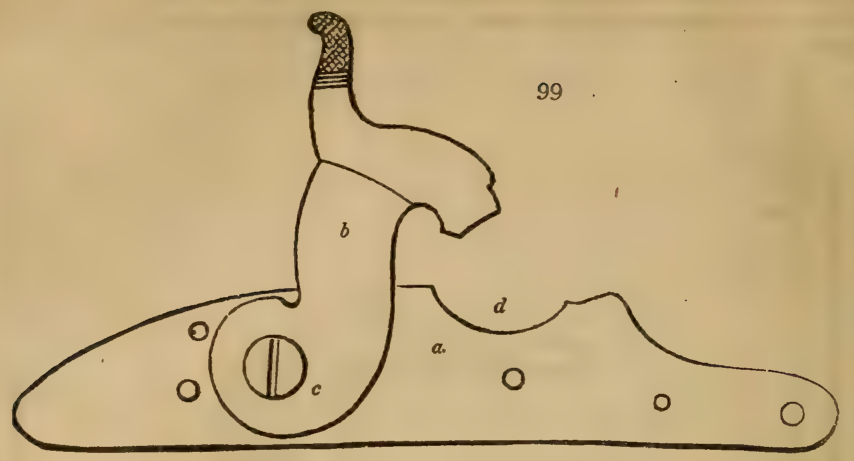

Fig. 100. The lock inside, half size, showing all the parts in their places with the cock down at bearer. $a$, the main-spring; $b$, the sear-spring; $c$, the sear; $d$, the tumbler;

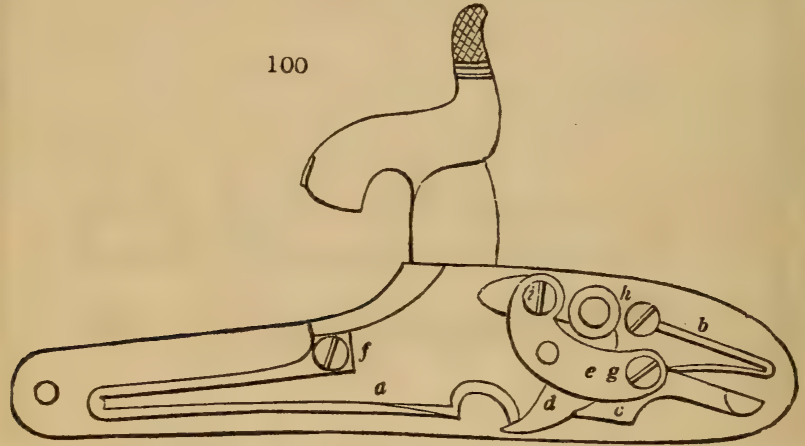

e, the bridle; $f$, the main-spring; $g$, the sear-pin; $h$, the sear-spring-pin; $i$, the bridle-pin.

$\mathrm{N}$.

NITRE (Saltpetre), and NITRATE OF SODA (Cubic nitre), imported for home consumption in $1839,314,543$ cwts.; in $1840,369,204$; duty $6 d$. per cwt.

NITROMETER. See Appendix.

NUTMEGS; imported for consumption in 1839, 133,470 lbs.; in 1840, 118,554; duty from Britısh possessions, $2 s .6 d$. ; from foreign, $3 s .6 d$. per lb.

O.

OIL, COCOA-NUT; imported for consumption in 1839, 15,153 cwts.; in 1840, 37,269 ; duty, 1 s. $3 d$. per ewt.

OIL, OLIVE; imported for consumption in 1839, 1,806,178 gallons; in 1840, $1,985,902$; duty $8 d$. per gallon.

OIL, PALM; imported for consumption in $1839,262,910$ cwts.; in 1840, 314,881; duty, 1 s. $3 d$. per cwt.

OIL, train, spermaceti, and blubber; imported for consumption in 1839, 21,438 tuns; in 1840, 19,955, of British fishing 1s. per tun; of foreign fishing, 26l. 12s. per tun.

The numerous uses of unctuous oils give importance to their preparation, as articles of food, or for burning in lamps, and for the manufacture of soaps, \&c. The seeds 
most productive of oil are those of colza (a species of cabbage, brassica arvensis), rape, mustard, sesamum, poppy, linseed, hemp, and beechmast. Nuts aftord an oil that is much esteemed for certain purposes, and may be easily obtained by pressure. The following table indicates the quantities of oil which can be extracted from different fruits, and some other substances:-

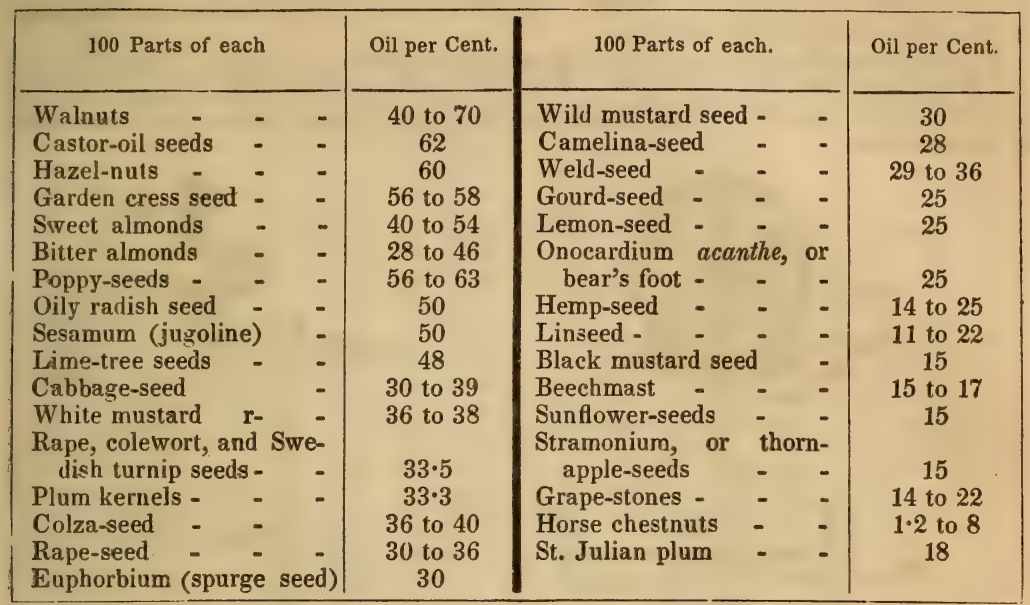

To obtain the above proportions of oil, the fruits must be all of good quality, deprived of their pods, coats, or involucra, and of all the parts destitute of oil, which also must be extracted in the best manner.

The following table is given by M. Dumas, as exhibiting the practical results of the French seed oil manufacturers :-

\begin{tabular}{|c|c|c|c|c|c|}
\hline & & & & Weight per Hectolitre. & Produce in Litres. \\
\hline Summer colza & - & & - & 54 to 65 kilogs. & 21 to 25 \\
\hline Winter colza & - & • & - & 56 to $70-$ & 25 to 28 \\
\hline Rape-seed & - & & - & 55 to $68-$ & 23 to 26 \\
\hline Camelina-seed & - & - & - & 53 to $60-$ & 20 to 24 \\
\hline Poppy-seed & - & - & - & 54 to $62-$ & 22 to 25 \\
\hline Madia Sativa & - & - & - & 40 to $50-$ & 12 to 15 \\
\hline Beechmast. & - & - & - & 42 to $50-$ & 12 to 15 \\
\hline Hemp-seed & - & - & - & 42 to $50-$ & 12 to 15 \\
\hline Linseed - & - & - & - & By sample, 67. & 10 to 12 \\
\hline Stripped walnuts & $3-$ & - & - & From 100 kilogs. & 46 to 50 \\
\hline Sweet almonds & - & - & - & $-100-$ & 44 to 48 \\
\hline Olives - & - & - & - & $-100-$ & 10 to 12 \\
\hline
\end{tabular}

Colza, rape-seed, and cameline oils are employed for lamps; poppy, madia sativa, are employed, when recent, as articles of food-or for soaps and paintings; hemp-seed and linseed for painting, soft soaps, and for printers' ink; walnut oil, for food, painting, and lamps; olive oil, for food, soaps, lamps.

In extracting oil from seeds, two processes are required-1st, trituration; $2 \mathrm{~d}$, expression; and the steps are as follows:-

1. Bruising under revolving heavy-edge millstones, in a circular bed, or trough of iron, bedded on granite.

2. Heating of the bruised seeds, by the heat either of a naked fire or of steam.

3. First pressure or crushing of the seeds, either by wedges, screw, or hydraulic presses.

4. Second crushing of the seed cakes of the first pressure.

5. Heating the bruised cakes; and 6. A final crushing.

The seeds are now very generally crushed, first of all between two iron cylinders revolving in opposite directions, and fed in from a hopper above them; after which they yield more completely to the triturating action of the edge stones, which are usually hooped round with a massive iron ring. A pair of edge millstones of about 7 or $7 \frac{1}{2}$ feet in diameter, and 25 or 26 inches thick, weighing from 7 to 8 tons, can crush, in 12 hours, from $2 \frac{1}{2}$ to 3 tons of seeds. The edge-millstones serve not merely to grind 
the seeds at first, but to triturate the cakes after they have been crushed in the press. Old dry seeds sometimes require to be sprinkled with a little water to make the oil come more freely away; but this practice requires great care.

The apparatus for heating the bruised seeds consists usually of cast-iron or copper pans, with stirrers moved by machinery. Figs. 101, 102, 103, 104, represent the heaters by naked fire, as mounted in Messrs. Maudsley and Field's excellent seed crushing mills, on the wedge or Dutch plan.

Fig. 101 is an elevation, or side view of the fireplace of a naked heater; fig. 102 is a plan, in the line UU of fig. 101. Fig. 103, is an elevation and section parallel to the line VV of fig. 102. Fig. 104, is a plan of the furnace, taken above the grate of the fireplace.
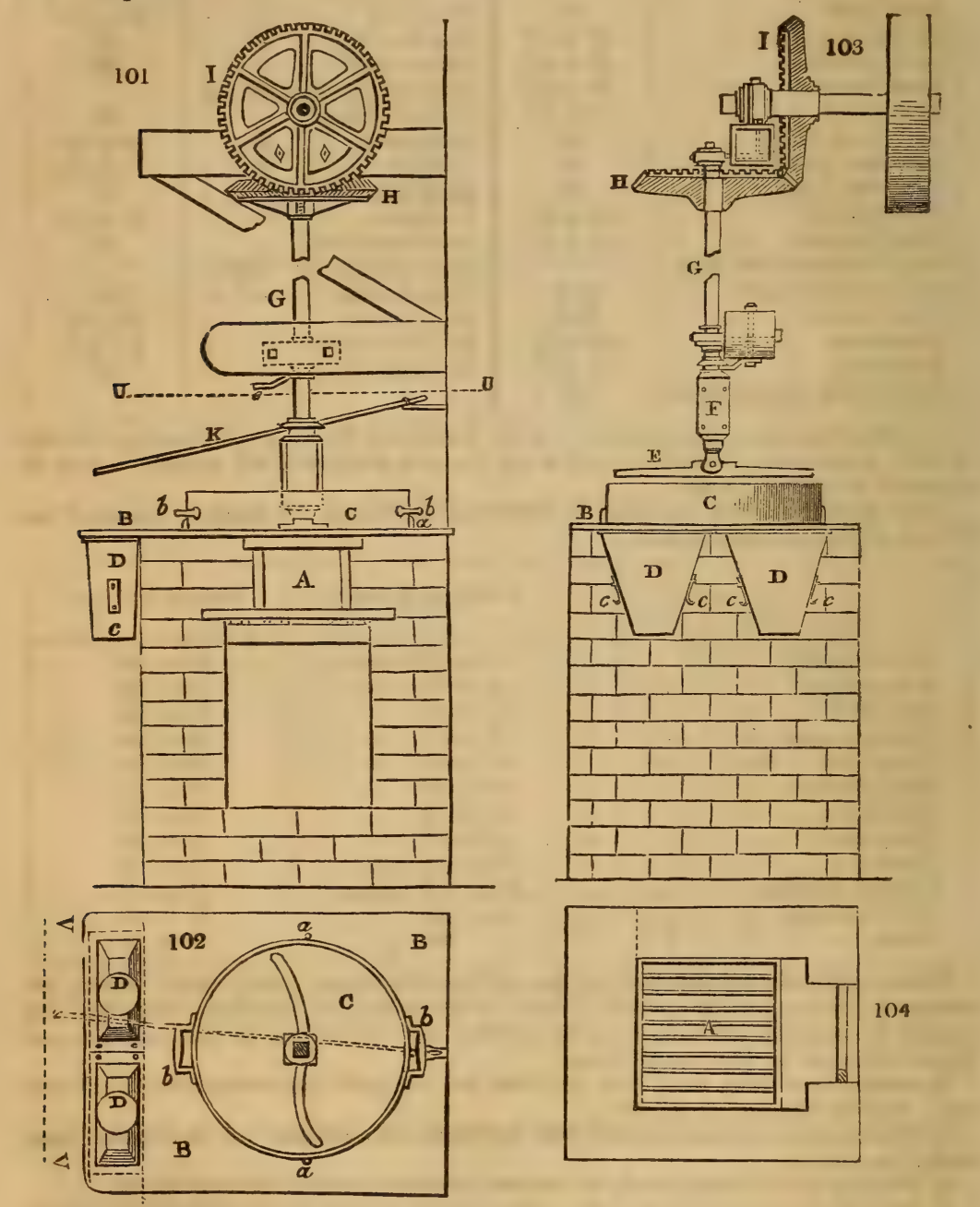

A, fireplace shut at top by the cast-iron plate B; called the fireplate.

C, iron ring-pan, resting on the plate $B$, for holding the seeds; which is kept in its place by the pins or bolts $a$.

$\mathrm{D}$, funnels, britchen, into which by pulling the ring-case $c$, by the handles $b, b$, the seeds are made to fall, from which they pass into bags suspended to the hooks $c$.

E, fig. 103, the stirrer which prevents the seeds from being lourned by continued contact with the hot plate. It is attached by a turning-joint to the collar $F$, which 
turns with the shaft $G$, and slides up and down upon it. $H$, a bevel wheel, in gear with the bevel wheel I, and giving motion to the shaft G.

$\mathbf{K}$, a lever for lifting up the agitator or stirrer $\mathrm{E}$. $e$, a catch for holding up the lever $\mathrm{K}$, when it has been raised to a proper height.

Fig. 105, front elevation of the wedge seed-crushing machine, or wedge-press. Fig. 106, section, in the line XX, of fig. 107.

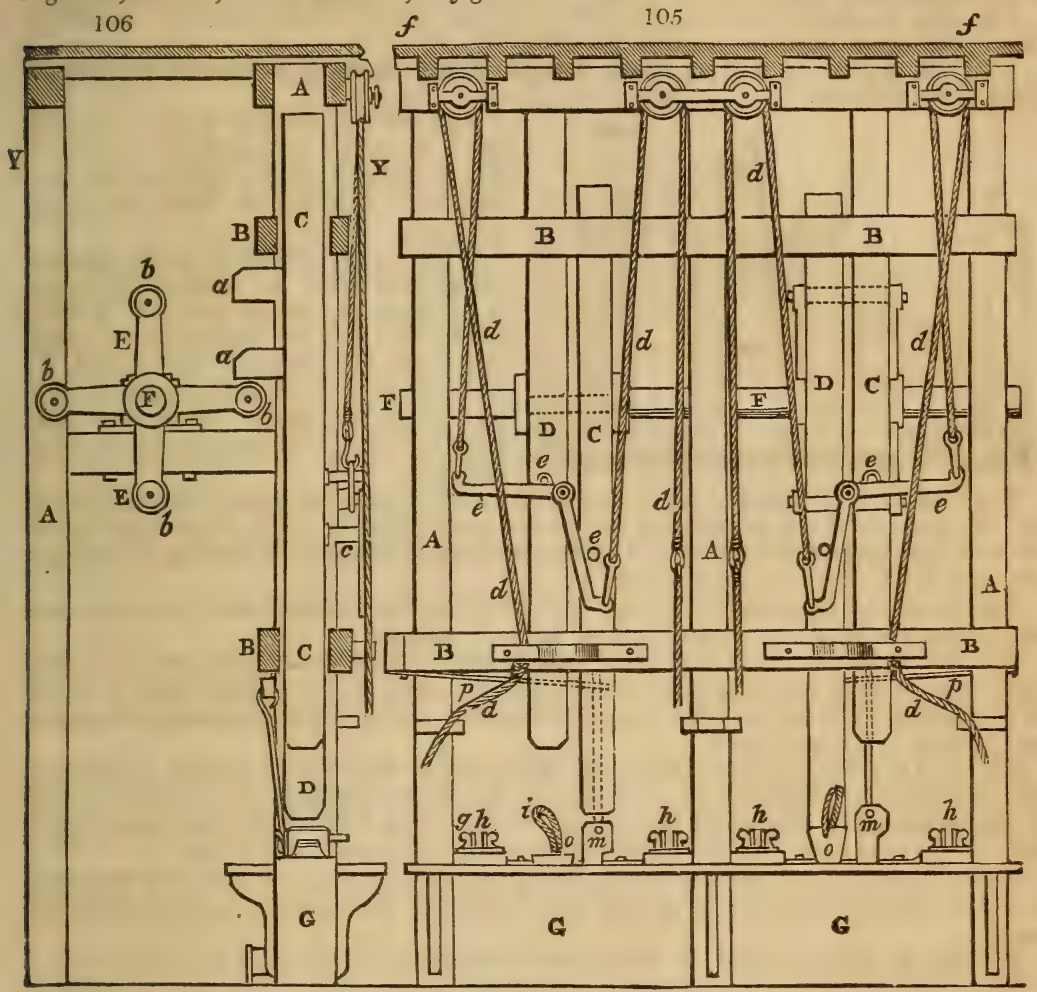

Fig. 107, horizontal section, in the line YY, of fig. 106.

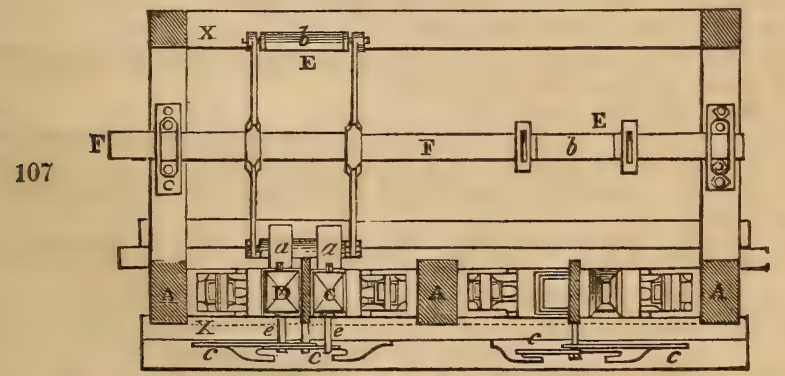

A, A, Upright guides, or frame-work of wood.

B, $B$, Side guide-rails.

D, Driving stamper of wood which presses out the oil; C, spring stamper, or relieving wedge to permit the bag to be taken out when sufficiently pressed. $\mathbf{E}$ is the lifting shaft, having rollers, $b, b, b, b$, fig. 106, which lift the stampers by the cams, $x, a, f i g .106$. F, is the shaft from the power-engine, on which the lifters are fixed.

$\mathrm{G}$, is the cast iron press-box, in which the bags of seed are placed for pressure, laterally by the force of the wedge. 
o, figs. 105 and 108; the spring, or relieving wedge.

$e$, lighter rail ; $d$, lifting-rope to ditto.

$f, f, f, f$, flooring overhead.

g, figs. 105 and 108 ; the back iron, or end-plate minutely perforated.

$h$, the horse-hair bags (called hairs), containing the flannel bag, charged with seed; $i$, the dam-block; $m$, the spring wedge.

Fig. 107, A, upright guides; C, and D, spring and driving stampers; E, lifting roller; $\mathrm{F}$, lifting shaft; $a, a$, cams of stampers.

Fig. 108, a view of one set of the wedge-boxes, or presses; supposing the front of them to be removed.

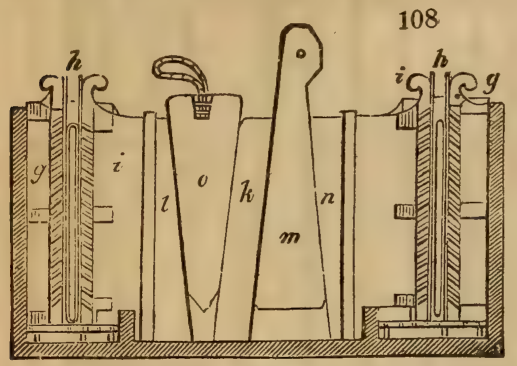

Fig. 108; 0, driving wedge; g, back iron ; $h$, hairs ; $i$, dam-block; $k$, speering or oblique block, between the twc stampers; $l$, ditto; $n$, ditto; $m$, spring wedge.

When in the course of a few minutes the bruised seeds are sufficiently heated in the pans, the double door FF is withdrawn, and they are received in the bags, below the aperture $\mathrm{G}$. These bags are made of strong twilled woollen cloth, woven on purpose. They are then wrapped in a hair-cloth, lined with leather.

The first pressure requires only a dozen blows of the stamper, after which the pouches are left alone for a few minutes till the oil has had time to flow out; in which interval the workmen prepare fresh bags. The former are then unlocked, by making the stamper fall upon the loosening wedge or key, $m$.

The weight of the stampers is usually from 500 to 600 pounds; and the height from which they fall upon the wedges is from 16 to 21 inches.

Such a mill as that now described, can produce a pressure of from 50 to 75 tons upon each cake of the following dimensions: 8 inches in the broader base, 7 inches in the narrower, 18 inches in the height; altogether nearly 140 square inches in surface, and about $\frac{3}{4}$ of an inch thick.

OILS, ADULTERATION OF. M. Heidenreich has found in the application of a few drops of sulphuric acid to a film of oil, upon a glass plate, a means of ascertaining its purity. The glass plate should be laid upon a sheet of white paper, and a drop of the acid let fall on the middle of ten drops of the oil to be tried.

With the oil of rape-seed and turnip-seed, a greenish blue ring is gradually formed at a certain distance from the acid, and some yellowish brown bands proceed from the centre.

With onl of black mustard, in double the above quantity, also a bluish green color.

With whale and cod-oil, a peculiar centrifugal motion, then a red color, increasing gradually in intensity; and after sometime, it becomes violet on the edges.

With oul of cameline, a red color, passing into bright yellow.

Olive-oil, pale yellow, into yellowish green.

Oil of poppies and sweet almonds, canary yellow, passing into an opaque yellow.

Of linseed, a brown magma, becoming black.

Of tallow or oleine, a brown color.

In testing oils, a sample of the oil imagined to be present should be placed alongside of the actual oil, and both be compared in their reactions with the acid. A good way of approximating to the knowledge of an oil is by heating it, when its peculiar odor becomes more sensible.

Specific gravity is also a good criterion. The following table is given by M. Heidenreich :-

\begin{tabular}{|c|c|c|c|c|}
\hline & & & Sp. Gr. & Gay-Lussac's Alcololm. \\
\hline Oleine or Tallow Oil & - & - & 0.9003 & 66 \\
\hline Oil of Turnip Seed & - & - & 0.9128 & $60 \cdot 75$ \\
\hline Rape Oil - & - & - & $0 \cdot 9136$ & $60 \cdot 20$ \\
\hline Olive Oil - & - & - & 0.9176 & $58 \cdot 40$ \\
\hline Purified Whale Oil & - & - & 0.9231 & $55 \cdot 80$ \\
\hline Oil of Poppies - & - & - & 0.9243 & $55 \cdot 25$ \\
\hline Oil of Camelina - & - & - & 0.9252 & $54 \cdot 75$ \\
\hline Linseed Oil - & - & - & 0.9347 & 50 \\
\hline Castor Oil - & - & - & 0.9611 & $33 \cdot 75$ \\
\hline
\end{tabular}


M. Laurot, a Parisian chemist, finds that colza oil (analogous to rapeseed oil) may be tested for sophistication with cheaper vegetable oils by the increase of density which it therefrom acquires, and which becomes very evident when the several oils are heated to the same pitch. The instrument, which he calls an oleometer, is merely a hydrometer, with a very slender stem. He plunges it into a tin cylinder, filled with the oil, and sets this cylinder in another containing boiling water. His oleometer is so graduated as to sink to zero in pure colza oil so heated; and he finds that it stops at $210^{\circ}$ in linseed oil, at $124^{\circ}$ in poppy-seed oil, at $83^{\circ}$ in fish oil, and at $136^{\circ}$ in hempseed oil-all of the same temperature. By the increase of density, therefore, or the ascent of the stem of the hydrometer in any kind of colza oil, he can infer its degree of adulteration.

The presence of a fish oil in a vegetable oil is readily ascertained by agitation with a little chlorine gas, which blackens the fish oil, but has little or no effect upon the vegetable oil.

I find that lard oil, and also hogs' lard, are not at all darkened by chlorine.

A specific gravity, bottle or globe, having a capillary tube-stopper, would make an excellent oleometer, on the above principle. The vessel should be filled with the oil, and exposed to the heat of boiling water, or steam at $212^{\circ}$, till it acquires that temperature, and then weighed. The vessel with the pure colza oil will weigh several grains less than with the other oils similarly treated. Such an instrument would serve to detect the smallest adulterations of sperm oil. Its specific gravity at $60^{\circ}$ when pure is only 0.875 ; that of southern whale oil is 0.922 , or 0.925 ; and hence their mixture will give a specific gravity intermediate, according to the proportion in the mixture. Thus I have been-enabled to detect sperm oil in pretended lard oil, in my examination of oils for the customs.

OPIUM. Imported for consumption in $1839,41,632 \mathrm{lbs}$; in 1840, 46,736; duty, 1s. per $\mathrm{lb}$.

\section{P.}

PAPER. The construction of wire-web cylinders for paper-making machines, and the combination of two such cylinders in one machine, by the use of which two distinct thicknesses of paper pulp are obtained, and applied face-wise, to form one thick sheet, were made the subject of a patent under the name of John Donkin. Two cylinders are so placed in a vat that their circumferences are nearly in contact, and by being turned in opposite directions, they bring two sheets of paper pulp into contact, and incorporate them into one, by what is technically termed couching.

An extensive patent for improvements in the manufacture of paper was granted to Charles Edward Amos in 1840. These consist, first, in gradually lowering the roll of the engine in which the rags are prepared and converted into pulp; secondly, in a mode of regulating the supply of pulp to the paper-making machine, in order to produce papers of any required thickness; thirdly, in an improved sifter or strainer through which the pulp is passed for clearing it of knobs and lumps; fourthly, in certain modifications of the parts of the machine in which the pulp is deposited and moulded into continuous lengths of paper; fifthly, in an improved method of heating the cylinders of the drying apparatus; and, sixthly, in improvements of the machinery for cutting the paper into sheets of any required dimensions. The details of these ingenious contrivances, illustrated with engravings, are given in Newton's Journal, xx., p. 153., C. S.

Henry Crossley purposes to manufacture paper from waste tan, and spent hopswith what success I have not heard. Joseph Hughes gives a higher finish to the long web of paper by friction between two cylinders, the one of which moves much quickez than the other, both being covered with felt or not, at pleasure.

Mr. John Dickinson, the eminent paper manufacturer, ubtained a patent in 1840 for a new mode of sizing paper continuously, in an air-tight vessel (partly exhausted of air), by unwinding a scroll of dried paper from a reel, and conducting it through heated size; then, after pressing out the superfluous size, winding the paper on to another reel.

A longitudinal section of the apparatus employed for this purpose is represented fig. 109; where $a$ is the air-tight vessel ; $b$, the reel upon which the paper to be sized is wound; whence it proceeds beneath the guide-roller $c$, and through the warm size :o another guide-roller $d$. It thence ascends between the press-rolls, $e, f$ (by whose revolution the paper is drawn from the reel $b$ ), and is wound upon the reel $g$. A float $\hat{h}$ is suspended from the cross-bar $i$, of the vessel $a$, for the purpose of diminishing the surface of size exposed to evaporation; and beneath the bottom of the vessel is an 
enclosed space $j$, into which steam or hot water is introduced for maintaining the tem nerature of the size,-Newton's Journal, xxiii. 20.

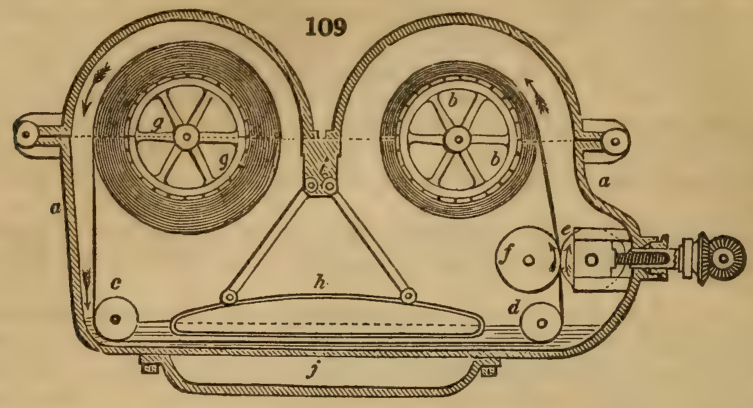

Messrs. Charles Cowan and Adam Ramage, paper-makers, patented, in 1840, im. proved rag machinery; in which a cylindrical sieve or strainer of wire-cloth, of a peculiar construction, is substituted for the ordinary strainers, by which the dirty water is separated from the pulp. They do not claim the cylindric form or sieve, but "the adding or applying, and combining within the interior of such drum, scoops, or buckets, for the purpose of elevating the water, which has entered into it through its wire circumference, so that the water when elevated may be able to run by its own gravity out of the hollow around the central axis of the drum into any suitable shoot or trough, and escape at a level above the surface of the water and rags or material contained in the paper-machine."

Thomas Barrett claims, in his patent of 1841, " a mode of drying paper by applying streams of air to its two surfaces, as it passes over the steam cylinders, whether in the state of engine size or water leaf, or after sizing; as also, the application of currents of air to the surfaces of paper, after sizing, in order to cool the size; as the paper is passing to the drying cylinders."

The improvements in paper-making, for which T. W. Wrigley, of Bridge Hall Mills, Bury, obtained a patent ln 1842 , relate to the rag engine, figs. $110,111,112$.
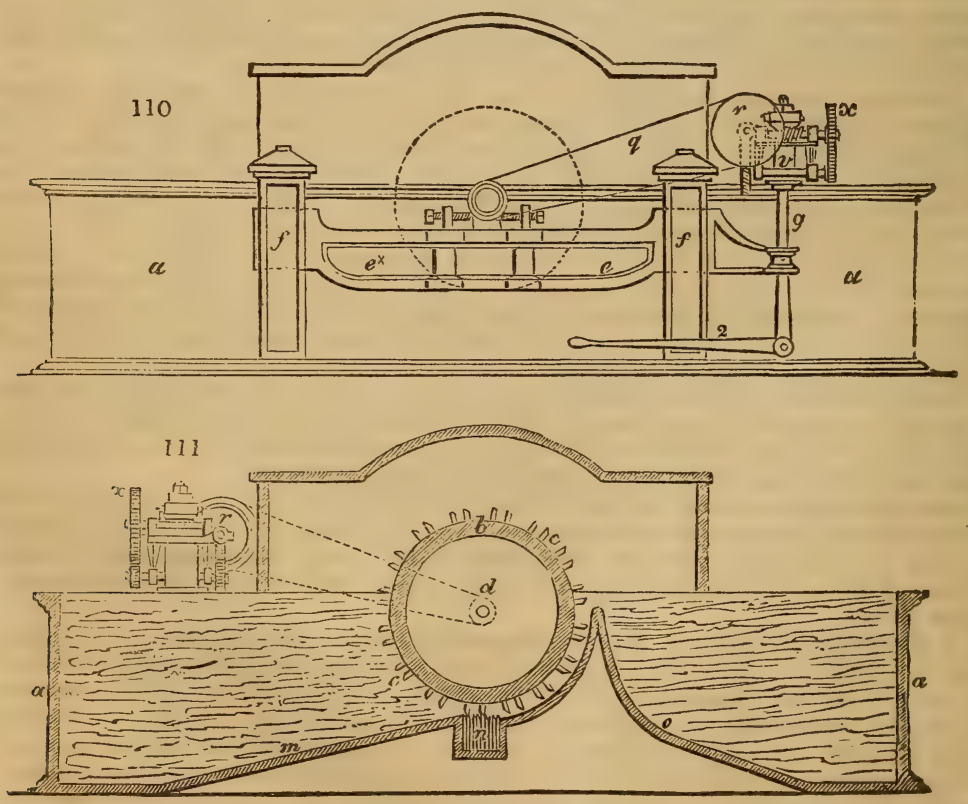
113. Fig. 110 is a side elevation; fig. 111, a transverse section, taken lengthwise through nearly its middle; fig. 112, a plan view of the apparatus detached upon a
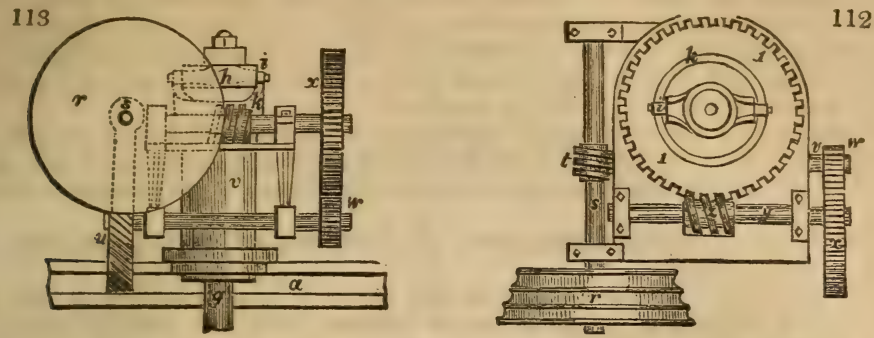

larger scale; and fig. 113 is an elevation. The vessel in which the rags are placed is shown at $a a$, and in about the centre of this vessel the beating or triturating roll, $b, b$, is placed: it is surrounded with the blades or roll bars, $c$ c, fig. 111. The roll is mounted upon a shaft, $d d$, one end of which is placed in a pedestal or bearing on the further side of the chamber $a$, and the other in a bearing upon the arm or level $e e^{*}$, fig. 110, which is supported by its fulcrum, at the end $e^{*}$, in one of the standards, $f f$, and at the other end by a pin fixed in the connecting rod, $g \mathrm{~g}$. At the upper end of this connecting rod there is a cross-piece, or head $h$, having turned pirots at each end upon which are placed small rollers, $i$, resting upon a horizontal cam, $k k$, which is made to revolve. This cam, $k k$, by means of its gearing, causes the roll $b$ first of all to wash the rags a short time, then to be lowered at whatever rate is desired for breaking the fibres; to be maintained at the lowest point for the required number of revolutions for beating; and to be raised and retained, as required, for the final purpose of clearing the pulp. The upper or working edge of this cam is to be shaped exactly according to the action required by the engine roll; as, for instance, suppose the previous operation of washing to be completed, and the time required for the operation of the rag machine to be three hours, one of which is required for lowering the roll, that, or the first division of the working surface of the cam, $k k$, must be so sloped or inclined, that, according to the speed at which it is driven, the rollers upon the crosshead shall be exactly that portion of the time descending the incline upon the cam, and consequently lowering the roll upon the plates $n, f i g .111$; and if the second hour shall be required for the roll to beat up the rags, the roll revolving all the time in contact with the plates, the second division of the cam, $k k$, must be so shaped (that is, made level), that the roll shall be allowed to remain, during that period, at its lowest point; and if the third portion of the time, or an hour, be required for raising the roll again, either gradually or interruptedly, then the third division of the cam, $k$, must be suitably shaped or inclined, so as to cause the cross-head to lift the roll during such interval or space of time; the particular shape of the inclined portions of the cam depending on the manner in which the manufacturer mas wish the roll to approach to or recede from the bottom plates, during its descent and ascent respectively.

Its mode of connexion and operation in the rag engine is as follows : supposing that the rags intended to be beaten up are placed in the vessel $a$, fig. 111, and motion is communicated, from a steam-engine or other power, to the farther end of the shaft $d$, the roll $b$, will thus be caused to revolve, and the rags washed, broken, and beaten up, as they proceed from the front weir $m$, over the bottom plates $n$, and again round by the back weir 0 . There is a small pulley $p$, upon the near end of the shaft $d$, round which a band $q$ passes, and also round another pulley $r$, upon the cross shaft $s$; upon this shaft is a worm $t$, gearing into a worm-wheel $u$, fixed upon another shaft $v$, below; upon the reverse end of which is a piniun $w$, gearing into a spur-wheel $x$, upon the end of a shaft $y$; and upon the centre of this shaft $y$, there is another worm $z$, gearing into a horizontal worm-wheel 1 , upon which the cam, $k k$, is fixed. Thus it will be seen, that the requisite slow motion is communicated to the cam, which may be made to perform half a revolution in three hours; or it will be evident, that half a revolution of the cam, $k k$, may be performed in any other time, according to the calculation of the gearing employed. The shaft may also be driven by hand, so as to give the required motion to the cam. Supposing, now, at the beginning of the operation, the cross head bearing the lever and roll, to be at the highest point upon the cam, $k k$, as its revolution commences, the roll will revolve for a short time on the level surface of the cam, and will then be lowered until the cam, $k k$, has arrived at that point which gorerns the time that the roll remains at the lowest point, for the purpose of beating the rags into pulp, and as the cam, $k k$, continues to revolve, and thus brings the opposite slope upon 
the third portion of its working surface into action upon the cross head, the roll will be raised, in order to clear the pulp from knots and other imperfections, and thus complete the operation of the engine. In order to raise the cross head and roll to the height from which it descended without loss of time, or to lift the cross-head entirely from off the cain when requisite; a lever 2 , or other suitable contrivance may be attached to the apparatus, also a shaft may be passed across the rag-engine, and both ends of the roll may be raised instead of one only, as above described.

The patentee does not claim as his invention the lowering and raising the roll of the rag engine, nor the lowering of it by mechanism, as this was effected in Mr. Amos's patent of 1840 ; but he claims the above peculiar apparatus for this purpose.-Newton's Journal, xxiii. 254. C. S.

PAPER-Gross produce of revenue from. In $1831,723,248 l$. ; 1836, 812,782l.; 1837, 555,943l.; 1840, 626,663l.

PAPER CLOTH. The preparation of this fabric is thus described in the specification of Mr. Henry Chapman's patent of January, 1843. A suitable quantity of canvass, gauze, muslin, calico, linen, \&c., is wound upon a roller, which is introduced between the third press felt of a Fourdrinier paper machine; and between the above roller and the endless felt a trough is introduced, containing a solution of gum, glue, \&c., with a roller partially immersed in it. Pulp being now allowed to flow upon the endless wire wheel of the machine, paper is made in the ordinary way; and when the endless sheet of paper has been led through the machine, the end of the cloth is brought over the upper part of the roller in the trough, and moved onward in the direction the paper is proceeding. The motion of the cloth causes the roller to revolve, and the adhesive material carried upon its surface is imparted to the cloth, which is then laid upon the paper, as it passes over the roller immediately preceding the third or last pressroller. By passing between these rollers, the cloth and paper are firmly united, and being dried by the steam cylinders, form the compound fabric. If required, a paper surface may be applied to the other side of the cloth, by repeating the operation. If the cloth be dressed with strong starch, the bath of adhesive solution may be dispensed with. The following prescription is given for making that solution :-

Dissolve in 15 parts of water, 4 of soda, and combine with this solution, by means of heat, 9 parts of yellow rosin; boil for an hour, adding a little linseed oil to prevent frothing, and add 1 part of glue to the mixture; after which dilute the whole with one and a half times its weight of water, and strain through flannel. Thirty parts of this composition are to be mixed with one part of flour-paste, and six parts of paper-pulp, which mixture is to be used warm.

PEARLS, ARTIFICIAL, and BEADS. The material out of which these are formed are small glass tubes like those with which thermometers are made. The tubes for the bright red pearls consist of two layers of glass, a white opaque one internally, and a red one externally; drawn from a ball of white enamel, coated in the Bohemian method with ruby-colored glass, either by dipping the white ball into a pot of red glass, and thus coating it, or by introducing the ball of the former into a cylinder of the latter glass, and then cementing them so soundly together as to prevent their separation in the subsequent pearl processes. These tubes are drawn in a gallery of the glasshouse to 100 paces in length, and cut into pieces about a foot long. These are afterward subdivided into cylindric portions of equal length and diameter, preparatory to giving them the spheroidal form. From 60 to 80 together are laid horizontally in a row upon a sharp edge, and then cut quickly and dexterously at once by drawing a knife over them. The broken fragments are separated from the regular pieces by a sieve. These cylinder portions are rounded into the pearl shape by softening them by a suitable heat, and stirring them all the time. To prevent them from sticking together, a mixture of gypsum and plumbago, or of ground clay and charcoal, is thrown in among them.

Figs. 114, 115, represent a new apparatus for rounding the beads; fig. 114, is a front view of the whole; fig. 115, is a section through the middle of the former figure, in the course of its operation. The brick furnace, strengthened with iron bands, 2, 3, $5,7,8$, has in its interior (see fig. 115), a nearly egg-shaped space B, provided with the following openings : beneath is the fire-hearth, $\mathrm{c}$, with a round mouth, and opposite are the smoke flue and chimney, $D$; in the slanting front of the furnace is a large opening, $\mathrm{E}, \mathrm{fig}$. 114. Beneath are two smaller oblong rectangular orifices, $F$, $G$, which extend somewhat obliquely into the laboratory, в. н serves for introducing the wood into the fireplace. All these four openings are, as shown in fig. 114, secured from injury by iron mouth-pieces. The wood is burned upon an iron or clay bottom piece, $r$. A semi-circular cover, $\mathrm{N}$, closes during the operation the large opening, $\mathrm{E}$, which at other times remains open. By means of a hook, $m$, and a chain, which rests upon a hollow arch, $h$, the cover, $\mathrm{N}$, is connected with the front end of the long iron lever, $\mathbf{R}, \boldsymbol{R}^{\prime}$. A prop supports at once the turning axis of this lever and the catch, $b, c$; the weight, 
Q, draws the arm B down, and thereby holds up $\mathrm{N}$; E therefore remains open. By rods on the back wall $\mathrm{T}, \mathrm{T}$, the hook $i$, in which $\mathrm{R}^{\prime}$ rests, proceeds from $f$. When $\mathbb{R}$

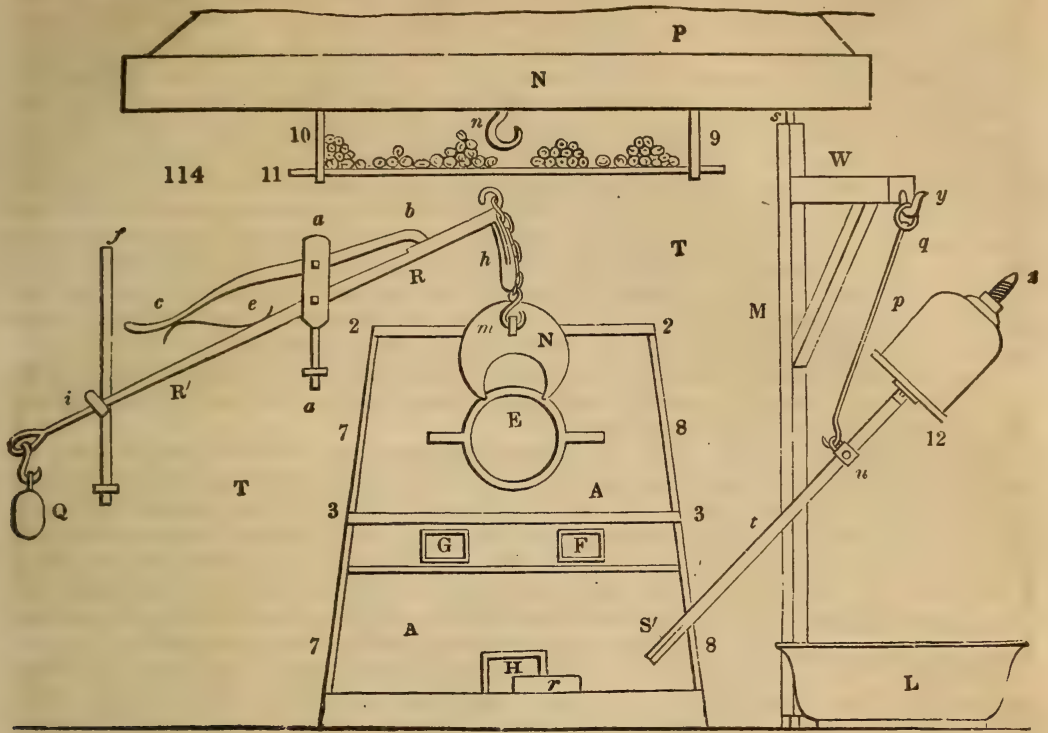

is raised $\mathrm{R}$ sinks. The catch, $c b$, enters with its front tooth into a slanting notch upon the upper edge of $\mathrm{R}$, spontaneously by the action of the spring $e$; whereby the opening $\mathrm{E}$, is shut. The small door, $\mathrm{N}$, rises again with the front arm of the lever by the operation of the weight $Q$ of itself, as soon as the catch is released by pressure upon $c$.

The most important part of the whole apparatus is the drum, $\mathbf{k}$, for the reception and rounding of the bits of glass. It may be made of strong copper, or of hammered

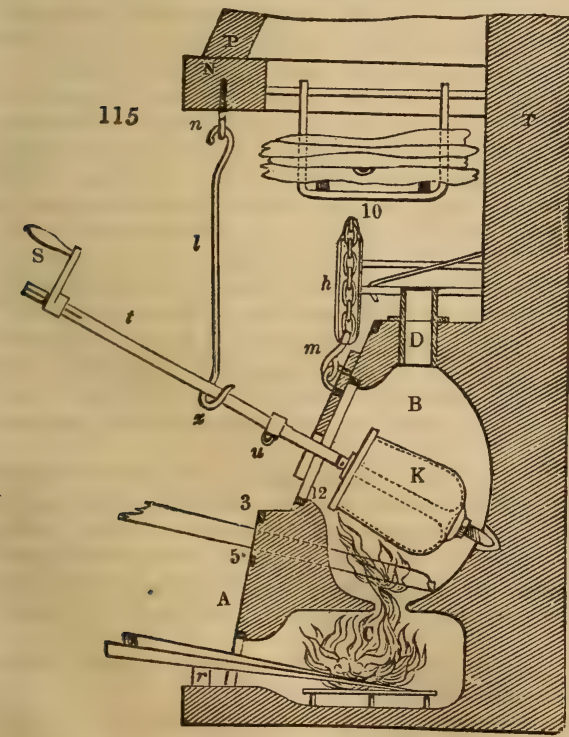

or cast iron, quite open above anci pierced at the bottom with a square hole, into which the lower end of the long rod, $t$, is exactly fitted, and secured in its place by a screwed collector nut. The blunt point, $x$, ( fig. 114) rests during the working in a conical iron step of the laboratory, fig. 115. On the mouth of the drum, $\mathrm{k}$, a strong iron ring is fixed, having a bar across its diameter, with a square hole in its middle point, fitted and secured by a pin to the rod $t$, and turned i its rotation. 'The vessel $\mathbf{K}$, and its axle, $t$, are laid in a slanting direction; the axle rests in the upper ring, $z$, at the lower end of the rod, $l$, of which the other end is hung to the hook, n, upon the mantel beam, N. On the upper end of $t$, the handle, $\mathrm{s}$, is fixed for turning round continuously the vessel, $\mathrm{K}$, while the fire is burning in the furnace, the fuel being put not only in it? 3 bottom chamber, but also into the holes, F, G (fig. 114). The fire-wood is made very dry before being used, by piling it in logs upon the ron bars, 9, 10, 11, under the mantelpiece, as shown in figs. 114, 115. 
After the operation is finished, and the cover, $\mathrm{N}$, is removed, the drum is emptied of its contents, as follows. Upon the axle, $t$, there is toward $\mathbf{K}$ a projection at $u$. Alongside the furnace ( $f \mathrm{ig} .114$ ) there is a crane, $M$, that turns upon the step $s$, $s$, on the ground. The upper pivot turns in a hole of the mantel-beam, $N$. Upon the perpendicuiar arm, $\mathrm{w}$, of the crane there is a hook, $y$, and a ring, $q$, in which the iron rod, $p$, is moveable in all directions. When the drum is to be removed from the furnace, the crane, with its arm, w, must be turned inward, the under hook of the rod, $p$, is to be hung in the projecting piece, $u$, and the rod, $l$, is lifted entirely out. After this, by means of the crane, the drum can be drawn with its rod, $t$, out of the furnace; and through the mobility of the crane, and its parts, $p, q$, any desired position can be given to the drum. Fig. 114, shows how the workman can with his hand applied to s' depress the axle, $t$, and thereby raise the drum, $\mathbf{K}$, so high that it will empty itself into the pot, $\mathbf{L}$, placed beneath. When left to itself, the drum on the contrary hangs nearly upright upon the crane by means of the rod, $p$, and may therefore be easily filled again in this position. The manner of bringing it into the proper position in the furnace by means of the crane and the rod, $l$, is obvious from fig. 115 .

The now well-rounded beads are separated from the pulverulent substance with which they were mixed, by careful agitation in sieves; and they are polished finally and cleaned by agitation in canvass-bags.

PENS, STEEL. When these have been punched out of the softened sheet of steel by the appropriate tool, fashioned into the desired form, and hardened by ignition in an oven, and sudden quenching with cold water, they are best tempered by being heated to the requisite spring elasticity in an oil bath. The heat of this bath is usually judged of by the appearance to the eye; but this point should be correctly determined by a thermometer, according to the scale (see Steel in the Drctionary); and then the pens would acquire a definite degree of flexibility or stiffness, adapted to the wants and wishes of the consumers. They are at present tempered too often at random.

PEPPER. The unripe grains or corns are known under the name of black pepper; the ripe ones, deprived of their epidermis, constitute white pepper. The latter are very generally bleached by steeping for a little while in a solution of chloride of lime, subsequent washing and drying; a process which improves their aspect, but not their flavor. I was recently led to examine the nature of this substance somewhat minutely, from being called professionally to investigate a sample of ground white pepper belonging to an eminent spice-house in the city of London, which pepper had been seized by the Excise on the charge of its being adulterated, or mixed with some foreign matter, contrary to law. I made a comparative analysis of that pepper and of genuine white pepper-corns, and found both to afford like results : viz. in 100 grains, a trace of volatile oil, in which the aroma chiefly resides; about $8 \frac{1}{2}$ grains of a pungent resin, containing a small fraction of a grain of piperine; about 60 grains of starch, with a little gum, and nearly 30 grains of matter insoluble in hot and cold water, which may be reckoned lignine. The two chemists in the service of the Excise made oath before the court of judicature, that the said pepper contained a notable proportion of sago, even to the amount of fully 10 per cent.; grounding their judgment upon the appearance of certain rounded particles in the pepper, and of the deep blue color which these assumed when moistened with iodine water. No allegation could be more frivolous. Bruised corns of genuine white pepper certainly acquire as deep a tint with iodine as any species of starch whatever. But the characters of sago, optical and chemical, are so peculiar, as to render the above surmise no less preposterous, than the prosecution of respectable merchants, for such a cause, was unjustifiable. A particle of sago appears in the microscope, by reflected light, to be a spherule of snow, studded round with brilliants; whereas the rounded particles of the seized pepper seem to be amorphous bits of gray clay. Had the pepper been adulterated with such a quantity of sago, or anything else, as was alleged, it could not have afforded me, by digestion in alcohol, as much of the spicy essence as the bruised genuine peppercorns did.

Moreover, sago, steeped for a short time in cold water, swells and softens into a pulpy consistence, whereas the particles of the seized pepper, rounded by attrition in the mill, retain, in like circumstances, their hardness and dimensions. Sago, being pearled by heating and stirring the fine starch of the sago palm in a damp state, upon iron or other plates, acquires its peculiar somewhat loose aggregation. and brilliant surface; while, in pepper, the starchy constituent is compactly condensed, and bound up with its ligneous matter.

The Excise laws are sufficiently odious and oppressive in themselves without being aggravated by the servile sophistry of pseudo-science.

Four pounds of black pepper yield only about one ounce of piperine, or one 636th part. It is an insipid crystalline substance, insoluble in water, but very soluble in boiling alcohol, and is extracted at first along with the resin, which may be separated from it afterward, by potash. 
PERFUMERY, INDIAN. The natives place on the ground a layer of the scented flowers, about 4 inches thick and 2 feet square; cover them with a layer 2 inches thick of $\mathrm{Tel}$ or Sesamum seed wetted; then lay on another 4-inch bed of flowers, and cover this pile with a sheet, which is pressed down by weights round the edges. After remaining in this state for 18 hours, the flowers are removed and replaced by a similar fresh layer, and treated as before; a process which is repeated a third time, if a very rich perfumed oil be required. The sesamum-seeds thus imbued with the essential oil of the plant, whether jasmine, Bela, or Chumbul, are placed in their swollen state in a mill, and subjected to strong pressure, whereby they give out their bland oil strongly impregnated with the aroma of the particular flower employed. The oil is kept in prepared skins called dubbers, and is largely used by the Indian women. The attar of roses is obtained by distillation at a colder period of the year.

PHOTOGRAPHY is the art of making pictorial impressions of objects by the action of light upon paper, \&c., prepared with certain substances, and exposed to the sun or in the focus of a camera obscura to the image of the object to be represented; which impressions are then fixed by other chemical re-agents. Photographic paper may be made by dipping Whatman's glazed post paper into brine containing 90 grains of common salt dissolved in an ounce of water, wiping it with a towel, brushing over one side of it with a broad camel-hair brush, a solution of nitrate of silver, containing 50 grains to the ounce of distilled water, and drying it in the dark. The paper may be rendered more sensitive by repeating the above operation; drying it between each step. It affords perfect images of leaves and petals laid upon it, and exposed simply to the sunbeams. A solution of 100 grains of bromide of potassium in an ounce of distilled water answers still better than brine. The paper, when dry, is to be brushed over on one side with a solution containing 100 grains of nitrate of silver to an ounce of water; the paper being brushed, and dried in the dark. If the application of the nitrate of silver be repeated, it will render the paper more sensitive. The silvered side should be marked. This paper laid flat under painted glass, lace, leaves, feathers, ferns, \&c., and exposed to the light of day, takes the impression of the objects. It is to be then washed with lukewarm water, and finally dipped in a solution containing one ounce of hyposulphite of soda, in about a pint of distilled water. The design of the object is necessarily reversed : the light parts forming the dark shades of the photogenic impression, and the dark parts the lighter ones. But a direct picture may be obtained by applying that paper, rendered transparent with white wax (see CALOTYPE), upon a sheet of white photogenic paper, and exposing it to the sunbeams, or bright daylight.

A modification of Photography, called Chrysotype by its inventor, Sir John Herschel, consists in washing the paper in a solution of ammonia-citrate of iron, drying it, and brushing it over with a solution of ferro-sesquicyanure of potassium. This paper, when dried in a perfectly dark room, is ready for use, the image being finally brought out by a neutral solution of silver.

Another modification by Sir John, called Cyanotype, is as follows: Brush the paper with the solution of the ammonia-citrate of iron, so strong as to resemble sherry-wine in color; expose the paper in the usual way, and pass over it very sparingly and evenly a wash made by dissolving common ferro-cyanide of potassium. As soon as this liquid is applied, the negative picture vanishes, and is replaced by the positive one, of a violet blue color, on a greenish yellow ground, which at a certain time possesses a high degree of sharpness, and singular beauty of tint.

The impreved process of photography recently contrived by Mr. Robert Hunt is performed by washing over good letter-paper with the following liquid :-

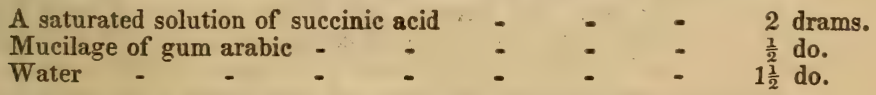

When the paper is dry, it is washed over once with a solution containing 1 dram of nitrate of silver in 1 ounce of distilled water. The paper is allowed to dry in the dark, and it is fit for use. It can be preserved in a portfolio, and employed at any time in the camera obscura, exposing it to the light from 2 to 8 minutes, according to its vivacity. When the paper is taken out of the camera, no trace of a picture can be seen. To produce this effect, mix 1 dram of a saturated solution of sulphate of iron, with 2 or 3 drams of mucilage of gum arabic, and brush over the paper evenly with this mixture. In a few seconds the latent images are seen to develop themselves, producing a negative photographic picture. The excess of the iron solution is to be washed off with a sponge whenever the best effect appears. The drawing is then to be soaked a short time in water, and is fixed by washing over with ammonia, or preferably with hyposnlphite of soda; taking care to wash out the excess of salt. From the pictures thus produced, any number of others, corrected in light and shadow, may be 
produced by using like succinated papers, in the common way of transfer in sunshine.Athenceum.

PICKLES are various kinds of vegetables and fruits preserved in vinegar. The substances are first well cleaned with water, then steeped for some time in brine, and afterward transferred to bottles, which are filled up with good vinegar. Certain fruits, like walnuts, require to be pickled with scalding-hot vinegar; others, as red cabbage, with cold vinegar; but onions, to preserve their whiteness, with distilled vinegar. Wood vinegar is never used by the principal pickle-manufacturers, but the best malt or whitewine vinegar, No. 22 or 24 . Kitchener says, that by parboiling the pickles in brine, they will be ready in half the time of what they require when done cold. Cabbage, however, cauliflowers, and such articles, would thereby become flabby, and lose that crispness which many people relish. When removed from the brine, they should be cooled, drained, and even dried, before being put into the vinegar. To assist the preservation of pickles, a portion of salt is often added, and likewise, to give flavor, various spices, such as long pepper, black pepper, white pepper, allspice, ginger, cloves, mace, garlic, mustard, horseradish, shallots, capsicum. When the spices are bruised, they are most efficacious, but they are apt to render the pickle turbid and discolored. The flavoring ingredients of Indian pickle are Curry powder mixed with a large proportion of mustard and garlic. Green peaches are said to make the best imitation of the Indian mango.

I have examined the apparatus in the great fish-sauce, pickle, and preserved-fruit establishment of Messrs. Crosse and Blackwell, Soho square, and found it arranged on the principles most conducive to economy, cleanliness, and salubrity; no material employed there is ever allowed to come into contact with copper. A powerful steam-boiler is placed in one corner of the ground floor of the factory, from which a steam-pipe issues, and is laid horizontally along the wall about 4 feet above the floor. Under this pipe a range of casks is placed, into the side of each of which a branch steam-pipe, furnished with a stop-cock, is inserted, while the mouth of the cask is exactly closed with a pan of salt-glazed earthenware, capable of resisting the action of every acid, and incapable of communicating any taint to its contents. These casks form, by their nonconducting quality as to heat, the best kind of steam-jackets. In these pans the vinegars with their compounds are heated, and the fish and other sauces are prepared. The waste steam at the farthest extremity of the pipe is conducted into a reservoir of clean water, so as to furnish a constant supply of hot water for washing bottles and utensils.

The confectionary and ham-smoking compartments are placed in a separate fireproof chamber on the same floor.

The floor above is occupied along the sides with a range of large rectangular cast iron cisterns, furnished with a series of steam-pipes, laid gridironwise along their bot toms, which pipes are covered with a perforated wooden shelf. These cisterns being filled up to a certain height above the shelf with water, the bottles full of green gooseberries, apricots, cherries, \&c., to be preserved, are set upon the shelf, and the steam being then admitted into the gridiron pipes, the superjacent water gets gradually heated to the boiling point ; the air in the bottles round the fruit is thus partly expelled by expansion, and partly disoxygenated by absorption of the green vegetable matter. In this state the bottles are tightly corked, and being subsequently sealed, preserve the fruit fresh for a very long period.

The sauces, pastes, and potted meats, prepared in the above-described apparatus, can seldom be riralled and probably not surpassed in the kitchens of the most fastidious gastronomes.

PITCOAL, ANALYSIS OF. The greater part of the analyses of coals hitherto published hare been confined to the proportions of carbon, hydrogen, and oxygen, to the neglect of the sulphur, which exists in many coals to a degree unwholesome for their domestic use, pernicious for the smelting of iron, and detrimental to the production of gas; since the sulphuretted hydrogen produced requires so much washing and purification as at the same time to impoverish the light, by condensing much of the olefiant gas, its most luminiferous constituent. In the numerous reports upon the composition of coals which I have been professionally called upon to make, I have always sought to determine the proportion of sulphur, which may be done readily to one part in a thousand; as also that of combustible gaseous matter, of coke, and of incombustible ashes.

The following coals have been found to be ox excllent quality, as containing very little sulphur, seldom much above 1 per cent., and little incombustible matter-hence well adapted as fuel, whether for steam-navigation, for iron-smelting, for household consumption, or for gas, according to their relative proportions of carbon and hydrogen; a relative excess of carbon constituting a coal best adapted for furnaces of 
various kinds, while a relative excess of hydrogen forms the best coal for tne common grates and gas-works.

1. Mr. Pouell's Duffry, or Steam Coal.-Specific gravity, $1 \cdot 32$; ashes, per cent., $2 \cdot 6$; gaseous products in a luted crucible, 14 ; brilliant coke, 86 ; not more than 1 per cent. of sulphur; while many of the Newcastle coals contain fiom 4 to 6 , and others which I have examined from 8 to 10 of the same noxious constituent; and which is a less powerful calorific constituent than hydrogen and carbon.

2. The Blackley Hurst Coal of Lancashire.-Specific gravity, 1.26; ashes, per cent., 1.2 ; combustible gases, 41.5 ; coke, 58.5 ; sulphur, 1 . Another specimen had a specific gravity of $1.244 ; 2$ per cent. of ashes ; 38.5 of combustible gases ; 1 of sulphur. This is a very good coal for gas and for domestic use.

3. The Varley Rock Vein Coal, near Pontypool; shipped by Mr. John Vipond.-Specific gravity, 1.296; ashes (whitish) 5 per cent. ; 32 of combustible gases; 68 of coke. Sulphur, from 2 to 3 per cent. A gond household coal.

4. The Llangennech Coal has a well-established reputation for the production of steam, and is much employed by the British government for steam navigation, as well as at Meux's, and others of the great breweries in London. It affords a very intense heat, with little or no smoke; and sufficiently diffusive for extending along the flues of the boilers; whereas the anthracite coal, containing very little hydrogen, yields, in common circumstances, a heat too much concentrated under the bottom of the boilers, and acting too little upon their sides. Specific gravity, $\mathbf{1 \cdot 3 3 7}$; intermediate between that of the Newcastle and the anthracite. Ashes per cent., from 3 to 3.5 ; combustible gases, 17 ; coke, 83 ; sulphur, only one half per cent. It is therefore a pure and very powerful fuel.

I have examined many coals with my calorimeter; of which some account is given under Fuel.

PLATING. See Electrotype.

PLATINUM MOHR. This interesting preparation, which so rapidly oxidizes alcohol into acetic acid, \&c., by what has been called in chemistry the catalytic or contact action, is most easily prepared by the following process of $\mathbf{M}$. Bœttger : The insoluble powder of potash-chlorure or ammonia-chlorure of platinum, is to be moistened with sulphuric acid (oil of vitriol), and a bit of zinc is to be laid in the mixture. The platinum becomes reduced into a black powder, which is to be washed first with muriatic acid, and then with water. The fineness of this powder depends upon that of the saline powders employed to make it ; so that if these be previously finely ground, the platinum-mohr will be also very fine, and proportionally powerful as a chemical agent.

POTTER'S OVEN. A patent was obtained in August, 1842, by Mr. W. Ridgway, for the following construction of oven, in which the flames from the fireplaces are conveyed by parallel flues, both horizontal and vertical, so as to reverberate the whole of the flame and heat upon the goods after its ascension from the flues. His oven is built square instead of round, a fire-proof partition wall being built across the middle of it, dividing it into two chambers, which are covered in by two parallel arches. The fireplaces are built in the two sides of the oven opposite to the partition wall; from which fireplaces narrow flues rise in the inner face of the wall, and distribute the flame in a sheet equally over the whole of its surface. The other portion of the heat is conveyed by many parallel or diverging horizontal flues, under and across the floor or hearth of the oven, to the middle or partition wall; over the surface of which the flame which ascends from the numerous flues in immediate contact with the wall is equally distributed. This sheet of ascending flame strikes the shoulder of the arch, and is reverberated from the seggars beneath, till it meets the flame reverberated from the opposite side of the arch, and both escape at the top of the oven. The same construction is also applied to the opposite chamber. In figs. 116, 117, $a$, represents the

116

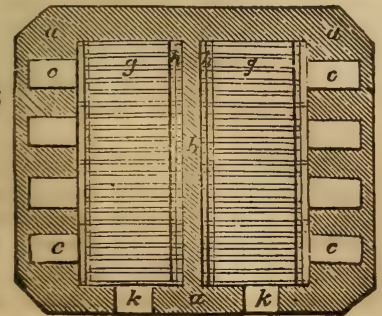

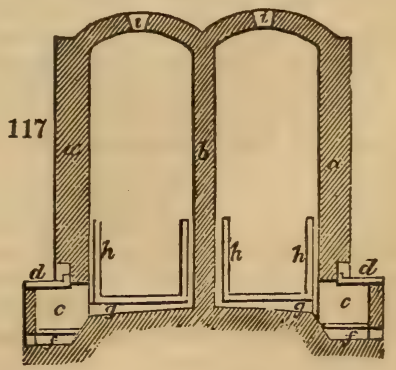


square walls or body of the oven; $b$, the partition wall ; $c$, the fireplaces or furnaces with their iron boilers; $d$, the mouths of the furnaces for introducing the fuel; $f$, the ash-pits; $g$, the horizontal flues under the hearth of the oven; $h$, the vertical flues; $i$, the vents in the top of the arches; and $k$, the entrances to the chambers of the ovens.

PRUSSIAN BLUE. The following process deserves peculiar notice, as the first in which this interesting compound has been made to any extent, independently of animal matter. Mr. Lewis Thompson, of the Old Barge House, Lambeth, received a well-merited medal from the Society of Arts for this invention. He justly observes, that in the common way of manufacturing prussiate of potash, the quantity of nitrogen furnished by a given weight of animal matter is not large, and seldom exceeds 8 per cent. ; and of this small quantity, at least one half appears to be dissipated during the ignition. It occurred to him that the atmosphere might be economically made to supply the requisite nitrogen, if caused to act in favorable circumstances upon a mixture of carbon and potash. He has found the following prescription to answer: Take of pearlash and coke, each 2 parts ; iron turnings, 1 part ; grind them together into a coarse powder; place this in an open crucible, and expose the whole for half an hour to a full red heat in an open fire, with occasional stirring of the mixture. During this process, little jets of purple flame will be observed to rise from the surface of the materials. When these cease, the crucible must be removed and allowed to cool. The mass is to be lixiviated ; the lixivium, which is a solution of ferrocyanide of potassium, with excess of potash, is to be treated in the usual way, and the black matter set aside for a fresh operation with a fresh dose of pearlash. Mr. Thompson states that 1 pound of pearlash, containing 45 per cent. of alkali, yielded 1,355 grains of pure Prussian blue, or ferrocyanide of iron; or about 3 ounces avoird.

PRUSSIATE OF POTASH. Leuch's Polytechnic Zeitung, June, 1837. Manufacture of Kalium Eisen Cyanure, by Hoffmayr and Prükner.-The potash must be free from sulphate, for each atom of sulphur destroys an atom of the Eisencyankalium. A very strong heat is advantageous. The addition of from 1 to $3 \frac{0}{0}$ of saltpetre is useful, when the mass is too long of fusing. A reverberatory furnace (flammofen) is recommended; but the flame must not beat too much upon the materials, for fear of oxygenating them. When the smoky red flame ceases, it is useful to throw in from time to time small portions of uncarbonized animal matter, particularly where the flame first beats upon the mass, whereby the resulting gases prevent oxidation by the air. The animal matters should not be too much carbonized, but left somewhat brown-colored, provided they be readily pulverized. Of uncarbonized animal matters, the proportions may be 100 parts dried blood, to from 28 to 30 of potash (carbonate), and from 2 to 4 of hammerschlag (smithy scales), or iron filings; 2, 100 parts of horns or hoofs ; from 33 to 35 potash; 2 to 4 iron; 3, 100 leather; 45 to 48 potash; and 2 to 4 iron. From blood, 8 to 9 per cent. of the prussiate are obtained; from horns, 9 to 10 ; and from leather, 5 to 6 . The potash should be mixed in coarse particles, like peas, with the carbonized animal matter, which may be best done in a revolving pot, containing cannon-balls. Of the animal coal and potash, equal parts may be taken, except with that from leather, which requires a few parts more potash per cent. On the average, blood and horn coal should afford, never less than 20 per cent. of prussiate, nor the leather than 8 ; but by good treatment, they may be made to yield, the first 25 , and the last from 10 to 11 .

A patent for a singular process and apparatus, for making this compound, was obtained by a foreigner not named, by Mr. Berry, patent agent, in January, 1840. The prescription is as follows :-

Reduce charcoal into bits of the size of a walnut, soak them with a solution of carbonate of potash in urine; and then pour over them a solution of nitrate or acetate of iron; dry the whole by a moderate heat, and introduce them into the cast-iron tubes, presently to be described. The following proportions of constituents have been found to answer : Ordinary potash, 30 parts ; nitre, 10; acetate of iron, 15; charcoal or coke, 45 to 55 ; dried blood, 50 . The materials, mixed and dried, are put into retorts similar to those for coal gas. The animal matter, however (the blood), is placed in separate compartments of pipes connected with the above retorts. The pipes containing the animal matter should be brought to a red heat before any fire is placed under the retorts.

In fig. 118, A, B, c, D, is a horizontal section of a furnace constructed to receive four elliptical iron pipes. The furnace is arched in the part A, C, B, in order to reverberate the heat, and drive it back on the pipes $w, w^{\prime}, w^{\prime \prime}, w^{\prime \prime \prime}$. These pipes are placed on the plane $\mathrm{E}, \mathrm{F}$, of the ellipsoid; $a$, represents the grating or bars of the furnace to be heated with coal or coke; I, I, is the pot or retort shown in figs. 119, 120, 121 .

This pot or retort is placed in a separate compartment, as seen in fig. 119, which is a 
vertical section taken through fig. 121, at the line G, H. K, is a connecting tube from the retort and the elliptical pipes w.
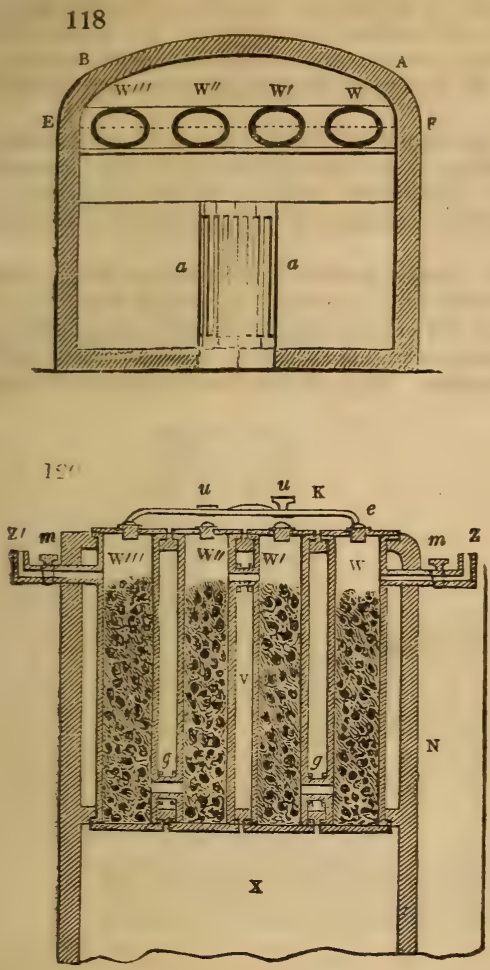

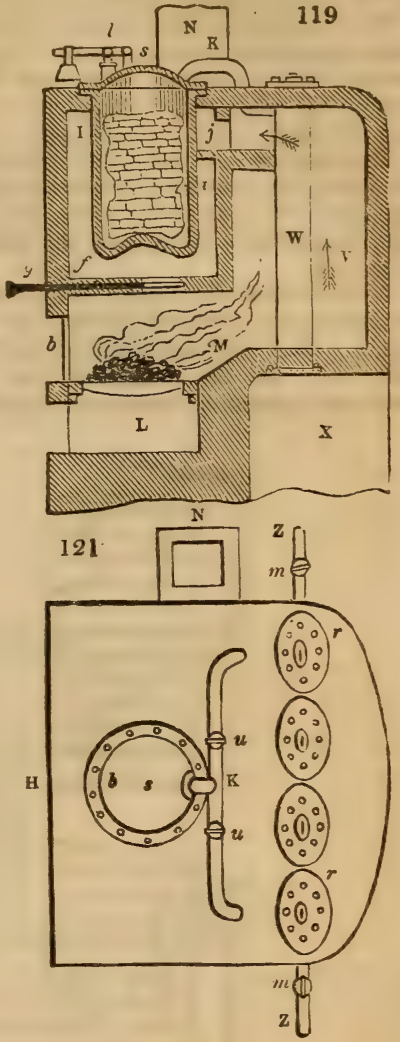

In the section, fig. 119, the shape of the tube $\mathrm{x}$, will be better seen; also its cocks $u$, and likewise its connexion with the pipes $w . l$, is a safety valve; $s$, the cover of the pot or retort; $\mathrm{L}$, is the ash-pit; and $b$, the door of the furnace; $\mathbf{x}$, is an open space, roofed over, or a kind of shed, close to the furnace, and under it the pipes are emptied.

The arrows indicate the direction of the current of heat. This current traverses the intervals left between the pipes, and ascends behind them, passing through the aperture $j$, in the brickwork, which is provided with a valve or damper, for closing it, as required. The heat passes through this aperture, and strikes against the sides of the pot when the valve is open. Another valve $f, g$, must also be open to expose the pot or retort to the direct action of the fire. The smoke escapes by a lateral passage into a chimney $\mathrm{N}$.

It must be remarked, that there is a direct communication between the chimney and that compartment of the furnace which contains the pipes, so that the heat, reflected from the part $\nabla$, strikes on the pot or retort only when the pipes $w, w^{\prime}, w^{\prime \prime}, w^{\prime \prime}$, are sufficiently heated.

In fig. 120 is shown an inclined plane $\mathrm{M}$ (also represented in $f i g, 119)$ and the junction-tubes which connect the four pipes with their gas-burners $\mathrm{z}, \mathrm{z}$, and the cocks $m, m^{\prime}$. $r, r$, are covers, closing the pipes, and having holes formed in them; these holes are shut by the stoppers $e$.

Whether the pipes are placed in the vertical or horizontal position, it is always proper to be able to change the direction of the current of gas; this is easily done by closing, during one hour (if the operation is to last two hours) the cocks $u, m^{\prime}$, and opening those $u^{\prime} m$; then the gas passes through $u^{\prime}$, into the branch $\mathrm{K}$, and entering $w^{\prime \prime}$. passes through $q$, into $w^{\prime \prime}$, through $p$, into $w^{\prime}$, and through $o$, and $w$, and finally 
escapes by the burner $z$. During the following or other hour, the cocks $u^{\prime} m$, mast be closed; the cocks $u, m^{\prime}$ being opened, the current then goes from $u$, into $\mathrm{K}, \mathrm{w}, \mathrm{w}^{\prime}$, $\mathrm{w}^{\prime \prime}, \mathrm{w}^{\prime \prime \prime}$, and escapes by the burner $\mathrm{z}^{\prime}$, where it may be ignited.

The changing of the direction of the current dispenses, to a certain degree, with the labor required for stirring, with a spatula, the matters contained in the pipes; nevertheless, it is necessary, from time to time, to pass an iron rod or poker among the substances contained in the pipes. It is for this purpose that apertures are formed, sc as to be easily opened and closed.

The patentee remarks, that although this operation is only described with reference to potash, for obtaining prussiate of potash, it is evident, that the same process is applicable to soda; and when the above mentioned ingredients are employed, soda being substituted for potash, the result will be prussiate of soda.-Newton's Journal, C. S. xxı. 96.

PUDDLING OF IRON. This is the usual process employed in Great Britain for converting cast iron into bar or malleable iron-a crude into a more or less pure metal. The following plan of a puddling furnace has been deemed economical, especially with res ect to fuel, as two furnaces are joined side by side together, and the workmen operate at doors on the opposite sides. Fig. 122 represents this twin furnace
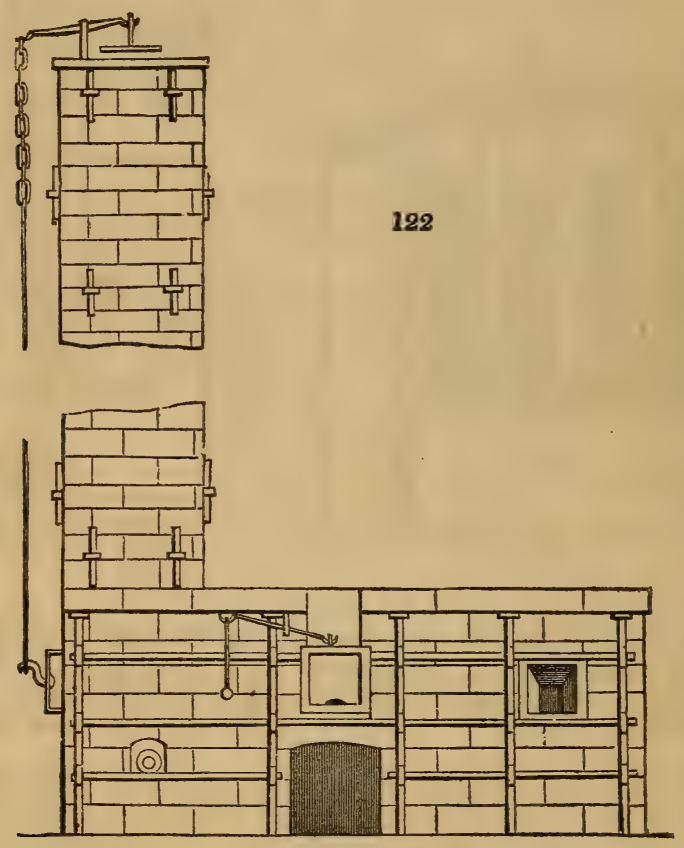

In a side elevation; fig. 123 in section, according to the line E F, in fig. 124, which exhibits a plan of the furnace. The various parts are so clearly shown in form and construction as to require no explanation. The total length outside is $14 \frac{3}{4}$ feet ; width, $12 \frac{8}{4}$ feet : from which the dimensions of the other parts may be measured.

Iron is puddled either from cast pigs, or from the plates of the refinery (finery) furnace. In several iron-works a mixture of these two crude metals is employed. In the refining process, the waste at the excellent establishment of Mr. Jessop, at Codner Park, is from $2 \frac{1}{2}$ to $2 \frac{\mathrm{s}}{\frac{1}{4}} \mathrm{cwt}$. per ton; on which process the wages are $1 \mathrm{~s}$. per ton; and the coke $\frac{1}{2}$ ton, worth $6 s$.; so that the total cost of refining per ton is $15 s_{\text {. }}$, when pig-iron is worth $3 l$. 10 s.

The puddling is accompanied with a loss of weight of $1 \frac{1}{2} \mathrm{cwt}$. per ton; it costs in wages, for puddling refinery plates, $6 s .6 d$., and for pigs, $8 s_{.}$; in which $18 \mathrm{cwt}$. of coal are consumed; value, 5 s. per ton.

Shingling (condensing the bloom by the heavy hammer) costs, in wages, 1s. 9d. per ton; and rough-rolling, $1 s .2 d$. Cutting and weighing these bars cost $9 d$. for wages, including their delivery to the mill furnace, where they are reheated and welded 
together. The mill furnace heating costs $1 s .6 d$. in wages, and consumes in fuel $12 \mathrm{cwt}$. of coals, at $5 s$. per ton. The rolling and straightening cost $5 s .6 d$; cropping the ends, weighing, and stocking in the warehouse, $1 \mathrm{~s}$. for wages. Wear and tear of power, $5 s$. Laborers for clearing out the ashes, \&c., 1s. $6 d$. per ton.
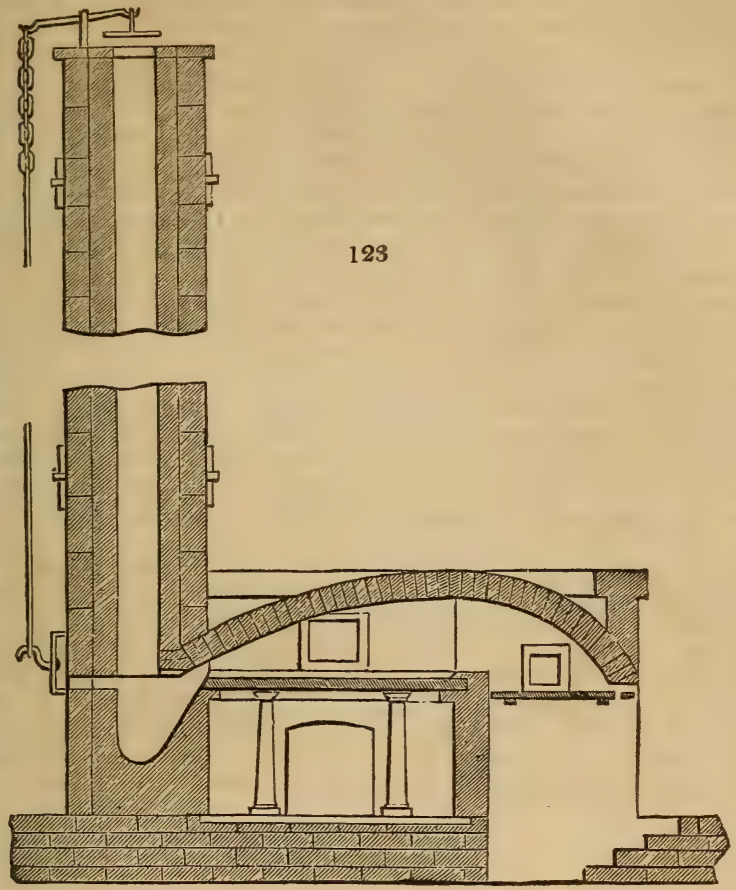

In Wales 4 tons of pig-iron afford upon an average only 3 tons of bars. From the above data a calculation may easily be made of the total expense of converting crude into cast iron at the respective iron works.

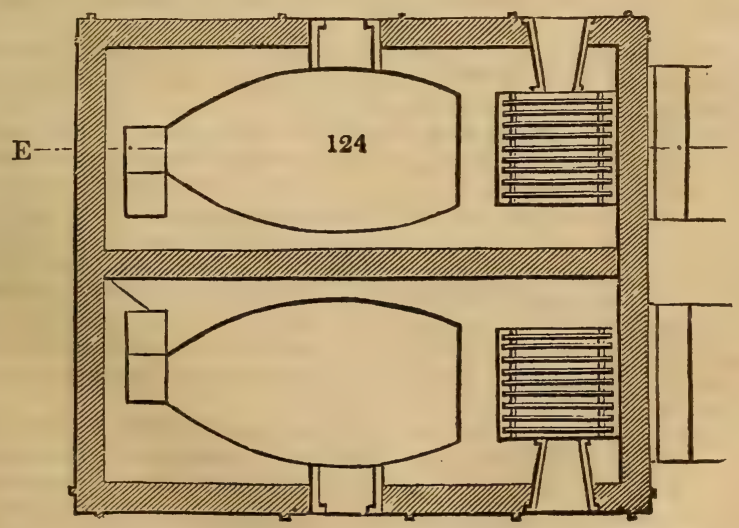

A great economy in the conversion of the cast into wrought metal seems about to be effected in our iron works, by the application of a current of voltaic electricity to the crude iron in a state of fusion, whether on the hearth of the blast furnace, on the fused pigs in the sand, or on the metal immediately on its being run from the finery furrace; the voltaic force of from 50 to 100 pairs of a powerful Smee's battery being previously arranged to act upon the whole train of the metal. This process, for which 
Mr. Arthur Wall has recently obtained a patent, is founded apon the well-establishee fact, that when a compound is subjected to an electrical current, its negative and positive elements are detached from one another. Crude iron contains more or less carbon, sulphur, phosphorus, arsenic, oxygen, and silicon-bodies all electro-negative in relation to iron, which is electro-positive. When the impure iron, as it flows from the blast furnaces, is subjected during its cooling and consolidation to a powerful stream of voltaic electricity, the chemical affinities by which its various heterogeneous components are firmly associated, are immediately subverted, whereby, in the case of crude iron, the sulphur, phosphorus, \&c., which destroy or imprair its tenacity and malleability, become readily separable in the act of puddling. On this principle, I would explain the extraordinary effect of Mr. Wall's patent electric process, as performed in my presence in the excellent iron-works of Mr. Jessop, at Codner Park, Derbyshire, where the electrised forge pigs discharged those noxious elements so copiously in the puddling furnace, as to become after a single reheating, without piling or fagoting, brilliant bars of the finest fibrous metal. The bars so made have been subjected, under my inspection, to the severest proofs by skilful London blacksmiths, and they have been found to bear piercing, hammering, bending, and twisting, as well as the best iron in the market. I have also analyzed the said iron with the utmost minuteness of chemical research, and have ascertained it to be nearly pure metal, containing neither sulphur nor phos phorus, and merely an inappreciable trace of assenic. I can therefore conscientiously recommend Mr. Wall's patent process to iron-masters as one of the greatest, easiest, and most economical improvements, which that important art has ever received.

The pecuniary adrantage of this process, in respect of saring of labor and waste of material, has been estimated by competent judges at from one pound to two pounds sterling per ton.

The effect of electrising iron is displayed in a singular manner by the conversion into steel of a soft rod, exposed in contact with cole, for a few hours, to a moderate red heat; a result which I have witnessed and can fully attest.

PURPLE OF CASSIUS is best made according to the French Pharmacopœia, by dissolring 10 parts of acid chloride of gold in 2,000 parts of distilled water; preparing in another vessel a solution of 10 parts of pure tin in 20 of muriatic acid, which is diluted with 1,000 of water, and adding this by degrees to the gold solution as long as a precipitate is formed. The precipitate is allowed to subside, and is to be washed by means of decantation : it is then filtered and dried at a very gentle heat.

\section{R.}

REFINING OF SILVER. In this process, as effected by sulphuric acid, the arrangements are so complete, that a two thousandth part, or even less, of gold is extracted free of charge to the bullion merchant, and the whole silver returned or accounted for. By mistake a one thousandth was stated in the article REFining of Silver in the Dictionary.

RESINS. An ingenious memoir upon the resins of dammar, copal, and animé, has lately been published by M. Guihourt, an eminent French pharmacien, from which the following extracts may be found interesting.

The hard copal of India and Africa, especially Madagascar, is the product of the Hymenca verrucosa; it is transparent and vitreous within, whatever may be its appearance outside; nearly colorless, or of a tawny yellow; without taste or smell in the cold, and almost as hard as amber, which it much resembles, but from which it may be distinguished, 1st, by its smelting and kindling at a candle-flame, and running down in drops, while amber burns and swells up without flowing ; 2dly, this hard copal or animé, when blown out and still hot, exhales a smell like balsam copaiva or capivi; while amber exhales an unpleasant bituminous odor; $3 \mathrm{dl}$, when moistened by alcohol of 85 per cent., copal becomes sticky, and shows after drying a glazed opaque surface, while amber is not affected by alcohol; 4thly, the copal affords no succenic acid, as amber does, on distillation.

When the pulverized copal is digested in cold alcohol of $0 \cdot 830$, it leaves a considerable residuum, at first pulverulent, but which swells afterward, and forms a slightly coherent mass. When this powder is treated with boiling alcohol, it assumes the consistence of a thick gluten, like crumbs of bread, but which does not stick to the fingers. Thus treated, it affords,

Resin soluble in cold alcohol -

Resin dissolved in boiling alcohol.

Resin insoluble in both

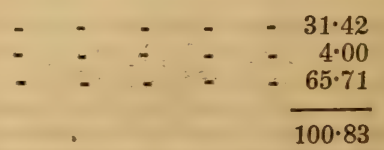

The small excess is due to the adhesion of some of the menstruum to the resins. 
Ether, boiling hot, dissolves $39 \cdot 17$ per cent. of copal.

Essence (spirits) of turpentine does not dissolve any of the copal, but it penetrates and combines with it at a heat of $212^{\circ} \mathrm{Fahr}$.

The property of swelling, becoming viscid and elastic, which Berzelius assigns to copal, belongs not to it, but to the American resin of courbaril, or the occidental unime; and the property of dissolving entirely in ether belongs to the aromatic dammar, a friable and tender resin.

2. Resin of courbaril of Rio Janeiro, the English gum-animé, and the semi-hard copal of the French. It is characterized by forming, in alcohol, a bulky, tenacious, elastic mass. It occurs in rounded tears, has a very pale, glassy aspect, transparent within, corered with a thin white powder, which becomes glutinous with alcohol. Another rariety is soft, and dissolves, for the most part, in alcohol; and a third resembles the oriental copal so much as to indicate that they may both be produced from the same tree. 100 parts of the oriental and the occidental animé yield respectively the following residua:-

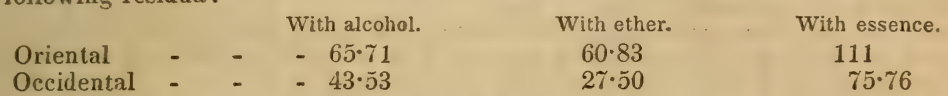

The hard and soft copals possess the remarkable property in common of becoming soluble in alcohol, after being oxygenated in the air.

3. Daminar puti, or dammar batu. - This resin, soft at first, becomes eventually like amber, and as hard. It is little soluble in alcohol and ether, but more so in essence of turpentine.

4. Aromatic dammar.-This resin occurs in large orbicular masses. It is pretty soluble in alcohol. Only small samples hare hitherto been obtained. Of 100 parts, 3 are insoluble in alcohol, none in ether, and 93 in essence of turpentine. M. Guibourt thinks that this resin comes from the Molucca islands. Its ready solubility in slcohol, and great hardness, render it valuable for varnish-making.

5. Austral dammar.-This resin is the product of the Dammara australis, one of the highest trees in New Zealand, where it is called Kaurè or Kouri. It resembles elemi in some measure. It flows from the trunk and branches in the form of a resinous juice called vare, and gum-coudee by the English settlers. The natives chew it continually, and with the soot obtained from its combustion they make the indelible black tatoo figures upon their faces. It comes home in lumps of considerable size. It possesses a certain toughness, which makes it difficult to break or to pulverize. It takes fire at a candle-flame, and continues to burn by itself. It melts in water, heated below the boiling point. Alcohol boiled with it, leaves 43.3 per cent. of insoluble matter; ether leaves 36.66 ; and essence of turpentine 80 per cent. This resin, in fact, resembles very closely the resin of courbaril.

6. Slightly aromatic dammar leaves, after alcohol, 37 per cent.; after ether, 17 per cent. ; and after essence, 87 per cent.

7. Tender and friable dammar selan.-This resin occurs in considerable quantity in commerce (at Paris). It is in round or oblong tears, vitreous, nearly colorless and transparent within, dull whitish on the surface. It exhales an agreeable odor of olibanum, or mastic, when it is heated. It crackles with the heat of the hand like roll-sulphur. It becomes fluid in boiling water, but brittle when cooled again. It sparkles and burns at the flame of a candle; but this being the effect of a volatile oil, the combustion soon ceases.
$\begin{array}{lllll}\text { Resin soluble in cold alcohol } & - & - & - & -75 \cdot 28 \\ \text { Resin insoluble in boiling alcohol } & - & - & - & -70.86\end{array}$

It dissolves readily and completely in cold essence of turpentine, and forms a good varnish. M. Guibnurt refers the origin of this resin to the Dammara selanica of Rumphius Of the preceding resins, 100 parts have left respectively.

\begin{tabular}{|c|c|c|c|}
\hline & Alcohol of 0.830 . & $\begin{array}{l}\text { Insoluble in } \\
\text { Ether. }\end{array}$ & Essence. \\
\hline $\mathrm{H}_{3 s 1}$ sopal, er animé & -65.71 & $60 \cdot 83$ & 111 \\
\hline Tender cupal - & - 43.53 & $27 \cdot 50$ & $75 \cdot 76$ \\
\hline Dammar puti & $-\quad-$ & - & - \\
\hline Dammar aromatic & $-\quad 3.0$ & - & 93 \\
\hline Dammar austral - & $-\quad 43 \cdot 33$ & $36 \cdot 66$ & 80 \\
\hline Dammar slightly aromati & - $\quad 37 \cdot 00$ & $17 \cdot 00$ & 87 \\
\hline Dammar friable - & -20.86 & $2 \cdot 00$ & - \\
\hline
\end{tabular}

RETORTS OF CLAY are now extensively used in gas-making, and they are well manufactured at Newcastle. See the article GAs. 


\section{S.}

SACCHAROMETER is the name of a hydrometer, adapted by its scale to poin out the proportion of sugar, or the saccharine matter of malt, contained in a solution of any specific gravity. Brewers, distillers, and the Excise, sometimes denote by the term gravity, the excess of weight of 1,000 parts of a liquid by volume above the weight of a like volume of distilled water; so that if the specific gravity be $1,045,1,070,1,090$, \&c., the gravity is said to be 45,70 , or 90 ; at others, they thereby denote the weight of saccharine matter in a barrel (36 gallons) of worts; and again, they denote the excess in weight of a barrel of worts over a barrel of water, equal to 36 gallons, or 360 pounds. This and the first statement are identical, only 1,000 is the standard in the first ease, and 360 in the second.

The saccharometer now used by the Excise, and by the trade, is that constructed by Mr. R. B. Bate, well known for the accuracy of his philosophical and mathematical instruments. The tables published by him for ascertaining the values of wort or wash, and low wines, are preceded by explicit drrections for their use. "The instrument is composed of brass; the ball or float being a circular spindle, in the opposite ends of which are fixed a stem and a loop. The stem bears a scale of divisions numbered downward from the first to 30 ; these divisions, which are laid down in an original manner, observe a diminishing progression according to true principles; therefore each division correctly indicates the one thousandth part of the specific gravity of water; and further, by the alteration made in the bulk of the saccharometer at every change of poise, each of the same divisions continues to indicate correctly the said one thousandth part throughout."

In my own practice, I prefer to take specific gravities of all liquids whatever with a glass globe containing 500 or 1,000 grains of distilled water at $60^{\circ} \mathrm{Fahr}$., when it is closed with a capillary-bored glass stopper; and with the gravity so taken, I look into a table constructed to show the quantity per cent. of sugar, malt, extract, or of any other solid, proportional to the density of the solution. By bringing the liquid in the gravity-bottle to the standard temperature, no correction on this account is needed. Mr. Bate's elaborate table contains all these equations correctly for solutions of sugar of every successive specific gravity. When employed in such researches by the Molasses Committee of the House of Commons in the year 1830, I found that the specific gravities of solutions of the concrete extract of malt differed somewhat from those of solutions of sugar, as given by Mr. Bate. (See page 100 of Dictionary.)

The following table shows the quantities of sugar contained in syrups of the annexed specific gravities. It was the result of experiments carefully made :-

\begin{tabular}{|c|c|c|c|}
\hline $\begin{array}{l}\text { Experimental spec. gravity. } \\
\text { of solution at } 60^{\circ} \mathrm{F} \text {. }\end{array}$ & $\begin{array}{l}\text { Sugar in } 100^{\circ} \text { by } \\
\text { weight. }\end{array}$ & $\begin{array}{l}\text { Experimental spec. gravity. } \\
\text { of solution, at } 60^{\circ} \mathrm{F} \text {. }\end{array}$ & $\begin{array}{l}\text { Sugar in } 100 . \text { by } \\
\text { weight. }\end{array}$ \\
\hline $1 \cdot 3260$ & $66 \cdot 666$ & $1 \cdot 1045$ & $25 \cdot 000$ \\
\hline $\mathbf{1} \cdot 2310$ & $50 \cdot 000$ & $1 \cdot 0905$ & $21 \cdot 740$ \\
\hline $1 \cdot 1777$ & $40 \cdot 000$ & $1 \cdot 0820$ & $20 \cdot 000$ \\
\hline $1 \cdot 4400$ & $33 \cdot 333$ & 1.0635 & $16 \cdot 666$ \\
\hline $1 \cdot 1340$ & $31 \cdot 250$ & $1 \cdot 0500$ & $12 \cdot 500$ \\
\hline $1 \cdot 1250$ & $29 \cdot 412$ & $1 \cdot 0395$ & $10 \cdot 000$ \\
\hline $1 \cdot 1110$ & $26 \cdot 316$ & & \\
\hline
\end{tabular}

N. B. The column in the opposite table, marked Solid extract by weight, is $\mathrm{Mr}$. Bate's; it may be compared with this short table, and also with the table of malt infusions in page 100 of the Dictionary.

If the decimal part of the number denoting the specific gravity of syrup be multiplied by 26 , the product will denote very nearly the quantity of sugar per gallon in pounds weight, at the given specific gravity.*

* This rule was annexed to an extensive table, representing the quantities of sugar per gallon cor responding to the specific ravities of the syrups constructed by the author, for the Excise, in subserviency to the Beet- $=0$ bil 
SACCHAROMETER.

Table exhibiting the Quantity of Sugar, in Pounds Avoirdupois, which is contained in One Gallon of Syrup, at successive Degrees of Density, at $60^{\circ} \mathrm{F}$.

\begin{tabular}{|c|c|c|c|c|c|c|c|c|c|}
\hline 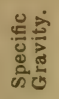 & 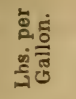 & 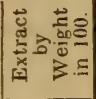 & 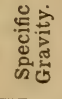 & 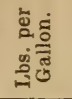 & 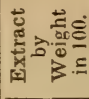 & 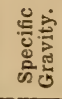 & 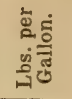 & 异: & 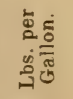 \\
\hline 1.000 & 0.0000 & $\cdot 0000$ & 1.077 & 2.0197 & $\cdot 1851$ & $1 \cdot 154$ & 4.0880 & $1 \cdot 231$ & 6.1474 \\
\hline 1.001 & 0.0255 & $\cdot 0026$ & 1.078 & $2 \cdot 0465$ & $\cdot 1873$ & $1 \cdot 155$ & $4 \cdot 1148$ & $1 \cdot 232$ & $6 \cdot 1743$ \\
\hline 1.002 & 0.0510 & .0051 & 1.079 & $2 \cdot 0734$ & $\cdot 1896$ & $1 \cdot 156$ & $4 \cdot 1319$ & $1 \cdot 233$ & 6.2012 \\
\hline 1.003 & $0.0 \div 65$ & .0077 & $1 \cdot 080$ & $2 \cdot 1006$ & $\cdot 1918$ & $1 \cdot 157$ & $4 \cdot 1588$ & $1 \cdot 234$ & $6 \cdot 2280$ \\
\hline 1.004 & 0.1020 & .0102 & $1 \cdot 081$ & $2 \cdot 1275$ & $\cdot 1941$ & $1 \cdot 158$ & $4 \cdot 1857$ & $1 \cdot 235$ & 6.2551 \\
\hline 1.005 & 0.1275 & $\cdot 0128$ & 1.082 & $2 \cdot 1543$ & $\cdot 1963$ & $1 \cdot 159$ & $4 \cdot 2128$ & $1 \cdot 236$ & 6. 2822 \\
\hline 1.006 & 0.1530 & 0153 & 1.083 & $2 \cdot 1811$ & $\cdot 1985$ & $1 \cdot 160$ & $4 \cdot 2502$ & $1 \cdot 237$ & 6 3093 \\
\hline $1.00 \tau$ & 0.1785 & .0179 & $1 \cdot 084$ & $2 \cdot 2080$ & 2007 & $1 \cdot 161$ & $4 \cdot 2 \% \div 1$ & 1.238 & $6 \cdot 3362$ \\
\hline 1.008 & 0.2040 & .0204 & 1.085 & $2 \cdot 2359$ & 2029 & $1 \cdot 162$ & $4 \cdot 3040$ & $1 \cdot 239$ & 6.3631 \\
\hline 1.009 & 0.2295 & $\cdot 0230$ & 1.086 & $2 \cdot 2627$ & 2051 & $1 \cdot 163$ & $4 \cdot 3309$ & $1 \cdot 240$ & 6.3903 \\
\hline 1.010 & 0.2550 & .0255 & 1.087 & $2 \cdot 2894$ & $\cdot 2073$ & $1 \cdot 164$ & $4 \cdot 35 \div 8$ & 1.241 & 6.4152 \\
\hline 1.011 & 0.2805 & .0280 & $1 \cdot 088$ & $2 \cdot 3161$ & $\cdot 2095$ & $1 \cdot 165$ & $4 \cdot 3847$ & $1 \cdot 242$ & 6.4401 \\
\hline 1.012 & 0.3060 & .0306 & $1 \cdot 089$ & $2 \cdot 3438$ & 2117 & $1 \cdot 166$ & $4 \cdot 4115$ & $1 \cdot 243$ & $6 \cdot 4650$ \\
\hline 1.013 & 0.3315 & .0331 & 1.090 & $2 \cdot 3 \div 10$ & .2139 & $1 \cdot 167$ & 4.4383 & 1.244 & 6.4902 \\
\hline 1.014 & 0.3570 & $\cdot 0356$ & 1091 & $2 \cdot 3987$ & .2161 & $1 \cdot 168$ & 4.4652 & 1.245 & 6.5153 \\
\hline 1.015 & 0.3825 & $\cdot 0381$ & 1.092 & 2.4256 & $\cdot 2183$ & $1 \cdot 169$ & $4 \cdot 4923$ & $1 \cdot 246$ & $6 \cdot 5402$ \\
\hline 1.016 & 0.4180 & .0406 & 1.093 & 2.4524 & $\cdot 2205$ & 1170 & 4.5201 & $1 \cdot 247$ & 6.5651 \\
\hline 1.017 & 0.4335 & .0431 & 1.094 & 2.4792 & .2227 & $1 \cdot 171$ & 4.5460 & 1248 & 6.5903 \\
\hline 1.018 & 0.4590 & .0456 & 1.095 & 2.5061 & .2249 & 1.172 & 4. 5722 & $1 \cdot 249$ & 6.6152 \\
\hline 1.019 & 0.4845 & .0481 & 1.096 & 2.5329 & $.22 \% 0$ & 1.173 & $4 \cdot 5983$ & $1 \cdot 250$ & 66402 \\
\hline 1.020 & 0.5100 & .0506 & 1.097 & 25598 & $\cdot 2292$ & $1 \cdot 174$ & 46242 & 1.251 & 6.6fi81 \\
\hline 1.021 & 0.5351 & .0531 & 1.098 & $2 \cdot 5866$ & .2314 & 1.175 & $4 \cdot 6505$ & 1.252 & 6.6960 \\
\hline 1.022 & 0.5602 & $\cdot 0555$ & 1.099 & $2 \cdot 6130$ & $\cdot 2335$ & 1.176 & 4.6764 & 1.253 & 6.7240 \\
\hline 1.023 & 0.5853 & $\cdot 0580$ & $1 \cdot 100$ & 26404 & $\cdot 2357$ & $1 \cdot 177$ & $4 \cdot 7023$ & $1 \cdot 254$ & $6 \cdot 7521$ \\
\hline 1.024 & 0.6104 & $\cdot 0605$ & $1 \cdot 101$ & $2 \cdot 6663$ & $\cdot 2378$ & 1.178 & $4 \cdot 7281$ & $1 \cdot 255$ & $6 \div 800$ \\
\hline 1.025 & 0.6355 & $\cdot 0629$ & $1 \cdot 102$ & $2 \cdot 6921$ & $\cdot 2400$ & $1 \cdot 179$ & $4 \cdot 7539$ & I. 256 & 6.8081 \\
\hline 1.026 & 0.6606 & $\cdot 0654$ & $\mathrm{j} \cdot 103$ & 27188 & -2421 & 1180 & 47802 & $1 \cdot 257$ & $6 \cdot 8362$ \\
\hline 1.027 & 0.6857 & $\cdot 0678$ & $1 \cdot 104$ & $2 \cdot 7446$ & $\cdot 2443$ & $1 \cdot 181$ & $4 \cdot 8051$ & $1 \cdot 258$ & $6 \cdot 8643$ \\
\hline 1028 & 0.7108 & .0703 & $1 \cdot 105$ & $2 \cdot 7704$ & $\cdot 2464$ & $1 \cdot 182$ & $4 \cdot 8303$ & $1 \cdot 259$ & 6.8921 \\
\hline 1.029 & 0.7359 & 0727 & $1 \cdot 106$ & $2 \cdot 7961$ & $\cdot 2486$ & $1 \cdot 183$ & 4.8554 & $1 \cdot 260$ & 6 9201 \\
\hline 1.030 & 0.7610 & .0752 & $1 \cdot 107$ & 2.8227 & .2507 & 1184 & $4 \cdot 8802$ & $1 \cdot 261$ & 6.9510 \\
\hline 1.031 & 0.7861 & $.0 \tau \tau 6$ & $1 \cdot 108$ & $2 \cdot 8485$ & $\cdot 2529$ & 1.185 & $4 \cdot 9051$ & 1.262 & 69822 \\
\hline 1.032 & 0.8112 & .0800 & $1 \cdot 109$ & $2 \cdot 8 \pi 40$ & $\cdot 2550$ & 1186 & $4 \cdot 9300$ & 1.263 & 7.0133 \\
\hline 1.033 & 0.8363 & .0825 & $1 \cdot 110$ & 29001 & $.25 \% 1$ & $1 \cdot 187$ & 4. 9552 & $1 \cdot 264$ & 7.0444 \\
\hline 1.034 & 0.8614 & . 0849 & $1 \cdot 111$ & $2 \cdot 9263$ & $\cdot 2593$ & 1.188 & 4.9803 & 1.265 & 7.0751 \\
\hline 1.035 & $0.8866^{\circ}$ & .0873 & 1.112 & $2 \cdot 9522$ & $\cdot 2614$ & 1189 & 5.0054 & $1 \cdot 266$ & $7 \cdot 1060$ \\
\hline $1 \cdot 036$ & 0.9149 & .0897 & 1.113 & 2.9780 & $\cdot 2635$ & 1190 & $5(1304$ & 1.267 & $7 \cdot 1369$ \\
\hline 1.037 & 09449 & .0921 & 1.114 & 3.0045 & -2656 & ]'191 & 5.0563 & $1 \cdot 268$ & $7 \cdot 1678$ \\
\hline 1.038 & 0.9768 & $\cdot 0945$ & 1.115 & 3.0304 & .2677 & 1192 & $5 \cdot 0822$ & 1269 & $7 \cdot 1988$ \\
\hline $1 \cdot 039$ & 1.0090 & .0969 & $1 \cdot 116$ & 3.0563 & $\cdot 2698$ & $1 \cdot 193$ & $5 \cdot 1080$ & 1.270 & $7 \cdot 2300$ \\
\hline 1040 & 1.0400 & .0993 & 1.117 & $3 \cdot 0821$ & $\cdot 2 \pi 19$ & 1194 & $5 \cdot 1341$ & $1 \cdot 271$ & $7 \cdot 2601$ \\
\hline 1.041 & $1 \cdot 0653$ & $\cdot 1017$ & 1.118 & $3 \cdot 1080$ & $\cdot 2740$ & 1.195 & $5 \cdot 1602$ & 1.272 & $\tau \cdot 2902$ \\
\hline 1.042 & 1.0906 & $\cdot 1041$ & $1 \cdot 119$ & $3 \cdot 1343$ & 2761 & 1196 & $5 \cdot 1863$ & 1.273 & $\div 3204$ \\
\hline 1.043 & $1 \cdot 1159$ & $\cdot 1065$ & 1.120 & $3 \cdot 1610$ & $\cdot 2782$ & 1.197 & 5.2124 & $1 \cdot 2 \tau 4$ & $7 \cdot 3506$ \\
\hline 1.044 & $1 \cdot 1412$ & $\cdot 1089$ & 1.121 & $3 \cdot 1871$ & 2803 & 1198 & 5.2381 & $1 \cdot 275$ & 73807 \\
\hline 1.045 & $1 \cdot 1665$ & .1113 & $1 \cdot 122$ & 3.2130 & $\cdot 2824$ & 1199 & $5 \cdot 2639$ & 1.276 & $7 \cdot 4109$ \\
\hline 1046 & I-1918 & $\cdot 1136$ & 1.123 & 3.2399 & $\cdot 2845$ & 1.200 & $5 \cdot 2901$ & 1.277 & 7.4409 \\
\hline 1047 & $1.21 \% 1$ & $\cdot 1160$ & 1.124 & 3.2658 & $\cdot 2865$ & 1.201 & 5.3160 & $1.2 \pi 8$ & $7 \cdot 4708$ \\
\hline 1.048 & 1.2424 & $\cdot 1184$ & 1.125 & 3.2916 & $\cdot 2886$ & $1 \cdot 202$ & $5 \cdot 3422$ & 1.279 & $7 \cdot 5007$ \\
\hline 1.049 & 1.2687 & $\cdot 1207$ & $1 \cdot 126$ & 3.3174 & $\cdot 2907$ & 1'203 & $5 \cdot 3681$ & $1 \cdot 280$ & 7.5307 \\
\hline 1050 & $1 \cdot 2940$ & $\cdot 1231$ & 1.127 & $3 \cdot 3431$ & $\cdot 2927$ & 1204 & 5 3941 & 1.281 & 7.5600 \\
\hline 1.051 & $1 \cdot 3206$ & $\cdot 1254$ & $1 \cdot 128$ & 3. 3690 & $\cdot 2948$ & 1.205 & 5. 4203 & 1.282 & 7.5891 \\
\hline 1.052 & $1 \cdot 34 \div 2$ & $\cdot 1278$ & $1 \cdot 129$ & $3 \cdot 3949$ & $\cdot 2969$ & 1206 & 5.4462 & $1 \cdot 283$ & 7.6180 \\
\hline 1.053 & $1 \cdot 3738$ & $\cdot 1301$ & $1 \cdot 130$ & 3.4211 & $\cdot 2989$ & 1207 & 5.4720 & $1 \cdot 284$ & $7 \cdot 6469$ \\
\hline 1.054 & 1.4004 & $\cdot 1325$ & $1 \cdot 131$ & 3.4490 & .3010 & 1.208 & 5.4979 & $1 \cdot 285$ & 7.6758 \\
\hline 1.055 & 1.4270 & $\cdot 1348$ & 1.132 & 3.4769 & $\cdot 3030$ & $1 \cdot 209$ & 5. 5239 & 1.286 & $7 \cdot 7048$ \\
\hline 1.056 & 1.4536 & $\cdot 1372$ & 1.133 & 3.5048 & $\cdot 3051$ & 1210 & 5. 5506 & 1.287 & 77331 \\
\hline 1.057 & 1.4802 & $\cdot 1395$ & $1 \cdot 134$ & 3.5326 & $\cdot 3071$ & 1211 & $5 \cdot 5786$ & 1.288 & $7 \cdot 7620$ \\
\hline 1.058 & $1 \cdot 5068$ & $\cdot 1418$ & $1 \cdot 135$ & 3.5605 & $\cdot 3092$ & 1.212 & 56071 & 1.289 & $7 \cdot 7910$ \\
\hline 1.059 & 1.5334 & $\cdot 1441$ & $1 \cdot 136$ & 3.5882 & ·3112 & $1 \cdot 213$ & $5 \cdot 6360$ & 1.290 & 7.8201 \\
\hline 1.060 & $1 \cdot 5600$ & $\cdot 1464$ & $1 \cdot 137$ & $3 \cdot 6160$ & 3132 & 1.214 & $5 \cdot 6651$ & 1.291 & 7.8482 \\
\hline 1.061 & 1.5870 & $\cdot 1487$ & $1 \cdot 138$ & 3.6437 & -3153 & 1.215 & 5. 6942 & $1 \cdot 292$ & $78 \% 63$ \\
\hline 1.062 & 1.6142 & $\cdot 1510$ & $1 \cdot 139$ & $3 \cdot 6716$ & $31 \% 3$ & 1.216 & 5. 7233 & 1.293 & $7 \cdot 9042$ \\
\hline 1.063 & 1.6414 & $\cdot 1533$ & 1.140 & 3. 7000 & $\cdot 3193$ & 1.217 & 5.7522 & 1.294 & $7 \cdot 9321$ \\
\hline $1 \cdot 064$ & 1.6688 & $\cdot 1556$ & $1 \cdot 141$ & 3.7281 & -3214 & 1.218 & 5.7814 & 1.295 & 7.9600 \\
\hline 1.065 & 1. 6959 & $\cdot 1579$ & $1 \cdot 142$ & $3 \div 562$ & $\cdot 3234$ & 1219 & 5.8108 & 1.296 & $7 \cdot 9879$ \\
\hline 1.066 & $1 \cdot 7228$ & $\cdot 1602$ & $1 \cdot 143$ & 3.7840 & $\cdot 3254$ & $1 \cdot 220$ & 5.8401 & 1297 & 8.0158 \\
\hline 1.067 & $1 * 7496$ & $\cdot 1625$ & $1 \cdot 144$ & 3.8119 & $\cdot 3274$ & 1.221 & $5.86 \times 0$ & 1.298 & 8.0448 \\
\hline 1.068 & 17764 & $\cdot 1647$ & 1145 & 3. 8398 & $\cdot 3294$ & 1.222 & $5 \cdot 8962$ & $1 \cdot 299$ & 8.0719 \\
\hline $1 \cdot 069$ & $1 \cdot 8033$ & $\cdot 1670$ & $1 \cdot 146$ & 3.8677 & $\cdot 3314$ & $1 \cdot 223$ & 5. 9242 & 1.300 & $8 \cdot 1001$ \\
\hline $1.0 \% 0$ & $1 \cdot 8300$ & $\cdot 1693$ & $1 \cdot 147$ & 3.8955 & $\cdot 3334$ & 1.224 & 5. 9523 & & \\
\hline 1.071 & 18571 & 1716 & $1 \cdot 148$ & 3.9235 & $\cdot 3354$ & $1 \cdot 225$ & 5.9801 & & \\
\hline 1.072 & $1 \cdot 8843$ & $\cdot 1738$ & $1 \cdot 149$ & 3.9516 & $\cdot 3374$ & 1.226 & 6.0081 & & \\
\hline 1.073 & 1.9116 & $\cdot 1761$ & $1 \cdot 150$ & 3.9801 & 3394 & $1 \cdot 227$ & 6.0361 & & \\
\hline $1 \cdot 074$ & $1 \cdot 9385$ & $\cdot 1783$ & $1 \cdot 151$ & 4.0070 & & 1228 & 6. 0642 & & \\
\hline 1.075 & 1.9653 & $\cdot 1806$ & $1 \cdot 152$ & $4 \cdot 0342$ & & 1.229 & 6.0425 & & \\
\hline 1.076 & 1.9928 & $\cdot 1828$ & $1 \cdot 153$ & $4 \cdot 0611$ & & $1 \cdot 230$ & $6 \cdot 1205$ & & \\
\hline
\end{tabular}


SAFETY LAMP. During a visit which I paid to Newcastle some time ago, I took pains to learn the opinion of the best judges of coal mining, upon the merits of the patent invention of Upton and Roberts, described in the Dictionary, and I found from the eoncurring testimony of that very able engineer, Mr. Buddle, since lost to his friends and the world, and of Mr. Sopwith, well known for the geological study of the coal formation, that the said lamp could not be safely used on account of its glass case, which, being most liable to break, would be apt to cut or rupture the meshes of the wire gauze within it, and thus to lay the flame open for explosions. It is not therefore in use.

SAGO. See PEPPER in this Supplement.

SAL AMMONIAC. A patent was obtained in 1840, for improvements in the manufacture of this article, by Mr. H. Waterton. Two modes of operating are described; the first consists in making a saturated solution of common salt in water, and mixing with it a quantity of finely pulverized carbonate of ammonia, about equal in weight to the salt contained in the solution. The mixture is agitated in a close vessel for six or eight hours, and as much carbonic acid gas is infused therein as it will absorb (but the introduction of the gas is not absolutely necessary, although the patentee prefers it); the liquid is then separated from the solid matter, by filtration and pressure. The solid matter is chiefly bi-carbonate of soda, and the liquid holds in solution muriate and carbonate of ammonia, and common salt, and sometimes a small portion of the bicarbonate of soda.

The liquid is now placed in a distilling vessel, and the carbonate of ammonia being distilled over into a suitable receiver, a solution of muriate of ammonia and common salt remains in the still. This solution is evaporated, by heat, to such a consistency as will cause the separation of the common salt, by crystallization, and the salt, thus crystallized, is evaporated from the liquid by any convenient method. The liquid is then evaporated until it attains the proper specific gravity for crystallizing, and it is transferred into suitable utensils for that purpose. The crystals, produced by these means, are nearly pure muriate of ammonia, and, when pressed and dried, may be brought to market without further preparation, or they may be sublimed into cake sal ammoniac.

The other mode of manufacturing sal ammoniac consists in taking a quantity of liquid, containing ammonia, either in the caustic state, or combined with carbonic, hydrosulphuric, or hydrocyanic acid (such as gas ammoniacal liquor, or bone ammoniacal liquor), and rectifying it, by distillation, until the distilled portion contains from twenty to twenty-five per cent. of carbonate of ammonia. If the liquid contains any other acids than those above mentioned, a sufficient quantity of lime is used in the distillation to decompose the ammoniacal salt.

The distilled liquid being now mixed with as large a quantity of powdered common salt as it will dissolve, is agitated for several hours, and as much carbonic acid gas is infused into it as it will absorb. The remainder of the operation is the same as before described in the first method of manufacturing sal ammoniac.-Newton's Journal, C. S. xxii. 35 .

SEMOULE. The name given in France, and used in this country, to denote the large hard grains of wheat flour retained in the bolting machine after the fine flour has been passed through its meshes. The best sémoule is obtained from the wheat of the southern parts of Europe. With the sémoule, the fine white Parisian bread called gruau is baked. Skilfnl millers contrive to produce a great proportion sémoule from the largegrained wheat of Naples and Odessa.

SILK. Several pieces of silk were put into my hands, for analysis, on the 18th of February, after I had, on the preceding 12 th of the month, visited the St. Katharine's Dock warehouses, in New street, Bishopsgate street, for the purpose of inspecting a large package of the Corahs, per Colonist. I was convinced, by this inspection, that, notwithstanding the apparent pains bestowed upon the tin plate and teakwood packingcases, certain fissures existed in them, through which the atmospheric air had found access, and had caused iron-mould spots upon the gunny wrapper, from the rusting or oxidizement of the tinned iron.

I commenced my course of analysis upon some of the pieces which were most damaged, as I thought they were most likely to lead me to an exact appreciation of the cause of the mischief; and I pursued the following general train of research :-

1. The piece of silk, measuring from 6 to 7 yards, was freely exposed to the air, then weighed, afterward dried near a fire, and weighed again, in order to determine its hygrometric property, or its quality of becoming damp by absorbing atmospheric vapor. Many of the pieces absorbed, in this way, from one tenth to one eighth of their whole weight; that is, from $1 \mathrm{oz}$ to $1 \frac{1}{2} \mathrm{oz}$. upon $13 \mathrm{oz}$. This fact is very instructive, an: shows that the goods had been dressed in the loom, or imbued subsequently, with some verv deliquescent pasty matter.

2. I next subjected the piece to the action of distilled water, at a boiling tempera- 
ture, till the whole glutinous matter was extracted; five pints of water were employed for this purpose, the fifth being used in rinsing out the residuum. The liquid wrung out from the silk was evaporated first over the fire, but toward the end over a steam bath, till it became a dry extract; which in the damaged pieces was black, like extract of liquorice, but in the sound pieces was brown. In all cases the extract so obtained absorbed moisture with great avidity. The extract was weighed in its driest state, and the weight noted, which showed the addition made, by the dressing to the weight of the silk. The piece of silk was occasionally weighed in its cleansed state, when dry, as a check upon the preceding experiment.

3. The dry extract was now subjected to a regular chemical analysis, which was morlified according to circumstances, as follows: 100 parts of it were carefully ignited in a platinum capsule; during which a considerable flame and fetid smoke were disengaged. The ashes or incombustible residuum were examined by the action of distilled water, filtration, as also by that of acids, and other chemical tests, whereby the constituents of these ashes were ascertained. In the course of the incineration or calcination of the extract from the several samples, I never observed any sparkling or scintillation; whence I inferred that no nitre had been used in the dressing of the goods, as some persons suggested.

4. Having, in the course of boiling some of the extract from two of the damaged pieces in a little distilled water, felt a urinous odor, I was induced to institute the following minute course of researches, in order to discover whether the urine of man had been introduced into the dressing paste of the silk webs. I digested a certain portion of the said extract in alcohol, 60 per cent. over proof, which is incapable of dissolving the rice water, or other starchy matter, which might be properly applied to the silk in the loom. The alcohol, however, especially when aided by a moderate heat, readily dissolves urea, a substance of a peculiar nature, which is the characteristic constituent of human urine. The alcohol took a yellow tint, and being, after subsidence of the sediment, decanted clear off into a glass retort, and exposed to the gentle heat of a water bath, it distilled over clear into the receiver, and left a residuum in the retort, which possessed the properties of urea. This substance was solid when cold, but melted at a heat of $220^{\circ}$ F.; and at a heat of about $245^{\circ}$ it decomposed with the production of water and carhonate of ammonia-the well-known products of urea at that temperature. The exhalation of ammonia was very sensible to the smell, and was made peculiarly manifest by its browning yellow turmeric paper, exposed in a moist state to the fumes, as they issued from the orifice of the glass tube, in which the decomposition was usually effected. I thus obtained perfect evidence that urine had been employed in India in preparing the paste with which a great many of the pieces had been dressed. It is known to every experienced chemist, that one of the most fermentative or putrefactive compositions which can be made, results from the mixture of human urine with starchy or gummy matter, such as rice water; a substance which, by the test of iodine water, these Corahs also contained, as I showed to the gentlemen present, at my visit to the Bonding Warehouse.

5. On incinerating the extract of the Corahs, I obtained, in the residuum, a notable quantity of free alkali ; which, by the test of chloride of platinum, proved to be potassa. But, as the extract itself was neutral to the tests of litmus and turmeric paper, I was consequently led to infer that the said extract contained some vegetable acid, probably produced by the fermentation of the weaver's dressing, in the hot climate of Hindostan. I, accordingly, examined the nature of this acid, by distilling a portion of the extract along with some very dilute sulphuric acid, and obtained in the receiver a notable quantity of the volatilized acid condensed. This acid might be the acetic (vinegar), the result of fermentation, or it might be the formic or acid of ants, the result of the action of sulphuric acid upon starchy matter. To decide this point, I saturated the said distilled acid with magnesia, and obtained on evaporation the characteristic gummy mass of acetate of magnesia, soluble in alcohol, but none of the crystals of formiate of magnesia, insoluble in alcohol. From the quantity of alkali (potassa) which I obtained from the incineration of the extract of one piece of the damaged silk, and which amounted to six grains at least, I was convinced that wood ashes had been added, in India, to the mixture of sour rice water and urine, which would therefore constitute a compound remarkably hygrometric, and well qualified to keep the warp of the web damp, even in that arid atmosphere, during the time that the Tanty or weaver was working upon it. The acetate of potassa, present in the said Corahs, is one of the most deliquescent salts known to the chemist : and, when mixed with fermented urine, forms a most active hygrometric dressing-one, likewise, which will readily generate nildew upon woven goods, with the aid of heat and the smallest portion of atmospheric exygen. By the above-mentioned fermentative action, the carbon, which is one of the chemical constituents of the rice or starchy matter, had been eliminated, so as to occa- 
sion the dark stains upon the silk, and the blackness of the extract taken out of it by distilled water.

6. That the dressing applied to the webs is not simply a decoction of rice, becomes very manifest, by comparing the incinerated residuum of rice with the incinerated residuum of the extract of the said Corahs. I find that 100 grains of rice, incinerated in a platinum capsule, leave only about one fifth of a grain, or 1 in 500 of incombustible matter, which is chiefly silicious sand; whereas, when 100 grains of an average extract of several of these Corahs were similarly incinerated, they left fully 17 parts of incombustible matter. This consisted chiefly of alumina or earth of clay, with silica, potassa, and a little common or culinary salt. (Has the clay been added, as is done in Manchester, to give apparent substance to the thin silk web ?)

From the above elaborate course of experiments, which occupied me almost constantly during a period of four weeks, I was fully warranted to conclude that the dam. age of the said goods had been occasioned by the vile dressing which had been put into them in India; which, as I have said, under the influence of heat and air, had caused them to become more or less mildewed, in proportion to their original dampness when packed at Calcutta, and to the accidental ingress of atmospheric air into the cases during the voyage from Calcutta to London.

The following is the list of Corahs which I chemically examined :-

1 and 2, per Colonist, from Calcutta, 2 pieces, sound.-These two pieces had been dressed with a sweet viscid matter, like jaggery or goor (molassy sugar), mixed with the rice water. This extract contained no urine, but emitted a smell of caramel or burned sugar, when ignited. It amounted to 270 grains in the one, and 370 in the other.

3, ditto, 1 piece, mildewed, 1st degree.-This piece had been dressed like No. 5 , and contained no trace of urine. It afforded 400 grains of a most deliquescent sweetish glutinous matter.

4, ditto, 1 piece, mildewed, 1 st degree, as No. 3 .

5, ditto, 1 piece, mildewed, $3 \mathrm{~d}$ degree.-This piece contained no trace of urine, but it afforded 210 grains of a light brown extract, being rice water, mixed with something like jaggery.

6 , ditto, 1 piece, $3 \mathrm{~d}$ degree, mildewed.-This piece afforded evidence of urine in it, by test of carbonate of ammonia. The extract amounted to 320 grains.

8 , ditto, 2 pieces, damaged in the 3 d degree.-The total weight of one of these pieces, after exposure to air, was 4,610 grains, and it lost 440 grains by drying. The total weight of the other was 4,950 grains, and it lost 320 grains by drying. The weight of extract was, in one piece, 210 grains; and both pieces contained abundant traces of urine, as well as of potash. These constituents, along with the rice water, accounted sufficiently for the great damage of these two pieces by mildew.

10, ditto, 2 pieces, sound.- These contained no urea. Each afforded from 300 to 500 grains of a light brown vegetable extract.

12 , ditto, 2 pieces.-The extract in the one amounted to 222 grains; and in the other to 330 . Both contained urea, and had, therefore, been imbued with urine.

14, ditto, 2 pieces, mildewed, $3 \mathrm{~d}$ degree.-There was no urea in the extracts from these two pieces; but they afforded, the one 300 grains of extract, and the other 750 . But this extract was a saccharine molassy matter, impossible to dry over a steam heat. The same quantity as the last, if dried by stronger means, would have weighed probably 600 grains. Its extraordinary deliquescence kept the pieces very moist, and thereby caused the mildewing of them. With the saccharine matter, four per cent. of culinary salt was mixed in one of these extracts.

16, ditto, 2 pieces, 3d degree of mildew.-The extract, about 200 grains, contained abun' ant evidence of urea, and, consequently of urine.

18 , ditto, 2 pieces, sound.-Both these contained some traces of urea; but the one yielded only 102 grains of extract, and the other 370 grains. They must have been well screened from the air to have resisted the action of the urine.

20 , ditto, 2 pieces, damaged, 1 st degree.-No urea. The extract of the one was 320 grains; of the other piece 380 ; and it had a light brown color, being a saccharine mucilage.

22 , ditto, 2 preces, $3 \mathrm{~d}$ degree mildew.-200 grains of extract in the one, and 210 in the other: they contained urea.

24,2 pieces, $3 \mathrm{~d}$ degree of mildew. -310 grains of extract in the one, and 180 grains in the other. Both were impregnated with urea, and consequently with urine.

Having in the preceding report demonstrated, by the clearest processes of chemical research, that the above mildewed Corahs had been damaged by the fermentative decomposition of the dressing paste with which they had been so abundantly impregnated, I would recommend the importers of such goods to cause the whole of the dressing to be washel out of them, and the pieces to be thoroughly dried, before being packed up. 
Lelieve that clean silk may be kept and transported, even in the most humid atmoirl.ere, without undergoing any change, if it be not imbued with fermentative paste.

examined eight other pieces of a different mark, imported by another mercantile - cuse, per Colonist, and they afforded results similar to the above.

SILVER, Extraction of from Leat; Pattinson's process.-The desilverizing apparatus of Locke, Blackett, and Co., consists of seven crystallizing pots, and one smaller pot for receiving the desilvered lead. They are all made of cast iron, and arranged in a straight line.

The lead in each pot varies in its contents of silver.

The first containing $85 \mathrm{cwts}$. lead, at about $60 \mathrm{oz}$. of silver, or 1544 ths per ton

Is divided into $55 \mathrm{cwts}$. crystals carried to second pot, at $35 \mathrm{oz}$. per ton - 96

$18 \mathrm{cwts}$. do. to be put in first pot again, at $64 \mathrm{oz}$. per ton 57 and 12 cwts. rich lead to be cupelled, at $170 \mathrm{oz}$. per ton - 102

The second pot containing $90 \mathrm{cwts}$. lead, at about $35 \mathrm{oz}$. silver per ton $\quad$ - $15 \%$

Is divided into $60 \mathrm{cwts}$. crystals carried to third pot, at $20 \mathrm{oz}$. per ton $\quad-60$ and $30 \mathrm{crts}$. lead put into first pot, at $65 \mathrm{oz}$. per ton _ $\quad$ - 97

The third pot containing $90 \mathrm{cwts}$. of lead, at about $20 \mathrm{oz}$. per ton -
Is divided into $55 \mathrm{cwts}$. crystals carried to fourth pot, at $10 \mathrm{oz}$. per ton $\quad-\quad 27$ and $25 \mathrm{cwts}$. lead put into second pot, at $36 \mathrm{oz}$. per ton - $\quad 63$

The fourth pot containing 80 cwts. lead, at about $10 \mathrm{oz}$. per ton $\quad$ - $\quad 40$

Is divided into $55 \mathrm{cwts}$. crystals, carried to fifth pot, at $5 \frac{1}{2} \mathrm{oz}$. per ton $\quad 15$ and $25 \mathrm{cwts}$. lead put into third pot, at $20 \mathrm{oz}$. per ton $\quad-\quad 25$

The fifth pot containing $80 \mathrm{cwts}$. lead, at about $5 \frac{1}{2} \mathrm{oz}$. silver per ton - $\quad 22$

Is divided into $55 \mathrm{cwts}$. crystals, put into sixth pot, at $3 \mathrm{oz}$. per ton - - $8 \frac{\mathrm{z}}{4}$ and 25 cwts. lead, put into fourth pot, at $11 \mathrm{oz}$. per ton - $\quad 13 \frac{3}{4}$

The sixth pot containing 80 cwts. lead, at about $3 \mathrm{oz}$. per ton - _ - 12

Is divided into 55 cwts. crystals, carried to seventh pot, at $1 \frac{1}{2}$ oz. per ton - $4 \frac{1}{2}$ and $25 \mathrm{cwts}$. lead, put into fifth pot, at $6 \mathrm{oz}$. per ton - $\quad-\quad 7 \frac{1}{2}$

The seventh pot containing $55 \mathrm{cwts}$. lead, at about $1 \frac{1}{2} \mathrm{oz}$. per ton - $\quad 4$

Is divided into 25 cwts. crystals, carried to small pot, at $1 \frac{1}{2} \mathrm{oz}$. per ton and $30 \mathrm{cwts}$. lead, put into sixth pot, at $2 \frac{1}{2} \mathrm{oz}$. per ton -

The above $25 \mathrm{cwts}$. of crystals are melted and cast into pigs and sent to the market. In operating upon lead containing about $10 \mathrm{oz}$. per ton, the fourth pot is filled with it; if it should contain $20 \mathrm{oz}$., or thereabouts, it is put into the third pot; and so of any other.

Fig. 125 represents the arrangement of the iron pots or caldrons, in their order.

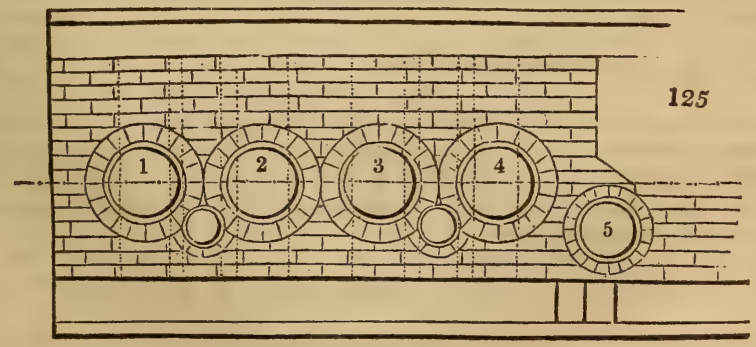

The desilvering apparatus represented in fig. 125 is composed of five caldrons of east iron, each heated by its own fire, besides two smaller pots, similarly heated. The caldrons rest by their upper flange and surface upon bricks properly formed and arranged. Their shape is not hemispherical; their mouth is 40 inches in length, but only 26 inches in width. Over the door of the fireplace, the mouth stands 8 feet 4 inches above the ground or bottom of the ash-pit, of which space, 18 inches intervene 
between the grate and the brim. The grate is 2 feet long and $8 \frac{8}{4}$ inches wide. All the caldrons have the same elliptic form, with a bottom like the small end of an egg. The fifth alone is smaller, but this one serves merely to melt the lead which has bee: stripped of its silver, in order to be cast into salmons or blocks.

The charge consists of 64 or 65 salmons, each weighing from 120 to $140 \mathrm{lbs}$. When they are well melted, the fire is removed from the grate, as well as the small film of litharge from the surface of the metal; and one or two salmons are added to accelerate the cooling, or sometimes, instead, a little soapy water is sprinkled into the caldron, whereby a crust of lead is formed, which being pushed down into the mass, melts with ebullition. This is repeated till the whole becomes sufficiently cool, that is, when crystals begin to form. The lead concreted round the sides being now detached, the whole is stirred with an iron bar, by a motion in a vertical plane, and varying its posture in this plane. During this operation, intended to establish a uniform temperature throughout the mass, a second workman heats in the smaller pot adjoining to No. 1 a large skimmer at the end of a long wooden handle, and next proceeds to fish out the crystals, taking care to let them drain off for a few seconds all the liquid lead among them, and then turns out the crystals slowly into the next caldron, No. 2; the second workman meanwhile adds the metal solidified round the sides, and stirs all together to equalise the temperature. These two-fold operations occupy about fifty minutes; by which time, there remains in the caldron about 16 salmons. The workman now lifts out the crystals, as before, with the drainer, and throws them upon the ground in two heaps. His assistant takes them up a little while afterward, and puts them away to make room for fresh crystals, which the first workman continues to throw down. This process goes on till only 8 salmons remain in the caldron, a point ascertained by gauging the height to the bath. The fire being at this time removed from cauldron No. 2 into the grate of No. 1 , the 8 salmons of lead enriched with silver, which remain at the bottom of the caldron, are run out into movable moulds; and the 8 salmons which were thrown upon the ground are put into it; the full charge being then made up with salmons of the same richness as those previously used.

While this mass is melting in No. 1 the process just finished in it is repeated in No. 2. About three fourths of the metallic mass is next separated in the state of crystals, which are transferred to No. 3 , and also one eighth of crystals thrown on the ground, after pouring the remaining one eighth at the bottom of caldron No. 2 not into moulds, but into No. 1 .

A like process is performed in caldrons 3 and 4 ; and the poor lead taken out of 4 is transferred to 5 to be melted, and run into salmons, which are submitted afresh to the preceding series of crystallizations, provided the lead still contains a sufficient proportion of silver.

The following Table will place the results of the above successive operations in a clear light :-

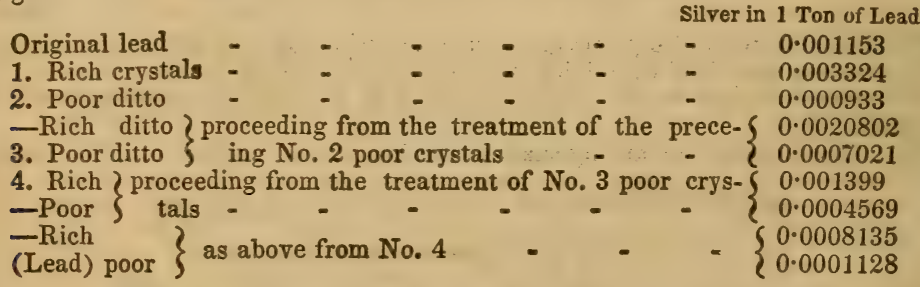

We thus see, that four crystallizations, repeated upon the original lead from the smelting furnace, of the above richness, will afford a lead ten times poorer. With a lead originally containing only 0.0002248 in silver, three crystallizations would suffice to make it ten times poorer. In general, the poorer the lead, within certain limits, the better adapted is it to this process.

SILVERING. See ELEctrotYPE.

SLIDES. The name given by the Cornish miners to clay veins of more modern formation.

SMELTING IRON FURNACES, commonly called BLAST FURNACES. Several of these furnaces, as mounted near Glasgow, deserve to be made known, on account of the economy of their construction, the advantage of their form, and the amount of their performance.

Fig. 126 represents one of the smallest of these, which measures from the line at the bottom to the top 48 feet, from which all the other dimensions may be estimated. It produces a soft cast iron for casting into moulds and for melting in the cupola. Figs. 127 and 128 represent a much larger furnace, being from the top, to the line A, B, C, D 
60 feet high. A few have been built still larger. This furnace has a double case, each of which consists of fire-bricks. This case is enclosed by common bricks, and these by a wall of stone masonry. The successive rows of bricks are laid stair-wise, having the

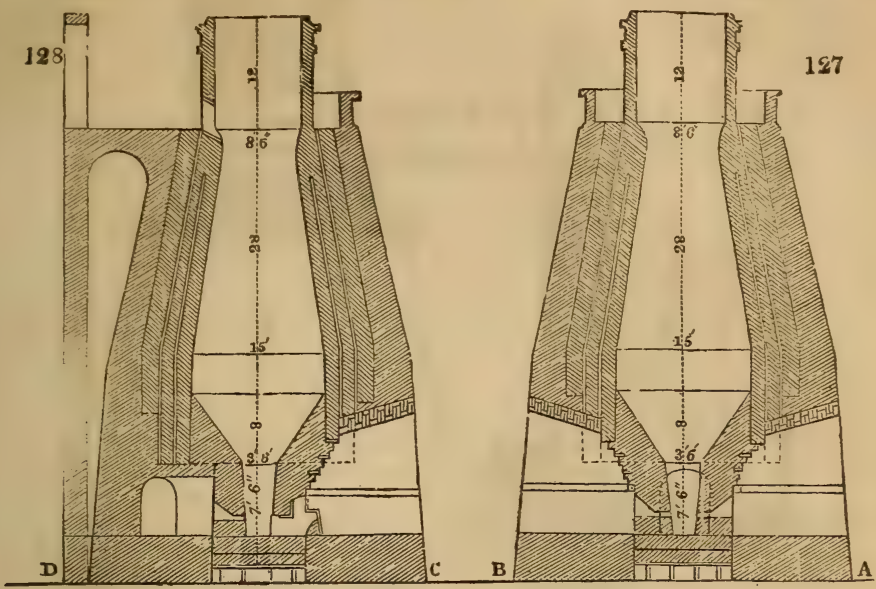

angular retreat filled up with fire-clay. Fig. 131 is a modern furnace of very large dimensions, as the numbers upon it show.
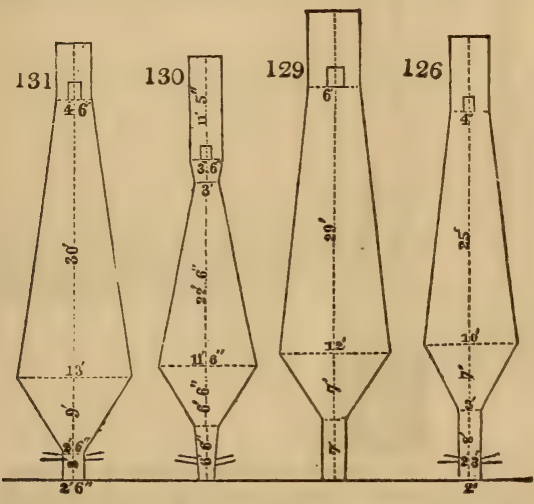

William Jessop, Esq., proprietor of the great iron works of Butterley and Codner Park in Derbyshire, has invented a very elegant and effective apparatus for feeding his blast furnaces with fuel, mine (calcined ironstone), and limestone in due proportions, and equally distributed round the inside of the furnace. Figs. 132, 133, represent this feed-apparatus. Fig. 132, shows at A, an outline of the furnace, and at B, the line of entrance into its throat. $\mathrm{C}$, is the feed mechanism. It consists of a long balance lever barrow, D, E; D, being an iron cylinder, open at top and bottom, 4 feet in diameter and $2 \frac{1}{2}$ feet in height, in the inside of which a hollow cone of iron is suspendea, with its apex uppermost, so that while the base of the cone is kept above the level of the bottom of the cylinder it shuts it; but on the cone being lowered below that level, it allows the charge of materials resting all round on the slant surface of the cone to fall down equally round the side of the cylinder into the furnace. In fig. 133 the barrow lever, $\mathrm{D}, \mathrm{E}$, is seen in profile or vertical section; $a$, is the fulcrum wheel, upon which the lever is in equilibrio when 9 cwt. of coals are put into the cylinder; then a weight is hung on, near the end, $\mathrm{E}$, of the lever, as an equipoise either to 9 or $12 \mathrm{cwt}$. of mine, according to circumstances; and next, a weight to balance one third of that weight of limestone. These weights of materials being introduced into the cylinder, while the barrow rests upon a level with the line $\mathrm{E} \mathbf{D}$, it is then rolled forward into its place, as shown in the figure, upon the wheels, $b, b$, upon a platform sustained on the top of an 
inverted cylinder within the cast-iron column, into which cylinder air is admitted (through a valve opened by the workman) from the furnace blast, the air passing up the

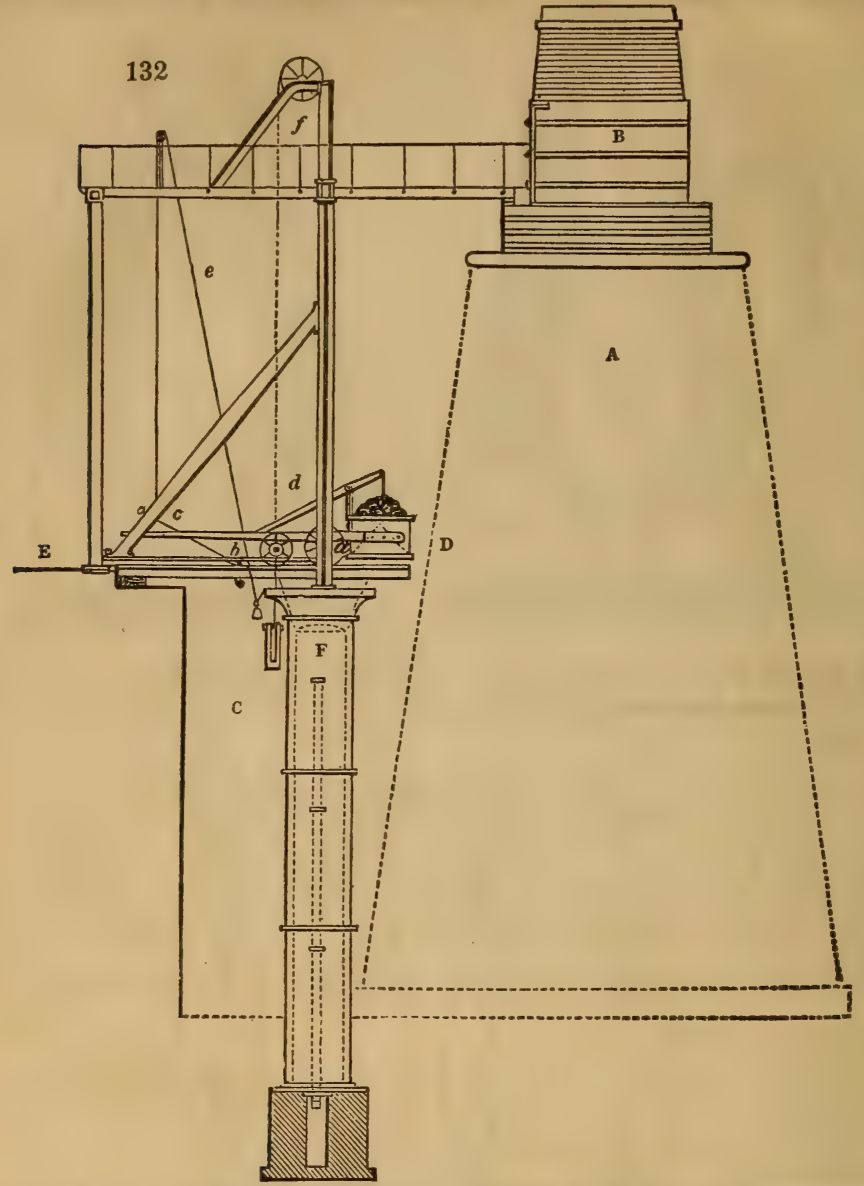

tube seen in the axis of F. The inverted air-cylinder is $3 \frac{1}{2}$ feet in diameter, 36 feet long, and rises 25 feet; being made air-tight with water, it ascends in its columnar case,

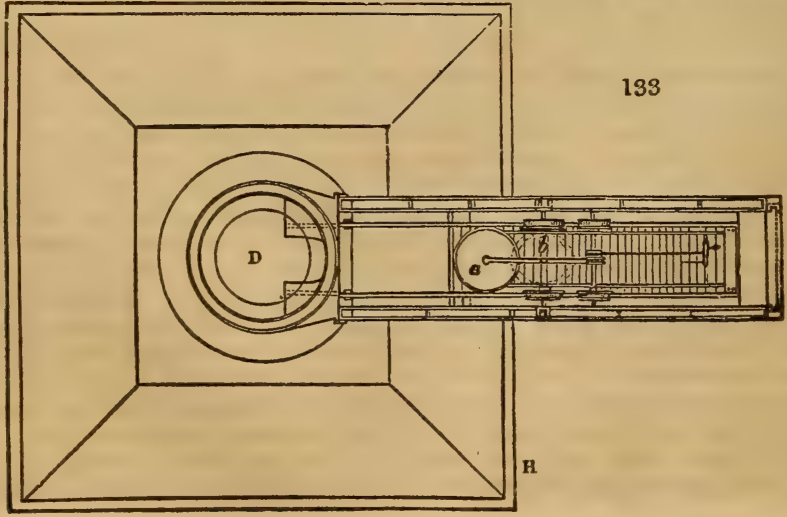


which is 4 feet in diameter, without friction. The space, G H, fig. 133, is 36 feet square.

The iron cone, which serves as a valve to the charging-drum or cylinder, is raised and lowered by means of a chain passing round a worm-wheel, which is turned round by an endless screw, acted upon by the long rod at $c$, which the workman can move by hand at pleasure, thereby lowering or raising the end of the short lever, $d$, to which the valve cone is suspended. The cord by which the workman opens or shuts the air piston-valve is seen at $e, f$. I have viewed with much pleasure the precise and easy movements of this feed-apparatus, at an excellent blast furnace in Codner Park iron works.

SMOKE PREVENTION. Among the fifty several inventions which have been patented for effecting this purpose, with regard to steam-boiler and other large furnaces, very few are sufficiently economical or effective. The first person who investigated this subject in a truly philosophical manner was Mr. Charles Wye Williams, managing director of the Dublin and Liverpool Steam Navigation company, and he also has had the merit of constructing many furnaces both for marine and land steam-engines, which thoroughly prevent the production of smoke, with increased energy of combustion, and a more or less considerable saving of fuel, according to the care of the stoker. The specific invention, for which he obtained a patent in 1840 , consists in the introduction of a proper quantity of atmospheric air to the bridges and flame-beds of the furnaces, through a great number of small orifices, connected with a common pipe or canal, whose aita can be increased or diminished, according as the circumstances of complete combustion may require, by means of an external valve. The operation of air thus entering in small jets into the half-burned hydro-carburetted gases over the fires, and in the first flue, is their perfect oxygenation, the development of all the heat which that can produce, and the entire prevention of smoke. One of the many ingenious methods in which Mr. Williams has carried out the principle of what he justly calls his Argand furnace, is represented in fig. 134, where $a$ is the ash-pit of a steam boiler furnace; $b$, is the

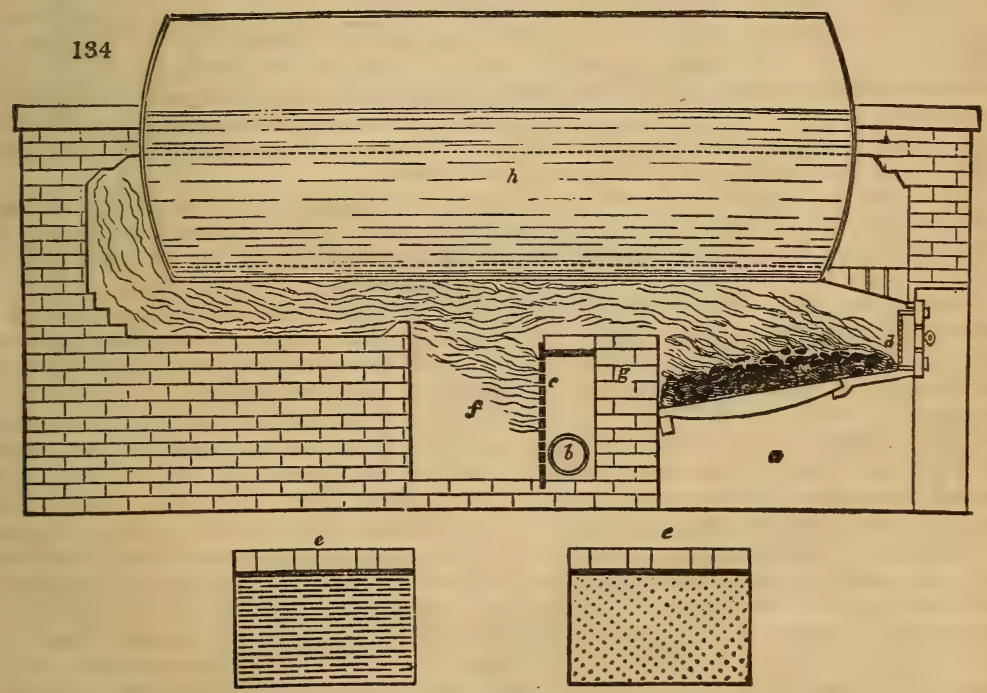

mouth of a tube which admits the external air into the chamber or iron box of distribution, $c$, placed immediately beyond the fire-bridge, $g$, and before the diffusion or mixing chamber, $f$. The front of the box is preforated either with round or oblong orifices, as shown in the two small figures $e$, $e$ beneath $f i g .134 ; d$, is the fire-door, which may have its fire-brick lining also perforated. In some cases, the fire-door projects in front, and it, as well as the sides and arched top of the fireplace, are constructed of perforated fire-tiles, enclosed in common brickwork, with an intermediate space, into which the air may be admitted in regulated quantity through a moveable valve in the door. I have seen a fireplace of this latter construction performing admirably, without smoke, with an economy of one seventh of the coals formerly consumed in producing a like amount of steam from an ordinary furnace; $h$ is the steam boiler.

Very ample evidence was presented last session to the Smoke Prevention committee of the house of commons of the successful application of Mr. Williams's patent inven- 
tion to many furnaces of the largest dimensions, more especially by Mr. Henry Houldsworth, of Manchester, who, mounting in the first flue a pyrometrical rod, which acted on an external dial index, succeeded in observing every variation of temperature, produced by varying the introduction of the air-jets into the mass of ignited gases passing out of the furnace. He thereby demonstrated, that 20 per cent. more heat could be easily obtained from the fuel, when Mr. Williams's plan was in operation, that when the fire was left to burn in the usual way, and with the production of the usual volumes of smoke. It is to be hoped, that a law will be enacted in the next session of parliament for the suppression, or at least abatement, of this nuisance, which so greatly disfigures and pollutes many parts of London, as well as all our manufacturing towns. while it acts injuriously on animal and vegetable life. Much praise is due to $\mathbf{M r}$ Williams for his indefatigable and disinterested labors in this difficult enterprise, and for his forbearance under much unmerited obloquy from narrow-minded prejudice and indocile ignorance.

SOAP. Several contrivances upon this subject have bustled over the patent stage within these few years ; such as Mr. Dunn's for making soap rapidly at a temperature of $310^{\circ} \mathrm{Fahr}$. under high steam pressure, by which many credulous shareholders were gulled into a belief that they would realize by this joint-stock project 200,000l. per annum. The soap so made was merely swelled in size and weight, by being surcharged with water, so that in a few weeks, the bars of it shrunk, rent, and twisted into mere skeletons; and being in this plight, returned to the company by their customers, caused that large soap bubble to burst.

Mr. Sheridan's silica soap had a somewhat longer career, but is now also nearly consigned to oblivion. C'austicity and abrasiveness were the chief characteristics, resulting from the mixture of a strong solution of silicate of soda, or liquor of flints, with soap made in the common way.

The invention for which Dr. Normandy obtained a patent merits a better fate. When yellow soap is made with the cheaper kinds of fat, it will hardly acquire a suffcient degree of firmness or hardness to satisfy the thrifty washerwoman. It melts away too rapidly in the hot water; a defect which may be well remedied by the introduction into the soap of a little fused sulphate of soda; and the salt concreting gives the soap a desirable hardness, while it improves its color, and renders it a more economical article for the washing-tub. In a trial recently before the court of common pleas, it was proved that the soap made according to Dr. Normandy's patent was worth fully $2 l$. a ton more than the original soap, without the sulphate of soda.

Mr. Dunn has recently obtained a patent for accelerating the process of soap-making; he promotes the combination of the alkali, fat, and water, by pumping streams of atmospheric air through the saponaceous materials, while exposed to the usual heat in the pan. This scheme is said to effect its purpose, and to save much time.

SODA. On the 30th of June, 1838, Messrs. Dyar and Hemmings obtained a patent for manufacturing soda by the decomposition of sea salt with sesqui-carbonate or bicarbonate of ammonia. Equal parts of the chloride of sodium and sesqui-carbonate are prescribed, being very nearly the equivalent decomposing proportions, and the ammonia salt is recommended to be added in powder to a saturated solution of the sea salt, and the mixture to be stirred and then set aside till the mutual action and decomposition be effected. Having been employed to examine this process for a gentleman who wished to adopt it upon a manufacturing scale, I obtained the following results: On making the prescribed mixture in the cold, brisk effervescence takes place, because the quantity of carbonic acid combined with the ammonia is greater than the resulting soda can readily absorb, even to form its bicarbonate, and this extrication of gas carries off with it more or less ammonia, amounting, in carefully conducted experiments, to no less than 27 per cent. of the sesqui-carbonate employed; though the magma deposited from the mixture was drained in vessels nearly close, and though the ammonia which adhered to it, as well as that in the drained mother liquors, was recovered by distillation in vessels connected with a Woulfe's apparatus. Moreover, the utmost amount of soda-ash (not pure carbonate) which was obtained, was only 37.5 for 100 of sea salt used, whereas 90 of carbonate should result from 100 of the sea salt, with the above equivalent dose of sesqui-carbonate of ammonia. This latter salt contains about one half more carbonic acid than is required by the soda to become a carbonate. A good illustration of the loss of ammonia in a similar case is afforded by the decomposition of chloride of calcium in solution, by adding to it the equivalent dose of pulverized ammonia carhonate; viz., 56 of the former and 59 of the latter. The rapid extrication of the carbonic acid on making this mixture, causes such a waste of ammonia, that more of the sesqui-carbonate must be afterward introduced, to complete the decomposition of the chloride; the stronger the solution of the chloride the greater is the loss of ammonia. 
In one of my experiments where were employed 3500 grains $=$ half a pound avoir duposs, of each ingredient, the following were the products :-

1. Ammonia recovered by distillation from the drained magma, Grains. equivalent in sesqui-carbonate to - _ . . . -

2. Ammonia as carbonate, from the remaining liquid, sucked into a

vacuous apparatus and distilled
3. Additional ammonia as carbonate, obtained from the cold mother liquors, by distillation with quicklime, and out of the sal ammoniac formed

Sesqui carbonate employed

or 27.4 per cent.

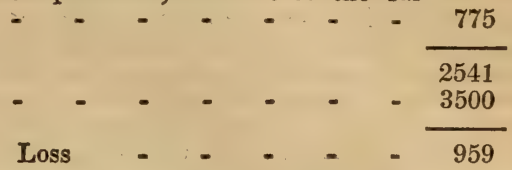

The product from this experiment in dry soda ash was only 1500 grains, which were

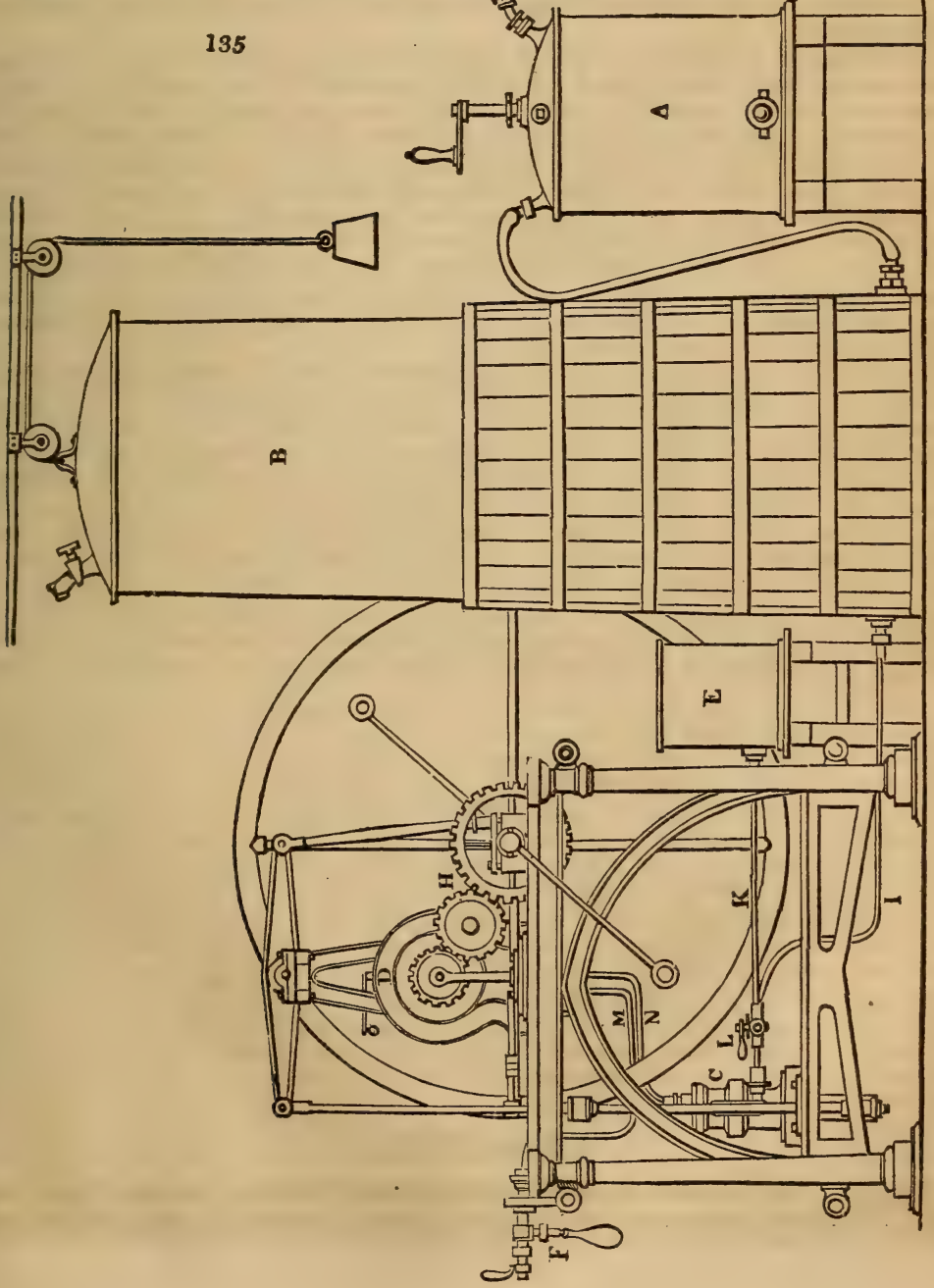


found to contain only 1,312 of pure carbonate, or $87 \cdot 5$ per cent. of the whole. Here is a deficiency of soda carbonate, upon the quantity of the chloride used, of no less than $58 \frac{1}{3}$ per cent., for only 1,312 grains are obtained instead of 3,150 .

Subsequently a method occured to me, whereby this process, elegant in a scientifie point of view, might possibly be executed with advantage upon the commercial scale; but it would require a very peculiar apparatus, though not nearly so costly as what was erected by Mr. Cooper under the direction of the patentees at Battersea, and in Brussels.

SODA-WATER. At page 21 of vol. x. of the conjoined series of Newton's Journal, the patent apparatus of Mr. F. C. Bakewell, of Hampstead, for making sodawater, is well described with illustrative figures. The patent was obtained in March, 1832 , but how far it has been introduced into practice I have not heard. Its arrangement discovers ingenuity, but it seems less likely to prove durable than the patent apparatus of Mr. Tyler, which fig. 135 in the preceding page represents according to his latest specification. A, is the gas generator, where the chalk and sulphuric acid are mixed; $\mathrm{B}$, the gasometer; $\mathrm{C}$, the soda-water pump, for forcing in the gas; $\mathrm{D}$, the condenser; $\mathrm{E}$, the solution (of soda) pan; F, the bottling cork; G, the acid bottle, at the right hand shoulder of $\mathrm{A}$; $\mathrm{H}$, the wheels, for working the agitator in the condenser; $\mathrm{I}$, the pipe, for conveying the gas to the pump; $\mathrm{K}$, pipe for conveying the solution to the pump; L, cocks for regulating the admission of the gas into the solution; $M$, drawing-off pipe leading to the bottling cork; $N$, the forcing pipe from the pump to the condenser.

The vessel in which the soda-water is condensed is lined with silver in order to resist corrosion.

SOLDERING OF LEAD, and other melals, is called by its inventor M. de Richemont, autogenous, because it takes place by the fusion of the two edges of the metals themselves, without interposing another metallic alloy, as a bond of union. He effects this purpose, by directing a jet of burning hydrogen gas, from a small movable beak, upon the two surfaces or edges to be soldered together. Metals thus joined are much less apt to crack asunder at the line of union, by differences of temperature, flexure, \&c., than when the common soldering processes are employed. The fusing together the edges of lead sheets, for making sulphuric acid chambers, has been long practised in this country, but it was performed by pouring some of the melted metal along the line of junction, and afterward removing its excess by means of a plumber's soldering iron. The method of M. Richemont is a great improvement upon that old practice. It is much quicker and more convenient.

SPINNING. The greatest improvement hitherto made in forming textile fabrics, since the era of Arkwright, is due to Mr. G. Bodmer, of Manchester. By his patent inventions the several organs of a spinning factory are united in one self-acting and self-supplying body - a system most truly automatic. His most comprehensive patent was obtained in 1824, and was prolonged by the Judicial Committee of the Privy Council, for 7 years after the period of 14 years was expired. It contained the first development of a plan by which fibres of cotton, flax, \&c., were lapped and unlapped through all the operations of cleaning and blowing, carding, drawing, roving, and spinning; in the latter, however, only as far as the operation of feeding is concerned. The lapping from the blower was then not new, but the lapping directly and in connexion with the carding engines was his invention, and was brought by him into operation at St. Blaize in the Black Forest, several years before he took out his patent

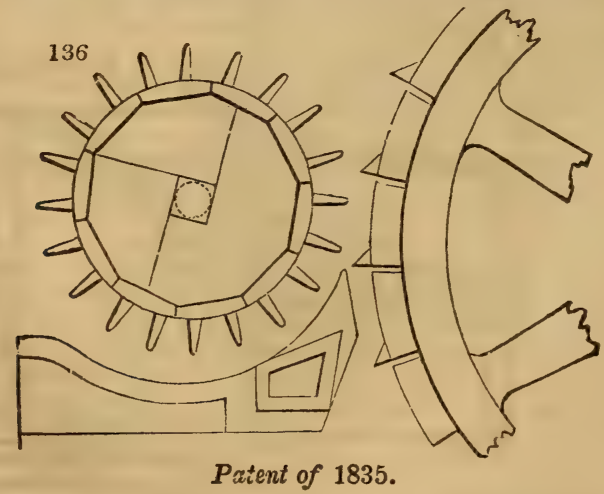

in Eingland. The method applied through all the following operations was then new. Mr. Dyer's and several other patents granted subsequently were decided and 
acknowledged infringements. The patent of 1824 was the beginning; the result of which was the several patents for improvements in $1835,1837,1838$, and 1842 , of Mr. Bodmer.

By a machine generally called a Devil or Opener ("Wolf," in German), which consists of a feeding-plate set with teeth and a roller covered with spikes (see fig. 136), the cotton is cleared from its heaviest dirt and opened. This machine delivers the cotton into a room or on to a travelling cloth, from which it is taken, weighed in certain portions, and spread upon cloth in equal portions : this is then rolled up, and placed behind the first blower.

The first blower has a feeding-plate like fig. 137, without teeth, and over this plate the cotton is delivered to the operation of the common beaters, from which it is received

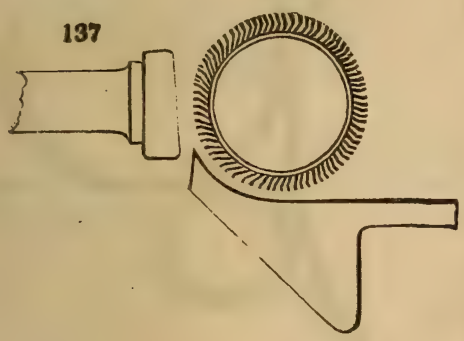

Patent of 1835.

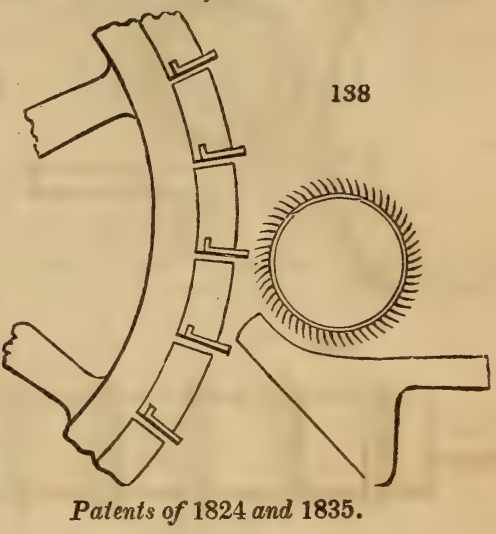

Patents of 1824 and 1835.

into a narrow compartment of $4 \frac{1}{2}$ or 5 inches broad, and wound, by means of his lapmachines, upon rollers in beautifully level and well-cleaned laps. Eight of these narrow laps are then placed behind a second blower, of a similar construction to the first. Instead of the common beater, however, a drum with tooth.ed straight edges is used (see $f \mathrm{~g} .138$ ), which opens the cotton still more, and separates the fibres from one another. The cotton is again formed into similar narrow laps, which are still more equal than the preceding ones, and eight of these laps are then placed behind the carding engines. It was only by applying his lap-machine, patented in 1842 , that he succeeded in forming small laps on the blower; without this he could not perform the doffing of the laps without stopping the wire-cloth, and in doing this, an irregular lap would be formed because of the accumulating of the falling cotton in one place while the wirecloth was standing.

Carding Engine.-His patent of 1824 showed a mode of coupling a number of carding engines, the product of which was delivered upon an endless belt or a trough, and at the end of this trough was wound upon a roller. This arrangement wants no description, as it is generally known. I have seen it in use on the Continent.

When a set of cards work together, any interruption or stoppage of a single carding engine causes a defect in the produce of the whole lap. Interruption occurred several times a day by the stripping of the main cylinder, and during this operation the missing band or sliver was supplied out of a can, being the produce of a single earding engine working into cans (a spare card). The more objectionable defect was, however, the difference of the product of the carding engine after the main cylinder had been stripped; the band or sliver from it will be thin and light until the cards of the main cylinder are again sufficiently filled with cotton, when the band will again assume its proper thickness. Another irregularity was caused by the stripping of the flats or top crris. but was not so fatal as the first one. These lefects were of course a serious arawoack in his system of working, the latter of which he provided against in his first patent by stripping the top cards by mechanism; the former, however, was only conquered by $r$ is invention of the self-strippers for the main cylinders; thus the carding engine may now work from Monday morning till Saturday night without interruption, the cylinders requiring only to be brushed out every evening; the consequence is, that much time is gained, and a very equal, clean, and clear product is obtained. Old carding engines to which he applied his feeders (see fig. 139), and main eylinder-clearers produce much superior work, and increase the production from 18 to 24 per cent.

The main cylinder-clearer consists of a very light cast iron cylinder upon which 
five, six, or more sets of wire brushes are fixed, which are caused to travel to and fre across the main cylinder; the surface or periphery of the brushes overrunning the surface or periphery of the main cylinder by 8 or 10 per cent., the brushes thus lifting the cotton out of the teeth of the cards of the main cylinder, and causing the dirt and lumps to fall.

As the brushes are not above a quarter-inch in breadth, and travel to and fro, it is clear that no irregularity can take place in the fleece which comes from the doffer; not more than 140 th part of the breadth of the cylinder being acted upon at the same time. Figs. 140, and 141, give an idea of the clearer : the mechanism within the clearer,

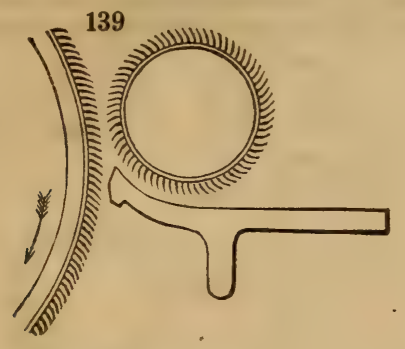

140

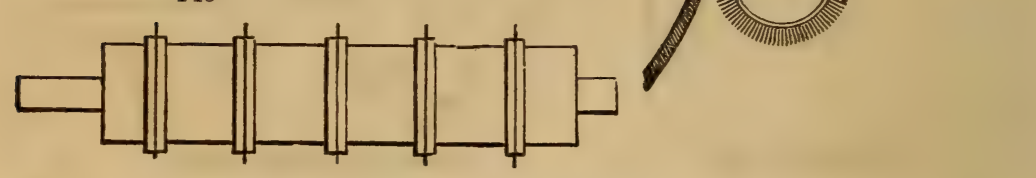

141

Patents of 1838 and 1842.

and by which the brushes, $a$, are caused to travel, is simple and solid. The main cylinders for the carding engines are made of cast iron, the two sets of arms and rim are cast in the same piece; when complete, they weigh $50 \mathrm{lbs}$. less than those made of wood.

The new lap machine connected with these engines is almost self-acting; a girl has only to turn a crank when the lap is full; by this turn, the full lap is removed and an empty roller put in its place, the band of cotton is cut, and no waste is made.

Drawing Frame.-The drawing frame of 1824 was improved, and the improvements patented, in 1835, and others again in 1842. That of 1824 is known in Germany and France, and generally in use. The laps from the carding engine lap-machine are put upon delivering rollers, behind a set of drawing rollers, and from them delivered upon a belt or trough, and again formed into laps similar to those from the carding engines. The next operation formed the laps into untwisted rovings, and the next again into smaller untwisted rovings, or rovings with false twist in them, as infringed upon by Dyer. The false twist was rather objectionable, and in his patent of 1835 he put a number of rovings on the same bobbin, with left and right permanent twist in them. This does very well; there is, however, a little objection to that place in which the twist changes from right to left when it comes to the last operation before spinning. In his patent of 1838 , and particularly in that of 1842 , he confined the left and righthand twist to the drawing frame, when he converts two laps into one roving, and forms a roller or bobbin of 14 inches diameter and 15 inches broad, with six separate and twisted rovings wound upon it. (See figs. 142 and 143.) The twist is given by tubes in two directions, so that it remains in it (see fig. 143), the tube turns in the same direction, while the roving advances 4 or 5 inches, and then turns in the other direction. These laps or bobbins are then placed behind a machine, which he calls a coil-frame, the most important arrangement of which he claimed already in his patent of 1835 . It consists of a slot with a travelling spout, without which the coils can not be formed under pressure. Coiling in cotton can not be claimed, as it was done in the first system of cotton spinning.

Coil Frame.-The bobbins (fig. 142), are placed behind this machine, and two ends from the bobbin are passed through the drawing rollers and formed into one untwisted sliver or roving in the following manner: When the cotton has passed through the drawing rollers (see fig. 144), and calender rollers, A, it is passed through the tube, B, and the finger, $C$; the spindle with its disc, $D$, revolves in such a proportion as to take 
up the cotton which proceeds from the calender rollers, A, and cause the roving 10 be laid down in a spiral line closely one by one, and as the rollers, A, work at a regular
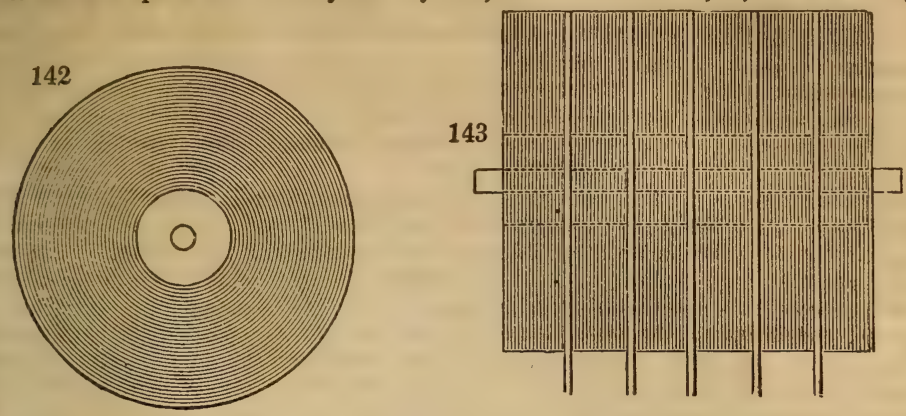

Patents of 1835,1838 , and 1842 ,

speed, it is evident that the motion of the finger, $\mathrm{C}$, and the speed of the tube, B, must vary accordingly. The coil, $\mathbf{E}$, is stationary, and is pressed by the lid or top, $\mathbf{F}$, which
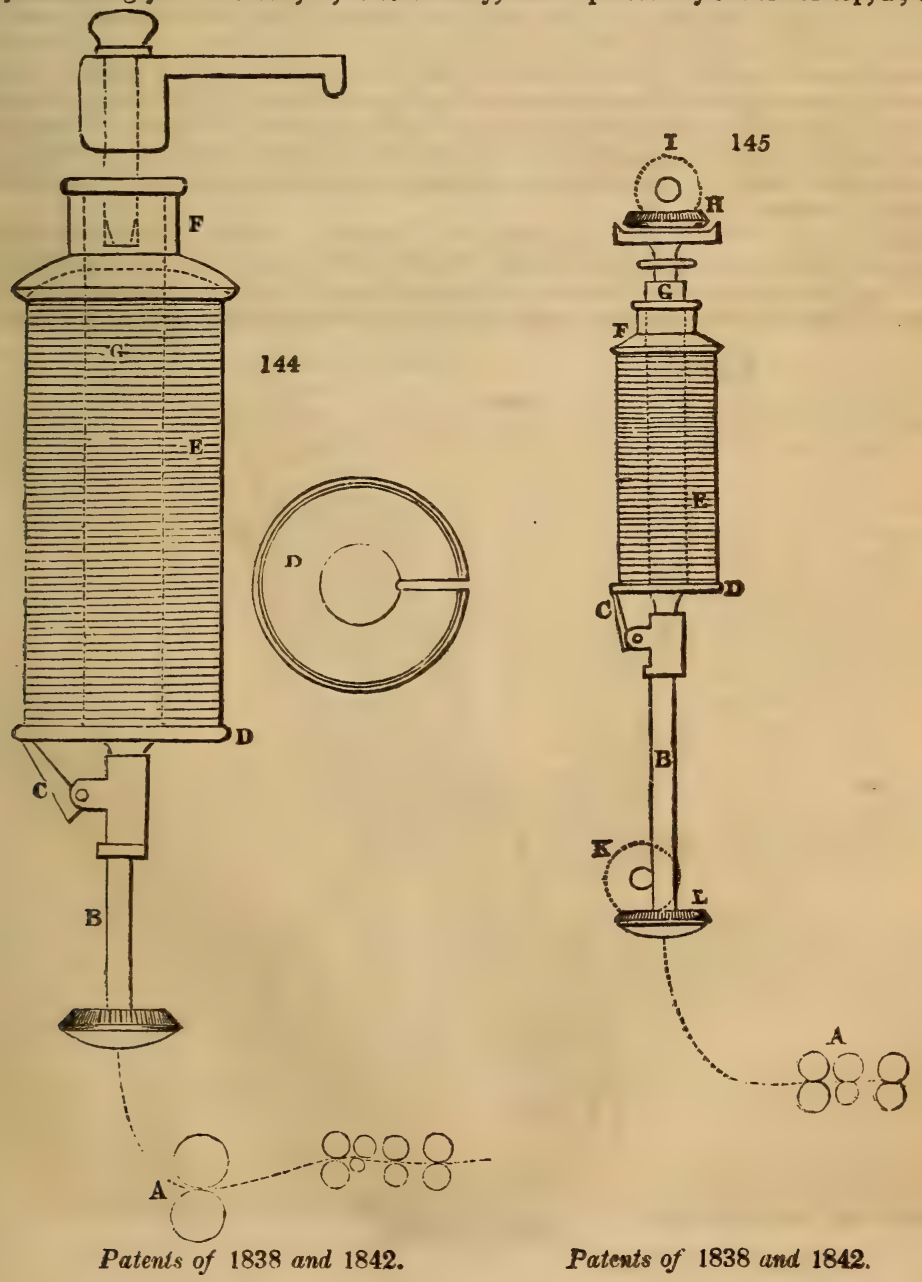
slides up the spindle, $G$, made of tin plate. The cotton enters, through the slot, $\mathbf{X}$, in fig. D. It is quite evident that the finger, $\mathbf{C}$, and spindle, $\mathbf{G}$, only perform one and the same varying motion, which is repeated at every fresh layer, and the coil is thus built from below; it is about 8 inches in diameter and 18 inches high when compressed, and contains $4 \frac{1}{2} \mathrm{lbs}$. of cotton. Mr. Bodmer has several modes of forming these coils, but one only is shown here. These coils are placed behind the twist coil frames in half cans or partly open ones or troughs, or behind a winding machine, where they are wound upon rollers side by side, like the lap or bobbin shown in the drawing frame, and placed behind the twist coil frame in this state.

Tuist Coil Frume. - This frame forms rovings into coils similar to those above explained, with this difference, that the rovings are fine, say, from 1 to 10 hanks per pound, and regularly twisted : their diameter varies from $2 \frac{1}{2}$ to 5 inches. The same machine produces rovings more or less fine, but the diameter of the coils does not differ. The difference of this machine from that above described consists in the dimensions of their parts, and in its having the spindle, G, and the lid or top, F, revolving, as well as the tube, B. (See fig. 145.) In this machine the motion of the spindle, B, is uniform: the spindle, $\mathrm{G}$, however, is connected by the bevel wheels, $\mathrm{H}$ and $\mathrm{l}$, with a differential motion at the end of the frame, with which the motion of the finger $\mathrm{C}$, corresponds. The skew wheels, $\mathrm{K}$ and $\mathrm{L}$, are connected with the drawing rollers, A. The speeds of the tube, $B$, and the spindle, $G$, are 80 proportioned, that while the spindle, $G$, performs one revolution, and therefore puts one twist into the roving, the tube, $B$, also performs one revolution, missing so much as will be required to pass through the slot in the cap or disc, D, and lay on it as much of the roving as proceeds from the rollers, A, and in which one twist is contained. Of course the twist of these rovings can be adapted to their fineness and varied; but it is evident that, on account of the regularity of the machine and its simplicity of movement, the rovings can never be stretched, and much less twist can be put into them than can be put in the common fly frames. These coils are put behind the spinning machines on shelves or in small cans, open in front; or they are wound from 24 to 72 ends upon bobbins, and placed upon unlap rollers behind the spinning frames.

Coiling Machine for Carding Engines and Drawing Frames.-These are simple machines, which may be applied to carding engines or drawing frames of any description. They form large coils, 9 inches in diameter and 22 inches long, when on the machire. There are two spindles, $a$ (see fig. 146), on each machine, for the purpose of

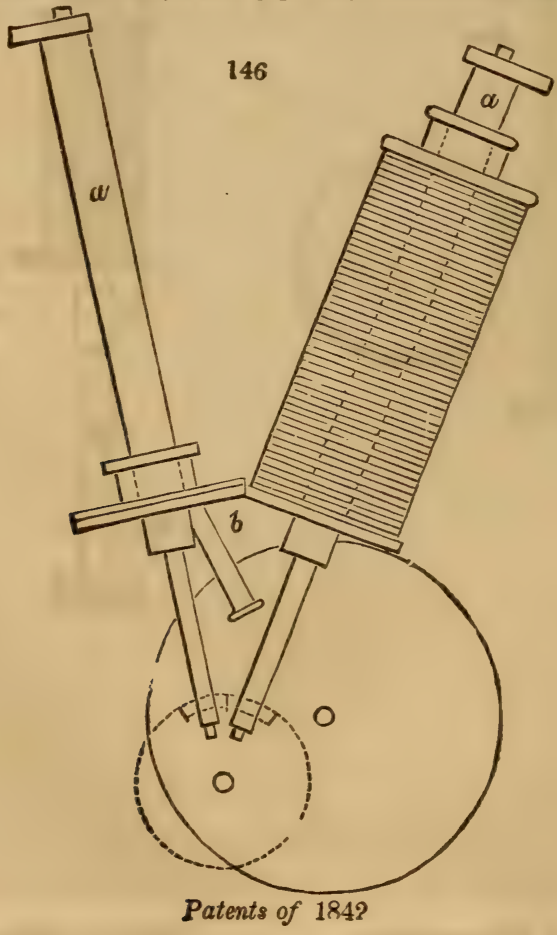


doffing without stopping the drawing frame or carding engines. When one coil is filled, the finger, $b$, is just brought over to the other spindle, so that the full coil is stopped, and the new one begins to be formed without the slightest interruption of the machine.

Mr. B. forms coils in various ways, also in cans; but this description is sufficient to show the application of this mode of winding up bands or rovings. Several of the abovedescribed machines are adopted with equal success to wool and flax. In his patents of 1835,1837 , and 1838 , he shows several modes of applying his system to cotton and other machinery. He winds directly from the carding engines the slivers separately upon long bobbins, and he gives them twist in two directions, for the purpose of uniting the fibres to some extent, so that they not only come off the bobbins without sticking to one another, but also that they may draw smoother. He also showed a machine, by which several rovings, say 4 or more, are put upon the same bobbin with conical ends; these bobbins are placed behind the mules or throstles, and are unwound by a belt or strap running parallel with the fluted rollers of the spinning machine, as seen in fig. 147 . The belt or band, A, is worked in a similar way to that described in his former patent, and the bobbins, B, rest upon and revolve upon their surface, exactly according to the speed of the belt. It is quite evident that the whole set of rovings must be unwound exactly at the same speed, and that no stretching can take place. He can put real and reversed twist in these rovings as well as false twist only. The most important feature in the roving machine is a metal plate, in which a slot is formed through which the rovings pass; this slot is seen in figs.148, 149, and 150 . The cotton, when cuning from
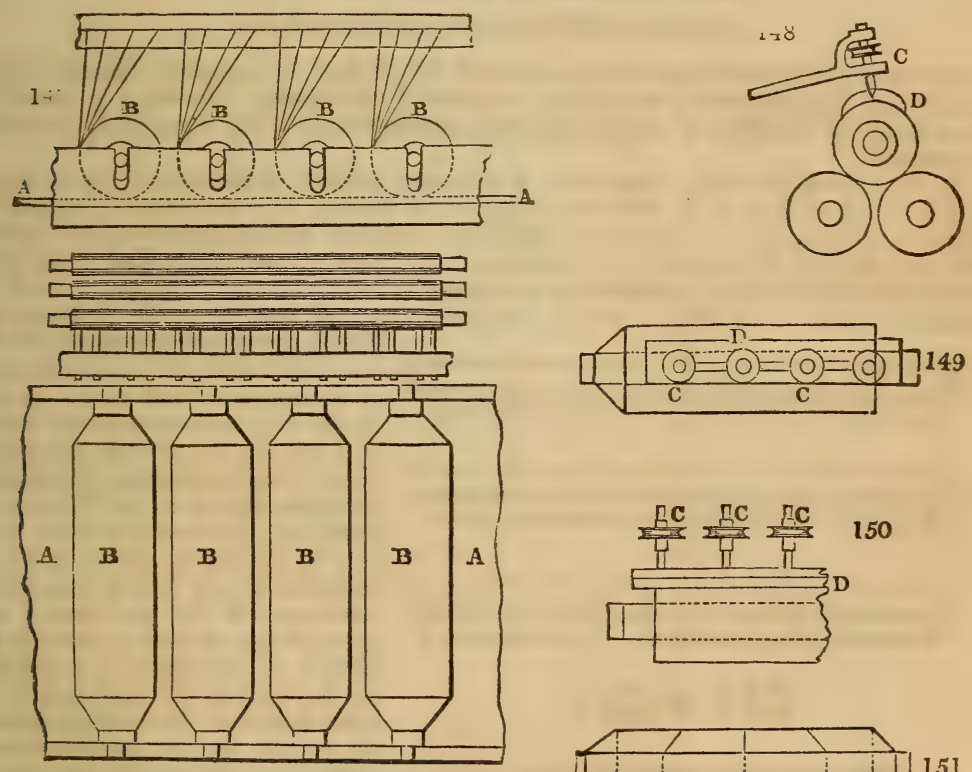

Patent of 1835.
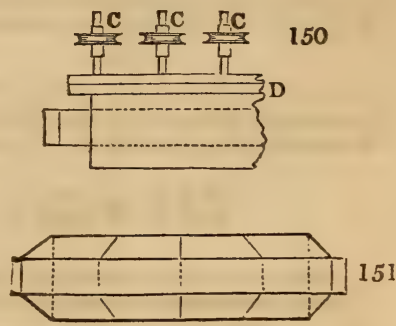

the drawing rollers, is passed through the twisters, C, and through the slot in the plate, $\mathbf{D}$. Thus he is enabled to put any convenient number of neatly formed and perfectly separate coils upon the wooden barrel or bobbin. The bobbin formed upon these machines is represented in fig. 151, and the conical ends are formed by a mechanism, by which the twisters, C, are caused to approach a little more to one another, after each layer of rovings has been coiled round the barrel: the section of the bobbin is therefore like that shown in fig. 151. He makes use of exactly the same arrangement, viz., a finger travelling along a slot in a plate, for the purpose of forming the coils, which has been already described.

Rovings wound upon bobbins by means of tubes revolving in one direction, are certainly not so fit for spinning as rovings into which a small degree of twist is put. The tube by which a twist is put in on one side and taken out at the other, curls or ruffes 
the cotton, and causes it to spread out as it passes between the rollers, while rovings with a little permanent twist in them are held together in the process of drawing, and thus produce smooth yarn. To remedy the evil above described, when untwisted rovings are used, he causes the spouts or guides, through which the rovings pass into or between the drawing rollers, to revolve slowly first in one, and then in the other direction, and thus puts a certain quantity of twist into the rovings while they are being prepared for spinning. Two modes of performing this operation are clearly described in his patent of 1835 .

There is a little defect in the working of the rovings with reversed twist when too much or too little twist is put in them, or when the winding machine is not kept in good order. This defect proceeds from the change in the twist of the roving seen at $A$, fig. 152; in this place the twist is not like that at B, and it would, in some parts of the 152

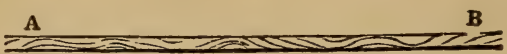

yarn, be detected under circumstances just described. In cases where double rovings are used, the twisters are so arranged as to put the twist in the rovings, as shown in fig. 153 : in this case the reversing place of one roving meets the twisted place of the other, and the fault is completely rectified.

153

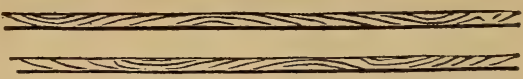

The preceding description given an idea of Mr. Bodmer's admirable system of pre paring and spinning cotton, wool, flax, \&c., and of the several processes ; it would be superfluous to describe the several machines, or the details of the same, as exhibited in his patents.

In his patent of 1838 , he specifies a self-actor, namely, a machine in itself, which can he attached to 2,3 , or even 4 mules of almost any convenient number of spindles. The mules are previously stripped of all their mechanism except the rollers and their wheels, the carriage and spindles; all the other movements ordinarily combined with the mule are contained in the machine, which is placed between a set of mules, as seen in fig. 154; $a$ and $b$, the self-actors, to each of which 3 mules are

154
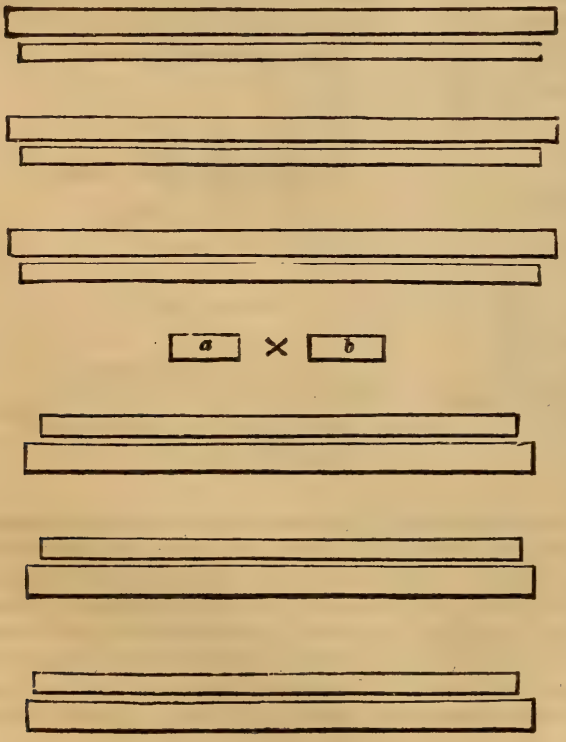
as it is necessary to have a girl of a certain age to watch over the piecers for a certain number of mules, he preferred the simplified machine; placing the girl near these machines, from whence the whole set of mules attached to the same can be overlooked onds and shafts with the self-actor, or rather partly self-actor. A girl of fifteen or sixteen years old stands at $X$ between $a$ and $b$, and never leaves her place except, perhaps, for aiding in doffing or in banding the spindles. The gearing of the room acts by means of straps upon the machines $a$ and $b$, and from these machines all the movements are given to the six mules, namely, the motion of the rollers, the spindles, the drawing out of the carriage, the after draft, \&c. When the carriages are to be put up, the girl takes hold of two levers of the machine $a$, and by moving them in certain proportions, acts upon two cones and pulleys, and thus causes, in the most easy and certain manner, the carriages to run in and the yarn to be wound on the spindles. The first machine Mr. B. made for this pur pose was completely self-acting, but he found very soon that the mechanism was more complicated and apt to go out of order than that of the 
as the creels behınd tne mules are not wanted in his system, this impediment to the sight of the girl would be removed. He schemed these machines for the purpose of altering, at a trifling expense, the common mules into self-actors; they are equally good for any numbers of yarn.

Bastard Frame.-In his patent of 1838 and 1842, we find the description of a very simple bastard frame, namely, a throstle with mule spindles, forming cops, as seen in fig. 155, and wound so hard that they can be handled about without any danger of 155 spoiling them; in the same dimensions they contain one third more yarn than the best cops of self-actors. The machine is extremely simple; but owing to some circumstances in the construction of the winders and plates, he has not been able to spin advantageously upon large machines above No. 20's. $\mathrm{He}$ has spun on it No. 56, and most beautiful yarn. The quantity this machinery produces is nearly one third more than the best self-actor, on an equal number of spindles, and the yarn and cops are much superior. Of course there is a copping motion connected with the machine: the winding, however, is continuous, as well

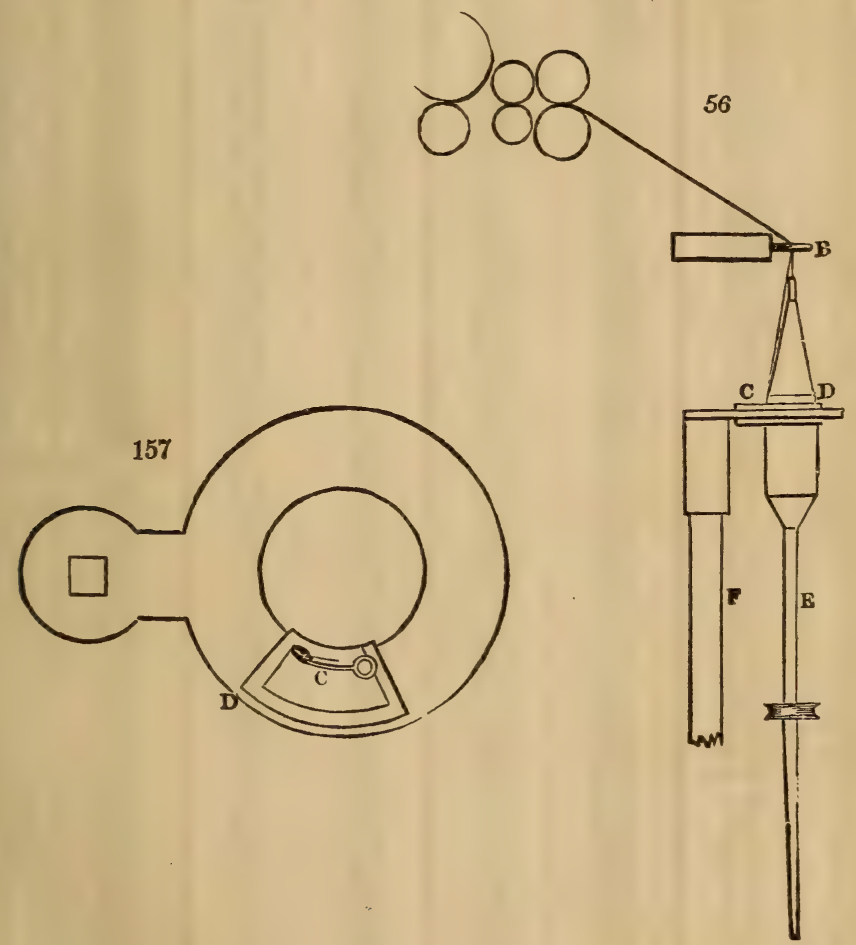

Patents of 1838, and 1842.

as the twisting, and figs. 156 and 157, will give the reader an idea of the frame. The yarn coming from the rollers, A, goes through an eye, $\mathbf{B}$, to the wire, $\mathbf{C}$, fixed in the flyer, D, and from thence on to the mule spindle, E: as the spindle revolves the flyer is dragged along, and by its centrifugal power winds the yarn tight upon the spindles. 
BPIRITS. Correspondence between Specific Gravity and per Cents. over Proof at $60^{\circ}$ is

\begin{tabular}{|c|c|c|c|c|c|c|c|}
\hline $\begin{array}{l}\text { Specific } \\
\text { Gravity. }\end{array}$ & \begin{tabular}{|} 
Per Cent. \\
Over Proof.
\end{tabular} & $\begin{array}{l}\text { Specific } \\
\text { Gravity. }\end{array}$ & $\mid \begin{array}{c}\text { Per Cent. } \\
\text { Over Proof. }\end{array}$ & $\begin{array}{l}\text { Specific } \\
\text { Gravity. }\end{array}$ & $\mid \begin{array}{c}\text { Per Cent. } \\
\text { Over Proof. }\end{array}$ & $\begin{array}{l}\text { Specific } \\
\text { Gravity. }\end{array}$ & \begin{tabular}{|c|} 
Per Cent. \\
Over Proof.
\end{tabular} \\
\hline 0.8856 & $67 \cdot 0$ & $\cdot 8455$ & $51 \cdot 7$ & .8748 & 33.4 & $\cdot 9056$ & $11 \cdot 4$ \\
\hline .8160 & 66.8 & $\cdot 8459$ & 51.5 & .8751 & 332 & $\cdot 9060$ & $11 \cdot 1$ \\
\hline$\cdot 8163$ & $66 \cdot 6$ & $\cdot 8462$ & $51 \cdot 3$ & .8755 & 32.9 & .9064 & 10.8 \\
\hline 8167 & $66 \cdot 5$ & $\cdot 8465$ & $51 \cdot 1$ & .8758 & $32 \cdot 7$ & .9067 & $10 \cdot 6$ \\
\hline $.81 \% 0$ & $66 \cdot 3$ & .8469 & 50.9 & 8762 & $32 \cdot 4$ & .9071 & $10 \cdot 3$ \\
\hline .8174 & $66 \cdot 1$ & $\cdot 8472$ & 50.7 & 8765 & $32 \cdot 2$ & .9075 & 10.0 \\
\hline 8178 & $65^{\circ} 6$ & $\cdot 8476$ & 50.5 & 8769 & $32 \cdot 0$ & •9079 & 9.7 \\
\hline$\cdot 8181$ & 65.8 & .8480 & $50^{\circ} 3$ & 8772 & $31 \cdot 7$ & .9082 & $9 \cdot 4$ \\
\hline .8185 & $65 \cdot 6$ & .8482 & 50.1 & .8776 & 31.5 & .9085 & $9 \cdot 2$ \\
\hline .8188 & 65.5 & $\cdot 8486$ & $49 \cdot 9$ & 8779 & $31 \cdot 2$ & .9089 & 89 \\
\hline .8192 & 65.3 & .8490 & 49.7 & .8783 & 31.0 & .9093 & 86 \\
\hline .8196 & 65.1 & .8493 & 49.5 & .8786 & 30.8 & .9097 & $8 \cdot 3$ \\
\hline$\cdot 8199$ & $65^{\circ} 0$ & 8496 & $49 \cdot 3$ & $8 \% 90$ & 30.5 & .9000 & 8.0 \\
\hline .8203 & 648 & $\cdot 8499$ & 49.1 & 8793 & 30.3 & .9104 & $7 \cdot 7$ \\
\hline$\cdot 8206$ & $64 \cdot 7$ & .8503 & $48 \cdot 9$ & 8797 & 30.0 & .9107 & $7 \cdot 4$ \\
\hline$\cdot 8210$ & $64 \cdot 5$ & 8506 & 487 & .8800 & $29 \cdot 8$ & •9111 & $7 \cdot 1$ \\
\hline .8214 & $64 \cdot 3$ & .8510 & $48 \cdot 5$ & $\cdot 8804$ & 29.5 & .9115 & $6 \cdot 8$ \\
\hline .8218 & $64 \cdot 1$ & .8513 & $48 \cdot 3$ & .8807 & $29 \cdot 3$ & •9118 & 6.5 \\
\hline .8221 & $64 \cdot 0$ & .8516 & 48.0 & 8811 & 29.0 & $\cdot 9122$ & $6 \cdot 2$ \\
\hline$\cdot 8224$ & 63.8 & $\cdot 8520$ & $47 \cdot 8$ & ·8814 & 28.8 & •9126 & 5.9 \\
\hline$\cdot 8227$ & 63.6 & $\cdot 8523$ & $47 \cdot 6$ & .8818 & 28.5 & .9130 & $5 \cdot 6$ \\
\hline .8231 & 63.4 & $\cdot 8527$ & $47 \cdot 4$ & .8822 & 283 & $\cdot 9134$ & $5 \cdot 3$ \\
\hline 8234 & $63 \cdot 2$ & $\cdot 8530$ & $47 \cdot 2$ & $\cdot 8825$ & $28 \cdot 0$ & .9137 & 5.0 \\
\hline$\cdot 8238$ & 63.1 & .8533 & $47 \cdot 0$ & $\cdot 8829$ & $27 \cdot 8$ & $\cdot 9141$ & 4.8 \\
\hline$\cdot 8242$ & $62 \cdot 9$ & .8537 & $46 \cdot 8$ & $\cdot 8832$ & $27 \cdot 5$ & .9145 & 4.5 \\
\hline .8245 & $62 \cdot 7$ & .8540 & 46.6 & 8836 & $27 \cdot 3$ & .9148 & $4 \cdot 2$ \\
\hline$\cdot 8249$ & 62.5 & .8543 & $46 \cdot 4$ & .8840 & $27^{\circ} 0$ & .9152 & 3.9 \\
\hline .8252 & $62 \cdot 3$ & .8547 & $46 \cdot 2$ & .8843 & 26.8 & .9156 & 3.6 \\
\hline .8256 & $62 \cdot 2$ & .8550 & 46.0 & .8847 & 26.5 & 9159 & $3 \cdot 3$ \\
\hline$\cdot 8259$ & 62.0 & $\cdot 8553$ & 45.8 & 8850 & $26 \cdot 3$ & .9163 & 3.0 \\
\hline$\cdot 8263$ & $61 \cdot 8$ & .8556 & $45 \cdot 6$ & 8854 & 26.0 & 9167 & $2 \cdot 7$ \\
\hline •8266 & 616 & $\cdot 8560$ & 45.4 & .8858 & 25.8 & $\cdot 9170$ & $2 \cdot 4$ \\
\hline .8270 & 61.4 & .8563 & $45 \cdot 2$ & .8861 & 255 & .9174 & $2 \cdot 1$ \\
\hline$\cdot 8273$ & $61 \cdot 3$ & $\cdot 8566$ & 45.0 & 8865 & $25 \cdot 3$ & .9178 & $1 \cdot 9$ \\
\hline 8277 & 61.1 & .8570 & $44 \cdot 8$ & 8869 & 250 & .9182 & 1.6 \\
\hline$\cdot 8280$ & 609 & .8573 & $44 \cdot 6$ & .8872 & $24 \cdot 8$ & .9185 & $1 \cdot 3$ \\
\hline •8284 & 60.7 & $\cdot 8577$ & 44.4 & 8876 & $24 \cdot 5$ & .9189 & 1.0 \\
\hline 8287 & 60.5 & 8581 & $44 \cdot 2$ & $\cdot 8879$ & $24 \cdot 3$ & .9192 & 0.7 \\
\hline 8291 & 60.4 & $\cdot 8583$ & 43.9 & .8883 & 24.0 & .9196 & 0.3 \\
\hline - 8294 & 60.2 & $\cdot 8587$ & $43 \cdot 7$ & .8886 & 23.8 & .9200 & Proof. \\
\hline$\cdot 8298$ & $60^{\circ} 0$ & $\cdot 8590$ & $43 \cdot 5$ & .8890 & 23.5 & Under & Proof. \\
\hline 8301 & 59.8 & .8594 & $43 \cdot 3$ & .8894 & $23 \cdot 2$ & .9204 & 0.3 \\
\hline$\cdot 8305$ & $59 \cdot 6$ & .8597 & $43 \cdot 1$ & .8897 & 23.0 & .9207 & 0.6 \\
\hline$\cdot 8308$ & 59.5 & .8601 & $42 \cdot 8$ & 8901 & 227 & .9210 & 0.9 \\
\hline 8312 & $59 \cdot 3$ & .8604 & $42 \cdot 6$ & 8904 & 225 & .9214 & $1 \cdot 3$ \\
\hline 8315 & $59 \cdot 1$ & .8608 & $42 \cdot 4$ & .8908 & $22^{\circ} 2$ & .9218 & 1.6 \\
\hline 8319 & $58 \cdot 9$ & .8611 & $42 \cdot 2$ & $\cdot 8912$ & 21.9 & .9222 & $1 \cdot 9$ \\
\hline '8322 & $58 \cdot 7$ & .8615 & $42 \cdot 0$ & .8915 & $21 \cdot 7$ & .9226 & $2 \cdot 2$ \\
\hline$\cdot 8326$ & $58 \cdot 6$ & $\cdot 8618$ & $41 \cdot 7$ & .8919 & 21.4 & 9229 & 25 \\
\hline $\begin{array}{r}8329 \\
\end{array}$ & 58.4 & $\cdot 8622$ & $41 \cdot 5$ & $\cdot 8922$ & $21 \cdot 2$ & .9233 & 2.8 \\
\hline$\cdot 8333$ & $58 \cdot 2$ & 8625 & $41 \cdot 3$ & .8926 & 20.9 & .9237 & $3 \cdot 1$ \\
\hline$\cdot 8336$ & 58.0 & .8629 & $41 \cdot 1$ & $\cdot 8930$ & 20.6 & .9241 & $3 \cdot 4$ \\
\hline$\cdot 8340$ & $57 \cdot 8$ & $\cdot 8632$ & 40.9 & .8933 & 20.4 & .9244 & $3 \cdot 7$ \\
\hline .8344 & $57 \cdot 7$ & .8636 & 40.6 & .8937 & $20 \cdot 1$ & .9248 & 4.0 \\
\hline 8347 & $57 \cdot 5$ & $\cdot 8639$ & $40 \cdot 4$ & $\cdot 8940$ & $19 \cdot 9$ & $\cdot 9252$ & $4 \cdot 4$ \\
\hline 8351 & $57 \cdot 3$ & .8643 & $40 \cdot 2$ & $\cdot 8944$ & 19.6 & .9255 & $4 \cdot 7$ \\
\hline$\cdot 8354$ & $57 \cdot 1$ & $\bullet 8646$ & 40.0 & $\cdot 8948$ & $19 \cdot 3$ & .9259 & $5 \cdot 0$ \\
\hline •8358 & 56.9 & $\cdot 8650$ & 39.8 & .8951 & 19.1 & .9263 & $5 \cdot 3$ \\
\hline 8362 & 56.8 & .8653 & $39 \cdot 5$ & .8955 & 18.8 & $\cdot 9267$ & $5 \cdot 7$ \\
\hline-8365 & $56 \cdot 6$ & $\cdot 8657$ & $39 \cdot 3$ & .8959 & $18 \cdot 6$ & .9270 & 6.0 \\
\hline$\bullet 8369$ & 56.4 & $\cdot 8660$ & $39 \cdot 1$ & $\cdot 8962$ & $18 \cdot 3$ & .9274 & 6.4 \\
\hline$\bullet 8372$ & 56.2 & 8664 & 389 & $\cdot 8966$ & 18.0 & $\cdot 9278$ & 6.7 \\
\hline 8376 & 56.0 & 8667 & $38 \cdot 7$ & .8970 & $17 \cdot 7$ & $\cdot 9282$ & $7 \cdot 0$ \\
\hline$\cdot 8379$ & $55 \cdot 9$ & 8671 & $38 \cdot 4$ & .8974 & 17.5 & $\cdot 9286$ & $7 \cdot 3$ \\
\hline 8383 & $55 \cdot 7$ & 8674 & $38 \cdot 2$ & .8977 & 172 & •9291 & $7 \cdot 7$ \\
\hline .8386 & 55.5 & 8678 & $38 \cdot 0$ & .8981 & $16^{\circ} 9$ & $\cdot 9295$ & $8 \cdot 0$ \\
\hline$\cdot 8390$ & $55 \cdot 3$ & 8681 & 37.8 & .8955 & $16^{\circ} 6$ & •9299 & $8 \cdot 3$ \\
\hline$\cdot 8393$ & $55 \cdot 1$ & .8685 & $37 \cdot 6$ & .8989 & 16.4 & .9302 & $8 \cdot 6$ \\
\hline$\cdot 8396$ & $55^{\circ} 0$ & ·8688 & $37 \cdot 3$ & $\cdot 8992$ & $16 \cdot 1$ & $\cdot 9306$ & 9.0 \\
\hline .8400 & $54 \cdot 8$ & .8692 & $37 \cdot 1$ & .8996 & $15^{\circ} 9$ & .9310 & $9 \cdot 3$ \\
\hline$\cdot 8403$ & $54 \cdot 6$ & .8695 & $36 \cdot 9$ & $\cdot 9000$ & 156 & $\cdot 9314$ & $9 \cdot 7$ \\
\hline$\cdot 8407$ & $54 \cdot 4$ & $\cdot 8699$ & 36.7 & .9004 & $15 \cdot 3$ & .9318 & 10.0 \\
\hline 8410 & $54 \cdot 2$ & .8702 & $36 \cdot 4$ & .9008 & 150 & $\cdot 9322$ & $10 \cdot 3$ \\
\hline 8413 & $54 \cdot 1$ & .8706 & $36 \cdot 2$ & .9011 & 14.8 & .9326 & 107 \\
\hline 8417 & 53.9 & 8709 & $35 \cdot 9$ & .9015 & $14 \cdot 5$ & $\cdot 9329$ & 110 \\
\hline '8420 & 53.7 & 8713 & $35 \cdot 7$ & .9019 & $14 \cdot 2$ & •9332 & $11 \cdot 4$ \\
\hline 8424 & 53.5 & 8716 & $35 \cdot 5$ & .9023 & 13.9 & $\cdot 9337$ & 11.7 \\
\hline 8427 & $53 \cdot 3$ & . 8720 & $35 \cdot 2$ & .9026 & 13.6 & $\cdot 9341$ & $12 \cdot 1$ \\
\hline$\cdot 8431$ & 53.1 & 8723 & 35.0 & $\cdot 9030$ & $13 \cdot 4$ & $\cdot 9345$ & $12 \cdot 4$ \\
\hline 8434 & $52 \cdot 9$ & 8727 & $34 \cdot 7$ & •9034 & $13 \cdot 1$ & $\cdot 9349$ & 12.8 \\
\hline 8438 & 527 & 8730 & $34 \cdot 5$ & $\cdot 9038$ & 12.8 & .9353 & $13 \cdot 1$ \\
\hline 8441 & 525 & 8734 & $34 \cdot 3$ & .9041 & 12.5 & $\cdot 9357$ & 13.5 \\
\hline - $E 445$ & $52 \cdot 3$ & 8737 & $34 \cdot 1$ & .9045 & $12 \cdot 2$ & $\cdot 9360$ & 13.9 \\
\hline 8448 & 521 & 8741 & 33.8 & .9049 & 120 & -9364 & $14 \cdot 2$ \\
\hline '8452 & 51.0 & 8744 & 38.6 & .9058 & $11^{\circ} 7$ & •9368 & $14: 6$ \\
\hline
\end{tabular}


STAINED GLASS.

Table-continued.

\begin{tabular}{|c|c|c|c|c|c|c|c|}
\hline $\begin{array}{l}\text { Specific } \\
\text { Gravity. }\end{array}$ & $\begin{array}{l}\text { Per Cent. } \\
\text { Under Prf. }\end{array}$ & $\begin{array}{l}\text { Specific } \\
\text { Gravity. }\end{array}$ & $\begin{array}{l}\text { Per Cent. } \\
\text { Under Prf. }\end{array}$ & $\begin{array}{l}\text { Specific } \\
\text { Gravity. }\end{array}$ & $\begin{array}{l}\text { Per Cent. } \\
\text { Under Prf. }\end{array}$ & $\begin{array}{l}\text { Specific } \\
\text { Gravity. }\end{array}$ & $\begin{array}{l}\text { Per Cent } \\
\text { Under Prf. }\end{array}$ \\
\hline$\cdot 9372$ & 14.9 & .9530 & $31 \cdot 0$ & $\cdot 9685$ & $52 \cdot 2$ & .9846 & $79 \cdot 2$ \\
\hline $9370^{\circ}$ & $15 \cdot 3$ & .9534 & $31 \cdot 4$ & $\cdot 9689$ & $52 \cdot 9$ & $\cdot 9850$ & 79.8 \\
\hline$\cdot 9380$ & $15 \cdot 7$ & $\cdot 9539$ & $31 \cdot 1$ & $\cdot 9693$ & $53 \cdot 3$ & .9854 & $80 \cdot 4$ \\
\hline .9384 & 16.0 & .9542 & $32 \cdot 3$ & 9697 & $54 \cdot 2$ & .9858 & $81 \cdot 1$ \\
\hline 9388 & 16.4 & .9546 & $32 \cdot 8$ & .9701 & 54.8 & .9862 & $81 \cdot 7$ \\
\hline .9392 & 16.7 & .9550 & $33 \cdot 2$ & .9705 & 55.5 & $\cdot 9866$ & $82 \cdot 3$ \\
\hline .9396 & $17 \cdot 1$ & .9553 & $33 \cdot 7$ & $\cdot 9709$ & $56 \cdot 2$ & .9870 & $82 \cdot 9$ \\
\hline .9399 & $17 \cdot 5$ & .9557 & $34 \cdot 2$ & .9713 & 56.9 & $98 \% 4$ & 83.5 \\
\hline$\cdot 9403$ & 178 & $\cdot 9561$ & $34 \cdot 6$ & .9718 & $57 \cdot 6$ & .9878 & 84.0 \\
\hline .9407 & 18.2 & $\cdot 9565$ & $35 \cdot 1$ & .9722 & $58 \cdot 3$ & $\cdot 98 \times 2$ & $84 \cdot 6$ \\
\hline .9411 & 18.5 & $\cdot 9569$ & $35 \cdot 6$ & $\cdot 9726$ & $59 \cdot 0$ & 9886 & $85 \cdot 2$ \\
\hline .9415 & $18 \cdot 9$ & .9573 & $36 \cdot 1$ & 9730 & $59 \cdot 7$ & $\cdot 9890$ & $85 \cdot 8$ \\
\hline .9419 & 193 & $\cdot 9577$ & $36 \cdot 6$ & 9734 & 60.4 & '9ऽ94 & 863 \\
\hline .9422 & 19.7 & $\cdot 9580$ & $37 \cdot 1$ & $9 \tau 38$ & $61 \cdot 1$ & $\cdot 9698$ & $86 \cdot 9$ \\
\hline 9426 & 200 & .9584 & $37 \cdot 6$ & $\begin{array}{r}.9742 \\
\end{array}$ & $61 \cdot 8$ & .9902 & $87 \cdot 4$ \\
\hline .9430 & 20.4 & .9588 & 38.1 & .9746 & 62.5 & $\cdot 9906$ & 88.0 \\
\hline .9434 & 20.8 & .9592 & 38.6 & .9750 & $63 \cdot 2$ & .9910 & 88.5 \\
\hline .9437 & $21 \cdot 2$ & .9596 & $39 \cdot 1$ & .9754 & 63.9 & .9914 & $89 \cdot 1$ \\
\hline 9441 & $21 \cdot 6$ & $\cdot 9599$ & $39 \cdot 6$ & .9758 & 64.6 & .9918 & $89 \cdot 6$ \\
\hline .9445 & 21.9 & .9603 & 40.1 & .9762 & $65 \cdot 3$ & $\cdot 9922$ & 90.2 \\
\hline $9+48$ & 222 & $\cdot 9607$ & 40.6 & $\cdot 9766$ & 66.0 & 9926 & 90.7 \\
\hline$\cdot 9452$ & 227 & $\cdot 9611$ & $41 \cdot 1$ & .9770 & $66^{\circ} 7$ & •9930 & $91 \cdot 2$ \\
\hline .9456 & $23 \cdot 1$ & $\cdot 9615$ & $41 \cdot 7$ & .9774 & $67 \cdot 4$ & $\cdot 9934$ & $91 \cdot 7$ \\
\hline$\cdot 9460$ & 23.5 & •9619 & $42 \cdot 2$ & .9778 & $68 \cdot 0$ & .9938 & $92 \cdot 3$ \\
\hline 9464 & 23.9 & .9623 & $42 \cdot 8$ & .9782 & $68 \cdot 7$ & $\cdot 9942$ & 92.8 \\
\hline $\begin{array}{r}-9468 \\
\end{array}$ & $24 \cdot 3$ & $\cdot 9627$ & $43 \cdot 3$ & .9786 & $69 \cdot 4$ & 9946 & $93 \cdot 3$ \\
\hline .9172 & $24 \cdot \pi$ & $\cdot 9631$ & 43.9 & 9790 & $70 \cdot 1$ & $\cdot 9950$ & $93 \cdot 8$ \\
\hline .9476 & $25 \cdot 1$ & $\cdot 9635$ & $44 \cdot 4$ & .9794 & $70 \cdot 8$ & $\cdot 9954$ & $94 \cdot 3$ \\
\hline .9480 & $25 \cdot 5$ & •9638 & 45.0 & .9798 & $71 \cdot 4$ & 9958 & $94 \cdot 9$ \\
\hline$\cdot 9484$ & $25 \cdot 9$ & $\cdot 9642$ & 45.5 & 9802 & $72 \cdot 1$ & •9962 & $95 \cdot 4$ \\
\hline .9488 & $26 \cdot 3$ & $\cdot 9646$ & $46 \cdot 1$ & $\cdot 9806$ & $72 \cdot 8$ & $\cdot 9966$ & $95 \cdot \overline{9}$ \\
\hline .9492 & 26.7 & $\cdot 9650$ & $46 \cdot 7$ & .9810 & 73.5 & -9970 & 96.4 \\
\hline .9496 & $27 \cdot 1$ & $\cdot 9654$ & $47 \cdot 3$ & .9814 & $74 \cdot 1$ & .9974 & 96.8 \\
\hline$\cdot 9+99$ & 27.5 & 9657 & 47.9 & .9816 & 74.8 & .9978 & $97 \cdot 3$ \\
\hline$\cdot 9503$ & $28 \cdot 0$ & .9661 & 48.5 & .9822 & $75 \cdot 4$ & $\cdot 9982$ & 977 \\
\hline .9507 & 28.4 & $\cdot 9665$ & $49 \cdot 1$ & .9826 & $76 \cdot 1$ & .9986 & $98 \cdot 2$ \\
\hline .9511 & 28.8 & $\cdot 9669$ & $49 \cdot 7$ & $\cdot 9830$ & 76.7 & •9990 & $98 \cdot 7$ \\
\hline $.95 । 5$ & $29 \cdot 2$ & $\cdot 96 \pi 4$ & $50 \cdot 3$ & $\cdot 9834$ & $77 \cdot 3$ & .9993 & $99 \cdot 1$ \\
\hline .9519 & $29 \cdot 7$ & $96 \pi 7$ & $51 \cdot 0$ & .9838 & $78 \cdot 0$ & 9997 & 91.6 \\
\hline $\begin{array}{l}.9522 \\
.9526\end{array}$ & $\begin{array}{l}30 \cdot 1 \\
30 \cdot 6\end{array}$ & $\cdot 9681$ & $51 \cdot 6$ & .9842 & $78 \cdot 6$ & 1.0000 & 100.0 \\
\hline
\end{tabular}

STAINED GLASS. The blues of vitrified colors are all obtained from the oxide of cobalt. Cobalt ore (sulphuret) being well roasted at a dull red heat, to dissipate all the sulphur and arsenic, is dissolved in somewhat dilute nitric acid, and after the addition of much water to the saturated solution, the oxide is precipitated by carbonate of soda, then washed upon a filter, and dried. The powder is to be mixed with thrice its weight of saltpetre; the mixture is to be deflagrated in a crucible, by applying a red hot cinder to it, then exposed to the heat of ignition, washed, and dried. Three parts of this oxide are to be mixed with a flux, consisting of white sand, borax, nitre, and a little chalk, subjected to fusion for an hour, and then ground down into an enamel powder for use. Blues of any shade or intensity may be obtained from the above, by mixing it with more or less flux.

The beautiful greenish yellow, of which color so many ornamental glass vessels have been lately imported from Germany, is made in Bohemia by the following process. Ore of uranium, Uran-ochre, or Uran-glimmer, in fine powder, being roasted, and dissolved in nitric acid; the filtered solution is to be freed from any lead present in it, by the cautious addition of dilute sulphuric acid. The clear green solution is to be evaporated to dryness, and the mass ignited till it becomes yellow. One part of this oxide is to be mixed with 3 or more parts of a flux, consisting of 4 parts of red lead and 1 of ground flints; the whole fused together and then reduced to powder.

Chrome Green. Triturate together in a mortar equal parts of chromate of potash and flowers of sulphur : put the mixture into a crucible and fuse. Pour out the fluid mass; when cool, grind and wash well with water to remove the sulphuret of potash and to leave the beautiful green oxide of chrome. This is to be collected upon a filter, dried, rubbed down along with thrice its weight of a flux, consisting of 4 parts of red lead and 1 part of ground flints fused into a transparent glass; the whole is now to be melted and afterward reduced to a fine powder.

Violet. One part of calcined black oxide of manganese, one of zaffre, ten parts of white glass pounded, and one of red lead, mixed, fused, and ground. Or gold purple (Cassius's purple precipitate) with chlorsilver previously fused, with ten times its weight of a flux, consisting of ground quartz, borax, and red lead, all melted together; or, solution of tin being dropped into a large quantity of water, solution of nitrate of 
silver may be first added, and then solution of gold in aqua regia, in proper proportions. The precipitate to be mixed with flux and fused.

STARCH. In January, 1839, M. Pierre Isidore Verduer obtained a patent tor making starch, the chief object of which was to obtain the gluten of the wheat in a pure state, as a suitable ingredient in making bread, biscuits, \&c. He works wheat flour into dough by a machine, kneads it, washes out the starch by streams of cold water, a process long known to the chemist, and purifies the starch by fermentation of the superjacent water. I can see nothing new in his specification.

Mr. Jones's patent, of date April, 1840, is based upon the purification of the starch of rice and other farinaceous matters, by means of caustic alkali. He macerates $10 \mathrm{C}$ lbs. of ground rice in 100 gallons of a solution composed of 200 grains of caustic soda or potash to a gallon of water, stirs it gradually, till the whole be well mixed; after 24 hours, draws off the superjacent liquid solution of gluten in alkali, treats the starchy deposite with a fresh quantity of weak caustic ley, and thus repeatedly, till the starch Decomes white and pure. The rice before being ground is steeped for sometime in a like caustic ley, drained, dried, and sent to the mill.

Starch is made from wheat flour in a like way. The gluten may be recovered for use, by saturating the alkaline solution with sulphuric acid, washing and drying the precipitate.

In June, 1841, Mr. W. T. Berger obtained a patent for manufacturing starch by the agency of an alkaline salt upon rice. He prefers the carbonates of potash and soda.

Mr. James Colman, by his patent invention of December, 1841, makes starch from ground maize or Indian corn, by the agency either of the ordinary process of steeping and fermenting, or of caustic or carbonated alkaline leys. He also proposes to employ dilute muriatic acid to purify the starchy matter from gluten, \&c.-See Newton's Journal, C. S. xix. 246; xx. 184, 188; and xxi. 173.

The manufacture of potato flour ( $f e c u l e)$ or starch in France and Holland has been economized to such a degree that they supply this country with it, at the rate of $8 \mathrm{~s}$. or 10s. a hundredweight. Fig. 158 represents in section the powerful and ingenious

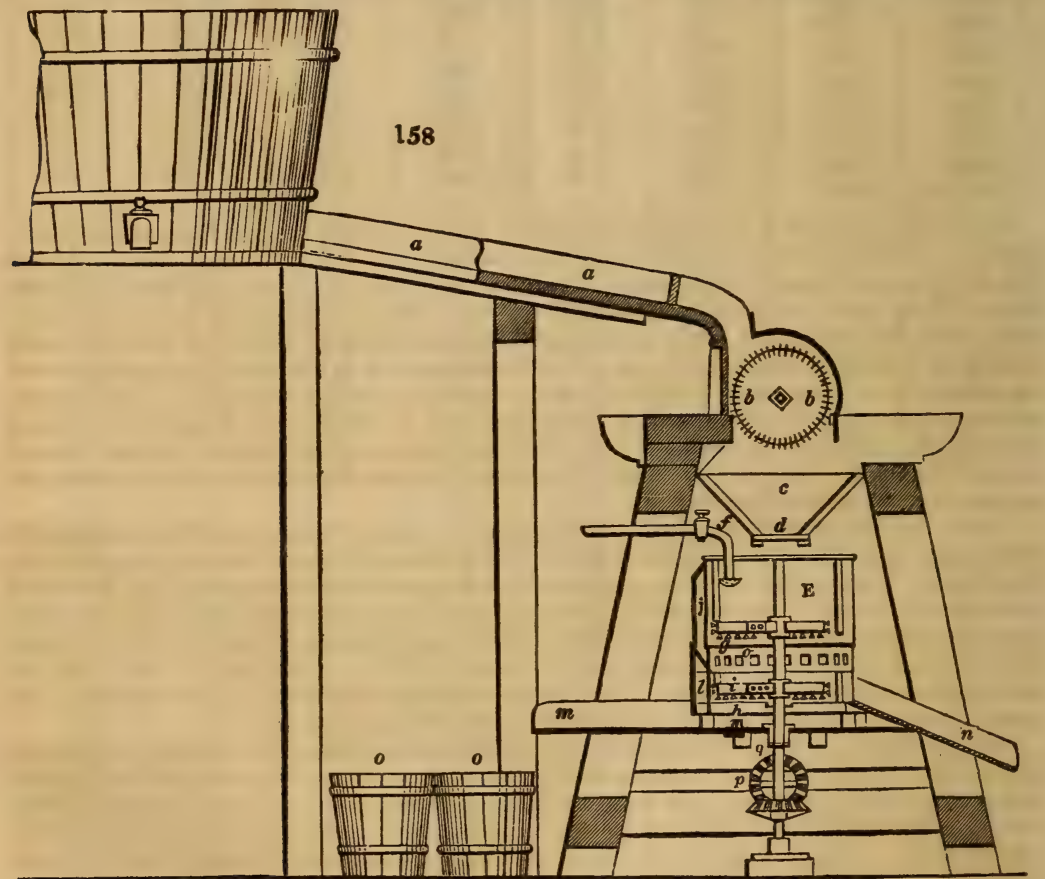

mechanical grater, or rasp (râpe), now used in France: $a \boldsymbol{a}$, is the canal, or spout, along which the previously well-washed potatoes descend; $b b$, is the grater, composed of a wooden cylinder, on whose round surface circular saw rings of steel, with short sharp teeth, are planted pretty close together. The greater the velocity of the cylinder the finer is the pulp. A cylinder 20 inches in diameter revolves at the rate of from 600 to 900 times in a minute, and it will convert into pulp from 14 to 15 hecto- 
litres (about $\mathbf{3 0 0}$ imperial gallons) of potatoes in an hour. Potatoes contain from $\mathbf{1 5}$ to 22 per cent. of dry fecula. The pulp, after leaving the rasp, passes directly into the apparatus for the preparation of the starch. $c c$, is a wooden hopper for receiving the falling pulp, with a trap door, $d$, at bottom. $\mathbf{E}$, is the cylinder-sieve of M. Etienne; $f$, a pipe ending in a rose spout, which delivers the water requisite for washing the pulp, and extracting the starch from it; $g \mathrm{~g}$, a diaphragm of wire cloth, with small meshes, on which the pulp is exposed to the action of the brushes $i i$, moving with great speed, whereby it gives out its starchy matter, which is thrown out by a side aperture into the spout $n$. The fecula now falls upon a second web of fine wire-cloth, and leaves upon it merely some fragments of the parenchyma or cellular matter of the potato, to be turned out by a side opening in the spout $n$. The sifting or straining of the starch likewise takes place through the sides of the cylinder, which consist also of wire-cloth; it is collected into a wooden spout, $m$, and is thence conducted into the tubes $o 0$, to be deoosited and washed. $p$, is a metre-toothed wheel-work placed on the driving-shaft, and gives motion to the upright axis or spindle, $q q$, which turns the brushes, $i$.

STEARINE. Fig. 159, is a view of both the exterior and interior of the saponi-

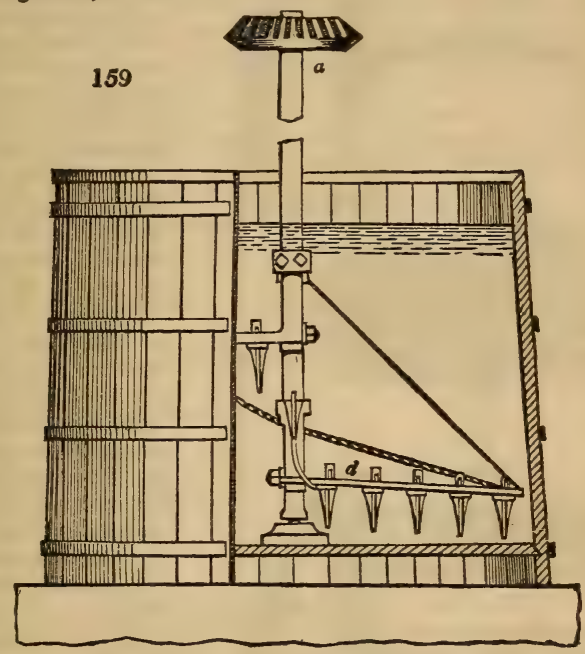

fying tun of a stearine factory; where the constituents of the tallow are combined with quicklime, by the intervention of water and steam: $a$, is the upright shaft of iron, turned by the bevel wheel above, in gear with another bevel wheel on the moving shaft, not shown in this figure. This upright shaft bears several arms $d$, furnished with large teeth. The tun is bound with strong hoops of iron, and its contents are heated by means of a spiral tube laid on the bottom, perforated with numerous holes, and connected by a pipe with a high-pressure steam-boiler.

Fig. 160, represents a longitudinal section of the horizontal hydraulic press for depriving stearic acid, as also spermaceti, of all their fluid oily impurities. $a$, is the

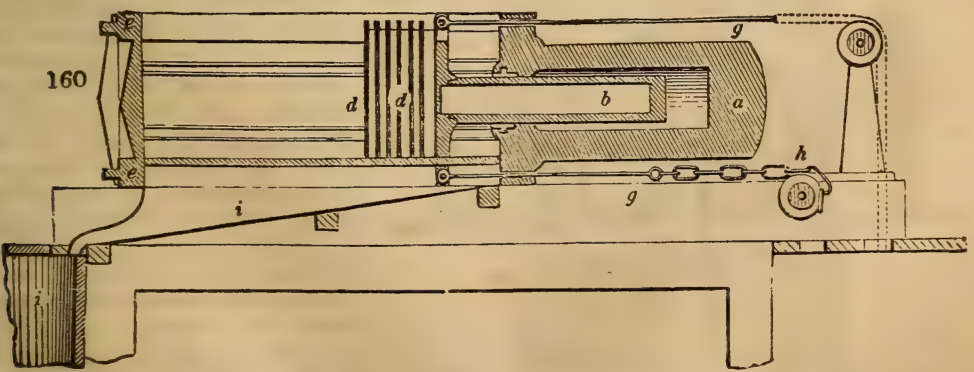

cylinder of the press; $b$, the ram or piston: $i, i, i, i$, hair and flannel bags enclosing the impure cakes to be exposed to pressure; $d, d, d, d$, iron plates previously heated, and placed between every two cakes to facilitate the discharge of their oily matter; $e_{,} e_{\text {. }}$ 
solid iron end of the press, made to resis'. great pressure; it is strongly bolted to the cylinder $\alpha$, so as to resist the force of the ram; $g, g$, iron rods, for bringing back the $\operatorname{ram} b$, into its place after the pressure is over, by means of counter weights suspended to a chain, which passes over the pulleys $h, h ; i, i$, a spout and a sheet-iron pan for receiving the oily fluid.

STEFL. One of the greatest improvements which this valuable modification of iron has ever received is due to Mr. Josiah M. Heath, who, after many elaborate and costly researches, upon both the small and the great scale, discovered that by the intruluction of a small portion, 1 per cent., and even less, of carburet of manganese into the melting-pot along with the usual broken bars of blistered steel, a cast steel was (ibtained, after fusion, of a quality very superior to what the bar steel would have yielded without the manganese, and moreover possessed of the new and peculiar property of being weldable either to itself or to wrought iron. He also found that a common bar-steel, made from an inferior mark or quality of Swedish or Russian iron,

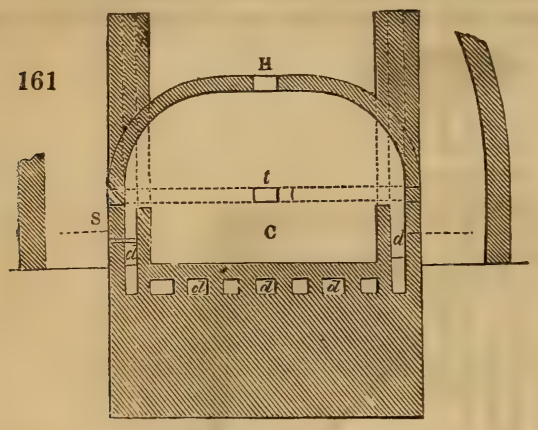
would, when so treated, produce an excellent cast coal. One im mediate consequence of this discovery has been the reduction of the price of good steel in the Sheffield market by from 30 to 40 per cent., and likewise the manufacture of table-knives of cast steel with iron tangs welded to them; whereas, till Mr. Heath's invention, table-knives were necessarily made of shear steel, with unseemly wavy lines in them, because cast steel could not be welded to the tangs. Mr. Heath obtained a patent for this and other kindred meritorious inventions on the 5th of April, 1839; but, strange and melancholy to
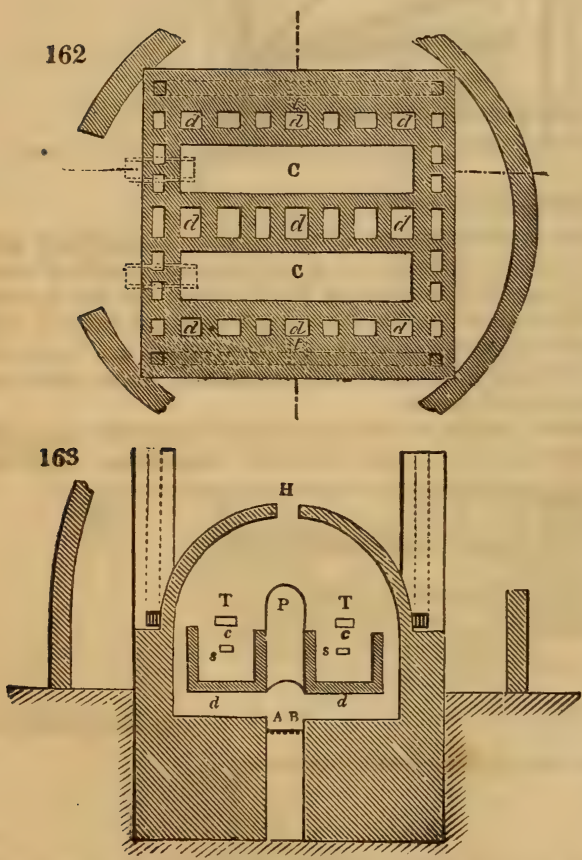
say, he has never derived an; thing from his acknowledged improvement but vexation and loss, in consequence of a numerous body of Sheffield steel manufacturers having banded together to pirate his patent, and to baffle him in our complex law courts. I hope, however, that eventually justice will have its own, and the ridiculously unfounded pretences of the pirates to the prior use of carburet of manganese will be set finally at rest. It is supposed that fifty persons at least are embarked in this pilfering conspiracy.

The furnace of cementation in which bar-iron is converted into bar or blistered steel is represented in figs. 161, 162, 163. It is rectangular and covered in by a groined or cloister arch : it contains two cementing chests, or sarcophaguses, c, c, made either of fire-stone or fire-bricks : each is $2 \frac{1}{2}$ feet wide, 3 feet deep, and 12 long; the one being placed on the one side, and the other on the other of the grate, $A$ B, which occupies the whole length of the furnace, and is from 13 to 14 feet long. The grate is 14 inches broad, and rests from 10 to 12 inches below the inferior plane or bottom level of the chests; the height of the top of the arch above the chests is $5 \frac{1}{2}$ feet; the bottom of the 
chests is nearly on a level with the ground, so that the bars do not need to be lifted high in charging the furnace. The flame rises between the two chests, passes also below and round them through horizontal and vertical flues, $d$, and issues from the furnace by an opening, $\mathrm{H}$, in the top of the vault, and by oririces, $t$, which communicate with the chimneys placed in the angles. The whole is placed within a large cone of bricks, 25 or 30 feet high, and open at top; this cone increases the draught, makes it nore regular, and carries off the smoke away from the establishment. The furnace has three doors; two, T ( $f g .162$ ), above the chests, serve to admit and to remove the bars; they are about 7 or 8 inches square; in each of them a piece of sheet-iron is put, folded back on its edges; upon which the bars are made to slide, so as to save the wall. A workman enters by the middle door, $\mathrm{P}$, to arrange the bars; the trial bars are taken out from time to time by the apertures, s ( $f \mathrm{~g} .16 \mathrm{l}$ ), left in the sides of the chests. The bars are laid in strata, along with wood charcoal in powder, in the said chests; they are about 3 inches broad, and one third of an inch thick; they must not be placed too near each other, lest they should get welded together; the last or uppermost layer is covered with a stratum of loamy matter from 4 to 5 inches thick. The furnace must be gradually heated, not reaching its maximum temperature before 8 or 9 days, and the cooling lasts 5 or 6 days; the whole operation 18 or 20 days, and sometimes more, according to the quality of the stee] to be cemented. About 13 tons of coals are consumed in this period. It is of consequence that the refrigeration be slow, to faror the crystallization of the metal. The grain of the steel varies with the rate of cooling, the largest and whitest grain denoting the most fusible steel.

STILL. The continuous system of distillation has been carried in France to a great pitch of perfection, by the ingenuity chiefly of M. Cellier Blumenthal, and M. Ch. Derosne. Fig. 164 is a general view of their apparatus ; $\mathrm{A}$ and $\mathrm{B}$ are boilers or alembics

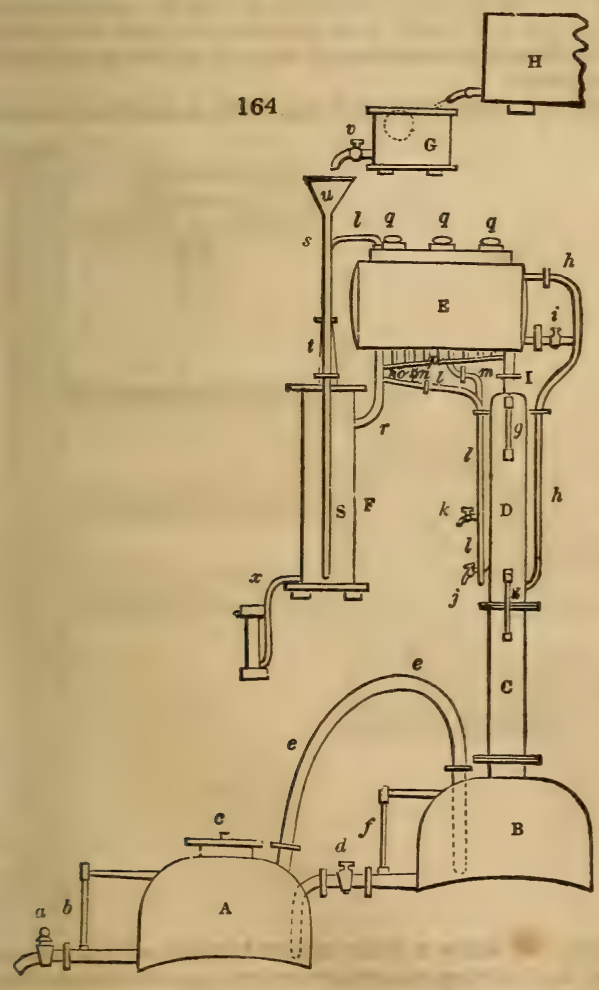
encased in brickwork, and receiving directly the action of the flame playing beneath them; in the copper, $A$, the vinasse, or spent wine, is finally exhausted of all its alcohol. c, is the column of distillation; $D$, the column of rectification; $\mathbf{E}$, the wine-heating condenser; $F$, the refrigerator; $G$, a vessel supplying vinasse to the cooler $F$, and feeding itself at the same time by means of a ball, stopcock placed in the vessel $\mathbf{H}$; $\mathrm{H}$, reservoir of vinasse; $\mathrm{I}$, tube of communication conducting the alcoholic vapors of the rectifying column, $\mathrm{D}$, up into the flat worm of the wineheater, $\mathrm{s} ; \boldsymbol{a}$, stop-cock of discharge of the alembic, $\mathbf{A}$; when the operation goes on, the spent vinasse runs aff continually by this stop-cock; $b$, a glass tube to show the height of the liquor in $\mathrm{A}$; $c$, a safety-valve; $d$, a stop-cock for passing the vinasse from the alembic, $B$, into the bottom of the alembic, $A ; e$, a tube to lead the alcoholic vapors, generated in $\mathbf{A}$, into the bottom of $B$, which vapors, in passing through the liquor in $\mathrm{B}$, heat it, and are partially condensed; $f$, glass tube to mark the level of the liquor in $\mathrm{B} ; \mathrm{g}$, and $\mathrm{g}$, level indicators; $h$, pipe conducting the vinasse from the lower part of the wine-heater, $E$, upnn the uppermost of the series of horizonta! dis $s$, wounted within the cuiumn of distillatinn: $i$, a stop-coci for emptying the wine-heater at the end of an operation; $l, l$, two tubes fitteu to the wist-heater, $\mathbf{E}$, of which the first descends into the last compartment of the rectifier, whence it rises 
to the fifth; and the second tube descends to the third compartment, whence it rises above the second. At the curvature of each of these two tubes a stop-cock, $l$, and $k$, is placed on them, for liawing at pleasure a sample of the liquor returned to the rectifier; $m, n$, and $o$, are tutses communicating on one side with the slanting tule, $p$, and on the other with the tube $l$. These three communications serve to furnish a spirit of greater or less strength. Thus if it be wished to obtain a very strong spirit, the alcoholic vapors which condense in the worm enclosed in $\mathrm{E}$, are all to be led back into the rectifier, $\mathrm{D}$, to effect which purpose, it is requisite merely to open the stop-cocks, $n$ and 0 ; again, weaker spirits may be had by closing the stop-cock, 0 , and still weaker by closing the stop-cock $n$; for in this case, the alcoholic vapors condensed in the worm within $\Sigma$, will flow off into the worm within the upright cooler $F$, and will get mixed with the richer vapors condensed in this refrigeratory. The interior of the columns $\mathrm{c}$, contains a series of movable concave scale pans (like those of balances), with spaces between, each alternate pan having the convex side turned reversely of the preceding one, for the purpose of prolonging the cascade descent of the vinasse through c, and exposing it more to the heating action of the ascending vapors; the edges of these pans are, moreover, furnished with projecting spiculæ of copper wires, to lead off the liquor from their surfaces in a fine shower. The interior of the rectifier column, $\mathrm{D}$, is mounted with a series of shelves, or floors, the passage from one compartment to that above it being through a short tube, bent at right angles, and open at either end; $p, p, p$, is a general tube, for receiving the vapors condensed in each of the turns of the large serpentine within $\mathrm{E}$. The axis of this worm is horizontal; $q, q q$, peep-holes in the top of the wine-heater; $r$, a tube to conduct the alcoholic vapors not condensed in the worm of $\mathrm{E}$, and also, if desired, those which have been condensed there, into the worm of the refrigeratory, $F$; $s$, a tube to bring the vinasse from the reservoir, $G$, into the lower part of the cooler, $\mathbf{F}$; $t$, a tube to lead the vinasse from the upper part of the cooler, $F$, into the upper part of the wine-heater, $\mathrm{E} ; u$, a funnel; $v$, a stop-cock to feed the tube, $t$, with vinasse; $x$, a tube of outlet for the spirits produced; it ends, as shown in the figure, in a test tube containing an hydrometer.

The still of Laugier is represented by a general view in fig. 165: $\mathrm{A}$ and $\mathrm{B}$ are alembics

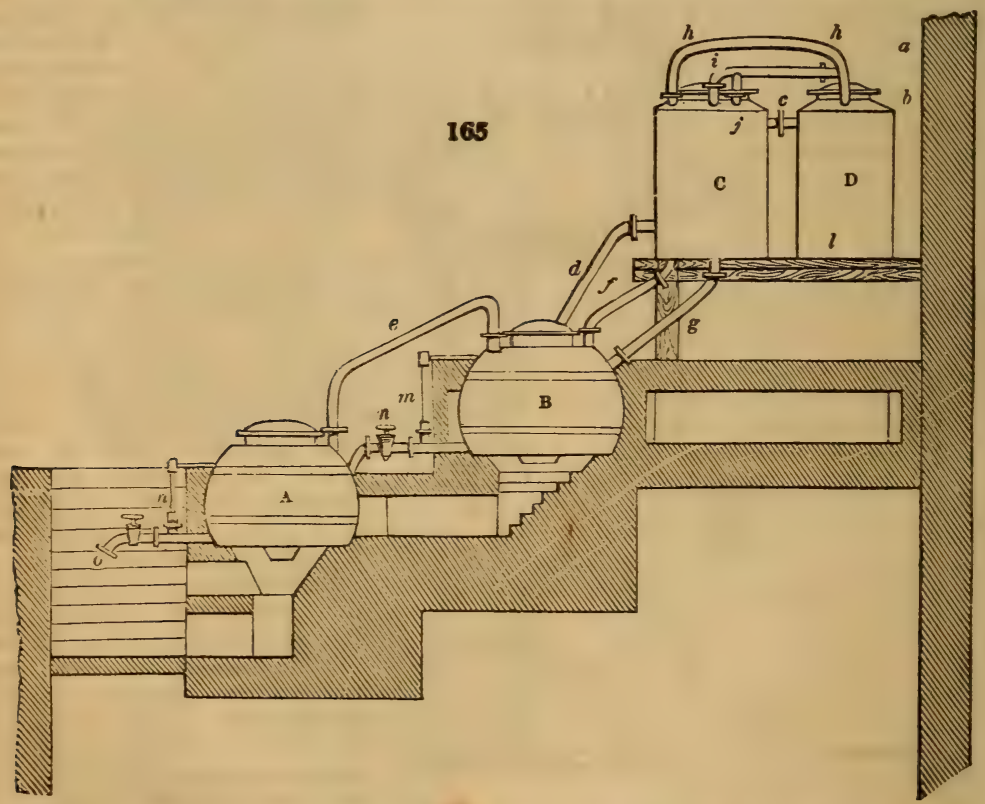

exposed to the direct action of the fire, and serve a like purpose to those of fig. 164 ; c, is a cylinder containing the rectifier, and serving as a wine-heater; $D$, is the condensing cylinder; $a$, a stop-cock communicating with the wine tun; $b$, a plunger-tube, furnished with a funnel, through which wine runs constantly into the condenser, $D ; c$, an overflow pipe of $\mathrm{D}$, between $\mathrm{c}$ and $\mathrm{D}$, communicating by a tube, dipping in the cylinder, c; $d$, a plunger equilibrium tube, supplying the alembics with hot wine; $e$, a tube leading the vapors of the first alembic, $A$, into the second one, $D$, into which it dips; 
$f$, a tube conducting the vapors of alcohol from the alembic, $B$, into the circles of the rectifier; $g$, a tube bringing back into the alembic, $\mathbf{B}$, the vapors condensed in the circles of the rectifier; $h$, a tube conducting the vapors not condensed into the worm of the condenser; $i$, a tube serving for the expulsion of the air when the wine comes into the vessel, $c$; it communicates with the tube, $h$, so as not to lose alcohol. $j$, is a prolongation of the tube $\mathbf{D}$, communicating with the tube $h$, so that it may be in contact with the external air; $l$, a stop-cock through which the alcohol condensed runs off into the serpentine; $m$, levels, indicating the height of the liquor in the alembics, $\mathrm{A}$ and $\mathrm{B}$; $n$, tube with a stop-cock, for feeding the alembic A; 0 , discharge stop-cock of the spent vinasse (wash).

A description of the operation of the first still will render that of the second intelli. gible.

The alembic A, being filled three fourths with vinasse, and $\mathrm{B}$ having only 4 or 5 inches of vinasse over its bottom, the liquor in $\mathbf{A}$ is made to boil, and the stop-cock, $r$, being at the same time opened, some of the wine to be distilled is allowed to fall into the funnel, $u$; this cold liquor runs to the bottom of the cooler, $F$, fills it, passes into the wineneater by the tube $l$, spreads into a perforated conduit along the top of $\mathrm{E}$, thence trickles down into this vessel till it fills it to the level of the tube $h$, by which it is conducted into the column, c, and, flowing down through all its compartments, it falls at last into the second alembic, $\mathbf{B}$.

During this progress, the liquor of A having begun to boil, the alcoholic vapor passes, by means of the tube $e, e$, into the second alembic, $\mathrm{B}$, which, being heated by those va. pors, and by the products of combustion issuing from the fireplace under the first alembic, is also soon made to boil. The vapor which it produces is disengaged into the column of distillation $\mathrm{c}$, meets there the wine which trickles through all its compartments, transfers to it a portion of its heat, and deprives it of alcohol, goes into the column $\mathrm{D}$. where it is alcoholized afresh, then enters into the worm within the wine-heater $\mathbf{E}_{\text {, }}$ glides through all its windings, gets stripped in part of the aqueous vapors which accompanied the alcohol, and which return first by the tube $p, p$, then by $l, l$, into the column of rectification; afterward the spirituous vapor passes into the worm enclosed in the cooler $F$, to issue finally condensed and deprived of all the water wished to be taken from it, by the tube $x$, into the gauge receiver.

When the indicator $f$, of the alembic в, shows it to be nearly full, the stop-cock $a$ of the alembic $\mathrm{A}$ is opened, and the vinasse is allowed to run out entirely exhausted of spirit; but as soon as there are only seven inches of liquor above the discharge pipe the cock $a$ is shut, and $d$ is opened to run off seven inches of liquor from $\mathbf{B}$.

It appears, therefore, that in reference to the discharge, the operation is not quite continuous; but this slight interruption is a real improvement introduced by $M$. Derosne into the working of M. Blumenthal's apparatus. It is impossible for any distiller, however expert, to exhaust entirely the liquor of the last alembic, if the discharge be not stopped for a short time. The above distilling apparatus requires from two to three hours to put it in full action. From 10 to 15 per cent. of spirit of $\frac{3}{6}$ are obtained from the average of French wine! and 600 litres of such spirit are run ${ }^{6}$ off with 150 kilogrammes of coals; or about two old English quarts of spirit for each pound of coals.

S'TONES, for building, and bricks, may be proved as to their power of resisting the action of frost, by the following method, first practised by M. Brard, and afterward by MM. Vicat, Billaudel, and Coarad, engineers of the bridges and highways in France. The operation of water in congealing within the pores of a stone may be imitated by the action of a salt, which can increase in bulk by a cause easily produced; such as efflorescence or crystallization, for example. Sulphate of soda or Glauber's salt answers the purpose perfectly, and it should be applied as follows :-

Average samples of the stones in their sound state, free from shakes, should be sawed into pieces 2 or 3 inches cube, and numbered with China ink or a graving tool. A large quantity of Glauber's salt should be dissolved in hot water, and the solution should be left to cool. The elear saturated solution being heaved to the boiling point in a saucepan, the several pieces of stone are to be suspended by a thread in the liquid for exactly one half-hour. They are then removed and hung up each by itself over a vessel containing some of the above cold saturated solution. In the course of 24 hours, if the air be not very damp or cold, a white efflorescence will appear upon the stones. Each piece must be then immersed in the liquor in the subjacent vessel, so as to cause the crystals to disappear, be once more hung up, and dipped again whenever the dry efflorescence forms. The temperature of the apartment should be kept as uniförm as possible during the progress of the trials. According to their tendency to exfoliate by frost, the several stones will show, even in the course of the first day, alterations on the edges and angles of the cubes; and in 5 days after effloresence begins, the result will be manifest, and may be estimated by the weight of disintegrated fragments, compared to the known weight of the piece in its original state, both taken equally dry. 
STREAM-WORKS. The name given by the Cornish miners to alluvial departes of tix ore, usually worked in the open air.

STRINGS. The name given by the Cornish miners to the small filamentuus ranifcations of a metallic vein.

SUGAR. The recent researches of the eminent French chemist, M. Casaseca, upon cane-juice at Havana, in Cuba, have demonstrated clearly the enormous loss which sugar-planters suffer by the imperfection of their manufacturing processes. His results confirm those previously obtained by M. Peligot in Paris, and show that canejuice evaporated in vacuo at the atmospheric temperature, yields, in 100 parts :-

\begin{tabular}{llllll} 
Crystalline white sugar & - & - & - & - & - \\
Water - - & - & - & - & - & -78.94 \\
Mineral substances & - & - & - & - & - \\
Organic matter, different from sugar & - & - & - & - \\
\hline
\end{tabular}

The cane from which the above was drawn is called canade la tierra in Cuba. The juice of the Otaheite cane is identical with the preceding. But the proportions of lig. neous fibres in the two canes are very different; that of la tierra containing, according to $\mathrm{M}$. Casaseca, $16 \cdot 4$ per cent., while that of Otaheite contains only 10 . Other canes, however, differ in this respect considerably from these two varieties. The average quantity of grained sugar obtained from cane-juice in our colonial plantations is probably not more than one third of the quantity of crystalline sugar in the juice which they boil.

The following analysis of cane-juice, performed by a French chemist, was given me by Mr. Forstall of New Orleans. In 10 English gallons, of 231 cubic inches each, of juice marking $8 \frac{1}{2} \mathrm{O}$ Baumé, there are $5 \frac{3}{4}$ ounces English of salts, which consist of-

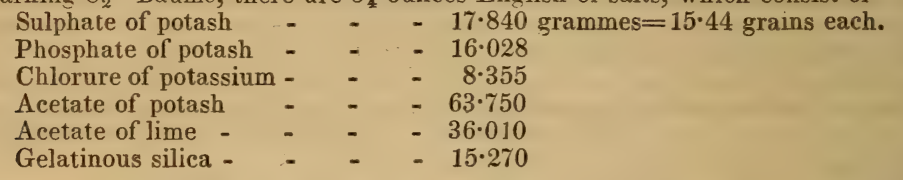

$157 \cdot 253=5 \cdot 57$ ounces avoirdupois.

To the large proportion of deliquescent saline matter, of which one half, he says, remains in the sugar, the analyst ascribes very properly the deliquescence and deterioration of the sugar when kept for some time or transported. It was probably the juice of the cane grown in the rich alluvial soil of Louisiana, and therefore more abundant in saline matter than the average soil of our West India islands. The Demerara canejuice has perhaps the above saline constitution, as it suffers much loss of weight by drainage in the home voyage.

SUGAR OF POTATOES, GRAPES, or STARCH. About two years ago a sample of sweet mucilaginous liquid was sent to me for analysis, by the honorable the commissioner of customs. It was part of a quanity imported in casks at Hull, from Rotterdam. It was called by the importers, "Vegetable Juice." I found it to be imperfectly saccharified starch or fecula; and, on my reporting it as such, it was admitted at a moderate rate of duty.

Three months since I received a sample of a similar liquid from the importer at Hull, with a request that I would examine it chemically. He informed me that an importation just made by him, of 30 casks of it, had been detained by orders of the excise, till the sugar duty of $25 \mathrm{~s}$. per cwt. of solid matter it contained was paid upon it. It was of specific gravity $1 \cdot 362$, and contained 80 per cent. of ill-saccharified fecula.

In the interval between the first importation and the second, an act of parliament had been obtained for placing every lind of sugar, from whatever material it was formed, under the provisions of the "Beet-root Sugar Bill." As the saccharometer tables, subservient to the levying of the excise duties, under this act, were constructed by me, at the request of the president of the board of trade, I well knew that 50 per cent. of the syrup of the beet-root was deducted as a waste product, because beet-root molasses is too crude an article for the use of man. Well saccharified starch paste, however, constitutes a syrup, poor indeed in sweetness when compared with cane syrup, or that of the beet-root; but then it does not spontaneously blacken into molasses, by evaporation, as solutions of ordinary sugar never fail to do when they are concentrated, even with great care. Hence the residuary syrups of saccharified fecula may be all worked up into a tolerably white granular mass, which, being crushed, is used by greedy grocers to mix with their dark-brown bastard sugars, to improve their color.

It is only within two years that sugar has been in this country manufactured from potato starch to any extent, though it has been long an object of commercial enterpris 
in France, Belgium, and Holland, where the large coarse potatoes are used for this purpose. The raw material must be very cheap there, as well as the labor; for potato flour or starch, for conversion into sugar, has been imported from the continent into this country in large quantities, and sold in London at the low price of $10 \mathrm{~s}$. per cwt.

The process usually followed by the potato-sugar makers, is to mix 100 gallons of boiling water with every $112 \mathrm{lbs}$. of the fecula, and $2 \mathrm{lbs}$. of the strongest sulphuric acid. This mixture is boiled about 12 hours in a large vat, made of white deal, having pipes laid along its bottom, which are connected with a high pressure steam-boiler. After being thus saccharified, the acid liquid is neutralized with chalk, filtered, and then evaporated to the density of about $1 \cdot 300$, at the boiling temperature, or exactly $1 \cdot 342$, when cooled to $60^{\circ}$. When syrup of this density is left in repose for some days. it concretes altogether into crystalline tufts, and forms an apparently dry solid, of spe. cific gravity $1 \cdot 39$. When this is exposed to the heat of $220^{\circ}$, it fuses into a liquid nearly as thin as water; on cooling to $150^{\circ}$, it takes the consistence of honey, and at $100^{\circ} \mathrm{F}$. it has that of a viscid varnish. It must be left a considerable time at rest before it recovers its granular state. When heated to $270^{\circ}$, it boils briskly, gives off one tenth of its weight of water, and concretes, on cooling, into a bright yellow, brittle, but very deliquescent mass, like barley sugar. If the syrup be concentrated to a much greater density than $1 \cdot 340$, as to $1 \cdot 362$, or if it be left faintly acidulous, in eithe ase it will not granulate, but will remain either a viscid magma or become a concrete mass, which may indeed be pulverized, though it is so deliquescent as to be unfit for the adulteration of raw sugar. The Hull juice is in this predicament, and is therefore, in my opinion, hardly amenable to the new sugar law, as it can not by any means be worked up into even the semblance of sugar.

Good Muscovado sugar, from Jamaica, fuses only when heated to $280^{\circ}$, but it turns immediately dark brown, from the disengagement of some of its carbon, at that temperature, and becomes, in fact, the substances called "caramel" by the French, which is used for coloring brandies, white wines, and liqueurs.

Thus we see that starch or grape-sugar is well distinguished from cane-sugar, by its fusibility, at a moderate heat, and its inalterability at a pretty high heat. Its sweetening power is only two fifths of that of ordinary sugar. A good criterion of incompletely formed starch-sugar is, its resisting the action of sulphuric acid, while perfectly saccharified starch or cane-sugar is readily decomposed by it. If, to a strong solution of imperfectly saccharified grape-sugar, nearly boiling hot, one drop of strong sulphuric acid be let fall, no perceptible change will ensue, but if the acid be dropped into solu. tions of either of the other two sugars, black carbonaceous particles will make thein appearance.

The article which was lately detained by the Excise, for the high duties, at Hull, is not affected by sulphuric acid, like the solutions of cane-sugar, and of the well-made potato-sugar of London; and for this reason I gave my opinion in favor of admitting the so-called vegetable juice at a moderate rate of duty.

I submitted the solid matter, obtained by evaporating the Hull juice, to ultimate analysis, by peroxide of copper, in a combustion tube, with all the requisite precautions, and obtained, in one experiment, $3 \pi$ per cent. of carbon; and in another 38 per cent., when the substance had been dried in an air bath, heated to $2 \pi 5^{\circ}$. The difference to 100 , is hydrogen and oxygen, in the proportion to form water. Now this is nearly the constitution of starch. Cane-sugar contains about 5 per cent. more carbon, whereby it readily evolves this black element, by the action of heat or sulphuric acid.

An ingenious memoir, by Mr. Trommer, upon the distinguishing criteria of gum, dextrine, grape-sugar, and cane-sugar, has been published in the 39 th rolume of the "Annalen der Chemie und Pharmacie." I have repeated his experiments, and find them to give correct results, when modified in a certain way. His general plan is to expose the hydrate of copper to the action of solutions of the above-mentioned regetable products. He first renders the solution alkaline, then adds solution of sulphate of copper to it, and either heats the mixture or leaves it for some time in the cold. By pursuing his directions, I encountered contradictory results; but, by the following method, I have secured uniform success, in applying the criteria, and have even arrived at a method of determining, by a direct test, the quantity of sugar in diabetic urine.

I dissolve a weighed portion of sulphate of copper in a measured quantity of water, and make the solution faintly alkaline, as tested with turmeric paper, by the addition of potash ley, in the cold; for if the mixture be hot, a portion of the disengaged green hydrate of copper is converted into black oxide. This mixture being always agitated before applying it, forms the test liquor. If a few drops of it be introduced into a solution of gum, no change ensues on the hydrate of copper, even at a boiling heat, which shows that a gummate of copper is formed, which resists decomposition : but the cupreous mixture, without the gum, is rapidly blackened at the boiling tem 
perature. I do not find that the gummate is redissolved by an excess of water, as Trommer affirms.

Starcn and tragacants comport like gum, in which respect I agree with Trommer. Starch, however, possesses already a perfect criterion, in iodine water. Mr. Trommer says, that solution of dextrine affords a deep glue-colored liquid, with sut a trace of precipitate; and that when his mixture is heated to $85^{\circ} \mathrm{C}$., it deposites red grains of protoxide of copper, soluble in muriatic acid. I think these phenomena are dependant, in some measure, upon the degree of alkaline excess in the mixture. I find, the solution of dextrine, treated in my way, hardly changes in the cold; but when heated slightly, it becomes green, and by brisk boiling an olive tint is produced. It thus betrays its tendency of transition into sugar.

Solution of cane-sugar, similarly treated, undergoes no change in the cold at the end of two days; and very little change of color even at a boiling heat, if not too concentrated. Cane-sugar, treated by Trommer in his way, becomes of a deep blue; it can be boiled by potash in excess, without any separation of orange-red oxide of copper.

Starch or grape-sugar has a marvellous power of reducing the green hydrate of copper to the orange oxide. I find, however, that it will not act upon the pure blue hydrate, even when recently precipitated; it needs the addition, in every case, of a small portion of alkali. Yet ammonia does not seem to serve the purpose; for, in using the ammonia-sulphate of copper, in solution, I obtained unsatisfactory results with the above vegetable products.

The black oxide of copper is not affected by being boiled in solution of starch-sugar.

"If solution of grape-sugar," says Trommer, "and potash, be treated with a solution of sulphate of copper, till the separated hydrate is redissolved, a precipitate of red oxide will soon take place, at common temperatures, but it immediately forms, if the mixture is heated. A liquid containing $\frac{1}{10} \overline{0} \overline{00}$ of grape-sugar, even one millionth part," says he, "gives a perceptible tinge (orange), if the light is let fall upon it." To obtain such a minute result, very great nicety must be used in the dose of alkali, which I have found it extremely difficult to hit. With my regulated alkaline mixture, however, I never fail of discovering an exceedingly small proportion of starch-sugar, even when mixed with Muscovado sugar; and thus an excellent method is afforded of detecting the frauds of the grocers.

I find that manna deoxidizes the green hydrate of copper slowly when heated, but not nearly to the same extent as grape-sugar, which reduces it rapidly to the orange oxide.

If an excess of the hydrate of copper test be used, there will be a deposite of green hydrate at the bottom of the vessel, under the orange oxide.

To apply these researches to the sugar of diabetic urine: This should first be boiled briskly to decompose the urea, and to dissipate its elements in the form of ammonia, as well as to concentrate the saccharine matter, whereby the test becomes more efficacious. Then add to the boiling urine, in a few drops at a time, the cupreous mixture, containing a known quantity of sulphate of copper, till the whole assumes a greenish tint, and continue the heat until the color becomes bright orange. Should it remain green, it is a proof that more hydrate of copper has been introduced than has been equivalent to the deoxidizing power of the starch-sugar. I have found that one grain of sulphate of copper in solution, supersaturated very slightly with potash, is decomposed with the production of orange protoxide, by about 3 grains of potato-sugar; or, more exactly, 30 parts of the said sulphate, in the state of an alkaline hydrate of copper, pass altogether into the state of orange oxide, by means of 100 parts of granular starch-sugar. Thus, for every 3 grains of sulphate so changed, 10 grains of sugar may be estimated to exist in diabetic urine.

Acetate of copper may be used in the above experiments, but it is not so good as the sulphate. The chloride of copper does not answer.

Specific gravity is also an important criterion, applied to sugars; that of the cane and beet-root is 1.577; that of starch-sugar, in crystalline tufts, is 1.39 , or perhaps $1 \cdot 40$, as it varies a little with its state of dryness. At $1 \cdot 342$, syrup of the cane contains 70 per cent. of sugar; at the same density, syrup of starch-sugar contains $75 \frac{1}{2}$ per cent. of concrete matter, dried at $260^{\circ} \mathrm{F}$, and therefore freed from the $10 \mathrm{per}$ cent. of water which it contains in the granular state. Thus, another distinction is obtained between the two sugars, in the relative densities of their solutions, at like saccharine contents per cent.

SULPHATE OF AMMONIA. This salt, now so extensively used in preparing artificial manures and imitations of guano, for farmers, is made of great purity, and at an economical rate, by the patent process of Mr. Croll, described under the article Gas. A mixture of 10 per cent. of this sulphate with 20 of bone-dust, some gypsum, and farmyard manure, will form a very fertilizing compost, applicable to a great variety of soils. 
SULPHURIC ACID. A valuable improvement of the process for manufacturing this fundamental chemical agent has been lately contrived by M. Gay Lussac, and made the subject of a patent in this country by his agent $M$. Sautter. It consists in causing the waste gas of the vitriol chamber to ascend through the chemical cascade of M. Clement Desornes, and to encounter there a stream of sulphuric acid of specific gravity 1.750. The nitrous acid gas, which is in a well-regulated chamber always slightly redundant, is perfectly absorbed by the said sulphuric acid; which, thus impregnated, is made to trickle down through another cascade, up through which passes a current of sulphurous acid, from the combustion of sulphur in a little adjoining chamber. The condensed nitrous acid gas is thereby immediately transformed into nitrous gas (deutoxide of azote) which is transmitted from this second cascade into the large vitriol chamber, and there exercises its well-known reaction upon its aeriform contents. The economy thus effected in the sulphuric acid manufacture is such that for 100 parts of sulphur 3 of nitrate of soda will suffice, instead of 9 or 10 as usually consumed.

Upon the formation of sulphated nitrous gas ( $\mathrm{N} \mathrm{O}_{2}, 3 \mathrm{~S} \mathrm{O}, 2 \mathrm{H} \mathrm{O}$ ), and its combination with oil of vitriol, the manufacture of hydrated sulphuric acid is founded. Either sulphur is burned in mixture with about one ninth of saltpetre; whence along with sulphurous acid gas, nitrous oxide gas is disengaged, while sulphate of potash remains; thus $\mathrm{K} \mathrm{O}, \mathrm{N} \mathrm{O}^{5}+\mathrm{S}=\mathrm{S} \mathrm{O}+\mathrm{N} \mathrm{O}_{2}, \mathrm{~K} \mathrm{O}$. 2. Or, nitric acid in the fluid or vaporous form may be present in the lead-chamber, into which the sulphurous acid gas passes, in consequence of placing in the flames of the sulphur a pan, charged with a mixture of sulphuric acid and nitre or nitrate of soda. This nitric acid being decomposed by a portion of the sulphurous acid, there will result sulphuric acid and nitrous gas. By the mutual reaction of the sulphurous and nitric acids, sulphuric acid and nitrous gas will be produced: $\mathrm{N} \mathrm{O}^{5}+3 \mathrm{~S} \mathrm{O}=\mathrm{N} \mathrm{O}_{2}+3 \mathrm{~S} \mathrm{O}_{3}$. 3. Or, by heating sugar or starch with nitric acid, the mixture of nitrous gas and nitrous acid vapor which results, may be thrown into the chamber among the sulphurous acid. In any one of these three cases, sulphurous acid gas, nitrous acid vapors (proceeding from the mixture of nitrous oxide and atmospherical oxygen) and steam are mingled together; whence arises the crystalline compound of sulphated nitrous oxide with sulphuric acid, which compound subsides in white clouds to the bottom of the chamber, and dissolves in the dilute oil of vitriol placed there, into sulphuric acid, with disengagement of nitrous gas. This gas now forms, with the remaining atmospherical oxygen, nitrous acid vapors once more, which condense a fresh portion of sulphurous acid gas into the above crystalline compound; and thus in perpetual alternation.

Sulphurous acid gas does not act upon nitrous gas, not even upon the nitrous acid vapor produced by the admission of oxygen, if water be absent; but the moment that a little steam is admitted, the crystalline compound is condensed. The presence of much sulphuric acid favors the formation of the sulphated nitrous gas. These crystals are decomposed by tepid water with disengagement of nitrous gas, which seizes the oxygen present and becomes nitrous acid (hyponitric of many chemists).

\section{T.}

TEA. This well-known plant has recently acquired peculiar interest among men of science, both in a chemical and physiological point of view. In its composition it approaches by the quantity of azote it contains to animalized matter, and it seems thereby qualified, according to Liebig, to exercise an extraordinary action on some of the funo tions of animals, especially the secretion of bile. The chemical principle characteristic of tea, coffee, and cocoa-beans, is one and the same when equally purified, from whichever of these substances it is extracted; and is called indifferently either theine or caffeine. Mulder takes it from tea, by treating the evaporated extract by hot water, with calcined magnesia, filtering the mixture, evaporating to dryness the liquor which passes through, and digesting the residuum in ether. This solution being distilled, the ether passes over, and the theine remains in the retort. This principle is extracted in t.e same wa? from ground raw coffee and from guarana, a preparation of the seeds of pawurnı, highly valued by the Brazilians. Theine, when pure, crystallizes in fine glossy needles, like white silk, which lose, at the heat of boiling water, 8 per .ent. of their weight, constituting its two atoms of water of crystallization. These needles are bitter tasted. They melt at $350^{\circ} \mathrm{F}$., and sublime at $543^{\circ}$ without decomposing. The crystals dried at $250^{\circ}$ dissolve in 98 parts of cold water, 97 of alcohol, and 194 parts of ether. In their ordinary state, they are but little more soluble in these menstrua. Theine is a feeble base, and is precipitable by tannin alone from its solution. 
Mr. Stenhouse prepares theine by precipitating a decoction of tea with solution of acetate of lead, evaporating the filtered liquor to a dry extract, and exposing this extract to a subliming heat in a shallow iron pan, whose mouth is covered flatly with porous paper luted round the edges, as a filter to the vapor, and surmounted with a cap of compact paper, as a receiver to the crystals. In this way he obtained, at a maximum, only 1.37 from 100.00 of tea. But M. Peligot, from the quantity of azote amounting to about 6 per cent., which he found in the tea leaves, being led to believe that much more theine existed in them than had hitherto been obtained, adopted the following improved process of extraction. To the hot infusion of tea, subacetate of lead and then ammonia were added; through the filtered liquor a current of sul phureted hydrogen was passed to throw down all the lead, and the elear liquid being evaporated at a gentle heat afforded, on cooling, an abundant crop of crystals. By re evaporation of the mother liquor, more crystals were procured, amounting altogether to from 5 to 6 out of 100 of tea.

The composition of theine may be represented by the chemical formula, $\mathrm{C}^{8}, \mathrm{H}^{5}, \mathrm{~N}$, 02; whence it appears to contain no less than 29 per cent. of nitrogen or azote.

Pehgot found, on an average, in 100 parts of -

\begin{tabular}{llllll} 
Dried black teas - & - & - & - & - & $-43 \cdot 2$ \\
green teas - & - & - & - & - & $-47 \cdot 1$ \\
Black teas, as sold & - & - & - & - & $-38 \cdot 4$ \\
Green teas, ditto - & - & - & - & - & - \\
\hline
\end{tabular}

Tea, by Mulder's general analysis, has a very complex constitution; 100 parts contain-

Essential oil (to which the flavor is due)
Chlorophyle (leaf-green matter)
Wax

Since the proportion of azote in theine and caffeine is so much greater than even in any animal compound, urea and uric acid excepted, and since so many different nations have been, as it were, instinctively led to the extensive use of tea, coffee, and chocolate or cocoa, as articles of food and enlivening beverage, which agree in no feature or property, but in the possession of one peculiar chemical principle, we must conclude that the constitution of these vegetable products is no random freak of nature, but that it has been ordained by Divine Wisdom for performing beneficial effects on the human race. Hitherto, indeed, medicine, a conjectural art, exercised too much by men superficially skilled in the science of nature, and the slaves or abettors of baseless hypotheses, has laid tea and coffee generally under its ban, equally infallible with the multitude, as that of the pope in the olden time, and has denounced their use, as causing a variety of nervous and other nosological maladies. But chemistry, advancing with her unquenchable torch into the darkest domains of nature, has now unveiled the mystery, and displayed those elemental transformations of the organic functions in the human body, to which tea and coffee contribute a salutary and powerful aid.

Liebig, in his admirable researches into the kingdoms of life, has been led to infer that the bile is one of the products resulting from the decomposition of the animal tissues, and that our animal food may be resolved by the action of oxygen, so amply applied to the lungs in respiration, into bile and urea, the characteristic constituent of urine.

When the consumption of tissue in man is small, as among mankind in the artificial state of life, with little exercise and consequently languid digestion, assimilation, and decomposition, the constant use of substances rich in azotized compounds, closely analogous to tbe chief principle of the bile, must assist powerfully in the production of this secretion, so essential to the healthy action of the bowels and other organs. Liebig has fully proved that the bile is not an exerementitious fluid, merely to be rejected, as

* This constituent is obviously much underrated. 
a prejudicial inmate of the system, but that it deserves, after secretion, some important purpose in the animal economy, being, in particular, subservient to respiration.

I shall conclude these remarks, perhaps more appropriate to a work on chemistry than to the present, by stating the relation between theine and the animal product taurine, the characteristic constituent of bile.

One atom of theine - $=\mathrm{C}_{8}, \mathrm{~N}_{2}, \mathrm{H}_{5}, \mathrm{O}_{2}>\quad$ Two atoms of taurine.

Nine atoms of water - $\left.=\mathrm{H}_{9}, \mathrm{O}_{9}\right\}=\mathrm{C}_{8}, \mathrm{~N}_{2}, \mathrm{H}_{14}, \mathrm{O}_{20}$.

Nine atoms of oxygen - $=\mathrm{O}_{9}$

The ietters $\mathrm{C}, \mathrm{N}, \mathrm{H}, \mathrm{O}$, denote carbon, nitrogen or azote, hydrogen, and oxygen; and the figures attached to each, the number of atoms; one atom of carbon being 6 , one of azote 14, one of hydrogen 1, and one of oxygen 8; from which the composition of the bodies, theine and taurine, may be easily computed for 100 parts. Now, supposing one tenth of the bile to consist of solid matter, and this solid matter to be choleic acid (resolrable into taurine but different from it), which contains 3.87 of nitrogen, then 2.8 grains of theine would afford to 480 grains of bile (supposed solid, or 4,800 grains in its ordinary state) all the nitrogen required for the constitution of laurine, its peculiar cyrstalline principle.

It may be remarked here, however, with regard to tea and coffee, that while they agree in the main feature, they differ in some others, and especially in the large pro. portion of tannin in the former, and its non-existence, according to my experiments, in the latter, notwithstanding the statement of its presence, in many chemical works. Hence, tea may act injuriously in persons of Cretian habits, ${ }^{*}$ while coffee has no constipating power, however much it may cause excitement and heat under certain idio. syncrasies.

A pure, agreeable, and convenient concentrated preparation of tea and coffee has been recently made the subject of an ingenious patent by Mra Staite, which I can recommend as being made from the best articles in the market, bj a perfectly wholesome apparatus and process. The patentee has printed a little explanatory pamphlet on the object of his improvement, from which the following extracts are taken :-

"The quantity of tea grown and consumed in China can not be ascertained, but the consumption of Europe and America may be taken as follows :-

Russia -
United States of America
France - -
Holland -
Other countries -
Great Britain -

- 6,500,000 lbs.

- $8,000,000$

- $2,000,000$

- $2,800,000$

- 2,000,000

- 50,000,000

$71,300,000$ lbs. or 31,830 tons.

"The number of tea-dealers in the year 1839 was, in England, 82, 794 ; in Scotland, 13,611; and in Ireland, 12, 744 ; maliing a total of 109,179. It is presumed that in consequence of the increased population their number at present must exceed 120,000 .

"The observations of Liebig afford a satisfactory explanation of the cause of the great partiality of the poor not only for tea, but for tea of an expensive and superior kind. He says, 'We shall never certainly be able to discorer how men were first led to the use of the hot infusion of the leaves of a certain shrub (tea), or of a decoction of certain roasted seeds (coffee). Some cause there must be, which will explain how the practice has become a necessary of life to all nations. But it is still more remarkable, that the beneficial effects of both plants on the health must be ascribed to one and the same substance (théine or caffeine, the presence of which in two regetables, belonging to natural families, the products of different quarters of the globe, could hardly have presented itself to the boldest imagination. Yet recent researches have shown, in such a manner as to exclude all doubt, that théine and caffeine are in all respects identical.' And he adds, that 'we may consider these vegetable compounds, so remarkable for their action on the brain, and the substance of the organs of motion, as elements of food for organs as yet unknown, which are destined to convert the blood into nervous substance, and thus recruit the energy of the moring and thinking faculties.' Such a discorery gives great importance to tea and coffee, in a physiological and medical point of view.

"At a meeting of the Academy of Sciences, in Paris, lately held, M. Peligot read a paper on the chemical combinations of tea. He stated that tea contained essential principles of nutrition, far exceeding in importance its stimulating properties; and showed that tea is, in evers respect, one of the most desirable articles of general use. 
One of his experiments on the nutritious qualities of tea, as compared with inose of soup, was decidedly in favor of the former.

"Coffee is grown in Brazil, Cuba, Hayti, Java, British West Indies, Dutch Guiana, states of South America, French West India colonies, Porto Rico, Sumatra, Ceylon, Bourbon, Manilla, and Mocha. Brazil produces the largest quantity, 72,000,000 pounds weight; and the other states and colonies according to the order in which they are enumerated, down to Mocha, which produces the least, or 1,000,000 pounds; making a total of $346,000,000$ pounds, equal to the consumption of the enormous quantity of 2,900 tons weekly, or 150,800 tons per annum.

"From the official returns, the quantities of coffee exported in one year from the different places of production were 154,550 tons :-

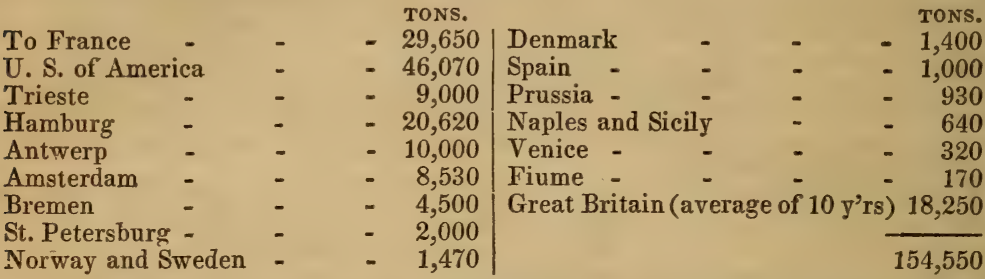

"Every reflecting man will admit, that articles of such vast consumption as tea and coffee (amounting together to more than 185,000 tons annually), forming the chief liquid food of a whole nation, must exercise a great influence upon the health of the people, and that any discovery which tends to the purification of these alimentary drinks, rendering them more wholesome, without rendering them less agreeable, is a great boon conferred upon society.

"The inventor and manufacturers of the 'Pure Concentrated Fluid Essence of Tea and Coffee,' hope that the convenient and portable form in which they are enabled to offer them to the public (in 'Rand's Patent Collapsible Tubes,' made of pure tin, whereby all the usual trouble and inconvenience of making tea and coffee are avoided), affords rational grounds (in addition to more important considerations) for anticipating an extensive sale."

TOBACCO. This important subject of our national revenue has been, during the last session of parliament, very fully investigated in reference to the smuggling and adulteration carried to an enormous extent, and hitherto but little checked by all the efforts of the officers of the customs and excise. Mr. Joseph Hume, M.P., who moved the appointment of the committee of the house of commons, and of which he was chairman, proposed a reduction of duty from $3 s .2 d$. a pound to $1 s$., as the only effectual remedy against these joint evils; but he was counteracted by Mr. Goulbourn, chancellor of the exchequer, and a majority of the members of the committee, on the score that the state of the national finances did not permit such a defalcation of income as that reduction would occasion. It would appear, from a great mass of evidence, that much more tobacco is introduced illicitly than what duty is paid upon, and that very great adulterations are practised. The following statement shows the temptations:-

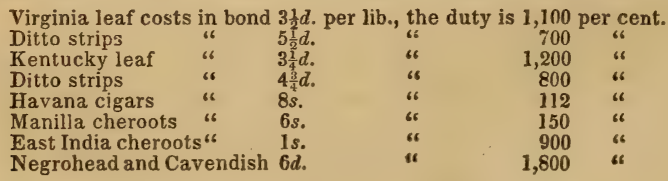

Rates of duty on tobacco in foreign countries :-

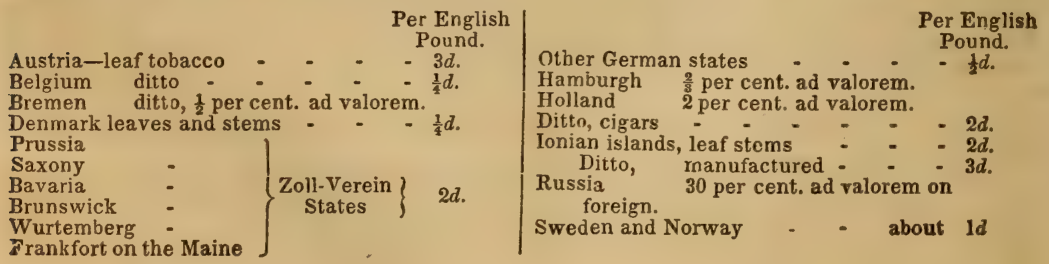


A strict royal monopoly (régie) exists in Austria Proper, France, Sardinia, the Dutchies of Parma and Lucca, and the Grand Dutchy of Tuscany; and in Portugal, Spain, Naples, and the states of the church, the license to manufacture is periodically sold to companies, which regulate the prices of tobacco as they please. It will be found that the situation of all these couniries where the monopolies and high prices are kept up, is nearly the same, as to illicit trade in tobacco, as in England.

In the years 1841,1842 , and 1843 , the average revenue in this country on tobacco, at 3 s. $2 d$. per lb., was $3,635,105 l$.

The greater part of the committee's report is occupied with the examination of witnesses as to the extent, modes, facilities, and chief localities of the illicit trade. It exhibits a great body of very curious and useful information, and demonstrates, beyond a doubt, that no measure short of a reduction of the duty to $1 s$. per lb. can put a stop to it.

The portion of the report most interesting to the readers of the present work will probably be found to be the scientific evidence as to the means of detecting adulteration.

The society of tobacco manufacturers in London, desirous of ascertaining how far chemistry could detect adulteration in tobacco, mixed four samples with different materials. No. 1 contained 5 per cent. of refined sugar; No. 210 per cent. of extraneous matter, viz., sugar and common salt; No. 315 per cent. of extraneous matter, the one half of it sugar, and the other half a certain powder used by the trade; No. 4 I5 per cent. also, consisting of about 3 parts sugar, and 1 part terra japonica (catechu). These four samples were placed in the hands of Mr. A. Garden, chemist, of Oxford street, for analysis, who made the following report upon their composition :-

"The leaves of the tobacco-plant, in addition to the vegetable principles of which they are chiefly composed, are known to contain a considerable number of saline and earthy substances, and which no doubt ought to be regarded as legitimate constituents of the plant. The proportion which these saline substances bear to the whole vegetable mass, is, it is true, but small, vet it becomes a question how far the presence of these salts, in quantities somewhat larger than those in which they are generally found to exist, can fairly be referred to a process of adulteration. The five samples of tobacco sent to me for examination do not appear to differ very widely from one another. After the removal of all the vegetable matter, they have, on an average, yielded 14 per cent. of solid substances, consisting of soluble and insoluble saline and earthy compounds, the principal of which are as follows: sulphate and phosphate of lime, carbonate of potash, muriate of ammonia and soda, sulphate of iron, malate of lime, and siliceous earth. The specimens marked 1,2 , and $4,{ }^{*}$ are exceedingly similar; that marked genuine contains about 2 per cent. less than the others, and the specimen No. 4 ( 3 as stated above) is remarkable for having yielded a proportion of oxide of iron, which would almost lead to the supposition that sulphate of iron had been introduced. The other samples, it is true, also afforded evidence of the presence of iron, but in such minute quantity as might easily be accounted for by the contact of iron vessels or instruments, in the transports or manipulations to which the articles may have been exposed.

\section{"A. Garden."}

Mr. Rogers, chairman of the Tobaconist's Society, who superintended the adulteration, states that no iron or copperas was put into any of them; that Mr. Garden had them some time under process, having received them in May or June, and having made his report on the 26th of August, 1843. "The manufacturers, in consequence of that report, came to the conclusion that the analyzation was not satisfactory, and could not be done so far as that process was concerned."

Mr. Joseph Hume, M. P., under instructions from the committee, requested two chemists, Mr. Heathfield and Mr. Edward Solly, Jun., each to prepare six samples in the presence of two members of the committee. That was done, and the samples were sent to the Excise Board, and during ten or twelve days were carefully analyzed by the three chemists employed by the board to detect adulteration. The evidence of these three gentlemen, viz., Professor Graham, Mr. Richard, and Mr. George Phillips, will explain the course they took to carry on the analysis; and the following are the results :-

* 6 in the original, but altered by me to 4 , as denoting the fourth of the adulterated samples. The fifth was genuine tobacco. 
Particulars of Six Samples of Tobacco, prepared as above, in the presence of Sir Charles Douglas, M. P., and Mr. Ewart, M. P., Members of the Committee.

\section{No. I., marked X.}

9 lbs. $8 \mathrm{oz}$. of tobacco, with $1 \mathrm{lib} .8 \mathrm{oz}$. of garden rhubarb-leaves, about 16 per cent., or $12 \frac{1}{2}$ per cent. in 100 parts.

\section{No. II., marked K.}

$9 \mathrm{lbs} .6 \mathrm{oz}$, of tobacco, with $1 \mathrm{lib}$. foxglove leaves. About 10 per cent. added, or 9.52 in 100 parts.

\section{No. III., marked $\mathrm{N}$}

$11 \mathrm{lbs} .11 \mathrm{oz}$, tobacco, mixed with $8 \mathrm{oz}$. brown

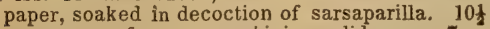
oz. syrup of sugar, combining solid sugar, 7 or 8 dwts. : 1 oz. of saltpetre, and $\frac{1}{2} \mathrm{oz}$. of alum ; in all, 18t oz. About 8 per cent. added, or 7.54 in 100 parts.

\section{No. IV., marked F.}

11 lbs. $14 \mathrm{oz}$. of tobacco, mixed with chickory root, dried and powdered, Irish moss glutenized, carbonate of potash, sulphate of potash, carbonate of magnesia, and carbonate of lime, about $9 \mathrm{oz}$., or about $5 \frac{1}{4}$ per cent., or 4 in 100 parts.

\section{No. V., marked $\mathrm{O}$}

13 lbs. 9 oz. of tobacco, mixed with 12 ox. of ground tobacco stalks.

No. VI. marked $\mathrm{R}$

11 libs, $4 \mathrm{oz}$. No adulteration.
Report of the Analysis of the first Series of SIz Samples by Messrs. Richard Phillips, Graham, and George Phillips, 7th June, 1844

No. I., marked X.

Adulterated with the leaves of garden rhubarb; the amount of the adulteration is estimated at 3.3 per cent.

\section{No. II., marked $\mathrm{K}$.}

Adulterated by a green leaf, not tobacco, which appears to belong to a plant of the same natura] family, probably the potato ; the amount of the adulteration estimated at $\mathbf{3 . 9}$ per cent.

\section{No. III., marked N.}

Adulterated with brown paper or millboard, and also with sugar; the amount of the first adulteration is estimated at 6 per cent., of the second $1 \frac{1}{2}$ or two per cent.

\section{No. IV., marked F.}

Adulterated with a vegetable matter, not tobacco, the nature of which we are not agreed upon The amount of this adulteration is estimated at 1.2 per cent. There is reason to suspect the addition to this tobacco of both sand and sugar, in small quantity.

\section{No. V., marked 0.}

Adulterated with sand and sugar; the amount of the first adulteration is estimated at 2 per cent. of the second at 3 per cent.

\section{No. VI., marked R.}

Genuine, but with a proportion of sand usually high.

Second Series of Six Samples, signed Rogers and Son, marked as under, analyzed by Messrs. Richard and George Phillips, and Thomas Graham.

No. I., marked C.

Adulterated with sugar of milk Terra japonica

Nitrate and sealed up at Mr. Rogers's, 392 Oxford street, in the presence of Sir Charles Douglas and Mr. Ewart, by Edward Solly, Jun., Esq.

\section{No. I., marked C.}

Adulterated with sugar, the adulteration estima ted at 1 per cent.

No. II., marked $\mathrm{I}$.

Adulterated with sugar, the adulteration estima. ted at 3 per cent.

Adulterated with refined sugar _. . $\quad$ - 3

Terra japonica - - - - - - 1

$\begin{aligned} & \text { Carbonate of potash } \\ & \text { Common salt }\end{aligned}-\div \quad: \quad: \quad-1$

Total per cent. - - . $\frac{-}{6}$

No. III., marked Q.

Adulterated with refined sugar

Crude nitrate of ammonia

Common salt .

Muriate of potash

Nitrate of potash

Alum

sh $-=-\quad=-0.5$

Total per cent. : - $\quad-\quad-9$

No. IV., marked P.

Adulterated with sugar of milk -
Refined sugar

Conimon salt

Carbonate of potash

Adulterated with sugar, the adulteration estima. ted at 4 per cent.

No. III., marked Q.

Adulterated with sugar, the adulteration estimated at 2 per cent.

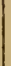

\section{No, V., marked B.}

Genuine ; grains of sugar were, however, found in it and picked out, but the quantity so small, that we allow their introduction to be accidental.

No. VI., ma ked M.

Not adulterated at all.

\section{No. VI., marked M.}

Adulterated with loaf-bread, which has been cut up in the same manner as the tobacco; the amount of this adulteration was not estimated, but is small; the sample contains also a little sugar. 
It appears from the preceding statement, that the chemists made no attempt at a true chemical analysis, but contented themselves with a physical examination of the appearances, and with the single process of fermentation to detect sugar, by the production of alcohol; and yet they declare the presence of sugar in the tobacco which contained none; as in samples F, O, and B. They did not search for saline matter, such as nitrate of potash, alum, common salt, nitrate of ammonia, muriate of potash, or carbonate of potash, and in fact do not seem to have made any analytical research for any such foreign budies; so that if the sugar had been left out (as a skilful adulterator would have done), and if the foxglove-leaves had been well browned by tobacco-juice, none of the adulterations would have been detected.

Mr. R. Phillips says, in his examination before the committee "generally speaking we did not employ chemical tests," but it was principally by mechanical analysis and examination of the plant that they judged. When asked if he could obtain alcohol from sugar of milk, Mr. R. Phillips replies, "I think not." Question 7,555.

" $7,59 \%$. Chairman.-Will you [Mr. R. Phillips] look at your report, letter C, adulterated with sugar estimated at 1 per cent.? I asked you the question, whether you could discover the adulteration, if made with sugar of milk; and your answer was, 'No, I believe not, for I think it would not ferment.'

" 7,598 . That specimen was mixed with 5 per cent. of sugar of milk, and 3 per cent. of other articles, earthy matters, making altogether 8 per cent.; your report is, ' adulterated with sugar, estimated at 1 per cent.;' how do you reconcile your detecting sugar there, when you said that you could not detect sugar of milk ?-I said that I spoke from the best of my recollection, and that is my recollection."

“ 7,632. Letter $\mathrm{O}$, you [Mr. R. Phillips] report as being ' adulterated with sand and sugar; the amount of the first estimated at 2 , and the second at 3 per cent.,' therefore that is reported as adulterated to the extent of 5 per cent. ?-Yes.

" 7,633 . That specimen is stated to be ' 13 pounds 9 ounces of tobacco mixed with 12 ounces of ground stalk ;' what should you say to that? Are you perfectly satisfied that the sugar in this specimen was foreign matter?-I have no doubt of it; and with respect to the increased quantity of sand, that might come in with the increased quantity of stalks, which always contain a larger quantity than leaf-tobacco."

" 7,637 . But are you the least shaken in your opinion as to this analysis, by its being stated to you, that that which was presented to you was tobacco mixed with 12 ounces of stalk? - Not the least, neither with respect to sugar nor sand; confirmed with respect to sand, and.not shaken with regard to sugar."

" 7,640 . Here is an addition of eight per cent. of stalk; do you imagine that that would give $l w o$ per cent. of sand upon the whole amount?-It seems a large amount, but I can not account for it in any other way; it would increase it, but I can not say to what amount."

" 7,645. Taking the specimen letter $\mathrm{Q}$, on which you were before examined, in which you have stated, 'adulteration at 2 per cent.;' if informed that eight per cent. of muriate of potash, and other soluble matters were mixed, do you not consider that tobacco might be adulterated by those matters, which you could not discover as adulterations, to the extent of 5 or 6 per cent., without the possibility of detection?-I think they might, with such matter us that, without the possibility of detection."

Mr. R. Phillips says, in answer to question 7,673, that he never saw nicotine, and " never saw anybody that saw it." Now he has seen me often, and I have a specimen of pure nicotine, which I showed to the committee in my examination, and which I shall be happy to show him at any time.

"Mr. George Phillips called in and examined.

« 7,767 . What situation do you hold in the excise ?-That of an examiner.

" 7,768 . What are the duties of an examiner ?-They are various, but I have been employed chemically.

" 7,769. How long have you been in that situation?-About two years."

"7,773. Have you been educated as a chemist ?-No, I am self-educated."

"7,775. Have you examined all those specimens which have been suspected to be

adulterated tobacco?-Yes, I have examined the whole of them.

" 7,776. And you were employed at Gainsborough, Liverpool, and Manchester, in those different seizures and trials which took place?-Yes, I was present at the whole of them.

" 7,777. You were also associated with Mr. Richard Phillips and Professor Graham, in making inquiries into the 12 samples submitted to you ?-Yes."

" 7,780 . What time did you take in making these experiments ?-I think we were about 12 days.

" 7,781 . In examining the 12 specimens ?-Yes.

"7,782. Did you sign the report ?-Yes." 
His process of examination consists in drying the tobacco thoroughly by a heat of from $176^{\circ}$ to $182^{\circ}$, in then weighing out 100 grains of it, in digesting that weight in $\$ \frac{1}{2}$ pints of water of the above temperature, stirring the mixture with a glass rod, leaving it to infuse for 50 minutes, and then separating the liquid from the insoluble matter by a fine strainer or filter. The insoluble part is gently pressed, dried at the above heat, and weighed. In this way Mr. Phillips found, that-

\begin{tabular}{|c|c|c|c|c|}
\hline \multicolumn{5}{|c|}{ Virginia hand, the entire leaf and stem gave } \\
\hline \multicolumn{5}{|c|}{ Virginia stripped, the stem being taken out } \\
\hline Do. & & do. & & - \\
\hline Kentucky hand & - & - & - & - \\
\hline \multirow{3}{*}{$\begin{array}{l}\text { Do. do. } \\
\text { Do. stripped } \\
\text { Do. do. }\end{array}$} & - & - & - & - \\
\hline & - & - & - & - \\
\hline & - & - & - & - \\
\hline \multicolumn{2}{|c|}{ Maryland, not stripped } & - & - & - \\
\hline \multicolumn{2}{|c|}{ Do. do. } & & - & - \\
\hline \multicolumn{4}{|c|}{ Mean of Virginia hand experiments } & - \\
\hline \multicolumn{4}{|c|}{ Do. do. stripped do. - } & - \\
\hline \multirow{2}{*}{\multicolumn{3}{|c|}{ Do. Kentucky hand }} & - & - \\
\hline \multirow{2}{*}{\multicolumn{3}{|c|}{$\begin{array}{l}\text { Do. } \\
\text { Do. }\end{array}$}} & - & - \\
\hline & & & - & - \\
\hline
\end{tabular}

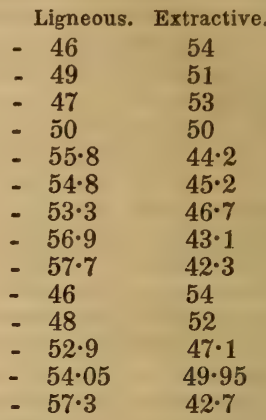

After experimenting upon between 500 and 600 different samples, he has never found any to exceed the highest of the above, which is 54 ; though he calls it, in his subsequent examination, 55 of extract.

\begin{tabular}{|c|c|c|c|c|c|c|}
\hline \multirow{2}{*}{\multicolumn{3}{|c|}{ Porto-Rico tobacco-leaf gave }} & \multicolumn{3}{|r|}{ Ligneous. } & \multirow{2}{*}{$\begin{array}{l}\text { Extractive } \\
30\end{array}$} \\
\hline & & & - & - & & \\
\hline Colombia & do. & - & - & - & -61.5 & $38 \cdot 5$ \\
\hline Do. & do. & - & - & - & $-60 \cdot 8$ & $39 \cdot 2$ \\
\hline Virginia stalks & - & - & - & - & -48.5 & $51 \cdot 5$ \\
\hline Kentucky do. & - & - & - & - & $-64 \cdot 1$ & $35 \cdot 9$ \\
\hline Do. do. & - & - & - & - & $-66 \cdot 4$ & $33 \cdot 6$ \\
\hline Turkey & - & - & - & - & $-46 \cdot 8$ & $53 \cdot 2$ \\
\hline
\end{tabular}

«7,865. Do you know to what natural family tobacco belongs ?-Mr. G. Phillips. I am not a botanist."

" 7,867 . Take the foxglove, for instance?-I can not say whether it belongs to the same family. I know it has not the same character; it is essentially different in character."

Yet the sample marked $\mathrm{K}$, which contained foxglove, maladroitly introduced in the green state, and thus easily distinguishable by the least experienced eye from tobacco, was pronounced by the three chemists to be "adulterated by a green leaf, not tobaccos which appears to belong to a plant of the same natural family, possibly the potato." Digitalis belongs to the family Scrophulariæ, not the Solaneæ, like tobacco.

By selecting such tobaccoes from the above tables as abound in ligneous matter, imbuing them with a quantity of some cheap vegetable extracts not fermentable into alcohol, drying and pressing them properly, it would be easy to adulterate tobaccoes to the amount of 10 or more per cent., and set at defiance this scheme of Mr. G. Phillips. The manufacturer has, of course, a stock of Virginia tobacco at hand, capable of yielding a like proportion of extract, to cover his deception, which he puts into their hands, pour leur donner le change.

"7,869. What is the power of microscope you employ ?-About 60,000 ."

Does Mr. G. Phillips mean to say that the linear magnifying power of his microscope is 60,000 ? There is no such instrument in existence. If he means the area is enlarged by his microscope 60,000 times, he ought to have said so, and the linear power in that case would be about 245 times.

" 7,877 . In what manner do you obtain saline matter from the tobacco?-I first ascertained the amount of ashes : I took 100 grains of Virginia leaf; I burned it to a red heat, not sufficiently high to drive off anything beyond the carbonaceous matter; those 100 grains of Virginia leaf produced 10.5 of ashes, and it contained 1.3 of sand or silica. The next experiment upon the same leaf gave ashes $11 \cdot 2$, and precisely the same amount of sand as the other, $1 \cdot 3$. The next was upon stalk alone, 100 grains of the same tobacco-stalk; it gave ashes $16 \cdot 5$, and $\cdot 4$ of sand. The next, stalk and leaf, was upon 62 grains, which gave ashes $8 \cdot 2$, and $\cdot 5$ of sand $(13.2$ and 0.8 per cent.) Now we come to the Kentucky : 100 grains of the Kentucky leaf gave ashes 19.5, sand 1.4 ; ditto 18.4 and $1.4 ; 47$ grains of stalk and leaf gave ashes 9.5 , and sand $\cdot 8$ (20 and 1.7 per cent.); 100 grains of stalk gave 20.4 and $\cdot 9$. Another experim ${ }^{2}$ t 
gave the same." Here we have nearly double the proportion of sand from leaf that there is from stalks.

Let us compare these experiments of Mr. G. Phillips with the unlucky counterstatement of Mr. R. Phillips. The former actually finds in the one set of stalks only 410 ths of 1 per ct. of sand, and 1 and 310 ths, or upward of three times as much, in the lear; while, in the other set, he finds about one half of sand in the stalks of what was in the leaf. Now Mr. R. Phillips defers to Mr. G. P. as his standard authority on this point. Thus-

"Question 7,580. You [Mr. R. Phillips] are not able to state the exact amount of those saline matters which exist in the different kinds of tobacco?-No, I have never tried."

" 7,582. You have not, up to this time, made that experiment in the various inquiries you have made? -No, I have not. I have not thought it necessary.

" 7,583 . You state that the earthy matter found" (in the ashes) "is silica; do you mean sand?-Yes.

" 7,584 . What is the greatest or the smallest quantity you have found in the genuine tobacco?-I have not got the proportion; I perhaps could find it, but I have it not with me.

" $\tau, 585$. You do not know the minimum or the maximum?-No, but I believe that can be stated by Mr. George Phillips very accurately."

" 7,633.—- - [Answer of Mr. R. Phillips.] With respect to the increased quantity of sand, that might come in with the increased quantity of stalks, which always contain a larger quantity than leaf-tobacco.

" 7,634 . Would the proportion of sand be accounted for, in your opinion, by the introduction of $12 \mathrm{oz}$. of stalks ? - I think it would."

Why did not the two Messrs. Phillips compare notes beforehand?

"7,878. Besides silica, what did you [Mr. G. Phillips] find ?-Number 36, which produced 19.5 ; gave carbonate of lime $7 \cdot 3$ grains, silica $1 \cdot 4$, carbonate of potash, and a trace of iron, 9.7 grains ; ${ }^{*}$ that is, it showed the presence of iron, but there was no appreciable quantity. The object of separating it into lime and potash was, that if there had been an introduction of 5 per cent. of potash, I should have had an increased amount of extractive matter" (soluble, he should say), "and an increased amount of ashes; in genuine tobacco, where the extractive proportion is high, the ashes are low." $\dagger$

" 7,885 . Are there any other articles found, besides the silica, and carbonate of lime, and potash ?-Not by my mode : both lime and potash exist in tobacco, in various quantities, malate and nitrate, and so on; but the amount of each has never been found by any one." $\ddagger$

" 7,918. - - Ans. The only two kinds of tobacco manufactured worth speaking of, are Virginia and Kentucky, and they come in strips principally. Now the tobaccomanufacturer might endeavor to defeat us in this way ; suppose we endeavor to tie him down, he might mix his tobacco not in the proportion that we expect."

The excise, though it has no power to tie the manufacturers down so hard and fast that they may not use any duty-paid tobacco which they please, yet it is too apt to give rise to a spirit of evasion. When a low-priced Kentucky, poor in extract, is laid down, it may be easily enriched with 10 per cent. of some vegetable extract to bring it up to Mr. G. Phillips's standard Virginia pitch of 55 .

" 7,924 . Have you subjected the extract to the process of combustion, in order to ascertain its amount of saline matter ?-Not the extract ; I make a point of preserving part, in order to experiment upon."

Yet he immediately afterward declares he makes no experiments upon it.

" 7,928 . What do the ashes of $\mathrm{C}$ consist of ?-We did not try that beyond the sand.

" 7,929 . You have got the ashes 16 by combustion?-Yes, we suspected this sample to contain sugar, and also tried for sand."

« 7,934. Could you not find alum?-Yes, but it would be impossible to try for the various salts that might be put in tobacco; you might be experimenting for years on one sample."

Relatively to Mr. R. Phillips's explanation of the rationale of the production of sand, question 7,961 to Mr G. Phillips affords a flat contradiction. "Could the introduction of 12 ounces of ground stalk into 13 pounds 9 ounces of tobacco, give-an adulteration of 3 per cent. of sand ?-It is perfectly impossible; the stalk contains less sand than the leaf."

" 8,032 . In any of those 12 specimens that you examined, were you able to distin.

* An extraordinary quantity.

† How easy to give this character to tobacco by adding extractive matter from different plants !

\$ What a rash assertion! He missed a uniform and main constituent, the phosphate of lime. 
guish any vegetable mixture besides the tobacco?-We readily distinguished in $\mathrm{K}$, the mixture of foxglove and rhubarb."

There was no rhubarb in $\mathrm{K}$; the foxglove was unluckily conspicuous by its light green color, and easily picked out. Answer to question 7,858: "So also as regards the rhubarb, the rhubarb is very full of massy strong hairs, which are something like bodlins rather round; they are larger at the end ; in fact, where the points of the hairs of tobacco are small, the rhubarb swell out and increase and cross each other in various directions, while those of the tobacco are flattened at the top."

Has the foxglove the same massy hairs as the rhubarb?

With regard to the production of alcohol from the samples of adulterated tobacco, Mr. E. Solly, in his examination before the committee, makes the following observations in answers to questions $8,350,8,352$ : "Then you do not consider the mode by which saccharine matter is said to be detected at present to be a good and sufficient test ? -I should receive it with very great caution."

" 8,352 .- - Ans. I believe that tobacco contains substances that may give rise to the formation of spirit; therefore I think it possible, until the contrary has been proved. that spirit may be formed from fermenting pure tobacco."

Seven samples of tobacco, mixed under the superintendence of the committee, as above described, were sent to me on the 18th of June last, for analysis, markied B B, $\mathrm{C} \mathrm{C}, \mathrm{F} \mathrm{F}, \mathrm{M} \mathrm{M}, \mathrm{O} O, \mathrm{Q}$, X X, which I now presume were of like composition to the parcels marked $\mathrm{B}, \mathrm{C}, \mathrm{F}, \mathrm{M}, \mathrm{O}, \mathrm{Q}, \mathrm{X}$, in those sent to the excise. As I operate always alone in my laboratory, I required naturally a considerable time to perform the several sets of experiments which I deemed requisite in trying to detect adulterations in so heterogereous a compound as tobacco. In my first examination before the committee, on the 15tri of July, I stated that my first line of research had been to determine the proportion of azote in each of the above specimens, and compare it with that in genuine tobacco, with the view of ascertaining into which of the parcels non-azotized plants, such as rhubarb and other indigenous leaves, might have been introduced. For this purpose, I took a weighed portion of each tobacco, dried it thoroughly by a gentle steam heat, and found thereby how much moisture it contained.-See second table below.

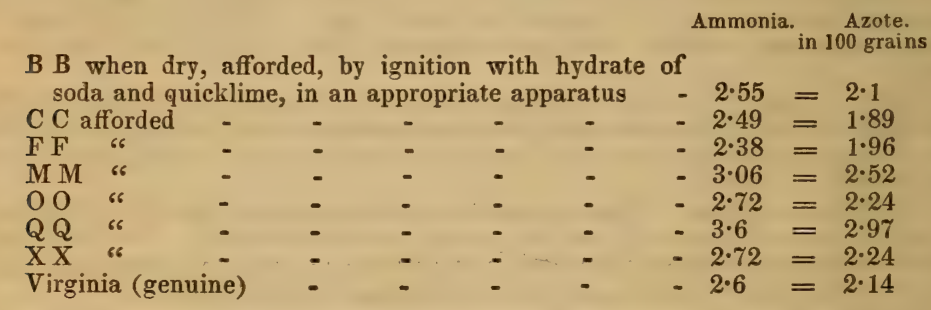

1 was now convinced that no good purpose could be served by this process of analysis, since it appeared that the differences in the proportions of azote resulting from the different proportions of nicotine, albumen, gluten, \&c., in the tobacco, were too slight, and most probably too variable, from soil, climate, and mode of culture, to be relied on as tests of purity ; and more especially when I found, on trial, that the dried leaves of foxglove afforded 1.96 , or nearly 2 per cent. of azote, and that this plant was one likely to be used in making the adulterations. Besides, it appears that $\mathrm{X} X$, which I now perceive to have contained 16 per cent. of rhubarb-leaves, afforded as much azote as $\mathrm{O} O$, which was altogether tobacco. Practical chemists are aware that each of the above experiments requires no little time, as well as nicety of manipulation. The results may, I believe, be depended on ; they were derived in each case from the decomposition of 50 grains of the dried tobacco. I next dried by a gentle heat, continued for several hours, as long as weight was lost, 200 grains of each sample.

\begin{tabular}{|c|c|c|c|c|c|c|}
\hline & & Dry. & $\begin{array}{l}\text { Water } \\
\text { at } 70^{\circ} \mathrm{F} \text {. }\end{array}$ & $\begin{array}{l}\text { Spec. grav. } \\
\text { of infusion. }\end{array}$ & $\begin{array}{l}\text { Residuum } \\
\text { dried. }\end{array}$ & $\begin{array}{c}\text { Soluble matter } \\
\text { or extract }\end{array}$ \\
\hline B B lost & $11 \cdot 75 \mathrm{p}$ & and $100 \mathrm{gr}$ & $+1,000$ & $=1.017$ & $59 \cdot 5$ & $40 \cdot 5$ \\
\hline C C " & $14 \cdot 5$ & "6 & 6 & $=1.0184$ & 54 & 46 \\
\hline F F & 15 & " & "6 & $=1 \cdot 017$ & 60 & 40 \\
\hline M M “ & $14 \cdot 75$ & " & “ & $=1.015$ & 71 & 29 \\
\hline $00 \%$ & $15 \cdot 5$ & “6 & c6 & $=1.018$ & 62 & 38 \\
\hline$Q Q \%$ & $17 \cdot 5$ & "6 & "6 & $=1.021$ & 72 & 28 \\
\hline X X " & $17 \cdot 75$ & "6 & "6 & $=1 \cdot 019$ & 67 & 33 \\
\hline Virginia & 6.5 & 66 & "6 & $=1.015$ & 53 & 47 \\
\hline
\end{tabular}


In a second series of experiments, where 100 grains of each of the dried tobaccoes, as under, were digested for two hours in 4,000 grains of distilled water, at the temperature of $176^{\circ} \mathrm{F}$., the following results were obtained :-

\begin{tabular}{|c|c|c|c|c|c|c|}
\hline$B B$ & & & & & residuum. & Soluble or extract. \\
\hline C C & - & - & - & - & $56 \cdot 7$ & $43 \cdot 3$ \\
\hline F F & - & - & - & . & $54 \cdot 7$ & $45 \cdot 3$ \\
\hline M M & - & - & - & - & $63 \cdot 7$ & $36 \cdot 3$ \\
\hline 00 & - & - & - & - & $58 \cdot 2$ & $41 \cdot 8$ \\
\hline Q Q & - & - & - & 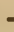 & $54 \cdot 0$ & $46 \cdot 0$ \\
\hline $\mathrm{x} x$ & - & - & - & - & $57 \cdot 7$ & $42 \cdot 3$ \\
\hline
\end{tabular}

Thus, by a longer digestion with heat, and a larger quantity of water, more soluble matter or extract is obtained, and also in different proportions, from the same samples :

\begin{tabular}{|c|c|c|c|c|c|c|}
\hline \multirow{2}{*}{\multicolumn{2}{|c|}{ Havana tobacco }} & & & \multicolumn{2}{|c|}{ Dried residuum. } & Soluble or extract \\
\hline & & - & - & - & $60 \cdot 1$ & \\
\hline Cuba & - & - & - & - & $62 \cdot 1$ & $37 \cdot 9$ \\
\hline Virginia & - & - & - & - & $53 \cdot 9$ & $46 \cdot 1$ \\
\hline Kentucky & - & - & - & - & $57 \cdot 2$ & $42 \cdot 8$ \\
\hline
\end{tabular}

It may, moreover, be remarked, that none of the tobaccoes, either adulterated or gen. uine, yielded so great a proportion of extract as Mr. G. Phillips asserts.

It will be observed from the table of the specific gravity of the infusion of 100 grains of the respective tobaccoes, in 1,000 grains of water, at $70^{\circ} \mathrm{F}$. (with trituration in a mortar), that $Q Q$ afforded the densest liquor, having a specific gravity of 1.021 . I was hence led to imagine that this sample was adulterated with some soluble substances, and possibly with sugar, of which such a handle had been made in the excise prosecutions. I therefore boiled 1,000 grains of that sample with 5,000 grains of distilled water, and evaporated the soluble matter to a solid extract, which weighed 400 grains. These were next digested in 3,000 grain-measures of alcohol of 0.834 , and they left 330 grains insoluble in this menstruum. The matter insoluble in alcohol should have contained the sugar if any were present; but when it was treated with nitric acid of proper density and temperature, it afforded no oxalic acid whatever; even by the test of chloride of calcium. Hence I inferred that if sugar had been mixed with that tobacco, it could not be discovered by probably the best test of sugar in common circumstances; and indeed, on looking now into the actual composition of sample $Q$, we find it to contain only 2 per cent. of sugar, mixed with 4 of nitrate of ammonia, 1 of common salt, 1 of the mixed nitrates of potash and muriate of potash, and one of alum. As the infusion of 100 ) grains of X X in 1,000 of water was next in density, being 1,019, I treated 1,000 grains of it as I had done with $\mathrm{Q} Q$, and obtained 600 grains of watery extract, which, being digested in alcohol, left 330 grains like the preceding. When this also was treated with nitric acid, it afforded no oxalic. I therefore abandoned this line of research as unprofitable.

It occurred to me that muriate or nitrate of ammonia might have been employed in adulterating some of the samples of tobacco. To ascertain this point, I distilled 100 grains of each sumple along with water and quicklime, condensing the vapors, and testing the distilled liquid for ammonia :-

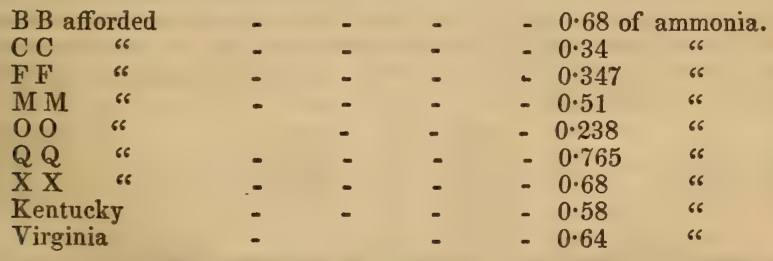

Ammonia exists in the saline state in all tobaccoes, but here in $Q Q$ in notable excess, corresponding to the 4 per cent. of crude nitrate of ammonia, which had been introduced by the mixers. So far this experiment has a positive result.

The filtered cold infusions of 100 grains of each dried sample in 1,000 grains of distilled water were examined by many chemical tests, as follows :-

1. Virginia taken as a standard:

Infusion pale brown; acid reaction with litmus paper; nitrate of barytes, $\mathrm{O}$; nitrate of silver, a faint opalescence, but no curdy precipitate; oxalate of ammonia, a faint cloud of calcareous matter; water of ammonia, $\mathrm{O}$ : chlorure of $\mathrm{un}$, a faint white precipitate-hence no sulphuretted hydrogen present; chloride of platinum, a crpious 
white precipitate, from the ammoniacal salt present; acetate of lead, an abundant whr tish precipitate, soluble in nitric acid; chloride of iron caused a green tint, and sulphate of copper an olive brown, both resulting from the yellow of the iron and blue of the eopper solutions with the brown of the tobacco.

B B afforded the same results with the above tests as the Virginia tobacco: hence it might be inferred to be free from soluble sulphates, muriates, carbonates, \&c.

C C, acid reaction like the preceding; nitrate of barytes, a precipitate which, being drained on a filter, washed, dried, and ignited, weighed $2 \cdot 2$ grains ; resulting, as it now appears, from the 1 per cent. of alum introduced into that sample.

$\mathrm{F} \mathrm{F}$ afforded 2.6 sulphate of barytes, resulting from the sulphate of potash introduced into this sample.

M M afforded 0.6 of sulphate of barytes, a quantity belonging to this description of tobacco, derived, no doubt, from the soil-indicating possibly the proportion that should be deducted from that afforded by $\mathrm{C} \mathrm{C}$ and $\mathrm{F} \mathrm{F}$.

$\mathrm{OO}$ afforded 0.5 of sulphate of barytes, indicating freedom from any added sulphate.

Q Q, 1 grain of sulphate of barytes, corresponding to the 1 per cent. of alum, which was possibly not uniformly diffused through the parcel; so that probably more of it existed in the portion taken for experiment of $\mathrm{C} C$, than in that taken of $Q \mathrm{Q}$.

$\mathrm{XX}$, sulphate of barytes, $0 \cdot 8$; the small excess here over pure tobacco due to the admixture of rhubarb-leaves. It is to be observed that all these barytic precipitates were insoluble in nitric acid.

After separation by the filter of the barytic precipitates, from the infusions made with heat, a definite quantity of solution of nitrate of silver was added to each at once, because it was found impossible to define the point at which that test ceased to produce a change. The phenomena here were singular and puzzling. The phials containing the infusions of B B, C C, F F, O O, had their sides coated with a lively green film; that with XX slightly; those containing $\mathrm{M} \mathrm{M}$ and $\mathrm{Q} \mathrm{Q}$, with a brown film; as also those from the Virginia, Kentucky, Havana, and Cuba tobaccoes; while the contents of $\mathrm{F} \mathrm{F}$ remained turbid after many days. From these phenomena, it appears that nitrate of silver can not be advantageously used as a test upon infusions of tobacco made with hot water.

When the infusions of the tobaccoes are made in the cold, those hydrogenated principles. which seem to reduce a portion of the oxide of the nitrate of silver, and render its precipitate insoluble in ammonia, are not apparently generated. 'The nitrate of silver in this case gave the following results :-

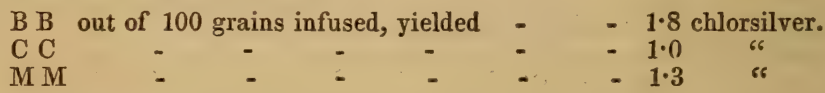

These quantities belong to genuine tobacco, as I found on trying the Virginia. The precipitates were insoluble in nitric acid.

My next series of experiments was instituted to determine, by fermentative action, with the addition of good yeast to the infusions of the respective samples, whether sugar could be detected in any of them by the production of alcohol. Accordingly, I infused half a pound avoird. $=3,500$ grains of each in 4,000 grains of boiling water, strained off the liquor into wide-mouthed phials, introduced into each 800 grains by weight of the best fresh porter-yeast from Messrs. Meux's brewery in my neighborhood, and enclosed them all in a space kept at the temperature of $80^{\circ} \mathrm{F}$. The specific gravity of each, before the yeast, was very nearly 1.08. After 40 hours, the specific gravities were found to be (at $80^{\circ}$ ) of-

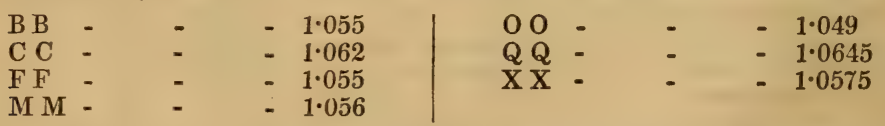

The contents of F F being distilled carefully in glass vessels, 700 water-grain measures of liquid were drawn off, which, at $70^{\circ} \mathrm{F}$., had the specific gravity, 0.9921 .

The contents of $\mathrm{OO}$ afforded 700 grain-measures of specific gravity, 0.9876 ; besides 500 grain-measures $\longrightarrow$ of 0.9974 .

Contents of B B afforded 700 grain-measures of 0.9946 , and 240 grain-measures of 0.998 .

From 2,400 grains by weight of the yeast, 700 grain-measures of liquor were distilled off, at specific gravity, 0.983 .

On the hypothesis that the liquor distilled from the infusions of the three samples of tobacco were alcoholic, the 700 grains of $F$ F would contain about 10 per cent. of oroof spixit or nearly 70 grains. 
Alcoholic spirits of 0.983 specific gravity contains 23.3 per cent. of proof spirit; hence $7 \times 23 \cdot 3=163 \cdot 1$ grains, of which one third $=54.4$ grains, being the product of 800 grains of yeast, had been introduced into each of the tobacco worts. The product of $F \mathrm{~F}$ is therefore $70-54 \cdot 4=15 \cdot 6$ grains of proof spirit, containing about 7 grains of alcohol-a paltry result, and much too fallacious, whereon to found a fiscal persecution, as we shall presently show.

O 0 , yielded 700 grain measures of 0.9876 , equivalent to 16 per cent. of proof spirit $=112$ grains, besides 500 grains of 0.9974 , equivalent to $3 \cdot 2$ per cent. of proof $=16$ grain ; of proof; together $=128$ grains, from which $54 \cdot 4$ being deducted on account of the yeast spirit, there remain 73.6 of apparent spirit, as the product of the tobacco wash of half, a pound of $\mathrm{O} O$.

B B afforded 760 grains at 0.9946 , representing 6.7 per cent. of proof $=50$ grains of proof; to which $8 \frac{1}{2}$ grains for 240 , at 0.998 , the sum $58 \frac{1}{2}$ represents the whole obtained for this wash, and $54 \frac{1}{2}$ being deducted for the yeast spirit, there will remain 4 grains of proof spirit, corresponding to 2 grains of alcohol and 4 grains of sugar in 3,500 grains of the tobacco.*

The only inference that can be drawn from these results of experiments carefully conducted on the principles assumed as certain by Professor Graham and the iAessrs. Phillips, is, that the sample $O \mathrm{O}$ contained 73.4 grains or thereby of sugar mixed in the half pound of tobacco; that F F sample contained about $15 \cdot 6$ grains, and B B 4 ; wriereas, as it appears in the published report that there was no sugar in any one of the three samples, the fallacy of the excise process is manifest.

It would therefore seem, that infusions of tobacco without sugar, when mixed with brisk yeast, and placed for 40 hours in a temperature of about $80^{\circ}$, undergo a certain degree of decomposition, attended with a diminution of their specific gravity, or, in the vulgar language of the excise, they suffer attenuation. This phenomenon offers no difficulty to any one conversant with organic chemistry. He knows that there are no fewer than twelve different species of fermentation, all involving a specific series of decompositions and recompositions, each occupied with its appropriate subject, and gen erating peculiar products. See Fermentation in this supplement. I shall advert, in this place, merely to that marvellous metamorphosis which bitter almonds experience by contact of pure water; during which, aided by heat alone, the solid inert matter of the kernel is converted into a volatile, pungent, poisonous, ethereal oil, mixed with hydrocranic or prussic acid, a fluid lighter than water. Such remarkable changes must be well known to Mr. R. Phillips and Professor Graham, and ought to have made them hesitate before they pronounced a distilled fluid, which is destitute of the smell and taste of alcohol, and which they do not say they had submitted to the requisite ordeal, to be this substance.

If by fermenting the infusion of 3,500 grains of tobacco, my distilled products were so slight and fallacious, what could the chemist get from 1,000 grains? or, as Mr. Graham is wont to operate, from 200 grains, or less than half an ounce? See Question 7,548. Have they ever converted their supposed alcohol into ether, have they made fulminating mercury by its means, or have they extracted olefiant gas out of it? If not, their testimony would have been scouted in any of our great courts of judicature.

If sugar be present in any notable proportion, I think that it should be found by evaporating the watery extract to dryness, digesting the extract in alcohol, and then treating the residuum properly with nitric acid. From the quantity of oxalic acid formed, the proportion of sugar might possibly be approximately estimated. I am not aware that there are any principles in tobacco itself which would give rise to the formation of oxalic acid; but this point could be easily set at rest by preliminary experiments. I tried this method, and obtained, as I have stated, no oxalic acid from the samples subjected to the process.

The last series of experiments which I made upon the samples of tobacco sent to me by the committee, was the incineration of 500 grains of each in a platinum basin, and the analysis of the ashes. The results per cent. were as follows :-

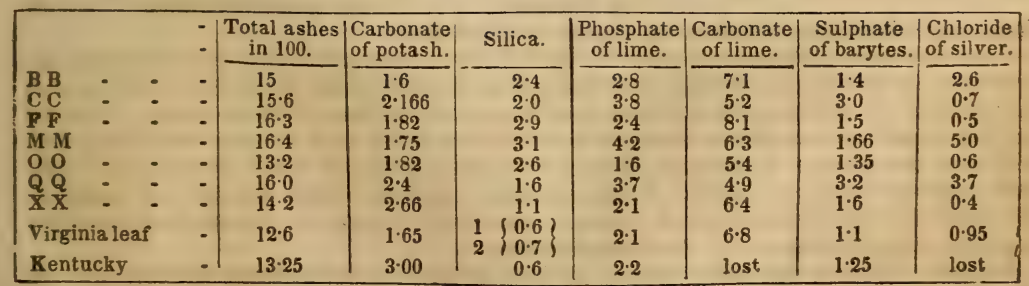

- I have not deemed it necessary to convert water-grain measures into weights, or vice versa, in this frivolous speculation. 
The results here stated may be relied on, as they were the mean of many very delib. erate experiments. They show that there are great variations in the proportions of the constituents, even in the five genuine tobaccos; B B, M M, O O, Virginia and Kentucky. But the alum in $\mathbf{C ~ C}$ and $\mathbf{Q}$, is indicated by the larger proportion of sulphate of barytes, obtained by precipitating the matter soluble in water, and acidulated with nitric acid, by means of nitrate of barytes. The sulphate of potash in F F bad been probably decomposed into carbonate during the ignition, along with carbonate of lime and carbonaceous matter; and has thereby escaped notice in the column of sulphate of barytes.

I tried each of the aqueous infusions of the fresh samples with solution of gelatine, but obtained no indication of tannin, as should have happened with $\mathbf{C ~ C}$, in consequence of the introduction into it, of 1 per cent. of terra japonica or catechu.

Finally, I regret exceedingly, that so short a space of time was allowed me for making and digesting all these various researches, prior to my examinations before the committee. Even my report supplementary to my oral evidence, was given in before I had finished my experiments on the action of nitric acid upon the tobacco extracts, and hence I mention there my having obtained crystals of oxalic acid, which turned out upon further examination to be no such thing.

The following analysis of 10,000 parts of fresh tobacco, by Posselt and Reimann, will show the exceeding complexity of this substance :-

Nicotine -
Eicotianine -
Gum with a little malate of lime
Green resin -
Vegetable albumen -
Substance analogous to gluten -
Malic acid -
Malate of ammonia -
Sulplate of potash -

\begin{tabular}{rr|}
- & 6 \\
- & 1 \\
- & 287 \\
- & 174 \\
- & 26.7 \\
- & 26.0 \\
- & 104.8 \\
- & 51.0 \\
- & 12.0 \\
- & 4.8
\end{tabular}

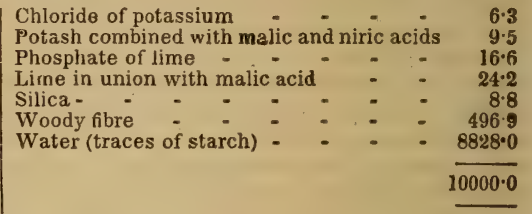

In Silliman's Journal, vol. vii., p. 2, a chemical examination of tobacco is given by Dr. Covell, which shows its components to have been but imperfectly represented in the above German analysis. He found, 1, gum; 2, a viscid slime, equally soluble in water and alcohol, and precipitable from both by subacetate of lead; $3, \operatorname{tannin}$; 4 , gallic acid; 5, chlorophyle (leaf-green); 6, a green pulverulent matter, which dissolves in boiling water, but falls down again when the water cools; 7, a yellow oil, possessing the smell, taste, and poisonous qualities of tobacco; 8 , a large quantity of a pale yellow resin; 9 , nicotine; 10, a white substance, analogous to morphia, soluble in hot, but hardly in cold, alcohol; 11, a beautiful orange-red dye stuff, soluble only in acids: it deflagrates in the fire, and seems to possess neutral properties; 12 , nicotianine. In the infusion and decoction of the leaves of tobacco, little of this substance is found; but after they are exhausted with ether, alcohol, and water, if they be treated with sulphuric acid, and evaporated nearly to dryness, crystals of sulphate of nicotianine are obtained. Ammonia precipitates the nicotianine from the solution in the state of a yellowish white, soft powdering matter, which may be kneaded into a lump, and is void of taste and smell, as all its neutral saline combinations also are : its most characteristic property is that of forming soluble and uncrystallizable compounds with vegetable acids.

According to Buchner, the seeds of tobacco yield a pale yellow extract to alcohol, which contains a compound of nicotine and sugar.-Repertorium für die Pharmacie, vol. xxxii.

MM. Henry and Boutron Charlard found in 1,000 parts of Cuba tobacco Maryland - Virginia - Ile et Vilaine Lot et Garonne

- 8.64 of nicotine;

$-5 \cdot 28$

$-10 \cdot 00$

$-11 \cdot 20$

- 8.20; quantities from 12 to 19

\section{times more than were obtained by Posselt and Reimann.}

I shall conclude this examination of the tobacco report with a few remarks upon the pretences of the Messrs. Phillips and Graham to botanical and microsenpic skill in distinguishing the minutest filaments of shag tobacco from those of other plants. Having applied a good achromatic microscope to this object along with my son, who is familiar with the use of that instrument, I must acknowledge that I would place exceedingly little reliance on the possibility of distinguishing such vegetable leaves as I could easily select for the adulteration of tobacco; and I will engage to set at fault even the vuperior accomplishments of Professor Lindley.

" 6,999 . When a vegetable fibrous addition is made to the ordinary tobacco, and so ground and minutely divided as not to allow an examination by the glass, could you 
distinguish it from tobacco ?"-Mr. Graham's answer : "It would be extremely diff cult to divide it so finely as not to present a sensible magnitude to the microscope. I have never met with tobacco manufactured for sale as shag-tobacco, in which I could not distinguish it."

Mr. R. Phillips, in reply to question 7,511 : "Generally speaking, we did not employ any chemical tests."

" 7,512 Then it was principally by mechanical analysis, and examination of the fibre of the plant, that you judged ?-Yes, certainly."

Answer to question 7,523: "You may distinguish it (tobacco) not by the naked eye, but by the microscope."

" 7,856. Can you [Mr. G. Phillips] distinguish the fibre of tobacco from the fibre of dock, or any other vegetable of the same family?-Yes.

" $7,85 \%$. In how small a quantity can you detect it?-However small the quantity, if you take pains, you can discover it : nothing can be finer than the sample $\mathrm{K}$, in which there was foxglove."

Professor Lindley and Mr. George Phillips distinguish tobacco from ither plants chiefly by the structure of its hairs. But in Geiger's "Pharmaceutische Botanik," the second edition, improved by Nees von Esenbeck, and Heinrich Dierbach, a book of standard authority, the Nicotiana tabucum of Linnæus, which is the Florida tobacco of the French botanists, is described as having smooth (glabra), somewhat glutinous leaves. Several varieties of this plant are said to be cultivated under peculiar provincial names, to which the Nicotiana petiolata, Nicotiana decurrens, \&c., belong; all with smooth and blistery leaves.

In my examination before the committee on the 15 th of July, in answer to question 8,569 , I said-" The conclusion to which I am led is this, that when the tobacco is brought in this shag state, it is next to impossible, by chemical means in most cases, or by physiological means, to determine the adulteration; the only case in which adulteration ean be detected, in my opinion, is when sugar is mixed.

" 8,570 . Does the presence of alcohol, by distillation from a fermented solution, give you an invariable test that sugar is present?-If sugar is present in any quantity above 5 per cent., I think alcohol may be produced from it."

But I would never content myself with the deceptious test of the specific gravity of a minute portion of the distilled liquid. I would take at least seven pounds of the suspected tobacoo, rinse it rapidly in cold water, in order to dissolve out the saccharine matter, with as little as possible of the tobacco extract; mix it with a certain quantity of yeast; take the specific gravity of the mixture; set it in a chamber heated to $80^{\circ} \mathrm{F}$., and watch the phenomena of fermentation, if any occur. At the end of 40 hours, or whenever the density of the mixture had sunk to the lowest point, I would note it; then distil, rectify the distilled liquor, and expose it to the appropriate tests of alcohol, as stated above. I am quite convinced that no certainty could be obtained by operating upon the infusion of 200 grains of a tobacco containing 5 or 10 per cent. of sugar, as Professor Graham, in his evidence before the magistrates in the Gainsborough prosecution, said he had done with the tobacco then in question. The total quantity of sugar that could be present was under 20 grains, and this being mixed with tobacco-juice, which counteracts the fermentative process, would afford a most unsatisfactory quantity of alcohol-a quantity most difficult of verification; one on which, in my humble apprehension, knowing, as I do, the fallacy of chemical experiments and experimenters, no person should ever venture to seek a verdict, or to levy a heavy fine.

" 8,589 . Then you are of opinion that it will be impossible, if care be taken, such as you state, by chemists, for detection to be within the power of the government ?Quite impossible. I will pledge my chemical character to make such specimens as the excise can not detect.

" 8,590 . Then to continue the system of alleged detection by analysis might subject individuals to punishment most unjustly.

"8,591. Have any cases come under your knowledge of errors in judgment upon 1 1at point?-There is a case which has lately occurred to me of a very unjustifiable kind on the part of the excise, aad I think I might mention it. It is a case of pepper.

" 8,592 . Will you describe the case?-About a year ago, the excise officers entered the premises of Messrs. Mayor and Dove, large spice merchants in Little Distaff Lane, and seized a quantity of ground white pepper, alleging it to be adulterated, and carried it off. I attended the court of excise. Professor Graham and Mr. George Phillips, the two witnesses as to the adulteration on the part of the excise, were first examined, and they swore that the seized pepper contained sago to the amount of 10 or 12 per cent., and they produced a few particles like sago in a very small pill-box." [For the other details, see the article PEPPER in this Supplement.]

" 8,596 . From the advance of chemical science, suppose the excise office to have your assistance, or the assistance of other experienced chemists, do you think that, wite 
all that assistance, they could detect an adulteration that might, with perfect facility, be introduced by chemists ?-I would say that adulteration may te made upon tobacco which may defy all the chemists in Europe to find out.

" 8,597. And not only chemists, but physiologists ?-Yes, and botanists."

It will be seen, from the vagueness of the results of the several series of experiments which I made on the seven samples of tobacco sent to me by the committee, with every possible attention in the short period allowed me, that it is no easy matter to detect adulterations in tobacco ; and a chemist should be extremely cautious in pronouncing a decided opinion upon such slender grounds as the professional gentlemen employed in the excise board have in many cases done. Supposing a tobacco poor in extractive matter, like the Kentucky, were skilfully imbued with juice of liquorice till it came to the standard of the Virginia, neither Mr. G. Phillips, by his plan of infusion, nor Professor Graham, by that of fermentation, could detect the adulteration. The liquorice-juice assimilates with tobacco better even than sugar, but " it is incapable of undergoing the spirituous fermentation."**

I offer this one example, out of many, merely as a hint to my brother chemists to be somewhat less confident and dogmatical in their decisions. Were the questions of tobacco adulteration referred to one of our law courts in Westminster or Guildhall, the evidence of the chemists for the prosecution would be weighed in a more ticklish balance than that of a provincial justice of the peace, or even of the honorable commissioners of excise, and it might possibly be found wanting.

How vexatiously inquisitorial, and how abhorrent from the genius of the British constitution, must the practice of the excise board be, when the following regulations are recommended by its most influential functionary! To question 8,005, Mr. George Phillips replies-"I would make the manufacturers responsible for the samples which they gave; for instance, we know very well there are only two tobaccoes used for general cutting tobaccoes, that is, Kentucky and Virginia ; we know the nature of those, and we very well know what description of tobacco the manufacturer must use to make it answer his purpose. A tobacco which will not yield 45 per cent. of extractive, is not fit for him to use. If he sent a sample which should be 35, such as Porto Rico, or got some rubbishing stuff from the sales at the tobacco warehouse, $I$ would not allow that sample to be used, or at any rate to be mixed with any other; if he used that, he should use that alone; he should be confined within a range, which experience has proved to be the general range." No choice for taste is to be allowed. Again, to question 8,023, he answers, "Sometimes the seizures are made before the tobacco is examined; sometimes seizures are made afterward, upon $m y$ report that it is adulterated. The officers send a sample up unknown to the manufacturer : they take a samiple unknown to the manufacturer, and then, after I have examined it, instructions are sent to the supervisor, that any tobacco of that sort that he can find on the manufacturer's premises, he should seize. If the tobacco is seized merely upon the examination of the sample, samples taken from the bulk of the seizure are then sent up and examined. I could mention cases where samples have been sent up by the supervisor or other officer, and have been examined, they have gone and seized, after the lapse of a fortnight, and it has turned out that the - tobacco has been pure when it has been examined; of course, that has been returned again." Question 8,024: "How small an amount would you report to be adulterated ? Two per cent."

Every intelligent reader of the experimental and other evidence detailed in the present article, must perceive the precariousness of decisions hased upon an adulteration of only 2 per cent., in so complex a substance as tobacco, that adulteration being sworn to in consequence of such unsatisfactory microscopic and chemical researches. What a servile spirit must be engendered among the tributaries to the excise, when thirteen eminent tobacconists of London could recently petition the House of Commons to aggravate the astringent administration of that tribunal, praying that adulterations detected by its officers should be prosecuted more rigorously; and the efficiency of the law be "further secured by the abolition of compromise, publicity by prosecution in the local courts or otherwise, and the substitution of personal for pecuniary penalties."

What powerful inducements are held out to Mr. George Phillips and his coadjutore to obtain convictions for adulterations of tobacco, may be inferred from the fact, that " all penalties and seizures are by law divisible in equal parts, between the crown and the informer ;" 1 , to the person by whom the information was communicated; 2 , to the officers by whose instrumentality, and subsequent aid, proceedings for penalties are brought to a successful termination. In all other cases, liberal means of remuneration are placed at the disposal of the board of excise.-See Memorandum as to rewards for information given to the Excise, p. 584, Tobacco Report. 
TORTOISE-SHELL is manufactured in various objects, partly by cutting out the shapes, and partly by agglutinating portions of the shell by heat. When the shell has become soft by dipping it in hot water, the edges are in the cleanest possible state, without grease, pressed together with hot flat tongs, and then plunged into cold water, to fix them in their position. The teeth of the larger combs are parted in their heated state, or cut out with a thin frame saw, while the shell, equal in size to two combs, with their teeth interlaced, as in fig. 166, is bent like an arch in the direction of the
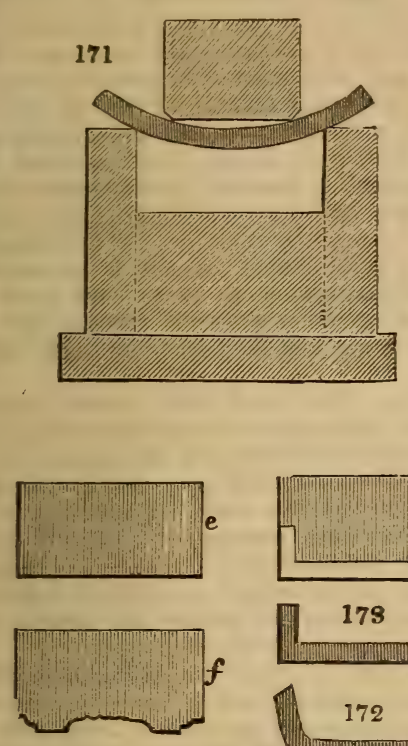
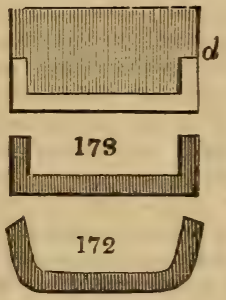

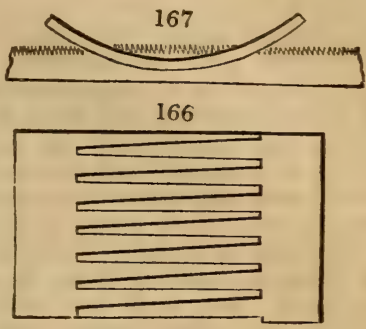

168
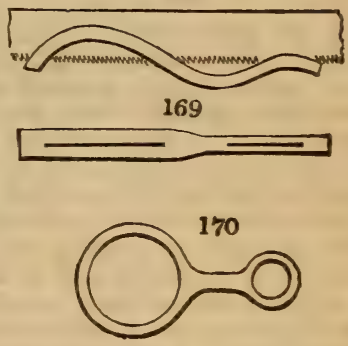

length of the teeth, as in fig. 16\%. The shell is then flattened, the points are separated with a narrow chisel or pricker, aud the two combs are finished, while flat, with coarse single-cut files and triangular scrapers. They are finally warmed, and bent on the knee over a wooden mould, by means of a strap passed round the foot, just as a shoemaker fixes his last. Smaller combs of horn and tortoise-shell are parted, while flat, by an ingenious machine, with two chisel-formed cutters placed obliquely, so that each cut produces one tooth. See Rogers' comb-cutting machine, Trans. Soc. Arts, vol. xlix., part 2, since improved by Mr. Kelly. In making the frames for eye-glasses, spectacles, \&c., the apertures for the glasses were formerly cut out to the circular form, with a tool something like a carpenter's centre-bit, or with a crown saw in the lathe. The dises so cut out were used for inlaying in the tops of boxes, sc. This required a piece of shell as large as the front of the spectacle; but a piece one third of the size will now suffice, as the eyes are strained or pulled. A long narrow piece is cut out, and two slits are made in it with a saw. The shell is then warmed, the apertures are pulled open, and fastened upon a taper triblet of the appropriate shape; as illustrated by figs. 168, 169, and 170. The groove for the edge of the glass is cut with a small circular cutter, or sharp-edged saw, about three eighths or half an inch in diameter; and the glass is sprung in when the frame is expanded by heat.

In making tortoise-shell boxes, the round plate of shell is first placed centrally over the edge of the ring, as in fig. 171: it is slightly squeezed with the small round edgeblock $\mathrm{g}$, and the whole press is then lowered into the boiling water: after immersion for a jout half an hour, it is transferred to the bench, and $g$ is pressed entirely down, so as $t 0$ bend the shell into the shape of a saucer, as at fig. 172, without cutting or injuring the material; and the press is then cooled in a water-trough. The same processes are repeated with the die $d$, which has a rebate turned away to the thickness of the shell, and completes the angle of the box to the section fig. 173, ready for finishing in the lathe. It is always safer to perform each of these processes at two successive boilings and coolings. Two thin pieces are cemented together by pressure with the die $e$, and a device may be given by the engraved die f.-See Holtzapffel's Turning and Mechanical Manipulation, vol. i., p. 129. 
TURPENTINE, SPIRITS, ESSENCE, OR OIL OF. Camphen is the now name given by the continental chemists to every ethcreous or volatile oil, which is com. posed of 5 atoms of carbon and 8 of hydrogen, and which coma bines directly with hydrochloric acid, either into a solid or a liquid compound, resembling camphor. Under this title the following oils are included: turpentine, citron, or lemon, orange-flower, copaiva, balsam-oil, juniper, cubebs, and pepper. Some add to this last-the oils of cloves, valerian, and bergamot. As the new patent lamps burn spirits of turpentine, they have been called Camphine. (See LAMPs.) Since that article was printed I have had occasion to test a variety of Camphine lamps during the preceding three months, and I am convinced the patent Vesta lamp of Mr. Young is not merely the best, but it is the only one hitherto made public, which can be used with comfort in closed apartments. It was the first spirit lamp constructed on right principles, keeping in view the peculiar nature of Camphine spirits, and being secured by a correct specification, leaves no room to expect another equally good. In this lamp the burner is completely insulated from the reservoir by a ring of wood, or other non-conducting material, placed between them, and as no metallic tube passes down from the flame into the volatile spirits, they remain eold; whereas, when such a tube passes down through the reservoir, for the admission of air to the inside of the flame (as in all other argand lamps), without being insulated from the flame, the spirits become 20 or 30 degrees hotter, so as to emit acrid and offensive fumes. The wick also, which embraces the heated tube becomes dry and resinous, loses its capillary power, coals at the flame, and then sends up smoke with a shower of lamp black.

The Vesta lamp is free from these defects, and when used with properly rectified spirits, never smokes nor smells ; it may be easily distinguished by the above characters, and by the circumstance of the air passing between the wicks to the interior of the flame. It affords, undoubtedly, the brightest, cleanliest, and most economical light, hitherto invented, when supplied with pure spirits free from rosin. I have lighted my drawing-rooms with the Vesta lainp for several evenings successively, without having its wick trimmed or its occasioning the slightest inconvenience. I therefore deem it due to the patentee's ingenuity, as well as to the public welfare, to give this deliberate opinion at a time when the volatile spirits of turpentine are getting into general use, and when, if burned in lamps on the argand plan, they must create danger.

Great care must be taken in the choice of the spirits of turpentine as the combustible. As those very generally sold in London contain rosin and other impurities, they are quite unfit for that purpose; but the spirits manufactured by Messrs. John Tall and Co. of Hull, to be had of their agents, Ratcliffe and Co., 103 Hatton Garden, London, answer perfectly. I have subjected these spirits to careful chemical examination, and I find them to be quite pure, and very different indeed from those on common sale here. Their specific gravity is only 0.864 at $62^{\circ} \mathrm{Fahr}$, while that of the average London article is from 0.874 to 0.882 , the greater density being due to rosin. Messrs. Tall's spirits may be boiled off in a retort without leaving any sensible residuum, and they also boil at a lower degree of heat; but the best proof of their excellence, in the present point of view, is exhibited in the preceding notice of the Vesta lamp, for it was Messrs. Tall's spirits which were used on that occasion.

\section{V.}

VENTILATION. There are two general plans in use for at once diffusing heat and renewing the air in extensive buildings, which plans differ essentially in their principles, modes of action, and effects. The oldest, and what may be called, the vulgar method, consists in planting stoves in the passages or rooms, to give warmth in cold weather, and in constructing large and lofty chimney-stalks, to draw air in hot weather out of the house, by suction, so to speak, whereby fresh air flows in, to maintain, though . imperfectly, an equilibrium of pressure. In apartments, thus warmed and ventilated, the atmosphere is necessarily rarer than it is out of doors, while, in cold weather, the external air rushes in at every opening and crevice of door, window, or chimney-the fruitful source of indisposition to the inmates.

The evils resulting from the stove-heating and air-rarefying system were, a few years ago, investigated by me, in a paper read before the Royal Society,* and afterward

* I had been professionally employed by a committee of the officers of the customhouse, to ex amine the nature of the malaria which prevailed there, but I had no concern in erecting the stoves which caused it. 
published in several scientific and technological journals. It is there said that the observations of Saussure, and other scientific travellers in mountainous regions, demon. strate how difficult and painful it is to make muscular or mental exertions in rarefied air. Even the slight rarefaction of the atmosphere, corresponding to a low state of the barometer, at the level of the sea, is sufficient to occasion languor, lassitude, and uneasiness, in persons of delicate nerves; while the opposite condition of increased pressure as indicated by a high state of the barouneter, has a bracing effect upon both body and mind. Thus, we see how ventilation, by the powerful draught of a high chimney-stalk, as it operates by pumping out, exhausting and attenuating the air, may prove detrimental to vivacity and health; and how ventilation, by forcing in air with a fan or a pump, is greatly to be preferred, not only for the reason above assigned, but because it prevents all regurgitation of foul air down the chimneys, an accident sure to happen in the former method. Genial air thrown in by a fan, in the basement story of a building, also prevents the stagnation of vapors from damp and miasmata, which lurk about the foundation of buildings and in sewers, and which are sucked in by the rarefying plan. Many a lordly mansion is rendered hardly tenantable from such a cause, during certain vicissitudes of wind and weather.

The condensing plan, as executed by the engineers, Messrs. Easton and Amos, at the Reform Club House, consists of a large fan, revolving rapidly in a cylindrical case, and is capable of throwing 11,000 cubic feet of air per minute, into a spacious subterranean tunnel, under the basement story. The fan is driven by an elegant steam-engine, worked on the expansion principle, of 5 horses' power. It is placed in a vault, under the flagparement, in front of the building; and as it moves very smoothly, and burns merely cinders from the house fires, along with some anthracite, it occasions no nuisance of any kind. The steam of condensation of the engine supplies 3 cast-iron chests with the requisite heat for warming the whole of the building. Each of these chests is a cube of 3 feet externally, and is distributed internally into 7 parallel cast-iron cases, each about 3 inches wide, which are separated by parallel alternate spaces, of the same width, for the passage of the air transversely, as it is impelled by the fan.

Fig. 174 is a transverse vertical section of the steam-chest, for heating the air; fig. 175 is a plan of the same; and fig. 176 is a perspective view, showing the outside casing. also the pipe $a$, for admitting the steam, and the stop-cock $b$, for allowing the condensed water to escape.

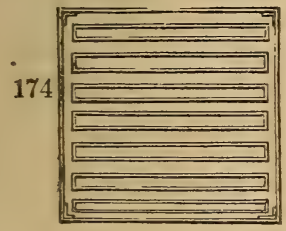

175

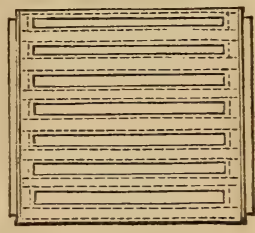

176

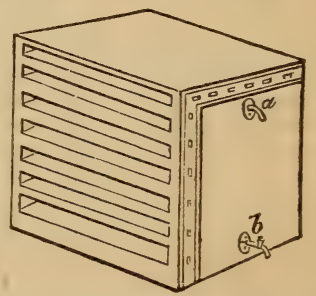

This arrangement is most judicious, economizing fuel to the utmost degree; because the steam of condensation which, in a Watt's engine, would be absorbed and carried off by the air-pump, is here turned to good account, in warming the air of ventilation during the winter months. Two hundred weight of fuel suffice for working this steamengine during twelve hours. It pumps water for household purposes, raises the coals to the several apartments on the upper flours, and drives the fan ventilator. The air, in flowing rapidly through the series of cells, placed alternately between the steamcases, can not be scorched, as it is generally with air stoves; but it is heated only to the genial temperature of from $75^{\circ}$ to $85^{\circ}$ Fahr., and it thence enters a common chamber of brickwork in the basement story, from which it is let off into a series of distinct flues, governed by dialled valves or registers, whereby it is conducted in regulated quantities to the sereral apartments of the building. I am of opinion that it would not be easy to devise a better plan for the purpose of warming and ventilating a large house; and I am only sorry to observe, that the plan projected by the engineers has been injudiciously counteracted in two particulars.

The first of these is, that the external air, which supplies the fan, is made to traverse a great heap of coke before it can enter that apparatus, whereby it suffers such friction as materially to obstruct the ventilation of the house. The following experiments, which I made recently upon this point, will place the evil in a proper light: Having fitted up Dr. Wollaston's differential barometer, as an anemometer, with oil, of specific gravity 0.900 in one leg of its syphon, and water of 1.000 in the other, covered with the said oil in the two eisterns at top, I found that the stream of air produced by the fan, in a ces 
tain part of the flue, had a velocity only as the number 8 , while the air was drawn through the coke, but that it had a velocity in the same place as the number 11 , whenever the air was freely admitted to the fan by opening a side door. Thus, three elevenths, both of the ventilating and warming eflect of the fan, are lost. I can not divine any good reason for making the members of the Reform Club breathe an atmosphere, certainly not improved, but most probably vitiated, by being passed in a moist state through a porous sulphurous carbon, whereby it will tend to generate the two deleterious gases, carbonic oxide and sulphuretted hydrogen, in a greater or less degree. It is vain to allege that these gases may not be discoverable by chemical analy. sis-can the gaseous matters, which generate cholera, yellow fever, or ague, be detected by chemical reagents? No, truly; yet every one admits the reality of their specific virus. I should propose that the air be transmitted through a large sheet of wire-cloth before it reaches the fan, whereby it would be freed from the grosser particles of soot that pollute the atmosphere of London. The wire-cloth should be brushed every morning.

The second particular, which counteracts in some measure the good effects of the fan in steam ventilation, is the huge stove placed in the top story of the building. This potent furnace, consuming, when in action, 3 cwt. of coals per day, tends to draw down foul air, for its own supply, from the chimneys of the adjoining rooms, and thus to impede the upward current created by the fan. I have measured, by Dr. Wollaston's differential barometer, the ventilating influence of the said furnace stove, and find it to be perfectly insignificant-nay, most absurdly so-when compared with the fan, as to the quantity of fuel which each requires per day. The rarefaction of air in the stove chamber, in reference to the external air, was indicated by a quarter of an inch difference of level in the legs of the oil and water syphon, and this when the door of the stove-room was shut, as it usually is; the tube of the differential barometer being inserted in a hole in the door. The fan indicates a ventilating force equal to 2 inches of the water syphon, which is 20 inches of the above oil and water syphon, and therefore 80 times greater than that of the stove furnace; so that, taking into view the smaller quantity of fuel which the fan requires, the advantage in ventilation, in favor of the fan, in the enormous ratio of 120 to 1 , at the lowest estimate. The said stove, in the attic, seems to me to be not only futile, but dangerous. It is a huge rectangular cast-iron chest, having a large hopper in front, kept full of coals, and it is contracted above into a round pipe, which discharges the burnt air and smoke into a series of horizontal pipes of cast-iron, about 4 inches diameter, which traverse the room under the ceiling, and terminate in a brick chimney. In consequence of this obstruction, the draught through the furnace is so feeble, that no rush of air can be perceived in its ashpit, even when this is contracted to an area of 6 inches square: nay, when the ash-pit was momentarily luted with bricks and clay, and the tube of the differential barometer was introduced a little way under the grate, the level of the oil and water syphon in that instrument was displaced by no more than one tenth of an inch, which is only one hundredth of an inch of water-a most impotent effect under a daily consumption of $3 \mathrm{cwt}$. of coals. In fact, this stove may be fitly styled an incendiary coal-devourer, as it has already set fire to the house; and though now laid upon a new floor of iron rafters and stone flags, it still offers so much danger from its outlet iron pipes, should they become ignited from the combustion of charcoal deposited in them, that I think no premium of insurance adequate to cover the imminent risk of fire. The stove being, therefore, a superfluous and dangerous nuisance, should be turned out of doors as speedily as possible. Its total cost, with that of its fellow in the basement story, can not be much less than the cost of the steam-engine, with all its truly effectual warming and ventilating appurtenances.

I take leave to observe, that the system of heating and ventilating apparatus, constructed by Messrs. Easton and Amos, in the Reform Club House, offers one striking and peculiar advantage. It may be modified at little expense, so as to become the ready means of introducing, during the sultriest dog-days, refreshing currents of air, at a temperature of 10,20,30, or even 40 degrees under that of atmosphere. An apparatus of this nature, attached to the houses of parliament and courts of law, would prove an inestimable blessing to our legislators, lawyers, judges, and juries. Of such conl air a very gentle stream would suffice to make the most crowded apartments comfortable, without endangering the health of their inmates with gusts of wind through the doors, windows, and floors.

It is lamentable to reflect how little has been done for the well-being of the sentient and breathing functions of man in the public buildings of the metropolis, notwithstanding our boasted march of intellect, and diffusion of useful knowledge. Almost all our churches are filled on Sundays with stove-roasted air ; and even the House of Commons has its atmosphere exhausted by the suction of a huge chimney-stalk, with a furnace equal, it is said, to that of a 40 -horse steam-boiler. To gentlemen plunged in air so 
attenuated, condensation of thought and terseness of expression can hardly be the order of the day.

Nearly seven years have elapsed since I endeavored to point public attention to this important subject in the following terms: "Our legislators, when bewailing, not long ago, the fate of their fellow-creatures, doomed to breathe the polluted air of a factory, were little aware how superior the system of ventilation adopted in many cotton-mills was to that employed for their own comfort in either house of parliament. The engineers of Manchester do not, like those of the metropolis, trust for a sufficient supply of fresh air into any crowded hall, to currents physically created in the atmosphere by the difference of temperature excited by chimney-draughts, because they know them to be ineffectual to remove, with requisite rapidity, the dense carbonic acid gas generated by many hundred powerful lungs." ${ }^{\prime *}$ At page 382 of the work just quoted, there is an exact drawing and description of the factory ventilating fan.

On the 6th of June, 1836, I took occasion again, in a paper read before the Royal Society, upon the subject of the malaria which then prevailed in the customhouse, to investigate the principles of ventilation by the fan, and to demonstrate, by a numerous train of experiments, the great preference due to it, as to effect, economy, and comfort, over chimney-draught ventilation. Yet at this very time, the latter most objectionable plan was in progress of construction, upon a colossal scale, for the House of Commons. About the same period, however, the late ingenious Mr. Oldham, engineer of the bank of England, mounted a mechanical ventilator and steam-chest heater, for supplying a copious current of warm air to the rooms of the engraving and printing departments of that establishment. Instead of a fan, Mr. Oldham employed a large pump to force the air through the alternate cells of his steam-chest. He had introduced a similar system into the bank of Ireland about ten years before, which is now in full action.

About two years ago, Messrs. Easton and Amos were employed to ventilate the letter carriers' and inland office departments of the general post-office, of which the atmosphere was rendered not only uncomfortable but insalubrious, by the numerous gaslights required there in the evenings. This task has been executed to the entire satisfaction of their employers, by means of fans driven by steam-engine power. The said engineers made, about the same time, a set of machinery similar to that erected at the bank of England, for warming and ventilating the bank of Vienna. They are justly encitled to the credit of having been the first to execute, in all its bearings, the system of heating and ventilating buildings, having special respect to the health of their inmates, which I urged upon the public mind many years ago.

As fans of sufficient size, driven by steam power with sufficient velocity to warm in winter, and ventilate at all times, the most extensive buildings, may be erected upon the principles above described, without causing any nuisance from smoke, it is to be hoped that the chapel of Henry VII. will not be desecrated by having a factory Vesurius reared in its classical precincts, and that the noble pile of architecture of the new houses of parliament will not be disfigured with such a foul phenomenon.

The cheering and bracing action of condensed air, and the opposite effects of rarefied air upon human beings, formed the subject of several fine physiological experiments, made a few years ago by $\mathbf{M}$. Junot, and described by him in the ninth volume of the Archives Générales de Médecine: "When a person is placed," says he, "in condensed air, he breathes with a new facility; he feels as if the capacity of his lungs was enlarged; his respirations become deeper and less frequent; he experiences, in the course of a short time, an agreeable glow in his chest, as if the pulmonary cells were becoming dilated with an elastic spirit, while the whole frame receives, at each inspiration, fresh vital impulsion. The functions of the brain get excited, the imagination becomes vivid, and the ideas flow with a delightful facility; digestion is rendered more active, as after gentle exercise in the air, because the secretory organs participate immediately in the increased energy of the arterial system, and there is therefore no thirst."

In rarefied air the effects on the living functions are just the reverse. The breathing is difficult, feeble, frequent, and terminates in an asthmatic paroxysm; the pulse is quick and most compressible; hœmorrhages often occur, with a tendency to fainting; the secretions are scanty or totally suppressed, and at lenth apathy supervenes.

These striking results obtained on one individual at a time, with a small experimental apparatus, have been recently reproduced, on a working scale, with many persons at once enclosed in a mining-shaft, encased with strong tubbing, formed of a series of large sheet-iron cylinders, riveted together, and sunk to a great depth through the bed of the river Loire, near Languin. The seams of coal, in this distriet of France, lie under a stratum of quicksand, from 18 to 20 metres thick (20 to 22 yards), and they had been found to be inaccessible by all the ordinary modes of mining previously practised. The obstacle had been regarded to be so perfectly insurmountable, that every 
portion of the great coa known for centuries, had remained untouched. To endeavor, by the usual workings, to penetrate through these semi-fluid quicksands, which communicate with the waters of the Loire, was, in fact, nothing less than to try to sink a shaft in that river, or to drain the river itself. But this difficulty has been successfully grappled with, through the resources of science, boldly applied by M. Triger, an able civil engineer.

By means of the above frame of iron tubbing, furnished with an air-tight antechamber at its top, he has contrived to keep his workmen immersed in air, sufficiently condensed by forcing-pumps, to repel the water from the bottom of the iron cylinders, and thereby to enable them to excavate the gravel and stones to a great depth. The compartment at top has a man-hole door in its cover, and another in its floor. The men, after being introduced into it, shut the door over their heads, and then turn the stop-cock upon a pipe, in connexion with the condensed air in the under shaft. An equilibrium of pressure is soon established in the ante-chamber, by the influx of the dense air from below, whereby the man-hole door in the floor may be readily opened, to allow the men to descend. Here they work in air, maintained at a pressure of three atmospheres, by the incessant action of leathern-valved pumps, driven by a steam-engine. While the dense air thus drives the waters of the quicksand, communicating with the Loire, out of the shaft, it infuses at the same time such energy into the miness, that they can easily excavate double the work without fatigue which they could do in the open air. Upon many of them the first sensations are painful, especially upon the ears and eyes, but ere loing they get quite reconciled to the bracing element. Old asthmatic men become here effective operatives; deaf persons recover their hearing, while others are sensible to the slightest whisper. The latter phenomenon proceeds from the stronger pulses of the dense air upon the membrane of the drum of the ear.

Much annoyance was at first experienced from the rapid combustion of the candles, but this was obviated by the substitution of flax for cotton thread in the wicks. The temperature of the air is raised a few degrees by the condensation.

Men who descend to considerable depths in diving-bells, experience an augmentation of muscular energy, similar to that above described. They thereby acquire the power of bending over their knees strong bars of iron, which they would find quite inflexibie by their utmost efforts when drawn up to the surface.

These curious facts clearly illustrate and strongly enforce the propriety of ventilating apartments by means of condensed air, and not by air rarefied with large chimneydraughts, as has been hitherto most injudiciously, wastefully, and filthily done, in too many cases.

VERMICELLI is made with most advantage from the flour of southern countries, which is richest in gluten. It may also be made from our ordinary flour, provided an addition of gluten be made to the flour paste. Vermicelli prepared from ordinary flour is apt to melt into a paste when boiled in soups. It may, however, be well made economically by the following prescription :-

\begin{tabular}{lllllll} 
Vermicelli or Naples flour & - & - & - & - & $-21 \mathrm{lbs}$. \\
$\begin{array}{l}\text { White potato flour } \\
\text { Boiling water - }\end{array}$ & - & - & - & - & - & -14 \\
& & - & - & - & $-12-$ \\
\hline
\end{tabular}

Affording $45 \mathrm{lbs}$. of dough, and 30 of dry vermicelli. With gluten, made from common flour, the proportions are-

$\begin{array}{lllllll}\text { Flour as above } & - & - & - & - & - & -30 \mathrm{lbs} . \\ \begin{array}{l}\text { Fresh gluten } \\ \text { Water - }\end{array} & - & - & - & - & - & -10- \\ & & - & - & - & - & -7 \\ \text { Total } & - & - & - & -47 \mathrm{lbs} .\end{array}$

Affording $30 \mathrm{lbs}$. of dry vermicelli or macaroni.

\section{W.}

WATERS, MINERAL. The following tables exhibit the nature and composition of the most celebrated mineral waters of Germany, according to the best analyses. The symbol $\mathrm{N}$ denotes nitrogen or azote; $\mathrm{O}$, oxygen; $\mathrm{CO}^{2}$, carbonic acid; $\mathrm{SH}$, sulphuretted hydrogen. Therm. ; cent. scale; if not, $\mathbf{R}$. for Reaumur. 


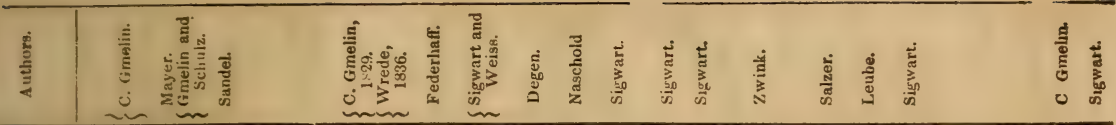

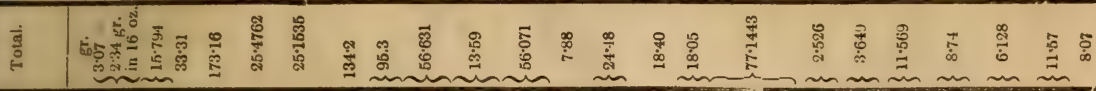

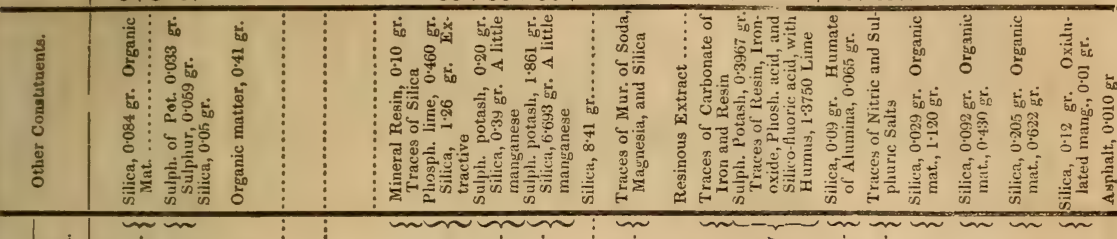

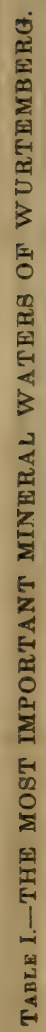

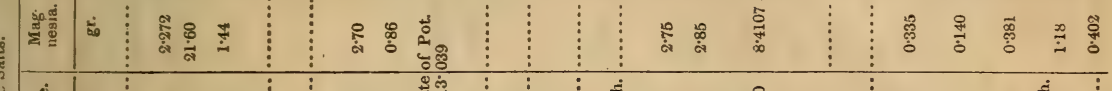

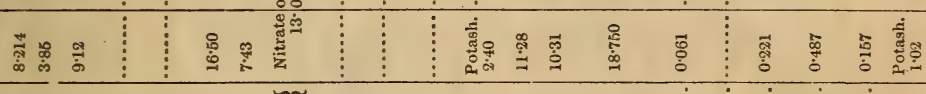

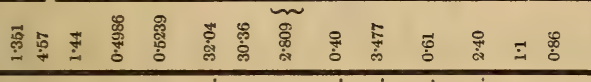

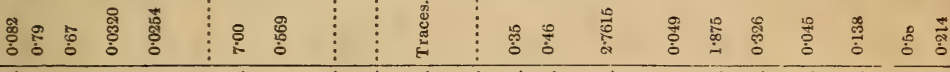

离

参

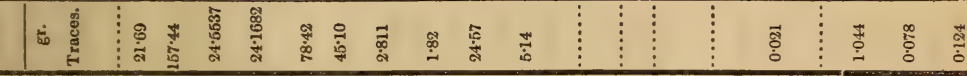

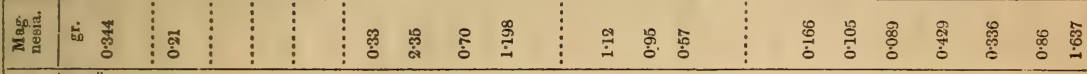

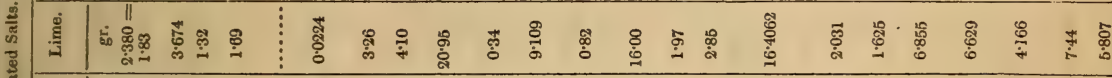

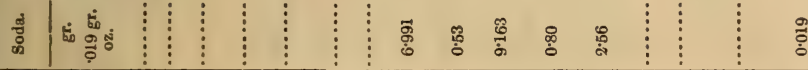

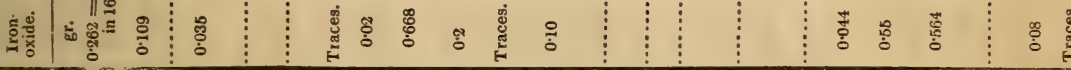

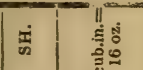

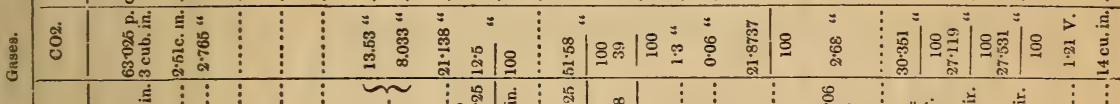

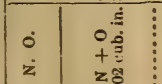

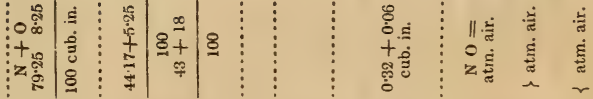

एक

110

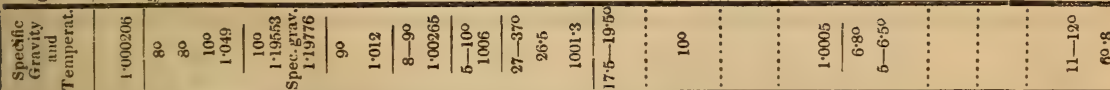

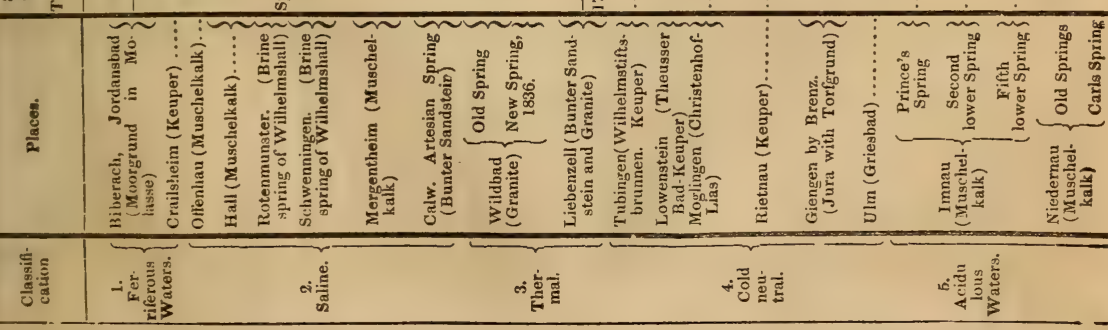




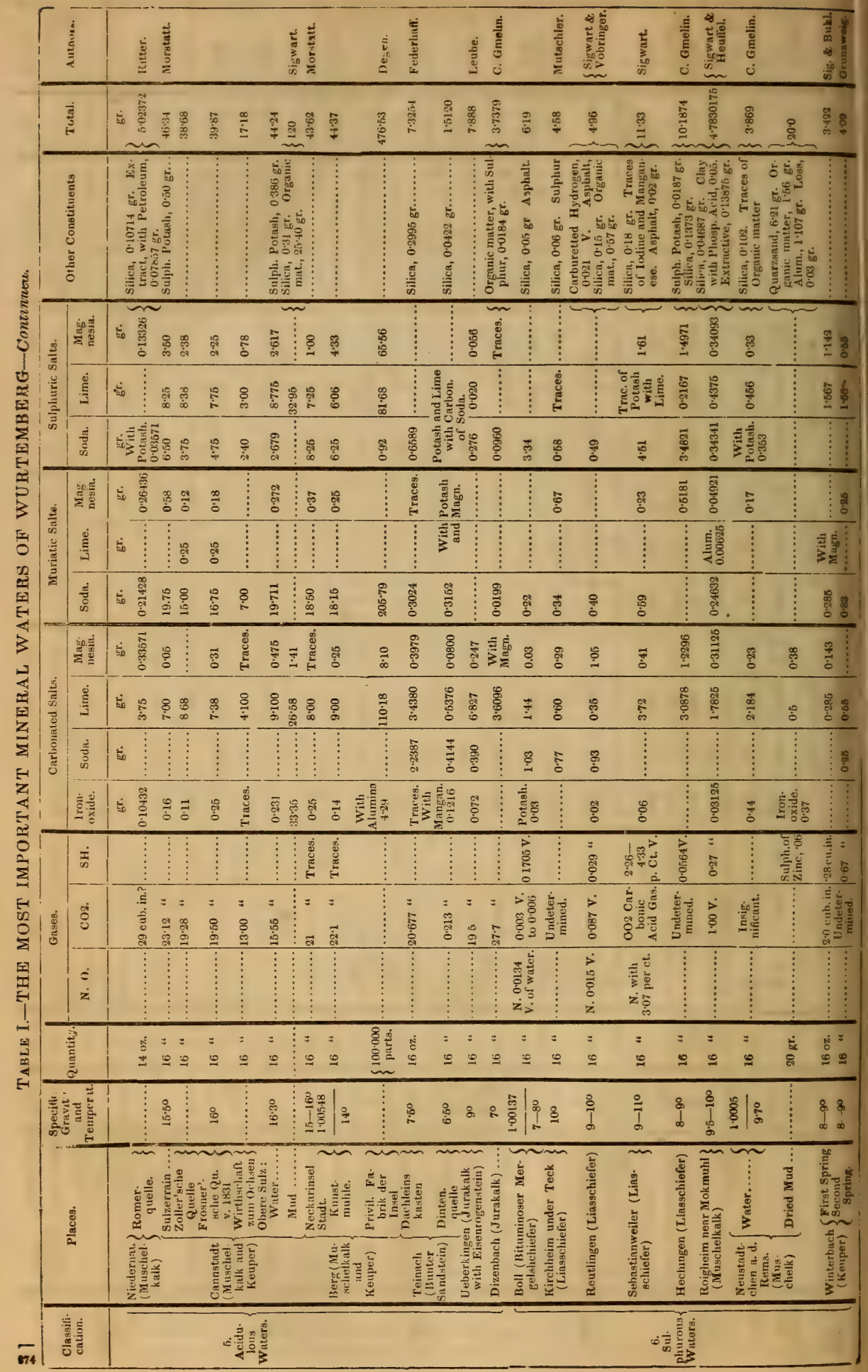




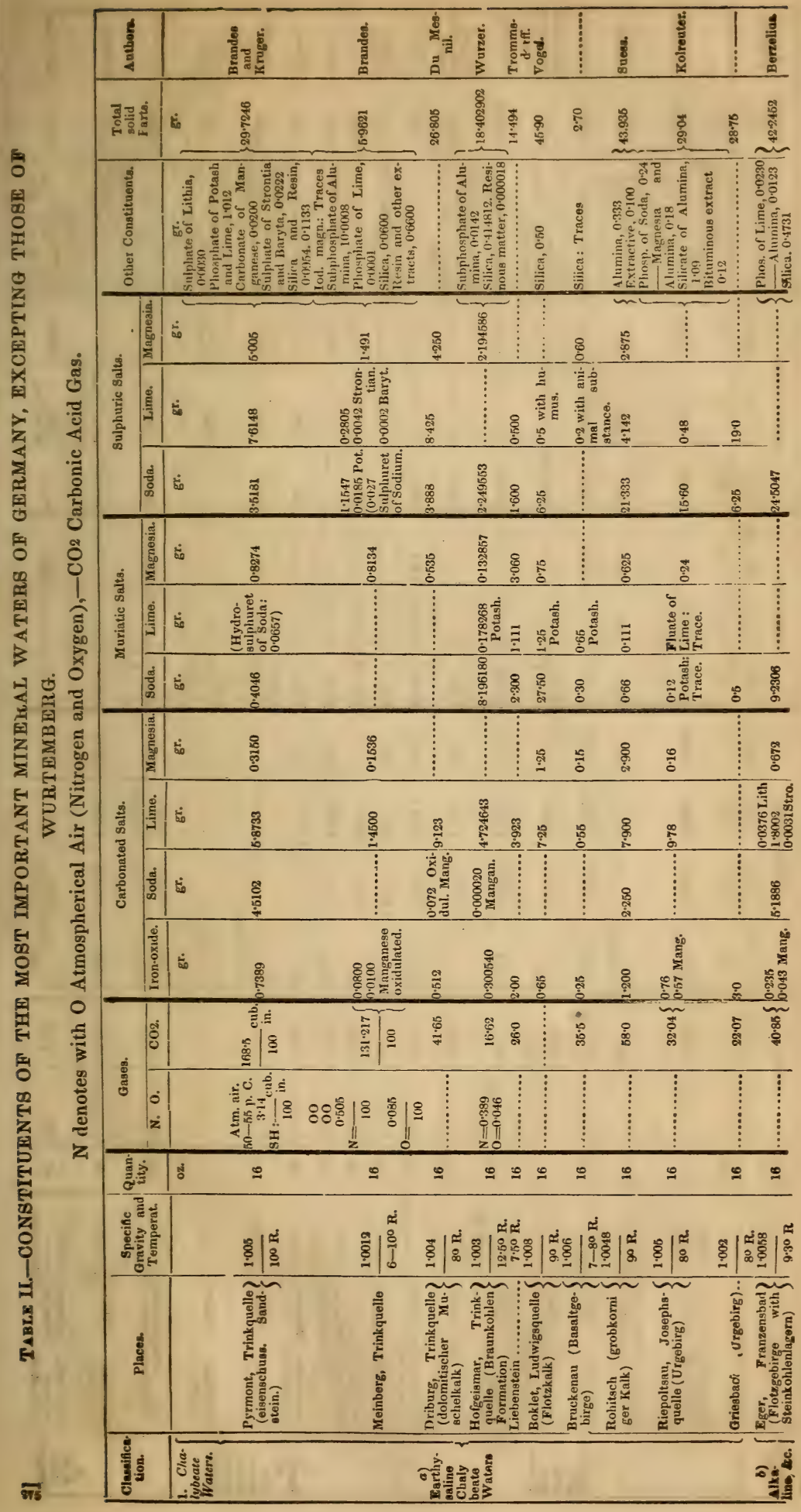




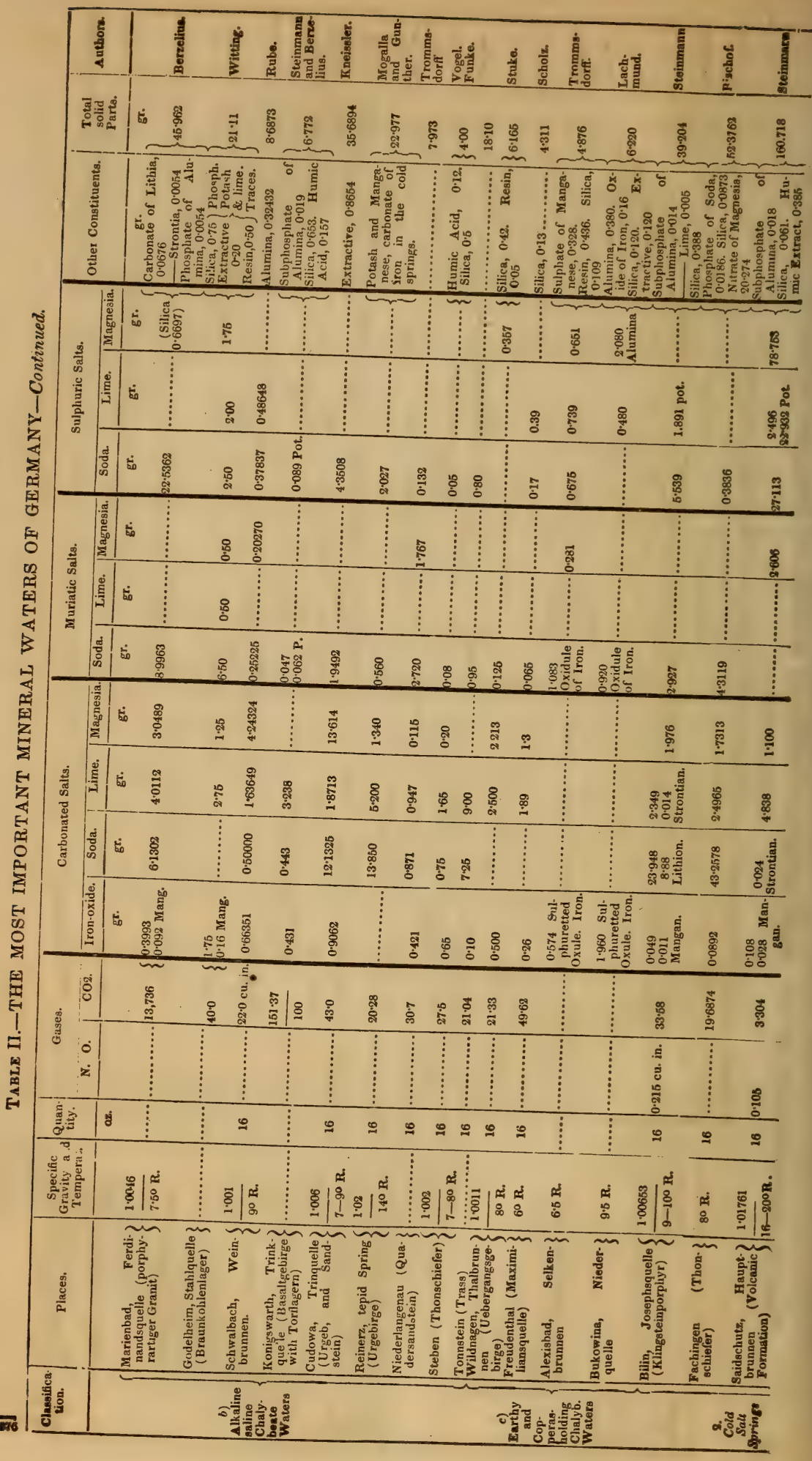




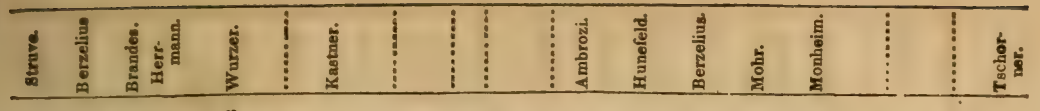

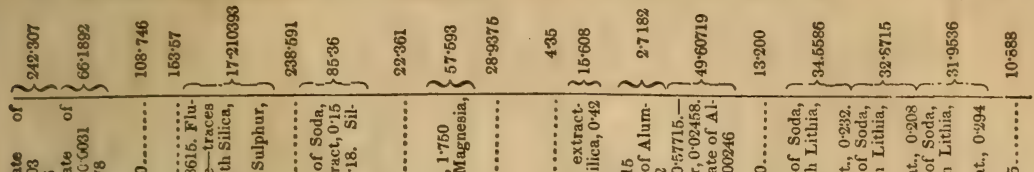

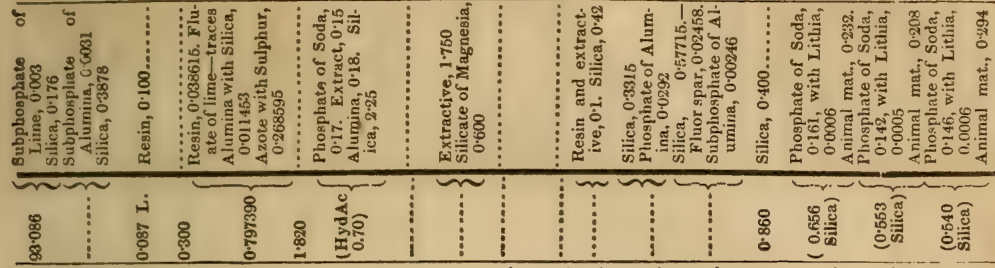

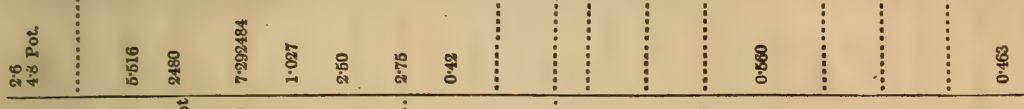

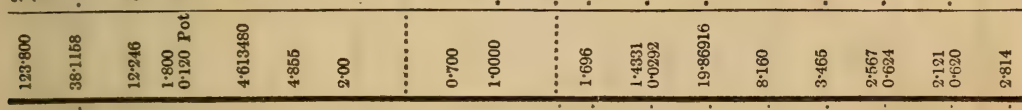

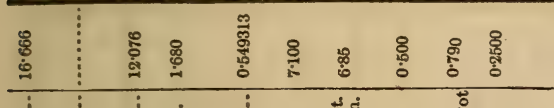

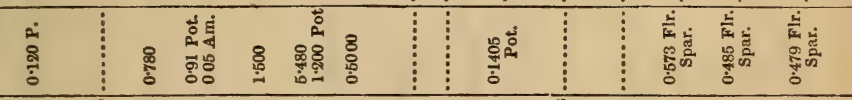

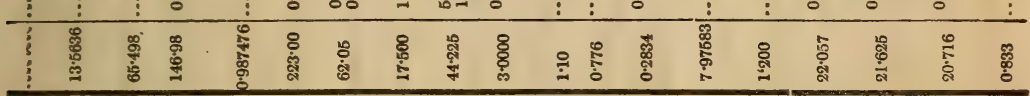

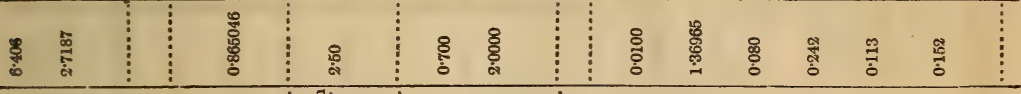

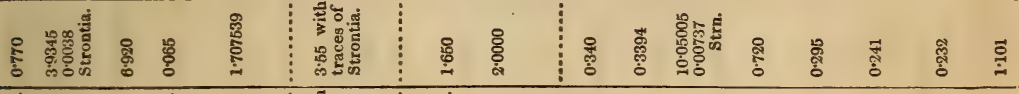

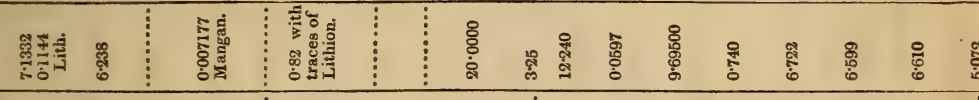

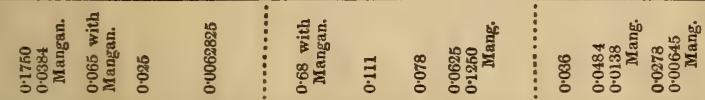

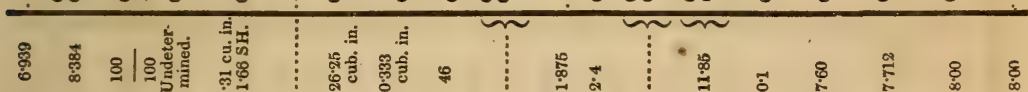

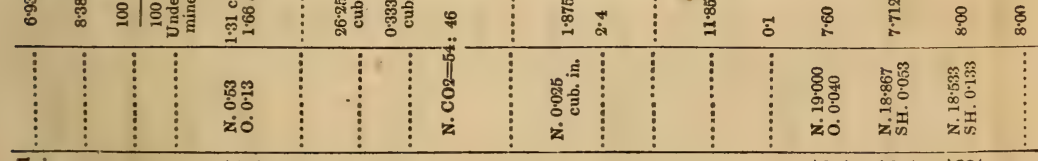

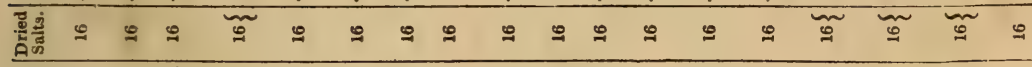

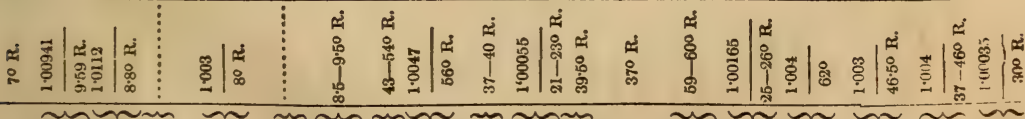

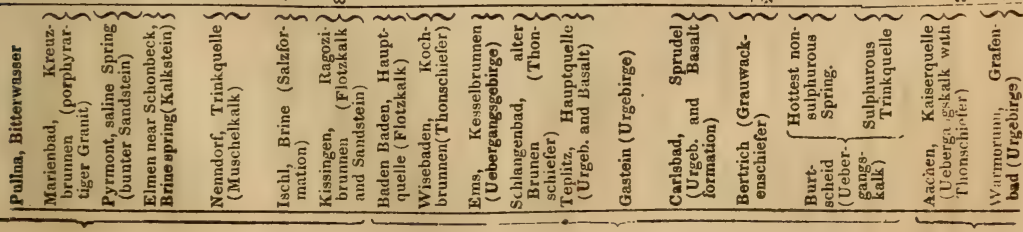




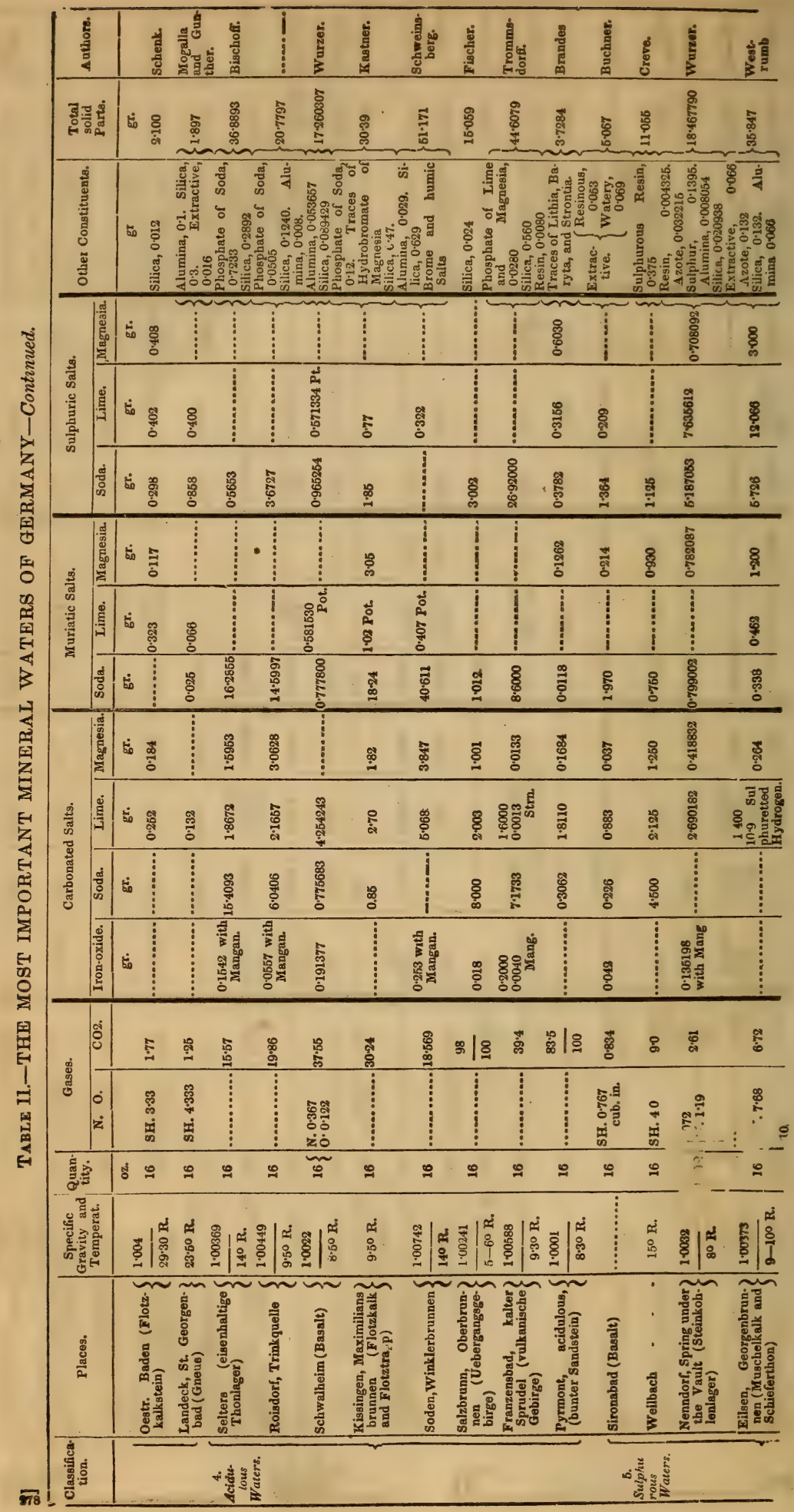




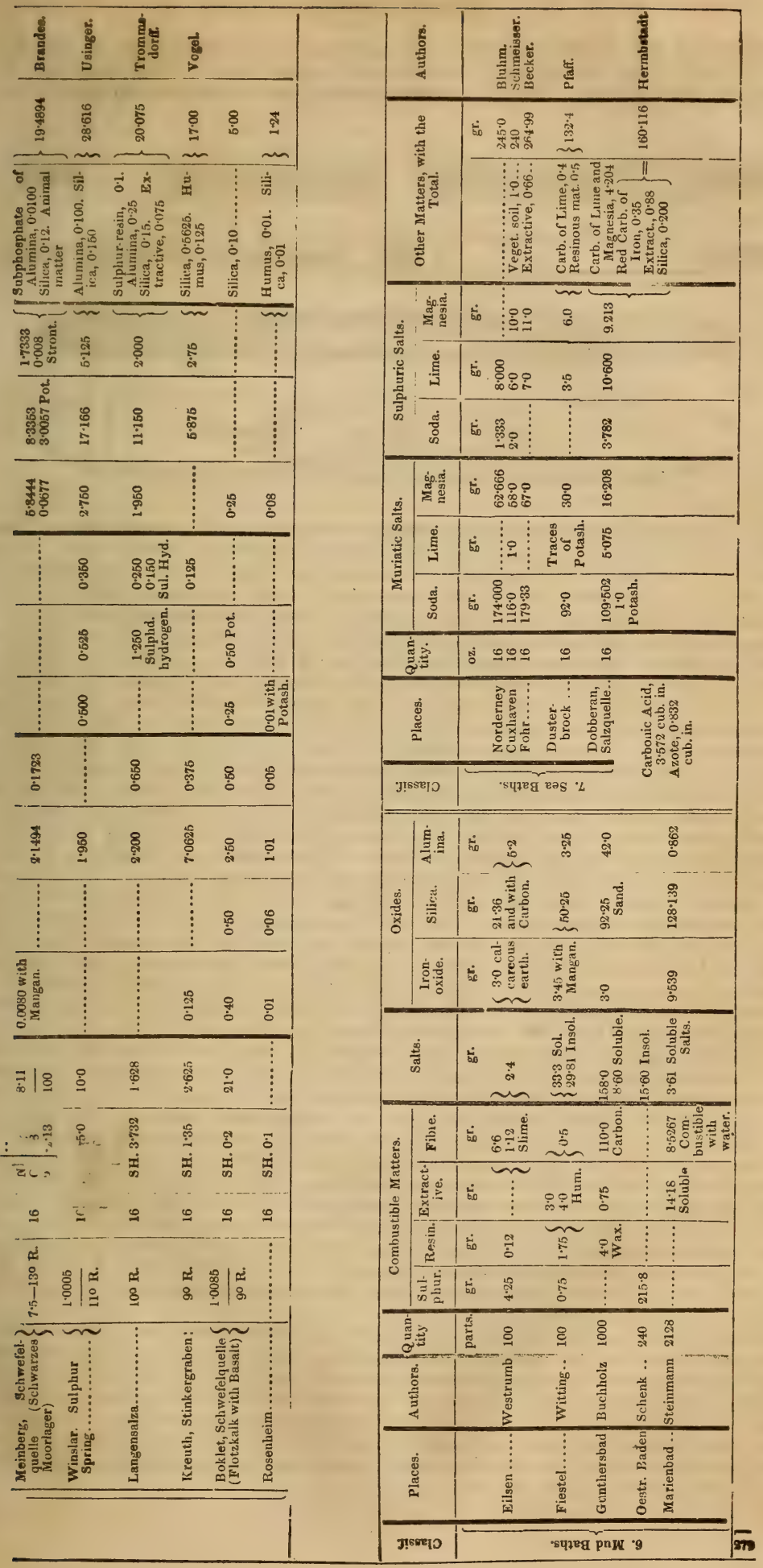


WEAVING OF HAIR CLOTH. In addition to the description of this arth under "Hair" in the Dictionary, I shall give here a short notice of the best kind of shuttle for weaving hair. Fig. 177, shows in plan A, and in longitudinal section B, a
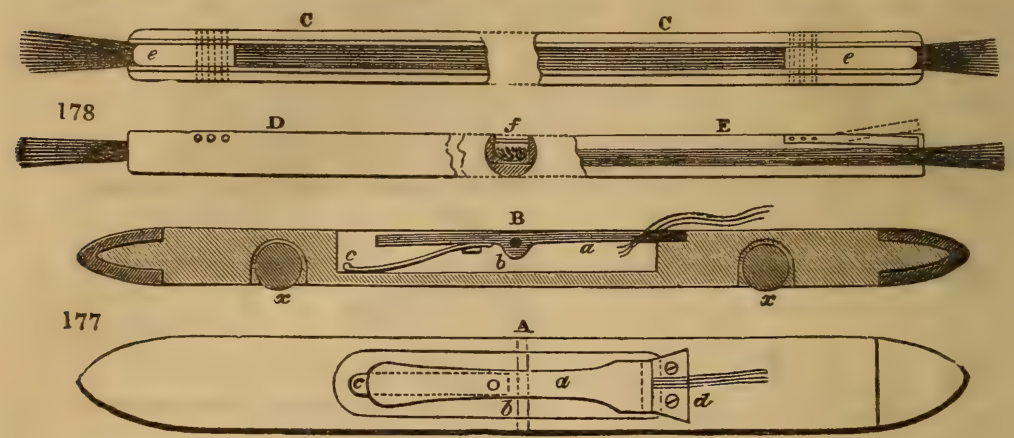

shuttle which differs from that of the common cloth weaver only in not having a pirn enclosed in the body of the box-wood, but merely an iron trap $a$, which turns in the middie upon the pin $b$. This trap-piece is pressed up at the one end, by the action of the spring $c$, so as to bear with its other end upon the cleft of the iron plate $d$, which is intended to hold fast the ends of the hair-weft : $d$ and $c$ together are called the jaw or mouth, whence the popular name of this shuttle. The workman opens this jaw by the pressure of his thumb upon the spring end of the trap $a$, introduces with the other hand one or more hairs (according to the description of hair cloth) into the mouth, and removing his thumb, lets the hair be seized by the force of the spring. The hairs having one end thus made fast are passed across the warp by the passage of the shuttle, which is received at the other end by the weaver's left hand. The friction rollers, $x$, $x$, are like those of fly-shuttles, but are used merely for convenience, as the shuttle can not be thrown swiftly from side to side. The hand which receives the shuttle opens at the same time the trap, in order to insert another hair, after the preceding has been drawn through the warp on both sides and secured to the list. A child attends to count and stretch the hairs. This assistant may, however, be dispensed with by means of the following implement, represented in fig. 178. C, C, is the view of it from above, or the plan; D, is a side view; E, a longitudinal section, and $\mathrm{F}$, an oblique section across. The chief part consists in a wooden groove, or chamfered slip of wood, open above, and rounded on the sides. It is about twenty-one inches in length, about as long nearly as the web is broad, therefore a little shorter than the horse-hairs inserted in it, which project about an inch beyond it at each end. They are therein pressed by elastic slips $e$, of Indian rubber, so that the others remain, when one or more are drawn out by the ends. The ends of the grooves are flat where the Indian rubber spring exerts its pressure, as shown by the dotted line at F. The spring is formed by cutting out a double piece from the curvature of the neck of a caoutchouc bottle or llask, fastening the one end of the piece by a wire staple in the groove of the shuttle, whereby the other end, which alone can yield, presses upon the inlaid hairs. Wire staples like $f$ (in the section $E$ ) are passed obliquely through two places of the groove or gutter, to present the hairs from springing up in the middle of the shuttle, which is suitably charged with them. The workman shoves the tool across the opened warp with the one hand, seizes with the other the requisite number of hairs by the projecting ends, and holds them fast, while he draws the shuttle once more through the warp. The remaining hairs are retained in the groove by the springs, and only those for the single decussation remain in the web, to be secured to the list on either side. A weaver with this tool can turn out a double length of cloth of what he could do with the mouth-shuttle.

WHALEBONE. A patent was granted to Mr. Laurence Kortright in March, 1841, for improvements in the treatment of whalebone, which consist in compressing the strips in width to increase their thickness, so as to render the material applicable for forming walking-sticks, whip handles, parasol and umbrella sticks, ramiods, archery bows, \&c. He accomplishes this purpose by bending the strips together, introducing them into a steam chest, thereby softening them, and in that state compressing them into a compact mass by appropriate machinery; for a description, with figures, of which, see Newton's Journal, C. S. xxi. 444. 
WHITE LEAD. Mr. Thomas Richardson of Newcastle, one of the most distinguished chemists of Liebig's school, obtained a patent in December, 1839, for a preparation of sulphate of lead, applicable to some of the purposes to which the carbonate is applied. His plan is to put 56 pounds of flake litharge into a tub, to mix it with one pound of acetic acid (and water) of specific gravity $1 \cdot 046$, and to agitate the mixture till the oxide of lead becomes an acetate. But whenever this change is partially effected, he pours into the tub, through a pipe, sulphuric acid of specific gravity 1.5975 , at the rate of about 1 pound per minute, until a sufficient quantity of sulphuric acid has been added to convert all the lead into a sulphate; being about 20 parts of acid to 112 of the litharge. The sulphate is afterward washed and dried in stoves for the market. I have examined the particles of this white lead with a good achromatic microscope, and found them to be semi-crystalline, and semi-transparent, like all the varieties of carbonate precipitated from saline solutions of the metal.

Mr. Leigh, surgeon in Manchester, prepares his patent white lead, by precipitating a carbonate from a solution of the chloride of the metal by means of carbonate of ammonia. On this process, in a commercial point of view, no remarks need be made. In Liebig and Woehler's Annalen for May, 1843, Chr. Link has communicated his investigation of two sorts of lead, prepared in the Dutch way, by the slow action of vinegar and carbonic acid upon metallic lead, under the heat of fermenting horse-dung. The one sort was manufactured by Sprenger, the other by Klagenfurth of Krems. He also examined 3 specimens of the Offenbach white lead. They all agreed in composition; affording $11 \cdot 29$ per cent. of carbonic acid, and 2.23 of water; corresponding to the formula, $2\left(\mathrm{PbO}, \mathrm{CO}_{2}\right)+\mathrm{PbO}, \mathrm{H}_{2} \mathrm{O}$; that is, in words, 2 atoms of carbonate of lead with 1 atom of oxide and 1 atom of water-in round numbers, thus, $\overline{2 \times 134}+112+9$.

Mulder observed specimens of white lead, of different atomic proportions of carbonate, oxide, and water, from the above, and discovered that the quality improved as the carbonate increased. The white lead by the Dutch process, as made by Messrs. Blacliett of Newcastle, is certainly superior as a covering oil pigment to all others. Its particles are amorphous and opaque.

A patent was granted to Mr. Hugh Lee Pattinson in September, 1811, for improvements in the manufacture of white lead, \&c. This invention consists in dissolving carbonate of magnesia in water impregnated with carbonic acid gas, by acting upon magnesian limestone, or other earthy substances containing magnesia in a soluble form, or upon rough hydrate of magnesia in the mode hereafter described, and in applying this solution to the manufacture of magnesia and its salts, and to the precipitation of carbonate of lead from any of the soluble salts of lead, but particularly the chloride of lead; in which latter case the carbonate of lead, so precipitated, is triturated with a solution of caustic potash or soda, by which a small quantity of chloride of lead contained in it is converted into hydrated oxide of lead, and the whole rendered similar in composition to the best white lead of commerce. The manner in which these improvements are carried into effect is thus described by the patentee: I take magnesian limestone, which is well known to be a mixture of carbonate of lime and carbonate of magnesia, in proportions varying at different localities; and on this account I am careful tc procure it from places where the stone is rich in magnesia. This I reduce to powder, and sift it through a sieve of forty or fifty apertures to the linear inch. I then heat it red hot, in an iron retort or reverberatory furnace, for two or three hours, when, the carbonic acid being expelled from the carbonate of magnesia, but not from the carbonate of lime, I withdraw the whole from the retort or furnace, and suffer it to cool. The magnesia contained in the limestone is now soluble in water impregnated with carbonic acid gas, and to dissolve it I proceed as follows: I am provided with an iron cylinder, lined with lead, which may be of any convenient size, say 4 feet long by $2 \frac{1}{2}$ feet in diameter; it is furnished with a safety-valve and an agitator, which latter may be an axis in the centre of the cylinder, with arms reaching nearly to the circumference, all made of iron and covered with lead. The cylinder is placed horizontally, and one extremity of this axis is supported within it by a proper carriage, the other extremity being prolonged, and passing through a stuffing-box at the other end of the cylinder, so that the agitator may be turned round by applying manual or other power to its projecting end. A pipe, leading from a force-pump, is connected with the under side of the cylinder, through which carbonic acid gas may be forced from a gasometer in communication with the pump, and a mercurial gauge is attached, to show at all times the amount of pressure within the cylinder, independently of the safety-valve. Into a cylinder of the size given I introduce from 100 to $120 \mathrm{lbs}$. of the calcined limestone, with a quantity of pure water, nearly filling the cylinder; I then pump in carbonic acid gas, constantly turning the agitator, and forcing in more and more gas, till absorption ceases, under a pressure of five atmospheres. I suffer it to stand in this condition three or four hours, and then run off the contents of the cvlinder into a 
cistern, and allow it to settle. The clear liquor is now a solution of carbonate of magnesia in water impregnated with earbonic acid gas, or, as I shall hereafter call it, a solution of bicarbonate of magnesia, having a specific gravity of about 1.028 , and containing about 1,600 grains of carbonate of magnesia to the imperial gallon.

I consider it the best mode of obtaining a solution of bicarbonate of magnesia from magnesian limestone, to operate upon the limestone after being calcined at a red heat in the way described; but the process may be varied by using in the cylinder the mixed hydrates of lime and magnesia, obtained by completely burning magnesian limestone in a kiln, as commonly practised, and slaking it with water in the usual manner; or, to lessen the expenditure of carbonic acid gas, the mixed hydrates may be exposed to the air a few weelis till the lime has become less caustic by the absorption of carbonic acid from the atmosphere. Or the mixed hydrates may be treated with water, as practised by some manufacturers of Epsom salts, till the lime is wholly or principally removed; after which the residual rough hydrate of inagnesia may be acted upon in the cylinder, as described; or hydrate of magnesia may be prepared for solution in the cylinder, by dissolving magnesian limestone in hydrochloric acid, and treating the solution, or a solution of chloride of magnesium, obtained from sea-water by salt-makers in the form of bittern, with its equivalent quantity of hydrate of lime, or of the mixed hydrates of lime and magnesia, obtained by completely burning magnesian limestone, and slaking it as above. When I use this solution of bicarbonate of magnesia for the purpose of preparing magnesia and its salts, I evaporate it to dryness, by which a pure carbonate of magnesia is at once obtained, without the necessity of using a carbonated alkali, as in the old process; and from this I prepare pure magnesia by calcination in the usual manner; or, instead of boiling to dryness, I merely heat the solution for some time to the boiling point, by which the excess of carbonic acid is partly driven off, and pure carbonate of magnesia is precipitated, which may then be collected, and dried in the same way as if precipitated by a carbonated alkali. If I require sulphate of mairnesia, I neutralize the solution of bicarbonate of magnesia with sulphuric acid, boil iown, and crystallize; or I mix the solution with its equivalent quantity of sulphate of iron, dissolved in water, heated to the boiling point, and then suffer the precipitated carbonate of iron to subside; after which I decant the clear solution of sulphate of magnesia, boil down, and crystallize as before. When using this solution of bicarbonate of magnesia for the purpose of preparing carbonate of lead, I make a saturated solution of chloride of lead in water, which, at the temperature of $50^{\circ}$ or $60^{\circ} \mathrm{Fahr}$, has a specifiz gravity of about $1 \cdot 008$, and consists of 1 part of chloride of lead dissolved in 126 parts of water. I then mix the two solutions together, when carbonate of lead is immdiately precipitated; but in this operation I find it necessary to use certain precautions, otherwise a considerable quantity of chloride of lead is carried down along with the carbonate. These precautions are, first, to use an excess of the solution of magnesia, and secondly, to mix the two solutions together as rapidly as possible. As to the first, when using a magnesian solution, containing 1,600 grs. of carbonate of magnesia per imperial gallon, with a solution of chloride of lead saturated at $55^{\circ}$ or $60^{\circ}$ Fahr., 1 measure of the former to $8 \frac{1}{2}$ of the latter is a proper proportion; in which case there is an excess of carbonate of magnesia employed, amounting to about an eighth of the total quantity contained in the solution. When either one or both the solvtions vary in strength, the proportions in which they are to be mixed must be determined by preliminary trials. It is not, however, necessary to be very exact, provided there is always an excess of carbonate of magnesia amounting to from one eighth to one twelfth of the total quantity employed. If the excess is greater than one eighth no injury will result except the unnecessary expenditure of the magnesian solution. As to the second precaution, of mixing the two solutions rapidly together, it may be accomplished variously; but I have found it a good method to run them in two streams, properly regulated in quantity, into a small cistern in which they are to be rapidly blended together by brisk stirring, before passing out, through a hole in the bottom, to a large cistern or tank, where the precipitate finally settles. The precipitate thus obtained is to be collected, washed and dried in the usual manner. It is a carbonate of lead, very nearly pure, and suitable for most purposes; but it always contains a small portion of chloride of lead, seldom less than from 1 to 2 per cent., the presence of which, even in so small a quantity, is somewhat injurious to the color and body of the white lead. I decompose this chloride, and convert it into a hydrated oxide of lead by grinding the dry precipitate with a solution of caustic alkali, in a mill similar to the ordinary mill used in grinding white lead with oil, adding just so much of the ley as may be required to convert the precipitate into a soft paste. I allow this paste to lie a few days, after which, the chloride of lea 1 being entirely, or almost entirely decomposed, I wash out the alkaline chloride formed by the reaction, and obtain a white lead, similar in composition to the best white lead of commerce. I prepare the caustic alkaline ley by boiling together, in a 
leaden vessel, for an hour or two, 1 part by weight of dry and recently-slaked lime, 2 parts of crystallized earbonate of soda (which, being cheaper than carbonate of potash, I prefer) and 8 parts of water. The clear and colorless caustic lie, obtained after subsidence, will have a specific gravity of about 1.090 , and, when drawn off from the sediment, must be kejt in a close vessel for use.

WINES. In a case tried before the court of exchequer, at the instance of the board of customs, in December, 1843 , of an attempt to obtain the drawback upon a large quantity of damaged claret offered for exportation, I had observed, in my examination of the wine, that on the addition to it of water of ammonia to super-saturate its acidity, a large flocculent precipitate of decomposed gluten fell, and the supernatant liquor lost its ruby color, and became yellow-brown. I have tried sound samples of genuine claret, very old, as well as new, by the same test, and I have found the ruby color to remain but little impaired; contrary to the allegation of the chemist of the defendants in the lawsuit. The wine was declared by the verdict of a jury and the decision of the judge, to be unworthy of being admitted for drawback, and therefore forfeited to the crown.

WINES, BRITISH, are made either from infusions of dried grapes (raisins) or from the juices of native fruits, properly fermented. These wines are called sweets in the language of the excise, under whose superintendence they were placed till 1834, when the duties upon them were repealed as onerous to the trade and unproductive to the revenue. The raisins called Lexias are said to produce a dry flavored wine; the Denias a sweet wine; the black Smyrnas a strong-bodied wine, and the red Smyrnas and Valencias a rich and full wine. The early spring months are the fittest time for the wine manufacture. The masses of raisins, on being taken out of the packages, are either beaten with mallets or crushed between rollers in order to loosen them, and are then steeped in water in large vats, between a perforated board at bottom and another at top. The water being after some time drawn off the swollen and softened fruit, pres sure 1s applied to the upper board to extract all the soluble sweet matter, which passes down through the false bottom, and flows off by an appropriate pipe into fermenting tuns. The residuary fruit is infused with additional water, and then squeezed; a process which is repeated till all the sweets are drained off, after which the "rape" is subjected to severe pressure in a screw or hydraulic press. The wine, in the process of the vinous fermentation, is occasionally passed through a great body of the rape to improve its flavor, and also to modify the fermentative action; it is afterward set to ripen in casks, clarified by being repeatedly racked off, and fined with isinglass.

WOOD PAVING. Among the numerous illustrations of the durability and resistance of wood paving, reference may be made to the specimens :-

At Whitehall, 1093 yards, laid in December, 1839 -

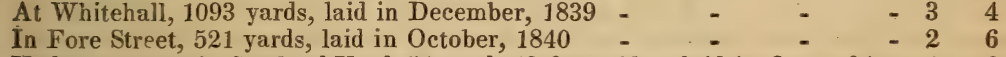
Under the arch in Scotland Yard, 54 yards (8 feet wide), laid in Oct., $1841 \quad 1 \quad 6$

The first, in every respect a perfect piece of pavement, has been more than 3 years subjected to a constant traffic, including the utmost amount of percussion from velocity, and the extremest pressure from the ponderous engines which have been transported over its surface. Scarce less may be said of that in Fore street, while the specimen in Scotland Yard has successfully withstood at least a like amount of pressure, the traffic from the wharfs in Great Scotland Yard being no less than 78,000 tons per annum; the passage, narrowed within the limit of a single carriage line, exposing the wood to the most critical test of resistance.

Slipperyness is not a natural defect in wood paving. The accumulations on wood pavement are drawn from the proximate areas of granite and macadam. In granite the imperfect structure admits of the constant oozing of dust and filth; in macadam the surface is always wearing into dirt and slop. In dry, hot, or cold weather, the stonepaved streets of London are proverbially as slippery as glass, while slipperyness on wood pavement may be altogether obviated by cleanliness ; and that may now be ensured by the use of Whitworth's cleansing machine, which has already been successfully tried in some of the principal streets-thanks to the commissioners of woods and forests.

It is impossible not to perceive the great amount of suffering and loss that may be saved in horses by the wood pavement. Cabmen and omnibus drivers assure us that, in the winter season, for a month or two only, there is any serious cause for complaint, and then there is as much or more danger on other pavements; whereas, during the summer months, the advantages of wood over all other pavements is immense; the great mortality of horses in the streets of London, from over-driving during the hot weather, is well known; so far as wood is concerned, the reduction of effort must necessarily decrease the destruction in a greater ratio than even 5 to 2 . 
WOOD-PRESERVING. Mr. Bethell's invention consists in impregna ing wood throughout with oil of tar and other bituminous matters, containing creosote, and also with pyrolignite of iron, which holds more creosote in solution than any other watery menstruum.

The wood is put in a close iron tank, like a high-pressure steam-boiler, which is then closed and filled with the tar oil or pyrolignite. The air is then exhausted by airpumps, and afterward more oil or pyrolignite is forced in by hydrostatic pumps, until a pressure equal to from 100 to 150 pounds to the inch is obtained. This pressure is kept up by the frequent working of the pumps during six or seven hours, whereby the wood becomes thoroughly saturated with the tar oil, or the pyrolignite of iron, and will be found to weigh from 8 to 12 pounds per cube foot heavier than before.

In a large tank, like one of those used on the Bristol and Exeter railway, 20 loads of timber per day can be prepared.

The effect produced is that of perfectly coagulating the albumen in the sap, thus preventing its putrefaction. For wood that will be much exposed to the weather, and alternately wet and dry, the mere coagulation of the sap is not sufficient; for although the albumen contained in the sap of the wood is the most liable and the first to putrefy, yet the ligneous fibre itself, after it has been deprived of all sap, will, when exposed in a warm damp situation, rot and crumble into dust. To preserve wood, therefore, that will be much exposed to the weather, it is not only necessary that the sap should be coagulated, but that the fibres should be protected from moisture, which is effectually done by this process.

The atmospheric action on wood thus prepared renders it tougher, and infinitely stronger. A post made of beech, or even of Scotch fir, is rendered more durable, and as strong as one made of the best oak; the bituminous mixture with which all its pores are filled acting as a cement to bind the fibres together in a close tough mass; and the more porous the wood is, the more durable and tough it becomes, as it imbibes a greater quantity of the bituminous oil, which is proved by its increased weight. The materials which are injected preserve iron and metals from corrosion; and an iron bolt driven into wood so saturated, remains perfectly sound and free from rust. It also resists the attack of insects ; and it has been proved by Mr. Pritchard, at Shoreham Harbor, that the teredo navalis, or naval worm, will not touch it.

Wood thus prepared for sleepers, piles, post, fencing, \&c., is not at all affected by alternate exposure to wet and dry ; it requires no painting, and after it has been exposed to the air for some days it loses every unpleasant smell.

This proeess has been adopted by the following eminent engineers, viz.: Mr. Robert Stephenson, Mr. Brunel, Mr. Bidder, Mr. Brathwaite, Mr. Buck, Mr. Harris, Mr. Wickstead, Mr. Pritchard, and others; and has been used with the greatest success on the Great Western railway, the Bristol and Exeter railway, the Manchester and Birmingham railway, the North Eastern, the South Eastern, the Stockton and Darlington, and at Shoreham Harbor; and lately, in consequence of the excellent appearance of the prepared sleepers, after three years' exposure to the weather, an order has been issued by Mr. Robert Stephenson, that the sleepers hereafter to be used on the London and Birningham railway are to be prepared with it before being put down.

The expense of preparing the wood varies from $10 \mathrm{~s}$. to $15 \mathrm{~s}$. per load, according to situation, and the distance from the manufactories where the material is made.

Mr. Bethell supplies the material at a low price from his manufactories, either at Nine Elms, Vauxhall; Bow Common; or Birmingham; and parties prepare the timber themselves.

For railway sleepers it is highly useful, as the commonest Scotch fir sleeper, when thus prepared, will last for centuries. Those which have been in use 3 years and upward, look much better now than when first laid down, having become harder, more consolidated, and perfectly waterproof; which qualities, combined with that of perfectly resisting the worm, render this process eminently useful for piles, and all other woodwork placed under water. Posts for gates or fencing, if prepared in this manner, may be made of Scotch fir, or the cheapest wood that can be obtained, and will not decay like oak posts, which invariably become rotten near the earth after a few years.

\section{Y.}

YEAST, ARTIFICIAL. Mix two parts, by weight, of the fine flour of pale barley malt with one part of wheat flour. Stir 50 pounds of this mixture gradually into 100 quarts of cold water, with a wooden spatula, till it forms a smooth pap. Put this pap into a copper over a slow fire; stir it well till the temperature rise to fully $155^{\circ}$ to $160^{\circ}$, when a partial formation to sugar will take place, but this sweetening must not be pushed too far; turn out the thinned paste into a flat cooler, and 
$x$ it from time to time. As soon as the wort has fallen to $59^{\circ} \mathrm{Fahr}$., transfer it to a tub, and add for every 50 quarts of it 1 quart of good fresh beer-yeast, which will throw the wort into brisk fermentation in the course of 12 hours. This preparation will be giod yeast, fit for bakers' and brewers' uses, and will continue fresh and active for days. It should be occasionally stirred.

When beer-barm has become old and flat, but not sour, it may be revived by mixing with every quart of it a small potato, boiled, peeled, and rubbed down into a paste. The mixture is to be placed in a warm situation, where it will speedily show its renewed activity, by throwing up a froth upon its surface. It must be forthwith incorporated with the dough, for the purpose of baking bread. When the barm has become sour, its acid should be neutralized with a little powdered carbonate of soda, and then treated as above, when it will, in like manner, be revived. A bottle of brisk small beer may furnish ferment enough to form, in this way, a supply of good yeast for a small baking.

The German yeast imported into this country in large quantities, and employed by our bakers, in baking cakes, and other fancy bread, is made by putting the unterhefe (see BEER, Bavarian), into thick sacks of linen or hempen yarn, letting the liquid part, or beer, drain away; placing the drained sacks between boards, and exposing them to a gradually increasing pressure, till a mass of a thin cheesy consistence is obtained. This cake is broken into small pieces, which are wrapped in separate linen cloths; these parcels are afterward enclosed in waxed cloth, for exportation. The yeast cake may also be rammed hard into a pitched cask, which is to be closed air-tight. In this state, if kept cool, it may be preserved active for a considerable time. When this is to be used for beer, the proportion required should be mixel with a quantity of worts at $60^{\circ}$ Fahr., and the mixture left for a little to work, and send up a lively froth; when it is quite ready for adding to the cooled worts in the fermenting back.

Yeast, Patent. Boil 6 ounces of hops in 3 gallons of water 3 hours ; strain it off, and let it stand 10 minutes; then add half a peck of ground malt, stir it well up, and sover it over; return the hops, and put the same quantity of water to them again, boiling them the same time as before, straining it off to the first mash; stir it up, and et it remain 4 hours, then strain it off, and set it to work at $90^{\circ}$, with 3 pints of patent feast; let it stand about 20 hours; take the scum off the top, and strain it through

hair sieve; it will be then fit for use. One pint is sufficient to make a bushel of bread.

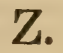

ZINC. Mr. Nicholas Troughton, of Swansea, obtained a patent in May, 1839, for improvements in the manufacture of this metal. His invention relates to the application of a peculiar apparatus in roasting the ores, and in smelting the zinc. Fig. 179,

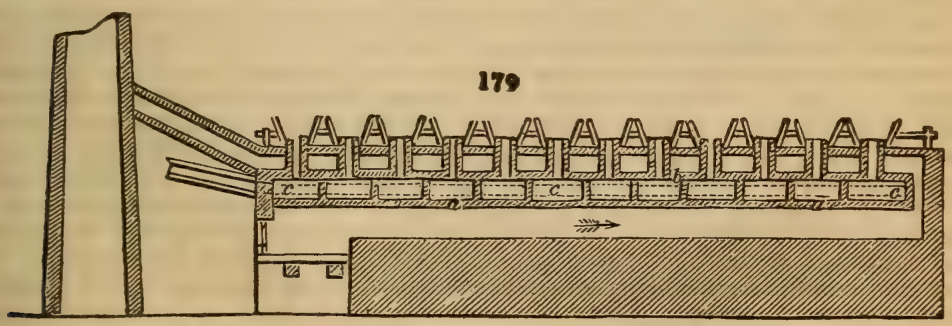

represents the section of a series of retorts for calcining zinc ores, arranged and constructed according to this invention. The retorts shown in this figure are composed of a series of fire-tiles or parallelograr: slabs. $a, a, a$, are the slabs or tiles, which constitute the bottoms of the retorts; $b, b$, are the slabs, which constitute the upper surfaces or tops of the retorts; and $c, c$, are slabs, placed vertically, to produce the sides of the retorts. The back ends of the retorts are closed by similar tiles or slabs, having a bole through them for the passage of the vapors evolved from the ores; these vapors 
are conveyed in any direction by the flue at that end, and being thus separated from the products of combustion, may be separately acted on, according to either of the patentee's former inventions, which treat of the separated vapors of copper ores in the process of - alcining or roasting such ores; or the separated products of the ore may be allowed to pass into the atmosphere. The patentee states, that by treating zinc. ores in furnaces or retorts, such as are above described, considerable saving of fuel will result, and the zinc ore will be more evenly roasted or calcined.

The front ends of the retorts are closed by means of tiles or doors, having a small hole or opening in each, for the passage of atmospheric air; and the holes may be closed, or more or less open, according to the object required. The retorts are charged through the hoppers above, which have proper slides to close the openings into the retorts; the quantity charged into each retort being sufficient to cover the lower surface thereof two or three inches deep. During the operation the ore must be raked from time to time, to change the surfaces, and the retorts should be kept to a moderate red heat.

The second part of this invention relates to an arrangement of apparatus cr furnace for calcining zinc ores, wherein the ore is subjected to the direct action of the products of combustion. Fig. 180, shows a longitudinal section of the furnace, which is so con-

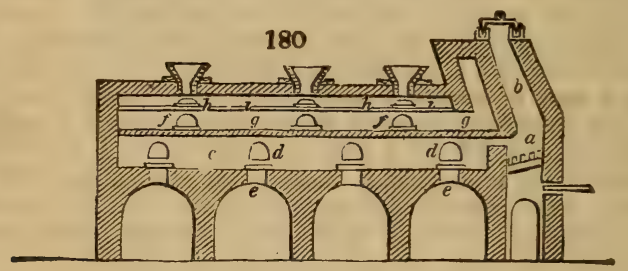

structed that while one portion of the zinc ore is being heated in a manner similar to the working of an ordinary calcining surface, other zinc ore is going through a preparatory process by the heat that has passed away from the ore which is undergoing the completing process of calcining. This furnace may be heated by a separate fire, to burn by blast or by draught; or the flue from the smelting furnace may be conducted into the entrance of this furnace, and the otherwise waste heat of the smelting furnace will be thus brought into useful application for calcining or roasting of zinc ore; and this part of the invention is applicable, whether it be applied to the furnace, or to the retorts herein-before explained, and will be found a means of saving much fuel in the processes of obtaining zinc from ore. $\alpha$, fig. 180, represents the furnace, which is suitable for blast, and a constant supply of fuel is kept up in the chamber $b$, there being a close cover, with a sand-joint. $c$, is the bed or floor on which the ore is spread, in like manner to an ordinary reverberatory furnace; the ore is stirred about on the floor by passing the ordinary rakes or instruments through the openings, $d, d$; and when the process has been sufficiently carried on, the ore is discharged through the openings $e, e$, which, at other times, remain closed by fire-tiles. The heat of the fire, and the flame thereof, passing in contact with the ore on the floor or bed, $c$, also acts on the roof, $f$, and that roof, $f$, being hot, reverberates the heat on to the floor or bed, at the same time the heat, which passes through the roof, heats the ore in the upper chamber, $g$; and, in addition to such heat passing through the roof, the flame and heat from the furnace, having passed over the zinc ore, in the lower compartment of the apparatus, enters into and passes over the ore in the chamber $\mathrm{g}$; and, in doing so, heats the roof $h$, of that chamber, and also the ore contained therein; and it will be seen that there is a third chamber, $i$; the heat, therefore, which passes through the roof $h$ : heats the ore in the chamber $i$. In working this arrangement of calcining furnace or apparatus, when the charge is withdrawn from the lower chamber, the charge in the chamber $g$ is to be raked into the lower chamber, through the openings for that purpose, which, at other times, are kept covered with fire-tiles, as shown in the drawing; and the charge in the chamber $i$ is to be raked into the chamber $g$, and a fresh supply of ore charged into the chamber $i$.

The third part of this invention relates to a mode of arranging a series of 'retorts side by side, and of applying heat thereto in the process of smelting or distilling zinc from the ore. According to the practice most generally pursued in smelting zinc, the ore is submitted to the action of heat in crucibles, having descending iron pipes, which enter into vessels containing water : all which is well understood, as well as the process of smelting or distilling zinc from the ores. Fig. 181, is a side elevation of two sets of 


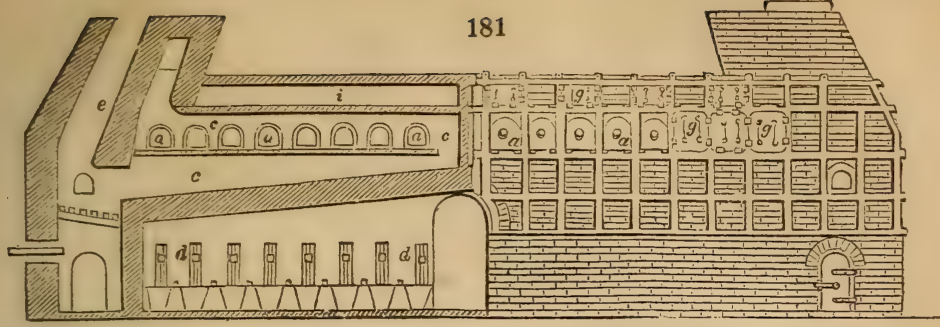

furnaces and retorts, arranged according to this invention, one of the furnaces being in section; and fig. 182, is a transverse section of the same. $a, a$, are a series of retorts of fire-clay, arranged, side by side, on a shelf of slabs or fire-tiles. These retorts are each closed at one end and open at each other, such open end being closed, when in operation, by a tile or door, $b$, fitting closely, and luted with fire-clay, as will readily be traced in the drawing. Each series of retorts is placed in a chamber, $c, c$, in such a manner that the heat and flame of the fire will pass from the fireplace or furnace, and act on one side

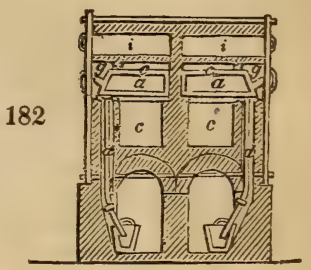
of the retorts; and having passed along all the series, will proceed to the upper part of the chamber, $c, c$, and heat the other side of the retorts; and as the fires are muintained and urged by means of blasts of atmospheric air, the heat may be maintained and regulated with great advantage, and at comparatively small cost. The blasts of air muy be produced by any ordinary blowing machinery, but rotatory blowers are prefexred, and the air may be cold or heated. When anthracite coal is used as the fuel, the patentee prefers adopting the hot blast, at a temperature of at least $500^{\circ} \mathrm{Fahr}$., and such heating may be performed by any of the well-known means now very generally resorted to for heating the blasts of air for smelting iron. $d, d$, are iron pipes, descending from the retorts and entering into vessels containing water, similar to the apparatus at present in use for like purposes. Each chamber, $c$, is heated by its separate furnace or fireplace, which have openings, to be closed when at work; and in order to keep up a supply of fuel to the fire, each fireplace has an inclined chamber, $e$, which is filled with fuel, and then closed air-tight by the cover, $f$, fitting into a sandbath or joint, in order to prevent draught upward. By this means the lower portion only of the fuel will be in an ignited state when at work. $g, g$, are a series of iron doors, one opposite the mouth of each retort; these doors are capable of being removed by sliding them upward, till the portions cut out at the sides come opposite the dips or holders, $h, h$, when the doors may be removed, in order to get at the retorts. $i$, is a chamber in which the ore is heated previous to its being placed in the retorts. The arrangement of the brickwork, the construction and setting of the furnaces, being clearly shown in the drawing, no further description need be given.

The patentee remarks, that he is aware attempts have been made to employ retorts in the smelting of zinc, and he does not, therefore, claim the same generally; but he does claim, in respect to the third part of this invention, the mode of placing a series of retorts in a chamber, $c$, and causing the heat and flame to pass along, under and over, such series of retorts, as above described; and he also claims the mode of smelting zinc by means of blast, whether the heat of the fuel is caused to act on a series of retorts or vesseis, in the manner shown, or on other arrangements of retorts or vessels, placed in a suitable chamber or chambers.-Newton's Journal, xxiii., p. 81. C. S. 



\section{A P P E N D I X.}

ALKALIMETRY. Twenty-eight years have elapsed since I was led, by peculiar circumstances, to construct a very simple method of testing alkalis, the principle of which I soon afterward applied to acids, bleaching powder, dye-stuffs, and inost other hemical substances extensively used in manufactures.* In 1814 and 1815 , during the summer vacation of my Glasgow classes, I was engaged in delivering courses of lectures on chemistry in the Belfast Academical Institution, and had many of the most eminent meinbers of the Linen Board of that town for my pupils. Being occasionally consulted upon the qualities of the alkalis, which were used to the value of $200,000 l$. by the linen bleachers of Ireland, I saw the importance to them of a simple alkalimetrical test, both for purchasing and for using their barillas and potashes. The following extract from the Belfast News Letter, of July 9th, 1816, will show the nature of $\mathrm{mv}$ contrivance :-

"This day one of the porters of the Linen Hall, Belfast, was called into the libraryroom at the request of Dr. Ure, who being quite unknown to Dr. Ure, and never having seen any experiments made with acids and alkalis, he took the instrument at our desire, which being filled with colored acid, by pouring it slowly on adulterated alkali, which we had previously prepared, he ascertained exactly the per-centage of genuine alkali in the mixture. Belfast, 25th June, 1816.

"John S. Ferguson, Chairman.

JAMES M`Donnei, M. D.

JoHN M. STOUPE.

S. Thomson, M. D."

Of these gentlemen, two were leading members of the Linen Board, and the others the two principal physicians of the town. The publication of the details of my method of alkalimetry was delayed till arrangements were made for its general introduction, under the direction of the Linen Board of Dublin, whose professor of chemistry, Mr. W. Higgins, as well as Dr. Barker, professor of chemistry in Trinity College, granted certificates of the "accuracy and the national importance" of the instrument. The alkaline matter then imported into Ireland was often largely contaminated with common salt, even to the extent of 80 or 90 per cent. During the procrastination of the Board, I lent my Treatise on Alkalimetry to Dr. Henry, of Manchester, who inadvertently published an account of it, though with reference to me, in the next edition of his Elements of Chemistry. Having, in the long interval since, contrived many modifications of the instrument, and having extended its principle to testing other articles $I$ am induced to offer it now to the world, in consequence of the recent appearance of a publication upon the same subject, by two very ingenious chemists of Liebig's school, Drs. R. Fresenius and H. Will. Of their system of alkalimetry, \&c., a copious abstract appeared in the Annalen der Chimie und Pharmacie for July last, and about the same time a pamphlet was published by Winter, at Heidelberg, under the title Neue Verfahrungsweisen zur Bestimmung des Werthes der Pottasche und Eoda, der Saüren, und des Braunstein; or "New Processes for determining the Value of Potash and Soda, of Acids, and Black Oxide of Manganese." However accurate these processes may be, and however apt for a German or French student of chemistry, they are, in my apprehension, not at all fitted for the familiar use of manufacturers and dealers in any country, and certainly not for those of the United Kingdom.

Descroizilles was the first person who contrived an instrument, called an alkalim. eter, to ascertain the alkaline strength of potash and soda, without much calculation. His method was described in the Annales de Chimie for 1806, tom. lx., and a translation of it appeared in our Philosophical Magazine, vol. xxviii., for July

* Among others to nitrate of potash, nitrate of soda, and to white lead, either in powder or in paint. My nitrometer enables a person not at all versant in chemistry to ascertain in a quarter of an hour, but by two distinct processes, the quantity of pure nitrate in either of these salts, to one part in 200 . The cerussa-meter is equally simple and expeditious. 
and August of the following year. His apparatus consisted of a glass tube, $\gamma$ or $y$ inches long, and 7 or 8 lines in diameter, closed at one end, but terminated at the other in a kind of small funnel (with a beak or spout), connected to the tube by a narrow neck, having a calibre of two lines and a half. Upon the shoulder, under the throat, there was a hole for admitting air to the long tube in the act of being emptied, by sloping its mouth downward. This cylindrical vessel was to contain 38 grammes of water, which space was divided into 76 equal parts, which it was extremely important to proportion accurately. The liquor was prepared by taking concentrated sulphuric acid, at $66^{\circ}$ Baumé (1.845 spec. grav.), and diluting it with nine times its weight of water. The instrument being poised in a balance, he introduced into it very exactly two grammes of the above test acid, and when the instrument stood upright, he scratched a line at the level of the liquor, and thus proceeded by addition of successive grammes to graduate the whole, till 36 were added, after which he subdivided these spaces by lines into 72 demi-gramme volumes. He then proceeds to describe eight different subsidiary articles required for his operations :-

"Alkalimetrical trials of potash.-Weigh exactly one demi-gramme of potash, put it inti a glass, and pour upon it about four fifths of a decilitre of water; facilitate the solution of the potash by stirring it with a small chip of wood, three or four times in an hour and a half, a minute at each time. When the solution is effected, pour it into the small tin measure, No. 4, which is to be then filled up with water; pour it back again into the glass, in which you must still pour a measure full of pure water; stir this new mixture also three or four times within half an hour, in order to facilitate the precipitation of a slight sediment, which soon falls down. This sediment being completely formed, slope the glass with caution, in order to fill with clear liquor the small measure; then empty this last into another large glass; after this place round the edges of a plate drops of syrup of violets; pour also into the alkalimeter test liquor until the line marks 0 ; take it afterward with the left hand, inclining it upon the glass which contains the moiety of the clean alkaline solution: the acid liquor will fall into it by hasty drops, or in a very small thread, which you may moderate at pleasure, by retarding the entrance of the air at the lateral hole or vent, upon which must be placed the end of the finger; at the same time, with a small stick or match, assist the mixture and facilitate the development of the carbonic acid which is manifested by effervescence. When you have emptied the alkalimeter to about the line 40 , try if the saturation approaches, by drawing your small stick from the mixture, and resting it upon the drops of syrup of violets, which should become green, if the potash is not of a very inferior quality. If, on the contrary, the violet color is not altered, or what would be worse, if it be changed into red, there would be, in the first case, an indication of saturation, and in the second a proof of super-saturation. But this is not the case with good potashes; at that line, the liquor tried can alter the syrup of violets into green only; or cause to return to the violet, and even to the green, the drops which had been changed into red at the time of a former trial; we must, therefore, in general add more acid, which occasions a new effervescence. This addition must always be made with caution, and we must touch every time a drop of syrup of violets in order to stop. When at last the latter assumes a red hue, then, after having restored the alkalimeter to a perpendicular position, in order to see at what line the testing liquor stops, you must reckon one degree less, in order to compensate the excess of saturation. The mean term of potashes is 56 ; this implies that they require for their saturation fifty-five hundredths of their weight of sulphurie acid."

For the analysis of commercial sodas of all kinds, M. Descroizilles prescribes using ten and a half deci-grammes of this alkali, instead of the ten deci-grammes for potashes, and proceeds as above detailed. In his table of results annexed, we find American potashes called $60^{\circ}$ to $63^{\circ}$.

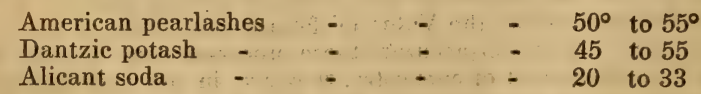

It is obvious, from these statements, that the alkalimeter so made and graduated denoted comparative, but not absolute, quantities of alkalis present in the com mercial samples. The rest of his very long memoir is occupied with what he calls the graduation of potashes and sodas, the economy of their graduation, the proportions of carbonic acid in them, the processes of caustification, the presence of potash in all lime which is burnt by a wood fire, origin of neutral soda, and probable origin of natrum; without any more explicit instructions. The instrument, as left in this vague state, never was employed, nor could it come into use, among English manufacturers and dealers 
The next alkalimeter, of which an account has been published, was my own. In constructing this instrument, I availed myself of the lights recently shed on chemical proportions by Dr. Dalton's atomic theory, and I thus made it to represent, not relative, but absolute measures of the amount of real alkali existing in any commercial sample. The test-liquor used at that time was sulphuric acid, which is most readily and accurately diluted to the requisite degree by means of a glass bead, very carefully made, of the specific gravity that the standard acid should have. In order to make the test-liquor, therefore, nothing more is requisite than to put the bead into distilled water, and to add to it somewhat dilute but pure sulphuric acid, slowly and with agitation, till the bead rises from the bottom, and floats in the middle of the liquor at the temperature of $60^{\circ}$ Fahr. The delicacy of this means of adjustment is so great, that a single degree of increase of heat will cause the bead to sink to the bottom-a precision which no hydrometer can rival. The test-tube, about 14 inches long, contains generally 1,000 grains of water, and is graduated into 100 equal parts by means of equal measures of mercury. The test-liquor is faintly tinged with red cabbage or litmus; so that the change of color, as it approaches to the saturating pitch, on adding it to 100 grains of the commercial alkali, becomes a sure guide in conducting the experiment to a succescful issue. One hundred measures of this test-liquor neutralize exactly 100 grains of absolute soda (oxide of sodium), and of course very nearly 150 of potash. A bead may also be adjusted for test-liquors, of which 1,000 grain measures neutralize 100 of potash, and therefore $66^{2} \frac{2}{3}$ of soda, as well as other proportions, for special purposes of greater minuteness of research. One may be so graduated as to indicate clearly a difference of $\frac{1}{100}$ of a grain of ammonia. In making such nice experiments, it is of course requisite to free the alkaline matter beforehand from sulphurets, sulphites, and hyposulphites, by igniting it in contact with chlorate of potash, as long since recommended by GayLussac. With such means in careful hands, all the problems of alkalimetry may be accurately solved by an ordinary operator.

On the same principle, my Acidimeter is constructed; pure water of ammonia is made of such a standard strength by an adjusted glass bead, as that 1,000 grain measures of it neutralize exactly a quantity of any one real acid, denoted by its atomic weight, upon either the hydrogen or oxygen scale or radix ; as for example, 40 grains of sulphuric acid. Hence it becomes a universal acidimeter; after the neutralization of 10 or 100 grains of any acid, as denoted by the well-defined color in the litmustinted ammonia, the test-tube measures of ammonia expended being multiplied by the atomic weight of the acid, the product denotes the quantity of it present in 10 or 100 grains. The proportion of any one free acid in any substance may thus be determined with precision, or to one fiftieth of a grain, in the course of five minutes. Like methuds are applied to Chlorometry, and other analytical purposes, with equal facility; adapting the test-liquor to the particular object in view. Instead of using beads for preparing the alkalimetric and acidimetric test-liquors, specific gravity bottles, or hydrometers, may of course be employed; but they furnish incomparably more tedious, and less delicate means of adjustment. To adapt the above methods to the French weights and measures, now used generally also by the German chemists, we need only. substitute I00 deci-grammes for 100 grains, and proceed in the graduation, \&c., as already described.

The possession of two reciprocal test-liquids affords ready and rigid means of verification. For microscopic analyses of alkaline and acid matter, a graduated tube of small bore, mounted in a frame with a valve apparatus at top, so as to let fall drops of any size, and at any interval, is desirable; and such I have employed for many years. Of this kind is my ammonia-meter, used in the ultimate analysis of guanos and other azotized products, in conjunction with a modified apparatus on the principle of that of Varrentrapp and Will. It may be remarked, that when the crude alkali contains some hyposulphite, it should not be calcined with chlorate of potash, because one atom of hyposulphurous acid is thereby converted into two atoms of sulphuric, which of course saturate double the quantity of alkali, previously in combination with the hyposulphurous acid. In such cases it is preferable to change the condition of the sulphurets, sulphites, and hyposulphites, by adding a little neutral chromate of potash to the alkaline solution, whence result sulphate of chromium, water, and sulphur, three bodies, which will not affect the accuracy of the above alkalimetrical process.

In the Annals of Philosophy for October, 1817, I described a new instrument for analyzing the earthy and alkaline carbonates, and for determining the quantity of base present in them from the volume of carbonic acid, disengaged by their solution in acids, upon the data of the atomic theory. . This method was applied to the analysis of the carbonates of ammonia, soda, potash, lime, magnesian limestone (dolomite), \&c.

"The indications of the above analytical instrument are so minute as to enable us, by the help of the old and well-known theorem for computing the proportions of two 
metals from the specific gravity of an alloy to deduce the proportions of the bases from the volume of gas disengaged by a given weight of a mixed carbonate."**

That small instrument consisted of a bent glass tube, open at one end, and terminated at the other with an egg-shaped bulb from two to three inches in diameter, and it required for operating with it, about five pounds of quicksilver. The following glass apparatus ( $f \mathrm{~g} .183$ ) will be found more generally convenient, and equally exact. $\mathrm{A}$ is

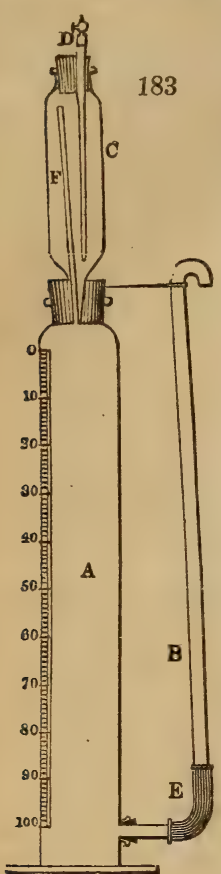
a cylinder, 2 inches in diameter, and 14 inches long. It contains 10,000 grains of water in the graduated portion; 0 , or zero being at the top. It has a tubulure in the side close to the bottom, through the cork of which a short tube passes tight, and is connected to a collar of caoutchouc, $E$, which serves for a joint to the upright tube, $\mathrm{B}$, resting near its open upper end in a hooked wire. Through the cork in the mouth of the cylinder, the taper tail of the flask c passes air-tight. The small tube F, open at both ends, is cemented at bottom into the tail of c, and rises to the shoulder of the flask. The cork of $\mathrm{c}$ is perforated, and receives air-tight the taper tube $\mathrm{r}$, which can also be closed with the stopcock.

In operating with this apparatus, proceed as follows :-

Fill the cylinder with water, and cover its surface with half an inch of oil. Insert the tail of the flask. Put into the flask c, $58 \cdot 6$ grains of carbonate of potash, or $45 \cdot 2$ of carbonate of soda, according as common pearl-ash or soda-ash is to be tested, along with as much water as will cover fully the lower end of $D$, and then introduce this tube. Have a bottle containing about 40 parts of oil of vitriol, previously mixed with 60 of water, and cooled. Take of this, in a pouring or dropping glass, 100 water grain measures, and suck this quantity gradually up into the tube $\mathrm{n}$, then shut the stopcock. On opening it slightly the acid will fall into c, and as slowly as may be prudent. The carbonic acid gas, forthwith disengaged, will depress the water in A, cause an overflow of it from the tube $B$, whieh, being held in the left hand, must have its swanbeak placed over a basin, and progressively lowered to the level of the descending water in the cylinder. When all the sulphuric acid has been introduced by the right hand, the orifice of $D$ is to be corked, and the tube $B$ continually lowered with the left, till the effervescence being finished, the water in A remains stationary. The number on the centigrade scale, opposite to the surface of the oil, deducting 100 grain measures for the bulk of dilute acid added, denotes the per-centage of pure carbonate of potash, or of soda, in the sample under examination. The above prescribed weights of these two carbonates, when pure, disengage each by the action of sulphuric acid (used here in small excess) 10,000 water grain measures of carbonic acid gas, or 100 measures of the scale on A. The cylinder which I employ contains about 12,000 water grain measures, so that the bottom of the centigrade scale is fully two inches above the level of the lower tubulure. This capacity and the graduation into 120 parts, will be found convenient in certain cases, particularly in analyzing bicarbonates of potash and soda. $t$

We may estimate 10,000 water grain measures of carbonic acid at $60^{\circ} \mathrm{Fahr}$, to weigh 18.4 grains, and we thus perceive what a magnified scale we should possess, if we applied the vernier contrivance here, as we div to barometers. At any rate, he must be an awkward operator who can not determine the value of an alkaline carbonate, by the above means, to one part in a thousand.

In operating upon limestones, marles, \&c., $42 \cdot 1$ grains should be taken as the standard weight of assay, because that weight of pure carbonate of lime should give out on solution in dilute muriatic acid 10,000 water grain measures of carbonic acid gas. Since 100 water grain measures of liquid hydrochloric acid, specific gravity $1 \cdot 14$, will supersaturate the lime in the above weight of carbonates, that quantity may be used in the experiment. The preceding instrument will be found more convenient in experimenting, as also the system of indication, than one on similar principles constructed by the ingenious Dr. Mohr, of Coblenz.

In examining bicarbonates of potash and of soda, the weights to be used in the above apparatus are 42 grains of the former, and $35 \frac{1}{4}$ grains of the latter, each of which

* Dictionary of Chemistry, 1821.

+ For the greatest precision hot acid may he used in the above experiment, by taking in a graduated test-tube seventy-five grains of water, and filling it up to the line 100 with concentrated sulphuric acid. This mixtrure being poured in successive portions into the flask c (represented much too large in pro. portion to the cylinder $\mathbf{A}$ ), will ensure the expulsion of all the carbonic acid from $\mathbf{c}$, which mav be afterward cooled by wrapping round it a towel dipped in cold water. 
quantities, if the salts be perfect, will disengage 10,000 water grain measures of carbonic acid gas, by the action of sulphuric acid. There will be no harm in taking the formerly prescribed measure of the sulphuric acid though considerably less would answer the purpose. The centigrade measures of gas obtained in A will indicate the carbonated state of the two alkalis respectively. Their alkaline force may be most readily ascertained by my old alkalimeter, with colored test acid. Since the bicarbonates usually sold in our shops, especially that of soda, are far from being exact atomic compounds, they should be always examined, both for their base and acid, which may also be well done in the following way, where the quantity of carbonic acid gas is determined by weight instead of by volume.

For this purpose, a small compact apparatus of the annexed form ( $f$ g. 184) will be

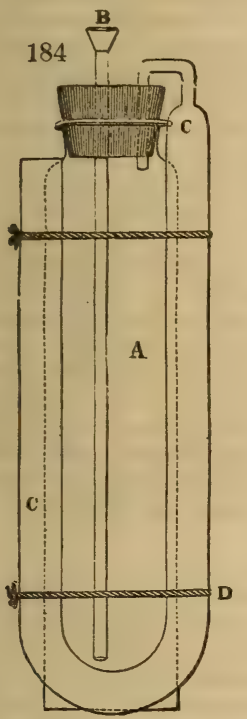
found convenient; it is to be used in conjunction with my alkalimeter. $A$ in the dotted line is the phial for receiving the carbonate to be tested. $\mathbf{B}$, the funnel into which the test acid is to be poured; c c, an inverted syphon filled with pieces of chloride of calcium for absorbing the aqueous vapors exhaled by the carbonic acid. The loss of weight in the phial above that in the tube of test acid shows the quantity of acid gas, and the indication of the alkalimeter tube, that of alkaline base, from which data the proportion of neutral carbonate and bicarbonate may be immediately deduced. Thus, 100 grains of bicarbonate of soda should give out $51 \frac{3}{4}$ grains of carbonic acid, and saturate $37 \cdot 6$ centigrade measures of the test acid, equivalent to $37 \cdot 6$ grains of real soda. But if neutral carbonate of soda be present, less gas will be given out, and more or less alkali may be indicated, according to the degree of dryness of the neutral soda. The amount of water in the bicarbonate may be determined by igniting 20 grains in a test tube, connected with the chlorealcium inverted syphon; $10 \frac{1}{3}$ grains of carbonic acid gas should be expelled, and $2 \frac{1}{8}$ of water, making a total loss of $12 \frac{1}{2} \frac{1}{4}$ grains; of which $2 \frac{1}{8}$ will be found as water absorbed by the chlorealcium. But since a very moderate heat suffices to expel the second atom of carbonic acid from the bicarbonate of soda, the readiest mode of estimating its quality is to heat, over a spirit lamp, in a small flask, or retort, connected air-tight by a tube with the mouth of the cylinder A, ( $f \mathrm{~g} .183), 70 \frac{3}{4}$ grains of the supposed bicarbonate. Of the perfect salt this quantity should give out pretty exactly 10,000 grain measures of gas; and whatever aliquot part of this volume is evolved will inaicate, without calculation, the relative value of the substance as a bisalt. Thus if 8,500 grain measures of gas are obtained, 85 parts of bicarbonate of soda are present in 100 . The crystalline form of bicarbonate of potash is a tolerably good criterion of its quality.

The quantity of caustic alkali mixed with carbonate may be readily determined, with sufficient accuracy, by the expert use of my alkalimeter; because, till the caustic portion be nearly neutralized, little or no carbonic gas is expelled. When the effervescence at length begins, the test measures already expended denote the percentage of caustic alkali. It is not right to disregard the alkali which is present in the state of sulphuret, because as such it is effective in many processes of the chemical arts; in the manufacture of yellow soap, crown glass, in the bleaching of linen and cotton goods, \&c. The alkalimeter, directly applied, will show the alkali present in this form, when compared with that indicated after ignition of the crude alkali with ch]orate of potash, or after its treatment with yellow chromate of potash.*

A few years ago I had the following apparatus made for the ready analysis of carbonates, by ascertaining the loss of weight they suffered from the disengagement of their carbonic acid gas, during their solution in an acid. A, B ( fig. 185) are two globes, of about two inches in diameter each; A has its inferior neck strangled into a bore nearly capillary ; B stands lower, with its centre line on a level with the narrow neck of B. The tubes of these globes are about one half inch in diameter. $\mathbf{c}$ is shut at top with a perforated cork, through which enters, air-tight, a small glass tube, which is bent across to the mouth of the tube $\mathrm{E}$, and then passes down into it a little below the centre line of

* If the alkaline carbonate contains sulphuret, sulphite, or hyposulphite, a teaspoonful of yellow chromate of potash may be added to it, wherefrom result sulphate of chromium, water, and sulphur, which remin in the apparatus without effecting its weight. The mutual action of neutral chromate of potash, and of sulphuret of potash, \&c., has been discussed in an ingenious paper published by Dopping, in the Annalen der Chimie for May, 1843, p. 172. 
the globe $\mathbf{8}$. This globe is rather more than half filled with sulphuric acid, when the instrument is employed in the analysis of the carbonates. 'The standard weight of carbonate of soda $=24 \frac{1}{8}$ grains, or of carbonate of potash $=$ $18531 \frac{1}{3}$ grains, is then put into $\mathrm{A}$, having previously laid a

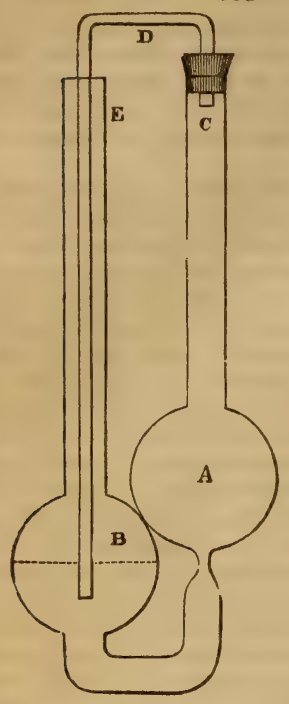
minute globe of glass over the lower orifice; the cork, with its small tube, is now firmly adjusted; and the apparatus is weighed in its upright position, either by suspension with a hook to the end of the beam, or by resting it on the scale in a light socket of any kind. It is next laid hold of, and inclined so as to cause a little of the acid in B to pass over into A. Effervescence ensues with greater or less vehemence, according to the nature of the carbonate and quantity of the acid introduced. Should it be too violent, and threaten an overflow by intumescence, it can be instantly abated to any degree by the slightest slope of the instrument. Now, this power of control forms the peculiar feature and advantage of this contrivance; whereas in all other forms of such apparatus that I know, whether by sucking over or pouring in, if a little too much acid comes upon the carbonate, the experiment is effectually marred. The gas disengaged in A must necessarily traverse the sulphuric acid in $B$, and be stripped of its moisture before escaping into the air. Having supersaturated the alkaline base, and cooled the apparatus, we weigh it again, and the loss of weight in grains and tenths denotes the per-centage of soda or potash, provided their neutral carbonates had been the subjects of experiment. For limestone, on the same plan of computation, $22 \frac{3}{4}$ grains may be taken. It deserves to be noted, that the present instrument has only one junction, and needs no chloride of calcium, a substance so apt by its swelling to burst the glass tubes that contain it.*

\section{ACIDIMETRY.}

I have already stated, that water of ammonia of standard strength, faintly tinted with litmus, affords a most exact and convenient acidimeter, when poured or let fall from a graduated dropping-tube. Bicarbonate of potash also, when dissolved in water, so that 1,000 grain measures contain one atom of the salt counted in grains, is a good test-liquor for the same purpose ; for if the centigrade measures expended in effecting neutralization are multiplied by the atomic weight of the given acid, the product is the quantity in grains of acid present.

Acidimetry may be likewise exactly performed by measuring in the cylindric gasmeter ( fig. 183) the volumes of carbonic acid gas disengaged from pure bicarbonate of potash or soda, by a given weight of any acid, taking care to use a small excess of the salt. Thus, for example, $16 \cdot 8$ grains of dry and $20 \frac{2}{3}$ of hydrated sulphuric acid disengage 10,000 water grain measures of gas from bicarbonate of potash. Therefore, if $20 \frac{2}{3}$ grains of a given sulphuric acid be poured into the flask of fig. 183, upon about 50 grains of the bicarbonate, powdered and covered with a little water, it will cause the evolution of a volume of gas proportioned to its strength. If the acid be pure oil of vitriol, that weight of it will disengage 10,000 grain measures of gas; but if it be weaker, so much less gas-the centigrade measures of which will denote the per-centage value of the acid. If the question be put, how much dry acid is present per cent. in a given sulphuric acid, then 16.8 grains of the acid under trial must be used; and the resulting volume of carbonic acid gas read on the scale will denote the per-centage of dry acid. $\dagger$

For nitric acid, we should take $22 \cdot 6$ grains; for hydrochloric or muriatic acid, $15 \cdot 34$; for acetic acid, 21.6 ; for citric acid, 24.6 ; for tartaric acid, 28 grains : then in each case we shall obtain a volume of carbonic acid gas proportioned to the strength and purity of these acids respectively. The nitric, hydrochloric, and acetic acids are referred to in their anhydrous state; the tartaric and citric in their crystalline. If the latter two acids be pure, a solution of 24.6 grains of the first and of 28 of the last

* 1,000 water grain measures of sulphuric acid of specific gravity $1 \cdot 032$, or 32 above water, neutralize 32 grains of soda, and, consequently, one atoin, on the hydrogen scale, of each of the other bascs. reckoned in grains.

Having in the course of many years subjected my tables of sulphuric, nitric, and muriatic acids, as well as of ammonia, to strict cross-examination, I have found them trustworthy for all alkalimetrica: and acidimetrical purposes.

$\dagger$ The bicarbonate must be free from carbonate, a point easily secured by washing its powder with cold water, and drying it in the air. 
will disengage from 50 grains of bicarbonate of potash 10,000 grain measures of carbonic acid gas.*

Acidimetrical operations may likewise be performed by determining the weight of carbonic acid gas expelled from the bicarbonate of potash or soda, by a given quantity of any acid, in the apparatus either fig. 184, or fig. 185. Here the weights to be taken are as follows, in reference to

$\begin{array}{llllc}\text { Dry Sulphuric acid } & - & - & - & 9 \cdot 127 \\ \text { "6 Nitric - - } & - & - & - & 12 \cdot 33 \\ \text { " Hydrochloric } & - & - & - & 8 \cdot 29 \\ \text { "Acetic - } & - & - & - & 11 \cdot 67 \\ \text { Crystallized Tartaric - } & - & - & 13 \cdot 31 \\ \text { 66 Citric - } & - & - & 15 \cdot 13\end{array}$

Each of these quantities of real acid, with 25 or 26 grains of bicarbonate of potash, will give off 10 grains of carbonic acid gas; and hence whatever weight the apparatus loses, being reckoned in grains and tenths of a grain, denotes the 186 per-centage of acid in the sample under trial; without the necessity of any arithmetical reduction. Persons accustomed to the French metrical system may use deci-grammes instead of grains, and they will arrive at the same per-centage results.

The preceding experiments, in reference to the weight of carbonic acid gas expelled for the purpose of either alkalimetry or acidimetry, may also be made by means of the ordinary apparatus represented in fig. 186. A is a small matrass which contains the acid or carbonated alkali at its bottom; and conversely the alkali or acid, for their mutual decomposition in the small test-tube, shown first at $b$ nearly upright and filled, but afterward at $a$, horizontal and emplied. B is a bulbous tube filled with fragments of chlorcalcium for absorbing the aqueous vapor that rises with the carbonic acid gas, and $d c$ is a small bent tube which dips into the liquid in the matrass. The weighings, \&c., may be conducted as already detailed; and when the effervescence is completed, the residuary gas is sucked up through B, while the atmospheric air enters to replace it at the orifice $d$ of the bent tube.

The NEw methods which pervade the whole treatise of Drs. Fresenius and Will are all based on the principle of estimating alkalinity, acidity, and the oxygen in manganese (or chlorometry) by the weight of carbonic acid gas evolved. As in taking these measures the gas must be discharged without carrying water off with it, an elegant and ingenious little piece of apparatus has been invented by the authors for effecting that purpose, and it will do it well. A and B ( $f$ ig. 187) are two flasks (wide-mouthed medicine-bottles may be employed). A must have a capacity of from 2 ounces to $2 \frac{1}{2}$

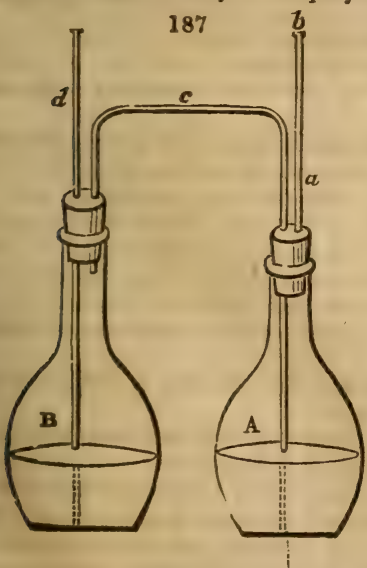
ounces of water; it is advisable that $\mathrm{B}$ should be somewhat smaller, say of a capacity of about 1 to $1 \frac{1}{2}$ ounces. Both flasks are closed by means of doubly perforated corks. These perforations serve for the reception of the tubes $a, c$, and $d . \quad c$ is a tube bent twice at right angles, which enters at its one end just into the flask $A$, but descends at its other end, near to the bottom of в. These tubes are open at both ends when operating; except the top end $b$ of the tube $a$, which is closed by means of a pellet of wax. The substance to be examined is weighed and put into the flask $A$, into which water is then poured to the extent of one third of its capacity. B is filled with common English sulphuric acid to about half its capacity. Both flasks are then corked (by which they become united by the rectangular tube), and the apparatus is weighed.

The air of the whole apparatus is next rarefied by applying suction to the tube $d$ : the consequence is, that the sulphuric acid contained in B ascends into

The expulsion of the gas may be completed by surrounding the flask with a towel dipped in hot water. 
the tube $c$, and thus a portion of it flows over into B. Immediately upon its coming into contact with the carbonate contained in A, carbonic acid gas is disengaged, and in its escape must necessarily traverse the oil of vitriol in $B$, and therein deposite all its aqueous vapor before issuing from $d$. The sulphuric acid in passing over into $\mathrm{A}$ heats the mixture at the same time, and thus promotes the expulsion of the gas. Whenever this ceases to flow, a little more sulphuric acid must be sent over into $\mathrm{A}$ by suction from $d$ (or rather from a recurved tube attached, pro tempore, to it); an artifice which may be repeated till no more gas can be expelled, even when the contents of $A$ are heated, as they must be at the end by the excess of oil of vitriol.

"From the aperture $b$ of the tube $a$, which has been all the time closed, the bit of wax is now to be removed, and to the tube connected with $d$, suction is to be applied, till all the carbonic acid lodged in the apparatus be replaced by atmospheric air. The whole is to be then cooled, wiped, and weighed; the loss of weight indicates exactly the quantity of carbonic acid which existed in the carbonate submitted to experiment The process is no less neat than it is simple, and does honor to the ingenuity of its in ventors. Their mode of deducing the per-centage of alkali from the quantity of carbonic acid discharged in the operation is also quite exact, and suitable for continental chemists familiar with gramme weights and calculations, but certainly not for persons conversant only with ounces, drams, and scruples, or even with grain subdivisions. The whole book, however excellent, needs, for the British public, transposition, before it can serve in this country the purpose intended by its scientific authors. Thus, in section 4, where several results of their analyses are given, the statements have a somewhat mysterious aspect. Should any one ask why the oracular number of 4.83 grammes of carbonate of soda is used as their standard weight for analysis, he can obtain no response in the book, either in a note or anywhere else. A German or French student, familiar with chemical computation, will probably be able to discover that $4 \cdot 83$ grammes of pure carbonate of soda contain, by Berzelius's tables of atomic weights, 2 grammes of carbonic acid; for 53.47 ( 1 atom of carbonate) : 22.15 (1 of carbonic acid) $:: 4 \cdot 83: 2 \cdot 00$. Such is the simple solution of this apparent enigma, and of some other similar puzzles in the book. Indeed, unless the reader is aware of that proportion, he can not see the grounds of the accordance in the results between experiment and theory, or why the numbers $2 \cdot 010,1.993$, and $2 \cdot 020$, are presented as specimens of great precision. This accordance gives satisfaction when it is known that these numbers, in experiments 1,2 , and 3 , oscillate on one side or other so near to the theoretical number $2 \cdot 00$. But 4 grammes and 83 centi-grammes, as also 1 gramme and 995 milli-grammes, are awkward weights for an ordinary English chemist or apothecary, which would require a month or two's residence in the laboratories of Giessen and Paris to manipulate with readiness.

Again, in testing carbonate of potash, our authors take 6.29 grammes as their unity of weight, undoubtedly, because, if pure, it should discharge, by saturation with the sulphuric acid, 2 grammes of carbonic acid. Here, however, they have not stuck so rigidly as the school of Giessen usually does to Berzelius's atomic numbers; for his atom of carbonate of potash is $69 \cdot 42$; whence, $22 \cdot 15: 69 \cdot 42:: 2 \cdot 00: 6 \cdot 68$, hydrogen $=1 \cdot 00$; or $276 \cdot 44: 866 \cdot 33:: 2 \cdot 00: 6 \cdot 268$ oxygen $=100$.

Admitting the value of the new method in testing neutral carbonates, it can not be directly applied to the mixed carbonate and bicarbonate of soda, so commonly sold in this country for bicarbonate; nor is it applicable to the case of a mixture of caustic and carbonated alkali, without the tedious process of previous treatment with carbonate of ammonia and heat.

The new German method of acidimetry consists in determining how much carbonic acid gas is disengaged from a standard bicarbonate of soda, by a given weight of any acid. The twin-flask apparatus ( fig. 187) is used. The weighed portion of acid is put into $\mathrm{A}$, and a sufficient quantity of the soda into a test-tube, which is suspended upright with a silk thread fastened by the pressure of the cork to the mouth of the flask. On letting the thread loose, the test-tube falls, and the cork being instantly replaced, the whole gas evolved is forced to pass through the sulphuric acid in B, and there to deposite its moisture. The experiment is conducted in other respects as already described for alkalimetry.

The following extract from Drs. Fresenius and Will's New Methods of Alkalimetry, \&c., will show the Giessen plan of calculating results :-

"The amount of anhydrous acid contained in the hydrated acid under examination is determined from the amount of carbonic acid escaped, as follows :-

"Two measures of carbonic acid bear the same proportion to one measure of the anhydrous acid in question, as the amount of carbonic acid expelled does to the amount sought of anhydrous acid. Thus, let us suppose, for instance, we have examined dilute sulphuric acid, and obtained 1.5 grammes of carbonic acid, the arrangement would be :- 


$$
\begin{aligned}
550(2 \times 275): 501 & =1 \cdot 5: x \\
x & =1 \cdot 36 .
\end{aligned}
$$

The amount of sulphuric acid operated upon consequently would contain 1.36 grammes of anhydrous acid. Let us suppose the weight of this amount to have been 15 grammes, the sulphuric acid under examination would contain a per-centage amount of 9.06 ; for

$$
\begin{aligned}
15: 1 \cdot 36 & =100: x \\
x & =9 \cdot 06 .{ }^{\prime \prime *}
\end{aligned}
$$

"SEcrion XXIX. Stating the Quantities of the various Acids to be used in their Examination.-To enable our readers at once, without the trouble of calculation, to determine from the weight of carbonic acid expelled, the exact amount of anhydrous acid contained in those acids which are of most frequent occurrence, we have subjoined lists of certain quantities to be taken of each acid for experiment, so that the number of centi-grammes of carbonic acid expelled will directly indicate the per-centage amount of anhydrous acid in the acid under examination.

"Multiples of those weights may of course be substituted for the numbers given, according to the degree of dilution of the acid under examination. In such cases the number of centi-grammes of the carbonic acid expelled must be divided by the same number, which has served as the multiplier.

"These numbers are obtained by dividing the atomic weight of the acid by 550 $(2 \times 275$, one eq. of carbon), $t$ as follows :-

"Two eq. of carbonic acid, corresponding to one eq. of the acid to be examined, how much should be taken of the latter to expel 1.00 grammes of carbonic acid?

"The arrangement of sulphuric acid, for instance, is as follows :-

$$
\begin{aligned}
550: 501 & =1 \cdot 00: x \\
x & =0.91 \text { (or, more correctly, } 0.911 \text { ). }
\end{aligned}
$$

"6 When examining acids, it is most advisable to use that multiple of the unity (according to the degree of concentration) which will expel from one to two grammes of

\begin{tabular}{|c|c|c|}
\hline $0.911=$ & $1 \cdot 822$ & ammes. \\
\hline $0.911=$ & $2 \cdot 733$ & 6 \\
\hline$\times 0.911=$ & $3 \cdot 644$ & 66 \\
\hline $5 \times 0.911=$ & $4 \cdot 555$ & c6 \\
\hline $6 \times 0.911=$ & $5 \cdot 466$ & “ \\
\hline$\cdot 911=$ & $6 \cdot 377$ & "6 \\
\hline $0.911=$ & $7 \cdot 288$ & «6 \\
\hline $0.911=$ & $8 \cdot 199$ & "s \\
\hline$\times 0.911=$ & $9 \cdot 110$ & "6 \\
\hline $0.911=$ & $13 \cdot 665$ & "6 \\
\hline $.911=$ & $18 \cdot 220$ & “ \\
\hline & & \\
\hline
\end{tabular}
carbonic acid.

$$
\text { "I. SULPHURIC ACID. }
$$

"Unity 0.91 grammes (or, more correctly, 0.911 grammes).

"Multiples :-

"Thus, knowing that 0.91 of anhydrous sulphuric acid will expel 1.00 of carbonic acid, it will be easy to determine what multiple ought to be used, according to the degree of concentration of the acid to be examined." $\ddagger$

\section{CHLOROMETRY,}

\section{And the testing of Black Oxide of Manganese for its available Oxygen.}

The value of manganese may be estimated very exactly by measuring the quantity of chlorine which a given weight of it produces with hydrochloric acid; the chlorine being at the same time estimated by the quantity of solution of green sulphate of iron, which it will peroxidize. A process of this kind was long ago practised with chloride of lime (bleaching powder or liquor) by Dr. Dalton; and it has been since improved by Mr. Waltercrum. As the conversion of two atoms of green sulphate of iron into red sulphate requires only one atom of oxygen, this change may be effectin by the reaction of one atom of chlorine in liberating one atom of oxygen, while this appropriates one of hydrogen from the hydrochloric acid. 
The weight of 2 atoms of green sulphate of iron is $278=(139 \times 2)$, consisting of 2 atoms of protoxide $=72, \times 2$ of sulphuric acid $=80, \times 14$ of water $=126$; in all $=278$; and this weight is equivalent to 36 of chlorine, to 8 of oxygen, and to 44 of peroxide of manganese.* Therefore, if we take a solution of copperas, containing 278 grains in 1,000 water grain measures, that volume of liquid will represent, by the conversion of its protoxide into peroxide, exactly one atom, either of peroxide of manganese $=44$ grains, or 1 atom of chlorine $=36$. Hence the following plan of research :-

Into the fla.sk or phial $\mathrm{c}$ of my chlorometric apparatus ( $\mathrm{fig.} \mathrm{188}$ ), put 100 grains of the

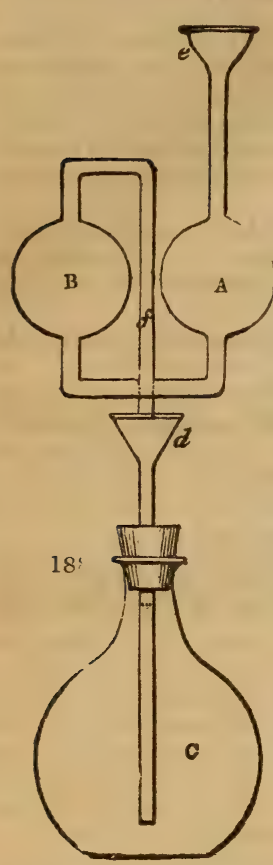
manganese to be tested, and into the globes $A, B$, pour out of an alkalimetrical tube charged with 1,000 grain measures of the above equivalent copperas solution, from 200 to 500 grain measures, according to the supposed quality of the manganese; then introduce through the funnel $d$, some hydrochloric acid of known specific gravity (suppose $1 \cdot 1$ ), containing nearly 20 per cent. of chlorine, also from a charged alkalimetrical tube, and apply gentle heat to the bottom of the flask by placing it in a capsule of water standing over a spirit-lamp. The chlorine evolved will rise up through the tube $f$, which passes merely beyond the cork, and will enter into the solution in $B$ and A, converting it into red sulphate. Have ready some dry paper imbued with solution of red ferrocyanide of potassium (red prussiate of iron). Dip a slip of whalebone into the liquor in the globe $A$, through the funnel $e$ (represented in the figure rather too high above the globe), and touch the paper with its point. As long as it forms a blue spot, some of the iron still exists as black oxide, and the process is to be urged by the addition of a little more hydrochloric acid to the manganese, as long as chlorine gas continues to be disengaged, and while it maintains the level of the liquor in $\mathbf{A}$ above that in $\mathbf{B}$. Whenever the liquor, by the reaction of the chlorine, ceases to stain the test-paper blue, more of the solution from the graduated tube must be added till it begins to do so. By the cautious administration of the hydrochloric acid on the one hand, and of the copperas liquor on the other, the term of saturation will be arrived at in a few minutes. The manganese has then produced all the chlorine which it can yield. The number of water grain measures, of the liquor, or degrees of its alkalimeter scale being multiplied by 44 , will give a product denoting the per-centage of pure manganese present in the sample; or being multiplied by 36 , a product which will denote the quantity of chlorine by weight which 100 grains of it can serve to generate.

Since one atom of pure manganese (44 grains), in producing 36 grains of chlorine, consumes 2 atoms $=74$ grains of hydrochloric acid, the quantity of this acid expended from the graduated tubes, beyond the due proportion of chlorine obtained, will show how much of the acid is unprofitably consumed by foreign substances in the manganese. In fact, every grain of chlorine should, with pyrolusite, be generated by an expenditure of little more than 2 grains of real muriatic acid, or 10 grains weight of the dilute acid, = about 9 grain measures of the graduated tube. Liquid hydrochloric acid of spec. grav. 1.093 contains in 1,000 grain measures exactly 200 grains of real acid. Hence 100 grains of pure pyrosulite should produce about 82 grains of chlorine, and consume about 169 of real muriatic acid $=845$ grain measures of liquid acid, spec. grav. 1.093. Instead of taking 100 grains of manganese as the testing dose, 10 or 20 grains may be taken, according to the dimensions of the apparatus and the exactness of the operator.

But if it be wished to obtain direct per-centage of manganese by the graduated tubes without the trouble of reduction, then for a dose of 10 grains take a solution of fresh green copperas (free from adhering moisture), containing 632 grains in 10,000 grain measures. Proceed as above directed. If the manganese be a pure peroxide, 10 grains of it

* Berzelius, in the 4th edition of his Lehrbuch, rates the atom of the green sulphate of iron (ferrous sulphate) at $129 \cdot 43$, hydrogen $=1$, and considers it, after-Mitscherlich, to contain only 6 atoms of water. I have ascertained, by the most careful experiments, that it contains 7 atoms of water; and that 139 grains of it, or 138.44 (Berzelius) are equivalent to 1 atom of chlorbarium, and to very nearly 40 grains of peroxide of iron.

This remarkable error has probably arisen from an attempt to measure the proportion of water in the salt from its loss of weight by desiccation. But I have found it impossible by this means to expel more than 6 atoms of water without causing partial decomposition of the salt by disengagement of sulphuric acid. The copperas so dried acquires such an affinity for water, that it absorbs fully one tenth of its weight of moisture from the atmosphere in the course of an hour. 
will generate as much chlorine as will peroxidize exactly 1,000 grain measures, or 100 degrees by the test-tube of the copperas solution. But if the manganese contain only 40 or 50 per cent. of peroxide, then 40 or 50 centigrade measures of the said solution will be equivalent to the chlorine evolved from it by the reaction of hydrochloric acid.

If the object is on the other hand to obtain direct indications as to chlorine, then a test solution of copperas, containing 772 grains in 10,000 grain measures, will serve to show, by the peroxidizement of each 10 grain measures, or of one degree of the centestimal scale of the test-tube, the reaction of one grain of chlorine available for bleaching, \&c., in the chloride of lime or of soda, \&c. The test solutions of copperas should be kept in well-corked bottles, containing a little powdered sulphuret of iron at their bottom, which is to be shaken up occasionally in order to preserve the iron in the state of protoxide.

The manganese should always be treated with dilute nitric acid before submitting it to the above-described ordeal; and if it exhibits effervescence, 100 grains of it should be digested with the acid for a sufficient time to dissolve out all the carbonates present, then thrown upon a filter, washed and dried before weighing it for the testing operation. The loss of weight thereby sustained denotes the per-centage of carbonates, and if calcareous it will measure the waste of acid that would ensue from that source alone, in using that manganese for the production of chlorine.

That manganese is most chlorogenous which contains no carbonates, the least proportion of oxide of iron, and of sesquioxide of manganese.

The plan of testing manganese with oxalic and sulphuric cids was originally practised by M. Berthier and Dr. Thomson, but is lately modified by Drs. Fresenius and Will, who employ oxalate of potash, as likely to afford more exact results. They prescribe a multiple by 3 of 993 milli-grammes $=2.979$ grammes, as the quantity of manganese best adapted to experiment; but this quantity will not be found convenient by ordinary British operators.

I, therefore, take leave to prescribe the following proportions: Into the vessel A of my twin-globe apparatus ( $f \mathrm{gg} .185$ ), put 100 grains of the ground manganese under trial, along with 250 grains of oxalate of potash and a little water; poise the whole in the scale of a balance; then, by gentle inclination, cause a little of the strong sulphuric acid to pass from B up into $A$. The oxygen thereby liberated from the manganese, reacting in its nascent state upon the oxalic acid, will convert it into carbonic acid gas ; which, in passing through $\mathrm{B}$, will deposite its moisture before escaping into the air. Whenever the extrication of gas ceases, after such a quantity of oil of vitriol has been introduced into the globe $\mathrm{A}$, as both to complete the decomposition of the oxalic acid and to heat the mixture, withdraw the cork for a moment, to replace the carbonic acid with air, then cool, and weigh the apparatus. The loss of weight, in grains, will denote the per-centage value of the manganese; that is, the proportion per cent. of perfect peroxide in the sample. If the manganese be pure no black powder should remain.

The preceding experiment is founded upon the following principle: One atom of peroxide of manganese $=44$, contains one atom of oxygen separable by sulphuric acid, and capable of converting one atom of oxalic acid into two atoms of carbonic acid, also $=44$, which fly off; and cause therefore a loss of weight equal to that of the whole peroxide. To one atom of oxalic acid, which consists of three atoms of oxygen, and two of carbon-if one atom of oxygen be added, the sum is obviously four atoms of oxygen and two of carbon $=2$ atoms of carbonic acid.

The apparatus ( $f \mathrm{~g} .187$ ) of Drs. Fresenius and Will will answer perfectly well for making the same experiment, the manganese being put into $\mathrm{A}$, with about two and a half times its weight of oxalate of potash, and the sulphuric acid being drawn over into the mixture by suction, as above described.

The economy of any sample of manganese in reference to its consumption of acid, in generating a given quantity of chlorine, may be ascertained also by the oxalic acid test : 44 grains of the pure peroxide, with 93 grains of neutral oxalate of potash, and 98 of oil of vitriol disengage 44 grains of carbonic acid, and afford a complete neutral solution; because the one half of the sulphuric acid, $=49$ grains, goes to form an atom of sulphate of manganese, and the other half to form an atom if sulphate of potash.

The deficiency in the weight of carbonic acid thrown off will show the deficiency of peroxide of manganese; the quantity of free sulphuric acid may be measured by a test solution of bicarbonate of potash, and the quantity neutralized, compared to the carbonic gas produced, will show, by the ratio of 98 to 44 , the amount of acid unprofitably consumed. 
In fig. 183 , the tube, $\mathrm{D}$, may also be graduated, and may contain the quantity of acid, for the purpose either of alkalimetry or acidimetry ; and if the lower orifice be capillary, it will allow none of its contents to flow out, till the stopcock in the top orifice is opened.

In fig. 184, such a tube as $\mathrm{I}$ ( $f$ ig. 183) may be substituted with advantage for the funnel, B; and as that tube, $D$, may be made of such dimensions as to contain enough of acid to supersaturate the bases of the carbonates in the phial, $\mathrm{A}$, there will be no necessity for a separate vessel to hold the decomposing acid. Thus the apparatus becomes very light, convenient, and may be placed in the small scale of a fine balance; whereas the twin matrasses of Drs. Fresenius and Will ( $f i g .187$ ), as furnished by Mr. Bullock, require a very large pan or scale to stand in. I flatter myself that the instrument, fig. 184, so mounted, will be found an acceptable present to practical chemists, and that it will enable them readily to examine, not only carbonates, but also manganese and bleaching substances, with great precision, by the weight of carbonic acid gas disengaged, on the principles above explained.

Into the twin globe apparatus ( $f \mathrm{~g} .185$ ), after the sulphuric acid is poured into $\mathbf{B}$, a little water should be poured into $\mathrm{C}$, before the carbonate is introduced into the latter. By this means, the capillary throat of the tube under $\mathrm{A}$ will not be apt to get choked with concrete salt.

The following quotations are from the work of Drs. Fresenius and Will, as edited by Mr. Bullock for the English reader. An accurate comparison may thus be made between the relative utility of their methods and mine to the practice of ordinary operators :-

"Sectron XXXIV. Examination of Manganese: having at the same time due regard to the amount of Acid required for its complete Decomposition.-We have stated, at Section 30, that it is not a matter of indifference, with regard to the amount of acid employed in the production of chlorine from manganese, what are the minerals which this substance contains in admixture with the peroxide. The following modification of our method will give the most correct information on this point :-

"Sulphuric acid of commerce is taken, and its amount of anhydrous acid determined, as directed at Section 26, or by means of an accurate hydrometer. Of this sulphuric acid as much is weighed into A ( $\mathrm{fig} .187$ ), as to give an amount of 5.47 grammes of anhydrous acid.

"The following table will show the amount which ought to be taken, acoording to the various degree of concentration of the acid :-

\begin{tabular}{|c|c|c|c|c|c|}
\hline $\begin{array}{l}\text { Specific weight } \\
\text { found. }\end{array}$ & $\begin{array}{l}\text { Per-centage } \\
\text { amount of } \\
\text { anhydrous } \\
\text { acid found. }\end{array}$ & $\begin{array}{l}\text { Amount to } \\
\text { be used for } \\
\text { the exami- } \\
\text { nation. }\end{array}$ & $\begin{array}{l}\text { Specific weight } \\
\text { found. }\end{array}$ & $\begin{array}{l}\text { Per-centage } \\
\text { amount of } \\
\text { anhydrous } \\
\text { acid found. }\end{array}$ & $\begin{array}{l}\text { Amount to } \\
\text { be used for } \\
\text { the exami- } \\
\text { nation. }\end{array}$ \\
\hline $1 \cdot 8485$ & $81 \cdot 54$ & $6 \cdot 708$ & $1 \cdot 8336$ & $76 \cdot 65$ & $7 \cdot 136$ \\
\hline $1 \cdot 8480$ & $81 \cdot 13$ & $6 \cdot 742$ & $1 \cdot 8313$ & $76 \cdot 24$ & $7 \cdot 174$ \\
\hline $1 \cdot 8475$ & $80 \cdot 72$ & $6 \cdot 776$ & 1.8290 & $75 \cdot 83$ & $7 \cdot 213$ \\
\hline $1 \cdot 8467$ & $80 \cdot 31$ & $6 \cdot 811$ & $1 \cdot 8261$ & $75 \cdot 42$ & $7 \cdot 252$ \\
\hline $1 \cdot 8460$ & $79 \cdot 90$ & $6 \cdot 846$ & $1 \cdot 8233$ & $75 \cdot 02$ & $7 \cdot 291$ \\
\hline $1 \cdot 8449$ & $79 \cdot 49$ & $6 \cdot 881$ & $1 \cdot 8206$ & $\tau 4 \cdot 61$ & $7 \cdot 331$ \\
\hline $1 \cdot 8439$ & $79 \cdot 09$ & $6 \cdot 916$ & $1 \cdot 8179$ & $74 \cdot 20$ & $7 \cdot 371$ \\
\hline $1 \cdot 8424$ & $78 \cdot 68$ & 6.951 & $1 \cdot 8147$ & $73 \cdot 79$ & $7 \cdot 412$ \\
\hline $1 \cdot 8410$ & $78 \cdot 28$ & $6 \cdot 987$ & $1 \cdot 8115$ & $73 \cdot 39$ & $7 \cdot 453$ \\
\hline 1.8393 & $77 \cdot 84$ & $7 \cdot 027$ & 1.8079 & 72.97 & $7 \cdot 495$ \\
\hline $1 \cdot 8376$ & $77 \cdot 40$ & $7 \cdot 067$ & $1 \cdot 8043$ & 72.57 & $7 \cdot 537$ \\
\hline $1 \cdot 8356$ & $77 \cdot 02$ & $7 \cdot 101$ & & & \\
\hline
\end{tabular}

"As much water is then poured into $A$ as will fill the flask to about one fourth; and, lastly, from 6.5 to 7 grammes of neutral oxalate of potash, or from 5.5 to 6 grammes of neutral oxalate of soda, are added; 2.98 grammes of the (finely-pounded) manganese to be examined are then weighed (the manganese must have been previously tested for carbonate alkaline earths: compare this section at the end) into a small glass tube, such as used in acidimetry, and described in Section 25. About the same quantity of pure pyrolusite, ${ }^{*}$ in powder, is then put into another similar tube. The tube, with the manganese to be examined, is then suspended in A ( $f \mathrm{~g} .187$ ), as described at Section 26, and the apparatus prepared, as directed at Section 3. The

\footnotetext{
* "Any variety of pyrolusite will serve this purpose, provided it be free from other manganese ores. If ${ }^{+}$contains heavy spar, it may be employed directly; but should it contain alumina or lime, it must be treated first with dilute nitric acid, at a gentle heat, until all soluble parts have been dissolved; it is then washed and dried. Artificially prepared, hydrated peroxide of manganese may be substitut od for pyrolusite
} 
apparatus is then placed on one scale of a balance, together with the other little tube containing the pyrosulite, and exactly weighed.

"The cork of $\mathrm{A}$ is then somewhat raised to allow the little tube with the manganese to fall into the flask. The evolution of carbonic acid commences immediately, and continues until all the manganese is decomposed. When the operation begins to get on more slowly, the flask, $\mathbf{A}$, is placed in boiling water, and allowed tu remain there until no more bubbles appear. The little wax-stopper is then removed* from $a$, the flask, A, taken out of the hot water, and suction applied to $d$, until the sucked air tastes no longer of carbonic acid. The apparatus, after having been allowed to cool, is wiped dry, and replaced in the original scale, where the little tube with the pyro. lusite still remains; weights are then substituted for the loss of carbonic acid. The number of centigrammes required, divided by three, directly indicates the per-centage amount of peroxide of manganese (vide Section 32). The centigrammes substituted for the loss of carbonic acid are then removed from the balance, and the little tube with the pyrosulite is thrown into A. (The little wax-stopper must of course previously be replaced on $a$ ). If no fresh evolution of carbonic acid takes place, the manganese examined consists of pure pyrosulite, and the experiment is at an end. But should a fresh evolution of carbonic acid take place, the operation must be further conducted, and brought to a close, exactly as just stated (vide suprà). The apparatus is then replaced on the balance, with an additional weight of three grammes on the same scale. If this is sufficient to restore a perfect equilibrium, no loss of acid has taken place; the manganese, indeed, contains other matters in admixture, but only such as do not consume any acid. But if the scale with the apparatus sinks, this is a certain sign that a portion of the acid has been lost by combining with the oxides which the manganese under examination contains. The number of centigrammes required to restore the perfect equilibrium of the balance, multiplied by 0.6114 , immediately indicates how much anhydrous sulphuric acid has been wasted in the decomposition of 100 parts of the manganese under examination. The same number, multiplied by D.333, indicates the amount of acid wasted in every 100 parts of sulphuric acid employed for the decomposition of the manganese in question. The same number, multiplied by 0.5552 , indicate how much anhydrous hydrochloric acid would be wasted in the decomposition of 100 parts of the manganese. The same number, multiplied by 0.333 , indicates also how much acid would be wasted in every 100 parts of hydrochloric acid employed for the decomposition of the manganese.

"These figures result from the following equations :-

"I. 275 (eq. of carbonic acid) : 501 (eq. of sulphuric acid) = the carbonic acid obtained minus (in proportion to the sulphuric acid used) $: x$.

$x=$ this carbonic acid $\times \frac{5}{2} \frac{0}{7} \frac{1}{5}, i$. e. $\times 1.822$.

Thus, the number obtained for $x$ indicates the amount of sulphuric acid corresponding to the amount of carbonic acid obtained minus.

"II. $2 \cdot 98$ of manganese: $100=x$ of equation I. $: x$. $x=x$ of $\mathrm{I} . \times \frac{10}{2} \frac{0}{8}, i$. e. $\times 0.33557$.

"The $x$ of the first equation tells us how much sulphuric acid has been wasted without contributing to the decomposition of 2.98 grammes of the manganese; the $x$ of the second equation tells us the same for 100 parts of manganese.

"If, therefore, the amount of carbonic acid obtained minus be directly multiplied by the product of the quotients of I. and II.,

$$
1 \cdot 822 \text { and } 0.33557 \text {, }
$$

$i$. e. with 0.61141 (the number given above), the amount of anhydrous sulphuric acid wasted in the decomposition of every 100 parts of manganese will immediately be found.

6III. 5.47 (the amount of sulphuric acid used): $100=$ the $x$ of I. $: x$.

$x=$ the $x$ of $\mathrm{I} . \times \frac{100}{5}, i . e . \times 0 \cdot 18282$.

" 0 5.47 of sulphuric acid, the $x$ of $\mathrm{I}$. has been wasted, 100 corresponds to the $x$ of III.

"T The $x$ of III. is, therefore, found directly by multiplying the amount of carbonic acid obtained minus with the product of the quotients, 1.822 and $0.18282, i$. $e_{0}=$ 0.33301.

"The figures for hydrochloric acid are found in the same manner (4.967 of hydrochloric acid must be taken instead of 5.47 of the sulphuric acid)."' $f$

* " This must of necessity be done while the flask is still standing in the hot water, or else the sulphuric acid will recede upon the apparatus being removed from the hot water."

+ New Methods of Alkalimetry, and of determining the Commercial Value of Acids and Manganese. By Drs. C. R. Fresenius and H Will. Edited by J Lloyd Bullock: pp. 123-128. 
ARTESIAN WELLS. Fig. 189, is that referred to in the foot-note of page 10.

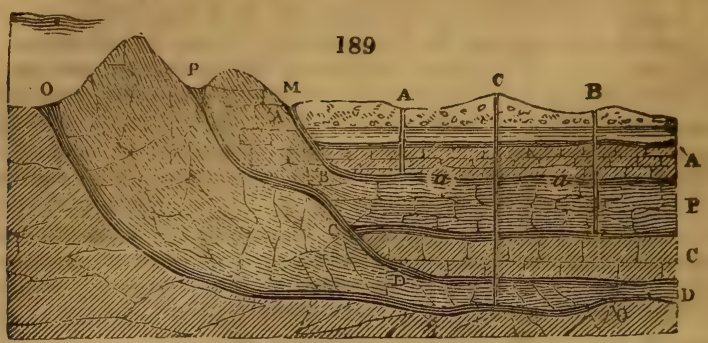

BEER. A gentleman well acquainted with the brewing of porter in London has favored me with the following information.

Essentia bina has been discontinued by the London porter brewers since the employment of black malt, which is prepared by roasting in such a cylinder as coffee is usually roasted in over a fire. A peculiar flavor has sometimes been imparted by using roasted barley instead of malt. The usual quantity of yeast employed in the Londen porter breweries is from 5 to 7 tenths per cent. The grist, as it is technically termed, or charge for a mash-tun, is composed of from $\frac{2}{3}$ to $\frac{4}{5}$ pale malt, and the rest of high dried malt, of which about from $\frac{1}{15}$ to $\frac{1}{2}$ black. The oil of birch bark is not used by any respectable brewers in this country. The proportion of hops for double stout is seldom more than 15 pounds to the 8 bushels of malt.

FLAX. The new roving machine, called by the ingenious inventor, Mr. W. K. Westley, of Leeds, the Slivfre Roving Frame, seems to be a philosophical induction happily drawn from the nature of the material itself, and accommodated to its peculiar constitution. It is remarkable for the simplicity or its construction, and, at the same time, for its comprehensiveness ; requiring no nicety of adjustment in its application, and no tedious apprenticeship to be able to work it.

It is known, that the glutinous matter of the plant may be softened by water, and hardened again by heat; of this fact advantage is taken, in order to produce a roving wholly without twist; that is, in the form of a riband or sliver, in which the fibres are held together by the glutinous matter which may be natural to them; or which may, for that purpose, he artificially applied. The sliver roving, as long as it remains dry, possesses all requisite tenacity, and freely unwinds fiom the bobbin, but on becoming again wetted in the spinning frame, it readily admits, with a slight force, of being drawn into yarn, preserving the fibres quite parallel.

The diagram, fig. 190, shows in explanation, that

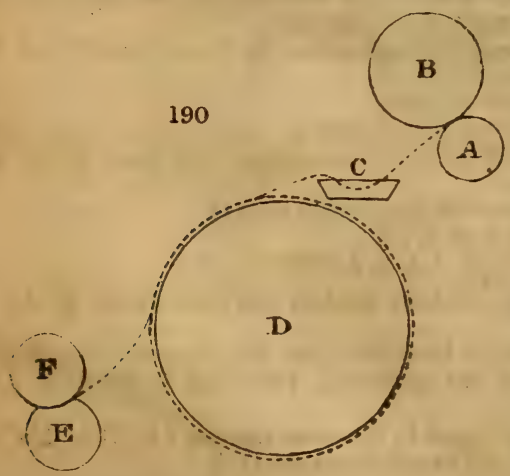

$A$, is the drawing roller of the roving frame in front of the usual comb. $B$, the pressing drawing roller. C, a shallow trough of water. $D$, a cylinder heated by steam. $\mathbf{E}$, a plain iron roller for winding. $\mathrm{F}$, a bobbin lying loose upon the winding roller, and revolving upon it, by the friction of its own weight.

The roving, or sliver, as shown by the dotted line, after leaving the drawing rollers $A$, passes through the water, in the trough $\mathbf{C}$, which softens the gluten of the fibres; and then it is carried round by the steam eylinder $\mathrm{D}$, which dries it, and delivers it hard and tenacious to the bobbin F, on which it is wound by the action of the roller $\mathbf{E}$.

This is the whole of the mechanism required in producing the sliver roving. All the complex arrangements of the common cone roving are superseded, and the machine at once becomes incomparably more durable, and easier to manage; requiring only half the motive power, and occupying only half the room. A frame of 48 bobbins is only 6 feet long, and affords rovings sufficient to supply 1,200 spinning spindles. 


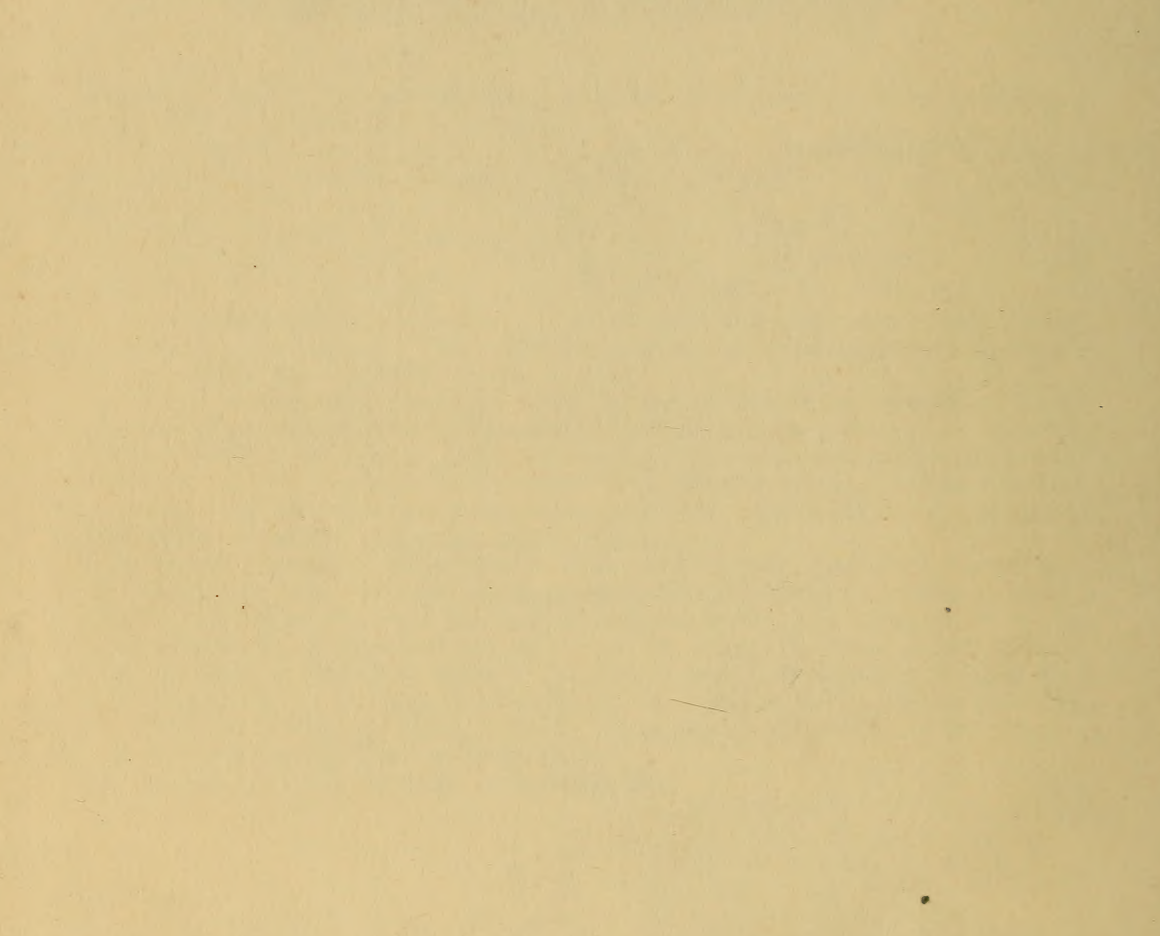

SMITHSONIAN INSTITUTION LIBRARIES 